ORNL/TM-2005/39

Version 6.2.2

\title{
SCALE Code System
}

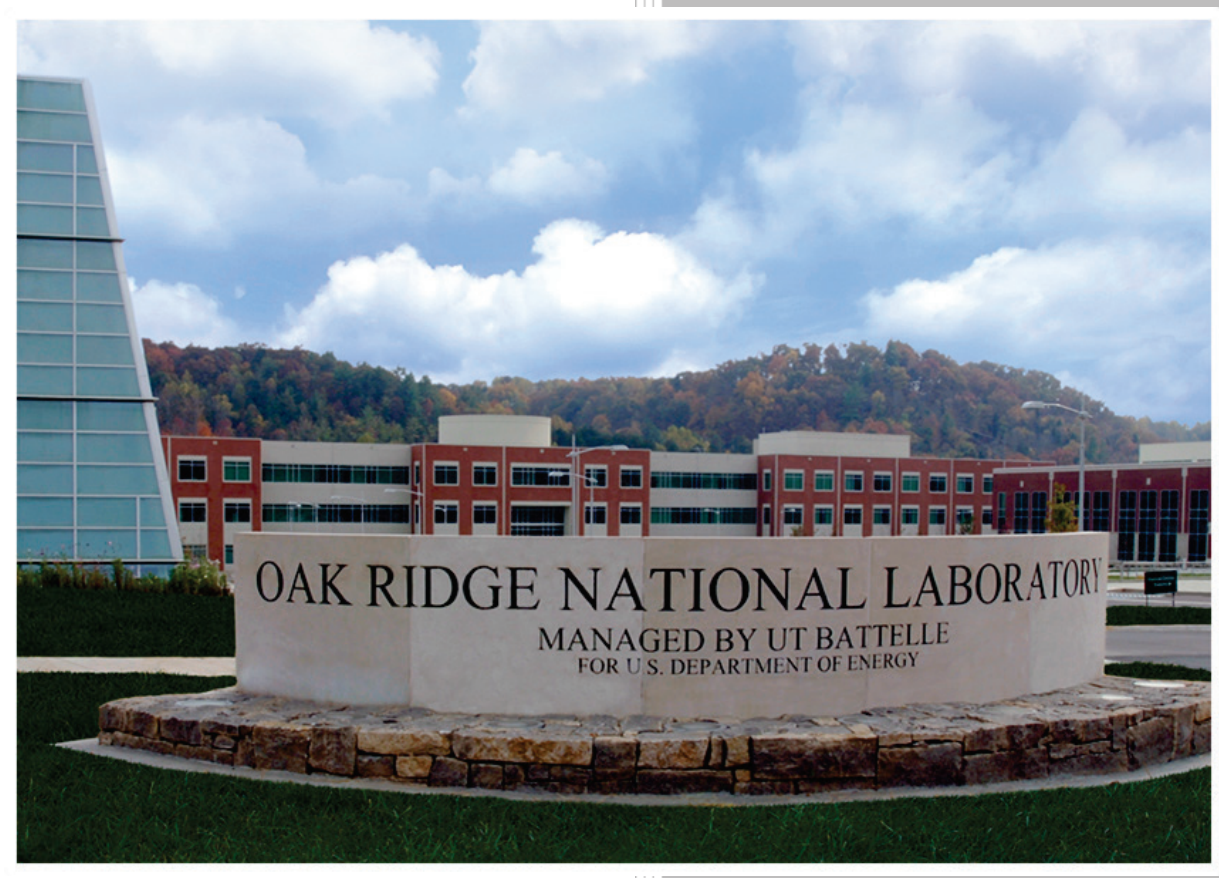

Approved for public release.

Distribution is unlimited.

B.T. Rearden

M.A. Jessee, Editors

February 2017 


\section{DOCUMENT AVAILABILITY}

Reports produced after January 1, 1996, are generally available free via US Department of Energy (DOE) SciTech Connect.

Website http://www.osti.gov/scitech/

Reports produced before January 1, 1996, may be purchased by members of the public from the following source:

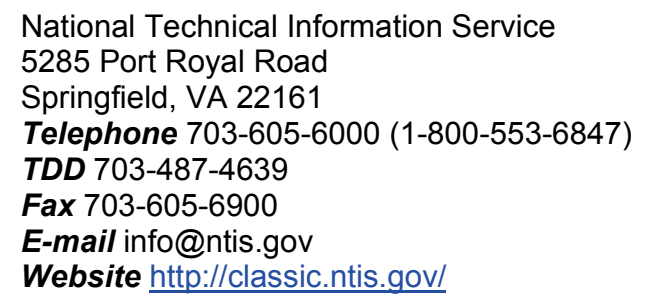

Reports are available to DOE employees, DOE contractors, Energy Technology Data Exchange representatives, and International Nuclear Information System representatives from the following source:

Office of Scientific and Technical Information

PO Box 62

Oak Ridge, TN 37831

Telephone 865-576-8401

Fax 865-576-5728

E-mail reports@osti.gov

Website http://www.osti.gov/contact.html

This report was prepared as an account of work sponsored by an agency of the United States Government. Neither the United States Government nor any agency thereof, nor any of their employees, makes any warranty, express or implied, or assumes any legal liability or responsibility for the accuracy, completeness, or usefulness of any information, apparatus, product, or process disclosed, or represents that its use would not infringe privately owned rights. Reference herein to any specific commercial product, process, or service by trade name, trademark, manufacturer, or otherwise, does not necessarily constitute or imply its endorsement, recommendation, or favoring by the United States Government or any agency thereof. The views and opinions of authors expressed herein do not necessarily state or reflect those of the United States Government or any agency thereof. 
Reactor and Nuclear Systems Division

\section{SCALE CODE SYSTEM}

B. T. Rearden

M. A. Jessee, Editors

Date Published: February 2017

Prepared by OAK RIDGE NATIONAL LABORATORY

Oak Ridge, TN 37831-6283

managed by

UT-BATTELLE, LLC

for the

US DEPARTMENT OF ENERGY

under contract DE-AC05-00OR22725 



\begin{abstract}
The SCALE Code System is a widely-used modeling and simulation suite for nuclear safety analysis and design that is developed, maintained, tested, and managed by the Reactor and Nuclear Systems Division (RNSD) of Oak Ridge National Laboratory (ORNL). SCALE provides a comprehensive, verified and validated, user-friendly tool set for criticality safety, reactor and lattice physics, radiation shielding, spent fuel and radioactive source term characterization, and sensitivity and uncertainty analysis. Since 1980, regulators, licensees, and research institutions around the world have used SCALE for safety analysis and design. SCALE provides an integrated framework with dozens of computational modules including three deterministic and three Monte Carlo radiation transport solvers that are selected based on the desired solution strategy. SCALE includes current nuclear data libraries and problem-dependent processing tools for continuous-energy (CE) and multigroup (MG) neutronics and coupled neutron-gamma calculations, as well as activation, depletion, and decay calculations. SCALE includes unique capabilities for automated variance reduction for shielding calculations, as well as sensitivity and uncertainty analysis. SCALE's graphical user interfaces assist with accurate system modeling, visualization of nuclear data, and convenient access to desired results.
\end{abstract}

SCALE 6.2 provides many new capabilities and significant improvements of existing features.

New capabilities include:

- ENDF/B-VII.1 nuclear data libraries CE and MG with enhanced group structures,

- Neutron covariance data based on ENDF/B-VII.1 and supplemented with ORNL data,

- Covariance data for fission product yields and decay constants,

- Stochastic uncertainty and correlation quantification for any SCALE sequence with Sampler,

- Parallel calculations with KENO,

- Problem-dependent temperature corrections for CE calculations,

- CE shielding and criticality accident alarm system analysis with MAVRIC,

- CE depletion with TRITON (T5-DEPL/T6-DEPL),

- CE sensitivity/uncertainty analysis with TSUNAMI-3D,

- Simplified and efficient LWR lattice physics with Polaris,

- Large scale detailed spent fuel characterization with ORIGAMI and ORIGAMI Automator,

- Advanced fission source convergence acceleration capabilities with Sourcerer,

- Nuclear data library generation with AMPX, and

- Integrated user interface with Fulcrum.

Enhanced capabilities include:

- Accurate and efficient CE Monte Carlo methods for eigenvalue and fixed source calculations,

- Improved MG resonance self-shielding methodologies and data,

- Resonance self-shielding with modernized and efficient XSProc integrated into most sequences,

- Accelerated calculations with TRITON/NEWT (generally 4x faster than SCALE 6.1),

- Spent fuel characterization with 1470 new reactor-specific libraries for ORIGEN,

- Modernization of ORIGEN (Chebyshev Rational Approximation Method [CRAM] solver, API for high-performance depletion, new keyword input format)

- Extension of the maximum mixture number to values well beyond the previous limit of 2147 to $\sim 2$ billion,

- Nuclear data formats enabling the use of more than 999 energy groups, 
- Updated standard composition library to provide more accurate use of natural abundances, and

- Numerous other enhancements for improved usability and stability.

The user documentation for SCALE has also been substantially updated and reorganized.

The appropriate citation to use when referencing SCALE is as follows:

B. T. Rearden and M. A. Jessee, Eds., SCALE Code System, ORNL/TM-2005/39, Version 6.2.2, Oak Ridge National Laboratory, Oak Ridge, Tennessee (2017). Available from Radiation Safety Information Computational Center as CCC-834. 


\section{CONTENTS}

Getting Started

$\begin{array}{ll}1.0 & \text { SCALE Introduction } \\ 2.0 & \text { Criticality Safety } \\ 3.0 & \text { Reactor Physics } \\ 4.0 & \text { Radiation Shielding } \\ 5.0 & \text { Depletion, Activation, and Spent Fuel Source Terms } \\ 6.0 & \text { Sensitivity and Uncertainty Analysis } \\ 7.0 & \text { Material Specification and Cross Section Processing } \\ 8.0 & \text { Monte Carlo Transport } \\ \text { 9.0 } & \text { Deterministic Transport } \\ 10.0 & \text { SCALE Nuclear Data Libraries } \\ 11.0 & \text { Utility Modules For SCALE Libraries }\end{array}$




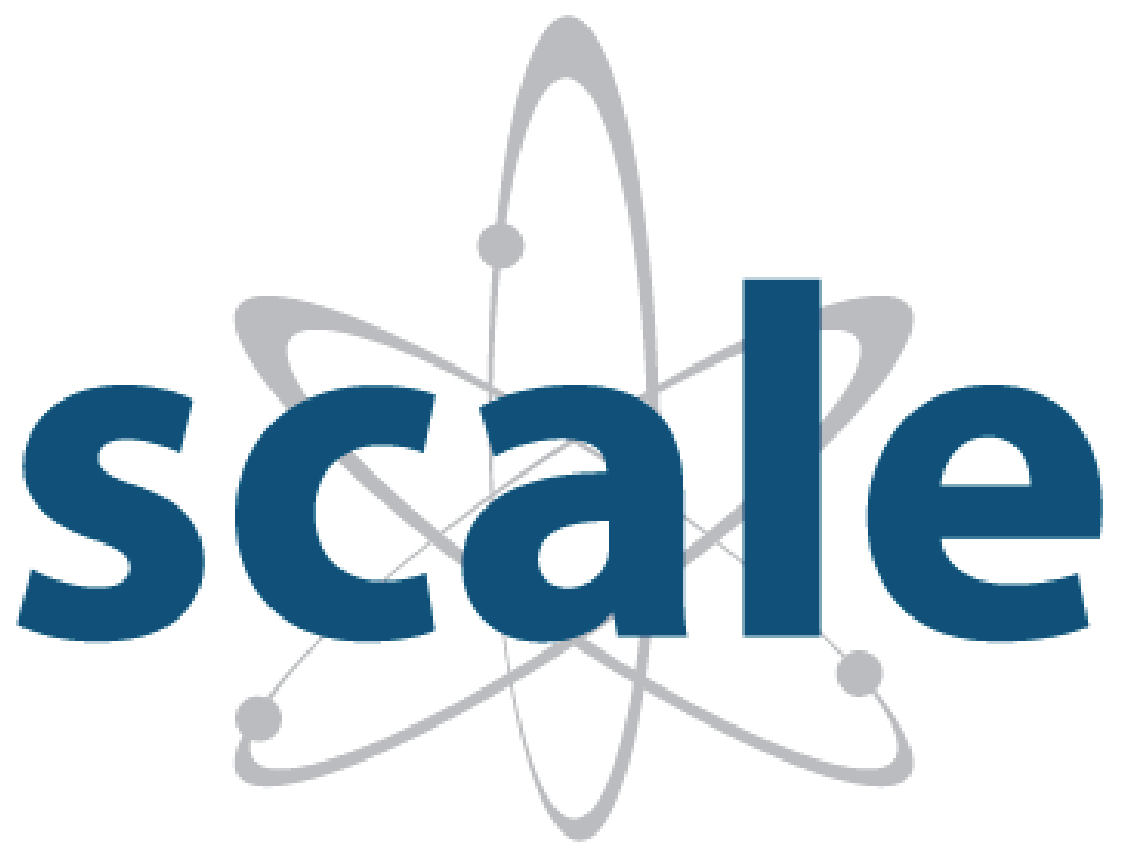

Getting Started with SCALE 6.2.2

February 2017 



\section{CONTENTS}

$\underline{\text { Page }}$

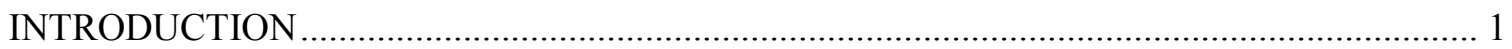

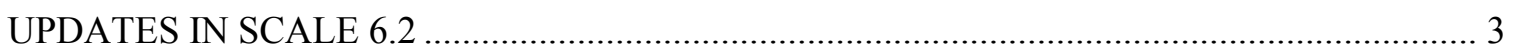

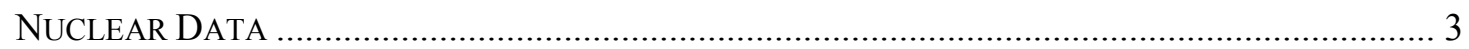

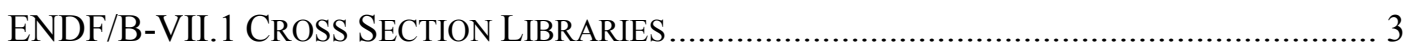

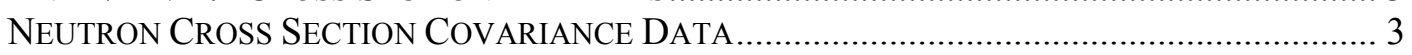

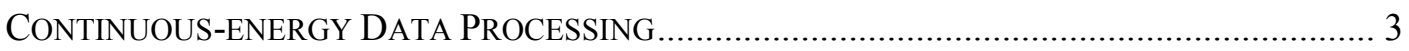

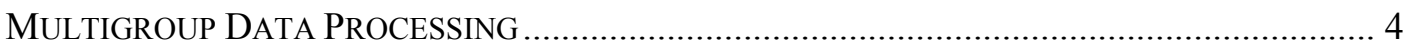

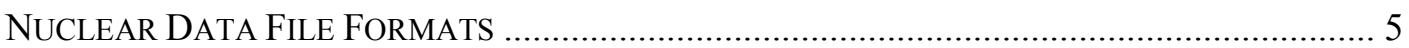

MODERNIZED MATERIAL INPUT PROCESSING AND RESONANCE SELF-SHIELDING..................... 6

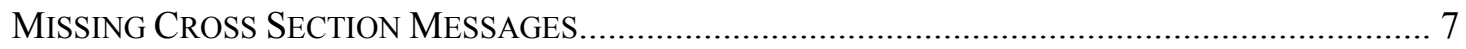

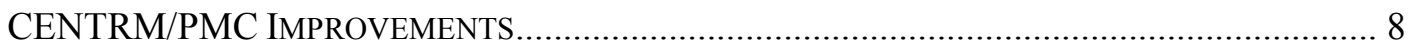

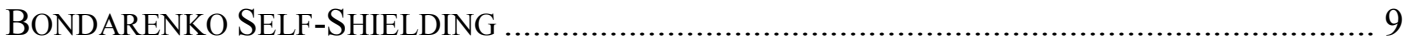

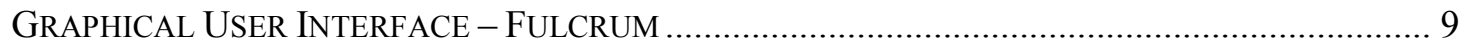

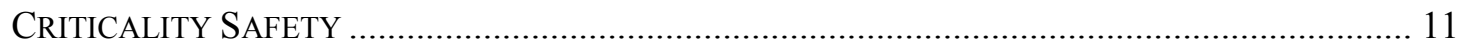

REDUCTION IN MEMORY REQUIREMENT OF CE INTERNAL STORAGE............................... 11

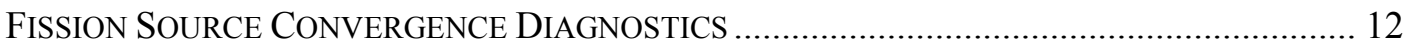

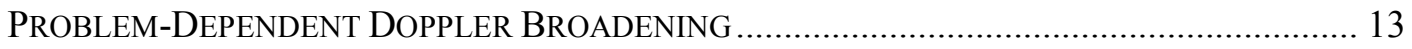

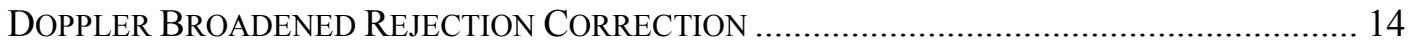

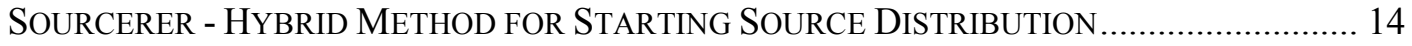

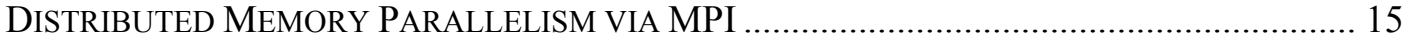

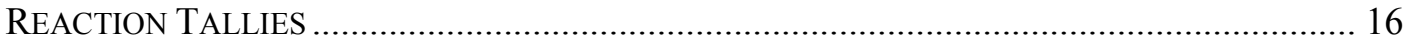

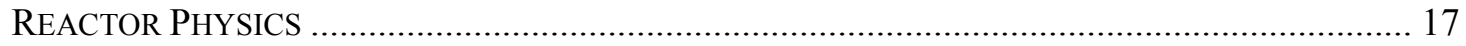

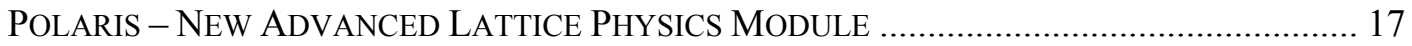

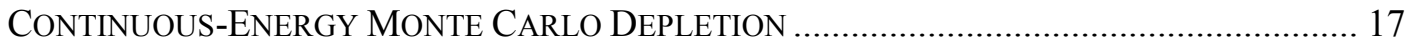

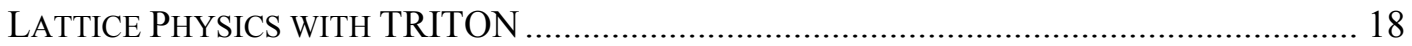

BIAS REDUCTION FOR DEPLETION

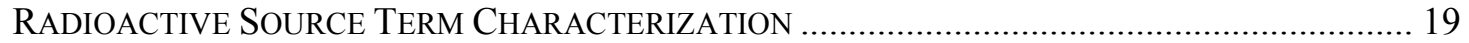

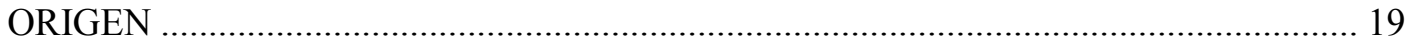

ORIGAMI - NEW ADVANCED USED FUEL ASSEMBLY CHARACTERIZATION.................... 21

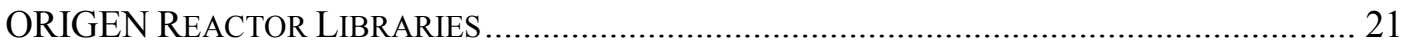

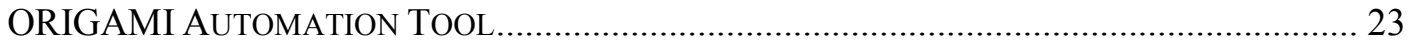

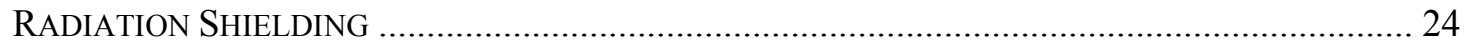

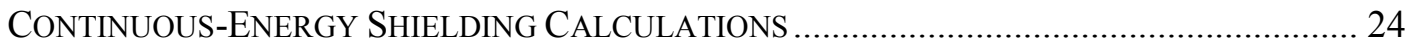

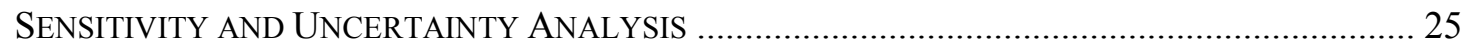

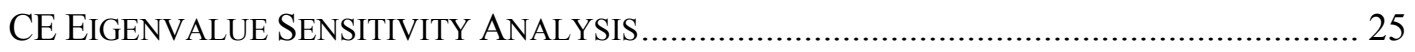

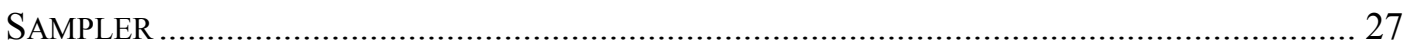

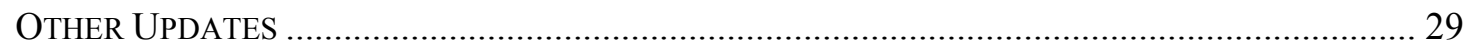

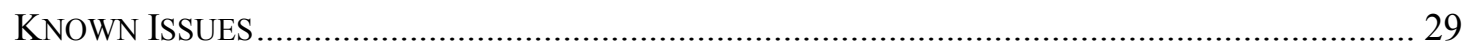

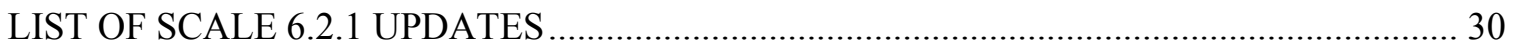

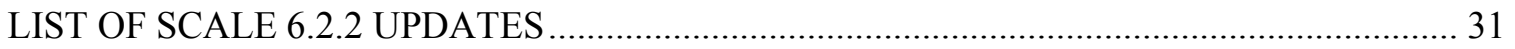

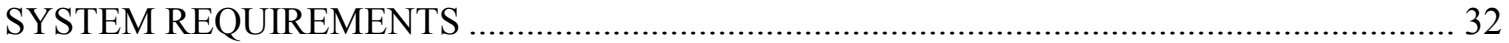

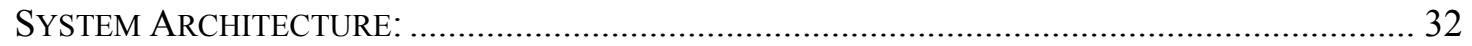

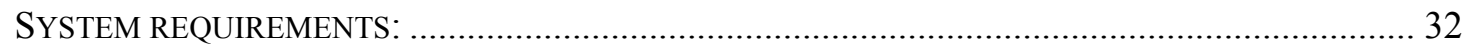

Getting Started — iii 


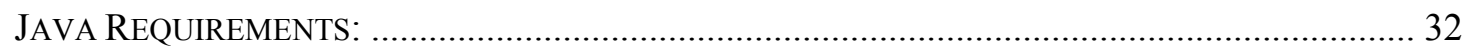

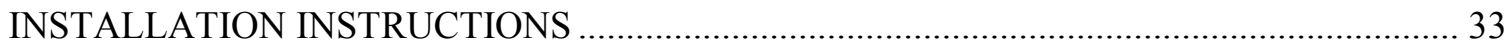

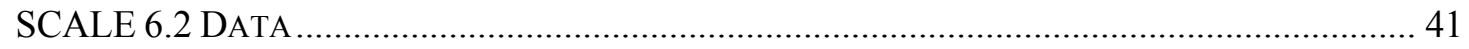

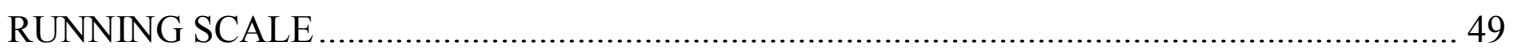

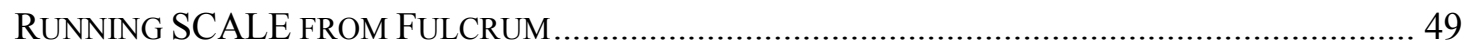

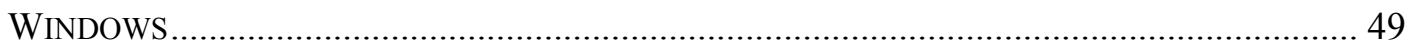

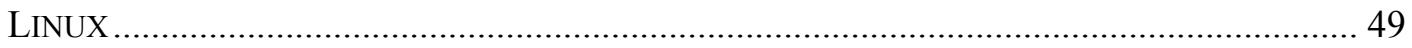

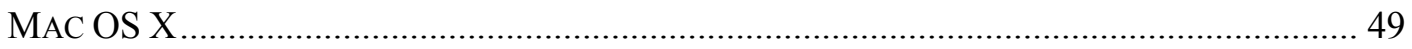

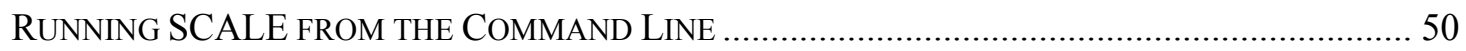

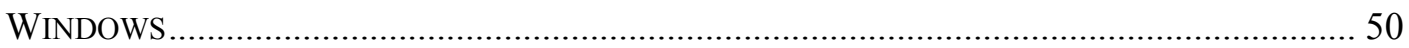

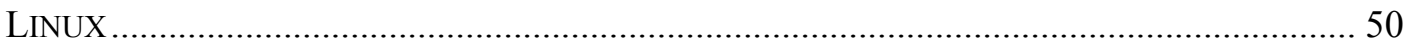

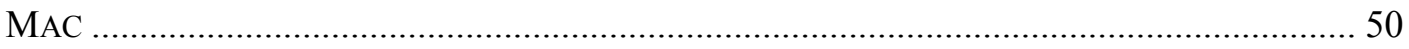

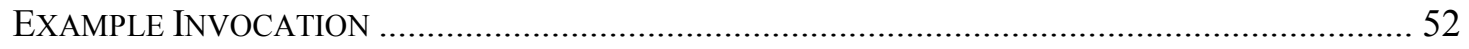

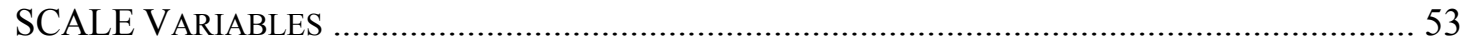

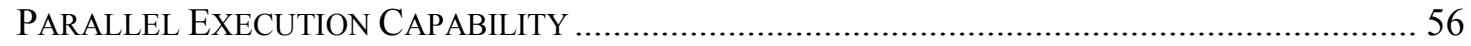

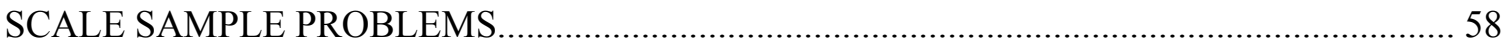

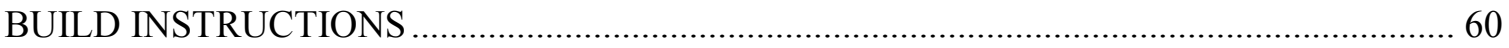

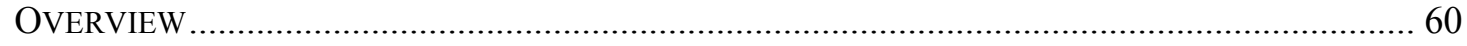

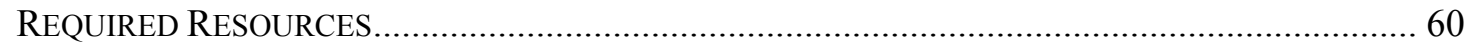

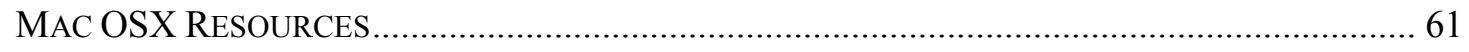

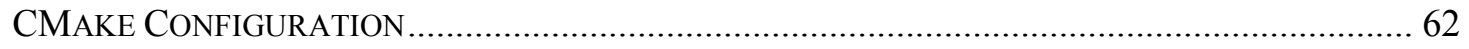

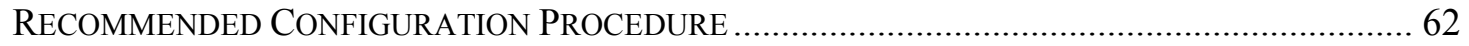

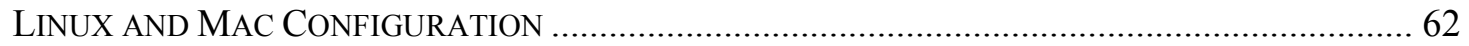

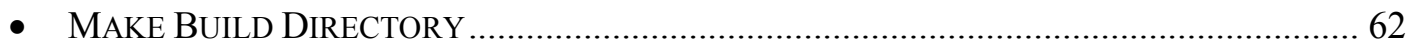

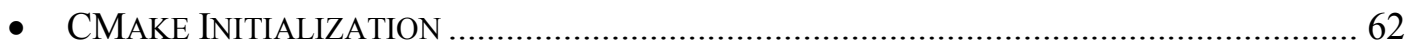

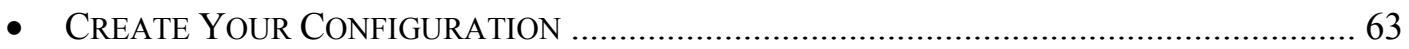

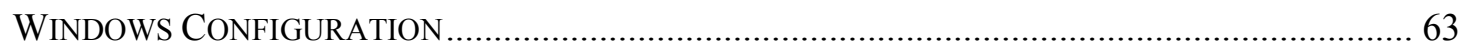

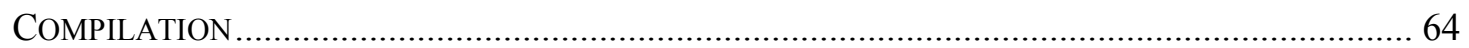

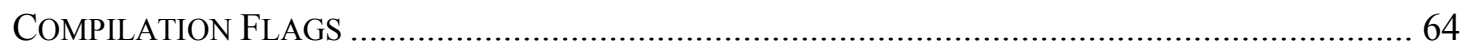

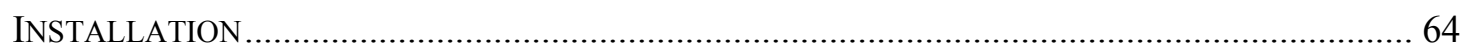

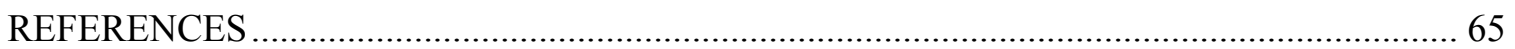

Getting Started - iv 


\section{List of Figures}

Page

Figure 1. SCALE calculated results for International Criticality Safety Benchmark Evaluation Project (ICSBEP) thermal mixed oxide critical systems.............. 4

Figure 2. $\mathrm{k}_{\mathrm{eff}}$ values for IEU-MET-FAST-005-001 with different MG options............ 9

Figure 3. SCALE graphical user interface - Fulcrum........................................ 10

Figure 4. Eigenvalues computed for a PWR pin cell with different temperature

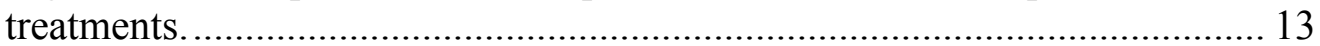

Figure 5. Fraction of failure to agree with the reference $k_{\text {eff }}$ value for KENO calculations with different starting sources (Figure 4 from Ref. 9)............... 15

Figure 6. Speedup for parallel KENO-VI calculations for a graphite-moderated reactor model. ............................................................................................. 16

Figure 7. Comparison of calculation times for 1,470 TRITON calculations................ 18

Figure 8. Comparison of computational biases as a function of burnup...................... 19

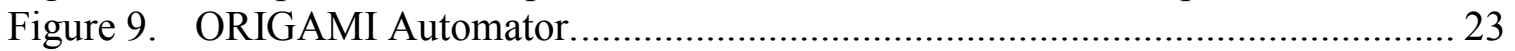

Figure 10. MIX-COMP-THERM-004-001 nuclide sensitivity figure of merit

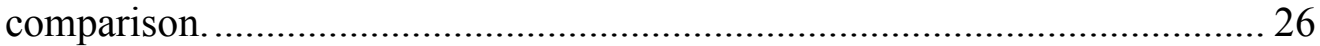

Figure 11. Uncertainty in plutonium isotopics as function of burnup.......................... 28

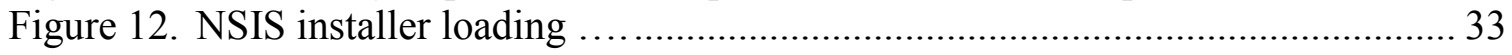

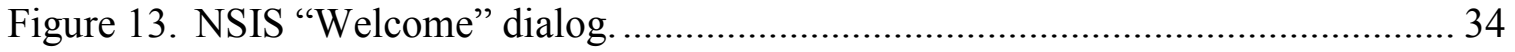

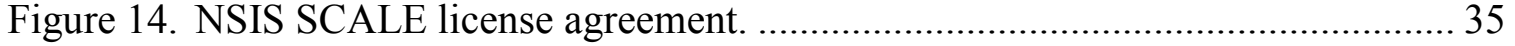

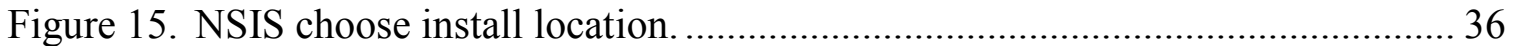

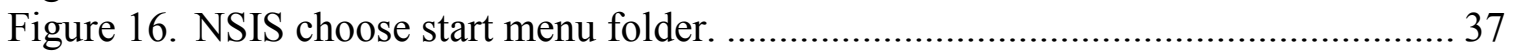

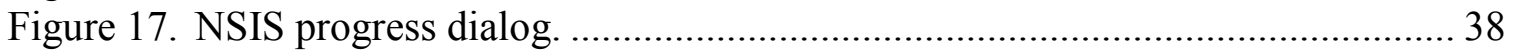

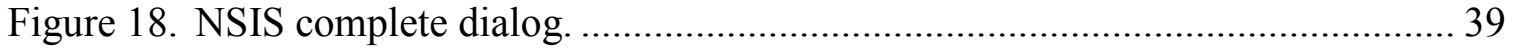

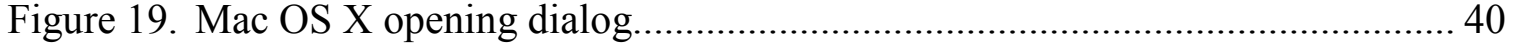

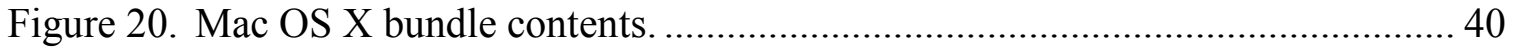

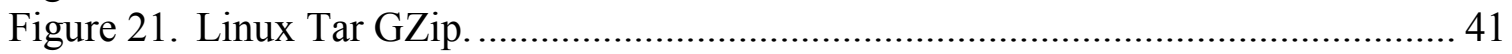

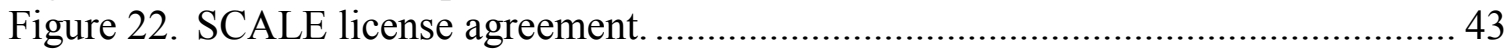




\section{List of Tables}

$\underline{\text { Page }}$

Table 1. SCALE Sequence Modernization ............................................................. 7

Table 2. Isotopes with No ENDF/B-VII.0 or -VII.1 Nuclear Data ............................. 8

Table 3. Effect of DBRC on $k_{\text {inf }}$ for a LWR Fuel Pin at $1200 \mathrm{~K}$................................ 14

Table 4. Assembly and Lattice Types with Available ORIGEN Reactor Libraries ..... 22

Table 5. Additional Information for ORIGEN Reactor Libraries.............................. 22

Table 6. MIX-COMP-THERM-004-001 Nuclide Sensitivity Coefficient Comparison ......................................................................................... 26

Table 7. Sensitivity Method Memory Usage. .......................................................... 27 


\section{Introduction}

The SCALE Code System is a widely used modeling and simulation suite for nuclear safety analysis and design that is developed, maintained, tested, and managed by the Reactor and Nuclear Systems Division (RNSD) of Oak Ridge National Laboratory (ORNL). SCALE provides a comprehensive, verified and validated, user-friendly tool set for criticality safety, reactor physics, radiation shielding, radioactive source term characterization, and sensitivity and uncertainty analysis. Since 1980, regulators, licensees, and research institutions around the world have used SCALE for safety analysis and design. SCALE provides an integrated framework with dozens of computational modules including 3 deterministic and 3 Monte Carlo radiation transport solvers that are selected based on the desired solution strategy. SCALE includes current nuclear data libraries and problem-dependent processing tools for continuous-energy (CE) and multigroup (MG) neutronics and coupled neutron-gamma calculations, as well as activation, depletion, and decay calculations. SCALE includes unique capabilities for automated variance reduction for shielding calculations, as well as sensitivity and uncertainty analysis. SCALE's graphical user interfaces assist with accurate system modeling, visualization of nuclear data, and convenient access to desired results. SCALE 6.2 represents one of the most comprehensive revisions in the history of SCALE, providing several new capabilities and significant improvements in many existing features.

New capabilities include:

- ENDF/B-VII.1 CE and MG nuclear data libraries with enhanced group structures,

- Neutron covariance data based on ENDF/B-VII.1 and supplemented with ORNL data,

- Covariance data for fission product yields and decay constants,

- Stochastic uncertainty and correlation quantification for any SCALE sequence with Sampler,

- Parallel calculations with KENO,

- Problem-dependent temperature corrections for CE calculations,

- CE shielding and criticality accident alarm system analysis with MAVRIC,

- CE depletion with T5-DEPL/T6-DEPL,

- CE sensitivity/uncertainty analysis with TSUNAMI-3D,

- Simplified and efficient LWR lattice physics with Polaris,

- Simplified spent fuel characterization with ORIGAMI and ORIGAMI Automator,

- Advanced fission source convergence acceleration capabilities with Sourcerer,

- Nuclear data library generation with AMPX,

- Integrated user interface with Fulcrum, and

- Many other new features.

Enhanced capabilities include:

- Accurate and efficient CE Monte Carlo methods for eigenvalue and fixed source calculations,

- Improved MG resonance self-shielding methodologies and data,

- Resonance self-shielding with modernized and efficient XSProc integrated into most sequences,

$$
\text { Getting Started - } 1
$$


- Accelerated calculations with TRITON (generally $4 \times$ faster than SCALE 6.1),

- Spent fuel characterization with 1,470 new reactor-specific libraries for ORIGEN,

- Keyword input for ORIGEN,

- Extension of the maximum mixture number to values well beyond the previous limit of 2,147 to $\sim 2$ billion,

- Expanded nuclear data formats enabling the use of more than 999 energy groups,

- Updated standard composition library to provide more accurate use of natural abundances, and

- Numerous other enhancements for improved usability and stability.

The user documentation for SCALE has also been substantially updated and reorganized around capabilities instead of the historical division of function modules, control modules, etc.

SCALE 6.2 was originally released in April 2016. Two minor updates have been released to provide numerous enhancements and increased stability. SCALE 6.2.1 was released in July 2016. The current version—SCALE 6.2.2—was released in February 2017.

This start-up guide provides an overview of many of the updated features of SCALE 6.2, instructions on how to install SCALE, instructions on using all the features of the SCALE runtime environment command line interface, and instructions on how to build SCALE. Because SCALE 6.2.1 and SCALE 6.2.2 are minor updates, references to the updated version are used only when necessary.

The SCALE user manual provides comprehensive documentation of all computational capabilities, nuclear data, input requirements, and output edits. Additional resources are available in the SCALE primers, located in the docs/primers directory after installation. These primers provide step-by-step instructions for running SCALE. However, at the time of this release, not all primers have been updated to reflect the latest features and user interface of SCALE 6.2. Additional resources are available on the SCALE website at http://scale.ornl.gov or by e-mailing scalehelp@ornl.gov. 


\section{Updates in SCALE 6.2}

\section{Nuclear Data}

\section{ENDF/B-VII.1 Cross Section Libraries}

ENDF/B-VII.1 nuclear data libraries are introduced in SCALE 6.2. CE data are available for general-purpose neutron, gamma, and coupled neutron/gamma calculations. MG neutron libraries in the 252- and 56-group structures are available, where the 252-group library is for general-purpose reactor physics and criticality safety applications and the 56-group library is intended for light water reactor analysis. Coupled neutron/gamma MG libraries for shielding are available in a fine 200-neutron/47-gamma group structure and a broad 28-neutron/19-gamma structure. An additional 8-group neutron library is available for code testing purposes but is not intended for production use.

\section{Neutron Cross Section Covariance Data}

Updated cross section covariance libraries are provided with SCALE 6.2 for use with the sensitivity and uncertainty modules. The data have been assembled from a variety sources, including high-fidelity covariance evaluations from ENDF/B-VII.1, other domestic and international evaluations, as well as approximate uncertainties obtained from a collaborative project performed by Brookhaven National Laboratory, Los Alamos National Laboratory, and ORNL. In addition, the covariance libraries now use a 56-group structure for broad group analysis, which is suitable for most applications, as well as the 252-group structure for fine group analysis, such as for energy-dependent reaction rates. These libraries are generated for compatibility with the ENDF/B-VII.1 56-group lattice physics library, 252-group criticality library, and CE analysis. The current SCALE covariance library spans the full energy range of the MG cross section libraries. The new 56-group and 252-group covariance libraries (56groupcov7.1 and 252groupcov7.1) are recommended for all applications. However, the previous library (44groupcov) distributed with SCALE 6.0 and SCALE 6.1 is retained for backwards compatibility. Covariance data are available for 456 materials, including some duplication for materials with multiple thermal scattering kernels. The SCALE 6.2 56-group covariance library (56groupcov7.1) is the default library for uncertainty calculations, but other libraries can be accessed by setting the appropriate parameter in the sensitivity/uncertainty analysis codes.

\section{Continuous-energy Data Processing}

Investigations into the CE data generated by the AMPX code system for deployment in SCALE 6.0 and 6.1 revealed a need for improvement in the $S(\alpha, \beta)$ treatment, especially for forward-peaked kinematics. The SCALE 6.2 ENDF/B-VII.0 and ENDF/B-VII.1 data libraries have been generated using new AMPX processing procedures, and the benchmark testing results with SCALE 6.2 show substantially improved results relative to SCALE 6.0 and 6.1. Select critical benchmark results for thermal mixed oxide (MOX) systems are provided in Figure 1, which shows that the bias in the SCALE 6.2 ENDF/B-VII.0 CE results is lower than SCALE 6.1. Additional testing has revealed that 
biases for burned light water reactor (LWR) fuel are resolved with the improved treatment. Additional details and further results are provide in Marshall et al. 2013. ${ }^{1}$

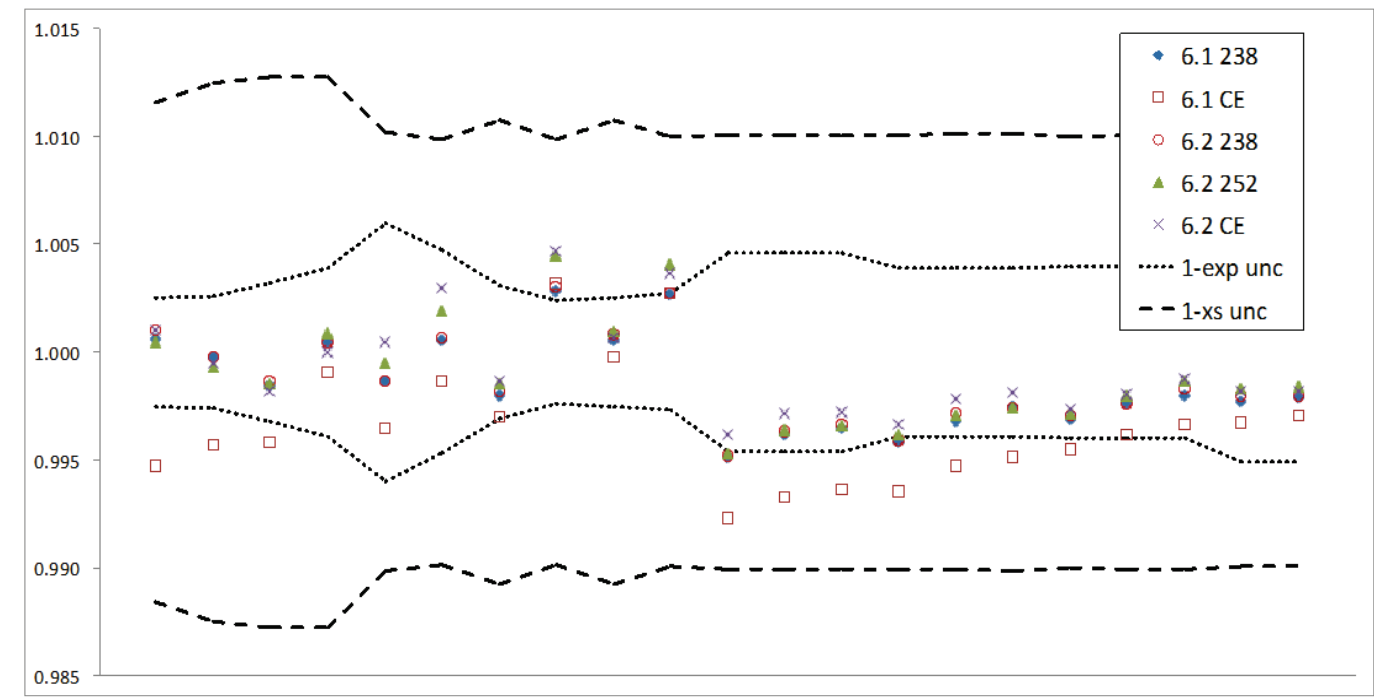

Figure 1. SCALE calculated results for International Criticality Safety Benchmark Evaluation Project (ICSBEP) thermal mixed oxide critical systems.

Additionally, the probability tables for $\mathrm{CE}$ reactions in the unresolved resonance range have been improved, primarily through the inclusion of additional resolution and error correction. Testing with the new probability tables has shown reduced biases for systems sensitive to the intermediate energy range. ${ }^{1}$

Historically, only neutron CE data libraries with a specific reaction subset of the ENDF libraries have been supported in KENO Monte Carlo criticality safety codes. To support CE depletion, sensitivity analysis, and coupled neutron-gamma shielding analysis, AMPX capabilities for the generation of CE neutron data have been improved and extended. Monaco now has the capability to model CE gamma interaction data and CE gamma yield data from neutron interactions. In addition, wide ranges of neutron reactions not needed for criticality calculations were also added to provide desired responses in Monaco.

The improved CE data have been comprehensively reviewed, verified, and validated, with approximately 5,000 infinite medium eigenvalue tests, 6,300 neutron and photon fixed-source transmission tests, and 400 criticality and shielding benchmark experiments to ensure robust and accurate calculations.

\section{Multigroup Data Processing}

ORNL has performed detailed comparisons between SCALE CE and MG results. Historically, a bias of 200-500 pcm has been observed where the earlier 238-group library is applied to LWR systems. AMPX has been used to develop a new 252- and 56-energy-group ENDF/B-VII.1 neutron cross section libraries. The new 252-group structure for SCALE 6.2 provides a more detailed representation of the ${ }^{238} \mathrm{U}$ resonance 
structure and reduces the previous bias. In addition to the new group structures, these libraries have been generated using a new weighting spectrum with improved resonance self-shielding procedures. For nuclides with atomic numbers of 40 and above, a CE flux spectrum computed with the CENTRM transport module for a PWR pin cell at $300 \mathrm{~K}$ was used as a weighting function. Temperature-dependent thermal scatter matrices for all actinides and $\mathrm{H}_{2} \mathrm{O}$ were processed with $\mathrm{CE}$ thermal flux at the corresponding temperatures. The standard AMPX weight function (i.e., fission spectrum $+1 / E+$ Maxwellian) was used for nuclides with $\mathrm{Z}<40$. Intermediate resonance parameters (lambdas) for all isotopes have been included in the library, which provides a capability for improved self-shielding with the Bondarenko method and Polaris. Bondarenko shielding factors, as a function of background cross section and temperature, were computed with three different approaches, depending on the nuclides. For nuclides with atomic numbers less than 40, the standard narrow resonance (NR) approximation was used. For all other actinides - except ${ }^{91} \mathrm{Zr},{ }^{96} \mathrm{Zr},{ }^{235} \mathrm{U},{ }^{238} \mathrm{U},{ }^{239} \mathrm{Pu},{ }^{240} \mathrm{Pu}$, and ${ }^{241} \mathrm{Pu}$ - the Bondarenko shielding factors were generated with CE spectra calculated by CENTRM for homogeneous mixtures of the resonance material and hydrogen, corresponding to the respective background cross section. Bondarenko factors for ${ }^{91} \mathrm{Zr},{ }^{96} \mathrm{Zr},{ }^{235} \mathrm{U},{ }^{238} \mathrm{U},{ }^{239} \mathrm{Pu}$, ${ }^{240} \mathrm{Pu}$, and ${ }^{241} \mathrm{Pu}$, are based on CENTRM CE calculations for heterogeneous pin cell models that span the range of anticipated self-shielding conditions. The thermal cutoff for bound and free-gas moderators has been raised from $3 \mathrm{eV}$ to $5 \mathrm{eV}$. The new library has been tested by analyzing a wide variety of critical benchmark experiments and by comparing results with CE Monte Carlo results. The 252-group results presented in Figure 1 demonstrate consistent performance with the SCALE 6.2 CE results for the thermal mixed oxide (MOX) critical benchmark experiments. Most results lie within the experimental uncertainty, and the outliers are believed to be caused by deficiencies in the ENDF/B-VII.1 data. Based on additional studies with the 252-group library, computational benchmark comparisons with $\mathrm{CE}$ results at room temperature and elevated temperatures show agreement within $100 \mathrm{pcm}$ in most cases.

\section{Nuclear Data File Formats}

A new binary data format has been introduced to SCALE and applied to modernize the format of the AMPX MG cross section data libraries, replacing the AMPX Working and Master formats that were designed in the 1960s. The previous formats were restricting the extension of capabilities desired to provide improved physics.

The binary object formatted file (BOFF) is a binary data format designed for flexibility and compactness and is intended to compete with native binary file formats that incorporate optimization techniques implicit to the developer and the data being stored. BOFF revolves around the steadfast data structures of primitive, array, and object data and can provide backward and forward data format compatibility. BOFF provides the capability to store data in a hierarchical manner using objects, arrays, and keyed-values. The BOFF format is implemented in the AMPX MG format to provide the ability to incorporate additional data in future updates. 


\section{Modernized Material Input Processing and Resonance Self-Shielding}

Most SCALE sequences now use the modern material and cross section processing module of SCALE, XSProc, which was developed for SCALE 6.2 to prepare data for CE and MG calculations. XSProc integrates and enhances the capabilities previously implemented independently in BONAMI, CENTRM, PMC, WORKER, ICE, and XSDRNPM, along with some additional capabilities provided by MIPLIB and SCALELIB in earlier releases of SCALE. XSProc expands material input from Standard Composition Library definitions into nuclide number densities; and for MG calculations, it also performs resonance self-shielding, energy group collapse, and spatial homogenization calculations for infinite homogeneous medium, lattice cell, multiregion, and double heterogeneous cell types.

For all but the double heterogeneous cells, a new capability is implemented for accelerated self-shielding using the intermediate resonance (IR) approximation for the full energy range (PARM=BONAMI), as well as the more rigorous option of a deterministic $\mathrm{CE}$ treatment in the resolved resonance range (PARM=CENTRM). Only the CENTRM path is available for double heterogeneous cell types. Sequence-specific control for generation of on-disk or in-memory self-shielded MG libraries for microscopic or macroscopic data containing selected reaction cross sections and scattering data is available with this modernized tool.

XSProc runtime and memory requirements are substantially improved compared to legacy computations, especially when performing calculations with many unit cells. Generally, speedups in self shielding of about $3 \times$ are realized, but in an extreme test case using an as-loaded spent nuclear fuel storage package with hundreds of unique materials, the cross section processing and cell homogenization time was reduced by $1000 \times$ while still obtaining equivalent results. XSProc is implemented across most SCALE sequences, as shown in Table 1, where modernized sequences with XSProc and older legacy sequences that run that standalone codes are identified. 
Table 1. SCALE Sequence Modernization

\begin{tabular}{|l|c|c|}
\hline \multicolumn{1}{|c|}{ Sequence } & Modern & Legacy \\
\hline CSAS-MG & $\checkmark$ & \\
\hline CSAS1 & $\checkmark$ & \\
\hline CSAS1X & $\checkmark$ & \\
\hline CSAS5 & $\checkmark$ & \\
\hline CSAS6 & $\checkmark$ & \\
\hline CSASI & $\checkmark$ & \\
\hline CSASIX & $\checkmark$ & \\
\hline MAVRIC & $\checkmark$ & \\
\hline STARBUCS & & $\checkmark$ \\
\hline T-NEWT & $\checkmark$ & \\
\hline T-XSDRN & $\checkmark$ & \\
\hline T-XSEC & $\checkmark$ & \\
\hline T5-DEPL & $\checkmark$ & \\
\hline T6-DEPL & $\checkmark$ & \\
\hline T-DEPL & $\checkmark$ & \\
\hline T-DEPL-1D & $\checkmark$ & \\
\hline TSUNAMI-1D & $\checkmark$ & \\
\hline TSUNAMI-2D & & $\checkmark$ \\
\hline TSUNAMI-3D_K5 & $\checkmark$ & \\
\hline TSUNAMI-3D_K6 & $\checkmark$ & \\
\hline
\end{tabular}

\section{Missing Cross Section Messages}

Several naturally occurring isotopes are not present in the SCALE nuclear data libraries. For example, the natural abundance for oxygen contains $0.20 \%{ }^{18} \mathrm{O}$, but ENDF evaluations do not provide cross sections for this isotope.

In previous versions of SCALE, the Standard Composition Library made several nonphysical adjustments to facilitate the use of nuclear data libraries with missing nuclides. In the case of ${ }^{18} \mathrm{O}$, the standard compositions $U O_{2}, O$, and others assumed oxygen to consist of $100 \%{ }^{16} \mathrm{O}$ instead of its true natural abundance. If the user entered the standard composition name $O X Y G E N$, the true natural abundances would be used, and the calculation would immediately stop on an error that ${ }^{18} \mathrm{O}$ was not present on the nuclear data library. For source terms calculations and nuclear heating analysis, the absence of ${ }^{17} \mathrm{O}$ can non-conservatively reduce results by several percent.

In SCALE 6.2, this behavior has been changed because natural abundances are consistently applied for all standard composition materials. When isotopes without nuclear data are present in the input, the calculation continues with the isotopes removed from the model, which produces identical radiation transport and similar activation/depletion results as those obtained when using a zero-valued cross section for each missing isotope. The isotopes that occur in the Standard Composition Library, but with no corresponding nuclear data in ENDF/B-VII.0 or -VII.1, are shown in Table 2.

$$
\text { Getting Started }-7
$$


In SCALE 6.2.2, an update has been introduced for processing ${ }^{18} \mathrm{O}$. If a composition contains ${ }^{18} \mathrm{O}$, either explicitly or implicitly (such as $\mathrm{UO}_{2}$ ), SCALE internally introduces a zero-value cross section for ${ }^{18} \mathrm{O}$, and a warning is provided in the message file. The radiation transport solution is unaltered, but output edits such as the KENO mixing table contain ${ }^{18} \mathrm{O}$. The inclusion of ${ }^{18} \mathrm{O}$ in composition output edits allow users to verify that the input composition density is consistent with the density computed from the isotope concentrations. For depletion calculations, the zero-valued cross section for ${ }^{18} \mathrm{O}$ is replaced by the cross sections included in the JEFF-3.0/A neutron activation files.

For compositions that contain isotopes in Table 2 other than ${ }^{18} \mathrm{O}$, a warning message is provided with explicit number densities of every nuclide in the composition so that the user has a choice of accepting the SCALE composition with decreased density or updating the composition input to compensate for the missing material in some other way such as increasing the density of some other nuclide. In most cases, the missing nuclides do not make up a large fraction of the natural abundance, and making some other adjustments will have a negligible impact on computed results.

Table 2. Isotopes with No ENDF/B-VII.0 or -VII.1 Nuclear Data

\begin{tabular}{|c|c|c|c|c|}
\hline Element & $\begin{array}{c}\text { SCALE } \\
\text { standard } \\
\text { composition ID }\end{array}$ & $\begin{array}{c}\text { Missing } \\
\text { Isotopes }\end{array}$ & ZA numbers & \% abundance \\
\hline oxygen & 8000 & 18 & 8018 & 0.20 \\
\hline neon & 10000 & 21,22 & 10021,10022 & $0.27,9.25$ \\
\hline ytterbium & 70000 & $\mathrm{All}^{(1)}$ & $(1)$ & \\
\hline osmium & 76000 & $\mathrm{All}^{(2)}$ & $(2)$ & \\
\hline platinum & 78000 & $\mathrm{All}^{(3)}$ & $(3)$ & 0.01 \\
\hline tantalum & 73000 & $180 \mathrm{~m}$ & 1073180 & \\
\hline
\end{tabular}

(I) no ENDF/B-VII data for any of the seven naturally occurring ytterbium isotopes

(2) no ENDF/B-VII data for any of the seven naturally occurring osmium isotopes

(3) no ENDF/B-VII data for any of the six naturally occurring platinum isotopes

\section{CENTRM/PMC Improvements}

A new two-dimensional unit cell geometry is available in CENTRM to explicitly treat the boundary of square-pitched reactor lattices using with a method-of-characteristics (MoC) $\mathrm{CE}$ transport option, typically resulting in approximately $100 \mathrm{pcm}$ reduction in bias between $\mathrm{MG}$ and $\mathrm{CE}$ calculations. This is now the default option for fuel lattice calculations using CENTRM through XSProc within SCALE sequences.

New options are available in CENTRM/PMC to address the effects of resonance self-shielding on scattering matrices using the $N 2 D=$ option in centrm data in celldata. $N 2 D=2$ corrects the higher order Legendre moments with corresponding higher order fluxes, based on the consistent $\mathrm{P}_{\mathrm{N}}$ approximation, $N 2 D=-2$ similarly treats the higher order Legendre moments and explicitly treats within-group elastic scattering removal. The impact of these options is demonstrated on the IEU-MET-FAST-005-001 fast system with a steel reflector, with $\mathrm{k}_{\text {eff }}$ results shown in Figure 2 . Here it is observed that the use

$$
\text { Getting Started }-8
$$


of the $\mathrm{N} 2 \mathrm{D}=2$ option produces $\mathrm{MG}$ results more consistent with the reference CE Monte Carlo results. However, these results are not universal for all calculations, so the $N 2 D=-2$ option should be applied after careful investigation of each system type. In particular, the use of this option for systems with large reflectors is not recommended.

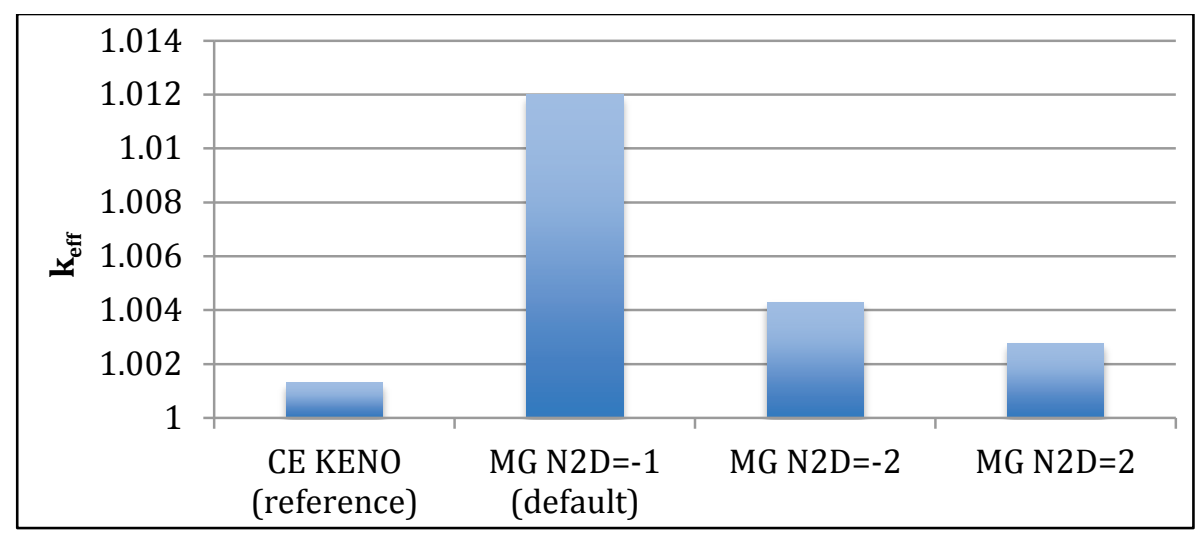

Figure 2. $k_{\text {eff }}$ values for IEU-MET-FAST-005-001 with different MG options.

\section{Bondarenko Self-Shielding}

BONAMI has been rewritten as part of the overall SCALE modernization effort. BONAMI now implements IR approximation theory, as well as the previous narrow resonance (NR) approximation. The IR approximation allows for improved accuracy and provides a rapid self-shielding option using only full-range Bondarenko factors. This technique produces accurate results and provides up to a $10 \times$ speedup relative to $\mathrm{CE}$ treatment with CENTRM. IR theory is described in the updated BONAMI section, Section 8.2. To enable this option, set $P A R M=B O N A M I$ on the sequence specification record (e.g. =t-depl $P A R M=B O N A M I$ ) and $I R O P T=1$ in the more data section of celldata.

\section{Graphical User Interface - Fulcrum}

Fulcrum is a cross platform graphical user interface designed to create, edit, validate and visualize SCALE input, output, and data files. Historically, SCALE has provided several special-purpose graphical user interfaces, which operate only on specific platforms and are loosely integrated with SCALE's computational and data components. Fulcrum, in contrast, is intended to provide a single user interface that directly integrates with SCALE's internal resources to provide a consistent experience between Fulcrum and SCALE's command line interface.

The concept of Fulcrum is based on decades of feedback from the user community through the release of numerous interfaces. In contrast to the SCALE 6.1 GeeWiz interface with many layers of dialog boxes, Fulcrum directly connects the user with the text form of the input file while providing inline features to assist with building correct inputs. Fulcrum provides input editing and navigation, interactive geometry visualization 
for KENO V.a, KENO-VI and NEWT, job execution, overlay of mesh results within a geometry view, and plotting of data from most SCALE file formats. An error checker interactively identifies poorly formed input with spelling errors or data entry omissions for all SCALE sequences. The Hierarchical Input Validation Engine (HIVE) identifies allowed data ranges and interdependencies in the input and reports inconsistencies to the user. Fulcrum interactively processes standard composition data to produce a mixing table, lists expanded input aliases for review, provides an internal list of input as required for Sampler material and geometry perturbation analysis, and launches the SCALE sample problems.

The layout of panels in Fulcrum is highly configurable to accommodate the preferences of many users, as shown in Figure 3.

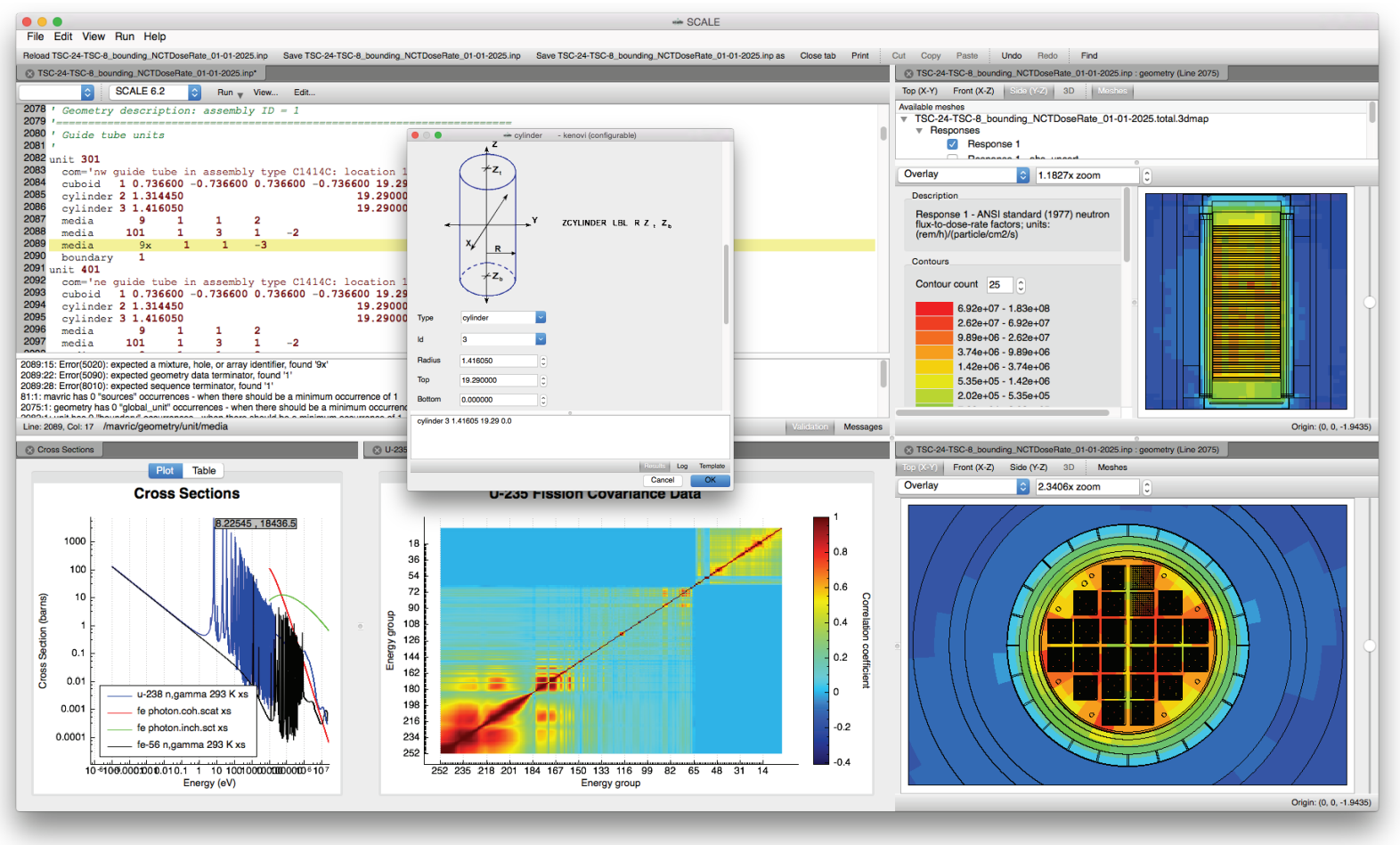

Figure 3. SCALE graphical user interface - Fulcrum.

The Fulcrum user interface provides numerous features, such as:

- The interface operates on all supported platforms-Linux, Mac and Windows.

- The user can edit, view, and run multiple inputs simultaneously.

- Context-aware auto-completion input generation menus are presented when $c t r l+$ space is pressed within an input file,

- Graphical forms-based input is available to assist users with the creation of sequence blocks and specific input components (press ctrl+space and select a configurable item).

- Input is validated as it is entered, and erroneous or missing input is identified in the Validation panel. 
- Geometry models can be visualized for sequences that use KENO V.a, KENO-VI, Monaco, and NEWT (View... menu shown in toolbar associated with relevant input file).

- Fulcrum is preconfigured to run SCALE 6.2 but can be custom configured for other codes.

- Associated files (such as .out, .msg, plot, or data files) are shown in contextual pop-up menus for each file in the navigation panel and can be opened by right clicking on the input file name in the navigation panel.

- Associated files not supported in Fulcrum open a local tool for appropriate viewing (e.g. .html files launch the system web browser).

- Mesh tallies stored in .3dmap files can be overlaid with geometry view (Meshes on toolbar in geometry view, right click in Available Meshes panel to load a .3dmap file).

- Data plotting is available for the following SCALE files:

- MG and CE cross section data

- Cross section covariance data

- ORIGEN gamma data

- UNF-STANDARDS time series

- $\quad$ ORIGEN concentration file ( $f 71)$ with integrated unit conversion (OPUS capability)

- Sensitivity data file (.sdf)

- $\quad$ KENO reaction rate and flux file $(. k m t)$

- $\quad$ Ptolemy plot file (.ptp) (previously Javapeño 2D plot, .plt)

- MAVRIC Chart Plot file (.chart)

- Data files with file extensions (.sdf, .kmt, etc.) are accessed through File/Open file. . .

- Other file types, especially SCALE nuclear data files, have a specific menu item in the File menu (e.g., Open continuous-energy library. . . ).

- Plots can now be saved in an editable format as a SCALE Plot File (.spf).

- Data tables are available for data shown in a plot.

- Geometry volumes can be computed for KENO and Monaco models.

- Online help is available.

- Many more features are included but are too numerous to list here.

As Fulcrum is a new user interface, users are directed to the Help menu within the application itself for Help documentation. A detailed introduction and tutorial to Fulcrum is available for download at http://scale.ornl.gov.

\section{Criticality Safety}

KENO has been substantially improved for SCALE 6.2, especially for the accuracy and efficiency of $\mathrm{CE}$ calculations, $\mathrm{CE}$ temperature corrections, source convergence diagnostics and acceleration, and parallel capabilities.

\section{Reduction in Memory Requirement of CE Internal Storage}

Numerous improvements were made to enhance the CE capabilities of KENO, especially to reduce the memory requirements of the calculations. CE calculations now require $40-$ $99 \%$ less memory than previous versions with no loss of accuracy in the results. Improved memory efficiency will be observed for all calculations, but especially for 
models with many materials and/or temperatures. Models that previously would have required hundreds of GB of memory with SCALE 6.0 and 6.1 can now be performed with only a few GB of memory.

User controllable options:

- UUM: Optional unionization of mixture-dependent cross section data results in a $50-90 \%$ reduction in memory requirements for a single material, with no additional memory required for the use of the same nuclide in more than one mixture. Previously burned fuel calculations that would have required hundreds of GB of memory can now be run with just a few GB through the use of the default setting of $U U M=n o$ in the KENO parameter data.

- $M 2 U$ : Optional nuclide level energy grid unionization (map2union) controls the unionization of all reaction types within a single nuclide. The default behavior, $M 2 U=y e s$, in KENO parameter data helps to reduce runtime and was the default behavior in SCALE 6.0 and 6.1. Optionally disabling map2union $(M 2 U=n o)$ results in $\sim 20 \%$ reduction in memory with $\sim 10 \%$ increase in runtime.

Other internal optimizations include:

- Redundant copies of temperature-independent data were removed to improve memory efficiency for models with multiple temperatures. Previously, each set of temperature-dependent cross sections also included a redundant copy of the temperature-independent data, which caused an almost linear increase in memory for each new temperature. With this update, the temperature-independent data are shared by all temperatures, resulting in $>50 \%$ memory savings for the addition of each temperature relative to the techniques implemented in SCALE 6.0 and 6.1.

- Kinematics (scattering) data structures were updated to optimize data storage, especially with an updated structure in the CE data itself. These updates result in $\sim 40 \%$ reduction in memory requirements using the same runtime.

- Internal data storage was changed from double to single precision where possible, resulting in a $15-45 \%$ reduction in memory requirements depending on the nuclides used in the model.

- Optional data loading was introduced by filtering data during reading instead of loading all available data whether it is needed or not. Filtering methods such as energy range, reaction types, data types, etc., results in $\sim 20 \%$ reduction in memory requirements.

\section{Fission Source Convergence Diagnostics}

Prior to SCALE 6.2, KENO provided only plots of $k_{\text {eff }}$ by generation and average $k_{\text {eff }}$ for visual inspection of source convergence, followed by a $\chi^{2}$ statistical assessment of convergence. With SCALE 6.2, Shannon Entropy fission source convergence diagnostic techniques have been implemented in KENO to provide improved confidence in the 
computed results, as well reduced simulation times in some cases. Confirming the convergence of the fission source distribution is especially useful to avoid the false convergence of $k_{\text {eff }}$ and neutron flux tallies that can be caused by insufficient sampling of important portions of the system. ${ }^{2}$ Source convergence diagnostics are enabled with $S C D=y e s$ (default) in the KENO parameter data.

\section{Problem-Dependent Doppler Broadening}

The CE data libraries distributed with SCALE are provided with only about five temperatures per isotope. The Doppler broadening temperature corrections using only a few temperatures may not match the desired temperature of the calculation. When temperatures of the KENO model are different from those present on the library, KENO selects the nearest temperature, which can be several hundred degrees from the desired temperature, producing results that can vary significantly from those that would be produced at the correct temperature.

New methods have been developed and implemented to provide problem-dependent temperature corrections by Doppler broadening the point-wise data in the resolved resonance region and the probability tables in the unresolved resonance region when the cross sections are loaded for the calculation. ${ }^{3}$ The thermal scattering data are also updated to requested temperature. The runtime penalty for this methodology is negligible, as all temperature corrections are performed as the calculation begins, typically requiring only a few seconds to a few minutes depending on the number of nuclides and temperatures used.

The eigenvalues computed for a typical fresh pressurized water reactor (PWR) pin cell using the nearest selected $\mathrm{CE}$ temperature, and problem-dependent $\mathrm{CE}$ temperature treatments are shown in Figure 4. Problem-dependent Doppler broadening is controlled with $D B X=0$ to select the nearest temperature, $D B X=1$ to perform problem-dependent corrections for the resolved and unresolved resonance ranges, and $D B X=2$ (default) to perform also corrections for the $S(\alpha, \beta)$ thermal scattering data.

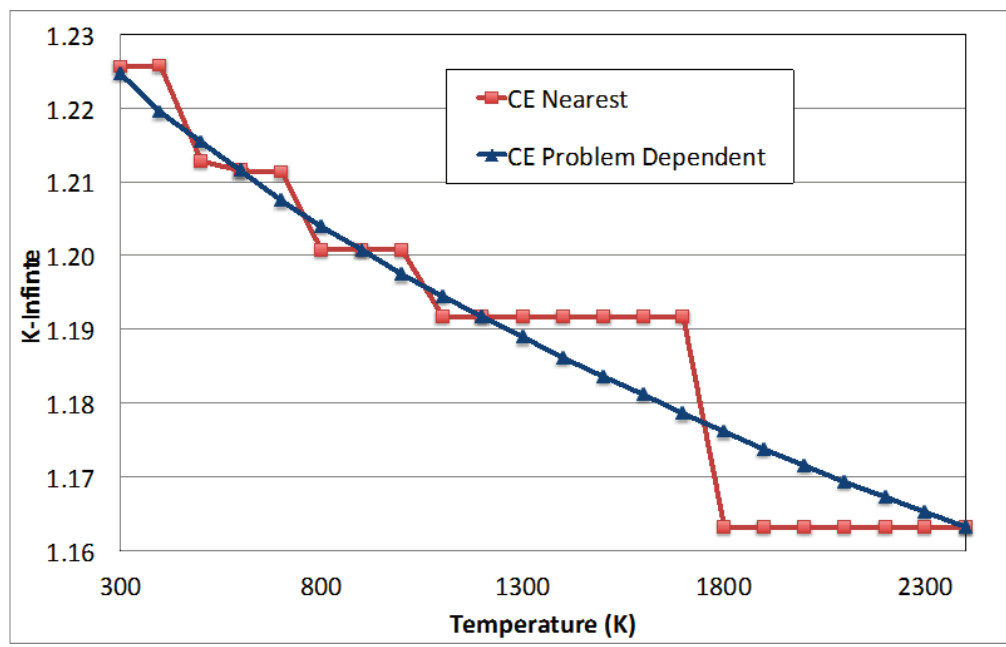

Figure 4. Eigenvalues computed for a PWR pin cell with different temperature treatments.

Getting Started - 13 


\section{Doppler Broadened Rejection Correction}

The implementation of Doppler Broadened Rejection Correction (DBRC) techniques provides further enhancements for calculations with elevated temperatures. ${ }^{4}$ As shown in Table 3, DBRC in KENO presents a reactivity correction of approximately $300 \mathrm{pcm}$ relative to the default methodology for a $1200 \mathrm{~K}$ LWR fuel pin, consistent with that predicted with MCNPX by the originators of the methodology. ${ }^{5}$ DBRC is enabled with $D B R=$ in the KENO parameter data. Available options are $D B R=0$ to disable the correction (default), $D B R=1$ to perform $\mathrm{DBRC}$ for ${ }^{238} \mathrm{U}$ only, and $D B R=2$ to perform DBRC for all major actinides. The use of DBRC only impacts calculations at elevated temperatures, and there is a runtime penalty for the use of this methodology. As such, it is not recommended for calculations near room temperature.

Table 3. Effect of DBRC on $\boldsymbol{k}_{\text {inf }}$ for a LWR Fuel Pin at 1200K

\begin{tabular}{|c|c|c|c|}
\hline Case & Default & DBRC & $\begin{array}{c}\text { Difference } \\
(\mathrm{pcm})\end{array}$ \\
\hline MCNPX & $1.31137+/-9 \mathrm{E}-5$ & $1.30791+/-9 \mathrm{E}-5$ & -346 \\
\hline KENO-VI & $1.31029+/-15 \mathrm{E}-5$ & $1.30730+/-15 \mathrm{E}-5$ & -299 \\
\hline
\end{tabular}

\section{Sourcerer - Hybrid Method for Starting Source Distribution}

The Sourcerer sequence introduced in SCALE 6.2 uses the Denovo ${ }^{6}$ discrete-ordinates code to generate a starting fission source distribution in a KENO Monte Carlo calculation. Initial studies ${ }^{7,8}$ have shown that using a starting fission distribution that is similar to the true fission distribution can both reduce the number of skipped generations required for fission source convergence and significantly improve the reliability of the final $k_{\text {eff }}$ result.

For many criticality safety applications, the additional step of performing a deterministic calculation to initialize the starting fission source distribution is not necessary. However, for the most challenging criticality safety analyses, such as spent nuclear fuel loaded transportation packages with a mixed loading of low- and high-burnup fuel, even a lowfidelity deterministic solution for the fission source produces more reliable results than the typical starting distributions of uniform or cosine functions over the fissionable regions, as demonstrated in a recent study. ${ }^{9}$ In that study, a cask holding 24 spent fuel assemblies was examined using a uniformly distributed starting source and a deterministically calculated starting source. Multiple clones of KENO were run (with different random number seeds) for different values of skipped cycles. The number of clones that gave an incorrect result for $k_{\text {eff }}$ was then tabulated. The results, presented in Figure 5, show that using a deterministic starting source significantly increases the $k_{\text {eff }}$ reliability. See the Sourcerer documentation in Section 2.4 for full details. 


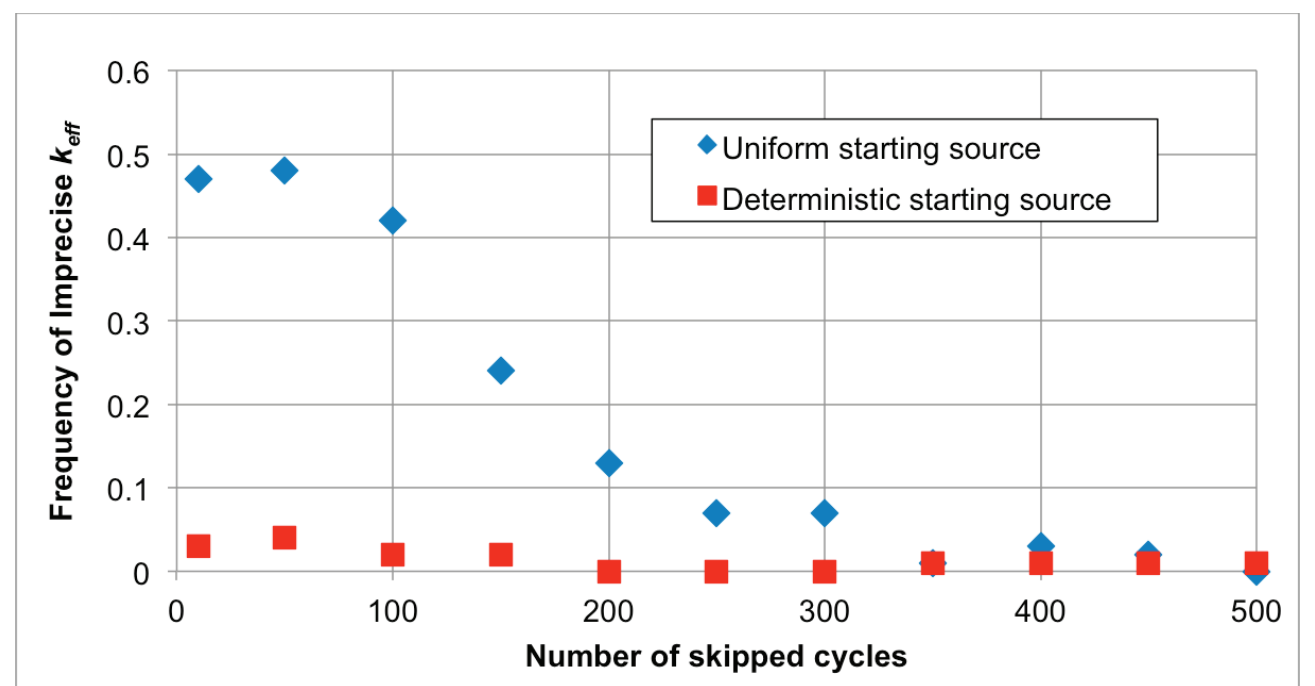

Figure 5. Fraction of failure to agree with the reference $\boldsymbol{k}_{\text {eff }}$ value for KENO calculations with different starting sources (Figure 4 from Ref. 9).

\section{Distributed Memory Parallelism via MPI}

In addition to the numerous improvements described that directly affect solution accuracy and efficiency, parallel computation capabilities, especially for Linux clusters, have been added to KENO to provide reductions in wall clock time, especially for $\mathrm{S} / \mathrm{U}$ analysis or Monte Carlo depletion on computer clusters. By introducing a simple master-slave approach via message passing interface (MPI), ${ }^{10}$ KENO runs different random walks concurrently on the replicated geometry within the same generation. The fission source and other tallied quantities are gathered at the end of each generation by the master process; then, they are processed either for final edits or next generations.

The parallel performance of KENO as used in a CE calculation for a graphite-moderated reactor model is shown in Figure 6. These tests were conducted on a heterogeneous Linux cluster in which the size of the nodes varies from 4 to 16 cores with differing processor speeds, much like SCALE users may encounter in practice. Tests were conducted with systematically increasing numbers of particles per generation, and various combinations of options were enabled to develop the distributions of speedups for each number of MPI processes shown in the figure. With larger numbers of particles per generation, KENO provides nearly linear speedup on the 64 processors tested here and has been successfully demonstrated on hundreds of processors. 


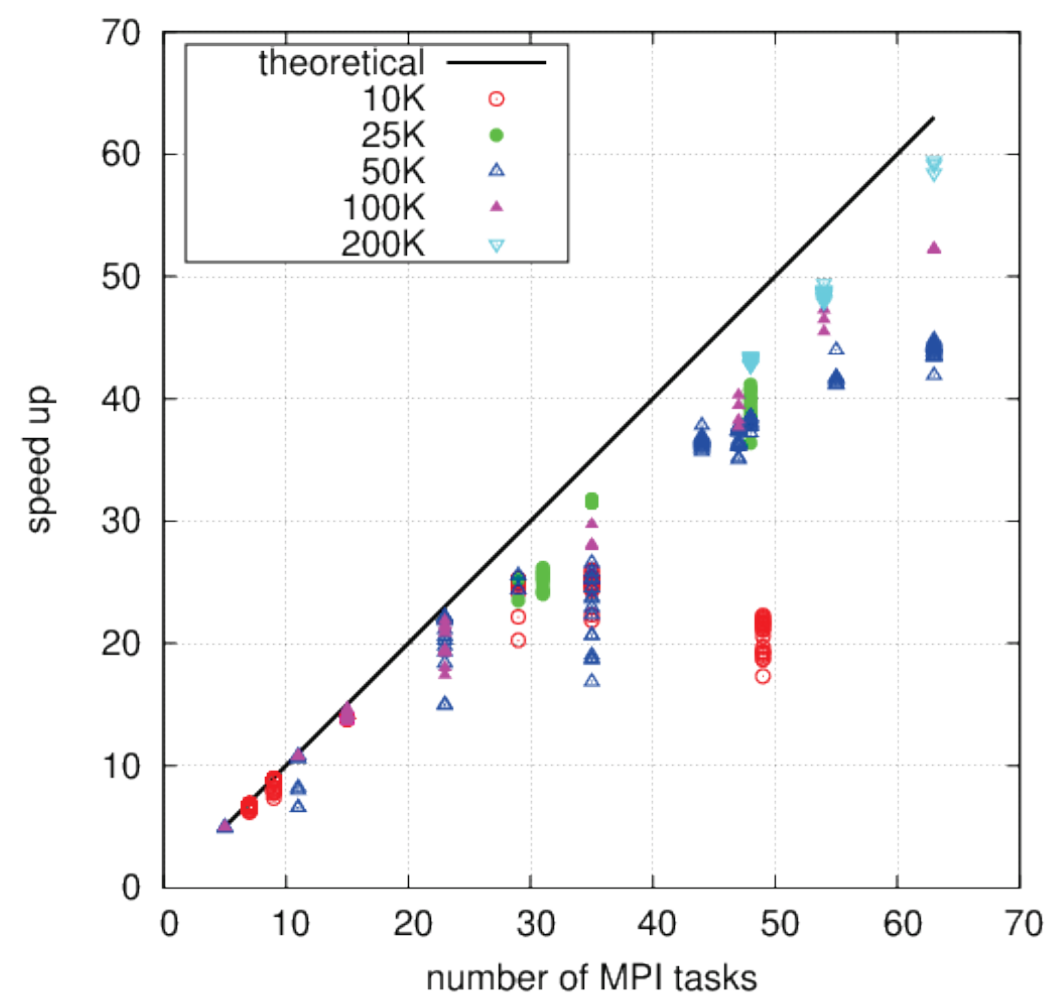

Figure 6. Speedup for parallel KENO-VI calculations for a graphite-moderated reactor model.

\section{Reaction Tallies}

User-configurable reaction rate tallies are now available within KENO CE calculations. The tallies are specified in the REACTION block of KENO. 


\section{Reactor Physics}

\section{Polaris - New Advanced Lattice Physics Module}

Polaris is a new 2D lattice physics code for SCALE that uses a new MG self-shielding method called the Embedded Self-Shielding Method (ESSM) ${ }^{11}$ and a new MoC transport solver. Polaris uses a simplified input, where only a few lines are required to describe the entire model. Polaris provides substantially reduced input requirements and improved runtime performance compared to TRITON (see Section 3.2).

The ESSM approach computes MG self-shielded cross sections using interpolation of the Bondarenko factors in the MG library. The background cross section used in the interpolation is determined by a series of $2 \mathrm{D} \mathrm{MoC}$ fixed-source calculations similar to the subgroup method. Polaris does not require explicit celldata input, as needed in TRITON. Additionally, heterogeneous lattices are explicitly treated without the need to externally compute Dancoff factors. Additional details on ESSM are provided in Ref. 11.

Polaris currently employs ESSM with either the 252- or 56-group ENDF/B-VII.1 libraries. Each library contains cross sections, IR parameters, and full-range Bondarenko factors for all nuclides.

Polaris uses the self-shielded cross sections to perform a MG 2D eigenvalue calculation with the new MoC transport solver developed for Denovo, a parallel 3D Cartesian mesh MG discrete ordinates $\left(\mathrm{S}_{\mathrm{N}}\right)$ code. ${ }^{6}$ Polaris also provides a critical spectrum calculation for correcting the flux distribution for computing both few-group homogenized cross section edits and depletion reaction rates.

Polaris is integrated with ORIGEN for depletion calculations. The depletion of each pin, or radial subregion of the pin, is based on the local normalized flux distribution. Cross section values in the ORIGEN transition matrix are updated from the MG self-shielded cross sections and the MG flux distribution for each depletion region. The criticalspectrum correction to the flux distribution for depletion is controlled by an input user option. Cross section updates are performed in-memory, as compared to the file-based approach utilized in the TRITON lattice physics sequence. Polaris supports branch calculations for the generation of few group constants for reactor core simulators.

\section{Continuous-Energy Monte Carlo Depletion}

SCALE 6.1 provided MG Monte Carlo depletion capability that coupled the SCALE MG cross section processing methodology with KENO and ORIGEN. A new CE-based KENO/ORIGEN Monte Carlo depletion capability has been developed for SCALE-6.2, which can be activated by simply changing the input library specification. CE depletion is especially useful for models with complex geometry that present difficulties in obtaining accurate resonance self-shielded MG data, and for models with many depletion regions where run-time to generate and store the resonance self-shielded cross section data for each material is prohibitive. 


\section{Lattice Physics with TRITON}

Two-dimensional lattice physics calculations with TRITON will realize substantial speedups due to the use of XSProc for resonance self-shielding as well as numerous optimizations within the NEWT code. Speedups of 2-6× faster calculations are common, and some models have realized speedups of $30 \times$ relative to previous versions of NEWT. Calculation times for 1,470 TRITON calculations used to generate the ORIGEN reactor libraries for SCALE 6.1.3 and SCALE 6.2 are shown in Figure 7. The average calculation time was reduced by a factor of 4 .

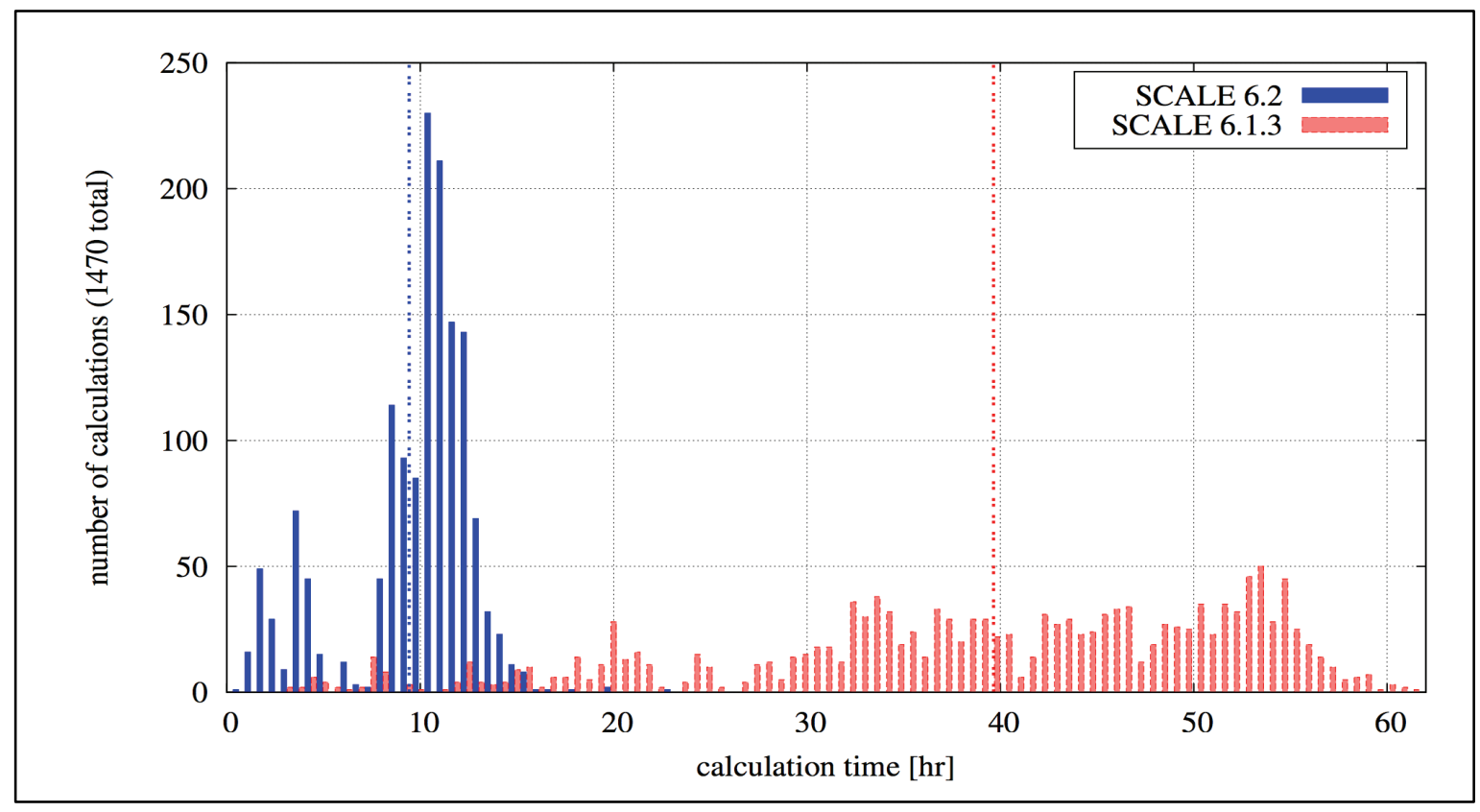

Figure 7. Comparison of calculation times for 1,470 TRITON calculations.

A material SWAP capability is now available within the TIMETABLE block of TRITON. This feature allows a user to switch the material present during a depletion calculation, which is especially useful for modeling removable absorber materials.

\section{Bias Reduction for Depletion}

Historical SCALE biases have been mitigated as a result of the numerous enhancements included in SCALE 6.2.

Figure 8 shows the results for a reference PWR pin cell burnup calculation using SCALE 6.1 and SCALE 6.2. Here, the SCALE 6.2 CE results are the reference, and the SCALE 6.1 CE results are eigenvalue statepoints using the isotopics provide by the SCALE 6.2 results, as CE depletion is not available in SCALE 6.1. The 1,000 pcm bias in the SCALE 6.1 CE results are due to erroneous treatment of thermal scattering data in the AMPX CE data distributed with SCALE 6.1. The SCALE 6.1 TRITON 238 group results using the default settings from SCALE 6.1 demonstrate the typical 400-500 pcm bias observed for SCALE 6.1 LWR calculations. The TRITON 252 group results apply the improved nuclear data library, the CENTRM MoC solver, and the enhanced within

$$
\text { Getting Started - } 18
$$


group treatment for CENTRM/PMC, $N 2 D=-2$. The SCALE 6.2 Polaris results apply the default settings in Polaris with the ESSM methodology and IR factors on the library. These calculations demonstrate the impact of improvements in both the MG libraries and resonance self-shielding techniques, as well as improvements in the CE treatments from SCALE 6.1 to SCALE 6.2.

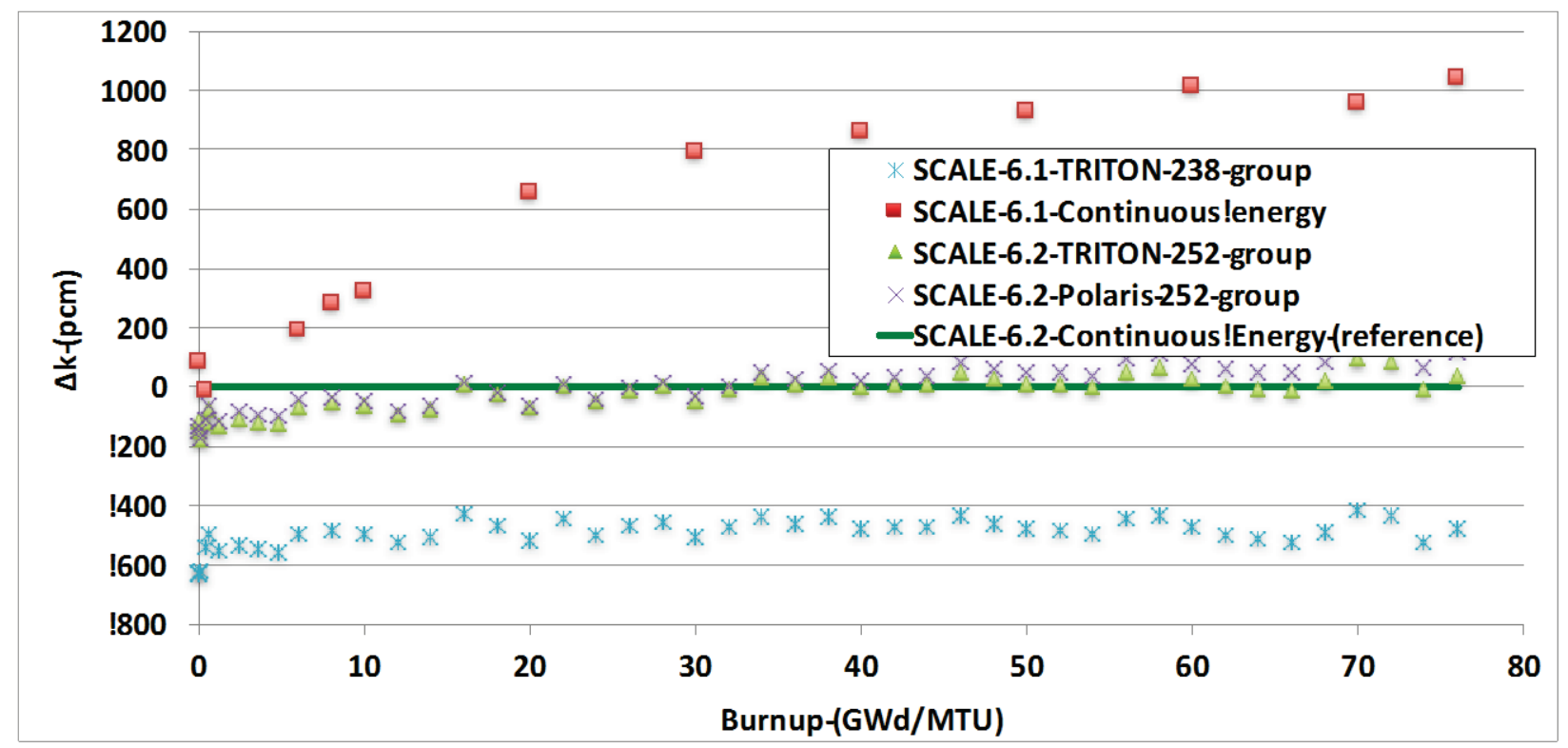

Figure 8. Comparison of computational biases as a function of burnup.

\section{Radioactive Source Term Characterization}

\section{ORIGEN}

SCALE includes the ORIGEN code with its comprehensive depletion, activation, decay, gamma-ray, and x-ray library for over 2,200 nuclides. ORIGEN and its nuclear data libraries have been updated in SCALE-6.2 to include convenient modular interfaces that provide easy access to ORIGEN's robust capabilities by other software packages.

The input for ORIGEN has been updated to provide a modern interface based on SCALE's Standard Object Notation (SON). The legacy FIDO input interface will continue to be supported for backwards compatibility, but users are encouraged to begin using the modern SON procedures through input files, as well as the forms-based input in Fulcrum. An excerpt from an example problem is included below to show this new structured, keyword-based input. 


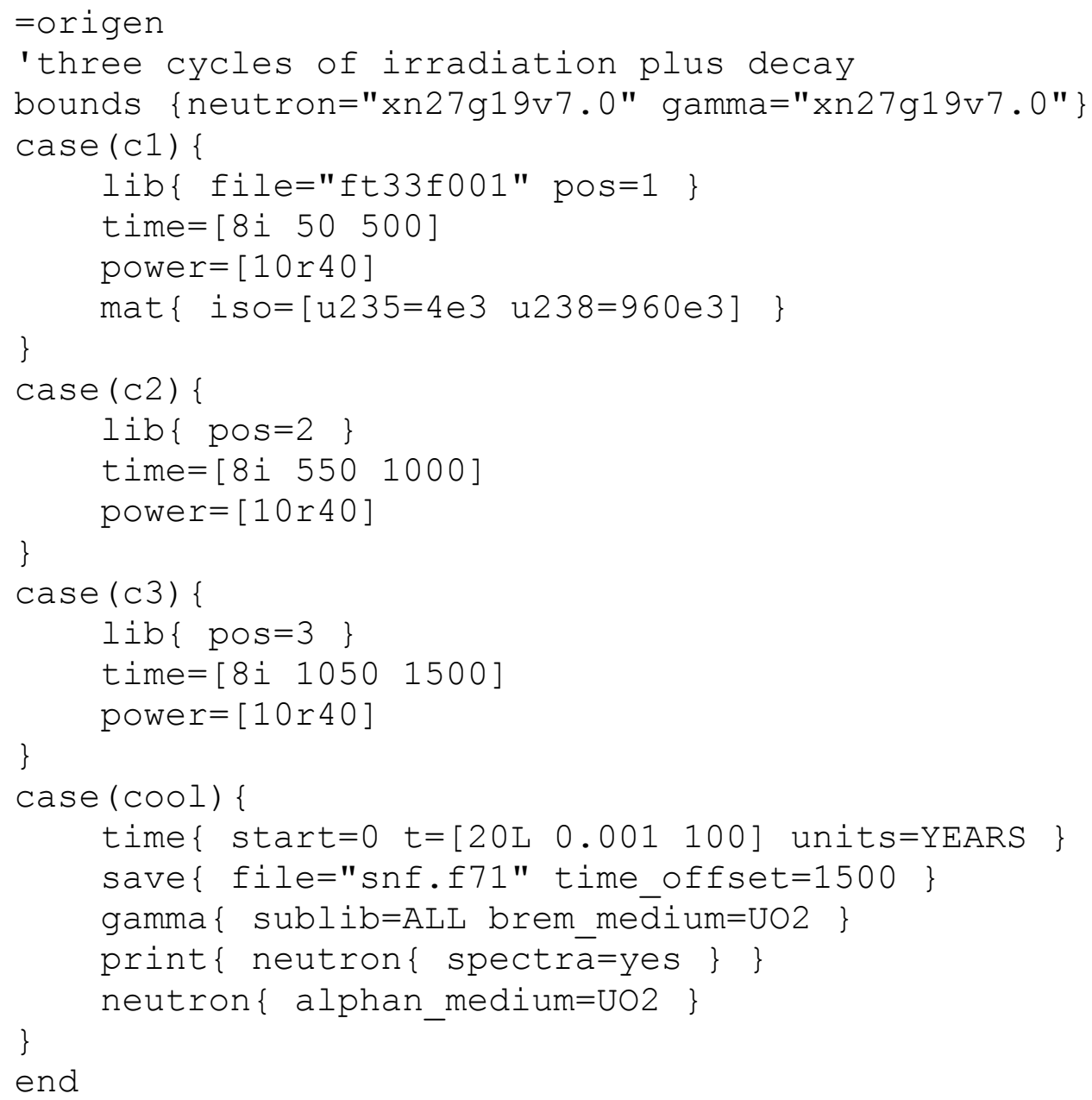

The ENDF/B-VII.0-based fission product yields used by ORIGEN are updated to provide improved agreement with burst fission experiments for ${ }^{235} \mathrm{U},{ }^{238} \mathrm{U},{ }^{239} \mathrm{Pu}$, and ${ }^{241} \mathrm{Pu}$. The changes correct inconsistencies in ENDF/B-VII.0 introduced by updating the nuclear decay data in ENDF/B VII.0, but not updating the independent and cumulative fission product yields. The yield adjustments improve the consistency between the direct fission yields, decay data, and the cumulative yields in ENDF/B-VII.0.

ORIGEN has been enhanced to provide an alternative solver based on the Chebyshev Rational Approximation Method (CRAM). When compared to a robust reference solution for a depletion problem, ORIGEN's traditional matrix power series supplemented by Bateman solutions for short-lived isotopes, predicts isotopic concentrations with an average relative error of about 10E-5, where the CRAM solver produces an average relative error of about 10E-15 for the same case. The CRAM solver can also handle essentially arbitrary step lengths through internal substepping with minimal computational cost, so the runtimes are generally faster for large depletion problems. The previously mentioned depletion case required $550 \mathrm{~ms}$ to execute 8 substeps with the traditional solver, where CRAM completed the more accurate calculation in $44 \mathrm{~ms}$. However, CRAM is slower than the traditional solver for decay problems or problems 
with small libraries. CRAM also allows for time-dependent source terms and adjoint calculations, enabling the extension of ORIGEN to new classes of calculations. CRAM is currently available as an optional solver in stand-alone ORIGEN calculations, ORIGAMI, and in the Polaris lattice physics code.

\section{ORIGAMI - New Advanced Used Fuel Assembly Characterization}

SCALE 6.2 includes the new ORIGAMI (ORIGEN Assembly Isotopics) tool to compute detailed isotopic compositions for LWR assemblies with $\mathrm{UO}_{2}$ fuel. ORIGAMI calls interfaces to the ORIGEN transmutation code, with pre-generated parameterized libraries (see next section) and a specified assembly power distribution. The assembly may be represented by a single lumped model with only an axial power distribution or by a square array of fuel pins with variable pin-powers as well as an axial distribution. In either case, ORIGAMI performs ORIGEN burnup calculations for each of the specified power regions to obtain the spatial distribution of isotopes in the burned fuel. Multiple cycles with varying burn-times and down-times may be used. ORIGAMI produces files containing SCALE and MCNP-formatted composition data for axial burnup distribution at the last time-step, as well as a file containing the axial decay-heat at the final time-step, and a file specifying the energy-dependent radioactive source for use in shielding calculations of the burned assembly.

\section{ORIGEN Reactor Libraries}

A new series of 1,470 pre-generated burnup libraries for use in ORIGEN and ORIGAMI are introduced to SCALE 6.2. These libraries are generated under quality assurance with TRITON using the ENDF/B-VII.1 252-group nuclear data library through the use of a new template and script system. These libraries are intended to replace the ORIGENARP libraries that were generated with a previous version of SCALE with varying levels of quality assurance. Libraries are available for a variety of fuel assemblies for commercial and research reactors, as detailed in Table 4 and Table 5. 
Table 4. Assembly and Lattice Types with Available ORIGEN Reactor Libraries

\begin{tabular}{|c|c|c|}
\hline \multicolumn{2}{|c|}{$\begin{array}{c}\text { Assembly type } \\
\text { (Number of Libraries) }\end{array}$} & Lattice types \\
\hline \multirow{4}{*}{ PWR } & Babcock \& Wilcox & $15 \times 15$ \\
\hline & Westinghouse & $14 \times 14,15 \times 15,17 \times 17,17 \times 17-$ OFA \\
\hline & Combustion Engineering & $14 \times 14,16 \times 16$ \\
\hline & Siemens & $14 \times 14,18 \times 18$ \\
\hline \multirow{4}{*}{ BWR } & $\mathrm{ABB}$ & $8 \times 8-1$ \\
\hline & Atrium & $9 \times 9-9,10 \times 10-9$ \\
\hline & General Electric & $8 \times 8-4,9 \times 9-7,7 \times 7-0,8 \times 8-1,8 \times 8-2,9 \times 9-2,10 \times 10-8$ \\
\hline & SVEA & $64(8 \times 8-1), 96(10 \times 10-4), 100(10 \times 10-0)$ \\
\hline \multirow[b]{2}{*}{ MOX } & BWR Lattices (75) & $\begin{array}{l}\text { ABB } 8 \times 8-1 \text {, Atrium } 9 \times 9-9,10 \times 10-9 ; \text { GE } 7 \times 7-0,8 \times 8- \\
1,8 \times 8-2,9 \times 9-2,10 \times 10-8 ; \text { SVEA- } 64,96,100\end{array}$ \\
\hline & PWR Lattices (15) & $\begin{array}{l}\text { Siemens } 14 \times 14,18 \times 18 \text {; CE } 14 \times 14,16 \times 16 \text {; } \\
\text { B\&W } 15 \times 15 \text {; } \\
\text { Westinghouse } 14 \times 14,15 \times 15,17 \times 17,17 \times 17 \text {-OFA }\end{array}$ \\
\hline \multirow{7}{*}{ Other } & AGR (6) & \\
\hline & CANDU (1) & 19-pin, 28-pin, 37-pin \\
\hline & IRT (12) & $2 \mathrm{M}, 3 \mathrm{M}, 4 \mathrm{M}$ \\
\hline & Magnox (4) & \\
\hline & RBMK (24) & \\
\hline & VVER-440 (3) & flat, radial enrichments $(3.82,4.25,4.38)$ \\
\hline & VVER-1000 (7) & flat enrichment \\
\hline
\end{tabular}

Table 5. Additional Information for ORIGEN Reactor Libraries

\begin{tabular}{|l|l|l|c|c|}
\hline $\begin{array}{c}\text { Assembly } \\
\text { type }\end{array}$ & \multicolumn{1}{|c|}{$\begin{array}{c}\text { Enrichments } \\
{[\%]}\end{array}$} & \multicolumn{1}{|c|}{$\left.\begin{array}{c}\text { Coolant/moderator } \\
\text { densities [g/cm }\end{array}\right]$} & $\begin{array}{c}\text { Maximum } \\
\text { burnup } \\
\text { [GWd/MTU] }\end{array}$ & $\begin{array}{c}\text { Number of } \\
\text { Libraries }\end{array}$ \\
\hline PWR LEU & $0.5,1.5,2,3,4,5,6$ & 0.73 & 70.5 & 7 \\
\hline BWR LEU & $0.5,1.5,2,3,4,5,6$ & $0.1,0.3,0.5,0.7,0.9$ & 70.5 & 35 \\
\hline PWR MOX & $*$ & $\sim 0.73$ & 70.5 & 15 \\
\hline BWR MOX & $*$ & $0.1,0.3,0.5,0.7,0.9$ & 70.5 & 75 \\
\hline AGR & $0.5,1.5,2,3,4,5$ & 1.65 & 48.7 & 6 \\
\hline CANDU & 0.711 & 0.8445 & 13.7 & 3 \\
\hline IRT & $19.75,36,80,90$ & 0.989 & 159 & 12 \\
\hline Magnox & $0.7,0.8,0.9,1$ & 1.628 & 13.7 & 4 \\
\hline RBMK & $1.8,2.2,2.6,3$ & $0.15,0.28,0.41,0.54,0.67,0.8$ & 24.3 & 24 \\
\hline VVER-440 & $1.6,2.4,3.6$, profiled & 0.73 & 70.5 & 6 \\
\hline VVER-1000 & $0.5,1.5,2,3,4,5,6$ & 0.7145 & 70.5 & 7 \\
\hline
\end{tabular}

${ }^{*} \mathrm{Pu}$ contents $[\%]: 4,7,10 ;{ }^{239} \mathrm{Pu}$ contents $[\%]: 50,55,60,65,70$ 


\section{ORIGAMI Automation Tool}

ORIGAMI Automator, a graphical user interface integrated with Fulcrum, facilitates the quantification of isotopics as a function of time for a large set of fuel assemblies. This tool was developed to support the Nuclear Regulatory Commission (NRC) in MELCOR analyses, but can be adapted to many other uses. The ORIGAMI Automator is an integrated graphical user interface that leverages many of modern components developed for SCALE 6.2 to enable the analyst to (1) easily create, view, and edit the reactor site and assembly information, (2) automatically create and run ORIGAMI inputs, and (3) analyze the results from ORIGAMI. The Automator uses the standard ORIGEN binary concentrations files produced by ORIGAMI, with concentrations available at all time points in each assembly's life. ORIGAMI Automator enables plotting of results such as mass, concentration, activity, and decay heat using an OPUS component. The ORIGAMI Automator Primer includes a description and user guide, a step-by-step tutorial for a simplified scenario, and appendices that document the file structures used.

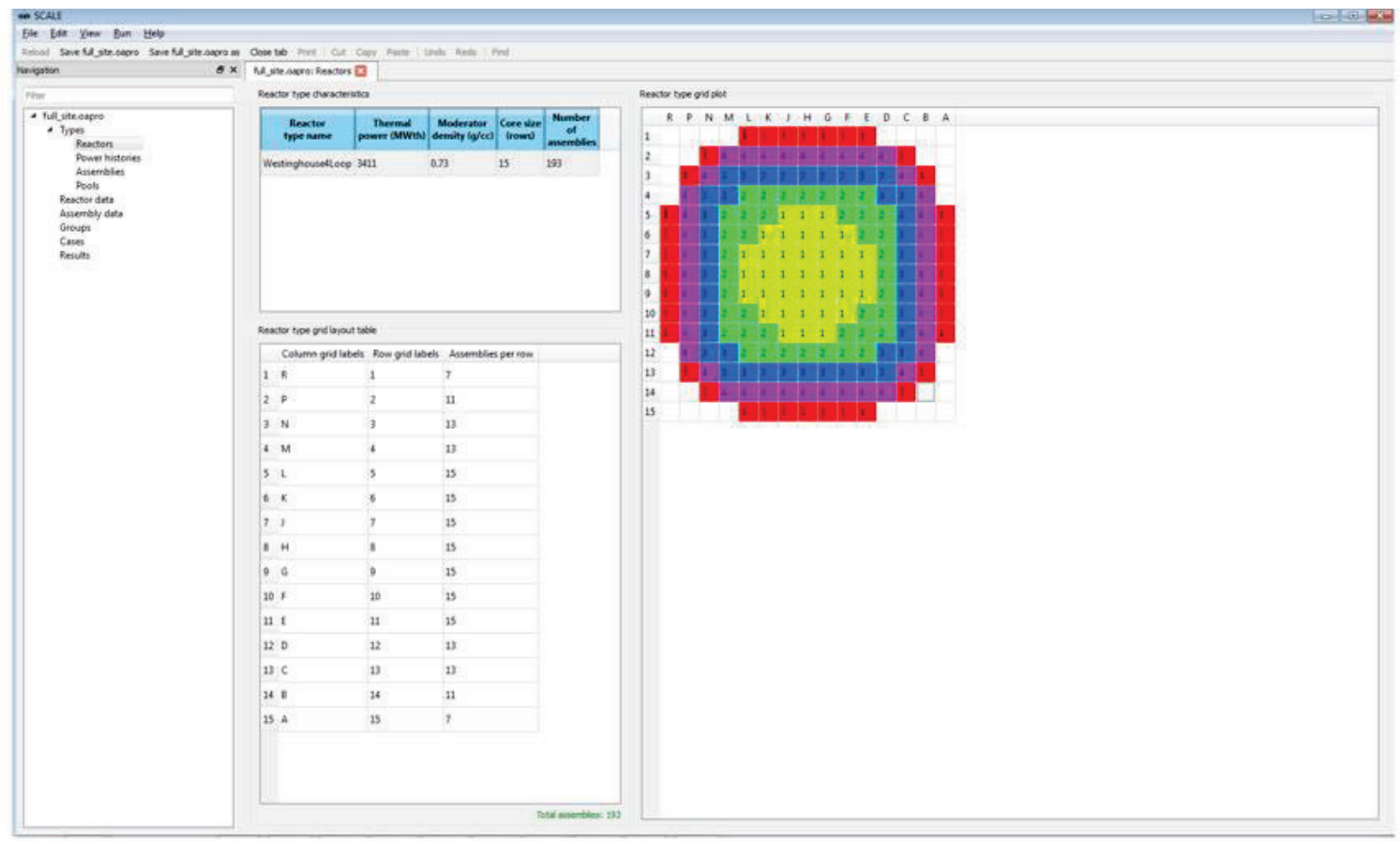

Figure 9. ORIGAMI Automator. 


\section{Radiation Shielding}

The SCALE fixed-source Monte Carlo capability with automated variance reduction has been enhanced to enable $\mathrm{CE}$ calculations. The new $\mathrm{CE}$ capabilities provide enhanced solution fidelity while still implementing the unique acceleration techniques of the Forward-Weighted Consistent Adjoint Driven Importance Sampling (FW-CADIS) methodology for deep penetration shielding and criticality accident alarm system modeling.

\section{Continuous-Energy Shielding Calculations}

Prior to SCALE 6.2, the MAVRIC/Monaco capabilities relied on the MG approach for radiation transport. The MG approach is suitable for many applications but can be problematic for others. For example, it is problematic for deep penetration shielding through iron, where the MG-averaged cross section for capture resonances may not accurately represent the true transmission of neutrons due to fine "windows" through (i.e., minima in) the cross section. Additionally, $\mathrm{MG}$ cannot adequately resolve discrete gamma emission lines such as those of ${ }^{60} \mathrm{Co}$. With the $\mathrm{CE}$ treatment introduced in SCALE 6.2, improved solution fidelity is available. The generation of CE nuclear data and the implementation of $\mathrm{CE}$ physics in Monaco are based on a first-principles approach, where the simulation is represented as realistically as possible. This approach will lead to higher-fidelity results, but runtimes may be substantially increased over more approximate methods.

Monaco also includes capabilities to import sources generated with ORIGAMI and to access gamma sources directly from ORIGEN data. 


\section{Sensitivity and Uncertainty Analysis}

\section{CE Eigenvalue Sensitivity Analysis}

With SCALE 6.2, the MG eigenvalue sensitivity and uncertainty analysis methods that use KENO for transport analysis are extended to provide CE capabilities through the implementation of the contributon-linked eigenvalue sensitivity/uncertainty estimation via tracklength importance characterization (CLUTCH) methodology and iterated fission probability (IFP) methods. ${ }^{12}$ CLUTCH is an efficient methodology that has been demonstrated to provide high-fidelity results with manageable run-times and memory requirements. State-of-the-art sensitivity methods make CE calculations easier to use than MG in several ways; for example, CE calculations do not require resonance self-shielding calculations to determine implicit sensitivity effects, the simulation of a separate adjoint transport calculation, or the use of a flux mesh for tallying fluxes and flux moments.

The new capabilities enable improvements in sensitivity coefficient accuracy compared to MG. Table 6 compares the accuracy of the total nuclide sensitivity coefficients estimated by the each sensitivity method for the MIX-COMP-THERM-004-001 system. ${ }^{13}$ The MG analysis produced a ${ }^{238} \mathrm{U}$ total nuclide sensitivity that disagreed with the reference direct perturbation sensitivity by 2.80 effective standard deviations $\left(\sigma_{\text {eff }}\right)$, while the CE methods (IFP and CLUTCH) sensitivities were within almost 1 standard deviation of the reference sensitivity coefficients for all of the nuclides examined.

Figure 10 compares the figures of merit for the nuclide sensitivity coefficients presented in Table 6. Figure 10 indicates that the CE sensitivity calculations are of a comparable efficiency to the MG calculations, and in some cases the CE CLUTCH method is more efficient than MG method. This is remarkable because of the use of CE physics, which can impose relatively long cross section lookup times, and indicates the efficiency of the CLUTCH method. 
Table 6. MIX-COMP-THERM-004-001 Nuclide Sensitivity Coefficient Comparison

\begin{tabular}{|c|c|c|c|c|}
\hline \multirow{2}{*}{ Sensitivity } & \multirow{2}{*}{ Reference } & \multirow{2}{*}{ MG TSUNAMI } & \multicolumn{2}{|c|}{ CE TSUNAMI } \\
\hline & & & IFP & CLUTCH \\
\hline $\mathrm{H}_{2} \mathrm{O}$ & $0.2935 \pm 0.0179$ & $\begin{array}{c}0.2805 \pm 0.0088 \\
\quad\left(-0.65 \sigma_{e f f}\right)\end{array}$ & $\begin{array}{l}0.2733 \pm 0.0052 \\
\quad\left(-1.08 \sigma_{e f f}\right)\end{array}$ & $\begin{array}{c}0.2793 \pm 0.0065 \\
\quad\left(-0.75 \sigma_{e f f}\right)\end{array}$ \\
\hline${ }^{238} \mathrm{U}$ & $-0.0061 \pm 0.0003$ & $\begin{array}{c}-0.0050 \pm 0.0002 \\
\left(2.80 \sigma_{e f f}\right)\end{array}$ & $\begin{array}{c}-0.0055 \pm 0.0003 \\
\left(1.16 \sigma_{e f f}\right)\end{array}$ & $\begin{array}{c}-0.0057 \pm 0.0001 \\
\left(1.14 \sigma_{e f f}\right)\end{array}$ \\
\hline${ }^{239} \mathrm{Pu}$ & $0.1262 \pm 0.0087$ & $\begin{array}{l}0.1264 \pm 0.0014 \\
\quad\left(0.02 \sigma_{e f f}\right)\end{array}$ & $\begin{array}{l}0.1188 \pm 0.0020 \\
\quad\left(-0.83 \sigma_{e f f}\right)\end{array}$ & $\begin{array}{l}0.1190 \pm 0.0001 \\
\quad\left(-0.83 \sigma_{e f f}\right)\end{array}$ \\
\hline${ }^{240} \mathrm{Pu}$ & $\begin{array}{c}-0.03777 \pm \\
0.00350\end{array}$ & $\begin{array}{c}-0.03750 \pm \\
0.00011 \\
\left(0.08 \sigma_{\text {eff }}\right) \\
\end{array}$ & $\begin{array}{c}-0.03738 \pm \\
0.00060 \\
\left(0.11 \sigma_{\text {eff }}\right) \\
\end{array}$ & $\begin{array}{c}-0.03743 \pm \\
0.00002 \\
\left(0.10 \sigma_{\text {eff }}\right) \\
\end{array}$ \\
\hline${ }^{241} \mathrm{Pu}$ & $\begin{array}{c}0.00589 \pm \\
0.00042\end{array}$ & $\begin{array}{c}0.00599 \pm 0.00004 \\
\left(0.24 \sigma_{e f f}\right)\end{array}$ & $\begin{array}{c}0.00567 \pm 0.00014 \\
\left(-0.50 \sigma_{e f f}\right)\end{array}$ & $\begin{array}{c}0.00579 \pm 0.00003 \\
\left(-0.24 \sigma_{e f f}\right)\end{array}$ \\
\hline
\end{tabular}

\section{Figure 10. MIX-COMP-THERM-004-001 nuclide sensitivity figure of merit comparison.}

Table 7 gives the amount of computational memory required by each method for the sensitivity coefficient calculations. These memory requirements were obtained by subtracting the memory requirements of each eigenvalue-only calculation from the memory requirements of each sensitivity coefficient calculation. As seen below, the CLUTCH method excels in terms of memory usage and can readily compute sensitivities for complex systems on a typical personal computer. 
Table 7. Sensitivity Method Memory Usage.

\begin{tabular}{|c|c|c|c|}
\hline \multirow{2}{*}{ Model } & \multirow{2}{*}{ MG TSUNAMI } & \multicolumn{2}{|c|}{ CE TSUNAMI } \\
\cline { 3 - 4 } & & IFP & CLUTCH \\
\hline MIX-COMP-THERM-004-001 & $13,785 \mathrm{MB}$ & $10,643 \mathrm{MB}$ & $63 \mathrm{MB}$ \\
\hline
\end{tabular}

TSUNAMI-3D also includes a Generalized Perturbation Theory (GPT) capability to compute the sensitivity of ratios of reaction rates to cross section data by introducing the Generalized Adjoint Responses in Monte Carlo (GEAR-MC) method. The two GEAR-MC implementations are available for use in CE Monte Carlo simulations.

GPT sensitivity coefficients now share the same input at the previous MG GPT methods implemented in TSUNAMI-1D and TSUNAMI-2D. The Definitions block is used to define reaction rates in terms of material, reactions, and energy ranges, and the SystemResponses block is used to define ratios for which sensitivity coefficients will be computed. A separate SDF is generated for each ratio defined in SystemResponses, detailing the sensitivity of the reaction rate ratio to the cross section data on an energydependent basis. The uncertainty in each ratio due to cross section covariance data is also provided in the output file.

\section{Sampler}

A new stochastic uncertainty quantification capability has been added with the SCALE 6.2 Sampler module that implements stochastic techniques to quantify the uncertainty in any computed result from any SCALE sequence, due to uncertainties in neutron cross sections, fission yield and decay data, and user input parameter, such as geometry, material density, isotopic composition, temperature, etc.

Sampler propagates these uncertainties through complex SCALE analysis sequences such as depletion calculations, and it provides the variation in the output quantities due to variations in any combination of input data, as shown in Figure $11 .{ }^{14}$ Correlations between systems with shared uncertainties are also computed, which is especially useful for quantifying correlated uncertainties in benchmark experiments, required for generalized linear least-squares techniques implemented by the SCALE module TSURFER. ${ }^{15}$ 


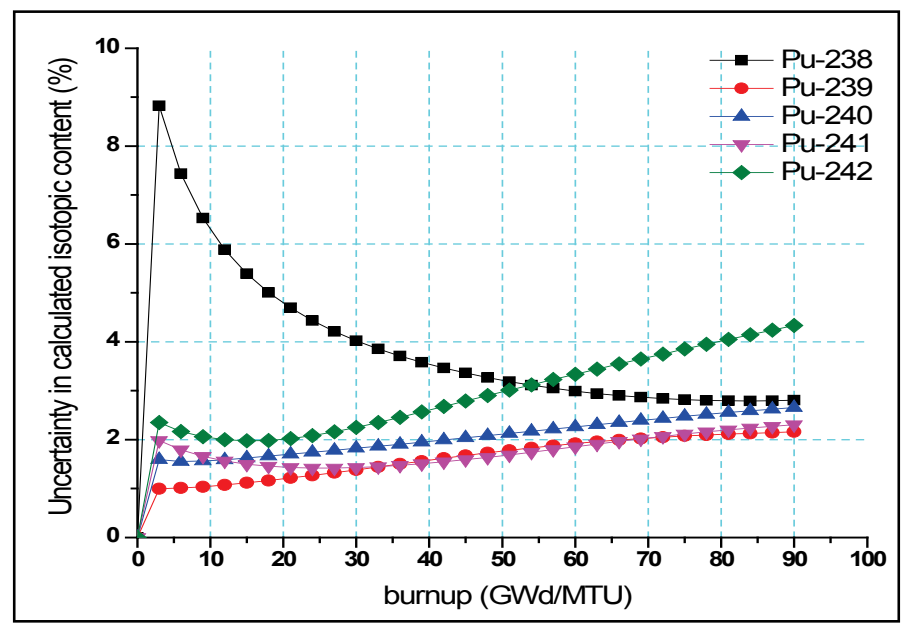

Figure 11. Uncertainty in plutonium isotopics as function of burnup.

The Medusa module of the XSUSA program ${ }^{16}$ is used to generate perturbation factors for the $1 \mathrm{D}$ cross sections on the MG library, assuming that the probability density functions are multivariate normal distributions with covariances given in the SCALE nuclear data covariance library. Uncertainty libraries for fission product yields are implemented from ENDF/B-VII.1 data for the major actinides ${ }^{235} \mathrm{U},{ }^{238} \mathrm{U},{ }^{239} \mathrm{Pu}$, and ${ }^{241} \mathrm{Pu}$, with independent correlations developed by ORNL using a Bayesian method applied to ensure that uncertainties in the cumulative fission yields are consistent with the independent yields in each chain. ${ }^{17}$ Multiplicative perturbation factors for fission product yields have been generated with XSUSA. The decay uncertainty data has 4 major components: decay constant uncertainty, q-value uncertainty, fraction of q value in photons, and branch ratio uncertainty. Perturbation factors for these 4 components are generated using uncertainties from the ENDF/B-VII.1 library. Correlations are defined for decays with multiple decay modes to ensure the sum of branching is unity. No uncertainties are applied for data that do not have associated uncertainty information. Perturbations are applied using the covariance information with XSUSA to provide 1,000 ORIGEN decay libraries. The uncertainty information has presently not been consolidated as a single file of perturbation factors.

Currently, decay data sampling is available for ORIGEN and ORIGAMI calculations but is not operational with TRITON and Polaris calculations without additional user-supplied preliminary calculations.

Sampler also provides the ability to perform multivariate parametric studies for variables with uniform distributions.

Note that Sampler can be built with MPI enabled for parallel computations (see "Build Instructions" below), but it can only sample the execution of serial codes. Threading is available with the default binary distribution (use the "-I" option of scalerte) when executing Sampler. 


\section{Other Updates}

SCALE 6.2 includes a number of other improvements, such as the extension of the maximum allowed number of materials from around 2,000 to around 2 billion. Some older features are removed such as ENDF/B-V and -VI nuclear data, the point kernel shielding capability, and a material optimization search sequence. Additionally, the default temperature for materials with no temperature explicitly specified has been modified from $300 \mathrm{~K}$ to $293 \mathrm{~K}$ for improved consistency between $\mathrm{MG}$ and $\mathrm{CE}$ calculations.

\section{Known Issues}

In this release, there are a few issues scheduled to be resolved in the future:

1. CELLMIX and DOUBLEHET treatments are not implemented for radiation shielding problems using MAVRIC; and

2. Parallel branch calculations in TRITON is currently not supported. 


\section{List of SCALE 6.2.1 Updates}

See http://scale.ornl.gov for details.

- The TRITON T-DEPL features ASSIGN and SWAP were updated for SCALE 6.2.1 to correct issues that could affect the results of SCALE 6.2 calculations. SCALE 6.2 T-DEPL calculations that use ASSIGN and/or SWAP should be reanalyzed with SCALE 6.2.1.

- Several new features were implemented into the Fulcrum user interface, along with updates to resolve minor issues identified in the original release.

- Improvements implemented for KENO output edits and resolved memory issues for some Windows calculations.

- Additional support for the SCALE 6.1 ORIGEN FIDO input format was implemented to enhance backwards compatibility.

- The Polaris $\mathrm{MoC}$ solver was updated to support anisotropic scattering.

- Improvements implemented in the few-group diffusion coefficient calculation in Polaris.

- A new multi-dimensional parametric study capability was enabled in Sampler.

- A new environment variable MISSING_NUCLIDE_MSG_LEVEL=QUIET was introduced to reduce the number of warning messages regarding missing nuclides that are not present in the Standard Composition library.

- Minor improvements implemented in TSUNAMI sensitivity coefficient output edits.

- Minor improvements implemented in XSProc and improved code stability for the CENTRM cross section processing module.

- A modernized version of the PUFF module in AMPX was enabled. 


\section{List of SCALE 6.2.2 Updates}

See http://scale.ornl.gov for details.

- The TRITON T-DEPL ASSIGN feature was enabled for problems with BRANCH and TIMETABLE definitions.

- A deficiency in ENDF/B-VII.0 and ENDF/B-VII.1 was addressed where ${ }^{18} \mathrm{O}$ has a natural abundance of $0.2 \%$, yet no cross section data are provided. A new feature was implemented for all SCALE sequences to add zero-valued cross section data for ${ }^{18} \mathrm{O}$ so that material masses are maintained when using abundances provided by the Standard Composition Library.

- The TRITON T6-DEPL depletion sequence for continuous-energy depletion calculations was updated with code enhancements and improved stability.

- Polaris was updated to support BWR geometry and operation history modeling.

- ORIGEN was updated to compute k-infinities correctly based on cross section values embedded in the ORIGEN data library. Calculations in the TRITON and Polaris sequences are not affected by this update.

- The CE KENO problem-dependent Doppler broadening techniques where enhanced for scattering data for certain thermal moderators such as zirconium hydride. Although models with $\mathrm{H}_{2} \mathrm{O}$ are not affected, the results for other moderators may be improved over those obtained SCALE 6.2 and SCALE 6.2.1.

- CE TSUNAMI-3D calculations using the IFP and GPT methods now use variance reduction technique of Monte Carlo particle splitting, which was disabled for these specific sensitivity methods in SCALE 6.2 and 6.2.1. The results for IFP and GPT calculations with SCALE 6.2.2 will vary from previous results within the stochastic uncertainty of the calculation, but the runtimes will be improved by $10-40 \%$. 


\section{System Requirements}

\section{System Architecture:}

- Linux 64-bit (RHEL 6 or newer)

- Mac OS X (Darwin) 10.11.6 or newer

- Windows 7, 64-bit or newer

\section{System requirements:}

Minimum requirements: 4 GB RAM per CPU, 40 GB of disk space + additional space to store output results

Recommended requirements: 8 GB RAM per CPU, 40 GB of disk space $+100 \mathrm{~GB}$ of scratch space + additional space to store output results

Production requirement for large models: 64 GB RAM, 40 GB of disk space +500 $\mathrm{GB}$ of scratch space + additional space to store output results

Java Requirements:

Java 1.6 or newer. 


\section{Installation Instructions}

The SCALE 6.2.2 installation has been divided into three components: 64-bit precompiled binaries, source code, and the data. The precompiled binaries are available in platform-familiar installers: NSIS on Windows, Drag-N-Drop bundle on Mac, and Tar GZip on Linux. For deployments that include source code, it is available as a zip file, and the data are available as a self-extracting IzPack installer. Other systems may require a custom build of SCALE from source code using the build instructions provided below.

Pre-Compiled Binaries

Note: After the Binaries are installed, the nuclear data still must be installed. After completing the binary installation according to the instructions in this section, please follow the instructions for SCALE 6.2 Data.

\section{Windows}

After double-clicking the SCALE-6.2.2-setup.exe a "loading..." dialog may briefly pop up.

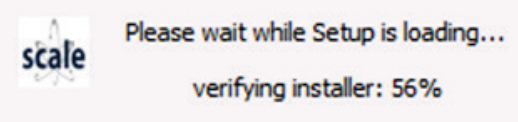

Figure 12. NSIS installer loading ....

Once loaded, a "Welcome" dialog will prompt the user to begin the installation. Click "Next $>$ " to continue. 


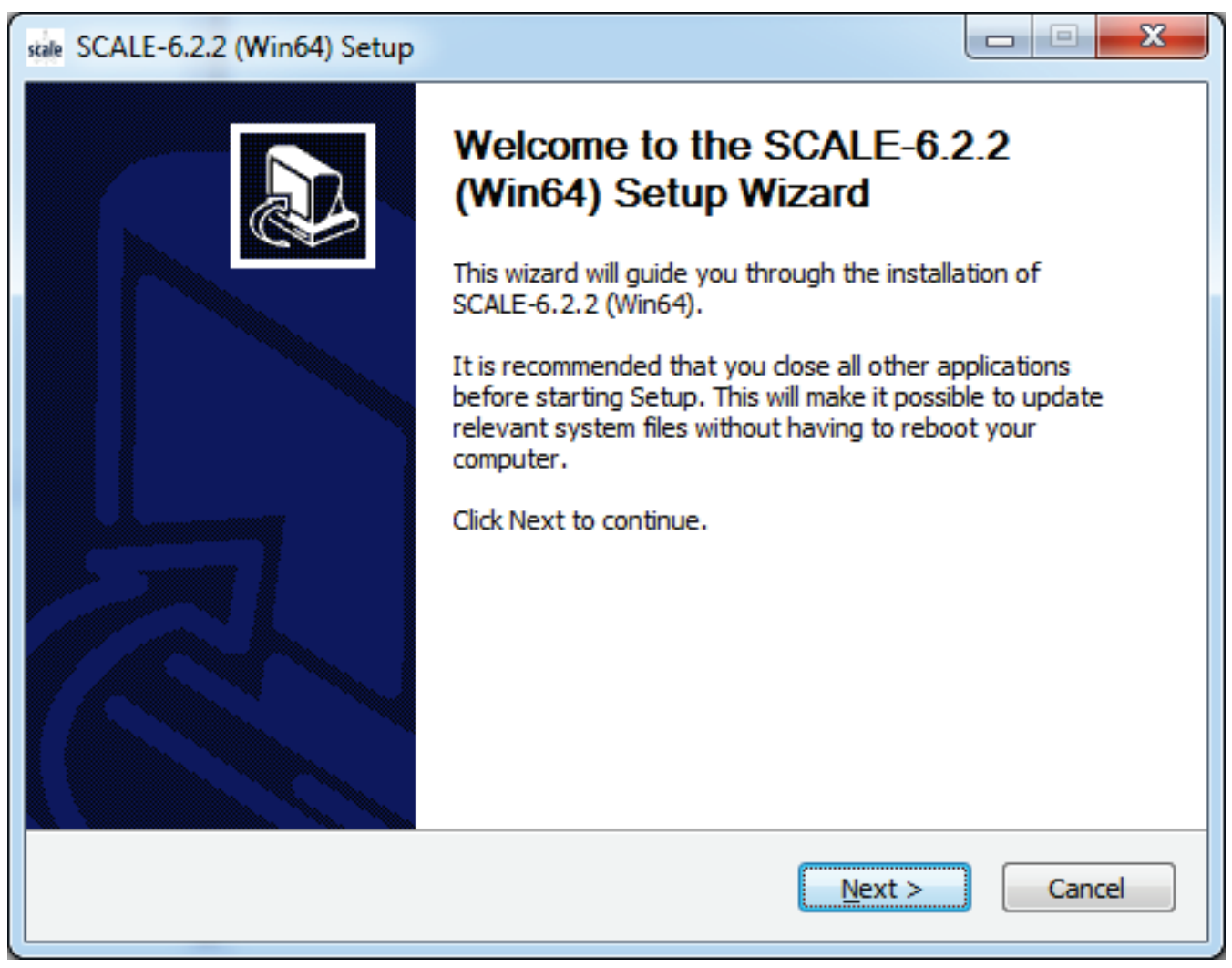

Figure 13. NSIS "Welcome" dialog.

Next, the installer will prompt the "License Agreement." Clicking "I Agree" will allow the user to continue. 


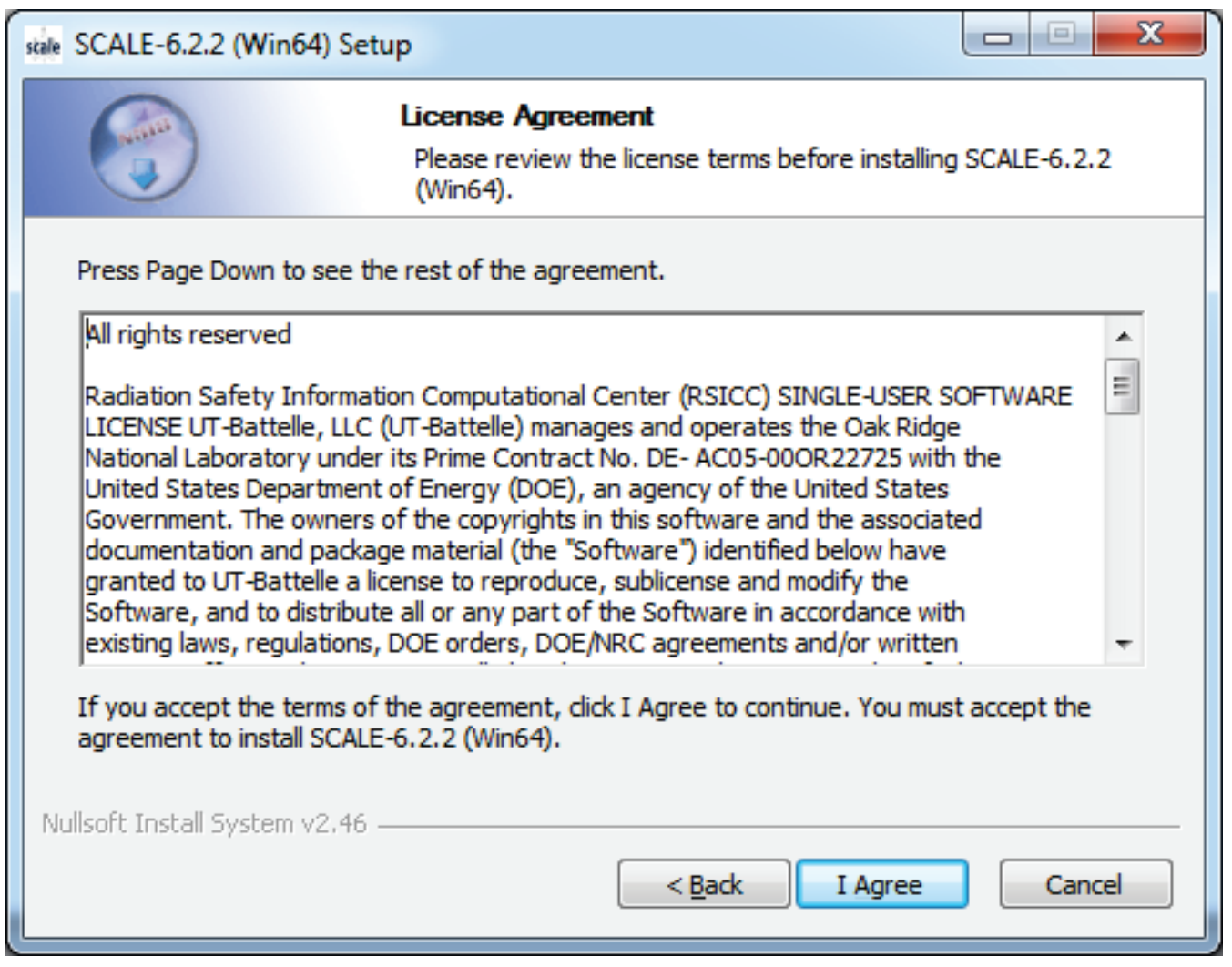

Figure 14. NSIS SCALE license agreement.

Next, the "Choose Install Location" dialog is displayed. Clicking "Next >" will allow the user to continue. 


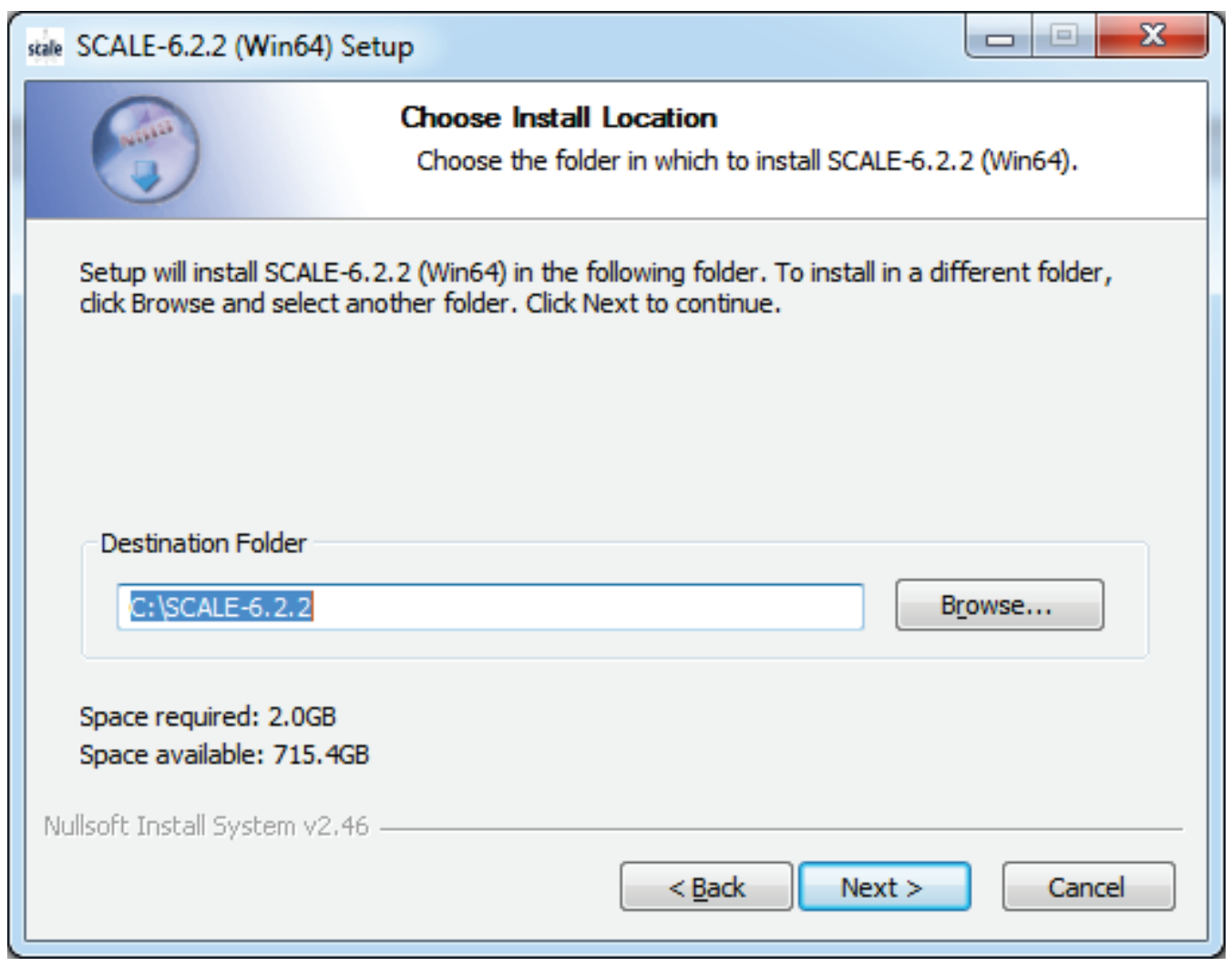

Figure 15. NSIS choose install location.

Next, the "Choose Start Menu Folder" is prompted. This defaults to installing Start Menu shortcuts. The user may click "Do not create shortcuts" to disable Start Menu shortcuts. Desktop shortcuts are always installed. Clicking "Install" will allow the user to continue. 


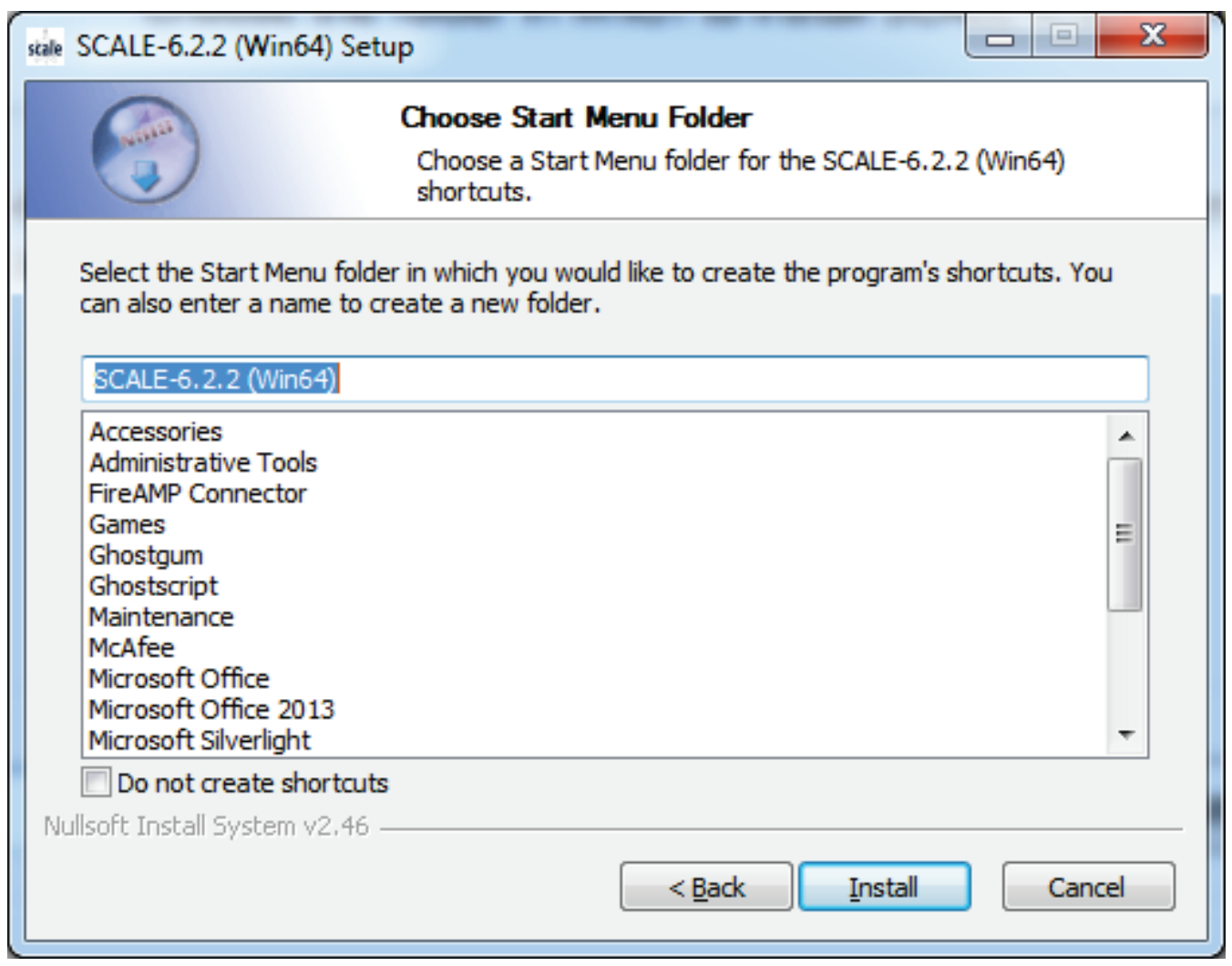

Figure 16. NSIS choose start menu folder.

Upon clicking "Install" the install starts, and progress will be shown. Once complete, clicking "Next $>$ " will allow the user to continue. 


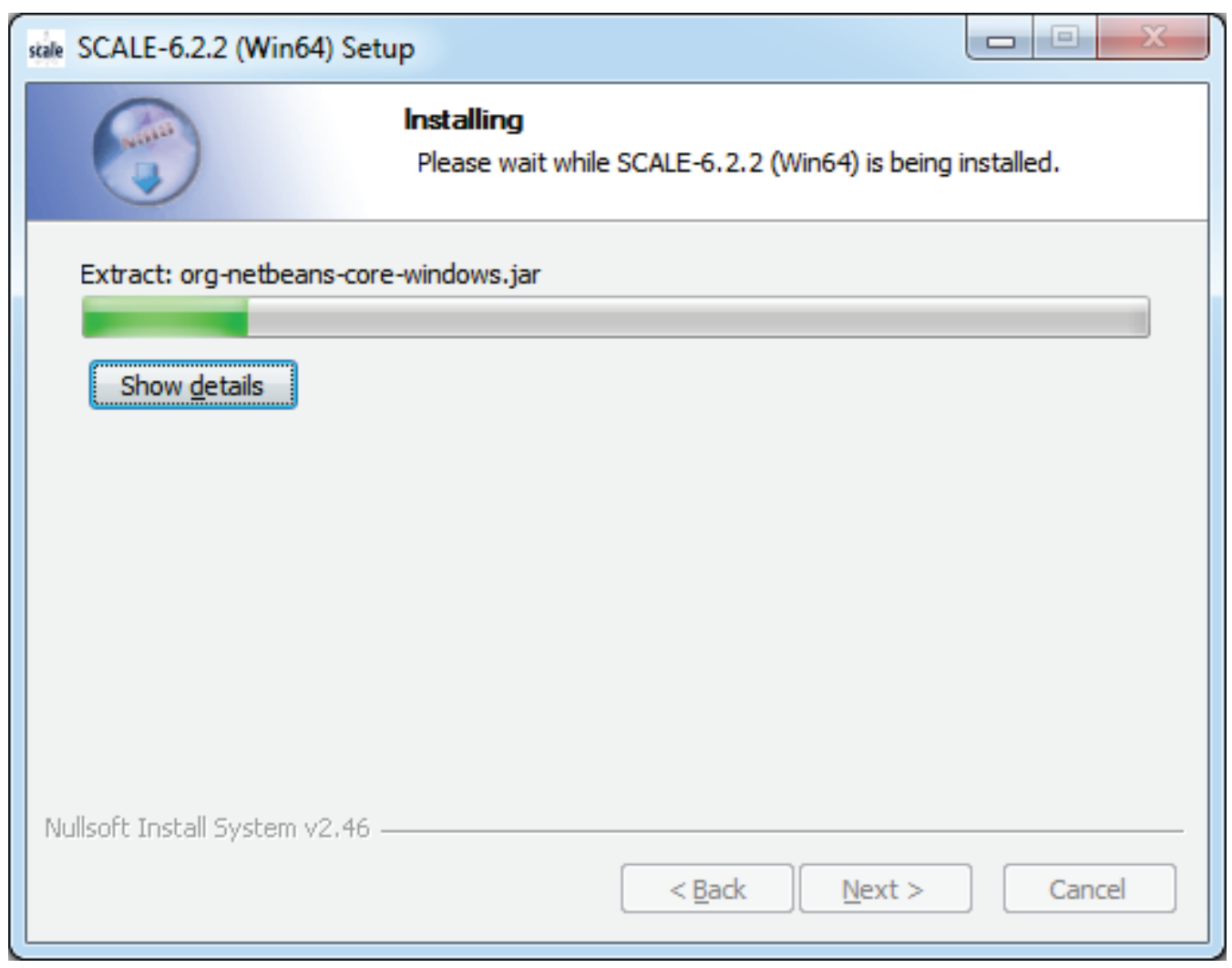

Figure 17. NSIS progress dialog.

Finally, installation is complete and the user may click "Finish" to exit the NSIS installer, and then follow the instructions to install SCALE 6.2 Data. 


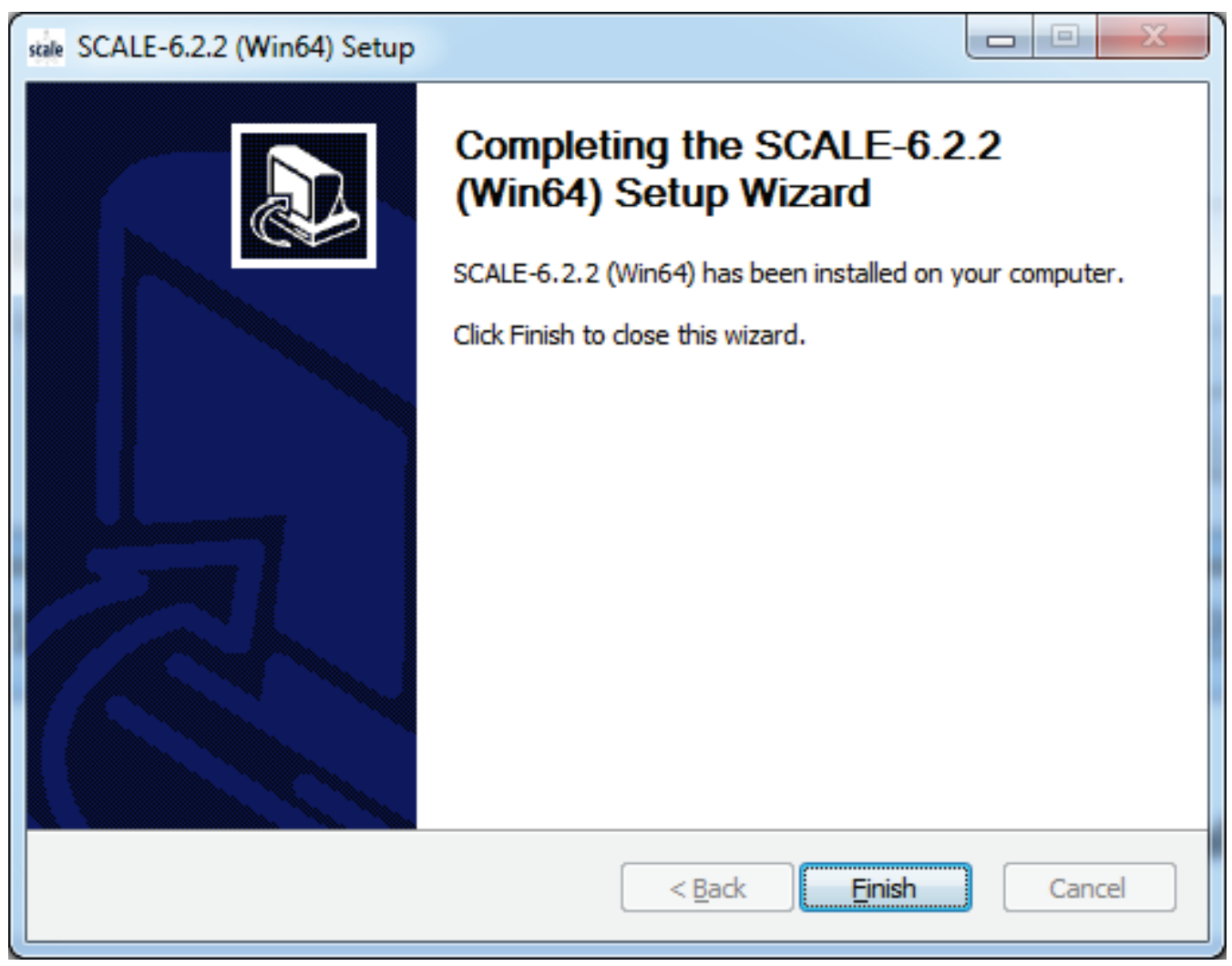

Figure 18. NSIS complete dialog.

Note that Norton Antivirus on Windows requires an exception for the SCALE Runtime Environment program, scalerte.exe. If using Norton Antivirus, please establish this exception prior to installing or SCALE 6.2.2 will not be able to execute.

\section{Mac OSX}

After double-clicking the SCALE-6.2.2-setup.dmg, you will see an opening dialog. 


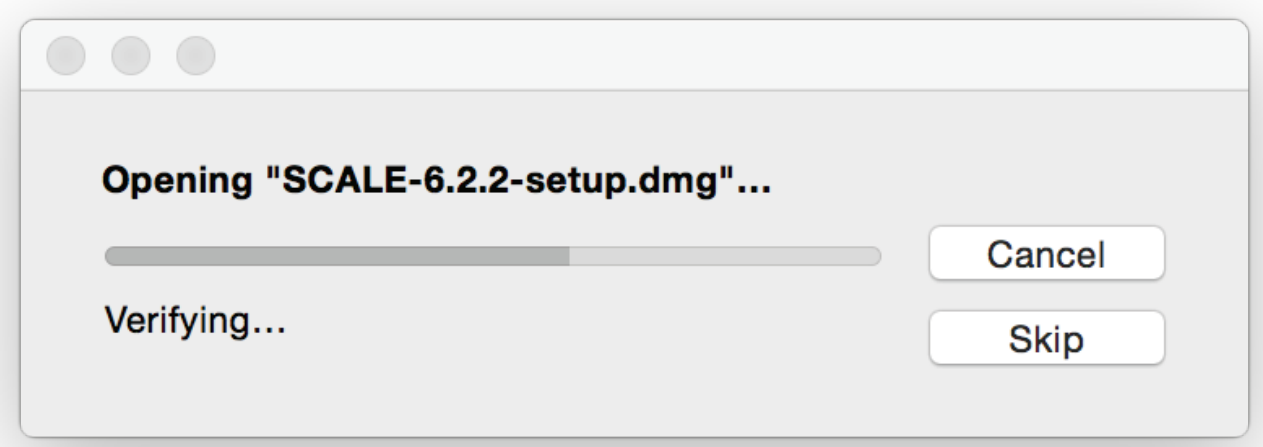

Figure 19. Mac OS X opening dialog.

Upon accepting, a Finder window will be raised showing "Applications" and "SCALE6.2.2.app". Simply drag "SCALE-6.2.2.app" onto "Applications" to install SCALE-6.2.2, and then follow the instructions given below to install SCALE 6.2 Data.

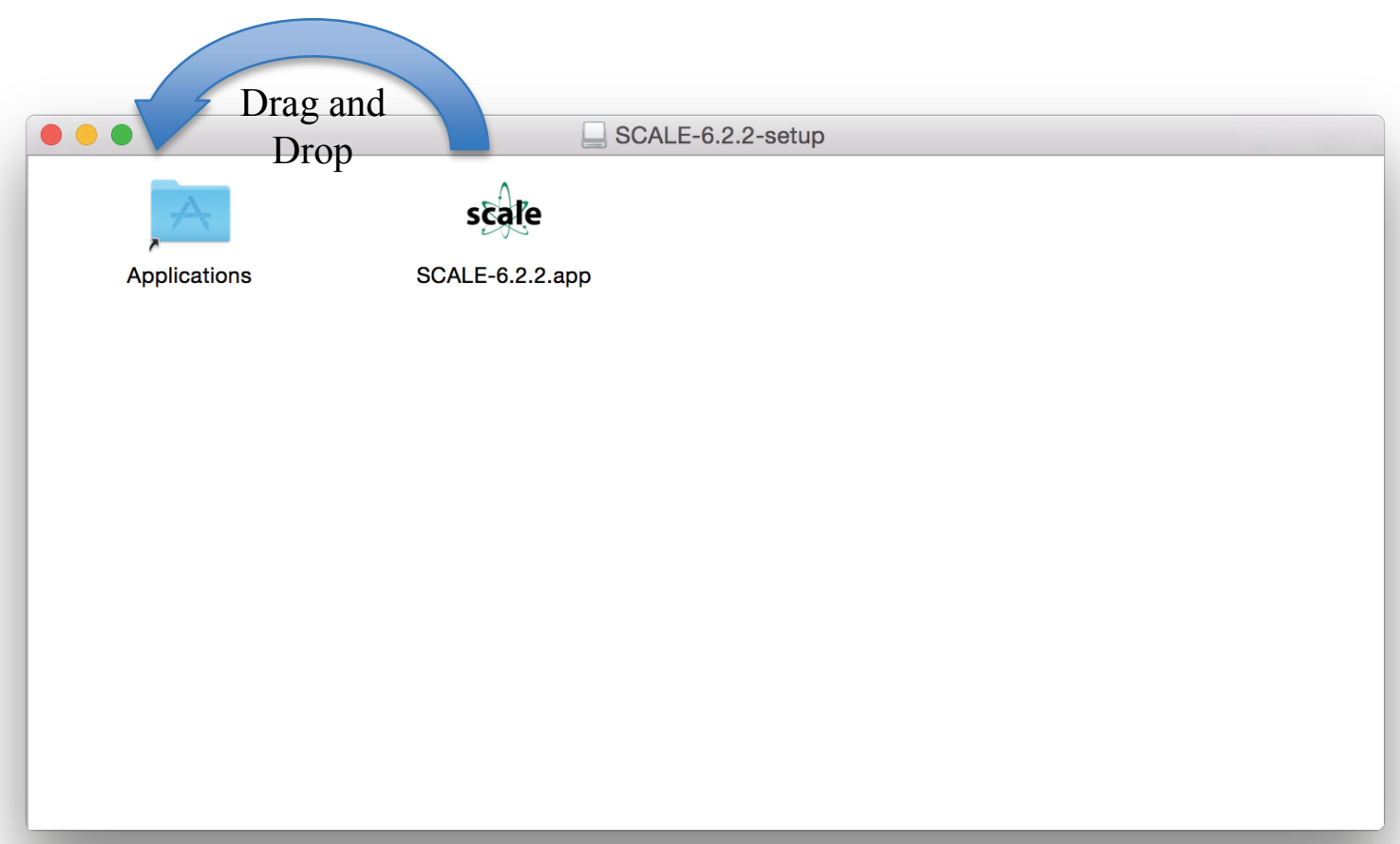

Figure 20. Mac OS X bundle contents. 


\section{Linux}

Copy the SCALE-6.2.2.tar.gz to the installation directory. Invoke 'tar -xzf SCALE6.2.2.tar.gz' to create the SCALE-6.2.2 directory. Follow the instructions given below to install SCALE 6.2 Data.

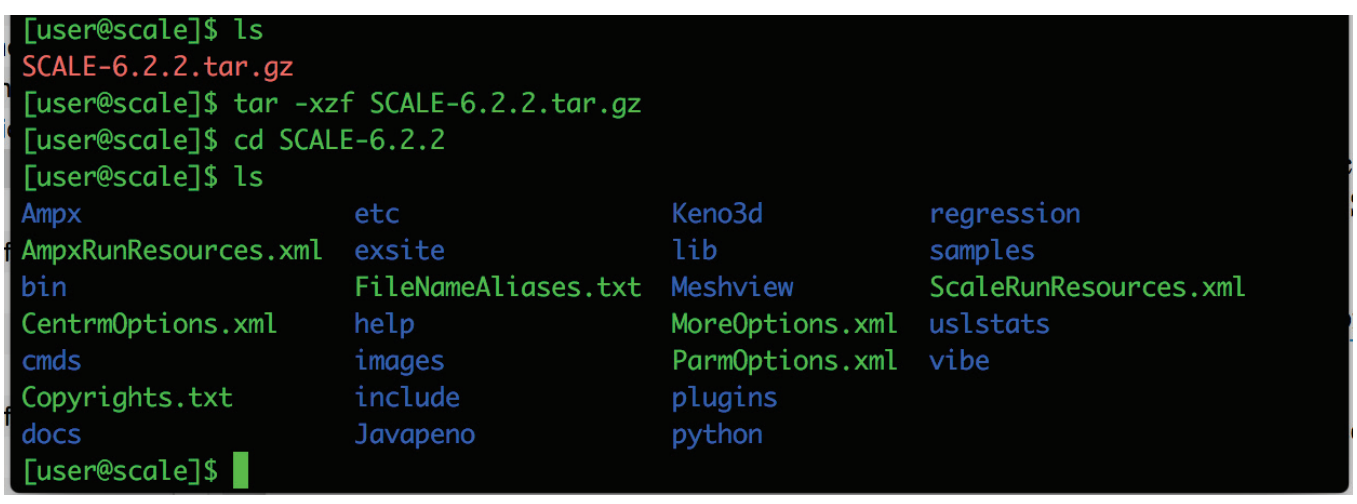

Figure 21. Linux Tar GZip.

\section{Source Code}

If you received the source code version, you can unzip SCALE-6.2-serial-6.2.2Source.zip file to any location on your computer.

For example, on a Windows platform, double click the SCALE-6.2-serial-6.2.2Source.zip file. This will extract the files into the "SCALE-6.2-serial-6.2.2-Source" directory.

On Linux and Mac OS X, create a destination directory for the source and copy the SCALE-6.2-serial-6.2.2-Source.zip into that directory.

Change to the directory and invoke 'unzip SCALE-6.2-serial-6.2.2-Source.zip' to deploy the source code.

\section{SCALE 6.2 Data}

Note that if you have SCALE 6.2 data installed from a prior SCALE 6.2 installation, you can copy or link the data into the SCALE 6.2.2 directory. To link preinstalled SCALE 6.2 data, open a command prompt and change the directory to the SCALE 6.2.2 installation directory:

(C:ISCALE-6.2.2, /Applications/SCALE-6.2.2/Contents/Resources, etc.).

On Windows, the following will link the SCALE 6.2 data into the SCALE 6.2.2 installation. (This may require administrative privileges.)

mklink /D data C:\SCALE-6.2\data 
On OS X, the following will link the SCALE 6.2 data into the SCALE 6.2.2 installation:

In -s /Applications/SCALE-6.2.app/Contents/Resources/data data

On Linux, the following will link the SCALE 6.2 data into the SCALE 6.2.2 installation:

In -s /scale/scale6.2/data data

To begin installation of SCALE 6.2 data, copy the scale-6.2-data-setup.jar to your local disk. Double-click this jar file. If the installer does not start then bring up a command prompt or terminal window and issue the following command: java -jar scale-6.2-data-setup.jar in the location where the installer jar file was copied.

After launching the installer, a dialog should appear as shown below.

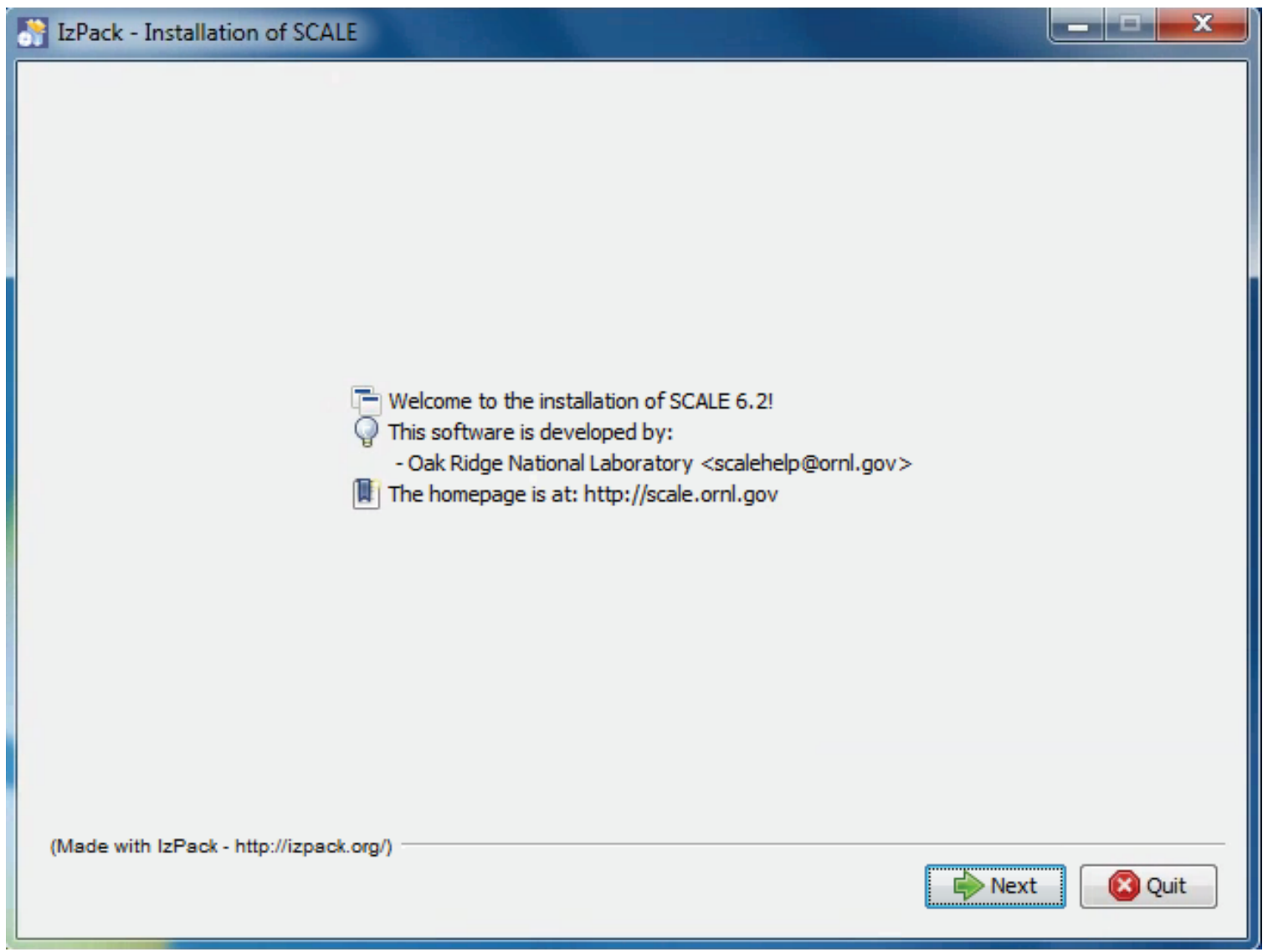

Figure 22. SCALE 6.2 data installer welcome dialog.

Continue by pressing Next. 
You will be prompted to review and accept the terms of the license agreement.

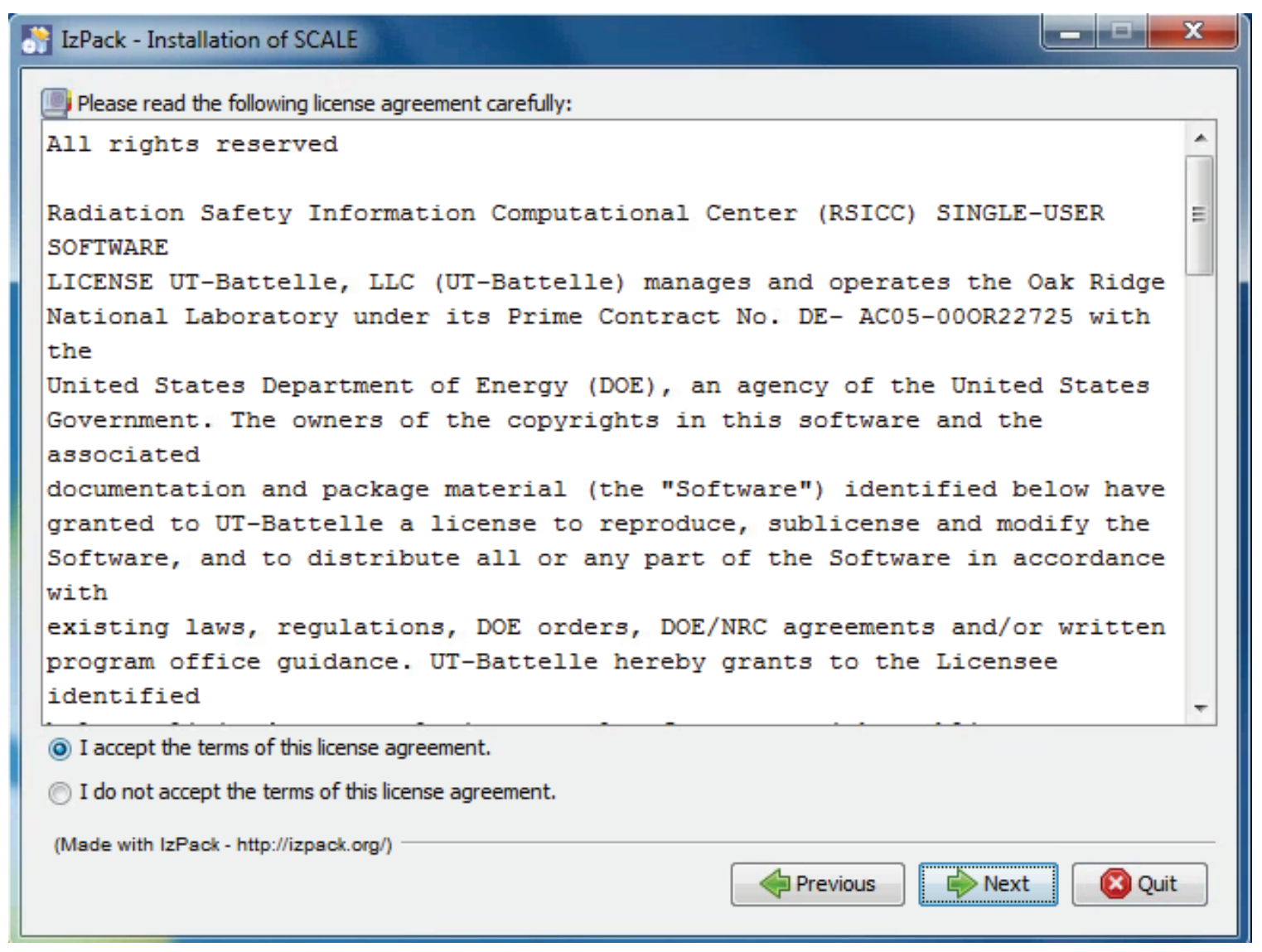

Figure 22. SCALE license agreement.

To proceed, check to indicate your acceptance of the terms, and press next.

You will be prompted to choose the destination of your installation.

For Windows users, the recommended installation path is inside your SCALE-6.2.2 directory:

$\mathrm{C}: \backslash \mathrm{SCALE}-6.2 .2$

For Linux, a typical location is:

/scale/scale6.2.2

For Mac, a typical location is:

/Applications/SCALE-6.2.2.app/Contents/Resources 


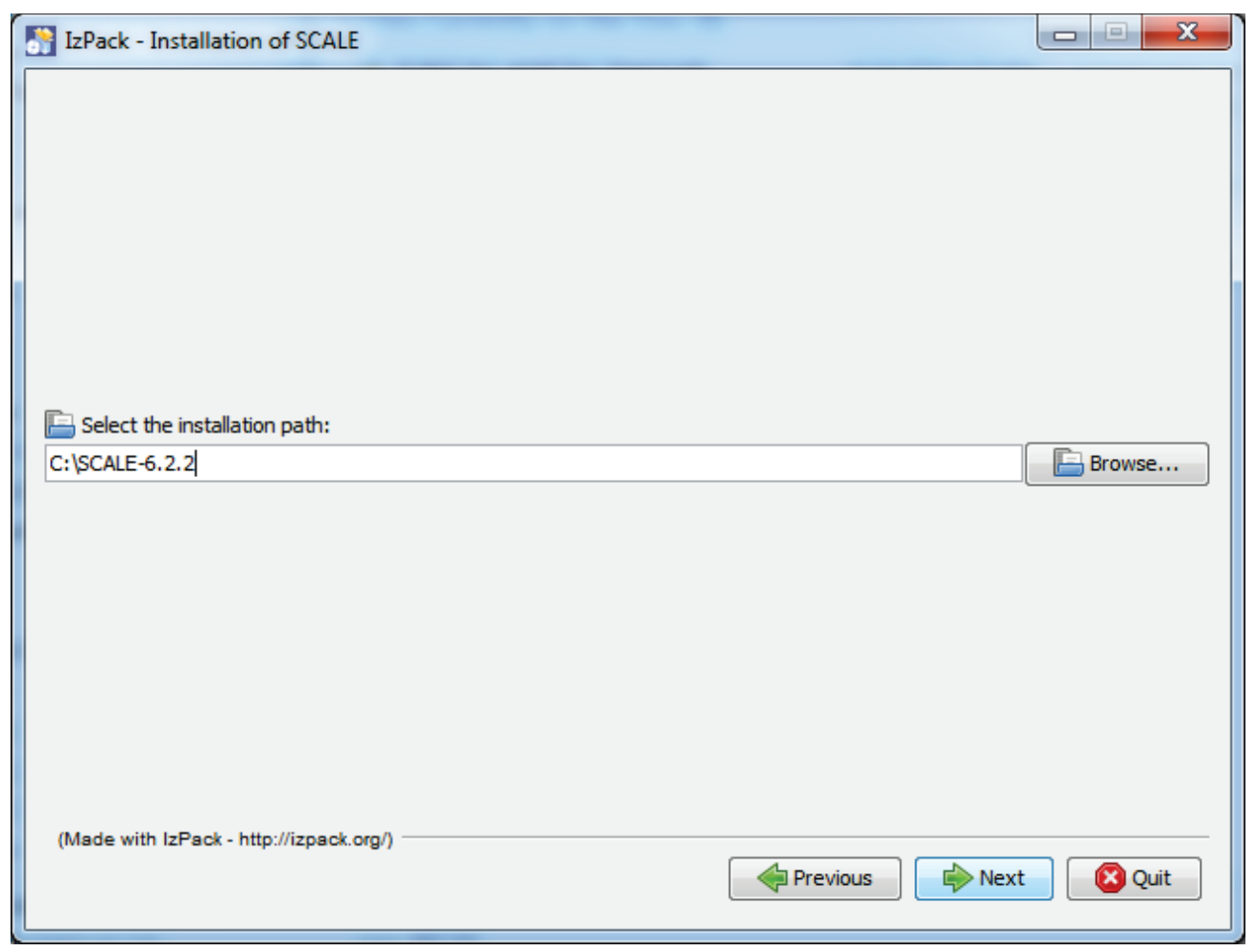

Figure 24. Setting typical data installation path on Windows.

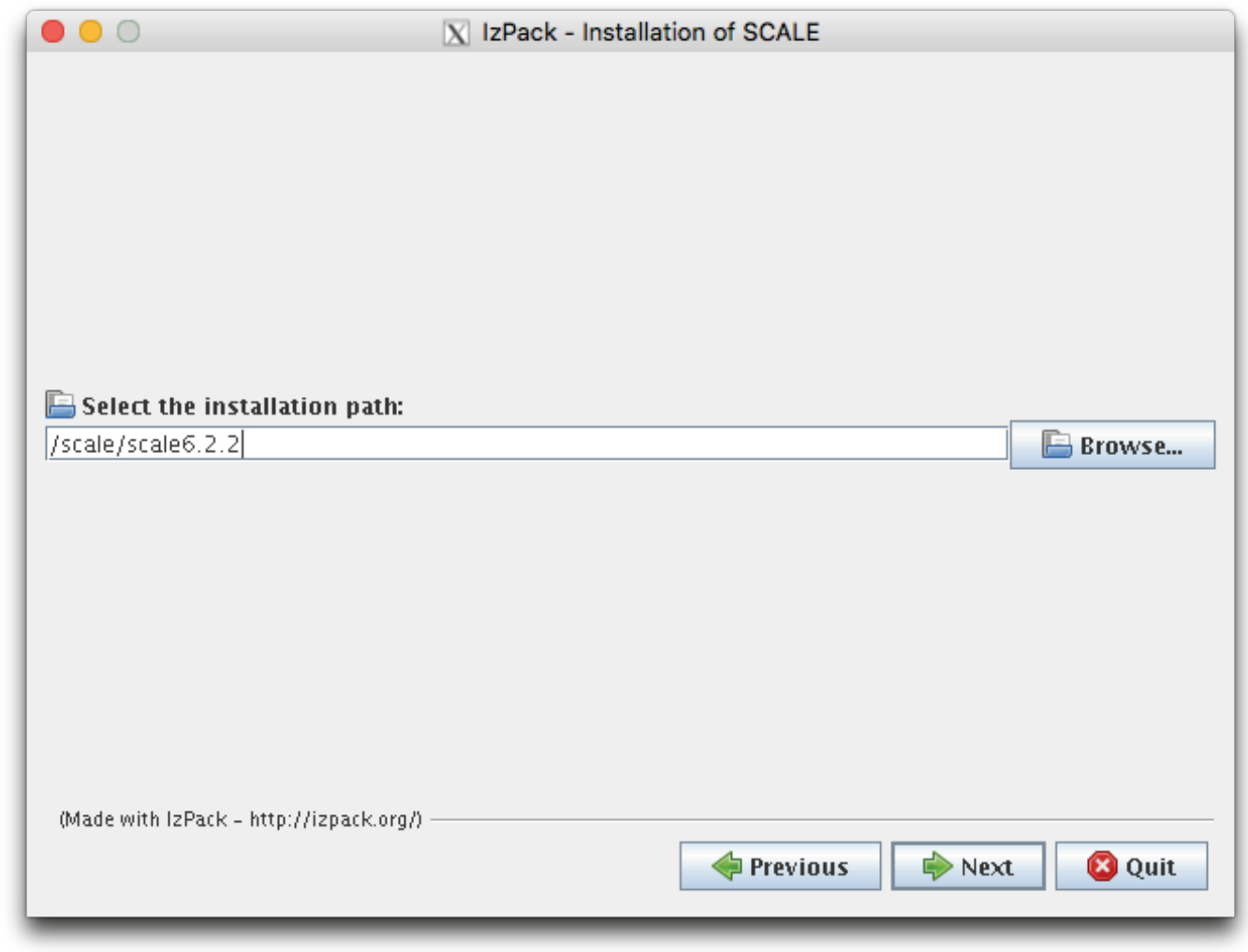

Figure 25. Setting a typical data installation path on Linux. Getting Started - 44 


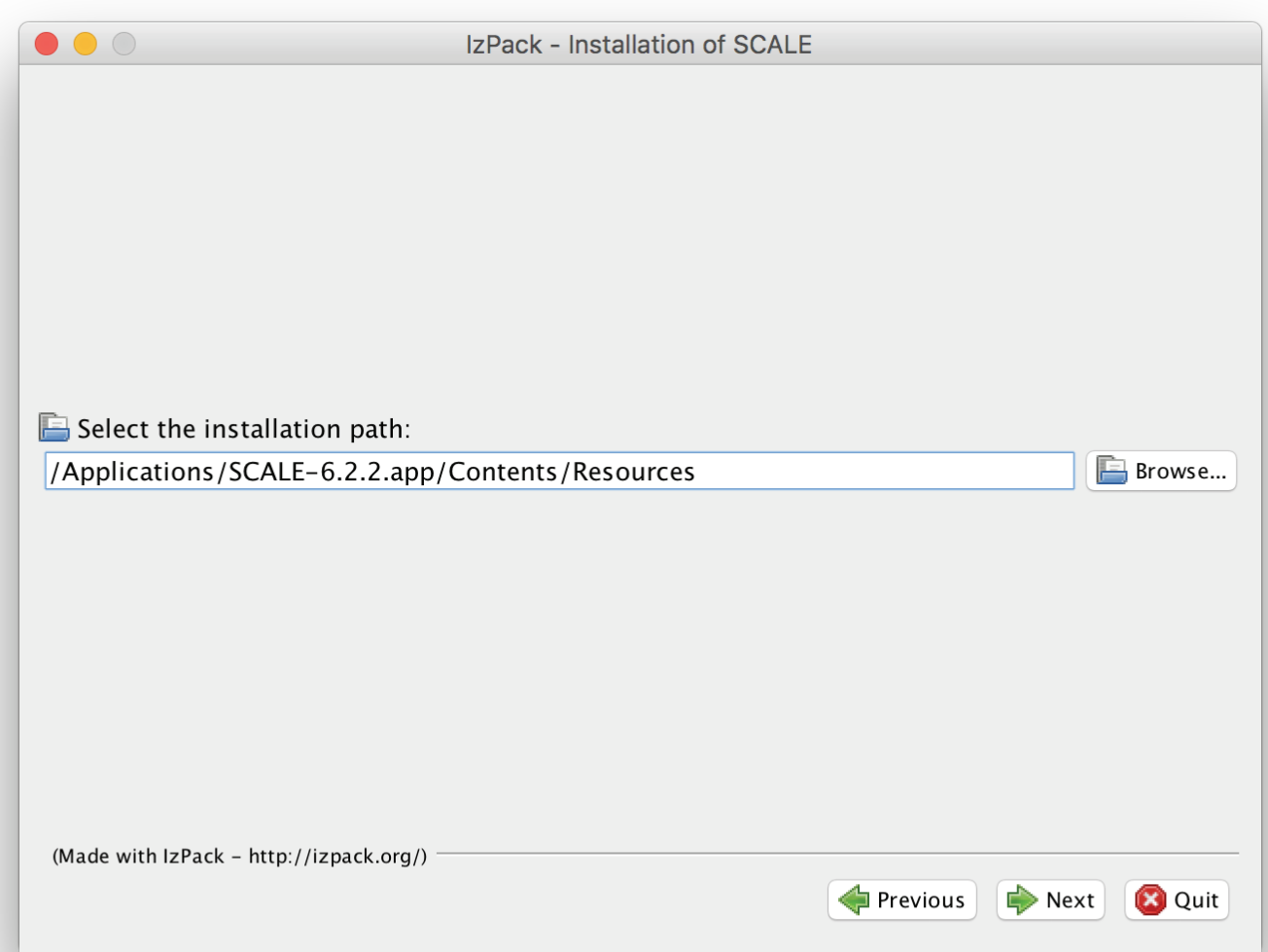

Figure 26. Setting a typical data installation path on Mac OS X.

For some systems, the installer will not be allowed to create a new directory. If you encounter a message like the one shown below, simply create the directory manually (e.g., using Windows Explorer), and then return to the installer and continue.

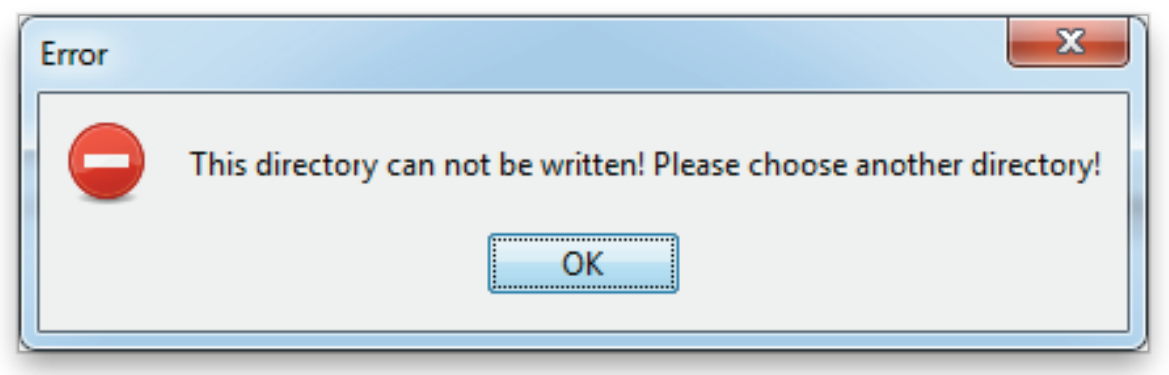

Figure 27. Data installer directory creation error message. 
In most cases, the installer will present a prompt to confirm the creation of a new directory; if that target directory is correct, then you can simply press $O K$.

If the directory was manually created, the installer will notify you that it is about to overwrite any previous contents. Press Yes to continue.

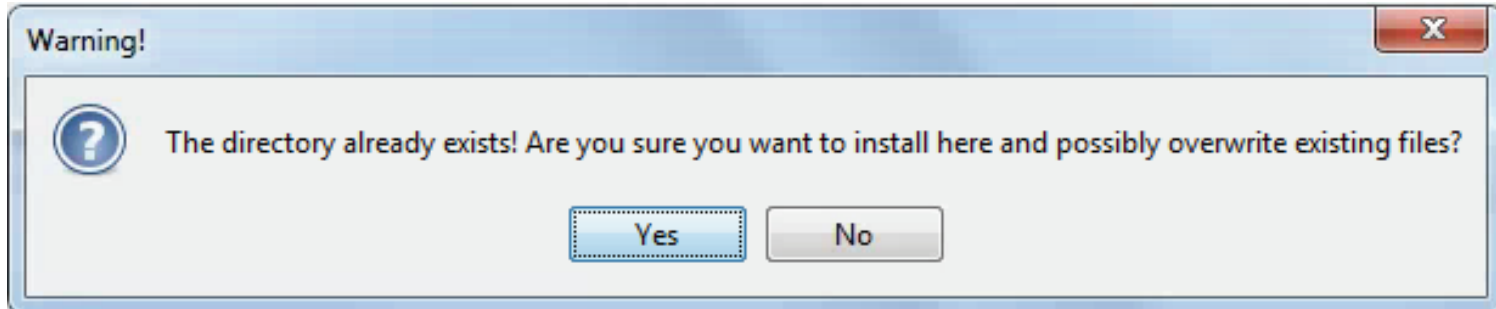

\section{Figure 28. Directory overwrite dialog.}

The installer then presents a dialog for available data sets you may wish to install.

IzPack - Installation of SCALE

s.s. Select the packs you want to install:

Note: Grayed packs are required.

V Basic Data

( ENDF/B-VII.1 Data

$19.22 \mathrm{~GB}$

$\checkmark$ Perturbation Data

$3.28 \mathrm{~GB}$

V ORIGEN Data

V ORIGEN-ARP Data

Composition data, physical constants, and other foundational data required for all SCALE calculations.

Figure 29. Data selection dialog. 
Please select the data needed to perform your desired SCALE calculations.

- Basic Data - Composition data, physical constants, and other foundational data required for all SCALE calculations

- ENDF/B-VII.0 Data - Nuclear Data from ENDF/B-VII.0 for CE and MG calculations, coupled n-gamma data from ENDF/B-VII.0 for MG calculations, and SCALE 6.0/6.1 44-group neutron covariance data (optional for most calculations, but provided for backwards compatibility)

- ENDF/B-VII.1 Data - Nuclear Data from ENDF/B-VII.1 for CE and MG calculations, coupled n-gamma data from ENDF/B-VII.1 for MG calculations, and ENDF/B-VII.1 neutron covariance data (recommended for most calculations; required to run the SCALE sample problems)

- Perturbation data - 1,000 samples of data each for ENDF/B-VII.1 neutron covariance data, SCALE 6.0/6.1 neutron covariance data, fission product yield covariance data, and radioactive decay covariance data (required for Sampler calculations)

- ORIGEN Data - Activation, depletion and decay data from ENDF/V-VII.1 and JEFF 3.1 (required for activation, depletion and decay calculations)

- ORIGEN-ARP Data - Pregenerated ORIGEN reactor libraries for many fuel types (required for spent fuel characterization and source terms calculations)

Next, the installer may ask for the location of the data .pak files that are unpacked during the installation process. Please direct the installer to the location of these data files, which are part of the SCALE distribution and may be on the distribution media or copied to a local directory as shown below.

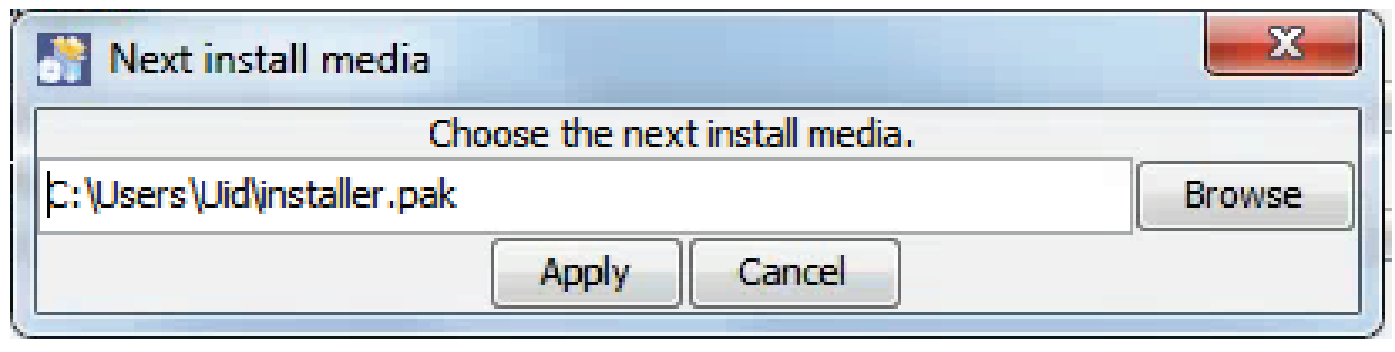

Figure 30. Media selection dialog.

The installer will display the progress of the installation. If installing from a DVD set, part way through the installation, you will be prompted for installer.pak.1. Please insert the PAK.1 disk and click Apply.

The installation from PAK.1 will complete and prompt for installer.pak.2. Please insert the PAK.2 disk and click Apply.

PAK.2 will finish. Proceed with all DVDs or files in the delivery until you have completed your installation of SCALE 6.2 data.

$$
\text { Getting Started }-47
$$


Getting Started — 48 


\section{Running SCALE}

SCALE is run by using the Fulcrum user interface or by invoking the SCALE Runtime Environment, scalerte, from the command line. Note that this release does not include the previous interface GeeWiz or OrigenArp for running SCALE on Windows, as Fulcrum should be used instead. Additionally, previous shortcuts like runscale are not implemented.

\section{Running SCALE from Fulcrum}

The most convenient way to run SCALE from a desktop is by launching Fulcrum. The Fulcrum executable is provided in the bin directory where SCALE was installed (e.g., $\mathrm{C}:$ SCALE-6.2.2|bin $\mid$ Fulcrum.exe). Fulcrum includes an online help document to assist users with its many features, and it includes links to the user manual and primers.

\section{Windows}

For a Windows installation, a Fulcrum shortcut is provided in the SCALE-6.2.2 shortcuts folder on the desktop.

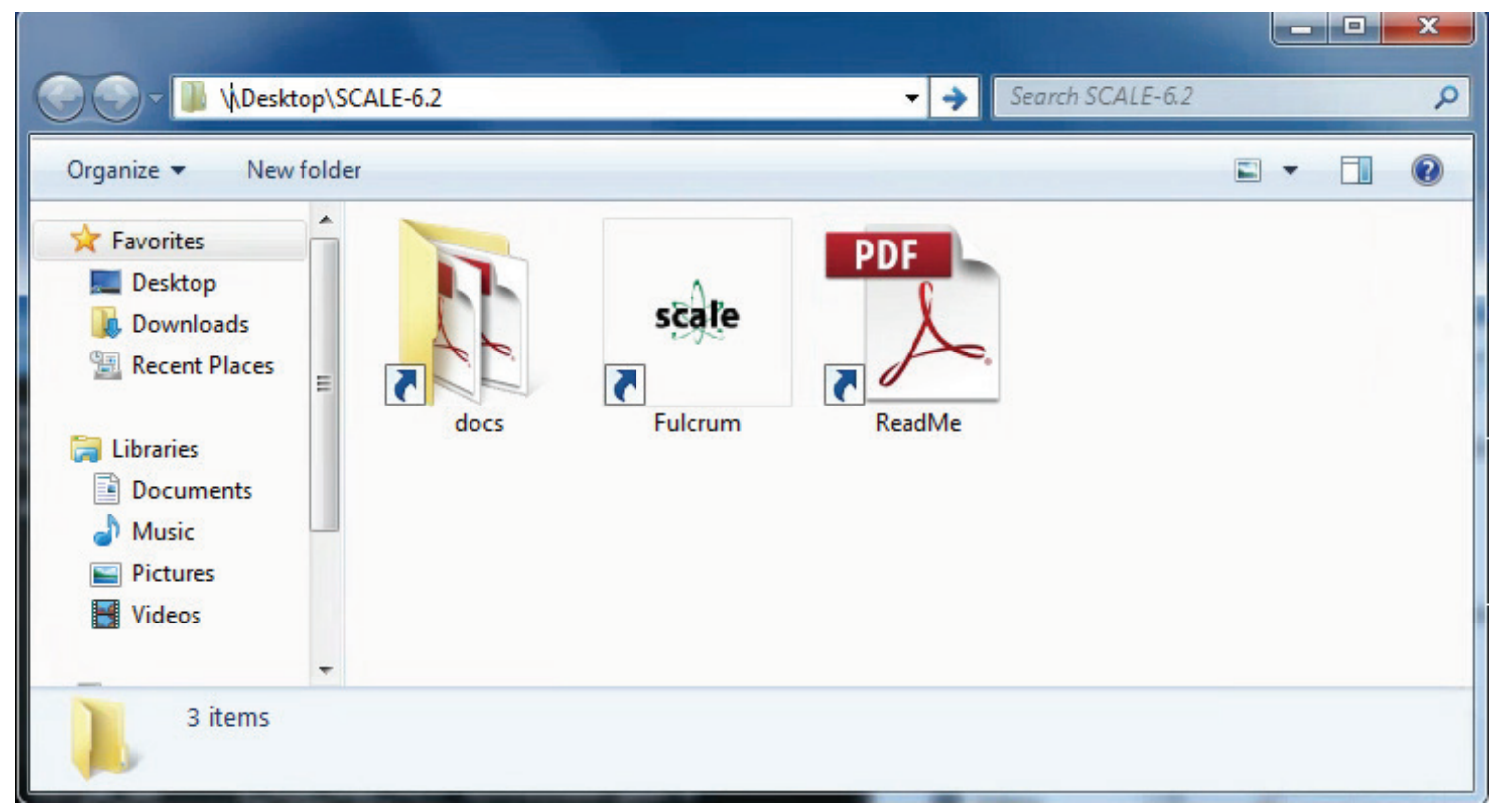

Figure 31. Windows desktop SCALE-6.2.2 shortcuts folder.

\section{Linux}

For Linux, launch Fulcrum from the bin directory of the SCALE installation (e.g., /scale/scale6.2.2/bin/Fulcrum).

\section{Mac OS X}

For a Mac installation, please use the $S C A L E-6.2 .2$ app in the Applications directory. 


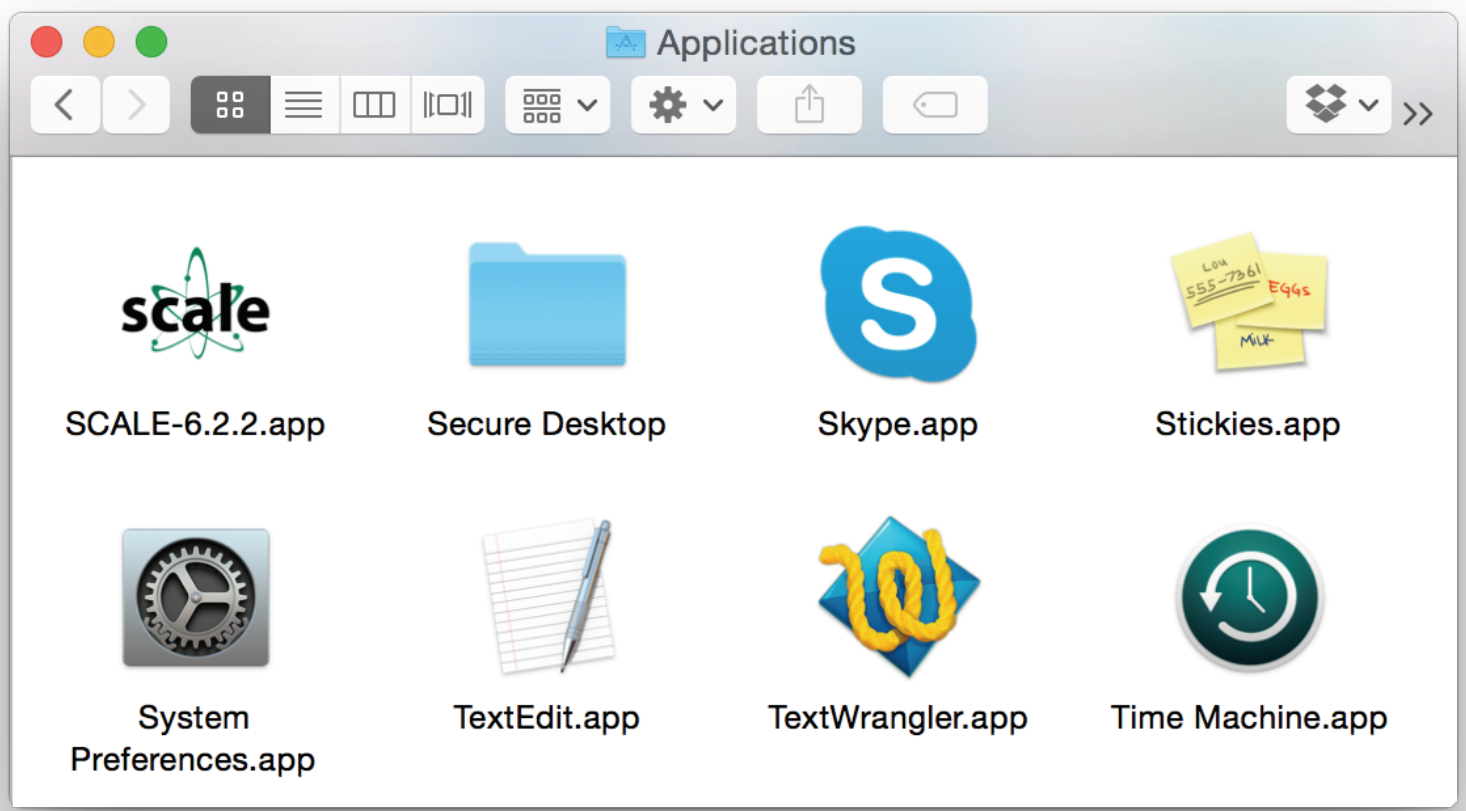

Figure 32. Mac SCALE-6.2.2 app in the applications folder.

\section{Running SCALE from the Command Line}

Using the command line, SCALE can be executed using the scalerte command from the bin directory inside the SCALE installation. Paths for each platform are shown below based on recommended installation directories. Your directory may differ based on the installation.

\section{Windows}

\section{$\mathrm{C}:$ SCALE-6.2.2|bin|scalerte.exe}

* Note that Norton Antivirus may require an exception for scalerte.exe on Windows to prevent the executable from being quarantined.

\section{Linux}

/scale/scale6.2.2/bin/scalerte

\section{Mac}

/Applications/SCALE-6.2.2.app/Contents/Resources/bin/scalerte

$$
\text { Getting Started - } 50
$$


The SCALE runtime environment provides several command line options. The usage is as follows.

scalerte [options] inputfile(s) [options] [inputfile(s)]

Where inputfile (s) are one or more files or file patterns (test.inp, or test*.inp, etc.).

Where options are:

-a: Specify alias file.

$$
\text { -a path/to/aliasesfile }
$$

-f: Add hostname to output filename. Produces inputfile.hostname.out

-h: Print this information as a help message.

- I: Number of threads to use for MPI/OpenMP directives. - I 4

-m: Print information messages as SCALE executes.

-M: Specify a machine names file for SCALE parallel capabilities.

$$
\text { -M /path/to/machine/names/file }
$$

-n: Nice level on Nix systems, ignored on Windows. Default: $-\mathrm{n} 2$

$-\mathrm{N}$ : Number of MPI processes to run. $-\mathrm{N} 20$

- : Overrides the default inputfile.out output name. The out extension is appended by scalerte, so there is no need to specify the extension.

-o path/to/outputfile

NOTE: If the path/to/outputfile already exists, it will be deleted. If this option is specified while in stack mode (multiple input files), the value provided is prepended to the inputfile's basename.

scalerte triton* -o myout results in output names myout.triton*.out

$-r$ : Keep the temporary working directory after execution.

-t: No new temporary working directory. Uses last temporary working directory, $-r$ is implied.

- T: Specify temporary working directory.

-T directory/path 
NOTE: If $-T$ is specified while in stack mode (multiple input files) the value provided is appended with the index of the file.

scalerte t0.inp t1.inp t2.inp -T mytmp

results in mytmp, mytmp1, and mytmp2 temporary directories.

$-\mathrm{v}$ : Turn on verbose activity printing for scalerte.

$-\mathrm{V}$ : Print the scalerte version date.

-x: Do not return XSDRNPM output in a .xsdrnfiles directory.

- z: Add date to the output filename.

Produces output files in the form of inputfile.YYYY.MM.ddThh.mm.s.s.out

Where:

- yyyy- is the year of execution.

- MM- is the month of execution.

- dd- is the day of execution.

- hh- is the hour of execution.

- mm- is the minute of execution.

- ss- is the second of execution.

\section{Example Invocation}

For users familiar with previous invocation of the SCALE batch script, this usage is no longer valid. The only valid entry point is scalerte. Scalerte can be executed from anywhere with a fully qualified path such as $\mathrm{c}: \backslash \mathrm{SCALE}-6.2 .2 \backslash \mathrm{bin} \backslash \mathrm{scalerte}$ or /scale/scale6.2.2/bin/scalerte, for example.

Invoke SCALE on a single input file named HelloWorld.inp.

scalerte HelloWorld

or

scalerte HelloWorld.inp

Invoke SCALE on all input files patterned HelloWorld*.inp.

scalerte HelloWorld*.inp

Invoke SCALE on all input files patterned HelloWorld*.inp and print runtime messages to the console.

scalerte HelloWorld*.inp $-\mathrm{m}$

$$
\text { Getting Started - } 52
$$


Invoke SCALE on all input files patterned HelloWorld*.inp and include hostname and date/time in the output file's name.

scalerte HelloWorld*.inp -fz

or

scalerte HelloWorld*.inp -f $-\mathrm{z}$

Invoke SCALE on HelloWorld.inp and rename output to be MyHello.out.

scalerte HelloWorld -o MyHello

or

scalerte HelloWorld.inp -o MyHello

Invoke SCALE on all files patterned HelloWorld*inp and rename output to be MyHelloWorld*.out.

NOTE: When SCALE is run in stack mode (multiple inputs), the output override is prepended to the input file's name.

scalerte HelloWorld*.inp -o My

Invoke SCALE on HelloWorld.inp and keep the working directory.

scalerte HelloWorld.inp -r

Invoke SCALE on HelloWorld.inp and override and keep the working directory.

scalerte HelloWorld.inp -r -T myHelloWorldTempDir

Invoke SCALE on HelloWorld.inp and specify the number of threads to be 4 .

scalerte HelloWorld.inp -I 4

\section{SCALE Variables}

This section describes the environment variable used within scalerte. These variables can be accessed through SCALE's shell module to populate the working directory, and/or to return SCALE-generated files not returned by scalerte.

Shell is used to perform system commands inside a problem either before, after, or between explicitly called modules. It is usually used in SCALE to link a file from one name and place to another name and place, delete files or directories, and move or copy files. The use of shell in an input has the following form:

$=$ shel $\mathrm{I}$

*** System Commands *** 
end

Where System Commands are any UNIX or DOS command(s). This is particularly useful in tandem with the following environment variables when files not automatically returned are desired. The following example shell command copies the $\mathrm{x} 16$ file back alongside the output file.

$=$ shell

$\mathrm{cp} \times 16 \$$ [OUTBASENAME\} . $\times 16$

end

Please note the syntax of Nix and Windows environment variables, $\$\{$ VAR $\}$ and $\% \mathrm{VAR} \%$ respectively, are interchangeable, as scalerte and shell understand both.

There are seven primary locations known by scalerte. These primary locations are:

- The user's home directory, HOME.

*Nix systems, \$ $\{\mathrm{HOME}\}$, /home/uid.

*Windows, \%HOME\%, C:|Users $\backslash$ uid.

- The directory of SCALE, SCALE.

*Nix systems, \$\{SCALE $\}$, location of user's installation. Typically/scale/scale\#.

*Windows, \%SCALE\%, location of user's installation. Typically C:ISCALE\#.

- The directory of the input file, INPDIR.

*Nix systems, \$ $\{$ INPDIR $\}$.

*Windows, \%INPDIR\%.

- The directory of the output file, OUTDIR, by default, this is the same as INPDIR, because the output file is written next to the input file.

*Nix systems, \$ $\{$ OUTDIR $\}$.

*Windows, \%OUTDIR\%.

- The directory from which SCALE was invoked, the return directory, RTNDIR. This is the directory your console will return to upon completion.

*Nix systems, \$ $\{$ RTNDIR $\}$.

*Windows, \%RTNDIR\%.

- The directory that contains the SCALE data, DATA.

Getting Started - 54 
*Nix systems, $\$\{$ DATA $\}$.

*Windows, \%DATA\%.

- The working directory for a given input file, TMPDIR, or shorthand TMP.

*Nix systems, $\$\{$ TMPDIR $\}, \$\{T M P\}$.

*Windows, \%TMPDIR\%, \%TMP\%.

There are several secondary locations located in the SCALE directory tree. These are as follows:

- The directory containing the platform-specific compiled programs, PGMDIR, or legacy PGM_DIR.

*Nix systems, \$ $\{$ PGMDIR $\}, \$\{$ PGM_DIR $\}$.

*Windows, \%PGMDIR\%, \%PGM_DIR\%.

Lastly, there are several environment variables provided for convenience and/or associated with output data that can be useful.

- The directory containing the ORIGEN data files, ORIGENDIR.

*Nix systems, $\$\{$ ORIGENDIR $\}$.

*Windows, \%ORIGENDIR\%.

- The base name of the input file, BASENAME. This is the name of the input file without both absolute path and extension.

*Nix systems, $\$\{$ BASENAME $\}$, or $\$\{$ CASE_NAME $\}$.

*Windows, \%BASENAME\%, or \%CASE_NAME\%.

- The base name of the output file, OUTBASENAME, or legacy CASE_NAME. This is the name of the output file without both absolute path and extension.

*Nix systems, $\$\{$ OUTBASENAME $\}$, or $\$\{$ CASE_NAME $\}$.

*Windows, \%OUTBASENAME\%, or \%CASE_NAME\%.

- The base name of the output file, OUTBASE. This is the absolute name of the output file without absolute file extension.

*Nix systems, $\$\{$ OUTBASE $\}$.

*Windows, \%OUTBASE\%.

- The absolute path to the input file, INPUTFILE.

*Nix systems, \$\{INPUTFILE $\}$.

$$
\text { Getting Started - } 55
$$


*Windows, \%INPUTFILE\%.

- The absolute path to the output file, OUTFILE.

*Nix systems, \$\{OUTFILE\}.

*Windows, \%OUTFILE\%.

- The directory containing USLSTATS output, USLDIR. If USLSTATS data were output, these data will be located in OUTDIR $\backslash$ OUTBASENAME.uslstats directory.

*Nix systems, $\$\{$ USLDIR $\}$.

*Windows, \%USLDIR\%.

- The directory containing CENTRM output, CENTRMDIR. If CENTRM data were output, these data will be located in OUTDIR $\backslash$ OUTBASENAME.centrmfiles directory.

*Nix systems, \$CENTRMDIR.

*Windows, \%CENTRMDIR\%.

- The directory containing XSDRNPM output, XSDRNDIR. If XSDRNPM data were output, these data will be located in OUTDIR $\backslash$ OUTBASENAME.xsdrnfiles directory.

*Nix systems, \$XSDRNDIR.

*Windows, \%XSDRNDIR\%.

- The platform specific file separator, FS. This is either backslash ( () on Windows, or forward slash (/) on Nix systems.

*Nix systems, $\$\{\mathrm{FS}\}$.

*Windows, $\% \mathrm{FS} \%$.

\section{Parallel Execution Capability}

SCALE 6.2 contains five modules and sequences that have distributed memory (MPI) parallelism; KENO V.a, KENO-VI, Sampler, and ORIGAMI. However, the binary executable files distributed with SCALE do not have MPI enabled. To run one of these codes in parallel, the user must first build SCALE with MPI enabled (see build instructions in this guide). Control modules like CSAS6, T6-DEPL, and TSUNAMI-3DK6 automatically initiate the parallel version of KENO-VI in a parallel SCALE build if the user provides the required arguments as summarized below. When running a standalone code in parallel (such as KENO-VI), a "\%" prefix is required on the sequence specification record in the input file (e.g., $=\%$ keno-vi). Parallel code execution is available on Linux and Mac systems but is not available on Windows PCs.

The executable binary code distributed with SCALE only enables serial calculations. If MPI parallelism is desired, the source code must be compiled with MPI support enabled 
for the platform and configuration where the code will be executed in parallel. The SCALE build configuration sets some variables (SCALEMPI, SCALECMDS, and MPIRUN) depending on the third party MPI package for the SCALE driver, which prepares two different execution environments with these variable sets for both serial and parallel code execution.

Executing SCALE in parallel is initiated by scalerte when the user provides the necessary MPI command line arguments with the two options, $-N$ and $-M$, which specify the number of MPI processes and machine names, respectively. To run an input called HelloWorld.inp from an MPI build on SCALE on two nodes specified in the machine file mach use the command:

scalerte -N $2-$ M mach HelloWorld.inp

With these options, scalerte sets NTASKS and MACHINEFILE variables depending on the user request and passes them to the SCALE driver. During the parallel code execution process, the driver invokes the MPIRUN wrapper to run the parallel functional module across MPI processes. An environment variable MPIARGS is available for the driver to pass some user-defined MPI options to MPIRUN wrapper. 


\section{SCALE Sample Problems}

The SCALE sample problems are carefully designed by the SCALE developers to verify the installation and functionality of SCALE relative to expected results. Users are urged to run the sample problems to verify the proper installation of SCALE. ORNL has provided a set of reference results from each sample problem against which the results of each installation can be compared.

The Sample Problems are most easily run through Fulcrum by selecting Run SCALE Verification from the Run menu on the main menu bar. This will run all of the Sample Problems sequentially and will present any differences from the ORNL-generated results.

The SCALE Runtime Environment, scalerte, which is described in the subsequent section, has a built-in scripting interface to allow for invocation of several groupings of sample problems, from individual problems, problems for a specific module, subsets of modules, or all samples. The sample problems will print a message indicating the sample problem currently running, followed by the differences between ORNL-generated results and the newly generated results for the sample problem(s). Note that there may be up to an hour's delay before messages are printed to the console. The final message will indicate that the "Process finished with a 0 return code ...." If no differences or minimal differences are noted for a particular sample problem, SCALE has been properly installed for the tested functionality.

The set of sample problems is located in the samples directory of the SCALE installation.

To run all sample problems, execute the following command.

scalerte @samples/samples

To invoke sample problems for a particular module or sequence, use

scalerte @samples/samples modulename

where modulename is the name of the module or sequence to test, e.g., centrm

To invoke a single sample problem, do the following.

scalerte dsamples/samples problemname

where problemname is the name of the specific sample problem to test, e.g., centrmpwr.inp

The above modulename and problemname examples can be combined and repeated to exercise sets of sample problems of interest. For example, run csas and origen sequence sample problems in addition to the centrm-pwr.inp sample.

scalerte @samples/samples csas centrm-pwr.inp origen

As the sample problems execute, feedback will be provided to the screen such as shown in the example below. Where no differences or only small differences are reported

Getting Started - 58 
between the ORNL results and the currently generated results for each sample, SCALE has been properly installed and configured for the tested functionality.

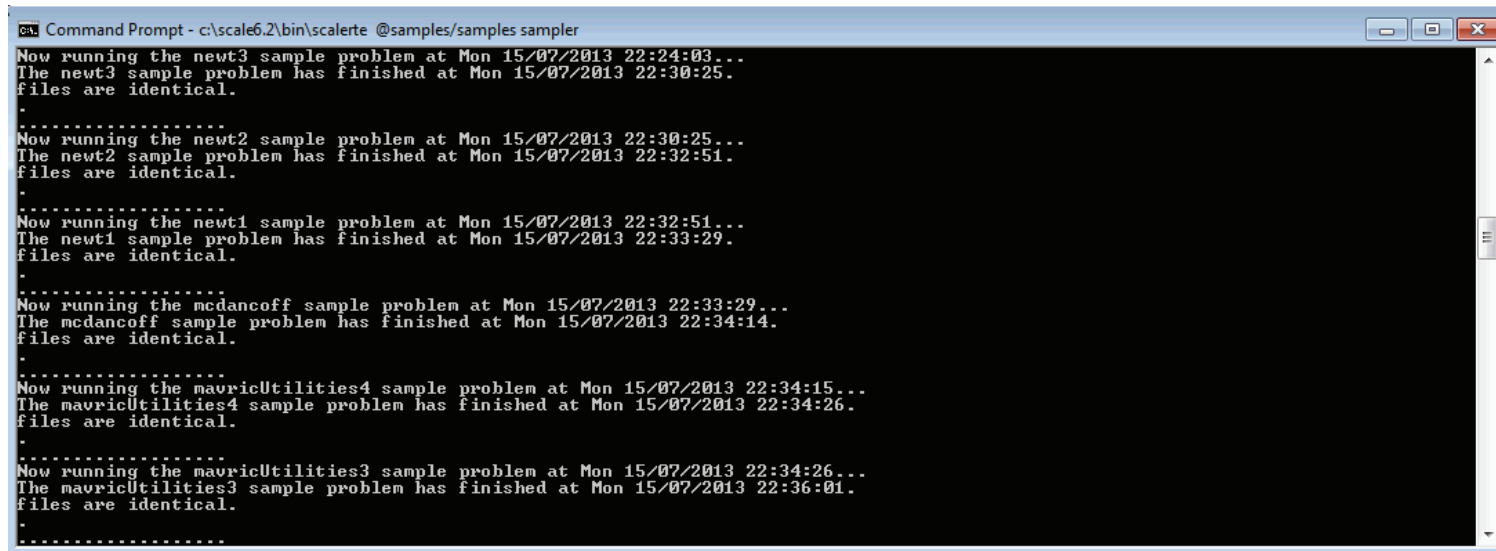

\section{Figure 33. Sample problem output messages.}

For any problems or questions, please contact scalehelp@ornl.gov. 


\section{Build Instructions}

These instructions are only for those who wish to recompile their SCALE binaries. If you are running SCALE using the precompiled binary executable files distributed with SCALE, please disregard this section.

For SCALE 6.2 and subsequent updates, the build configuration has been completely renewed relative to all previous versions. The SCALE build is now based on CMake from KitWare, which supports a consistent experience on Linux, Mac, and Windows.

\section{Overview}

There are four main steps to create and install SCALE binaries

1. Installation of compilers and third-party libraries (TPLs)

2. CMake configuration to generate a native build tree

3. Compilation of all executables and libraries

4. Installation to deploy all executables into a configuration ready for execution

\section{Required Resources}

SCALE requires the following programs in order to compile:

1. Fortran Compiler

i. Windows - Intel Fortran compiler 15.0.

ii. Linux - Intel Fortran compiler 14.1+/GNU gfortran 4.8.3+ compiler.

iii. Mac OS - GNU gfortran 4.8.5+ compiler.

2. $\mathrm{C} / \mathrm{C}++$ Compiler

i. Windows - Intel $\mathrm{C} / \mathrm{C}++$ compiler 15.0.

ii. Linux - Intel C/C++ compiler 14.1+ / GNU g++/gec 4.8.3+ compiler.

iii. $\mathrm{Mac}$ OS $-\mathrm{GNU} \mathrm{g}++/$ gcc $4.8 .5+$ compiler.

3. CMake 2.8.12.2+ - Platform independent build configuration for Linux and Darwin, and CMake 3.3.2+ for Windows.

SCALE requires the following libraries, which are not distributed with SCALE:

LAPACK libraries:module

a. lapack

b. blas

You must modify the LAPACK variable in configure_scale_gnu.sh to point to your installation of LAPACK prior to configuration.

The following optional package is required by SCALE if MPI support is desired: 
OpenMPI-1.8.1+ - Optional for SCALE build with MPI support on Linux and Mac OS (currently, SCALE does not support MPI for Windows)

\section{Mac OSX Resources}

Mac OSX does not come preloaded with the necessary resources for compiling SCALE. Macports provides a simple mechanism for retrieving almost all of the required resources necessary to compile SCALE on a Mac. This process has only been tested on Mac OSX 10.11.6.

You can download the latest macports from http://www.macports.org/. You will need $\mathrm{XCode}$ (Free from App Store), with commandline tools installed for macports to work.

\section{You will need administrative privileges to install macports and the necessary resources.}

Please see macports instructions for xcode installation/requirements.

http://guide.macports.org/\#installing.xcode

After installing these resources, a new shell session is necessary to access the new tools.

NOTE if your rsync port is blocked by the firewall (as is the case at ORNL), you may synchronize over http by updating your/opt/local/etc/macports/sources.conf file to change the line:

rsync://rsync.macports.org/release/tarballs/ports.tar [default]

to

http://www.macports.org/files/ports.tar.gz [default]

The following commands will install all necessary resources.

If rsync port is blocked, use

$\$>$ sudo port $-d$ sync

If rsync port is open, use

\$>sudo port selfupdate

Regardless of rsync status, execute the following commands:

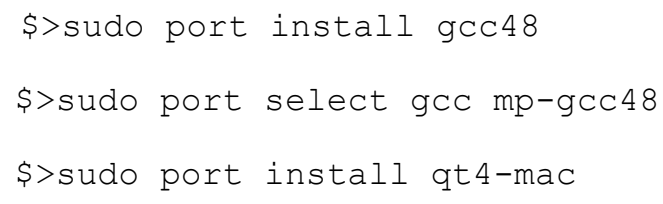

These commands upgrade the default compilers from gcc-4.2.2 to gcc-4.8.3, and they install qt4.8.6. 


\section{CMake Configuration}

'CMakeLists.txt' files can be found throughout SCALE. From the SCALE root directory, these CMakeLists.txt files create a tree of included directories called the SOURCE TREE. Namely, the source directories are

packages/AmpxLib

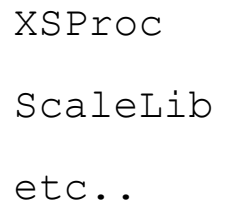

To configure a build, call 'cmake' on the root CMakeLists.txt file, namely scale_dir/CMakeLists.txt. CMake takes your source tree and creates a BUILD TREE. The build tree contains or will contain the build configuration, the Make or NMake files, and all compilation output: object files, archive libraries, and binary executables.

SCALE requires several TPLs, specifically, QT and Lapack. These TPLs must be specified at the time of configuration. For ease of use, configuration scripts for every supported platform are available in the 'scripts' directory of the source code. These scripts describe the necessary variables to define. A user's modifications to these scripts should be limited to the path to the root directories for the TPLs.

\section{Recommended Configuration Procedure}

After unpacking the source code, navigate to the root scale directory $(<$ SCALE_SRC_ROOT $>$. You will see CMakeLists.txt, PackagesList.cmake, and CTestConfig.cmake. This is the root of the source tree, to which you will point CMake. This example demonstrates creating build trees for multiple configurations for your working copy.

\section{Linux and Mac Configuration}

\section{- Make Build Directory}

mkdir build

mkdir -p build/intel **this could be any directory

or

mkdir -p build/gcc

\section{- CMake Initialization}

Copy the cmake script from the 'scripts' directory to your build directory. Update the cmake script with your TPL specifications.

$$
\text { Getting Started - } 62
$$


- Serial SCALE (without MPI support)

$\mathrm{CP} \quad<$ SCALE_SRC_ROOT>/script/configure_scale_gnu.sh build/gcc

chmod u+x build/gcc/configure_scale_gnu.sh

- Parallel SCALE (with MPI support)

cp script/configure_scale_mpi.sh build/gcc

chmod u+x build/gcc/configure_scale_mpi.sh

\section{- Create Your Configuration}

Create your configuration by pointing this script at the source tree root

cd build/gcc

Edit the CMakelists.txt file in

$<$ SCALE_SRC_ROOT>/Trilinos/packages/anasazi/srC/ so as

to comment out the following line:

ASSERT_DEFINED (Anasazi_ENABLE_Tpetra)

- Serial SCALE (without MPI support)

./configure_scale_gnu.sh.../.

- Parallel SCALE (with MPI support)

./configure_scale_mpi.sh .../.

/*** Configuration Output....***/

**If you add or remove source files from the source tree, CMake will NOT see these modifications unless a CMake file is modified (CMakelists.txt, PackagesList.cmake, etc...).

CMake will re-evaluate the entire source tree when any CMake file has been modified. If CMake does not pick up the addition/removal of sources files, the easiest way to update the build tree is to 'touch' any CMake file in the source tree.

\section{Windows Configuration}

Instructions are not documented here. Please contact scalehelp@ornl.gov for assistance. 


\section{Compilation}

Every library and executable is a TARGET. Calling 'make' on Linux and Mac and 'nmake' on Windows from the root of your build tree $(<$ SCALE_SRC_ROOT $>/$ build/gCC from the previous example) will build ALL targets. There are two options when building specific targets. For example MavricUtilities contains a number of executables: mtadder, mtaverager, and so on.

You may invoke 'make mtadder' to build mtadder alone. Alternatively, you can build it from build/gcc, 'cd packages/Mavricutilities' and 'make' to compile ALL targets in mavricUtilities.

\section{Compilation Flags}

You may modify CMAKE_Fortran_FLAGS, CMAKE_C_FLAGS, CMAKE_CXX_FLAGS on the cmake command invocation line.

\section{Installation}

CMake provides the 'install' target, which installs all binaries from the current directory down. For example, 'make install' from build/gcc will install any targets, which would install aim, mavric, mtadder, mtaverager, etc., while 'cd packages/Mavric' and 'make install' will only install targets declared in the Mavric directory.

***Note that 'make install' re-evaluates the build for all dependencies. For example, package A depends on packages $\mathrm{B}$ and D. Package D depends on package E. 'make install' for package A would result in packages E, D, and B being re-evaluated and rebuilt if necessary. Thus, if you know you want to build and install, you can save time building by simply doing a 'make install'. An alternative is 'make install/fast' which will skip the dependency checking and only execute the install operations. 


\section{References}

1) W. J. Marshall, D. Wiarda, C. Celik, B. T. Rearden, and D. R. Wentz, "Validation of Criticality Safety Calculations with SCALE 6.2," Proceedings of ANS NCSD 2013 Criticality Safety in the Modern Era: Raising the Bar, Wilmington, NC, September 29October 3, 2013, on CD-ROM, American Nuclear Society, LaGrange Park, IL (2013).

2) T. Ueki and F. B. Brown, "Stationarity Modeling and Informatics-Based Diagnostics in Monte Carlo Criticality Calculations," Nucl. Sci. Eng., 149, 38-50 (2005).

3) N. Larson, Updated User's Guide for Sammy: Multilevel R-Matrix Fits to Neutron Data Using Bayes' Equations, ORNL/TM-9179/R8, Oak Ridge National Laboratory, Oak Ridge, TN, October 2008.

4) S. Hart, "Implementation of the Doppler Broadening Rejection Correction in KENO," Trans. Am. Nucl. Soc., 108, 423-426 (June 2013).

5) B. Becker, R. Dagan, G. Lohnert, "Proof and Implementation of the Stochastic Formula for Ideal Gas, Energy Dependent Scattering Kernel," Ann. Nucl. Energy, 36, 470-474 (2009).

6) T. M. Evans, A. S. Stafford, R. N. Slaybaugh, and K. T. Clarno, "Denovo: A New ThreeDimensional Parallel Discrete Ordinates Code in SCALE," Nucl. Technol., 171, 171-200 (2010).

7) A. M. Ibrahim, D. E. Peplow, J. C. Wagner, S. W. Mosher, and T. M. Evans, "Acceleration of Monte Carlo Criticality Calculations Using Deterministic-Based Starting Sources," Trans. Am. Nucl. Soc., 105, 539-541 (2011).

8) A. M. Ibrahim, D. E. Peplow, J. C. Wagner, S. W. Mosher, and T. M. Evans, "Acceleration of Monte Carlo Criticality Calculations Using Deterministic-Based Starting Sources," PHYSOR 2012, Knoxville, TN, April 15-20, 2012.

9) A. M. Ibrahim, D. E. Peplow, K. B. Bekar, C. Celik, D. Ilas, J. M. Scaglione, and J. C. Wagner, "Hybrid Technique in SCALE for Fission Source Convergence Applied to Used Nuclear Fuel Analysis," in preparation for the 2013 Topical Meeting on Nuclear Criticality Safety (NCSD 2013), Wilmington, NC, September 29-October 3, 2013.

10) E. Gabriel et al., "Open MPI: Goals, Concept, and Design of a Next Generation MPI Implementation," in Proceedings, 11th European PVM/MPI Users' Group Meeting," 97104, Budapest, Hungary (2004).

11) M. L. Williams, K. S. Kim, "The Embedded Self-Shielding Method," PHYSOR 2012, Knoxville, Tennessee, USA, April 15-20, 2012.

12) C. M. Perfetti and B. T. Rearden, "Development of a SCALE Tool for Continuous-Energy Eigenvalue Sensitivity Coefficient Calculations," Proceedings of Joint International Conference on Supercomputing in Nuclear Applications and Monte Carlo 2013 (SNA + MC 2013) La Cité des Sciences et de l'Industrie, Paris, France, October 27-31, 2013.

13) International Handbook of Evaluated Criticality Safety Benchmark Experiments NEA/NSC/DOC(95)03, Organization for Economic Co-operation and DevelopmentNuclear Energy Agency (OECD-NEA) (September 2012).

14) M. L. Williams, F. Havlůj, D. Wiarda, M. T. Pigni, I. C. Gauld, "SCALE Uncertainty Quantification Methodology for Criticality Safety Analysis of Used Nuclear Fuel,"

Getting Started - 65 
Proceedings of ANS NCSD 2013 - Criticality Safety in the Modern Era: Raising the Bar, Wilmington, NC, September 29-October 3, 2013, on CD-ROM, American Nuclear Society, LaGrange Park, IL (2013).

15) B. T. Rearden, K. J. Dugan, and F. Havlůj, "Quantification of Uncertainties and Correlations in Criticality Experiments," Proceedings of ANS NCSD 2013 - Criticality Safety in the Modern Era: Raising the Bar, Wilmington, NC, September 29-October 3, 2013, on CD-ROM, American Nuclear Society, LaGrange Park, IL (2013).

16) B. Krzykacz, E. Hofer, M. Kloos, "A Software System for Probabilistic Uncertainty and Sensitivity Analysis of Results from Computer Models," Proc. International Conference on Probabilistic Safety Assessment and Management (PSAM-II), San Diego, CA (1994).

17) M.T. Pigni, M.W. Francis, I.C. Gauld, "Investigation of Inconsistent ENDF/B-VII.1 Independent and Cumulative Fission Product Yields with Proposed Revisions," Nuclear Data Sheets 123, 231 (2015). 


\subsection{INTRODUCTION}

The SCALE code system is a widely used modeling and simulation suite for nuclear safety analysis and design that is developed, maintained, tested, and managed by the Reactor and Nuclear Systems Division (RNSD) of the Oak Ridge National Laboratory (ORNL). SCALE provides a comprehensive, verified and validated, user-friendly tool set for criticality safety, reactor physics, radiation shielding, radioactive source term characterization, and sensitivity and uncertainty analysis. Since 1980, regulators, licensees, and research institutions around the world have used SCALE for safety analysis and design. SCALE provides an integrated framework with dozens of computational modules, including three deterministic and three Monte Carlo radiation transport solvers selected based on the user's desired solution strategy. SCALE includes current nuclear data libraries and problem-dependent processing tools for continuous energy and multigroup neutronics and coupled neutron-gamma calculations, as well as activation, depletion, and decay calculations. SCALE includes unique capabilities for automated variance reduction for shielding calculations, as well as sensitivity and uncertainty analysis. SCALE's graphical user interfaces assist with accurate system modeling and convenient access to desired results.

This manual documents version 6.2 of the SCALE code system, released in 2016. Users are directed to the README file for details on updates available in version 6.2 relative to 6.1 , as well as instructions on installing or building SCALE, testing the configuration with the sample problems, and beginning analysis. This introduction provides historical information on SCALE, an overview of current features, information on technical assistance, code availability, and acknowledgments.

\subsection{BACKGROUND}

The history of the SCALE code system dates back to 1969, when ORNL began providing the transportation package certification staff at the US Atomic Energy Commission (AEC) with computational support in the use of the new KENO code. KENO was used to perform criticality safety assessments with the statistical Monte Carlo method. From 1969 to 1976, the AEC certification staff relied on ORNL personnel to assist them in the correct use of codes and data for criticality, shielding, and heat transfer analyses of transportation packages. However, the certification staff learned that occasional users had difficulty in becoming proficient in performing the calculations often needed for an independent safety review. Thus, shortly after the certification staff was moved to the US Nuclear Regulatory Commission (NRC), the NRC proposed development of an easy-to-use analysis system that provided the technical capabilities of the individual modules with which they were familiar. With this proposal, the concept of SCALE as a comprehensive modeling and simulation suite for nuclear safety analysis and design was born.

The NRC staff provided ORNL with some general development criteria for SCALE: (1) focus on applications related to nuclear fuel facilities and package designs, (2) use well-established computer codes and data libraries, (3) design an input format for the occasional or novice user, (4) prepare standard analysis sequences (control modules) to automate the use of multiple codes (functional modules) and data to perform a system analysis, and (5) provide complete documentation and public availability. With these criteria, the ORNL staff laid out the framework for the SCALE system and began development efforts. The initial version of SCALE (Version 0) was distributed in July 1980. Although the capabilities of the system continue to evolve, the philosophy established with the initial release still serves as the foundation of SCALE 6.2 more than 35 years later.

In July 1980, the initial version of SCALE was made available to the Radiation Safety Information Computational Center (RSICC) at ORNL. This system was packaged and released by RSICC as CCC-288/SCALE 0. Subsequent additions and modifications resulted in the following releases: CCC-424/SCALE 1 in 1981; CCC-450/SCALE 2 in 1983; CCC-466/SCALE 3 in 1985; 
CCC-545/SCALE 4.0 in 1990; SCALE 4.1 in 1992; SCALE 4.2 in 1994; SCALE 4.3 in 1995; SCALE 4.4 in 1998; SCALE 4.4a in 2000; CCC-725/SCALE 5 in 2004; CCC-732/SCALE 5.1 in 2006; CCC-750/SCALE 6.0 in 2009; CCC-785/SCALE 6.1 in 2011; CCC-834/SCALE 6.2 in 2016.

\subsection{SYSTEM OVERVIEW}

A primary goal of SCALE is to provide robust calculations while reducing requirements for user input and knowledge of the intricacies of the underlying code and data architecture. As such, SCALE provides standardized sequences to integrate many modern and advanced capabilities into a seamless calculation that the user controls from a single input file. Additional utility modules are provided primarily for post processing data generated from the analysis sequences for advanced studies. Input for SCALE sequences is provided in the form of text files using free-form input with extensive use of keywords and engineering-type input requirements. A graphical user interface is provided to assist in the creation of input files, visualization of geometry and nuclear data, execution of calculations, viewing output, and visualization of results. An overview of the major SCALE capabilities and the analysis areas they serve is provided in Table 1 with additional descriptions provided below.

Table 1. Summary of major SCALE capabilities

\begin{tabular}{|c|c|c|c|}
\hline Analysis area & $\begin{array}{l}\text { Modules/ } \\
\text { libraries }\end{array}$ & Analysis function(s) & Documentation section \\
\hline \multirow{3}{*}{ Criticality safety } & $\begin{array}{l}\text { CSAS5/ } \\
\text { CSAS6 }\end{array}$ & $\begin{array}{l}\text { 3D multigroup and continuous } \\
\text { energy eigenvalue Monte Carlo } \\
\text { analysis and criticality search } \\
\text { capability }\end{array}$ & \multirow{3}{*}{2} \\
\hline & STARBUCS & $\begin{array}{l}\text { Burnup credit analysis using } \\
\text { 3D Monte Carlo }\end{array}$ & \\
\hline & Sourcerer & $\begin{array}{l}\text { Hybrid 3D deterministic/Monte } \\
\text { Carlo analysis with optimized } \\
\text { fission source distribution }\end{array}$ & \\
\hline \multirow{4}{*}{ Reactor physics } & \multirow{3}{*}{ TRITON } & $\begin{array}{l}1 \mathrm{D} \text { and } 2 \mathrm{D} \text { general purpose } \\
\text { lattice physics depletion } \\
\text { calculations and generation of } \\
\text { few-group cross section data } \\
\text { for use in nodal core simulators }\end{array}$ & \multirow{4}{*}{3} \\
\hline & & $\begin{array}{l}\text { 3D multigroup and continuous } \\
\text { energy Monte Carlo depletion } \\
\text { analysis }\end{array}$ & \\
\hline & & $\begin{array}{l}\text { 2D eigenvalue and reaction rate } \\
\text { sensitivity analysis }\end{array}$ & \\
\hline & Polaris & $\begin{array}{l}\text { 2D streamlined light water } \\
\text { reactor lattice physics depletion } \\
\text { calculations and generation of } \\
\text { few-group cross section data } \\
\text { for use in nodal core simulators }\end{array}$ & \\
\hline
\end{tabular}


Table 1. Summary of major SCALE Capabilities (continued)

\begin{tabular}{|c|c|c|c|}
\hline Analysis area & $\begin{array}{c}\text { Sequences/ } \\
\text { modules/ } \\
\text { libraries }\end{array}$ & Analysis function(s) & Documentation section \\
\hline Radiation shielding & MAVRIC & $\begin{array}{l}\text { 3D continuous energy and } \\
\text { multigroup fixed-source Monte } \\
\text { Carlo analysis with automated } \\
\text { variance reduction }\end{array}$ & 4 \\
\hline \multirow{3}{*}{$\begin{array}{l}\text { Activation, depletion and } \\
\text { decay }\end{array}$} & ORIGEN & $\begin{array}{l}\text { General purpose point } \\
\text { depletion and decay code to } \\
\text { calculate isotopic } \\
\text { concentrations, decay heat, } \\
\text { radiation source terms, and } \\
\text { curie levels }\end{array}$ & \multirow{3}{*}{5} \\
\hline & ORIGAMI & $\begin{array}{l}\text { Simulated 3D analysis for light } \\
\text { water reactor spent fuel } \\
\text { assemblies (isotopic activation, } \\
\text { depletion, and decay for light } \\
\text { water reactor fuel assemblies) }\end{array}$ & \\
\hline & $\begin{array}{l}\text { ORIGEN } \\
\text { reactor } \\
\text { libraries }\end{array}$ & $\begin{array}{l}\text { Pregenerated burnup libraries } \\
\text { for a variety of fuel assemblies } \\
\text { for commercial and research } \\
\text { reactors }\end{array}$ & \\
\hline \multirow{4}{*}{$\begin{array}{l}\text { Sensitivity and uncertainty } \\
\text { analysis }\end{array}$} & \multirow{3}{*}{ TSUNAMI } & $\begin{array}{l}\text { 1D and 2D multigroup } \\
\text { eigenvalue and reaction rate } \\
\text { sensitivity analysis }\end{array}$ & \multirow{4}{*}{6} \\
\hline & & $\begin{array}{l}\text { 3D multigroup and continuous } \\
\text { energy eigenvalue and reaction } \\
\text { rate sensitivity analysis }\end{array}$ & \\
\hline & & $\begin{array}{l}\text { Determination of experiment } \\
\text { applicability and biases for use } \\
\text { in code and data validation }\end{array}$ & \\
\hline & Sampler & $\begin{array}{l}\text { Stochastic uncertainty } \\
\text { quantification in results based } \\
\text { on uncertainties in nuclear data } \\
\text { and input parameters }\end{array}$ & \\
\hline
\end{tabular}


Table 1. Summary of major SCALE Capabilities (continued)

\begin{tabular}{|c|c|c|c|}
\hline Analysis area & $\begin{array}{c}\text { Sequences/ } \\
\text { modules/ } \\
\text { libraries }\end{array}$ & Analysis function(s) & Documentation section \\
\hline \multirow{3}{*}{$\begin{array}{l}\text { Material specification and } \\
\text { Cross section processing }\end{array}$} & XSProc & $\begin{array}{l}\text { Temperature correction, } \\
\text { resonance self-shielding, and } \\
\text { flux weighting to provide } \\
\text { problem-dependent } \\
\text { microscopic and macroscopic } \\
\text { multigroup cross section data } \\
\text { integrated with computational } \\
\text { sequences, but also available } \\
\text { for stand-alone analysis }\end{array}$ & \multirow{3}{*}{7} \\
\hline & $\begin{array}{l}\text { Standard } \\
\text { composition } \\
\text { library }\end{array}$ & $\begin{array}{l}\text { Library used throughout } \\
\text { SCALE that provides } \\
\text { individual nuclides; elements } \\
\text { with tabulated natural } \\
\text { abundances; compounds, } \\
\text { alloys, mixtures, and fissile } \\
\text { solutions commonly } \\
\text { encountered in engineering } \\
\text { practice }\end{array}$ & \\
\hline & MCDancoff & $\begin{array}{l}\text { 3D Monte Carlo calculation of } \\
\text { Dancoff factors }\end{array}$ & \\
\hline \multirow{2}{*}{ Monte Carlo transport } & $\begin{array}{l}\text { KENO V.a/ } \\
\text { KENO-VI }\end{array}$ & $\begin{array}{l}\text { Eigenvalue Monte Carlo codes } \\
\text { applied in many computational } \\
\text { sequences for multigroup and } \\
\text { continuous energy neutronics } \\
\text { analysis }\end{array}$ & \multirow{2}{*}{8} \\
\hline & Monaco & $\begin{array}{l}\text { Fixed source Monte Carlo code } \\
\text { applied in the MAVRIC } \\
\text { sequence for multigroup and } \\
\text { continuous energy analysis }\end{array}$ & \\
\hline \multirow[b]{2}{*}{ Deterministic transport } & XSDRNPM & $\begin{array}{l}\text { 1D discrete ordinates transport } \\
\text { applied for neutron, gamma, } \\
\text { and coupled neutron/gamma } \\
\text { analysis }\end{array}$ & \multirow[b]{2}{*}{9} \\
\hline & NEWT & $\begin{array}{l}\text { 2D extended step characteristic } \\
\text { transport with flexible } \\
\text { geometry applied to neutronics } \\
\text { analysis, especially within the } \\
\text { TRITON sequences }\end{array}$ & \\
\hline
\end{tabular}


Table 1. Summary of major SCALE Capabilities (continued)

\begin{tabular}{|c|c|c|c|}
\hline Analysis area & $\begin{array}{c}\text { Sequences/ } \\
\text { modules/ } \\
\text { libraries }\end{array}$ & Analysis function(s) & Documentation section \\
\hline & Denovo & $\begin{array}{l}\text { 3D Cartesian geometry discrete } \\
\text { ordinates transport applied for } \\
\text { neutron, gamma, and coupled } \\
\text { neutron/gamma analysis, } \\
\text { especially to generate biasing } \\
\text { parameters within the } \\
\text { MAVRIC and Sourcerer } \\
\text { sequences (not generally run as } \\
\text { stand-alone code in SCALE) }\end{array}$ & \\
\hline \multirow{3}{*}{ Nuclear Data } & $\begin{array}{c}\text { Cross Section } \\
\text { Data }\end{array}$ & $\begin{array}{l}\text { Recent neutron, gamma and } \\
\text { coupled neutron/gamma } \\
\text { nuclear data libraries in } \\
\text { continuous-energy and several } \\
\text { multigroup structures for use in } \\
\text { all transport modules }\end{array}$ & \multirow{3}{*}{10} \\
\hline & ORIGEN Data & $\begin{array}{l}\text { Recent nuclear decay data, } \\
\text { neutron reaction cross sections, } \\
\text { energy-dependent neutron- } \\
\text { induced fission product yields, } \\
\text { delayed gamma ray emission } \\
\text { data, neutron emission data, } \\
\text { and photon yield data }\end{array}$ & \\
\hline & $\begin{array}{l}\text { Covariance } \\
\text { Data }\end{array}$ & $\begin{array}{l}\text { Recent uncertainties in nuclear } \\
\text { data for neutron interaction, } \\
\text { fission product yields, and } \\
\text { decay data for use in } \\
\text { TSUNAMI tools and Sampler }\end{array}$ & \\
\hline Utilities & Various & $\begin{array}{l}\text { Numerous pre- and post- } \\
\text { processing utilities for data } \\
\text { introspection and format } \\
\text { conversion }\end{array}$ & 11 \\
\hline
\end{tabular}

\subsubsection{Criticality Safety}

SCALE provides a suite of computational tools for criticality safety analysis that is primarily based on the KENO Monte Carlo codes for eigenvalue neutronics calculations. Two variants of KENO provide identical solution capabilities with different geometry packages. KENO V.a uses a simple, efficient geometry package sufficient for modeling many systems of interest to criticality safety and reactor physics analysts. KENO-VI uses the SCALE Generalized Geometry Package, which provides a quadraticbased geometry system with much greater flexibility in problem modeling but with slower runtimes. Both versions of KENO perform eigenvalue calculations for neutron transport primarily to calculate multiplication factors $\left(k_{e f f}\right)$ and flux distributions of fissile systems in continuous energy and multigroup 
modes and are typically accessed through Criticality Safety Analysis Sequence with KENO V.a (CSAS5) and Criticality Safety Analysis Sequence with KENO-VI (CSAS6). The CSAS sequences implement XSProc to process material input and provide a temperature and resonance-corrected cross section library based on the physical characteristics of the problem being analyzed. If a continuous energy cross section library is specified, no resonance processing is needed, and the continuous energy cross sections are used directly in KENO, with temperature corrections provided as the cross sections are loaded.

A search capability is available with CSAS5 to find desired values of $k_{\text {eff }}$ as a function of dimensions or densities. The two basic search options offered are (1) an optimum search seeking a maximum or minimum value of $k_{\text {eff }}$ and (2) a critical search seeking a fixed value of $k_{\text {eff. }}$ For continuous energy calculations, reaction rate tallies can be requested within the CSAS input, and for multigroup calculations, reaction rate calculations are performed using the KMART (KENO Module for Activity-Reaction Rate Tabulation) post-processing tools. A conversion tool is provided to up-convert KENO V.a input to KENO-VI either as a direct KENO input K5toK6, or more commonly, as a CSAS sequence C5toC6.

The Standardized Analysis of Reactivity for Burnup Credit using SCALE (STARBUCS) performs criticality calculations for spent fuel systems employing burnup credit. STARBUCS automates the criticality safety analysis of spent fuel configurations by coupling the depletion and criticality aspects of the analysis, thereby eliminating the need to manually process the spent fuel nuclide compositions into a format compatible with criticality safety codes. For burnup loading curve iterative calculations, STARBUCS employs the search algorithm from CSAS5 to determine initial fuel enrichments that satisfy a convergence criterion for the calculated $k_{\text {eff }}$ value of the spent fuel configuration.

The Sourcerer sequence applies the Denovo discrete ordinates code to generate the starting fission source distribution in a KENO Monte Carlo calculation. This sequence is especially applied to burnup credit transportation and storage analysis of as-loaded canister of used fuel.

\subsubsection{Reactor Physics}

The Transport Rigor Implemented with Time-dependent Operation for Neutronic depletion (TRITON) control module provides flexible capabilities to meet the challenges of modern reactor designs by providing 1D pin-cell depletion capabilities using XSDRNPM, 2D lattice physics capabilities using the NEWT flexible mesh discrete ordinates code, or 3D Monte Carlo depletion using KENO V.a or KENO-VI, including continuous energy treatment with problem-dependent temperature corrections. For multigroup analysis, TRITON implements XSProc to process material input and provide a temperature and resonancecorrected cross section library. TRITON allows users to input Dancoff factors to account for nonuniform lattices. In all cases, ORIGEN is implemented for depletion and decay calculations. Additionally, TRITON can produce assembly-averaged few group cross sections for use in core simulators. There are few limitations to the types of systems that can be modeled with TRITON, but the input complexity and long runtimes can be burdensome on users for detailed analysis.

Polaris is an optimized tool that produces assembly-averaged few group cross sections for light water reactor (LWR) analysis with core simulators. Polaris provides simplified input; only a few lines are required to describe the entire model. Polaris uses a multigroup self-shielding method called the Embedded Self-Shielding Method (ESSM) and Method-of-Characteristics (MoC) transport solver. The ESSM approach computes multigroup self-shielded cross sections using Bondarenko interpolation. The background cross section used in the interpolation is determined by a series of $2 \mathrm{D}$ MoC fixed-source calculations similar to the subgroup method that does not require explicit celldata input. Additionally, heterogeneous lattices are explicitly treated without the need to externally compute Dancoff factors. Like TRITON, Polaris implements ORIGEN for depletion and decay calculations. 


\subsubsection{Radiation Shielding}

The Monaco with Automated Variance Reduction using Importance Calculations (MAVRIC) fixedsource radiation transport sequence is designed to apply the multigroup and continuous-energy fixedsource Monte Carlo code, Monaco, to solve problems too challenging for standard, unbiased Monte Carlo methods. The intention of the sequence is to calculate fluxes and dose rates with low uncertainties in reasonable times, even for deep penetration problems. MAVRIC is based on the Consistent Adjoint Driven Importance Sampling (CADIS) methodology, which uses an importance map and a biased source that are derived to work together. MAVRIC generates problem-dependent cross section data, and then it automatically performs a coarse mesh 3D discrete ordinates transport calculation using Denovo to determine the adjoint flux as a function of position and energy, and apply the information to optimize the shielding calculation in Monaco. In the Forwarded-Weighted CADIS (FW-CADIS) methodology, an additional Denovo calculation is performed to further optimize the Monaco model to obtain uniform uncertainties for multiple tally locations. Several utility modules are also provided for data introspection and conversion.

\subsubsection{Activation, Depletion, and Decay}

The Oak Ridge Isotope Generation (ORIGEN) code calculates time-dependent concentrations, activities, and radiation source terms for a large number of isotopes simultaneously generated or depleted by neutron transmutation, fission, and radioactive decay. Provisions are made to include continuous nuclide feed rates and continuous chemical removal rates that can be described with rate constants for application to reprocessing or other systems that involve nuclide removal or feed. ORIGEN includes the ability to use multigroup cross sections processed from standard ENDF/B evaluations. Within SCALE, transport codes can be used to model user-defined systems, and the COUPLE code can be applied to calculate problemdependent neutron-spectrum-weighted cross sections representative of conditions within any given reactor or fuel assembly and then convert these cross sections into a library to be used by ORIGEN. Time-dependent cross section libraries can be produced to reflect fuel composition variations during irradiation. An alternative sequence for depletion/decay calculations is ORIGEN-ARP, which interpolates pregenerated ORIGEN cross section libraries versus enrichment, burnup, and moderator density.

ORIGEN Assembly Isotopics (ORIGAMI) computes detailed isotopic compositions for LWR assemblies containing $\mathrm{UO}_{2}$ fuel by using the ORIGEN code with pregenerated ORIGEN libraries for a specified assembly power distribution. The assembly may be represented by a single lumped model with only an axial power distribution or by a square array of fuel pins with variable pin powers, as well as an axial distribution. Multiple cycles with varying burn times and down times may be used. ORIGAMI produces files containing SCALE and MCNP composition input for material in the burnup distribution, files containing decay heat for use in thermal analysis, and energy-dependent radioactive source for use in shielding calculations.

A series of 1470 pregenerated burnup libraries for use in ORIGEN and ORIGAMI are provided with SCALE for 61 fuel assemblies for commercial and research reactors.

\subsubsection{Sensitivity and Uncertainty Analysis}

SCALE provides a suite of computational tools for sensitivity and uncertainty analysis to (1) identify important processes in safety analysis and design, (2) provide a quantifiable basis for neutronics validation for criticality safety and reactor physics analysis based on similarity assessment, and (3) quantify the effects of uncertainties in nuclear data and physical parameters for safety analysis.

The TSUNAMI-1D, TSUNAMI-2D and TSUNAMI-3D analysis sequences compute the sensitivity of $k_{\text {eff }}$ and reaction rates to energy-dependent cross section data for each reaction of each nuclide in a system 
model. The one-dimensional (1D) transport calculations are performed with XSDRNPM, the twodimensional (2D) transport calculations are preformed using NEWT, and the three-dimensional (3D) calculations are performed with KENO V.a or KENO-VI. The Monte Carlo capabilities of TSUNAMI-3D provide for $\mathrm{S} / \mathrm{U}$ analysis from either continuous energy or multigroup neutron transport, where the deterministic capabilities of TSUNAMI-1D and TSUNAMI-2D only operate in multigroup mode. The Sensitivity Analysis Module for SCALE (SAMS) is applied within each analysis sequence to provide the requested $\mathrm{S} / \mathrm{U}$ data. Whether performing a continuous-energy or multigroup calculation, energydependent sensitivity data are stored in group form in a sensitivity data file (SDF) for subsequent analysis. These sequences use the energy-dependent cross section covariance data to compute the uncertainty in the response value due to the cross section covariance data.

Tool for Sensitivity Analysis of Reactivity Responses (TSAR) computes the sensitivity of the reactivity change between two $k_{\text {eff }}$ calculations using SDFs from TSUNAMI-1D, TSUNAMI-2D, and/or TSUNAMI-3D. TSAR also computes the uncertainty in the reactivity difference due to the cross section covariance data.

TSUNAMI Indices and Parameters (TSUNAMI-IP) computes correlation coefficients that determine the amount of shared uncertainty between each target application and each benchmark experiment considered in the analysis. TSUNAMI-IP offers a wide range of options for more detailed assessment of system-tosystem similarity. Additionally, TSUNAMI-IP can generate input for the Upper Subcritical Limit Statistical Software (USLSTATS) trending analysis and compute a penalty or additional margin needed for the gap analysis.

Tool for S/U Analysis of Response Functions Using Experimental Results (TSURFER) is a bias and bias uncertainty prediction tool that implements the generalized linear least-squares (GLLS) approach to data assimilation and cross section data adjustment that also uses the SDFs generated from TSUNAMI-1D, $-2 \mathrm{D},-3 \mathrm{D}$, or TSAR. The data adjustments produced by TSURFER are not used to produce adjusted cross section data libraries for subsequent use; rather, they are used only to predict biases in application systems.

The graphical user interface Extensible SCALE Intelligent Text Editor (ExSITE) facilitates analysis with TSUNAMI-IP, TSURFER, TSAR, and USLSTATS, and the Validation, Interpretation and Bias Estimation (VIBE) interface is applied to examine SDF files, create sets of benchmark experiments for subsequent analysis, and gather additional information about each benchmark experiment.

Sampler is a super-sequence that performs general uncertainty analysis by stochastically sampling uncertain parameters that can be applied to any type of SCALE calculation, propagating uncertainties throughout a computational sequence. Sampler treats uncertainties from two sources: (1) nuclear data and (2) input parameters. Sampler generates the uncertainty in any result generated by any computational sequence through stochastic means by repeating numerous passes through the computational sequence, each with a randomly perturbed sample of the requested uncertain quantities.

\subsubsection{Material Specification and Cross Section Processing}

Cross (X) Section Processing (XSProc) provides material input and multigroup cross section preparation for most SCALE sequences. XSProc allows users to specify problem materials using easily remembered and easily recognizable keywords associated with mixtures, elements, nuclides, and fissile solutions provided in the SCALE Standard Composition Library. For multigroup calculations, XSProc provides cross section temperature correction and resonance self-shielding, as well as energy group collapse and spatial homogenization for systems that can be represented in celldata input as infinite media, finite 1D systems, or repeating structures of 1D systems such as uniform arrays of fuel units. Improved resonance self-shielding treatment for nonuniform lattices can be achieved through the use the Monte Carlo Dancoff (MCDancoff) code that generates Dancoff factors for generalized 3D geometries for subsequent use in 
XSProc. Cross sections are generated on a microscopic and/or macroscopic basis as needed. Although XSProc is most often used as part of an integrated sequence, it can be run without subsequent calculations to generate problem-dependent multigroup data for use in other tools.

\subsubsection{Nuclear Data}

The cross section data provided with SCALE include comprehensive continuous energy neutron and coupled neutron-gamma data based on ENDF/B-VII.0 and ENDF/B-VII.1. These data have been generated with the AMPX codes. The multigroup data are provided in several energy-group structures optimized for different application areas, including criticality safety, lattice physics, and shielding analysis. The comprehensive ORIGEN data libraries are based on ENDF/B-VII.1 and recent JEFF evaluations, and they include nuclear decay data, neutron reaction cross sections, neutron-induced fission product yields, delayed gamma ray emission data and neutron emission data for over 2,200 nuclides. The photon yield data libraries are based on the most recent ENSDF nuclear structure evaluations. The libraries used by ORIGEN can be coupled directly with detailed and problem-dependent physics calculations to obtain self-shielded, problem-dependent cross sections based on the most recent evaluations. There are no limitations with regard to compositions or energy spectra. SCALE also contains a comprehensive library of neutron cross section covariance data for neutron interactions, fission product yields, and decay data for use in sensitivity and uncertainty analysis with the TSUNAMI codes as well as Sampler.

The full suite of AMPX codes for generating multigroup and continuous energy neutron, gamma and coupled neutron/gamma libraries and covariance data are also included in the SCALE distribution, allowing users to create their own nuclear data libraries using differing sources of data and energy group structures than those provided with SCALE.

\subsubsection{Graphical User Interfaces}

Fulcrum is a cross platform graphical user interface designed to create, edit, validate and visualize SCALE input, output, and data files. Historically, SCALE has provided several special purpose graphical user interfaces which operate only on specific platforms and are loosely integrated with SCALE's computational and data components. Fulcrum is intended to provide a single user interface that directly integrates with SCALE's internal resources to provide a consistent experience between Fulcrum and SCALE's command line interface.

Fulcrum provides input editing and navigation, interactive geometry visualization for KENO V.a, KENO-VI, and NEWT, job execution, overlay of mesh results within a geometry view, and plotting of data from most SCALE file formats. An error checking parser interactively identifies poorly constructed input with spelling errors or data entry omissions for all SCALE sequences. The Hierarchical Input Validation Engine (HIVE) will identify allowed data ranges and interdependencies in the input and report inconsistencies to the user. Fulcrum will interactively process standard composition data to produce a mixing table, list expanded input aliases for review, provide an internal listing of input as is required for Sampler material and geometry perturbation analysis, and launch the SCALE sample problems. The layout of panels in Fulcrum is highly configurable to accommodate the preferences of many users.

ORIGAMI Automator, a graphical user interface integrated with Fulcrum, facilitates the quantification of isotopics as a function of time for a large set of fuel assemblies such as the complete inventory of a spent fuel pool. This tool was developed to support the NRC in severe accident analyses, but it can be adapted to many other uses.

Additional user interfaces include the KENO3D interactive visualization program for Windows for solidbody rendering of KENO geometry models, as well as the previously mentioned ExSITE and VIBE 
interfaces for sensitivity and uncertainty analysis. Several codes provide HTML-formatted output, in addition to the standard text output, to provide convenient navigation using most common Web browsers through the computed results with interactive color-coded output and integrated data visualization tools.

\subsection{USER GUIDANCE AND TECHNICAL ASSISTANCE}

This SCALE Manual serves as the primary reference for SCALE users. The fundamental theory and all code options are documented herein. Several SCALE primers are available to serve as step-by-step guides for new users performing common calculations using the graphical user interfaces. SCALE training courses are presented several weeks each year, where users can interact directly with the software developers and expert users from ORNL.

Additional technical information on SCALE can be found at http://scale.ornl.gov, including training course schedules, a link to an online user forum, newsletters, benchmark reports, and downloads. Technical assistance is also provided via e-mail to scalehelp@ornl.gov.

\subsection{AVAILABILITY}

The SCALE code system is packaged and distributed by the Radiation Safety Information Computational Center (RSICC) and is also distributed through the Organization for Economic Cooperation and Development (OECD) Nuclear Energy Agency (NEA) Data Bank in France and the Research Organization for Information Science and Technology (RIST) in Japan.

\subsection{ACKNOWLEDGEMENTS}

The SCALE team consists of over 40 staff members across RNSD, as well as several external collaborators. SCALE 6.2 is the culmination of many years of this team's effort under the guidance of the SCALE Leadership Team:

Brad Rearden - Manager, SCALE Code System

Matt Jessee - Deputy Manager, SCALE Code System

Steve Bowman - Group Leader, Reactor Physics

Mike Dunn - Group Leader, Nuclear Data and Criticality Safety

Bob Grove - Group Leader, Radiation Transport

Rob Lefebvre - SCALE Software Development Coordinator

Mark Williams - Distinguished Developer, Nuclear Data and Reactor Physics

Most team members are credited for their authorship of the sections in this manual that correspond to their work. A few individuals have been essential to the development and maintenance of SCALE but are not credited by authorship. These individuals include S. Y. Walker for user interactions and documentation; J. P. Lefebvre and T. D. Walsh, who provide essential computer science infrastructure for building, testing, and deploying SCALE; and B. J. Ade, M. L. Baird, R. J. Ellis, A. T. Godfrey, J. Hu, D. Ilas, G. Ilas, K. S. Kim, B. J. Marshall, U. Mertyurek, D. E. Mueller, T. M. Miller, J. L. Peterson, and J. M. Risner for extensive testing and recommendations for improvements to provide a better-quality product to the end users. 
The SCALE system is maintained and modernized at ORNL to keep pace with technical advancements in computing, nuclear data, and analysis areas of interest to our sponsors and the user community. Over the years, numerous individuals within these sponsoring organizations have played key roles in ensuring that the SCALE system remained a readily available, reliable system for the analysis of nuclear fuel facilities and packages. The individuals who have worked with the ORNL staff to coordinate maintenance and development activities include R. H. Odegaarden (NRC, ret.), G. H. Bidinger (NRC, ret.), C. Mauck (DOE, ret.), E. P. Easton (NRC), W. H. Lake (NRC and DOE, ret.), M. E. Wangler (DOE), M. G. Bailey (NRC), C. J. Withee (NRC, ret.), A. B. Barto (NRC), M. Aissa (NRC), D. R. Algama (NRC), T. D. Powell (NRC), J. N. McKamy (DOE), and J. M. Shuler (DOE).

Special acknowledgement is also due to R. M. Westfall and G. E. Whitesides (ret.) who worked with R. H. Odegaarden of the NRC to develop the concept and long-range goals of the SCALE system in the late 1970s. The current management team is grateful to previous SCALE project leaders: C. V. Parks, who served in this role for the first 15 years of SCALE, and S. M. Bowman, who served in this role for the following 14 years. Additional acknowledgements are due to L. M. Petrie, who for nearly 50 years has consistently provided consultation and advice on nearly every module and cross section library in the present system.

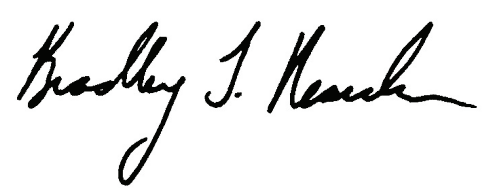

Bradley T. Rearden, Ph.D.

Manager, SCALE Code System 


\title{
2.0 CRITICALITY SAFETY
}

\author{
Introduction by B. T. Rearden
}

SCALE provides a suite of computational tools for criticality safety analysis that is primarily based on the KENO Monte Carlo code for eigenvalue neutronics calculations. ${ }^{1}$ Two variants of KENO provide identical solution capabilities with different geometry packages. KENO V.a uses a simple and efficient geometry package sufficient for modeling many systems of interest to criticality safety and reactor physics analysts. KENO-VI uses the SCALE Generalized Geometry Package, which provides a quadraticbased geometry system with much greater flexibility in problem modeling but with slower runtimes. Both versions of KENO perform eigenvalue calculations for neutron transport primarily to calculate multiplication factors $\left(k_{e f f}\right)$ and flux distributions of fissile systems in both continuous energy and multigroup modes. They are typically accessed through the integrated SCALE sequences described below. KENO's grid geometry capability extends region-based features for accumulating data for source or biasing parameter specifications, as well as for tallying results from a calculation for visualization or communication of data into or out of a calculation. Criticality safety analysts may also be interested in the sensitivity and uncertainty analysis techniques that can be applied for code and data validation as described elsewhere in this document.

\section{Criticality Safety Analysis Sequences}

The Criticality Safety Analysis Sequences (CSAS) with KENO V.a (CSAS5) and KENO-VI (CSAS6) provide a reliable, efficient means of performing $k_{\text {eff }}$ calculations for systems routinely encountered in engineering practice. The CSAS sequences implement XSProc to process material input and provide a temperature and resonance-corrected cross section library based on the physical characteristics of the problem being analyzed. If a continuous energy cross section library is specified, no resonance processing is needed, and the continuous energy cross sections are used directly in KENO, with temperature corrections provided as the cross sections are loaded.

A search capability is available with CSAS5 to find desired values of $k_{\text {eff }}$ as a function of dimensions or densities. The two basic search options offered are (1) an optimum search seeking a maximum or minimum value of $k_{\text {eff }}$ and (2) a critical search seeking a fixed value of $k_{\text {eff. }}$.

For continuous energy calculations, reaction rate tallies can be requested within the CSAS input, and for multigroup calculations, reaction rate calculations are performed using the KENO Module for ActivityReaction Rate Tabulation (KMART) post-processing tools. A conversion tool is provided to up-convert KENO V.a input to KENO-VI either as a direct KENO input (K5toK6) or, more commonly, as a CSAS sequence (C5toC6).

\section{STARBUCS: Burnup-Credit Analysis Sequence}

The Standardized Analysis of Reactivity for Burnup Credit using SCALE (STARBUCS) ${ }^{2,3}$ is a control module to perform criticality calculations for spent fuel systems employing burnup credit. STARBUCS automates the criticality safety analysis of spent fuel configurations by coupling the depletion and criticality aspects of the analysis, thereby eliminating the need to manually process the spent fuel nuclide compositions into a format compatible with criticality safety codes.

STARBUCS performs a depletion analysis calculation for each spatially varying burnup region (if an axial or horizontal burnup profile is specified) of a spent fuel assembly using the ORIGEN-ARP methodology of SCALE. If a multigroup calculation is to be performed in KENO, the spent fuel compositions are then used to generate resonance self-shielded cross sections for each burnup-dependent fuel region. Finally, a KENO criticality calculation is performed to determine the neutron multiplication factor for the system. 
The STARBUCS input format has been designed around the existing depletion analysis and criticality safety sequences of SCALE. Only a minimal amount of input beyond that typically required for a freshfuel calculation is needed to perform a burnup-credit calculation.

STARBUCS was developed to facilitate studies of major burnup-credit phenomena, such as those identified in the US Nuclear Regulatory Commission's Interim Staff Guidance $8{ }^{4}$ but it is restricted to modeling one assembly type with the same starting enrichment loaded throughout the transportation or storage model. Greater flexibility is available by computing individual assembly burnup compositions with the ORIGAMI code and then creating a KENO model to implement these compositions.

For burnup loading curve iterative calculations, STARBUCS employs the search algorithm from CSAS5 to determine initial fuel enrichments that satisfy a convergence criterion for the calculated $k_{\text {eff }}$ value of the spent fuel configuration.

\section{Sourcerer: Hybrid Method for Starting Source Distribution}

As the fidelity of criticality models continues to increase, especially for storage and transportation systems, the ability of the Monte Carlo codes to consistently provide a converged fission source can be challenging. Studies have shown that using a starting fission distribution similar to the true fission distribution can reduce the number of skipped generations required for fission source convergence, and it can significantly improve the reliability of the final $k_{\text {eff }}$ result. ${ }^{5,6}$ The Sourcerer sequence applies the Denovo $^{7}$ discrete ordinates code to generate a starting fission source distribution in a KENO Monte Carlo calculation. The discrete ordinates calculation is performed on a user-defined Cartesian grid geometry where macroscopic material definitions are automatically created from the Monte Carlo model and multigroup group cross sections are appropriately generated.

For many criticality safety applications, the additional step of performing a deterministic calculation to initialize the starting fission source distribution is not necessary. However, for challenging criticality safety analyses such as as-loaded spent nuclear fuel transportation packages with a mixed loading of lowand high-burnup fuel, even a low-fidelity deterministic solution for the fission source produces more reliable results than the typical starting distributions of uniform or cosine functions over the fissionable regions, as demonstrated in a recent study. ${ }^{8}$

\section{Criticality Accident Alarm System Analysis with KENO and MAVRIC}

Criticality accident alarm systems (CAAS) safety analyses modeling presents challenges because the analysis consists of a criticality problem and a deep-penetration shielding problem. ${ }^{9}$ Modern codes are typically optimized to handle one of these types of problems, but not both. The two problems also differ in size - the criticality problem depends on materials relatively close to the fissionable materials, whereas the shielding problem can cover a much larger range.

CAAS analysis can be performed using the CSAS6 criticality sequence and the MAVRIC shielding sequence. First, the fission distribution (in space and energy) is determined via CSAS6. This information is collected on a grid geometry that overlies the physical geometry model and is saved as a Monaco mesh source file. The mesh source is then used as the source term in MAVRIC. The absolute source strength is set by the user to the total number of fissions (based on the total power released) during the criticality excursion. MAVRIC can be optimized to calculate a specific detector response at one location or to calculate multiple responses/locations with roughly the same relative uncertainty. 


\section{References}

1. S. Goluoglu, L. M. Petrie, Jr., M. E. Dunn, D. F. Hollenbach, and B. T. Rearden, "Monte Carlo Criticality Methods and Analysis Capabilities in SCALE," Nucl. Technol. 174(2), pp. 214-235 (May 2011).

2. I. C. Gauld and S. M. Bowman, STARBUCS: A Prototypic SCALE Control Module for Automated Criticality Safety Analyses Using Burnup Credit, NUREG/CR-6748 (ORNL/TM-2001/33), prepared for the US Nuclear Regulatory Commission by Oak Ridge National Laboratory (October 2001).

3. G. Radulescu and I. C. Gauld, "Enhancements to the Burnup Credit Criticality Safety Analysis Sequence in SCALE," NCSD 2009: Nuclear Criticality Safety: Realism, Robustness and the Nuclear Renaissance, Richland, Washington (September 13-17, 2009).

4. Spent Fuel Storage and Transportation Division Interim Staff Guidance-8, Rev. 3-Burnup Credit in the Criticality Safety Analyses of PWR Spent Fuel in Transport and Storage Casks, US Nuclear Regulatory Commission (September 26, 2012).

5. A. M. Ibrahim, D. E. Peplow, J. C. Wagner, S. W. Mosher, and T. M. Evans, "Acceleration of Monte Carlo Criticality Calculations Using Deterministic-Based Starting Sources," Trans. Am. Nucl. Soc. 105, pp. 539-541 (2011).

6. A. M. Ibrahim, D. E. Peplow, J. C. Wagner, S. W. Mosher, and T. M. Evans, “Acceleration of Monte Carlo Criticality Calculations Using Deterministic-Based Starting Sources," PHYSOR 2012, Knoxville, Tennessee, April 15-20 (2012).

7. T. M. Evans, A. S. Stafford, R. N. Slaybaugh, and K. T. Clarno, "Denovo: A New Three-Dimensional Parallel Discrete Ordinates Code in SCALE," Nucl. Technol. 171(2), pp. 171-200 (2010).

8. A. M. Ibrahim, D. E. Peplow, K. B. Bekar, C. Celik, D. Ilas, J. M. Scaglione, and J. C. Wagner, "Hybrid Technique in SCALE for Fission Source Convergence Applied to Used Nuclear Fuel Analysis," ANS NCSD Topical Meeting on Nuclear Criticality Safety (NCSD 2013), Wilmington, North Carolina (September 29-October 3, 2013).

9. D. E. Peplow and L. M. Petrie, Jr., "Criticality Accident Alarm System Modeling with SCALE," Proc. 2009 International Conference on Advances in Mathematics, Computational Methods, and Reactor Physics' Saratoga Springs, New York (May 3-7, 2009). 


\title{
2.1 CSAS5: Control Module For Enhanced Criticality Safety Analysis Sequences With KENO V.a
}

\author{
L. M. Petrie, K. B. Bekar, S. Goluoglu, ${ }^{*}$ D. F. Hollenbach, ${ }^{*}$ N. F. Landers ${ }^{*}$
}

\begin{abstract}
The Criticality Safety Analysis Sequences with KENO V.a (CSAS5) provides reliable and efficient means of performing $k_{\text {eff }}$ calculations for systems that are routinely encountered in engineering practice. In the multigroup calculation mode, CSAS5 uses XSProc to process the cross sections for temperature corrections and problem-dependent resonance self-shielding and calculates the $k_{\text {eff }}$ of a three-dimensional (3-D) system model. If the continuous energy calculation mode is selected no resonance processing is needed and the continuous energy cross sections are used directly in KENO V.a, with temperature corrections provided as the cross sections are loaded. The geometric modeling capabilities available in KENO V.a coupled with the automated cross-section processing within the control sequences allow complex, 3-D systems to be easily analyzed. A search capability is achieved by repeatedly activating the control module MODIFY, to alter either the system dimensions or densities, and the functional module KENO V.a to calculate the $k_{\text {eff }}$ for the modified dimensions or densities.
\end{abstract}




\section{TABLE OF CONTENTS}

$\underline{\text { Page }}$

2.1 CSAS5: Control Module for Enhanced Criticality Safety Analysis Sequences with

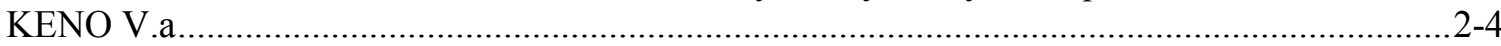

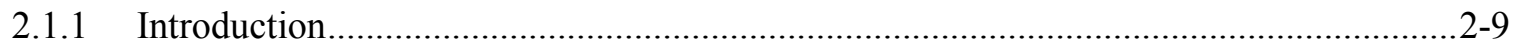

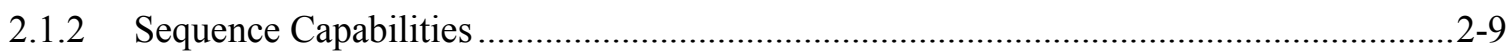

2.1.2.1 Optimum (minimum/maximum) search ................................................2-10

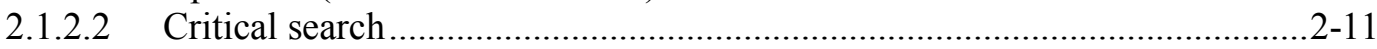

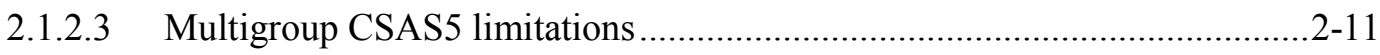

2.1.2.4 Continuous energy CSAS5 limitations...................................................2-12

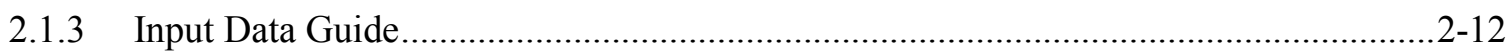

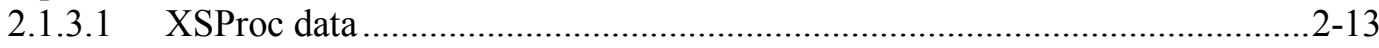

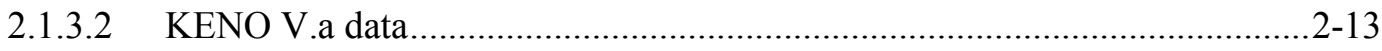

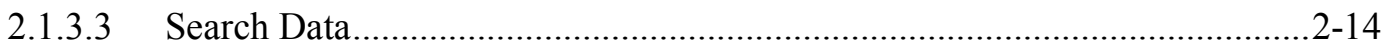

2.1.4 Example Problems ...............................................................................................2-24

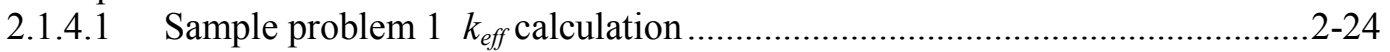

2.1.4.2 Sample problem 2 optimum pitch search using detailed geometry ...............2-26

2.1.4.3 Sample problem 3 optimum pitch search using homogenized geometry ......2-27

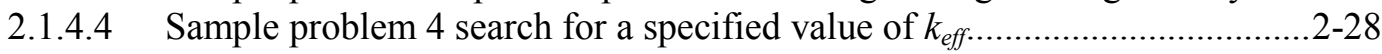

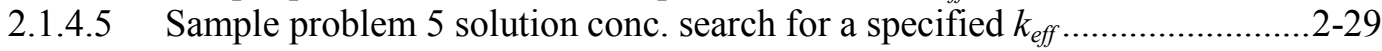

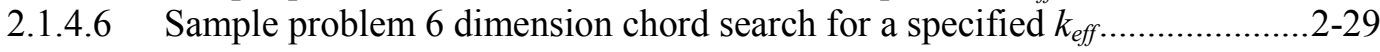

2.1.4.7 Sample problem 7 two material conc. search for a specified $k_{\text {eff }}$....................2-30

2.1.4.8 Sample problem $8 \mathrm{k}_{\infty}$ for a pebble bed fuel..............................................2-31

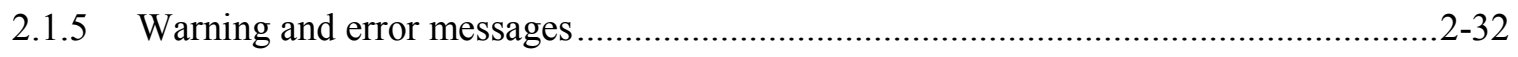

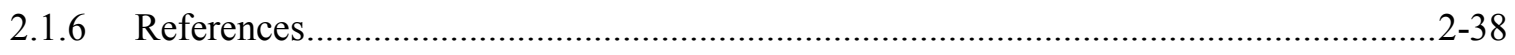

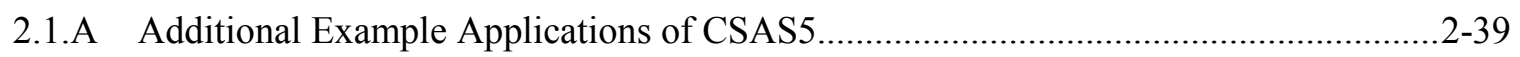




\section{LIST OF FIGURES}

$\underline{\text { Page }}$

Figure 2.1.4.1. Critical assembly of four solution units and four metal units....................................2-25 


\section{LIST OF TABLES}

$\underline{\text { Page }}$

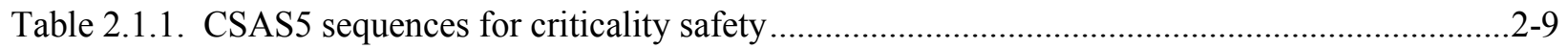

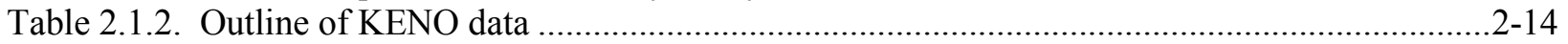

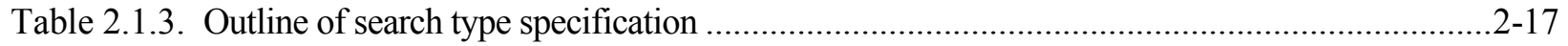

Table 2.1.4. Outline of auxiliary search commands and constraints...............................................2-22 


\section{ACKNOWLEDGMENTS}

CSAS5 and its related Criticality Safety Analysis Sequences are based on the old CSAS2 control module (no longer in SCALE) and the KENO V.a functional module described in the KENO V.a chapter. Therefore, special acknowledgment is made to J. A. Bucholz, R. M. Westfall, and J. R. Knight who developed CSAS2. G. E. Whitesides is acknowledged for his contributions through early versions of KENO. Appreciation is expressed to C. V. Parks and S. M. Bowman for their guidance in developing CSAS5.

Special appreciation is expressed to S. J. Poarch and S. Y. Walker for their efforts in formatting this document. 


\subsubsection{Introduction}

Criticality Safety Analysis Sequence with KENO V.a (CSAS5) provides reliable and efficient means of performing $k_{\text {eff }}$ calculations for systems that are routinely encountered in engineering practice, especially in the calculation of $k_{\text {eff }}$ of three-dimensional (3-D) system models. CSAS5 implements XSProc to process material input and provide a temperature and resonance-corrected cross-section library based on the physical characteristics of the problem being analyzed. If a continuous energy cross-section library is specified, no resonance processing is needed and the continuous energy cross sections are used directly in KENO V.a, with temperature corrections provided as the cross sections are loaded. A search capability is available to find a desired values of $k_{\text {eff }}$ as a function of dimensions or densities. The two basic search options offered are (1) an optimum search seeking a maximum or minimum value of $k_{\text {eff }}$ and (2) a critical search seeking a fixed value of $k_{\text {eff }}$.

All the control sequences in the CSAS5 control module are listed in Table 2.1.1 with the modules they invoke. The first four sequences are subsets of the CSAS5 sequence.

Table 2.1.1. CSAS5 sequences for criticality safety

\begin{tabular}{llrl}
\hline $\begin{array}{c}\text { Control } \\
\text { sequence }\end{array}$ & Function & & $\begin{array}{c}\text { Functional modules executed by the control sequence } \\
\text { (for multigroup libraries) }\end{array}$ \\
\hline CSAS5 & $k_{\text {eff }}(3-D)$ & XSProc & KENO V.a \\
CSAS5S & $k_{e f f}(3-D)$ search XSProc & KENO V.a $\quad$ MODIFY \\
\hline
\end{tabular}

\subsubsection{Sequence Capabilities}

In order to minimize human error, the SCALE data handling is automated as much as possible. CSAS5 and many other SCALE sequences apply a standardized procedure to provide appropriate number densities and cross sections for the calculation. XSProc is responsible for reading the standard composition data and other engineering-type specifications, including volume fraction or percent theoretical density, temperature, and isotopic distribution as well as the unit cell data. XSProc then generates number densities and related information, prepares geometry data for resonance self-shielding and flux-weighting cell calculations, if needed, and (if needed) provides problem-dependent multigroup cross-section processing. Sequences that execute KENO V.a include a KENO V.a Data Processor to read and check the KENO V.a data. Sequences that execute a search use a Search Data Processor to read and check the search data. When the data checking has been completed, the control sequence executes XSProc to prepare a resonance-corrected microscopic cross-section library in the AMPX working library format if a multigroup library has been selected.

For each unit cell specified as being cell-weighted, XSProc performs the necessary calculations and produces a cell-weighted microscopic cross-section library. KENO V.a may be executed to calculate the $k_{\text {eff }}$ or neutron multiplication factor using the cross-section library that was prepared by the control sequence. MODIFY may be invoked to perform a search ${ }^{1}$ by repeatedly altering the unit cell (multigroup mode only) and KENO V.a data prior to executing the next pass through the calculation. Cross sections are updated at the 
beginning of each search pass with the modified data. If unit cell data is altered as part of the search, i.e., pitch or material search, the cross-sections are correctly processed with the updated data.

The search capability is implemented by the control module MODIFY. It performs operations according to the specified search data to determine (1) the maximum or minimum value of $k_{\text {eff }}$ as a function of pitch, dimensions or densities or (2) the pitch, dimensions, or densities corresponding to a specified value of $k_{\text {eff. An }}$ iterative procedure is used, making use of all previous information to modify the dimensions or densities to achieve the desired result. The procedures for conducting optimum and critical searches are summarized in the following sections.

\subsubsection{Optimum (minimum/maximum) search}

Because only an initial value of $k_{\text {eff }}$ and a set of boundary constraints are available, four initial points are generated spanning the range defined by the constraints. The search package identifies the type of cubic equation [i.e., a cubic with no local extrema (type A) or a cubic with two local extrema (type B)] and utilizes this knowledge in determining the pitch, dimensions, or material densities corresponding to the maximum (or minimum) value of $k_{\text {eff. }}$. The optimum search procedure is summarized as follows:

1. Calculate $k_{\text {eff }}$ for the specified problem.

2. Calculate $k_{\text {eff }}$ for the minimum constraint.

3. Calculate $k_{\text {eff }}$ for the maximum constraint.

4. Calculate $k_{\text {eff }}$ for a fourth point that lies approximately equidistant between the initial guess and the constraint that is farthest from it.

5. Utilize a weighted least-squares fit to a cubic polynomial on the data points.

6. Determine the type of cubic. For a type A cubic, go to step 11.

7. Take the first derivative of the least-squares cubic.

8. Solve the quadratic for its roots.

9. Take the second derivative of the least-squares cubic to determine which root is the maximum (or minimum), and if it falls within the constraints, use this root as the next guess. Otherwise, convergence has been defined as occurring at the constraint with the maximum (or minimum) $k_{\text {eff. }}$

10. Calculate the $k_{\text {eff }}$ corresponding to the next guess. Go to step 5. Repeat this procedure until convergence is achieved.

11. If the cubic equation is a type A cubic, the optimum lies on one of the boundaries. If the fit shows that the cubic is actually a type B cubic, go to step 7 and continue.

Convergence is defined as occurring when a $k_{\text {eff }}$ has been calculated for a point on the curve where the value of the curve is within epsilon of the maximum (or minimum) of the curve. Additionally, the calculated $k_{\text {eff }}$ must be within two standard deviations of the value of the curve at that point. The search is terminated when convergence is achieved, when the code determines there is no local maximum within the constraints, or the maximum number of search iterations is reached. 
NOTE: At the beginning of each search pass, the cross sections are updated using the updated values of pitch, dimensions, or material densities. Also, the unit or material being modified can be directly tied to a unit cell, so that unit cell is updated during the search. Therefore, the final result should be consistent with the results obtained by running a non-search problem using the data from the last pass.

\subsubsection{Critical search}

The critical search option searches for the pitch, dimensions, or material densities corresponding to a specified value of $k_{\text {eff. }}$. If the calculated value of $k_{\text {eff }}$ is within the specified search tolerance (EPS) of the desired $k_{\text {eff }}$, the search is considered to be complete. The critical search procedure is summarized as follows:

1. Calculate $k_{\text {eff }}$ for the specified problem. If it is within EPS of the specified $k_{\text {eff }}$, convergence has been achieved.

2. Calculate $k_{\text {eff }}$ for one of the constraints. If the specified $k_{\text {eff }}$ of the system does not fall between the initial value and the $k_{\text {eff }}$ of the constraint, calculate the $k_{\text {eff }}$ of the other constraint. If the calculated $k_{e f f}$ is within EPS of the specified $k_{\text {eff }}$, convergence has been achieved.

3. Calculate $k_{\text {eff }}$ for a point chosen from a linear fit of the two existing points closest to the specified $k_{\text {eff: }}$

4. Repeat step 3 until convergence has been achieved, the program determines that the specified value lies outside the constraints, or the maximum number of search iterations is reached. Convergence has been achieved when the calculated $k_{\text {eff }}$ is within EPS of the specified $k_{\text {eff. }}$.

5. If convergence is achieved, calculate $k_{\text {eff }}$ for a point determined from fitting the previous points to a cubic and solving the cubic for the point closest to the desired $k_{\text {eff }}$. If all roots lie outside the constraints, the problem is terminated and an appropriate message is written. If the maximum number of iterations is reached without the problem converging, the problem is terminated and an appropriate message is written.

NOTE: At the beginning of each search pass, the cross sections are updated using the updated values of pitch, dimensions, or material densities. Also, the unit or material being modified can be directly tied to a unit cell, so that unit cell is updated during the search. Therefore, the final result should be consistent with the results obtained by running a non-search problem using the data from the last pass.

\subsubsection{Multigroup CSAS5 limitations}

The CSAS5 control module was developed to use simple input data and prepare problem-dependent cross sections for use in calculating the effective neutron multiplication factor of a 3-D system using KENO V.a. An attempt was made to make the system as general as possible within the constraints of the standardized methods chosen to be used in SCALE. Standardized methods of data input were adopted to allow easy data entry and for quality assurance purposes. Some of the limitations of the CSAS5 multigroup sequences are a result of using preprocessed multigroup cross sections. Inherent limitations in multigroup CSAS5 calculations are as follows: 
1. Two-dimensional (2-D) effects such as fuel rods in assemblies where some positions are filled with control rod guide tubes, burnable poison rods and/or fuel rods of different enrichments. The cross sections are processed as if the rods are in an infinite lattice of identical rods. If the user inputs a Dancoff factor for the cell (such as one computed by MCDancoff), XSProc can produce an infinite lattice cell, which reproduces that Dancoff. This can mitigate some two dimensional lattice effects

\subsubsection{Continuous energy CSAS5 limitations}

When continuous energy KENO calculations are desired, none of the resonance processing capabilities of XSProc are applicable or needed. The continuous energy cross sections are directly used in KENO. An existing multigroup input file can easily be converted to a continuous energy input file by simply specifying the continuous energy library. In this case, all cell data is ignored. However, the following limitations exist:

1. If CELLMIX is defined in the cell data, the problem will not run in the continuous energy mode. CELLMIX implies new mixture cross sections are generated using XSDRNPM-calculated cell fluxes and therefore is not applicable in the continuous energy mode.

2. Only VACUUM, MIRROR, PERIODIC, and WHITE boundary conditions are allowed. Other albedos, e.g., WATER, CARBON, POLY, etc. are for multigroup only.

3. Problems with DOUBLEHET cell data are not allowed as they inherently utilize CELLMIX feature.

\subsubsection{Input Data Guide}

This section describes the input data required for CSAS5. Several subsets of the CSAS5 sequences listed in Table 2.1.1 are available to achieve several different levels of processing.

The input data for these CSAS5 sequences are composed of three broad categories of data. The first is XSProc, including Standard Composition Specification Data and Unit Cell Geometry Specification. This first category specifies the cross-section library and defines the composition of each mixture and optionally unit cell geometry that may be used to process the cross sections. This data block is necessary for all CSAS5 sequences. The second category of data, the KENO V.a input data, is used to specify the geometric and boundary conditions that represent the physical 3-D configuration of a KENO V.a problem. Both data blocks are necessary for CSAS5 and CSAS5S. The last category of data is the search data and is required only for CSAS5S.

All data are entered in free form, allowing alphanumeric data, floating-point data, and integer data to be entered in an unstructured manner. Up to 252 columns of data entry per line are allowed. Data can usually start or end in any column with a few exceptions. As an example, the word END beginning in column 1 and followed by two blank spaces or a new line will end the problem and any data following will be ignored. Each data entry must be followed by one or more blanks to terminate the data entry. For numeric data, either a comma or a blank can be used to terminate each data entry. Integers may be entered for floating-point values. For example, 10 will be interpreted as 10.0. Imbedded blanks are not allowed within a data entry unless an E precedes a single blank as in an unsigned exponent in a floating-point number. For example, $1.0 \mathrm{E} 4$ would be correctly interpreted as $1.0 \times 10^{4}$. 
The word "END" is a special data item. An "END" may have a name or label associated with it (e.g., "END DATA"). The name or label associated with an "END" is separated from the "END" by a single blank and is a maximum of 12 characters long. At least two blanks or a new line MUST follow every labeled and unlabeled "END." It is the user's responsibility to ensure compliance with this restriction. Failure to observe this restriction can result in the use of incorrect or incomplete data without the benefit of warning or error messages.

Multiple entries of the same data value can be achieved by specifying the number of times the data value is to be entered, followed by either $\mathrm{R}, *$, or $\$$, followed by the data value to be repeated. Imbedded blanks are not allowed between the number of repeats and the repeat flag. For example, 5R12, $5 * 12,5 \$ 12$, or $5 \mathrm{R} 12$, etc., will enter five successive 12s in the input data. Multiple zeros can be specified as $\mathrm{nZ}$ where $\mathrm{n}$ is the number of zeroes to be entered.

The purpose of this section is to define the input data in discrete subsections relating to a particular type of data. Tables of the input data are included in each subsection, and the entries are described in more detail in the appropriate sections.

Resonance-corrected cross sections are generated using the appropriate boundary conditions for the unit cell description (i.e., void for the outer surface of a single unit, white for the outer surface of an infinite array of cylinders). As many unit cells as needed may be specified in a problem. A unit cell is cellweighted by using the keyword "CELLMIX=" followed by a unique user specified mixture number in the unit cell data.

To check the input data without actually processing the cross sections, the words "PARM=CHECK" or "PARM=CHK" should be entered, as shown below.

=CSAS5 PARM $=\mathrm{CHK}$
or
\#CSAS5 PARM $=\mathrm{CHK}$

This will cause the input data for CSAS5 to be checked and appropriate error messages to be printed. If plots are specified in the data, they will be printed. This feature allows the user to debug and verify the input data while using a minimum of computer time.

\subsubsection{XSProc data}

The XSProc reads the standard composition specification data and the unit cell geometry specifications. It then produces the mixing table and unit cell information necessary for processing the cross sections if needed. The XSProc section of this manual provides a detailed description of the input data and processing options.

\subsubsection{KENO V.a data}

If the problem utilizes a sequence that contains KENO V.a as a functional module, the input to KENO V.a comes after the XSProc input. Table 2.1.2 contains the outline for the KENO V.a input and the SEARCH input, which is required for a search case (i.e., CSAS5S). The KENO V.a input is divided into 13 data blocks and CSAS5S includes an additional block of search data. A brief outline of commonly used data blocks is shown in Table 2.1.2. Note that parameter data must precede all other 
KENO data blocks. Information on all KENO V.a input is provided in the KENO chapter of this document and will not be repeated here.

Table 2.1.2. Outline of KENO data

\begin{tabular}{llll}
\hline \multicolumn{1}{c}{ Type of data } & \multicolumn{1}{c}{ Starting flag } & \multicolumn{1}{c}{ Comments } & \multicolumn{1}{c}{ Termination flag } \\
\hline $\begin{array}{l}\text { Parameters* } \\
\text { Geometry }\end{array}$ & READ PARAMETER & Enter desired parameter data & END PARAMETER \\
Array data & READ GEOMETRY & Enter desired geometry data & END GEOMETRY \\
Boundary conditions & READ ARRAY & Enter desired array data & END ARRAY \\
Energy group boundaries & READ BOUNDS & $\begin{array}{l}\text { Enter desired boundary } \\
\text { conditions }\end{array}$ & END BOUNDS \\
& READ ENERGY & $\begin{array}{l}\text { Enter desired neutron energy } \\
\text { group boundaries }\end{array}$ & END ENERGY \\
Start data or initial source & READ START & Enter desired start data & END START \\
$\begin{array}{l}\text { Plot data } \\
\text { Grid geometry data }\end{array}$ & READ PLOT & Enter desired plot data & END PLOT \\
Reaction & READ GRID & Enter desired mesh data & END GRID \\
KENO V.a data terminus & READ REACTION & $\begin{array}{l}\text { Enter desire reaction tallies (CE } \\
\text { mode only) }\end{array}$ & END REACTION \\
Search data & READ DATA & Enter to signal the end of all & \\
\hline
\end{tabular}

*Must precede all other data blocks in this table.

\subsubsection{Search Data}

Search data must be entered for CSAS5S. The search data enable the code to perform a search according to the instructions specified by the user. The code begins reading search data when it encounters the words READ SEARCH and continues reading search data until it encounters the words END SEARCH. Search data consist of the search type specification and auxiliary search commands.

\subsection{Search type specification}

These data are used to define the type of search and to set the parameters that provide limits for the search. The search type specification data consist of (a) a search descriptor, (b) the search type, and (c) optional search parameters as described below.

\subsection{SEARCH DESCRIPTOR is used to define the search mode.}

Use OPTIMUM if the maximum value of $k_{\text {eff }}$ is to be determined.

Use CRITICAL if a specified value of $k_{e f f}$ is to be obtained.

Use MINIMUM if the minimum value of $k_{\text {eff }}$ is to be determined.

\subsection{SEARCH TYPE is used to specify the variable that is to be changed during the search procedure.}

Use PITCH to alter the center-to-center spacing between the units at the lowest array level. By default only the spacing in the $\mathrm{X}$ and $\mathrm{Y}$ directions will be altered. Use DIMENSION to alter the dimensions of one or more geometry regions in one or more units. Use CONCENTRATION to alter the concentration of one or more standard compositions in one or more mixtures. 
The combination of the search descriptor and the search type defines the search method. Each search type has a set of predefined defaults and the ability to change the default settings and expand the scope of the search. Only one SEARCH DESCRIPTOR and one SEARCH TYPE are allowed in a problem.

An OPTIMUM PITCH search determines the pitch that gives the maximum value of $k_{\text {eff. }}$ By default the $\mathrm{X}$ spacing will be altered for slab arrays, the $\mathrm{X}$ and $\mathrm{Y}$ spacing will be altered for arrays of cylinders, and the $\mathrm{X}, \mathrm{Y}$, and $\mathrm{Z}$ spacing will be altered for spherical arrays. Auxiliary search commands can be used to instruct the code to change any of these defaults.

An OPTIMUM DIMENSION search determines the maximum value of $k_{\text {eff }}$ by altering the dimensions of one or more geometry regions in one or more units in accordance with the specified auxiliary search commands. Only the dimensions specified in the search commands will be modified. The relative variations in dimensions are determined by the search constants specified for each dimension.

An OPTIMUM CONCENTRATION search determines the maximum value of $k_{\text {eff }}$ by altering the concentration of standard compositions in mixtures in accordance with specified search commands. Only the standard compositions in the materials specified are altered. The relative variations in concentrations are determined by the search constants specified for each composition.

A CRITICAL PITCH search alters the spacing between units in the same manner as an optimum pitch search to achieve the specified value of $k_{\text {eff. }}$ By default the X spacing will be altered for slab arrays, the X and $\mathrm{Y}$ spacing will be altered for arrays of cylinders, and the $\mathrm{X}, \mathrm{Y}$, and $\mathrm{Z}$ spacing will be altered for spherical arrays. Auxiliary search commands can be used to instruct the code to change any of these defaults.

A CRITICAL DIMENSION search alters the dimensions of one or more geometry regions in accordance with the specified auxiliary search commands to achieve the specified value of $k_{\text {eff }}$. Only the dimensions specified in the search commands will be modified. The relative variations in dimensions are determined by the search constants specified for each dimension.

A CRITICAL CONCENTRATION search alters the concentration of standard compositions in mixtures in accordance with the specified auxiliary search commands to achieve the specified value of $k_{\text {eff. }}$ Only the standard compositions in the materials specified are altered. The relative variations in concentrations are determined by the search constants specified for each composition.

A MINIMUM PITCH search determines the pitch that gives the minimum value of $k_{\text {eff. }}$ By default the $\mathrm{X}$ spacing will be altered for slab arrays, the $\mathrm{X}$ and $\mathrm{Y}$ spacing will be altered for arrays of cylinders, and the $\mathrm{X}$, $\mathrm{Y}$, and $\mathrm{Z}$ spacing will be altered for spherical arrays. Auxiliary search commands can be used to instruct the code to change any of these defaults.

A MINIMUM DIMENSION search determines the minimum value of $k_{\text {eff }}$ by altering the dimensions of one or more geometry regions in one or more units in accordance with the specified auxiliary search commands. Only the dimensions specified in the search commands will be modified. The relative variations in dimensions are determined by the search constants specified for each dimension.

A MINIMUM CONCENTRATION search determines the minimum value of $k_{\text {eff }}$ by altering the concentration of standard compositions in mixtures in accordance with specified search commands. Only the standard compositions in the materials specified are altered. The relative variations in concentrations are determined by the search constants specified for each composition. 
OPTIONAL SEARCH PARAMETERS are entered after the SEARCH DESCRIPTOR AND SEARCH TYPE and are used to alter the default values of the optional search parameters. Only one set of optional search parameters can be entered for a problem. The optional search parameters are listed below.

$\mathrm{PAS}=\mathrm{nn} \quad$ is used to set the maximum number of times the search will calculate $k_{\text {eff: }}$ The first pass calculates the $k_{\text {eff }}$ corresponding to the initial geometry dimensions. The second pass calculates the $k_{\text {eff }}$ corresponding to one of the constraints, and the third pass often corresponds to the other constraint. After the third pass, the search dimensions or concentrations are changed based on a fit to a quadratic or cubic equation. The default value of $n n$ is 10 .

$\mathrm{NPM}=\mathrm{nn} \quad$ is used to set the number of search parameters. The default value of $\mathrm{nn}$ is 1 and should not be overridden.

$\mathrm{EPS}=\mathrm{ff} \quad$ is used to set the search convergence tolerance (the amount by which $k_{\text {eff }}$ is allowed to vary from the desired $k_{\text {eff }}$. An optimum or minimum search is terminated when the calculated $k_{\text {eff }}$ is within EPS of the optimum or minimum value as indicated by the mathematical fit to the calculated points. A critical search is terminated when the calculated $k_{e f f}$ is within EPS of the specified $k_{\text {eff. }}$ The default value of $\mathrm{ff}$ is 0.005 .

$\mathrm{KEF}=\mathrm{ff} \quad$ is used only for a CRITICAL search. The default value of $\mathrm{ff}$ is 1.000 .

MINPITCH=ff is allowed ONLY for a PITCH search. It is used to specify the minimum allowed pitch (center-to-center spacing in the $\mathrm{X} ; \mathrm{X}, \mathrm{Y}$; or $\mathrm{X}, \mathrm{Y}, \mathrm{Z}$ directions depending on array type) between the units in an array. The search will terminate if the pitch becomes smaller than the specified minimum pitch. The default value of $\mathrm{ff}$ is the pitch at which the region immediately inside the outer most region of the unit touches the same region in an adjacent unit. It is much easier to specify the minimum allowed pitch than to calculate the appropriate value of the minimum constraint.

MAXPITCH=ff is allowed ONLY for a PITCH search. It is used to specify the maximum allowed pitch (center-to-center spacing in the X; X, Y; or X, Y, Z directions depending on array type) between units in an array. The search will terminate if the specified pitch is exceeded. The default value of $\mathrm{ff}$ is the pitch corresponding to -5 times the parameter that corresponds to the minimum pitch. It is much easier to specify a maximum allowed pitch than to calculate the appropriate value of the maximum constraint.

MORE is used to terminate the optional search parameters and initiate the auxiliary search commands. Do not enter MORE unless auxiliary search commands are to be entered. This command may only be entered once, immediately prior to the auxiliary search commands.

The input data for the CSAS5S search sequence are provided in Table 2.1.3. 
Table 2.1.3. Outline of search type specification

\begin{tabular}{|c|c|c|c|}
\hline $\begin{array}{l}\text { Entry } \\
\text { No. }\end{array}$ & Type of data & Data entry & Comments \\
\hline \multirow[t]{3}{*}{1} & Search descriptor & OPTIMUM & Initiates a search for the maximum value of $k_{\text {eff }}$. \\
\hline & & CRITICAL & Initiates a search for a specified value of $k_{\text {eff }}$. \\
\hline & & MINIMUM & Initiates a search for the minimum value of $k_{\text {eff }}$ \\
\hline \multirow[t]{3}{*}{2} & Search type & PITCH & Vary the pitch of an array. \\
\hline & & DIMENSION & $\begin{array}{l}\text { Vary one or more dimensions in one or more regions } \\
\text { of one or more units. }\end{array}$ \\
\hline & & CONCENTRATION & $\begin{array}{l}\text { Vary the concentration of one or more standard } \\
\text { compositions in one or more mixtures. }\end{array}$ \\
\hline 3 & $\begin{array}{l}\text { Optional search } \\
\text { parameters }\end{array}$ & & $\begin{array}{l}\text { Optional search parameters allow changing default } \\
\text { values. Any or all may be entered in any order. }\end{array}$ \\
\hline $3 a$ & $\begin{array}{l}\text { No. of search } \\
\text { passes }\end{array}$ & $\mathrm{PAS}=$ & $\begin{array}{l}\text { Enter the keyword PAS }=\text { followed by the desired } \\
\text { number of search passes. Default }=10 \text {. }\end{array}$ \\
\hline $3 b$ & $\begin{array}{l}\text { No. of search } \\
\text { parameters }\end{array}$ & $\mathrm{NPM}=$ & $\begin{array}{l}\text { Enter the keyword } \mathrm{NPM}=\text { followed by the number of } \\
\text { search parameters. Present capability is limited to } \\
1 .\end{array}$ \\
\hline $3 \mathrm{c}$ & $\begin{array}{l}\text { Search } \\
\text { convergence } \\
\text { tolerance }\end{array}$ & $\mathrm{EPS}=$ & $\begin{array}{l}\text { Enter the keyword EPS }=\text { followed by the desired } \\
\text { convergence tolerance. Default }=0.005 \text {. }\end{array}$ \\
\hline $3 d$ & $\begin{array}{l}\text { Desired value of } \\
k_{\text {eff }}\end{array}$ & $\mathrm{KEF}=$ & $\begin{array}{l}\text { Enter the keyword } \mathrm{KEF}=\text { followed by the desired } \\
\text { value of } k_{\text {eff }} \text {. The default value is } 1.000 \text {. } \\
\text { DO NOT ENTER FOR OPTIMUM OR MINIMUM } \\
\text { SEARCHES. }\end{array}$ \\
\hline $3 e$ & $\begin{array}{l}\text { Maximum allowed } \\
\text { pitch }\end{array}$ & MAXPITCH= & $\begin{array}{l}\text { Enter the keyword MAXPITCH }=\text { followed by the } \\
\text { maximum allowed pitch for a search whose search } \\
\text { type, entry } 2 \text { above, is PITCH. The default value is } \\
\text { the pitch corresponding to }-5.0 \text { times the parameter } \\
\text { at the minimum possible pitch. }\end{array}$ \\
\hline $3 f$ & $\begin{array}{l}\text { Minimum allowed } \\
\text { pitch }\end{array}$ & MINPITCH= & $\begin{array}{l}\text { Enter the keyword MINPITCH= followed by the } \\
\text { minimum allowed pitch for a search whose search } \\
\text { type, entry } 2 \text { above, is PITCH. The default value is } \\
\text { the minimum possible pitch (i.e., the pitch at which } \\
\text { the shapes in the array touch). }\end{array}$ \\
\hline 4 & $\begin{array}{l}\text { Additional search } \\
\text { data }\end{array}$ & MORE & $\begin{array}{l}\text { Enter the delimiter MORE. This delimiter ends the } \\
\text { optional search commands and initiates the } \\
\text { auxiliary search commands found in Table 2.1.4. }\end{array}$ \\
\hline
\end{tabular}

\subsection{Auxiliary search commands and constraints}

Auxiliary search commands are entered only if MORE, item 4, of the search type specification data is entered (see Table 2.1.3). Individual search commands are used to specify search constraints and to communicate to the search program. Searches can alter geometric dimensions (PITCH or DIMENSION Search) or alter standard composition number densities (CONCENTRATION Search). 
A PITCH or DIMENSION search may require the user to specify the units that will be altered, the regions that will be altered within those units, and the faces or surfaces of those regions that will be altered. For a PITCH search, the program automatically assigns the units in the arrays to a unit cell if possible. If multiple units are contained in the array, each unit could be assigned a unit cell if the data in the unit cells match the geometry data of the units in the array. This data may be overridden in the MORE section of the search data. For a DIMENSION search, if the user wishes to tie a unit to unit cell this must be explicitly done in the MORE section of the search data. Several examples of search problems are provided in Sect. 2.1.1.

A CONCENTRATION search requires the user to specify the mixture, standard composition name, and the search constant for the component being altered. For a CONCENTRATION search, the program automatically assigns the material being changed to a unit cell. This data may be overridden in the MORE section of the search data. Several examples of search problems are provided in Sect. 2.1.1.

The data comprising the auxiliary search commands are listed in Table 2.1.4. All data except items 1a, $1 \mathrm{~b}$, and $1 \mathrm{c}$ are keyworded (i.e., the data are entered by specifying a keyword, followed by a value). An explanation of each individual search command follows the table.

1 Command definition. A command definition tells the code what action is to be taken. A new search command is initiated whenever an item 1a through 1c is encountered. The code will vary the geometry according to subsequent commands.

1a. ALTER CHANGE MODIFY

1b. MAINTAIN

1c. KEEP HOLD

$2 \quad \mathrm{PAR}=$

$3+\mathrm{CON}=$
Alter geometry regions. These words specify that modifications will be made to the geometry according to subsequent commands.

Maintain the thickness. The thickness of the specified geometry region(s) will be maintained when the interior regions grow or shrink (i.e., the specified region will grow or shrink in conjunction with the interior region in such a way as to maintain the original distance between the two regions). This means that the original thickness of the region is preserved. For instance, the inner radius of a pipe can be altered and the wall thickness can be preserved by applying the MAINTAIN command to the region defining the outer radius of the pipe.

Keep the original specification. This command causes the specified geometry region(s) to be reset to their original input value for every search pass. Therefore they go through the entire search process unchanged.

Parameter number. The search parameter number is not functional. The default number is 1 and should not be overridden.

Maximum constraint. Enter the maximum value you wish to allow the search parameter to obtain. The maximum constraint must be larger than the minimum constraint. For a DIMENSION or PITCH search the default value of the maximum constraint is $10^{11}$. For a CONCENTRATION search the default value of the maximum constraint is as follows:

$$
\begin{aligned}
& +\mathrm{CON}=\min (-1 / \mathrm{FACTOR}), \text { if any FACTOR }<0 \\
& +\mathrm{CON}=-5^{*}(-\mathrm{CON}), \text { if all FACTOR }>0
\end{aligned}
$$


NOTE: For a PITCH search, the maximum constraint is redefined and is calculated from the data entered for MAXPITCH. +CON should not be entered if a value was entered for MAXPITCH. If constraints, $+\mathrm{CON}=$ and $/$ or $-\mathrm{CON}=$ are not entered as data, the code computes the minimum constraint corresponding to the pins touching, and the maximum constraint is then negative five times the magnitude of the minimum constraint.

Minimum constraint. Enter the minimum value you wish to allow the search parameter to obtain. The minimum constraint must be smaller than the maximum constraint but need not be a negative number. The default value of the minimum constraint for a dimension search is $-10^{11}$. The default value of the minimum constraint for a pitch search is redefined to correspond to the pins in the lattice touching. For a CONCENTRATION search the default value of the minimum constraint is as follows:

$$
\begin{aligned}
& -\mathrm{CON}=-5(+\mathrm{CON}), \text { if all FACTOR }<0 \\
& -\mathrm{CON}=\max (-1 / \mathrm{FACTOR}), \text { if any FACTOR }>0
\end{aligned}
$$

$5 \quad \mathrm{CELL}=$

Unit Cell Number. This is the unit cell to which the unit or mixture will be tied. It needs to follow either the UNIT= or MIX= keyword data. Tying a unit cell to a unit or mixture ensures the unit cell data gets changed as the geometry or mixture data gets changed thus ensuring the cross sections are properly processed.

$6 \quad \mathrm{UNIT}=$

Geometry unit number. This is the geometry unit to which the previously entered command definition (item $1 \mathrm{a}, 1 \mathrm{~b}$, or $1 \mathrm{c}$ ) is applied. Items 7,8 , and 9 specify the region(s) within the unit and the surfaces of the region(s) to be altered.

First region to be altered. This is used to specify the first or only region in the unit (specified by item 6) that is to be altered according to the search command (item 1a, 1b, or 1c). The region(s) are altered according to the search constants (items 9a, 9b, 9c, and/or 9d).

Last region to be altered. This item is entered to specify the last region to be altered, starting with the region specified by $\mathrm{REG}=$. For example, assume unit 3 contains eight regions and you wish to make changes to regions $4,5,6,7$, and 8 . These regions are identified by entering the following data. UNIT $=3$ REG $=4$ TO 8.

Geometric search constants. A search constant is the proportionality factor utilized to alter a geometry region. A search constant must be entered for each surface of a region that is to be altered. A nonzero search constant will cause the region dimension for that surface to be changed. A search constant of 0.0 will cause the region dimension to remain unchanged. The default value of the search constant is 0.0 . 
$9 \quad \mathrm{ALL}=$

9a. $+X=$

$-\mathrm{X}=$

$+\mathrm{Y}=$

$-\mathrm{Y}=$

$+\mathrm{Z}=$

$-\mathrm{Z}=$

9b. RADIUS $=$

9c. + HEIGHT $=$

-HEIGHT=

9d. $\mathrm{CHORD}=$

10 MIX $=$

11 SCNAME $=$
Search constant for all surfaces. All of the surfaces in a region are altered simultaneously by using this search command.

Search constant for $+X$ face. This parameter is used to specify the value of the search constant for the $+\mathrm{X}$ face of a cuboid.

Search constant for $-\mathrm{X}$ face. This parameter is used to specify the value of the search constant for the $-\mathrm{X}$ face of a cuboid.

Search constant for $+Y$ face. This parameter is used to specify the value of the search constant for the $+Y$ face of a cuboid.

Search constant for $-\mathrm{Y}$ face. This parameter is used to specify the value of the search constant for the $-\mathrm{Y}$ face of a cuboid.

Search constant for $+Z$ face. This parameter is used to specify the value of the search constant for the $+Z$ face of a cuboid.

Search constant for $-Z$ face. This parameter is used to specify the value of the search constant for the $-Z$ face of a cuboid.

NOTE: If it is desirable to change all the faces of a cuboid except the $-Z$ face by some amount (search constant of 1.0), items $9 \mathrm{a}$ and $9 \mathrm{~b}$ can be used together as follows: $\mathrm{ALL}=1.0-\mathrm{Z}=0.0$. This is the same as entering $+\mathrm{X}=1.0-\mathrm{X}=1.0+\mathrm{Y}=1.0-\mathrm{Y}=1.0+\mathrm{Z}=1.0$. If both $\mathrm{Z}$ faces are to remain unchanged, items $8 \mathrm{a}$ and $8 \mathrm{~b}$ can be entered as: $A L L=1.0$ $+\mathrm{Z}=0.0-\mathrm{Z}=0.0$ or as $+\mathrm{X}=1.0-\mathrm{X}=1.0+\mathrm{Y}=1.0-\mathrm{Y}=1.0$.

Search constant for radius. This parameter is used to specify the value of the search constant for the radius of a sphere, hemisphere, cylinder, or hemicylinder.

Search constant for theight. This parameter is used to specify the value of the search constant for the theight of a cylinder or hemicylinder.

Search constant for -height. This parameter is used to specify the value of the search constant for the -height of a cylinder or hemicylinder.

Search constant for chord. This parameter is used to specify the value of the search constant for the chord of a hemisphere or hemicylinder.

Search constant for mixture. This parameter is the mixture number containing the standard composition that is to be changed during the search.

Search constant for the standard composition name. This parameter is the standard composition name associated with the material that is to be changed during the search. Only compositions listed in the Standard Composition Library section of the Standard Composition Library chapter are allowed. Standard compositions beginning with SOLN cannot be altered directly. 
NOTE: If the standard composition name specified in the Material Information Data begins with SOLN and the Concentration Search Data specifies SCNAME $=\mathrm{UO} 2(\mathrm{NO} 3) 2$, the amount of $\mathrm{UO} 2(\mathrm{NO} 3) 2$ in the solution is altered but the amount of $\mathrm{H} 2 \mathrm{O}$ and nitric acid is not altered during the search. The resulting mixture, when the search is finished, may no longer meet the criteria associated with the SOLN specification.

12 FACTOR $=\quad$ Concentration search factor used to specify the value of the search constant used in the concentration search. It is a proportionality factor used to alter the specified mixture standard composition. A search constant must be entered for each standard composition that is altered. A non-zero search constant will cause the concentration of the associated standard composition to be altered. The default value of the search constant is 1.0. A set of concentration search data consists of the mixture to be altered, the standard composition to be altered in the mixture, and the search factor. Keywords are used to enter the data. Each set of data consists of items 10,11, and 12. The keywords used in this data may be entered using terse notation. 
Table 2.1.4. Outline of auxiliary search commands and constraints

\begin{tabular}{|c|c|c|c|}
\hline $\begin{array}{c}\text { Entry } \\
\text { no. }\end{array}$ & $\begin{array}{c}\text { Keyword } \\
\text { name }\end{array}$ & Type of data & Comments \\
\hline \multicolumn{4}{|c|}{ GENERIC SEARCH DATA - May be used with all Search Types - } \\
\hline $1 \mathrm{a}$ & $\begin{array}{l}\text { ALTER } \\
\text { CHANGE } \\
\text { MODIFY }\end{array}$ & $\begin{array}{l}\text { Begin a new search } \\
\text { command }\end{array}$ & $\begin{array}{l}\text { These words are used to specify that modifications will be } \\
\text { made to the geometry or concentration according to } \\
\text { subsequent commands (entries } 3 \text { through } 12 \text { as required } \\
\text { to specify the desired changes). }\end{array}$ \\
\hline $1 b$ & MAINTAIN & $\begin{array}{l}\text { Begin a new search } \\
\text { command }\end{array}$ & $\begin{array}{l}\text { The spacing (thickness) of the specified geometry regions } \\
\text { will be maintained when the interior regions grow or } \\
\text { shrink. }\end{array}$ \\
\hline $1 \mathrm{c}$ & $\begin{array}{l}\text { KEEP } \\
\text { HOLD }\end{array}$ & $\begin{array}{l}\text { Begin a new search } \\
\text { command }\end{array}$ & $\begin{array}{l}\text { This command resets the specified geometry to the original } \\
\text { input specifications. }\end{array}$ \\
\hline 2 & $\mathrm{PAR}=$ & Parameter number & $\begin{array}{l}\text { Enter the parameter number that the current command } \\
\text { (ALTER, MAINTAIN, KEEP) applies to. Default }=1 \text { and } \\
\text { should not be changed. }\end{array}$ \\
\hline 3 & $+\mathrm{CON}=$ & Maximum constraint & $\begin{array}{l}\text { Enter the maximum constraint for the current parameter. } \\
\text { Default }=+10 \mathrm{E} 10 \text {. }\end{array}$ \\
\hline 4 & $-\mathrm{CON}=$ & Minimum constraint & $\begin{array}{l}\text { Enter the minimum constraint for the current parameter. } \\
\text { Default }=-10 \mathrm{E} 10 \text {. }\end{array}$ \\
\hline 5 & $\mathrm{CELL}=$ & $\begin{array}{l}\text { Unit Cell Associated } \\
\text { with search data }\end{array}$ & $\begin{array}{l}\text { Default values are assigned for PITCH and } \\
\text { CONCENTRATION searches. Associated Unit Cells } \\
\text { must be entered for a DIMENSION search if desired. }\end{array}$ \\
\hline
\end{tabular}

PITCH \& DIMENSION SEARCH DATA — Defines Geometric Changes -

$6 \quad$ UNIT $=\quad$ Unit to which the Enter the unit in which regions are to be altered. current command applies

7 REGION $=$ First region to be Enter the first or only region in the unit that the search altered in the unit constants (entry $9 \mathrm{a}, \mathrm{b}, \mathrm{c}$ and/or d) apply to. Default is the first region.

8 TO

Last region to be Enter the last region in the unit to which the search constants altered in the unit apply (entry 9a, b, c and/or d).

Default is the first region.

NOTE: Entry 7 must be entered in order to alter a single region in a unit. Entries 7 and 8 must both be entered in order to alter more than one region in a unit. 
Table 2.1.4. Outline of auxiliary search commands and constraints (continued)

\begin{tabular}{|c|c|c|c|}
\hline $\begin{array}{c}\text { Entry } \\
\text { no. }\end{array}$ & $\begin{array}{c}\text { Keyword } \\
\text { name }\end{array}$ & Type of data & Comments \\
\hline 9 & $\mathrm{ALL}=$ & $\begin{array}{l}\text { Search constant for all } \\
\text { surfaces (faces) of } \\
\text { the region(s) }\end{array}$ & $\begin{array}{l}\text { Enter a value for the search constants for the specified } \\
\text { regions. This value will be applied to all surfaces of the } \\
\text { region(s). }\end{array}$ \\
\hline \multirow[t]{6}{*}{$9 \mathrm{a}$} & $+X=$ & $\begin{array}{l}\text { Search constant for } \\
+\mathrm{X} \text { face of cuboid }\end{array}$ & $\begin{array}{l}\text { Enter a value for the search constant for the }+X \text { face of a } \\
\text { cuboid. }\end{array}$ \\
\hline & $-X=$ & $\begin{array}{l}\text { Search constant for } \\
-X \text { face of cuboid }\end{array}$ & $\begin{array}{l}\text { Enter a value for the search constant for the }-\mathrm{X} \text { face of a } \\
\text { cuboid. }\end{array}$ \\
\hline & $+\mathrm{Y}=$ & $\begin{array}{l}\text { Search constant for } \\
+\mathrm{Y} \text { face of cuboid }\end{array}$ & $\begin{array}{l}\text { Enter a value for the search constant for the }+Y \text { face of a } \\
\text { cuboid. }\end{array}$ \\
\hline & $-\mathrm{Y}=$ & $\begin{array}{l}\text { Search constant for } \\
-Y \text { face of cuboid }\end{array}$ & $\begin{array}{l}\text { Enter a value for the search constant for the }-Y \text { face of a } \\
\text { cuboid. }\end{array}$ \\
\hline & $+\mathrm{Z}=$ & $\begin{array}{l}\text { Search constant for }+Z \\
\text { face of cuboid }\end{array}$ & $\begin{array}{l}\text { Enter a value for the search constant for the }+Z \text { face } \\
\text { of a cuboid. }\end{array}$ \\
\hline & $-Z=$ & $\begin{array}{l}\text { Search constant for }-Z \\
\text { face of cuboid }\end{array}$ & $\begin{array}{l}\text { Enter a value for the search constant for the }-\mathrm{Z} \text { face } \\
\text { of a cuboid. }\end{array}$ \\
\hline $9 \mathrm{~b}$ & RADIUS $=$ & $\begin{array}{l}\text { Search constant for } \\
\text { radius }\end{array}$ & $\begin{array}{l}\text { Enter a value for the search constant for the radius of a sphere } \\
\text { or a cylinder. }\end{array}$ \\
\hline \multirow[t]{2}{*}{$9 \mathrm{c}$} & $+\mathrm{HEIGHT}=$ & $\begin{array}{l}\text { Search constant for } \\
\text { +height }\end{array}$ & $\begin{array}{l}\text { Enter a value for the search constant for the theight of a } \\
\text { cylinder. }\end{array}$ \\
\hline & $-\mathrm{HEIGHT}=$ & $\begin{array}{l}\text { Search constant for } \\
\text {-height }\end{array}$ & $\begin{array}{l}\text { Enter a value for the search constant for the -height } \\
\text { of a cylinder. }\end{array}$ \\
\hline $9 \mathrm{~d}$ & $\mathrm{CHORD}=$ & $\begin{array}{l}\text { Search constant for } \\
\text { chord }\end{array}$ & $\begin{array}{l}\text { Enter a value for the search constant for the chord face of a } \\
\text { hemisphere or hemicylinder. }\end{array}$ \\
\hline \multicolumn{4}{|c|}{ CONCENTRATION SEARCH DATA - Defines concentration changes - } \\
\hline 10 & $\mathrm{MIX}=$ & $\begin{array}{l}\text { Search constant for } \\
\text { mixture }\end{array}$ & $\begin{array}{l}\text { Enter the mixture number containing the standard } \\
\text { composition to be changed. }\end{array}$ \\
\hline 11 & SCNAME $=$ & $\begin{array}{l}\text { Search constant for } \\
\text { Standard } \\
\text { Composition }\end{array}$ & $\begin{array}{l}\text { Enter the standard composition name whose density is to be } \\
\text { changed. }\end{array}$ \\
\hline 12 & $\mathrm{FACTOR}=$ & $\begin{array}{l}\text { Search proportionality } \\
\text { constant }\end{array}$ & $\begin{array}{l}\text { Enter the value of the search constant for the concentration } \\
\text { search. }\end{array}$ \\
\hline
\end{tabular}




\subsubsection{Example Problems}

This section contains example problems to demonstrate some of the options available in CSAS5 and its associated sequences. A brief problem description and the associated input data for multigroup mode of calculation are included for each problem. The same sample problems may be executed in the continuous energy mode by changing the library name from "v7-238" to "ce-v7". Note that sample problems 3, 7, and 8 do not run in the continuous energy mode because they use CELLMIX or DOUBLEHET cell type. See Appendix A for additional and historical examples.

\subsubsection{Sample problem $1 \boldsymbol{k}_{\text {eff }}$ calculation}

The purpose of this problem is to calculate the k-effective of a system. This problem is the same as the KENO V.a sample problem 12 in Appendix B except the cross-section library and KENO V.a mixing table are prepared by CSAS. The problem represents a critical experiment consisting of a composite array $^{2,3}$ of four highly-enriched (93.2\%) uranium metal cylinders having a density of $18.76 \mathrm{~g} / \mathrm{cc}$ and four 5.0677-L Plexiglas containers filled with uranyl nitrate solution. The uranium metal cylinders have a radius of $5.748 \mathrm{~cm}$ and a height of $10.765 \mathrm{~cm}$. The uranyl nitrate solution has a specific gravity of 1.555 and contains $415 \mathrm{~g}$ of uranium per liter. The ID of the Plexiglas bottle is $19.05 \mathrm{~cm}$ and the inside height is $17.78 \mathrm{~cm}$. The Plexiglas is $0.635 \mathrm{~cm}$ thick. The center-to-center spacing between the metal units is $13.18 \mathrm{~cm}$ in the $\mathrm{Y}$ direction and $13.45 \mathrm{~cm}$ in the $\mathrm{Z}$ direction. The center-to-center spacing between the solution units is $21.75 \mathrm{~cm}$ in the $Y$ direction and $20.48 \mathrm{~cm}$ in the $Z$ direction. The spacing between the $\mathrm{Y}-\mathrm{Z}$ plane that passes through the centers of the metal units and the Y-Z plane that passes through the centers of the solution units is $17.465 \mathrm{~cm}$ in the $\mathrm{X}$ direction.

The metal units in this experiment are designated in Table II of Ref. 2 as cylinder index 11 and reflector index 1. A photograph of the experiment, Fig. 9 in Ref. 3, is given in Figure 2.1.4.1.

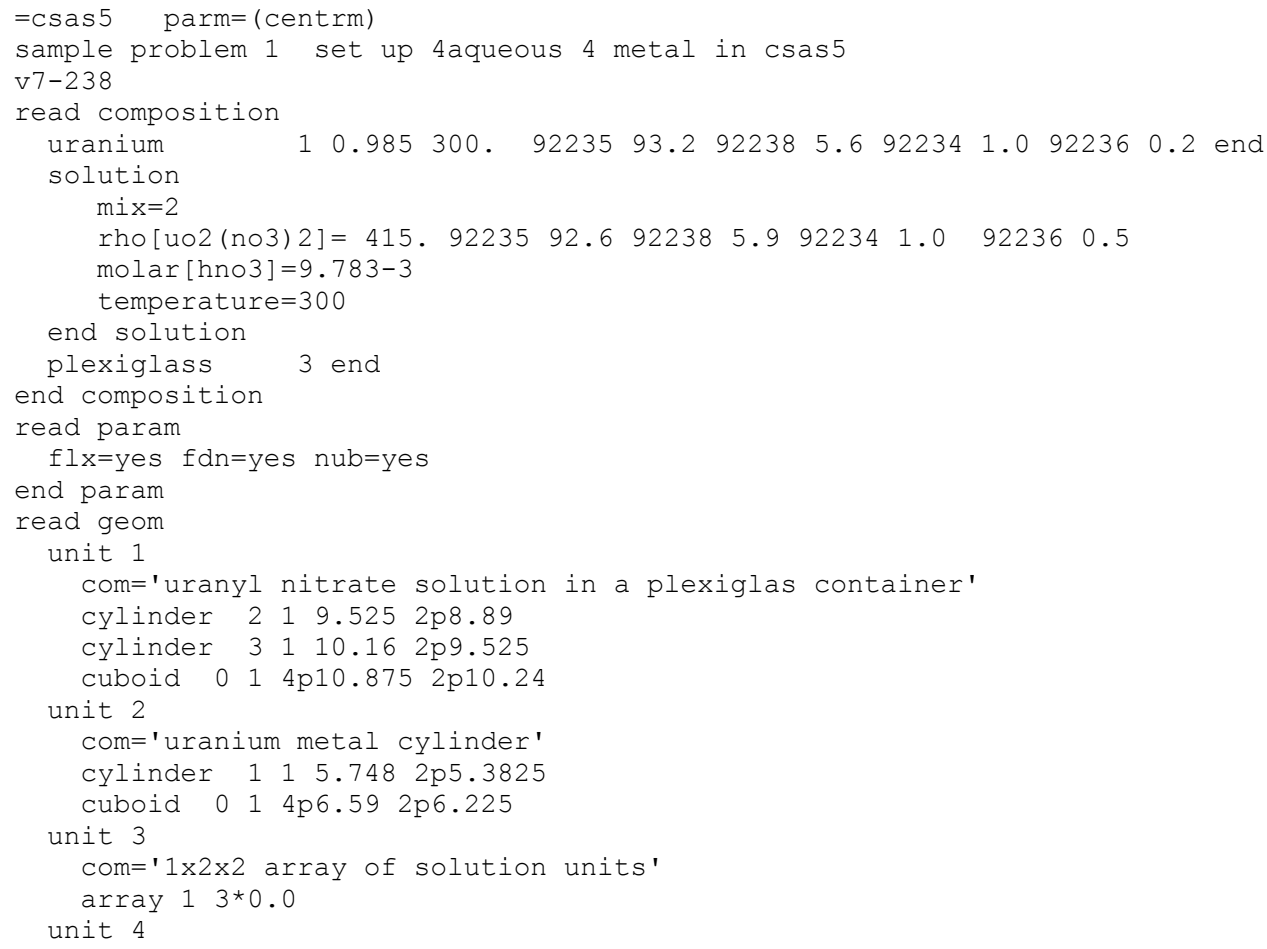


com=' $1 \times 2 \times 2$ array of metal units padded to match solution array'

array $23 * 0.0$

replicate $0 \quad 12 * 0.02 * 8.572 * 8.03 \quad 1$

global unit 5

array $3 * 0.0$

end geom

read array

ara=1 nux=1 nuy=2 nuz=2 fill fl end fill

ara=2 nux=1 nuy=2 nuz=2 fill f2 end fill

gbl=3 ara=3 nux=2 nuy=1 nuz=1

com='composite array of solution and metal units'

fill 43 end fill

end array

end data

end

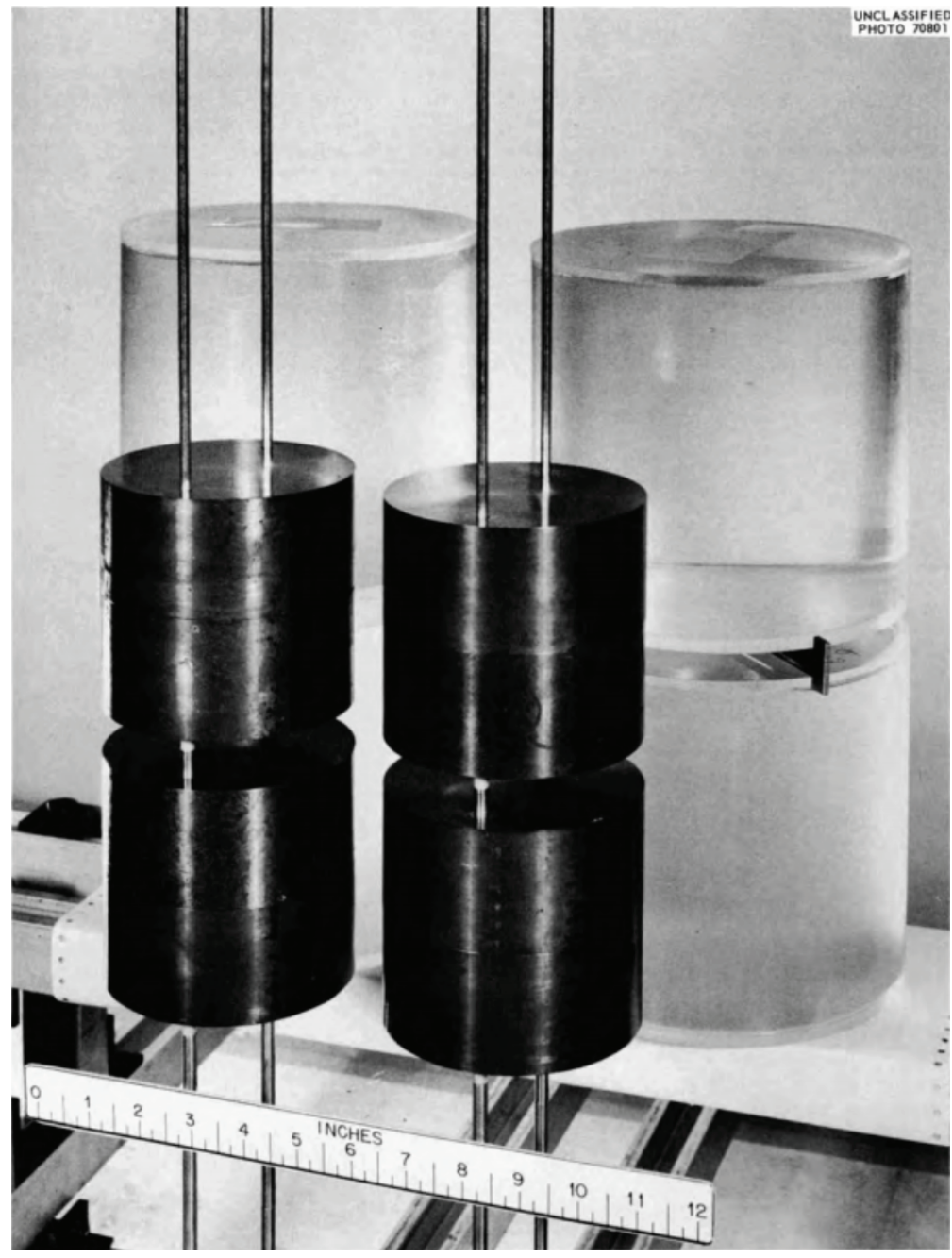

Figure 2.1.4.1. Critical assembly of four solution units and four metal units. 


\subsubsection{Sample problem 2 optimum pitch search using detailed geometry}

This problem represents an attempt to optimize the reactivity of PWR-like fuel bundles in a storage pool. The storage array is an infinite planar array of flooded fuel bundles. Each bundle consists of a $17 \times 17 \times 1$ array of $2.35 \%$-enriched $\mathrm{UO}_{2}$ pins, density $9.21 \mathrm{~g} / \mathrm{cc}$, clad with Zircaloy- 2 . The fuel is $0.823 \mathrm{~cm}$ in diameter, the clad diameter is $0.9627 \mathrm{~cm}$, and the length of each pin is $366 \mathrm{~cm}$. Each fuel bundle is encased in a Boral sheath. There is a 1/4-in. gap flooded with water between the bundle and the sheath. The Boral sheath is $3 / 8$ in. thick. One inch of water separates the fuel bundle sheaths in the horizontal plane, and $15 \mathrm{~cm}$ of water is present on the top and bottom of the array.

The KENO V.a geometry represents each fuel pin in the bundle discretely. The search should determine the optimum pitch within the fuel bundle. The gap between the bundle and the Boral remains fixed, as does the thickness of the sheath and the spacing between sheaths.

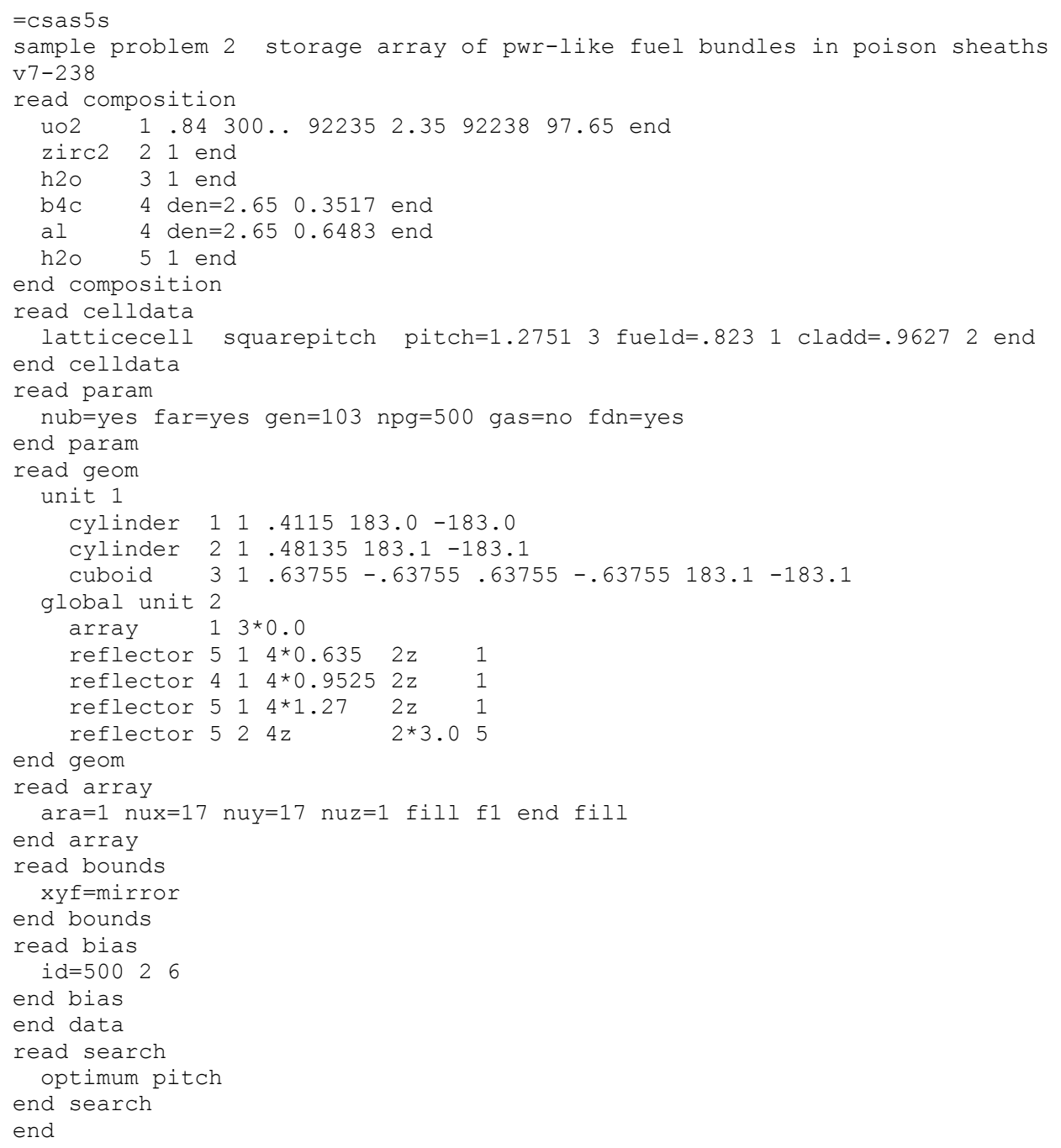




\subsubsection{Sample problem 3 optimum pitch search using homogenized geometry}

This problem illustrates the use of a cell-weighted mixture to represent a PWR-like fuel bundle. The cask contains a $2 \times 2 \times 1$ array of fuel bundles. Each fuel bundle consists of a $17 \times 17 \times 1$ array of Zircaloy- 2 clad, $2.35 \%$-enriched $\mathrm{UO}_{2}$ fuel pins with a density of $9.21 \mathrm{~g} / \mathrm{cc}$ arranged in a square pitch. The pin diameter is $0.823 \mathrm{~cm}$, and its length is $366 \mathrm{~cm}$. The clad is $0.06985 \mathrm{~cm}$ thick, and the pitch is $1.275 \mathrm{~cm}$. Each fuel bundle is contained in a $0.6625-\mathrm{cm}$-thick Boral sheath. The bundles are separated by $1 \mathrm{~cm}$ of water, representing a flooded cask. The square aluminum cask is $10-\mathrm{cm}$ thick on all faces and is reflected by $15 \mathrm{~cm}$ of water.

By using CELLMIX=, a cell-weighted cross section is created to represent the fuel bundle. The KENO V.a geometry utilizes the cell-weighted mixture (500) and the overall dimensions of the fuel bundle to represent the entire fuel bundle as a single homogeneous region. The first reflector entry represents the fuel cask, and the second reflector entry represents the $15-\mathrm{cm}$ reflector. Because this problem utilizes a cell-weighted mixture, which is not applicable in the continuous energy mode, the problem will end with an error message in the continuous energy mode.

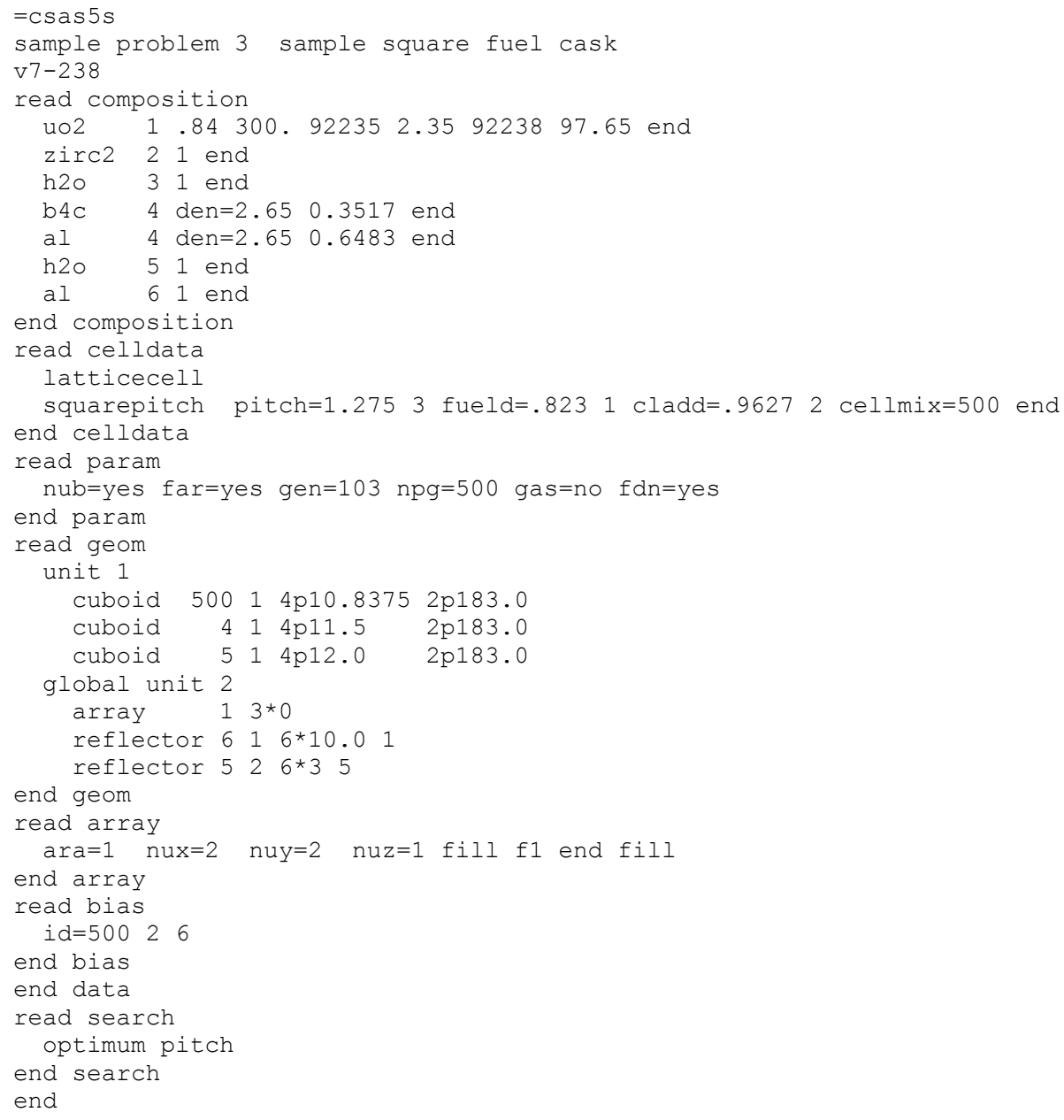




\subsubsection{Sample problem 4 search for a specified value of $\boldsymbol{k}_{\text {eff }}$}

Find the pitch at which a $2 \times 2 \times 2$ array of cylinders of highly enriched (93.2\%) uranium metal with a density of $18.76 \mathrm{~g} / \mathrm{cc}$ are critical. Each cylinder has a radius of $5.748 \mathrm{~cm}$ and a height of $10.765 \mathrm{~cm}$. The surface-to-surface spacing between the units is the same in all directions. The initial guess for the critical surface-to-surface spacing was $3.0 \mathrm{~cm}$. The experimentally critical surface-to-surface separation for this system is $2.248 \mathrm{~cm}$. The input data for this problem are given below.

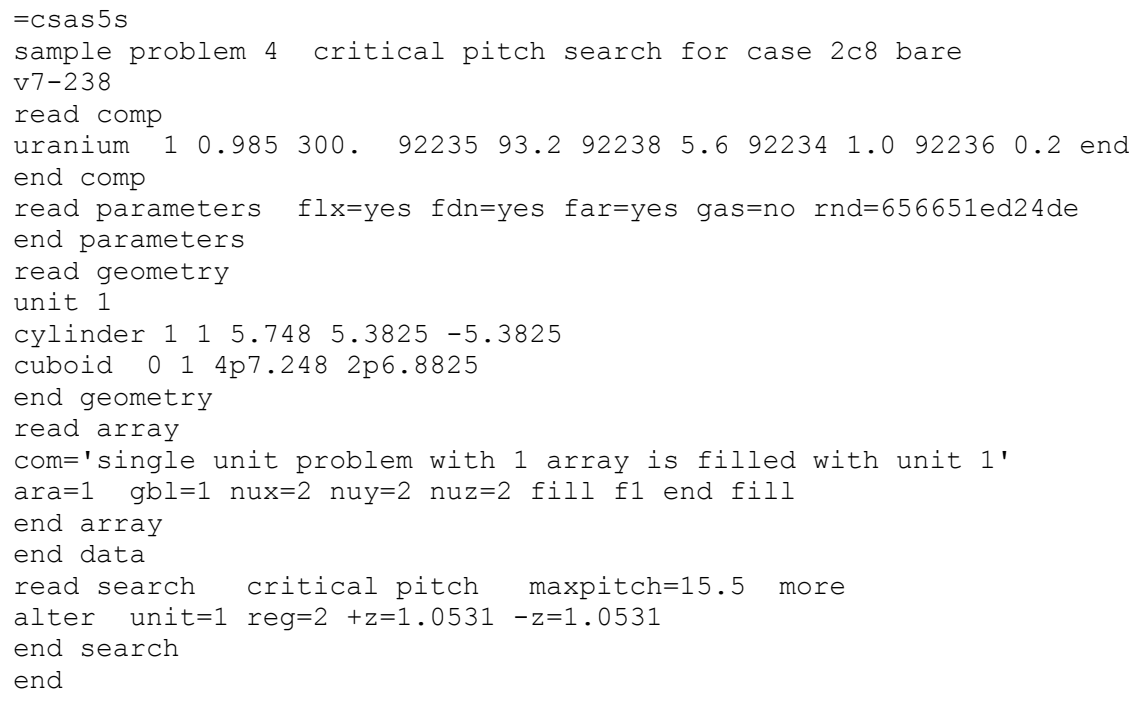




\subsubsection{Sample problem 5 solution conc. search for a specified $\boldsymbol{k}_{\text {eff }}$}

Consider a large spherical tank partially filled with $\mathrm{UO}_{2} \mathrm{~F}_{2}$ solution. The tank has a radius of $34.6 \mathrm{~cm}$ and is filled with solution to a height of $30.0 \mathrm{~cm}$ above the midpoint. The tank is composed of a $0.759 \mathrm{~cm}$ thick $\mathrm{Al}$ shell. The $\mathrm{UO}_{2} \mathrm{~F}_{2}$ solution is composed of three standard compositions: UO2F2, $\mathrm{HF}$ acid, and $\mathrm{H} 2 \mathrm{O}$. The code combines these using a set algorithm. This may or may not produce a solution at the desired density. If the density of the solution is known it should be entered. Also, extra acid can be added to the solution by specifying a non-zero acid molarity.

A critical concentration search is performed on the solution yielding system $k_{\text {eff }}=1.0$ for various densities of $\mathrm{UO}_{2} \mathrm{~F}_{2}$ in the solution. In the MORE search data, MIX=1 and SCNAME=UO2F2 specify that the $\mathrm{UO}_{2} \mathrm{~F}_{2}$ component of the mixture 1 solution is to be altered during the search. The code calculates the density of the solution. The initial uranium fuel density is $300 \mathrm{gm} /$ liter. The maximum allowed uranium density is $600 \mathrm{gm} /$ liter. The minimum allowed uranium density is $150 \mathrm{gm} / \mathrm{liter}$.

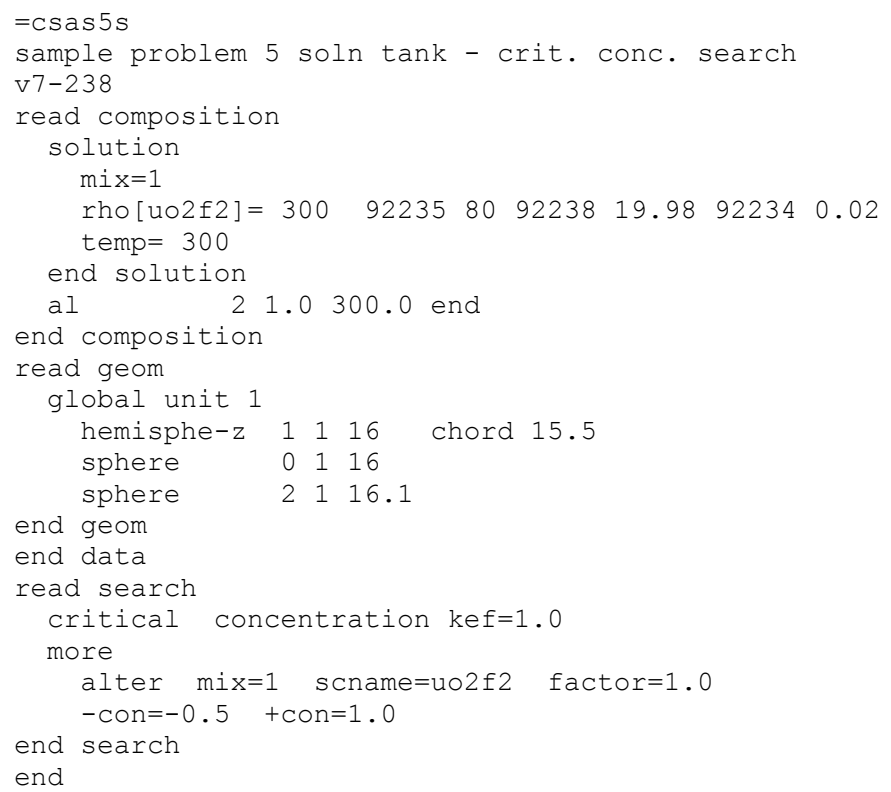

\subsubsection{Sample problem 6 dimension chord search for a specified $\boldsymbol{k}_{\text {eff }}$}

Consider a large spherical tank partially filled with $\mathrm{UO}_{2} \mathrm{~F}_{2}$ solution. The tank has a radius of $34.6 \mathrm{~cm}$ and is filled with solution to an initial height of $10.0 \mathrm{~cm}$ above the midpoint. The tank is composed of a $0.759 \mathrm{~cm}$ thick $\mathrm{Al}$ shell. The $\mathrm{UO}_{2} \mathrm{~F}_{2}$ solution is composed of three standard compositions: UO2F2, HF acid, and $\mathrm{H} 2 \mathrm{O}$. The code combines these using a set algorithm. This may or may not produce a solution at the desired density. If the density of the solution is known it should be entered. Also, extra acid can be added to the solution by specifying a non-zero acid molarity.

A critical dimension search is performed on the chord length yielding system $k_{\text {eff }}=0.98$. In the MORE search data, UNIT=1 REG=1 CHORD=1.0 specify that the chord length in region 1 of unit 1 is to be altered during the search. The constraints are set so the chord length varies from $-10.0 \mathrm{~cm}$ to just under $34.6 \mathrm{~cm}$. 


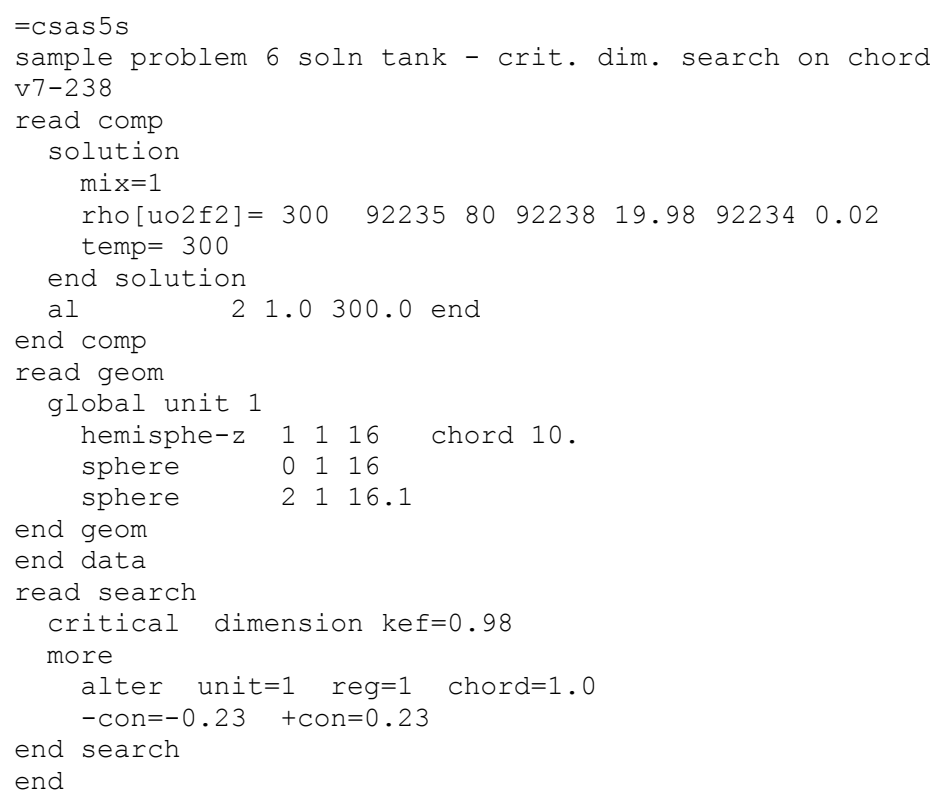

\subsubsection{Sample problem 7 two material conc. search for a specified $\boldsymbol{k}_{\text {eff }}$}

The fuel bundles in this problem represent $17 \times 17$ PWR fuel assemblies. The fuel pin lattice is homogenized, making a cell-weighted mixture 100. Because this problem utilizes a cell-weighted mixture, which is not applicable in the continuous energy mode, the problem will end with an error message in the continuous energy mode. The fuel pins consist of $4.35 \mathrm{wt} \%{ }^{235} \mathrm{U}$ having a diameter of $0.823 \mathrm{~cm}$, zirconium cladding having an outer diameter of $0.9627 \mathrm{~cm}$, and a pitch of $1.275 \mathrm{~cm}$. The fuel bundle is represented as a $10.8375 \mathrm{~cm} \times 10.8375 \mathrm{~cm} \times 366 \mathrm{~cm}$ cuboid of mixture 100 surrounded by Boral and then water. The Boral has a density of $2.61 \mathrm{~g} / \mathrm{cm}^{3}$ and has an initial composed of $50.0 \mathrm{wt} \%$ $\mathrm{B}_{4} \mathrm{C}$ and $50.0 \mathrm{wt} \% \mathrm{Al}$. The fuel bundles are at a fixed pitch of $13.0 \mathrm{~cm}$. Boral plates surrounding the $\mathrm{X}$ and $\mathrm{Y}$ sides of each fuel assembly are $0.1625 \mathrm{~cm}$ thick. Full density water is between the Boral plates.

A critical concentration search is performed on the Boral plates searching for a system $k_{\text {eff }}=0.95$. The Boral plates are at a fixed density of $2.61 \mathrm{gm} / \mathrm{cc}$. As the density of the $\mathrm{B}_{4} \mathrm{C}$ changes, the density of the $\mathrm{Al}$ changes in the opposite direction maintaining a constant Boral density. There are two entries in the MORE search data. The first entry, MIX $=4$ SCNAME $=b 4 c$ factor=1.0 specifies that the $\mathrm{B}_{4} \mathrm{C}$ of mixture 4 is to be altered during the search. The second entry, MIX=4 SCNAME=al factor=-1.0 specifies that aluminum is to be changed in the opposite direction and proportionally to $\mathrm{B}_{4} \mathrm{C}$ during the search. Both $\mathrm{B}_{4} \mathrm{C}$ and $\mathrm{Al}$ have the same initial density of $0.5 * 2.61=1.305 \mathrm{gm} / \mathrm{cc}$.

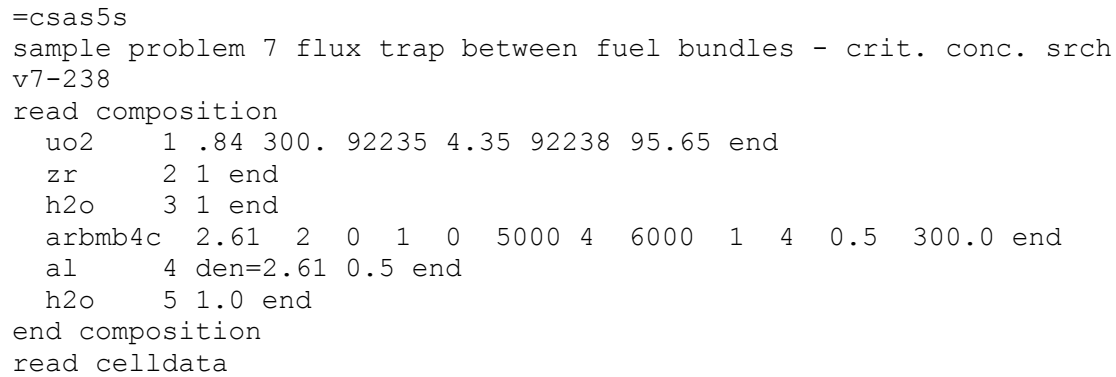




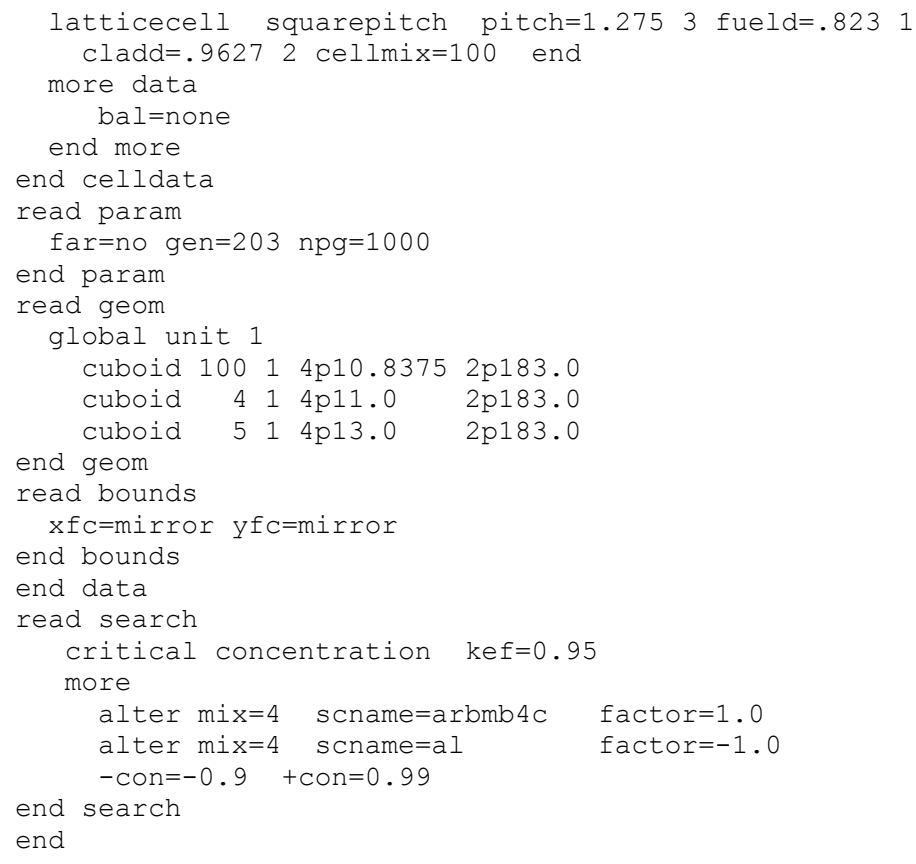

\subsubsection{Sample problem $8 k_{\infty}$ for a pebble bed fuel}

This problem demonstrates setting up a fuel pebble from a pebble bed reactor, and calculating its $\mathrm{k}_{\infty}$. The pebble consists of a fuel grain of $\mathrm{UO}_{2} 0.025 \mathrm{~cm}$ in radius, coated with $0.003 \mathrm{~cm}$ of pyrolitic carbon, a further coat of $0.0035 \mathrm{~cm}$ thick silicon carbide, with a final coat of $0.004 \mathrm{~cm}$ thick pyrolitic carbon. 15000 grains are packed with graphite into an internal fuel sphere of $2.5 \mathrm{~cm}$ radius clad with a $0.5 \mathrm{~cm}$ thick covering of carbon and surrounded by helium. The fuel is $8.2 \%$ enriched ${ }^{235} \mathrm{U}$. The pebbles are stacked into an infinite square pitched array with a pitch of $6 \mathrm{~cm}$.

This problem uses DOUBLEHET cell type, which is applicable only in the multigroup mode of KENO calculations. Therefore, the continuous energy version of this problem will end with an error message.

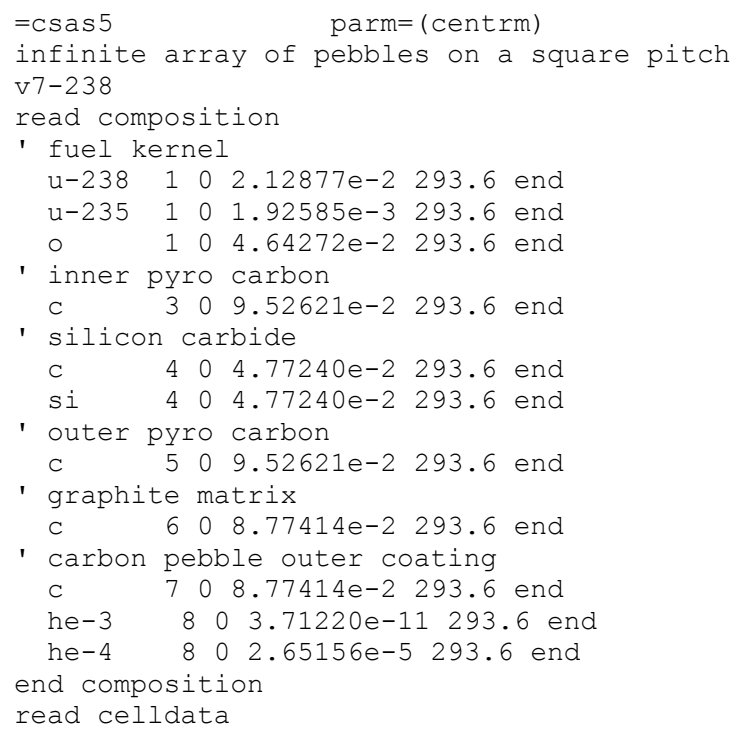




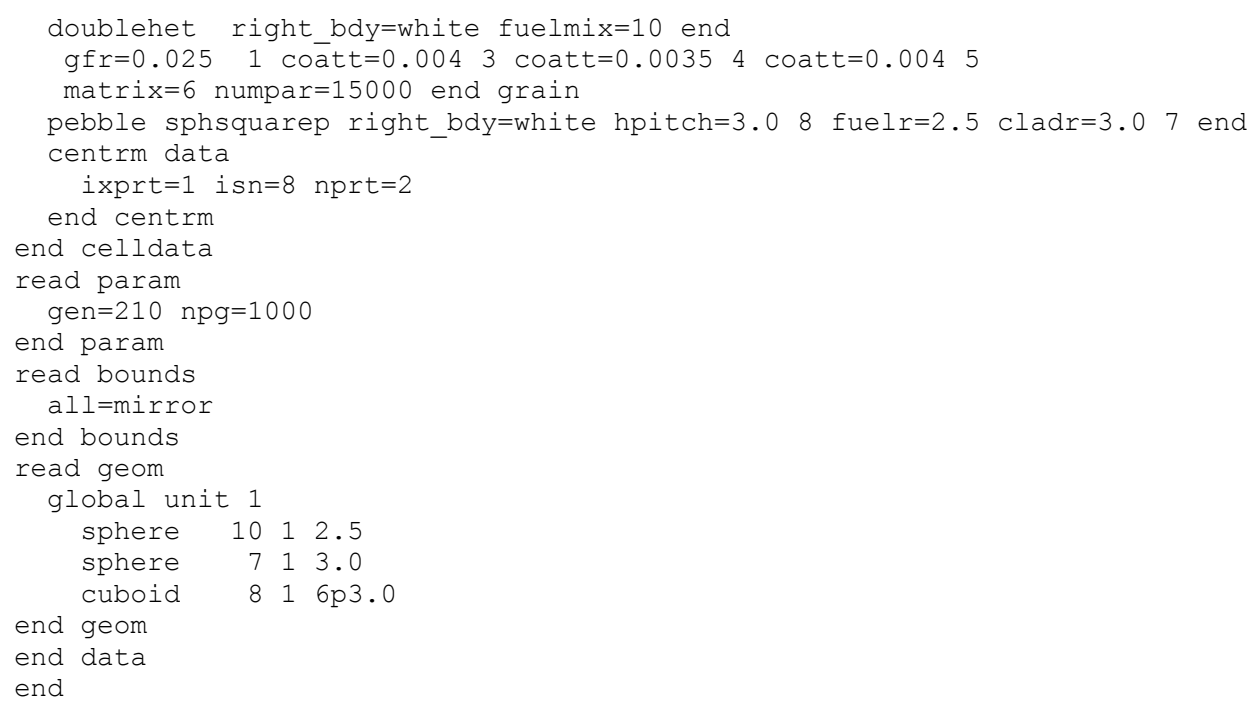

\subsubsection{Warning and error messages}

CSAS5 contains two types of warning and error messages. Warning messages appear when a possible error is encountered. It is the responsibility of the user to verify whether the data are correct when a warning message is encountered. The functional modules activated by CSAS5 sequences will be executed if no error messages are generated and a warning message has been generated.

When an error is recognized, an error message is written and an error flag is set so the functional modules will not be activated. The code stops immediately if the error is too severe to allow continuation of input. However, it will continue to read and check the data if it is able. When the data reading is completed, execution is terminated if an error flag was set when the data were being processed. If the error flag has not been set, execution continues. When error messages are present in the output, the user should focus on the first error message, because subsequent messages may have been caused by the error that generated the first message.

The following messages originate in the part of CSAS5 that reads, checks, and prepares data for KENO V.a and the search module MODIFY.

\section{CS-10 $* * *$ ERROR $* * *$ CONCENTRATION SEARCH MATERIAL WAS NOT SPECIFIED IN THE STANDARD COMPOSITION.}

This self-explanatory message from subroutine $\mathrm{CNCN}$ indicates that the indicated material is not in the standard composition data. Recheck the standard composition data and the search data, correct the input, and resubmit the problem.

\section{CS-11 *** ERROR *** CONCENTRATION SEARCH DATA HAS BEEN DESTROYED. $\mathrm{I}=\quad \mathrm{ICMND}=\quad \mathrm{IPNUM}=\quad \mathrm{MIXUR}=\quad \mathrm{SCNAME}=$}

This message from subroutine CNCSRH indicates that the search data could not be read properly. This usually indicates either: search data that is required for the specified search type is missing or search data 
inappropriate for the specified search type is present. Recheck the search data, correct the input, and resubmit the problem.

\section{CS-16 ***WARNING*** READ FLAG NOT FOUND. ASSUME KENO V PARAMETER DATA FOLLOWS.}

This message from subroutine CPARAM indicates that the word READ is not the first word of KENO V.a data following the Material Information Processor input data. If parameter data is to be entered, the code expects the words READ PARAMETERS to precede the parameter input data. If the word READ is not the first word, the code assumes the data are parameter input data.

CS-21 A UNIT NUMBER WAS ENTERED FOR THE CROSS-SECTION LIBRARY. (LIB= IN PARAMETER DATA.) THE DEFAULT VALUE SHOULD BE USED IN ORDER TO UTILIZE THE CROSS SECTIONS GENERATED BY CSAS5. MAKE CERTAIN THE CORRECT CROSS-SECTION LIBRARY IS BEING USED.

This message is from subroutine CPARAM. It indicates that a value has been entered for the crosssection library in the KENO V.a parameter data. The cross-section library created by the analytical sequence should be used. MAKE CERTAIN THAT THE CORRECT CROSS SECTIONS ARE BEING USED.

CS-50 $* * *$ ERROR $* * *$ SEARCH COMMAND NUMBER IS UNABLE TO PERFORM A PITCH SEARCH BECAUSE THE DIMENSIONS OF $\overline{\text { REGION }}$ NOT EXPLICITLY DEFINED. OF UNIT ARE

This message from subroutine PCHSRH indicates that the specified search command is not valid for the specified region. An ARRAY or CORE region cannot be altered; nor can a REPLICATE or REFLECTOR region immediately following an ARRAY or CORE region.

\section{CS-55 *** ERRORS WERE ENCOUNTERED IN PROCESSING THE CSAS-KENO5 DATA. EXECUTION IS IMPOSSIBLE. ***}

This message from subroutine SASSY is printed if errors were found in the KENO V.a input data for CSAS5. If a search is being made, data reading will continue until all the data have been entered or a fatal error terminates the data reading. When the data reading and checking have been completed, the problem will terminate without executing. Check the printout to locate the errors responsible for this message.

CS-62 *** ERROR *** MIXTURE IN THE GEOMETRY WAS NOT CREATED IN THE STANDARD COMPOSITIONS SPECIFICATION DATA.

This message from subroutine MIXCHK indicates that a mixture specified in the KENO V.a geometry was not created in the standard composition data. 
CS-68 *** ERROR *** AN INPUT DATA ERROR HAS BEEN ENCOUNTERED IN THE DATA ENTERED FOR THIS PROBLEM.

This message from the main program, CSAS5, is printed if the subroutine library routine LRDERR returns a value of "TRUE," indicating that a reading error has been encountered in the "KENO PARAMETER" data or the CSAS5 "SEARCH" data. The appropriate data type is printed in the message. Locate the unnumbered message stating “***** ERROR IN INPUT. CARD IMAGE PRINTED ON NEXT LINE *****." Correct the data and resubmit the problem.

CS-69 ***ERROR*** MIXTURE

IS AN INAPPROPRIATE MIXTURE NUMBER FOR USE IN THE KENO GEOMETRY DATA BECAUSE IT IS A COMPONENT OF THE CELLWEIGHTED MIXTURE CREATED BY XSDRNPM.

This message from subroutine CMXCHK indicates that a mixture that is a component of a cell-weighted mixture has been used in the KENO V.a geometry data.

CS-70 ***** ERROR ***** SEARCH OR OPTIMIZATION DATA MUST BE ENTERED FOR CSAS5. NO SEARCH DATA WAS ENTERED.

This message from subroutine RDOPT is self-explanatory. If the user does not desire to run a search, another sequence such as CSAS5 should be chosen.

CS-71 ***ERROR $* * *$ IS NOT A VALID SEARCH TYPE.

This message is from subroutine RDOPT. The allowed search types include PITCH, DIMENSION, and CONCENTRATION. The first four characters of the search data after the words READ SEARCH must be PIT, PITC, DIM, DIME, DMSN, CON, or CONC. The data may be misspelled or out of order.

\section{CS-72 $* * *$ ERROR $* * *$ THE SEARCH TYPE IS INVALID. I=}

This message is from subroutine RDOPT. The numerical index, I, should be 1 for an optimum pitch search, 2 for a dimension search, and 3 for a concentration search. If it is none of these, the search type has been incorrectly specified or a code error has been introduced.

\section{CS-73 ***** AN END OF FILE WAS ENCOUNTERED BEFORE ALL THE SEARCH DATA WAS READ.}

This self-explanatory message is from subroutine RDOPT. Check the input data to be sure nothing was omitted or misspelled. 
CS-74 ***** AN END SEARCH FLAG WAS READ BEFORE ALL THE SEARCH DATA WAS READ.

This self-explanatory message is from subroutine RDOPT. Check the input data for omissions and correct order.

CS-75 *** ERROR *** READ SEARCH FLAG WAS NOT FOUND. INSTEAD.

This self-explanatory message is from subroutine RDOPT. READ SEARCH was expected but was not found. Check the input data for omissions and correct order.

CS-76 *** ERROR *** END SEARCH FLAG WAS NOT FOUND. WAS READ INSTEAD.

This self-explanatory message from subroutine RDOPT indicates that an end of file was encountered when looking for READ SEARCH. Check the input data for omissions, correct order, and spelling.

\section{CS-77 *** ERROR *** AN END OF FILE WAS FOUND WHEN THE READ SEARCH FLAG WAS EXPECTED.}

This self-explanatory message is from subroutine RDOPT. Check the input data for omissions, correct order, and spelling.

CS-78 *** ERROR ***__ IS NOT A VALID SEARCH TYPE.

This message is from subroutines SRCHTYP. It indicates that an invalid search type was read. The valid search names include PITCH, CONCENTRATION, and DIMENSION. Either the data was entered improperly or a code error has been introduced. A STOP 215 is executed when this message is printed.

CS-80 *** ERROR *** SEARCH DATA HAS BEEN DESTROYED. I= $\mathrm{ICMND}=$ IPNUM= $\mathrm{II}=$ IGEOM=

This message from subroutines DIMSRH indicates that the search data cannot be interpreted. This usually indicates either: search data that is required for the specified search type is missing or search data inappropriate for the specified search type is present. Recheck the search data, correct the input, and resubmit the problem.

\section{CS-82 *** AN ERROR WAS ENCOUNTERED IN ONE OF THE FUNCTIONAL MODULES.}

This message from CSAS5 or MODIFY indicates that an error was encountered during execution of one of the functional modules such as CRAWDAD, BONAMI, CENTRM, PMC, XSDRNPM, or KENO V.a. Check the printout to locate and correct the error. 
This message comes from the MODIFY search package, and indicates that it is unable to find a solution to the problem as presented. This usually occurs when a solution is not within the range specified. For this case the user must decide what to change to improve the possibility of a solution. Fairly rarely, for an optimum problem, there may be 2 maximums within the range specified, and the package has found the wrong one. For this case, the user would tighten the range to be searched to eliminate the unwanted peak. The package uses least mean square fitted cubic polynomials to make guesses as to where a solution is. If the Keffectives have too much variance, the polynomials may not be a good representation of the actual behavior of the system. For this case the user could rerun the problem using more histories per pass to reduce the variance.

\section{CS-85 *** ERROR $* * *$ ALL OF THE ROOTS FOR K=__ LIE OUTSIDE THE PARAMETER CONSTRAINTS}

This message from MODIFY indicates that the polynomial fit to the Keffectives already calculated only has solutions for the Keffective asked for outside the parameter constraints specified. Keffectives with large variances can lead to polynomials which fit the actual behavior of a system poorly. The user could rerun the case with more histories to reduce the variance, if this is the problem. If the variance is not the problem, then extending the parameter range, or making some other change to the problem definition to change the Keffective range will be necessary.

\section{CS-89 *** ERROR ***__ IS AN INVALID DIMENSION SEARCH COMMAND.}

This message from subroutine DMSN indicates that the dimension search data are out of order, a search command is spelled incorrectly, or the search data are specified incorrectly.

CS-90 *** ERROR ***__ IS AN INVALID SEARCH PARAMETER.

This message printed from subroutines CNCTYP, DIMTYP, and PCHTYP indicates that a parameter entered in the search type specification data is not valid. The data could be misspelled or out of order. Omission of the keyword MORE before entering the individual search commands can cause this error.

CS-91 *** ERROR *** IS AN INVALID CONCENTRATION SEARCH COMMAND.

This message from subroutine $\mathrm{CNCN}$ is caused by a misspelled or illegal search command when attempting to do a concentration search.

\section{CS-94 *** ERROR *** REG $=$ IS AN INVALID SEARCH DATA ENTRY. THE REGION NUMBER MUST BE GREATER THAN ZERO AND NO LARGER THAN THE NUMBER OF REGIONS IN THE UNIT.}

This message from subroutine DMSN indicates that the region to be altered is incorrectly specified. For example, if the unit being altered contains five geometry regions, the value specified for $\mathrm{REG}=\mathrm{can}$ be as small as 1 and as large as 5 . 
CS-95 *** ERROR *** AN ERROR WAS ENCOUNTERED IN THE SEARCH DATA. THE LAST REGION NUMBER MUST BE AT LEAST AS LARGE AS THE FIRST REGION NUMBER. CHECK THE SEARCH DATA PRINTED BELOW. keyword UNIT REGIONS TO PARAMETER $=$ SEARCH CONSTANTS ARE

This message from subroutine DMSN indicates that the search data specified an invalid region number for the final region to be altered. Check the printed data and correct as appropriate.

CS-96 $* * *$ ERROR $* * *$ AN ERROR WAS ENCOUNTERED IN THE SEARCH DATA. THE REGION NUMBERS MUST BE GREATER THAN ZERO AND NO LARGER THAN THE NUMBER OF REGIONS IN THE UNIT. CHECK THE SEARCH DATA PRINTED BELOW. keyword UNIT REGIONS TO PARAMETER $=$ SEARCH CONSTANTS ARE

This message from subroutine DMSN indicates that one of the specified region numbers is incorrect. Check the printed data and correct as appropriate.

CS-97 *** ERROR *** NO VALID SEARCH COMMANDS WERE FOUND IN THE DATA.

This message is accompanied by a STOP 235 and is printed from subroutines PCHSRH, CNCSRH, and DIMSRH. A common cause of this error is the omission of the MORE command before the individual search commands are entered.

CS-99 *** ERROR *** THIS PROBLEM WILL NOT BE RUN BECAUSE PARM=CHECK WAS ENTERED IN THE ANALYTICAL SEQUENCE SPECIFICATION.

This message from subroutine CSAS5 indicates that the problem data were read and checked and no errors were found. To execute the problem, remove the PARM=CHECK or PARM=CHK from the analytical sequence indicator data entry.

CS-100*** ERROR *** THIS PROBLEM WILL NOT BE RUN BECAUSE ERRORS WERE ENCOUNTERED IN THE INPUT DATA.

This message from subroutine CSAS5 is self-explanatory. Examine the printout to locate the error or errors in the input data. Correct them and resubmit the problem. 
CS-101*** ERROR *** THE CONSTRAINTS ARE NOT VALID FOR PARAMETER SET. $-\mathrm{CON}=$ $+\mathrm{CON}=$

This message is printed from either subroutine CNCN or DMSN if the parameter set is invalid. For a parameter set to be valid, $-\mathrm{CON}$ must be less than 0.0 and $+\mathrm{CON}$ must be greater than 0.0 . They can be explicitly set or calculated using default or provided data.

\section{CS-102*** ERROR *** THE UNIT CELL SPECIFICATION FOR UNIT IS OUT OF BOUNDS. CELL NUMBER WAS SPECIFIED. THE SPECIFIED CELL MUST BE BETWEEN 1 AND THE NUMBER OF CELLS}

This self-explanatory message from subroutine DMSN indicates that the unit cell specified is not between 1 and the number of unit cells present in the problem. Recheck the unit cell number specified in the search data, correct the input, and resubmit the problem.

\section{CS-103 $* * *$ ERROR $* * *$ THE UNIT CELL SPECIFICATION FOR MIXTURE IS OUT OF BOUNDS. CELL NUMBER WAS SPECIFIED. THE SPECIFIED CELL MUST BE BETWEEN 1 AND THE NUMBER OF UNIT CELLS}

This self-explanatory message from subroutine $\mathrm{CNCN}$ indicates that the unit cell specified for the indicated mixture is not between 1 and the number of unit cells present in the problem. Recheck the unit cell number specified in the search data, correct the input, and resubmit the problem.

\subsubsection{References}

1. M. J. Lorek, Improved Criticality Search Techniques for Low- and High-Enriched Systems, NUREG/CR-2122 (ORNL/NUREG/CSD/TM-13), Union Carbide Corporation (Nuclear Division), Oak Ridge National Laboratory, 1981.

2. J. T. Thomas, "Critical Three-Dimensional Arrays of U(93.2)-Metal Cylinders," Nucl. Sci. Eng. 52, 350 (November 1973).

3. J. T. Thomas, Critical Three-Dimensional Arrays of Neutron-Interacting Units, Part II, ORNL/TM-868, Union Carbide Corporation (Nuclear Division), Oak Ridge National Laboratory (July 1964). 


\subsection{A Additional Example Applications of CSAS5}

Several example uses of CSAS5 are shown in this section for a variety of applications. Note that many of these examples have been provided since the earliest versions of the CSAS sequences and output data shown may not represent the most current version.

\subsection{A.1 Typical Search}

EXAMPLE 1. CSAS5S - Determine the optimum pitch for an array of Example 4 fuel assemblies

Consider a $4 \times 4 \times 1$ array of fuel assemblies in a square aluminum cask. Each assembly consists of a $17 \times 17 \times 1$ array of zirconium-clad, $2.35 \%$-enriched $\mathrm{UO}_{2}$ fuel pins in a square pitched array. The $\mathrm{UO}_{2}$ has a density of $9.21 \mathrm{~g} / \mathrm{cc}$. The pin diameter is $0.8 \mathrm{~cm}$ and is $366 \mathrm{~cm}$ long. The clad is $0.07 \mathrm{~cm}$ thick, and the pitch is $1.3 \mathrm{~cm}$. Each fuel bundle is contained in a $0.65-\mathrm{cm}$-thick Boral sheath. The bundles are separated by an edge-to-edge spacing of $1 \mathrm{~cm}$. The array of bundles is centered in a 10-cm-thick aluminum cask whose inside dimensions are $0.5 \mathrm{~cm}$ beyond the outer edges of the fuel bundles. Search for the assembly spacing that yields the maximum value of $k_{\text {eff. }}$. Because the spacing between the assemblies is to be altered, the last region of unit 2 will be altered. In order to do this, the fuel assembly shroud cannot be defined as a replicate, because the code does not know the size of the array until later. In order to perform a pitch or dimension search, the code calculates the distance between the outermost region of the unit and the region interior to it. Therefore, the fuel assembly gap must be defined as a cuboid, and the water gap between assemblies can be entered as either a cuboid or a replicate. This search has been defined as an optimum dimension search. The input data for this problem follow.

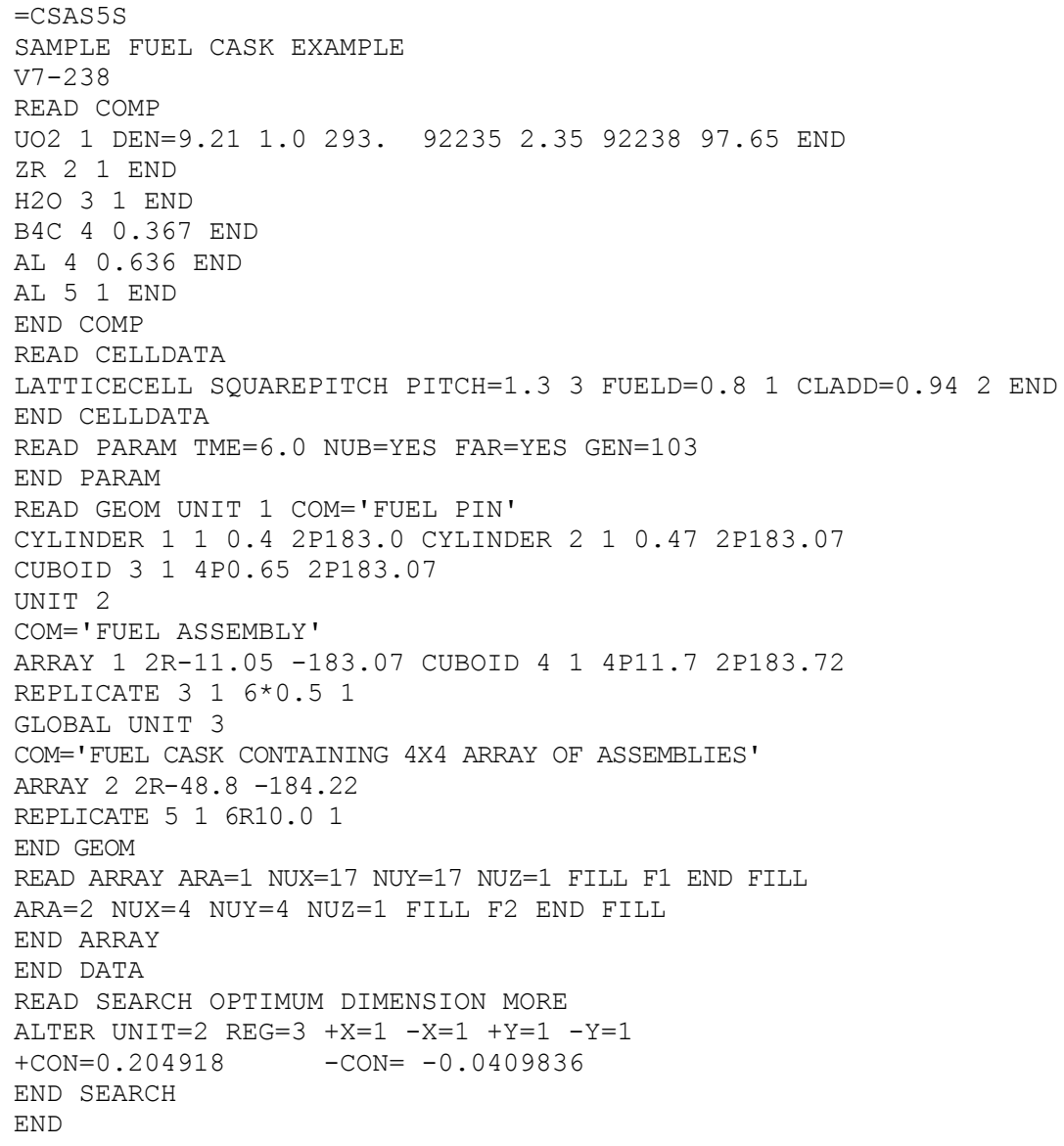


An alternative method of entering the search data for this problem is to define the search as an optimum pitch search and require that the spacing of the fuel pin cells remain unchanged. Because a pitch search is always conducted at the lowest array level, in this case the spacing between the pins in the fuel assembly (the outer region of unit 1), it is necessary to countermand the automatic alteration of the outer region of unit 1 by entering the KEEP command. Search constants must be entered for the X and Y faces to instruct the code to KEEP those dimensions unchanged. It is easier to use the keyword ALL, which applies the KEEP command to all of the faces since the optimum pitch search would have changed only the $\mathrm{X}$ and $\mathrm{Y}$ faces leaving the $\mathrm{Z}$ faces unchanged. The ALTER command must then be entered to instruct the search to alter the spacing between the fuel assemblies (region 3 of unit 2). Only the $\mathrm{X}$ and $\mathrm{Y}$ dimensions are to be altered; so the search constants are entered individually for those dimensions. It is acceptable to enter ALL=1 $+\mathrm{Z}=0-\mathrm{Z}=0$ rather than $+\mathrm{X}=1-\mathrm{X}=1+\mathrm{Y}=1-\mathrm{Y}=1$. It is not necessary to enter the constraints for an optimum pitch search $(+\mathrm{CON}=$ and $-\mathrm{CON}=)$. They were entered in this case to ensure that the alternative data more nearly duplicate the optimum dimension search data from the previous example. These alternative search data are:

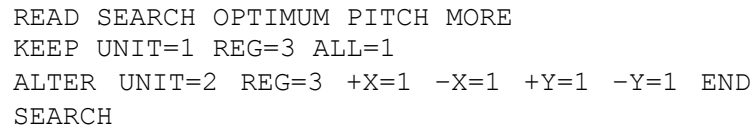

\subsection{A.2 Auxiliary search commands}

Auxiliary search commands are entered ONLY if the word MORE was entered in the search type specification data. These data are used to define the method the search will use to alter the pitch, geometry or concentration data and to set the constraints for the parameter search. The auxiliary search commands consist of (1) INDIVIDUAL SEARCH COMMANDS and (2) SEARCH PARAMETER CONSTRAINTS.

\section{EXAMPLE 1}

Consider an example in which region 2 of unit 1 is a cuboid and all of the dimensions of the cuboid are to be altered. The search data could be entered as:

ALTER UNIT=1 REG=2 ALL=1.0

or

ALTER UNIT=1 REG=2 +X=1.0 - X=1.0 +Y=1.0 - Y=1.0 +Z=1.0 - Z=1.0

Because all of the search constants are nonzero, all of the dimensions will be changed. Because the search constants are identical, the original relationship between the dimensions will be preserved as they are altered.

For example, if region 2 of unit 1 is a cylinder and if all the dimensions of the cylinder are to be altered, and if unit 1 is tied to unit cell 1 the search data could be entered as:

ALTER UNIT=1 REG=2 ALL=1.0 CELL=1

or

ALTER UNIT=1 REG=2 RADIUS=1.0 +H=1.0 ?H=1.0 CELL=1

Because all of the search constants are nonzero, all of the dimensions will be changed. Because the search constants are identical, the original relationship between the dimensions will be preserved as they 
are altered. If the original height-to-diameter ratio is 1.5 , that ratio will be preserved throughout the search only if the search constants for the radius and + and - height are identical.

Search constants can be entered sequentially with each new entry overriding only identical previous entries. For example, if region 2 of unit 1 is a cuboid and if all of the dimensions except the $-\mathrm{Z}$ dimension are to be altered, the search data could be entered as:

ALter UNIT=1 REG=2 $+\mathrm{X}=1.0 \quad-\mathrm{X}=1.0 \quad+\mathrm{Y}=1.0 \quad-\mathrm{Y}=1.0 \quad+\mathrm{Z}=1.0$

or

ALTER UNIT=1 REG=2 ALL=1.0 $-\mathrm{Z}=0.0$

In the second example, all of the search constants corresponding to the cuboid's dimensions are set to 1.0 by using the $A L L=$ command. This includes the $-Z$ dimension. To reset the search constant for the $-\mathrm{Z}$ dimension to zero, $-\mathrm{Z}=0.0$ is added following the $\mathrm{ALL}=1.0$ command. If the search commands are reversed, $-\mathrm{Z}=0.0 \mathrm{ALL}=1.0$, the $-\mathrm{Z}$ dimension will also be altered because the $-\mathrm{Z}$ portion of the $\mathrm{ALL}=$ command will override the previously entered $-Z=0.0$ command.

\section{EXAMPLE 2}

A search command must be entered for each unit and region specification. Consider a problem having units 1, 2 , and 3. Unit 1 consists of three concentric spheres in a cuboid. Unit 2 consists of a single sphere in a cuboid, and unit 3 contains three concentric cuboids. A search is to be made that changes the inner sphere and outer cuboid of unit 1 , and the sphere and cuboid of unit 2, and the exterior cuboid of unit 3 . The thicknesses of the outer spheres of unit 1 are to be maintained, and the two inner cuboids of unit 3 are to remain unchanged. The search data for this problem can be entered as follows:

ALTER UNIT=1 REG=1 ALL=1.0

ALTER UNIT=1 REG=4 ALL=1.0 MAINTAIN UNIT=1

REG=2 TO 3 ALL=1.0 ALTER UNIT=2 REG=1 TO 2

ALL=1.0 ALTER UNIT=3 REG=3 ALL=1.0

\subsection{A.3 Search constraints for dimension or pitch searches}

SEARCH PARAMETER CONSTRAINTS set the parameter limits for the search. The minimum constraint is the minimum value of the parameter allowed in the search. The maximum constraint is the maximum value of the parameter allowed in the search. The initial geometry configuration corresponds to a parameter value of 0.0 . A physical limit occurs when the value of the parameter causes geometry intersections. Constraints should be entered for a DIMENSION search. Only one set of constraints (i.e., min and max) are allowed per problem. These constraints apply to all the dimensions that are being altered.

For a DIMENSION search, the constraints are given by Eqs. (2.1.1) and (2.1.2):

$$
\begin{gathered}
\mathrm{C}_{\text {min }}=\left(\left(\mathrm{D}_{\min } / \mathrm{D}_{\mathrm{i}}\right)-1.0\right) / \mathrm{SC}, \\
\mathrm{C}_{\max }=\left(\left(\mathrm{D}_{\max } / \mathrm{D}_{\mathrm{i}}\right)-1.0\right) / \mathrm{SC},
\end{gathered}
$$

where

$$
\begin{aligned}
& \mathrm{C}_{\min } \text { is the minimum constraint for the search } \\
& \mathrm{C}_{\max } \text { is the maximum constraint for the search }
\end{aligned}
$$


$\mathrm{D}_{\text {min }}$ is the minimum allowed dimension for the search [For a chord, $\mathrm{D}_{\min }=$ (Radius $_{\min }+$ Chord $\left._{\min }\right) / 2$ Radius ] min

$\mathrm{D}_{\max }$ is the maximum allowed dimension for the search [For a chord, $\mathrm{D}_{\max }=$ (Radius $_{\max }+$ Chord $\left._{\max }\right) / 2$ Radius $\left._{\max }\right]$

$\mathrm{D}_{\mathrm{i}} \quad$ is the initial dimension [For a chord, $\mathrm{D}_{\mathrm{i}}=\left(\right.$ Radius $_{\text {initial }}+$ Chord $\left._{\text {initial }}\right) / 2$ Radius $\left._{\text {initial }}\right]$

SC is the search constant for that dimension (i.e., $+\mathrm{X},-\mathrm{X}$, RADIUS, etc.)

For example, the initial radius of a sphere is $6 \mathrm{~cm}$, and a search is to be conducted to determine the radius at which the sphere is critical. The minimum radius the user wishes to allow is $3 \mathrm{~cm}$, and the maximum radius to be allowed is $9 \mathrm{~cm}$. A nonzero search constant must be entered to cause the radius to be changed. A search constant of 1.0, will be used for the radius (RADIUS=1.0). The constraints calculated from Eqs. (2.1.1) and (2.1.2) are:

$$
\begin{aligned}
& \mathrm{C}_{\min }=((3.0 / 6.0)-1.0) / 1.0=-0.5 \\
& \mathrm{C}_{\max }=((9.0 / 6.0)-1.0) / 1.0=0.5
\end{aligned}
$$

The constraints would be entered in the problem by entering the following data:

$$
-\mathrm{CON}=-0.5+\mathrm{CON}=0.5
$$

For a PITCH search, the minimum constraint defines the limit for shrinking the system, and the maximum constraint defines the limit for expanding the system:

$$
\begin{gathered}
\mathrm{C}_{\min }-\underset{\mathrm{i}=\text { number of faces }}{\mathrm{MAX}\left[\mathrm{D}_{+}, \mathrm{D}_{-}\right] \text {for shrinking, }} \\
\left.\mathrm{C}_{\max }-\underset{\substack{\mathrm{i}=\text { number of faces } \\
\mathrm{i}=1}}{\mathrm{MIN}} \mathrm{D}_{+}, \mathrm{D}_{-}\right] \text {for expanding, }
\end{gathered}
$$

where
$\mathrm{C}_{\min }$ is the minimum constraint,
$\mathrm{C}_{\max }$ is the maximum constraint,
$\mathrm{D}_{+} \quad$ are face constraints for the positive dimensions,
D_ are face constraints for the negative dimensions. 
Face constraints must be calculated for each face using Eqs. (2.1.3) and (2.1.4):

$$
\begin{gathered}
\mathrm{D}_{+}=\frac{\left(\mathrm{X}_{\mathrm{d}+}-\mathrm{X}_{\mathrm{i}+}\right)\left(\mathrm{SC}_{+} \times \mathrm{del}_{+} \mathrm{SC}_{-} \times \mathrm{del}_{-}\right)}{\left(\mathrm{X}_{\mathrm{i}+}-\mathrm{X}_{\mathrm{i}-}\right)\left(\mathrm{SC}_{+} * * 2 \times \mathrm{del}_{+}\right)}, \\
\mathrm{D}_{-}=\frac{\left(\mathrm{X}_{\mathrm{d}-}-\mathrm{X}_{\mathrm{i}-}\right)\left(\mathrm{SC}_{+} \times \mathrm{del}_{+} \mathrm{SC}_{-} \times \mathrm{del}_{-}\right)}{\left(\mathrm{X}_{\mathrm{i}+}-\mathrm{X}_{\mathrm{i}-}\right)\left(\mathrm{SC}_{-} * 2 \times \mathrm{del}_{-}\right)},
\end{gathered}
$$

where

$\mathrm{X}_{\mathrm{d}+}$ is the desired limit of the positive dimension of the spacing cuboid in that direction (positive dimension of $\mathrm{X}, \mathrm{Y}$, or $\mathrm{Z}$, whichever dimension is under consideration)

$\mathrm{X}_{\mathrm{i}+} \quad$ is the initial positive dimension of the spacing cuboid in that direction (positive dimension of $\mathrm{X}, \mathrm{Y}$, or $\mathrm{Z}$, whichever dimension is under consideration)

$\mathrm{X}_{\mathrm{d}-}$ is the desired limit of the negative dimension of the spacing cuboid in that direction (negative dimension of $\mathrm{X}, \mathrm{Y}$, or $\mathrm{Z}$, whichever dimension is under consideration)

$\mathrm{X}_{\mathrm{i}^{-}}$is the initial negative dimension of the spacing cuboid in that direction (negative dimension of $\mathrm{X}, \mathrm{Y}$, or $\mathrm{Z}$, whichever dimension is under consideration)

$\mathrm{SC}_{+}$is the search constant for the positive dimension of the spacing cuboid in that direction (positive dimension of $\mathrm{X}, \mathrm{Y}$, or $\mathrm{Z}$, whichever dimension is under consideration)

$\mathrm{SC}_{-}$is the search constant for the negative dimension of the spacing cuboid in that direction (negative dimension of $\mathrm{X}, \mathrm{Y}$, or $\mathrm{Z}$, whichever dimension is under consideration)

NOTE: Using a search constant of 1.0 simplifies the determination of $\mathrm{C}_{\max }$ and $\mathrm{C}_{\min }$ when the dimensions are to change proportionately.

$\mathrm{del}_{+} \quad$ is the initial distance from the spacing cuboid to the closest interior region in the positive direction (positive dimension of $\mathrm{X}, \mathrm{Y}$, or $\mathrm{Z}$, whichever dimension is under consideration)

del ${ }_{-} \quad$ is the initial distance from the spacing cuboid to the closest interior region in the negative direction (negative dimension of $\mathrm{X}, \mathrm{Y}$, or $\mathrm{Z}$, whichever dimension is under consideration)

The search parameter constraints are entered using the following keywords:

$-\mathrm{CON}=\mathrm{pp} \quad$ is used to set the minimum constraint for the current parameter. The value of $\mathrm{pp}$ is defaulted to $-10 \mathrm{E} 10$ for a dimension search. The value of $\mathrm{pp}$ is defaulted to a value that allows geometry regions to touch for a pitch search unless a value was entered for MINPITCH, in which case the parameter corresponding to that pitch is calculated and used.

$+\mathrm{CON}=\mathrm{rr} \quad$ is used to set the maximum constraint for the current parameter. The value of $\mathrm{rr}$ is defaulted to $+10 \mathrm{E} 10$ for a dimension search and to $-5 \times \mathrm{pp}$ for a pitch search. 
NOTE: A search will reset a constraint (entered using the keyword $+\mathrm{CON}=$ or $-\mathrm{CON}=$ ) that falls outside the default range to the default value. If a PITCH search is specified and if a value has been entered for MAXPITCH and/or MINPITCH, values should not be entered for the constraints. If values are entered for $+\mathrm{CON}=$ and/or $-\mathrm{CON}=$ for a PITCH search and if MAXPITCH and/or MINPITCH were specified in the optional search parameters, the maximum and minimum constraints will be set to the values corresponding to MAXPITCH and MINPITCH, even though the value of MINPITCH may result in an intersection.

\subsection{A.3.1.1 Individual concentration search commands}

An INDIVIDUAL SEARCH COMMAND for a concentration search consists of (1) a command definition, (2) the mixture number of the mixture to be altered, (3) the name of the standard composition to be altered, (4) a search constant, and (5) a unit cell containing the mixture. A series of individual search commands can be entered to govern the search process. A new search command is initiated whenever a command definition, item (1) above, is encountered.

The COMMAND DEFINITION defines the action to be taken for the specified mixture and standard composition component. ALTER, CHANGE, or MODIFY are used to cause the concentration (number densities) of the specified standard composition in the specified mixture to be modified.

$\begin{array}{ll}\text { ALTER } & \text { The commands to the left are command definitions, item (1) above. These commands } \\ \text { CHANGE } & \text { instruct the code to modify the specified concentration data. } \\ \text { MODIFY } & \end{array}$

The MIXTURE NUMBER, item (2) above, defines the mixture that contains the standard composition whose concentration is to be varied during the search. The keyword MIX= is entered, followed by the mixture number, nn.

$\mathbf{M I X}=\mathrm{nn} \quad$ is used to define the mixture number associated with the component that is to be changed. The keyword $\mathrm{MIX}=$ is entered, followed by the mixture number, nn. There is no default value of nn.

The STANDARD COMPOSITION NAME, item (3) above, defines the standard composition whose concentration will be changed in the defined mixture. Only standard compositions listed in the Standard Composition Library chapter can be entered.

SCNAME $=\mathrm{mm}$ is used to specify the standard composition name of the component that is to be altered. The keyword SCNAME= is entered, followed by the mixture number, mm. There is no default value for $\mathrm{mm}$.

NOTE: If the standard composition component name specified in the Material Information Data (item 1 of the Standard Composition Specification Data) is a solution, for example, SOLNUO2(NO3)2, and the Concentration Search Data specifies SCNAME=UO2(NO3)2, the amount of UO2(NO3)2 in the solution will be altered, but the amount of water and nitric acid will not be changed. Thus, the resultant mixture may no longer meet the criteria associated with the SOLN specification. 
The SEARCH CONSTANT for a concentration search, item (4) above, is a proportional factor that applies to the standard composition being altered. The keyword FACTOR= followed by a proportionality search constant is used to specify the search constant for a concentration search.

FACTOR $=$ pc is used to specify the search constant. The keyword FACTOR= is entered, followed by the value of the search constant or proportionality factor, $\mathrm{pc}$.

The UNIT CELL NUMBER, item (5) above, defines the unit cell containing the mixture to be varied during the search. The keyword CELL $=$ is entered, followed by the unit cell number, $\mathrm{mm}$.

$\mathbf{C E L L}=\mathrm{mm} \quad$ is used to link mixture $\mathrm{nn}$ to unit cell $\mathrm{mm}$. The keyword CELL $=$ is entered, followed by the unit cell number, $\mathrm{mm}$. The code defaults the cell number to the unit cell containing the mixture specified using $\mathrm{MIX}=$.

\section{ENTERING AUXILIARY SEARCH COMMANDS IN THE CONCENTRATION SEARCH DATA}

\section{EXAMPLE 1}

Consider an example in which the density of water is to be varied and is contained in unit cell 2. Full density water, mixture 1, was specified in the standard composition specification data as: $\mathrm{H} 2 \mathrm{O} 1$ END The auxiliary search data could be entered as follows:

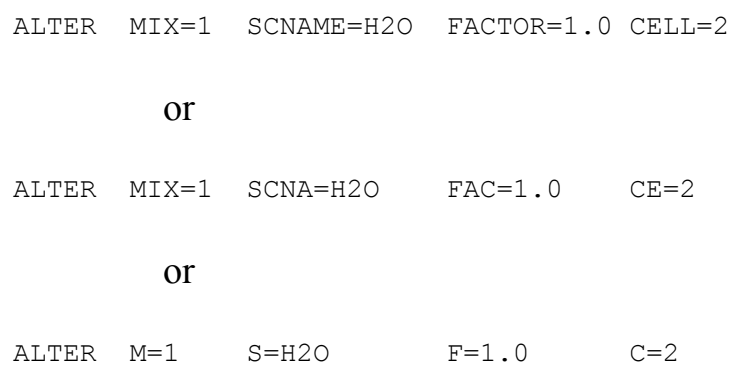

Note that terse input allows truncation of the keywords.

\section{EXAMPLE 2}

Consider an example in which the density of $\mathrm{UO}_{2} \mathrm{~F}_{2}$, is to be varied in mixture 2 contained in unit cell 4, a uranyl fluoride solution. The uranyl fluoride solution was specified as:

$\begin{array}{lllllllllll}\text { SOLNUO2F2 } & 2 & 300 & 0 & 1 & 293 & 92235 & 5 & 92238 & 95 & \text { END }\end{array}$

The auxiliary search data could be entered as follows:

ALTER MIX=2 SCNAME=UO2F2 FACTOR=1 CELL=4

or

ALTER M=2 S=UO2F2 $\quad \mathrm{F}=1 \quad \mathrm{C}=4$


The terse input option allows truncation of the keywords.

\subsection{A.4 Search constraints for concentration searches}

A SEARCH PARAMETER CONSTRAINT for concentration searches sets the parameter limits for the search. The minimum constraint is the minimum value of the parameter allowed in the search. The maximum constraint is the maximum value of the parameter allowed in the search. The initial concentration corresponds to a parameter value of 0.0. A physical limit occurs when the value of the parameter causes the density of the specified standard composition to become negative. The search can produce an unrealistically high density. Users should manually eliminate those results or set constraints to avoid them. Only one set of constraints (i.e., min and max) are allowed per problem. These constraints apply to all the standard compositions that are being altered.

For a CONCENTRATION search, the constraints are given by Eqs. (2.1.5) and (2.1.6). The maximum constraint must be larger than the minimum constraint.

$$
\begin{aligned}
& \mathrm{C}_{\min }=\left(\left(\frac{\mathrm{D}_{\min }}{\mathrm{D}_{\mathrm{i}}}\right)-1\right) / \text { FACTOR, } \\
& \mathrm{C}_{\max }=\left(\left(\frac{\mathrm{D}_{\max }}{\mathrm{D}_{\mathrm{i}}}\right)-1\right) / \text { FACTOR, }
\end{aligned}
$$

where

$$
\begin{aligned}
& \mathrm{C}_{\min } \quad \text { is the minimum constraint for the search, } \\
& \mathrm{C}_{\max } \quad \text { is the maximum constraint for the search, } \\
& \mathrm{D}_{\min } \quad \text { is the minimum allowed density for the specified standard composition, } \\
& \mathrm{D}_{\max } \quad \text { is the maximum allowed density for the specified standard composition, } \\
& \mathrm{D}_{\mathrm{i}} \quad \text { is the initial density of the specified standard composition, }
\end{aligned}
$$

FACTOR is the search constant for the standard composition that is being varied. 
Default search constraints are calculated if $+\mathrm{CON}$ and $-\mathrm{CON}$ are not entered. The default concentration search constraints are calculated as follows:

$$
\begin{aligned}
& +\mathrm{CON}=\left\{\begin{array}{l}
\min (1 / \mathrm{FACTOR}), \text { if any FACTOR }<0 \\
-\mathrm{S} \times(-\mathrm{CON}), \text { if all FACTOR }>0
\end{array}\right\}, \\
& -\mathrm{CON}=\left\{\begin{array}{l}
-\mathrm{S}(+\mathrm{CON}), \text { if all FACTOR }<0 \\
\max (-1 / \mathrm{FACTOR}), \text { if any FACTOR }>0
\end{array}\right\} .
\end{aligned}
$$

\subsection{A.5 Search considerations}

DIMENSION, PITCH, and CONCENTRATION searches can be performed using CSAS5S. A DIMENSION search alters only those regions specified in the search data. A PITCH search alters the center-to-center spacing of units in an array and any other dimensions specified in the auxiliary data. A CONCENTRATION search alters the density of the standard compositions in the mixtures specified.

By default a pitch search is performed at the lowest array level and changes the spacing in either the $\mathrm{X}$ dimension for a slab, the $\mathrm{X}$ and $\mathrm{Y}$ dimensions for an array of cylinders, or the $\mathrm{X}, \mathrm{Y}$ and $\mathrm{Z}$ dimensions for an array of spheres. For example, if an array of fuel assemblies is described in the geometry, the lowest array level is the array of fuel pins comprising an assembly. Therefore, an optimum pitch search would alter the spacing between the fuel pins within the assembly. The spacing can be expanded until the array intersects the first region external to it. The exterior size of the fuel pin array would grow or shrink within the confines of the exterior region (fuel assembly shroud). If the external regions are described using replicate regions, the array can grow or shrink within the confines of the maximum and minimum constraints.

If replicate regions are used outside an altered region or an array whose spacing units are altered, the dimensions of the replicate regions are recalculated (maintaining the thickness) at each search pass without having to enter search data for those regions. However, geometry regions specified by a geometry shape (sphere, cuboid, cylinder, etc.) that exist outside an altered region or an array whose spacing units are being altered will remain unchanged unless search data are provided for them. In other words, REPLICATE regions will grow and shrink in response to changes in the dimensions of the interior region, but other geometry shapes will not.

Some of the limitations applicable to a pitch or dimension search are:

1. A pitch search is performed only at the lowest array level unless search commands are entered to keep the lowest array level unit unchanged and other commands are entered to cause other units to be altered.

2. A pitch search alters the spacing in either the $X$ dimension for a slab, the $X$ and $Y$ dimensions for an array of cylinders, or the $\mathrm{X} \mathrm{Y}$ and $\mathrm{Z}$ dimensions for an array of spheres. Entering a search constant of zero for $+\mathrm{X},-\mathrm{X},+\mathrm{Y},-\mathrm{Y},+\mathrm{Z}$, and/or $-\mathrm{Z}$ will keep the corresponding dimension from being altered.

3. A pitch search alters only the outer region of the unit(s) used in the array at the lowest array level unless the search data specifying otherwise is input.

4. A search cannot alter a region whose boundaries are set by the code (i.e., an ARRAY, CORE BOUNDARY, or REPLICATE following an ARRAY). If the dimensions of a replicate region are to be altered, the dimensions of the region interior to it must be explicitly defined. For example, the interior region can be a standard geometry shape (sphere, cylinder, cuboid, etc.), but cannot be a replicate following an array or core boundary. 
5. All searches allow auxiliary search data.

Concentration searches can also be performed using CSAS5S. A concentration search alters only those standard compositions specified in the search data. Care must be taken when searching on standard compositions beginning with SOLN, ATOM, WTPT, or ARBM (predecessor to ATOM and WTPT in earlier SCALE versions). One or more of their components can be altered but this will not directly affect the other components. For example, if the standard composition component name specified in the material information data is SOLN (SOLNUO2F2) and the concentration search data specifies $\mathrm{SCNAME}=\mathrm{UO} 2 \mathrm{~F} 2$, the amount of uranyl fluoride salt, $\mathrm{UO} 2 \mathrm{~F} 2$, in the solution will be altered, but the amount of water and hydrofluoric acid in the solution will remain unchanged. Therefore, the resultant mixture may no longer meet the criteria for a solution (SOLN) specification.

Physically, the concentration can vary from zero to some upper limit. The code will prevent the concentration from falling below zero, but the user is responsible for setting constraints that prevent the concentration from exceeding reasonable values. The theoretical density is a reasonable upper limit.

\subsection{A.6 Optimum pitch search}

An optimum pitch search searches for the pitch that yields the highest value of $k_{\text {eff }}$. An optimum pitch search is activated by entering "OPTIMUM PITCH" in the search data. By default, the search is performed at the lowest array level and only the spacing in the X-direction for slabs, the $\mathrm{X}$ and Y-directions for cylindrical arrays, and the $\mathrm{X}, \mathrm{Y}$, and Z-directions for spherical arrays. The search constants are defaulted to 1.0 for the applicable $+\mathrm{X},-\mathrm{X},+\mathrm{Y},-\mathrm{Y},+\mathrm{Z}$, and $-\mathrm{Z}$ dimensions of the outermost region. The dimensions of other geometry regions will not be changed (their search constants are defaulted to 0.0) unless additional search data containing appropriate instructions are supplied.

The limits for an optimum pitch search can be set using either the MAXPITCH= and MINPITCH= options in the optional search parameters or the $+\mathrm{CON}=$ and $-\mathrm{CON}=$ options in the search parameter constraints of the auxiliary search commands. MAXPITCH= and MINPITCH= are used to enter a value of the maximum allowed pitch and minimum allowed pitch respectively. $+\mathrm{CON}=$ and $-\mathrm{CON}=$ are used to enter values for the parameter constraints (i.e., the maximum and minimum allowed value of the search parameter). Typically, it is easier to set the maximum allowed pitch and minimum allowed pitch using $\mathrm{MAXPITCH}=$ and $\mathrm{MINPITCH}=$ than it is to calculate the value of the parameter corresponding to those pitches. The default minimum constraint corresponds to MINPITCH, the pitch at which the largest interior region of a unit used in the array is in contact with the spacing cuboid of that unit. The maximum constraint is defaulted to -5 times the minimum constraint. In the first search pass, the code calculates $k_{\text {eff }}$ for the initial problem geometry. The next two passes calculate the $k_{\text {eff }}$ for the dimensions corresponding to the minimum constraint and maximum constraint. Subsequent passes fit the previous results to a cubic equation to select new dimensions.

Consider a $10 \times 10 \times 10$ array of uranium spheres surrounded by water having a "square" pitch. The uranium spheres are $2 \mathrm{~cm}$ in radius, and the center-to-center spacing is $8 \mathrm{~cm}$. The uranium spheres and their associated spacing are defined to be unit 1, and the $10 \times 10 \times 10$ array is defined to be array 1 . Search data and results for some optimum pitch searches using this example are given in inputs 1 through 6 below.

\section{Default Search — Array of Centered Spheres}

This problem contains search data for a default optimum pitch search. The problem consists of a $10 \times 10 \times 10$ array of uranium spheres surrounded by low density water. The spheres are $90 \%{ }^{235} \mathrm{U}$ and $10 \%{ }^{238} \mathrm{U}$ and have a radius of $2.0 \mathrm{~cm}$. The spheres are originally centered on an $8.0 \mathrm{~cm} \mathrm{X,} \mathrm{Y,} \mathrm{and} \mathrm{Z} \mathrm{pitch}$ 
with interstitial low density $(0.01 \mathrm{gm} / \mathrm{cc})$ water. The problem searches for the pitch that will produce the maximum $k_{\text {eff }}$ for the system.

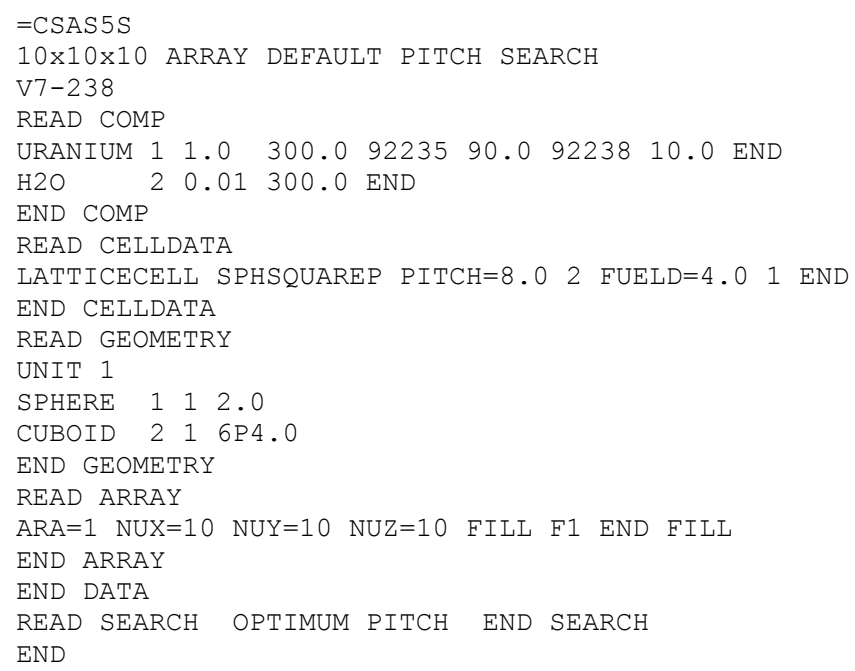

This data will cause the code to alter the $+\mathrm{X},-\mathrm{X},+\mathrm{Y},-\mathrm{Y},+\mathrm{Z}$, and $-\mathrm{Z}$ dimensions of the outer region of unit 1 and search for the dimensions that give the maximum $k_{\text {eff. }}$. Because the next to last outer dimension of the unit contained in the array are spheres, all six dimensions are altered. The original relationship between the dimensions is preserved (i.e., the original ratio of the $\mathrm{X}$ to $\mathrm{Y}$ to $\mathrm{Z}$ dimensions of the cuboid is preserved throughout the search). Because this is an optimum pitch search, the sphere dimensions will not be changed. For this particular example, results for six passes are given. A final search results for the problem follow:

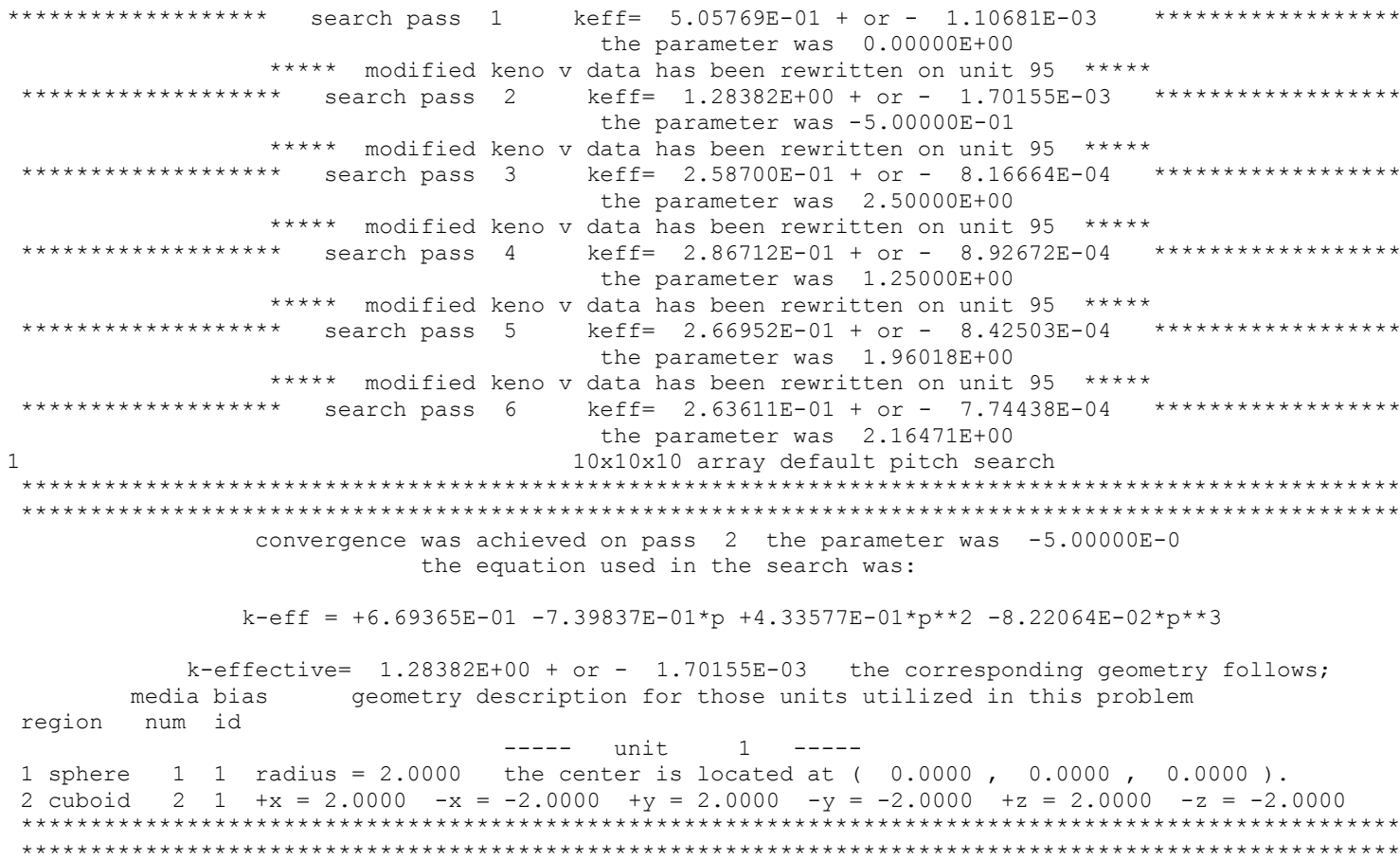




\section{Default Search - Array of Off-Centered Spheres}

This is the same problem as described above except the spheres are now off-centered in the $\mathrm{X}$ and Y dimensions. The input data and the final search results for this problem follow:

$=\operatorname{CSAS} 5 \mathrm{~S}$

10×10x10 ARRAY DEFAULT PITCH SEARCH- OFFSET

$\mathrm{V} 7-238$

READ COMP

URANIUM $11.0 \quad 300.0 \quad 9223590.092238 \quad 10.0$ END

$\begin{array}{lllll}\mathrm{H} 2 \mathrm{O} & 2 & 0.01 & 300.0 & \mathrm{END}\end{array}$

END COMP

READ CELLDATA

LATTICECELL SPHSQUAREP PITCH=8.0 2 FUELD=4.0 1 END

END CELLDATA

READ GEOMETRY

UNIT 1

SPHERE $\quad 1 \quad 1 \quad 2.0$

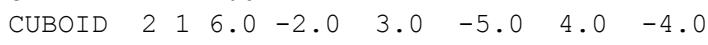

END GEOMETRY

READ ARRAY

ARA=1 NUX=10 NUY=10 NUZ=10 FILL F1 END FILL

END ARRAY

END DATA

READ SEARCH OPTIMUM PITCH END SEARCH

END

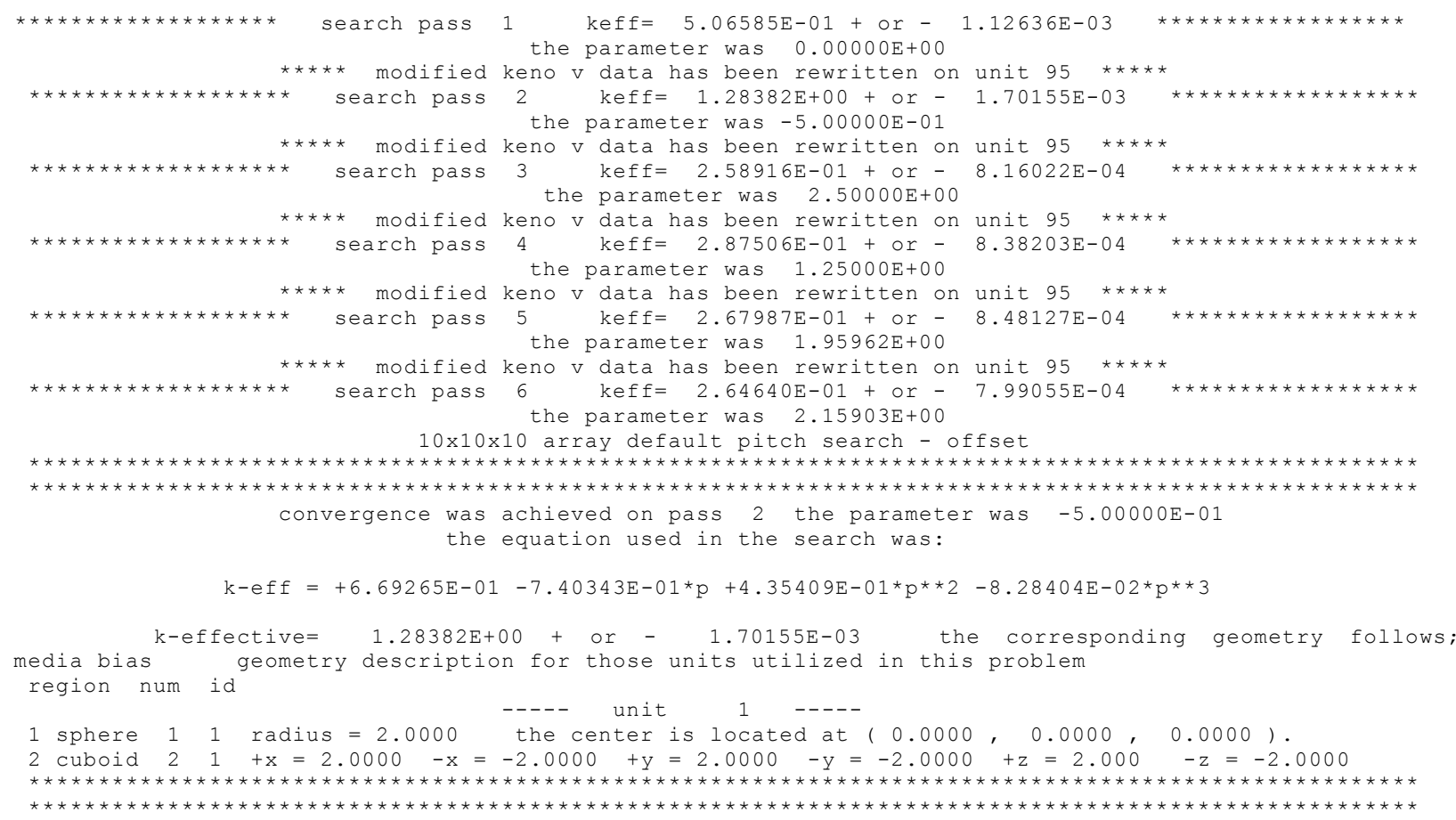

\section{Array of Centered Spheres — Constant Z Spacing}

This is the same problem as the first problem described above except the array does not change in the $\mathrm{Z}$ dimensions. This is done by using MORE and specifying a KEEP command. The input data and the final search results for this problem follow:

$=\operatorname{CSAS} 5 \mathrm{~S}$

10X10X10 ARRAY DEFAULT PITCH SEARCH - CHANGE IN X \& Y ONLY

$\mathrm{V} 7-238$

READ COMP

URANIUM $11.0 \quad 300.09223590 .092238 \quad 10.0$ END 
END COMP

READ CELLDATA

LATTICECELL SPHSQUAREP PITCH=8.0 2 FUELD=4.0 1 END

END CELLDATA

READ GEOMETRY

UNIT 1

SPHERE 1112.0

CUBOID $216 \mathrm{p} 4.0$

END GEOMETRY

READ ARRAY

$A R A=1 \quad N U X=10 \quad N U Y=10 \quad N U Z=10$ FILL F1 END FILL

END ARRAY

END DATA

READ SEARCH OPTIMUM PITCH MORE

KEEP UNIT=1 REG=2 $+\mathrm{Z}=1.0 \quad-\mathrm{Z}=1.0$

END SEARCH

END

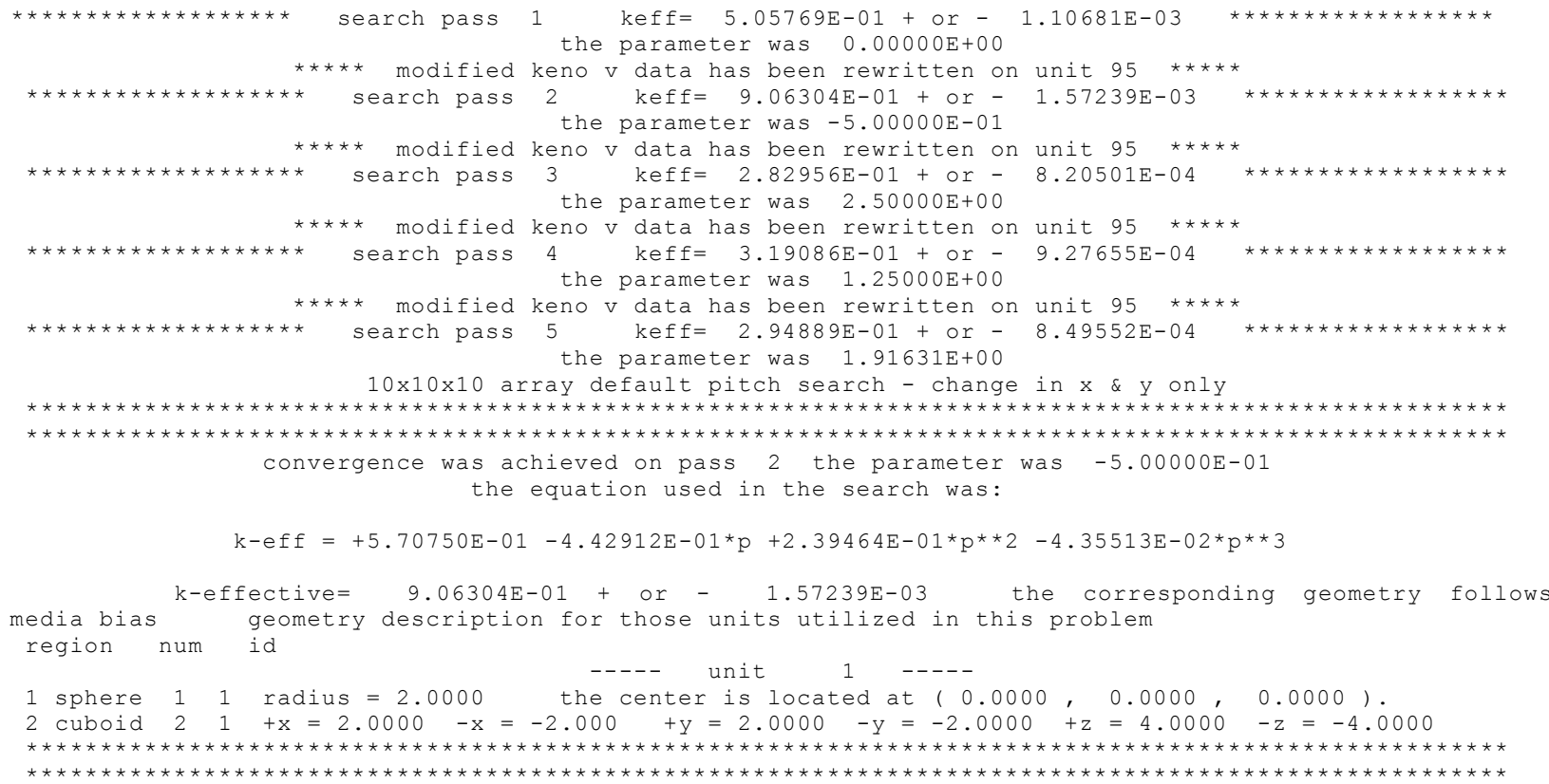

\section{Default Search — Array of Centered Cylinders}

This problem contains search data for a default optimum pitch search. The problem consists of a $10 \times 10 \times 10$ array of uranium cylinders surrounded by low density water. The cylinders are $90 \%{ }^{235} \mathrm{U}$ and $10 \%{ }^{238} \mathrm{U}$ and have a radius of $2.0 \mathrm{~cm}$ and a length of $20 \mathrm{~cm}$. The cylinders are originally centered on an $8.0 \mathrm{~cm} \mathrm{X}$ and $\mathrm{Y}$ pitch and a $24 \mathrm{~cm} \mathrm{Z}$ pitch with interstitial low density $(0.01 \mathrm{gm} / \mathrm{cc})$ water. The problem searches for the pitch that will produce the maximum $k_{\text {eff }}$ for the system. Because this is an array of cylinders, by default only the $\mathrm{X}$ and $\mathrm{Y}$ dimensions are modified during the search. The input data and the final search results for this problem follow:

$=\mathrm{CSAS} 5 \mathrm{~S}$

10X10x10 ARRAY DEFAULT PITCH SEARCH - CHANGE IN X \& Y ONLY

$\mathrm{V} 7-238$

READ COMP

$\begin{array}{llllllllll}\text { URANIUM } & 1 & 1.0 & 300.0 & 92235 & 90.0 & 92238 & 10.0 & \text { END }\end{array}$

H2O 20.01300 .0 END

END COMP

READ CELLDATA

LATTICECELL SQUAREPITCH PITCH=8.0 2 FUELD=4.0 1 END

END CELLDATA

READ GEOMETRY 
UNIT 1

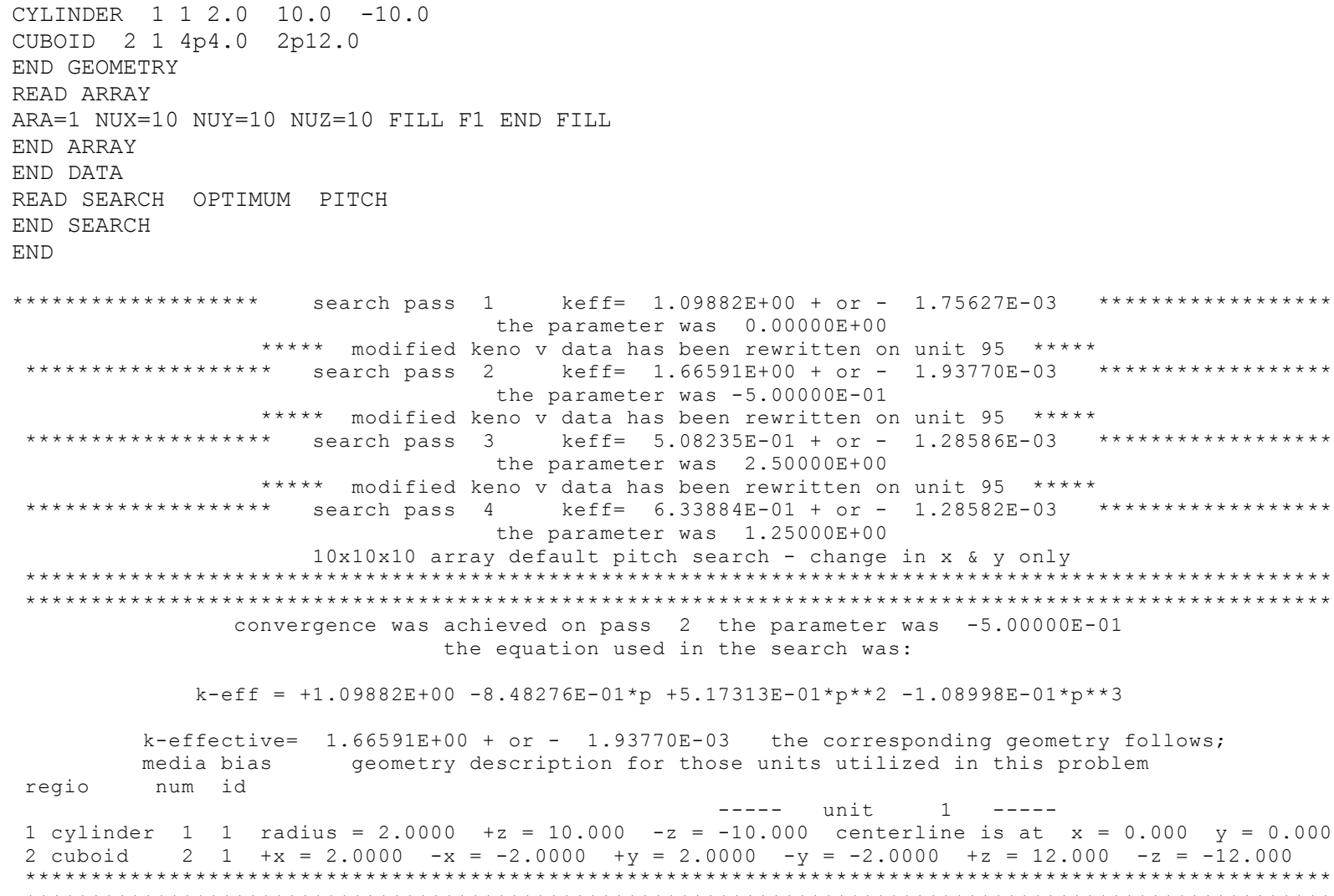

\section{Array of Centered Cylinders - Search Extended to Z Dimension}

This is the same problem as given above except the search has been extended to include the $Z$ dimension. The input data causes the code to alter the $+\mathrm{X},-\mathrm{X},+\mathrm{Y},-\mathrm{Y},+\mathrm{Z}$, and $-\mathrm{Z}$ dimensions of the outer region of unit 1 and search for the dimensions that give the maximum $k_{\text {eff. }}$ The $+Z$ and $-Z$ search constants were chosen to maintain the same spacing between cylinder surfaces in the $\mathrm{X}, \mathrm{Y}$ and $\mathrm{Z}$ dimensions throughout the search. Because this is an optimum pitch search, the cylinder dimensions will not be changed. The input data and the final search results for this problem follow:

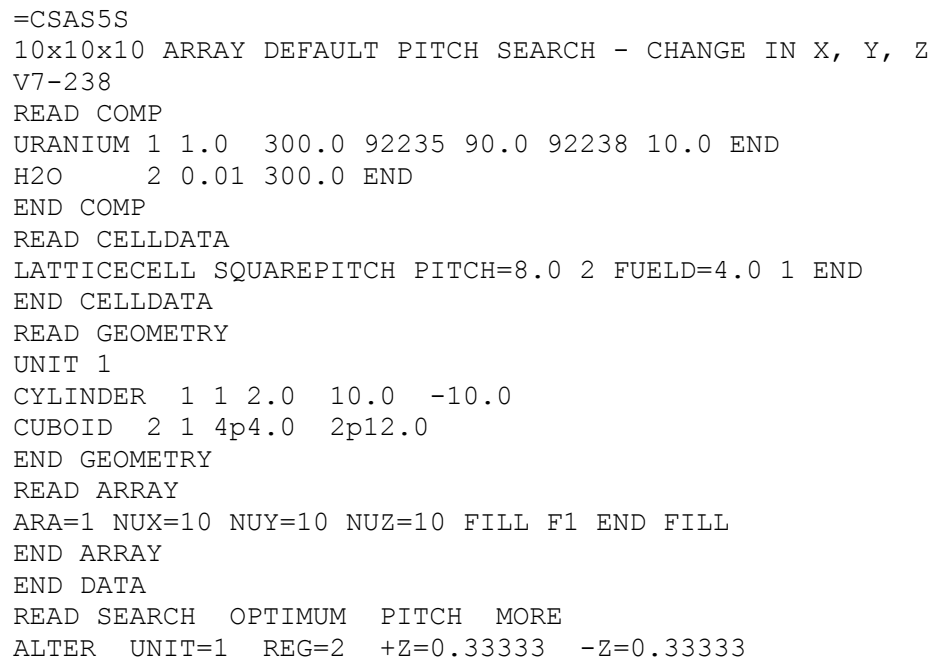


END

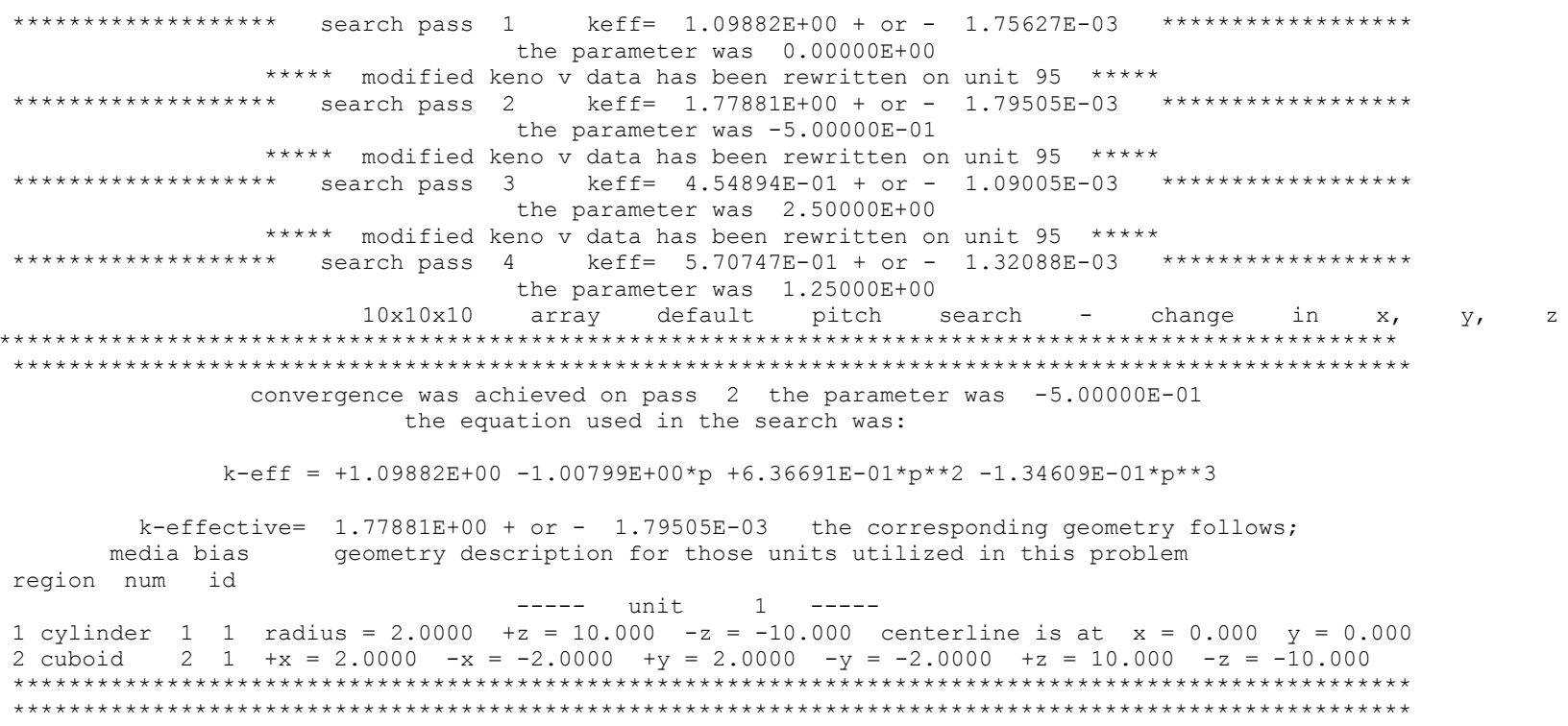

\subsection{A.7 Minimum pitch search}

A minimum pitch search searches for the pitch that yields the lowest value of $k_{\text {eff: }}$ A minimum pitch search is activated by entering "MINIMUM PITCH" in the search data. By default, the search is performed at the lowest array level and only the spacing in the $\mathrm{X}$-direction for slabs, the $\mathrm{X}$ and $\mathrm{Y}$ directions for cylindrical arrays, and the $\mathrm{X}, \mathrm{Y}$, and $\mathrm{Z}$ directions for spherical arrays. The search constants are defaulted to 1.0 for the applicable $+\mathrm{X},-\mathrm{X},+\mathrm{Y},-\mathrm{Y},+\mathrm{Z}$, and $-\mathrm{Z}$ dimensions of the outermost region. The dimensions of other geometry regions will not be changed (their search constants are defaulted to 0.0) unless additional search data containing appropriate instructions are supplied.

The limits for a minimum pitch search are the same as an optimum pitch search as described in the previous section.

\section{Array of Centered Uranium Slabs}

Consider a $10 \times 1 \times 1$ array of uranium metal slabs. The uranium slabs are $2 \mathrm{~cm}$ in thick in the $\mathrm{X}$ dimension and $200 \mathrm{~cm}$ thick in the $\mathrm{Y}$ and $\mathrm{Z}$ dimensions. On each side of the uranium slab in the $\mathrm{X}$ dimension is $1 \mathrm{~cm}$ of $\mathrm{H}_{2} \mathrm{O}$, then $1 \mathrm{~cm}$ of Boral, then $1 \mathrm{~cm}$ of $\mathrm{H}_{2} \mathrm{O}$ resulting in an initial center-to-center spacing is $8.0 \mathrm{~cm}$. The slabs are $90 \%{ }^{235} \mathrm{U}$ and $10 \%{ }^{238} \mathrm{U}$, the water is full density, and the Boral is $36.7 \% \mathrm{~B}_{4} \mathrm{C}$. The uranium slabs and their associated materials are defined to be unit 1 , and the $10 \times 1 \times 1$ array is defined to be array 1 . A minimum pitch of $6.01 \mathrm{~cm}$ is specified, MINPITCH=6.01, and a maximum pitch of $14.0 \mathrm{~cm}$ is specified, MAXPITCH=14.0. The input data and the final search results for this problem follow:

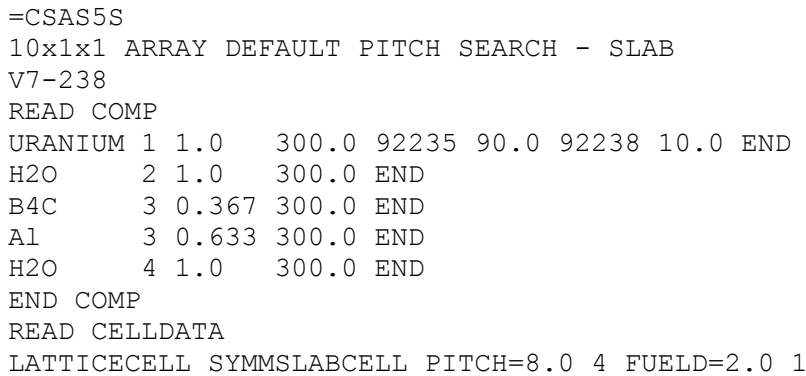




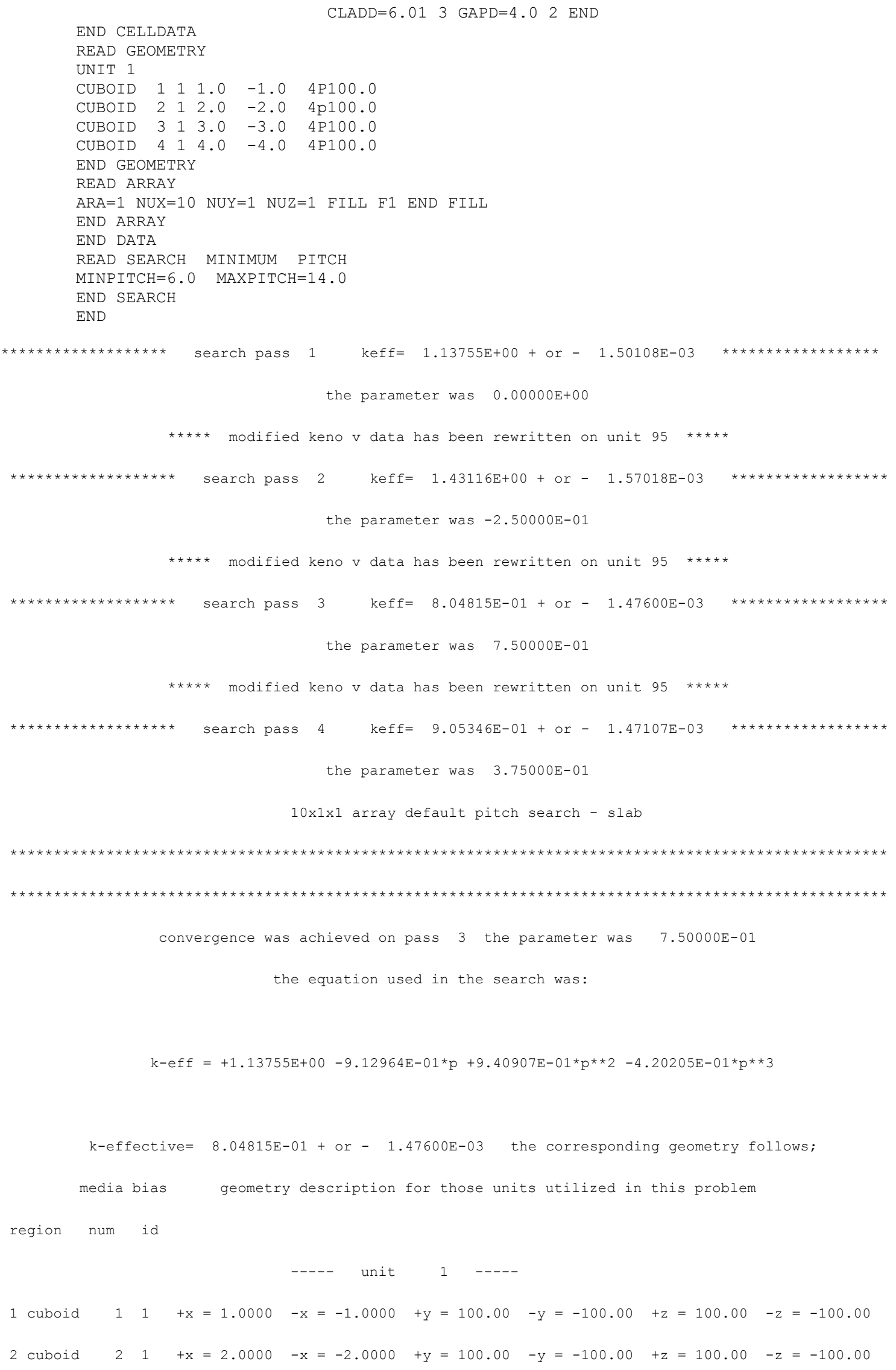




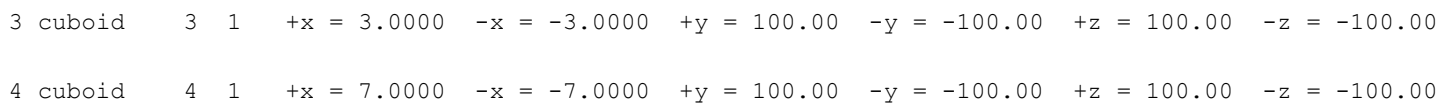

\subsection{A.8 Critical pitch search}

A critical pitch search alters the outer region of the unit or units at the lowest array level in search of a specified value of $k_{\text {eff. }}$ A critical pitch search is activated by entering "CRITICAL PITCH" in the search data. By default, the search is performed at the lowest array level, and only the spacing in the X and $\mathrm{Y}$ directions (the $\mathrm{X}$ and $\mathrm{Y}$ dimensions of the outermost region of the unit(s) used in the array at the lowest array level) will be changed. The search constants are defaulted to 1.0 for the $+\mathrm{X},-\mathrm{X},+\mathrm{Y}$, and $-\mathrm{Y}$ dimensions of the region. The dimensions of other geometry regions will not be changed (their search constants are defaulted to 0.0 ) unless additional search data containing appropriate instructions are supplied.

The limits for a critical pitch search can be set using either MAXPITCH= and MINPITCH= in the optional search parameters or $+\mathrm{CON}=$ and $-\mathrm{CON}=$ in the search parameter constraints of the auxiliary search commands. MAXPITCH= and MINPITCH= are used to enter a value of the maximum allowed pitch and minimum allowed pitch respectively. Parameters $+\mathrm{CON}=$ and $-\mathrm{CON}=$ are used to enter values for the parameter constraints (i.e., the maximum and minimum allowed value of the search parameter). Typically, it is easier to set the maximum allowed pitch and minimum allowed pitch using MAXPITCH $=$ and MINPITCH= than it is to calculate the value of the parameter corresponding to those pitches. The default minimum constraint corresponds to MINPITCH, the pitch at which the largest interior region of a unit used in the array is in contact with the spacing cuboid of that unit. The maximum constraint is defaulted to -5 times the minimum constraint. The code calculates $k_{\text {eff }}$ for the initial geometry first. Then the dimensions corresponding to the minimum constraint and maximum constraint are calculated.

\section{Default Search — Array of Centered Spheres}

This problem contains search data for a default critical pitch search with $k_{\text {eff }}$ specified to be 0.95 . The problem consists of a $10 \times 10 \times 10$ array of uranium spheres surrounded by low density water. The spheres are $90 \%{ }^{235} \mathrm{U}$ and $10 \%{ }^{238} \mathrm{U}$ and have a radius of $2.0 \mathrm{~cm}$. The spheres are originally centered on an $8.0 \mathrm{~cm} \mathrm{X}, \mathrm{Y}$, and $\mathrm{Z}$ pitch with interstitial low density $(0.01 \mathrm{gm} / \mathrm{cc})$ water. The problem searches for the pitch that will produce a $k_{\text {eff }}=0.95$ for the system.

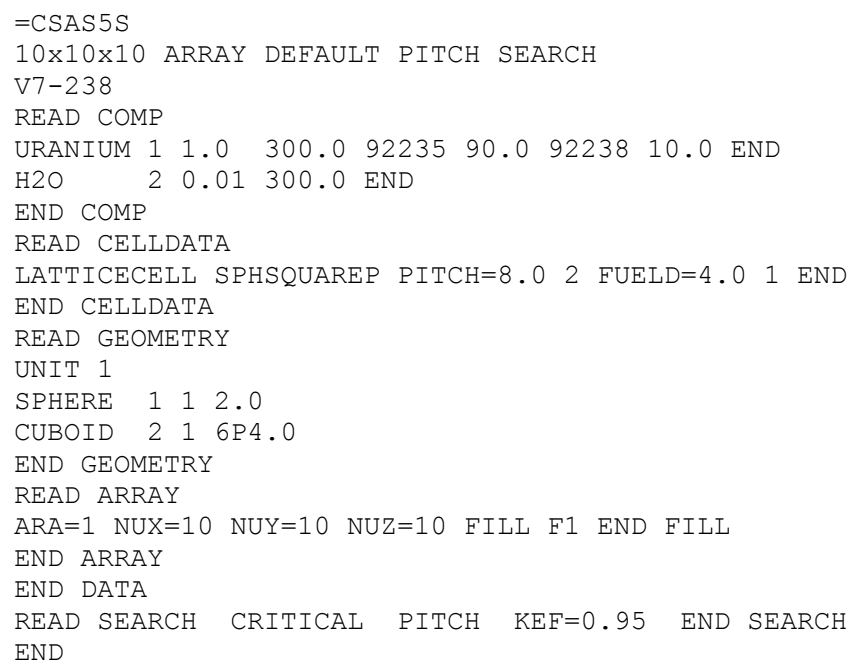




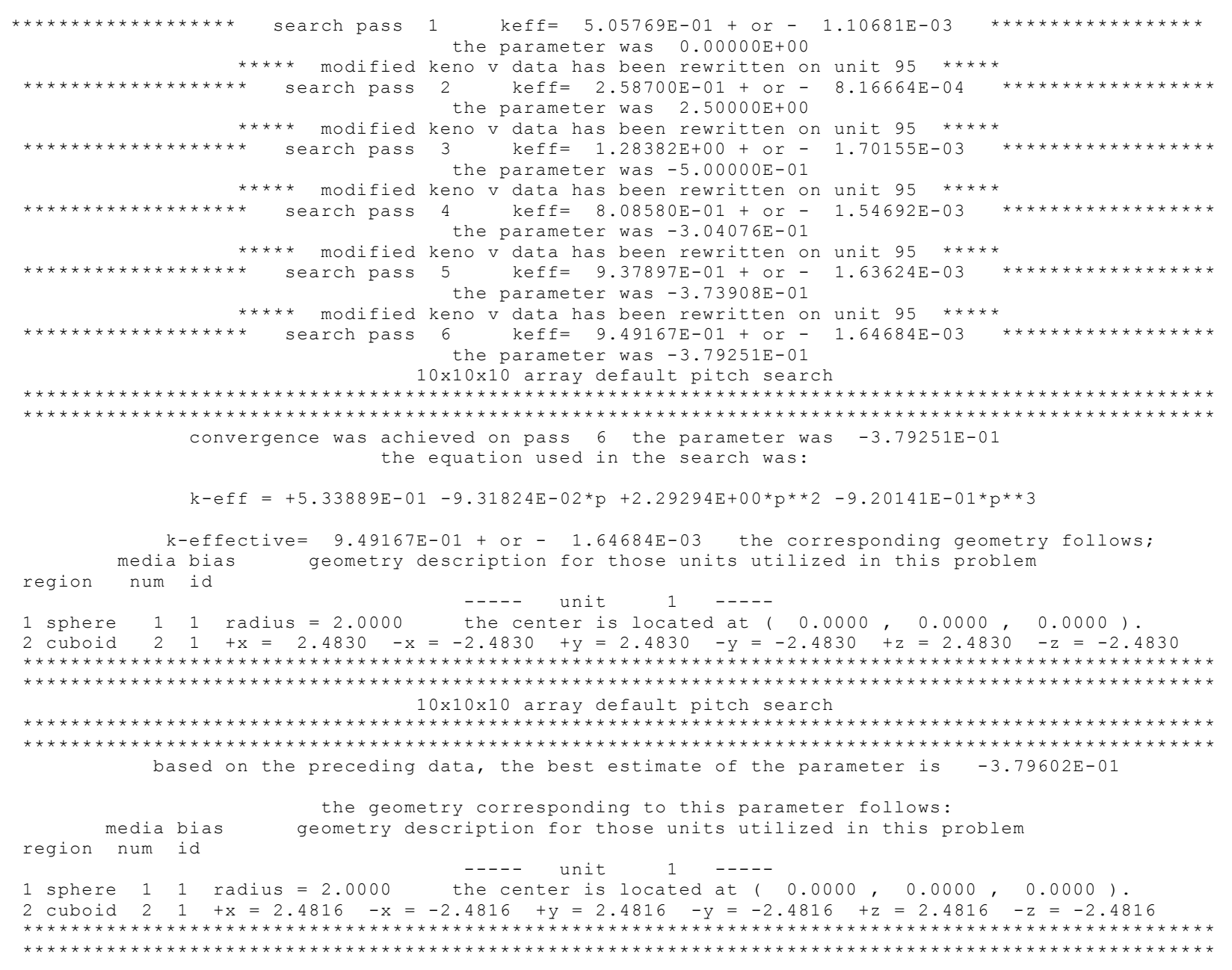

\section{Array of Centered Cylinders - Search Extended to Z Dimension}

This problem contains search data for a default critical pitch search with $k_{\text {eff }}$ specified to be 0.95 . The problem consists of a $10 \times 10 \times 10$ array of uranium cylinders surrounded by low density water. The cylinders are $90 \%{ }^{235} \mathrm{U}$ and $10 \%{ }^{238} \mathrm{U}$ and have a radius of $2.0 \mathrm{~cm}$ and a length of $20 \mathrm{~cm}$. The cylinders are originally centered on an $8.0 \mathrm{~cm} \mathrm{X}$ and $\mathrm{Y}$ pitch and a $24 \mathrm{~cm} \mathrm{Z}$ pitch with interstitial low density $(0.01 \mathrm{gm} / \mathrm{cc})$ water. The problem searches for the pitch that will produce a $k_{\text {eff }}=0.95$ for the system. Because this is an array of cylinders, by default only the $\mathrm{X}$ and $\mathrm{Y}$ dimensions are modified during the search. The $+Z$ and $-Z$ search constants were included and chosen such that the same spacing is maintained between cylinder surfaces in the $\mathrm{X}, \mathrm{Y}$ and $\mathrm{Z}$ dimensions throughout the search. The input data and the final search results for this problem follow:

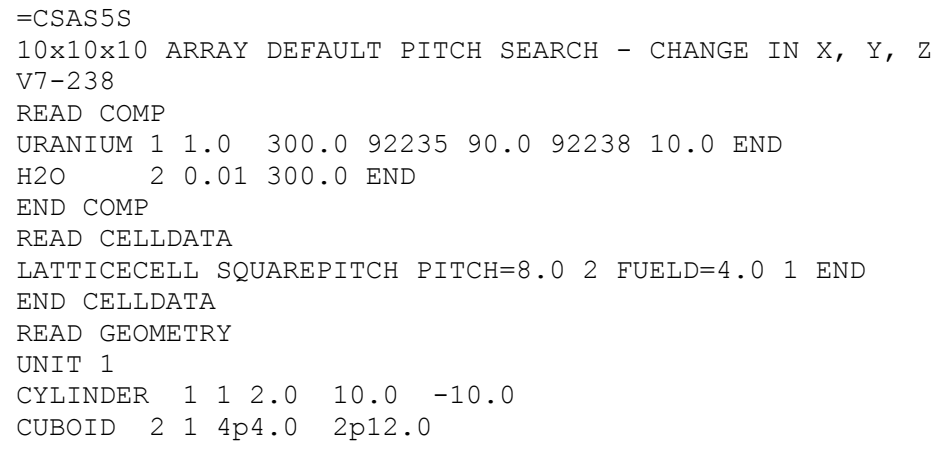


END GEOMETRY

READ ARRAY

ARA=1 NUX=10 NUY=10 NUZ=10 FILL F1 END FILL

END ARRAY

END DATA

READ SEARCH CRITICAL PITCH KEFF=0.95 MORE

ALTER UNIT=1 REG=2 $+\mathrm{Z}=0.33333 \quad-\mathrm{Z}=0.33333$

END SEARCH

END

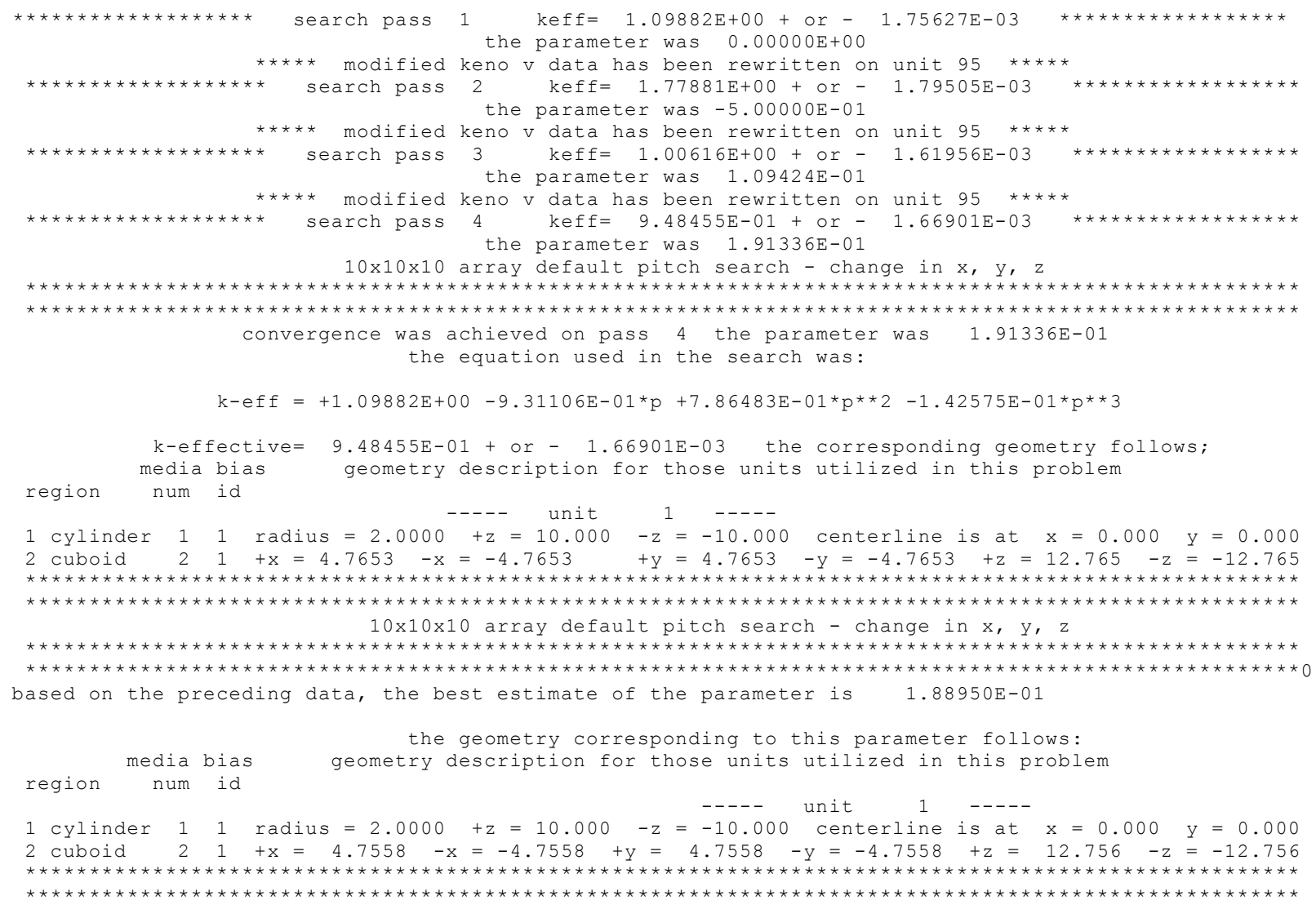

\section{Array of Centered Uranium Slabs}

Consider a $10 \times 1 \times 1$ array of uranium metal slabs. The uranium slabs are $2 \mathrm{~cm}$ thick in the $\mathrm{X}$ dimension and $200 \mathrm{~cm}$ thick in the $\mathrm{Y}$ and $\mathrm{Z}$ dimensions. On each side of the uranium slab in the $\mathrm{X}$ dimension is $1 \mathrm{~cm}$ of $\mathrm{H}_{2} \mathrm{O}$, then $1 \mathrm{~cm}$ of Boral, then $1 \mathrm{~cm}$ of $\mathrm{H}_{2} \mathrm{O}$ resulting in an initial center-to-center spacing of $8.0 \mathrm{~cm}$. The slabs are $90 \%{ }^{235} \mathrm{U}$ and $10 \%{ }^{238} \mathrm{U}$, the water is full density, and the Boral is $36.7 \% \mathrm{~B}_{4} \mathrm{C}$. The uranium slabs and their associated materials are defined to be unit 1 , and the $10 \times 1 \times 1$ array is defined to be array 1. A minimum pitch of $6.0 \mathrm{~cm}$ is specified, $\mathrm{MINPITCH}=6.0$, and a maximum pitch of $14.0 \mathrm{~cm}$ is specified, MAXPITCH=14.0. The code searches for the default value of $k_{\text {eff. }}$ The input data and the final search results for this problem follow: 
$=\mathrm{CSAS} 5 \mathrm{~S}$

10x1X1 ARRAY DEFAULT PITCH SEARCH - SLAB

$\mathrm{V} 7-238$

READ COMP

URANIUM $11.0 \quad 300.09223590 .092238 \quad 10.0$ END

$\begin{array}{lllll}\mathrm{H} 2 \mathrm{O} & 2 & 1.0 & 300.0 \mathrm{END}\end{array}$

$\begin{array}{lllllll}B 4 C & 3 & 0.367 & 300.0 & \text { END }\end{array}$

Al $\quad 30.633 \quad 300.0$ END

H2O $41.0 \quad 300.0$ END

END COMP

READ CELLDATA

LATTICECELL SYMMSLABCELL PITCH=8.0 4 FUELD=2.0 1

CLADD $=6.0 \quad 3 \mathrm{GAPD}=4.02 \mathrm{END}$

END CELLDATA

READ GEOMETRY

UNIT 1

CUBOID $\begin{array}{llllll}1 & 1 & 1.0 & -1.0 & 4 \mathrm{P} 100.0\end{array}$

CUBOID $\begin{array}{llllll}2 & 1 & 2.0 & -2.0 & 4 \mathrm{p} 100.0\end{array}$

CUBOID $\begin{array}{llllll}3 & 1 & 3.0 & -3.0 & 4 \mathrm{P} 100.0\end{array}$

CUBOID $4 \begin{array}{lllll} & 4 & 4.0 & -4.0 & 4 \mathrm{P} 100.0\end{array}$

END GEOMETRY

READ ARRAY

ARA=1 GBL=1 NUX=10 NUY=1 NUZ=1 FILL F1 END FILL

END ARRAY

END DATA

READ SEARCH CRITICAL PITCH

MINPITCH $=6.0 \quad$ MAXPITCH $=14.0$

END SEARCH

END

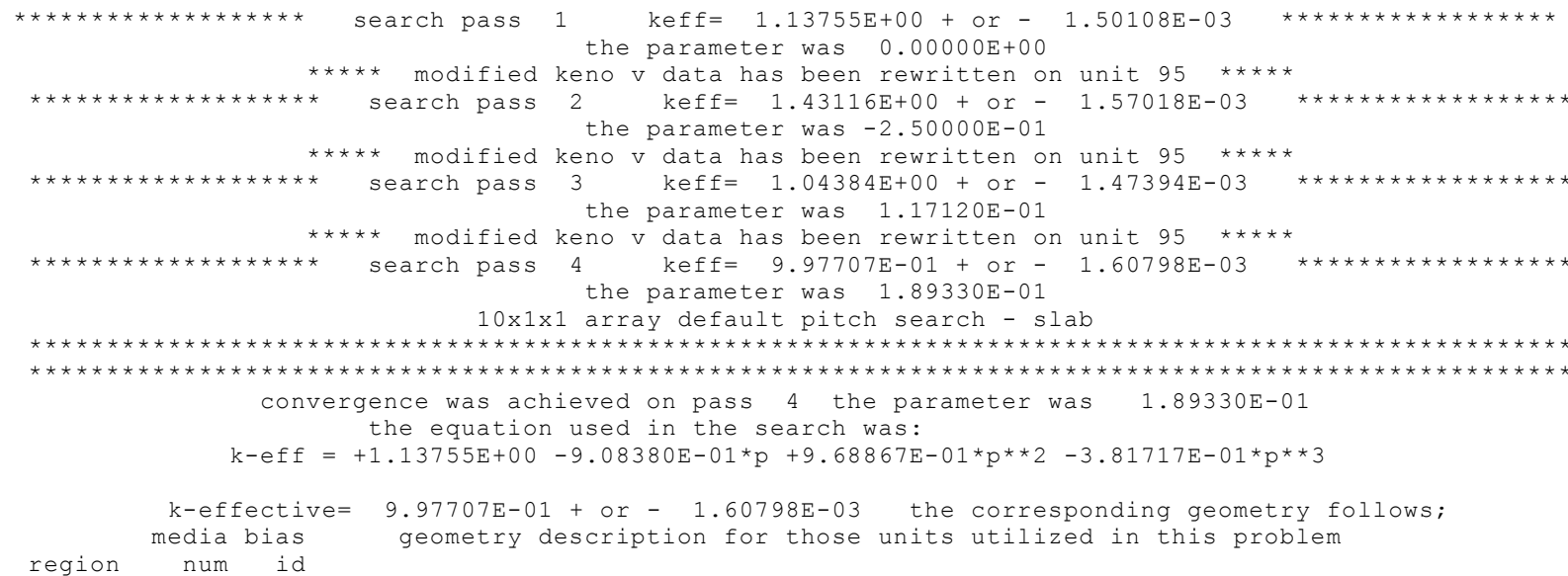

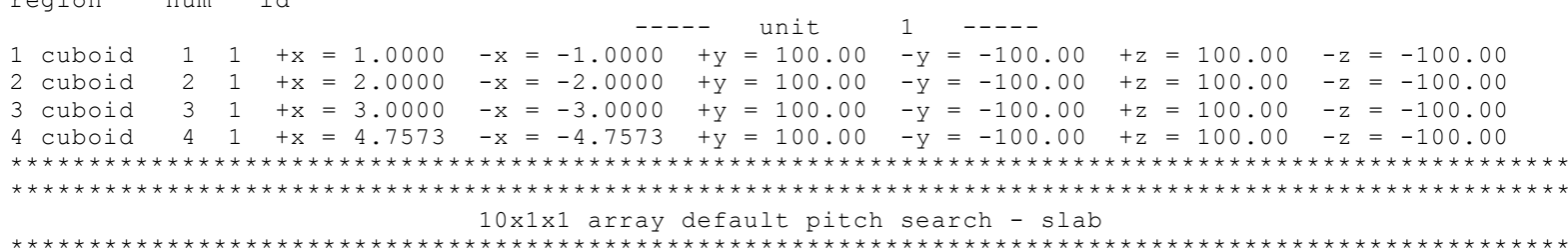

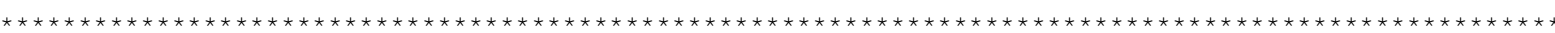
based on the preceding data, the best estimate of the parameter is $1.85414 \mathrm{E}-01$

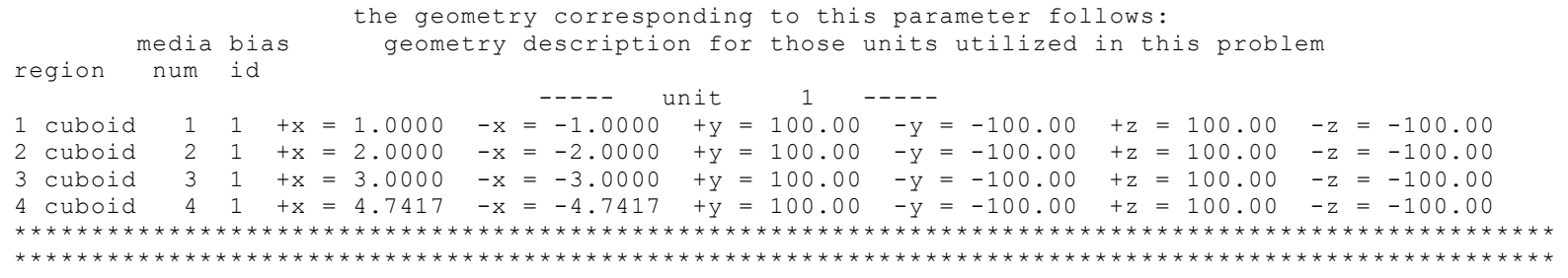




\subsection{A.9 Optimum dimension search}

An optimum dimension search searches for the geometry dimensions that yield the highest value of $k_{\text {eff: }}$ An optimum dimension search is activated by entering "OPTIMUM DIMENSION" in the search data. There are no defaulted search data in a dimension search. The user must specify the dimensions to be changed and the manner in which they will be changed as described in the auxiliary search commands .

A dimension search is performed by altering the regions having nonzero search constants specified in the auxiliary search commands portion of the search data. By default, the search constants for every dimension in the problem are zero. Only those dimensions having a nonzero search constant are altered by the code.

By default, a dimension search sets the minimum constraint to $-10 \mathrm{E} 10$ and the maximum constraint to $+10 \mathrm{E} 10$. The relationship between the constraints and the search constants are given in Eqs. (2.1.1) and (2.1.2). If the default values of the constraints are used, appropriate search constants must be calculated using these equations. It may be simpler to set the search constants to 1.0 and calculate the corresponding maximum and minimum constraints. Several dimension search examples are shown below.

\section{Array of Centered Spheres — Search in X, Y, and Z Dimensions}

Consider a $10 \times 10 \times 10$ array of uranium spheres arranged in an array having a "square" pitch. The uranium spheres are $2 \mathrm{~cm}$ in radius, and the center-to-center spacing is $8 \mathrm{~cm}$. The uranium spheres and their associated spacing are defined to be unit 1 , and the $10 \times 10 \times 10$ array is defined to be array 1 . The constraints are set to be consistent with those of this optimum pitch search case. The search data and results for an optimum dimension search that alters the $\mathrm{X}, \mathrm{Y}$, and $\mathrm{Z}$ dimensions of region 2 of unit 1 are given as follows.

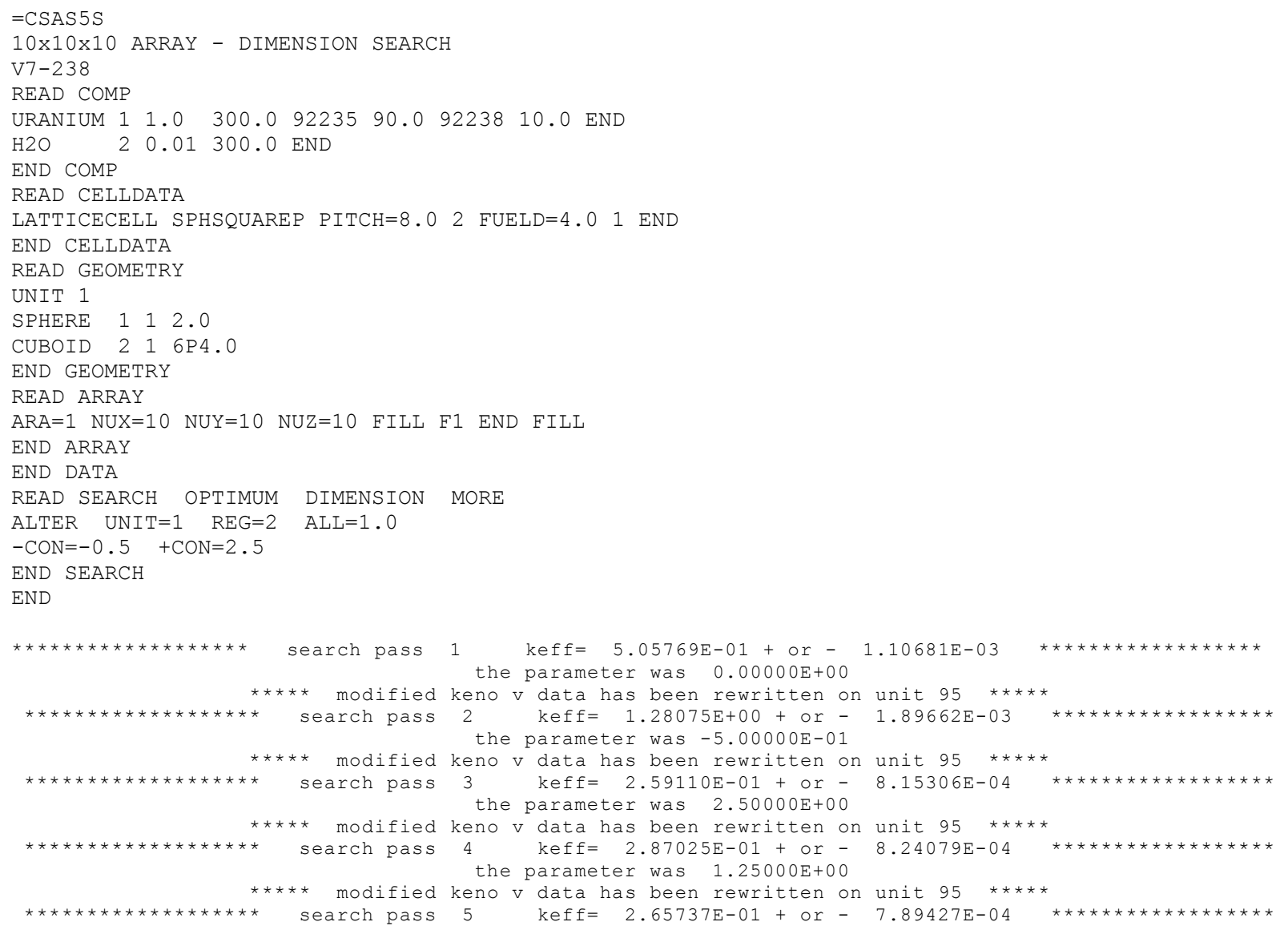




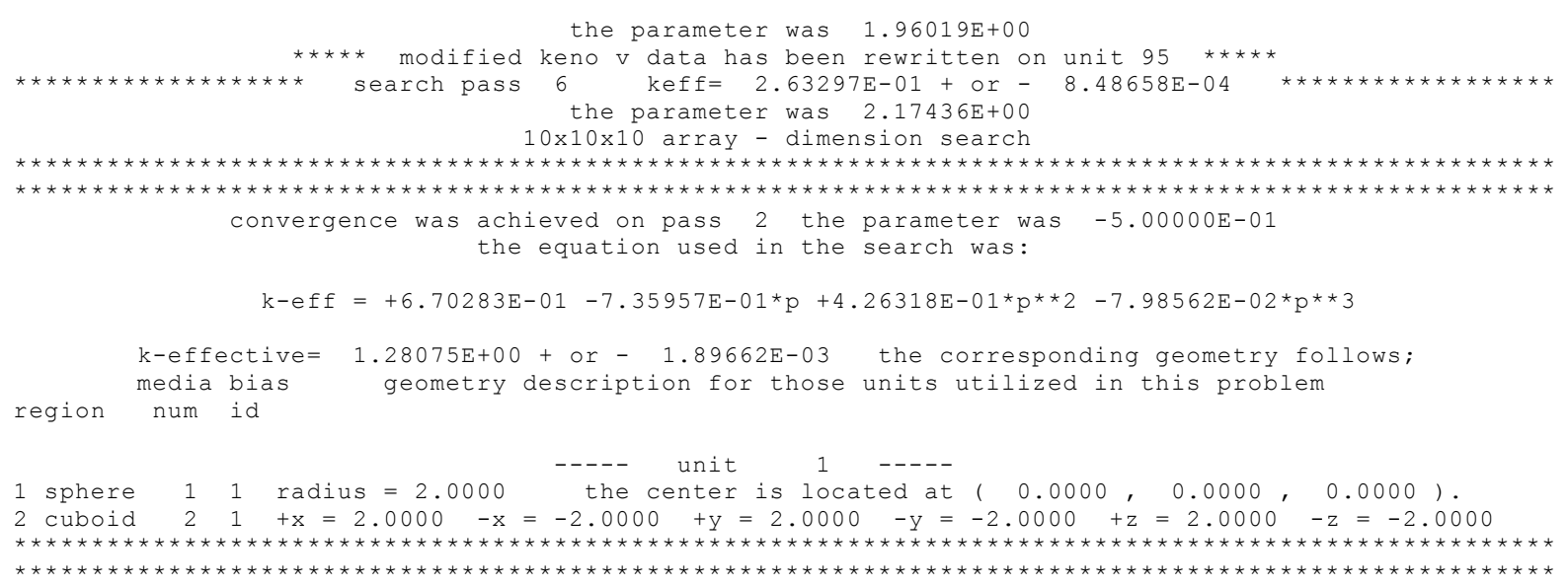

These MORE search data will cause the code to alter the $+\mathrm{X},-\mathrm{X},+\mathrm{Y},-\mathrm{Y},-\mathrm{Z}$, and $+\mathrm{Z}$ dimensions of the outer region (region 2) of unit 1 and search for the dimensions that give the maximum $k_{\text {eff. }}$ Since all the UNIT 1 outer dimensions are the same $(4$ or -4$)$ and all the search constants are the same $(A L L=1.0)$ the code will alter each dimension the same amount for a search pass thus preserving the original relationship between dimensions. Because this is an optimum pitch search, the sphere dimensions will not be changed. The optimum dimension for this problem is when the spheres touch as shown in the output data above.

\section{Array of Centered Spheres — Search in X and Y Dimensions}

This is the same problem as described above except the spacing is only varied in the $\mathrm{X}$ and $\mathrm{Y}$ dimensions. Since only changes in the $\mathrm{X}$ and $\mathrm{Y}$ dimensions of the outer region are desired, the $+\mathrm{X},-\mathrm{X},+\mathrm{Y}$, and $-\mathrm{Y}$ search constants must be set. Since all dimensions are to change at the same rate and have the same initial value, the search constants for the changing surfaces must be the same. The $+Z$ and $-Z$ search constants by default are zero so these dimensions will not change. The input data and the final search results for this problem follow:

$=\mathrm{CSAS} 5 \mathrm{~S}$

10X10X10 ARRAY - X \& Y DIMENSION OPTIMUM SEARCH

$\mathrm{V} 7-238$

READ COMP

URANIUM $11.0 \quad 300.09223590 .092238 \quad 10.0$ END

$\begin{array}{lllll}\mathrm{H} 2 \mathrm{O} & 2 & 0.01 & 300.0 & \mathrm{END}\end{array}$

END COMP

READ CELLDATA

LATTICECELL SPHSQUAREP PITCH=8.0 2 FUELD=4.0 1 END

END CELLDATA

READ GEOMETRY

UNIT 1

SPHERE $\quad 1 \quad 1 \quad 2.0$

CUBOID 2 I 16 P 4.0

END GEOMETRY

READ ARRAY

ARA=1 NUX=10 NUY=10 NUZ=10 FILL F1 END FILL

END ARRAY

END DATA

READ SEARCH OPTIMUM DIMENSION MORE

ALTER UNIT=1 REG=2 +X=1.0 -X=1.0 +Y=1.0 - $\mathrm{Y}=1.0$

$-\mathrm{CON}=-0.5+\mathrm{CON}=2.5$

END SEARCH

END

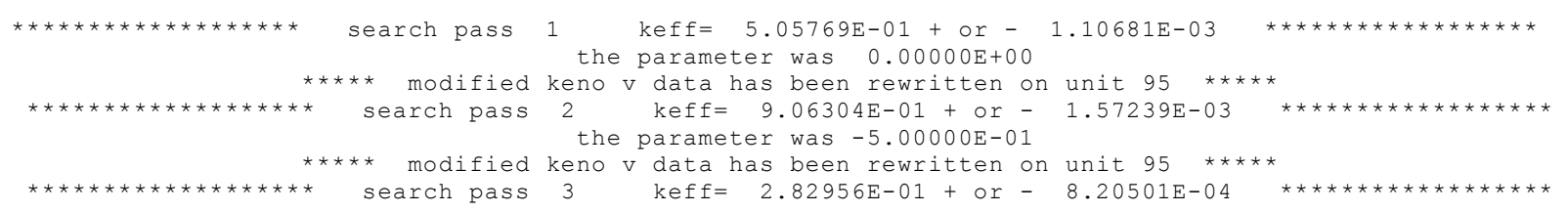




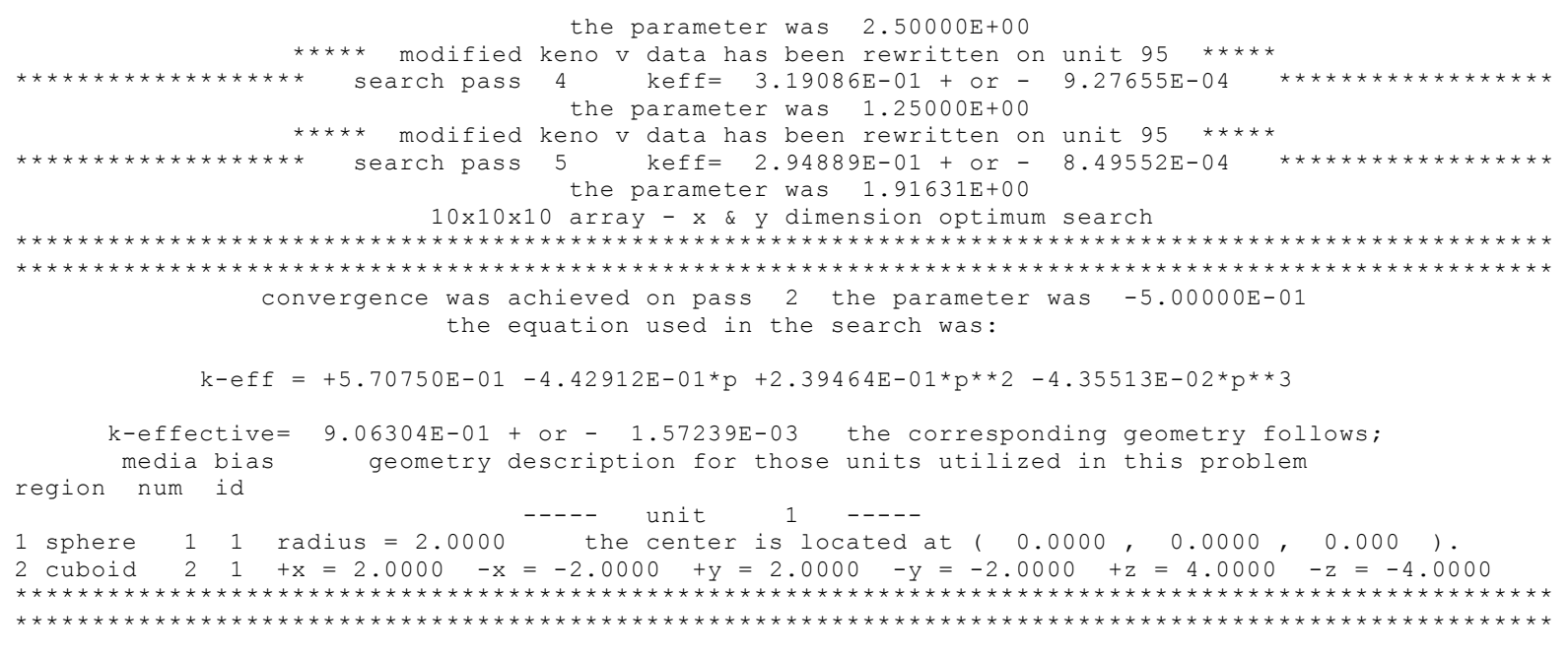

\section{Array of Offset Spheres — Search in X and Y Dimensions}

This is the same problem as described above except the spheres are now off-centered in the $\mathrm{X}$ and $\mathrm{Y}$ dimensions. Since only changes in the $\mathrm{X}$ and $\mathrm{Y}$ dimensions of the outer region are desired, the $+\mathrm{X},-\mathrm{X},+\mathrm{Y}$, and $-\mathrm{Y}$ search constants must be set. All dimensions are to change at the same rate but they have different initial values. The search constants specify how surfaces change relative to each other. For this case the search constants were chosen so that the moderator spacing for the $\mathrm{X}$ and $\mathrm{Y}$ dimensions will change at the same rate. The $+Z$ and $-Z$ search constants by default are zero so these dimensions will not change.

The input data and the final search results for this problem follow:

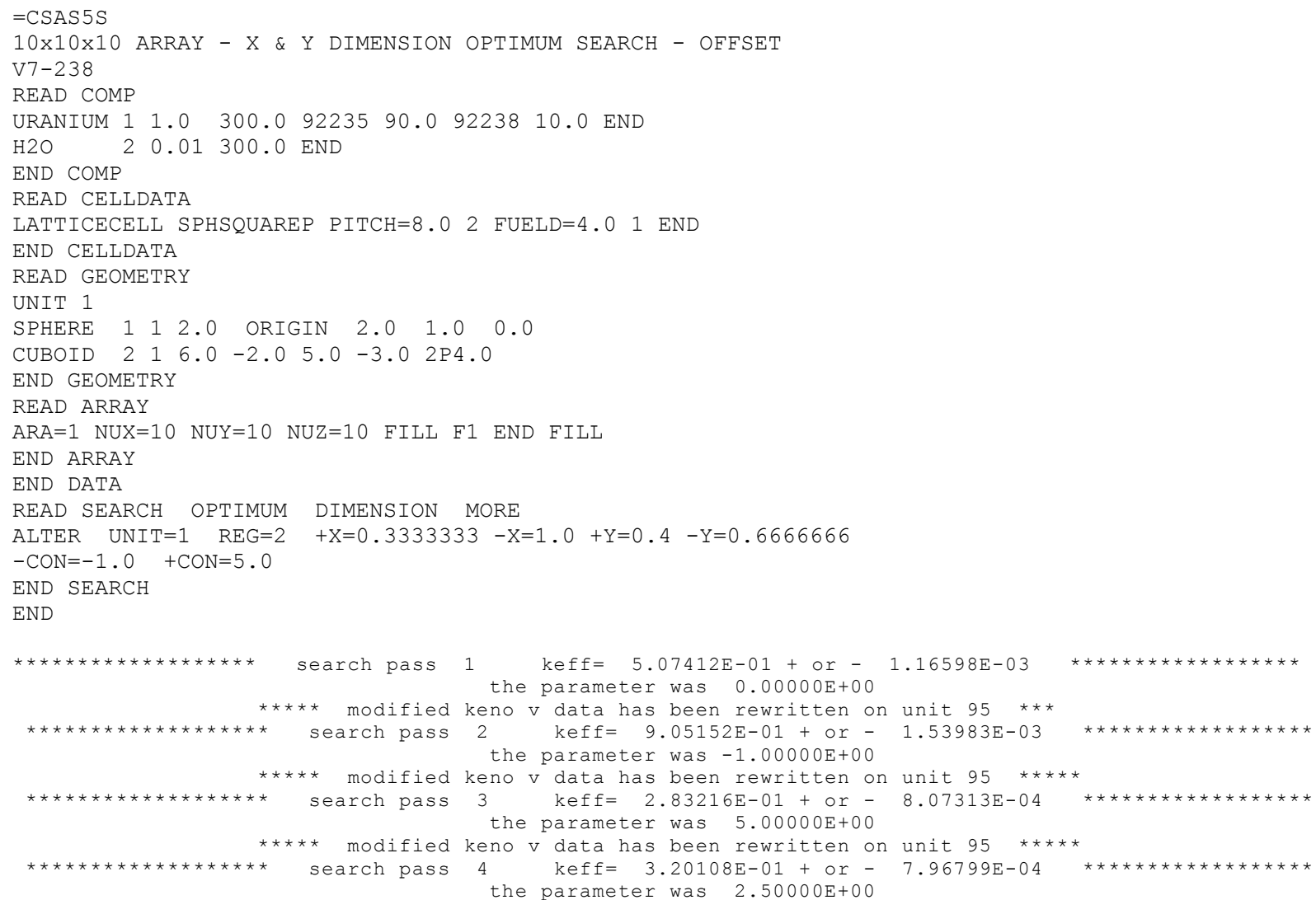




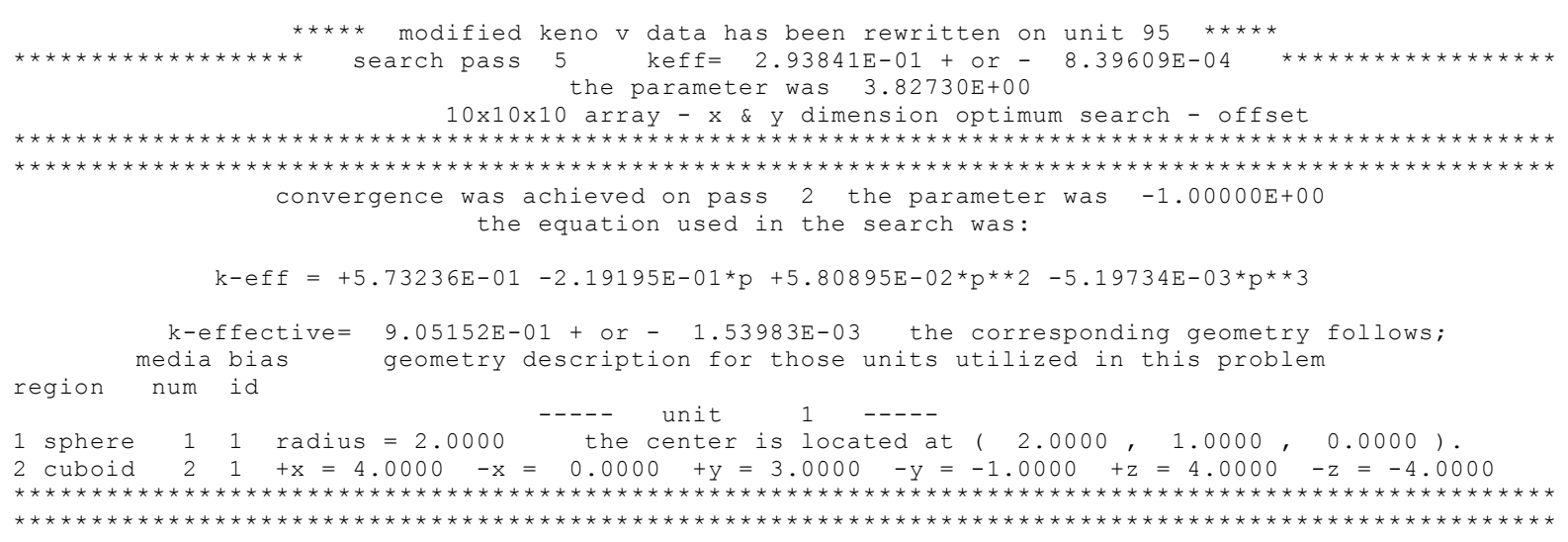

\section{Array of Cylinders - Search in X, Y, and Z Dimensions}

This is the same array of cylinder problem as described in the previous section, except the search is an optimum dimension search. Since changes in the $X, Y$, and $Z$ dimensions of the outer region are desired, the $+\mathrm{X},-\mathrm{X},+\mathrm{Y},-\mathrm{Y},+\mathrm{Z}$, and $-\mathrm{Z}$ search constants must be set. The search data ALL $=1.0$ sets all the outer region search constants to 1.0. However, a constant ratio for the center-to-center spacing of the units in desired so the $\mathrm{Z}$ dimensions will need to be reset to a lower value of 0.333333 . This will ensure that the $\mathrm{Z}$ dimensions change at $1 / 3$ the rate of the $\mathrm{X}$ and $\mathrm{Y}$ dimensions relative to their initial values. The input data and the final search results for this problem follow:

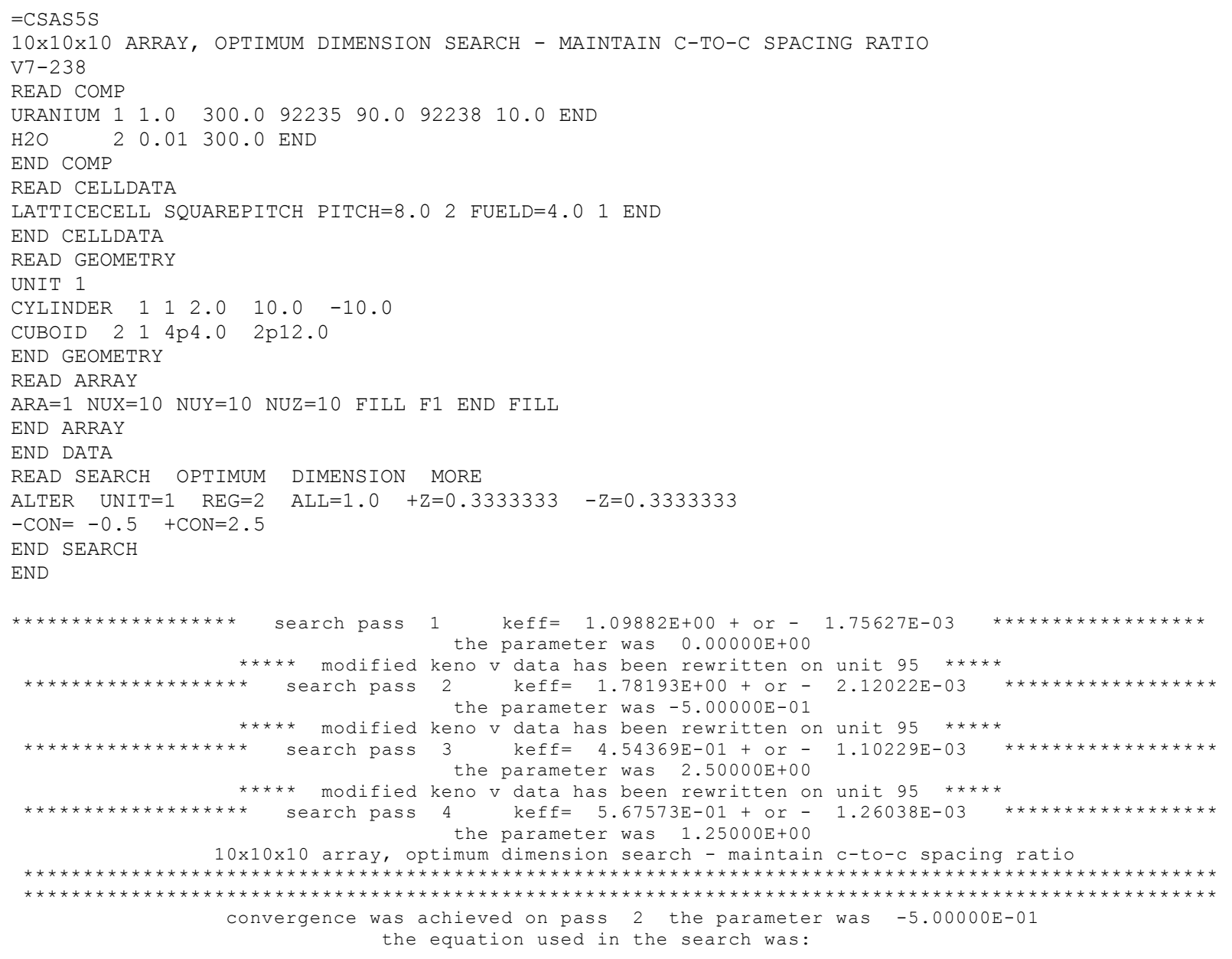




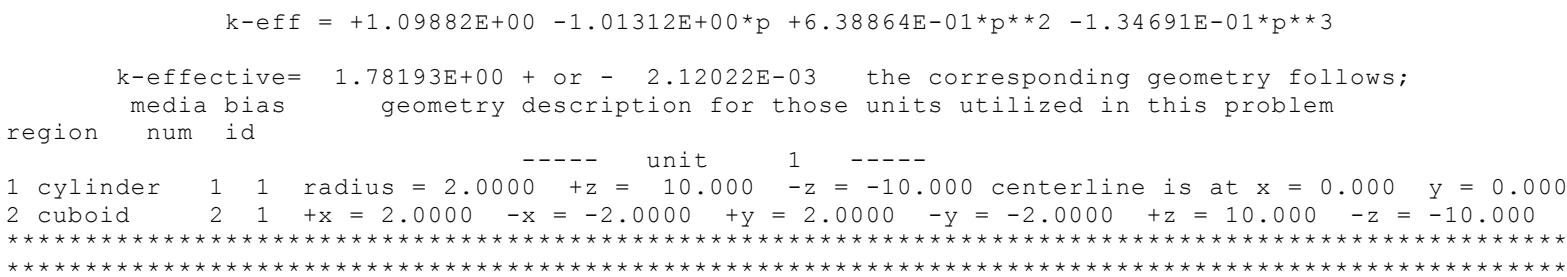

\subsection{A.10 Minimum dimension search}

A minimum dimension search searches for the geometry dimensions that yield the lowest value of $k_{\text {eff. }}$ A minimum dimension search is activated by entering "MINIMUM DIMENSION" in the search data. There are no defaulted search data in a dimension search. The user must specify the dimensions to be changed and the manner in which they will be changed as described in the auxiliary search commands.

A dimension search is performed by altering the regions having nonzero search constants specified in the auxiliary search commands portion of the search data. By default, the search constants for every dimension in the problem are zero. Only those dimensions having a nonzero search constant are altered by the code.

By default, a dimension search sets the minimum constraint to $-10 \mathrm{E} 10$ and the maximum constraint to $+10 \mathrm{E} 10$. The relationship between the constraints and the search constants are given in Eqs. (2.1.1) and (2.1.2). If the default values of the constraints are used, appropriate search constants must be calculated using these equations. In some cases it may be simpler to set the search constants to 1.0 and calculate the corresponding maximum and minimum constraints. Several dimension search examples are shown below.

\section{Infinite Array of Fuel Bundles Separated by Flux Traps — Search in X and Y-Dimensions}

The fuel bundles in this problem represent $17 \times 17$ PWR fuel assemblies. The fuel pins are smeared together, making a mixture 100. The fuel pins consist of $2.35 \mathrm{wt} \%{ }^{235} \mathrm{U}$ having a diameter of $0.823 \mathrm{~cm}$, zirconium cladding having an outer diameter of $0.9627 \mathrm{~cm}$, and a pitch of $1.275 \mathrm{~cm}$. The fuel bundle is represented as a $10.8375 \mathrm{~cm} \times 10.8375 \mathrm{~cm} \times 366 \mathrm{~cm}$ cuboid of mixture 100 surrounded by Boral and then water. The Boral has a density of $2.65 \mathrm{~g} / \mathrm{cm}^{3}$ and is composed of $35.17 \mathrm{wt} \% \mathrm{~B}_{4} \mathrm{C}$ and $64.83 \mathrm{wt} \% \mathrm{Al}$. The fuel bundles are at a fixed pitch of $13.0 \mathrm{~cm}$. The problem searches for the thickness of Boral that will produce the lowest system $k_{\text {eff. }}$. The input data and the final search results for this problem follow: 
$=\operatorname{csas} 5 \mathrm{~s}$

array of fuel bundles with flux trap

$\mathrm{v} 7-238$

read comp

uo2 $\quad 1.84300 .922352 .359223897 .65$ end

zr $\quad 21$ end

h20 31 end

b4c 4 den=2.65 0.3517 end

al 4 den=2.65 0.6483 end

h2o 51 end

end comp

read celldata

latticecell squarepitch pitch=1.275 2 fueld=0.823 1 gapd=0.9627 2 cellmix=100 end end celldata

read param far=yes gen=203 npg=1000 end param

read geom

global unit 1

cuboid $10014 \mathrm{p} 10.8375$ 2p183.0

cuboid $414 \mathrm{p} 11.0 \quad 2 \mathrm{p} 183.0$

cuboid $514 p 13.0 \quad 2 p 183.0$

end geom

read bounds $\mathrm{xfc}=\mathrm{mirror}$ yfc=mirror end bounds

end data

read search minimum dimension more

alter unit=1 reg=2 $+x=1.0-x=1.0+y=1.0-y=1.0$

$-\mathrm{con}=-0.009+\mathrm{con}=0.181818$

end search

end

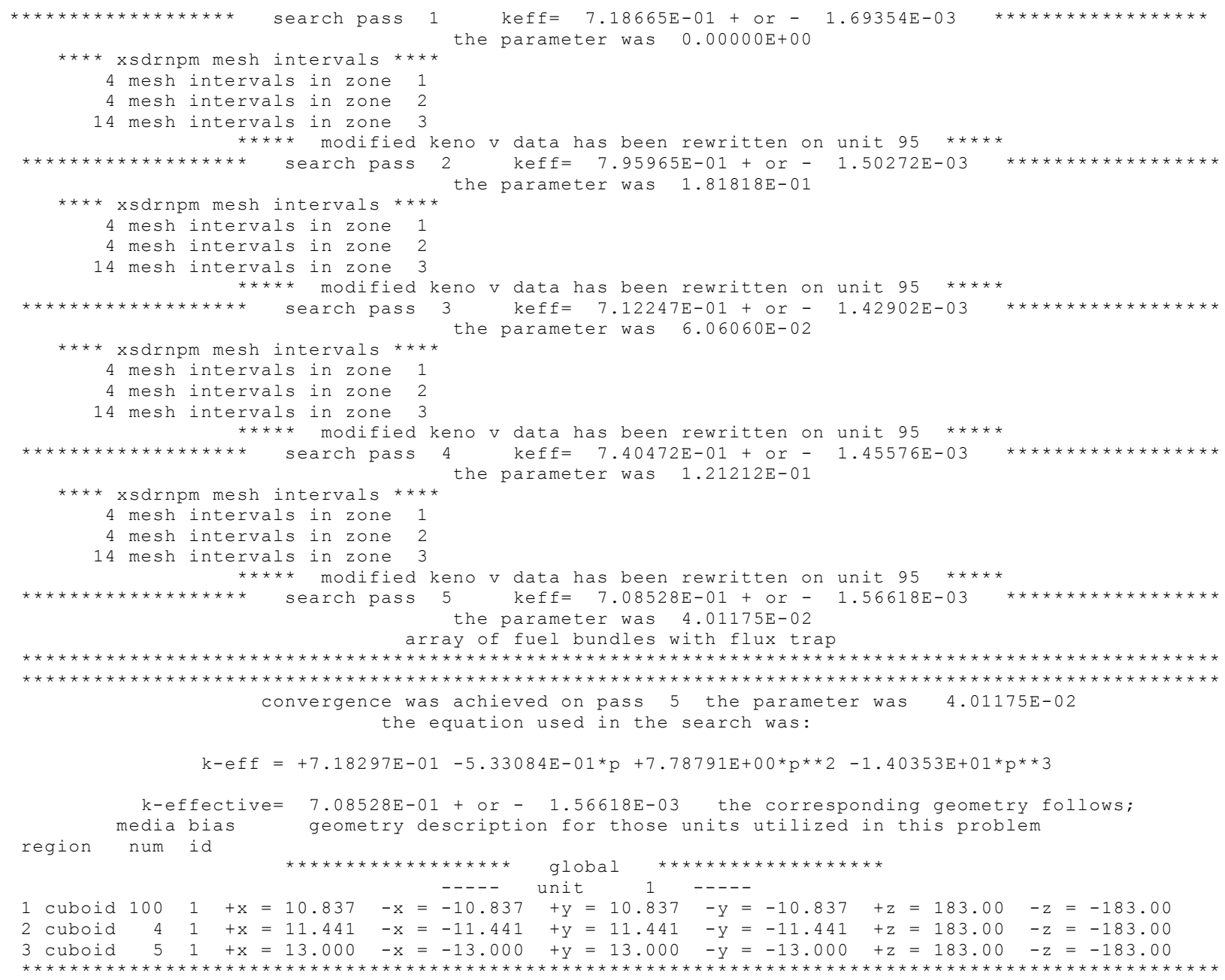




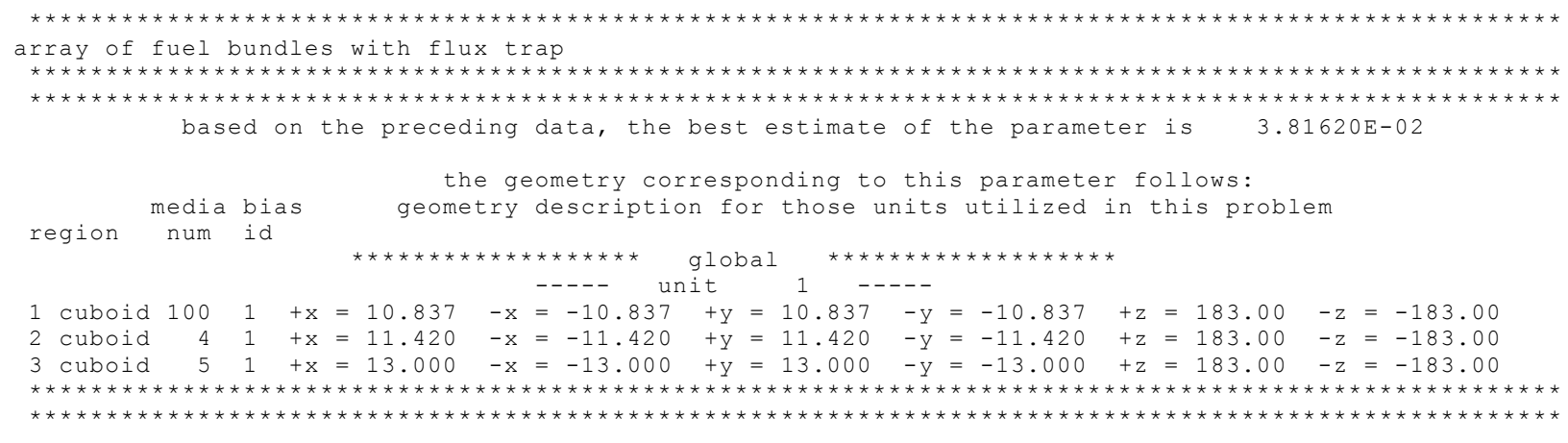

\section{Two Uranium Slabs Separated by a Flux Trap — Search in +X Dimensions}

This problem consists of two $90 \mathrm{wt} \%$ enriched uranium metal slabs separated by a flux trap. Each uranium slab is $3.0 \mathrm{~cm}$ thick. The spacing between the slabs is $4 \mathrm{~cm}$. Between the slabs is a Boral plate at each slab surface and water in between the two Boral plates. This problem searches for the thickness of the Boral plates and separating water that produces the lowest system $k_{\text {eff. }}$ The input data and the final search results for this problem follow:

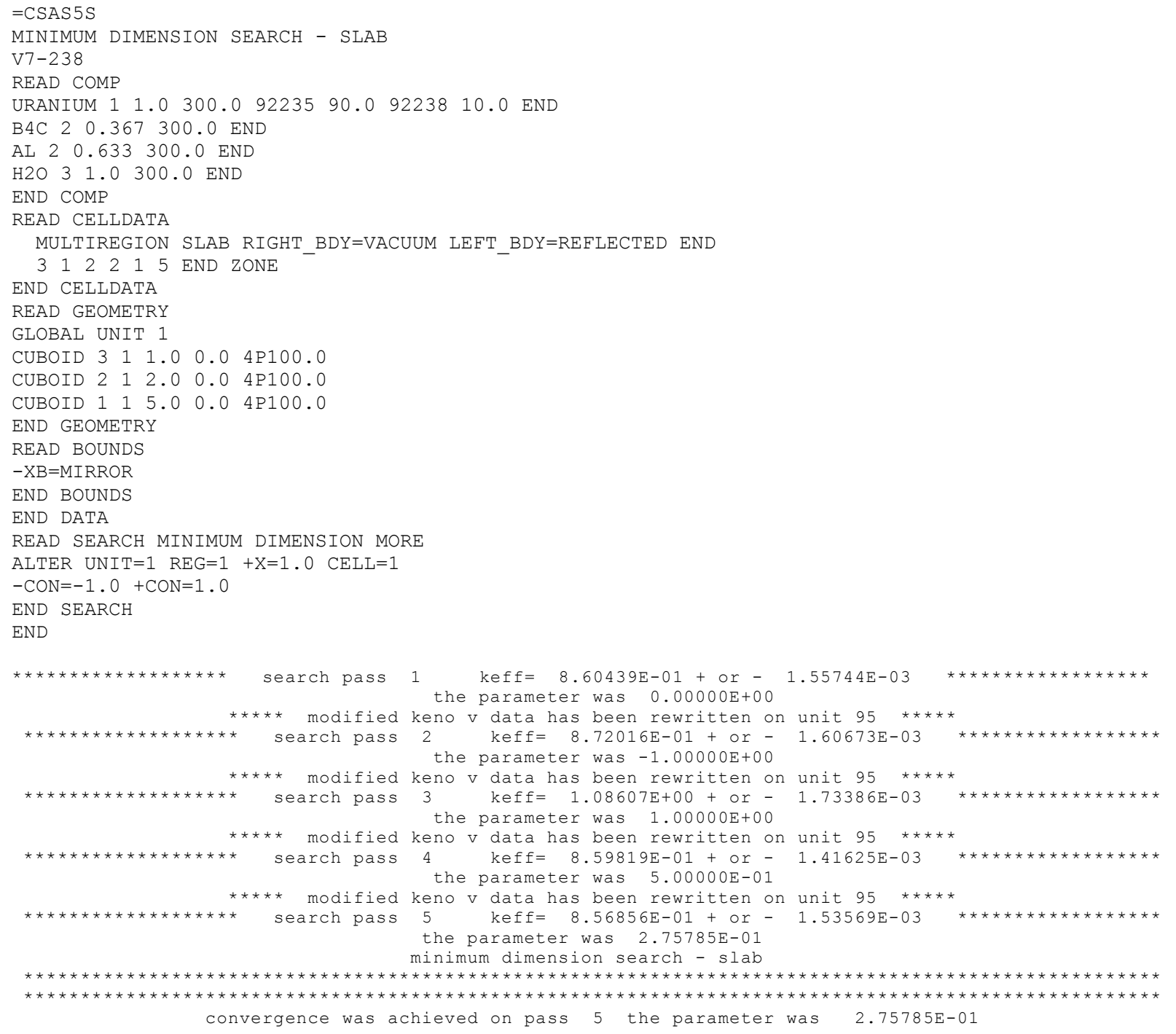




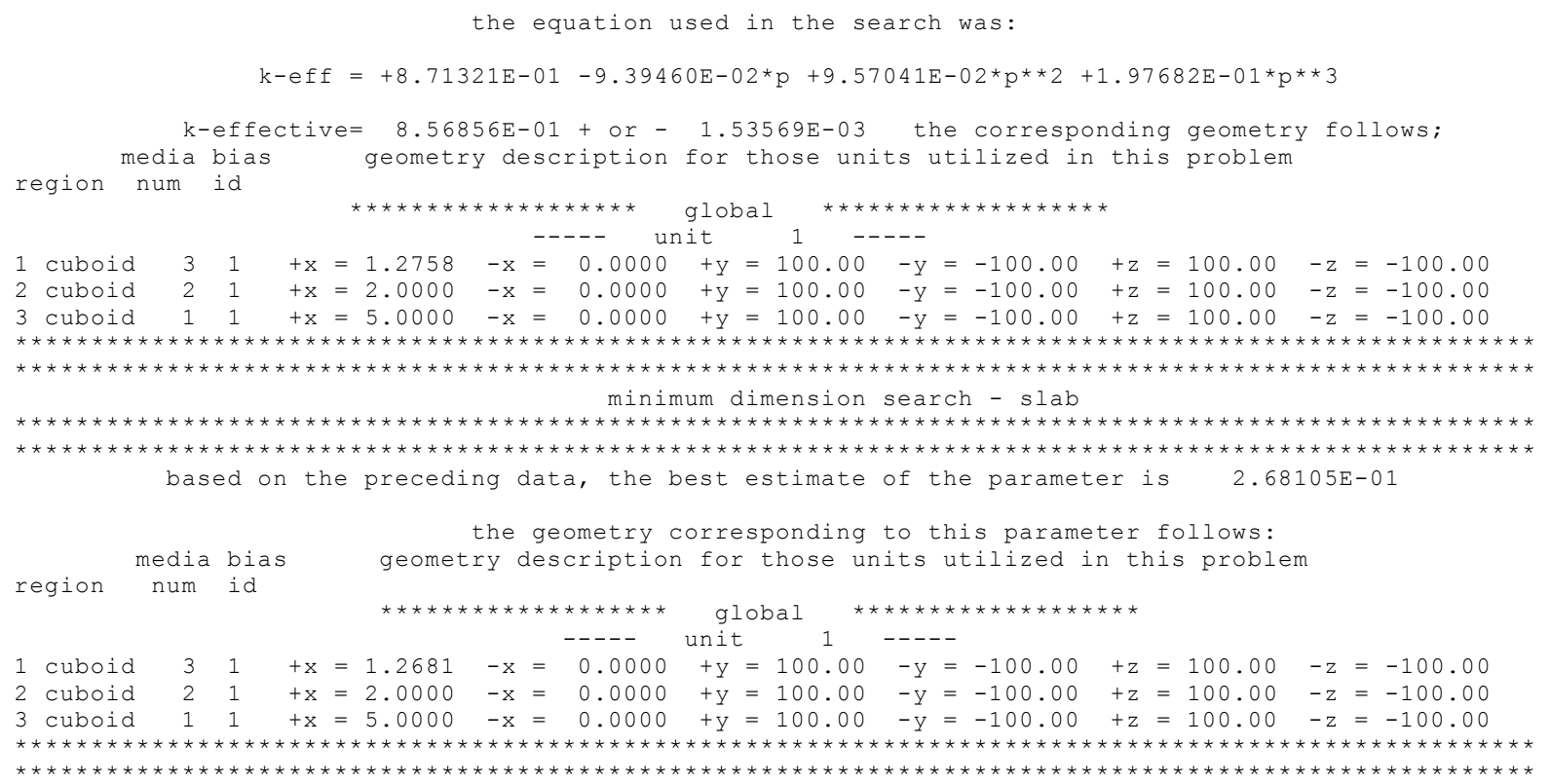

\subsection{A.11 Critical dimension search}

A critical dimension search searches for the geometry dimensions that yield a specified value of $k_{\text {eff. }}$ A critical dimension search is activated by entering "CRITICAL DIMENSION" in the search data. No defaulted search data are in a dimension search. The user must specify the dimensions to be changed and the manner in which they will be changed.

A dimension search is performed by altering the regions having nonzero search constants specified in the individual search commands portion of the search data. By default, the search constants for every dimension in the problem are zero. Only those dimensions having a nonzero search constant are altered by the code.

By default, a dimension search sets the minimum constraint to $-10 \mathrm{E} 10$ and the maximum constraint to +10E10. The relationship between the constraints and the search constants is given in Eqs. (2.1.1) and (2.1.2). If the default values of the constraints are used, appropriate search constants must be calculated using these equations. It may be simpler to set the search constants to 1.0 and calculate the corresponding maximum and minimum constraints. Several critical dimension search examples are shown below.

\section{Array of Centered Spheres - Search in X, Y, and Z Dimensions}

Consider a $10 \times 10 \times 10$ array of uranium spheres arranged in an array having a "square" pitch. The uranium spheres are $2 \mathrm{~cm}$ in radius, and the center-to-center spacing is $8 \mathrm{~cm}$. The uranium spheres and their associated spacing are defined to be unit 1 , and the $10 \times 10 \times 10$ array is defined to be array 1 . A critical dimension search is performed on the outer dimension of the cuboid that will produce a system $k_{\text {eff }}$ of 1.0. In the MORE search data, All=1.0 on region 2 of unit 1 specifies all six surfaces of the cuboid change identically. The search constraints are set to search from a sphere center-to-center spacing of $4 \mathrm{~cm}$ to $20 \mathrm{~cm}$. The search data and results are given below.

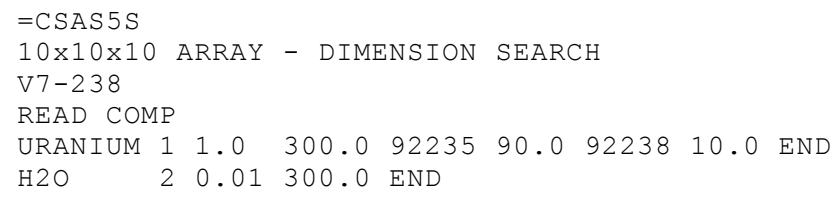


END COMP

READ CELLDATA

LATTICECELL SPHSQUAREP PITCH=8.0 2 FUELD=4.0 1 END

END CELLDATA

READ GEOMETRY

UNIT 1

SPHERE 1112.0

CUBOID 2 1 6 P 4.0

END GEOMETRY

READ ARRAY

ARA=1 NUX=10 NUY=10 NUZ=10 FILL F1 END FILL

END ARRAY

END DATA

READ SEARCH CRITICAL DIMENSION MORE

ALTER UNIT=1 REG=2 ALL=1.0 CELL=1

$-\mathrm{CON}=-0.5+\mathrm{CON}=2.5$

END SEARCH

END

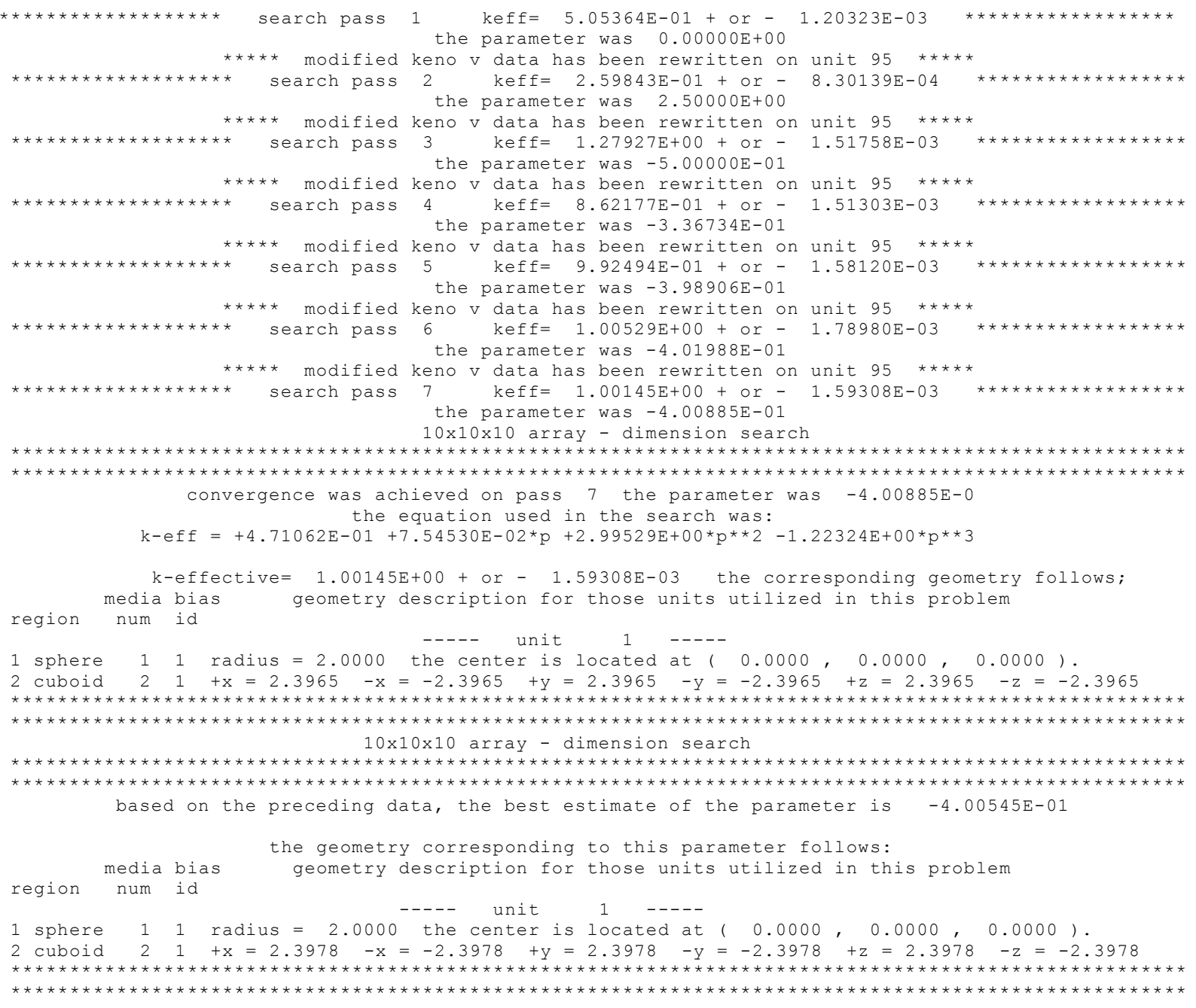

\section{Array of Centered Spheres - Search in X, Y, and Z Dimensions}

Consider a $10 \times 10 \times 10$ array of uranium spheres arranged in an array having a "square" pitch. The uranium spheres are $2 \mathrm{~cm}$ in radius, and the center-to-center spacing is $8 \mathrm{~cm}$. The uranium spheres and their associated spacing are defined to be unit 1 , and the $10 \times 10 \times 10$ array is defined to be array 1 . A critical dimension search is performed on the radius of the uranium sphere that will produce a system $k_{\text {eff }}$ of 1.0. In the MORE search data, RADIUS $=1.0$ on region 1 of unit 1 specifies the sphere's radius is to be 
altered. The search constraints are set to search from a radius of $0.5 \mathrm{~cm}$ to $4.0 \mathrm{~cm}$. The search data and results are given below.

$=$ CSAS $5 \mathrm{~S}$

10X10x10 ARRAY - DIMENSION SEARCH

$\mathrm{V} 7-238$

READ COMP

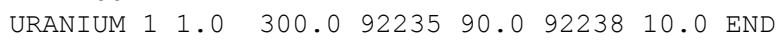

$\begin{array}{llllll}\mathrm{H} 2 \mathrm{O} & 2 & 0.01 & 300.0 & \mathrm{END}\end{array}$

END COMP

READ CELLDATA

LATTICECELL SPHSQUAREP PITCH=8.0 2 FUELD=4.0 1 END

END CELLDATA

READ GEOMETRY

UNIT 1

SPHERE 1112.0

CUBOID 2 I 6 P 4.0

END GEOMETRY

READ ARRAY

ARA=1 NUX=10 NUY=10 NUZ=10 FILL F1 END FILL

END ARRAY

END DATA

READ SEARCH CRITICAL DIMENSION MORE

ALTER UNIT $=1$ CELL=1 REG=1 RADIUS $=1.0$

$-\mathrm{CON}=-0.75+\mathrm{CON}=1.0$

END SEARCH

END

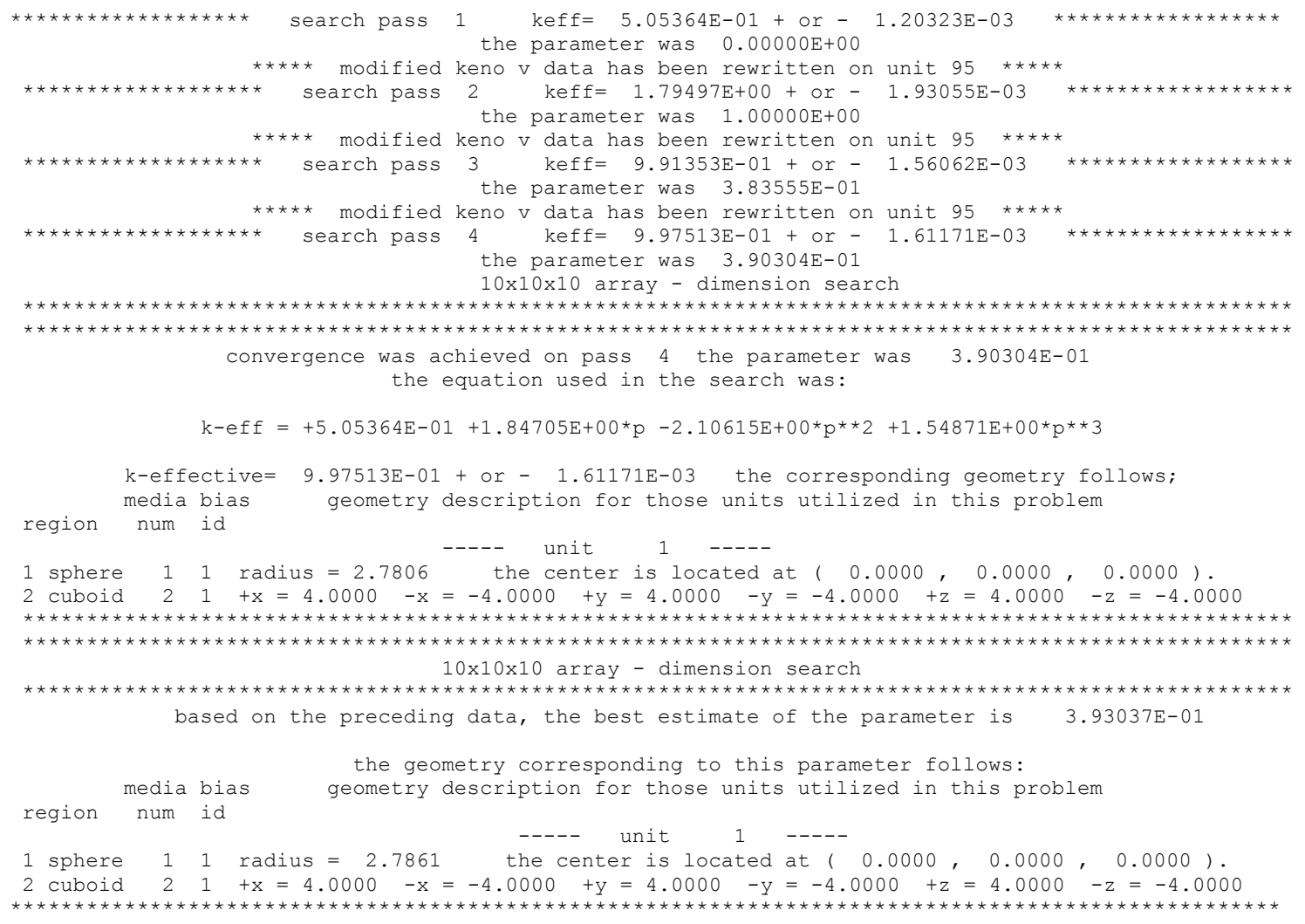

\section{Array of Centered Cylinders — Search in X and Y Dimensions}

This problem contains search data for a critical dimension search with $k_{\text {eff }}$ specified to be 0.95 . The problem consists of a $10 \times 10 \times 2$ array of uranium cylinders surrounded by low density water. The cylinders are $90 \%{ }^{235} \mathrm{U}$ and $10 \%{ }^{238} \mathrm{U}$ and have a radius of $2.0 \mathrm{~cm}$ and a length of $20 \mathrm{~cm}$. The 
cylinders are originally centered on an $8.0 \mathrm{~cm} \mathrm{X}$ and $\mathrm{Y}$ pitch and a $24 \mathrm{~cm} \mathrm{Z}$ pitch with interstitial low density $(0.01 \mathrm{gm} / \mathrm{cc})$ water. The problem searches for the cylinder center-to-center spacing that will produce a $k_{\text {eff }}=0.95$ for the system. Because this is a dimension search, all search data must be specified. The $+\mathrm{X},-\mathrm{X},+\mathrm{Y}$, and $-\mathrm{Y}$ search constants are specified as well as the $-\mathrm{CON}$ and $+\mathrm{CON}$ search constraints. The search data is set up to alter the cylinder center-to-center spacing from $4.0 \mathrm{~cm}$ to $16.0 \mathrm{~cm}$ during the search. The MORE search data $C E L L=1$ ties the search data to the first unit cell thus ensuring they change in unison properly modifying the cross-section processing. The input data and the final search results for this problem follow:

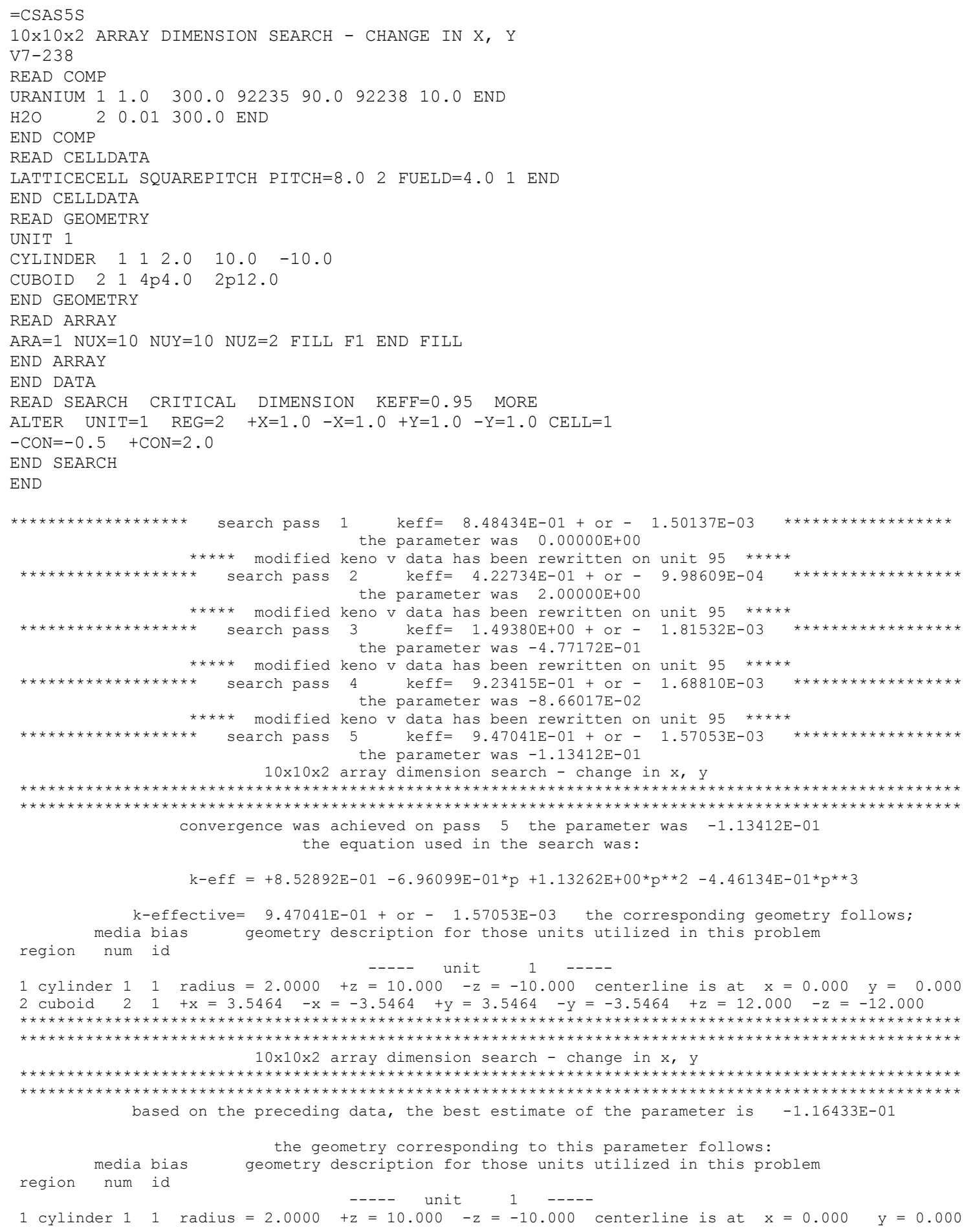




\section{Array of Centered Uranium Slabs}

Consider a $10 \times 1 \times 1$ array of uranium metal slabs. The uranium slabs are $2 \mathrm{~cm}$ thick in the $X$ dimension and $200 \mathrm{~cm}$ thick in the $\mathrm{Y}$ and $\mathrm{Z}$ dimensions. On each side of the uranium slab in the $\mathrm{X}$ dimension is $1 \mathrm{~cm}$ of $\mathrm{H}_{2} \mathrm{O}$, then $1 \mathrm{~cm}$ of Boral, then $1 \mathrm{~cm}$ of $\mathrm{H}_{2} \mathrm{O}$ resulting in an initial center-to-center spacing of $8.0 \mathrm{~cm}$. The slabs are $90 \%{ }^{235} \mathrm{U}$ and $10 \%{ }^{238} \mathrm{U}$, the water is full density, and the Boral is $36.7 \% \mathrm{~B}_{4} \mathrm{C}$. The uranium slabs and their associated materials are defined to be Unit 1 , and the $10 \times 1 \times 1$ array is defined to be array 1 . The problem searches for the slab center-to-center spacing that will produce a $k_{e f f}=1.0$ for the system, which is the default search value. Because this is a dimension search all search data must be specified. The $+\mathrm{X}$ and $-\mathrm{X}$ search constants are specified as well as the $-\mathrm{CON}$ and $+\mathrm{CON}$ search constraints. The search data is set up to alter the cylinder change the water slab thickness between the Boral plates from $0.8 \mathrm{~cm}$ to $10.0 \mathrm{~cm}$ thick during the search. The MORE search data CELL=1 ties the search data to the first unit cell thus ensuring they change in unison properly modifying the cross-section processing. The input data and the final search results for this problem follow:

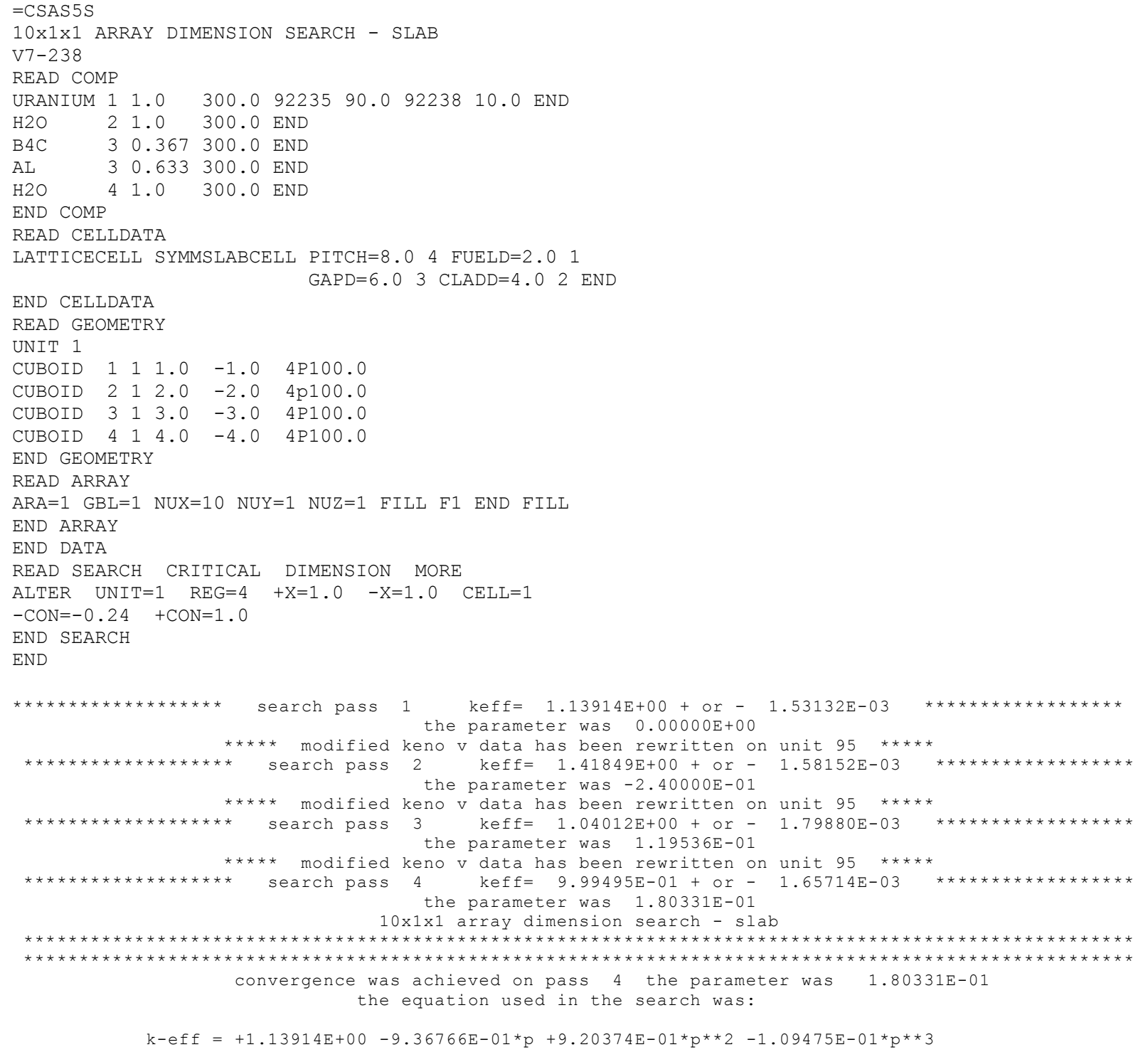




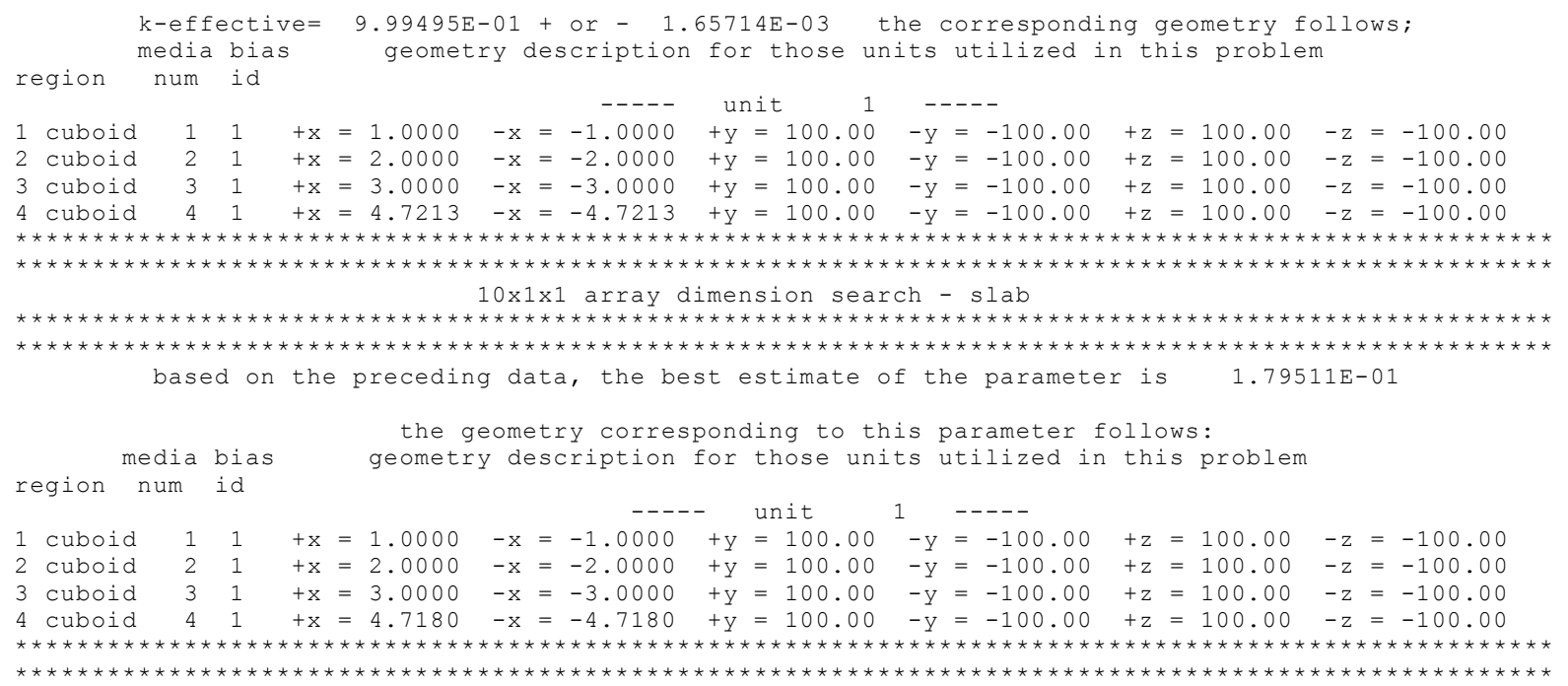

\section{Search on Multiple Units and Regions}

Consider a problem having Units 1, 2, and 3. Unit 1 consists of three concentric spheres in a cuboid. Unit 2 consists of a single sphere in a cuboid, and Unit 3 contains three concentric cuboids. A search is to be made that changes the exterior dimensions of the three units, the inner sphere of Unit 1, and the sphere of Unit 2. The thicknesses of the outer spheres of Unit 1 are to be maintained, and the two inner cuboids of Unit 3 are to remain unchanged. The search data and results for this problem are the following:

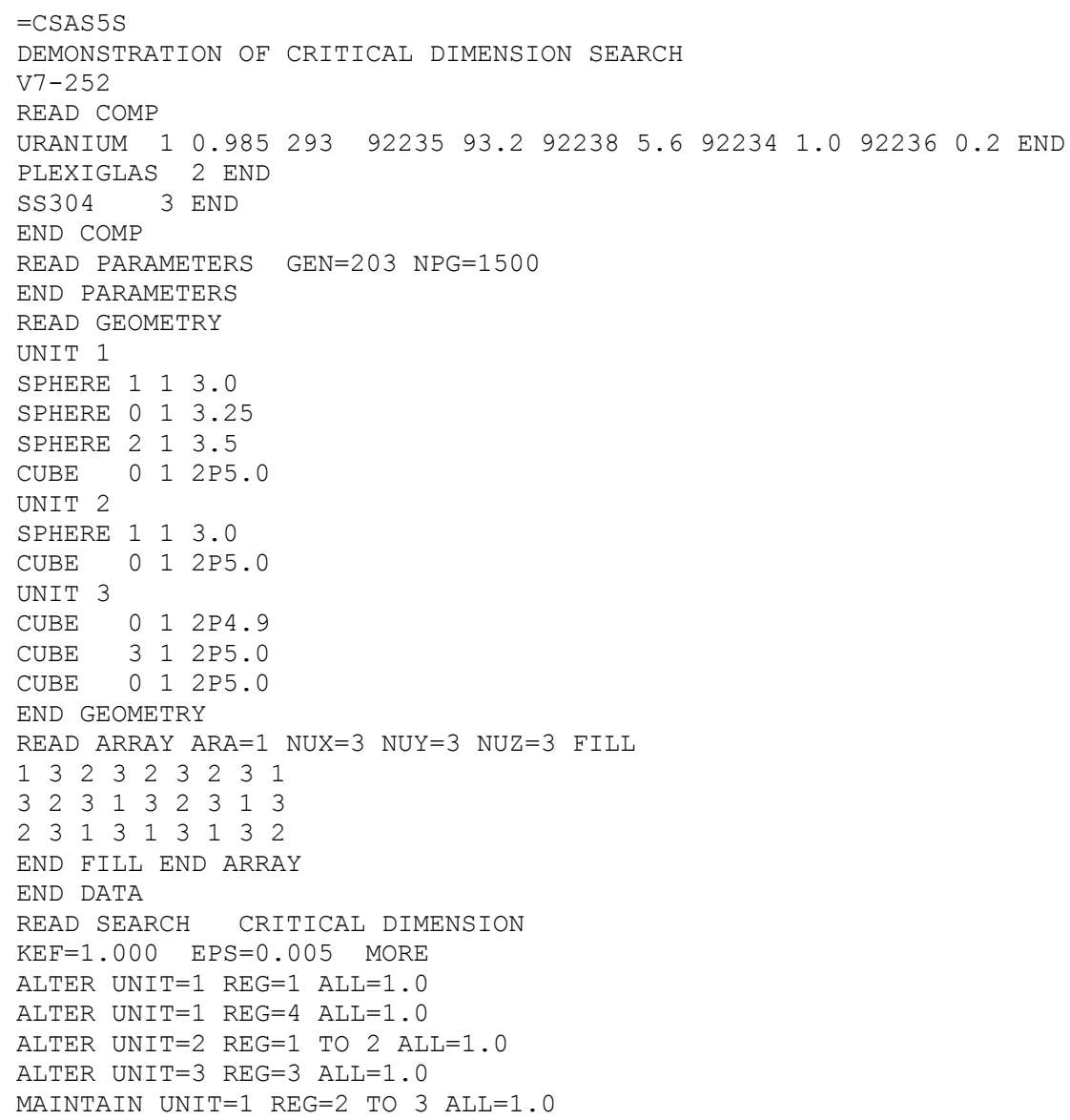


$+\mathrm{CON}=2.0 \quad-\mathrm{CON}=-0.5$

END SEARCH

END

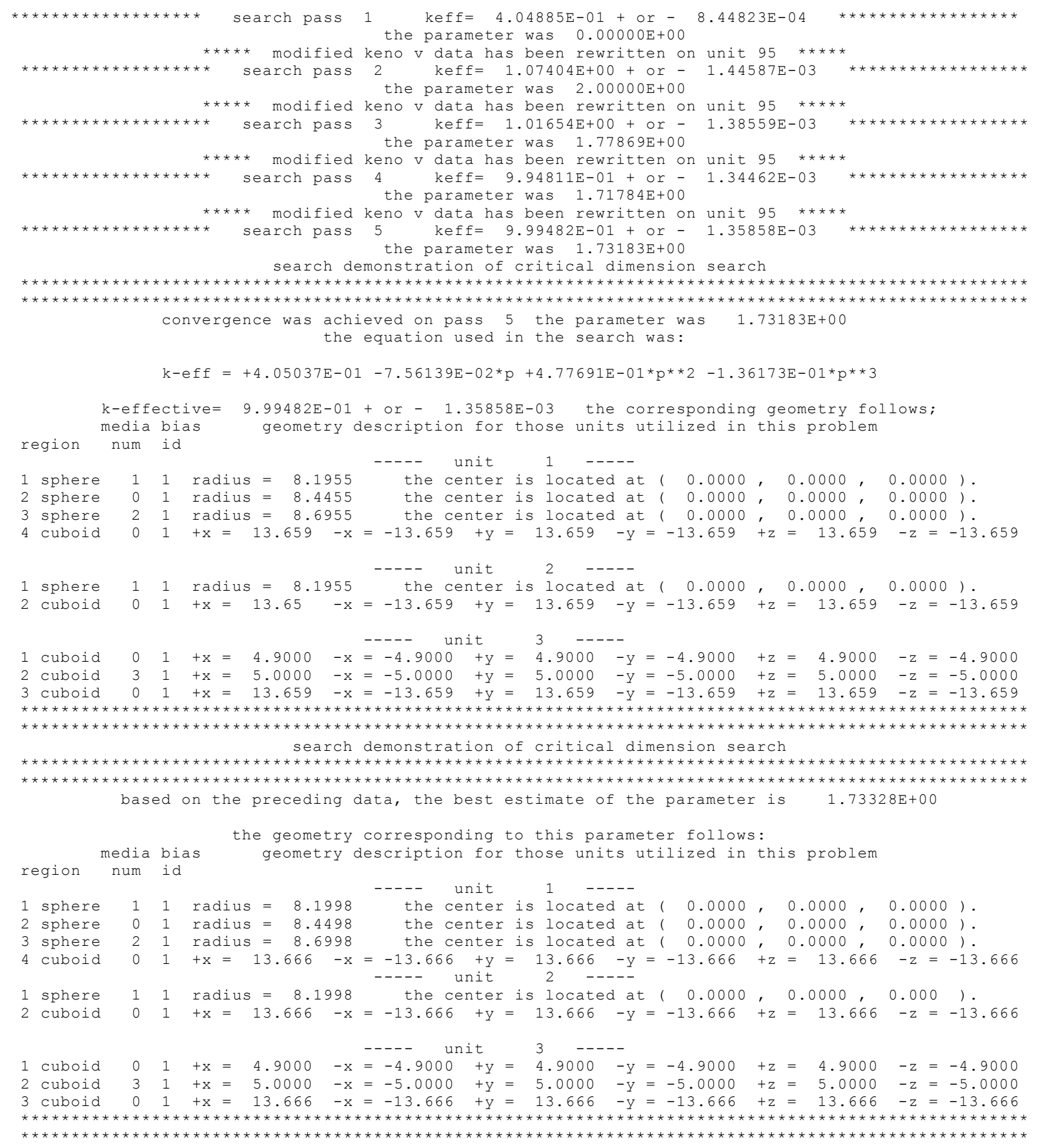

\section{$\mathrm{UO}_{2} \mathrm{~F}_{2}$ Solution Tank - Critical Search on Chord Length}

Consider a large spherical tank partially filled with $\mathrm{UO}_{2} \mathrm{~F}_{2}$ solution. The tank has a radius of $34.6 \mathrm{~cm}$ and is initially filled with solution to a height of $10.0 \mathrm{~cm}$ above the midpoint. The tank is composed of a 0.759-cm-thick $\mathrm{Al}$ shell. The $\mathrm{UO}_{2} \mathrm{~F}_{2}$ solution is composed of three standard compositions: $\mathrm{UO}_{2} \mathrm{~F}_{2}$, $\mathrm{HF}$ acid, and $\mathrm{H}_{2} \mathrm{O}$. The code combines these using a set algorithm. This may or may not produce a 
solution at the desired density. If the density of the solution is known it should be entered. Also, extra acid can be added to the solution by specifying a non-zero acid molarity.

A critical dimension search is performed on the length of the chord yielding system $k_{\text {eff }}=0.98$. In the MORE search data, UNIT=1 REG=1 CHORD=1 specifies that the chord length of region 1 in Unit 1 is to be altered with the limits defined by the search constraints. The minimum search constraint is set so region 1 consists of the part of the sphere below an X-Y plane $10.0 \mathrm{~cm}$ below the sphere's midpoint. The maximum search constraint is set so region 1 consists of almost the entire sphere.

The constraints are calculated as follows:

$$
\begin{aligned}
& \left.\mathrm{D}_{\mathrm{i}}=\left(\operatorname{Radius}_{\mathrm{i}}+\text { Chord }_{\mathrm{i}}\right) /\left(2 * \operatorname{Radius}_{\mathrm{i}}\right)=(34.6+10.0) / 2 * 34.6\right)=0.6445 \\
& \left.\mathrm{D}_{\text {min }}=\left(\operatorname{Radius}_{\min }+\text { Chord }_{\min }\right) /\left(2 * \operatorname{Radius}_{\min }\right)=(34.6+-10.0) / 2 * 34.6\right)=0.3555 \\
& \left.\mathrm{D}_{\max }=\left(\operatorname{Radius}_{\max }+\text { Chord }_{\max }\right) /\left(2 * \text { Radius }_{\max }\right)=(34.6+34.6) / 2 * 34.6\right)=1.0
\end{aligned}
$$

The maximum constraint is calculated according to:

$$
+\mathrm{CON}=\left[\left(\mathrm{D}_{\max } / \mathrm{D}_{\mathrm{i}}\right)-1.0\right] / \mathrm{CHORD}=[(1.0 / 0.6445)-1.0] / 1.0=0.5515
$$

The minimum constraint is calculated according to:

$$
-\mathrm{CON}=\left[\left(\mathrm{D}_{\min } / \mathrm{D}_{\mathrm{i}}\right)-1.0\right] / \mathrm{CHORD}=[(0.3555 / 0.6445)-1.0] / 1.0=-0.4484
$$

The search data and final search results for this problem follow:

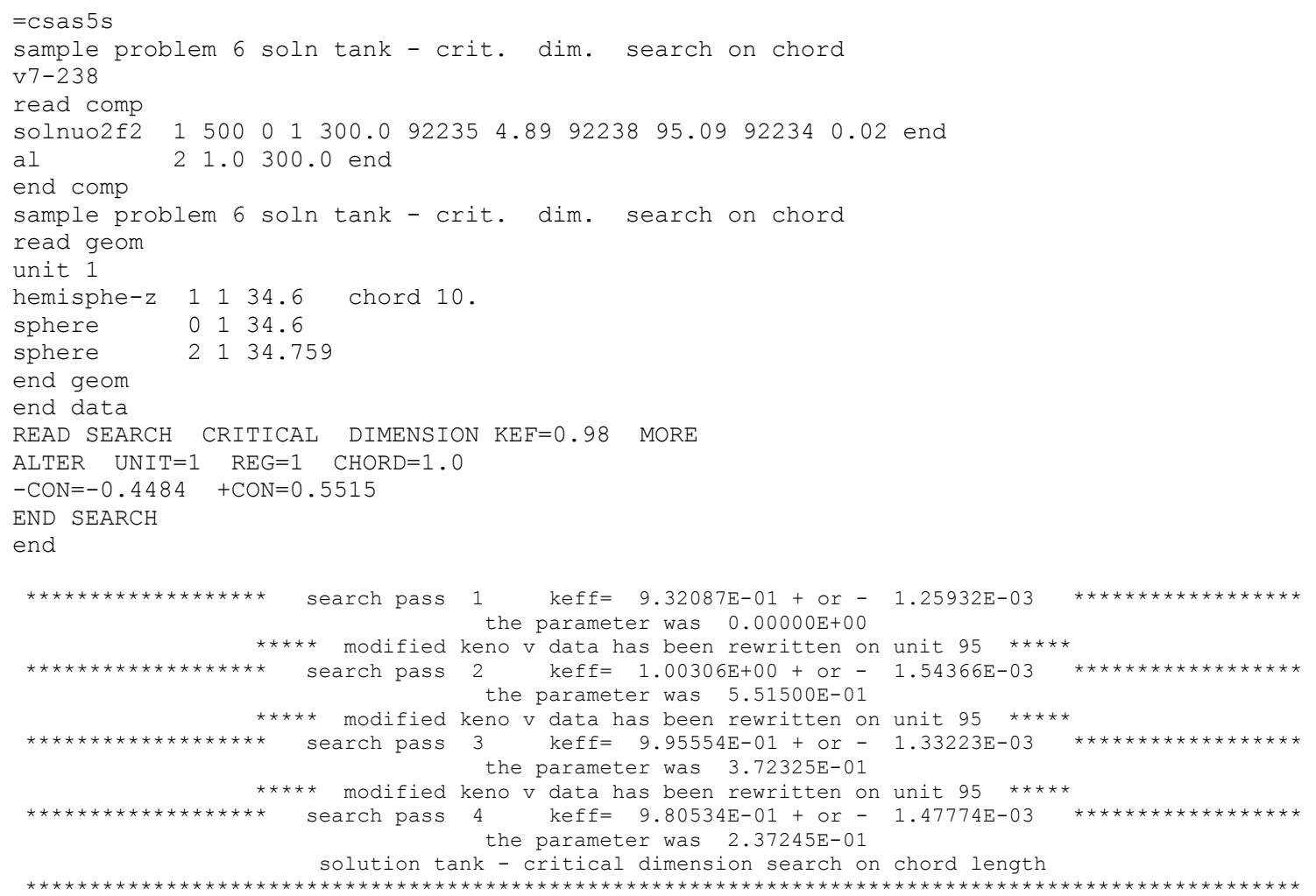




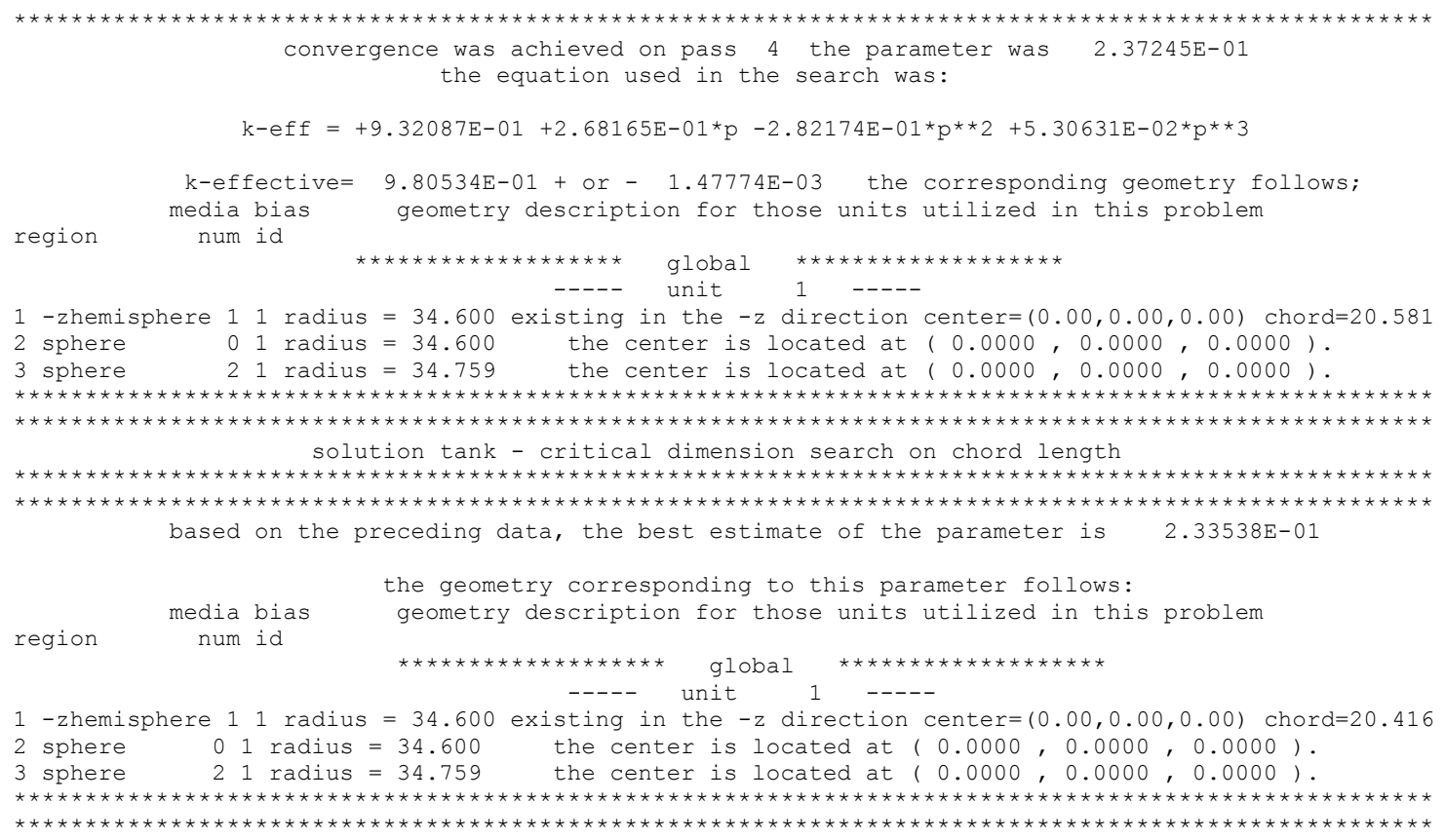

\subsection{A.12 Optimum concentration search}

An optimum concentration search alters the concentration of the specified standard composition in the specified mixture to determine the highest value of $k_{\text {eff. }}$. The limits for an optimum concentration search are governed by the values entered for the parameter constraints. An optimum concentration search is activated by entering "OPTIMUM CONCENTRATION" in the search data. No defaulted search data are in a concentration search. The user must specify the material and standard composition name to be changed and the manner in which they will be changed.

A concentration search is performed by altering the atom densities of the specified standard compositions in the specified materials. The ratio of how the standard compositions change relative to each other is controlled using FACTOR. If a material and standard composition is not listed in the search data it remains unchanged. The concentration search can vary from zero to some upper limit. The code will prevent the concentration from falling below zero, but the user is responsible for setting constraints that prevent the concentration from exceeding reasonable values. In most cases the theoretical density is a reasonable upper limit.

\section{Array of Spheres in $\mathrm{H}_{2} \mathrm{O}$ - Search on $\mathrm{H}_{2} \mathrm{O}$ Density}

Consider a $10 \times 10 \times 10$ array of uranium spheres arranged in an array having a "square" pitch. The uranium spheres are $2 \mathrm{~cm}$ in radius, and the center-to-center spacing is $8 \mathrm{~cm}$. The uranium spheres and their associated spacing are defined to be unit 1 , and the $10 \times 10 \times 10$ array is defined to be array 1 . The spheres are initially moderated by $1 / 2$ density water.

An optimum concentration search is performed on the water yielding the maximum system $k_{\text {eff }}$ for various densities of water. In the MORE search data, MIX=2 and SCNAME=H2O specify that the water component of mixture 2 is to be altered during the search. The maximum allowed density is full density water. The minimum allowed density is 0.05 density water.

The maximum constraint is calculated according to: 


$$
+\mathrm{CON}=\left(\frac{\mathrm{D}_{\max }}{\mathrm{D}_{\text {initial }}}-1\right) / \text { FACTOR } \quad+\mathrm{CON}=\left(\frac{1.0}{0.5}-1\right) / 1=1 / 0
$$

The minimum constraint is calculated according to:

$$
-\mathrm{CON}=\left(\frac{\mathrm{D}_{\text {min }}}{\mathrm{D}_{\text {initial }}}-1\right) / \text { FACTOR } \quad-\mathrm{CON}=\left(\frac{0.05}{0.5}-1\right) / 1=-0.9 .
$$

The search data and final search results for this problem follow:

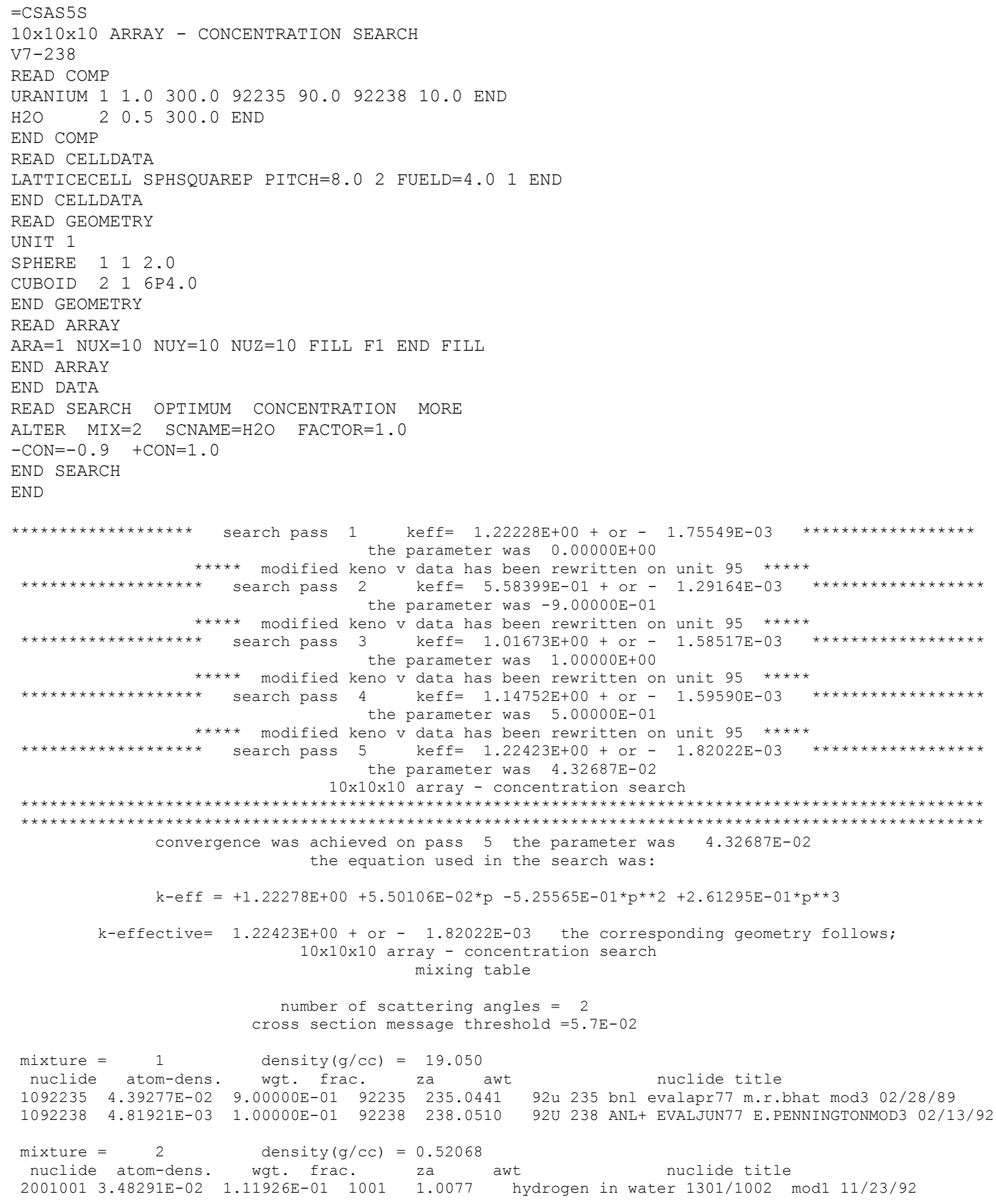




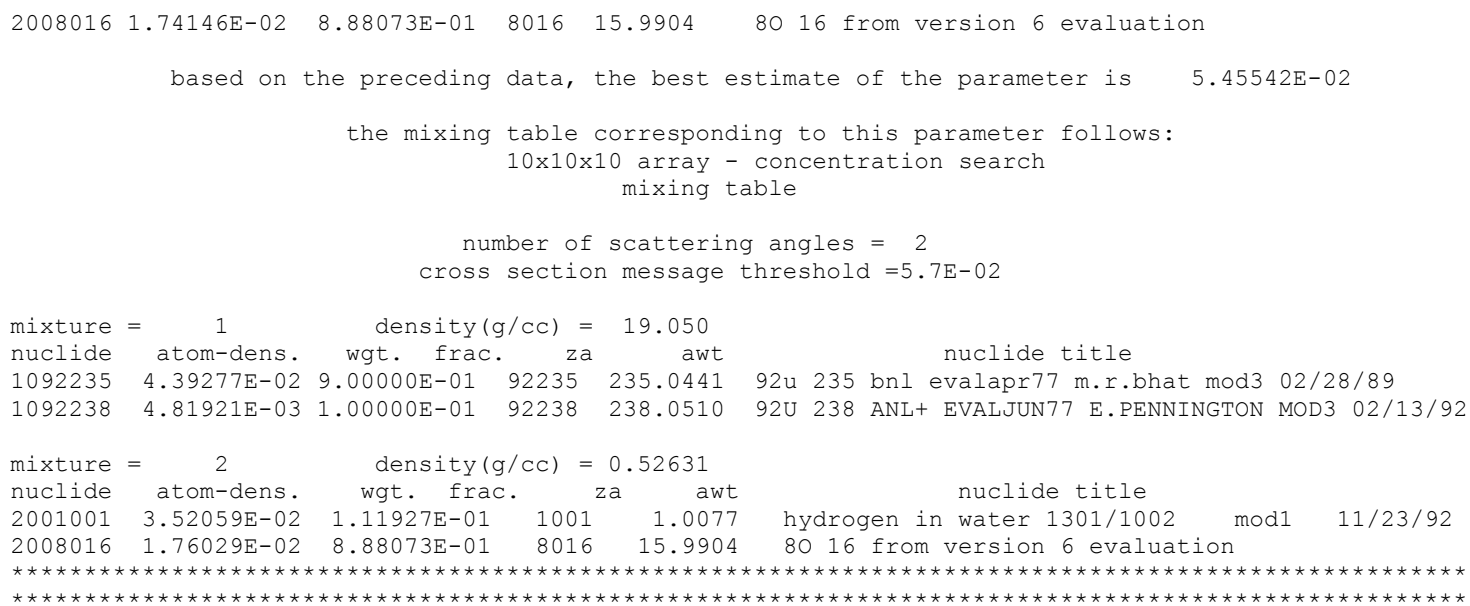

\section{Array of Cylinders in $\mathrm{H}_{2} \mathrm{O}$ - Search on $\mathrm{H}_{2} \mathrm{O}$ Density}

This problem contains search data for an optimum concentration search. The problem consists of a $10 \times 10 \times 1$ array of uranium cylinders surrounded initially by $0.5 \mathrm{gm} / \mathrm{cc}$ density water. The cylinders are $90 \%{ }^{235} \mathrm{U}$ and $10 \%{ }^{238} \mathrm{U}$ and have a radius of $2.0 \mathrm{~cm}$ and a length of $200 \mathrm{~cm}$. The cylinders are originally centered on an $8.0 \mathrm{~cm} \mathrm{X}$ and $\mathrm{Y}$ pitch with interstitial low density $(0.5 \mathrm{gm} / \mathrm{cc})$ water. The problem searches for the $\mathrm{H}_{2} \mathrm{O}$ density that will produce the maximum system $k_{\text {eff. }}$ In the MORE search data, $\mathrm{MIX}=2$ and SCNAME$=\mathrm{H} 2 \mathrm{O}$ specify that the water component of mixture 2 is to be altered during the search. The maximum allowed density is full density water. The minimum allowed density is 0.05 density water.

The maximum constraint is calculated according to:

$$
+\mathrm{CON}=\left(\frac{\mathrm{D}_{\max }}{\mathrm{D}_{\text {initial }}}-1\right) / \text { FACTOR } \quad+\mathrm{CON}=\left(\frac{1.0}{0.5}-1\right) / 1=-1.0 .
$$

The minimum constraint is calculated according to:

$$
-\mathrm{CON}=\left(\frac{\mathrm{D}_{\text {min }}}{\mathrm{D}_{\text {initial }}}-1\right) / \text { FACTOR } \quad-\mathrm{CON}=\left(\frac{0.05}{0.5}-1\right) / 1=-0.9
$$

The search data and final search results for this problem follow:

$=\operatorname{CSAS} 5 \mathrm{~S}$

10x10x1 ARRAY CONCENTRATION SEARCH - CHANGE H2O

$\mathrm{V} 7-238$

READ COMP

URANIUM $111.0 \quad 300.0 \quad 9223590.0 \quad 92238 \quad 10.0 \quad$ END

$\begin{array}{lllll}\mathrm{H} 2 \mathrm{O} & 2 & 0.5 & 300.0 & \mathrm{END}\end{array}$

END COMP

READ CELLDATA

LATTICECELL SQUAREPITCH PITCH=8.0 2 FUELD=4.0 1 END

END CELLDATA

READ GEOMETRY

UNIT 1

$\begin{array}{lllllll}\text { CYLINDER } & 1 & 1 & 2.0 & 100.0 & -100.0\end{array}$

CUBOID 21 4p 4.0 2p100.0

END GEOMETRY

READ ARRAY

ARA=1 NUX=10 NUY=10 NUZ=1 FILL F1 END FILL

END ARRAY 
END DATA

READ SEARCH OPTIMUM CONCENTRATION MORE

ALTER MIX $=2 \quad$ SCNAME $=\mathrm{H} 2 \mathrm{O} \quad$ FACTOR $=1.0$

$-\mathrm{CON}=-0.9+\mathrm{CON}=1.0$

END SEARCH

END

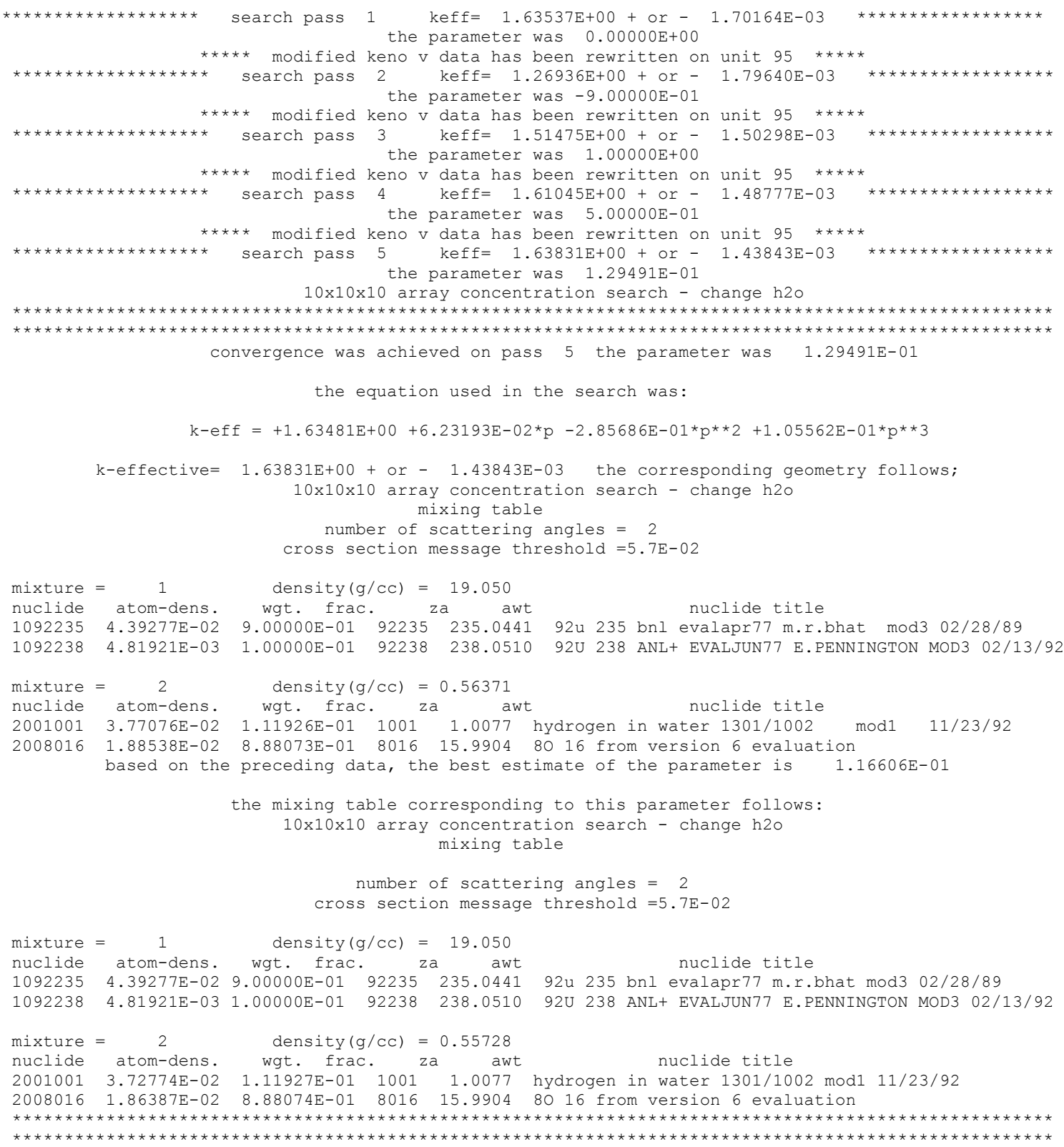

\section{Cell-Weighted Array of Uranium Cylinders in $\mathrm{H}_{2} \mathrm{O}$ - Search on $\mathrm{H}_{2} \mathrm{O}$ Density}

This problem contains search data for an optimum concentration search. The problem consists of a single material representing a $10 \times 10 \times 1$ array of uranium cylinders surrounded initially by $0.5 \mathrm{gm} / \mathrm{cc}$ density water. The cylinders are $90 \%{ }^{235} \mathrm{U}$ and $10 \%{ }^{238} \mathrm{U}$ and have a radius of $2.0 \mathrm{~cm}$ and a length of $200 \mathrm{~cm}$. The cylinders are originally centered on an $8.0 \mathrm{~cm} \mathrm{X}$ and Y-pitch with interstitial low density $(0.5 \mathrm{gm} / \mathrm{cc})$ water. The keyword CELL=100 in the unit cell data indicates this will be a cell-weighted problem with mixture 100 representing the array of uranium rods in water. The problem searches for the $\mathrm{H}_{2} \mathrm{O}$ density 
that will produce the maximum system $k_{\text {eff. }}$ In the MORE search data, MIX $=2$ and SCNAME $=\mathrm{H}_{2} \mathrm{O}$ specify that the water component of mixture 2 is to be altered during the search. On each pass the water density is altered, XSDRNPM is run to produce a new mixture 100, and mixture 100 is used in KENO V.a to calculate a new system $k_{\text {eff. }}$ The cylinder and cuboid in unit 1 and the array contained in the above problem are replaced by a cuboid of the same size as the array containing mixture 100 . The maximum allowed density is full density water. The minimum allowed density is 0.05 density water.

The maximum constraint is calculated according to:

$$
+\mathrm{CON}=\left(\frac{\mathrm{D}_{\text {max }}}{\mathrm{D}_{\text {initial }}}-1\right) / \text { FACTOR } \quad+\mathrm{CON}=\left(\frac{1.0}{0.5}-1\right) / 1=-1.0 .
$$

The minimum constraint is calculated according to:

$$
-\mathrm{CON}=\left(\frac{\mathrm{D}_{\text {min }}}{\mathrm{D}_{\text {initial }}}-1\right) / \text { FACTOR } \quad-\mathrm{CON}=\left(\frac{0.05}{0.5}-1\right) / 1=-0.9
$$

The search data and final search results for this problem follow:

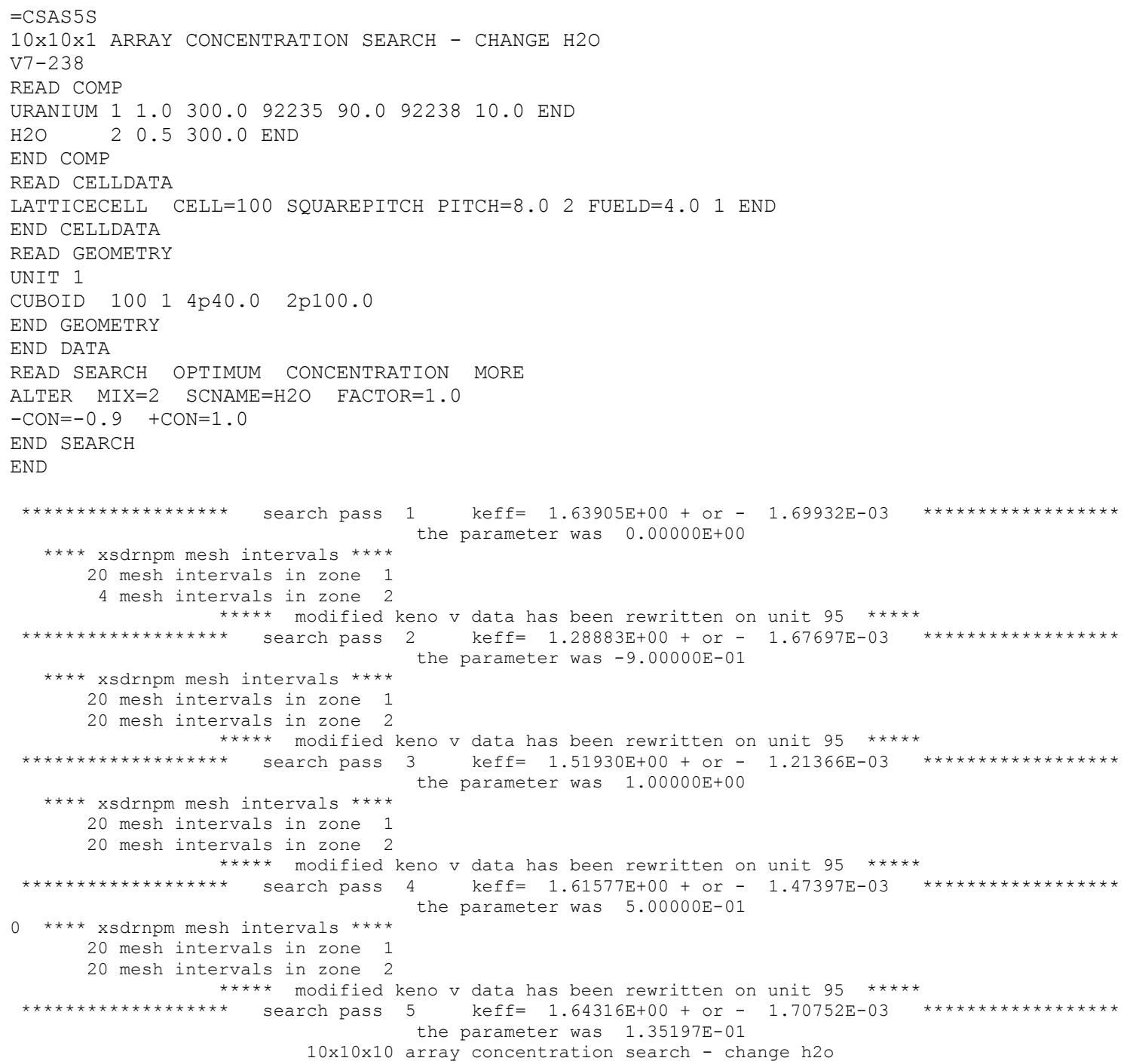




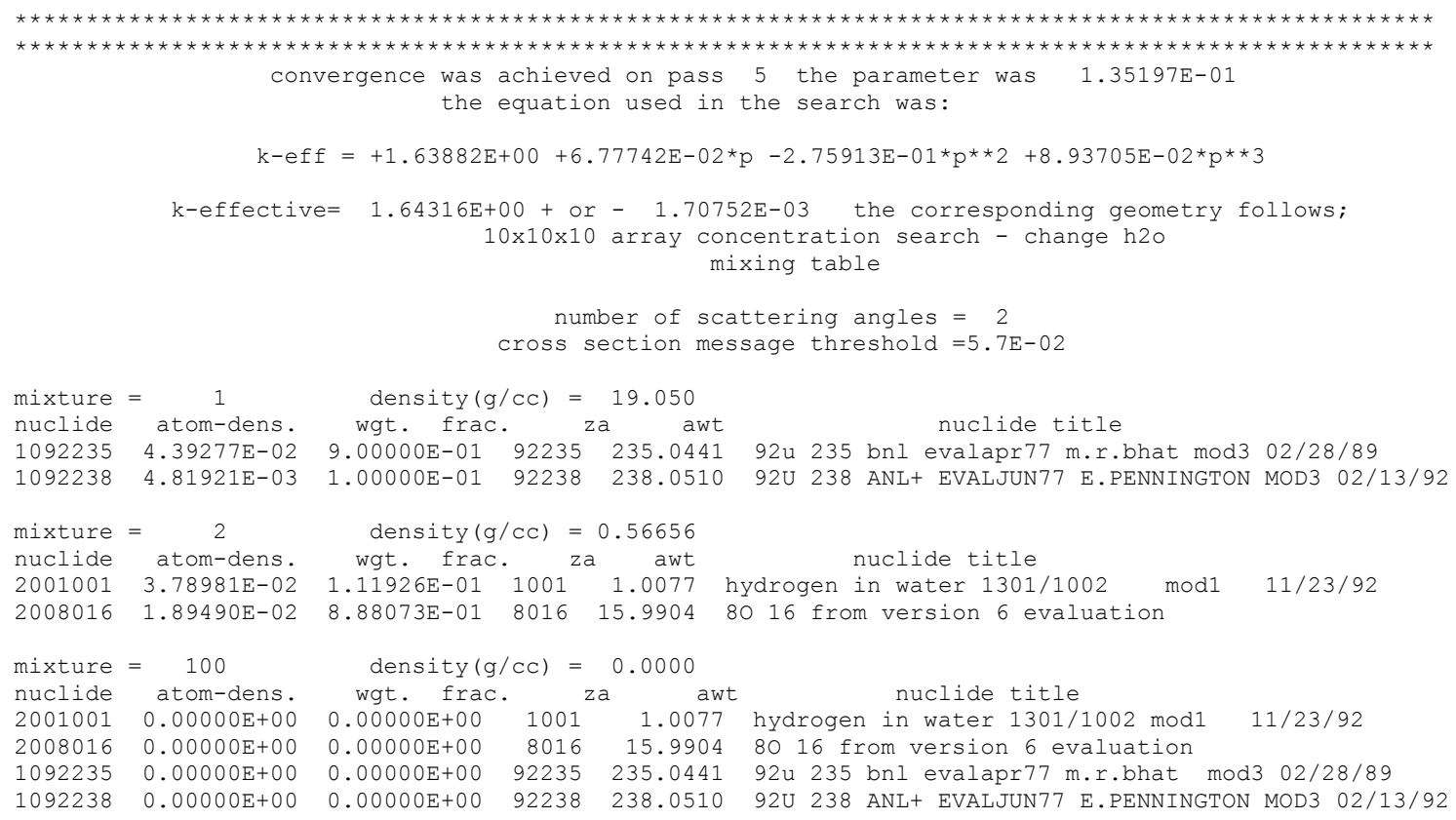

\subsection{A.13 Minimum concentration search}

A minimum concentration search searches for the standard composition density that yields the lowest value of $k_{\text {eff. }}$ A minimum concentration search is activated by entering "MINIMUM CONCENTRATION" in the search data. There are no defaulted search data in a concentration search. The user must specify the material and standard composition name to be changed and the manner in which they will be changed as described in the auxiliary search commands.

A concentration search is performed by altering the atom densities of the specified standard compositions in the specified materials. The ratio of how the standard compositions change relative to each other is controlled using FACTOR. If a material and standard composition is not listed in the search data it remains unchanged. The concentration search can vary from zero to some upper limit. The code will prevent the concentration from falling below zero, but the user is responsible for setting constraints that 
prevent the concentration from exceeding reasonable values. In most cases the theoretical density is a reasonable upper limit.

\section{Infinite Array of Fuel Bundles Separated by Flux Traps — Search on $\mathrm{H}_{2} \mathrm{O}$ Density in Trap}

The fuel bundles in this problem represent $17 \times 17$ PWR fuel assemblies. The fuel pins are smeared together, making a mixture 100. The fuel pins consist of $2.35 \mathrm{wt} \%{ }^{235} \mathrm{U}$ having a diameter of $0.823 \mathrm{~cm}$, zirconium cladding having an outer diameter of $0.9627 \mathrm{~cm}$, and a pitch of $1.275 \mathrm{~cm}$. The fuel bundle is represented as a $10.8375 \mathrm{~cm} \times 10.8375 \mathrm{~cm} \times 366 \mathrm{~cm}$ cuboid of mixture 100 surrounded by Boral and then water. The Boral has a density of $2.65 \mathrm{~g} / \mathrm{cm}^{3}$ and is composed of $35.17 \mathrm{wt} \% \mathrm{~B}_{4} \mathrm{C}$ and $64.83 \mathrm{wt} \% \mathrm{Al}$. The fuel bundles are at a fixed pitch of $13.0 \mathrm{~cm}$. This is the same problem as described for a minimum dimension search above with the water component of the trap size optimized, thus producing a minimum system $k_{\text {eff. }}$ The input data and the final search results for this problem follow:

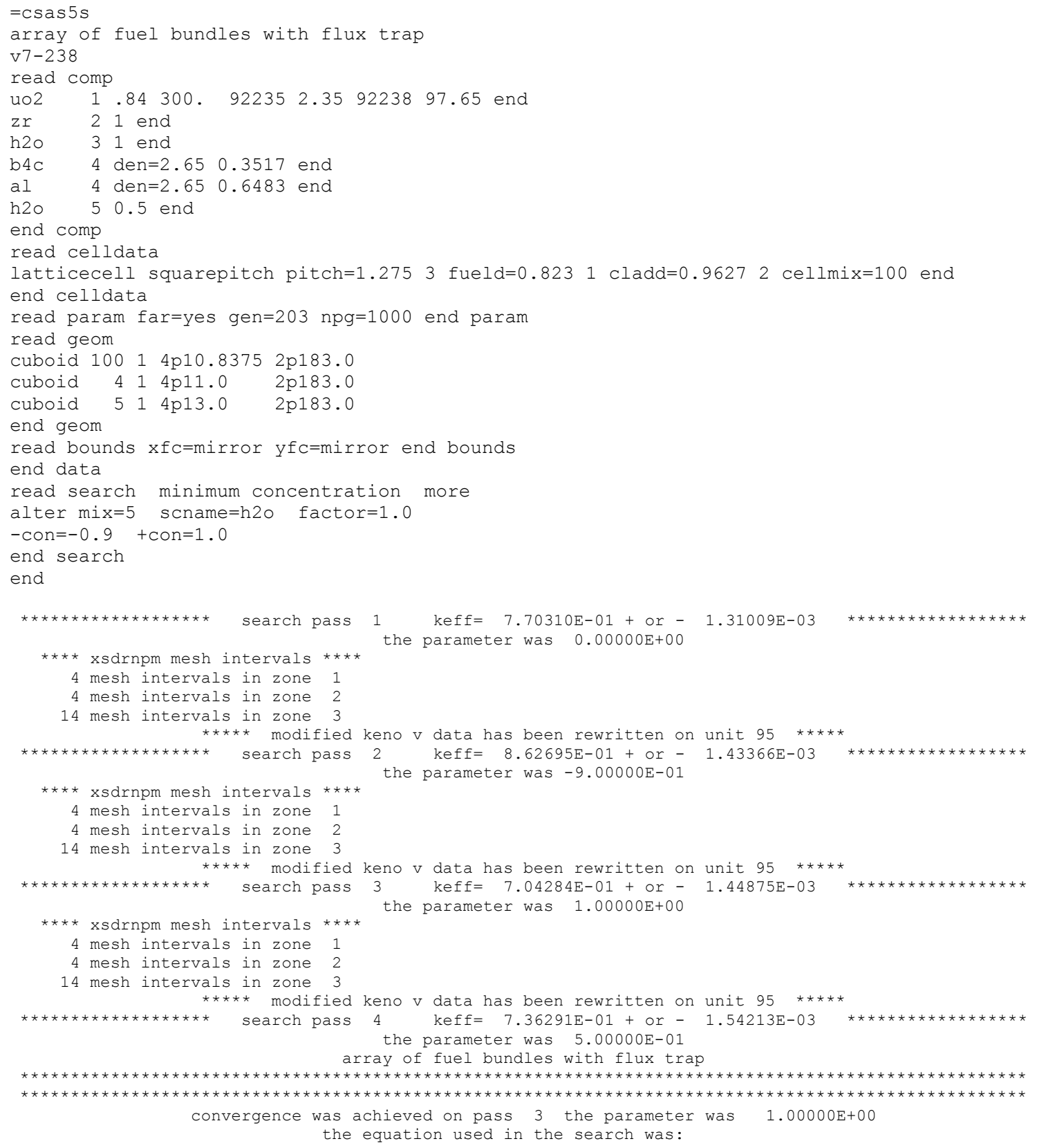




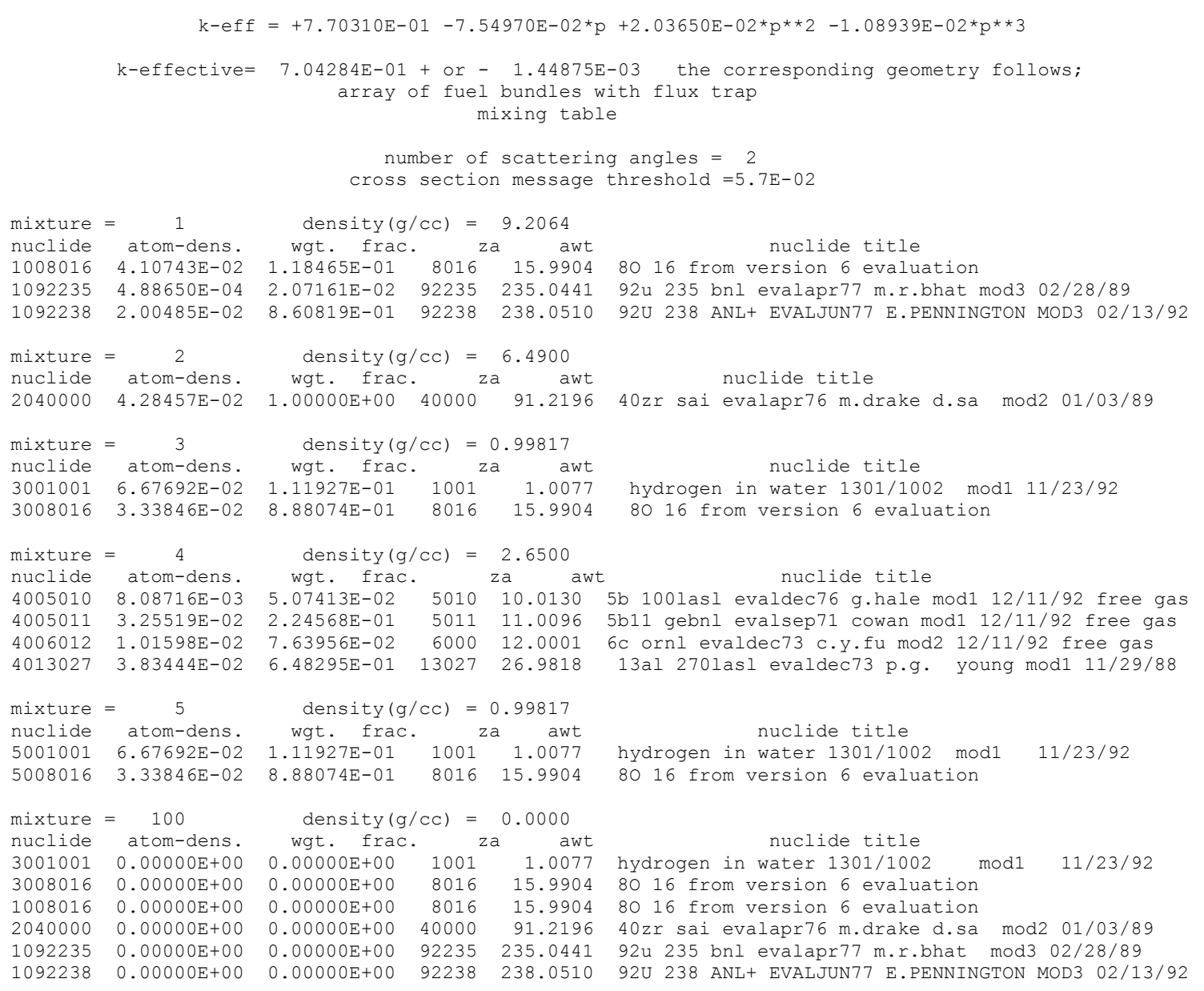

\subsection{A.14 Critical concentration search}

A critical concentration search alters the concentration of the specified standard composition in the specified mixture to obtain a specified value of $k_{\text {eff. }}$ A critical concentration search is activated by entering "CRITICAL CONCENTRATION" in the search data. There are no defaulted search data in a critical concentration search except for the value of $k_{\text {eff. }}$. If something other than $k_{\text {eff }}=1.0$ is desired the user must specify $\mathrm{KEF}=* *$, where $* *$ is the desired value of $k_{\text {eff. }}$ The remaining data is entered after the keyword MORE in the search data block. The user must specify the material and standard composition name to be changed and the manner in which they will be changed as described in the auxiliary search commands.

A concentration search is performed by altering the atom densities of the specified standard compositions in the specified materials. The ratio of how the standard compositions change relative to each other is controlled using FACTOR. If a material and standard composition is not listed in the search data it remains unchanged. The concentration search can vary from zero to some upper limit. The code will prevent the concentration from falling below zero, but the user is responsible for setting constraints that prevent the concentration from exceeding reasonable values. In most cases the theoretical density is a reasonable upper limit.

\section{Array of Spheres in $\mathrm{H}_{2} \mathrm{O}$ - Search on $\mathrm{H}_{2} \mathrm{O}$ Density}

Consider a $10 \times 10 \times 10$ array of uranium spheres arranged in an array having a "square" pitch. The uranium spheres are $2 \mathrm{~cm}$ in radius, and the center-to-center spacing is $8 \mathrm{~cm}$. The uranium spheres 
and their associated spacing are defined to be unit 1 , and the $10 \times 10 \times 10$ array is defined to be array $1.0 \mathrm{~cm}$. The spheres are initially moderated by $1 / 2$ density water.

A critical concentration search is performed on the water yielding system $k_{\text {eff }}=1.0$ for various densities of water. In the MORE search data, MIX $=2$ and $\mathrm{SCNAME}=\mathrm{H} 2 \mathrm{O}$ specify that the water component of mixture 2 is to be altered during the search from an initial density of $0.5 \mathrm{gm} / \mathrm{cc}$. The maximum allowed density is full density water $(1.0 \mathrm{gm} / \mathrm{cc})$. The minimum allowed water density is $0.0005 \mathrm{gm} / \mathrm{cc}$.

The maximum constraint is calculated according to:

$$
+\mathrm{CON}=(1.0 / 0.5-1) / 1.0=1.0
$$

The minimum constraint is calculated according to:

$$
-\mathrm{CON}=(0.0005 / 0.5-1) / 1.0=-0.999
$$

The search data and final search results for this problem follow:

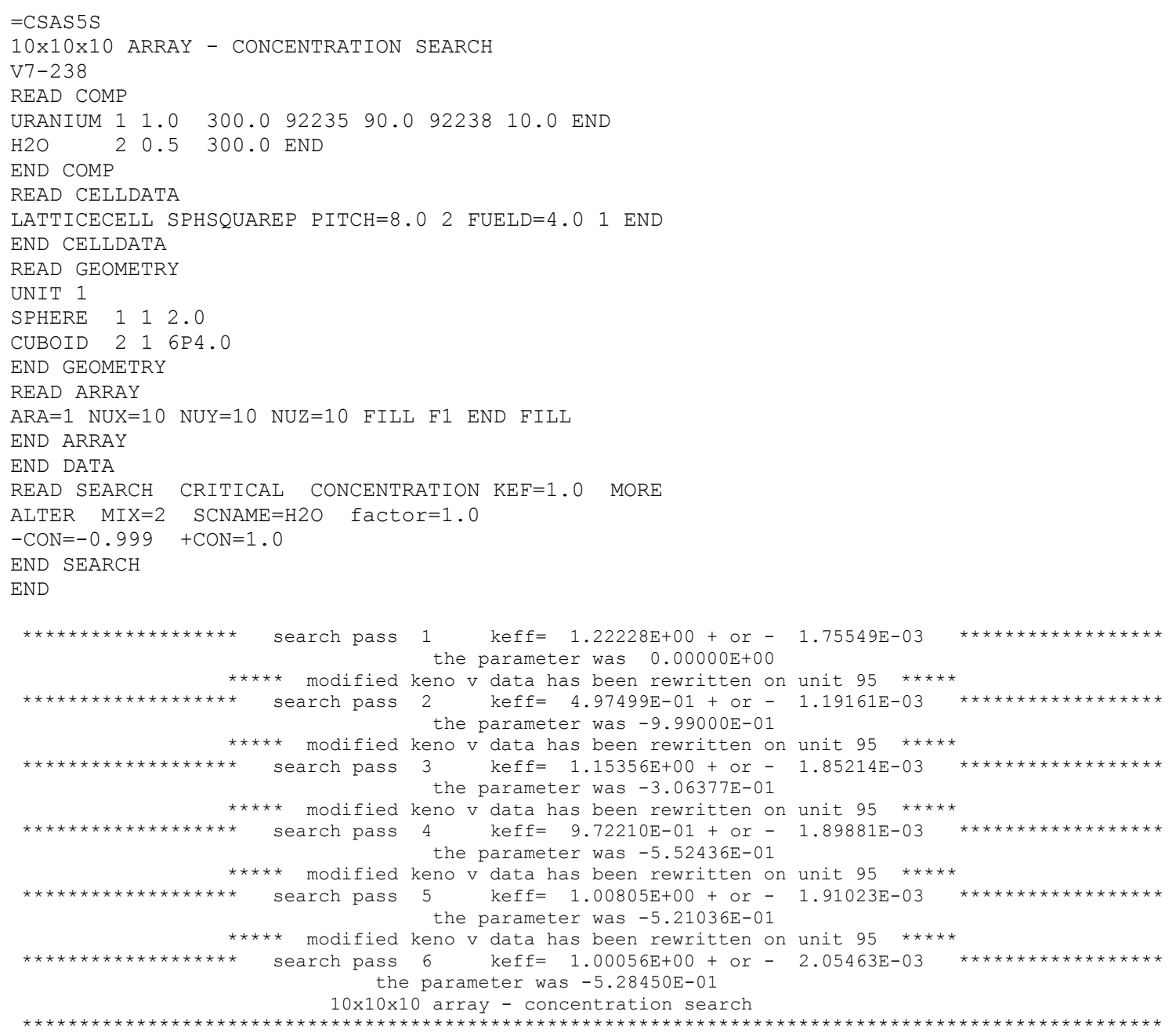




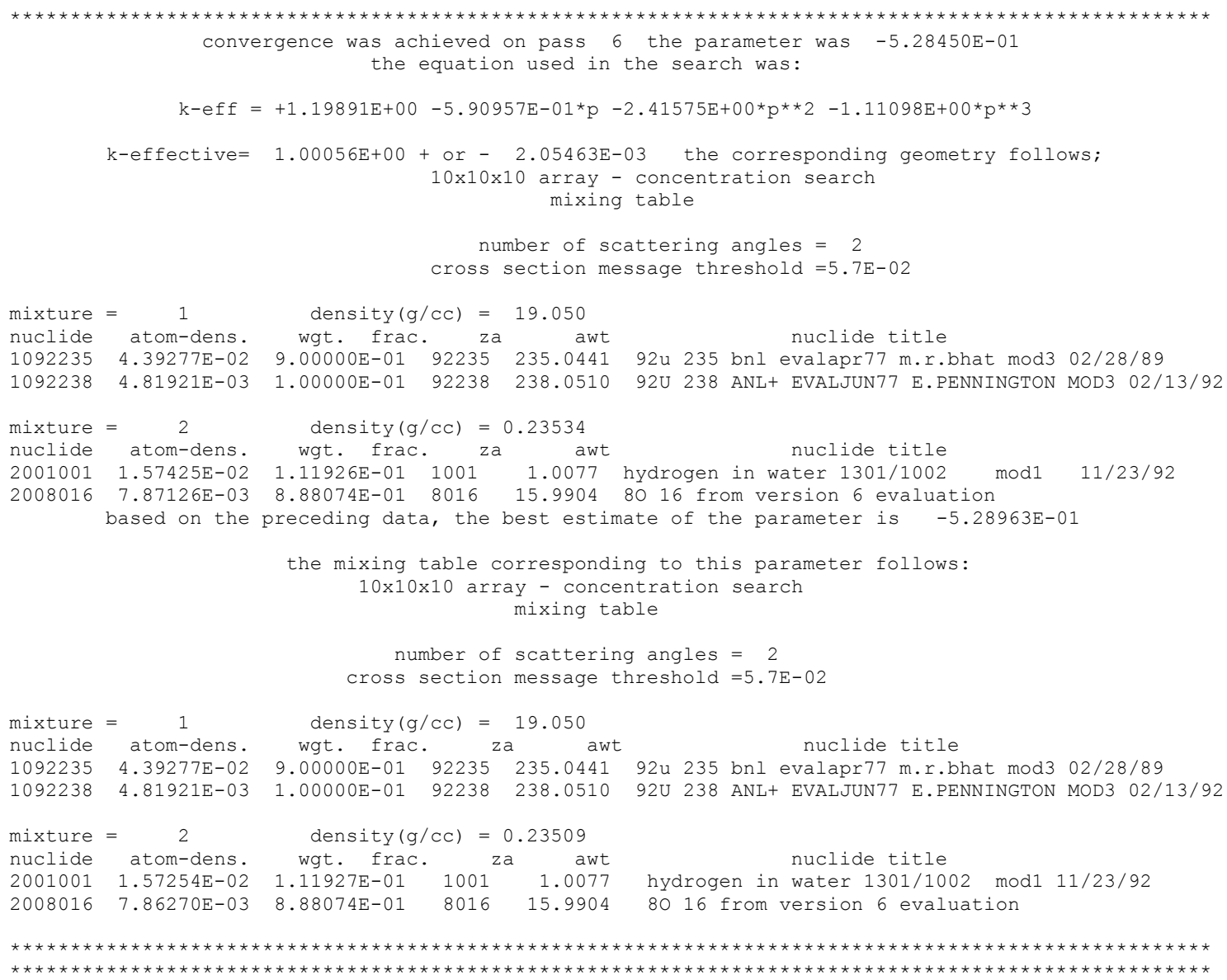

\section{Array of Spheres in $\mathrm{H}_{2} \mathrm{O}$ - Search on $\mathrm{H}_{2} \mathrm{O}$ Density}

This is the same problem as described above with the exception of the initial water density, which is now $0.25 \mathrm{gm} / \mathrm{cc}$. A critical concentration search is performed on the water yielding a system $k_{\text {eff }}=1.0$ for various densities of water. In the MORE search data, MIX $=2$ and SCNAME $=\mathrm{H} 2 \mathrm{O}$ specify that the water component of mixture 2 is to be altered during the search from an initial value of $0.25 \mathrm{gm} / \mathrm{cc}$. The maximum allowed density is $1 / 2$ density water $(0.5 \mathrm{gm} / \mathrm{cc})$. The minimum allowed water density is $0.1 \mathrm{gm} / \mathrm{cc}$.

The maximum constraint is calculated according to:

$$
+\mathrm{CON}=(5.0 / 0.25-1) / 1.0=1.0
$$

The minimum constraint is calculated according to:

$$
-\mathrm{CON}=(0.1 / 0.25-1) / 1.0=-0.6
$$

The search data and final search results for this problem follow: 


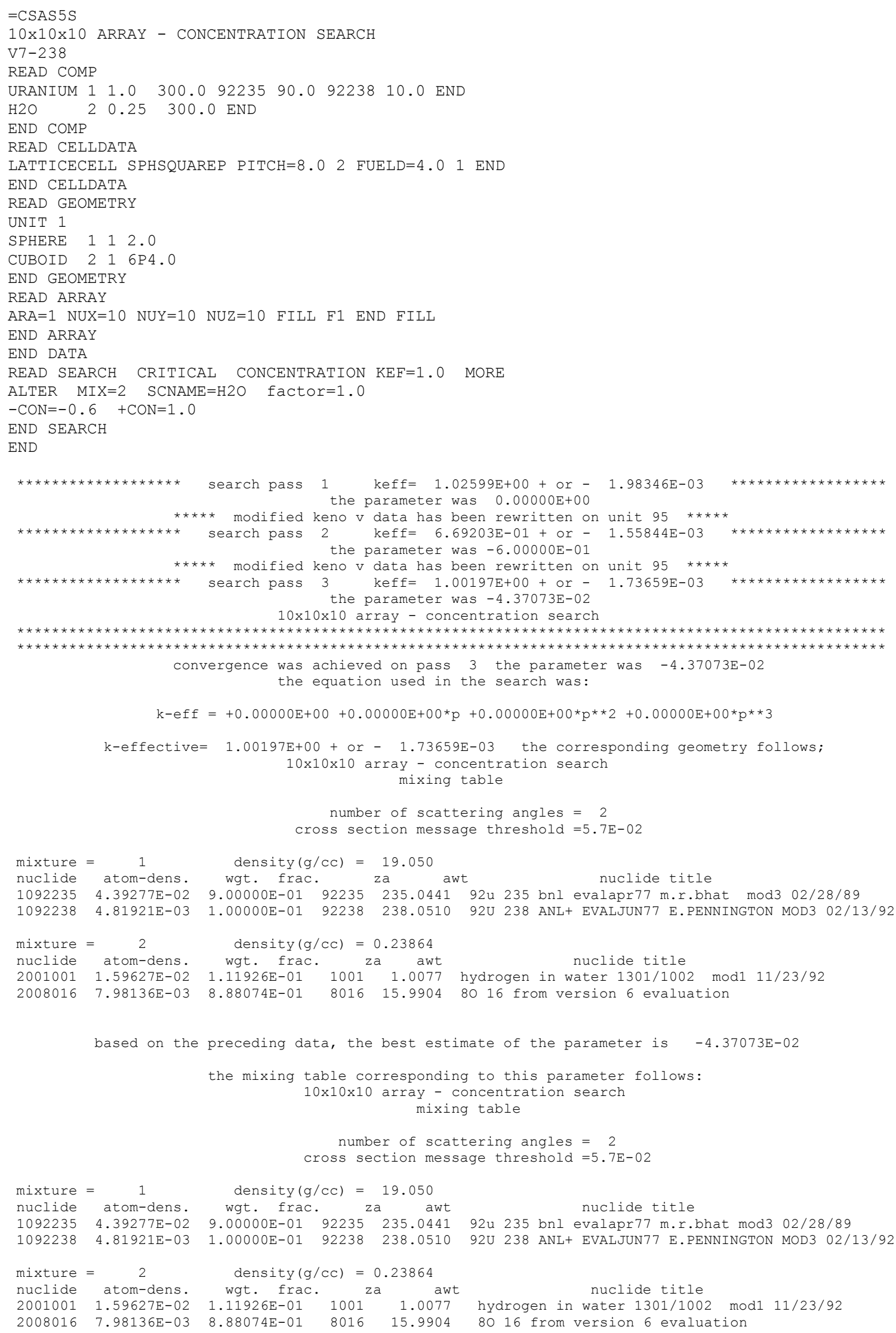




\section{$\mathrm{UO}_{2} \mathrm{~F}_{2}$ Solution Tank - Critical Search on $\mathrm{UO}_{2} \mathbf{F}_{2}$ Density}

Consider a large spherical tank partially filled with $\mathrm{UO}_{2} \mathrm{~F}_{2}$ solution. The tank has a radius of $34.6 \mathrm{~cm}$ and is filled with solution to a height of $30.0 \mathrm{~cm}$ above the midpoint. The tank is composed of a $0.759 \mathrm{~cm}$ thick $\mathrm{Al}$ shell. The $\mathrm{UO}_{2} \mathrm{~F}_{2}$ solution is composed of three standard compositions: UO2F2, $\mathrm{HF}$ acid, and $\mathrm{H} 2 \mathrm{O}$. The code combines these using a set algorithm. This may or may not produce a solution at the desired density. If the density of the solution is known it should be entered. Also, extra acid can be added to the solution by specifying a non-zero acid molarity.

A critical concentration search is performed on the water yielding system $k_{\text {eff }}=1.0$ for various densities of $\mathrm{UO}_{2} \mathrm{~F}_{2}$ in the solution. In the MORE search data, $\mathrm{MIX}=1$ and SCNAME=UO2F 2 specify that the $\mathrm{UO}_{2} \mathrm{~F}_{2}$ component of the mixture 1 solution is to be altered during the search. The code calculates the density of the solution. The initial uranium fuel density is $300 \mathrm{gm} /$ liter. The maximum allowed uranium density is $600 \mathrm{gm} /$ liter. The minimum allowed uranium density is $150 \mathrm{gm} / \mathrm{liter}$.

The maximum constraint is calculated according to:

$$
+\mathrm{CON}=(600 / 300-1) / 1.0=1.0
$$

The minimum constraint is calculated according to:

$$
-\mathrm{CON}=(150 / 300-1) / 1.0=-0.5
$$

The search data and final search results for this problem follow:

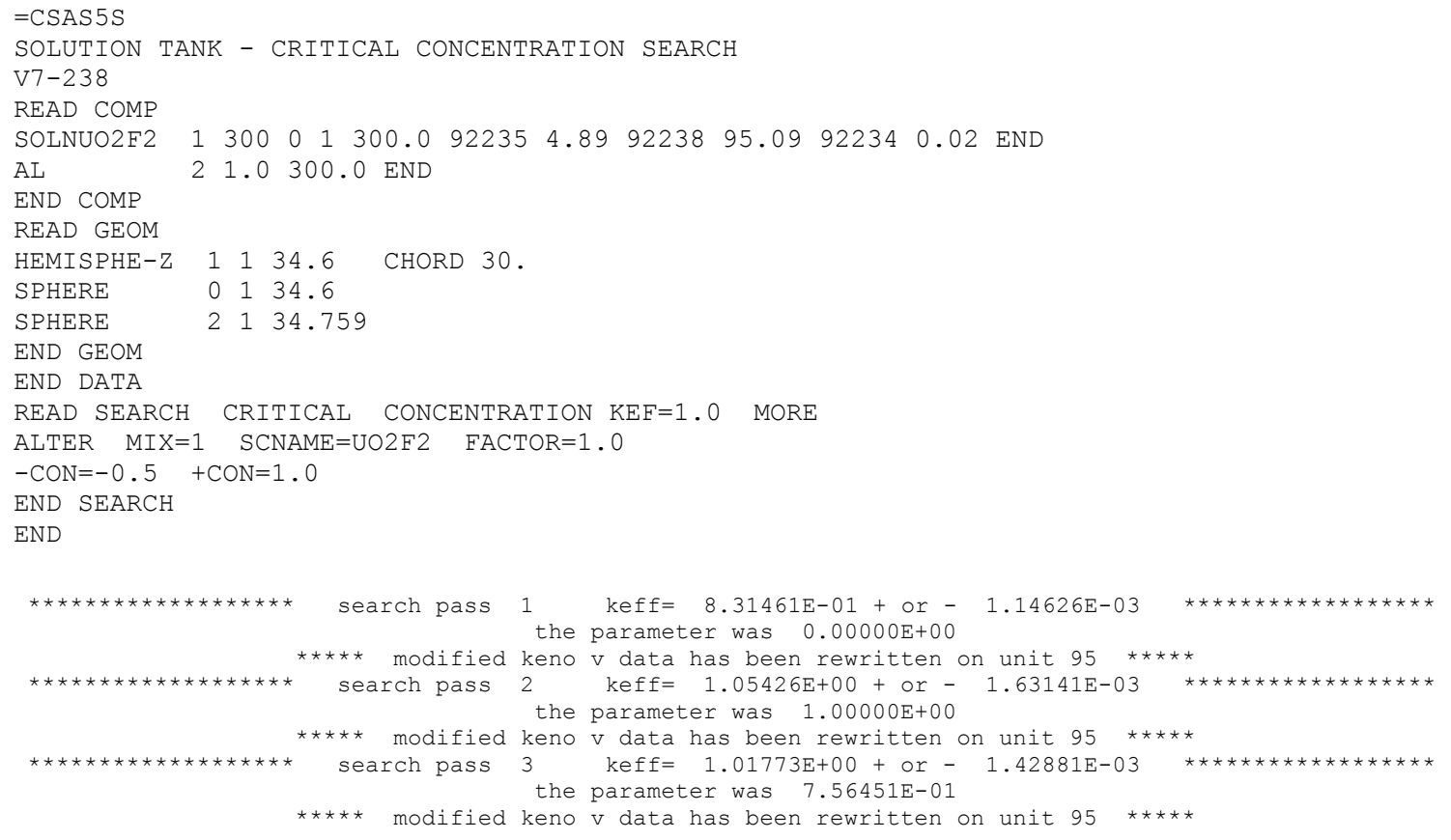




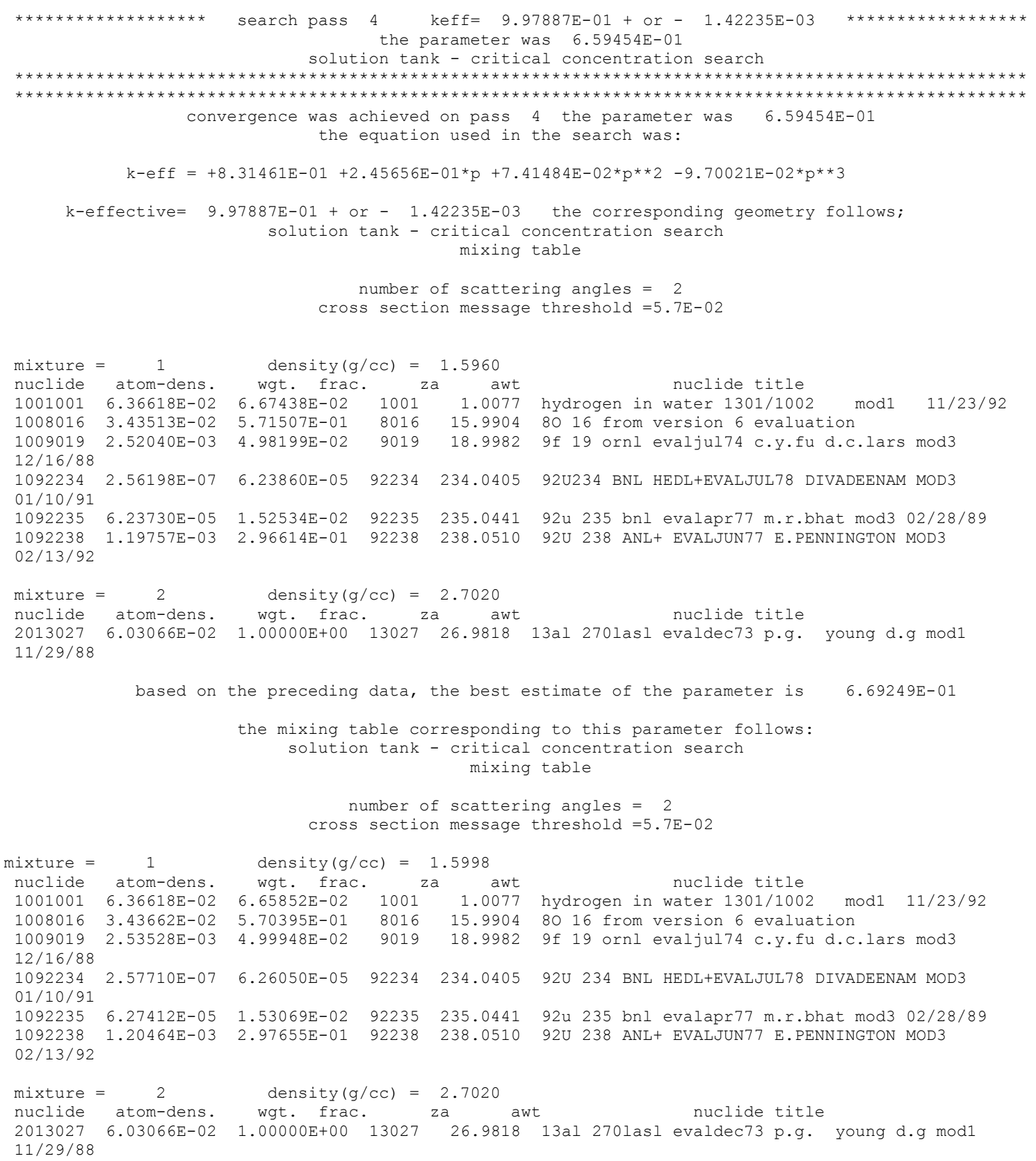

The final solution for this search contains $500 \mathrm{gm} /$ liter of uranium. The search, however, did not change the amount of acid or water in the solution. To get a better estimation of the solution density and $k_{\text {eff }}$ for this concentration of uranium the problem should be run again with an initial uranium density of $500 \mathrm{gm} /$ liter as shown below.

\section{$\mathrm{UO}_{2} \mathrm{~F}_{2}$ Solution Tank - Critical Search on $\mathrm{UO}_{2} \mathrm{~F}_{2}$ Density, Check}

This is the same $\mathrm{UO}_{2} \mathrm{~F}_{2}$ solution tank problem examined above except the initial uranium density is $500 \mathrm{gm} / \mathrm{liter}$. This problem is run again so the code will calculate water and acid densities associated with this density of uranium. The search data has also been modified to account for the new density of uranium. 
A critical concentration search is performed on the water yielding system $k_{\text {eff }}=1.0$ for various densities of $\mathrm{UO}_{2} \mathrm{~F}_{2}$ in the solution. In the MORE search data, $\mathrm{MIX}=1$ and $\mathrm{SCNAME}=\mathrm{UO} 2 \mathrm{~F} 2$ specify that the $\mathrm{UO}_{2} \mathrm{~F}_{2}$ component of the mixture 1 solution is to be altered during the search. The code calculates the density of the solution. The initial uranium fuel density is $500 \mathrm{gm} /$ liter. The maximum allowed uranium density is $600 \mathrm{gm} /$ liter. The minimum allowed uranium density is $400 \mathrm{gm} / \mathrm{liter}$.

The maximum constraint is calculated according to:

$$
+\mathrm{CON}=(600 / 500-1) / 1.0=0.2
$$

The minimum constraint is calculated according to:

$$
-\mathrm{CON}=(400 / 500-1) / 1.0=-0.2
$$

The search data and final search results for this problem follow:

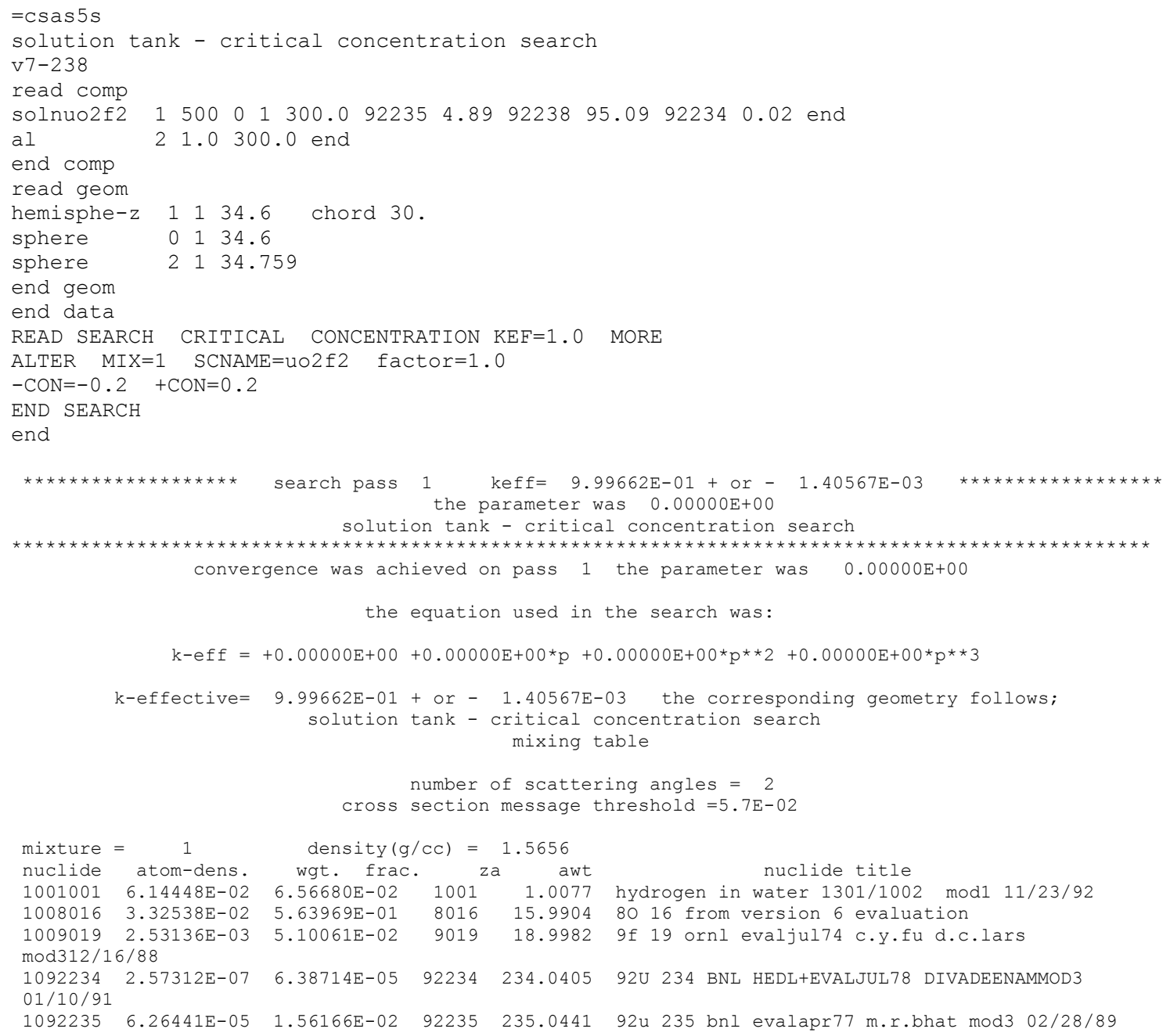




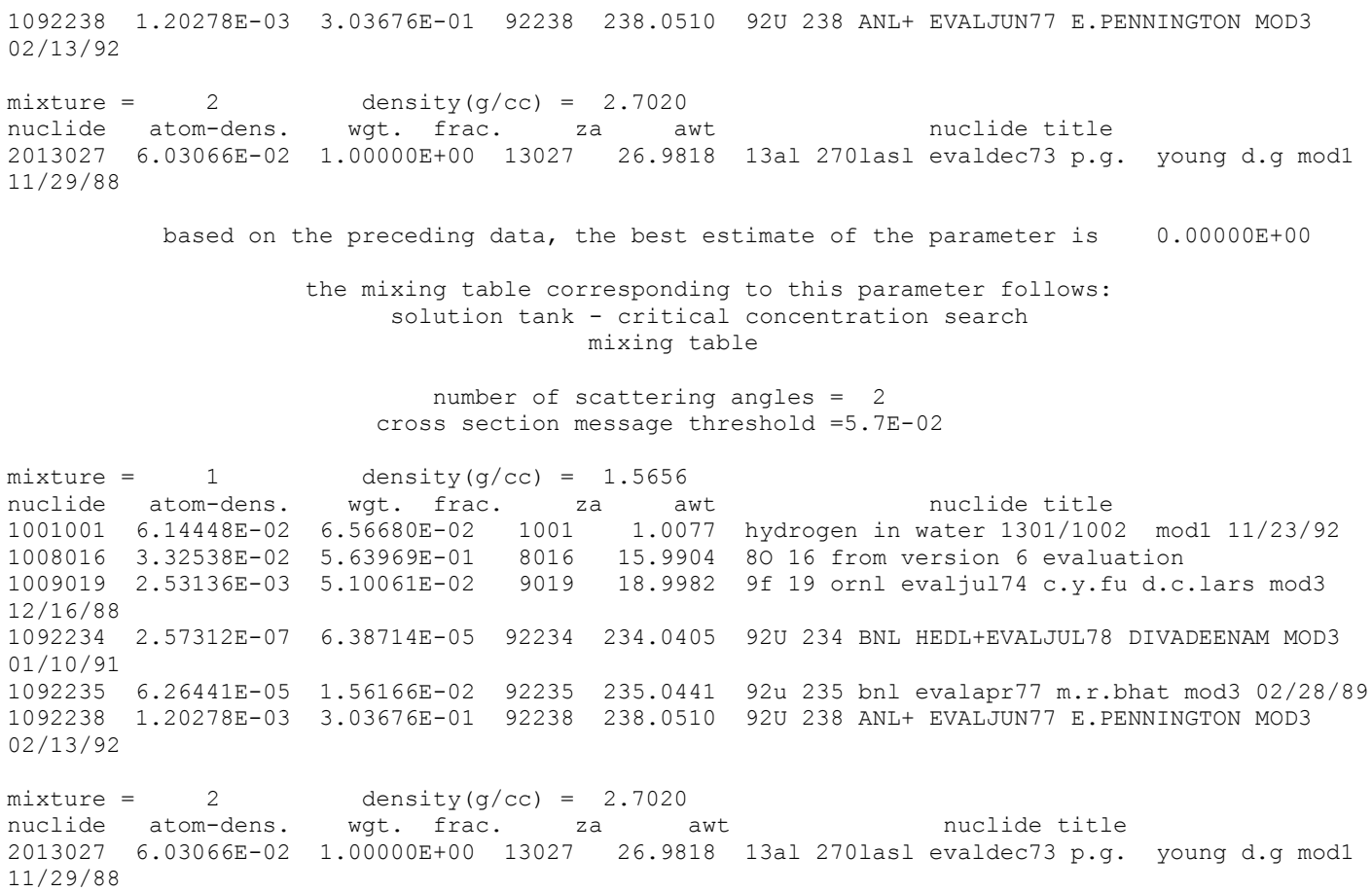

This problem converged on the first pass, so the amount of uranium, HF acid, and water in the solution were reasonably good estimates. For this problem the $\mathrm{HF}$ acid and $\mathrm{H}_{2} \mathrm{O}$ only changed marginally and, therefore, had very little effect on the system $k_{\text {eff. }}$ This is not always the case; therefore if a search is being done on a solution, the problem should always be rerun with the final search densities.

\section{Fuel Bundles Separated by Flux Traps - Critical Search Boron and Al Densities}

The fuel bundles in this problem represent $17 \times 17$ PWR fuel assemblies. The fuel pins are smeared together, making a mixture 100. The fuel pins consist of $4.35 \mathrm{wt} \%{ }^{235} \mathrm{U}$ having a diameter of $0.823 \mathrm{~cm}$, zirconium cladding having an outer diameter of $0.9627 \mathrm{~cm}$, and a pitch of $1.275 \mathrm{~cm}$. The fuel bundle is represented as a $10.8375 \mathrm{~cm} \times 10.8375 \mathrm{~cm} \times 366 \mathrm{~cm}$ cuboid of mixture 100 surrounded by Boral and then water. The Boral has a density of $2.61 \mathrm{~g} / \mathrm{cm}^{3}$ and has an initial composed of $50.0 \mathrm{wt} \% \mathrm{~B}_{4} \mathrm{C}$ and $50.0 \mathrm{wt} \% \mathrm{Al}$. The fuel bundles are at a fixed pitch of $13.0 \mathrm{~cm}$. Boral plates surrounding the $\mathrm{X}$ and $\mathrm{Y}$ sides of each fuel assembly are $0.1625 \mathrm{~cm}$ thick. Full density water is between the Boral plates.

A critical concentration search is performed on the Boral plates searching for a system $k_{\text {eff }}=0.95$. The Boral plates are at a fixed density of $2.61 \mathrm{gm} / \mathrm{cc}$. As the density of the $\mathrm{B}_{4} \mathrm{C}$ changes, the density of the $\mathrm{Al}$ changes in the opposite direction maintaining a constant Boral density. There are two entries in the MORE search data. The first entry, MIX $=4$ SCNAME $=b 4 c$ factor $=1.0$ specifies that the $\mathrm{B}_{4} \mathrm{C}$ of mixture 4 is to be altered during the search. The second entry, MIX $=4$ SCNAME $=$ al factor $=-1.0$ specifies that aluminum is to be changed in the opposite direction and proportionally to $\mathrm{B}_{4} \mathrm{C}$ during the search. Both $\mathrm{B}_{4} \mathrm{C}$ and $\mathrm{Al}$ have the same initial density of $0.5 * 2.61=1.305 \mathrm{gm} / \mathrm{cc}$.

The maximum constraint is calculated according to:

$$
+\mathrm{CON}=(2.59695 / 1.305-1) / 1.0=0.99
$$


The minimum constraint is calculated according to:

$-\mathrm{CON}=(0.1305 / 1.305-1) / 1.0=-0.9$

The search data and final search results for this problem follow:

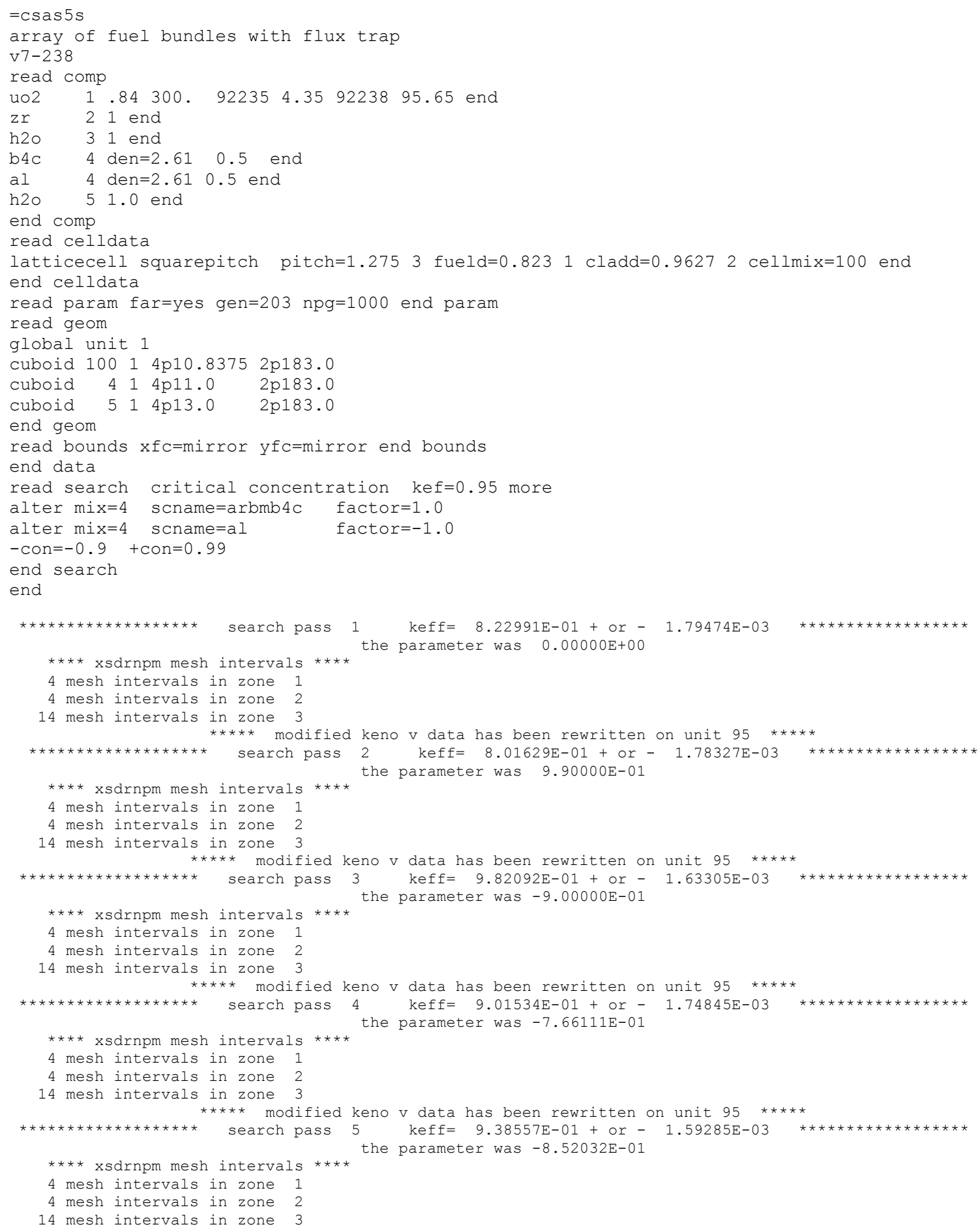




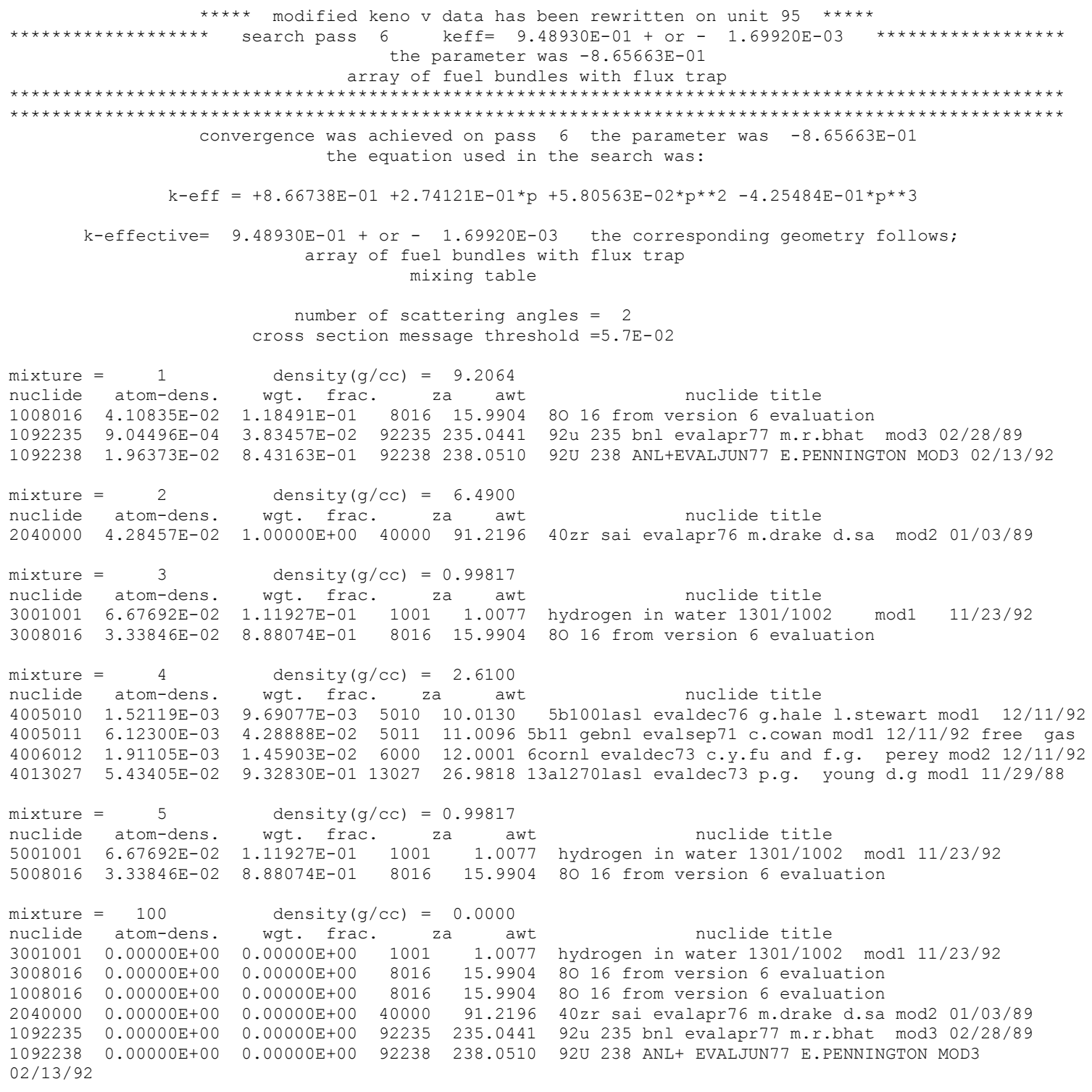

based on the preceding data, the best estimate of the parameter is $-8.66988 \mathrm{E}-01$

the mixing table corresponding to this parameter follows:

array of fuel bundles with flux trap

mixing table

number of scattering angles $=2$

cross section message threshold $=5.7 \mathrm{E}-02$

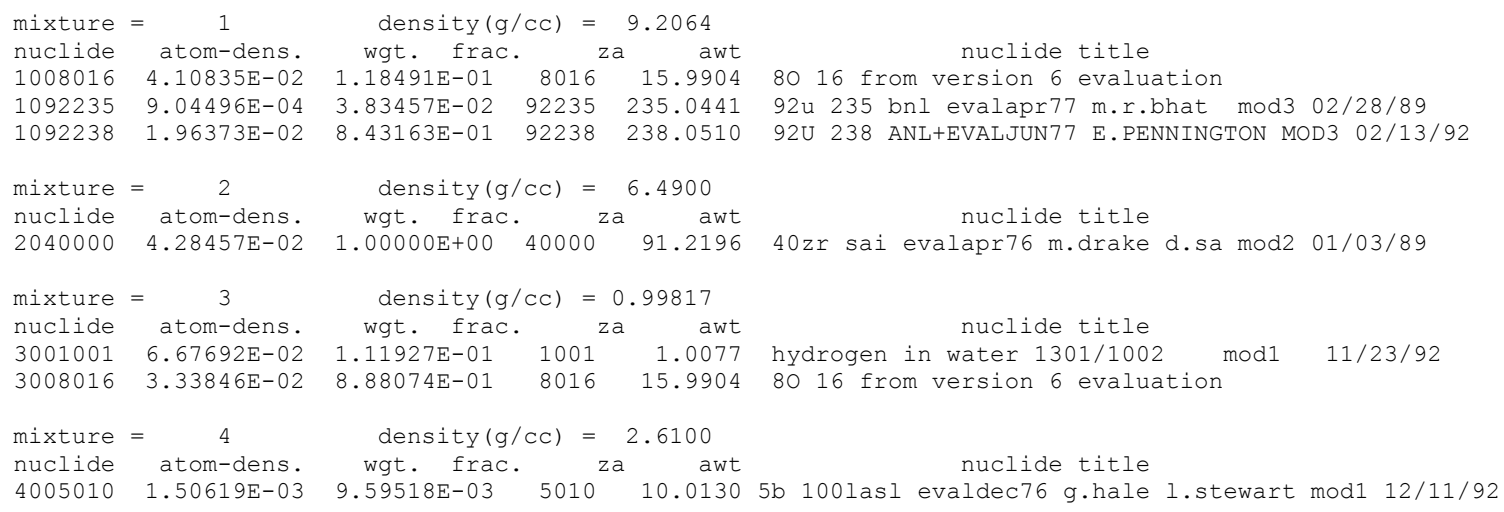




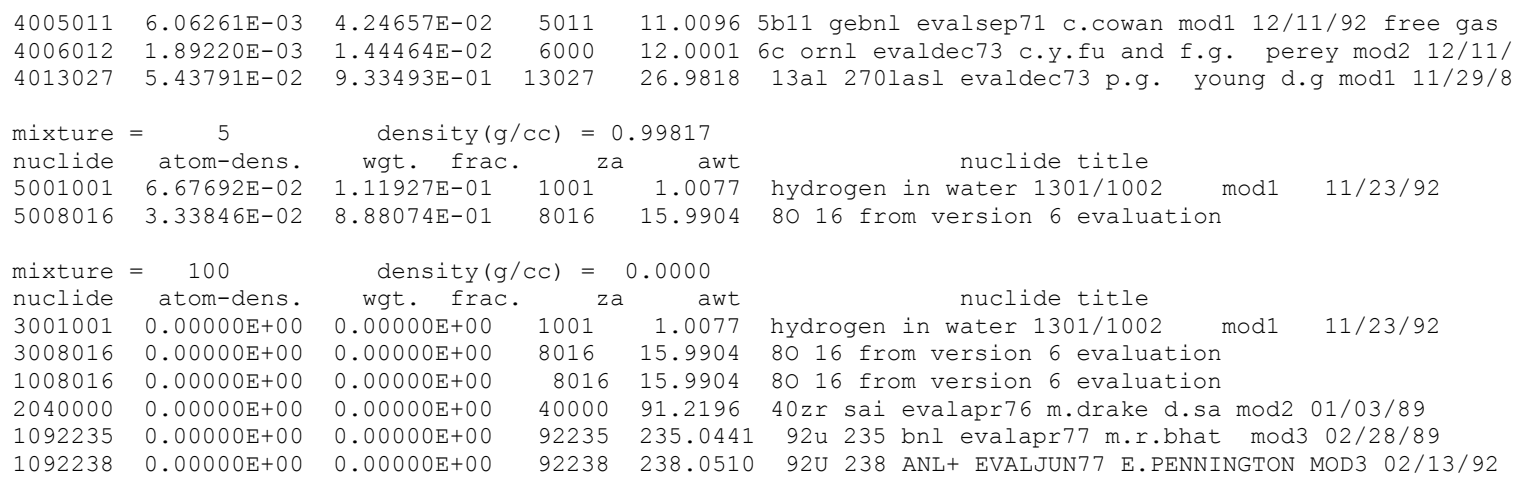

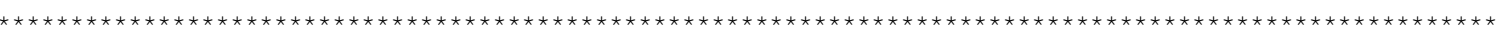

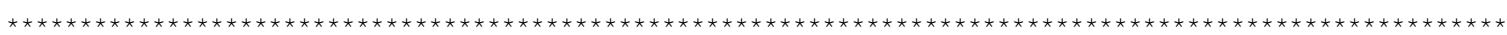




\title{
2.2 CSAS6: CONTROL MODULE FOR ENHANCED CRITICALITY SAFETY ANALYSIS WITH KENO-VI
}

\author{
L. M. Petrie, K. B. Bekar, D. F. Hollenbach, ${ }^{1}$ S. Goluoglu ${ }^{1}$
}

\begin{abstract}
The Criticality Safety Analysis Sequence with KENO-VI (CSAS6) provides reliable and efficient means of performing $k_{\text {eff }}$ calculations for systems that are routinely encountered in engineering practice. In the multigroup calculation mode, CSAS6 uses XSProc to process the cross sections for temperature corrections and problem-dependent resonance self-shielding and calculates the $k_{\text {eff }}$ of three-dimensional (3-D) system models. If the continuous energy calculation mode is selected no resonance processing is needed and the continuous energy cross sections are used directly in KENO-VI, with temperature corrections provided as the cross sections are loaded. The geometric modeling capabilities available in KENO-VI coupled with the automated crosssection processing within the control sequences allow complex, 3-D systems to be easily analyzed.
\end{abstract}

${ }^{1}$ Formerly with Oak Ridge National Laboratory. 
2.2 CSAS6: Control Module for Enhanced Criticality Safety Analysis with KENO-VI ...............2-92

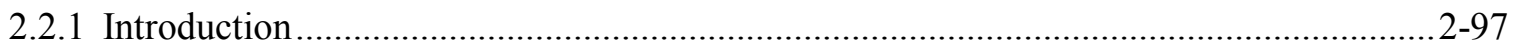

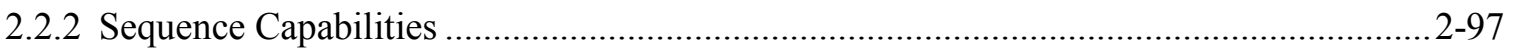

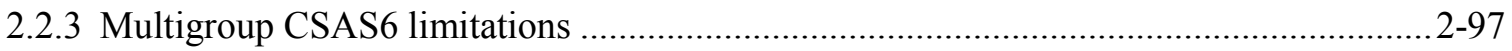

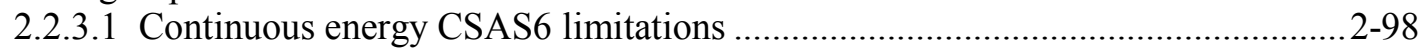

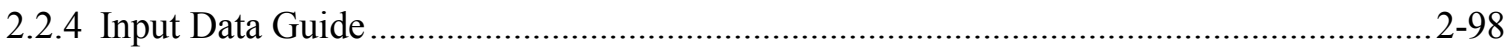

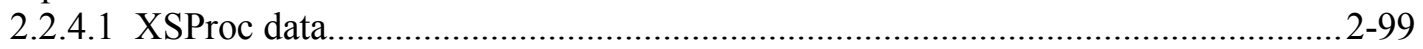

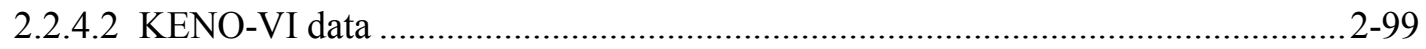

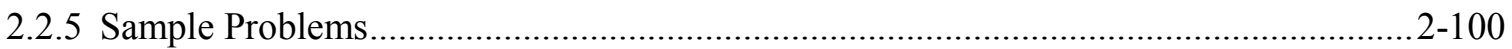

2.2.5.1 Sample Problem 1 Aluminum 30 Degree Pipe Angle Intersection....................2-101

2.2.5.2 Sample Problem 2 Plexiglas Cross ...........................................................2-102

2.2.5.3 Sample Problem 3 Sphere ........................................................................2-105

2.2.5.4 Sample Problem 4 Sphere Models Using Chords and Mirror Albedos ..............2-105

2.2.5.5 Sample Problem 5 Sphere Models Using Chords and Mirror Albedos ..............2-106

2.2.5.6 Sample Problem 6 Sphere Models Using Chords and Mirror Albedos ..............2-106

2.2.5.7 Sample Problem 7 Grotesque without the Diaphragm......................................2-106

2.2.5.8 Sample Problem 8 Infinite Array of MOX and UO2 Assemblies.......................2-110

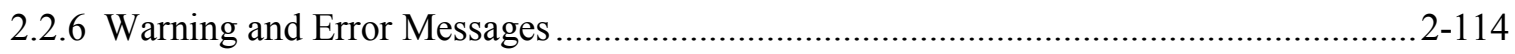

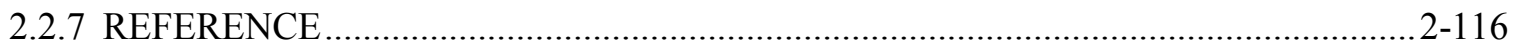

2.2.A Additional Example Applications of CSAS6..........................................................2-118

2.2.A.1 Run KENO-VI using CSAS6 ................................................................. 2-118

2.2.A.2 Run KENO-VI containing cell-weighted mixtures ...................................... 2-119

2.2.A.3 Run KENO-VI containing multiple unit cells............................................. 2-121 


\section{LIST OF FIGURES}

Page

Figure 2.2.1. Critical assembly of $\mathrm{UO}_{2} \mathrm{~F}_{2}$ solution in a $30^{\circ}-\mathrm{Y}$ aluminum pipe................................2-101

Figure 2.2.2. Critical assembly of $\mathrm{UO}_{2} \mathrm{~F}_{2}$ solution in a Plexiglas cross...........................................2-103

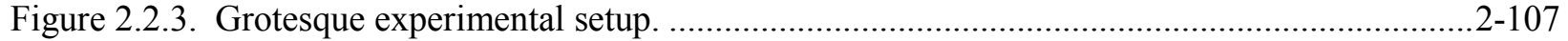

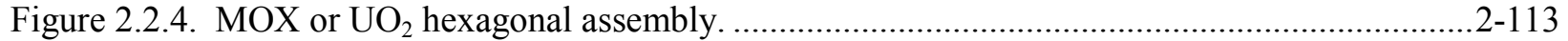

Figure 2.2.5. Infinite array of MOX assemblies interspersed between $\mathrm{UO}_{2}$ assemblies. ..................2-114 


\section{LIST OF TABLES}

$\underline{\text { Page }}$

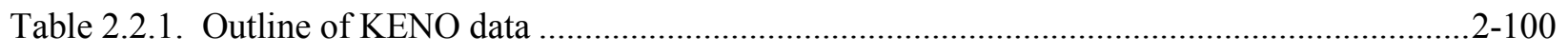




\section{ACKNOWLEDGMENTS}

The CSAS6 Criticality Safety Analysis Sequence is based on the CSAS control module, and the KENO-VI functional module, described in their respective chapters. G. E. Whitesides is acknowledged for his contributions through early versions of KENO. Appreciation is expressed to C. V. Parks and S. M. Bowman for their guidance in developing CSAS6. 


\subsubsection{Introduction}

Criticality Safety Analysis Sequence with KENO-VI (CSAS6) provides reliable and efficient means of performing $k_{\text {eff }}$ calculations for systems that are routinely encountered in engineering practice, especially in the calculation of $k_{\text {eff }}$ of three-dimensional (3-D) system models. CSAS6 implements XSProc to process material input and provide a temperature and resonance-corrected cross-section library based on the physical characteristics of the problem being analyzed. If a continuous energy cross-section library is specified, no resonance processing is needed and the continuous energy cross sections are used directly in KENO-VI, with temperature corrections provided as the cross sections are loaded.

\subsubsection{Sequence Capabilities}

CSAS6 is designed to prepare a resonance-corrected cross-section library for subsequent use in KENO-VI. In order to minimize human error, the SCALE data handling is automated as much as possible. CSAS6 and many other SCALE sequences apply a standardized procedure to provide appropriate number densities and cross sections for the calculation. XSProc is responsible for reading the standard composition data and other engineering-type specifications, including volume fraction or percent theoretical density, temperature, and isotopic distribution as well as the unit cell data. XSProc then generates number densities and related information, prepares geometry data for resonance self-shielding and flux-weighting cell calculations, if needed, and (if needed) provides problem-dependent multigroup cross-section processing. CSAS6 invokes a KENO-VI Data Processor to read and check the KENO-VI data. When the data checking has been completed, the control sequence executes XSProc to prepare a resonance-corrected microscopic cross-section library in the AMPX working library format if a multigroup library has been selected.

For each unit cell specified as being cell-weighted, XSProc performs the necessary calculations and produces a cell-weighted microscopic cross-section library. KENO-VI may be executed to calculate the $k_{\text {eff }}$ or neutron multiplication factor using the cross-section library that was prepared by the control sequence.

\subsubsection{Multigroup CSAS6 limitations}

The CSAS6 control module was developed to use simple input data and prepare problem-dependent cross sections for use in calculating the effective neutron multiplication factor of a 3-D system using KENO-VI and possibly XSDRNPM. An attempt was made to make the system as general as possible within the constraints of the standardized methods chosen to be used in SCALE. Standardized methods of data input were adopted to allow easy data entry and for quality assurance purposes. Some of the limitations of the CSAS6 sequence are a result of using preprocessed multigroup cross sections. Inherent limitations in CSAS6 are as follows:

1. Two-dimensional (2-D) effects such as fuel rods in assemblies where some positions are filled with control rod guide tubes, burnable poison rods and/or fuel rods of different enrichments. The cross sections are processed as if the rods are in an infinite lattice of rods. If the user inputs a Dancoff factor for the cell (such as one computed by MCDancoff), XSProc can produce an infinite lattice cell, which reproduces that Dancoff. This can mitigate some two dimensional lattice effects.

It is strongly recommended that the user perform CSAS6 calculations of benchmark experiments similar to the problem of interest to demonstrate the validity of the cross-section data and processing for that type of problem. 


\subsubsection{Continuous energy CSAS6 limitations}

When continuous energy KENO calculations are desired, none of the resonance processing modules are applicable or needed. Moreover, the MG limitations noted in the previous section are eliminated. The continuous energy cross sections are directly used in KENO. An existing multigroup input file can easily be converted to a continuous energy input file by simply specifying the continuous energy library. In this case, all cell data is ignored. However, the following limitations exist:

1. If CELLMIX is defined in the cell data, the problem will not run in the continuous energy mode. CELLMIX implies new mixture cross sections are generated using XSDRNPM-calculated cell fluxes and therefore is not applicable in the continuous energy mode.

2. Only VACUUM, MIRROR, PERIODIC, and WHITE boundary conditions are allowed. Other albedos, e.g., WATER, CARBON, POLY, etc. are for multigroup only.

3. Problems with DOUBLEHET cell data are not allowed as they inherently utilize CELLMIX feature.

\subsubsection{Input Data Guide}

The input data for CSAS6 are composed of two broad categories of data. The first is XSProc, including Standard Composition Specification Data and Unit Cell Geometry Specification. This first category specifies the cross-section library and defines the composition of each mixture and optionally the unit cell geometry that may be used to process the cross sections. The second category of data, the KENO-VI input data, is used to specify the geometric and boundary conditions that represent the physical 3-D configuration of the problem. Both data blocks are necessary for CSAS6.

All data are entered in free form, allowing alphanumeric data, floating-point data, and integer data to be entered in an unstructured manner. Up to 252 columns of data entry per line are allowed. Data can usually start or end in any column with a few exceptions. As an example, the word END beginning in column 1 and followed by two blank spaces or a new line will end the problem and any data following will be ignored. Each data entry must be followed by one or more blanks to terminate the data entry. For numeric data, either a comma or a blank can be used to terminate each data entry. Integers may be entered for floating values. For example, 10 will be interpreted as 10.0. Imbedded blanks are not allowed within a data entry unless an E precedes a single blank as in an unsigned exponent in a floating-point number. For example, 1.0E 4 would be correctly interpreted as $1.0 \times 10^{4}$.

The word "END" is a special data item. An "END" may have a name or label associated with it. The name or label associated with an "END" is separated from the "END" by a single blank and is a maximum of 12 characters long. At least two blanks or a new line MUST follow every labeled and unlabeled "END." It is the user's responsibility to ensure compliance with this restriction. Failure to observe this restriction can result in the use of incorrect or incomplete data without the benefit of warning or error messages.

Multiple entries of the same data value can be achieved by specifying the number of times the data value is to be entered, followed by either $\mathrm{R}, *$, or $\$$, followed by the data value to be repeated. Imbedded blanks are not allowed between the number of repeats and the repeat flag. For example, 5R12, $5 * 12,5 \$ 12$, or $5 \mathrm{R} 12$, etc., will enter five successive $12 \mathrm{~s}$ in the input data. Multiple zeros can be specified as $\mathrm{nZ}$ where $\mathrm{n}$ is the number of zeroes to be entered. 
The purpose of this section is to define the input data in discrete subsections relating to a particular type of data. Tables of the input data are included in each subsection, and the entries are described in more detail in the appropriate sections.

Resonance-corrected cross sections are generated using the appropriate boundary conditions for the unit cell description (i.e., void for the outer surface of a single unit, white for the outer surface of an infinite array of cylinders, spheres, or planes). As many unit cells as needed may be specified in a problem. A unit cell is cell-weighted by using the keyword CELLMIX = followed by a unique user specified mixture number in the unit cell data.

To check the input data without actually processing the cross sections, the words "PARM=CHECK" or "PARM=CHK" should be entered, as shown below.

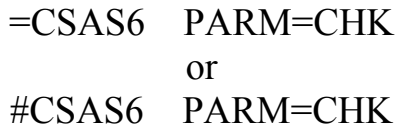

This will cause the input data for CSAS6 to be checked and appropriate error messages to be printed. If plots are specified in the data, they will be printed. This feature allows the user to debug and verify the input data while using a minimum amount of computer time.

\subsubsection{XSProc data}

The XSProc reads the standard composition specification data and the unit cell geometry specifications. It then produces the mixing table and unit cell information necessary for processing the cross sections if needed. The XSProc section of this manual provides a detailed description of the input data and processing options.

\subsubsection{KENO-VI data}

Table 2.2.1 contains the outline for the KENO-VI input. The KENO-VI input is divided into 13 data blocks. A brief outline of commonly used data blocks is shown in Table 2.2.1. Note that parameter data must precede all other KENO data blocks. Information on all KENO-VI input is provided in the KENO chapter of this document and will not be repeated here. 
Table 2.2.1. Outline of KENO data

\begin{tabular}{|c|c|c|c|}
\hline Type of data & Starting flag & Comments & Termination flag \\
\hline Parameters* & READ PARAMETER & Enter desired parameter data & END PARAMETER \\
\hline Geometry & READ GEOMETRY & Enter desired geometry data & END GEOMETRY \\
\hline Array data & READ ARRAY & Enter desired array data & END ARRAY \\
\hline Boundary conditions & READ BOUNDS & $\begin{array}{l}\text { Enter desired boundary } \\
\text { conditions }\end{array}$ & END BOUNDS \\
\hline Energy group boundaries & READ ENERGY & $\begin{array}{l}\text { Enter desired neutron energy } \\
\text { group boundaries }\end{array}$ & END ENERGY \\
\hline Start data or initial source & READ START & Enter desired start data & END START \\
\hline Plot data & READ PLOT & Enter desired plot data & END PLOT \\
\hline Grid geometry data & READ GRID & Enter desired mesh data & END GRID \\
\hline Reaction & READ REACTION & $\begin{array}{l}\text { Enter desire reaction tallies (CE } \\
\text { mode only) }\end{array}$ & END REACTION \\
\hline KENO-VI data terminus & END DATA & $\begin{array}{l}\text { Enter to signal the end of all } \\
\text { KENO-VI data }\end{array}$ & \\
\hline
\end{tabular}

*Must precede all other data blocks in this table.

\subsubsection{Sample Problems}

This section contains sample problems to demonstrate some of the options available in CSAS6. A brief problem description and the associated input data for multigroup mode of calculation are included for each problem. The same sample problems may be executed in the continuous energy mode by changing the library name to an continuous-energy library. See Appendix A for additional examples. 


\subsubsection{Sample Problem 1 Aluminum 30 Degree Pipe Angle Intersection}

The purpose of this problem is to calculate the k-effective of a system composed of intersecting aluminum pipes, in the shape of a $\mathrm{Y}$, filled with a $5 \%$ enriched $\mathrm{UO}_{2} \mathrm{~F}_{2}$ solution. The $\mathrm{UO}_{2} \mathrm{~F}_{2}$ solution at $299^{\circ} \mathrm{K}$ contains $907.0 \mathrm{gm} / 1$ of uranium, no excess acid, and has a specific gravity of $2.0289 \mathrm{gm} / \mathrm{cm}^{3}$. The assembly is composed of a $212.1 \mathrm{~cm}$ long vertical pipe and a second pipe that intersects the vertical pipe $76.7 \mathrm{~cm}$ from the outside bottom at an angle of 29.26 degrees with the upper vertical pipe. Both pipes have $13.95 \mathrm{~cm}$ inner diameters and $14.11 \mathrm{~cm}$ outer diameters. The vertical pipe is open on the top and $1.3 \mathrm{~cm}$ thick on the bottom. The Y-leg pipe, in the YZ-plane, is $126.04 \mathrm{~cm}$ in length with the sealed end $0.64 \mathrm{~cm}$ thick. The assembly is filled with solution to a height $129.5 \mathrm{~cm}$ above the outside bottom of the vertical pipe. From the point where the pipes intersect the assembly is surrounded by water $37.0 \mathrm{~cm}$ in the $\pm X$ directions, $100 \mathrm{~cm}$ in the $+Y$ direction, $-37 \mathrm{~cm}$ in the $-Y$ direction, to the top of the assembly in the $+\mathrm{Z}$ direction, and $-99.6 \mathrm{~cm}$ in the $-\mathrm{Z}$ direction.

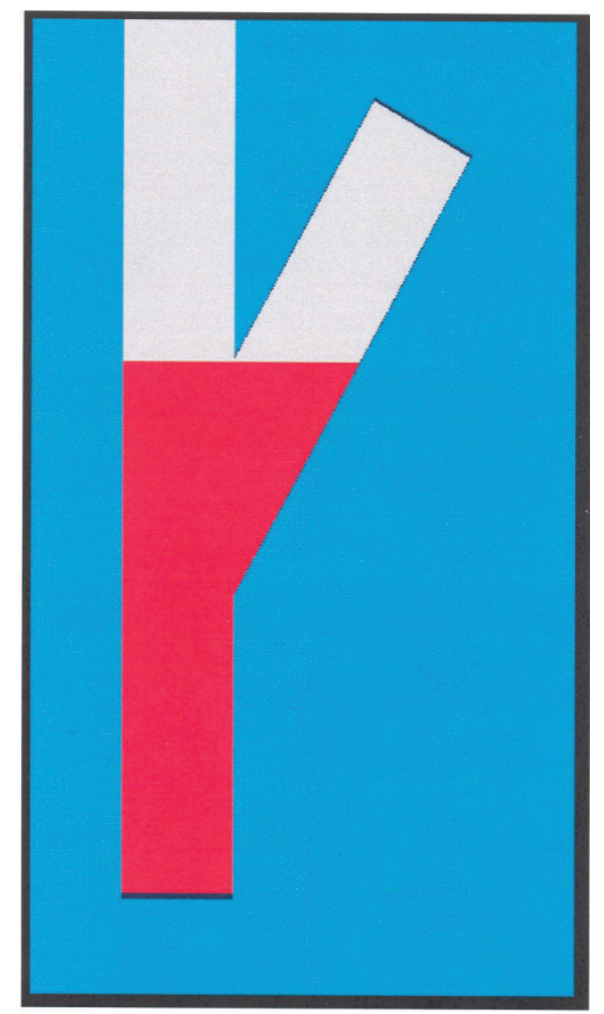

Figure 2.2.1. Critical assembly of $\mathrm{UO}_{2} \mathrm{~F}_{2}$ solution in a $30^{\circ}-\mathrm{Y}$ aluminum pipe.

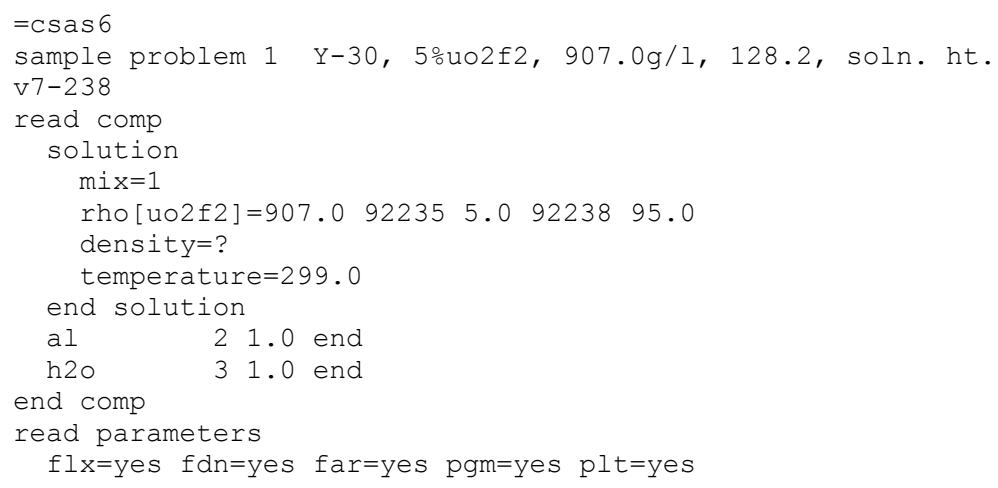




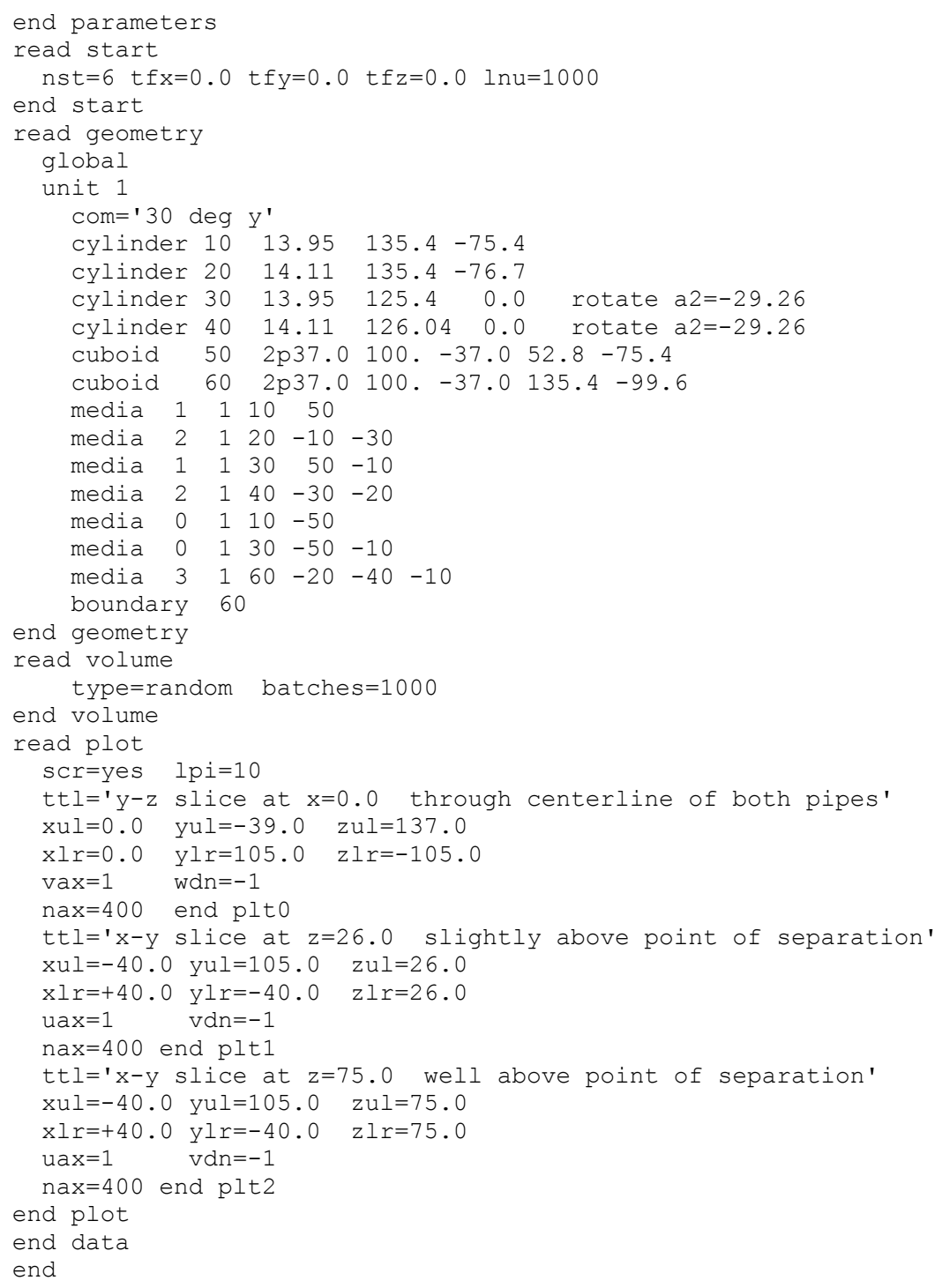

\subsubsection{Sample Problem 2 Plexiglas Cross}

The purpose of this problem is to calculate the k-effective of a system composed of intersecting Plexiglas pipes, in the shape of a cross, filled with a $5 \%$ enriched $\mathrm{UO}_{2} \mathrm{~F}_{2}$ solution. The room temperature $\mathrm{UO}_{2} \mathrm{~F}_{2}$ solution contains $896.1 \mathrm{gm} / 1$ of uranium, no excess acid, and has a specific gravity of $2.015 \mathrm{gm} / \mathrm{cm}^{3}$. The pipes have a $13.335 \mathrm{~cm}$ inner diameter and $16.19 \mathrm{~cm}$ outer diameter. The vertical pipe is open on the top and $3.17 \mathrm{~cm}$ thick on the bottom. The horizontal pipe ends are 3.17 thick. The vertical pipe is $210.19 \mathrm{~cm}$ in length and filled with solution to a height of $117.2 \mathrm{~cm}$. The two horizontal legs, positioned in the XZ-plane, intersect the vertical pipe $91.44 \mathrm{~cm}$ from the outside bottom at an 89 degree angle with the upper section of the pipe. Each horizontal is $91.44 \mathrm{~cm}$ in length and filled with the above specified $\mathrm{UO}_{2} \mathrm{~F}_{2}$ solution. A water reflector surrounding the solution filled pipes extends out from the point where the pipes intersect $111.76 \mathrm{~cm}$ in the $\pm X$ directions, $20.64 \mathrm{~cm}$ in the $\pm Y$ directions, $29.03 \mathrm{~cm}$ in the $+\mathrm{Z}$ direction, and $-118.428 \mathrm{~cm}$ in the $-\mathrm{Z}$ direction. 


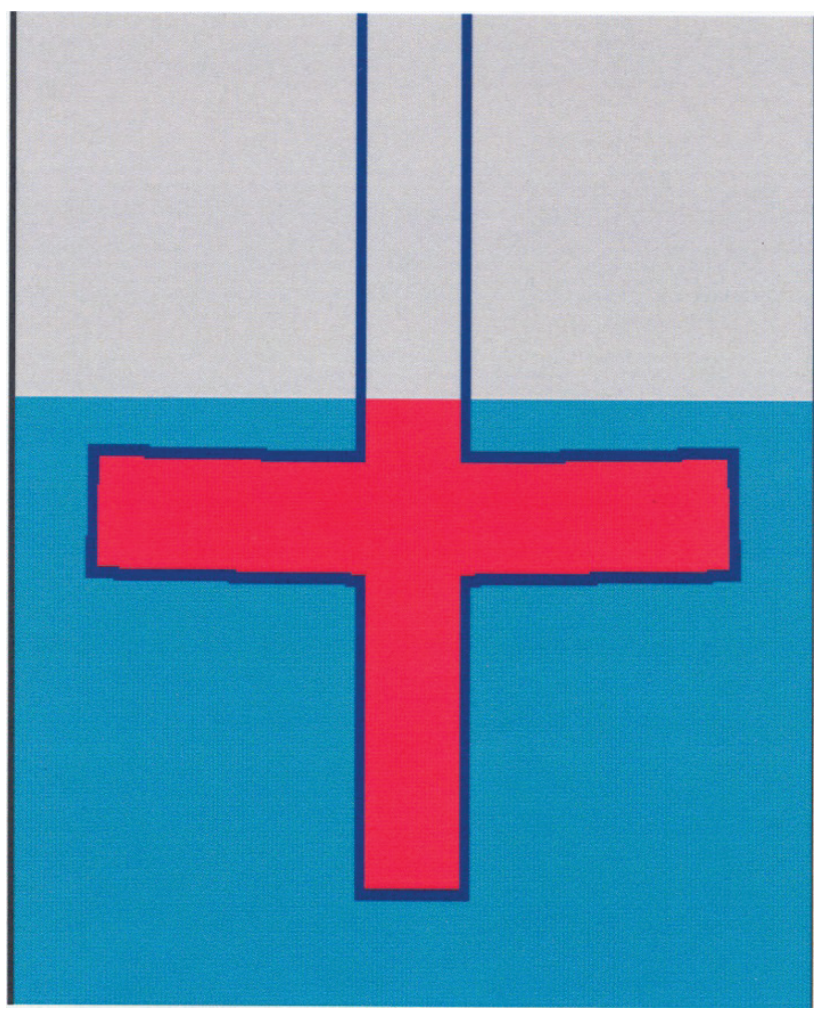

Figure 2.2.2. Critical assembly of $\mathrm{UO}_{2} \mathrm{~F}_{2}$ solution in a Plexiglas cross. 


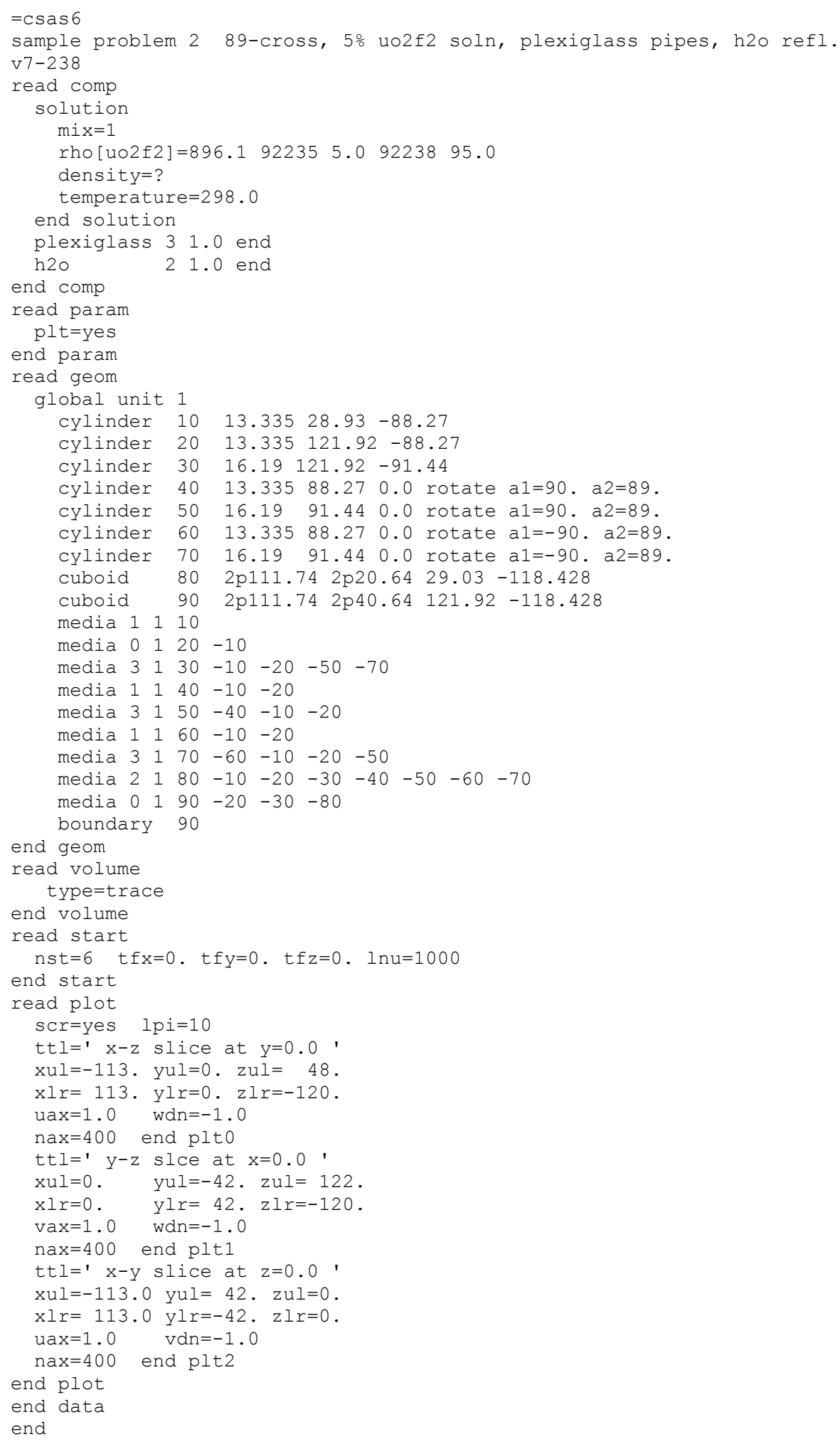




\subsubsection{Sample Problem 3 Sphere}

This problem models an assembly consisting of a 93.2\% enriched bare uranium sphere, $8.741 \mathrm{~cm}$ in radius, having a density of $18.76 \mathrm{gm} / \mathrm{cm}^{3}$. Problem 3 models the assembly as a single bare sphere. The second problem models the assembly as a hemisphere with mirror reflection on the flat surface. The next three problems model the sphere using chords. This set of four problems is designed to illustrate the use of multiple chords in a problem.

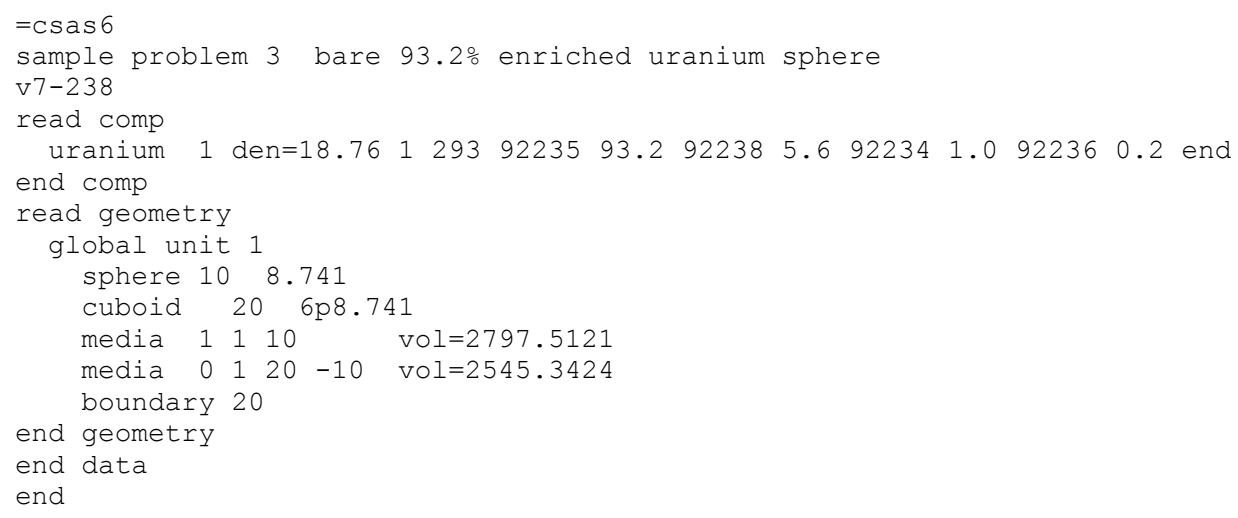

\subsubsection{Sample Problem 4 Sphere Models Using Chords and Mirror Albedos}

This problem models an assembly consisting of a $93.2 \%$ enriched bare uranium sphere, $8.741 \mathrm{~cm}$ in radius, having a density of $18.76 \mathrm{gm} / \mathrm{cm}^{3}$. The problem models the assembly as a hemisphere with mirror reflection on the flat surface.

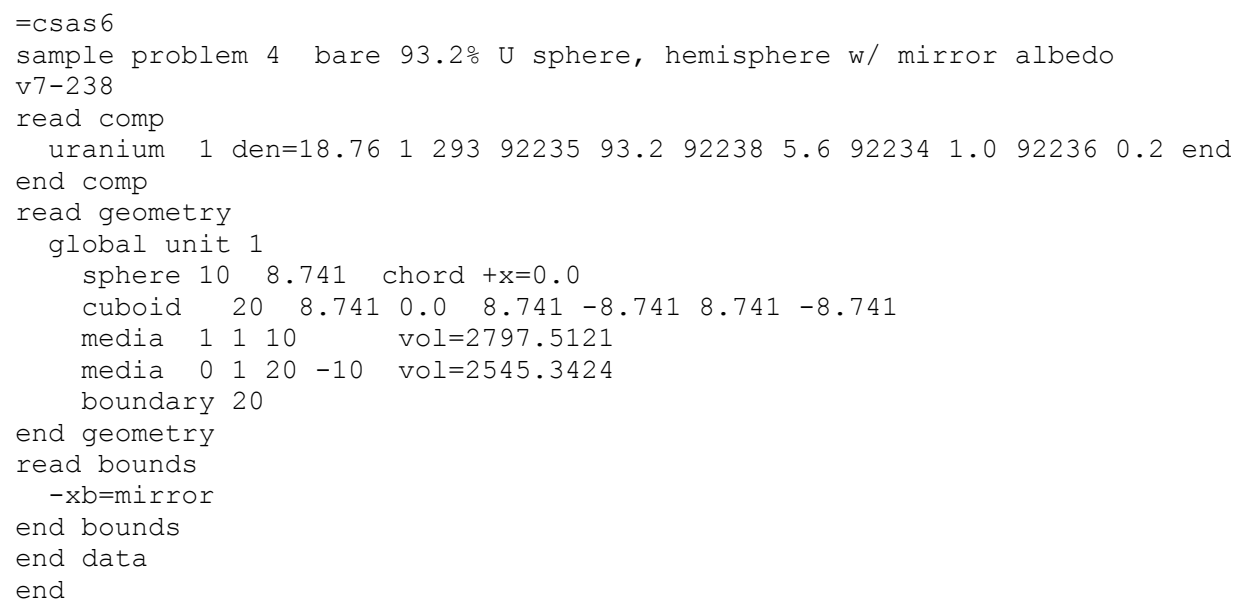




\subsubsection{Sample Problem 5 Sphere Models Using Chords and Mirror Albedos}

This problem models an assembly consisting of a $93.2 \%$ enriched bare uranium sphere, $8.741 \mathrm{~cm}$ in radius, having a density of $18.76 \mathrm{gm} / \mathrm{cm}^{3}$. The problem models the assembly as a quarter sphere with mirror reflection on the two flat surfaces.

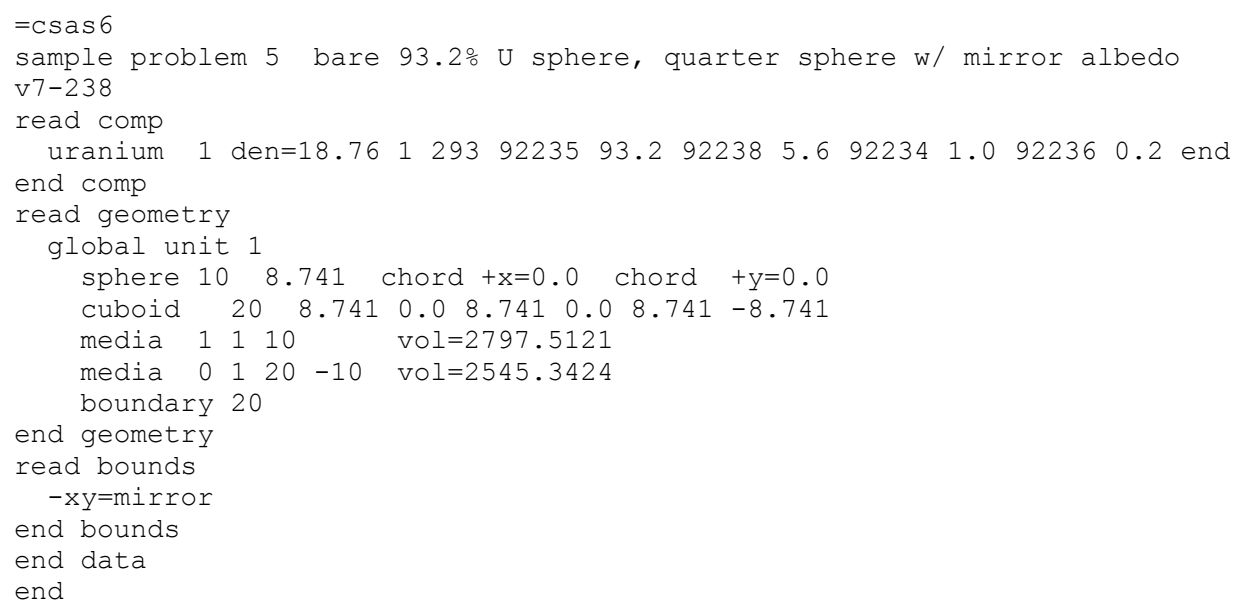

\subsubsection{Sample Problem 6 Sphere Models Using Chords and Mirror Albedos}

This problem models an assembly consisting of a 93.2\% enriched bare uranium sphere, $8.741 \mathrm{~cm}$ in radius, having a density of $18.76 \mathrm{gm} / \mathrm{cm}^{3}$. The problem models the assembly as an eighth sphere with mirror reflection on the three flat surfaces.

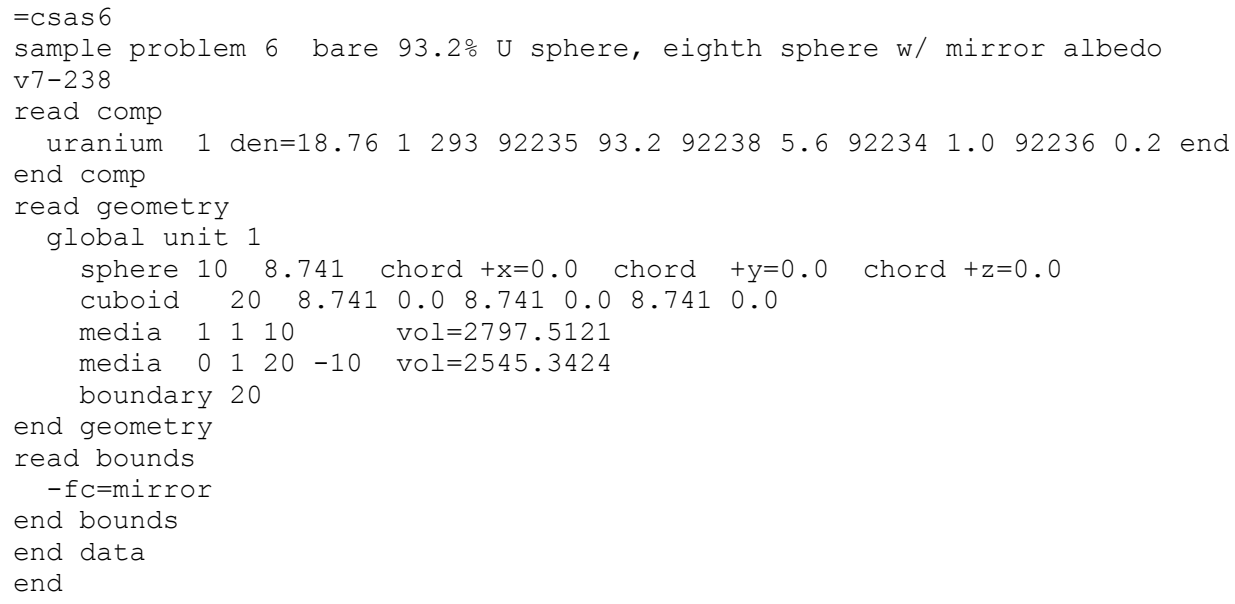

\subsubsection{Sample Problem 7 Grotesque without the Diaphragm}


The purpose of this problem is to calculate the $k_{\text {eff }}$ of a system composed of eight enriched uranium units placed on a diaphragm, with an irregularly shaped centerpiece positioned in the center hole of the diaphragm. ${ }^{1}$ The assembly and centerpiece are shown in Figure 2.2.3, which is Fig. 4 from Ref. 1. The eight units consist of an approximate parallelepiped with an irregular top, a parallelepiped, and six cylinders of various sizes. The centerpiece, which penetrates the hole in the diaphragm, consists of a cylinder topped by a parallelepiped topped by a hemisphere. The diaphragm is not modeled in this example.

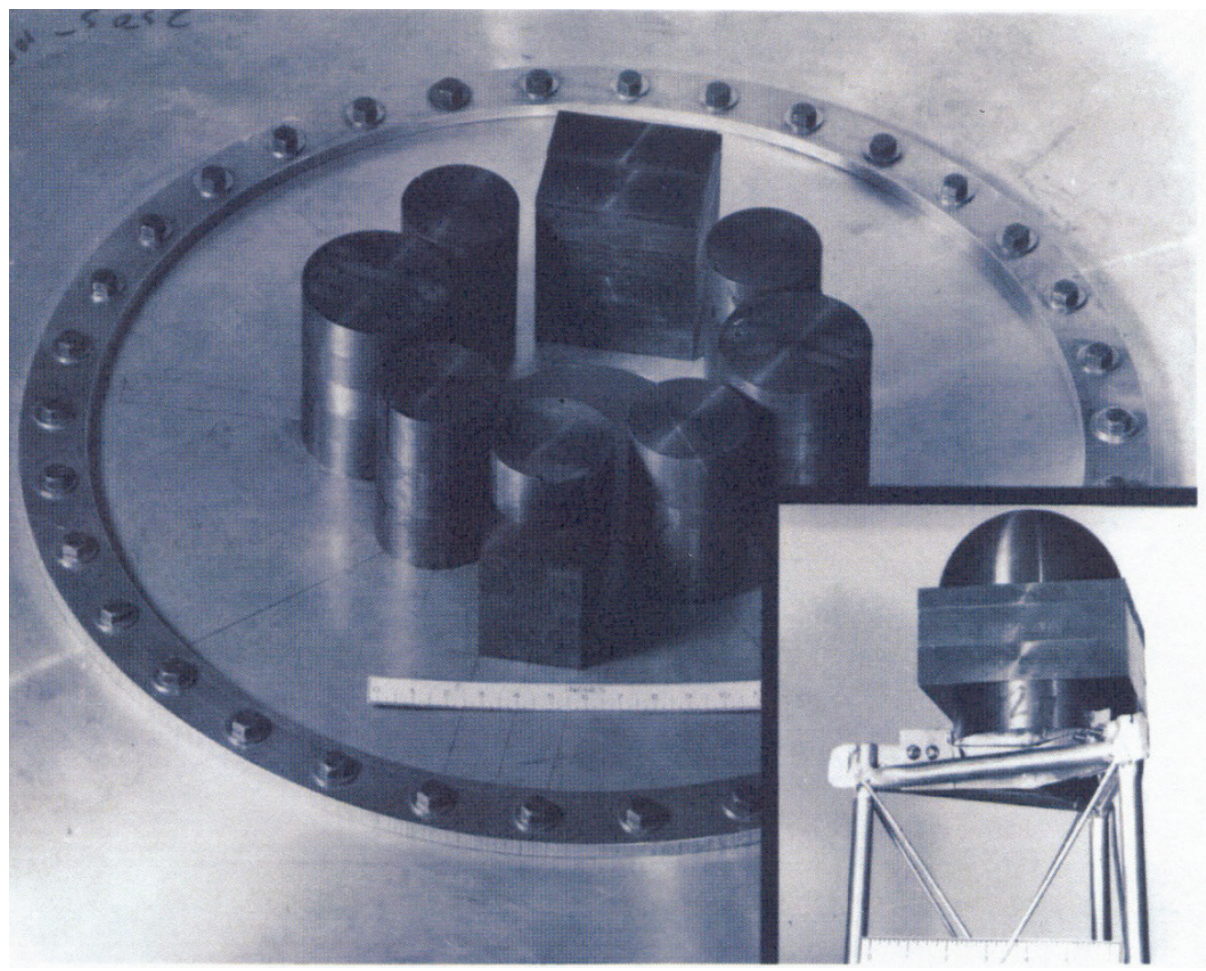

Figure 2.2.3. Grotesque experimental setup. 


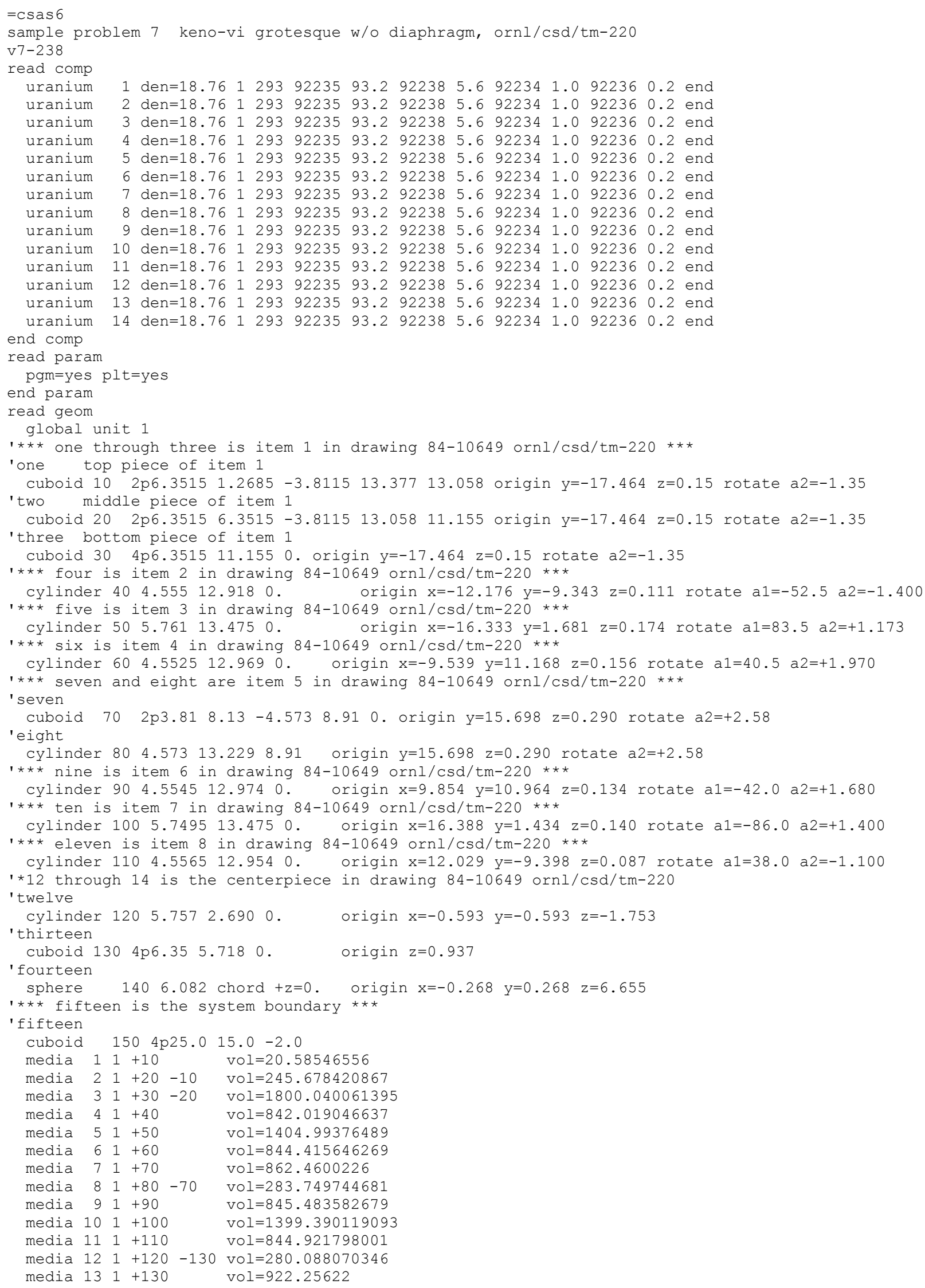




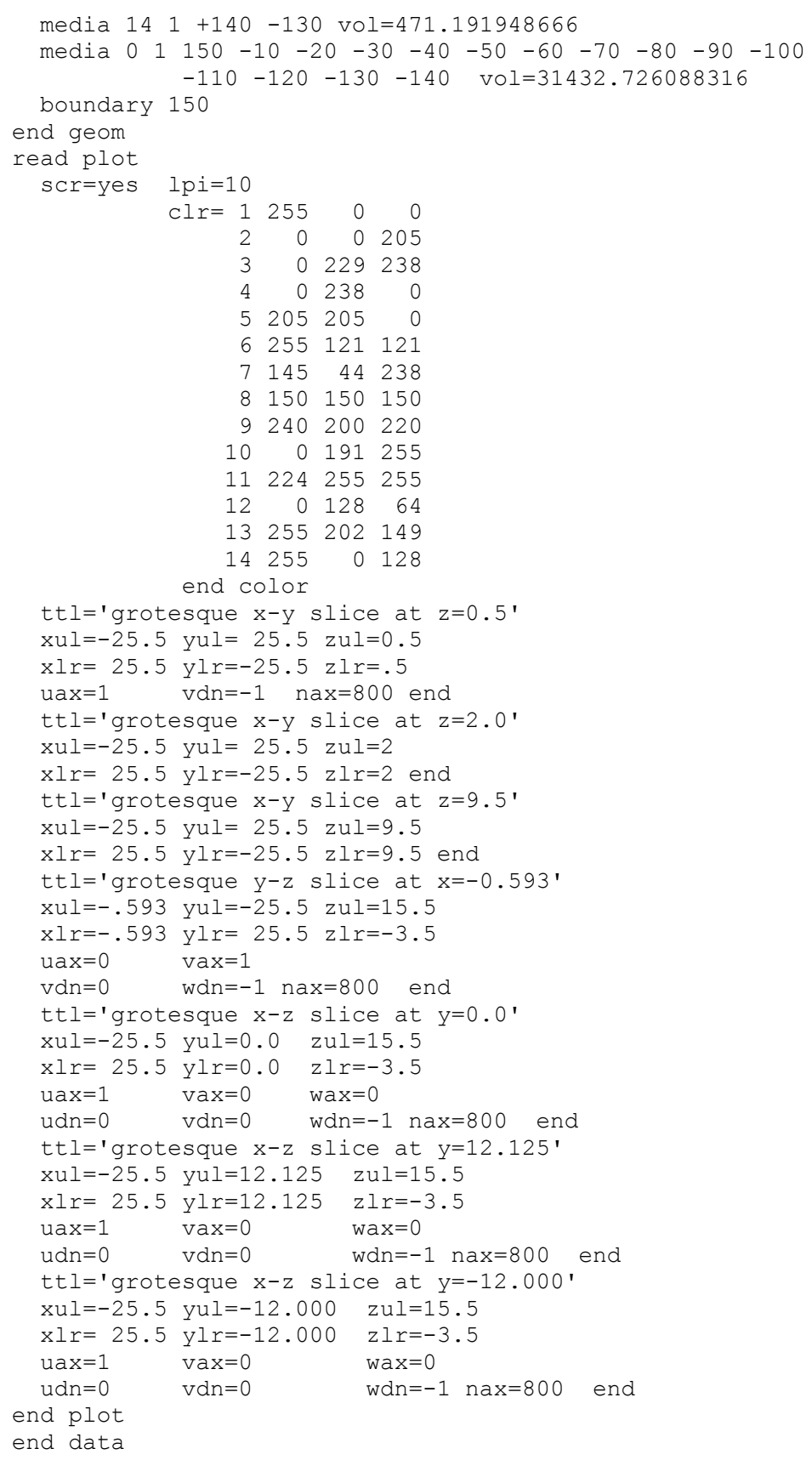




\subsubsection{Sample Problem 8 Infinite Array of MOX and UO2 Assemblies}

The purpose of this problem is to calculate the $k_{\text {eff }}$ of a system composed of an infinite array of MOX assemblies interspersed between $\mathrm{UO}_{2}$ assemblies. Both assembly types contain 331 pins in a hexagonal lattice with a pin pitch of $1.275 \mathrm{~cm}$ and an assembly pitch of $23.60 \mathrm{~cm}$ as shown in Figure 2.2.4. The moderator is borated water at $306^{\circ} \mathrm{C}$ having a density of $0.71533 \mathrm{gm} / \mathrm{cc}$ and composed of $99.94 \mathrm{wt} \% \mathrm{H}_{2} \mathrm{O}$ and $0.06 \mathrm{wt} \%$ natural boron. Each fuel rod is $355 \mathrm{~cm}$ in length, has a radius of $0.3860 \mathrm{~cm}, 0.722-\mathrm{cm}$-thick $\mathrm{Zr}$ cladding with no gap, and is at a temperature of $754^{\circ} \mathrm{C}$.

The $\mathrm{UO}_{2}$ fuel consists of $4.4 \mathrm{wt} \%{ }^{235} \mathrm{U}$ and $95.6 \mathrm{wt} \%{ }^{238} \mathrm{U}$ at a density of $8.7922 \mathrm{gm} / \mathrm{cc}$. The $\mathrm{UO}_{2}$ fuel also contains $9.4581 \mathrm{E}-9$ atoms $/ \mathrm{b}-\mathrm{cm}$ of ${ }^{135} \mathrm{Xe}$ and $7.3667 \mathrm{E}-8$ atoms $/ \mathrm{b}-\mathrm{cm}$ of ${ }^{149} \mathrm{Sm}$.

The MOX fuel consists of $96.38 \mathrm{wt} \% \mathrm{UO}_{2}$ and $3.62 \mathrm{wt} \% \mathrm{PuO}_{2}$ at a density of $8.8182 \mathrm{gm} / \mathrm{cc}$. The $\mathrm{UO}_{2}$ fuel is composed of $2.0 \mathrm{wt} \%{ }^{235} \mathrm{U}$ and $98.0 \mathrm{wt} \%{ }^{238} \mathrm{U}$. The $\mathrm{PuO}_{2}$ fuel is composed of $93.0 \mathrm{wt} \%{ }^{239} \mathrm{Pu}$, $6.0 \mathrm{wt} \%{ }^{240} \mathrm{Pu}$ - and $1.0 \mathrm{wt} \%{ }^{241} \mathrm{Pu}$. The MOX fuel also contains $9.4581 \mathrm{E}-9$ atoms $/ \mathrm{b}-\mathrm{cm}$ of ${ }^{135} \mathrm{Xe}$ and $7.3667 \mathrm{E}-8$ atoms $/ \mathrm{b}-\mathrm{cm}$ of ${ }^{149} \mathrm{Sm}$.

These two assemblies are placed so they represent an infinite array in the $\mathrm{X}$ and $\mathrm{Y}$ dimensions as shown in Figure 2.2.5. There is $20 \mathrm{~cm}$ of water above and below fuel assemblies. This problem uses CENTRM/PMC as the resolved resonance processor cross section. Since an infinite array cannot be explicitly modeled, a section of the array is modeled and the $\mathrm{X}$ and $\mathrm{Y}$ sides have mirror reflection.

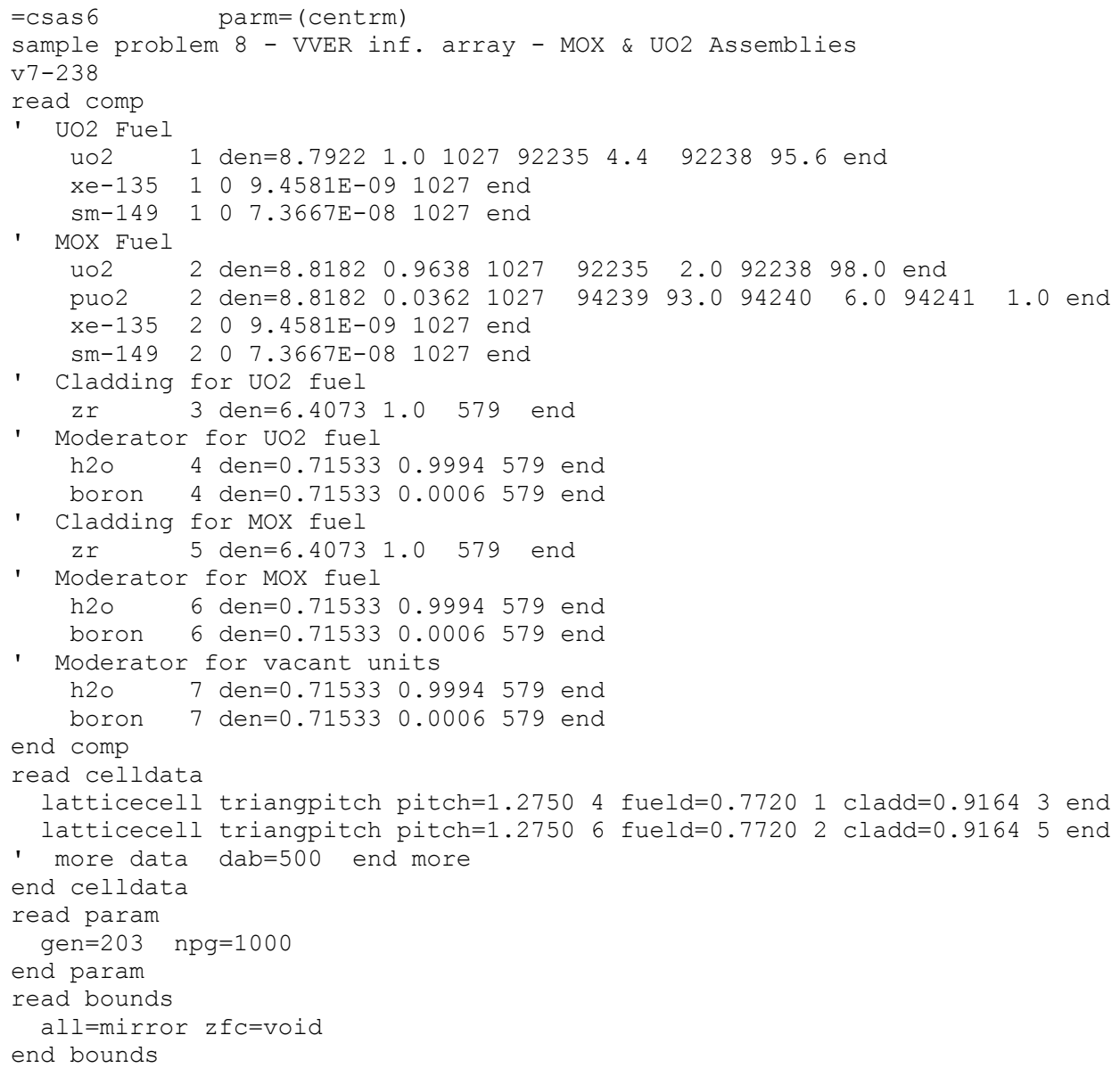




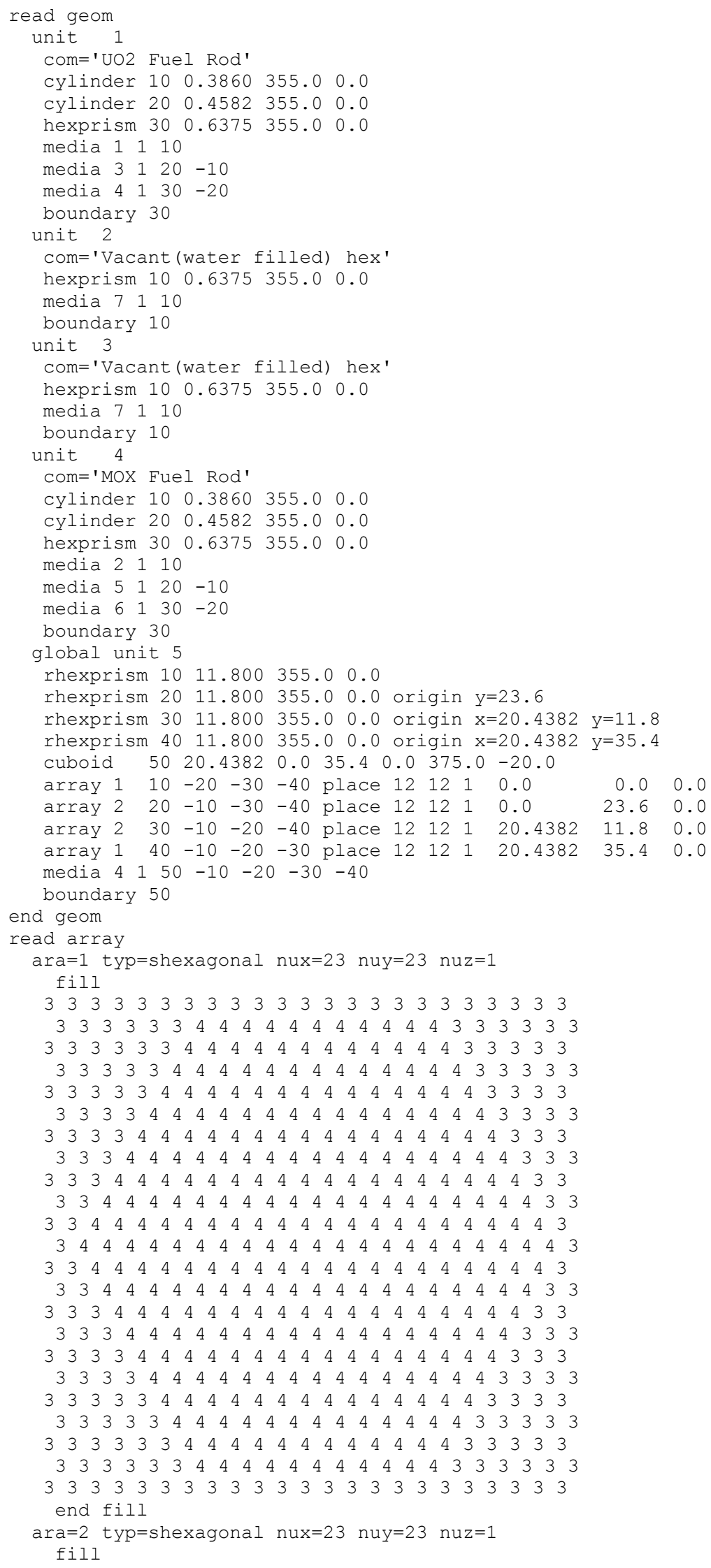




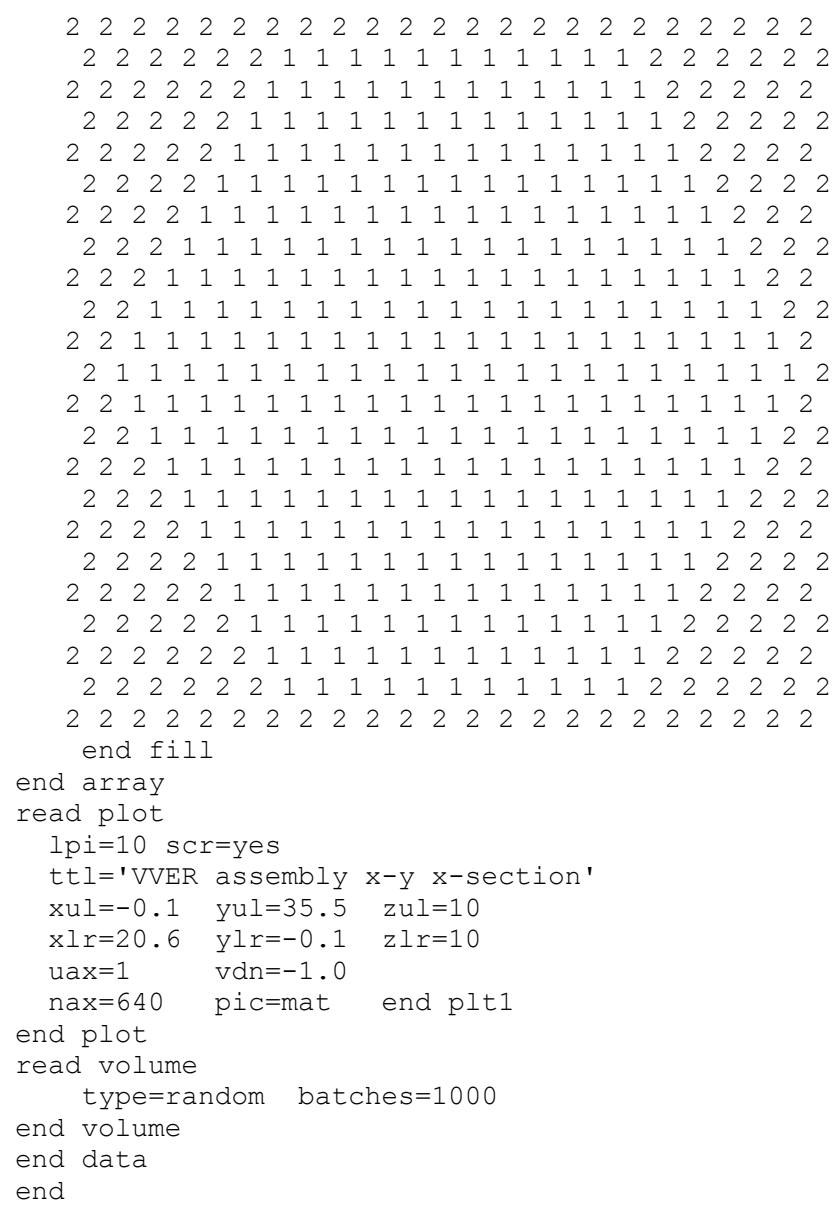




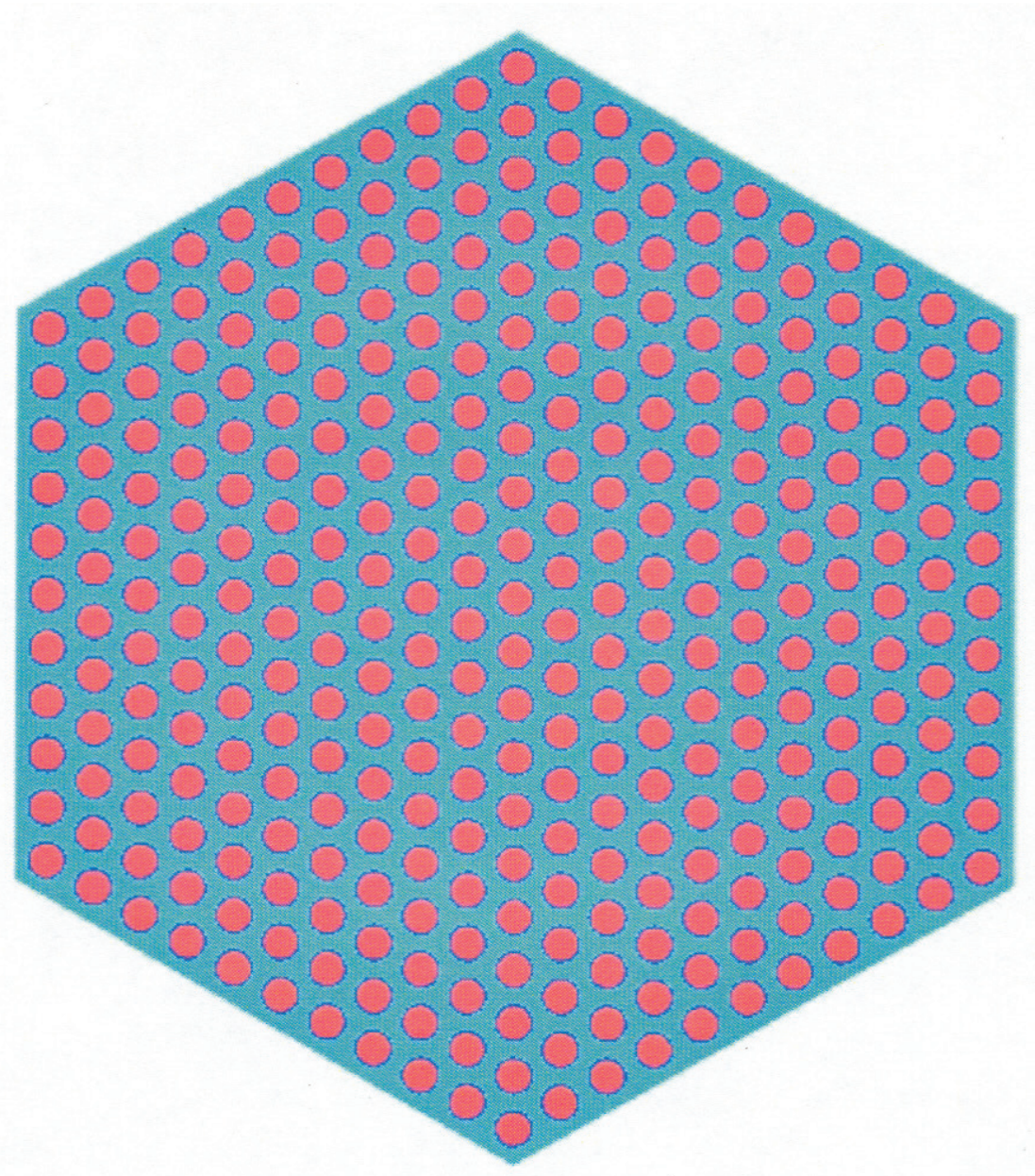

Figure 2.2.4. $\mathrm{MOX}$ or $\mathrm{UO}_{2}$ hexagonal assembly. 


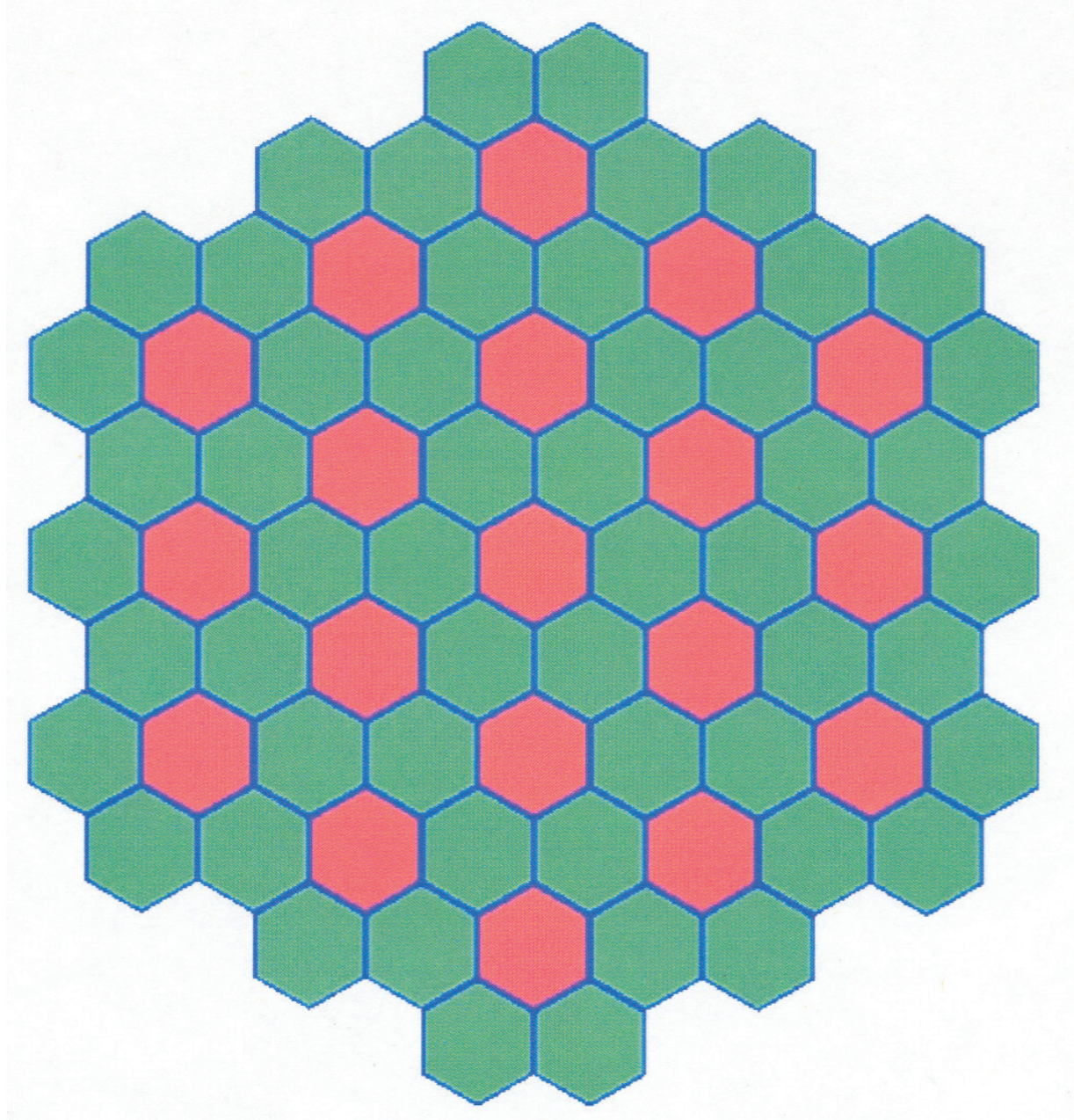

Figure 2.2.5. Infinite array of MOX assemblies interspersed between $\mathrm{UO}_{2}$ assemblies.

\subsubsection{Warning and Error Messages}

CSAS6 contains two types of warning and error messages. The first type of message is from XSProc is common to many of the SCALE analytical sequences. The second type of message is from the CSAS6 subroutines and is identified by CS- followed by a number. These messages are listed in numerical order in Sect. 0. For additional information concerning a message, simply look up the number in this section.

Warning messages appear when a possible error is encountered. It is the responsibility of the user to verify whether the data are correct when a warning message is encountered. The functional modules activated by CSAS6 and related sequences will be executed even though a warning message has been generated.

When an error is recognized, an error message is written and an error flag is set so the functional modules will not be activated. The code stops immediately if the error is too severe to allow continuation of input. However, it will continue to read and check the data if it is able. When the data reading is completed, 
execution is terminated if an error flag was set when the data were being processed. If the error flag has not been set, execution continues. When error messages are printed, the user should focus on the first error message, because subsequent messages may have been caused by the error that generated the first message.

The following messages originate in the part of CSAS6 that reads, checks, and prepares data for KENO-VI. The same set of error messages are also used for CSAS5 that reads, checks, and prepares data for KENO V.a and MODIFY. CSAS6 is not capable of performing searches at this time. An error message referring to a SEARCH routine, from a CSAS6 problem, indicates a code error.

\section{CS-16 ***WARNING*** READ FLAG NOT FOUND. ASSUME KENO V PARAMETER DATA FOLLOWS.}

This message from subroutine CPARAM indicates that the word READ is not the first word of KENO-VI data following the Material Information Processor input data. If parameter data is to be entered, the code expects the words READ PARAMETERS to precede the parameter input data. If the word READ is not the first word, the code assumes the data are parameter input data.

CS-21 A UNIT NUMBER WAS ENTERED FOR THE CROSS-SECTION LIBRARY. (LIB= IN PARAMETER DATA.) THE DEFAULT VALUE SHOULD BE USED IN ORDER TO UTILIZE THE CROSS SECTIONS GENERATED BY CSAS. MAKE CERTAIN THE CORRECT CROSS-SECTION LIBRARY IS BEING USED.

This message is from subroutine CPARAM. It indicates that a value has been entered for the crosssection library in the KENO-VI parameter data. The cross-section library created by the analytical sequence should be used. MAKE CERTAIN THAT THE CORRECT CROSS SECTIONS ARE BEING USED.

\section{CS-55 *** ERRORS WERE ENCOUNTERED IN PROCESSING THE CSAS-KENO6 DATA. EXECUTION IS IMPOSSIBLE. ***}

This message from subroutine SASSY is printed if errors were found in the KENO-VI input data for CSAS. If a search is being made, data reading will continue until all the data have been entered or a fatal error terminates the data reading. When the data reading and checking have been completed, the problem will terminate without executing. Check the printout to locate the errors responsible for this message.

CS-62 *** ERROR *** MIXTURE IN THE GEOMETRY WAS NOT CREATED IN THE STANDARD COMPOSITIONS SPECIFICATION DATA.

This message from subroutine MIXCHK indicates that a mixture specified in the KENO-VI geometry was not created in the standard composition data.

\section{CS-68 $* * *$ ERROR $* * *$ AN INPUT DATA ERROR HAS BEEN ENCOUNTERED IN THE DATA} ENTERED FOR THIS PROBLEM.

This message from the main program, CSAS6, is printed if the subroutine library routine LRDERR returns a value of "TRUE," indicating that a reading error has been encountered in the "KENO PARAMETER" data. The appropriate data type is printed in the message. Locate the unnumbered message stating “***** ERROR IN INPUT. CARD IMAGE PRINTED ON NEXT LINE *****." Correct the data and resubmit the problem. 
This message from subroutine CMXCHK indicates that a mixture that is a component of a cell-weighted mixture has been used in the KENO-VI geometry data.

\section{CS-82 *** AN ERROR WAS ENCOUNTERED IN ONE OF THE FUNCTIONAL MODULES.}

This message from CSAS6 indicates that an error was encountered during execution of one of the functional modules such as CRAWDAD, BONAMI, CENTRM, PMC, XSDRNPM, or KENO-VI. Check the printout to locate and correct the error.

\section{CS-99 THIS PROBLEM WILL NOT BE RUN BECAUSE PARM=CHECK WAS ENTERED IN THE ANALYTICAL SEQUENCE SPECIFICATION.}

This message from subroutine CSAS indicates that the problem data were read and checked and no errors were found. To execute the problem, remove the PARM=CHECK or PARM=CHK from the analytical sequence indicator data entry.

\section{CS-100 THIS PROBLEM WILL NOT BE RUN BECAUSE ERRORS WERE ENCOUNTERED IN THE INPUT DATA.}

This message from subroutine CSAS is self-explanatory. Examine the printout to locate the error or errors in the input data. Correct them and resubmit the problem.

\subsubsection{REFERENCE}

1. J. T. Mihalczo, Brief Summary of Unreflected and Unmoderated Cylindrical Critical Experiments with Oralloy at Oak Ridge, ORNL/TM-1999/302, Oak Ridge National Laboratory (1999). 


\subsection{A Additional Example Applications of CSAS6}

Several example uses of CSAS6 are shown in this section for a variety of applications.

\subsection{A.1 Run KENO-VI using CSAS6}

CSAS6 creates a microscopic working format library and a mixing table that is passed to KENO-VI. The library is created using CENTRM/PMC/WORKER to process the cross section data in the resolved resonance regions of the isotopes contained in the library. CSAS6 then executes KENO-VI, which calculates $k_{\text {eff }}$ for the problem. The following examples are for using the multigroup mode of calculation for KENO-VI. Using the continuous energy mode can be accomplished by simply changing the library name to one of the continuous energy libraries.

EXAMPLE 1. CSAS6 - Determine the $k_{\text {eff }}$ of a system.

Consider a problem consisting of eight uranium metal cylinders that are $93.2 \% \mathrm{wt}$ enriched, having a density of $18.76 \mathrm{~g} / \mathrm{cm}^{3}$. The cylinders are arranged in a $2 \times 2 \times 2$ array. Each has a radius of $5.748 \mathrm{~cm}$ and a height of $10.765 \mathrm{~cm}$. The center-to-center spacing in the horizontal (X-Y) plane is $13.74 \mathrm{~cm}$ and the vertical center-to-center spacing is $13.01 \mathrm{~cm}$. Because the cross section processing will be done assuming an infinite homogeneous medium and no cell mixtures are used, there is no unit cell data. The input data for this problem follow.

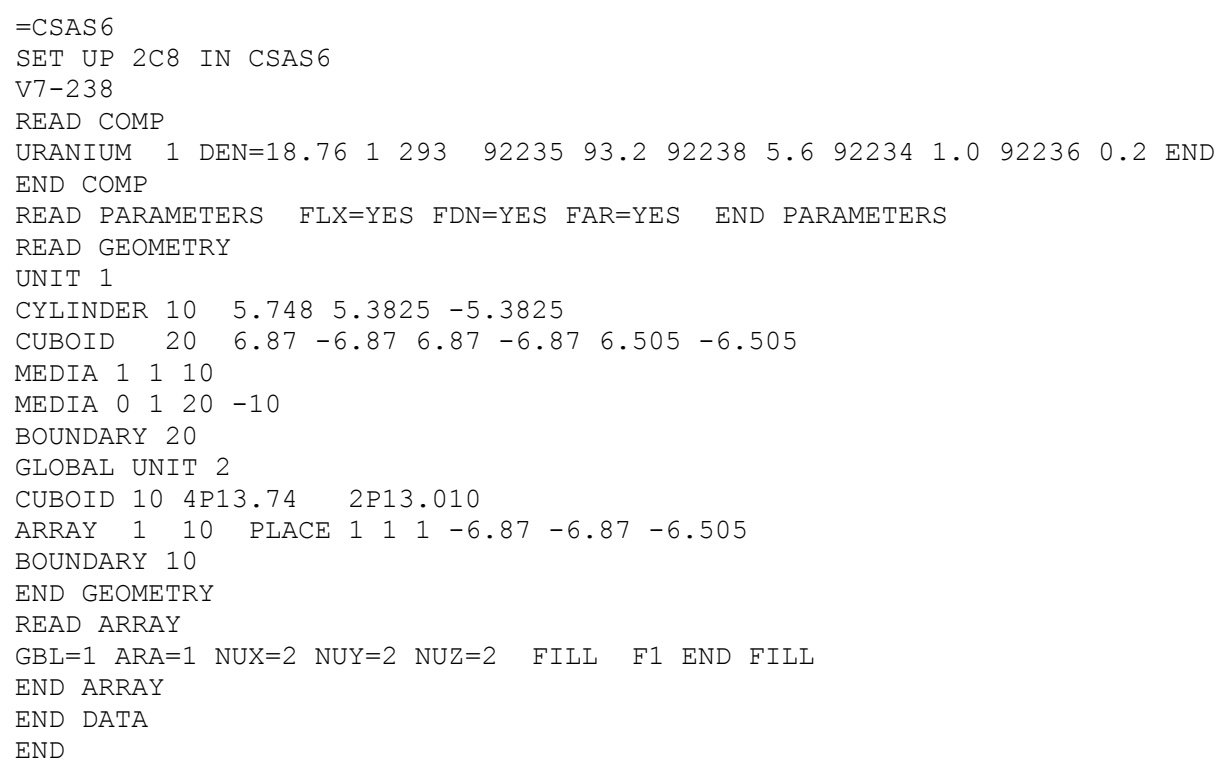

EXAMPLE 2. CSAS6 - Determine the $k_{\text {eff }}$ of an array of fuel pellets in a $\mathrm{UO}_{2} \mathrm{~F}_{2}$ solution.

Consider a $60 \mathrm{~cm}$ inside diameter cylindrical tank filled with 5.0\%-enriched $\mathrm{UO}_{2}$ fuel rods and $5.0 \%$-enriched $\mathrm{UO}_{2} \mathrm{~F}_{2}$ solution at $295 \mathrm{gm} /$ liter. A $51 \times 51 \times 1$ array of fuel rods is centered on the bottom of the tank. The fuel rods are $366 \mathrm{~cm}$ long, $0.45 \mathrm{~cm}$ in radius, clad with $0.01-\mathrm{cm}$-thick Al, and at a pitch of $1.5 \mathrm{~cm}$. The fuel rods sit on the bottom of the container and the container and solution rise $5.0 \mathrm{~cm}$ above the top of the rods. The container is $10 \mathrm{~cm}$ thick in the side and bottom and open at the top. Determine the $k_{\text {eff }}$ of the system. Input data for this problem follow. 


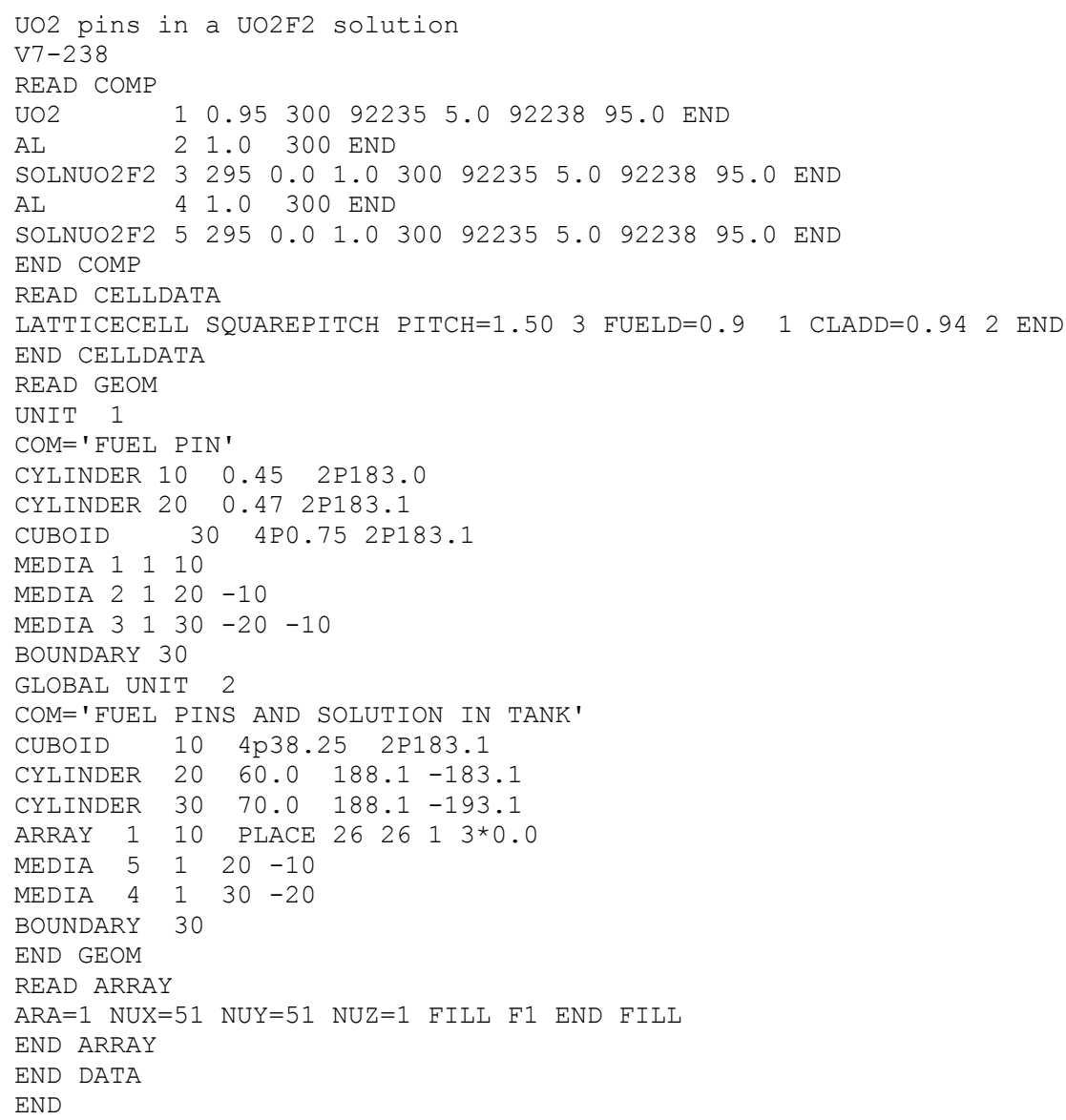

\subsection{A.2 Run KENO-VI containing cell-weighted mixtures}

CSAS6 creates a microscopic working format library and a mixing table that is passed to KENO-VI. The microscopic cross sections of the nuclides used in the unit cell geometry description are cell-weighted by specifying CELLMIX = followed by a unique mixture number. This mixture number utilizes the cellweighted cross sections that represent the heterogeneous system. CSAS6 executes KENO-VI and calculates $k_{\text {eff }}$ for the problem.

EXAMPLE 1. CSAS6 - Calculate the $k_{\text {eff }}$ of an array of fuel assemblies using cell-weighted cross sections.

Consider the $4 \times 4 \times 1$ array of fuel assemblies in a square aluminum cask described in Sect. 2.2.A.1.1, Example 2. Calculate the $k_{\text {eff }}$ of the system by using the cell-weighted mixture 200 to represent the fuel pins in the fuel assembly. Note that mixtures 1, 2, and 3, representing $\mathrm{UO}_{2}$, zirconium, and water, respectively, are used in the unit cell description. Cell-weighting is applied to the microscopic cross sections that are used in the cell, making them incorrect for use elsewhere. Because water is used both inside the cell and between the fuel assemblies, an additional mixture, mixture 6 , has been added to represent the water between the fuel assemblies. The input data for this problem follow.

$=$ CSAS 6

SQUARE FUEL CASK EXAMPLE USING HOMOGENEOUS MOCKUP

$\mathrm{V} 7-238$

READ COMP

UO2 1 DEN=9.21 $1.0 \quad 293.92235 \quad 2.359223897 .65$ END 
ZR $\quad 2 \quad 1$ END

$\mathrm{H} 2 \mathrm{O} \quad 3 \quad 1$ END

$\begin{array}{llllll}B 4 C & 4 & 0.367 & \text { END }\end{array}$

AL $\quad 4 \quad 0.636$ END

AL $\quad 5 \quad 1$ END

H2O 61 END

END COMP

READ CELLDATA

LATTICECELL SQUAREPITCH PITCH=1.3 3 FUELD=0.8 1 CLADD=0.94 2 CELLMIX=200 END

END CELLDATA

READ PARAM FAR=YES GEN=253 END PARAM

READ GEOM

UNIT 2

COM= 'FUEL ASSEMBLY'

CUBOID $10 \quad 4 \mathrm{P} 11.05 \quad 2 \mathrm{P} 183.07$

CUBOID $20 \quad 4 \mathrm{P} 11.70 \quad 2 \mathrm{P} 183.72$

CUBOID $30 \quad 4 \mathrm{P} 12.20 \quad 2 \mathrm{P} 184.22$

MEDIA $200 \quad 1 \quad 10$

$\begin{array}{lllll}M E D I A & 4 & 1 & 20 & -10\end{array}$

$\begin{array}{llllll}\text { MEDIA } & 6 & 1 & 30 & -20 & -10\end{array}$

BOUNDARY 30

GLOBAL UNIT 3

COM='FUEL CASK CONTAINING $4 \mathrm{X} 4$ ARRAY OF ASSEMBLIES'

CUBOID $\quad 10 \quad 4 \mathrm{P} 48.8 \quad 2 \mathrm{P} 184.22$

CUBOID $20 \quad 4 \mathrm{P} 58.8 \quad 2 \mathrm{P} 194.22$

$\begin{array}{lllllllllll}\text { ARRAY } & 1 & 10 & \text { PLACE } & 1 & 1 & 1 & -36.6 & -36.6 & 0.0\end{array}$

$\begin{array}{lllll}\text { MEDIA } & 5 & 1 & 20 & -10\end{array}$

BOUNDARY 20

END GEOM

READ ARRAY

$A R A=1 \quad N U X=4 \quad N U Y=4 \quad N U Z=1$ FILL F2 END FILI

END ARRAY

END DATA

END 
EXAMPLE 2. CSAS6 - Determine the $k_{\text {eff }}$ of an array of fuel pellets in a $\mathrm{UO}_{2} \mathrm{~F}_{2}$ solution using cell-weighted cross sections.

This is the same problem as described in Sect. 2.2.A.1.1, Example 2. However, the rods and solutions have been replaced with a cell-weighted mixture 50. Determine the $k_{\text {eff }}$ of the container. Input data for this problem follow.

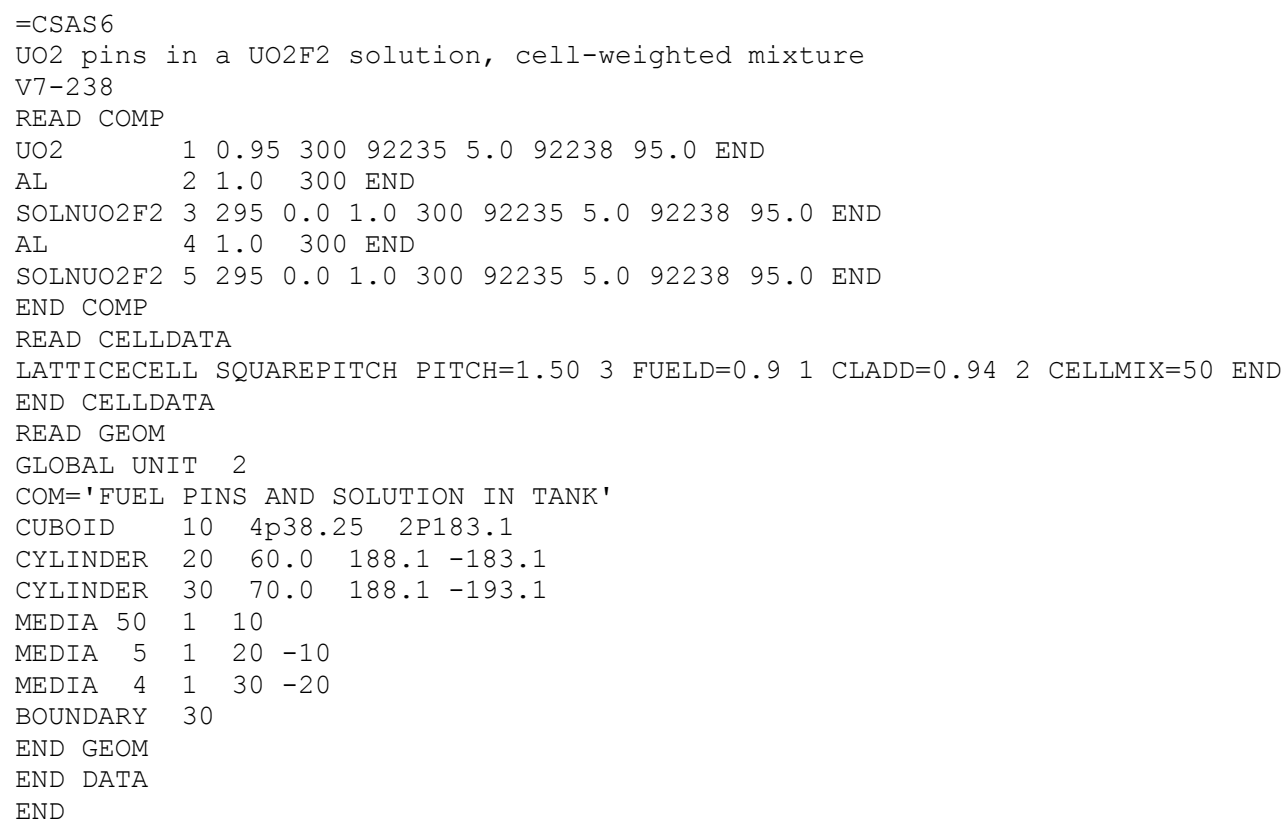

\subsection{A.3 Run KENO-VI containing multiple unit cells}

CSAS6 can create a microscopic working format library and a mixing table that contains more than one unit cell. Each unit cell is explicitly defined in the CELLDATA section of the standard composition data. Materials may appear in only one unit cell. All materials in the standard composition that are not contained in a unit cell are processed assuming infinite homogeneous media. CSAS6 passes the created working library to KENO-VI which calculates $k_{\text {eff }}$ for the problem.

EXAMPLE 1. CSAS6 - Calculate the $k_{\text {eff }}$ of a system using two unit cell descriptions.

Consider an infinite XY-array composed of two types of fuel assemblies in a checkerboard pattern moderated by water. Each assembly consists of a $17 \times 17 \times 1$ array of zirconium-clad, enriched $\mathrm{UO}_{2}$ fuel pins in a square pitched array. In one array the uranium is $3.5 \%$-enriched and in the other array the uranium is $2.9 \%$-enriched. The $\mathrm{UO}_{2}$ has a density of $9.21 \mathrm{~g} / \mathrm{cm}^{3}$. The pin diameter is $0.8 \mathrm{~cm}$ and is $366 \mathrm{~cm}$ long. The clad is $0.07 \mathrm{~cm}$ thick, and the pitch is $1.3 \mathrm{~cm}$. Each fuel bundle is contained in a $0.65-$ $\mathrm{cm}$-thick Boral sheath. The bundles are separated by an edge-to-edge spacing of $1.0 \mathrm{~cm}$. The water and zirconium is input in the standard composition data once for every unit cell in which it appears because a material may appear in only one unit cell. Determine the $k_{\text {eff }}$ of the infinite array. Note that periodic boundary conditions are required to obtain an infinite checkerboard array. Input data for this problem follow. 


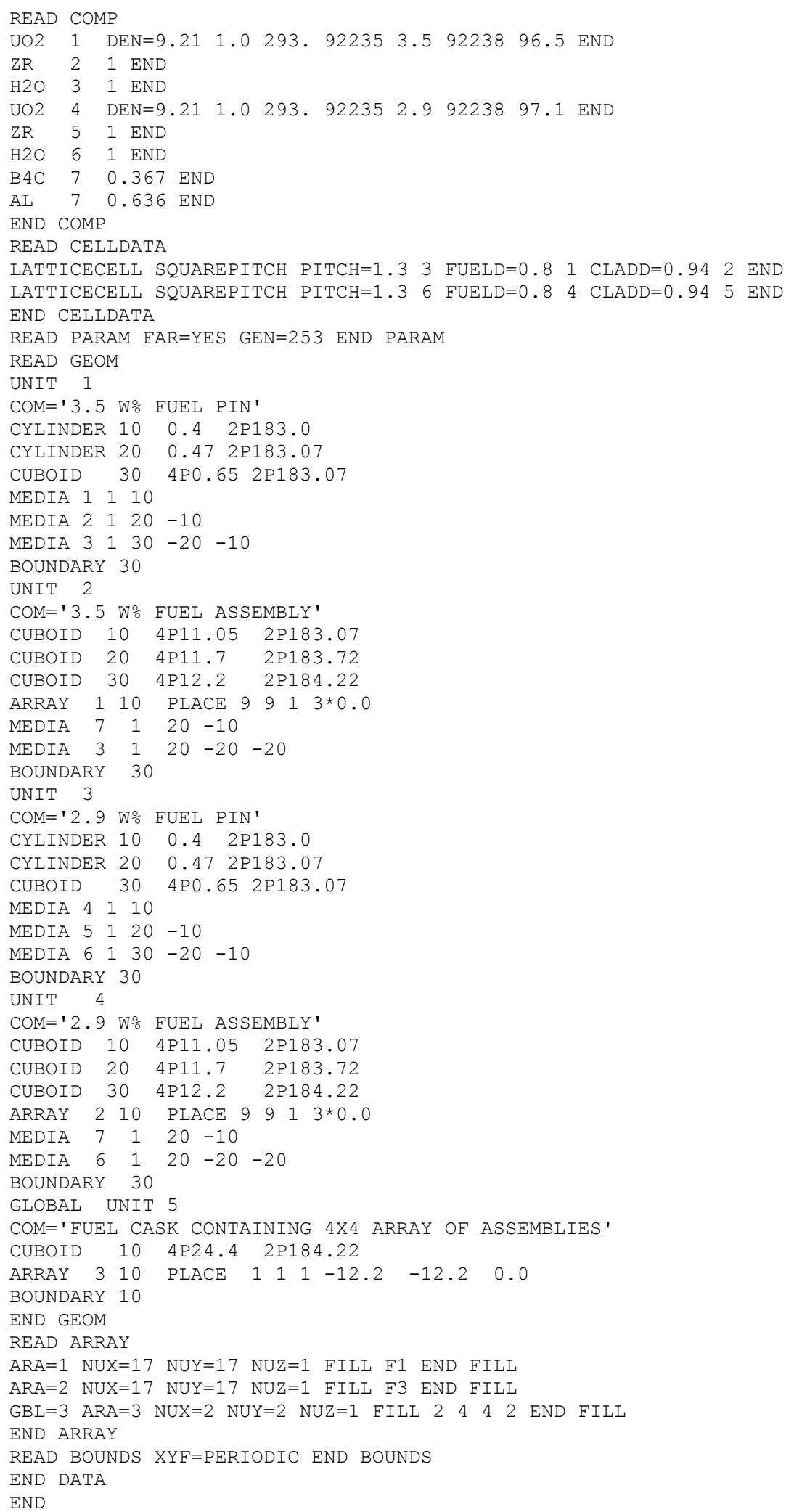

EXAMPLE 2. CSAS6 - Calculate the $k_{\text {eff }}$ of a system using two unit cell descriptions and cell-weighted mixtures. 
Consider a problem in which a stainless steel cylinder with an inner diameter of $56 \mathrm{~cm}$ and an inside height of $91 \mathrm{~cm}$ is filled with pellets of $\mathrm{UO}_{2}$ in borated water. The steel is $0.125 \mathrm{~cm}$ thick. The spherical $2.57 \%$-enriched $\mathrm{UO}_{2}$ pellets have a diameter of $1.07 \mathrm{~cm}$ and are arranged in a triangular pitch array with a pitch of $1.13 \mathrm{~cm}$. The spherical $2.96 \%$-enriched $\mathrm{UO}_{2}$ pellets have a diameter of $1.07 \mathrm{~cm}$ and are arranged in a triangular pitch array with a pitch of $1.12 \mathrm{~cm}$. The cylindrical tank is filled half full of the $2.96 \%$ pellets in borated water, and the remainder is filled with the $2.57 \%$-enriched pellets in borated water.

Mixture 100 is the cell-weighted mixture containing the $2.57 \%$-enriched uranium pellets and mixture 200 is the cell-weighted mixture containing the $2.96 \%$-enriched uranium pellets. Determine the $k_{\text {eff }}$ of this system. Input data for this problem follow.

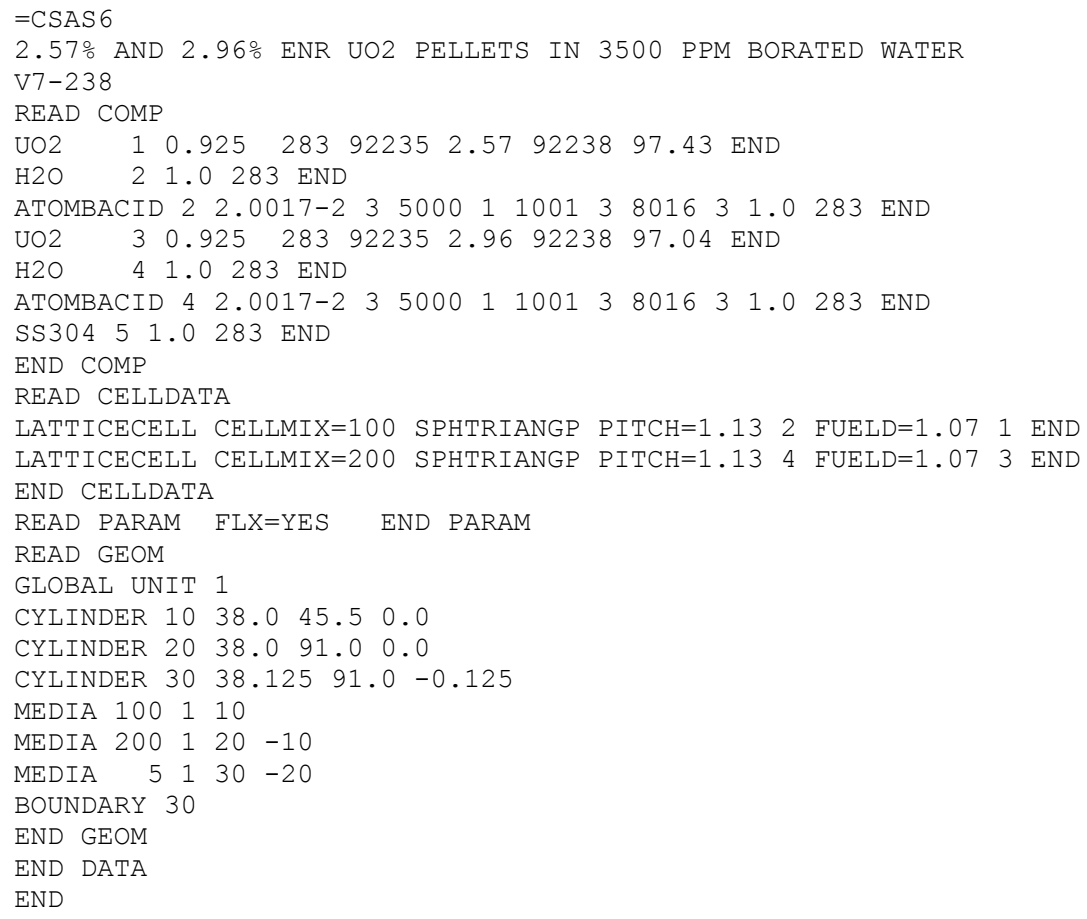




\title{
2.3 STARBUCS: A SCALE CONTROL MODULE FOR AUTOMATED CRITICALITY SAFETY ANALYSES USING BURNUP CREDIT
}

\author{
G. Radulescu and I. C. Gauld
}

\begin{abstract}
STARBUCS is an analysis sequence in SCALE for automating criticality safety and burnup loading curve analyses of spent fuel systems employing burnup credit. STARBUCS requires only the fresh fuel composition, an irradiation history, and the KENO model for a spent fuel configuration to be provided in an input file. It automatically performs all necessary calculations to determine spent fuel compositions, self-shielded cross sections, and the $k_{\text {eff }}$ of the spent fuel configuration. In addition, for burnup loading curve analyses, STARBUCS performs iterative calculations to search for initial fuel enrichments that result in an upper subcritical limit. STARBUCS allows the user to simulate axial- and horizontal-burnup gradients in a spent fuel assembly, select the specific actinides and/or fission products that are to be included in the criticality analysis, and apply isotopic correction factors to the predicted spent fuel nuclide inventory to account for calculational bias and uncertainties. A depletion analysis calculation for each of the burnup-dependent regions of a spent fuel assembly, or any other system containing spent nuclear fuel, is performed using the ORIGEN-ARP sequence of SCALE. For criticality safety calculations employing multigroup cross-section data, the spent fuel compositions are used to generate resonance self-shielded cross sections for each region of the problem. The region dependent nuclide concentrations and cross sections are applied in a three-dimensional criticality safety calculation using the KENO code. Both KENO V.a and KENO-VI criticality codes are supported for single criticality safety calculations using burnup credit, but only KENO V.a can be used in criticality calculations for burnup loading curve analyses. Although STARBUCS was developed specifically to address the burnup-credit analysis needs for spent fuel transport and storage applications, it provides sufficient flexibility to allow criticality safety assessments involving many different potential configurations of $\mathrm{UO}_{2}$ spent nuclear fuel.
\end{abstract}




\section{TABLE OF CONTENTS}

Page

2.3 STARBUCS: A SCALE Control Module for Automated Criticality Safety Analyses

Using Burnup Credit 2-124

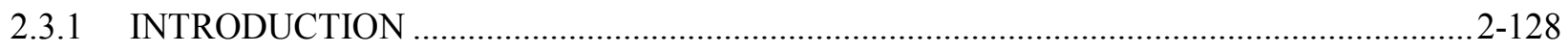

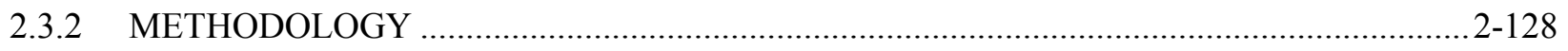

2.3.3 CAPABILITIES AND LIMITATIONS _........................................................................ 2-131

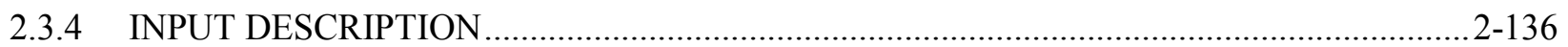

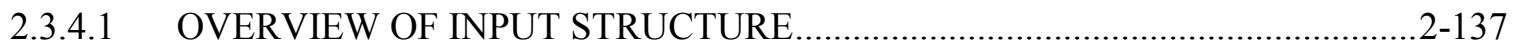

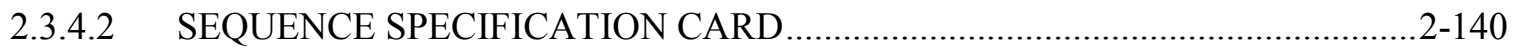

2.3.4.3 OPTIONAL SEQUENCE PARAMETERS ...........................................................2-140

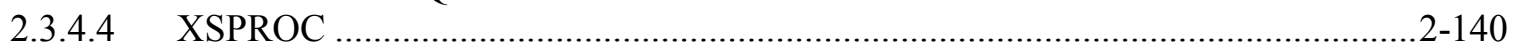

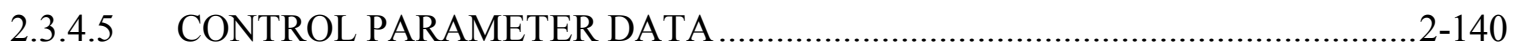

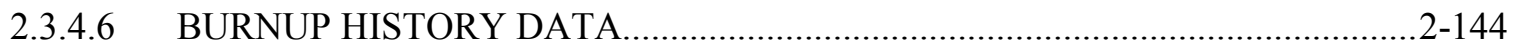

2.3.4.7 SEARCH PARAMETER DATA …................................................................. 2-147

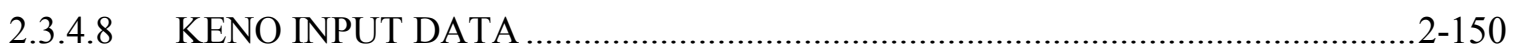

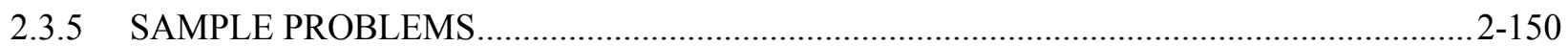

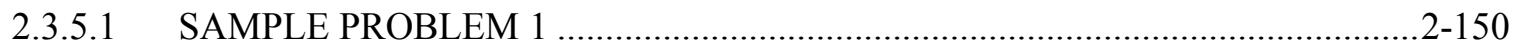

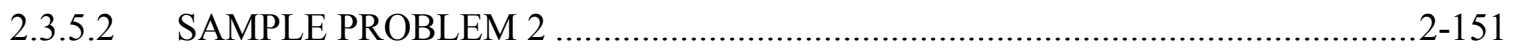

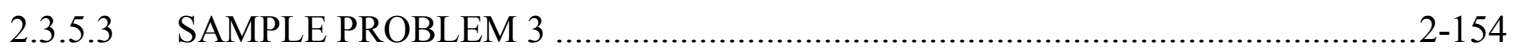

2.3.5.4 SAMPLE PROBLEM 4 …........................................................... $2-158$

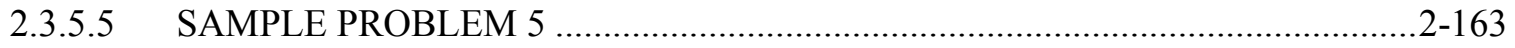

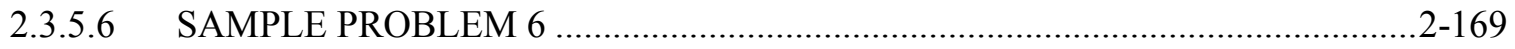

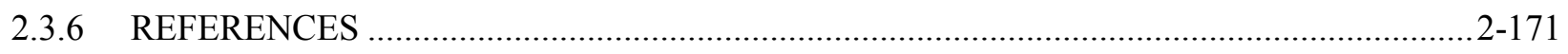




\section{LIST OF FIGURES}

Figure 2.3.1. Modules and flow of STARBUCS sequence for criticality calculations........................2-131

Figure 2.3.2. Modules and flow of STARBUCS sequence for burnup loading curve calculations......2-131

Figure 2.3.3. Fuel and material mixture numbering convention used in STARBUCS......................2-134

Figure 2.3.4. Fuel and material mixture numbering convention used in STARBUCS ......................2-134

Figure 2.3.5. Example of mixture numbering scheme used in STARBUCS..................................2-135

Figure 2.3.6. Plot of the CE $14 \times 14$ assembly array geometry in sample problem 3.......................2-157

Figure 2.3.7. Cutaway view of the generic 32 -assembly burnup-credit cask ...................................2-158

Figure 2.3.8. Plot of the $2 \times 2$ array of CE $14 \times 14$ assemblies with burnup gradient........................2-168

Figure 2.3.9. Cutaway 3 -D view of the hexagonal VVER assembly model with water hidden..........2-171 


\section{LIST OF TABLES}

Table 2.3.1. Valid data block keywords for a single criticality safety calculation using burnup credit.....

Table 2.3.2. Valid data block keywords for burnup loading curve calculations

Table 2.3.3. Outline of input data for the STARBUCS sequence

Table 2.3.4. Table of control parameter data

Table 2.3.5. Built-in burnup-dependent axial profiles, NAX $=-18$ from Ref. 2)

Table 2.3.6. Table of power history data

Table 2.3.7. Table of search data

Table 2.3.8. STARBUCS input listing for sample problem 1

Table 2.3.9. STARBUCS input listing for sample problem 2

Table 2.3.10. STARBUCS input listing for sample problem 3

Table 2.3.11. STARBUCS input listing for sample problem 4

Table 2.3.12. STARBUCS input listing for sample problem 5

Table 2.3.13. STARBUCS input listing for sample problem 6 


\subsubsection{Introduction}

The U.S. Nuclear Regulatory Commission (NRC) issued Revision 2 of the Interim Staff Guidance 8 (ISG-8) (Ref. 1) on burnup credit in September, 2002. ISG-8 provides guidance on the application of burnup-credit in criticality safety analyses for pressurized-water reactor (PWR) spent fuel in transportation and storage casks. Burnup credit is the concept of taking credit for the reduction in reactivity in spent fuel due to burnup. The reduction in reactivity that occurs with fuel burnup is due to the change in concentration (net reduction) of fissile nuclides and the production of actinide and fissionproduct neutron absorbers. In contrast to criticality safety analyses that employ a fresh-fuel assumption (i.e., conservatively assuming unirradiated fuel compositions), credit for burnup requires the prediction of both fissile material and absorber nuclide concentrations in spent nuclear fuel (SNF) and consideration of many burnup-related phenomena, in addition to the criticality issues.

Consideration of the depletion aspects in the criticality assessment of SNF places an increasing reliance on computational tools and methods, and significantly increases the overall complexity of the criticality safety analysis. The use of spent fuel nuclide concentrations in the criticality evaluation also necessitates consideration of many additional sources of uncertainty associated with fuel depletion. ISG-8 highlights, for example, the need for applicants employing burnup credit in criticality safety assessments to address the axial and horizontal variation of the burnup within a spent fuel assembly, uncertainties and bias in the nuclide predictions, and the additional reactivity margin available from fission products and actinides not credited in the licensing basis.

To assist in performing and reviewing criticality safety assessments of transport and storage casks that apply burnup credit, a new control sequence called STARBUCS (Standardized Analysis of Reactivity for Burnup Credit using SCALE) was developed in SCALE 5. STARBUCS automates the generation of spatially-varying nuclide compositions in a spent fuel assembly, and applies the assembly compositions in a three-dimensional (3-D) Monte Carlo analysis of the system. STARBUCS automatically prepares input files for each of the modules in the sequence, executes the modules through the SCALE driver, and performs all flow control, module interface, and data management functions. The STARBUCS sequence uses well-established code modules currently available in SCALE. STARBUCS also performs iterations over a range of initial fuel enrichments to determine the initial enrichments below which $\mathrm{UO}_{2}$ commercial spent fuel may be loaded in a transport/storage cask for specified burnup values. With this capability, STARBUCS assists in generating burnup loading curves for criticality safety analyses of spent fuel in transport and storage casks.

The STARBUCS sequence automates the depletion calculations using the ORIGEN-ARP methodology to perform a series of cross-section preparation and depletion calculations to generate a comprehensive set of spent fuel isotopic inventories for each spatially-varying burnup region of an assembly. The spent fuel nuclide concentrations are subsequently input to either CSAS5 or CSAS6 to and perform a criticality calculation of the system using the KENO V.a or KENO-VI code, respectively, to determine the neutron multiplication factor $\left(k_{\text {eff }}\right)$ for the system. Only minimal input is required by the user to perform a typical burnup-credit analysis. The user can specify the assembly-average irradiation history, the axial density variation of the reactor moderator, the axial- and horizontal-burnup profile, and the nuclides that are to be applied in the criticality safety analysis. Nuclide correction factors may also be applied to the predicted concentrations to account for known bias and/or uncertainty in the predicted SNF compositions.

\subsubsection{Methodology}

The STARBUCS control module is a burnup-credit sequence designed to perform 3-D Monte Carlo criticality safety calculations that include the effects of spatially-varying burnup in SNF configurations. 
STARBUCS offers two options: either perform a single criticality safety calculation with burnup credit or perform iterative calculations for burnup loading curve analyses of commercial $\mathrm{UO}_{2}$ spent fuels. The sequence contains a set of instructions designed to automatically process input data, execute code modules currently available in SCALE for depletion, resonance cross section, and criticality calculations. In addition, for burnup loading curve analyses, STARBUCS checks whether $k_{\text {eff }}$ converges to a userprovided upper subcritical limit, adjusts the initial fuel enrichment using the least squares method, and repeats the sequence until either convergence is achieved or determine that no solution can be found. The overall program structures and flow for a single criticality calculation and for burnup loading curve calculations are illustrated in Figure 2.3.1 and Figure 2.3.2, respectively.

The sequence uses well-established code modules currently available in the SCALE code system. These modules include ARP and ORIGEN to perform the depletion analysis phase of the calculations. ORIGEN-ARP is a sequence within the SCALE system that serves as a faster alternative to the TRITON depletion sequence of SCALE to perform point-irradiation calculations with the ORIGEN code using problem-dependent cross sections. ARP uses an algorithm that enables the generation of cross-section libraries for the ORIGEN code by interpolation over pregenerated cross-section libraries. The ORIGEN code performs isotopic generation and depletion calculations to obtain the spent fuel nuclide compositions. For criticality safety calculations using multigroup cross-section data, problem dependent cross sections are processed with the resonance self-shielding capabilities of XSProc using the regiondependent compositions from the depletion analyses. Finally, the region dependent nuclide concentrations and cross sections are applied in a 3-D criticality calculation for the system using either KENO V.a or KENO-VI to calculate the $k_{\text {eff }}$ value.

The ORIGEN-ARP depletion analysis methodology represents a significant increase in computational speed as compared to equivalent calculations performed using the SCALE depletion analysis sequences that use two-dimensional transport methods, with virtually no sacrifice in accuracy. ARP uses an algorithm that enables the generation of cross-section libraries for the ORIGEN code by interpolating on cross sections available in pre-generated data libraries. For uranium-based fuels the interpolation parameters available are initial fuel enrichment, burnup and, optionally, moderator density. STARBUCS creates input files for ARP and ORIGEN for each burnup-dependent region of an assembly and calculates the spent fuel nuclide concentrations for the region using a user-specified assembly irradiation history, cooling time, and burnup profiles. The ARP cross-section libraries must be available in advance of a STARBUCS burnup-credit calculation. These libraries may be created using TRITON. The ARP libraries include the effects of assembly design and operating conditions on the neutron cross sections used in the burnup analysis. Several ARP libraries are distributed in the SCALE code system and can be applied in a STARBUCS analysis. Alternatively, a user may create a specific ARP library for other assembly types or operating conditions not available in the default libraries. The generation of ARP libraries is discussed in ORIGENARP's Appendix A.

The depletion phase of the analysis is performed using ARP and ORIGEN to calculate the compositions of each discrete fuel region (axial or horizontal). After a single ORIGEN-ARP depletion calculation is completed, control is passed back to the STARBUCS module which reads the spent fuel nuclide inventories generated by ORIGEN, saves them, prepares the ARP and ORIGEN input files for the next burnup region, and executes the codes in sequence. This cycle continues until the fuel compositions for all axial and horizontal regions have been calculated and saved, completing the depletion phase of the analysis. The depletion calculations for each axial and radial zone are performed using an initial fuel basis of 1 MTHM $\left(10^{6} \mathrm{~g}\right.$ heavy metal).

After all depletion calculations are completed, STARBUCS reads the spent fuel nuclide inventories for all regions and prepares input for the criticality calculation. The concentrations of all nuclides in the 
ORIGEN depletion analysis are converted from gram-atom units (per MTU) to units of atoms/b-cm applied in the criticality calculation. The criticality calculation is performed using the capabilities in the CSAS5 or CSAS6 control module of SCALE. Specifically, STARBUCS prepares input for the CSAS5 module when criticality calculations are to be performed using KENO V.a, and for the CSAS6 sequence when using KENO-VI. Note that only the criticality safety sequence CSAS5 of SCALE can be used for burnup loading curve calculations.

For burnup loading curve iterative calculations, STARBUCS employs the search algorithm described in CSAS5 section on Optimum (Minimum/Maximum) Search to determine initial fuel enrichments that satisfy a convergence criterion for the $\mathrm{k}_{\text {eff }}$ of the spent fuel configuration. If convergence is not achieved in a search pass, the initial fuel enrichment is automatically adjusted. This sequence repeats until either $\mathrm{k}_{\text {eff }}$ converges to an upper subcritical limit or until the algorithm determines that a solution is not possible. The procedure is repeated for each requested burnup value. The maximum allowable iterations, upper subcritical limit, tolerance for convergence, and a range of initial fuel enrichments can be set by the user. The lower and upper enrichment bounds as well as the burnup values for spent fuel regions must be contained within the range of enrichment and burnup values used to generate the applicable ORIGENARP library. The control module prepares a STARBUCS input file for each search pass requesting a single criticality calculation using the calculated spent fuel compositions. In this input file, the burnup history data block and/or the fuel mixture compositions are updated based on the outcome of the search sequence. The pre-burnup compositions for the two minor uranium isotopes, ${ }^{234} \mathrm{U}$ and ${ }^{236} \mathrm{U}$, are updated in the STARBUCS input file for a new pass only if they were included in the initial input file prepared by the user. Their updated weight percentages are based on the assumption that the mass ratios ${ }^{234} \mathrm{U} /{ }^{235} \mathrm{U}$ and ${ }^{236} \mathrm{U} /{ }^{235} \mathrm{U}$ do not change with fuel enrichment.

\section{STARBUCS}

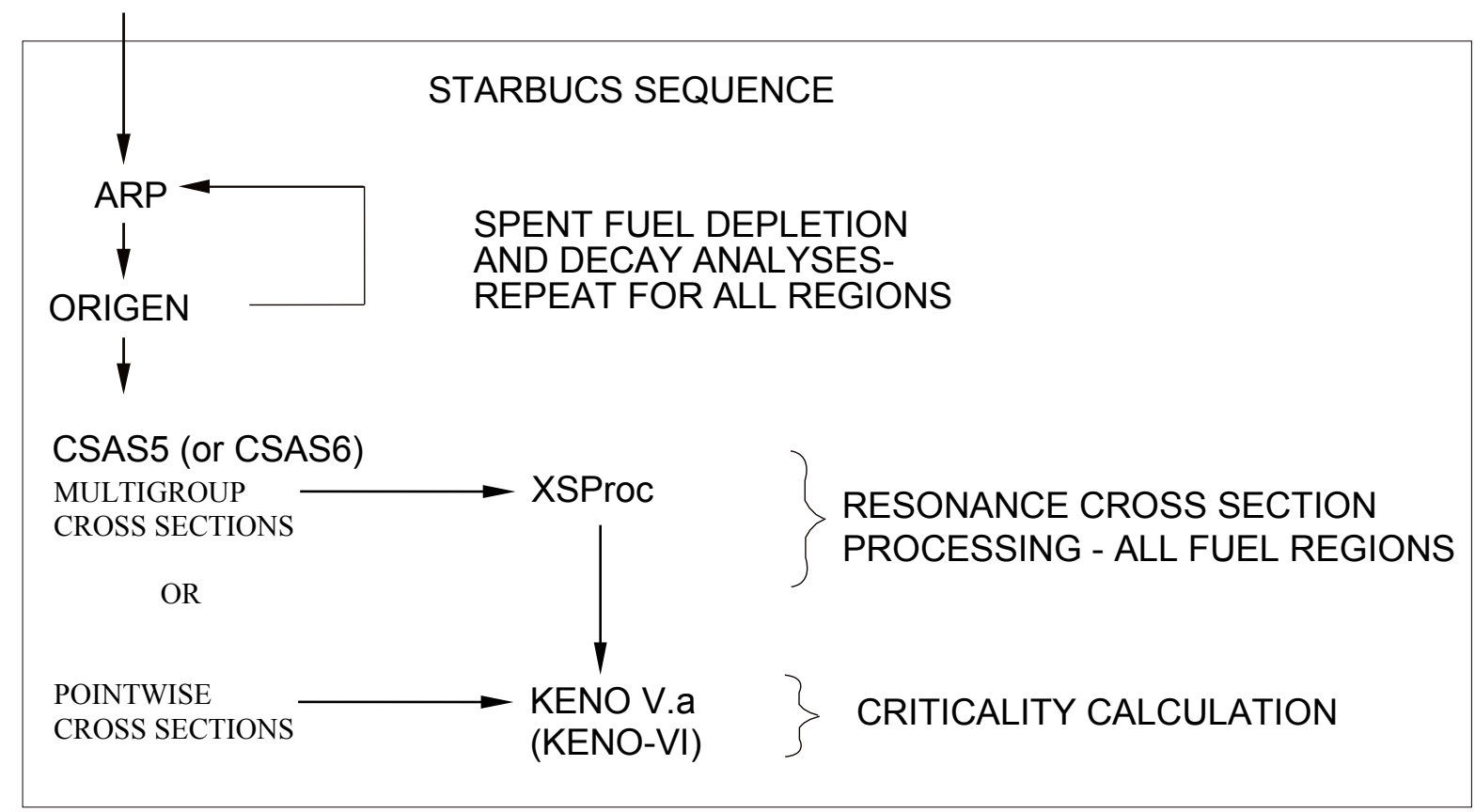


Figure 2.3.1. Modules and flow of STARBUCS sequence for criticality calculations.

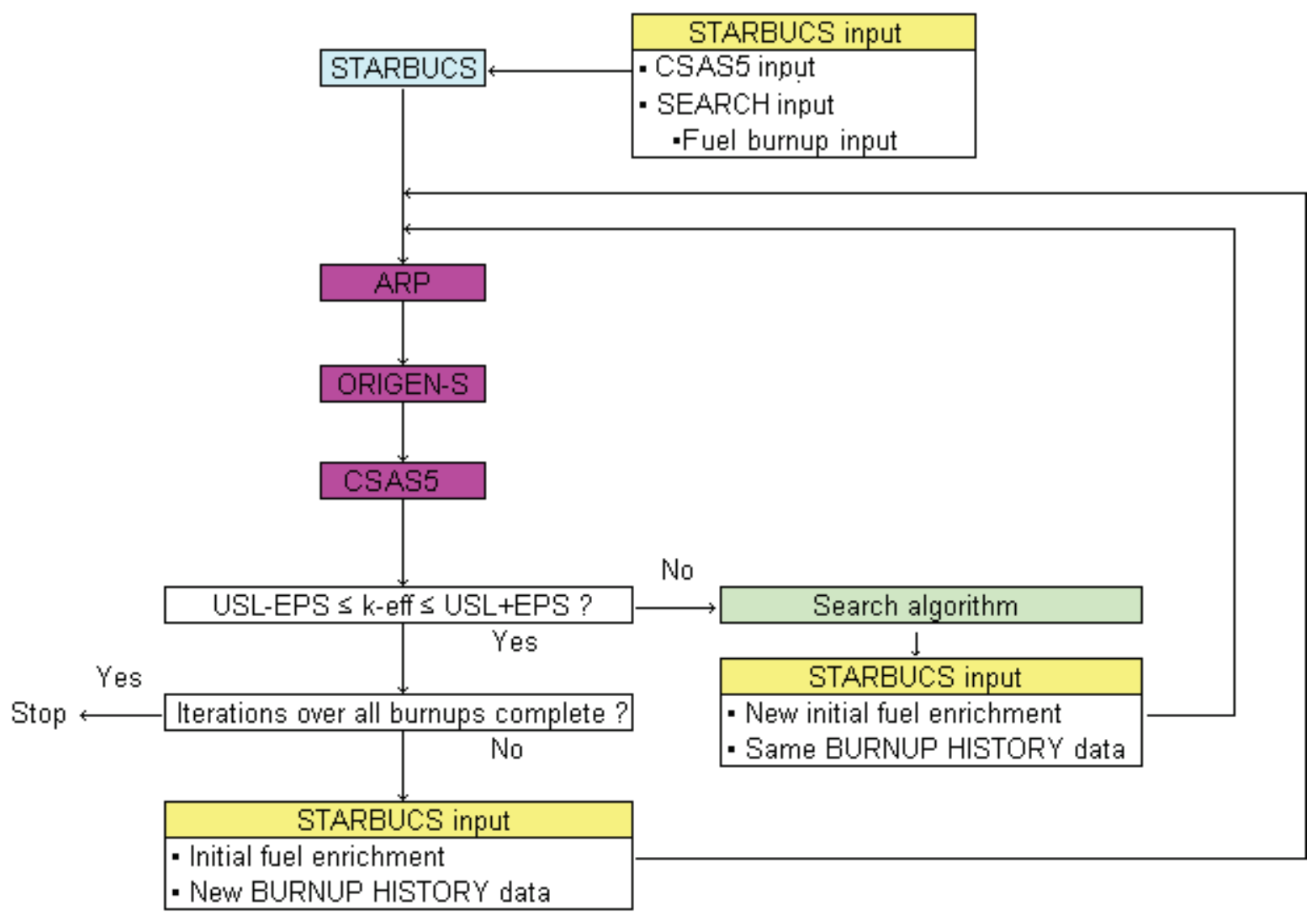

Figure 2.3.2. Modules and flow of STARBUCS sequence for burnup loading curve calculations.

\subsubsection{Capabilities and Limitations}

STARBUCS is designed to facilitate criticality safety analyses employing burnup credit by automating and linking the depletion and criticality calculations. The STARBUCS sequence has been designed to readily allow analysts and reviewers to assess the subcritical margins associated with many of the important phenomena that need to be evaluated in the context of the current regulatory guidance on burnup credit. However, STARBUCS is sufficiently general to allow virtually any configuration involving irradiated nuclear material to be analyzed. Limitations and some of the key capabilities of the STARBUCS sequence are described below.

1. STARBUCS limitations include the use of a single $\mathrm{UO}_{2}$ fuel type and, for analyses employing multigroup cross-section data, the use of geometry configurations consisting of spent fuel rod arrays. However, the type of spent fuel configurations that can be analyzed is entirely general. STARBUCS can be used to perform criticality safety assessments of individual fuel assemblies, a spent fuel cask, a spent fuel storage pool, or any nuclear system containing $\mathrm{UO}_{2}$ irradiated nuclear fuel. 
2. Only the criticality safety sequence CSAS5 of SCALE can be used for burnup loading curve calculations; therefore KENO V.a geometry description must be available in a STARBUCS input file for burnup loading curve calculations.

3. Burnup calculations can incorporate any desired operating history. The user may enter the specific power, cycle lengths, cycle down time, post-irradiation cooling time, etc. The axial-water-moderator density variation may also be specified in the depletion analysis, provided the ARP cross-section library contains such data.

4. The effects of assembly design, soluble boron concentrations, burnable poison exposure, reactor operating conditions, etc., are accounted for in the ARP (Automatic Rapid Processing) cross-section libraries used in the ORIGEN depletion calculations. Libraries for several fuel assembly designs are distributed with SCALE. These libraries can also be readily created for any reactor and fuel assembly design that can be represented in the depletion analysis sequences of the SCALE system.

5. The user can select the specific actinide and/or fission product nuclides to be included in the criticality safety analysis. The user also has the option to perform a criticality calculation employing all nuclides for which cross-section data exist.

6. Isotopic correction factors may be input to adjust the calculated nuclide inventories to account for known bias and/or uncertainties associated with the depletion calculations.

Minimal user input is required to perform many types of analyses. Default values are supplied for many of the input parameter keywords. The user may select from built-in burnup-dependent 18-axial-zone profiles taken from Ref. 2, or the user may input an arbitrary user-defined burnup distribution with up to 100 -axial zones and up to 7-horizontal zones. The depletion analysis calculations for each zone are performed for all nuclides (the ORIGEN data libraries contain cross-section and decay data for more than 1000 unique actinides, fission products, and structural activation products). The specific nuclides to be considered in the $k_{\text {eff }}$ analysis may be input by the user. If no nuclide set is explicitly selected, then all nuclides that have cross-section data in the ORIGEN library are automatically applied in the criticality analysis, resulting in a "full" burnup-credit criticality assessment. A capability to adjust the calculated isotopic inventories using correction factors that can account for biases and/or uncertainties in the calculated isotopic concentrations is also provided.

An appropriate ARP cross-section library for $\mathrm{UO}_{2}$ fuel must be available for the depletion analysis using STARBUCS. The user may use the libraries distributed with SCALE (e.g., ge7 $\times 7-0$, ge $8 \times 8-4$, ce14 $\times 14$, w1 $15 \times 15$, w17 $\times 17$ ofa) or the user may generate their own problem-specific libraries using the TRITON depletion analysis sequence available in SCALE. A complete list of ARP libraries distributed with SCALE and methods for generating ARP libraries are both described in ORIGENARP's Appendix A. The range of initial fuel enrichment and requested burnup values to be used in the STARBUCS calculations must be contained within the range of the enrichments and burnups used to generate the applicable ARP cross-section library.

The user is required to provide a complete KENO V.a model of the spent fuel configuration for burnup loading curve calculations and a complete KENO V.a or KENO-VI model of the spent fuel configuration for single criticality calculations using burnup credit. The initial material composition information is defined in a standard composition data block. The fuel material is automatically depleted in the sequence for each of the burnup-dependent regions or zones in the problem. The nuclide concentrations after irradiation and decay are automatically applied to the KENO criticality analysis. The mixture numbers for each of the fuel regions are identified by unique mixture numbers assigned automatically by 
STARBUCS based on the axial and horizontal region in the problem (see Figure 2.3.3). The user is required to specify the geometry/extent of the axial and horizontal zones in the KENO model and apply the appropriate mixture numbers for the desired configuration based on the mixture identifying scheme. STARBUCS performs no checking of the criticality model to verify that all mixtures in the problem have been used or that the order of the mixture numbers in the KENO model corresponds to the corresponding order of the input burnup profile. This provides the user a great deal of flexibility in setting up problems. However, it also requires that the user accurately prepare the input files to ensure that the spent fuel zone mixtures are assigned to the correct KENO V.a or KENO-VI geometry regions. For instance, the user could (intentionally) reverse the order of the axial-material identifiers in the KENO model to simulate inverted fuel, or zone mixtures could be omitted to simulate a problem using only a subset of the available fuel zones that were simulated in the depletion analysis. 


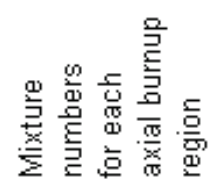
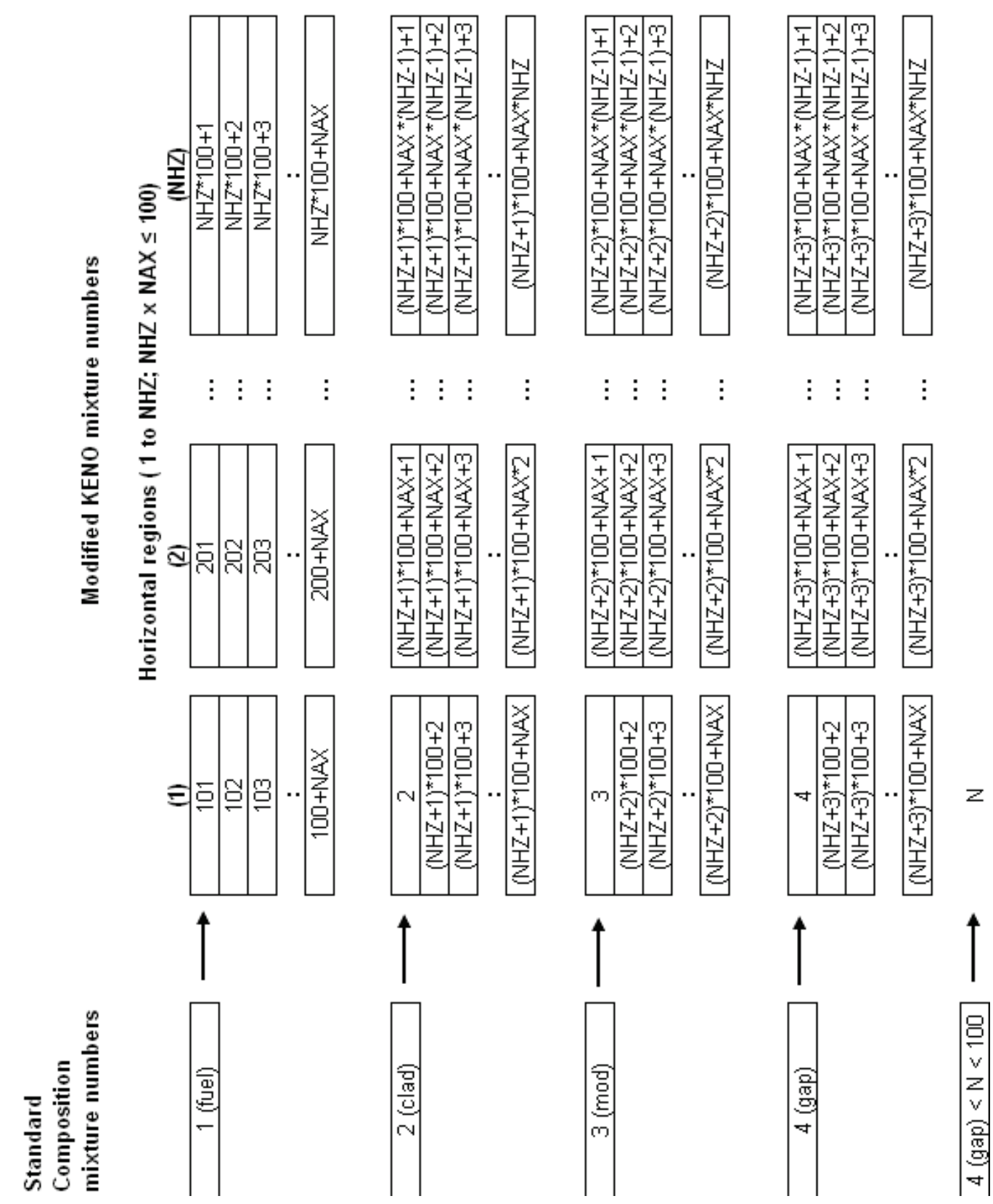

Figure 2.3.4. Fuel and material mixture numbering convention used in STARBUCS 

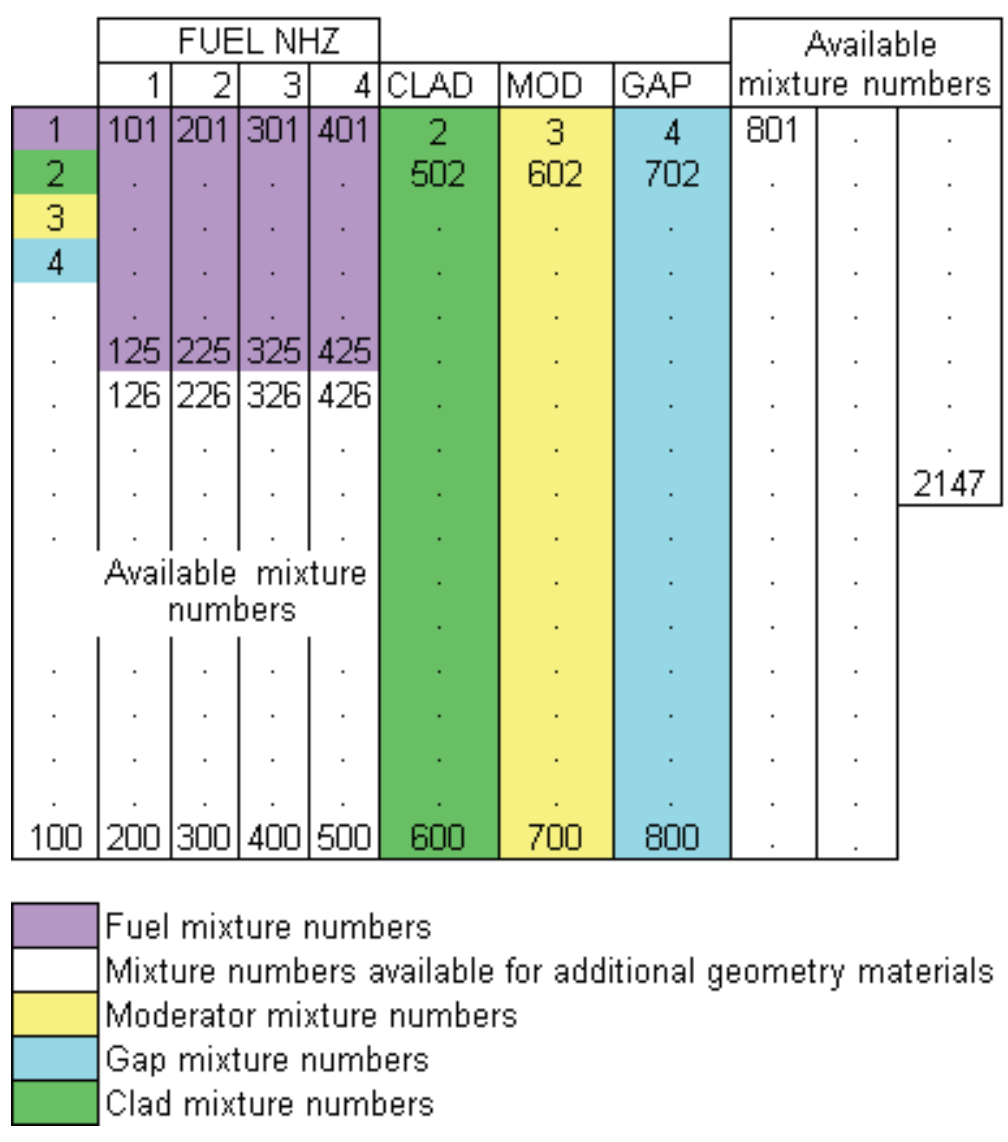

Figure 2.3.5. Example of mixture numbering scheme used in STARBUCS.

There are several conventions that must be followed when using STARBUCS. In general, these relate to the specification of materials and mixture numbering of the cross-section mixing table.

1. The maximum number of horizontal zones is restricted to seven if there is no gap or second moderator mixture, six if a gap or second moderator mixture is defined, and five if both a gap and a second moderator are defined. The number of axial-fuel zones is limited such that the product of horizontal zones $*$ axial zones is less than or equal to 100 . These limits constrain the maximum mixture number used for burned fuel in the KENO criticality calculation to less than 1000 and assign unique mixture numbers to clad, moderator, and gap mixtures for lattice cell descriptions. The convention used to number the depleted fuel zones is to start at mixture 101 and increment by 1 for each axial-burnup region. Thus, for a case with 10 axial-burnup regions, the fuel mixtures used in the criticality analysis would range from 101 to 110 . For a similar case having two horizontal zones in addition to the axial zones, the mixture numbers would also include mixtures 201 to 210 .

2. Mixture numbers for the clad, gap (if applicable), and moderator may also be used directly in the KENO model. Additional unique mixture numbers are required by the code for the lattice cell 
descriptions for each separate fuel zone (except for mixture 0 for void). These additional mixtures are assigned automatically by the code and are shown in Figure 2.3.3 for a lattice cell consisting of fuel, gap, clad, and moderator. The additional mixture numbers may also be used directly in the KENO model. Mixture number allocation is illustrated in Figure 2.3.5 for an example case where the number of different horizontal zones is four and the maximum number of axial zones is limited to 25 .

3. All structural materials in the problem must have mixture numbers different from the numbers automatically generated by the code (see Figure 2.3 .5 for an example of available mixture numbers). For the example shown in Figure 2.3.5, mixtures 5-100, 126-200, 226-300, 326-400, 501, 601, 701, 426-500, and 801-2147 are not allocated by STARBUCS and may be defined by the user in the composition data block and used in the geometry model. If the constraints in paragraph 1 are followed, mixture numbers less than 100 that were not used for fuel, gap, clad, moderator and mixture numbers from 1001 to 2147 are always available for structural materials. Note that STARBUCS does not provide a warning or stop program execution if a mixture number assigned to a structural material has also been generated internally by the computer code. The mixture numbers for structural materials are not changed and are thus applied in the KENO model in a one-to-one correspondence with the standard composition mixture as done for typical CSAS calculations. Therefore, the use of a mixture number for structural materials that is identical to one of the mixture numbers automatically generated by the code results in the combination of both materials in the composition for the mixture number.

4. Not all SCALE standard composition alphanumeric names (see the Standard Composition Library chapter) are currently recognized by STARBUCS. The use of special materials (e.g., C-GRAPHITE, NIINCONEL, H-POLY), particularly as fuel materials, that have nuclide identifiers that are not readily translated to ORIGEN ZA numbers should be avoided since these materials cannot be depleted.

5. A single STARBUCS calculation is limited to a single initial fuel type (composition, enrichment, assembly design, etc.). Configurations involving multiple fuel types may be solved by running a separate STARBUCS case for each type, saving the corresponding CSAS cases generated by STARBUCS that contain the irradiated fuel nuclide compositions, and manually merging the cases in such a way that all required fuel types are represented in the final case.

\subsubsection{Input Description}

STARBUCS input is divided into different data blocks containing related types of information. The standard composition data block used to define initial (fresh) fuel composition and all other materials in the criticality analysis problem, is read and processed by the Material Information Processor of SCALE and conforms to the standard input conventions (see Chapter M7). In addition to the standard composition data, three more input data blocks are required by STARBUCS. The data blocks are entered in the form

\section{READ XXXX input data END XXXX}

where $\mathbf{X X X X}$ is the data block keyword for the type of data being entered. The types of data blocks that are entered include general control parameter information, irradiation history and decay data or search parameter data, and the KENO V.a or KENO-VI input specifications. The valid block keywords for a single criticality safety calculation using burnup credit and for burnup loading curve calculations are listed in Table 2.3.2 and Table 2.3.3, respectively. A minimum of four characters is required for most keywords. The exception is the criticality model input data block READ KENOVA or READ KENOVI 
in which case the code must check additional character positions to determine the CSAS control sequence to be executed. The keywords can be up to twelve characters long, the first four of which must be input exactly as listed in the table. Entering the words READ XXXX followed by one or more blanks activates the data block input. All input data pertinent to block XXXX are then entered. Entering END XXXX followed by two or more blanks terminates data block XXXX.

Table 2.3.1. Valid data block keywords for a single criticality safety calculation using burnup credit

\begin{tabular}{ll}
\hline \multicolumn{1}{c}{ Data block type } & \multicolumn{1}{c}{ Block keyword } \\
\hline Control parameters & CONTROL \\
Burnup history & HISTORY or BURNDATA \\
KENO V.a input & KENOVA or KENO5 \\
KENO-VI input & KENOVI or KENO6 \\
\hline
\end{tabular}

Table 2.3.2. Valid data block keywords for burnup loading curve calculations

\begin{tabular}{ll}
\hline Data block type & \multicolumn{1}{c}{ Block keyword } \\
\hline Control parameters & CONTROL \\
Search parameters & SEARCH \\
KENO V.a input & KENOVA or KENO5 \\
\hline
\end{tabular}

All input within a data block is entered using keywords and is free format. Keyword entries may be of variable or array type. Variable keyword entries include the keyword plus the "=", followed by the value. Array keywords are usually followed by a series of entries, each separated by a blank or comma, and must always be terminated with an END that does not begin in column one. In some instances a single value may be input as an array entry; however, the word END is still always required. Within a given input data block the keyword entries may be in any order.

A single data entry may be entered anywhere on a line but cannot be divided between two lines; however, array data entries may be divided over many lines. The code identifies data keywords using only the first four (maximum) characters in the keyword name. Beyond the first four characters, the user may enter any alphanumeric or special character acceptable in FORTRAN, including single blanks, before the "=" character. Floating-point data may be entered in various forms; for example, the value 12340.0 may be entered as: $12340,12340.0,1.234+4,1.234 \mathrm{E}+4,1.234 \mathrm{E} 4$, or $1.234 \mathrm{E}+04$. Also, the value 0.012 may be entered as $12 \mathrm{E}-3,12-3,1.2-2$, etc. Numeric data must be followed immediately by one or more blanks or a comma.

\subsubsection{Overview of input structure}

An overview of the input to the STARBUCS sequence is given in Table 2.3.3. This table provides an outline of the input data block structure. The input data in positions 1 to 5 (see Table 2.3.3) are read and processed by the Material Information Processor of SCALE. These are the first data read by the code and 
must be in the order indicated. Data positions 6,7 or 8 , and 9 are read directly by STARBUCS and may be entered in any order. 
Table 2.3.3. Outline of input data for the STARBUCS sequence

\begin{tabular}{|c|c|c|c|}
\hline $\begin{array}{c}\text { Data } \\
\text { position }\end{array}$ & Type of data & Data entry & Comments \\
\hline & Sequence name & $=$ STARBUCS & Start in column one \\
\hline 1 & TITLE & Enter a title & 80 characters \\
\hline 2 & $\begin{array}{l}\text { Standard SCALE } \\
\text { pointwise or } \\
\text { multigroup cross- } \\
\text { section library name } \\
\text { or } \\
\text { the name of a user- } \\
\text { supplied multigroup } \\
\text { cross-section library }\end{array}$ & Library name & $\begin{array}{l}\text { The currently available standard SCALE cross- } \\
\text { section libraries are listed in the Standard } \\
\text { Comp. Lib. chapter, table Standard SCALE } \\
\text { Cross-Section Libraries. } \\
\text { STARBUCS allows a non-standard SCALE } \\
\text { multigroup cross-section library to be used } \\
\text { in a criticality calculation. }\end{array}$ \\
\hline 3 & $\begin{array}{l}\text { Standard Composition } \\
\text { specification data }\end{array}$ & $\begin{array}{l}\text { Enter the appropriate } \\
\text { data }\end{array}$ & $\begin{array}{l}\text { Begins this data block with READ COMP and } \\
\text { terminate with END COMP. See Standard } \\
\text { Composition chapter for details. }\end{array}$ \\
\hline 4 & Type of calculation & LATTICECELL & $\begin{array}{l}\text { Begins this data block with READ CELL and } \\
\text { terminate with END CELL. This is the } \\
\text { available option. See XSProc chapter for } \\
\text { details. }\end{array}$ \\
\hline 5 & $\begin{array}{l}\text { Unit cell geometry } \\
\text { specification }^{a}\end{array}$ & $\begin{array}{l}\text { Enter the appropriate } \\
\text { data }\end{array}$ & See XSProc chapter for LATTICECELL. \\
\hline 6 & Control parameter data & Enter the desired data & $\begin{array}{l}\text { Begins this data block with READ CONT and } \\
\text { terminate with END CONT. } \\
\text { See Sect. } 2.3 .5 .5 \text {. }\end{array}$ \\
\hline $7^{b}$ & $\begin{array}{l}\text { Burnup history } \\
\text { specification }\end{array}$ & $\begin{array}{l}\text { Enter the desired data } \\
\quad \text { for each cycle }\end{array}$ & $\begin{array}{l}\text { Begins this data block with READ HISTORY } \\
\text { (or BURNDATA) and terminate with } \\
\text { END HISTORY (or BURNDATA). } \\
\text { See Sect. 2.3.5.6. }\end{array}$ \\
\hline $8^{b}$ & Search parameter data & Enter the desired data & $\begin{array}{l}\text { Begins this data block with READ SEARCH } \\
\text { and terminate with END SEARCH. } \\
\text { See Sect. 2.3.5.7. }\end{array}$ \\
\hline 9 & KENO data & $\begin{array}{l}\text { Enter KENO } \\
\quad \text { criticality model }\end{array}$ & $\begin{array}{l}\text { Begins this data block with READ KENOVA } \\
\text { (or KENO5) and terminate with } \\
\text { END KENOVA (or KENO5). } \\
\text { For KENO-VI use block keyword KENOVI } \\
\text { (or KENO6) in place of KENOVA } \\
\text { (or KENO5). See Sect. } 2.3 .5 .8 \text {. }\end{array}$ \\
\hline & Terminate input & END & Must begin in column 1 . \\
\hline
\end{tabular}




\subsubsection{Sequence specification card}

The STARBUCS analytical sequence is initiated with "=STARBUCS" beginning in column 1 of the input. This instructs the SCALE driver module to execute the STARBUCS sequence. The input data are then entered in free-format. The input is terminated with the word "END" starting in column 1. An "END" is a special data item, which may be used to delimit an input data block, end an array of input items, and terminate the input for the case. In the context of input data blocks, the "END" has a name or label associated with it. An "END" used to terminate an array of entries must not begin in column 1 as this instructs the SCALE driver to terminate input to the sequence.

\subsubsection{Optional sequence parameters}

To check the input data, run STARBUCS and specify PARM=CHECK or PARM=CHK after the analytical sequence specification as shown below.

\section{$=$ STARBUCS $\quad$ PARM $=$ CHK}

Other optional input for the PARM field to control multigroup resonance self-shielding calculations are described in the XSProc section of this manual.

\subsubsection{XSProc}

The XSProc is used to read and process the standard composition specification data that define the initial compositions of the fuel and all structural materials in the problem, into mixing tables and unit cell geometry information that are used by STARBUCS. All composition data required for the problem are entered as standard composition entries. A detailed description of this portion of the input can be found in the section on XSProc. Only one $\mathrm{UO}_{2}$ fuel type is permitted in STARBUCS. Therefore, a single fuel mixture defining the fresh fuel composition and, for criticality safety calculations employing multigroup cross sections, the geometry description of a single fuel lattice cell are required in a STARBUCS input file.

\subsubsection{Control parameter data}

The control parameter data block allows the user to specify control parameters and array data related to many of the burnup-credit analysis parameters to be used in the problem. All input is by keyword entry. All keywords are three-character identifiers that must be followed immediately by an equals sign ("="). The keywords may be in any order within a data block. Input to the parameter data block is initiated with the data block keywords READ CONTROL (only first four characters of block name are required). The data block is terminated by the keywords END CONTROL.

The types of control parameter data that may be input are summarized in Table 2.3.4. The individual keyword entries are described below.

1. $\mathrm{ARP}=$ NAME OF THE ARP LIBRARY TO BE USED. A character string with the name of the ARP library to be used in the depletion calculation. This is a required entry. The library must be defined in the SCALE text file ARPDATA.TXT that contains the cross-section library names and interpolation data used by ARP. A description of an ARP input and the location of the ARP cross-section libraries are provided in ARP Input Description 
located in the ORIGENARP chapter. STARBUCS calculations are limited to $\mathrm{UO}_{2}$ spent fuels.

2. $\mathrm{NAX}=$ NUMBER OF AXIAL ZONES. This is the number of axial-burnup subdivisions. For a user-input profile the value of NAX is determined automatically by the code, and the NAX keyword is optional, provided the $\mathrm{AXP}=$ array has been entered. The maximum value of NAX must be chosen such that due product of NAX * NHZ is less than or equal to 100 (i.e., $\mathrm{NAX}_{\max }$ is $100,50,33,25,20,16$, or 14 when the number of horizontal zones is $1,2,3,4,5,6$, or 7 , respectively). By default, the profile is automatically normalized to unity by the code unless NPR=NO. Built-in burnup-dependent 18 -axial-zone profiles may be selected with an entry of -18 . These built-in profiles and the burnup range over which they are applied, are listed in Table 2.3.5. These profiles have been proposed elsewhere (Ref. 2) as bounding axial profiles and are included as options for convenience only. The default value of NAX is -18 (use built-in profiles).

3. $\mathrm{NHZ}=$ NUMBER OF HORIZONTAL ZONES. This is the number of horizontal-burnup subdivisions in the assembly. An optional entry if no horizontal profile is requested. The maximum value is seven zones. The exact limit is determined by the number of mixtures defined in the lattice cell description. If a gap and second moderator type are used the number of horizontal zones is limited to five.

4. NUC $=$ BURNUP-CREDIT NUCLIDES used in the criticality calculation. A list of actinides and/or fission products that are to be included in the KENO criticality safety calculation. This is an array entry keyword and is delimited by the keyword END. The nuclides are entered using their standard composition alphanumeric names, as listed in the Standard Composition Library chapter of the SCALE manual. Isotopic correction factors may be entered, optionally, immediately following the nuclide name. The isotopic correction factors will be multiplied times the spent fuel nuclide concentrations to account for isotopic composition bias. The concentration of any nuclide that does not have a correction factor is not adjusted. To select all available actinide and fission product nuclides (with cross-section data and atom densities greater than 1.0E-29) for the criticality calculation, the user may select $\mathrm{NUC}=\mathrm{ALL}$, without an END terminator. This is the only situation where an array entry does not require an END. The available nuclides for this entry are included in the ORIGEN data library and the format of the library is documented in Decay Data Library of the ORIGENLIB chapter.

5. FLE $=$ FUEL LIGHT ELEMENT NUCLIDES. A user-provided list of light element nuclides that are to be included in the irradiated fuel compositions for a CSAS5 or a CSAS6 calculation. This is an array entry keyword and is delimited by the keyword END. The nuclides are entered using their standard composition alphanumeric names, as listed in Standard Composition Library chapter of the SCALE manual. To select all available light element nuclides (with cross-section data and atom densities greater than 1.0E-29) for the criticality calculation, the user may specify FLE= ALL, without an END terminator. This is the only situation where an array entry does not require an END. The available nuclides for this entry are included in the ORIGEN data library and the format of the library is documented in Decay Data Library of the ORIGENLIB chapter. The use of the keyword FLE is not required if only o-16 is to be included in the composition of irradiated uranium oxide fuel pellets. For these material mixtures, o-16 will be automatically included in irradiated fuel compositions due to its significant concentration. Isotopic correction factors are not allowed for light element nuclides. 
6. $\mathrm{AXP}=$ AXIAL-BURNUP PROFILE. The user-supplied axial-burnup profile of the assembly to be used in the analysis. This entry is required unless use of the built-in burnup-dependent axial profiles shown in Table 2.3.5 is requested $(\mathrm{NAX}=-18)$. If NAX is set to anything other than -18 , the AXP array must contain NAX entries. Otherwise, the value of NAX is determined automatically by the code. By default (NPR=YES), the profile is automatically normalized by the code; this may be disabled by setting NPR $=$ NO. If the burnup profile is normalized, it is implicitly assumed that the height/volume of each axial region is uniform when determining the average fuel burnup (i.e., the burnup of each axial region is equally weighted). The user is cautioned that if fuel region subdivisions of unequal volume are used, normalization should not be applied and the user must ensure a correct correspondence between the axial-profile input and the axial regions specified in the criticality calculation. AXP is an array entry and must be delimited by an END that must not start in the first column.

7. $\mathrm{HZP}=$

HORIZONTAL-BURNUP PROFILE. An optional array entry used to specify a burnup gradient across assemblies. The elements of the array are the ratios of the burnups of horizontal subdivisions in the assembly to average assembly burnup (entry for the POWER = keyword described in Sect. 2.3.5.6). If NHZ is input, the HZP array must contain NHZ entries delimited by an END that must not start in the first column. Otherwise, the value of NHZ is determined automatically by the code. The profile will be normalized if NPR=YES (default). Sample problem 5 illustrates use of this option.

8. FIX $=$ FIXED ASSEMBLY POWER OPTION. Option to select a constant specific power level for the depletion analysis for all axial and horizontal zones of the assembly. For FIX=YES, the depletion analysis for all zones is performed using the specific power input in the power history data block for the POWER=keyword. The irradiation time is adjusted to achieve the desired burnup. The default of FIX=NO applies a variable power for all zones and a constant irradiation time as defined by the BURN= keyword.

9. $\quad$ NPR $=$ NORMALIZE PROFILE. Option to control whether the user input axial- and horizontalburnup profiles will be normalized. The input profiles are automatically normalized using NPR=YES (default). If fuel region subdivisions of unequal volume are used, $\mathrm{NPR}=\mathrm{NO}$ should be specified.

10. $\mathrm{MOD}=$ AXIAL MODERATOR DENSITY. This is an array entry keyword and is delimited by the keyword END. The array dimension is equal to the number of axial zones (NAX entry) and the array values are provided in the same order as the AXP array elements. This input array is required only if the applicable ARP library contains variable moderator density cross sections.

11. $\mathrm{BUG}=$ DEBUG PRINT OPTION. BUG $=$ YES will print program debugging variables and arrays in STARBUCS. The default is $\mathrm{BUG}=\mathrm{NO}$. 
Table 2.3.4. Table of control parameter data

\begin{tabular}{|c|c|c|c|}
\hline $\begin{array}{l}\text { Keyword } \\
\text { name }\end{array}$ & $\begin{array}{l}\text { Data } \\
\text { type }\end{array}$ & $\begin{array}{c}\text { Default } \\
\text { value }\end{array}$ & Comments \\
\hline READ CONTROL & & & Initiate reading the control parameter block of data \\
\hline $\mathrm{ARP}=$ & Character & None & $\begin{array}{l}\text { Name of the ARP library to be used. Required. Library must be } \\
\text { defined in SCALE text file ARPDATA.TXT. }\end{array}$ \\
\hline $\mathrm{NAX}=$ & Integer & -18 & $\begin{array}{l}\text { Number of axial-burnup subdivisions in fuel assembly. The value of } \\
\text { NAX is determined automatically if an axial profile is input using } \\
\text { AXP }=\text { entries. The maximum value of NAX is } 100 \text {. Default } \\
\text { value }(-18) \text { applies a built-in 18-axial-region-burnup profile. }\end{array}$ \\
\hline $\mathrm{NHZ}=$ & Integer & 1 & $\begin{array}{l}\text { Number of horizontal-burnup subdivisions. Maximum value of } \\
5-7 \text { zones (see Sect. 2.3.5.5). No entry is required if horizontal } \\
\text { profile is not used. }\end{array}$ \\
\hline $\mathrm{NUC}=$ & $\begin{array}{l}\text { Character and real } \\
\text { mixed array }{ }^{a}\end{array}$ & None & $\begin{array}{l}\text { List of burnup-credit nuclides, and optionally the corresponding } \\
\text { isotopic correction factors, to be included in the criticality } \\
\text { calculation. Array entry generally delimited by END, unless } \\
\text { ALL is selected. Nuclides are input using their standard } \\
\text { composition alphanumeric identifiers. }\end{array}$ \\
\hline $\mathrm{FLE}=$ & Character array $^{a}$ & $0-16$ & $\begin{array}{l}\text { List of light element nuclides to be included in the criticality } \\
\text { calculation. Array entry generally delimited by END, unless } \\
\text { ALL is selected. Nuclides are input using their standard } \\
\text { composition alphanumeric identifiers. }\end{array}$ \\
\hline $\mathrm{AXP}=$ & Real array ${ }^{a}$ & See NAX & $\begin{array}{l}\text { Axial-burnup-profile array. Required if NAX }>0 \text {. NAX entries that } \\
\text { define the axial-burnup shape. The profile is automatically } \\
\text { normalized if NPR=YES (default). Delimited by END. }\end{array}$ \\
\hline $\mathrm{HZP}=$ & Real array ${ }^{a}$ & None & $\begin{array}{l}\text { Horizontal-burnup-profile array. Required if NHZ }>1 \text {. } \\
\text { Array containing NHZ entries that define the horizontal, } \\
\text { or radial, burnup profile for the analysis. Array is automatically } \\
\text { normalized by the code. Delimited by END. }\end{array}$ \\
\hline $\mathrm{MOD}=$ & Real array ${ }^{a}$ & None & $\begin{array}{l}\text { Axial-moderator density, applied in the fuel depletion analysis. } \\
\text { Note that } \mathrm{MOD}=\text { is required only if the ARP library contains } \\
\text { variable moderator density cross sections. NAX entries ordered } \\
\text { as AXP= array. Delimited by END. Moderator density default } \\
\text { values are not available in STARBUCS for variable moderator } \\
\text { density cross sections. }\end{array}$ \\
\hline $\mathrm{FIX}=$ & Character & NO & $\begin{array}{l}\text { Option to select a constant specific power level for all axial and } \\
\text { horizontal zones of the assembly using FIX=YES. }\end{array}$ \\
\hline $\mathrm{NPR}=$ & Character & YES & $\begin{array}{l}\text { Option to normalize user-input axial- and horizontal-burnup profiles. } \\
\text { Default is to automatically normalize profiles. }\end{array}$ \\
\hline $\mathrm{BUG}=$ & Character & NO & Optional debug printout with $\mathrm{BUG}=\mathrm{YES}$. \\
\hline END CONTROL & & & End of the control parameter block of data \\
\hline
\end{tabular}

\footnotetext{
${ }^{a}$ Terminate array data entries with END. Do not place this END in column 1.
} 
Table 2.3.5. Built-in burnup-dependent axial profiles, $N A X=-18$ from Ref. 2)

\begin{tabular}{|c|c|c|c|c|}
\hline \multirow{2}{*}{$\begin{array}{c}\text { Axial } \\
\text { zone no. }\end{array}$} & \multirow{2}{*}{$\begin{array}{l}\text { Fraction of } \\
\text { core height }\end{array}$} & $\begin{array}{c}\text { Burnup } \\
<18 \mathrm{GWd} / \mathrm{MTU} \\
\end{array}$ & $\begin{array}{c}18 \leq \text { Burnup } \\
<30 \text { GWd/MTU }\end{array}$ & $\begin{array}{c}\text { Burnup } \\
\geq 30 \mathrm{GWd} / \mathrm{MTU} \\
\end{array}$ \\
\hline & & 1 & 2 & 3 \\
\hline 1 & 0.0278 & 0.649 & 0.668 & 0.652 \\
\hline 2 & 0.0833 & 1.044 & 1.034 & 0.967 \\
\hline 3 & 0.1389 & 1.208 & 1.150 & 1.074 \\
\hline 4 & 0.1944 & 1.215 & 1.094 & 1.103 \\
\hline 5 & 0.2500 & 1.214 & 1.053 & 1.108 \\
\hline 6 & 0.3056 & 1.208 & 1.048 & 1.106 \\
\hline 7 & 0.3611 & 1.197 & 1.064 & 1.102 \\
\hline 8 & 0.4167 & 1.189 & 1.095 & 1.097 \\
\hline 9 & 0.4722 & 1.188 & 1.121 & 1.094 \\
\hline 10 & 0.5278 & 1.192 & 1.135 & 1.094 \\
\hline 11 & 0.5833 & 1.195 & 1.140 & 1.095 \\
\hline 12 & 0.6389 & 1.190 & 1.138 & 1.096 \\
\hline 13 & 0.6944 & 1.156 & 1.130 & 1.095 \\
\hline 14 & 0.7500 & 1.022 & 1.106 & 1.086 \\
\hline 15 & 0.8056 & 0.756 & 1.049 & 1.059 \\
\hline 16 & 0.8611 & 0.614 & 0.933 & 0.971 \\
\hline 17 & 0.9167 & 0.481 & 0.669 & 0.738 \\
\hline 18 & 0.9722 & 0.284 & 0.373 & 0.462 \\
\hline
\end{tabular}

\subsubsection{Burnup history data}

The burnup history data block defines the irradiation history for the assembly. These data are entered by keyword. The keywords are summarized in Table 2.3.6. Only the first four characters of the keywords are required (i.e., any characters after the first four characters are optional). A minimum of two entries are required for each cycle, (1) the average assembly power (POWER=) and (2) the irradiation time $(\mathrm{BURN}=)$. The decay time $(\mathrm{DOWN}=)$, if any, at the end of the cycle, and the number of cross-section libraries $(\mathrm{NLIB}=)$ are optional. The word END is required to delimit the entries for each cycle. The entries within a given cycle may be in any order.

The burnup history data block reading is initiated with the keywords READ HISTORY (or BURNDATA) and terminated by END HISTORY (or BURNDATA).

POWER $=$ THE AVERAGE SPECIFIC POWER OF THE ASSEMBLY FOR THIS CYCLE. The units of the specific power are in MW/MTU (W/g) of initial uranium. The axial and horizontal profiles are multiplied by the specific power to achieve the desired spatially-dependent 
burnup profiles for the assembly when FIX=NO (default). If FIX=YES, the specific power input using this keyword is assumed to be uniform over all fuel regions (axial and horizontal) and the code will adjust the irradiation time to obtain the desired burnup for each region.

BURN $=\quad$ THE IRRADIATION TIME FOR THIS CYCLE. The cycle irradiation time in days.

DOWN $=$ CYCLE DOWN TIME. An optional entry to specify the down time, in days, at the end of an irradiation cycle. The down time is simulated as an irradiation time step of effectively zero power after the irradiation cycle. The down time for the last cycle is simulated as a separate ORIGEN decay case with nine equally-spaced time steps. If a negative down time is input, the time steps are spaced logarithmically.

$\mathrm{NLIB}=\quad$ LIBRARIES PER CYCLE. An optional entry to request multiple cross-section libraries during a depletion cycle. If requested, the code automatically subdivides the cycle in NLIB segments of uniform duration and generates a separate library for the depletion analysis for each segment using ARP. Generating multiple libraries provides a more accurate representation of the time-dependent cross-section variation during the burnup analysis. Each segment of the cycle is assumed to have the same specific power, and no down time is assumed between each segment of the cycle.

END The word END is required to terminate the input for each cycle.

Repeat the above entries for each cycle to define the complete assembly power history. 
Table 2.3.6. Table of power history data

\begin{tabular}{|c|c|c|c|}
\hline $\begin{array}{l}\text { Keyword } \\
\text { name }\end{array}$ & $\begin{array}{l}\text { Data } \\
\text { type }\end{array}$ & $\begin{array}{c}\text { Default } \\
\text { value }\end{array}$ & Comments \\
\hline $\begin{array}{l}\text { READ } \\
\text { HISTORY (or }^{\text {BURNDATA) }}{ }^{\text {a }}\end{array}$ & & & Start of burnup history data block \\
\hline POWER $=$ & Real variable & None & Average assembly power for this cycle (MW/MTU) \\
\hline $\mathrm{BURN}=$ & Real variable & None & Cycle irradiation time (days) \\
\hline DOWN= & Real variable & 0 & $\begin{array}{l}\text { End-of-cycle decay time (days). Optional. A negative } \\
\text { down time may be used to select logarithmic decay } \\
\text { time intervals for the last decay case. }\end{array}$ \\
\hline NLIB/CYCLE $=$ & Integer variable & 1 & $\begin{array}{l}\text { Number of libraries to be applied in this cycle. Optional. } \\
\text { If multiple libraries are requested for this cycle, the } \\
\text { cycle is subdivided into equal time segments, and an } \\
\text { updated library is generated for each segment. No } \\
\text { down time is simulated between segments. }\end{array}$ \\
\hline END & & & $\begin{array}{l}\text { Required. Defines the end of the data for the current } \\
\text { cycle. Repeat the above entries for each cycle in the } \\
\text { irradiation history. An END, not to begin in } \\
\text { column } 1 \text {, must terminate each cycle definition. }\end{array}$ \\
\hline $\begin{array}{l}\text { END } \\
\text { HISTORY (or } \\
\text { BURNDATA) }^{a}\end{array}$ & & & End block \\
\hline
\end{tabular}

${ }^{a}$ Only the first four characters are required, i.e., HIST (or BURN). 


\subsubsection{Search parameter data}

The search parameter data block defines input data for burnup loading curve analyses for commercial $\mathrm{UO}_{2}$ spent fuels. Burnup history input data are not allowed in an input file that supplies search parameters. A burnup history data block is generated in STARBUCS for subsequent iterative calculations using the initial user-supplied search parameter data. STARBUCS sample problem starbucsl.input contains a search data block to request burnup loading curve analyses for spent fuel at various burnups. The search data block reading is initiated with the keywords READ SEARCH and terminated by END SEARCH. The keywords are summarized in Table 2.3.7. These keywords may be in any order.

USL $=\quad$ THE UPPER SUBCRITICAL LIMIT FOR BURNUP LOADING.

EPS $=$ TOLERANCE ON CONVERGENCE. The convergence criterion used in the search for initial fuel enrichment so that user-specified $k_{\text {eff }}$ value is within USL \pm EPS. The tolerance value must be greater that the standard deviation of the calculated $\mathrm{k}_{\text {eff }}$ for the solution to converge.

ITMAX $=$ MAXIMUM ITERATIONS ALLOWED FOR EACH ENRICHMENT SEARCH. The search for initial fuel enrichment stops when the number of iterations exceeds this parameter and a warning message is provided to the user.

$\mathrm{ECL}=\quad$ LOWER ENRICHMENT CONSTRAINT. The unit for this parameter is $w \mathrm{t} \%{ }^{235} \mathrm{U}$. The lower enrichment constraint must be within the enrichment interval used in the ARP library specified in READ CONTROL data block.

$\mathrm{ECH}=\quad$ UPPER ENRICHMENT CONSTRAINT. The unit for this parameter is $\mathrm{wt} \%{ }^{235} \mathrm{U}$. The upper enrichment constraint must be within the enrichment interval used in the ARP library specified in READ CONTROL data block.

$\mathrm{BU}=\quad$ ARRAY OF REQUESTED BURNUP VALUES (GWd/MTU). The word END is required to terminate this array. The user inputs a series of discharge burnup values for which the initial fuel enrichments that result in a desired $k_{\text {eff }}$ value (USL \pm EPS) are to be determined.

AVGBU $=$ AVERAGE BURNUP PER CYCLE (GWd/MTU). An optional entry used to determine the number of irradiation cycles as the ratio of a burnup value in the BU array to AVGBU.

POWER $=$ THE AVERAGE SPECIFIC POWER OF THE ASSEMBLY. The units of the specific power are in MW/MTU (W/g) of initial uranium. This entry has the same function as the entry for POWER= keyword in the HISTORY data block (see Sect. 2.3.5.6). It is also used to determine cycle irradiation time as the ratio of a burnup value in the BU array to average assembly power.

FDT $=\quad$ FRACTIONAL DOWNTIME. An optional entry used to determine down time between irradiation cycles (the entry for DOWN= keyword in the HISTORY data block) if fuel irradiation requires two or more cycles. For example, for a cycle with 365 days of irradiation followed by a 30 -day downtime, FDT $=30 / 395=0.07595$. STARBUCS uses the userprovided FDT to compute cycle downtime as the irradiation time per cycle multiplied by FDT and divided by (1-FDT). 
$\mathrm{DEC}=$ DECAY TIME AFTER IRRADIATION. An optional entry to specify the decay time, in days, after fuel discharge. A negative value may be used to select logarithmic decay time intervals.

$\mathrm{NLIB}=\quad$ NUMBER OF LIBRARIES PER CYCLE. An optional entry to request multiple crosssection libraries during a depletion cycle. Generating multiple libraries provides a more accurate representation of the time-dependent cross-section variation during the burnup analysis. Each segment of the cycle is assumed to have the same specific power.

$\mathrm{FFE}=\quad$ FRESH FUEL ENRICHMENT. The purpose of this option is to help in reducing the total number of iterations needed to achieve convergence. There are two options implemented in STARBUCS for the fresh fuel enrichment value to be used in the first inner iterations over fuel enrichment, FFE=SEARCH (default) and FFE=INPUT. With the default option $(\mathrm{FFE}=\mathrm{SEARCH})$, the lower enrichment bound and the starting fresh fuel enrichment at the beginning of a search are adjusted based on the results of the previous outer iteration over burnup. The procedure includes the following steps. First, the user requested burnup values are sorted in ascending order so that STARBUCS outer iterations over burnup proceed from the lowest to the highest burnup value. Then, the initial fresh fuel for the lowest burnup is changed to the mid-value of the enrichment interval, $(\mathrm{ECL}+\mathrm{ECU}) / 2$, and the search for the fresh fuel enrichment corresponding to the lowest burnup is initiated and completed. Suppose that a solution for this burnup step exists. This solution becomes the lower enrichment constraint (ECL) in the search passes for the next burnup value and the initial fresh fuel enrichment is chosen as the middle point of the enrichment interval. The procedure is applied for the entire set of the requested burnups. The average number of iterations for each burnup step with this option is approximately 4. The alternate option (FFE=INPUT) starts a search for fuel enrichment with the user supplied fresh fuel enrichment.

PRT $=\quad$ OUTPUT PRINT OPTION FOR BURNUP LOADING CURVE CALCULATIONS. The STARBUCS output file for default option $(\mathrm{PRT}=\mathrm{LONG})$ provides ARP, ORIGEN, and CSAS5 outputs for the last search pass for each of the burnup values in BU array in addition to STARBUCS summary information. The STARBUCS output file for option PRT=SHORT provides only summary information prepared by STARBUCS, such as fresh fuel enrichments for search passes, spent fuel compositions and the CSAS5 $k_{\text {eff }}$ result for each of the search passes, and a summary table providing burnup loading curve data, i.e., the fresh fuel enrichments that satisfy the user supplied convergence criterion for the requested burnup values. 
Table 2.3.7. Table of search data

\begin{tabular}{|c|c|c|c|}
\hline $\begin{array}{c}\text { Keyword } \\
\text { Name }\end{array}$ & $\begin{array}{l}\text { Data } \\
\text { type }\end{array}$ & $\begin{array}{c}\text { Default } \\
\text { value }\end{array}$ & Comments \\
\hline READ SEARCH $^{a}$ & & & Initiate reading the search block of data. \\
\hline $\mathrm{USL}=$ & Real & 1.0 & Upper subcritical limit. \\
\hline $\mathrm{EPS}=$ & Real & 0.005 & Tolerance on convergence. \\
\hline ITMAX = & Integer & 10 & Iteration limit. \\
\hline $\mathrm{ECL}=$ & Real & 1.5 & Lower initial fuel enrichment constraint (U-235 wt\%). \\
\hline $\mathrm{ECH}=$ & Real & 5.0 & Upper initial fuel enrichment constraint (U-235 wt\%). \\
\hline BU & Real $^{b}$ & None & Array entry of requested burnup values $(\mathrm{GWd} / \mathrm{MTU}) .^{\mathrm{c}}$ \\
\hline AVGBU $=$ & Real & 20.0 & Average burnup per cycle. \\
\hline POWER $=$ & Real & 25.0 & Average specific power $(\mathrm{W} / \mathrm{g})$. \\
\hline $\mathrm{FDT}=$ & Real & 0.2 & Fractional downtime. \\
\hline $\mathrm{DEC}=$ & Real & 1825.0 & Decay time (days). \\
\hline $\mathrm{NLIB}=$ & Integer & 2 & Libraries per cycle. \\
\hline $\mathrm{FFE}=$ & Character & SEARCH & $\begin{array}{l}\text { Fresh fuel option. FFE=INPUT starts the outer iterations over the } \\
\text { burnup values with user supplied fresh fuel composition. } \\
\text { FFE=SEARCH helps in reducing the number of search passes } \\
\text { (approximately } 4 \text { in average). }\end{array}$ \\
\hline $\mathrm{PRT}=$ & Character & LONG & $\begin{array}{l}\text { Print option. PRT=LONG generates STARBUCS summary output, } \\
\text { ARP, ORIGEN, and CSAS outputs for the last search passes. } \\
\text { PRT=SHORT generates only STARBUCS summary output. }\end{array}$ \\
\hline END SEARCH & & & End of the search data \\
\hline
\end{tabular}

${ }^{a}$ Only the first four characters are required.

${ }^{\mathrm{b}}$ Terminate array data entries with END. Do not place this END in column 1.

${ }^{c}$ There are no restraints on the maximum number of the burnup values requested in burnup loading curve calculations. A user may consider computer time and resources in assessing the maximum number of burnup values in this array. 


\subsubsection{KENO input data}

The KENO input for the problem is specified in the KENO data block. Input to the data block is initiated with the data block keywords READ KENO or READ KENOVA and is terminated by the keywords END KENO or END KENOVA for criticality calculations using KENO V.a. Input to the data block is initiated with the data block keywords READ KENOVI or READ KENO6 and is terminated by the keywords END KENOVI or END KENO6 for criticality calculations using KENO VI. STARBUCS performs no error checking of the KENO input. The data within the data block delimiters is copied, without change, to the CSAS input file and executed. The user is therefore advised to ensure that the KENO input is free of errors by first running the case within CSAS5 or CSAS6 before applying the input in STARBUCS.

The input requirements for KENO V.a and KENO-VI are not described in this section, but are described in detail in the KENO chapter of this manual. This section describes only the input requirements as related to the execution of KENO within STARBUCS and the conventions used for module compatibility.

The mixture numbers for each of the non-fuel materials applied to the material regions of the KENO model are defined as the mixture numbers (MX) specified in the standard composition input. STARBUCS automatically defines the MIXTURE ID for each of the fuel regions according to the axial and/or horizontal zones defined by the NAX and NHZ entries in the burnup-profile arrays. The first axial-zone mixture is assigned $\mathrm{MX}=101$, and is incremented by one for each additional axial zone. Therefore, in a problem that defines 18 axial zones, spent fuel mixtures will be generated with identifiers that range from 101 to 118 . The correspondence of these mixtures to the assembly locations is determined by the ordering of the $\mathrm{AXP}=$ input array that defines the axial-burnup profile for the assembly. If the $\mathrm{AXP}=$ array orders the burnup profile from the bottom of the assembly to the top of the assembly, the resulting $\mathrm{MX}=101$ will correspond to the bottom axial-zone segment, and $\mathrm{MX}=118$ would correspond to the top axial zone. If multiple horizontal zones are defined, then the numbering sequence of the second horizontal zone will start at $\mathrm{MX}=201$ and, in the example given here, would range up to $\mathrm{MX}=218$. Refer to Sect. 2.3.3 for limitations in the mixture-numbering scheme. The mixture-numbering scheme is illustrated in Figure 2.3.3.

\subsubsection{Sample Problems}

A series of example problems are presented to illustrate the application of STARBUCS to burnup-credit criticality safety and burnup loading curve analyses. Sample problem 1 is a simple pin-cell problem for burnup loading curve iterative calculations. The fuel pin contains a single axial-burnup zone (i.e., uniform-axial burnup). It is useful to illustrate the main features of the system and demonstrate functionality of the system modules within SCALE. Problem 2 illustrates the same problem with 18-axial burnup-dependent zones. Problem 3 extends the pin-cell model to an array of spent fuel assemblies residing in a water-filled pool. The models apply 18-axial-burnup-dependent zones. Problem 4 is a generic cask model, and this problem exercises more of the burnup credit options available in STARBUCS. Problem 5 illustrates the use of the horizontal-burnup option for a simple $4 \times 4$ array of spent fuel assemblies residing in water. Sample problem 6 uses KENO-VI to model a hexagonal VVER-440 fuel assembly.

\subsubsection{Sample problem 1}

Sample problem 1, listed in Table 2.3.8, defines a simple infinite $\mathrm{UO}_{2}$ pin-cell model with uniform-axial burnup for burnup loading curve calculations. The initial fuel enrichment is $2.0 \mathrm{wt} \%$. The control 
parameter data block specifies that the standard Westinghouse (W) $17 \times 17$ ARP library is to be used for the depletion analysis. The burnup-credit criticality calculation uses a subset of the major actinides as defined in the NUC= array. The sample problem contains a "read search" data block, which provides an upper limit for subcriticality, usl, a tolerance value for the search algorithm, eps, the lower and upper enrichment bounds, ecl and ech, respectively, the maximum number of iterations for each burnup value requested, imaxl, average specific power in $\mathrm{W} / \mathrm{g}$, power, decay time after irradiation in days, dec, number of libraries per cycle, $n l i b$, average burnup per cycle in GWd/MTU, avgbu, fractional downtime, $f d t$, and a set of burnup values, $b u$ array.

\subsubsection{Sample problem 2}

Sample problem 2, listed in Table 2.3.9, illustrates a simple pin-cell model using 18-axial-burnupdependent zones. In this example, the built-in axial profiles for three burnup ranges are applied using the NAX $=-18$ option (see profiles in Table 2.3.5). STARBUCS determines the average assembly burnup from the power history data input, and automatically selects the appropriate profile based on the discharge assembly burnup. The axial-profile data were developed for a predefined axial-zoning structure (i.e., fraction of the assembly height). It is important that the KENO V.a geometry model therefore also reflect this axial-zone structure. That is, the height of each axial zone in the criticality model must conform to the axial zones for the profile applied in the analysis. In this example, the total pin height is $365.7 \mathrm{~cm}$ (144 in.), which is subdivided into 18 equal-height segments of $20.32 \mathrm{~cm}$ each.

The burnup-dependent cross sections generated for the criticality analysis have material identifiers ranging from 101 (bottom) to 118 (top). There is no constraint on how the fuel materials can be applied in the KENO V.a model. For example, the order of the material numbers could easily be reversed, which would effectively invert the profile and could be used to simulate an assembly loaded upside down. It is also not necessary to use all of the materials in the problem. For instance, all fuel regions in the KENO V.a model could be assigned the same fuel mixture number to represent a flat axial profile having a burnup value equal to that of the particular mixture used. The average assembly burnup would also be equal to that of the particular mixture used, and not that defined by the power history data block.

\section{Table 2.3.8. STARBUCS input listing for sample problem 1}

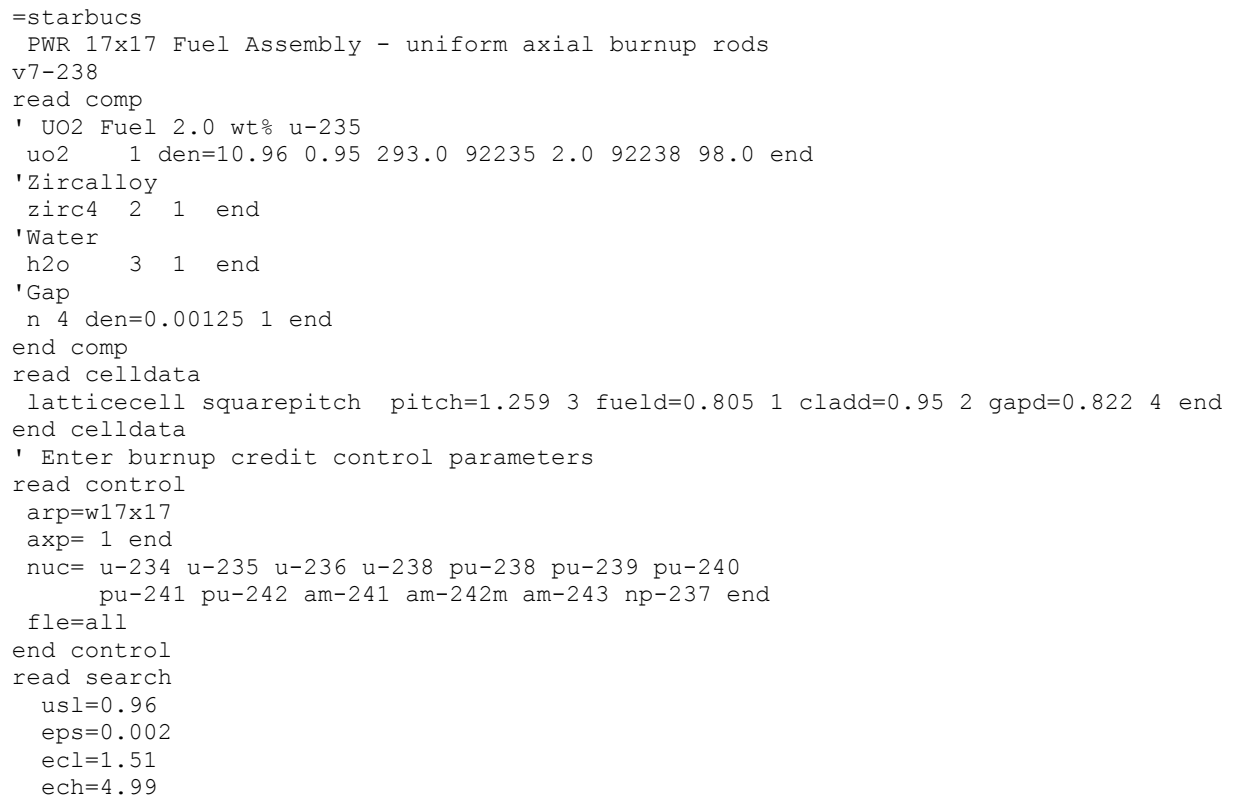




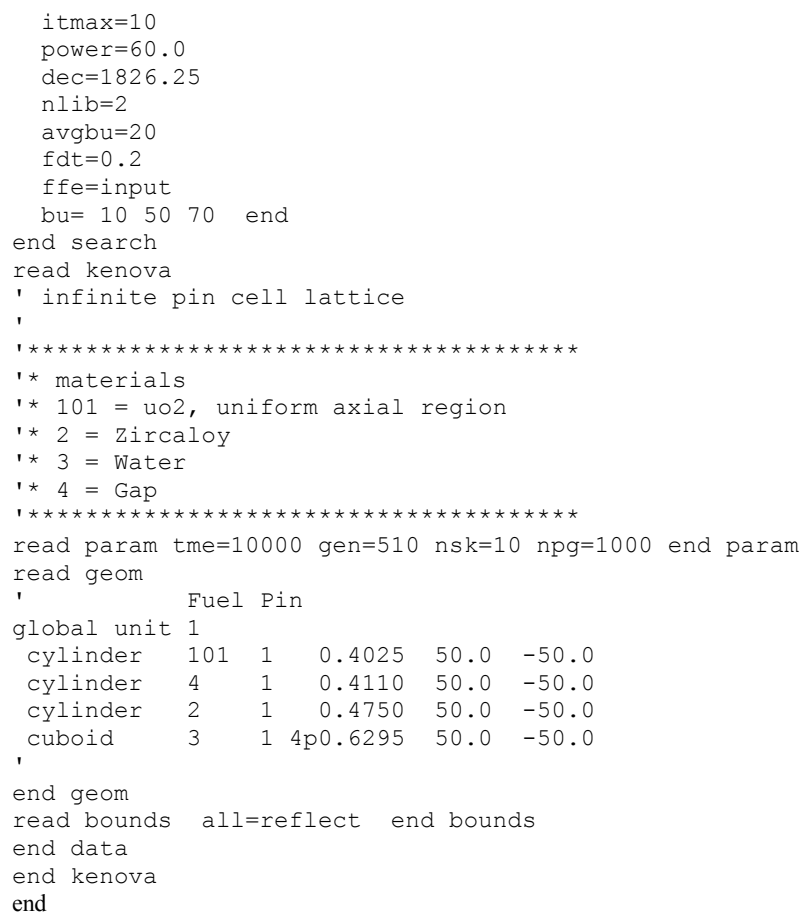


Table 2.3.9. STARBUCS input listing for sample problem 2

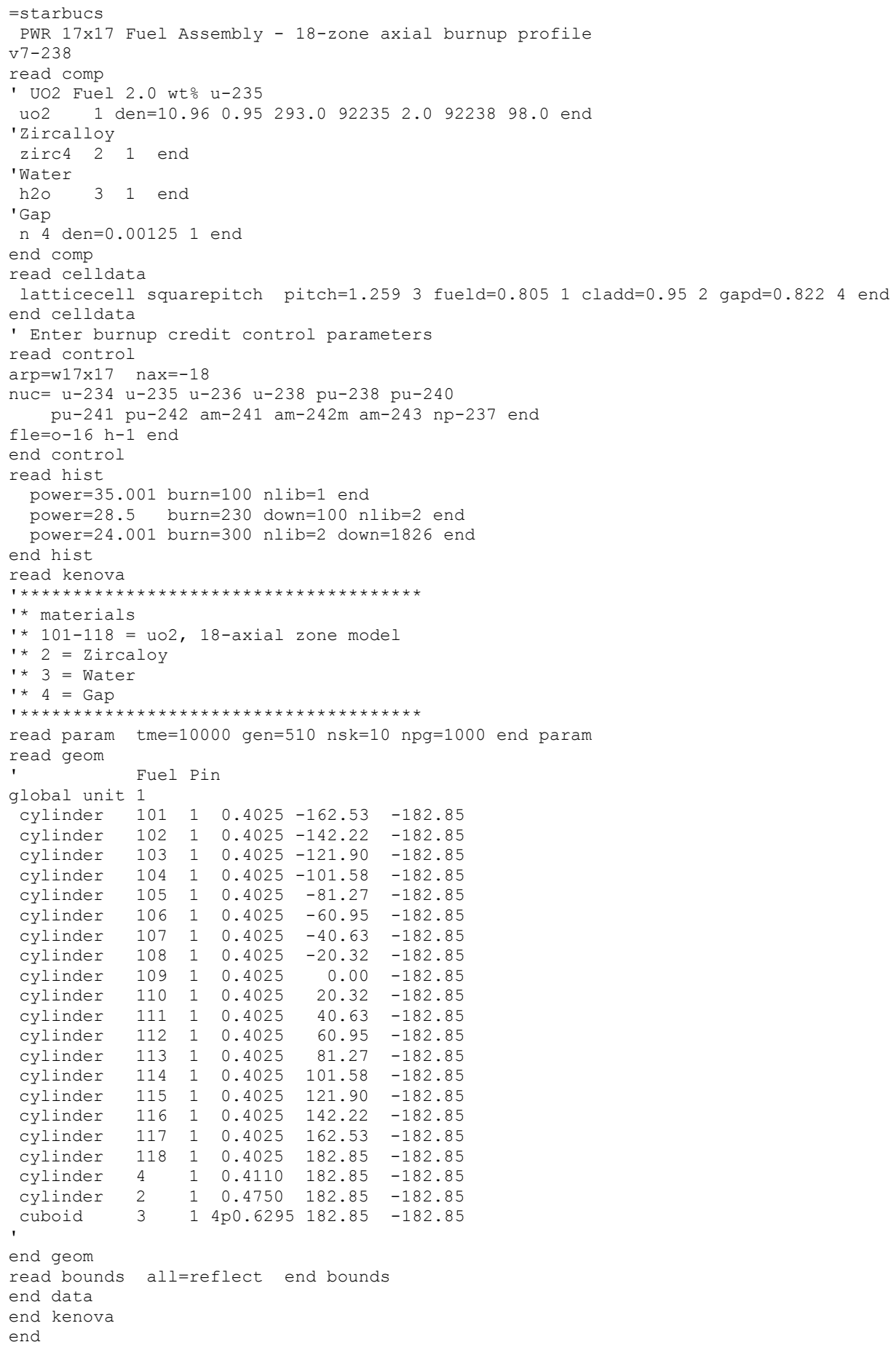




\subsubsection{Sample problem 3}

Sample problem 3, listed in Table 2.3.10, performs a burnup-credit criticality safety calculation using the SCALE 238-group ENDF/B-VI cross-section library (V6-238) for an array of Combustion Engineering (CE) $14 \times 14$ spent fuel assemblies in water. A subset of burnup-credit actinides and fission products are included in the criticality calculation. A user-supplied 18-axial-region-burnup profile of the assemblies is input. This profile was obtained from the axial-burnup-profile database ${ }^{3}$ for Maine Yankee assembly N863. Note that the axial profile will be normalized automatically by the code using NPR=YES (default). The normalization is performed such that the average value of the profile values is unity (i.e., the sum of the profile values is equal to the number of axial zones). The $3.3 \mathrm{wt} \%$ enriched $\mathrm{UO}_{2}$ fuel is assumed to achieve a discharge burnup of $37,626 \mathrm{MWd} / \mathrm{MTU}$ in three cycles of approximately $12.5 \mathrm{GWd} / \mathrm{MTU}$ per cycle and a downtime per cycle of 80 days, followed by a cooling time of 5 years after discharge (1826 days). An average assembly power level of $32 \mathrm{MW} / \mathrm{MTU}$ is used for the depletion calculation. Two libraries per cycle are requested during the depletion. Note that by increasing the number of libraries generated per cycle, the cross sections used in the burnup analysis are updated more frequently to reflect the changes that occur with burnup. The nominal CE $14 \times 14$ assembly design specifications were obtained from Ref. 4 . The assembly pitch in the criticality calculations is $22.78 \mathrm{~cm}$. A cross-section view of the assembly geometry, a $2 \times 8$ array of water reflected assemblies, is illustrated in Figure 2.3.6. 
Table 2.3.10. STARBUCS input listing for sample problem 3

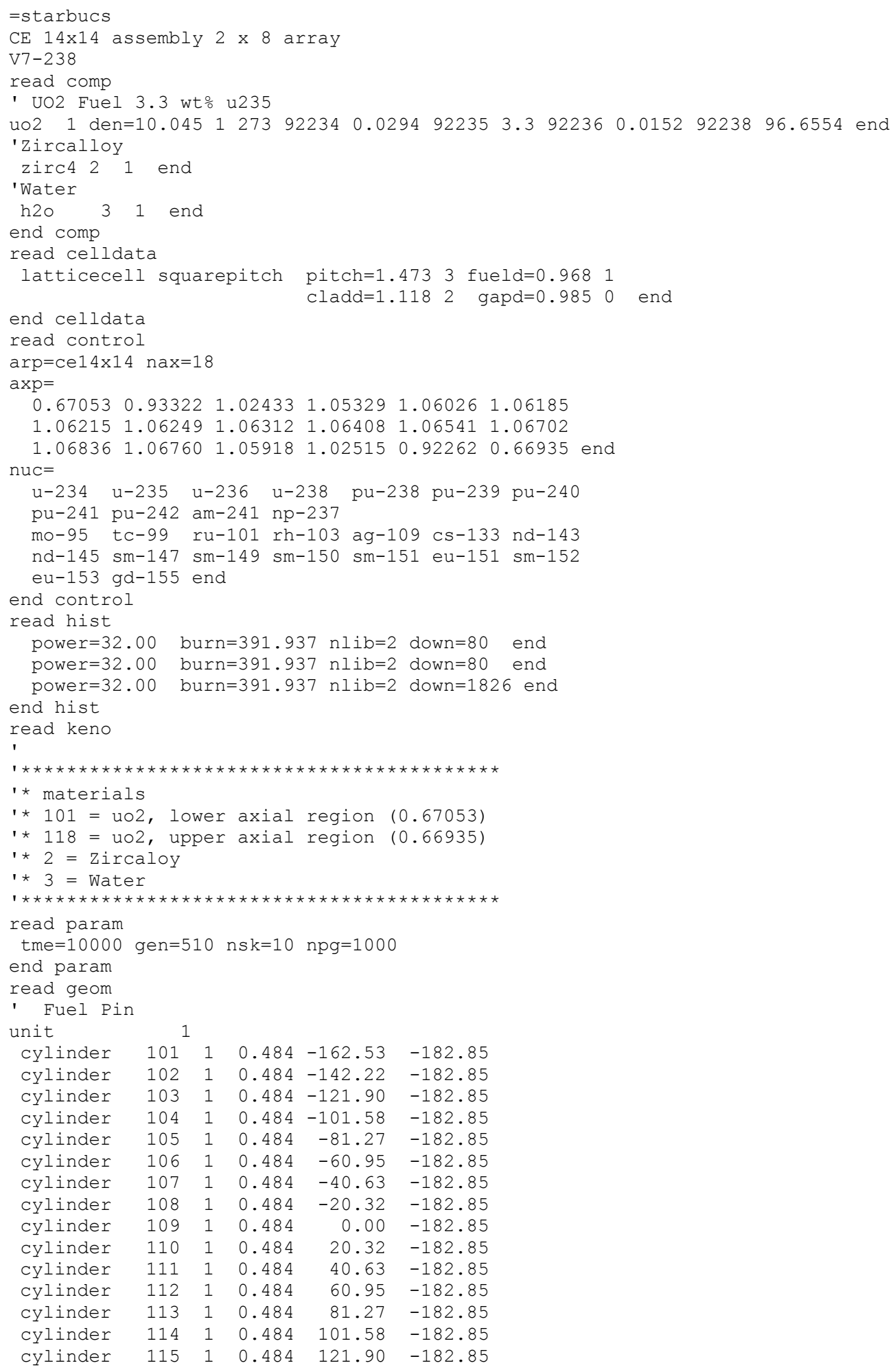


Table 2.3.11. STARBUCS input listing for sample problem 3 (continued)

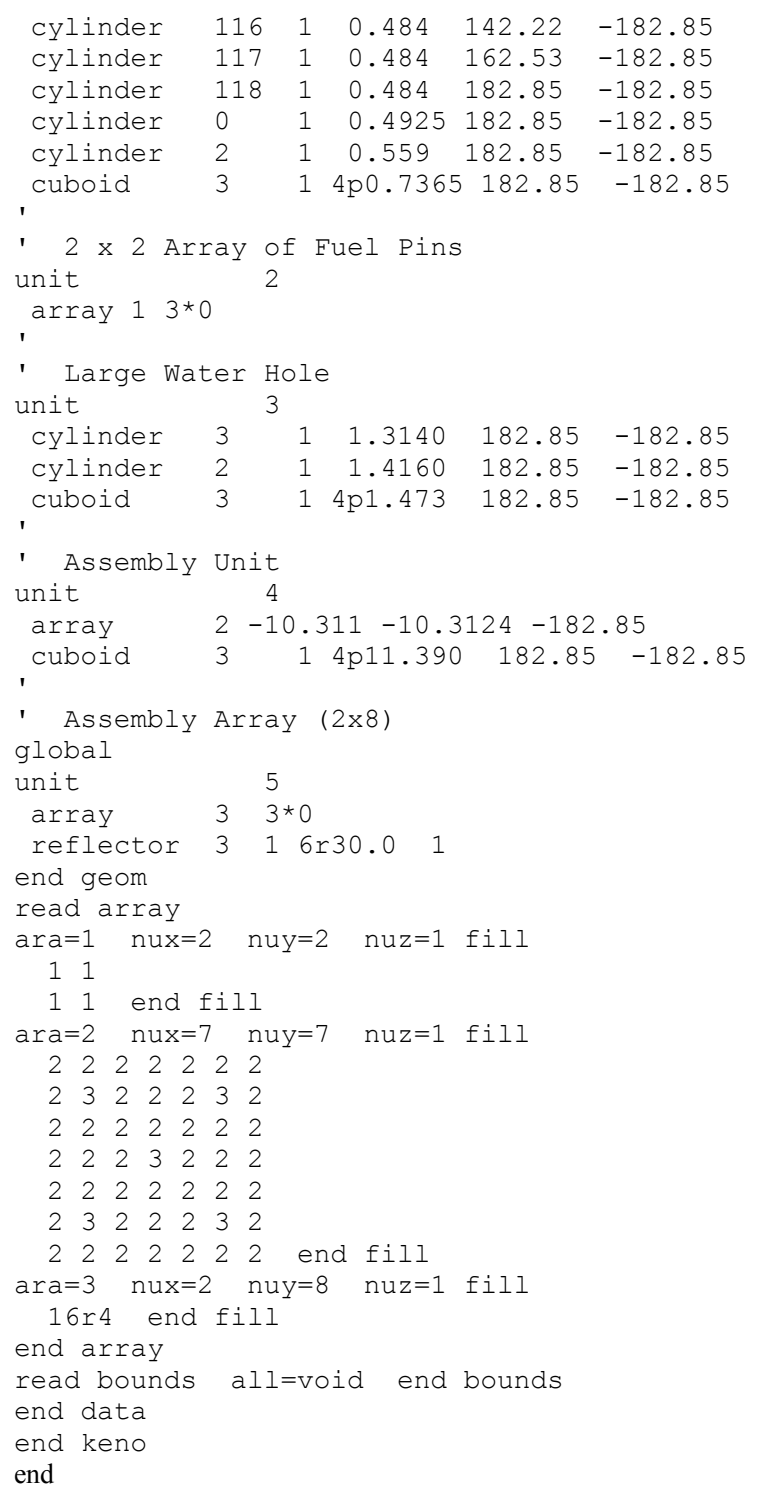




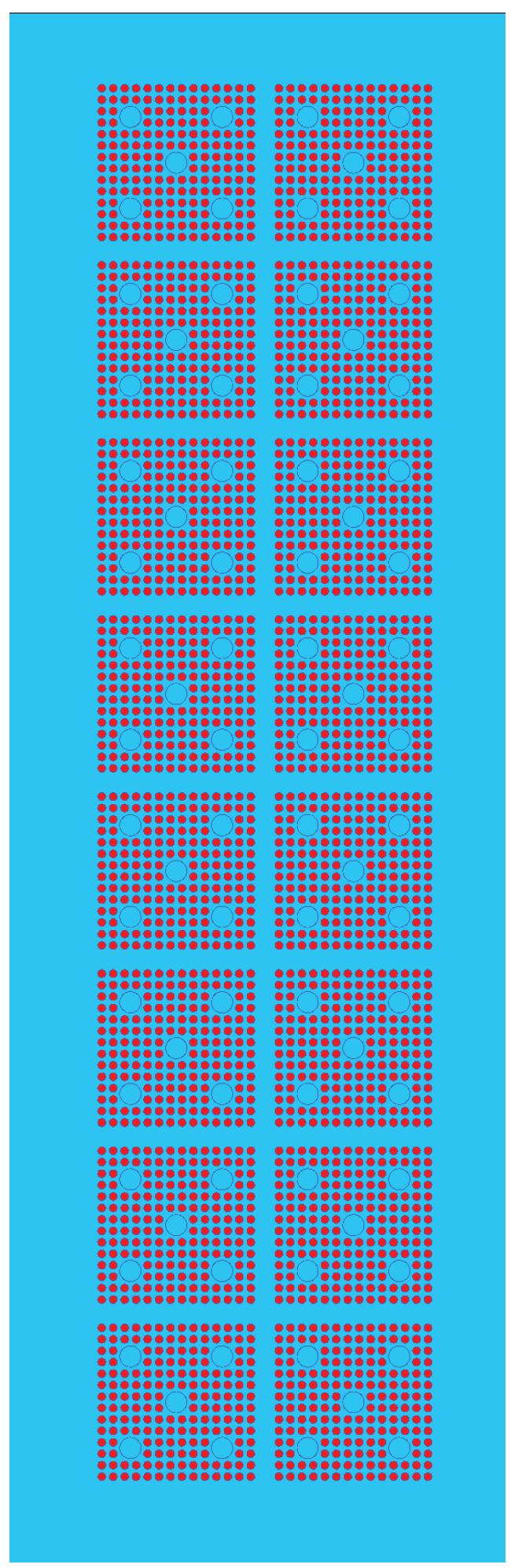

Figure 2.3.6. Plot of the CE $14 \times 14$ assembly array geometry in sample problem 3 . 


\subsubsection{Sample problem 4}

Sample problem 4, listed in Table 2.3.11, illustrates the application of STARBUCS for a criticality safety analysis of a burnup-credit cask. The cask geometry in this example is based on a 32-assembly generic burnup-credit cask model and is illustrated in Figure 2.3.7.

The assemblies are assumed to be W $17 \times 17$ OFA assemblies with an initial enrichment of 4.98 wt $\%$. The standard composition description for this problem includes the fuel assembly and all cask structural material definitions. The analysis applies built-in 18-axial-zone profiles, and actinide-only burnup credit (i.e., only a subset of actinides and no fission products). The assembly is irradiated to an average burnup of about $50 \mathrm{GWd} / \mathrm{MTU}$. The axial-burnup profile is automatically selected by the code based on the average assembly burnup. Isotopic correction factors are applied to the calculated actinide inventories. The correction factors were obtained from Ref. 4. An axial-moderator density is also applied. Note that actual entries in the MOD= array are not realistic for a PWR and are only intended to illustrate the use of this feature. Since the ARP library applied in this calculation does not have variable moderator density, the values in the $\mathrm{MOD}=$ array have no effect on the calculation. The criticality evaluation of the cask is performed following a cooling time of 1826 days (5 years).

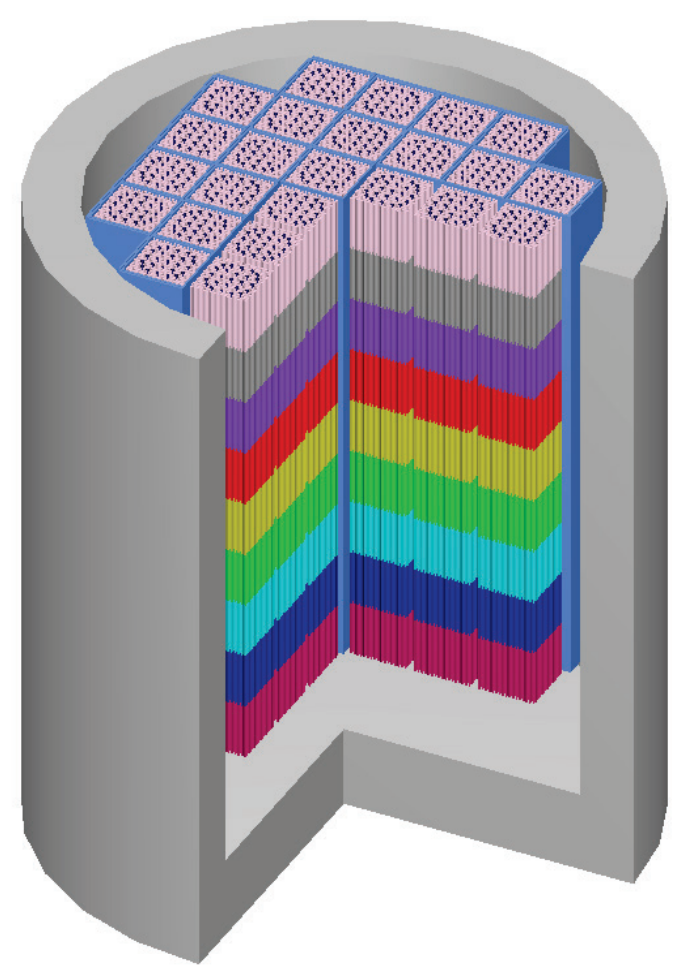

Figure 2.3.7. Cutaway view of the generic 32-assembly burnup-credit cask showing the cask bottom half with a quarter of the model removed. 


\section{Table 2.3.11. STARBUCS input listing for sample problem 4}

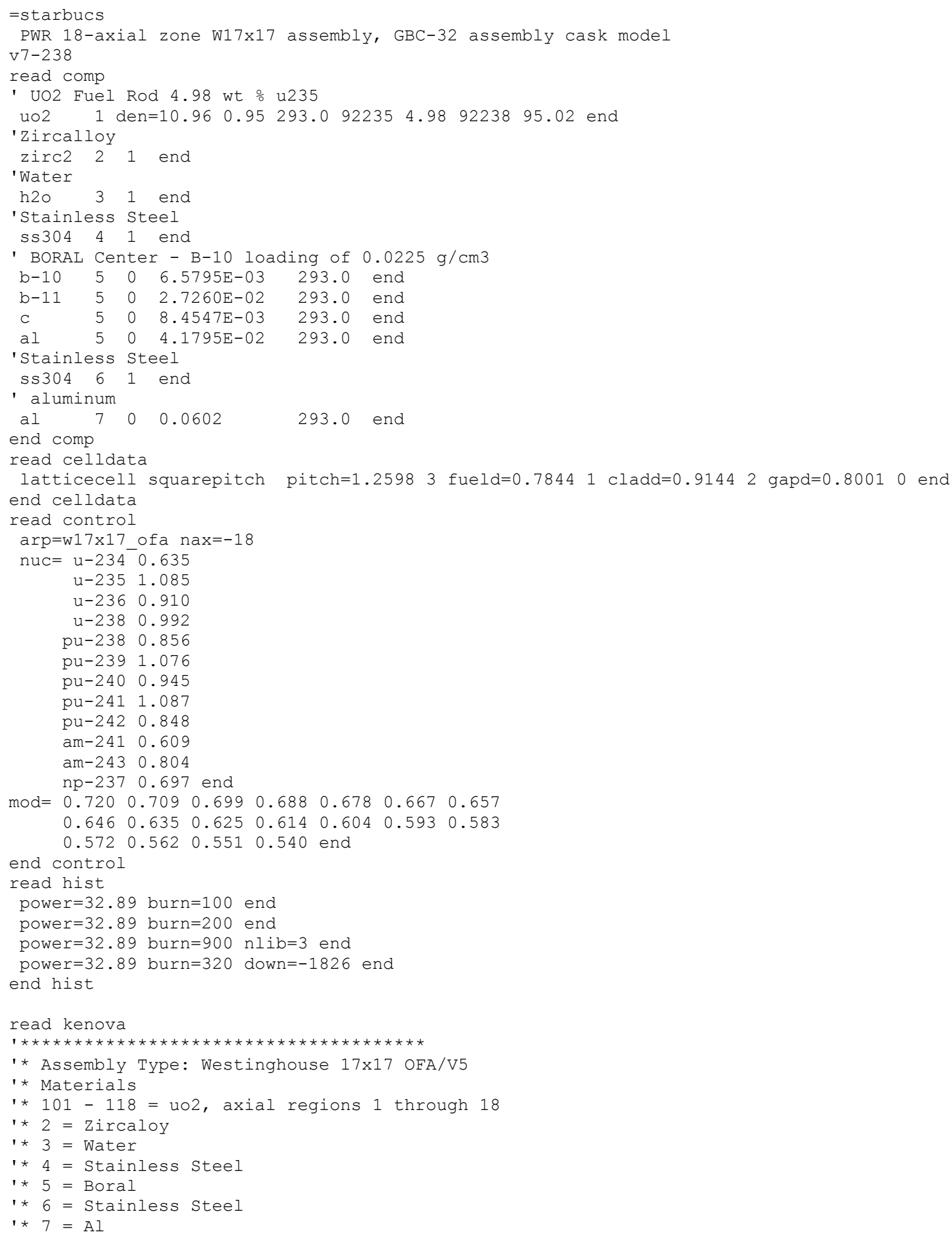


Table 2.3.12. STARBUCS input listing for sample problem 4 (continued)

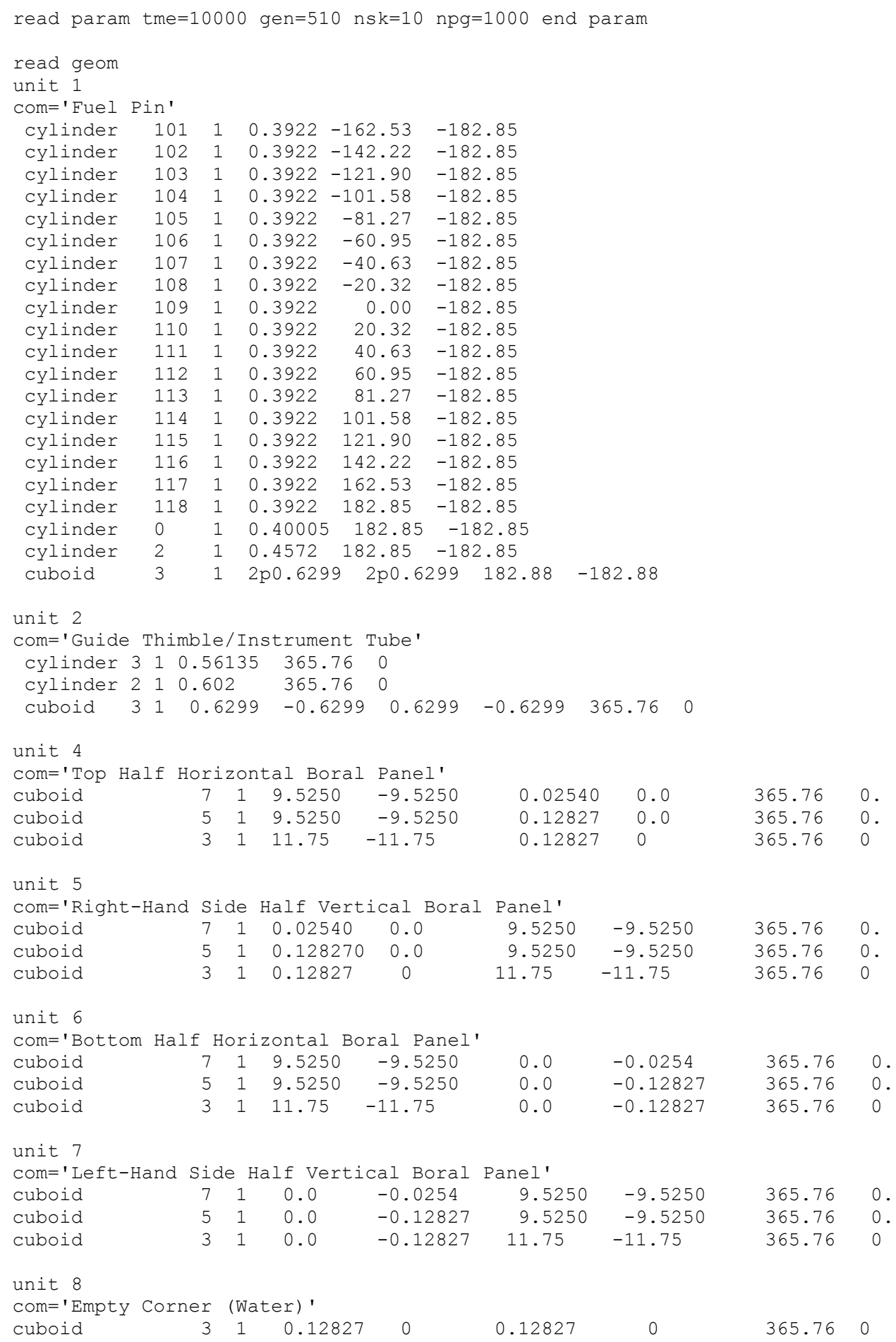


Table 2.3.12. STARBUCS input listing for sample problem 4 (continued)

\begin{tabular}{|c|c|c|c|c|c|c|c|}
\hline \multicolumn{2}{|c|}{ com='Top Boral/Basket } & t Plate with & \multicolumn{2}{|c|}{ h water added to fit } & \multicolumn{2}{|c|}{ array dimensions' } & \multirow[b]{2}{*}{0} \\
\hline cuboid & $5:$ & 19.525 & -9.525 & -0.7754 & -0.87827 & 365.76 & \\
\hline cuboid & 7 & 9.525 & -9.525 & -0.75 & -0.87827 & 365.76 & 0 \\
\hline cuboid & 3 & 111.7500 & -11.75 & -0.75 & -0.87827 & 365.76 & 0 . \\
\hline cuboid & 4 & 11.7500 & -11.75 & 0.0 & -0.87827 & 365.76 & 0 . \\
\hline cuboid & 3 & 111.87827 & -11.87827 & 0.12827 & -0.87827 & 365.76 & 0 \\
\hline \multicolumn{8}{|l|}{ unit 11} \\
\hline com='Bottom Bor & $\mathrm{al} / \mathrm{B}$ & Basket Plate & with water ac & added to $\mathrm{f}$ & fit array din & mensions & \\
\hline cuboid & 5 & 9.525 & -9.525 & 0.87827 & 0.7754 & 365.76 & 0 \\
\hline cuboid & 7 & 9.525 & -9.525 & 0.87827 & 0.75 & 365.76 & 0 \\
\hline cuboid & 3 & 111.7500 & -11.75 & 0.87827 & 0.75 & 365.76 & 0 . \\
\hline cuboid & 4 & 11.7500 & -11.75 & 0.87827 & 0.0 & 365.76 & 0 . \\
\hline cuboid & 3 & 11.87827 & -11.87827 & 0.87827 & -0.12827 & 365.76 & 0 \\
\hline \multicolumn{8}{|l|}{ unit 12} \\
\hline $\mathrm{com}=$ ' Left-Hand & Side & Boral/Basket & t Plate with & water ad & dded to fit a & array di & mensions' \\
\hline cuboid & 5 & 0.87827 & 0.7754 & 9.525 & -9.525 & 365.76 & 0 \\
\hline cuboid & 7 & 0.87827 & 0.75 & 9.525 & -9.525 & 365.76 & 0 \\
\hline cuboid & 3 & 0.87827 & 0.75 & 11.75 & -11.75 & 365.76 & 0 . \\
\hline cuboid & 4 & 0.87827 & 0.0 & 11.75 & -11.75 & 365.76 & 0 . \\
\hline cuboid & 3 & 0.87827 & -0.12827 & 11.87827 & -11.87827 & 365.76 & 0 . \\
\hline \multicolumn{8}{|l|}{ unit 13} \\
\hline com='Right-Hand & Sid & de Boral/Baske & set Plate with & ch water a & added to fit & array d & limensions' \\
\hline cuboid & & $1-0.7754$ & -0.87827 & 9.525 & -9.525 & 365.76 & 0 \\
\hline cuboid & 7 & $1-0.75$ & -0.87827 & 9.525 & -9.525 & 365.76 & 0 \\
\hline cuboid & 3 & -0.75 & -0.87827 & 11.75 & -11.75 & 365.76 & 0 . \\
\hline cuboid & 4 & 0.0 & -0.87827 & 11.75 & -11.75 & 365.76 & 0 . \\
\hline cuboid & 3 & 0.12827 & -0.87827 & 11.87827 & $7-11.87827$ & 365.76 & 0 \\
\hline \multicolumn{8}{|l|}{ unit 20} \\
\hline com='Top Boral/ & Bask & ret Plate' & & & & & \\
\hline cuboid & $5:$ & 9.525 & -9.525 & $-0.7754-$ & -0.87827 & 365.76 & 0 \\
\hline cuboid & 7 & 9.525 & -9.525 & -0.75 & -0.87827 & 365.76 & 0 \\
\hline cuboid & 3 & 111.7500 & -11.75 & -0.75 & -0.87827 & 365.76 & 0 . \\
\hline cuboid & 4 & 111.7500 & -11.75 & 0.0 & -0.87827 & 365.76 & 0 . \\
\hline \multicolumn{8}{|l|}{ unit 21} \\
\hline \multicolumn{8}{|c|}{ com='Bottom Boral/Basket Plate' } \\
\hline cuboid & 5 & 9.525 & -9.525 & 0.87827 & 0.7754 & 365.76 & 0 \\
\hline cuboid & 7 & 9.525 & -9.525 & 0.87827 & 0.75 & 365.76 & 0 \\
\hline cuboid & 3 & 111.7500 & -11.75 & 0.87827 & 0.75 & 365.76 & 0 . \\
\hline cuboid & 4 & 111.7500 & -11.75 & 0.87827 & 0.0 & 365.76 & 0 . \\
\hline \multicolumn{8}{|l|}{ unit 22} \\
\hline $\mathrm{com}=$ 'Left-Hand & Side & Eoral/Basket & t Plate' & & & & \\
\hline cuboid & 5 & 0.87827 & 0.7754 & 9.525 & -9.525 & 365.76 & 0 \\
\hline cuboid & 7 & 0.87827 & 0.75 & 9.525 & -9.525 & 365.76 & 0 \\
\hline cuboid & 3 & 0.87827 & 0.75 & 10.9999 & -10.9999 & 365.76 & 0 . \\
\hline cuboid & 4 & 0.87827 & 0.0 & 10.9999 & -10.9999 & 365.76 & 0 . \\
\hline \multicolumn{8}{|l|}{ unit 23} \\
\hline $\mathrm{com}=$ 'Right-Hand & Side & de Boral/Baske & zet Plate' & & & & \\
\hline cuboid & 5 & $1-0.7754$ & -0.87827 & 9.525 & -9.525 & 365.76 & 0 \\
\hline cuboid & 7 & $1-0.75$ & -0.87827 & 9.525 & -9.525 & 365.76 & 0 \\
\hline cuboid & 3 & $1-0.75$ & -0.87827 & 10.9999 & -10.9999 & 365.76 & 0 . \\
\hline cuboid & 4 & 0.0 & -0.87827 & 10.9999 & -10.9999 & 365.76 & 0 . \\
\hline
\end{tabular}


Table 2.3.12. STARBUCS input listing for sample problem 4 (continued)

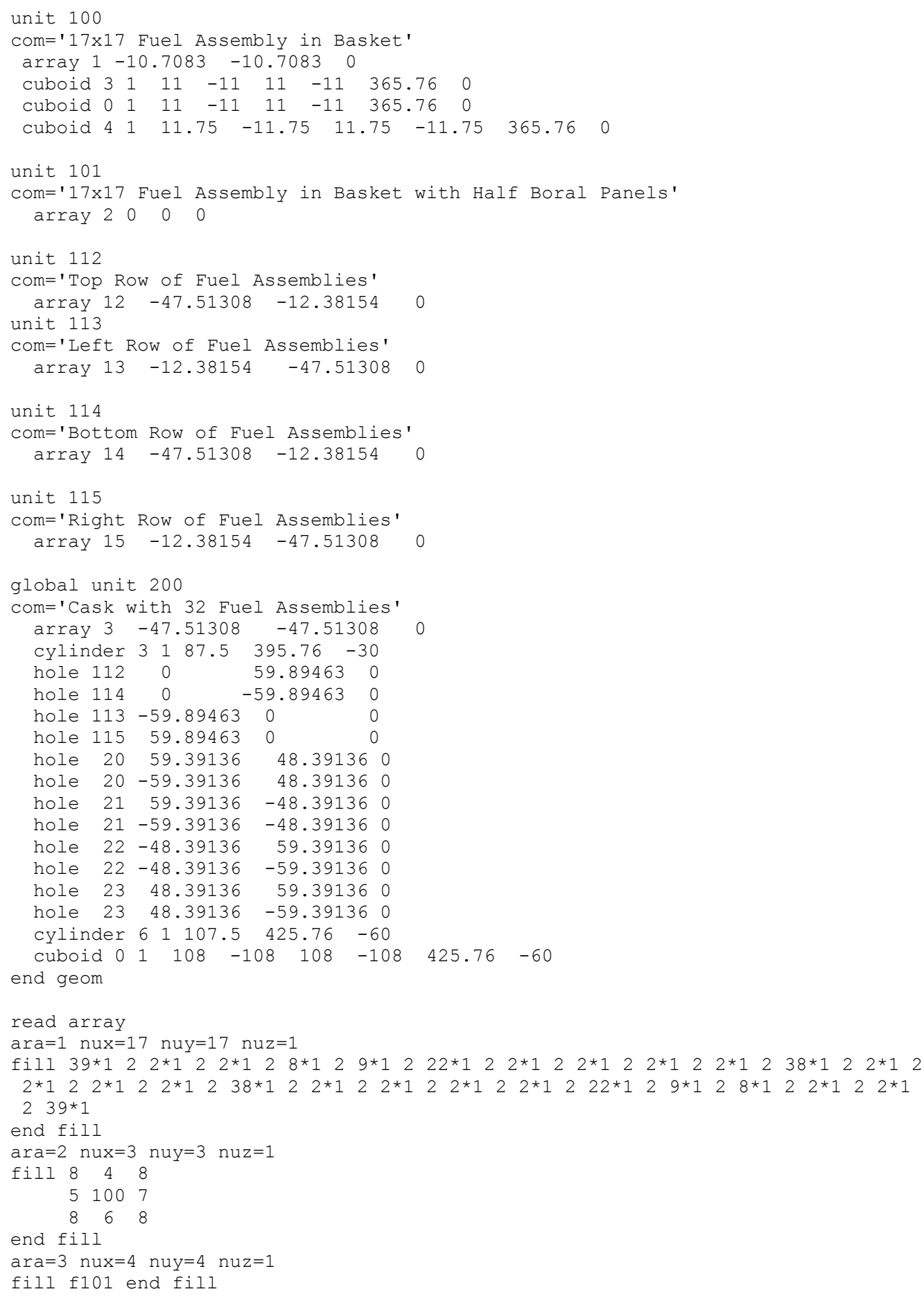


Table 2.3.12. STARBUCS input listing for sample problem 4 (continued)

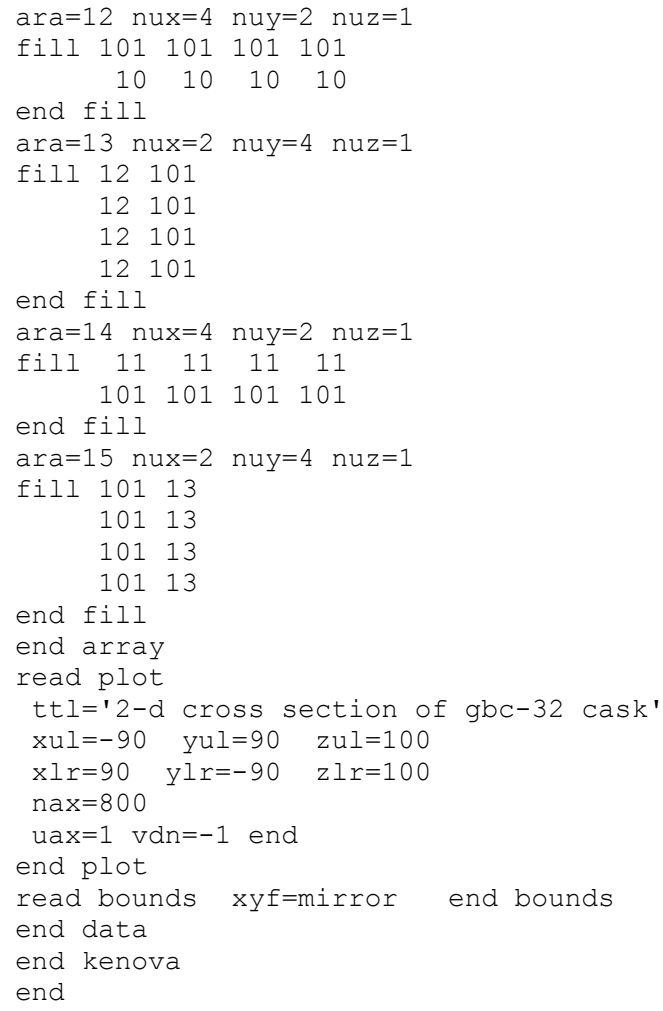

\subsubsection{Sample problem 5}

Sample problem 5, listed in Table 2.3.12, uses the CE $14 \times 14$ assembly design from problem 3, and performs a burnup-credit calculation using the horizontal burnup-profile option. The assembly configuration is taken to be a simple $2 \times 2$ assembly array with water reflection. This problem is only designed to illustrate the basic features of the horizontal profile option. In this example, it is assumed that there is a burnup gradient across the assemblies, such that half the fuel pins have a burnup exceeding the average assembly burnup by $10 \%$ and half the pins have a burnup of $10 \%$ less than the average, with the two burnup regions separated by the assembly diagonal. The input card required to simulate the two horizontal burnup regions in an assembly is

$$
h z p=0.91 .1 \text { end }
$$

STARBUCS applies these factors to calculate compositions for each of the horizontally-varying burnup regions in each zone of the problem. It is important to note that the option inherently assumes that there is an equal volume/mass of fuel in each of the horizontal (or axial) zones since the code weights all regions equally when determining the average assembly burnup. To illustrate this, consider modeling an assembly with only one quadrant having a burnup that is $10 \%$ higher than the other three quadrants. The user would enter data for each of the four horizontal assembly quadrants or zones, e.g.,

$$
\mathrm{hzp}=0.97660 .97660 .9766 \quad 1.0700 \text { end }
$$

such that the average of the HZP array entries is unity. This ensures that the average assembly burnup will be that specified in the power history data block. Note that this array is automatically normalized if 
NPR=YES (default). However, the user could substantially reduce the computational time involved by specifying only two fuel regions, e.g.,

$$
\mathrm{hzp}=0.97661 .0700 \text { end }
$$

and turning off the normalization option (e.g., NPR=NO). The normalization option must be turned off to prevent the profile from being altered (since the sum is not equal to 2). This allows the user to account for the fact that, in this scenario, there are three quadrants having a lower burnup (and consequently three times the mass) and just one quadrant having an elevated burnup compared to the average. However, it is the responsibility of the user to ensure that the profiles and the KENO V.a problem description produce the desired average burnup.

In this sample problem the four assemblies are aligned so the lower burnup regions of the assemblies are adjacent to one another to maximize the system reactivity. The assembly geometry showing the different burnup regions of the assemblies is illustrated in Figure 2.3.8. The criticality calculation is performed using the SCALE ENDF/B-VII continuous cross-section library (CE_V7).

Following the STARBUCS calculation, the KENO V.a geometry model could be readily altered to simulate other assembly configurations (e.g., shuffle the fuel assembly locations). The CSAS5 case could subsequently be executed as a standalone case since all of the material compositions have already been created during the initial STARBUCS run. This facilitates the rapid evaluation of different fuel configurations without the need to regenerate the material compositions using STARBUCS.

Table 2.3.12. STARBUCS input listing for sample problem 5

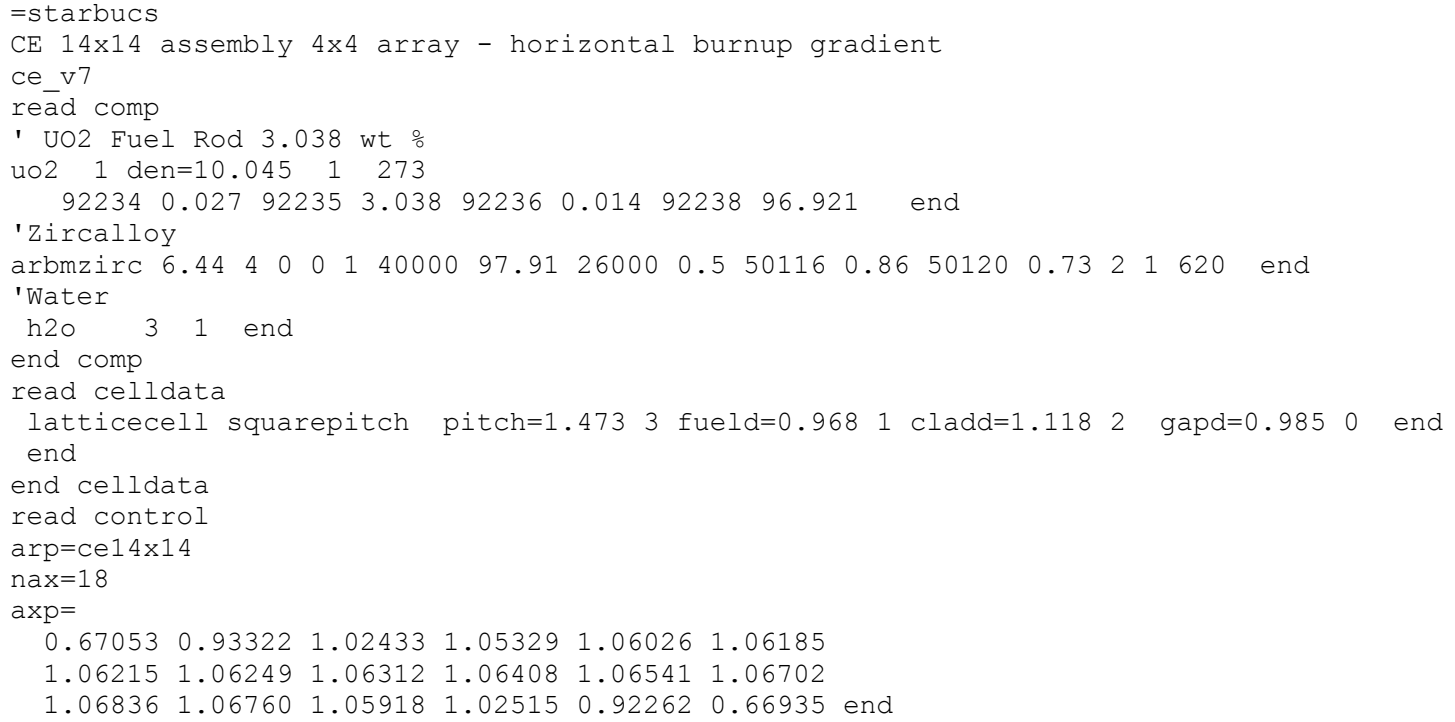


Table 2.3.13. STARBUCS input listing for sample problem 5 (continued)

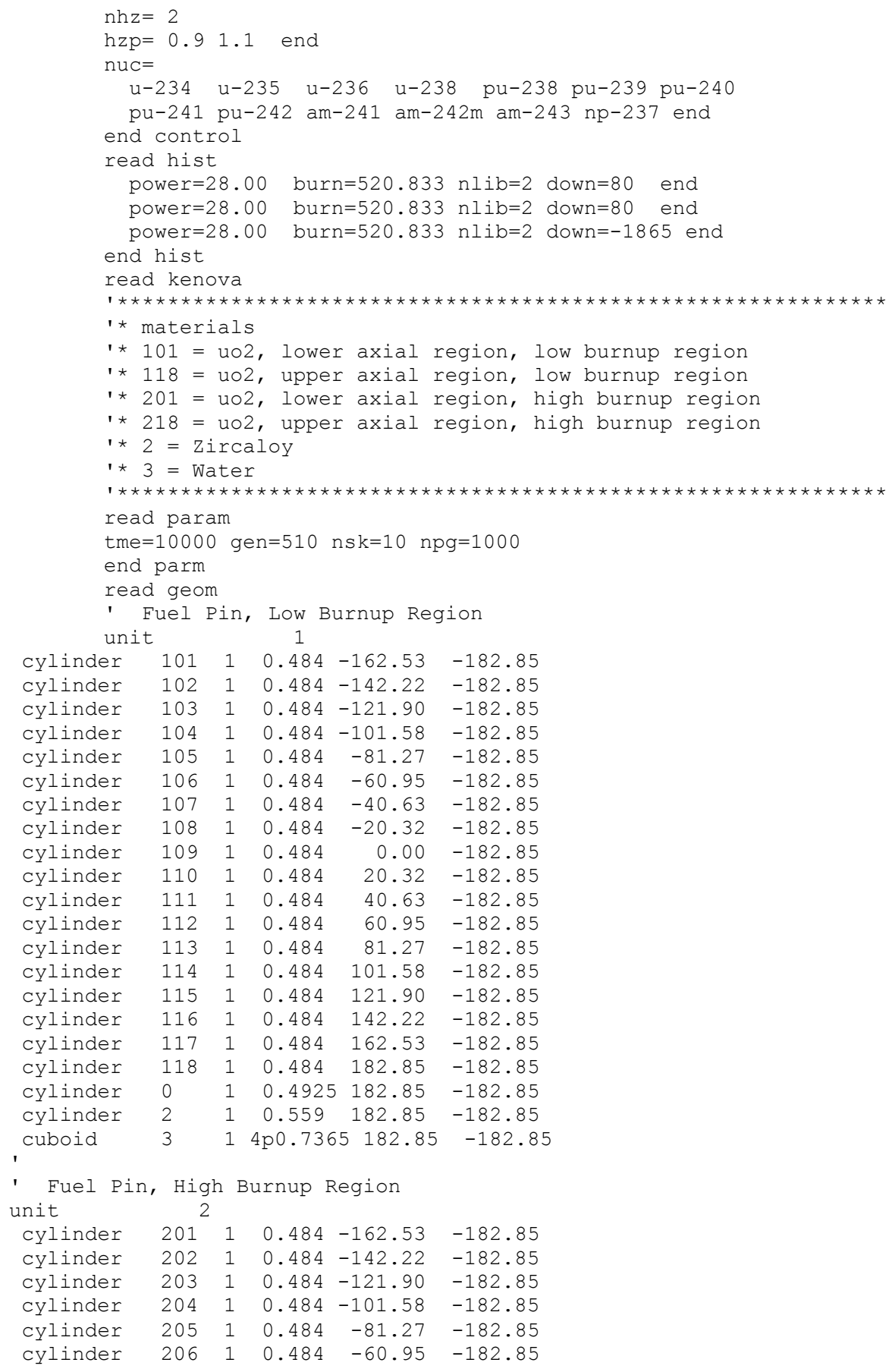


Table 2.3.13. STARBUCS input listing for sample problem 5 (continued)

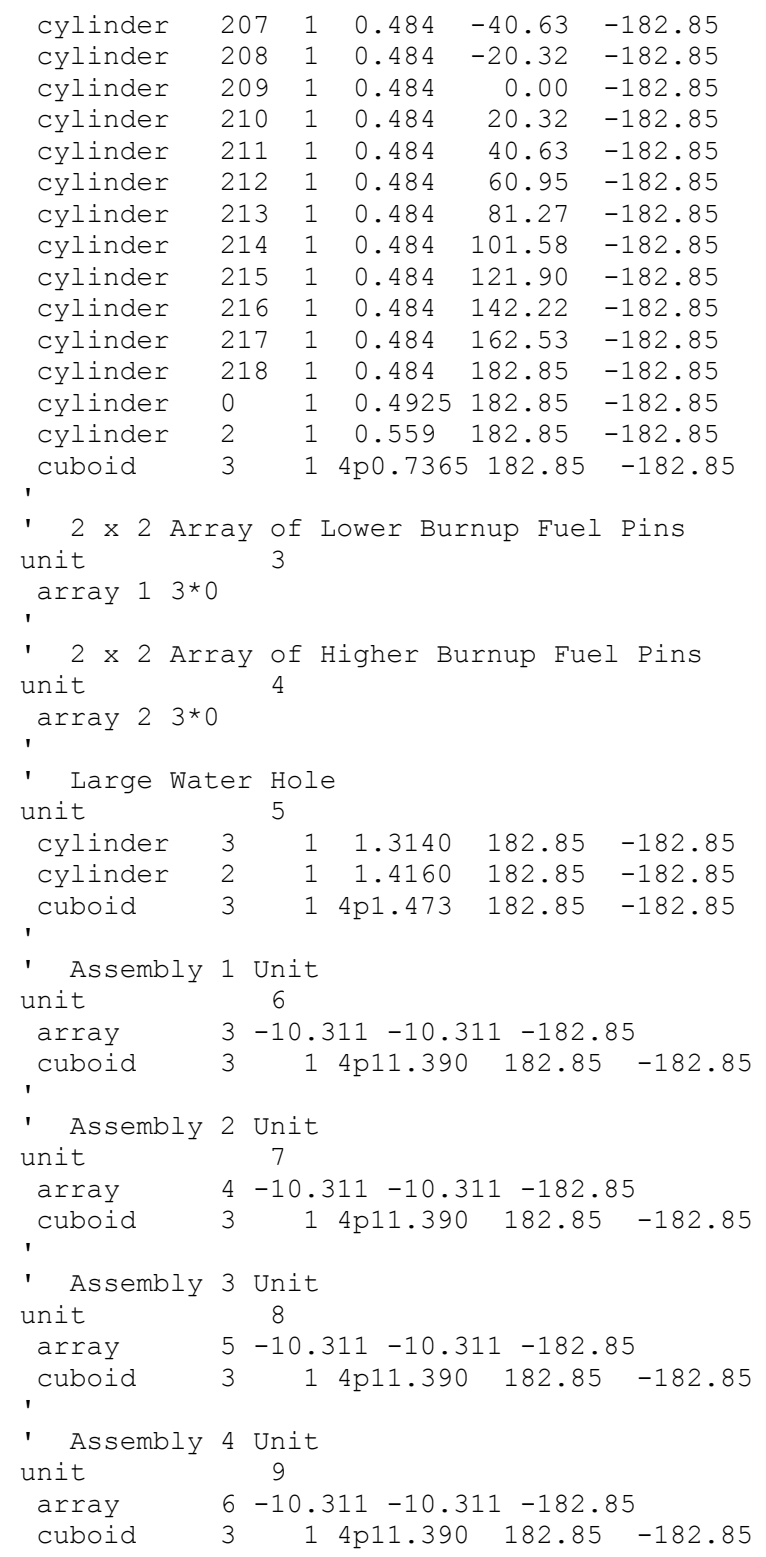


Table 2.3.13. STARBUCS input listing for sample problem 5 (continued)

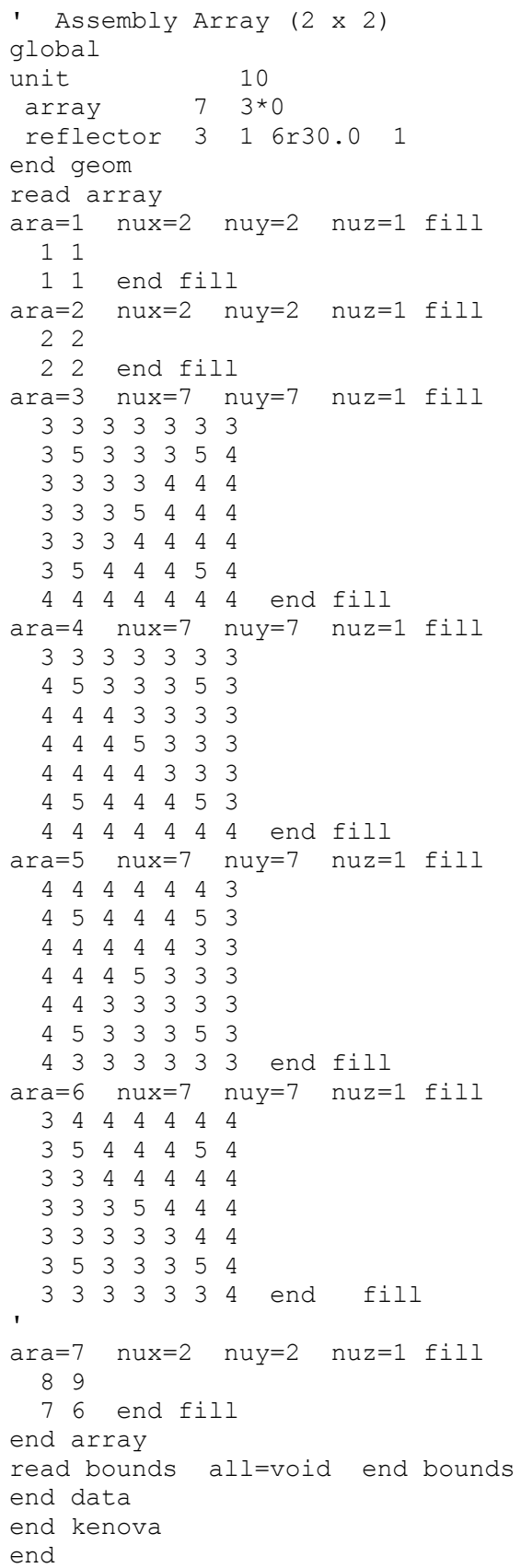


Higher burnup

region

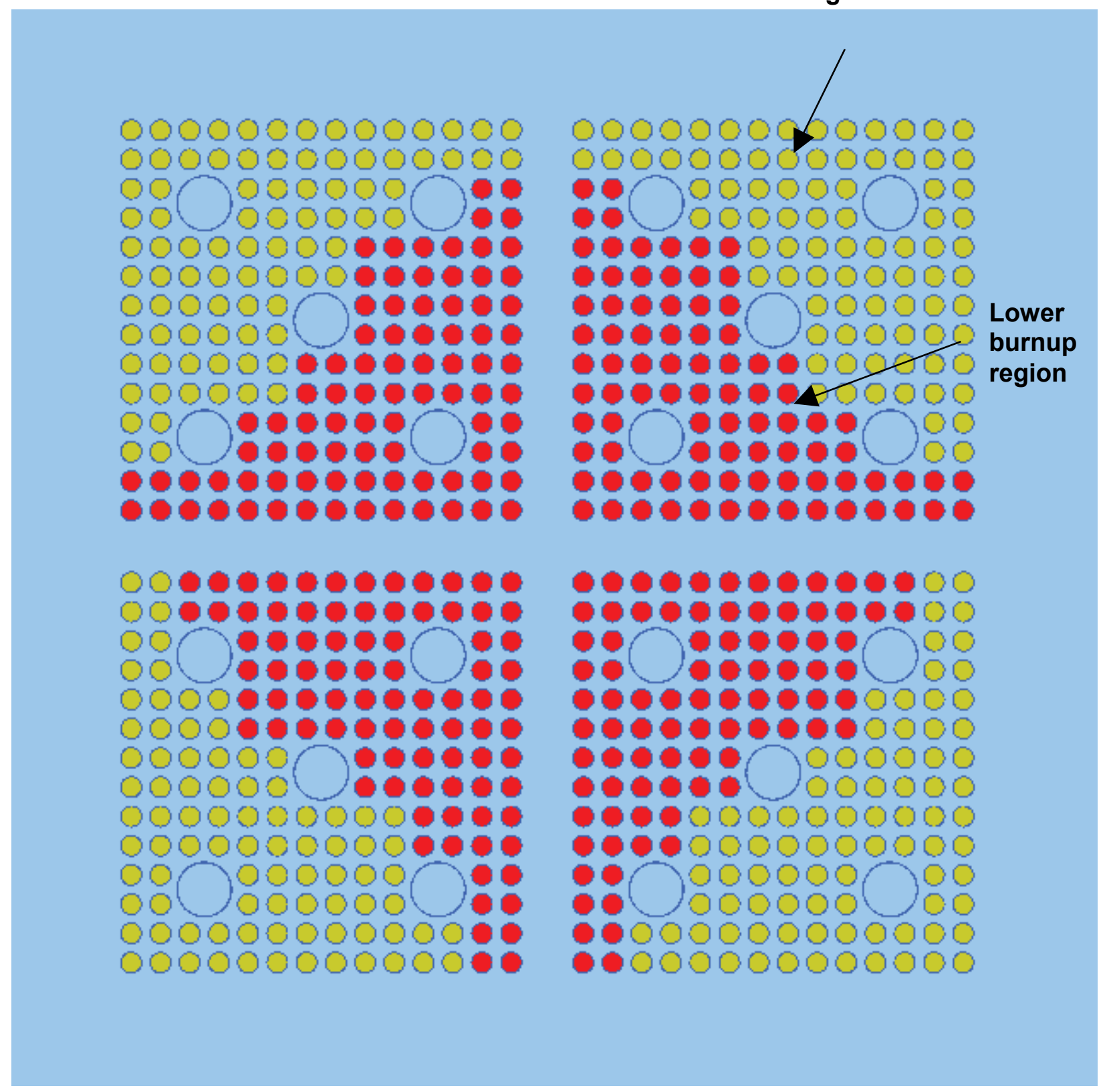

Figure 2.3.8. Plot of the $2 \times 2$ array of CE $14 \times 14$ assemblies with burnup gradient. 


\subsubsection{Sample problem 6}

The last sample problem uses KENO-VI to model a hexagonal VVER-440 fuel assembly. In this example the axial burnup profile is simulated using five axial regions of non-uniform volume (height). In this case the profile input in the $\mathrm{AXP}=$ array is not normalized by the code (i.e., NPR=NO). The criticality calculation is performed using actinide credit only. The input file is listed in Table 2.3.13 and is the geometry is illustrated in Figure 2.3.9.

\section{Table 2.3.13. STARBUCS input listing for sample problem 6}

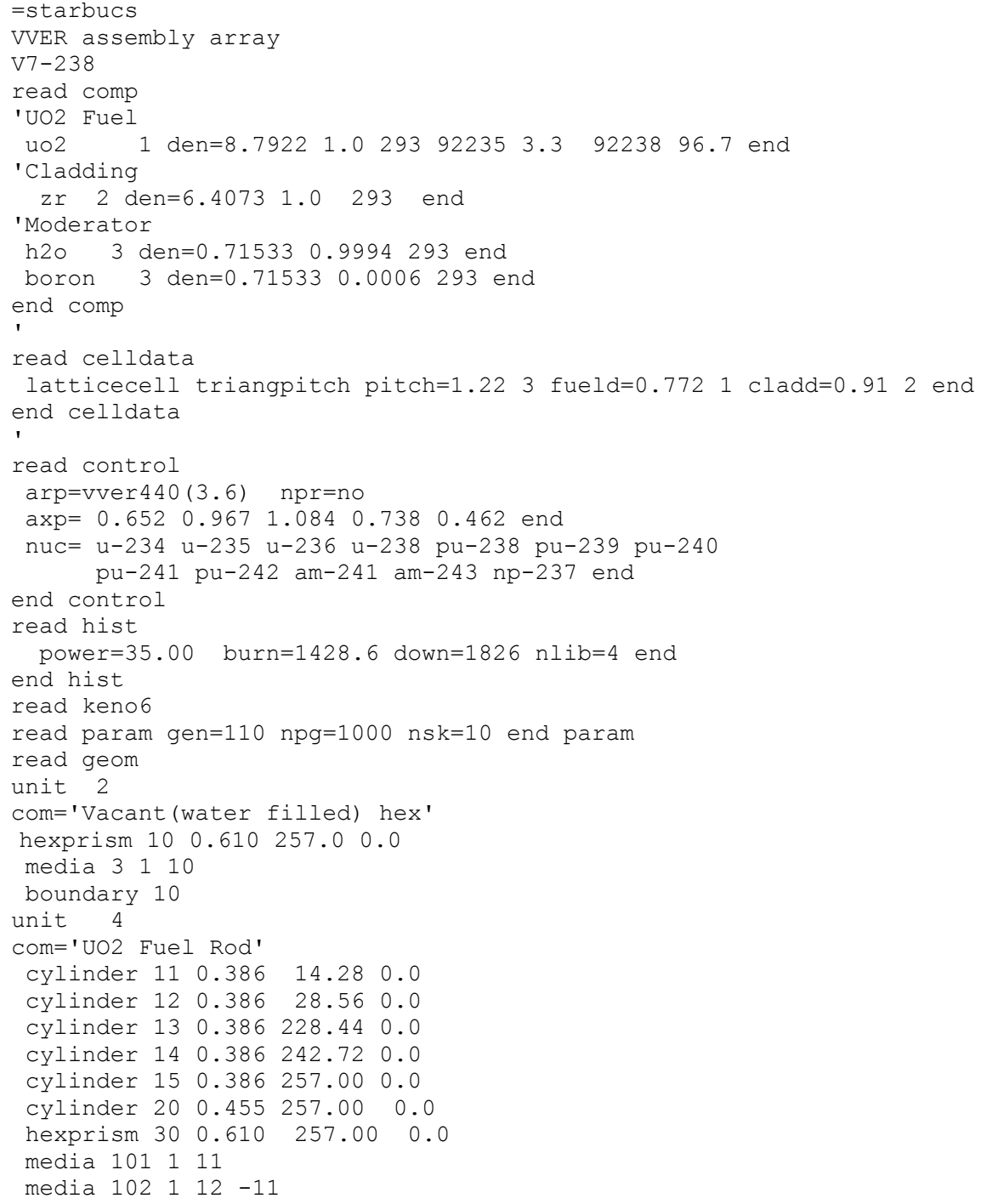


Table 2.3.13. STARBUCS input listing for sample problem 6 (continued)

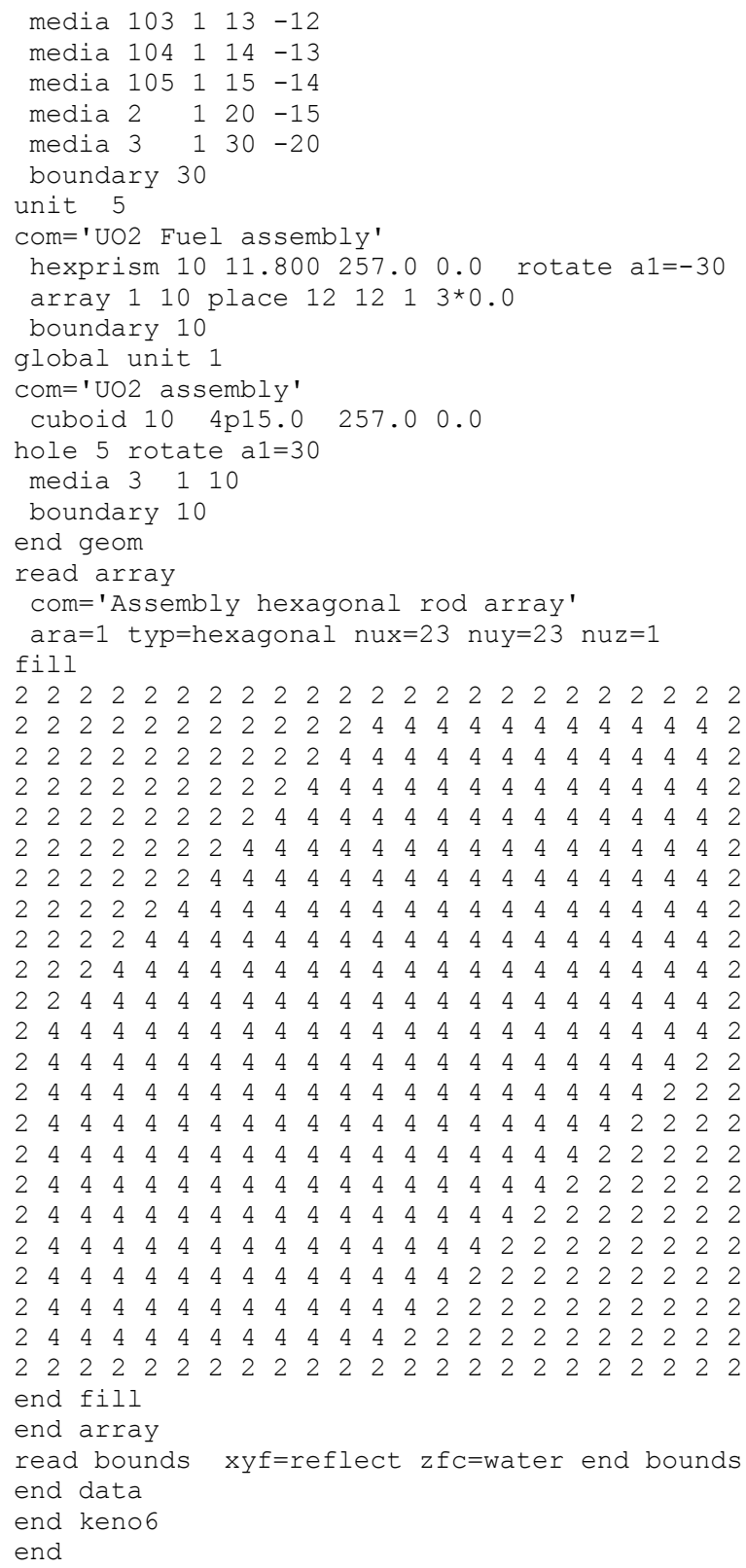




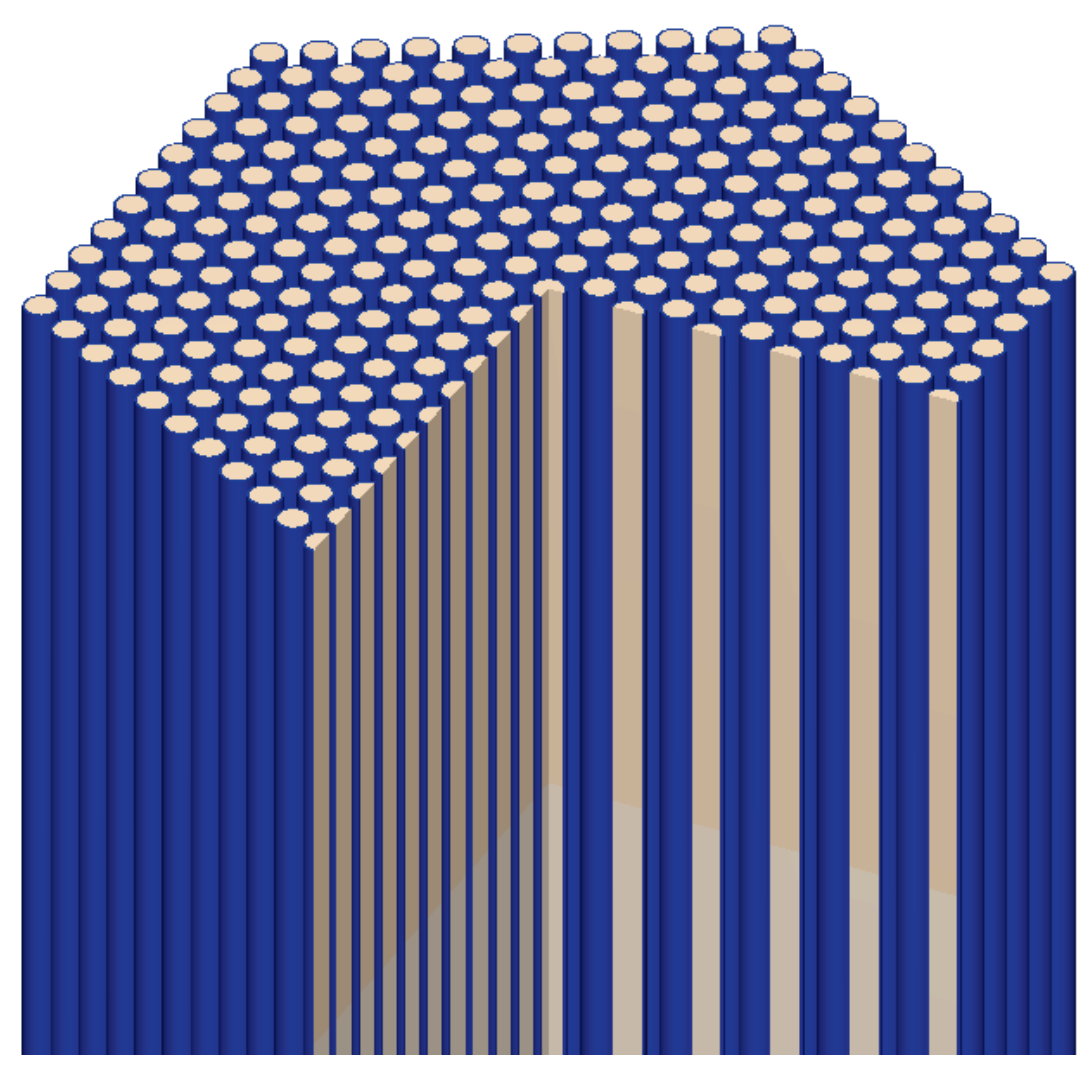

Figure 2.3.9. Cutaway 3-D view of the hexagonal VVER assembly model with water hidden.

\subsubsection{References}

1. "Spent Fuel Project Office Interim Staff Guidance - 8, Revision 2 - Burnup Credit in the Criticality Safety Analyses of PWR Spent Fuel in Transportation and Storage Casks," USNRC, September 27, 2002.

2. Office of Civilian Radioactive Waste Management, Topical Report on Actinide-Only Burnup Credit for PWR Spent Nuclear Fuel Packages, DOE/RW-0472, Rev. 2, U.S. Department of Energy, Office of Civilian Radioactive Waste Management, September 1998.

3. R. J. Cacciapouti and S. Van Volkinburg, Axial Burnup Profile Database for Pressurized Water Reactors, YAEC-1937, Yankee Atomic Electric Company, May 1997.

4. M. D. DeHart and O. W. Hermann, An Extension of the Validation of SCALE (SAS2H) Isotopic Predictions for PWR Spent Fuel, ORNL/TM-13317, Lockheed Martin Energy Research Corp., Oak Ridge National Laboratory, September 1996. 


\title{
2.4 SOURCERER: DETERMINISTIC STARTING SOURCE FOR CRITICALITY CALCULATIONS
}

\author{
D. E. Peplow, A. M. Ibrahim, K. B. Bekar, C. Celik, and B. T. Rearden
}

\begin{abstract}
The Sourcerer sequence in SCALE deterministically computes a fission distribution and uses it as the starting source in a Monte Carlo eigenvalue criticality calculation. Using a reasonably accurate starting source, developed from the Denovo discrete-ordinates code through the DEVC sequence, Sourcerer improves the KENO/CSAS Monte Carlo calculation in two ways. First, the number of skipped generations required to converge the fission source distribution in the KENO solution is reduced. Second, for problems with loosely coupled fissionable areas, the reliability of the final eigenvalue $\left(k_{\text {eff }}\right)$ is increased. Several convergence diagnostic capabilities available in the KENO codes help the user better measure when the fission source actually convergences.
\end{abstract}




\section{TABLE OF CONTENTS}

$\underline{\text { Page }}$

2.4 Sourcerer: Deterministic Starting Source for Criticality Calculations........................2-172

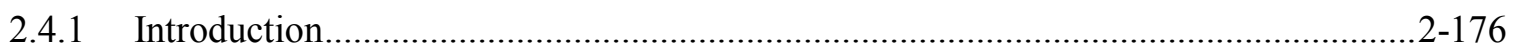

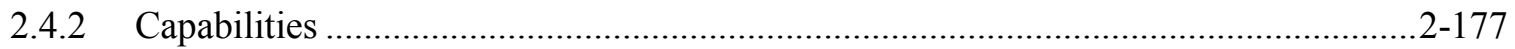

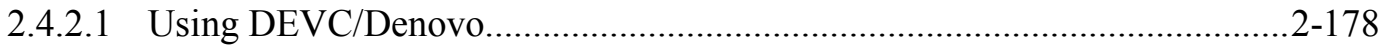

2.4.2.2 Convergence Metrics in KENO ..............................................................2-179

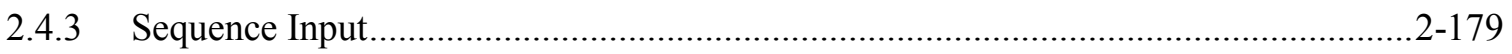

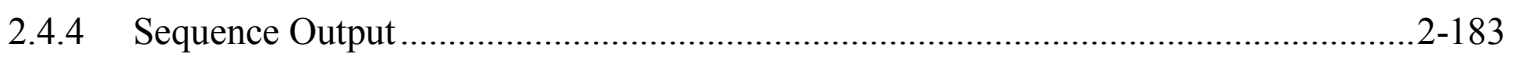

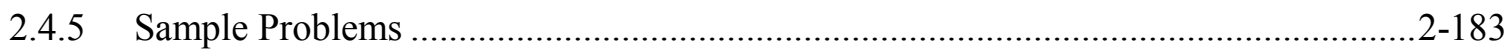

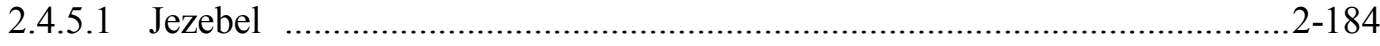

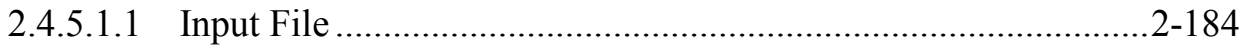

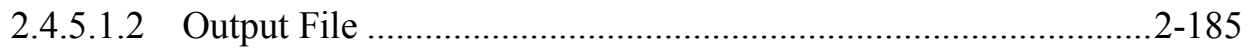

2.4.5.1.3 Other Variations ..................................................................... 2-186

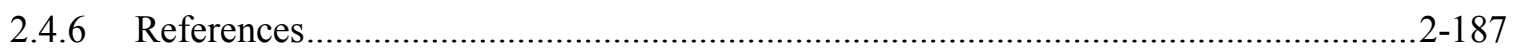

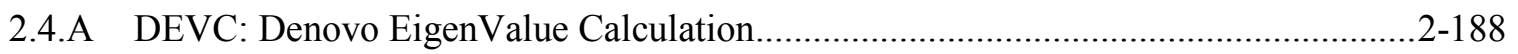

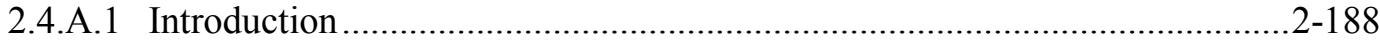

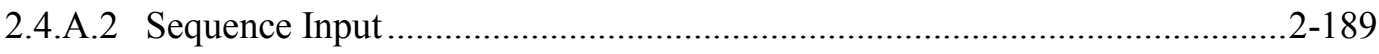

2.4.A.2.1 Parameters Block ............................................................2-189

2.4.A.2.2 Grid Geometry Block ...........................................................2-191

2.4.A.2.3 Macromaterial Block ...........................................................2-192

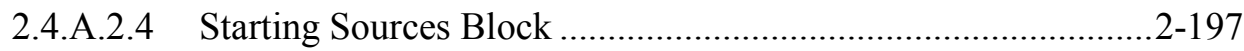

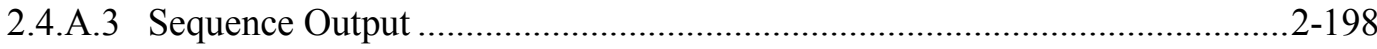

2.4.A.3.1 Using the Mesh File Viewer..................................................2-199

2.4.A.3.2 Viewing the Starting Source.................................................2-200

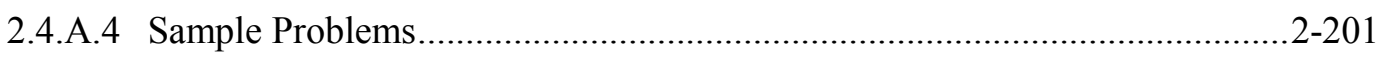

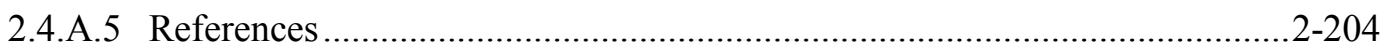




\section{LIST OF FIGURES}

$\underline{\text { Page }}$

Figure 2.4.1. Fraction of failure to agree with the reference $k_{\text {eff }}$ value for KENO calculations with different starting sources (Figure 4 from Ref. 6)................................................2-177

Figure 2.4.2. Fission source distribution from Denovo (left) and KENO (right). ..............................2-186

Figure 2.4.A.1 Ray positions within a voxel with different mmSubCells parameters. ...................2-193

Figure 2.4.A.2 Geometry model (left) and the Denovo representation (right) of an assembly using macromaterials determined by ray tracing....................................................2-194

Figure 2.4.A.3 Ray tracing (in two dimensions) through a voxel................................................2-195

Figure 2.4.A.4 Progression of the recursive bisection method (from upper left to lower right).......2-196

Figure 2.4.A.5 Cask geometry model (upper left) and the Denovo representation using

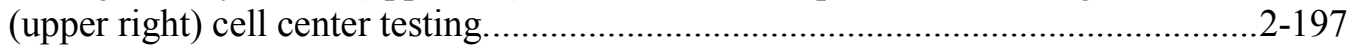

Figure 2.4.A.6 Viewing material information with the Mesh File Viewer....................................2-199

Figure 2.4.A.7 Viewing material information from a Denovo input file......................................2-200

Figure 2.4.A.8 Fine-mesh version of sample problem 8, showing the materials using macromaterial blending of $\mathrm{UO}_{2}, \mathrm{MOX}$, clad, and water (left) and the starting source strength limited to cells with fissionable material (right).

Figure 2.4.A.9 Denovo geometry (left) and starting source distribution (right) for the sample

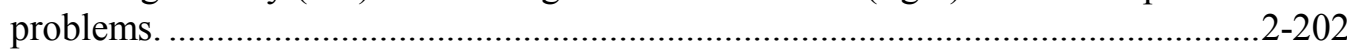

Figure 2.4.A.10 Fission source distributions computed by CSAS6 (left) and Denovo (right)..........2-203 


\section{LIST OF TABLES}

$\underline{\text { Page }}$

Table 2.4.1. Steps in Sourcerer for an input file named input.inp................................................2-178

Table 2.4.2. Input file for a Sourcerer calculation (and differences with a CSAS input file, where black text is the same as CSAS and green text is new for Sourcerer sequence)...............2-180

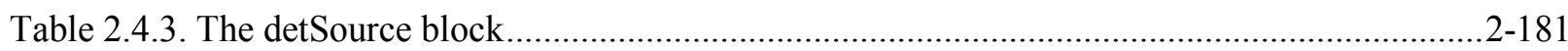

Table 2.4.4. Common Denovo parameters in the eigenValParams sub-block ...................................2-182

Table 2.4.5. Boundary conditions in the eigenValParams sub-block...............................................2-182

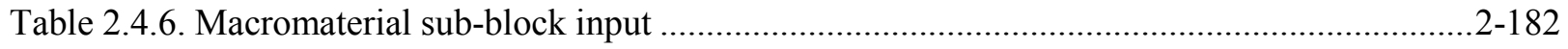

Table 2.4.7. Files created by Sourcerer for an input file named input.inp .......................................2-183

Table 2.4.8. Eigenvalue results for the Jezebel problem............................................................2-185

Table 2.4.A.1 Steps in DEVC for an input file named input.inp................................................2-188

Table 2.4.A.2 Input file for a DEVC calculation (and differences with a CSAS6 input file, where black text is the same as CSAS6 and green text is new for DEVC

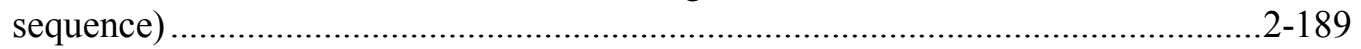

Table 2.4.A.3 Denovo parameters in the parameters block........................................................2-190

Table 2.4.A.4 Boundary conditions and what type of file to save .............................................2-191

Table 2.4.A.5 Grid geometry input keywords .........................................................................2-192

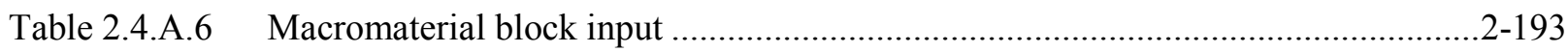

Table 2.4.A.7 Starting source types (within the fissile areas of the listed shape) .........................2-198

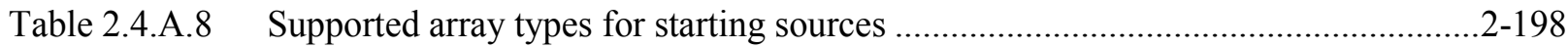

Table 2.4.A.9 Files created by DEVC for an input file named input.inp ...................................2-199

Table 2.4.A.10 Sample problem results .....................................................................................2-204 


\subsubsection{Introduction}

Monte Carlo eigenvalue calculations have been used in evaluating critical and sub-critical systems for decades. Calculations are typically done iteratively - starting with a set of fission neutrons, transporting them through the geometry until they leak or are absorbed, and then tabulating the fission sites for the next iteration. Each iteration corresponds to a generation in a chain reaction, and the eigenvalue, $k_{\mathrm{eff}}$, is the ratio of the number of fissions in one generation to the number in the previous generation. Once the fission source distribution (the eigenfunction) has converged, many generations are simulated to obtain more estimates of $k_{\text {eff }}$ with lower statistical uncertainty.

Two common questions that concern practitioners are (1) how many generations (skipped generations) are required before the fission source distribution is sufficiently converged that generational estimates of $k_{\text {eff }}$ can be included in the final average of the eigenvalue (the active generations) and (2) has the fission source converged to the correct distribution, such that the final value of the eigenvalue will be correct? For most calculations, the final value of the $k_{\text {eff }}$ eigenvalue is all that matters, so the convergence of the generational value of $k_{\text {eff }}$ is used to determine the number of skipped generations. Fluxes and reaction rates computed during the active generations are more sensitive to the entire fission distribution and should only be accumulated when the fission distribution is sufficiently converged - which is not necessarily as soon as the generational value of $k_{\text {eff }}$ has converged. To address this concern, tools such as Shannon entropy ${ }^{1}$ can be used to measure the convergence of the fission source distribution eigenfunction.,

For the second question, the reliability in the final $k_{\text {eff }}$ eigenvalue depends on whether the fission source converges to the correct distribution. Because no tool currently exists in SCALE to verify that the fission distribution is correct, models are often run for many generations using many histories per generation to ensure that the result does not change. Another approach to verify eigenvalue accuracy is to run several clones of the same problem, but each starting with different random numbers seed, and ensure that they all predict the same value for $k_{\text {eff }}$. Addressing this concern relies heavily on the engineering judgment of the practitioner.

Initial studies ${ }^{4,5}$ have shown that the use of a starting fission distribution that is similar to the true fission distribution can both reduce the number of skipped generations required for fission source convergence and significantly improve the reliability of the final $k_{\text {eff }}$ result. A recent study ${ }^{6}$ focusing on criticality calculations of a spent nuclear fuel cask showed that the chance of a low eigenvalue result due to undersampling from an unconverged source was dramatically reduced when using a deterministic starting source. In that study, a cask holding 24 assemblies was examined using a uniform starting source, a deterministic starting source with loose convergence criteria, and a deterministic starting source with tight convergence criteria. Multiple clones of KENO were run (with different random number seeds) for different values of skipped cycles. The number of clones that gave an incorrect result for $k_{\text {eff }}$ was then tabulated. The results from that study, presented in Figure 2.4.1, show that using a deterministic starting source significantly increases the $k_{\text {eff }}$ reliability.

The Sourcerer sequence in SCALE uses the solution from the Denovo ${ }^{7}$ discrete-ordinates code (through the DEVC sequence) as that starting fission source distribution in a CSAS/KENO Monte Carlo ${ }^{8}$ calculation. For challenging criticality safety analyses, such as as-loaded spent nuclear fuel transportation packages with a mixed loading of low- and high-burnup fuel, even a low-fidelity deterministic solution for the fission source should be more accurate than the typical starting guesses of uniform or cosine shape over the fissionable regions. The Sourcerer sequence is fairly automated and uses an input very similar to standard CSAS (KENO V.a or KENO-VI) inputs, along with a short description of the mesh and other parameters for the Denovo calculation. 


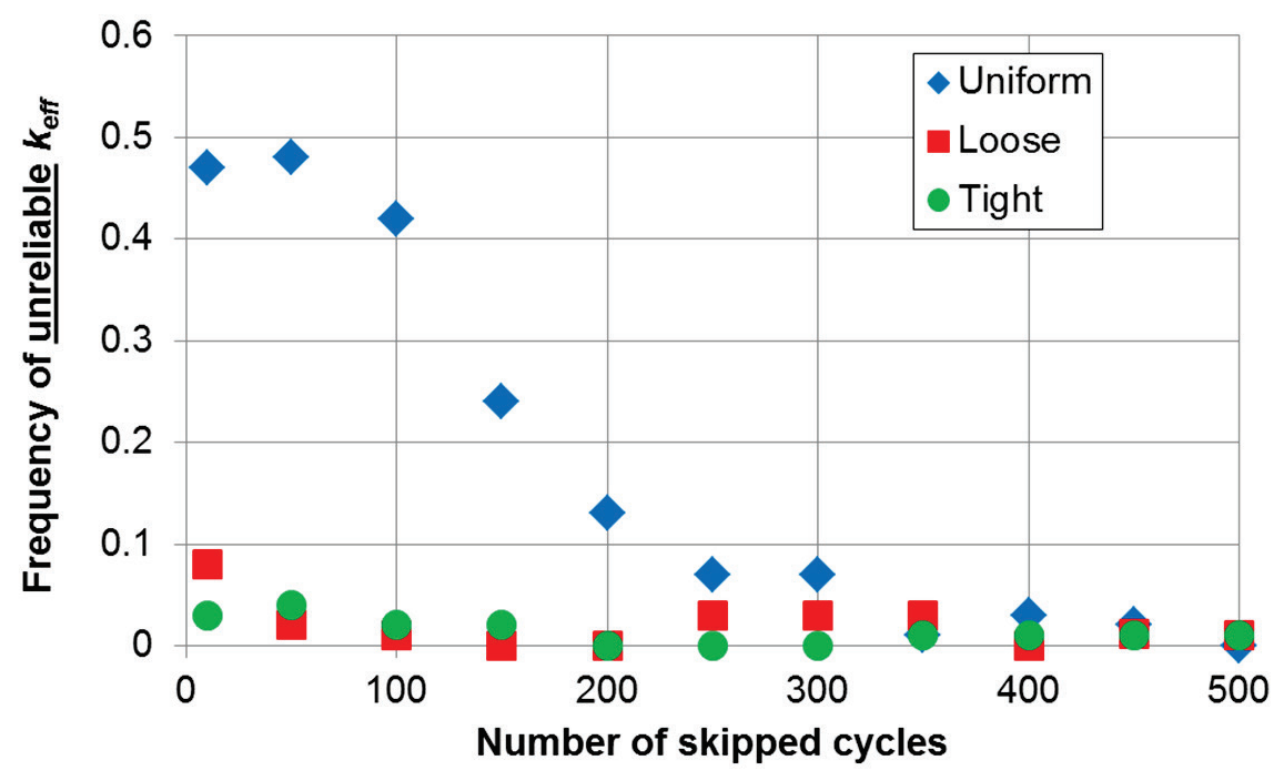

Figure 2.4.1. Fraction of failure to agree with the reference $\boldsymbol{k}_{\text {eff }}$ value for KENO calculations with different starting sources (Figure 4 from Ref. 6).

\subsubsection{Capabilities}

The Sourcerer sequence calls a series of other sequences and utilities in SCALE - most importantly DEVC (for Denovo) and one of the CSAS sequences. Because DEVC can only use KENO-VI geometry, the utility c5toc6 geometry converter is used for KENO V.a geometries. The utility dso2msl is used to convert the Denovo spatial output (*.dso file) into a mesh source lite $(* . m s l)$ file that can be read as a starting source in KENO. All of the steps in Sourcerer are described in Table 2.4.1.

The sequence can be terminated at several points throughout the calculation using the "parm=" control on the "=sourcerer" line that starts the sequence. This capability can be used to stop the sequence between steps to ensure that the problem is progressing correctly or used to make a single source distribution that can be used with many variants of the final CSAS problem. Also when running several versions of a problem, if a file that is normally created by the Sourcerer sequence is supplied, then that step will be skipped, thus saving time. Note that files that use the name of the input file (input.inp) will be copied back to the user's working directory automatically when the sequence finishes. Files that do not use the input file name can be copied back to the user's working area with an extra "=shell" directive in the input. inp input file.

The Sourcerer sequence can be used with KENO V.a or KENO-VI geometries. Either multi-group (MG) or continuous energy cross-section libraries can be used for the final CSAS calculation. Denovo only uses multi-group libraries, and self-shielding can be done like any MG sequence in SCALE. For efficient calculations, the user should understand the basics of Denovo eigenvalue calculations, how to use macromaterials, and how to use the KENO convergence metrics. 
Table 2.4.1. Steps in Sourcerer for an input file named input.inp

\begin{tabular}{|c|c|c|c|}
\hline Step & Module/Task & Creates file & To stop after \\
\hline 0 & Check user input & & parm $=$ check \\
\hline 1 & $\begin{array}{l}\text { c5toc } 6 \text { - For KENO V.a sequences, the geometry is } \\
\text { translated into KENO-VI geometry. }\end{array}$ & $\begin{array}{l}\text { input.geom0... } \\
\text { 00.inp }\end{array}$ & \\
\hline $2 \mathrm{a}$ & Create Denovo input and AMPX cross sections & $\begin{array}{l}\text { xkba_b.inp } \\
\text { ft02f001 } \\
\text { input.mmt }\end{array}$ & parm=deninp \\
\hline $2 b$ & $\begin{array}{l}\text { devc - Denovo eigenvalue calculation to compute a } \\
\text { fission source distribution }\end{array}$ & input.dso & parm=denovo \\
\hline 3 & $\begin{array}{l}\text { dso2ms } 1 \text { - Convert the fission source distribution file } \\
\text { into a mesh source lite file }\end{array}$ & input.msl & \\
\hline $4 a$ & Create the CSAS input & & parm=csasinp \\
\hline $4 \mathrm{~b}$ & $\begin{array}{l}\text { csasX - Run the specific CSAS sequence using the } \\
\text { mesh source lite as the starting source }\end{array}$ & & \\
\hline
\end{tabular}

\subsubsection{Using DEVC/Denovo}

Some discussion is required about the extent and level of detail needed in the grid geometry that will be used in the Denovo calculation and the mesh-based starting source. When using discrete-ordinates transport $\left(\mathrm{S}_{\mathrm{N}}\right)$ methods alone for solving radiation transport problems, a good rule of thumb is to use mesh cell sizes on the order of a mean-free path of the particle. For complex problems, this could lead to an extremely large number of mesh cells, especially when considering the size of the mean-free path of the lowest energy neutrons.

In Sourcerer, the goal is to use the $\mathrm{S}_{\mathrm{N}}$ calculation for a quick estimate. Accuracy is not paramount-just getting an approximation of the overall shape of the true fission source distribution will benefit the CSAS Monte Carlo calculation. With a more accurate starting source, fewer skipped generations may be required. At some point there is a time trade-off where calculating the starting source guess requires more time than the saved skipped generations would have used. Large numbers of mesh cells, as a result of using very small mesh sizes, for $\mathrm{S}_{\mathrm{N}}$ calculations also use a great deal of computer memory.

Because the $\mathrm{S}_{\mathrm{N}}$ calculation is only used to establish the initial distribution of source neutrons, the runtime and memory requirements for Sourcerer can be reduced by using larger/coarser mesh cell sizes than is typical for a stand-alone $\mathrm{S}_{\mathrm{N}}$ analysis. Some general guidelines to consider when creating a mesh for the Denovo eigenvalue calculation/mesh-based starting source are as follows.

- All fissionable areas of the geometry and areas where neutrons can reasonably affect the eigenvalue should be included in the mesh.

- More detail should be used in the fissionable areas.

- Mesh planes should be placed at significant material boundaries.

- Neighboring cell mesh sizes should not be drastically different. 


\subsubsection{Convergence Metrics in KENO}

KENO provides several tools that can be used to examine the convergence of $k_{\text {eff }}$ and the fission source distribution. These include tools based on Shannon Entropy ${ }^{1,3}$ and tools based on the mesh tally metrics described in Ref. 9. Running Sourcerer should accelerate Shannon Entropy convergence for KENO calculations, and Sourcerer/KENO users should always check that the calculation Shannon Entropy has converged before active generations begin.

\subsubsection{Sequence Input}

The input file for a Sourcerer calculation is similar to a CSAS input, as shown in Table 2.4.2. There are two major differences between Sourcerer and CSAS: the beginning/end syntax of the CSAS input and the presence of the "read detSource" block for specifying the deterministic starting source. The CSAS input appears as one block in the Sourcerer sequence - instead of "=csasXX" use "read csasXX" and instead of "end data" use "end csasXX". If any parm= parameters are required for the CSAS sequence, they can be listed as "read csasX parm=(...)". Because Sourcerer runs both the DEVC and CSAS sequences, which will most likely use different cross-section data libraries (coarse group for $\mathrm{S}_{\mathrm{N}}$ and fine group or continuous energy for Monte Carlo), the library for DEVC is listed in the new "read detSource" block, along with other parameters used by the Sourcerer sequence.

One important note about the geometry in Sourcerer is that for KENO V.a geometries, an outer boundary region should be added for an accurate internal conversion to a KENO-VI geometry prior to ray tracing. Also note that when using CSAS methods with the search capability, the Denovo mesh must encompass any changes to the size of the geometry.

Parameters used for building the deterministic starting source and establishing the CSAS sequence are specified in the detSource block. The library name for the Denovo calculation and the grid for the Denovo calculations are required. Many optional parameters are available for controlling the Denovo solver and applying boundary conditions (in the eigenValParams sub-block). The grid geometry is defined in a sub-block, or the keyword "gridGeometryID= $n$ " can be used to point to a grid geometry defined in its own input block. The use of macromaterials to construct a more representative mesh model from the Monte Carlo geometry is controlled with the "mmSubCell=" and "mmTolerance=" parameters in the macromaterial sub-block.

The overall layout of the detSource block is shown in Table 2.4.3. The more common keywords for the eigenValParams sub-block are shown in Table 2.4.4 and Table 2.4.5. A full list of the Denovo parameters appears in Appendix A. Macromaterials are explained in detail in the DEVC manual, and a list of keywords is given in Table 2.4.6. 
Table 2.4.2. Input file for a Sourcerer calculation (and differences with a CSAS input file, where black text is the same as CSAS and green text is new for Sourcerer sequence)

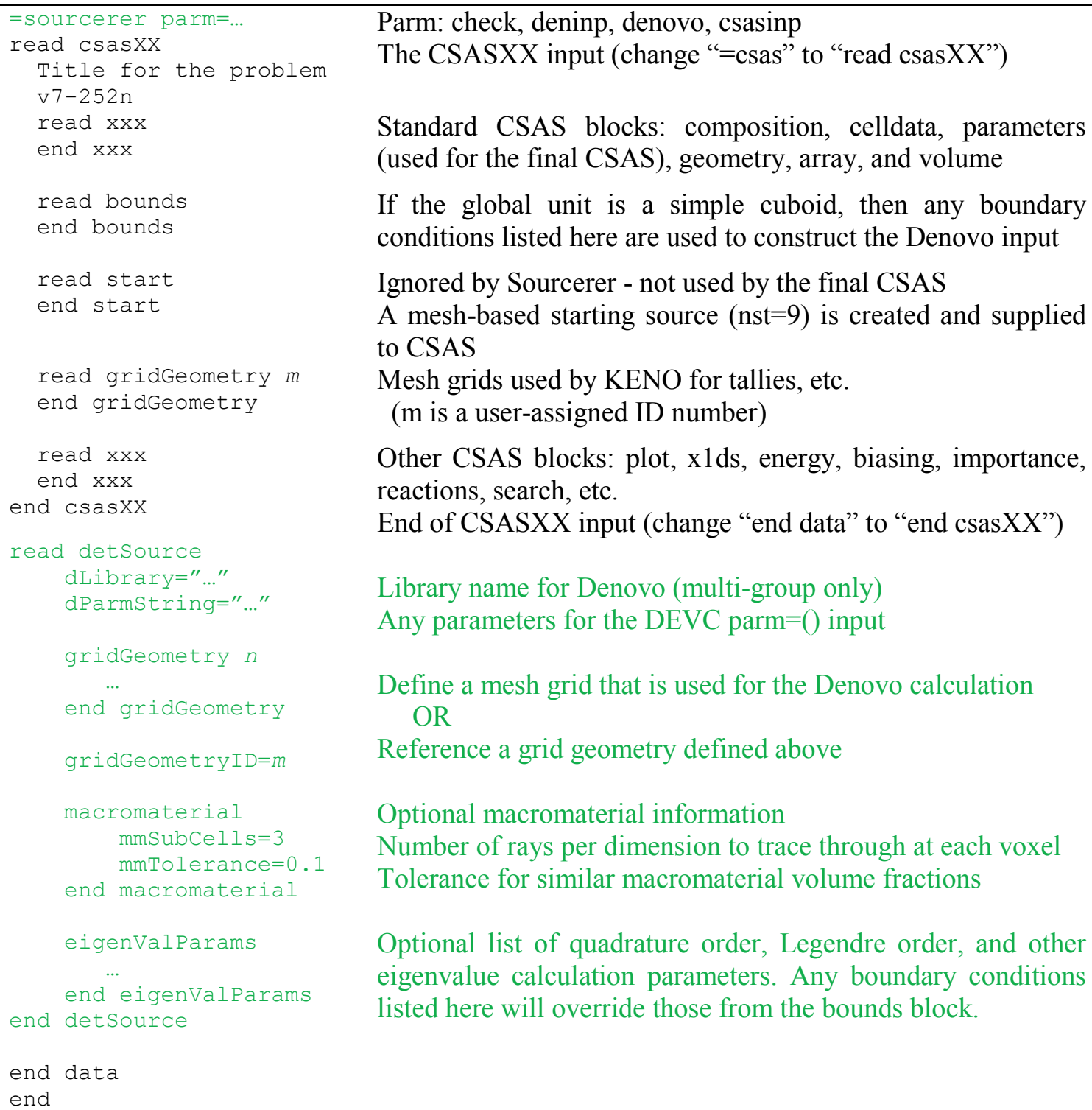

Parm: check, deninp, denovo, csasinp

The CSASXX input (change "=csas" to "read csasXX")

Standard CSAS blocks: composition, celldata, parameters (used for the final CSAS), geometry, array, and volume

If the global unit is a simple cuboid, then any boundary conditions listed here are used to construct the Denovo input

Ignored by Sourcerer - not used by the final CSAS

A mesh-based starting source (nst=9) is created and supplied to CSAS

Mesh grids used by KENO for tallies, etc.

( $\mathrm{m}$ is a user-assigned ID number)

Other CSAS blocks: plot, x1ds, energy, biasing, importance, reactions, search, etc.

End of CSASXX input (change "end data" to "end csasXX")

Library name for Denovo (multi-group only)

Any parameters for the DEVC parm=() input

Define a mesh grid that is used for the Denovo calculation OR

Reference a grid geometry defined above

Optional macromaterial information

Number of rays per dimension to trace through at each voxel

Tolerance for similar macromaterial volume fractions

Optional list of quadrature order, Legendre order, and other eigenvalue calculation parameters. Any boundary conditions listed here will override those from the bounds block. 
Table 2.4.3. The detSource block

\begin{tabular}{|c|c|c|c|}
\hline keyword & type & required & restrictions/comments \\
\hline \multirow{2}{*}{\multicolumn{4}{|c|}{$\begin{array}{l}\text { read detSource } \\
\quad \text { For the preliminary Denovo calculation }\end{array}$}} \\
\hline & & & \\
\hline dLibrary= & character & yes & multi-group library for Denovo calculation \\
\hline dParmString $=$ & character & no & any parameters for the DEVC parm=(). \\
\hline $\begin{array}{l}\text { gridGeometry id } \\
\text { title }= \\
x \text { planes ... end } \\
x \text { Linear } n \text { min max } \\
y \text { planes ... end } \\
y \text { Linear } n \text { min max } \\
z \text { planes ... end } \\
\text { z Linear } n \text { min max } \\
\text { tolerance }= \\
\text { make3dmap }\end{array}$ & $\begin{array}{l}\text { character } \\
\text { real array } \\
\text { int real real } \\
\text { real array } \\
\text { int real real } \\
\text { real array } \\
\text { int real real } \\
\text { real }\end{array}$ & yes $^{*}$ & $\begin{array}{l}\text { covers a cuboid where all points are in defined regions } \\
\text { contained in double quotes (title="yada yada yada") } \\
\text { adds list of } x \text { plane values (order not important) } \\
\text { adds } n+1 x \text { planes ( } n \text { cells) evenly from min to max } \\
\text { adds list of } y \text { plane values (order not important) } \\
\text { adds } n+1 \text { y planes ( } n \text { cells) evenly from min to max } \\
\text { adds list of } z \text { plane values (order not important) } \\
\text { adds } n+1 z \text { planes ( } n \text { cells) evenly from min to max } \\
\text { for removing duplicate planes from *planes or *Linear } \\
\text { makes } a * \text { *.3dmap file showing the grid geometry }\end{array}$ \\
\hline xdivide $=$ & integer & no & once all xplanes are entered, further divide them by this \\
\hline ydivide $=$ & integer & no & once all yplanes are entered, further divide them by this \\
\hline $\begin{array}{l}\text { zdivide }= \\
\text { end gridGeometry }\end{array}$ & integer & no & once all zplanes are entered, further divide them by this \\
\hline gridGeometry $I D=$ & integer & yes $^{*}$ & $\begin{array}{l}\text { *either define a grid geoemtry in a sub block or refer to } \\
\text { a grid geometry defined in its own block }\end{array}$ \\
\hline $\begin{array}{l}\text { macroMaterial } \\
\quad \ldots\end{array}$ & & no & $\begin{array}{l}\text { parameters for the macro material calculation to } \\
\text { create a more representative Denovo model }\end{array}$ \\
\hline end macroMaterial & & & \\
\hline $\begin{array}{l}\text { eigenValParams } \\
\quad \ldots \\
\text { end eigenValParams } \\
\text { end detSource }\end{array}$ & & no & $\begin{array}{l}\text { parameters for eigenvalue Denovo calculation, including } \\
\text { legendre }=\text {, quadrature }=\text {, tolerance }=\text {, } \\
\text { kTolerance }=\text {, etc. }\end{array}$ \\
\hline
\end{tabular}


Table 2.4.4. Common Denovo parameters in the eigenValParams sub-block

\begin{tabular}{llrl}
\hline \multicolumn{1}{c}{ keyword } & type & default & restrictions/comments \\
\hline eigenValParams & & & \\
quadType $=$ & integer & 2 & 0-level symmetric, 1-Gauss-Legendre product, 2-QR \\
legendre $=$ & integer & 0 & $\mathrm{P}_{\mathrm{L}}, \mathrm{L}=$ highest Legendre polynomial, $\mathrm{L}=0,1,2,3, \ldots$ \\
quadrature $=$ & integer & 8 & level symmetric $\mathrm{S}_{\mathrm{N}}$ quadrature, $\mathrm{N}=2,4,6,8,10,12,14,16$ \\
polarsPerOct $=$ & integer & 2 & Gauss-Legendre product quadrature or $\mathrm{QR}$ \\
azimuthsPerOct $=$ & integer & 2 & Gauss-Legendre product quadrature or QR \\
diagnostics $=$ & integer & 0 & 0-no diganostics, 1-all diagnostics \\
output $=$ & integer & 0 & 0-no ouput, 1-all output \\
tolerance $=$ & double & $1 . \mathrm{E}-04$ & tolerance used in convergence test \\
toleranceMG $=$ & double & $1 . \mathrm{E}-04$ & tolerance used in convergence test \\
kTolerance $=$ & double & $1 . \mathrm{E}-05$ & tolerance on $\mathrm{k}$ \\
diagnosticLevel= $=$ & integer & 0 & 0-off, 1-on \\
I2Tolerance $=$ & double & 0.01 & tolerance on the L-2 norm \\
linfTolerance $=$ & double & 0.001 & tolerance on L-inf \\
end eigenValParams & & & \\
\hline
\end{tabular}

Table 2.4.5. Boundary conditions in the eigenValParams sub-block

\begin{tabular}{llcc}
\hline keyword & type & default & restrictions/comments \\
\hline eigenValParams & & & \\
xmin $=$ & integer & 0 & boundary conditions on the six faces: \\
xmax $=$ & integer & 0 & 0 - vacuum \\
ymin $=$ & integer & 0 & 1 - reflective \\
ymax $=$ & integer & 0 & 2 - periodic \\
zmin $=$ & integer & 0 & 3 - white \\
zmax $=$ & integer & 0 & (these will override the "read bounds" block if specified here.) \\
end eigenValParams & & & \\
\hline
\end{tabular}

Table 2.4.6. Macromaterial sub-block input

\begin{tabular}{|c|c|c|c|c|}
\hline keyword & type & length & default & restrictions/comments \\
\hline $\begin{array}{l}\text { macromaterial } \\
\text { mmSubCell }= \\
\text { mmTolerance }=\end{array}$ & $\begin{array}{l}\text { integer } \\
\text { real }\end{array}$ & & $\begin{array}{r}1 \\
0.01\end{array}$ & $\begin{array}{l}\text { rays per dimension to throw at each voxel } \\
\text { smallest volume fraction for macromaterial }\end{array}$ \\
\hline $\begin{array}{l}\text { mmSubCells } \\
\text { mmPointTest } \\
\text { mmRayTest }\end{array}$ & integer & 6 & & $\begin{array}{l}\text { rays per dimension to throw (x:ny,nz; y:nx,nz; z: nx,ny) } \\
\text { use recursive bisection point testing method } \\
\text { use ray tracing method }\end{array}$ \\
\hline $\begin{array}{c}\text { mmRTSpeed } \\
\text { mmRTMemory } \\
\text { end macromaterial }\end{array}$ & & & & $\begin{array}{l}\text { optimize ray-tracing method for speed } \\
\text { optimize ray-tracing method for memory conservation }\end{array}$ \\
\hline
\end{tabular}




\subsubsection{Sequence Output}

In addition to the data contained in the main Sourcerer text output file, many other files are created containing the intermediate data used by the sequence. These files are listed in Table 2.4.7. Some of the files produced can be viewed using the Java Mesh File Viewer, which is distributed with SCALE.

Note that files that use the name of the input file (input.inp) will be copied back to the user's working directory automatically when the sequence finishes. Files that do not use the input file name can be copied back to the user's working area with an extra "=shell" directive in the input.inp input file.

Instructions on how to use the Java Mesh File Viewer to view the various output files listed in Table 2.4.7 as well as how to use the macromaterial table file are located in the DEVC manual and the Mesh File Viewer help file, which is accessible through the Help/Help menu.

Table 2.4.7. Files created by Sourcerer for an input file named input.inp

\begin{tabular}{|c|c|c|}
\hline Filename & Viewer & Description \\
\hline \multicolumn{3}{|l|}{ Output Summary } \\
\hline input.out & & main text output file, contains results summary \\
\hline input.msg & & messages file \\
\hline \multicolumn{3}{|l|}{ Geometry Conversion } \\
\hline i_c5toc6 & & input file for c 5 toc 6 module \\
\hline input.geom $000 \ldots .$. inp & & KENO-VI version of a KENO V.a geometry, if applicable \\
\hline \multicolumn{3}{|l|}{ Denovo } \\
\hline i_devc & & input file for DEVC sequence \\
\hline xkba_b.inp & $\mathrm{V}^{a}$ & $\begin{array}{l}\text { input file for Denovo - if this file is renamed to have } \\
\mathrm{a}^{*} \text {.dsi extension (Denovo simple input), it is viewable } \\
\text { in the Mesh File Viewer }\end{array}$ \\
\hline $\mathrm{ft} 02 \mathrm{f001}$ & & AMPX formatted cross sections for Denovo \\
\hline input.dso & $\mathrm{V}$ & Denovo fission source distribution \\
\hline input.mmt & $\mathrm{V}$ & macromaterial table, use with *.dso or *.dsi file \\
\hline \multicolumn{3}{|l|}{ Mesh Source Conversion } \\
\hline i_util & & input file for dso $2 \mathrm{msl}$ utility \\
\hline input.msl & $\mathrm{V}$ & mesh source lite file, the starting source distribution \\
\hline \multicolumn{3}{|l|}{ CSAS } \\
\hline i_csasXX & & input file for the final CSASXX sequence \\
\hline $\begin{array}{l}\text { input.fissionSource. } 3 \mathrm{dmap} \\
\text { input.kenoNuBar.txt }\end{array}$ & $\mathrm{V}$ & $\begin{array}{l}\text { mesh tally of fission source distribution from KENO } \\
\text { text file containing value of nu-bar }\end{array}$ \\
\hline
\end{tabular}

\subsubsection{Sample Problems}

In addition to the sample problems described in this section (with input files included with SCALE), the reader is referred to the paper by Ibrahim et al. ${ }^{10}$ for a detailed study using a real used nuclear fuel transport and storage canister containing assemblies with a range of initial enrichments and burnups. 


\subsubsection{Jezebel}

Consider the Jezebel critical assembly [PU-MET-FAST-001 in Volume I of the International Handbook of Evaluated Criticality Safety Benchmark Experiments, NEA/NSC/DOC(95)03, Organisation for Economic Cooperation and Development, Nuclear Energy Agency (OECD-NEA), September 2012]. This is a very simple problem (a single sphere) to solve with CSAS and can be useful as a way to demonstrate the Sourcerer sequence.

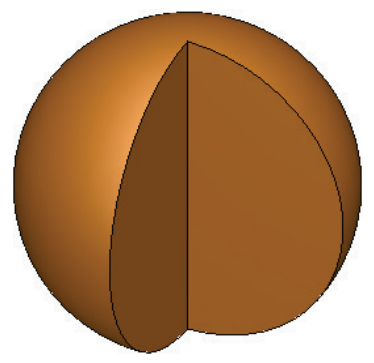

\subsection{Input File}

The standard CSAS inputs for Jezebel are shown below using both KENO V.a and KENO-VI geometries.

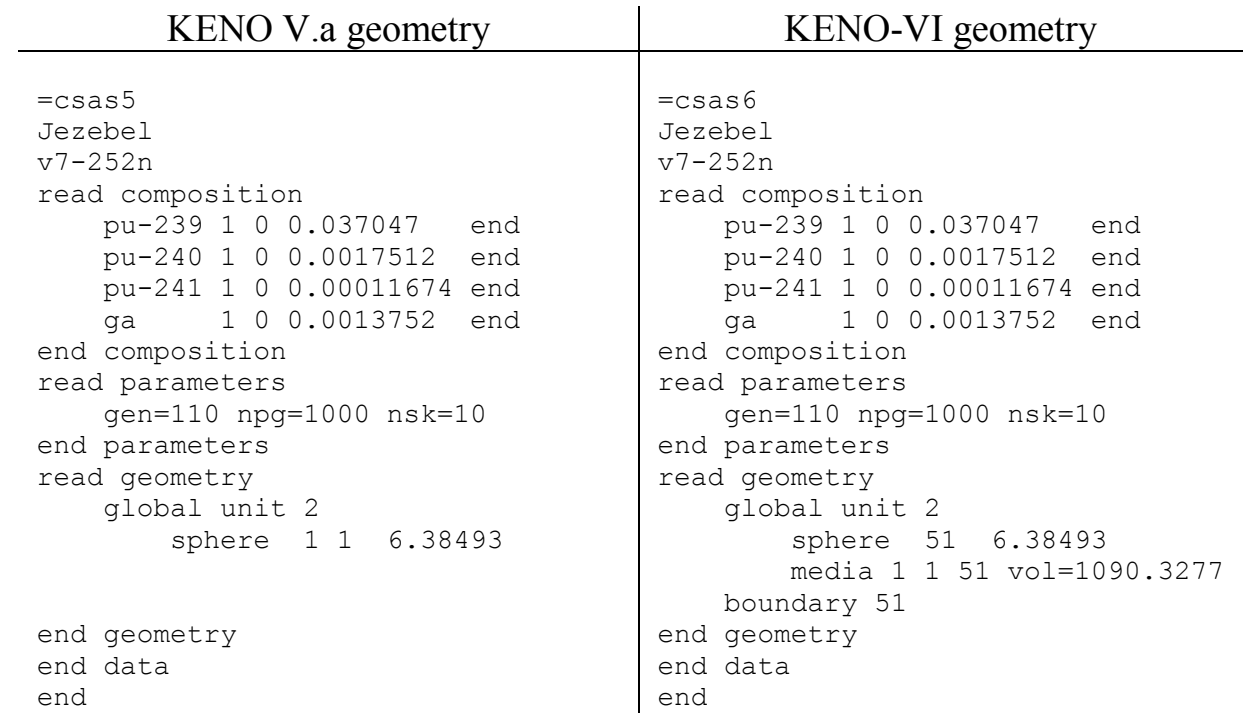

The above inputs can be easily changed into the following Sourcerer inputs (with geometry additions in blue and extra Sourcerer input in green).

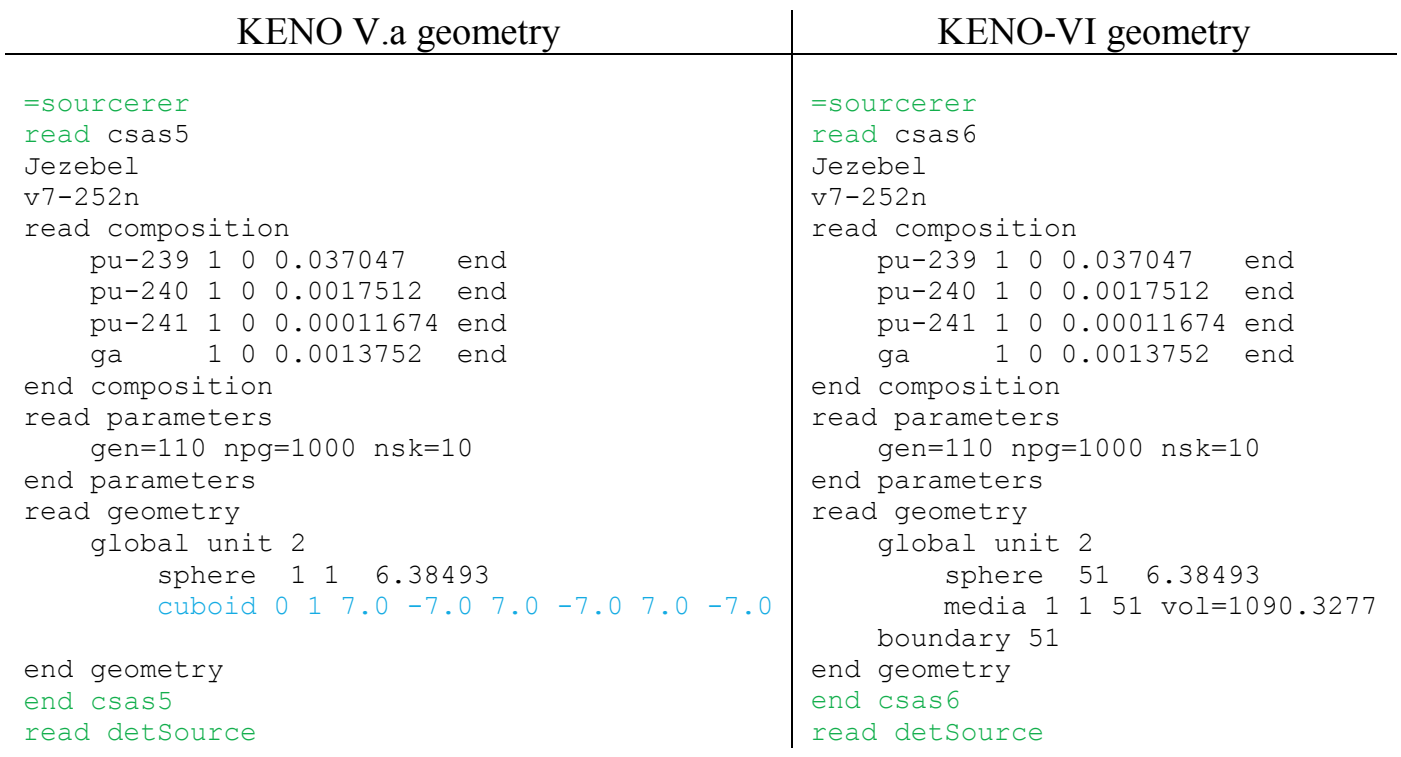



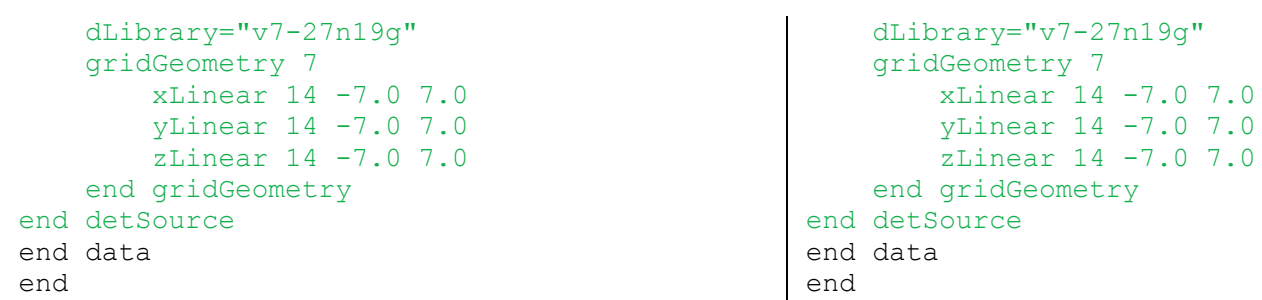

With either of these variations of the Jezebel problem, the fission source distribution can be tallied by KENO and saved to a mesh tally (*.3dmap) file by adding the following to the input:

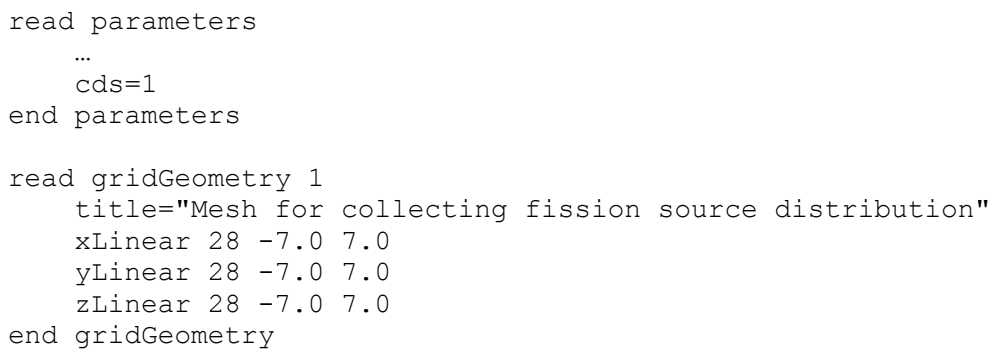

Note that the mesh grid used for the KENO mesh tally can be different from the mesh grid used by Denovo to create a starting source in the Sourcerer sequence. Also note that more total histories (more particles per generation or more active generations) would be required to produce a KENO fission source tally with low relative uncertainties in every voxel.

\subsection{Output File}

The results for the standard CSAS calculations and the Sourcerer results are shown in Table 2.4.8 for calculations with the 252-energy-group and continuous-energy cross sections.

Table 2.4.8. Eigenvalue results for the Jezebel problem

\begin{tabular}{ll|cc}
\hline & Sample Problem & CSAS & Sourcerer \\
\hline 1. & KENO V.a geometry, v7-252 & $1.0045 \pm 0.0017$ & $1.0054 \pm 0.0018$ \\
2. & KENO-VI geometry, v7-252 & $0.9998 \pm 0.0018$ & $1.0007 \pm 0.0020$ \\
3. & KENO V.a geometry, ce_v7 & $1.0058 \pm 0.0027$ & $1.0026 \pm 0.0017$ \\
3. & KENO-VI geometry, ce_v7 & $0.9990 \pm 0.0023$ & $1.0041 \pm 0.0016$ \\
\hline
\end{tabular}

The Denovo fission source provides a reliable starting source that is similar to the actual fission source distribution computed by KENO (using npg=250000), as shown in Figure 2.4.2. 

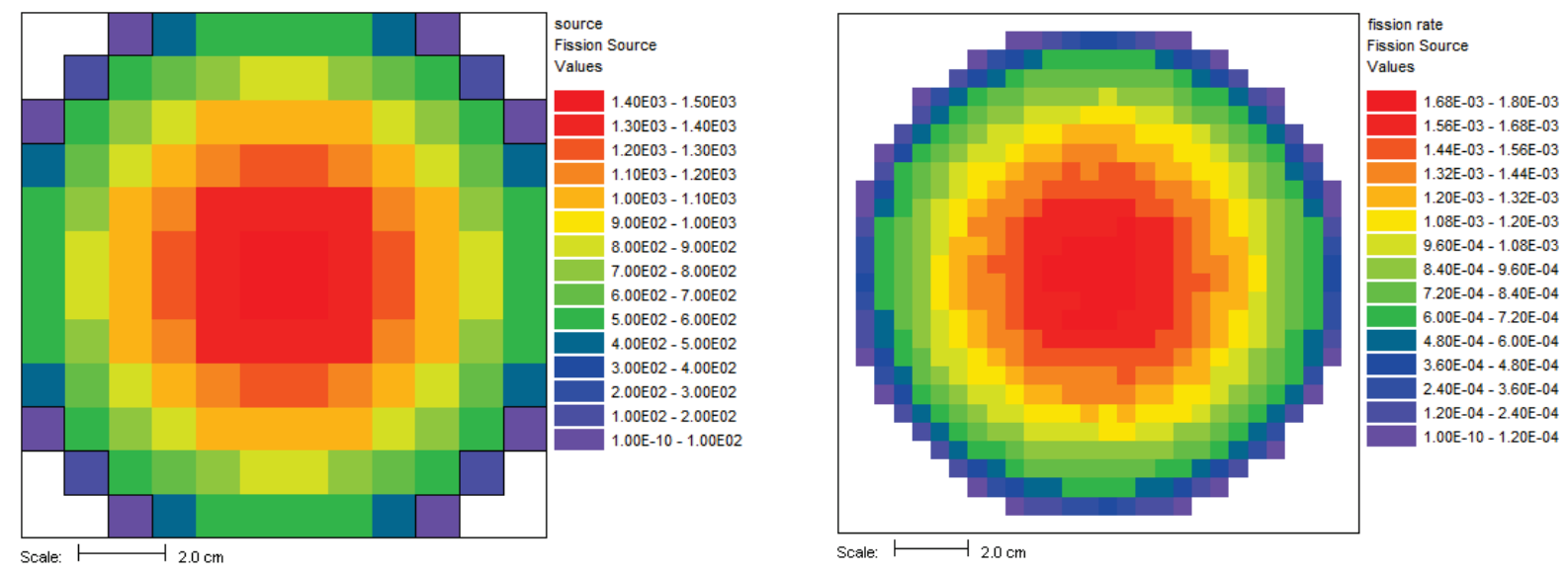

Figure 2.4.2. Fission source distribution from Denovo (left) and KENO (right).

\subsection{Other Variations}

To increase the Denovo calculation speed (but decrease the fidelity of the fission source result), the discretization in angle (quadrature= or polarsPerOct=/azimuthsPerOct=) can be coarsened and/or the tolerance parameters can be loosened. To reduce the amount of memory required by Denovo, the number of Legendre moments in the scattering cross-section expansion can be reduced (legendre=). Using macromaterials can help increase the fidelity of the Denovo calculation with only a small increase in model setup time. Macromaterials do not impact the Denovo run time. Denovo diagnostic messages can be turned on and will print to the messages file.

\begin{tabular}{|c|c|c|c|}
\hline $\begin{array}{l}\text { Macromaterials } \\
\text { (higher fidelity) }\end{array}$ & $\begin{array}{l}\text { Looser tolerances } \\
\text { (faster Denovo) }\end{array}$ & Screen messages & $\begin{array}{l}\text { Higher fidelity } \\
\text { (slower Denovo) }\end{array}$ \\
\hline read detSource & read detSource & read detSource & read sequence \\
\hline$\ldots$ & $\ldots$ & $\ldots$ & $\ldots$ \\
\hline $\begin{array}{r}\text { macromaterial } \\
\text { mmSubCell=3 }\end{array}$ & eigenValParams & eigenValParams & eigenValParams \\
\hline $\begin{array}{l}\text { mmTolerance }=0.001 \\
\text { end macromaterial }\end{array}$ & & & $\ldots$ \\
\hline end detSource & $\begin{array}{l}\text { tolerance }=1.0 \mathrm{e}-2 \\
\text { kTolerance }=1.0 \mathrm{e}-3 \\
\text { end eigenValParams } \\
\text { end detSource }\end{array}$ & $\begin{array}{l}\text { diagnostics=1 } \\
\text { output=1 } \\
\text { diagnosticLevel=1 } \\
\text { end eigenValParams } \\
\text { end detSource }\end{array}$ & $\begin{array}{l}\text { quadType }=2 \\
\text { polarsPerOct }=4 \\
\text { azimuthsPerOct }=4 \\
\text { legendre }=3\end{array}$ \\
\hline & & & $\begin{array}{l}\text { end eigenValParams } \\
\text { end sequence }\end{array}$ \\
\hline
\end{tabular}

Sample problems sourcerer.smp101.inp, sourcerer.smp102.inp, sourcerer.smp103.inp, and sourcerer.smpl04.inp in the SCALE sample problems directory are the four cases for Jezebel using combinations of KENO V.a/KENO-VI geometry and v7-252 multi-group/continuous-energy v7 cross sections. 


\subsubsection{References}

1. C. E. Shannon, "A Mathematical Theory of Communication," The Bell System Technical Journal 27, pp. 379-423, July; 623-656, October 1948.

2. T. Ueki and Forrest B. Brown, "Stationarity and Source Convergence Diagnostics in Monte Carlo Criticality Calculation," Nuclear Mathematical and Computational Sciences Conference (M\&C 2003), Gatlinburg, TN, April 6-10, 2003.

3. T. Ueki and F. B. Brown, "Stationarity Modeling and Informatics-Based Diagnostics in Monte Carlo Criticality Calculations," Nuclear Science and Engineering 149, 38-50 (2005).

4. A. M. Ibrahim, D. E. Peplow, J. C. Wagner, S. W. Mosher, and T. M. Evans, "Acceleration of Monte Carlo Criticality Calculations Using Deterministic-Based Starting Sources," Transactions of the American Nuclear Society 105, 539-541 (2011).

5. A. M. Ibrahim, D. E. Peplow, J. C. Wagner, S. W. Mosher, and T. M. Evans, "Acceleration of Monte Carlo Criticality Calculations Using Deterministic-Based Starting Sources," Physor 2012, Knoxville, TN, April 15-20, 2012.

6. A. M. Ibrahim, D. E. Peplow, K. B. Bekar, C. Celik, D. Ilas, J. M. Scaglione, and J. C. Wagner, "Hybrid Technique in SCALE for Fission Source Convergence Applied to Used Nuclear Fuel Analysis," in preparation for the 2013 Topical Meeting on Nuclear Criticality Safety (NCSD 2013), Wilmington, NC, September 29 - October 3, 2013.

7. T. M. Evans, A. S. Stafford, R. N. Slaybaugh, and K. T. Clarno, "Denovo: A New Three-Dimensional Parallel Discrete Ordinates Code in SCALE,” Nuclear Technology 171, 171-200 (2010).

8. S. Goluoglu, L. M. Petrie, Jr., M. E. Dunn, D. F. Hollenbach, and B. T. Rearden, "Monte Carlo Criticality Methods and Analysis Capabilities in SCALE," Nuclear Technology 174, 214-335 (2011).

9. B. C. Kiedrowski and C. J. Solomon, "Statistical Assessment of Numerous Monte Carlo Tallies," International Conference on Mathematics and Computational Methods Applied to Nuclear Science and Engineering (M\&C 2011), Rio de Janeiro, RJ, Brazil, May 8-12, 2011, on CD-ROM. 


\subsection{A DEVC: Denovo EigenValue Calculation}

Douglas E. Peplow and Cihangir Celik

\subsection{A.1 Introduction}

The DEVC (Denovo EigenValue Calculation) sequence is an interface to the Denovo discrete-ordinates package $^{1}$ for calculating criticality eigenvalue problems. This sequence reads an input file very similar to a CSAS6 input file ${ }^{2}$ that contains an extra block of input for describing the Denovo mesh grid and calculational parameters. Many of the subroutines are shared from the MAVRIC routines that interface with Denovo for fixed-source calculations.

This manual assumes that the user is familiar with the discrete-ordinates method for radiation transport and the Denovo package. DEVC provides an easy way for users to modify existing CSAS6 inputs and use them to run Denovo. The DEVC sequence also provides a way to create mesh geometry for Denovo from the combinatorial solid geometry description used by KENO-VI.

The steps in the DEVC sequence are listed in Table 2.4.A.1.

Table 2.4.A.1. Steps in DEVC for an input file named input.inp

\begin{tabular}{|c|c|c|c|}
\hline Step & Module/Task & Creates file & To stop after \\
\hline 0 & Check user input & & \\
\hline 1 & Self-shielding (celldata/cellmix) calculations & & \\
\hline 2 & $\begin{array}{l}\text { Produces optional *.png plots } \\
\text { Produces optional *.3mdap files (to visualize grid in } \\
\text { MeshFileViewer) }\end{array}$ & & parm $=$ check \\
\hline 3 & Creates AMPX cross sections for the "real" materials & fto $2 £ 001$ & parm $=$ cross \\
\hline 4 & $\begin{array}{l}\text { Creates Denovo binary stream input file and the } \\
\text { macromaterial table file }\end{array}$ & $\begin{array}{l}\text { xkba_b.inp } \\
\text { input.mmt }\end{array}$ & parm=input \\
\hline 5 & $\begin{array}{l}\text { Runs Denovo to compute } k_{\text {eff }} \text { and either the fluxes or the } \\
\text { fission source }\end{array}$ & $\begin{array}{l}\text { input.dff } \\
\text { or } \\
\text { input.dso }\end{array}$ & \\
\hline
\end{tabular}

The DEVC sequence uses KENO-VI geometry. Users can specify what output Denovo will generate: fluxes by space and energy in a binary *.dff (Denovo flux file) file or the space-only fission source distribution in a binary *.dso (Denovo spatial output) file. The eigenvalue is printed in the main output text file.

Some of the more common KENO starting source types are supported. Other starting source types may be added or extended to all of the different array types in the future. Currently, starting sources are not sent to Denovo because the Arnoldi solver does not use it. This may change in the future. 


\subsection{A.2 Sequence Input}

The input file for a DEVC calculation looks similar to a CSAS6 input file, as shown in Table 2.4.A.2. The major difference is that the parameter block contains information for the Denovo calculation, not the KENO Monte Carlo calculation. A macromaterial block is used to describe how the KENO-VI materials are mapped onto the Denovo mesh grid. Only multi-group cross-section libraries can be used with Denovo.

Table 2.4.A.2. Input file for a DEVC calculation (and differences with a CSAS6 input file, where black text is the same as CSAS6 and green text is new for DEVC sequence)

\begin{tabular}{|c|c|}
\hline $\begin{array}{l}\text { =deve parm }=. . . \\
\text { Title for the problem }\end{array}$ & $\begin{array}{l}\text { Parm: check, cross, input } \\
\text { Multi-groun cross-section library name }\end{array}$ \\
\hline $\begin{array}{l}\text { read } x x x \\
\text { end } x x x\end{array}$ & $\begin{array}{l}\text { Standard CSAS6 blocks used by DEVC: composition, } \\
\text { celldata, geometry, array, plot and gridGeometry. }\end{array}$ \\
\hline $\begin{array}{l}\text { read bounds } \\
\text { end bounds }\end{array}$ & Bounds are used if the boundary of the global unit is a cuboid. \\
\hline $\begin{array}{l}\text { read start } \\
\text { end start }\end{array}$ & $\begin{array}{l}\text { Most KENO start types are supported for some array types. } \\
\text { Currently, Denovo does not make use of a starting source. }\end{array}$ \\
\hline $\begin{array}{l}\text { read } x \times x \\
\text { end } x \times x\end{array}$ & $\begin{array}{l}\text { Not used by DEVC: volume, x1ds, energy, biasing, } \\
\text { importance, reactions, and search }\end{array}$ \\
\hline $\begin{array}{l}\text { read parameters } \\
\text { gridGeometry } 7 \\
\quad \cdots \\
\text { end gridGeometry }\end{array}$ & $\begin{array}{l}\text { Denovo discrete-ordinates parameters } \\
\text { Mesh grid - list of planes in each dimension }\end{array}$ \\
\hline $\begin{array}{l}\text { quadType }=2 \\
\text { polarsperoct }=4 \\
\text { azimuthsperoct }=4 \\
\text { ktolerance }=1.0 e-5\end{array}$ & $\begin{array}{l}\text { List of quadrature order, Legendre order, upscatter, and } \\
\text { other eigenvalue calculation parameters }\end{array}$ \\
\hline $\begin{array}{l}\quad \cdots \\
\text { xmin }=0 \\
\cdots \\
\text { fissionSource } \\
\text { end parameters }\end{array}$ & $\begin{array}{l}\text { Boundary conditions - these override conditions listed in } \\
\text { the bounds block } \\
\text { Save fission source instead of fluxes }\end{array}$ \\
\hline $\begin{array}{l}\text { read macromaterial } \\
\text { mmSubCell }=3 \\
\text { mmTolerance }=0.001 \\
\text { end macromaterial }\end{array}$ & How to create materials for each voxel in the mesh grid \\
\hline $\begin{array}{l}\text { read } x \times x \\
\text { end } x \times x\end{array}$ & \\
\hline $\begin{array}{l}\text { end data } \\
\text { end }\end{array}$ & \\
\hline
\end{tabular}

\subsection{A.2.1 Parameters Block}

This block contains the parameters for the Denovo eigenvalue calculation, the grid geometry, and the macromaterials. Boundary conditions listed in the parameters block will override those listed in the bounds block (using CSAS6 syntax). Table 2.4.A.3 lists the Denovo calculation parameters and their default values, and Table 2.4.A.4 lists the keywords for the setting the boundary conditions and file 
saving options. The grid geometry is defined in a sub-block in the parameters block, or the keyword "gridGeometryID=n" can be used to point to a grid geometry defined in its own input block.

Table 2.4.A.3. Denovo parameters in the parameters block

\begin{tabular}{|c|c|c|c|}
\hline keyword & type & default & restrictions/comments \\
\hline \multicolumn{4}{|l|}{ read parameters } \\
\hline discretization= & integer & 4 & $\begin{array}{l}\text { 0-diamond difference, 1-DD with flux fix-up:lin0, } \\
\text { 2-theta-weighted DD, 3-linear discontinuous finite element, } \\
\text { 4-step characteristics, 5-trilinear discontinuous finite element }\end{array}$ \\
\hline quadType= & integer & 2 & 0-level symmetric, 1-Gauss-Legendre product, 2-QR \\
\hline legendre $=$ & integer & $3^{*}$ & $P_{L}, L=$ highest Legendre polynomial, $L=0,1,2,3, \ldots$ \\
\hline \multirow[t]{2}{*}{ tportcorrection= } & integer & 1 & $\begin{array}{l}\text { *default is to use min(the highest available in the data,3) } \\
\text { transport correction: } 0 \text {-none, 1-diagonal, 2-Cesaro }\end{array}$ \\
\hline & & & ${ }^{*} \mathrm{P}_{2}$ or higher is required for Cesaro \\
\hline upScatter= & integer & 1 & upscatter iterations: 0-none, 1-yes, 2-ignore \\
\hline xblocks $=$ & integer & 1 & parallel calcs - how many divisions in $x$ \\
\hline yblocks = & integer & 1 & parallel calcs - how many divisions in y \\
\hline zblocks= & integer & 1 & parallel calcs - how many commication layers in z \\
\hline numSets $=$ & integer & 1 & parallel calcs - how many energy sets \\
\hline partUpscatter= & integer & 1 & partition upscatter (0-no, 1-yes) \\
\hline quadrature $=$ & integer & 8 & level symmetric $S_{N}$ quadrature, $N=2,4,6,8,10,12,14,16$ \\
\hline polarsPerOct $=$ & integer & 3 & Gauss-Legendre product quadrature or QR \\
\hline azimuthsPerOct $=$ & integer & 3 & Gauss-Legendre product quadrature or $\mathrm{QR}$ \\
\hline maxlters $=$ & integer & 1000 & maximum number of iterations \\
\hline diagnostics $=$ & integer & 0 & 0-no diganostics, 1-all diagnostics \\
\hline output $=$ & integer & 0 & 0-no ouput, 1-all output \\
\hline krylovSpaceSize= & integer & 25 & size in memory for Krylov space \\
\hline tolerance $=$ & double & 1.E-04 & tolerance used in convergence test \\
\hline krylovType= & integer & 0 & 0-GMRES, 1-BiCGStab \\
\hline eigenSolver= & integer & 1 & 0-power iteration, 1-Arnoldi, 2-shifted inverse \\
\hline multiGSolover= & integer & 1 & 0-Gauss-Seidel, 1-Krylov \\
\hline withinGSolver= & integer & 0 & 0-Krylov, 1-residual Krylov, 2-source iteration \\
\hline mgSettings $=$ & integer & 0 & 0-user supplied, 1-automatic by Denovo \\
\hline upGroupSolver= & integer & 0 & $\begin{array}{l}\text { 0-same as within-group solver, 1-Krylov, 2-residual Krylov, } \\
\text { 3-source iteration, 4-single source iteration }\end{array}$ \\
\hline acceleration $=$ & integer & 0 & 0-none, 1-two grid \\
\hline maxItersMG= & integer & 1000 & maximum number of iterations \\
\hline toleranceMG= & double & 1.E-04 & tolerance used in convergence test \\
\hline keff= & double & 1 & starting guess of $k$ \\
\hline kTolerance $=$ & double & 1.E-05 & tolerance on $\mathrm{k}$ \\
\hline diagnosticLevel= & integer & 0 & 0-off, 1-on \\
\hline eigenSettings $=$ & integer & 0 & 0-user supplied, 1 -automatic by Denovo \\
\hline I2Tolerance $=$ & double & 0.01 & tolerance on the L-2 norm \\
\hline linfTolerance= & double & 0.001 & tolerance on L-inf \\
\hline powerlterAcc $=$ & integer & 0 & 0-none, 1-rebalance, 2-RQI \\
\hline arnoldiKSpace= & integer & 25 & \\
\hline arnoldiRestarts $=$ & integer & 100 & \\
\hline $\begin{array}{l}\text { calcMoments }= \\
\text { end parameters }\end{array}$ & integer & 1 & 0-off, 1-on \\
\hline
\end{tabular}


Table 2.4.A.4. Boundary conditions and what type of file to save

\begin{tabular}{llll}
\hline keyword & type & default & restrictions/comments \\
\hline read parameters & & & \\
xmin $=$ & integer & 0 & boundary conditions on the six faces: \\
xmax $=$ & integer & 0 & 0 - vacuum \\
ymin $=$ & integer & 0 & 1 - reflective \\
ymax $=$ & integer & 0 & 2- periodic \\
zmin= & integer & 0 & 3 - white \\
zmax $=$ & integer & 0 & (these will override the "read bounds" block if specified here.) \\
fluxes & & & save space/energy fluxes (default) \\
fissionSource & & & save the space-only fission source \\
end parameters & & &
\end{tabular}

\subsection{A.2.2 Grid Geometry Block}

Grid geometries ("gridGeometry $i d$ ") require an identification number and then a description of a threedimensional rectangular mesh by specifying the bounding planes of the cells in each of the $x, y$, and $z$ dimensions. The keyword "xPlanes ... end" can be used to list plane values (in any order). The keyword "xLinear $n a b$ " can be used to specify $n$ cells between $a$ and $b$. The keywords "xPlanes" and "xLinear" can be used together and multiple times - they will simply add planes to any already defined for that dimension. Any duplicate planes will be removed. Similar keywords are used for the $y$ and $z$ dimensions.

When using multiple instances of the keywords * Linear and ${ }^{*} \mathrm{Pl}$ lanes for a given dimension, duplicates should be removed from the final list. In some cases, double precision math will leave two planes that are nearly identical but not removed (e.g., 6.0 and 5.9999999). To prevent this, a default tolerance is set to remove planes that are within $10^{-6} \mathrm{~cm}$ of each other. The user is free to change this by using the keyword "tolerance=" and specifying something else. Note that the tolerance can be reset to a different value in between each use of *inear or *Planes.

The keyword "make3dmap" for a particular grid geometry definition will create a file called "outputName.gridid.3dmap", which can be visualized using the Java Mesh File Viewer. These files will contain crude geometry information (unit, region, material) that corresponds to the center of each voxel.

Keywords for the grid geometry block are listed in Table 2.4.A.5. 
Table 2.4.A.5. Grid geometry input keywords

\begin{tabular}{|c|c|c|}
\hline keyword & type & restrictions/comments \\
\hline \multicolumn{3}{|l|}{ read parameters } \\
\hline $\begin{array}{l}\text { gridGeometry id } \\
\text { title= }\end{array}$ & character & $\begin{array}{l}\text { covers a cuboid where all points are in defined regions } \\
\text { contained in double auotes (title="vada vada vada") }\end{array}$ \\
\hline$x$ planes $\ldots$ end & real array & a list of $x$ plane values (order not important) \\
\hline$x$ Linear $n$ min $\max$ & int real real & adds $n+1 \times$ planes ( $n$ cells) evenly from min to max \\
\hline$y$ planes $\ldots$ end & real array & a list of $y$ plane values (order not important) \\
\hline$y$ Linear $n \min \max$ & int real real & adds $n+1$ y planes ( $n$ cells) evenly from min to max \\
\hline z planes ... end & real array & a list of $z$ plane values (order not important) \\
\hline$z$ Linear $n \min \max$ & int real real & adds $n+1 z$ planes ( $n$ cells) evenly from min to max \\
\hline $\begin{array}{l}\text { tolerance }= \\
\text { make3dmap }\end{array}$ & real & $\begin{array}{l}\text { for removing duplicate planes from }{ }^{*} \text { planes or }{ }^{*} \text { Linear } \\
\text { makes a }{ }^{*} .3 \mathrm{dmap} \text { file showing the grid geometry }\end{array}$ \\
\hline xdivide $=$ & integer & once all xplanes are entered, further divide them by this \\
\hline ydivide $=$ & integer & once all yplanes are entered, further divide them by this \\
\hline zdivide $=$ & integer & once all zplanes are entered, further divide them by this \\
\hline \multicolumn{3}{|l|}{ end gridGeometry } \\
\hline gridGeometry $I D=$ & integer & $\begin{array}{l}\text { Either define a grid geoemtry in a sub block or refer to } \\
\text { a grid geometry defined in its own block }\end{array}$ \\
\hline end parameters & & \\
\hline
\end{tabular}

\subsection{A.2.3 Macromaterial Block}

In order to get more accurate solutions from a coarse-mesh discrete-ordinates calculation, Denovo can represent the material in each voxel of the mesh as a volume-weighted mixture of the real materials in the problem. When constructing the Denovo input, DEVC can estimate the volume fraction taken by each real material in each voxel by a sampling method. The user can specify parameters for how to sample the geometry. Note that finer sampling makes more accurate estimates of the material fraction but requires more setup time to create the Denovo input. Users should understand how the macromaterials are sampled and consider that when constructing a mesh grid. This is especially important for geometries that contain arrays. Careful consideration should be given when overlaying a mesh on a geometry that contains arrays of arrays.

Because the list of macromaterials could become large, the user can also specify a tolerance for how close two different macromaterials can be to be considered the same, thereby reducing the total number of macromaterials. The macromaterial tolerance, "mmTolerance=", is used for creating a different macromaterial from the ones already created by looking at the infinity norm between two macromaterials. The number of macromaterials does not appreciably impact Denovo run time or memory requirements.

Keywords for the macromaterial block are listed Table 2.4.A.6. Two different sampling methods are available - ray tracing ${ }^{3}$ with the keyword mmRayTest and point testing ${ }^{4}$ with the keyword mmPointTest. 
Table 2.4.A.6. Macromaterial block input

\begin{tabular}{|c|c|c|c|c|}
\hline keyword & type & length & default & restrictions/comments \\
\hline $\begin{array}{c}\text { read macromaterial } \\
\text { mmSubCell= } \\
\text { mmTolerance }=\end{array}$ & $\begin{array}{l}\text { integer } \\
\text { real }\end{array}$ & & $\begin{array}{r}1 \\
0.01\end{array}$ & $\begin{array}{l}\text { rays per dimension to throw at each voxel } \\
\text { smallest volume fraction for macromaterial }\end{array}$ \\
\hline mmSubCells & integer & 6 & & rays per dimension to throw (x:ny,nz; y:nx,nz; z: nx,ny) \\
\hline $\begin{array}{l}\text { mmPoint Test } \\
\text { mmRayTest }\end{array}$ & & & & $\begin{array}{l}\text { use recursive bisection point testing method } \\
\text { use ray tracing method }\end{array}$ \\
\hline $\begin{array}{c}\text { mmRTSpeed } \\
\text { mmRTMemory } \\
\text { end macromaterial }\end{array}$ & & & & $\begin{array}{l}\text { optimize ray-tracing method for speed } \\
\text { optimize ray-tracing method for memory conservation }\end{array}$ \\
\hline
\end{tabular}

\subsection{A.2.3.1 Ray Tracing}

This method estimates the volume of different materials in the Denovo mesh grid elements by throwing rays through the KENO-VI geometry and computing the average track lengths through the each material. Rays are traced in all three dimensions to better estimate the volume fractions of materials within each voxel. The mmSubCell parameter controls how many rays to trace in each voxel in each dimension. For example, if mmSubCell $=n$, then when tracing rays in the $z$ dimension, each column of voxels uses a set of $n \times n$ rays starting uniformly spaced in the $x$ and $y$ dimensions. With rays being cast from all three orthogonal directions, then a total of $3 n^{2}$ rays are used to sample each voxel. One can think of subcells as an equally spaced sub-mesh with a single ray positioned at each center. The number of subcells in each direction, and hence the number of rays, can be explicitly given with mmsubcells ny $\mathrm{nz} \mathrm{nx} \mathrm{nz} \mathrm{nx}$ ny end keyword for rays parallel to the $x$ axis, $y$ axis, and $z$ axis. Figure 2.4.A.1 shows different subcell configurations (in two dimensions) for a given voxel.

Ray tracing is a more robust method compared to the simple point testing method used in previous versions of SCALE/MAVRIC; however, it requires more

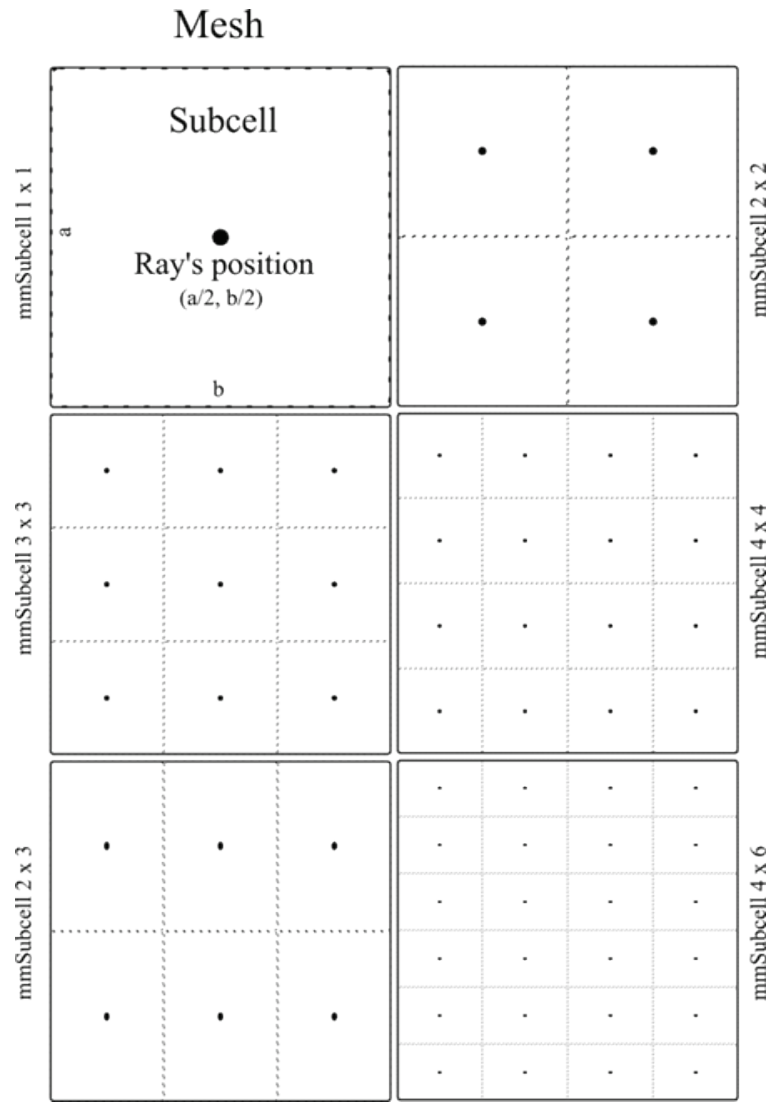

Figure 2.4.A.1. Ray positions within a voxel with different mmSubCells parameters.

memory than point testing. Ray tracing gives more accurate estimates of volume fractions because track lengths across a voxel give more information than a series of test points. Ray tracing is also much faster than point testing because the particle tracking routines are optimized for quickly determining lists of materials and distance along a given ray. 
Ray tracing operates on the grid geometry supplied by the user and shoots rays in all three directions starting from the lower bounds of the mesh grid. An example of arbitrary assembly geometry is shown in Figure 2.4.A.2. A ray consists of a number of steps that corresponds to crossing a different material along the path of the ray. Ratios of each step's length to the voxel length in the ray's direction determine the material volume fraction of that step in that voxel, and summation of the same material volume fractions gives the material volume fraction of that material in that voxel. Ray tracing through a single voxel that contains a fuel pin is illustrated in Figure 2.4.A.3.
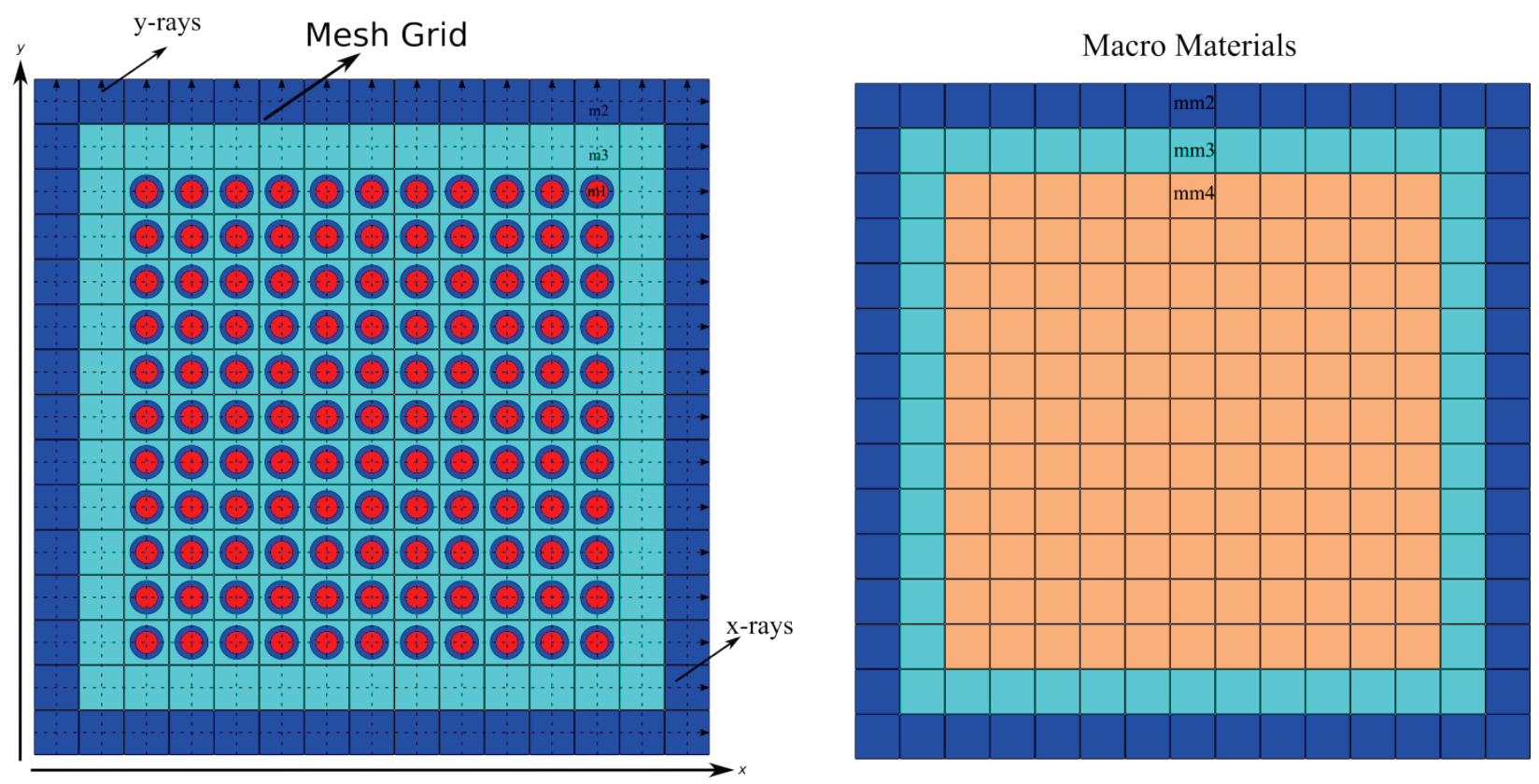

Figure 2.4.A.2. Geometry model (left) and the Denovo representation (right) of an assembly using macromaterials determined by ray tracing.

The final constructed macromaterials for this model are also shown in Figure 2.4.A.2. Voxels that contain only a single material are assigned that original material number in the constructed macromaterials. For the voxels that contain a fuel pin with three different materials, the result is a new macromaterial consisting of the volume weighted fractions of each original material.

After the rays are shot in all three directions, the material volume fractions are updated and macromaterials are created by using these material volume fractions. Material volume fraction calculations for a single voxel, as shown in Figure 2.4.A.3, are given by

$$
F_{m}=\sum_{d=x, y, z} \sum_{r=1}^{N_{r}} \sum_{s=1}^{N_{s}}\left\{\begin{array}{cc}
\frac{L_{d, r, s}}{L_{d}}, & m_{s}==m \\
0, & \text { otherwise }
\end{array} \quad \text { and } \quad V_{m}=\frac{F_{m}}{\sum_{n=1}^{N_{m}} F_{n}},\right.
$$

where $F_{m}=$ sampled fraction of material $m$ in the voxel,

$d=$ direction of the rays $(x, y, z)$,

$r=$ ray number,

$N_{r}=$ total number of rays in the voxel for direction of $d$, 
$s=$ step number,

$N_{s}=$ total number of steps for ray $r$ in the voxel for direction of $d$,

$L_{d, r, s}=$ length of the steps $s$ for ray $r$ in the voxel for direction of $d$,

$L_{d}=$ length of the voxel along direction of $d$,

$m_{s}=$ material of step $s$,

$m=$ material number,

$N_{m}=$ total number of materials in the voxel, and

$V_{m}=$ volume fraction of material $m$ in the voxel.

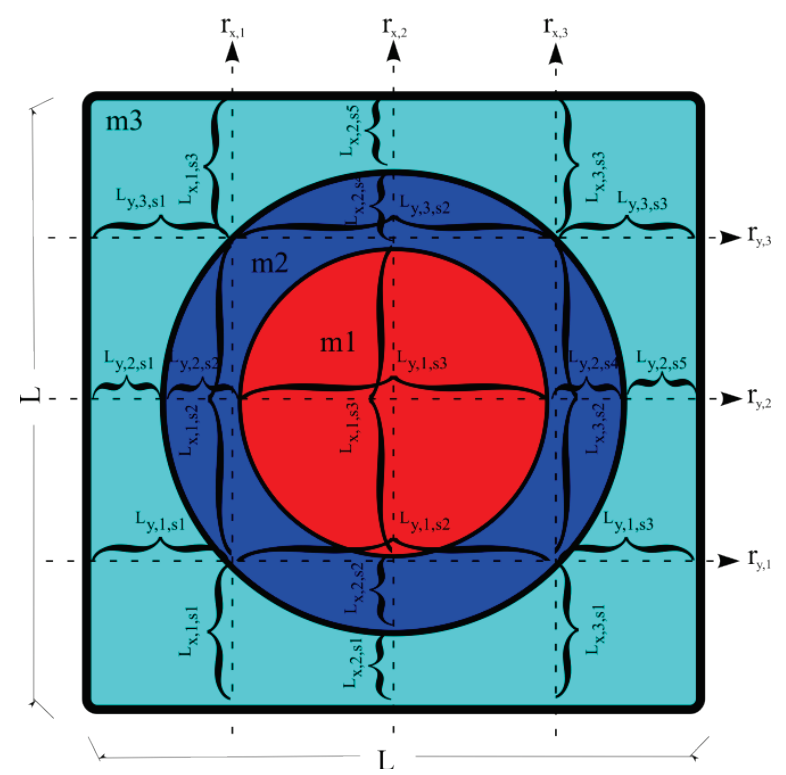

Figure 2.4.A.3. Ray tracing (in two dimensions) through a voxel.

\subsection{A.2.3.2 Point Testing}

The recursive bisection method uses a series of point tests to determine the macromaterial fractions. For a given voxel, the material at the center is compared to the material at the eight corners. If they are all the same, the entire volume is considered to be made of that material. If different, the volume is divided into two in each dimension. Each subvolume is tested, and the method is then applied to the subvolumes that are not of a single material. When the ratio of the volume of the tested region to the original voxel becomes less than a user-specified tolerance (in the range of $10^{-1}$ to $10^{-4}$ ), then further subdivision and testing are stopped. This is illustrated in Figure 2.4.A.4.
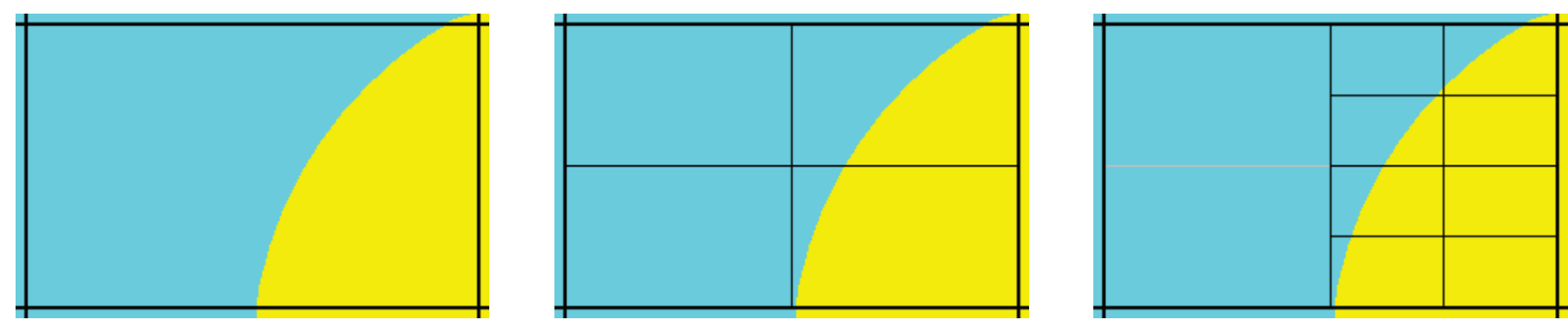

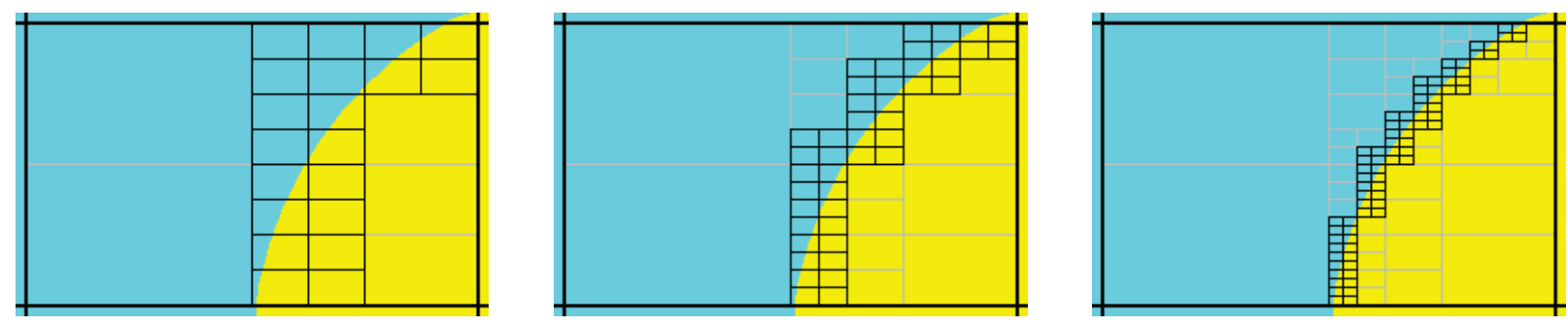

Figure 2.4.A.4. Progression of the recursive bisection method (from upper left to lower right).

In point testing, the keyword "mmTolerance $f$ " is interpreted to be where $f$ is the smallest fraction of the voxel volume to consider. This same tolerance $f$ is also used to limit the number of macromaterials. Before a new macromaterial is created, if one already exists where the fraction of each actual material matches to within the given tolerance, then the existing material will be used. If using only a single point at the center of each voxel, use "mmTolerance=1". The mmSubCell keyword is not used in point testing.

\subsection{A.2.3.3 Example}

Figure 2.4.A.5 shows an example of a cask geometry with two types of spent fuel (yellows), steel (blue), resin (green), and other metals (gray). When the Denovo geometry is set up by testing only the center of each mesh cell, the curved surfaces are not well represented (upper right). By applying the ray-tracing method and defining a new material made of partial fractions of the original materials, an improved Denovo model can be made. In the lower left of the figure, the Denovo model was constructed using one ray (in each dimension) per voxel and a tolerance of 0.1 . This gives 20 new materials that are a mixture of the original 13 actual materials and void. With mmsubCells=3 and an mmTolerance=0.01, 139 macromaterials are created.

A macromaterial table listing the fractions of each macromaterial is saved to a file called "outputName.mmt", where outputName is the name the user chose for his or her output file. This file can be used by the Mesh File Viewer to display the macromaterials as mixtures of the actual materials, as seen in lower row of Figure 2.4.A.5. See the Mesh File Viewer help pages for more information on how to use colormap files and macromaterial tables. 

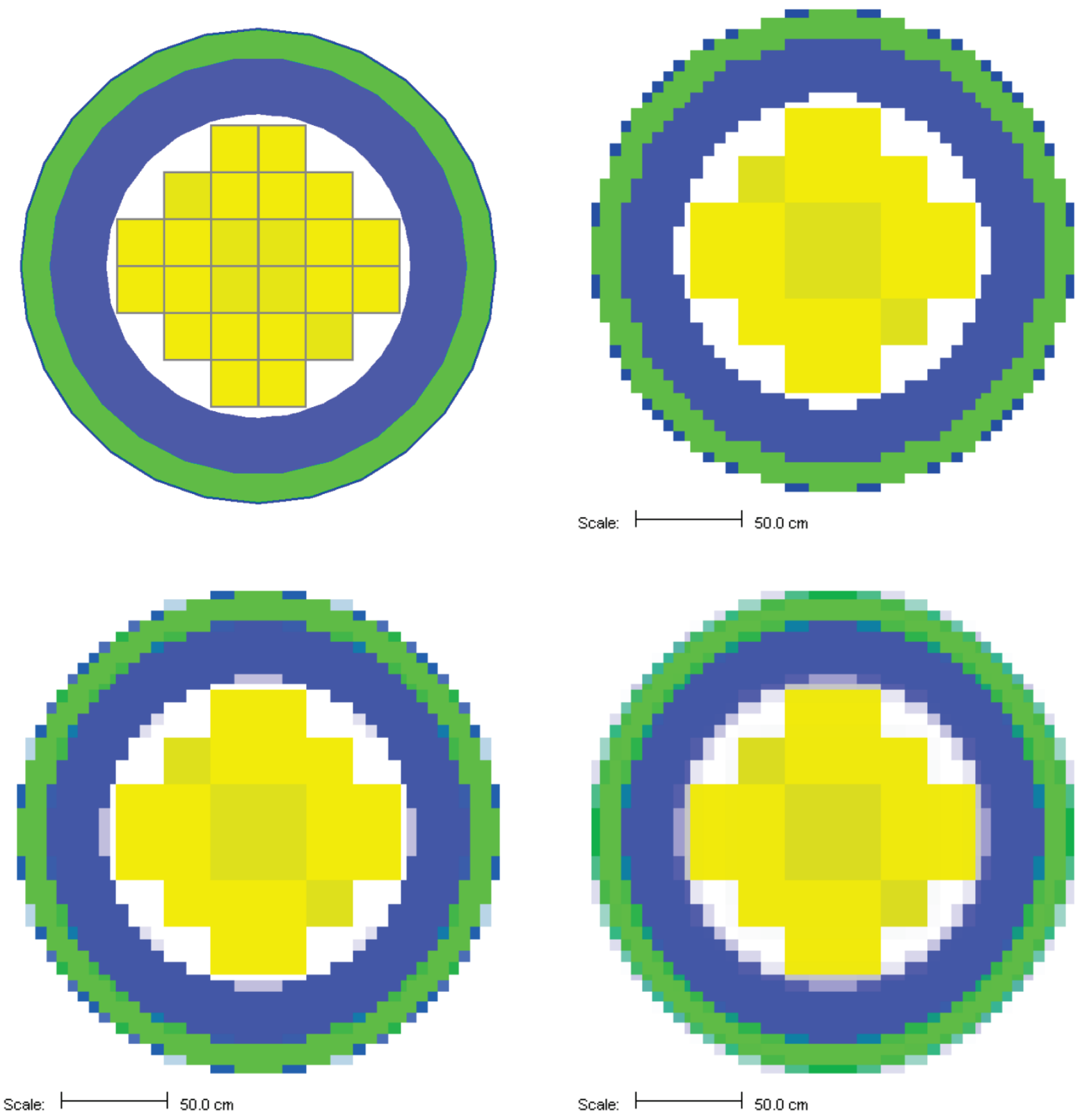

Figure 2.4.A.5. Cask geometry model (upper left) and the Denovo representation using (upper right) cell center testing. Representations using macromaterials determined by ray tracing are shown for (lower left) $\mathrm{mmSubCell}=1 / \mathrm{mm}$ Tolerance $=0.1$ and (lower right) $\mathrm{mmSubCell}=3 / \mathrm{mm}$ Tolerance $=0.01$.

\subsection{A.2.4 Starting Sources Block}

The default KENO-VI starting source is "flat over the volume specified by the unrotated, untranslated geometry record specified in the first position of the global unit boundary record in fissile material only". For DEVC, the default starting source strength is uniform in the fissile voxels contained within the bounding box of the global unit (uniform density). If macromaterials are used, the amounts in each voxel 
are volume averaged between fissile and non-fissile materials. Table 2.4.A.7 and Table 2.4.A.8 describe the starting sources available in the DEVC interface to Denovo.

Table 2.4.A.7. Starting source types (within the fissile areas of the listed shape)

\begin{tabular}{|c|c|c|}
\hline & KENO-VI start type & DEVC \\
\hline nst $=0$ & the first surface of boundary (default) & The bounding box of global unit \\
\hline $\mathrm{nst}=0$ & Within boundary of global array having a reflector key set to false & Not supported \\
\hline nst $=0$ & A cuboid defined by XSM, XSP, YSM, YSP, ZSM, and ZSP & Supported \\
\hline nst $=1$ & $\begin{array}{l}\text { A cuboid defined by XSM, XSP, YSM, YSP, ZSM, and ZSP with } \\
\text { cosine distributions }\end{array}$ & Supported \\
\hline nst $=2$ & $\begin{array}{l}\text { Arbitrary fraction (FCT) in element NXS, NYS, NZS of the global } \\
\text { array with the remainder in a cuboid defined by XSM, XSP, YSM, } \\
\text { YSP, ZSM, and ZSP with cosine distributions }\end{array}$ & $\begin{array}{l}\text { Supported for some array types } \\
\text { (see Table 2.4.A.8) }\end{array}$ \\
\hline nst $=3$ & $\begin{array}{l}\text { At the location TFX, TFY, TFZ in the element NXS, NYS, NZS of the } \\
\text { global array }\end{array}$ & $\begin{array}{l}\text { Supported for some array types } \\
\text { (see Table 2.4.A.8) }\end{array}$ \\
\hline nst $=4$ & At the location TFX, TFY, TFZ in units NBX of the global array & Supported \\
\hline nst $=5$ & Across units NBX in the global array & Not supported \\
\hline nst $=6$ & List of points TFX, TFY, TFZ in global coordinates & Limited to 1 point \\
\hline $\mathrm{nst}=6$ & $\begin{array}{l}\text { List of points TFX, TFY, TFZ in element NXS, NYS, NZS of the } \\
\text { global array }\end{array}$ & $\begin{array}{l}\text { Limited to } 1 \text { point and only for } \\
\text { some array types (see } \\
\text { Table } 2.4 . \text { A.8) }\end{array}$ \\
\hline nst $=7$ & $\begin{array}{l}\text { Flat distributions in } \mathrm{X} \text { and } \mathrm{Y} \text { with }\left[1-\cos ^{2}(\mathrm{z})\right] \text { in } \mathrm{Z} \text { for a cuboid defined } \\
\text { by XSM, XSP, YSM, YSP, ZSM, and ZSP }\end{array}$ & Supported \\
\hline nst $=8$ & $\begin{array}{l}\text { Flat distributions in } \mathrm{X} \text { and } \mathrm{Y} \text { with a segmented distribution in } \mathrm{Z} \text { for a } \\
\text { cuboid defined by XSM, XSP, YSM, YSP, ZSM, and ZSP }\end{array}$ & Not supported \\
\hline nst $=9$ & Use a mesh source lite file & Not supported \\
\hline
\end{tabular}

Table 2.4.A.8. Supported array types for starting sources

\begin{tabular}{ll}
\hline no arrays & supported \\
cuboid & supported \\
hexagonal & supported \\
shexagonal & no \\
rhexagonal & no \\
dodecahedral & no \\
\hline
\end{tabular}

The starting source initialized in Denovo is always a volumetric source - DEVC does not create point sources (which would activate the first collision option in Denovo).

\subsection{A.3 Sequence Output}

The main text output file consists of the output from the cross-section processing codes and Denovo. The user should examine the output and pay attention to any warnings or errors. Table 2.4.A.9 lists the files generated during the DEVC sequence. 
Table 2.4.A.9. Files created by DEVC for an input file named input.inp

\begin{tabular}{ccl}
\hline \multicolumn{1}{c}{ Filename } & Viewer & \multicolumn{1}{c}{ Description } \\
\hline $\begin{array}{l}\text { Output Summary } \\
\text { input.out } \\
\text { input.msg }\end{array}$ & & $\begin{array}{l}\text { main text output file, contains } k_{\text {eff }} \\
\text { messages file }\end{array}$ \\
$\begin{array}{l}\text { Denovo } \\
\text { xkba_b.inp }\end{array}$ & $\mathrm{V}^{a}$ & $\begin{array}{l}\text { input file for Denovo - if this file is renamed to have } \\
\text { a.dsi extension (Denovo simple input), it is viewable } \\
\text { in the Mesh File Viewer } \\
\text { AMPX formatted cross sections for Denovo } \\
\text { ft02f001 } \\
\text { input.mmt }\end{array}$ \\
$\begin{array}{l}\text { macromaterial table, use with *.dso or *.dsi file } \\
\text { input.dff }\end{array}$ & $\mathrm{V}$ & $\begin{array}{l}\text { Denovo fission fluxes } \\
\text { Denovo fission source distribution }\end{array}$ \\
\hline
\end{tabular}

${ }^{a} \mathrm{~V}$ - can be displayed with the Mesh File Viewer.

\subsection{A.3.1 Using the Mesh File Viewer}

The Mesh File Viewer is a Java utility shipped with SCALE that is used for viewing mesh tallies from Monaco, as well as importance maps and mesh-based sources in MAVRIC. The Mesh File Viewer can be used with DEVC to view the Denovo input file (*.dsi, showing the starting source), the Denovo output fission source distribution $(*$.dso) file, or the Denovo output flux (*.dff) file.

With any Denovo file, material information for each voxel can be displayed. Users can use the graphical user interface to select colors for each material in the DEVC input and save them to a colormap text file (*.cmp) for later use. For example, Figure 2.4.A.6 shows a cask model that has 13 materials with the default colors assigned by the viewer. A better color map (uranium is yellow, steel is blue, etc.) and an image using that color map are also shown in Figure 2.4.A.6.

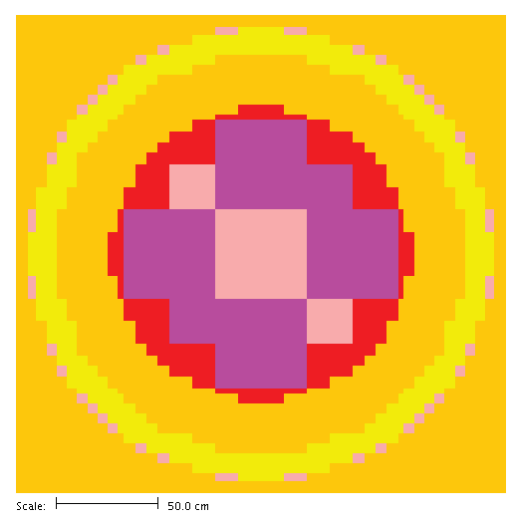

Default Colors

$\begin{array}{rrrrr}1 & 225 & 225 & 0 & 255 \\ 2 & 255 & 255 & 0 & 255 \\ 3 & 128 & 128 & 128 & 255 \\ 4 & 0 & 0 & 255 & 255 \\ 5 & 64 & 64 & 255 & 255 \\ 6 & 0 & 255 & 0 & 255 \\ 7 & 128 & 128 & 128 & 255 \\ 8 & 128 & 128 & 128 & 255 \\ 9 & 0 & 0 & 255 & 255 \\ 10 & 0 & 0 & 255 & 255 \\ 11 & 0 & 0 & 255 & 255 \\ 12 & 225 & 225 & 0 & 255 \\ 13 & 255 & 255 & 255 & 255\end{array}$

Colormap text file

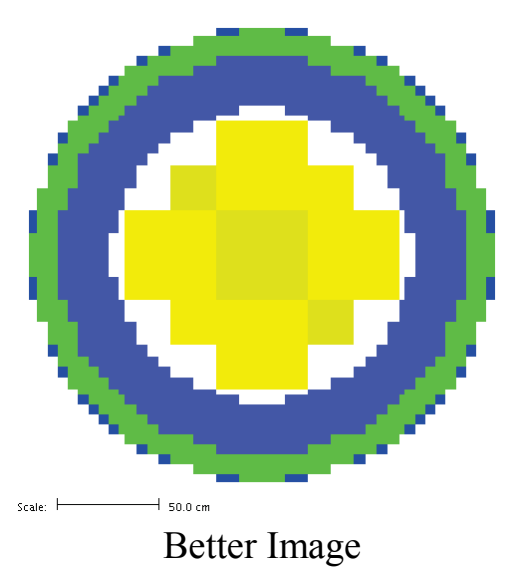

Figure 2.4.A.6. Viewing material information with the Mesh File Viewer. 
If the Denovo input was made using the mixed macromaterials, that can also be shown with the Mesh File Viewer. When one of the mesh files is loaded and the geometry is displayed (e.g., the materials for a cask model shown in Figure 2.4.A.7), there will be many values since each macromaterial is treated by the Java viewer as a separate material. Redefining the color map using the GUI is not realistic.

A colormap needs to be defined for the original materials of the problem. For this example, there are 13 original materials, but when using macromaterials, 73 macromaterials are used in the discrete-ordinates model (as listed in the *.mmt file). The colormap file should only contain values for the 13 original materials. When loading the colormap, a corresponding *.mmt file can also be loaded. This will display mixed colors for the macromaterials, as shown in Figure 2.4.A.7.

The final result is an image where the colors for the different values of the geometry attribute (which in the above example is material) are mixed in the ratio of the macromaterial definition.

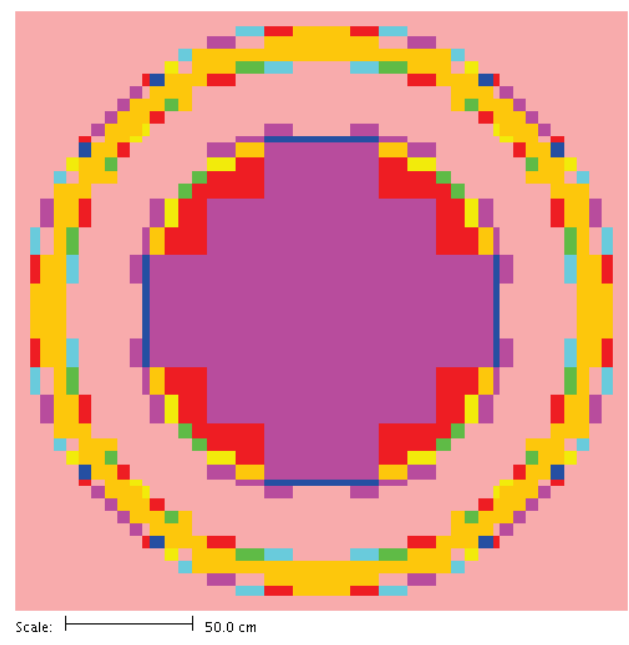

Default material coloring

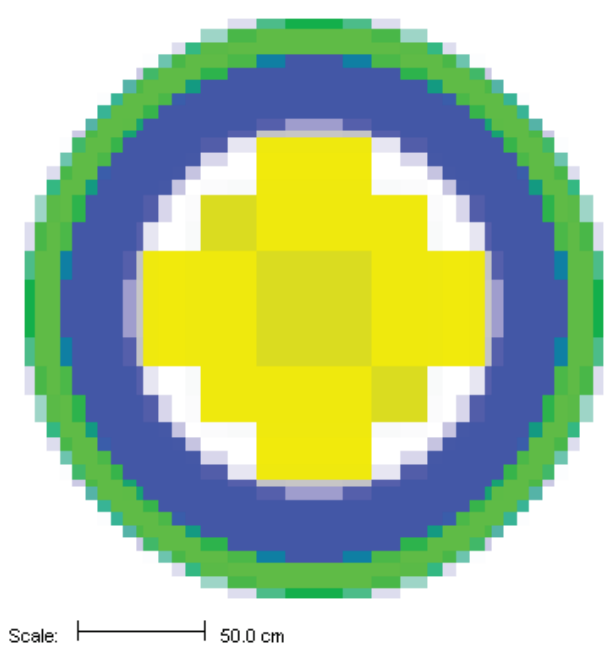

With colormap and *.mmt file both used

Figure 2.4.A.7. Viewing material information from a Denovo input file.

\subsection{A.3.2 Viewing the Starting Source}

The Java MeshFileViewer (scale/cmds/meshview) can be used to visualize the Denovo binary stream input file to show materials and starting source strengths. An example is shown in Figure 2.4.A.8.

\section{Material Numbering}

The cross sections produced by the SCALE cross-section mixing module ICE typically have a number of materials equal to the maximum material ID specified by the user in the "read composition" block. For a user input that contains materials 1, 3, and 5, the GIP-formatted cross-section file will contain five entries. For each lattice cell calculation that uses a cellmix = parameter, one more entry is contained in the GIP file. Hence, the GIP file does not contain the user's value from the "cellmix=" parameter but instead numbers the cellmix materials sequentially starting from 1+maximum(material ID). The Denovo geometry input and macroMixTable file produced by DEVC are modified to match the GIP cross-section file. When viewing the Denovo binary input file with the Mesh File Viewer, the GIP numbering for materials will be seen. 

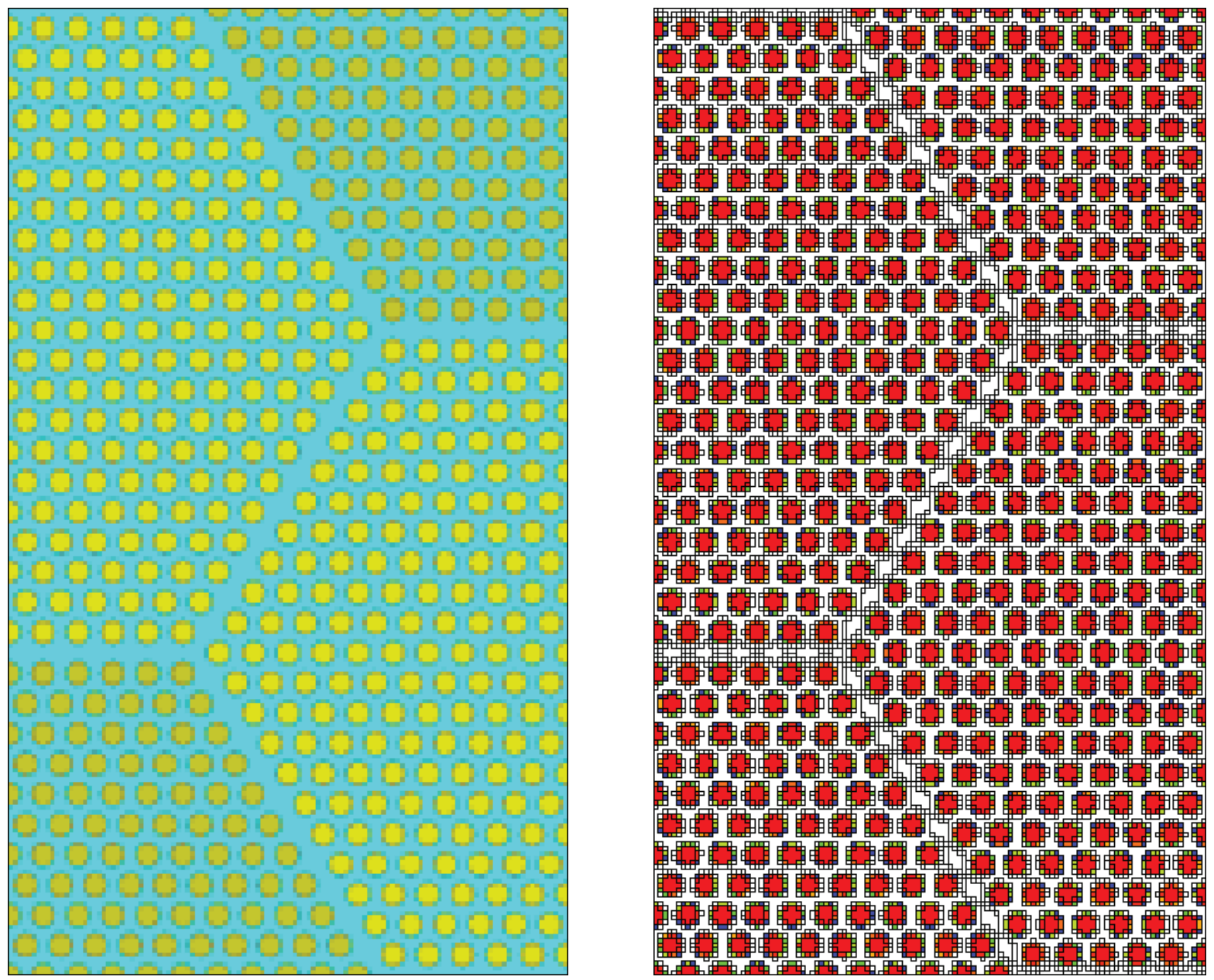

Figure 2.4.A.8. Fine-mesh version of sample problem 8, showing the materials using macromaterial blending of $\mathrm{UO}_{2}$, MOX, clad, and water (left) and the starting source strength limited to cells with fissionable material (right).

\subsection{A.4 Sample Problems}

Sample problems have been made that correspond to the eight CSAS6 sample problems. In each problem, the KENO parameters block was commented out, a parameters block containing Denovo calculation parameters was added, and a grid geometry block was added. These sample problems use a coarse discretization and loose tolerances to obtain a short runtime. Users will typically use much finer discretization (mesh, quadrature) and higher fidelity parameter settings for real eigenvalue calculations. The voxelized geometry and starting source distribution are shown below in Figure 2.4.A.9.

Results for the sample problems are displayed in Table 2.4.A.10. The sample problems used QR 1/1, a $\mathrm{P}_{0}$ scattering expansion, a $\mathrm{k}$ tolerance of 0.001 and coarse meshes for speed. The higher fidelity runs used finer spatial meshes, default parameters of QR 2/2, $P_{0}$ scattering expansion, and the default $k$ tolerance $\left(10^{-5}\right)$. Results for the longer-time CSAS6 and higher fidelity Denovo calculations are shown in Figure 2.4.A.10. 


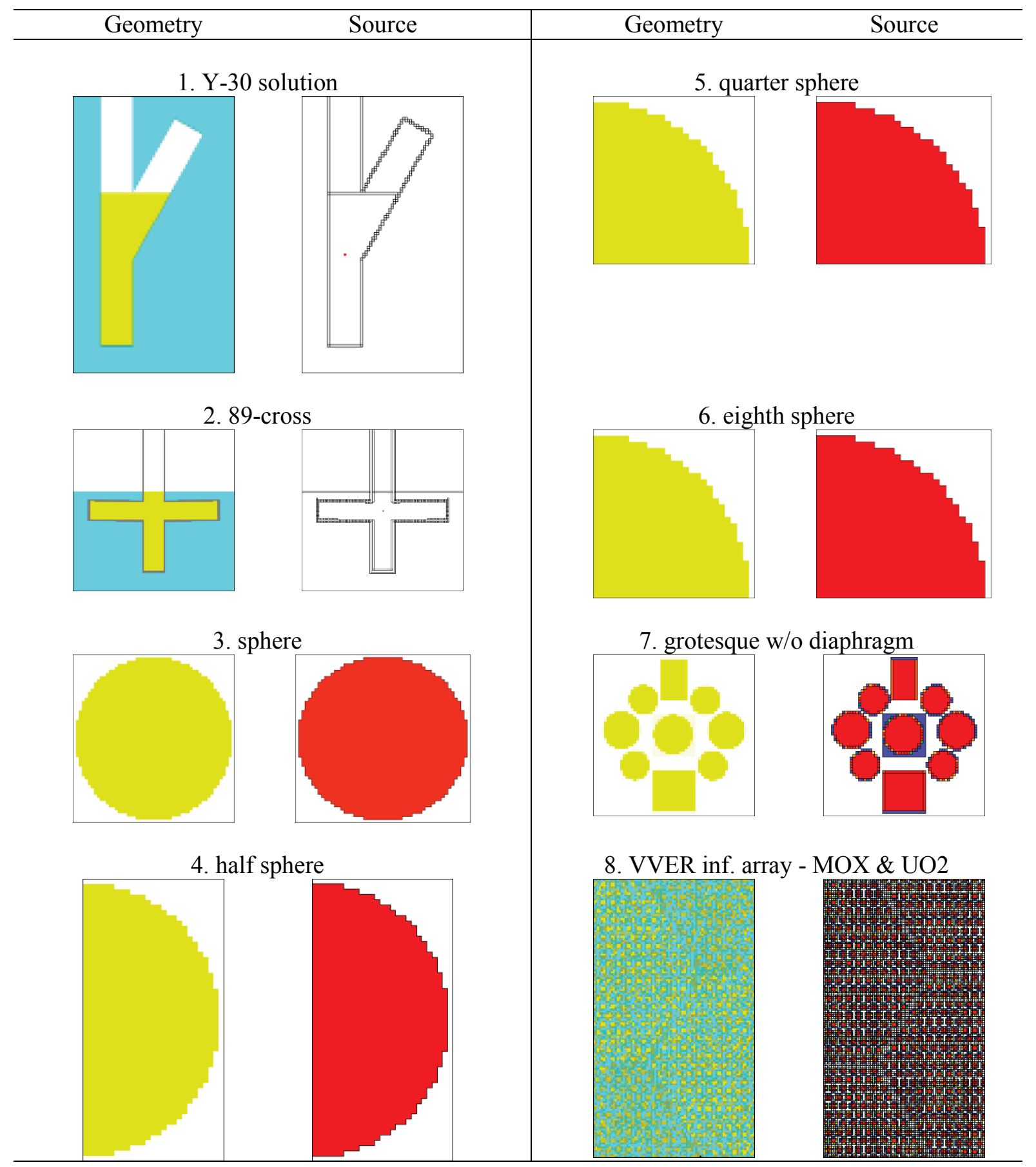

Figure 2.4.A.9. Denovo geometry (left) and starting source distribution (right) for the sample problems. 


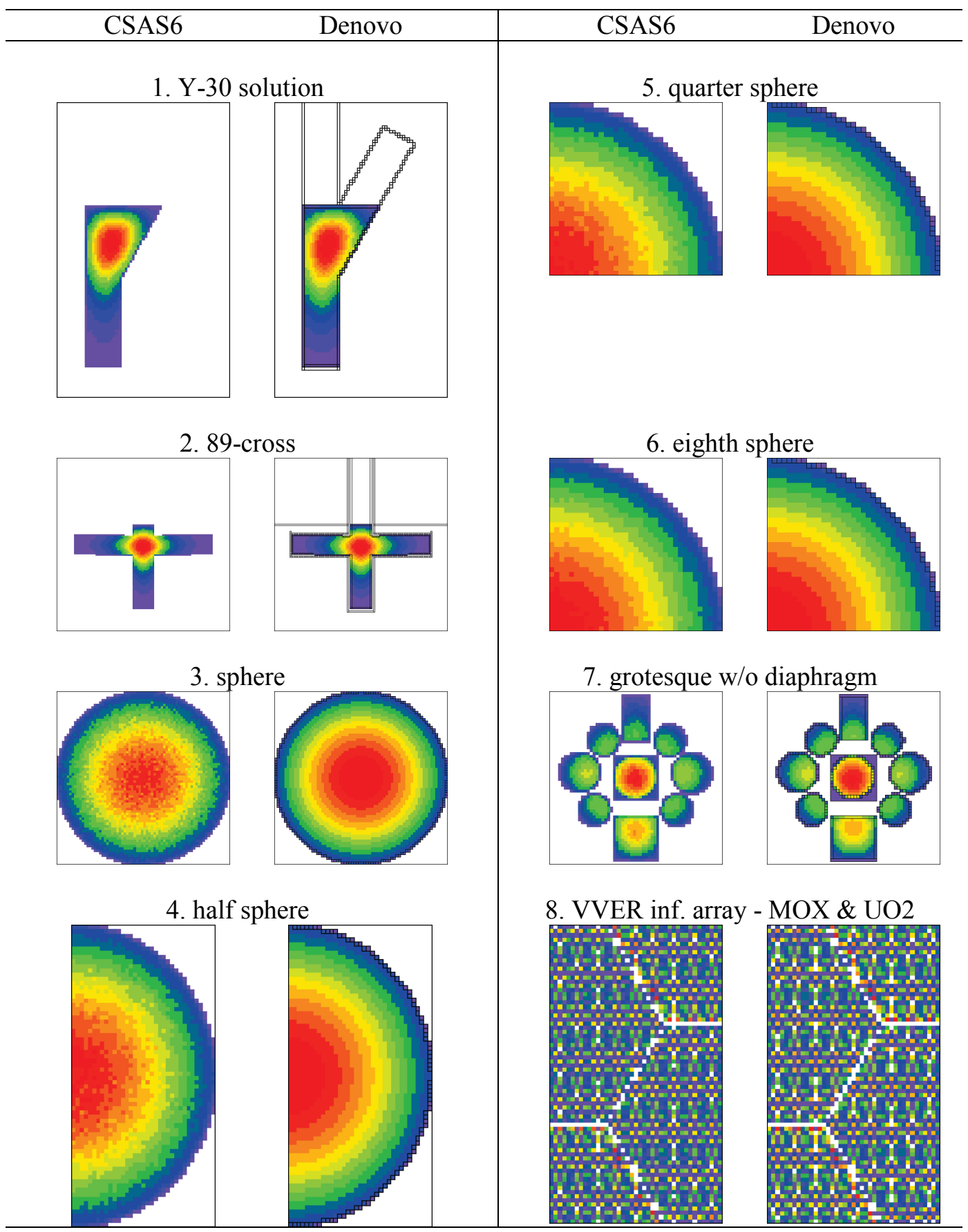

Figure 2.4.A.10. Fission source distributions computed by CSAS6 (left) and Denovo (right). 
Table 2.4.A.10. Sample problem results

\begin{tabular}{r|rrr|rrr|rr|rrr}
\hline & \multicolumn{7}{|c|}{ CSAS6 } & \multicolumn{3}{c}{ Denovo using DEVC } \\
\hline & \multicolumn{3}{|c}{ Short time } & \multicolumn{3}{c|}{ Long time } & \multicolumn{2}{c}{ Sample problem } & \multicolumn{2}{c}{ High Fidelity } \\
& $k_{\text {eff }}$ & uncert & min & $k_{\text {eff }}$ & uncert & \multicolumn{1}{c|}{ min } & $k_{\text {eff }}$ & min & \multicolumn{2}{c}{$k_{\text {eff }}$} & \multicolumn{1}{c}{ min } \\
\hline 1 & 1.0048 & 0.0023 & 1.94 & 1.00362 & 0.00018 & 380.18 & 0.889 & 10.97 & 1.00220 & 334.13 \\
2 & 1.0048 & 0.0016 & 1.80 & 1.00421 & 0.00017 & 377.52 & 0.859 & 6.96 & 0.99816 & 245.34 \\
3 & 0.9997 & 0.0017 & 0.45 & 0.99831 & 0.00018 & 178.69 & 0.982 & 3.30 & 0.99412 & 75.00 \\
4 & 1.0013 & 0.0014 & 0.53 & 0.99841 & 0.00018 & 106.02 & 0.982 & 2.01 & 0.99412 & 30.91 \\
5 & 0.9987 & 0.0019 & 0.46 & 0.99869 & 0.00020 & 63.18 & 0.982 & 1.40 & 0.99413 & 16.78 \\
6 & 0.9998 & 0.0019 & 0.53 & 0.99830 & 0.00019 & 42.03 & 0.982 & 1.21 & 0.99412 & 8.98 \\
7 & 0.9955 & 0.0017 & 1.43 & 0.99776 & 0.00019 & 101.56 & 0.882 & 8.98 & 0.99988 & 38.19 \\
8 & 1.2668 & 0.0013 & 2.22 & 1.26784 & 0.00013 & 279.69 & 1.198 & 5.70 & 1.22295 & 204.08 \\
\hline
\end{tabular}

\subsection{A.5 References}

1. T. M. Evans, A. S. Stafford, R. N. Slaybaugh, and K. T. Clarno, "Denovo: A New Three-Dimensional Parallel Discrete Ordinates Code in SCALE," Nuclear Technology 171, 171-200 (2010).

2. S. Goluoglu, L. M. Petrie, Jr., M. E. Dunn, D. F. Hollenbach, and B. T. Rearden, "Monte Carlo Criticality Methods and Analysis Capabilities in SCALE," Nuclear Technology 174, 214-335 (2011).

3. A. M. Ibrahim, D. E. Peplow, T. M. Evans, J. C. Wagner, and P. P. H. Wilson, "Improving the Mesh Generation Capabilities in the SCALE Hybrid Shielding Analysis Sequence," Transactions of the American Nuclear Society 100, 302-304 (2009).

4. S. R. Johnson, "Fast Mix Table Construction for Material Discretization," 2013 International Conference on Mathematics \& Computational Methods Applied to Nuclear Science and Engineering (M\&C 2013), Sun Valley, Idaho, May 5-9, 2013. 


\title{
2.5 KMART5 AND KMART6: POSTPROCESSORS FOR KENO V.A AND KENO-VI
}

L. M. Petrie

\begin{abstract}
KMART5 and KMART6 (Keno Module for Activity-Reaction Rate Tabulation) are modules whose primary purpose is to postprocess a KENO V.a or KENO-VI restart file with the corresponding working cross-section library to generate nuclide activity tables. It also allows collapsing and printing fluxes calculated by KENO. The KENO problem must have a mixing table, must calculate the fluxes, and must write a restart file containing the calculated data. KMART calculations are generally imbedded within a CSAS5 or CSAS6 input file immediately following the CSAS input as a stacked input case.
\end{abstract}




\section{TABLE OF CONTENTS}

$\underline{\text { Page }}$

2.5 KMART5 and KMART6: Postprocessors for KENO V.A and KENO-VI .........................2-205

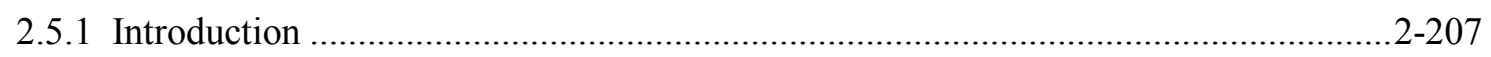

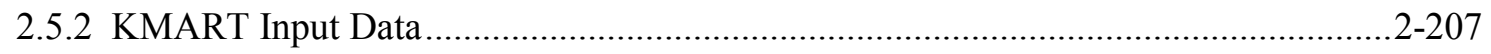

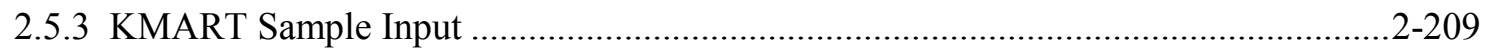




\subsubsection{Introduction}

KMART5 and KMART6 (Keno Module for Activity-Reaction Rate Tabulation) are modules whose primary purpose is to postprocess a KENO V.a or KENO-VI restart file with the corresponding working cross-section library to generate nuclide activity tables. It also allows collapsing and printing fluxes calculated by KENO. The KENO problem must have a mixing table, must calculate the fluxes, and must write a restart file containing the calculated data.

\subsubsection{KMART Input Data}

Input data for KMART is read into the program using free form blocked input similar to KENO. The data blocks are started with a READ BLOCK NAME and ended with an END BLOCK NAME. There are three data blocks that KMART can read. The first data block is named INITIAL, and the input starts with the keywords READ INITIAL. There are ten possible keyworded entries in this block that may be entered in any order.

Keyword $\quad \underline{\text { Variable }}$

PRTVOLS PRINT_VOLUMES

KUNIT $=\quad$ KUNIT

$\mathrm{FNI}=\quad$ RESTART

XUNIT $=\quad$ XUNIT

ACTBYGRP ACTIVITIES_BY_GROUP

RRPVOL REACTION_RATES_PER_ UNIT_VOLÜME

KENO3D NK3D

K3DFILE

NOPRINT PRINT_RESULTS

\section{Description}

A flag to cause the volumes calculated by KENO to be printed by KMART. Off by default, turn on by entering.

The logical unit number of the KENO restart file. The default is 35

Mode in extra field in the input restart file name [restart_mode_in.keno_input] and [restart_mode_in.keno_calculated]. The default is an empty field.

The logical unit number of the cross-section library. The default is 4

A flag which turns on printing activities by group. If the fluxes are collapsed, the activities will be by broad group, otherwise they will be by fine group. Off by default, turn on by entering

A flag causing the activities to be printed per unit volume rather than integrated over the volume of the region. Off by default, turn on by entering

Unit number on which to write data for plotting with KENO3D.

File name of the KENO input file (minus the trailing extension). The plot data file will be named K3DFILE.kmt.

A flag allowing suppressing printing results. 


$\begin{array}{lll}\underline{\text { Keyword }} & \underline{\text { Variable }} & \begin{array}{l}\text { Description } \\ \text { FLUXBIN }\end{array} \\ \text { FLUX_BIN } & \begin{array}{l}\text { A flag to turn on generating a collapsed cross section } \\ \text { file for TRITON. }\end{array} \\ \text { WUNIT } & \text { WGTD } & \begin{array}{l}\text { The logical unit number on which to write an AMPX } \\ \text { weighted library of the 1-D neutron cross sections. }\end{array}\end{array}$

A sample data block is given below.

READ INITIAL KUNIT=35 XUNIT=4 END INITIAL

One of the next two blocks is required, but both can be specified if desired. If both are entered, either one can be first. The next data block specified is named ACTIVITY, and the input starts with the keywords READ ACTIVITY. It contains the data specifying which activities are to be calculated. The activities are specified by pairs of numbers giving the nuclide identifier and the reaction type identifier desired. A list of reaction types, also known as MT numbers, can be found in Appendix A of the XSLib chapter. These pairs are repeated until all the desired activities have been specified. If the nuclides are identified by the SCALE scheme, then the nuclide can be specified most explicitly by following the nuclide by the keyword MIX= and the mixture number the nuclide is in. By specifying a mixture of zero the activity will be calculated for each region in which the nuclide occurs. If the nuclide specifies a natural element identifier $(1000 * \mathrm{Z})$ and individual isotopes occur on the cross-section library, the isotope activities will be summed to produce the total activity for the element. If the nuclide is a special nuclide, i.e., identified with a prefix id times a million $+\mathrm{a} Z \mathrm{ZA}$, then MIX= must be specified as a mixture the nuclide occurs in. The data pair is described below.

$\begin{array}{lll}\text { Keyword } & \text { Variable } & \text { Description } \\ \text { NUCLIDE } & \begin{array}{l}\text { The nuclide identification number on the cross-section library } \\ \text { for this activity request. }\end{array} \\ \text { MIX } & \text { MIXTURE } & \begin{array}{l}\text { Mixture number of the nuclide for this activity request. This is } \\ \text { an optional entry, and defaults to } 0 .\end{array}\end{array}$

REACTION The reaction type identifier for this activity request.

If no activities are desired, then the block can be omitted. A sample block is given below.

READ ACTIVITY 92235189223527922351452 END ACTIVITY

The other input block is named COLLAPSE, and starts with the keywords READ COLLAPSE. There are two keyword entries that may be input in this block. A flux factor to normalize the fluxes by can be specified. It defaults to 1 . The last fine group in the current broad group is the other entry. The broad groups are specified sequentially starting with group one. If the flux factor is specified more than once, the last value given is used. The data is specified as below.

$\begin{array}{lll}\text { Keyword } & \text { Variable } & \underline{\text { Description }} \\ \text { FACTOR } & \text { FACTOR } & \begin{array}{l}\text { A flux multiplier used to scale the fluxes before } \\ \text { printing (default 1.0). }\end{array} \\ \text { LASTG }= & \text { LAST_GROUP } & \begin{array}{l}\text { The last fine group to be included in the current } \\ \text { broad group. The broad groups are input sequentially } \\ \text { starting with group one. }\end{array}\end{array}$


If no collapsed fluxes are desired, then the block can be omitted. A sample block is given below.

READ COLLAPSE FACTOR 1.0 LASTG=10 LASTG=20 LASTG=30 LASTG=56

END COLLAPSE

\subsubsection{KMART Sample Input}

Sample input data for KMART5 is given in Figure 2.5.1.

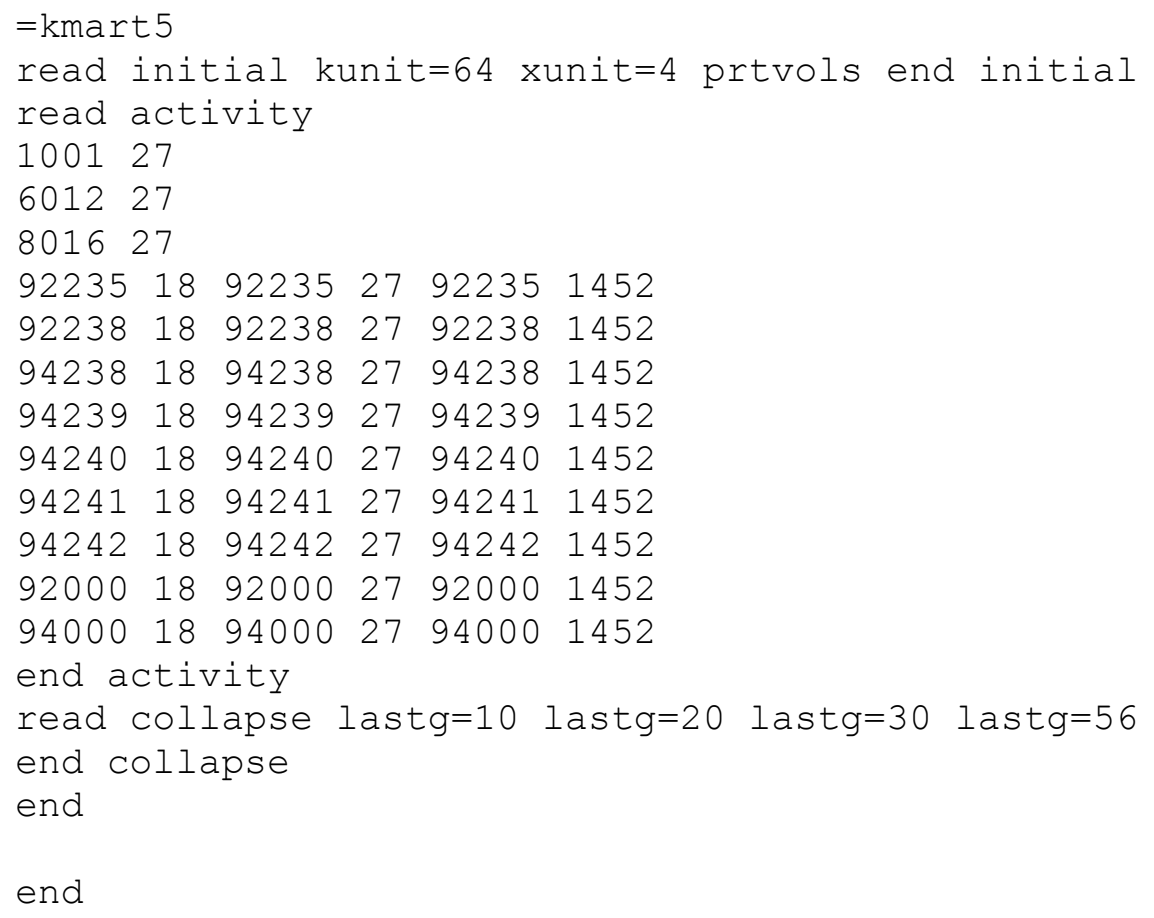

Figure 2.5.1. KMART5 sample input. 
2.6 K5TOK6 AND C5TOC6: INPUT FILE CONVERSION PROGRAMS FOR KENO AND CSAS 


\section{TABLE OF CONTENTS}

$\underline{\text { Page }}$

2.6 KtoK6 and C5toC6: Input File Conversion Programs for KENO and CSAS....................2-210

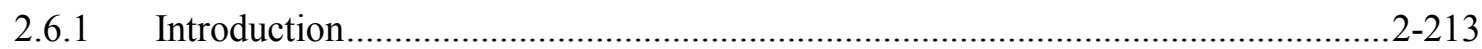

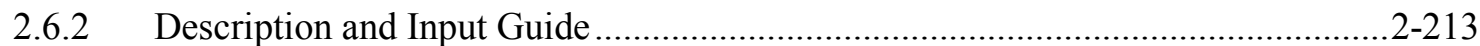




\section{TABLE OF FIGURES}

$\underline{\text { Page }}$

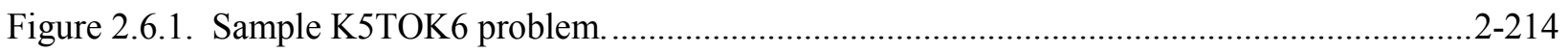

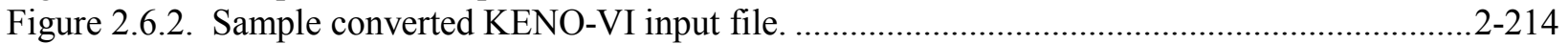

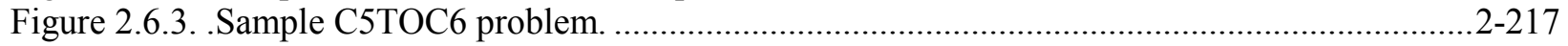

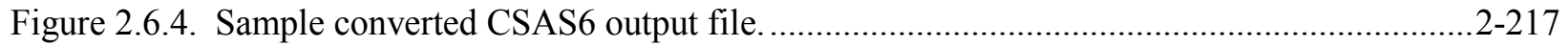




\subsubsection{Introduction}

Program K5TOK6 can be used to automatically convert a KENO V.a input file to a KENO-VI input file. Program C5TOC6 can be used to automatically convert a CSAS5 input file to a CSAS6 input file. This functionality can be useful when converting a KENO V.a validation sequence to a KENO-VI validation sequence. It removes the problem of introducing a mistake or inadvertently changing the data when remodeling a geometry to the KENO-VI format. For some cases, however, the converted model may be a very inefficient KENO-VI model.

\subsubsection{Description and Input Guide}

Program K5TOK6 is a utility program that can be used to automatically convert a KENO V.a input file to a KENO-VI input file. Program C5TOC6 is a utility program that can be used to automatically convert a CSAS5 input file to a CSAS6 input file. For program K5TOK6, the "=KENOVA" record in the input stream is replaced by "=K5TOK6". The output file is then named_geomnnnnnnn where nnnnnn is a unique 7-digit number. This allows a string of KENO V.a problems to be converted in one job. For program C5TOC6 the "=CSASBB" record in the input stream (where the $B B$ is 5,25 , or $2 \mathrm{x}$ ) is replaced by "=C5TOC6 PARM=CSAS5." The output file is named_geomnnnnnn where nnnnnnn is a unique 7-digit number.

For large problems, the output file may need to be edited to specify an increased value for parameter $N B 8$ in KENO-VI and also an increased value for parameter $D A B$ in CSAS6. The conversion makes no attempt to optimize the output file, so it almost surely will be inefficient in its use of storage, and in its use of bodies. This can lead to models that are very inefficient in their running time also.

The input/output (I/O) units for K6TOK6 and C5TOC6 are given below. Note that K5TOK6 requires the cross-section library designated by the " $\mathrm{LIB}=$ " parameter in the KENO V.a input file.

\begin{tabular}{cll}
\cline { 1 - 2 } Unit Number & & Function \\
5 & KENO V.a (or CSAS5) input file \\
6 & Output \\
7 & Input file generated for KENO-VI (or CSAS6)
\end{tabular}

Sample K5TOK6 input file is shown in Figure 2.6.1 and the corresponding converted KENO-VI input file is shown in Figure 2.6.2. Likewise, a C5TOC6 sample input file and corresponding converted CSAS6 input file are shown in Figure 2.6.3 and Figure 2.6.4, respectively. 


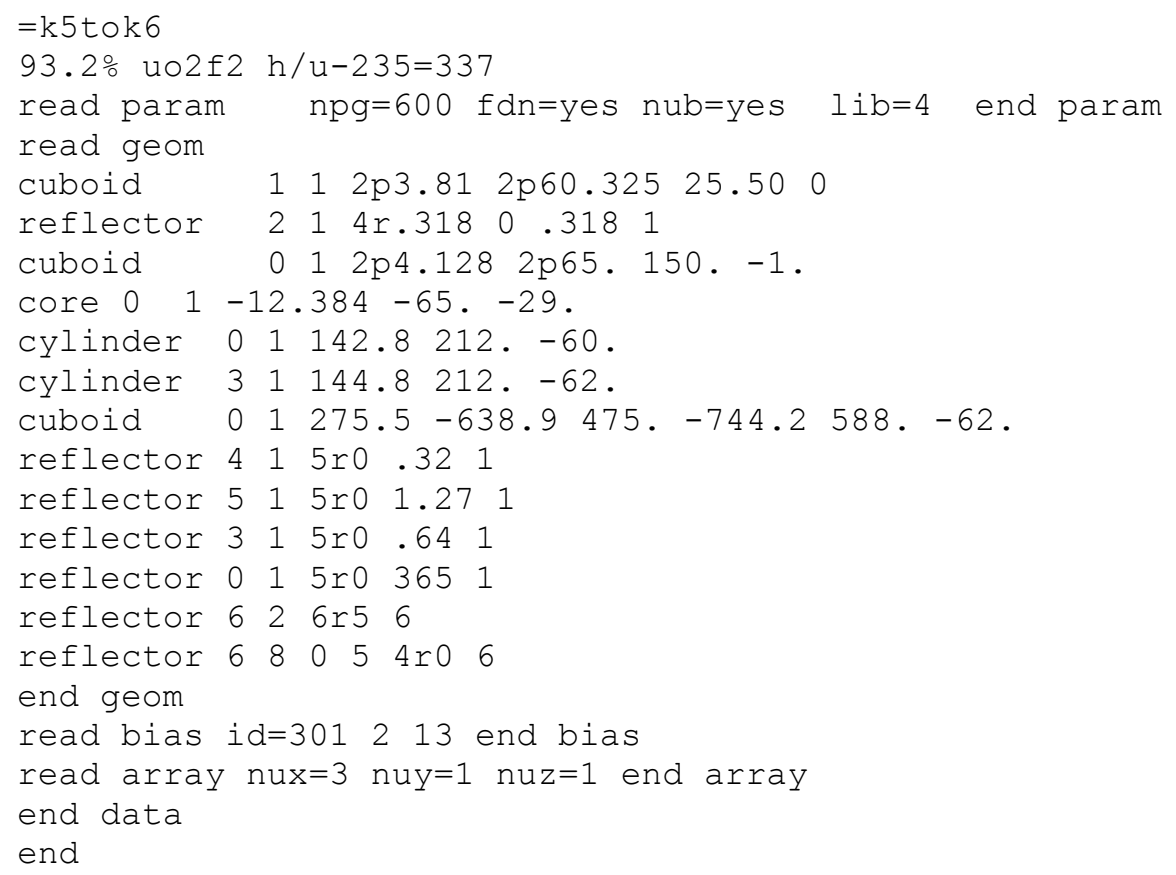

Figure 2.6.1. Sample K5TOK6 problem.

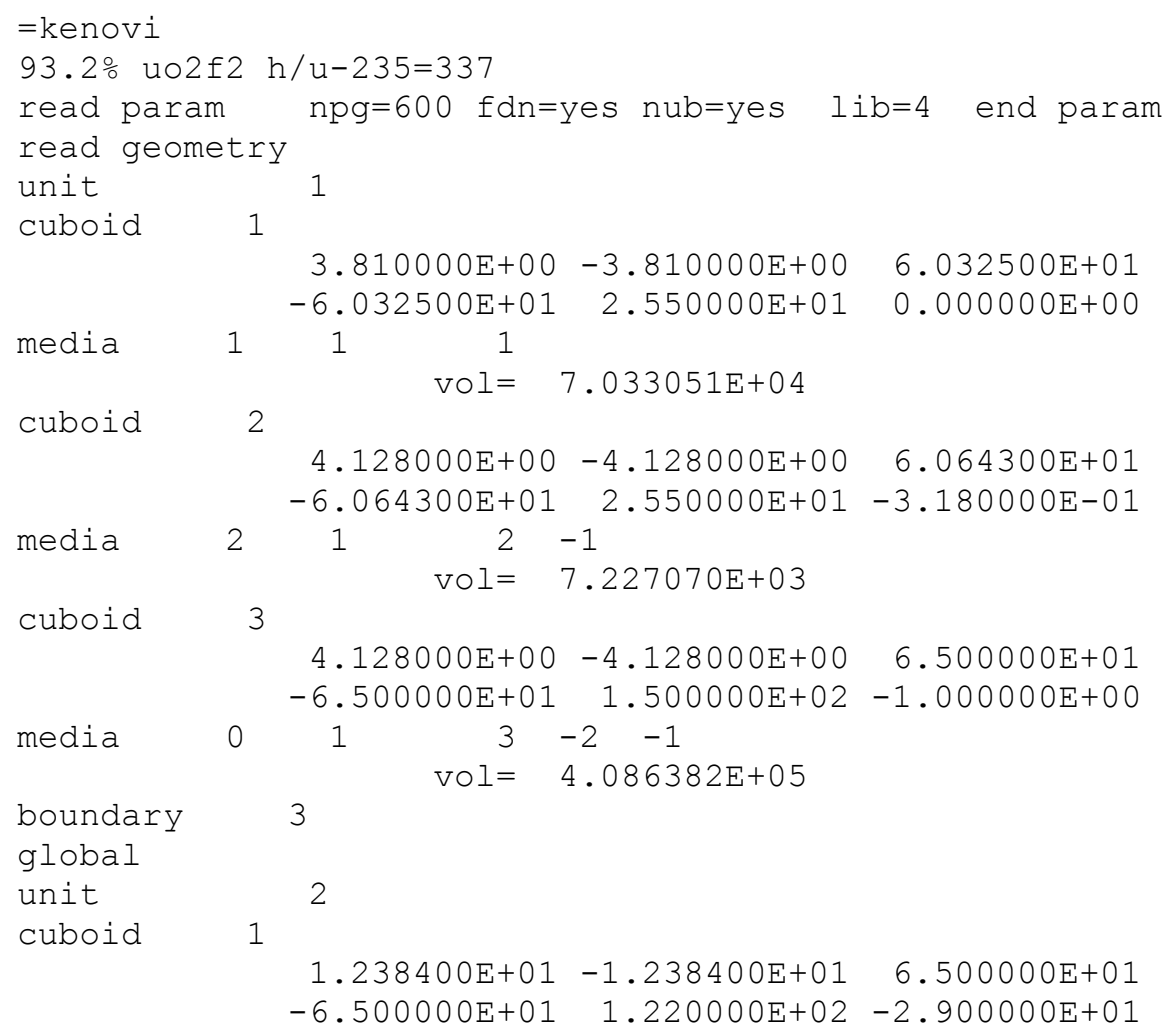

Figure 2.6.2. Sample converted KENO-VI input file. 


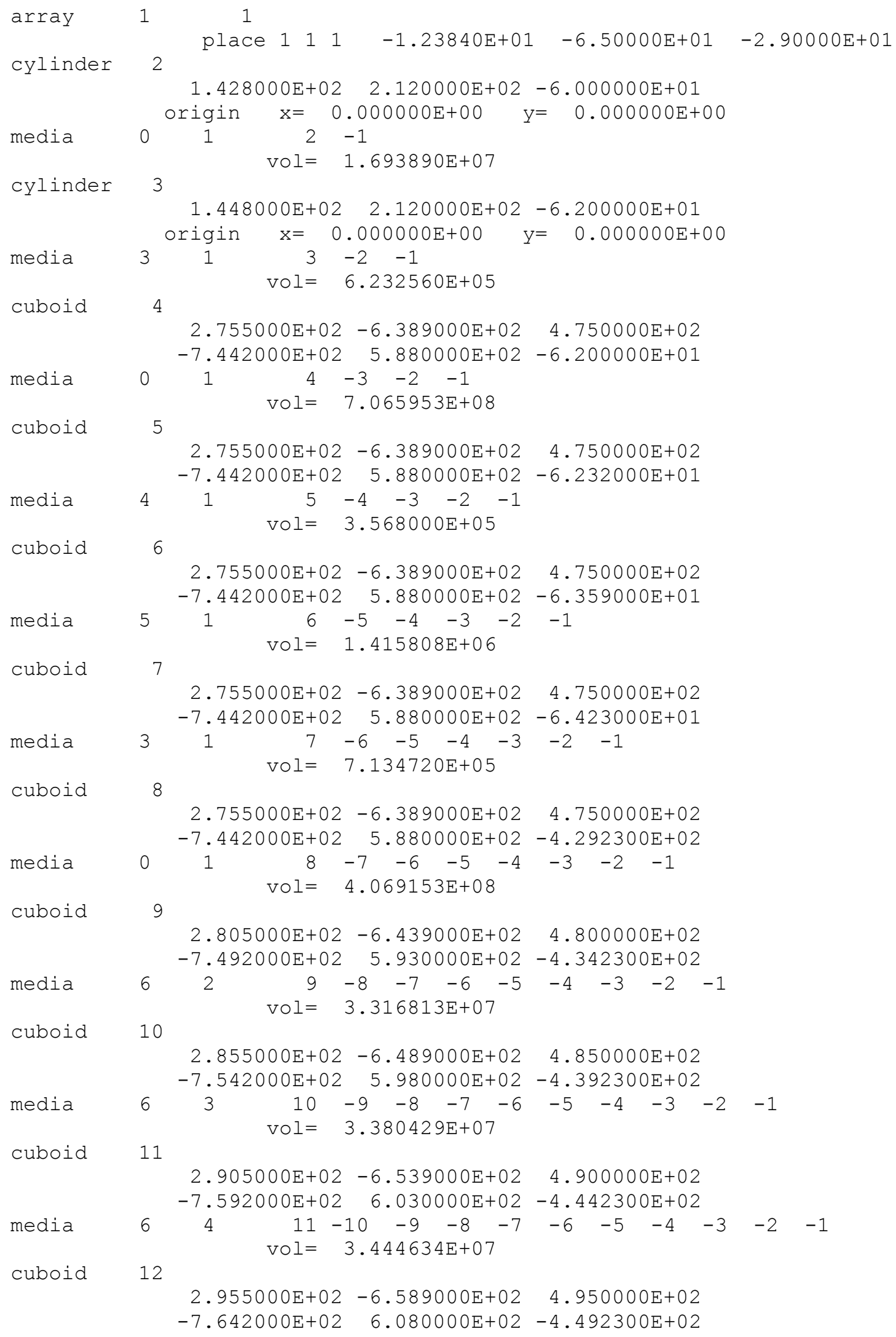

Figure 2.6.2. Sample converted KENO-VI input file. (continued) 


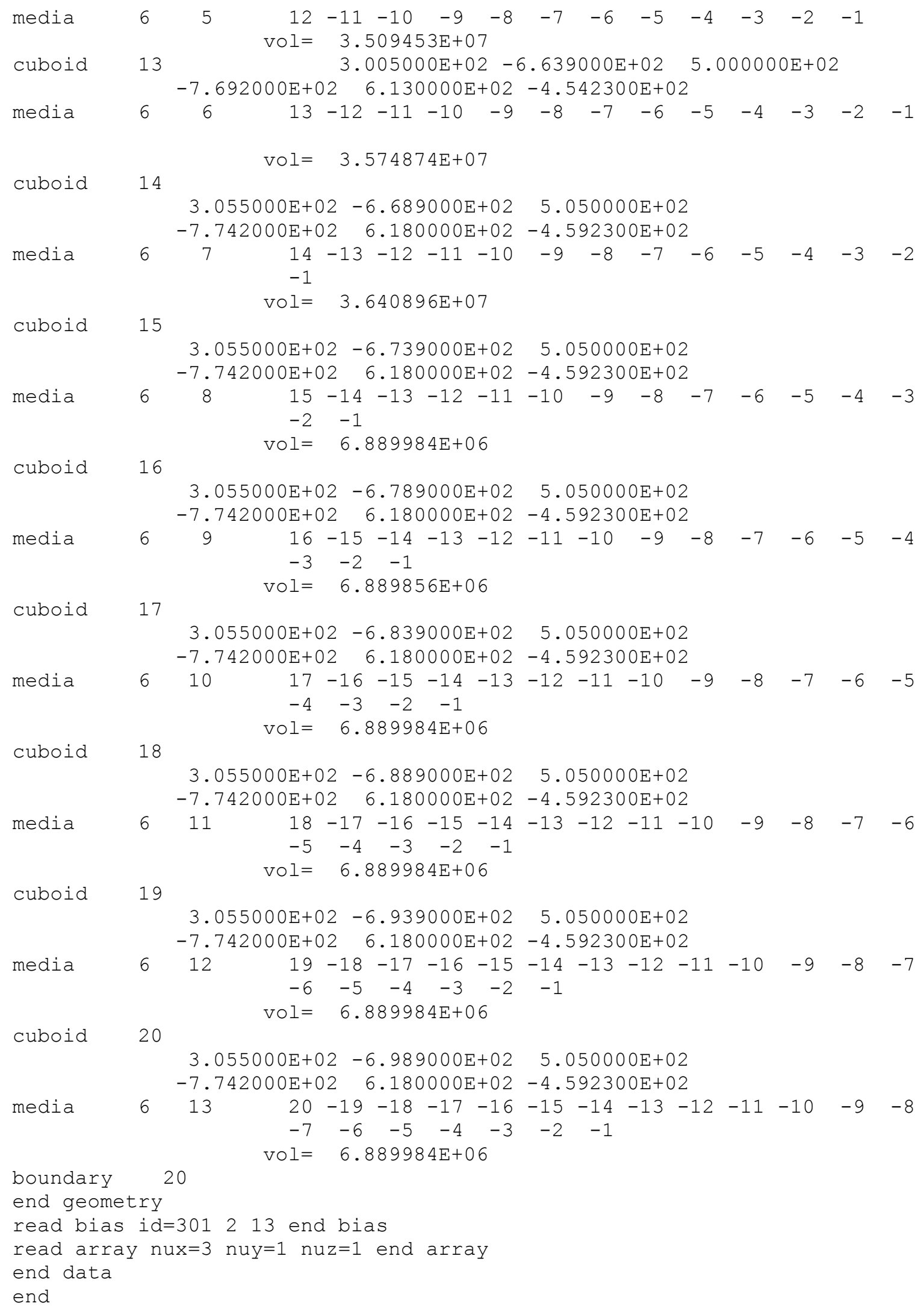

Figure 2.6.2. Sample converted KENO-VI input file. (continued) 


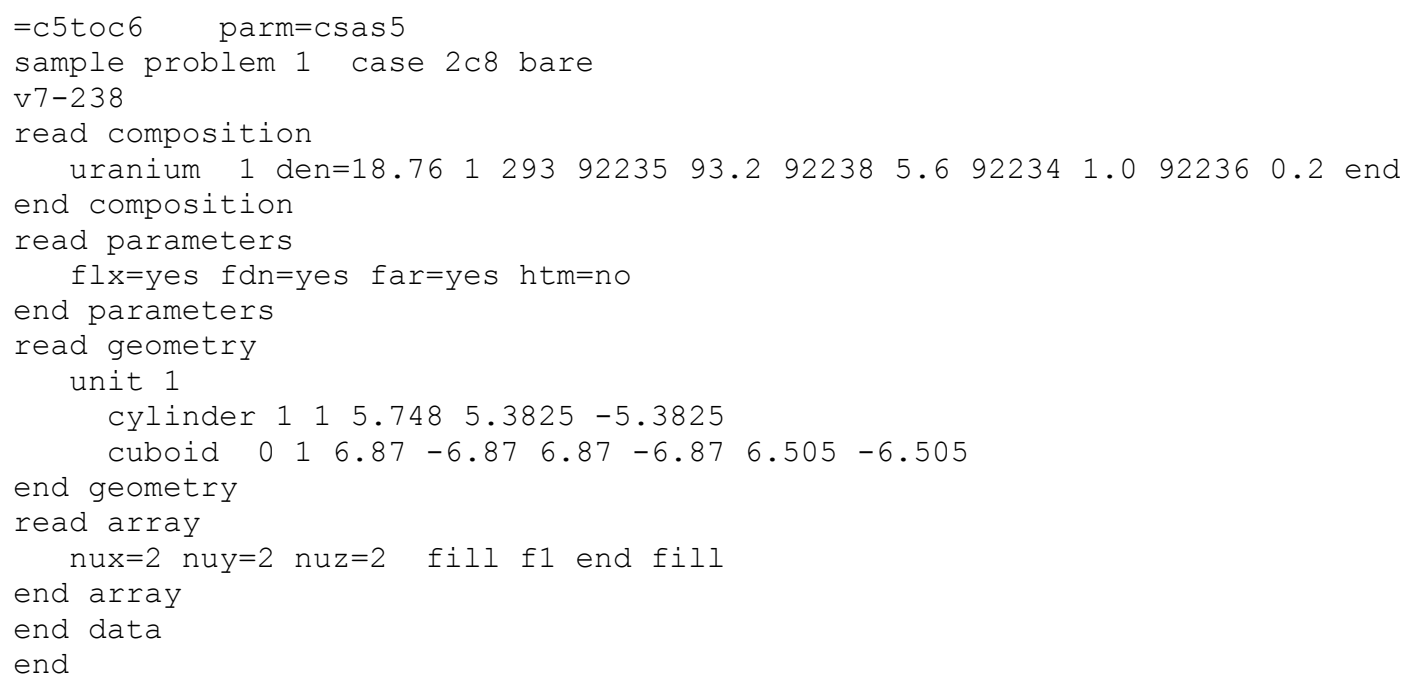

Figure 2.6.3. .Sample C5TOC6 problem.

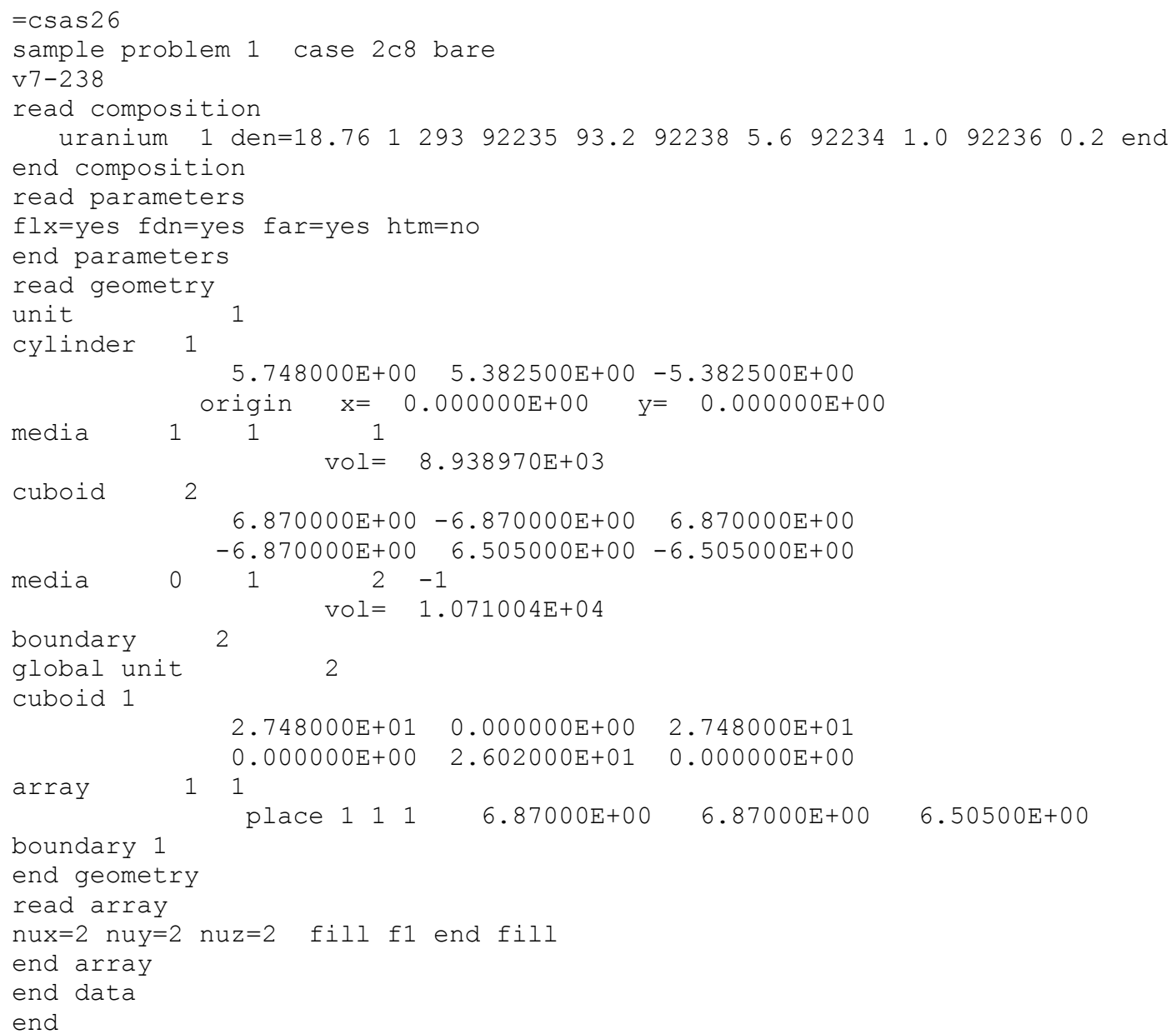

Figure 2.6.4. Sample converted CSAS6 output file. 


\subsection{REACTOR PHYSICS}

\section{Introduction by M. A. Jessee}

SCALE supports a wide range of reactor physics analysis capabilities. SCALE reactor physics calculations couple neutron transport calculations with ORIGEN to simulate the time-dependent transmutation of various materials of interest. The two reactor physics analysis tools within SCALE are TRITON and Polaris. TRITON is SCALE's modular reactor physics sequence for a wide variety of system types; Polaris is SCALE's light water reactor (LWR) lattice physics sequence.

The primary function of TRITON is to simulate the time-dependent evolution of nuclide inventories of a reactor system through a series of multigroup transport calculations and depletion/decay calculations. Before each multigroup transport calculation, TRITON executes the XSProc module to calculate multigroup cross sections for each user-designated depletion material. The XSProc calculation is performed based on the time-dependent material compositions and the user-defined cross section processing definitions. TRITON provides maximum modeling flexibility, supporting the full range of cross section processing options in XSProc along with support for four different multigroup transport modules available in SCALE. These transport modules include the following:

- XSDRN: one-dimensional discrete ordinates $\left(\mathrm{S}_{\mathrm{N}}\right)$ transport module for modeling simple slab, cylindrical, and spherical geometries,

- NEWT: two-dimensional (2D) $\mathrm{S}_{\mathrm{N}}$ polygon-mesh deterministic transport module with arbitrary geometry model definitions, and

- KENO-V.a and KENO-VI: three-dimensional Monte Carlo transport modules with arbitrary geometry model definitions.

In addition to the multigroup-based calculation sequences, TRITON supports continuous-energy (CE) transport calculations with KENO-V.a and KENO-VI. For each depletion material, the CE Monte Carlo calculation tallies energy-integrated nuclide-dependent reaction rates to couple the transport solution to the ORIGEN depletion calculation. Both the multigroup- and CE-based KENO depletion calculations are parallelizable and can run on an arbitrary number of processors.

TRITON provides easy-to-use input options to define the time-dependent reactor condition, including power history, material temperatures, and material compositions. TRITON also provides the option to perform lattice physics calculations, with input options to define branch calculations, homogenization edits, few-group energy structures, and assembly discontinuity factors. The homogenized few-group cross sections are archived onto auxiliary data files for subsequent in-reactor core calculations.

TRITON generates several data files for follow-on SCALE analysis. It creates the ORIGEN binary concentration file (.f71 extension) that stores all of the material inventories at each depletion/decay step. This file can be used as input to ORIGEN, ORIGAMI, and MAVRIC to support spent fuel characterization and shielding analysis. TRITON also creates the ORIGEN binary library file (.f33 extension) that stores the ORIGEN transition matrix for each depletion material at each depletion/decay step. The ORIGEN library files can be used as input to ORIGEN or ORIGAMI for rapid assessment of spent fuel inventories. Finally, TRITON also generates the aforementioned lattice physics few-group cross section archive (xfile016) for reactor core calculations.

In addition to the TRITON reactor physics sequence, SCALE supports an alternative easy-to-use LWR depletion sequence for generating lattice physics data for full-core reactor calculations. The Polaris lattice physics sequence couples 2D multigroup transport calculations with ORIGEN depletion to simulate LWR fuel assemblies. Polaris provides easy-to-use input definitions for defining the primary inputs necessary for lattice physics analysis, namely the pin and lattice geometry, the material compositions, and specifications for the power history and branch calculations. Polaris supports 
systematic input defaults for analysis of both fuel and reflector models for both pressurized water reactor (PWR) and boiling water reactor (BWR) geometries. It uses a new approach for cross section processing called the Embedded Self Shielding Method (ESSM). The hallmark feature of ESSM is that the self-shielding calculation is performed on the 2D fuel assembly geometry, eliminating the need for user-defined cross section processing definitions. The ESSM calculation and the $\mathrm{k}_{\text {eff }}$ calculation are performed with a new deterministic transport module based on the Method of Characteristics (MOC) approach. The Polaris sequence generates the lattice physics archive file (.t16 and .x16 extension), the ORIGEN binary concentration file (.f71 extension), and a geometry plot file (.png extension). 


\title{
3.1 TRITON: A MULTIPURPOSE TRANSPORT, DEPLETION, AND SENSITIVITY AND UNCERTAINTY ANALYSIS MODULE
}

M. A. Jessee, D. Wiarda, K. T. Clarno, U. Mertyurek, K. Bekar

\begin{abstract}
The TRITON computer code is a multipurpose SCALE control module for transport, depletion, and sensitivity and uncertainty analysis. TRITON can be used to provide automated, problem-dependent cross section processing followed by multigroup transport calculations for one-, two-, and three-dimensional (1D, 2D, and 3D) configurations. Additionally, this functionality can be used in tandem with the ORIGEN depletion module to predict isotopic concentrations, source terms, and decay heat, as well as to generate few-group homogenized cross sections for nodal core calculations.

TRITON has been designed using the modular approach that is a hallmark of SCALE functionality. TRITON provides the capability to perform deterministic transport analysis for 1D geometries using XSDRNPM and for 2D geometries using NEWT. TRITON also includes 3D Monte Carlo depletion capabilities using KENO V.a and KENO-VI. For Monte Carlo depletion calculations, TRITON supports both multigroup and continuous-energy options.

The TSUNAMI-2D sequence in TRITON provides calculation of 2D forward and adjoint transport solutions in NEWT, calculation of sensitivity coefficients, and calculation of the uncertainty in $k_{\text {eff }}$ and other responses due to cross section covariance data. The SAMS module is used to determine the sensitivity of the calculated value of responses to the nuclear data used in the calculation as a function of nuclide, reaction type, and energy. The uncertainty in the calculated value of the response, resulting from uncertainties in the basic nuclear data used in the calculation, is estimated using energy-dependent cross section covariance matrices. The implicit effects of the cross section processing calculations are also treated.
\end{abstract}




\section{TABLE OF CONTENTS}

$\underline{\text { Page }}$

3.1 TRITON: A Multipurpose Transport, Depletion, And Sensitivity and Uncertainty Analysis

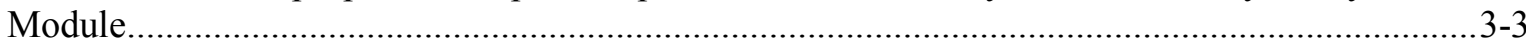

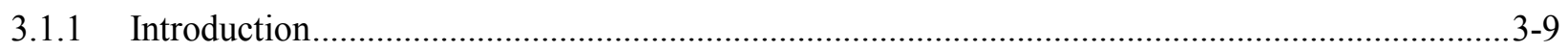

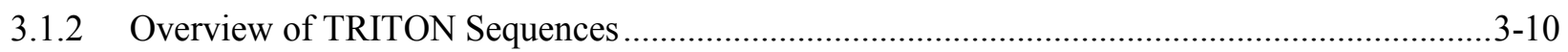

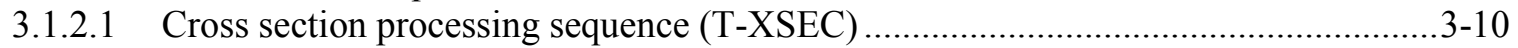

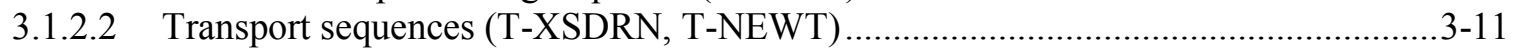

3.1.2.3 Depletion sequences (T-DEPL, T-DEPL-1D, T5-DEPL, T6-DEPL) ......................... $3-11$

3.1.2.4 S/U analysis sequences (TSUNAMI-2D, TSUNAMI-2DC) .....................................

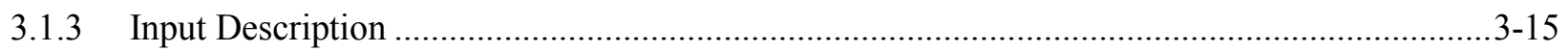

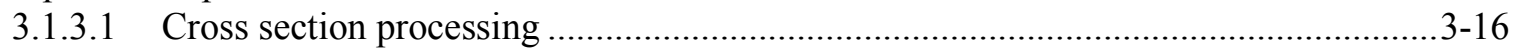

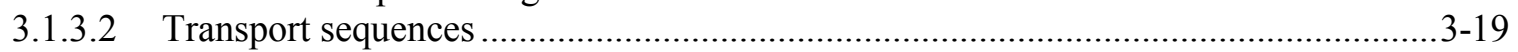

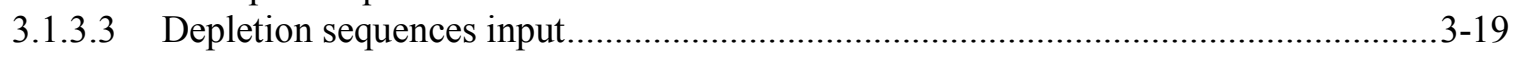

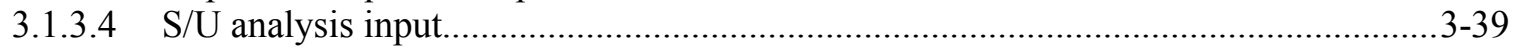

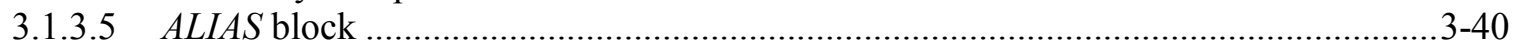

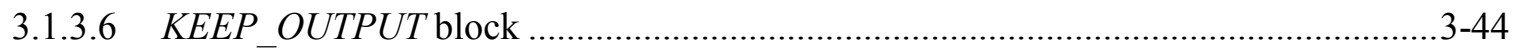

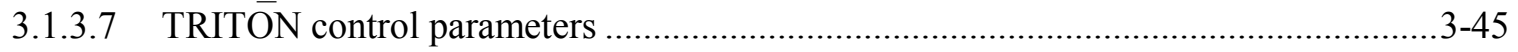

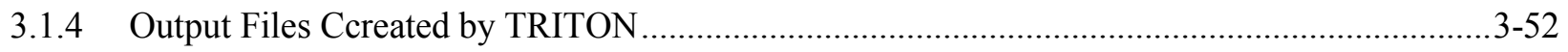

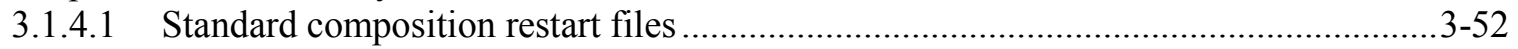

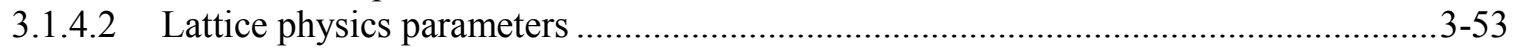

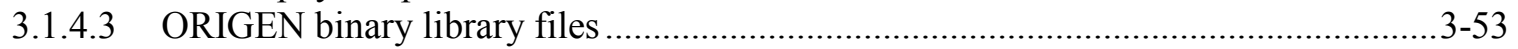

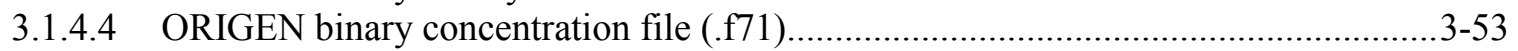

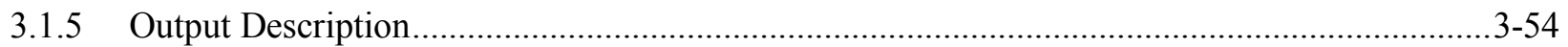

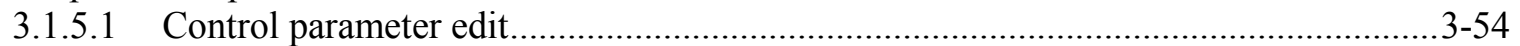

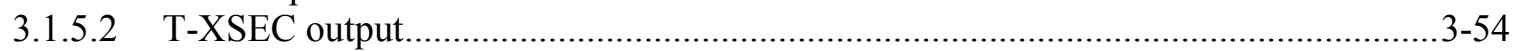

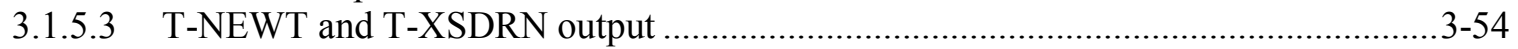

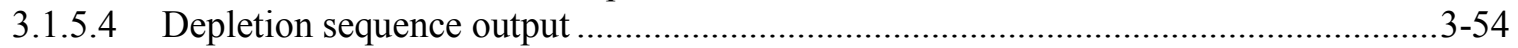

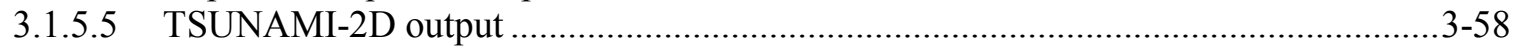

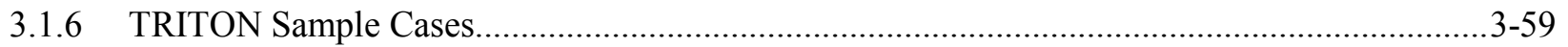

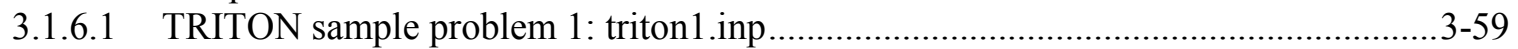

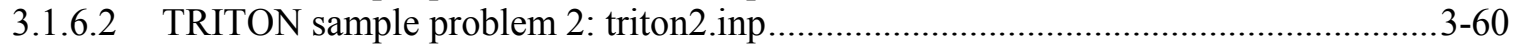

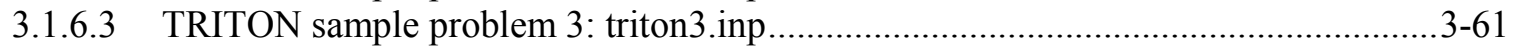

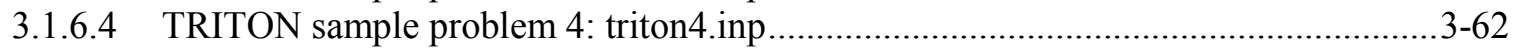

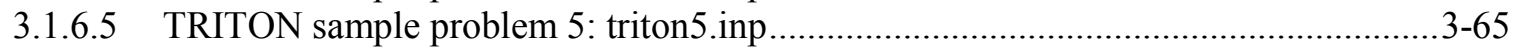

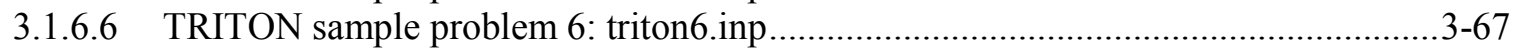

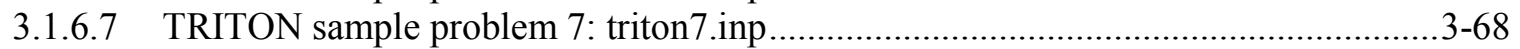

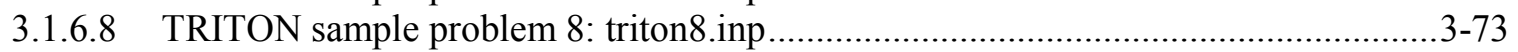

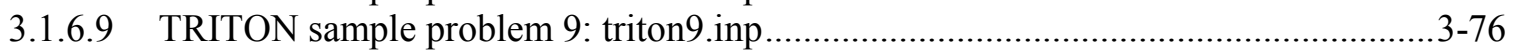

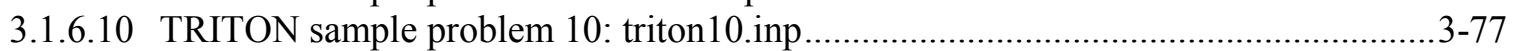

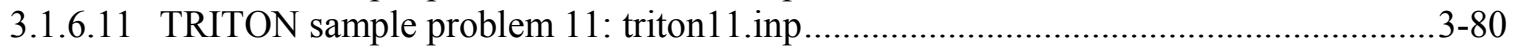

3.1.6.12 TRITON sample problem 12: triton12.inp......................................................... $3-87$

3.1.6.13 TRITON6 sample problem 1: triton6-1.inp ..................................................... 3-90

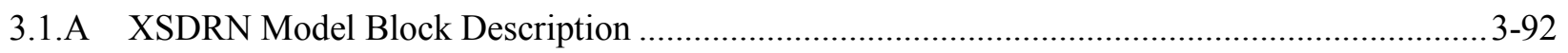




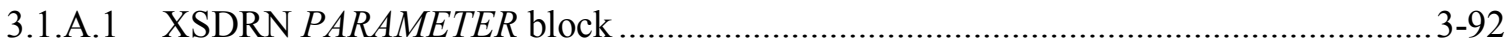

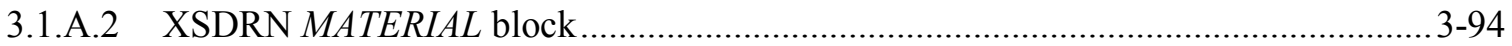

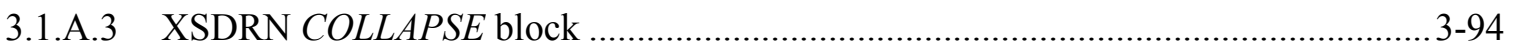

3.1.B Data Structure for Cross Section Database File xfile016 ....................................................... 3-94

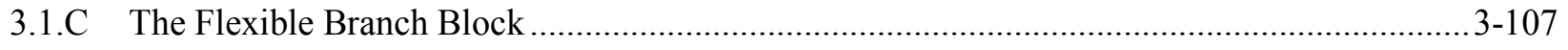




\section{LIST OF FIGURES}

Figure 3.1.1. Predictor/corrector depletion algorithm used by TRITON.......................................... $3-13$

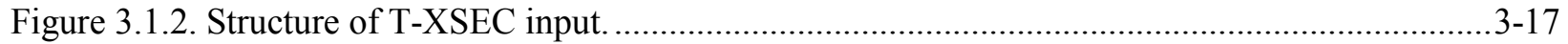

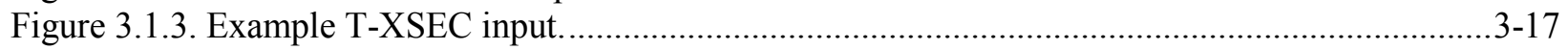

Figure 3.1.4. T-XSEC input with multiple cross section processing options. ....................................... 3-18

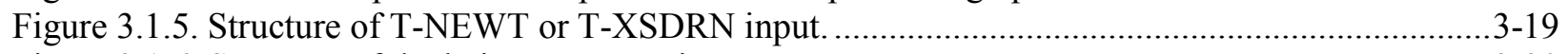

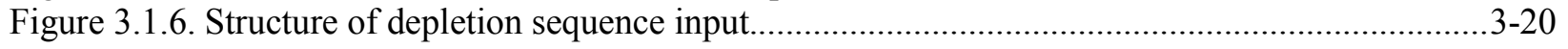

Figure 3.1.7. Example BURNDATA block input..............................................................................22

Figure 3.1.8. Schematic illustration of T-DEPL branch calculations during depletion. ..........................3-23

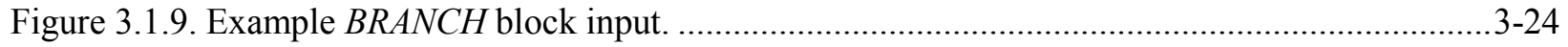

Figure 3.1.10. Example BRANCH block input with Dancoff factors. ............................................... 3-27

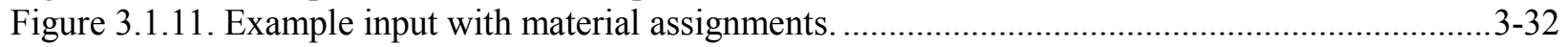

Figure 3.1.12. Example 2D model plot of material assignments. ..................................................3-32

Figure 3.1.13. Eigenvalue comparison of simplified cross section processing example.......................3-33

Figure 3.1.14. Example temperature and density TIMETABLE block input.........................................3-35

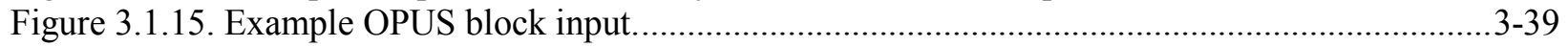

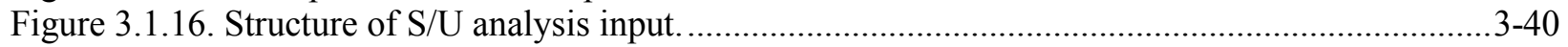

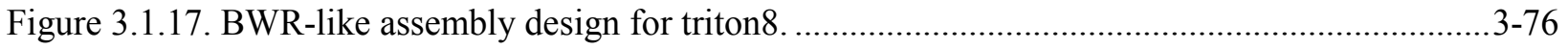

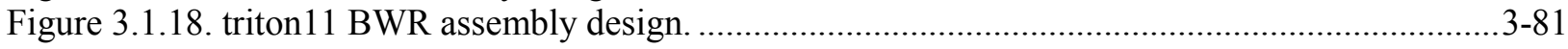




\section{LIST OF TABLES}

$\underline{\text { Page }}$

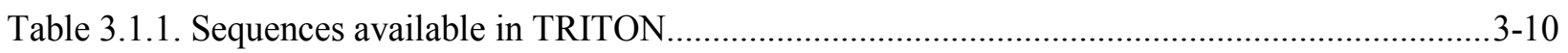

Table 3.1.2. Effects of different power basis specifications .............................................................28

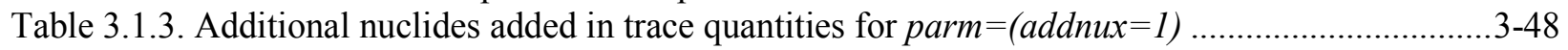

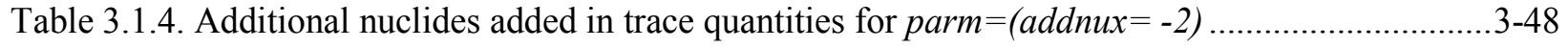

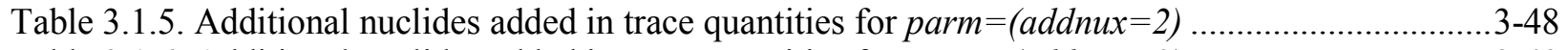

Table 3.1.6. Additional nuclides added in trace quantities for $\mathrm{parm}=(a d d n u x=3)$...............................3-49

Table 3.1.7. Additional nuclides added in trace quantities for $\mathrm{parm}=($ addnux $=4)$.................................5 30

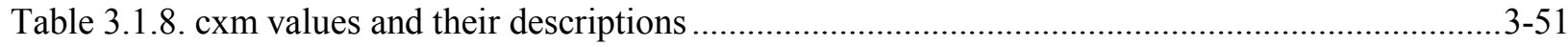




\section{ACKNOWLEDGMENTS}

The authors express gratitude to B. T. Rearden for supervision of the SCALE project and review of the manuscript. The authors acknowledge R. Y. Lee and M. Aissa of the U.S. Nuclear Regulatory Commission (NRC) for their support of this project and Mark DeHart, as the original developer of TRITON. 


\subsubsection{Introduction}

TRITON (Transport Rigor Implemented with Time-dependent Operation for Neutronic depletion) is a multipurpose SCALE control module for transport, depletion, and sensitivity and uncertainty analysis for reactor physics applications. TRITON can be used to provide automated, problem-dependent cross section processing followed by multigroup (MG) neutron transport calculations for one-, two-, and threedimensional (1D, 2D, and 3D) configurations. Additionally, this functionality can be used in tandem with the ORIGEN depletion module to predict isotopic concentrations, source terms, and decay heat.

Most notable improvements to TRITON in this latest release are the reduced run-time due to the integration of the XSProc module for cross section processing coupled with improvements in the NEWT transport module and the capability to perform continuous energy (CE) transport calculations using Monte Carlo transport codes KENO-V.a and KENO-VI (MG Monte Carlo depletion existed in previous versions).

TRITON has been designed using the modular approach that is a hallmark of SCALE functionality. TRITON provides the capability to perform deterministic transport analysis for 1D geometries using XSDRNPM and for 2D geometries using NEWT. TRITON also includes 3D Monte Carlo depletion capabilities using KENO V.a and KENO-VI.

The sensitivity and uncertainty (S/U) analysis sequence in TRITON, TSUNAMI-2D, provides calculation of the 2D forward and adjoint transport solutions in NEWT; calculation of sensitivity coefficients; and calculation of the uncertainty in $k_{\text {eff }}$ and other responses due to cross section covariance data. The SAMS module is used to determine the sensitivity of the calculated value of the response to the nuclear data used in the calculation as a function of nuclide, reaction type, and energy. The uncertainty in the calculated value of the response, resulting from uncertainties in the basic nuclear data used in the calculation, is estimated using energy-dependent cross section covariance matrices. The implicit effects of the cross section processing calculations are predicted using SENLIB and BONAMIST. The energy-dependent sensitivity data computed with TSUNAMI-2D are stored in a sensitivity data file (.sdf file) that is suitable for assessing system similarity for code validation purposes using TSUNAMI-IP or for data assimilation using TSURFER.

As a SCALE control module, TRITON automates execution of SCALE functional modules and manages data transfer and input/output processes for multiple analysis sequences. Each of TRITON's nine calculational sequences is provided in Table 3.1.1, which lists the sequence name keyword, the sequence description, and the function modules invoked within each sequence. The method for cross section processing is selected using a separate "parm=" keyword, which is described in more detail in the next section. 
Table 3.1.1. Sequences available in TRITON

\begin{tabular}{|c|c|c|c|}
\hline $\begin{array}{l}\text { Sequence } \\
\text { keyword }\end{array}$ & $\begin{array}{c}\text { Primary SCALE } \\
\text { modules }\end{array}$ & parm $=$ options & Sequence function \\
\hline \multicolumn{4}{|c|}{ Cross section processing sequences } \\
\hline$=\mathrm{T}-\mathrm{XSEC}$ & XSProc & $\begin{array}{l}\text { bonami } \\
\text { centrm }^{a} \\
\text { xslevel=1/2/3/4 }\end{array}$ & $\begin{array}{l}\text { Preparation of multigroup } \\
\text { (MG) cross section } \\
\text { library. }\end{array}$ \\
\hline \multicolumn{4}{|c|}{ Transport sequences } \\
\hline$=\mathrm{T}-\mathrm{XSDRN}$ & XSProc, XSDRNPM & \multirow{2}{*}{$\begin{array}{l}\text { bonami } \\
\text { centrm }^{a} \\
\text { xslevel=1/2/3/4 } \\
\text { weight }^{b}\end{array}$} & $\begin{array}{l}\text { 1D MG deterministic transport } \\
\text { calculation. }\end{array}$ \\
\hline$=\mathrm{T}-\mathrm{NEWT}$ & XSProc, NEWT & & $\begin{array}{l}\text { 2D MG deterministic transport } \\
\text { calculation. }\end{array}$ \\
\hline \multicolumn{4}{|c|}{ Depletion sequences } \\
\hline$=\mathrm{T}-\mathrm{DEPL}-1 \mathrm{D}$ & $\begin{array}{l}\text { XSProc, XSDRNPM, } \\
\text { ORIGEN, OPUS }\end{array}$ & \multirow{4}{*}{$\begin{array}{l}\text { bonami } \\
\text { centrm } \\
\text { xslevel }=1 / 2 / 3^{a} / 4 \\
\text { addnux }=0 / 1 / 2^{a} / 3 / 4 \\
\text { weight }^{b}\end{array}$} & $\begin{array}{l}\text { 1D MG deterministic transport, } \\
\text { coupled with ORIGEN depletion. }\end{array}$ \\
\hline$=\mathrm{T}-\mathrm{DEPL}$ & $\begin{array}{l}\text { XSProc, NEWT, } \\
\text { ORIGEN, OPUS }\end{array}$ & & $\begin{array}{l}\text { 2D MG deterministic transport, } \\
\text { coupled with ORIGEN depletion. }\end{array}$ \\
\hline$=\mathrm{T} 5-\mathrm{DEPL}$ & $\begin{array}{l}\text { XSProc }{ }^{c}, \text { KENO-V.a, } \\
\text { ORIGEN, OPUS }\end{array}$ & & $\begin{array}{l}\text { 3D, Monte Carlo transport (KENO- } \\
\text { V.a), coupled with ORIGEN depletion. }\end{array}$ \\
\hline$=\mathrm{T} 6-\mathrm{DEPL}$ & $\begin{array}{l}\text { XSProc }{ }^{c}, \text { KENOVI, } \\
\text { ORIGEN, OPUS }\end{array}$ & & $\begin{array}{l}\text { 3D, Monte Carlo transport (KENO- } \\
\text { VI), coupled with ORIGEN depletion. }\end{array}$ \\
\hline \multicolumn{4}{|c|}{ Sensitivity and Uncertainty Analysis sequences } \\
\hline $\begin{array}{l}=\mathrm{TSUNAMI}-2 \mathrm{D} \\
=\mathrm{TSUNAMI}-2 \mathrm{DC}\end{array}$ & $\begin{array}{l}\text { XSProc, NEWT, } \\
\text { SAMS, BONAMIST }\end{array}$ & $\begin{array}{l}\text { bonamist } \\
\text { bonami } \\
\text { centrm }^{a}\end{array}$ & $\begin{array}{l}\text { 2D forward and adjoint transport } \\
\text { calculations, followed by S/U analysis } \\
\text { with SAMS }\end{array}$ \\
\hline \multicolumn{4}{|c|}{$\begin{array}{l}{ }^{a} \text { Default parm value. Note that centrm is equivalent to xslevel=4. See Sect. 3.1.3.7.2 for details. } \\
{ }^{b} \text { parm=weight is used to generate a broad group cross section library. This parm option is only available } \\
\text { for the T-DEPL sequence. } \\
{ }^{c} \text { T5-DEPL and T6-DEPL is also available in CE-mode, which does not invoke XSProc for cross section } \\
\text { processing. }\end{array}$} \\
\hline
\end{tabular}

\subsubsection{Overview of TRITON Sequences}

The TRITON control module supports nine calculational sequences, each with its own design and applications. Each of these sequences is described in the following subsections.

The first subsection covers the basic cross section processing sequence T-XSEC. The T-XSEC sequence prepares problem-dependent multigroup cross sections for subsequent transport analysis. The second subsection covers TRITON's transport analysis sequences, while the third subsection discusses TRITON's depletion analysis sequences. The final subsection is dedicated to the TSUNAMI-2D sensitivity and uncertainty analysis sequences in TRITON.

\subsubsection{Cross section processing sequence (T-XSEC)}

The T-XSEC sequence provides the ability to prepare a problem-dependent multigroup cross section library using SCALE cross section processing modules to appropriately account for spatial and energy self-shielding effects. The problem-dependent cross section library contains microscopic cross sections for each nuclide for each material composition defined in the TRITON input. SCALE provides several unit cell types (e.g., a lattice of pins, an infinite medium, a multiregion problem, or a doubly heterogeneous cell) to correct the cross sections for spatial and energy self-shielding. Multiple cell 
calculations can be used in the same calculation. The calculation of multigroup cross sections is executed by XSProc.

\subsubsection{Transport sequences (T-XSDRN, T-NEWT)}

The TRITON transport sequences build upon the cross section processing sequence by automating a transport calculation after cross section processing. Both $1 \mathrm{D}$ and $2 \mathrm{D}$ discrete-ordinates transport calculations can be performed using XSDRNPM and NEWT, respectively. The T-XSDRN sequence calls XSDRNPM for transport analysis in slab, sphere, or cylindrical geometries, while the T-NEWT sequence calls NEWT for analyses in 2D $x y$-geometries. In addition to the input necessary for cross section processing, an XSDRN or NEWT input model is also required. The XSDRN model input is discussed in Appendix A of TRITON; the NEWT model input requirements are described in the NEWT chapter. Similar capabilities and applications for KENO-V.a and KENO-VI are handled through the CSAS5 and CSAS6 sequences, respectively.

\subsubsection{Depletion sequences (T-DEPL, T-DEPL-1D, T5-DEPL, T6-DEPL)}

The TRITON depletion sequences build upon the transport sequences by automating depletion/decay calculations after the transport calculations for each material designated for depletion. One or more materials in the model can be designated for depletion. Each designated material is depleted using regionaveraged reaction rates, accounting for all regions in the model associated with a given depletion material. The TRITON depletion calculation procedure is described further in the next subsection. TRITON automates the various computational processes - cross section processing, transport, and depletion - over a series of depletion and decay intervals supplied by the user. The depletion procedure is discussed in Sect. 3.1.2.3.1. The 2D TRITON depletion sequence (T-DEPL), which uses NEWT for the transport calculations, also provides the capability to generate lattice-physics data for nodal core calculations. These lattice physics capabilities are discussed in Sect. 3.1.2.3.2.

Within TRITON depletion calculations, TRITON invokes the ORIGEN depletion module for the timedependent transmutation of each user-defined material. TRITON provides ORIGEN the neutron flux space-energy distribution, the multigroup cross sections, material concentrations, and material volumes. ORIGEN performs the flux normalization, cross section collapse, and multi-material depletion/decay operations to determine new isotopic concentrations for the next calculation.

\subsection{Predictor-corrector depletion process}

For all depletion sequences, TRITON automates cross section processing, transport, and depletion calculations over a series of depletion-decay intervals supplied by the user. A depletion interval represents a time interval in which the model power level is assumed constant. A depletion model that exhibits various power level changes will require multiple depletion intervals to accurately model the changes in power. Each depletion interval can be followed by a decay calculation over a user-specified decay interval.

Within a given depletion interval (e.g., an LWR operating at constant power for a 12-month fuel cycle), the isotope concentrations of different depletion materials change, which induces changes in the problemdependent multigroup cross sections (through spatial and energy self-shielding effects) as well as the neutron flux distribution, leading to different power distributions and transmutation rates in depletion materials. This requires TRITON to represent each depletion interval as a series of smaller time intervals in which cross section processing and transport solutions are recomputed to accurately model these timedependent effects. A depletion subinterval represents a time interval in which TRITON performs cross 
section processing and transport calculations to determine cross sections and flux distributions used in the depletion calculations. All depletion subintervals for a given depletion interval have the same length-for example, one 12-month depletion interval can be represented as a series of 12 one-month depletion subintervals, or as 6 two-month depletion subintervals. Alternatively, the 12-month depletion interval can be modeled as two consecutive 6-month depletion intervals, each one having a different number of subintervals. Therefore the formulation of a depletion scheme in TRITON is highly flexible. A depletion scheme is the set of user-defined depletion and decay intervals with associated power levels and number of subintervals. TRITON does not provide automated means to determine the appropriate depletion scheme for a given application. The user must determine the accurate depletion scheme specific to his or her application.

TRITON uses a predictor-corrector approach to process the user-defined depletion scheme. The predictorcorrector approach performs cross section processing and transport calculations based on anticipated isotope concentrations at the midpoint of a depletion subinterval. Depletion calculations are then performed over the full subinterval using cross sections and flux distributions predicted at the midpoint. Depletion calculations are then extended to the midpoint of the next subinterval (possibly through a decay interval and into a new depletion interval), followed by cross section processing and transport calculations at the new midpoint. The iterative process is repeated until all depletion subintervals are processed. In order to start the calculation, a "bootstrap case" is required using initial isotope concentrations for the initial cross section processing and transport calculation. The bootstrap calculation is used to determine the anticipated isotope concentrations at the midpoint of the first depletion subinterval.

The predictor-corrector approach is best explained by an example. Figure 3.1.1 illustrates the predictorcorrector process for a hypothetical depletion scheme with two depletion intervals. The first depletion interval contains two subintervals, followed by a decay interval. The second depletion interval contains one subinterval and is also followed by a decay interval. In Figure 3.1.1, cross section processing and transport calculations are represented by the " $T$ " label, and depletion calculations are represented by the "D" label. For this example, four sets of calculations would be necessary: one for each of the three depletion subintervals, and one for the initial "bootstrap case." These calculations are represented in the following eight steps.

Step $1 \mathrm{~T}_{0}$ : Cross section processing and transport calculation using initial (i.e., time-zero) isotope concentrations.

Step $2 D_{1}$ : $\quad$ Depletion calculation from time-zero to the midpoint of the first depletion subinterval. The dashed horizontal arrow denotes a "predictor" depletion step.

Step $3 \mathrm{~T}_{1}$ : Cross section processing and transport calculation at the midpoint of the first depletion subinterval.

Step $4 D_{1}$ : Depletion calculation for the first depletion subinterval. The solid horizontal arrow across the subinterval denotes a "corrector" depletion step. Corrector steps use cross sections and flux distribution computed at the subinterval midpoint. This is represented by a solid arrow from $T_{1}$ to $D_{1}$.

$\mathrm{D}_{2}$ : $\quad$ Predictor depletion calculation for the second depletion subinterval. Predictor steps use cross sections and flux distribution computed at the previous subinterval midpoint. This is represented as the dashed arrow from $T_{1}$ to $D_{2}$.

Step $5 \mathrm{~T}_{2}$ : Cross section processing and transport calculation at the midpoint of the second depletion subinterval. 
Step $6 \mathrm{D}_{2}$ : Corrector depletion calculation for the second depletion subinterval, followed by the decay calculation at the end of the first depletion interval.

$\mathrm{D}_{3}$ : $\quad$ Predictor depletion calculation for the third depletion subinterval. The third depletion subinterval is the first and only subinterval associated with the second depletion interval.

Step $7 \mathrm{~T}_{3}$ : Cross section processing and transport calculation at the midpoint of the third depletion subinterval.

Step $8 \quad \mathrm{D}_{3}: \quad$ Corrector depletion calculation for the third depletion subinterval. This calculation is followed by a second decay calculation.

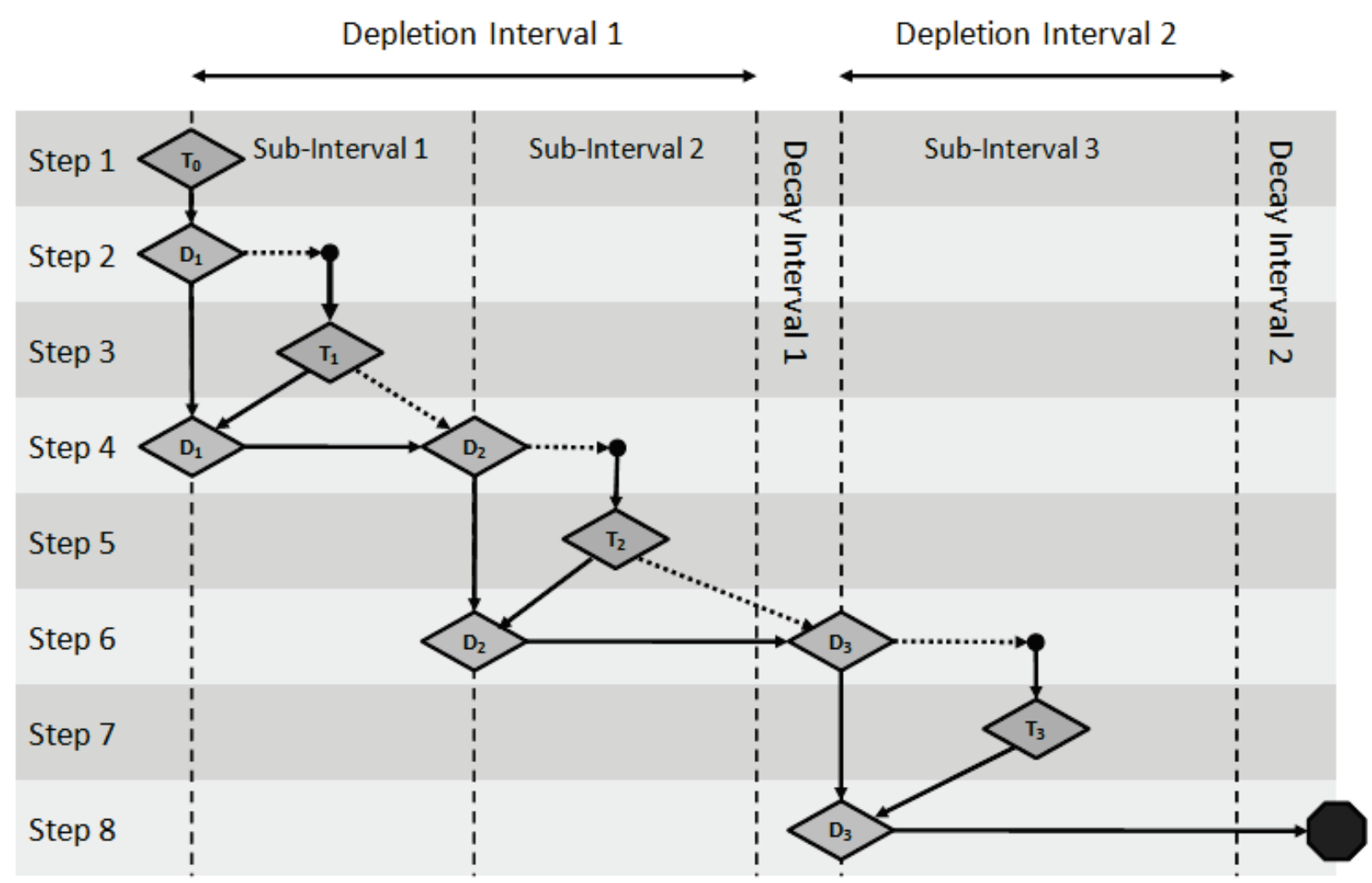

Figure 3.1.1. Predictor/corrector depletion algorithm used by TRITON.

The depletion calculations are performed by ORIGEN and span either the first half of a subinterval (predictor step) or the full subinterval (corrector step). ORIGEN performs these depletion calculations and possible decay calculations over a series of smaller time intervals. The ORIGEN time intervals are automatically determined by TRITON depending on the length of the depletion subinterval and decay interval. Additionally, TRITON will automatically adjust the number of subintervals per depletion interval if the time length of the user-defined subinterval is large (i.e., $>400$ days). TRITON writes the utilized depletion scheme near the top of the output file. The depletion scheme output edit is further described in Sect. 3.1.5.4.1. 


\subsection{Lattice physics analysis}

The 2D depletion sequence (T-DEPL) may be used to generate lattice physics data for subsequent core analysis calculations using core simulator software. Core simulators typically employ few-group nodal diffusion theory for neutronic calculations, coupled with other calculation methods for thermal hydraulics, fuel performance, and plant operation (e.g., soluble boron letdown or control rod movement). Core simulation requires the use of pretabulated lattice physics data for the neutronic calculations - that is, few-group homogenized cross sections, with appropriate discontinuity factors, pin powers, and kinetic parameters, functionalized in terms of burnup and other system conditions such as fuel temperature and moderator density.

To support lattice physics database preparation, the NEWT transport module contains flexible input options to define the few-group energy structure, spatial homogenization regions, and discontinuity factors. After the transport calculation at the midpoint of each depletion subinterval, NEWT computes the lattice physics data and stores this data on a temporary file. TRITON reads the temporary file and archives the lattice physics data onto a separate database file. In addition, the $T$ - $D E P L$ sequence supports branch calculations in which perturbations may be applied to certain system conditions such as fuel temperatures and moderator density. TRITON automates the cross section processing and transport calculations for each branch condition at the midpoint of the depletion subinterval. NEWT computes the lattice physics data for the branch calculations, and TRITON archives this data onto the lattice physics database file.

The TRITON input options for branch calculations are described in Sect. 3.1.3.3.2, and the file format of the lattice physics database is provided in the Appendix B of TRITON. The TRITON input options for branch calculations are designed to be highly flexible to support a large range of core analyses; therefore, TRITON does not provide automated means to determine the branch calculations. The user must determine the necessary branch calculations for his or her core analysis and be knowledgeable of the capabilities and limitations of the cross section treatment of the core simulator. The TRITON Lattice Physics Primer has been developed to provide guidance on appropriate TRITON branch calculations for LWR core analysis (NUREG/CR-7041) and in "Cross Section Generation Guidelines for TRACEPARCS” (NUREG/CR-7164).

\subsubsection{S/U analysis sequences (TSUNAMI-2D, TSUNAMI-2DC)}

TRITON supports a 2D sequence (TSUNAMI-2D) to support cross section sensitivity and uncertainty $(\mathrm{S} / \mathrm{U})$ analysis. The TSUNAMI-2D sequence is similar in function to the $2 \mathrm{D}$ transport sequence $\mathrm{T}$ NEWT, except that TRITON sets up additional calculations to perform $\mathrm{S} / \mathrm{U}$ analysis. After the initial transport calculation, a second transport calculation is performed to compute the adjoint flux solution. Both the forward and adjoint fluxes are saved to different files that are read by the SAMS module to compute sensitivity coefficients and the uncertainty in $k_{\text {eff. }}$ In addition to $\mathrm{S} / \mathrm{U}$ analysis for $k_{\text {eff }}$, the TSUNAMI-2D sequence allows for $\mathrm{S} / \mathrm{U}$ analysis of user-defined ratios of forward flux responses, such as flux-weighted cross sections, reaction rate ratios, and power-peaking factors. For each user-defined response ratio, TRITON automates additional fixed-source adjoint transport calculations in NEWT, followed by S/U calculations in SAMS. TRITON iteratively calls NEWT and SAMS for each response ratio definition. TRITON also automatically sets up the fixed-source input for NEWT, which is implicitly defined by the response ratio specification.

For TSUNAMI-2D calculations, the TRITON input is similar to 2D transport calculations (T-NEWT) with some additional input options. Input data blocks are available to define response ratios for $\mathrm{S} / \mathrm{U}$ analysis. Additional input data blocks are available for the SAMS module to control various aspects of the 
sensitivity and uncertainty calculations and output formatting. The S/U input options are further discussed in the TSUNAMI-1D manual. Examples of TSUNAMI-2D models are provided in Sect. 3.1.6.

Although TSUNAMI-2D is similar to the T-NEWT sequence, enhanced versions of the cross section processing codes are used to compute sensitivity data necessary for the SAMS calculation. In place of the BONAMI code used by T-NEWT, TSUNAMI-2D utilizes a sensitivity version called BONAMIST. This enhanced code computes the problem-dependent multigroup cross sections along with their sensitivities to the input data, the so-called "implicit sensitivities." Implicit sensitivity effects from ENDF/B-VII cross sections are now treated with full-range Bondarenko factors present on the multigroup library with BONAMIST.

The NEWT-based TSUNAMI-2D sequence is functionally similar to the TSUNAMI-1D and TSUNAMI3D_K5/TSUNAMI-3D_K6 sequences of SCALE, in which cross section processing, forward and adjoint transport calculations, and S/U calculations are automated using XSDRN and KENO V.a/KENO-VI, respectively. Like these S/U sequences, TRITON supports an auxiliary sequence, TSUNAMI-2DC, which does not perform the additional adjoint transport calculations and SAMS calculations. The TSUNAMI2DC sequence does not employ the sensitivity version of the cross section processing code, BONAMIST, as the sensitivity data from this code is not used in the calculation sequence. The TSUNAMI-2DC sequence is identical to the T-NEWT sequence with one exception: if user-defined response ratios are provided in the input, then TSUNAMI-2DC will compute the response ratios and edit their values in the output file.

During the development of SCALE 6.2, the cross section processing routines were significantly modernized to use XSProc for all of the TRITON sequence except TSUNAMI-2D; therefore, the run times will be slower for each T-NEWT calculation and the solution will be somewhat different. However, the other TSUNAMI sequences do use XSProc.

\subsubsection{Input Description}

TRITON input is free-form and keyword based, similar in form to many other modules in SCALE. With a few exceptions, the following formatting rules apply:

- Data is limited to 255 columns but may wrap into as many lines as are needed.

- Comment lines start with a tick mark ( ' ) in the first column of a line and may be placed anywhere in the input.

- The keyword-based input is case insensitive.

- TRITON input is organized into blocks of data. Each data block begins with read blockname and terminates with end blockname.

- Blocks of data may appear in any order. Each block of data may appear only once in the input.

- Input can be redirected from an auxiliary file by using the open angle bracket $(<)$ and the name of the file_-for example, </path/to/auxiliary_input_file.

The first three lines of input and the last line of the input are unique. The first line of input contains the TRITON sequence name along with parameter specifications, e.g., parm=centrm. The second line contains the case title (up to 80 characters), and the third line contains the cross section library identifier. The last line of the input contains the end keyword and terminates the input file. An example TRITON input is as follows: 


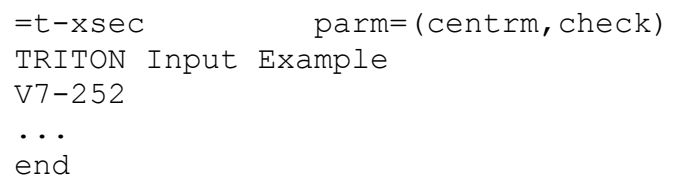

In this example, the first line of input declares this calculation to use the T-XSEC sequence. The name of the sequence is preceded by the "=" sign. After the sequence name, two parameter options are specified. Parameters are optional. If specified, the keyword parm $=$ must precede the parameter options. Multiple parameter options can be provided in a comma-separated list enclosed in parentheses. In this example, the centrm option specifies the CENTRM-based discrete-ordinates sequence is used by default. The check option implies that TRITON will read all input and ensure that no input errors are present, without running additional calculations. The second input line provides the case title: TRITON Input Example. The third input line provides the cross section library: V7-252. This example input file is terminated at the end keyword. The end keyword must appear by itself at the beginning of the final line of the input file.

The TRITON input section is organized by sequences. The first section summarizes the input requirements for the cross section processing sequence T-XSEC, which includes discussion of the COMPOSITION and CELLDATA block. The second section summarizes the input requirements for the TRITON transport sequences T-XSDRN and T-NEWT. The XSDRN MODEL block is described in Appendix B of TRITON. The third section summarizes the input for TRITON depletion sequences: T-DEPL-1D, T-DEPL, T5-DEPL, and T6-DEPL. The depletion sequence input section includes discussion of the DEPLETION, BURNDATA, TIMETABLE, BRANCH, and OPUS blocks. The fourth section summarizes the input requirements for the TRITON S/U sequences TSUNAMI-2D and TSUNAMI-2DC.

The input requirements for the depletion sequences and the $\mathrm{S} / \mathrm{U}$ sequences build upon the input requirements for the cross section processing sequence and the transport sequences, so the user should be familiar with these first two sections. However, the input requirements for the depletion and $\mathrm{S} / \mathrm{U}$ sequences are independent, so the user can skip over these sections as needed.

The fifth and sixth section of the input description is dedicated to two TRITON-specific blocks of data to simplify model development and output control: the ALIAS block and the KEEP_OUTPUT block, respectively. The final section describes TRITON control parameters used in the parm $=$ specification.

\subsubsection{Cross section processing}

An example input structure for a cross section processing sequence calculation is provided in Figure 3.1.2.

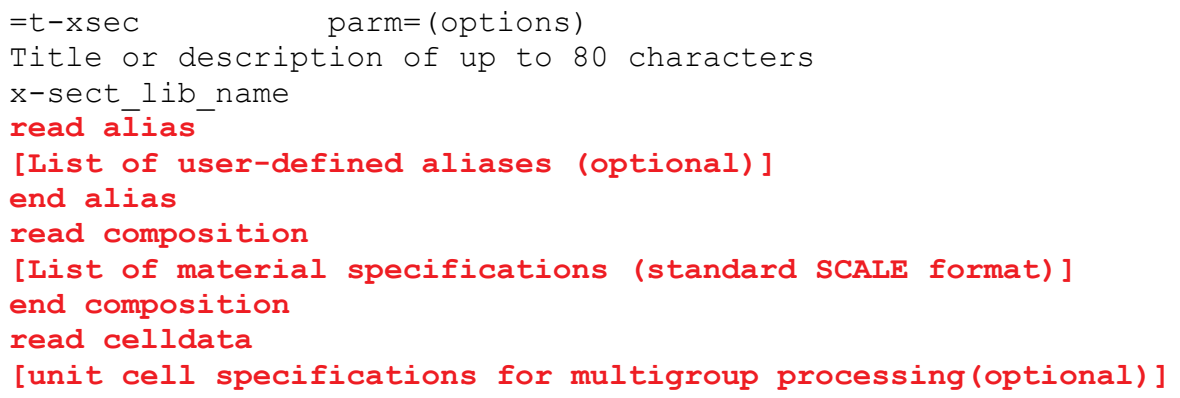


end celldata

end

Figure 3.1.2. Structure of T-XSEC input.

In this input, the title can be any descriptive title, and the cross section library $x$-sect lib name can be any multigroup SCALE cross section library (or continuous-energy library if KENO is used). The three blocks of data highlighted in red-ALIAS, COMPOSITION, and CELLDATA-must appear in the order shown above. However, the ALIAS and CELLDATA blocks are optional. If the ALIAS block is not used, the COMPOSITION block follows the cross section library line. If the CELLDATA block is not used, the input is terminated after the COMPOSITION block.

The input requirements for the ALIAS block are deferred to Sect. 3.1.3.5 as the ALIAS block impacts many different blocks of data for all TRITON sequences. The COMPOSITION block is used to define material compositions and temperatures. The CELLDATA block is used to specify unit cell calculations used to generate problem-dependent multigroup cross sections. The input requirements for the COMPOSITION and CELLDATA blocks are comprehensively described in the XSProc manual and are not repeated here. Figure 3.1.3 shows an example input for a cross section processing calculation. In this input file, cross section processing calculations are performed for two different square-pitched $\mathrm{UO}_{2}$ fuel pins surrounded by Zircaloy-4 cladding and borated $\mathrm{H}_{2} \mathrm{O}$ moderator. The first fuel pin (material 1) is $2.5 \%$ enriched in ${ }^{235} \mathrm{U}$. The second fuel pin (material 4 ) is $4.5 \%$ enriched in ${ }^{235} \mathrm{U}$. These materials are used in two separate unit cell definitions in the CELLDATA block.

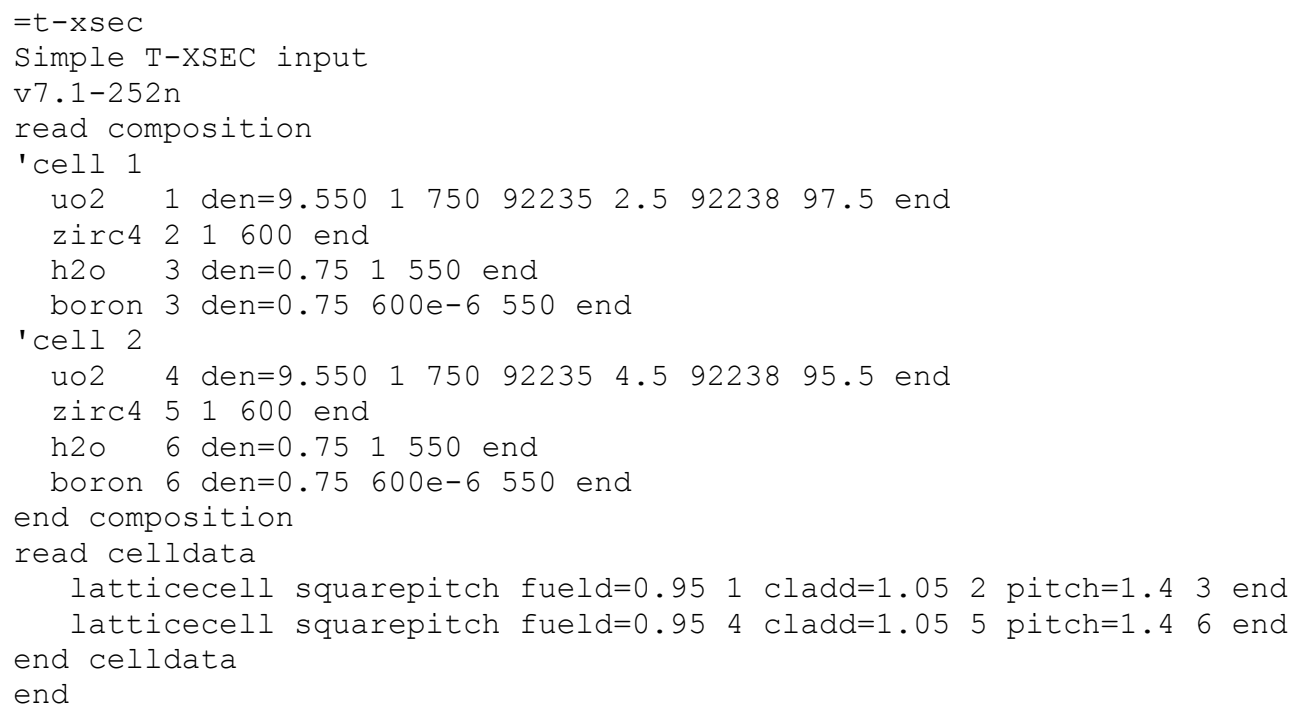

Figure 3.1.3. Example T-XSEC input.

One key observation in this example is the duplicate definitions for the clad material (materials 2 and 5) and the moderator material (materials 3 and 6). For practical use in subsequent transport calculations, only four material compositions need to be defined: one each for the different fuel pin enrichments and one definition each for the clad and moderator material compositions. However, as described in the XSProc manual, the same material identifier cannot be used in multiple unit cell definitions. Because this example requires two separate unit cell definitions to appropriately generate cross sections for each fuel pin enrichment, duplicate definitions are required for the clad and moderator compositions. The unique mixture number input requirement can lead to many duplicate definitions of clad and moderator materials, depending on model complexity. To simplify model development, duplicate material compositions and 
similar unit cell definitions can be defined simultaneously through the use of aliases. The ALIAS block is discussed further in Sect. 3.1.3.5.

\subsection{Combined two-region and $S_{N}$ cross section processing}

It is possible to use the both the CENTRM-based two-region method and the CENTRM-based $\mathrm{S}_{\mathrm{N}}$ method within the same input file. Figure 3.1.4 shows a modified input file of the previous example in which the first unit cell uses $\mathrm{S}_{\mathrm{N}}$ cross section processing and the second unit cell uses two-region cross section processing. Each unit cell contains a centrmdata keyword specification after the latticecell specification. The centrmdata specification contains a set of additional keyword specifications used to identify the $\mathrm{S}_{\mathrm{N}}$ and the two-region options in CENTRM.

The input centrmdata npxs=1 end centrmdata instructs TRITON to use $\mathrm{S}_{\mathrm{N}}$ cross section processing, whereas the input centrmdata npxs $=5$ end centrmdata instructs TRITON to use tworegion cross section processing. These keyword options are described in detail in the XSProc manual. The default cross section option for TRITON is $\mathrm{S}_{\mathrm{N}}$; therefore, the first centrmdata specification is not needed (but still acceptable). If parm =centrm was specified, the first centrmdata specification would not be needed (but still acceptable), whereas the second centrmdata specification would be required to activate the two-region option. Conversely, if parm=2region was specified, the second centrmdata specification is not needed (but still acceptable), whereas the first centrmdata specification would be required to activate the $\mathrm{S}_{\mathrm{N}}$ option.

The centrmdata specifications may also be applied to other unit cell types (e.g., multiregion); however, the two-region method is only valid for specific unit cell configurations described in the XSProc manual. The user should determine the applicability of the two-region method by comparing calculation results with continuous-energy calculations or multigroup calculations using the CENTRM-based $\mathrm{S}_{\mathrm{N}}$ method.

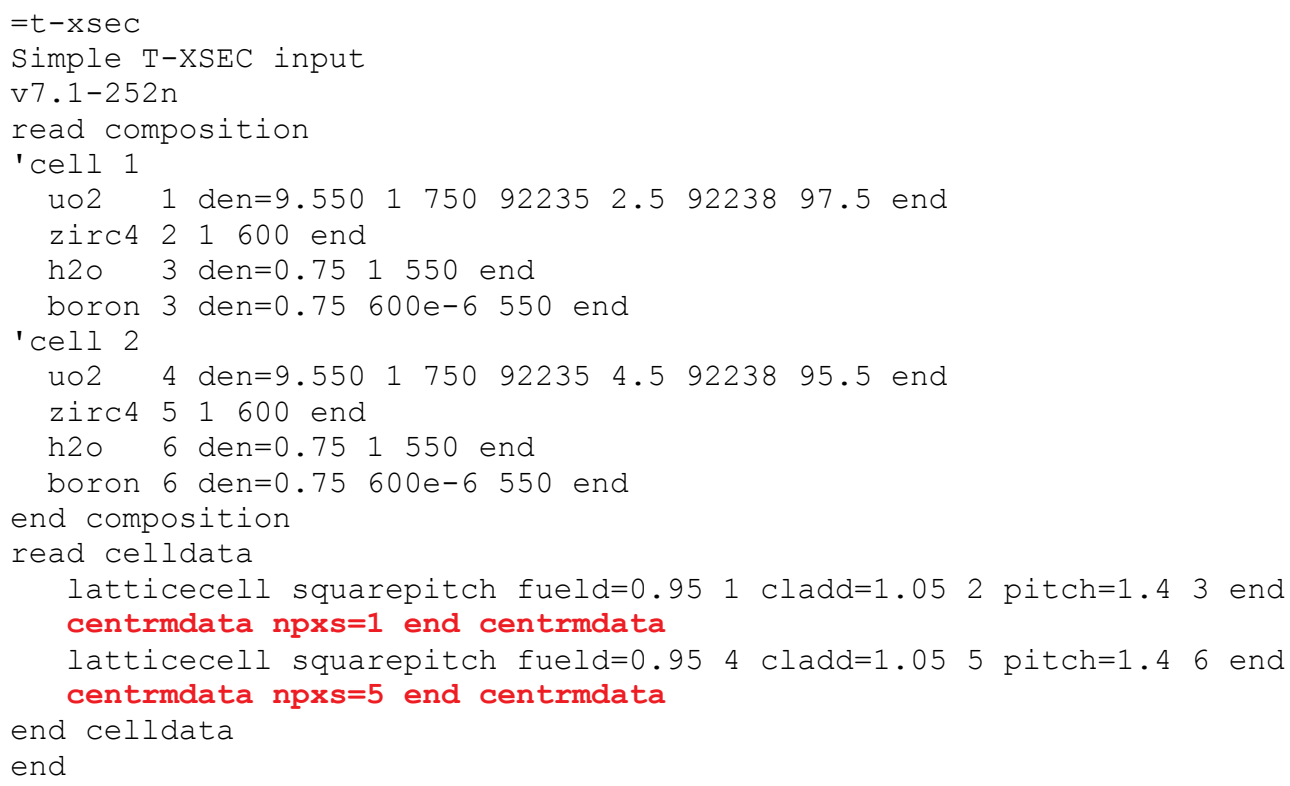

Figure 3.1.4. T-XSEC input with multiple cross section processing options. 


\subsection{User-defined Dancoff factors}

Like other SCALE calculations, TRITON uses Dancoff factors as part of its cross section processing calculations. The user can specify Dancoff factors for various materials by using the centrmdata specification and the dan2pitch keyword. Here is an example.

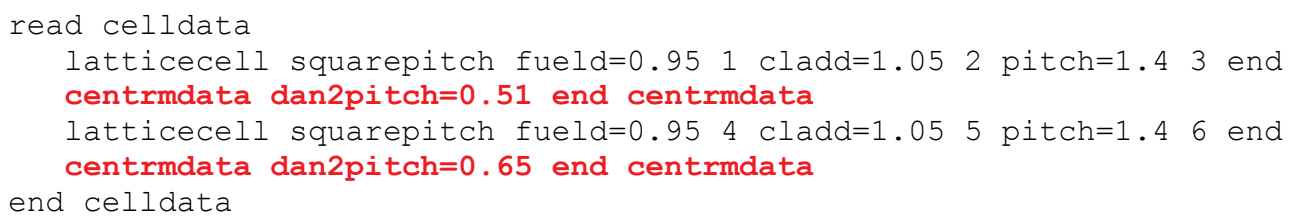

In this example, fuel materials 1 and 4 were assigned a Dancoff factor of 0.51 and 0.65 , respectively. These Dancoff factor values can be computed using the SCALE MCDANCOFF sequence. Only one dan2pitch keyword is allowed for a given centrmdata specification.

\subsubsection{Transport sequences}

An example input structure for a transport sequence calculation is provided in Figure 3.1.5.

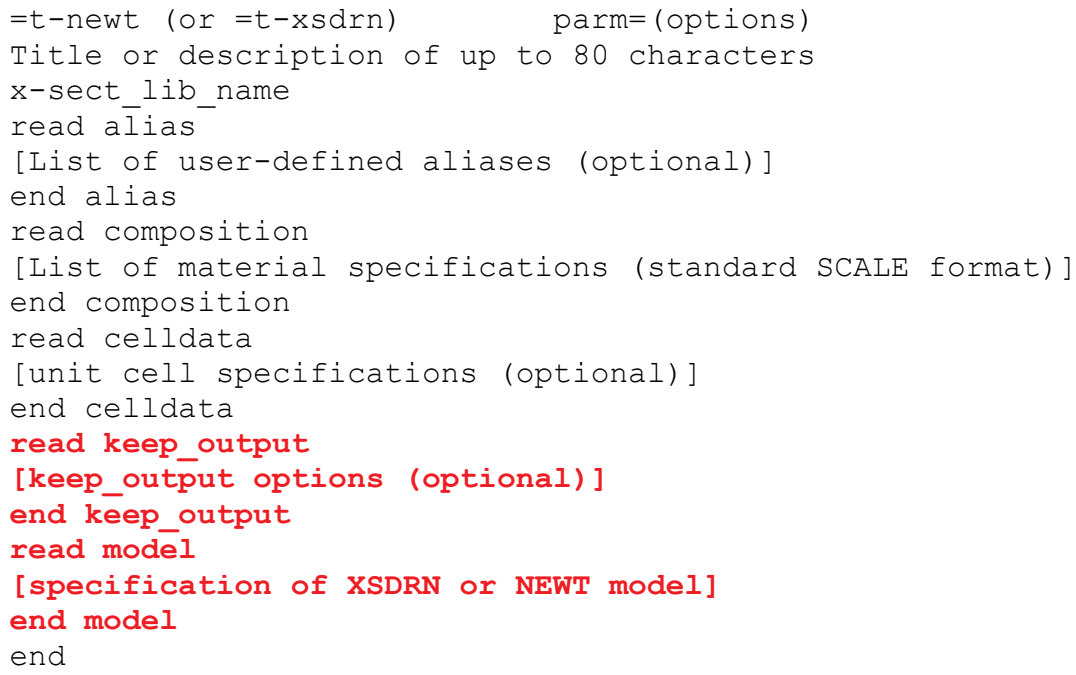

Figure 3.1.5. Structure of T-NEWT or T-XSDRN input.

The MODEL block contains a full transport model input description and is required for both the T-NEWT and T-XSDRN sequences. The MODEL block must be the last block of data in the input file. The MODEL block provides the physical layout of the configuration for which the transport calculation is to be performed, along with general control parameters. The nature of data embedded within the MODEL block depends on the sequence selected. For the T-NEWT sequence, the MODEL block contains a complete NEWT input listing. NEWT input is fully described in the NEWT chapter and is not repeated here. For the T-XSDRN sequence, the MODEL block is described in the Appendix B of TRITON. Sample problems for both the T-NEWT and T-XSDRN sequences are provided in Sect. 3.1.6. The optional KEEP_OUTPUT block is described in Sect. 3.1.3.6.

\subsubsection{Depletion sequences input}

An example input structure for a depletion calculation is provided in Figure 3.1.6. 


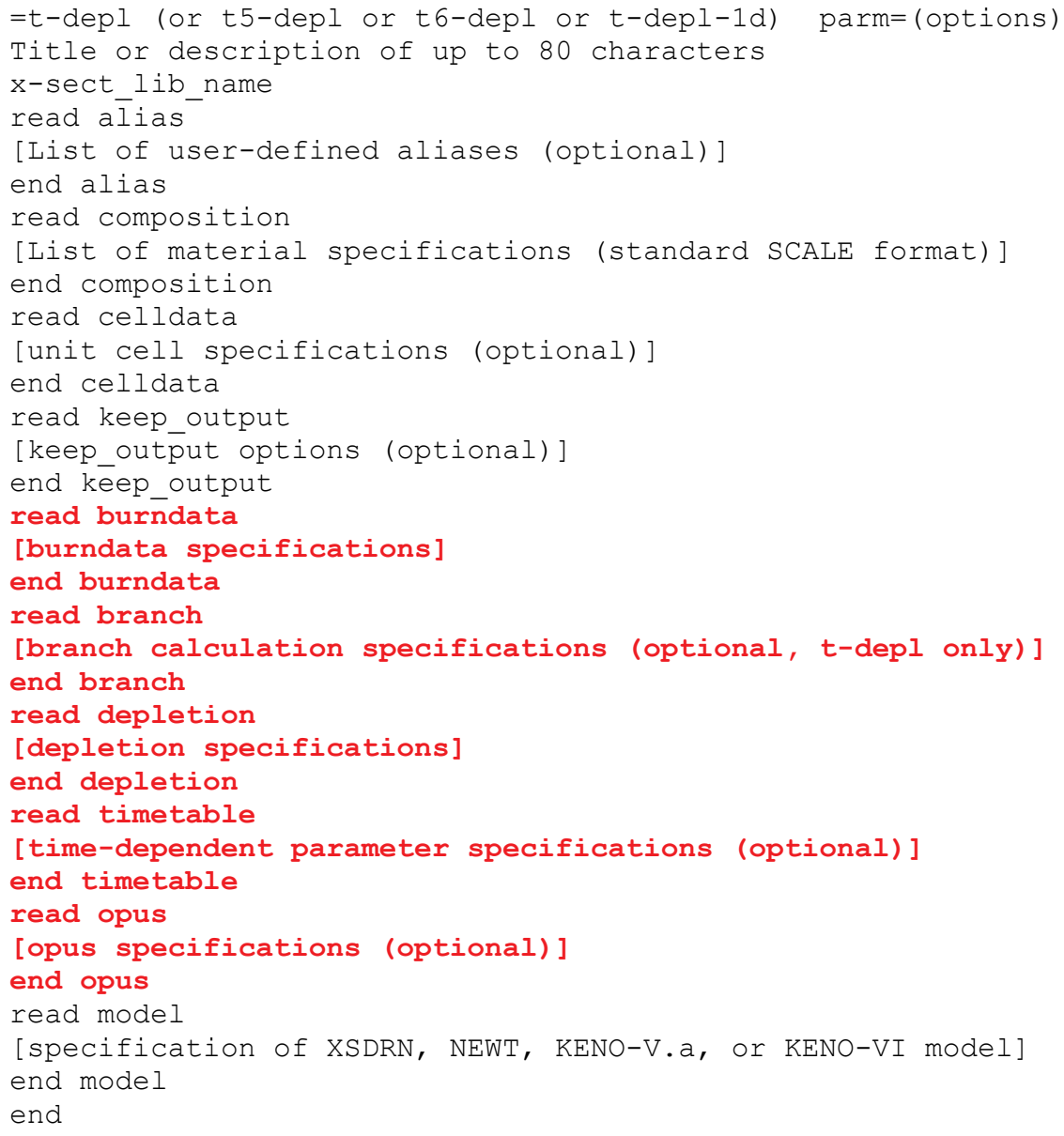

Figure 3.1.6. Structure of depletion sequence input.

The TRITON depletion sequences support the blocks of data highlighted in red: the DEPLETION, BURNDATA, OPUS, BRANCH, and TIMETABLE data blocks. These data blocks, along with the KEEP_OUTPUT block, may appear only once, in any order, and must follow the COMPOSITION and $C E L L \bar{D} A T A$ blocks and must precede the MODEL block. The DEPLETION and BURNDATA blocks are always required for depletion calculations.

The MODEL block contains a full transport model input description and is required for all depletion sequences. For the $T-D E P L$ sequence, the MODEL block contains a complete NEWT input listing. NEWT input is fully described in NEWT chapter and is not repeated here. For the $T$-DEPL- $1 D$ sequence, the MODEL block is described in Appendix A of TRITON. For T5-DEPL and T6-DEPL sequences, the $M O D E L$ block contains input for KENO V.a and KENO-VI, respectively. The details of KENO V.a and KENO-VI input formats are described in the KENO V.a and KENO-VI chapters and are not repeated here.

TRITON reads the MODEL block at the beginning of the sequence to process the input and save data to appropriate data in memory (or on a restart file for KENO). Reading the MODEL block at the beginning of the sequence allows TRITON to check all transport module data and to terminate immediately if errors are found in the model input. When the transport module is eventually invoked by the sequence, TRITON uses the processed data in memory (or reads it from the restart file), allowing for transport iterations 
(XSDRN, NEWT) or neutron histories (KENO) to begin immediately, eliminating the need for recalculation of geometry data each time the transport module is invoked.

\subsection{BURNDATA block}

The BURNDATA data block allows specification of the depletion scheme for the model and is used only by the four depletion sequences in TRITON. As described in Sect. 3.1.2.3.1, the depletion scheme consists of a series of depletion intervals - time intervals of constant power operation-which may be partitioned into many depletion subintervals - intervals over which cross section processing and transport calculations are performed to update cross sections and flux distributions used in the depletion calculation. Moreover, depletion intervals may be optionally followed by a decay interval-a time interval for zero-power decay.

The depletion intervals that define the depletion scheme are specified in the BURNDATA block in chronological order within the BURNDATA block, with the following format.

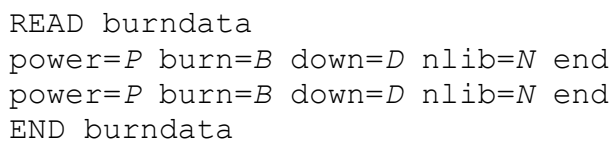

where

$P=$ average specific power in the basis material(s), in megawatts per metric tonne of initial heavy metal (MW/MTHM) (typically MW/MTU for uranium-only models);

$B=$ length of depletion interval in days;

$D=$ length of decay interval in days following the depletion interval (optional, default $=0.0$ );

$N=$ number of depletion subintervals for the depletion interval (optional, default $=1$ ).

The average specific power is provided for the basis material(s). In other words, localized power distributions are uniformly scaled accordingly in the transport solution such that the average power in the basis material(s) matches the power specified in input. By default, the basis consists of all materials in the model, so that local powers are scaled to obtain a problem-wide average power matching the power specified in input. The basis can be set as a single material or set of materials in the DEPLETION data block. The DEPLETION data block is described in Sect. 3.1.3.3.4.

Each depletion interval specification must be terminated by an end keyword. As many depletion intervals as necessary may be entered to model the depletion scheme. At least one depletion interval must always be entered; hence, the BURNDATA block is always required in depletion calculations. The number of depletion subintervals can be used to refine the temporal discretization to force more cross section processing and transport calculations per depletion interval, as discussed in Sect. 3.1.2.3.1.

An example of a BURNDATA block is shown in Figure 3.1.7. The example case in Figure 3.1.7 contains three depletion intervals, with the first interval at power 26.54 MW/MTHM in the basis materials (the basis is defined in the DEPLETION block), for an interval of 121 days. This is followed by a second depletion interval at power $38.01 \mathrm{MW} / \mathrm{MTHM}$ for 201.5 days and then 30 days of zero-power operation. In the third depletion interval, the basis materials are depleted at a $31.44 \mathrm{MW} / \mathrm{MTHM}$ power level for 386.25 days, followed by 5 years (1826.25 days) of decay. In this model, three, two, and one depletion subintervals are used for the first, second, and third depletion intervals, respectively. 


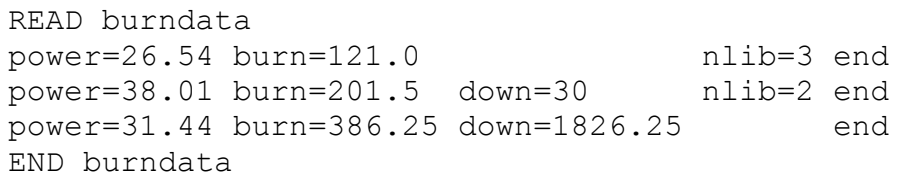

Figure 3.1.7. Example BURNDATA block input.

\subsection{BRANCH block}

The T-DEPL sequence in TRITON supports the ability to perform branch calculations during depletion calculations. Branch calculations are not supported for the $3 \mathrm{D}$ depletion sequences, nor are branch calculations supported for problems that require doubly heterogeneous cross section processing. A branch calculation is a recalculation of cross section processing and transport calculations with one or more of a limited set of input parameters modified. These calculations are performed at the same location in the depletion scheme as in the nominal cross section processing and transport calculations - that is, at $t=0$ and at the midpoint of the depletion subintervals (see Sect. 3.1.2.3.1 for more details on the TRITON predictor-corrector depletion scheme). Branch calculations allow for the quantification of changes in system responses of interest (eigenvalue, pin powers, homogenized few-group cross sections, and kinetic parameters) due to changes in system parameters. TRITON saves the responses of interest for the nominal and each perturbed (branch) state, for each evaluation within the TRITON depletion scheme. These responses of interest - in particular, homogenized cross sections - may be subsequently extracted for use in nodal core simulation calculations.

Branch calculations represent a branch from the primary depletion scheme at each depletion subinterval. With branching enabled, selected properties or conditions (fuel temperature, moderator temperature, moderator density, soluble boron concentration, and control rod insertion, or any combination thereof) can be varied from the reference state for as many branches as are desired. Depletion calculations, however, are performed for reference-state conditions only. Figure 3.1.8 illustrates the branch loop during a T-DEPL sequence calculation. Although not technically a branch state, the reference state is considered to be branch 0 for numbering purposes within TRITON. For each branch calculation $>0$, TRITON updates the appropriate parameters and re-executes the cross section processing and transport calculations. Responses of interest are saved to a database file (i.e., the txtfile16 file) for both the nominal and perturbed-state conditions, and TRITON reverts to cross sections and fluxes from the reference branch 0 to proceed with the depletion calculation. The process repeats following each depletion subinterval, until all depletion subintervals are simulated. Responses of interest are added to the database file for all branches at each depletion subinterval. 


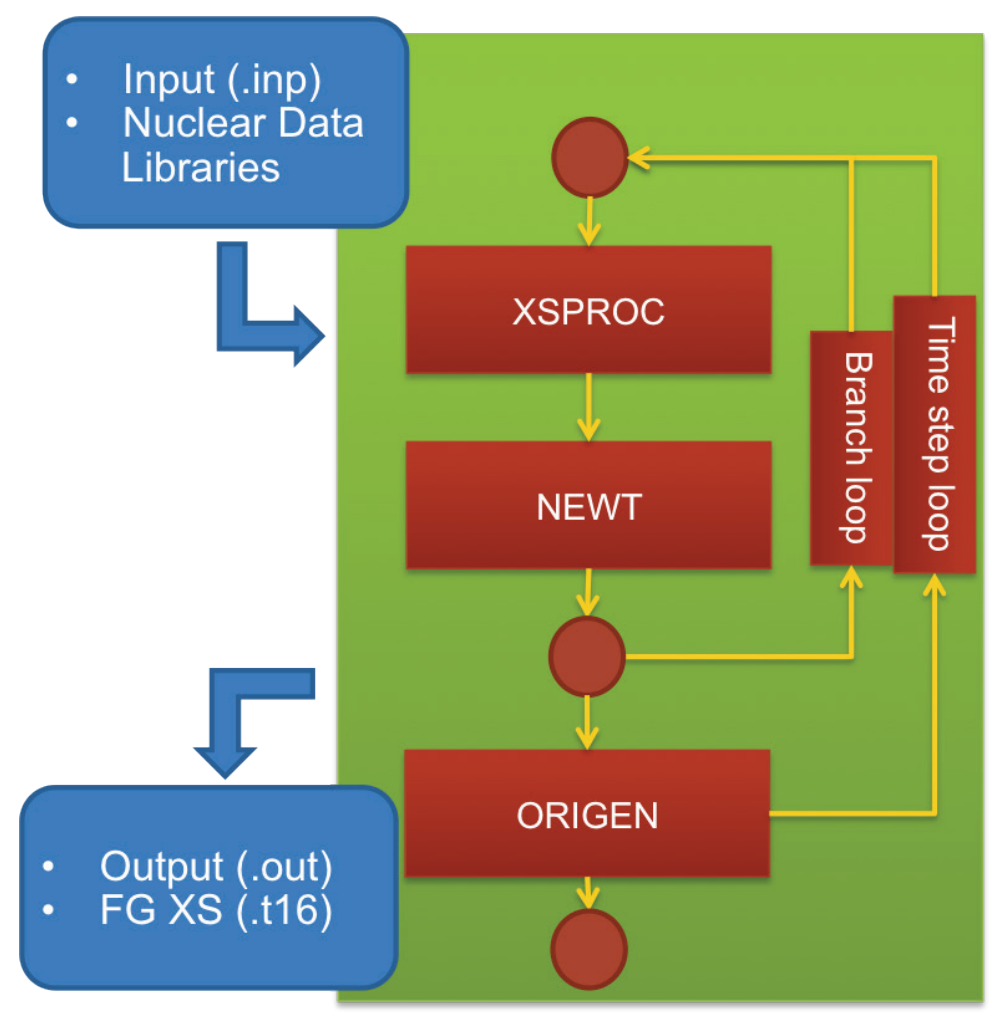

Figure 3.1.8. Schematic illustration of T-DEPL branch calculations during depletion.

Branch perturbations may be applied to any of the following five parameters: fuel temperature, moderator temperature, moderator density, moderator soluble boron concentration, and control rod insertion. These properties may be varied individually or simultaneously. Branch calculations are specified in the TRITON $B R A N C H$ data block. The BRANCH data block has the following form.

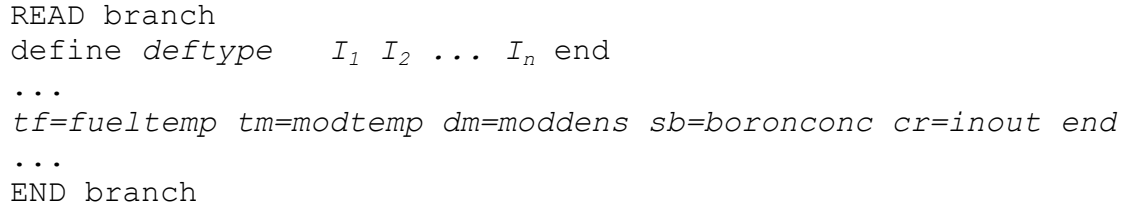

where

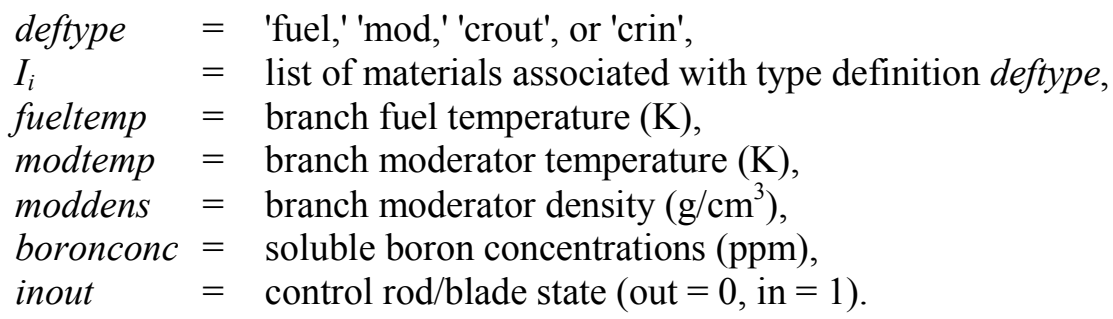

The type definitions must come first within the $B R A N C H$ block, and at least one definition is always required. The 'fuel' type definition is used to specify which of the problem materials are considered to be fuel during branch calculations; similarly, the 'mod' type definition specifies the material or materials that are to be considered moderator. The 'crout' definition specifies the materials that are in place in the transport model when control structures are withdrawn, while the 'crin' definition specifies the materials that are present in the transport model when a control structure is inserted. The 'fuel' definition must be 
present if any fuel temperature branches are performed. The 'mod' type definition must be present whenever moderator temperature, moderator density, or soluble boron branches are performed. Both the 'crout' and 'crin' definitions must be present if control rod branches are requested. Definitions may not be repeated - for example, 'define fuel' may occur only once.

Type definitions are followed by branch specifications. For each branch, one or more branch specifications may be given; if a particular property is omitted, then the reference conditions of the original model and material specifications are used. The first branch specification must describe the nominal conditions, and all parameters must be specified for this branch. Each branch specification can optionally define up to five branch keywords before terminating with the end keyword. The five branch keywords are as follows.

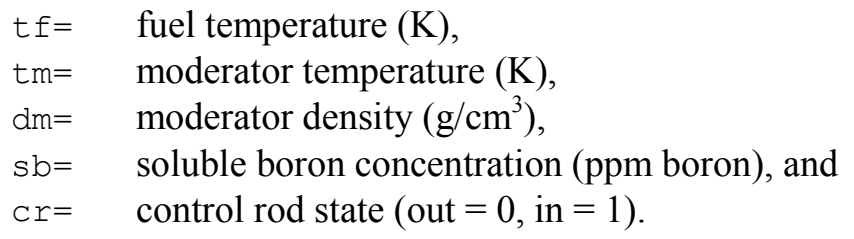

The format of a $B R A N C H$ block is best illustrated by an example. Figure 3.1.9 shows a complete branch data block for a five-branch calculation, with embedded descriptions of each branch. Note that there are six entries; the first branch is the reference or branch 0 state.

In this example, materials 11 and 12 are specified as 'fuel', and fuel temperature perturbations will be applied to only these materials. The nominal temperature for both materials is determined from the branch 0 input $(901 \mathrm{~K})$. The nominal fuel temperature must be the same for all materials in the definition and must be consistent with the initial standard composition input. Similarly, materials 13 and 14 are defined as the moderator materials. The temperature $(559 \mathrm{~K})$, density $\left(0.76 \mathrm{~g} / \mathrm{cm}^{3}\right)$, and soluble boron concentrations $(655 \mathrm{ppm})$ for the reference state must be identical to those of the initial material specifications and must be identical for all materials defined as moderator.

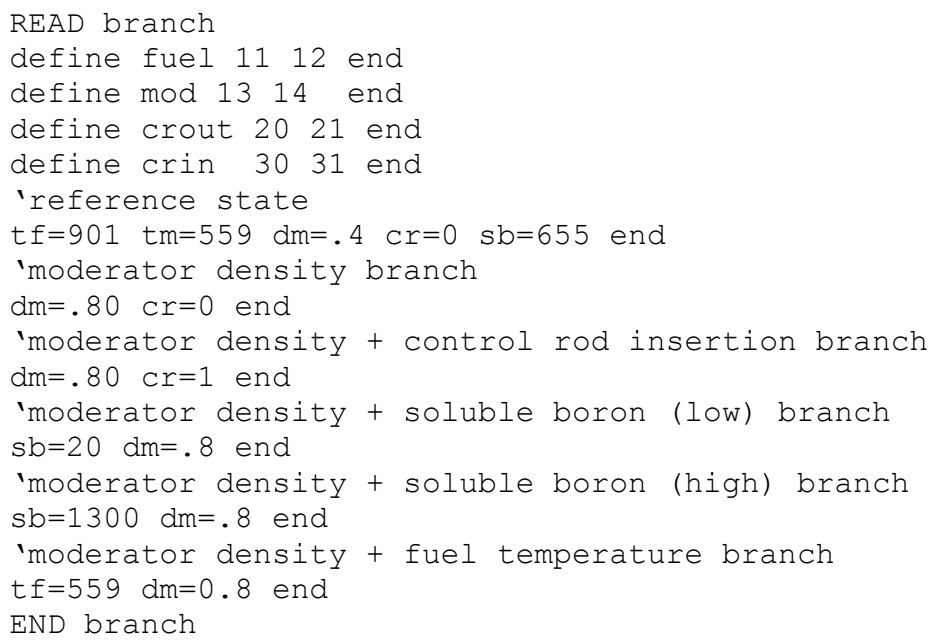

Figure 3.1.9. Example BRANCH block input.

In a reactor core, when a control structure (rod, blade, etc.) is withdrawn, the volume occupied by the structure is replaced by something else. Thus, in a branch calculation with rod insertion and withdrawal, the material(s) present for both states must be specified. If the reference condition is defined as control 
rods withdrawn (i.e., $\mathrm{cr}=0$ ), the NEWT geometry model must contain the materials defined by 'crout'. For a control rod insertion branch $(\mathrm{cr}=1)$, TRITON exchanges the materials specified in the 'crin' definition $(30,31)$ with corresponding materials in the 'crout' definition $(20,21)$. Conversely, if the reference condition is defined as control rods inserted (i.e., $\mathrm{cr}=1$ ), the NEWT geometry model must contain the materials defined by 'crin'. For a control rod withdrawal branch $(\mathrm{cr}=0)$, TRITON exchanges the materials specified in the 'crout' definition with corresponding materials in the 'crin' definition. For this reason, unique material numbers must be paired between crin and crout definitions. For example, consider a zirc-clad $\mathrm{B}_{4} \mathrm{C}$ control rod inserted during a control rod insertion branch, with materials 30 and 31 representing the clad and rod materials, respectively. In the withdrawn position, both the clad and poison materials are replaced by the moderator. To have consistent definitions of 'crin' and 'crout', two moderator materials must be defined for the withdrawn state: one corresponding to the clad material and one corresponding to the rod material.

As mentioned earlier, only one condition keyword is required per branch, but all five may be used. However, the reference state (branch 0) entry must specify all five conditions. For subsequent branches, when a specific branch state is not specified, the reference state is used. In the above example, the first entry, branch zero, specifies the reference state with a fuel temperature of $901 \mathrm{~K}$, moderator temperature of $559 \mathrm{~K}$, moderator density of $0.4 \mathrm{~g} / \mathrm{cm}^{3}$, control rod withdrawn, and a soluble boron concentration of $655 \mathrm{ppm}$. The second entry (branch 1) specifies a moderator density of $0.80 \mathrm{~g} / \mathrm{cm}^{3}$ and the control rod state as withdrawn. Since the reference state is for a withdrawn control rod, the statement $\mathrm{cr}=0$ is redundant (but completely acceptable). The next branch is identical to the previous branch, except that in this case the control rod is inserted. For both cases, reference fuel and moderator temperatures were used. In the following branch, the soluble boron concentration is changed to $20 \mathrm{ppm}$, and the moderator density is again set to a value of $0.8 \mathrm{~g} / \mathrm{cm}^{3}$. In fact, this moderator density is applied to all five branches. Along with the moderator density change, the soluble boron concentration is changed to $1300 \mathrm{ppm}$ for the next branch. And finally, in the last branch, in addition to the moderator density change, the fuel temperature is changed to $559 \mathrm{~K}$. For this case, reference conditions are used for boron concentration, moderator temperature, and control rod state.

Note that TRITON compares the reference values of fuel temperature, moderator temperature, moderator density, and soluble boron concentration with the data entered in the COMPOSITION block. TRITON prints warning messages if the data in the COMPOSITION block and BRANCH block are inconsistent. Also note that each branch calculation is independent of other branch calculations. Thus, the order in which branch calculations are computed is not important.

Branch calculations are usually requested for lattice physics analysis, where the objective is to generate a database of few-group homogenized cross sections for nodal core calculations. Thus, BRANCH blocks are used in tandem with the NEWT's COLLAPSE, HOMOGENIZATION, and ADF blocks. With these blocks of data, TRITON will archive lattice physics data-few-group homogenized cross sections, assembly discontinuity factors (ADFs), homogenized kinetic parameters, pin powers, and form factorsto a binary file called xfile 016 in the SCALE temporary working directory. An auxiliary text-formatted data file called txtfile16 is also created in the SCALE temporary working directory. This file format is documented in Appendix A of TRITON.

\subsection{BRANCH block with user-defined Dancoff factors}

As previously mentioned in Sect. 3.1.3.1.2, TRITON uses Dancoff factors as part of its cross section processing calculations. Dancoff factors play an important role in characterizing spatial self-shielding effects. The XSProc module computes the Dancoff factors based on the CELLDATA input. For a squarepitched lattice cell example, Dancoff factors are computed by DANCOFF by assuming that the fuel pin is 
within an infinite lattice of identical fuel pins. The assumption of an infinite uniform lattice of fuel pins may lead to inaccurate Dancoff factors for certain configurations such as BWR assembly designs, leading to inappropriate problem-dependent multigroup cross sections. Moreover, the Dancoff factors may change significantly for certain branch conditions, such as changing the in-channel moderator density in a BWR assembly.

The TRITON BRANCH block allows the user to specify material-dependent Dancoff factors for various branch conditions. Branch-specific Dancoff factors may be utilized by defining a new set of materialdependent Dancoff factors using the $d 2 p s e t$ type definition. The set of Dancoff factors may be included in a branch specification by using the $d 2 p=$ keyword. The $B R A N C H$ block now has the following format.

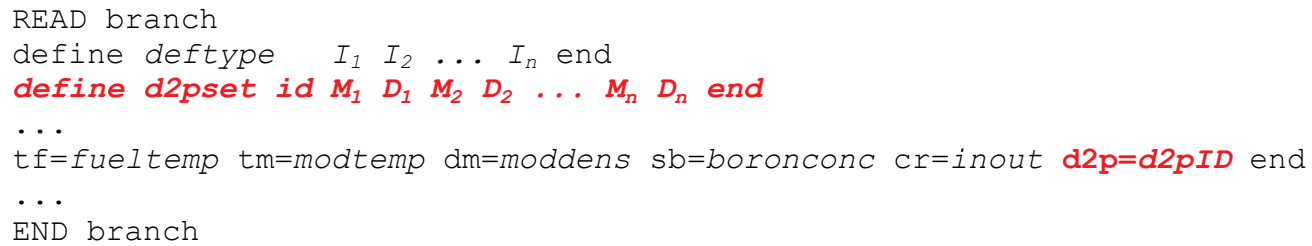

In the type definition section, the $d 2$ pset keyword is followed by a positive integer identifier, which is subsequently followed by pairs of material identifiers and their user-defined Dancoff factor value. Multiple material/Dancoff pairs may be entered for a particular set definition, as long as the material identifiers are unique. Multiple set definitions are allowed, as long as the set identifiers are unique.

The $d 2 p=$ keyword in the branch specification can be assigned to any set identifier defined in the branch definition section. If $d 2 p=$ is utilized, the material/Dancoff pairs in the set definition are applied for the given branch condition. The values $d 2 p=0$ and $d 2 p=-1$ have special meaning. If $d 2 p=$ is set to 0 , the material/Dancoff pairs defined in the CELLDATA block are utilized. If $d 2 p=$ is set to -1, the default MIPLIBcomputed Dancoff factors will be utilized, even if material/Dancoff pairs are defined in the CELLDATA block using the dan2pitch keyword available there. The nominal (branch 0) condition must use the material/Dancoff pairs (if defined) in the CELLDATA block; therefore, the first branch specification must not set the $d 2 p$ keyword to anything other than zero. (Note: $d 2 p=0$ need not be defined for the first branch condition since this is always the case.)

In Figure 3.1.10, the BRANCH block from the previous example has been modified to use branch-specific Dancoff factors. In this example, the nominal branch defines the reference moderator density to be $0.4 \mathrm{~g} / \mathrm{cm}^{3}$, and five branches use a higher moderator density of $0.8 \mathrm{~g} / \mathrm{cm}^{3}$. The Dancoff factors for the higher moderator density condition are different from the reference moderator density. To account for the different Dancoff factors at the higher moderator density condition, a set of material/Dancoff pairs are defined with the set identifier of 400. In the set, fuel material 11 has a Dancoff factor of 0.4 , and fuel material 12 has a Dancoff factor of 0.5. The set of Dancoff factors is used for the five branch states through the specification of the $d 2 p=$ keyword to 400 .

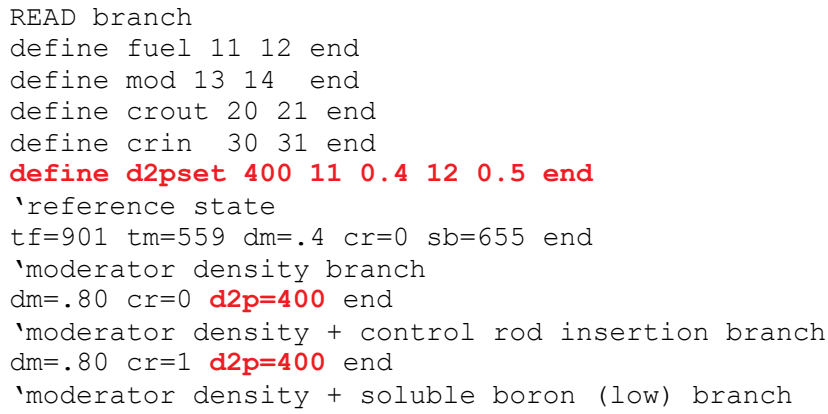




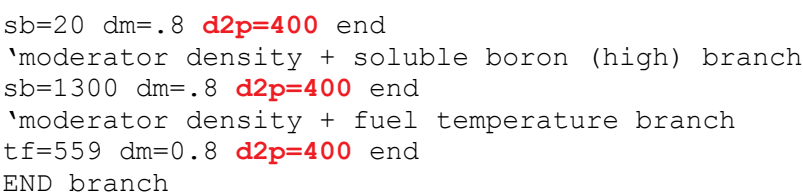

Figure 3.1.10. Example $B R A N C H$ block input with Dancoff factors.

\subsection{DEPLETION block}

The DEPLETION block, used by the four depletion sequences, is simple in concept but performs four important functions. First, this block specifies the materials for which depletion calculations are to be performed. In general, it is desirable to perform depletion calculations only for fuel and target materials of interest. Calculating the depletion of gas gaps, cladding, moderator, or coolant is usually of little value unless the material contains components that will be significantly depleted with burnup. Additionally, it is not usually desirable to deplete soluble poisons in reactor coolants. Therefore, the DEPLETION block requires that the user specify the materials to be depleted. There are no defaults; hence, the block is required for all depletion sequences.

The second function of the DEPLETION block is to specify the basis to which the model power is normalized. In general, the average time-dependent power to which an irradiated object is exposed is known. For example, an LWR fuel assembly discharged from a reactor is known to have operated at certain power levels for one or more time periods. The individual pins in the assembly will have varying power levels depending on position and assembly design. In such a case, the basis for the input power is the full assembly. Fluxes computed in the transport solution will be normalized by TRITON based on reaction rates and energies in all problem materials (depleted and nondepleted materials) such that the assembly-wide power will match the power given in BURNDATA block. However, it is often the case in radiochemical assay analysis that the burnup history of a specific pin is known and isotopic concentrations for that pin are desired. It is still necessary to model the full assembly in order to properly characterize the fluxes in that pin. In such a case, it would be advantageous to specify the operating history for the pin instead of the full assembly. When this is done, the average specific power of the full assembly will be different from that of the pin and will be computed automatically based on power distributions calculated for the assembly. In other words, powers for other materials in the assembly will be normalized such that the power in the pin of interest matches that specified in the BURNDATA block. The material of that pin becomes the basis for power normalization.

Section 3.1.3.3.4.1 below describes the general format of the DEPLETION block that is available to all four depletion sequences. The third function of the DEPLETION block is an optional function used to specify ORIGEN solver options and ORIGEN depletion mode for each depletion material. These options are further described in Sect. 3.1.3.3.4.2. The fourth function of the DEPLETION block is to define optional deletion instructions used to simplify cross section processing using the ASSIGN function. Special provisions have been made in the $1 \mathrm{D}$ and 2D depletion sequence (T-DEPL-1D and T-DEPL) to reduce the number of cross section processing calculations in order to decrease calculation run-time. The ASSIGN functionality is further described in Sect. 3.1.3.3.4.3.

\subsection{Basic DEPLETION block format}

The basic format of the DEPLETION block is as follows:

READ depletion $M_{1} \quad M_{2} M_{3} \ldots M_{n}$ END depletion 
where $\mathrm{M}_{\mathrm{i}}$ represents the SCALE material numbers for materials to be depleted. As discussed above, the DEPLETION block can also be used to specify the basis for the input power. Power normalization is accomplished by prefixing the material number(s) with a negative sign $(-)$. For example, consider a problem in which materials 1,2 , and 3 are being depleted, but the power for material 1 is known. The $D E P L E T I O N$ block for this case is

READ depletion -1 23 END depletion

In this case, powers for all materials will be normalized such that the power in material 1 matches the input power specification in the BURNDATA block.

Note that multiple materials can be used as a power basis. Consider a fuel assembly with three fuel types represented by materials 1,2, and 3, and also containing cladding as material 4 and water as material 5 . Table 3.1.2 illustrates multiple ways that the power basis for this assembly might be specified and describes the effect of each specification.

Table 3.1.2. Effects of different power basis specifications

\begin{tabular}{|l|l|}
\hline $\begin{array}{l}\text { READ depletion } \\
123 \\
\text { END depletion }\end{array}$ & The assembly-averaged power is normalized to match the input specific power \\
\hline $\begin{array}{l}\text { READ depletion } \\
-123\end{array}$ END depletion & $\begin{array}{l}\text { The assembly-averaged power is normalized such that the power of material 1 } \\
\text { matches the input specific power }\end{array}$ \\
$\begin{array}{l}\text { READ depletion } \\
-1-23 \\
\text { END depletion }\end{array}$ & $\begin{array}{l}\text { The assembly-averaged power is normalized such that the average power in materials } \\
1 \text { and } 2 \text { matches the input specific power }\end{array}$ \\
\hline $\begin{array}{l}\text { READ depletion } 235 \\
\text { END depletion }\end{array}$ & $\begin{array}{l}\text { The assembly-averaged power is normalized to match input specific powers. } \\
\text { TRITON will attempt to do depletion in cladding and moderator materials too. (Note } \\
\text { that cladding and moderator materials should be depleted using the deplete-by-flux } \\
\text { option described in the next subsection) }\end{array}$ \\
\hline $\begin{array}{l}\text { READ depletion } \\
-1-2-3 \\
\text { END depletion }\end{array}$ & $\begin{array}{l}\text { The assembly-averaged power is normalized such that the average power in materials } \\
1-3 \text { matches the input specific power. This is not the same as the normalizing } \\
\text { specification for an assembly average, because it neglects contributions of n- } \gamma \text { sources } \\
\text { in moderator and cladding materials }\end{array}$ \\
\hline
\end{tabular}

\subsection{ORIGEN depletion options}

ORIGEN provides two input options for the flux used in the depletion calculation: direct specification of fluxes (i.e., deplete by flux) or indirect specification of fluxes in terms of power (i.e., deplete by power). The ORIGEN depletion is based on a known flux; however, it is more often the case that one knows the specific power in a depletion region rather than the actual flux. When ORIGEN is used in deplete-bypower mode, ORIGEN will internally determine the corresponding flux from the input-specific power and internal tables of fission and capture energy releases for the nuclides present and the macroscopic cross sections of those nuclides. Additionally, at each ORIGEN time interval, ORIGEN recalculates the material power density as nuclide inventories change. Hence, the deplete-by-power mode will result in a time-varying flux, whereas the deplete-by-flux mode will result in a constant flux over the calculation time interval. Since reactors typically operate at a constant (or nearly so) power level, with varying local fluxes, the deplete-by-power option is closer to reality. However, the choice of approach is generally not an issue. Significant differences between calculation results between the two depletion modes would indicate that the TRITON depletion subintervals are too large. 
By default, all TRITON depletion materials use the deplete-by-power mode. However, there exist some circumstances where deplete-by-flux is more appropriate. In deplete-by-power mode, ORIGEN will often halt when an attempt to maintain constant power results in a large change in flux between ORIGEN time intervals. Large changes in flux can occur in media where isotope contents are changing rapidly with time, such as in a gadolinium-bearing burnable absorber rod, where gadolinium is being rapidly depleted with time. Another circumstance pertains to activation analysis of nonfuel materials. The flux for these materials is typically governed by external power sources (i.e., fuel materials located elsewhere in the problem domain) rather than by internal power sources. Therefore, the deplete-by-flux option is recommended for these materials.

TRITON provides the option to specify deplete-by-flux mode for selected depletion materials using a modified form of the depletion specification:

READ depletion $M_{1} \quad M_{2} \quad M_{3} \ldots M_{i-1}$ flux $M_{i} M_{i+1} \ldots$ Mn END depletion

Materials preceding the flux keyword are depleted using the deplete-by-power mode; materials following the flux keyword are depleted using the deplete-by-flux mode. For example, consider a problem in which materials 1-6 are to be depleted, but materials 3 and 4 represent nonfuel materials that do not contribute significantly to the total power and are therefore to be depleted assuming constant flux. The DEPLETION block for this situation could be specified as follows.

READ depletion 12556 flux 34 END depletion

The DEPLETION block also supports the specification of the ORIGEN calculation method. The default option is solver=matrex, which represents the matrix exponential option. The other option is solver $=$ cram, which represents the new CRAM solver option in ORIGEN. An example depletion specification for the cram solver is as follows.

READ depletion 12556 flux 34 solver=cram END depletion

\subsection{Cross section processing simplification using ASSIGN}

When depleting a large number of fuel materials, considerable time may be spent in the cross section processing calculations prior to the multigroup transport calculation. Fuel assembly designs may require 20-200 unique depletion materials across the different fuel pins in the assembly. In such cases, an assembly model may require hours of run-time for each pin-wise cross section processing calculation in order to perform a 10-minute transport solution.

Although highly rigorous, such a cross section processing process is extremely burdensome for depletion calculations, especially if branch calculations are requested. To reduce run-time, the 2D depletion sequence $(T-D E P L)$ provides the option to group depletion materials together such that they are tracked independently in the depletion calculation but use a common set of microscopic cross sections. The microscopic cross sections for a given depletion group are computed using the average composition of all the depletion materials within the group. Typically, this grouping is applied to fuel pins of identical initial composition. Although the nuclide number densities of such pins will diverge with burnup as a function of location within an assembly, the cross sections of these pins are well represented by a single pin cell calculation with an average composition representative of all these pins.

Although the material grouping option introduces approximations in the cross section processing calculations, which in turn affects the transport and depletion calculations, internal investigations have 
shown that solution accuracy can be maintained for a wide range of assembly designs while significantly improving the run-time.

The alternate format of the DEPLETION block for simplified cross section processing is as follows.

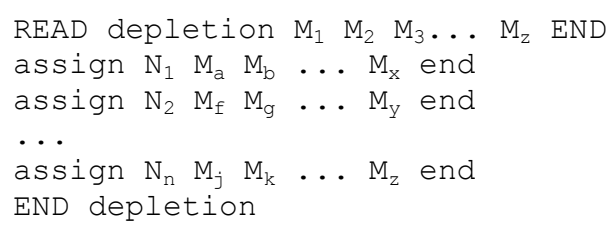

Similar to the basic format, each material designated for depletion $\left(\mathrm{M}_{\mathrm{i}}\right)$ is listed after $R E A D$ depletion and before the END keyword. Each designated depletion material must be present in the 2D NEWT model. After the first END keyword, the alternate format contains a list of material "assignments" used to simplify cross section processing for a group of depletion materials. The material assignments begin with the assign keyword and terminate with the end keyword. After the assign keyword, a unique representative material identifier $\left(\mathrm{N}_{\mathrm{j}}\right)$ is defined. The representative material is associated with the group of depletion materials that immediately follows in the assign definition. The representative material identifier is used in the COMPOSITION and CELLDATA blocks to define the initial composition, temperature, and cell definition for the group of depletion materials. Thus, the assign definitions in TRITON are currently constrained such that each depletion material group must have the same initial composition. After the last assign definition, the depletion block is terminated with END depletion.

Only depletion materials may be assigned to representative materials. The group of depletion materials assigned to a particular representative material must not appear in the COMPOSITION and CELLDATA blocks.

The use of material assignments is best illustrated by an example. Figure 3.1.11 shows a complete T-DEPL input that uses material assignments. A 2D plot of the model is shown in Figure 3.1.12. In this example, two fuel materials are defined as materials 1 and 2 in the COMPOSITON block. In the DEPLETION block, the list of depletion materials includes materials 1, 20,30, and 40. Depletion materials 20, 30, and 40 are "assigned" to representative material 2. Material 2 does not appear in the depletion list or the transport model; materials 20,30, and 40 do. But only material 2 is defined in the COMPOSITION and CELLDATA blocks. In the transport model, four units are defined, one for each material. An array is used to place each unit in its own location.

The initial calculation uses material 2 to define the compositions of materials 20,30, and 40, since all are initially identical. Microscopic cross sections computed for material 2 are used for each of the three assigned depletion materials during the transport calculation and the depletion calculation. After the first depletion calculation, materials 20,30, and 40 will have different isotopic concentrations because of different locations in the nonsymmetric transport model. At this time, the number densities in each of these three materials are averaged and used to update the concentration of representative material 2. A new set of cell calculations will be performed for materials 1 and 2; this will be followed by a transport calculation that uses the microscopic cross sections for material 2 along with local nuclide number densities for materials 20,30, and 40 to calculate new and unique macroscopic cross sections for each. The transport and subsequent depletion calculation are then run. The iterative process will continue until all depletion steps have been completed. 


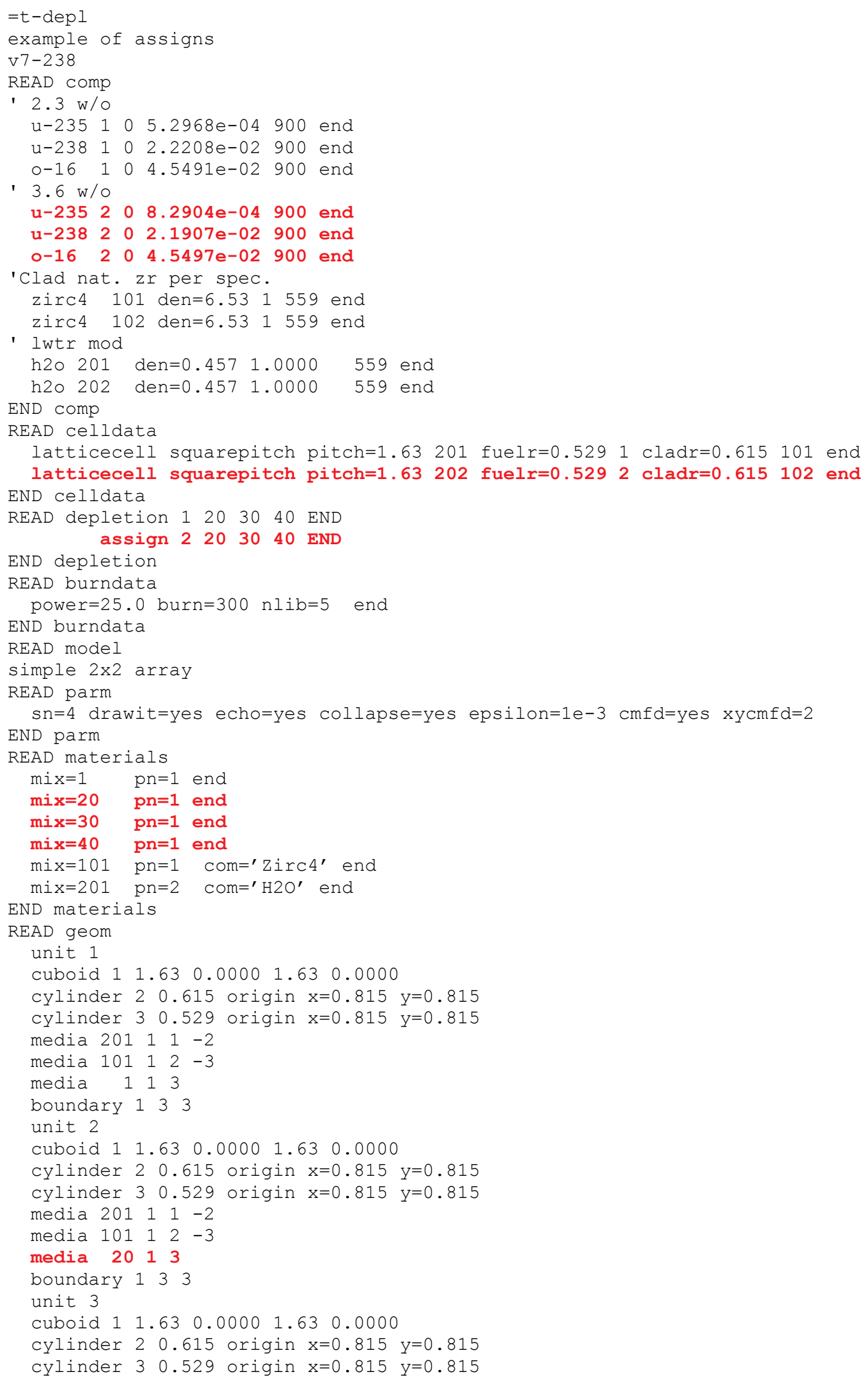




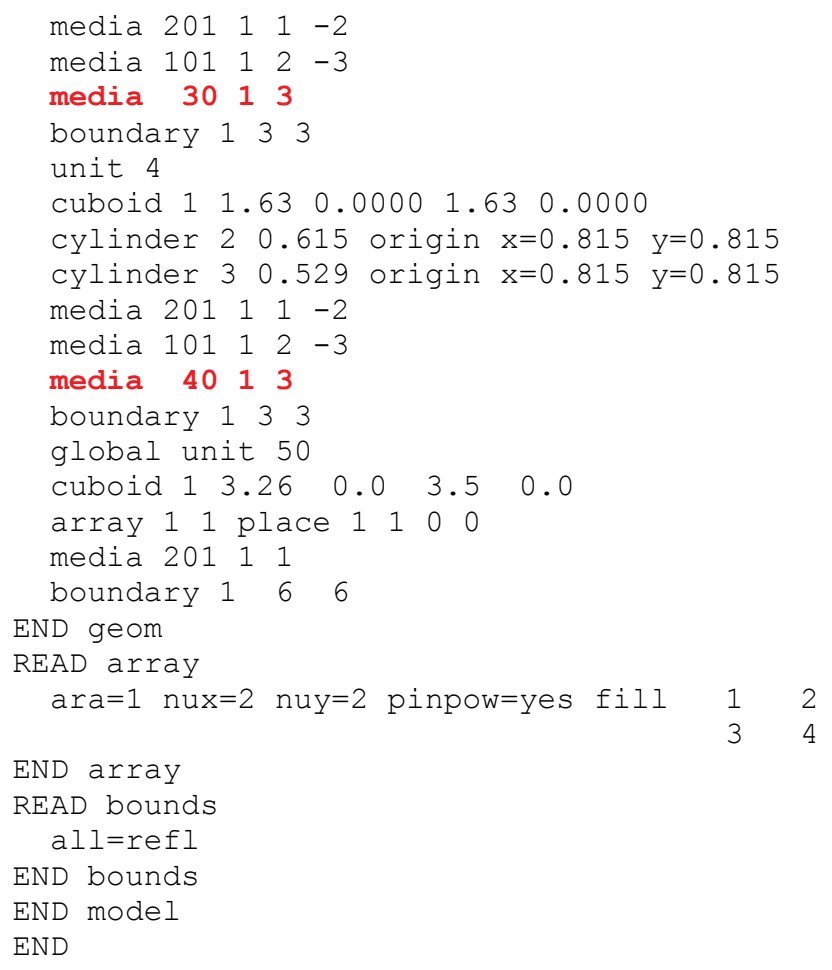

Figure 3.1.11. Example input with material assignments.
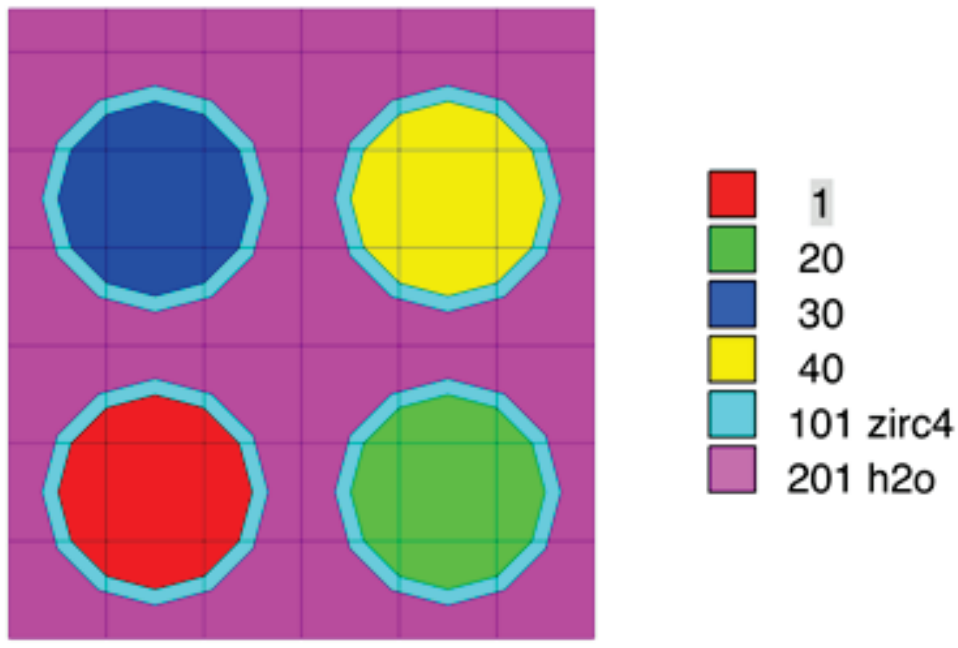

Figure 3.1.12. Example 2D model plot of material assignments.

The use of assignments can make a considerable difference in run-time performance with minimal sacrifice in accuracy. The above example ran 1.8 times faster using the assignment of three similar pins to one initial specification. A larger BWR calculation, in which 41 pin positions were depleted independently, was run in an assessment of the accuracy of the method. Using this approach, the simplified representation ran 20 depletion steps in $20 \%$ of the time required for the explicitly modeled cells. Figure 3.1.13 shows a comparison of the eigenvalues using the simplified (with assignments) and 
explicit (without assignments) models. Also shown on the figure is the percent difference between the approximate and explicit models. For this model, the error in $k_{\text {eff }}$ remains well below $0.05 \%$.

Note that one can combine depletion mode control with material assignments, as follows.
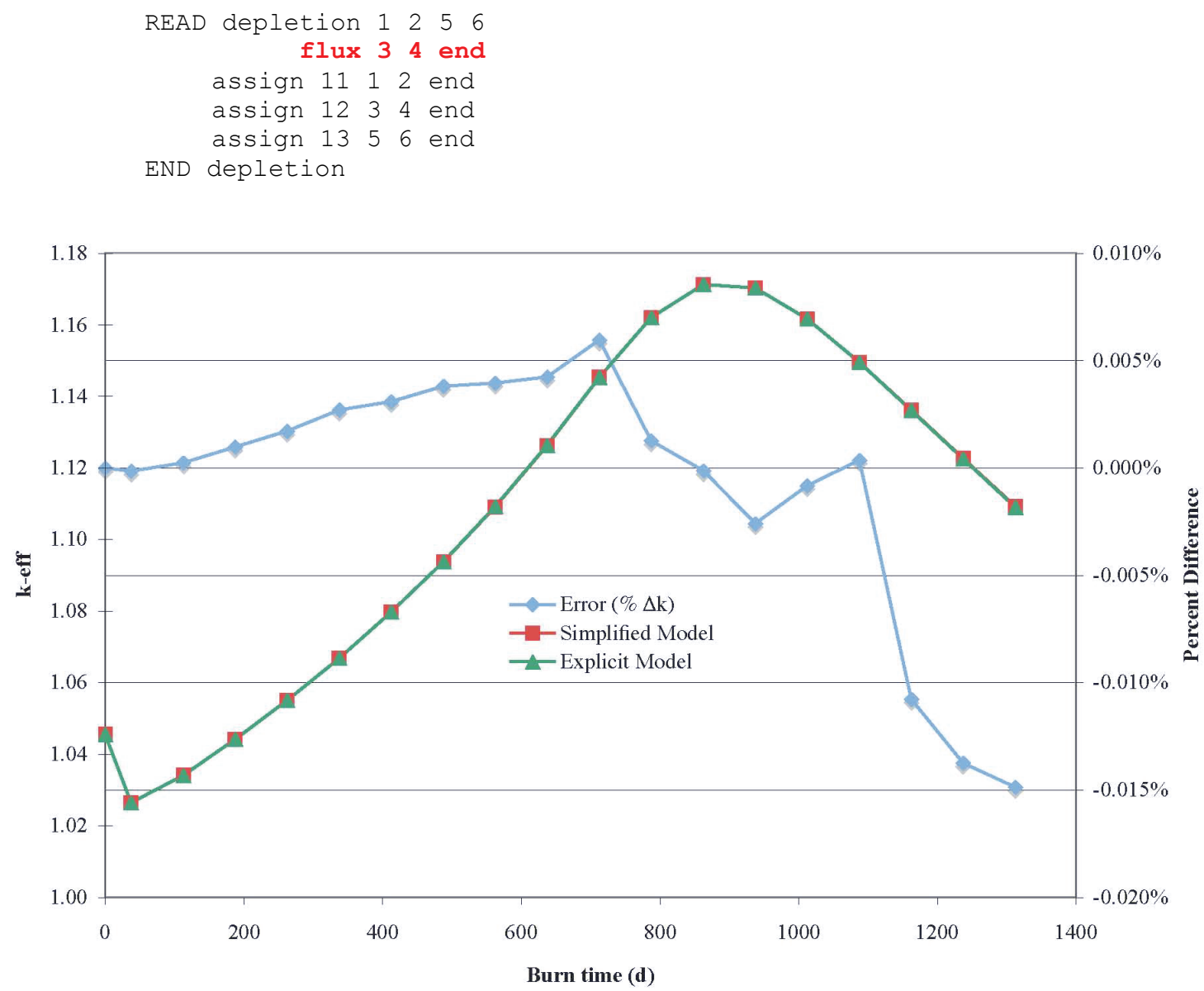

Figure 3.1.13. Eigenvalue comparison of simplified cross section processing example.

\subsection{TIMETABLE block}

In many depletion analyses, material properties can change due to influences outside the depletion process (e.g., boron letdown in pressurized water reactors [PWRs], the insertion or removal of poisons during or between fuel cycles, or changes in temperatures of materials with time). The TIMETABLE block has been provided to allow modification of properties during a depletion calculation. Timetables may be entered for any material or for select nuclides within a material and allow changes in number densities or temperatures. Timetables may also be entered to swap a material in and out of the geometry during depletion.

The TIMETABLE block takes the following general format.

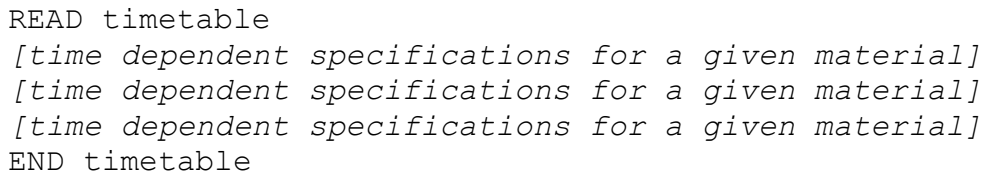


Three different material specifications are allowed to modify temperature, density, or swap materials.

Temperature timetable entries are specified in the format

temperature I t $1 \mathrm{k} 1 \mathrm{t} 2 \mathrm{~K} 2 \mathrm{t} 3 \mathrm{~K} 3 \ldots \mathrm{t} C \mathrm{KC}$ end

where
$I=$ material ID number;
$t_{i}=$ time (days) in calculation where temperature $\mathrm{K}_{i}$ is set, $i=1$ to $\mathrm{C}$;
$K_{i}=$ temperature (in K) of specified materials at time $t_{i}, i=1$ to C;
$C=$ number of time steps.

Density entries have an analogous specification, with the addition of a couple of extra terms:

$$
\text { density } \quad I \quad M \quad N_{1} \quad N_{2} N_{3} \ldots N_{M} \quad t_{1} D_{1} t_{2} \quad D_{2} t_{3} D_{3} \ldots t_{C} D_{C} \text { end }
$$

where
$I=$ material ID number;
$M=$ number of nuclides to which this change is applied;
$N_{i}=$ nuclide ID for the ith nuclide in the list, $i=1$ to $\mathrm{M}$;
$t_{j}=$ time (days) in calculation where density multiplier $\mathrm{D}_{\mathrm{j}}$ is set, $i=1$ to $\mathrm{C}$;
$D_{j}=$ density multiplier (fractional change) of specified nuclides at time $t_{j}, i=1$ to C;
$C=$ number of time steps.

In both formats, time and data (temperature or density multiplier) must be entered in pairs. Note that density changes may be applied to specific nuclides, while for temperature the change must be applied to all nuclides within the material simultaneously. If $M$ (the number of nuclides for which the density is to be modified) is specified as 0 and no nuclide IDs are entered, then the timetable values are applied to all nuclides in the material.

Note that timetable entries are specified at distinct times in the calculation. These times are measured relative to the beginning of the calculation and are continuous (as opposed to BURNDATA entries, which give burn times or down times in increments per depletion interval). The initial timetable entry should always begin at $\mathrm{t}=0$ days. To allow for time-dependent changes in properties, TRITON applies linear interpolation between data pairs. To hold a parameter constant over a time interval, that parameter should be specified at the same value at both the beginning and the end of this time interval.

The application of timetable entries is best illustrated by example. Consider the depletion scheme described by the BURNDATA block of Figure 3.1.7, which contains three depletion intervals. Assume that the moderator, material 3 , has temperatures and boron concentrations that vary over the three depletion intervals in the following manner: 


\begin{tabular}{cccc}
\hline \multirow{2}{*}{ Interval } & \multicolumn{2}{c}{$\begin{array}{c}\text { Boron concentration } \\
(\mathbf{p p m})\end{array}$} & $\begin{array}{c}\text { Temperature } \\
\mathbf{( K )}\end{array}$ \\
\cline { 2 - 3 } & BOC & $\mathbf{E O C}$ & 615 \\
\hline 1 & 1000 & 100 & 685 \\
2 & 1250 & 130 & 610 \\
\hline 3 & 980 & 100 & \\
\hline
\end{tabular}

Figure 3.1.14 demonstrates a TIMETABLE format that could be used to provide this information to TRITON. This is based on an initial input concentration of $1000 \mathrm{ppm}$ boron in the standard composition specification for material 3. The temperature of all nuclides in material 3 is constant over each depletion interval, while the density of only the soluble boron in the moderator changes linearly over each depletion interval.
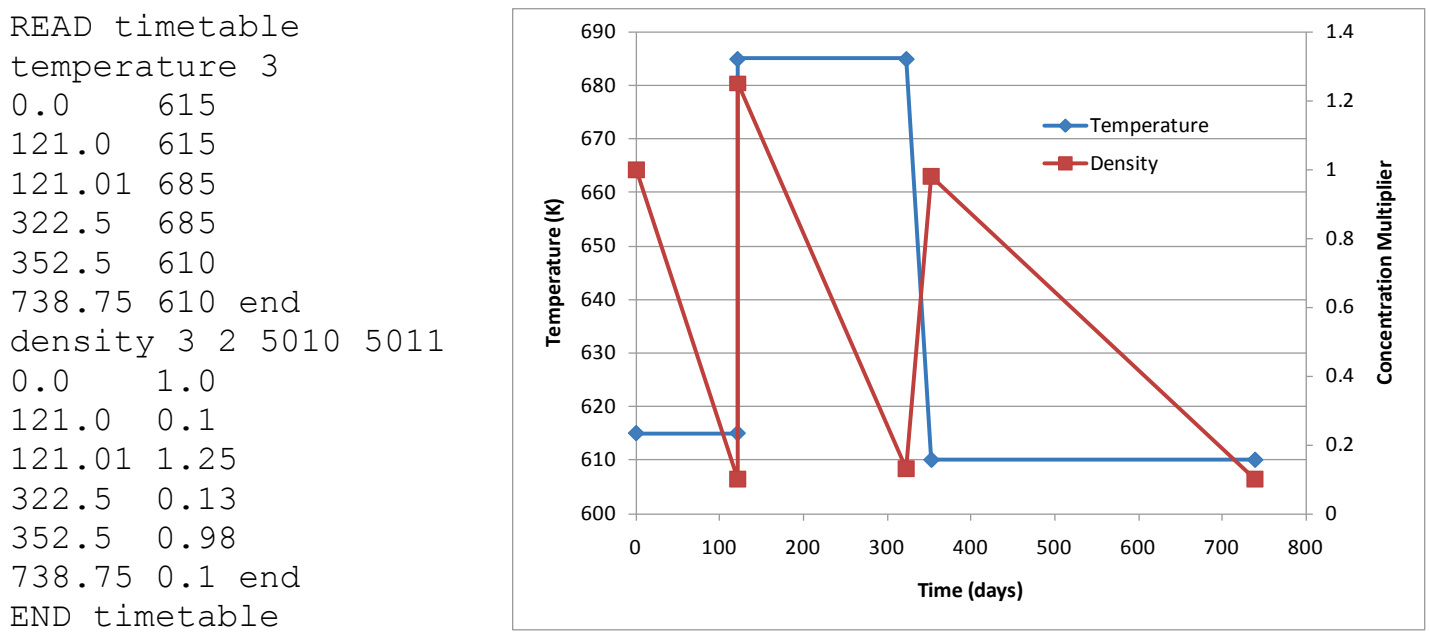

Figure 3.1.14. Example temperature and density TIMETABLE block input.

It is important to note that time-dependent changes to temperatures and number densities are not applied continuously over the depletion calculation but instead are applied only at the times at which cross section processing and transport calculations are performed - that is, the midpoint of the depletion subintervals. The user must determine the accurate depletion scheme specific to his or her application to accurately model time-dependent changes in system properties.

Density timetable specifications can be used to effectively exchange compositions of a single material. One may construct a compound material comprised of two distinct materials at their design densities; a timetable specification can be used to set the density multiplier to 1.0 for the nuclides initially present and to use a multiplier of 0.0 for all nuclides in materials that are not intended to be present at time zero. The timetable can then affect the exchange by changing the multipliers from 0 to 1 , and from 1 to 0 , at the time of the material exchange. One must bear in mind that timetable processing within TRITON performs linear interpolation between time points; if the exchange is intended to occur at a specific moment in time, then the timetable should be set up with the exchange occurring within a very short period. Moreover, it is important to note that material exchanges for two materials that have common nuclides are more difficult to model. For example, a $\mathrm{B}_{4} \mathrm{C}$ absorber material and borated $\mathrm{H}_{2} \mathrm{O}$ moderator material both contain boron nuclides in common. In order to exchange the $\mathrm{B}_{4} \mathrm{C}$ absorber material and the borated $\mathrm{H}_{2} \mathrm{O}$ moderator 
material, the carbon, oxygen, and hydrogen density multipliers would be 0 or 1 , but the boron density multipliers would need to be derived from the boron concentrations in both materials.

Material exchange timetables offer another option to users to exchange one material with another material during depletion calculations.

Material exchange timetable has a similar format to temperature timetables:

$$
\text { swap I1 I2 t1 s1 t2 s2 t3 s3...tC SC end }
$$

where

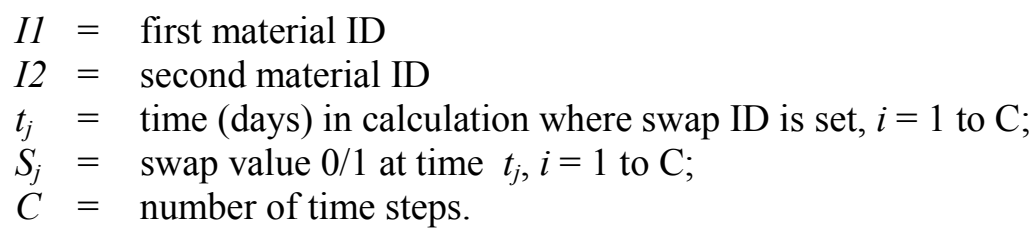

The first two entries in the timetable specify the material IDs for swap materials. The remaining entries are entered in pairs: the first pair value is a time value, the second pair value is either " 0 " or " 1 ". " 0 " instructs TRITON to model the swap materials as defined in the nominal model. "1" instructs TRITON to swap the materials (swap every $I 1$ for every $I 2$ and swap every $I 2$ for every $I 1$ ). The swap state persists until the next time entry in the timetable. For the last time entry in the timetable, the swap state persists for the duration of the calculation. For the example:

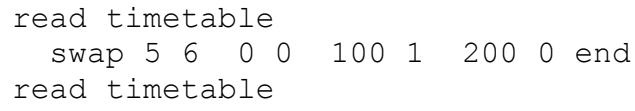

- Do not perform the material swap on the interval $[0,100]$,

- Perform the material swap on the interval [100, 200], and

- Return to the nominal state at time 200 days until the duration of the calculation.

Depending on the BURNDATA specification, there may be one or more depletion/decay steps between timetable entries. Moreover, for accurate depletion modeling, material exchanges must not occur during a depletion subinterval. If a material exchange occurs during a depletion interval, TRITON will subdivide the depletion subinterval at the time of the material exchange. Extending the example above, assume the $B U R N D A T A$ block is as follows:

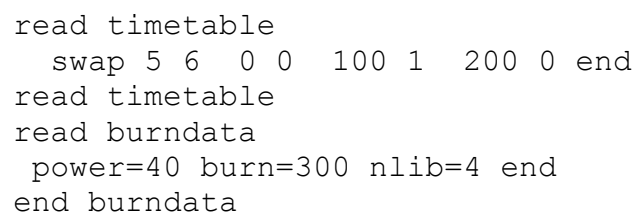

Without the material exchange table, the depletion subintervals are $[0,75],[75,150],[150,225]$, and $[225,300]$. With the material exchange table, the subintervals are:

- $[0,75]-$ Swap value is 0

- $\quad[75,100]-$ Swap value is 0

- $\quad[100-150]-$ Swap value is 1 , i.e. materials 5 and 6 swap definitions 
- $\quad[150-200]-$ Swap value is 1 , i.e. materials 5 and 6 swap definitions

- [200-225] - Swap value is 0 , materials 5 and 6 return to their original definitions

- $\quad[225-300]-$ Swap value is 0

As a limitation of the material exchange timetable, if a depletion material is removed from the geometry, the isotope concentrations at the time of removal are stored in-memory, and then reused upon re-entry into the geometry. In other words, the depletion material does not undergo radioactive decay for the period of time outside the problem geometry.

\subsection{OPUS block}

The OPUS module of SCALE is fully documented in the OPUS chapter of the SCALE manual. OPUS provides the ability to extract specific data from ORIGEN output libraries, perform unit conversions, and generate plot data for post-calculation analysis. In essence, OPUS is an ORIGEN post-processor that provides data in the desired form for a desired subset of nuclides. TRITON by default calls OPUS to extract nuclide concentrations for selected nuclides for all depletion materials and for the most important nuclides. TRITON provides the capability to specify the full set of OPUS commands to tailor OPUS calculations to obtain specific information. TRITON allows a stacked set of OPUS calculations in order to retrieve selected data for selected nuclides.

The content of the OPUS block is based on standard OPUS input parameters, as described in the OPUS chapter; the details of OPUS control and use are not repeated here. However, additional input is necessary to support TRITON operations with OPUS, because TRITON enables additional capabilities beyond those provided for in standard OPUS input. For example, OPUS is designed to process the output file from a single ORIGEN calculation; because ORIGEN is a point depletion solver, the output represents data from a single material. TRITON is typically used to perform multiple depletion calculations at each depletion step - one calculation for each material being depleted. Hence, multiple OPUS calculations are needed to obtain results from multiple materials. The OPUS calculations are performed automatically by TRITON but require the user to specify the materials for which OPUS processing is desired. Additionally, TRITON supports stacked OPUS cases within the READ OPUS data block; hence, keywords are introduced to separate stacked cases.

There are two alternatives available to SCALE users that are complimentary to the OPUS block within TRITON. First, standalone OPUS case(s) can be used to post process the ORIGEN binary concentration file (.f71 extension). This file is automatically saved in the output directory with the file name \$ \{OUTBASENAME\}.f71. (e.g. if the input file is reactor.inp, the concentration file is saved in the output directory as reactor.f71) Second, the user may also open the concentration file within Fulcrum to enable similar post-processing capabilities.

\subsection{Selection of materials for OPUS processing}

Beyond standard OPUS input keywords (see OPUS chapter), TRITON reads a matl= keyword to allow specification of ID number(s) for the material(s) in the problem for which outputs are desired. The matl $=\ldots$ end input keyword accepts one or more materials from the DEPLETION data block for which OPUS processing is desired. If omitted, OPUS processing will be performed for all materials in the DEPLETION block. For example, consider the following DEPLETION and OPUS data blocks:

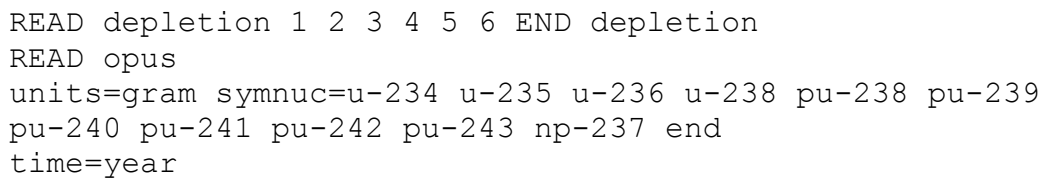


In this example, OPUS processing will be performed for all depletion materials, 1-6. Adding a subset of materials using the matl= keyword, for example.

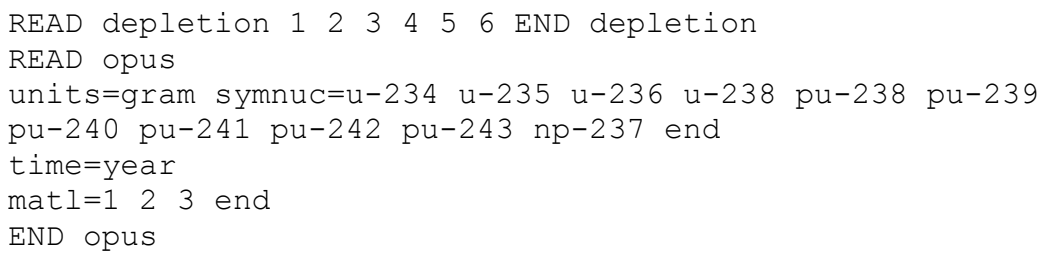

will result in OPUS calculations for materials 1,2 , and 3 only.

Although ORIGEN calculations are performed only for individual materials, TRITON provides the capability of combining the results of all or a subset of all depletion materials to get a multimaterial average set of ORIGEN responses. TRITON provides two special ID numbers for combining material results. Specification of material ID 0 will return system-averaged results for the entire set of depletion materials (typically, all fuel elements in a depletion model). Specification of material -1 returns the average of only those materials with ID $>0$ present in the matl=list. Again, this is best illustrated by example. Specification of the data blocks

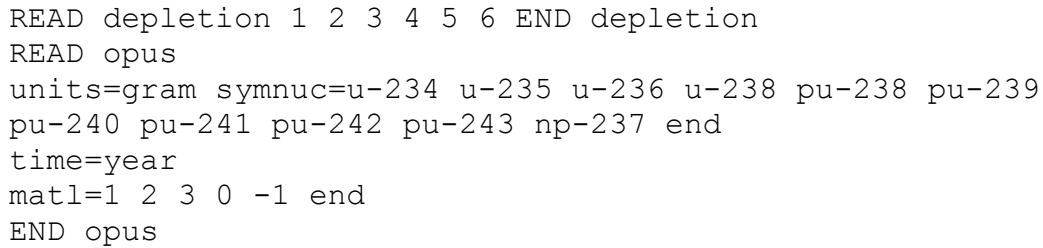

will result in five OPUS calculations and five sets of results-one for each of materials 1,2 , and 3, one for the average of materials $1-6$ (due to input of material ID 0 ), and one for the average of materials $1-3$ (due to input of material ID -1 ).

\subsection{Specification of stacked OPUS cases}

In a given calculation, multiple output units may be desired (e.g., grams, curies, and watts), or multiple time scales (e.g., seconds and years), or a combination of these or other parameters. TRITON provides the ability to stack inputs such that multiple cases may be run within a single TRITON calculation. In order to stack cases, the keywords new case are entered in the input stream. Any parameters following these keywords are used to define a new OPUS case.

There is no limit on the number of stacked cases that may be input; however, the matl= specification may be used only in the first case. OPUS calculations are run for each of the materials in this list, for all cases.

Consider a depletion calculation where gadolinium pins are present in the assembly design. One may wish to determine the quantities of gadolinium nuclides from the initial poison rods (tracked as a light element by ORIGEN within TRITON) and from fission (tracked as a fission product by ORIGEN). One may also need masses of selected actinides as well as the total $(\alpha, n)$ reaction rate. Figure 3.1.15 shows how the new case keyword set is used to define unique OPUS calculations. In this example, the new case keywords are shown in upper case and on a line by themselves, but this has been done only for readability. The text may be entered in lower case and on the same line as other keywords. Note, however, that the matl= specification is given only in the first case. All OPUS calculations will be performed for materials 1, 2, and 3 and for the average of these three materials. 


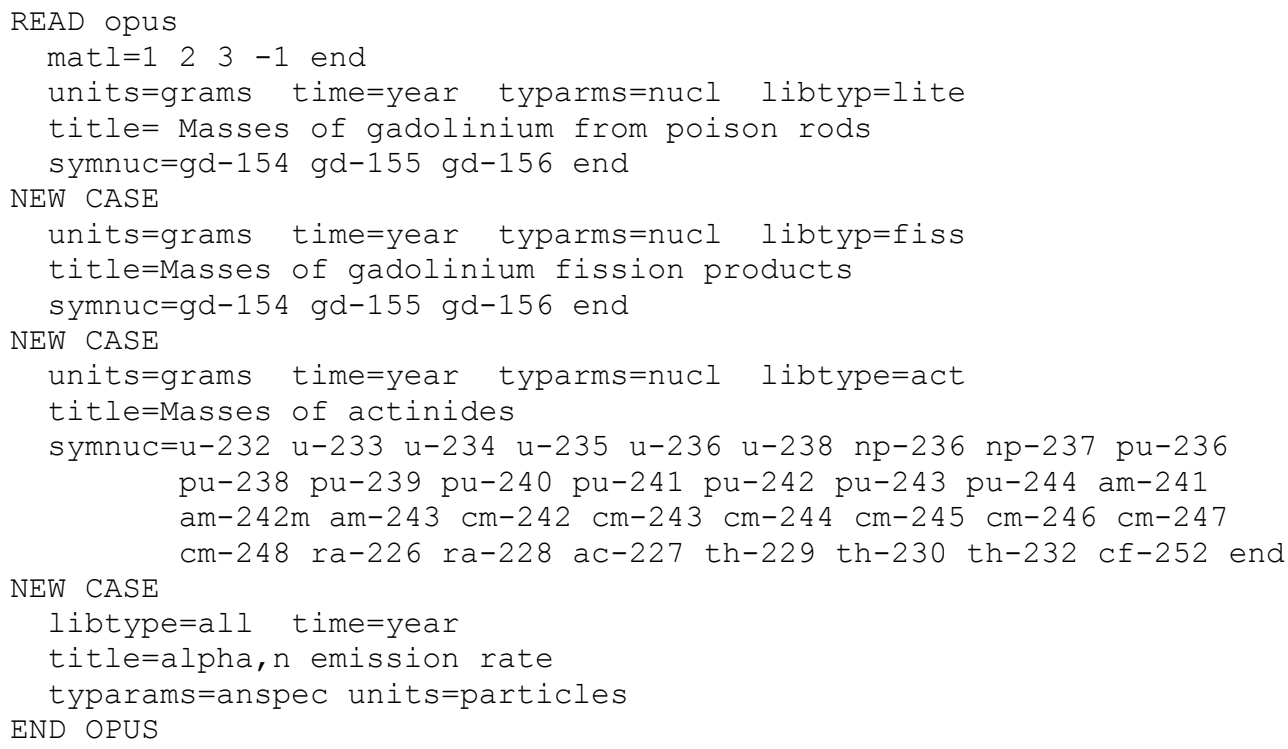

Figure 3.1.15. Example OPUS block input.

\subsubsection{S/U analysis input}

An example input structure for the sensitivity and uncertainty $(\mathrm{S} / \mathrm{U})$ analysis sequences is provided in Figure 3.1.16.

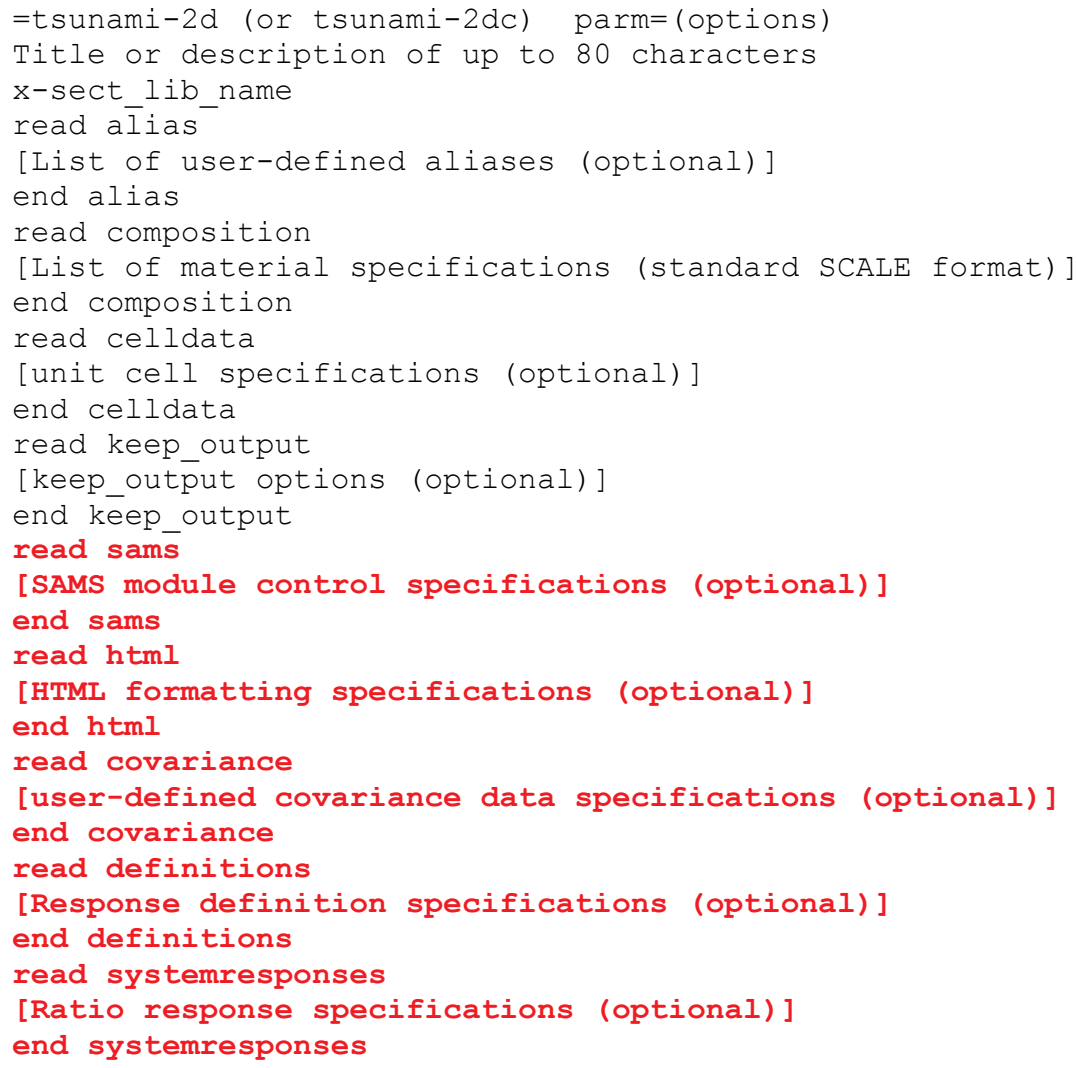


read model

[specification of NEWT model]

end model

end

Figure 3.1.16. Structure of $S / U$ analysis input.

The TRITON S/U sequences support the blocks of data highlighted in red: the SAMS, HTML, COVARIANCE, DEFINITIONS, and SYSTEMRESPONSES data blocks. These data blocks, along with the KEEP OUTPUT block, may appear only once, in any order, and must follow the COMPOSITION and $C E L L \bar{D} A T A$ blocks and must precede the MODEL block. If specified, the HTML and COVARIANCE blocks must follow the $S A M S$ block.

These five blocks of data serve the same function as described in the TSUNAMI-1D manual, and the information is not repeated here.

The MODEL block contains a full NEWT transport model input description and is required for both $\mathrm{S} / \mathrm{U}$ analysis sequences. The MODEL block must be the last block of data in the input file. The MODEL block provides the physical layout of the configuration for which the transport calculation is to be performed, along with general control parameters. The NEWT input is described fully in the NEWT chapter and is not repeated here.

\subsubsection{ALIAS block}

The optional ALIAS block may be used to simplify model development within TRITON by defining a set of material numbers that will be inserted in place of the alias when that alias is used in subsequent data blocks. Aliases function as variables for which a user-defined set of materials are inserted; they are identified by a dollar character (\$) preceding a single-word alphanumeric label. The ALIAS block is used to preprocess an input, creating a new, modified input deck with all alias variable substitutions included. TRITON then processes the modified input deck before proceeding with the calculation.

The use of an alias variable is best illustrated by a brief example. Assume that the alias $\$ f u e l$ is defined as materials 1,2, and 3, and $\$$ mod as materials 4, 5, and 6. (The input format for defining aliases is described below.) The user wishes to create three identical sets of materials and use them in three identical pin cell specifications. In the COMPOSITION data block, specifications could be written in the following form

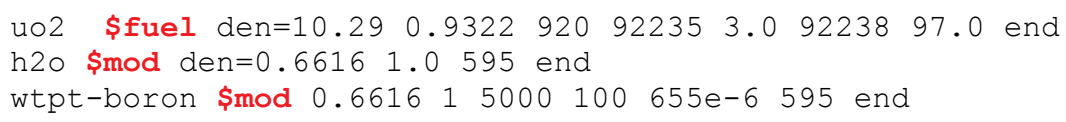

TRITON would create a modified input with the alias expanded as follows:

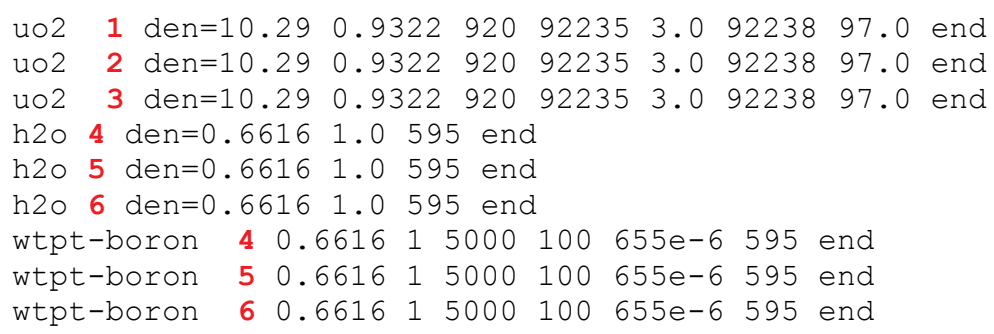

Similarly, if the alias were used in the CELLDATA block as 
latticecell squarepitch pitch=1.26 \$mod fuelr=0.4095 \$fuel end

then TRITON would expand the aliases to

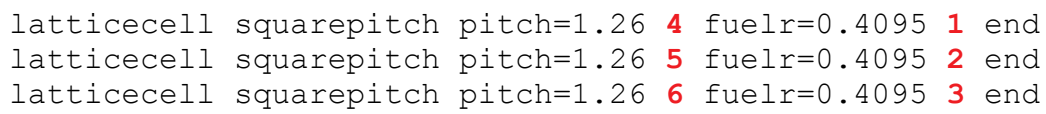

In a depletion calculation, one may wish to deplete a large number of fuel rods independently because of different geometric locations in a fuel assembly. Even though each fuel rod may have the same initial composition, each must be specified as a unique material composition in order to be depleted independently. Furthermore, multiple cell specifications must all use unique material identifiers for each cell component. Thus, if one desired to deplete 25 fuel materials in a fuel/clad/moderator pin cell, one would need to set up material composition definitions for 25 fuels, 25 moderators, and 25 clads. Then one would need to provide 25 pin cell specifications. By using aliases, one need only specify the material identifiers corresponding to each alias and then provide only one material composition specification for each alias type, and then one pin cell specification. TRITON will automatically expand the aliases and create a revised input with all materials and cell specifications explicitly defined. Note that although this will simplify the pin cell input in the CELLDATA, 25 pin cell calculations would still be required. The number of pin cell calculations can be reduced by using the ASSIGN function described in Sect. 3.1.3.3.4.3.

The purpose of the $A L I A S$ block is to define a set of alias variables to be used in subsequent data blocks. The ALIAS block is optional, but aliases may not be used in other blocks if an ALIAS block is not present to define the aliases. An $A L I A S$ block may contain as many aliases as desired. Each alias specification consists of three parts: the alias name, consisting of a dollar sign followed by up to 11 alphanumeric characters with no embedded spaces; the material number or numbers; and an end keyword. Material numbers may be entered in any order and may be separated by spaces or commas (or both). Material numbers may also be separated by a dash (-), but this represents an inclusive list. In other words, a material specification of 1-3 (or 1 - 3) indicates materials 1, 2, and 3. The example ALIAS block below illustrates the various means for assigning a set of materials for an alias definition.

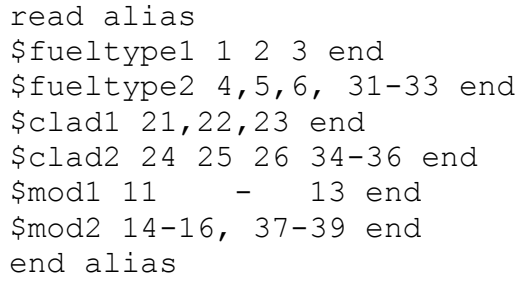

The ALIAS block simply serves to assign material identifiers to specific variables, and the variables are used in subsequent data blocks. The same material identifier can be used in more than one alias if desired. As indicated earlier, TRITON will preprocess any input deck containing an ALIAS block and replace instances of alias variables with the appropriate material identifiers. The following subsections describe how aliases are implemented in TRITON's various input blocks, as the form of alias variable substitution is block dependent. Aliases are processed only in these input blocks; aliases used in other blocks will result in an error. 


\subsection{COMPOSITION block aliases}

The COMPOSITION block uses aliases to create multiple copies of each standard composition specification, replacing the alias variable with each material identifier associated with the alias definition. For example, consider the following alias definition in an $A L I A S$ block:

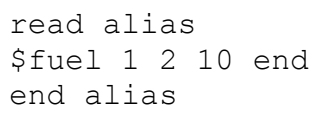

and the standard composition specification:

uo2 \$fuel den=10.045 1800922352.59223897 .5 end

A modified TRITON input would be created with the standard composition specification replaced by

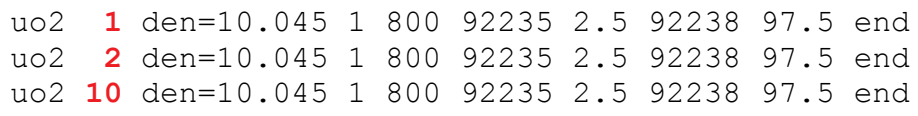

\subsection{CELLDATA block aliases}

CELLDATA block latticecell specifications typically contain more than one material; therefore, multiple aliases are permitted in each cell specification. However, this constrains the set of aliases used in the cell specification to have the same number of material identifiers associated with it.

Consider the ALIAS block:

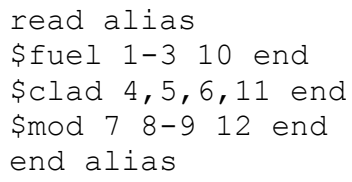

All three aliases contain four materials each. One could then create a single cell specification that uses one or more of these alias variables, such as

latticecell squarepitch pitch=1.26 \$mod fuelr=0.41 \$fuel cladr=0.50 \$clad end

This would result in the following alias expansion by TRITON:

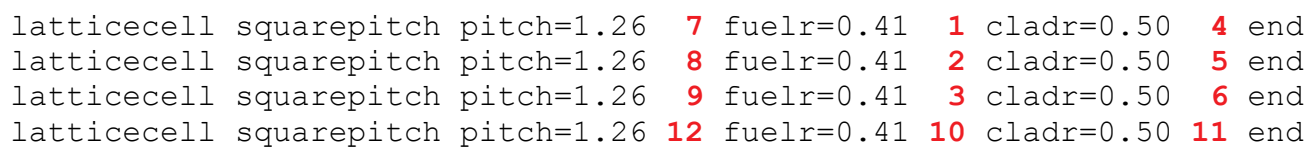

Material identifiers are substituted according to their position in the alias definition (i.e., the first substitution will use the first material associated with each alias, and the second expansion will use the second material associated with each alias, etc.)

Material numbers should not be entered manually in a cell specification; for example,

latticecell triangpitch pitch=1.26 \$mod fuelr=0.4095 1 end 
TRITON would allow this to occur and would create a set of cell specifications as follows:

latticecell triangpitch pitch=1.26 2 fuelr=0.4095 1 end

latticecell triangpitch pitch=1.26 3 fuelr=0.4095 1 end

where \$mod was defined as materials 2 and 3. However, SCALE does not allow the same material identifier to occur in two different cell specifications, and the fact that material 1 occurs in two different cell specifications would result in TRITON ending with an error. Note that alias expansions for multiregion and doublehet cell specifications are not supported. Also note that TRITON will not copy centrmdata and moredata specifications that follow a cell specification that uses an alias variable.

\subsection{DEPLETION block aliases}

Aliases in the TRITON DEPLETION are simply replaced by the set of materials associated with the alias. For example, the $A L I A S$ block

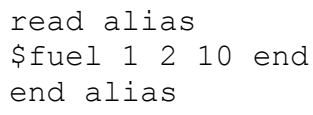

and DEPLETION block

read depletion 789 \$fuel end depletion

would be expanded to

read depletion 7891210 end depletion

Aliases may be mixed with actual material numbers in the depletion block, along with the flux and assign keywords. However, the negative sign-used to define the basis for power normalization-cannot precede an alias definition.

\subsection{TIMETABLE block aliases}

TIMETABLE block alias expansion is similar to that of the COMPOSITION block: TRITON will create a new timetable entry for each material associated with the alias used in the TIMETABLE definition. For the TIMETABLE block below, using the alias \$allmod, unique timetables will be created for each material identifier associated with this alias variable. Note that alias expansion of density timetable entries is not yet supported.

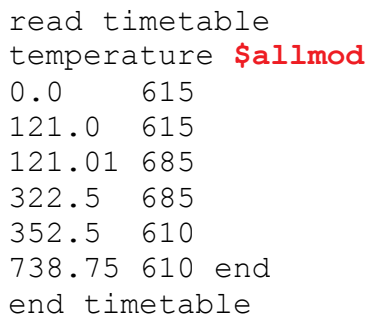




\subsection{BRANCH block aliases}

Aliases may be used within the define keyword definitions of the BRANCH block. Aliases are simply replaced by the list of materials associated with the alias, as is done for the DEPLETION block. Hence,

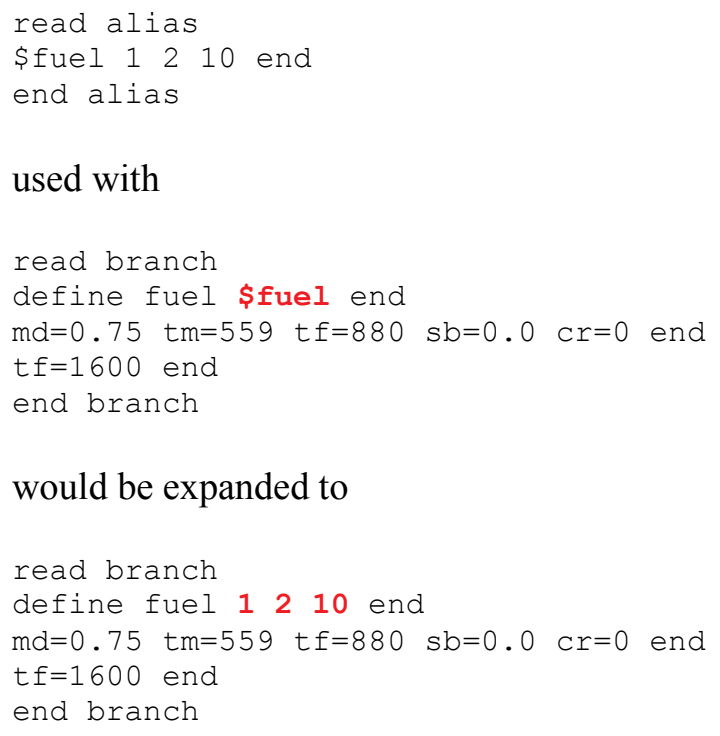

used with

read branch

\subsection{NEWT MATERIAL block aliases}

The MATERIAL block within the NEWT model section of a TRITON input can also use aliases. As with COMPOSITION and TIMETABLE entries, TRITON will create a new material specification for each material represented by an alias. For the sample material block below, using the alias $\$$ fuel, unique material block entries will be created for each material associated with the alias variable.

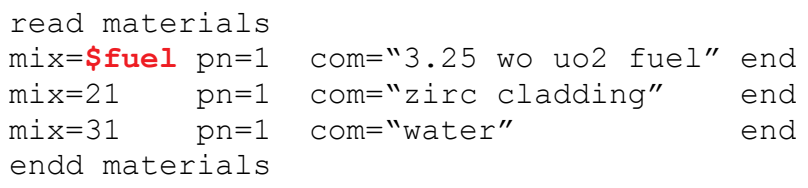

If an alias were defined as

\$fuel 101112 end

then the MATERIAL block would be expanded to

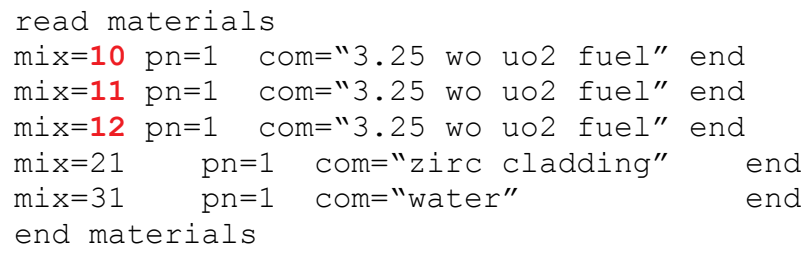

\subsubsection{KEEP_OUTPUT block}

When performing depletion calculations for a number of different materials, TRITON output can become quite voluminous. Often, much of that output is not needed for calculations that seek only eigenvalues, 
sources, or concentrations as a function of irradiation history. TRITON provides the ability to trim output to only those portions for which output is desired. Output produced directly by the TRITON module is always provided and cannot be disabled, but output from any other code in the sequence can be automatically removed from the output listing. Retaining certain output is accomplished using the KEEP_OUTPUT data block.

The KEEP_OUTPUT data block provides the ability to preserve only selected outputs. The format of this data block is

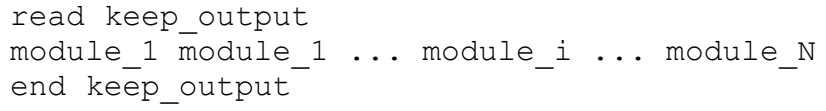

where module_i represents any valid module name from the list of modules invoked by TRITON, as listed here:

xsproc xsdrn newt kenova kenovi couple origen

By default, the output from all these modules is retained with the exception of XSProc COUPLE and ORIGEN. SAMS and OPUS output is always retained.

\subsubsection{TRITON control parameters}

TRITON supports the following of control parameter options:

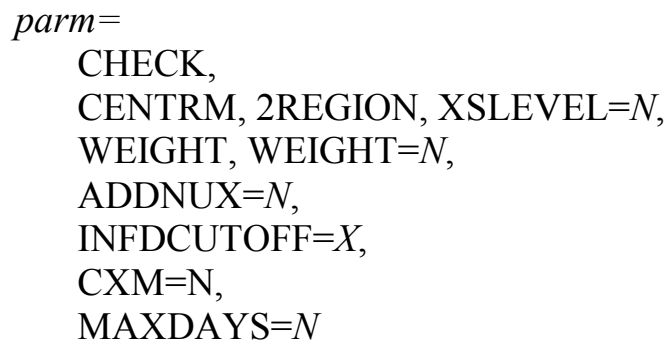

If an invalid control parameter option is specified, including misspelled keywords, an error message will be generated and execution terminated. TRITON also provides the ability to nest several control parameter keywords together; to combine keywords (where appropriate), a list may be entered, enclosed in parentheses, and separated by commas. For example, to specify CHECK, 2REGION, and ADDNUX=1 at the same time, input would begin with

=t-depl parm=(check, 2region, addnux=1)

The following subsections provide more detail on each of the control parameters listed above.

\subsection{Check mode: parm $=$ check}

Specification of parm $=$ check will request that TRITON read all input and ensure that no input errors are present, without running additional calculations. In this mode, all input is set up as if a full calculation will be run, but the sequence exits without any functional module execution. The check mode is useful for debugging or obtaining processed standard composition data, without actually running a calculation. It can also be used to generate plot files for embedded NEWT and KENO inputs for additional review and checking of input specifications. Of course, some errors may be uncovered only by dynamically executing 
the functional modules; hence, there are rare occasions where a parm=check run will complete with no errors but will fail when run outside of check mode as the problem begins to run.

\subsection{Multigroup cross section processing options}

The most common use of parm= sequence control is in the selection of an alternate multigroup cross section processing mode.

By default, XSProc enables both the BONAMI and CENTRM modules for cross section processing. BONAMI-only XSProc calculations can be performed using the control parameter parm=bonami.

TRITON also supports the control parameter parm $=(x$ slevel $=N)$. The $x$ slevel parameter option initializes various CENTRM options for the XSProc calculations. The xslevel option is equivalent to initializing all unit cell calculations with the following centrmdata specifications:

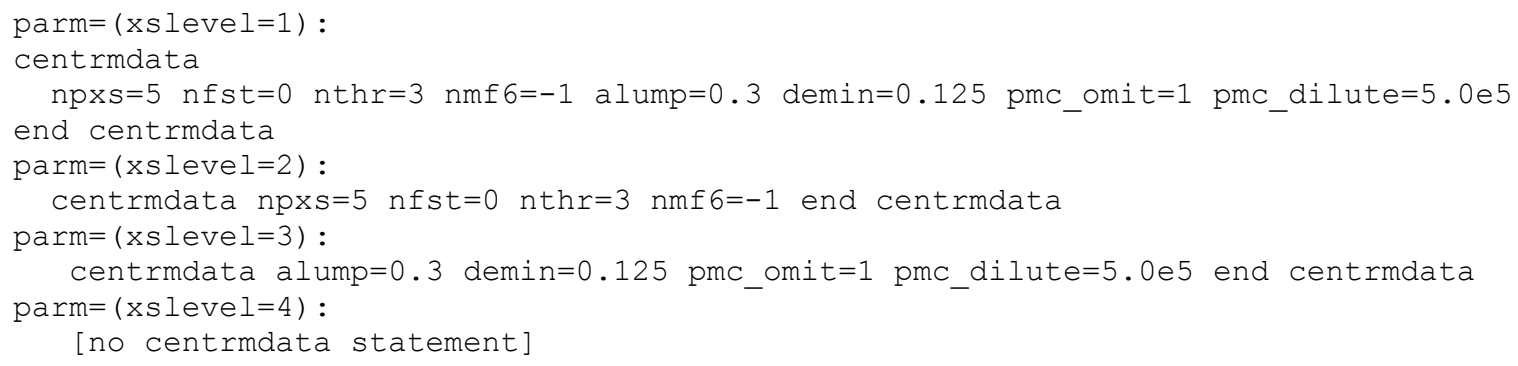

The option parm $=(x$ slevel $=4)$ is equivalent to parm $=$ centrm. The option parm $=(x$ slevel $=3)$ is the default for depletion sequences and is equivalent to parm=centrm but with some minor approximations to decrease run time. The option parm $=(x$ slevel $=2)$ is equivalent to parm $=2$ region for all sequences.

Note that the $x$ slevel $=1$ and $x$ slevel $=3$ options have additional specifications for keywords alump, demin, pmc_omit, and pmc_dilute. These keywords are further discussed in the XSProc chapter. The additional keyword specifications are used to decrease run-time for the CENTRM and PMC calculations. Internal investigations have shown that the approximations introduced by the additional keyword specifications have minimal impact on solution accuracy for a wide range of calculations. Therefore the additional keyword specifications are used by default for depletion calculations, where several CENTRM and PMC calculations are necessary. The additional keyword values are not used by default for nondepletion calculations to be consistent with the SCALE CSAS5 and CSAS6 criticality sequences.

The TSUNAMI-2D sequence also invokes the BONAMIST module, a modified version of BONAMI to support sensitivity calculations. TSUNAMI-2D calculations may use parm=centrm, 2region, bonami, or xslevel. However, these cross section processing options will not utilize the sensitivity-enabled version of BONAMI.

\subsection{Creating a broad group library: parm $=$ weight, parm $=($ weight $=N)$}

Used in tandem with the TRITON T-NEWT sequence, the specification parm=weight extends the sequence by setting up and executing the MALOCS2 module to generate a weighted broad-group crosslibrary (AMPX master format). The spectrum generated in the NEWT transport calculation is used as the weighting function for the collapse. Additionally, the broad-group library energy structure is defined by the NEWT COLLASPE block. 
The parm=weight option uses the problem-averaged flux spectrum for the weighting function in the collapse. The problem may be a simple pin cell or a full assembly. However, there may be cases where the flux in a specific region or material is most appropriate for the spectral collapse. TRITON allows identification of a specific material from which the collapsing spectrum should be used. When specified in the form parm $=($ weight $=N)$, the average flux determined for material $\mathrm{N}$ is used in place of the total domain spectrum to perform the collapse.

TRITON sample problem 1 (Sect. 3.1.6.1) provides an example of the use of T-NEWT to produce a new broad-group library. Note that the broad-group library produced in this calculation will reside in the SCALE temporary working directory with the name newxnlib at the end of the calculation. If the library will be needed for future calculations, the user should use a shell script to copy the library back to a more permanent location, and perhaps give it a more meaningful name. In sample problem 1, the SCALE 252group master library is collapsed to 56 energy groups.

The process for creating a broad-group master library is also supported in the $2 \mathrm{D}$ depletion sequence T-DEPL. When parm $=$ weight or parm $=($ weight $=N)$ is specified in a depletion calculation, the input cross section library must be one of the SCALE 238-group or 252-group libraries, which will automatically be collapsed to the SCALE 49-group or 56-group structure, respectively. An initial fine group calculation is performed for the input configuration, and the flux from the solution is used to create the broad group library. The initial calculation is then repeated with the new broad group library, followed by the remainder of the depletion calculation. Note that for lattice physics calculations, the NEWT COLLAPSE block will be based on the 49-group (or 56-group) energy structure, not the fine group structure.

It is important to note that the 252-group library contains intermediate resonance parameters and other data that cannot be accurately collapsed into 56-group data with the collapsing procedures available in MALOCS2. These parameters are important for bonami-only cross section processing calculations, i.e., parm=bonami. Therefore, the parm=centrm option is recommended for follow-on application of the collapsed 56-group collapsed library. The 238-group and 49-group libraries do not contain intermediate resonance parameter data, and bonami-only processing is available, provided that this cross section processing option and group structure is suitable for the intended application.

\subsection{Inclusion of additional nuclides for depletion: parm $=(\operatorname{addnux}=N)$}

For depletion calculations, it is important to add trace quantities $\left(1 \times 10^{-20} \mathrm{at} / \mathrm{b}-\mathrm{cm}\right)$ of certain nuclides to the inventories of depletion materials in order to accurately track the nuclides' impact on cross section processing and transport calculations as a function of burnup. By default, TRITON automatically adds to all fuel materials trace quantities of a set of nuclides that have been determined to be important in the characterization of spent fuel. TRITON recognizes fuel materials as any material containing quantities of heavy metals $(Z>89)$ in the standard composition specification.

TRITON provides user control of the set of nuclides added to a fuel material through the parm $=($ addnux $=N)$ control parameter, where $\mathrm{N}$ is an integer value. For $\mathrm{N}=0$, no nuclides are added, which is generally a very poor approximation and should only be used when the ramifications are fully understood. For $\mathrm{N}=1$, a bare minimum set of 15 nuclides (actinides) are added; this will generate improved number density estimates for actinides in low-burnup fuels but will not update cross sections for fission products of primary importance. Again, use of this option is discouraged unless it addresses special modeling needs. For $\mathrm{N}=2$, the default setting for the TRITON depletion sequences, 95 nuclides are added. $\mathrm{N}=3$ and $\mathrm{N}=4$ add 231 and 388 nuclides, respectively. Note that in previous versions of TRITON, $\mathrm{N}=2$ would add 64 nuclides. The set of 64 nuclides is still supported by specifiying 
parm $=($ addnux $=-2)$ in the input. The default in the SCALE 6.1 release remains parm=(addnux=2). Table 3.1.3 through Table 3.1.7 list the set of nuclides added in trace quantities for each value of addnux.

Table 3.1.3. Additional nuclides added in trace quantities for $\mathbf{p a r m}=(\operatorname{addnux}=1)$

\begin{tabular}{cccc}
\hline & ${ }^{234} \mathrm{U}$ & ${ }^{235} \mathrm{U}$ & ${ }^{236} \mathrm{U}$ \\
${ }^{238} \mathrm{U}$ & ${ }^{237} \mathrm{Pu}$ & ${ }^{238} \mathrm{Pu}$ & ${ }^{239} \mathrm{Pu}$ \\
${ }^{242} \mathrm{Am}$ & ${ }^{241} \mathrm{Pu}$ & ${ }^{242} \mathrm{Pu}$ & $\mathrm{Am}$ \\
& ${ }^{243} \mathrm{Am}$ & ${ }^{242} \mathrm{Cm}$ & ${ }^{243} \mathrm{Cm}$ \\
\hline
\end{tabular}

*15 nuclides total.

Table 3.1.4. Additional nuclides added in trace quantities for parm $=(\operatorname{addnux}=-2)$

\begin{tabular}{cccc}
\hline${ }^{1} \mathrm{H}$ & ${ }^{10} \mathrm{~B}$ & ${ }^{11} \mathrm{~B}$ & \\
${ }^{14} \mathrm{~N}$ & ${ }^{83} \mathrm{O}$ & $\mathrm{Kr}$ & ${ }^{93} \mathrm{Nb}$ \\
${ }^{94} \mathrm{Zr}$ & ${ }^{95} \mathrm{Mo}$ & ${ }^{99} \mathrm{Tc}$ & ${ }^{103} \mathrm{Rh}$ \\
${ }^{105} \mathrm{Rh}$ & ${ }^{106} \mathrm{Ru}$ & ${ }^{126} \mathrm{Ag}$ & $\mathrm{Sn}$ \\
${ }^{135} \mathrm{I}$ & ${ }^{131} \mathrm{Xe}$ & ${ }^{133} \mathrm{Cs}$ \\
${ }^{134} \mathrm{Cs}$ & ${ }^{135} \mathrm{Cs}$ & ${ }^{137} \mathrm{Cs}$ & ${ }^{143} \mathrm{Pr}$ \\
${ }^{147} \mathrm{Ne}$ & ${ }^{143} \mathrm{Nd}$ & ${ }^{145} \mathrm{Nd}$ & $\mathrm{Nd}$ \\
${ }^{148} \mathrm{Nd}$ & ${ }^{147} \mathrm{Pm}$ & ${ }^{148} \mathrm{Pm}$ & ${ }^{149} \mathrm{Pm}$ \\
${ }^{151} \mathrm{Sm}$ & ${ }^{147} \mathrm{Sm}$ & $\mathrm{Sm}$ & ${ }^{153} \mathrm{Sm}$ \\
${ }^{154} \mathrm{Eu}$ & ${ }^{152} \mathrm{Sm}$ & ${ }^{151} \mathrm{Eu}$ & ${ }^{154} \mathrm{Gd}$ \\
${ }^{155} \mathrm{Gd}$ & ${ }^{155} \mathrm{Eu}$ & ${ }^{152} \mathrm{Gd}$ & \\
${ }^{160} \mathrm{Gd}$ & ${ }^{156} \mathrm{Gd}$ & & \\
\hline
\end{tabular}

*49 additional nuclides in addition to the 15 nuclides added in addnux=1, for a total of 64 .

Table 3.1.5. Additional nuclides added in trace quantities for $\mathbf{p a r m}=(\operatorname{addnux}=2)$

\begin{tabular}{cccc}
\hline${ }^{91} \mathrm{Zr}$ & ${ }^{93} \mathrm{Zr}$ & ${ }^{96} \mathrm{Zr}$ & $\mathrm{Zr}$ \\
${ }^{95} \mathrm{Nb}$ & ${ }^{97} \mathrm{Mo}$ & ${ }^{98} \mathrm{Mo}$ \\
${ }^{100} \mathrm{Mo}$ & ${ }^{101} \mathrm{Ru}$ & ${ }^{102} \mathrm{Ru}$ & ${ }^{103} \mathrm{Ru}$ \\
${ }^{104} \mathrm{Ru}$ & ${ }^{105} \mathrm{Pd}$ & ${ }^{107} \mathrm{Pd}$ & ${ }^{108} \mathrm{Pd}$ \\
${ }^{113} \mathrm{Cd}$ & ${ }^{115} \mathrm{In}$ & ${ }^{127} \mathrm{I}$ & ${ }^{141} \mathrm{Ce}$ \\
${ }^{133} \mathrm{Xe}$ & ${ }^{139} \mathrm{La}$ & ${ }^{140} \mathrm{Ba}$ & ${ }^{144} \mathrm{Nd}$ \\
${ }^{142} \mathrm{Ce}$ & ${ }^{143} \mathrm{Ce}$ & ${ }^{141} \mathrm{Pr}$ & \\
${ }^{153} \mathrm{Sm}$ & ${ }^{156} \mathrm{Eu}$ & ${ }^{242 m} \mathrm{Am}$ & \\
\hline
\end{tabular}

*31 additional nuclides in addition to the 15 nuclides in Table 3.1.3 and 49 nuclides in Table 3.1.4, for a total of 95 . 
Table 3.1.6. Additional nuclides added in trace quantities for $\mathbf{p a r m}=(\operatorname{addnux}=3)$

\begin{tabular}{|c|c|c|c|}
\hline${ }^{72} \mathrm{Ge}$ & ${ }^{73} \mathrm{Ge}$ & ${ }^{74} \mathrm{Ge}$ & ${ }^{76} \mathrm{Ge}$ \\
\hline${ }^{75} \mathrm{As}$ & ${ }^{79} \mathrm{Br}$ & ${ }^{76} \mathrm{Se}$ & ${ }^{77} \mathrm{Se}$ \\
\hline${ }^{78} \mathrm{Se}$ & ${ }^{80} \mathrm{Se}$ & ${ }^{82} \mathrm{Se}$ & ${ }^{81} \mathrm{Br}$ \\
\hline${ }^{80} \mathrm{Kr}$ & ${ }^{82} \mathrm{Kr}$ & ${ }^{84} \mathrm{Kr}$ & ${ }^{85} \mathrm{Kr}$ \\
\hline${ }^{86} \mathrm{Kr}$ & ${ }^{85} \mathrm{Rb}$ & ${ }^{86} \mathrm{Rb}$ & ${ }^{87} \mathrm{Rb}$ \\
\hline${ }^{84} \mathrm{Sr}$ & ${ }^{86} \mathrm{Sr}$ & ${ }^{87} \mathrm{Sr}$ & ${ }^{88} \mathrm{Sr}$ \\
\hline${ }^{89} \mathrm{Sr}$ & ${ }^{90} \mathrm{Sr}$ & ${ }^{89} \mathrm{Y}$ & ${ }^{90} \mathrm{Y}$ \\
\hline${ }^{91} \mathrm{Y}$ & ${ }^{90} \mathrm{Zr}$ & ${ }^{92} \mathrm{Zr}$ & ${ }^{92} \mathrm{Mo}$ \\
\hline${ }^{94} \mathrm{Mo}$ & ${ }^{96} \mathrm{Mo}$ & ${ }^{94} \mathrm{Nb}$ & ${ }^{96} \mathrm{Ru}$ \\
\hline${ }^{98} \mathrm{Ru}$ & ${ }^{99} \mathrm{Ru}$ & ${ }^{100} \mathrm{Ru}$ & ${ }^{105} \mathrm{Ru}$ \\
\hline${ }^{102} \mathrm{Pd}$ & ${ }^{104} \mathrm{Pd}$ & ${ }^{106} \mathrm{Pd}$ & ${ }^{110} \mathrm{Pd}$ \\
\hline${ }^{107} \mathrm{Ag}$ & ${ }^{111} \mathrm{Ag}$ & ${ }^{106} \mathrm{Cd}$ & ${ }^{108} \mathrm{Cd}$ \\
\hline${ }^{110} \mathrm{Cd}$ & ${ }^{111} \mathrm{Cd}$ & ${ }^{112} \mathrm{Cd}$ & ${ }^{114} \mathrm{Cd}$ \\
\hline${ }^{115 \mathrm{~m}} \mathrm{Cd}$ & ${ }^{116} \mathrm{Cd}$ & ${ }^{140} \mathrm{Ce}$ & ${ }^{113} \mathrm{In}$ \\
\hline${ }^{140} \mathrm{La}$ & ${ }^{112} \mathrm{Sn}$ & ${ }^{114} \mathrm{Sn}$ & ${ }^{115} \mathrm{Sn}$ \\
\hline${ }^{{ }^{116} \mathrm{Sn}}$ & ${ }^{117} \mathrm{Sn}$ & ${ }^{118} \mathrm{Sn}$ & ${ }^{119} \mathrm{Sn}$ \\
\hline${ }^{120} \mathrm{Sn}$ & ${ }^{122} \mathrm{Sn}$ & ${ }^{123} \mathrm{Sn}$ & ${ }^{124} \mathrm{Sn}$ \\
\hline${ }^{125} \mathrm{Sn}$ & ${ }^{121} \mathrm{Sb}$ & ${ }^{123} \mathrm{Sb}$ & ${ }^{124} \mathrm{Sb}$ \\
\hline${ }^{125} \mathrm{Sb}$ & ${ }^{126} \mathrm{Sb}$ & ${ }^{120} \mathrm{Te}$ & ${ }^{122} \mathrm{Te}$ \\
\hline${ }^{123} \mathrm{Te}$ & ${ }^{124} \mathrm{Te}$ & ${ }^{125} \mathrm{Te}$ & ${ }^{126} \mathrm{Te}$ \\
\hline${ }^{127 \mathrm{~m}} \mathrm{Te}$ & ${ }^{128} \mathrm{Te}$ & ${ }^{129 \mathrm{~m}} \mathrm{Te}$ & ${ }^{130} \mathrm{Te}$ \\
\hline${ }^{132} \mathrm{Te}$ & ${ }^{130} \mathrm{I}$ & ${ }^{131} \mathrm{I}$ & ${ }^{124} \mathrm{Xe}$ \\
\hline${ }^{126} \mathrm{Xe}$ & ${ }^{128} \mathrm{Xe}$ & ${ }^{129} \mathrm{Xe}$ & ${ }^{130} \mathrm{Xe}$ \\
\hline${ }^{132} \mathrm{Xe}$ & ${ }^{134} \mathrm{Xe}$ & ${ }^{136} \mathrm{Xe}$ & ${ }^{134} \mathrm{Ba}$ \\
\hline${ }^{135} \mathrm{Ba}$ & ${ }^{136} \mathrm{Ba}$ & ${ }^{137} \mathrm{Ba}$ & ${ }^{138} \mathrm{Ba}$ \\
\hline${ }^{136} \mathrm{Cs}$ & ${ }^{142} \mathrm{Pr}$ & ${ }^{142} \mathrm{Nd}$ & ${ }^{150} \mathrm{Nd}$ \\
\hline${ }^{151} \mathrm{Pm}$ & ${ }^{144} \mathrm{Sm}$ & ${ }^{148} \mathrm{Sm}$ & ${ }^{154} \mathrm{Sm}$ \\
\hline${ }^{152} \mathrm{Eu}$ & ${ }^{157} \mathrm{Eu}$ & ${ }^{232} \mathrm{U}$ & ${ }^{233} \mathrm{U}$ \\
\hline${ }^{159} \mathrm{~Tb}$ & ${ }^{160} \mathrm{~Tb}$ & ${ }^{160} \mathrm{Dy}$ & ${ }^{161} \mathrm{Dy}$ \\
\hline${ }^{162} \mathrm{Dy}$ & ${ }^{163} \mathrm{Dy}$ & ${ }^{164} \mathrm{Dy}$ & ${ }^{165} \mathrm{Ho}$ \\
\hline${ }^{166} \mathrm{Er}$ & ${ }^{167} \mathrm{Er}$ & ${ }^{175} \mathrm{Lu}$ & ${ }^{176} \mathrm{Lu}$ \\
\hline${ }^{181} \mathrm{Ta}$ & ${ }^{182} \mathrm{~W}$ & ${ }^{183} \mathrm{~W}$ & ${ }^{184} \mathrm{~W}$ \\
\hline${ }^{186} \mathrm{~W}$ & ${ }^{185} \mathrm{Re}$ & ${ }^{187} \mathrm{Re}$ & ${ }^{197} \mathrm{Au}$ \\
\hline${ }^{231} \mathrm{~Pa}$ & ${ }^{233} \mathrm{~Pa}$ & ${ }^{230} \mathrm{Th}$ & ${ }^{232} \mathrm{Th}$ \\
\hline
\end{tabular}

*136 additional nuclides in addition to the 15 nuclides in Table 3.1.3, 49 nuclides in Table 3.1.4, and 31 nuclides in Table 3.1.5, for a total of 231. 
Table 3.1.7. Additional nuclides added in trace quantities for $\mathbf{p a r m}=(\operatorname{addnux}=4)$

\begin{tabular}{|c|c|c|c|}
\hline${ }^{2} \mathrm{H}$ & ${ }^{3} \mathrm{H}$ & ${ }^{3} \mathrm{He}$ & ${ }^{4} \mathrm{He}$ \\
\hline${ }^{6} \mathrm{Li}$ & ${ }^{7} \mathrm{Li}$ & ${ }^{7} \mathrm{Be}$ & ${ }^{9} \mathrm{Be}$ \\
\hline${ }^{15} \mathrm{~N}$ & ${ }^{17} \mathrm{O}$ & ${ }^{19} \mathrm{~F}$ & ${ }^{23} \mathrm{Na}$ \\
\hline${ }^{24} \mathrm{Mg}$ & ${ }^{25} \mathrm{Mg}$ & ${ }^{26} \mathrm{Mg}$ & ${ }^{27} \mathrm{Al}$ \\
\hline${ }^{28} \mathrm{Si}$ & ${ }^{29} \mathrm{Si}$ & ${ }^{30} \mathrm{Si}$ & ${ }^{31} \mathrm{P}$ \\
\hline${ }^{32} \mathrm{~S}$ & ${ }^{33} \mathrm{~S}$ & ${ }^{34} \mathrm{~S}$ & ${ }^{36} \mathrm{~S}$ \\
\hline${ }^{35} \mathrm{Cl}$ & ${ }^{37} \mathrm{Cl}$ & ${ }^{36} \mathrm{Ar}$ & ${ }^{38} \mathrm{Ar}$ \\
\hline${ }^{40} \mathrm{Ar}$ & ${ }^{39} \mathrm{~K}$ & ${ }^{40} \mathrm{~K}$ & ${ }^{41} \mathrm{~K}$ \\
\hline${ }^{40} \mathrm{Ca}$ & ${ }^{42} \mathrm{Ca}$ & ${ }^{43} \mathrm{Ca}$ & ${ }^{44} \mathrm{Ca}$ \\
\hline${ }^{46} \mathrm{Ca}$ & 每8 $\mathrm{Ca}$ & ${ }^{45} \mathrm{Sc}$ & ${ }^{46} \mathrm{Ti}$ \\
\hline${ }^{47} \mathrm{Ti}$ & ${ }^{48} \mathrm{Ti}$ & ${ }^{49} \mathrm{Ti}$ & ${ }^{50} \mathrm{Ti}$ \\
\hline${ }^{50} \mathrm{Cr}$ & ${ }^{52} \mathrm{Cr}$ & ${ }^{53} \mathrm{Cr}$ & ${ }^{54} \mathrm{Cr}$ \\
\hline${ }^{55} \mathrm{Mn}$ & ${ }^{54} \mathrm{Fe}$ & ${ }^{56} \mathrm{Fe}$ & ${ }^{57} \mathrm{Fe}$ \\
\hline${ }^{58} \mathrm{Fe}$ & ${ }^{58} \mathrm{Co}$ & ${ }^{58 \mathrm{~m}} \mathrm{Co}$ & ${ }^{59} \mathrm{Co}$ \\
\hline${ }^{58} \mathrm{Ni}$ & ${ }^{59} \mathrm{Ni}$ & ${ }^{60} \mathrm{Ni}$ & ${ }^{61} \mathrm{Ni}$ \\
\hline${ }^{62} \mathrm{Ni}$ & ${ }^{64} \mathrm{Ni}$ & ${ }^{63} \mathrm{Cu}$ & ${ }^{65} \mathrm{Cu}$ \\
\hline${ }^{70} \mathrm{Ge}$ & ${ }^{69} \mathrm{Ga}$ & ${ }^{71} \mathrm{Ga}$ & ${ }^{74} \mathrm{As}$ \\
\hline${ }^{74} \mathrm{Se}$ & ${ }^{79} \mathrm{Se}$ & ${ }^{78} \mathrm{Kr}$ & ${ }^{110 m} \mathrm{Ag}$ \\
\hline${ }^{113} \mathrm{Sn}$ & ${ }^{123} \mathrm{Xe}$ & ${ }^{130} \mathrm{Ba}$ & ${ }^{132} \mathrm{Ba}$ \\
\hline${ }^{133} \mathrm{Ba}$ & ${ }^{136} \mathrm{Ce}$ & ${ }^{138} \mathrm{Ce}$ & ${ }^{139} \mathrm{Ce}$ \\
\hline${ }^{138} \mathrm{La}$ & ${ }^{148 \mathrm{~m}} \mathrm{Pm}$ & ${ }^{153} \mathrm{Gd}$ & ${ }^{156}$ Dy \\
\hline${ }^{158} \mathrm{Dy}$ & ${ }^{166 \mathrm{~m}} \mathrm{Ho}$ & ${ }^{162} \mathrm{Er}$ & ${ }^{164} \mathrm{Er}$ \\
\hline${ }^{168} \mathrm{Er}$ & ${ }^{170} \mathrm{Er}$ & ${ }^{174} \mathrm{Hf}$ & ${ }^{176} \mathrm{Hf}$ \\
\hline${ }^{177} \mathrm{Hf}$ & ${ }^{178} \mathrm{Hf}$ & ${ }^{179} \mathrm{Hf}$ & ${ }^{180} \mathrm{Hf}$ \\
\hline${ }^{182} \mathrm{Ta}$ & ${ }^{191} \mathrm{Ir}$ & ${ }^{193} \mathrm{Ir}$ & ${ }^{196} \mathrm{Hg}$ \\
\hline${ }^{198} \mathrm{Hg}$ & ${ }^{199} \mathrm{Hg}$ & ${ }^{200} \mathrm{Hg}$ & ${ }^{201} \mathrm{Hg}$ \\
\hline${ }^{202} \mathrm{Hg}$ & ${ }^{204} \mathrm{Hg}$ & ${ }^{204} \mathrm{~Pb}$ & ${ }^{206} \mathrm{~Pb}$ \\
\hline${ }^{207} \mathrm{~Pb}$ & ${ }^{208} \mathrm{~Pb}$ & ${ }^{209} \mathrm{Bi}$ & ${ }^{223} \mathrm{Ra}$ \\
\hline${ }^{224} \mathrm{Ra}$ & ${ }^{225} \mathrm{Ra}$ & ${ }^{225} \mathrm{Ac}$ & ${ }^{226} \mathrm{Ac}$ \\
\hline${ }^{227} \mathrm{Ac}$ & ${ }^{226} \mathrm{Ra}$ & ${ }^{227} \mathrm{Th}$ & ${ }^{228} \mathrm{Th}$ \\
\hline${ }^{229} \mathrm{Th}$ & ${ }^{233} \mathrm{Th}$ & ${ }^{234} \mathrm{Th}$ & ${ }^{232} \mathrm{~Pa}$ \\
\hline${ }^{235} \mathrm{~Np}$ & ${ }^{236} \mathrm{~Np}$ & ${ }^{238} \mathrm{~Np}$ & ${ }^{239} \mathrm{~Np}$ \\
\hline${ }^{237} \mathrm{U}$ & ${ }^{239} \mathrm{U}$ & ${ }^{240} \mathrm{U}$ & ${ }^{241} \mathrm{U}$ \\
\hline${ }^{236} \mathrm{Pu}$ & ${ }^{237} \mathrm{Pu}$ & ${ }^{243} \mathrm{Pu}$ & ${ }^{244} \mathrm{Pu}$ \\
\hline${ }^{246} \mathrm{Pu}$ & ${ }^{244} \mathrm{Am}$ & ${ }^{244 m} \mathrm{Am}$ & ${ }^{241} \mathrm{Cm}$ \\
\hline${ }^{245} \mathrm{Cm}$ & ${ }^{246} \mathrm{Cm}$ & ${ }^{247} \mathrm{Cm}$ & ${ }^{248} \mathrm{Cm}$ \\
\hline${ }^{249} \mathrm{Cm}$ & ${ }^{250} \mathrm{Cm}$ & ${ }^{249} \mathrm{Bk}$ & ${ }^{250} \mathrm{Bk}$ \\
\hline${ }^{249} \mathrm{Cf}$ & ${ }^{250} \mathrm{Cf}$ & ${ }^{251} \mathrm{Cf}$ & ${ }^{252} \mathrm{Cf}$ \\
\hline${ }^{253} \mathrm{Cf}$ & ${ }^{254} \mathrm{Cf}$ & ${ }^{253} \mathrm{Es}$ & ${ }^{254} \mathrm{Es}$ \\
\hline${ }^{255}$ Es & & & \\
\hline
\end{tabular}

*158 additional nuclides in addition to the 15 nuclides in Table 3.1.3, 49 nuclides in Table 3.1.4, 30 nuclides in Table 3.1.5, and 136 nuclides in Table 3.1.6, for a total of 388.

\subsection{Few-group reaction cross section calculation control for continuous energy depletion: parm $=(\mathbf{c x m}=N)$}

In continuous energy depletion calculations, few group reaction cross sections are computed by KENO directly rather than using a post-processing approach that TRITON uses for multigroup mode. In addition to these region averaged multigroup reaction cross sections, KENO also provides problem-dependent region-averaged multigroup fluxes to TRITON that will be used by COUPLE to generate one-group cross section library for each depletion material. 
Option parm $=(\mathrm{cxm}=N)$ is used to setup continuous-energy depletion calculation with different modes of calculation, which tells KENO the details of the tallying process for the reaction cross sections and mixture fluxes. Available calculations modes and their descriptions are presented in Table 3.1.8.

Table 3.1.8. cxm values and their descriptions

\begin{tabular}{|c|c|c|c|c|}
\hline \multirow[t]{2}{*}{$\mathrm{cxm}$} & \multicolumn{2}{|c|}{ cross sections } & flux & \multirow[t]{2}{*}{ description } \\
\hline & reactions & $\begin{array}{l}\text { number } \\
\text { of } \\
\text { energy } \\
\text { groups }\end{array}$ & $\begin{array}{l}\text { number } \\
\text { of } \\
\text { energy } \\
\text { groups }\end{array}$ & \\
\hline 1 & All & $\mathrm{NGP}^{1}$ & $\mathrm{NGP}^{1}$ & $\begin{array}{l}\text { KENO uses default NGP-group energy group } \\
\text { boundaries to generate region-averaged reaction } \\
\text { cross sections for all available reactions of the } \\
\text { nuclides in each depletion mixture. } \\
\text { KENO also computes region-averaged multigroup } \\
\text { fluxes using the default NGP-group energy bins. }\end{array}$ \\
\hline $2^{2}$ & $\begin{array}{l}\text { Transmutation } \\
(\mathrm{MT}=16-18, \\
102-125)\end{array}$ & $\mathrm{NGP}^{1}$ & $\mathrm{NGP}^{1}$ & $\begin{array}{l}\text { KENO uses default NGP-group energy group } \\
\text { boundaries to generate region-averaged reaction } \\
\text { cross sections for only transmutation reactions of } \\
\text { the nuclides in each depletion mixture. } \\
\text { KENO also computes region-averaged multigroup } \\
\text { fluxes using the default NGP-group energy bins. }\end{array}$ \\
\hline 3 & All & 1 & $\mathrm{NGP}^{1}$ & $\begin{array}{l}\text { KENO uses 1-group energy group boundaries to } \\
\text { generate region-averaged reaction cross sections for } \\
\text { all available reactions of the nuclides in each } \\
\text { depletion mixture. } \\
\text { KENO also computes region-averaged multigroup } \\
\text { fluxes using the default NGP-group energy bins. }\end{array}$ \\
\hline 4 & $\begin{array}{l}\text { Transmutations } \\
(\mathrm{MT}=16-18, \\
102-125)\end{array}$ & 1 & $\mathrm{NGP}^{1}$ & $\begin{array}{l}\text { KENO-VI uses 1-group energy group boundaries to } \\
\text { generate region-averaged reaction cross sections for } \\
\text { only transmutation reactions of the nuclides in each } \\
\text { depletion mixture. } \\
\text { KENO-VI also computes region-averaged } \\
\text { multigroup fluxes using the default NGP-group } \\
\text { energy bins. }\end{array}$ \\
\hline
\end{tabular}

${ }^{1}$ Energy group structure in KENO and associated number of energy groups, NGP, should be consistent with those from the ORIGEN library used in the problem.

${ }^{2} \mathrm{cxm}=2$ is the default mode for reaction cross section calculations.

\subsection{Infinite dilution cutoff control: $p a r m=($ infdcutoff $=X)$}

The addition of nuclides to depletion materials as described in the previous section can lead to increased run-times for CENTRM-based XSProc calculations. However, many nuclides (e.g., low-density nuclides) are effectively infinitely dilute and can be treated as such and omitted from the expensive point-wise cross section collapse operation. For the option parm $=($ infdcutoff=sigma0) sequence option, XSProc will compute an effective background microscopic cross section for each nuclide. If the computed background cross section is greater than the cutoff value sigma0, recommended as $5 \times 10^{5}$ barns, then the nuclide is considered infinitely dilute and the infinitely dilute multigroup cross section is utilized from the cross section library.

In general, a sigma0 cutoff value of $5 \times 10^{5}$ barns will be acceptable for most applications. However, TRITON and the centrmdata card in the CELLDATA block provide a means for the user to control the 
cutoff value. The cutoff value may be assigned in either of two ways. A single global value may be assigned to all cells using the TRITON parm= specifier with the keyword infdcutoff, for example, parm $=($ infdcutoff $=1$ e10 $)$. Addition of the specifier with a value of $1 \times 10^{10}$ will set the cutoff value to $1 \times 10^{10}$ for all cells in the problem, which is generally appropriate for most calculations. However, a provision is made to specify a unique cutoff value to each cell using the pmc_dilute keyword in a centrmdata specification. An example of this is shown in the description of parm=xslevel in Sect. 3.1.3.7.2.

The default value of sigma0 depends on the sequence and cross section processing option. For nondepletion sequences that use parm=centrm, the default is 0 . The default value of 0 instructs PMC to include all nuclides for PMC processing. For depletion sequences that use parm $=$ centrm or for any sequence that uses parm $=2$ region, the default value is $5 \times 10^{5}$ barns.

\subsection{Override of the maximum number of days per depletion subinterval: $P A R M=(M A X D A Y S=N)$}

TRITON is set to limit ORIGEN time intervals to no more than 40 days to avoid potential numerical error that would be introduced if depletion were performed over a long time interval. For depletion subintervals of more than 400 days (10 time intervals of 40 days), TRITON will automatically increase the number of depletion subintervals in a depletion interval. The depletion subinterval is based on a rule of thumb for ORIGEN depletion. However, the rule breaks down when burning at very low powers for extended time intervals. Thus, TRITON allows the user to override the default behavior by specifying a new value for the maximum number of days per ORIGEN time interval. A 100-day limit per ORIGEN time interval may be set using parm $=($ maxdays $=100)$. In overriding the default behavior, the user must be aware of any potential errors introduced in the approximation.

\subsubsection{Output Files Ccreated by TRITON}

TRITON produces a variety of output files that may be of use in related calculations. Of those files, only certain files are copied back to the return directory: the TRITON output file (.out); plot files generated by NEWT, KENO, or OPUS (.plt); SAMS sensitivity data files (.sdf), in the case of an S/U calculation; ORIGEN binary concentration files (.f71) and HTML-formatted output (.html), where available. The TRITON output file is a concatenated listing of outputs from TRITON and all modules for which output is kept. Other files of potential interest are not copied, and the user should be aware of these files and their names so that they may be retrieved using a SHELL script after TRITON execution is complete. The following subsections list those files and their purposes.

\subsubsection{Standard composition restart files}

At the end of all depletion calculations, standard composition files are automatically produced for each material, listing the nuclides and number densities of the materials at the time the transport calculation (i.e., XSDRN, NEWT, KENO) is performed. Only nuclides for which cross section data are available in the master cross section library are saved in these files. Files are saved using the file naming convention StdCmpMixNNNNN, where $N N N N N$ is the material identifier. The file contains compositions at the final time of the calculation. Additional files are saved with the file naming convention StaCmpMixNNNNN_MMMMM, where $M M M M M$ is an index to a particular time step in the depletion calculation. For example, if a calculation is completed with materials 1 and 40 for two depletion steps, then the following files will be created in the temporary working directory.

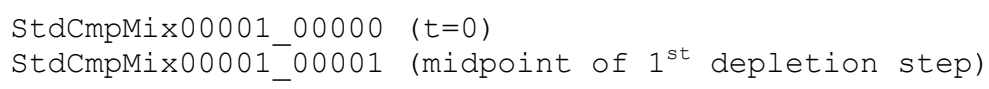




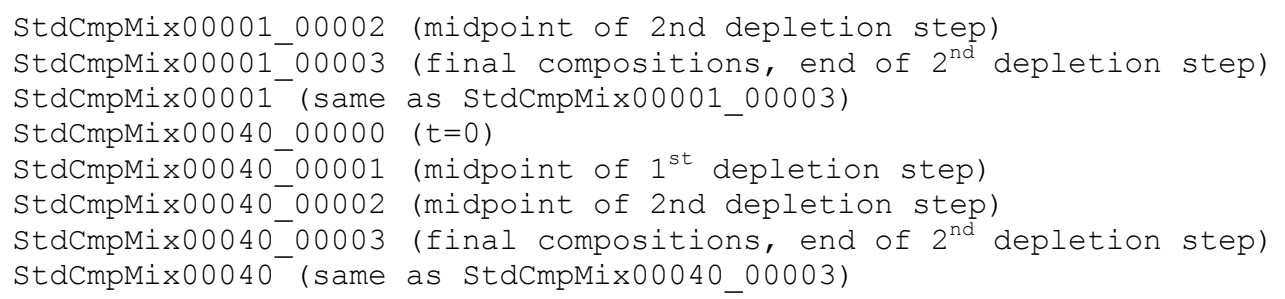

The contents of these files will be a standard composition description of each material by atomic contents - that is, SCALE standard nuclide IDs (e.g., U-235), number density, and temperature (using the temperature of the original material). Using SCALE's external file read capability, these outputs may be automatically included in a follow-on calculation that relies on depleted/decayed number densities. TRITON sample problem 7 (Sect. 3.1.6.7) provides an example of the use of these restart files. Standard composition restart files should be used only for follow-on criticality or shielding calculations.

\subsubsection{Lattice physics parameters}

During T-DEPL depletion calculations that use branch states and homogenization, a database of fewgroup cross sections is saved for each branch state and at each depletion step containing homogenized cross section data and other lattice physics parameters (e.g., discontinuity factors, pin power peaking factors, diffusion coefficients, etc.). The $x$ file016 file is intended for post-processing, to be read and written in the desired format for subsequent nodal diffusion core simulator calculations. The $x$ file016 file is a binary-formatted file, which is described in detail in Appendix A of TRITON. An auxiliary textformatted database file (txtfile 16) is also created that contains the same data as the binary-formatted file.

\subsubsection{ORIGEN binary library files}

During depletion calculations, ORIGEN binary library files are created to archive cross sections for each depletion material at each depletion subinterval. These files can be used in future depletion calculations in ORIGEN, ORIGAMI, and ARP. For each depletion material, the ORIGEN binary library file is named $f t 33 f 001 . m i x \mathrm{NNNN}$, where $N N N N$ is the material number for each depleted material. Additionally, the combined cross section file is saved with the name ft33f001.cmbined. Here is an example of a script to retrieve cross section files.

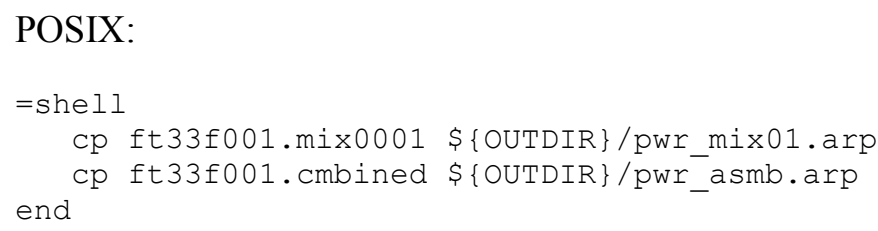

Windows:

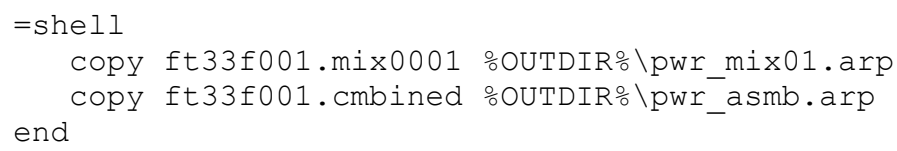

\subsubsection{ORIGEN binary concentration file (.f71)}

During depletion calculations, TRITON creates the ORIGEN binary concentration file (.f71). This file is created in the temporary directory as $f t 71 f 001$ and is copied back at the end of the SCALE calculation to the return directory with the name $\$$ \{OUTBASENAME\}.f71. TRITON archives computed concentrations 
for each depletion material at the beginning and end of each depletion subinterval or decay interval. These files include concentrations and also decay heat term, photon and neutron data, and other quantities or interest computed by ORIGEN. These data may be post-processed by the OPUS module.

The .f71 file contains concentrations for each individual material, and it also contains the combined concentrations of the individual material results (i.e., the net response for the entire system). The TRITON output contains an index of the contents of this file (see Sect. 3.1.5.4.5).

\subsubsection{Output Description}

This section contains a brief description and explanation of TRITON output. As with any SCALE module, TRITON output begins with the SCALE header, the job information, the input file, and the program verification information. These outputs are common to all SCALE modules. Likewise, all SCALE calculations report a run-time summary at the end of the output file.

\subsubsection{Control parameter edit}

When TRITON control parameters are specified using the parm= command (see Sect.3.1.3.7), all specified parameters are echoed following the above output, with an explanation of the meaning of the parameter, as shown below. If no parameters are specified, no edit is provided.

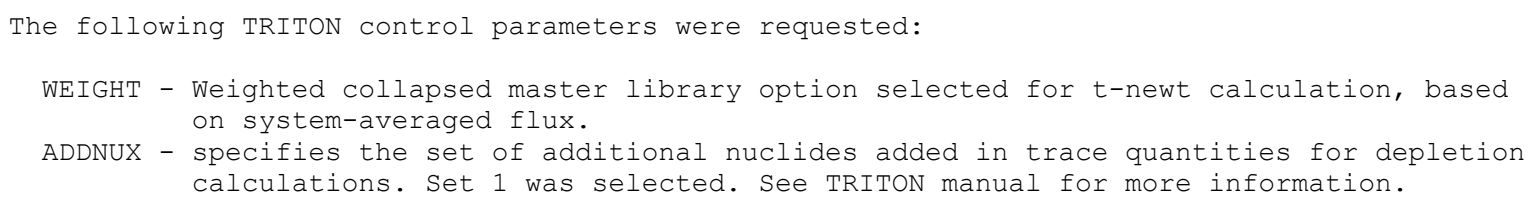

\subsubsection{T-XSEC output}

The T-XSEC sequence performs only cross section processing functions. The XSProc output is written to the output file as the calculation proceeds.

\subsubsection{T-NEWT and T-XSDRN output}

By default, the T-NEWT and T-XSDRN outputs include only the NEWT and XSDRN output respectively. The XSProc output can be included by using the KEEP_OUTPUT block (see Sect. 3.1.3.6).

\subsubsection{Depletion sequence output}

The output of TRITON depletion sequences contains several depletion edits. The edits are described in the following subsections. These output edits are written to the output file in the order in which they are computed during the calculation. 


\subsection{Burnup history summary (all depletion sequences)}

TRITON generates the burnup history summary table after processing the BURNDATA block. An example of this table is as follows.

\begin{tabular}{|c|c|c|c|c|c|c|}
\hline \multicolumn{7}{|c|}{$\begin{array}{l}\text { Based on the supplied burnup history, triton w } \\
\text { depletion calculations. This breakdown has bee } \\
\text { no more than } 40 \text { days, and decay times using th } \\
\text { period of no more than } 75 \text { days. } \\
8 \text { time-dependent libraries will be created }\end{array}$} \\
\hline $\begin{array}{c}\text { Sub-Interval } \\
\text { No. }\end{array}$ & $\begin{array}{l}\text { Depletion } \\
\text { Interval }\end{array}$ & $\begin{array}{l}\text { Sub-interval } \\
\text { in interval }\end{array}$ & $\begin{array}{c}\text { Specific } \\
\text { Power (MW/MTIHM) }\end{array}$ & $\begin{array}{l}\text { Burn Length } \\
\text { (d) }\end{array}$ & $\begin{array}{l}\text { Decay Length } \\
\text { (d) }\end{array}$ & $\begin{array}{c}\text { Library Burnup } \\
\text { (MWd/MTIHM) }\end{array}$ \\
\hline 0 & $\star \star \star \star$ Initial & Bootstrap Calch & 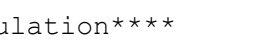 & & & $0.00000 \mathrm{E}+00$ \\
\hline 1 & 1 & 1 & 30.0 & 357. & 0.00 & $5.35714 \mathrm{E}+03$ \\
\hline 2 & 1 & 2 & 30.0 & 357. & 0.00 & $1.60714 \mathrm{E}+04$ \\
\hline 3 & 1 & 3 & 30.0 & 357. & 0.00 & $2.67857 \mathrm{E}+04$ \\
\hline 4 & 1 & 4 & 30.0 & 357. & 0.00 & $3.75000 \mathrm{E}+04$ \\
\hline 5 & 1 & 5 & 30.0 & 357. & 0.00 & $4.82143 \mathrm{E}+04$ \\
\hline 6 & 1 & 6 & 30.0 & 357. & 0.00 & $5.89286 \mathrm{E}+04$ \\
\hline 7 & 1 & 7 & 30.0 & 357. & 0.00 & $6.96429 \mathrm{E}+04$ \\
\hline
\end{tabular}

NOTE: Library Burnup is the cumulative burnup computed at the midpoint of the depletion sub-interval. Specific Power and Library Burnup depend on basis material(s) selected in DEPLETION block.

This table shows the results of a burnup history using one depletion interval with seven depletion subintervals. Column 1 is the cumulative depletion subinterval number. Column 2 is the depletion interval number, and column 3 is the depletion subinterval number within the current depletion interval. Columns 4-6 echo the specific power, depletion interval, and decay interval specified in the BURNDATA block. The final column shows the cumulative burnup at the midpoint of each depletion subinterval. 


\subsection{Embedded transport model output}

The output from the initial transport calculation follows the burnup history edit. The output edits for NEWT, XSDRN, KENO-V.a, and KENO-VI are described in their respective manual sections.

\subsection{System mass balance table}

After the initial transport calculation output, a summary of system mass information is printed, an example of which is provided as follows.

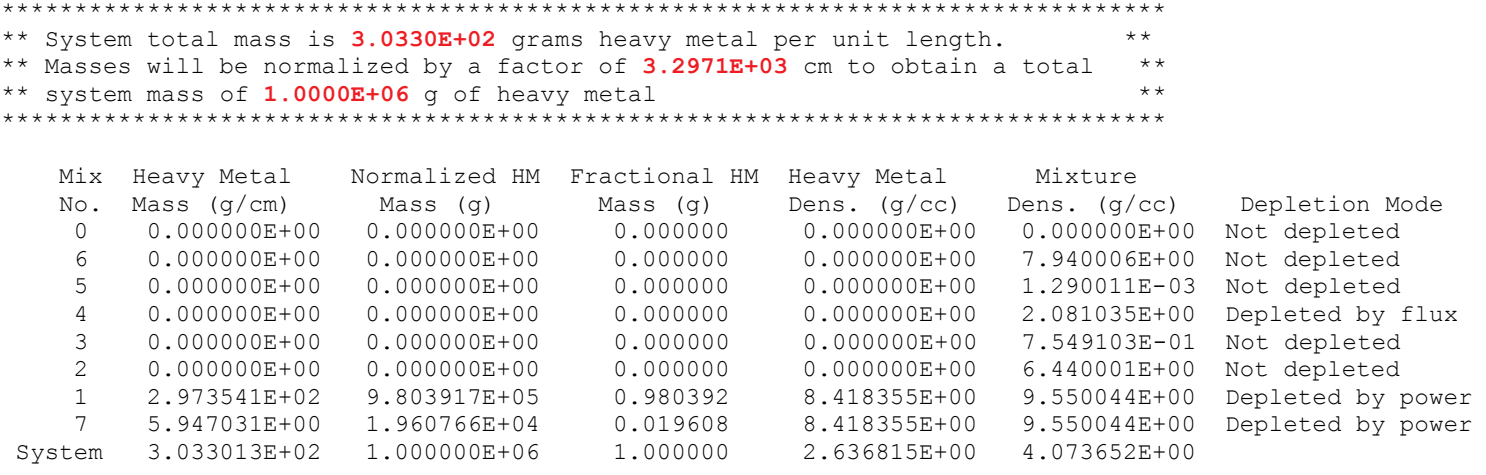

This table provides mass and density data for each material used in the transport model. Column 1 provides the material identifier, and columns 5 and 6 provide the material density and material heavy metal density, respectively, in units of grams per cubic centimeter. Heavy metal mass is determined from masses of all nuclides with an atomic number greater than 89 . The final column provides the depletion mode for each material (see Sect. 3.1.3.3.4.2). Column 2 provides the "prenormalized" heavy metal mass of each material. The units for this mass value depend on the transport model. For 2D $x y$ NEWT models, the units are grams per centimeter since there is no $z$ - dimension in the model. Similarly, the units are grams per centimeter for 1D cylinder XSDRN models, grams per square centimeter for 1D slab XSDRN models, and grams for 1D spherical XSDRN models and 3D KENO models. The total prenormalized heavy metal mass is printed in the final row of the table as well as in the table header (highlighted in red). The heavy metal mass is normalized such that a total system mass of 1 MTHM is present. The volume scaling factor used to normalize the system mass is also printed in the table banner (highlighted in red). The units of the volume scaling factor depend on the transport model. Column 3 prints the normalized material heavy metal mass in units of grams, which is equal to the prenormalized material heavy metal mass in column 2 multiplied by the volume scaling factor in the table header. The total normalized mass is printed in the final row and also in the table header (highlighted in red). The fourth column shows the fractional heavy metal mass of all materials, which is equal to the normalized heavy metal mass in column 3, divided by the total normalized system heavy metal mass in the table header.

\subsection{Power balance tables}

As the TRITON calculation proceeds, the results of the cross section processing and transport calculations are used to calculate fluxes and powers in each material. The output segment listed below shows the results for the first calculation based on the initial material compositions. The total power (column 2) represents the material-specific power in units of MW/MTHM of initial system mass. The fractional power (column 3 ) is equal to the total power for a material divided by the total system power (highlighted in red). The mixture power (column 4) represents material-specific power in units of MW/MTHM of initial material mass. The mixture power is equal to the total power of the material divided by the 
fractional heavy metal mass of the material, which is provided in the system mass balance table (Sect. 3.1.5.4.3). If the material does not contain heavy metal, then "N/A" is printed in the mixture power column. Columns 5 and 6 show the material thermal and total flux values, respectively, in units of neutrons $/ \mathrm{cm}^{2}$-sec. The thermal flux is determined by integrating multigroup flux values for energy groups below $0.625 \mathrm{eV}$. If the specific power is normalized to the total system power, the summation of the material powers in column 1 should match the input specification in the BURNDATA block (in the example given here, 20.86 MW/MTHM).

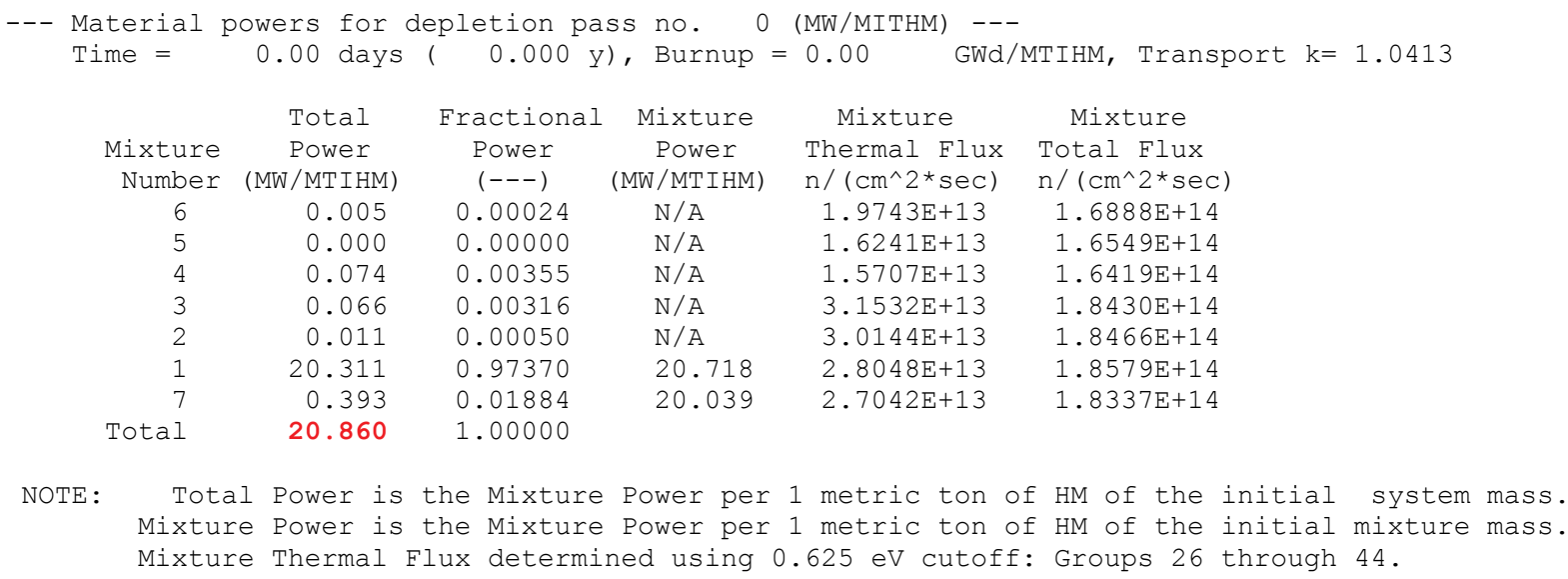

NOTE: Total Power is the Mixture Power per 1 metric ton of HM of the initial system mass. Mixture Power is the Mixture Power per 1 metric ton of HM of the initial mixture mass. Mixture Thermal Flux determined using $0.625 \mathrm{eV}$ cutoff: Groups 26 through 44.

The form of the output changes if the specific power is normalized to the power to one or more specific materials. For the case above, if depletion was performed with input power normalized to material 7, the power output table would have the following form.

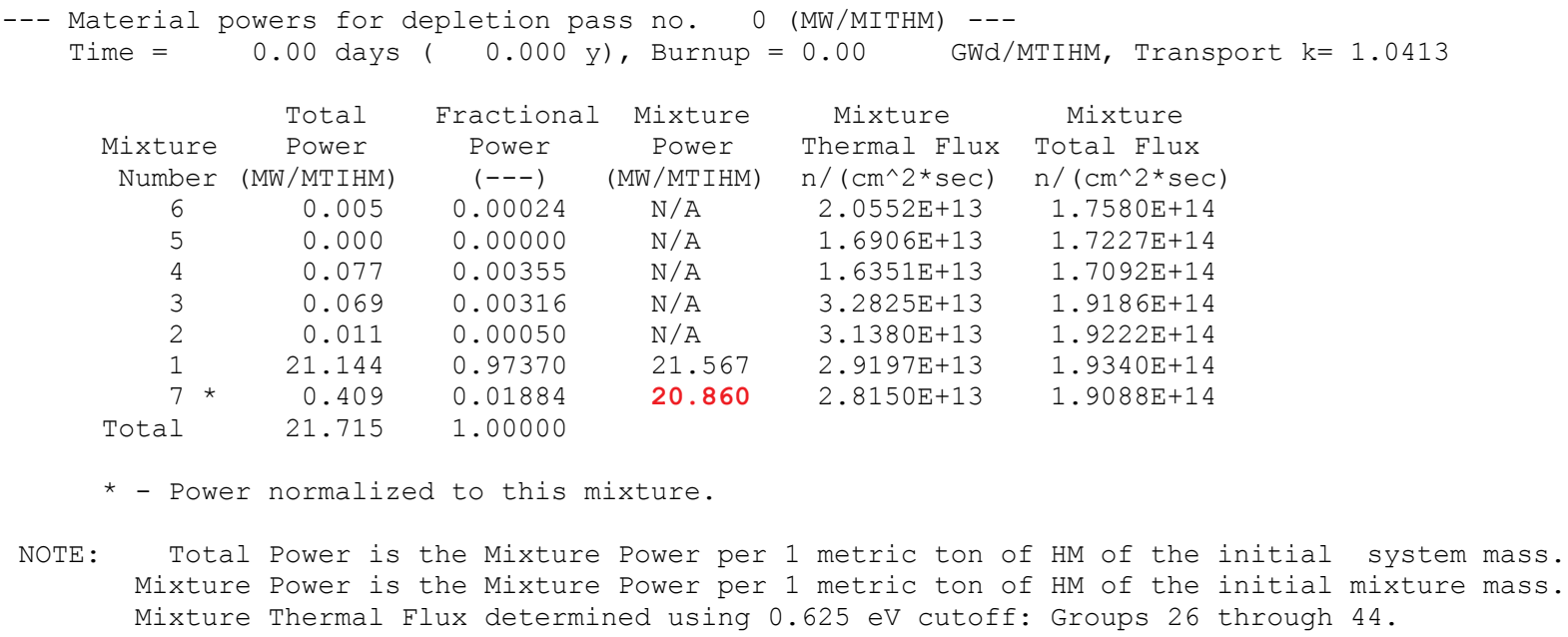

In this example, material 7 has the input-specified power (20.86 MW/MTHM, highlighted in red), and the power in the remainder of the model materials is normalized according to this basis material.

\subsection{ORIGEN binary concentration file listing}

After all depletion calculations are completed, TRITON creates an ORIGEN binary concentration file (.f71) with isotopic concentrations for each depletion material. The order and content of the .f71 file is provided in the TRITON output. An example of this edit is shown below. For each depletion material, the 
output gives the location in the file, the ORIGEN time interval number, the depletion interval time in days, the cumulative time in years, and a title. After all materials are added to the library, the system average of all libraries is computed and added to the library. In this case, with only one depletion material, the system average would be the same as the single material.

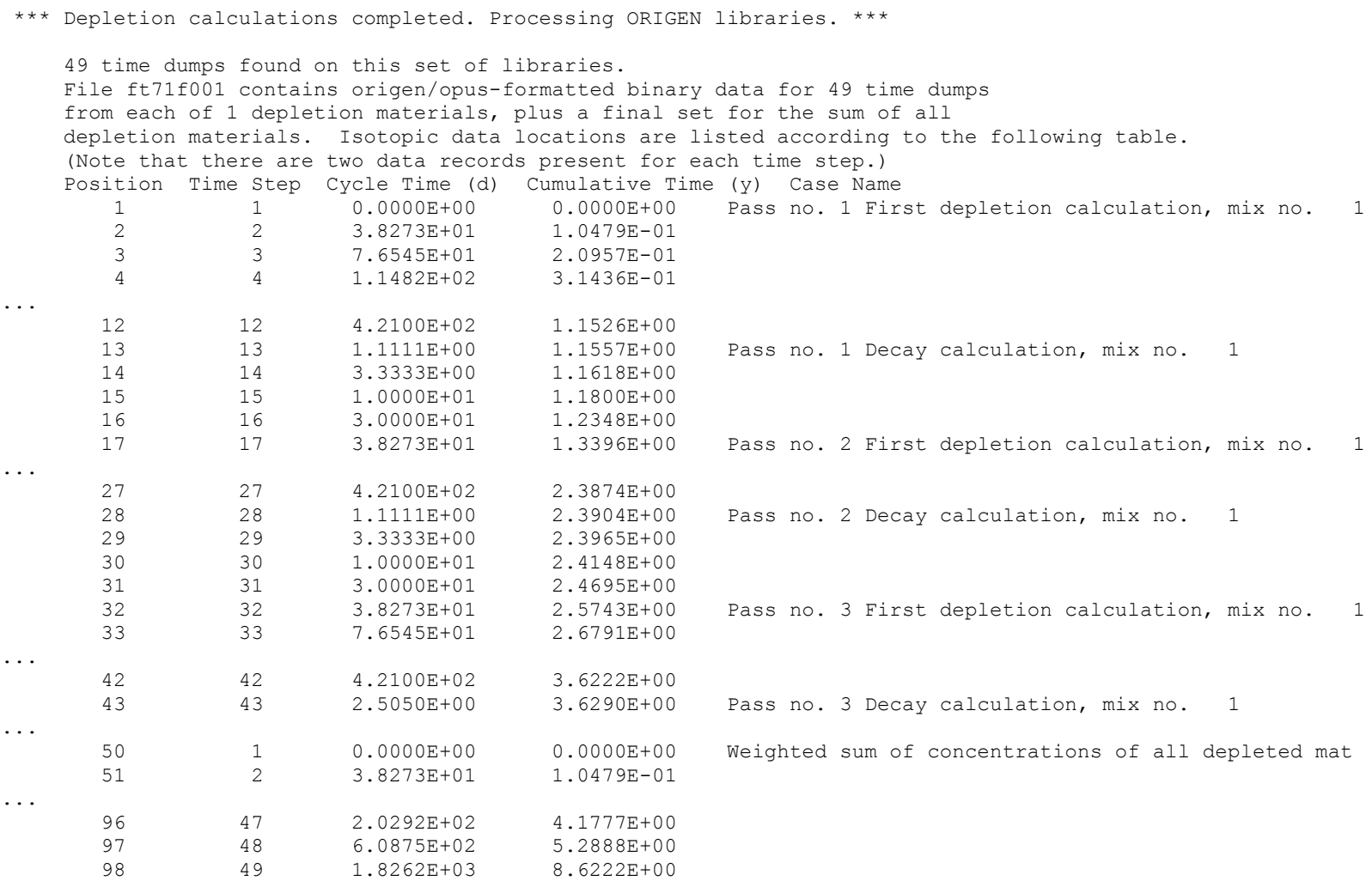

The requested OPUS output edits follow the .f71 file summary edit.

\subsubsection{TSUNAMI-2D output}

Output from the TSUNAMI-2D and TSUNAMI-2DC sequence is similar to that of the T-NEWT sequence, with the addition of the SAMS module output. If the user requests sensitivity and uncertainty analysis for non- $k_{\text {eff }}$ responses via the DEFINITIONS and SYSTEMRESPONSES blocks, the TRITON output will edit out the computed values of these responses. An example of this is shown here for sample problem 9 in Sect. 3.1.6.9.

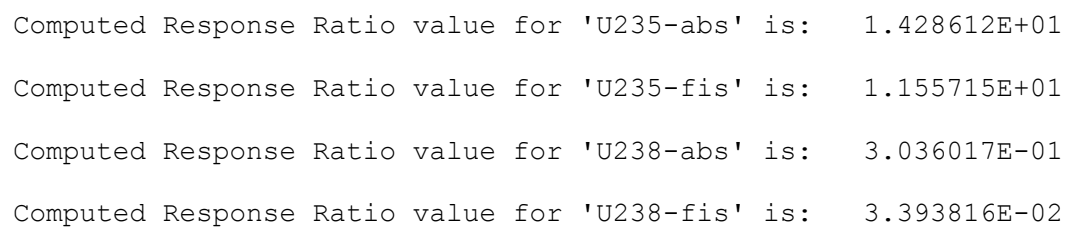

In addition to the text-formatted output, TRITON generates HTML-formatted output for TSUNAMI-2D calculations. The HTML output is fully described in the SAMS chapter. 


\subsubsection{TRITON Sample Cases}

This section provides descriptions of the 13 TRITON sample problems included with SCALE. Note that all of these problems (along with all other SCALE sample problems) are typically executed in the initial SCALE installation to test the performance of various codes and options, for validation of the installation process. Because of the number of problems that are executed, these sample problems are adjusted to run as fast as possible so that all test problems may be completed in relatively short order. To accomplish this, crude modeling approximations (reduced convergence, few histories, simplified cross section processing, low-order quadrature and scattering approximations, coarse computational grids, reduced numbers of libraries per depletion cycle, etc.) may be used. Hence, although these problems provide guidance in setting up and executing TRITON problems, it is generally a good idea to review all control settings to ensure sufficient accuracy in one's own calculations.

Additional TRITON input files for several reactor types can be generated with the SCALE/ORIGEN Library Generator (SLIG). The SLIB documentation is available as Appendix B of Chapter 5 (i.e. the ORIGEN chapter).

\subsubsection{TRITON sample problem 1: triton1.inp}

Sample problem tritonl.inp is an example of a T-NEWT transport calculation sequence. Input begins (as with all SCALE sequences) with a title card and cross section library specification; this calculation is performed using the 252-group ENDF/B-7.1 library. After the library specification, three materials are defined in the composition block, followed by a cell specification and the NEWT transport model.

This example includes an axial height of $37.1 \mathrm{~cm}$ and will therefore do a buckled calculation based on this height. The geometric model consists of a simple pin cell, with cylindrical fuel and clad regions inside a square moderator region, with a $6 \times 6$ base grid. The NEWT BOUNDS block specifies that periodic boundary conditions are used for this model.

This simple problem also demonstrates the use of TRITON's automatic cross section collapse capability-parm=weight. For T-NEWT calculations, TRITON uses the NEWT COLLAPSE block to define the broad-group energy structure. For this sample problem, the cross sections are collapsed to a 56-group format. The new broad-group library will be identified as filename newxnlib in the temporary working directory, which can be used in follow-up SCALE calculations.

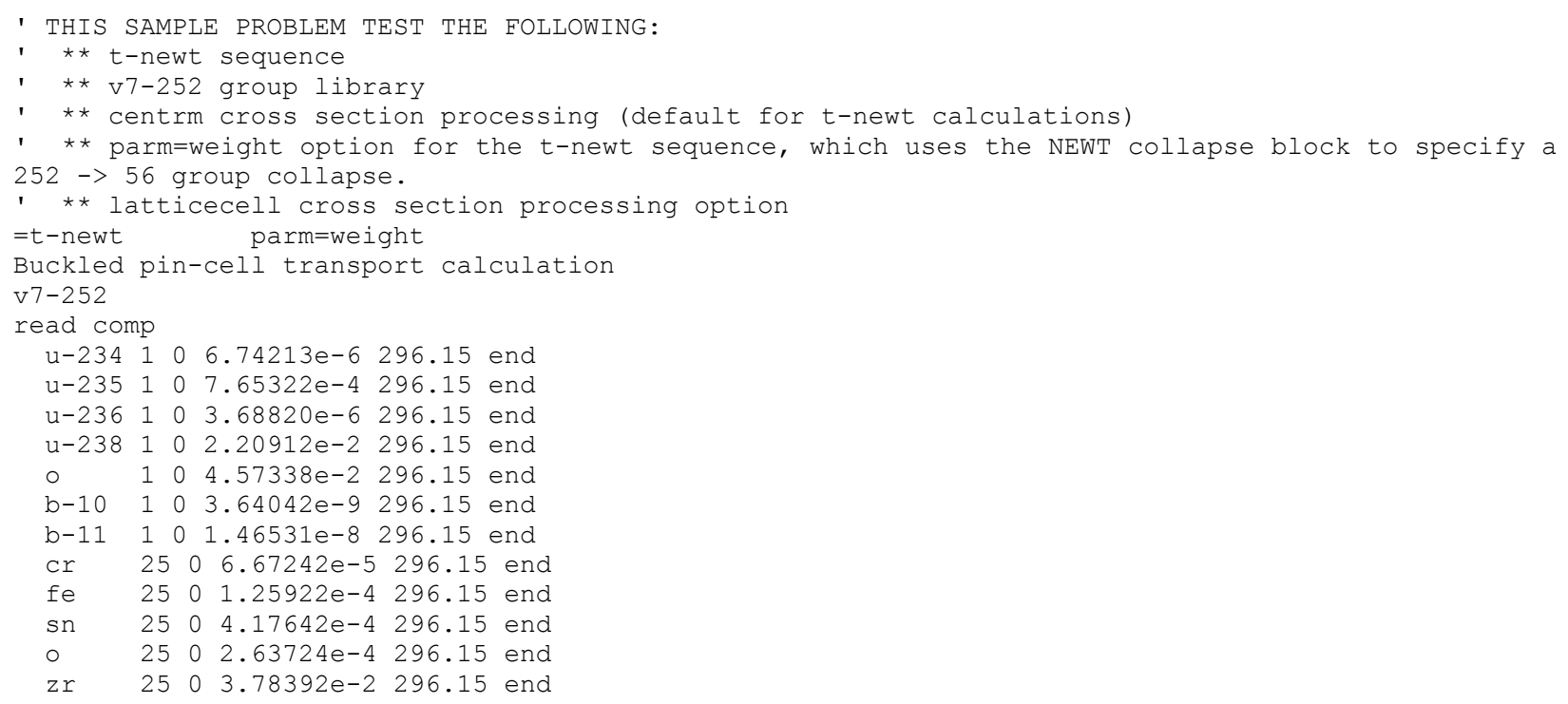




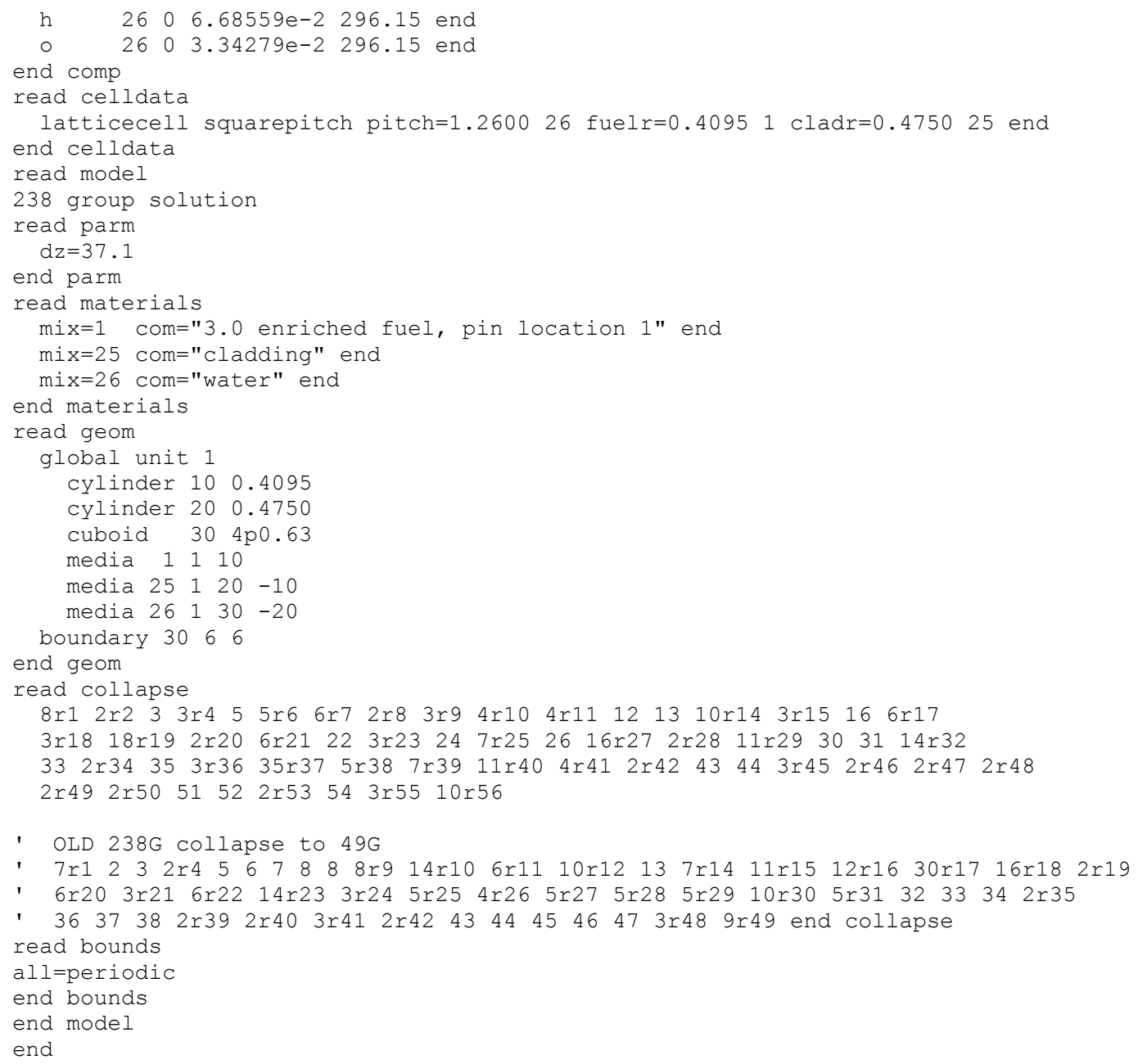

\subsubsection{TRITON sample problem 2: triton2.inp}

Sample problem triton2.inp is an example of a T-XSDRN transport calculation sequence. In this case, the parameter specification parm=2region instructs TRITON to perform cross section processing using the CENTRM-based two-region option in place of the default CENTRM-based $\mathrm{S}_{\mathrm{N}}$ option (see Sect. 3.1.2.1). As in sample problem 1, a simple square-pitched pin cell is modeled but in this case using an XSDRN model block rather than the NEWT model block. The moderator radius was defined in order to preserve the volume of the moderator region.

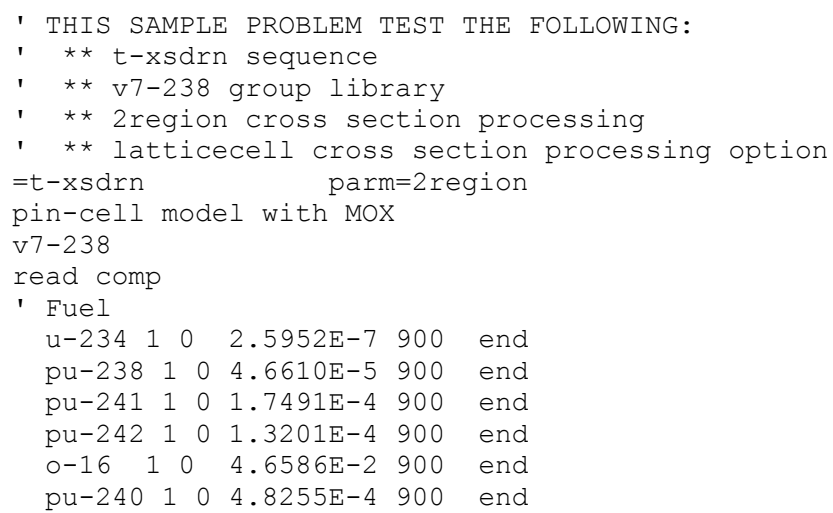




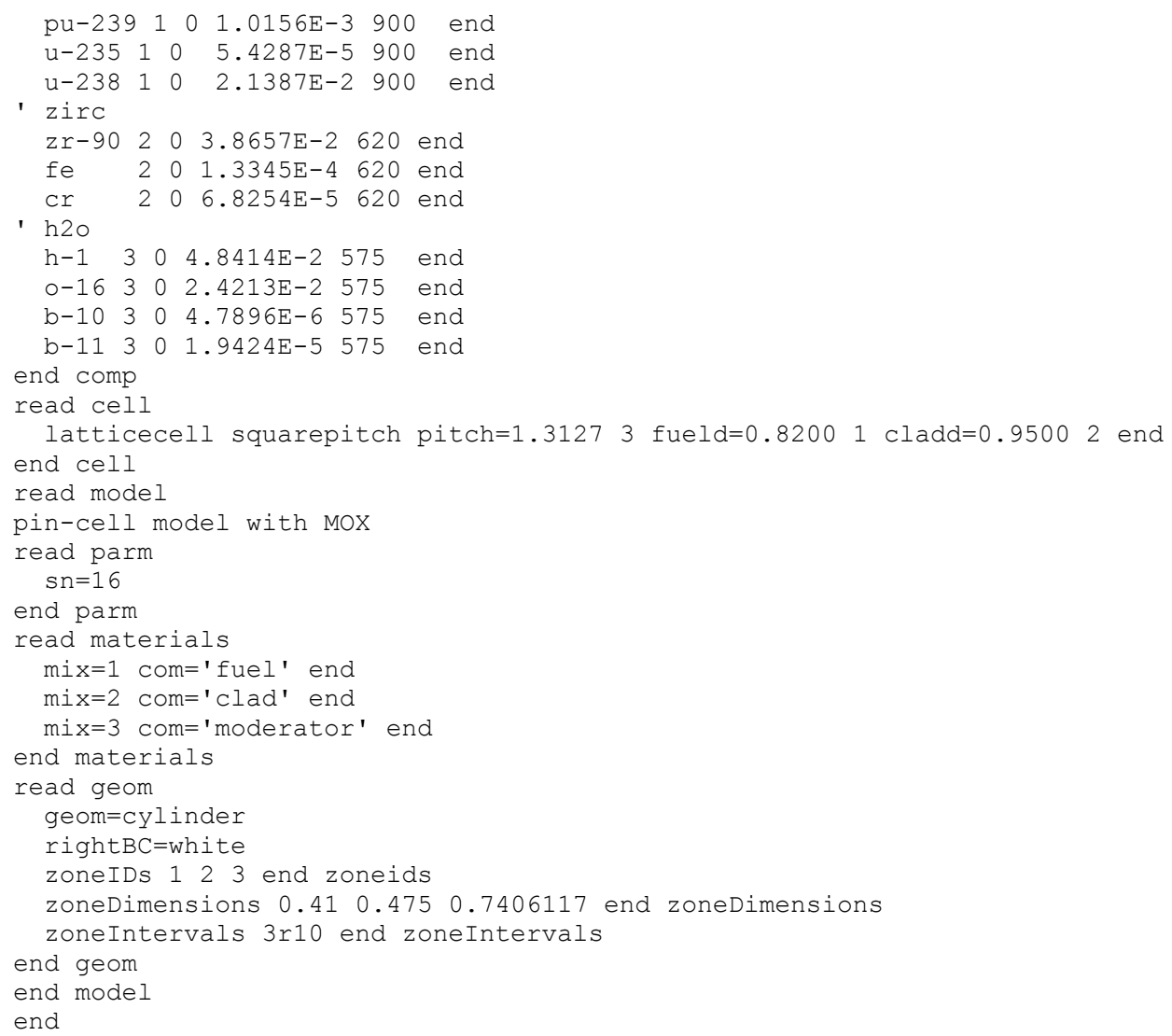

\subsubsection{TRITON sample problem 3: triton3.inp}

Sample problem 3 illustrates the input format for a T-DEPL-1D depletion calculation. In this case, a single square-pitched pin-cell model is depleted, where the fuel composition is comprised of $\mathrm{UO}_{2}$ fuel homogenized with aluminum and $\mathrm{B}_{4} \mathrm{C}$. Although this is not representative of real fuel, it does allow one to observe the effect of boron depletion during burnup; results will show an increasing multiplication factor as boron is depleted, followed by a decreasing eigenvalue after the fuel depletion becomes the dominant contributor to reactivity change. Three depletion intervals are specified with the same power and no decay intervals. Two depletion subintervals are specified for the first two depletion intervals, with only one depletion subinterval for the final depletion interval. Note that this may be insufficient to capture the effect of boron depletion early in life; fewer depletion subintervals are used here only to reduce the run-time for this sample problem. In this model, power is normalized such that material 1 has a power density of 21.22 MW/MTHM (or MT/MTU for $\mathrm{UO}_{2}$ fuel), and OPUS output is requested for 35 nuclides. The problem is run using the addnux $=3$ option set to add trace quantities of 231 nuclides to depletion materials.

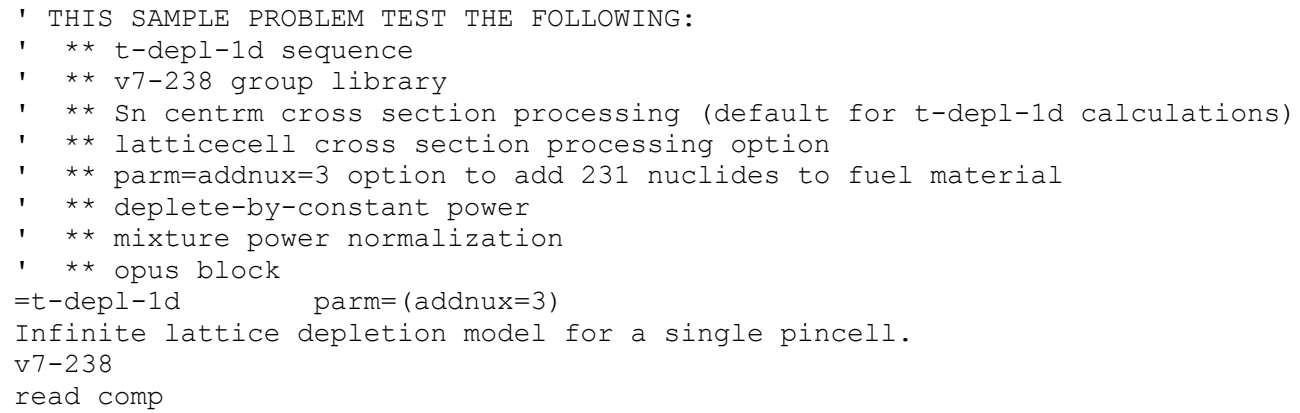




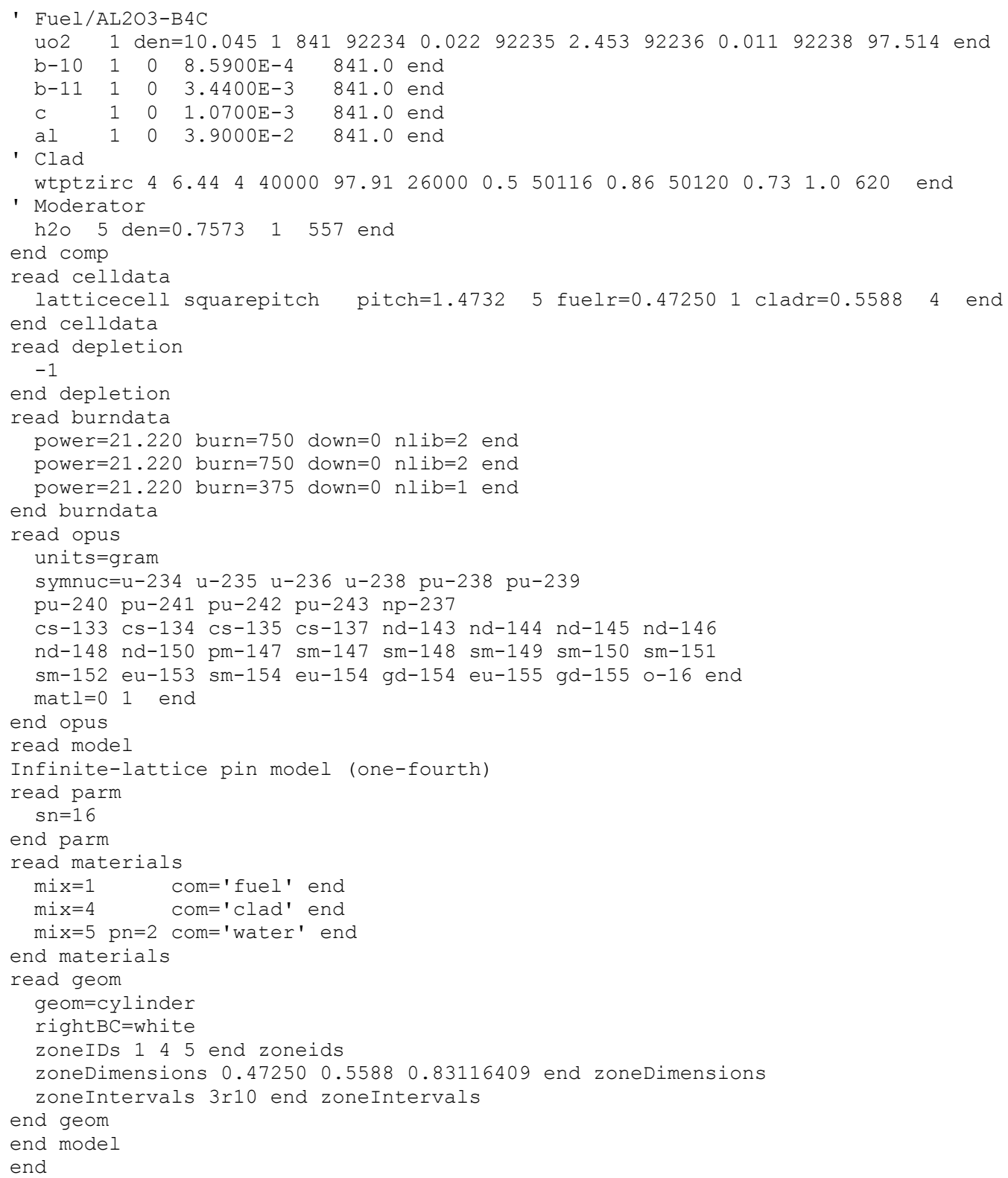

\subsubsection{TRITON sample problem 4: triton4.inp}

Sample problem triton4.inp performs a large-scale depletion calculation for a one-fourth PWR assembly, taking advantage of symmetry to reduce the problem size. The same fuel material is used in each fuel rod, which will result in assembly-averaged isotopic compositions for all fuel rods. If one wanted to obtain an isotopic estimate for one or more unique fuel rod locations, then different materials would be specified for different rod positions. Even though all fuel is identical at the beginning of life, unique materials must be specified if one desires to perform tracking of the unique response of each unique fuel position.

The problem parameter specification parm $=($ weight $)$ instructs TRITON to perform an automated cross section library collapse. For library collapse automation within depletion calculations (see Sect. 3.1.3.7.3), TRITON will perform a single 252-group calculation at $t=0$ to generate the 56-group cross section library. TRITON will restart the depletion calculation at $\mathrm{t}=0$ using the broad-group library after it is created. Because parm=weight is specified, the NEWT COLLAPSE block must comply with the 
56-group energy structure and not the 252-group energy structure. The COLLAPSE block input is slightly different for the library collapse automation for T-NEWT calculations, where the NEWT COLLAPSE block must comply with the 252-group energy structure.

Problem 4 also uses a timetable to specify boron letdown in the moderator. The initially specified boron concentration in the COMP (or COMPOSITION) data block is multiplied by a density multiplier at the time of each cross section processing and transport calculation (i.e., the midpoint of depletion subinterval). Linear interpolation is performed between values on the timetable to obtain the multiplier for a given time. Typically a multiplier of 1.0 is used for $\mathrm{t}=0$, and the beginning-of-life boron concentration is input in the COMPOSITION block, but this example demonstrates that this is not necessary. For this calculation, a $500 \mathrm{ppm}$ boron concentration is specified in the standard composition description, and a concentration of $(500 \mathrm{ppm}) *(1.832)$, or $916 \mathrm{ppm}$, would be used in the $t=0$ transport calculation.

Problem 4 is also an example of a lattice physics calculation for a full fuel assembly. The NEWT model employs coarse-mesh finite-difference acceleration, whole-assembly homogenization, 2-energy-group collapse, and a pin-power print, and computes assembly discontinuity factors. Although this sample problem will create the cross section database file for core calculations, this sample problem does not contain branching calculations, nor do lattice physics calculations typically use boron letdown curves. Additional guidance for TRITON lattice physics calculations can be found in the lattice physics primer.

Because only one fuel material is used, only one cell specification is necessary. If multiple fuel materials were used, then a corresponding cell specification would be required for each fuel, with a unique clad and moderator material identifier for each cell. To apply boron letdown properly, the moderator present in each cell specification would need to have the same letdown curve specified. Hence, a letdown timetable would need to be specified for each moderator (even if the moderators are not all used in the NEWT model block). If multiple fuel materials are used, requiring corresponding multiple clad, moderation, cell, and timetable specifications, the use of an alias specification can simplify input. Aliases are described in Sect. 3.1.3.5; sample problems triton6.inp (Sect. 3.1.6.6), triton8.inp (Sect. 3.1.6.8), and triton12.inp (Sect. 3.1.6.12) demonstrate the use of aliases.

This case also illustrates the use of stacked OPUS cases within a single TRITON input file. Here, an OPUS calculation is requested to obtain the mass in grams of 26 actinides and fission products for material 1 and for the entire system; since material 1 is the entire set of depletion materials, the system output will be identical to the material 1 output. A second OPUS calculation is also specified, which requests a ranked output of the top 20 nuclides in terms of decay heat (in watts).

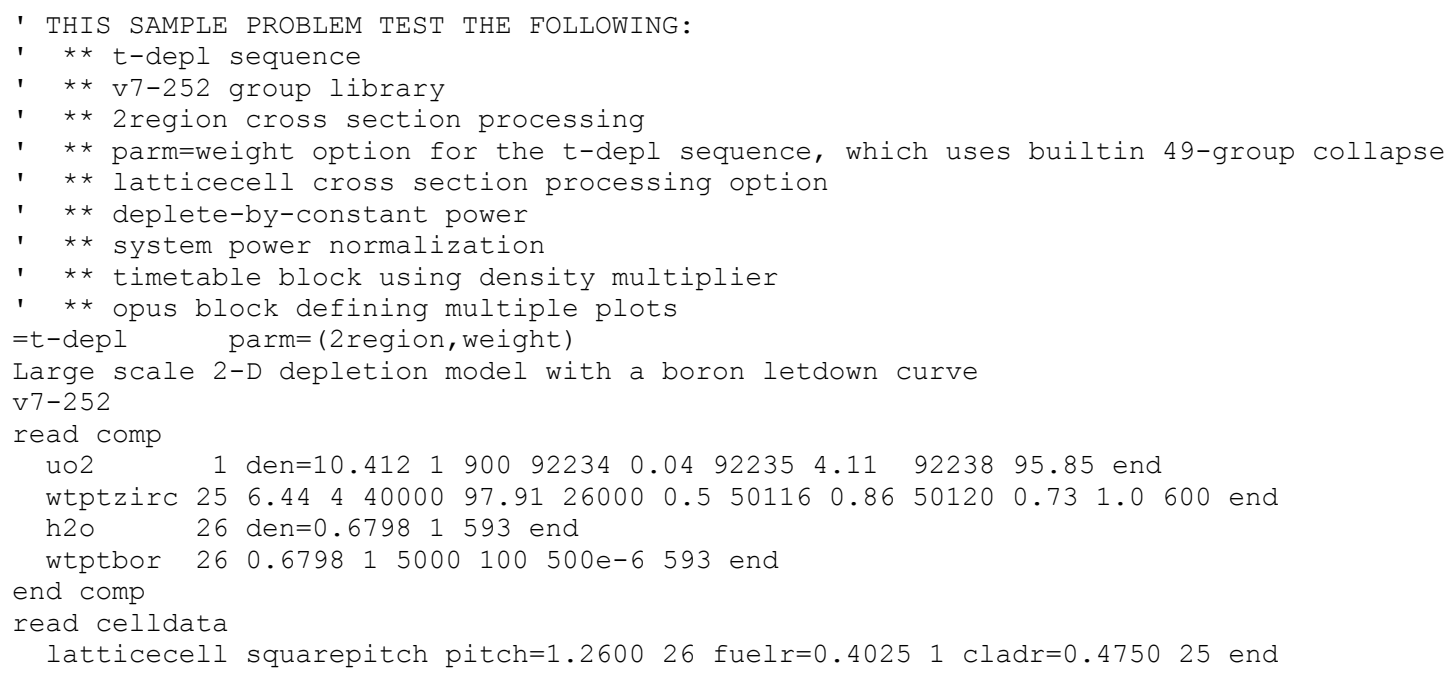




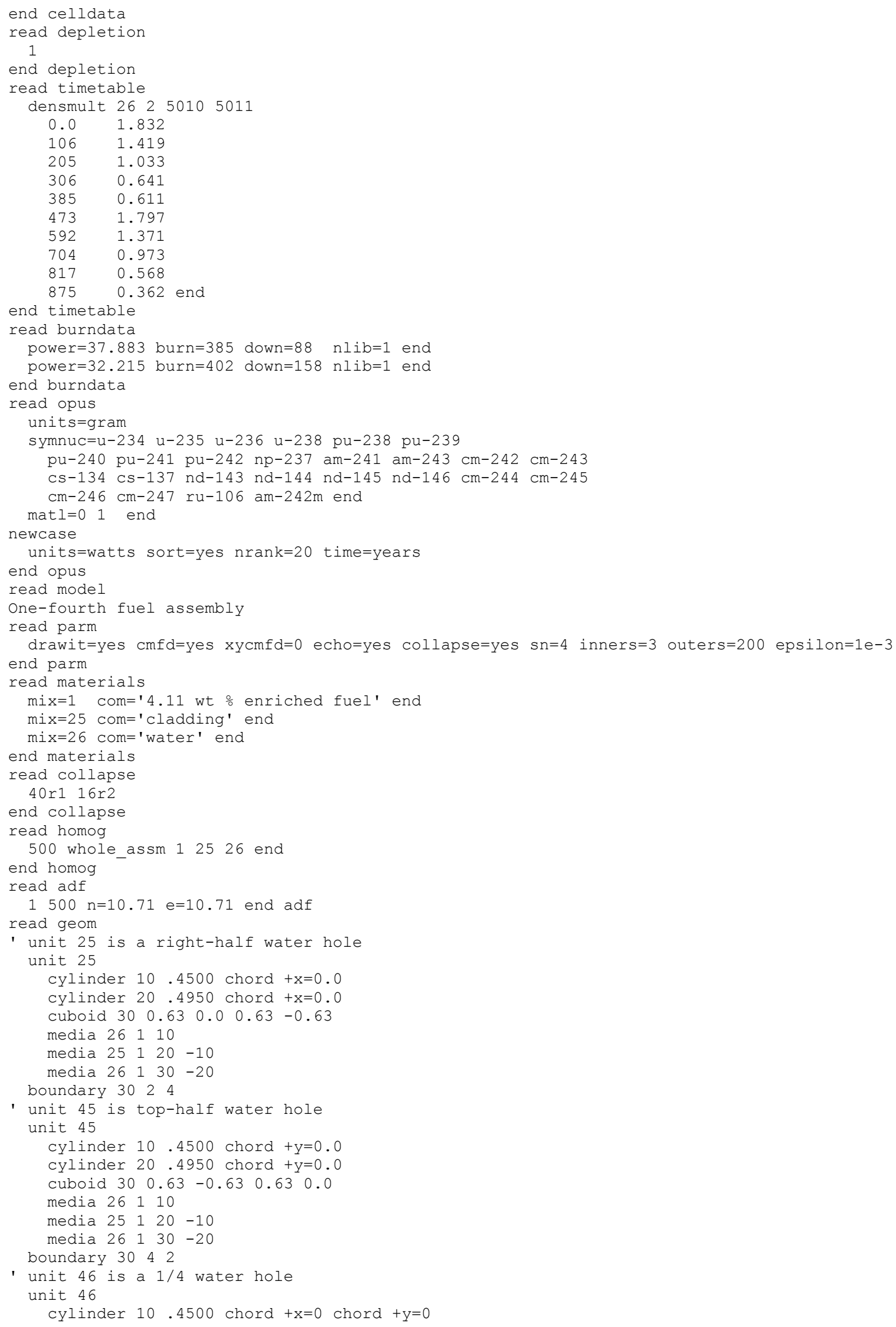




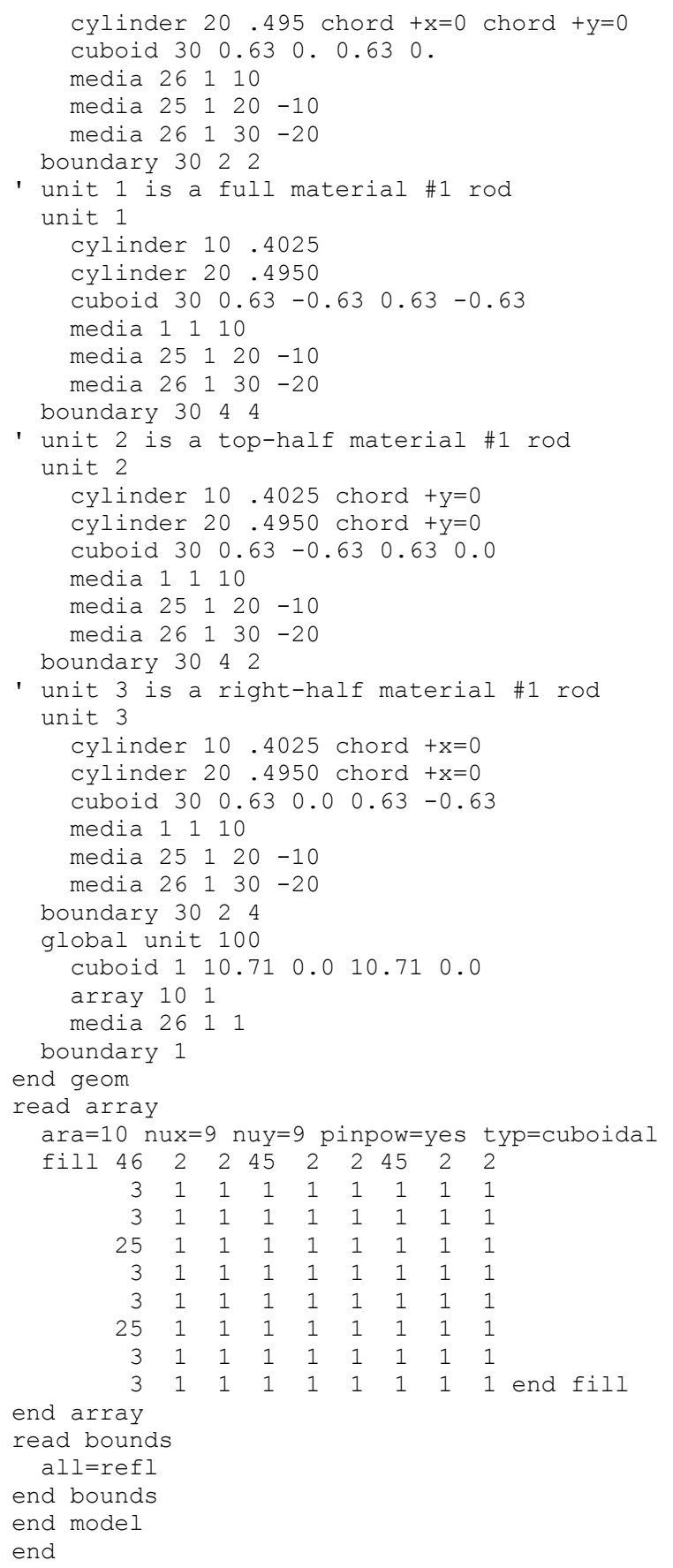

\subsubsection{TRITON sample problem 5: triton5.inp}

Sample problem triton5.inp is similar to triton4.inp, except that it is a T5-DEPL case; thus, a KENO V.a transport model is used in place of the NEWT model of the earlier case. The KENO V.a model, although $3 \mathrm{D}$, is axially uniform with reflecting boundary conditions, so it is effectively the same model as the $2 \mathrm{D}$ model of triton4.inp. Moreover, the KENO V.a model represents the full assembly rather than a onefourth model. Hence, both cases will generate similar results. In the KENO model, only 300,000 neutron histories are retained, which is somewhat low to obtain good statistics on fluxes. The 238 ENDF/B-VII library is used for this sample problem compared to the 252 ENDF/B-VII.1 library utilized in triton4.inp. 


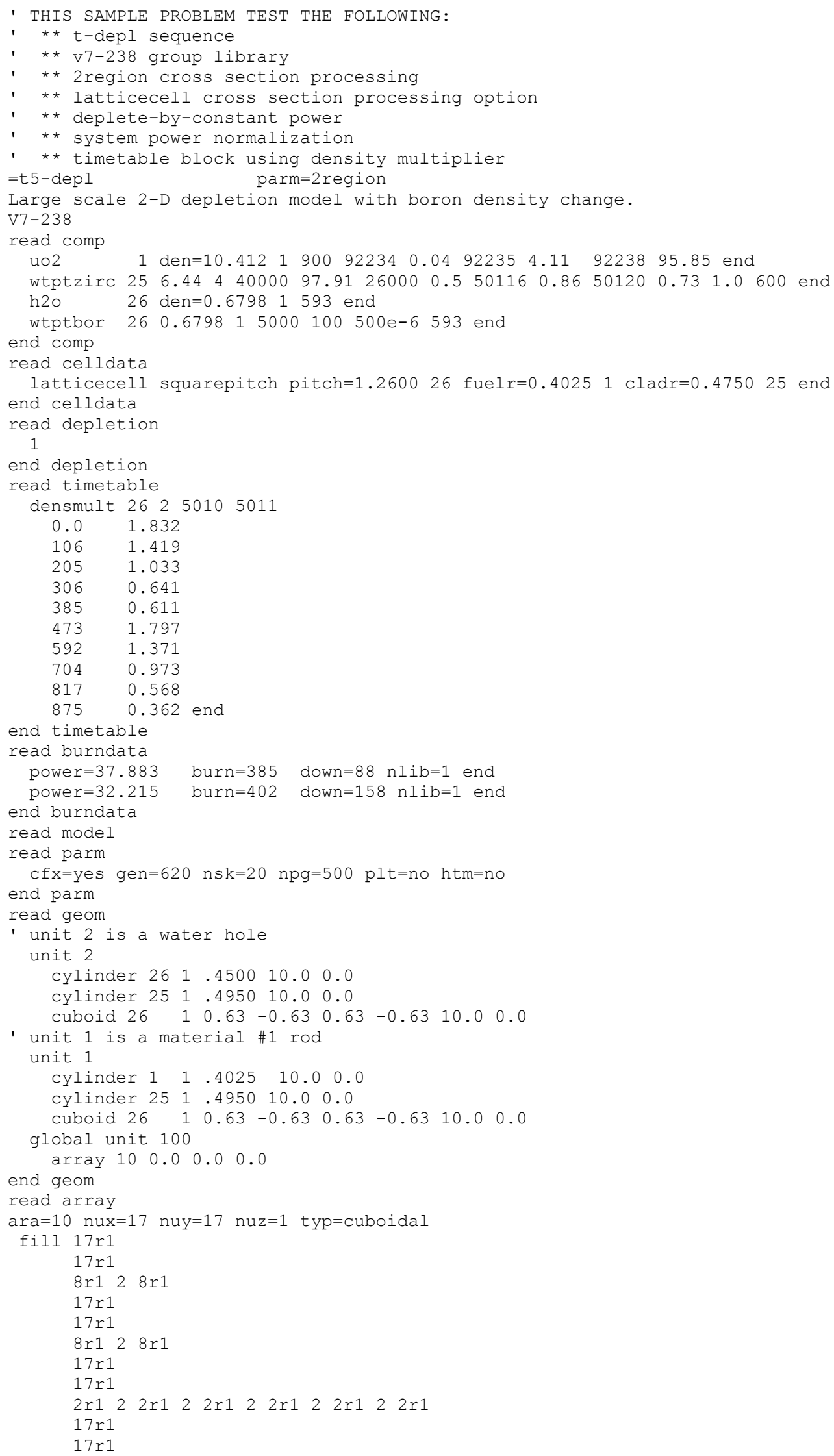




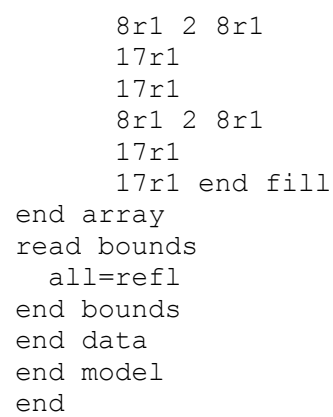

\subsubsection{TRITON sample problem 6: triton6.inp}

Sample problem triton6.inp performs T-DEPL depletion in a pin cell model; however, the pin is discretized into five equal-volume rings of fuel. Thus, CENTRM-based $S_{N}$ cross section processing is necessary to capture the radial burnup of the pin cell. A multiregion cell specification is given to allow specification of the varying radii for the fuel regions. Because the multiregion cell is cylindrical, the moderator volume is represented in terms of a radius that corresponds to the volume associated with the pin pitch. The right boundary condition for the cell is set to white; this is important, as the default right boundary condition for a multiregion cylinder is vacuum. In this case, addnux $=1$ is also requested in the parameter specification, simply for a faster (but less accurate) calculation. Material aliases are used to simplify input. The calculation is performed with the 238 ENDF/B-VII library. The TRITON TIMETABLE block is used to demonstrate time-dependent temperature changes to the moderator material.

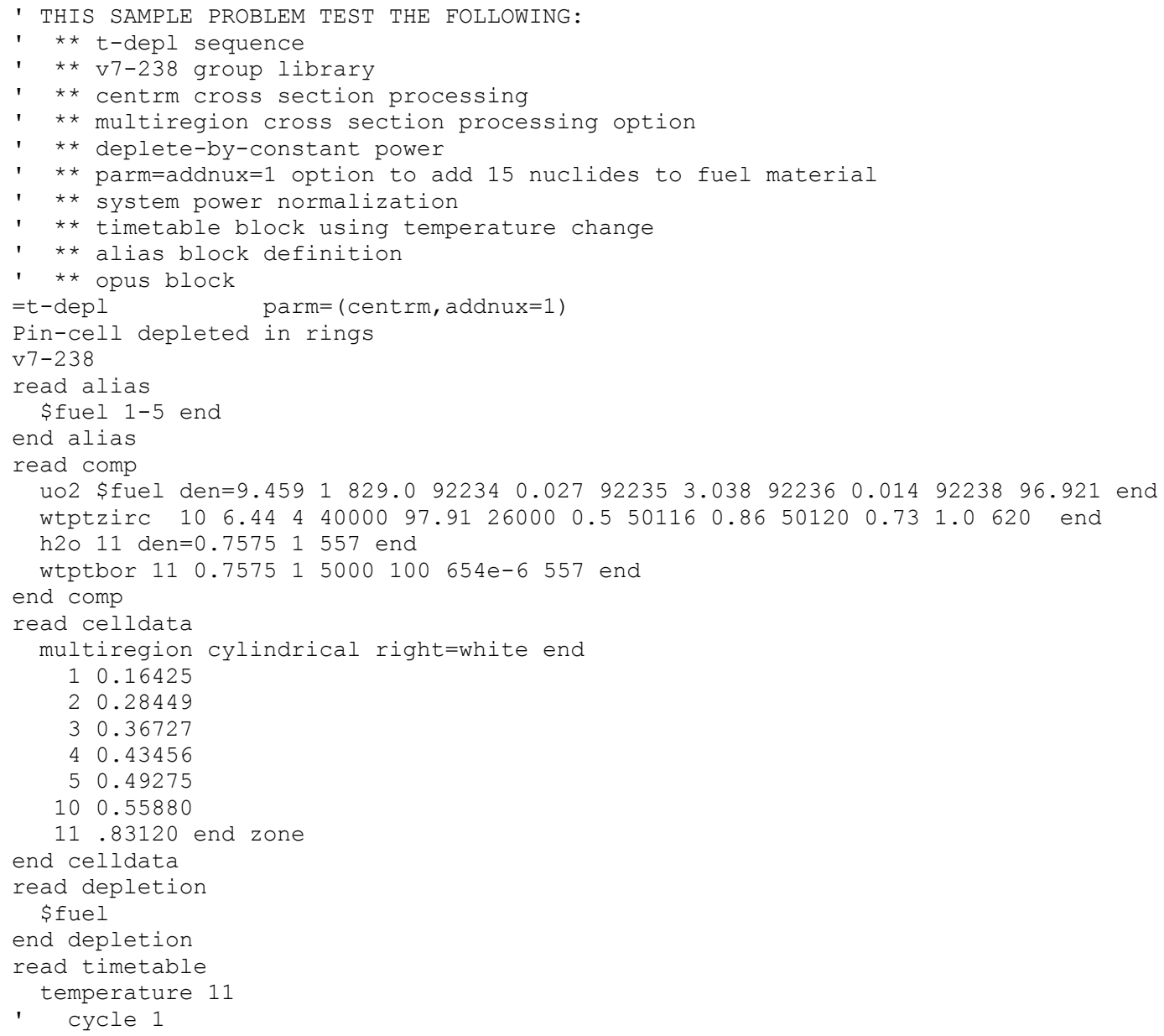




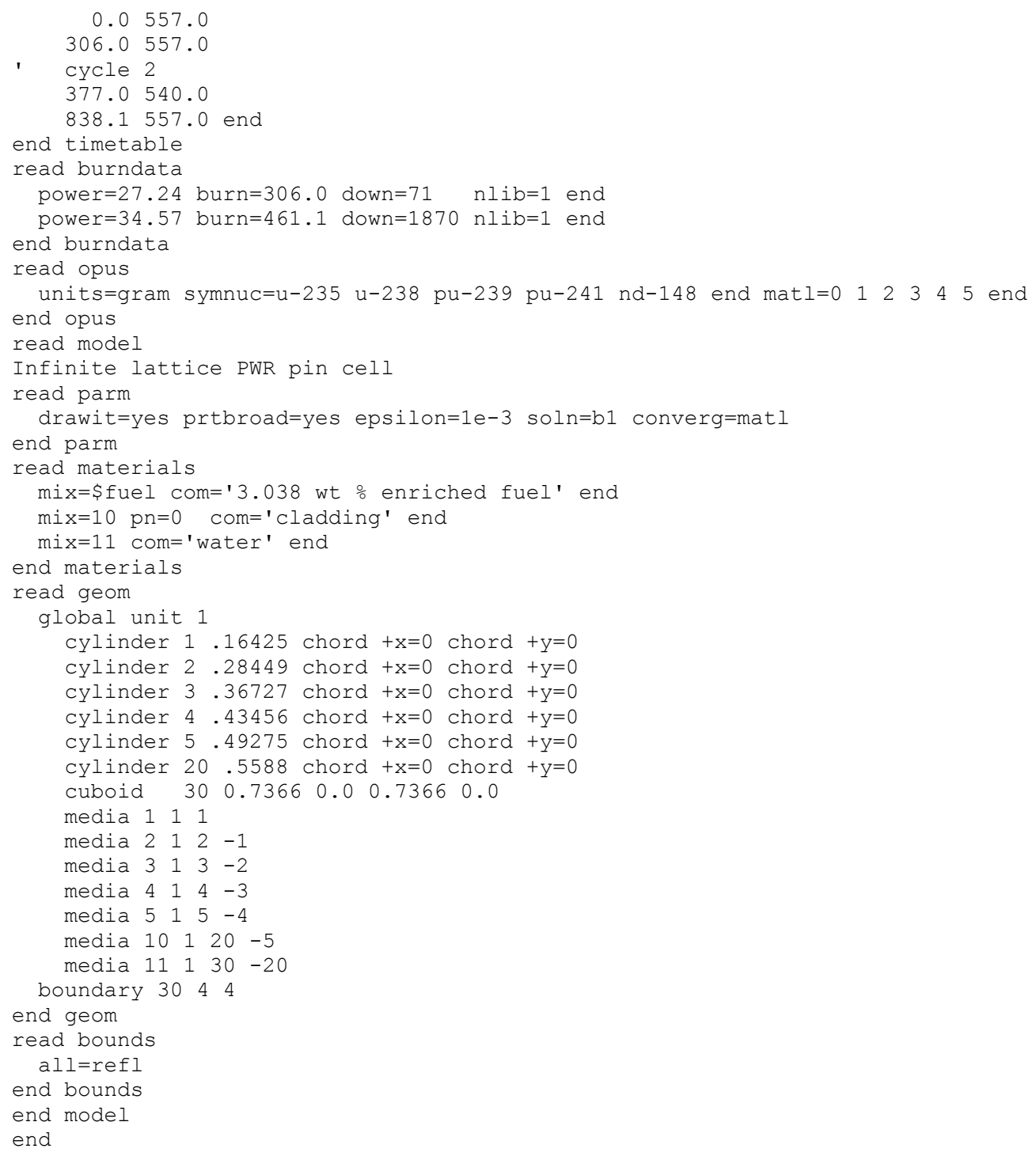

\subsubsection{TRITON sample problem 7: triton7.inp}

Sample problem triton7.inp is an example of a T-DEPL depletion calculation for a full PWR fuel assembly model. Depletion is performed on the basis of material 7, which is located in a single fuel pin for which destructive assay measurements were performed. All other fuel is modeled as a single (average) material, material 1 . The parameter specification parm $=(2$ region, $a d d n u x=1$, weight $)$ was chosen to reduce the run-time of the sample problem.

This sample problem also demonstrates the use of TRITON's standard composition restart files and SCALE external file reading capabilities to represent the time-dependent behavior of an assembly in which burnable poisons are removed after the first cycle of operation.

This problem consists of two TRITON 2D depletion cases. In the first case, the full assembly model contains borosilicate glass burnable poison rods (BPRs), material 4, which are included in the list of materials to be depleted. The calculation is run for the entirety of the first operational cycle, which included a 40-day mid-cycle decay interval. The model also includes a 64-day decay interval after the end of the operational cycle. When this calculation is completed, TRITON creates in the temporary working 
directory a standard composition file for each material containing the isotopic inventories for each depletion material at the end of the 64-day decay interval. The second TRITON calculation reads the standard composition specifications for materials 1 and 7 as part of the input to provide the fuel state for the second calculation. In the second model, the BPRs are removed and replaced with the moderator in the embedded NEWT model. The initial depletion calculation uses the 252 ENDF/B-VII.1 library. With the parm $=(. . .$, weight $)$ option, a 56 group library is created in the temporary directory called newxnlib. This library is used for the second $T-N E W T$ calculation.

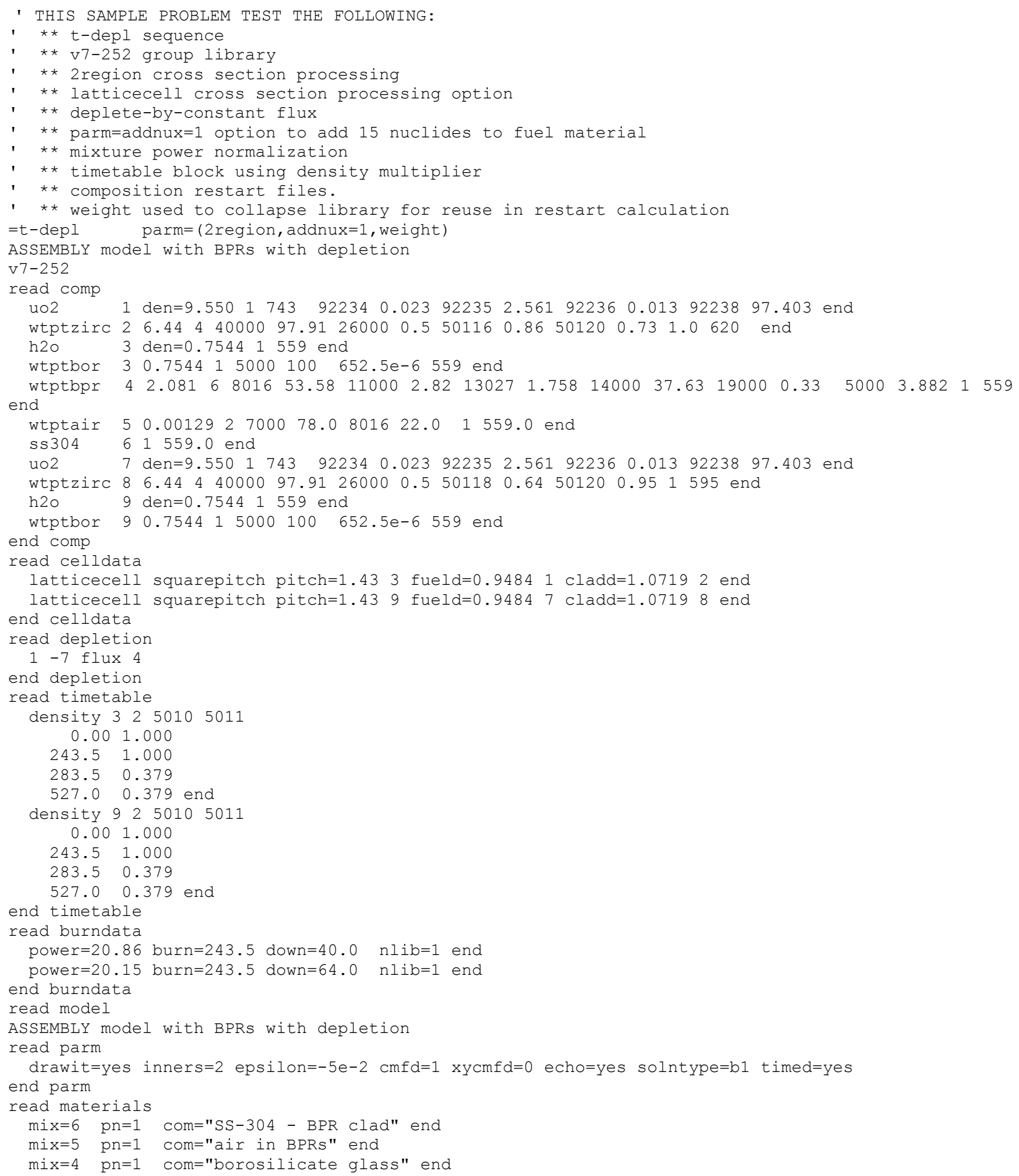




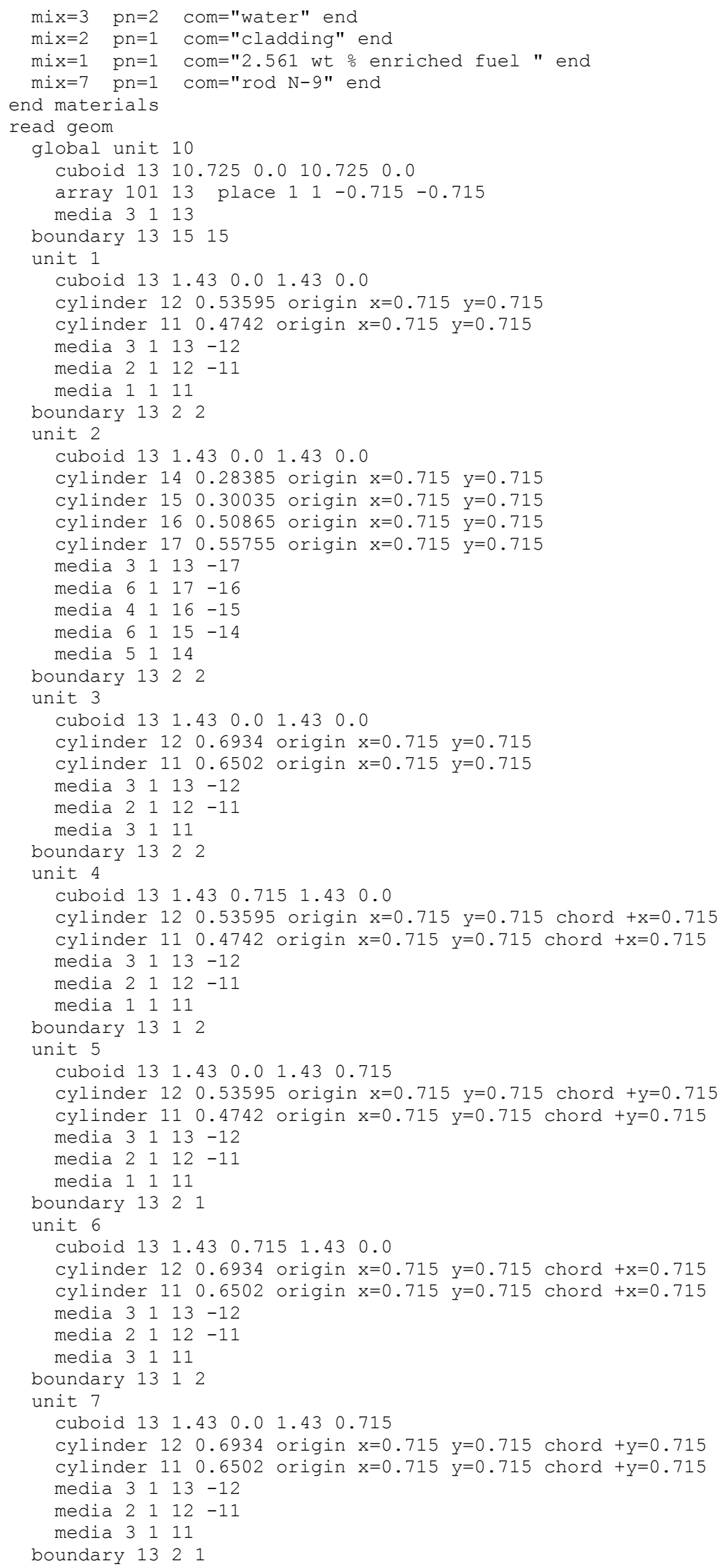




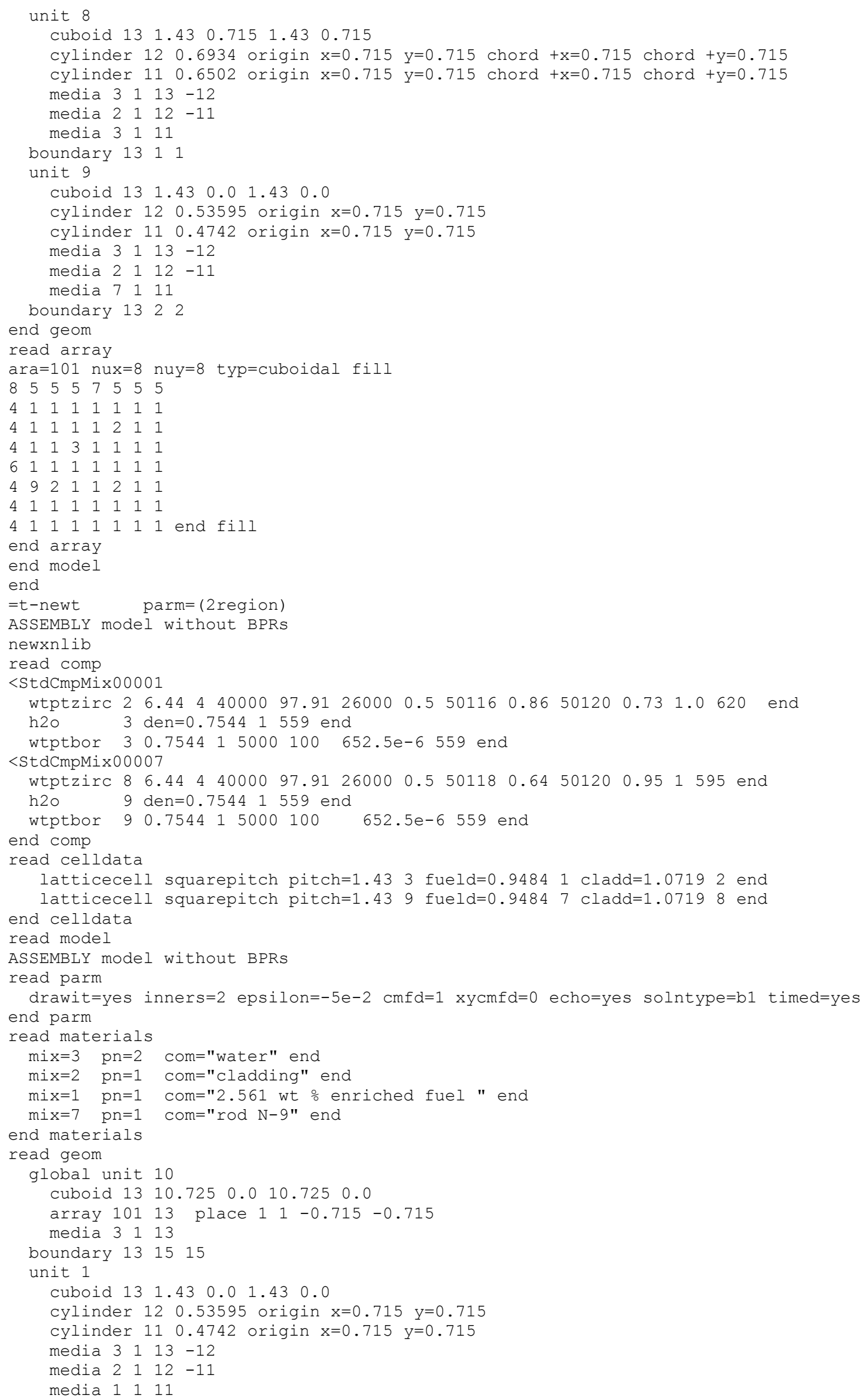




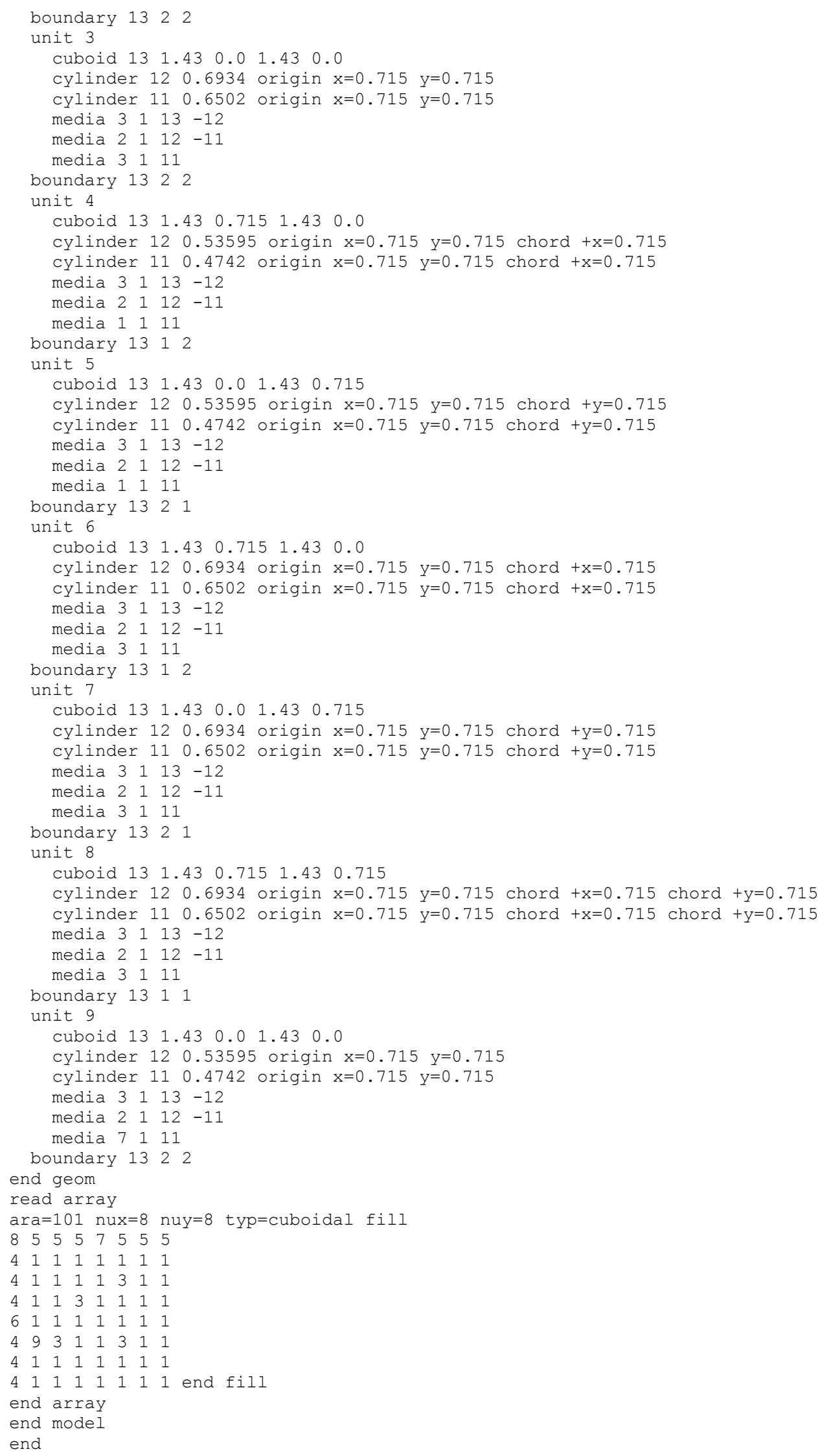




\subsubsection{TRITON sample problem 8: triton8.inp}

Sample problem triton8.inp is an example of TRITON's simplified cross section processing scheme in a BWR-like configuration. It uses the T-DEPL extended format for the DEPLETION block to allow material assignments to be made to reduce the number of cross section processing calculations required in a multimaterial depletion model. In this sample problem, the lower-left quadrant of a $6 \times 6$ fuel assembly is modeled (see Figure 3.1.17). The fuel pin layout is as follows: one $2.3 \%{ }^{235} \mathrm{U}$-enriched fuel pin in the southwest corner (red pin), one-fourth of a water rod in the northeast corner, five $3.6 \%{ }^{235} \mathrm{U}$-enriched fuel pins ( 2 green, 1 yellow, and 2 blue pins), and two gadolinium-bearing pins each modeled with three fuel rings. (Gadolinium-bearing pins are typically modeled with multiple fuel rings due to the strong spatial dependence of the flux.) Due to diagonal symmetry, only seven depletion material regions need to be defined: the red, green, yellow, and blue pins along with three regions for the gadolinium-bearing pins. Although seven depletion materials are defined, only three cell specifications are used: one for the gadolinium-bearing pin cell and one each for the $2.3 \%$ and $3.6 \%{ }^{235} \mathrm{U}$-enriched pin cells. This model makes extensive use of aliases. The sequence is run with parm $=($ addnux $=0, \ldots)$, which includes no extra nuclides, for an accelerated solution; however, this is an extremely poor approximation unless important nuclides are manually specified. This example also illustrates the use of the keyword flux in the DEPLETION data block to force flux-based ORIGEN calculations in place of power-based calculation for all three rings of the gadolinium-bearing fuel pins. Finally, this case uses the parm $=(\ldots$, weight,...) directive to request the automatic collapse of the input 238 ENDF/B-VII library to a 49 group library (collapsed using the 238-group system-averaged flux), which is then used for depletion calculations.

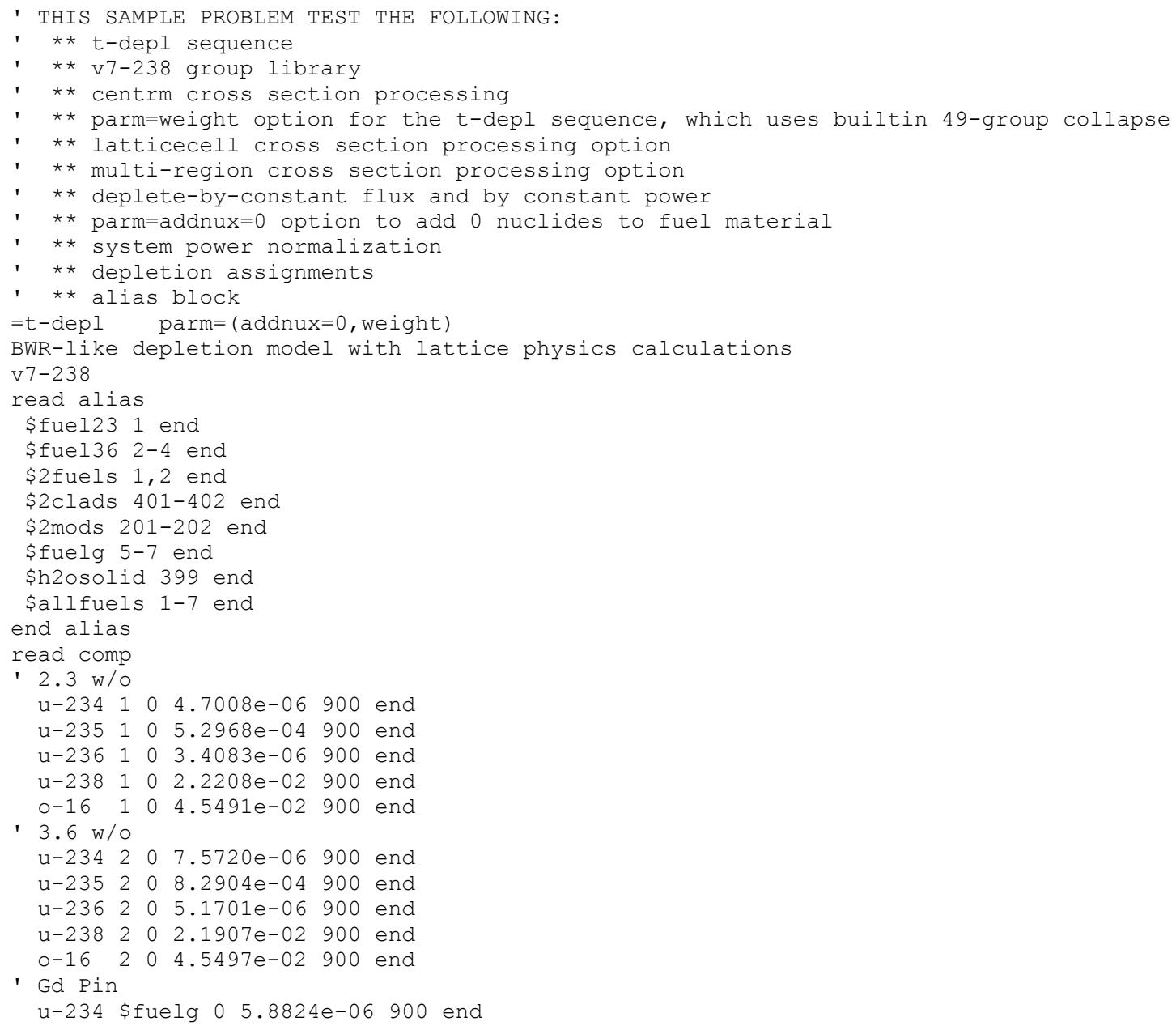




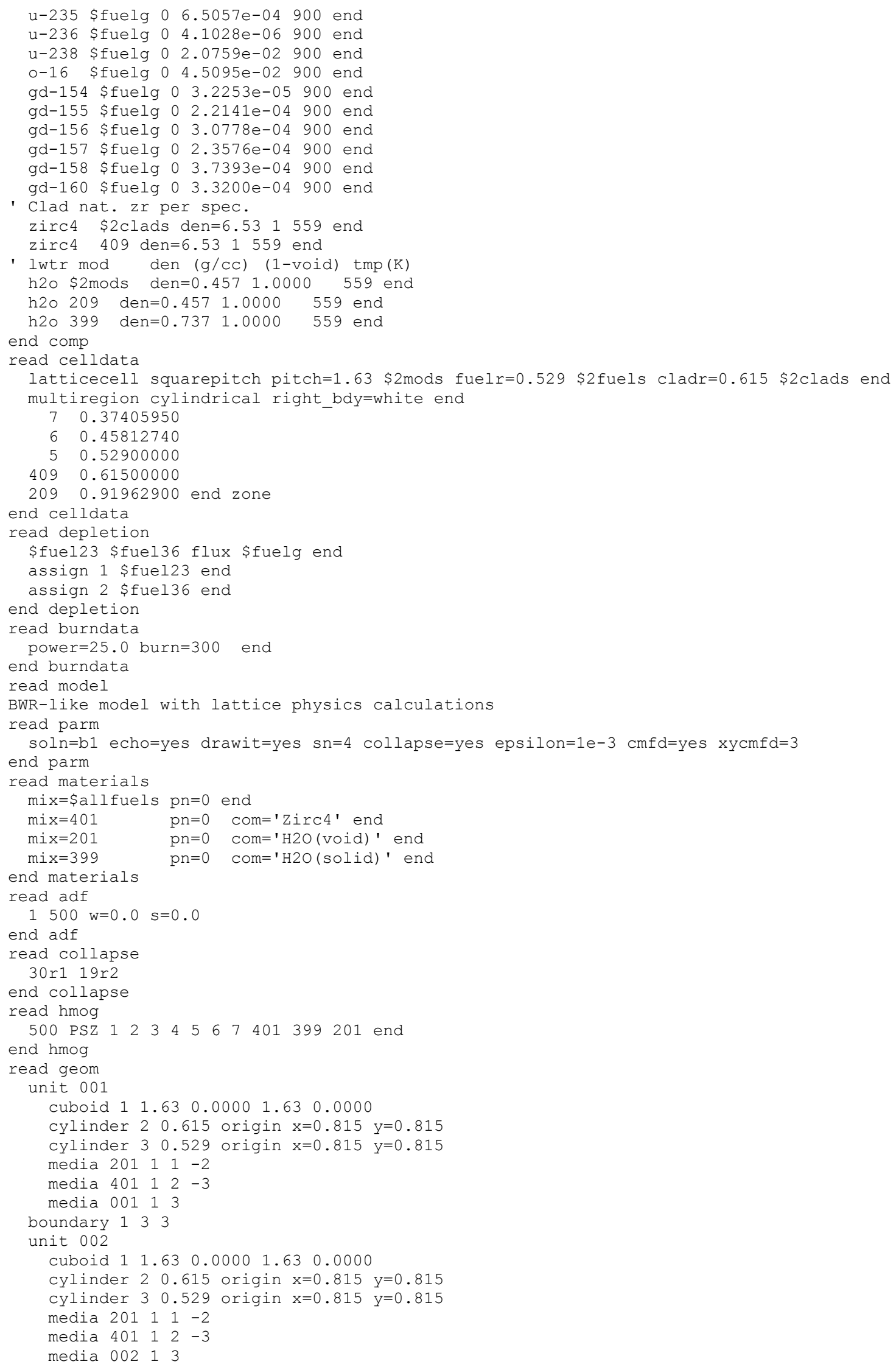




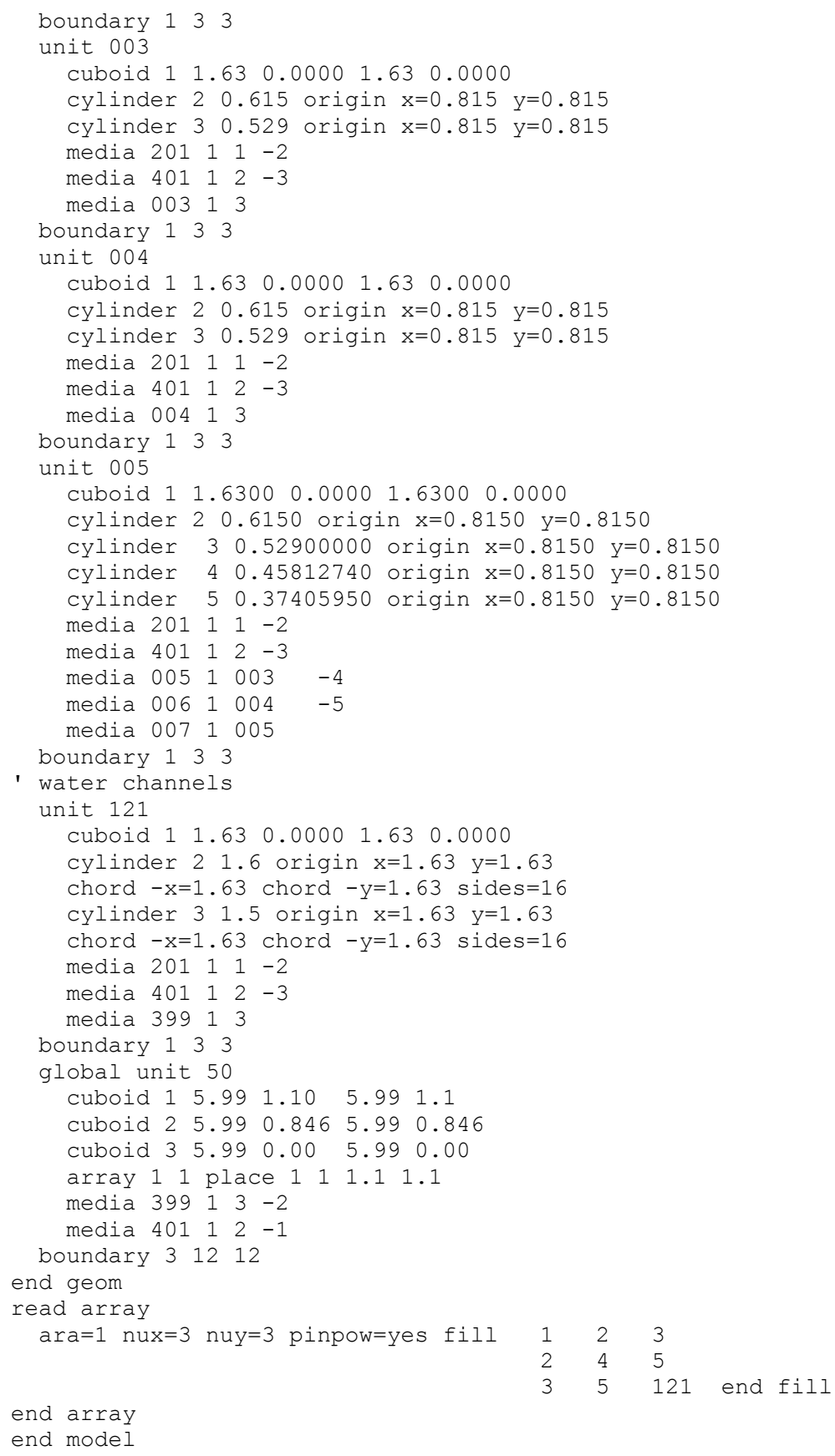




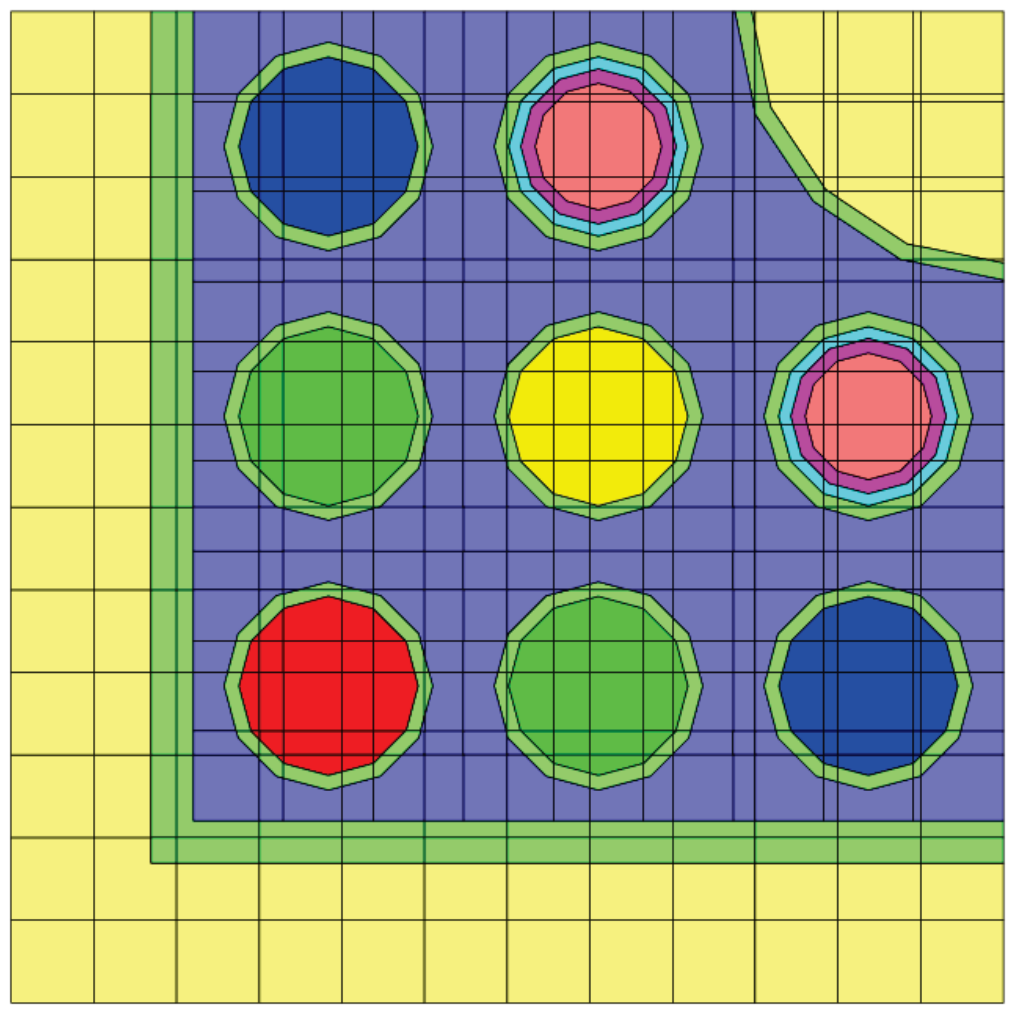

Figure 3.1.17. BWR-like assembly design for triton8.

\subsubsection{TRITON sample problem 9: triton9.inp}

Sample problem triton9.inp illustrates the use of the TSUNAMI-2D sequence for sensitivity and uncertainty (S/U) analysis of a PWR pin cell. The SCALE ENDF/B-VII 238-group library is used, with cross section processing performed by BONAMIST and CENTRM/PMC. In this sample problem, the DEFINITIONS and SYSTEMRESPONSES blocks are used to define four additional responses in addition to $k_{\text {eff }}$ for $\mathrm{S} / \mathrm{U}$ analysis: ${ }^{235} \mathrm{U}$ one-group absorption cross section, ${ }^{235} \mathrm{U}$ one-group fission cross section, ${ }^{238} \mathrm{U}$ one-group absorption cross section, and ${ }^{238} \mathrm{U}$ one-group fission cross section. The SAMS block is also used to disable the HTML-formatted output for this model.

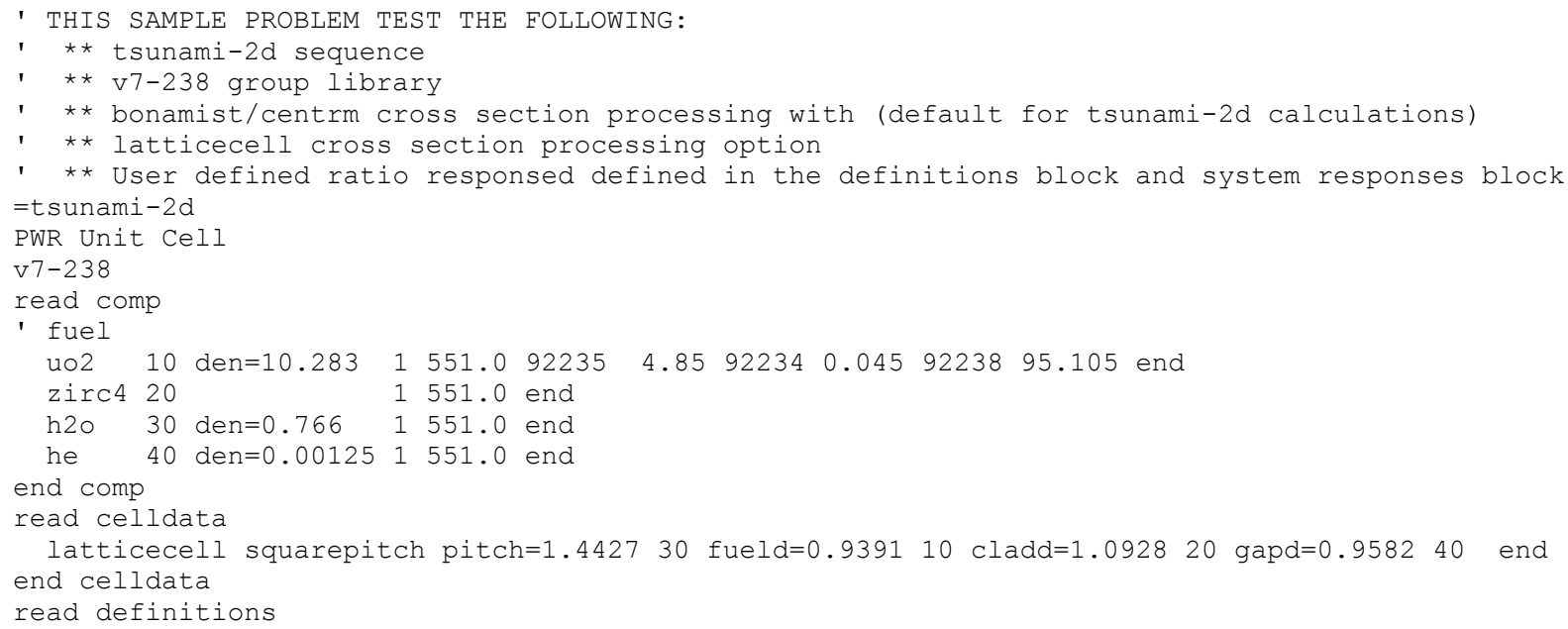




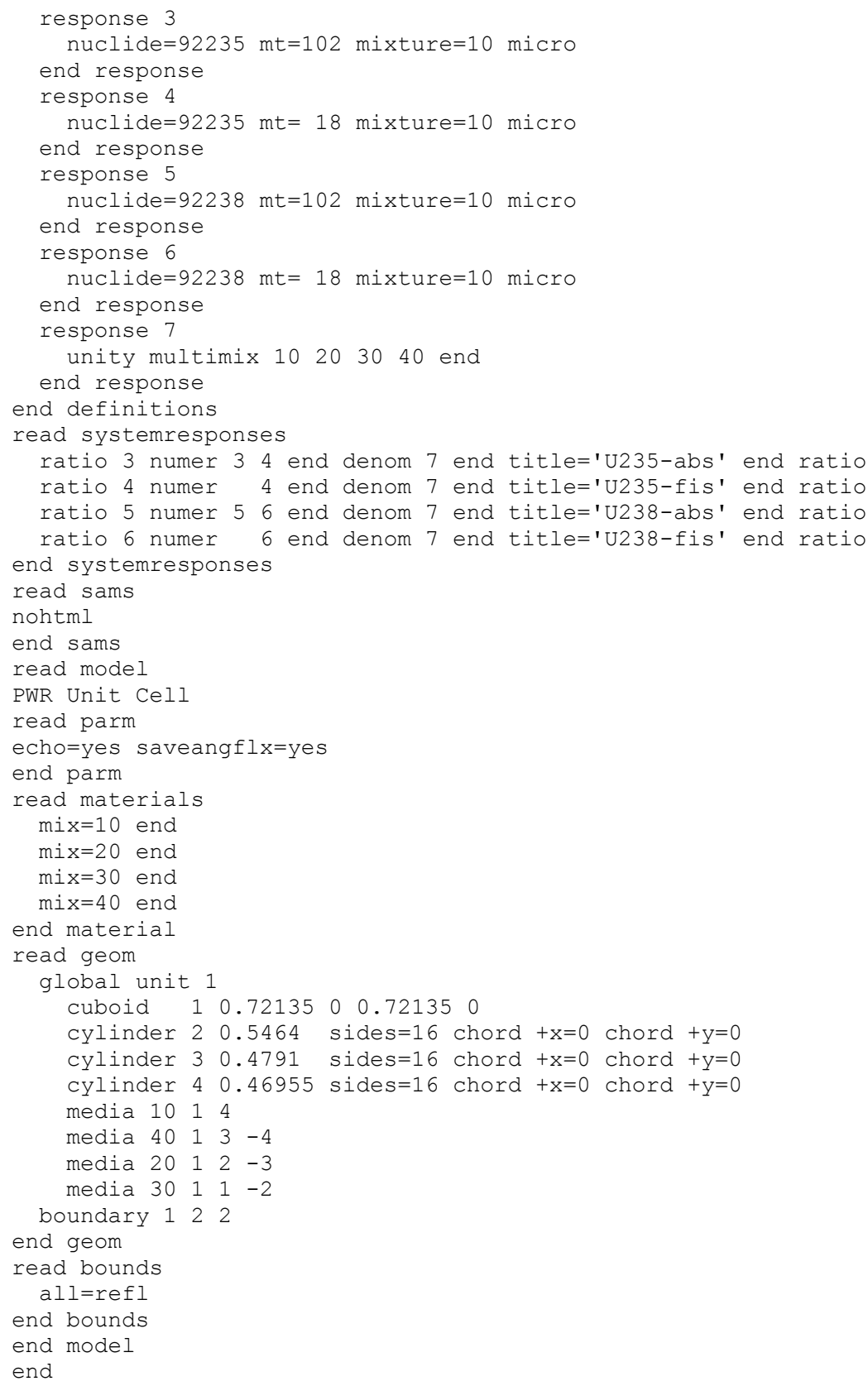

\subsubsection{TRITON sample problem 10: triton10.inp}

Sample problem triton10.inp performs NEWT-based depletion of a one-fourth symmetric assembly model. The primary intent of this sample problem is to test and to demonstrate the use of branches and archival of computed cross sections for a depletion case. The model includes two branch calculations-in addition to the nominal condition - that demonstrate the different perturbation outputs available in the $B R A N C H$ block. A two-group collapse is requested in the NEWT input, along with homogenization over all materials. (Note that the parameter specification parm $=(a d d n u x=1)$ is only used to reduce run-time of the sample problem.) At the end of the calculation, the binary file "xfile016" and text file "txtfile16" will exist in the temporary working directory and will contain all lattice physics parameters for all branches at all depletion states. These files are often copied back from the SCALE temporary working directory to another more permanent directory for subsequent post-processing. 


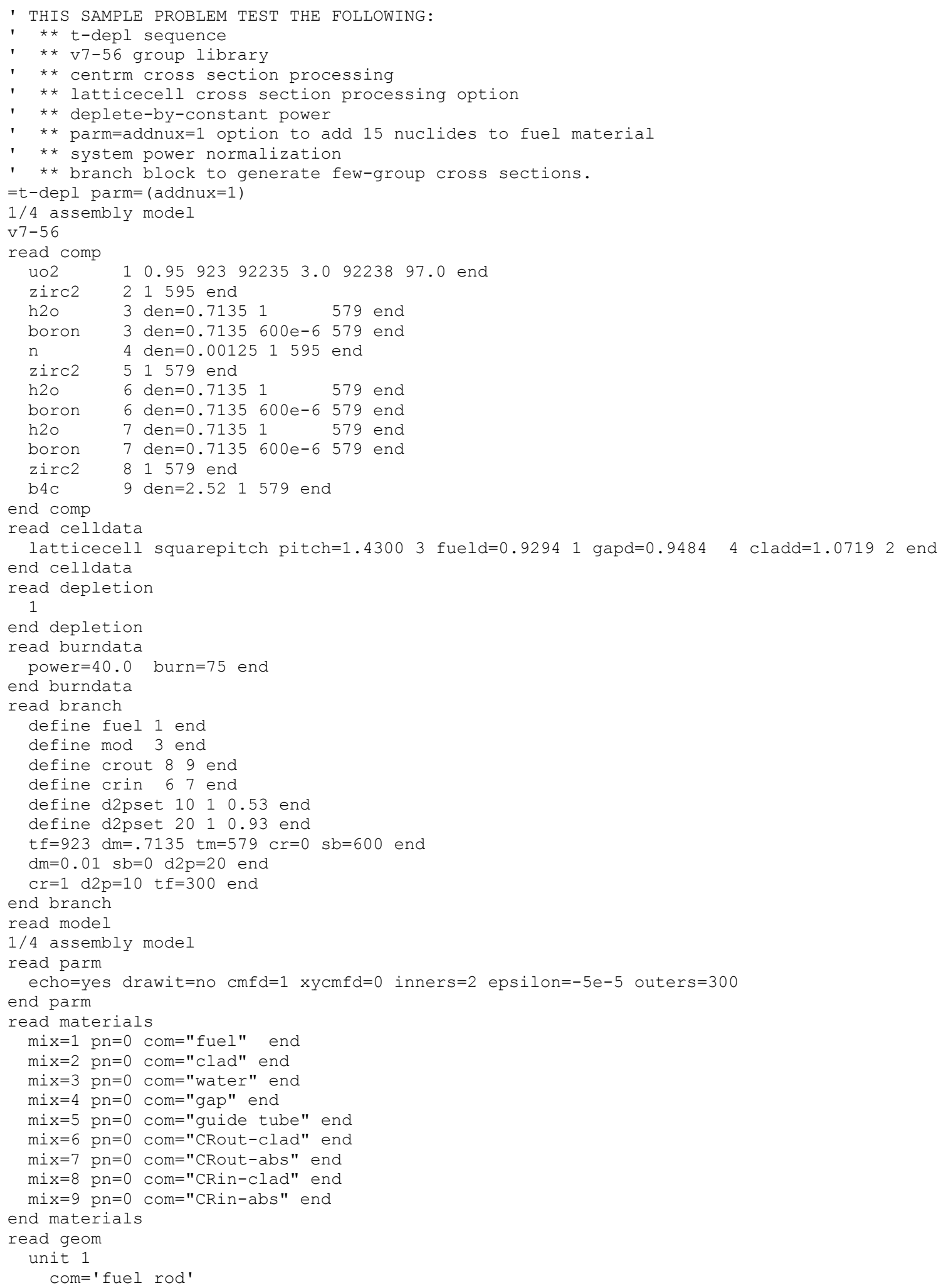




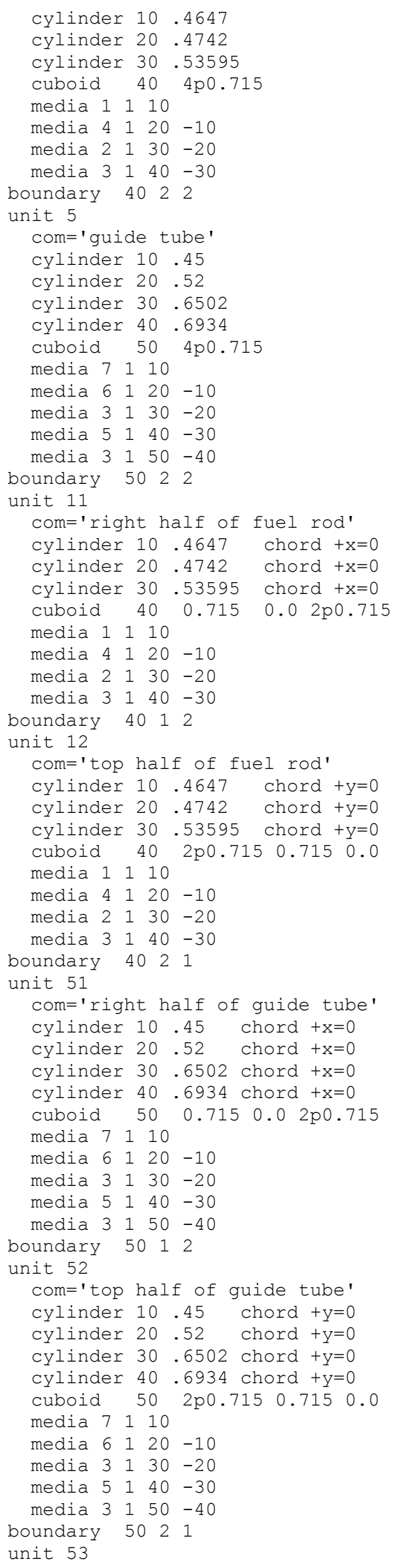




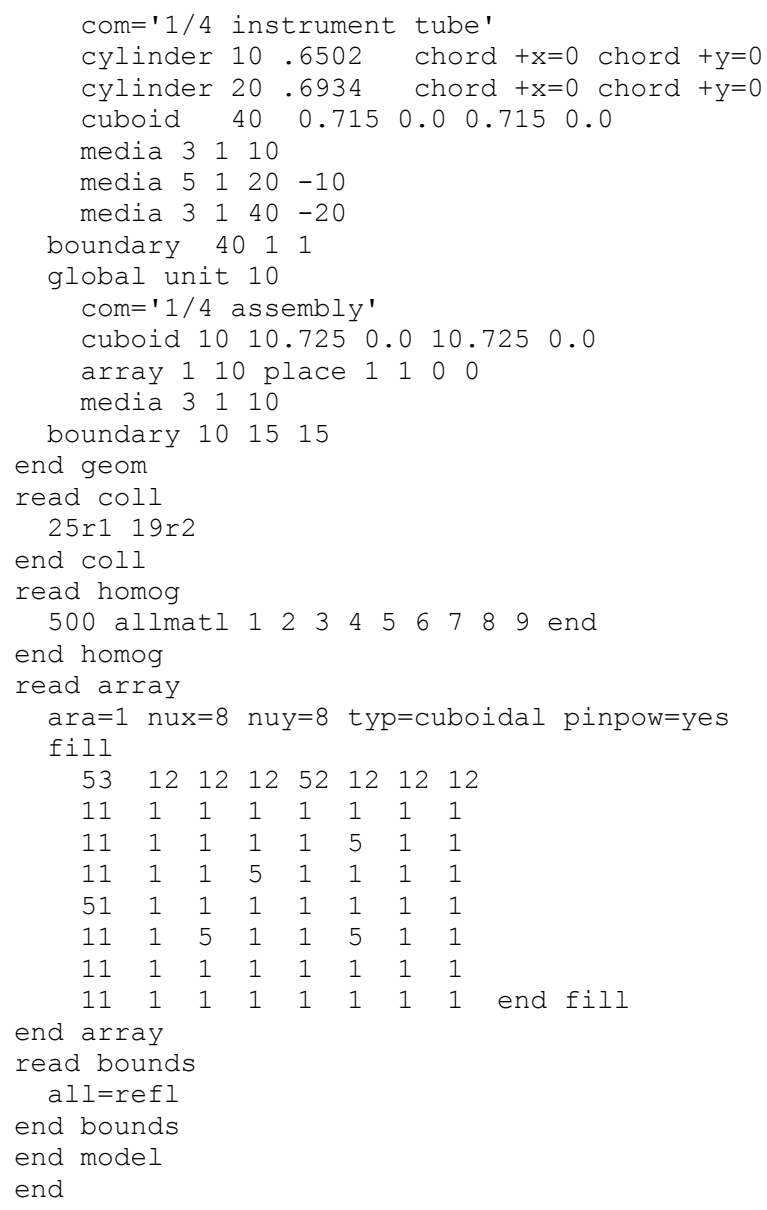

\subsubsection{TRITON sample problem 11: triton11.inp}

Sample problem triton11.inp demonstrates the use of determining Dancoff factors for a BWR fuel assembly. The BWR assembly design contains a $7 \times 7$ array of fuel pins enclosed by a channel box (see Figure 3.1.18). The in-channel moderator void fraction is $40 \%$, and the bypass moderator void fraction is $0 \%$. The input file contains an MCDANCOFF input file that calculates the Dancoff factors for each fuel pin (See the MCDANCOFF chapter). The MCDANCOFF input is essentially the equivalent of the KENO-VI model of the 2D assembly design. Following the MCDANCOFF input, the T-NEWT input is provided that shows how the computed Dancoff factors are inserted into the TRITON model. The Dancoff factors are inserted into the model via the centrmdata keyword entry in the CELLDATA block. 


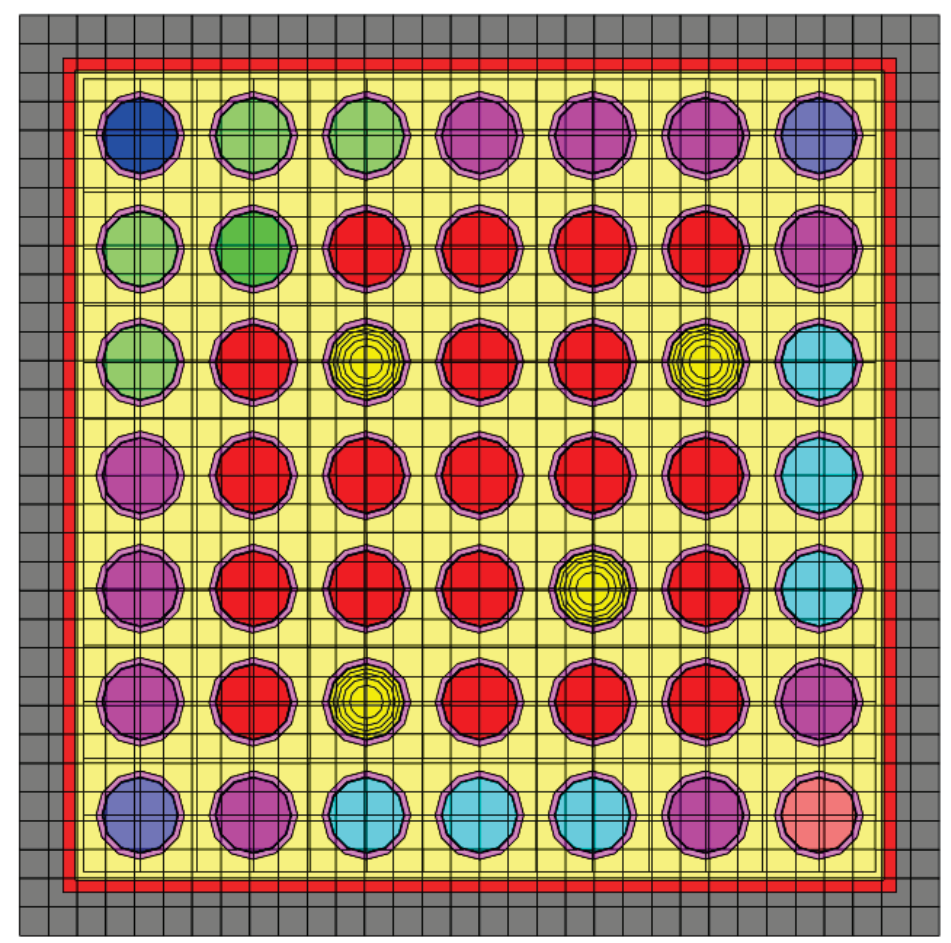

Figure 3.1.18. triton 11 BWR assembly design.

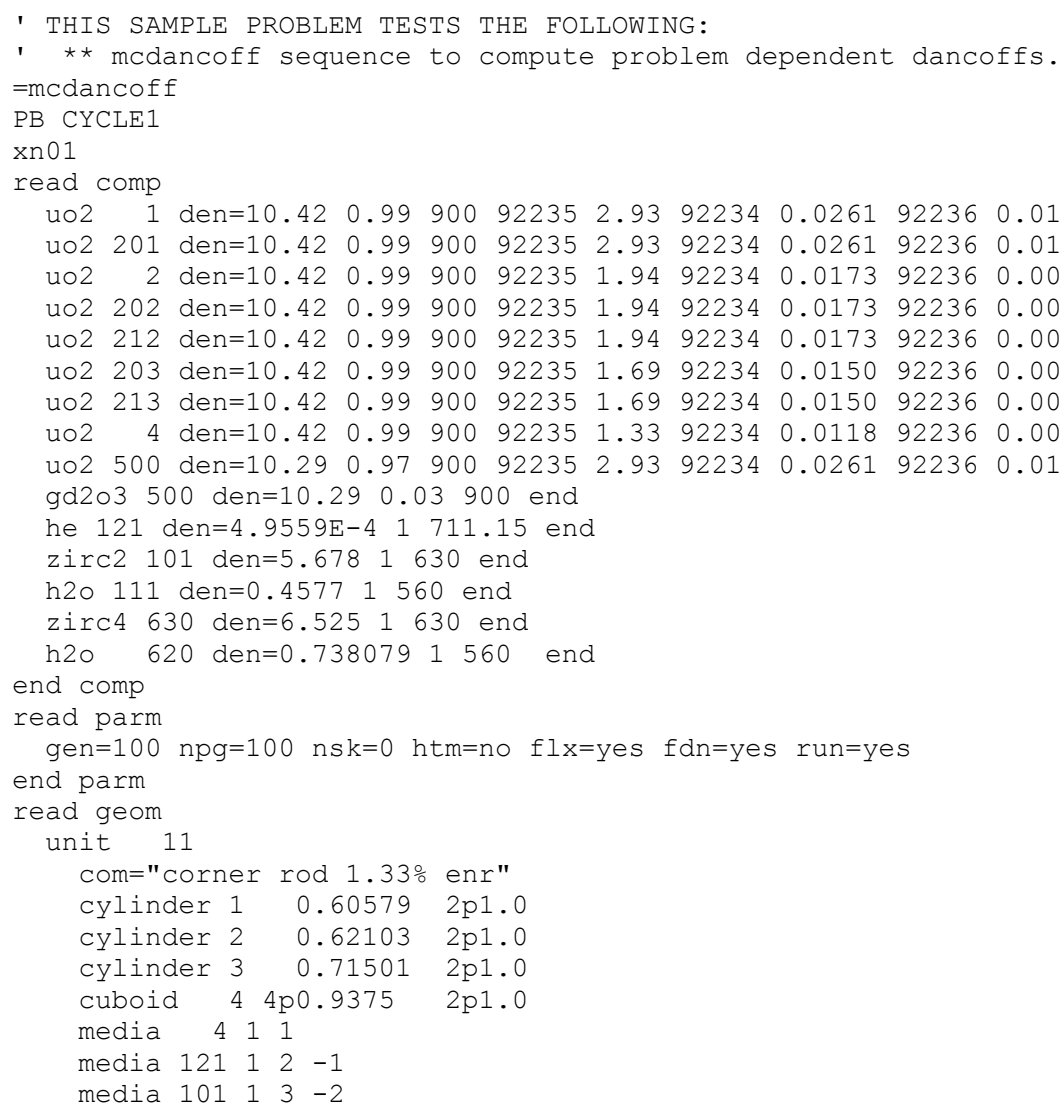




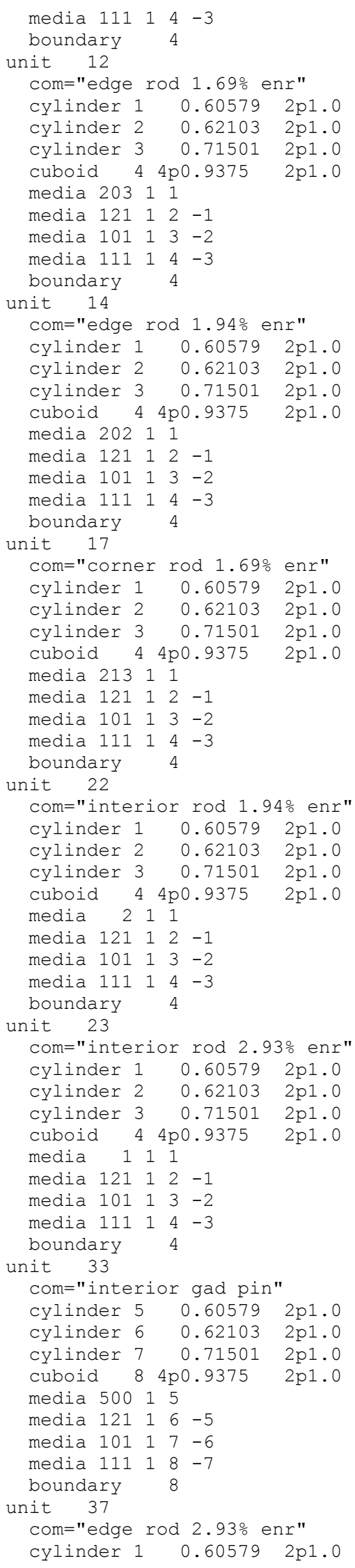




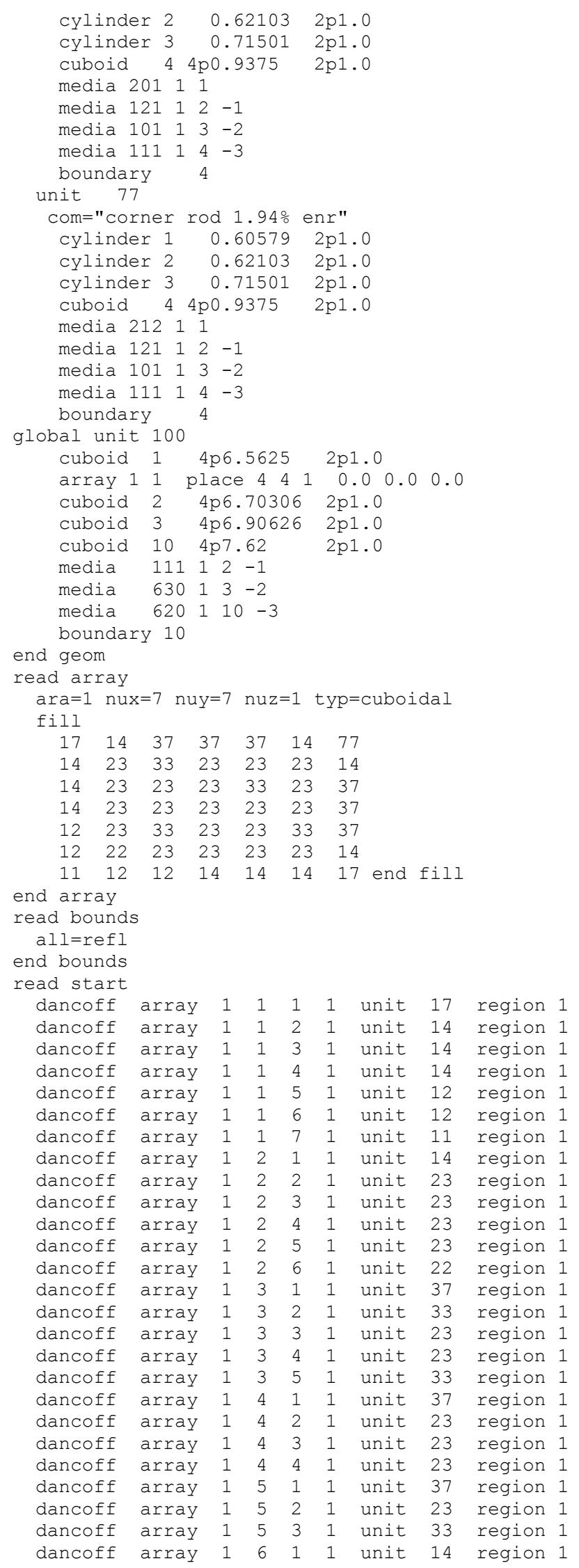




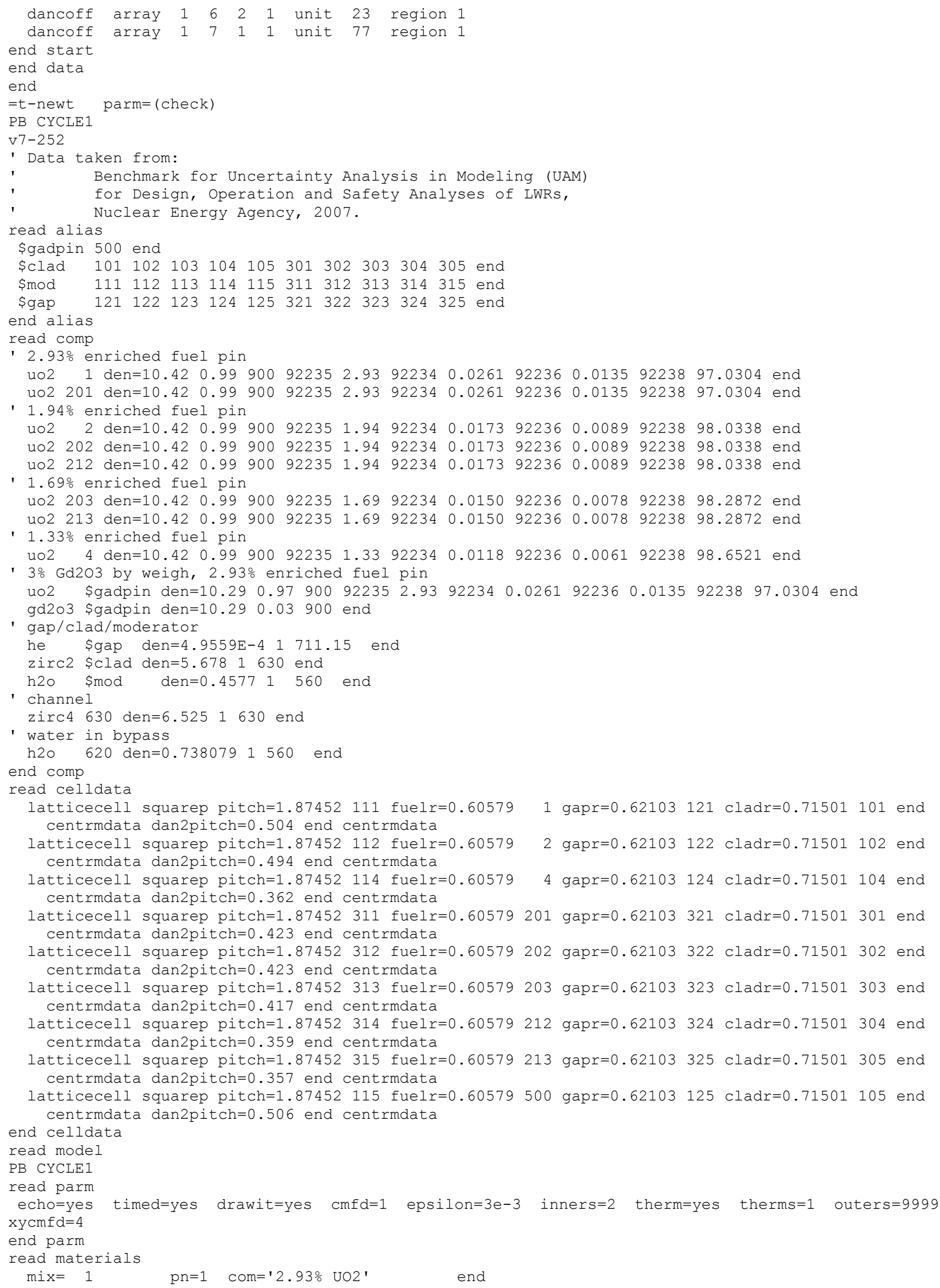




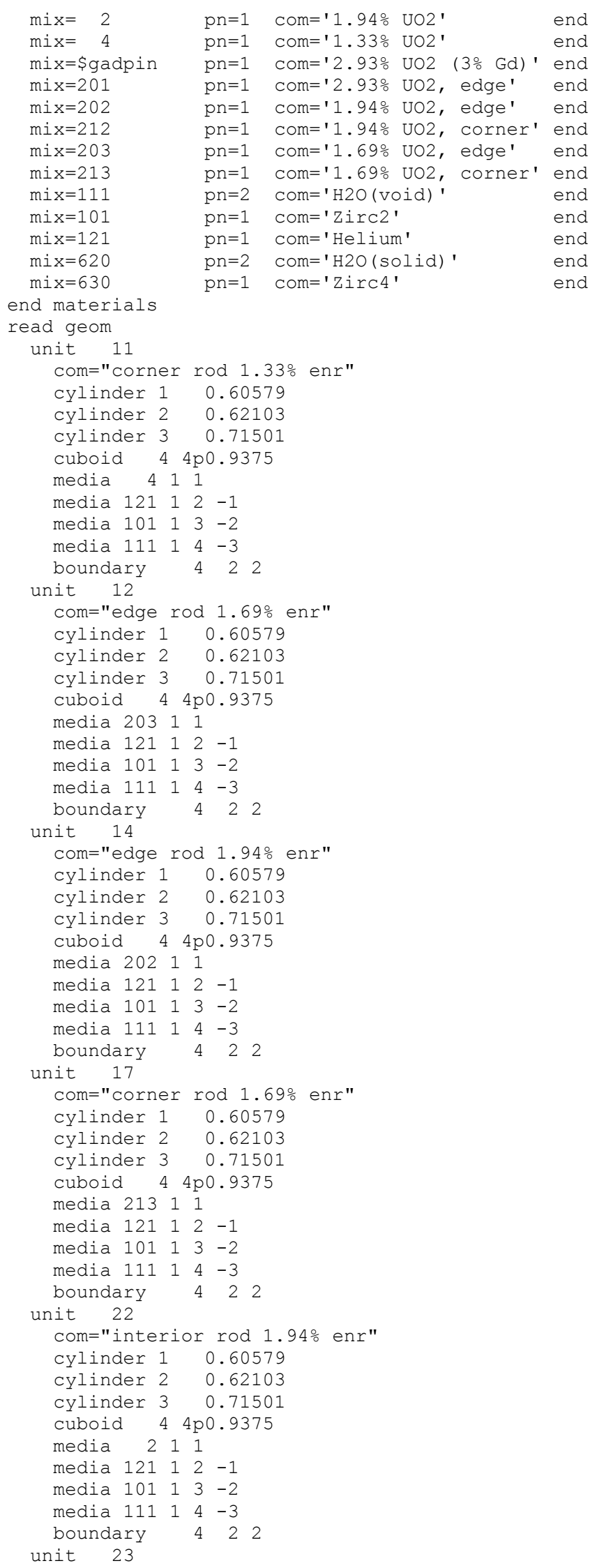




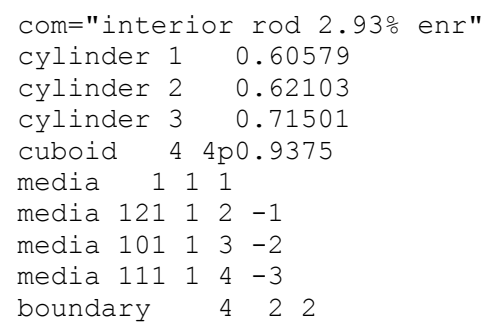


end array

read bounds

all=refl

end bounds

end model

end

\subsubsection{TRITON sample problem 12: triton12.inp}

Sample problem triton12.inp illustrates the use of the TSUNAMI-2D sequence for sensitivity and uncertainty (S/U) analysis of the BWR fuel assembly. The sample problem builds on the output of the previous sample problem. Sample problem 11 demonstrates how MCDANCOFF is used to compute fuel pin Dancoff factors for designs where nonuniform lattice effects play a critical role in cross section processing. The Dancoff factors are inserted into the follow-on T-NEWT model through the centrmdata keyword in the CELLDATA block. The output for the T-NEWT model in sample problem 11 provides an adjusted moderator pitch needed to preserve the user-specified Dancoff factor. These adjusted pitch values are used in triton 12.inp for the $\mathrm{S} / \mathrm{U}$ analysis of $k_{\text {eff }}$ for this assembly. As common in other sample problems, several input options were used to reduce the run-time - and therefore solution accuracy - of the sample problem.

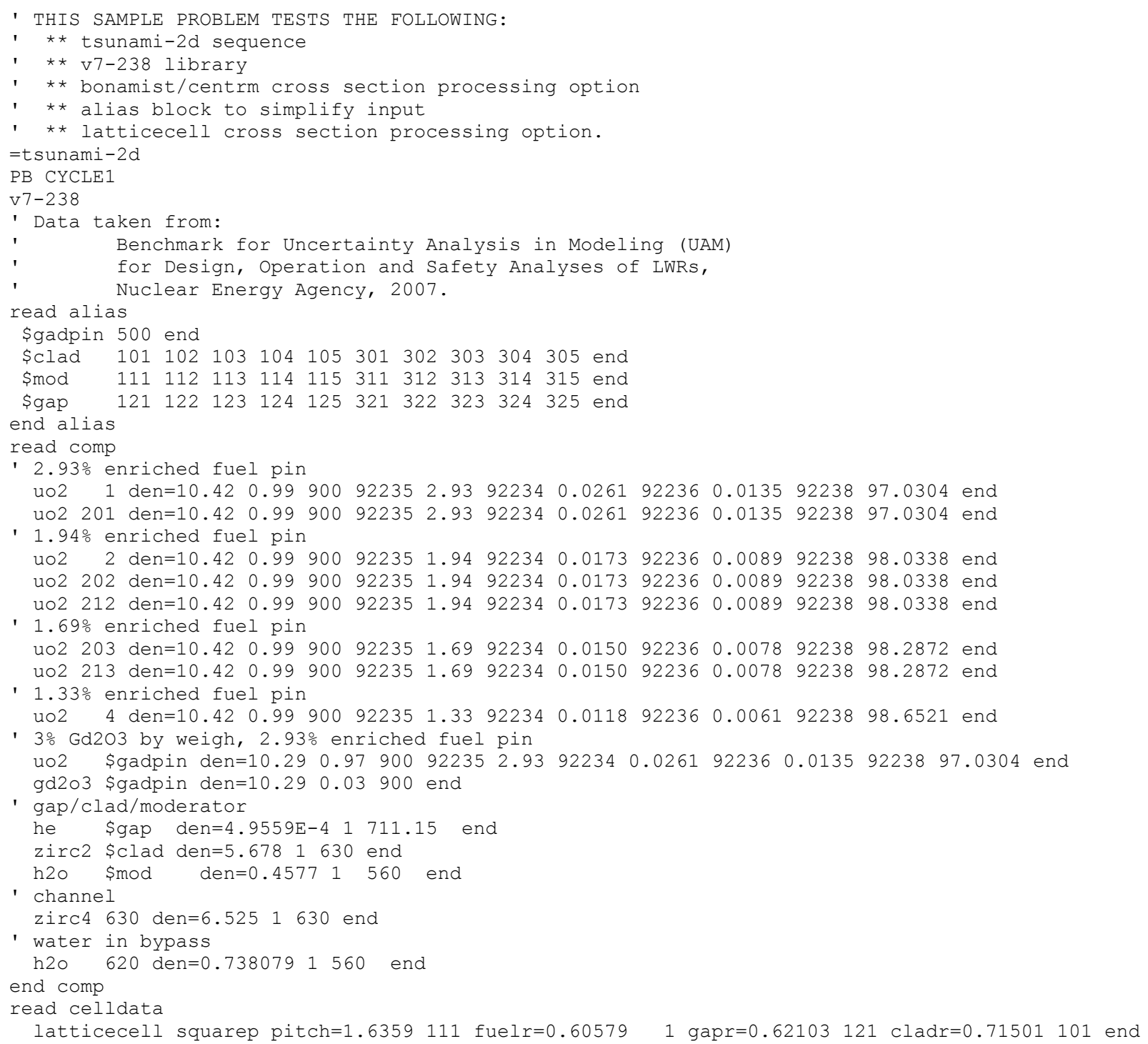




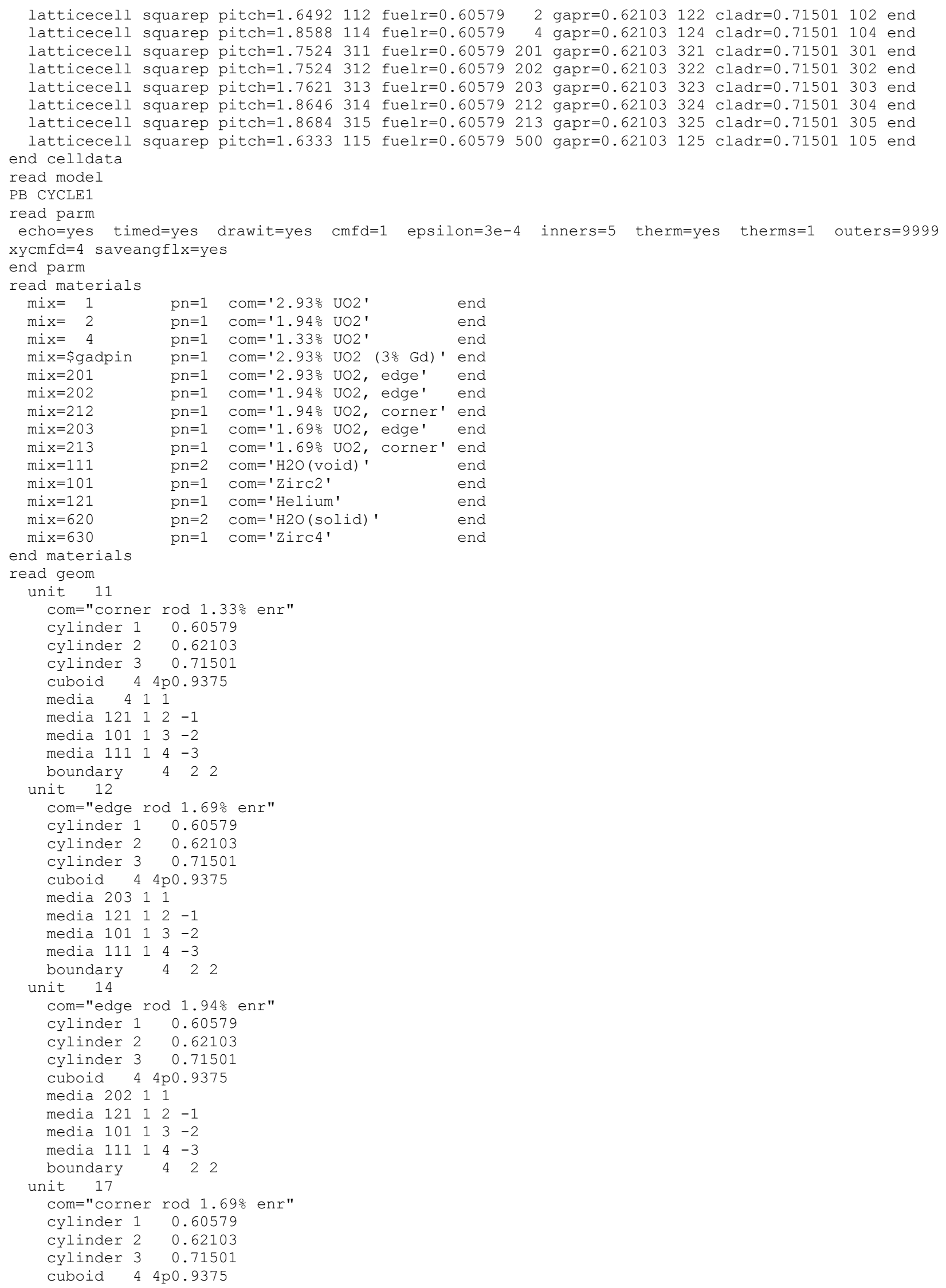




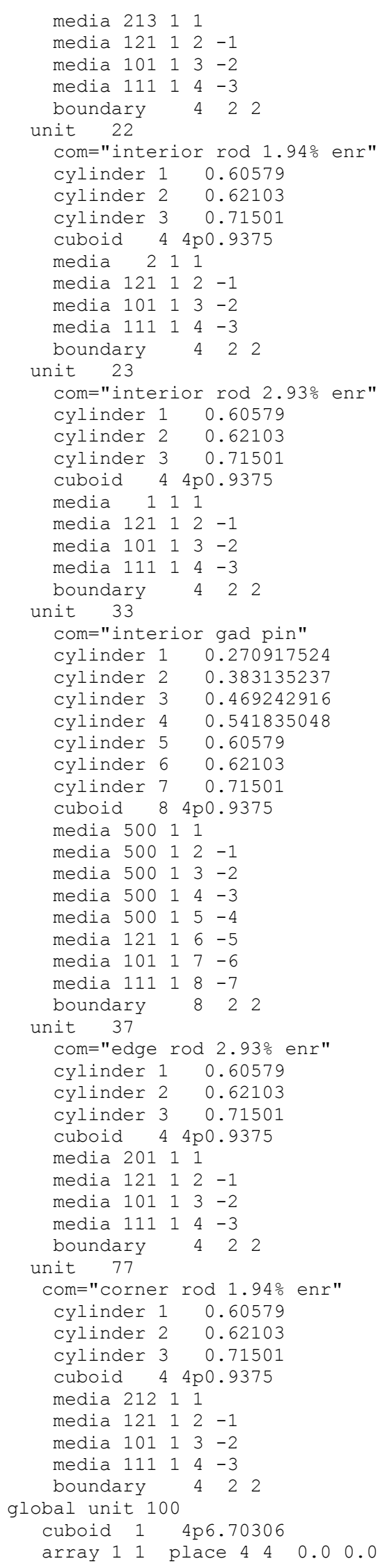




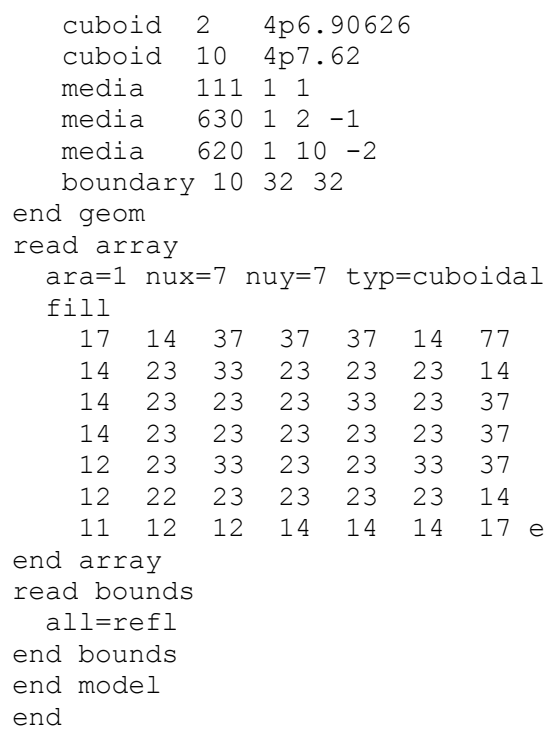

\subsubsection{TRITON6 sample problem 1: triton6-1.inp}

Sample problem triton6-1.inp is an example of KENO-VI-based depletion for an infinite lattice of cylinders fabricated with particulate TRISO fuel dispersed in a graphite matrix. This provides an example of the cross section processing specification of a doubly heterogeneous (DOUBLEHET) media and use of the resultant homogenized media in a depletion calculation.

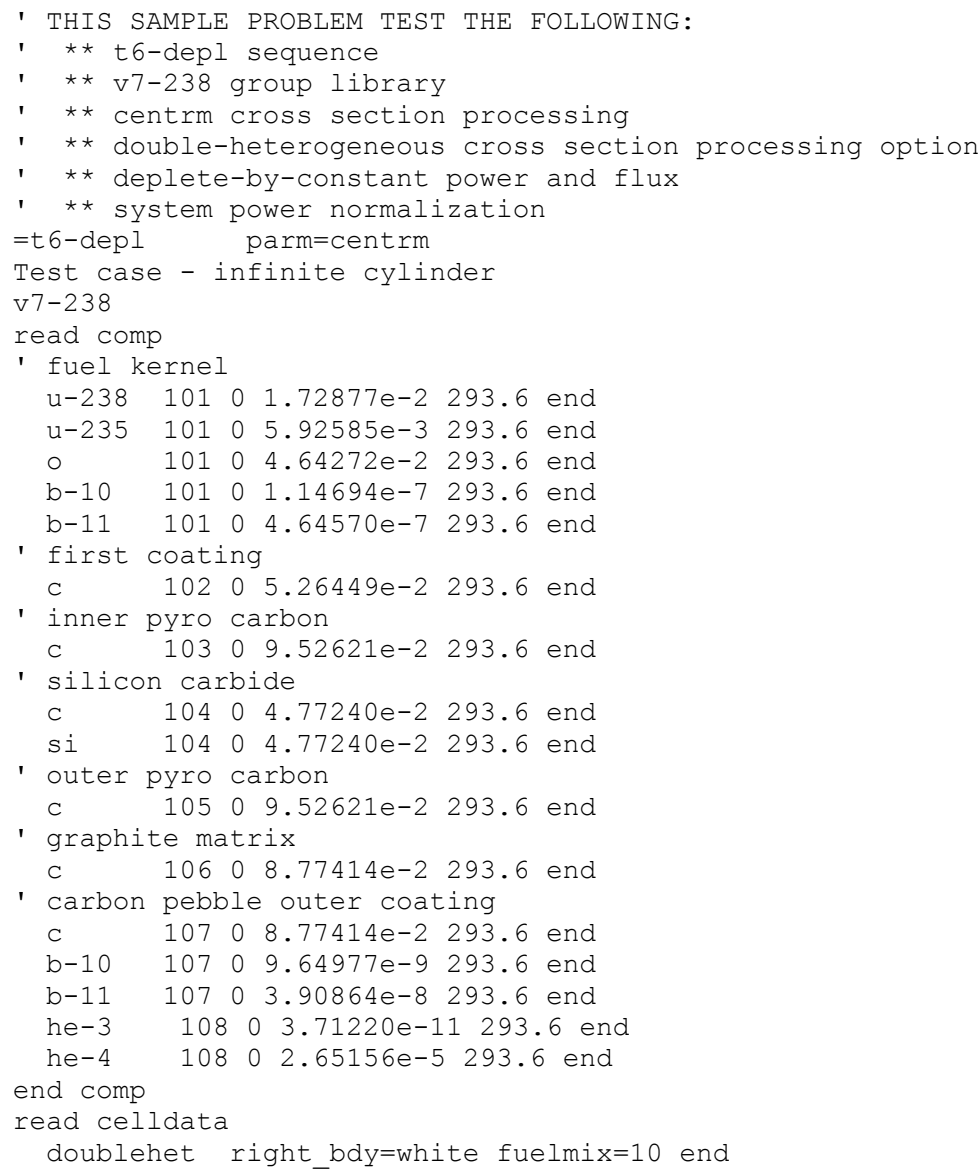




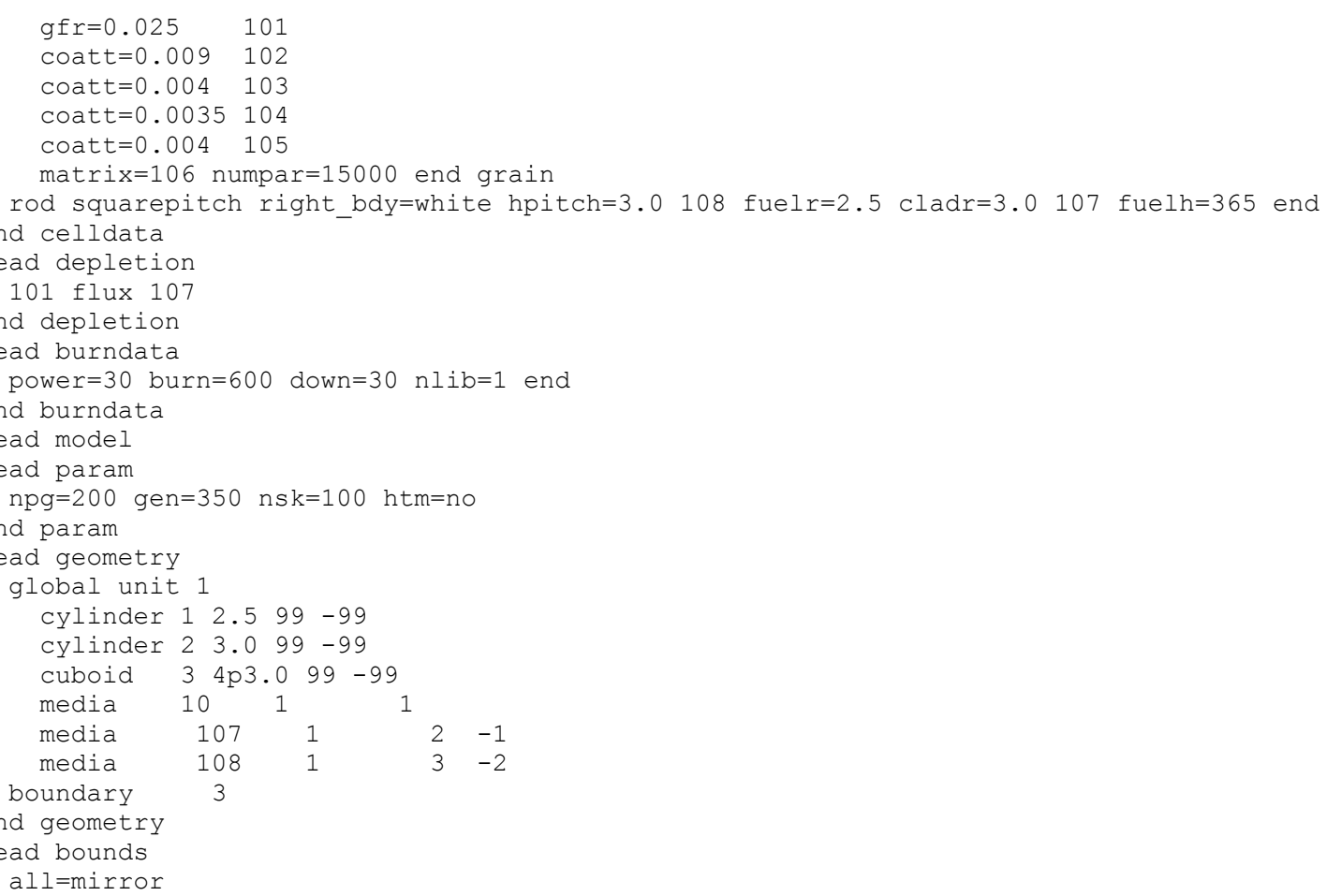




\subsection{A XSDRN Model Block Description}

The model data block for $T-X S D R N$ and $T-D E P L-1 D$ calculations allows specification of the $1 \mathrm{D}$ geometry model and various control parameters used in the transport solution. The XSDRN MODEL block input is arranged in blocks of data that are similar to the NEWT MODEL block input described in Chapter 9.2. The XSDRN model input starts with an optional 80-character title, followed by a PARAMETER block, and then the following three data blocks in any order: the GEOMETRY data block, the MATERIAL data block, and the optional COLLAPSE data block. If the PARAMETER, GEOMETRY, and MATERIAL data block are not specified, an error message is printed and the problem is terminated. Sample input files for $T-X S D R N$ and $T$-DEPL-1D calculations are provided in TRITON sample problem 2 and sample problem 3 , respectively, in the TRITON chapter.

\subsection{A.1 XSDRN PARAMETER block}

PARAMETER Block keyword = parm, para, parameter, or parameters

Valid PARAMETER block specifications are described below. For each keyword, allowable values are listed in parentheses, and the default is listed in brackets. Input that can take an arbitrary integer value is indicated by an IN; similarly, any parameter that can take an arbitrary real/floating point value is indicated by $\mathrm{RN}$ as the allowable value. SCALE read routines allow the input of integers for real numbers, and vice versa, and the number will be converted accordingly. The order of the parameters within the block is arbitrary and may be skipped if a default value is desired for that parameter. If a parameter is listed multiple times, the final specified value is used.

$\mathbf{b f}=(\mathrm{RN})$ - Buckling factor, equal to twice the extrapolation distance multiplier used to determine the zero point of the asymptotic flux. [1.420892]

collapse $=($ yes $/$ no $)$ - If collapse $=$ yes is specified, a flux-weighted collapse is performed by material number; cross sections for each nuclide in each material in the problem are collapsed to a specified (or default) group structure based on the average flux in that material. If collapse=yes, TRITON will look for the COLLAPSE block; if not found, TRITON will generate cross sections based on the original group structure. [no]

deltay $=(\mathrm{RN})$ - The first transverse dimension in centimeters used in a buckling correction to calculate leakage normal to the principal calculation direction (i.e., the height of a slab or a cylinder).

deltaz $=(\mathrm{RN})$ - The second transverse dimension in centimeters used in a buckling correction (i.e., the width of a slab).

difftreatment $=\left(\mathrm{mg} \_1 \mathrm{~d} \_\right.$sigtr/mg_0d_diff/mg_0d_sigtr/1g_0d_sigtr $)$- Diffusion treatment option for transverse leakage corrections. The $\mathrm{mg} 1 \mathrm{~d}$ sigtr option uses zone-dependent transport cross-sections for the transverse leakage correction. The mg_0d_diff option uses flux-volume-weighted homogenized diffusion coefficients. The mg_0d_sigtr option uses flux-volume weighted homogenized transport crosssections. The 1g_0d_sigtr option uses a one-group homogenized transport cross-section. [1g_0d_sigtr]

epsglobal=(RN) - Overall problem convergence criteria. [1.0e-6]

epsouter $=(\mathrm{RN})$ - Scalar flux convergence criteria. $[1.0 \mathrm{e}-6]$

inners $=(\mathrm{IN})$ - Maximum number of inner iterations in an energy group. [20] 
outers $=(\mathrm{IN})$ - Maximum number of outer iterations. [100]

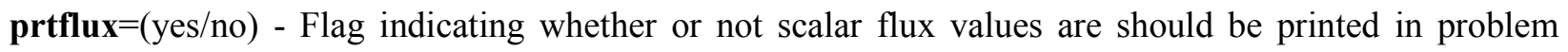
output. [no]

prtangflux $=($ yes/no $)$ - Flag indicating whether or not angular flux values should be printed in problem output. [no]

prtbalnc $=($ yes $/$ no $)$ - Flag indicating whether or not fine-group material balance tables should be printed in problem output. [no]

prtmxsec $=($ yes/no/1d $)$ - Flag indicating whether or not material macroscopic cross sections should be printed in problem output. The 1D option indicates that 2D scattering tables are not to be printed. [no]

$\mathbf{s n}=(2 / 4 / 6 / 8 / 16 / 32)-$ Sn quadrature order for the transport calculations.

\section{XSDRN GEOMETRY block}

GEOMETRY Block keyword = geom, geometry

The GEOMETRY block is used to specify the geometry type (e.g., slab, cylinder, or sphere), the boundary conditions, the 1D material mesh (i.e., zone mesh), and the 1D spatial mesh used in the transport calculation. The order of the parameters entered in the GEOMETRY block is arbitrary and can be any of the following supported keyword specifications or keyword array specifications.

geom $=($ slab/cylinder/sphere $)$ - Problem geometry. Keywords geometry $=$, ige $=$, and cyl for cylinder are also allowed. [slab]

leftbc $=($ vaccum/periodic/white/albedo/mirror $)$ - Left-hand boundary condition. Keywords $i b l=$, vac for vacuum, refl for mirror, and reflected for mirror are also allowed. [mirror]

rightbc $=($ vaccum/periodic/white/albedo/mirror $)$ - Right-hand boundary condition. Keywords ibr=, vac for vacuum, refl for mirror, and reflected for mirror are also allowed. [mirror]

left_albedo RN1 RN2 ... RNN end left_albedo - The left-hand boundary albedo values as a function of energy group. The left_albedo array is ignored if leftbc $=$ is vacuum, periodic, white, or mirror. If the left_albedo array is omitted and leftbc=albedo, white boundary conditions are used. If the number of entries in the left albedo array does not equal the number of energy groups in the cross-section library, an error message is printed and the problem is terminated.

right_albedo RN1 RN2 ... RNN end right_albedo - The right-hand boundary albedo values as a function of energy group. The right_albedo array is ignored if rightbc= is vacuum, periodic, white, or mirror. If the right_albedo array is omitted and rightbc=albedo, white boundary conditions are used. If the number of entries in the right_albedo array does not equal the number of energy groups in the cross-section library, an error message is printed and the problem is terminated.

zoneids IN1 IN2 … INN end zoneids - Material composition number by zone. The number of entries in the zoneids array defines the number of zones for the problem. If the zoneids array is not defined, an error message is printed and the problem is terminated. 
zonedimensions RN1 RN2 ... RNN end zonedimensions - The right-hand boundary for each zone is given in centimeters. Note that the left-hand boundary of the first zone is 0.0 and must not be entered. If the zonedimensions array is not defined or the number of entries does not equal the number of entries in the zoneids array, then an error message is printed and the problem is terminated.

zoneintervals IN1 IN2 ... INN end zoneintervals - Number of spatial mesh of constant width per each problem zone. If specified, the number of entries of the zonedimensions array must equal the number of entries in the zoneids array. Otherwise, an error message is printed and the problem is terminated.

mesh RN1 RN2 ... RNN end mesh - The right-hand boundary for each spatial mesh in centimeters. The spatial mesh is the discretization used in the transport calculation. Note that the left-hand boundary of the first spatial mesh is 0.0 and must not be entered. The zone boundaries in the zonedimensions array must be a subset of the spatial mesh boundaries in the mesh array. Otherwise, an error message is printed and the problem is terminated. The mesh array is optional and is not used if the zoneintervals array is specified. If neither the zoneintervals array nor the mesh array is specified, an error message is printed and the problem is terminated.

\subsection{A.2 XSDRN MATERIAL block}

MATERIAL Block keyword = matl, mat, material, materials

The MATERIAL block is used to specify the material numbers for each material used in the calculation in the order of scattering cross section to be used for each material. The format of the MATERIAL block is identical to the NEWT MATERIAL block that is described in detail in (Chapter 9.2). Although source and description specifications are allowed, these options are not used by XSDRN.

\subsection{A.3 XSDRN COLLAPSE block}

COLLAPSE Block keyword = collapse, coll

The COLLAPSE block is used to define the energy group collapsing operation to calculate broad group cross-section libraries using the XSDRN flux solution. The format of the COLLAPSE block is identical to the NEWT COLLAPSE block that is described in detail in the NEWT chapter

\subsection{B Data Structure for Cross Section Database File xfile016}

When branch calculations are performed, TRITON archives collapsed homogenized cross sections in an unformatted, direct-access FORTRAN file called xfile016. The contents and format of this file are described in this appendix.

TRITON uses a library of SCALE subroutines to read and write blocks of data to direct-access FORTRAN files. The SCALE subroutine library allows the blocks of data to have variable length, even though direct-access FORTRAN files have a fixed record length. The data blocks can be retrieved from the file at random, provided the block length and block starting record position are known. The block length is expressed in terms of 4-byte words. For example, a block of 3-group macroscopic cross sections that contained the total, fission, capture, chi, and nubar cross sections would have a block length of 15 $(3 \times 5)$, assuming that the cross sections are stored in single precision 4-byte format. 
The $x$ file016 file supports 11 different block types. The first seven block types appear only once in the file, each block type occupying one of the first seven record positions. The remaining four block types, types 8-11, are repeated for each branch, at each depletion step, starting at the eighth record position.

Branch-specific blocks, i.e., block types 8-11, are written in the following order, for $\mathrm{N}$ branch calculations over M depletion steps:

First $(\mathrm{t}=0)$ transport calculation, branch 0 (reference state)

First $(\mathrm{t}=0)$ transport calculation, branch 1

First $(\mathrm{t}=0)$ transport calculation, branch 2

$\cdots$

First $(\mathrm{t}=0)$ transport calculation, branch $\mathrm{N}$

Second transport calculation, branch 0 (reference state)

Second transport calculation, branch 1

Second transport calculation, branch 2

...

Second transport calculation, branch N

...

$\cdots$

$(\mathrm{M}+1)^{\text {th }}$ transport calculation, branch 0 (reference state)

$(\mathrm{M}+1)^{\text {th }}$ transport calculation, branch 1

$(\mathrm{M}+1)^{\text {th }}$ transport calculation, branch 2

...

$(\mathrm{M}+1)^{\text {th }}$ transport calculation, branch $\mathrm{N}$

Note that $(\mathrm{M}+1) \times(\mathrm{N}+1)$ sets are saved for $\mathrm{M}$ depletion steps and $\mathrm{N}$ branches. For each set, block types 8 and 9 are always written, whereas block types 10 and 11 are written only if pin data output was requested $(\mathrm{nx} \neq 0)$. 


\section{Block Type 1: block length data}

Length: 13

Position: 1

Type: integer.

Data: datlen(13)

datlen(1) Length of block type 1 (this array), which is 13.

datlen(2) Number of blocks allocated for this file (1000). Currently not used.

datlen(3) Length of FORTRAN record for this file (512).

datlen(4) Length of block type 2: general dimensioning data.

datlen(5) Length of block type 3: depletion data.

datlen(6) Length of block type 4: branching data.

datlen(7) Length of block type 5: branching data for advanced branch block (not yet supported).

datlen(8) Length of block type 6: currently not used.

datlen(9) Length of block type 7: energy group boundaries.

datlen(10) Length of block type 8: cross sections and misc data.

datlen(11) Length of block type 9: corner discontinuity factors.

datlen(12) Length of block type 10: pin power factors.

datlen(13) Length of block type 11: groupwise form factors.

\section{Block Type 2: general dimensioning data}

Length: datlen(4)

Position: 2

Type: integer, unless specified otherwise

Data: brnchdepl, nobranch, nsets, igm, iftg, ndelay, nadf, ncdf, ipin, nxpin, nypin, ivers, adftype, branchflag

brnchdepl Number of depletion steps +1 .

nobranch Number of branches.

nsets Number of cross-section sets on library (typically 1).

igm Number of energy groups in collapsed cross sections.

iftg First thermal energy group (max upscatter group).

ndelay Number of delayed neutron precursor groups (6).

nadf Number of assembly discontinuity factors (ADFs).

ncdf Number of corner discontinuity factors (CDFs).

ipin $\quad$ Flag for pin data $(0=$ no pin data, $1=$ pin data included $)$.

nxpin $\quad$ Number of pins in $x$-direction $(0$ if ipin $=0)$.

nypin $\quad$ Number of pins in y-direction $(0$ if ipin $=0)$.

ivers Format version number. This appendix describes version 5 of the database structure.

adftype $\quad$ ADF type: (1= single-assembly, $2=$ reflector, $3=$ single-assembly on arbitrary grid lines).

branchflag (logical) TRUE for simple BRANCH block format, FALSE for advanced format. 


\section{Block Type 3: depletion data}

Length: datlen(5)

Position: 3

Type: real

Data: burnup(brnchdepl), time(brnchdepl), power(brnchdepl), sysHMdens

burnup(brnchdepl) Burnup (GWd/MTHM) at each transport step.

time(brnchdepl) Cumulative cycle time (days) at each transport step.

power(brnchdepl) Specific power (MW/MTHM) at each transport step.

sysHMdens $\quad$ System heavy metal mass density $\left(\mathrm{g} / \mathrm{cm}^{3}\right)$.

\section{Block Type 4: branching data}

Length: datlen(6)

Position: 4

Type: integer, unless specified otherwise

Data: fuelused, modused, crused, fuelcount, modcount, crcount, crref, tfref, tmref, mdref, sbref, fuelmix(fuelcount), modmix(modcount), crinmix(crcount), croutmix(crcount), crstate(nobranch), tfuel(nobranch), tmod(nobranch), dmod(nobranch), sboron(nobranch)

$\begin{array}{ll}\begin{array}{l}\text { fuelused } \\ \text { modused }\end{array} & \text { (logical) TRUE if fuel mixtures were specified for branches. } \\ \text { crused } & \text { (logical) TRUE if moderator mixtures were specified for branches. } \\ \text { fuelcount } & \text { (logical) TRUE if control rod mixtures were specified for branches. } \\ \text { modcount } & \text { Number of mixtures in fuel definition. } \\ \text { crcount } & \text { Number of mixtures in moderator definition. } \\ \text { crref } & \text { Number of mixture pairs in control rod definition. } \\ \text { tfref } & \text { Reference control rod state }(0 / 1) . \\ \text { tmref } & \text { (real) Reference fuel temperature }(\mathrm{K}) . \\ \text { mdref } & \text { (real) Reference moderator temperature }(\mathrm{K}) . \\ \text { sbref } & \text { (real) Referenced moderator density }\left(\mathrm{g} / \mathrm{cm}^{3}\right) . \\ \text { fuelmix(fuelcount) } & \text { (real) Reference soluble boron concentration }(\mathrm{ppm}) . \\ \text { modmix(modcount) } & \text { Mixtures defined as fuel. } \\ \text { crinmix(crcount) } & \text { Mixtures defined as moderator. } \\ \text { croutmix(crcount) } & \text { Mixtures defined for the control-rod in state. } \\ \text { crstate(nobranch) } & \text { Mixtures defined for the control-rod out state. } \\ \text { tfuel(nobranch) } & \text { Control rod state }(0=\text { withdrawn } / 1=\text { inserted) for each branch. } \\ \text { (real) Fuel temperature }(\mathrm{K}) \text { for each branch. } \\ \text { tmod(nobranch) } & \text { (real) Moderator temperature }(\mathrm{K}) \text { for each branch. } \\ \text { dmod(nobranch) } & \text { (real) Moderator density }\left(\mathrm{g} / \mathrm{cm}^{3}\right) \text { for each branch. } \\ \text { sboron(nobranch) } & \text { (real) Soluble boron concentration }(\mathrm{ppm}) \text { for each branch. }\end{array}$

\section{Block Type 5: advanced branching data}

Length: datlen(7)

Position: 5

Type: integer

Data: Stores data for advanced branch block (not yet supported)

\section{Block Type 6: currently not used}




\section{Block Type 7: energy group boundary data}

\section{Length: datlen(9)}

Position: 7

Type: real

Data: ebnds(igm +1$)$

ebnds(igm+1) Energy group boundaries

Blocks 1-7 are written only once. Blocks 8 and 9 (plus 10 and 11 if pin power data is output) are written for each branch case at each depletion step.

\section{Block Type 8: cross-section data}

Length: datlen(10)

Position: $8+($ igm +3$)[i *($ nobranch +1$)+j]), i=0, \ldots$, brnchdepl, $j=0, \ldots$, nobranch

Type: real

Data: $\{\operatorname{kinf}(i)$, beta_eff(1:ndelay, i), lam_eff(1:ndelay, i) , y_i135(i), y_xe135(i), y_pm149(i), id(i),

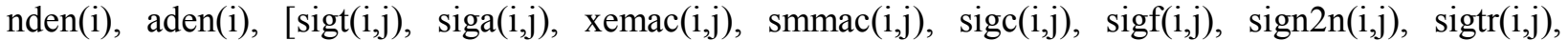
nusigf(i,j), kappaf(i,j), nu(i,j), chi(i,j), diffcoef(i,j), flux(i,j), sigselas(i,j), sig_xe(i,j), sig_sm (i,j), $\operatorname{detfis}(i, j), \operatorname{detflx}(i, j), \operatorname{invvel}(i, j), \operatorname{sigtr} 2(i, j), \operatorname{sigtr}(i, j),[(\operatorname{adf}(i, j, k), k=1, \operatorname{nadf}),(0, k=\operatorname{nadf}+1,12),(\operatorname{current}(i, j, k)$, $\mathrm{k}=1$, nadf $),(0, \mathrm{k}=$ nadf $+1,12)]$, ( $\operatorname{sigs}(\mathrm{i}, \mathrm{j}, \mathrm{k}), \mathrm{k}=1, \mathrm{igm}), \mathrm{j}=1, \mathrm{igm}], \mathrm{i}=1, \mathrm{nsets}\}$

Data is saved for $i=1$,nsets (number of homogenized regions):

kinf(i)
beta_eff(1:ndelay,i)
lam_eff(1:ndelay,i)
y_i135(i)
y_xe135(i)
y_pm149(i)

k-infinity

Approximate delayed neutron fractions.

Approximate delayed neutron decay constants $\left(\mathrm{sec}^{-1}\right)$.

Fission product yield for ${ }^{135} \mathrm{I}$.

Fission product yield for ${ }^{135} \mathrm{Xe}$.

Fission product yield for ${ }^{149} \mathrm{Pm}$.

Data is saved for $\mathrm{j}=1$, igm (number of energy groups):

$\operatorname{sigt}(i, j)$
$\operatorname{siga}(i, j)$
xemac(i,j)
$\operatorname{smmac}(i, j)$
$\operatorname{sigc}(i, j)$
$\operatorname{sigf}(i, j)$
$\operatorname{sign} 2(i, j)$
$\operatorname{sigtr}(i, j)$
nusigf(i,j)
kappaf(i,j)
nu(i,j)
chi(i,j)
diffcoef(i,j)
flux(i,j)
sigselas(i,j)
sig_xe(i,j)
sig_sm $(i, j)$

Total cross section $\left(\mathrm{cm}^{-1}\right)$.

Effective absorption cross section $\left(\mathrm{cm}^{-1}\right)$.

Macroscopic ${ }^{135} \mathrm{Xe}$ cross section $\left(\mathrm{cm}^{-1}\right)$

Macroscopic ${ }^{149} \mathrm{Sm}$ cross section $\left(\mathrm{cm}^{-1}\right)$.

Capture cross section $\left(\mathrm{cm}^{-1}\right)$.

Fission cross section $\left(\mathrm{cm}^{-1}\right)$.

Effective $\mathrm{n} 2 \mathrm{n}$ cross section $\left(\mathrm{cm}^{-1}\right)$.

Transport cross section $\left(\mathrm{cm}^{-1}\right)$, determined by outscatter approximation.

Average total number of neutrons/fission $\times$ fission cross section $\left(\mathrm{cm}^{-1}\right)$.

Energy released per capture $\times$ capture cross section +

Energy released per fission $\times$ fission cross section $(\mathrm{J} / \mathrm{cm})$.

Average total number of neutrons released per fission (delayed + prompt).

Fission spectrum (delayed + prompt).

Diffusion coefficient $(\mathrm{cm}), 1 /(3 \times \operatorname{sigtr}(\mathrm{i}, \mathrm{j}))$.

Average flux $\left(\mathrm{n} / \mathrm{cm}^{2}-\mathrm{sec}\right)$.

Total elastic scattering cross section $\left(\mathrm{cm}^{-1}\right)$.

Microscopic cross section for ${ }^{135} \mathrm{Xe}$ (barns).

Microscopic cross section for ${ }^{149} \mathrm{Sm}$ (barns). 
$\operatorname{detfis}(\mathrm{i}, \mathrm{j})$

$\operatorname{detflx}(\mathrm{i}, \mathrm{j})$

invvel(i,j)

$\operatorname{sigtr} 2(i, j)$

$\operatorname{sigtr}(\mathrm{i}, \mathrm{j})$

$\operatorname{adf}(1:$ nadf,i,j)

current(1:nadf,i,j)

$\operatorname{sigs}(\mathrm{i}, \mathrm{j}, \mathrm{k}), \mathrm{k}=1, \mathrm{igm}$
Microscopic ${ }^{235} \mathrm{U}$ cross section at detector location (barns).

Average flux in detector mixture $\left(\mathrm{n} / \mathrm{cm}^{2}-\mathrm{sec}\right)$.

Inverse neutron velocity $(\mathrm{sec} / \mathrm{cm})$.

Transport cross section $\left(\mathrm{cm}^{-1}\right)$, determined by inscatter approximation.

Transport cross section $\left(\mathrm{cm}^{-1}\right)$, determined by outscatter approximation. Assembly discontinuity factors for up to 12 faces.

Net current for up to 12 faces $\left(\mathrm{n} / \mathrm{cm}^{2}\right.$-sec), adftype $=3$ only.

Macroscopic scattering cross section, $\mathrm{j} \mathrm{k}\left(\mathrm{cm}^{-1}\right)$.

End of data saved for $\mathrm{j}=1$, igm

End of data saved for $i=1$, nsets

\section{Block Type 9: corner discontinuity factors}

Length: datlen(11)

Position: $9+($ igm +3$)[i *($ nobranch +1$)+j]), i=0, \ldots$, brnchdepl, $j=0, \ldots$, nobranch

Type: real

Data: $((\operatorname{cdf}(i, j), i=1, n c d f), j=1$, igm $)$

Data is saved for $\mathrm{i}=1, \operatorname{ncdf}$ (number of "corner" discontinuity factors):

Data is saved for $\mathrm{j}=1$, igm (number of energy groups):

$\operatorname{cdf}(\mathrm{i}, \mathrm{j}) \quad$ Corner discontinuity factors

End of data saved for $\mathrm{j}=1$, igm

End of data saved for $i=1$, ncdf

\section{Block Type 10: pin power peaking factors}

Length: datlen(12)

Position: $10+($ igm +3$)[i *($ nobranch +1$)+j]), i=0, \ldots$, brnchdepl, $j=0, \ldots$, nobranch Type: double precision

Data: $((\operatorname{ppf}(\mathrm{i}, \mathrm{j}), \mathrm{i}=1, n x), \mathrm{j}=1, n y)$

Data is saved for $\mathrm{j}=1$, ny (number of pins in y direction):

Data is saved for $\mathrm{i}=1, \mathrm{nx}$ (number of pins in $\mathrm{x}$ direction):

$\operatorname{ppf}(\mathrm{i}, \mathrm{j}) \quad$ Pin power (peaking) factors

End of data saved for $\mathrm{i}=1, \mathrm{nx}$

End of data saved for $\mathrm{j}=1$, ny

\section{Block Type 11: group form factors}

Length: datlen(13)

Position: $10+k+($ igm +3$)[i *($ nobranch +1$)+j])$,

$\mathrm{k}=1, \ldots$, igm, $\mathrm{i}=0, \ldots$, brnchdepl, $\mathrm{j}=0, \ldots$, nobranch

Type: double precision

Data: (( gff(i,j), i=1,nx), j=1,ny) 
Data is saved for $\mathrm{j}=1$,ny (number of pins in $\mathrm{y}$ direction):

Data is saved for $\mathrm{j}=1, \mathrm{nx}$ (number of pins in $\mathrm{x}$ direction):

gff(i,j,k) Groupwise form factors

End of data saved for $\mathrm{i}=1, \mathrm{nx}$

End of data saved for $\mathrm{j}=1$, ny

NOTE: Block Type 11 is repeated igm times where igm is the number of energy groups.

It is recommended that code written to process $x$ file016 include the SCALE subroutine library. Although possible to link in the appropriate files in the scalelib object library in SCALE, it may be more practical to copy the appropriate SCALE routines into a new FORTRAN code used in reading xfile016. All directaccess operations needed to operate on this file are contained in the file direct_access_M.f90 in the scale $\mathrm{src} / \mathrm{scalelib}$ directory. This file has dependencies and requires the following additional subroutines, all in the src/scalelib directory, in order to compile:

Error_functions_M.f90

common_unit_C.f90

Vast_kind_param_M.f90

separator_character_M.f90

Y0trns_M. .90

f_exit.c

The single $\mathrm{C}$ routine can be eliminated by eliminating the call to f_exit in subroutine stop of Error_function.f90, e.g., change

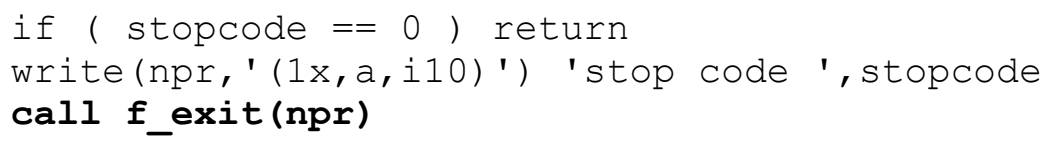

to

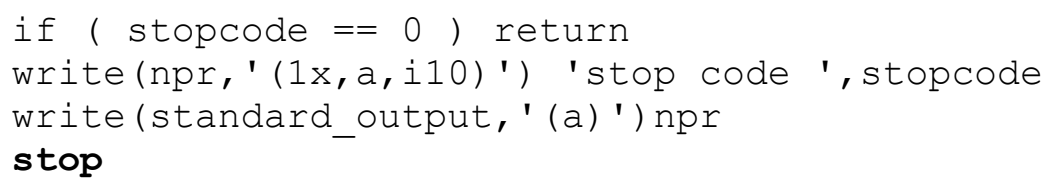

Alternatively, one can utilize the module listed on the following pages, developed by Mr. Benjamin Collins of the University of Michigan, which includes all necessary coding wrapped into a single Fortran module. Although developed from SCALE 5.1 routines, the format of SCALE direct access does not change and this source should remain compatible with later versions of SCALE. 


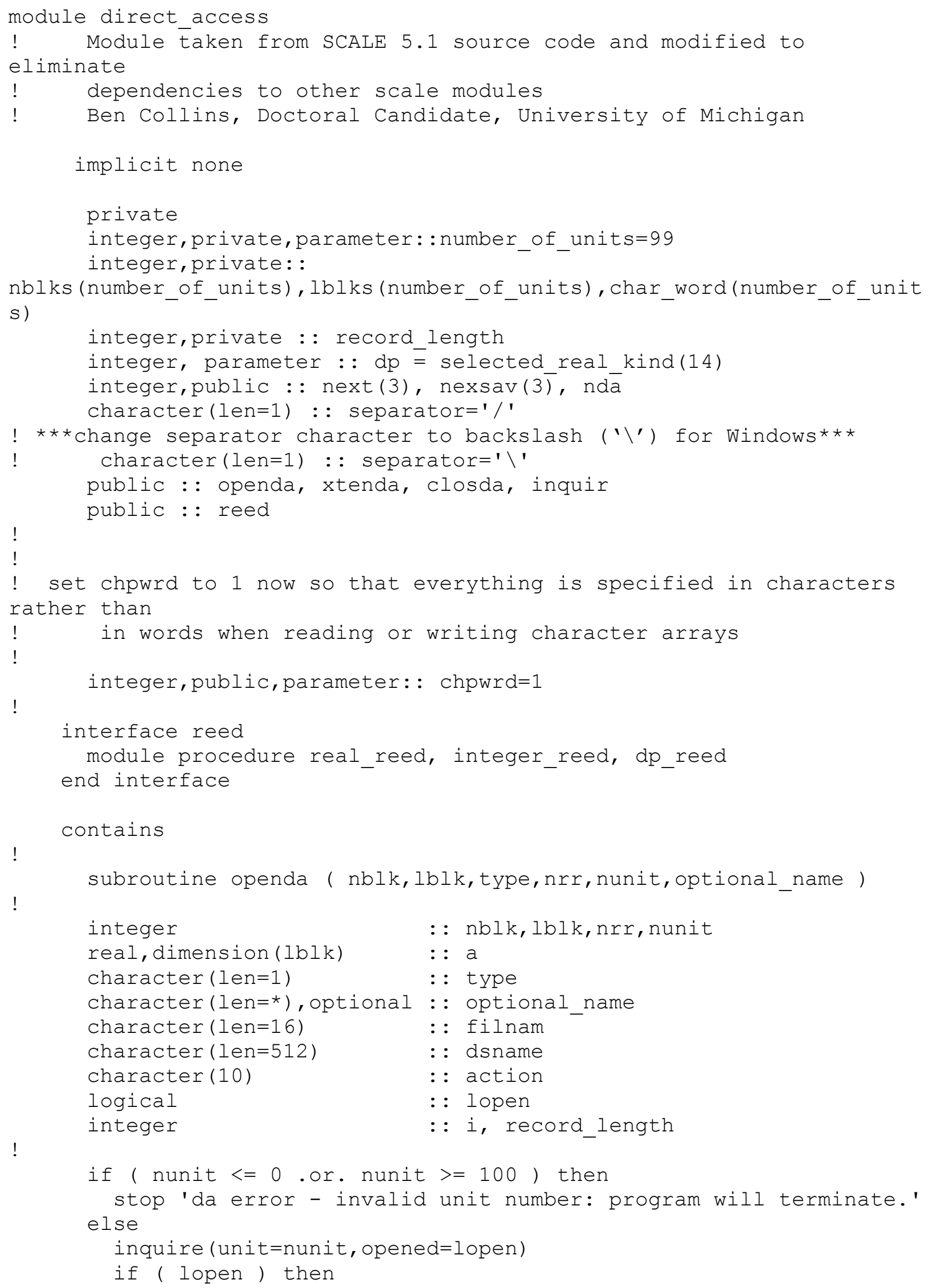




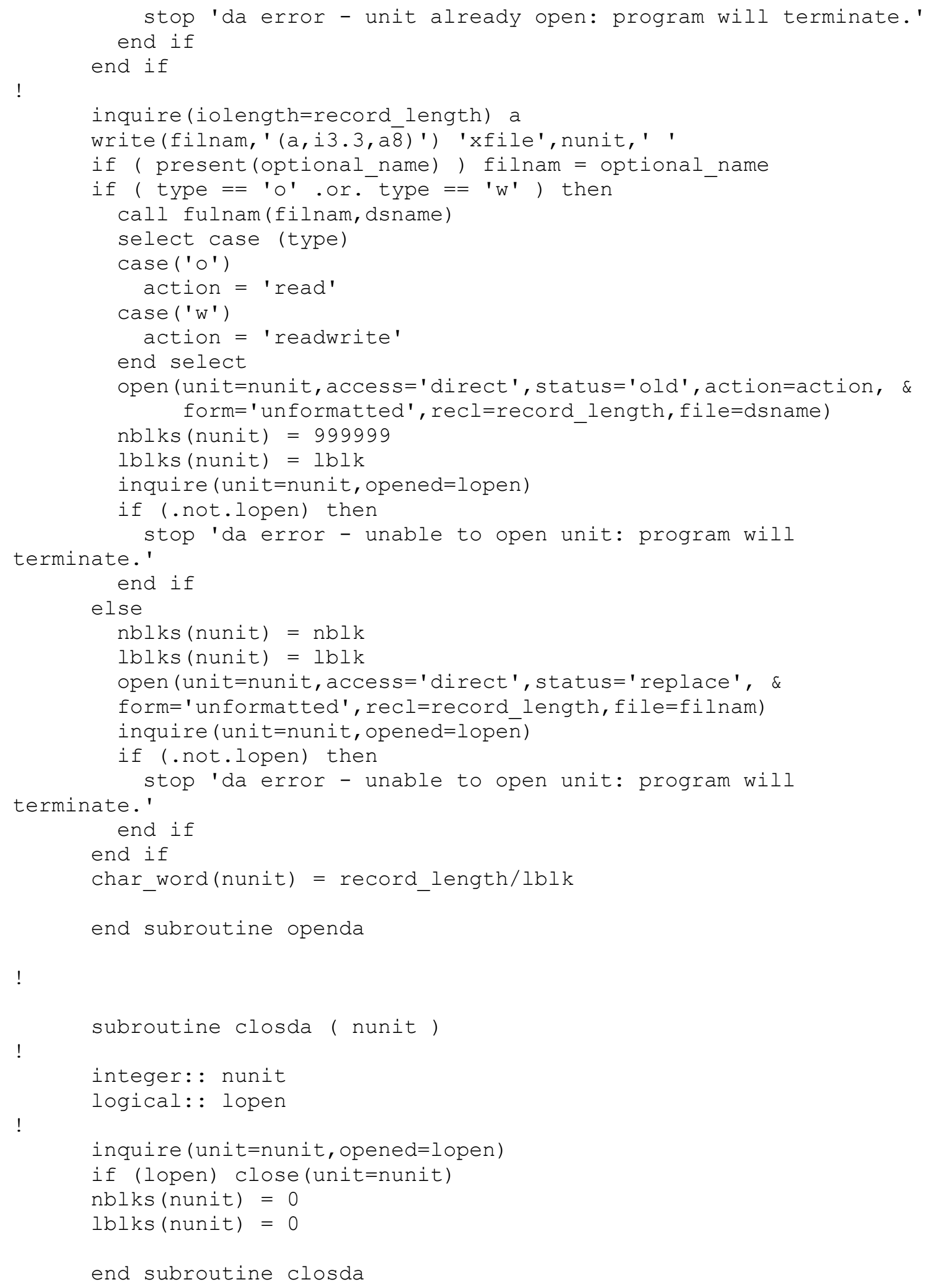




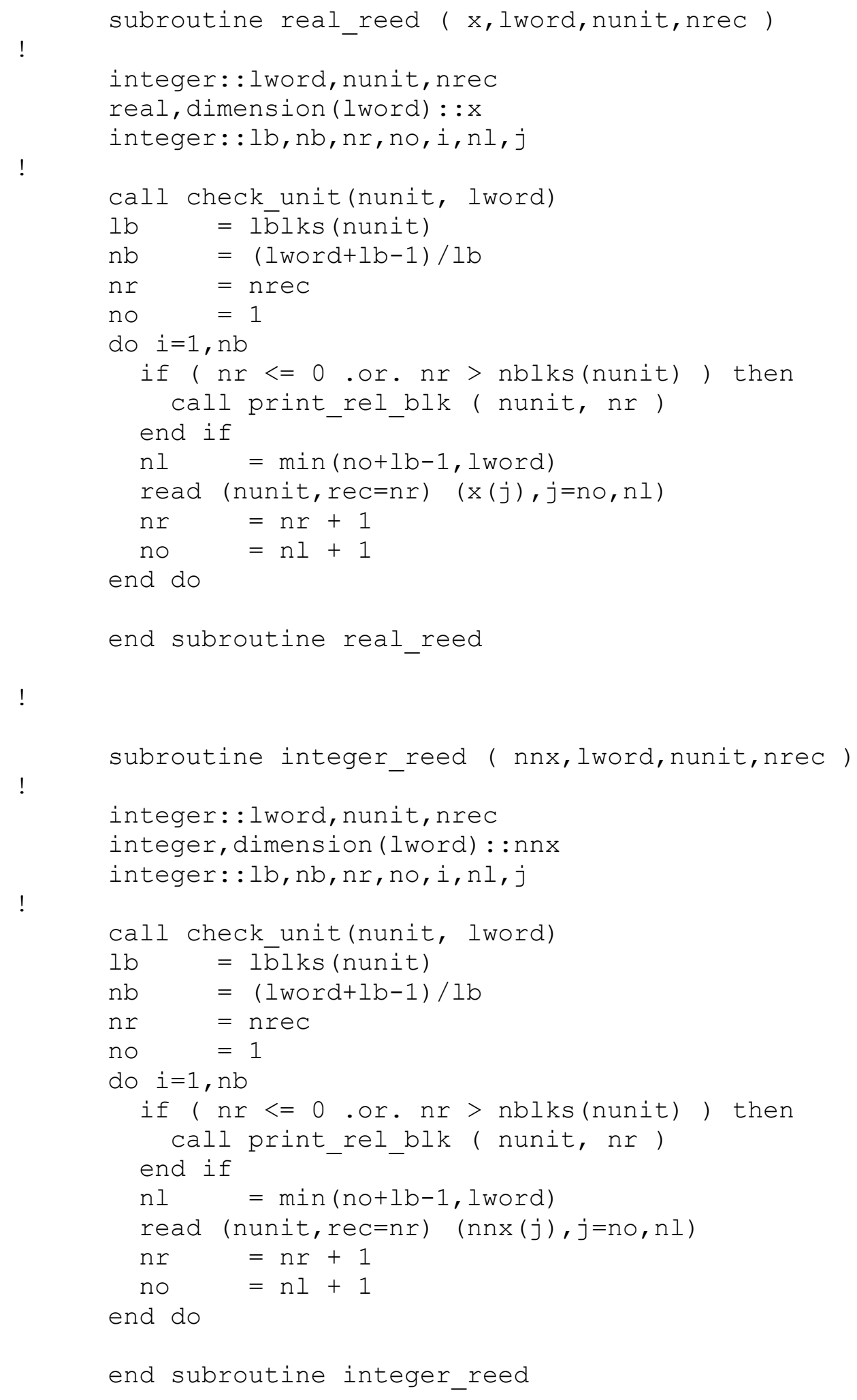




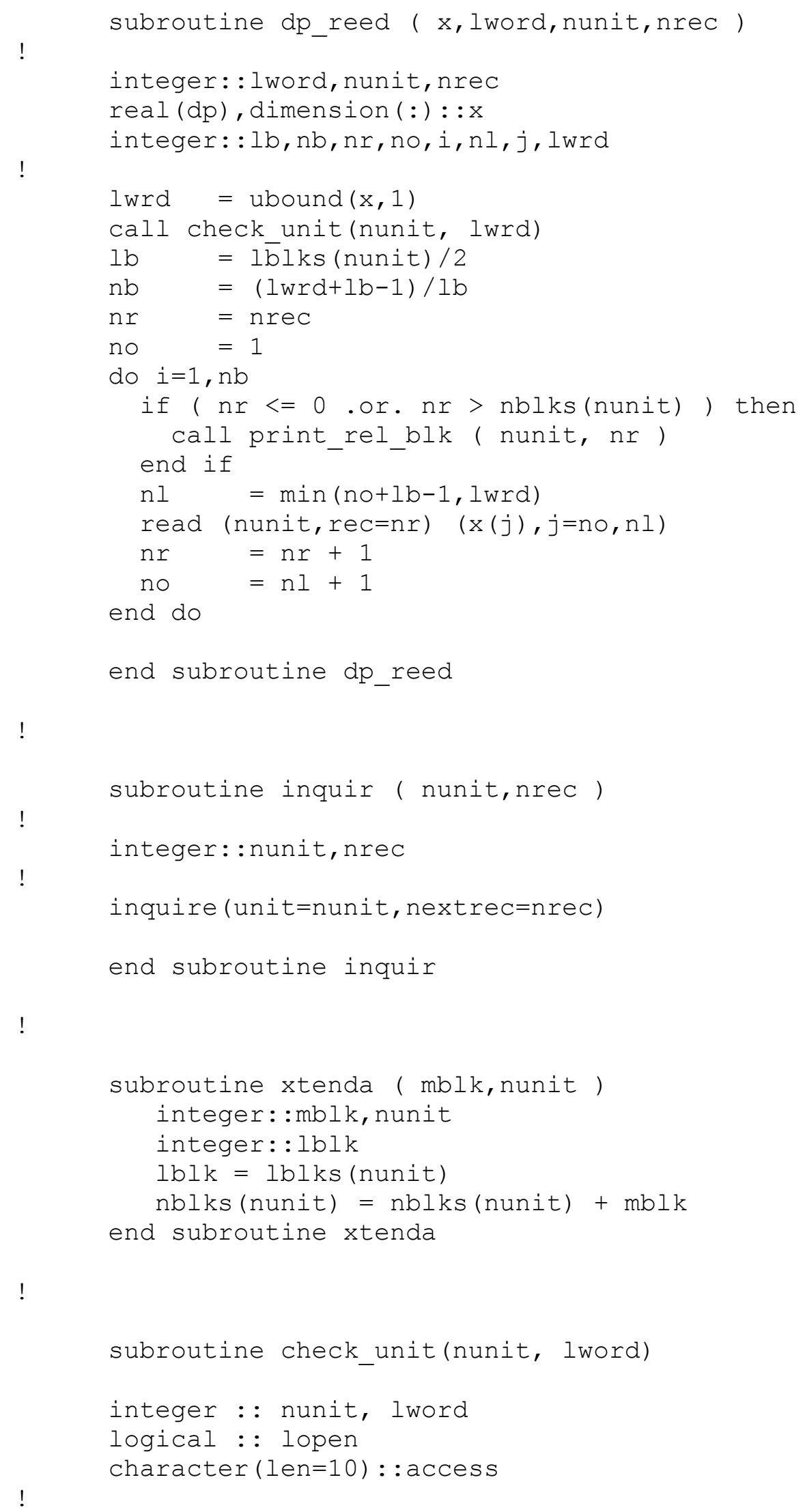




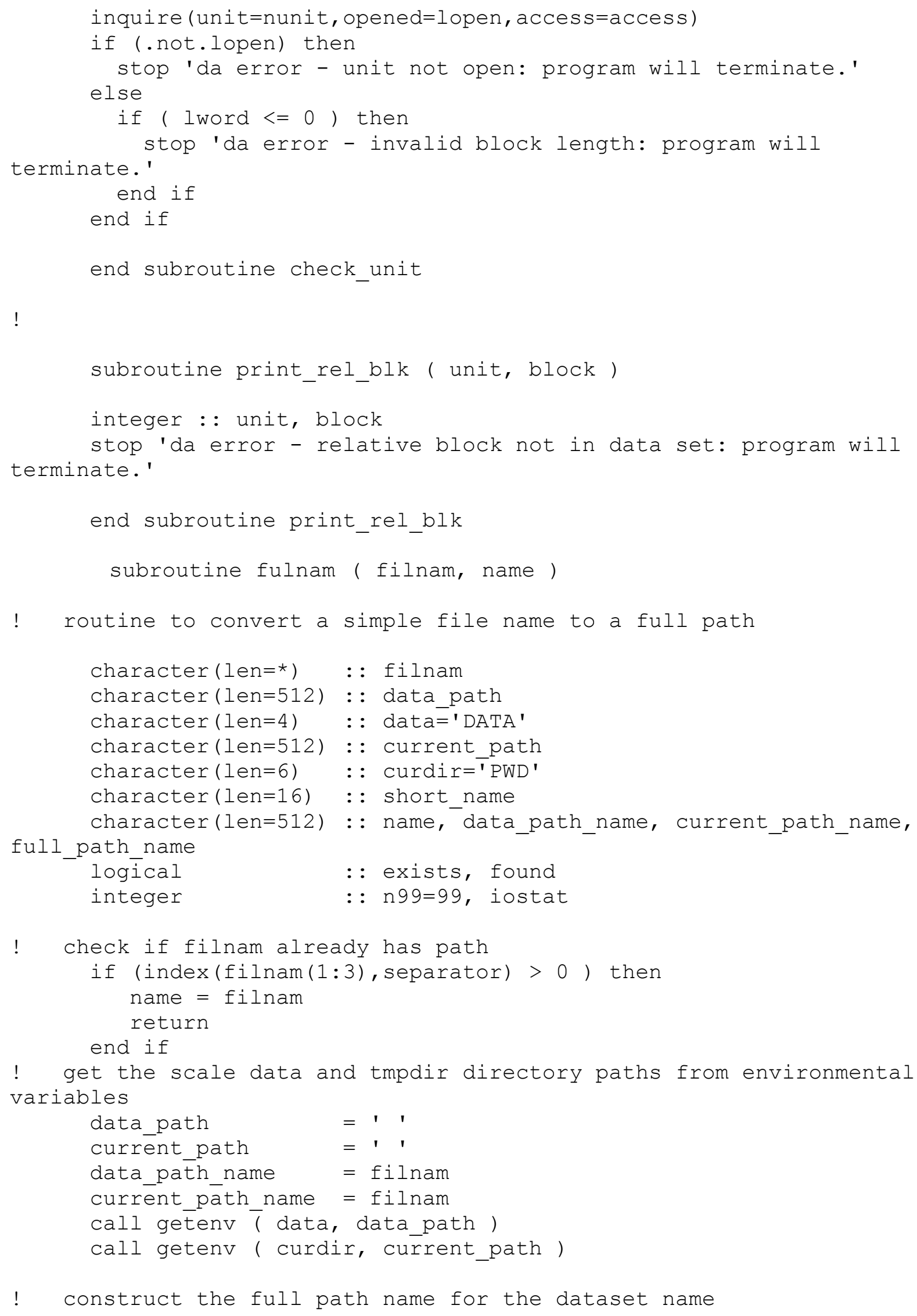




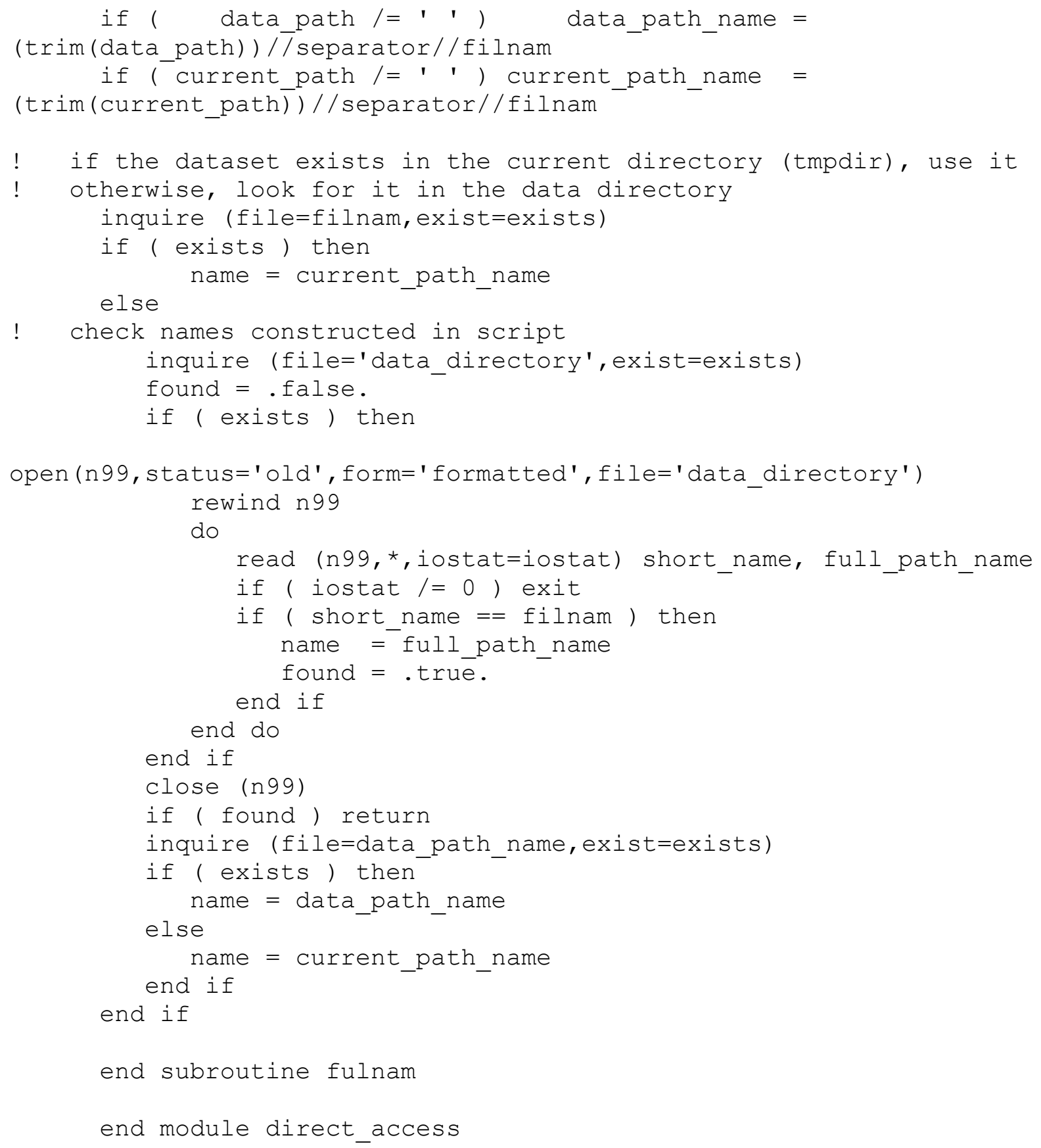




\subsection{The Flexible Branch Block}

In support of various projects, the "flexible branch block" was developed to enable a broader set of perturbations than are available in the typical TRITON branch capability. The typical branch block allows the user to define a single set of mixtures for 'fuel,' 'mod,' 'crout', or 'crin'. Having only four material set definitions limits user's ability to specify more complex perturbations that may be possible in some reactors, especially under transient conditions. The flexible branch block was developed such that the user can specify any number of material sets, and then apply separate perturbations to those sets. This capability, for example, enables specification of bypass flow density branches in BWRs in which the inchannel coolant and out-channel moderator can set to different densities in the same branch calculation.

The flexible branch block was developed in the SCALE 6.1 implementation of TRITON and was not modernized for SCALE 6.2. As a result, the flexible branch block is available in SCALE 6.1 and in the legacy mode in SCALE 6.2. The legacy mode can be accessed using $t$ - $d$ as the sequence name, rather than the more typical $t$-depl. The flexible branch block can be accessed using branchblock as the block name, rather than branch that is used for the typical branch block.

The following section of the manual explains the syntax of the branchblock and contains short examples of each element within the the branchblock. At the end of this section, a full example of a branchblock is provided so that users can gain an understanding of how to use all of the parts of the branchblock in order to define needed calculation branches.

SYNTAX:

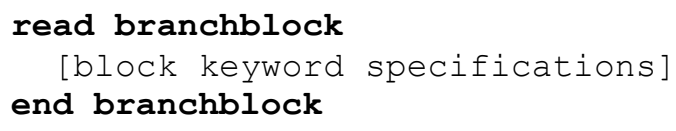

The advanced branchblock supports five different keyword specifications described below.

- mixset - used to define a set of mixtures which can be used in swap and perturbset definition,

- systemchange - used to define a system change to, temperatures, nuclide concentrations, and Dancoff factors,

- swap - used to define a set of mixtures to swap,

- $\quad$ perturbset - used to define a set of perturbations which apply the system changes defined by systemchange to a set of mixtures, and

- $\quad$ branch - used to define a branch calculation, composed of various swaps and perturbsets. Additional perturbations may also be defined.

NOTE: Several keywords in the branchblock are defined using strings. These strings must be must be delimited, i.e. starts and ends with an identifying marker. (Examples: title="cold", tit le=\#hot Doppler\#, title=!40\%void!, title=(80\%void)). As shown in the following examples, the string can optionally start with open angle bracket < and end with a closing angle bracket > (Example: tit $I e=\langle\operatorname{cold}>)$. All string-value inputs in the branchblock are delimited, alphanumeric strings with a maximum length of 80 characters. It is recommended that users choose a single type of delimiter, and then use that delimiter throughout the branchblock. 


\section{systemchange}

SYNTAX:

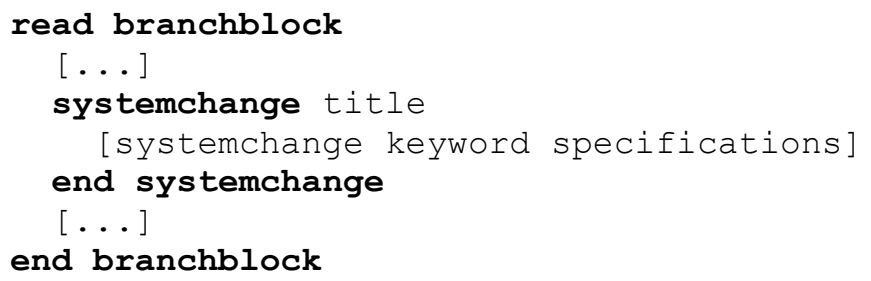

systemchange supports the following keyword specifications:

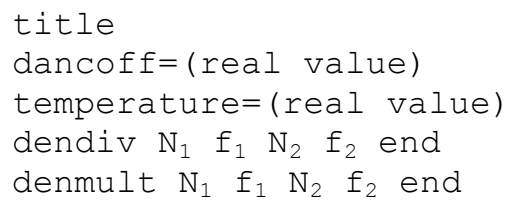

title is required string input and must follow systemchange. Only one title keyword may be specified. Multiple systemchange specifications are allowed, so each specification must have a unique title.

dancoff is optional and is used to set a dancoff factor value in the interval $[0,1]$. Only one dancoff specification is allowed and can appear anywhere in the systemchange specification following the title.

temperature is optional and is used to set a system temperature in Kelvin. It must be nonnegative. Only one temperature specification is allowed and can appear anywhere in the systemchange specification following the title.

dendiv and denmult are keyword arrays used to define nuclide concentration dividers and multipliers respectively. The arrays must be terminated with the end keyword. Each array is defined by a series of nuclide/factor pairs where nuclide is the ZZZAAA identifier and factor is either a multiply or divide factor applied to that nuclide concentration (Note that the particular mixture for which the factor is applied is defined in the perturb specification described below). Multiply factors must be $>=0$. Divide factors must be $>0$. A nuclide identifier set to zero implies that the factor is applied to all nuclides that are not explicitly listed in the array. Multiple dendiv and denmult arrays are allowed and can appear anywhere in the systemchange specification following the title. TRITON applies the concentration factors in the order in which they are entered in the systemchange specification.

Multiple systemchange specifications are allowed in the branch block. They can appear in any order, but must have a unique title.

EXAMPLE:

Define a temperature change to 60 kelvin. (The temperature change will be applied to a set of mixtures defined in the perturbset specification defined later.)

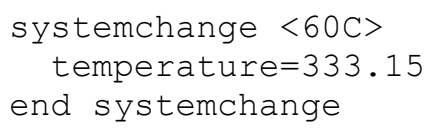


swap

SYNTAX:

read branchblock

[...]

swap title

[swap keyword specifications]

end swap

[... ]

end branchblock

swap supports the following keyword specifications:

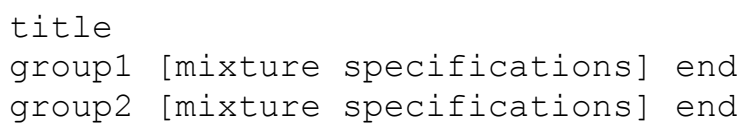

title is required string and must follow swap. Only one title keyword may be specified. Multiple swap specifications are allowed, so each specification must have a unique title.

group1 and group2 are used to define a set of mixtures to exchange. group1 must follow the swap title. group2 must follow group1. Only one specification for each group is allowed and they must have the same number of mixtures.

The group1 and group2 keywords support the following keyword specifications:

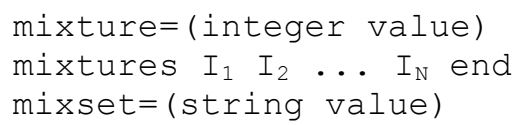

mixture is used to define a single mixture. mixtures is used to define an array of mixtures and is terminated with the end keyword. mixset is used to substitute a mixset specification defined elsewhere in the branchblock. Multiple mixture, mixtures, and mixset are allowed and can be placed in any order. TRITON will remove any duplicated mixture identifier, however each mixture must be defined in the model input.

EXAMPLES:

Exchange material 1 for 4.

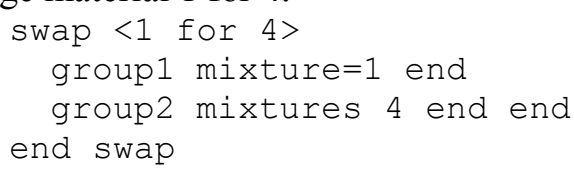

Exchange a set of mixtures:

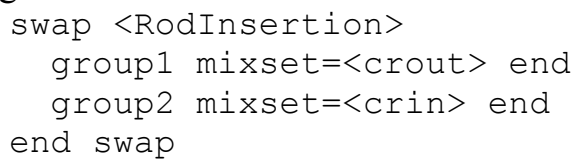

\section{branch}

SYNTAX:

read branchblock 


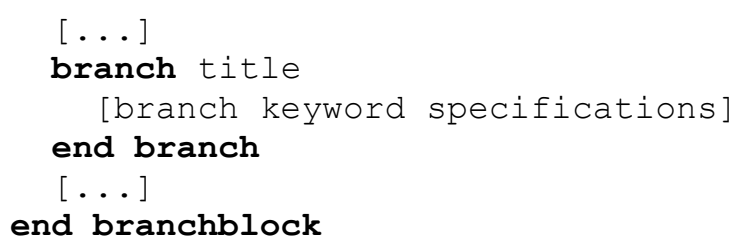

branch supports the following keyword specifications.

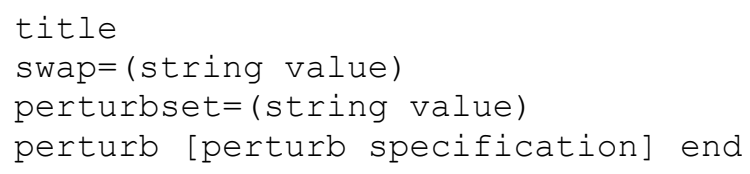

title is required string and must follow branch. Only one title keyword may be specified. Multiple branch specifications are allowed, so each specification must have a unique title.

swap is used to swap different sets of mixtures. The swap value is a string which is the title of a swap specification defined elsewhere in the branchblock. (The swap specification is described below). Multiple swap specifications are allowed and can appear anywhere in the branch specification following the title.

perturbset is used to apply a series of system perturbations. The perturbset value is a string which is the title of a perturbset specification defined elsewhere in the branchblock. (The perturbset specification is described below). Multiple perturbset specifications are allowed and can appear anywhere in the branch specification following the title.

perturb is used to apply a system perturbation that is not defined through the use of a perturbset specification. perturb specifications must terminate with the end keyword.

perturb supports the following keyword specifications.

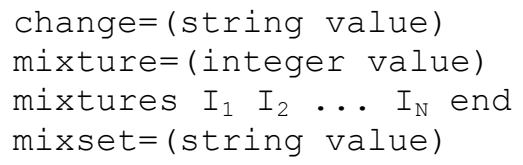

change is a string which is the title of a systemchange specification defined elsewhere in the branchblock. Only one change specification is allowed and may appear anywhere in the perturb specification.

The system change is applied to a set of mixtures defined by the mixture, mixtures, and mixset specifications. Only one of each of these keywords is allowed (however all three may be used in the same perturb specification). mixture, mixtures, and mixset may be placed in any order. TRITON will remove any duplicated mixture, however each mixture must be defined in the model input. TRITON will perform swap and perturb operations in the order they appear in the input.

\section{EXAMPLES:}

Define a branch to charactize the rodded, cold-zero-power condition. This requires the use of mixture swap entitled $<$ CRodIn $>$ along with the perturbset definition $<$ ColdMod $>$ which perturbs all of the moderator mixtures to a cold temperature and density. The fuel mixtures (defined as $<$ FuelMix $>$ ) must also be set to a temperature of $300 \mathrm{~K}$. 


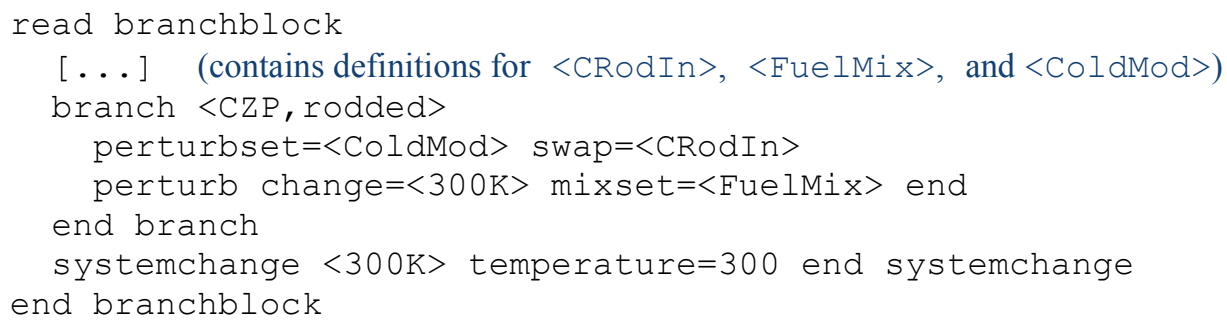

Define a branch to characterize the BWR instantaneous $100 \%$ void branch. This requires that:

- in-channel moderator mixtures $(<$ ChannelMod $>$ ) are perturbed from $40 \%$ void to $100 \%$ void (defined by systemchange $<40 \mathrm{vf}-100 \mathrm{vf}>$ ).

- Water-rod moderator mixtures ( $<$ WaterRodMod $>$ ) are perturbed from $0 \%$ void to $5 \%$ void ( $<0 \mathrm{vf}$ $5 \mathrm{vf}>$ )

- Bypass moderator mixtures ( $<$ BypassMod $>$ ) are perturbed from $0 \%$ void to $3 \%$ void $(<0 \mathrm{vf}-3 \mathrm{vf}>$ )

- Corner Rod Fuel mixture (mixture 1) dancoff factor changes (described by $<100 \mathrm{vf-cornerDF}>$ )

- Edge Fuel Rod Mixtures $(3,4,5,6,7,10)$ dancoff factor changes (described by $<100 v f$-edgeDF $>$ )

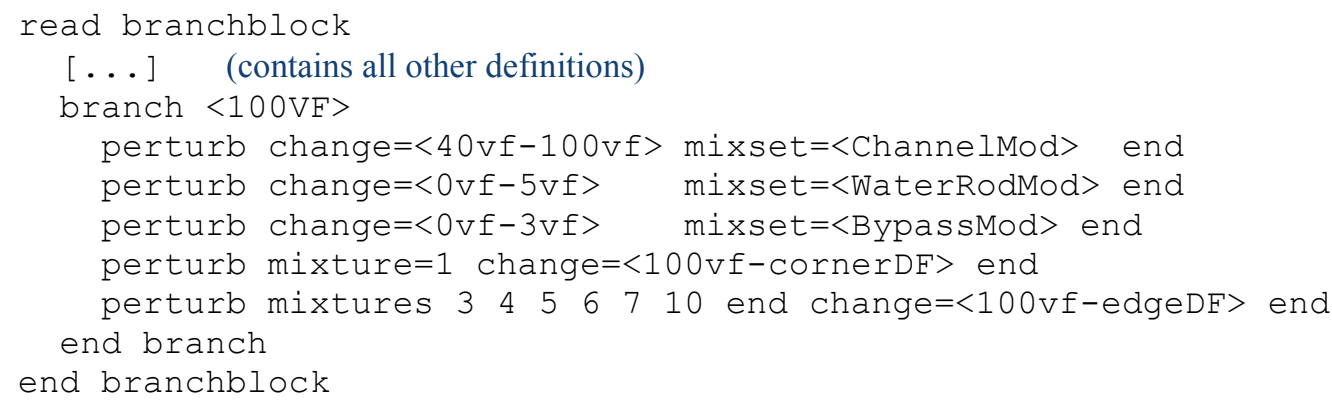

mixset - used to define a set of mixtures used in swap, perturbset, and perturb specifications.

SYNTAX:

read branchblock

[...]

mixset title

[mixset keyword specifications]

end mixset

[... ]

end branchblock

mixset supports the following keyword specifications:

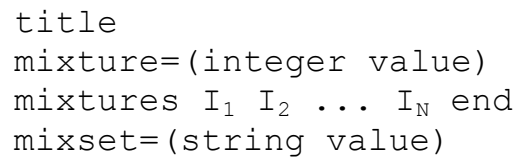

title is required string and must follow mixset. Only one title keyword may be specified. Multiple mixset specifications are allowed, so each specification must have a unique title.

mixture is used to define a single mixture. mixtures is used to define an array of mixtures and is terminated with the end keyword. mixset is used to substitute a mixset specification defined elsewhere in 
the branchblock. Multiple mixture, mixtures, and mixset are allowed and can be placed in any order. TRITON will remove any duplicated mixture identifier, however each mixture must be defined in the model input. If mixset is used to, the mixture set must be previously defined in the branchblock.

\section{EXAMPLE:}

In previous example for $100 \%$ void fraction, define a mixture set to be used for the edge rod dancoff factor perturbation.

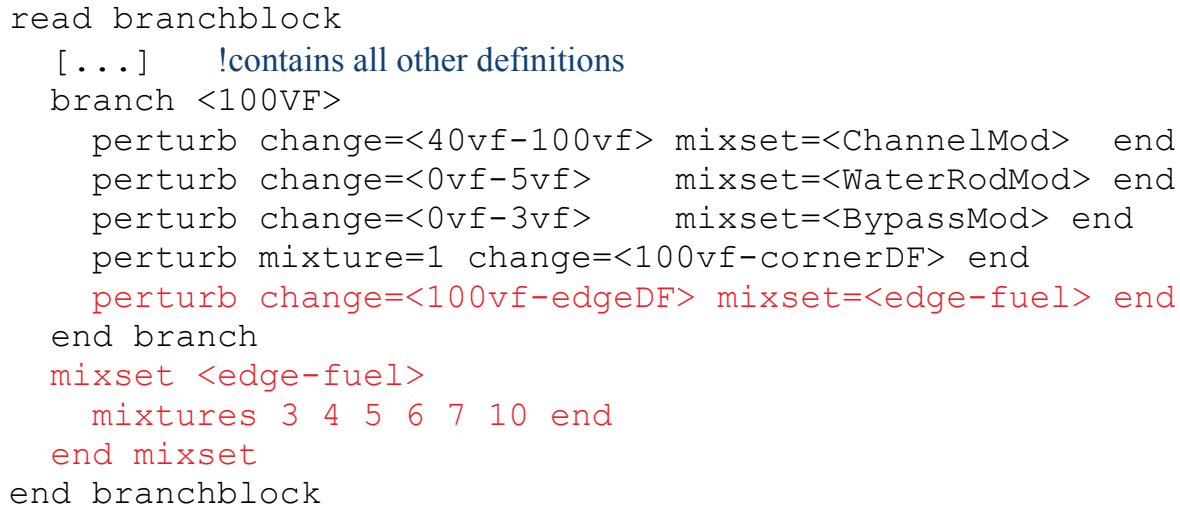

perturbset - used to define a set of system perturbations that can be used in branch specifications.

SYNTAX:

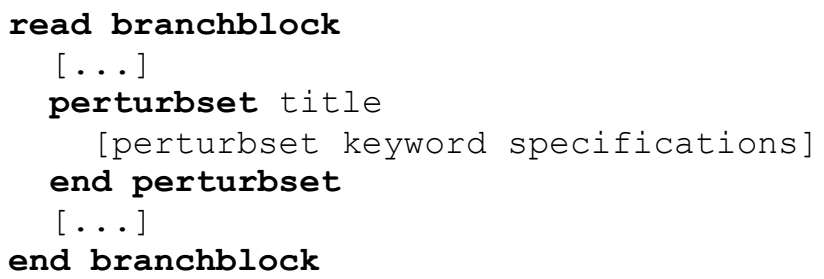

perturbset supports the following keyword specifications:

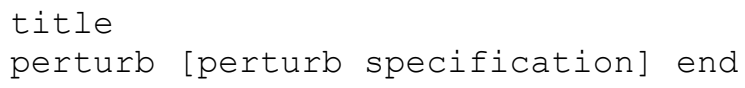

title is required string and must follow perturbset. Only one title keyword may be specified. Multiple perturbset specifications are allowed, so each specification must have a unique title.

After title, multiple perturb specifications can be used to defined a set of perturbations. The perturbset can then be used in branch specifications to simplify the branch input. TRITON will apply the perturbations in the order in which they appear in the perturbset specification.

EXAMPLE:

In previous example for $100 \%$ void fraction, define a perturbset for the moderator perturbations, and a separate perturbset for the fuel perturbations.

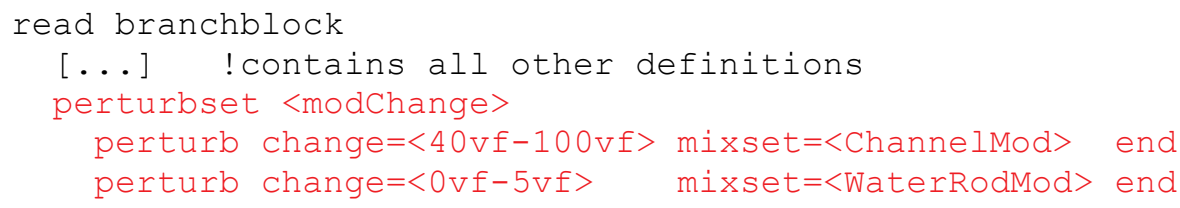


Also note that in the example provided, no soluble boron changes have been specified (as this is a BWR example). However, soluble poisons (boron or other), are also fairly straightforward to specify using the density divisors and density multipliers.

\section{BWR branchblock Example}

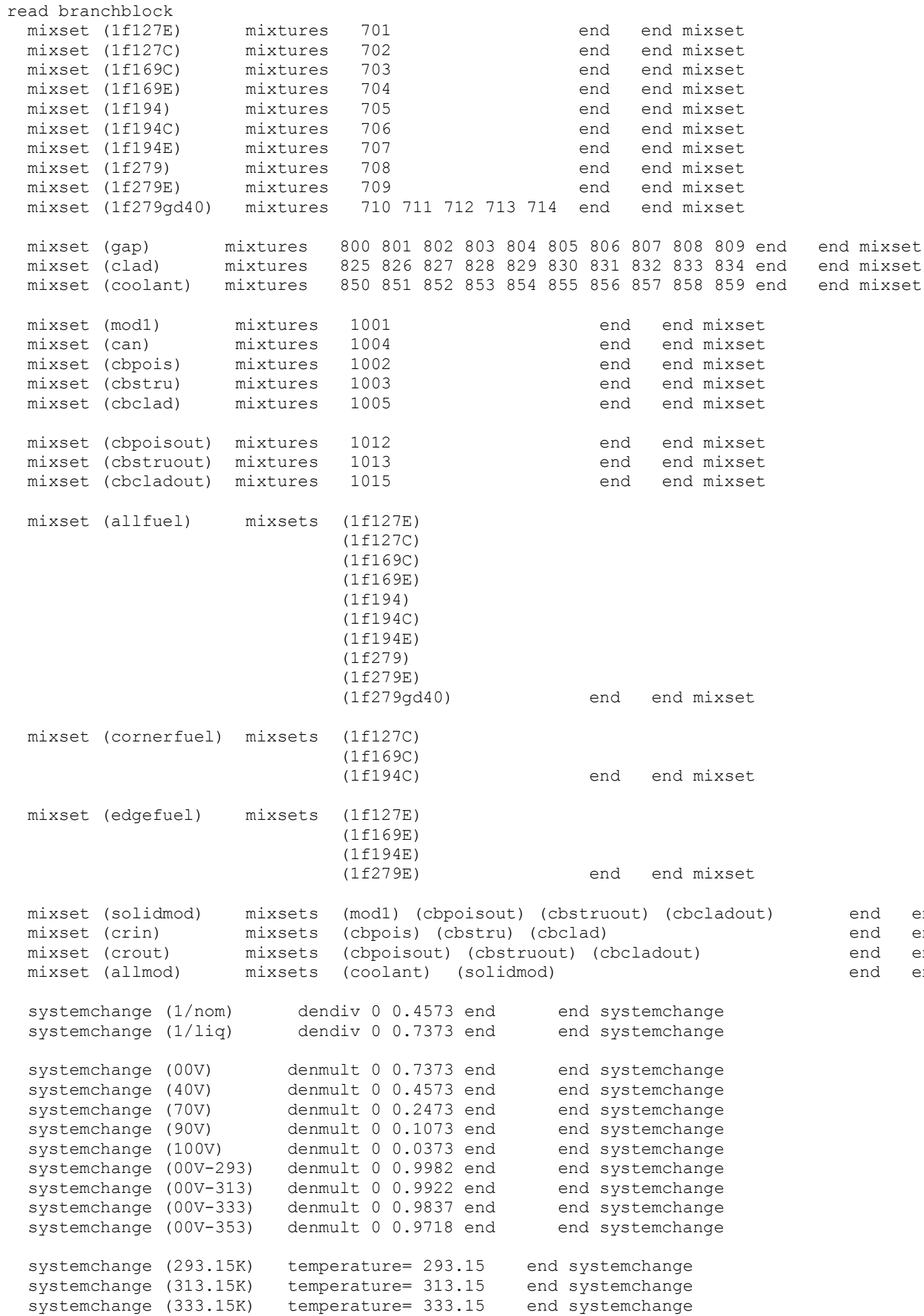

end end mixset

denmult $0 \quad 0.7373$ end denmult 00.4573 end denmult 00.2473 end denmult 00.1073 end denmult 00.0373 end denmult 00.9982 end denmult 0 0.9922 end denmult $0 \quad 0.9837$ end denmult 00.9718 end

temperature $=293.15$ temperature $=313.15$ temperature $=333.15$

end mixset

end mixset

end mixset

end end mixset

end end mixset

end mixse

end end mixset

end end mixset end end mixset end end mixset

end systemchange end systemchange end systemchange end systemchange end systemchange end systemchange end systemchange end systemchange end systemchange

end systemchange end systemchange end systemchange 


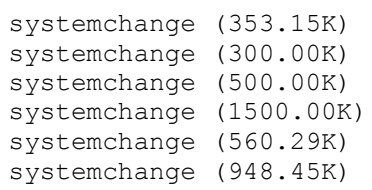

' 0\% void, cold Dancoff Factors

systemchange (00VCold-dfCO) dancoff $=0.084$

systemchange (00VCold-dfEO) dancoff $=0.125$

' 0\% void, Dancoff Factors

systemchange (OOV-dfCO)

systemchange (00V-dfEO)

- 40\% void, Dancoff Factors

systemchange (40V-dfCO)

systemchange (40V-dfEO)

' 70\% void, Dancoff Factors

systemchange (70V-dfCO)

systemchange (70V-dfEO)

' 90\% void, Dancoff Factors

systemchange (90V-dfCO)

systemchange (90V-dfEO)

dancoff $=0.116$

dancoff $=0.171$

dancoff $=0.180$

dancoff $=0.256$

dancoff $=0.281$

dancoff $=0.376$

dancoff $=0.421$

dancoff $=0.524$ end systemchange

end systemchange

end systemchange

end systemchange

end systemchange end systemchange

end systemchange end systemchange

end systemchange end systemchange

end systemchange end systemchange

end systemchange end systemchange

end systemchange end systemchange

swap (cr)

groupl mixset $=$ (crout) end group2 mixset $=($ crin) end end swap

perturbset (00\%Void293)

perturb mixset $=($ coolant $)$

perturb mixset $=($ coolant $)$

perturb mixset $=($ solidmod)

perturb mixset $=($ solidmod $)$

perturb mixset $=($ cornerfuel

perturb mixset $=($ edgefuel)

end perturbset

perturbset (00\%Void313)

perturb mixset $=($ coolant $)$

perturb mixset $=($ coolant $)$

perturb mixset $=($ solidmod $)$

perturb mixset $=($ solidmod $)$

perturb mixset $=($ cornerfuel $)$

perturb mixset $=($ edgefuel $)$

end perturbset

perturbset (00\%Void333)

perturb mixset $=($ coolant $)$

perturb mixset $=($ coolant $)$

perturb mixset $=($ solidmod $)$

perturb mixset $=($ solidmod)

perturb mixset $=($ cornerfuel $)$

perturb mixset $=($ edgefuel $)$

end perturbset

perturbset (00\%Void353)

perturb mixset $=($ coolant $)$

perturb mixset $=($ coolant $)$

perturb mixset $=($ solidmod $)$

perturb mixset $=($ solidmod)

perturb mixset $=($ cornerfuel $)$

perturb mixset $=($ edgefuel $)$

change $=(1 /$ nom $)$

change $=(00 \mathrm{~V}-293)$

change $=(1 /$ liq $)$

change $=(00 \mathrm{~V}-293)$

change $=(0 \mathrm{VCold}-\mathrm{dfCO})$

change $=(0$ VCold - dfEO $)$

end

end

end

end

end

end

change $=(1 / \mathrm{nom})$

change $=(00 \mathrm{~V}-313)$

change $=(1 /$ liq $)$

change $=(00 \mathrm{~V}-313)$

change $=(0$ VCold $-\mathrm{dfCO})$

change $=(0$ VCold $-d f E O)$

end

end

end

end

end

end

change $=(1 /$ nom $)$

change $=(00 \mathrm{~V}-333)$

change $=(1 / 1 i q)$

change $=(00 \mathrm{~V}-333)$

change $=(0$ VCold $-\mathrm{dfCO})$

change $=(0$ VCold-dfEO $)$

change $=(1 /$ nom $)$

change $=(00 \mathrm{~V}-353)$

change $=(1 / 1 i q)$

change $=(00 \mathrm{~V}-353)$

change $=(00 \mathrm{VCol} d-\mathrm{dfCO})$

change $=($ OVVold-dfEO $)$

end

end

end

end

end

end

end perturbset

perturbset (00\%Void)

perturb mixset $=($ coolant $)$

perturb mixset $=($ coolant $)$

change $=(1 / \mathrm{nom})$

change $=(00 \mathrm{~V})$

perturb mixset $=($ cornerfuel $)$

perturb mixset $=($ edgefuel $)$

end perturbset

perturbset (40\%Void)

perturb mixset $=($ coolant $)$

perturb mixset $=($ coolant $)$

change $=(0 \mathrm{OV}-\mathrm{dfCO})$

change $=(0 \mathrm{OV}-\mathrm{dfEO})$

end

end

end

end

end

end

perturb mixset $=($ cornerfuel $)$

perturb mixset $=($ edgefuel $)$

end perturbset

perturbset ( $70 \%$ Void)

perturb mixset $=($ coolant $)$

perturb mixset $=($ coolant $)$

change $=(1 /$ nom $)$

change $=(40 \mathrm{~V})$

change $=(40 \mathrm{~V}-\mathrm{dfCO})$

change $=(40 \mathrm{~V}-\mathrm{dfEO})$

end

end

end

end

change $=(1 /$ nom $)$

change $=(70 \mathrm{~V})$

end

end

end

end

end

end 


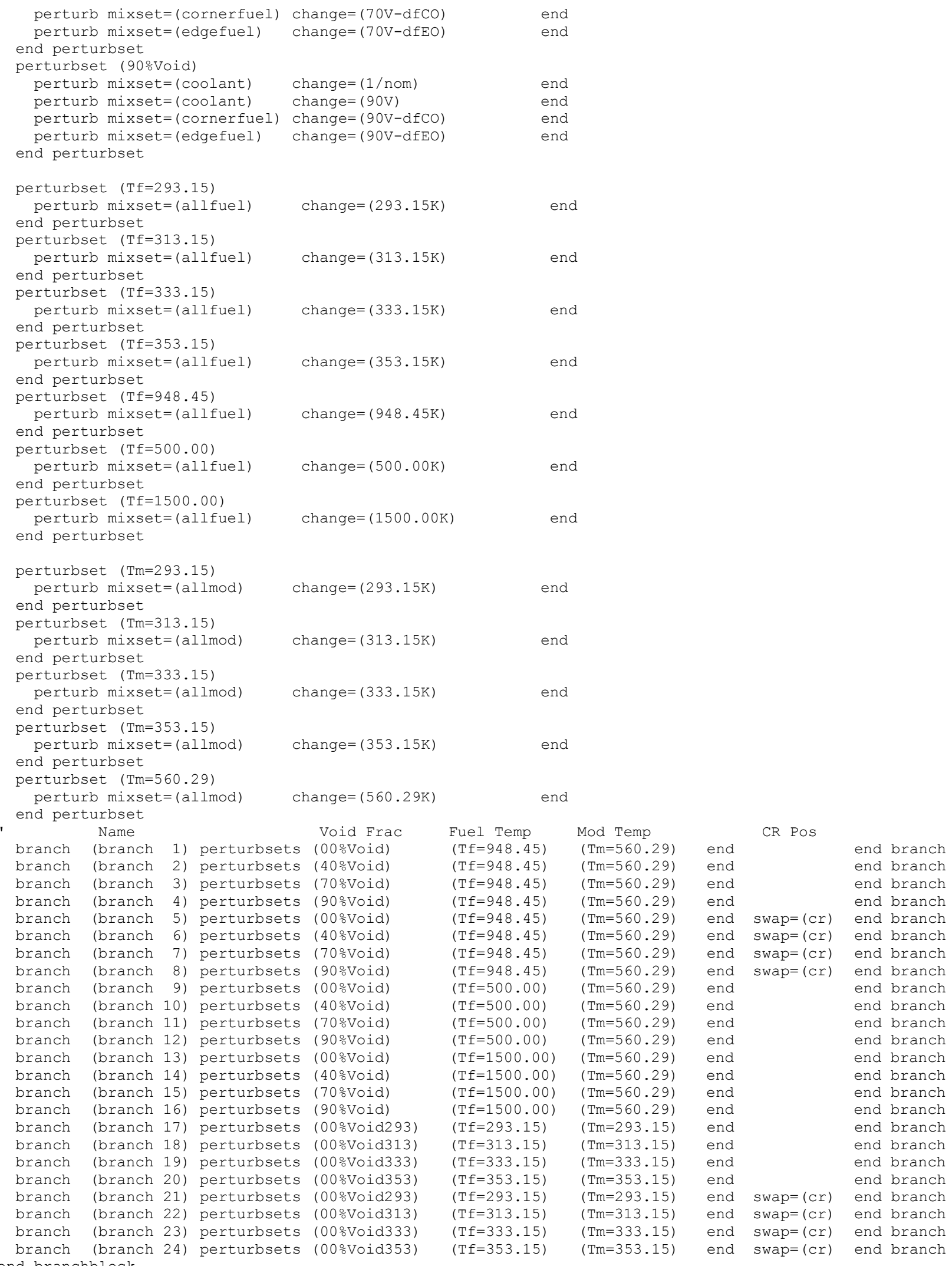




\title{
3.2 POLARIS - 2D LIGHT WATER REACTOR LATTICE PHYSICS MODULE
}

M. A. Jessee, J. J. Jarrell, W. A. Wieselquist, M. L. Williams, K. S. Kim, T. M. Evans, S. P. Hamilton, C. A. Gentry

\begin{abstract}
Polaris is a new module for SCALE 6.2 that provides 2D lattice physics analysis capability for light water reactor (LWR) fuel designs. Polaris uses a new multigroup self-shielding method called the Embedded Self Shielding Method (ESSM) and a new transport solver based on the Method of Characteristics (MoC). The ESSM computes multigroup self-shielded cross sections using Bondarenko interpolation methods. The background cross section used in the interpolation is determined by iterative 2D MoC fixed source transport calculations. Polaris is integrated with ORIGEN for depletion calculations. Each pin —or each radial subregion of the pin-is depleted based on the local power distribution. An optional critical spectrum calculation is incorporated into the depletion calculation and the output edits of few-group homogenized cross sections. Few-group cross sections are archived to an xfile016 file, which can be used in subsequent core simulator calculations. Polaris provides an easy-to-use input format to allow users to set up lattice models with minimal lines of input.
\end{abstract}




\section{TABLE OF CONTENTS}

3.2 POLARIS - 2d light water reactor lattice physics module.................................................... $3-117$

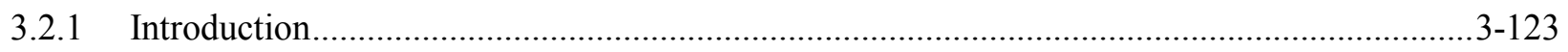

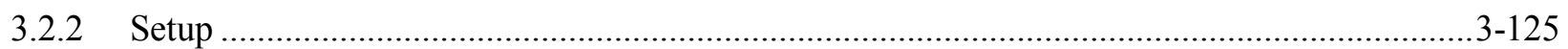

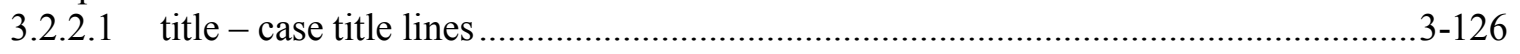

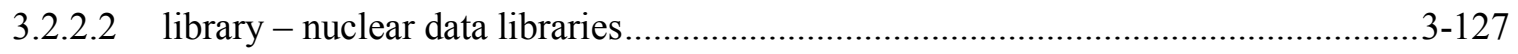

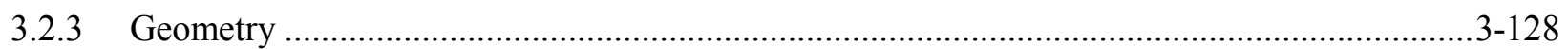

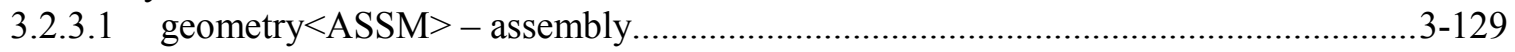

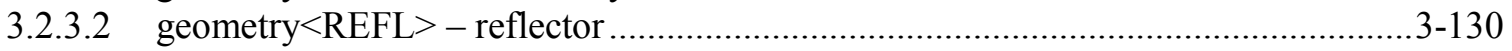

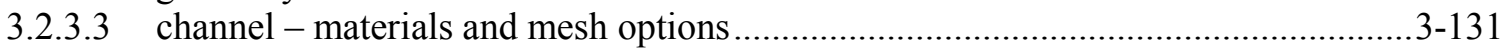

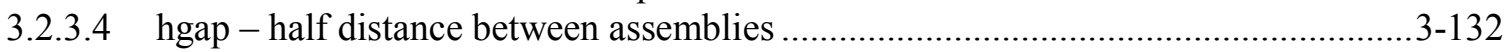

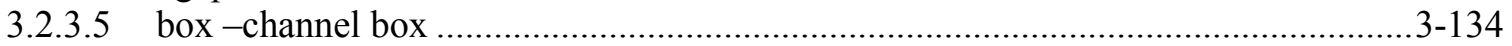

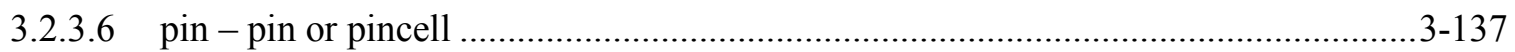

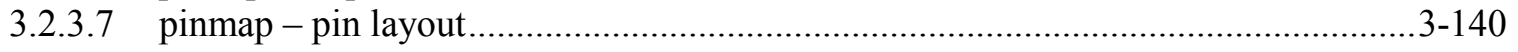

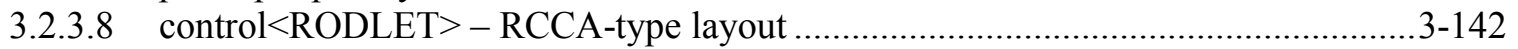

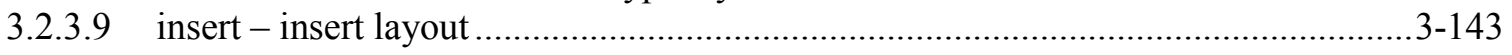

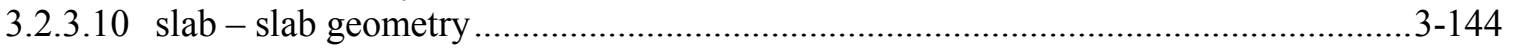

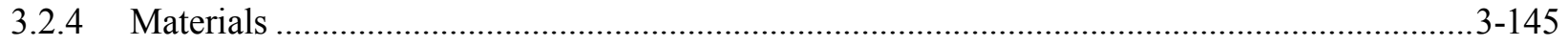

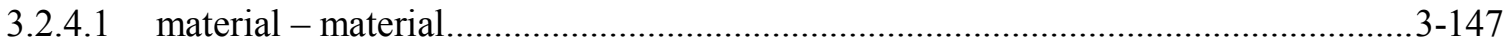

3.2.4.2 composition $<\mathrm{NUM} \mid \mathrm{WT}>-$ general atom/wt fraction ..........................................

3.2.4.3 composition<FORM> - general chemical formula.................................................

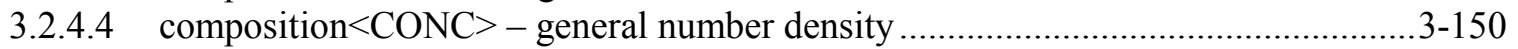

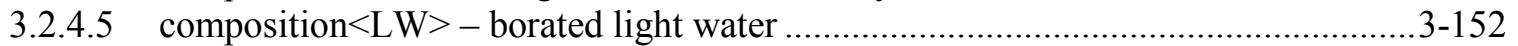

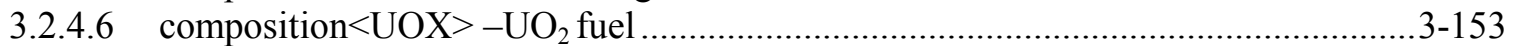

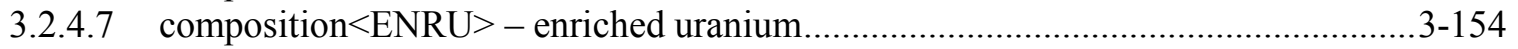

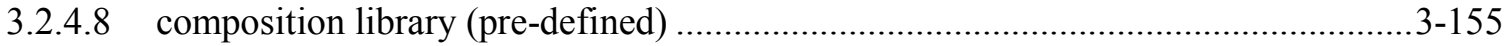

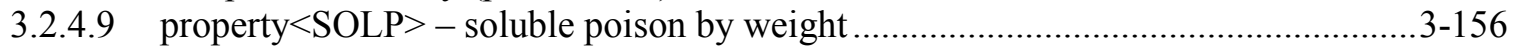

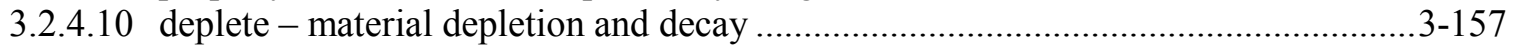

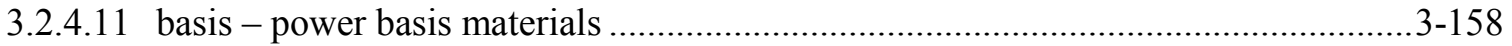

3.2.4.12 shield - cross section self-shielding expansion specification..................................3-159

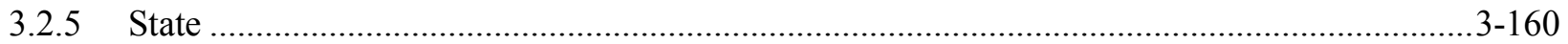

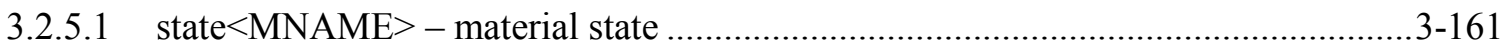

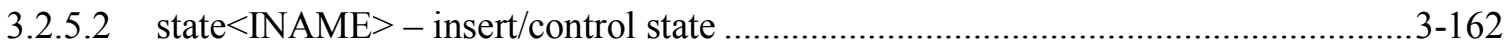

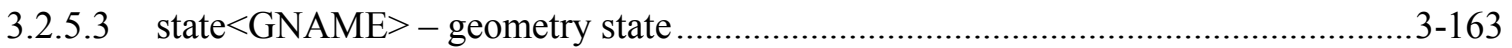

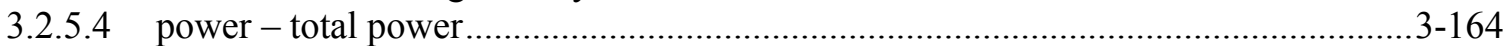

3.2.5.5 $\mathrm{bu}$ - initiate calculation with cumulative burnups..................................................... $3-165$

3.2.5.6 dbu - initiate calculation with incremental burnups........................................... 3-166

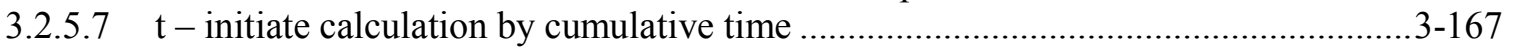

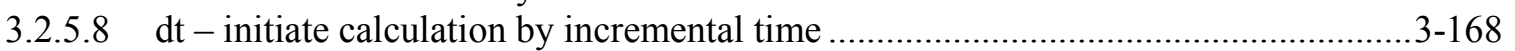

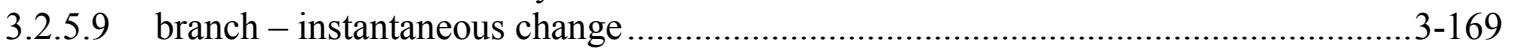

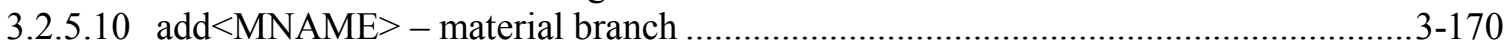

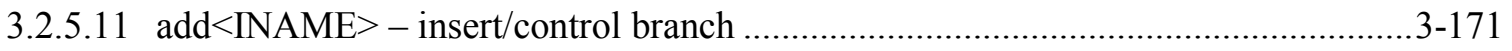

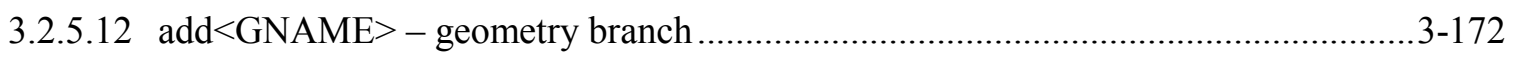

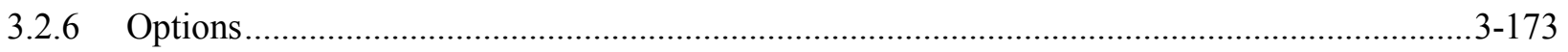


3.2.6.1 option $<\mathrm{KEFF}>-$ eigenvalue

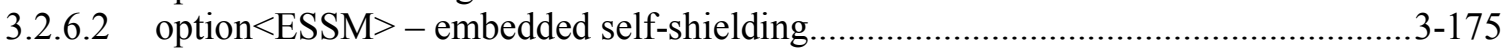

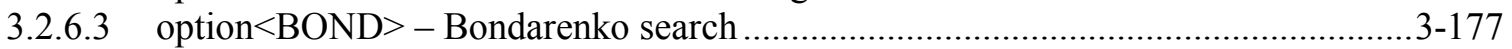

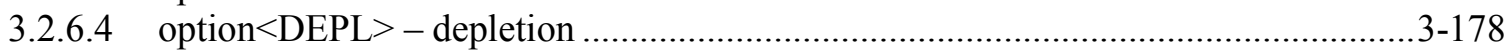

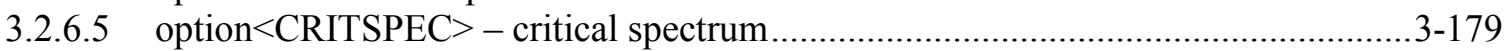

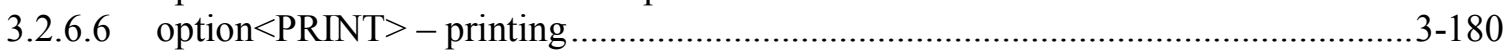

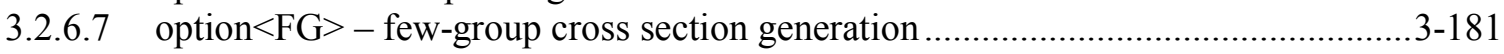

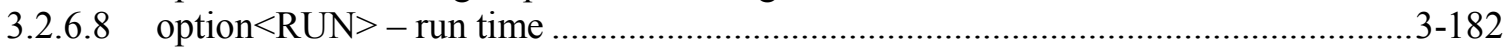

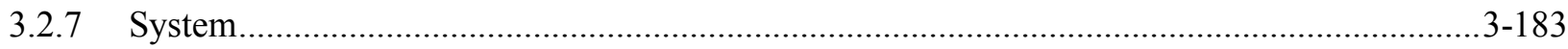

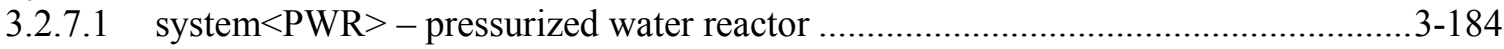

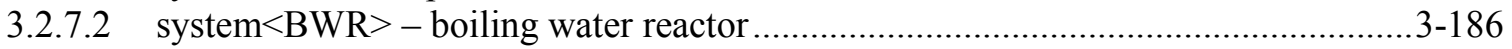

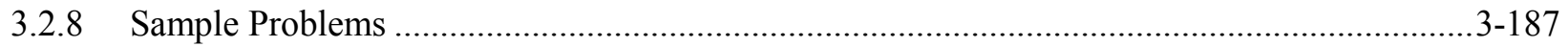

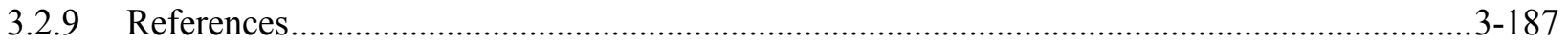

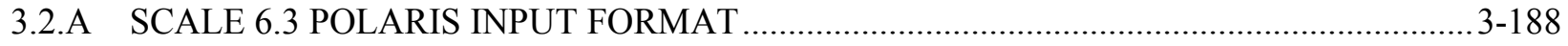

3.2.a.1 box (version 6.3) - channel box geometry ............................................................. 3-189

3.2.a.2 pin (version 6.3) - pincell comprised of nested geometry zones of variable

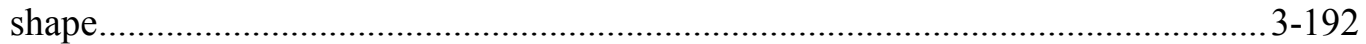

3.2.a.3 mesh (version 6.3) - advanced material dependent meshing options ....................... 3-195

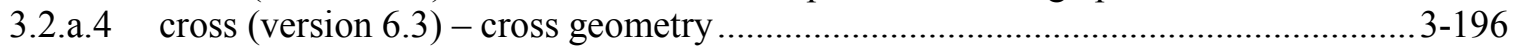

3.2.a.5 dxmap and dymap (version 6.3) - pin-by-pin displacement maps........................... 3-200

3.2.a.6 control<blade $>$ (version 6.3) - bwr control blade ..................................................3-201

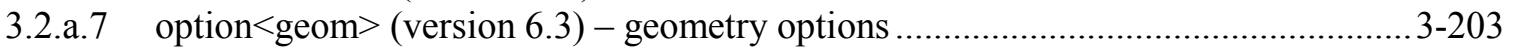

3.2.a.8 bu (version 6.3) - initiate calculation with cumulative burnups ...............................3-204

3.2.a.9 bui (version 6.3) - initiate calculation with cumulative burnups (with restart) ........ 3-205

3.2.a.10 dbu (version 6.3) - initiate calculation with incremental burnups ........................... 3-206

3.2.a.11 t (version 6.3) - initiate calculation by cumulative time ......................................... 3-207

3.2.a.12 ti (version 6.3) - initiate calculation by cumulative time (with restart) .................... 3-208

3.2.a.13 dt (version 6.3) - initiate calculation by incremental time ...................................... 3-209

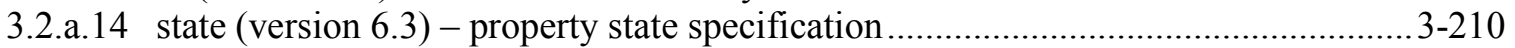

3.2.a.15 history (version 6.3) - time-dependent history ...................................................... 3-212 


\section{LIST OF FIGURES}

$\underline{\text { Page }}$

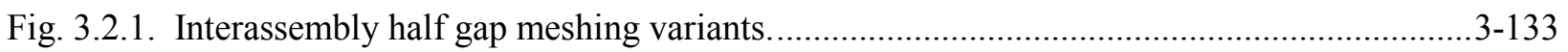

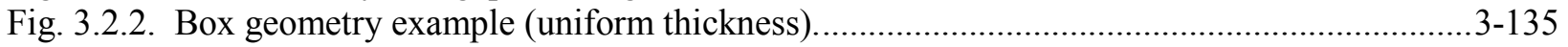

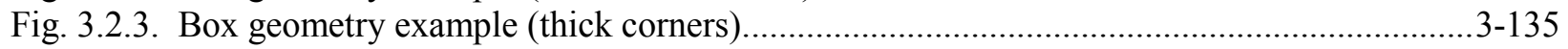

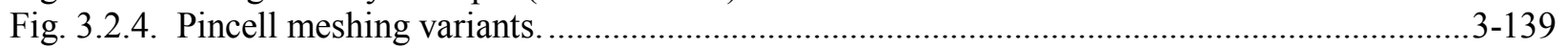

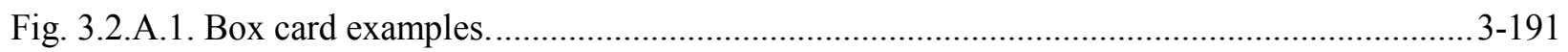

Fig. 3.2.A.2. Pin examples with different shape geometries............................................................. 3-193

Fig. 3.2.A.3. Pin examples with noninteger pin size........................................................................... 3-194

Fig. 3.2.A.4. Construction of the BWR cross geometry (full example shown later)...........................3-197

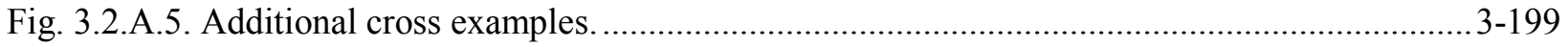

Fig. 3.2.A.6. Control blade example ........................................................................................... 3-202 


\section{LIST OF TABLES}

$\underline{\text { Page }}$

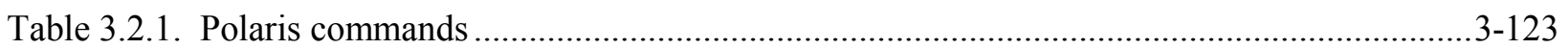

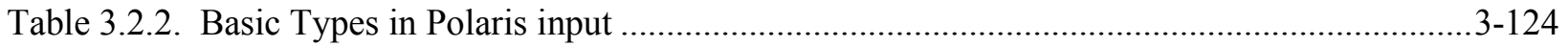

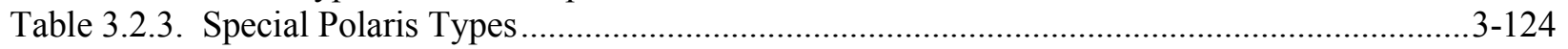




\section{ACKNOWLEDGMENTS}

The authors express gratitude to Brad Rearden and Stephen Bowman for their supervision of Polaris development for the US Nuclear Regulatory Commission (NRC). The author acknowledges Don Algama and Mourad Aissa of the NRC for their support of the project. Appreciation is extended to the ATLAS development team - Jordan Lefebvre, Rob Lefebvre, and Adam Thompson — for their development of the ATLAS Ray Tracing Geometry package used in the Polaris MoC solver. Appreciation is also extended to

Ugur Mertyurek, Brian Ade, Ben Betzler, Scott Palmtag, and Andrew Godfrey for testing and benchmarking efforts and also Sheila Walker for finalizing the publication of this document. 


\subsubsection{Introduction}

Polaris is a new module for SCALE 6.2 that provides 2D lattice physics analysis capability for light water reactor (LWR) fuel designs. For multigroup cross section processing, Polaris uses the Embedded SelfShielding Method (ESSM) [1]. Unlike SCALE multigroup sequences that use XSProc, ESSM does not require user-defined unit cell definitions. ESSM computes multigroup cross sections using Bondarenko interpolation methods. The background cross section used in the interpolation is determined by iterative 2D fixed source transport calculations. Both the ESSM fixed source calculations and the keff calculation utilize a new Method of Characteristics (MoC) transport solver developed in the Exnihilo computational package.

Polaris is integrated with ORIGEN for depletion calculations. Each pin-or each radial subregion of the pin -is depleted based on the local power distribution. An optional critical spectrum calculation is incorporated into the depletion calculation and the output edits of few-group homogenized cross sections. Few-group cross sections are archived to an xfile016 file, which can be used in subsequent core simulator calculations. A complete description of the Polaris computational methods is provided in [2].

Polaris provides an easy-to-use input format to allow users to set up lattice models with minimal lines of input. All recognized Polaris commands are shown in Table 3.2.1. Note that many commands support short and long forms. The allowed basic Types for input are described in Table 3.2.2. The special Polaris TYPES are shown in Table 3.2.3. The convention used in this manual is that basic types appear italicized and capitalized (Type), while special Polaris types appear in all caps (TYPE).

Table 3.2.1. Polaris commands

\begin{tabular}{lll}
\hline \multicolumn{1}{c}{ card } & \multicolumn{1}{c}{ long } & \multicolumn{1}{c}{ short } \\
command & command(s) \\
\hline system & system & sys \\
geometry & geometry & geom \\
composition & composition & comp \\
property & property & prop \\
material & material & mat \\
burnup & bu or dbu & - \\
power & power & pow \\
options & option & opt \\
time & t or dt & - \\
state & state & - \\
branch block & branch & - \\
pin geometry component & pin & - \\
assembly pin map & pinmap & - \\
assembly channel & channel & - \\
assembly half gap & hgap & - \\
channel box & box & - \\
shield & shield & - \\
deplete & deplete & - \\
slab geometry component & slab & - \\
power basis materials & basis & - \\
assembly inserts & insert & - \\
assembly control elements & control & - \\
\hline
\end{tabular}


Table 3.2.2. Basic Types in Polaris input

\begin{tabular}{|c|c|c|c|}
\hline $\begin{array}{l}\text { basic } \\
\text { Type }\end{array}$ & description & examples & incorrect examples \\
\hline Word & $\begin{array}{l}\text { starts with a character } \mathrm{A}-\mathrm{Z} \text { or } \mathrm{a}-\mathrm{z} \\
\text { and includes characters, numbers, } \\
\text { underscores }\end{array}$ & $\begin{array}{l}\text { uox } \\
\text { bor_water_500ppm } \\
\text { FUEL }\end{array}$ & $\begin{array}{l}\text { uox_enr5.1 } \\
316 \text { SS } \\
\text { uox-3.1 }\end{array}$ \\
\hline Int & integer & $\begin{array}{l}17 \\
92235 \\
2 \\
565 \\
\end{array}$ & $\begin{array}{l}31.4 \\
\text { uox }\end{array}$ \\
\hline Bool & boolean/logical & $\begin{array}{l}\text { yes } \\
\text { false }\end{array}$ & $\begin{array}{l}\text { TRUE } \\
\text { No }\end{array}$ \\
\hline Real & any number & $\begin{array}{l}565 \\
10.257 \\
1.5 \mathrm{e}-6\end{array}$ & $\begin{array}{l}\text { yes } \\
\text { bor_water }\end{array}$ \\
\hline String & a single or double quoted string & $\begin{array}{l}\text { "INFMED" } \\
\text { "Includes spaces" } \\
\text { 'NONE' }\end{array}$ & Includes spaces \\
\hline Value & any non-word & & Int|Bool|Real|String \\
\hline
\end{tabular}

Table 3.2.3. Special Polaris Types

\begin{tabular}{lll}
\hline Polaris Type & \multicolumn{1}{c}{ description } & \multicolumn{1}{c}{ Variants } \\
\hline STYPE & system type & $P W R \mid B W R$ \\
GTYPE & geometry type & ASSM $\mid R E F L$ \\
CTYPE & composition type & NUM $|W T| F O R M|C O N C| L W \mid$ UOX \\
PTYPE & property type & SOLP \\
ETYPE & control element type & RODLET \\
OTYPE & option type & KEFF|BOND|ESSM|CRITSPEC $|F G| D E P L|R U N| P R I N T$ \\
\hline
\end{tabular}

The Polaris input supports a very flexible input scheme that allows some elements to be suppressed for better readability. With key=value type input, when the standard order of keys is used, the keys may be suppressed. Consider the following input specification as an example.

geometry GNAME : ASSM npins=Int ppitch=Rea7 [sym=FULL|SE]

The geometry card requires a geometry name (GNAME) in the first group, then a geometry type (GTYPE) which is ASSM here indicating an assembly geometry. The remaining arguments have keys: "npins" with an integer value, "ppitch" with a real value, and the optional "sym" with either FULL or $S E$ values (optional arguments are always shown in square brackets: [sym $=\underline{F U L L} \mid S E]$. The default value is underlined: $(\underline{F U L L)}$. The pipe "|" shows an or relation i.e., FULL or $S E$ is an acceptable value ). With the flexible input processing, the following inputs are all valid and identical.

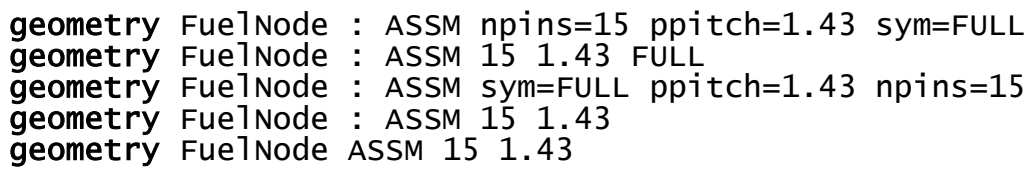


The group separator ":" is suppressed in the last variant. This is possible in any situation where (1) the group is implicitly terminated by running out of arguments or (2) the next type does not match the expected type in the current group. For example, consider the hgap card:

$$
\text { hgap }[d][: M]
$$

In this card, $\mathrm{d}$ and $\mathrm{M}$ are values (without keys) defined as Real and material name (MNAME), respectively. The following form would automatically bypass the Real value, which allows a default, and set the interassembly gap material name as COOL.2.

$$
\text { hgap COOL. } 2
$$

\subsubsection{Setup}

The cards in this section generally appear at the beginning of an input file. Note that the manual is organized with each card starting a new page. This is especially convenient when printing a few cards across different sections. 


\subsubsection{1 title - case title lines}

title Line $_{1}$ Line $_{2} \ldots$ Line $_{\mathrm{i}} \ldots$ Line $_{\mathrm{N}}$

\begin{tabular}{lllll}
\hline param & type & name & details & default \\
\hline Line $_{i}$ & String & line & used in output file headers & "DEFAULT TITLE" \\
\hline
\end{tabular}

\section{Examples:}

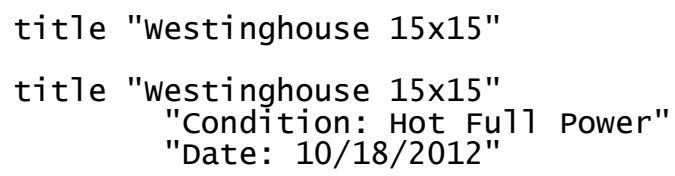

\section{Comments:}

The title card gives a title to this Polaris case, which appears as a descriptive header on the output file. The additional lines may be used to document a subcase or to embed additional information in the output file in an orderly way (e.g., author, date, project identifier).

The title card is optional.

See also:

lib 


\subsubsection{2 library - nuclear data libraries}

lib $[\mathrm{mg}=$ String $]$

\begin{tabular}{lllll}
\hline param & type & name & details & default \\
\hline $\mathrm{mg}$ & String & multigroup library & multigroup cross section library & "fine_n" \\
\hline
\end{tabular}

\section{Examples:}

$\%$ a name of library in the DATA directory

$\%$ use SCALE 252g ENDF/B-VII.1 1ibrary

1ib "fine_n" \% SAME AS "v7-252"

$\%$ use SCALE $56 \mathrm{~g}$ ENDF/B-VII.1 1ibrary

lib mg="broad_n" \% SAME AS mg="v7-56"

$\%$ a name of a local 1ibrary in the temporary working directory

$\%$ (useful in SAMPLER calculations)

1ib "perturbed_xs_1ibrary"

\% fully specified path

1ib "c: \scale6.2\data\scale. rev04.xn252v7.1"

\section{Comments:}

The lib card specifies the multigroup library location. See SCALE's FileNameAliases.txt file in the installation directory for up-to-date library aliases for the fine and broad group libraries provided in SCALE's data directory. Only the 252-group and the 56-group cross section libraries can be used in Polaris. Full specification of the file path is acceptable, as in the final example shown above.

The lib card is optional.

\section{See also:}

title 


\subsubsection{Geometry}

The highest level structures in the model are named and defined with a geometry card. The general outline for a geometry definition is shown below. Two types of geometry are currently supported, ASSM for pressurized water reactor (PWR) or boiling water reactor (BWR) assemblies with fuel elements in a square-pitch, and REFL for an assembly-adjacent reflector.

geom GNAME : GTYPE arguments

\begin{tabular}{|c|c|c|c|c|}
\hline argument & type & name & details & default \\
\hline GNAME & Word & geometry name & & \\
\hline \multirow[t]{3}{*}{ GTYPE } & - & geometry type & & \\
\hline & ASSM & assembly & see pin \& pinmap & \\
\hline & $R E F L$ & reflector & see slab & \\
\hline arguments & - & remaining arguments & depends on GTYPE & \\
\hline
\end{tabular}

The control element geometry is also enumerated with types, as shown below. To model PWR-type rod cluster control assemblies (RCCAs), the RODLET element type is used in conjunction with pin definitions. In future releases of Polaris, other control element types, such as BWR-type control blades will be supported..

control INAME : ETYPE arguments

\begin{tabular}{llll}
\hline Argument & type & name & details \\
\hline INAME & Word & $\begin{array}{l}\text { control elault } \\
\text { name }\end{array}$ \\
ETYPE & - & $\begin{array}{l}\text { control element type } \\
\text { PWR-type RCCA }\end{array}$ & \\
& RODLET & requires PINIDs & \\
arguments & - & remaining arguments depends on ETYPE & \\
\hline
\end{tabular}

For accurate solutions to lattice physics problems using the method of characteristics (MoC), the input geometry must be subdivided into smaller regions called cells. Although the defaults should be applicable in most cases, the capability to change the number of cells is provided through meshing options appearing at the end of many geometry cards. See the slab, pin, box, and hgap cards for details. 


\subsubsection{1 geometry $<$ ASSM $>-$ assembly}

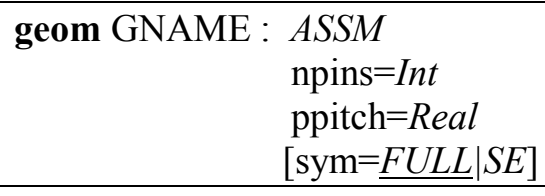

\begin{tabular}{|c|c|c|c|c|}
\hline param & type & name & details & default \\
\hline GNAME & Word & assembly name & & \\
\hline GTYPE & ASSM & & & \\
\hline npins & Int & number of pins & $\begin{array}{l}\text { on each side of } \\
\text { the assembly }\end{array}$ & \\
\hline ppitch & Real & pin pitch & units: cm & \\
\hline sym & FULL|SE & symmetry & $\begin{array}{l}\text { assembly symmetry } \\
\text { FULL: no symmetry } \\
\text { SE: south-east quarter }\end{array}$ & FULL \\
\hline
\end{tabular}

\section{Examples:}

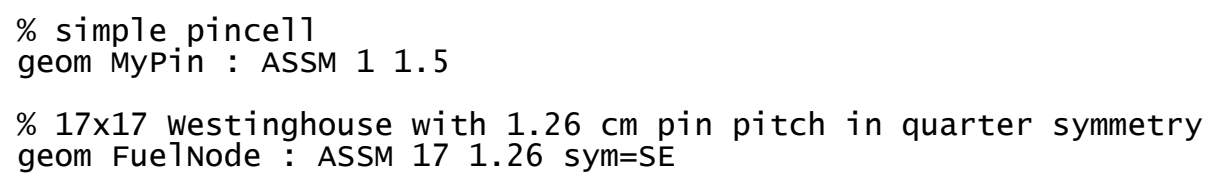

\section{Comments:}

The assembly geometry describes the basic elements of an assembly. The pin and pinmap cards are required to finalize the assembly geometry. The hgap card specifies the interassembly half gap, and the channel specifies the channel material for the assembly.

See also:

pinmap, pin, hgap, box, channel, control, insert 


\subsubsection{2 geometry $<$ REFL $>-$ reflector}

geom GNAME : REFL

thick $=R E A L$

\begin{tabular}{lllll}
\hline param & type & name & details & default \\
\hline GNAME & Word & reflector name & & \\
GTYPE & REFL & & & \\
thick & Real & thickness & units: $\mathrm{cm}$ & \\
\hline
\end{tabular}

\section{Examples:}

$\%$ defines a $20 \mathrm{~cm}$ reflector

geom ReflectorNode : REFL 20.0

\section{Comments:}

The reflector geometry describes the basic elements of a simple slab-type reflector. The slab card can be used to define geometric dimensions and mesh for the reflector geometry.

\section{See also:}

slab 


\subsubsection{3 channel - materials and mesh options}

\section{channel $\left[\mathrm{M}_{\mathrm{chan}}=\mathrm{MCLASS}\right]$}

\begin{tabular}{lllll}
\hline param & type & name & details & default \\
\hline $\mathrm{M}_{\text {chan }}$ & $\mathrm{MCLASS}$ & material class & initializes materials in outermost pin zone & $*$ \\
\hline *By default, $\mathrm{M}_{\text {chan }}$ will be set to COOL by “system PWR" and "system BWR." Otherwise, $\mathrm{M}_{\text {chan }}$ is required.
\end{tabular}

\section{Examples:}

$\%$ define the channel material class to be COOL channel COOL

\section{Comments:}

The channel card is used to set the default channel material class for the outermost region of each pin, typically containing reactor coolant. See the material card for a description of material classes.

\section{See also:}

pin, material, geometry $<$ ASSM $>$ 


\subsubsection{4 hgap - half distance between assemblies}

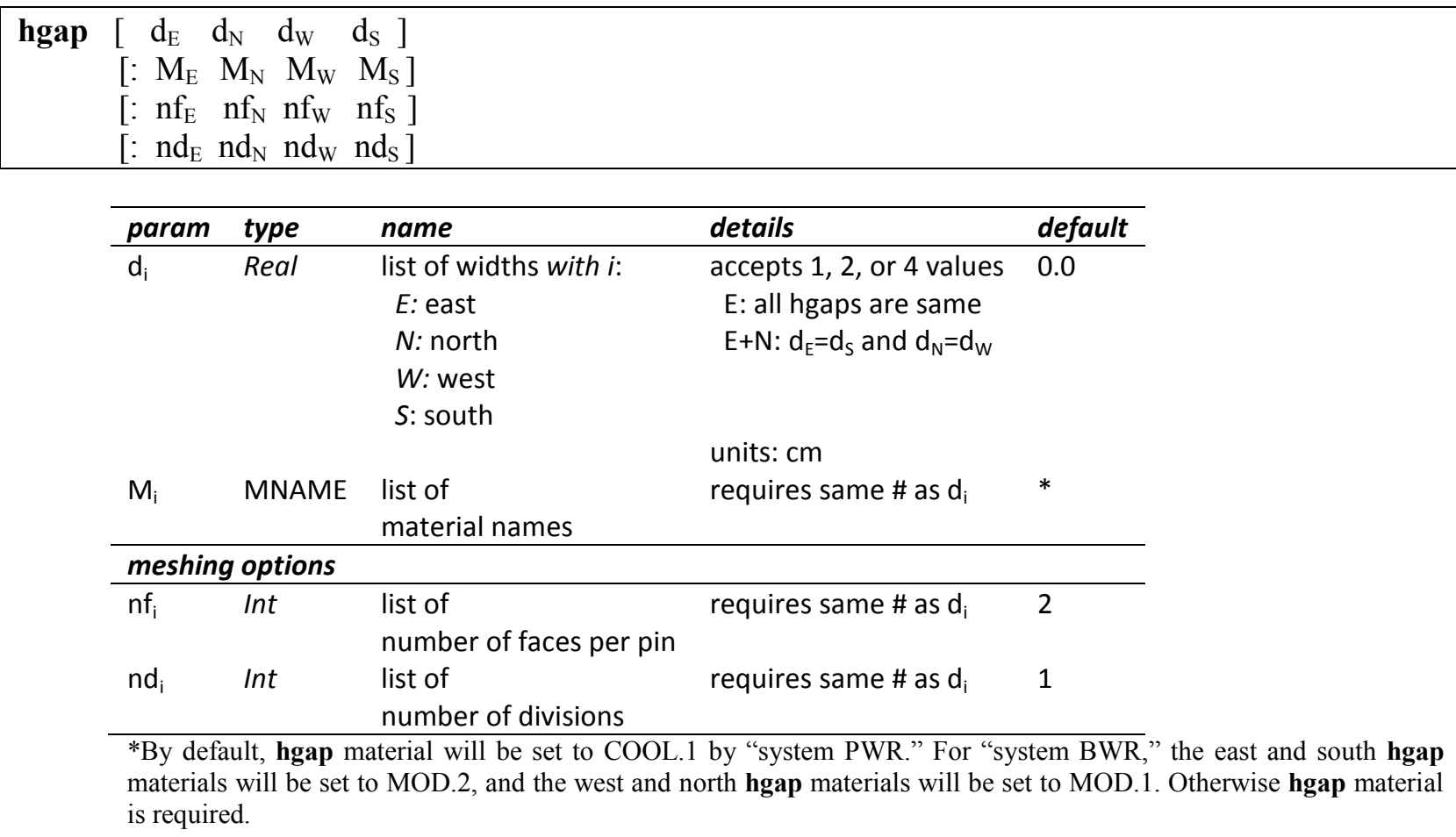

\section{Examples:}

$\%$ defines a $17 \times 17$ westinghouse assembly with $1.26 \mathrm{~cm}$ pin pitch

$\%$ with $0.04 \mathrm{~cm}$ half-gap filled with material COOL.1

geom w17x17 : ASSM 171.26 sym=SE

hgap 0.04 COOL. 1

$\%$ defines a GE $7 \times 7$ assembly with $1.88 \mathrm{~cm}$ pin pitch

$\% \quad 0.48 \mathrm{~cm}$ narrow gap on east and south edge

$\% \quad 0.95 \mathrm{~cm}$ wide gap on north and west edge

$\%$ narrow gap mesh is 3

$\%$ wide gap mesh is 4

\% faces per pin is 2 for both narrow and wide gap

geom ge7x7 : ASSM 71.88

hgap 0.480 .95 : MOD.1 MOD. $1: 22: 34$

\section{Comments:}

The hgap specifies the outermost geometry region in an assembly. If a channel box exists, then hgap specifies the material and mesh from outer channel box edge to the problem boundary. Otherwise, hgap specifies the material and mesh from the edge of the fuel array to the problem boundary. In both cases, hgap represents the half-distance between adjacent assemblies for single assembly calculations. Fig. 3.2.1 shows some of the hgap meshing options. Referring to the south edge of the assembly, the number of faces per pin refers to the extra cells introduced by "splitting" the pin cell boundary, and the number of divisions refers to extra horizontal lines dividing half gap into smaller width cells.

\section{See also:}

pinmap, control, insert, geometry<ASSM $>$, channel, box 


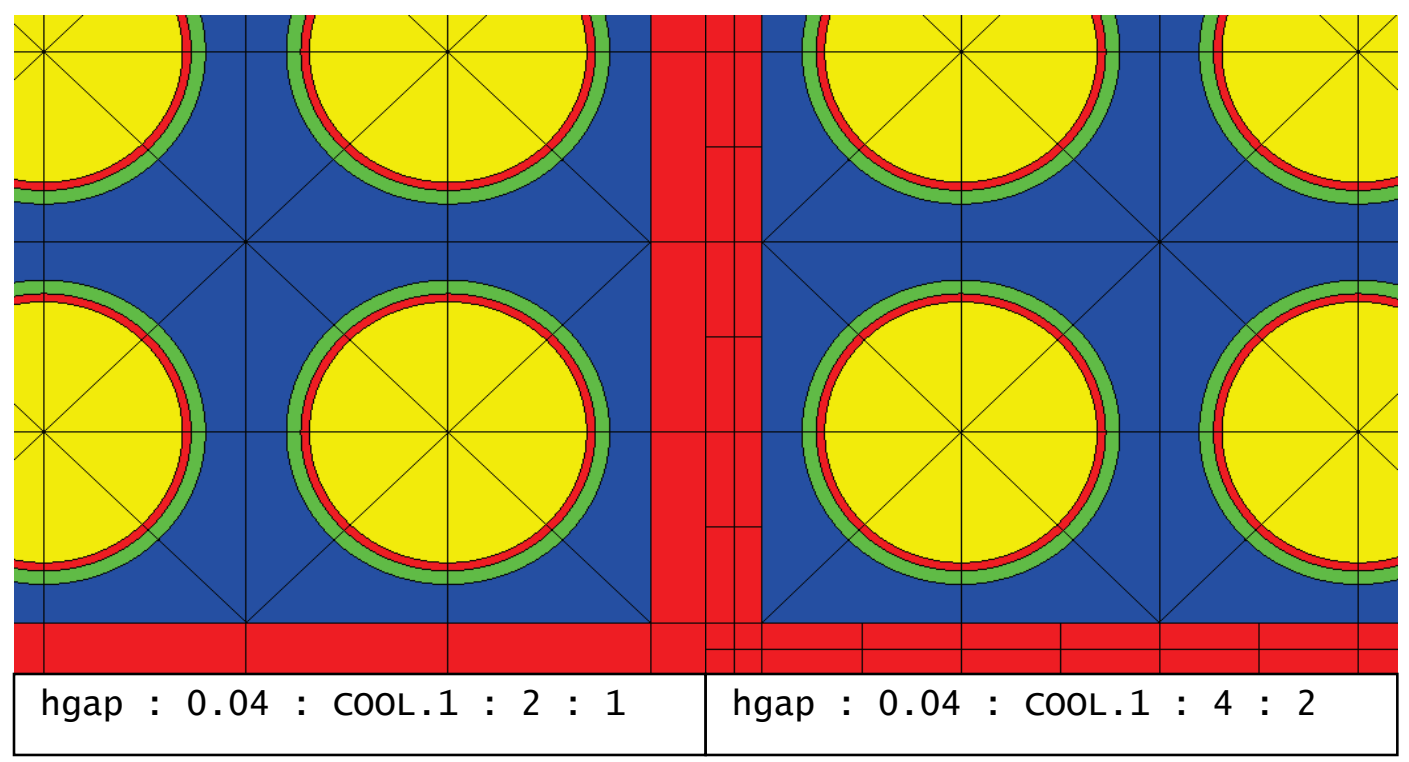

Fig. 3.2.1. Interassembly half gap meshing variants. 


\subsubsection{5 box -channel box}

box $[$ thick=Real $][\mathrm{rad}=$ Real $][\mathrm{icdist}=$ Real $][\mathrm{xrad}=$ Real $][\mathrm{xlen}=$ Real $][\mathrm{Mbox}=\mathrm{MNAME}]$

\begin{tabular}{|c|c|c|c|c|}
\hline param & type & name & details & default \\
\hline thick & Real & nominal thickness $(\mathrm{cm})$ & must be $>0$ & none \\
\hline $\mathrm{rad}$ & Real & inner corner radius $(\mathrm{cm})$ & $\begin{array}{l}\text { must be }>0 \\
\text { additional constraints listed below }\end{array}$ & none \\
\hline icdist & Real & in-channel distance $(\mathrm{cm})$ & must be $>0$ & none \\
\hline xrad & Real & extra corner thickness $(\mathrm{cm})$ & must be $>=0$ & 0 \\
\hline xlen & Real & extra corner length $(\mathrm{cm})$ & excludes rounded corner length & 0 \\
\hline$M_{\text {box }}$ & MNAME & box material & & $*$ \\
\hline
\end{tabular}

*By default, box material will be set to CAN.1 by "system BWR." Otherwise box material is required.

\section{Examples:}

$\%$ GE $7 \times 7$ assembly with $1.88 \mathrm{~cm}$ pin pitch

$\% \quad 0.48 \mathrm{~cm}$ narrow gap

$\% \quad 0.95 \mathrm{~cm}$ wide gap

system BWR

geom ge $7 \times 7$ : ASSM 71.88

hgap 0.480 .95

$\%$ Box geometry
$\% \quad 0.2$ thickness
$\% \quad 0.97$ inner corner radius
$\% \quad 0.14$ in-channel distance
box 0.20 .970 .14

\% same example, all variables

box $0.20 .97 \quad 0.14 \quad 0 \quad 0$ CAN. 1

\section{Comments:}

The box specifies the channel box geometry and material that surround the array of fuel pins.

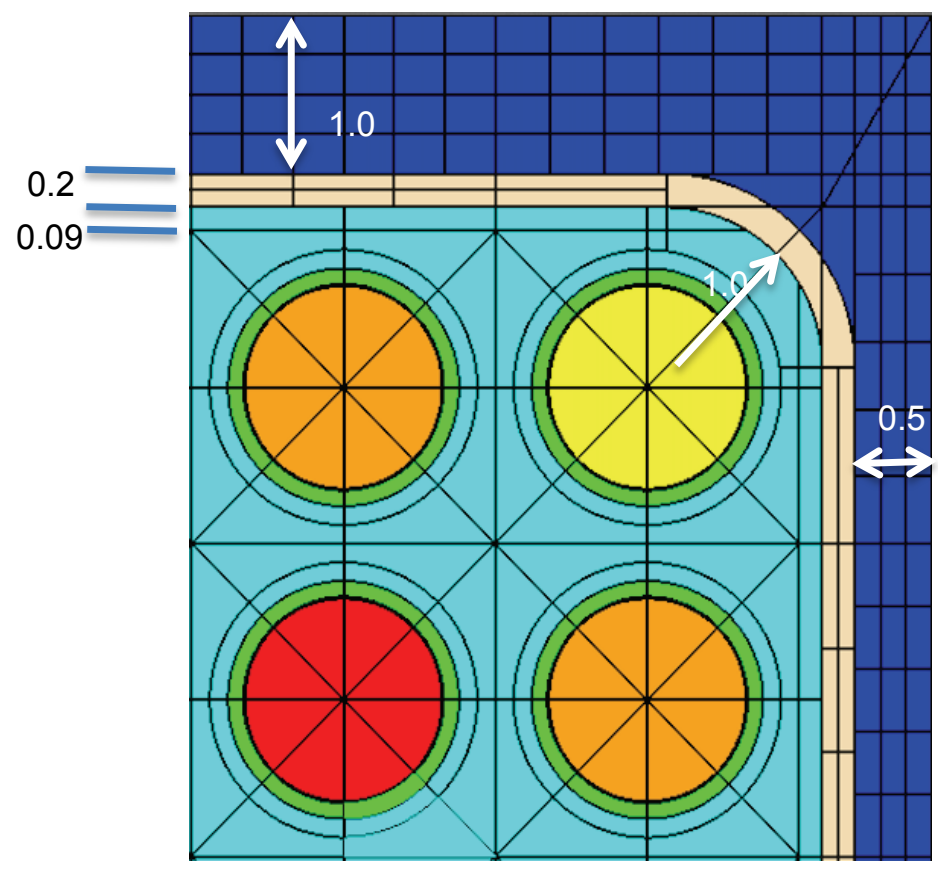


Fig. 3.2.2 and Fig. 3.2.3 show different box geometries, with and without thick corners respectively. See also:

\section{hgap}

geom ge $7 \times 7$ : ASSM 71.88

hgap 0.51 .0

: MOD.1 MOD.1

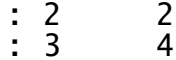

box 0.21 .00 .09

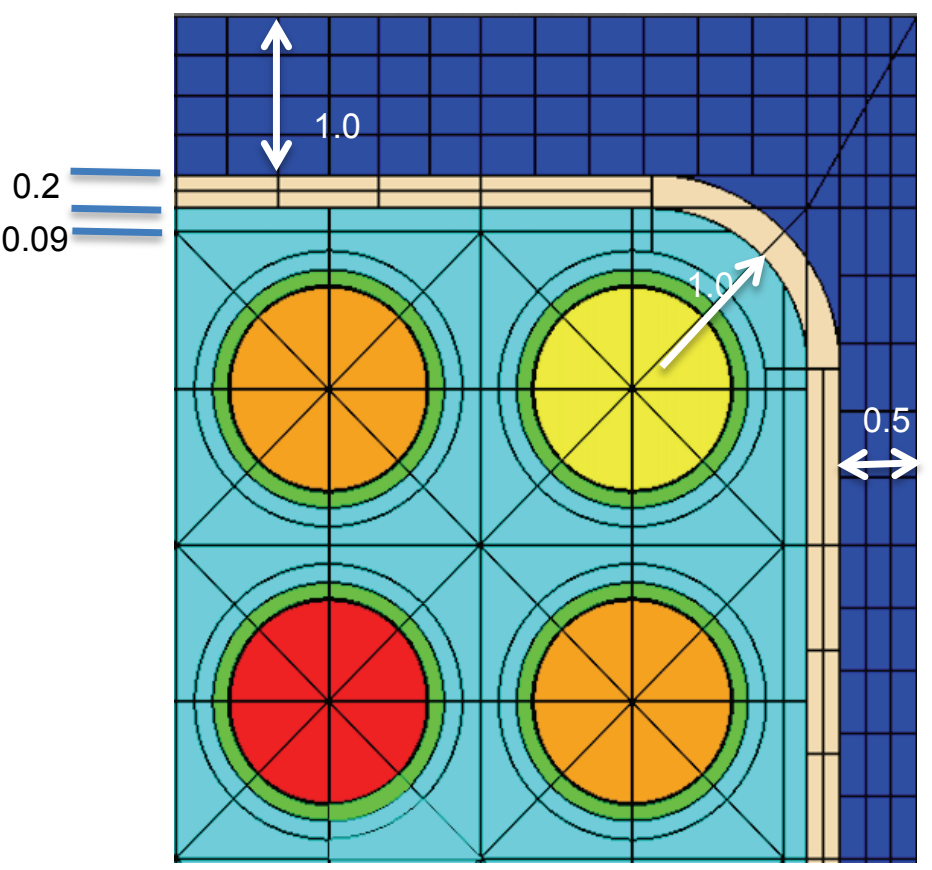

Fig. 3.2.2. Box geometry example (uniform thickness).

geom bwr10x10 : ASSM 101.3

hgap $0.51 .0: 22: 34$

box thick $=0.2 \mathrm{rad}=0.9$

icdist $=0.2$ xrad $=0.1$

$\mathrm{x}$ len $=3.5$

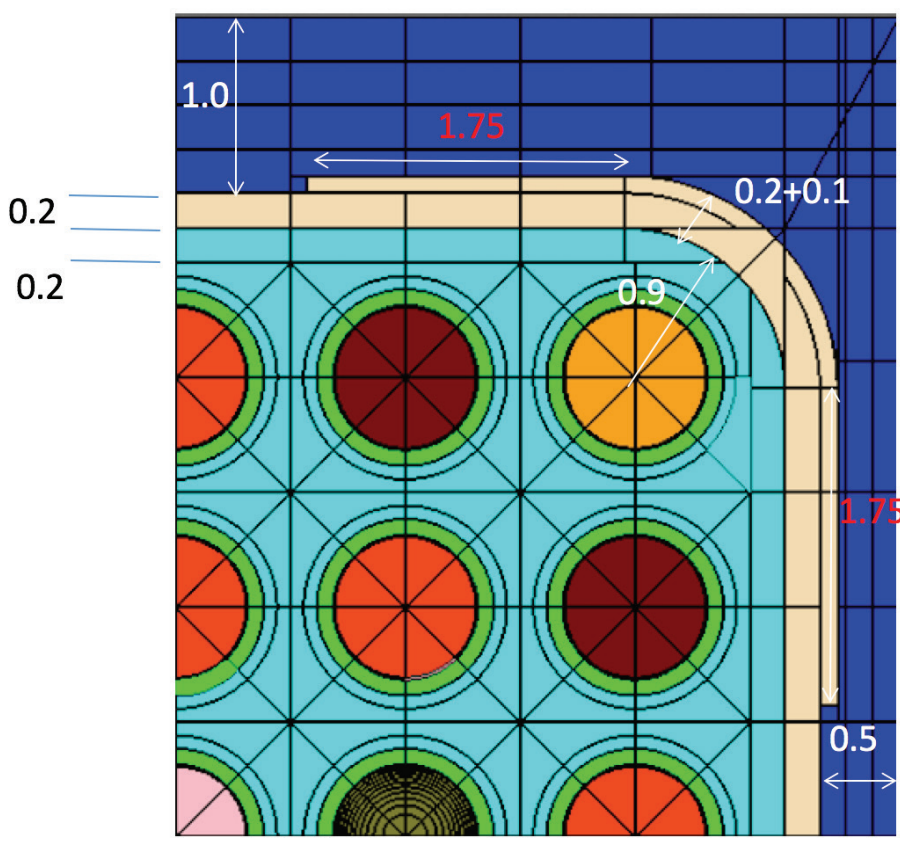

Fig. 3.2.3. Box geometry example (thick corners). 


\subsubsection{6 pin - pin or pincell}

\begin{tabular}{|c|c|c|c|c|}
\hline \multicolumn{5}{|c|}{ 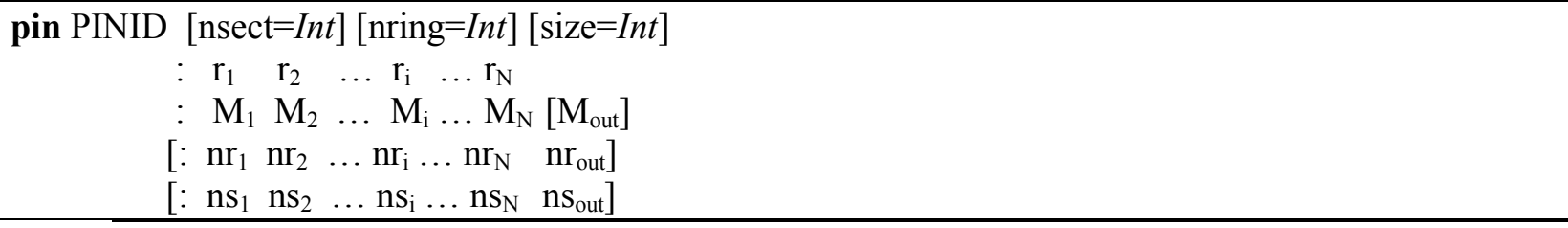 } \\
\hline param & type & name & details & default \\
\hline PINID & Word|Int & pin identifier & & \\
\hline size & Int & $\begin{array}{l}\text { pin will be placed in a } \\
\text { size } x \text { size pincell grid }\end{array}$ & $\begin{array}{l}\text { used to create large water rods in CE PWRs and } \\
\text { GE BWRs (see pinmap) }\end{array}$ & \\
\hline$r_{i}$ & Real & $\begin{array}{l}\text { list of } \\
\text { radii }\end{array}$ & $\begin{array}{l}\text { for each radial zone from center out } \\
\text { units: } \mathrm{cm}\end{array}$ & \\
\hline$M_{i}$ & MNAME & $\begin{array}{l}\text { list of } \\
\text { pin materials }\end{array}$ & $\begin{array}{l}\text { material in each radial zone } \\
.1 \text { added if given MCLASS, e.g., FUEL } \rightarrow \text { FUEL.1 }\end{array}$ & \\
\hline$M_{\text {out }}$ & MNAME & $\begin{array}{l}\text { material } \\
\text { in outermost zone }\end{array}$ & $\begin{array}{l}\text { material in outermost zone } \\
.1 \text { added if given MCLASS, e.g., FUEL } \rightarrow \text { FUEL. } 1\end{array}$ & $*$ \\
\hline \multicolumn{5}{|c|}{ meshing options } \\
\hline nsect & Int & number of sectors & $\begin{array}{l}\text { azimuthal sections of the pin cell, value applies } \\
\text { to all } n s_{i} \text { and } n s_{\text {out }}\end{array}$ & \\
\hline nring & Int & number of rings & value applies to all $n r_{i}$ and $n r_{\text {out }}$ & \\
\hline $\mathrm{nr}_{\mathrm{i}}$ & Int & $\begin{array}{l}\text { list of } \\
\text { number of rings }\end{array}$ & total rings in each radial zone & 1 \\
\hline $\mathrm{nr}_{\text {out }}$ & Int & $\begin{array}{l}\text { number of rings } \\
\text { in outermost zone }\end{array}$ & $\begin{array}{l}\text { because the outermost zone is not a cylindrical } \\
\text { region, the default number of rings is } 0 \text {, and a } \\
\text { value }>0 \text { introduces extra rings in this zone }\end{array}$ & 0 \\
\hline $\mathrm{ns}_{\mathrm{i}}$ & Int & number of sectors & number of sectors in each radial zone & 1 \\
\hline $\mathrm{ns}_{\text {out }}$ & Int & $\begin{array}{l}\text { number of sectors } \\
\text { in outermost zone }\end{array}$ & number of sectors in the outermost zone & 1 \\
\hline
\end{tabular}

*If not specified, the material class MCLASS is taken from the channel card $\left(\mathrm{M}_{\text {chan }}\right)$ and set to the first member of that class, " $\mathrm{M}_{\text {chan. }} 1$. ." For example if $\mathrm{M}_{\text {chan }}=$ "COOL," then $\mathrm{M}_{\text {out }}=$ "COOL.1."

\section{Examples:}

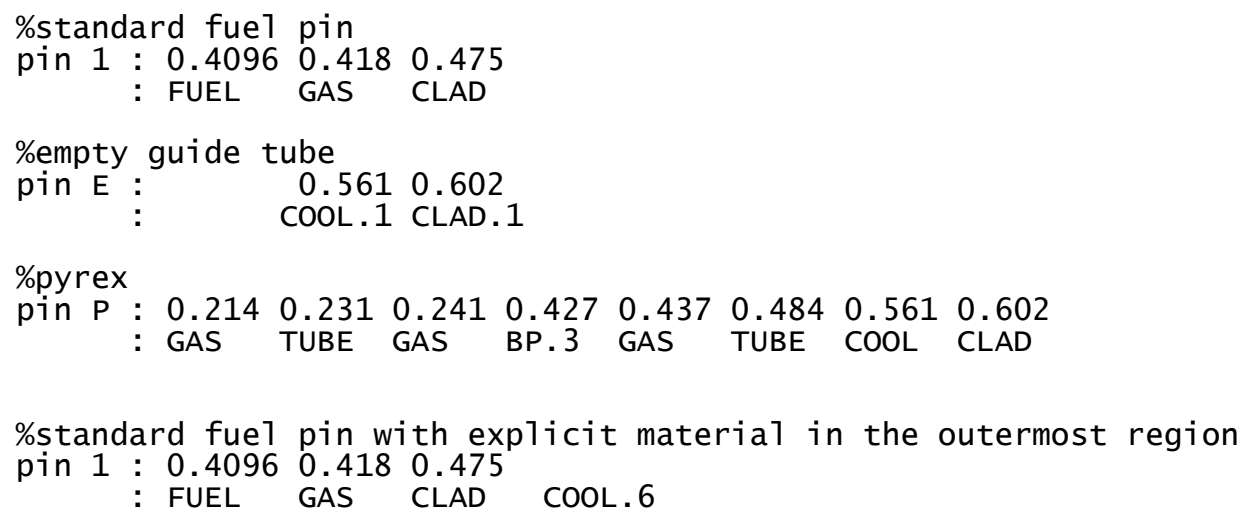




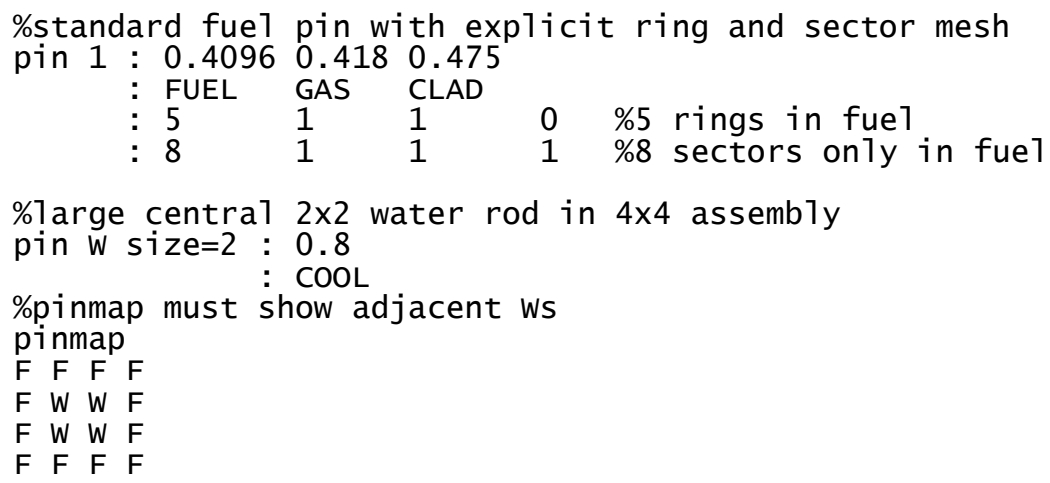

\section{Comments:}

The pin card is one of the basic building blocks of the assembly model. It is the only geometry component which allows an integer (Int) identifier as well as a Word-all other geometric identifiers use Word. Note that the materials are required, except for the last $\mathrm{M}_{\text {out }}$, which can be used to overwrite the material given by a channel for the outermost region in the pincell. The various pin cell meshing options are displayed in

Fig. 3.2.4. Note that extra rings in the radial zones create equal area regions, whereas extra rings in the outermost region create equal distance divisions between the last radius and the pincell boundary. Extra sectors create additional azimuthal divisions.

The total number of cells used in the transport calculation is determined from both the number of rings and the number of sectors. With the MoC transport solver, the fidelity of the solution is also dictated by the number of azimuthal and polar angles and ray spacing. These parameters are changed on the option $<$ KEFF $>$ card.

Due to self-shielding and depletion, each cell could be modeled as a unique material with its own cross section data. However, this is prohibitively memory intensive and typically not necessary. The shield card provides the mechanism to control the additional self-shielded materials introduced.

\section{See also:}

pinmap, control, insert, channel, system, option $<$ KEFF $>$, shield 

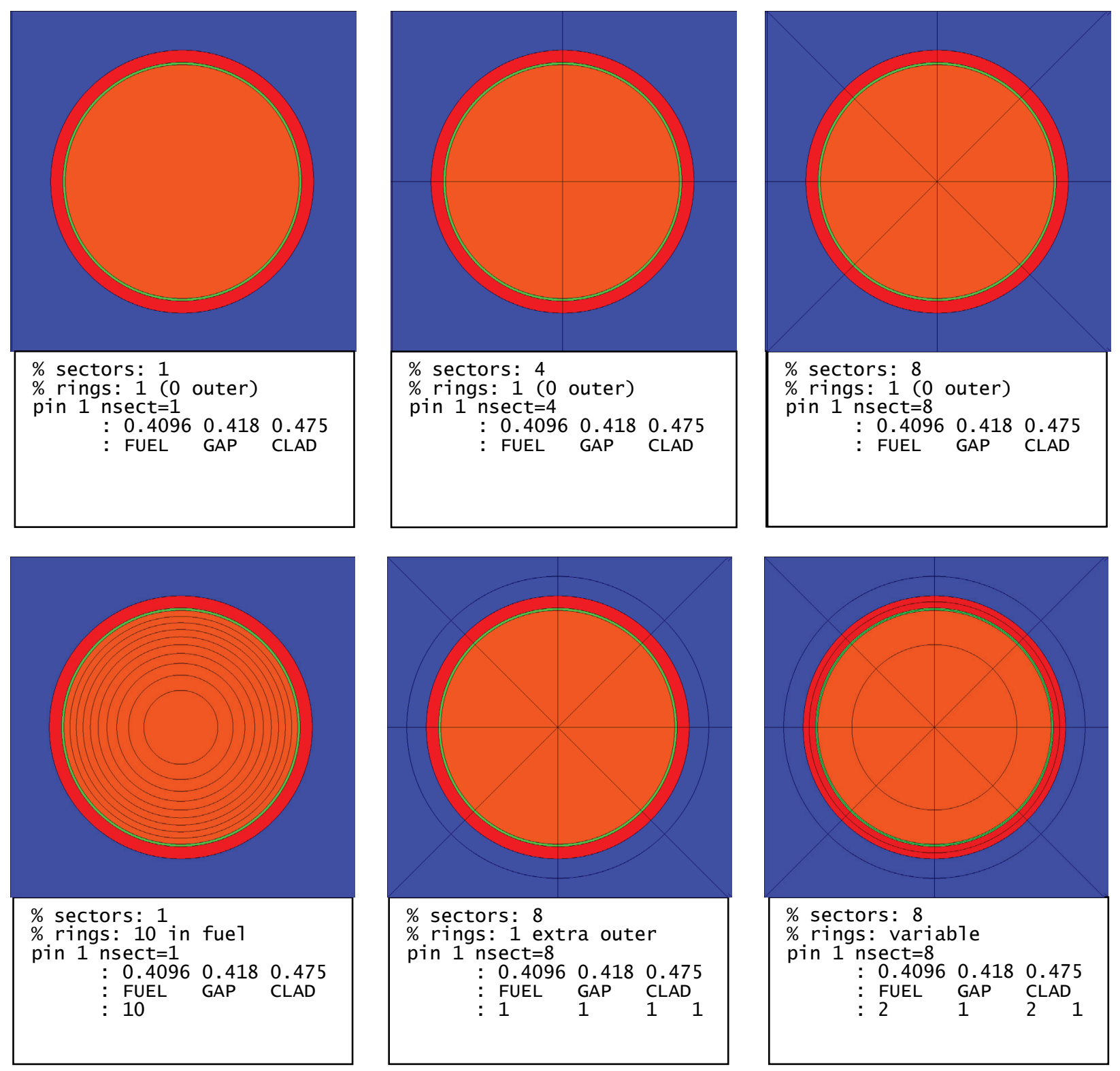

Fig. 3.2.4. Pincell meshing variants. 


\subsubsection{7 pinmap - pin layout}

\begin{tabular}{|c|c|c|c|c|}
\hline \multicolumn{5}{|c|}{ 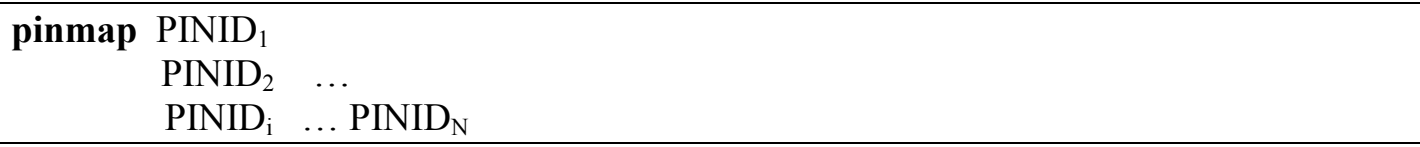 } \\
\hline param & type & name & details & default \\
\hline PINID $_{\mathrm{i}}$ & Word|Int & $\begin{array}{l}\text { list of } \\
\text { pin identifiers }\end{array}$ & $\begin{array}{l}\text { supports full, quarter, or octant symmetry } \\
\text { quarter: assumes southeast (SE) } \\
\text { octant: assumes south-by-southeast (SSE) }\end{array}$ & \\
\hline
\end{tabular}

\section{Examples:}

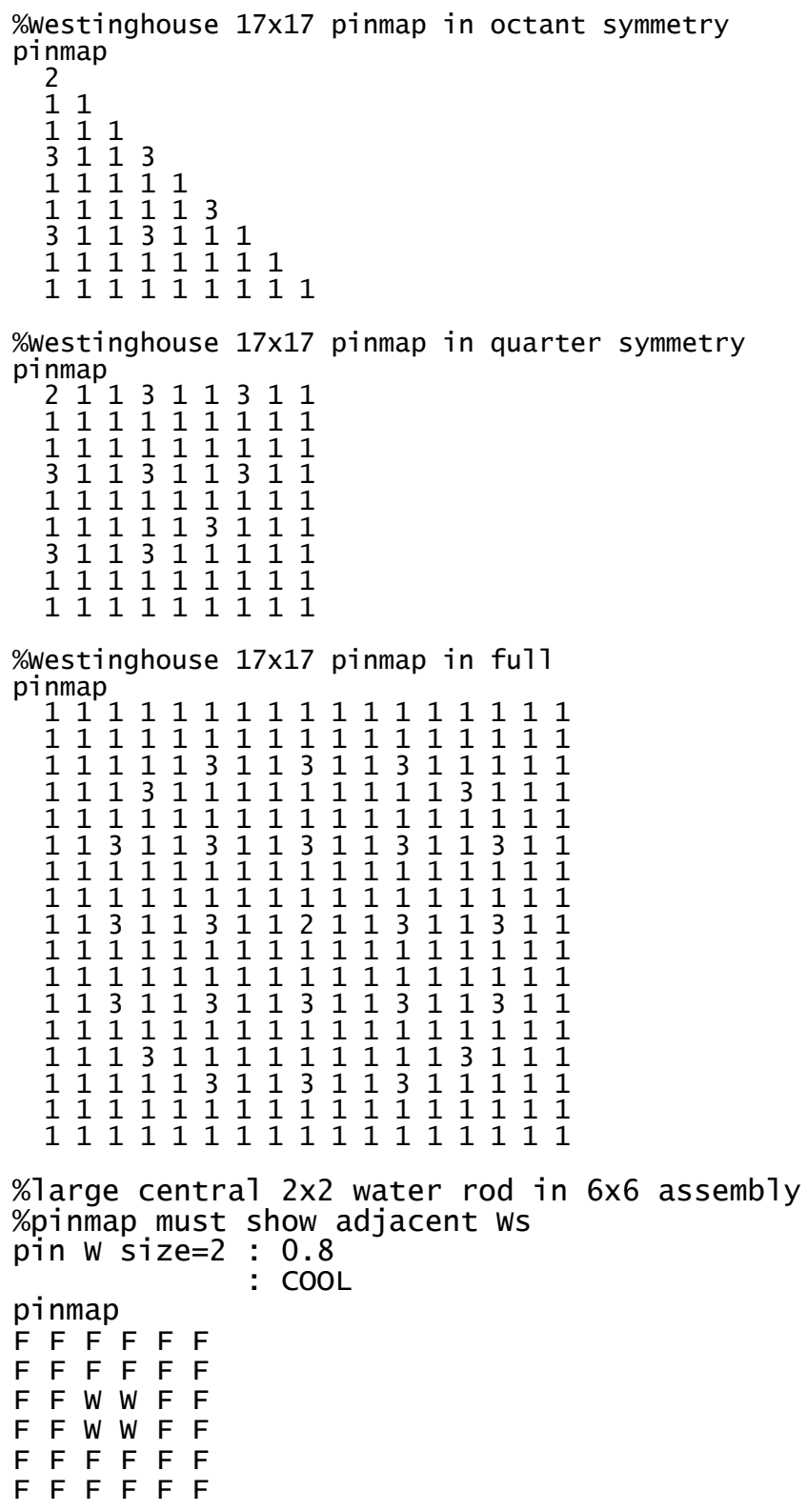




\section{Comments:}

The pinmap card defines the layout of pin cells in the assembly. The symmetry is determined by the number of pin identifiers given on the card and must not be more general than the symmetry option given on the assembly geometry card (i.e., do not define a full pin map for a $s y m=$ SE assembly model).. If the pin has a large size specifier, size $>1$, then the pinmap must reflect that with those pins occurring in blocks of size $\times$ size.

See also:

pin, control, insert 


\subsubsection{8 control $<$ RODLET $>-$ RCCA-type layout}

\begin{tabular}{|c|c|c|c|c|}
\hline $\begin{array}{r}\text { control INAME } \\
\text { PINII } \\
\text { PINII } \\
\text { PINII }\end{array}$ & 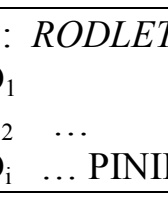 & & & \\
\hline param & type & name & details & default \\
\hline $\begin{array}{l}\text { INAME } \\
\text { FTYPF }\end{array}$ & Word & insert name & & \\
\hline $\begin{array}{l}\text { PIYPE } \\
\text { PINID }_{\mathrm{i}}\end{array}$ & Word|Int & $\begin{array}{l}\text { list of } \\
\text { pin identifiers }\end{array}$ & $\begin{array}{l}\text { same format as pinmap } \\
\text { "_" indicate empty locations }\end{array}$ & \\
\hline
\end{tabular}

\section{Examples:}

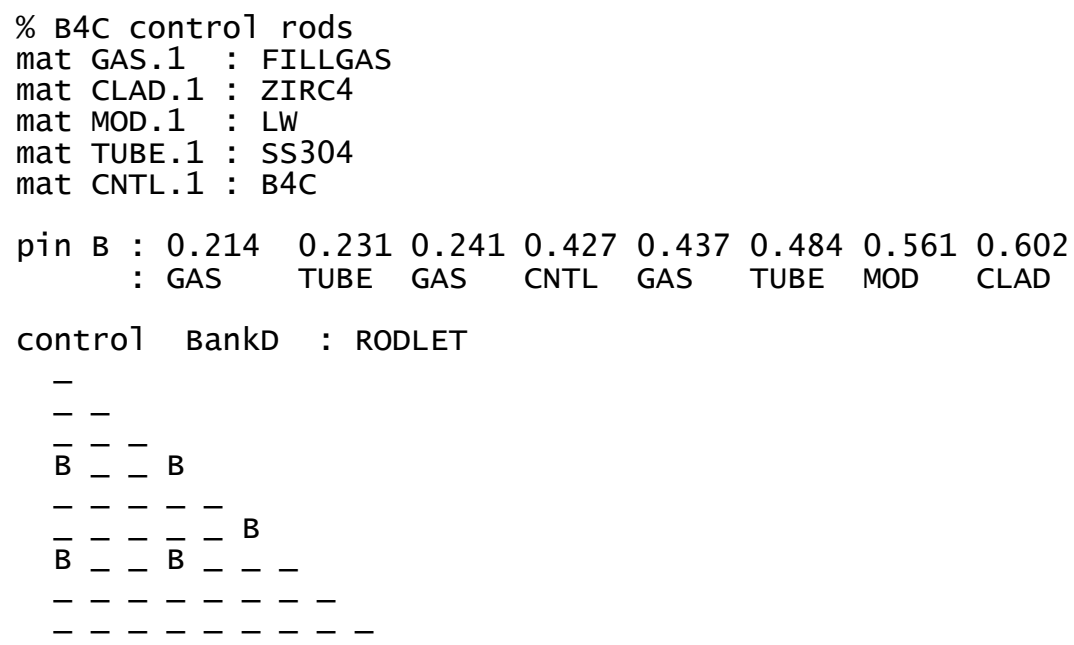

\section{Comments:}

Note that control elements and inserts share the INAME identifiers, so an insert and a control element cannot have the same name. Different control rod banks may be included in a single input file using more than one control card with unique INAMEs. The main difference between the inserts defined by control element and insert cards is that by default, control element materials are not depleted, whereas insert materials are depleted.

The outer dimensions of the tube must be included in the pin card that is inserted.

\section{See also:}

pinmap, control, insert, state 


\subsubsection{9 insert - insert layout}

\begin{tabular}{|c|c|c|c|c|}
\hline $\begin{array}{r}\text { insert INAME : } \\
\text { PINII } \\
\text { PINIL } \\
\text { PINII }\end{array}$ & $\begin{array}{ll}\partial_{1} & \\
\partial_{2} & \ldots \\
\partial_{i} & \ldots \\
\end{array}$ & & & \\
\hline param & type & name & details & default \\
\hline INAME & Word & insert name & & \\
\hline PINID $_{i}$ & Word|Int & $\begin{array}{l}\text { list of } \\
\text { pin identifiers }\end{array}$ & $\begin{array}{l}\text { same format as pinmap } \\
\text { "_" indicate empty locations }\end{array}$ & \\
\hline
\end{tabular}

\section{Examples:}

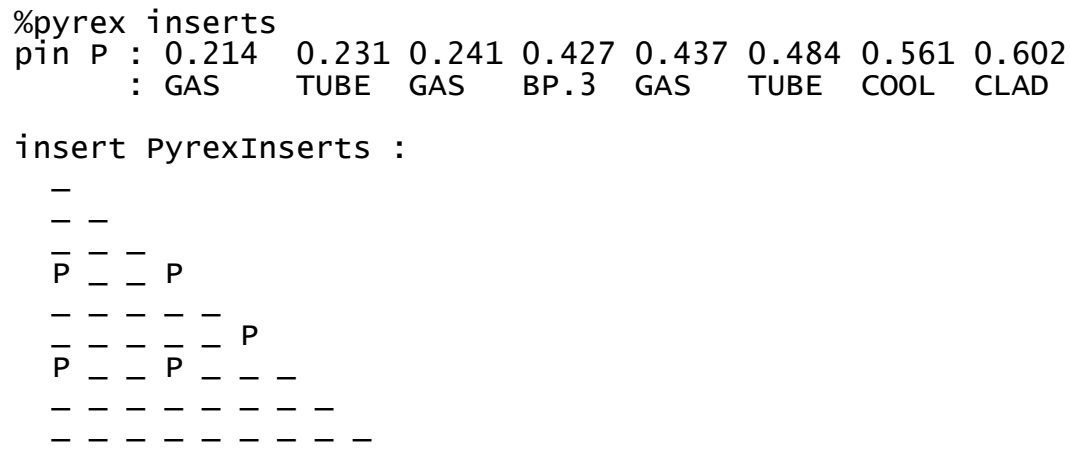

\section{Comments:}

The insert card defines a set of pins to be used to model inserts such as WABA. When the insert is "in," the insert pins replace overlapping regions of the pins defined on the assembly pinmap. An underscore $(\subset)$ is used to indicate locations without inserts. See the notes on the control<RODLET $>$ card for additional guidelines.

See also:

pinmap, control, insert 


\subsubsection{0 slab - slab geometry}

\begin{tabular}{|c|c|c|c|c|}
\hline \multicolumn{5}{|l|}{ slab [SLABID] } \\
\hline \multicolumn{5}{|c|}{$\left.\begin{array}{ccccccc}: & \mathrm{t}_{1} & \mathrm{t}_{2} & \ldots & \mathrm{t}_{\mathrm{i}} & \ldots & \mathrm{t}_{\mathrm{N}} \\
: & \mathrm{M}_{1} & \mathrm{M}_{2} & \ldots & \mathrm{M}_{\mathrm{i}} & \ldots & \mathrm{M}_{\mathrm{N}} \\
{[:} & \mathrm{nx}_{1} & \mathrm{nx}_{2} & \ldots & \mathrm{nx}_{\mathrm{i}} & \ldots & \mathrm{nx}_{\mathrm{N}} \\
{[:} & \mathrm{ny}_{1} & \mathrm{ny}_{2} & \ldots & \mathrm{ny}_{\mathrm{i}} & \ldots & \mathrm{ny}_{\mathrm{N}}\end{array}\right]$} \\
\hline param & type & name & details & default \\
\hline $\begin{array}{l}\text { SLABID } \\
t_{i}\end{array}$ & $\begin{array}{l}\text { Word } \\
\text { Real }\end{array}$ & $\begin{array}{l}\text { slab geometry identifier } \\
\text { list of }\end{array}$ & & reflector GNAME \\
\hline$M_{i}$ & MNAME & $\begin{array}{l}\text { slab thicknesses } \\
\text { list of } \\
\text { material names }\end{array}$ & units: $\mathrm{cm}$ & \\
\hline \multicolumn{5}{|c|}{ meshing options } \\
\hline$n x_{i}$ & Int & $\begin{array}{l}\text { list of } \\
\text { number of } x \text {-divisions }\end{array}$ & & 1 \\
\hline$n y_{i}$ & Int & $\begin{array}{l}\text { list of } \\
\text { number of } y \text {-divisions }\end{array}$ & & 1 \\
\hline
\end{tabular}

\section{Examples:}

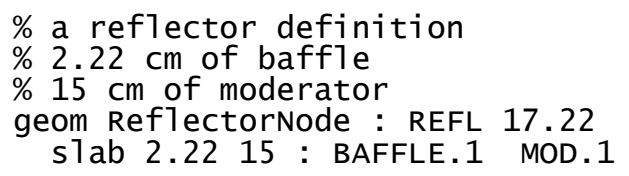

\section{Comments:}

The slab card may be used to define three things: (1) the materials and thicknesses of a reflector initiated on a geometry card, (2) slabs in a control blade, and (3) spacer grids. If the first argument identifier is not present, then the first purpose of describing the various material thicknesses in a reflector is assumed. The meshing options allow each material slab to be spatially refined in $\mathrm{x}$ and $\mathrm{y}$, increasing the number of cells in the transport problem. The meshing option for the number of $\mathrm{x}$ divisions creates the equivalent of additional "sub-slabs" in each user-defined slab thickness. The y-divisions create additional cells vertically. The default of one y-division corresponds to the entire assembly.

See also:

geometry $<$ REFL $>$ 


\subsubsection{Materials}

A material contains two main types of information: (1) the composition, or distribution of nuclides, and (2) the properties which include basic (required) properties like density and temperature, and as well as (optional) properties like soluble poison content, void, or grid spacer smearing. The composition is defined by a composition card. The basic specification for a material is shown below.

mat MNAME : CNAME [dens=Real] [temp=Real] [: properties]

\begin{tabular}{|c|c|c|c|c|}
\hline argument & type & name & details & default \\
\hline MNAME $=$ & Word.Int & material name & used to reference & \\
\hline MCLASS.MSUB & & & this material & \\
\hline CNAME & Word & $\begin{array}{l}\text { composition } \\
\text { name }\end{array}$ & & \\
\hline dens & Real & density & $\begin{array}{l}\text { basic property } \\
\text { units: } \mathrm{g} / \mathrm{cm}^{3}\end{array}$ & $\begin{array}{l}\text { composition } \\
\text { reference } \\
\text { density, if } \\
\text { defined }\end{array}$ \\
\hline temp & Real & temperature & $\begin{array}{l}\text { basic property } \\
\text { units: } \mathrm{K}\end{array}$ & 293 \\
\hline properties & Word=Value & properties & $\begin{array}{l}\text { extra properties } \\
\text { are defined with } \\
\text { property cards }\end{array}$ & $\begin{array}{l}\text { no extra } \\
\text { properties }\end{array}$ \\
\hline
\end{tabular}

A material name has two parts, the material class, or MCLASS, and a member identifier, or MSUB. For example, FUEL. 2 has an MCLASS=FUEL and an MSUB=2. All properties are defined by MCLASS. The composition referenced by CNAME is created with a composition card, as shown below.

comp CNAME : CTYPE arguments

\begin{tabular}{|c|c|c|c|c|}
\hline argument & type & Name & details & default \\
\hline CNAME & Word & composition name & $\begin{array}{l}\text { used to reference this } \\
\text { composition later in materials } \\
\text { and property definitions }\end{array}$ & \\
\hline \multirow[t]{9}{*}{ CTYPE } & - & composition type & & \\
\hline & \multicolumn{2}{|c|}{ General Composition Constructors } & & \\
\hline & NUM & number fraction & & \\
\hline & $W T$ & weight fraction & & \\
\hline & FORM & Formula & & \\
\hline & CONC & Concentrations & & \\
\hline & \multicolumn{2}{|c|}{ Reactor Composition Constructors } & & \\
\hline & $L W$ & borated light water & & \\
\hline & UOX & uranium oxide fuel & & \\
\hline arguments & - & remaining arguments & depends on CTYPE & \\
\hline
\end{tabular}


Additional properties are defined with the property card, which defines the property PNAME for a material class MCLASS. The property type, PTYPE, determines the remaining arguments.

prop PNAME MCLASS : PTYPE arguments

\begin{tabular}{lllll}
\hline argument & type & name & details & default \\
\hline PNAME & Word & property name & $\begin{array}{l}\text { used to reference this } \\
\text { property }\end{array}$ \\
\hline PTYPE & PTYPE & $\begin{array}{l}\text { property type } \\
\text { soluble poison }\end{array}$ & $\begin{array}{l}\text { used to define soluble boron } \\
\text { content }\end{array}$ \\
\hline arguments & - & remaining arguments depends on PTYPE \\
\hline
\end{tabular}




\subsubsection{1 material- material}

mat MNAME : CNAME $[$ dens $=$ Real $][$ temp $=$ Real $]$

$\left[\begin{array}{llllll}: \mathrm{p}_{1}=\mathrm{val}_{1} & \mathrm{p}_{2}=\mathrm{val}_{2} & \ldots & \mathrm{p}_{\mathrm{i}}=\mathrm{val}_{\mathrm{i}} & \ldots & \mathrm{p}_{\mathrm{N}}=\mathrm{val}_{\mathrm{N}}\end{array}\right]$

\begin{tabular}{|c|c|c|c|c|}
\hline argument & type & name & details & default \\
\hline MNAME & Word.Int & material name & $\begin{array}{l}\text { uses form } \\
\text { MCLASS.MSUB }\end{array}$ & \\
\hline CNAME & Word & composition name & & \\
\hline dens & Real & density & $\begin{array}{l}\text { basic property } \\
\text { units: } \mathrm{g} / \mathrm{cm}^{3}\end{array}$ & $\begin{array}{l}\text { composition } \\
\text { reference density }\end{array}$ \\
\hline temp & Real & temperature & $\begin{array}{l}\text { basic property } \\
\text { units: } \mathrm{K}\end{array}$ & 293 \\
\hline $\mathrm{p}_{\mathrm{i}}=\mathrm{val}_{\mathrm{i}}$ & PNAME=Value & properties & additional properties & 0 \\
\hline
\end{tabular}

\section{Examples:}

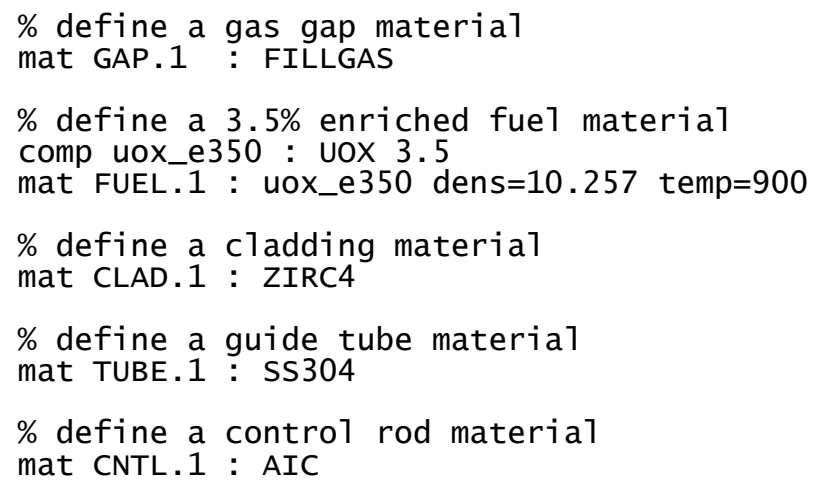

\section{Comments:}

Material properties may be set on either a material card or on a state card. If a temperature is specified rather than a density, the "temp=" key must be used to skip over the density argument.

\section{See also:}

state, composition, property 


\subsubsection{2 composition $<\mathrm{NUM} \mid \mathrm{WT}>-$ general atom/wt fraction}

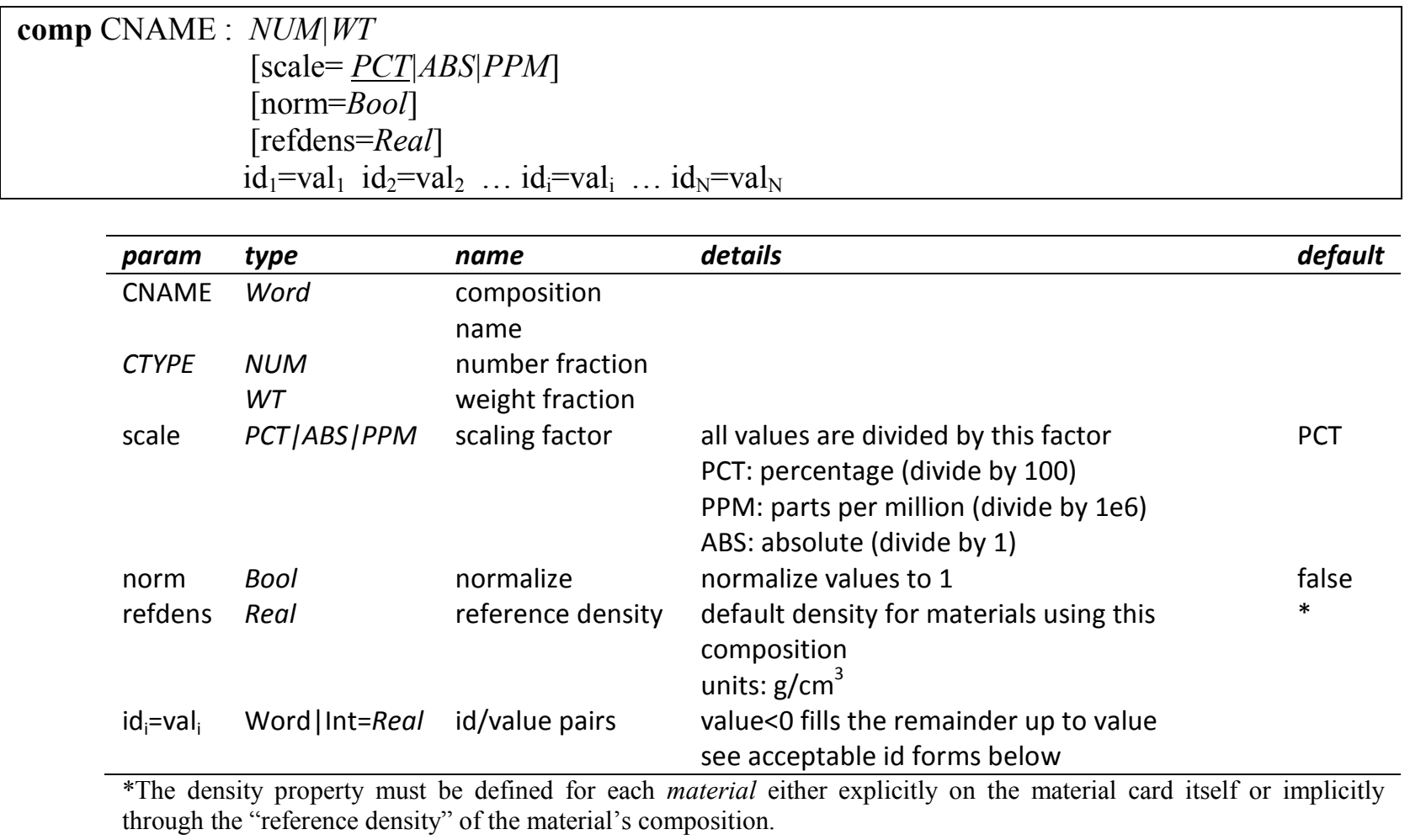

\section{Examples:}

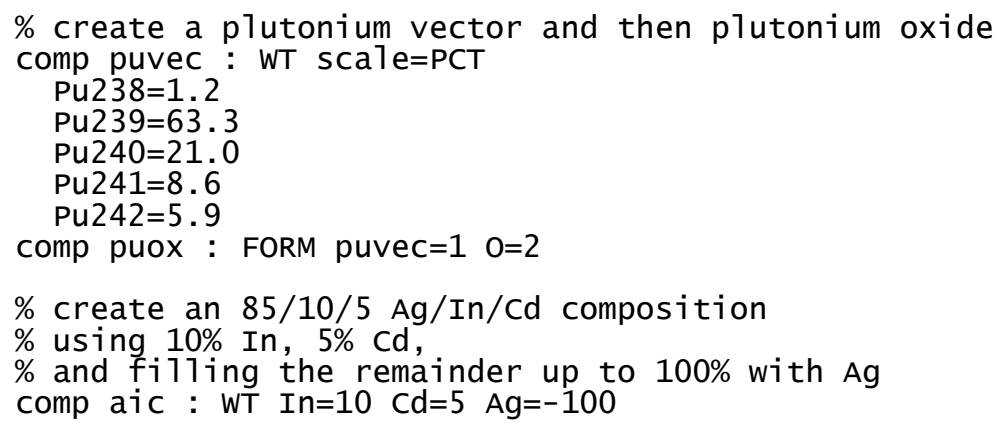

\section{Comments:}

IDs in weight or number fraction-based compositions may be any of the following:

- $\quad$ nuclide IDs (Int), e.g., 92235,

- nuclide names (Word), e.g., U235 or u235,

- $\quad$ element $Z$ numbers (Int), e.g., 92,

- element names (Word), e.g., U or u, or

- other composition names (CNAME).

\section{See also:}

\section{composition <FORM>}




\subsubsection{3 composition $<$ FORM $>-$ general chemical formula}

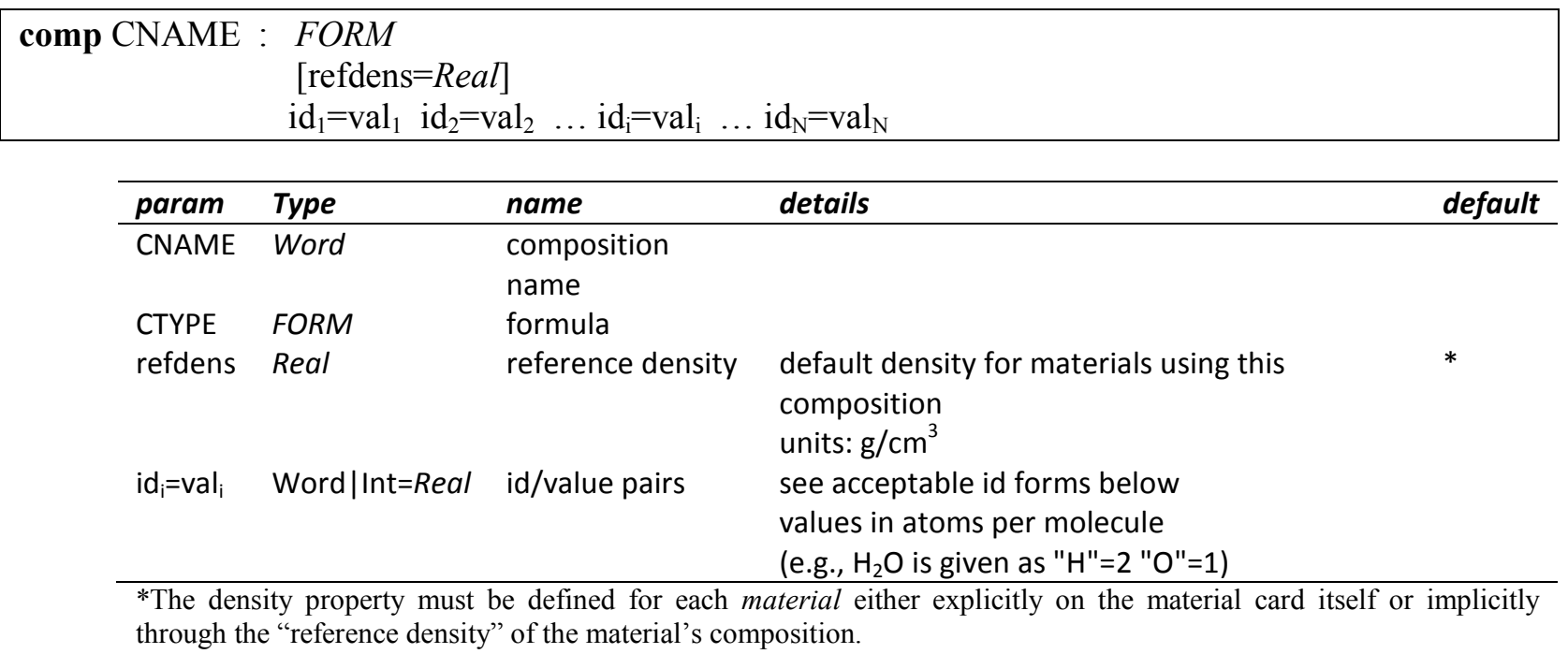

\section{Examples:}

\% define Gd203 using element names

\% (using elements implies natural abundances used in isotopics)

comp gd2o3 : FORM $\mathrm{Gd}=2 \mathrm{O}=3$

\% define Gd203 using 100\% Gd 155

comp gd203 : FORM Gd155=2 $0=3$

\% define D20 using nuclide IDs and element names

comp d2o : FORM 1002=2 8000=3

comp d2O : FORM H2 $=2 \quad \mathrm{O}=3$

\section{Comments:}

IDs in formula-based composition may be any of the following:

- nuclide IDs (Int), e.g., 92235,

- $\quad$ nuclide names (Word), e.g., U235 or u235,

- element $Z$ numbers (Int), e.g., 92,

- element names (Word), e.g., U or u, or

- other composition names (CNAME).

See also:

composition $<\mathrm{NUM} \mid \mathrm{WT}>$, composition $<\mathrm{CONC}>$ 


\subsubsection{4 composition $<$ CONC $>-$ general number density}

comp CNAME : $C O N C$ [refdens $=$ Real] $\mathrm{id}_{1}=\mathrm{val}_{1} \quad \mathrm{id}_{2}=\mathrm{val}_{2} \ldots \mathrm{id}_{\mathrm{i}}=\mathrm{val}_{\mathrm{i}} \ldots \mathrm{id}_{\mathrm{N}}=\mathrm{val}_{\mathrm{N}}$

\begin{tabular}{|c|c|c|c|c|}
\hline param & type & name & details & default \\
\hline CNAME & Word & $\begin{array}{l}\text { composition } \\
\text { name }\end{array}$ & & \\
\hline CTYPE & CONC & concentration & & \\
\hline refdens & Real & reference density & $\begin{array}{l}\text { default density for materials using this composition } \\
\text { units: } \mathrm{g} / \mathrm{cm}^{3}\end{array}$ & $* *$ \\
\hline $\mathrm{id}_{\mathrm{i}}=\mathrm{val}_{\mathrm{i}}$ & Int=Real & id/value pairs & see acceptable id forms below & \\
\hline & & & $\begin{array}{l}\text { note: cannot use other CNAMEs for IDs in CONC } \\
\text { input } \\
\text { units: \#/barn-cm }\end{array}$ & \\
\hline
\end{tabular}

\footnotetext{
**A reference density is automatically calculated from concentrations input. If specified, it will simply scale up concentrations linearly.
}

\section{Examples:}

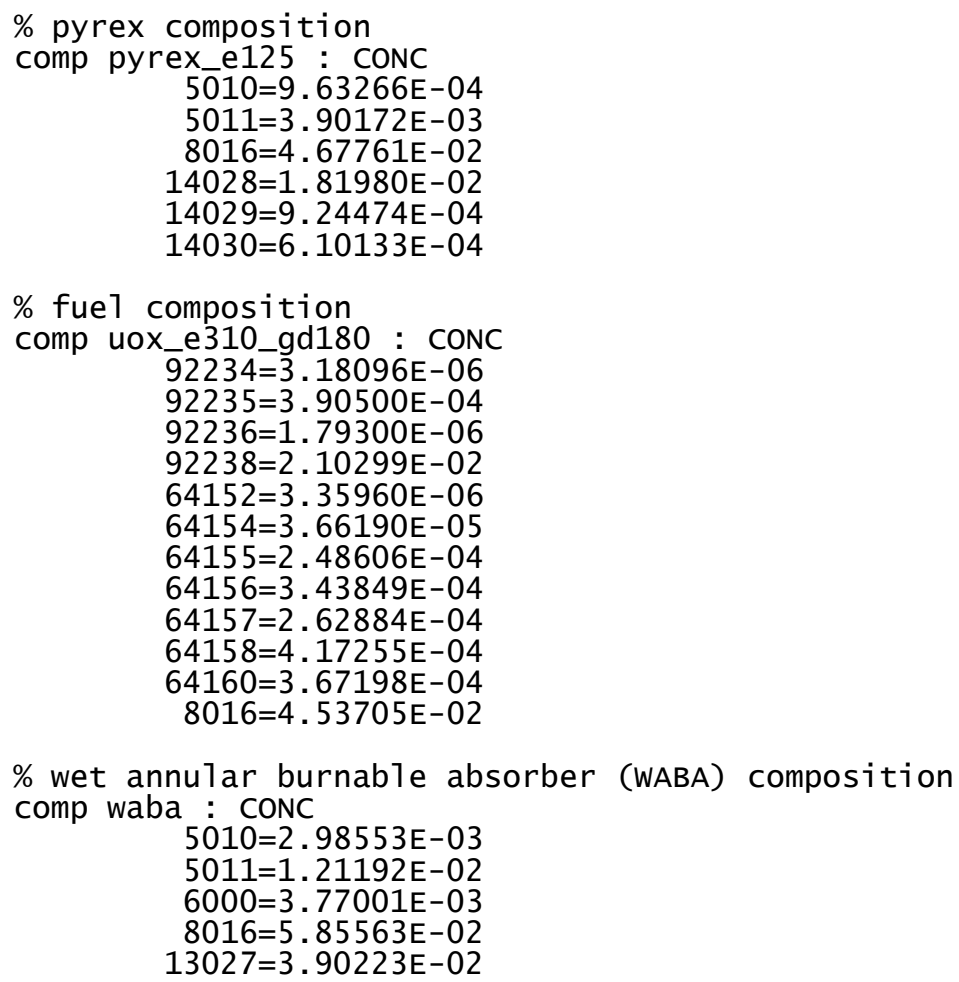

\section{Comments:}

IDs in concentration-based composition may be any of the following:

- $\quad$ nuclide IDs (Int), e.g., 92235,

- nuclide names (Word), e.g., U235 or u235,

- element $Z$ numbers (Int), e.g., 92, and

- element names (Word), e.g., U or u. 
Other composition names (CNAME) cannot be used in a concentration definition. To easily ensure consistency of input when comparing codes, the composition input should be used in the concentrations described here. In all other cases, the other composition constructors are recommended because they are much simpler and easier to use.

See also:

composition $<$ FORM $>$, composition $<$ NUM $\mid$ WT $>$ 


\subsubsection{5 composition $<\mathbf{L W}>-$ borated light water}

comp CNAME : $L W[$ borppm=Real $][$ refdens=Real $]$

\begin{tabular}{|c|c|c|c|c|}
\hline param & Type & name & details & default \\
\hline CNAME & Word & composition name & & \\
\hline CTYPE & $L W$ & light water & & \\
\hline borppm & Real & boron & $\begin{array}{l}\text { parts per million by weight of } \\
\text { natural boron }(B) \text { in } \\
\text { light water }\left(\mathrm{H}_{2} \mathrm{O}\right)\end{array}$ & $*$ \\
\hline refdens & Real & reference density & $\begin{array}{l}\text { default density for materials using this composition } \\
\text { units: } \mathrm{g} / \mathrm{cm}^{3}\end{array}$ & 0.0 \\
\hline
\end{tabular}

\section{Examples:}

$\%$ define a 600ppm boron moderator composition

comp mod_600ppm : LW 600

\% same composition using FORM and WT

comp mod : FORM $\mathrm{H}=2 \mathrm{O}=1$

comp mod_600ppm : WT scale=PPM norm=yes

$\bmod =1$ e $6 \quad B=600$

\section{Comments:}

Internally, the borated light water composition is built from composition<FORM $>$ and composition $<\mathbf{W T}>$ cards assuming natural boron. To use different boron isotopics such as depleted or enriched boron, the more general composition cards should be used.

See also:

composition $<$ FORM $>$, composition $<\mathrm{WT}>$ 


\subsubsection{6 composition $<\mathrm{UOX}>-\mathrm{UO}_{2}$ fuel}

comp CNAME : UOX enr=Real $[\mathrm{bu}=$ Real $][\mathrm{refdens}=$ Real $]$

\begin{tabular}{|c|c|c|c|c|}
\hline param & type & name & details & default \\
\hline CNAME & Word & composition name & & \\
\hline CTYPE & UOX & uranium dioxide & & \\
\hline enr & Real & enrichment & $\begin{array}{l}\text { U-235 wt. \% } \\
\text { (see composition<ENRU> for formula) }\end{array}$ & \\
\hline bu & Real & burnup & $\begin{array}{l}\text { only available for } 0 \leq b u \leq 100 \\
\text { units: GWd/MTU }\end{array}$ & $0 *$ \\
\hline refdens & Real & reference density & $\begin{array}{l}\text { default density for materials using this composition } \\
\text { units: } \mathrm{g} / \mathrm{cm}^{3}\end{array}$ & $* *$ \\
\hline
\end{tabular}

\section{Examples:}

\% define a $4.95 \%$ enriched fuel composition with a reference density comp uox_495: UOX 4.95 refdens $=10.25$

$\%$ same result as above

comp u_495 : ENRU 4.95

comp uox_495 : FORM u_495=1 $0=2$

\section{Comments:}

Internally, the $\mathrm{UO}_{2}$ composition is built from composition $<$ FORM $>$ and composition $<$ ENRU $>$ cards .

\section{See also:}

composition $<$ ENRU $>$, composition $<$ FORM $>$ 


\subsubsection{7 composition $<$ ENRU $>-$ enriched uranium}

comp CNAME : ENRU enr=Real [refdens=Real]

\begin{tabular}{lllll}
\hline param & type & name & details & default \\
\hline CNAME & Word & composition name & & - \\
CTYPE & UOX & uranium dioxide & & - \\
enr & Real & enrichment & $* \mathrm{U}-235 \mathrm{wt.} \%$ & $\begin{array}{l}\text { default density for materials using this composition } \\
\text { refdens }\end{array}$ \\
Real & reference density & $\begin{array}{l}* * \\
\text { units: } \mathrm{g} / \mathrm{cm}^{3}\end{array}$ & \\
\hline
\end{tabular}

*The following formula from [3] is used to determine the ${ }^{234} \mathrm{U}$ and ${ }^{236} \mathrm{U} w \mathrm{t} \%$ from the ${ }^{235} \mathrm{U}$ enrichment. Note that this formula is only valid for U-235 enrichments less than $10 \mathrm{wt} \%$.

$\mathrm{W}_{\mathrm{u} 234}=0.007731 *(\mathrm{enr})^{1.0837}$

$\mathrm{w}_{\mathrm{u} 236}=0.0046 * \mathrm{enr}$

$\mathrm{w}_{\mathrm{u} 238}=100-\mathrm{w}_{\mathrm{u} 234}-\mathrm{enr}-\mathrm{w}_{\mathrm{u} 236}$

**The density property must be defined for each material either explicitly on the material card itself or implicitly through the "reference density" of the material's composition.

\section{Examples:}

$\% 5 \%$ enriched metal fuel

comp umetal : ENRU 5

\section{Comments:}

This composition for enriched uranium is used internally to create $\mathrm{UO}_{2}$ using the composition $<\mathbf{U O X}>$ card.

\section{See also:}

composition $<\mathrm{UOX}>$ 


\subsubsection{8 composition library (pre-defined)}

The Polaris composition library contains predefined compositions that may be used without a constructor by simply referencing the CNAME below. Each predefined library composition has a reference density, so it can be used directly on a material card.

\begin{tabular}{ll} 
& \multicolumn{1}{c}{ Standard molecular compositions } \\
\hline CNAME & Description \\
\hline H2O & light water \\
B4C & Boron carbide burnable poison material \\
ER2O3 & Erbium oxide burnable poison material \\
GD2O3 & Gadolinium oxide burnable poison material \\
FILLGAS & Helium gas \\
\hline \multicolumn{2}{c}{ Standard reactor mixtures and alloys } \\
\hline CNAME & Description \\
\hline AIC & Ag-In-Cd control rod absorber material \\
PYREX & Pyrex glass \\
ZIRC2 & Zircaloy-2 clad material \\
ZIRC4 & Zircaloy-4 clad material \\
SS304 & Stainless Steel 304 \\
SS316 & Stainless Steel 316 \\
INC718 & Inconel 718 \\
WATER & Light water with trace amount of boron \\
\hline
\end{tabular}




\subsubsection{9 property $<$ SOLP $>-$ soluble poison by weight}

prop PNAME M1 ...: SOLP poison $[$ scale $=\underline{P P M}|P C T| A B S]$

\begin{tabular}{|c|c|c|c|c|}
\hline param & Type & name & details & default \\
\hline PNAME & Word & property name & property value $p \geq 0$ & \\
\hline M1 ... & MCLASS & material class & $\begin{array}{l}\text { one or more material classes to gain this } \\
\text { property }\end{array}$ & \\
\hline PTYPE & SOLP & soluble poison & & \\
\hline poison & CNAME & $\begin{array}{l}\text { soluble poison } \\
\text { composition name }\end{array}$ & & \\
\hline scale & $P C T / A B S / P P M$ & scaling factor & $\begin{array}{l}\text { all values are divided by this factor } \\
\text { PCT: percentage (divide by 100) } \\
\text { PPM: parts per million (divide by 1e6) } \\
\text { ABS: absolute (divide by } 1 \text { ) }\end{array}$ & PPM \\
\hline
\end{tabular}

\section{Examples:}

$\%$ define a soluble boron property for moderator

$\%$ and coolant material classes

$\%$ using naturat boron

prop boron MOD COOL : SOLP B

$\%$ investigate coolant crud/impurity activation

$\%$ 1. define a general impurity property to mix in coolant, comp crud : NUM $\mathrm{Ni}=12.7 \mathrm{Cr}=2.3 \mathrm{Fe}=-100$ \%mostly $\mathrm{Fe}$

prop impurity COOL : SOLP crud

$\% 2$. create coolant material with $100 \mathrm{ppm}$ of crud

mat COOL.1 : LW dens $=0.75$ : impurity $=100$

\% 3. make sure to "deplete" coolant so crud gets activated deplete $\mathrm{COOL}=$ true

\section{Comments:}

None

See also:

pinmap, control, insert 


\subsubsection{0 deplete - material depletion and decay}

deplete $\mathrm{M}_{1}=$ Bool $\mathrm{M}_{2}=$ Bool $\ldots \mathrm{M}_{\mathrm{i}}=$ Bool $\ldots \mathrm{M}_{\mathrm{N}}=$ Bool

\begin{tabular}{lllll}
\hline param & type & name & details & default \\
\hline $\mathrm{M}_{\mathrm{i}}$ & MNAME|MCLASS & list of & use ALL for all materials \\
& material & \\
& names or & \\
& material \\
& classes & \\
& &
\end{tabular}

NOTE: Only one deplete card is allowed in an input.

NOTE: ALL only applies in the first position

Examples:

$\%$ turn on depletion/decay for two new materials

sys PWR

deplete MyMaterial=true MyotherMaterial=true

\% activate/deplete/decay every material

deplete $A L L=t r u e$

\% impose strict conditions

sys PWR

deplete $A L L=$ false $F U E L=$ true $C L A D=$ true

\section{Comments:}

The deplete card not only instructs Polaris to deplete a material, but also to solve the Bateman equations with ORIGEN for that material. Thus if the flux/power is zero, only materials that are flagged to "deplete" will undergo decay. The deplete card modifies the depletables included in a system card to avoid the situation in which "deplete MyMaterial=true" would make only MyMaterial depletable. Thus to completely re-specify the depletable materials, "ALL=false" should be used as the first argument. This is in contrast to the basis card, which completely specifies a new power basis.

See also:

material, shield, basis 


\subsubsection{1 basis - power basis materials}

basis $\mathrm{M}_{1}=$ Bool $\mathrm{M}_{2}=$ Bool $\ldots \mathrm{M}_{\mathrm{i}}=$ Bool $\ldots \mathrm{M}_{\mathrm{N}}=$ Bool

\begin{tabular}{lllll}
\hline param & type & name & details & default \\
\hline $\mathrm{M}_{\mathrm{i}}$ & MNAME|MCLASS & $\begin{array}{l}\text { list of } \\
\text { material names or } \\
\end{array}$ & use ALL for all materials & $\mathrm{ALL}$ \\
& material classes & & \\
\hline
\end{tabular}

NOTE: Only one basis card is allowed per input.

NOTE: ALL is only allowed in the first position.

\section{Examples:}

$\%$ use only FUEL materials as the basis

basis $A L L=$ no $F U E L=Y E S$

\% specify FUEL.3 as the basis

basis $A L L=$ no FUEL. $3=$ YES

\section{Comments:}

The basis card is used to specify the materials to use in power normalization. By default, the energy release from all materials is taken into account, including (n,gamma) reactions in structural materials such as cladding. It is not recommended to change the default of ALL in most situations. Exceptions include (1) when comparing results to other codes that only use fuel in the basis and (2) fixing the power in a specific pin is known - a material should be created only for that pin, and the power basis should be specified for that material only. The basis card overrides any power basis imposed by a system card. Thus it behaves differently than a deplete card, which is combined with depletable materials imposed by a system card.

\section{See also:}

material, shield, deplete 


\subsubsection{2 shield - cross section self-shielding expansion specification}

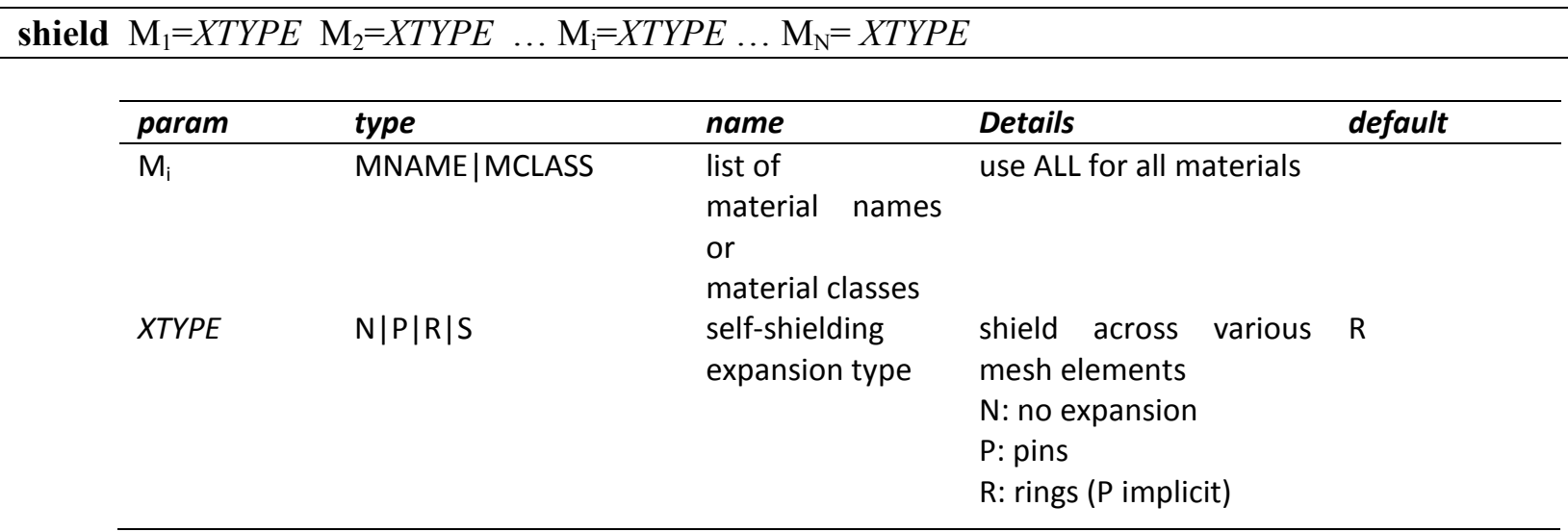

NOTE: Only one shield card is allowed per input.

NOTE: ALL is only allowed in the first position.

\section{Examples:}

\%create a unique self-shielded FUEL cross sections in each pin

\%consider all other materials to have a single self-shielded cross section

shield $A L L=N$ FUEL=P

\%assess effect of self-shielding each pin's cladding

shield $C L A D=P$

\%re-specify self-shielding to be $\mathrm{P}$ by default, $\mathrm{R}$ for the FUEL shield $A L L=P$ FUEL=R

\section{Comments:}

The shield card controls how materials are internally expanded for self-shielding purposes. By default, Polaris expands all materials across pins and rings $(\mathrm{R})$. For example, a fuel region defined on a pin card as having 10 rings will be expanded internally to have 10 different self-shielded cross sections. Because the $\mathrm{R}$ option also implicitly includes the $\mathrm{P}$ option, each instance of that pin will also get different cross sections.

When using specific systems (e.g., system $P W R$ ), this card is generally not needed. The shield card modifies the self-shielding options included in a system card. Thus, to completely re-specify the expansion, use " $\mathrm{ALL}=\mathrm{N}$ " as the first argument. This is in contrast to the basis card, which completely specifies a new power basis.

See also:

material, deplete, system 


\subsubsection{State}

The idea of a "state" or "statepoint" is a standard concept in lattice physics calculations. In Polaris, the concept of state is mostly tied to the values of material properties. The base state for a calculation is determined as follows:

1. The base state is initialized with any property values set on material cards.

2. The base state is updated with any state cards that apply to ALL.

3. The base state is updated with any other state cards, and the power card is used to set the base state power.

This sequence ensures that the state does not change, even if the order of inputs changes. A time or burnup card is then used to initiate a calculation as a function of time or burnup, thus producing a sequence of states. A branch block is used to perform branches off the base state at specific times or burnups. 


\subsubsection{1 state $<$ MNAME $>-$ material state}

state MNAME|MCLASS :

$\mathrm{p}_{1}=\mathrm{val}_{1} \mathrm{p}_{2}=\mathrm{val}_{2} \ldots \mathrm{p}_{\mathrm{i}}=\mathrm{val}_{\mathrm{i}} \ldots \mathrm{p}_{\mathrm{N}}=\mathrm{val}_{\mathrm{N}}$

\begin{tabular}{lllll}
\hline param & type & name & details & default \\
\hline MNAME IMCLASS & - & material name or & use ALL for all materials & \\
& & material class & & \\
$\mathrm{p}_{\mathrm{i}}$ & PNAME & property name & & \\
$\mathrm{val}_{\mathrm{i}}$ & Value & property value & & \\
\hline
\end{tabular}

\section{Examples:}

$\%$ reset to hot zero power conditions

state ALL : temp $=565$

state COOL : dens $=0.75$

$\%$ set channe1/bypass materials to different ppm boron

state $\mathrm{COOL}$ : boron $=0$

state BYP : boron $=600$

$\%$ set all materials with a boron property

state ALL : boron $=300$

\section{Comments:}

The state card declares the base state for materials and the base state of possible control elements or insert elements.

See also: material, deplete, system 


\subsubsection{2 state $<$ INAME $>-$ insert/control state}

state INAME : in $=$ Bool

\begin{tabular}{lllll}
\hline param & type & name & details & default \\
\hline INAME & - & $\begin{array}{l}\text { insert name or } \\
\text { control element name }\end{array}$ & \\
& insertion & "in=" is required & \\
in & Bool & insen
\end{tabular}

\section{Examples:}

$\%$ insert bank $\mathrm{D}$ control rods

state BankD : in=true

$\%$ remove inserts named Ins6A

state Ins6A false

$\%$ perform reflector calculation

state ReflectorNode : in=true

\section{Comments:}

This form of the state card is required to insert any control element or inserts. By default, inserts and control elements are out when defined.

\section{See also:}

material, deplete, system 


\subsubsection{3 state $<$ GNAME $>-$ geometry state}

state GNAME : pres $=$ Bool

\begin{tabular}{lllll}
\hline param & type & name & details & default \\
\hline GNAME & - & geometry name & & \\
pres & Bool & present & "pres=" is required &
\end{tabular}

\section{Examples:}

\% disable reflector calculation even though

$\%$ reflector geometry is present

state ReflectorNode : pres=false

\section{Comments:}

The geometry version of the state card is used to declare which geometric elements are present in the system. The geometry version of the add card is useful for performing branch calculations for reflector nodes. Note that an assembly geometry must be present to perform a calculation.

See also:

geometry $<$ REFL $>$, state $<$ MNAME $>$, state $<$ INAME $>$ 


\subsubsection{4 power - total power}

pow $\left[: \mathrm{p}_{1} \mathrm{p}_{2} \ldots \mathrm{p}_{\mathrm{i}} \ldots \mathrm{p}_{\mathrm{N}}\right]$

\begin{tabular}{|c|c|c|c|c|}
\hline param & type & Name & details & default \\
\hline$p_{i}$ & Real & list of specific powers in W/g initial heavy metal & & 0 \\
\hline
\end{tabular}

\section{Examples:}

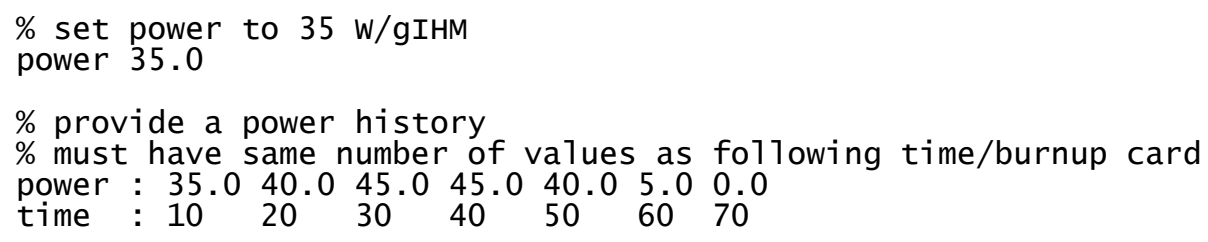

\section{Comments:}

The power card specifies the total power of the basis materials specified by a basis card. The power value may be specified only once.

\section{See also:}

t, bu, history, basis, state $<$ MNAME $>$ 


\subsubsection{5 bu - initiate calculation with cumulative burnups}

\begin{tabular}{|c|c|c|c|c|}
\hline \multicolumn{5}{|c|}{ 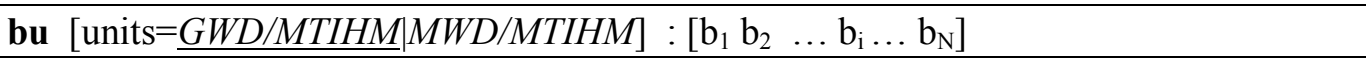 } \\
\hline param & Type & name & details & default \\
\hline units & $\begin{array}{l}\text { GWD/MTIHM| } \\
\text { MWD/MTIHM }\end{array}$ & burnup units & & GWD/MTIHM \\
\hline$b_{i}$ & Real & $\begin{array}{l}\text { list of } \\
\text { absolute burnups }\end{array}$ & & 0 \\
\hline
\end{tabular}

\section{Examples:}

\% simple depletion case with constant power and absolute/cumulative burnups power 40

bu $0 \begin{array}{lllllllll}5 & 10 & 15 & 20 & 30 & 40 & 50 & 60 & 80\end{array}$

$\%$ using MWd/MTIHM units with variable power

$\% 40 \mathrm{~W} / \mathrm{gIHM}$ for $0 \rightarrow 5000 \mathrm{MWD} / \mathrm{MTIHM}$, then $30 \mathrm{~W} / \mathrm{gIHM}$ for $5000 \rightarrow 10000 \mathrm{MWD} / \mathrm{MTIHM}$

power $40 \quad 30$

bu MWD/MTIHM: 0500010000

$\%$ combine burn/time cards

$\% 20 \mathrm{~W} / \mathrm{gIHM}$ for $0 \rightarrow 5$ then $5 \rightarrow 10 \mathrm{GWD} / \mathrm{MTIHM}$ steps, then

$\% 40 \mathrm{~W} / \mathrm{gIHM}$ for a 5 -day step then $30 \mathrm{~W} / \mathrm{gIHM}$ for a 5 -day step

power 20

bu GWD/MTIHM : 510 GWD/MTIHM

power 4030

$\mathrm{dt}$ DAYS : 55

\section{Comments:}

The bu card initiates a calculation for a given sequence of cumulative burnups. A burnup or time card usually follows a power card, the two effectively specifying the power history. If multiple burnups are given, then the power card must have either a single power or a list of powers the same size as the list times. A value of 0 is implicit at the beginning of the first burnup list. Multiple burnup/time cards may be specified in an input. This can be convenient for switching units or changing from burnup-based to timebased depletion. Internal automatic substeps are always in effect unless modified with the option $<$ DEPL $>$ card.

\section{See also:}

t, dt, dbu, power, option<DEPL $>$, branch, deplete 


\subsubsection{6 dbu - initiate calculation with incremental burnups}

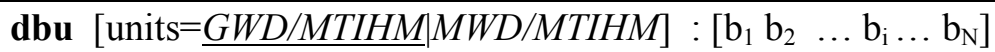

\begin{tabular}{|c|c|c|c|c|}
\hline param & Type & name & details & default \\
\hline units & $\begin{array}{l}\text { GWD/MTIHM| } \\
\text { MWD/MTIHM }\end{array}$ & burnup units & & GWD/MTIHM \\
\hline$b_{i}$ & Real & $\begin{array}{l}\text { list of } \\
\text { incremental burnups }\end{array}$ & & 0 \\
\hline
\end{tabular}

\section{Examples:}

\% incremental burnups equivalent to

$\%$ power 40

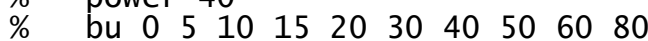

power 40

dbu $5 \begin{array}{llllllll}5 & 5 & 5 & 10 & 10 & 10 & 10 & 20\end{array}$

\section{Comments:}

The dbu card initiates a calculation for a given sequence of incremental burnups. Otherwise, it is identical to the bu card for specifying cumulative burnups.

See also:

t, dt, bu, power, option<DEPL $>$, branch, deplete 


\subsubsection{7 t $\mathrm{t}$ - initiate calculation by cumulative time}

t [units=SECONDS|MINUTES|HOURS|DAYS|YEARS $]:\left[\mathrm{t}_{1} \mathrm{t}_{2} \ldots \mathrm{t}_{\mathrm{i}} \ldots \mathrm{t}_{\mathrm{N}}\right]$

\begin{tabular}{lllll}
\hline param & Type & name & details & default \\
\hline units & SECONDS|MINUTES $\mid$ & time units & & DAYS \\
& $\begin{array}{l}\text { HOURS|DAYS|YEARS } \\
\mathrm{t}_{\mathrm{i}}\end{array}$ & $\begin{array}{l}\text { Real } \\
\text { list of } \\
\text { times }\end{array}$ & 0 & 0 \\
\hline
\end{tabular}

\section{Examples:}

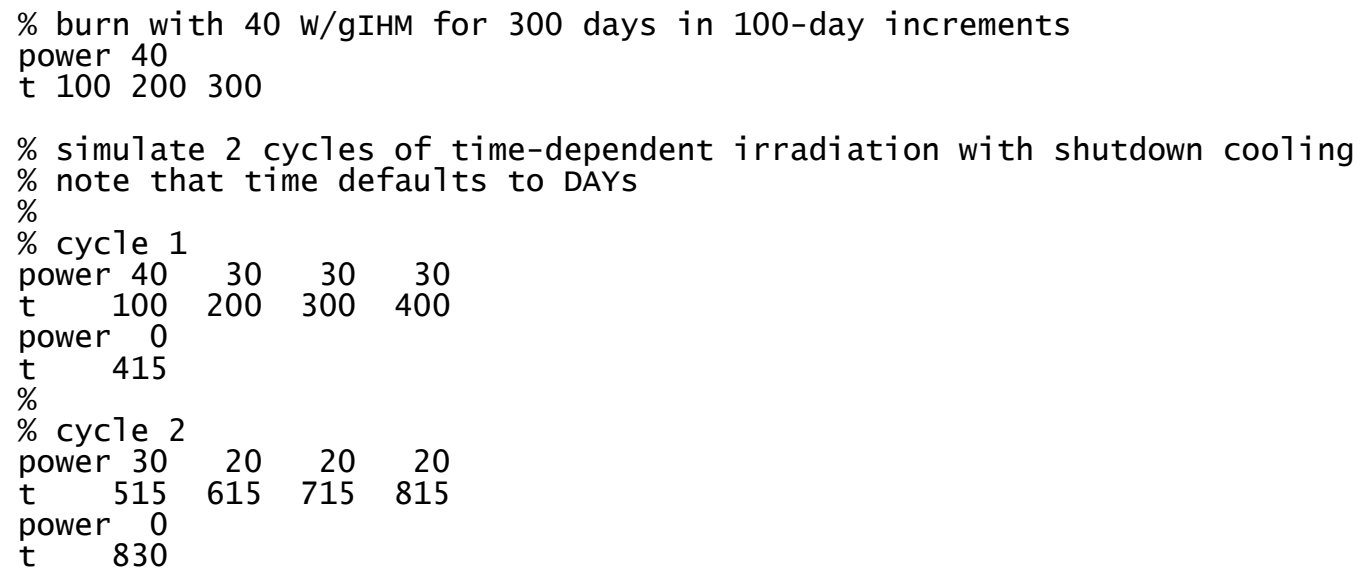

\section{Comments:}

The $\mathbf{t}$ card initiates a calculation for a given sequence of cumulative/absolute times. One of the time cards ( $\mathbf{t}, \mathbf{d t}$, or ti) is required to model periods of decay in conjunction with power 0 . Otherwise, the time card $\mathbf{t}$ is similar in functionality to the burnup bu card but with different units.

See also:

dt, bu, dbu, power, option<DEPL $>$, branch, deplete 


\subsubsection{8 dt - initiate calculation by incremental time}

dt $[$ units $=S E C O N D S|M I N U T E S| H O U R S|\underline{D A Y S}|$ YEARS $]:\left[\mathrm{t}_{1} \mathrm{t}_{2} \ldots \mathrm{t}_{\mathrm{i}} \ldots \mathrm{t}_{\mathrm{N}}\right]$

\begin{tabular}{|c|c|c|c|c|}
\hline param & Type & name & details & default \\
\hline \multirow[t]{2}{*}{ units } & SECONDS|MINUTES| & time units & & DAYS \\
\hline & HOURS|DAYS|YEARS & & & \\
\hline$t_{i}$ & Real & $\begin{array}{l}\text { list of } \\
\text { times }\end{array}$ & 0 & 0 \\
\hline
\end{tabular}

\section{Examples:}

$\%$ burn with $40 \mathrm{~W} / \mathrm{gIHM}$ for 300 days in 100-day increments equivalent to

$\%$ power 40

$\% \quad \mathrm{t} 100200300$

power 40

dt 100100100

\% decay for 30 minutes

power 0

$\mathrm{dt} 30$ MINUTES

\section{Comments:}

The dt card is identical to the cumulative time card $\mathbf{t}$ except that the values given are incremental.

See also:

t, bu, dbu, power, option<DEPL $>$, branch, deplete 


\subsubsection{9 branch - instantaneous change}

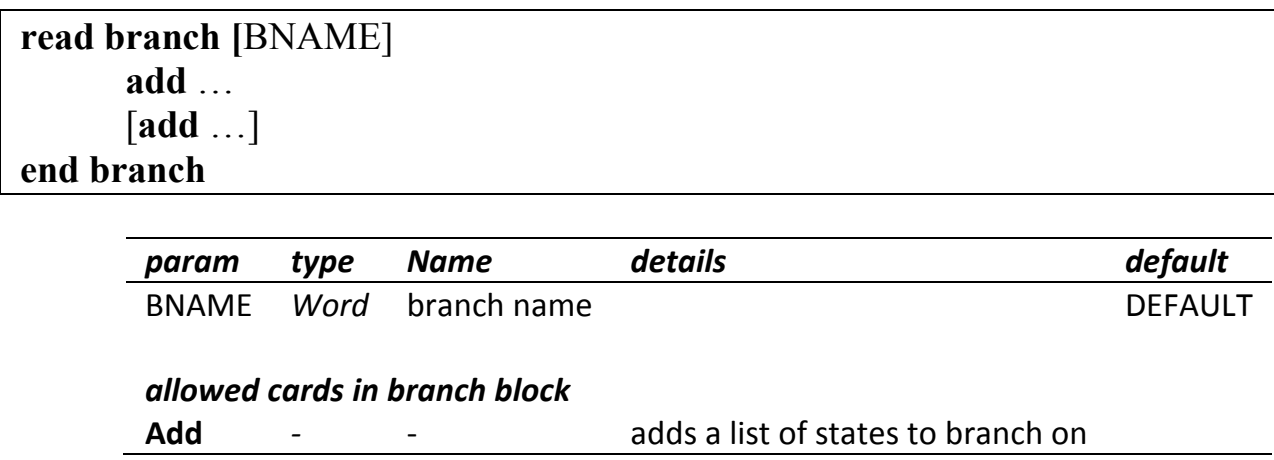

\section{Examples:}

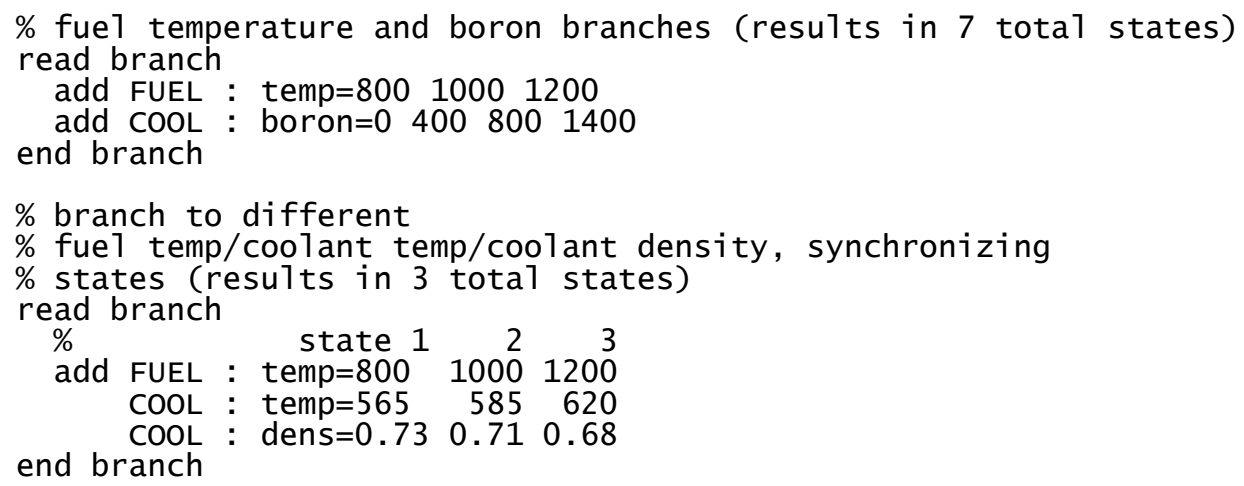

\section{Comments:}

The branch card initiates so-called "branch" calculations, i.e., instantaneous changes of state at specific burnups/times during the base depletion sequence of calculations. The syntax for the add card is identical to the state card except, instead of taking a list of different properties and their values, it takes a single property and a list of values. Note that a time or burnup card is not necessary-if not found, branches will be performed at every burnup/time specified in the base state. The initial state for any branch card is the base state as specified in the main file. This means branch cards have no knowledge of one another.

\section{See also:}

add, bu, t, title 


\subsubsection{0 add $<$ MNAME $>-$ material branch}

\begin{tabular}{|c|c|c|c|c|}
\hline $\begin{array}{c}\text { add } \\
{[\text { incr }=\text { Bool }]} \\
{[\text { scale }=A B S \mid P C T]:} \\
\text { PNAME }=\text { val }_{1} \text { val }_{2} \text { :MCLASS } \\
{[\text { MNAME } \mid \text { MCLASS }} \\
\ldots]\end{array}$ & $\ldots \operatorname{val}_{\mathrm{i}} \ldots$ & $\operatorname{val}_{\mathrm{N}}$ & & \\
\hline param & type & name & details & default \\
\hline MNAME|MCLASS & - & $\begin{array}{l}\text { material name or } \\
\text { material class }\end{array}$ & use ALL for all materials & \\
\hline incr & Bool & increment & values are added to reference value & false \\
\hline scale & $A B S \mid P C T$ & Scaling & $\begin{array}{l}\text { scaling } \\
\text { ABS: absolute units } \\
\text { PCT: percentage units }\end{array}$ & $A B S$ \\
\hline $\begin{array}{l}\text { PNAME } \\
\text { val }_{i}\end{array}$ & - & $\begin{array}{l}\text { property name } \\
\text { list of } \\
\text { property values }\end{array}$ & & \\
\hline
\end{tabular}

\section{Examples:}

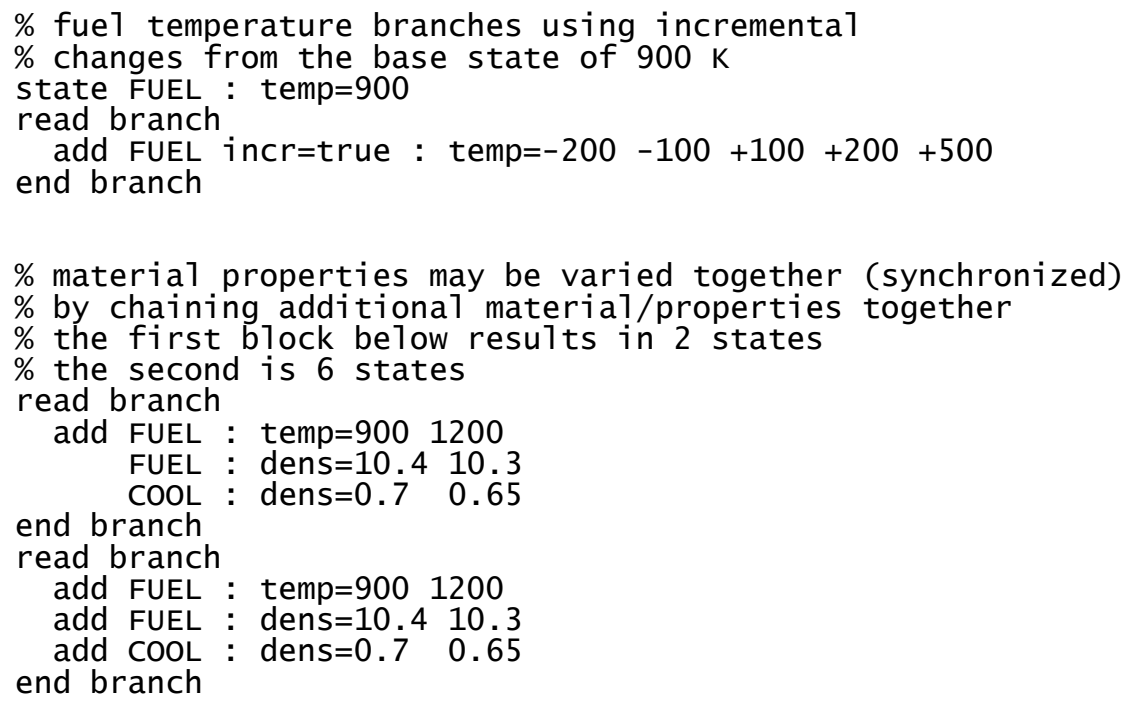

\section{Comments:}

The add card is only valid inside a branch block. This version adds a set of branches for a specific material name (MNAME) or class (MCLASS). Branches are always with respect to the base state. Although similar to the state card, the add card has a single property name and a list of values. The state card has a list of property=value pairs.

See also:

material, state $<$ MNAME $>$ 


\subsubsection{1 add $<$ INAME $>$ - insert/control branch}

add INAME : in $=\mathrm{Bool}_{l}\left[\mathrm{Bool}_{2}\right]$

\begin{tabular}{|c|c|c|c|c|}
\hline param & type & name & details & default \\
\hline INAME & - & $\begin{array}{l}\text { insert name or } \\
\text { control element name }\end{array}$ & & \\
\hline in & Bool & list of insertion states & "in=" is required & \\
\hline
\end{tabular}

\section{Examples:}

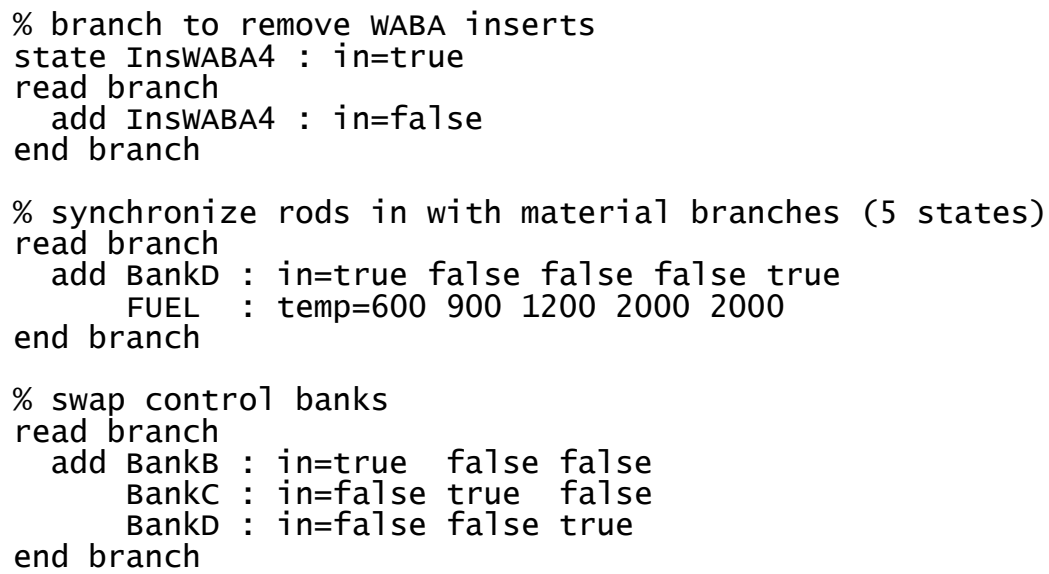

\section{Comments:}

This form of the add card is required to add branches to insert/remove control elements or inserts. Given that only two possible states exist, specifying "true false" will result in a calculation at the other state not specified by the base state.

See also:

insert, control, state $<$ INAME $>$ 


\subsubsection{2 add $<$ GNAME $>-$ geometry branch}

add GNAME : pres $^{-B_{0 o l}}\left[\mathrm{Bool}_{2}\right]$

\begin{tabular}{lllll}
\hline param & type & name & details & default \\
\hline GNAME & - & geometry name & & \\
pres & Bool & list of geometry states & "pres=" is required & \\
\hline
\end{tabular}

\section{Examples:}

\% perform a reflector calculation on a branch

state ReflectorNode : pres=no

read branch

add ReflectorNode : pres=yes

end branch

\section{Comments:}

This form of the add card is required to add branches for new geometry, such as reflector calculations. Given that only two possible states exist, specifying "true false" will result in a calculation at the other state not specified by the base state.

See also:

geometry, state $<$ GNAME $>$ 


\subsubsection{Options}

An extensive set of options is provided for manipulating the solvers and output. Most option cards support a key=value style of input, with reasonable defaults in place for all parameters. 


\subsubsection{1 option $<$ KEFF $>$ - eigenvalue}

opt $K E F F\left[\begin{array}{lllll}\mathrm{key}_{1}=\mathrm{val}_{1} & \mathrm{key}_{2}=\mathrm{val}_{2} & \ldots \mathrm{key}_{\mathrm{i}}=\mathrm{val}_{\mathrm{i}} & \left.\ldots \mathrm{key}_{\mathrm{N}}=\mathrm{val}_{\mathrm{N}}\right]\end{array}\right.$

\begin{tabular}{llll}
\hline key & $\begin{array}{l}\text { value } \\
\text { type }\end{array}$ & details & default \\
\hline NumAzim & Int & number of azimuthal angles per octant & 20 \\
NumPolar & Int & number of polar angles per octant & 3 \\
PnOrder & Int & scattering order & 2 \\
RaySpacing & Real & spacing between MoC rays & $0.04 \mathrm{~cm}$ \\
\hline
\end{tabular}

developer options (generally should not change)

\begin{tabular}{|c|c|c|c|}
\hline PolarScheme & String & $\begin{array}{l}\text { polar quadrature } \\
\text { "TY": Tabuchi-Yamamoto quadrature } \\
\text { "DECART": DeCART quadrature } \\
\text { "LO": Leonard optimal quadrature } \\
\text { "CACTUS": Cactus quadrature }\end{array}$ & "TY" \\
\hline EigSolver & String & $\begin{array}{l}\text { eigenvalue solver type } \\
\text { "CMFD" (single-assembly default) } \\
\text { "POWER" (reflector default) }\end{array}$ & \\
\hline UpscatterSolver & String & $\begin{array}{l}\text { upscatter solver type } \\
\text { "GAUSS_SEIDEL", (default if EigSolver="CMFD") } \\
\text { "BICGSTAB" (default if EigSolver="POWER") } \\
\text { "GMRES" }\end{array}$ & \\
\hline $\begin{array}{l}\text { DownscatterSolver, } \\
\text { UpscatterInnerSolver }\end{array}$ & String & $\begin{array}{l}\text { within-group solver type } \\
\text { "SOURCE", (default if EigSolver="CMFD") } \\
\text { "BICGSTAB" (default if EigSolver="POWER") } \\
\text { "GMRES" }\end{array}$ & \\
\hline $\begin{array}{l}\text { EigMaxIterations } \\
\text { DownscatterMaxIterations, } \\
\text { UpscatterMaxIterations, } \\
\text { UpscatterInnerMaxIterations }\end{array}$ & $\begin{array}{l}\text { Int } \\
\text { Int }\end{array}$ & $\begin{array}{l}\text { maximum number of eigenvalue iterations } \\
\text { maximum iterations for multigroup solves } \\
\text { default is } 1 \text { for EigSolver="CMFD" } \\
\text { default is } 1000 \text { for EigSolver="POWER" }\end{array}$ & 1000 \\
\hline EigTolerance & Real & eigenvector $L_{2}$ residual tolerance & 1.0 \\
\hline EigFissionTolerance & Real & eigenvector $L_{\infty}$ relative fission error tolerance & $3 e-4$ \\
\hline $\begin{array}{l}\text { DownscatterTolerance, } \\
\text { UpscatterTolerance, } \\
\text { UpscatterInnerTolerance }\end{array}$ & Real & $\mathrm{L}_{2}$ residual tolerance for multigroup solve & $3 e-6$ \\
\hline EigVerbosity & String & $\begin{array}{l}\text { msg file print level for eigenvalue iterations } \\
\text { "NONE", "LOW", "HIGH" }\end{array}$ & “LOW" \\
\hline $\begin{array}{l}\text { DownscatterVerbosity, } \\
\text { UpscatterVerbosity, } \\
\text { UpscatterInnerVerbosity }\end{array}$ & String & $\begin{array}{l}\text { msg file print level for multigroup iterations } \\
\text { "NONE", "LOW", "HIGH" }\end{array}$ & “NONE” \\
\hline CmfdRelaxation & Real & relaxation parameter for CMFD solution update & 1.0 \\
\hline UpscatterSuperGroup & Int & $\begin{array}{l}\text { number of energy groups in the inner iteration in the } \\
\text { upscatter calculation }\end{array}$ & 1 \\
\hline DownscatterSuperGroup & Int & $\begin{array}{l}\text { number of energy groups in the inner iteration in the } \\
\text { downscatter calculation }\end{array}$ & 1 \\
\hline PartitionUpscatter & Bool & $\begin{array}{l}\text { Partition problem into downscatter and upscatter } \\
\text { energy regions } \\
\text { default is true for EigSolver="POWER" } \\
\text { default is false for EigSolver="CMFD" }\end{array}$ & \\
\hline ReverseRay & Bool & true - store attenuation coefficients from forward & true \\
\hline
\end{tabular}




\begin{tabular}{|c|c|c|}
\hline & & $\begin{array}{l}\text { ray calculation and reuse in reverse ray calculation } \\
\text { false - recompute attenuation coefficients for } \\
\text { reverse ray }\end{array}$ \\
\hline CyclicRay & Bool & $\begin{array}{l}\text { true - continue particle tracks after reaching the } \\
\text { boundary } \\
\text { false - separate particle tracks }\end{array}$ \\
\hline Accumulator & String & $\begin{array}{l}\text { angle-to-moment flux calculation } \\
\text { "SEGMENT": during sweep ( } P_{0} \text { default) } \\
\left.\text { "SWEEP": after sweep ( } P_{\mathrm{N}} \text { default }\right)\end{array}$ \\
\hline TransportCorrection & Bool & $\begin{array}{l}\text { true: use } \mathrm{P} 1 \text { transport correction } \\
\text { false: do not perform correction } \\
\text { (Only applies for } \mathrm{P}_{0} \text { calculation) }\end{array}$ \\
\hline
\end{tabular}

\section{Examples:}

\% change the MOC ray spacing for the

\% eigenvalue calculation to $0.01 \mathrm{~cm}$

opt KEFF Rayspacing $=0.01$

\%P3 scattering

opt KEFF PnOrder=3

\subsubsection{2 option $<$ ESSM $>-$ embedded self-shielding}

opt $\operatorname{ESSM}\left[\mathrm{key}_{1}=\mathrm{val}_{1} \mathrm{key}_{2}=\mathrm{val}_{2} \ldots \mathrm{key}_{\mathrm{i}}=\mathrm{val}_{\mathrm{i}} \ldots \mathrm{key}_{\mathrm{N}}=\mathrm{val}_{\mathrm{N}}\right]$

\begin{tabular}{|c|c|c|c|}
\hline key & $\begin{array}{l}\text { value } \\
\text { type }\end{array}$ & details & default \\
\hline \multicolumn{4}{|c|}{ developer options (generally should not change) } \\
\hline Solver & String & $\begin{array}{l}\text { solver method } \\
\text { "BICGSTAB": Bi-conjugate } \\
\text { gradient stabilized } \\
\text { "GMRES": general minimized } \\
\text { residual } \\
\text { "SOURCE": source iteration }\end{array}$ & "BICGSTAB" \\
\hline Maxlterations & Int & maximum number of solver iterations & 20 \\
\hline NumAzim & Int & number of azimuthal angles per octant & 8 \\
\hline NumPolar & Int & number of polar angles per octant & 3 \\
\hline RaySpacing & Real & spacing between MoC rays & $0.02 \mathrm{~cm}$ \\
\hline Tolerance & Real & solver tolerance & $3 e-7$ \\
\hline EscapeXSConvergenceCriteria & Real & & $1 e-3$ \\
\hline ZeroEscapeXsTolerance & Real & & $1 e-10$ \\
\hline FluxMaxValue & Real & & 0.999999 \\
\hline LargeEscapeXs & Real & & $1 \mathrm{e} 9$ \\
\hline ReverseRay & Bool & $\begin{array}{l}\text { true - store attenuation coefficients } \\
\text { from forward ray calculation and reuse } \\
\text { in reverse ray calculation } \\
\text { false - recompute attenuation } \\
\text { coefficients for reverse ray }\end{array}$ & true \\
\hline CyclicRay & Bool & $\begin{array}{l}\text { true - continue particle tracks after } \\
\text { reaching the boundary } \\
\text { false - separate particle tracks }\end{array}$ & true \\
\hline
\end{tabular}




$\begin{array}{llc}\text { Accumulator } & \text { String } & \text { angle-to-moment flux calculation } \\ & \text { "SEGMENT": during sweep } & \text { "SEGMENT" } \\ & \text { "SWEEP": after sweep } & \\ \text { PolarScheme } & \text { polar quadrature } & \text { "TY": Tabuchi-Yamamoto quadrature } \\ & \text { "DECART": DeCART quadrature } \\ & \text { "LO": Leonard optimal quadrature } \\ & \text { "CACTUS": Cactus quadrature } & \\ & \end{array}$

\section{Examples:}

\% change the solver method to source iteration opt ESSM

Solver="SOURCE" 


\subsubsection{3 option $<$ BOND $>-$ Bondarenko search}

opt $B O N D\left[\mathrm{key}_{1}=\mathrm{val}_{1} \mathrm{key}_{2}=\mathrm{val}_{2} \ldots \mathrm{key}_{\mathrm{i}}=\mathrm{val}_{\mathrm{i}} \ldots \mathrm{key}_{\mathrm{N}}=\mathrm{val}_{\mathrm{N}}\right]$

\begin{tabular}{llll}
\hline key & $\begin{array}{l}\text { value } \\
\text { type }\end{array}$ & details & default \\
\hline Maxlterations & Int & number of Bondarenko iterations (0 is disabled) & $0^{*}$ \\
ConvergenceXS & String & $\begin{array}{l}\text { cross section used in Bondarenko convergence } \\
\text { iterations } \\
\end{array}$ & "SIGA": absorption \\
& "SIGT": total & \\
& & \\
&
\end{tabular}

developer options (generally should not change)

\begin{tabular}{lll}
\hline IterationCriteria & Real & $1 \mathrm{e}-3$ \\
TempSearchMaxIterations & Int & 20 \\
TempSearchCriteria & Real & $1 \mathrm{e}-8$ \\
TempSearchEqualTolerance & Real & $1 \mathrm{e}-3$ \\
TempSearchMaxPower & Real & 35.0 \\
SigOSearchMaxlterations & Int & 20 \\
SigOSearchCriteria & Real & $1 \mathrm{e}-8$ \\
SigOSearchEqualTolerance & Real & $1 \mathrm{e}-3$ \\
SigOSearchMaxPower & Real & 35.0 \\
\hline * By setting the Bondarenko iterations >0, resonance interference effects may be taken into account. The default \\
MaxIterations=0 effectively disables the Bondarenko resonance interference model. \\
**In SCALE, the transport cross section ("SIGT") has historically been used in Bondarenko iterations.
\end{tabular}

\section{Examples:}

$\%$ introduce Bondarenko iterations on total cross section

opt BOND

MaxIterations $=10$

ConvergenceXS="SIGT" 


\subsubsection{4 option $<$ DEPL $>-$ depletion}

opt DEPL $\left[\begin{array}{llllll}\mathrm{key}_{1}=\mathrm{val}_{1} & \mathrm{key}_{2}=\mathrm{val}_{2} & \ldots \mathrm{key}_{\mathrm{i}}=\mathrm{val}_{\mathrm{i}} & \ldots & \mathrm{key}_{\mathrm{N}}=\mathrm{val}_{\mathrm{N}}\end{array}\right]$

\begin{tabular}{|c|c|c|c|}
\hline key & value type & details & default \\
\hline TrackingSet & String & $\begin{array}{l}\text { set of nuclides tracked in depletion } \\
\text { calculations } \\
\text { "None": set of nuclides present in user } \\
\text { input } \\
\text { "Complete": complete set of all nuclides } \\
\text { available on ORIGEN data libraries }\end{array}$ & "Complete" \\
\hline Solver & String & $\begin{array}{l}\text { "MATREX": legacy ORIGEN } \\
\text { method } \\
\text { "CRAM": }\end{array}$ & “MATREX" \\
\hline Method & String & $\begin{array}{l}\text { Approximation Method } \\
\text { "PREDICTOR" } \\
\text { "PREDICTOR_CORRECTOR" }\end{array}$ & “PREDICTOR_CORRECTOR” \\
\hline StepRefinement & Int & $\begin{array}{l}\text { divide the user input steps by this } \\
\text { factor }>0 \text {, i.e., refinement of } 2 \text { divides all } \\
\text { steps by } 2 \text { (NOT ENABLED) }\end{array}$ & 1 \\
\hline NumSubsteps & Int & $\begin{array}{l}\text { Number of internal substeps for } \\
\text { depletion calculations }\end{array}$ & 4 \\
\hline DepleteMode & String & $\begin{array}{l}\text { "BOSS" or "MOSS." The depletion power } \\
\text { renormalization is done at the beginning } \\
\text { of each substep (BOSS) or the middle of } \\
\text { each substep (MOSS) }\end{array}$ & “BOSS" \\
\hline
\end{tabular}

\section{Examples:}

$\%$ set the number of origen substeps per time steps to 2

$\%$ (may be useful for convergence studies)

opt DEPL

NumSubsteps $=2$

$\%$ disable the addition of depletion nuclides to input materials

opt DEPL TrackingSet="NONE"

$\%$ use CRAM solver

opt DEPL Solver="CRAM" 


\subsubsection{5 option $<$ CRITSPEC $>-$ critical spectrum}

opt $C R I T S P E C\left[\mathrm{key}_{1}=\mathrm{val}_{1} \mathrm{key}_{2}=\mathrm{val}_{2} \ldots \mathrm{key}_{\mathrm{i}}=\mathrm{val}_{\mathrm{i}} \ldots \mathrm{key}_{\mathrm{N}}=\mathrm{val}_{\mathrm{N}}\right]$

\begin{tabular}{|c|c|c|c|}
\hline key & value type & details & default \\
\hline Mode & String & $\begin{array}{l}\text { critical spectrum mode } \\
\text { "SEARCH": search for critical mode (k-eff=1) } \\
\text { "SPECIFIED": provide B2 below } \\
\text { "NONE": do not use critical spectrum }\end{array}$ & "SEARCH" \\
\hline B2 & Real & $\begin{array}{l}\text { value of critical buckling if Mode="SPECIFIED" } \\
\text { units: } \mathrm{cm}^{-2}\end{array}$ & 0.0 \\
\hline Method & String & $\begin{array}{l}\text { critical spectrum system } \\
\text { "B1": solve the } B_{1} \text { equations } \\
\text { "P1": solve the } P_{1} \text { equations }\end{array}$ & "P1" \\
\hline
\end{tabular}

\section{Examples:}

\% enable critical buckling search using B1 equations for a buck1ing of 1e-3 opt CRITSPEC

Mode="SPECIFIED"

$\mathrm{B} 2=1 \mathrm{e}-3$

Method="B1" 


\subsubsection{6 option $<$ PRINT $>-$ printing}

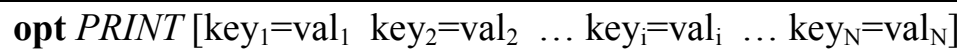

\begin{tabular}{llll}
\hline key & value type & details & default \\
\hline $\begin{array}{l}\text { XSSummary } \\
\text { CritSpecSummary }\end{array}$ & $\begin{array}{l}\text { Bool } \\
\text { String }\end{array}$ & $\begin{array}{l}\text { print a cross section summary in the output file } \\
\text { print critical spectrum summary } \\
\text { "NONE": no print out } \\
\text { "BUCKLING": limited buckling info } \\
\text { "SPECTRUM": full spectrum } \\
\text { XFile16 }\end{array}$ & $\begin{array}{l}\text { "BUCKLING" } \\
\text { InputDataContainer }\end{array}$ \\
\hline
\end{tabular}

\section{Examples:}

$\%$ print the $x f i 1 e 016$

$\%$ if input file is polaris.inp, file name will be polaris.x16

opt PRINT XFile16=true 


\subsubsection{7 option $<$ FG $>-$ few-group cross section generation}

\begin{tabular}{|c|c|c|c|}
\hline \multicolumn{4}{|c|}{ 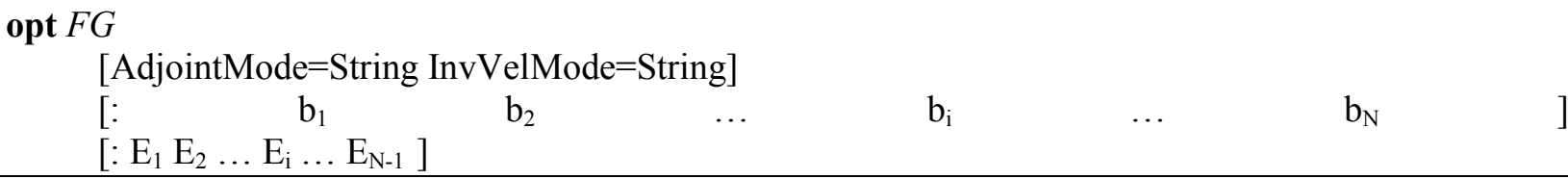 } \\
\hline param & type & details & default \\
\hline AdjointMode & String & $\begin{array}{l}\text { type of adjoint calculation to use in few-group data } \\
\text { generation } \\
\text { "INFMED": infinite medium adjoint } \\
\text { "CRITICAL": critical spectrum adjoint } \\
\text { "UNIFORM": uniform adjoint }\end{array}$ & "INFMED" \\
\hline InvVelMode & String & $\begin{array}{l}\text { weighting option for few-group inverse velocities } \\
\text { "FORWARD": forward flux weighting } \\
\text { "ADJOINT": adjoint flux weighting }\end{array}$ & “FORWARD” \\
\hline$b_{i}$ & Real & $\begin{array}{l}\text { list of burnups to include in output few-group cross } \\
\text { section database, e.g., XFile16 output } \\
\text { units: GWd/MTHM }\end{array}$ & all burnups available \\
\hline$E_{i}$ & Real & $\begin{array}{l}\text { note descending order and only } \mathrm{N}-1 \text { divisions are } \\
\text { needed for an } \mathrm{N} \text { group structure } \\
\mathrm{E}_{0} \text { is maximum energy (typically } 2 \mathrm{e} 7 \mathrm{eV} \text { ) } \\
\mathrm{E}_{\mathrm{N}} \text { is minimum (typically } 1 \mathrm{e}-5 \mathrm{eV} \text { ) } \\
\text { units: } \mathrm{eV}\end{array}$ & $\begin{array}{l}0.625 \mathrm{eV} \text { division (two } \\
\text { groups) }\end{array}$ \\
\hline
\end{tabular}

\section{Examples:}

$\%$ enable the critical spectrum adjoint opt FG AdjointMode="CRITICAL"

\%on1y include $0,10,15,20 \mathrm{GWd} / \mathrm{MTHM}$ burnups in few-group outputs, including XFile16 opt FG : 0101520

\%redefine group energy divisions for 3 groups with divisions at 10 and $0.625 \mathrm{eV}$ opt FG : : 100.625 


\subsubsection{8 option $<$ RUN $>-$ run time}

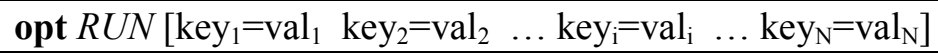

\begin{tabular}{llll}
\hline key & value type & details & default \\
\hline CheckOnly & Bool & check input and terminate & true \\
\hline
\end{tabular}

\section{Examples:}

$\%$ check input

opt RUN Checkonly=true 


\subsubsection{System}

The system cards provide a way to initialize a set of defaults to simplify input and add robustness for a well-known and well-characterized system.

system STYPE

\begin{tabular}{llll}
\hline argument & Type & name & details default \\
\hline STYPE & - & system type & \\
& $P W R$ & pressurized water reactor & \\
& $B W R$ & boiling water reactor & \\
\hline
\end{tabular}

The system card performs the following actions:

1. defines a set of materials and properties, imposing standard names for the materials and properties;

2. warns user of potential mistakes; and

3. uses heuristics to modify unspecified mesh and solver options for robust results. 


\subsubsection{1 system $<$ PWR $>-$ pressurized water reactor}

\section{sys PWR}

\section{Definitions}

\begin{tabular}{|c|c|c|}
\hline type & purpose & introduced \\
\hline channel & channel material & channel COOL \\
\hline \multicolumn{3}{|c|}{ properties } \\
\hline PNAME & purpose & introduced \\
\hline boron & soluble boron in $\mathrm{ppm}$ & $\begin{array}{l}\text { prop boron COOL : SOLP B } \\
\text { prop boron MOD : SOLP B }\end{array}$ \\
\hline \multicolumn{3}{|c|}{ materials } \\
\hline MCLASS & purpose & introduced \\
\hline COOL & light water in channel & $\begin{array}{ll}\text { mat COOL } .1 & : \text { WATER dens }=0.743 \\
\text { mat COOL } .2 & \text { (same) }\end{array}$ \\
\hline MOD & $\begin{array}{l}\text { light water elsewhere } \\
\text { (guide/instrument tubes) }\end{array}$ & $\begin{array}{l}\text { mat MOD. } 1 \text { : WATER dens }=0.743 \\
\text { mat MOD. } 2 \text { (same) }\end{array}$ \\
\hline FUEL & fuel & no default members \\
\hline CLAD & fuel cladding & mat CLAD.1: ZIRC4 \\
\hline GRID & $\begin{array}{l}\text { grid spacers (Zircaloy-4 and } \\
\text { Inconel) }\end{array}$ & $\begin{array}{l}\text { mat GRID.1 : ZIRC4 } \\
\text { mat GRID. } 2: \text { INC718 }\end{array}$ \\
\hline TUBE & guide/instrument tubes & mat TUBE.1 : ZIRC4 \\
\hline STRUCT & structural material & mat STRUCT.1 : SS304 \\
\hline GAP & fuel/clad gap fill gas & mat GAP.1 : FILLGAS \\
\hline CNTL & control elements & $\begin{array}{l}\text { mat CNTL. } 1: \text { AIC } \\
\text { mat CNTL. } 2: B 4 C\end{array}$ \\
\hline $\mathrm{BP}$ & burnable poison & no default members \\
\hline \multicolumn{3}{|c|}{ miscellaneous } \\
\hline type & purpose & introduced \\
\hline deplete & depletion materials & deplete $A L L=N \quad F U E L=R \quad B P=R$ \\
\hline shield & material self-shielding & 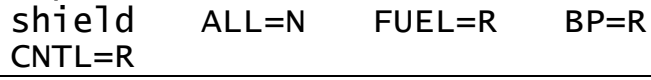 \\
\hline
\end{tabular}

\section{Description}

Twelve reactor materials are initialized with compositions and densities from the predefined composition set. In most cases, all that remains is to define fuel materials, all material temperatures, and properties such as COOL/MOD soluble boron and density. Note that some rules are based on naming conventions. For example, burnable poisons (the material class BP) are declared to be depletable materials, whereas the CNTL (control elements) class of materials is not. 


\section{Examples:}

\% a complete input file for a PWR pincel1 model =polaris

system PWR

geom MyPin : ASSM 11.5

comp f35 : UOX 3.5

mat FUEL. 1 : f 35 dens $=10.25$

pin $1: 0.50 .6$ : FUEL CLAD

state ALL : temp $=565$

state MOD : dens $=0.743$

state COOL: dens $=0.743$

state ALL : boron $=600$

power 40

burn $0 \begin{array}{lllllllll}0.1 & 0.2 & 0.5 & 1 & 5 & 10 & 15\end{array}$

end $\begin{array}{llllllllll}20 & 25 & 30 & 35 & 40 & 45 & 50 & 55 & 60\end{array}$ 


\subsubsection{2 system $<$ BWR $>-$ boiling water reactor}

\section{sys BWR}

\section{Definitions}

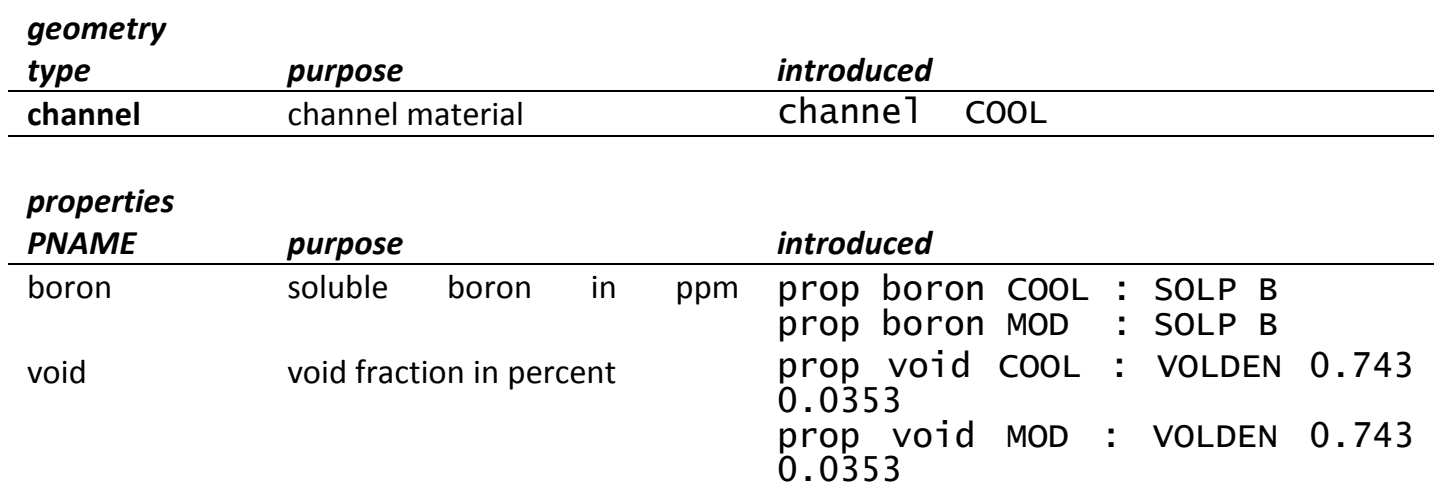

\begin{tabular}{|c|c|c|}
\hline MCLASS & purpose & introduced \\
\hline $\mathrm{COOL}$ & light water in channel & 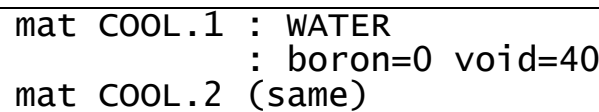 \\
\hline MOD & $\begin{array}{l}\text { light water elsewhere (water } \\
\text { rods/bypass) }\end{array}$ & $\begin{array}{l}\text { mat MOD.1 } \begin{array}{l}\text { : WATER } \\
\text { boron=0 void=0 } \\
\text { mat MOD. } 2 \text { (same) }\end{array}\end{array}$ \\
\hline FUEL & fuel & no default members \\
\hline CLAD & fuel cladding & mat CLAD.1 : ZIRC2 \\
\hline TUBE & guide/instrument tubes & mat TUBE.1 : ZIRC2 \\
\hline STRUCT & structural material & mat STRUCT.1 : SS304 \\
\hline GAP & fuel/clad gap fill gas & mat GAP.1 : FILLGAS \\
\hline CNTL & control elements & $\begin{array}{l}\text { mat CNTL.1 }: \text { AIC } \\
\text { mat CNTL.2 }\end{array}$ \\
\hline CAN & channel box material & mat CAN.1 : ZIRC2 \\
\hline \multicolumn{3}{|c|}{ miscellaneous } \\
\hline type & purpose & introduced \\
\hline $\begin{array}{l}\text { deplete } \\
\text { shield }\end{array}$ & $\begin{array}{l}\text { depletion materials } \\
\text { material self-shielding }\end{array}$ & $\begin{array}{l}\text { deplete } A L L=N \text { FUEL }=R \\
\text { shield } A L L=N \quad F U E L=R \quad C N T L=R\end{array}$ \\
\hline
\end{tabular}




\subsubsection{Sample Problems}

Within the SCALE distribution, 27 Polaris sample problems are provided to demonstrate the differences in calculation and geometry options, and 21 sample problems consider the Consortium for Advanced Simulation of Light Water Reactors (CASL) Virtual Environment for Reactor Applications (VERA) benchmark problems for pin cell and lattice configurations described in [4]. The VERA pin cell problems are identified as polaris_1a_252g.inp through polaris_1e_252g.inp. The VERA lattice problems are identified as polaris_2a_252g.inp through polaris_2k_252g.inp, polaris_21_56g.inp through polaris_2m_56g.inp, polaris_2o_252g.inp, and polaris_2p_ $252 \mathrm{~g}$.inp.

The remaining six sample problems are described as follows:

- polaris_TMI1_Cycle1-2.inp - $15 \times 15$ PWR geometry model with branch block definition for lattice physics calculations

- polaris_bench_taka3_sf97-4_assm.inp, polaris_bench_taka3_sf97-4_pin.inp - Takahama UOX depletion benchmark for radiochemical assay NT3G24-SF97-4 described in [5].

- polaris_bwr10x10.inp, polaris_bwr7 $\times$ 7.inp - example BWR geometry models for $10 \times 10$ and $7 \times 7$ füel.

- $\quad$ polaris_dvla.inp - simple PWR pin cell depletion calculation.

\subsubsection{References}

1. M. L. Williams and K. S. Kim. "The Embedded Self-Shielding Method.” PHYSOR 2012, Knoxville, Tennessee, USA (2012).

2. M. A. Jessee, W. A. Wieselquist, et al. "Polaris: A New Two-Dimensional Lattice Physics Analysis Capability for the SCALE Code System." PHYSOR 2014, Kyoto, Japan (2014).

3. S. M. Bowman and T. Suto, "Analysis of Pressurized Water Reactor Critical Configurations: Volume 5 - North Anna Unit 1 Cycle 5,” ORNL/TM-12294/V5, October 1996.

4. A. T. Godfrey, "VERA Core Physics Benchmark Progression Problem Specification." Available Online. URL: http://www.casl.gov/docs/CASL-U-2012-0131-004.pdf. CASL Technical Report, CASL-U-2012-0131-002 (2013).

5. B. Roque and M. Erlund, "International Comparison of a Depletion Calculation Benchmark on Fuel Cycle Issues," OECD/NEA/NSC report. URL: https://www.oecd-nea.org/science/docs/2013/nsc-doc2013-1.pdf. 


\subsection{A SCALE 6.3 Polaris input format}

For the release of SCALE 6.2.2, several new input cards were implemented into Polaris to model boiling water reactor (BWR) geometries. Moreover, improvements to existing input cards were implemented, along with the ability to specify time-dependent state properties and the ability to specify one or more depletion histories. This appendix describes the new and modified input cards that will be included in the Polaris input format for SCALE 6.3, which are accessible as part of the release of SCALE 6.2.2.

To maximize backwards compatibility for input files developed with SCALE 6.2 and SCALE 6.2.1, the new and modified input cards are not available by default with SCALE 6.2.2. The new and modified input cards are activated if the input file begins with =polaris_6.3 rather than =polaris. The suffix "_6.3" is an indicator to the Polaris input processor to use the SCALE 6.3 input format. For the future release of SCALE 6.3, the original input cards supported in the SCALE 6.2 input format will be available if the input file begins with =polaris_6.2.

The new input cards to model BWR geometries include:

- cross - define the interior water cross geometry of SVEA assembly designs;

- dxmap (or dymap) - define displacement maps that indicate that translation of the pin center in the $\mathrm{x}$ - (or $\mathrm{y}$-) direction;

- control $<$ BLADE $>$ - define the control blade geometry;

- $\quad$ mesh - define advanced spatial meshing options for different materials; and

- option $<$ GEOM $>$ - define geometry tolerances, advance meshing options, and plotting options.

The modified input cards to model BWR geometries include:

- $\quad$ pin - define circular and square-based geometry zones, as well as arbitrarily sized pins, e.g. size $=1.5$ water rod in some 9x9 BWR lattice designs; and

- $\quad$ box - define channel box geometry with arbitrary number of zones and cutout regions.

The new input cards for time-dependent modeling include:

- $\quad$ history - define one or more operating histories in the input file; and

- bui (or ti) - define restart cumulative burnup (or time) values.

The modified input cards for time-dependent modeling include:

- state - define one or more time-independent or time-dependent state properties;

- bu (or t t) - define cumulative burnup (or time) values; and

- dbu (or dt) - define incremental burnup (or time) values.

Example input files are included in the $\$\{$ SCALE $\} /$ regression/input directory:

- polaris.6.3.atrium9x9.inp and polaris.6.3 atrium10x10.inp - prototypic ATRIUM models;

- $\quad$ polaris.6.3.blade1.inp and polaris.6.3.blade2.inp - control $<$ BLADE $>$ examples;

- polaris.6.3.ge7x7.inp through polaris.6.3.ge10x10.inp - prototypic GE models;

- polaris.6.3.svea100.inp and polaris.6.3.svea64.inp - prototypic SVEA models; and

- polarisHistory.inp: history example. 


\subsection{A.1 box (Version 6.3) - channel box geometry}

\begin{tabular}{|c|c|c|c|c|}
\hline \multicolumn{5}{|c|}{ 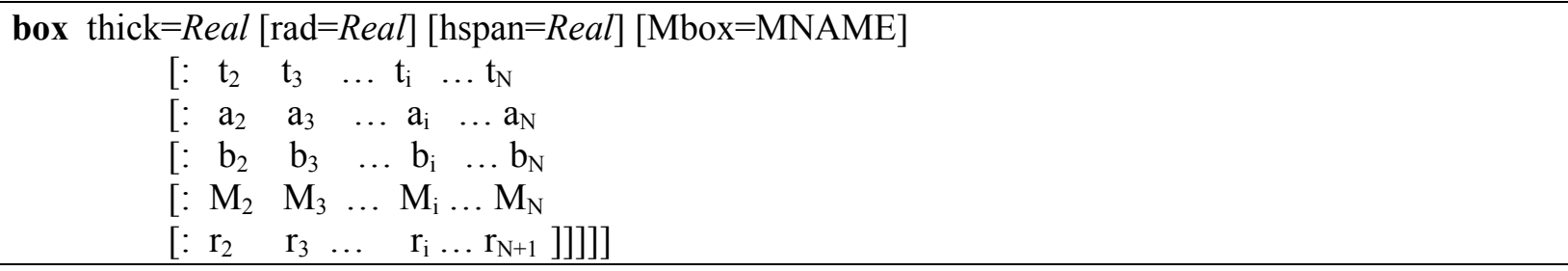 } \\
\hline param & type & name & details & default \\
\hline thick & Real & nominal thickness $(\mathrm{cm})$ & must be $>0.0$ & \\
\hline & Real & inner corner radius $(\mathrm{cm})$ & must be $\geq 0.0$ & 0.0 \\
\hline hspan & Real & half inner span $(\mathrm{cm})$ & -------See com & nts------- \\
\hline Mbox & $M N A M E$ & box material & & * \\
\hline \multicolumn{5}{|c|}{ options for additional box zones } \\
\hline $\mathrm{t}_{\mathrm{i}}$ & Real & zone thickness $(\mathrm{cm})$ & \multicolumn{2}{|l|}{ must be $\geq 0$} \\
\hline $\mathrm{a}_{\mathrm{i}}$ & Real & $\begin{array}{l}\text { distance from box centerline to bottom of zone cutout } \\
\text { region }(\mathrm{cm})\end{array}$ & \multicolumn{2}{|c|}{-----See comments----- } \\
\hline$b_{i}$ & Real & $\begin{array}{l}\text { distance from box centerline to top of zone cutout } \\
\text { region }(\mathrm{cm})\end{array}$ & \multicolumn{2}{|c|}{-----See comments----- } \\
\hline $\mathrm{M}_{\mathrm{i}}$ & $M N A M E$ & zone material & \multirow{2}{*}{\multicolumn{2}{|c|}{$\begin{array}{l}\text {-----See comments----- } \\
\text {-----See comments----- }\end{array}$}} \\
\hline ri & Real & zone inner corner radius $(\mathrm{cm})$ & & \\
\hline
\end{tabular}

\section{Examples:}

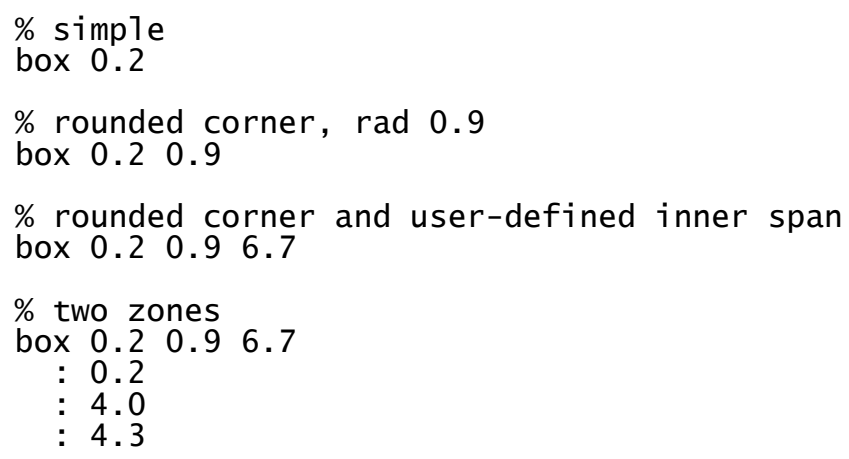

\section{Comments:}

The box specifies the channel box geometry that surrounds the pinmap. The three primary dimensions of the channel box are the thickness (thick), the inner corner radius (rad), and the half inner span (hspan). Several additional dimensions for both box and cross are defined with respect to the channel box center. The channel box center is not to be confused with the lattice center: the former is the centroid of the inner channel box square boundary and the latter will depend on the wide and narrow gap dimensions provided on the hgap card. By default, the half inner span is equal to the half pin pitch multiplied by the number of pins on each side of the assembly (see npins and ppitch on the geometry $<$ ASSM $>$ card). If a cross card is applied, the default half inner span is increased by the half width of the interior cross buffer region (see hwidth on the cross card).

Additional channel box zones can be specified on the box card. The additional zones are useful for defining thick corner regions of the channel box. Each additional zone must have a user-defined thickness 
$\left(t_{i}, i=2\right.$ to $\left.N\right)$. Note that the starting index begins at " 2 " rather than " 1 " because the zone 1 thickness has already been defined by the "thick" input field.

"Cutout regions" may be defined in which a portion of the channel box zone is replaced by the corresponding hgap material along the horizontal and vertical centerlines of the channel box. The cutout region is defined by the distance from the channel box centerline to the bottom additional channel box zone $\left(a_{i}\right)$ and the top of the channel box zone $\left(b_{i}\right)$. The values of $a_{i}$ and $b_{i}$ determine the size of trapezoidal cutout region centered along each face of the channel box. The $b_{i}$ value must be greater than or equal to the $a_{i}$ value. The $a_{i}$ value must be greater than or equal to the previous zone's $b_{i}$ value, i.e., $b_{i-1}$. By default, $a_{2}$ and $b_{2}$ are zero. If only $M$ cutout regions are specified for $N$ additional zones, i.e., $M<N$, both $a_{i}$ and $b_{i}$ is set to $b_{M}$ for $i=M+1$ to $N$.

Additional zones can also have a different inner corner radius $\left(r_{2} \ldots r_{N}\right)$. The outer corner radius of the last zone may also be specified $\left(\mathrm{r}_{\mathrm{N}+1}\right)$. By default, $\mathrm{r}_{2}$ is zero if rad is zero. If rad is greater than zero, the default value of $r_{2}$ is rad+thick. Similar rules apply for determining the default corner radii for additional zones if they are omitted in the input specification.

Additional zones can also have a different material $\left(\mathrm{M}_{\mathrm{i}}\right)$. By default, $\mathrm{M}_{2}$ is $\mathrm{M}_{\mathrm{box}}$. If additional materials are omitted in the input, the default value of $\mathrm{M}_{\mathrm{i}}$ is $\mathrm{M}_{\mathrm{i}-1}$ for $\mathrm{i}=3$ to $\mathrm{N}$.

The spatial mesh along each face of the channel box will be determined by the nf values specified on the hgap card.

The four examples listed above are displayed in Fig. 3.2.A.1. For additional examples, see the polaris.6.3 regression input files described at the beginning of 3.2.A.

\section{See also:}

geometry $<$ ASSM $>$, hgap, cross (Version 6.3) 

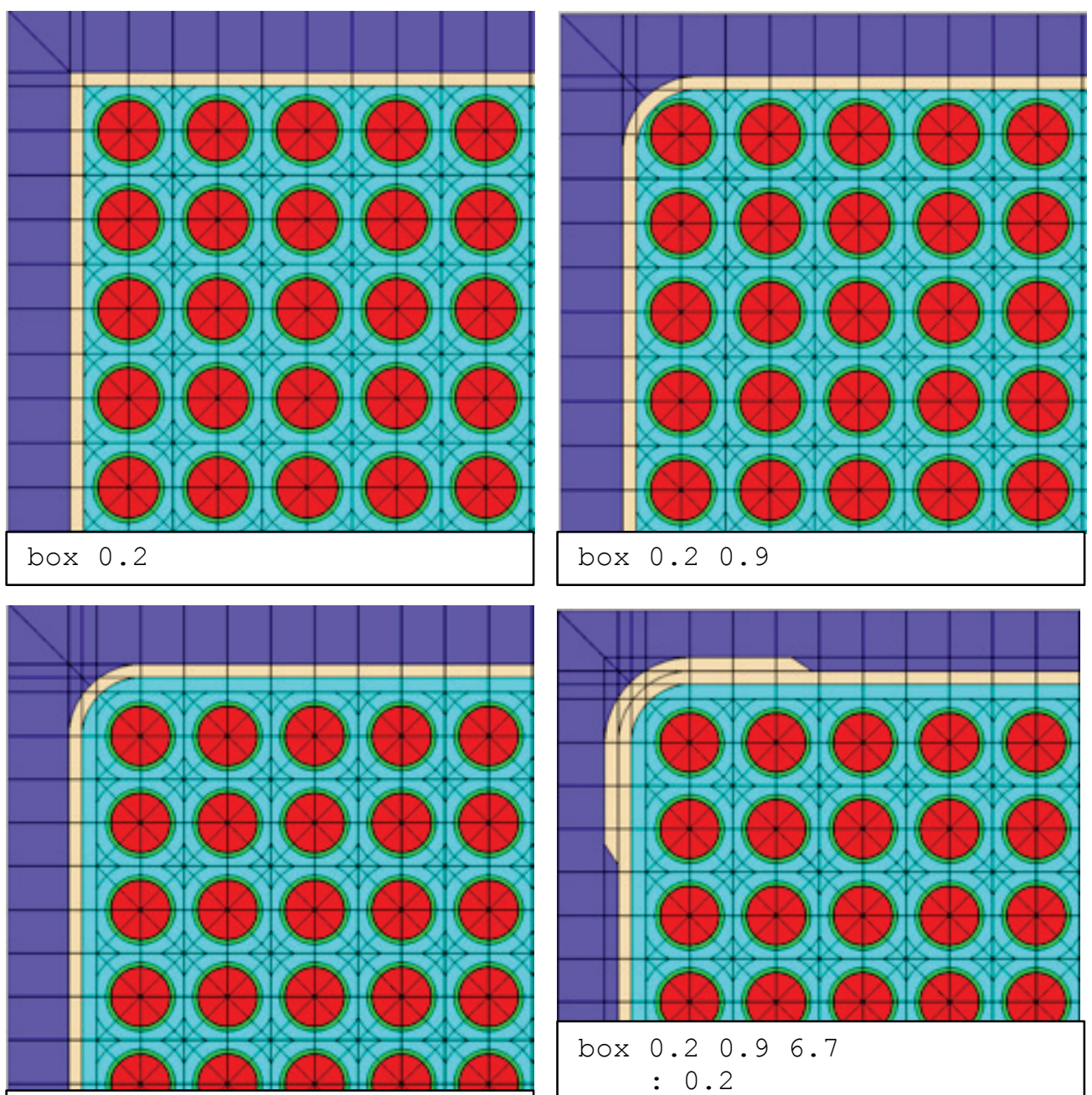

odefault hspan $=6.475 \mathrm{~cm}$ box $0.20 .9 \quad 6.7$

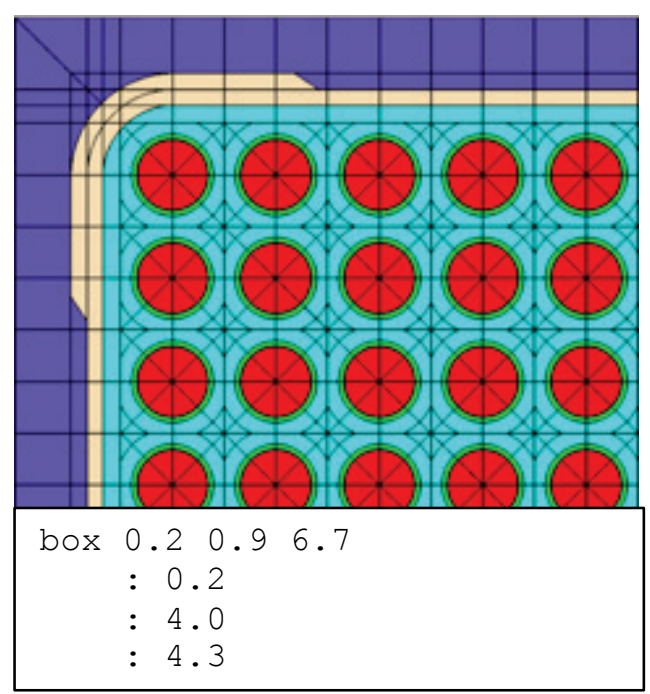

Fig. 3.2.A.1. Box card examples. 


\subsection{A.2 pin (Version 6.3) - pincell comprised of nested geometry zones of variable shape}

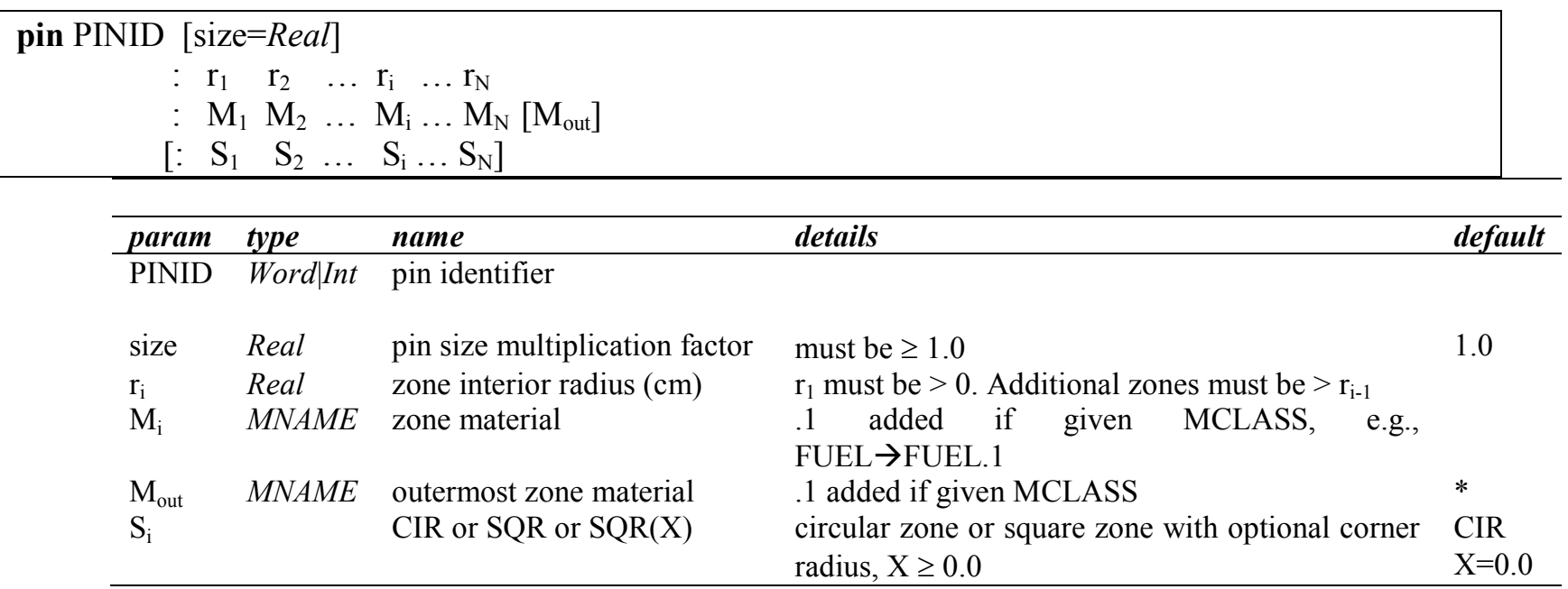

*If not specified, the material class MCLASS is taken from the channel card $\left(\mathrm{M}_{\text {chan }}\right)$ and set to the first member of that class, " $\mathrm{M}_{\text {chan }}$.1." For example if $\mathrm{M}_{\text {chan }}=$ "COOL," then $\mathrm{M}_{\text {out }}=$ "COOL.1."

\section{Examples:}

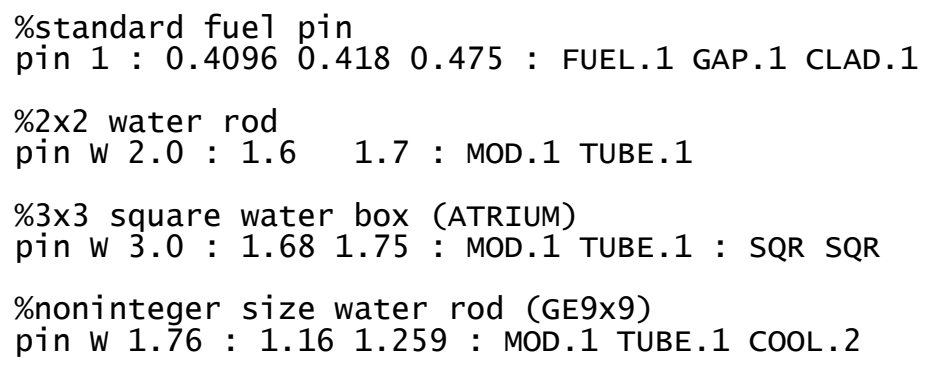

\section{Comments:}

The pin card is one of the basic building blocks of the assembly model. pin and slab are the only geometry components which allows an integer (Int) identifier as well as a Word-all other geometric identifiers use Word. Note that the materials are required, except for the last $\mathrm{M}_{\text {out }}$, which can be used to overwrite the material given by a channel for the outermost region in the pincell. The pin geometry is constructed from the inside out, using either circle zones (defined by the radius) or square zones (defined by the half-width, and optional corner radius). Different examples of pin geometries are displayed in Fig. 3.2.A.2. All meshing options for the pin are provided through the mesh card.

If the pin size is an integer value, the pin consumes a size $\times$ size subarray in the pinmap (e.g. $1 \times 1,2 \times 2$, $3 \times 3$, etc). If the pin size is noninteger, the pin consumes a ceil(size $\times$ ceil(size) subarray in the pinmap. ceil $(x)$ represents the ceiling function to round the value of $\mathrm{x}$ to the nearest integer greater than or equal to $\mathrm{x}$. For size equal to 1.3 , each instance of the pin will consume a $2 \times 2$ subarray in the pinmap. Each instance of a noninteger-sized pin must share a location with another instance of a noninteger-sized pin, but not necessarily the same pin. The shared location must be set to "_" in the pinmap. The identification of the shared location is necessary to determine the center of each pin. The pin center is at a distance of size*half pitch*sqrt(2) from the opposite corner of the shared location, along the diagonal of the pin boundary. An example of an integer-sized pins is displayed in Fig. 3.2.A.2. An example of nonintegersized pins is displayed in Fig. 3.2.A.3. 


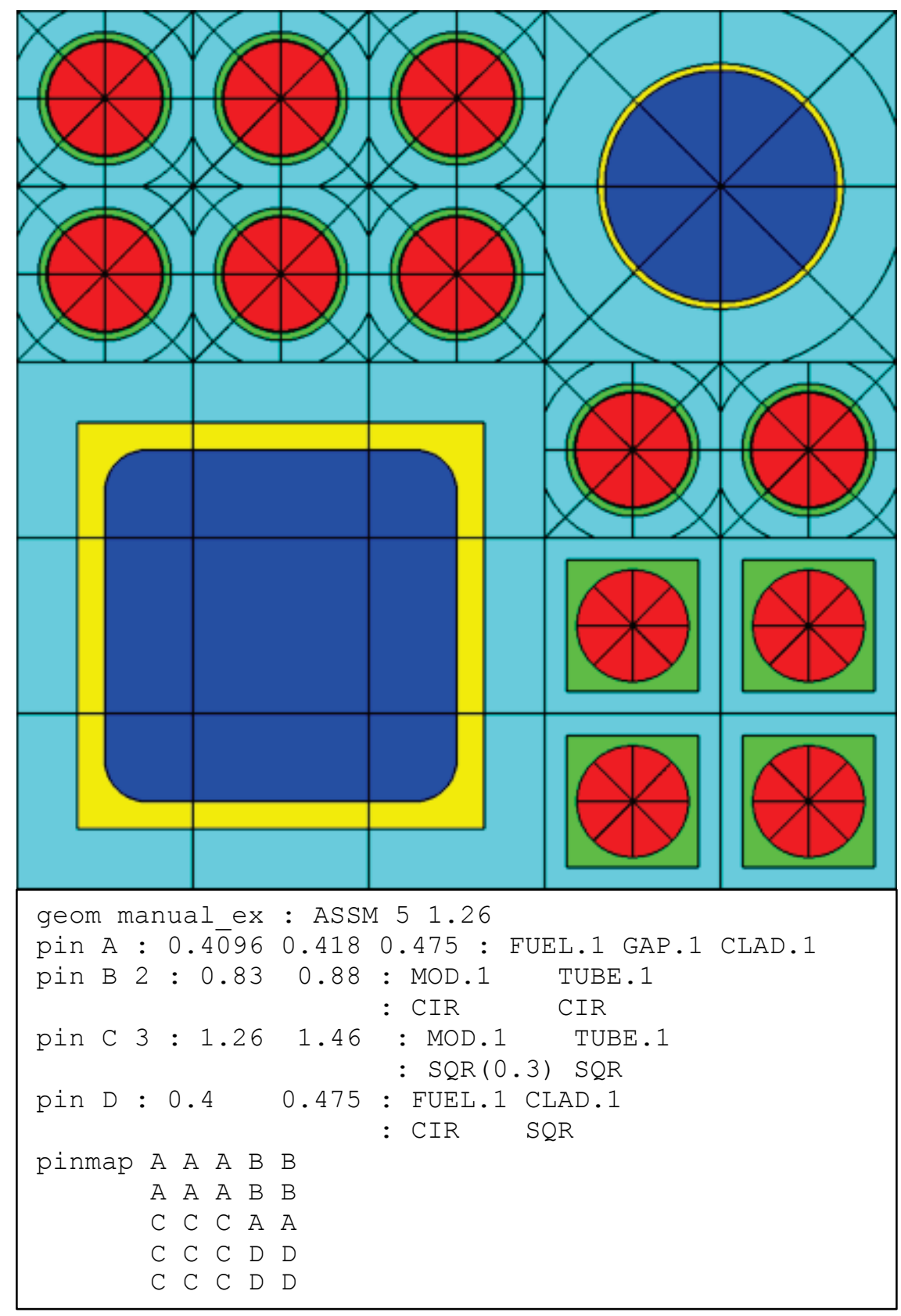

Fig. 3.2.A.2. Pin examples with different shape geometries. 


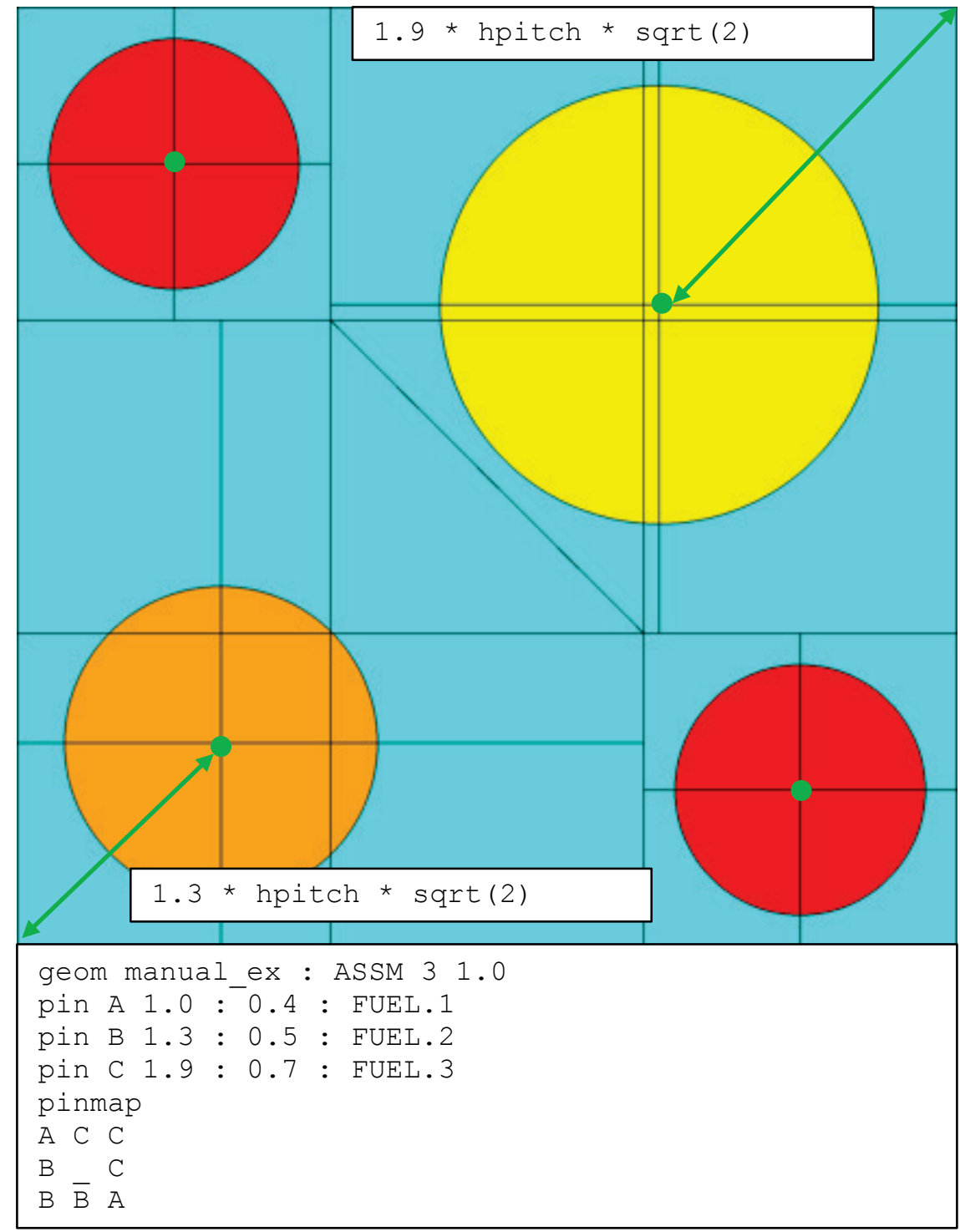

Fig. 3.2.A.3. Pin examples with noninteger pin size.

For additional examples, see the polaris.6.3 regression input files described at the beginning of 3.2.A.

See also:

slab, pinmap, channel, mesh (Version 6.3) 


\subsection{A.3 mesh (Version 6.3) - advanced material dependent meshing options}

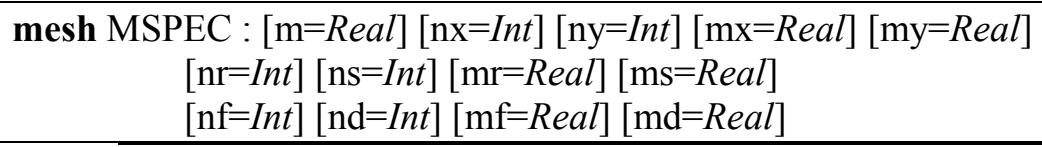

\begin{tabular}{lllll}
\hline param & type & name & details & default \\
\hline MSPEC & MCLASS $\mid$ MNAME & material identifier & & \\
& & & & \\
$\mathrm{nx}$ & Int & \# of x divisions & must be $>0$ & MeshNumX \\
$\mathrm{ny}$ & Int & \# of y divisions & must be $>0$ & MeshNumY $^{*}$ \\
$\mathrm{nr}$ & Int & \# of radial rings & must be $>0$ & MeshNumRings \\
$\mathrm{ns}$ & Int & \# of radial sectors & must be nonzero & MeshNumSectors \\
$\mathrm{nf}$ & Int & \# of faces/pin & must be $>0$ & $2^{* *}$ \\
$\mathrm{nd}$ & Int & \# of gap divisions & must be $>0$ & 1 \\
\hline meshing multipliers & & & \\
\hline $\mathrm{m}$ & Real & global multiplier & must be $>0$ & 1.0 \\
$\mathrm{mx}$ & Real & x divisions & must be $>0$ & 1.0 \\
$\mathrm{my}$ & Real & y divisions & must be $>0$ & 1.0 \\
$\mathrm{mr}$ & Real & radial rings & must be $>0$ & 1.0 \\
$\mathrm{~ms}$ & Real & radial sectors & must be $>0$ & 1.0 \\
$\mathrm{mf}$ & Real & faces/pin & must be $>0$ & 1.0 \\
$\mathrm{md}$ & Real & gap divisions & must be $>0$ & 1.0 \\
\hline
\end{tabular}

*The global mesh default values are set on the option $<$ GEOM $>$ card using the parameter name in the table above. **Default number of faces per pin is 1 . Default is 2 for system BWR or system PWR.

\section{Examples:}

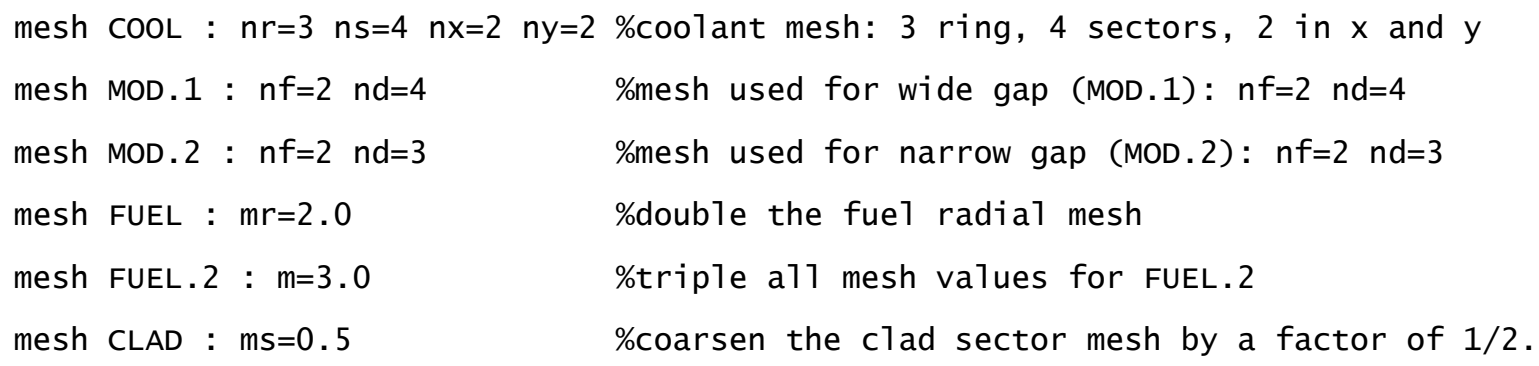

\section{Comments:}

Polaris supports three different mesh types: 1) cylindrical mesh for CIR shapes in the pin card, 2) Cartesian mesh for SQR shapes in the pin card, and 3) a special Cartesian mesh for the region external to the pinmap region. As shown in the examples above, the mesh card is used to define, refine, or coarsen the mesh parameters for one or more of the mesh types associated with a given material class or material name. The default values for mesh parameters are defined through the option $<$ GEOM $>$ card and the system card. The default values on the option $<\mathbf{G E O M}>$ card are $\mathrm{nr}=1, \mathrm{~ns}=1, \mathrm{nx}=1, \mathrm{ny}=1, \mathrm{nf}=1, \mathrm{nd}=1$, and MeshMult=1.0. The "MeshMult" multiplier from the option $<\mathbf{G E O M}>$ is a global mesh multiplier applied in conjunction with any material-specific multiplier (see option $<$ GEOM $>$ example for details). If system BWR or system PWR is applied, new default values include $n f=2, n s=8$, and $n r=2$ (only for the channel material class). If the final mesh value is noninteger, Polaris rounds down to determine the applied value.

See also: pin, system, option $<$ GEOM $>$ 


\subsection{A.4 cross (Version 6.3) - cross geometry}

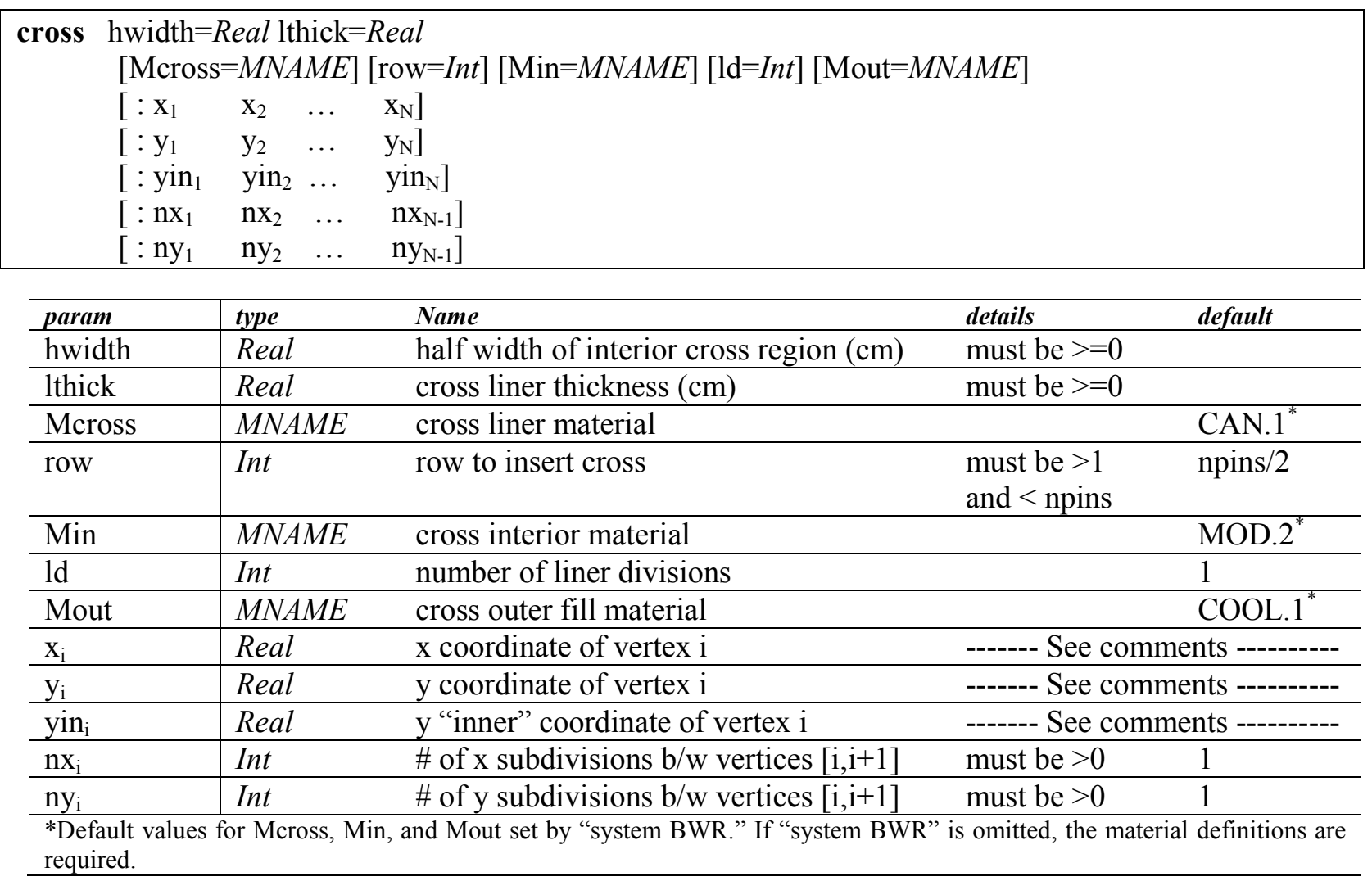

\section{Comments:}

The cross card performs two tasks. First, it subdivides the pinmap into four subarrays, optionally adding a horizontal and vertical gap between the subarrays. The row parameter is uses to subdivide the pinmap. If the pinmap is $10 \times 10$ and row $=5$, each of the four subarrays is $5 \times 5$. If the pinmap is $10 \times 10$ and row $=4$, the northwest subarray is $4 \times 4$, the northeast subarray is $4 \times 6$, the southwest subarray is $6 \times 4$, and the southeast subarray is $6 \times 6$. The hwidth parameter controls the half-spacing of the horizontal and vertical gap in between the subarrays. The hwidth parameter must be $\geq 0.0$ and if hwidth is $>0.0$, the gap is filled with material $\mathrm{M}_{\text {out }}$ (default is COOL.2 with system BWR).

The second task is the insertion of the cross structure into the lattice geometry. The process is described with reference to the example in Fig. 3.2.A.4. In the example, the pinmap is $9 \times 9$ and row $=3$, hwidth $=1.5$, and hspan=10.5. The top left plot contains the four following lines:

1. the line in the center of the vertical cross gap,

2. the line in the center of the horizontal cross gap,

3. the diagonal line from the northwest (NW) channel box corner to the southeast (SE) corner, and

4. the diagonal line, perpendicular to line 3, passing through the intersection of line 1 and line 2 .

These four lines intersect and form 8 separate regions, i.e., octants, within the channel box interior. The intersection point, i.e., cross center, is not necessarily equal to the box center as shown in this example. In the top left plot, the red triangle represents the WNW octant. In the bottom left plot, the red triangle represents the SSE octant. 


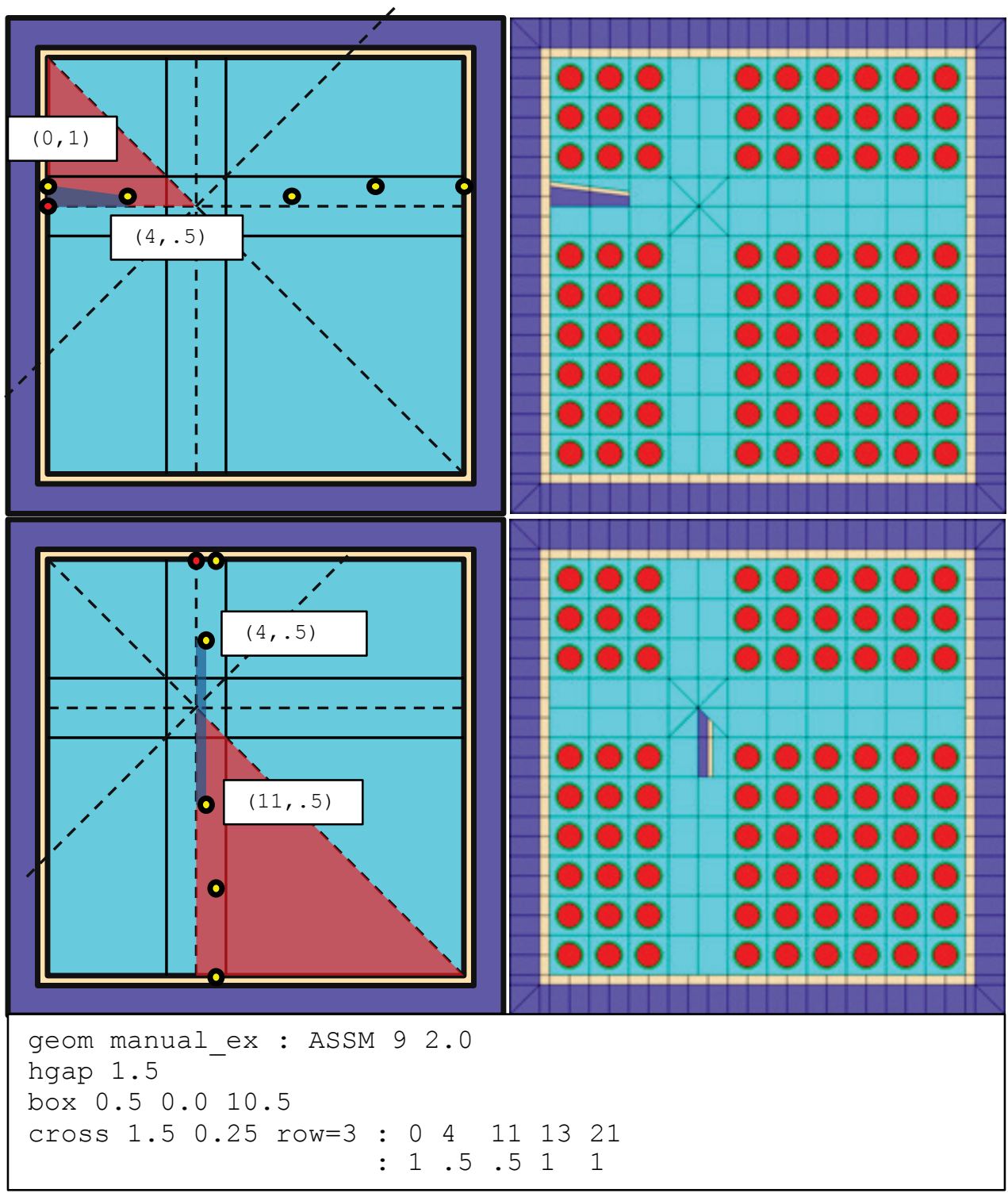

Fig. 3.2.A.4. Construction of the BWR cross geometry (full example shown later).

The cross structure is defined be a series of vertices $\left(\mathrm{x}_{\mathrm{i}}, \mathrm{y}_{\mathrm{i}}\right)$. Shown as yellow points in the top left plot, the cross vertices are defined based on an origin displayed as the red point, which is the intersection of the inner west edge of the channel box and the horizontal line in that passes through the cross center.

The top plots demonstrate how Polaris inserts a section of the cross into the WNW octant. In the top left plot, the blue polygon is constructed based on the first two vertices defined on the cross card: $(0.0,1.0)$ and $(4.0,0.5)$. The intersection of the blue polygon and red polygon is inserted into the lattice and filled with cross interior material $\left(\mathrm{M}_{\mathrm{in}}\right)$. The liner is then inserted above the blue polygon, padded by the liner thickness (lthick), and clipped by WNW red polygon if needed.

Similarly, the bottom plots demonstrate insertion into the SSE octant. For SSE insertions, the origin and the cross vertices are rotated 90 degrees about the cross center. The blue polygon is constructed from the second and third vertices on the cross card: $(4.0,0.5)$ and $(11,0.5)$. The intersection of the blue polygon and red polygon is inserted into the lattice and filled with cross interior material $\left(\mathrm{M}_{\mathrm{in}}\right)$. The liner is then 
inserted above the blue polygon, padded by the liner thickness (lthick), and clipped by SSE red polygon if needed.

For each consecutive set of cross vertices, Polaris inserts a polygonal region into each of the 8 octants. The cross vertices are entered in the input as an $x$-values list followed by a y-values list of the same length. The coordinate system of the $\mathrm{x}$ - and $\mathrm{y}$ - lists is displayed in the top left plot of Fig. 3.2.A.4. The coordinate system is transformed based on the following rules for each octant:

- WNW, ENE: no transform,

- NNW, SSW: reflected across the diagonal line from NW to SE channel box corners,

- NNE, SSE: rotated 90 degrees about the cross center, and

- ESE, WSW: reflected across the line in the center of the horizontal cross gap.

The cross liner is inserted above the cross vertex values. The liner has a uniform thickness (lthick) and uniform material $\left(\mathrm{M}_{\text {cross }}\right)$. The uniform liner thickness is constructed with a miter joint at each cross vertex as shown in the following diagram:

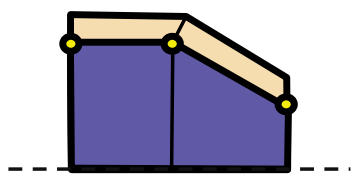

After the set of y-values on the cross card, an optional list of interior $y$-values can be specified. The length of the interior $y$-values list must be equal to the length of the $\mathrm{x}$ - and $\mathrm{y}$ - lists. The optional interior $\mathrm{y}$-values list is used to split the polygons into two material regions as shown in the following diagram:

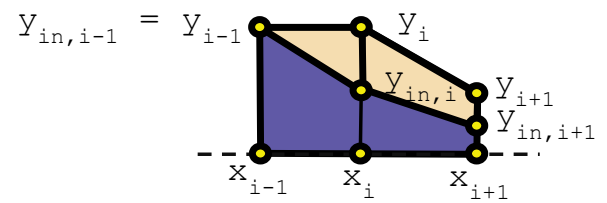

For the left-hand polygon, $\mathrm{y}_{\mathrm{in}, \mathrm{i}-1}$ is the same as $\mathrm{y}_{\mathrm{i}-1}$, but $\mathrm{y}_{\mathrm{in}, \mathrm{i}}$ is less than $\mathrm{y}_{\mathrm{i}}$. In this scenario, the trapezoid region is filled with $\mathrm{M}_{\mathrm{in}}$, and the triangular region is filled with $\mathrm{M}_{\text {cross. }}$. Similarly for the right-hand polygon, the lower trapezoid is filled with $\mathrm{M}_{\text {in }}$ and the upper trapezoid is filled with $\mathrm{M}_{\text {cross. }}$. Note that the uniform liner above the $y$-values is not shown for simplicity.

The interior y-list values can be specified in one of two ways. First, a positive value may be entered that is greater than or equal to zero and less than or equal to the corresponding y-value. Second, a negative value may be entered that represents the relative distance of the interior y-value below the corresponding $y-$ value. Note the Polaris input processor interprets "-0" different than "0". "-0" implies that the internal yvalue is the same as the y-value. " 0 " implies that the internal y-value is zero. If the interior y-list is omitted, the default for all internal y-values is "-0", i.e., the polygon regions defined are completely filled with $\mathrm{M}_{\text {in }}$.

In addition to the interior $\mathrm{y}$-values list, two additional lists can be used to refine the spatial mesh in the $\mathrm{x}$ and $\mathrm{y}$ - directions. The list of nx- and ny- values subdivide the polygon regions along the $\mathrm{x}-$ and $\mathrm{y}-$ directions respectively. Both lists must have one less entry than the $x_{-}, y_{-}$, and $y_{i n}-$ lists. If omitted, the default values for both the nx- and ny- lists are 1, i.e., no additional spatial refinement is applied to the polygon regions. For refinement in the y-direction, only the $M_{\text {in }}$ material is refined. The following diagram shows $n x=2$ refinement for the left polygon and $n y=2$ refinement for the right polygon: 


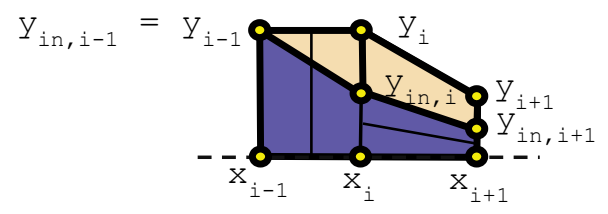

The full cross example from Fig. 3.2.A.4 is displayed in the top left plot of

Fig. $\quad 3.2$. A.5. The bottom plot shows a centered cross structure with a diamond water box and empty pins surrounding the water box.

For additional examples, see the polaris.6.3 regression input files described at the beginning of 3.2.A.

\section{See also:}

\section{box (Version 6.3), system BWR, pinmap}

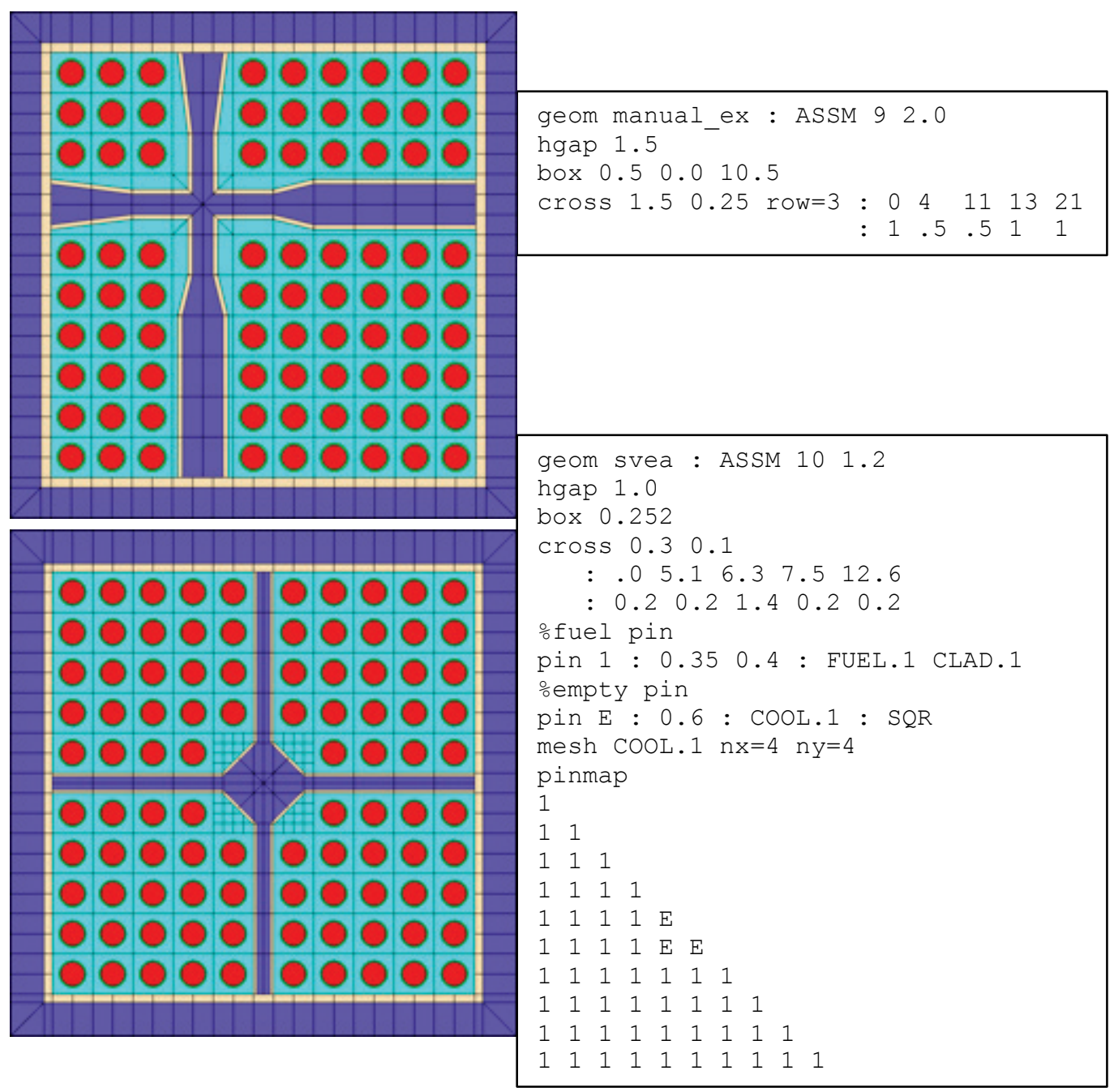

Fig. 3.2.A.5. Additional cross examples. 


\subsection{A.5 dxmap and dymap (Version 6.3) - pin-by-pin displacement maps}

$$
\begin{array}{lllllll}
\operatorname{dxmap} & d_{1} & d_{2} & \ldots & d_{i} & \ldots & d_{N} \\
\operatorname{dymap} & d_{1} & d_{2} & \ldots & d_{i} & \ldots & d_{N}
\end{array}
$$

\begin{tabular}{llll}
\hline param & type & details & default \\
\hline $\mathrm{d}_{\mathrm{i}}$ & Real & pin center displacement value in the $\mathrm{x}$ or $\mathrm{y}$ direction $(\mathrm{cm})$ & 0.0 \\
\hline
\end{tabular}

\section{Comments:}

The dxmap and dymap cards displace pins from their natural position in the geometry (see comments for the pin card). If displacement maps are required, both the dxmap and dymap must be specified in the input and they must have the same length. However, the length of the displacement maps does not have to equal of length of the pinmap if the displacement maps have reduced symmetry. For integer-sized pins greater than 1 , the displacement value should be entered in the northwest corner element of the size $\times$ size subarray. For noninteger-sized pins, the displacement value should be in the corner element opposite of shared corner location. Note the following symmetry restrictions for the displacement maps:

- $\mathrm{dy}_{\mathrm{i}}$ value must be zero on a horizontal symmetry line for an odd $\times$ odd pinmap,

- $\mathrm{dx}_{\mathrm{i}}$ value must be zero on a vertical symmetry line for an odd $\times$ odd pinmap, and

- $\mathrm{dx}_{\mathrm{i}}$ must equal $\mathrm{dy}_{\mathrm{i}}$ for an element on a diagonal symmetry line.

\section{Examples:}

$\begin{array}{rrrrr}\text { dxmap } & & & & \\ 0.0 & 0.0 & 0.0 & 0.0 & 0.0 \\ 0.0 & 0.2 & 0.0 & 0.0 & -0.2 \\ 0.0 & 0.0 & 0.0 & 0.0 & 0.0 \\ 0.0 & 0.0 & 0.0 & 0.0 & 0.0 \\ 0.0 & 0.0 & 0.0 & 0.0 & 0.0 \\ \text { dymap } & & & & \\ 0.0 & 0.0 & 0.0 & 0.0 & 0.0 \\ 0.0 & 0.0 & 0.0 & 0.0 & 0.0 \\ 0.0 & 0.0 & 0.0 & 0.0 & 0.0 \\ 0.0 & 0.2 & 0.0 & 0.0 & 0.0 \\ 0.0 & 0.0 & 0.0 & -0.1 & 0.0\end{array}$

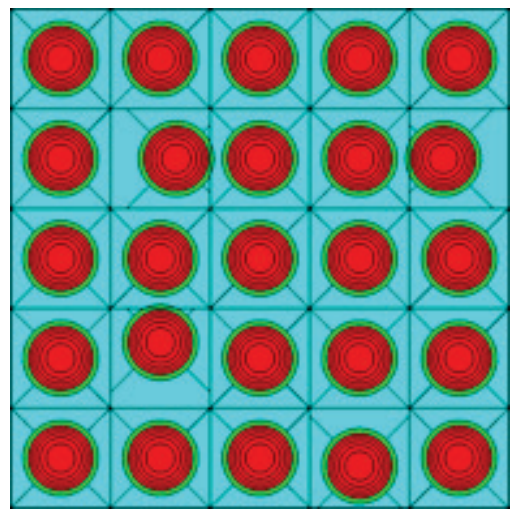

See also:

pinmap 


\subsection{A.6 control $<$ BLADE $>($ Version 6.3) - BWR control blade}

\begin{tabular}{|c|c|c|c|c|}
\hline \multicolumn{5}{|c|}{$\begin{array}{l}\text { control INAME : BLADE hwgthck=Real sththck=Real cslnth=Real } \\
\text { [sthmat=MNAME] [csmat=MNAME] [hcsthck=Real }[\text { wgcrv=Real }] \\
: \mathrm{ID}_{1} \mathrm{ID}_{2} \ldots \mathrm{ID}_{\mathrm{N}} \\
: \mathrm{L}_{1} \mathrm{~L}_{2} \ldots \mathrm{L}_{\mathrm{N}} \\
{\left[: \mathrm{N}_{1} \mathrm{~N}_{2} \ldots \mathrm{N}_{\mathrm{N}}\right]}\end{array}$} \\
\hline param & type & Name & details & default \\
\hline hwgthck & Real & half blade wing thickness $(\mathrm{cm})$ & must be $>0$ & \\
\hline sththck & Real & sheath thickness $(\mathrm{cm})$ & must be $>=0$ & \\
\hline cslnth & Real & central support length $(\mathrm{cm})$ & must be $>=\mathrm{h}$ & \\
\hline sthmat & $M N A M E$ & sheath material & & STRUCT.1 ${ }^{*}$ \\
\hline csmat & $M N A M E$ & central support material & & STRUCT.1 ${ }^{*}$ \\
\hline hesthck & Real & half central support thickness $(\mathrm{cm})$ & must be $>0$ & hwgthck \\
\hline wgerv & Real & wing tip radius $(\mathrm{cm})$ & must be $>=0$ & 0 \\
\hline $\mathrm{ID}_{\mathrm{i}}$ & Word $\mid$ Int & pin or slab identifier & ------- See co & -------- \\
\hline $\mathrm{L}_{\mathrm{i}}$ & Real & length of section $\mathrm{i}$ & ------ See co & $\begin{array}{l}----- \\
-\cdots-1\end{array}$ \\
\hline $\mathrm{N}_{\mathrm{i}}$ & Real & \# of pins or slabs in section $\mathrm{i}$ & ------- See co & ------- \\
\hline
\end{tabular}

\section{Comments:}

The blade card defines a control blade geometry. The control blade identifier (INAME) can be used to insert the control blade using state or add statements to define histories or branches respectively. INAME $=$ yes inserts the control blade into the northwest corner of the lattice.

The control blade geometry is described with reference to the blade wing on the northern edge of the lattice in Fig. 3.2.A.6. The blade wing on the west edge is a reflection of the northern edge wing along the diagonal symmetry line that extends from the northwest corner to the southeast corner of the lattice.

The two primary regions of the blade are the central support and the active blade wing. The central support has a length (cslnth), half width (hcsthck), and material (csmat). The central support half width is the vertical distance between the north face of the lattice and the south boundary of the central support. The central support length is the horizontal distance from west face of the lattice to the east boundary of the central support. See Fig. 3.2.A.6 for details.

The active portion of the blade wing begins at the east boundary of the central support. The active portion has a half width (hwgthck), sheath thickness (sththck), sheath material (sthmat), and wing tip radius (wgcrv). The half width is the vertical distance between the north face of the lattice and the southern boundary of the active blade wing, including the sheath. The wing tip radius can be any nonnegative number. If the radius is zero, the wing tip is a straight edge.

The active portion of the blade wing is subdivided into sections. Each section has a length $\left(\mathrm{L}_{\mathrm{i}}\right)$, and identifier associated with a pin or slab $\left(\mathrm{ID}_{\mathrm{i}}\right)$, and the number of pins or slabs for each section $\left(\mathrm{N}_{\mathrm{i}}\right)$. The list of section lengths and section identifiers is required and must have consistent list lengths. The final list for number of pin/slabs per section is optional. If omitted, the default number of pin or slabs per section is one. 


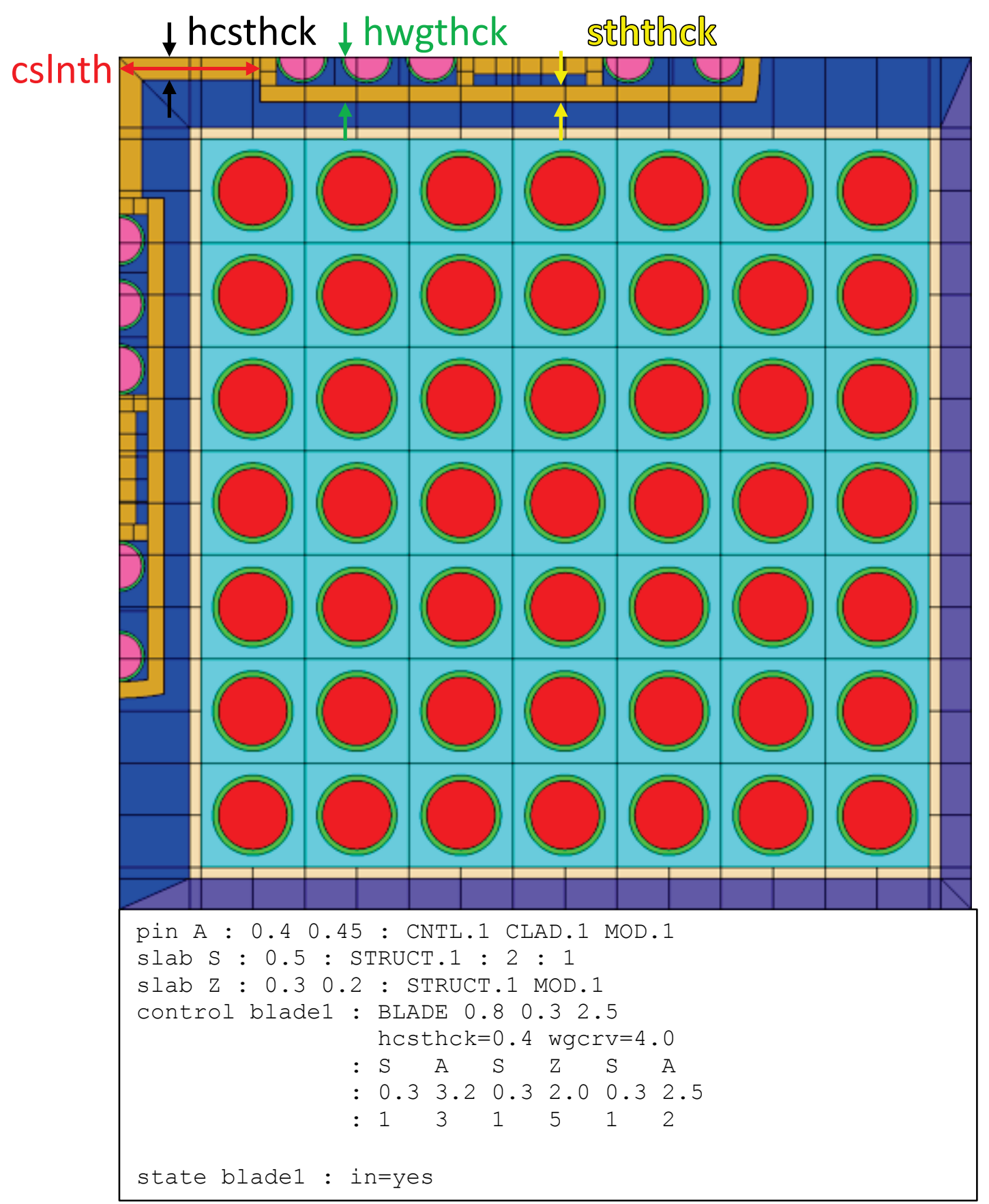

Fig. 3.2.A.6. Control blade example.

If there is only one pin in a pin section, the pin is placed in the section center. If there are multiple pins, the first and last pin are positioned flush against the west and east section boundary respectively, and the interior pins are uniformly spaced between the two edge pins. Slab sections are built from the blade centerline in the vertical direction towards the interior sheath boundary. Each slab zone has width equal to the section length. Each slab zone can be subdivided in the vertical direction by the zone nx parameter on the slab card. The slab zone can be subdivided in the horizontal direction by the product of the zone ny parameter and the number of slabs in the blade wing section. 
The blade is further subdivided by the mesh nf/nd settings for hgap material in the north and west bypass region, typically MOD.1 for models that include system BWR.

See also: pin (Version 6.3), slab, mesh (Version 6.3), system BWR

\subsection{A.7 option $<$ GEOM $>($ Version 6.3) - geometry options}

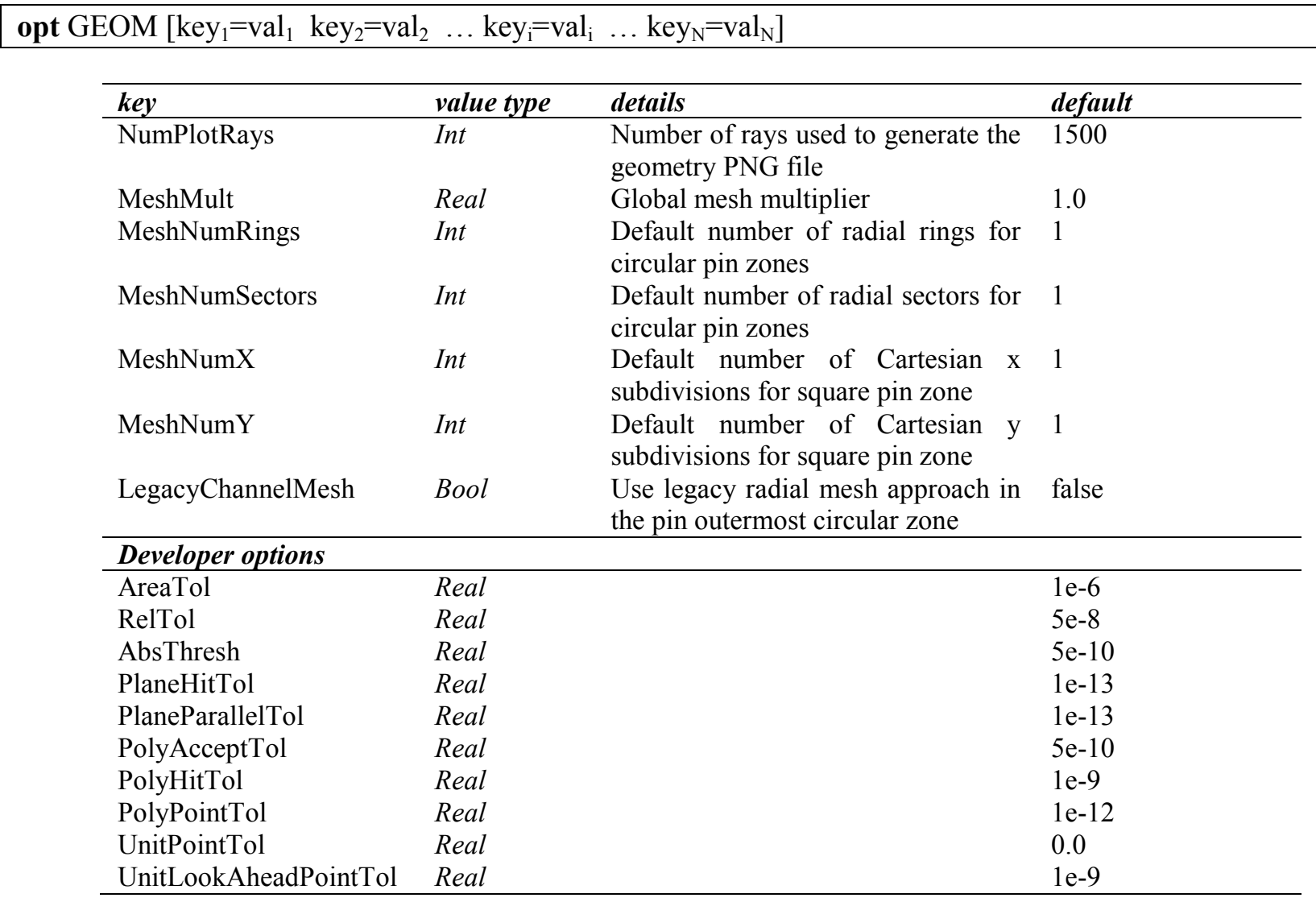

\section{Examples:}

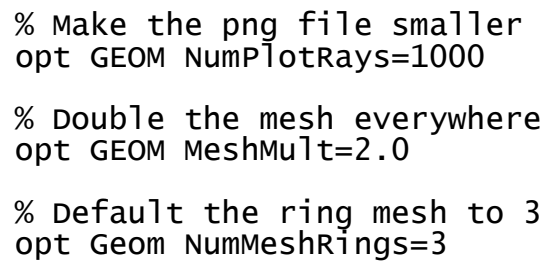

See also: mesh (Version 6.3) 


\subsection{A.8 bu (Version 6.3) - initiate calculation with cumulative burnups}

\begin{tabular}{|c|c|c|c|}
\hline \multicolumn{4}{|c|}{ 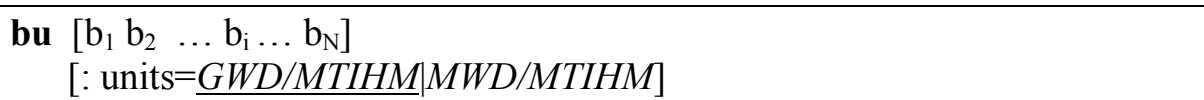 } \\
\hline param & Type & name & default \\
\hline$b_{i}$ & Real & $\begin{array}{l}\text { list of } \\
\text { burnups }\end{array}$ & 0 \\
\hline units & $\begin{array}{l}\text { GWD/MTIHM| } \\
\text { MWD/MTIHM }\end{array}$ & burnup units & GWD/MTIHM \\
\hline
\end{tabular}

\section{Examples:}

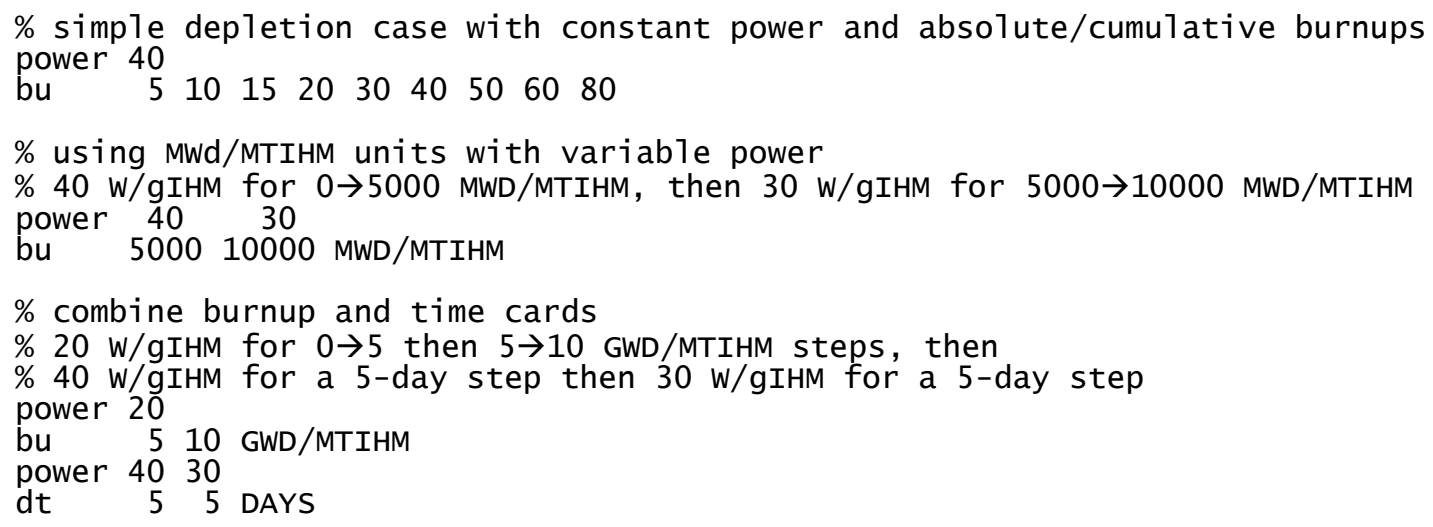

\section{Comments:}

The bu card initiates a calculation for a given sequence of cumulative/absolute burnups. A burnup or time card usually follows a power card, the two effectively specifying the power history. If multiple burnups are given, then the power card must have either a single power or a list of powers the same size as the list times. A value of 0 is implicit at the beginning of the first burnup list. Multiple burnup/time cards may be specified in an input. This can be convenient for switching units or changing from burnup-based to timebased depletion. Internal automatic substeps are always in effect unless modified with the option $<$ DEPL $>$ card.

See also:

t, dt, ti, bui, dbu, power, option $<$ DEPL $>$, branch, deplete 


\subsection{A.9 bui (Version 6.3) - initiate calculation with cumulative burnups (with restart)}

bui $\left[b_{1} b_{2} \ldots b_{i} \ldots b_{N}\right]$ $[:$ units $=\underline{G W D / M T I H M} \mid M W D / M T I H M]$

\begin{tabular}{llll}
\hline param & Type & name & default \\
\hline $\mathrm{b}_{\mathrm{i}}$ & Real & $\begin{array}{l}\text { list of } \\
\text { burnups } \\
\text { burnup units }\end{array}$ & GWD/MTIHM \\
units & $\begin{array}{l}\text { GWD/MTIHM } \\
\text { MWD/MTIHM }\end{array}$ & & \\
\hline
\end{tabular}

\section{Examples:}

power 30

bui 510 \%equivalent to: bu 510

power 40

bui 510 \%equivalent to: bu 1520

\section{Comments:}

The bui card initiates a calculation for a given sequence of cumulative burnups. If only one burnup list is provided, the bui card is identical to the bu card. For any subsequent burnup list, the bui card specifies cumulative burnups that restart at zero at the beginning of each list (see example above).

See also:

t, dt, ti, bu, dbu, power, option<DEPL $>$, branch, deplete 


\subsection{A.10 dbu (Version 6.3) - initiate calculation with incremental burnups}

dbu $\left[b_{1} b_{2} \ldots b_{i} \ldots b_{\mathrm{N}}\right]$

$[:$ units $=\underline{G W D / M T I H M} \mid M W D / M T I H M]$

\begin{tabular}{|c|c|c|c|c|}
\hline param & Type & name & details & default \\
\hline $\mathrm{b}_{\mathrm{i}}$ & Real & $\begin{array}{l}\text { list of } \\
\text { incremental burnups }\end{array}$ & & 0 \\
\hline units & $\begin{array}{l}\text { GWD/MTIHM| } \\
\text { MWD/MTIHM }\end{array}$ & burnup units & & GWD/MTIHM \\
\hline
\end{tabular}

\section{Examples:}

$\%$ incremental burnups equivalent to

$\%$ power 40

\% bu $0 \begin{array}{lllllllll}5 & 10 & 15 & 20 & 30 & 40 & 50 & 60 & 80\end{array}$

power 40

dbu $5 \begin{array}{llllllll}5 & 5 & 5 & 10 & 10 & 10 & 10 & 20\end{array}$

\section{Comments:}

The dbu card initiates a calculation for a given sequence of incremental burnups. Otherwise, it is identical to the bu card for specifying cumulative burnups.

See also:

t, dt, ti, bu, bui, power, option $<$ DEPL $>$, branch, deplete 


\subsection{A.11 t (Version 6.3) - initiate calculation by cumulative time}

$\mathbf{t}\left[\mathrm{t}_{1} \mathrm{t}_{2} \ldots \mathrm{t}_{\mathrm{i}} \ldots \mathrm{t}_{\mathrm{N}}\right]$

[: units $=S E C O N D S|M I N U T E S| H O U R S|\underline{D A Y S}|$ YEARS $]$

\begin{tabular}{lllll}
\hline param & Type & name & details & default \\
\hline $\mathrm{t}_{\mathrm{i}}$ & Real & $\begin{array}{l}\text { list of } \\
\text { times }\end{array}$ & 0 & 0 \\
\multirow{2}{*}{ units } & SECONDS $\mid$ MINUTES $\mid$ & time units & DAYS \\
& HOURS $\mid$ DAYS $\mid$ YEARS & & \\
\hline
\end{tabular}

\section{Examples:}

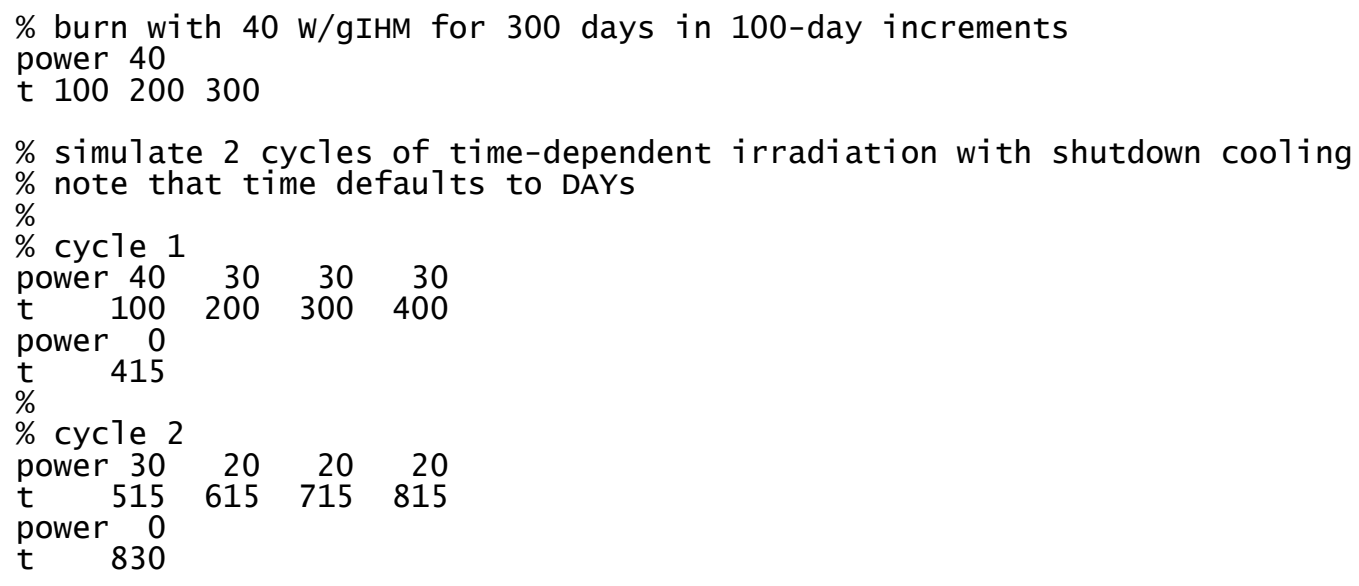

\section{Comments:}

The $\mathbf{t}$ card initiates a calculation for a given sequence of cumulative/absolute times. One of the time cards (t, dt, or ti) is required to model periods of decay in conjunction with power 0 . Otherwise, the time card $\mathbf{t}$ is similar in functionality to the burnup bu card but with different units.

See also:

ti, dt, bu, bui, dbu, power, option $<$ DEPL $>$, branch, deplete 


\subsection{A.12 ti (Version 6.3) - initiate calculation by cumulative time (with restart)}

$\mathbf{t}\left[\begin{array}{llll}\mathrm{t}_{1} \mathrm{t}_{2} & \ldots & \mathrm{t}_{\mathrm{i}} \ldots \mathrm{t}_{\mathrm{N}}\end{array}\right]$

[: units=SECONDS|MINUTES|HOURS|DAYS $\mid$ YEARS]

\begin{tabular}{lllll}
\hline param & Type & name & details & default \\
\hline $\mathrm{t}_{\mathrm{i}}$ & Real & $\begin{array}{l}\text { list of } \\
\text { times }\end{array}$ & 0 & 0 \\
\multirow{2}{*}{ units } & SECONDS $\mid$ MINUTES $\mid$ & time units & DAYS \\
& HOURS $\mid$ DAYS $\mid$ YEARS & & \\
\hline
\end{tabular}

\section{Example with t card:}

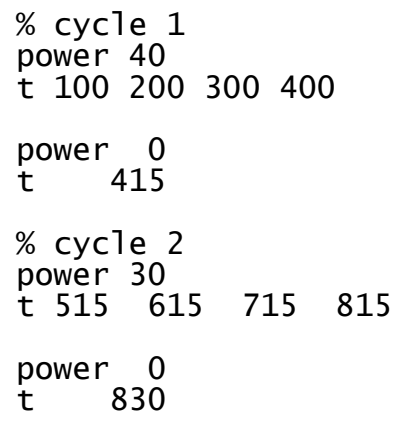

Equivalent example with ti card:

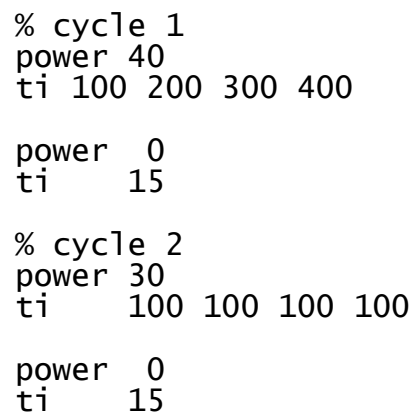

\section{Comments:}

The ti card initiates a calculation for a given sequence of cumulative times. If only one time list is provided, the ti card is identical to the $\mathbf{t}$ card. For any subsequent time list, the ti card specifies cumulative times that restart at zero at the beginning of each list (see example above).

See also:

t, dt, bu, bui, dbu, power, option $<$ DEPL $>$, branch, deplete 
3.2.A.13 dt (Version 6.3) - initiate calculation by incremental time

$\mathbf{t}\left[\mathrm{t}_{1} \mathrm{t}_{2} \ldots \mathrm{t}_{\mathrm{i}} \ldots \mathrm{t}_{\mathrm{N}}\right]$

[: units=SECONDS|MINUTES|HOURS|DAYS $\mid$ YEARS $]$

\begin{tabular}{|c|c|c|c|}
\hline param & Type & details & default \\
\hline $\mathrm{t}_{\mathrm{i}}$ & Real & $\begin{array}{l}\text { list of } \\
\text { times }\end{array}$ & 0 \\
\hline units & $\begin{array}{l}\text { SECONDS|MINUTES } \mid \\
\text { HOURS|DAYS|YEARS }\end{array}$ & time units & DAYS \\
\hline
\end{tabular}

\section{Examples:}

$\%$ burn with $40 \mathrm{~W} /$ gIHM for 300 days in 100-day increments equivalent to

$\%$ power 40

$\% \quad \mathrm{t} 100200300$

power 40

dt 100100100

\% decay for 30 minutes

power 0

dt 30 MINUTES

\section{Comments:}

The $\mathbf{d t}$ card is identical to the cumulative time card $\mathbf{t}$ except that the values given are incremental.

See also:

t, ti, bu, bui, dbu, power, option<DEPL $>$, branch, deplete 


\subsection{A.14 state (Version 6.3) - property state specification}

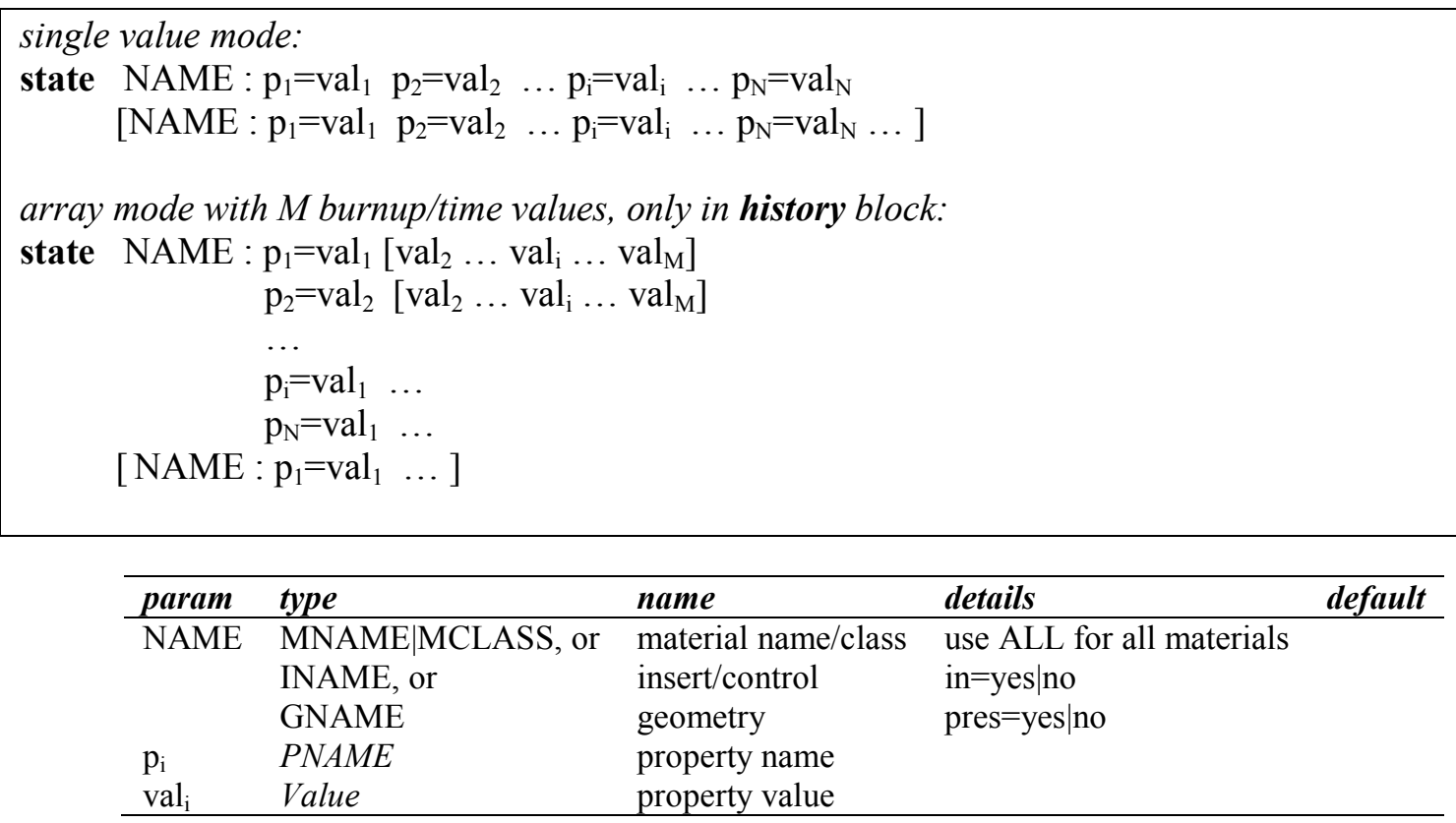

\section{Examples:}

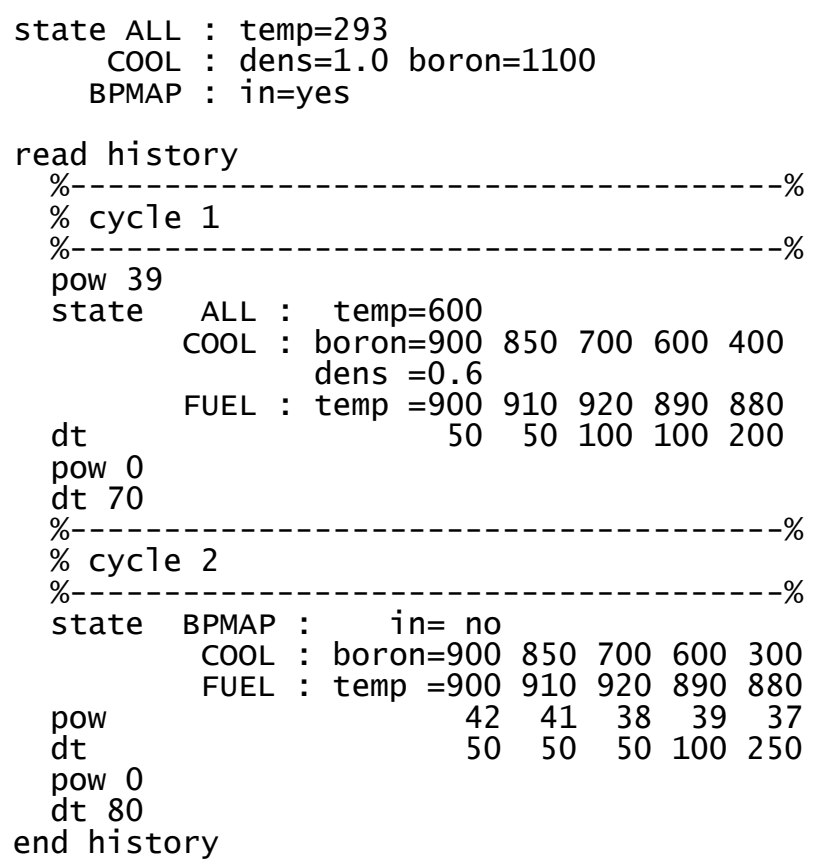

\section{Comments:}

The state card is used to specify properties for different materials, control maps, control blades, and insert maps. The state card can specify one or multiple properties simultaneously. The property specifications can either be a single value or multiple values, each value corresponding to a burnup/time step in the burnup/time input card (bu|bui| $\mathbf{d} \mathbf{b u}|\mathbf{t}| \mathbf{t i} \mid \mathbf{d t})$. 
The state card can be used outside a history block or inside a history block. Outside the history block, i.e., at the "root" input level, a single state card can initialize property values to a single value. If one or more power histories are defined at the root input level, the state properties are constant throughout the calculation. The state properties are only modified for branch calculations.

Time-dependent state properties are allowed through the history block. One or more power histories are allowed inside a history block. Each power history contains a power card (single value or array value) and a burnup/time card (bu|bui|dbu|t|ti|dt). Before the burnup/time card, a state card can be used to define one or more state properties. The property specifications are either a single value or an array of values that correspond to each burnup/time step. If a property is omitted in a state card, the value is defined based on the following precedence:

- the last value specified through the closest preceding state card in a given history block, or

- the value specified in the state card at the root input level, or

- the default property value.

By default, all control or insert geometries are not inserted (in=no). Material property defaults are defined on the material card or through the system card.

See also:

history, system 


\subsection{A.15 history (Version 6.3) - time-dependent history}

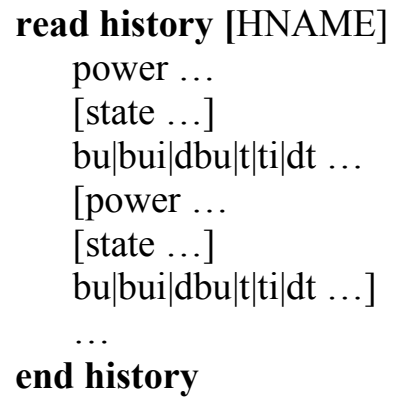

\begin{tabular}{lllll}
\hline param & type & Name & details & default \\
\hline HNAME & Word & history name & DEFAULT
\end{tabular}

allowed cards in history block

$\begin{array}{llll}\text { power } & - & - & \text { specific power } \\ \text { state } & - & - & \text { state properties } \\ \text { bu|bui|dbu|t|ti|dt } & - & - & \text { burnup or time }\end{array}$

\section{Examples:}

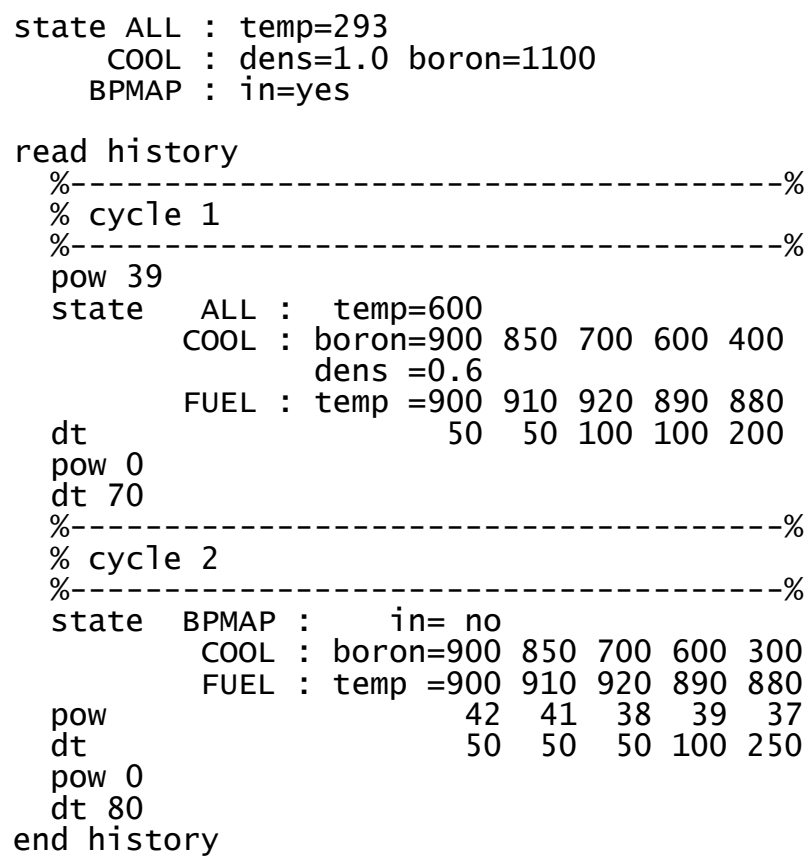

\section{Comments:}

The history card initiates a time-dependent calculation with user defined power history and timedependent material or geometry properties. Each history block is independent from one another. Each history calculation generates an ORIGEN binary concentration file with the name filename_hname.f71 where filename is the root name of the input file and hname is the name for the history. Similarly, if fewgroup cross section files are requested, the filenames are filename_hname.t16. 
History calculations are also allowed in conjunction with branch calculation. At this time, all history calculations will perform all branch calculations defined in the input file. Selection of the burnup values for branch calculations is available on the option $<$ FG $>$ card.

See also:

state, branch, option<FG>, power, bu, bui, dbu, t, ti, dt 


\subsection{RADIATION SHIELDING}

\section{Introduction by D. E. Peplow and B. T. Rearden}

The Monaco with Automated Variance Reduction using Importance Calculations (MAVRIC) fixedsource radiation transport sequence is designed to apply the multigroup and continuous energy fixedsource Monte Carlo code Monaco to solve problems too challenging for standard, unbiased Monte Carlo methods. ${ }^{1}$ The intention of the sequence is to calculate fluxes and dose rates with low uncertainties in reasonable times even for deep penetration problems. MAVRIC is based on the Consistent Adjoint Driven Importance Sampling (CADIS) methodology, which uses an importance map and biased source that are derived to work together. MAVRIC generates problem-dependent cross section data and then automatically performs a coarse mesh 3D discrete ordinates transport calculation using Denovo to determine the adjoint flux as a function of position and energy. MAVRIC then applies the information to optimize the shielding calculation in Monaco. In the Forward-Weighted CADIS (FW-CADIS) methodology, an additional Denovo calculation is performed to further optimize the Monaco model to obtain uniform uncertainties for multiple tally locations. Sources can be input using many spatial and energy distributions, or they can be imported from a previously generated mesh file. Responses can be generated from cross section reaction rates, flux-to-dose conversion factors, or user-defined functions; responses are tallied on geometry regions, Cartesian or cylindrical grids, or as point detectors.

MAVRIC enables users to import spent fuel isotopics from ORIGEN binary concentrations files such as those generated with ORIGAMI and to import gamma emission spectral data directly from the ORIGEN nuclear data files to streamline analysis and reduce the opportunity for human error.

Several utility modules are also provided for data introspection and conversion.

\section{Reference}

1. D. E., Peplow, "Monte Carlo Shielding Analysis Capabilities with MAVRIC," Nucl. Technol., 174(2), 289-313 (2011). 


\title{
4.1 MAVRIC: MONACO WITH AUTOMATED VARIANCE REDUCTION USING IMPORTANCE CALCULATIONS
}

D. E. Peplow and C. Celik

\begin{abstract}
The MAVRIC sequence (Monaco with Automated Variance Reduction using Importance Calculations) will perform radiation transport on problems that are too challenging for standard, unbiased Monte Carlo methods. The intention of the sequence is to calculate fluxes and dose rates with low uncertainties in reasonable times even for deep penetration problems. MAVRIC is based on the CADIS (Consistent Adjoint Driven Importance Sampling) and FW-CADIS (Forward-Weighted CADIS) methodologies, which use an importance map and biased source that are derived to work together. MAVRIC uses XSProc to generate cross sections for the materials input by the users. Using these multigroup cross sections and a user-specified spatial mesh, MAVRIC performs a three-dimensional, discrete-ordinates calculation using Denovo to determine the adjoint flux as a function of position and energy. This adjoint flux information is then used by MAVRIC to construct a space and energy-dependent importance map (i.e., weight windows) to be used for biasing during particle transport and a mesh-based biased source distribution. MAVRIC then passes the importance map and biased source distribution to the functional module Monaco, a threedimensional, fixed-source, multigroup, and continuous-energy Monte Carlo radiation transport code. In addition to materials input, the user will also supply the geometry description using the SCALE General Geometry Package; source description as a function of position, energy, and direction; tally descriptions (fluxes in which regions, at what point detectors, or over what mesh grids); response functions (function of energy); planes for the mesh used by Denovo; and which tally or tallies to use as the Denovo adjoint source. Output consists of tables detailing the region and point detector fluxes (and their responses), as well as files for mesh tallies.
\end{abstract}




\section{TABLE OF CONTENTS}

$\underline{\text { Page }}$

4.1 MAVRIC: MONACO with Automated Variance Reduction Using Importance Calculations ....4-3

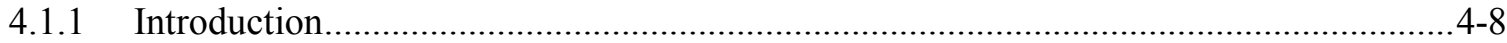

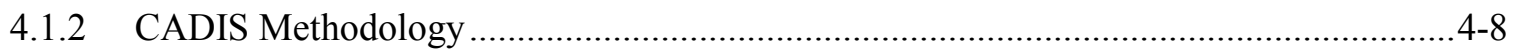

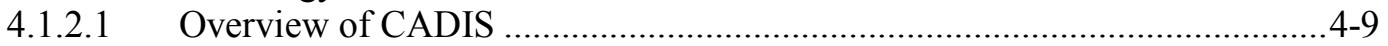

4.1.2.2 Multiple sources with CADIS .......................................................... $4-10$

4.1.2.3 Multiple tallies with CADIS ..................................................................4-11

4.1.2.4 Forward-weighted CADIS ................................................................ $4-12$

4.1.2.5 MAVRIC implementation of CADIS …................................................ $4-14$

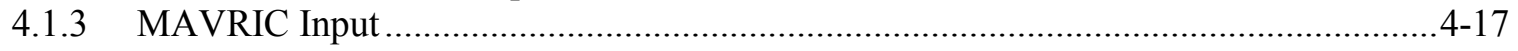

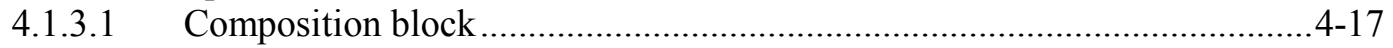

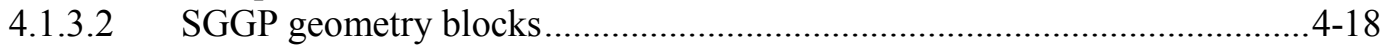

4.1.3.3 Other blocks shared with Monaco ............................................................ 4 -19

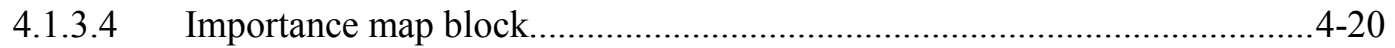

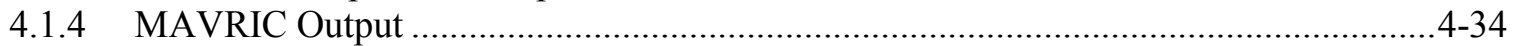

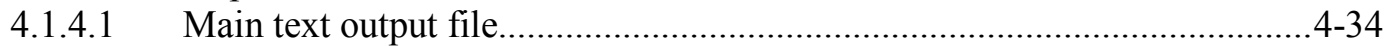

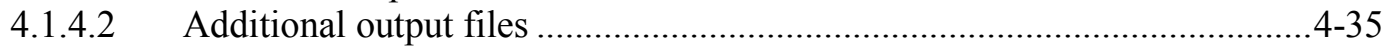

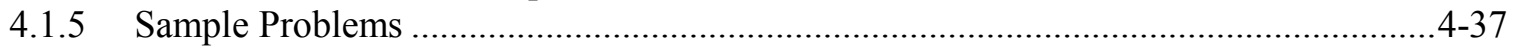

4.1.5.1 Graphite shielding measurements with CADIS ......................................4-37

4.1.5.2 Dose rates outside of a simple cask ...........................................................4-40

4.1.5.3 Gamma-ray litho-density logging tool using FW-CADIS ...........................4-60

4.1.5.4 AOS-100 using FW-CADIS and continuous-energy transport....................4-66

4.1.5.5 Independent spent fuel storage installation...............................................4-71

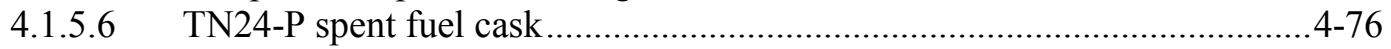

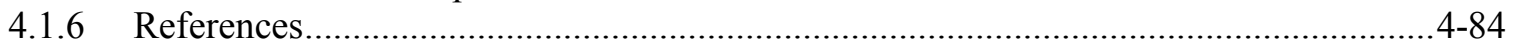

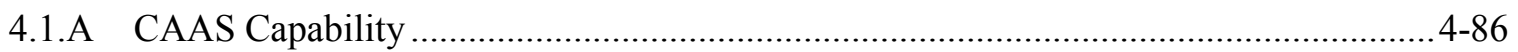

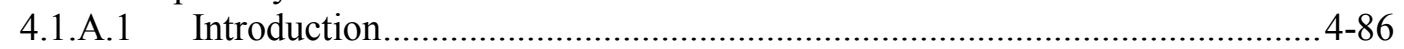

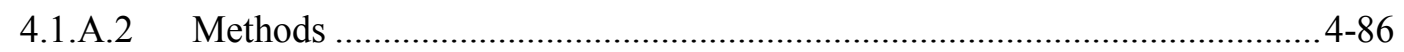

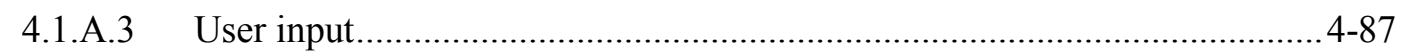

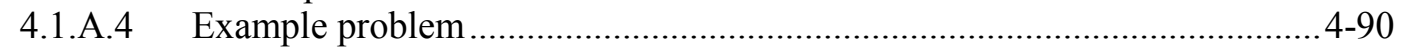

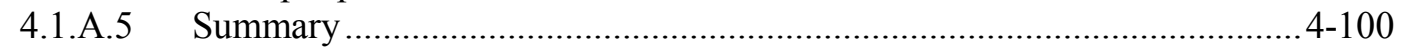

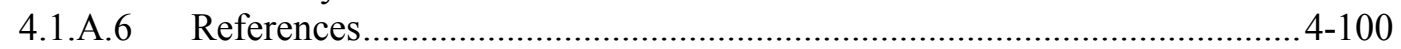

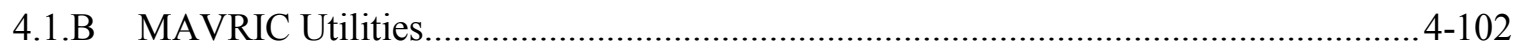

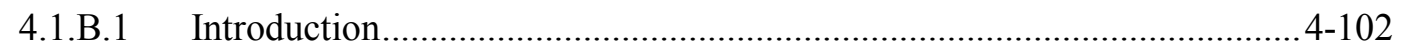

4.1.B.2 Utilities working with Monaco mesh tally (*.3DMAP) files..................... 4-102

4.1.B.3 Utilities for working with DENOVO binary flux (*.dff) files ................... 4-112

4.1.B.4 Utilities for working with DENOVO *.varscl (a TORT format) files....... 4-119

4.1.B.5 Miscellaneous utilities ..................................................................... 4-124

4.1.C Advanced Features...................................................................................... 4-128

4.1.C.1 Alternate normalization of the importance map and biased source ...........4-128

4.1.C.2 Importance maps with directional information..................................... 4-129

4.1.C.3 University of Michigan methods for global variance reduction ................. 4-134

4.1.C.4 Using MAVRIC to run fixed-source Denovo calculations ........................ 4-144

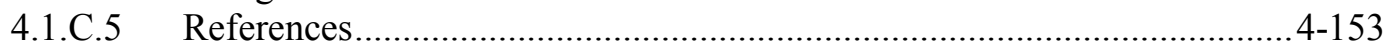




\section{LIST OF FIGURES}

Page

Figure 4.1.1. Ray positions within a voxel with different mmSubCells parameters.........................4-22

Figure 4.1.2. Geometry model (left) and the Denovo representation (right) of an assembly using

macromaterials determined by ray tracing...............................................................4-23

Figure 4.1.3. Ray tracing (in two dimensions) through a voxel...................................................4-23

Figure 4.1.4. Successive steps in the recursive macro-material method. ..........................................4-25

Figure 4.1.5. Cask geometry model (upper left) and the Denovo representation using cell center

testing (upper right). .......................................................................................... 4-26

Figure 4.1.6. Mesh tally showing neutron dose rate (rem/hr) and uncertainties for the analog

case and the CADIS case.......................................................................................4-40

Figure 4.1.7. Cask geometry and detector locations. .......................................................................4-41

Figure 4.1.8. Spent fuel neutron source spectrum, with strength $8.577 \times 10^{9} /$ second. ............................4-42

Figure 4.1.9. ANSI-1977 neutron flux-to-dose-rate factors $(\mathrm{rem} / \mathrm{hr}) /\left(\right.$ neutrons $\left./ \mathrm{cm}^{2} / \mathrm{sec}\right) \ldots \ldots \ldots \ldots \ldots \ldots . . . . .42$

Figure 4.1.10. Spent fuel photon source spectrum, with strength $7.155 \times 10^{16} /$ second........................4-42

Figure 4.1.11. ANSI-1977 photon flux-to-dose-rate factors $(\mathrm{rem} / \mathrm{hr}) /\left(\right.$ photons $\left./ \mathrm{cm}^{2} / \mathrm{sec}\right)$.................4-42

Figure 4.1.12. Convergence plot for the neutron dose rate at point detector 1. Error bars show the

1-sigma tally uncertainties. ..........................................................................................4-4

Figure 4.1.13. Importance map mesh planes in the $x$ and $z$ dimensions for detector positions $1 / 4$,

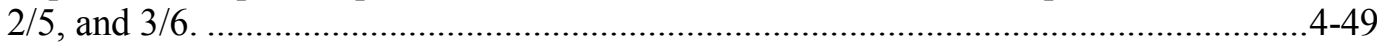

Figure 4.1.14. Importance map mesh planes in the $x$ and $y$ dimensions for detector positions $1 / 4$, $2 / 5$, and $3 / 6$.

Figure 4.1.15. Adjoint neutron fluxes $\left(/ \mathrm{cm}^{2} / \mathrm{s}\right)$ for groups $5(0.9-1.4 \mathrm{MeV}), 10(0.58-3.0 \mathrm{keV})$, and $19(0.8-1 \mathrm{eV})$ calculated by Denovo...............................................................4-52

Figure 4.1.16. Neutron target weights from the importance map and source weights (at birth) for neutron group 5 (0.9 to $1.4 \mathrm{MeV})$.

Figure 4.1.17. Biased source sampling probability $\left(\right.$ neutrons $\left./ \mathrm{cm}^{3}\right)$ for neutron groups 5 (0.9-1.4 MeV), $10(0.58-3.0 \mathrm{keV})$, and $19(0.8-1 \mathrm{eV})$.

Figure 4.1.18. Adjoint photon fluxes $\left(/ \mathrm{cm}^{2} / \mathrm{s}\right)$ for groups $2(8-10 \mathrm{MeV}), 12(0.8-1.0 \mathrm{MeV})$, and $18(45-100 \mathrm{keV})$ calculated by Denovo.

Figure 4.1.19. Photon target weights from the importance map and source weights (at birth) for photon group $12(0.8-1.0 \mathrm{MeV})$.

Figure 4.1.20. Biased source sampling probability (photons $\left./ \mathrm{cm}^{3}\right)$ for groups $2(8-10 \mathrm{MeV}), 12$

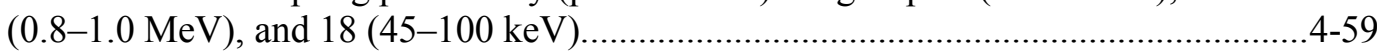

Figure 4.1.21. Cutaway view of the litho-density tool in a rock formation. ......................................4-60

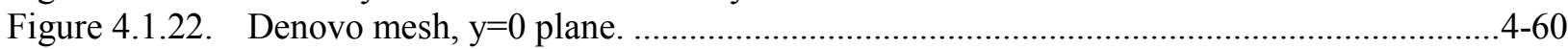

Figure 4.1.23. Denovo mesh, $z=20$ plane (through near detector). ................................................4-60

Figure 4.1.24. Mesh tallies showing total photon flux in $\mathrm{cm}^{2} / \mathrm{s}$ (left column) and its relative error (right column) in the $\mathrm{y}=0$ plane ..............................................................................4-65

Figure 4.1.25. Simple AOS-100 cask geometry showing tungsten (brown) and steel (gray)..............4-66

Figure 4.1.26. Dose rates $(\mathrm{mrem} / \mathrm{hr} / \mathrm{Ci})$ and relative uncertainties along the $\boldsymbol{y}=\mathbf{0}$ midplane of the cask as calculated by an analog calculation.

Figure 4.1.27. Dose rates $(\mathrm{mrem} / \mathrm{hr} / \mathrm{Ci})$ and relative uncertainties from the CE, FW-CADIS calculation, showing the midplane views of the cask $(\boldsymbol{z}=\mathbf{0}$ above and $\boldsymbol{y}=\mathbf{0}$ below).

Figure 4.1.28. Flux $10 \mathrm{~cm}$ above the cask 4-70

Figure 4.1.29. Ratio of the 47 group MG computed dose rates to the CE dose rates $(\boldsymbol{y}=\mathbf{0}$ left and $\boldsymbol{z}=\mathbf{0}$ right). 
Figure 4.1.30. Ratio of the 19 group MG computed dose rates to the CE dose rates $(\boldsymbol{y}=\mathbf{0}$ left and $\mathbf{z}=\mathbf{0}$ right).

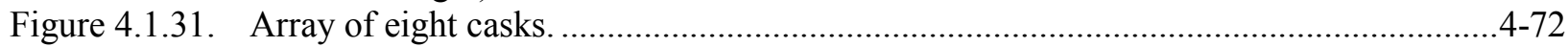

Figure 4.1.32. Cask detail..............................................................................................4-72

Figure 4.1.33. Photon dose rate values in $\mathrm{rem} / \mathrm{hr}$ (left) and the relative uncertainty (right) for the area around the cask array (1 hr Monaco).....

Figure 4.1.34. Photon dose rate values in $\mathrm{rem} / \mathrm{hr}$ (left) and the relative uncertainty (right) for the area around the cask array (64 hr Monaco).....

Figure 4.1.35. Distribution of relative uncertainties for different run times of mavric.isfsi.inp.

Figure 4.1.36. MAVRIC model of the TN24-P cask. Materials: spent fuel (light and dark yellow), steels (blues), resin (green), and other metals (gray).

Figure 4.1.37. Neutron (left) and photon (right) dose rate response functions.

Figure 4.1.38. Distributions in the TN24-P model.

Figure 4.1.39. Rectilinear mesh tally of total dose rate (rem/hr) and its relative uncertainty along the $\mathrm{x}=0$ plane

Figure 4.1.40. Cylindrical mesh tally of total dose rate (rem/hr) and its relative uncertainty along the $\theta=0^{\circ}$ plane.

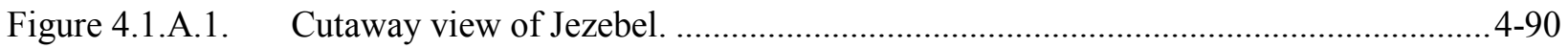

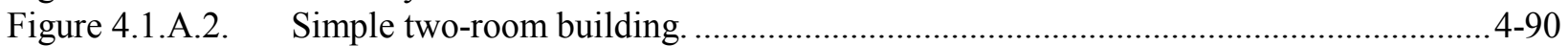

Figure 4.1.A.3. Fission source spatial distribution for the center horizontal slice........................... 4-92

Figure 4.1.A.4. Fission source energy distribution for the center voxel. .......................................4-92

Figure 4.1.A.5. Coordinates of the floor, walls, ceiling, and door of the simple block building

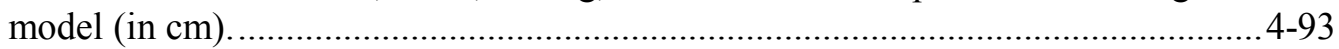

Figure 4.1.A.6. Dose (rem) results for the $\mathrm{z}=100 \mathrm{~cm}$ plane (containing the source)......................4-99

Figure 4.1.A.7. Relative uncertainties in the dose, most less than $1 \%$, for the $\mathrm{z}=100 \mathrm{~cm}$ plane...... $4-100$

Figure 4.1.C.1. Importance map computed using standard CADIS............................................. 4-128

Figure 4.1.C.2. Targets weights using the "shiftNormPos" keyword...........................................4-129

Figure 4.1.C.3. Mesh tally results for the photon dose over the entire building use five different methods. ......................................................................................... 4-142

Figure 4.1.C.4. The fraction of mesh tally voxels with less than a given amount of relative uncertainty for the five different methods.......................................................... 4-143

Figure 4.1.C.5. Forward Denovo source (left) and mesh geometry (right).................................. $4-150$

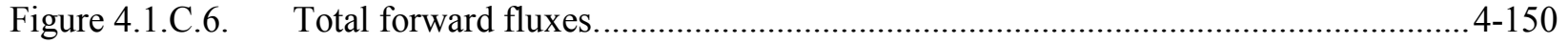

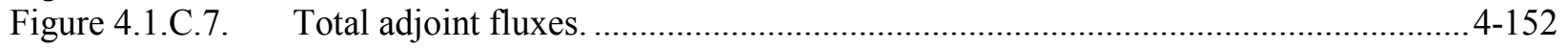




\section{LIST OF TABLES}

Page

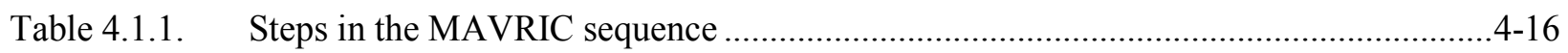

Table 4.1.2. Parameters for the MAVRIC command line ("parm=...")........................................4-16

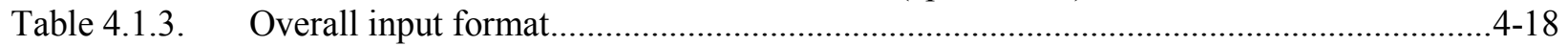

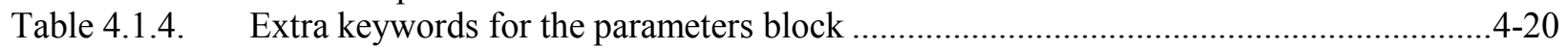

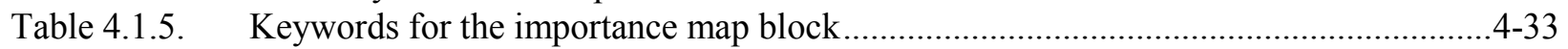

Table 4.1.6. Denovo options for the importance map block..............................................................4-34

Table 4.1.7. Output files that are copied back to user's area when the sequence finishes ${ }^{a}$...............4-36

Table 4.1.8. Other intermediate files - available in the temporary directory. ................................4-37

Table 4.1.9. Source and response data using the SCALE 27-group energy structure for neutrons and the 19 group energy structure for photons ..........................................................4-43

Table 4.1.10. Analog Monaco results for the simplified cask model—neutron source/neutron

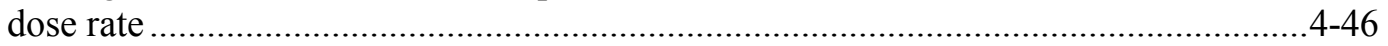

Table 4.1.11. Analog Monaco results for the simplified cask model—photon source/photon dose

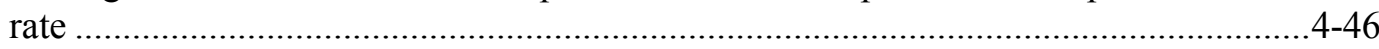

Table 4.1.12. SAS4 results, using radial biasing (361 minutes) and axial biasing (361 minutes), for the simplified cask model — neutron source/neutron dose rate...............................4-47

Table 4.1.13. SAS4 results, using radial biasing (361 minutes) and axial biasing (361 minutes), for the simplified cask model — photon source/photon dose rate.................................4-48

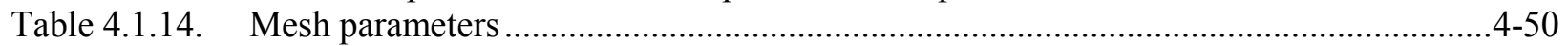

Table 4.1.15. Listing of the various sets of mesh planes used for the importance calculations for six different point detectors ...................................................................................... $4-50$

Table 4.1.16. Final MAVRIC results (rem/hr) for each point detector in the neutron

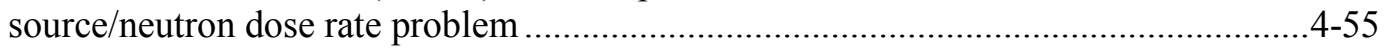

Table 4.1.17. Comparison of neutron dose rates (rem/hr) to other SCALE codes ............................4-55

Table 4.1.18. Ratio of the figure-of-merit (speed-up) of MAVRIC and SAS4 compared to analog Monaco ........................................................................................................... $4-55$

Table 4.1.19. Final MAVRIC results (rem/hr) for each point detector in the photon source/photon dose rate problem ...........................................................................4-57

Table 4.1.20. Comparison of the photon dose rates (rem/hr) to other SCALE codes.......................4-58

Table 4.1.21. Ratio of the figure-of-merit (speed-up) of MAVRIC and SAS4 compared to

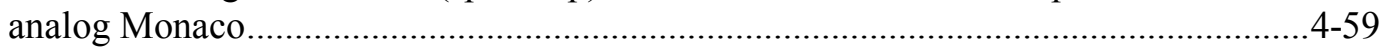

Table 4.1.22. Comparison of different CADIS techniques for the litho-density problem...................4-64

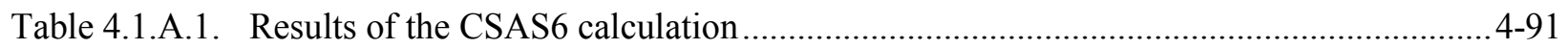

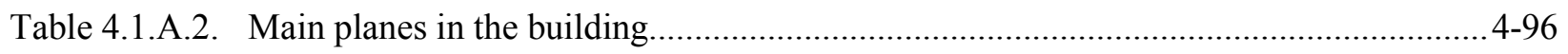

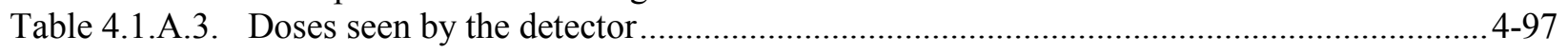

Table 4.1.C.1. Results of the Ueki Shielding Problem (35 cm graphite shield) ..............................4-134

Table 4.1.C.2. Results of the Ueki Shielding Problem (35 cm graphite shield) ................................ $4-141$

Table 4.1.C.3. Comparison of the five different methods .............................................................. $4-143$

Table 4.1.C.4. Optional Denovo parameters for the MAVRIC Importance Map Block.................... 4-146 


\subsubsection{Introduction}

Monte Carlo particle transport calculations for deep penetration problems can require very long run times in order to achieve an acceptable level of statistical uncertainty in the final answers. Discrete-ordinates codes can be faster but have limitations relative to the discretization of space, energy, and direction. Monte Carlo calculations can be modified (biased) to produce results with the same variance in less time if an approximate answer or some other additional information is already known about the problem. If an importance can be assigned to different particles based on how much they will contribute to the final answer, more time can be spent on important particles with less time devoted to unimportant particles. One of the best ways to bias a Monte Carlo code for a particular tally is to form an importance map from the adjoint flux based on that tally. Unfortunately, determining the exact adjoint flux could be just as difficult as computing the original problem itself. However, an approximate adjoint can still be very useful in biasing the Monte Carlo solution. ${ }^{1}$ Discrete ordinates can be used to quickly compute that approximate adjoint. Together, Monte Carlo and discrete ordinates can be used to find solutions to thick shielding problems in reasonable times.

The MAVRIC (Monaco with Automated Variance Reduction using Importance Calculations) sequence is based on the CADIS (Consistent Adjoint Driven Importance Sampling) and FW-CADIS (ForwardWeighted CADIS) methodologies..$^{2,3,4,7}$ MAVRIC automatically performs a three-dimensional, discreteordinates calculation using Denovo ${ }^{5}$ to compute the adjoint flux as a function of position and energy. This adjoint flux information is then used to construct an importance map (i.e., target weights for weight windows) and a biased source distribution that work together - particles are born with a weight matching the target weight of the cell into which they are born. The fixed-source Monte Carlo radiation transport Monaco $^{6}$ then uses the importance map for biasing during particle transport and the biased source distribution as its source. During transport, the particle weight is compared with the importance map after each particle interaction and whenever a particle crosses into a new importance cell in the map.

For problems that do not require variance reduction to complete in a reasonable time, execution of MAVRIC without the importance map calculation provides an easy way to run Monaco. For problems that do require variance reduction to complete in a reasonable time, MAVRIC removes the burden of setting weight windows from the user and performs it automatically with a minimal amount of additional input. Note that the MAVRIC sequence can be used with the final Monaco calculation as either a multigroup (MG) or a continuous-energy (CE) calculation.

Monaco has a wide variety of tally options: it can calculate fluxes (by group) at a point in space, over any geometrical region, or for a user-defined, three-dimensional, rectangular grid. These tallies can also integrate the fluxes with either standard response functions from the cross section library or user-defined response functions. All of these tallies are available in the MAVRIC sequence.

While originally designed for CADIS, the MAVRIC sequence is also capable of creating importance maps using both forward and adjoint deterministic estimates. The FW-CADIS method ${ }^{7}$ can be used for optimizing several tallies at once, a mesh tally over a large region, or a mesh tally over the entire problem. Several other methods for producing importance maps are also available in MAVRIC and are explored in Appendix C.

\subsubsection{CADIS Methodology}

MAVRIC is an implementation of CADIS (Consistent Adjoint Driven Importance Sampling) using the Denovo $S_{N}$ and Monaco Monte Carlo functional modules. Source biasing and a mesh-based importance map, overlaying the physical geometry, are the basic methods of variance reduction. In order to make the best use of an importance map, the map must be made consistent with the source biasing. If the source 
biasing is inconsistent with the weight windows that will be used during the transport process, source particles will undergo Russian roulette or splitting immediately, wasting computational time and negating the intent of the biasing.

\subsubsection{Overview of CADIS}

CADIS has been well described ${ }^{1-4}$ in the literature, so only a brief overview is given here. Consider a class source-detector problem described by a unit source with emission probability distribution function $q(\vec{r}, E)$ and a detector response function $\sigma_{d}(\vec{r}, E)$. To determine the total detector response, $R$, the forward scalar flux $\phi(\vec{r}, E)$ must be known. The response is found by integrating the product of the detector response function and the flux over the detector volume $V_{d}$.

$$
R=\int_{V_{d}} \int_{E} \sigma_{d}(\vec{r}, E) \phi(\vec{r}, E) d E d V
$$

Alternatively, if the adjoint scalar flux, $\phi^{+}(\vec{r}, E)$, is known from the corresponding adjoint problem with adjoint source $q^{+}(\vec{r}, E)=\sigma_{d}(\vec{r}, E)$, then the total detector response could be found by integrating the product of the forward source and the adjoint flux over the source volume, $V_{S}$.

$$
R=\int_{V_{S}} \int_{E} q(\vec{r}, E) \phi^{+}(\vec{r}, E) d E d V
$$

Unfortunately, the exact adjoint flux may be just as difficult to determine as the forward flux, but an approximation of the adjoint flux can still be used to form an importance map and a biased source distribution for use in the forward Monte Carlo calculation.

Wagner $^{1}$ showed that if an estimate of the adjoint scalar flux for the corresponding adjoint problem could be found, then an estimate of the response $R$ could be made using Eq. (4.1.2). The adjoint source for the adjoint problem is typically separable and corresponds to the detector response and spatial area of tally to be optimized: $q^{+}(\vec{r}, E)=\sigma_{d}(E) g(\vec{r})$, where $\sigma_{d}(E)$ is a flux-to-dose conversion factor and $g(\vec{r})$ is 1 in the tally volume and 0 otherwise. Then, from the adjoint flux $\phi^{+}(\vec{r}, E)$ and response estimate $R$, a biased source distribution, $\hat{q}(\vec{r}, E)$, for source sampling of the form

$$
\hat{q}(\vec{r}, E)=\frac{1}{R} q(\vec{r}, E) \phi^{+}(\vec{r}, E)
$$

and weight window target values, $\bar{w}(\vec{r}, E)$, for particle transport of the form

$$
\bar{w}(\vec{r}, E)=\frac{R}{\phi^{+}(\vec{r}, E)}
$$

could be constructed, which minimize the variance in the forward Monte Carlo calculation of $R$.

When a particle is sampled from the biased source distribution $\hat{q}(\vec{r}, E)$, to preserve a fair game, its initial weight is set to 


$$
w_{0}(\vec{r}, E)=\frac{q(\vec{r}, E)}{\hat{q}(\vec{r}, E)}=\frac{R}{\phi^{+}(\vec{r}, E)}
$$

which exactly matches the target weight for that particle's position and energy. This is the "consistent" part of CADIS - source particles are born with a weight matching the weight window of the region/energy they are born into. The source biasing and the weight windows work together.

CADIS has been applied to many problems - including reactor ex-core detectors, well-logging instruments, cask shielding studies, and independent spent fuel storage facility models - and has demonstrated very significant speed-ups in calculation time compared to analog simulations.

\subsubsection{Multiple sources with CADIS}

For a typical Monte Carlo calculation with multiple sources (each with a probability distribution function $q_{i}(\vec{r}, E)$ and a strength $S_{i}$, giving a total source strength of $\left.S=\sum S_{i}\right)$, the source is sampled in two steps. First, the specific source $i$ is sampled with probability $p(i)=S_{i} / S$, and then the particle is sampled from the specific source distribution $q_{i}(\vec{r}, E)$.

The source sampling can be biased at both levels: which source to sample from and how to sample each source. For example, the specific source can be sampled using some arbitrary distribution, $\hat{p}(i)$, and then the individual sources can be sampled using distributions $\hat{q}_{i}(\vec{r}, E)$. Particles would then have a birth weight of

$$
w_{0} \equiv(p(i) / \hat{p}(i))\left(q_{i}(\vec{r}, E) / \hat{q}_{i}(\vec{r}, E)\right) .
$$

For CADIS, a biased multiple source needs to be developed so that the birth weights of sampled particles still match the target weights of the importance map. For a problem with multiple sources (each with a distribution $q_{i}(\vec{r}, E)$ and a strength $\left.S_{i}\right)$, the goal of the Monte Carlo calculation is to compute some response $R$ for a response function $\sigma_{d}(\vec{r}, E)$ at a given detector.

$$
R=\int_{V} \int_{E} \sigma_{d}(\vec{r}, E) \phi(\vec{r}, E) d E d V
$$

Note that the flux $\phi(\vec{r}, E)$ has contributions from each source. The response, $R_{i}$, from each specific source $\left(S_{i}\right.$ with $\left.q_{i}(\vec{r}, E)\right)$ can be expressed using just the flux from that source, $\phi_{i}(\vec{r}, E)$, as

$$
R_{i}=\int_{V} \int_{E} \sigma_{d}(\vec{r}, E) \phi_{i}(\vec{r}, E) d E d V
$$

The total response is then found as $R=\sum_{i} R_{i}$.

For the adjoint problem, using the adjoint source of $q^{+}(\vec{r}, E)=\sigma_{d}(\vec{r}, E)$, the response $R$ can also be calculated as 


$$
R=\int_{V} \int_{E}\left[\sum_{i} S_{i} q_{i}(\vec{r}, E)\right] \phi^{+}(\vec{r}, E) d E d V
$$

with response contribution from each specific source being

$$
R_{i}=\int_{V} \int_{E} S_{i} q_{i}(\vec{r}, E) \phi^{+}(\vec{r}, E) d E d V
$$

The target weights $\bar{w}(\vec{r}, E)$ of the importance map are found using

$$
\bar{w}(\vec{r}, E)=R / S / \phi^{+}(\vec{r}, E) .
$$

Each biased source $\hat{q}_{i}(\vec{r}, E)$ pdf is found using

$$
\hat{q}_{i}(\vec{r}, E)=\frac{S_{i}}{R_{i}} q_{i}(\vec{r}, E) \phi^{+}(\vec{r}, E),
$$

and the biased distribution used to select an individual source is $\hat{p}(i)=R_{i} / \sum R_{i}=R_{i} / R$.

When using the biased distribution used to select an individual source, $\hat{p}(i)$, and the biased source distribution, $\hat{q}_{i}(\vec{r}, E)$, the birth weight of the sampled particle will be

$$
\begin{aligned}
& w_{0} \equiv(p(i) / \hat{p}(i))\left(q_{i}(\vec{r}, E) / \hat{q}_{i}(\vec{r}, E)\right) \\
& =\left(\frac{S_{i}}{S} / \frac{R_{i}}{R}\right)\left(q_{i}(\vec{r}, E) / \frac{S_{i}}{R_{i}} q_{i}(\vec{r}, E) \phi^{+}(\vec{r}, E)\right) \\
& =\quad R / S / \phi^{+}(\vec{r}, E){ }^{\prime}
\end{aligned}
$$

which matches the target weight, $\bar{w}(\vec{r}, E)$.

\subsubsection{Multiple tallies with CADIS}

The CADIS methodology works quite well for classic source/detector problems. The statistical uncertainty of the tally that serves as the adjoint source is greatly reduced since the Monte Carlo transport is optimized to spend more simulation time on those particles that contribute to the tally, at the expense of tracking particles in other parts of phase space. However, more recently, Monte Carlo has been applied to problems where multiple tallies need to all be found with low statistical uncertainties. The extension of this idea is the mesh tally - where each voxel is a tally where the user desires low statistical uncertainties. For these problems, the user must accept a total simulation time that is controlled by the tally with the slowest convergence and simulation results where the tallies have a wide range of relative uncertainties. 
The obvious way around this problem is to create a separate problem for each tally and use CADIS to optimize each. Each simulation can then be run until the tally reaches the level of acceptable uncertainty. For more than a few tallies, this approach becomes complicated and time-consuming for the user. For large mesh tallies, this approach is not reasonable.

Another approach to treat several tallies, if they are in close proximity to each other, or a mesh tally covering a small portion of the physical problem is to use the CADIS methodology with the adjoint source near the middle of the tallies to be optimized. Since particles in the forward Monte Carlo simulation are optimized to reach the location of the adjoint source, all the tallies surrounding that adjoint source should converge quickly. The drawback to this approach is the difficult question of "how close." If the tallies are too far apart, certain energies or regions that are needed for one tally may be of low importance for getting particles to the central adjoint source. This may under-predict the flux or dose at the tally sites far from the adjoint source.

MAVRIC has the capability to have multiple adjoint sources with this problem in mind. For several tallies that are far from each other, multiple adjoint sources could be used. In the forward Monte Carlo, particles would be drawn to one of those adjoint sources. The difficulty with this approach is that typically the tally that is closest to the true physical source converges faster than the other tallies-showing the closest adjoint source seems to attract more particles than the others. Assigning more strength to the adjoint source further from the true physical source helps, but finding the correct strengths so that all of the tallies converge to the same relative uncertainty in one simulation is an iterative process for the user.

\subsubsection{Forward-weighted CADIS}

In order to converge several tallies to the same relative uncertainty in one simulation, the adjoint source corresponding to each of those tallies needs to be weighted inversely by the expected tally value. In order to calculate the dose rate at two points - say one near a reactor and one far from a reactor-in one simulation, then the total adjoint source used to develop the weight windows and biased source needs to have two parts. The adjoint source far from the reactor needs to have more strength than the adjoint source near the reactor by a factor equal to the ratio of the expected near dose rate to the expected far dose rate.

This concept can be extended to mesh tallies as well. Instead of using a uniform adjoint source strength over the entire mesh tally volume, each voxel of the adjoint source should be weighted inversely by the expected forward tally value for that voxel. Areas of low flux or low dose rate would have more adjoint source strength than areas of high flux or high dose rate.

An estimate of the expected tally results can be found by using a quick discrete-ordinates calculation. This leads to an extension of the CADIS method: forward-weighted CADIS (FW-CADIS). ${ }^{7}$ First, a forward $S_{N}$ calculation is performed to estimate the expected tally results. A total adjoint source is constructed where the adjoint source corresponding to each tally is weighted inversely by those forward tally estimates. Then the standard CADIS approach is used - an importance map (target weight windows) and a biased source are made using the adjoint flux computed from the adjoint $\mathrm{S}_{\mathrm{N}}$ calculation.

For example, if the goal is to calculate a detector response function $\sigma_{d}(E)$ (such as dose rate using fluxto-dose-rate conversion factors) over a volume (defined by $g(\vec{r})$ ) corresponding to mesh tally, then instead of simply using $q^{+}(\vec{r}, E)=\sigma_{d}(E) g(\vec{r})$, the adjoint source would be

$$
q^{+}(\vec{r}, E)=\frac{\sigma_{d}(E) g(\vec{r})}{\int \sigma_{d}(E) \phi(\vec{r}, E) d E},
$$


where $\phi(\vec{r}, E)$ is an estimate of the forward flux and the energy integral is over the voxel at $\vec{r}$. The adjoint source is nonzero only where the mesh tally is defined $(g(\vec{r}))$, and its strength is inversely proportional to the forward estimate of dose rate.

The relative uncertainty of a tally is controlled by two components: first, the number of tracks contributing to the tally and, second, the shape of the distribution of scores contributing to that tally. In the Monte Carlo game, the number of simulated particles, $m(\vec{r}, E)$, can be related to the true physical particle density, $n(\vec{r}, E)$, by the average Monte Carlo weight of scoring particles, $\bar{w}(\vec{r}, E)$, by

$$
n(\vec{r}, E)=\bar{w}(\vec{r}, E) m(\vec{r}, E)
$$

In a typical Monte Carlo calculation, tallies are made by adding some score, multiplied by the current particle weight, to an accumulator. To calculate a similar quantity related to the Monte Carlo particle density would be very close to calculating any other quantity but without including the particle weight. The goal of FW-CADIS is to make the Monte Carlo particle density, $m(\vec{r}, E)$, uniform over the tally areas, so an importance map needs to be developed that represents the importance to achieving uniform Monte Carlo particle density. By attempting to keep the Monte Carlo particle density more uniform, more uniform relative errors for the tallies should be realized.

Two options for forward weighting are possible. For tallies over some area where the entire group-wise flux is needed with low relative uncertainties, the adjoint source should be weighted inversely by the forward flux, $\phi(\vec{r}, E)$. The other option, for a tally where only an energy-integrated quantity is desired, is to weight the adjoint inversely by that energy-integrated quantity, $\int \sigma_{d}(E) \phi(\vec{r}, E) d E$. For a tally where the total flux is desired, then the response in the adjoint source is simply $\sigma_{d}(E)=1$.

To optimize the forward Monte Carlo simulation for the calculation of some quantity at multiple tally locations or across a mesh tally, the adjoint source needs to be weighted by the estimate of that quantity. For a tally defined by its spatial location $g(\vec{r})$ and its optional response $\sigma_{d}(E)$, the standard adjoint source would be $q^{+}(\vec{r}, E)=\sigma_{d}(E) g(\vec{r})$. The forward-weighted adjoint source, $q^{+}(\vec{r}, E)$, depending on what quantity is to be optimized, is listed below.

\begin{tabular}{|c|c|c|}
\hline \multicolumn{2}{|c|}{ For the calculation of } & Adjoint source \\
\hline Energy and spatially dependent flux & $\phi(\vec{r}, E)$ & $\frac{g(\vec{r})}{\phi(\vec{r}, E)}$ \\
\hline Spatially dependent total flux & $\phi(\vec{r}, E) d E$ & $\frac{g(\vec{r})}{\int \phi(\vec{r}, E) d E}$ \\
\hline Spatially dependent total response & $\sigma_{d}(E) \phi(\vec{r}, E) d E$ & $\frac{\sigma_{d}(E) g(\vec{r})}{\int \sigma_{d}(E) \phi(\vec{r}, E) d E}$ \\
\hline
\end{tabular}

The bottom line of FW-CADIS is that in order to calculate a quantity at multiple tally locations (or across a mesh tally) with more uniform relative uncertainties, an adjoint source needs to be developed for an objective function that keeps some non-physical quantity — related to the Monte Carlo particle density and similar in form to the desired quantity - constant. FW-CADIS uses the solution of a forward discreteordinates calculation to properly weight the adjoint source. After that, the standard CADIS approach is used. 


\subsubsection{MAVRIC implementation of CADIS}

With MAVRIC, as with other shielding codes, the user defines the problem as a set of physical modelsthe material compositions, the geometry, the source, and the detectors (locations and response functions) - as well as some mathematical parameters on how to solve the problem (number of histories, etc.). For the variance reduction portion of MAVRIC, the only additional inputs required are (1) the mesh planes to use in the discrete-ordinates calculation(s) and (2) the adjoint source description-basically the location and the response of each tally to optimize in the forward Monte Carlo calculation. MAVRIC takes this information and constructs a Denovo adjoint problem. (The adjoint source is weighted by a Denovo forward flux or response estimate for FW-CADIS applications.) MAVRIC then uses the CADIS methodology: it combines the adjoint flux from the Denovo calculation with the source description and creates the importance map (weight window targets) and the mesh-based biased source. Monaco is then run using the CADIS biased source distribution and the weight window targets.

\subsection{Denovo}

Denovo is a parallel three-dimensional $\mathrm{S}_{\mathrm{N}}$ code that is used to generate adjoint (and, for FW-CADIS, forward) scalar fluxes for the CADIS methods in MAVRIC. For use in MAVRIC/CADIS, it is highly desirable that the $\mathrm{S}_{\mathrm{N}}$ code be fast, positive, and robust. The phase-space shape of the forward and adjoint fluxes, as opposed to a highly accurate solution, is the most important quality for Monte Carlo weightwindow generation. Accordingly, Denovo provides a step-characteristics spatial differencing option that produces positive scalar fluxes as long as the source (volume plus in-scatter) is positive. Denovo uses an orthogonal, nonuniform mesh that is ideal for CADIS applications because of the speed and robustness of calculations on this mesh type.

Denovo uses the highly robust GMRES (Generalized Minimum Residual) Krylov method to solve the $\mathrm{S}_{\mathrm{N}}$ equations in each group. GMRES has been shown to be more robust and efficient than traditional source (fixed-point) iteration. ${ }^{8}$ The in-group discrete $\mathrm{S}_{\mathrm{N}}$ equations are defined as

$$
\boldsymbol{L} \psi=\boldsymbol{M S} \phi+q
$$

where $\boldsymbol{L}$ is the differential transport operator, $\boldsymbol{M}$ is the moment-to-discrete operator, $\boldsymbol{S}$ is the matrix of scattering cross-section moments, $q$ is the external and in-scatter source, $\phi$ is the vector of angular flux moments, and $\psi$ is the vector of angular fluxes at discrete angles. Applying the operator $\boldsymbol{D}$, where $\phi=\boldsymbol{D} \psi$, and rearranging terms casts the in-group equations in the form of a traditional linear system, $\boldsymbol{A} x=b$,

$$
\left(I-D L^{-1} M S\right)=D L^{-1} q
$$

The operation $\boldsymbol{L}^{-1} v$, where $v$ is an iteration vector, is performed using a traditional wave-front solve (transport sweep). The parallel implementation of the Denovo wave-front solver uses the well-known Koch-Baker-Alcouffe (KBA) algorithm, which is a two-dimensional block-spatial decomposition of a three-dimensional orthogonal mesh. ${ }^{9}$ The Trilinos package is used for the GMRES implementation. ${ }^{10}$ Denovo stores the mesh-based scalar fluxes in a double precision binary file (*.dff) called a Denovo flux file. Past versions of SCALE/Denovo used the TORT ${ }^{11} *$.varscl file format (DOORS package ${ }^{12}$ ), but this was limited to single precision. Since the rest of the MAVRIC sequence has not yet been parallelized, Denovo is currently used only in serial mode within MAVRIC. 


\subsection{Monaco}

The forward Monte Carlo transport is performed using Monaco, a fixed-source, shielding code that uses the SCALE General Geometry Package (SGGP, the same as used by the criticality code KENO-VI) and the standard SCALE material information processor. Monaco can use either MG or CE cross section libraries. Monaco was originally based on the MORSE Monte Carlo code but has been extensively modified to modernize the coding, incorporate more flexibility in terms of sources/tallies, and read a userfriendly block/keyword style input.

Much of the input to MAVRIC is the same as Monaco. More details can be found in the Monaco chapter of the SCALE manual.

\subsection{Running MAVRIC}

The objective of a SCALE sequence is to execute several codes, passing the output from one to the input of the next, in order to perform some analysis - things that users typically had to do in the past. MAVRIC does this for difficult shielding problems by running approximate discrete-ordinates calculations, constructing an importance map and biased source for one or more tallies that the user wants to optimize in the Monte Carlo calculation, and then using those in a forward Monaco Monte Carlo calculation. MAVRIC also prepares the forward and adjoint cross sections when needed. The steps of a MAVRIC sequence are listed in Table 4.1.1. The user can instruct MAVRIC to run this whole sequence of steps or just some subset of the steps-in order to verify the intermediate steps or to reuse previously calculated quantities in a new analyses.

The MAVRIC sequence can be stopped after key points by using the "parm=parameter" operator on the "=mavric" command line, which is the first line of the input file. The various parameters are listed in Table 4.1.2. These parameters allow the user to perform checks and make changes to the importance map calculation before the actual Monte Carlo calculation in Monaco.

MAVRIC also allows the sequence to start at several different points. If an importance map and biased source have already been computed, they can be used directly. If the adjoint scalar fluxes are known, they can quickly be used to create the importance map and biased source and then begin the forward Monte Carlo. All of the different combinations of starting MAVRIC with some previously calculated quantities are listed in the following section detailing the input options.

When using MG cross-section libraries that do not have flux-to-dose-rate conversion factors, use "parm=nodose" to prevent the cross section processing codes from trying to move these values into the working library.

MAVRIC creates many files that use the base problem name from the output file. For an output file called "c:|path1 \path2\outputName.out" or "/home/path1/path2/outputName.inp", spaces in the output name will cause trouble and should not be used. 
Table 4.1.1. Steps in the MAVRIC sequence

\begin{tabular}{ll}
$\begin{array}{l}\text { Cross section calculation } \\
\begin{array}{c}\text { Forward Denovo (optional) } \\
\text { Cross section calculation } \\
\text { Forward flux calculation }\end{array}\end{array}$ & $\begin{array}{l}\text { XSProc is used to calculate the forward cross sections for Denovo } \\
\text { Denovo calculates the estimate of the forward flux }\end{array}$ \\
$\begin{array}{c}\text { Adjoint Denovo (optional) } \\
\text { Cross section calculation } \\
\text { Adjoint flux calculation }\end{array}$ & $\begin{array}{l}\text { XSProc is used to calculate the adjoint cross sections for Denovo } \\
\text { Denovo calculates the estimate of the adjoint flux }\end{array}$ \\
CADIS (optional) & $\begin{array}{l}\text { The scalar flux file from Denovo is then used to create the biased source } \\
\text { distribution and transport weight windows }\end{array}$ \\
Monte Carlo calculation & $\begin{array}{l}\text { Monaco uses the biased source distribution and transport weight } \\
\text { windows to calculate the various tallies }\end{array}$ \\
\hline
\end{tabular}

Table 4.1.2. Parameters for the MAVRIC command line ("parm=...")

\begin{tabular}{ll}
\hline Parameter & MAVRIC will stop after \\
\hline check & Input checking \\
forinp & Forward Denovo input construction (makes $x \mathrm{kba} \_\mathrm{b}$. inp in the tmp area) \\
forward & The forward Denovo calculation \\
adjinp & Adjoint Denovo input construction (makes $\times \mathrm{kba} b$. inp in the tmp area) \\
adjoint & The adjoint Denovo calculation \\
impmap & Calculation of importance map and biased source \\
\hline
\end{tabular}




\subsubsection{MAVRIC Input}

The input file for MAVRIC consists of three lines of text ("=mavric" command line with optional parameters, the problem title, and SCALE cross section library name) and then several blocks, with each block starting with "read $\mathrm{xxxx}$ " and ending with "end $\mathrm{xxxx".} \mathrm{There} \mathrm{are} \mathrm{three} \mathrm{required} \mathrm{blocks} \mathrm{and} \mathrm{nine}$ optional blocks. Material and geometry blocks must be listed first and in the specified order. Other blocks may be listed in any order.

Blocks (must be in this order):

- Composition - (required) SCALE standard composition, list of materials used in the problem

- Celldata - SCALE resonance self-shielding

- Geometry - (required) SCALE general geometry description

- Array - optional addition to the above geometry description

- Volume - optional calculation or listing of region volumes

- Plot - create 2D slices of the SGGP geometry

Other Blocks (any order, following the blocks listed above):

- Definitions - defines locations, response functions, and grid geometries used by other blocks

- Sources - (required) description of the particle source spatial, energy, and directional distributions

- Tallies - description of what to calculate: point detector tallies, region tallies, or mesh tallies

- Parameters - how to perform the simulation (random number seed, how many histories, etc.)

- Biasing - data for reducing the variance of the simulation

- ImportanceMap - instructions for creating an importance map based on a discrete-ordinates calculation

The material blocks (Composition and Celldata) and the physical model blocks (Geometry, Array, Volume, and Plot) follow the standard SCALE format. See the other SCALE references as noted in the following sections for details. The Biasing block and ImportanceMap block cannot both be used.

For the other six blocks, scalar variables are set by "keyword=value", fixed-length arrays are set with "keyword value ${ }_{1} \ldots$ value $_{N}$ ", variable-length arrays are set with "keyword value ${ }_{1} \ldots$ value $_{N}$ end", and some text and filenames are read in as quoted strings. Single keywords to set options are also used in some instances. The indention, comment lines, and upper/lowercase shown in this document are not requiredthey are used in the examples only for clarity. Except for strings in quotes (like filenames), SCALE is case insensitive.

After all input blocks are listed, a single line with "end data" should be listed. A final "end" should also be listed, to signify the end of all MAVRIC input. Nine of the blocks are the same input blocks used by the functional module Monaco, with a few extra keywords only for use with MAVRIC. These extra keywords are highlighted here, without relisting all of the standard Monaco keywords for those blocks. See Table 4.1.3 for an overview of MAVRIC input file structure.

\subsubsection{Composition block}

Material information input follows the standard SCALE format for material input. Basic materials known to the SCALE library may be used as well as completely user-defined materials (using isotopes with known cross sections). Input instructions are located in the XSProc chapter in the SCALE manual. The 
Standard Composition Library chapter lists the different cross section libraries and the names of standard materials. An example is as follows:

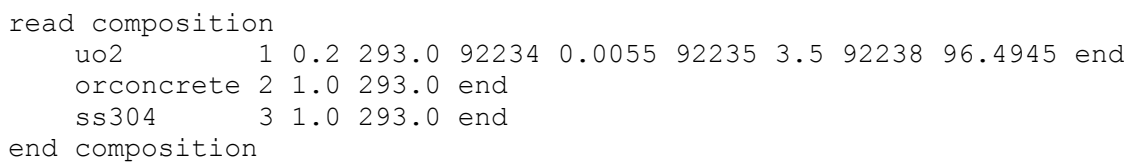

Details on the cell data block are also included in the XSProc chapter. When using different libraries for the importance map production (listed at the top of the input) and the final Monte Carlo calculation (listed in the parameters block, if different), make sure that the materials are present in both libraries.

Table 4.1.3. Overall input format

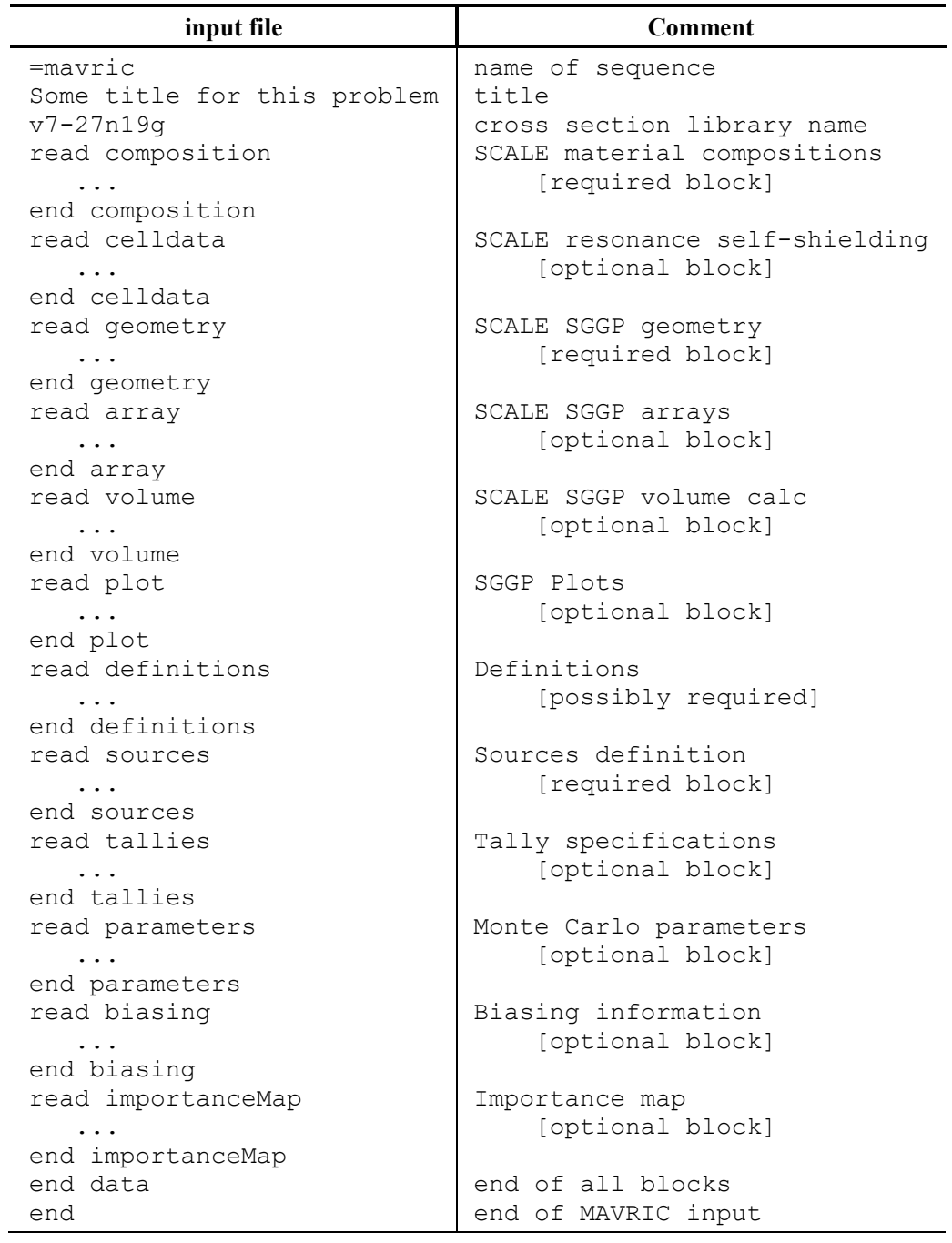

\subsubsection{SGGP geometry blocks}

MAVRIC uses the functional module Monaco for the forward Monte Carlo calculation. Monaco tracks particles through the physical geometry described by the SGGP input blocks as well as through the mesh importance map and any mesh tallies, which are defined in the global coordinates and overlay the 
physical geometry. Because Monaco must track through all of these geometries at the same time, users should not use the reflective boundary capability in the SGGP geometry.

For more details on each SGGP Geometry block, see the following sections of the KENO-VI chapter of the SCALE Manual.

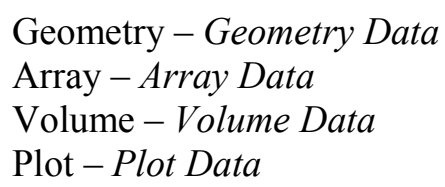

\subsubsection{Other blocks shared with Monaco}

The definitions, sources, tallies, and biasing blocks are all the same as Monaco. They are all fully described in the Monaco chapter of the SCALE Manual.

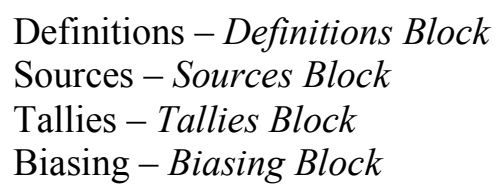

In the parameters block, there are several extra keywords compared to Monaco (see the Parameter Block section of the Monaco chapter) which are used when the cross section library used in the importance calculations is different from the library used in the final forward Monaco Monte Carlo calculation. The library listed at the beginning of the MAVRIC input file will be used for the importance calculations (forward and adjoint Denovo calculation, formation of the importance map, and biased sources). To use a different MG library in the final Monaco simulation, use the keyword "library=" with the cross section library name in quotes. A cross section library for Monaco will be made using csas-mg. If there are any extra parameters to use ("parm=" in the "=csas-mg" line of the csas-mg input), they can be passed along using the keyword "parmString=" with the extra information in quotes. For example, the following input file would use a coarse-group library for the importance calculations and a fine-group library for the final Monaco, each with CENTRM processing.

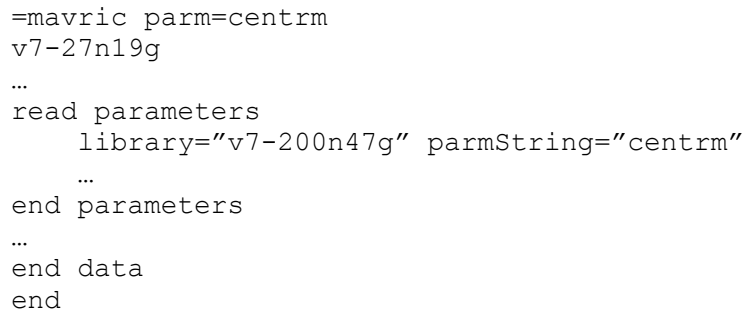

To use a CE cross section in the final Monaco step, use the keyword "ceLibrary=" with the cross section library name in quotes. When using the "library=" or "ceLibrary=" keywords, they should precede the "neutron", "photon", "noNeutron", and "noPhoton" keywords. Table 4.1.4 summarizes all of the keywords in the MAVRIC parameter block.

When using two different cross section libraries, be sure that the responses and distributions are defined in ways that do not depend on the cross section library. For example, any response that is just a list of $n$ values (corresponding to a cross section library of $n$ groups) needs to have the group energies specifically listed so that it can be evaluated properly on the other group structure. 
Table 4.1.4. Extra keywords for the parameters block

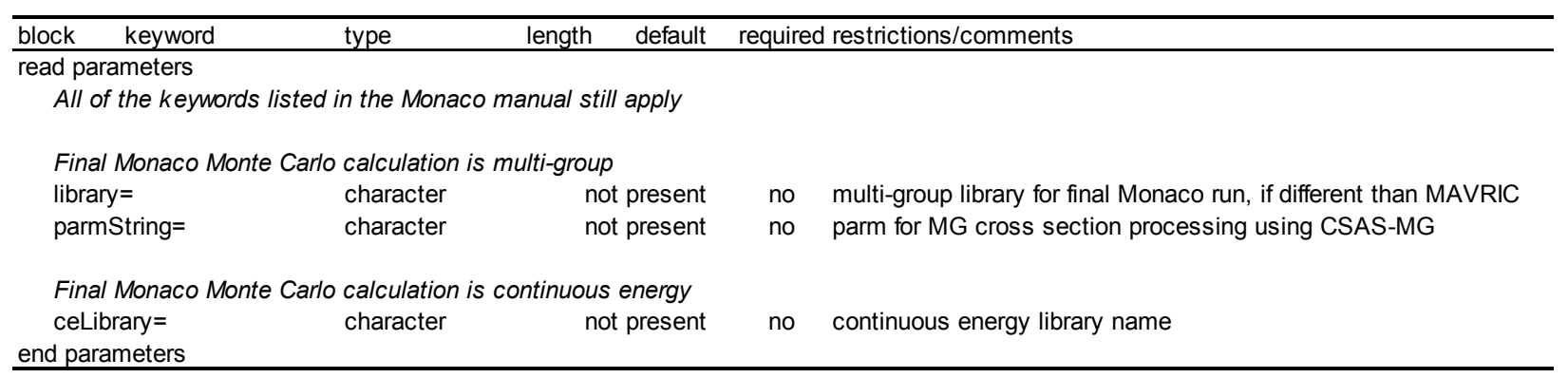

\subsubsection{Importance map block}

The importance map block is the "heart and soul" of MAVRIC. This block lists the parameters for creating an importance map and biased source from one (adjoint) or two (forward, followed by adjoint) Denovo discrete-ordinates calculations. Without an importance map block, MAVRIC can be used to run Monaco and use its conventional types of variance reduction. If both the importance map and biasing blocks are specified, only the importance map block will be used. There are a variety of ways to use the importance map block, as explained in the subsections below. Keywords for this block are summarized at the end of this section, in Table 4.1.5.

\subsection{Constructing a mesh for the $S_{N}$ calculation}

All of the uses of the importance map block that run the discrete-ordinates code require the use of a grid geometry that overlays the physical geometry. Grid geometries are defined in the definitions block of the MAVRIC input. The extent and level of detail needed in a grid geometry are discussed in the following paragraphs.

When using $\mathrm{S}_{\mathrm{N}}$ methods alone for solving radiation transport in shielding problems, a good rule of thumb is to use mesh cell sizes on the order of a meanfree path of the particle. For complex shielding problems, this could lead to an extremely large number of mesh cells, especially when considering the size of the meanfree path of the lowest energy neutrons and photons in common shielding materials.

In MAVRIC, the goal is to use the $\mathrm{S}_{\mathrm{N}}$ calculation for a quick approximate solution. Accuracy is not paramount - just getting an idea of the overall shape of the true importance map will help accelerate the convergence of the forward Monte Carlo calculation. The more accurate the importance map, the better the forward Monte Carlo acceleration will be. At some point there is a time trade-off when the computational time for calculating the importance map followed by the Monte Carlo calculation exceeds that of a standard analog Monte Carlo calculation. Large numbers of mesh cells, coming from using very small mesh sizes, for $\mathrm{S}_{\mathrm{N}}$ calculations also use a great deal of computer memory.

Because the deterministic solution(s) for CADIS and FW-CADIS can have moderate fidelity and still provide variance reduction parameters that substantially accelerate the Monte Carlo solution, mesh cell sizes in MAVRIC applications can be larger than what most $\mathrm{S}_{\mathrm{N}}$ practioners would typically use. The use of relatively coarse mesh reduces memory requirements and the run time of the deterministic solution(s). Some general guidelines to keep in mind when creating a mesh for the importance map/biased source are:

- The true source regions should be included in the mesh with mesh planes at their boundaries.

- For point or very small sources, place them in the center of a mesh cell, not on the mesh planes.

- Any region of the geometry where particles could eventually contribute to the tallies (the "important" areas) should be included in the mesh. 
- Point adjoint sources (corresponding to point detector locations) in standard CADIS calculations do not have to be included inside the mesh. For FW-CADIS, they must be in the mesh and should be located at a mesh cell center, not on any of the mesh planes.

- Volumetric adjoint sources should be included in the mesh with mesh planes at their boundaries.

- Mesh planes should be placed at significant material boundaries.

- Neighboring cell sizes should not be drastically different.

- Smaller cell sizes should be used where the adjoint flux is changing rapidly, for example, toward the surfaces of adjoint sources and shields (rather than their interiors).

Another aspect to keep in mind is that the source in the forward Monaco Monte Carlo calculation will be a biased, mesh-based source. Source particles will be selected by first sampling which mesh cell to use and then sampling a position uniformly within that mesh cell that meets the user criteria of "unit=", "region=", or "mixture=" if specified. The mesh should have enough resolution that the mesh source will be an accurate representation of the true source.

The geometry for the Denovo calculation is specified using the keyword "gridGeometryID=" and the identification number of a grid geometry that was defined in the definitions block. The material assigned to each voxel of the mesh is determined by testing the center point in the SGGP geometry (unless the macro-material option is used - see below).

\subsection{Macromaterials for $S_{N}$ geometries}

Part of the advantage of the CADIS method is that the adjoint discrete-ordinates calculation only needs to be approximate in order to form a reasonable importance map and biased source. This usually means that the mesh used is much coarser than the mesh that would be used if the problem were to be solved only with a discrete-ordinates code. This coarse mesh may miss significant details (especially curves) in the geometry and produce a less-than-optimal importance map.

In order to get more accurate solutions from a coarse-mesh discrete-ordinates calculation, Denovo can represent the material in each voxel of the mesh as a volume-weighted mixture of the real materials, called macromaterials, in the problem. When constructing the Denovo input, the Denovo EigenValue Calculation (DEVC, see section 2.4.A) sequence can estimate the volume fraction occupied by each real material in each voxel by a sampling method. The user can specify parameters for how to sample the geometry. Note that finer sampling makes more accurate estimates of the material fraction but requires more setup time to create the Denovo input. Users should understand how the macromaterials are sampled and consider that when constructing a mesh grid. This is especially important for geometries that contain arrays. Careful consideration should be given when overlaying a mesh on a geometry that contains arrays of arrays.

Because the list of macromaterials could become large, the user can also specify a tolerance for how close two different macromaterials can be to be considered the same, thereby reducing the total number of macromaterials. The macromaterial tolerance, "mmTolerance=", is used for creating a different macromaterial from the ones already created by looking at the infinity norm between two macromaterials. The number of macromaterials does not appreciably impact Denovo run time or memory requirements.

Two different sampling methods are available — point testing ${ }^{13}$ with the keyword mmPointTest and ray tracing ${ }^{14}$ with the keyword mmRayTest.

\section{Ray Tracing}


This method estimates the volume of different materials in the Denovo mesh grid elements by tracing rays through the SGGP geometry and computing the average track lengths through the each material. Rays are traced in all three dimensions to better estimate the volume fractions of materials within each voxel. The mmSubCell parameter controls how many rays to trace in each voxel in each dimension. For example, if mmSubCell $=\mathrm{n}$, then when tracing rays in the $\mathrm{z}$ dimension, each column of voxels uses a set of $\mathrm{n} \times \mathrm{n}$ rays starting uniformly spaced in the $\mathrm{x}$ and $\mathrm{y}$ dimensions. With rays being cast from all three orthogonal directions, a total of $3 n^{2}$ rays are used to sample each voxel. One can think of subcells as an equally spaced sub-mesh with a single ray positioned at each center. The number of subcells in each direction, and hence the number of rays, can be explicitly given with mmSubCells ny nz nx nz nx ny end keyword for rays parallel to the $\mathrm{x}$ axis, $\mathrm{y}$ axis, and $\mathrm{z}$ axis. Figure 4.1.1 shows different subcell configurations (in two dimensions) for a given voxel.

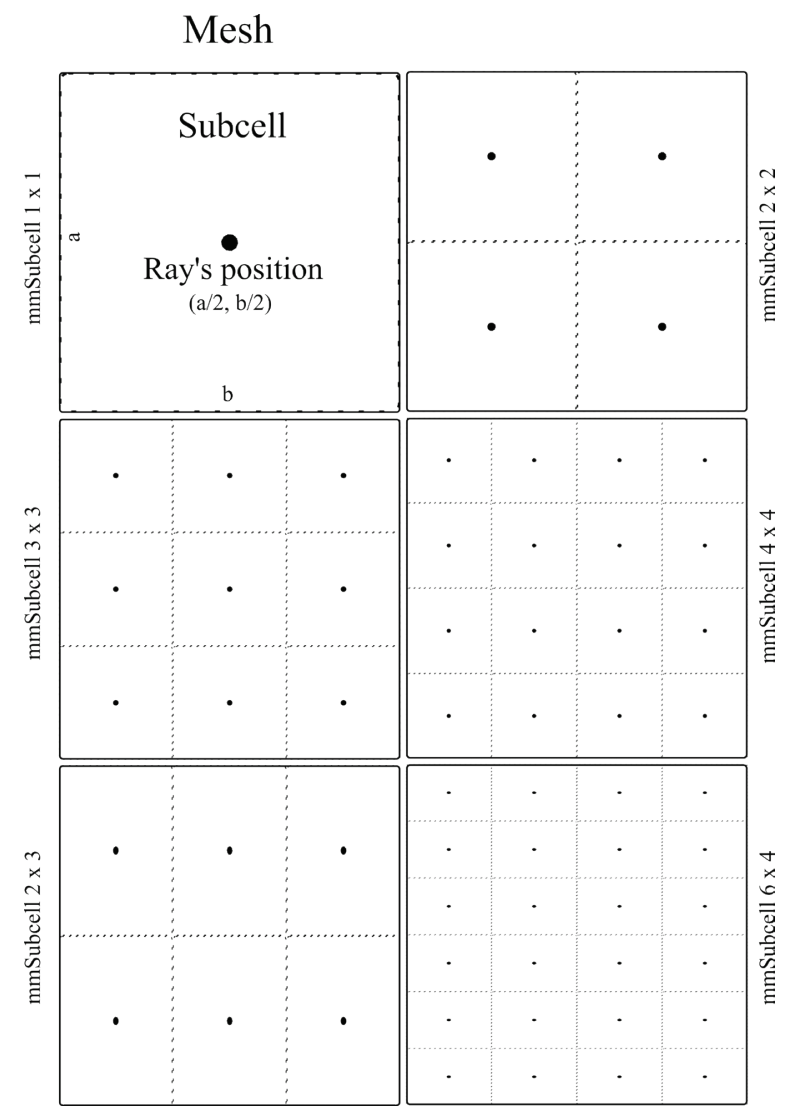

Figure 4.1.1. Ray positions within a voxel with different mmSubCells parameters.

Ray tracing is a more robust method compared to the simple point testing method used in previous versions of SCALE/MAVRIC; however, it requires more memory than point testing. Ray tracing gives more accurate estimates of volume fractions because track lengths across a voxel give more information than a series of test points. Ray tracing is also much faster than point testing because the particle tracking routines are optimized for quickly determining lists of materials and distance along a given ray.

Ray tracing operates on the grid geometry supplied by the user and shoots rays in all three directions starting from the lower bounds of the mesh grid. An example of an arbitrary assembly geometry is shown in Figure 4.1.2. A ray consists of a number of steps that each correspond to crossing a material boundary along the path of the ray. Ratios of each step's length to the voxel length in the ray's direction determine 
the material volume fraction of that step in that voxel, and summation of the same material volume fractions gives the material volume fraction of that material in that voxel. Ray tracing through a single voxel that contains a fuel pin is illustrated in Figure 4.1.3.
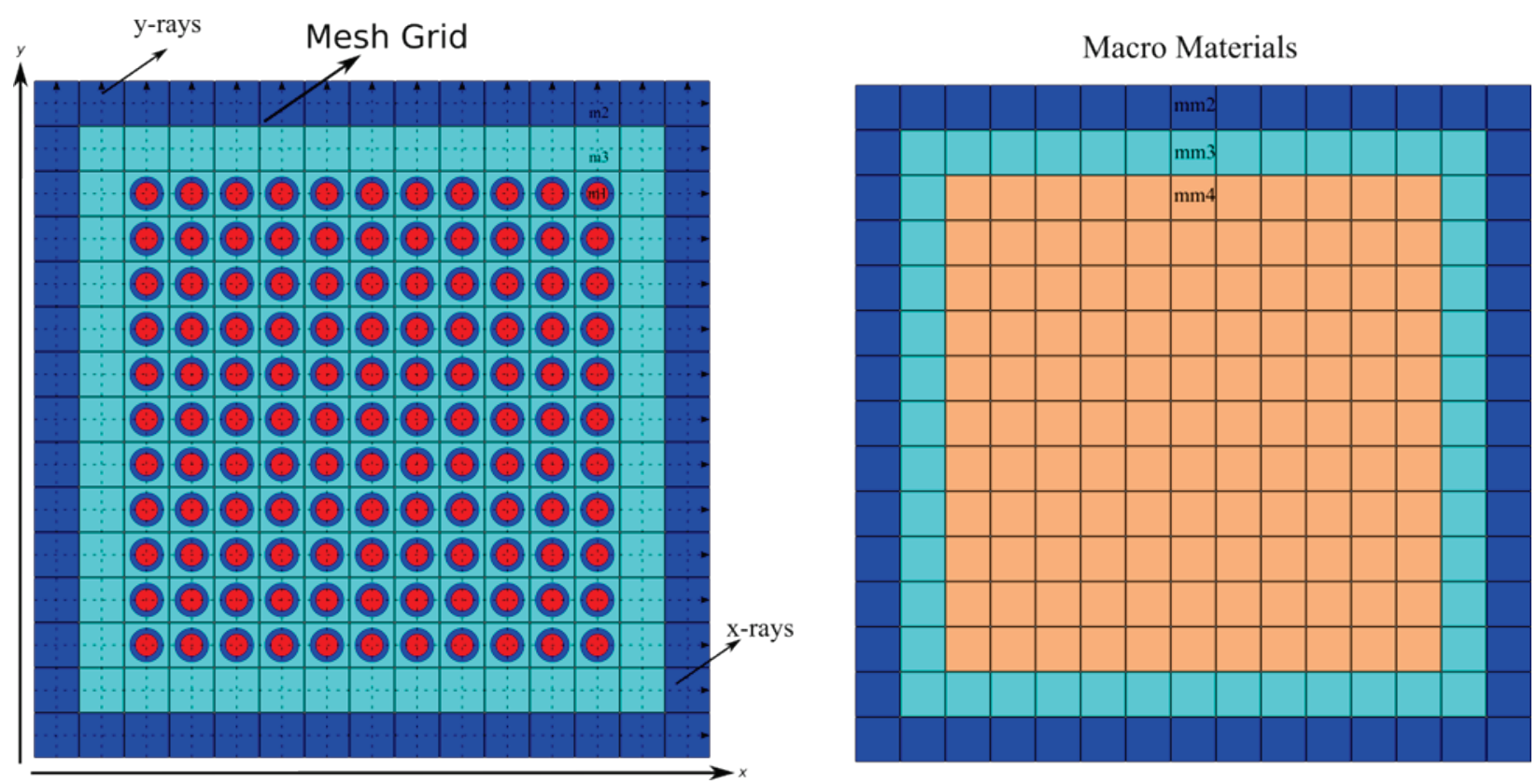

Figure 4.1.2. Geometry model (left) and the Denovo representation (right) of an assembly using macromaterials determined by ray tracing.

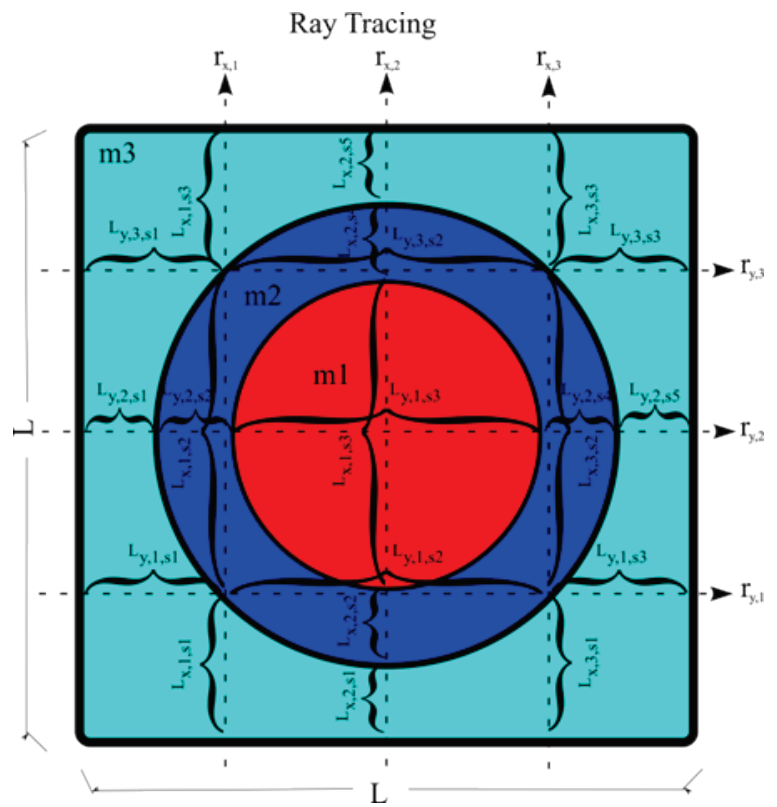

Figure 4.1.3. Ray tracing (in two dimensions) through a voxel.

The final constructed macromaterials for this model are also shown in Figure 4.1.2. Voxels that contain only a single material are assigned the original material number in the constructed macromaterials. For 
the voxels that contain a fuel pin with three different materials, the result is a new macromaterial consisting of the volume weighted fractions of each original material.

After the rays are shot in all three directions, the material volume fractions are updated and macromaterials are created by using these material volume fractions. Material volume fraction calculations for a single voxel, as shown in Figure 4.1.3, are given by

$$
F_{m}=\sum_{d=x, y, z} \sum_{r=1}^{N_{r}} \sum_{s=1}^{N_{s}}\left\{\begin{aligned}
\frac{L_{d, r, s}}{L_{d}}, & m_{s}==m \\
0, & \text { otherwise }
\end{aligned} \quad \text { and } \quad V_{m}=\frac{F_{m}}{\sum_{n=1}^{N_{m} F_{n}}}\right.
$$

where $F_{m}=$ sampled fraction of material $m$ in the voxel,

$d=$ direction of the rays $(x, y, z)$,

$r=$ ray number,

$N_{r}=$ total number of rays in the voxel for direction of $d$,

$s=$ step number,

$N_{s}=$ total number of steps for ray $r$ in the voxel for direction of $d$,

$L_{d, r, s}=$ length of the steps s for ray $\mathrm{r}$ in the voxel for direction of $d$,

$L_{d}=$ length of the voxel along direction of $d$,

$m_{s}=$ material of step $s$,

$m=$ material number,

$N_{m}=$ total number of materials in the voxel, and

$V_{m}=$ volume fraction of material $\mathrm{m}$ in the voxel.

\section{Point Testing}

The recursive bisection method is utilized in point testing and uses a series of point tests to determine the macromaterial fractions. For a given voxel, the material at the center is compared to the material at the eight corners. If they are all the same, the entire volume is considered to be made of that material. If different, the volume is divided into two in each dimension. Each subvolume is tested, and the method is then applied to the subvolumes that are not of a single material. When the ratio of the volume of the tested region to the original voxel becomes less than a user-specified tolerance (in the range of 10-1 to 10-4), then further subdivision and testing are stopped. This is illustrated in Figure 4.1.4. 

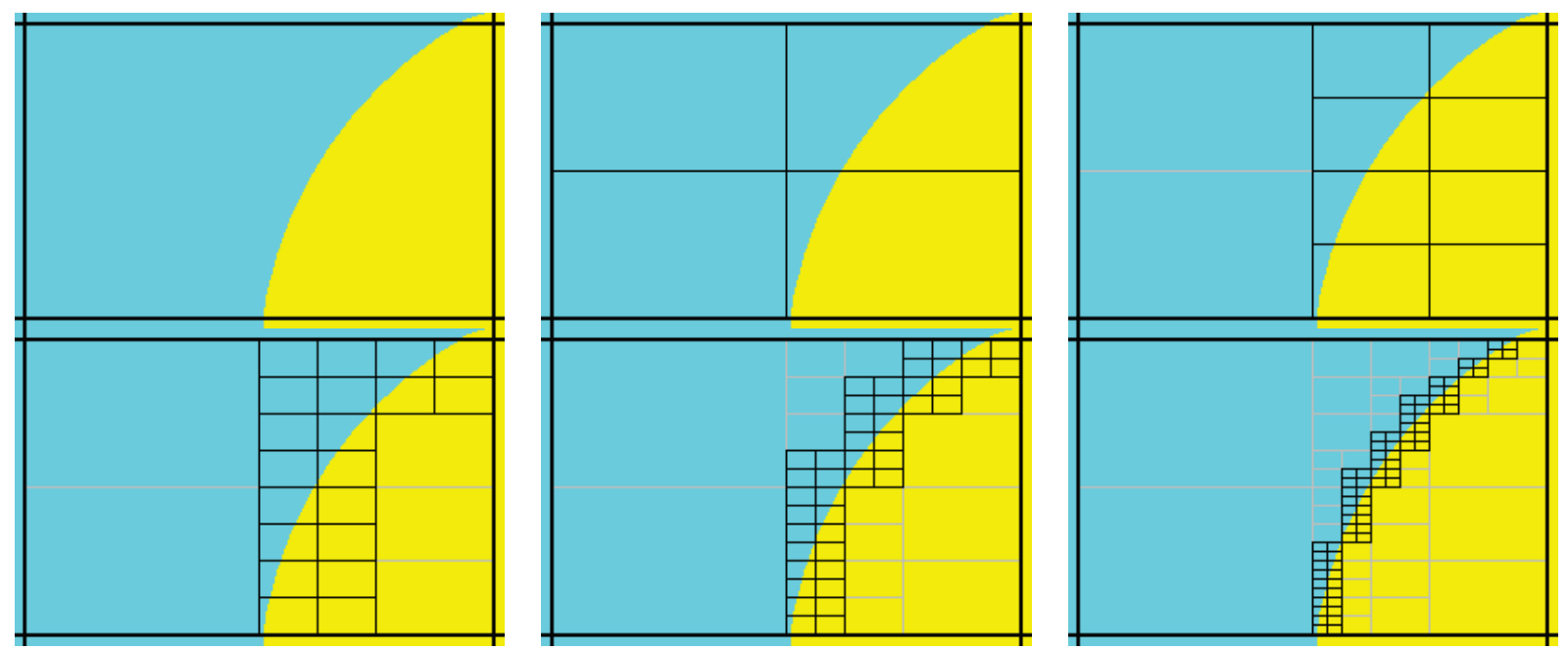

Figure 4.1.4. Successive steps in the recursive macro-material method.

In point testing, the keyword "mmTolerance $=\mathrm{f}$ " is interpreted to be where $\mathrm{f}$ is the smallest fraction of the voxel volume that can be achieved by bisection method and hence the limiting factor for dividing the voxel. This same tolerance $f$ is also used to limit the number of macromaterials. Before a new macromaterial is created, if one already exists where the fraction of each actual material matches to within the given tolerance, then the existing material will be used. If using only a single point at the center of each voxel, use "mmTolerance $=1$ ". The mmSubCell keyword is not used in point testing.

\section{Example}

Figure 4.1.5 shows an example of a cask geometry with two types of spent fuel (yellows), steel (blue), resin (green), and other metals (gray). When the Denovo geometry is set up by testing only the center of each mesh cell, the curved surfaces are not well represented (upper right). By applying the ray-tracing method and defining a new material made of partial fractions of the original materials, an improved Denovo model can be made. In the lower left of the figure, the Denovo model was constructed using one ray (in each dimension) per voxel and a tolerance of 0.1 . This gives 20 new materials that are a mixture of the original 13 actual materials and void. With mmSubCells $=3$ and an mmTolerance $=0.01,139$ macromaterials are created.

A macromaterial table listing the fractions of each macromaterial is saved to a file called "outputName.mmt", where outputName is the name the user chose for his or her output file. This file can be used by the Mesh File Viewer to display the macromaterials as mixtures of the actual materials, as seen in the lower row of Figure 4.1.5. See the Mesh File Viewer help pages for more information on how to use colormap files and macromaterial tables. 

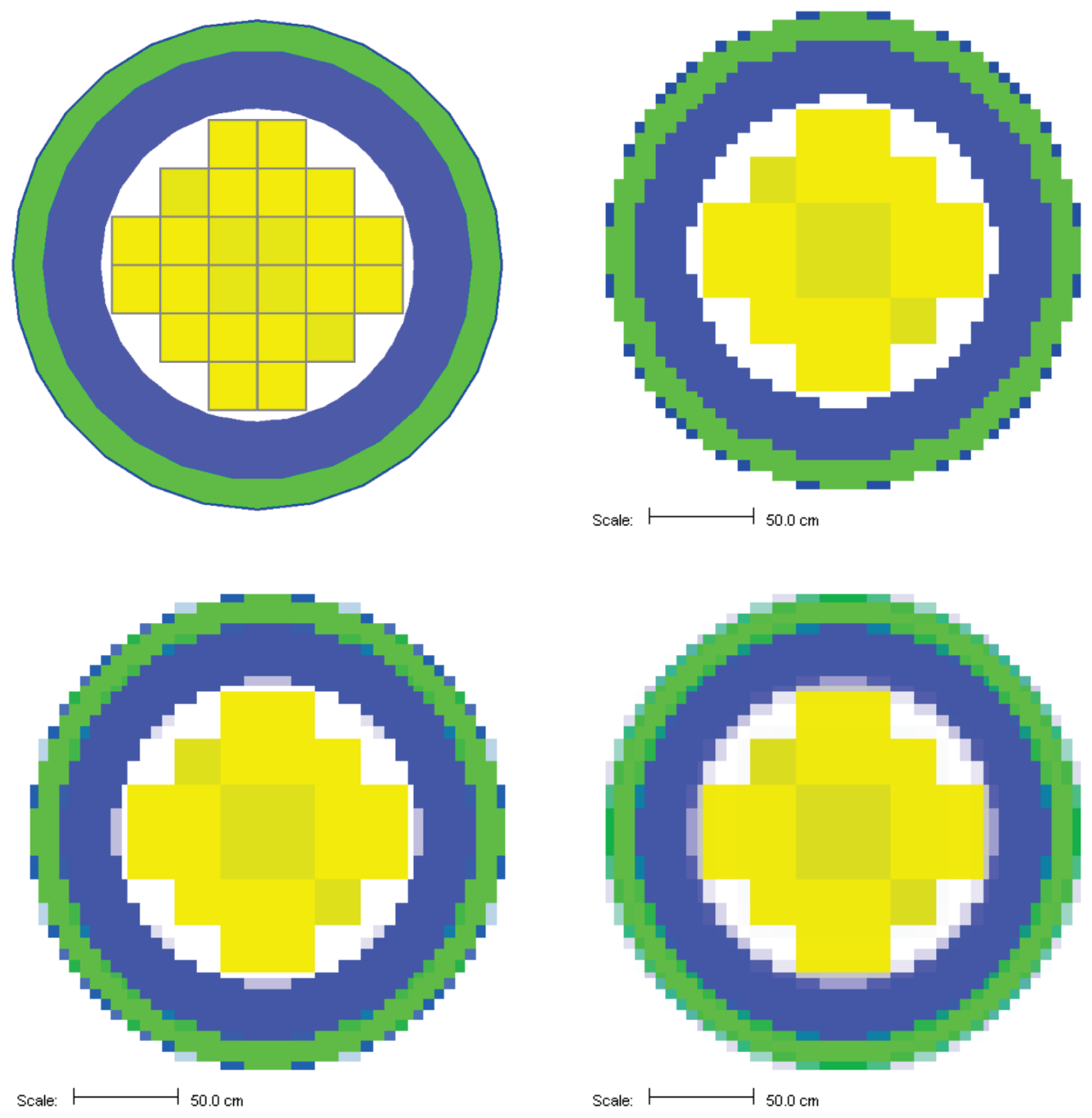

Figure 4.1.5. Cask geometry model (upper left) and the Denovo representation using cell center testing (upper right). Representations using macromaterials determined by ray tracing are shown for mmSubCell $=1 / \mathrm{mm}$ Tolerance $=0.1$ (lower left) and $\mathrm{mmSubCell}=3 / \mathrm{mmTolerance}=0.01$ (lower right). 


\subsection{Optimizing source/detector problems}

For standard source/detector problems where one tally is to be optimized in the forward Monte Carlo calculation, an adjoint source based on that tally needs to be constructed. An adjoint source requires a unique and positive identification number, a physical location, and an energy spectrum. The adjoint source location can be specified either by (1) a point location ("locationID=" keyword) or (2) a volume described by a box ("boundingBox" array). A bounding box is specified by maximum and minimum extent in each dimension- $\begin{array}{llllll}x_{\max } & x_{\min } & y_{\max } & y_{\min } & z_{\max } & z_{\min } \text { in global coordinates. The boundingBox should }\end{array}$ not be degenerate (should have volume $>0$ ) but can be optionally limited to areas matching a given unit number ("unit="), a given region number ("region="), or a given material mixture number ("mixture="). A mixture and a region cannot both be specified since that would either be redundant or mutually exclusive. The energy spectrum of an adjoint source is a response function ("responseID=") listing one of the ID numbers of the responses defined in the definitions block. An optional weight can be assigned to each adjoint source using the "weight=" keyword. If not given, the default weight is 1.0.

For example, to optimize a region tally, the user would construct an adjoint source located in the same place as the tally, with an adjoint source spectrum equal to the response function that the tally is computing. Note that the grid geometry 1 and response function 3 need to already be defined in the definitions block.

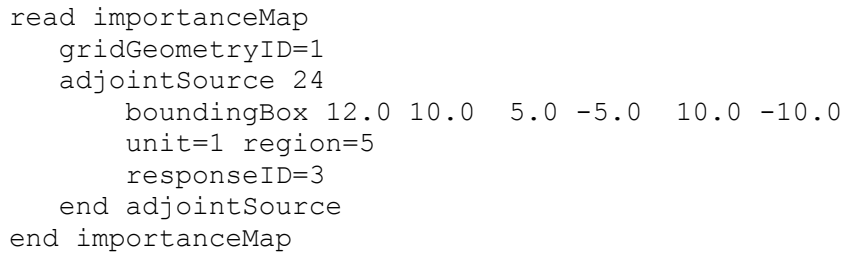

For optimizing a point detector for the calculation of total photon flux, the importance map block would look like the following:

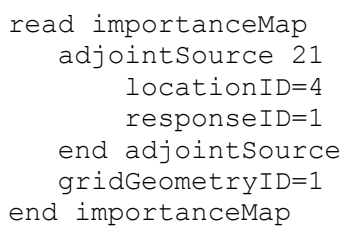

where location 4 is the same location used by the point detector. Response function 1, to calculate total photon flux, must be defined in the definitions block similar to this response

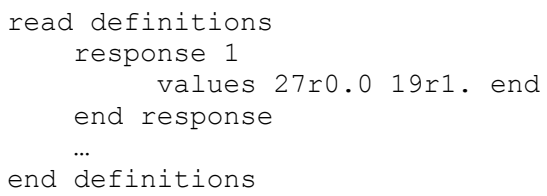

used for computing total photon flux for the 27-neutron/19-photon group coupled cross section library or like this response 


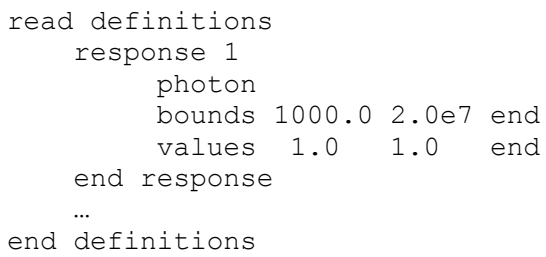

which is independent of cross section library.

\subsection{Multiple adjoint sources}

In cases where there are several tallies in very close proximity and/or several different responses being calculated by the tallies, multiple adjoint sources can be used.

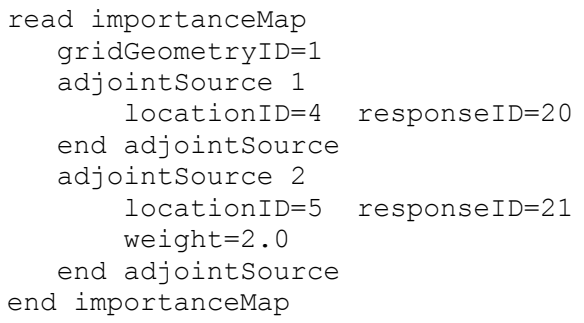

Note that adjoint sources using point locations can be mixed with volumetric adjoint sources (using bounding boxes).

\subsection{Options for Denovo $S_{N}$ calculations}

While the default values for various calculational parameters and settings used by Denovo for the MAVRIC sequence should cover most applications, they can be changed if desired. The two most basic parameters are the quadrature set used for the discrete ordinates and the order of the Legendre polynomials used in describing the angular scattering. The default quadrature order that MAVRIC uses is a level symmetric $\mathrm{S}_{8}$ set, and the default scattering order is $\mathrm{P}_{3}$ (or the maximum number of coefficients contained in the cross-section library if less than 3$)$. $\mathrm{S}_{8} / \mathrm{P}_{3}$ is an adequate choice for many applications, but the user is free to changes these. For complex ducts or transport over large distances at small angles, $\mathrm{S}_{12}$ may be required. $\mathrm{S}_{4} / \mathrm{P}_{1}$ or even $\mathrm{S}_{2} / \mathrm{P}_{0}$ would be useful in doing a very cursory run to confirm that the problem was input correctly, but would likely not be adequate for weight window generation for a problem that is complex enough to require advanced variance reduction.

These options, as well as the other Denovo options, are applied to both the forward and the adjoint calculations that are required from the inputs given in the importance map block.

In problems with small sources or media that are not highly scattering, discrete ordinates can suffer from "ray effects" 15,16 where artifacts of the discrete quadrature directions can be seen in the computed fluxes. To help alleviate the ray effects problem, Denovo has a first-collision capability to help alleviate ray effects. This method computes the uncollided flux in each mesh cell from a point source. The uncollided fluxes are then used as a distributed source in the main discrete-ordinates solution. At the end of the main calculation, the uncollided fluxes are added to the fluxes computed with the first collision source, forming the total flux. While this helps reduce ray effects in many problems, the first-collision capability can take a significant amount of time to compute on a mesh with many cells or for many point sources.

Adjoint sources that use point locations will automatically use the Denovo first-collision capability. Volumetric adjoint sources (that use a boundingBox) will be treated without the first-collision capability. 
The keywords "firstCollision" and "noFirstCollision" will be ignored by MAVRIC for adjoint calculations. Keywords for Denovo options in the importance map block are summarized at the end of this section, in Table 4.1.6.

\subsection{Starting with an existing adjoint flux file}

An importance map can be made from an existing Denovo flux file by using the keyword "adjointFluxes=" with the appropriate file name in quotes. The file must be a binary file using the *.dff file format, and the number of groups must match the number of groups in the MAVRIC cross section library (i.e., the library entered on the third line of the MAVRIC input file). Instead of performing an adjoint calculation, the fluxes read from the file will be used to create both the mesh-based importance map and the biased mesh source.

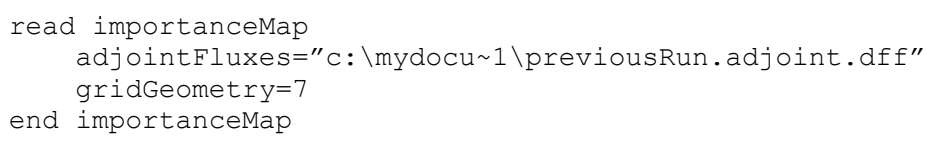

If the "adjointFluxes=" keyword is used and any adjoint sources are defined, an error will result. If a forward flux file is supplied for forward-weighting the adjoint source (see below), then an adjoint flux file cannot be specified.

The grid geometry is not required when using a pre-existing flux file. If grid geometry is not supplied, one will be created from the mesh planes that are contained in the Denovo flux file (which were used to compute the fluxes in that file).

\subsection{Forward-weighting the adjoint source}

To optimize a mesh tally or multiple region tallies/point detector tallies over a large region, instead of a uniform weighting of the adjoint source, a weighting based on the inverse of the forward response can be done. This requires an extra discrete-ordinates calculation but can help the forward Monte Carlo calculation compute the mesh tally or group of tallies with more uniform statistical uncertainties.

The same grid geometry will be used in both the forward calculation and the adjoint calculation, so the user needs to ensure that the mesh covers all of the forward sources and all of the adjoint sources, even if they are point sources.

To use forward-weighted CADIS, specify either of the keywords - "respWeighting" or "fluxWeighting". For either, MAVRIC will run Denovo to create an estimate of the forward flux, $\phi(\vec{r}, E)$. For response weighting ("respWeighting"), each adjoint source is inversely weighted by the integral of the product of the response function used in that adjoint source and the estimate of the forward flux. For an adjoint source described by the geometric function $g(\vec{r})$ and the response function $\sigma_{d}(E)$ (note that $\sigma_{d}(E)=1$ for computing total fluxes), the forward-weighted adjoint source becomes

$$
q_{i}^{+}(\vec{r}, E)=\frac{\sigma_{d}(E) g(\vec{r})}{\int \sigma_{d}(E) \phi(\vec{r}, E) d E}
$$

Response weighting will calculate more uniform relative uncertainties of the integral quantities of the tallies in the final Monte Carlo calculation. 
To optimize the calculation of the entire group-wise flux with more uniform relative uncertainties in each group, the adjoint source should be weighted inversely by the forward flux, $\phi(\vec{r}, E)$, using the "fluxWeighting" keyword. For an adjoint source described by the geometric function $g(\vec{r})$ and the response function $\sigma_{d}(E)=1$, the forward-weighted adjoint source becomes

$$
q_{i}^{+}(\vec{r}, E)=\frac{\sigma_{d}(E) g(\vec{r})}{\phi(\vec{r}, E)}
$$

For example, consider a problem with a single source and two detectors, one near the source that measures flux and one far from the source that measures some response. In a standard Monte Carlo calculation, it is expected that since more Monte Carlo particles cross the near detector, it will have a much lower relative uncertainty than the far detector. Standard CADIS could be used to optimize the calculation of each in separate simulations:

\begin{tabular}{|c|c|}
\hline To optimize the flux in the near detector & To optimize the response in the far detector \\
\hline $\begin{array}{l}\text { read importanceMap } \\
\text { gridGeometryID }=1 \\
\text { adjointSource } 1 \\
\quad \text { boundingBox } \quad x_{1} \quad x_{2} y_{1} \quad y_{2} \quad z_{1} z_{2} \\
\text { responseID } 1 \\
\text { end adjointSource } \\
\text { end importanceMap }\end{array}$ & $\begin{array}{l}\text { read importanceMap } \\
\text { gridGeometryID }=1 \\
\text { adjointSource } 2 \\
\text { boundingBox } \mathrm{u}_{1} \quad \mathrm{u}_{2} \mathrm{v}_{1} \quad \mathrm{v}_{2} \quad \mathrm{w}_{1} \mathrm{w}_{2} \\
\text { responseID } 6 \\
\text { end adjointSource } \\
\text { end importanceMap }\end{array}$ \\
\hline
\end{tabular}

where response 1 was defined as $\sigma_{1}(E)=1$ and response 6 was defined as $\sigma_{6}(E)=$ flux-to-response conversion factors. The two options for forward-weighting allow the tallies for both detectors to be calculated in the same MAVRIC simulation. Using "fluxWeighting", the importance map and biased source will be made to help distribute Monte Carlo particles evenly through each energy group and every voxel in each both detectors, making the relative uncertainties close to uniform. With "respWeighting", the importance map and biased source will optimize the total integrated response of each tally.

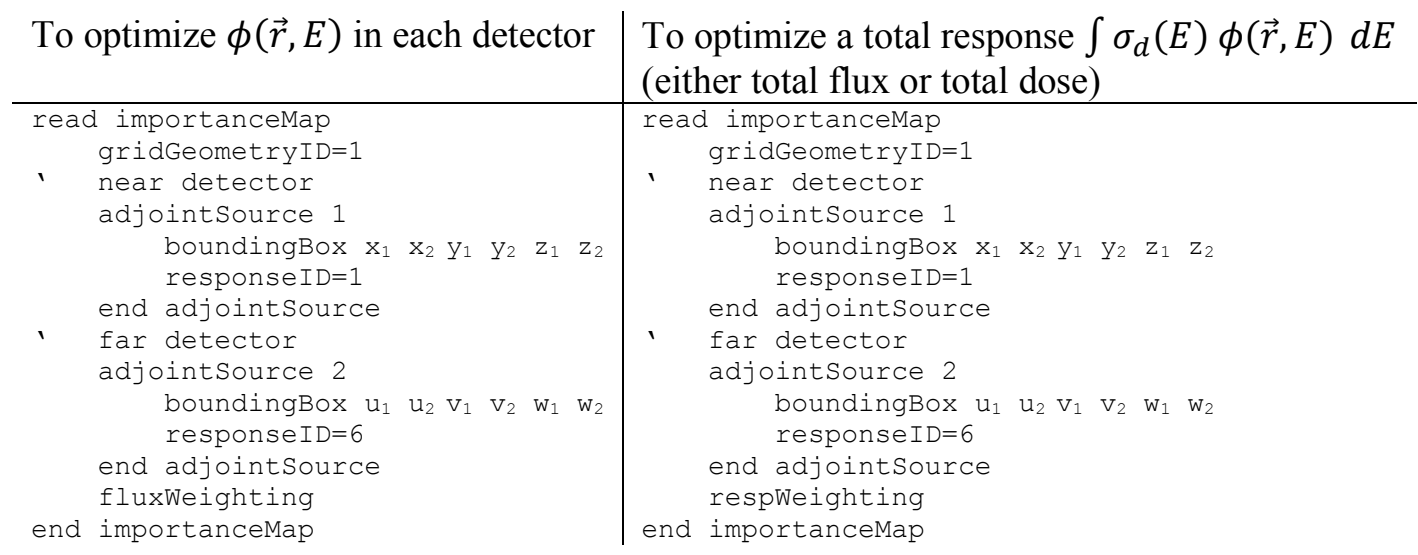

Using flux weighting, the adjoint source will be

$$
q^{+}(\vec{r}, E)=\frac{\sigma_{1}(E) g_{\mathrm{near}}(\vec{r})}{\phi(\vec{r}, E)}+\frac{\sigma_{6}(E) g_{\mathrm{far}}(\vec{r})}{\phi(\vec{r}, E)}
$$

or using response weighting, the adjoint source will be 


$$
q^{+}(\vec{r}, E)=\frac{\sigma_{1}(E) g_{1}(\vec{r})}{\int \sigma_{1}(E) \phi(\vec{r}, E) d E}+\frac{\sigma_{6}(E) g_{2}(\vec{r})}{\int \sigma_{6}(E) \phi(\vec{r}, E) d E}
$$

This implementation is slightly different from the original MAVRIC in SCALE 6. The current approach is simpler for the user and allows the importance parameters to optimize the final Monte Carlo calculation for the calculation of two different responses in two different areas.

If the number of mesh cells containing the true source is less than 10, then MAVRIC will convert these source voxels to point sources and Denovo will automatically use its first-collision capability to help reduce ray effects in the forward calculation. The user can easily override the MAVRIC defaults - to force the calculation of a first-collision source no matter how many voxels contain source - by using the keyword "firstCollision". To prevent the calculation of a first-collision source, the keyword "noFirstCollision" can be used. If the keywords "firstCollision" or "noFirstCollision" are used, they will only apply to the forward calculation, not the subsequent adjoint calculation.

The keyword "saveExtraMaps" will save extra files that can be viewed by the Mesh File Viewer. The source used by the forward Denovo calculation is stored in "outputName.dofs.3dmap", where outputName is the name the user chose for his output file.

\subsection{Forward-weighting with an existing forward flux file}

Similar to the capability of using pre-existing adjoint flux files, MAVRIC can use a pre-existing forward flux file to create a forward-weighted adjoint source without performing the forward Denovo calculation. The user may specify the *.dff file containing the forward fluxes using the keyword "forwardFluxes=". The filename should be enclosed in quotes, and the file must be a binary file using the Denovo flux file format. The number of groups must match the number of groups in the MAVRIC cross section library (i.e., the library entered on the third line of the MAVRIC input file).

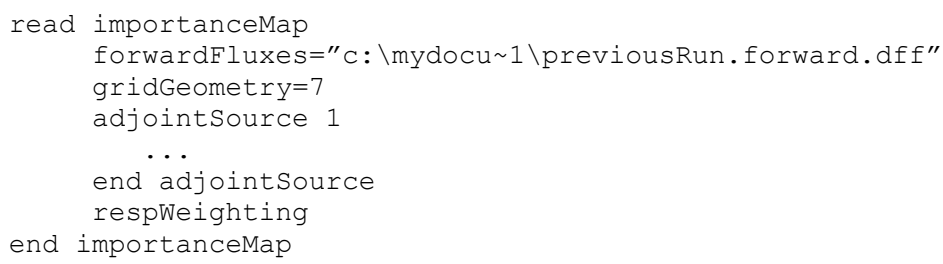

When using a pre-existing forward flux file, either "respWeighting" or "fluxWeighting" must still be specified.

\subsection{Using the importance map}

An importance map produced by the importance map block consists of the target weight values as a function of position and energy. The upper weight window used for splitting and the lower weight window used for Russian roulette are set by the window ratio. The window ratio is simply the ratio of the weight window upper bound to the weight window lower bound, with the target weight being the average of the upper and lower.

The keyword "windowRatio=" can be used within the importance map block to specify what window ratio will be used with the importance map that is passed to the Monaco forward Monte Carlo calculation. For a windowRatio of $r$, the upper weights for splitting, $w_{\max }$, and the lower weights for Russian roulette, $w_{\min }$, are set as 


$$
w_{\min }=\frac{2}{r+1} \bar{w}
$$

and

$$
w_{\max }=\frac{2 r}{r+1} \bar{w}
$$

for the target weight $\bar{w}$ in each mesh cell and for each energy of the importance map. The default value for the windowRatio is 5.0.

\subsection{Other notes on importance map calculations}

Since the importance map calculations all take place using mesh geometry, one of the first steps that occurs is to create a mesh representation of the true source (the forward source) on the same grid. This procedure uses the same two methods as the Monaco mesh source saver routine. Mesh cells can be subdivided and tested to see if they are within the defined source, or a set number of points can be sampled from the source. The keywords "subCells=" and "sourceTrials=" are used in the importance map block to change the default settings for constructing the mesh representation of the forward source.

If macromaterials are used ("mmTolerance $<1$ ") and the adjoint source is limited to a particular material, the amount of adjoint source in a mesh voxel will be weighted by the material amount in that voxel.

In SCALE/MAVRIC, Denovo is called as a fixed-source $\mathrm{S}_{\mathrm{N}}$ solver and cannot model multiplying media. Neither forward nor adjoint neutron calculations from Denovo will be accurate when neutron multiplication is a major source component. If neutron multiplication is not turned off in the parameters block of the MAVRIC input (using "fissionMult=0"), a warning will be generated to remind the user of this limitation.

By default, MAVRIC instructs Denovo not to perform outer iterations for neutron problems if the crosssection library contains upscatter groups. This is because the time required calculating the fluxes using upscatter can be significantly longer than without. For problems where thermal neutrons are an important part of the transport or tallies, the user should specify the keyword "upScatter=1" in the importance map block. This will instruct Denovo to perform the outer iterations for the upscatter groups, giving more accurate results but taking a much longer time for the discrete-ordinates calculation.

When doing a MAVRIC calculation using a coarse-group energy structure for Denovo (for example with the 27/19 library) but a fine-group energy structure (with the 200/47 library) for the final Monaco calculation, the source biasing parameters are determined on the coarse-group structure. The importance map (*.mim) file and the biased mesh source $(* . \mathrm{msm})$ files all use the coarse-group structure. The source biasing information is then applied to fine-group mesh versions of the sources, resulting in the *.sampling.*.msm files. This way, the biased sources used in the final Monaco calculation retain their fine-group resolution. This can be especially important in representing the high-energy portion of the fission neutron distribution for example. When using CE-Monaco, the source sampling routines first use the *.msm files to determine the source particle's voxel and energy group. From that voxel and energy group, the user-given source distributions are used to sample the specific starting location and specific energy of the source particle. This way, the CE-Monaco calculation samples the true CE distributions. 
Table 4.1.5. Keywords for the importance map block

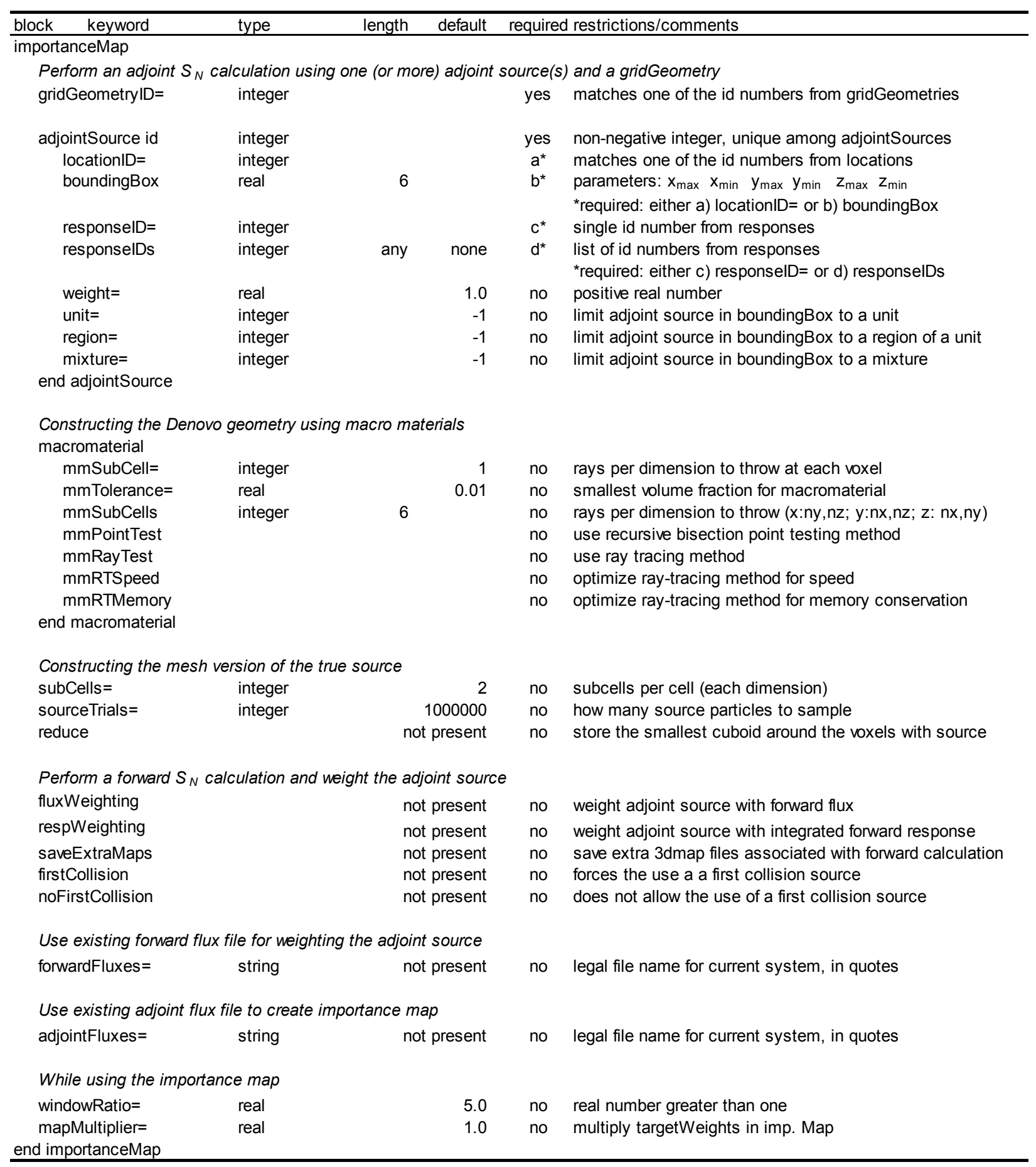


Table 4.1.6. Denovo options for the importance map block

\begin{tabular}{|c|c|c|c|c|}
\hline \multirow{2}{*}{$\begin{array}{l}\text { block keyword } \\
\text { importanceMap }\end{array}$} & type & length & \multicolumn{2}{|c|}{ required restrictions/comments } \\
\hline & & & & \\
\hline \multicolumn{5}{|c|}{ Optional Denovo $S_{N}$ code parameters } \\
\hline discretization $=$ & integer & 4 & no & $\begin{array}{l}\text { 0-diamond-difference, 1-diamond-difference with flux fix-up:lin0, } \\
\text { 2-theta-weighted diamond-difference, 3-linear-discontinuous finite } \\
\text { element, 4-step characteristics, 5-trilinear discontinuous finite elen }\end{array}$ \\
\hline quadType= & integer & 0 & no & 0-level symmetric, 1-Gauss-Legendre product, 2-QR \\
\hline legendre $=$ & integer & $3^{*}$ & no & $\begin{array}{l}P_{L}, L=\text { highest Legendre polynomial, } L=0,1,2,3, \ldots \\
{ }^{*} \text { default is to use min(the highest available in the data,3) }\end{array}$ \\
\hline tportcorrection= & integer & $1^{*}$ & no & $\begin{array}{l}\text { transport correction: } 0 \text {-none, 1-diagonal, 2-Cesaro } \\
{ }^{*} \text { For } \mathrm{P}_{2} \text { or higher, the default is } 2 \text { (Cesaro) }\end{array}$ \\
\hline upScatter= & integer & 0 & no & upscatter iterations: 0-none, 1-yes, 2-ignore \\
\hline $\mathrm{xblocks}=$ & integer & 1 & no & parallel stuff \\
\hline yblocks= & integer & 1 & no & parallel stuff \\
\hline zblocks= & integer & 1 & no & parallel stuff \\
\hline numSets $=$ & integer & 1 & no & $?$ \\
\hline partUpscatter= & integer & 1 & no & partition upscatter (0-no, 1-yes) \\
\hline quadrature $=$ & integer & 8 & no & level symmetric $S_{N}$ quadrature, $N=2,4,6,8,10,12,14,16$ \\
\hline polarsPerOct $=$ & integer & 0 & no & Gauss-Legendre product quadrature or QR \\
\hline azimuthsPerOct= & integer & 0 & no & Gauss-Legendre product quadrature or $\mathrm{QR}$ \\
\hline maxlters $=$ & integer & 20 & no & maximum number of iterations \\
\hline diagnostics $=$ & integer & 0 & no & 0-no diganostics, 1 -all diagnostics \\
\hline output= & integer & 0 & no & 0 -no ouput, 1 -all output \\
\hline krylovSpaceSize= & integer & 10 & no & size in memory for Krylov space \\
\hline tolerance $=$ & double & 1.E-03 & no & tolerance used in convergence test \\
\hline krylovType= & integer & 0 & no & 0-GMRES, 1-BiCGStab \\
\hline eigenSolver= & integer & 0 & no & 0-power iteration, 1-Arnoldi, 2-shifted inverse \\
\hline multiGSolover= & integer & 0 & no & 0-Gauss-Seidel, 1-Krylov \\
\hline withinGSolver= & integer & 0 & no & 0-Krylov, 1-residual Krylov, 2-source iteration \\
\hline mgSettings $=$ & integer & 1 & no & 0-user supplied, 1 -automatic \\
\hline upGroupSolver= & integer & 0 & no & $\begin{array}{l}\text { 0-same as within-group solver, 1-Krylov, 2-residual Krylov, } \\
\text { 3-source iteration, 4-single source iteration }\end{array}$ \\
\hline acceleration $=$ & integer & 0 & no & 0-none, 1-two grid \\
\hline maxltersMG= & integer & 20 & no & maximum number of iterations \\
\hline $\begin{array}{l}\text { toleranceMG= } \\
\text { end importanceMap }\end{array}$ & double & 0.001 & no & tolerance used in convergence test \\
\hline
\end{tabular}

\subsubsection{MAVRIC Output}

\subsubsection{Main text output file}

Similar to other SCALE sequences, MAVRIC returns a text output file containing the output from the SCALE driver, the sequence itself, and all of the functional modules called. The SCALE driver output first displays the problem input file then the first reading of the input file by the MAVRIC sequence is shown (which includes some material processing information). If there are any errors or warnings about the input file, they will be shown next. Next in the output file are the different passes through the MAVRIC sequence, up to 10 parts. If any errors or warning messages (such as lack of memory) are generated during processing, they will be displayed here. Finally, the output files from each functional module are concatenated to the above output and shows the files returned to the user.

The Monaco section of output first reviews the input it received. First is a review of the geometryshowing which materials are used in each region and the volume of that region, if input or calculated. Then there is a detailed list of other Monaco input: cross section parameters, data definitions, the source description, the tallies, the Monte Carlo parameters, and the biasing parameters. For MAVRIC calculations, if an importance map is used, then its summary is also given. The "Mesh Importance Map 
Characterization" shows where the importance map may be changing too rapidly and may require more refinement.

For each Monaco batch, the output file lists the batch time and the starting random number for the next batch, which may be useful in rerunning just a portion of a problem. Once all of the batches are completed, a list of the various tally files that have been created is given. Finally, the tallies are summarized in a section titled "Final Tally Results Summary." For each point detector, the total neutron and photon fluxes (uncollided and total) are given as well as the final response values for each response function. For each region tally, the total neutron and photon fluxes (both track-length and collision density estimates) are listed, followed by the final response values for each response function. Group-by-group details are saved to separate files for each tally.

\subsubsection{Additional output files}

In addition to the generous amount of data contained in the MAVRIC text output file, many other files are created containing the intermediate data used by the sequence and the final tally data. Many of the files produced can be viewed using the Mesh File Viewer or the Interactive Plotter capabilities of Fulcrum, which is distributed with SCALE. (Note that most of the images in this document were taken from the Mesh File Viewer from SCALE 6.1.) Table 4.1.7 lists the other output files, based on the name of the main output file (here called outputName), that are available to the user. These files will be copied back to the directory where the input file was located. Many of the files come from Monaco and are discussed in the Monaco chapter of the SCALE manual.

Other files that the user may be interested in are listed in Table 4.1.8. These files are kept in the temporary directory where SCALE executes and are not copied back to the directory where the input file was located, unless specifically requested using a SCALE "shell" command. Curious users may also be interested in viewing the various input files $\left(i_{-}{ }^{*}\right)$ that the MAVRIC sequence writes in order to run the SCALE functional modules. 
Table 4.1.7. Output files that are copied back to user's area when the sequence finishes ${ }^{a}$

\begin{tabular}{|c|c|c|}
\hline Filename & Viewer & Description \\
\hline \multicolumn{3}{|l|}{ Output Summary } \\
\hline outputName.out & & main text output file, contains results summary \\
\hline \multicolumn{3}{|l|}{ Diagnostic Files } \\
\hline outputName.respid.chart & $\mathrm{P}$ & response input and MG representation for response $i d$ \\
\hline outputName.gridid.3dmap & V & mesh version of geometry using grid geometry $i d$ \\
\hline outputName.cylid.3dmap & $\mathrm{V}$ & $\begin{array}{l}\text { mesh version of geometry using cylindrical geometry } \\
\text { id }\end{array}$ \\
\hline outputName.distid.chart & $\mathrm{P}$ & distribution input and sampling test for distribution $i d$ \\
\hline
\end{tabular}

Mesh Source Saver

filename.msm

$\mathrm{V}$ mesh representation of a single source or total source

filename.id.msm

filename.sampling.msm

$\mathrm{V}$ mesh representation of multiple sources

filename.sampling.id.msm

$\mathrm{V}$ biased representation of a single source or total source

V biased representation of multiple sources

Importance Map Generation

outputName.geometry.3dmap

$\mathrm{V} \quad$ voxelized geometry (cell-center testing only)

outputName.forward.dff

$\mathrm{V} \quad$ scalar forward flux estimate, $\phi(x, y, z, E)$

outputName.adjoint.dff

$\mathrm{V} \quad$ scalar adjoint flux estimate, $\phi^{+}(x, y, z, E)$

outputName.mim

outputName.msm

$\mathrm{V} \quad$ Monaco mesh importance map, $\bar{w}(x, y, z, E)$

outputName.mmt

$\mathrm{V} \quad$ Monaco mesh source, $\hat{q}(x, y, z, E)$

$\mathrm{V}$ macro-material table

Tally Files

outputName.pdid.txt
outputName.pdid.chart
outputName.rtid.txt
outputName.rtid.chart
outputName.mtid.3dmap
outputName.mtid.respxx.3dmap
outputName.mtid.flux.txt
outputName.mtid.tfluxtxt

$\mathrm{P} \quad$ batch convergence data for point detector tally $i d$ detailed results for region tally id

$\mathrm{P} \quad$ batch convergence data for region tally id

$\mathrm{V}$ mesh tally for meshTally id

$\mathrm{V}$ mesh tally of response by group for meshTally $i d$, response $x x$

detailed results for the group-wise flux of meshTally id

detailed results for total flux of meshTally $i d$ 
${ }^{a} \mathrm{~V}$ - can be displayed with the Mesh File Viewer capabilities of Fulcrum. P - can be displayed with the 2D plotting capabilities of Fulcrum.

Table 4.1.8. Other intermediate files-available in the temporary directory.

These may be useful for testing and debugging

\begin{tabular}{ll}
\hline Filename & \multicolumn{1}{c}{ Description } \\
\hline ft02f001 & AMPX formatted cross sections for Denovo \\
fort.51 & text file, listings of the mixing table for Monaco \\
fort.52 & text file, review of MAVRIC sequence input variables \\
fort.54 & energy bin boundaries for the current cross section library \\
xkba_b.inp & binary input file for Denovo - rename to have \\
& a *.dsi extension (Denovo simple input) to be viewed via Mesh File Viewer \\
\hline
\end{tabular}

\subsubsection{Sample Problems}

\subsubsection{Graphite shielding measurements with CADIS}

As shown in the Monaco sample problem for simulating the Ueki shielding experiments (Monaco chapter Graphite Shielding Measurements), as the amount of shielding material between a source and detector increases, the time required to reach a certain level of relative uncertainty increases quickly. This example will use the MAVRIC automated variance reduction capability to optimize the calculation of the dose rate at the detector location by specifying an importance map block with an adjoint source made from the detector response function and the detector location.

\subsection{Input file}

The following is a listing of the file mavric.graphiteCADIS.inp located in the SCALE samples \input directory. This calculation will use the coarse-group shielding library $(27 \mathrm{n} 19 \mathrm{~g})$ for all of the importance map calculations and the fine-group library $(200 \mathrm{n} 47 \mathrm{~g}$ ) for the final Monaco step. Additions, compared to the file monaco.graphite.inp, include a grid geometry for the Denovo computational mesh, a mesh tally to better visualize the particle flow, and the importance map block to optimize the Monte Carlo calculation of the point detector.

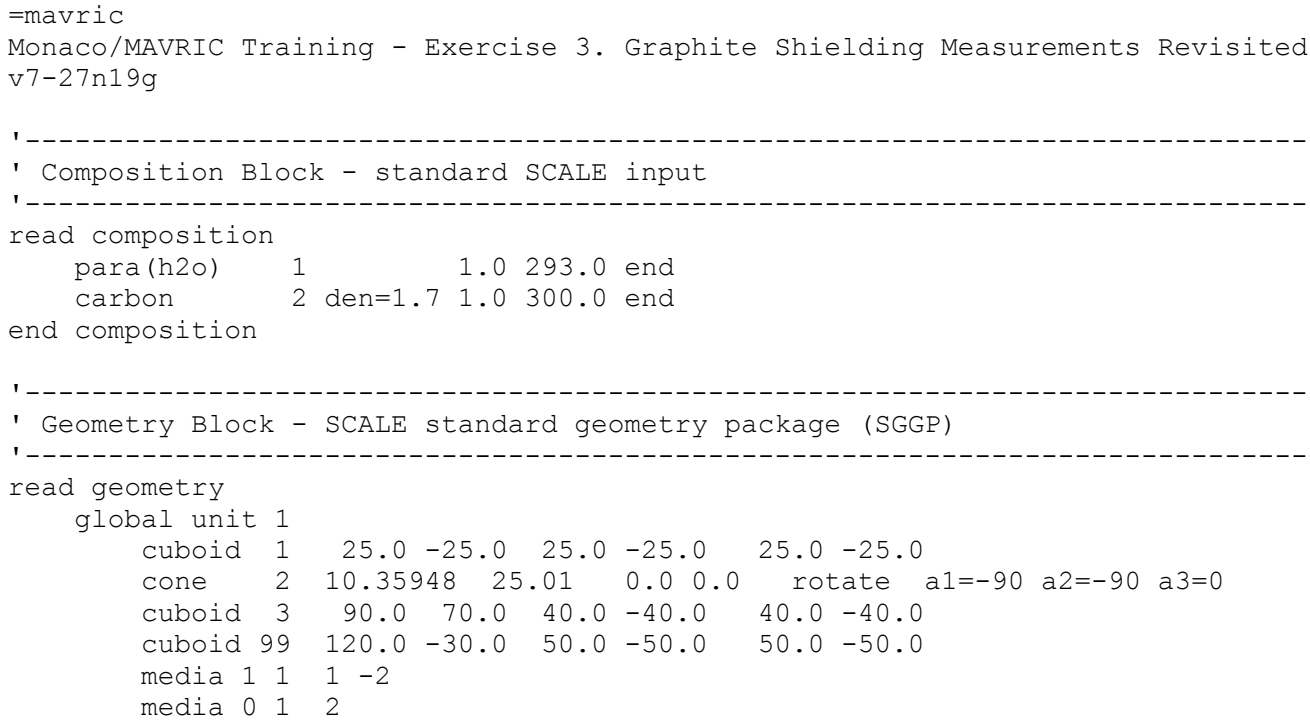


media $2 \quad 1 \quad 3$

$\begin{array}{lllllll}\text { media } & 0 & 1 & 99 & -1 & -2 & -3\end{array}$

boundary 99

end geometry

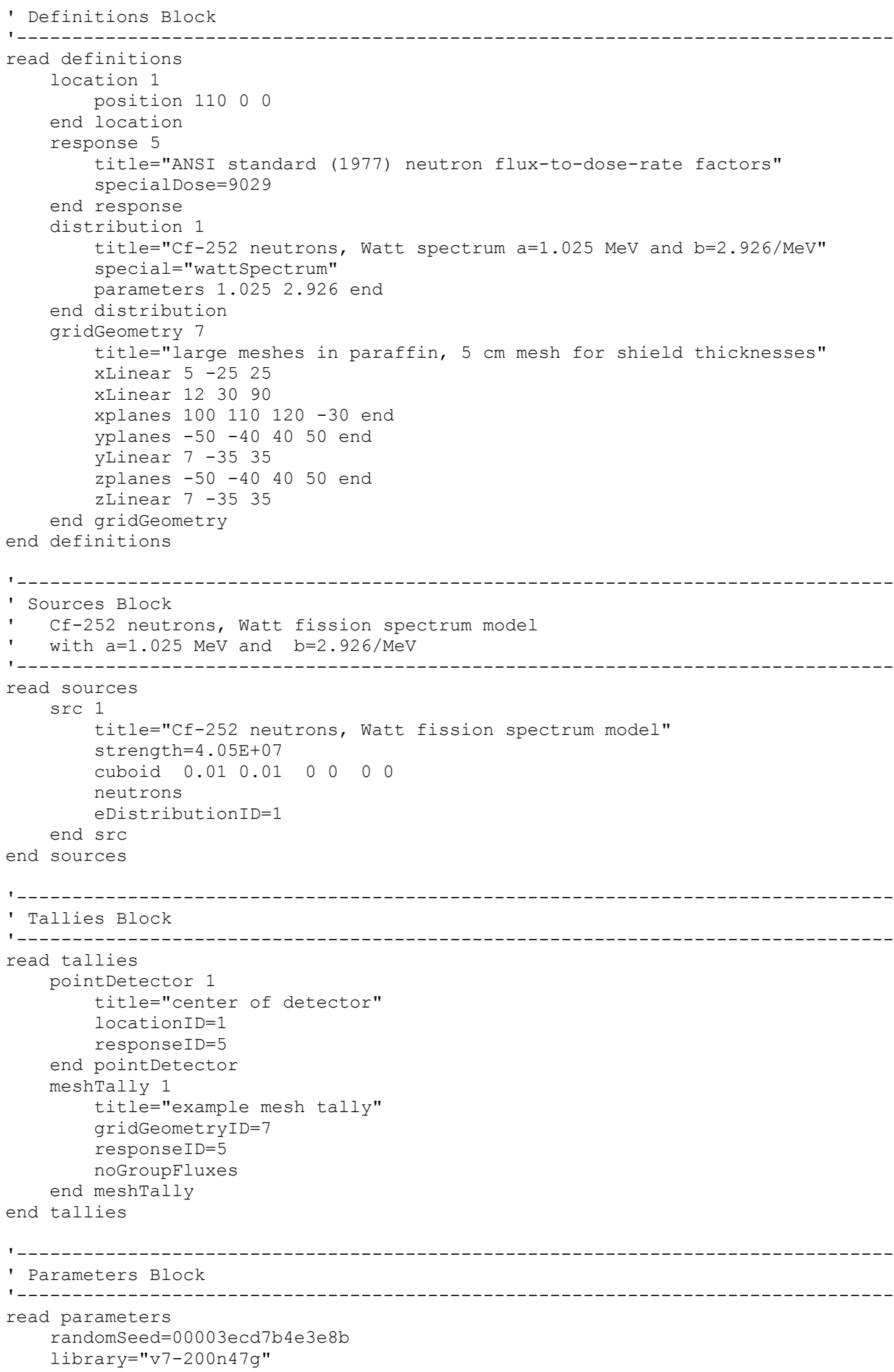



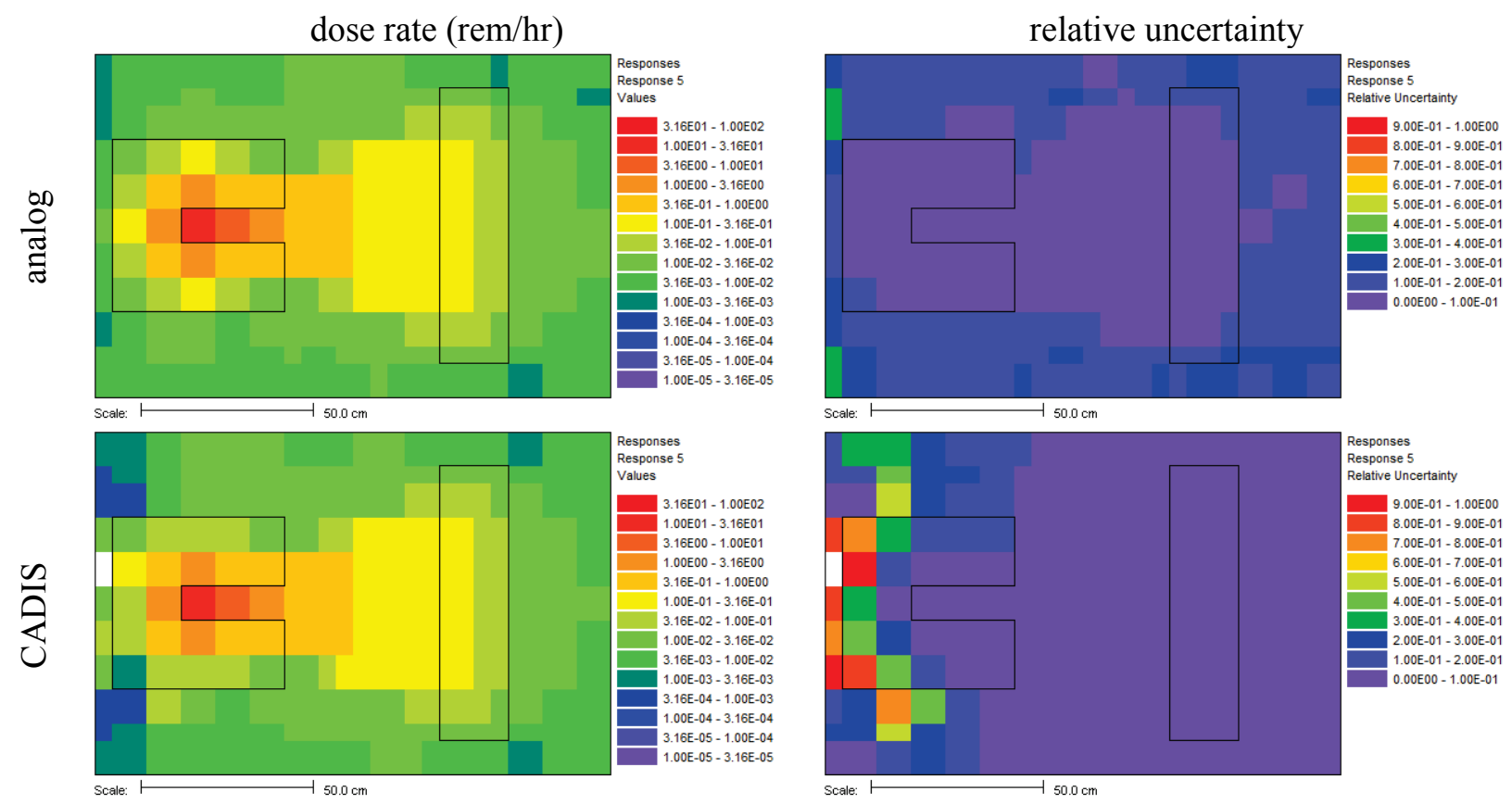

Figure 4.1.6. Mesh tally showing neutron dose rate (rem/hr) and uncertainties for the analog case and the CADIS case.

\subsubsection{Dose rates outside of a simple cask}

This example problem is a full-size cylindrical cask model, which consists of an inner steel liner, a thick section of concrete, and an outer steel cover. This problem is intended to be used as a tool to teach users how to build MAVRIC input files. This is not a completely realistic shipping cask but has been simplified greatly for this purpose. The goal of this example it to show how to quickly calculate neutron and photon does rates at six points outside of the cask, including in front of the vent port.

\subsection{Geometry and materials}

The simple model of a cask is shown in Figure 4.1.7. Vent ports at the top and bottom of the cask are modeled as void all of the way around the cask. The interior of the cask was modeled using materials from about 20 typical pressurized-water reactor (PWR) fuel assemblies (including the $\mathrm{UO}_{2}, \mathrm{Zr}, \mathrm{Fe}, \mathrm{Ni}, \mathrm{Cr}$, $\mathrm{Sn}$, and other constituents), homogenized over the interior volume. The total mass of the fuel/assembly hardware in this region is 10.6 metric tonnes. Separate end regions of the assemblies are not modeled in this simple example. Also note that the fuel material is based on fresh fuel, not spent fuel with its hundreds of fission products. 


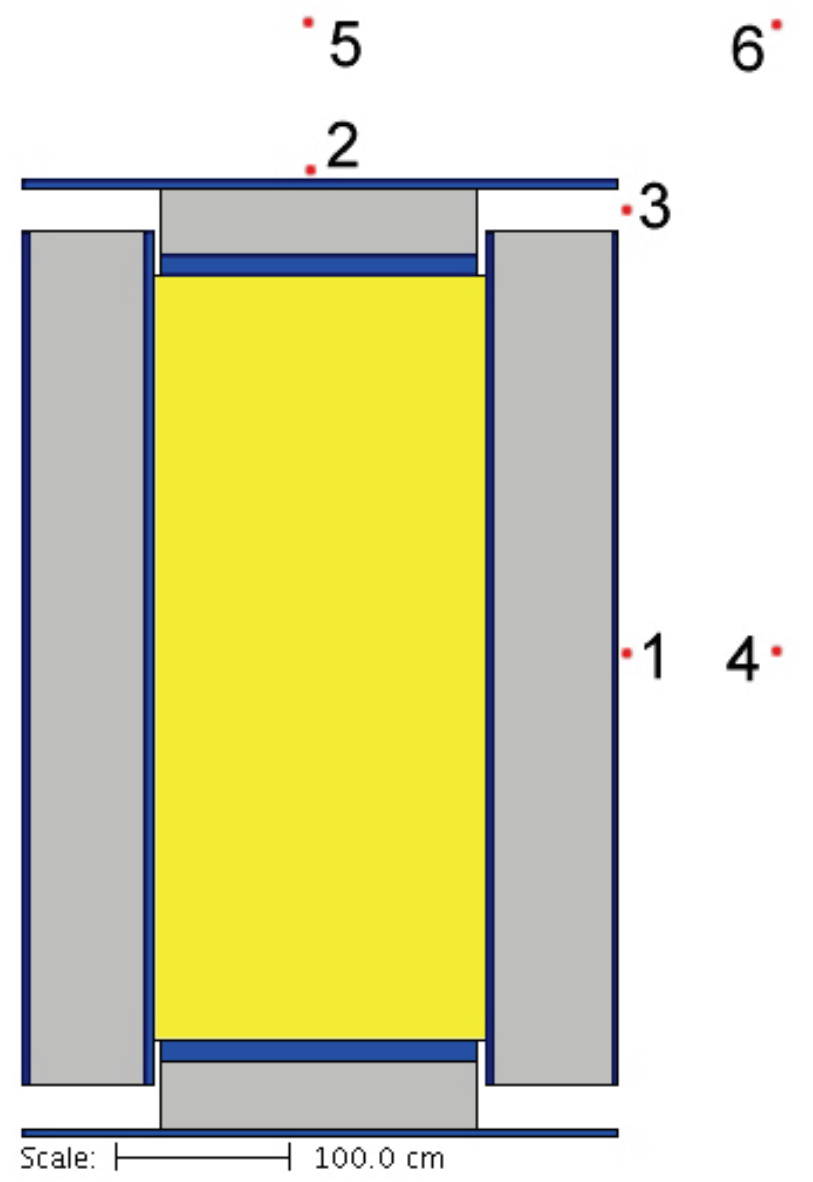

Model Specifics:

Materials:

fuel - $\mathrm{UO}_{2}, \mathrm{Zr}$, others, $\rho=0.9137 \mathrm{~g} / \mathrm{cm}^{3}$

stainless steel - SCALE ss $304, \rho=7.94 \mathrm{~g} / \mathrm{cm}^{3}$

concrete - SCALE orconcrete, $\rho=2.2994 \mathrm{~g} / \mathrm{cm}^{3}$

Regions: $\left(\mathrm{r}_{\min }, \mathrm{r}_{\max }, \mathrm{z}_{\min }, \mathrm{z}_{\max }\right.$, all in $\left.\mathrm{cm}\right)$

yellow - 0, 95, -228.6, 228.6; fuel

blue $-95,100,-255.2$ 255.2; inner steel liner

blue - 0, 90, 228.6, 240.6; inner steel liner

blue - 0, 90, -240.6, -228.6; inner steel liner

gray $-100,168,-255.2,255.2$; concrete

gray $-0,90,240.6,280.6$; concrete

gray $-0,90,-280.6,-240.6$; concrete

blue $-168,170,-255.2$ 255.2; outer steel liner

blue $-0,170,280.6 .2,285.6$; outer steel liner

blue $-0,170,-285.6,-280.6 .2$; outer steel liner

Detector locations: (coordinates in $\mathrm{cm}$ )

$1-180,0,0$; midplane, $10 \mathrm{~cm}$ from surface

$2-0,0,295.6$; on axis, $10 \mathrm{~cm}$ from surface

$3-180,0,267.9$; center of gap, $10 \mathrm{~cm}$ from $\mathrm{r}_{\max }$

4 - 270, 0, 0; midplane, $100 \mathrm{~cm}$ from surface

$5-0,0,385.6$; on axis, $100 \mathrm{~cm}$ from surface

$6-270,0,385.6 ; 100 \mathrm{~cm}$ from each surface

Figure 4.1.7. Cask geometry and detector locations.

\subsection{Sources and responses}

Spent fuel from a typical mid-sized PWR was used to determine the source term. ORIGEN was used to deplete a full core (46.1 metric tonnes of uranium, $4.2 \%$ enriched, with $\mathrm{O}, \mathrm{Zr}, \mathrm{Fe}, \mathrm{Ni}, \mathrm{Cr}$, Sn, and other constituents) to 55,000 MWdays/MTU. The contents of the modeled fuel represent typical values for PWR fuel. ORIGEN then computed the neutron and photon spectra in 27 group and 19 group energy structures for the fuel following a 10 year cooling period after the last irradiation. The total neutron source strength for the cask $(1 / 6$ of a full core - about 20 assemblies $)$ was $8.577 \times 10^{9}$ neutrons/s. The total photon source strength was $7.155 \times 10^{16}$ photons/s.

Two cases will be done for this example: one for calculating the neutron dose rates from the spent fuel neutrons and the other for calculating the photon dose rates from the spent fuel photons. The source spectra and response functions are shown in Figure 4.1.8 through Figure 4.1.11 and listed in Table 4.1.9. Note that in this example, the neutron source shown in Figure 4.1.8 and Table 4.1.9 is considered the final neutron source - no further neutron multiplication is considered. 


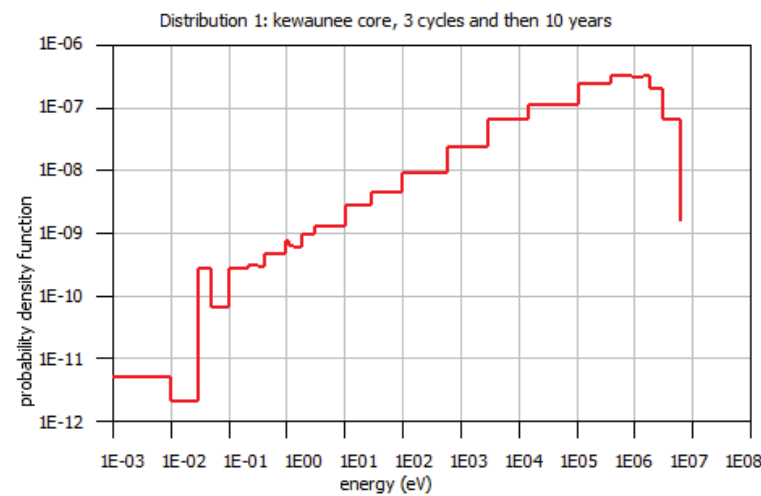

Figure 4.1.8. Spent fuel neutron source spectrum, with strength $8.577 \times 10^{9} /$ second.

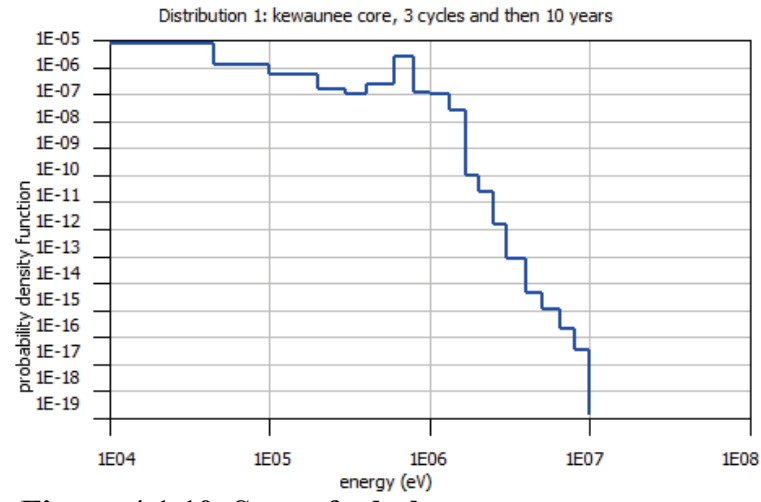

Figure 4.1.10. Spent fuel photon source spectrum, with strength $7.155 \times 10^{16} /$ second.

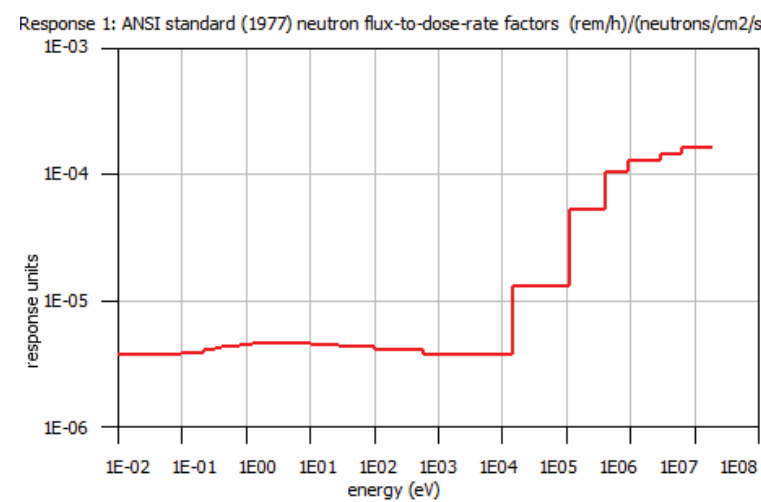

Figure 4.1.9. ANSI-1977 neutron flux-to-dose-rate factors $(\mathrm{rem} / \mathrm{hr}) /\left(\right.$ neutrons $\left./ \mathrm{cm}^{2} / \mathrm{sec}\right)$.

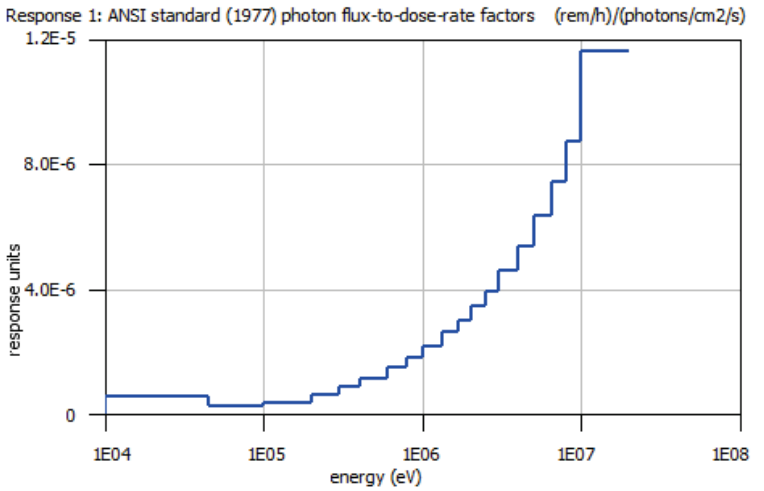

Figure 4.1.11. ANSI-1977 photon flux-to-dose-rate factors $(\mathrm{rem} / \mathrm{hr}) /\left(\right.$ photons $\left./ \mathrm{cm}^{2} / \mathrm{sec}\right)$. 
Table 4.1.9. Source and response data using the SCALE 27-group energy structure for neutrons and the 19 group energy structure for photons

\begin{tabular}{c|cc}
\hline $\begin{array}{c}\text { Energy } \\
(\mathrm{eV})\end{array}$ & $\begin{array}{c}\text { neutron } \\
\text { source }\end{array}$ & $\begin{array}{c}\text { response } \\
9029\end{array}$ \\
\hline $2.0000 \mathrm{E}+07$ & $2.040 \mathrm{E}-02$ & $1.615 \mathrm{E}-04$ \\
$6.3763 \mathrm{E}+06$ & $2.147 \mathrm{E}-01$ & $1.445 \mathrm{E}-04$ \\
$3.0119 \mathrm{E}+06$ & $2.365 \mathrm{E}-01$ & $1.270 \mathrm{E}-04$ \\
$1.8268 \mathrm{E}+06$ & $1.267 \mathrm{E}-01$ & $1.281 \mathrm{E}-04$ \\
$1.4227 \mathrm{E}+06$ & $1.586 \mathrm{E}-01$ & $1.298 \mathrm{E}-04$ \\
$9.0718 \mathrm{E}+05$ & $1.587 \mathrm{E}-01$ & $1.034 \mathrm{E}-04$ \\
$4.0762 \mathrm{E}+05$ & $7.281 \mathrm{E}-02$ & $5.266 \mathrm{E}-05$ \\
$1.1109 \mathrm{E}+05$ & $1.073 \mathrm{E}-02$ & $1.286 \mathrm{E}-05$ \\
$1.5034 \mathrm{E}+04$ & $7.688 \mathrm{E}-04$ & $3.736 \mathrm{E}-06$ \\
$3.0354 \mathrm{E}+03$ & $5.694 \mathrm{E}-05$ & $3.720 \mathrm{E}-06$ \\
$5.8295 \mathrm{E}+02$ & $4.479 \mathrm{E}-06$ & $4.009 \mathrm{E}-06$ \\
$1.0130 \mathrm{E}+02$ & $3.148 \mathrm{E}-07$ & $4.295 \mathrm{E}-06$ \\
$2.9023 \mathrm{E}+01$ & $4.983 \mathrm{E}-08$ & $4.473 \mathrm{E}-06$ \\
$1.0677 \mathrm{E}+01$ & $9.864 \mathrm{E}-09$ & $4.566 \mathrm{E}-06$ \\
$3.0590 \mathrm{E}+00$ & $1.117 \mathrm{E}-09$ & $4.560 \mathrm{E}-06$ \\
$1.8554 \mathrm{E}+00$ & $3.286 \mathrm{E}-10$ & $4.521 \mathrm{E}-06$ \\
$1.3000 \mathrm{E}+00$ & $1.060 \mathrm{E}-10$ & $4.487 \mathrm{E}-06$ \\
$1.1253 \mathrm{E}+00$ & $9.203 \mathrm{E}-11$ & $4.466 \mathrm{E}-06$ \\
$1.0000 \mathrm{E}+00$ & $9.135 \mathrm{E}-11$ & $4.434 \mathrm{E}-06$ \\
$8.0000 \mathrm{E}-01$ & $1.755 \mathrm{E}-10$ & $4.332 \mathrm{E}-06$ \\
$4.1399 \mathrm{E}-01$ & $2.590 \mathrm{E}-11$ & $4.203 \mathrm{E}-06$ \\
$3.2500 \mathrm{E}-01$ & $3.024 \mathrm{E}-11$ & $4.097 \mathrm{E}-06$ \\
$2.2500 \mathrm{E}-01$ & $3.451 \mathrm{E}-11$ & $3.840 \mathrm{E}-06$ \\
$1.0000 \mathrm{E}-01$ & $3.269 \mathrm{E}-12$ & $3.675 \mathrm{E}-06$ \\
$5.0000 \mathrm{E}-02$ & $5.447 \mathrm{E}-12$ & $3.675 \mathrm{E}-06$ \\
$3.0000 \mathrm{E}-02$ & $4.089 \mathrm{E}-14$ & $3.675 \mathrm{E}-06$ \\
$1.0000 \mathrm{E}-02$ & $4.916 \mathrm{E}-14$ & $3.675 \mathrm{E}-06$ \\
$1.0000 \mathrm{E}-05$ & & \\
\hline & & \\
\hline
\end{tabular}

\begin{tabular}{c|cc}
\hline $\begin{array}{c}\text { Energy } \\
(\mathrm{eV})\end{array}$ & $\begin{array}{c}\text { photon } \\
\text { source }\end{array}$ & $\begin{array}{c}\text { response } \\
9504\end{array}$ \\
\hline $2.00 \mathrm{E}+07$ & $1.320 \mathrm{E}-12$ & $1.162 \mathrm{E}-05$ \\
$1.00 \mathrm{E}+07$ & $7.185 \mathrm{E}-11$ & $8.745 \mathrm{E}-06$ \\
$8.00 \mathrm{E}+06$ & $3.281 \mathrm{E}-10$ & $7.460 \mathrm{E}-06$ \\
$6.50 \mathrm{E}+06$ & $1.672 \mathrm{E}-09$ & $6.351 \mathrm{E}-06$ \\
$5.00 \mathrm{E}+06$ & $4.167 \mathrm{E}-09$ & $5.400 \mathrm{E}-06$ \\
$4.00 \mathrm{E}+06$ & $8.086 \mathrm{E}-08$ & $4.602 \mathrm{E}-06$ \\
$3.00 \mathrm{E}+06$ & $7.937 \mathrm{E}-07$ & $3.952 \mathrm{E}-06$ \\
$2.50 \mathrm{E}+06$ & $1.164 \mathrm{E}-05$ & $3.459 \mathrm{E}-06$ \\
$2.00 \mathrm{E}+06$ & $3.331 \mathrm{E}-05$ & $3.013 \mathrm{E}-06$ \\
$1.66 \mathrm{E}+06$ & $8.160 \mathrm{E}-03$ & $2.620 \mathrm{E}-06$ \\
$1.33 \mathrm{E}+06$ & $3.511 \mathrm{E}-02$ & $2.194 \mathrm{E}-06$ \\
$1.00 \mathrm{E}+06$ & $2.478 \mathrm{E}-02$ & $1.827 \mathrm{E}-06$ \\
$8.00 \mathrm{E}+05$ & $4.827 \mathrm{E}-01$ & $1.515 \mathrm{E}-06$ \\
$6.00 \mathrm{E}+05$ & $4.641 \mathrm{E}-02$ & $1.160 \mathrm{E}-06$ \\
$4.00 \mathrm{E}+05$ & $9.735 \mathrm{E}-03$ & $8.705 \mathrm{E}-07$ \\
$3.00 \mathrm{E}+05$ & $1.514 \mathrm{E}-02$ & $6.219 \mathrm{E}-07$ \\
$2.00 \mathrm{E}+05$ & $5.182 \mathrm{E}-02$ & $3.708 \mathrm{E}-07$ \\
$1.00 \mathrm{E}+05$ & $7.015 \mathrm{E}-02$ & $2.688 \mathrm{E}-07$ \\
$4.50 \mathrm{E}+04$ & $2.560 \mathrm{E}-01$ & $5.933 \mathrm{E}-07$ \\
$1.00 \mathrm{E}+04$ & & \\
\hline & &
\end{tabular}

Energies listed are the bin upper energies. Source units are particles/s, normalized to a total of 1 particle/s. Response units are (rem $/ \mathrm{hr}) /\left(\right.$ particle $\left./ \mathrm{cm}^{2} / \mathrm{s}\right)$.

\subsection{Analog calculation}

The analog model for this problem starts with the problem title and the cross-section library name, which in this example is the ENDF/B-VII.0 27 neutron group/19 photon group library. This is in the SCALE samples \input directory as mavric.caskAnalogn.inp and mavric.caskAnalogp.inp.

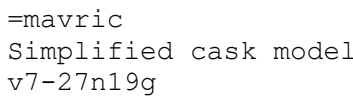

Then the material compositions are listed for fresh fuel, concrete, and steel.

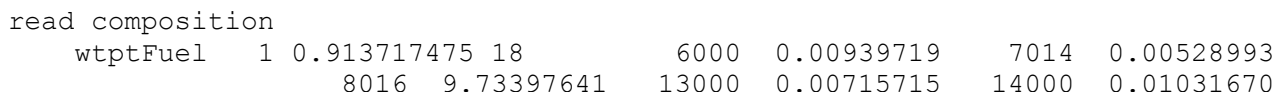




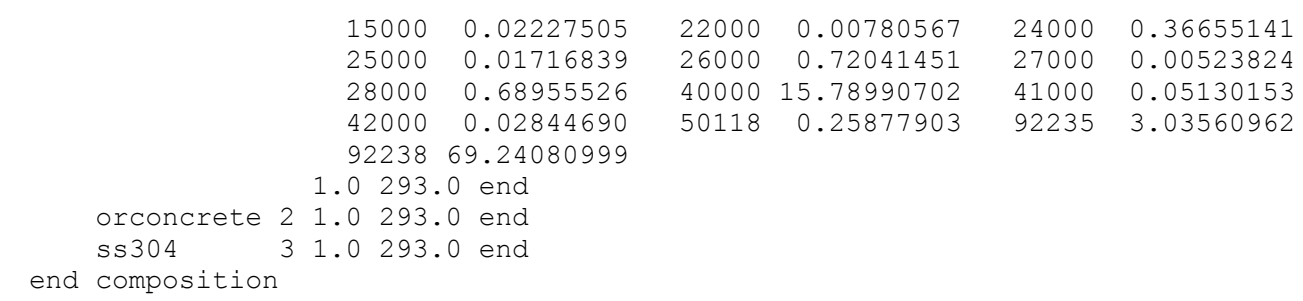

Then SGGP geometry is listed, with the origin of the coordinate system at the center of the cask.

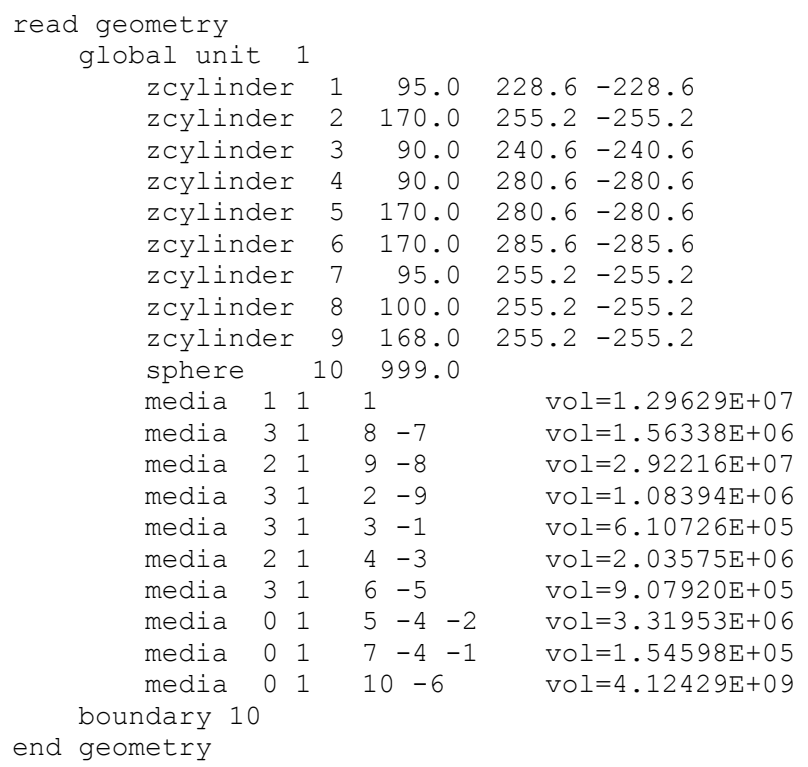

The definitions block contains locations, response functions, grid geometries, and a distribution used by the source input block. For the neutron source/neutron dose problem, the definitions block is listed below.

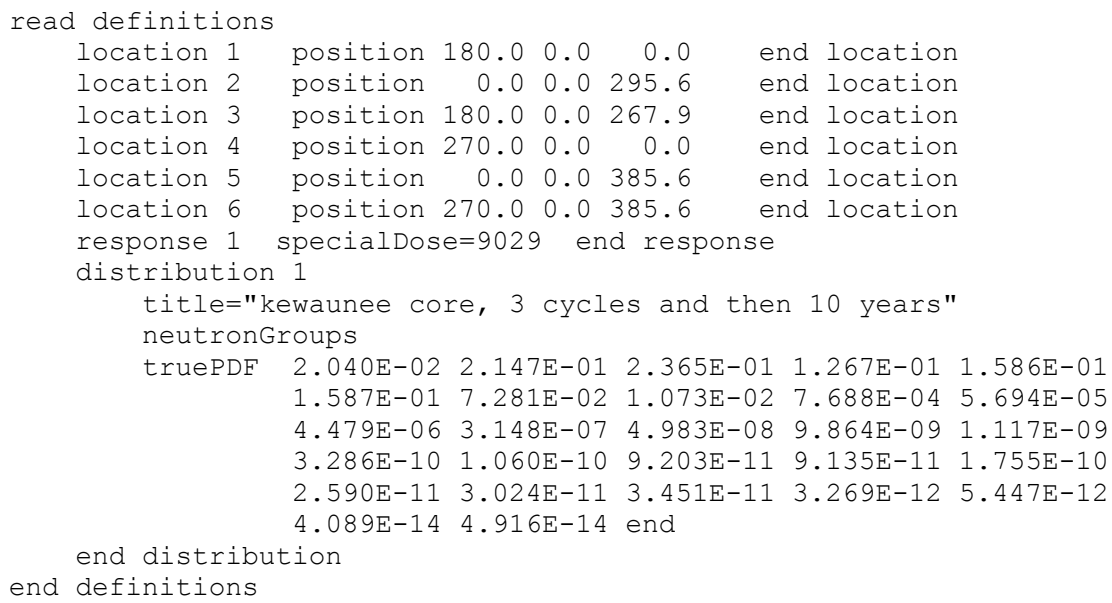


The neutron source from the spent fuel is then listed.

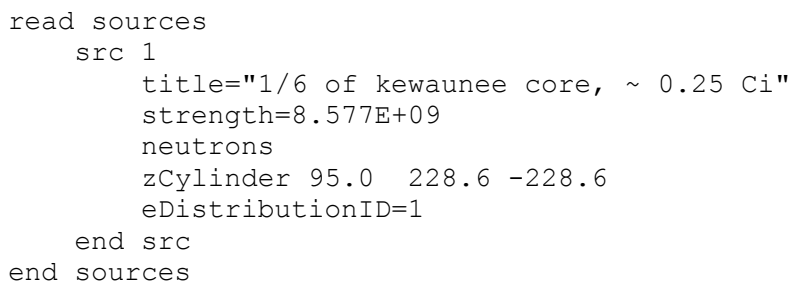

Six point detectors are used to evaluate dose rates radially, axially, and near the vent port.

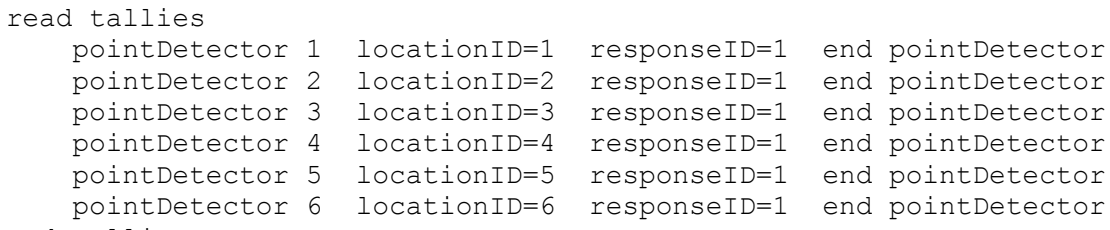

The Monte Carlo parameters were tailored for the neutron problem to be 1 minute batches on a $2 \mathrm{GHz}$ Linux computer. For the photon problem, the number per batch would be 91000 for 1 minute batches.

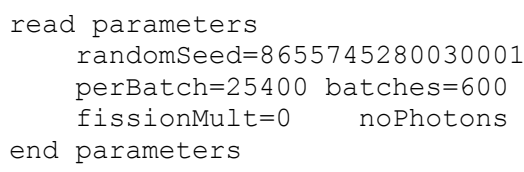

No biasing is specified, which will use the default weight window target value of 1 for every energy group in every region. In order to allow the neutrons to penetrate into the cask wall before being rouletted, a larger window ratio is used, making the lower weight window bound to be 0.01 .

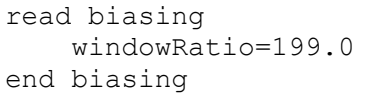

The Monaco input is then ended.

$$
\text { end data }
$$

end

For the photon source/photon dose rate problem, the definitions block would instead contain a photon flux-to-dose-rate response function and the energy distribution for the source.

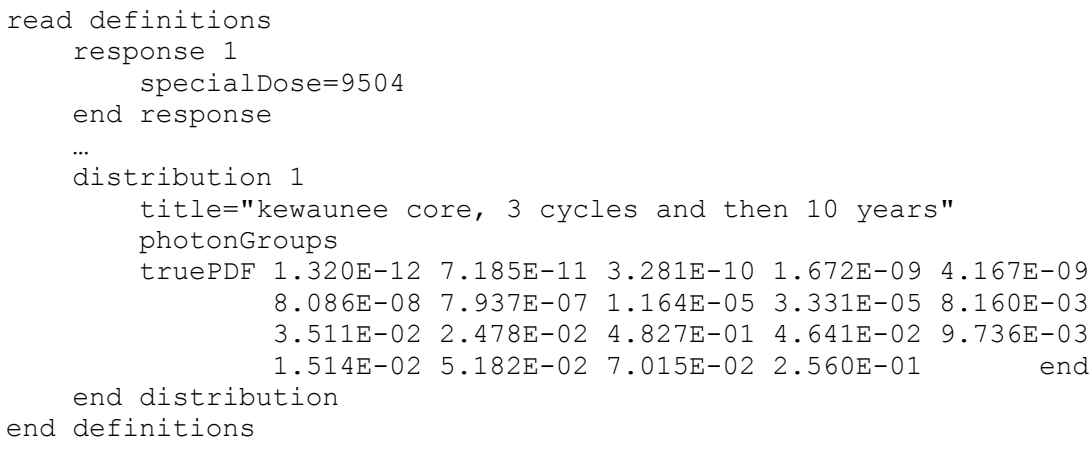


The sources block would contain the photon source information.

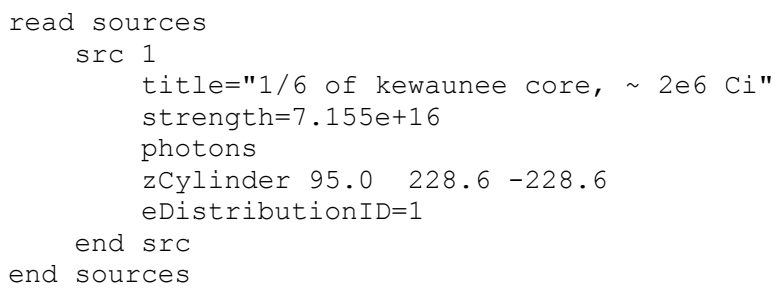

Each of the two analog problems in the samples $\backslash$ input directory run for about 10 minutes. In this time, no meaningful results will be generated due to the difficulty of the problem. Analog results for each case running $110 \mathrm{hr}$ are listed in Table 4.1.10 for the neutron source/neutron dose problem, while results for the photon problem are listed in Table 4.1.11. Note that after $110 \mathrm{hr}$, some of the relative uncertainties in the point detector tallies are still quite high and only one of the six tallies in each problem passed all of the statistical checks. Figure 4.1.12 is the convergence plot for the neutron dose rate at point detector 1, showing that the tally is not well converged and that some batches contain rare events that change the tally value a great deal.

Table 4.1.10. Analog Monaco results for the simplified cask model—neutron source/neutron dose rate

\begin{tabular}{c|cc}
\hline & $\begin{array}{c}\text { Dose Rate } \\
\text { detector }\end{array}$ & $\begin{array}{c}\text { relative } \\
\text { uncertainty }\end{array}$ \\
\hline 1 & $8.78 \mathrm{E}-04$ & 0.1853 \\
2 & $7.35 \mathrm{E}-03$ & 0.0408 \\
3 & $1.54 \mathrm{E}-02$ & 0.0124 \\
4 & $4.47 \mathrm{E}-04$ & 0.0311 \\
5 & $1.36 \mathrm{E}-02$ & 0.0058 \\
6 & $2.92 \mathrm{E}-03$ & 0.0073 \\
\hline
\end{tabular}

Table 4.1.11. Analog Monaco results for the simplified cask model-photon source/photon dose rate

\begin{tabular}{c|cc}
\hline detector & $\begin{array}{c}\text { Dose Rate } \\
\text { (rem/hr) }\end{array}$ & $\begin{array}{c}\text { relative } \\
\text { uncertainty }\end{array}$ \\
\hline 1 & $2.66 \mathrm{E}-02$ & 0.2713 \\
2 & $3.05 \mathrm{E}-01$ & 0.0787 \\
3 & $2.97 \mathrm{E}+00$ & 0.0199 \\
4 & $2.65 \mathrm{E}-02$ & 0.1345 \\
5 & $3.26 \mathrm{E}+00$ & 0.0133 \\
6 & $3.30 \mathrm{E}-01$ & 0.0244 \\
\hline
\end{tabular}




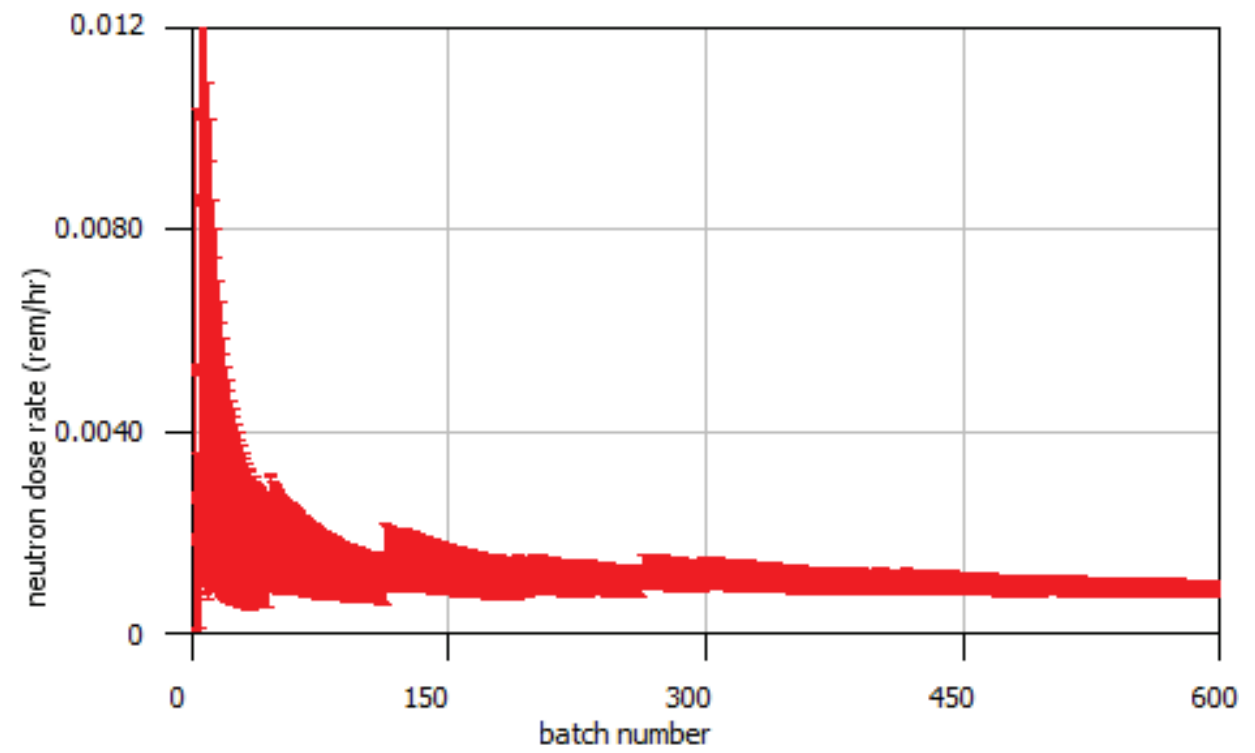

Figure 4.1.12. Convergence plot for the neutron dose rate at point detector 1. Error bars show the 1-sigma tally uncertainties.

\subsection{SAS4 calculations}

Calculations for these two problems were also done using the SAS4 sequence in SCALE 5.1. SAS4 was specifically designed for cask geometries and used a one-dimensional discrete-ordinates calculation (either radially or axially) to determine weight windows. Results for the neutron problem are shown in Table 4.1.12, and results for the photon problem are shown in Table 4.1.13. Note that SAS4 using radial biasing is only expected to do well for the two radial point detector locations. Similarly, only the two axial point detectors are expected to do well when using axial biasing. SAS4 was not intended to do well for the points near the vent port, but the results using the axial biasing seem reasonable.

Table 4.1.12. SAS4 results, using radial biasing (361 minutes) and axial biasing (361 minutes), for the simplified cask modelneutron source/neutron dose rate

\begin{tabular}{c|cc|cc}
\hline & \multicolumn{2}{|c|}{ Radial Biasing } & \multicolumn{2}{c}{ Axial Biasing } \\
detector & $\begin{array}{cccc}\text { Dose Rate } \\
\text { (rem/hr) }\end{array}$ & $\begin{array}{c}\text { relative } \\
\text { uncertainty }\end{array}$ & $\begin{array}{c}\text { Dose Rate } \\
\text { (rem/hr) }\end{array}$ & $\begin{array}{c}\text { relative } \\
\text { uncertainty }\end{array}$ \\
\hline 1 & $7.67 \mathrm{E}-04$ & 0.0081 & $1.32 \mathrm{E}-05$ & 0.4453 \\
2 & $2.67 \mathrm{E}-02$ & 0.8623 & $7.80 \mathrm{E}-03$ & 0.0040 \\
3 & $1.27 \mathrm{E}-02$ & 0.1425 & $1.53 \mathrm{E}-02$ & 0.0079 \\
4 & $4.54 \mathrm{E}-04$ & 0.0079 & $2.34 \mathrm{E}-04$ & 0.6780 \\
5 & $1.43 \mathrm{E}-02$ & 0.1349 & $1.35 \mathrm{E}-02$ & 0.0044 \\
6 & $2.81 \mathrm{E}-03$ & 0.1274 & $2.86 \mathrm{E}-03$ & 0.0052 \\
\hline
\end{tabular}


Table 4.1.13. SAS4 results, using radial biasing (361 minutes) and axial biasing ( 361 minutes), for the simplified cask modelphoton source/photon dose rate

\begin{tabular}{|c|c|c|c|c|}
\hline \multirow[b]{2}{*}{ detector } & \multicolumn{2}{|c|}{ Radial Biasing } & \multicolumn{2}{|c|}{ Axial Biasing } \\
\hline & $\begin{array}{c}\text { Dose Rate } \\
\text { (rem/hr) }\end{array}$ & $\begin{array}{c}\text { relative } \\
\text { uncertainty }\end{array}$ & $\begin{array}{c}\text { Dose Rate } \\
\text { (rem/hr) }\end{array}$ & $\begin{array}{c}\text { relative } \\
\text { uncertainty }\end{array}$ \\
\hline 1 & 4.54E-02 & 0.0118 & $7.07 \mathrm{E}-04$ & 0.5375 \\
\hline 2 & $6.74 \mathrm{E}-02$ & 0.3295 & $2.45 \mathrm{E}-01$ & 0.0630 \\
\hline 3 & $5.63 \mathrm{E}-01$ & 0.3818 & $2.07 \mathrm{E}+00$ & 0.2289 \\
\hline 4 & $2.76 \mathrm{E}-02$ & 0.0039 & $2.48 \mathrm{E}-03$ & 0.4570 \\
\hline 5 & $1.85 \mathrm{E}+00$ & 0.1699 & $2.70 \mathrm{E}+00$ & 0.0664 \\
\hline 6 & $1.46 \mathrm{E}-01$ & 0.1493 & $2.73 \mathrm{E}-01$ & 0.0838 \\
\hline
\end{tabular}

\subsection{Calculations using CADIS}

In the analog calculations, the dose rates at all six points could be calculated at the same time. With MAVRIC, using CADIS, the importance map will optimize the transport of particles towards only the selected detector. Hence, each detector will have a separate calculation with an importance map tailored to reduce the variance for just that detector. Close detectors could be done at the same time. For example, detectors 1 and 4 both need to push particles out of the cask in the positive $x$ direction, towards the $z=0$ plane. In this example, all six detectors will use separate importance maps.

For the importance map, the user lists in the input what planes to use for the adjoint discrete-ordinates calculation. These planes define cells, which are treated as homogenous parallelpipeds by Denovo, made of a macro material corresponding to a mixture of materials that are in the cell in the true geometry. Users should try to bound as many materials as possible with their selection of mesh planes. More mesh planes should be used where the importance (adjoint flux) varies quickly, for example near the adjoint sources (the detector positions). It is also important to have planes on the true source bounding box.

In this example problem, different sets of mesh planes will be used for the different detector positions. For detector positions 1 and 4, the mesh planes are shown in Figure 4.1.13 and Figure 4.1.14. Note that there are more planes closer to the detectors. Also note that in the $z$ dimension, it is quite easy to place mesh planes at every material boundary, but it is a bit harder to do so in the $x$ and $y$ dimensions due to the curved surfaces. Users need not worry about getting things perfect - an approximate importance map can still reduce Monte Carlo variances a great deal. The meshes used for detector positions 2/5 and positions 3/6 are also shown in Figure 4.1.13 and Figure 4.1.14. Mesh parameters are listed in Table 4.1.14, and the mesh planes are listed in Table 4.1.15. 

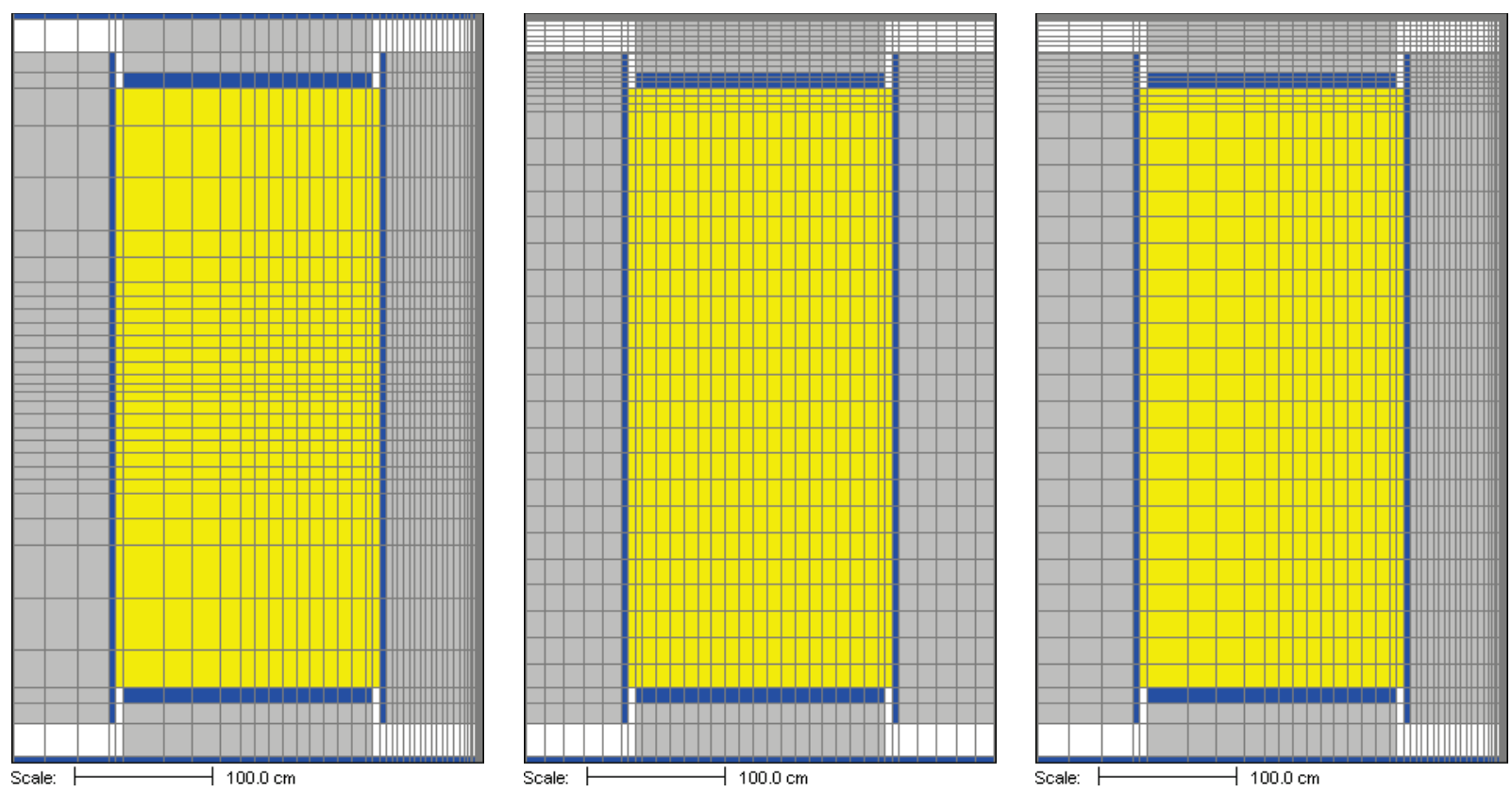

Figure 4.1.13. Importance map mesh planes in the $x$ and $z$ dimensions for detector positions $1 / 4,2 / 5$, and $3 / 6$.
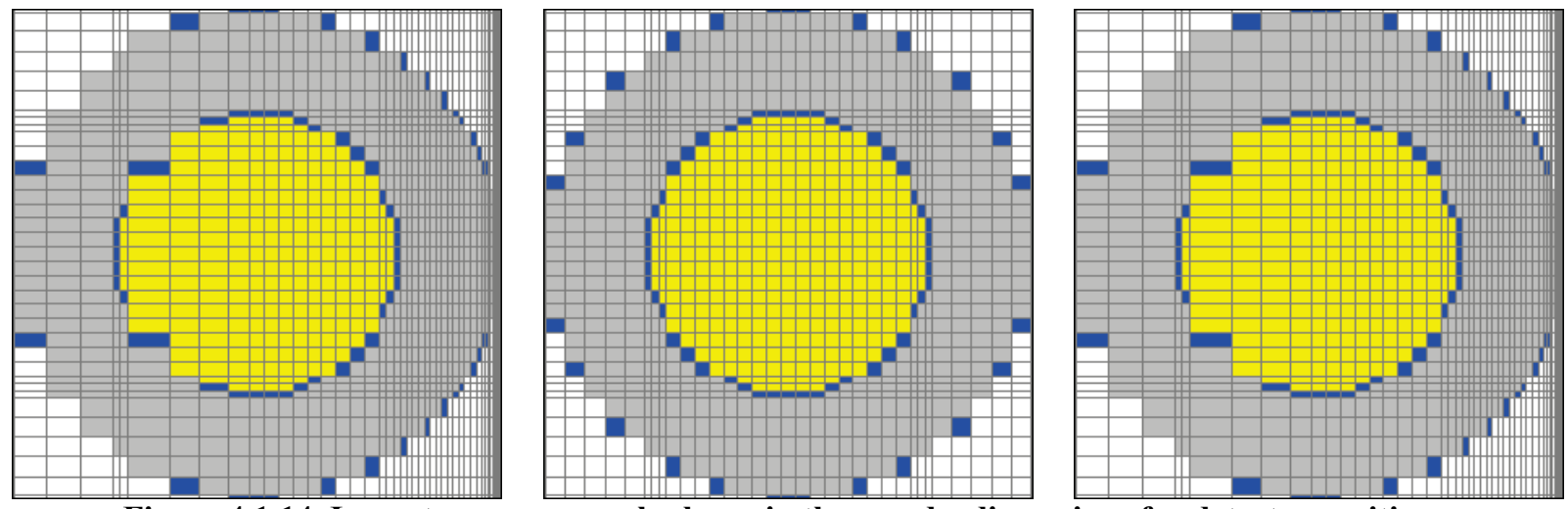

Figure 4.1.14. Importance map mesh planes in the $x$ and $y$ dimensions for detector positions $1 / 4,2 / 5$, and $3 / 6$. 
Table 4.1.14. Mesh parameters

\begin{tabular}{c|ccc|c}
\hline \multirow{2}{*}{$\begin{array}{c}\text { Detector } \\
\text { position }\end{array}$} & \multicolumn{3}{|c|}{$\begin{array}{c}\text { Number of } \\
\text { cells }\end{array}$} & \multirow{2}{*}{$\begin{array}{c}\text { Total } \\
\text { cells }\end{array}$} \\
\cline { 2 - 4 } & $x$ & $y$ & $z$ & \\
\hline $1 / 4$ & 46 & 35 & 35 & 56350 \\
$2 / 5$ & 35 & 35 & 49 & 60025 \\
$3 / 6$ & 46 & 35 & 49 & 78890 \\
\hline
\end{tabular}

Table 4.1.15. Listing of the various sets of mesh planes used for the importance calculations for six different point detectors

\begin{tabular}{|c|c|c|c|c|c|c|c|c|c|}
\hline \multicolumn{2}{|c|}{$\begin{array}{c}x \\
1,3,4,6\end{array}$} & \multicolumn{2}{|c|}{$\begin{array}{c}x \\
2,5\end{array}$} & \multicolumn{2}{|c|}{$\begin{array}{c}y \\
1,2,3,4,5,6\end{array}$} & \multicolumn{2}{|c|}{$\begin{array}{c}z \\
1,4 \\
\end{array}$} & \multicolumn{2}{|c|}{$\begin{array}{c}z \\
2,3,5,6 \\
\end{array}$} \\
\hline-170 & 108 & -170 & 5 & -170 & 5 & -285.6 & 3 & -285.6 & 190 \\
\hline-168 & 112 & -168 & 15 & -168 & 15 & -280.6 & 10 & -280.6 & 210 \\
\hline-146 & 116 & -155 & 25 & -155 & 25 & -255.2 & 20 & -255.2 & 216.2 \\
\hline-122 & 120 & -141 & 35 & -141 & 35 & -240.6 & 30 & -240.6 & 222.4 \\
\hline-100 & 124 & -127 & 45 & -127 & 45 & -228.6 & 40 & -228.6 & 228.6 \\
\hline-95 & 128 & -113 & 55 & -113 & 55 & -200 & 50 & -210 & 232.6 \\
\hline-90 & 132 & -100 & 65 & -100 & 65 & -160 & 60 & -190 & 236.6 \\
\hline-60 & 136 & -95 & 75 & -95 & 75 & -120 & 70 & -170 & 240.6 \\
\hline-40 & 140 & -90 & 85 & -90 & 85 & -100 & 80 & -150 & 245.1 \\
\hline-20 & 144 & -85 & 90 & -85 & 90 & -80 & 100 & -130 & 249.7 \\
\hline-5 & 148 & -75 & 95 & -75 & 95 & -70 & 120 & -110 & 254.2 \\
\hline 5 & 152 & -65 & 100 & -65 & 100 & -60 & 160 & -90 & 255.2 \\
\hline 15 & 156 & -55 & 113 & -55 & 113 & -50 & 200 & -70 & 256.2 \\
\hline 25 & 158 & -45 & 127 & -45 & 127 & -40 & 228.6 & -50 & 260.1 \\
\hline 35 & 160 & -35 & 141 & -35 & 141 & -30 & 240.6 & -30 & 264 \\
\hline 45 & 162 & -25 & 155 & -25 & 155 & -20 & 255.2 & -10 & 267.9 \\
\hline 55 & 164 & -15 & 168 & -15 & 168 & -10 & 280.6 & 10 & 271.8 \\
\hline 65 & 165 & -5 & 170 & -5 & 170 & -3 & 285.6 & 30 & 275.7 \\
\hline 75 & 166 & & & & & & & 50 & 279.6 \\
\hline 85 & 167 & & & & & & & 70 & 280.6 \\
\hline 90 & 168 & & & & & & & 90 & 281.6 \\
\hline 95 & 169 & & & & & & & 110 & 282.6 \\
\hline 100 & 170 & & & & & & & 130 & 283.6 \\
\hline 104 & & & & & & & & 150 & 284.6 \\
\hline & & & & & & & & 170 & 285.6 \\
\hline
\end{tabular}

\subsection{MAVRIC input files}

With two sources and six detectors, this example problem will require 12 separate input files. Starting with the two input files for the analog calculations, these 12 input files will share most of the same features and differ only in blocks related to the importance map calculation: the location of the adjoint source and the planes used in the grid geometry.

To change the input for the neutron problem from an analog calculation to one using CADIS, the user first adds the mesh planes for the discrete-ordinates calculation as a grid geometry to the definitions block. This set of planes is tailored for the vent port direction, towards detectors 3 and 6 .

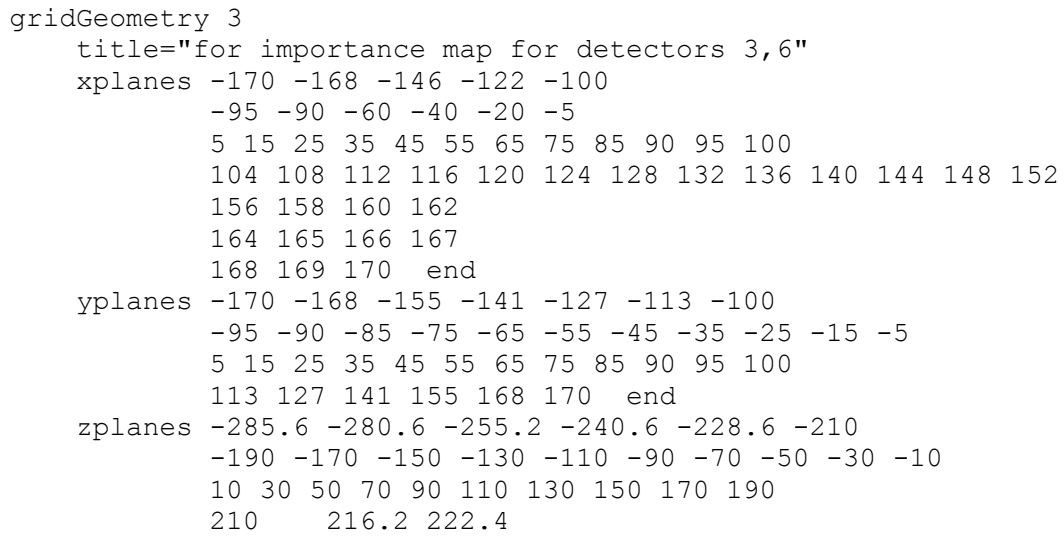




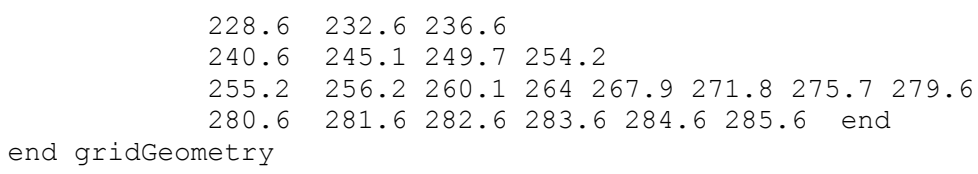

To help the mesh-based biased source represent the true source, "mixture=1" can be added to the source definition. This will ensure that particles sampled from the mesh source that are not in the fuel are rejected. Then an importance map block replaces the standard biasing block. In this case, the importance map will optimize the flow of particles to location 3 (where point detector 3 is defined).

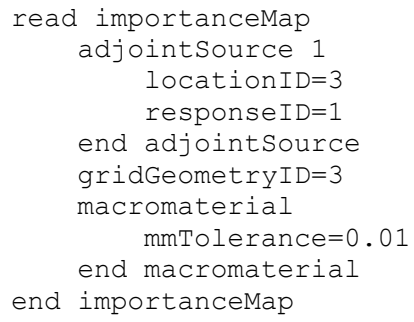

A mesh tally could be added with the following in the tallies block.

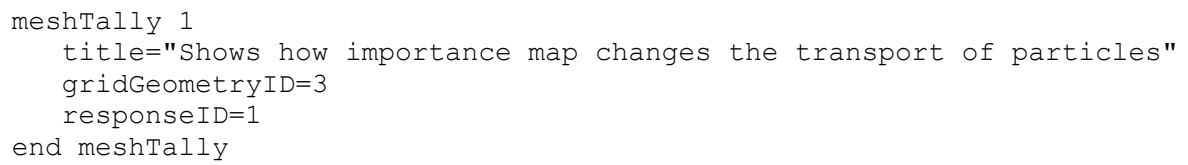

The above mesh tally uses the same grid geometry as the CADIS calculations, but a different grid (or grids) could be used. The files mavric. caskCADISn. inp and mavric. caskCADISp. inp are available in the SCALE samples $\backslash$ input directory. These are for calculating the dose rates at detector position 3 but can be modified for the other five positions (by changing the geometry grid planes and the adjoint source location).

\subsection{Neutron source/neutron response results}

The above MAVRIC input file first performed the discrete-ordinates calculation to determine the adjoint flux from detector position 3. The adjoint Denovo flux file (*.adjoint.dff) produced can be viewed using the Mesh File Viewer and is shown in Figure 4.1.15 for several of the neutron energy groups.

MAVRIC then combined a mesh representation of the true source (space and energy) with the adjoint fluxes to create the importance map and mesh-based biased source. These are shown in Figure 4.1.16 for the fifth neutron group, covering the energy range of 0.9 to $1.4 \mathrm{MeV}$. Notice how the most important region (lowest target weights) is right around the vent port near detector position 3 . This is something we know qualitatively, but quantitative values for exactly how the importance changes with space and energy are difficult to guess. Also notice the "consistent" part of CADIS-the source particles are born with a weight that matches the target weight for the position they are born into. The biased source sampling distribution is shown in Figure 4.1.17, showing how the source particles nearest to detector 3 will be sampled more often.

The biased source distribution and the importance map were then used by Monaco to compute the dose equivalent rate response at detector 3 . 

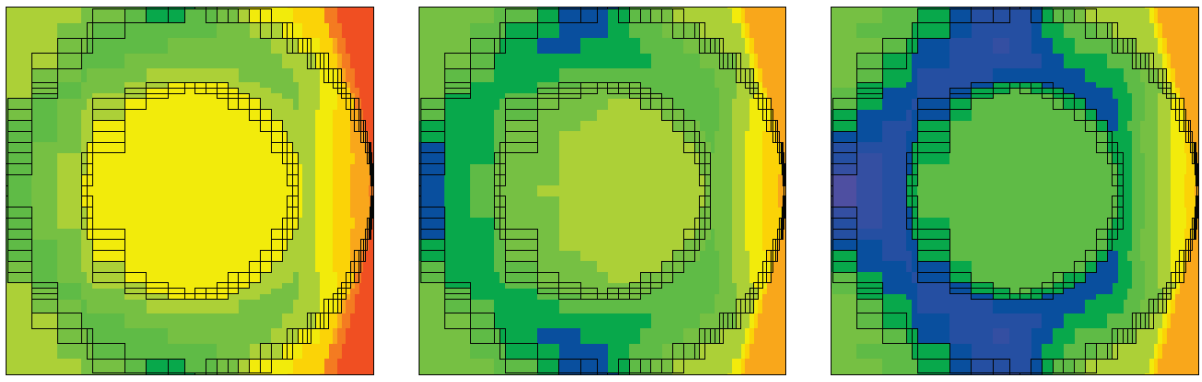

$1.00 \mathrm{E}-08-1.00 \mathrm{E}-07$ $1.00 \mathrm{E}-09-1.00 \mathrm{E}-08$ $1.00 \mathrm{E}-10-1.00 \mathrm{E}-09$ 1.00E-11 - $1.00 \mathrm{E}-10$ 1.00E-12 - 1.00E-11 1.00E-13 - 1.00E-12 $1.00 \mathrm{E}-14-1.00 \mathrm{E}-13$ 1.00E-15-1.00E-14 1.00E-16 - 1.00E-15 1.00E-17 - 1.00E-16 1.00E-18 - $1.00 \mathrm{E}-17$ 1.00E-19-1.00E-18 $1.00 \mathrm{E}-20-1.00 \mathrm{E}-19$

1.00E-21 - 1.00E-20

1.00E-22 - 1.00E-21

1.00E-23 - 1.00E-22

Scale

Group 5

Group 19

and $19(0.8-1 \mathrm{eV})$ calculated by Denovo. 

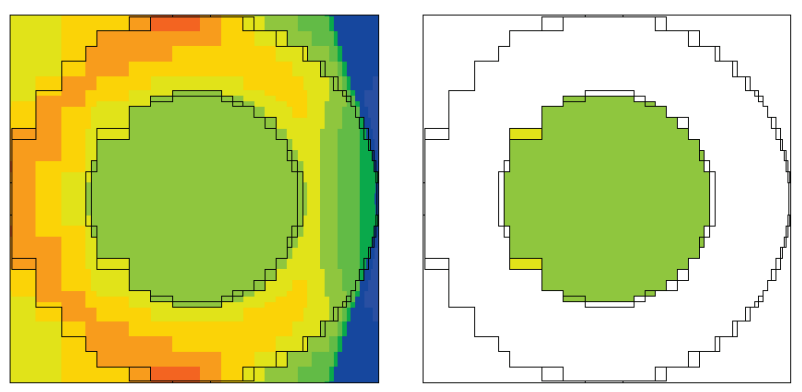

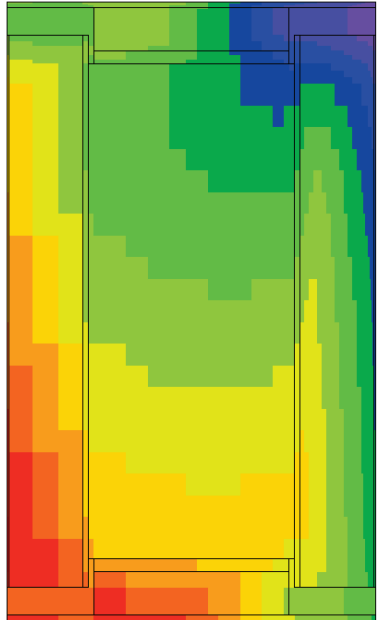

Target weights

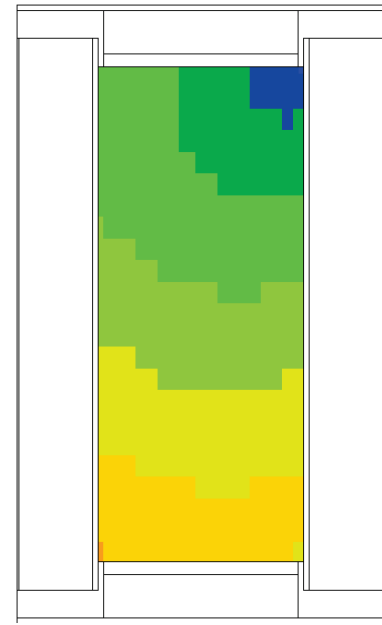

Source weights

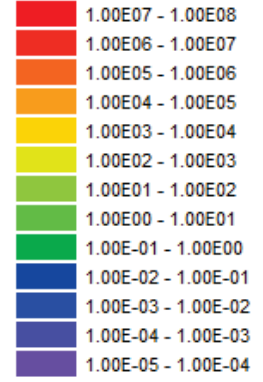

Scale

Figure 4.1.16. Neutron target weights from the importance map and source weights (at birth) for neutron group 5 (0.9 to $1.4 \mathrm{MeV})$. 

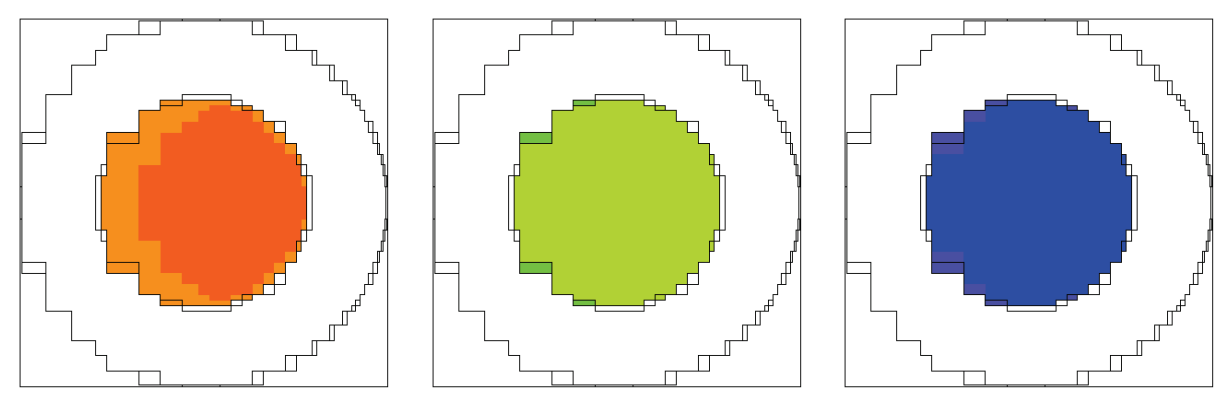

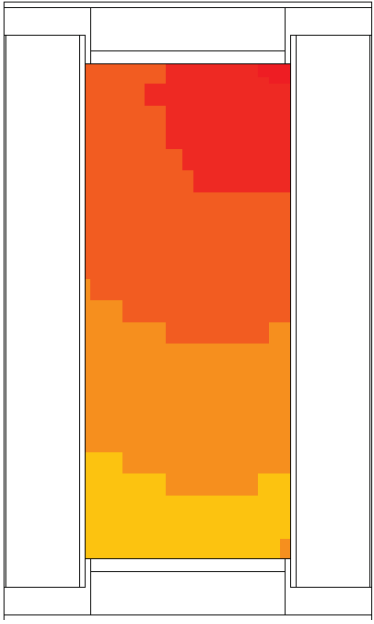

Group 5

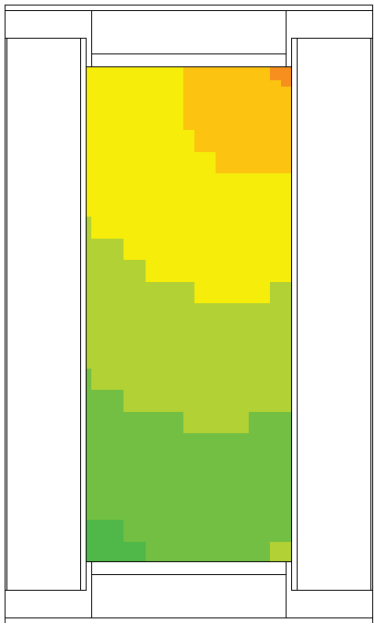

Group 10

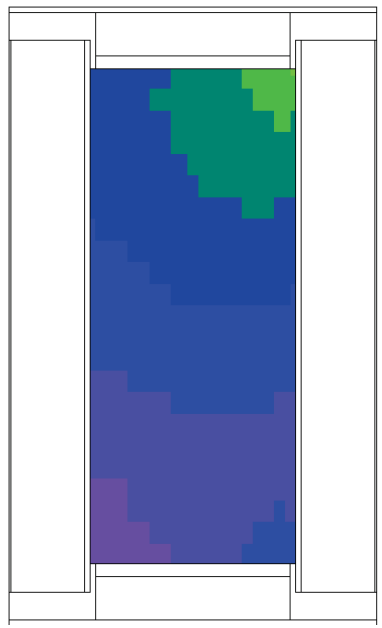

Group 19
3.16E03 - 1.00E05 1.00E02 - 3.16E03 3.16E00 - 1.00E02 1.00E-01 - 3.16E00 3.16E- $03-1.00 \mathrm{E}-01$ $1.00 \mathrm{E}-04-3.16 \mathrm{E}-03$ 3.16E-06 - $1.00 \mathrm{E}-04$ 1.00E- $07-3.16 \mathrm{E}-06$ 3.16E-09 - 1.00E-07 1.00E-10 - 3.16E-09 3.16E-12 - 1.00E-10 1.00E-13 - 3.16E-12 $3.16 \mathrm{E}-15-1.00 \mathrm{E}-13$ 1.00E-16 - 3.16E-15

Scale

Figure 4.1.17. Biased source sampling probability (neutrons $\left./ \mathrm{cm}^{3}\right)$ for neutron groups $5(0.9-1.4 \mathrm{MeV}), 10$ (0.58-3.0 keV), and $19(0.8-1 \mathrm{eV})$.

The results for all six neutron cases, each using their own importance map and biased source, are shown in Table 4.1.16.

The point of this example is to show that MAVRIC using CADIS gets the right answer much faster than the analog calculations. This is shown with a comparison to the results from the analog Monaco and SAS4 calculations, all of which are listed in Table 4.1.17.

To account for the time it takes to achieve a given level of uncertainties, the calculation figure-of-merit $\left.(\mathrm{FOM}=1 / \text { time/(relative uncertainty })^{2}\right)$ can be calculated for each of the codes. The ratios of each code FOM to the FOM of analog Monaco (speedup) are listed in Table 4.1.18 to show how much faster MAVRIC and SAS4 are compared to analog Monaco. The FOMs for MAVRIC include the Denovo calculation times. The FOMs for analog Monaco and SAS4 were modified to account for calculating all six detectors at once. 
Table 4.1.16. Final MAVRIC results (rem/hr) for each point detector in the neutron source/neutron dose rate problem

\begin{tabular}{|c|c|c|c|c|}
\hline \multirow[b]{2}{*}{ detector } & \multicolumn{2}{|c|}{ Time (minutes) } & \multirow{2}{*}{$\begin{array}{c}\text { Dose Rate } \\
(\mathrm{rem} / \mathrm{hr})\end{array}$} & \multirow{2}{*}{$\begin{array}{c}\text { relative } \\
\text { uncertainty }\end{array}$} \\
\hline & Denovo & Monaco & & \\
\hline 1 & 6.9 & 131 & $7.65 \mathrm{E}-04$ & 0.0081 \\
\hline 2 & 7.8 & 64 & $7.83 \mathrm{E}-03$ & 0.0030 \\
\hline 3 & 9.8 & 65 & $1.54 \mathrm{E}-02$ & 0.0029 \\
\hline 4 & 6.4 & 120 & $4.57 \mathrm{E}-04$ & 0.0029 \\
\hline 5 & 7.3 & 64 & $1.36 \mathrm{E}-02$ & 0.0028 \\
\hline 6 & 9.5 & 65 & $2.93 \mathrm{E}-03$ & 0.0023 \\
\hline
\end{tabular}

Table 4.1.17. Comparison of neutron dose rates (rem/hr) to other SCALE codes

\begin{tabular}{c|c|c|c|c}
\hline detector & $\begin{array}{c}\text { Analog Monaco } \\
6595 \mathrm{~min}\end{array}$ & $\begin{array}{c}\text { SAS4 radial } \\
360 \mathrm{~min}\end{array}$ & $\begin{array}{c}\text { SAS4 axial } \\
361 \mathrm{~min}\end{array}$ & $\begin{array}{c}\text { MAVRIC } \\
556 \mathrm{~min}\end{array}$ \\
\hline 1 & $8.78 \mathrm{E}-04 \pm 19 \%$ & $7.67 \mathrm{E}-04 \pm 0.8 \%$ & $1.32 \mathrm{E}-05 \pm 45 \%$ & $7.65 \mathrm{E}-04 \pm 0.8 \%$ \\
2 & $7.35 \mathrm{E}-03 \pm 4.1 \%$ & $2.67 \mathrm{E}-02 \pm 86 \%$ & $7.80 \mathrm{E}-03 \pm 0.4 \%$ & $7.83 \mathrm{E}-03 \pm 0.3 \%$ \\
3 & $1.54 \mathrm{E}-02 \pm 1.2 \%$ & $1.27 \mathrm{E}-02 \pm 14 \%$ & $1.53 \mathrm{E}-02 \pm 0.8 \%$ & $1.54 \mathrm{E}-02 \pm 0.3 \%$ \\
4 & $4.47 \mathrm{E}-04 \pm 3.1 \%$ & $4.54 \mathrm{E}-04 \pm 0.8 \%$ & $2.34 \mathrm{E}-04 \pm 68 \%$ & $4.57 \mathrm{E}-04 \pm 0.3 \%$ \\
5 & $1.36 \mathrm{E}-02 \pm 0.6 \%$ & $1.43 \mathrm{E}-02 \pm 13 \%$ & $1.35 \mathrm{E}-02 \pm 0.4 \%$ & $1.36 \mathrm{E}-02 \pm 0.3 \%$ \\
6 & $2.92 \mathrm{E}-03 \pm 0.7 \%$ & $2.81 \mathrm{E}-03 \pm 12.7 \%$ & $2.86 \mathrm{E}-03 \pm 0.5 \%$ & $2.93 \mathrm{E}-03 \pm 0.2 \%$ \\
\hline
\end{tabular}

Table 4.1.18. Ratio of the figure-of-merit (speed-up) of MAVRIC and SAS4 compared to analog Monaco

\begin{tabular}{c|c|ccc}
\hline & & $\begin{array}{c}\text { SAS4 } \\
\text { radial }\end{array}$ & $\begin{array}{c}\text { SAS4 } \\
\text { axial }\end{array}$ & MAVRIC \\
\hline 1 & 1 & 9498 & 3.2 & 4178 \\
2 & 1 & 0.0 & 1874 & 2858 \\
3 & 1 & 0.1 & 45 & 270 \\
4 & 1 & 287 & 0.0 & 1002 \\
5 & 1 & 0.0 & 32 & 65 \\
6 & 1 & 0.1 & 35.8 & 146 \\
\hline
\end{tabular}

\subsection{Photon source/photon response results}

The results for the photon source/photon response are similar to the results of the neutron source/neutron response. For the MAVRIC calculation using the photon importance map made from an adjoint source located at detector position 3, Figure 4.1.18 details the adjoint photon flux. Figure 4.1.19 compares the 
target weights from the importance map and the source birth weights. Figure 4.1.20 shows the distribution of the sampled source positions from the biased source.

Table 4.1.19 shows the results from all six photon MAVRIC runs, each using their own importance map and biased source.

The MAVRIC results of the photon problem compare well against SAS4 and analog Monaco, as shown in Table 4.1.20 and Table 4.1.21.
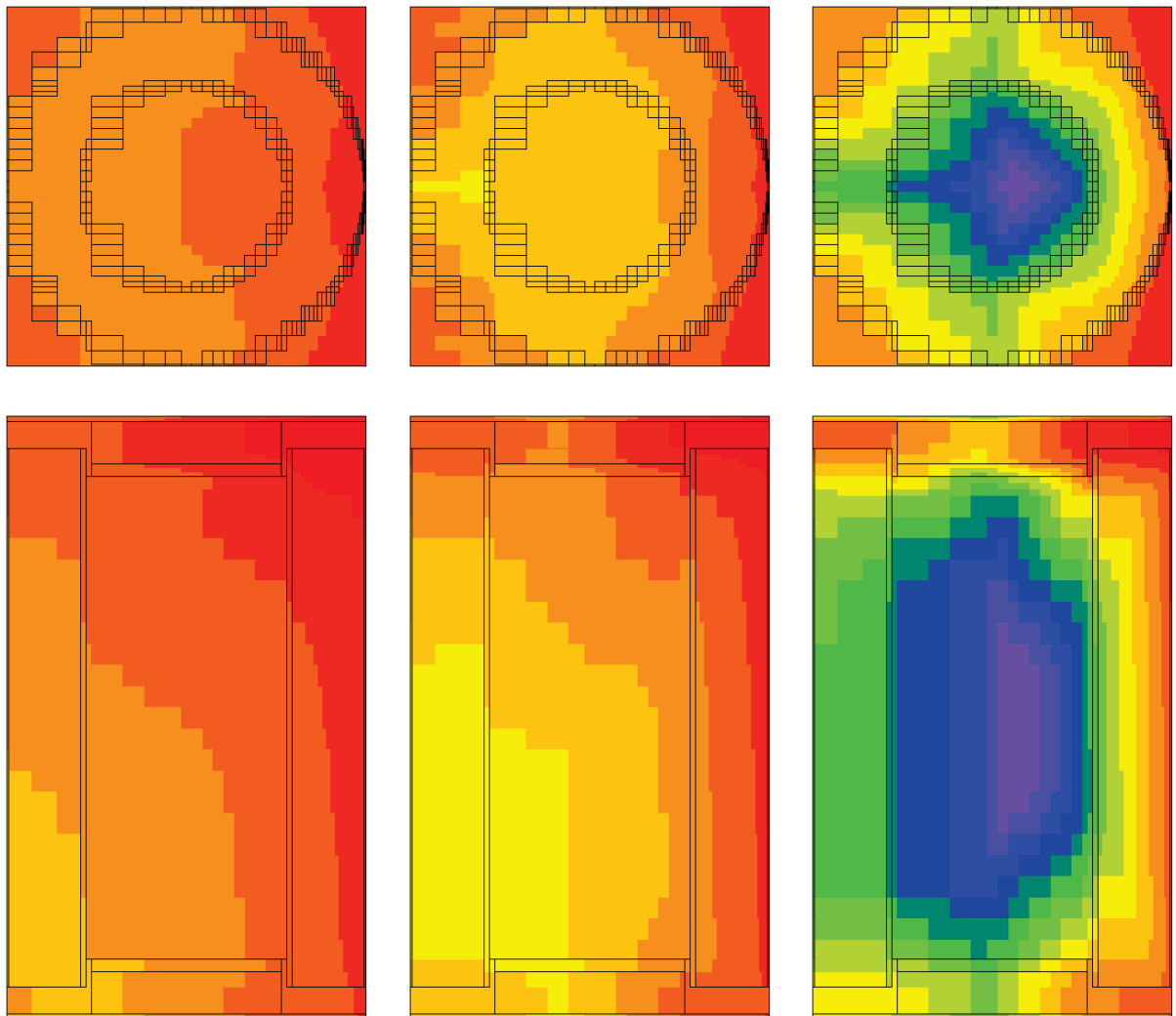

Group 2

Group 12

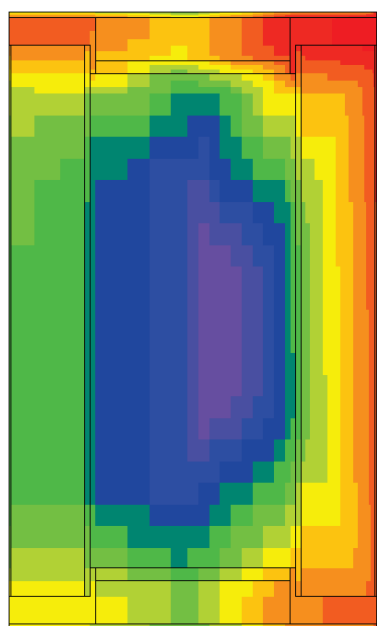

Group 18
$1.00 \mathrm{E}-11-1.00 \mathrm{E}-08$ $1.00 \mathrm{E}-14-1.00 \mathrm{E}-11$ 1.00E-17 - 1.00E-14 $1.00 \mathrm{E}-20-1.00 \mathrm{E}-17$ $1.00 \mathrm{E}-23-1.00 \mathrm{E}-20$ 1.00E-26-1.00E-23 $1.00 \mathrm{E}-29-1.00 \mathrm{E}-26$ 1.00E-32 - 1.00E-29 $1.00 \mathrm{E}-35-1.00 \mathrm{E}-32$ $1.00 \mathrm{E}-38-1.00 \mathrm{E}-35$ 1.00E-41 - 1.00E-38 $1.00 \mathrm{E}-44-1.00 \mathrm{E}-41$ $1.00 E-47-1.00 E-44$ 1.00E-50 - 1.00E-47

scale

Figure 4.1.18. Adjoint photon fluxes $\left(/ \mathrm{cm}^{2} / \mathrm{s}\right)$ for groups $2(8-10 \mathrm{MeV}), 12(0.8-1.0 \mathrm{MeV})$, and $18(45-100 \mathrm{keV})$ calculated by Denovo. 
Table 4.1.19. Final MAVRIC results (rem/hr) for each point detector in the photon source/photon dose rate problem

\begin{tabular}{|c|c|c|c|c|}
\hline \multirow[b]{2}{*}{ detector } & \multicolumn{2}{|c|}{ Time (minutes) } & \multirow{2}{*}{$\begin{array}{c}\text { Dose Rate } \\
(\mathrm{rem} / \mathrm{hr})\end{array}$} & \multirow{2}{*}{$\begin{array}{c}\text { relative } \\
\text { uncertainty }\end{array}$} \\
\hline & Denovo & Monaco & & \\
\hline 1 & 2.6 & 121 & $5.02 \mathrm{E}-02$ & 0.0024 \\
\hline 2 & 3.1 & 65 & $3.03 \mathrm{E}-01$ & 0.0177 \\
\hline 3 & 3.6 & 64 & $2.89 \mathrm{E}+00$ & 0.0105 \\
\hline 4 & 2.6 & 130 & $3.18 \mathrm{E}-02$ & 0.0024 \\
\hline 5 & 3.1 & 59 & $3.07 \mathrm{E}+00$ & 0.0156 \\
\hline 6 & 3.8 & 63 & $3.14 \mathrm{E}-01$ & 0.0137 \\
\hline
\end{tabular}



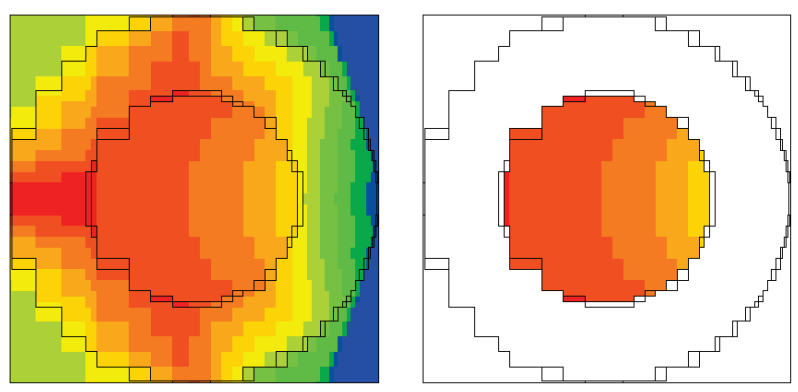

1.00E07-1.00E08 $1.00 E 06-1.00 E 07$ $1.00 \mathrm{E} 05-1.00 \mathrm{E} 06$

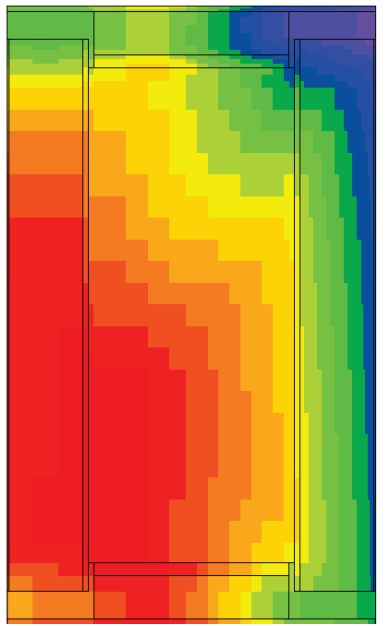

Target weights

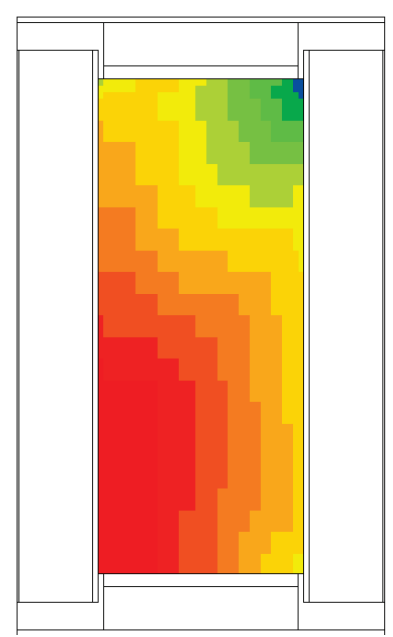

Source weights
.00E04 - 1.00E05

1.00E03 - 1.00E04 $1.00 \mathrm{E} 02-1.00 \mathrm{E} 03$ 1.00E01 - 1.00E02 1.00E00 - 1.00E01 $1.00 \mathrm{E}-01-1.00 \mathrm{E} 00$ 1.00E-02 - $1.00 \mathrm{E}-01$ $1.00 \mathrm{E}-03-1.00 \mathrm{E}-02$ $1.00 \mathrm{E}-04-1.00 \mathrm{E}-03$ $1.00 \mathrm{E}-05-1.00 \mathrm{E}-04$ $1.00 \mathrm{E}-06-1.00 \mathrm{E}-05$ 1.00E-07 - 1.00E-06 $1.00 \mathrm{E}-08-1.00 \mathrm{E}-07$

Scale

Figure 4.1.19. Photon target weights from the importance map and source weights (at birth) for photon group $12(0.8-1.0 \mathrm{MeV})$.

Table 4.1.20. Comparison of the photon dose rates (rem/hr) to other SCALE codes

\begin{tabular}{c|c|c|c|c}
\hline detector & $\begin{array}{c}\text { Analog Monaco } \\
6678 \mathrm{~min}\end{array}$ & $\begin{array}{c}\text { SAS4 radial } \\
361 \mathrm{~min}\end{array}$ & $\begin{array}{c}\text { SAS4 axial } \\
361 \mathrm{~min}\end{array}$ & $\begin{array}{c}\text { MAVRIC } \\
520 \mathrm{~min}\end{array}$ \\
\hline 1 & $2.66 \mathrm{E}-02 \pm 27 \%$ & $4.54 \mathrm{E}-02 \pm 1.2 \%$ & $7.07 \mathrm{E}-04 \pm 54 \%$ & $5.02 \mathrm{E}-02 \pm 0.2 \%$ \\
2 & $3.05 \mathrm{E}-01 \pm 7.9 \%$ & $6.74 \mathrm{E}-02 \pm 33 \%$ & $2.45 \mathrm{E}-01 \pm 6.3 \%$ & $3.03 \mathrm{E}-01 \pm 1.8 \%$ \\
3 & $2.97 \mathrm{E}+00 \pm 2.0 \%$ & $5.63 \mathrm{E}-01 \pm 38 \%$ & $2.07 \mathrm{E}+00 \pm 22.9 \%$ & $2.89 \mathrm{E}+00 \pm 1.0 \%$ \\
4 & $2.65 \mathrm{E}-02 \pm 13.4 \%$ & $2.76 \mathrm{E}-02 \pm 0.4 \%$ & $2.48 \mathrm{E}-03 \pm 46 \%$ & $3.18 \mathrm{E}-02 \pm 0.2 \%$ \\
5 & $3.26 \mathrm{E}+00 \pm 1.3 \%$ & $1.85 \mathrm{E}+00 \pm 17 \%$ & $2.70 \mathrm{E}+00 \pm 6.6 \%$ & $3.07 \mathrm{E}+00 \pm 1.6 \%$ \\
6 & $3.30 \mathrm{E}-01 \pm 2.4 \%$ & $1.46 \mathrm{E}-01 \pm 14.9 \%$ & $2.73 \mathrm{E}-01 \pm 8.4 \%$ & $3.14 \mathrm{E}-01 \pm 1.4 \%$ \\
\hline
\end{tabular}



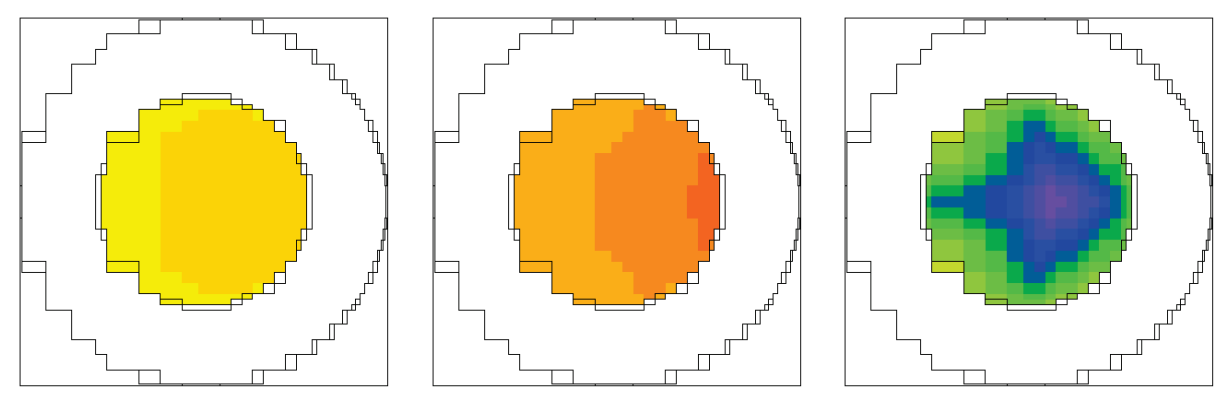

1.00E11 - $1.00 E 13$ 1.00E09 - 1.00E11 $1.00 \mathrm{E} 07-1.00 \mathrm{E} 09$ 1.00E05 - $1.00 E 07$ 1.00E03 - 1.00E05 $1.00 \mathrm{E} 01-1.00 \mathrm{E} 03$

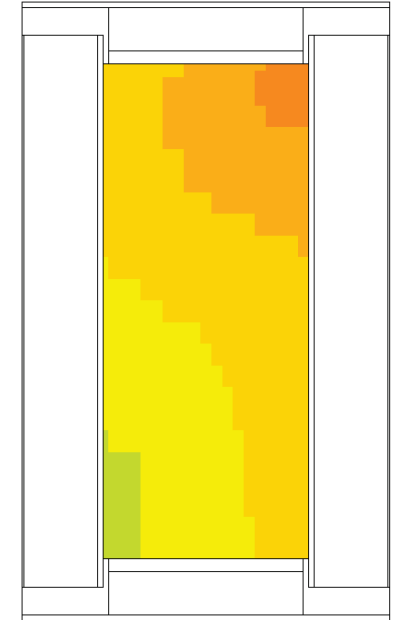

Group 2

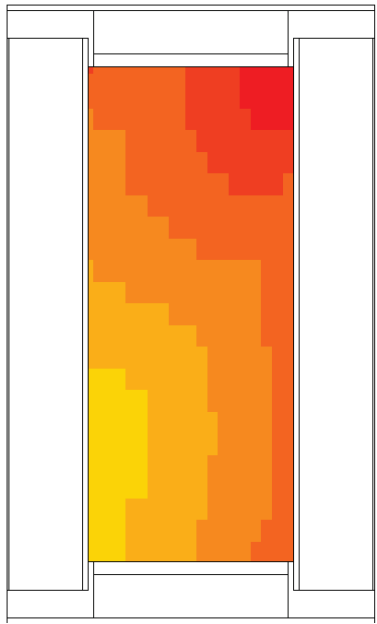

Group 12

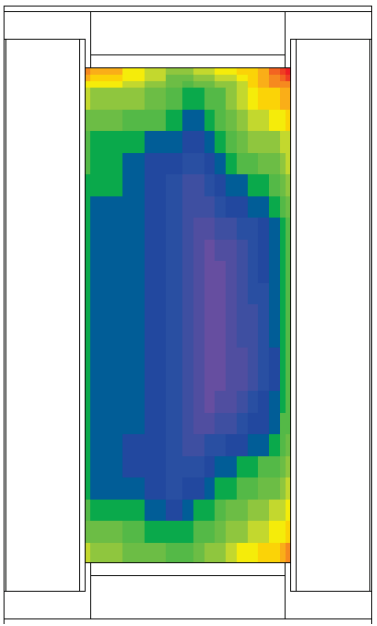

Group 18
1.00E-03 - 1.00E-01

$1.00 \mathrm{E}-05-1.00 \mathrm{E}-03$

1.00E-07 - 1.00E-05

$1.00 \mathrm{E}-09-1.00 \mathrm{E}-07$

$1.00 \mathrm{E}-11-1.00 \mathrm{E}-09$

1.00E-13 - 1.00E-11

1.00E-15 - 1.00E-13

1.00E-17 - 1.00E-15

1.00E-19 - $1.00 \mathrm{E}-17$

1.00E-21 - 1.00E-19

1.00E-23 - 1.00E-21

1.00E-25 - 1.00E-23

\section{Scale}

Figure 4.1.20. Biased source sampling probability $\left(\right.$ photons $\left./ \mathrm{cm}^{3}\right)$ for groups $2(8-10 \mathrm{MeV}), 12(0.8-1.0 \mathrm{MeV})$, and 18 (45-100 keV).

Table 4.1.21. Ratio of the figure-of-merit (speed-up) of MAVRIC and SAS4 compared to analog Monaco

\begin{tabular}{c|c|ccc}
\hline detector & Monaco & $\begin{array}{c}\text { SAS4 } \\
\text { radial }\end{array}$ & $\begin{array}{c}\text { SAS4 } \\
\text { axial }\end{array}$ & MAVRIC \\
\hline 1 & 1 & 9759 & 4.7 & 114619 \\
2 & 1 & 1.1 & 29 & 325 \\
3 & 1 & 0.1 & 0 & 60 \\
4 & 1 & 21570 & 1.6 & 26329 \\
5 & 1 & 0.1 & 1 & 13 \\
6 & 1 & 0.5 & 1.6 & 53 \\
\hline
\end{tabular}




\subsubsection{Gamma-ray litho-density logging tool using FW-CADIS}

Consider a simple model of a gamma-ray litho-density too ${ }^{17,18}$ used in well-logging studies, shown in Figure 4.1.21. This model uses a $10 \mathrm{~cm}$ diameter tool made of iron (with a tungsten density) in a $20 \mathrm{~cm}$ borehole filled with water. The near detector is a $2 \mathrm{~cm}$ diameter cylinder, $2 \mathrm{~cm}$ in length, located $20 \mathrm{~cm}$ from the source. The far detector is a $4 \mathrm{~cm}$ by $4 \mathrm{~cm}$ cylinder located $40 \mathrm{~cm}$ from the source. Each detector is made of $\mathrm{NaI}$ and collimated to look out into the formation. The source is contained in an angled collimator, aiming upward and into the formation. The collimators are filled with oxygen. The extent of the modeled formation is $100 \times 100 \times 140 \mathrm{~cm}$. The source is an isotropic ${ }^{137} \mathrm{Cs}$ source emitting $10^{11}$ photons $/ \mathrm{s}(661.7 \mathrm{keV})$. For these calculations, no response function is used-the goal of this example is to calculate the total photon flux within each detector volume.

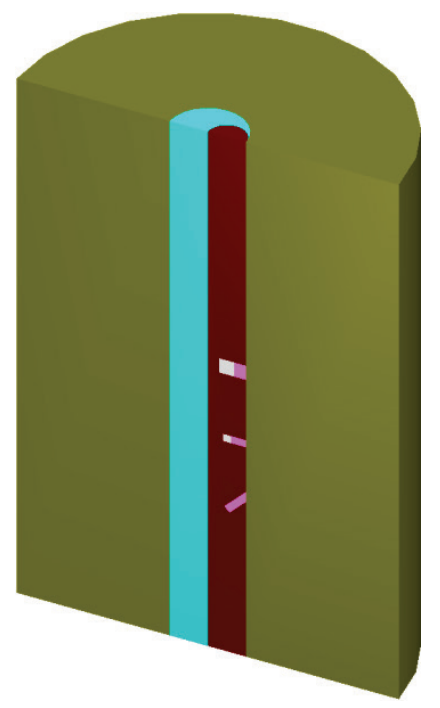

Figure 4.1.21. Cutaway view of the litho-density tool in a rock formation.

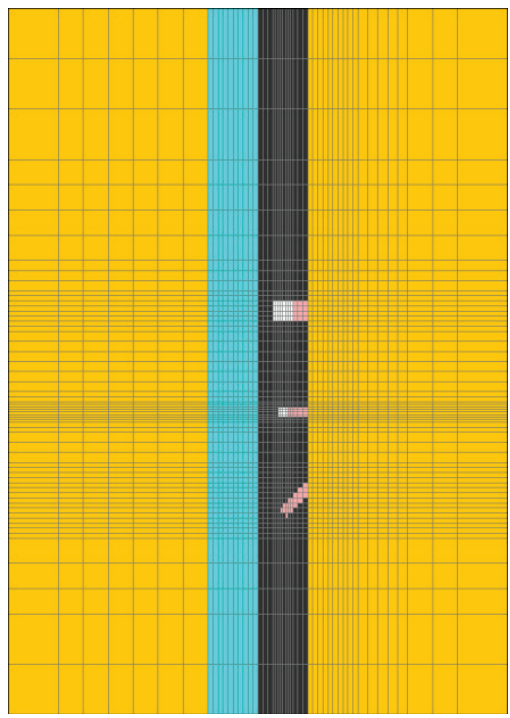

Figure 4.1.22. Denovo mesh, $y=0$ plane.

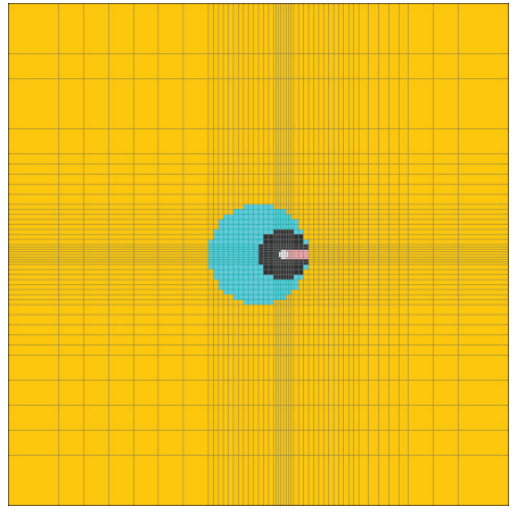

Figure 4.1.23. Denovo mesh, $z=20$ plane (through near detector).

\subsection{Input file}

The following input file represents the simple model of the litho-density tool. The following is a listing of the file mavric.lithoFW.inp located in the SCALE samples \input directory. The 27 neutron group/19 photon group library based on ENDF/B-VII.0 data was used for the discrete-ordinates calculations, while the final Monte Carlo calculation used the 200 neutron group/47 photon group library. 
The input file starts with the problem title, the library for the importance calculations, and the materials.

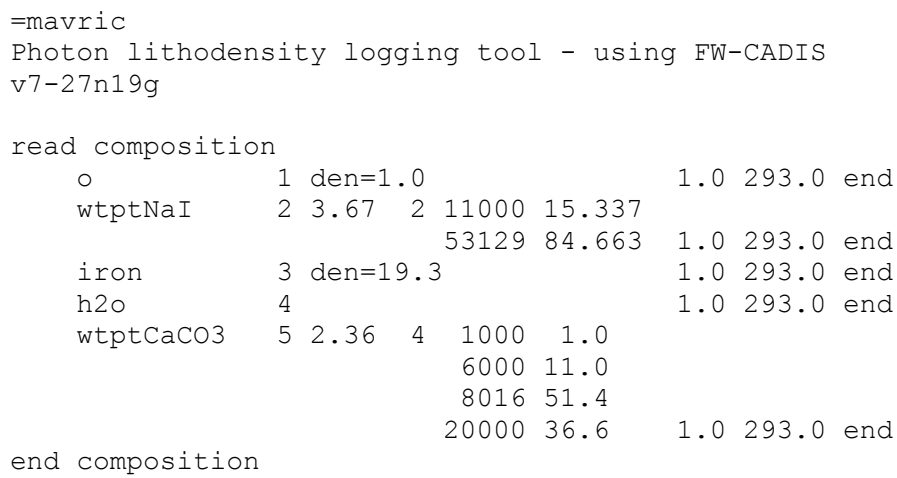

The geometry is fairly simple. Volumes are only needed for the regions where tallies will be made.

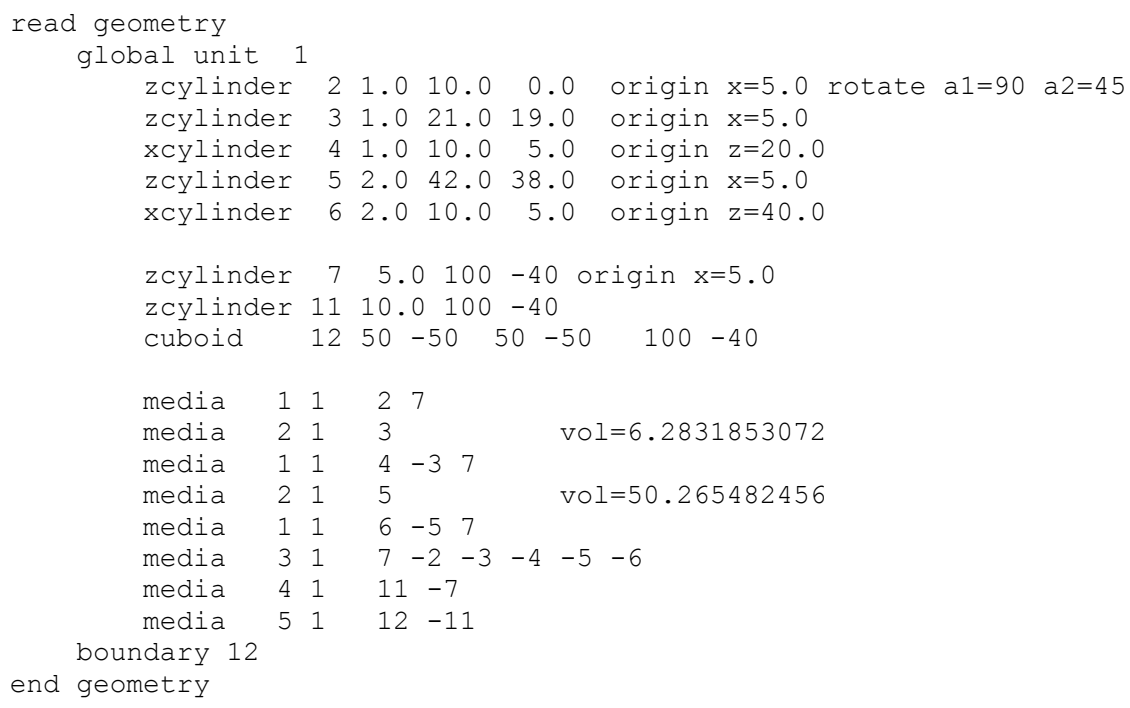

The definitions block lists the response (total photon flux) in a way that can be understood by both libraries used in the problem. The mesh grid for the importance calculations used $49 \times 43 \times 59=124,313$ mesh cells, with particular emphasis on geometric representation of the collimators to ensure accurate importance maps. This mesh grid is shown in Figure 4.1.22 and Figure 4.1.23. The source is represented by a distribution in which the most probable bin is located right near the $661.7 \mathrm{keV}$ line, which can be translated into one photon group in either library (from 600 to $800 \mathrm{keV}$ in the $27 / 19$ library or from 600 to $700 \mathrm{keV}$ in the $200 / 47$ library).

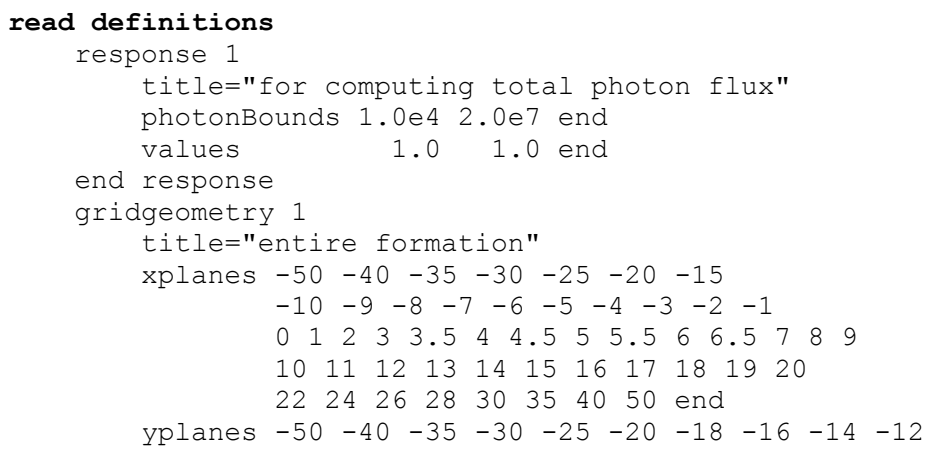




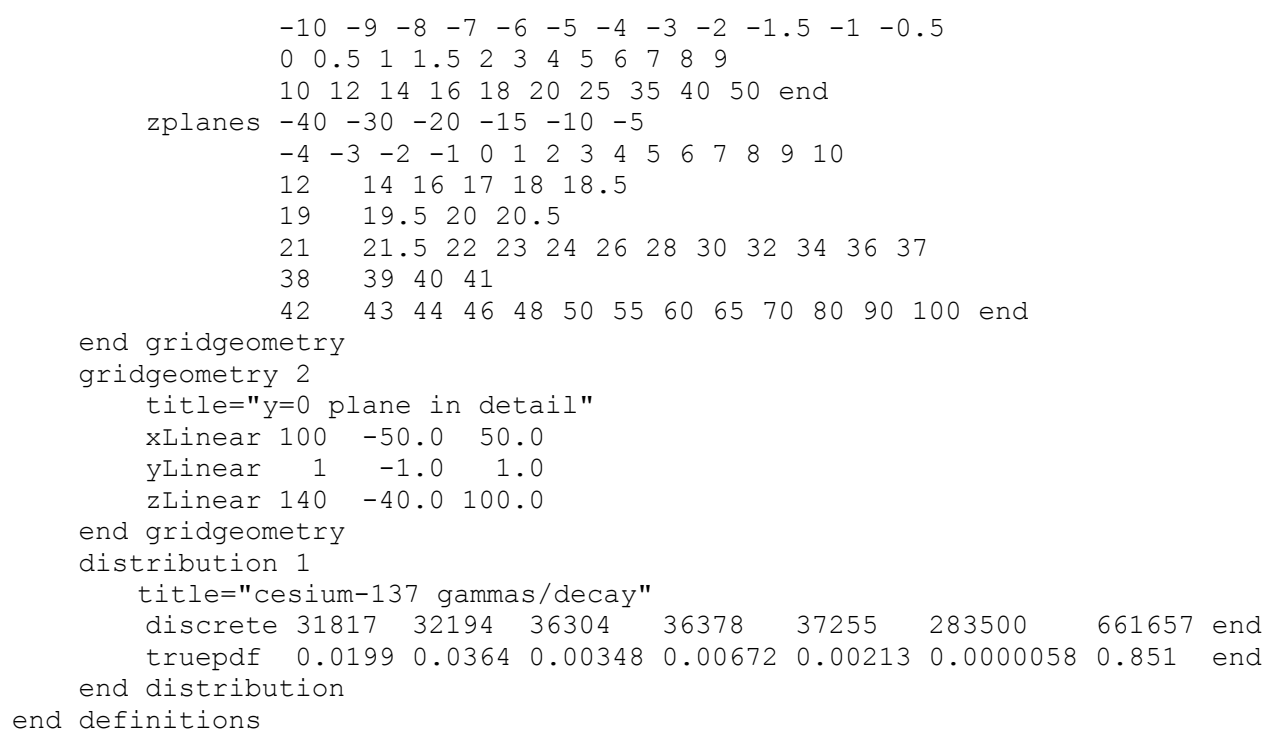

The source is a simple point source. For more realistic calculations, the source strength could be modified so that the total energy emitted is preserved, $S^{\prime}=S(661.7 / 0.5(700+600))$, which uses the ratio of the line energy to the energy at the center of the group in the 200/47 library.

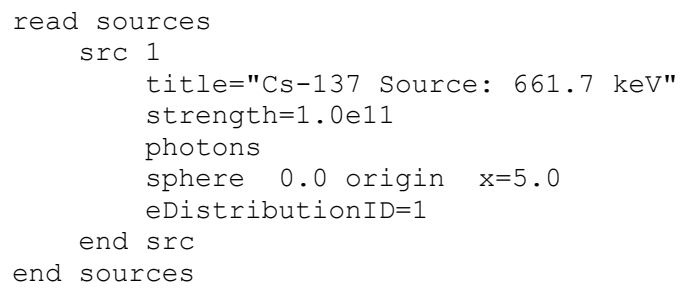

Each detector is represented by a region tally. A mesh tally is made for one slice in $y$ for visualization.

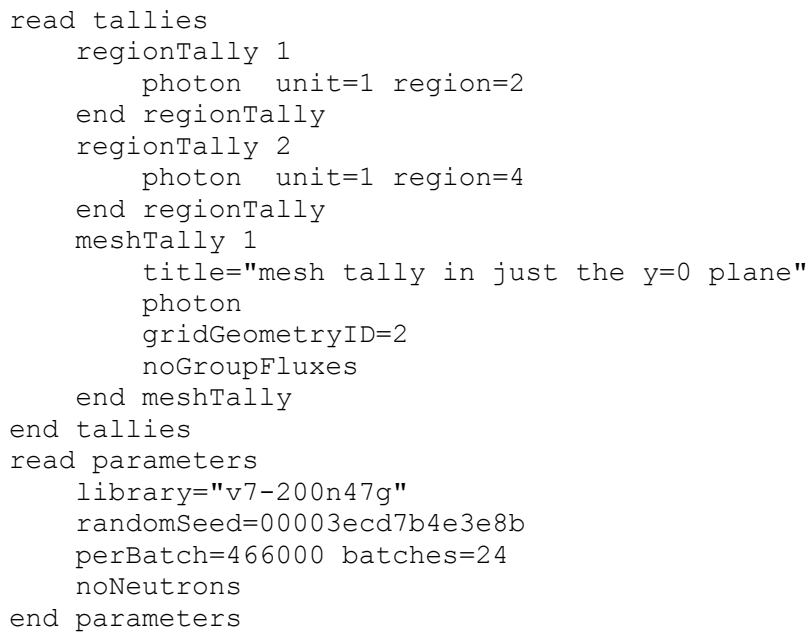

The importance map defines two adjoint sources, corresponding to the two tallies. Forward weighting, based on the response integrated over energy ("respWeighting"), is used. Because the true source is a point source, the subcell method of making a mesh source will fail, so the number of source trials is input. 
This number is small since the source is a monoenergetic point source. The Denovo calculations used the default $\mathrm{S}_{8}$ quadrature and $\mathrm{P}_{3}$ Legendre order.

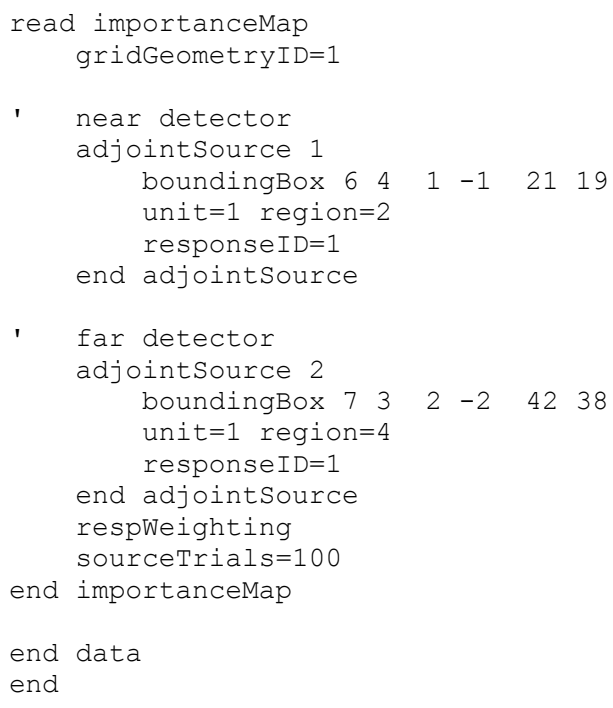

\subsection{Output}

The results for the two region tallies, the first for the near detector and the second for the far detector after 60 minutes of computations (3 forward Denovo, 4 adjoint Denovo and 52 Monaco), were as follows.

\begin{tabular}{|c|c|c|c|c|c|}
\hline loton Region Tally & average & standard & relat & FOM & stat checks \\
\hline tally/quantity & value & deviation & uncert & $(/ \mathrm{min})$ & 123456 \\
\hline ----------------- & ----------- & ----------- & ------- & -------- & ----------- \\
\hline total flux (tl) & $1.47918 \mathrm{E}+03$ & $1.57461 \mathrm{E}+01$ & 0.01065 & $1.70 \mathrm{E}+02$ & $x-x \times x x$ \\
\hline total flux (cd) & $1.47936 \mathrm{E}+03$ & $1.56963 \mathrm{E}+01$ & 0.01061 & $1.71 \mathrm{E}+02$ & $x-x-x x$ \\
\hline-------------------- & ----------- & ----------- & ------- & -------- & ----------- \\
\hline \multicolumn{6}{|l|}{ Photon Region Tally 2.} \\
\hline tally/quantity & $\begin{array}{c}\text { average } \\
\text { value }\end{array}$ & $\begin{array}{l}\text { standard } \\
\text { deviation }\end{array}$ & $\begin{array}{l}\text { relat } \\
\text { uncert }\end{array}$ & $\begin{array}{c}\text { FOM } \\
(/ \mathrm{min})\end{array}$ & $\begin{array}{l}\text { stat checks } \\
\begin{array}{llllll}1 & 2 & 3 & 4 & 5 & 6\end{array}\end{array}$ \\
\hline--------------- & ----------- & ----------- & ------- & -------- & ----------- \\
\hline total flux (tl) & $4.57691 \mathrm{E}+01$ & $2.81778 E-01$ & 0.00616 & $5.07 \mathrm{E}+02$ & $\mathrm{X} X \mathrm{X} X \mathrm{X} X$ \\
\hline total flux (cd) & $4.57825 E+01$ & $2.80714 E-01$ & 0.00613 & $5.11 \mathrm{E}+02$ & $\mathrm{X} X \mathrm{X} X \mathrm{X}$ \\
\hline--------------- & ----------- & ----------- & ------- & --- & - - \\
\hline
\end{tabular}

Note that both detectors have similarly low relative uncertainties (about $1 \%$ ) even though the tally values differ by a factor of 30 . These results should be compared to analog results (no biasing at all) and optimizations of each detector in separate input files, as shown in Table 4.1.22. The CADIS calculations for each detector (near or far) do exactly what they were supposed to do - optimize the Monte Carlo calculation for one tally or the other. The FOMs for the FW-CADIS calculation were about half of the FOMs for the single-detector CADIS calculations. Hence, for this two-detector problem, two CADIS calculations are just as efficient as one FW-CADIS calculation. For modern well-logging tools with up to a dozen detectors, a single FW-CADIS would be much more efficient and manageable. Note that the near detector still needs more time to pass the second (uncertainty fit) and the fourth (VOV fit) statistical checks. Neither of the single-detector CADIS calculations passed the fourth statistical check within an hour.

Figure 4.1.24 shows a mesh tally of total photon flux in the $\mathrm{y}=0$ plane for all four of the simulations: analog, CADIS for the near detector, CADIS for the far detector, and the FW-CADIS calculation for both. 
Table 4.1.22. Comparison of different CADIS techniques for the litho-density problem

\begin{tabular}{|c|c|c|c|c|c|c|c|c|c|}
\hline & \multirow{2}{*}{\multicolumn{3}{|c|}{$\begin{array}{l}\text { Time } \\
\text { (min) }\end{array}$}} & \multicolumn{3}{|c|}{ Near Detector } & \multicolumn{3}{|c|}{ Far Detector } \\
\hline & & & & flux & relative & FOM & flux & relative & FOM \\
\hline & For & Adj & $\mathrm{MC}$ & $\left(/ \mathrm{cm}^{2} / \mathrm{s}\right)$ & uncert. & $(/ \mathrm{min})$ & $\left(/ \mathrm{cm}^{2} / \mathrm{s}\right)$ & uncert. & $(/ \mathrm{min})$ \\
\hline Analog & & & 60.1 & $1.19 \mathrm{E}+03$ & 0.3765 & 0.12 & $1.17 \mathrm{E}+02$ & 0.7098 & 0.03 \\
\hline CADIS - Near & & 4.8 & 57.2 & $1.49 \mathrm{E}+03$ & 0.0064 & 390 & $0.00 \mathrm{E}+00$ & 0.0000 & ---- \\
\hline CADIS - Far & & 4.7 & 57.0 & $8.28 \mathrm{E}+02$ & 0.4162 & 0.09 & $4.56 \mathrm{E}+01$ & 0.0048 & 703 \\
\hline FW-CADIS & 2.8 & 4.4 & 52.1 & $1.48 \mathrm{E}+03$ & 0.0107 & 149 & $4.58 \mathrm{E}+01$ & 0.0062 & 444 \\
\hline
\end{tabular}



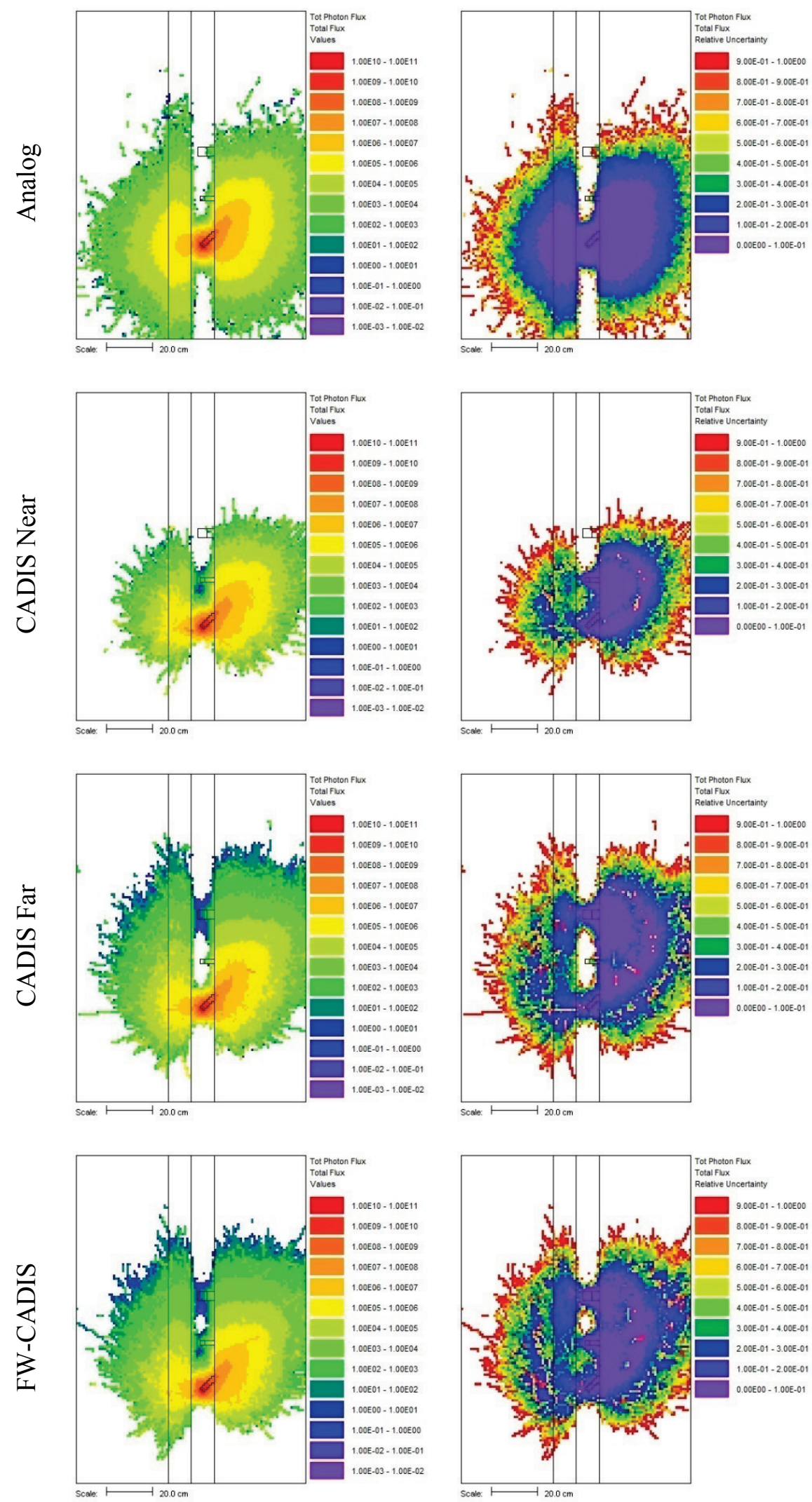

Figure 4.1.24. Mesh tallies showing total photon flux in $\mathrm{cm}^{2} / \mathrm{s}$ (left column) and its relative error (right column) in the $\mathbf{y}=\mathbf{0}$ plane. 


\subsubsection{AOS-100 using FW-CADIS and continuous-energy transport}

The advanced variance reduction in MAVRIC can also be used when the final Monaco Monte Carlo transport calculation is performed using a CE cross section library. Simulations involving discrete-energy photon sources are best handled with CE. Consider the AOS-100, one of several radioactive material transport packaging systems developed by Alpha Omega Services Inc., and a cobalt-60 source. (International Isotopes Inc. of Idaho Falls, Idaho, distributes the AOS Radioactive Material Transport Packaging Systems.)

A simple model of the AOS-100 package, which is constructed primarily of steel and tungsten, is shown in Figure 4.1.25. The diameter is $71.12 \mathrm{~cm}$ and the height is $91.44 \mathrm{~cm}$. The innermost cylinder $(16.51 \mathrm{~cm}$ diameter and $50.8 \mathrm{~cm}$ height) typically contains the material to be transported, but in this study, this region is simply modeled as an air region containing a uniform source of $1 \mathrm{Ci}$ of cobalt- 60 . This is conservative - it assumes the radioactive material containing the cobalt provides no selfshielding.

The objective in this study is to compute the dose rates around the cask. This can be done via using a mesh tally. Note that the dose rates inside the package are not of concern - only the dose rates outside the package are.

The results of a 15-hour analog calculation (using only implicit capture with a lower weight limit of $10^{-7}$ ) are shown in Figure 4.1.26. Note that this calculation does not show many photons that have escaped the package into the air, which is similar to reality for this heavily shielded cask. In order to compute dose rates outside the package, variance reduction is

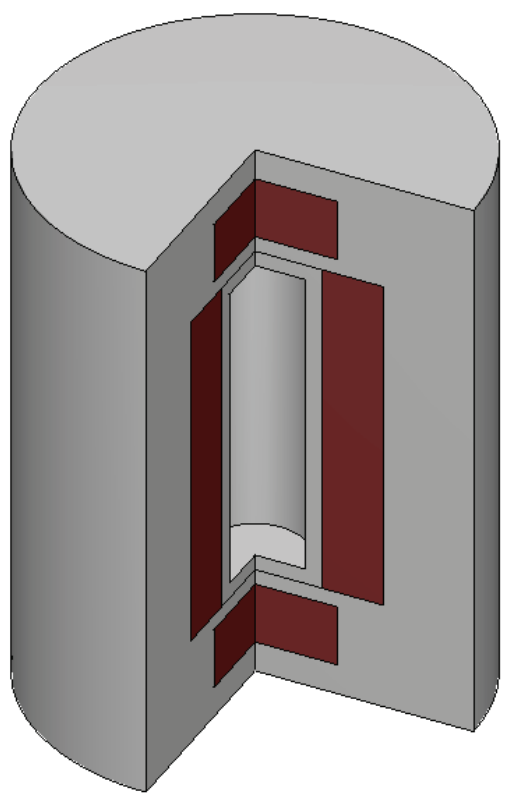

Figure 4.1.25. Simple AOS-100 cask geometry showing tungsten (brown) and steel (gray). needed.
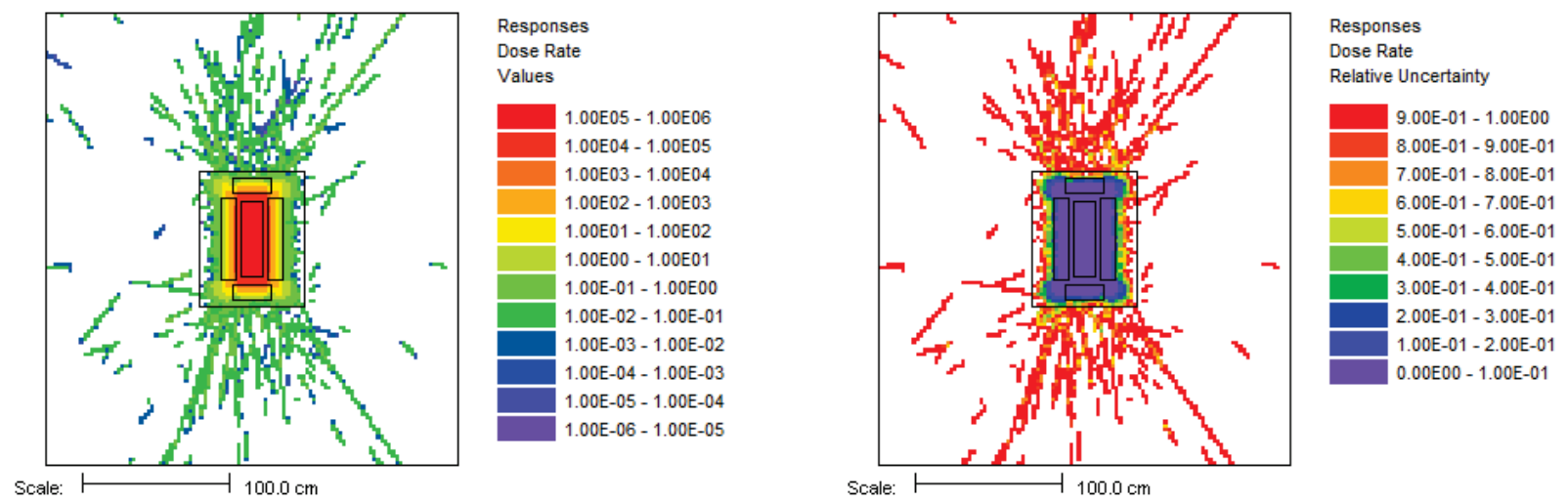

Figure 4.1.26. Dose rates $(\mathrm{mrem} / \mathrm{hr} / \mathrm{Ci})$ and relative uncertainties along the $y=0$ midplane of the cask as calculated by an analog calculation. 


\subsection{Input file}

The sample problem file mavric.aos100. inp is located in the samples \input directory. For this demonstration, a very simplified geometry is used, with a few tungsten regions inside a steel cylinder. The library given at the beginning of the input is the MG library for the importance calculations. The final Monaco Monte Carlo calculation will use the CE library, which is specified in the parameters block. About 1 meter of air is modeled around the cask.

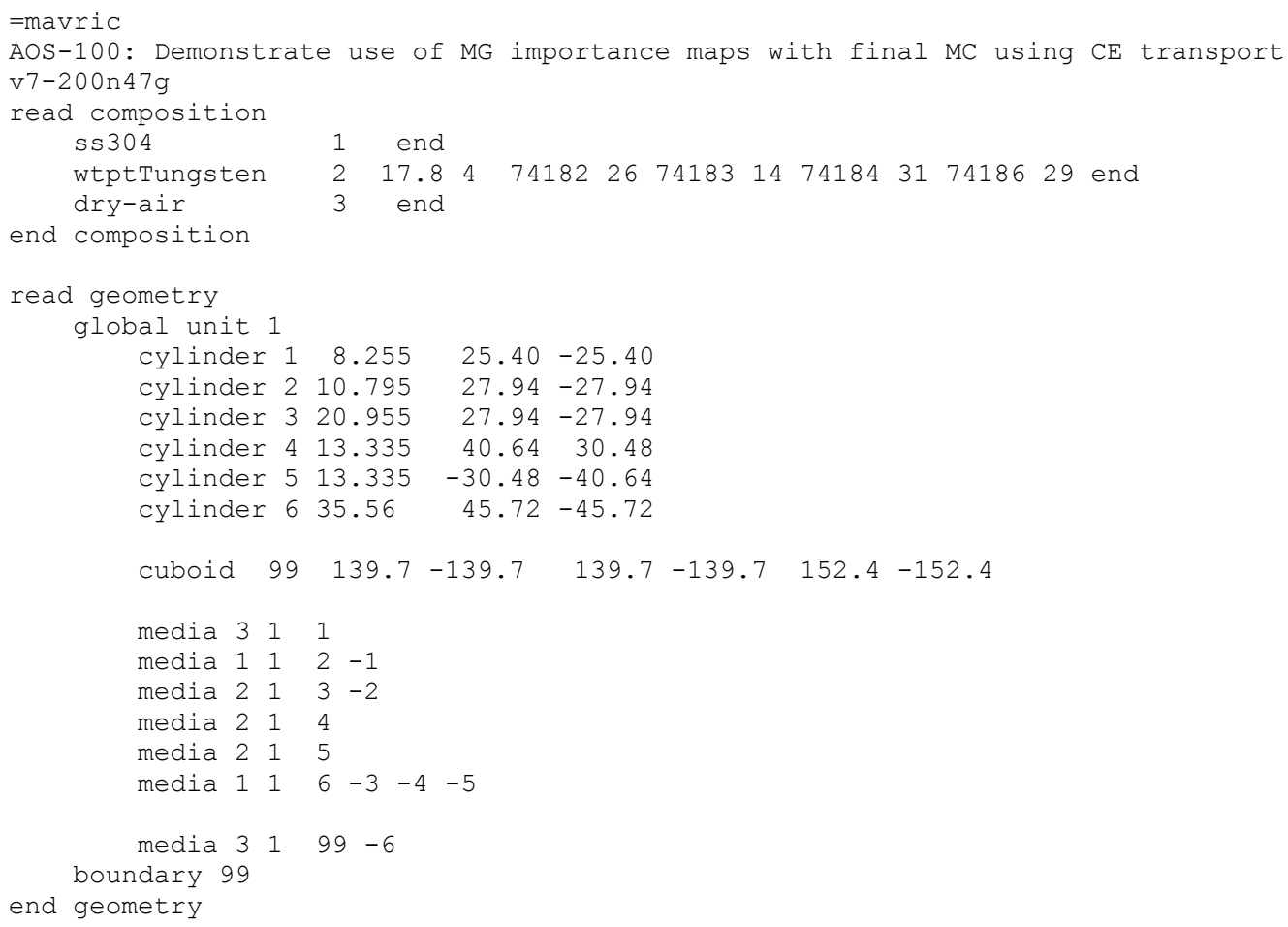

In the definitions block, the photon dose response function, the cobalt-60 line spectrum, two grid geometries, and an energy boundaries structure are all defined. The first grid geometry is used for the deterministic calculation, the importance map, and biased source. The second grid geometry is for the high-resolution ( 1 in.) mesh tally. The energyBounds defined here has a base structure of 30 bins that are $50 \mathrm{keV}$ wide, with three bins that are $2 \mathrm{keV}$ wide at the dominant cobalt line energies and the 511-keV annihilation gamma energy.

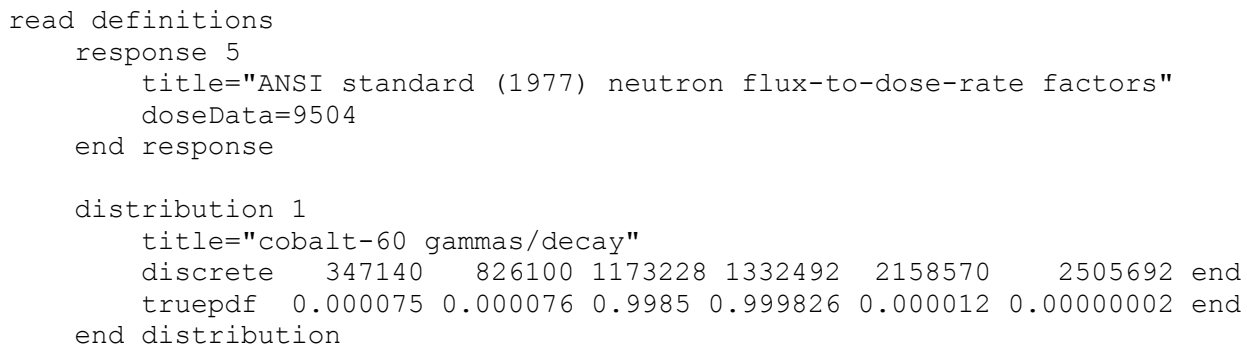




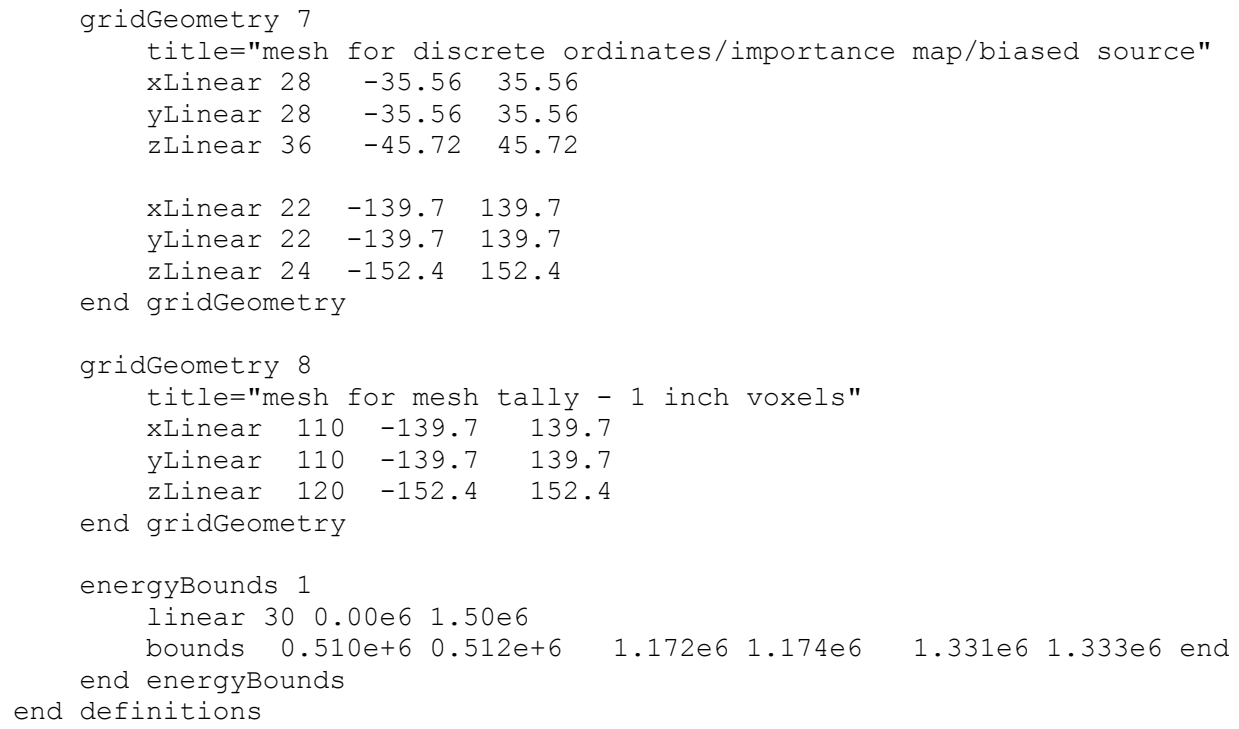

The source is modeled as a uniform volumetric source in the center region of the cask. Because the distribution of cobalt gamma rays was entered as gammas per decay, the keyword "useNormConst" will set the source strength to be the total of the energy distribution - about 2 photons/decay. The "multiplier" keyword is used to multiply that strength by $37 \mathrm{e} 9$ decays/sec to get $1 \mathrm{Ci}$.

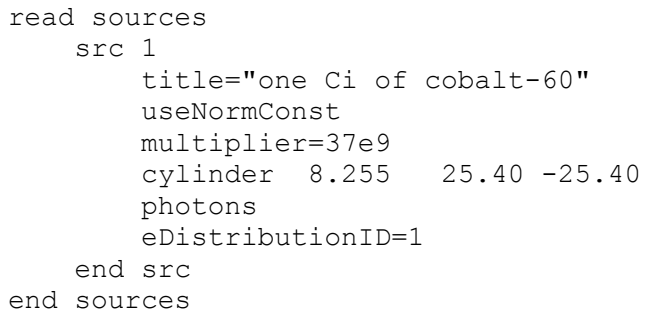

A single mesh tally is defined and is limited to the air region outside of the cask. A multiplier of 1000 is used to convert the response values from $\mathrm{rem} / \mathrm{hr}$ to $\mathrm{mrem} / \mathrm{hr}$.

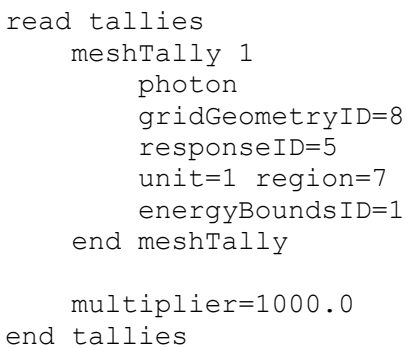

In this problem, the importance calculations will use the 200/47 MG library, which will transport all particles contained in the library by default. The keyword "noNeutrons" is used to turn off neutron transport during the Denovo calculations, saving time. The final Monaco calculation will use the CE library ("ceLibrary="), which only transports the particles specifically requested by the user (to avoid loading large amounts of cross section from disk to memory). Thus, the keyword "photons" is included to specifically tell the CE Monaco to transport photons. 


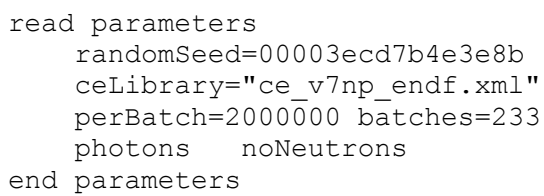

The importance map uses FW-CADIS to construct a map and biased source that will optimize the photon dose rate in the air outside the cask. Since photon scatter is typically forward peaked, an $\mathrm{S}_{12}$ quadrature and $\mathrm{P}_{5}$ Legendre scattering expansion are used.

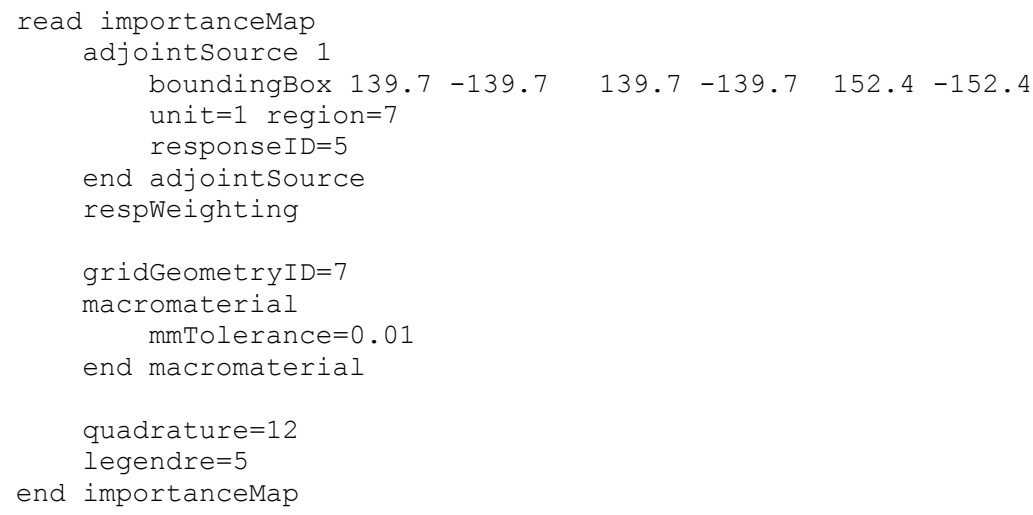

\subsection{Output file}

Results for the mesh tally after 958 minutes (27 forward Denovo, 31 adjoint Denovo, and 900 CEMonaco) are shown in Figure 4.1.27. Note that since dose rates inside the package are not of concern, that region was excluded from the mesh tally. Due to the optimization that focused the Monte Carlo calculation on dose rates outside the cask, values of the dose rate inside the cask are underestimated and should not be used. Also note that voxels along the outer cylindrical edge of the package could show low dose rates, since the voxel value is an average and only part of the voxel is actually outside the package. The resolution of the mesh tally is $2.54 \mathrm{~cm}$. 


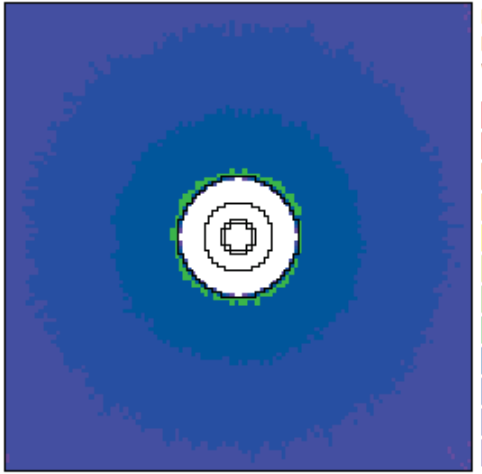

Scale: $\longmapsto 50.0 \mathrm{~cm}$

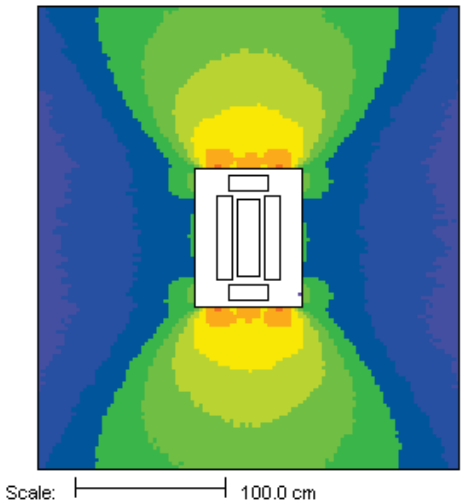

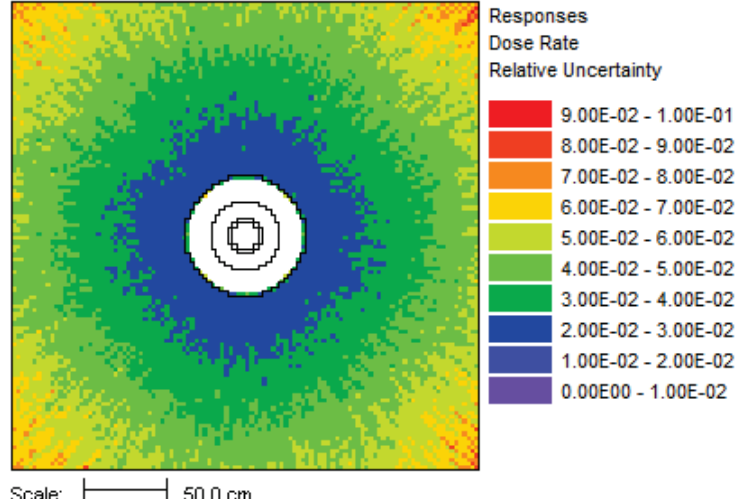

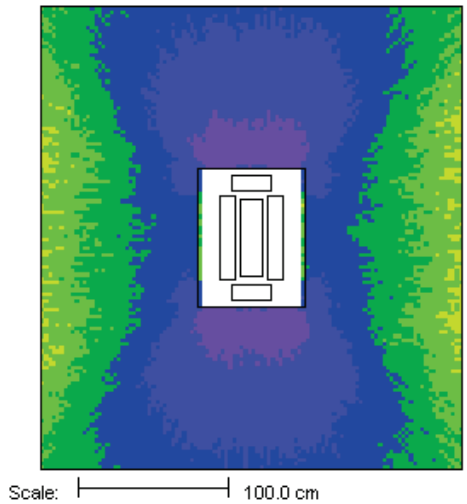

Responses

Dose Rate

Relative Uncertainty

$9.00 \mathrm{E}-02-1.00 \mathrm{E}-01$ $8.00 \mathrm{E}-02-9.00 \mathrm{E}-02$ $7.00 \mathrm{E}-02-8.00 \mathrm{E}-02$ $6.00 \mathrm{E}-02-7.00 \mathrm{E}-02$ $5.00 \mathrm{E}-02-6.00 \mathrm{E}-02$ $4.00 \mathrm{E}-02-5.00 \mathrm{E}-02$ 3.00E-02 - 4.00E-02 2.00E-02 - 3.00E-02 $1.00 \mathrm{E}-02-2.00 \mathrm{E}-02$ $0.00 E 00-1.00 \mathrm{E}-02$

Figure 4.1.27. Dose rates $(\mathrm{mrem} / \mathrm{hr} / \mathrm{Ci})$ and relative uncertainties from the $\mathrm{CE}, \mathrm{FW}$-CADIS calculation, showing the midplane views of the cask $(z=0$ above and $y=0$ below).

The flux using the user-defined energy bin boundaries for a point $10 \mathrm{~cm}$ above the top of the package (along the axis) is shown in Figure 4.1.28. Note that since the importance map was made to optimize the photon dose rate, some of the lowenergy bins (that do not contribute to dose much) may have larger uncertainties.

Figure 4.1.29 and Figure 4.1.30 show the ratios of the dose rates computed using a MG calculation to the $\mathrm{CE}$ calculation. Dose rates inside the cask should not be compared because the importance map is focused on

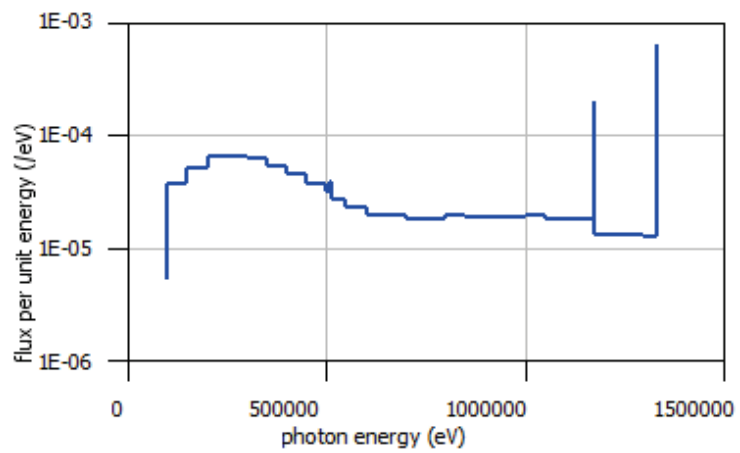

Figure 4.1.28. Flux $10 \mathrm{~cm}$ above the cask. dose outside the cask, so low-energy photons are not simulated inside. The 47 group MG calculation is fairly close to the CE-calculation in dose rate $(10 \%$ high axially, 20\% high radially), but the 19 group MG dose rates are much higher than the CE. Neither of the MG calculations shows the 1.17 and $1.33 \mathrm{MeV}$ lines in the energy spectra. 

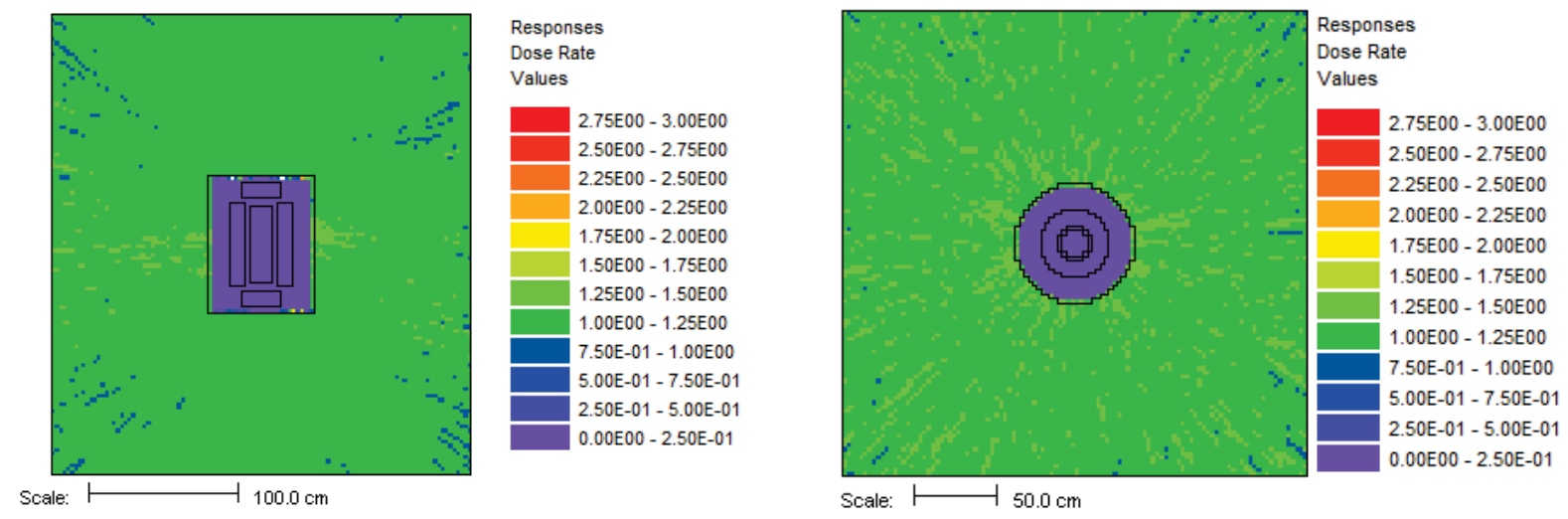

Figure 4.1.29. Ratio of the 47 group MG computed dose rates to the $\mathrm{CE}$ dose rates $(y=0$ left and $z=0$ right $)$.
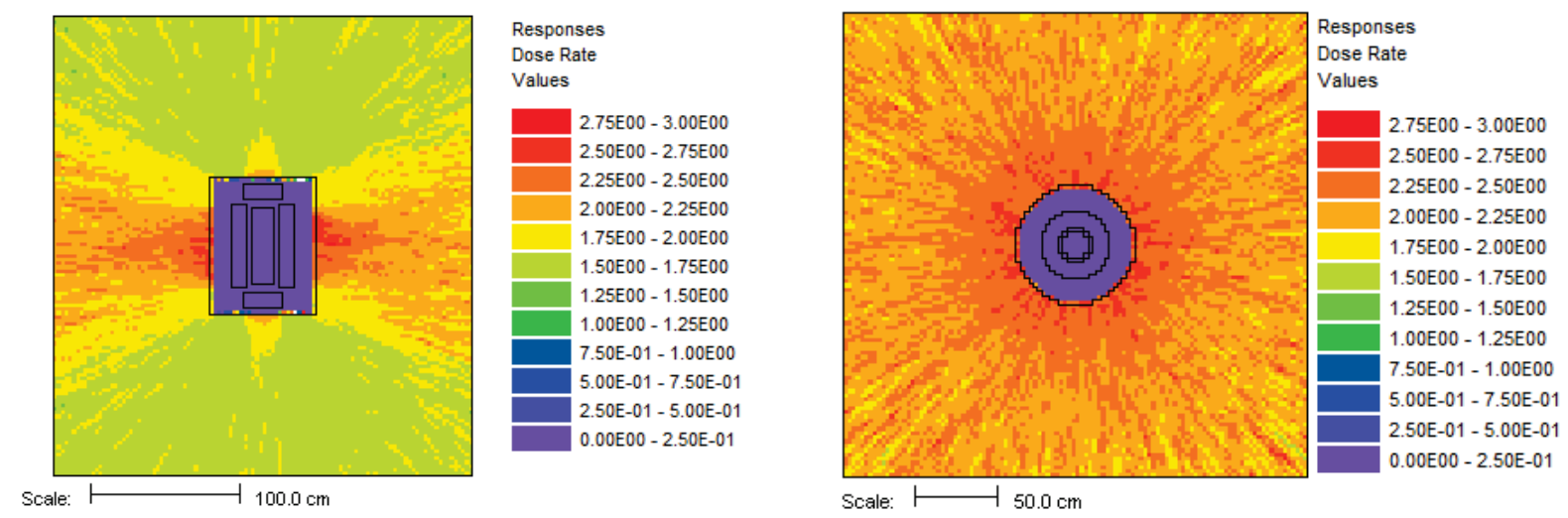

Figure 4.1.30. Ratio of the 19 group MG computed dose rates to the $\mathrm{CE}$ dose rates $(y=0$ left and $z=0$ right $)$.

\subsubsection{Independent spent fuel storage installation}

A good example of a problem where the dose rate needs to be known well everywhere-in low dose areas and high dose areas - is an independent spent fuel storage installation. The dose rates around these arrays of spent fuel casks need to be evaluated over a large area of ground in order to determine the boundary of the controlled area. This sample problem uses a simplified model of a cask array, shown in Figure 4.1.31 and Figure 4.1.32, to demonstrate the FW-CADIS method for obtaining the dose rates with reasonably low relative uncertainties over a mesh tally which covers a very large area. 


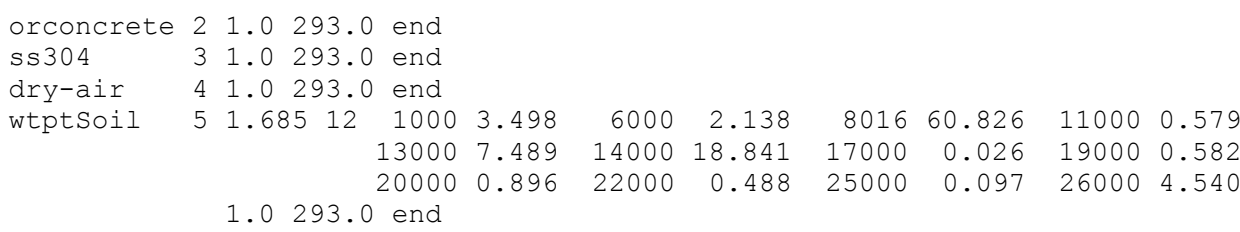

The geometry models one cask and then repeats it eight times using an array.

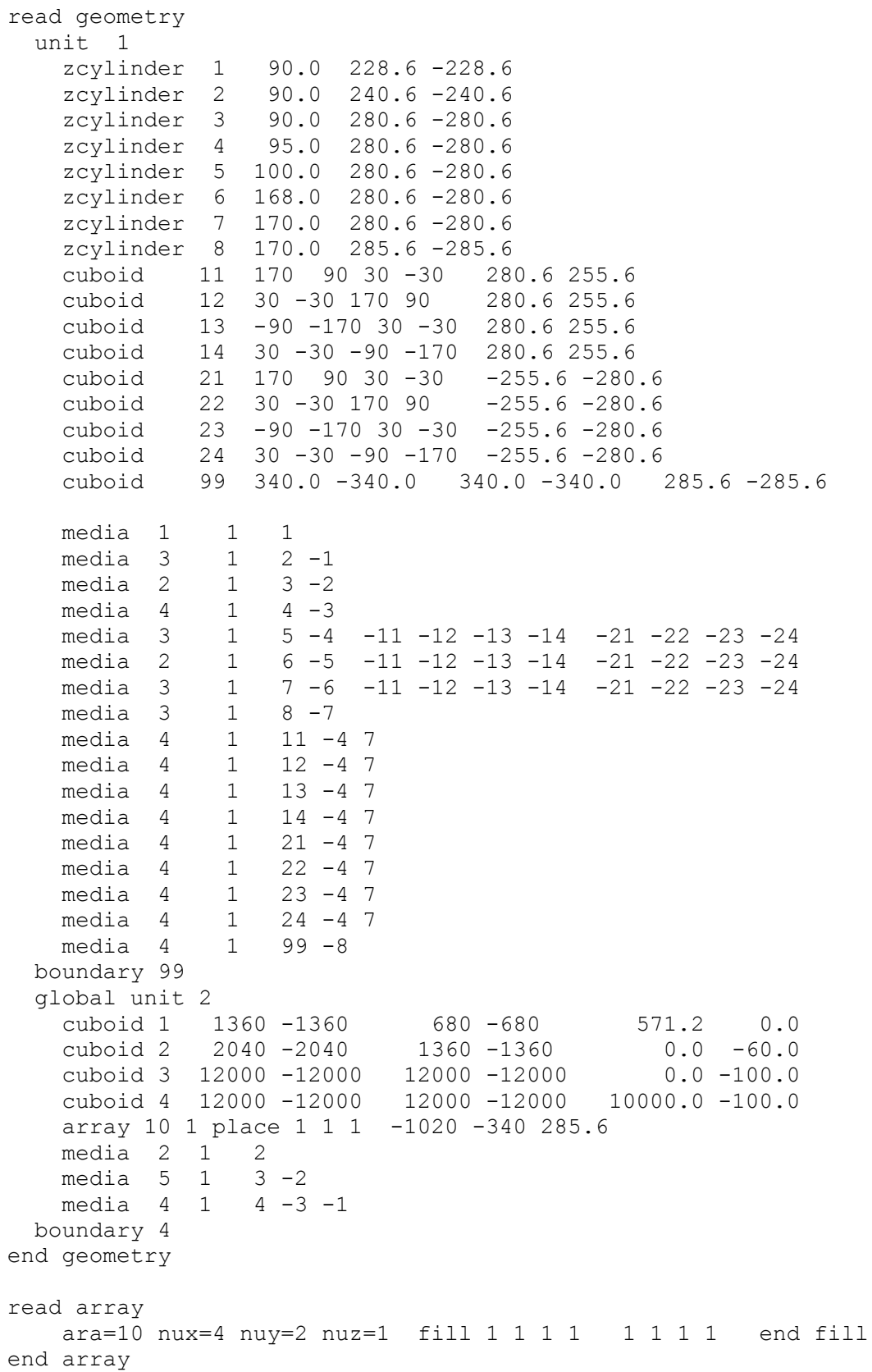

The definitions block contains the photon dose response, a mesh geometry for the discrete-ordinates calculations, a uniform mesh for the mesh tally, and the photon energy distribution read from the binary concentration file (located on unit 71, the last time step case 64). 


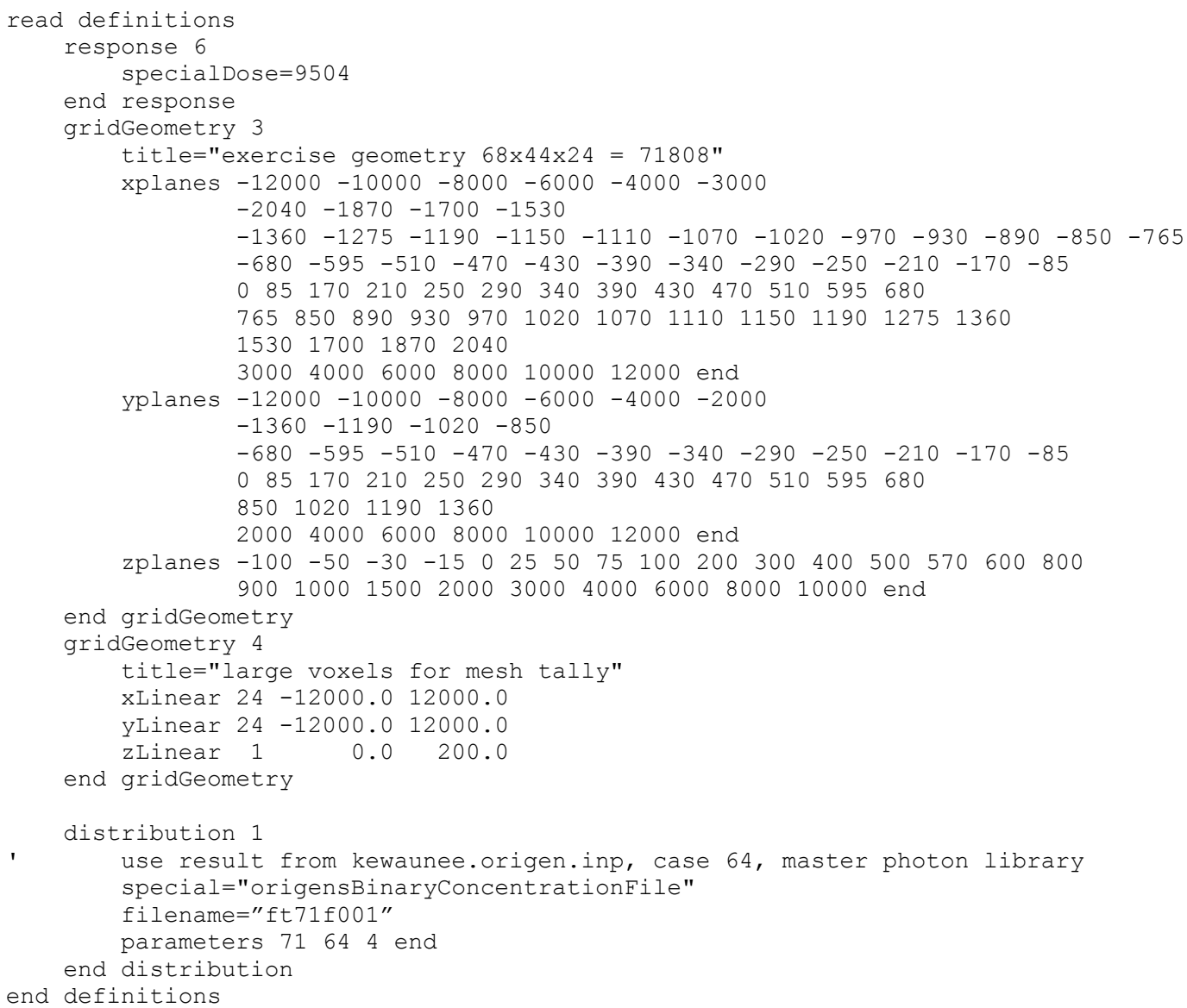

The source can be done in two ways. Since there are eight casks, eight sources could be defined, each over a cylinder with a strength of $7.155 \times 10^{16}$ photon/s. In this example of eight identical sources, one source region can be defined and then limited to only exist in the spent fuel material (the first material) but with a strength for all the casks of $5.724 \times 10^{17}$ photon/s.

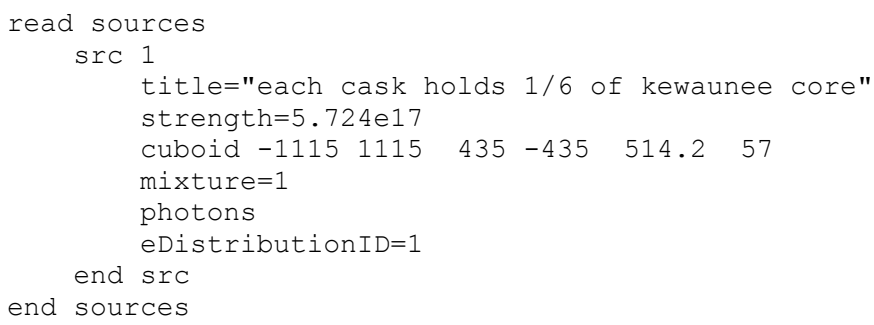

The mesh tally covers only the first 2 meters above the ground, since the dose rate above that would not impact a person.

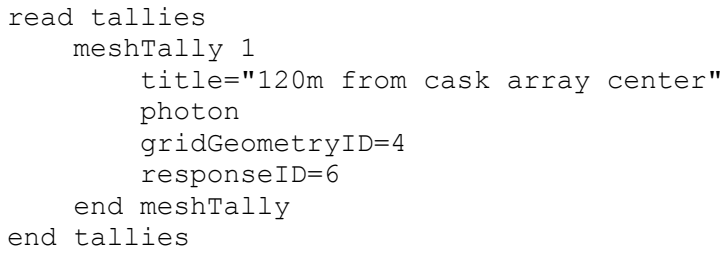




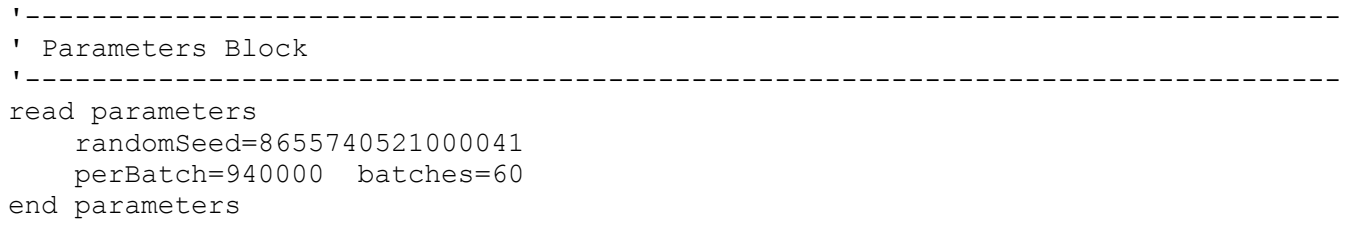

The adjoint source is placed everywhere that the dose rate is desired-near the ground but not very close to or in between the casks (where people will not be). The macro material option is used here to help the discrete-ordinates calculation be more representative of the problem.

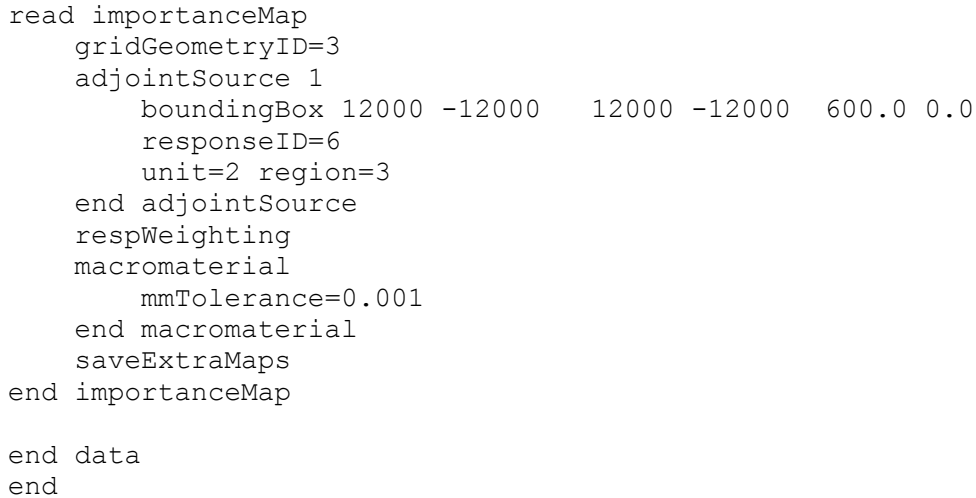

\subsection{Output}

The MAVRIC calculational times were 3 minutes for the forward Denovo, 3.5 minutes for the adjoint Denovo, and 60 minutes for the Monaco forward Monte Carlo. Results for the mesh tally of dose rates are shown in Figure 4.1.33. For a longer Monaco calculation $(64 \mathrm{hr})$, the mesh tally results are shown in Figure 4.1.34. Note that the scale for the dose rate has been adjusted to only show the values outside of the cask array.

Using the Mesh File Viewer, the distribution of relative uncertainty in a mesh tally can be plotted. For this $1 \mathrm{hr}$ calculation, $50 \%$ of the voxels in the mesh tally had less than $20 \%$ relative uncertainty. Figure 4.1.35 shows how the distribution of relative errors changes with longer run times. For the $64 \mathrm{hr}$ run, $90 \%$ of the voxels had less than $10 \%$ relative uncertainty.

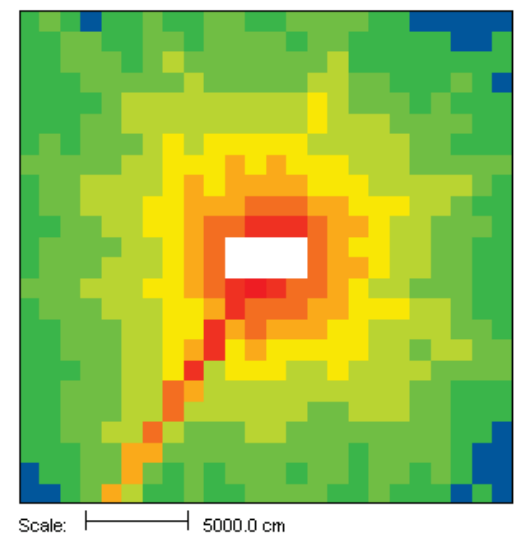

Figure 4.1.33. Photon dose rate values in $\mathrm{rem} / \mathrm{hr}$ (left) and the relative uncertainty (right) for the area around the cask array (1 hr Monaco). 

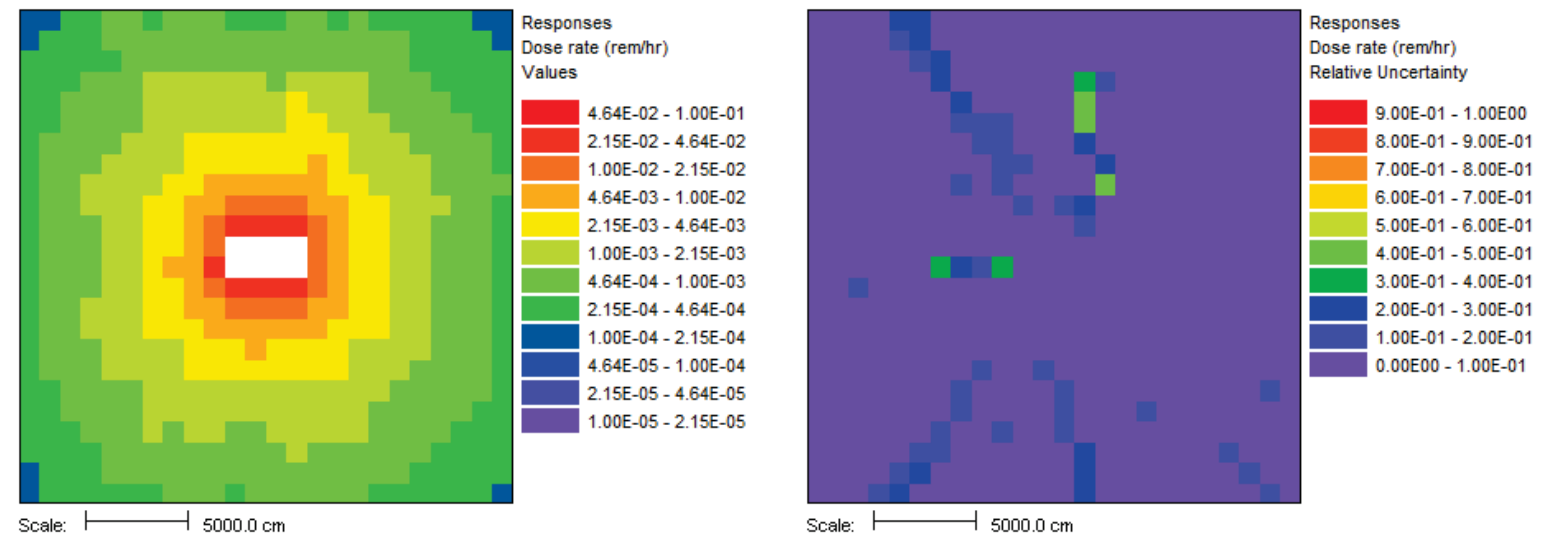

Figure 4.1.34. Photon dose rate values in $\mathbf{r e m} / \mathrm{hr}$ (left) and the relative uncertainty (right) for the area around the cask array (64 hr Monaco). For the uncertainty plot, purple represents $<=5 \%$.

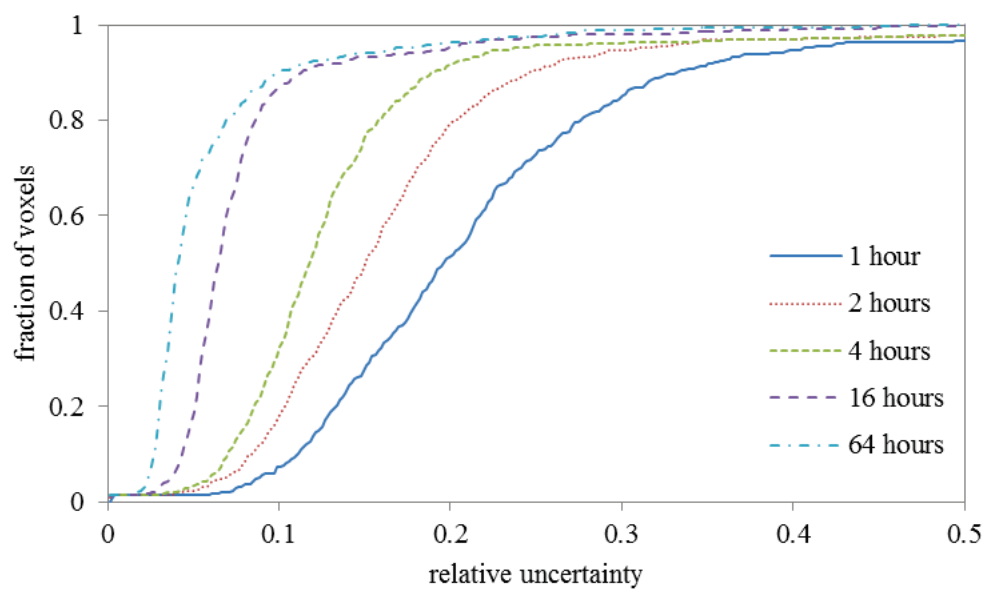

Figure 4.1.35. Distribution of relative uncertainties for different run times of mavric. isfsi. inp. This plot shows what fraction of the mesh tally voxels had less than a given amount of relative uncertainty.

\subsubsection{TN24-P spent fuel cask}

As an example that uses multiple sources, user-defined distributions in those sources, macromaterials for improved $\mathrm{S}_{\mathrm{N}}$ calculations, and the automated variance reduction capabilities in MAVRIC, consider the model for the TN-24P cask, as used in previous SCALE shielding reports ${ }^{19}$ and shown in Figure 4.1.36. This model contains two types of PWR spent fuel assemblies (Types V and W, both Westinghouse $15 \times 15$ assemblies of different starting enrichments and burnups), each with specified neutron and photon sources, in an aluminum/boron fuel basket. The cask is made of forged steel for photon shielding with a resin layer for neutron shielding. Also included in the model are three activated hardware regions (bottom nozzle, top nozzle, and top plenum), which consist of specified amounts of ${ }^{60} \mathrm{Co}$ (a photon source). The task for this example is to calculate the total dose rate within 2 meters of the cask surface. 

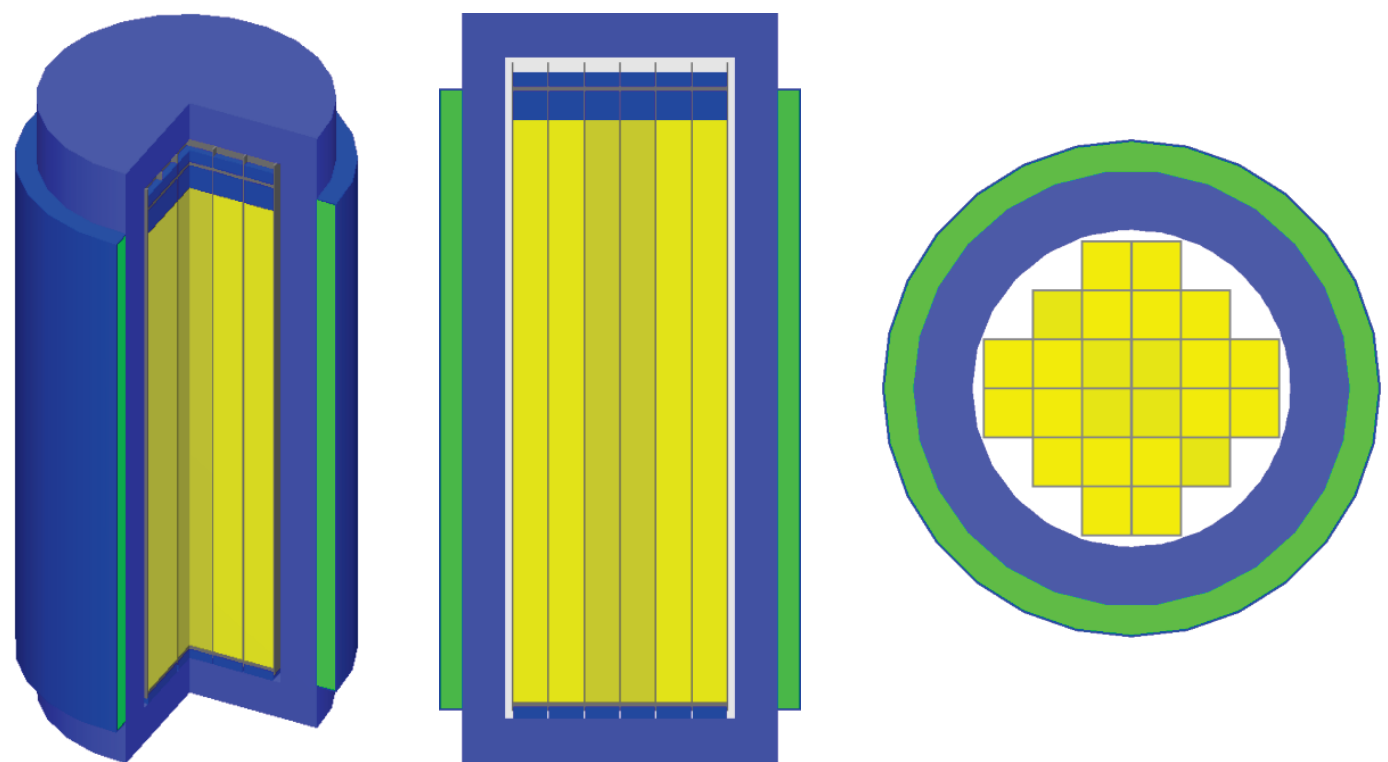

Figure 4.1.36. MAVRIC model of the TN24-P cask. Materials: spent fuel (light and dark yellow), steels (blues), resin (green), and other metals (gray).

For MAVRIC, this means the calculation of a dose rate mesh tally using FW-CADIS to ensure that each voxel has low relative uncertainty, independent of the dose rate. Without MAVRIC, the calculation of dose rate everywhere in three dimensions would be too challenging. Most likely, the dose rate would be evaluated with reasonable uncertainty at only a few locations. In fact, with analog calculations, this example would be a very difficult problem since most source particles never leave the cask, just as in the real-life situation. This type of problem really benefits from the CADIS-biased source distribution, in which source particles deep inside the cask are sampled very rarely since they do not contribute significantly to the response.

\subsection{Input file}

The following is a partial listing of the file mavric.tn24p. inp located in the SCALE samples \input directory. This calculation will use the coarse-group shielding library for all of the importance map calculations but then use the fine-group library for the final Monaco step. The full geometry and source distributions are not printed here due to their length.

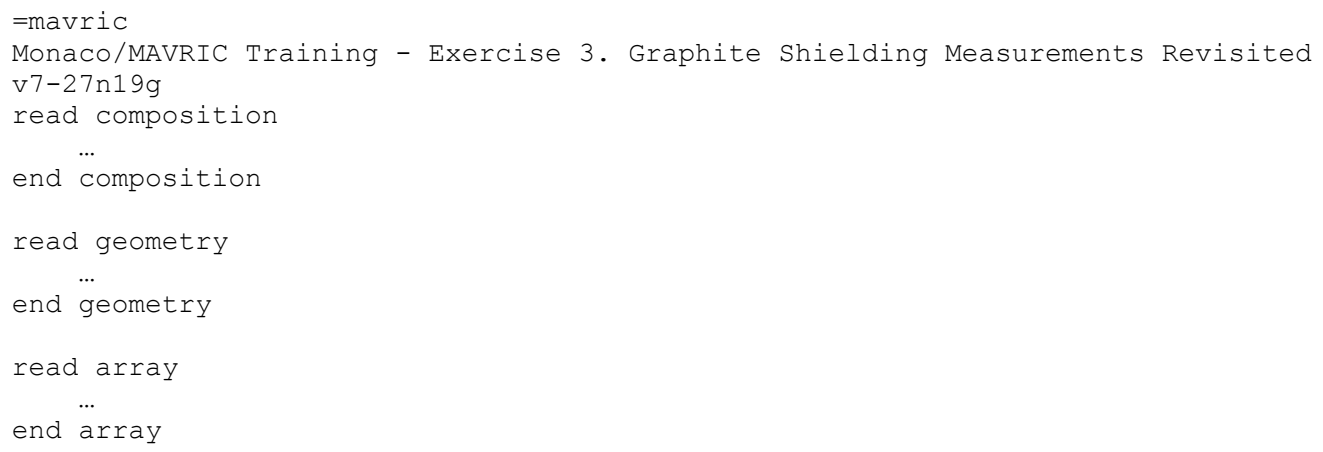

The definitions block includes three responses (neutron, photon, total), two grid geometries (one for the importance map calculations and one for a mesh tally), one cylindrical mesh for a mesh tally, five distributions for the energy spectra of the sources, and two distributions for the spatial distributions of the fuel assembly sources. 


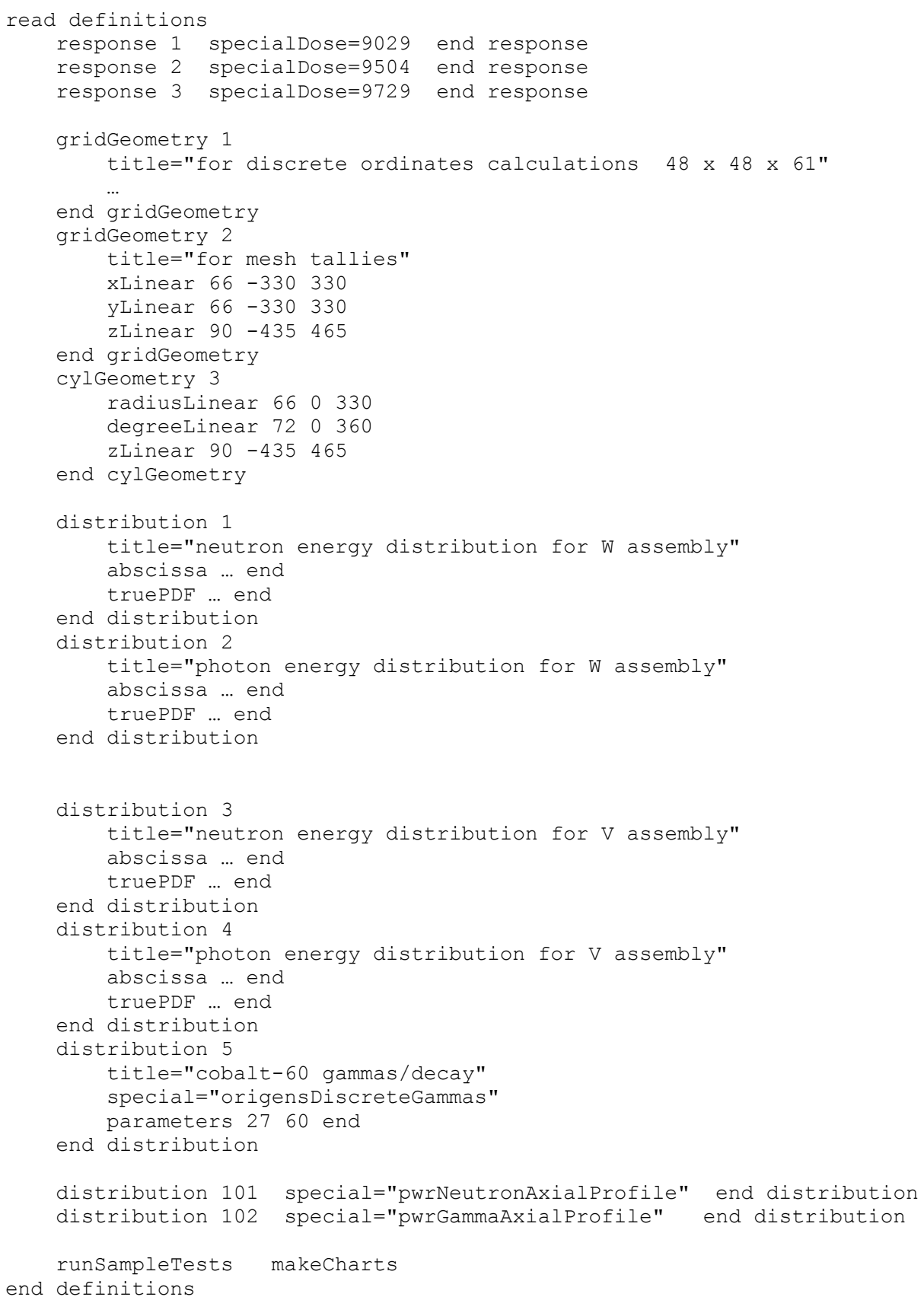

Seven sources are defined - a neutron and photon source for each type of fuel assembly and three activated hardware regions.

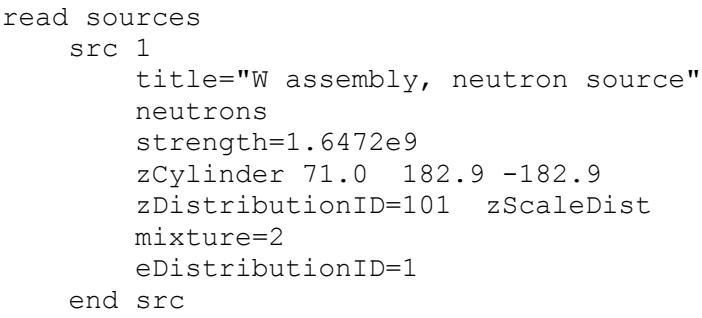




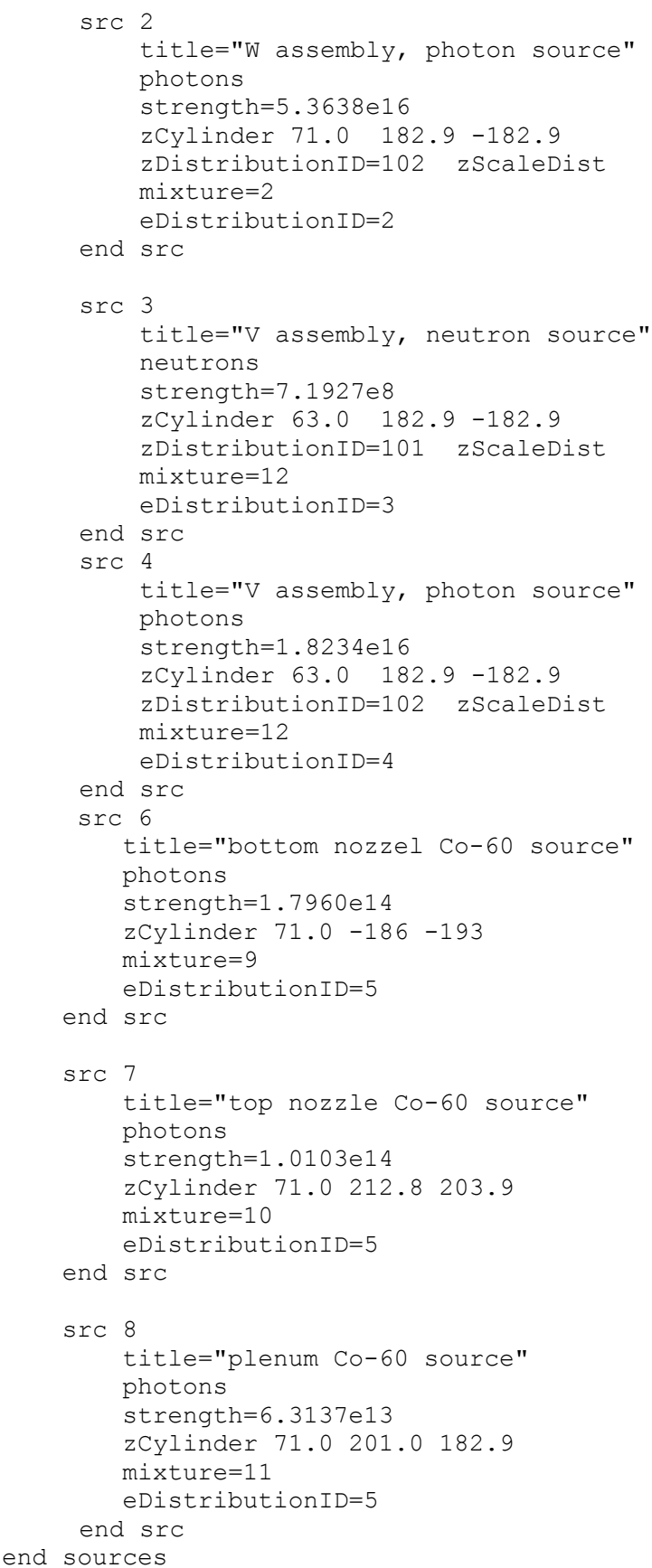

Four mesh tallies are used to collect the neutron dose rate, the photon dose rate, and the total dose rate.

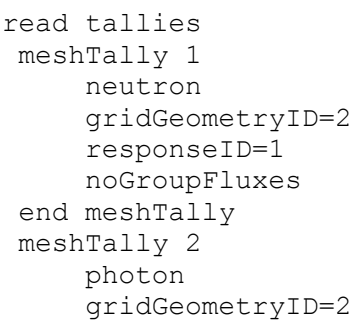




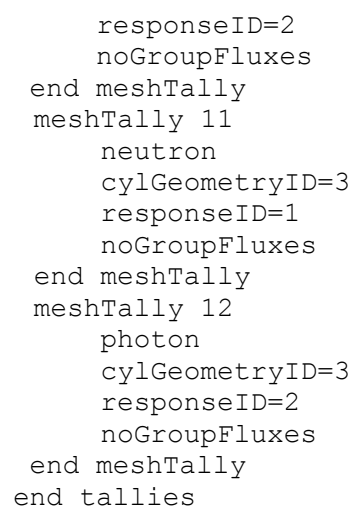

The goal of this example is to calculate the total dose outside the cask. Hence, the adjoint source uses the total dose rate response function for its energy component, while for the spatial component, it uses a large block around the cask. Note the "mixture=" keyword, which restricts the adjoint source to only exist where the material is air (13). There is no need to put adjoint source deep in the cask since the dose rates inside the cask are not of interest. Response weighting is used to put more adjoint source in the low dose areas outside the cask. Note that since this area is air, not many interactions/scatter take place, so we should not expect a great balance in relative uncertainties near and far from the cask. Macromaterials are used to improve the results from the discrete-ordinates calculations, which should improve the FOM of the final Monaco calculation. The geometry images in Section 4.1.3.4.2 are taken from this problem.

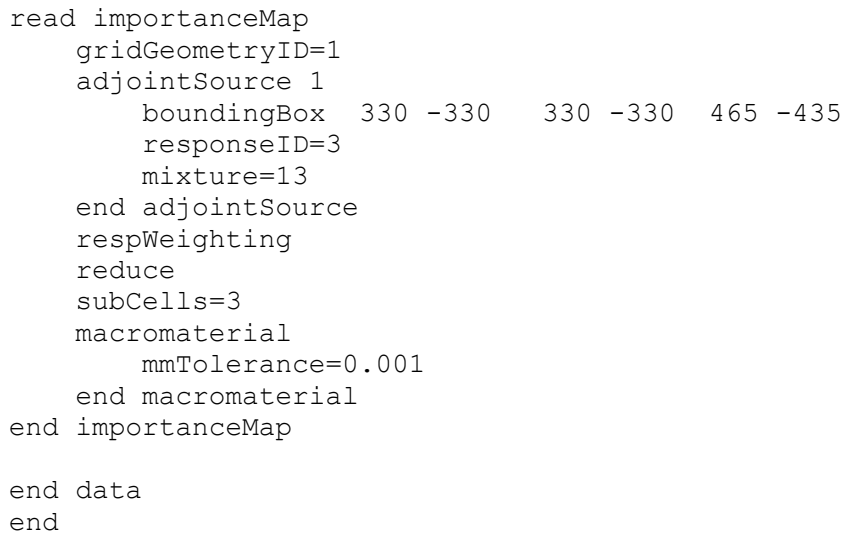

\subsection{Output}

The distributions used by the source descriptions of the TN24-P model are shown in Figure 4.1.38, and the responses used in this problem are shown in Figure 4.1.37. Figure 4.1.39 and Figure 4.1.40 show the total (neutron + photon) dose rate outside the TN24-P cask, up to 2 meters from each surface using a rectilinear and a cylindrical mesh tallies with $10 \mathrm{~cm}$ voxels. Uncertainties in the computed dose rates were 3-4\% after this $16 \mathrm{hr}$ calculation. The scale of the figure was adjusted to only show the dose rate outside the cask; dose rates inside the cask went as high as $6 \times 10^{5} \mathrm{rem} / \mathrm{hr}$. Likewise, the scale of the uncertainties ranged from 0 to $10 \%$ to highlight the uncertainties outside the cask. Relative uncertainties inside the cask were much higher (white areas) since those areas were deemed unimportant by the chosen adjoint source. Obtaining dose rates on a $10 \mathrm{~cm}$ mesh would not be achievable without the variance reduction capabilities of MAVRIC. 

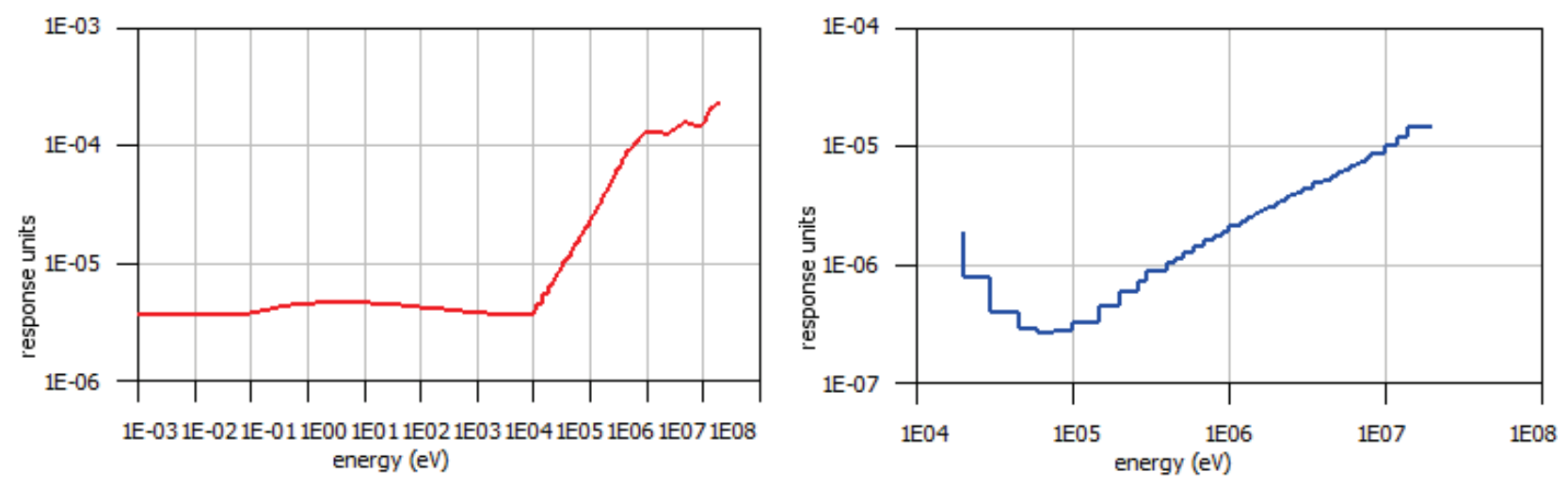

Figure 4.1.37. Neutron (left) and photon (right) dose rate response functions.

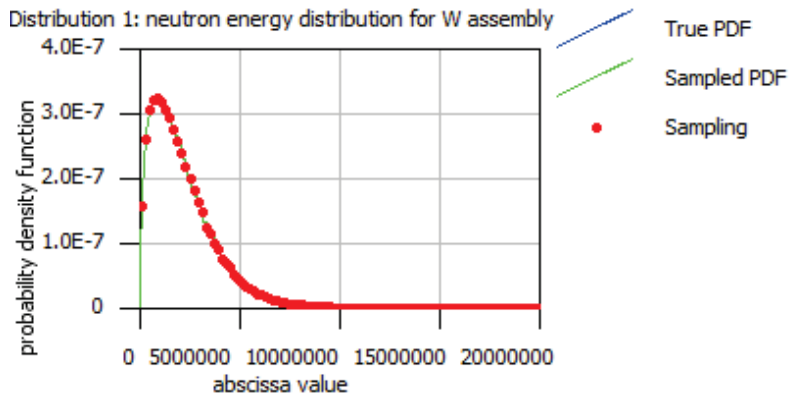

W assembly, neutron source

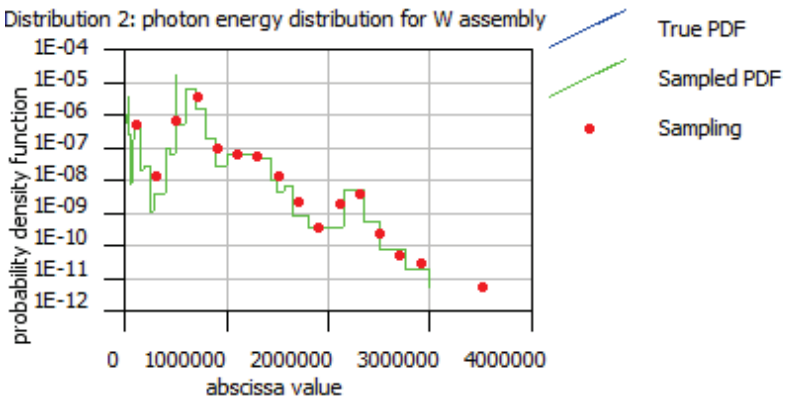

W assembly, photon source

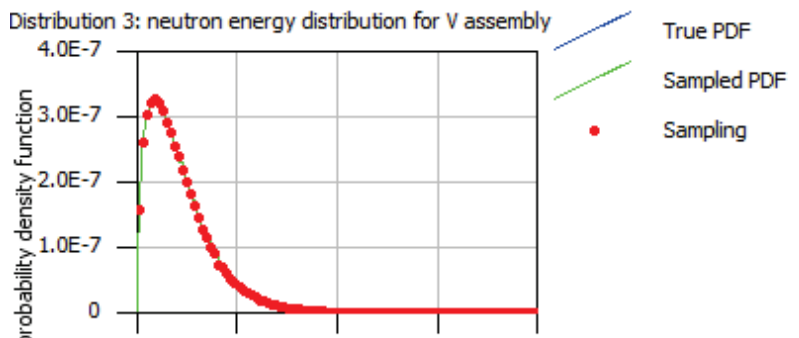

05000000100000001500000020000000 abscissa value

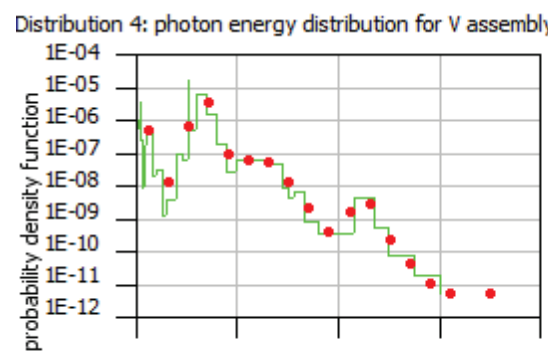

True PDF

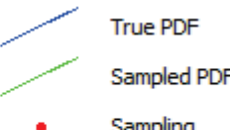

- $\quad$ Sampling

$\mathrm{V}$ assembly, neutron source

V assembly, photon source 


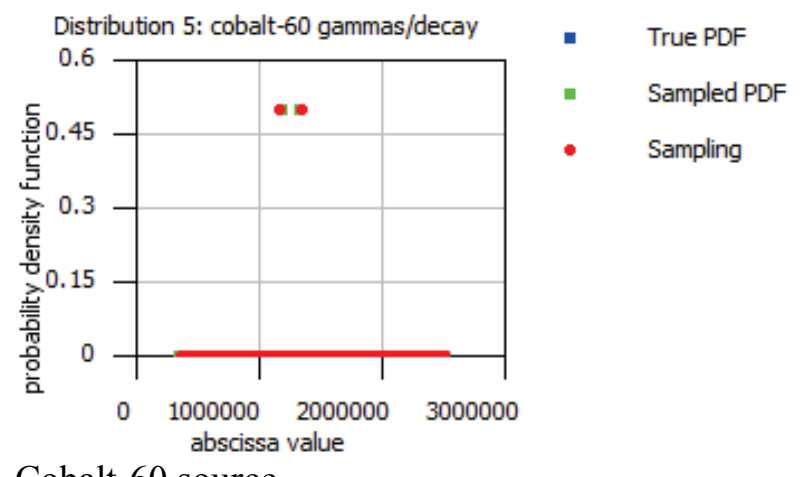

Cobalt-60 source
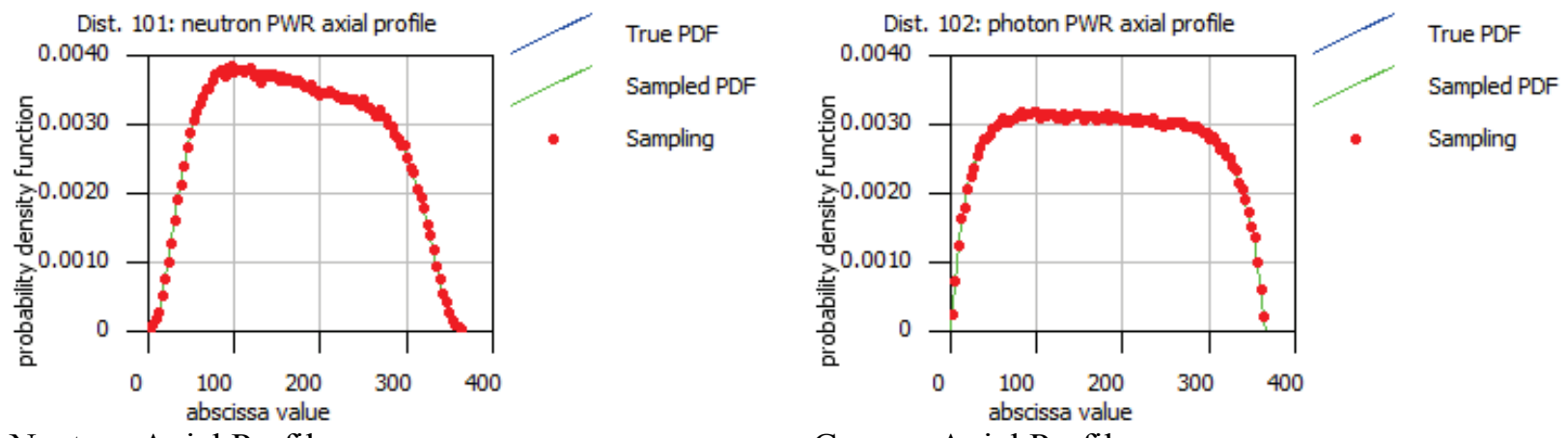

Neutron Axial Profile

Gamma Axial Profile

Figure 4.1.38. Distributions in the TN24-P model.
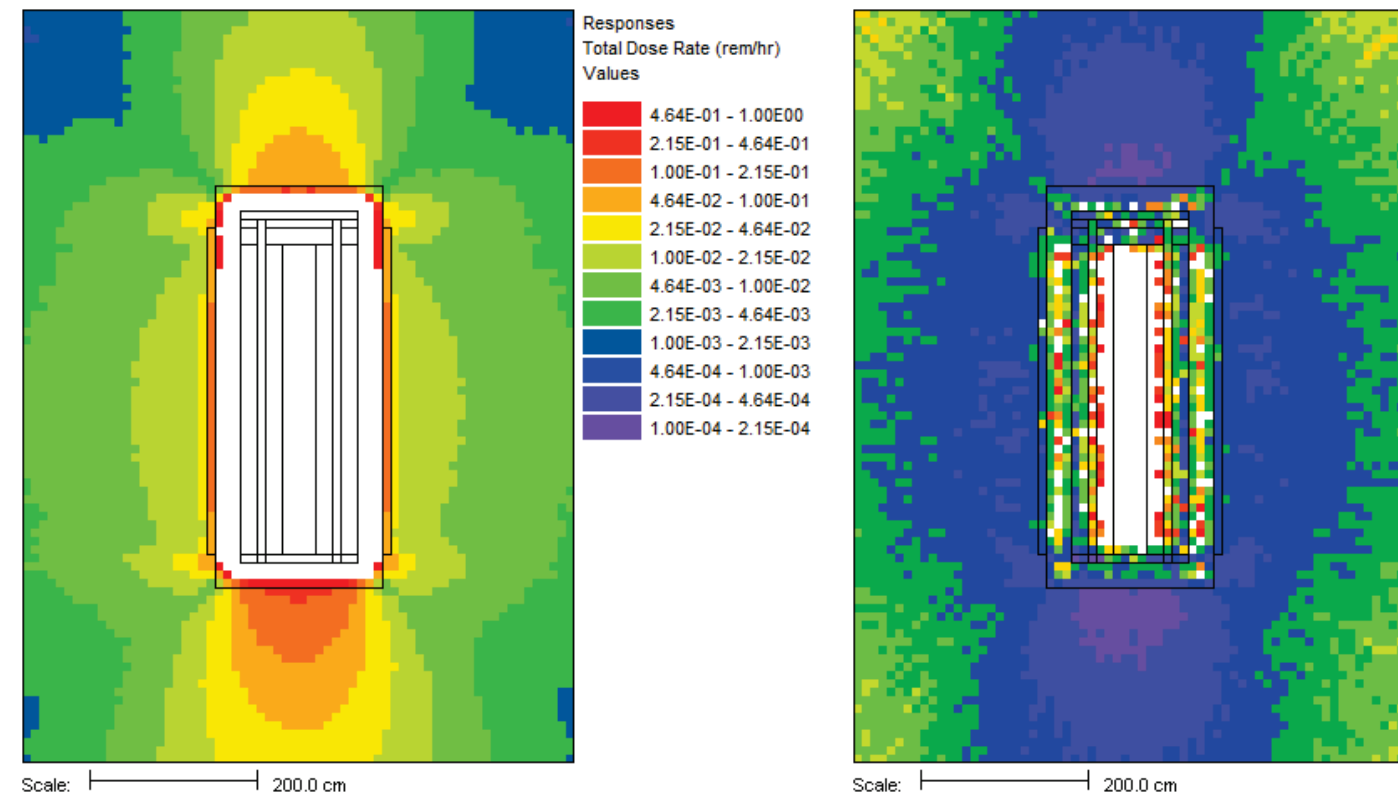

Responses

Total Dose Rate (rem/hr) Relative Uncertainty

$9.00 \mathrm{E}-02-1.00 \mathrm{E}-01$ $8.00 \mathrm{E}-02-9.00 \mathrm{E}-02$ $7.00 \mathrm{E}-02-8.00 \mathrm{E}-02$ $6.00 \mathrm{E}-02-7.00 \mathrm{E}-02$ $5.00 \mathrm{E}-02-6.00 \mathrm{E}-02$ 4.00E-02 - 5.00E-02 $3.00 \mathrm{E}-02-4.00 \mathrm{E}-02$ $2.00 \mathrm{E}-02-3.00 \mathrm{E}-02$ $1.00 \mathrm{E}-02-2.00 \mathrm{E}-02$ $0.00 \mathrm{E} 00-1.00 \mathrm{E}-02$

Figure 4.1.39. Rectilinear mesh tally of total dose rate $(\mathrm{rem} / \mathrm{hr})$ and its relative uncertainty along the $x=0$ plane. 

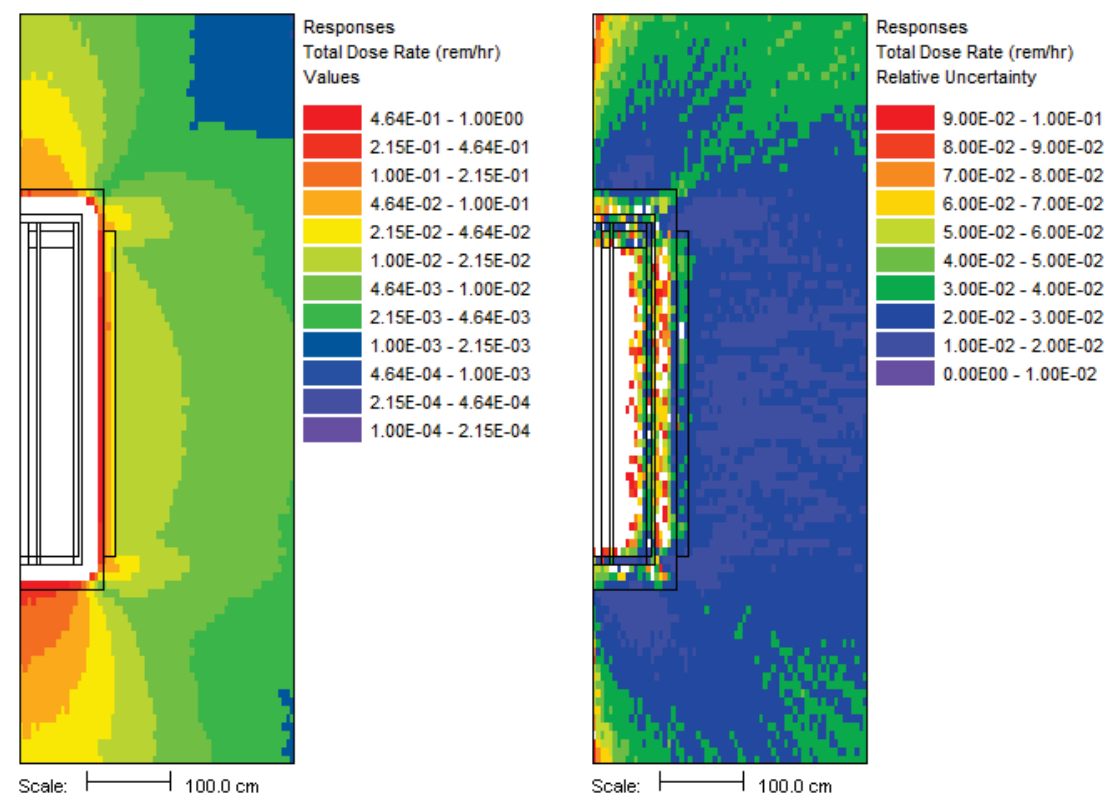

Figure 4.1.40. Cylindrical mesh tally of total dose rate ( $\mathrm{rem} / \mathrm{hr})$ and its relative uncertainty along the $\theta=0^{\circ}$ plane. 


\subsubsection{References}

1. J. C. Wagner, "Acceleration of Monte Carlo Shielding Calculations with an Automated Variance Reduction Technique and Parallel Processing," Ph.D. Dissertation, Pennsylvania State University, University Park, Pennsylvania, 1997.

2. J. C. Wagner and A. Haghighat, "Automated Variance Reduction of Monte Carlo Shielding Calculations Using the Discrete Ordinates Adjoint Function," Nucl. Sci. Eng., 128, 186-208 (1998).

3. J. C. Wagner, "An Automated Deterministic Variance Reduction Generator for Monte Carlo Shielding Applications," Proc. of the American Nuclear Society $12^{\text {th }}$ Biennial Radiation Protection and Shielding Division Topical Meeting, April 14-18, 2002, Santa Fe, New Mexico.

4. A. Haghighat and J. C. Wagner, "Monte Carlo Variance Reduction with Deterministic Importance Functions," Prog. Nucl. Energy, 42(1), 25-53, (2003).

5. T. M. Evans, A. S. Stafford, R. N. Slaybaugh, and K. T. Clarno, "Denovo: A New ThreeDimensional Parallel Discrete Ordinates Code In SCALE,” Nucl. Technol., 171, 171-200 (2010).

6. D. E. Peplow, "Monte Carlo Shielding Analysis Capabilities with MAVRIC," Nucl. Technol., 174, 289-313 (2011).

7. J. C. Wagner, D. E. Peplow, and S. W. Mosher, "FW-CADIS Method for Global and Semi-Global Variance Reduction of Monte Carlo Radiation Transport Calculations," submitted to Nuclear Science and Engineering, 2012.

8. J. S. Warsa, T. A. Wareing, and J. E. Morel, "Krylov Iterative Methods and the Degraded Effectiveness of Diffusion Synthetic Acceleration for Multidimensional $S_{N}$ Calculations in Problems with Material Discontinuities," Nucl. Sci. Eng., 147, 218 (2004).

9. R. S. Baker and K. R. Koch, "An $\mathrm{S}_{\mathrm{N}}$ Algorithm for the Massively Parallel CM-200 Computer," Nucl. Sci. Eng., 128, 312 (1998).

10. M. A. Heroux and J. M. Willenbring, Trilinos Users Guide, SAND2003-2952, Sandia National Laboratories (2003).

11. W. A. Rhoades and R. L. Childs, The TORT Three-Dimensional Discrete Ordinates Neutron/Photon Transport Code, ORNL-6268, Martin Marietta Energy Systems, Oak Ridge National Laboratory (1987).

12. DOORS 3.2A: One, Two, and Three Dimensional Discrete Ordinates Neutron/Photon Transport Code System, Oak Ridge National Laboratory, RSICC ID CCC-650 (2003).

13. A. M. Ibrahim, D. E. Peplow, T. M. Evans, J. C. Wagner, and P. P. H. Wilson, "Improving the Mesh Generation Capabilities in the SCALE Hybrid Shielding Analysis Sequence," Transactions of the American Nuclear Society 100, 302-304 (2009).

14. S. R. Johnson, "Fast Mix Table Construction for Material Discretization," 2013 International Conference on Mathematics \& Computational Methods Applied to Nuclear Science and Engineering (M\&C 2013), Sun Valley, Idaho, May 5-9, 2013.

15. K. D. Lathrop, "Ray Effects in Discrete Ordinate Equations," Nucl. Sci. Eng., 32, 357-369 (1968).

16. K. D. Lathrop, "Remedies for Ray Effects," Nucl. Sci. Eng., 45, 255-268 (1971).

17. R. P. Gardner and K. Verghese, "Monte Carlo Nuclear Well Logging Benchmark Problems with Preliminary Intercomparison Results," Nucl. Geophys. 5(4), 429-438 (1991). 
18. J. C. Wagner, D. E. Peplow, and T. M. Evans, "Automated Variance Reduction Applied to Nuclear Well-Logging Problems," Nucl. Technol. 168, 799-809 (2009).

19. D. Wiarda, M. E. Dunn, D. E. Peplow, T. M. Miller, and H. Akkurt, Development and Testing of ENDF/B-VI.8 and ENDF/B-VII.0 Coupled Neutron-Gamma Libraries for SCALE 6, NUREG/CR-6990 (ORNL/TM-2008/047), prepared for the U.S. Nuclear Regulatory Commission by Oak Ridge National Laboratory, Oak Ridge, Tenn., February 2009. 


\subsection{A CAAS Capability}

\subsection{A.1 Introduction}

Modeling criticality accident alarm systems (CAAS) presents challenges since the analysis consists of both a criticality problem and a deep-penetration shielding problem. ${ }^{1,2}$ Modern codes are typically optimized to handle one of those types of problems but usually not both. The two problems also differ in scale - the criticality problem depends on materials relatively close to the fissionable materials while the shielding problem can cover a much larger range. SCALE now contains fully three-dimensional tools to perform both parts of a CAAS analysis.

CAAS analysis can be performed with SCALE using the KENO-VI criticality code and the MAVRIC shielding sequence. First, the fission distribution (in space and energy) is determined by KENO-VI. This distribution is saved to a file using a user-specified three-dimensional mesh grid and an energy structure from the cross section library (or a user-defined energy structure). MAVRIC then uses the fission distribution as the source for a shielding calculation. MAVRIC is designed to implement advanced variance reduction methods to calculate dose rates or detector responses for difficult shielding problems.

For different types of shielding and different combinations of sources and detector locations, different strategies can be used within SCALE. ${ }^{3,4}$ Due to the way that cross sections for neutron reactions that create photons are stored in ENDF, some of the parameters used in the CAAS capability have changed since SCALE 6.1. Please be sure to understand and follow the new guidance for the correct accounting of secondary photons from neutron reactions. ${ }^{5,6,7}$

\subsection{A.2 Methods}

The CAAS capability in SCALE is a two-step approach using KENO-VI and MAVRIC. The first step is the determination of the source distribution, done with the CSAS6 sequence which uses the KENO-VI functional module. Along with calculating the system $k_{\text {eff, }}$ KENO-VI has been modified to now accumulate the fission distribution over the non-skip generations. This information is collected on a threedimensional Cartesian mesh that overlays the physical geometry model and is saved as a Monaco mesh tally file. A utility program is used to convert the mesh tally into a Monaco mesh source.

The mesh source is then used in the second step as the source term in MAVRIC. The absolute source strength is set by the user based on the total number of fissions (based on the total power released) during the criticality excursion. Further neutron multiplication should be prevented in the MAVRIC transport calculation. Because the fission neutrons have already been accounted for in the KENO-VI calculation, failure to suppress neutron multiplication in the MARVIC sequence would lead to incorrect flux estimates. In addition, if further fissions were allowed, Monaco would add neutrons to its particle bank faster than they could be removed (since the system is at or above critical) and the simulation may never finish.

For the transport part of a CAAS analysis, MAVRIC can be optimized to calculate one specific detector response at one location using CADIS or can be optimized to calculate multiple responses/locations with roughly the same relative uncertainty using FW-CADIS. For calculating mesh tallies of fluxes or dose rates, MAVRIC also uses FW-CADIS to help balance the Monaco Monte Carlo calculation such that low flux voxels are computed with about the same relative uncertainty as high flux voxels.

With this two-step approach, users will have a great deal of flexibility in modeling CAAS problems. The CSAS6 step and the MAVRIC step could both use the same geometry and materials definitions or could 
have different levels of detail included in each. The fission source distribution from one CSAS6 calculation could be used in a number of different MAVRIC building/detector models, with each MAVRIC calculation being optimized for a given type of detector.

\subsection{A.3 User input}

The user can create either one input file containing both the CSAS6 and MAVRIC calculations or can create two input files - one for each sequence. The materials and geometry for these two models could be the same but do not have to be. For example, the CSAS6 sequence might only contain the materials and geometry important to the criticality calculation. Note, however, that the critical source geometry and materials should be modeled identically in both problems. This allows users greater flexibility in modeling their specific problems.

\subsection{A.3.1 KENO-VI input}

For the criticality problem, the only extra input a user needs to supply is the keyword "cds=yes" in the parameter block and a spatial mesh around all of the fissionable materials of the problem in its own gridGeometry block. Standard input for KENO-VI is described in the KENO-VI chapter and CSAS6 chapter. The mesh used for the fission source distribution is input using the "read gridGeometry $i d$ " block, where $i d$ is an identification number for that grid. Note that only one grid can be specified, but that may change in the future. The cells of the mesh are specified in each dimension separately by either (1) listing all of the planes bounding the cells (keyword "xplanes" followed by an "end"), (2) using keyword "xLinear $n a b$ " to specify $n$ cells between $a$ and $b$, or by (3) specifying the minimum plane, the maximum plane, and how many cells to make in that dimension ("xmin=", "xmax=", "numXCells="). The keywords "xplanes" and "xLinear" can be used together and multiple times. Similar keywords are used for the $y$ - and $z$-dimensions. An example CSAS6 input file that collects the fission distribution information would be as follows:

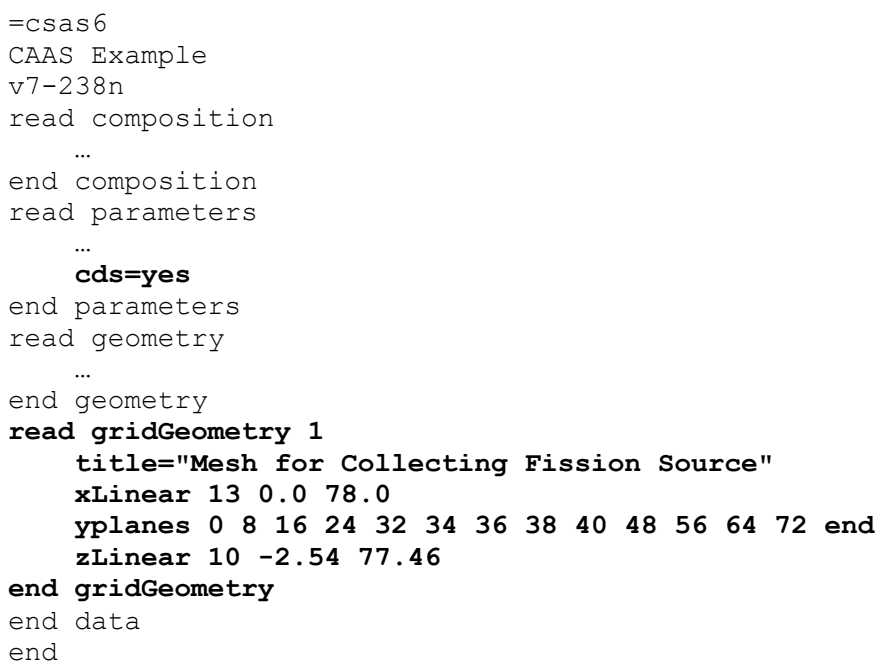

The fission source distribution collected by KENO-VI is saved to a Monaco mesh tally file and copied back to the home area with the name "problemName.fissionSource.3dmap". This file can be viewed with the Mesh File Viewer capability of Fulcrum that comes with SCALE. Note that the finer the mesh spacing is the more generations/histories will have to be simulated by the criticality calculation in order to reduce the stochastic uncertainty in each mesh voxel of the distribution. Regardless of the mesh size, creation of a fission mesh source file will take more iterations than the number required to find $k_{e f f}$. 
KENO-VI also saves the value of the system $\bar{v}$, the average number of neutrons per fission, in a file called "problemName.kenoNuBar.txt". This value is needed to properly determine the source strength.

\subsection{A.3.2 Mesh tally to mesh source conversion}

A utility program is used to convert the Monaco mesh tally file into a Monaco mesh source file. It can be part of the CSAS6 input file. The user then needs to copy the resulting *.msm file back to his home area.

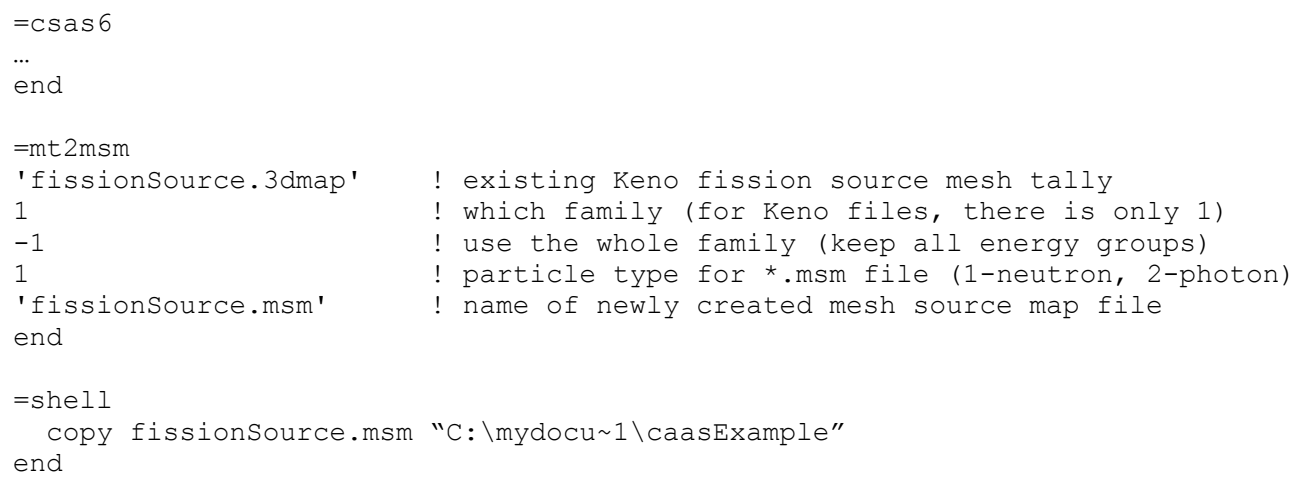

Details on the conversion utility program are contained in Appendix B of the MAVRIC manual.

In SCALE 6.1, the fission source distribution mesh tally produced by KENO contained data representing the number of fissions in each mesh cell in each energy group. In SCALE 6.2, the data stored was changed to be the fissions per unit volume - the fission density. This is more consistent with other mesh tallies from Monaco which store flux or dose rates that represent averages over the mesh cells. This change also allows the Mesh File Viewer to display the KENO fission source distribution better. The $\mathrm{mt} 2 \mathrm{msm}$ utility program also changed from SCALE 6.1 to SCALE 6.2 to account for the change in what is stored in the Keno mesh tally file. Therefore, KENO-produced fission source mesh tallies and the mt2msm utility should not be mixed-and-matched across versions of SCALE. Doing so would result in the final Monaco mesh source file being improperly normalized, which would not represent the KENO fission source distribution and would give incorrect results in subsequent MAVRIC calculations. Because there is not a specific 'version flag' in a mesh tally file or mesh source map file, the user must ensure that they have used the same version of SCALE for both the CSAS6 and MAVRIC sequences any time the CAAS capability is used.

\subsection{A.3.3 MAVRIC input}

The input for the MAVRIC portion of the CAAS problem should include the materials and geometry of the criticality model, use the fission distribution as a source, set the source strength, and set any optional modifiers to the source to change its location or add fission photons. The cross section library used by the MAVRIC calculation does not need to have the same group structure as the fission distribution. MAVRIC will automatically convert the fission source group structure to match the group structure of its cross section library.

The shielding calculation needs to specify that the source is the fission distribution file, which is typically "fissionSource.msm". The total source strength can be specified by either the number of fissions in the criticality accident (fission rate or total number) or by the number of released neutrons (the fission rate multiplied by $\bar{v}$ per fission). The value of $\bar{v}$ will be read from the file "kenoNuBar.txt" in the SCALE 
temporary directory if it is not given in the source input with the keyword "nu-bar=". The mesh source can also be placed at different coordinates in the geometry using the "origin $\mathrm{x}=x \mathrm{y}=y \mathrm{z}=z$ " keywords, if a different reference frame was used with the criticality geometry model that created the mesh source. Rotations of mesh sources are not available at this time. It is also recommended to use filters in the source block to define the source, such as the 'mixture=' filter to only allow source sampling from a specific mixture since the mesh source can be transformed from it's original origin or meshes can cover nonfissionable materials.

For example, using a KENO-VI fission distribution, placing it somewhere in the MAVRIC model and setting the source strength (in neutrons/s) to correspond to $10^{17}$ fission/s would look like

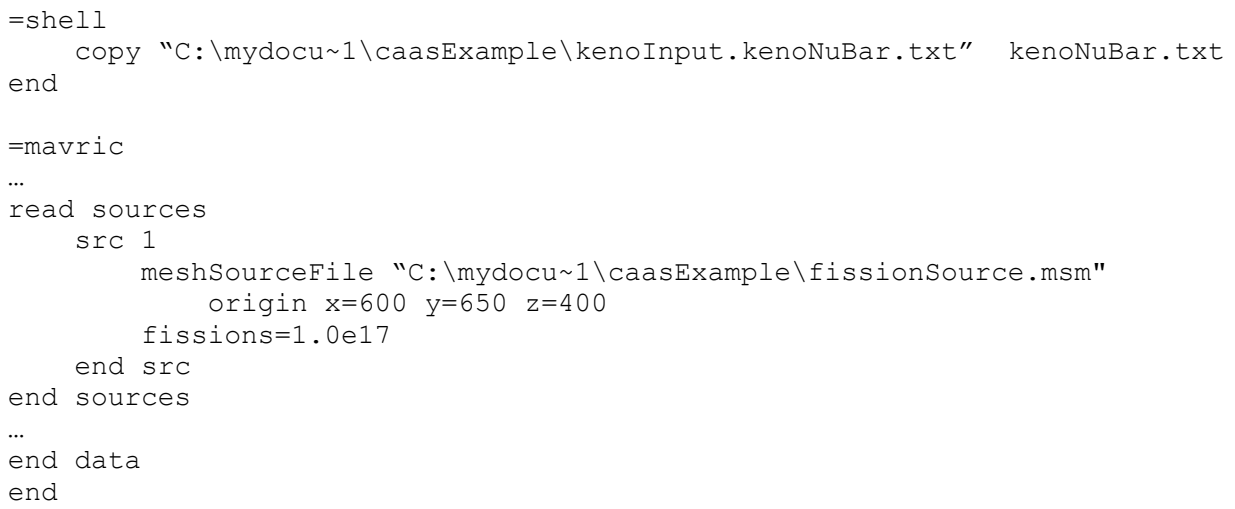

The source strength in neutrons/s will be calculated by MAVRIC to be the fission rate multiplied by the value of $\bar{v}$ read from the "kenoNuBar.txt" file. The neutron strength could have alternatively been specified using the standard source strength keyword "strength $=2.5 \mathrm{e} 17$ " (for an example with the average number of neutrons per fission of 2.5).

The Monte Carlo functional module used by MAVRIC, Monaco, is a fixed-source code. Unless told otherwise, neutrons will multiply in fissionable materials. Since all of the neutrons were part of the source, neutron multiplication should not be allowed and MAVRIC should be run with the keyword "fissionMult $=0$ " in the parameters block. For systems at or near critical without the "fissionMult=0" keyword, Monaco simulations may not end since neutrons will be added to the particle bank at the same rate they leave the system or get killed.

The shielding calculation can be run using standard variance reduction methods (such as path length stretching, user-defined weight windows based on geometry regions, and user-defined source biasing) or using the automated tools which employ approximate discrete-ordinates calculations to determine the space/energy weight windows as well as a biased source distribution in space and energy. The automated tools can be used to optimize the shielding calculation to determine one specific tally using CADIS or several separate tallies or a mesh tally over a large volume of the problem space using FW-CADIS. When using these advanced variance reduction methods, remember to include planes in the discrete-ordinates mesh definition that correspond to the planes in the fission distribution that the source is based on. If they are not included, MAVRIC will resample the fission source on the discrete-ordinates mesh it is using for the importance map, possibly smearing or reducing the original resolution of the fission distribution. 


\subsection{A.4 Example problem}

Consider the Jezebel critical plutonium sphere experiment, shown in Figure 4.1.A.1, taking place inside a simple fictitious building, shown in Figure 4.1.A.2. The building has two rooms: an experiment room and a control room. In the control room there is a criticality alarm detector, and it is positioned furthest from the entry to the experiment room. For this example, assume that a criticality excursion results in a total of $10^{18}$ fissions. This example will calculate the neutron and photon doses seen by a detector in the control room, as well as calculate a dose map for the entire building.

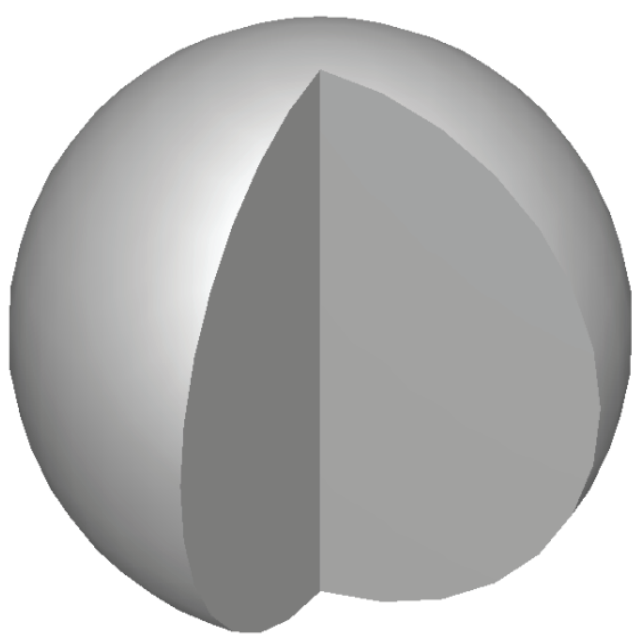

Figure 4.1.A.1. Cutaway view of Jezebel.

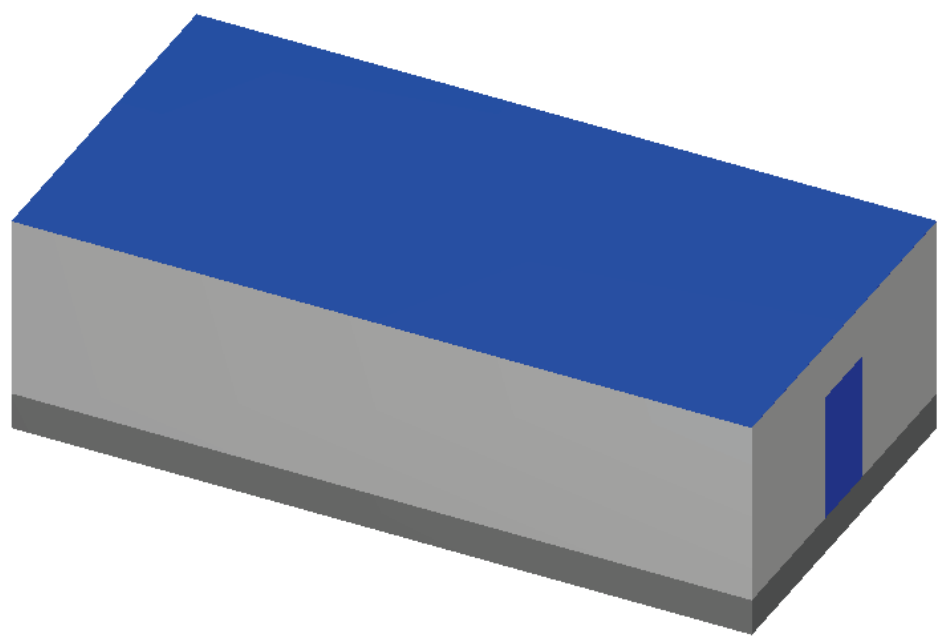

Figure 4.1.A.2. Simple two-room building.

\subsection{A.4.1 KENO-VI criticality and fission source distribution}

For the criticality calculation, consider just a bare sphere of plutonium, with a radius of $6.38493 \mathrm{~cm}$. Atom densities (atoms $/ \mathrm{b} \cdot \mathrm{cm}$ ) for the sphere material are ${ }^{239} \mathrm{Pu} 0.037047 ;{ }^{240} \mathrm{Pu} 0.0017512 ;{ }^{241} \mathrm{Pu}$ 0.00011674; and $\mathrm{Cu} 0.0013752$. This can be easily modeled as a sphere at the origin. For collecting the fission distribution, a uniform mesh grid can be constructed around the sphere, extending $7 \mathrm{~cm}$ in each direction, with a $1 \times 1 \times 1 \mathrm{~cm}$ voxel size. The first portion of the input files mavric.caasA.inp and mavric.caasB. inp looks like the following:

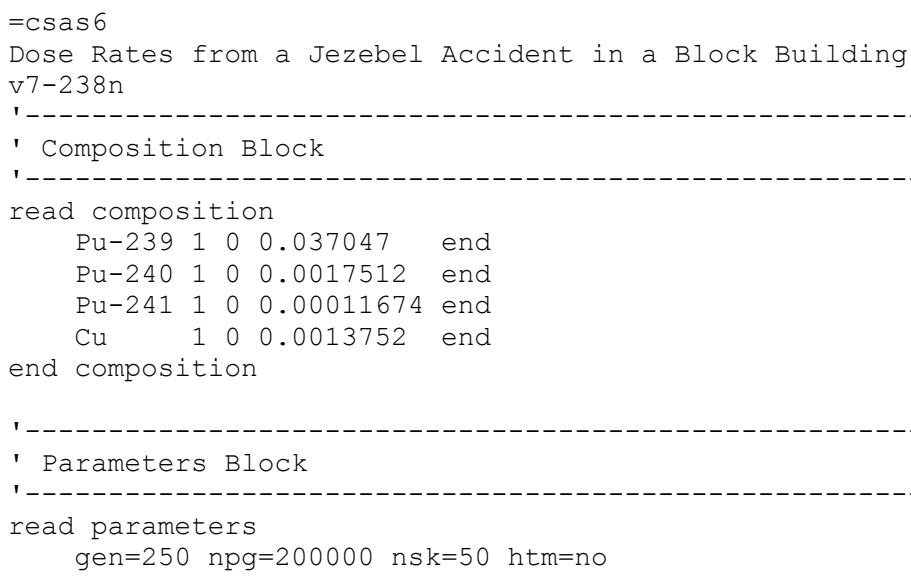




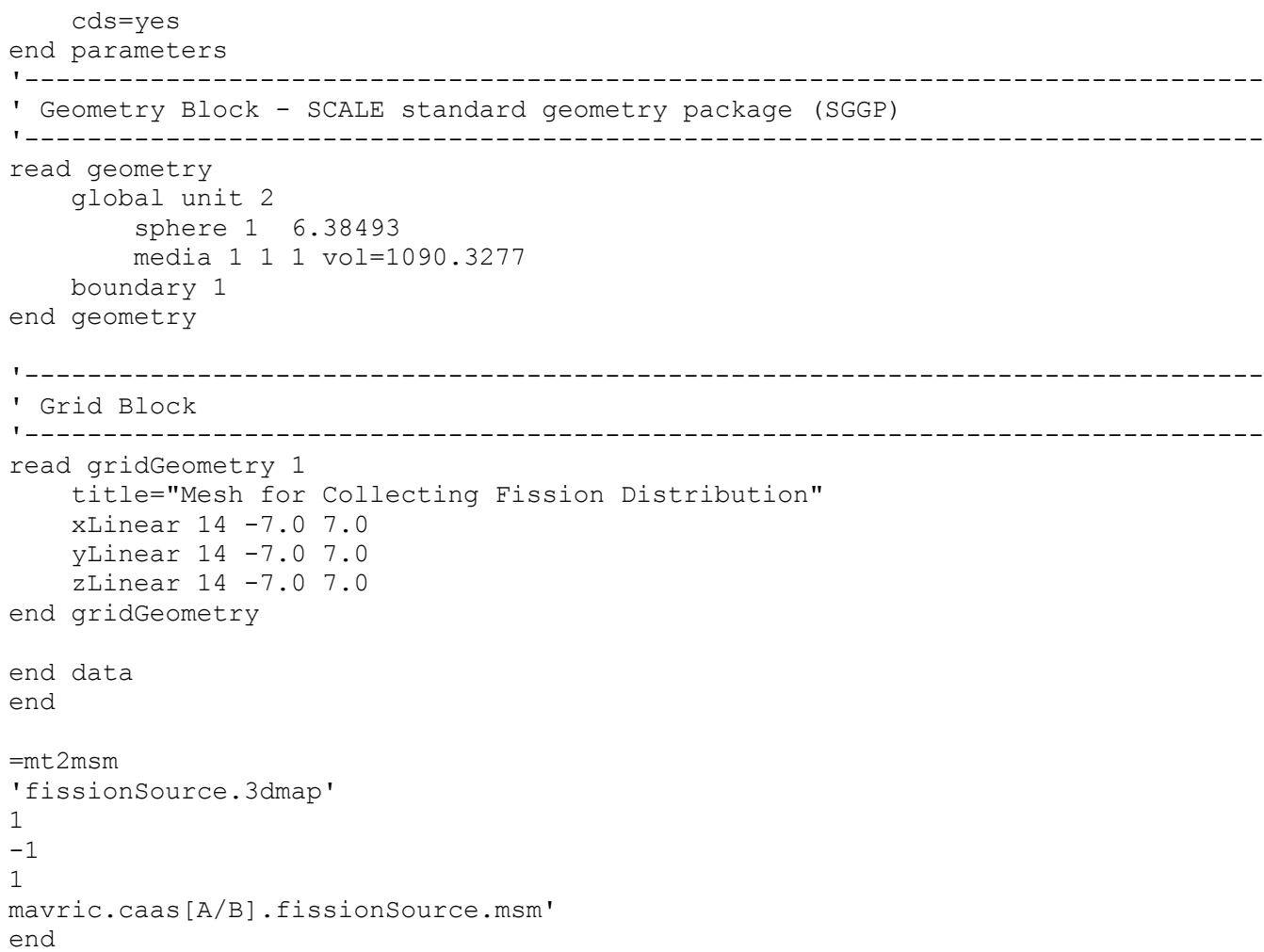

The results of this 26 minute calculation are shown in Table 4.1.A.1, and details about the calculated fission distribution are shown in Figure 4.1.A.3 and Figure 4.1.A.4.

Table 4.1.A.1. Results of the CSAS6 calculation

\begin{tabular}{clcc}
\hline & \multicolumn{1}{c}{ Quantity } & Value & Uncertainty \\
\hline$k_{\text {eff }}$ & best estimate system k-eff & 1.00024 & 0.00014 \\
$\bar{v}$ & system nu bar & 3.15671 & $4.77938 \mathrm{E}-05$ \\
\hline
\end{tabular}




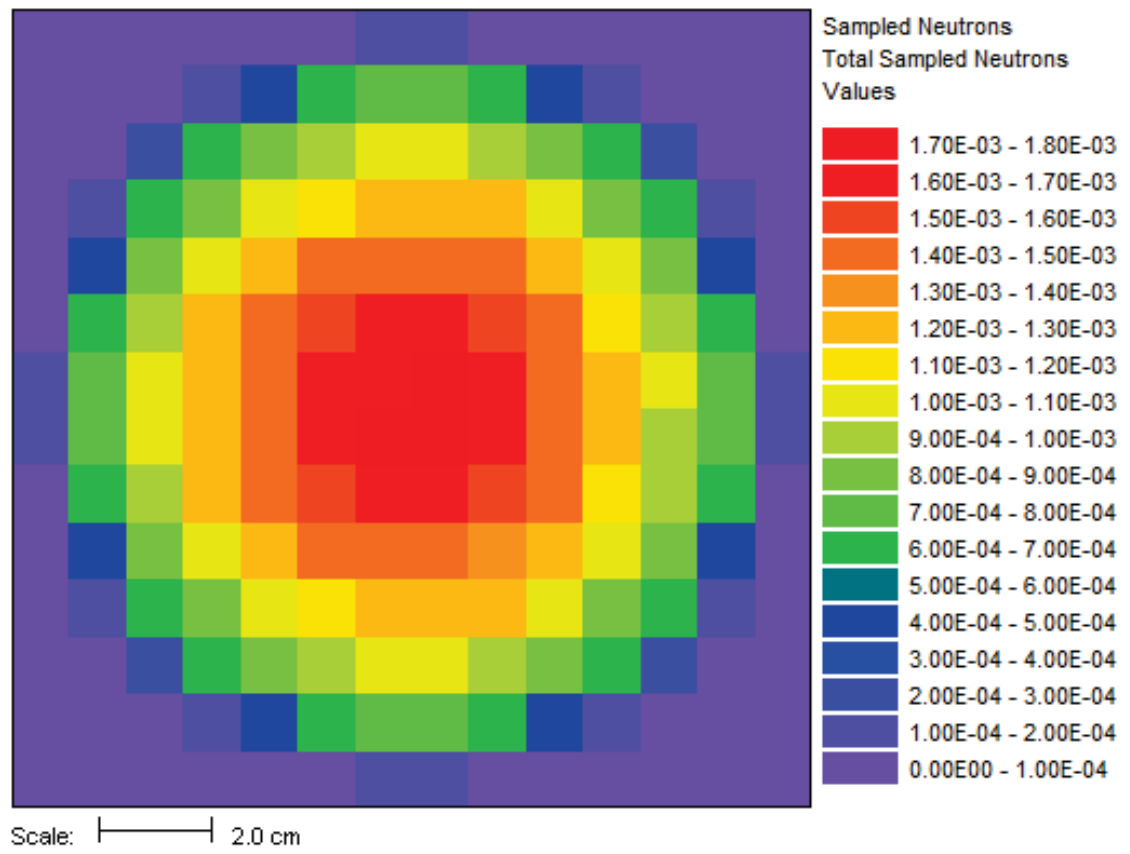

Figure 4.1.A.3. Fission source spatial distribution for the center horizontal slice.

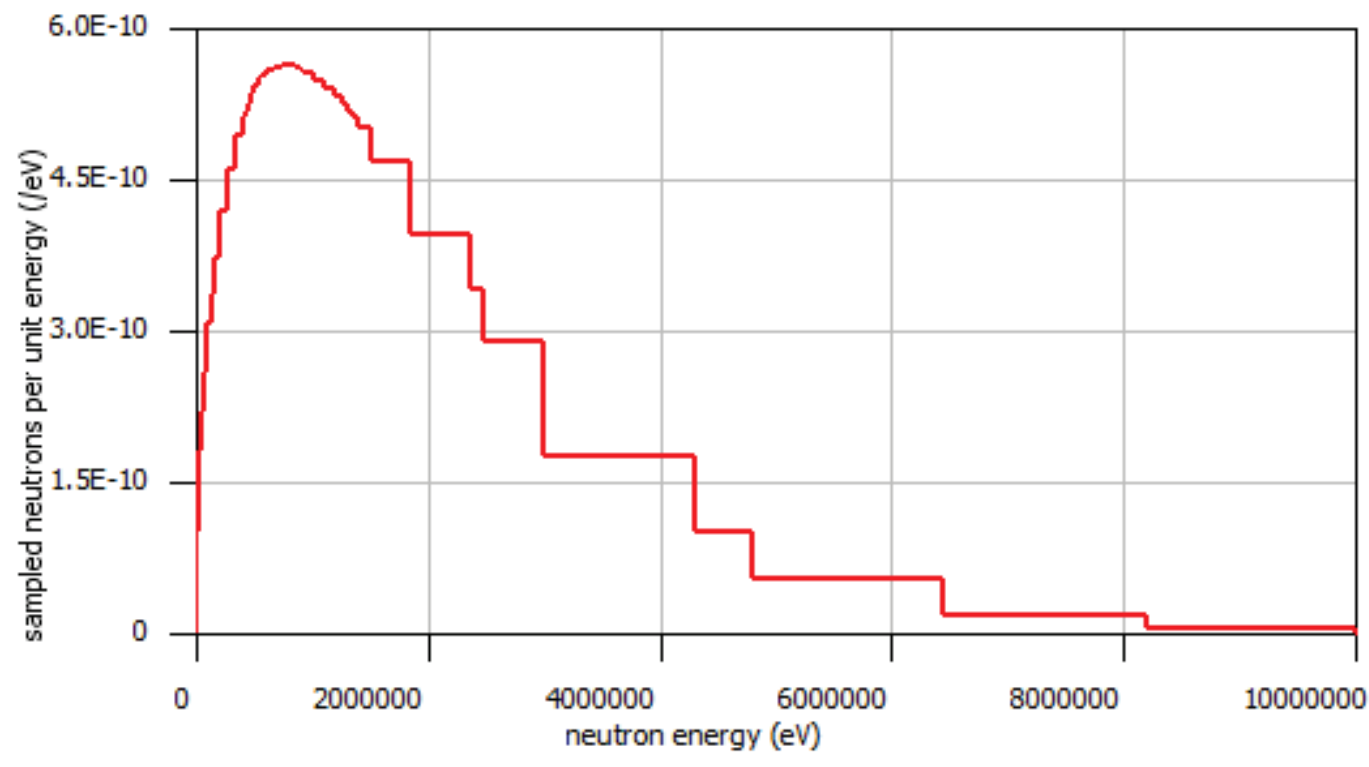

Figure 4.1.A.4. Fission source energy distribution for the center voxel. 


\subsection{A.4.2 MAVRIC transport calculations}

Two MAVRIC calculations will be done-one that calculates the doses seen at the detector and one that computes mesh tallies of doses over the entire building. They will share the same materials, geometry, and source but will have different tally and variance reduction options.

The two-room building will be a simple model using concrete-block walls, a concrete floor, and a steel roof, with dimensions shown in Figure 4.1.A.5. The building exterior dimensions are $1200 \mathrm{~cm}$ long, $600 \mathrm{~cm}$ wide, and $300 \mathrm{~cm}$ high above the ground. The exterior and interior walls are all made of a double layer of typical concrete blocks (total of $40 \mathrm{~cm}$ thick.) Concrete blocks are typically $39 \times 19 \times 19 \mathrm{~cm}$ and weigh $\sim 13.5 \mathrm{~kg}$, since they have a volume fraction of $33.2 \%$. The floor is made of poured concrete, extending $60 \mathrm{~cm}$ into the ground. The roof and the exterior door $(120 \mathrm{~cm}$ wide and $210 \mathrm{~cm}$ tall $)$ are made of $1 / 8$ in. $(0.3175 \mathrm{~cm})$ thick steel. The experiment room on the left connects to the control room on the right through a maze that prevents radiation streaming. Assume that the critical experiment was in the center of the experiment room, $100 \mathrm{~cm}$ above the floor. Assume the detector in the control room is a $30 \mathrm{~cm}$ diameter sphere located at position $(1145,55,285)$.
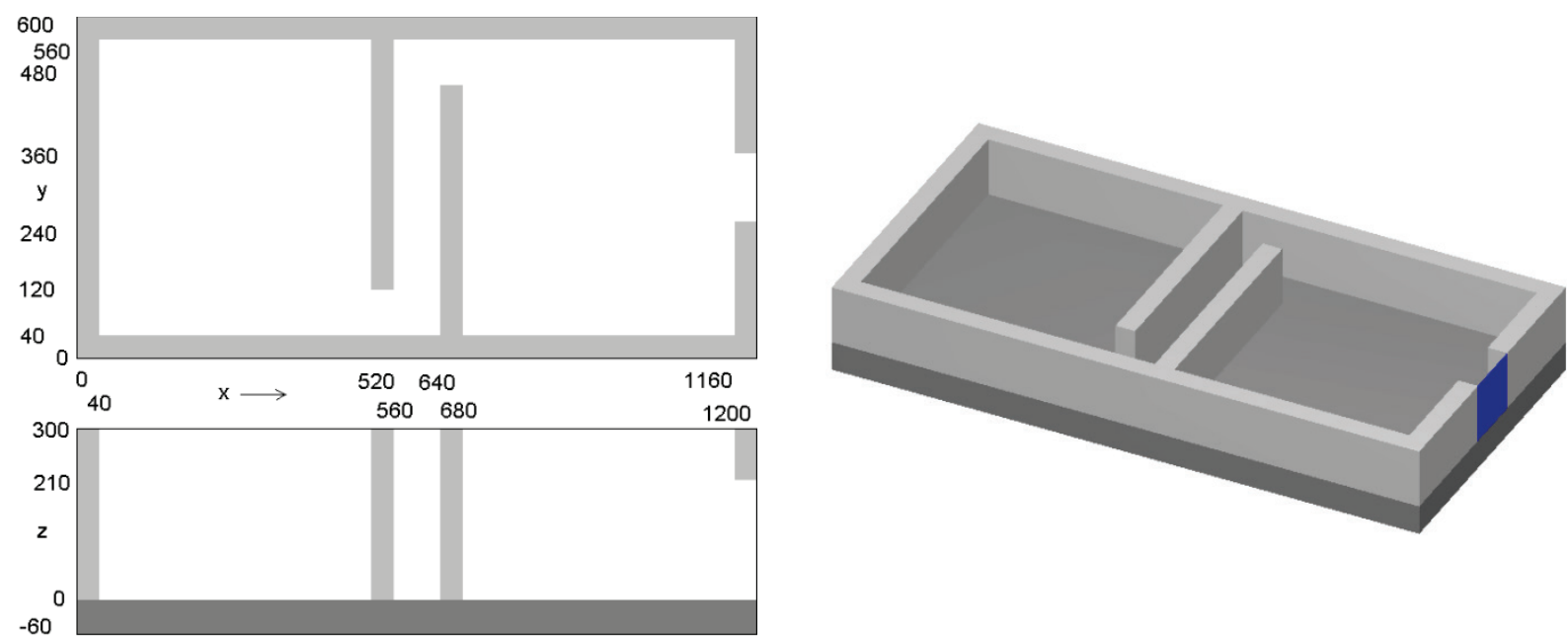

Figure 4.1.A.5. Coordinates of the floor, walls, ceiling, and door of the simple block building model (in cm).

If the MAVRIC transport calculation is not in the same file as the CSAS6 calculation, the MAVRIC input would start by moving the KENO-VI results into the SCALE temporary area:

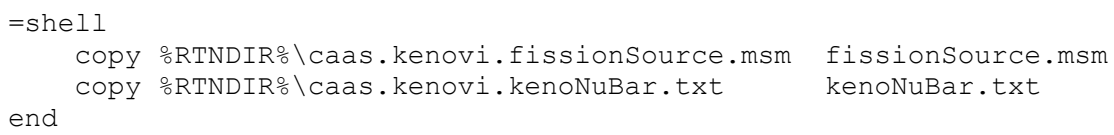

The materials and geometry blocks of the two MAVRIC input files for each of the two calculations, smplprbs/caas.mavricA. inp and smplprbs/caas.mavricB.inp, look like the following: 


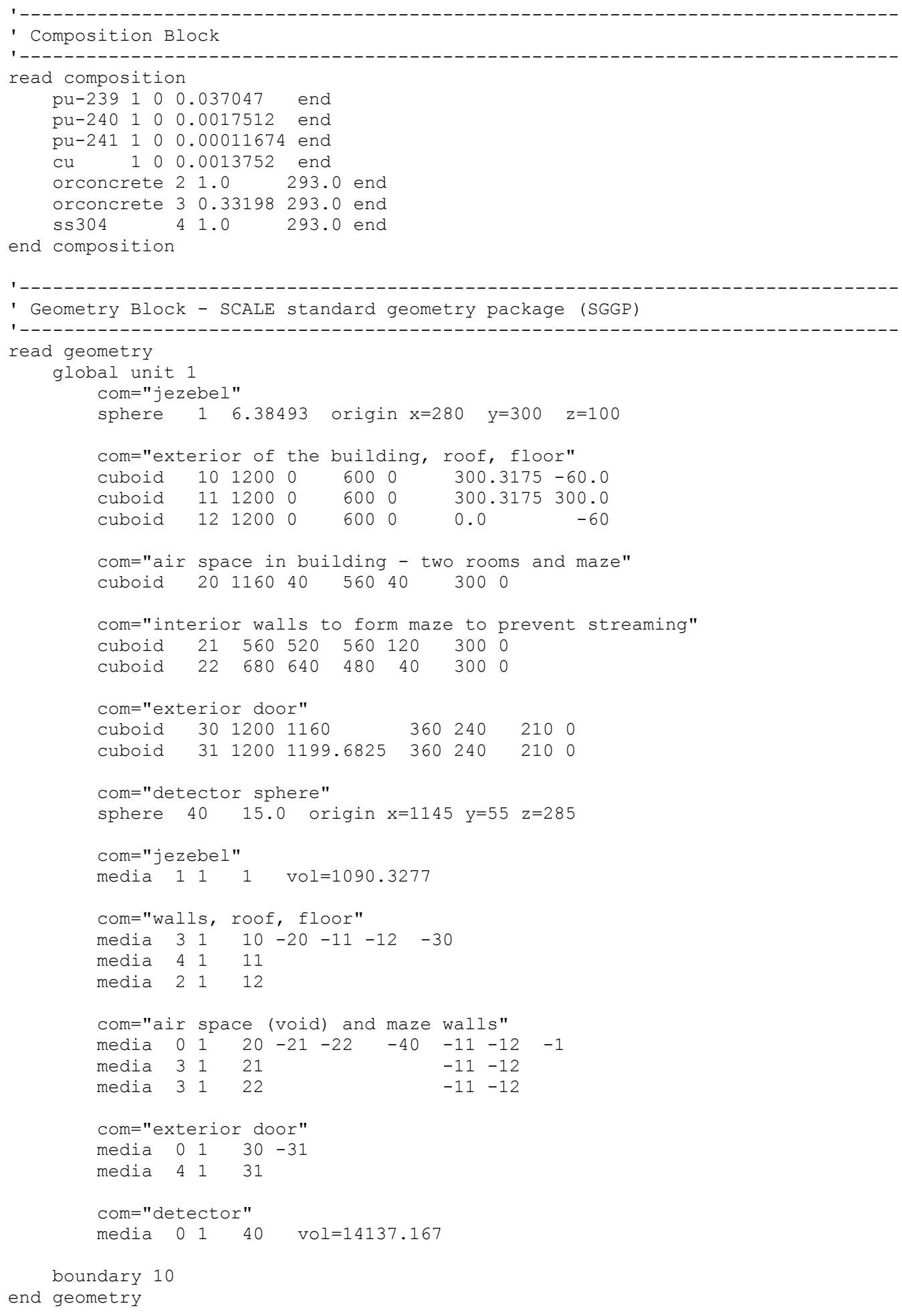

The response functions used to compute the doses will be the standard flux-to-dose rate conversion factors for neutrons and photons. These are defined in the definitions block. Note that these responses have units of $(\mathrm{rem} / \mathrm{hr}) /\left(/ \mathrm{cm}^{2} / \mathrm{s}\right)$. 


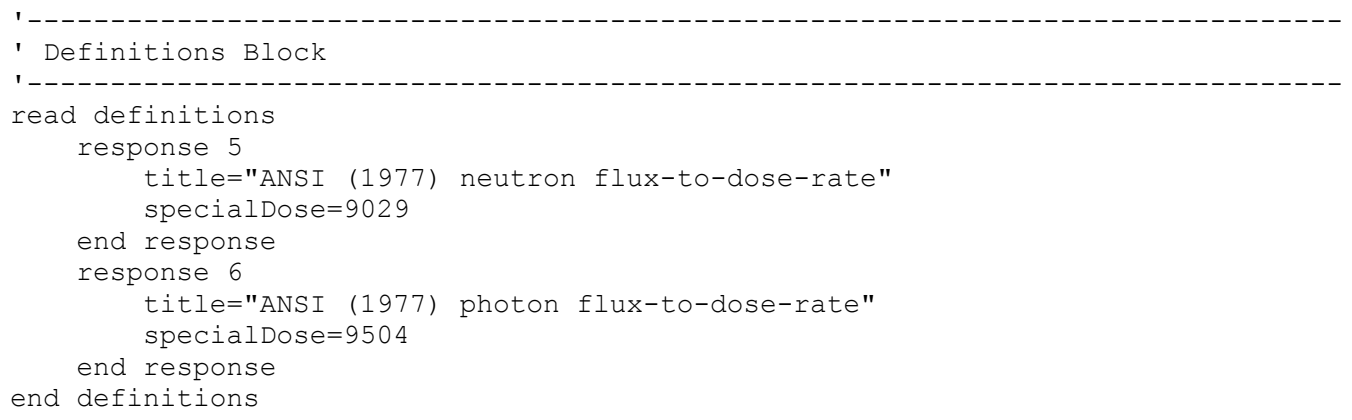

The source used by each MAVRIC simulation will be based on the fission distribution mesh source determined by KENO-VI. The strength of the source can be specified by the total number of fissions that occurred in the criticality event. Fission photons will be added for ${ }^{239} \mathrm{Pu}$. MAVRIC will determine the total source strength, including the fission photons, from the value of $\bar{v}$ saved by KENO-VI and the multiplicity data from the fission photon data file.

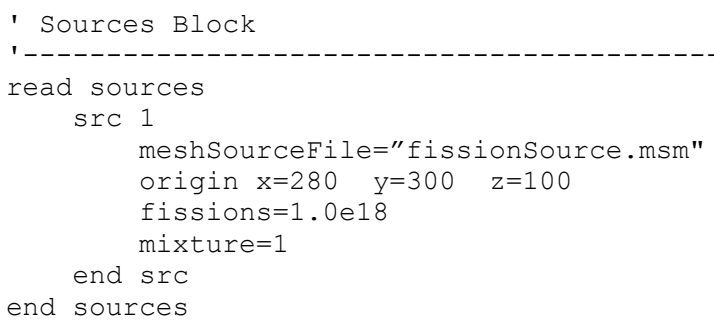

Note that further multiplication needs to be turned off in MAVRIC using the "fissionMult $=0$ " keyword in the parameter block as shown below.

For the responses from the tallies, MAVRIC usually calculates dose rates (rem/hr) using a source strength in particles/s. For this example problem, instead of a source rate, we used a total number of particles (by specifying the number of fissions). Hence, the computed fluxes will have units of particles $/ \mathrm{cm}^{2}$ and the computed responses using the standard dose responses from the cross section libraries will have units of $\mathrm{rem} \mathrm{s} / \mathrm{hr}$. To get a dose in rem, the responses need to be multiplied by $(3600 \mathrm{~s} / \mathrm{hr})^{-1}$. This can be done using the MAVRIC tally multiplier keyword.

Each MAVRIC simulation will need a discrete-ordinates mesh. The planes in each dimension where there are material changes are listed in Table 4.1.A.2. In addition to these planes, the discrete-ordinates mesh should also subdivide the thick shields in the direction of particle travel. For example, the walls of the maze should be divided to better model the radiation attenuation through the walls in the Denovo calculation. The interior walls of the building will reflect particles, so the first few centimeters are the most important to capture in the importance map. Mesh planes should also be added that correspond to the mesh source after it is placed into the geometry model. 
Table 4.1.A.2. Main planes in the building

\begin{tabular}{crc}
\hline $\boldsymbol{x}$ & \multicolumn{1}{c}{$\boldsymbol{y}$} & $\boldsymbol{z}$ \\
\hline 0 & 0 & -60 \\
40 & 40 & 0 \\
520 & 120 & 210 \\
560 & 240 & 300 \\
640 & 360 & 300.318 \\
680 & 480 & \\
1160 & 560 & \\
1199.68 & 600 & \\
1200 & & \\
\hline
\end{tabular}

\subsection{A.4.2.1 Detector doses using CADIS}

The grid geometry for this calculation should also include planes that bound the adjoint source, which is the detector area (these values are shown in boldface below). The definitions block in smplprbs/caas. mavricA. inp also includes the location of the center of the detector, which is used in the adjoint source description.

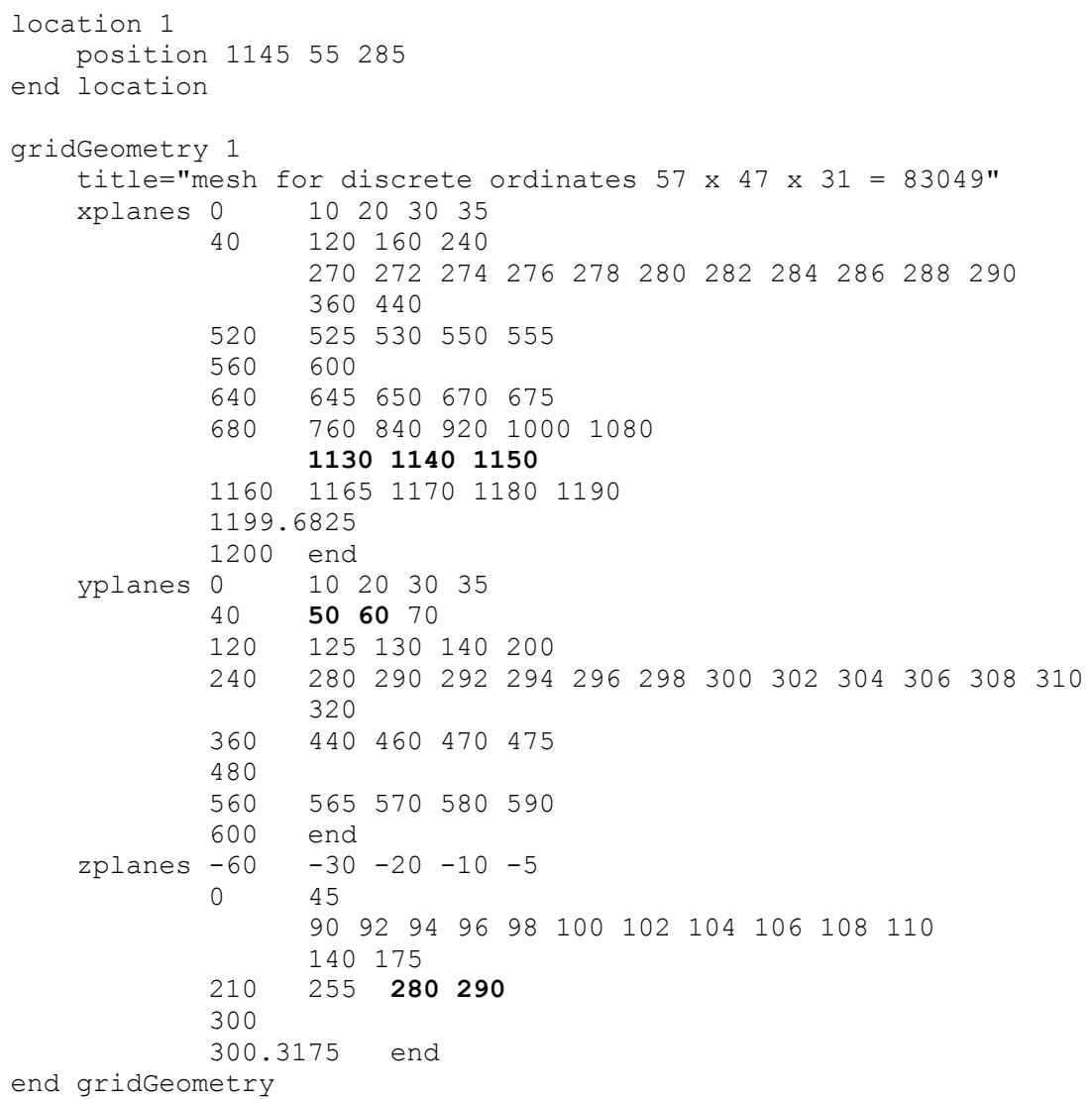

The tallies are region tallies over the detector region (the 10th media card in unit 1) using the appropriate response function for the particle type of the tally. The volume of the detector sphere needs to be listed in 
the geometry block so that the fluxes and tallies will be correctly computed. The importance map uses standard CADIS to bias the particles towards the detectors, optimizing the calculation of the total dose (by listing both response functions together, the total response will be used in the adjoint source).

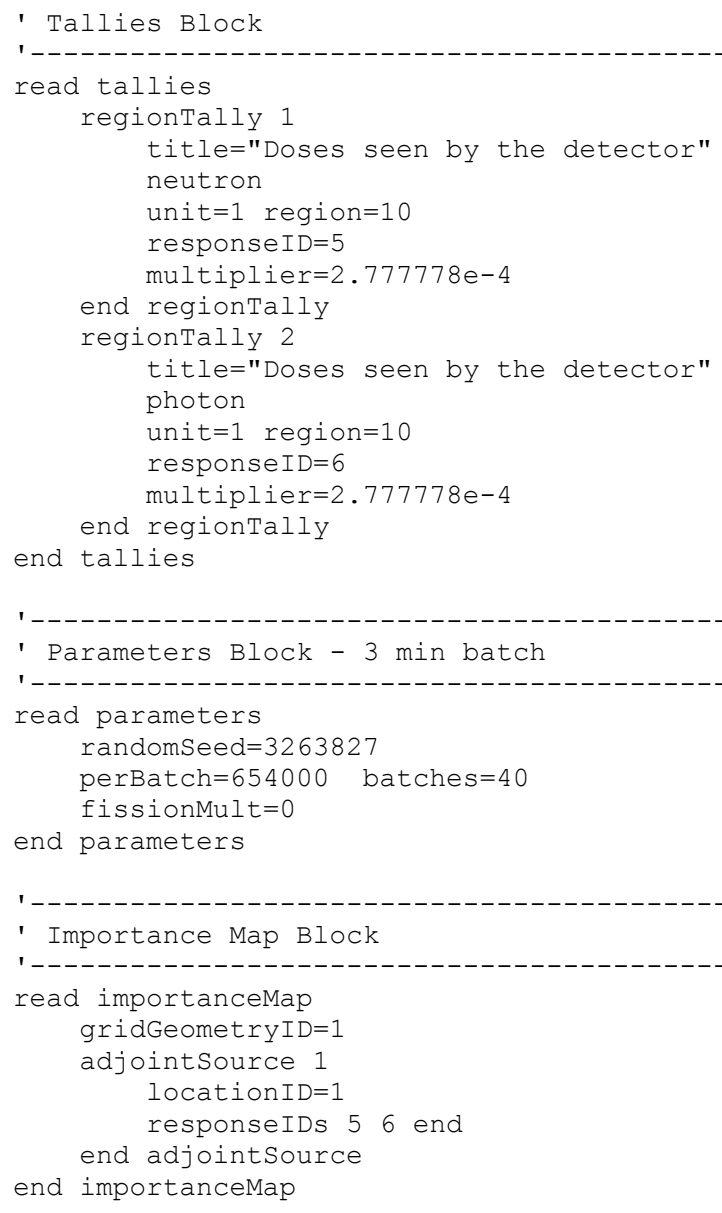

The results of this example problem are shown in Table 4.1.A.3. Calculation times were 12 minutes for Denovo and 135 minutes for Monaco. Note that the uncertainty for the photon dose is much higher than the neutron dose uncertainty. This is because the simulation was optimized for the calculation of total dose, and the photon component of the total dose is less than $2 \%$. Had a separate calculation been done that used an adjoint source of just the photon response, the photon dose rate uncertainty would have been much smaller but at the expense of the neutron dose rate uncertainty. A single calculation could have also been performed using two adjoint sources, one using the neutron dose response and one using the photon dose response, and forward weighting to help calculate each component of dose with more uniform relative uncertainties.

Table 4.1.A.3. Doses seen by the detector

\begin{tabular}{lcc}
\hline Dose & $\begin{array}{c}\text { Value } \\
\text { (rem) }\end{array}$ & $\begin{array}{l}\text { Rel. } \\
\text { Unc. }\end{array}$ \\
\hline neutron & 1539 & $0.78 \%$ \\
photon & 30.0 & $8.00 \%$ \\
\hline
\end{tabular}




\subsection{A.4.2.2 Dose map using FW-CADIS}

The grid geometry for this calculation does not need extra planes around the detector. The grid geometry in smplprbs/caas.mavricB. inp looks like the following:

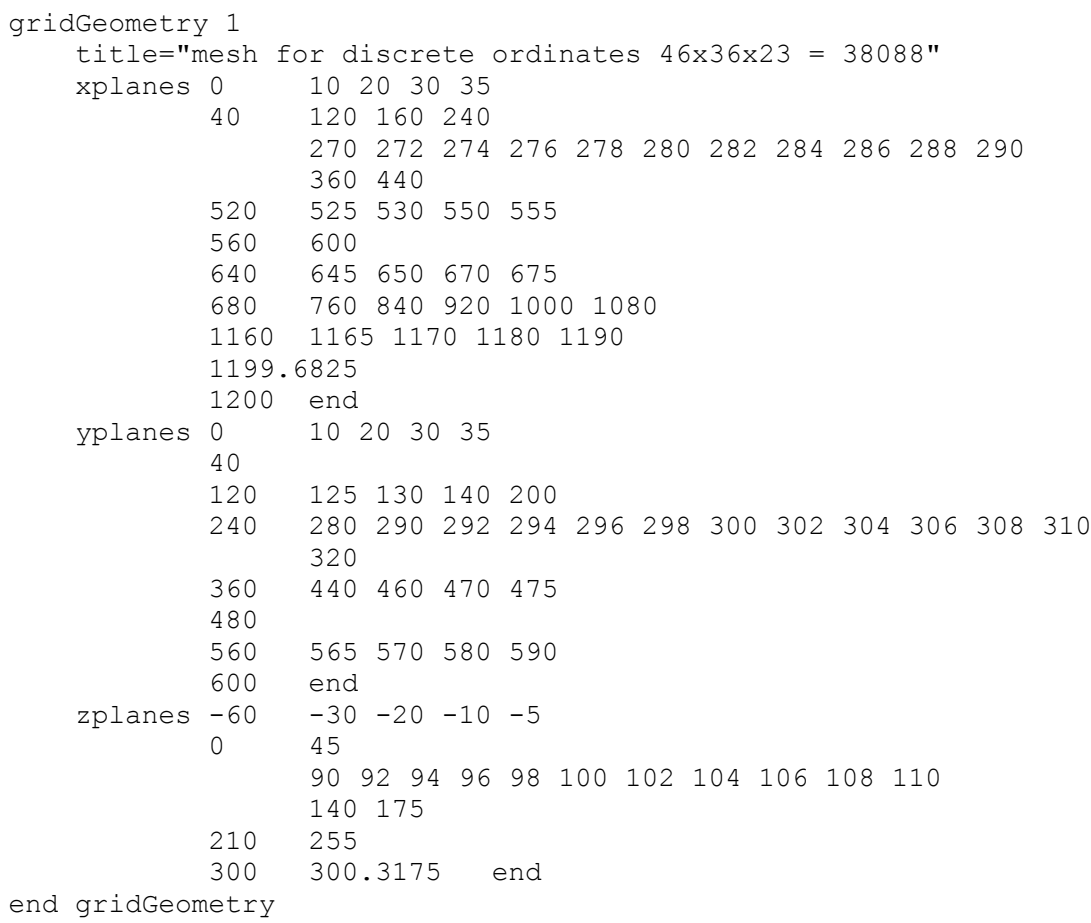

A second grid geometry also needs to be added to the definitions block for the mesh tally to use.

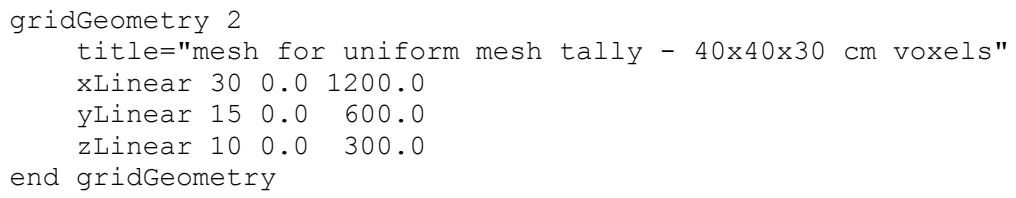

The mesh tallies for each particle type are listed, along with the appropriate response function. The importance map uses FW-CADIS to better spread the particles out over the entire geometry, optimized for the calculation of total dose in the void regions.

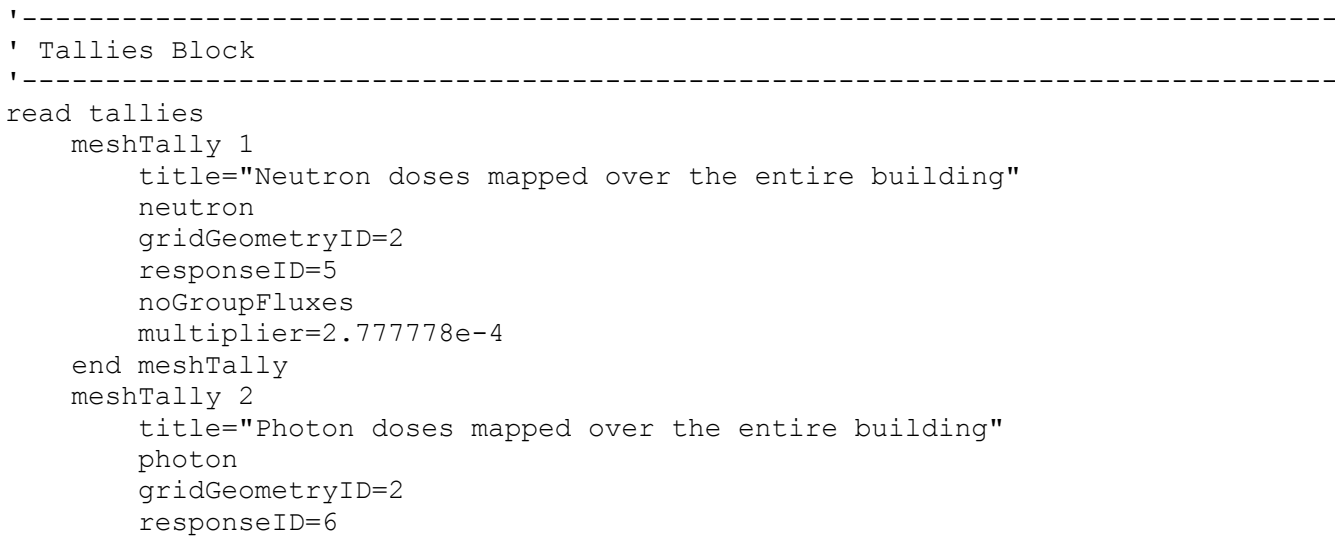




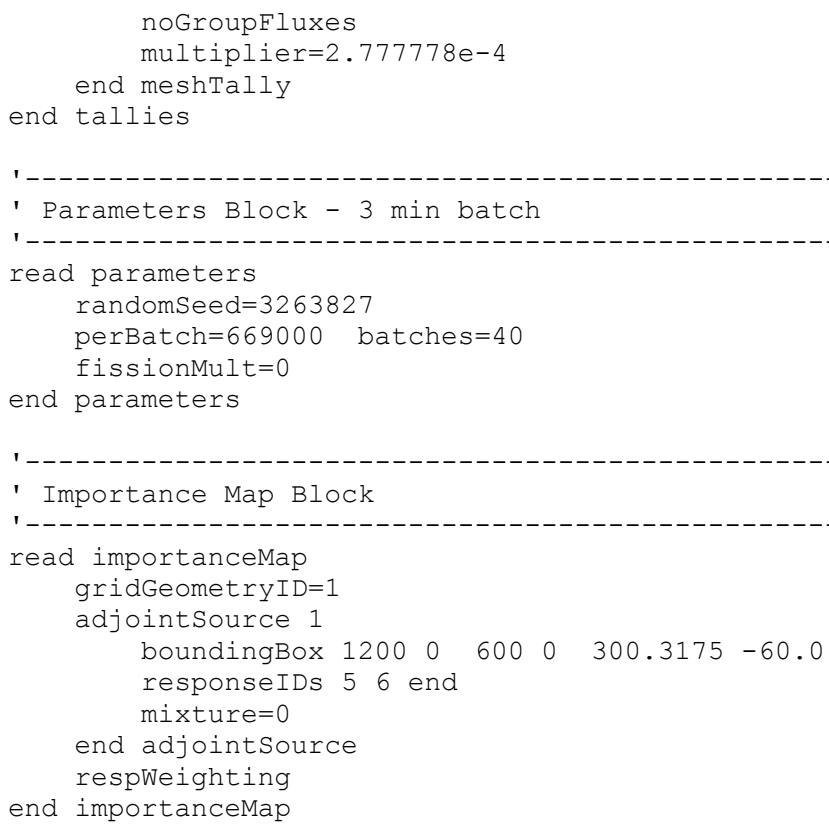

This calculation used 5 minutes for the forward Denovo $\mathrm{S}_{\mathrm{N}}$ calculation, 6 minutes for the adjoint Denovo, and 120 minutes for the Monaco Monte Carlo. The resulting mesh tally is shown in Figure 4.1.A.6. The uncertainties for each voxel are shown in Figure 4.1.A.7. Note that the dose in the voxel containing the detector (not shown in the figures) is $1.552 \times 10^{3}$ rem with a relative uncertainty of $3.8 \%$, closely matching the value calculated with the first MAVRIC simulation.

Similar to the detector doses above, a single calculation could have also been performed for the dose maps using two adjoint sources, one using the neutron dose response and one using the photon dose response, and forward weighting to help calculate each component of dose with more uniform relative uncertainties.

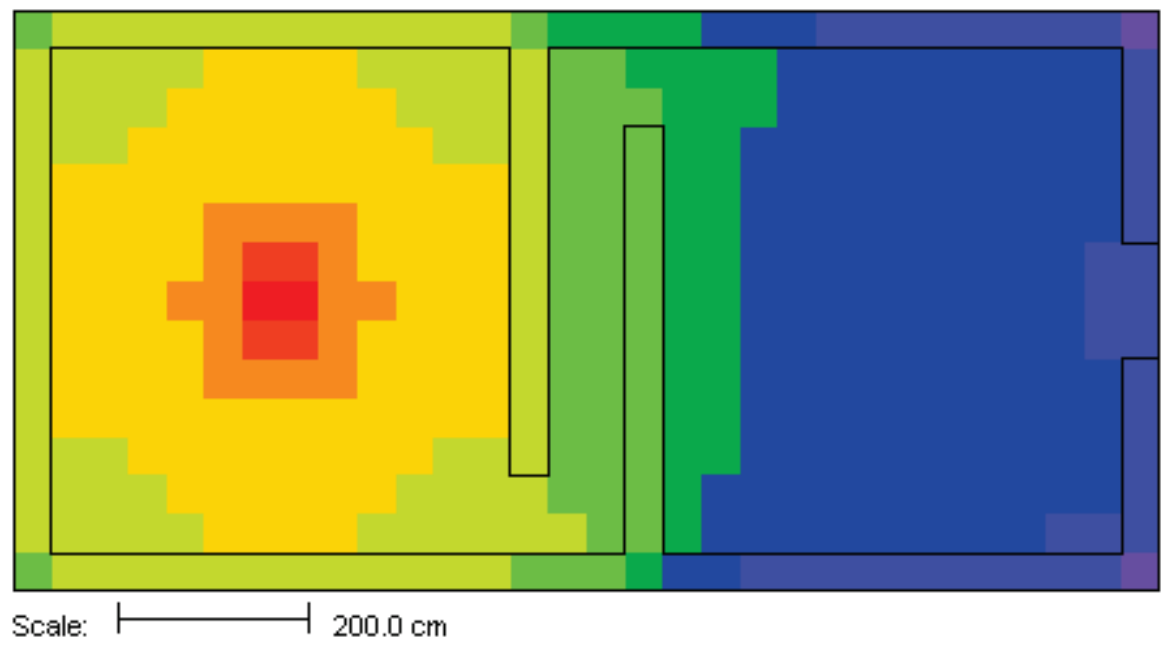

Responses

Total Dose (rem)

Values

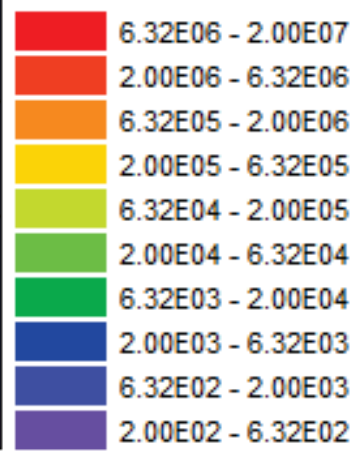

Figure 4.1.A.6. Dose (rem) results for the $\mathrm{z}=100 \mathrm{~cm}$ plane (containing the source). 


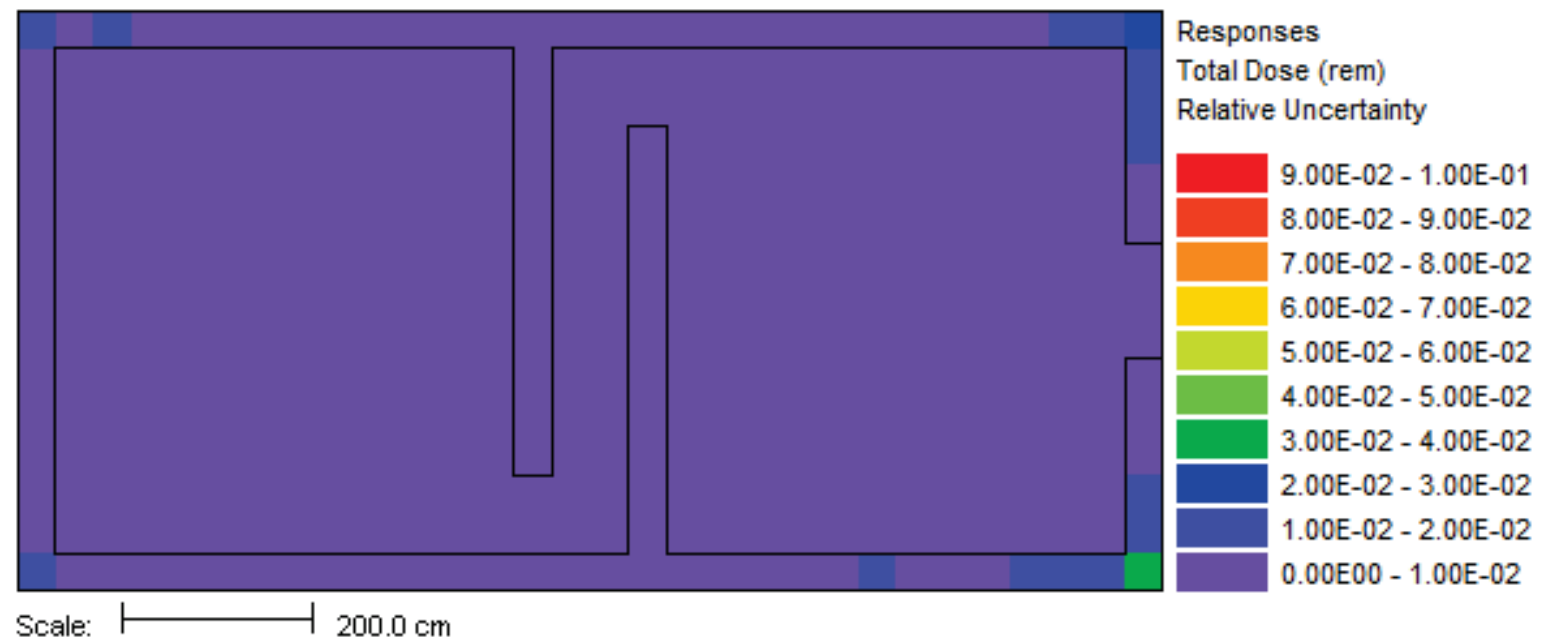

Figure 4.1.A.7. Relative uncertainties in the dose, most less than $1 \%$, for the $z=100 \mathrm{~cm}$ plane.

\subsection{A.5 Summary}

SCALE now has the capability to do detailed simulations of criticality accident alarm systems. The advanced variance reduction capabilities of the MAVRIC radiation transport sequence allow for the full three-dimensional analysis of CAAS problems in reasonable amounts of computer time. This enables the use of more realistic source definitions, such as a detailed spatial/energy dependent fission source distribution determined by the KENO-VI criticality code, and the critical assembly itself can be included in the transport model.

\subsection{A.6 References}

1. D. E. Peplow and Lester M. Petrie, Jr., "Criticality Accident Alarm System Modeling with SCALE," in Proceedings of the 2009 International Conference on Advances in Mathematics, Computational Methods, and Reactor Physics, Saratoga Springs, New York, May 3-7, 2009.

2. D. E. Peplow and L. M. Petrie, Jr. "Modeling Criticality Accident Alarm Systems with SCALE 6.1," Transactions of the American Nuclear Society 102, 297-299 (2010).

3. D. E. Peplow and L. L. Wetzel, "Methods for Detector Placement and Analysis of Criticality Accident Alarm System," Transactions of the American Nuclear Society 106, 497-500 (2012).

4. L. L. Wetzel, B. O'Donnell, and W. D. Newmyer, "CAAS Detector Placement Using MAVRIC," Transactions of the American Nuclear Society 107, 644-647 (2012).

5. T. M. Miller and D. E. Peplow, "Guidance Detailing Methods To Calculate Criticality Accident Alarm Detector Response and Coverage," Nuclear Criticality Safety Topical Meeting, Wilmington, NC, September 29-October 3, 2013, on CD-ROM, American Nuclear Society, LaGrange Park, IL (2013). 
6. T. M. Miller and D. E. Peplow, Guide to Performing Computational Analysis of Criticality Accident Alarm Systems, ORNL/TM-2013/211, August 2013.

7. T. M. Miller and D. E. Peplow, "Corrected User Guidance to Perform Three-Dimensional Criticality Accident Alarm System Modeling with SCALE," Transactions of the American Nuclear Society 108, 498-501 (2013). 


\subsection{B MAVRIC Utilities}

\subsection{B.1 Introduction}

Several utilities are provided to aid users in dealing with some of the output files produced by Monaco and MAVRIC. These utilities were developed at ORNL for specific projects and have been added to SCALE so that all users can benefit. More utilities have been added that deal with Denovo, including the older *.varscl files for flux output used in SCALE 6.1 and the current binary *.dff file for flux output used in SCALE 6.2. These tools do not have the modern block/keyword input structure but instead have a fixed format, which is fairly simple since each utility is made for a very specific function.

Each is described in the following sections. Five sample problems, mavricutilitiesl.inp, mavricUtilities2.inp, mavricutilities3.inp, mavricutilities4.inp and mavricutilities5.inp demonstrate the use of some of these. For all of these utilities, filenames should be enclosed in quotes.

\subsection{B.2 Utilities working with Monaco mesh tally (*.3DMAP) files}

Mesh tally files (*.3dmap) are binary files describing the mesh, optional integer data sets and optional real data sets with their uncertainties organized into related 'families'. The integer data sets are generally used to describe aspects of the geometry or materials of the problem. An example of a family of real data sets would be flux by energy group. Each group flux could have an uncertainty and all of the groups can be added together to make the total flux. These utilities include:

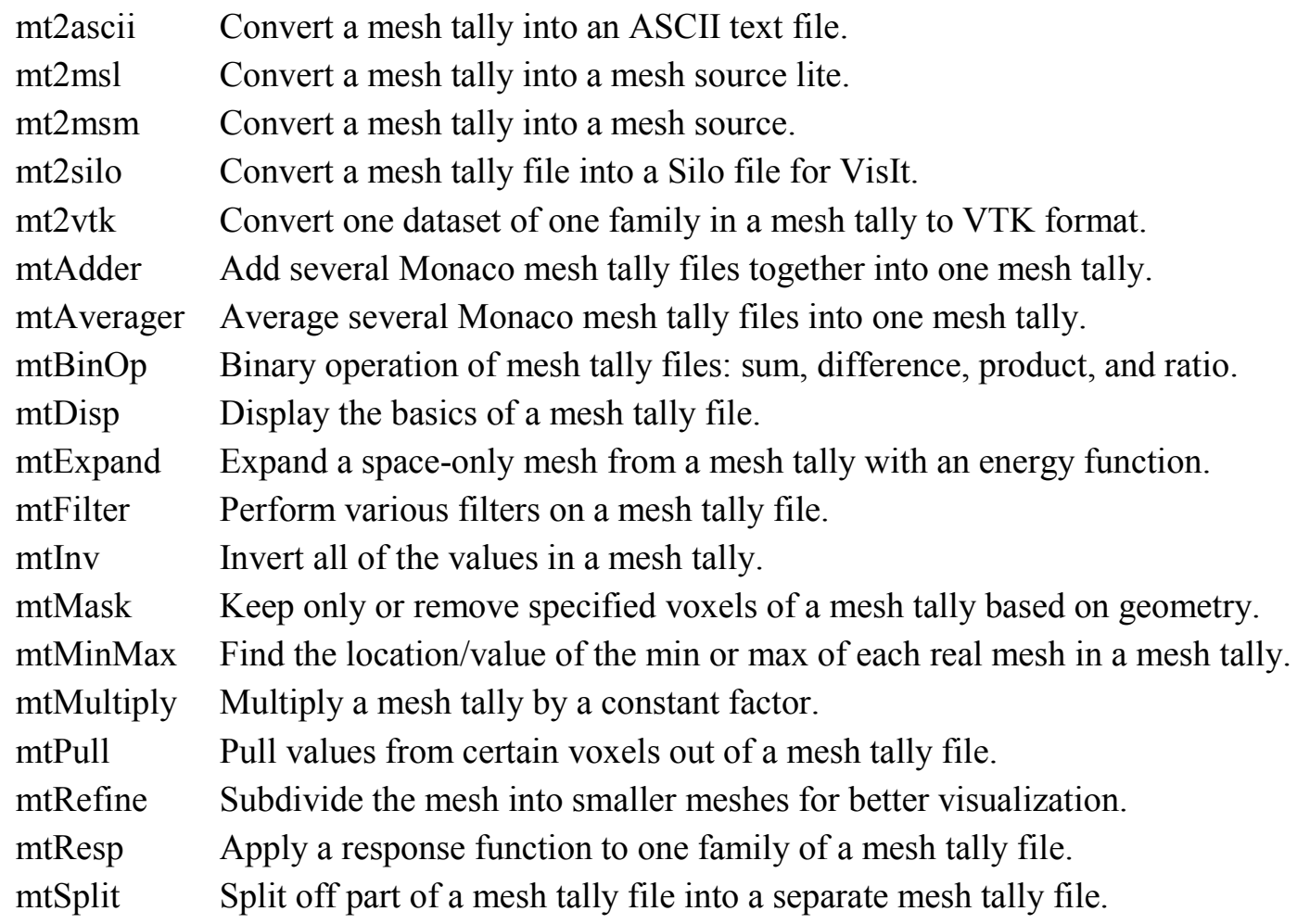

Intended use: Since mesh tally files are in binary, the viewer can be used to list mesh values. To get the values from the entire file, this utility can be used to create an ASCII text version. 
Input: The mesh tally file name and the filename for the resulting ASCII file

Output: An ASCII formatted file

Example:

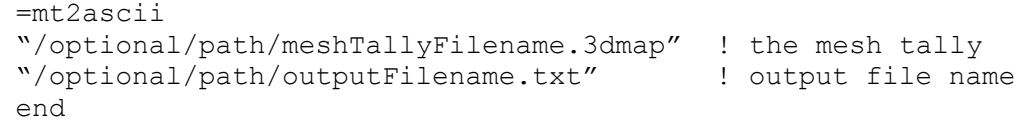

mt2msl - Convert a mesh tally into a mesh source lite.

Intended use: Convert a fissionSource.3dmap mesh tally computed by KENO into a meshSoureLite (*.msl) file that can be used by a subsequent KENO run using starting source type nst $=9$.

Input: Name of mesh tally (*.3dmap) file

Output: A mesh source lite (*.msl) file

Example:

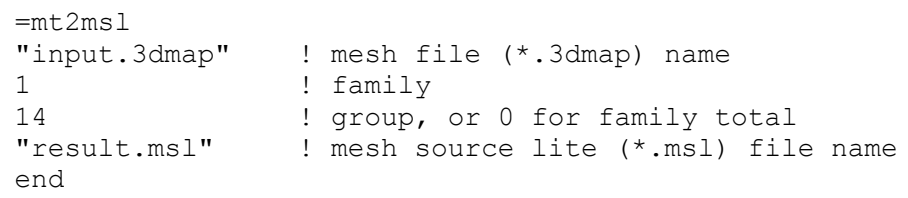

mt2msm - Convert a mesh tally into a mesh source.

Intended use: Turn a tally of fission rate data into a mesh source file. Mesh tallies are stored in a generic *.3dmap format, which consist of several families, each with one or more datasets. A typical mesh tally (without the "noGroupFluxes" keyword) contains three families: the neutron fluxes with each energy group as a dataset, the photon fluxes with each energy group as a data set, and the responses with each response as a dataset. This program uses the spatial information of the mesh tally and combines a usergiven energy distribution for all voxels. A second way to use this program is to use a whole family (all the energy groups) without a user-given energy distribution.

Input:

Line 1: filename of mesh tally

Line 2: which family of tally

Line 3: which dataset of that family (or 0 for sum of family)

Line 4: source type: 1-neutron, 2-photon

Line 5: number of bins for mesh source

Lines : energy $(\mathrm{eV})$ and pdf values

Lines : energy $(\mathrm{eV})$ and pdf values

Lines : energy $(\mathrm{eV})$ and pdf values

Line : energy (eV)

Line last: desired output name

Input:

Line 1: filename of mesh tally 
Line 2: which family of tally

Line 3: -1 (meaning use the whole family)

Line 4: source type: 1-neutron, 2-photon

Line 5: desired output name

Output: The resulting mesh source stored with the desired filename

Notes: Statistics of mesh tally are discarded.

Example:

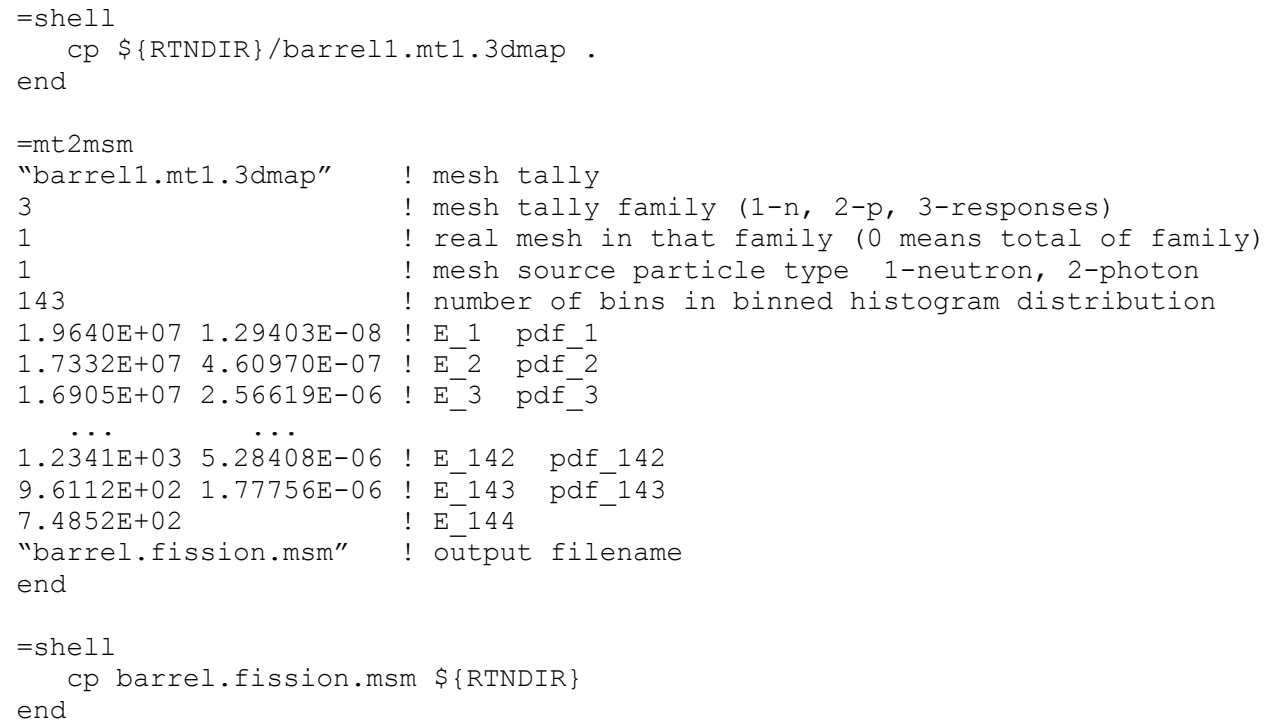

Example:

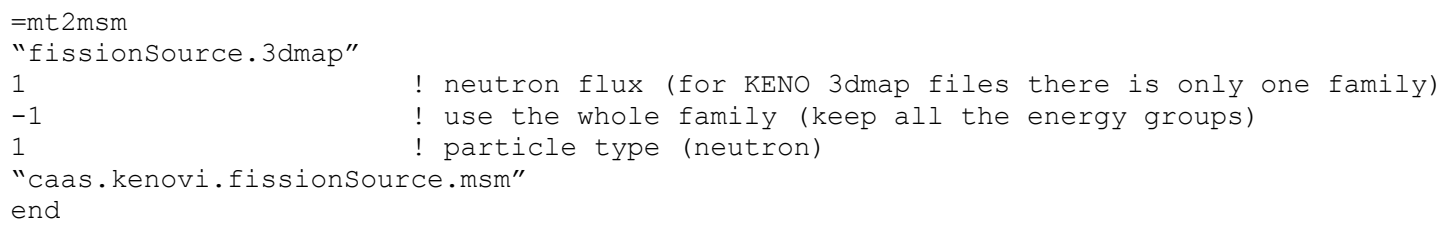

In SCALE 6.1, the fission source distribution mesh tally produced by KENO contained data representing the number of fissions in each mesh cell in each energy group. In SCALE 6.2, the data stored was changed to be the fissions per unit volume - the fission density. This is more consistent with other mesh tallies from Monaco which store flux or dose rates that represent averages over the mesh cells. This change also allows the MeshFileViewer to display the KENO fission source distribution better. The $\mathrm{mt} 2 \mathrm{msm}$ utility program also changed from SCALE 6.1 to SCALE 6.2 to account for the change in what is stored in the KENO mesh tally file. Therefore, KENO-produced fission source mesh tallies and the mt2msm utility should not be mixed-and-matched across versions of SCALE. Doing so would result in the final Monaco mesh source file being improperly normalized, which would not properly represent the KENO fission source distribution and would give incorrect results in subsequent MAVRIC calculations. Because there is not a specific 'version flag' in a mesh tally file or mesh source map file, the user must ensure that they have used the same version of SCALE for both the CSAS6 and MAVRIC sequences any time the CAAS capability is used. 
mt2silo - Convert a mesh tally file into a Silo file for VisIt.

Input: Name of mesh file (*.3dmap), name of a Silo file, and a format

Output: A new Silo file

Notes: For format, use either 2 (PDB) or 7 (HDF5).

Example:

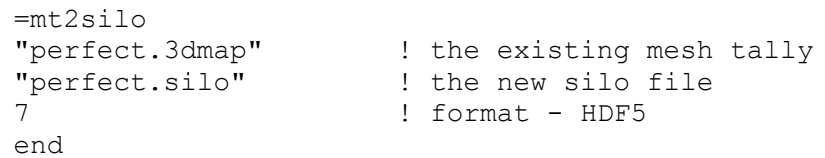

\section{mt2vtk - Convert one dataset of one family in a mesh tally to VTK format.}

Intended use: This is a way to transfer Monaco mesh tally data into a common format that can be used by many data visualization packages, including VisIt. Mesh tallies are stored in a generic *.3dmap format, which consist of several families, each with one or more datasets. A typical mesh tally contains three families: the neutron fluxes with each energy group as a dataset, the photon fluxes with each energy group as a data set, and the responses with each response as a dataset. This program selects one dataset of one family and saves the data (and optionally the absolute uncertainties) in an ASCII file using a VTK file format.

Input: The mesh file name, which family, which dataset of that family, whether or not to include absolute uncertainties and the filename for the resulting VTK file

Output: An ASCII VTK-formatted file

Example:

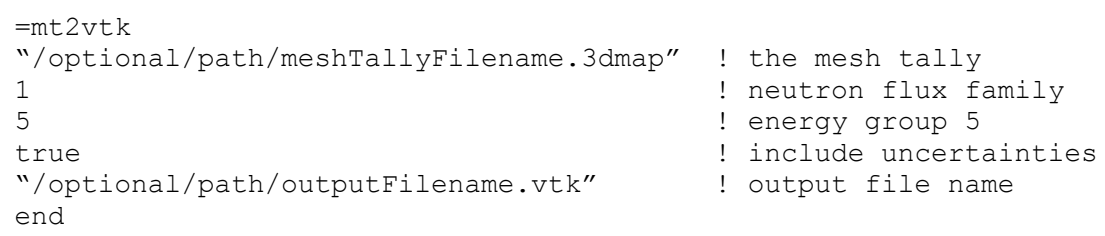

Example:

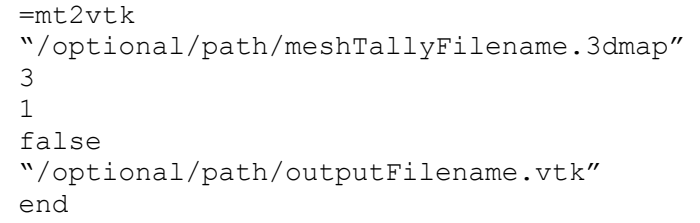

mtAdder - Add several Monaco mesh tally files together into one mesh tally.

Intended use: Add mesh tally results from different sources into one tally. The resulting mesh tally is the sum of all the components in the several mesh tallies - fluxes are added and responses are added. For 
example, two runs of MAVRIC from two different sources can be made. The mesh tally results can then be added together, getting the total fluxes and total responses from each.

Input: The number of files, followed by the list of mesh tally filenames to add, then the name of the total mesh tally

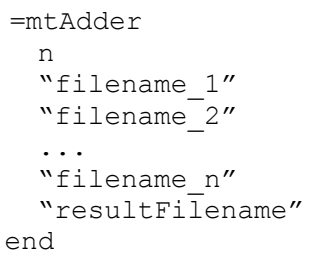

Output: A new mesh tally file

Notes: All of the mesh tally files must be the same size and shape (number of families, $\mathrm{x}$ cells, $\mathrm{y}$ cells, $\mathrm{z}$ cells, and energy groups in each family) and have the same number of responses. Responses (if any) must be consistent to calculate meaningful results.

Example:

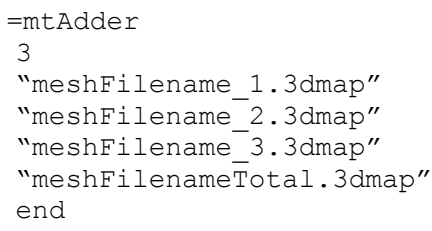

mtAverager - Average several Monaco mesh tally files into one mesh tally.

Intended use: Combine (average) separate runs of the same problem with different random number seeds into one tally. For example, if a user does 10 separate runs of the same problem (poor man's parallel) and wants to combine the results as if they were from one run, an average is needed. The average and uncertainties are weighted by the number of histories in each run, to maintain proper statistics.

Input: The number of files, each filename and how many histories, then the name of the total mesh tally

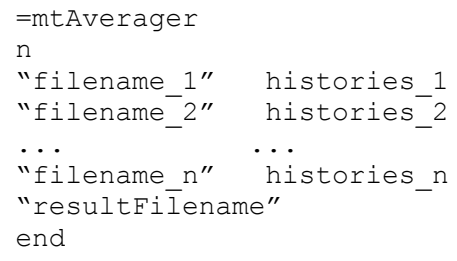

Output: A new mesh tally file

Notes: All of the mesh tally files must be the same size and shape (number of families, $\mathrm{x}$ cells, $\mathrm{y}$ cells, $\mathrm{z}$ cells, and energy groups in each family) and have the same number of responses. Responses (if any) must be consistent to calculate meaningful results.

Example:

=mtAverager 


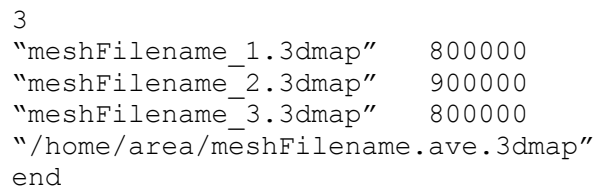

mtBinOp - Binary operation of mesh tally files: sum, difference, product, and ratio.

Intended use: Apply simple math to the results stored in mesh tally files Input: The first mesh tally, the operator: add (or sum, +), subtract (or difference, -), multiply (or product, $\mathrm{x}, *)$ and divide (or ratio, $\div, "$ "'), the second mesh tally name and then name of the resulting mesh tally file.

Output: A new mesh tally file

Notes: Uncertainties are propagated assuming the two mesh tallies are uncorrelated, which may not always be a good assumption. Mesh tallies need to have the same grid structure and number of families and groups. Dataset names in the results are inherited from the first mesh tally and may not make sense after the operation. When using the / (slash) for division, enclose it in quotes ("“").

Example:

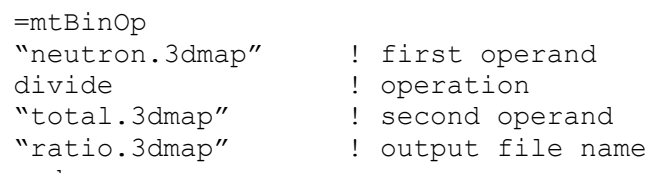

mtDisp - Display the basics of a mesh tally file.

Input: A mesh tally (*.3dmap) file

Output: Some of the basic details of mesh file

Example:

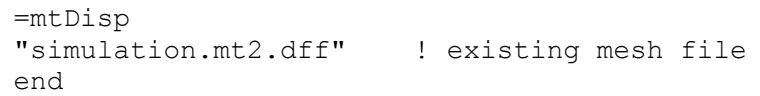

mtExpand - Expand a space-only mesh from a mesh tally with an energy function

Input: A mesh tally (*.3dmap) file and some parameters

Output: A mesh file similar to a mesh source but with uncertainty 


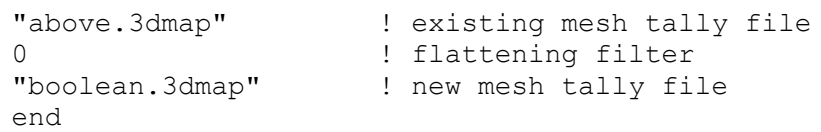

mtInv - Invert all of the values in a mesh tally.

Intended use: Invert non-zero values in a mesh tally to be used in further processing.

Input: The original mesh tally, the name of the resulting mesh tally file

Output: A new mesh tally file

Notes: Uncertainties are propagated (the relative uncertainty of the reciprocal of a value is the same as the relative uncertainty of the value).

Example:

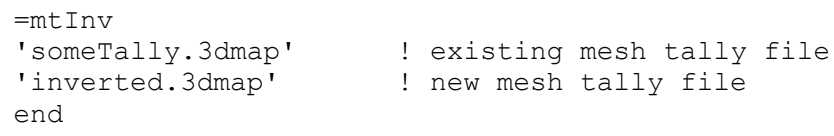

mtMask - Keep only or remove specified voxels of a mesh tally based on geometry.

Intended use: Only keep or remove certain portions of a mesh tally based on the unit, media, or mixture at the center of the voxel.

Input: A mesh tally file, an action (keeponly or remove), an operation (intersection or union) of the unit $=\mathrm{u}$, media $=\mathrm{r}$ and mixture $=\mathrm{m}$, a replacement value for voxels not kept and the file name of the resulting mesh tally file. User can specify things such as 1) keep only the voxels that have unit=2 and mixture $=5,2$ ) keep only the voxels that have media $=3$ or mixture $=4,3$ ) remove voxels that have unit $=2$ and mixture $=5,4$ ) remove voxels that have media $=3$ or mixture $=4$. To not include the unit, media, or mixture in the specification, use a value of -1 .

Output: A new mesh tally file.

Notes: When processing a file before finding the maximum, make the replacement value something very low. If mtMask is being used before finding the minimum, then set the replacement value high. Media is the SGGP media number within the unit.

Examples:

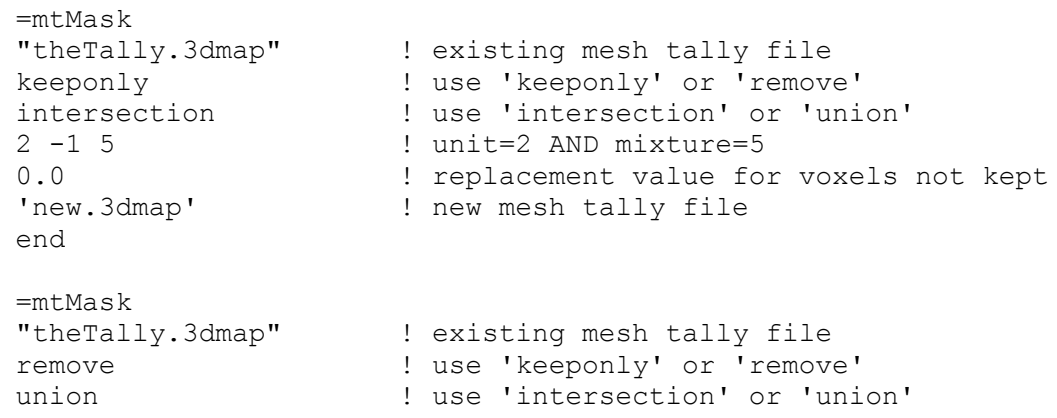




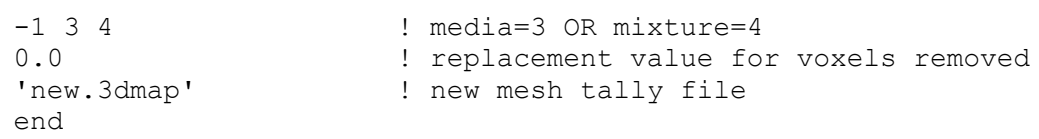

mtMinMax - Find the location/value of the min or max of each real mesh in a mesh tally.

Intended use: Determine the minimum or maximum values in a mesh tally.

Input: The mesh tally, what to find (minimums or maximums), how many mins/maxs for each real mesh in the mesh tally, and the name of the text output file to store the results

Output: A text output containing the values and locations of the minimums or maximums of each real mesh in a tally file

Notes: The same information is also in the main SCALE output file.

Example:

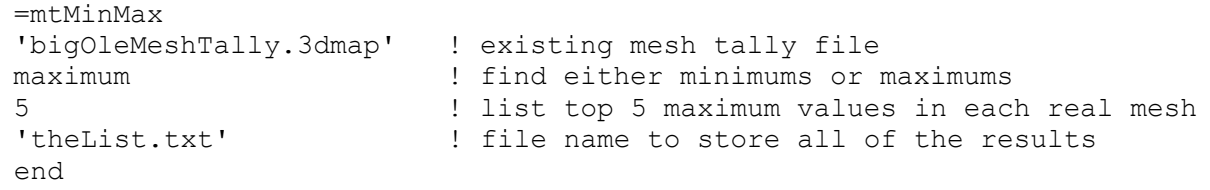

mtMultiply - Multiply a mesh tally by a constant factor.

Intended use: Multiply every group of every family in a mesh tally for either a change in source strength or a change in units.

Input: The original mesh tally, the multiplier, and the name of the resulting mesh tally file

Output: A new mesh tally file

Example:

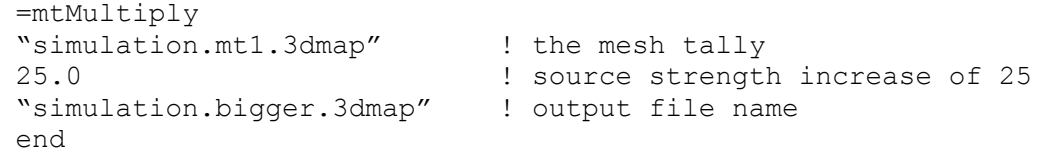

mtPull - Pull values from certain voxels out of a mesh tally file.

Intended use: Get energy-dependent fluxes for certain locations from a mesh file.

Input: A mesh file (*.3dmap) file and a list of positions and/or voxels

Output: Listing of energy-dependent fluxes from each desired location to an ASCII text file

Notes: Can pull fluxes either by a physical coordinate position or by voxel indices. Positions should be entered as a set of $\mathrm{x}, \mathrm{y}, \mathrm{z}$ for Cartesian coordinate system and $\mathrm{r}, \theta$, $\mathrm{z}$ for cylindrical coordinate system. 
Example:

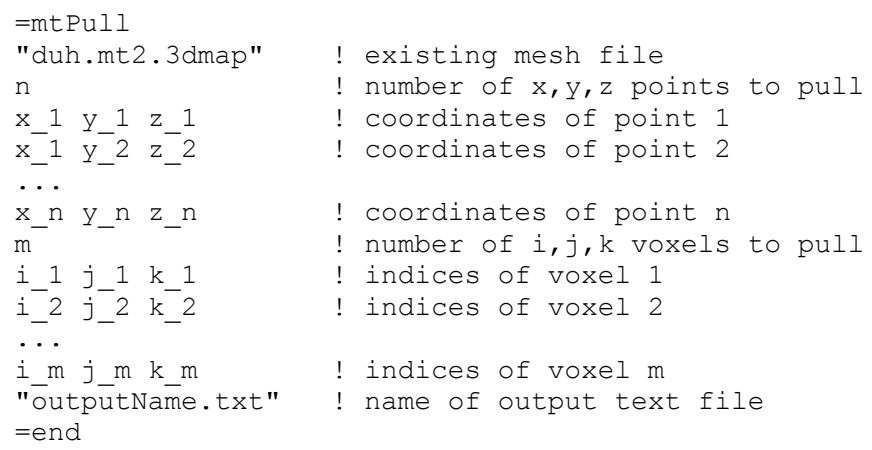

\section{mtRefine - Subdivide the mesh into smaller meshes.}

Input: A *.3dmap mesh tally file with geometry mesh size $(\mathrm{I}, \mathrm{J}, \mathrm{K})$ and three integers describing how many subdivisions of each voxel to create in each dimension

Output: A*.3dmap mesh tally file with geometry mesh size $\left(I^{*} n x, J^{*} n y, K^{*} n z\right)$

Example:

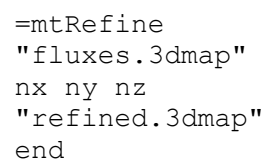

mtResp - Apply a response function to one family of a mesh tally file.

Intended use: Compute group-wise dose or reaction rates by combining a response function with the scalar fluxes.

Input: $\mathrm{A} * .3 \mathrm{dmap}$ mesh tally file and a group-wise response function

Output: A*.3dmap mesh tally file containing one family

\section{Example:}

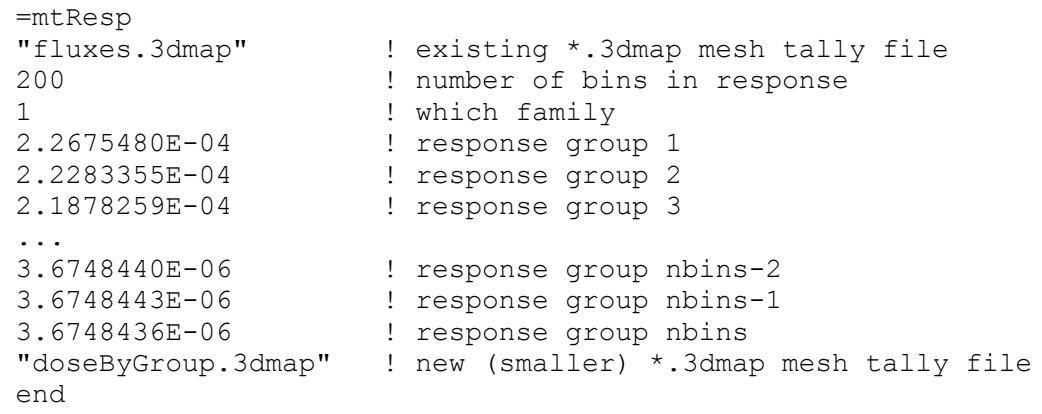

mtSplit - Split off part of a mesh tally file into a separate mesh tally file. 
Intended use: Some mesh tallies may become so large that the MeshFileViewer cannot load the entire file to view. This utility allows users to split off one family or just one group of one family into a separate mesh tally file.

Input: The original mesh tally, which family (neutron, photon, or responses), and which dataset (usually a group). Instead of a dataset, users may specify 0 to get the total of a family or -1 to get all datasets for that family. The name of the resulting mesh tally also needs to be given.

Output: A new, smaller, mesh tally file

Example:

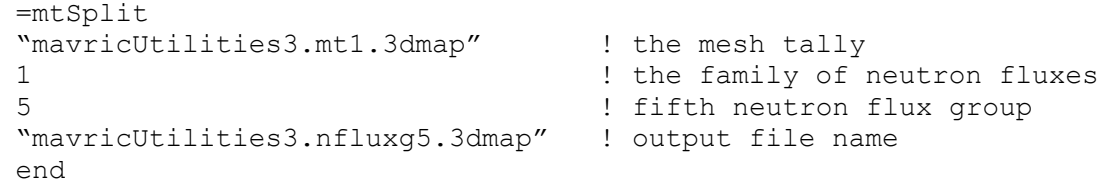

\subsection{B.3 Utilities for working with DENOVO binary flux (*.dff) files}

These utilities include the following:

dff2dso Convert a Denovo flux file into a Denovo spatial output file.

dff2mai Convert a Denovo flux file into a mesh angular information file.

dff2mim Invert a Denovo flux file and store as a mesh importance map.

dff2ms1 Convert a Denovo flux file into a mesh source lite.

dffBinOp Binary operation of Denovo flux files: sum, difference, product, and ratio.

dffDisp Display the basics of a Denovo flux file.

dffExpand Expand a space-only Denovo flux file by an energy function.

dffFilter Perform various filters on a Denovo flux file.

dffFix Fix the zero and negative values in a Denovo flux file.

dffInt Integrate a single particle type from a Denovo flux file.

dffInv Invert the values in a Denovo flux file.

dffMult Multiply a Denovo flux file by a constant factor.

dffPull Pull fluxes from certain voxels out of a Denovo flux file.

dffResp Apply a response function to scalar fluxes in a Denovo flux file.

dffSplit Split off a single particle type from a Denovo flux file.

\section{dff2dso - Convert a Denovo flux file into a Denovo spatial output file.}

Input: A binary (stream) Denovo flux file and which particle types to convert

Output: A binary (stream) Denovo Spatial Output file

Notes: For particle type, use 1 for neutron, 2 for photon, and 0 for all types.

Example: 


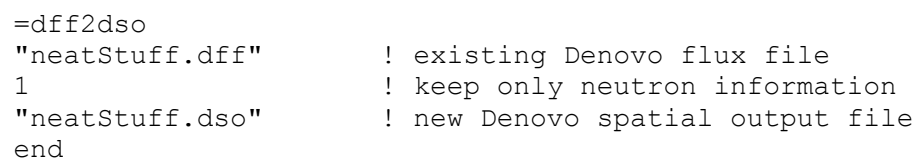

dff2mai - Convert a Denovo flux file into a mesh angular information file.

Intended use: Take the optional net current information from a Denovo flux file and create the adjoint current unit vectors and lambda parameters required for directional CADIS. This is stored in a mesh angular information (*.mai) file.

Input: A binary (stream) denovoFluxFile

Output: A binary (stream) meshAngularInfoFile, a mesh angular information file

Example:

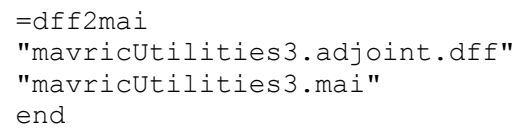

\section{dff2mim - Invert a Denovo flux file and store as a mesh importance map.}

Intended use: Make weight targets without a consistent biased mesh source.

Input: A Denovo flux (*.dff) file, a scalar constant, and the name of Monaco mesh importance map (*.mim) file.

Output: A Monaco mesh importance map (*.mim) file.

Example:

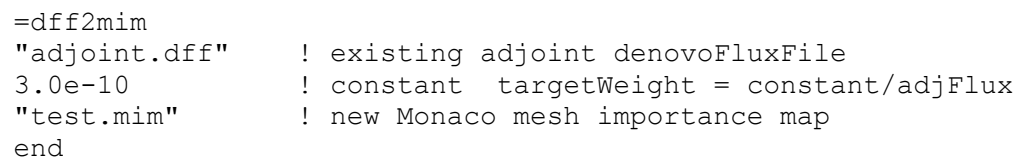

dff2msl- Convert a Denovo flux file into a mesh source lite.

Intended use: Take Denovo fission source information stored in a *.dff file and convert it to a mesh source lite file $(* . \mathrm{msl})$ to be used as a KENO starting source, nst $=9$.

Input: A Denovo flux (*.dff) file

Output: A mesh source lite (*.msl) file 
Example:

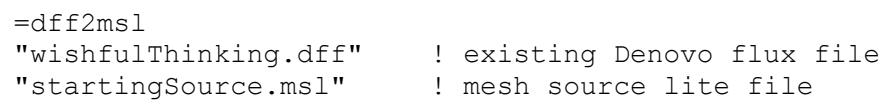

dffBinOp - Binary operation of Denovo flux files: sum, difference, product and ratio.

Intended use: Apply simple math to the results stored in Denovo flux files.

Input: The first flux file, the operator: add (or sum, +), subtract (or difference, -), multiply (or product, $\mathrm{x}$, $*$ ), or divide (or ratio, $\div, " / "$ ), the second flux file name, and the name of the resulting flux file

Output: A Denovo flux file

Notes: Flux files need to have the same grid structure and number of groups. When using the / (slash) for division, enclose it in quotes ("/").

Example:

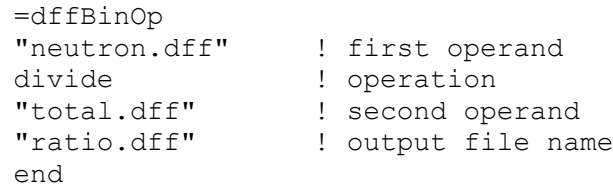

dffDisp - Display the basics of a Denovo flux file.

Input: A Denovo flux (*.dff) file

Output: Some of the basic details of the Denovo flux file

Example:

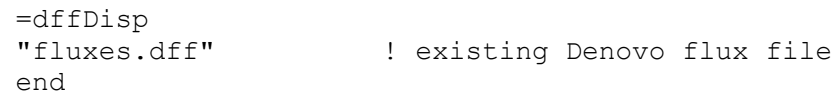

dffExpand - Expand a space-only Denovo flux file by an energy function.

Input: A Denovo flux (*.dff) file (with a single group - a space-only function), one or more particle types, and an energy function for each

Output: A full space/energy Denovo flux file 


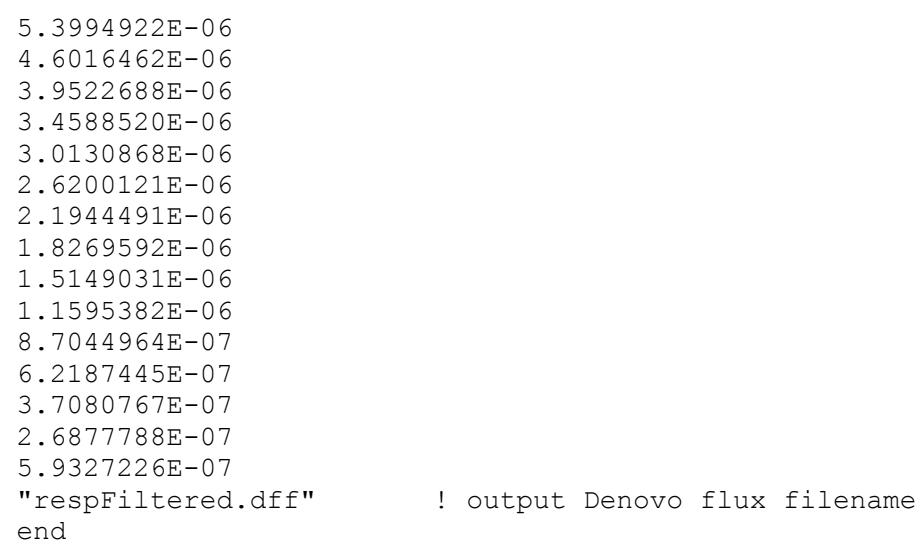

dffFix - Fix the zero and negative values in a Denovo flux file.

Intended use: Replace zero or negative values with nearest good neighboring value. Checks previous group, previous x voxel, previous y voxel, then previous $\mathrm{z}$ voxel.

Input: A Denovo flux file

Output: A new Denovo flux file

Example:

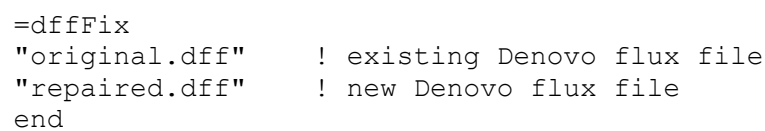

dffInt - Integrate a single particle type from a Denovo flux file.

Input: A Denovo flux file, which particle type to integrate (1-neutron, 2-photon), and the filename of the resulting integrated file

Output: A single-group Denovo flux file

Example:

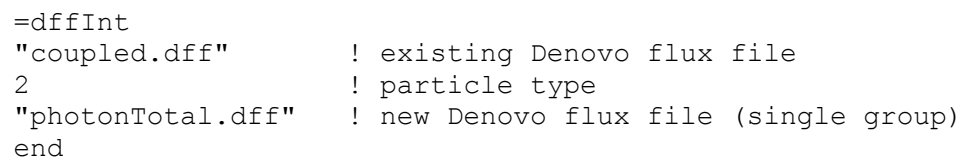

dffInv - Invert the values in a Denovo flux file.

Input: A Denovo flux file

Output: A Denovo flux file

Notes: Only non-zero values are inverted

Example: 


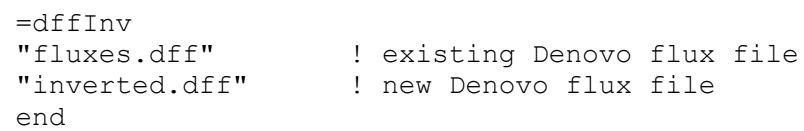

\section{dffMult - Multiply a Denovo flux file by a constant factor.}

Intended use: source strength change, change in units, etc.

Input: A Denovo flux file and a constant factor

Output: A Denovo flux file

Example:

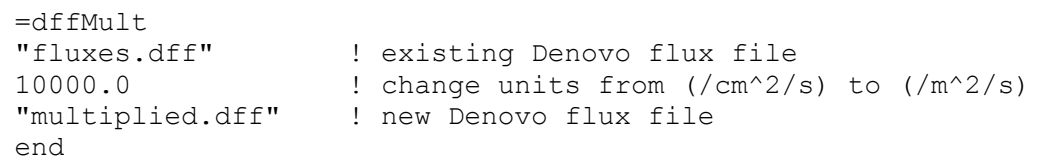

dffPull - Pull fluxes from certain voxels out of a Denovo flux file.

Intended use: Get energy-dependent fluxes for certain locations from a flux file.

Input: A Denovo flux file and a list of positions and/or voxels

Output: Listing of energy-dependent fluxes from each desired location to an ASCII text file

Notes: Can pull fluxes either by a physical coordinate position or by voxel indices.

Example:

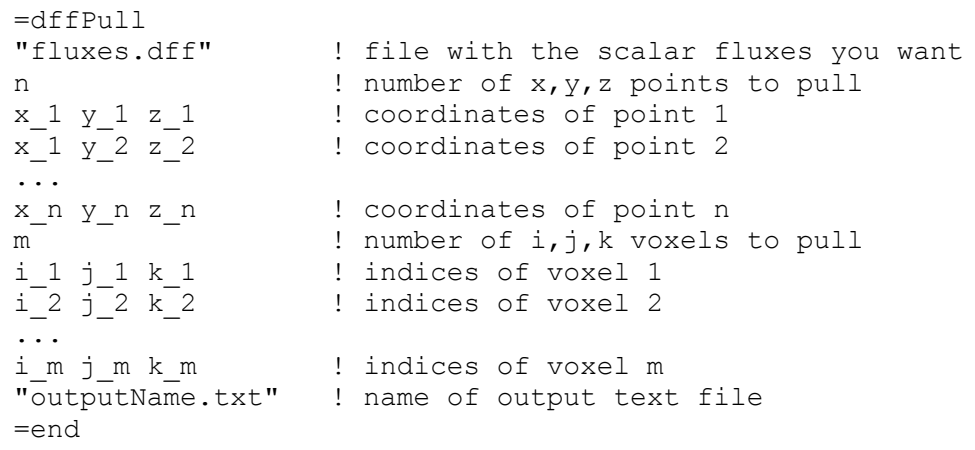

dffResp - Apply a response function to scalar fluxes in a Denovo flux file.

Intended use: Compute group-wise dose or reaction rates by combining a response function with the scalar fluxes. This can be done for every particle type in the flux file or a single specific particle type.

Input: A Denovo flux file, particle indicator and a group-wise response function

Output: A Denovo flux file 


\subsection{B.4 Utilities for working with DENOVO *.varscl (a TORT format) files}

These utilities include the following:

$\begin{array}{ll}\text { vs2dff } & \text { Convert a varscl file into a Denovo flux file. } \\ \text { vsAdder } & \text { Add two TORT *.varscl files together into one *.varscl file. } \\ \text { vsBinOp } & \text { Binary operation of TORT *.varscl files: sum, difference, product and ratio. } \\ \text { vsDisp } & \text { Display the basic contents of a TORT *.varscl file. } \\ \text { vsFilter } & \text { Perform various filters on a TORT *.varscl file. } \\ \text { vsInt } & \text { Integrate a single particle type from a TORT *.varscl file. } \\ \text { vsInv } & \text { Invert the values in a TORT *.varscl file. } \\ \text { vsMult } & \text { Multiply a TORT *.varscl file by a constant factor. } \\ \text { vsPull } & \text { Pull fluxes from certain voxels out of a TORT *.varscl file. } \\ \text { vsReGrp } & \text { Regroup a TORT *.varscl file. } \\ \text { vsResp } & \text { Apply a response function to scalar fluxes in a TORT *.varscl file. } \\ \text { vsSplit } & \text { Split off part of a TORT *.varscl file into a separate *.varscl file. }\end{array}$

These utilities work with the *.varscl files produced with SCALE 6 and SCALE 6.1. The *.varscl format (a TORT format) is a single precision, binary format that has been replaced with the double precision, binary *.dff file (Denovo flux file) in SCALE 6.2. SCALE 6 and SCALE 6.1 users can request the executable binaries for these utilities by sending an email to scaleHelp@ornl.gov.

vs2dff - Convert a varscl file into a Denovo flux file.

Intended use: Convert a varscl file (used in previous versions of MAVRIC) into a Denovo flux file (introduced in SCALE 6.2).

Input: The *varscl file name, whether or not it is an adjoint flux, and the filename for the resulting denovoFluxfile

Output: A binary (stream) denovoFluxFile

Example:

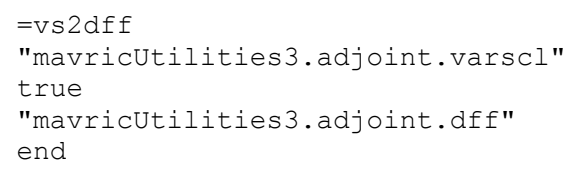

vsAdder - Add two TORT *.varscl files together into one *.varscl file.

Intended use: Beta versions of MAVRIC used TORT and GRTUNC-3D and could add the *.varscl files from each together before using them to create importance maps. MAVRIC now uses Denovo and no longer needs to add separate GRTUNC/TORT files. This utility is designed for people wishing to use the older files with the latest MAVRIC. 
Input: Two *.varscl file names, typically one from GRTUNC-3D and the other from TORT, the filename of the added file, whether you want lots of output displayed ("true" or "false") and whether or not there is a minimum value of flux to use. If so, it is then listed.

Output: A single *.varscl with the specified name

Notes: Addition is commutative, but not all varscl files are created equal. Do not mix up the GRTUNCL and the TORT files. GRTUNCL3D does not fill in the header info quite right, so the added varscl file takes header info only from the TORT varscl file.

Example:

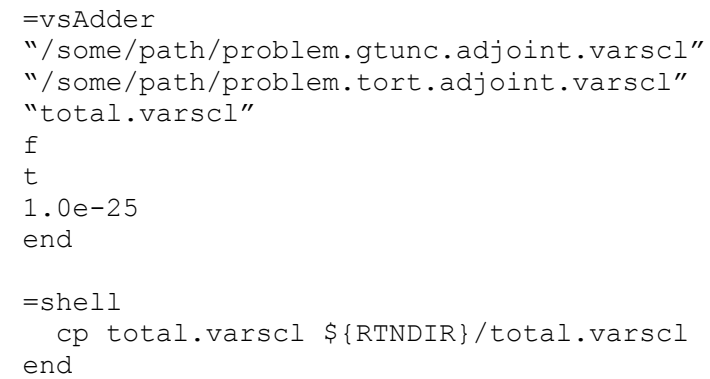

vsBinOp - Binary operation of TORT *.varscl files: sum, difference, product and ratio.

Intended use: Apply simple math to the results stored in TORT *.varscl files.

Input: The first flux file, the operator: add (or sum, + ), subtract (or difference, -), multiply (or product, $\mathrm{x}$, $*$ ), and divide (or ratio, $\div, " / "$ ), the second flux file name, and the name of the resulting flux file

Output: A TORT *.varscl file

Notes: Flux files need to have the same grid structure and number of groups. When using the / (slash) for division, enclose it in quotes ("/").

Example:

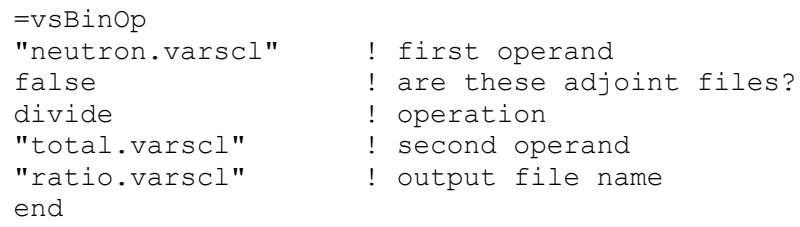

vsDisp - Display the basic contents of a TORT *.varscl file.

Input: A TORT *.varscl file name and adjoint flag

Output: Text display

Examples:

$=\mathrm{vsDisp}$

"some.varscl" ! input TORT *.varscl filename 


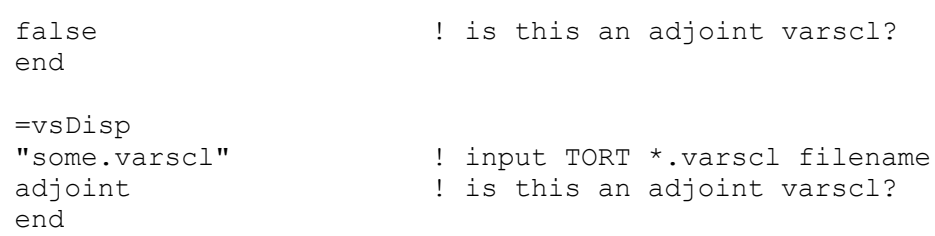

vsFilter - Perform various filters on a TORT *.varscl file.

Intended use: Keep fluxes in a *.varscl file where the flux or response meets a specified criterion.

Input: A TORT *.varscl file name, filter type, filter options, the output file name

Output: A TORT *.varscl file

Notes: There are three basics types of filters: 0) flattening filter, 1) high-pass filter, 2) low-pass filter. For types 1 and 2, the criteria could be a computed response. The input list changes depending on the filter type and whether a response function is included. For no response function, use 0 for the number of groups.

Examples:

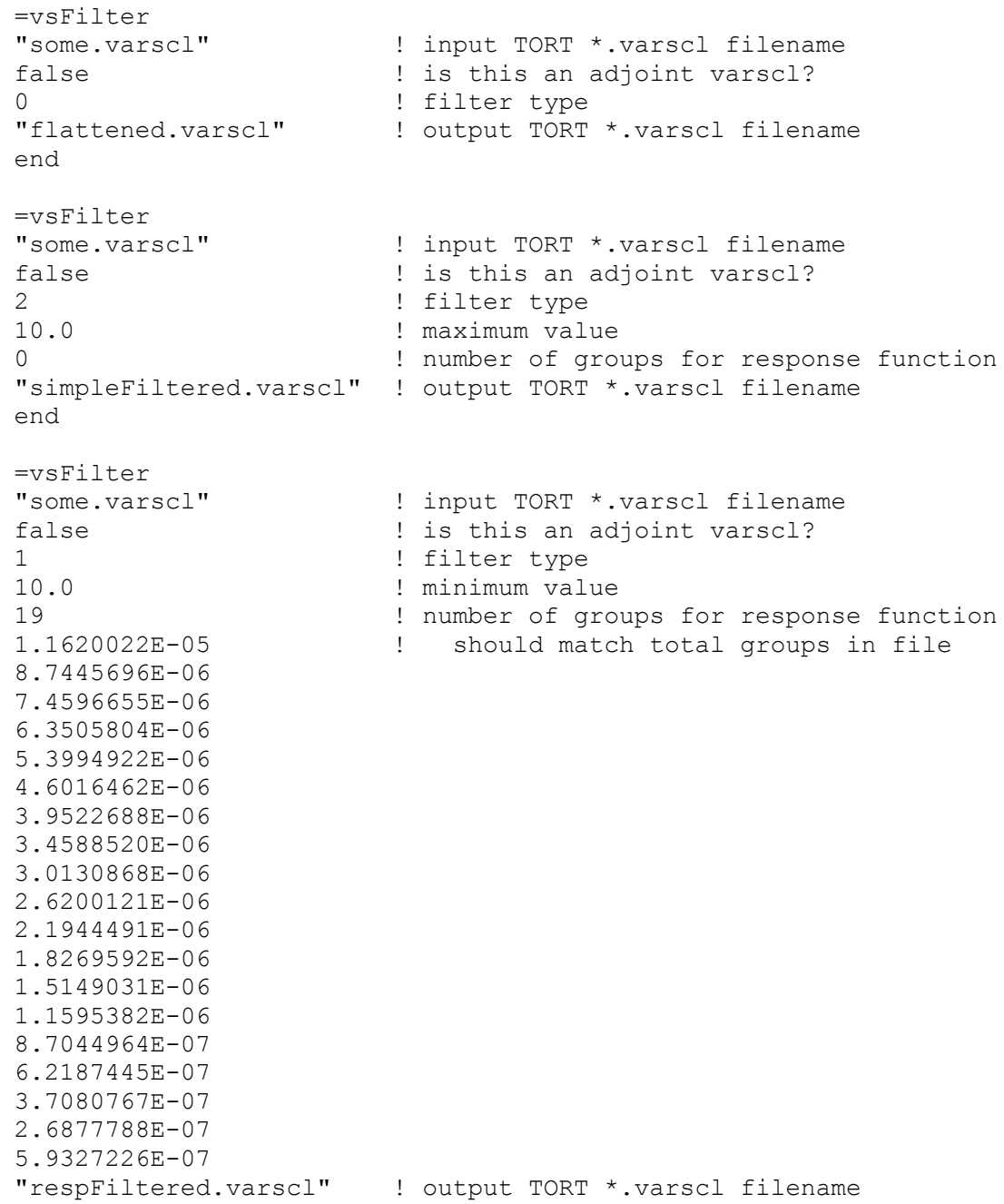


vsInt - Integrate a single particle type from a TORT *.varscl file.

Input: A TORT *.varscl file

Output: A single-group TORT *.varscl file

Example:

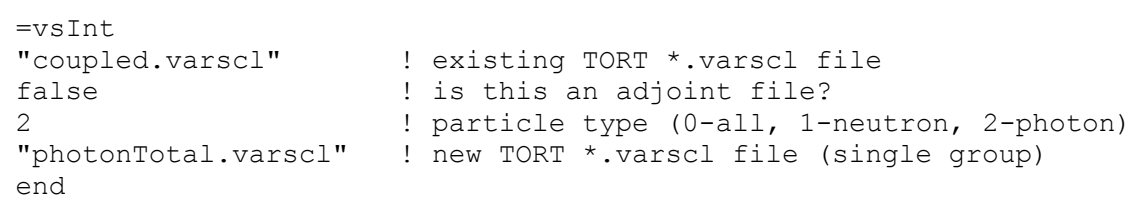

vsInv - Invert the values in a TORT *.varscl file.

Input: A TORT *.varscl file

Output: A TORT *.varscl file

Notes: Only non-zero values are inverted

Example:

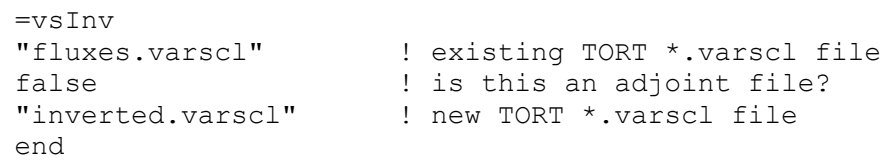

vsMult - Multiply a TORT *.varscl file by a constant factor.

Intended use: source strength change, change in units, etc.

Input: A TORT *.varscl file and a constant factor

Output: A TORT *.varscl file

Example:

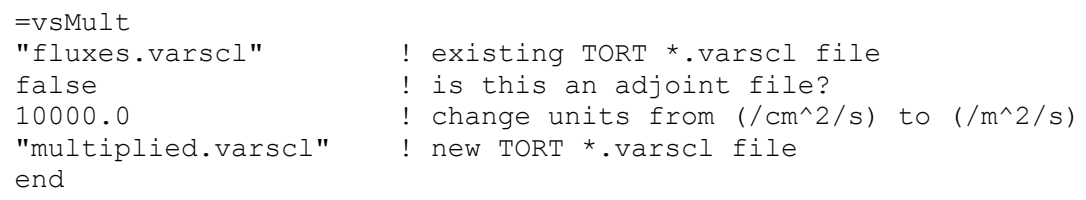

vsPull - Pull fluxes from certain voxels out of a TORT *.varscl file.

Intended use: Get energy-dependent fluxes for certain locations from a flux file. 
Output: Human-readable form of the Denovo input file

Example:

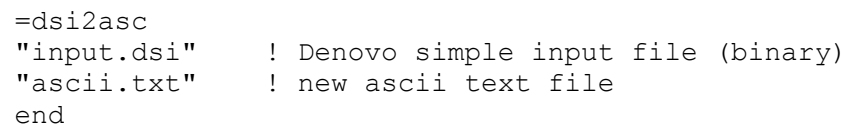

dsiDisp - Display the basics of a Denovo simple input file.

Input: A Denovo simple input (*.dsi) file

Output: Some of the basic details of the Denovo simple input file

Example:

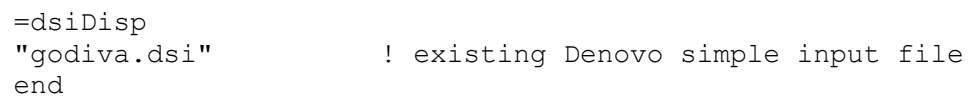

dso2msl - Use a Denovo spatial output to create a mesh source lite.

Input: $A^{*}$.dso file is made of three-dimensional data sets called fields. Which field to convert? 1-n: convert that field 0 : convert sum of all fields

Output: A mesh source lite (*.msl) file for KENO-VI.

Example:

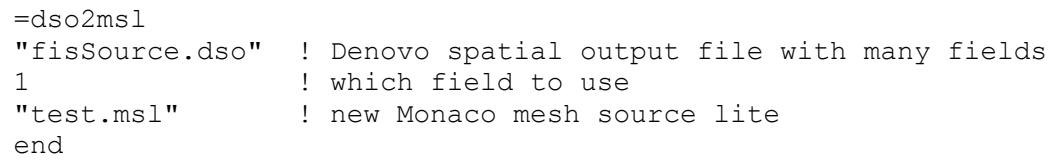

dsoDisp - Display the basics of a Denovo spatial output file.

Input: A Denovo spatial output (*.dsi) file

Output: Some of the basic details of the Denovo spatial output file

Example:

$=\mathrm{dsoDisp}$

"godiva.dso" ! existing Denovo spatial output file

end

mim2wwinp - Convert a mesh importance map into an MCNP weight window file.

Intended Use: To create an MCNP weight window file from a Monaco mesh importance map file outside of a MAVRIC calculation. Monaco mesh importance map files store target weights, but MCNP wwinp files store lower weight bounds. To convert, the user needs to supply the windowRatio, $r$ (the ratio of the upper weight bound for splitting to the lower weight bound for roulette). Target weights, $t$, are the average 
of the upper, $u$, and lower, $l$, weight window bounds, so $l=2 t /(r+1)$. For example, for a Monaco target weight of 1.0 and a windowRatio of 10.0, the MCNP lower weight bound will be $l=2(1.0) /(10.0+1)=0.1818$. To reduce the size of the map, the user can specify which neutron and photon groups to store in the new file. If the last group is less than the first group, no groups of that particle will be stored.

Input:

Line 1: filename of the Monaco mesh importance map file

Line 2: windowRatio $(>1.0)$

Line 3: first_neutron_group last_neutron_group

Line 4: first_photon_group last_photon_group

Line 5: filename of the MCNP weight window input file

Output: The resulting weight window input file stored with the desired filename

Notes: Geometry information in the Monaco mesh importance map file is lost since the MCNP wwinp format does not support it.

Example:

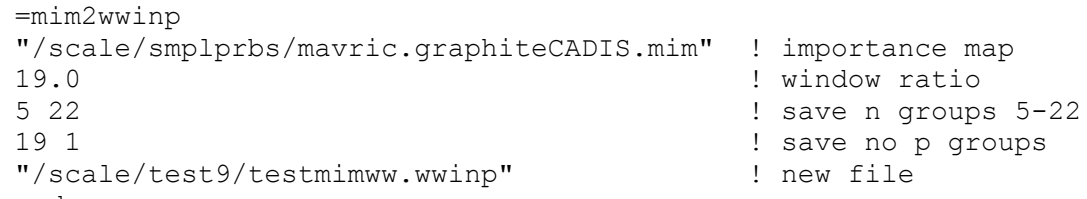

mimDisp - Display the basics of a mesh importance map file.

Input: A mesh importance map (*.mim) file

Output: Some of the basic details of mesh importance map file

Example:

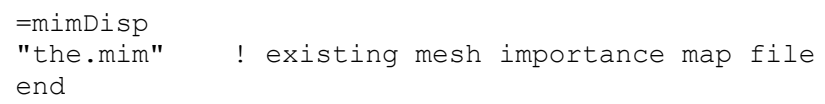

mimNorm - Normalize a mesh importance map to a given location/energy.

Input: A mesh importance map (*.mim) file, a location ( $\mathrm{x}, \mathrm{y}, \mathrm{z})$, a particle type and energy, and a filename for the normalized map file. Use 1 for neutron and 2 for photon. Energy should be in $\mathrm{eV}$. The new importance map file will be normalized such that the given location/energy has a target weight of 1 . If a particle type or energy is 0 , then the energy group with the minimum non-zero target value at the given location will be the group that is set to 1.0 in the new file. (This option is similar to the MCNP weight window generator.)

Output: A mesh importance map file 
Example:

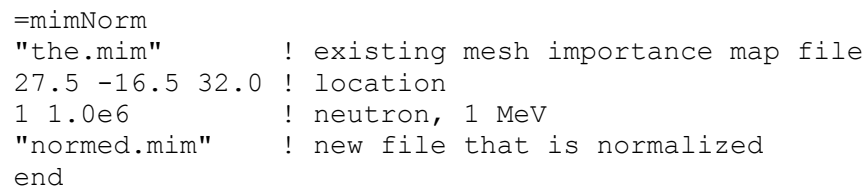

msmDisp - Display the basics of a mesh source map file.

Input: A mesh source map (*.msm) file

Output: Some of the basic details of mesh source map file

Example:

$=\mathrm{msmDisp}$

"the.msm" ! existing mesh source map file 


\subsection{Advanced Features}

This appendix contains information on several advanced features that are still under development or are non-standard use of the MAVRIC sequence.

\subsection{C.1 Alternate normalization of the importance map and biased source}

The importance map and biased source implemented in MAVRIC are only functions of space and energy only. The importance for a specific location and energy represents the average over all directions. For applications involving a collimated beam source, a space/energy importance map may not be representative of the true importance of the particles as they stream away from the source.

As an example, consider a $14.1 \mathrm{MeV}$ active interrogation beam source 1 meter from a small spherical boat containing illicit nuclear material. The objective is to compute the fission rate in the nuclear material. To create the biasing parameters, an adjoint source is located within the nuclear material and the resulting importance map is shown in Figure 4.1.C.1. Note that in both the air and water, the importances change with distance from the ship, but for the beam source, the importance (to causing a fission in the nuclear material) anywhere along the beam should be the same, since there is little chance a $14.1 \mathrm{MeV}$ neutron will interact with the air before striking the ship.

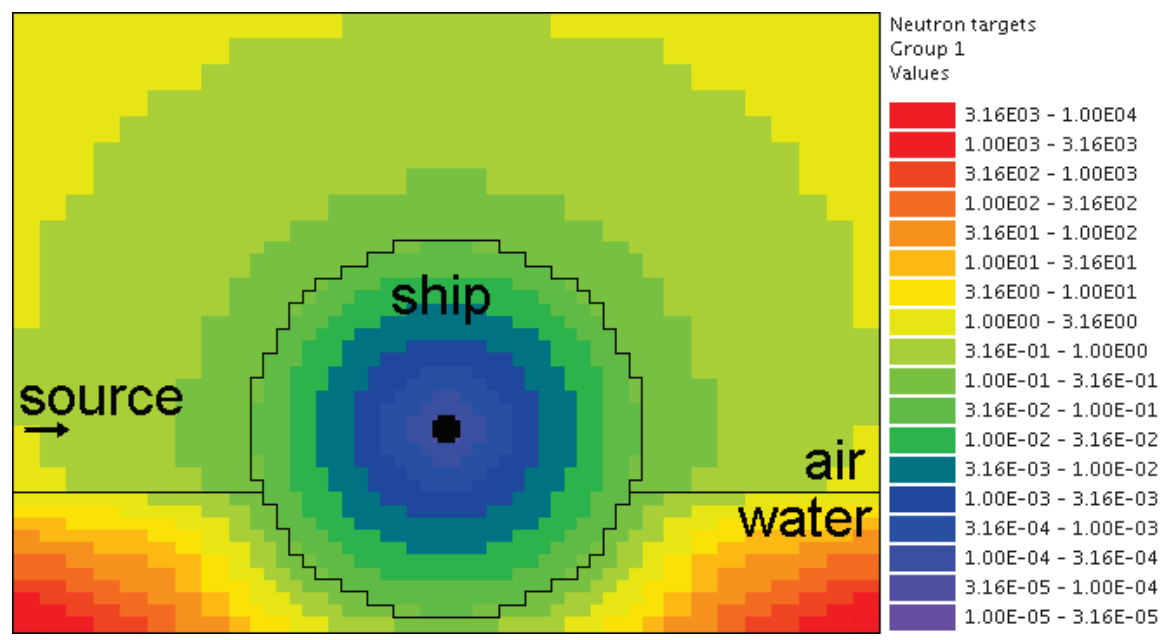

Figure 4.1.C.1. Importance map computed using standard CADIS.

The CADIS algorithm has done exactly what it was supposed to: it made a space/energy importance map and normalized it such that the target weight where the $14.1 \mathrm{MeV}$ source particles are born is 1 . The problem with this is that the source particles will stream towards the ship and strike the hull where the target weight is 0.092 . Since source particles have little chance of interacting in the air, the weight windows are not used to split the particle as they travel towards the ship. When source particles cross into the ship, they are split by a factor of 11 to match the target weight. For this example, splitting each particle by a factor of 11 once they strike the ship is not so bad, but for longer distances, this will result in much larger splits. For a polyenergetic source, this could lead to undersampling of the source and could result in higher variances. 
To remedy this problem when using beam sources, the normalization of the importance map and biased source should not be done at the source location but instead at the point where the source particles first interact with the ship. The keyword "shiftNormPos $\Delta \mathrm{x} \Delta \mathrm{y} \Delta \mathrm{z}$ " will shift the source normalization position by the amounts $\Delta \mathrm{x}, \Delta \mathrm{y}$, and $\Delta \mathrm{z}$ when the biased source and importance map are developed. For the Monaco Monte Carlo calculation, the source is returned to its normal position. The source input for the above problem would then be

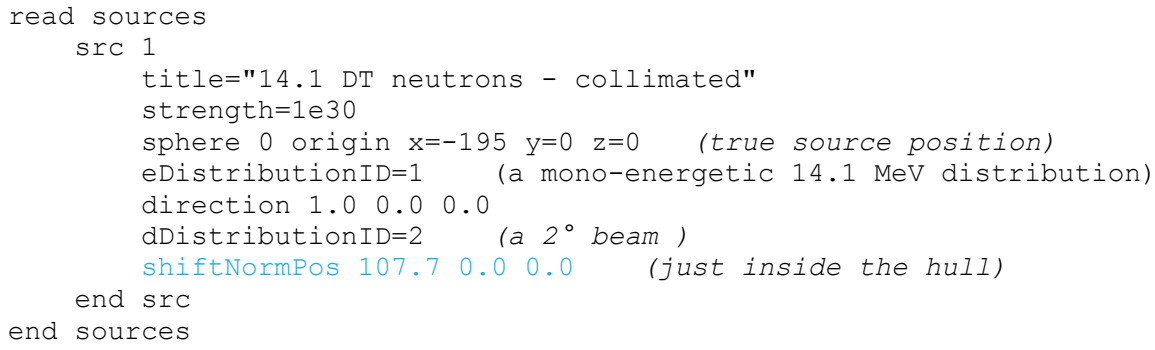

where the shift moves the source position from $\mathrm{x}=-195$ to $\mathrm{x}=-87.3$, just inside the hull. The resulting target weights are shown in Figure 4.1.C.2. The source particles are born with weight 1 in a location with a target weight 10.9. The particle weight is not checked until the particle crosses into the hull, where the target weight is 1.0 .
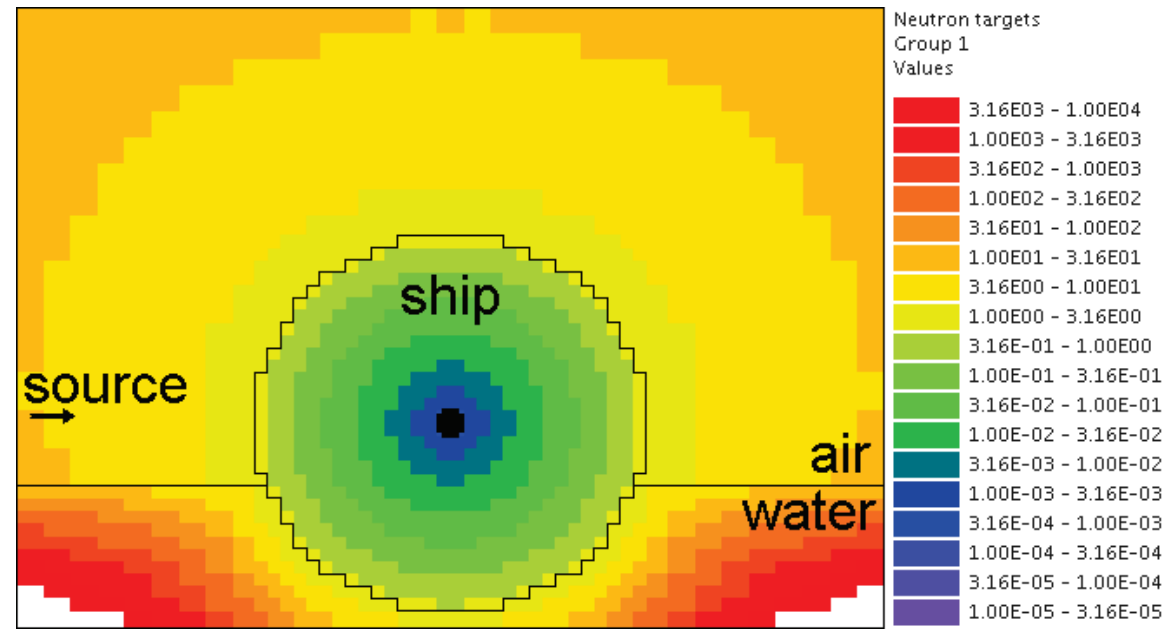

Figure 4.1.C.2. Targets weights using the "shiftNormPos" keyword.

Other options to manipulate the importance map for special situations include the "mapMultiplier=f" keyword (in the importanceMap block or the biasing block), which will multiply every target weight by the factor $f$, and the keyword "noCheckAtBirth" in the parameters block will prevent the weight windows from being applied to source particles when they are started. When used in the MAVRIC sequence, the "shiftNormPos" capability automatically adds "noCheckAtBirth" to the Monaco input that is created.

\subsection{C.2 Importance maps with directional information}

In MAVRIC, the CADIS method is implemented in space and energy, but in general, it could also include particle direction as well. This formulation would be the following. 
True source:

Desired response:

Adjoint angular flux using

$q^{+}(\vec{r}, E, \widehat{\Omega})=\sigma(\vec{r}, E, \widehat{\Omega}):$

Estimate of detector response:

Biased source:

Target weight windows:

$$
q(\vec{r}, E, \widehat{\Omega})
$$

$$
\sigma(\vec{r}, E, \widehat{\Omega})
$$

$$
\psi^{+}(\vec{r}, E, \widehat{\Omega})
$$

$$
R=\iiint q(\vec{r}, E, \widehat{\Omega}) \psi^{+}(\vec{r}, E, \widehat{\Omega}) d \Omega d E d V
$$

$$
\hat{q}(\vec{r}, E, \widehat{\Omega})=\frac{1}{R} q(\vec{r}, E, \widehat{\Omega}) \psi^{+}(\vec{r}, E, \widehat{\Omega})
$$

$$
\bar{w}(\vec{r}, E, \widehat{\Omega})=\frac{R}{\psi^{+}(\vec{r}, E, \widehat{\Omega})}
$$

For a system using a deterministic method to compute the adjoint fluxes, this completely general, space/energy/angle, approach presents many difficulties in implementation, namely,

a. dealing with the amount of memory required for a $(\vec{r}, E, \widehat{\Omega})$ importance map in memory,

b. interpolating the importance for particle directions in between quadrature angles, and

c. expressing the biased source in a form suitable for a general MC code since the above biased source is, in general, not separable.

\subsection{C.2.1 Approaches incorporating directional information}

Completely general space/energy/angle CADIS is most likely too difficult to implement and may not be necessary for most applications. In most real problems that involve directionally dependent source distributions, the directional dependence is azimuthally symmetric about some reference direction, $\hat{d}$. The angular distribution, $q_{i}(\widehat{\Omega})$, can be expressed as the product of the uniform azimuthal distribution and a polar distribution about reference direction $\hat{d}_{i}$ giving $\frac{1}{2 \pi} q_{i}\left(\widehat{\Omega} \cdot \hat{d}_{i}\right)$. The geometric size of these sources tends to be small, allowing each source distribution to be expressed as the product of two separable distributions: $q_{i}(\vec{r}, E, \widehat{\Omega}) \cong q_{i}(\vec{r}, E) q_{i}(\widehat{\Omega})$.

What is needed is a CADIS method that (1) can account for the importance of a particle traveling in a certain direction; (2) can be cast as a simple modification of the space/energy CADIS method using $\bar{w}(\vec{r}, E)$ and $\hat{q}(\vec{r}, E)$; and (3) is simpler than the full space/angle/energy approach. This can be done starting with the approximation that the angular component of the adjoint flux $\psi^{+}(\vec{r}, E, \widehat{\Omega})$ is separable and symmetric about the average adjoint current direction $\hat{n}(\vec{r}, E)$, such that

$$
\psi^{+}(\vec{r}, E, \widehat{\Omega}) \cong \phi^{+}(\vec{r}, E) \frac{1}{2 \pi} f(\widehat{\Omega} \cdot \hat{n})
$$

This is similar to the AVATAR approach ${ }^{1}$ but with explicitly including the azimuthal distribution so that the standard definition $\int \phi^{+}(\vec{r}, E) \frac{1}{2 \pi} f(\widehat{\Omega} \cdot \hat{n}) d \widehat{\Omega}=\phi^{+}(\vec{r}, E)$ applies. The probability distribution function $f(\mu)$ describing the shape of the azimuthally symmetric current at $(\vec{r}, E)$ has the form of

$$
f(\mu)=\frac{\lambda e^{\lambda \mu}}{2 \sinh (\lambda)}
$$


with the single parameter $\lambda(\vec{r}, E)$ determined from $\vec{\mu}(\vec{r}, E)$, the average cosine of scatter.

From this, we can propose that weight window targets be developed that are inversely proportional to the approximation of the adjoint angular flux:

$$
\bar{w}(\vec{r}, E, \widehat{\Omega})=\frac{2 \pi k}{\phi^{+}(\vec{r}, E) f(\widehat{\Omega} \cdot \hat{n})},
$$

where $k$ is the constant of proportionality that will be adjusted to make the importance map consistent with the biased source(s). Two methods will be examined here, one without and one with biasing of the source directional dependence.

For both of the methods, the $\mathrm{S}_{\mathrm{N}}$ code Denovo was modified to report not only the adjoint scalar fluxes, $\phi^{+}(\vec{r}, E)$, but also the adjoint net currents in $x, y$, and $z$ directions: $J_{x}(\vec{r}, E), J_{y}(\vec{r}, E)$, and $J_{z}(\vec{r}, E)$. These currents are used to find $\hat{n}(\vec{r}, E)$ and $\lambda(\vec{r}, E)$. The following methods have been developed so that the standard CADIS routines can be used to compute space/energy quantities of the response per unit source $R$, the weight window target values $\bar{w}(\vec{r}, E)$, and biased source $\hat{q}(\vec{r}, E)$ with just the adjoint scalar fluxes. These quantities are then modified by the directional information.

\subsection{C.2.2 Directionally dependent weight windows without directional source biasing}

It is proposed that the biased source $\hat{q}(\vec{r}, E, \widehat{\Omega})$ should be proportional to both the true source distribution and the space/energy component of the adjoint flux:

$$
\hat{q}(\vec{r}, E, \widehat{\Omega})=\frac{1}{R}\left[q(\vec{r}, E) \frac{1}{2 \pi} q(\widehat{\Omega} \cdot \hat{d})\right] \phi^{+}(\vec{r}, E),
$$

where the constant of proportionality, $R$, is determined by forcing $\hat{q}(\vec{r}, E, \widehat{\Omega})$ to be a pdf. Since the angular component of the adjoint flux is not included, the directional distribution of the biased source will be exactly the same as the true source. Note that this approach would be exact for cases where no directional biasing could be applied - beam sources.

For multiple sources (each with a probability distribution function $q_{i}(\vec{r}, E)$ and a strength $S_{i}$, giving a total source strength of $\left.S=\sum S_{i}\right)$, the user is required to provide one point in phase space $\left(\vec{r}_{i}, E_{i}, \widehat{\Omega}_{\mathrm{i}}\right)$ for each source $i$ that is representative of that entire source where the biased source will match the target weight windows. For each source, a vector $\hat{n}_{i}=\hat{n}\left(\vec{r}_{i}, E_{i}\right)$ is computed using that point. For the general case of multiple sources, the biased source sampling distribution, the biased source distributions, and the weight windows are computed using

$$
\begin{array}{rlr}
R_{i} & =\iint q_{i}(\vec{r}, E) \phi^{+}(\vec{r}, E) d E d r & \\
\hat{p}(i) & =\frac{S_{i} R_{i} f\left(\widehat{\Omega}_{\mathrm{i}} \cdot \hat{n}_{i}\right)}{\sum S_{i} R_{i} f\left(\widehat{\Omega}_{\mathrm{i}} \cdot \hat{n}_{i}\right)} & \text { (estimated response from source } i \text { ) } \\
\hat{q}_{i}(\vec{r}, E, \widehat{\Omega}) & =\frac{1}{R_{i}} q_{i}(\vec{r}, E) \phi^{+}(\vec{r}, E) \frac{1}{2 \pi} q_{i}\left(\widehat{\Omega} \cdot \hat{d}_{i}\right)=\hat{q}_{i}(\vec{r}, E) \frac{1}{2 \pi} q_{i}\left(\widehat{\Omega} \cdot \hat{d}_{i}\right)
\end{array}
$$




$$
\bar{w}(\vec{r}, E, \widehat{\Omega})=\quad \frac{\sum S_{i} R_{i} f\left(\widehat{\Omega}_{\mathrm{i}} \cdot \hat{n}_{i}\right)}{S \phi^{+}(\vec{r}, E)} \frac{1}{f(\widehat{\Omega} \cdot \hat{n})} \quad=\frac{\sum S_{i} R_{i} f\left(\widehat{\Omega}_{\mathrm{i}} \cdot \hat{n}_{i}\right)}{\sum S_{i} R_{i}} \bar{w}(\vec{r}, E) \frac{1}{f(\widehat{\Omega} \cdot \hat{n})}
$$

\subsection{C.2.3 Directionally dependent weight windows with directional source biasing}

Here it is proposed that the biased source be proportional to both the true source distribution and the approximation of the adjoint angular flux. With a small geometric source, it is also assumed that there is one vector, $\hat{n}_{0}=\hat{n}\left(\vec{r}_{0}, E_{0}\right)$, evaluated at a specific location and energy, which represents the adjoint current direction over that source. The biased source then looks like

$$
\begin{aligned}
\hat{q}(\vec{r}, E, \widehat{\Omega}) & =\frac{1}{R c} q(\vec{r}, E, \widehat{\Omega}) \psi^{+}(\vec{r}, E, \widehat{\Omega}) \\
& =\frac{1}{R c}\left[q(\vec{r}, E) \frac{1}{2 \pi} q\left(\widehat{\Omega} \cdot \hat{d}_{i}\right)\right]\left[\phi^{+}(\vec{r}, E) \frac{1}{2 \pi} f\left(\widehat{\Omega} \cdot \hat{n}_{0}\right)\right],
\end{aligned}
$$

where the constant $R c$ is used to make $\hat{q}$ a pdf. Note that if either the original source directional distribution $q(\widehat{\Omega})$ or the adjoint angular flux distribution at the source is isotropic, then $c=1 / 4 \pi$.

For the general case of multiple sources, the biased source sampling distribution, the biased source distributions and the weight windows are

$$
\begin{aligned}
R_{i} & =\iint q_{i}(\vec{r}, E) \phi^{+}(\vec{r}, E) d E d r \\
c_{i} & =\int \frac{1}{2 \pi} q_{i}\left(\widehat{\Omega} \cdot \hat{d}_{\mathrm{i}}\right) \frac{1}{2 \pi} f\left(\widehat{\Omega} \cdot \hat{n}_{i}\right) d \widehat{\Omega} \\
\hat{p}(i) & =\frac{S_{i} R_{i} c_{i}}{\sum S_{i} R_{i} c_{i}} \\
\hat{q}_{i}(\vec{r}, E, \widehat{\Omega}) & =\left[\frac{1}{R_{i}} q_{i}(\vec{r}, E) \phi^{+}(\vec{r}, E)\right]\left[\frac{1}{c_{i}} q_{i}(\widehat{\Omega}) f(\widehat{\Omega})\right]=\hat{q}_{i}(\vec{r}, E) \frac{1}{c_{i}} \frac{1}{2 \pi} q_{i}\left(\widehat{\Omega} \cdot \hat{d}_{\mathrm{i}}\right) \frac{1}{2 \pi} f\left(\widehat{\Omega} \cdot \hat{n}_{i}\right) \\
\bar{w}(\vec{r}, E, \widehat{\Omega}) & =\frac{\sum S_{i} R_{i} c_{i}}{S \phi^{+}(\vec{r}, E)} \frac{2 \pi}{f(\widehat{\Omega} \cdot \hat{n})} \\
& =\frac{\sum S_{i} R_{i} c_{i}}{\sum S_{i} R_{i}} \bar{w}(\vec{r}, E) \frac{2 \pi}{f(\widehat{\Omega} \cdot \hat{n})} .
\end{aligned}
$$

More details on the development of these methods and their application for several problems have been presented. ${ }^{2,3}$

\subsection{C.2.4 Using space/energy/angle CADIS in MAVRIC}

The two angular CADIS methods that use the AVATAR-type approximation of adjoint flux are specified in MAVRIC with the "angularBiasing=" keyword in the importanceMap block. Values for this keyword are 1 or 2.

Space/Energy/Angle CADIS without directional biasing (for beam sources) - This method uses one specific location, $\vec{r}_{0}$, energy, $E_{0}$, and direction, $\widehat{\Omega}_{0}$, which is the reference direction of the source $\hat{d}$, where the weight of the biased source matches the weight window. 
Space/Energy/Angle CADIS with directional biasing (for general sources) - This method uses one specific energy, $E_{0}$, to determine the adjoint current vector $\hat{n}_{0}$ and the $\lambda_{0}$ parameter for the biased angular distribution for each source.

With each method, the user must specify at what energy the importance map and the biased sources should be made consistent. The particle type must also be specified. This is done with the keywords "angBiasParType=" ( 1 for neutron or 2 for photon) and "angBiasEnergy=" (with a value in $\mathrm{eV}$ ), also in the importanceMap block.

Note that all sources should have a direction $\hat{d}$ set, using "direction $u v w$ " within each source, even if the angular distribution for a given source is isotropic. The direction is used for source biasing and for aligning the weight windows and biased sources. Also note that for either angular biasing method, the Denovo $\mathrm{S}_{\mathrm{N}}$ calculation must use a Legendre order greater than 0 .

With angular biasing, a mesh angular information (*.mai) file is produced which can be visualized with the MeshFileViewer. This file contains the space/energy-dependent $\lambda(\vec{r}, E)$ values and components of the average adjoint current direction $\hat{n}(\vec{r}, E)=\left\langle n_{x}, n_{y}, n_{z}\right\rangle$. An existing mesh angular information (*.mai) file can be used in a separate MAVRIC problem by using the "meshAngInfoFile=" keyword in the biasing block.

\subsection{C.2.5 Example problem}

Consider the Ueki shielding problem used as sample problems in the Monaco and MAVRIC manuals. The goal is to calculate the neutron dose on one side of a shield from a partially collimated ${ }^{252} \mathrm{Cf}$ source on the other side of the shield. Both of the angular approaches discussed above can be compared to analog and standard space/energy CADIS calculations. For the analog calculations, no importanceMap block is used. For the other cases, the importance map blocks are shown below.

\begin{tabular}{|c|c|c|}
\hline CADIS & $\begin{array}{l}\text { Angular CADIS } 1 \text { - without a } \\
\text { biased source angular dist. }\end{array}$ & $\begin{array}{l}\text { Angular CADIS } 2 \text { - with a } \\
\text { biased source angular } \\
\text { dist. }\end{array}$ \\
\hline $\begin{array}{c}\text { read importanceMap } \\
\text { adjointsource } 1 \\
\text { locationID=1 } \\
\text { responseID=5 } \\
\text { end adjointsource } \\
\text { gridGeometryID=7 } \\
\text { macromaterial } \\
\text { mmTolerance=0.01 } \\
\text { end macromaterial } \\
\text { end importanceMap }\end{array}$ & $\begin{array}{c}\text { read importanceMap } \\
\text { adjointSource } 1 \\
\text { locationID=1 } \\
\text { responseID=5 } \\
\text { end adjointSource } \\
\text { gridGeometryID=7 } \\
\text { macromaterial } \\
\text { mmTolerance=0.01 } \\
\text { end macromaterial } \\
\text { angularbiasing=1 } \\
\text { angBiasParType=1 } \\
\text { angBiasEnergy }=2.0 \text { e6 } \\
\text { end importanceMap }\end{array}$ & $\begin{array}{c}\text { read importanceMap } \\
\text { adjointSource } 1 \\
\text { locationID=1 } \\
\text { responseID=5 } \\
\text { end adjointSource } \\
\text { gridGeometryID=7 } \\
\text { macromaterial } \\
\text { mmTolerance }=0.01 \\
\text { end macromaterial } \\
\text { angularbiasing=2 } \\
\text { angBiasParType=1 } \\
\text { angBiasEnergy }=2.0 \text { e } 6 \\
\text { end importanceMap }\end{array}$ \\
\hline
\end{tabular}

Note that the energy at which to tie the importance map to the biased source, $2 \mathrm{MeV}$, is about the average energy of the source energy distribution. The figure-of-merit (FOM) of the calculation could change as this parameter is varied.

Results, shown in Table 4.1.C.1, demonstrate that the two directional approaches improved the FOM for this problem by more than a factor of 2 . A larger boost is seen in method 2 where biasing is applied to the source directional distribution. In this case, the biased source distribution was an exponential distribution 
particles, $m(\vec{r})$, is constant. The physical particle density, $n(\vec{r})$, is related by the average weight, $\bar{w}(\vec{r})$, to the Monte Carlo particle density by

$$
n(\vec{r})=\bar{w}(\vec{r}) m(\vec{r})
$$

To make $m(\vec{r})$ constant over the geometry, the weight window targets $\bar{w}(\vec{r})$ need to be proportional to the physical particle density. Cooper and Larsen calculate the weight window targets from an estimate of the forward scalar flux $\phi(\vec{r})$ to be

$$
\bar{w}(\vec{r})=\frac{\phi(\vec{r})}{\max (\phi(\vec{r}))} .
$$

Two approaches have since been developed, ${ }^{5}$ based on what global information the user desires from the simulation: global flux weight windows, for obtaining every energy group at every location, and global response weight windows, for obtaining an energy-integrated response at every spatial location. Both of these methods are designed for calculating the "global solution" - everywhere in the geometry of the problem - with nearly uniform statistics.

Note that none of the University of Michigan methods discussed here included the development of biased sources. These methods have all been extended to include a consistent biased source by ORNL during their implementation in the MAVRIC sequence of SCALE. The methods have also been extended by ORNL for multiple sources.

\subsection{C.3.1 Weight windows using only forward estimates of flux}

\section{Global flux weight windows}

This method keeps the Monte Carlo particle distribution uniform in space and energy. Note that this is a space/energy version of the original space-only Cooper's Method. The target weight windows, $\bar{w}(\vec{r}, E)$, should be proportional to the estimate of the forward scalar flux, $\phi(\vec{r}, E)$.

$$
\bar{w}(\vec{r}, E)=c \phi(\vec{r}, E) .
$$

A biased source distribution, $\hat{q}(\vec{r}, E)$, that is consistent with the target weight windows can be found from the true source distribution, $q(\vec{r}, E)$, and the forward flux to be

$$
\hat{q}(\vec{r}, E)=\frac{1}{c} \frac{q(\vec{r}, E)}{\phi(\vec{r}, E)}
$$

where the constant $c$ can be determined so that the biased source distribution is a probability distribution function,

$$
c=\int_{V} \int_{E} \frac{q(\vec{r}, E)}{\phi(\vec{r}, E)} d E d \vec{r} .
$$

Thus, using the estimate of forward flux, we can first compute $c$ and then form a consistent set of weight window target values and a biased source distribution. 


\section{Global response weight windows}

For an energy-integrated response (such as dose) desired at all spatial locations, this method keeps the Monte Carlo particle distribution proportional to the integral of the product of the response function, $\sigma_{d}(\vec{r}, E)$, and the estimate of the forward flux, $\phi(\vec{r}, E)$. The energy-integrated response, $D(\vec{r})$, is found from the estimate of the forward flux as

$$
D(\vec{r})=\int_{E} \sigma_{d}(\vec{r}, E) \phi(\vec{r}, E) d E
$$

The target weight windows, $\bar{w}(\vec{r}, E)$, should be

$$
\bar{w}(\vec{r}, E)=c \frac{D(\vec{r})}{\sigma_{d}(\vec{r}, E)} .
$$

A biased source distribution, $\hat{q}(\vec{r}, E)$, that is consistent with the target weight windows can be found from the true source distribution, $q(\vec{r}, E)$, and the forward dose estimate to be

$$
\hat{q}(\vec{r}, E)=\frac{1}{c} \frac{q(\vec{r}, E) \sigma_{d}(\vec{r}, E)}{D(\vec{r})}
$$

using a proportionality constant of

$$
c=\int_{V} \frac{1}{D(\vec{r})} \int_{E} q(\vec{r}, E) \sigma_{d}(\vec{r}, E) d E d \vec{r}
$$

\section{Implementation in MAVRIC}

The global flux weight windows (GFWW) approach and the global response weight windows (GRWW) are both triggered by specifying an importance map block without any adjoint sources. For the GRWW approach, the response of interest is listed in the importance map block. If none is listed, GFWW is used.

For problems with multiple sources (each with probability distribution function $q_{i}(\vec{r}, E)$ and strength $S_{i}$, giving a total source strength of $S=\sum S_{i}$ ), the GWW methods require a biased mesh source for each. This was implemented in MAVRIC in a manner similar to the multiple source CADIS routines. Each biased source, $\hat{q}_{i}(\vec{r}, E)$, is developed as

$$
\hat{q}_{i}(\vec{r}, E)=\left\{\begin{array}{cc}
\frac{1}{c_{i}} \frac{q_{i}(\vec{r}, E)}{\phi(\vec{r}, E)} & \text { global flux weight windows } \\
\frac{1}{c_{i}} \frac{q_{i}(\vec{r}, E) \sigma_{d}(\vec{r}, E)}{D(\vec{r})} & \text { global response weight windows }
\end{array},\right.
$$

where $c_{i}$ is a normalization constant. The weight windows are then set to

$$
\bar{w}(\vec{r}, E)=\left\{\begin{array}{lc}
\frac{\sum c_{i}}{\sum S_{i}} \phi(\vec{r}, E) & \text { global flux weight windows } \\
\frac{\sum c_{i}}{\sum S_{i}} \frac{D(\vec{r})}{\sigma_{d}(\vec{r}, E)} & \text { global response weight windows }
\end{array} .\right.
$$


In the final Monte Carlo, the specific source $i$ is sampled with probability $p(i)=S_{i} / S$, and then the particle is sampled from the biased mesh source $\hat{q}_{i}(\vec{r}, E)$. Unlike the CADIS method for multiple sources, there is no way to develop a biased probability distribution for which source to sample without knowing the contribution to the global estimate from each source separately.

For multiple source problems where the expected contribution from each source is very different from the true strengths of those sources, it may be more efficient to run a series of problems with one source each for different amounts of time. The resulting mesh tallies can then be added together using the mesh tally adder (part of the MAVRIC utilities).

\subsection{C.3.2 Methods using forward and adjoint estimates}

Becker $^{6}$ proposed three methods for developing weight windows based on both forward and adjoint deterministic solutions. These three methods correspond to the portion of the phase space over which uniform relative uncertainties are desired: a small "detector" region, a region comprising a significant portion of the entire problem, and the global problem. In this discussion, only a brief outline of each method, focusing on its implementation into MAVRIC, will be given.

\section{Source/detector problems}

For a small detector of volume $V_{D}$ where we want to optimize the $\mathrm{MC}$ calculation of the detector response

$$
R=\int_{V_{D}} \int_{0}^{\infty} \sigma(\vec{r}, E) \phi(\vec{r}, E) d E d V
$$

or optimize for the energy dependent flux at the detector, the following is used:

$$
\begin{aligned}
& \text { forward flux } \\
& \text { estimate } \\
& \text { adjoint source } \\
& \text { for flux } \\
& \text { or } \\
& \text { for response } \\
& \text { adjoint flux } \\
& \phi(\vec{r}, E) \\
& q^{+}(\vec{r}, E)=1 /_{\phi(\vec{r}, E)} \\
& q^{+}(\vec{r}, E)=\sigma(\vec{r}, E) \\
& \phi^{+}(\vec{r}, E) \\
& \phi^{c}(\vec{r}, E)=\phi(\vec{r}, E) \phi^{+}(\vec{r}, E) \\
& C_{\text {norm }}=V_{D} / \int_{V_{D}} \int_{0}^{\infty} \phi^{c}(\vec{r}, E) d E d V \\
& \tilde{\phi}^{c}(\vec{r})=C_{\text {norm }} \int_{0}^{\infty} \phi^{c}(\vec{r}, E) d E \\
& \alpha(\vec{r})=\left[1+\exp \left(\frac{\tilde{\phi}_{\max \in V_{D}}^{c}}{\tilde{\phi}^{c}(\vec{r})}-\frac{\tilde{\phi}^{c}(\vec{r})}{\tilde{\phi}_{\max \in V_{D}}^{c}}\right)\right]^{-1} \\
& B(\vec{r})=\alpha(\vec{r}) \tilde{\phi}^{c}(\vec{r})+1-\alpha(\vec{r}) \\
& \bar{w}(\vec{r}, E)=\frac{B(\vec{r})}{\phi^{+}(\vec{r}, E)}
\end{aligned}
$$




\section{Source-region problems}

For a detector of volume $V_{D}$ and surface area $A_{D}$ (smaller than the entire problem) where we want to optimize the $\mathrm{MC}$ calculation of the detector response

$$
R(\vec{r})=\int_{0}^{\infty} \sigma(\vec{r}, E) \phi(\vec{r}, E) d E \quad \vec{r} \in V_{D}
$$

or optimize for the energy dependent flux in the region, the following is used:

$$
\begin{aligned}
& \text { forward flux } \\
& \text { estimate } \\
& \text { adjoint source } \\
& \text { for flux } \\
& \text { or } \\
& \text { for response } \\
& \phi(\vec{r}, E) \\
& q^{+}(\vec{r}, E)=1 / \phi(\vec{r}, E) \\
& q^{+}(\vec{r}, E)=\sigma(\vec{r}, E) / \int_{0}^{\infty} \sigma(\vec{r}, E) \phi(\vec{r}, E) d E \\
& \phi^{+}(\vec{r}, E) \\
& \phi^{c}(\vec{r}, E)=\phi(\vec{r}, E) \phi^{+}(\vec{r}, E) \\
& C_{\text {norm }}=A_{D} / \int_{A_{D}} \int_{0}^{\infty} \phi^{c}(\vec{r}, E) d E d A \\
& \tilde{\phi}^{c}(\vec{r})=C_{\text {norm }} \int_{0}^{\infty} \phi^{c}(\vec{r}, E) d E \\
& \alpha(\vec{r})=\left[1+\exp \left(\frac{\tilde{\phi}_{\max \in V_{D}}^{c}}{\tilde{\phi}^{c}(\vec{r})}-\frac{\tilde{\phi}^{c}(\vec{r})}{\tilde{\phi}_{\max \in V_{D}}^{c}}\right)\right]^{-1} \\
& B(\vec{r})=\left\{\begin{array}{cc}
\tilde{\phi}^{c}(\vec{r}) & \vec{r} \in V_{D} \\
\alpha(\vec{r}) \tilde{\phi}^{c}(\vec{r})+1-\alpha(\vec{r}) & \text { otherwise }
\end{array}\right. \\
& \bar{w}(\vec{r}, E)=\frac{B(\vec{r})}{\phi^{+}(\vec{r}, E)}
\end{aligned}
$$

Note that $A_{D}$ does not include surfaces of $V_{D}$ which are on the boundary of the problem.

\section{Global response problem}

For optimizing the Monte Carlo calculation of a detector response everywhere in phase space

$$
R(\vec{r})=\int_{0}^{\infty} \sigma(\vec{r}, E) \phi(\vec{r}, E) d E
$$

or optimizing for the energy-dependent flux everywhere, the following is used:

forward flux

estimate

$$
\phi(\vec{r}, E)
$$




$$
\begin{aligned}
& \text { adjoint source } \\
& \text { for flux } \\
& q^{+}(\vec{r}, E)=1 / \phi(\vec{r}, E) \\
& \text { or } \\
& \text { for response } \\
& q^{+}(\vec{r}, E)=\sigma(\vec{r}, E) / \int_{0}^{\infty} \sigma(\vec{r}, E) \phi(\vec{r}, E) d E \\
& \phi^{+}(\vec{r}, E) \\
& \phi^{c}(\vec{r}, E)=\phi(\vec{r}, E) \phi^{+}(\vec{r}, E) \\
& \phi^{c}(\vec{r})=\int_{0}^{\infty} \phi^{c}(\vec{r}, E) d E \\
& B(\vec{r})=\phi^{c}(\vec{r}) \\
& \bar{w}(\vec{r}, E)=\frac{B(\vec{r})}{\phi^{+}(\vec{r}, E)}
\end{aligned}
$$

\subsection{C.3.3 Implementation in MAVRIC}

Like CADIS and FW-CADIS, the Denovo $\mathrm{S}_{\mathrm{N}}$ code is used to calculate the forward flux estimate, $\phi(\vec{r}, E)$, and the estimate of the adjoint flux, $\phi^{+}(\vec{r}, E)$, for all of the Michigan weight window methods.

None of the above discussions of the University of Michigan methods include information on how the weight window target values were adjusted to match the source sampling. When implemented into MAVRIC, each of the above problem types will compute a biased source, $\hat{q}(\vec{r}, E)$, along with the target weight, $\bar{w}(\vec{r}, E)$, that are produced. For a problem with a single source of strength $S$ and distribution $q(\vec{r}, E)$, the biased source distribution $\hat{q}(\vec{r}, E)$ is found by using

$$
\hat{q}(\vec{r}, E)=q(\vec{r}, E) / \bar{w}(\vec{r}, E) .
$$

The weight windows are multiplied by a factor of $R / S$, where $R$ is defined as

$$
R=\iint \hat{q}(\vec{r}, E) \phi^{+}(\vec{r}, E) d E d V
$$

Sampled source particles will then be born with a weight that matches the weight window of the phase space where they are born.

For multiple sources, each with strength $S_{i}$ and distribution $q_{i}(\vec{r}, E)$, each biased source distribution $\hat{q}_{i}(\vec{r}, E)$ is found by using

$$
\hat{q}_{i}(\vec{r}, E)=q_{i}(\vec{r}, E) / \bar{w}(\vec{r}, E)
$$

and the response from each source being

$$
R_{i}=\iint \hat{q}_{i}(\vec{r}, E) d E d V .
$$


The individual sources are sampled with a biased probability of $\hat{p}(i)=R_{i} / \sum R_{i}$. The weight windows are then multiplied by a factor of

$$
\frac{\sum_{i} R_{i}}{\sum_{i} S_{i}}
$$

to match the source birth weights.

To use one of the Becker methods in MAVRIC, the keyword "beckerMethod=" is used with values of 1, 2 , or 3 for the source/detector, source/region, or global method. Adjoint sources are described just like standard MAVRIC CADIS and FW-CADIS problems. To switch between optimizing flux in every group or optimizing a response, the keywords "fluxWeighting" and "respWeighting" are used. Just like FW-CADIS, the response listed in each adjoint source is the response that is optimized. Note that even when starting a calculation with known forward and adjoint flux files, the adjoint source(s) still need to be listed since they are used in the final normalization of the weight windows.

\subsection{C.3.4 Example problems}

\section{A source/detector problem}

The first Becker method (source/detector) is demonstrated using the Ueki shielding problem (used as sample problems in the Monaco and MAVRIC manuals and above in the space/energy/angle CADIS example). The goal is to calculate the neutron dose on one side of a shield from a partially collimated ${ }^{252} \mathrm{Cf}$ source on the other side of the shield. For the analog calculations, no importanceMap block is used. For the other cases, the importance map blocks are shown below.

\begin{tabular}{|c|c|c|}
\hline CADIS & Becker 1 - flux optimization & $\begin{array}{l}\text { Becker } 2 \text { - response } \\
\text { optimization }\end{array}$ \\
\hline $\begin{array}{c}\text { read importanceMap } \\
\text { adjointsource } 1 \\
\text { locationID }=1 \\
\text { responseID }=5 \\
\text { end adjointSource } \\
\text { gridGeometryID=7 } \\
\text { macromaterial } \\
\text { mmTolerance }=0.01 \\
\text { end macromaterial }\end{array}$ & $\begin{array}{c}\text { read importanceMap } \\
\text { adjointSource } 1 \\
\text { locationID=1 } \\
\text { responseID=5 } \\
\text { end adjointSource } \\
\text { gridGeometryID=7 } \\
\text { macromaterial } \\
\text { mmTolerance=0.01 } \\
\text { end macromaterial } \\
\text { beckerMethod=1 } \\
\text { fluxWeighting }\end{array}$ & $\begin{array}{c}\text { read importanceMap } \\
\text { adjointsource } 1 \\
\text { locationID=1 } \\
\text { responseID }=5 \\
\text { end adjointsource } \\
\text { gridGeometryID=7 } \\
\text { macromaterial } \\
\text { mmTolerance=0.01 } \\
\text { end macromaterial } \\
\text { beckerMethod=1 } \\
\text { respWeighting }\end{array}$ \\
\hline end importanceMap & end importanceMap & end importanceMap \\
\hline
\end{tabular}

The results are shown in Table 4.1.C.2. The Becker response-optimized source/detector method, which requires two deterministic estimates, does slightly better for this problem than standard CADIS, which only requires one deterministic estimate. 
Table 4.1.C.2. Results of the Ueki Shielding Problem (35 cm graphite shield)

\begin{tabular}{|c|c|c|c|c|c|c|}
\hline Calculation Method & \begin{tabular}{|c} 
For. $S_{\wedge}$ \\
$(\mathrm{min})$
\end{tabular} & $\begin{array}{l}\text { Adj. } S_{N} \\
(\min )\end{array}$ & $\begin{array}{c}\mathrm{MC} \\
\text { (min) }\end{array}$ & $\begin{array}{c}\text { dose rate } \\
(\mathrm{rem} / \mathrm{hr})\end{array}$ & $\begin{array}{r}\text { relative } \\
\text { uncert }\end{array}$ & $\begin{array}{c}\text { MC FOM } \\
(/ \mathrm{min})\end{array}$ \\
\hline Analog & 0.0 & 0.0 & 152.7 & $3.998 \mathrm{E}-03$ & 0.0101 & 64.7 \\
\hline CADIS & 0.0 & 0.2 & 9.9 & $3.988 \mathrm{E}-03$ & 0.0081 & 1550 \\
\hline Becker, source/detector, flux & 0.2 & 0.2 & 9.9 & $4.027 \mathrm{E}-03$ & 0.0099 & 1040 \\
\hline Becker, source/detector, resp & 0.2 & 0.2 & 10.1 & 4.025E-03 & 0.0075 & 1760 \\
\hline
\end{tabular}

\section{A global problem}

For an example of a global problem, consider a two-room block building with a criticality accident in one room. The objective is to find the photon dose everywhere in order to see the locations where criticality alarms would trigger. The building is 12 meters long, 6 meters wide, and 3 meters tall. A comparison will be made between an analog calculation, an FW-CADIS calculation (using response weighting), GRWW, Becker's source/region method (response optimization), and Becker's global method (response optimization). The MAVRIC importance map block of each calculation is as follows.

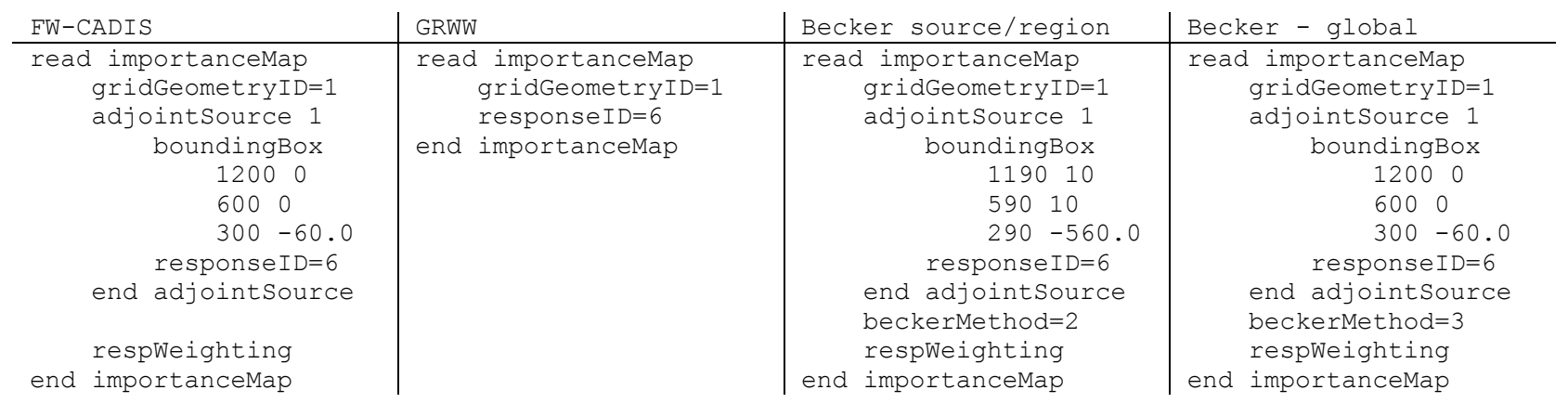

Note that Becker's source/region method is designed for regions smaller than the entire problem, so this is not a fair comparison, just a demonstration on how to use it in MAVRIC. The bounding box of the adjoint source in this case is set slightly smaller than the extent of the entire problem.

Results for the photon dose and its relative uncertainty using the five different methods of calculation are shown in Figure 4.1.C.3. Information on the distribution of relative uncertainties is shown in Figure 4.1.C.4. and listed in Table 4.1.C.3.

A more detailed comparison of the different hybrid methods for representative shielding problems can be found in Ref. 7 and Ref. 8. 

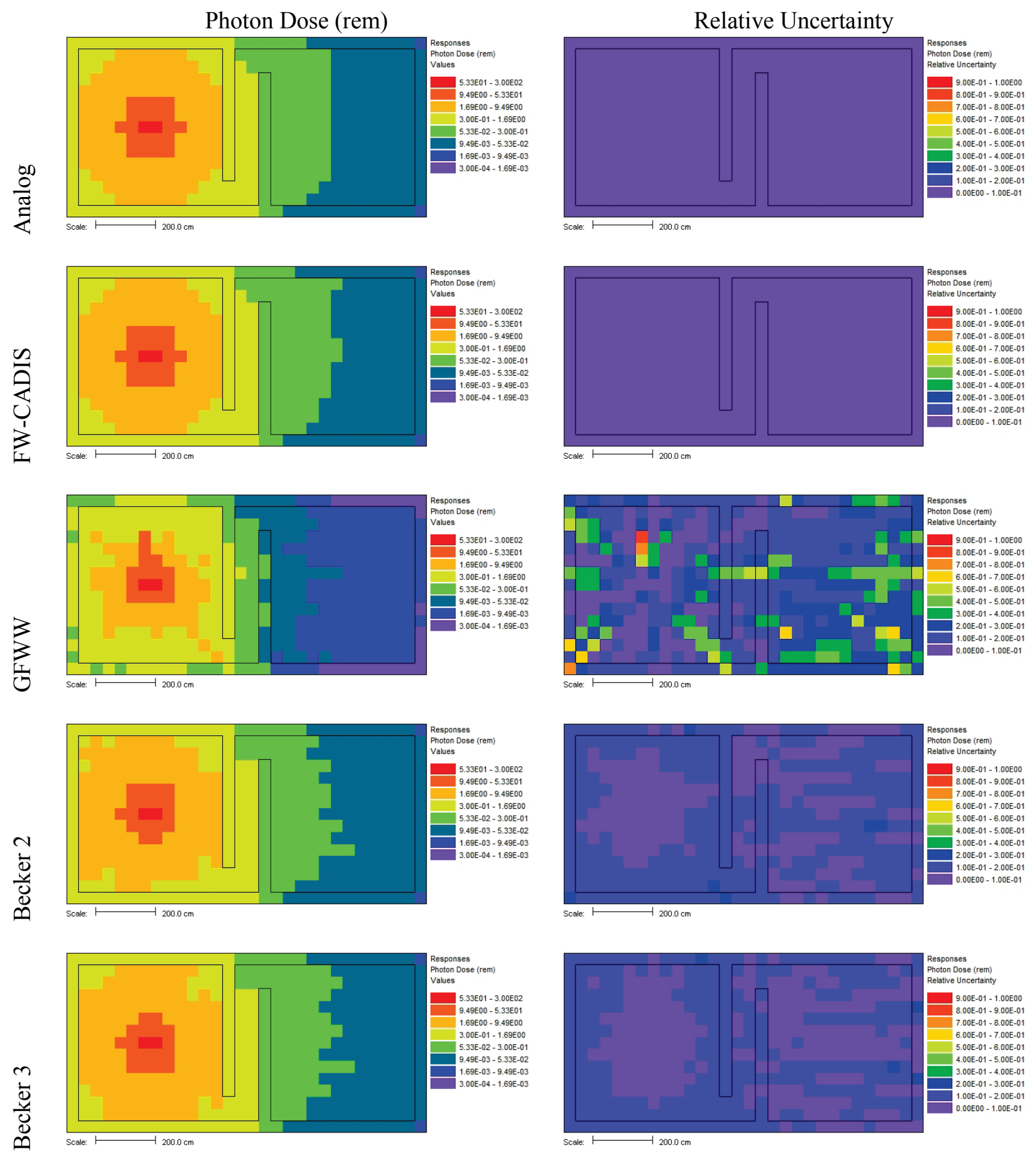

Figure 4.1.C.3. Mesh tally results for the photon dose over the entire building use five different methods. 


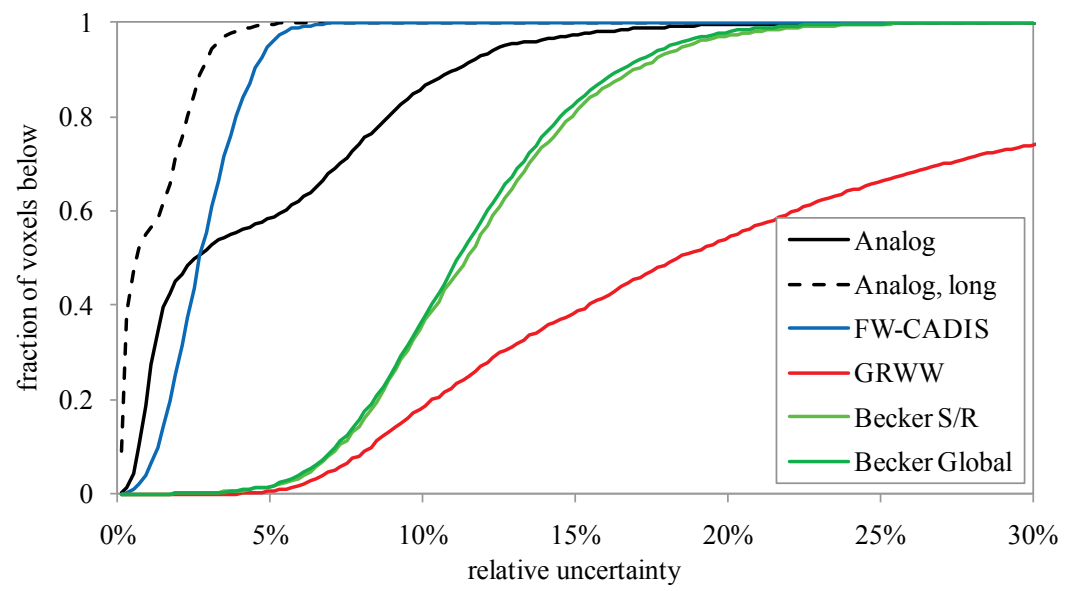

Figure 4.1.C.4. The fraction of mesh tally voxels with less than a given amount of relative uncertainty for the five different methods.

Table 4.1.C.3. Comparison of the five different methods

\begin{tabular}{|c|c|c|c|c|c|c|}
\hline \multirow[b]{2}{*}{ Calculation Method } & \multirow{2}{*}{$\begin{array}{c}\text { Forward } \\
\mathrm{S}_{\mathrm{N}} \\
(\mathrm{min}) \\
\end{array}$} & \multirow{2}{*}{$\begin{array}{l}\text { Adjoint } \\
\mathrm{S}_{\mathrm{N}} \\
(\mathrm{min})\end{array}$} & \multirow{2}{*}{$\begin{array}{l}\text { Monte } \\
\text { Carlo } \\
\text { (min) }\end{array}$} & \multicolumn{3}{|c|}{$\begin{array}{c}\text { fraction of voxels with } \\
\text { relative uncertainties less than }\end{array}$} \\
\hline & & & & $5 \%$ & $10 \%$ & $15 \%$ \\
\hline Analog & & & 20.1 & 0.583 & 0.857 & 0.973 \\
\hline Analog, long & & & 308.1 & 0.996 & 1.000 & 1.000 \\
\hline FW-CADIS, response weighting & 3.5 & 3.4 & 20.3 & 0.946 & 1.000 & 1.000 \\
\hline GRWW & 4.0 & & 20.2 & 0.005 & 0.180 & 0.387 \\
\hline Becker, source/region & 4.0 & 3.6 & 19.0 & 0.016 & 0.350 & 0.800 \\
\hline Becker, global response & 4.2 & 4.4 & 20.4 & 0.016 & 0.358 & 0.832 \\
\hline
\end{tabular}

\subsection{C.3.5 Other special options for the importance map block input}

In MAVRIC, the above methods have been extended to use biased sources. To turn off the use of a biased source, use the keyword "selfNormalize". For forward flux-based methods, this will scale the target weights in the importance map so that the largest value is 1 , which typically is at the source location. To use the forward-based Michigan methods (Cooper, GFWW, GRWW) or the van Wijk ${ }^{9}$ methods as they were presented (without the MAVRIC biased source), use this keyword.

Use the keyword "spaceOnly $=n$ " to create an importance map that contains one energy group over the entire range of energy. Use a value of 1 for neutron problems or a value of 2 for photon problems. This option is not designed for coupled problems. Both of the methods that van Wijk presents use importance maps that are space only.

Use the keyword "forwardParam= $p$ " to allow the target weights to be proportional to the forward estimate (using Denovo) of flux raised to any power $p$. 


$$
\bar{w}(\vec{r})=\frac{\phi^{p}(\vec{r})}{\max \left(\phi^{p}(\vec{r})\right)} .
$$

For Cooper's method or van Wijk's first method, $p=1$, which is the default if "forwardParam" is not specified. For van Wijk's second method, based on the relative error estimate $\operatorname{Re}(\vec{r}) \propto 1 / \sqrt{\phi(\vec{r})}$, set this parameter to $p=0.5$. Note that with a power less than 1 , the weight window targets will span a smaller range than the expected Monte Carlo flux values. Particles may be rouletted before they reach the lowest flux areas of the problem.

When applying FW-CADIS for a semi-global problem, the adjoint source should cover the area where the lowest uncertainties are desired. Sometimes the area where this is desired is not a fixed geometric area but instead an area that has a final answer of a certain range. For example, when finding dose rate maps, the user may only be interested in getting low uncertainties for areas below the dose limit - areas above the dose limit may not matter as much since people will not be allowed in those areas. Another example may be that the dose map should be optimized in those areas above the background dose rate, since dose rates below that are of no concern. To allow the adjoint source area to be tailored in this way, the keywords "minForwardValue=" and/or "maxForwardValue="can be used. Default values are 0 and $10^{100}$, respectively. For flux weighting, only the voxels within the adjoint source bounding box and containing a forward flux estimate between the min and max are included as adjoint sources.

A comparison ${ }^{10}$ of FW-CADIS to the University of Michigan methods and the Van Wijk methods showed that FW-CADIS performs better and is more straightforward to use than the other methods. These other methods were added to SCALE so that a fair comparison could be made and have been left in the code base for academic use in case some types of problems could benefit from them.

\subsection{C.4 Using MAVRIC to run fixed-source Denovo calculations}

The MAVRIC sequence of SCALE can be used to run the new discrete-ordinates code Denovo. ${ }^{11}$ The sequence is designed to use Denovo to compute an importance map for the Monte Carlo code Monaco but can be stopped without starting the final Monaco calculation. The full version of Denovo has a pythonbased user interface where the user must construct the geometry, source, and cross sections on their own. MAVRIC provides an easy interface to run the serial-only version of Denovo within SCALE (called 'xkba'). Denovo inputs made using MAVRIC can also be imported into the python interface for the HPC version of Denovo.

To create Denovo inputs or run Denovo using MAVRIC, input the composition, geometry (including arrays if needed), definitions (grid geometry and response information), and sources block as if an actual full MAVRIC case were being run. The sequence should then be started with a parm=xxx flag to indicate to MAVRIC to stop after making Denovo inputs or running Denovo.

\begin{tabular}{ll} 
parm $=$ & MAVRIC stops after \\
\hline $\begin{array}{l}\text { Forward Calculations } \\
\text { forinp }\end{array}$ & $\begin{array}{l}\text { Making cross sections and Denovo input } \\
\text { forward }\end{array}$ \\
$\begin{array}{l}\text { Adjoint Calculations } \\
\text { adjinp }\end{array}$ & $\begin{array}{l}\text { Making cross sections and Denovo input } \\
\text { adjoint }\end{array}$ \\
\end{tabular}


For forward Denovo simulations, the importance map block should contain which grid geometry to use and any of the optional Denovo parameters:

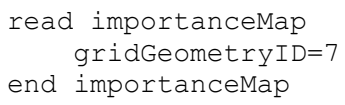

For adjoint Denovo calculations, the importance map block should contain which grid geometry to use, one or more adjoint sources, and any of the optional Denovo parameters:

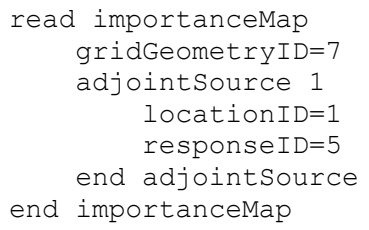

For either forward or adjoint, the following files will be produced and should be copied out of the SCALE temporary directory back to your home area:

$\mathrm{ft} 02 \mathrm{f001}$ the AMPX mixed working library cross section file

xkba_b.inp binary input file for Denovo

*.dff $\quad$ Denovo flux file (binary) - scalar fluxes

The scalar flux file is automatically copied back to the home area when SCALE completes. The others can be copied back if desired using a shell command at the end of the MAVRIC input file (windows example shown below):

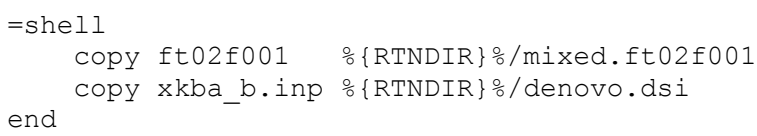

The xkba_b.inp file and the *.dff file can both be viewed with the Java utility, Mesh File Viewer, that is shipped with SCALE. The xkba_b.inp file shows the Denovo source distribution (space and energy), and the *.dff shows the final computed scalar fluxes. The binary Denovo input file should be renamed with an extension of *.dsi (Denovo simple input) so that the viewer can properly interpret the data.

\subsection{C.4.1 Optional Denovo parameters}

The default values for various calculational parameters and settings used by Denovo for the MAVRIC sequence should cover most applications. These settings can be changed by the interested user in the importance map block. The two most basic parameters are the quadrature set used for the discrete ordinates and the order of the Legendre polynomials used in describing the angular scattering. The default quadrature order that MAVRIC uses is $\mathrm{S}_{8}$, and for the order of Legendre polynomials, the default is $\mathrm{P}_{3}$ (or the maximum number of coefficients contained in the cross section library, if less than 3 ). $\mathrm{S}_{8} / \mathrm{P}_{3}$ should be a good choice for many applications, but the user is free to changes these. For complex ducts or transport over large distance at small angles, $\mathrm{S}_{12}$ may be required. $\mathrm{S}_{4} / \mathrm{P}_{1}$ would be useful in doing a very cursory run just to check if the problem was input correctly. Other parameters that can be set by the user in the importance map block for Denovo calculations are listed in Table 4.1.C.4. 
Table 4.1.C.4. Optional Denovo parameters for the MAVRIC Importance Map Block

\begin{tabular}{|c|c|c|c|c|c|}
\hline keyword & type & length & default & \multicolumn{2}{|c|}{ required restrictions/comments } \\
\hline \multicolumn{6}{|l|}{ importanceMap } \\
\hline \multicolumn{6}{|c|}{ Optional Denovo $S_{N}$ code parameters } \\
\hline discretization= & integer & & 4 & no & $\begin{array}{l}\text { 0-diamond-difference, 1-diamond-difference with flux fix-up:lin0, } \\
\text { 2-theta-weighted diamond-difference, 3-linear-discontinuous finite } \\
\text { element, 4-step characteristics, 5-trilinear discontinuous finite elem }\end{array}$ \\
\hline quadType= & integer & & 0 & no & 0-level symmetric, 1-Gauss-Legendre product, 2-QR \\
\hline legendre= & integer & & $3^{*}$ & no & $\begin{array}{l}P_{L}, L=\text { highest Legendre polynomial, } L=0,1,2,3, \ldots \\
\text { *default is to use min(the highest available in the data, } 3 \text { ) }\end{array}$ \\
\hline tportcorrection= & integer & & $1^{*}$ & no & $\begin{array}{l}\text { transport correction: } 0 \text {-none, 1-diagonal, 2-Cesaro } \\
{ }^{*} \text { For } \mathrm{P}_{2} \text { or higher, the default is } 2 \text { (Cesaro) }\end{array}$ \\
\hline upScatter= & integer & & 0 & no & upscatter iterations: 0-none, 1-yes, 2-ignore \\
\hline xblocks $=$ & integer & & 1 & no & parallel stuff \\
\hline yblocks= & integer & & 1 & no & parallel stuff \\
\hline zblocks= & integer & & 1 & no & parallel stuff \\
\hline numSets $=$ & integer & & 1 & no & $?$ \\
\hline partUpscatter= & integer & & 1 & no & partition upscatter (0-no, 1-yes) \\
\hline quadrature $=$ & integer & & 8 & no & level symmetric $S_{N}$ quadrature, $N=2,4,6,8,10,12,14,16$ \\
\hline polarsPerOct $=$ & integer & & 0 & no & Gauss-Legendre product quadrature or QR \\
\hline azimuthsPerOct= & integer & & 0 & no & Gauss-Legendre product quadrature or QR \\
\hline maxlters $=$ & integer & & 20 & no & maximum number of iterations \\
\hline diagnostics $=$ & integer & & 0 & no & 0-no diganostics, 1-all diagnostics \\
\hline output= & integer & & 0 & no & 0-no ouput, 1-all output \\
\hline krylovSpaceSize= & integer & & 10 & no & size in memory for Krylov space \\
\hline tolerance= & double & & 1.E-03 & no & tolerance used in convergence test \\
\hline krylovType= & integer & & 0 & no & 0-GMRES, 1-BiCGStab \\
\hline eigenSolver= & integer & & 0 & no & 0-power iteration, 1-Arnoldi, 2-shifted inverse \\
\hline multiGSolover= & integer & & 0 & no & 0-Gauss-Seidel, 1-Krylov \\
\hline withinGSolver= & integer & & 0 & no & 0-Krylov, 1-residual Krylov, 2-source iteration \\
\hline mgSettings $=$ & integer & & 1 & no & 0-user supplied, 1-automatic \\
\hline upGroupSolver= & integer & & 0 & no & $\begin{array}{l}\text { 0-same as within-group solver, 1-Krylov, 2-residual Krylov, } \\
\text { 3-source iteration, 4-single source iteration }\end{array}$ \\
\hline acceleration= & integer & & 0 & no & 0-none, 1-two grid \\
\hline maxltersMG= & integer & & 20 & no & maximum number of iterations \\
\hline $\begin{array}{l}\text { toleranceMG= } \\
\text { end importanceMap }\end{array}$ & double & & 0.001 & no & tolerance used in convergence test \\
\hline
\end{tabular}

In problems with small sources or media that are not highly scattering, discrete ordinates can suffer from "ray effects"-where artifacts of the quadrature directions can be seen in the computed fluxes. To help alleviate the ray effects problem, Denovo has a first-collision capability. This computes the amount of uncollided flux in each mesh cell from a point source. These uncollided fluxes are then used as a distributed source in the main discrete-ordinates solution. At the end of the main calculation, the uncollided fluxes are added to the fluxes computed with the first-collision source, forming the total flux. While this helps reduce ray effects in many problems, the first-collision capability can take a long time to compute on a mesh with many cells or for many point sources.

The macromaterials option ("mmTolerance=") can be used to better represent the geometry in Denovo. Refer to the main MAVRIC manual for details on macromaterials.

\subsection{C.4.2 Forward source preparations}

The user-entered source description is converted to a mesh-based source for Denovo. To create the mesh source, MAVRIC determines if the defined source exists within each cell. This is done by dividing each mesh cell into $n \times n \times n$ subcells (from the keyword "subCells=n" in the importance map block with a 
default of $n=2$ ) and testing each subcell center. For every subcell center that is a valid source position (within the spatial solid and meets optional unit, region, or mixture requirements), an amount of source proportional to the subcell volume is assigned to the mesh cell. The keyword "subCells=" can be used to better refine how much source is computed for the mesh cells at the boundary of a curved source region. Of course, more subcell testing takes more time.

The above process may miss small sources or degenerate sources (surfaces, lines, points) that do not lie on the tested subcell centers. If none of the mesh cells contain any source after the subcell method, then random sampling of the source is used. A number of source positions are sampled from the source (set by the "sourceTrials=" keyword, default of 1000000) and then placed into the proper mesh cell. If this method is used, the resulting Denovo input file should be visualized to ensure that the statistical nature of the source trials method does not unduly influence the overall mesh source.

For forward calculations, if the number of mesh cells containing the true source is less than 10, then MAVRIC will convert these source voxels to point sources, to allow Denovo to use its first-collision capability to help reduce ray effects. The user can easily override the MAVRIC defaults - to force the calculation of a first-collision source no matter how many voxels contain source-by using the keyword "firstCollision." To prevent the calculation of a first-collision source, the keyword "noFirstCollision" can be used.

For coupled problems, there are two ways to make Denovo compute photon fluxes from a neutron source: 1) include a tally using a photon response or 2) manually specify the "startGroup=" and the "endGroup=" to cover the particles/energy groups that are desired in the final Denovo output.

\subsection{C.4.3 Adjoint source preparation}

For adjoint calculations, adjoint sources that use point locations will use the Denovo first-collision capability. Volumetric adjoint sources (that use a boundingBox) will be treated without the first-collision capability. The keywords "firstCollision" and "noFirstCollision" will be ignored by MAVRIC for adjoint calculations.

Note that for an adjoint Denovo calculation, the MAVRIC input must still list a forward source. Otherwise, the sequence will report an error and stop. The forward source is not used for an adjoint Denovo calculation, but it must be present to make a legal MAVRIC input. For a coupled problem using an adjoint photon source, using a neutron forward source will make Denovo compute both photon and neutron adjoint fluxes. If the forward source(s) and adjoint source(s) are all photon, then only photon adjoint fluxes will be computed. The keywords "startGroup=" and "endGroup=" can also be used to manually set the particles/energy groups that are desired in the final Denovo output.

\subsection{C.4.4 Other notes}

Denovo (in SCALE 6) is a fixed-source $\mathrm{S}_{\mathrm{N}}$ solver and cannot model multiplying media. Neither forward nor adjoint neutron calculations from Denovo will be accurate when neutron multiplication is a major source component. If neutron multiplication is not turned off in the parameters block of the MAVRIC input (using keyword "noFissions"), a warning will be generated to remind the user of this limitation.

By default, MAVRIC instructs Denovo not to perform outer iterations for neutron problems if the crosssection library contains upscatter groups. This is because the time required to calculate the fluxes using upscatter can be significantly longer than without. For problems where thermal neutrons are an important part of the transport or tallies, the user should specify the keyword "upScatter=1" in the importance map 
block. This will instruct Denovo to perform the outer iterations for the upscatter groups, giving more accurate results but taking a longer time.

\subsection{C.4.5 MAVRIC utilities for Denovo}

Denovo simply calculates scalar fluxes for every mesh cell and energy group - it does not compute responses based on those fluxes. Several utilities have been added to the collection of MAVRIC utilities in order to allow the user to further process Denovo scalar flux results. The full details and input descriptions for these utilities can be found in the MAVRIC Utilities description in Appendix B.

$\begin{array}{ll}\text { dff2dso } & \text { Convert a Denovo flux file into a Denovo spatial output file. } \\ \text { dff2mai } & \text { Convert a Denovo flux file into a mesh angular information file. } \\ \text { dff2mim } & \text { Invert a Denovo flux file and store as a mesh importance map. } \\ \text { dff2msl } & \text { Convert a Denovo flux file into a mesh source lite. } \\ \text { dffBinOp } & \text { Binary operation of Denovo flux files: sum, difference, product, and ratio. } \\ \text { dffDisp } & \text { Display the basics of a Denovo flux file. } \\ \text { dffFilter } & \text { Perform various filters on a Denovo flux file. } \\ \text { dffFix } & \text { Fix the zero and negative values in a Denovo flux file. } \\ \text { dffInt } & \text { Integrate a single particle type from a Denovo flux file. } \\ \text { dffInv } & \text { Invert the values in a Denovo flux file. } \\ \text { dffMult } & \text { Multiply a Denovo flux file by a constant factor. } \\ \text { dffPull } & \text { Pull fluxes from certain voxels out of a Denovo flux file. } \\ \text { dffResp } & \text { Apply a response function to scalar fluxes in a Denovo flux file. } \\ \text { dffSplit } & \text { Split off a single particle type from a Denovo flux file. }\end{array}$

There are also two utility programs that look at the Denovo simple input file (binary) that MAVRIC creates. In the SCALE temporary directory, the file is called xkba_b.inp. In order to display in the MeshFileViewer, Denovo simple input files need to be renamed with $\bar{a}{ }^{*}$. dsi extension. The utilities are as follows.

dsi2asc Convert a Denovo simple input (*.dsi) from binary to ASCII (so the user can see every detail).

dsiDisp Display the basics of a Denovo simple input file.

\subsection{C.4.6 Example problem}

\section{Forward}

As an example, consider a simulation based on the Ueki shielding experiments (see Monaco manual). A ${ }^{252} \mathrm{Cf}$ neutron source was placed in the center of a $50 \mathrm{~cm}$ cube of paraffin which had a $45^{\circ}$ cone cut-out. The goal is to calculate the neutron dose at a detector $110 \mathrm{~cm}$ from the source.

The input file (denovo1.inp) needs the compositions of the paraffin and graphite

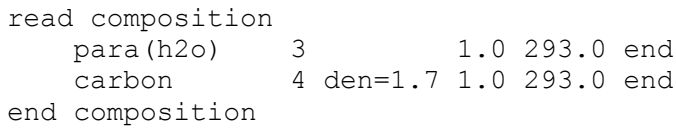

then the geometry 


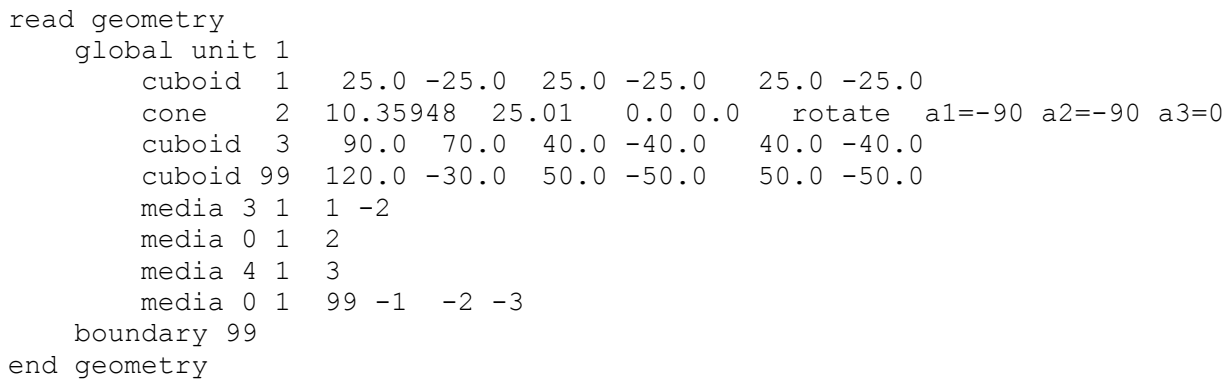

then the position of the detector, the response of the detector and the mesh to use for Denovo

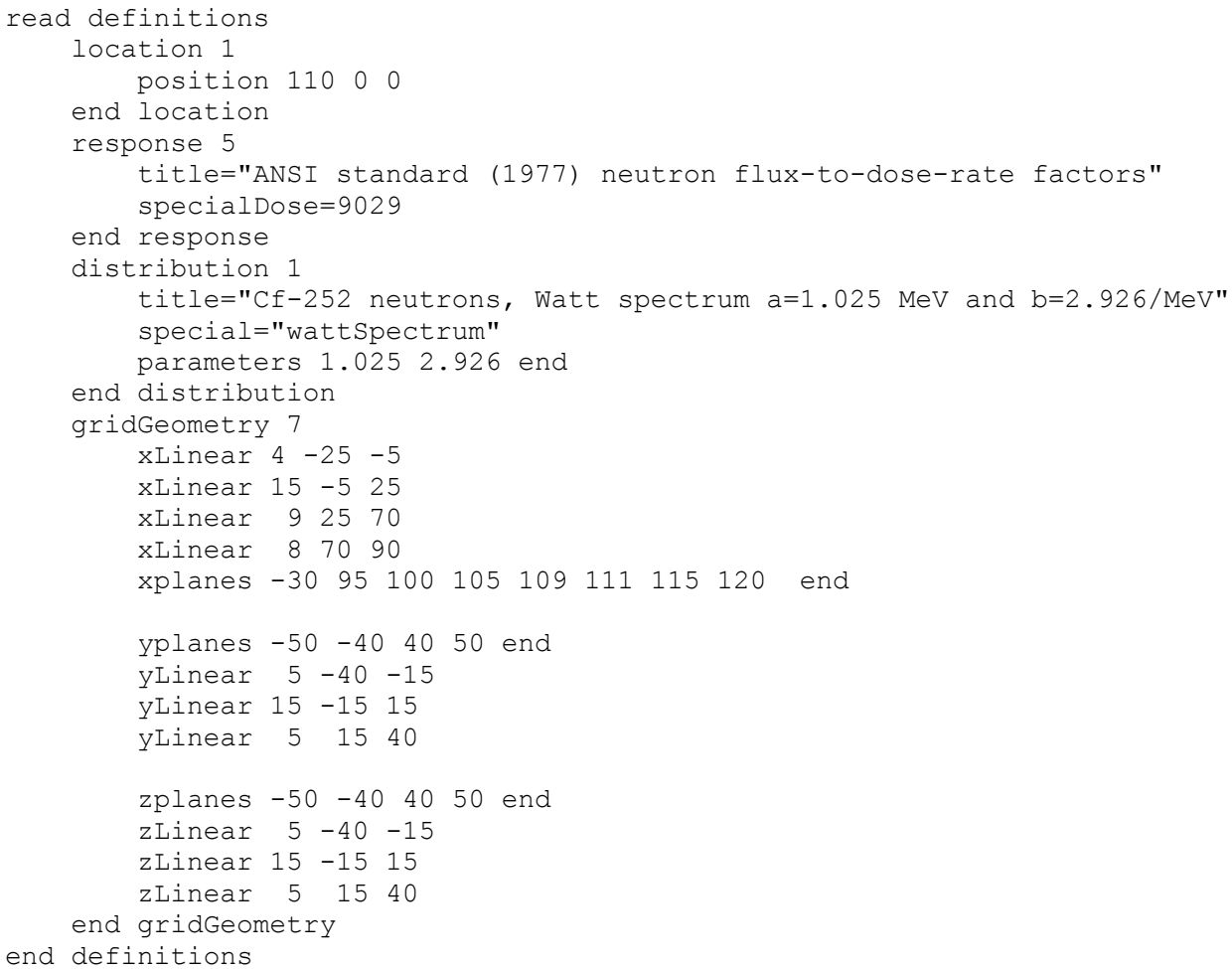

the description of the true source

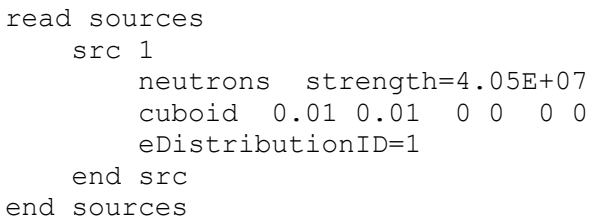

and finally the importance map block to trigger the Denovo calculation

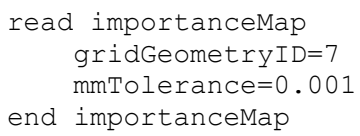


An optional shell command can be used to retrieve the cross section file and the Denovo input file (linux example shown below)

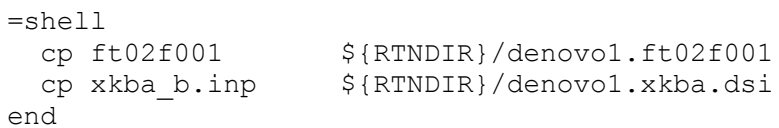

The denovol.xkba.dsi file (the Denovo simple input) contains both the source and mesh geometry that MAVRIC prepared for Denovo, as shown in Figure 4.1.C.5.
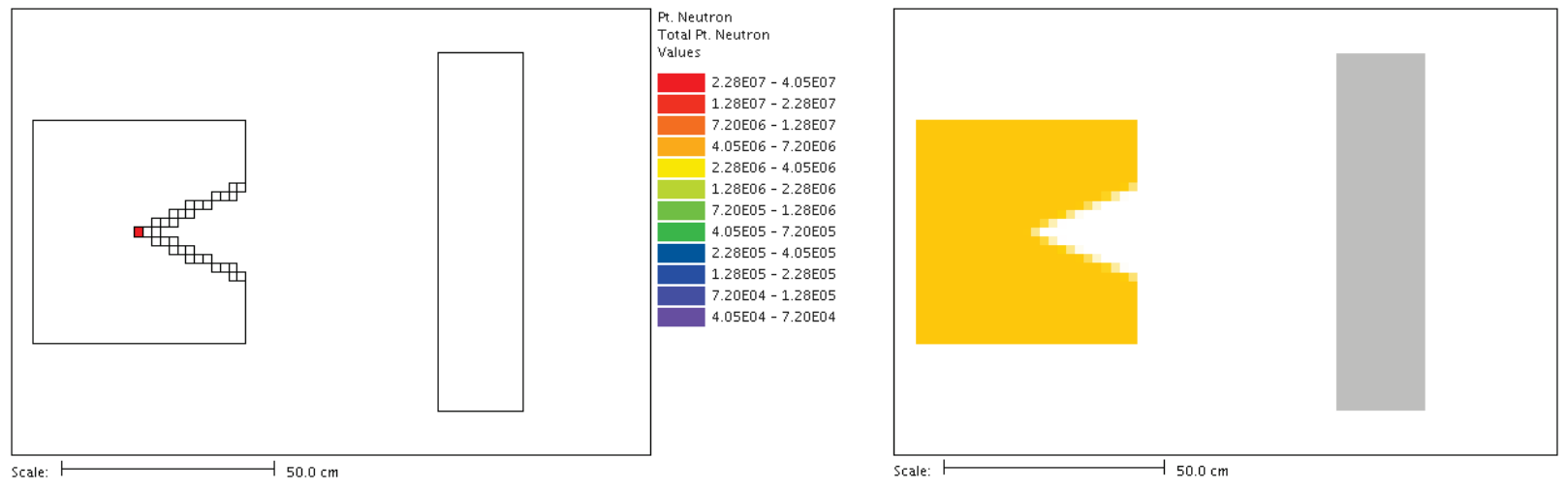

Figure 4.1.C.5. Forward Denovo source (left) and mesh geometry (right).

The result of the Denovo-only MAVRIC calculation is a file, denovo1. forward.dff, containing the scalar fluxes for each energy group. The Mesh File Viewer can be used to display each group or the total scalar flux, which is shown in Figure 4.1.C.6.

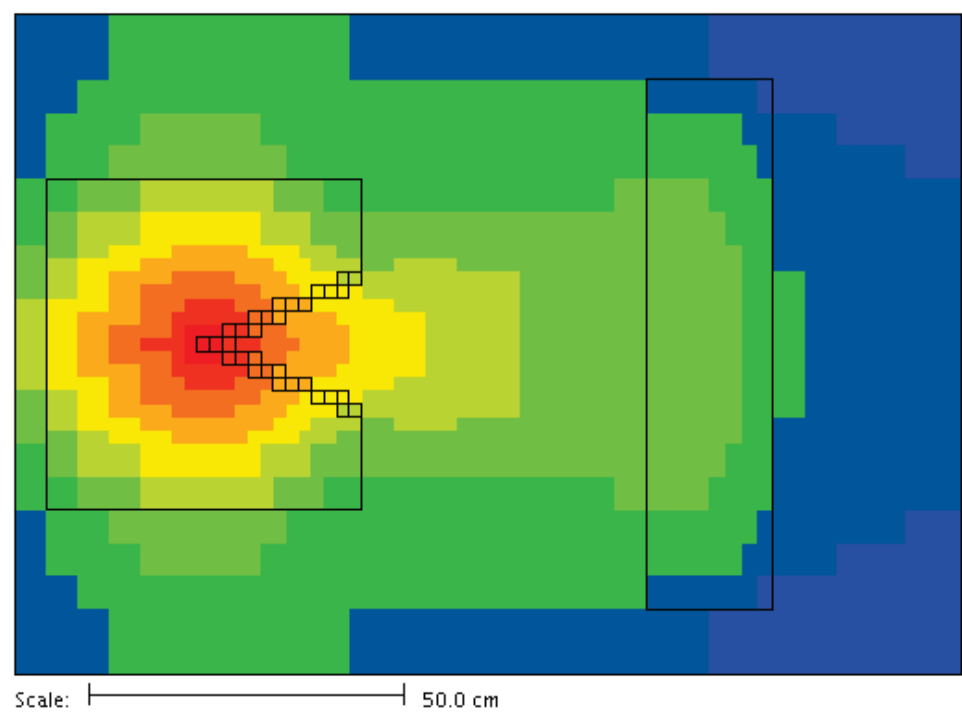

Forward Neutron Flux Total Fonward Neutron Flux Values

Figure 4.1.C.6. Total forward fluxes. 
The results of the example problems above used a fairly coarse mesh $(44 \times 27 \times 27)$ and the default values of $\mathrm{S}_{8}$ and $\mathrm{P}_{3}$. Run times were 2 minutes for the forward case and 2.5 minutes for the adjoint. With a finer mesh, larger quadrature order and larger numbers of Legendre moments for the scattering representation, the values calculated using Denovo should converge towards the Monte Carlo solution of $1.494 \times 10^{-2} \mathrm{rem} / \mathrm{hr} \pm 1.2 \%$.

\subsection{C.5 References}

1. K. A. Van Riper, T. J. Urbatsch, P. D. Soran, D. K. Parsons, J. E. Morel, G. W. Mckinney, S. R. Lee, L. A. Crotzer, F. W. Brinkley, T. E. Booth, J. W. Anderson, and R. E. Alcouffe, "AVATAR - Automatic Variance Reduction in Monte Carlo Calculations," Proceedings of the Joint International Conference on Mathematical Methods and Supercomputing in Nuclear Applications 1, Saratoga Springs, New York (October 6-10, 1997), p. 661.

2. D. E. Peplow, S. W. Mosher, and T. M. Evans, "Hybrid Monte Carlo/Deterministic Methods for Streaming/Beam Problems," the proceedings of the American Nuclear Society Joint Topical Meeting of the Radiation Protection and Shielding Division Isotopes and Radiation Division Biology and Medicine Division Las Vegas, NV, April 19-23, 2010.

3. D. E. Peplow, S. W. Mosher, and T. M. Evans, Consistent Adjoint Driven Importance Sampling using Space, Energy and Angle, in press, ORNL/TM-2012/7.

4. M. A. Cooper and E. W. Larsen, "Automated Weight Windows for Global Monte Carlo Particle Transport Calculations," Nuclear Science and Engineering 137, 1-13 (2001).

5. T. L. Becker and E. W. Larsen, "The Application of Weight Windows to 'Global' Monte Carlo Problems," International Conference on Mathematics, Computational Methods \& Reactor Physics (M\&C 2009), Saratoga Springs, New York, May 3-7, 2009, on CD-ROM, American Nuclear Society, LaGrange Park, IL (2009).

6. T. L. Becker, Hybrid Monte Carlo/Deterministic Methods for Deep-Penetration Problems, Doctoral Dissertation, University of Michigan (2009).

7. D. E. Peplow, "Comparison of Hybrid Methods for Global Variance Reduction in Shielding Calculations," accepted by the Transactions of the American Nuclear Society 107, November 2012, San Diego, CA.

8. D. E. Peplow, "Comparison of Hybrid Methods for Global Variance Reduction in Shielding Calculations," in preparation for 2013 International Conference on Mathematics \& Computational Methods Applied to Nuclear Science and Engineering, Sun Valley, ID, May 5-9, 2013.

9. A.J. van Wijk, G. Van den Eynde, and J.E. Hoogenboom, "An Easy to Implement Global Variance Reduction Procedure for MCNP,” Annals of Nuclear Energy 38, 2496-2503 (2011).

10. D. E. Peplow, "Comparison of Hybrid Methods for Global Variance Reduction in Shielding Calculations," 2013 International Conference on Mathematics \& Computational Methods Applied to Nuclear Science and Engineering (M\&C 2013), Sun Valley, ID, May 5-9, 2013.

11. T. M. Evans, A. S. Stafford, and K. T. Clarno, "Denovo-A New Three-Dimensional Parallel Discrete Ordinates Code in SCALE," Nuclear Technology 171, 171-200 (2010). 


\subsection{DEPLETION, ACTIVATION, AND SPENT FUEL SOURCE TERMS}

\section{Introduction by W. A. Wieselquist}

SCALE's general depletion, activation, and spent fuel source terms analysis capabilities are enabled through a family of modules related to the main ORIGEN depletion/irradiation/decay solver. The nuclide tracking in ORIGEN is based on the principle of explicitly modeling all available nuclides and transitions in the current fundamental nuclear data for decay and neutron-induced transmutation and relies on fundamental cross section and decay data in ENDF/B VII. Cross section data for materials and reaction processes not available in ENDF/B-VII are obtained from the JEFF-3.0/A special purpose European activation library containing 774 materials and 23 reaction channels with 12,617 neutroninduced reactions below $20 \mathrm{MeV}$. Resonance cross section corrections in the resolved and unresolved range are performed using a continuous-energy treatment by data modules in SCALE. All nuclear decay data, fission product yields, and gamma-ray emission data are developed from ENDF/B-VII.1 evaluations. Decay data include all ground and metastable state nuclides with half-lives greater than 1 millisecond. Using these data sources, ORIGEN currently tracks 174 actinides, 1149 fission products, and 974 activation products.

The purpose of this chapter is to describe the stand-alone capabilities and underlying methodology of ORIGEN - as opposed to the integrated depletion capability it provides in all coupled neutron transport/depletion sequences in SCALE, as described in other chapters. Through the stand-alone capabilities, there is generality to handle arbitrary systems (e.g., fast reactor fuel depletion or structural activation) by providing arbitrary flux spectra and arbitrary one-group cross sections to the module COUPLE, which in turn creates ORIGEN library (.f33) files containing the problem-dependent, onegroup reaction coefficients required to solve the actual equations governing depletion/decay. These libraries are required input for the ORIGEN module, along with the initial isotopics and irradiation history, in terms of either a time-dependent power or flux level. Two high-performance equation solvers are available: the hybrid linear chains and matrix exponential method and a new Chebyshev Rational Approximation Method (CRAM). Typical execution times are on the order of a few seconds for a multi-step solution, with each individual solution (step) taking approximately 10 milliseconds. ORIGEN also includes capabilities for continuous feed and removal by element. Output capabilities include isotopics (moles or grams), source spectra (alpha, beta, gamma, and neutron), activity (becquerels or curies), decay heat (total watts or gamma only), and radiological hazard factors (maximum permissible concentrations in air or water). These results can be displayed in the output file (.out extension) and/or archived in an ORIGEN binary results file (.f71 extension).

The use of current, fundamental data resources is a key feature of ORIGEN, including nuclear decay data, multigroup neutron reaction cross sections, neutron-induced fission product yields, and decay emission data for photons, neutrons, alpha particles, and beta particles. The nuclear decay data are based primarily on ENDF/B-VII.1 evaluations. The multigroup nuclear reaction cross section libraries now include evaluations from the JEFF-3.0/A neutron activation file containing data for 774 target nuclides, more than 12,000 neutron-induced reactions, and more than 20 different reaction types below $20 \mathrm{MeV}$, provided in various energy group structures. Energy-dependent ENDF/B-VII.0-based fission product yields are available for 30 fissionable actinides. Gamma-ray and x-ray emission data libraries are based on ENDF/B-VII.1. The photon libraries contain discrete photon line energy and intensity data for decay gamma-ray and $\mathrm{x}$-rays emission for 1,132 radionuclides, prompt and delayed continuum spectra for spontaneous fission, $(\alpha, n)$ reactions in oxide fuel, and bremsstrahlung from decay beta (electron and positron) particles slowing down in either a $\mathrm{UO}_{2}$ fuel or water matrix. Methods and data libraries used to calculate the neutron yields and energy spectra for spontaneous fission, $(\alpha, n)$ reactions, and delayed neutron emission are adopted from the SOURCES4C code. Capabilities to calculate the beta and alpha particle emission source and spectra have also been added. 
The ORIGEN reactor libraries distributed with SCALE include a set of pre-calculated ORIGEN libraries (with TRITON) for a variety of fuel assembly designs:

- $\quad$ BWR $7 \times 7,8 \times 8-1,8 \times 8-2,9 \times 9-2,9 \times 9-9,10 \times 10-9,10 \times 10-8$, SVEA-64, SVEA-96, and SVEA-100;

- $\quad$ PWR $14 \times 14,15 \times 15,16 \times 16,17 \times 17,18 \times 18$;

- $\quad$ CANDU reactor (19-, 28-, and 37-element bundle designs);

- $\quad$ Magnox graphite reactor;

- $\quad$ Advanced Gas-Cooled Reactor (AGR);

- $\quad$ VVER 440 and VVER 1000;

- RBMK;

- IRT;

- $\quad$ MOX BWR 7×7, 8×8-1, 8×8-2, 9×9-2, 9×9-9, 10×10-9, 10×10-8, SVEA-64, SVEA-96, and SVEA-100;

- $\quad$ MOX PWR $14 \times 14,15 \times 15,16 \times 16,17 \times 17,18 \times 18$.

These libraries may be used to rapidly assess spent fuel isotopics and source terms in these systems for arbitrary burnups and decay times. For $\mathrm{UO}_{2}$-based assembly isotopics, the new ORIGAMI sequence provides a very convenient, easy-to-use interface. The most general capability, and requiring more user input, is available using the ARP interpolator module in conjunction with the ORIGEN solver module.

Finally, with regards to user interfaces, ORIGEN has a new keyword-based input in SCALE 6.2 but also maintains the ability to read SCALE 6.1 input. Both ORIGEN and ORIGAMI are tightly integrated with the SCALE graphical user interface, Fulcrum, which includes syntax highlighting, input checking with immediate feedback, and (.f71) output viewing. Additionally, Fulcrum provides an ORIGAMI Automator project interface to characterize the fuel inventory for an entire reactor site and generate data needed for severe accident analysis. ORIGAMI Automator is not documented in this chapter, but a primer is available with step by step instructions on its use. 


\title{
5.1 ORIGEN: NEUTRON ACTIVATION, ACTINIDE TRANSMUTATION, FISSION PRODUCT GENERATION, AND RADIATION SOURCE TERM CALCULATION
}

W. Wieselquist, S. Hart, A. Isotalo ${ }^{1}$, F. Havlůj ${ }^{2}$, S. Skutnik ${ }^{3}$, R. Lefebvre, I. Gauld, D. Wiarda, J. Lefebvre, G. $\mathrm{Hu}^{4}$, N. $\mathrm{Sly}^{3}$, and D. Lago ${ }^{5}$

\begin{abstract}
ORIGEN (Oak Ridge Isotope Generation code) calculates time-dependent concentrations, activities, and radiation source terms for a large number of isotopes simultaneously generated or depleted by neutron transmutation, fission, and radioactive decay. ORIGEN is used internally within SCALE's TRITON and Polaris sequences to perform depletion and decay. As a stand-alone SCALE module, ORIGEN provides additional unique capabilities to (1) simulate continuous nuclide feed and chemical removal, which can be used to model reprocessing or liquid fuel systems, and (2) generate alpha, beta, neutron and gamma decay emission spectra. A standard decay library is provided to perform decay calculations. For neutron activation and fuel depletion problems, neutron spectrum-dependent ORIGEN libraries are required and may be created from (1) user-defined spectrum and self-shielded cross sections using the COUPLE module or (2) interpolation of existing ORIGEN reactor libraries (precalculated by TRITON) using the Automated Rapid Processing (ARP) module. Post-processing using the OPUS module allows calculated isotopics and spectra to be sorted, ranked, and converted to other units.
\end{abstract}

\footnotetext{
${ }^{1}$ Aalto University, Finland

${ }^{2}$ ÚJV Řež, a. s., Czech Republic

${ }^{3}$ University of Tennessee, Knoxville

${ }^{4}$ University of Illinois, Urbana-Champaign

${ }^{5}$ Georgia Tech
} 


\section{TABLE OF CONTENTS}

Page

5.1 ORIGEN: Neutron Activation, Actinide Transmutation, Fission Product Generation, and Radiation Source Term Calculation ................................................................................... 5-3

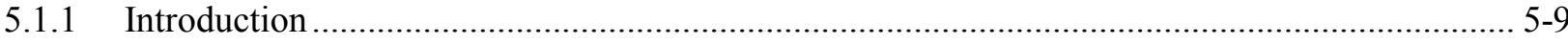

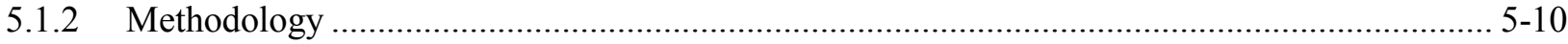

5.1.2.1 Generation of Problem-dependent Transition Matrix .............................................. 5-10

5.1.2.2 Solution of the Depletion/Decay Equations............................................................. 5-11

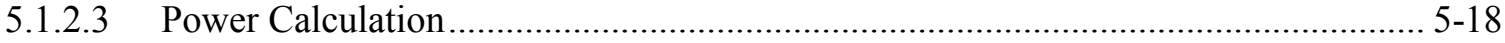

5.1.2.4 Decay Emission Sources Calculation ……............................................................ 5-19

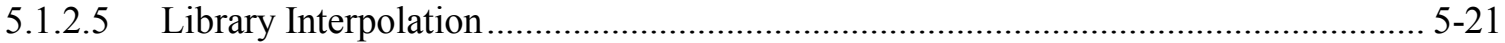

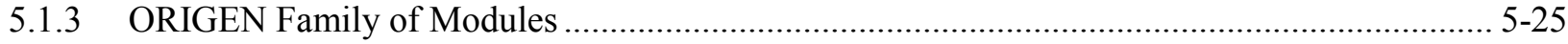

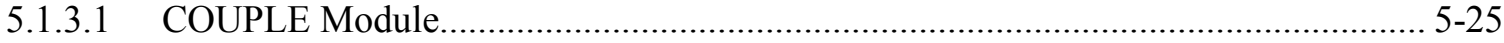

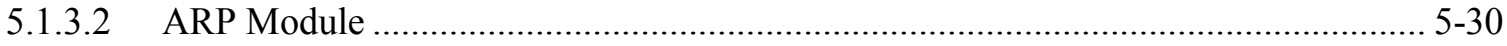

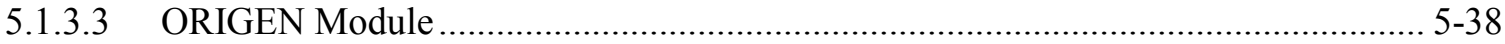

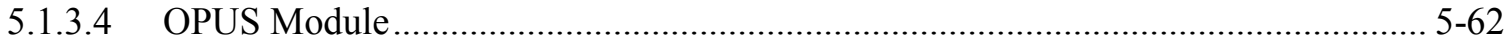

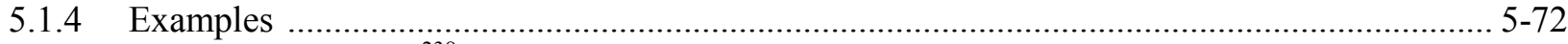

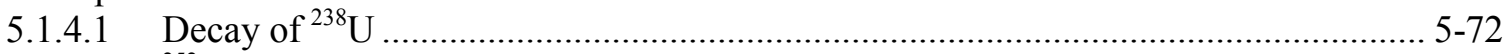

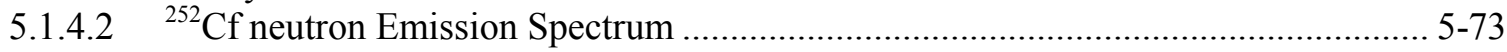

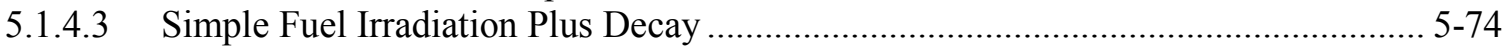

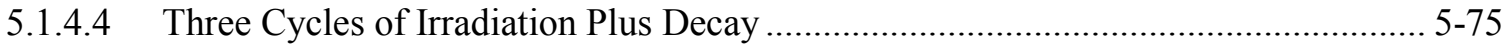

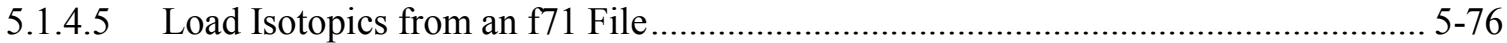

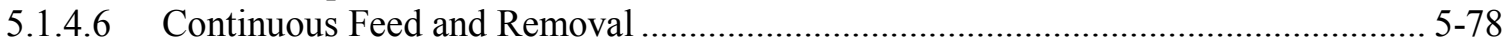

5.1.4.7 Calculate Fuel $(\alpha, N)$ Emissions in a Glass Matrix ................................................. 5-80

5.1.4.8 Create an ORIGEN Decay Library from a Decay Resource..................................... 5-83

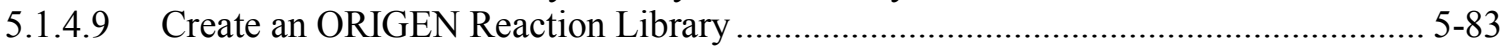

5.1.4.10 Create an ORIGEN Activation Library ................................................................... 5-84

5.1.4.11 Create an ORIGEN Library with User-Supplied Cross Sections.............................. 5-86

5.1.4.12 Print the Cross Section Values on an ORIGEN Library in COUPLE ....................... 5-87

5.1.4.13 Print the Cross Section Values on an ORIGEN Library in ORIGEN ....................... 5-88

5.1.4.14 Ranking Contribution to Toxicity ...................................................................... 5-88

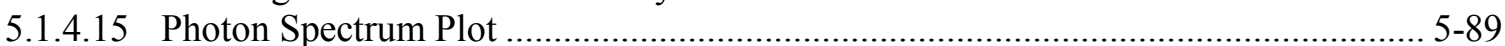

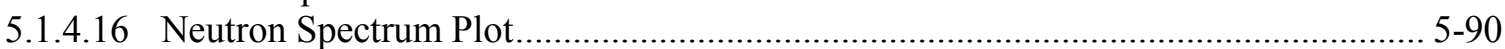

5.1.4.17 Isotopic Weight Percentages for Uranium and Plutonium During Decay ................. 5-90

5.1.4.18 User-Specified Response Function in OPUS ............................................................ 5-90

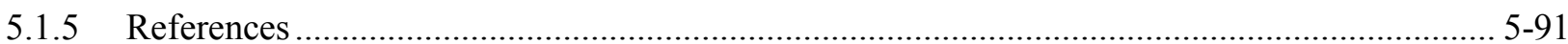




\section{LIST OF FIGURES}

$\underline{\text { Page }}$

Figure 5.1.1. Values of terms in Series for various values of the matrix norm................................5-14

Figure 5.1.2. Relative removal cross section as a function of burnup for key actinides (Westinghouse $17 \times 17$ assembly with $5 \%$ enrichment).

Figure 5.1.3. ${ }^{240} \mathrm{Pu}-240$ removal cross section as a function of burnup for various enrichments (GE $10 \times 10$ assembly).

Figure 5.1.4. ${ }^{240} \mathrm{Pu}$ removal cross section as a function of burnup for various moderator densities (GE $10 \times 10$ assembly).

Figure 5.1.5. ${ }^{240} \mathrm{Pu}$ removal cross section as a function of initial enrichment for various burnups (GE $10 \times 10$ assembly).

Figure 5.1.6. ${ }^{240} \mathrm{Pu}$ removal cross section as a function of moderator density for various burnups (GE $10 \times 10$ assembly).

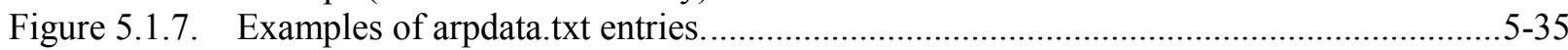

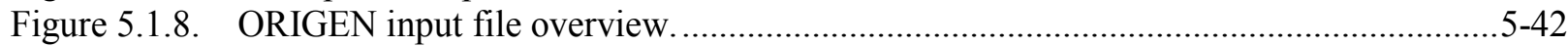

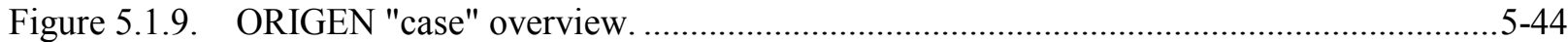

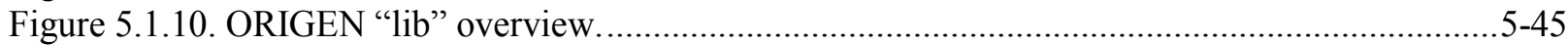

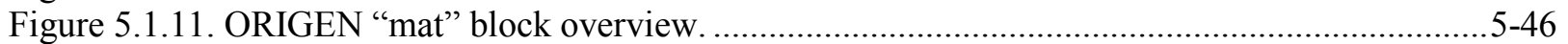

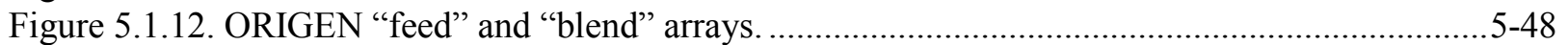

Figure 5.1.13. ORIGEN operating history blocks ("time," "flux," and "power"). .............................5-49

Figure 5.1.14. Example of ORIGEN operating history and power-to-flux conversion. .......................5-50

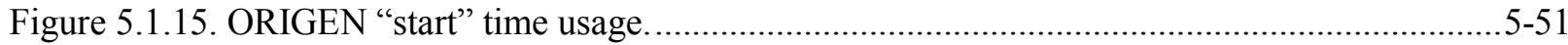

Figure 5.1.16. ORIGEN nuclide and element "print" blocks. .......................................................5-52

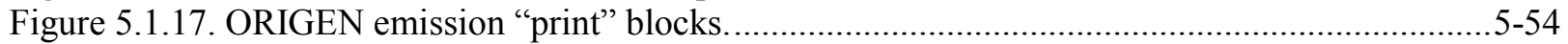

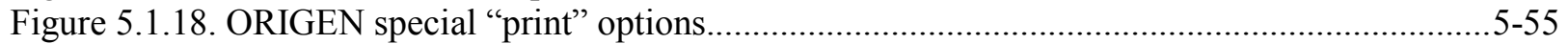

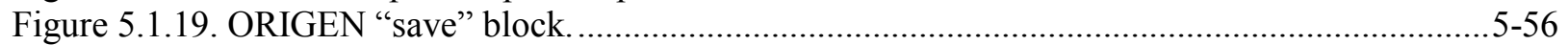

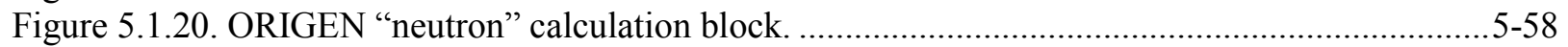

Figure 5.1.21. ORIGEN "gamma" calculation block.....................................................................5-59

Figure 5.1.22. ORIGEN "alpha" and "beta" calculation blocks. ….................................................5-59

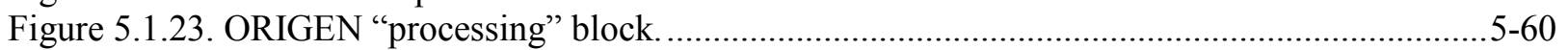

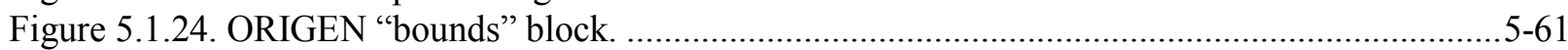

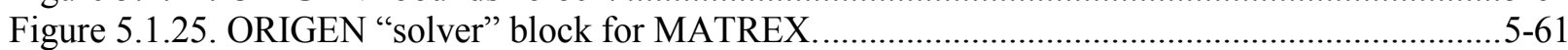

Figure 5.1.26. ORIGEN "solver” block for CRAM.................................................................. 5-61

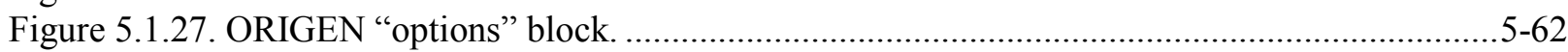

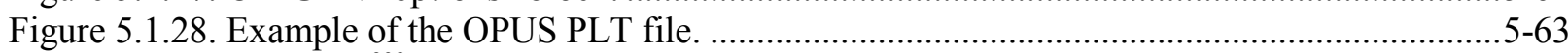

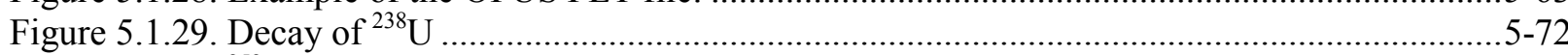

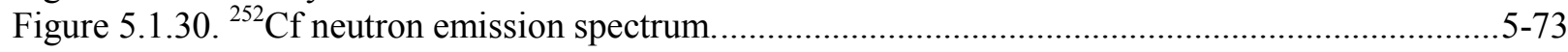

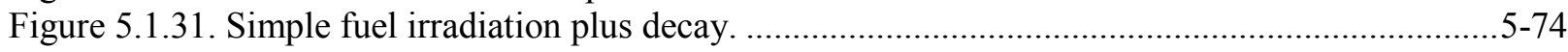

Figure 5.1.32. Three cycles of irrradiation plus decay.....................................................................5-76

Figure 5.1.33. Load isotopics from an $\mathrm{f} 71$ file using the "mat" block. ...........................................5-77

Figure 5.1.34. Table of contents printed when accessing the $\mathrm{f} 71$ file ...............................................5-77

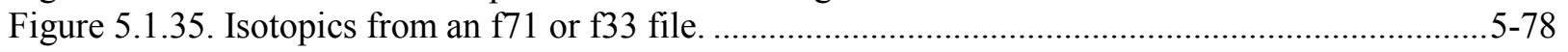

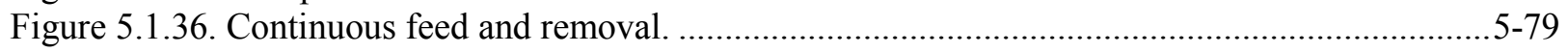

Figure 5.1.37. Continuous feed and removal - history overview. ........................................... $5-80$

Figure 5.1.38. Continuous feed and removal - blending option. .................................................5-82

Figure 5.1.39. ORIGEN decay library from decay resource..................................................... $5-83$

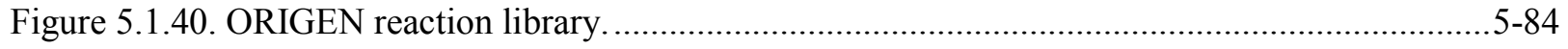

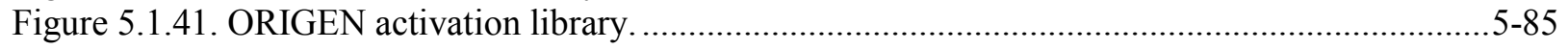


Figure 5.1.42. ORIGEN activation library - average energy per fission calculated...........................5-86

Figure 5.1.43. ORIGEN Library with user-supplied cross sections...............................................5-87

Figure 5.1.44. Cross section values on an ORIGEN library in COUPLE ........................................5-88

Figure 5.1.45. Cross Section values on an ORIGEN library in ORIGEN ........................................5-88

Figure 5.1.46. Ranking contribution to toxicity. .......................................................................... $5-89$

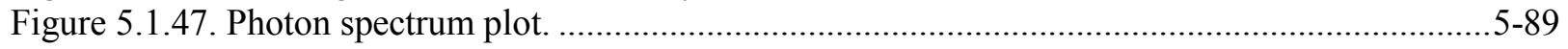

Figure 5.1.48. Photon spectrum plot - output of the time-dependent gamma spectrum......................5-90

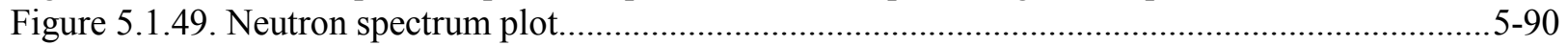

Figure 5.1.50. Isotopic weight percentages for uranium and plutonium during decay........................5-90

Figure 5.1.51. User-specified response function in OPUS.........................................................5-91

\section{LIST OF TABLES}

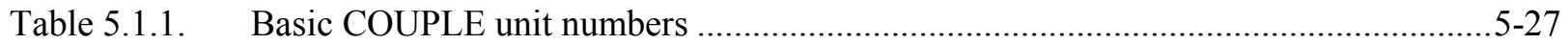

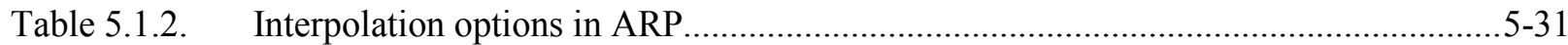

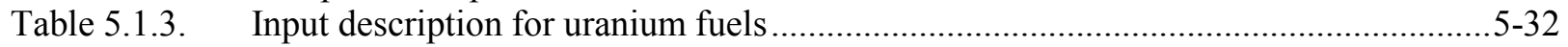

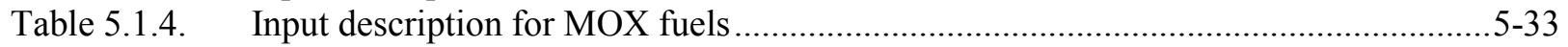

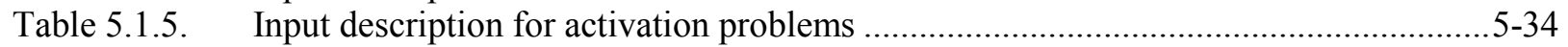

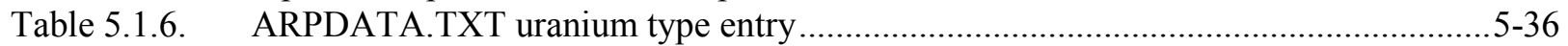

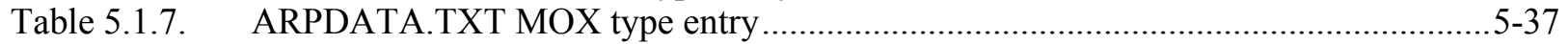

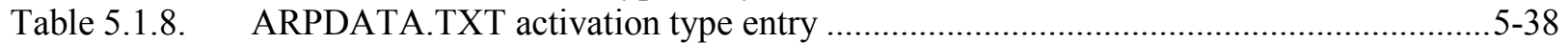

Table 5.1.9. Nuclide/Element Specification in ORIGEN ........................................................5-39

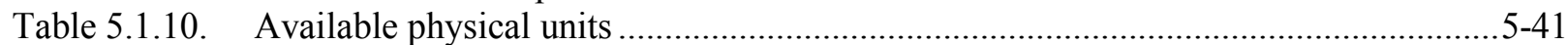

Table 5.1.11. List of all available ORIGEN input commands ......................................................5-43

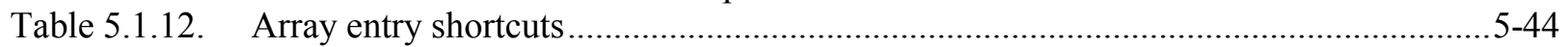

Table 5.1.13. Borosilicate glass composition used in the borosilicate glass option..........................5-57

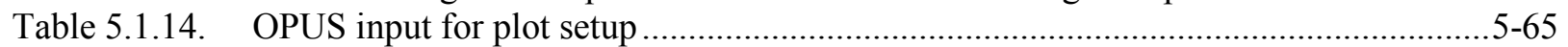

Table 5.1.15. OPUS input for response units and nuclide selection ..............................................5-66

Table 5.1.16. OPUS Input keywords for data set selection.............................................................5-69 


\section{ACKNOWLEDGMENTS}

Development and maintenance of ORIGEN and related codes and methods have been sponsored by many organizations including the US Nuclear Regulatory Commission (NRC), the US Department of Energy (DOE), and nuclear power and research institutions.

\section{VERSION INFORMATION}

The ORIGEN (Oak Ridge Isotope Generation) $\operatorname{code}^{1}$ was developed at Oak Ridge National Laboratory (ORNL) to calculate nuclide compositions and radioactivity of fission products, activation products, and products of heavy metal transmutation. Since 1991, ORIGEN has been developed as the depletion/decay module in SCALE with support from the NRC. ORIGEN in SCALE is the only version supported at ORNL, and it supersedes all earlier versions. The following is a brief description of the major enhancements in each version. Data are described in the ORIGEN Data Resources chapter.

6.2 (2016)

Code Responsible(s): W. A. Wieselquist

A major modernization effort for ORIGEN was initiated by I. Gauld in 2011 and has resulted in approximately 5 person-years of effort refactoring the ORIGEN and related codes to be more efficient and easily testable. The major enhancements and responsible parties are listed below.

- Extensive refactor and modernization of Fortran 77 to Fortran $90+$ performed by F. Havlůj, including substantial extension of the output capability

- Implementation of an alpha and beta spectrum calculation by F. Havlůj and I. Gauld

- Introduction of $\mathrm{C}++$ core data structures with Fortran bindings, implemented by S. Skutnik using R. Lefebvre's $\mathrm{C}++/ \mathrm{C} /$ Fortran binding generator created for this purpose

- Testing suite developed by S. Skutnik, W. Wieselquist, D. Lago, and N. Sly

- Standardization of codebase while developing application programming interface (API) for high-performance depletion in the Consortium for Advanced Simulation of Light Water Reactors (CASL) and Nuclear Energy Advanced Modeling and Simulation (NEAMS) projects performed by W. Wieselquist

- Unification of readers/writers for ORIGEN data files developed by W. Wieselquist

- Improvement of binary formats for the ORIGEN library (f33) and ORIGEN concentration file (f71) by J. Lefebvre, R. Lefebvre, and W. Wieselquist

- Implementation of Chebyshev Rational Approximation Method (CRAM) solver by A. Isotalo

- Development of new input format (ORIGEN sequence only) by S. Hart and W. Wieselquist using the SCALE Object Notation (SON) syntax developed by R. Lefebvre

- Improvement of cubic spline interpolation scheme for ARP by S. Skutnik and W. Wieselquist with monotonicity fix-up determined by $\mathrm{G}$. $\mathrm{Hu}$

- Major revision of manuals by W. Wieselquist, combining ORIGEN, ARP, COUPLE, and OPUS into a single manual

Additional guidance provided by D. Wiarda and I. Gauld with testing by J. W. Hu. 


\section{$6.1(2011)$}

The following section acknowledgements appeared in the SCALE 6.1 manual.

ORIGEN

Code Responsible(s): I. C. Gauld

The ORIGEN code was first developed by M. J. Bell with contributions from J. P. Nichols and other members of the Chemical Technology Division at ORNL. Development of the ORIGEN code as a depletion module of the SCALE code system was performed by O. W. Hermann with contributions from R. M. Westfall, supported by the NRC.

COUPLE

Code Responsible(s): D. Wiarda and I. C. Gauld

The COUPLE code was originally developed by O. W. Hermann with guidance from staff members including L. M. Petrie, N. M. Greene, W. E. Ford III, and R. M. Westfall, who contributed greatly to formulation of the methods, design of the data library interface with other modules, and testing. Many valuable suggestions concerning code applications were received from J. C. Ryman, J. R. Knight, and E. J. Allen.

$A R P$

Code Responsible(s): I. C. Gauld, S. M. Bowman, and J. E. Horwedel.

The authors thank S. B. Ludwig for his support in earlier stages of this work. The authors are grateful for the technical advice received from B. L. Broadhead, M. D. DeHart, N. M. Greene, O. W. Hermann, C. V. Parks, L. M. Petrie, and J. C. Ryman. The authors thank Germina Ilas and Georgeta Radulescu for reviewing the manual and Willena Carter for preparation of the manuscript.

\section{OPUS}

Code Responsible(s): I. C. Gauld and J. E. Horwedel

The work of O. W. Hermann in developing the PLORIGEN program, from which OPUS was later developed, and the work of D. L. Barnett in developing the original version of PlotOPUS, are acknowledged. Appreciation is extended to J. C. Ryman for his review and testing of the program. Finally the authors thank S. J. Poarch for formatting the manuscript. 


\subsubsection{Introduction}

ORIGEN solves the system of ordinary differential equations (ODEs) that describe nuclide generation, depletion, and decay,

where

$$
\frac{d N_{i}}{d t}=\sum_{j \neq i}\left(l_{i j} \lambda_{j}+f_{i j} \sigma_{j} \Phi\right) N_{j}(t)-\left(\lambda_{i}+\sigma_{i} \Phi\right) N_{i}(t)+S_{i}(t),
$$

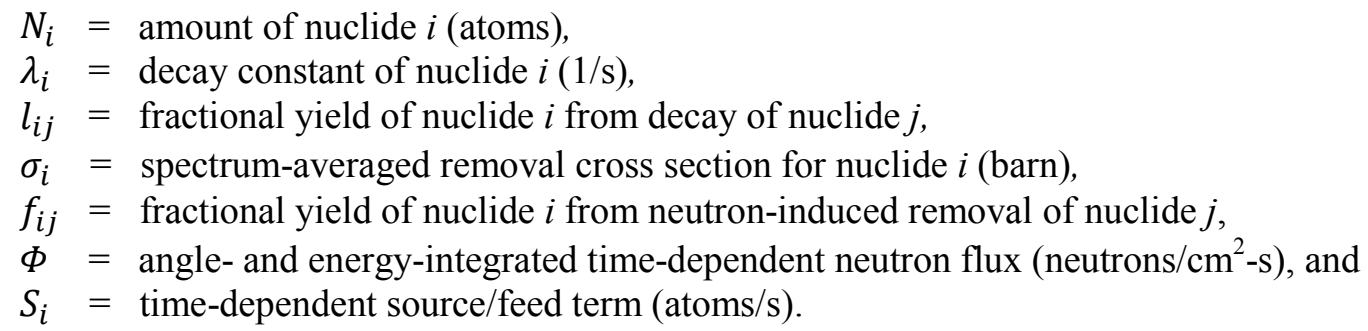

Note that Eq. (5.1.1) has no spatial dependence and can be interpreted as either a solution at a point in space or the spatial average over some volume. The latter interpretation is preferred here, such that $\Phi$ is the spatially averaged neutron flux magnitude, and all energy-dependence is embedded in the one-group flux-weighted average cross sections $\sigma_{i}$ and reaction yields $f_{i j}$. Eq. (5.1.1) is conveniently written in matrix form as

$$
\frac{d \vec{N}}{d t}=A \vec{N}(t)+\vec{S}(t)
$$

with a $\boldsymbol{A}$ commonly referred to as the "transition matrix." The representation of the transition matrix as $\boldsymbol{A}=\boldsymbol{A}_{\sigma} \Phi+\boldsymbol{A}_{\lambda}$, where $\boldsymbol{A}_{\sigma}$ is the part of the transition matrix containing reaction terms and $\boldsymbol{A}_{\lambda}$ is the part containing decay terms, is convenient, as the numerical solution of this system of ODEs holds the reaction, flux, and feed terms constant over step $n$,

$$
\begin{gathered}
\frac{d \vec{N}}{d t}=\left(\boldsymbol{A}_{\sigma, n} \Phi_{n}+\boldsymbol{A}_{\lambda}\right) \vec{N}(t)+\vec{S}_{n} \\
\quad \text { over time step } t_{n-1} \leq t \leq t_{n}
\end{gathered}
$$

Adding a continuous removal process described with rate constant $\lambda_{i \text {,rem }}$ simply modifies the decay constant, $\lambda_{i} \rightarrow \lambda_{i}+\lambda_{i, r e m}$, whereas a continuous feed process defines a nonzero component of the $\vec{S}$ vector.

ORIGEN can also compute the alpha, beta, neutron, and gamma emission spectra during decay. For the "stand-alone" ORIGEN calculations described here, the transition matrix is loaded from an ORIGEN binary library file (f33), which uses sparse-matrix storage to store one or more transition matrices. The f33s may be created using COUPLE, saved from TRITON depletion calculations, or interpolated using ARP from a set of precompiled $\mathrm{f} 33$ s distributed with SCALE.

Results from ORIGEN calculations may be stored on a binary concentration file (f71), which facilitates transfer of isotopics to other codes in SCALE. The f71 file can also store calculated emission spectra. Within ORIGEN, the $\mathrm{f} 71 \mathrm{can}$ be used to restart calculations from an existing set of compositions. 


\subsubsection{Methodology}

This section describes the methodology used in performing the following main functions:

- generation of problem-dependent transition matrices,

- solution of the system of depletion/decay equations,

- conversion from power to flux (important for reactor applications),

- calculation of emission spectra, and

- interpolation of pregenerated sets of transition matrices.

\subsubsection{Generation of Problem-dependent Transition Matrix}

In the transition matrix $\boldsymbol{A}$ from Eq. (5.1.2), each matrix element $a_{i j}$ is the first-order rate constant for the formation of nuclide $i$ from nuclide $j$ given below.

$$
a_{i j}=\left\{\begin{array}{rr}
l_{i j} \lambda_{j}+f_{i j} \sigma_{j} \Phi & i \neq j \\
-\lambda_{i}-\sigma_{i} \Phi & \text { otherwise }
\end{array}\right.
$$

The transition matrix coefficients for decay and reaction transitions are stored separately and reaction transitions are always stored with $\Phi=1$ and later during solution of the system, depending on the stepaverage flux level the actual transition matrix $\boldsymbol{A}_{n}=\boldsymbol{A}_{\sigma, n} \Phi_{n}+\boldsymbol{A}_{\lambda}$ is reconstructed using step-average flux, $\Phi_{n}$.

The decay coefficients $l_{i j} \lambda_{j}$ and $\lambda_{i}$ are generated directly from ORIGEN decay resource data. The reaction coefficients $f_{i j} \sigma_{j}$ and $\sigma_{i}$ are generated using the following two-stage procedure.

1. Calculate all removal cross sections $\sigma_{i}$ and yields $f_{i j}$, including isomeric branching ratios and fission yields, by folding provided flux spectrum $\phi^{g}$ with multigroup cross sections from the ORIGEN reaction resource and energy-dependent fission yield data from the ORIGEN yield resource.

2. Overwrite specific removal cross sections and yields based on a provided multigroup cross section library [SCALE Cross Section Libraries chapter] and/or user-provided one-group cross sections and yields.

The second stage is optional, but it is important for cases which there is significant self-shielding because ORIGEN's reaction resource assumes infinite dilution for its multigroup data. The decay, reaction, and yeld resources mentioned here are described in the ORIGEN Data Resources chapter. The collapse to a one-group cross section in either stage is given by

$$
\sigma_{r i}=\frac{\sum_{g} \sigma_{r i}^{g} \phi^{g}}{\sum_{g} \phi^{g}}
$$

for reaction type $r$, nuclide $i$, and provided multigroup flux $\phi^{g}$. Different reaction types are recognized by their ENDF MT numbers [SCALE Cross Section Libraries chapter] on the appropriate data resource For example, $\mathrm{MT}=16$ is $(n, 2 n)$, and $\mathrm{MT}=107$ is $(n, \alpha)$. The removal cross section $\sigma_{i}$ is simply calculated as the sum over all relevant reactions for a particular nuclide, $\sigma_{i}=\sum_{r} \sigma_{r i}$. This type of reaction-dependent multigroup data may be contained in either the data sources available in stage 1 or 2 above. However, only two types of data are expected to be available in stage 1 reaction resource data: (1) isomeric branching and (2) fission yields. 
The energy-dependent isomeric branching that describes the yield of each excited level (metastable state) of a daughter nucleus is calculated in a similar way,

$$
f_{\text {rim }}=\frac{\sum_{g} f_{r i m}^{g} \sigma_{r i}^{g} \phi^{g}}{\sum_{g} \sigma_{r i}^{g} \phi^{g}}
$$

where $m$ indicates the possible metastable states and the fractions always satisfy $\sum_{m} f_{\text {rim }}=1$.

Fission product yields are typically tabulated at discrete neutron energies such as thermal $(0.0253 \mathrm{eV})$, fission $(500 \mathrm{keV})$, and high energy $(14 \mathrm{MeV})$. The yield for each fissionable nuclide is calculated in stage 1 by linearly interpolating the tabulated data using the computed average energy of fission,

$$
\bar{E}_{f i}=\frac{\sum_{g} \bar{E}^{g} \sigma_{f i}^{g} \phi^{g}}{\sum_{g} \sigma_{f i}^{g} \phi^{g}}
$$

where $\sigma_{f i}^{g}$ is the multigroup fission cross section, and $\bar{E}^{g}$ is the average energy in the group (simple midpoint energy used). In addition to generating transition data for daughter/residual nuclides, the coefficients for byproducts such as He-4/ $\alpha$ byproducts from $(n, \alpha)$ reactions are also retained in the transition matrix and associated to an appropriate nuclide in the system: hydrogen, deuterium, tritium, ${ }^{3} \mathrm{He}$, or ${ }^{4} \mathrm{He}$.

\subsubsection{Solution of the Depletion/Decay Equations}

ORIGEN includes two solver kernels that can solve the depletion/decay equations of Eq. (5.1.3):

1. a hybrid matrix exponential/linear chains method (MATREX) and

2. a Chebyshev Rational Approximation Method (CRAM).

They are described in the following sections.

\subsection{MATREX}

Referring to the system of ODEs shown in Eq. (5.1.2) and setting the external feed/source $S(t)=0$, there is a formal solution by matrix exponential (an analog to the solution of a single ODE of this type by exponential),

$$
\vec{N}(t)=\exp (\boldsymbol{A} t) \vec{N}(0)
$$

where $\vec{N}(0)$ is a vector of initial nuclide concentrations, by defining the series expansion of $\exp (\boldsymbol{A} t)$ to be

$$
\exp (\boldsymbol{A} t)=\boldsymbol{I}+\boldsymbol{A} t+\frac{(\boldsymbol{A} t)^{2}}{2}+\cdots=\sum_{k=0}^{\infty} \frac{(\boldsymbol{A} t)^{k}}{k !}
$$

with $\boldsymbol{I}$ the identity matrix. Eqs. (5.1.8) and (5.1.9) describe the matrix exponential method, which yields a complete solution to the problem. However, in certain instances related to limitation in computer precision, difficulties occur in generating accurate values of the matrix exponential function. Under these 
circumstances, alternative procedures using either the generalized Bateman equations ${ }^{2}$ or Gauss-Seidel iterative techniques are applied. These alternative procedures will be discussed in further sections.

A straightforward solution of Eqs. (5.1.8) through (5.1.9) would require storage of the complete transition matrix. To avoid excessive memory storage requirements, a recursion relation has been developed. Substituting Eq. (5.1.9) into Eq. (5.1.8),

$$
\vec{N}(t)=\left[\boldsymbol{I}+\boldsymbol{A} t+\frac{(\boldsymbol{A} t)^{2}}{2}+\cdots\right] \vec{N}(0),
$$

one may recognize a recursion relation for a particular nuclide, $N_{i}(t)$.

$$
\begin{aligned}
N_{i}(t) & =N_{i}(0)+t \sum_{j} a_{i j} N_{j}(0)+\frac{t}{2} \sum_{k}\left[a_{i k} t \sum_{j} a_{k j} N_{j}(0)\right] \\
& +\frac{t}{3} \sum_{m}\left\{a_{i m} \frac{t}{2} \sum_{k}\left[a_{m k} t \sum_{j} a_{k j} N_{j}(0)\right]\right\}+\cdots
\end{aligned}
$$

where the range of indices, $j, k, m$, is 1 to $M$ for matrix $\boldsymbol{A}$ of size $M \times M$. The result is a series of terms that arise from the successive post-multiplication of the transition matrix by the vector of nuclide concentration increments produced from the computation of the previous terms. Within the accuracy of the series expansion approximation, physical values of the nuclide concentrations are obtained by summing a converged series of these vector terms. By defining the terms $C_{i}^{n}(t)$ as

$$
C_{i}^{0}=N_{i}(0), \quad C_{i}^{n+1}=\frac{t}{n+1} \sum_{j} a_{i j} C_{j}^{n},
$$

the solution for $N_{i}(t)$ is given as

$$
N_{i}(t)=\sum_{n=0}^{\infty} C_{i}^{n}
$$

The use of Eqs. (5.1.12) and (5.1.13) requires storage of only two vectors $-\vec{C}^{n}$ and $\vec{C}^{n+1}$-in addition to the current value of the solution. However, the series summation solution in Eq. (5.1.13) is not valid until a finite limit is identified which can achieve a reasonable accuracy, i.e.,

$$
N_{i}(t)=\sum_{n=0}^{n_{\text {term }}} C_{i}^{n}+\epsilon_{\text {trunc }}
$$

where $n_{\text {term }}$ is the number of terms and $\epsilon_{\text {trunc }}$ is the truncation error. The key is to split the nuclides into two sets: those that are long-lived and permit a rapid, accurate solution via Eq.(5.1.14), and those that are short-lived and require an alternate solution. 


\subsection{Solution for Long-Lived Nuclides}

This section describes the various tests used to ensure that the summations indicated in Eq. (5.1.14) do not lose accuracy due to large changes in magnitudes or small differences between positive and negative rate constants. Nuclides with large rate constants (short-lived) are removed from the transition matrix and treated separately. For example, in the decay chain $\mathrm{A} \rightarrow \mathrm{B} \rightarrow \mathrm{C}$, if the decay constant for $\mathrm{B}$ is large, a new rate constant is inserted in the matrix for $\mathrm{A} \rightarrow \mathrm{C}$. This technique was originally employed by Ball and Adams. ${ }^{3}$ The key to determining which transitions should be removed involves calculation of the matrix norm. The norm of matrix $\boldsymbol{A}$ is defined by Lapidus and Luus ${ }^{4}$ as being the smaller of the maximum-row absolute sum and the maximum-column absolute sum,

$$
[\boldsymbol{A}]=\min \left\{\max _{\mathrm{j}} \sum_{i}\left|a_{i j}\right|, \max _{\mathrm{i}} \sum_{j}\left|a_{i j}\right|\right\}
$$

To maintain precision in performing the summations of Eq. (5.1.14), the matrix norm is used to balance the user-specified time step, $t$, with the precision associated with the word length employed in the machine calculation. The constraint on the matrix norm has been chosen as

$$
[A] t \leq-2 \ln (0.001)=13.8155 .
$$

The remainder of this section shows that this constraint serves two purposes.

- It allows reasonable accuracy for a reasonable number (20-60) of matrix exponential terms.

- It defines what "short-lived" means over a particular time step, dictating which concentrations must be solved by alternate means.

A relationship between $m$ digits of machine precision and $p$ significant digits required in all results can be stated by the following inequality: (Largest term in series) $\times 10^{-\mathrm{m}} \leq$ (Series result) $\times 10^{-\mathrm{p}}$.

In this particular series, the relationship may be represented as

$$
\max _{n} \frac{|[A] t|^{n}}{n !} 10^{-m} \leq e^{-[A] t} 10^{-p},
$$

or alternatively,

$$
\max _{n} \frac{|[A] t|^{n}}{n !} e^{[A] t} \leq 10^{m-p} .
$$

Lapidus and Luus have shown that the maximum term in the summation for any element in the matrix exponential function cannot exceed $\frac{([A] t)^{n}}{n !}$, where $n$ is the largest integer not larger than $[\boldsymbol{A}] t$. For the constraint in Eq. (5.1.16), this yields $n=13$ and yields limit $\frac{([A] t)^{n}}{n !} \approx 10^{5}$. With $e^{[A] t} \approx 10^{6}$ and standard double precision with $m=16$, Eq. (5.1.18) evaluates to $10^{11} \leq 10^{16-j}$, which indicates that five significant figures will be maintained in values as small as $10^{-6}$. The number of terms required to converge the matrix exponential series can be investigated by a plot of the $\frac{|[A] t|^{n}}{n !} e^{[A] t}$ as a function of term index $n$ in Eq. (5.1.18), as shown in Figure 5.1.1. 


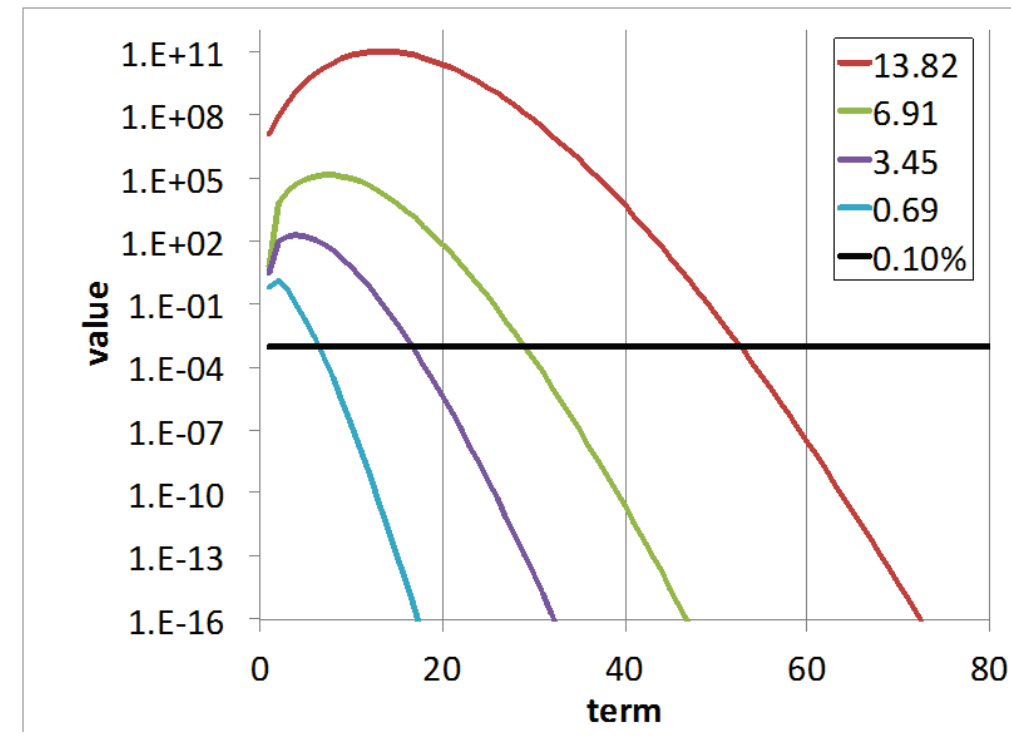

Figure 5.1.1. Values of terms in Series for various values of the matrix norm.

The intersection between the black line in Figure 5.1.1 and the various curves indicates the number of terms needed to achieve $\epsilon_{\text {trunc }} \leq 0.1 \%$. For example, with $[A] t=13.8155, n_{\text {term }}=54$ is required, and with $[\boldsymbol{A}] t=13.8155 / 2$, approximately $n_{\text {term }}=29$ is required. This behavior has been used to develop the heuristic

$$
n_{\text {term }}=7[\boldsymbol{A}] t / 2+6 .
$$

Thus it has been shown that the limit imposed in Eq. (5.1.16) leads to a maximum of $n_{\text {term }}=54$ terms with $\epsilon_{\text {trunc }} \leq 0.1 \%$.

It remains to be shown that any arbitrary system can be modified so that it does not violate Eq. (5.1.16). Because the time step $t$ is provided and fixed, $[\boldsymbol{A}] t \leq-2 \ln (0.001)$ cannot be satisfied unless the system is modified. The physical nature of the system leads to $\max _{j} \sum_{i}\left|a_{i j}\right| \leq \max _{j} 2\left|a_{j j}\right|$ based on production rates equal to loss rates when both parent and daughter nuclide are included in the system. The maximum column sum in Eq. (5.1.15) can then be bounded by twice the maximum diagonal term, $\max _{j} 2\left|a_{j j}\right|$. Using this upper limit as the matrix norm and substituting into Eq. (5.1.16) yields

$$
[A] t \leq 2 \max _{j}\left|a_{j j}\right| \leq-2 \ln (0.001) .
$$

Rearranging Eq. (5.1.20) leads to the condition

$$
e^{-\left|a_{j j}\right| t}<0.001
$$

which is used to mark nuclide $j$ as a short-lived nuclide for this time step, to be solved with linear chains instead of the series-based matrix exponential. An alternative interpretation of the short-lived condition can be made by rewriting Eq. (5.1.21) in terms of an effective half-life, $t_{1 / 2}=\frac{\ln (2)}{\left|a_{j j}\right|}$, which results in 
$t_{1 / 2}<\frac{-\ln (2)}{\ln (0.001)} t \approx 0.1 t$. In other words, when a nuclide's effective half-life (including destruction by both decay and reaction mechanisms) is less than $10 \%$ of the time step, it can be considered short-lived.

Finally, as a note, in applications where the nuclides of interest are in long transmutation chains, it has been found that the above algorithm may not yield accurate concentrations for those nuclides near the end of the chain that are significantly affected by those near the beginning of the chain. In these applications, specifying the minimum $n_{\text {term }}$ as

$$
n_{\text {term }} \geq|\Delta Z|+|\Delta A|+5
$$

where $\Delta Z$ is the atomic number difference and $\Delta A$ is the mass number difference, has been found to ameliorate the issue.

\subsection{Solution for Short-Lived Nuclides}

The condition in Eq. (5.1.21) forms the basis for declaring a nuclide short-lived, and its solution is found via solution of the nuclide chain equations. In conjunction with maintaining the transition matrix norm below the prescribed level, a queue is formed of the short-lived precursors of each long-lived isotope. These queues extend back up the several chains to the last preceding long-lived precursor. According to Eq. (5.1.21), the queues will include all nuclides whose effective half-lives are less than $10 \%$ of the time interval. A generalized form of the Bateman equations developed by Vondy ${ }^{5}$ is used to solve for the concentrations of the short-lived nuclides at the end of the time step. For an arbitrary forward-branching chain, Vondy's form of the Bateman solution is given by

$$
N_{i}(t)=N_{i}(0) e^{-d_{i} t}+\sum_{k=1}^{i-1} N_{k}(0)\left[\sum_{j=k}^{i-1} \frac{e^{-d_{j} t}-e^{-d_{i} t}}{d_{i}-d_{j}} a_{j+1, j} \prod_{\substack{n=k \\ n \neq j}}^{i-1} \frac{a_{n+1, n}}{d_{n}-d_{j}}\right]
$$

where $N_{1}(0)$ is the initial concentration of the first precursor, $N_{2}(0)$ is that of the second precursor, etc. As in Eq. (5.1.4), $a_{i j}$ is the first-order rate constant, and $d_{i}=-a_{i i}$ which is the magnitude of the diagonal element. Bell recast Vondy's form of the solution through multiplication and division by $\prod_{n=k}^{i-1} d_{n}$ and rearranged to obtain

$$
N_{i}(t)=N_{i}(0) e^{-d_{i} t}+\sum_{k=1}^{i-1} N_{k}(0) \prod_{n=k}^{i-1} \frac{a_{n+1, n}}{d_{n}}\left[\sum_{j=k}^{i-1} d_{j} \frac{e^{-d_{j} t}-e^{-d_{i} t}}{d_{i}-d_{j}} \prod_{\substack{n=k \\ n \neq j}}^{i-1} \frac{d_{n}}{d_{n}-d_{j}}\right] .
$$

The first product over isotopes $n$ is the fraction of atoms that remains after the $k^{\text {th }}$ particular sequence of decays and captures. If this product becomes less than $10^{-6}$, the contribution of this sequence to the concentration of nuclide $i$ is neglected. Indeterminate forms that arise when $d_{i}=d_{j}$ or $d_{n}=d_{j}$ are evaluated using L'Hôpital's rule. These forms occur when two isotopes in a chain have the same diagonal element.

Equation (5.1.24) is applied to calculate all contributions to the "queue end-of-interval concentrations" of each short-lived nuclide from the initial concentrations of all others in the queue described above. It is also applied to calculate contributions from the initial concentrations of all short-lived nuclides in the queue to the long-lived nuclide that follows the queue, in addition to the total contribution to its daughter products. These values are appropriately applied either before or after the matrix expansion calculation is performed to correctly compute concentrations of long-lived nuclides and the long-lived or short-lived 
daughters. Equation (5.1.24) is also used to adjust to certain elements of the final transition matrix, which now excludes the short-lived nuclides. The value of the element must be determined for the new transition between the long-lived precursor and the long-lived daughter of a short-lived queue. The element is adjusted so that the end-of-interval concentration of the long-lived daughter calculated from the single link between the two long-lived nuclides (using the new element) is the same as what would be determined from the chain including all short-lived nuclides. The method assumes zero concentrations for precursors to the long-lived precursor. The computed values asymptotically approach the correct value with successive steps through time. For this reason, at least five to ten time intervals during the decay of discharged fuel is reasonable, because long-lived nuclides have built up by that time.

If a short-lived nuclide has a long-lived precursor, an additional solution is required. First, the amount of short-lived nuclide $i$ due to the decay of the initial concentration of long-lived precursor $j$ is calculated as

$$
N_{j \rightarrow i}(t)=N_{j}(0) a_{i j} \frac{e^{-d_{j} t}}{d_{i}-d_{j}}
$$

from Eq. (5.1.23), assuming $e^{-d_{i} t} \ll e^{-d_{j} t}$. However, the total amount of nuclide $i$ produced depends on the contribution from the precursors of precursor $j$, in addition to that given by Eq. (5.1.25). The quantity of nuclide $j$ not accounted for in Eq. (5.1.25) is denoted by $N_{j}{ }^{\prime}(t)$, the end-of-interval concentration, minus the amount that would have remained had there been no precursors to nuclide $j$ :

$$
N_{j}^{\prime(t)}=N_{j}(t)-N_{j}(0) e^{-d_{j} t}
$$

Then the short-lived daughter and subsequent short-lived progeny are assumed to be in secular equilibrium with their parents, which implies that the time derivative is zero,

$$
\frac{d N_{i}}{d t}=\sum_{j} a_{i j} N_{j}(t)=0 .
$$

The queue end-of-interval concentrations of all the short-lived nuclides following the long-lived precursor are augmented by amounts calculated with Eq. (5.1.24). The concentration of the long-lived precursor used in Eq. (5.1.26) is that given by Eq. (5.1.25). The set of linear algebraic equations given by Eq. (5.1.27) is solved by the Gauss-Seidel iterative technique. This algorithm involves an inversion of the diagonal terms and an iterated improvement of an estimate for $N_{i}(t)$ through the expression

$$
N_{i}^{k+1}=-\frac{1}{a_{i i}} \sum_{j} a_{i j} N_{j}^{k} .
$$

Since short-lived isotopes are usually not their own precursors, this iteration often reduces to a direct solution.

\subsection{Solution of the Nonhomogeneous Equation}

The previous sections have presented the solution of the homogeneous equation in Eq. (5.1.8), applicable to fuel burnup, activation, and decay calculations. However, the solution of a nonhomogeneous equation is required to simulate reprocessing or other systems that require an external feed term, $S(t) \neq 0$. The nonhomogeneous equation is given in matrix form (assumed constant over a step $n$ ) as

$$
\frac{d \vec{N}}{d t}=A \vec{N}(t)+\vec{S}
$$


for a fixed feed or removal rate, $\vec{S}$. A particular solution of Eq. (5.1.29) will be determined and added to the solution of the homogeneous equation given by Eq. (5.1.10). As before, the matrix exponential method is used for the long-lived nuclides, and solution by linear chains is used for the short-lived nuclides. Assume $\vec{C}$ an arbitrary vector with which to test a particular solution of the form

$$
\vec{N}(t)=\sum_{k=0}^{\infty} \frac{(\boldsymbol{A} t)^{k}}{(k+1) !} \vec{C} t .
$$

Substituting Eq. (5.1.30) into Eq. (5.1.29) yields

$$
\sum_{k=0}^{\infty} \frac{A^{k} t^{k}}{k !} \vec{C}=\sum_{k=0}^{\infty} \frac{A^{k+1} t^{k+1}}{(k+1) !} \vec{C}+\vec{S}
$$

in which the $k=0$ term may be extracted from the LHS,

$$
\vec{N}(t)=\sum_{k=0}^{\infty} \frac{(\boldsymbol{A} t)^{k}}{k !} \vec{N}(0)+\sum_{k=0}^{\infty} \frac{(\boldsymbol{A} t)^{k}}{(k+1) !} \vec{S} t
$$

which allows the summations on the left and right to be easily shown equal. This proves the particular solution is indeed valid if the arbitrary vector is in fact the feed term $\vec{C}=\vec{S}$. The solution to the nonhomogeneous problem is therefore (as a series),

$$
\vec{N}(t)=\sum_{k=0}^{\infty} \frac{(\boldsymbol{A} t)^{k}}{k !} \vec{N}(0)+\sum_{k=0}^{\infty} \frac{(\boldsymbol{A} t)^{k}}{(k+1) !} \vec{S} t
$$

For the second term in Eq. (5.1.33), a new recursion relation is developed for the particular solution in the same manner as was done for the homogeneous solution,

$$
N_{i}^{P}(t)=\sum_{n=1}^{\infty} D_{i}^{n}
$$

where

$$
D_{i}^{1}=S_{i} t ; D_{i}^{n+1}=\frac{1}{n+1} \sum_{j} a_{i j} D_{j}^{n}
$$

For the short-lived nuclides, the secular equilibrium equations are modified to become

$$
\frac{d N_{i}}{d t}=\sum_{j} a_{i j} N_{j}(t)+S_{i}=0 .
$$

The Gauss-Seidel iterative method is applied to determine the solution. The complete solution to the nonhomogeneous equation in Eq. (5.1.29) is given by the sum of the homogeneous solutions described in previous sections and the particular solutions described here. 


\subsection{CRAM}

The solver kernel based on the Chebyshev Rational Approximation Method (CRAM) is described in detail in references ${ }^{6,7,8,9}$. Compared to the MATREX solver, CRAM generally has similar runtimes but is more accurate and robust on a larger range of problems. CRAM relies on the lower upper (LU) decomposition, so the SuperLU library has been used. The accuracy of CRAM is related to the order, with an order 16 solution having a truncation error less than $0.01 \%$ for all nuclides in most problems.

Unlike many methods for solving this type of system of ODEs, the length of a step does not significantly affect the accuracy of CRAM. However, any significant errors from CRAM will shrink rapidly over multiple steps as long as there are no large changes in reaction rates. The CRAM solver has an efficient internal substepping algorithm that can perform multiple same-sized substeps (with the same transition matrix) very efficiently by reusing the LU decomposition. When using internal substepping, 2-4 substeps are typical, with a large gain in accuracy for marginal increase in runtime.

\subsubsection{Power Calculation}

The following is formula is used to calculate power during irradiation $(\Phi>0)$,

$$
P(t)=\sum_{i}\left(\kappa_{f i} \sigma_{f i}+\kappa_{c i} \sigma_{c i}\right) \Phi N_{i}(t)
$$

where $\kappa_{f i}$ and $\kappa_{c i}$ are nuclide-dependent energy released per fission and "capture," with capture defined as removal minus fission: $\sigma_{c i}=\sigma_{i}-\sigma_{f i}$. The $\sigma_{f i}$ and $\sigma_{c i}$ terms are extracted from the transition matrix itself, whereas the $\kappa_{f i}$ and $\kappa_{c i}$ are available from a separate ORIGEN energy resource (see ORIGEN Data Resources chapter). If the flux $\Phi$ is specified, then the power can be calculated at any time according to Eq. (5.1.37). However in reactor fuel systems, it is convenient to be able to specify the power produced by the system and internally to the depletion code, to convert the power to an equivalent flux. Solving Eq.(5.1.37) for the flux, however,

$$
\Phi(t)=\frac{P}{\sum_{i}\left(\kappa_{f i} \sigma_{f i}+\kappa_{c i} \sigma_{c i}\right) N_{i}(t)}
$$

it is apparent that a fixed power over a time step $n$ does not lead to a fixed flux, due to changing isotopics that produce different amounts of power per fission and capture. ORIGEN performs a fluxcorrection calculation to obtain an estimate of the average flux over the step. The beginning-of-step flux is first calculated for the initial compositions: Eq. (5.1.38) is evaluated as $\Phi\left(t_{n-1}\right)$, and then Eq. (5.1.38) is solved with that flux. The flux is then recalculated at the end of step $\Phi\left(t_{n}\right)$ using the estimated end-ofstep isotopics, and the step-average flux $\Phi_{n}$ is estimated as the simple average of the beginning and endof-step fluxes,

$$
\Phi_{n}=0.5\left[\Phi\left(t_{n}\right)+\Phi^{\text {pred }}\left(t_{n+1}\right)\right]
$$

noting that the "predicted" flux at end-of-step $\Phi^{\text {pred }}\left(t_{n+1}\right)$ is based on "predicted" end-of-step isotopics, based on a beginning-of-step flux level. 


\subsubsection{Decay Emission Sources Calculation}

ORIGEN can calculate the emission sources (and spectra) during decay for alpha, beta, neutron, and photon particles according to

$$
R_{x}^{g}(t)=\sum_{i} \lambda_{i} N_{i}(t) \int_{E^{g}}^{E^{g-1}} w_{i, x}(E) d E
$$

where $w_{i, x}(E)$ is the number of particles of type $x$ emitted per disintegration of nuclide $i$ at energy $E$, using provided energy bins defined by energy bounds $E^{g}$ to $E^{g-1}$, where $g$ is an energy index. The fundamental data resources for performing emission source calculations are described in the ORIGEN Data Resources chapter.

\subsection{Neutron Sources}

Computed neutron sources include neutrons spontaneous fission, $(\alpha, n)$ reactions, and delayed $\left(\beta^{-}, n\right)$ neutron emission,

$$
w_{i, n}(E)=w_{i, S F n}(E)+w_{i,(\alpha, n)}(E)+w_{i, D n}(E)
$$

with components that will be described below. The method of computing the spontaneous fission and delayed neutron source is independent of the medium containing the fuel. However, $(\alpha, n)$ production varies significantly with the composition of the medium. The homogeneous medium $(\alpha, n)$ calculation methodology has been adopted from the Los Alamos code SOURCES 4B. ${ }^{10,11}$

The total yield of spontaneous fission neutrons from decay of nuclide $i$ is

$$
Y_{i, S F n}=\frac{\lambda_{i, S F n}}{\lambda_{i}}
$$

where $\frac{\lambda_{i, S F n}}{\lambda_{i}}$ is the fraction of decays which undergo spontaneous fission. The distribution of spontaneous fission neutrons, $w_{i, S F n}(E)$ is given by a Watt fission spectrum,

$$
w_{i, S F n}(E)=Y_{i, S F n} C_{i} e^{-E / A_{i}} \sinh \sqrt{B_{i} E},
$$

where $A_{i}, B_{i}$, and $C_{i}$ are model parameters.

The $(\alpha, n)$ neutron source is strongly dependent on the low-Z content of the medium containing the alphaemitting nuclides and requires modeling the slowing down of the alpha particles and the probability of neutron production as the $\alpha$ particle slows down. The calculation assumes (1) a homogeneous mixture in which the alpha-emitting nuclides are uniformly intermixed with the target nuclides and (2) that the dimensions of the target are much larger than the range of the alpha particles. Thus, all alpha particles are stopped within the mixture. The yield of a particular $\alpha$ is given by

$$
Y_{\ell i, \alpha}=f_{\ell i, \alpha} \frac{\lambda_{i, \alpha}}{\lambda_{i}}
$$

where $\frac{\lambda_{i, \alpha}}{\lambda_{i}}$ is the relative probability of $\alpha$-decay, and $f_{\ell i, \alpha}$ is the fraction of those $\alpha$-decays producing an $\alpha$ particle with initial energy $E_{\ell i, \alpha}$, and is considered fundamental data. The total neutron yield from an alpha particle $\ell$ emitted by nuclide $i$ and interacting with target $k$ is given by the following, 


$$
Y_{\ell i k,(\alpha, n)}=Y_{\ell i, \alpha} \frac{N_{k}}{N} \int_{0}^{E_{\ell i, \alpha}} \frac{\sigma_{k,(\alpha, n)}\left(E_{\alpha}\right)}{S\left(E_{\alpha}\right)} d E_{\alpha}
$$

where $S\left(E_{\alpha}\right)$ is the total stopping power of the medium, $\sigma_{k,(\alpha, n)}\left(E_{\alpha}\right)$ is the $(\alpha, n)$ reaction cross section for target nuclide $k$, and $\frac{N_{k}}{N}$ is the fraction of atoms in the medium composed of nuclide $k$. This expression is used to calculate the neutron yield for each target nuclide and from each discrete-energy alpha particle emitted by all alpha-emitting nuclides in the material. The stopping power for compounds, rather than pure elements, is approximated using the Bragg-Kleeman additivity rule. The energydependent elemental stopping cross sections are determined as parametric fits to evaluated data. Eq. (5.1.45) is solved for the total neutron yields from the alpha particle, as it slows down in the medium by subdividing the maximum energy $E_{\ell i, \alpha}$ into a number of discrete energy bins and evaluating stopping power and $(\alpha, n)$ reaction cross section at the midpoint energy of the bin. The distribution of $(\alpha, n)$ neutrons as required Eq. (5.1.40) is

$$
w_{i,(\alpha, n)}(E)=\sum_{k} \sum_{\ell \in i} Y_{\ell i k,(\alpha, n)} X_{\ell i k,(\alpha, n)}(E),
$$

with the distribution of $(\alpha, n)$ neutrons in energy, $X_{\ell i k,(\alpha, n)}(E)$, calculated using nuclear reaction kinematics, assuming that the $(\alpha, n)$ reaction emits neutrons with an isotropic angular distribution in the center-of-mass system. The maximum and minimum permissible energies of the emitted neutron are determined by applying mass, momentum, and energy balance for each product's nuclide energy level. The product nuclide levels, the product level branching data, the $(\alpha, n)$ reaction $\mathrm{Q}$ values, the excitation energy of each product nuclide level, and the branching fraction of $(\alpha, n)$ reactions result in the production of product levels. A more detailed discussion of the theory and derivation of the kinematic equations can be found in Ref. 10.

Delayed neutrons are emitted by decay of short-lived fission products. The observed delay is due to the decay of the precursor nuclide. The total yield of delayed neutrons from decay of nuclide $i$ is

$$
Y_{i, D n}=\frac{\lambda_{i, D n}}{\lambda_{i}}
$$

where $\frac{\lambda_{i, D n}}{\lambda_{i}}$ is the fraction of decays which emit delayed neutrons. The delayed neutrons emitted per decay of nuclide $i$ at energy $E$ is given by

$$
w_{i, D n}(E)=Y_{i, d l n} X_{i, D n}(E),
$$

where the spectrum $X_{i, D n}(E)$ is fundamental library data. Delayed neutrons are not important in typical spent fuel applications due to the very short half-lives of the parent nuclides, dropping off significantly after $\sim 10$ seconds, but they may be of value in specialized applications where calculating time-dependent delayed neutron source spectra is important.

\subsection{Alpha Sources}

An $\alpha$ slowing down calculation is performed as part of the $(\alpha, n)$ neutron calculation. However, the alpha source (i.e. without considering slowing down in the media) is also available, simply as the sum of delta functions at the discrete initial alpha particle energies $w_{i, \alpha}(E)=\sum_{\ell \in i} Y_{\ell i, \alpha} \delta\left(E-E_{\ell i, \alpha}\right)$ with yields $Y_{\ell i, \alpha}$, as required by Eq. (5.1.40). 


\subsection{Beta Sources}

The beta source (i.e. without considering slowing down in the media) is available as the sum of the continuous emission spectra for each $\beta_{-}$decay in nuclide $i$. The total yield of beta particles from decay of nuclide $i$ is

$$
Y_{i, \beta}=\frac{\lambda_{i, \beta}}{\lambda_{i}}
$$

where $\frac{\lambda_{i, \beta}}{\lambda_{i}}$ is the fraction of decays which emit betas. The betas emitted by nuclide $i$ at energy $E$ is given by

$$
w_{i, \beta}(E)=Y_{i, \beta} X_{i, \beta}(E) \text {, }
$$

where the spectrum $X_{i, \beta}(E)$ is fundamental data, independent of the media. The spectrum includes betas from allowed transitions and first, second, and third forbidden transitions.

\subsection{Photon Sources}

The total yield of photons from decay of nuclide $i$ is

$$
Y_{i, \gamma}=\frac{\lambda_{i, \gamma}}{\lambda_{i}}
$$

where $\frac{\lambda_{i, \gamma}}{\lambda_{i}}$ is the fraction of decays which emit photons. The photons emitted by nuclide $i$ at energy $E$ is given by

$$
w_{i, \gamma}(E)=Y_{i, \gamma} X_{i, \gamma}(E)
$$

where the spectrum $X_{i, \gamma}(E)$ is fundamental data and includes both line data from x-rays, gamma-rays and continuum data from Bremsstrahlung, spontaneous fission gamma rays, and gamma rays accompanying $(\alpha, n)$ reactions. The Bremsstrahlung component of the photon source has been tabulated for various media and no on-the-fly slowing down calculation is performed.

\subsubsection{Library Interpolation}

Accurate solution of fuel depletion with Eq. (5.1.1) requires coupling to self-shielding and neutron transport to accurately capture the time-dependent change in space and energy flux distribution and 1 -group cross sections with isotopic change. This is in generally a fairly computationally intensive problem compared to stand-alone depletion. In typical assembly design and analysis, the same basic assembly problem must be solved repeatedly with variations in power history, different periods of decay/burnup, different moderator density, etc. A question naturally arises: could the isotopics from numerous well-constructed cases be saved and interpolated to the actual system? Interpolating the isotopics themselves is fraught with difficulty. For example, consider two cases with the same burnup but different periods of decay between cycles. A better approach-the ORIGEN Automated Rapid Processing (ORIGEN-ARP) — was developed with the key realization that one can reconstruct very accurate isotopics from stand-alone depletion calculations by interpolating transition matrices rather than isotopics.

The accuracy of the interpolation methodology compared to the coupled transport/depletion solution (e.g., with TRITON) depends on the suitability of the interpolation parameters and the deviation of the desired system from the systems used to create the library. For example, for thermal systems with uranium-based fuels, it was found that enrichment, water density, and burnup were the dominant 
independent variables and thus were best suited for interpolation. An example of the variation of removal cross sections for key actinides is shown in Figure 5.1.2 for a Westinghouse $17 \times 17$ pressurized water reactor (PWR) assembly type with $5 \%$ initial enrichment in ${ }^{235} \mathrm{U}$. Each cross section has been divided by its initial value at zero burnup to show the variation more clearly. ${ }^{240} \mathrm{Pu}$ has been observed to have the most variation with spectral changes, with $\sim 60 \%$ reduction in cross section from beginning to end of life. The variations in ${ }^{240} \mathrm{Pu}$ with respect to enrichment and moderator density are shown in Figure 5.1.3, Figure 5.1.4, Figure 5.1.5, and Figure 5.1.6.

Currently there are two interpolation methods: a Lagrangian based on low-order polynomials and a cubic spline with an optional monotonicity fix-up.

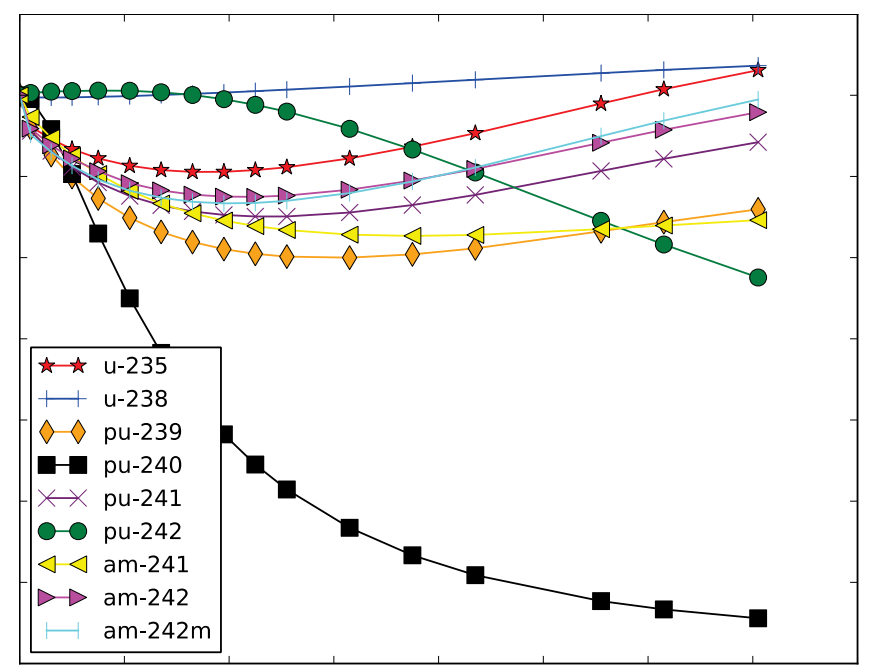

Figure 5.1.2. Relative removal cross section as a function of burnup for key actinides (Westinghouse $17 \times 17$ assembly with $5 \%$ enrichment).

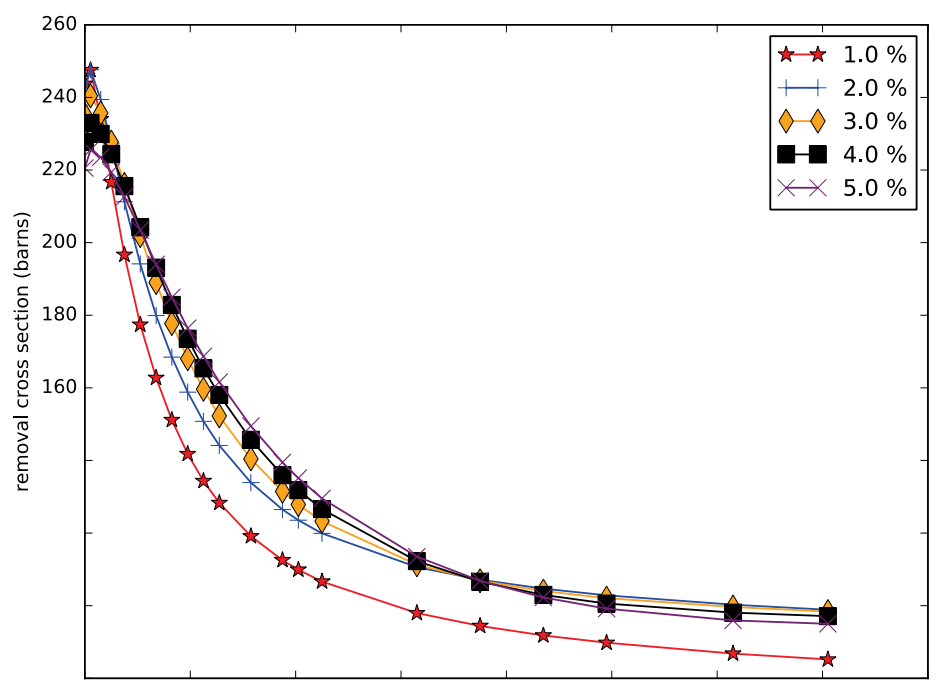

Figure 5.1.3. ${ }^{240} \mathrm{Pu}-240$ removal cross section as a function of burnup for various enrichments (GE $10 \times 10$ assembly). 


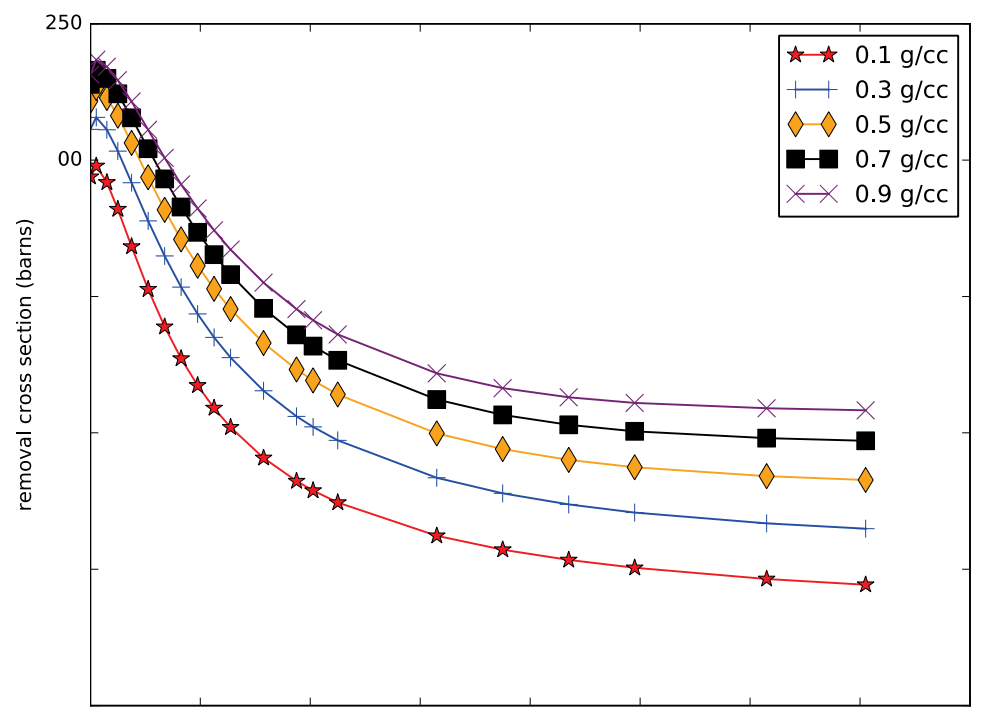

Figure 5.1.4. ${ }^{240} \mathrm{Pu}$ removal cross section as a function of burnup for various moderator densities (GE $10 \times 10$ assembly).

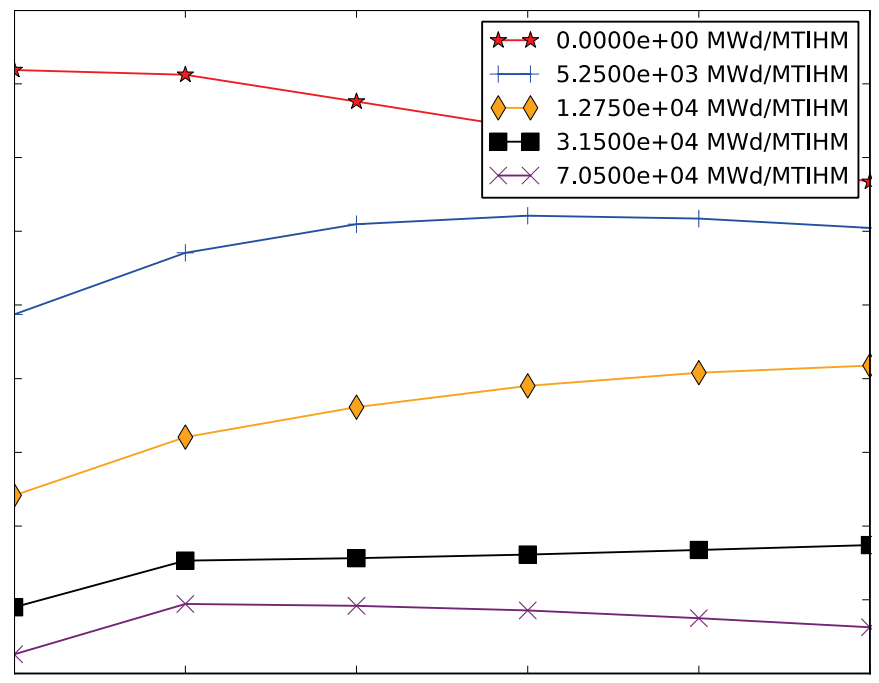

Figure 5.1.5. ${ }^{240} \mathrm{Pu}$ removal cross section as a function of initial enrichment for various burnups (GE $10 \times 10$ assembly). 


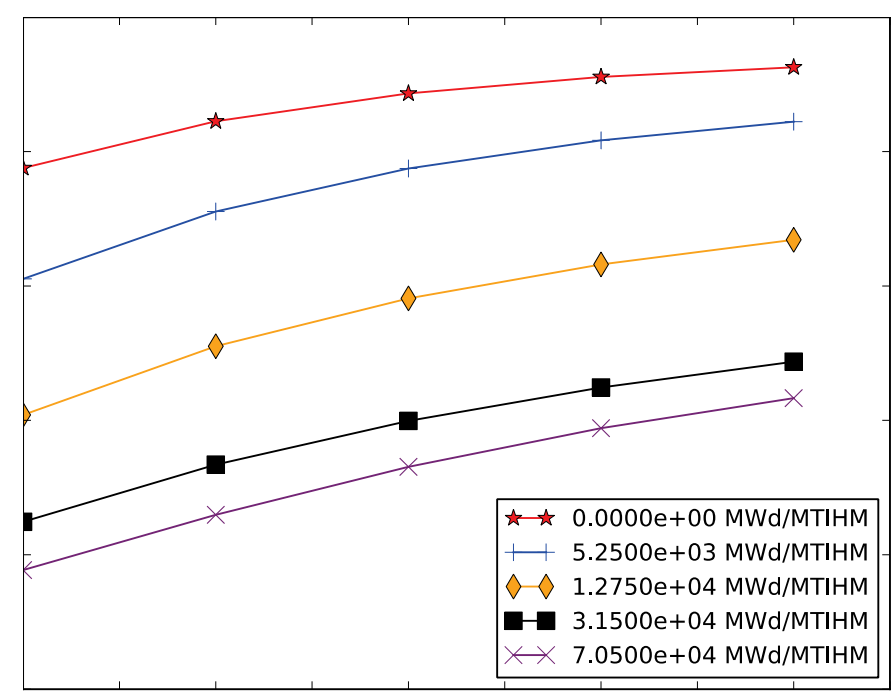

Figure 5.1.6. ${ }^{240} \mathrm{Pu}$ removal cross section as a function of moderator density for various burnups (GE $10 \times 10$ assembly).

\subsection{Lagrangian Interpolation}

Lagrangian interpolation ${ }^{12}$ seeks the unique $n-1$ order polynomial that will pass through $n$-points of the function and then interpolating to the desired point by evaluating the polynomial,

$$
y(x)=\prod_{i=1}^{n}\left(x-x_{i}\right) \sum_{k=1}^{n} \frac{y_{k}}{\left(x-x_{k}\right) \prod_{\substack{i=1 \\ i \neq k}}^{n}\left(x_{k}-x_{i}\right)},
$$

where $x_{i}$ and $y_{i}$ are the known $\mathrm{x}$ - and y-values in the neighborhood of the desired x-value $x$, with $n$ the number of data points/order of Lagrangian interpolation. Note that Lagrangian interpolation is by definition local, involving only points in the neighborhood of the desired value. Global alternatives such as Hermite cubic splines use the entire data set to construct the interpolants. Common interpolation methods based on polynomials can have difficulty with data that vary quickly and have uneven $x$-spacing, as is expected with transition data. Polynomials tend to produce unphysical oscillations in these cases. In cases with very small $y$-values $\left(\sim 10^{-10}\right)$, oscillations of the interpolant can produce negative interpolated values.

\subsection{Cubic Spline Interpolation}

Cubic spline interpolation has been observed to produce fewer, lower frequency oscillations. Oscillations can be effectively eliminated by enforcing monotonicity on the interpolation: that is, additional max maxima or minima are not introduced by the interpolant between known values of the function. Monotonic cubic splines ${ }^{13}$ have shown particularly stable behavior and have been implemented as an interpolation option. 


\subsubsection{ORIGEN Family of Modules}

This section describes how to perform the calculations and evaluations described in Sect. 5.1.2 using the ORIGEN family of modules in SCALE. These modules are summarized briefly below.

1. The COUPLE module is used to create ORIGEN libraries. The ORIGEN library contains transition matrices $\boldsymbol{A}$ and other relevant data in order to solve the depletion/decay equation of Eq. (5.1.1). COUPLE requires an input flux spectrum in order to perform the multigroup cross section collapse. Optionally, one-group self-shielded cross sections can be provided. Generally, in order to solve a non-decay problem, a library must be created with either COUPLE or ARP.

2. The ARP module is also used to create an ORIGEN library, but it is created by way of a special interpolation scheme on a set of existing libraries rather than by specifying a flux spectrum and one-group cross sections as in COUPLE. A set of ORIGEN libraries is distributed with SCALE for use with ARP and is described in ORIGEN Reactor Libraries chapter.

3. The ORIGEN module is used to solve depletion, decay, activation, and feed problems described by Eq. (5.1.1), as well as the decay emission calculations described by Eq. (5.1.40). For spent fuel calculations using the ARP interpolation methodology, it may be more convenient to use ORIGAMI, as described in ORIGAMI chapter.

4. The OPUS module is used to perform post processing and analysis on ORIGEN results contained in ORIGEN concentrations files, including sorting, ranking, and unit conversion.

Two types of files are an integral part of the ORIGEN family of modules: the library file and the concentrations files.

- The library file is a binary file, usually either with the complete filename "ft33f001" or with extension ".f33," and it contains a collection of transition matrices $\boldsymbol{A}$, usually corresponding to different burnups. It is typically called an "ORIGEN library," "ft33," or "f33" file.

- The concentrations file is also a binary file, usually either with the complete filename "ft71f001" or with extension ".f71." The f71 is a solution archive containing isotopics vectors $\vec{N}$ corresponding to different materials or different points in time.

\subsubsection{COUPLE Module}

COUPLE is a coupling code that prepares the transition matrix $\boldsymbol{A}$ from Eq. (5.1.2), which contains the decay and cross section transition rate constants according to the procedures defined in Sect. 5.1.2.1. The transition matrix and other important data are stored on an ORIGEN library (f33) file for use by other modules. COUPLE has two distinct modes of operation:

1. to create a new decay-only ORIGEN library from an ORIGEN decay resource, and

2. to add new or to update existing reaction transitions yield resource, reaction resource, and optionally an AMPX working library containing multigroup cross sections.

Details on the decay, yield, and reaction resources may be found in the ORIGEN Data Resources chapter. 


\subsection{Key Features}

This section briefly highlights some key features in COUPLE and describes how they are used.

\subsection{AMPX multigrouplibraries}

AMPX multigroup libraries contain multigroup cross sections by nuclide and material-zone identifiers. If the working library is the result of a multiregion transport calculation, then it is important to specify the correct zone identifier, e.g. corresponding to the fuel in a problem with moderator, clad, and fuel zones. The neutron flux is also stored on the AMPX library associated with a nuclide and a zone as are the cross sections. An AMPX library flux can be used to perform the cross section collapse as an alternative to providing a flux spectrum in the COUPLE input. New transitions may be added to the ORIGEN binary library for all reactions for which there are data in the weighted AMPX library if both the target and product nuclides are present in the ORIGEN library.

\subsection{Nuclide Specification}

In COUPLE, the following nuclide identifier is used:

Nuclide identifier $=\mathrm{Z} * 10000+\mathrm{A} * 10+\mathrm{I}$, where

$$
\begin{aligned}
& \mathrm{Z}=\text { atomic number, } \\
& \mathrm{A}=\text { mass number, } \\
& \mathrm{I}=\text { metastable/isomeric state }(0 \text { is ground } / 1 \text { is first metastable })
\end{aligned}
$$

Examples include 922350 for ${ }^{235} \mathrm{U}$ and 952421 for ${ }^{242 \mathrm{~m}} \mathrm{Am}$. Note that this varies from the identifiers used in other ORIGEN-related modules in which the isomeric state $I$ comes first, as in 1095242 for ${ }^{242 \mathrm{~m}} \mathrm{Am}$.

\subsection{Adding new transitions and user-defined transitions}

The use of a transition matrix in ORIGEN allows any nuclide to transition to any other nuclide. By default, when the reaction data on the library is updated, then the transition matrix's sparse storage is expanded to include the new reaction transition if both the target and the reaction product nuclide are in the library. The user may request that the code does not add new transitions by setting Block1 $1 \$ \$$ $\mathrm{JADD}=0$. This option ensures that the matrix structure on the input library is identical to that of the output library. The user may explicitly set one-group transition coefficients by setting Block1 $1 \$ \$$ LBUP $=1$ and entering Block6 and Block8 data.

\subsection{Unit numbers and Aliases}

In COUPLE, a unit number is used instead of a full file name to specify files, where unit number XY links to the data file "ftXYf001" in the working directory. For example, unit number 33 means file $\mathrm{ft} 33 \mathrm{f001}$. There are several predefined unit numbers that are controlled by a special "origen_filenames" file, which creates an alias for the local file "ftXYf001" to a file in the data directory. Table $\overline{5} .1 .1$ shows the basic COUPLE unit numbers, their aliases, and a description of the file. 
Table 5.1.1. Basic COUPLE unit numbers

\begin{tabular}{lll}
\hline Unit & Alias & Description \\
\hline 17 & YIELDS & ORIGEN Yield Resource \\
21 & END7DEC & ORIGEN library \\
& & ENDF/B-VII-based decay transitions only \\
27 & DECAY & ORIGEN Decay Resource \\
80 & JEFF252G & ORIGEN Reaction Resource (252 groups) \\
\hline
\end{tabular}

An "origen_filenames" list which maps unit number 21 to alias "END7DEC" could link unit 21 to the file "\$ $\{\mathrm{DAT} A\}$ /origen.rev04.end7dec," where $\$\{\mathrm{DATA}\}$ is the path to the SCALE data directory. To override this association, COUPLE must find a file named " $\mathrm{ft} 21 \mathrm{f} 001$ " in the working directory. The entire set of unit numbers is given in the ORIGEN Data Resources chapter.

\subsection{Input Description}

COUPLE uses the FIDO input system, except for title entries. The input is arranged in blocks, with each block containing one or more arrays, followed by the FIDO block terminator "t." Each input parameter is named and defined below in the order in which it appears, with the index of the parameter in the array. Some options have been deprecated over time and thus the first available entry may not correspond to index " 1 " and some indices may be skipped. Default values are given in parentheses. In the SCALE code system, COUPLE input appears between "=couple" and "end."

\subsection{Block1: titles, unit numbers, and case controls.}

TITLE - Title lines

Title lines can provide information about the ORIGEN library created and printed when the library is used. The input Block1 $1 \$ \$$ NUMA allows title lines to be copied from the input library to the output library.

The first blank line terminates the title.

A maximum of 40 lines can be included in the library.

A special title of "DONE" in the first four columns marks the completion of a COUPLE input case.

0\$\$ Array - Logical Unit Assignments

1. NOUT - Printed output unit number (6)

2. LIBDEC - Unit number of ORIGEN decay resource (27)

Only used if $1 \$ \$ L B I N=1$

3. JD - Unit number of ORIGEN reaction resource (80)

4. ND - Input ORIGEN binary library file (21)

Only used if $1 \$ \$ L B I N=0$ 
5. LD - Unit number of AMPX multigroup library file (0) Only used if LD>0; energy group structure must be consistent with that on ORIGEN reaction resource (JD)

6. MD - Unit number for output ORIGEN library file (33)

8. NY - Unit number of ORIGEN yield resource (17)

$1 \$ \$$ Array - Control Constants [19 entries]

1. LBIN $-1 / 0$ - Decay library creation/reaction update mode $(0)$

In decay library creation mode with $L B I N=1$, the reaction resource $(0 \$ \$ \mathrm{JD})$ is not used, any input associated with reaction processing is ignored, and Block2 and Block8 may not be entered. In reaction update mode with LBIN=0, Block3 may not be entered.

2. PRT $-1 / 0$ - Suppress all informational output / print informational output (0).

3. LBUP - 1/0 - Update from user input cross sections (Block6 and Block8 Arrays) / no user update (0).

4. JADD $-1 / 0-$ Add/do not add new transitions to the library (1).

5. JEDT $-1 / 0-$ Edit input library only/normal library generation case $(0)$.

6. NXX $-1 / 0-$ Allow/do not allow transitions with zero cross section (0).

7. NMO - Current month (as integer) for output library (0).

8. NDAY - Current day for output library (0).

9. NYR - Current year for output library (two digits) (0).

12. IDREF - Nuclide ID in AMPX multigroup library (0\$ LD) containing neutron flux weighting spectrum to use in cross section collapse (0). If IDREF $=0$, uses first nuclide found in NZONE. Only used if $N W G T=0$.

13. NZONE - Zone ID (usually a mixture ID) in AMPX multigroup library (0\$ LD) from which to add/update transitions (0). If $N Z O N E=0$, the AMPX library must not contain zone IDs.

14. IEDOU $-1 / 0-$ Edit/no edit of transition cross sections ( 0 )

15. NFISW - Number of nuclides with fission yields (-1)

$-1 \quad$ fission yields included for all fissionable nuclides

0 no yields added

$\mathrm{N}$ input $\mathrm{N}$ nuclides with fission yields (Block2 7\$\$ Array)

16. NUMA - Number of title lines to copy from the input ORIGEN library (0\$\$ ND) to the output ORIGEN library (0\$\$ MD) (0).

18. NWGT - Flux spectrum source (0).

0 flux spectrum from AMPX multigroup library (IDREF)

$\mathrm{N}$ input $\mathrm{N}$-group flux spectrum (Block2, 9** Array)

$\mathrm{T}-\quad$ Block1 terminator. 


\subsection{Block2: nuclides with fission yields and weighting flux spectrum}

This block is only read if in reaction update mode (Block1 $0 \$ \$ \mathrm{LBIN}=0)$.

$7 \$ \$$ Array - Nuclide IDs with fission yields [Block1 1\$\$ NFISW entries]

9** Array - Weighting flux spectrum [Block1 1\$\$ NWGT entries]

The flux spectrum must be given in order of descending neutron energy according to the convention that group 1 is the highest energy group. The group structure (number of groups and group boundaries) must be consistent with the ORIGEN reaction resource (Block1 0\$\$ JD).

$\mathrm{T}-$ Block2 terminator.

\subsection{Block3: array dimensions for decay library creation}

This block is only read if in decay library creation mode (Block1 0\$ LBIN=1). The default values usually apply. The values are used only internally for memory allocation and may be set to a larger value than is required.

$3 \$$ Array - Library constants

18. ITMAX - Total number of nuclides in library (2600)

19. ILMAX - Number of activation product nuclides (1000)

-1 , omits light-element library

20. IAMAX - Number of actinide nuclides (200)

-1 , omits actinide library

21. IFMAX - Number of fission-product nuclides (1400)

-1 , omits fission-product library

22. IXMAX - Total number of decay transitions from one nuclide to another $(40,000)$

$\mathrm{T}-$ Block3 terminator.

\subsection{Block6: number of user-defined transition coefficients}

This block is only read if user-defined transition coefficients have been specified in decay library creation mode (Block1 1\$\$ LBUP=1).

$15 \$ \$$ Array - Number of user update nuclides

1. LBU - Total number of transitions to be entered in Block8 71\$\$, 72\$\$, and $73 * *$ Arrays (0)

$\mathrm{T}$ - Block6 terminator

\subsection{Block8: user-defined transition coefficients}

Block8 is only required only if a nonzero value is entered in the Block6 $15 \$ \$$ array. The three arrays $\left(71 \$ \$, 72 \$ \$\right.$, and $\left.73^{* *}\right)$ represent the parent, daughter, and coefficients for Block6 LBU user-defined transitions, or the quantity $f_{i j} \sigma_{j}$ for a given parent $j$ and daughter $i$ from Eq. (5.1.4). 
$71 \$ \$$ Array - Parent Nuclides [LBU entries]

ISN1 - Parent Nuclide ID

$72 \$ \$$ Array - Daughter Nuclides or Reaction MT number [LBU entries]

ISN2 - Daughter Nuclide ID for the reaction product of the corresponding entry in ISN1 or reaction MT number

NOTE: The reaction transition will be added if it does not already exist by setting Block1 $1 \$ \$ J A D D=1$. Otherwise, new transitions are omitted.

73** Array - Reaction Cross Sections [LBU entries].

SIGMA - Reaction cross section (in barns) for the reaction described by ISN1 and ISN2

There are two special rules to facilitate modifying fission cross sections $\sigma_{f j}$ and removal cross sections $\sigma_{j}$.

if $I S N 1=I S N 2$, the removal cross section is set equal to the corresponding SIGMA. Note that this overrides the automatic calculation of the removal cross section as the sum of all transition cross sections.

if $I S N 1=-I S N 2$, the fission cross section is set equal to the corresponding SIGMA.

$\mathrm{T}-$ Block8 terminator.

This concludes the input for a single case in COUPLE. COUPLE allows for multiple cases in a single input and will automatically begin processing the next case's Block1 TITLE unless "DONE" (without quotes) is entered as the TITLE entry.

\subsubsection{ARP Module}

The ARP module performs multidimensional interpolation on a set of specially prepared ORIGEN libraries using interpolation methods discussed in Sect. 5.1.2.5, with available interpolators listed in Table 5.1.2. The ORIGEN reactor libraries distributed with this version of SCALE are described in the ORIGEN Reactor Libraries chapter, as well as details on how users can generate their own libraries. The ARP module has been validated extensively for light water reactor (LWR) spent fuel. ${ }^{14}$ Benchmarking studies for MOX fuel were also conducted. ${ }^{15}$ 
Table 5.1.2. Interpolation options in ARP

\begin{tabular}{|c|c|c|}
\hline Type & $\begin{array}{l}\text { Interpolation } \\
\text { keyword }\end{array}$ & Comments \\
\hline Nearest value & nearest & Searches for closest value to desired value. \\
\hline Linear interpolation & linear & Uses nearest two values bounding the desired value. \\
\hline $\begin{array}{l}\text { Lagrangian } \\
\text { interpolation }\end{array}$ & $\begin{array}{l}\text { lagrange }(\mathrm{N}) \\
\text { with order } N \text { from } 1 \\
\text { to } 4 \\
\text { lagrange } \\
\text { same as lagrange(4) }\end{array}$ & $\begin{array}{l}\text { Uses } \mathrm{N} \text { points near desired value and creates polynomial of } \\
\text { order } \mathrm{N}-1 \text { using Eq. }(5.1 .53) \text {. The specification of } \\
\text { lagrange(1) is equivalent to nearest and lagrange( } 2) \text { to linear. }\end{array}$ \\
\hline Standard cubic spline & stdspline & Standard, natural cubic spline (without monotonicity fix-up). \\
\hline $\begin{array}{l}\text { Monotonic cubic } \\
\text { spline }\end{array}$ & spline & $\begin{array}{l}\text { Natural cubic spline with a monotonicity fix-up designed to } \\
\text { prevent nonphysical oscillations that in some cases cause } \\
\text { negative interpolated cross sections. }\end{array}$ \\
\hline
\end{tabular}

Parametrizations for three types of problems have been developed: uranium-based fuel, mixed-oxide (MOX) fuel, and general activation.

- The parametrization for uranium-based fuel (e.g., $\mathrm{UO}_{2}$ ), as would be found in most LWRs, can interpolate on

○ fuel enrichment,

$\circ$ moderator density, and

○ burnup.

- The parametrization for MOX fuel contains a mixture of plutonium and uranium oxide and can interpolate on

○ total plutonium content in the heavy metal,

○ plutonium isotopic vector ( $\mathrm{Pu}$ vector) that defines the relative concentrations of the $\mathrm{Pu}$ isotopes,

- moderator density, and

○ burnup.

- The parametrization for general activation problems has only one-dimensional interpolation on fluence.

Variation of the absorption cross sections was observed to be near linear as a function of Pu content. Interpolation on the $\mathrm{Pu}$ vector is more complex than the uranium enrichment for $\mathrm{UO}_{2}$ fuel since the vector is composed of five different isotopes: ${ }^{238} \mathrm{Pu},{ }^{239} \mathrm{Pu},{ }^{240} \mathrm{Pu},{ }^{241} \mathrm{Pu}$, and ${ }^{242} \mathrm{Pu}$. Furthermore, the elements in the vector depend on one another and can therefore not be evaluated independently of one another since the entire vector must sum to $100 \%$. The scheme developed for the Pu vector was based on an evaluation of a large database of plutonium compositions from actual MOX fuel assemblies of European origin. It might be expect edthat the parametrization would need to include all Pu isotopes. However, an evaluation of the MOX fuel database indicated that there is a strong correlation between ${ }^{239} \mathrm{Pu}$ and the other isotopes in the vector that permits cross sections for the MOX fuel to be determined to sufficient accuracy using only the ${ }^{239} \mathrm{Pu}$ concentration. 


\subsection{Input Description}

ARP has a simple input scheme, a different line-by-line input expected for each of the three problem types - uranium, MOX, or activation-with the input required for each type shown in Table 5.1.3, Table 5.1.4, and Table 5.1.5. Available input depends on what is available in the relevant arpdata.txt file and the arplibs directory.

Table 5.1.3. Input description for uranium fuels

\begin{tabular}{|c|c|c|c|}
\hline $\begin{array}{l}\text { Entry } \\
\text { no. }\end{array}$ & Data type & $\begin{array}{l}\text { Entry } \\
\text { requirements }\end{array}$ & Comment \\
\hline 1 & Data set name & $\begin{array}{l}\text { Line } 1 \\
\text { always } \\
\text { required }\end{array}$ & $\begin{array}{l}\text { Enter a uranium CONFIGNAM from the active } \\
\text { arpdata. txt (see } \\
\text { Table 5.1.6Table 5.1.6). }\end{array}$ \\
\hline 2 & Enrichment & $\begin{array}{l}\text { New line } \\
\text { always }\end{array}$ & Enter the wt $\%{ }^{235} \mathrm{U}$ in total $\mathrm{U}$. \\
\hline 3 & Number of cycles & Always & Enter the number of irradiation cycles $N$. \\
\hline 4 & Fuel irradiation period & Always & $\begin{array}{l}\text { Enter the irradiation time for each cycle in days } \\
\qquad \Delta T_{i} \text {, for } i=1,2, \ldots, N \text {. }\end{array}$ \\
\hline 5 & Average power & Always & $\begin{array}{l}\text { Enter the specific fission power (MW/MTHM) } \\
\quad \text { for each cycle, } P_{i} \text {, for } i=1,2, \ldots, N \text {. }\end{array}$ \\
\hline 6 & Data interpolations per cycle & Always & $\begin{array}{l}\text { Enter the number of cross section sets to } \\
\text { interpolate during each cycle, } m_{i} \text {, for } \\
i=1,2, \ldots, N \text {. }\end{array}$ \\
\hline 7 & Moderator density & Always & $\begin{array}{l}\text { Enter the moderator density }\left(\mathrm{g} / \mathrm{cm}^{3}\right) \text {. Enter only } \\
\text { one value. }\end{array}$ \\
\hline 8 & New library name & $\begin{array}{l}\text { New line } \\
\text { always }\end{array}$ & $\begin{array}{l}\text { Enter the file name of the new ORIGEN library } \\
\text { created from interpolation. }\end{array}$ \\
\hline 9 & Interpolation keyword & Optional & $\begin{array}{l}\text { Enter the interpolation algorithm which will be } \\
\text { used from Table 5.1.2 (DEFAULT: spline) }\end{array}$ \\
\hline
\end{tabular}


Table 5.1.4. Input description for MOX fuels

\begin{tabular}{|c|c|c|c|}
\hline $\begin{array}{l}\text { Entry } \\
\text { no. }\end{array}$ & Data type & $\begin{array}{l}\text { Entry } \\
\text { requirements }\end{array}$ & Comment \\
\hline 1 & $\begin{array}{l}\text { Data set name } \\
\text { (starts with MOX) }\end{array}$ & $\begin{array}{l}\text { Line } 1 \\
\text { always } \\
\text { required }\end{array}$ & $\begin{array}{l}\text { Enter a MOX CONFIGNAM from the active } \\
\text { arpdata. txt (see } \\
\text { Table 5.1.7). }\end{array}$ \\
\hline 2 & Plutonium content & $\begin{array}{l}\text { New line } \\
\text { always }\end{array}$ & $\begin{array}{l}\text { Enter the } \mathrm{Pu} \text { content as wt } \% \mathrm{Pu} \text { in total heavy } \\
\text { metal. }\end{array}$ \\
\hline 3 & ${ }^{239} \mathrm{Pu}$ isotopic vector & Always & $\begin{array}{l}\text { Enter the }{ }^{239} \mathrm{Pu} \text { isotopic concentration as wt } \% \\
\text { of }{ }^{239} \mathrm{Pu} \text { in total } \mathrm{Pu} \text {. }\end{array}$ \\
\hline 4 & Reserved parameter (not used) & Always & Enter a dummy value (e.g., 1.0). \\
\hline 5 & Number of cycles & Always & Enter the number of irradiation cycles $N$. \\
\hline 6 & Fuel irradiation period & Always & $\begin{array}{l}\text { Enter the irradiation time for each cycle in days } \\
\qquad \Delta T_{i} \text {, for } i=1,2, \ldots, N .\end{array}$ \\
\hline 7 & Average power & Always & $\begin{array}{l}\text { Enter the specific fission power (MW/MTHM) } \\
\text { for each cycle, } P_{i} \text {, for } i=1,2, \ldots, N \text {. }\end{array}$ \\
\hline 8 & Data interpolations per cycle & Always & $\begin{array}{l}\text { Enter the number of cross section sets to } \\
\text { interpolate during each cycle, } m_{i} \text {, for } \\
i=1,2, \ldots, N \text {. }\end{array}$ \\
\hline 9 & Moderator density & Always & $\begin{array}{l}\text { Enter the water-moderator density }\left(\mathrm{g} / \mathrm{cm}^{3}\right) \text {. } \\
\text { Enter only one value. }\end{array}$ \\
\hline 10 & New library name & $\begin{array}{l}\text { New line } \\
\text { always }\end{array}$ & $\begin{array}{l}\text { Enter the character name of the new interpolated } \\
\text { library created by ARP. }\end{array}$ \\
\hline 11 & Interpolation keyword & Optional & $\begin{array}{l}\text { Enter the interpolation algorithm which will be } \\
\text { used from Table 5.1.2 (DEFAULT: spline). }\end{array}$ \\
\hline
\end{tabular}


Table 5.1.5. Input description for activation problems

\begin{tabular}{|c|c|c|c|}
\hline $\begin{array}{l}\text { Entry } \\
\text { no. }\end{array}$ & Data type & $\begin{array}{l}\text { Entry } \\
\text { requirements }\end{array}$ & Comment \\
\hline 1 & $\begin{array}{l}\text { Data set name } \\
\text { (starts with } A C T \text { ) }\end{array}$ & $\begin{array}{l}\text { Line } 1 \\
\text { always } \\
\text { required }\end{array}$ & $\begin{array}{l}\text { Enter an activation CONFIGNAM from the } \\
\text { active arpdata.txt (see } \\
\text { Table 5.1.8). }\end{array}$ \\
\hline 2 & Dummy parameter & Always & Enter 1. \\
\hline 3 & Number of cycles & Always & Enter the number of irradiation cycles $N$. \\
\hline 4 & Fuel irradiation period & Always & $\begin{array}{l}\text { Enter the irradiation time for each cycle in days } \\
\qquad \Delta T_{i} \text {, for } i=1,2, \ldots, N .\end{array}$ \\
\hline 5 & Average neutron flux & Always & $\begin{array}{l}\text { Enter the average flux level }\left(\mathrm{n} / \mathrm{cm}^{2} \text {-s }\right) \text { for each } \\
\text { cycle, } \Phi_{i}, \text { for } i=1,2, \ldots, N .\end{array}$ \\
\hline 6 & Data interpolations per cycle & Always & $\begin{array}{l}\text { Enter the number of cross section sets to } \\
\text { interpolate during each cycle, } m_{i} \text {, for } \\
i=1,2, \ldots, N \text {. }\end{array}$ \\
\hline 7 & Flux type (flag) & Always & Enter 1. \\
\hline 8 & New library name & $\begin{array}{l}\text { New line } \\
\text { always }\end{array}$ & $\begin{array}{l}\text { Enter the character name of the new } \\
\text { interpolated library created by ARP. }\end{array}$ \\
\hline 9 & Interpolation keyword & Optional & $\begin{array}{l}\text { Enter the interpolation algorithm which will be } \\
\text { used from } \\
\text { Table 5.1.2 (DEFAULT: spline). }\end{array}$ \\
\hline
\end{tabular}




\subsection{ARPDATA.TXT listing file}

In addition to the user input file, ARP also reads a file named arpdata.txt when it runs. This file describes the parametrization of the ORIGEN libraries. The file is required because the cross section libraries contain no imbedded information on the reactor type, fuel type, or irradiation conditions. Both the file arpdata.txt and the directory of ORIGEN libraries named arplibs is searched for, first in the working directory so that a user can override the default libraries, and then to the SCALE data directory. An example arpdata. txt file is shown in Figure 5.1.7.

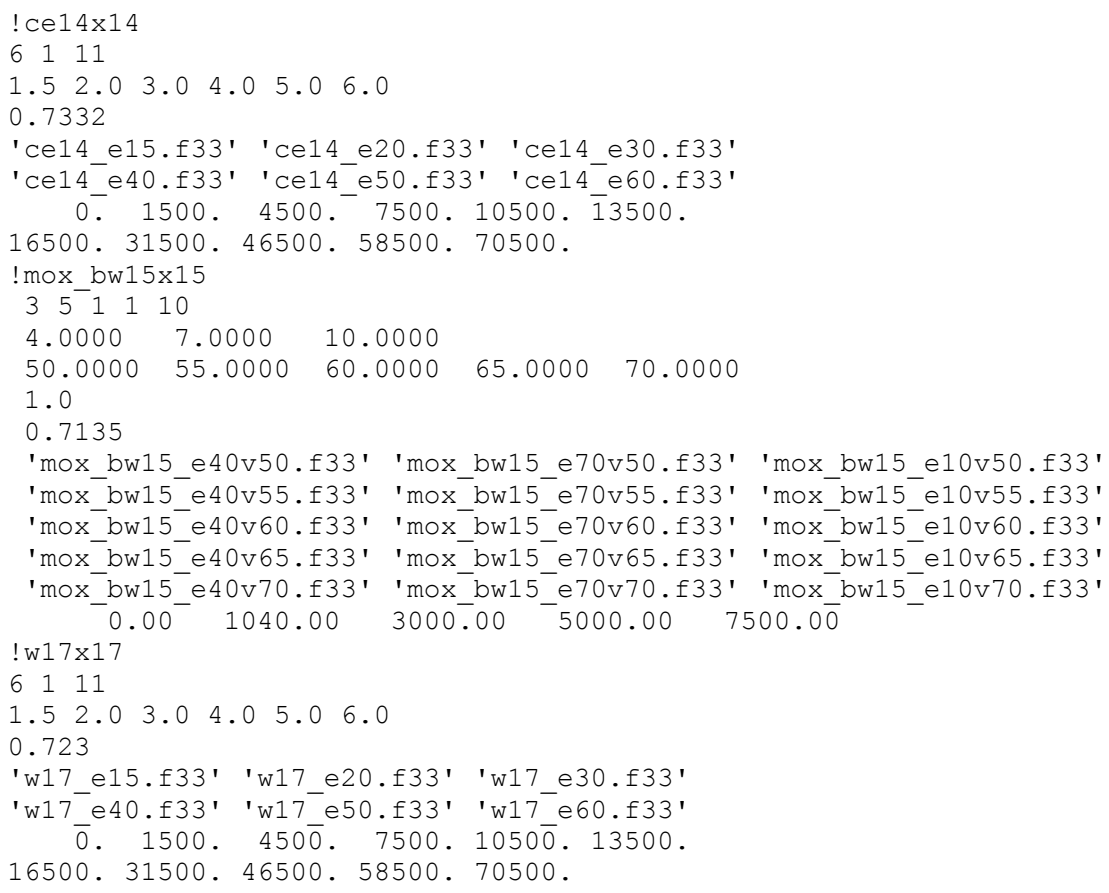

Figure 5.1.7. Examples of arpdata.txt entries.

As shown in Figure 5.1.7, the arpdata.txt is simply a list of entries, each beginning with a "!CONFIGNAM," where CONFIGNAM is the name to be used to reference the entire data set. Whether the entry is for a uranium, MOX, or activation problem is dictated by the actual CONFIGNAM. If it begins with MOX, it is a MOX entry, and if it begins with ACT, it is an activation entry. Otherwise it is uranium. The ORIGEN libraries listed must reside next to arpdata.txt, in a directory called arplibs. Each type of entry is described fully in Table 5.1.6, Table 5.1.7, and Table 5.1.8 for uranium, MOX, and activation, respectively. 
Table 5.1.6. ARPDATA.TXT uranium type entry

\begin{tabular}{|c|c|c|c|}
\hline $\begin{array}{l}\text { Line } \\
\text { no. }\end{array}$ & Data name & Description & Comments \\
\hline 1 & CONFIGNAM & $\begin{array}{l}\text { Data set name } \\
\text { (40-character maximum) }\end{array}$ & $\begin{array}{l}\text { Must begin with "!" in column one, } \\
\text { followed by the alphanumeric } \\
\text { name this data set will be } \\
\text { referenced by. } \\
\text { May not begin with ACT or MOX. }\end{array}$ \\
\hline 2 & $\begin{array}{l}\text { N1 } \\
\text { N2 } \\
\text { N3 }\end{array}$ & $\begin{array}{l}\text { Number of enrichments } \\
\text { Number of water densities } \\
\text { Number of burnup steps }\end{array}$ & $\begin{array}{l}\text { Entries pertain to the number of } \\
\text { parameterized cross section data } \\
\text { for each variable type. }\end{array}$ \\
\hline 3 & ENR & $\begin{array}{l}\text { Enrichment values }\left(\mathrm{wt} \%{ }^{235} \mathrm{U}\right) \\
\text { values at which ARP libraries } \\
\text { were generated }\end{array}$ & $\begin{array}{l}\text { N1 entries defining the discrete } \\
\text { enrichment values for each library }\end{array}$ \\
\hline 4 & DENS & Water density values $\left(\mathrm{g} / \mathrm{cm}^{3}\right)$ & $\begin{array}{l}\mathrm{N} 2 \text { entries defining the discrete } \\
\text { moderator density values for each } \\
\text { library }\end{array}$ \\
\hline 5 & FILES & $\begin{array}{l}\text { Filenames of ORIGEN libraries for } \\
\text { this fuel assembly type (Enclose } \\
\text { each filename in single quotes } \\
\text { with at least one space between } \\
\text { each name.) }\end{array}$ & $\begin{array}{l}\mathrm{N} 1 \times \mathrm{N} 2 \text { entries } \\
\text { Filenames are ordered first by } \\
\text { density values, then by } \\
\text { enrichment values. }\end{array}$ \\
\hline 6 & BURN & $\begin{array}{l}\text { Burnups (MWd/MTU) } \\
\text { corresponding to each position on } \\
\text { the ORIGEN library }\end{array}$ & $\begin{array}{l}\text { N3 entries } \\
\text { Each set of burnup-dependent } \\
\text { cross sections is stored within a } \\
\text { single ORIGEN binary library file } \\
\text { (the first burnup is usually zero) }\end{array}$ \\
\hline
\end{tabular}

NOTE: Repeat all of the above entries for each fuel assembly configuration type. 
Table 5.1.7. ARPDATA.TXT MOX type entry

\begin{tabular}{|c|c|c|c|}
\hline $\begin{array}{l}\text { Line } \\
\text { no. }\end{array}$ & Data name & Description & Comments \\
\hline \multirow[t]{2}{*}{1} & CONFIGNAM & $\begin{array}{l}\text { Data set name } \\
\text { (40-character maximum) }\end{array}$ & $\begin{array}{l}\text { Must begin with "!" in column one, } \\
\text { followed by the alphanumeric } \\
\text { name by which this data set will be } \\
\text { referenced. }\end{array}$ \\
\hline & & & $\begin{array}{l}\text { Must begin with MOX (e.g., } \\
\text { !mox_bw15x15). }\end{array}$ \\
\hline 2 & $\begin{array}{l}\text { N1 } \\
\text { N2 } \\
\text { N3 } \\
\text { N4 } \\
\text { N5 }\end{array}$ & $\begin{array}{l}\text { Number of Pu content values } \\
\text { Number of }{ }^{239} \mathrm{Pu} \text { values } \\
\text { Not used (enter } 1 \text { ) } \\
\text { Number of water densities } \\
\text { Number of burnup steps }\end{array}$ & $\begin{array}{l}\text { Entries pertain to the number of } \\
\text { separate cross section sets } \\
\text { generated for each parameter. }\end{array}$ \\
\hline 3 & PU & $\begin{array}{l}\mathrm{Pu} \quad \text { content } \\
\text { (wt \% Pu in heavy metal) }\end{array}$ & N1 entries \\
\hline 4 & VECT & $\begin{array}{l}{ }^{239} \mathrm{Pu} \quad \text { vector } \\
\left(\text { wt } \%{ }^{239} \mathrm{Pu} / \mathrm{Pu}\right)\end{array}$ & $\mathrm{N} 2$ entries \\
\hline 5 & RESRV & Not used (enter 1) & N3 entries; dummy entry required. \\
\hline 6 & DENS & $\begin{array}{l}\text { Water } \\
\left(\mathrm{g} / \mathrm{cm}^{3}\right)\end{array}$ & N4 entries \\
\hline 7 & FILE & $\begin{array}{l}\text { Filenames of ORIGEN libraries for } \\
\text { this fuel assembly type. Enclose } \\
\text { each filename in single quotes with } \\
\text { at least one space between each } \\
\text { name. }\end{array}$ & $\begin{array}{l}\mathrm{N} 1 \times \mathrm{N} 2 \times \mathrm{N} 3 \times \mathrm{N} 4 \text { entries } \\
\text { Increment FILE names in the order of } \\
\mathrm{N} 1 \text {, then } \mathrm{N} 2 \text {, then } \mathrm{N} 3 \text {, and then } \\
\mathrm{N} 4 \text { values }\end{array}$ \\
\hline 8 & BURN & $\begin{array}{l}\text { Burnups }(\mathrm{MWd} / \mathrm{MTU}) \text { corresponding } \\
\text { to each position on the ORIGEN } \\
\text { library }\end{array}$ & $\begin{array}{l}\text { N5 entries } \\
\text { (first burnup is usually zero) }\end{array}$ \\
\hline
\end{tabular}

NOTE: Repeat all of the above entries for each fuel assembly configuration type. 
Table 5.1.8. ARPDATA.TXT activation type entry

\begin{tabular}{|c|c|c|c|}
\hline $\begin{array}{l}\text { Line } \\
\text { no. }\end{array}$ & Data name & Description & Comments \\
\hline 1 & CONFIGNAM & $\begin{array}{l}\text { Data set name } \\
\text { (40-character maximum) }\end{array}$ & $\begin{array}{l}\text { Must begin with "!" in column } \\
\text { one followed by the } \\
\text { alphanumeric name by which } \\
\text { this data set will be referenced. } \\
\text { Must begin with ACT (e.g., } \\
\text { ! actcntlrod). }\end{array}$ \\
\hline 2 & $\begin{array}{l}\text { N1 } \\
\text { N2 } \\
\text { N3 }\end{array}$ & $\begin{array}{l}\text { Reserved (enter 1) } \\
\text { Not used (enter 1) } \\
\text { Number of fluence values }\end{array}$ & $\begin{array}{l}\text { The first two entries pertain to the } \\
\text { number of separate cross } \\
\text { section sets generated for each } \\
\text { variable parameter. These are } \\
\text { usually set to 1. The variable } \\
\mathrm{N} 3 \text { corresponds to the number } \\
\text { of fluence-dependent cross } \\
\text { section sets available in the } \\
\text { library. }\end{array}$ \\
\hline 3 & RESRV & Not used (enter 1) & Enter 1. \\
\hline 4 & FTYPE & Neutron flux type (flag) & Enter 1. \\
\hline 5 & FILES & $\begin{array}{l}\text { Filenames of ORIGEN library. } \\
\text { Enclose filename in single } \\
\text { quotes. }\end{array}$ & $\begin{array}{l}\text { Generally only one library name } \\
\text { is required. }\end{array}$ \\
\hline 6 & FLUENCE & $\begin{array}{l}\text { Neutron fluence values }\left(\mathrm{n} / \mathrm{cm}^{2}\right) \text { at } \\
\text { each position on the ORIGEN } \\
\text { libraries }\end{array}$ & $\begin{array}{l}\text { N3 entries } \\
\text { The fluence values are reduced by } \\
\text { the factor } 10^{-24} \text { to avoid } \\
\text { numerical problems during the } \\
\text { interpolation. (First value is } \\
\text { usually zero.) }\end{array}$ \\
\hline
\end{tabular}

NOTE: Repeat all of the above entries for each fuel assembly configuration type.

\subsubsection{ORIGEN Module}

The ORIGEN module drives depletion, decay, and activation calculations as described in Sect. 5.1.2.4, including the conversion of generated powers to fluxes described in Sect. 5.1.2.3, as well as alpha, beta, gamma, and neutron source calculations described in Sect. 5.1.2.4.

\subsection{Key Features}

This section briefly highlights some key features in ORIGEN and describes how they are used.

\subsection{Nuclide Specification and ORIGEN Sub-libraries}

The nuclide identifiers in ORIGEN are more flexible than those in other modules of SCALE and even within the ORIGEN family. Table 5.1.9 shows the possible ways to specify nuclides (and elements). 
Table 5.1.9. Nuclide/Element Specification in ORIGEN

\begin{tabular}{llll}
\hline Identifier Form & Comments & $\begin{array}{l}\text { Examples } \\
\text { nuclide }\end{array}$ & $\rightarrow$ input id \\
\hline IZZZAAA & Standard numeric identifier with one & ${ }^{235} \mathrm{U}$ & $\rightarrow 92235$ \\
I- isomeric state & optional digit of isomeric state, three & ${ }^{235 \mathrm{~m}} \mathrm{U}$ & $\rightarrow 1092235$ \\
ZZZ - atomic number & digits of atomic number, three digit & ${ }^{135} \mathrm{Xe}$ & $\rightarrow 54135$ \\
AAA - mass number & of mass number; elements have & ${ }^{1} \mathrm{H}$ & $\rightarrow 1001$ \\
& mass number of 000. & ${ }^{10} \mathrm{~B}$ & $\rightarrow 5010$ \\
& & $\mathrm{Fe}$ & $\rightarrow 54000$ \\
EAm & Standard symbolic identifier with & ${ }^{235} \mathrm{U}$ & $\rightarrow \mathrm{u} 235$ \\
E- element symbol & element symbol followed by mass & ${ }^{235 \mathrm{~m}} \mathrm{U} \rightarrow \mathrm{u} 235 \mathrm{~m}$ \\
$\mathrm{~A}-$ mass number & number, followed by optional & ${ }^{135} \mathrm{Xe}$ & $\rightarrow \mathrm{xe} 135$ \\
$\mathrm{~m}-$ metastable indicator & metastable indicator; can include a & ${ }^{1} \mathrm{H}$ & $\rightarrow \mathrm{h} 1$ \\
& dash between E and A (E-Am); case & ${ }^{10} \mathrm{~B}$ & $\rightarrow \mathrm{b} 10$ \\
& insensitive. & $\mathrm{Fe}$ & $\rightarrow \mathrm{fe}$ \\
\hline
\end{tabular}

One important aspect ORIGEN users must be aware of is that the ORIGEN library (f33) being used dictates the set of nuclides available in a calculation and that there may be more than one version of a nuclide in a library. The duplicates arise in large part from the need to analyze fission products separately. For example, a gadolinia-doped uranium oxide fuel with burnup will have some ${ }^{155} \mathrm{Gd}$ from the initial gadolinia loading and some ${ }^{155} \mathrm{Gd}$ generated as a fission product. Although these fuels physically behave the same way, it is sometimes important to be able to analyze them separately. These groups, versions, or categories are referred to as sublibraries because in an ORIGEN library, they appear almost like three separate, smaller ORIGEN libraries. The three libraries are for

1. naturally occurring, light nuclides, sometimes called "light elements" or "activation products,"

2. actinides and their reaction and decay products, and

3. fission products.

Called "sublibs" for short, they are identified by a number or 2-character specifier:

1. light nuclides with "LT" or 1 ,

2. actinides with "AC" or 2 , and

3. fission products with "FP" or 3.

The production of fission products from actinides $(2 / \mathrm{AC} \rightarrow 3 / \mathrm{FP})$ is the only type of transition in a typical ORIGEN library that spans sublibs. The sublib is optional in a nuclide specification and is indicated in parentheses after the identifier-IZZZAAA(S), EAm(S). If the sublib for a nuclide/element is not provided, it is guessed in the following manner:

1. If the nuclide is in fact an element, then it is placed in sublib $=1 / \mathrm{LT}$.

2. If the atomic number $\mathrm{Z}<26$, an attempt is made to place it in sublib=1/LT.

3. Otherwise ( $Z \geq 26$ or attempt fails), sublibs are searched in reverse order, from 3/FP, 2/AC, and then $1 / \mathrm{LT}$.

The third rule, which is to search sublibs in reverse order, correctly handles spent reactor fuel, a common and important scenario. The other two conditions can be interpreted as exceptions. The first exception correctly handles activation scenarios where it is most convenient to specify the initial elemental 
constituents. The second exception handles light nuclides that could not be real fission products, as fission products have $\mathrm{Z} \geq 26$ by definition. The byproducts ${ }^{1} \mathrm{H},{ }^{2} \mathrm{H},{ }^{3} \mathrm{H},{ }^{3} \mathrm{He}$, and ${ }^{4} \mathrm{He}$ actually exist in all sublibs, but FP and AC byproducts have a reduced set of transitions compared to the LT version, which has full decay and activation chains. Thus when a user specifies one of the byproduct nuclides as input, it is best to associate it to the LT version.

\subsection{Physical Units in Calculations}

A variety of units can be used in the input and specified for the output of an ORIGEN calculation. The input allows for initial concentrations in

1. grams,

2. moles (or gram-atoms),

3. number density in atoms/barn-cm, and

4. curies.

Time may be expressed in seconds, minutes, hours, days, years, or a user-defined unit. Irradiation may be expressed in terms of neutron flux $\left(\mathrm{n} / \mathrm{cm}^{2}-\mathrm{s}\right)$ or power $(\mathrm{W})$. The allowed units for output include those for input, as well as the following decay quantities:

1. total decay heat power (W),

2. gamma decay heat power (W),

3. airborne toxicity $\left(\mathrm{m}^{3}\right)$ required to dilute activities to the Radiation Concentration Guide (RCG) limit for air,

4. ingestion toxicity $\left(\mathrm{m}^{3}\right)$ required to dilute activities to the RCG limit for water, and

5. alpha, beta, neutron, photon sources (particles/s or MeV/s).

Table 5.1.10 summarizes the available units in ORIGEN. During irradiation cases, the following can also be returned:

1. absorption rates (absorptions/s),

2. fission rates (fissions/s), and

3. infinite neutron multiplication constant, $\mathrm{k}_{\infty}$. 
Table 5.1.10. Available physical units

\begin{tabular}{|c|c|c|c|c|}
\hline Unit name & Description & Input & $\begin{array}{l}\text { Output } \\
\text { (irrad.) }\end{array}$ & $\begin{array}{l}\text { Output } \\
\text { (decay) }\end{array}$ \\
\hline GRAMS & Mass in grams & $\checkmark$ & $\checkmark$ & $\checkmark$ \\
\hline $\begin{array}{l}\text { MOLES or } \\
\text { GRAM-ATOMS }\end{array}$ & $\begin{array}{l}\text { Number in moles (or legacy } \\
\text { equivalent of gram-atoms) }\end{array}$ & $\checkmark$ & $\checkmark$ & $\checkmark$ \\
\hline ATOMS-PER-BARN-CM & $\begin{array}{l}\text { Density in atoms } / \text { barn-cm } \\
\left(10^{-24} \mathrm{~cm} / \text { barn } \times \text { density in }\right. \\
\left.\text { atoms } / \mathrm{cm}^{3}\right) \text {; requires volume input }\end{array}$ & $\checkmark$ & $\checkmark$ & $\checkmark$ \\
\hline CURIES & Activity in curies & $\checkmark$ & $\checkmark$ & $\checkmark$ \\
\hline BECQUERELS & Activity in becquerels & $\checkmark$ & $\checkmark$ & $\checkmark$ \\
\hline ATOMS_PPM & Atom fractions $\times 10^{6}$ & & $\checkmark$ & $\checkmark$ \\
\hline WEIGHT_PPM & Weight fractions $\times 10^{6}$ & & $\checkmark$ & $\checkmark$ \\
\hline WATTS & Total decay heat in watts & & & $\checkmark$ \\
\hline G-WATTS & $\begin{array}{l}\text { Total decay heat from photons in } \\
\text { watts }\end{array}$ & & & $\checkmark$ \\
\hline M3_AIR & Radiotoxicity in $\mathrm{m}^{3}$ for inhalation & & & $\checkmark$ \\
\hline M3 WATER & Radiotoxicity in $\mathrm{m}^{3}$ for ingestion & & & $\checkmark$ \\
\hline
\end{tabular}

\subsection{Saving Results}

ORIGEN can save any results (isotopics and source spectra) in a special ORIGEN binary concentrations file (f71). The file is a simple sequence of solutions, and new results are simply appended on to the end of an existing file. Note that no matter how initial isotopics are entered or what units are asked for in the output file, the ORIGEN f71 contains moles (gram-atoms) of each isotope and an optional volume to permit unit conversions to number density (atoms/barn-cm). Isotopics for an ORIGEN calculation can be initialized from any position on this file in an ORIGEN calculation. The $\mathrm{f} 71 \mathrm{can}$ also be read by OPUS to perform various post-processing tasks. 


\subsection{Input Description}

ORIGEN uses the Scale Object Notation (SON) language for its input, although it can also read FIDObased input for backwards compatibility with SCALE $6.1^{16}$. The basic structure of an ORIGEN input is shown in Figure 5.1.8.

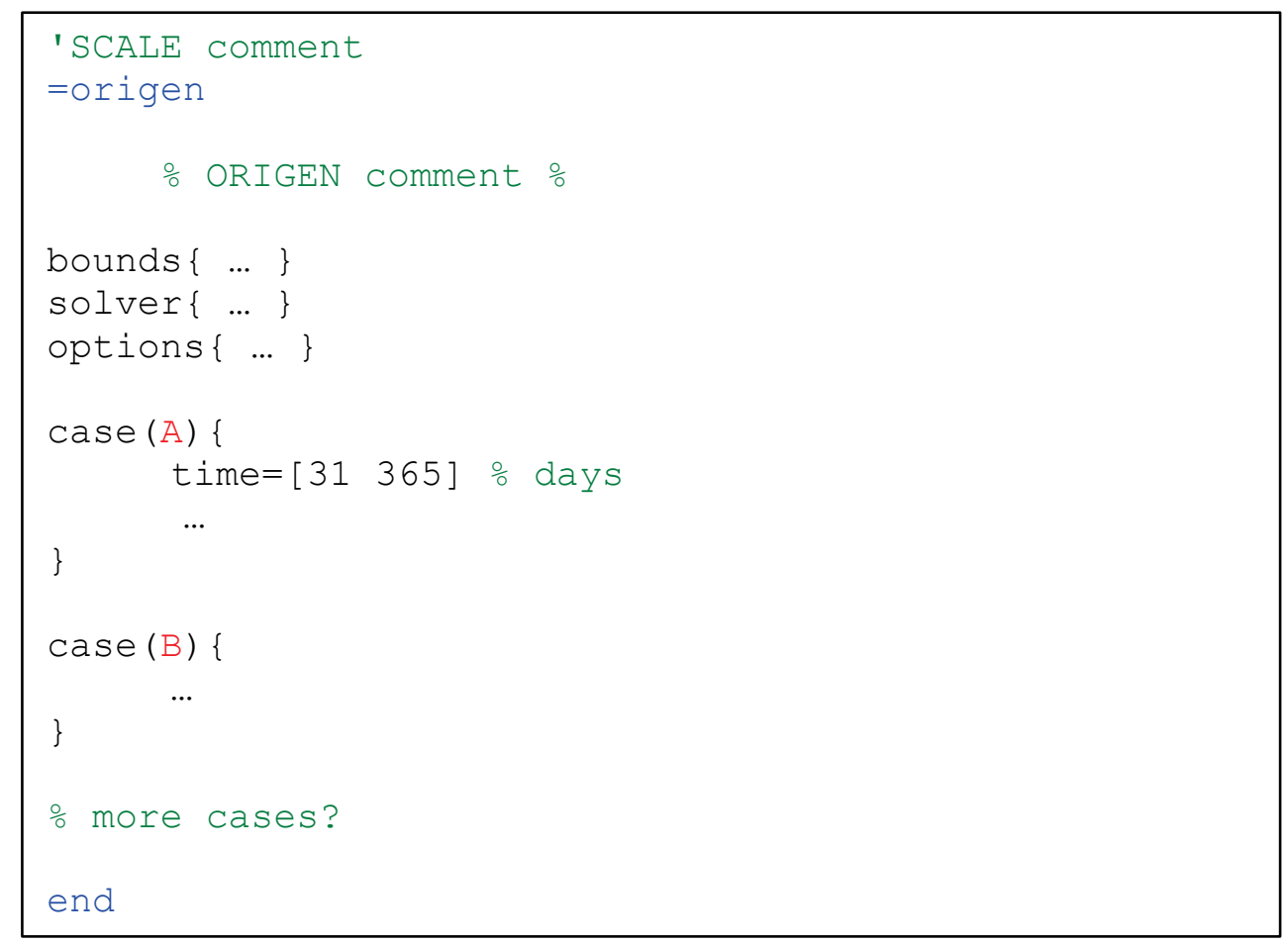

Figure 5.1.8. ORIGEN input file overview.

The ORIGEN input is hierarchical, containing four levels, where level 0 is the "root" level, allowed between "=origen" and "end." The complete set of keywords is shown in Table 5.1.11, with arrays denoted with "=[]", blocks with " \{\} ". Referring to the overview in Figure 5.1.11, at the root level, there is a "solver" block for changing solver options, a "bounds" block for entering the energy boundaries for various particle emissions, and an "options" block for altering the miscellaneous global options. These blocks may only appear once. The remainder of the input is a sequence of "case" blocks (in the above examples there are two cases with identifiers "A" and "B"), which each case is executed in order, with each case possibly depending on one or more of the previous cases. 
Table 5.1.11. List of all available ORIGEN input commands

\begin{tabular}{|c|c|c|c|}
\hline Level 0 & Level 1 & Level 2 & Level 3 \\
\hline \multirow[t]{18}{*}{ case \{\}} & title & & \\
\hline & time \{\} & $\begin{array}{l}\text { start, } \mathrm{t}=[] \text {, units, custom_name, } \\
\text { custom_length }\end{array}$ & \\
\hline & $\operatorname{lib}\{\}$ & file, pos & \\
\hline & flux $=[]$ & & \\
\hline & power $=[]$ & & \\
\hline & \multirow[t]{6}{*}{$\operatorname{print}\{\}$} & $\begin{array}{l}\text { cutoff_step, absfrac_step, } \\
\text { rel_cutoff, cutoffs, absfrac_sublib, } \\
\text { fisrate, kinf }\end{array}$ & \\
\hline & & nuc \{\}, ele \{\} & sublibs $=[]$, total, units $=[]$ \\
\hline & & neutron \{\} & summary, spectra, detailed \\
\hline & & $\operatorname{gamma}\{\}$ & $\begin{array}{l}\text { summary, spectra, } \\
\text { principal_step, } \\
\text { unbinned_warning, } \\
\text { principal_cutoff }\end{array}$ \\
\hline & & alpha \{\} & summary, spectra \\
\hline & & beta \{\} & $\begin{array}{l}\text { summary, spectra, } \\
\text { principal_step, principal_cutoff }\end{array}$ \\
\hline & \multirow[t]{2}{*}{$\operatorname{mat}\{\}$} & $\begin{array}{l}\text { iso }=[], \text { feed }=[], \text { units, previous, } \\
\text { volume, blend }=[]\end{array}$ & \\
\hline & & $\operatorname{load}\{\}$ & file, pos \\
\hline & save \{\} & $\begin{array}{l}\text { steps=[], file, time_offset, } \\
\text { time_units }\end{array}$ & \\
\hline & neutron \{\} & $\begin{array}{l}\text { alphan_medium, alphan_bins, } \\
\text { alphan_cutoff, alphan_step }\end{array}$ & \\
\hline & gamma \{\} & $\begin{array}{l}\text { sublib, adjust_for_missing, } \\
\text { conserve_line_energy, } \\
\text { split_near_boundary, continuum, } \\
\text { immediate, brem_medium, spont }\end{array}$ & \\
\hline & alpha \{\} & & \\
\hline & beta \{\} & sublib & \\
\hline \multirow[t]{4}{*}{ bounds \{\}} & alpha $=[]$ & & \\
\hline & beta $=[]$ & & \\
\hline & gamma $=[]$ & & \\
\hline & neutron $=[]$ & & \\
\hline \multirow[t]{2}{*}{ solver \{\}} & type & & \\
\hline & opt \{\} & $\begin{array}{l}\text { terms, maxp, abstol, reltol, } \\
\text { calc_type, order, substeps }\end{array}$ & \\
\hline options \{\} & $\begin{array}{l}\text { print_xs, digits, } \\
\text { fixed_fission_energy }\end{array}$ & & \\
\hline
\end{tabular}

The percent sign (\%) is the comment character inside the ORIGEN sequence, between "=origen" and "end." The \% is a very flexible comment that may be placed almost anywhere in the input and continues until the end of the line. Outside the ORIGEN sequence, the SCALE comment character of a single quote ' at the beginning of a line must be used. Arrays in SON begin with "[" and end with "]" and 
support the following special shortcuts inherited from FIDO. Note that the interpolation shortcuts (I and L) insert values between two specified values so that there will be $\mathrm{N}+2$ values in the final expanded array section.

Table 5.1.12. Array entry shortcuts

\begin{tabular}{|c|c|c|}
\hline Shortcut & Format & $\begin{array}{l}\text { Examples } \\
\text { shortcut } \rightarrow \text { expansion }\end{array}$ \\
\hline Repeat (R) & $N \mathbf{R} X$ & $\begin{array}{l}3 \mathrm{r} 1 \mathrm{e} 14 \rightarrow 1 \mathrm{e} 141 \mathrm{e} 141 \mathrm{e} 14 \\
6 \mathrm{r} 3 \rightarrow 333333\end{array}$ \\
\hline Linear interpolation (I) & $N \mathbf{I} X Y$ & $\begin{array}{l}\text { 3i } 51 \rightarrow 5 \underline{432} 1 \\
9 \text { i } 0.01 .0 \rightarrow 0.0 \underline{0.1} 0.20 .30 .40 .50 .60 .70 .80 .91 .0\end{array}$ \\
\hline Log interpolation $(\mathbf{L})$ & $N \mathbf{L} X Y$ & $\begin{array}{l}3115 \rightarrow \\
511 e-9 \text { 1e-3 } \rightarrow \text { 1e-9 1e-8 1e-7 1e-6 1e-5 1e-4 1e-3 }\end{array}$ \\
\hline
\end{tabular}

As an alternative to manually creating an ORIGEN input file via a text editor, the user may use the SCALE graphical user interface (GUI) Fulcrum to create ORIGEN input files. Advantages to using Fulcrum include syntax highlighting, autocomplete, immediate feedback when input is incorrect, and one-click running of calculations.

\subsection{Calculation Case (case)}

A single ORIGEN sequence may contain an unlimited number of case blocks. Each case block is processed in order and can represent either an independent calculation or continuation of a previous case. The complete contents of a single case block are shown in Figure 5.1.9.

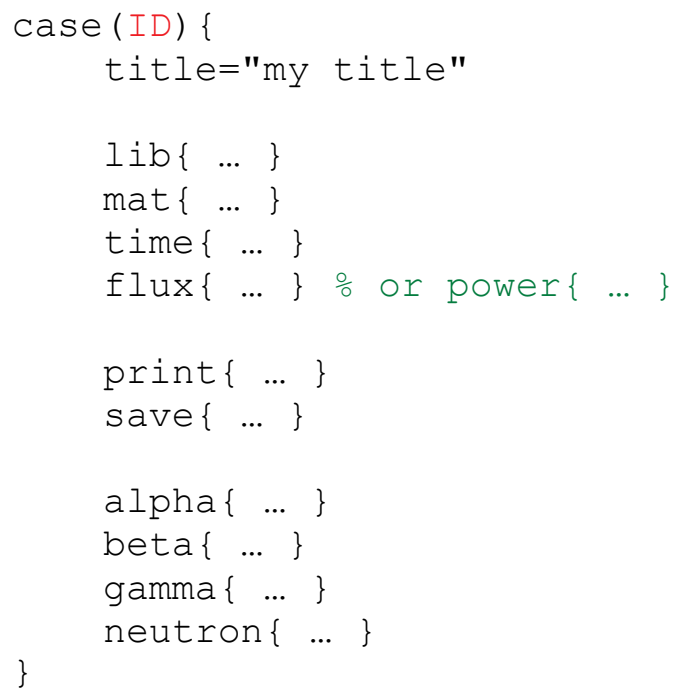

Figure 5.1.9. ORIGEN "case" overview.

The most important three components are the lib, mat, and time/power/flux inputs: 
1. an ORIGEN library and the transition matrix data set on it to use (lib),

2. initial amounts of nuclides (mat), and

3. a power or flux history (time/power or time/flux).

The case identifier and case title string (shown as ID and title="my title" in Figure 5.1.9) are echoed in the output file and can be a convenient way to differential cases. Both are optional, with the ID defaulting to the case index, with " 1 " for the first case, " 2 " for the second, etc. The "print" and "save" blocks represent two ways to analyze the output from a calculation. The "print" block prints tables directly to the output file, and the "save" block saves the solution in a special ORIGEN binary concentration file (f71), e.g., for later post-processing. Finally, the "alpha," "beta," "gamma," and "neutron" blocks control the emission source calculations for alpha, beta, gamma, and neutron particles, respectively. The remaining subsections will describe the input for each of these blocks.

\section{Transition Matrix Specification (lib)}

The transition matrix to use in a case is controlled by the "lib" shown in Figure 5.1.10.

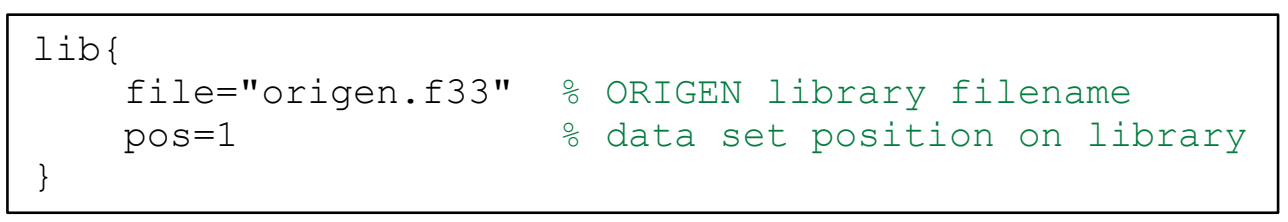

Figure 5.1.10. ORIGEN "lib” overview.

A "lib" must be present in the first case with a defined ORIGEN library file. The default position is "pos=1". The "lib" may be omitted in subsequent cases, and if so, the previous case's "lib" is used. The position refers to the set of transition coefficients (transition matrix $\boldsymbol{A}$ ) to load. To load another position on the same library file, the "lib" block with "pos=X" can be used to load position X. When ARP generates an ORIGEN library, it will contain a set of transition coefficients for each requested burnup. When COUPLE generates an ORIGEN library, it will contain a single position. For decay calculations, file="end7dec" can be used to load a decay-only library.

\section{Material Specification (mat)}

The initial isotopics for a case a controlled by the "mat" shown in Figure 5.1.11. Note that the material specification has a few different variants, with only one allowed to specify the material in a given case. 


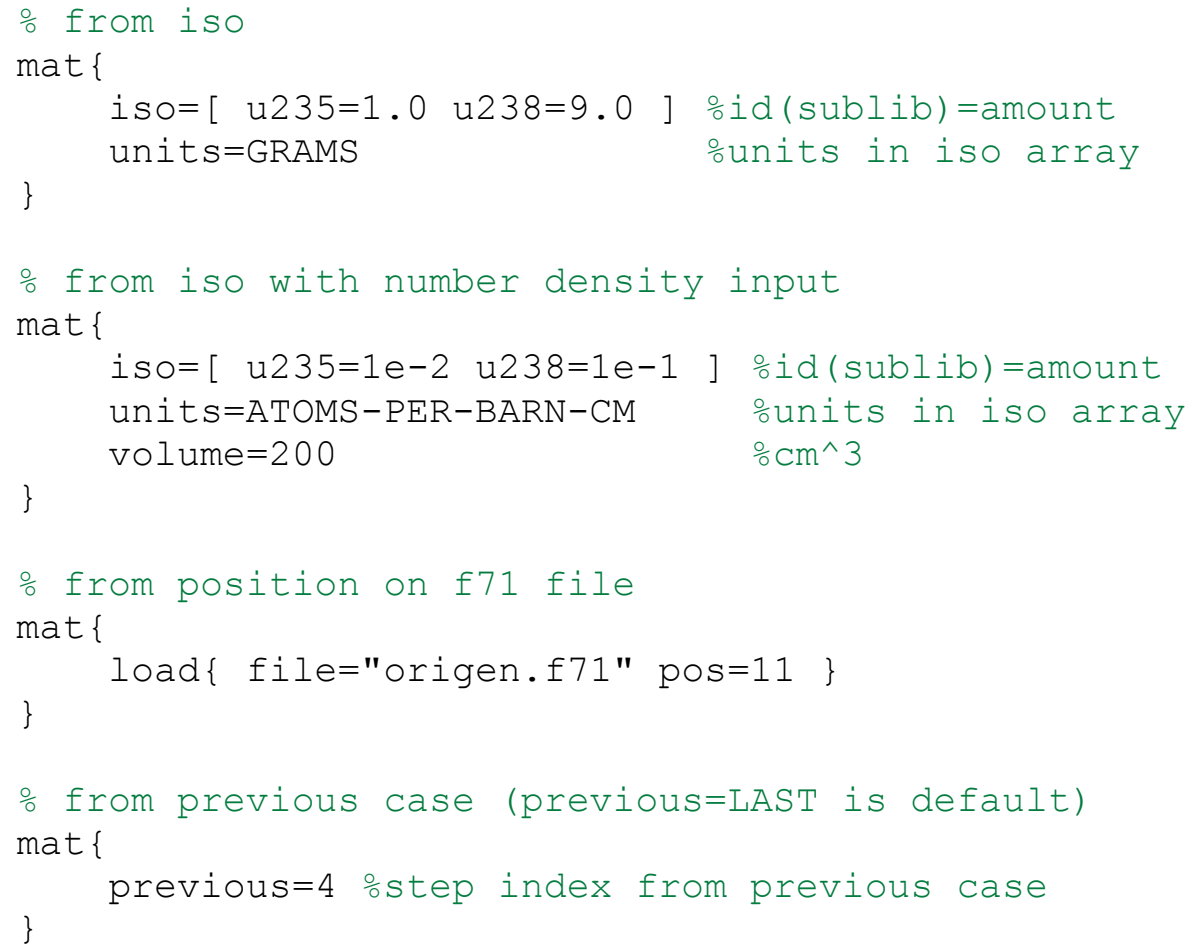

Figure 5.1.11. ORIGEN "mat" block overview.

In the first variant in Figure 5.1.11, the isotopic distribution "iso" is used with "units." The "iso" array contains a sequence of "id=amount" pairs, where "id" is a nuclide identifier in the format described in Sect. 5.1.3.3.1.1, and the units of the amount are given by the "units" keyword, one of the unit names listed in the third column of 
Table 5.1.10. Default units are MOLES.

In the second variant, the number density (ATOMS-PER-BARN-CM) is specified which requires an additional specification of the "volume" in $\mathrm{cm}^{3}$. Internally, the number density will be converted to MOLES using that volume. For any type of units specified internally for calculations, isotopics are always converted to MOLES and then reconverted to the output units required.

In the third variant, the isotopics are loaded from a specific position on the $\mathrm{f} 71$ file. Note that the position index starts at one (not zero) and because the $\mathrm{f} 71$ is always appended to, it may contain multiple materials, cases, timelines, etc. In the fourth and final variant, the isotopics are loaded from end of step 4 from the previous case ("previous=4"). The index zero (e.g., "previous=0") corresponds to the initial isotopics of the previous case. The keyword "LAST" may be used to load the isotopics from the end of the last step, "previous=LAST". This is the default behavior, used when a "mat" block is not present.There are two additional special material specifications shown in Figure 5.1.12: (1) with a feed rate term, $\vec{S}(t)$ in Eq. (5.1.3), or (2) the blend array. The feed specified is in the units specified per second and constant for the entire case. It is possible to perform a calculation with feed but with zero initial isotopics by specifying "iso=0". Feed can be negative, however, the calculation becomes undefined and will abort when the number of atoms of any nuclide becomes negative. 
The blend array allows a fraction of each result from the previous cases to be loaded. The identifier is the case name, or the index of the case if a case name is not provided and the fraction is the atom fraction. The step index for the isotopics can be specified in parentheses. For example, $B(2)=0.9$ indicates that $90 \%$ of the case(B) isotopics should be taken at the end of step 2. The default step index is the final step for the case. Only one blend is allowed in an ORIGEN input (between "=origen" and "end"). Multiple blends currently requires saving isotopics to an $\mathrm{f} 71$ file and reloading in a subsequent calculation.

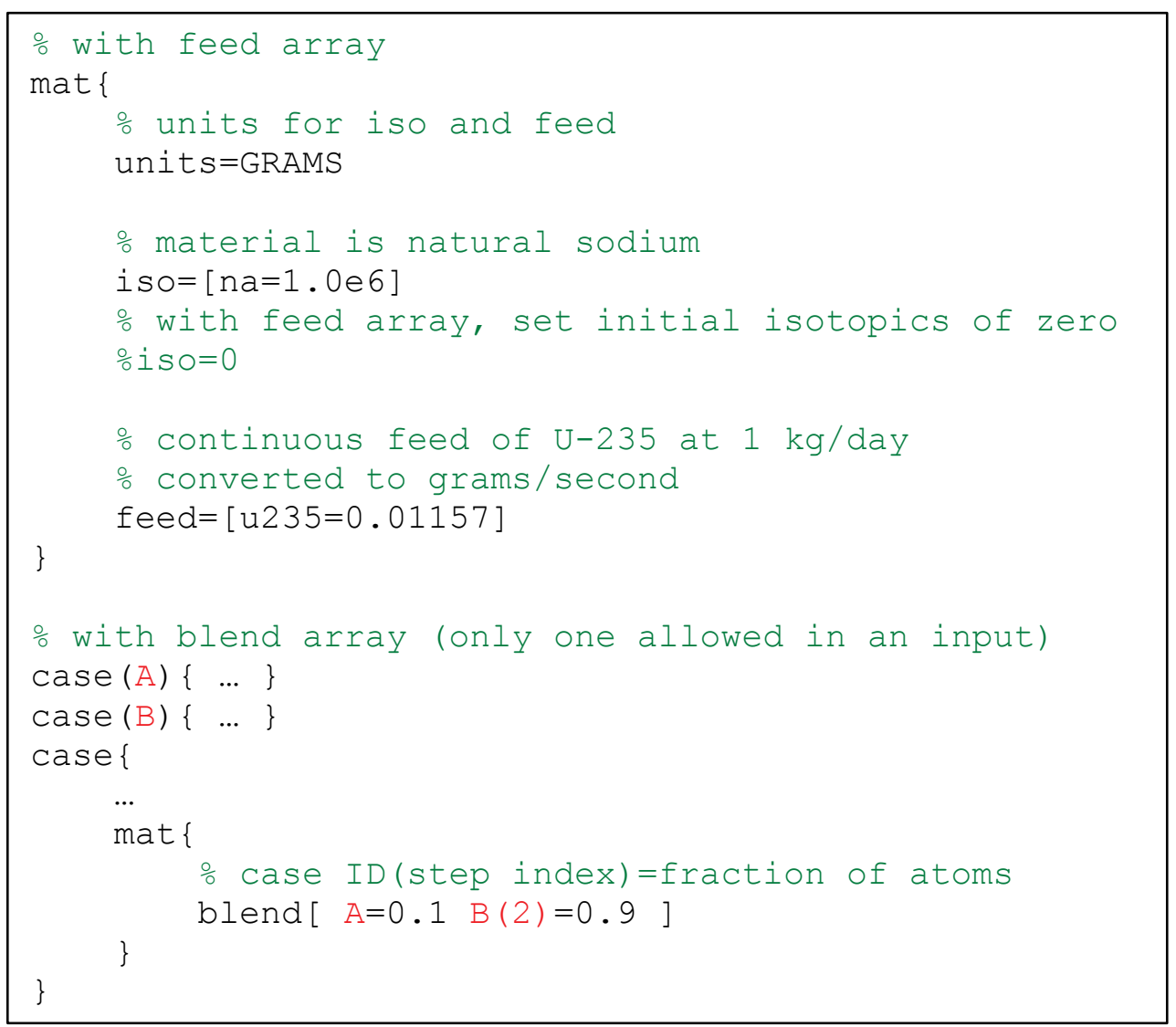

Figure 5.1.12. ORIGEN “feed" and "blend" arrays.

\section{Operating History (power, flux, time)}

The operating history is specified using "time," "power," and "flux," with examples shown in Figure 5.1.13. For decay cases, only the "time" array in units of days is required. For irradiation cases, either "power" or "flux" may be provided. When flux is used, it is the step-wise flux $\Phi_{n}\left(\frac{n}{\mathrm{~cm}^{2} s}\right)$ appearing directly in the depletion equations of Eq. (5.1.4). When power is used, it is the total stepaverage power $-P_{n}(M W)$ - converted to step-wise average flux using Eq. (5.1.39). With irradiation cases using flux or power, the same number of entries must be specified on the time and flux/power array. The start time corresponding to the initial conditions is not included in the array of time values. Additionally, the time specification allows time units (including a custom unit) and a start time in which the block form of "time" must be used "time $\{\ldots\}$. ." 


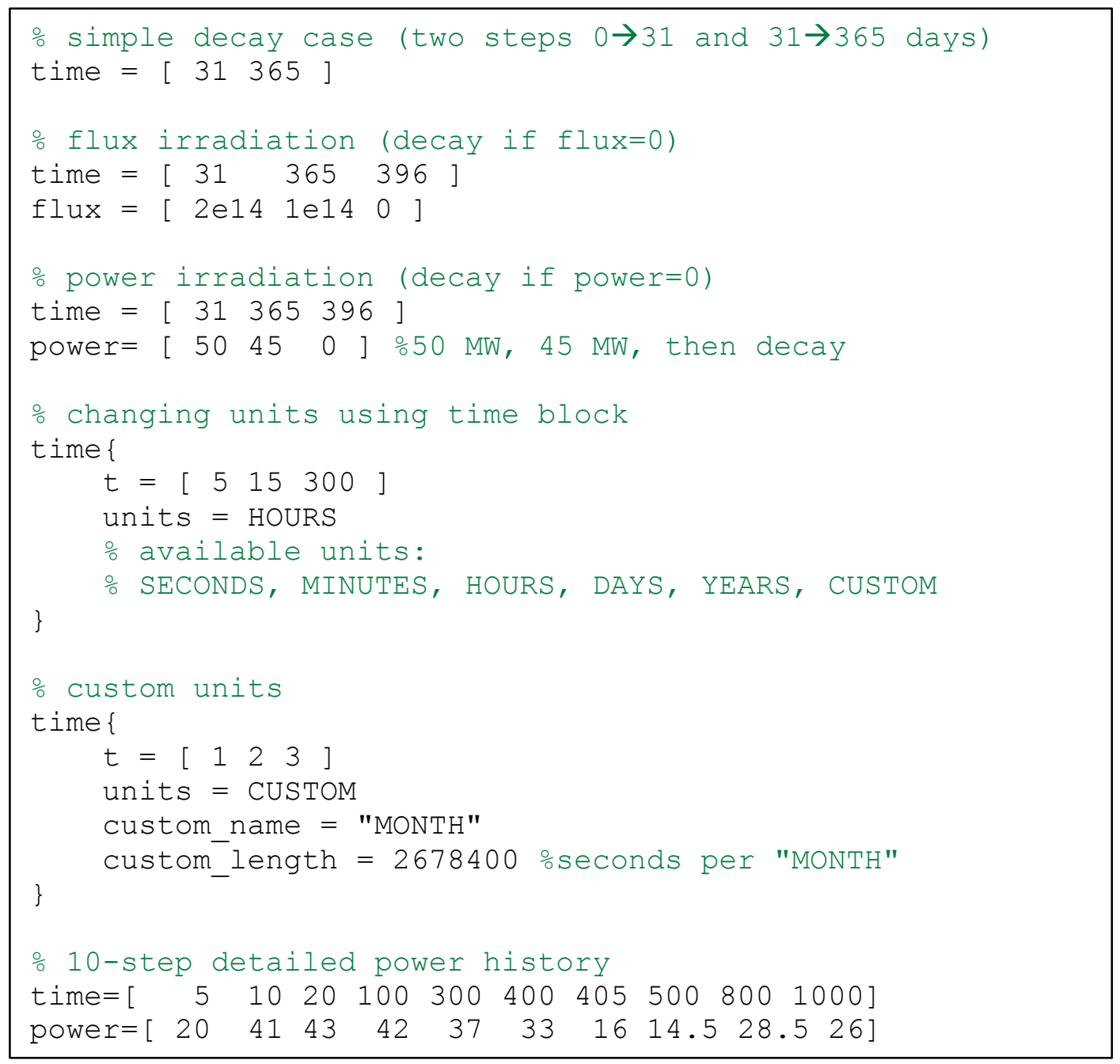

Figure 5.1.13. ORIGEN operating history blocks ("time," "flux," and "power").

To illustrate some aspects of specifying a power history, refer to Figure 5.1.14, where the black line ("actual power") shows a piecewise linear power history that is translated to a possible step-wise constant power history shown by the red line ("step-wise constant power"), with input shown in Figure 5.1.13 labeled "10-step detailed power history". The secondary (right) y-axis shows the step-wise flux, calculated from the step-wise power via the predictor-corrector approach of Eq. (5.1.39). The dependence of the power-to-flux conversion on the actual material composition is shown in the comparison of flux results for an initial composition with $6 \%$ fissile ${ }^{235} \mathrm{U}$ (blue dotted line) versus $2 \%$ fissile ${ }^{235} \mathrm{U}$ (purple dashed line). The flux at the beginning of the irradiation is a factor of 3 higher with the $2 \%$ fissile case, due to approximately a factor of 3 lower fissile content. With time, fissile plutonium build-up closes the gap to a factor of 1.5. 


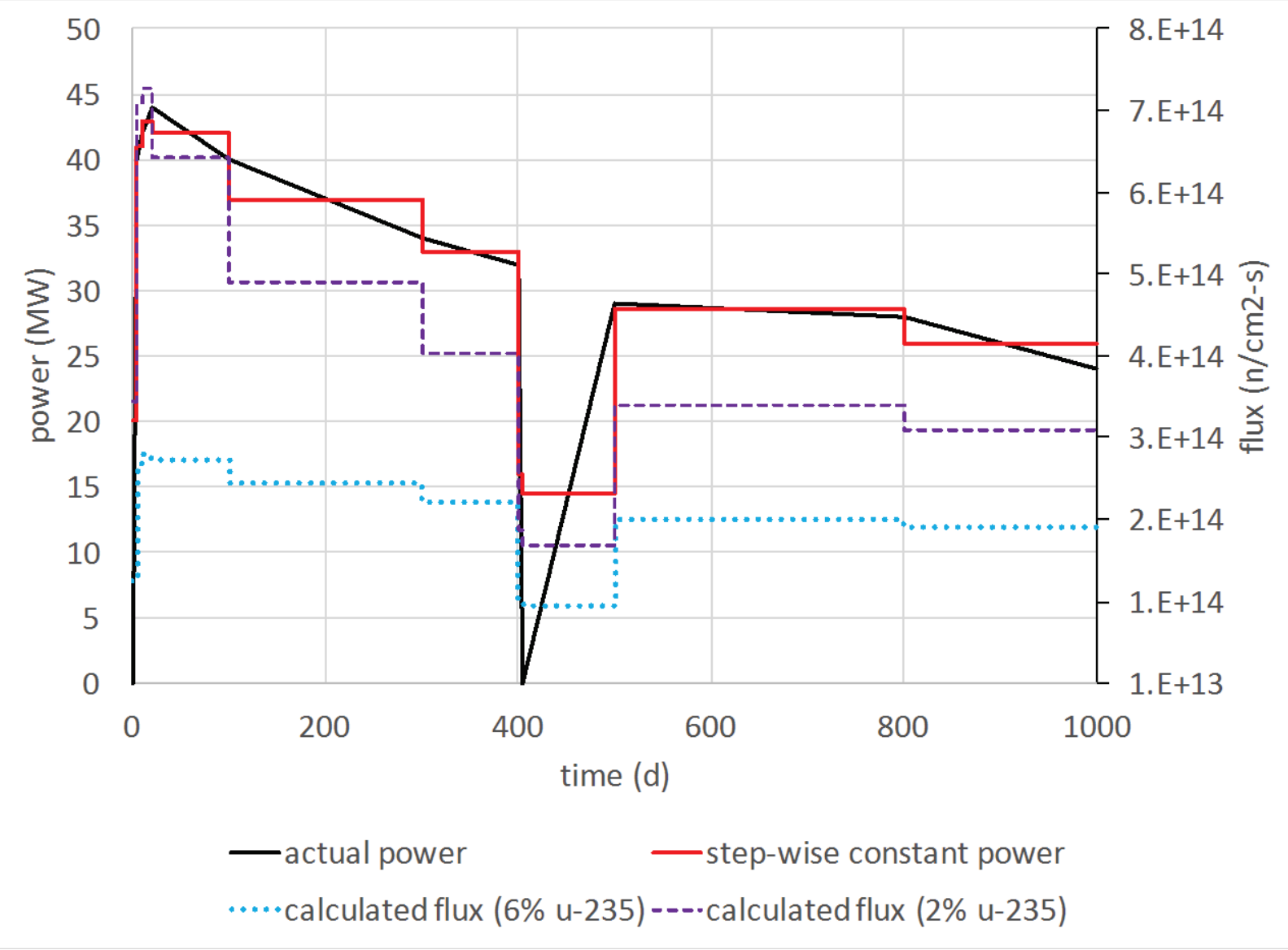

Figure 5.1.14. Example of ORIGEN operating history and power-to-flux conversion. 


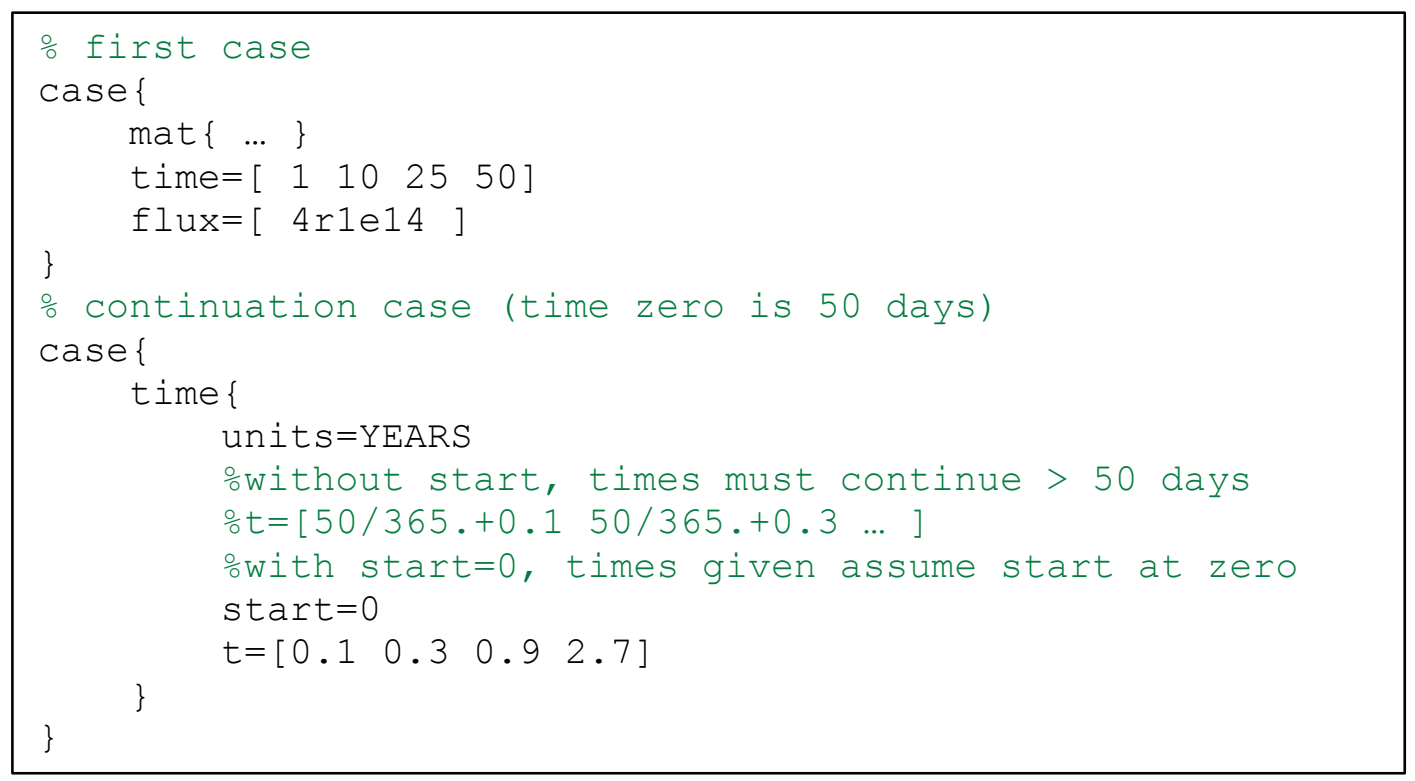

Figure 5.1.15. ORIGEN"start" time usage.

By default, subsequent cases that continue operations on a material, continue the timeline of that material. Using "start=0" is convenient when switching time unitsfrom irradiation in days to decay time in years, for example. Otherwise, the final time must be converted to years.

\section{Printing Options (print)}

The "print" command is one of the most complex inputs, with options to set printing cutoffs and control the concentrations returned, broken down by nuclides and elements and emission sources for gamma, neutron, alpha, and beta particles. Additionally, there are options to print fission rates, absorption rates, and the ratio of fission rate to absorption rate. Each case is allowed a print block. The options for printing nuclides and elements are shown in Figure 5.1.16. The print block allows the "nuc" block and "ele" block for printing nuclide and element results, respectively.

Inside the "nuc" or "ele" blocks, there are three possible entries:

- a "sublibs" array (a list from LT, AC, FP, ALL),

- a "total" (yes or no), and

- a "units" array (see column 1of Table 5.1.10 for possible units).

The "total" is whether to sum over all "sublibs," i.e., if Gd-155 occurs in both LT and FP sublibs, then the total will be the sum of the two. It is possible to have "sublibs=[LT AC FP] total=yes," which results in four output tables, one for each of the sublibs and one for the total. The specification of "sublibs=ALL" is the same as "sublibs=[LT AC FP]."

Three parameters set the cutoff for printing a nuclide or element:

- "cutoff_step" sets the index on which to base the cutoff (default -1 means use an average over all steps),

- "rel_cutoff" determines whether to treat the cutoff as a percent of the total (default/yes) or an absolute amount (no), and 
- the "cutoffs" array allows one to specify the cutoff for each unit in Table 5.1.10 as a sequence of "unit=cutoff" pairs.

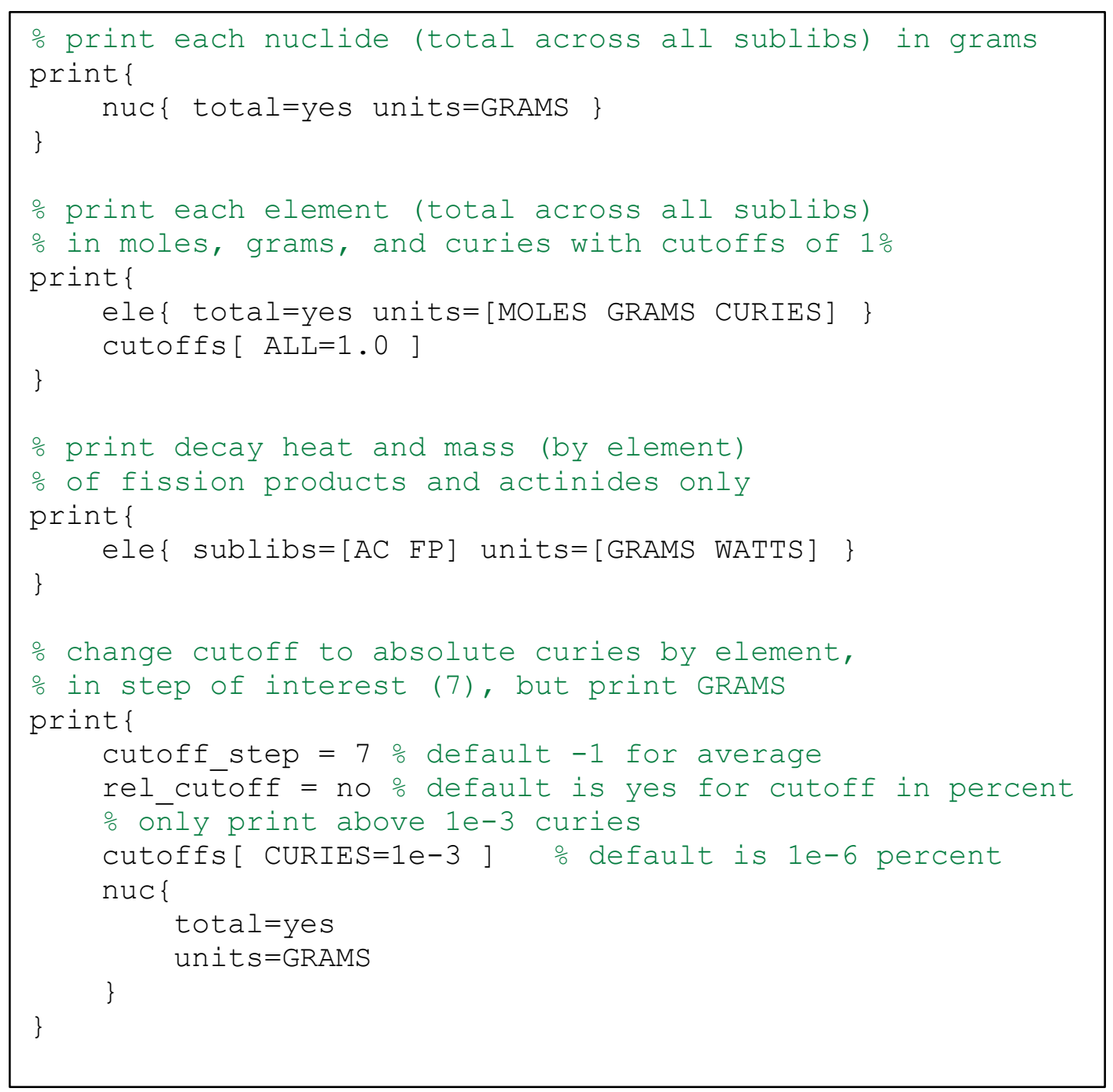

Figure 5.1.16. ORIGEN nuclide and element "print" blocks. 
The emission printing options are controlled by the "alpha," "beta," "gamma," and "neutron" emission blocks inside a "print" block (examples shown in Figure 5.1.17).

The "neutron" print options are

- "summary" (yes/no) controls the printing of a source strength summary,

- "spectra" (yes/no) controls the printing of the spectra (energy group-wise), and

- "detailed" (yes/no) controls the printing of extra details about the neutron calculation.

The "gamma" print options are

- "summary" (yes/no) controls the printing of a source strength summary and

- "spectra" (yes/no) controls the printing of the spectra (energy group-wise).

The gamma print allows a special output of the principal emitters in each energy group, controlled by setting the "principal_step" keyword to a specific step index in the case, with the "principal_cutoff" keyword used to set the minimum percent of the total a nuclide must have to be considered a principal emitter. For the gamma print there is a warning that can be enabled with "unbinned_warning=yes" if some gamma lines fall outside the user group structure and thus are not included.

The "alpha" print options are

- "summary" (yes/no) controls the printing of a source strength summary and

- "spectra" (yes/no) controls the printing of the spectra (energy group-wise).

The "beta" print options are

- "summary" (yes/no) controls the printing of a source strength summary and

- "spectra" (yes/no) controls the printing of the spectra (energy group-wise).

The beta print also allows a special output of the principal emitters by setting the "principal_step" keyword to a specific step index in the case, with the "principal_cutoff" keyword used to set the minimum percent of the total a nuclide must have to be considered a principal emitter.

The special printing options are shown in Figure 5.1.18. 


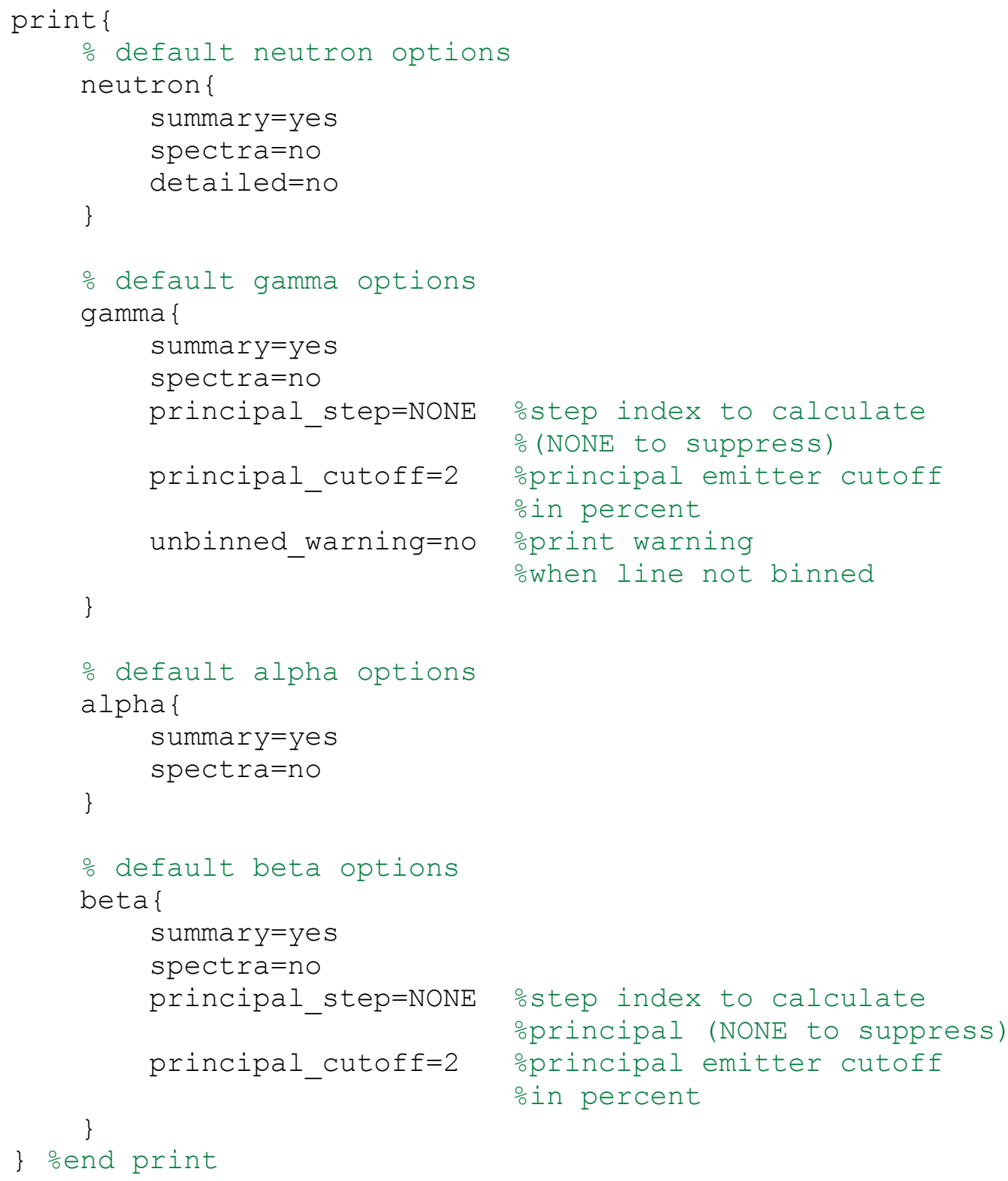

Figure 5.1.17. ORIGEN emission "print" blocks. 


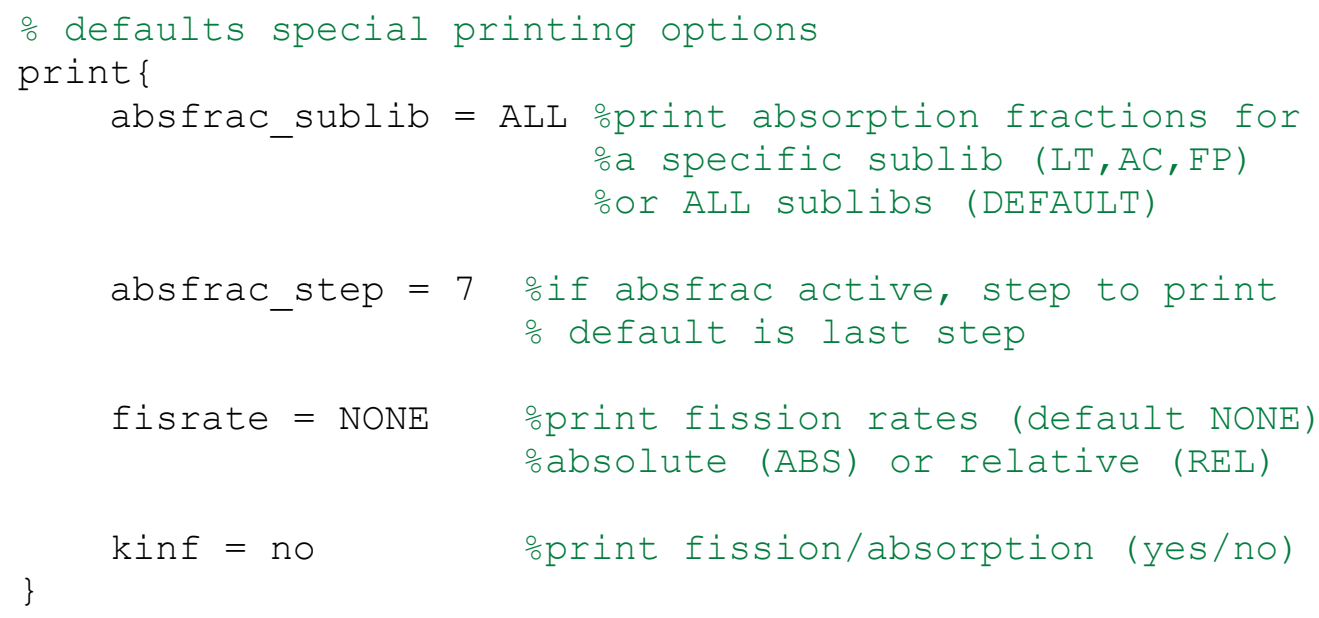

Figure 5.1.18. ORIGEN special "print" options.

\section{Saving Results (save)}

Saving the results to an ORIGEN binary file (f71) is requested with the "save" block, which specifies both the name of the file and the step indices to save, as shown in Figure 5.1.19. The default for the filename is "file=ft71f001" and default for the steps is the special "steps=ALL" which saves all isotopics and spectra as a shortcut to having to specify "steps=[ $\left.\begin{array}{llllll}0 & 1 & 2 & 3 & \ldots & \text { LAST }\end{array}\right]$ ". The step index "0" corresponds to the initial isotopics and the step index "LAST" may be used as a shortcut for the last case index. There is a special rule for copying 771 files from SCALE's temporary/working directory. If the file name "ft71f001" exists in the directory when SCALE finishes, it is copied to the user's "\$\{OUTDIR $\}$ " as "\$ $\{$ BASENAME $\} . f 71 "$, e.g. if my.inp produces " $\mathrm{ft} 71 \mathrm{f001"}$ " in the temporary/working directory then it will be copied to the same location as the main output file ("my.out") as "my.f71". Note that $\mathrm{f71}$ files are always appended to by ORIGEN. To save with the defaults, the shortcut "save=yes" is provided. The default is "save=no".

In order to change the time values written to the $\mathrm{f} 71$ file, use "time_offset $=\mathrm{T}_{0}$ " which will write the current cumulative time minus $\mathrm{T}_{0}$ to the file. The "time_offset" is convenient, for example, when time since discharge is desired instead of the absolute, cumulative time. The "time_units" entry specifies the units of the "time_offset", with the same units available in the "time" block. The default is "time_units=DAYS". 


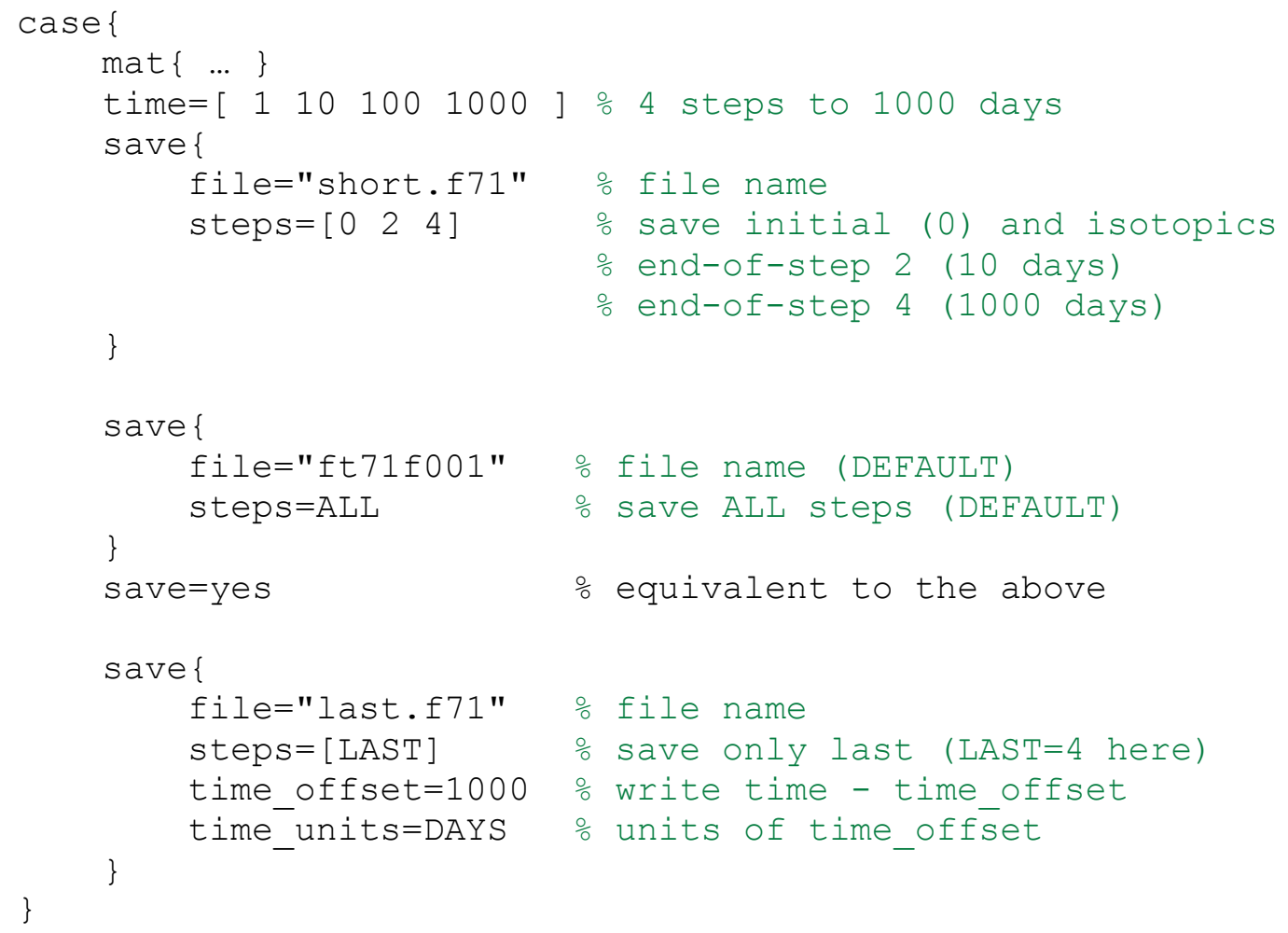

Figure 5.1.19. ORIGEN "save" block.

Decay Emission Calculations (alpha, beta, gamma, neutron)

A decay emission calculation is initiated with the appropriate block inside the calculation "case." The group structure for any emission spectra result is determined by the energy bounds provided as described in Sect. 5.1.3.3.2.2. Each type of emission calculation is activated by the existence of a calculation block named "alpha", "beta", "gamma", or "neutron" for those respective types of calculations. Alternatively, to turn on an emission calculation with defaults, use "alpha=yes", "beta=yes", "gamma=yes", or "neutron=yes" in a "case" block.

The neutron calculation (Sect. 5.1.2.4.1) is activated by the "neutron" calculation block. All neutron calculation options are to control the $(\alpha, n)$ calculation.

Three $(\alpha, n)$ options can be indicated with the "alphan_medium": a $\mathrm{UO}_{2}$ fuel matrix (alphan_medium=UO2), a borosilicate glass matrix (alphan_medium=BOROSILICATE), and the problem-dependent matrix defined by the user input compositions (alphan_medium=CASE). The numeric options 0,1 , and 2 are also valid for the UO2, BOROSILICATE, and CASE options, respectively. Note that the $\mathrm{UO}_{2}$ and borosilicate glass matrix options assume that the neutron source nuclides reside in these respective matrices, regardless of the actual composition of the material in the problem.

For oxide fuels, a significant neutron source can be produced from ${ }^{17} \mathrm{O}(\alpha, n)$ and ${ }^{18} \mathrm{O}(\alpha, n)$ reactions in the oxygen compounds of the fuel. For this reason, the $\mathrm{UO}_{2}$ matrix option (enabled by alphan_medium=UO2) is provided with natural isotopic distribution of ${ }^{17} \mathrm{O}$ and ${ }^{18} \mathrm{O}$. This includes the impact of oxygen isotopes on the neutron source without having to include oxygen in the initial composition. 
Another common use case is fuel storage in a borosilicate glass matrix (enabled by alphan_medium=BOROSILICATE), listed in Table 5.1.13.

Table 5.1.13. Borosilicate glass composition used in the borosilicate glass option

\begin{tabular}{clcc}
\hline Atomic number & Element symbol & $\mathbf{W t}^{\mathbf{a}}$ & Atom \% \\
\hline 3 & $\mathrm{Li}^{b}$ & 2.18 & 6.296 \\
5 & $\mathrm{~B}^{b}$ & 2.11 & 3.913 \\
8 & $\mathrm{O}^{b}$ & 46.4 & 58.138 \\
9 & $\mathrm{~F}^{b}$ & 0.061 & 0.0644 \\
11 & $\mathrm{Na}^{b}$ & 7.65 & 6.671 \\
12 & $\mathrm{Mg}^{b}$ & 0.49 & 0.404 \\
13 & $\mathrm{Al}^{b}$ & 2.18 & 1.620 \\
14 & $\mathrm{Si}^{b}$ & 25.4 & 18.130 \\
17 & $\mathrm{Cl}^{b}$ & 0.049 & 0.0277 \\
20 & $\mathrm{Ca}$ & 1.08 & 0.540 \\
25 & $\mathrm{Mn}$ & 1.83 & 0.668 \\
26 & $\mathrm{Fe}$ & 8.61 & 3.091 \\
28 & $\mathrm{Ni}$ & 0.70 & 0.239 \\
40 & $\mathrm{Zr}$ & 0.88 & 0.193 \\
82 & $\mathrm{~Pb}$ & 0.049 & 0.0047 \\
Total & & 99.669 & 100.000 \\
\hline
\end{tabular}

${ }^{a}$ Obtained from Ref. 11.

${ }^{b}$ Elements with $(\alpha, n)$ yields.

In the last most rigorous option, the $(\alpha, n)$ neutron source and spectra are calculated using the source, target, and constituents determined using the material compositions in the problem at a particular time, dictated by "alphan_step" in the "neutron" print options. For spent fuel neutron source calculations, there are a large number of potential source, target, and constituent nuclides in the $(\alpha, n)$ calculation and in order to remove low-importance nuclides from the calculation, the "alphan_step" and "alphan_cutoff" parameters are used. Only those nuclides with an $\alpha$-decay activity exceeding the product of "alphan_cutoff" times the total $\alpha$ activity are included as source nuclides in the $(\alpha, n)$ neutron calculation. Additionally, only those nuclides with a constituent or target atom fraction less than "alphan_cutoff" are included unless the concentration is greater than $1 \mathrm{ppm}$, in which case it will be retained regardless of the cutoff. The "blend" array is particularly useful for creating a problem-dependent medium composed of fuel and another material.

The gamma (photon) calculation described in Sect. 5.1.2.4.4 is activated with the "gamma" block, as shown in Figure 5.1.21. The gamma block includes a host of options where the default should be appropriate in most cases.

- The "sublib" option (default "ALL") affects the sublibraries included in the gamma emission calculation. The "immediate" option (default "yes") includes immediate gamma and x-rays.

- The "spont" option (default "yes") includes photons emitted during spontaneous fission and $(\alpha, n)$.

- The "continuum" option (default "yes") enables mapping of continuum data stored artificially as lines to a continuum across the user-defined energy bins. 
The following options may need to be modified in some scenarios.

- The "brem_medium" option (default "UO2") includes the photons emitted by beta particles as they slow down in a medium (Bremsstrahlung) in the gamma calculation. A problem-dependent medium is not available for "brem." The only options are "NONE," "H2O" (for Bremsstrahlung in water) and "UO2" (for uranium dioxide).

- The "conserve_line_energy" option (default "no") modifies the intensity of each gamma line within a group to conserve energy according to $I_{g}=I_{a} E_{a} / E_{g}$, where $I_{a}$ is the original line intensity, $E_{a}$ the original line energy and $E_{g}$ is the group energy (simple midpoint energy). Note that this option modifies intensities and thus results in number of particles not being conserved.

- The "adjust_for_missing" option (default "no") accounts for the scenario where a nuclide has a known gamma decay mode with known recoverable energy release, but the spectral data do not exist in the available emission resource. If "adjust_for_missing=yes," the entire spectrum is scaled up to include the missing energy. If the missing energy is more than $1 \%$ of the total, a warning is printed.

- The "split_near_boundary" option (default "no") enables a splitting of a line when it appears very close to a bin boundary. If "split=yes" and a photon line is within $3 \%$ of an interior energy boundary, then the intensity is split equally between the adjacent groups.

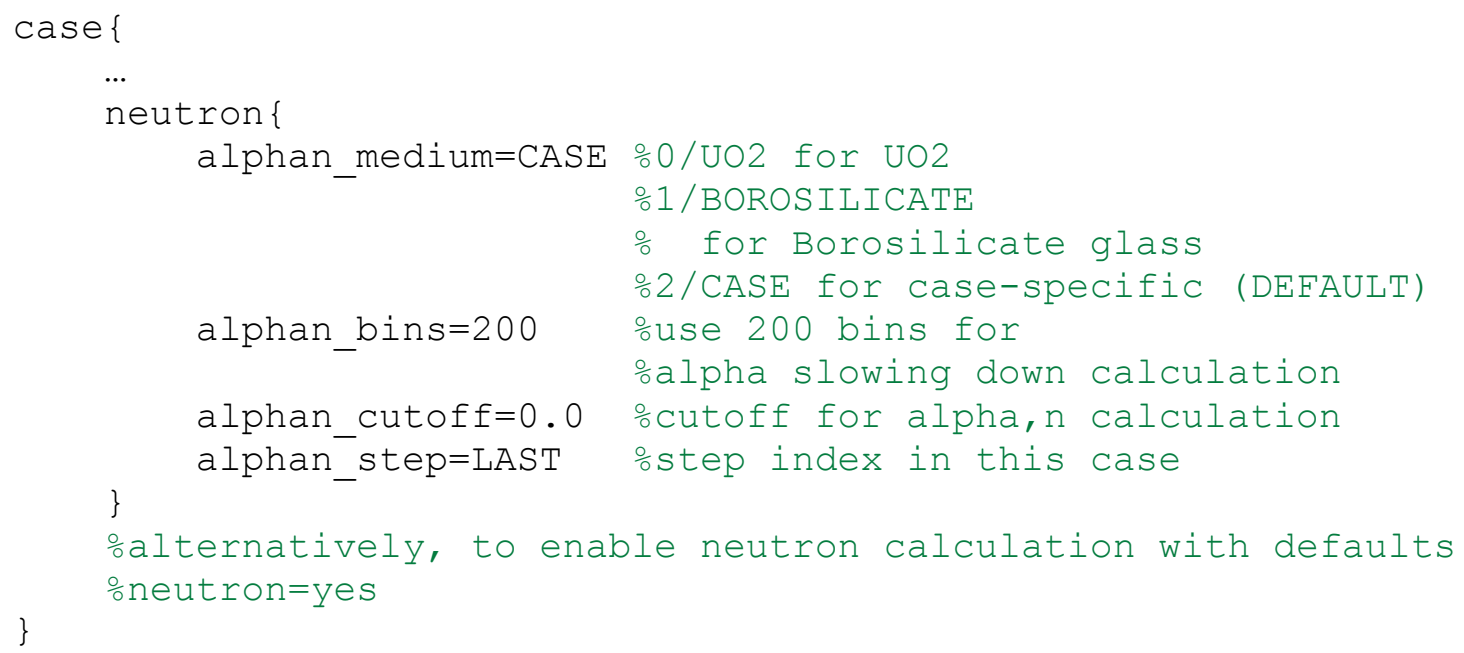

Figure 5.1.20. ORIGEN “neutron” calculation block. 


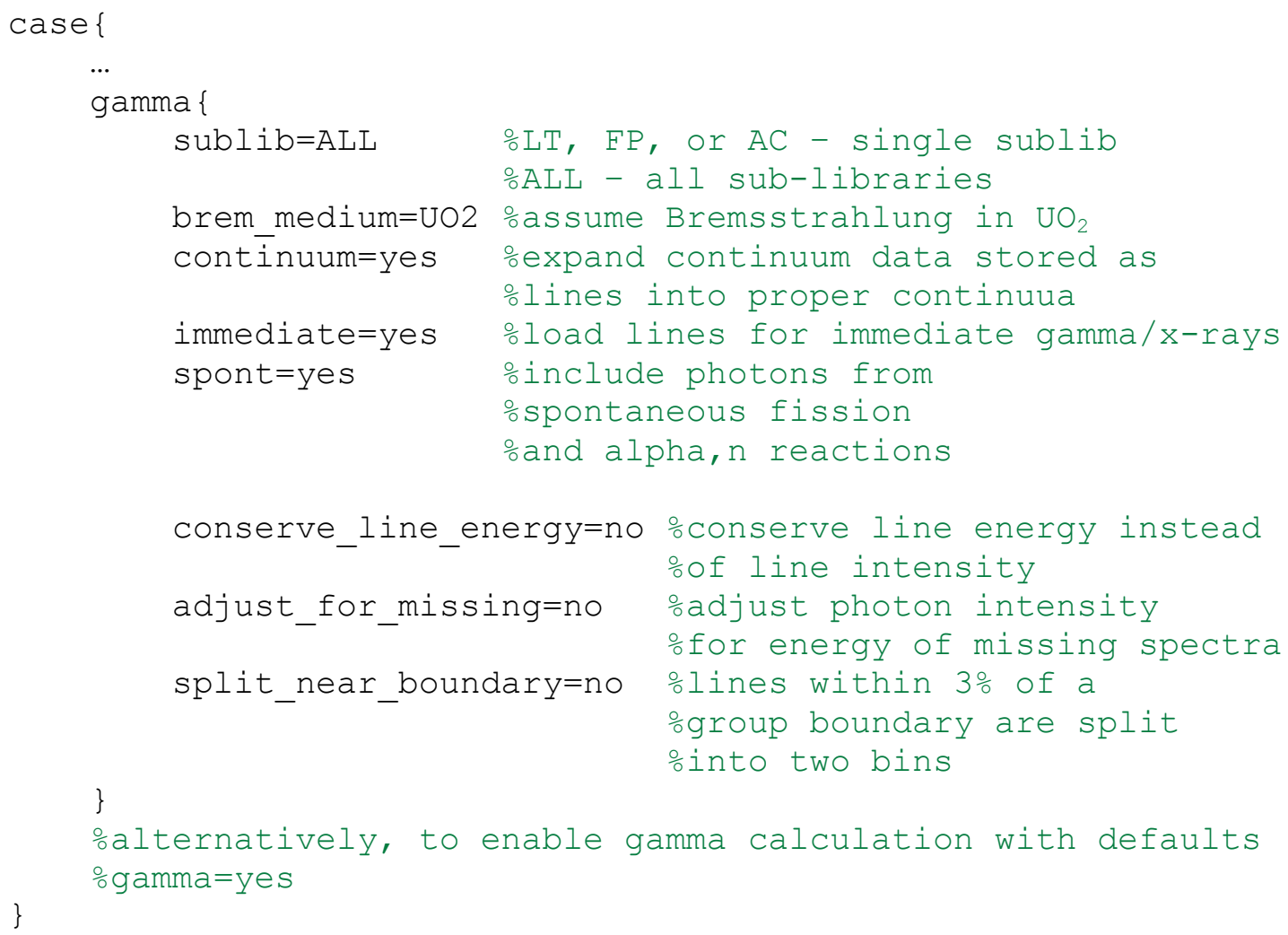

Figure 5.1.21. ORIGEN "gamma" calculation block.

The alpha calculation (Sect. 5.1.2.4.2) has no options and the beta calculation (Sect. 5.1.2.4.3) only has a single option to choose the nuclide sublibraries included (see Figure 5.1.22). Note that the alpha and beta calculations determine sources and do not include any slowing down physics for charged particles.

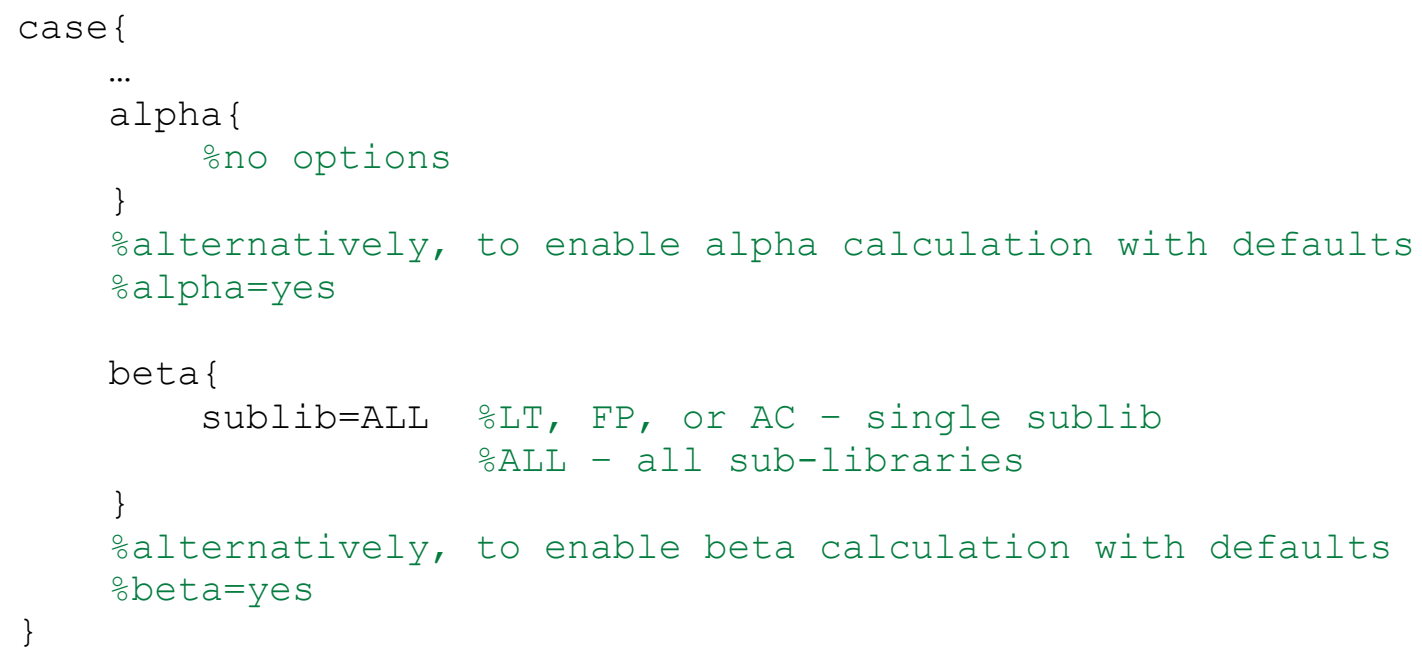

Figure 5.1.22. ORIGEN "alpha" and "beta" calculation blocks. 


\section{Processing Options (processing)}

The processing options are contained inside the "processing" block (inside a "case") and have two ways to modify the isotopics vector: the "removal" block and the "retained" array (see Figure 5.1.23). Both operate on elements instead of nuclides.

- Each "removal" block specifies a list of elements and a rate of continuous removal in units $(1 / \mathrm{s})$. This removal is an artificial increase of the decay constant, from Eq. (5.1.4), and it can be used to model any continuous system of removal, such as filtration. Note that more than one removal block may be specified.

- The "retained" array specifies a list of elements and the mole/atom fraction to be retained at the start of this case. Note that all unspecified elements become zero.

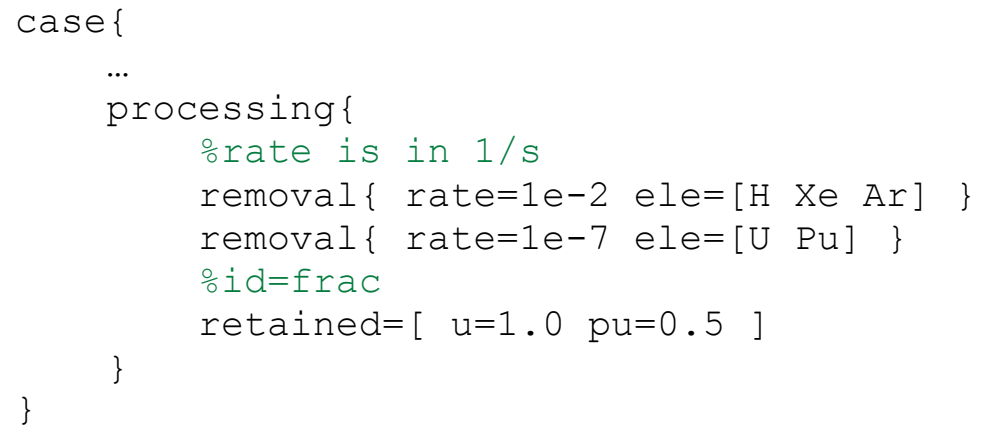

Figure 5.1.23. ORIGEN “processing” block.

\subsection{Bounds Block}

The "bounds" block appears outside all cases and is valid for the entire input, dictating the energy bins (i.e., groups) for emission spectra and source calculations (see Figure 5.1.24). The array shortcuts-"L" for logarithmically spaced intervals and "I" for linearly spaced intervals - can be particularly useful for specifying the bounds (see Table 5.1.12). The energy bounds are in units (eV) and can be given in increasing or decreasing order with the output convention of decreasing order. Additionally, neutron and gamma energy bounds can be read from a standard SCALE cross section library file by specifying the path to the file instead of the array bounds.

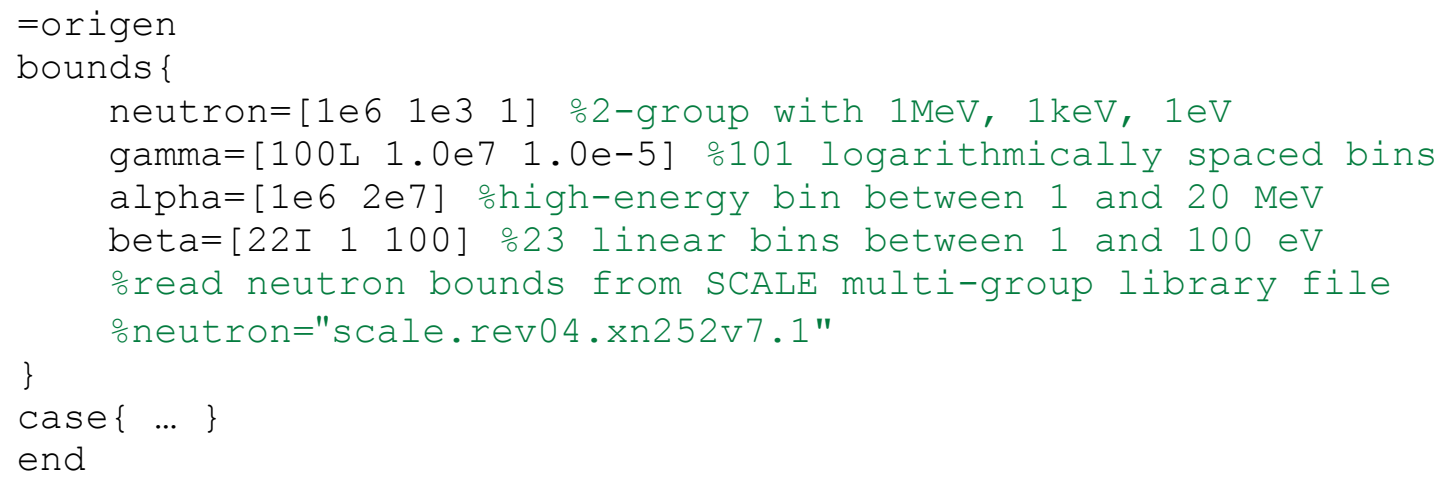


Figure 5.1.24. ORIGEN “bounds" block.

\subsection{Solver Block}

The solver block controls high-level solver options, the most important of which is the actual solver kernel used, either the default MATREX (Sect. 5.1.2.2.1, Figure 5.1.25) or CRAM (5.1.2.2.2, Figure 5.1.26).

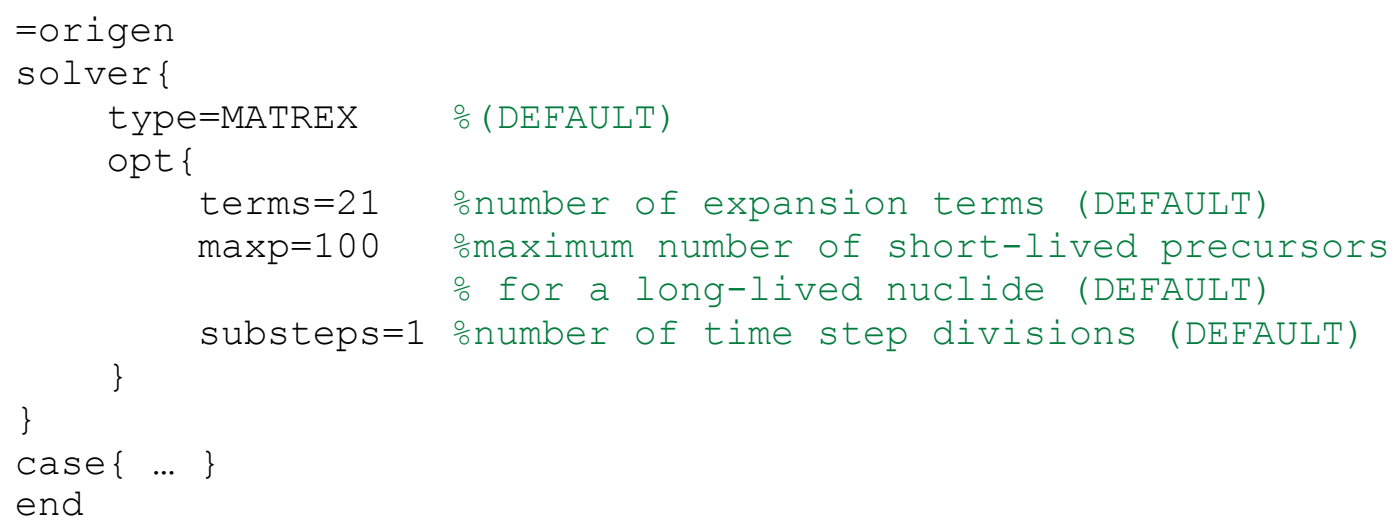

Figure 5.1.25. ORIGEN "solver" block for MATREX.

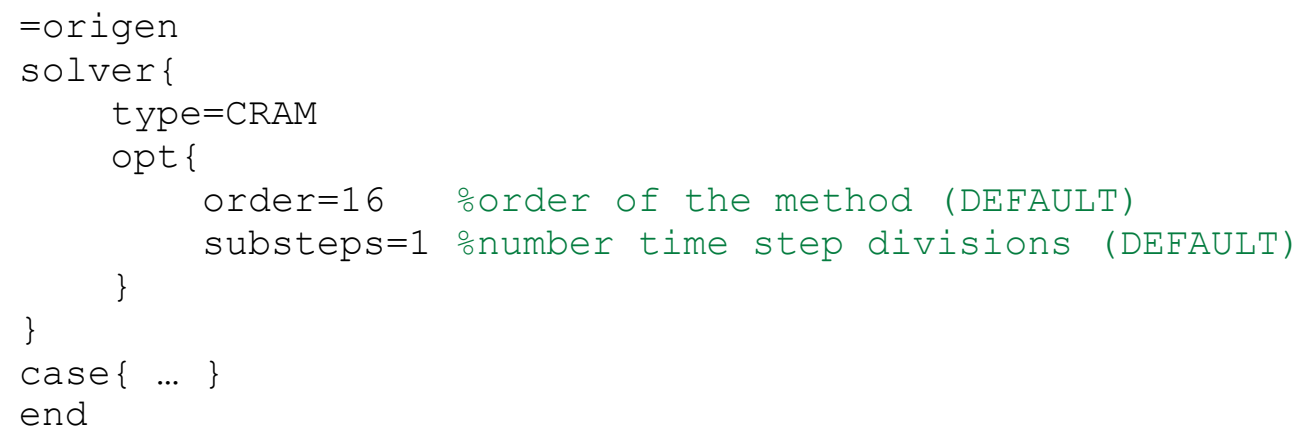

Figure 5.1.26. ORIGEN “solver” block for CRAM. 
The MATREX kernel contains two parameters that are generally sufficient with the default values. The minimum number of "terms" is $n_{\text {terms }}=21$, which overrides the heuristic in Eq. (5.1.19). Experience indicates that high flux levels (e.g., greater than $10^{16} \mathrm{n} / \mathrm{cm}^{2}-\mathrm{s}$ ) may require more terms. The second parameter, "maxp," is generally sufficient, describing the amount of storage available for the short-lived precursors of a long-lived isotope.

The CRAM kernel has a single parameter that impacts the numerical error: the "order." Both the CRAM and MATREX solver include the "substeps" parameter that adds substeps to each user-defined time step. Testing the same input with a different number of substeps (e.g., "substeps=1," "substeps=2," "substeps $=4$ ") can be a simple way to check that the time grid is sufficient.

\subsection{Options Block}

The "options" block contains miscellaneous global options that apply to all cases, as shown in

Figure 5.1.27. The "print_xs" option enables output of the transition matrix $\boldsymbol{A}$ used in each case, the "digits" option enables high-precision output when set to 6, and the "fixed_fission_energy" allows 200 $\mathrm{MeV}$ per fission to be used everywhere instead of the nuclide-dependent energy release being used by default.

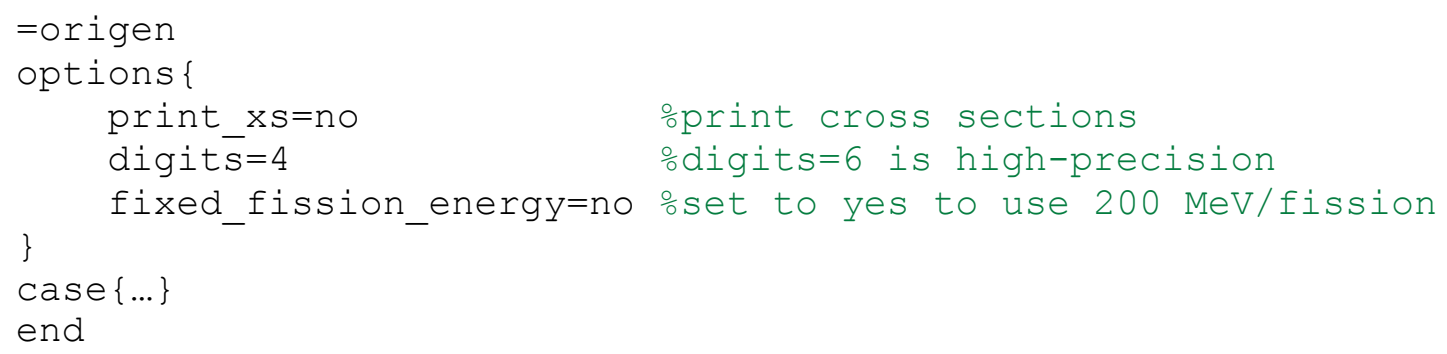

Figure 5.1.27. ORIGEN “options” block.

\subsubsection{OPUS Module}

The analysis of ORIGEN results often involves evaluating large amounts of output data that may include time-dependent nuclide and element inventories, radiological decay properties, and neutron, alpha, beta and photon source spectra. Visualization of the output data from ORIGEN often provides a means of rapidly evaluating the dominant nuclides in a problem, identifying important trends in the results, and providing insight into the problem that is not easily obtained otherwise. The OPUS utility program has been developed to read and process ORIGEN binary concentration results file (f71) into a format easily entered into graphics-plotting packages.

\subsection{Key Features}

The output of OPUS is a PLT file with extension ".plt" which can be easily read by most graphics packages, including the SCALE GUI Fulcrum. An example of the PLT file is shown in Figure 5.1.28. 


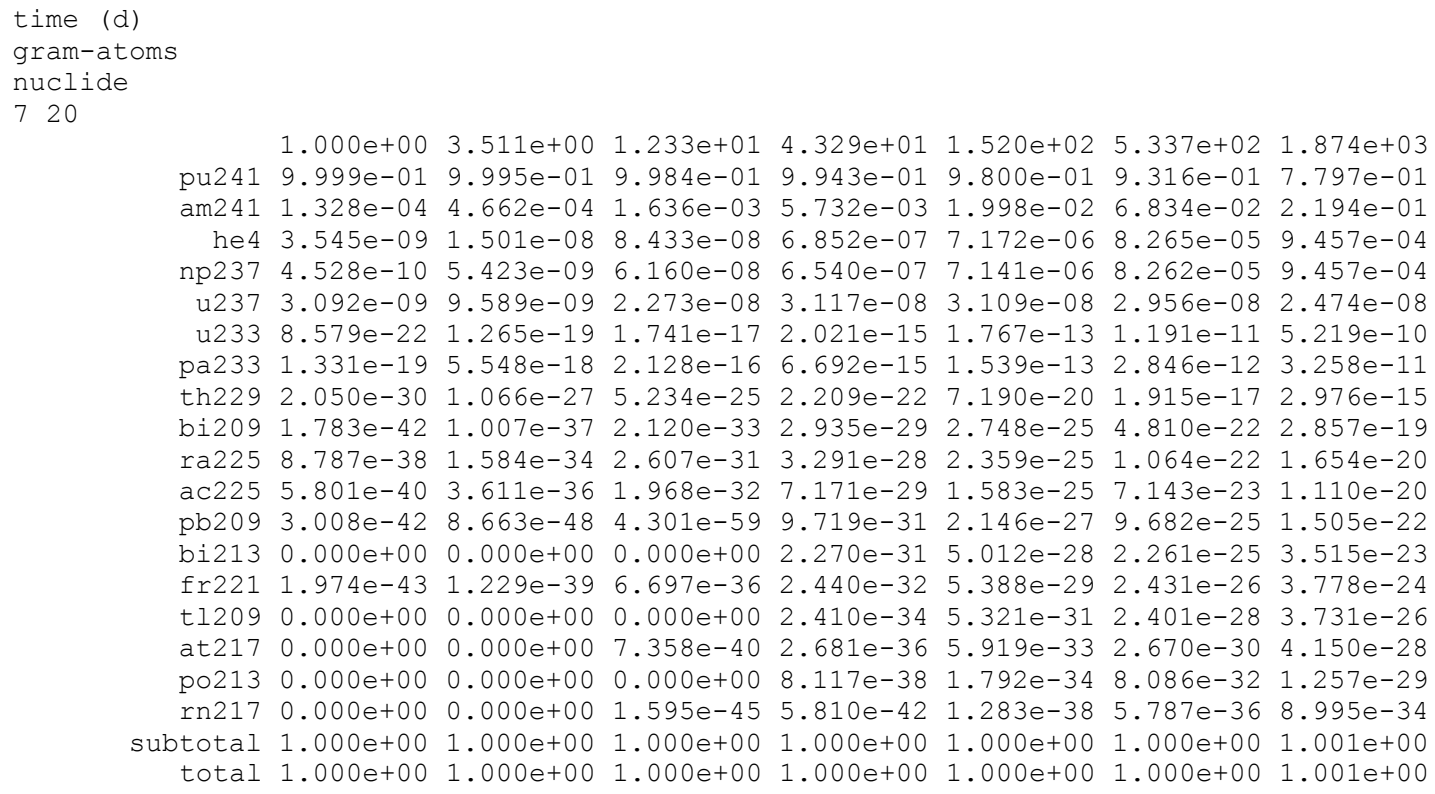

Figure 5.1.28. Example of the OPUS PLT file.

The various types of data that can be returned by OPUS fall into two separate classes, briefly described as (1) dominant or selected isotopes or elements and (2) photon, alpha, beta and neutron source spectra. These requested data may be extracted for either irradiation or decay time periods of interest. One key feature of the program is that by default, it will automatically extract the 40 most dominant nuclides or elements in the problem, rank them using the output response and time periods of interest as specified by the user, and give the total response. The returned data may be selected, ranked, and plotted according to the group (types) of nuclides in the problem. The groups include the actinides, fission products, activation products, fission products and actinides, or all nuclides in the problem. The totals are printed for all nuclides in the specified group, and a subtotal is generated for all nuclides in the printed list. The user may also specify any other nuclide or element to be included in the output, regardless of its importance to the problem. The nuclides may be ranked using different output units of mass, density, atomic density, activity, toxicity, decay heat, or isotopic fractions.

Default values are assigned to most input variables, allowing a comprehensive visual analysis of returned data with relatively little user input.

\subsection{Input Description}

OPUS input records are limited to a maximum of 80 columns. A single data entry may be entered anywhere in a record but cannot be divided between two records; however, array-data entries may be divided over many records. A data entry (from left to right) is composed of a keyword, an equal sign (=), and numeric or alphanumeric data. A data entry is illustrated in the following example:

title="PWR 33 GWD/MTU 3.3 wt U-235" 
The program identifies keywords either by using only the first four characters in the keyword name or the full keyword name. In the example above, "titl" may be used, omitting the "e." Alphanumeric data (as titles or axis labels) must be enclosed in double quotes, with nuclide or element symbols being the notable exception.

Floating-point data may be entered in various forms; for example, the value 12340.0 may be entered as $12340,12340.0,1.234+4,1.234 \mathrm{E}+4,1.234 \mathrm{E} 4$, or $1.234 \mathrm{E} 04$. Also, the value 0.012 may be entered as $12 \mathrm{E}-3,12-3,1.2-2$, etc. Numeric data must be followed immediately by one or more blanks.

Array data (alphanumeric floating-point and integer) for an array-type parameter must always be terminated with an END that does not begin in column one.

Input parameters in a single OPUS sequence input (i.e., between "=opus" and "end") may be entered in any order. Usually each sequence input produces a single plot file with the name "\$\{OUTBASENAME\}N.plt" where \$\{OUTBASENAME\} is the base of the input file name, e.g., my.inp has the base name "my," and "N" is an 18-digit integer that is incremented with each opus plot.

The OPUS input uses keywords. Keywords, descriptions, and allowed choices for each entry are listed in Table 5.1.14 for plot setup options and Table 5.1.15 for response units and nuclide selection. Input keywords and their choices occupy the first column. The next two columns indicate the type of plot in which the keyword may be entered. An " $X$ " in these columns indicates the keyword is an available entry for that type of plot. An "NA" in these columns indicates it is not a keyword for that type of plot. The last column is a description of the input keyword.

\footnotetext{
${ }^{6}$ Unlike in the previous OPUS versions, it is not permitted to enter four characters followed by any alphanumeric
} characters: only full keyword names or four-letter short-hands are allowed. 
Table 5.1.14. OPUS input for plot setup

\begin{tabular}{|c|c|c|c|}
\hline Input keywords & 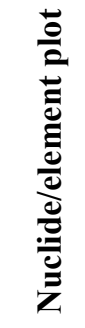 & 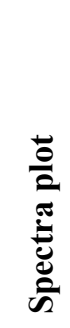 & Keyword description \\
\hline LIBRARY $=(“ “)$ & $\checkmark$ & $\checkmark$ & $\begin{array}{l}\text { ORIGEN binary cross section library (f33). Only } \\
\text { required in order to perform reaction rate calculations } \\
\text { (see ABSO and FISS units below). }\end{array}$ \\
\hline DATA $=$ (“ft71 f001”) & $\checkmark$ & $\checkmark$ & $\begin{array}{l}\text { ORIGEN concentrations file (f71). If entered, must be } \\
\text { enclosed in double quotes. }\end{array}$ \\
\hline TITLE $=$ & $\checkmark$ & $\checkmark$ & $\begin{array}{l}\text { Title of plot. If entered, must be enclosed in double } \\
\text { quotes. }\end{array}$ \\
\hline $\begin{array}{l}\text { TYPARAMS }=(\text { NUCLIDES) } \\
\text { NUCLIDES } \\
\text { ELEMENTS } \\
\text { ASPECTRUM } \\
\text { BSPECTRUM } \\
\text { GSPECTRUM } \\
\text { NSPECTRUM } \\
\text { SFSPEC } \\
\text { ANSPEC } \\
\text { DNSPEC }\end{array}$ & $\begin{array}{l} \\
\checkmark \\
\checkmark \\
\text { NA } \\
\text { NA } \\
\text { NA } \\
\text { NA } \\
\text { NA } \\
\text { NA } \\
\text { NA }\end{array}$ & $\begin{array}{l} \\
\text { NA } \\
\text { NA } \\
\checkmark \\
\checkmark \\
\checkmark \\
\checkmark \\
\checkmark \\
\checkmark \\
\checkmark\end{array}$ & $\begin{array}{l}\text { The type of parameter to be plotted } \\
\text { Request data for the comparison of individual nuclides } \\
\text { Request data for the comparison of elements } \\
\text { Request for alpha spectrum } \\
\text { Request for beta spectrum } \\
\text { Request for photon spectrum } \\
\text { Request total neutron spectrum from spontaneous fission } \\
\text { plus (alpha,n) reactions plus delayed neutrons } \\
\text { Request neutron spectrum for only spontaneous fission } \\
\text { Request neutron spectrum for only (alpha,n) reactions } \\
\text { Request neutron spectrum for only delayed neutrons }\end{array}$ \\
\hline $\begin{array}{l}\text { XLABEL }= \\
\text { YLABEL }=\end{array}$ & $\checkmark$ & $\checkmark$ & $\begin{array}{l}\mathrm{X}-\text { and Y-axis labels. If this parameter is not entered, a } \\
\text { label is generated automatically. If entered, must be } \\
\text { enclosed in double quotes. }\end{array}$ \\
\hline $\begin{array}{l}\text { COLS }=(\text { YES }) \\
\text { YES } \\
\text { NO }\end{array}$ & $\checkmark$ & NA & Print nuclide edits by columns (one nuclide per column). \\
\hline $\begin{array}{l}\text { ROWS }=(\text { YES }) \\
\text { YES } \\
\text { NO }\end{array}$ & $\checkmark$ & NA & Print nuclide edits by rows (one nuclide per row). \\
\hline VOLUME $=$ & $\checkmark$ & NA & $\begin{array}{l}\text { Material volume in } \mathrm{cm} 3 \text {; required when plotting in units } \\
\text { of grams } / \mathrm{cm} 3 \text { or atoms } / \text { barn-cm. }\end{array}$ \\
\hline FACTOR $=$ & $\checkmark$ & NA & User-defined multiplicative factor for all concentrations \\
\hline $\begin{array}{l}\text { TIME }=(\text { DAYS }) \\
\text { SECONDS } \\
\text { MINUTES } \\
\text { HOURS } \\
\text { DAYS } \\
\text { YEARS }\end{array}$ & $\begin{array}{l}\checkmark \\
\checkmark \\
\checkmark \\
\checkmark \\
\checkmark \\
\checkmark\end{array}$ & $\begin{array}{l}\text { NA } \\
\text { NA } \\
\text { NA } \\
\text { NA } \\
\text { NA } \\
\text { NA }\end{array}$ & $\begin{array}{l}\text { Unit of time to be plotted } \\
\text { Time plotted in seconds } \\
\text { Time plotted in minutes } \\
\text { Time plotted in hours } \\
\text { Time plotted in days } \\
\text { Time plotted in years }\end{array}$ \\
\hline
\end{tabular}


Table 5.1.15. OPUS input for response units and nuclide selection

\begin{tabular}{|c|c|c|c|}
\hline Input keywords & 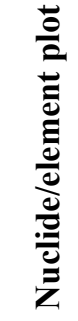 & 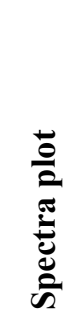 & Keyword description \\
\hline NRANK $=(40)$ & $\checkmark$ & NA & $\begin{array}{l}\text { Total number of nuclides or elements to be returned for } \\
\text { the plot. If user-selected nuclides or elements are } \\
\text { requested (see SYMNUC), output will include these } \\
\text { nuclides plus any remaining ones with the highest } \\
\text { rankings for the quantity specified. The total and } \\
\text { subtotal are always printed. Default is not used if } \\
\text { SYMNUC is entered. }\end{array}$ \\
\hline $\begin{aligned} \text { UNITS }= & (\text { GATO }) \text { nuclide/element } \\
& (\text { INTENSITY }) \text { spectrum }\end{aligned}$ & & & $\begin{array}{l}\text { Requested data units. Gram-atoms are default for } \\
\text { nuclide/element plots, and particles/s are default for }\end{array}$ \\
\hline ABSORPTIONS & $\checkmark$ & NA & $\begin{array}{l}\text { Absorption/removal reaction rate (using removal XS on } \\
\text { library). }\end{array}$ \\
\hline $\operatorname{AIRM}^{* * 3}$ & $\checkmark$ & NA & Toxicity, cubic meters of air to dilute to $\mathrm{RCG}_{\mathrm{a}}$ \\
\hline APELEM & $\checkmark$ & NA & $\begin{array}{l}\text { Atom \% of element; isotopic atom percentages of all } \\
\text { elements specified in SYMNUC. }\end{array}$ \\
\hline ATOMS / B-CM & $\checkmark$ & NA & Atoms/barn-cm; requires VOLUME entry \\
\hline BECQUERELS & $\checkmark$ & NA & Radioactivity, Bq. \\
\hline CAPTURES & $\checkmark$ & NA & $\begin{array}{l}\text { Capture reaction rate (removal minus fission; it is not } \\
\text { the radiative capture) }\end{array}$ \\
\hline CURIES & $\checkmark$ & NA & Radioactivity, $\mathrm{Ci}$ \\
\hline FISSIONS & $\checkmark$ & NA & Fission reaction rate (using fission XS on library) \\
\hline GAMWATTS & $\checkmark$ & NA & Gamma-ray thermal power, watts \\
\hline GATOMS & $\checkmark$ & NA & Gram atoms, gram-atomic weights or moles \\
\hline GPERCM $* * 3$ & $\checkmark$ & NA & Partial density, grams $/ \mathrm{cm}^{3}$; requires VOLUME entry \\
\hline GRAMS & $\checkmark$ & NA & Mass, grams \\
\hline H2OM**3 & $\checkmark$ & NA & Toxicity, cubic meters of water to dilute to $R_{C} G_{w}$ \\
\hline KILOGRAMS & $\checkmark$ & NA & Mass, kilograms \\
\hline WPELEM & $\checkmark$ & NA & $\begin{array}{l}\text { Weight \% of element; isotopic weight percentags of all } \\
\text { elements specified in SYMNUC }\end{array}$ \\
\hline WATTS & $\checkmark$ & NA & Total thermal power, watts \\
\hline PARTICLES & NA & $\checkmark$ & Print spectra in units of particles/s \\
\hline INTENSITY & NA & $\checkmark$ & $\begin{array}{l}\text { Print spectra in units of particles/s/MeV (normalized to } \\
\text { energy bin width) }\end{array}$ \\
\hline ENERGY & NA & $\checkmark$ & $\begin{array}{l}\text { Print spectra in units of } \mathrm{MeV} / \mathrm{s} / \mathrm{MeV} \text { (normalized to } \\
\text { energy bin width; multiplied by mean bin energy) }\end{array}$ \\
\hline LIBTYPE $=($ ALL) & $\checkmark$ & NA & $\begin{array}{l}\text { Identifies sublibrary to include for nuclide/element } \\
\text { plots. Does not apply to spectrum plots. }\end{array}$ \\
\hline LITE & $\checkmark$ & NA & $\begin{array}{l}\text { Include only nuclides in the light-nuclide/activation } \\
\text { product sub-library }\end{array}$ \\
\hline ACT & $\checkmark$ & NA & Include only nuclides in the actinide sub-library \\
\hline FISS & $\checkmark$ & NA & Include only nuclides in the fission product sub-library \\
\hline
\end{tabular}


Table 5.1.15. OPUS input for response units and nuclide selection (continued)

\begin{tabular}{|c|c|c|c|}
\hline Input keywords & 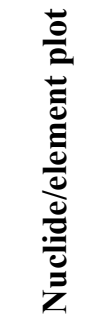 & 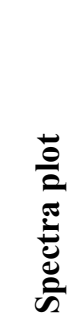 & Keyword description \\
\hline $\begin{array}{l}\text { FISACT } \\
\text { ALL }\end{array}$ & $\checkmark$ & NA & $\begin{array}{l}\text { Include nuclides in both fission product and actinide } \\
\text { sublibraries } \\
\text { Include all nuclides across all sublibraries }\end{array}$ \\
\hline SYMNUC $=$ & $\checkmark$ & NA & $\begin{array}{l}\text { Symbolic notation of nuclides or elements requested in } \\
\text { the plot data. The list is an array terminated with an } \\
\text { END. Nuclide entry is by chemical symbol and mass } \\
\text { number, separated by a dash "-." Metastable states } \\
\text { are indicated with an "cm" immediately following the } \\
\text { mass number (e.g., } 242 \mathrm{~m} \text { Am). Element entry is by } \\
\text { chemical symbol only (e.g., Pu). } \\
\text { Also, for nuclide entry it is allowed to use element } \\
\text { identifier; in such case, all isotopes of the element } \\
\text { found in the ORIGEN library are used. }\end{array}$ \\
\hline RESPONSE= & $\checkmark$ & NA & $\begin{array}{l}\text { Option to sort user-requested nuclides input via the } \\
\text { SYMNUC array by descending order of importance. } \\
\text { If SORT is not given or is SORT=NO, the order of } \\
\text { the nuclides in the plot table is the same as the input } \\
\text { order. Options that can be given to SORT are the } \\
\text { same as for UNITS. If SORT=YES, the same units } \\
\text { provided in the UNITS entered are used. } \\
\text { Optional user-specified arbitrary response conversion } \\
\text { factor array. These factors are applied to the } \\
\text { individual nuclides after conversion to quantities in } \\
\text { the units requested by UNITS. Entries are in nuclide } \\
\text { ID - response factor pairs. Nuclide identifiers } \\
\text { entered as legacy ORIGEN ZZAAAI 6-digit integer } \\
\text { IDs, where the isomeric state is the last digit. The } \\
\text { array must be terminated with an END. }\end{array}$ \\
\hline
\end{tabular}

The default values are given in parentheses after the input keyword definitions. Only the first four characters of any keyword (the letters are in bold) plus the "=," are required; they can be used instead of the full keyword name. However, unlike in previous versions of OPUS, the only two options accepted are the full keyword name or its first four letters. The keyword default value is listed in parentheses, and values are provided for almost all numeric input and axis labels. For example, the user has the option to enter axis labels using XLABEL $=$ and $\mathrm{YLABEL}=$ entries, or "built-in" axis labels based on the time units and the type of data units can be used.

The remaining sections give more detail on the nuclide/element plots, the spectra plots, and time range, and case selection to further filter the results on an $\mathrm{f} 71$ file. 


\subsection{Nuclidelelement plots}

Various responses may be returned either by nuclide or by element, as a function of time, for a specified time interval. By default, the top nuclides/elements contributing to the response of interest over the time range are returned. An explicit list of nuclides/elements can also be entered, in which case these nuclides will always be included, regardless of their ranking. Results for nuclides in specific sublibraries may be filtered (e.g., to only see output of ${ }^{155} \mathrm{Gd}$ the fission product (FP) sublib and not the light nuclide version [LT] sublib. The default behavior is to sum over all sublibs.

Response totals are always generated for each plot. These totals represent the sum over all nuclides or elements in the specified library type. In addition, a subtotal is printed (both in the printout and the plot file) that represents the sum over the printed nuclides or elements. The subtotal can be very useful in identifying whether the nuclides in the printed list include all of the dominant nuclides in the problem.

OPUS allows the user to sort using any of the provided concentration units. For each nuclide/element, approximate integral value over all selected time steps is calculated in the selected units; nuclides/elements are then ranked according to this value. The NRANK cutoff is applied after the sort has been performed.

It is permitted to enter SORT=YES, which is equivalent to setting SORT $=$ to the same value as UNITS $=$.

If the SORT key is not entered, nuclides/elements are sorted according to their position in the SYMNUC array.

\subsection{Spectra plots}

Alpha, beta, photon and neutron energy spectra calculated by ORIGEN may also be stored on the f71 file, and plotted by OPUS. One difference in the units of the spectra returned by OPUS and those printed by ORIGEN is that the intensities are typically converted from units of photons/s (stored on f71 file) to particles/s- $\mathrm{MeV}$ by dividing by the energy-group width. This conversion produces intensities that are more easily comparable across different energy groups and energy-group structures. Energy-intensity spectra in units of $\mathrm{MeV} / \mathrm{s}-\mathrm{MeV}$ and intensity spectra in units of particles/s may also be requested.

\subsection{Selection of plotting data}

OPUS provides four basic options for selecting a subset of the data on an f71 file:

1. a range of times with TMIN and TMAX (floating point numbers),

2. a range of positions on the $\mathrm{f} 71$ file with MINPOSITION and MAXPOSITION (integers),

3. a single CASE selection, and

4. a list of positions in the NPOSITION list.

A full overview of keywords that can be entered for time point selection is given in Table 5.1.16.

If no selection inputs are used, all available steps on the concentration file are printed. Range bounds (CASE, TMIN, TMAX, MINPOSITION, MAXPOSITION) are used as independent constraints, so any combination of those can be supplied, in which case all of the specified constraints are applied.

In case invalid positions are specified in the NPOSITION array, an error is raised, and no valid output is produced; the same happens if no steps fit into the specified selection. 
The case number is useful for ORIGEN stacked cases. Furthermore, TRITON stores the mixture number (with 0 for the sum of all depletion materials and -1 for the sum of selected materials) as the case number, so this option can be used to obtain a plot of the desired depletion mixture without actually knowing the time step numbers $=$. When an ORIGEN f71 file is accessed, a table of contents is printed in the output (.out) file. This can be very useful for understanding what is contained on the f71 at each position.

Table 5.1.16. OPUS Input keywords for data set selection

\begin{tabular}{|c|c|c|}
\hline Input keywords & Type & Keyword description \\
\hline NPOSITION= & Array & $\begin{array}{l}\text { List of all requested position numbers of data (on NUMUNIT), } \\
\text { in ascending order. No other entries are required since } \\
\text { NPOSITION is a unique data set identifier. Terminate with } \\
\text { END. } \\
\text { NPOSITIONS cannot be specified together with any of TMIN, } \\
\text { TMAX, MINPOSITION or MAXPOSITION. } \\
\text { If none of the position selectors is entered, all available } \\
\text { positions on the data file will be returned. }\end{array}$ \\
\hline CASE $=$ & Variable & $\begin{array}{l}\text { Number of ORIGEN case (or, a mixture number for TRITON- } \\
\text { produced f } 71 \text { files). }\end{array}$ \\
\hline $\begin{array}{l}\text { MINPOSITION= } \\
\text { MAXPOSITION= }\end{array}$ & $\begin{array}{l}\text { Variable } \\
\text { Variable }\end{array}$ & $\begin{array}{l}\text { Minimum position number, used to request a range of positions } \\
\text { Maximum position number } \\
\text { All positions between MINPOSITION and MAXPOSITION, } \\
\text { inclusive, are requested. } \\
\text { MINPOSITION and/or MAXPOSITION cannot be specified } \\
\text { together with NPOSITIONS, but they can be specified at the } \\
\text { same time as TMIN and/or TMAX. Up to all four conditions } \\
\text { (TMIN, TMAX, MINPOSITION, MAXPOSITION) will be } \\
\text { used to constraint the selected time points. } \\
\text { If none of the position selectors is entered, all available } \\
\text { positions on the data file will be returned. }\end{array}$ \\
\hline $\begin{array}{l}\text { TMAX= } \\
\text { TMIN= }\end{array}$ & $\begin{array}{l}\text { Variable } \\
\text { Variable }\end{array}$ & $\begin{array}{l}\text { Minimum and maximum time for selection of positions on the } \\
\text { data file. The units of TMAX and TMIN are the same as for } \\
\text { TIME. } \\
\text { TMIN and/or TMAX cannot be specified together with } \\
\text { NPOSITIONS, but they can be specified at the same time as } \\
\text { MINPOSITION and/or MAXPOSITION. Up to all four } \\
\text { conditions (TMIN, TMAX, MINPOSITION, } \\
\text { MAXPOSITION) will be used to constraint the selected time } \\
\text { points. } \\
\text { If none of the position selectors is entered, all available } \\
\text { positions on the data file will be returned. }\end{array}$ \\
\hline
\end{tabular}




\subsection{Plot File Formats}

The plot file produced by OPUS is a text file that contains plot information, including the title, axis labels, plot type, and the time-dependent data for inventory-type plots or energy-dependent data for spectral-type plots. The plot file is a free-format reading, so the column positions have no particular significance. However, the plot files created by OPUS are aligned by column to facilitate reading of the files by other graphics programs.

\subsection{File header information}

The format of the first five records of the plot file is common to all plot types. They are:

Record 1: TITL - problem title (maximum 32 characters)

Record 2: XHEA - X-axis label (maximum 20 characters)

Record 3: $\quad$ YHEA. y-axis label (maximum 20 characters)

Record 4: Plot type - The type of plot is selected by the words "nuclide," "element," "case," or "spec." The nuclide and element entries have the same effect and produce time-dependent plots of individual nuclides or elements in the specified units. The "case" entry is used to identify case comparisons of total quantities or individual nuclides. The "spec" entry identifies spectral-type plots.

The remaining records and formats depend on the plot type specified in Record 4.

\subsection{Nuclide and element plot types}

Record 5: NPTONC, KTOT - the number of time points (x-axis) for the plot data KTOT - the number of nuclides or elements in the plot; includes the last record that contains the totals, if present

Record 6: TIMES(NPTONC) - NPTONC entries for the times associated with each data point; units are in the units specified in Record 2 (XHEA)

Record 7: SYMBOL, (X(N,I), I=1, NPTONC) - SYMBOL is the alphanumeric symbol for the nuclide or element. No spaces are permitted in PlotOPUS. This symbol entry is followed by NPTONC entries containing the plot data for the nuclide or element SYMBOL with index N.

Record 8: Record 7 is repeated for all KTOT nuclides or elements.

Record 7+KTOT: Last record - contains the totals or other related quantity for the plot. The record has the same format as Record 7. 


\subsection{Spectral plot types}

Record 5: NPTONC, NGP - the number of entries that follow to construct the spectral histogram, where $\mathrm{NGP}=(\mathrm{NG}+1) * 2$ and $\mathrm{NG}$ is the number of energy groups. The histogram is made using a series of connecting lines, and therefore does not require any special histogram plotting capability in the graphics code.

Record 6: EN, YY - Each record contains a pair of entries: EN is the energy (MeV), and YY is the value for energy EN.

\section{Repeat Record 6 NGP times.}

\subsection{Case comparison plot types}

Record 5: NCAS - the negative value of the total number of cases in the plot

Record 6: NPTONC - the number of time points (x-axis) for the plot data for the case (non-userentered data)

Record 7: INPOIN the number of time points (x-axis) for the plot data for user-entered data

Record 8: XINP(INPOIN) - INPOIN entries for the times associated with each data point of the userentered data

Record 9: $\quad$ YINP(INPOIN) - INPOIN entries containing the plot data for the user-entered data

Record 10: LEGEND - legend for the user-entered data case.

The remaining records contain the case comparison data for the non-user-input data. Repeat records 7,8 , 9, and 10 for the case comparison plots, NPTONC times (same as case comparisons with no user-entered data). 


\subsubsection{Examples}

The main problems solvable by the ORIGEN family of codes are enumerated below (with relevant components in parentheses).

1. Decay (ORIGEN)

2. Activation (COUPLE+ORIGEN)

3. Fuel irradiation (ARP+ORIGEN or ORIGAMI)

4. Emission spectra from decay (ORIGEN)

5. Processing, including batch/continuous chemical removal, isotopic feed, and stream blending (ORIGEN)

6. Unit conversions (OPUS)

Examples of the six variations above are contained in the following sections, except for fuel irradiation problems with ORIGAMI, as described in its own chapter.

\subsubsection{Decay of ${ }^{238} \mathrm{U}$}

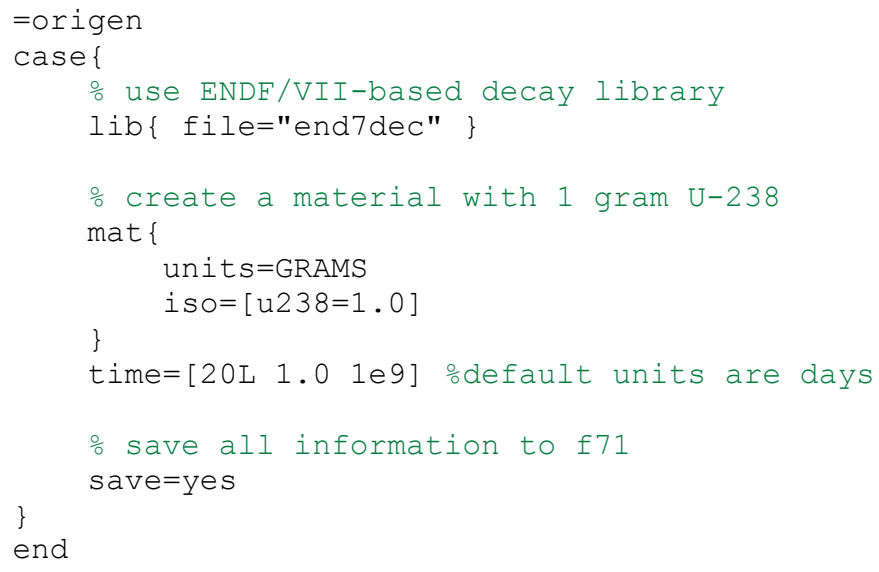

Figure 5.1.29. Decay of ${ }^{238} \mathrm{U}$

This example illustrates using the "end7 dec" binary decay library and decaying one gram of ${ }^{238} \mathrm{U}$ for $10^{9}$ days using the logarithmic array shortcut to put 20 logarithmically spaced values between 1.0 and 1 e9 days. 


\subsubsection{2 $\quad{ }^{252}$ Cf neutron Emission Spectrum}

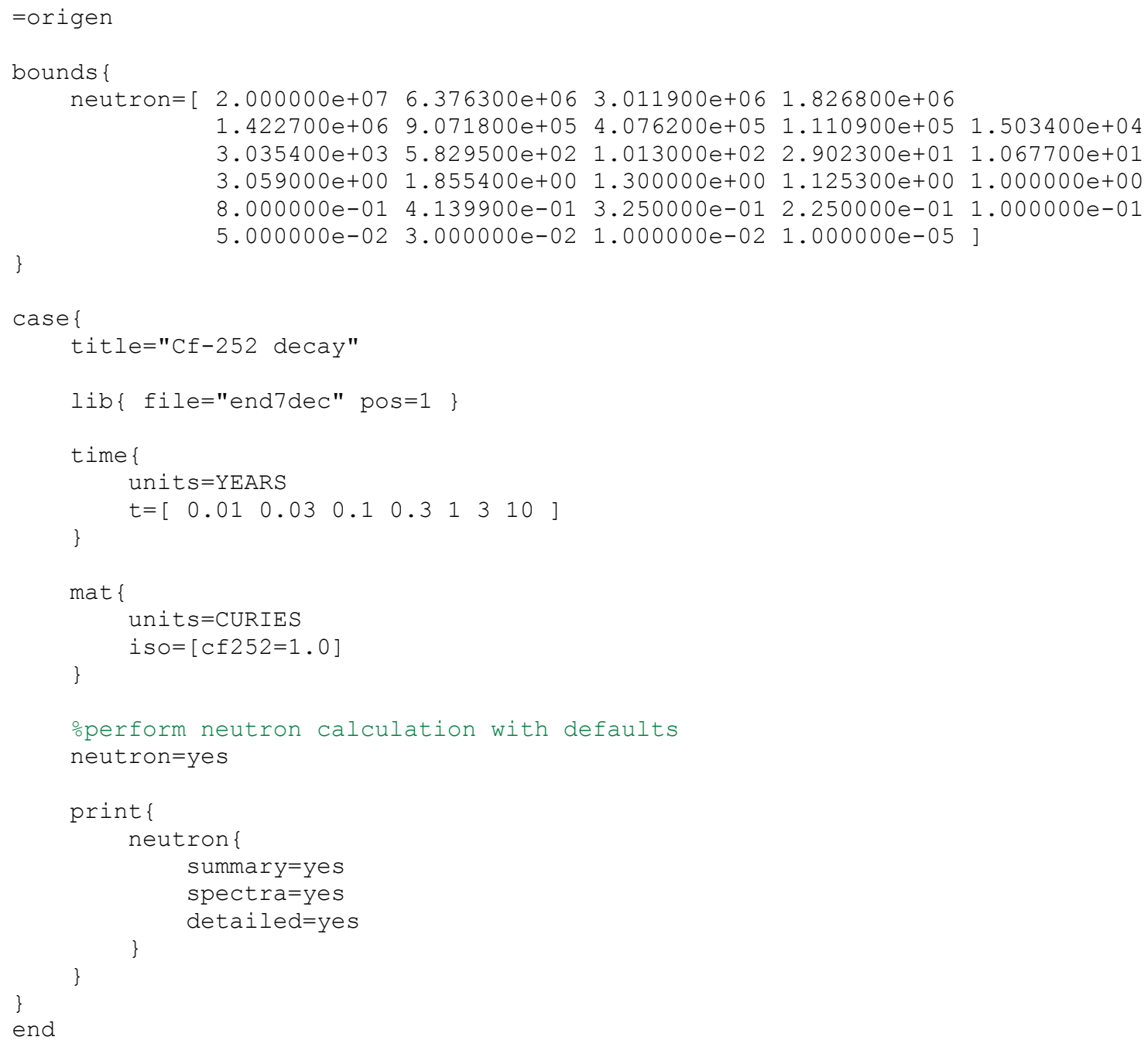

Figure 5.1.30. ${ }^{252} \mathrm{Cf}$ neutron emission spectrum.

This example illustrates decay of $1 \mathrm{Ci}$ of ${ }^{252} \mathrm{Cf}$ for ten years with calculation of the time- and energydependent neutron source. The case uses the binary decay library "end $7 \mathrm{dec}$." The neutron energy group structure is defined in the bounds block with array "neutron." 


\subsubsection{Simple Fuel Irradiation Plus Decay}

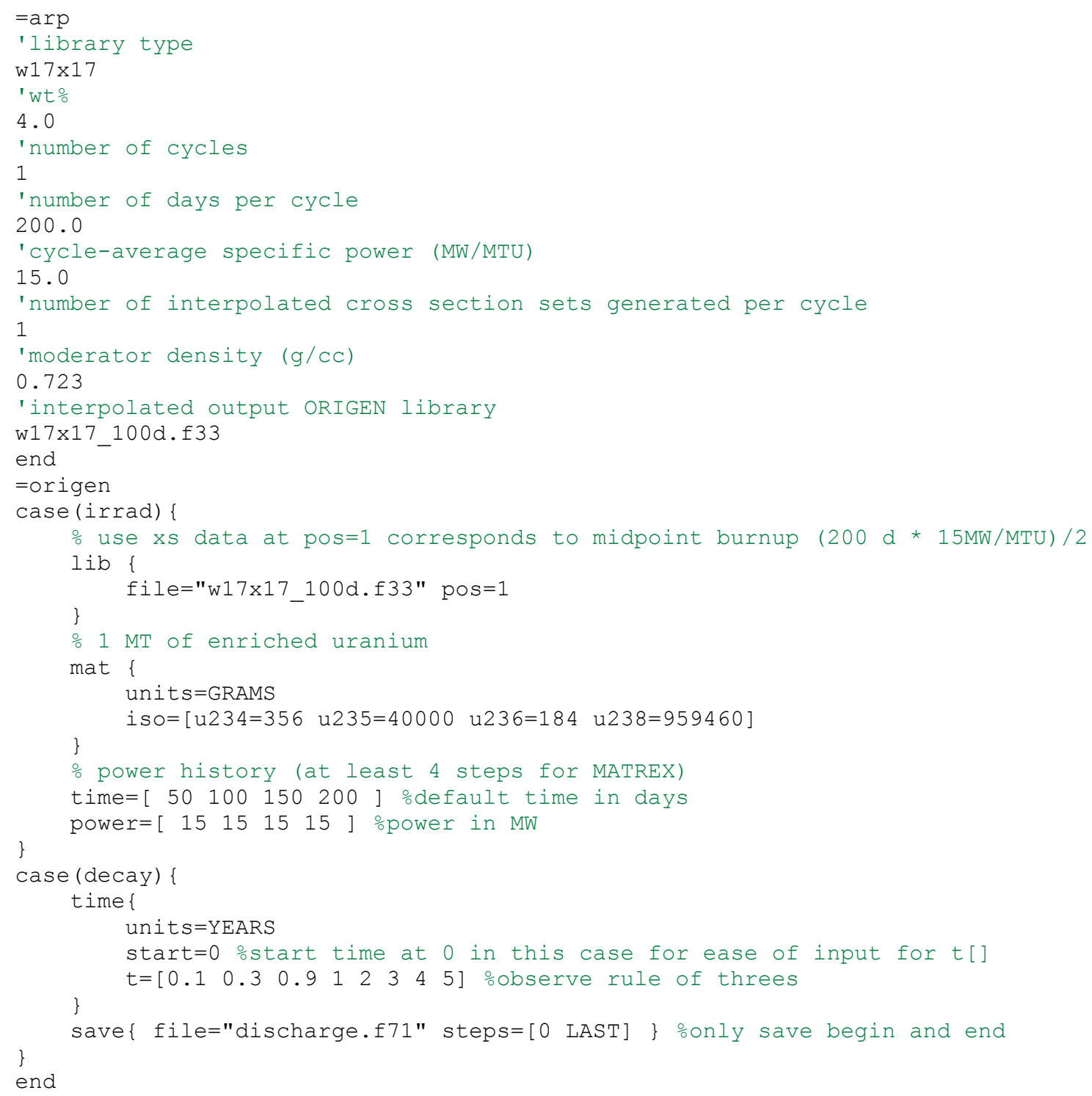

Figure 5.1.31. Simple fuel irradiation plus decay.

This example illustrates the input for irradiation of a fuel assembly for 200 days at $15 \mathrm{MW}$ in case "irrad," followed by decay for 5 years in the next case, "decay." The ORIGEN library (f33) was prepared by ARP to have a single position with a burnup of $1500 \mathrm{MWd} / \mathrm{MTU}$. The input material has been specified to have 1 MTU total with an enrichment of $4.0 \%$ in ${ }^{235} \mathrm{U}$. Note that there are consistency requirements between ARP and ORIGEN that cannot be checked by either module. Namely, the enrichment specified in ARP should be equivalent to the effective enrichment specified in the ORIGEN input, and the burnup-dependent cross section data on the ORIGEN library should be for the midpoint burnup of the case, which requires consistency between the operating history (power and time) and the initial isotopics heavy metal loading. In typical fuel depletion calculations, it is most convenient to specify a metric ton $\left(10^{6}\right.$ grams $)$ of initial heavy metal, such that the power in MW may be interpreted as 
MW/MTIHM. In the above example, the power history does not need to be constant but when combined with the time values should produce an average burnup of $1500 \mathrm{MWd} / \mathrm{MTU}$ in order that the cross sections interpolated by ARP in position 1 are valid.

It is important to note that with the MATREX solver, used by default, the recommendation for irradiation and decay of spent fuel is to use no fewer than four steps for the irradiation and begin the decay period with a time step on the order of weeks or a month, increasing the interval for each subsequent step by no more than a factor of three. In many continuation cases, such as the decay case described here, it is convenient to specify times starting from zero for the case with "start $=0$ " in the time block.

\subsubsection{Three Cycles of Irradiation Plus Decay}

This example is similar to the previous one, except there are three sets of burnup-dependent transition cross sections (positions) generated by ARP and used in ORIGEN, and there are three cases, one corresponding to each cycle. Neutron and gamma sources are generated and saved to the $\mathrm{f} 71$ file for the final decay case. The maximum burnup achieved is $60 \mathrm{GWd} / \mathrm{MTIHM}$. 


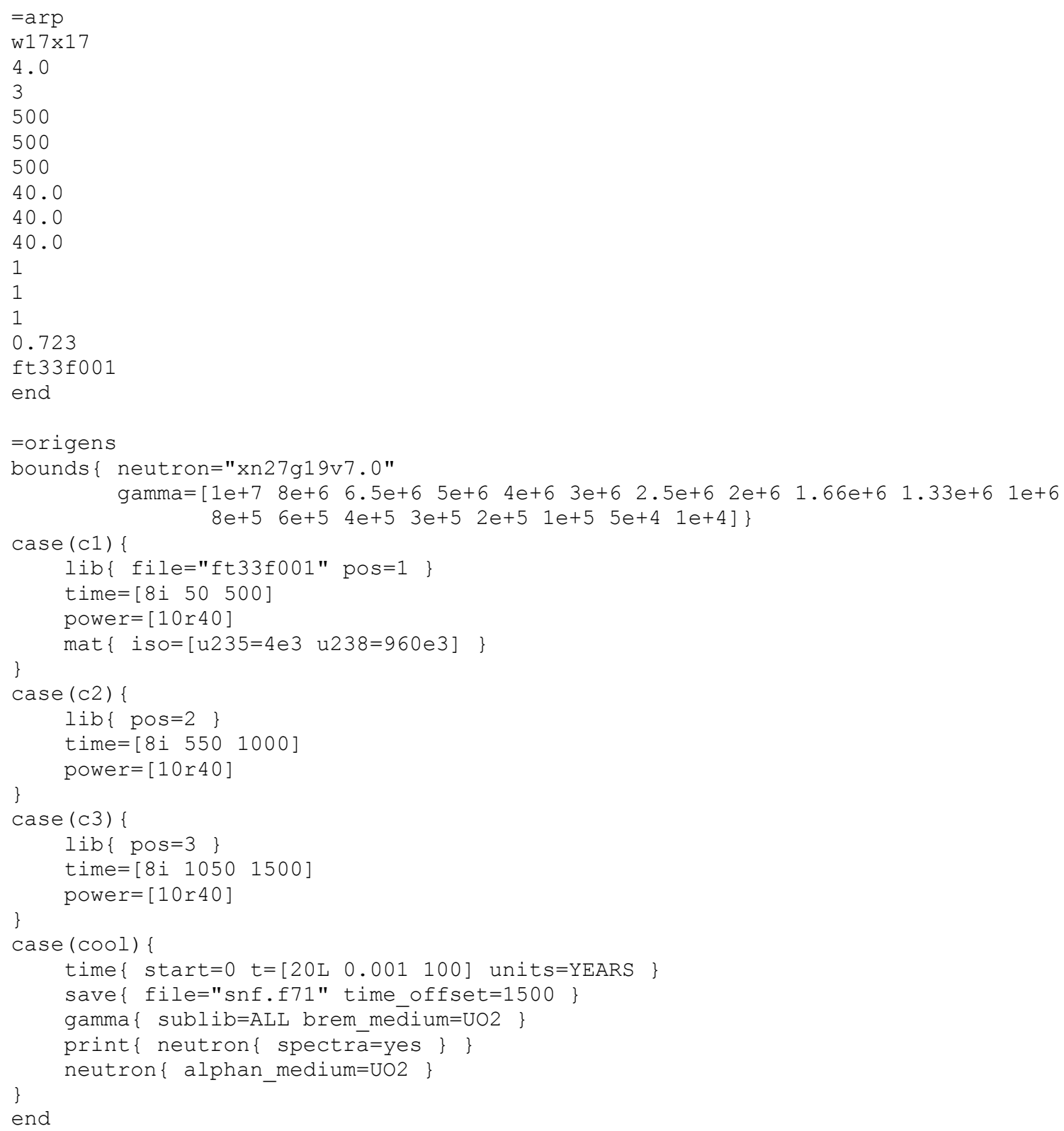

Figure 5.1.32. Three cycles of irrradiation plus decay.

\subsubsection{Load Isotopics from an f71 File}

The "mat" block allows isotopics to be loaded from any position on an existing f71 file. A table of contents is printed in the output file every time an $\mathrm{f} 71$ is read or written. Inspection of this table can help identify the appropriate position on the $\mathrm{f71}$, as shown in the table below, extracted from the output file. 


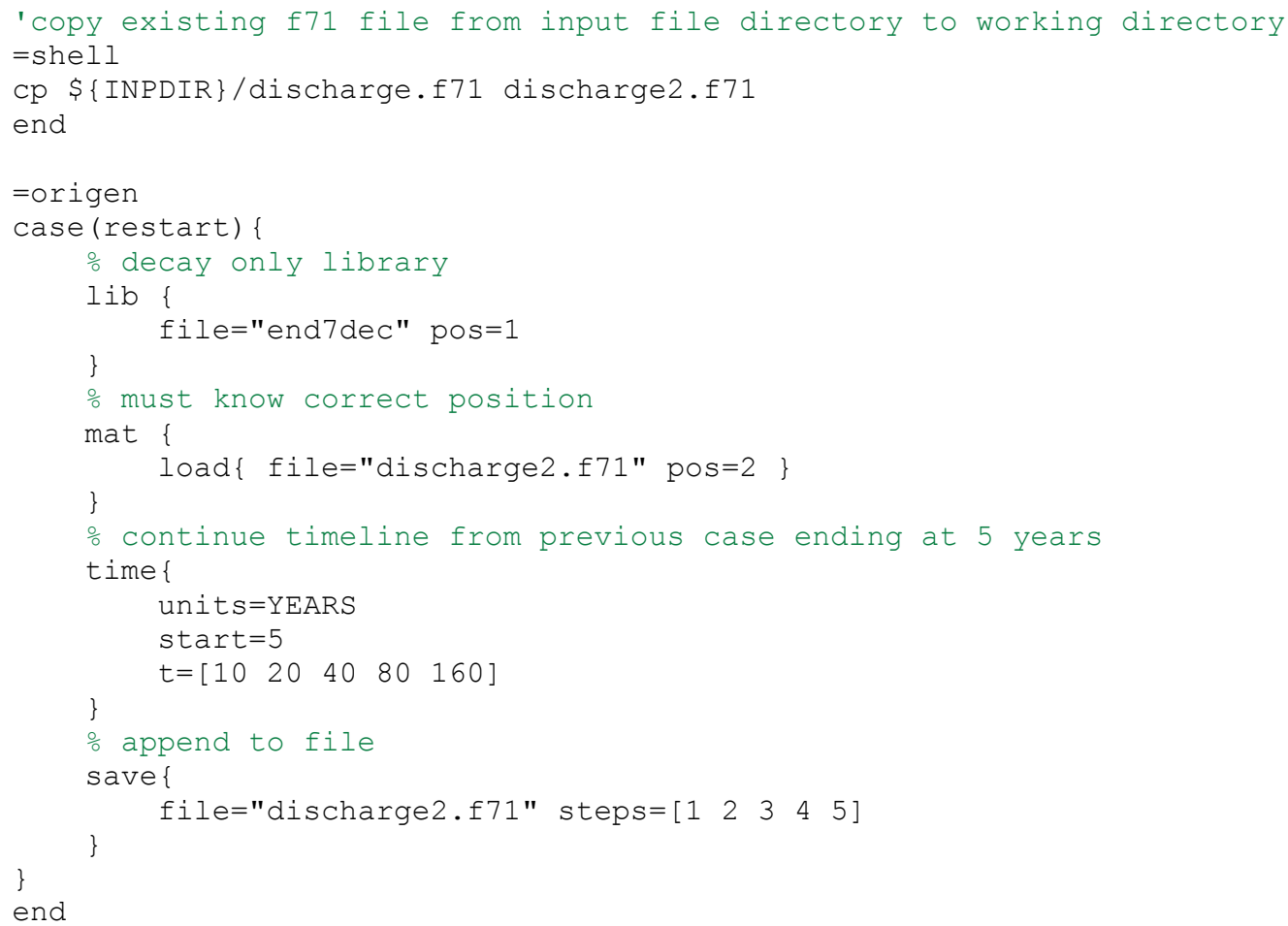

Figure 5.1.33. Load isotopics from an f71 file using the "mat" block.

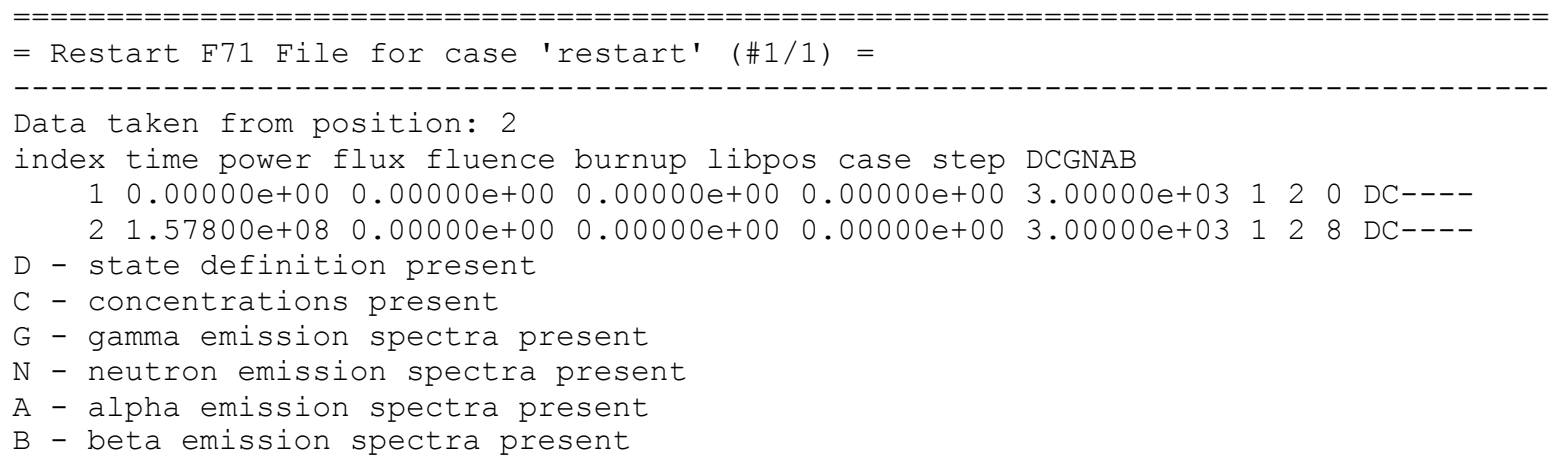

Figure 5.1.34. Table of contents printed when accessing the f71 file.

The simple input below can be used to print contents of an existing f71 file. The file is renamed so that it does not have extension ".f71." This prevents the automatic copy back from the working directory to the input file directory. Note that the same is true with any ORIGEN libraries that have the extension ".f33." There is a rule in place that any file in the working directory with extension ".f71" or ".f33" is copied back to the input file's directory. To prevent this from occurring, the ".f71" or ".f33" extensions must not be used in the filename, or unneeded files with a shell command at the end must be explicitly deleted. 


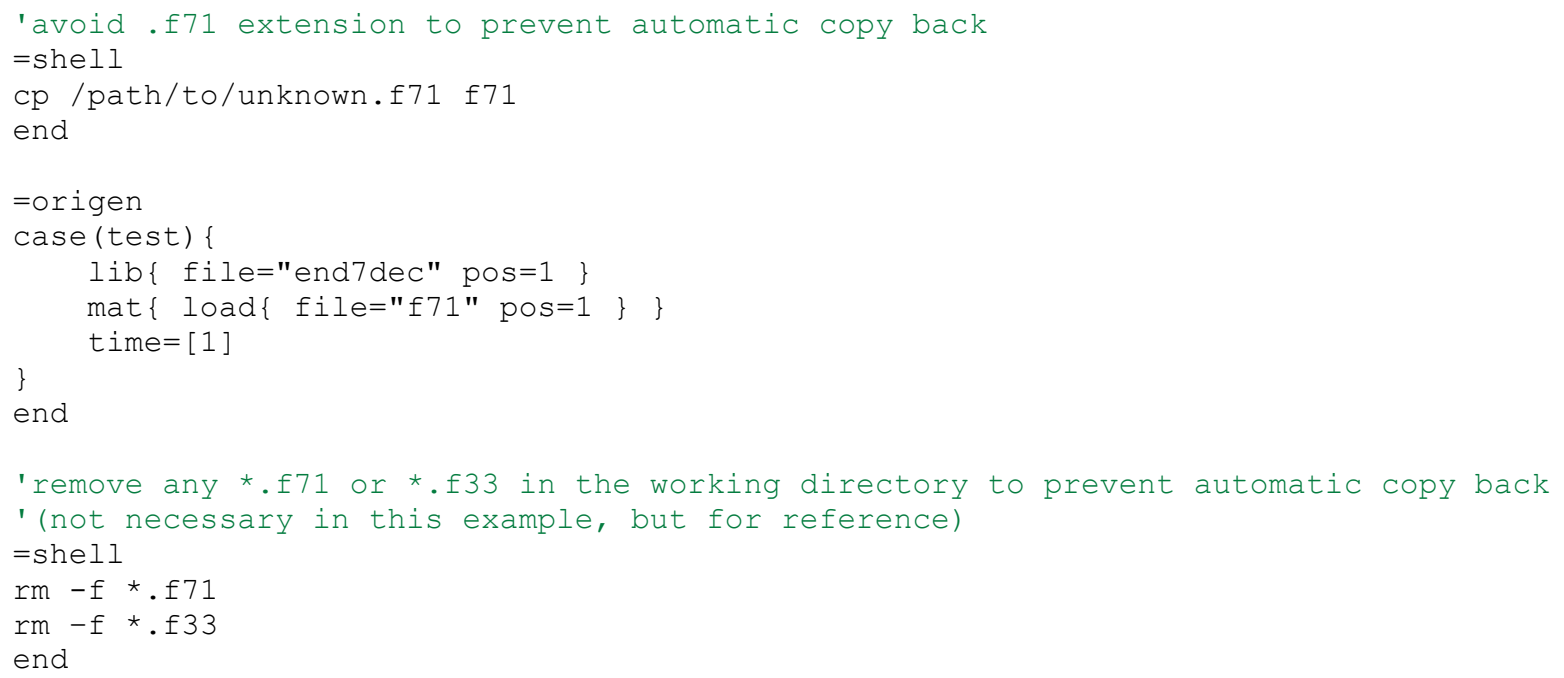

Figure 5.1.35. Isotopics from an f71 or f33 file.

\subsubsection{Continuous Feed and Removal}

Both continuous feeding of nuclides into a system and chemical removal of elements from a system are required to simulate molten fuel systems such as the molten salt reactor (MSR). Simulation of removal can be used to represent other physical processes such as purification systems (i.e., removal of chemical species by filtration or ion-exchange columns) and ventilated systems in which the removal can be represented using a rate constant $(1 / \mathrm{s})$. The following example applies to simulation of a molten salt reactor system, and it uses both continuous feed of ${ }^{232} \mathrm{Th}$ and simultaneous removal of fission products according to their removal process. To actually simulate this system properly, an ORIGEN reactor library appropriate for the MSR must be generated.

There are 11 groups of nuclides in this example, each with the same removal constant. The groups, by element and removal rate in units of $(1 / \mathrm{s})$, are

- Group 1: Ca to As $\left(3.37 \times 10^{-9}\right)$;

- Group 2: Y, La, Ce, Pr, Nd, Pm, Sm, Gd $\left(2.31 \times 10^{-7}\right)$;

- Group 3: Eu $\left(2.31 \times 10^{-8}\right)$;

- Group 4: Se, Nb, Mo, Tc, Ru, Rh, Pd, Ag, Sb, Te $\left(5 \times 10^{-2}\right)$;

- Group 5: Zr, Cd, In, Sn $\left(5.79 \times 10^{-8}\right)$;

- Group 6: Kr, Xe $\left(5 \times 10^{-2}\right)$;

- Group 7: Br, I $\left(1.93 \times 10^{-7}\right)$;

- Group 8: Rb, Sr, Cs, Ba $\left(3.37 \times 10^{-9}\right)$;

- Group 9: Th, Li, Be, F $\left(3.37 \times 10^{-9}\right)$;

- Group 10: $\mathrm{Pa}\left(3.86 \times 10^{-6}\right)$; and

- Group 11: Np, Pu, Am, Cm, Bk, Cf $\left(1.98 \times 10^{-9}\right)$.

$\mathrm{A}^{232} \mathrm{Th}$ feed rate of $2.0 \times 10^{-2}$ grams/s is used. 


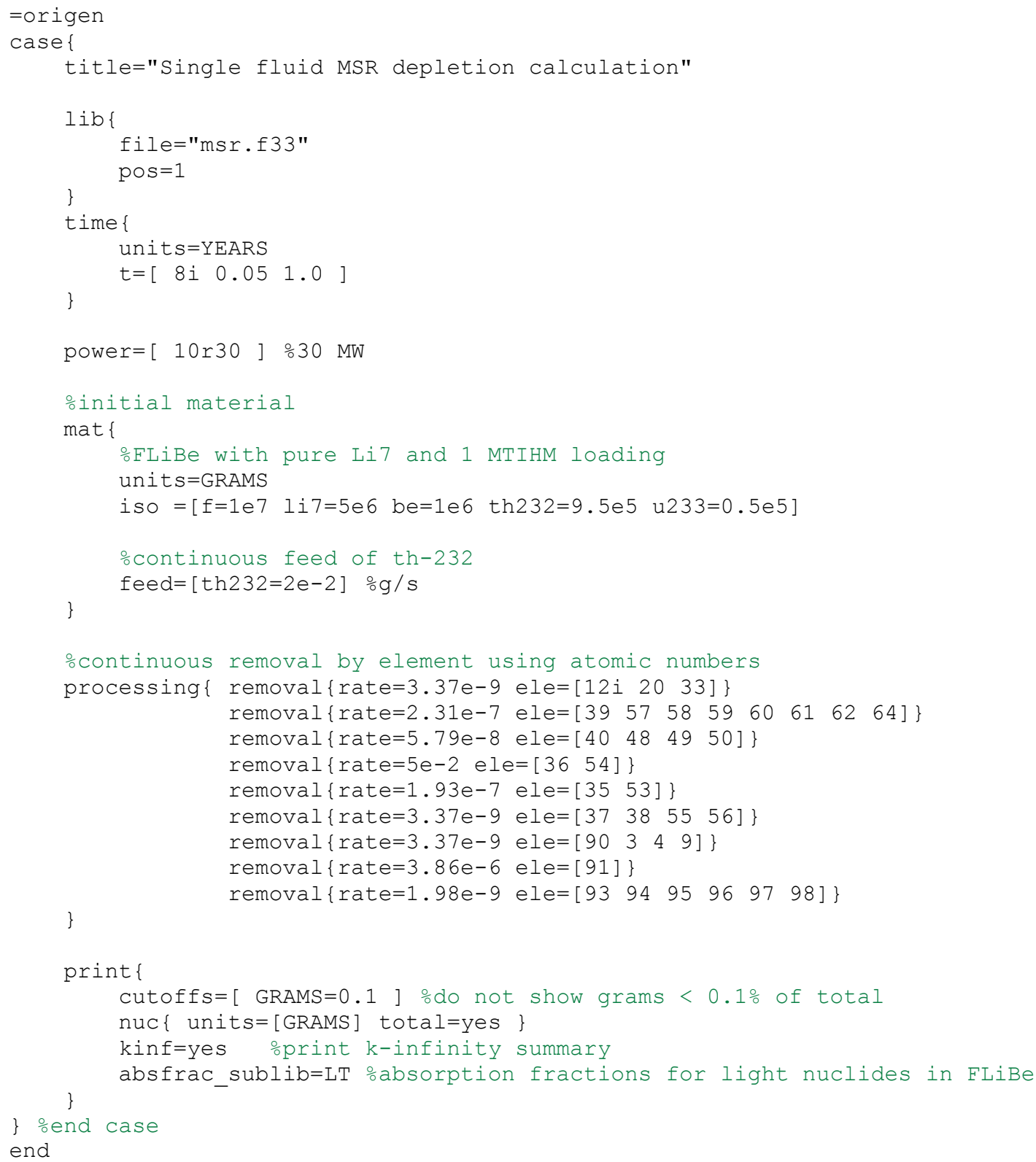

Figure 5.1.36. Continuous feed and removal.

The absorption rates, fission rates, and k-infinity values are printed during irradiation, which can be used to evaluate the influence of the feed rates and removal constants on the time-dependent reactor performance. For the MSR in particular, the ability to self-sustain can be assessed from the "k-infinity" summary output, enabled by "kinf=yes" in print block (as well as the absorption fractions in the light nuclides in FLiBe), and enabled by "absfrac_sublib=LT" in the print block. This example uses pure Li-7, whereas if natural Li is used, one will see a much larger fraction of absorptions in Li-6 and much lower "k-infinity." 


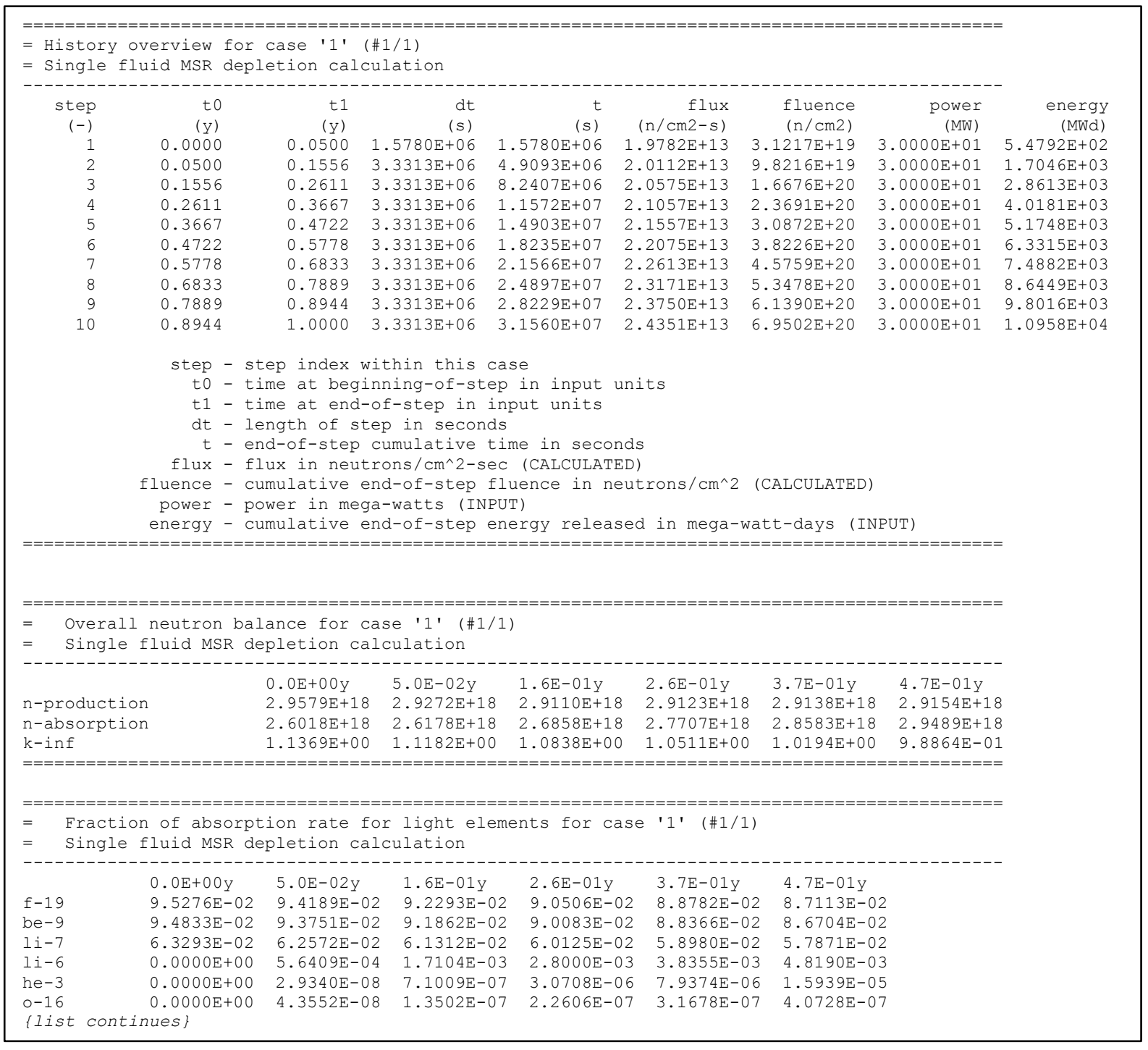

Figure 5.1.37. Continuous feed and removal - history overview.

\subsubsection{Calculate Fuel $(\alpha, N)$ Emissions in a Glass Matrix}

Batch processing options are provided to separate various components of the nuclide compositions into different streams and to recombine the streams to form new compositions. This example applies to the irradiation of typical commercial fuel and subsequent storage of separated fission products and actinides from fuel in a glass matrix. The matrix is important in determining the $(\alpha, n)$ component of the neutron source because the alpha particles interact with the light element constituents in the matrix, with $(\alpha, n)$ yields corresponding to the medium containing the $\alpha$-emitting nuclides. Therefore, an accurate calculation of the neutron source in a glass matrix requires combining the oxide fuel compositions after irradiation with the defined glass matrix. The calculation could be performed by creating a case by manually entering the calculated nuclide activities and the matrix composition. However, this is only practical if the number of source nuclides is small. This example applies batch processing and blending options to generate the required compositions. 
The blending option is used to combine two streams: one stream from irradiated fuel, and the other stream defining the glass matrix composition.

An irradiation case - "irrad"-is performed first to generate spent nuclear fuel compositions. The next case "spent" decays the results for one year. At the start of the decay, only selected elements are retained in the stream by performing processing with the "retained" array. In this example, all elements are removed except for Se (99.8\%); Rb, Sr, Te, Cs, Ba, Dy (77.8\%); and U, Np, Pu, Am, Cm (1\%).

The glass matrix compositions are then defined in the third case, "glass."

The final case - "blend"-blends $10 \%$ of each the last step's isotopics from the "spent" and "glass" cases. To test the dependence on the time when the blend is performed, the blend can be changed to "blend=[ spent (N)=0.1 glass=0.1 ]," where $N$ is the index of the step from which to take isotopics from the spent case. 


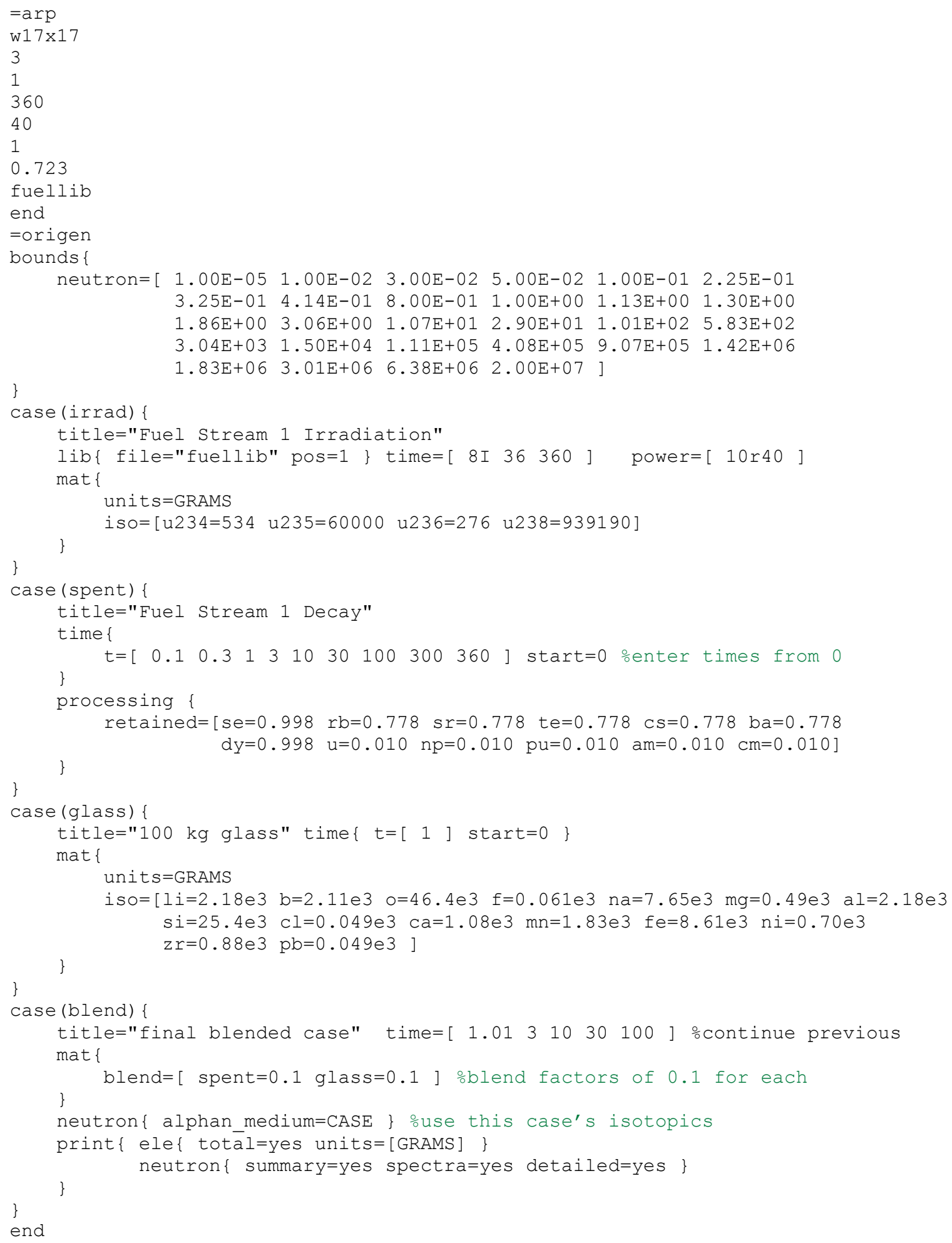

Figure 5.1.38. Continuous feed and removal - blending option. 


\subsubsection{Create an ORIGEN Decay Library from a Decay Resource}

The following COUPLE input is all that is required to produce a binary decay library from the ORIGEN decay resource file in the SCALE data directory, which is linked by default to unit number 27 . This means that to create a new decay library based on another decay resource, it could be copied to the working directory as "ft27f001." COUPLE, when looking for unit 27, will first look for file "ft27f001." If it is not found, then COUPLE will it look to the special file "origen_filenames" in the SCALE data directory and find that unit 27 is associated with "decay," shorthand for the decay resource.

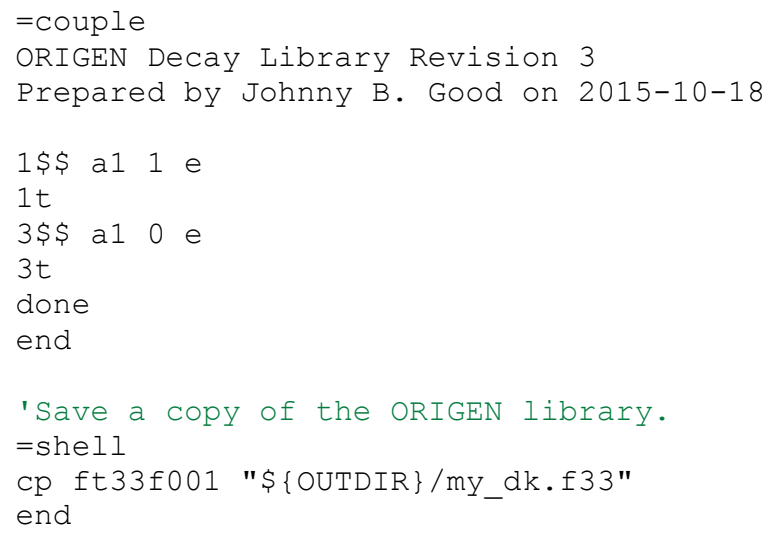

Figure 5.1.39. ORIGEN decay library from decay resource.

\subsubsection{Create an ORIGEN Reaction Library}

The following input example creates an ORIGEN reaction library from an AMPX library created using the SCALE csas1x sequence. After COUPLE completes, the ORIGEN library created (on default unit 33 or file "ft33f001") is copied to the input directory. There are two very important factors to verify when using COUPLE in this capacity.

1. The " $0 \$ \$$ a5 4" and " $1 \$ \$$ a13 6" entries are very important, as they dictate that the cross sections will be read from "ft04f001" for AMPX library zone 6, which in this case is mixture ID "6" (related input highlighted in red). There are some AMPX libraries which do not contain zones, e.g. " $\mathrm{ft} 03 \mathrm{f} 001$ " produced by some sequences, representing the system-average cross sections. In this case the default should be used for " $1 \$ \$$ a13" of " 0 " indicating that a specific zone ID is not desired. By accessing different zones with "1\$\$ a13", an ORIGEN library can be created for clad activation or boron depletion in water by choosing a different mixture.

2. The group structure of the reaction resource (" $0 \$ \$$ a3 75 ") should be the same as the AMPX library produced (for example, "v7-56" in the csas1x input [related input highlighted in blue]). This requires inspection of the "origen_filenames" file to verify that unit 75 is associated with a 56-group library. 


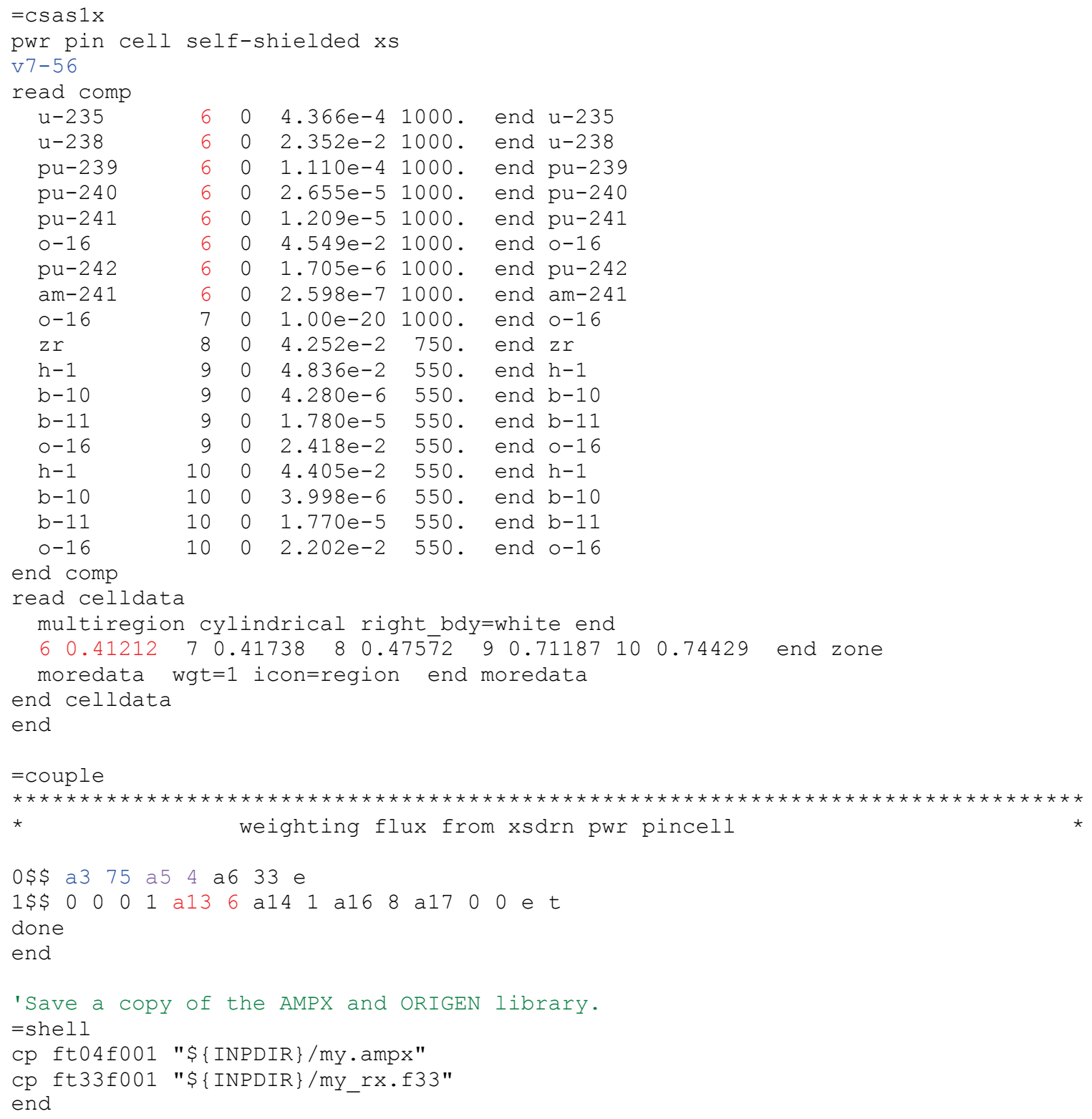

Figure 5.1.40. ORIGEN reaction library.

\subsubsection{Create an ORIGEN Activation Library}

Activation calculations typically do not require self-shielding, so an ORIGEN "activation" library is a means to refer to a library that is using infinitely dilute cross sections from the reaction resource. An ORIGEN activation library can be created very easily as long as the flux spectrum is available in one of the reaction resource group structures. In the example below, a 238-group structure is used with reaction resource on unit 18 ( $0 \$ \$$ a3 80). Additionally, this case requests explicit fission yields only for ${ }^{235} \mathrm{U}$, ${ }^{238} \mathrm{U},{ }^{239} \mathrm{Pu}$, and ${ }^{241} \mathrm{Pu}$ in the $7 \$ \$$ array. Other nuclides with fission cross sections will not generate fission products. In a library meant only for activation of standard structural materials, the library could be made much smaller by not including any fission yields without any impact on the calculation as long as it did 
not include fissionable isotopes. Since the reaction resource contains 238 neutron groups, the userdefined weighting flux given in the $9 * *$ array has 238 entries (in order of descending energy).

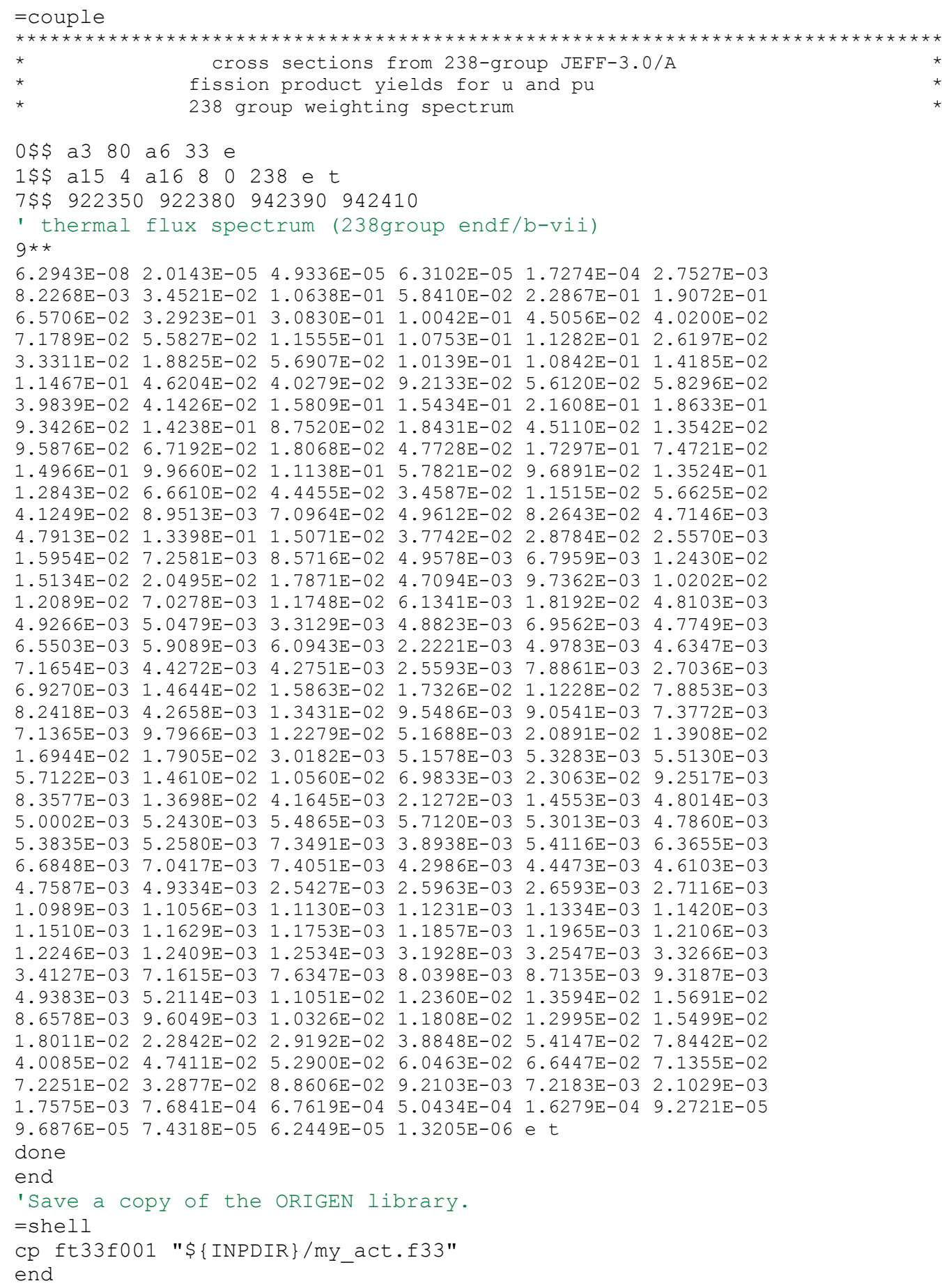

Figure 5.1.41. ORIGEN activation library.

The following excerpts from the output file show average energy of the neutrons causing fission that are used for the fission yield interpolation, as well as a partial listing of the one-group cross sections computed from the weighting spectrum and folded into the ORIGEN binary library. In the one-group 
cross section list, the units are barns and the lines labeled "tot-cap" correspond to the total removal cross section.

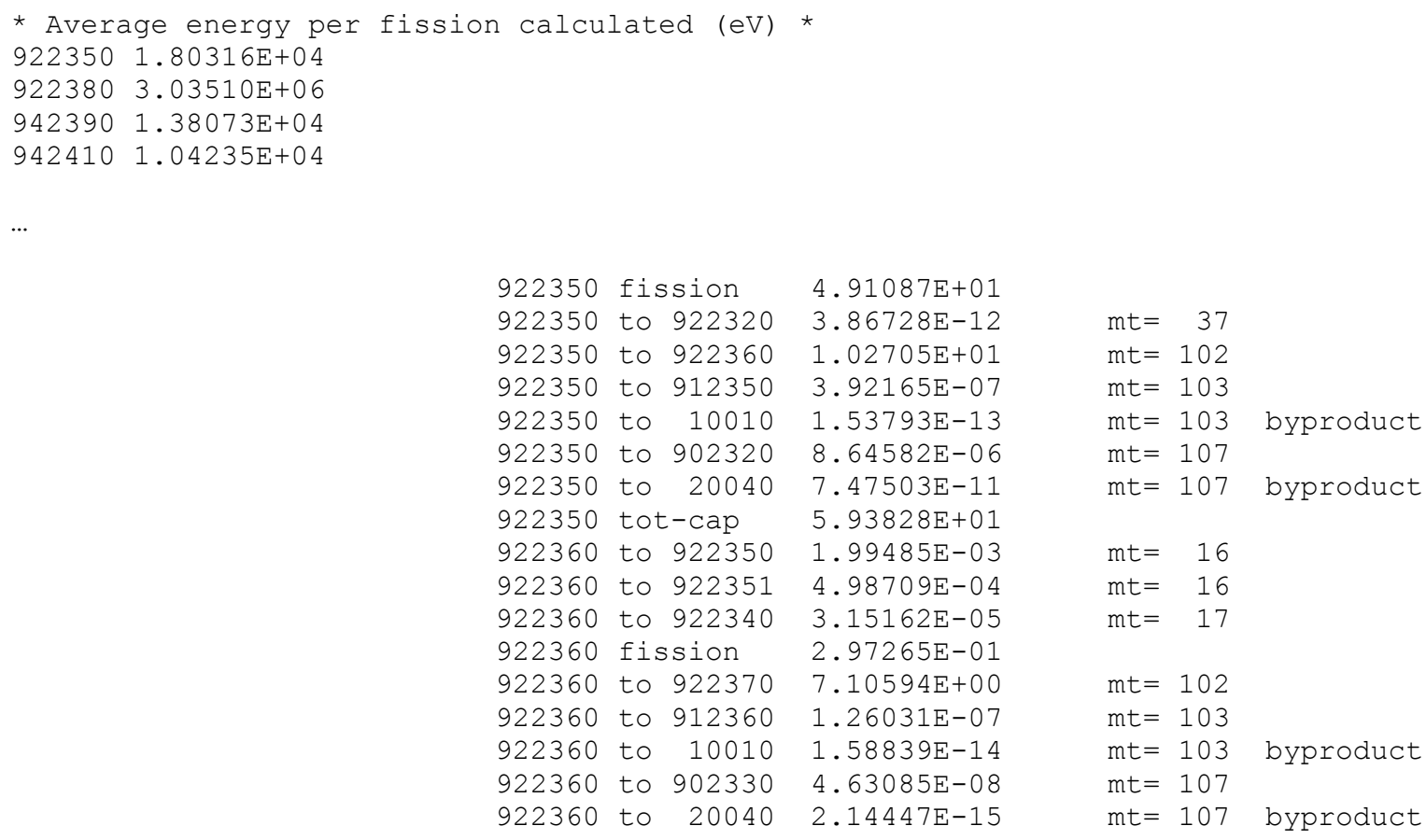

Figure 5.1.42. ORIGEN activation library - average energy per fission calculated.

\subsubsection{Create an ORIGEN Library with User-Supplied Cross Sections}

It is possible to set the fission and removal cross sections directly for a nuclide and for individual reaction transitions from nuclide to nuclide if the daughter nuclide is known. The following example is the same as the previous but with additional inputs for " $1 \$ \$$ a3 1, , $15 \$ \$, 71 \$ \$, 72 \$ \$$, and $73 * *$ arrays (highlighted in red). 


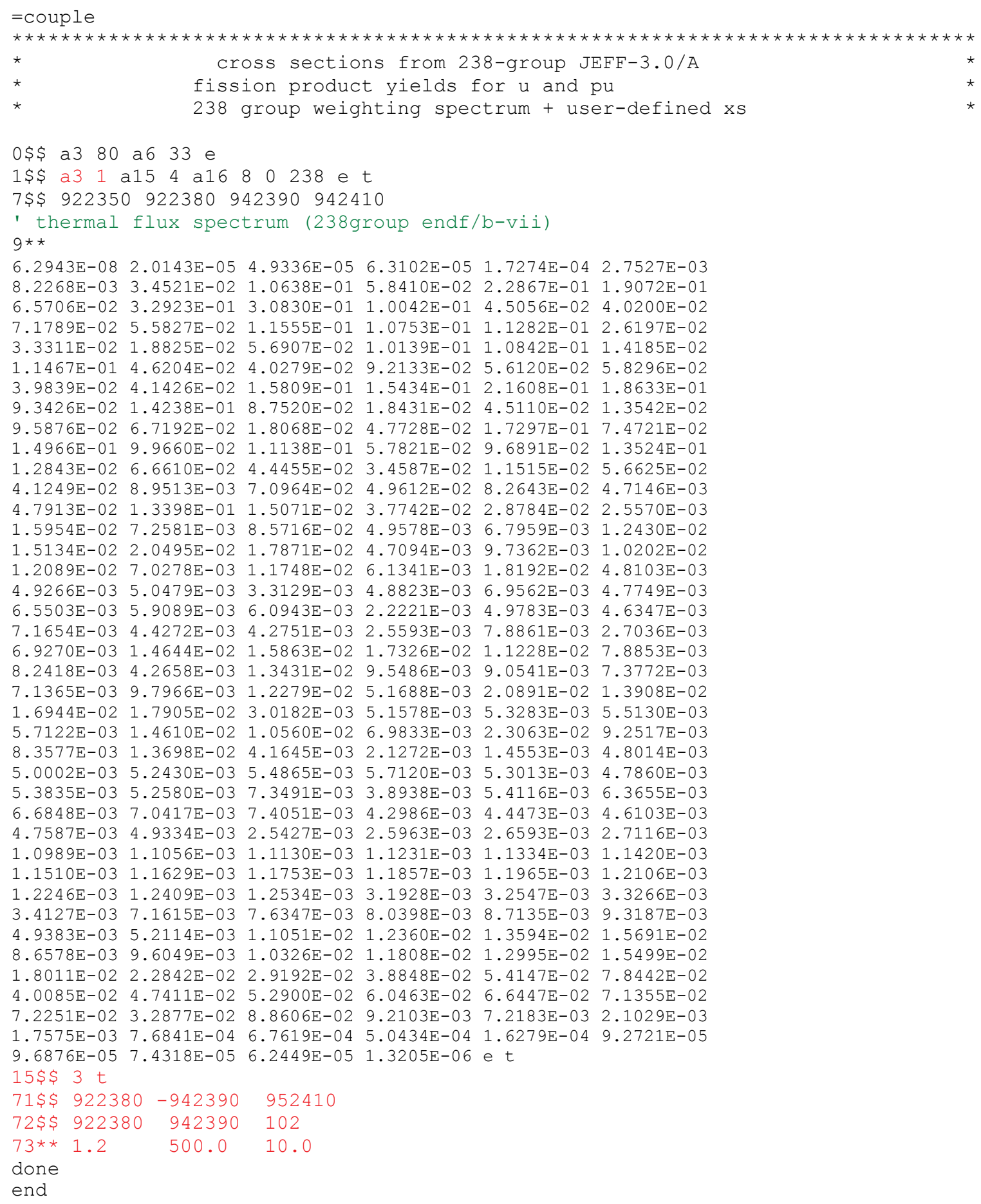

Figure 5.1.43. ORIGEN Library with user-supplied cross sections.

\subsubsection{Print the Cross Section Values on an ORIGEN Library in COUPLE}

Cross sections can be printed when a library is generated in COUPLE by setting the $1 \$$ array entry 14 , $\mathrm{IEDOU}=1$. 


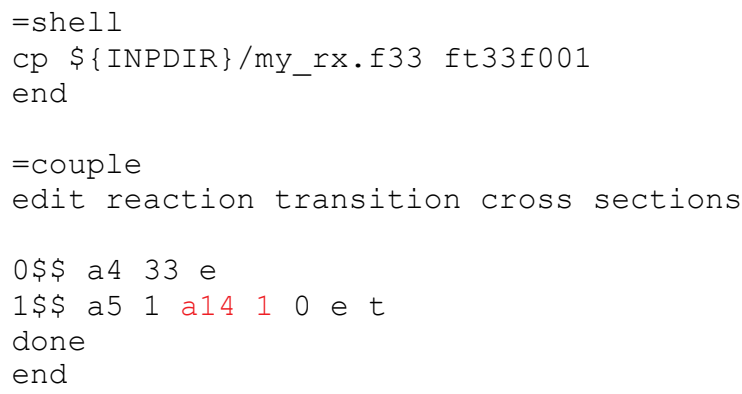

Figure 5.1.44. Cross section values on an ORIGEN library in COUPLE.

\subsubsection{Print the Cross Section Values on an ORIGEN Library in ORIGEN}

Cross sections can also be printed when the library is used in ORIGEN by setting "print_xs=yes" in the options block.

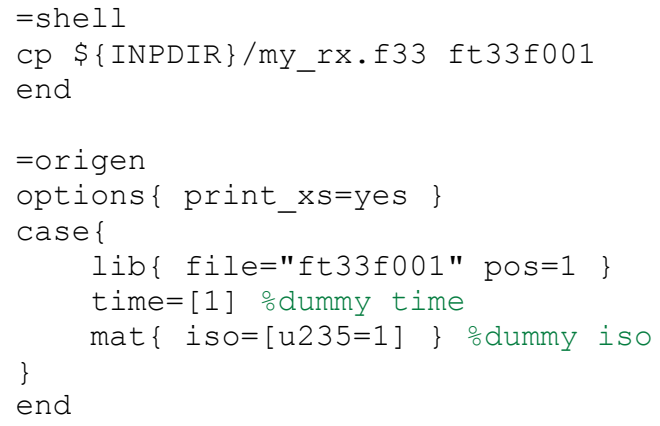

Figure 5.1.45. Cross Section values on an ORIGEN library in ORIGEN.

\subsubsection{Ranking Contribution to Toxicity}

The following OPUS input creates a plot of the volume of various nuclides with the maximum permissible concentration (MPC) in water using the "snf.f71" produced by the "Three cycle plus decay" example (5.1.4.4). Only positions 6-16 are plotted using "minposition" and "maxposition." Three nuclides $\left({ }^{242} \mathrm{Cm},{ }^{137 \mathrm{~m}} \mathrm{Ba}\right.$, and $\left.{ }^{99} \mathrm{Tc}\right)$ are forced to be included via the "symnuc" list, with 17 more nuclides (for total "nrank=20") included according to their average rank in terms of the MPC in water. The total number of nuclides requested is 20. The x-axis label is set to "TIME (YEARS)," and the title is "SPENT FUEL AT 60 GWD/MTHM." 


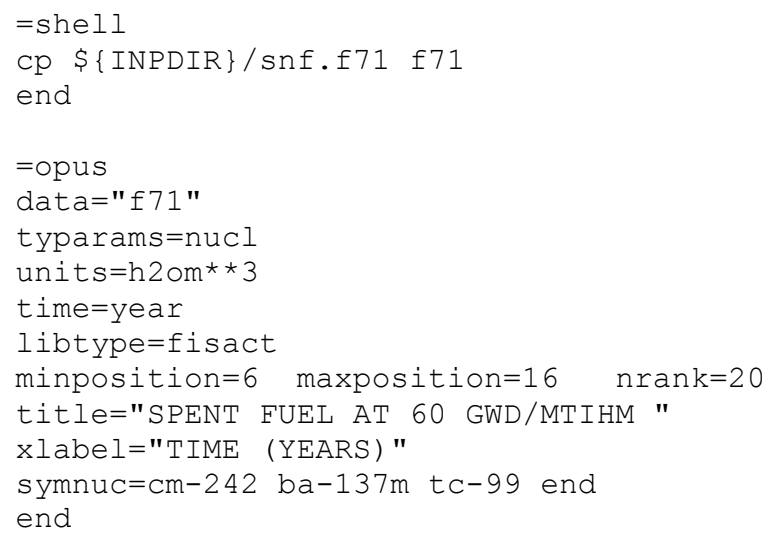

Figure 5.1.46. Ranking contribution to toxicity.

\subsubsection{Photon Spectrum Plot}

The following OPUS input creates a plot of the photon spectrum for all times between 1 and 5 years, using "tmin=1" and "tmax=5" with "time=years."

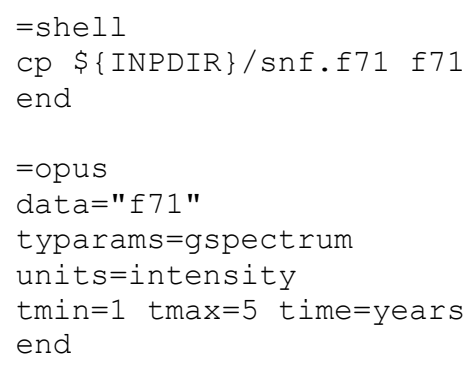

Figure 5.1.47. Photon spectrum plot.

The following shows the output of the time-dependent gamma spectrum extracted from the f71 file by OPUS. 


\begin{tabular}{|c|c|c|c|c|c|}
\hline & $1 /(\mathrm{s} \cdot \mathrm{MeV})$ & | & $1.25 y$ & $2.15 y$ & $3.73 y$ \\
\hline $1.00 e+01$ & $--8.00 e+00$ & 1 & $2.7961 e+11$ & $3.0661 e+03$ & $3.0661 e+03$ \\
\hline $8.00 e+00$ & $--6.50 e+00$ & | & $7.1201 e+13$ & $1.9436 e+04$ & $1.9436 e+04$ \\
\hline $6.50 e+00$ & $--5.00 e+00$ & | & $8.9725 e+15$ & $2.5300 e+10$ & $5.3041 e+09$ \\
\hline $5.00 e+00$ & $--4.00 e+00$ & | & $3.7075 e+16$ & $9.1643 e+12$ & $1.9001 e+12$ \\
\hline $4.00 e+00$ & $--3.00 e+00$ & i & $1.1734 \mathrm{e}+17$ & $1.3562 e+14$ & $2.8824 e+13$ \\
\hline $3.00 e+00$ & $--2.50 e+00$ & | & $3.4321 e+17$ & $5.5945 e+15$ & $4.8216 e+15$ \\
\hline $2.50 e+00$ & $--2.00 e+00$ & 1 & $5.2847 e+17$ & $1.1338 e+16$ & $5.9433 e+15$ \\
\hline $2.00 e+00$ & $--1.66 e+00$ & | & $8.3528 e+17$ & $3.3015 e+16$ & $1.8382 e+16$ \\
\hline $1.66 \mathrm{e}+00$ & $--1.33 e+00$ & | & $1.8060 e+18$ & $2.7233 e+17$ & $2.4658 e+17$ \\
\hline $1.33 e+00$ & $--1.00 e+00$ & | & $2.4156 e+18$ & $1.4238 e+17$ & $9.2264 e+16$ \\
\hline $1.00 e+00$ & $--8.00 e-01$ & 1 & $5.0662 e+18$ & $3.2193 e+17$ & $2.6845 e+17$ \\
\hline $8.00 e-01$ & $--6.00 e-01$ & | & $6.4083 e+18$ & $2.1010 e+18$ & $1.8903 e+18$ \\
\hline $6.00 e-01$ & $--4.00 e-01$ & | & $7.1803 e+18$ & $1.1822 \mathrm{e}+18$ & $1.0301 e+18$ \\
\hline $4.00 e-01$ & $--3.00 e-01$ & | & $8.7297 e+18$ & $1.7856 e+18$ & $1.6414 e+18$ \\
\hline $3.00 e-01$ & $--2.00 e-01$ & | & $1.9481 e+19$ & $1.0658 e+19$ & $9.7018 e+18$ \\
\hline $2.00 e-01$ & $--1.00 e-01$ & | & $2.9105 e+19$ & $1.5198 e+19$ & $1.3996 e+19$ \\
\hline $1.00 e-01$ & $--5.00 e-02$ & | & $6.2160 e+19$ & $9.1756 e+18$ & $8.3611 e+18$ \\
\hline $5.00 e-02$ & $--1.00 e-02$ & i & $1.1219 e+20$ & $3.4628 e+19$ & $3.1458 e+19$ \\
\hline
\end{tabular}

Figure 5.1.48. Photon spectrum plot - output of the time-dependent gamma spectrum.

\subsubsection{Neutron Spectrum Plot}

The following OPUS input creates a plot of the total neutron spectrum at all times.

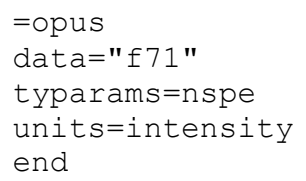

Figure 5.1.49. Neutron spectrum plot.

\subsubsection{Isotopic Weight Percentages for Uranium and Plutonium During Decay}

The isotopic distributions in uranium and plutonium, in weight percent, may be plotted with the following OPUS input.

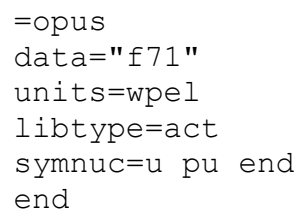

Figure 5.1.50. Isotopic weight percentages for uranium and plutonium during decay.

\subsubsection{User-Specified Response Function in OPUS}

The response conversion factor data are entered in the RESPONSE = array as nuclide-response factor pairs, with the nuclide id being the legacy nuclide ID (ZZAAAI), with ZZ two digits of atomic number, 
AAA three digits of mass number, and I one digit of isomeric state. For example, ${ }^{235 \mathrm{~m}} \mathrm{U}$ would be given as 922351 and H-1 as 10010. In this example, it is assumed that the response factors are activity based (response/Bq of nuclide), so the units of Becquerels are requested using the UNITS keyword. The usersupplied response conversion factors are applied to the Becquerel units for all nuclides in the RESPONSE = array, and the results for any nuclide for which no response factors are provided are zeroed.

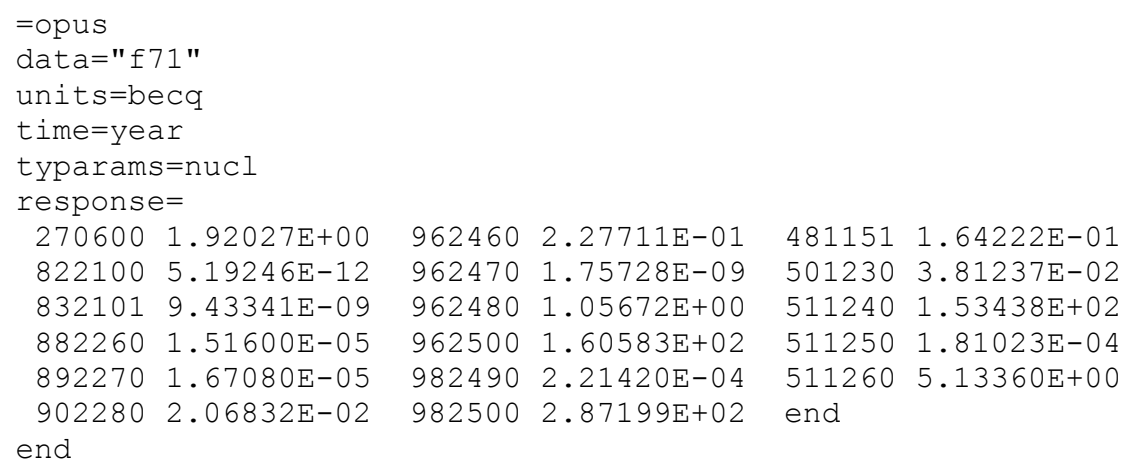

Figure 5.1.51. User-specified response function in OPUS.

\subsubsection{References}

1. M. J. Bell, ORIGEN B-The ORNL Isotope Generation and Depletion Code, ORNL-4628 (CCC-217), Union Carbide Corporation (Nuclear Division), Oak Ridge National Laboratory, May 1973.

2. H. Bateman, "The Solution of Differential Equations Occurring in the Theory of Radioactive Transformations," Proc. Cambridge Phil. Soc. 15, 423 (1910).

3. S. J. Ball and R. K. Adams, MATEXP: A General Purpose Digital Computer Program for Solving Ordinary Differential Equations by the Matrix Exponential Method, ORNL/TM-1933, Union Carbide Corporation (Nuclear Division), Oak Ridge National Laboratory, August 1967.

4. L. Lapidus and R. Luus, Optimal Control of Engineering Processes, Blaisdell Publishing Co., Waltham, Mass., 1967, pp. 45-49.

5. D. R. Vondy, Development of a General Method of Explicit Solution to the Nuclide Chain Equations for Digital Machine Calculations, ORNL/TM-361, Union Carbide Corporation (Nuclear Division), Oak Ridge National Laboratory, October 1962.

6. M. Pusa, J. Leppänen, "Computing the Matrix Exponential in Burnup Calculations," Nucl. Sci. Eng. 164, 140 (2010).

7. A. E. Isotalo, P. A. Aarnio, "Comparison of depletion algorithms for large systems of nuclides," Ann. Nucl. Energy. 38, 261 (2011).

8. M. Pusa, "Rational Approximations to the Matrix Exponential in Burnup Calculations," Nucl. Sci. Eng. 169, 155 (2011).

9. M. Pusa, Numerical Methods for Nuclear Fuel Burnup Calculations. Doctoral Dissertation, Aalto University (2013). 
10. E. F. Shores, Data Updates for the SOURCES-4A Computer Code, Los Alamos National Laboratory, LA-UR-00-5016, October 2000. SOURCES-4B available from the Radiation Safety Information Computational Center (RSICC) as code package CCC.

11. W. B. Wilson, R. T. Perry, W. S. Charlton, T. A. Parish, G. P. Estes, T. H. Brown, E. D. Arthur, M. Bozoian, T. R. England, D. G. Madland, and J. E. Stewart, SOURCES 4A: Code for Calculating $(\alpha, n)$, Spontaneous Fission, and Delayed Neutron Sources and Spectra, LA-13639-MS, Los Alamos National Laboratory, September 1999.

12. R. E. Funderlic, Ed., The Programmer's Handbook-A Compendium of Numerical Analysis Utility Programs, AEC Research and Development Report, K-1729, Oak Ridge National Laboratory, February 9, 1968.

13. D. J. Pellarin, W. L. Matney, and N. E. Bibler, "( $\alpha, n)$ Neutron Emission from DWPF Glass," George Wolberg, Itzik Alfy, "An energy-minimization framework for monotonic cubic spline interpolation," Journal of Computational and Applied Mathematics, Volume 143, Issue 2, 15 June 2002, pp. 145-188.

14. L. C. Leal, O. W. Hermann, S. M. Bowman, and C. V. Parks, ARP: Automatic Rapid Process for the Generation of Problem-Dependent SAS2H/ORIGEN-S Cross-Section Libraries, ORNL/TM-13584, Lockheed Martin Energy Research Corporation, Oak Ridge National Laboratory, April 1998.

15. I. C. Gauld, MOX Cross-Section Libraries for ORIGEN-ARP, ORNL/TM-2003/2, UT-Battelle, LLC, Oak Ridge National Laboratory, July 2003.

16. SCALE: A Comprehensive Modeling and Simulation Suite for Nuclear Safety Analysis and Design," ORNL/TM-2005/39, Version 6.1, Oak Ridge National Laboratory, Oak Ridge, Tennessee (June 2011). 


\title{
5.2 ORIGEN DATA RESOURCES
}

\author{
I. C. Gauld, D. Wiarda, M. Pigni, W. Wieslequist
}

\begin{abstract}
ORIGEN data resources include nuclear decay data, multigroup neutron reaction cross sections, neutroninduced fission product yields, and decay emission data for photons, neutrons, alpha particles and beta particles. The nuclear decay data are based primarily on ENDF/B-VII.1 evaluations. The multigroup nuclear reaction cross section libraries now include evaluations from the JEFF-3.0/A neutron activation file containing data for 774 target nuclides, more than 12,000 neutron-induced reactions, and more than 20 different reaction types below $20 \mathrm{MeV}$, provided in various energy group structures. Energydependent ENDF/B-VII.0-based fission product yields are available for 30 fissionable actinides. Gammaray and x-ray emission data libraries are based on ENDF/B-VII.1. The photon libraries contain discrete photon line energy and intensity data for decay gamma and x-rays emission for 1,132 radionuclides, prompt and delayed continuum spectra for spontaneous fission, $(\alpha, n)$ reactions in oxide fuel, and bremsstrahlung from decay beta (electron and positron) particles slowing down in either a $\mathrm{UO}_{2}$ fuel or water matrix. Methods and data libraries used to calculate the neutron yields and energy spectra for spontaneous fission, $(\alpha, n)$ reactions, and delayed neutron emission are adopted from the SOURCES4C code. Capabilities to calculate the beta and alpha particle emission source and spectra have also been added.
\end{abstract}




\section{TABLE OF CONTENTS}

$\underline{\text { Page }}$

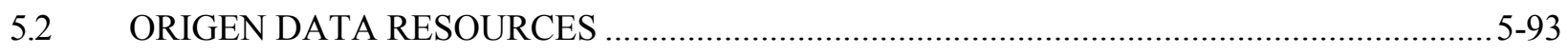

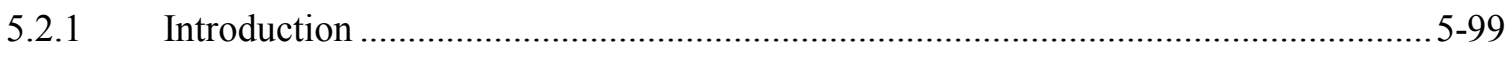

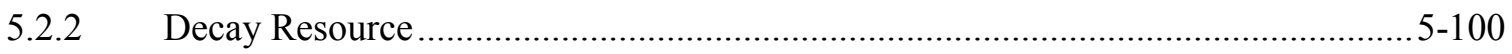

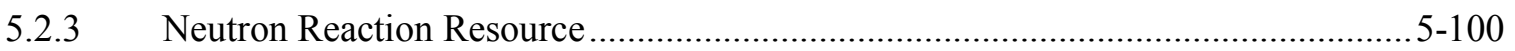

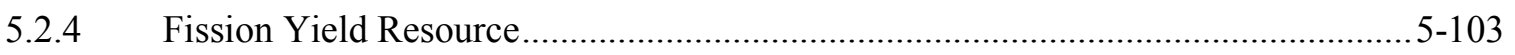

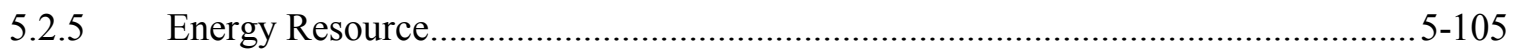

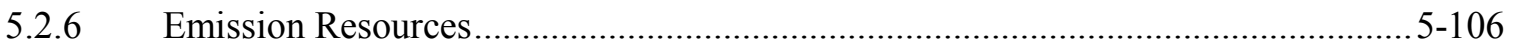

5.2.6.1 Gamma Emission ............................................................................... 5-106

5.2.6.2 Neutron Emission ................................................................................ 5-108

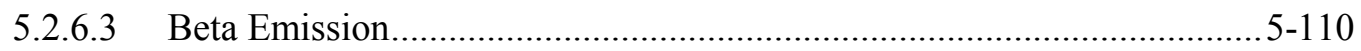

5.2.6.4 Alpha Emission .................................................................... 5-110

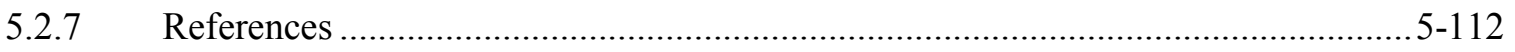

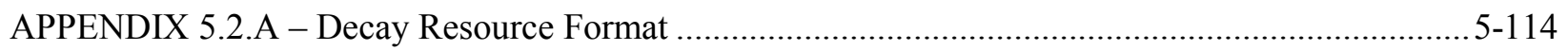

APPENDIX 5.2.B - Fission Yield Resource Format ................................................................... 5-117

APPENDIX 5.2.C - Gamma Resource Format …............................................................................ 5-118

APPENDIX 5.2.D - ORIGEN “end7dec” Nuclide Set ..................................................................... 5-119

APPENDIX 5.2.E - Decay Resource Contents ............................................................................... 5-154

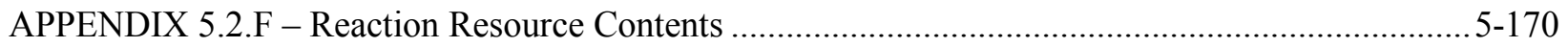




\section{LIST OF FIGURES}

$\underline{\text { Page }}$

Figure 5.2.1. Pointwise flux spectrum used to generate collapsed cross section libraries....................5-101

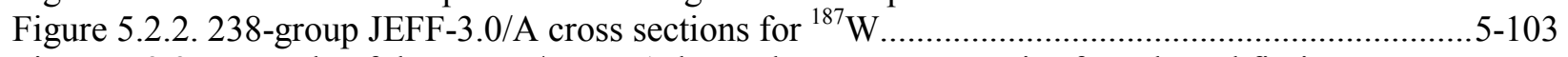

Figure 5.2.3. Example of the ENDF/B-VII.1 decay data resource entries for selected fission products.

Figure 5.2.4. Fission yield format example showing a portion of ${ }^{227} \mathrm{Th}$.

Figure 5.2.5. Gamma resource format example showing ${ }^{140} \mathrm{La}$ decay photon emission.......................5-118

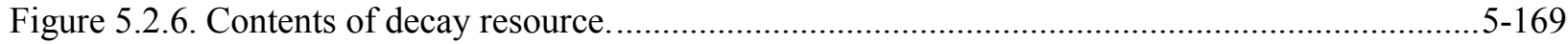

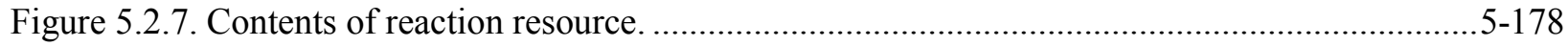




\section{LIST OF TABLES}

$\underline{\text { Page }}$

Table 5.2.1. Available resources in ORIGEN ..............................................................................5-99

Table 5.2.2. Fissionable isotopes having explicit fission yields ..................................................5-104

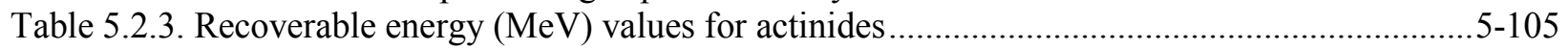

Table 5.2.4. Recoverable energy $(\mathrm{MeV})$ values for activation and fission products ..........................5-106

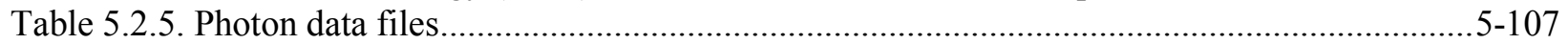

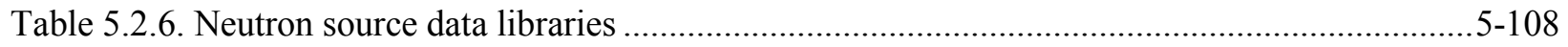

Table 5.2.7. Nuclides with spontaneous fission data and spectral parameters.....................................5-109

Table 5.2.8. Nuclides with delayed neutron emission spectral data ..................................................5-109

Table 5.2.9. Nuclides with $\alpha$-particle emission data for neutron yield calculations............................5-111

Table 5.2.10. Target $(\alpha, n)$ cross section and branching level isotopes and sources ...........................5-111

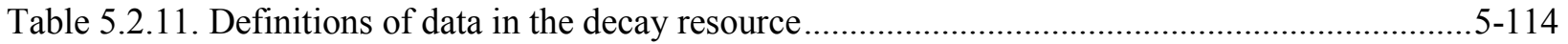

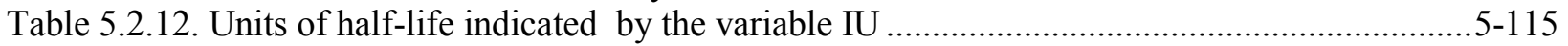

Table 5.2.13. Nuclide listing for "end7dec" ORIGEN library...........................................................5-119 


\section{ACKNOWLEDGMENTS}

Development and testing of ORIGEN data resources, libraries, and methods have been sponsored by many organizations including the US Nuclear Regulatory Commission (NRC), the US Department of Energy (DOE), and nuclear power and research institutions.

\section{VERSION INFORMATION}

Following is a description of the data resources available for use with ORIGEN in different SCALE versions. Methodologies and algorithms used in applying the data are described in the ORIGEN chapter.

\section{2 (2016)}

Data lead(s): I. C. Gauld, D. Wiarda, M. Pigni, and W. Wieselquist

Nuclear data in ORIGEN are unchanged from SCALE 6.1.3 except for the modification of independent fission yields for thermal fission of ${ }^{235} \mathrm{U}$ and ${ }^{241} \mathrm{Pu}$ and fast fission of ${ }^{238} \mathrm{U}$ to provide greater compatibility between the direct and cumulative fission yields when using the updated decay data from ENDF/B-VII.1. Additionally, ORIGEN no longer has its own independent source of nuclide mass and abundance data and now relies on the SCALE Standard Composition library so that there is consistency in this data across SCALE. D. Mueller and W. Wieselquist are acknowledged for testing of the new yield data. W. Wieselquist and S. Hart are acknowledged for the revision of this chapter.

\subsection{3 (2011)}

Data lead (s): I. C. Gauld and D. Wiarda

SCALE 6.1 represented a complete revision and update of the nuclear data available in ORIGEN. The following is a summary from the SCALE 6.1 manual.

The ORIGEN data libraries include nuclear decay data, neutron reaction cross sections, neutron induced fission product yields, delayed gamma-ray emission data, and neutron emission data. The nuclear decay data libraries have been updated based on ENDF/B-VII evaluations and expanded to include 903 activation products and structural materials, 174 actinides, and 1149 fission products. The cross section libraries have been revised using evaluations from the JEFF3.0/A neutron activation file, containing data for 774 target nuclides, more than 12,000 neutroninduced reactions, and more than 20 different reaction types below $20 \mathrm{MeV}$. The JEFF-3.0/A activation file is processed into several multigroup cross section libraries, from 44 groups to 238 groups, that can be used to determine the neutron reaction transition rates in ORIGEN. Energydependent ENDF/B-VII fission product yields are provided for 30 fissionable actinides. Photon yield data libraries have been updated based on the most recent ENSDF nuclear structure evaluations processed using the NuDat program. The photon libraries contain discrete photon line energy and intensity data for decay gamma and $x$-rays emission for 982 radionuclides, prompt and equilibrium continuum fission product spectra from spontaneous fission, $(\alpha, n)$ reactions in oxide fuel, and bremsstrahlung from decay beta (negatron and positron) particles slowing down in either $\mathrm{UO}_{2}$ fuel or water matrix. Methods and data libraries used to calculate the neutron yields and energy spectra for spontaneous fission, $(\alpha, n)$ reactions in any matrix, and delayed neutron emission are adopted from the SOURCES code. The libraries used by ORIGEN can be coupled directly with detailed and problem-dependent physics calculations to obtain selfshielded problem-dependent cross sections based on the most recent evaluations of ENDF/B-VII. 
In addition, the library formats allow multiple sets of cross section data to be stored on a library to represent the changes in cross sections during irradiation. 


\subsubsection{Introduction}

ORIGEN data resources include nuclear decay data, multigroup neutron reaction cross sections, neutroninduced fission product yields, and decay emission data for photons, neutrons, alpha particles and beta particles. The available resources are summarized in Table 5.2.1 and described in greater detail in the subsequent sections. The "Unit" column shows the corresponding unit number for use with FIDO input systems (e.g. with COUPLE).

Table 5.2.1. Available resources in ORIGEN

\begin{tabular}{|c|c|c|c|c|}
\hline Description & Alias & Unit & Category & Location in SCALE data directory \\
\hline ENDF/B-VII.1 decay data & decay & 27 & Decay & origen_data/origen.rev03.decay.data \\
\hline $\begin{array}{l}\text { ENDF/B-VII.0 based fission } \\
\text { yield data }\end{array}$ & yields & 17 & Yield & origen_data/origen.rev05.yields.data \\
\hline JEFF-3.0/A - 44g & $\mathrm{n} 44$ & 79 & Reaction & origen.rev03.jeff44g \\
\hline $\mathrm{JEFF}-3.0 / \mathrm{A}-47 \mathrm{~g}$ & $\mathrm{n} 47$ & 22 & Reaction & origen.rev03.jeff $47 \mathrm{~g}$ \\
\hline JEFF-3.0/A - 49g & $\mathrm{n} 49$ & 77 & Reaction & origen.rev03.jeff $49 \mathrm{~g}$ \\
\hline JEFF-3.0/A $-56 \mathrm{~g}$ & $\mathrm{n} 56$ & 75 & Reaction & origen.rev01.jeff56g \\
\hline JEFF-3.0/A $-200 \mathrm{~g}$ & $\mathrm{n} 200$ & 78 & Reaction & origen.rev03.jeff $200 \mathrm{~g}$ \\
\hline JEFF-3.0/A $-238 g$ & $\mathrm{n} 238$ & 80 & Reaction & origen.rev03.jeff $238 \mathrm{~g}$ \\
\hline JEFF-3.0/A $-252 \mathrm{~g}$ & $\mathrm{n} 252$ & 74 & Reaction & origen.rev01.jeff $252 \mathrm{~g}$ \\
\hline JEFF-3.0/A - 999g & n999 & 76 & Reaction & origen.rev01.jeff999g \\
\hline Energy per fission and capture & & & Energy & $\mathrm{n} / \mathrm{a}$ \\
\hline $\begin{array}{l}\text { Master photon (x-ray and } \\
\text { gamma) emission data }\end{array}$ & & & Emission & origen_data/origen.rev04.mpdkxgam.data \\
\hline $\begin{array}{l}\text { Spontaneous fission and }(\alpha, n) \\
\text { reaction gamma rays }\end{array}$ & & & Emission & origen_data/origen.rev00.mpsfangm.data \\
\hline $\begin{array}{l}\text { Bremsstrahlung from beta } \\
\text { particles slowing down in water }\end{array}$ & & & Emission & origen_data/origen.rev00.mpbrh2om.data \\
\hline $\begin{array}{l}\text { Bremsstrahlung from positrons } \\
\text { slowing down in water }\end{array}$ & & & Emission & origen_data/origen.rev00.mpbrh2op.data \\
\hline $\begin{array}{l}\text { Bremsstrahlung from beta } \\
\text { particles slowing down in } \mathrm{UO}_{2}\end{array}$ & & & Emission & origen_data/origen.rev00.mpbruo $2 \mathrm{~m}$.data \\
\hline $\begin{array}{l}\text { Bremsstrahlung from positrons } \\
\text { slowing down in } \mathrm{UO}_{2}\end{array}$ & & & Emission & origen_data/origen.rev00.mpbruo2p.data \\
\hline $\begin{array}{l}\text { Neutron source emission and } \\
\text { alpha decay data }\end{array}$ & & & Emission & origen_data/origen.rev01.alphdec.data \\
\hline $\begin{array}{l}\text { Alpha particle stopping cross } \\
\text { section expansion coefficients }\end{array}$ & & & Emission & origen_data/origen.rev00.stcoeff.data \\
\hline $\begin{array}{l}\text { Target }(\alpha, n) \text { product excited } \\
\text { level branching data }\end{array}$ & & & Emission & origen_data/origen.rev00.alphyld.data \\
\hline Target $(\alpha, n)$ cross section data & & & Emission & origen_data/origen.rev00.alphaxs.data \\
\hline Beta source emission data & & & Emission & $\begin{array}{l}\text { origen_data/ } \\
\text { origen.rev00.ensdf95beta.data }\end{array}$ \\
\hline
\end{tabular}




\subsubsection{Decay Resource}

The nuclear data stored on the decay resource is based on ENDF/B-VII.1 evaluations, ${ }^{1}$ including halflives, decay modes and branching fractions, and recoverable energy per disintegration. Decay modes include beta $\left(\beta^{-}\right)$, positron $\left(\beta^{+}\right)$and electron capture (EC), isomeric transition (IT), alpha $(\alpha)$, spontaneous fission (SF), delayed neutron ( $\beta^{-}$n) emission, neutron emission (n), double beta decay $\left(\beta^{-} \beta^{-}\right)$, and decay by beta and alpha emission $\left(\beta^{-} \alpha\right)$. The decay resource also includes radiotoxicity factors based on the radioactivity concentration guides (RCGs) for air and water as defined in Part 10, Title 20, of the Code of Federal Regulations $^{2}$ (10CFR20). RCGs specify the maximum permissible concentrations of an isotope in soluble and insoluble forms for both ingestion and inhalation and for occupational and unrestricted exposure. The radiotoxicity is calculated as the dilution volume of a nuclide for cases of direct ingestion or inhalation. The values are defined to be the smaller (i.e., more toxic) of the values for soluble and insoluble forms of the isotope. The maximum permissible RCGs for air and water are the public exposure limits for adult ingestion and inhalation dose coefficients of ICRP Publication 72. ${ }^{3}$ External exposure dose coefficients for noble gases were obtained from the Environmental Protection Agency (EPA) Federal Guidance Report 12. ${ }^{4}$

Recoverable energy includes the delayed energy from all electron-related radiations (e.g., $\beta^{-}, \beta^{+}$, Auger electrons), all gamma rays, x-rays, annihilation radiations, and the average energy of all heavy charged particles and delayed neutrons. The average alpha energy includes the energy of the recoil nucleus. A part of the recoverable energy per decay not included in the ENDF/B-VII.1 values is the additional contribution from spontaneous fission. This energy was calculated as the product of the spontaneous fission branching fraction and recoverable energy per fission using a value of $200 \mathrm{MeV}$ per fission and then added to the ENDF/B-VII.1 recoverable Q energy. A value of $12.56 \mathrm{MeV}$ gamma energy per fission was used in computing the fraction of recoverable spontaneous fission energy from gamma rays. External Bremsstrahlung radiation is not included in the Q-value since the Bremsstrahlung spectrum depends on electron interactions with the medium that contains the decay nuclide. The energy from capture gamma rays accompanying $(\alpha, n)$ reactions is not included either since it also depends on the medium.

Appendix A describes the decay resource file format. It is important to note that the decay resource not only defines fundamental decay data, but also the complete ORIGEN nuclide set, including the "duplicates" of nuclides across sublibraries. For example, a version of ${ }^{155} \mathrm{Gd}$ is contained in both the light nuclide/activation product and fission product sublibraries. Appendix D includes the full list of the nuclides on the ORIGEN decay library "end7dec" created by COUPLE based on the current decay resource, including duplicates. Appendix E contains a list of the fundamental decay data only, without duplicates. To consider a different set of nuclides in an ORIGEN calculation, the current process is to alter the decay resource and then regenerate the "end7dec" decay library with COUPLE. By default, all subsequent libraries created from COUPLE using problem-dependent reaction transitions are based on the "end7dec" decay library and will therefore include the modified nuclide set.

\subsubsection{Neutron Reaction Resource}

The neutron cross sections defining the nuclear reaction transmutation rates use a comprehensive collection of nuclear data evaluations compiled from the JEFF-3.0/A neutron activation files. ${ }^{5}$ The JEFF3.0/A files contain continuous energy neutron data for 774 target nuclei, including ground and metastable excited states, and 12,617 neutron-induced reactions below $20 \mathrm{MeV}$. The JEFF-3.0/A cross section data are developed directly from the European Activation File (EAF-2003) ${ }^{6}$ formatted as standard ENDF-6 format data. JEFF-3.0/A cross sections are stored using File 3, multiplicities on File 10, and isomeric branching to different metastable levels using File 9. 
The evaluations include many reactions that may be important for modeling fast fission and other highenergy systems. Neutron reactions are available for 23 reaction types, including $\left(n, n^{\prime}\right),(n, 2 n),(n, 3 n)$, $(n, f),\left(n, n^{\prime} \alpha\right),(n, 2 n \alpha),(n, 3 n \alpha),\left(n, n^{\prime} p\right),(n, n 2 \alpha),\left(n, n^{\prime} d\right),\left(n, n^{\prime} t\right),\left(n, n^{\prime}{ }^{3} H e\right),(n, 4 n),(n, 2 n p),(n, \gamma),(n, p)$, $(\mathrm{n}, \mathrm{d}),(\mathrm{n}, \mathrm{t}),\left(\mathrm{n},{ }^{3} \mathrm{He}\right),(\mathrm{n}, \alpha),(\mathrm{n}, 2 \alpha),(\mathrm{n}, 2 \mathrm{p})$, and $(\mathrm{n}, \mathrm{p} \alpha)$.

The JEFF-3.0/A evaluations also include extensive compilations of energy-dependent branching fractions that define neutron reaction transitions to ground and metastable energy states. Energy-dependent branching is fully implemented in the ORIGEN cross section libraries.

Implementation of the JEFF-3.0/A cross sections as ORIGEN multigroup data was accomplished by processing and collapsing the JEFF-3.0/A pointwise cross sections into a standard multigroup AMPX format using ENDF data-processing modules of the $\mathrm{AMPX}^{7}$ cross section processing code system. The collapse is performed using a thermal Maxwellian-1/E-fission-1/E weighting spectrum (see Figure 5.2.1) to provide infinite dilution multigroup cross sections.

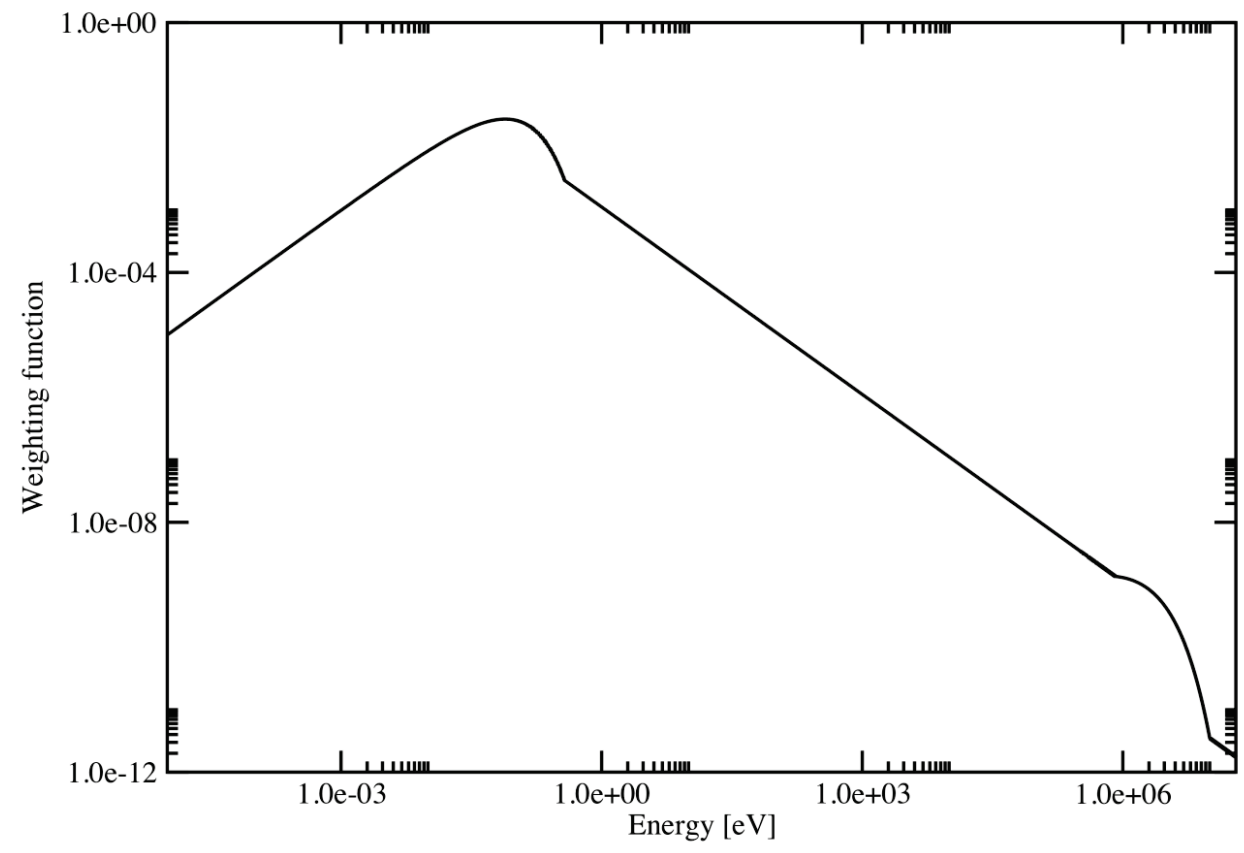

Figure 5.2.1. Pointwise flux spectrum used to generate collapsed cross section libraries.

Neutron reactions with transitions to multiple states of the daughter product are represented using separate cross sections to the ground and metastable states. A special reaction identifier $\left(\mathrm{MT}^{\prime}\right)$ is defined for this implementation of metastable transitions as

$$
\mathrm{MT}^{\prime}=\mathrm{MT}^{*} 10000+100 * \mathrm{LP}+\mathrm{LT}
$$

where MT is the reaction identifier, LP is the product metastable state, and LT is the target metastable state. Using the ${ }^{187} \mathrm{~W}(\mathrm{n}, 3 \mathrm{n}){ }^{185} \mathrm{~W}$ cross section $(\mathrm{MT}=17)$ as an example, the reaction identifier 170000 defines the partial cross section to the ground state of ${ }^{185} \mathrm{~W}$, and 170100 defines the cross section to metastable ${ }^{185 \mathrm{~m}} \mathrm{~W}$. 
Cross section data from the JEFF-3.0/A neutron activation file are first converted to point-wise cross section data, are Doppler broadened to $900 \mathrm{~K}$, and then they are collapsed to different group structures. The following group strucures are available in SCALE:

- 238-group neutron (thermal applications),

- 252-group neutron (thermal applications),

- 56-group neutron (thermal applications),

- 200-group neutron (fast applications and shielding),

- 47-group neutron (applications using the BUGLE shielding transport library),

- 49-group neutron (collapsed version of 238 groups),

- 44-group neutron (collapsed version of 238 groups), and

- 999-group neutron (multipurpose).

Several minor modifications were made to the JEFF-3.0/A data:

- $\quad$ The ${ }^{239} \mathrm{~Np}$ radiative neutron capture cross section was replaced with data from ENDF/B-VII.0. Neutron capture using JEFF-3.0/A cross sections was significantly larger than ENDF/B-VII.0 due to differences in the resonance cross section region. Although experimental resonance parameters are not available for ${ }^{239} \mathrm{~Np}$, comparisons of ${ }^{240} \mathrm{Pu}$ production during irradiation ${ }^{8}$ obtained using the two evaluations showed that better agreement with the experiment was obtained using the ENDF/B-VII.0 evaluation.

- The ${ }^{241} \mathrm{Am}(\mathrm{n}, \gamma)$ branching fraction to the ${ }^{242} \mathrm{Am}$ ground and metastable states was replaced by the evaluation from ENDF/B-VII.0 to yield better agreement with the results of destructive radiochemical assay measurements of irradiated fuels. The branching fraction of ${ }^{241} \mathrm{Am}$ to ${ }^{242 \mathrm{~m}} \mathrm{Am}$ for thermal neutron capture changed from $8.2 \%$ in JEFF-3.0/A to $10.0 \%$ in ENDF/B-VII.0.

The cross section library header record information and a complete list of nuclides in JEFF-3.0/A libraries developed for ORIGEN are provided in Appendix E.

Because JEFF-3.0/A-based libraries are formatted as standard AMPX working libraries, they can be accessed and/or manipulated using standard AMPX utility modules in SCALE. For example, multigroup cross sections may be listed using the PALEALE module. Additionally, the data may be visualized using the Fulcrum user interface. Cross section plots of the 238-group JEFF-3.0/A library are illustrated in Figure 5.2.2 for $(n, \gamma),(n, \alpha),(n, 2 n)$, and $(n, 3 n)$ cross sections to the ground and metastable states.

Before the cross sections in ORIGEN can be used, they must be collapsed with a user-defined multigroup flux to a one-group cross section and added to the ORIGEN binary library (see the COUPLE input description). 


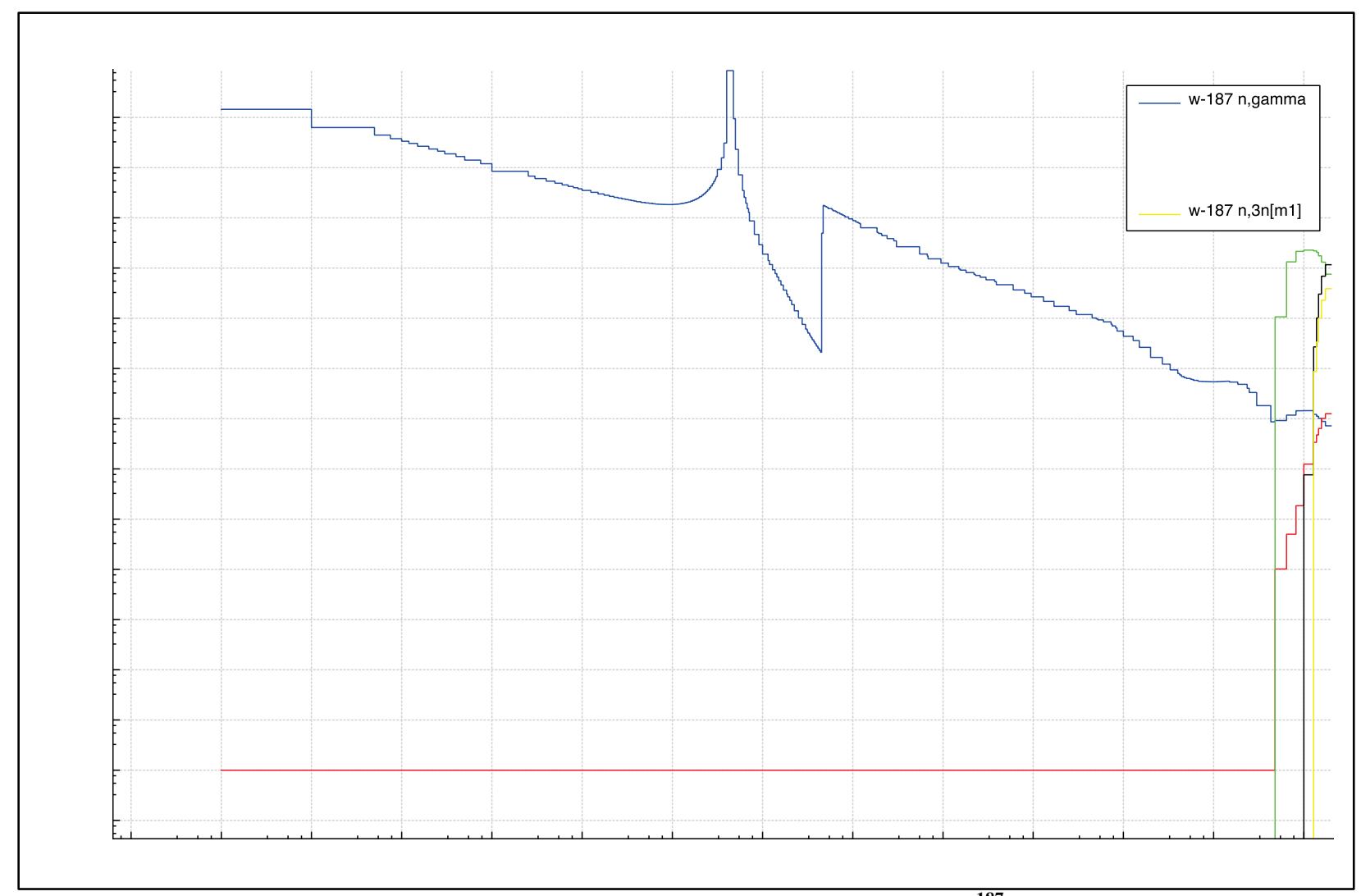

Figure 5.2.2. 238-group JEFF-3.0/A cross sections for ${ }^{187} \mathrm{~W}$.

\subsubsection{Fission Yield Resource}

The fission-yield resource contains the energy-dependent direct yields of each fission product for 30 fissionable actinides, including ${ }^{227,228,232} \mathrm{Th},{ }^{231} \mathrm{~Pa},{ }^{232-238} \mathrm{U},{ }^{238-242} \mathrm{Pu},{ }^{241,242 \mathrm{~m}, 243} \mathrm{Am},{ }^{237,238} \mathrm{~Np}$, ${ }^{242-246,248} \mathrm{Cm},{ }^{249,252} \mathrm{Cf}$, and ${ }^{254} \mathrm{Es}$. Independent (direct) fission product yields are stored as atom percent per fission, and except for ${ }^{235} \mathrm{U}$ (thermal), ${ }^{238} \mathrm{U}$ (fast), and ${ }^{241} \mathrm{Pu}$ (thermal), they are obtained from ENDF/B-VII. $0^{9}$ File 8 and MT $=454$.

Revised independent fission product yields for ${ }^{235} \mathrm{U}$ (thermal), ${ }^{238} \mathrm{U}$ (fast), and ${ }^{241} \mathrm{Pu}$ (thermal) were adopted to address inconsistencies between the direct and cumulative fission yields in ENDF/B-VII.0 caused by the use of updated nuclear decay schemes in the decay sublibrary. ${ }^{10-11}$ Namely, recent changes in the decay data, particularly the delayed neutron branching fractions, result in calculated fission product concentrations that do not agree with the cumulative fission yields in the ENDF/B-VII.0 library. These issues were particularly evident for the three cited isotopes because their fissioning systems result in a preferential formation of fragments that are sensitive to the changes in the decay data. For example, a study on ${ }^{239} \mathrm{Pu}$ (thermal) showed negligible differences between cumulative yields calculated (using the recent decay data sublibrary) and the cumulative yields in ENDF/B-VII.0. Energy-dependent product yields are available for thermal, fast, and high-energy incident neutron energies. For fast fission, the value of the energy of incident neutron was modified from the value of $500 \mathrm{keV}$ tabulated in ENDF/B-VII.0 to more accurately represent the relationship between the energy distribution of the neutrons causing fission and the and the fission neutron spectrum energy. For this implementation of the yield data, the effective incident neutron energy for fast fission was adjusted from $500 \mathrm{keV}$ to $2.0 \mathrm{MeV}$ to better reflect the average fission energy of most nuclides. The neutron energies for thermal fission $(0.0253 \mathrm{eV})$ and high energy fission (14 MeV) are unchanged. 
The fission product yields also include cumulative ternary yields from the JEF-2.2 fission yield library ${ }^{12}$ for ${ }^{3} \mathrm{H}$ and ${ }^{4} \mathrm{He}$. The nuclide ${ }^{3} \mathrm{He}$ was also added to the fission product library since it is a decay product of tritium.

Note that inclusion of fission yields for each actinide in an ORIGEN library can be controlled by the user through COUPLE. Actinides not assigned with explicit yields do not produce fission products during fission.

Table 5.2.2. Fissionable isotopes having explicit fission yields

\begin{tabular}{|c|c|c|c|}
\hline Nuclide & \multicolumn{3}{|c|}{ Neutron-induced fission energies ${ }^{a}$} \\
\hline${ }^{227} \mathrm{Th}$ & Thermal & & \\
\hline${ }^{229} \mathrm{Th}$ & Thermal & & \\
\hline${ }^{232} \mathrm{Th}$ & & Fast & High energy \\
\hline${ }^{231} \mathrm{~Pa}$ & & Fast & \\
\hline${ }^{232} \mathrm{U}$ & Thermal & & \\
\hline${ }^{233} \mathrm{U}$ & Thermal & & \\
\hline${ }^{234} \mathrm{U}$ & & Fast & High energy \\
\hline${ }^{235} \mathrm{U}$ & Thermal & Fast & High energy \\
\hline${ }^{236} \mathrm{U}$ & & Fast & High energy \\
\hline${ }^{237} \mathrm{U}$ & & Fast & \\
\hline${ }^{238} \mathrm{U}$ & & Fast & High energy \\
\hline${ }^{237} \mathrm{~Np}$ & Thermal & Fast & High energy \\
\hline${ }^{238} \mathrm{~Np}$ & & Fast & \\
\hline${ }^{238} \mathrm{Pu}$ & & Fast & \\
\hline${ }^{239} \mathrm{Pu}$ & Thermal & Fast & High energy \\
\hline${ }^{240} \mathrm{Pu}$ & Thermal & Fast & High energy \\
\hline${ }^{241} \mathrm{Pu}$ & Thermal & Fast & \\
\hline${ }^{242} \mathrm{Pu}$ & Thermal & Fast & High energy \\
\hline${ }^{241} \mathrm{Am}$ & Thermal & Fast & High energy \\
\hline${ }^{242 m} \mathrm{Am}$ & Thermal & & \\
\hline${ }^{243} \mathrm{Am}$ & & Fast & \\
\hline${ }^{242} \mathrm{Cm}$ & & Fast & \\
\hline${ }^{243} \mathrm{Cm}$ & Thermal & Fast & \\
\hline${ }^{244} \mathrm{Cm}$ & & Fast & \\
\hline${ }^{245} \mathrm{Cm}$ & Thermal & & \\
\hline${ }^{246} \mathrm{Cm}$ & & Fast & \\
\hline${ }^{248} \mathrm{Cm}$ & & Fast & \\
\hline${ }^{249} \mathrm{Cf}$ & Thermal & & \\
\hline${ }^{251} \mathrm{Cf}$ & Thermal & & \\
\hline${ }^{254} \mathrm{Es}$ & Thermal & & \\
\hline
\end{tabular}

${ }^{a}$ Neutron energy causing fission 


\subsubsection{Energy Resource}

The energy resource is a set of data defined internally to ORIGEN to compute the total power during irradiation if the flux is known, or the total flux if the power is known. The data include the energy contributed by fission and capture. The recoverable energy values taken primarily from $\mathrm{ENDF} / \mathrm{B}$ evaluations are listed in Table 5.2.3 and Table 5.2.4. The recoverable energy for fission and neutron capture for nuclides not listed in the tables are assumed to be $200 \mathrm{MeV}$ and $5.0 \mathrm{MeV}$, respectively.

Table 5.2.3. Recoverable energy (MeV) values for actinides

\begin{tabular}{lcc}
\hline Nuclide & Fission & Capture \\
\hline${ }^{230} \mathrm{Th}$ & 190.00 & 5.010 \\
${ }^{232} \mathrm{Th}$ & 189.21 & 4.786 \\
${ }^{233} \mathrm{Th}$ & 190.00 & 6.080 \\
${ }^{231} \mathrm{~Pa}$ & 190.00 & 5.660 \\
${ }^{233} \mathrm{~Pa}$ & 189.10 & 5.197 \\
${ }^{232} \mathrm{U}$ & 200.00 & 5.930 \\
${ }^{233} \mathrm{U}$ & 191.29 & 6.841 \\
${ }^{234} \mathrm{U}$ & 190.30 & 5.297 \\
${ }^{235} \mathrm{U}$ & 194.02 & 6.545 \\
${ }^{236} \mathrm{U}$ & 192.80 & 5.124 \\
${ }^{238} \mathrm{U}$ & 198.12 & 4.804 \\
${ }^{237} \mathrm{~Np}$ & 195.10 & 5.490 \\
${ }^{239} \mathrm{~Np}$ & 200.00 & 4.970 \\
${ }^{238} \mathrm{Pu}$ & 197.80 & 5.550 \\
${ }^{239} \mathrm{Pu}$ & 200.05 & 6.533 \\
${ }^{240} \mathrm{Pu}$ & 199.79 & 5.241 \\
${ }^{241} \mathrm{Pu}$ & 202.22 & 6.301 \\
${ }^{242} \mathrm{Pu}$ & 200.62 & 5.071 \\
${ }^{243} \mathrm{Pu}$ & 200.00 & 6.020 \\
${ }^{241} \mathrm{Am}$ & 202.30 & 5.529 \\
${ }^{242 \mathrm{~m}} \mathrm{Am}$ & 202.29 & 6.426 \\
${ }^{243} \mathrm{Am}$ & 202.10 & 5.363 \\
${ }^{244} \mathrm{Cm}$ & 200.00 & 6.451 \\
${ }^{245} \mathrm{Cm}$ & 200.00 & 6.110 \\
\hline
\end{tabular}


Table 5.2.4. Recoverable energy (MeV) values for activation and fission products

\begin{tabular}{cc}
\hline${ }^{\text {Nuclide }}$ & Capture \\
\hline${ }^{1} \mathrm{H}$ & 2.225 \\
${ }^{10} \mathrm{~B}$ & 2.790 \\
${ }^{16} \mathrm{O}$ & 4.143 \\
${ }^{56} \mathrm{Fe}$ & 7.600 \\
${ }^{58} \mathrm{Ni}$ & 9.020 \\
${ }^{90} \mathrm{Zr}$ & 7.203 \\
${ }^{91} \mathrm{Zr}$ & 8.635 \\
${ }^{92} \mathrm{Zr}$ & 6.758 \\
${ }^{96} \mathrm{Zr}$ & 5.571 \\
${ }^{95} \mathrm{Mo}$ & 9.154 \\
${ }^{95} \mathrm{Tc}$ & 7.710 \\
${ }^{101} \mathrm{Ru}$ & 9.216 \\
${ }^{103} \mathrm{Rh}$ & 6.999 \\
${ }^{105} \mathrm{Rh}$ & 7.094 \\
${ }^{109} \mathrm{Ag}$ & 6.825 \\
${ }^{131} \mathrm{Xe}$ & 8.936 \\
${ }^{135} \mathrm{Xe}$ & 7.880 \\
${ }^{133} \mathrm{Cs}$ & 6.704 \\
${ }^{134} \mathrm{Cs}$ & 6.550 \\
${ }^{143} \mathrm{Nd}$ & 7.817 \\
${ }^{145} \mathrm{Nd}$ & 7.565 \\
${ }^{147} \mathrm{Pm}$ & 5.900 \\
${ }^{148} \mathrm{Pm}$ & 7.266 \\
${ }^{148 m} \mathrm{Pm}$ & 7.266 \\
${ }^{147} \mathrm{Sm}$ & 8.140 \\
${ }^{149} \mathrm{Sm}$ & 7.982 \\
${ }^{150} \mathrm{Sm}$ & 5.596 \\
${ }^{151} \mathrm{Sm}$ & 8.258 \\
${ }^{152} \mathrm{Sm}$ & 5.867 \\
${ }^{153} \mathrm{Eu}$ & 6.444 \\
${ }^{154} \mathrm{Eu}$ & 8.167 \\
${ }^{155} \mathrm{Eu}$ & 6.490 \\
\hline
\end{tabular}

\subsubsection{Emission Resources}

The two main groups for emission resources are the photon (gamma) resource, which includes beta particle emission data, and the neutron resource, which includes alpha emission data.

\subsubsection{Gamma Emission}

The resources for gamma emission are stored as separate files (see Table 5.2.5) containing the photon data associated with different modes of decay or photon production. The photon data sets include decay gamma and x-ray line-energy data, gamma rays accompanying spontaneous fission, gamma rays accompanying $(\alpha, n)$ reactions in oxide fuels, and Bremsstrahlung spectra from decay electrons/positrons slowing down in $\mathrm{UO}_{2}$ and water. The photon energy spectra can be generated in any energy group structure for all activation products, actinides, and fission product nuclides with photon yield data. 
Table 5.2.5. Photon data files

\begin{tabular}{ll}
\hline \multicolumn{1}{c}{ File name } & \multicolumn{1}{c}{ Description } \\
\hline MPDKXGAM & x-ray and gamma emissions line data \\
MPSFANGM & spontaneous fission and $(\alpha, \mathrm{n})$ reactions \\
MPBRH2OM & bremsstrahlung from beta particles slowing down in water \\
MPBRH2OP & bremsstrahlung from positrons slowing down in water \\
MPBRUO2M & bremsstrahlung from beta particles slowing down in $\mathrm{UO}_{2}$ \\
MPBRUO2P & bremsstrahlung from positrons slowing down in $\mathrm{UO}_{2}$ \\
\hline
\end{tabular}

All photon data sets are constructed with the same format (see Appendix C). The majority of the photon emissions are discrete energy lines. Photon continuum data, used to represent Bremstrahlung and some other gamma-ray emission spectra, are stored at discrete energies and approximately expanded to a continuum, as needed.

Gamma and x-ray yields are directly from ENDF/B-VII.1 decay files containing spectral data for decay transitions of 1,132 nuclides. A separate file contains emission spectra for gamma rays accompanying spontaneous fission and for gamma rays accompanying $(\alpha, n)$ reactions in oxide fuels. ${ }^{13}$ The spontaneous fission spectra combine prompt and equilibrium fission product gamma-ray components. The prompt spectrum is similar to that of ${ }^{235} \mathrm{U}$, and the delayed fission product gamma intensity at equilibrium is about 0.75 of that from the prompt fission gamma rays. Based on measured prompt fission gamma spectra from ${ }^{235} \mathrm{U}$, spontaneous fission spectra are computed from the following approximation:

$$
N(E) \cong\left\{\begin{array}{cc}
11.5 & \text { for } 0.1 \leq E<0.6 \mathrm{MeV} \\
35.4 e^{-1.78 E} & \text { for } 0.6 \leq E<1.5 \mathrm{MeV} \\
12.6 e^{-1.09 E} & \text { for } 1.5 \leq E \leq 10.5 \mathrm{MeV} \\
0 & \text { otherwise }
\end{array}\right.
$$

where

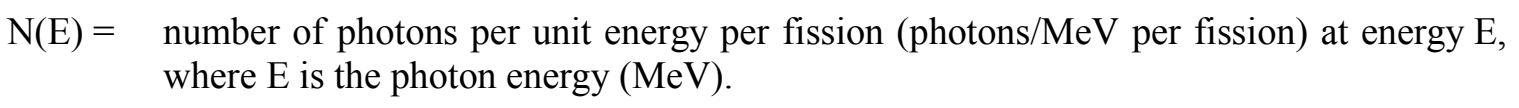

For medical and industrial spontaneous fission source applications, a more accurate simulation of the source may be desirable. Work has been performed on ${ }^{252} \mathrm{Cf}$ source modeling to explicity represent the fission product generation from fission and the delayed gamma emission. In this application, the equilibrium spontaneous fission gamma spectrum was replaced with an evaluation of the ${ }^{252} \mathrm{Cf}$ prompt gamma spectrum, and the delayed fission product gamma rays was modeled explicitly in ORIGEN by generating the time-dependent fission products using ${ }^{252} \mathrm{Cf}$ spontaneous fission product yields from ENDF/B-VII.0. ${ }^{14}$ This was performed by adding decay transitions to the ORIGEN library from the actinides to the fission products.

The spectrum of gamma rays accompanying $(\alpha, n)$ reactions is based on reaction data for alpha interactions on ${ }^{18} \mathrm{O}$ and from studies for ${ }^{238} \mathrm{PuO}_{2}$ systems. The spectrum is computed from the approximation:

$$
N(E) \cong 2.13 \times 10^{-8} e^{-1.38 E}
$$


where

$$
\begin{array}{ll}
\mathrm{N}(\mathrm{E})= & \text { number of photons per unit energy per alpha decay (photons/MeV per disintegration) at } \\
\text { energy } \mathrm{E}(\mathrm{MeV}) .
\end{array}
$$

The photon yields in this data set are continuum spectra represented by discrete lines with an energy width of $500 \mathrm{keV}$ and range from $250 \mathrm{keV}$ to $10.25 \mathrm{MeV}$.

Two photon data sets contain bremsstrahlung spectra from decay electrons and positrons slowing down in a $\mathrm{UO}_{2}$ fuel matrix. The yields are in the form of continuum spectra represented in the data sets as discrete lines using up to 70 quasi-logarithmic spaced energy points over the energy range between 0 and 13.5 MeV. Two libraries contain bremsstrahlung spectra from decay electrons and positrons slowing down in water. Bremsstrahlung spectra were calculated using a computer program developed by Dillman et al. ${ }^{15}$ using beta spectra derived from Evaluated Nuclear Structure Data Files (ENSDF) decay data with a computer program written by Gove and Martin. ${ }^{16}$

\subsubsection{Neutron Emission}

There are four neutron emission resources used by ORIGEN to calculate the neutron intensities and spectra: (1) neutron decay data, (2) an $\alpha$-particle stopping power, (3) a target $(\alpha, n)$ cross section, and (4) a target $(\alpha, n)$ product level branching. All of the neutron data are stored in a text format with names and descriptions given in Table 5.2.6. The neutron decay data contain basic decay information for decay processes that lead to direct and indirect emission of neutrons, including spontaneous fission branching fractions, alpha decay branching fractions, delayed neutron branching fractions, $\alpha$-particle decay energies, watt fission spectrum parameters, and delayed neutron spectra. The stopping cross sections, $(\alpha, n)$ target cross sections, and product-level branching data are used in calculating the neutron yield and spectra from $(\alpha, n)$ reactions.

The neutron data were obtained directly from the updated SOURCES-4B code package. The sources of the neutron data are described in Shores $2000 .{ }^{17}$ An update was made to correct an error in the ${ }^{250} \mathrm{Cf}$ spontaneous fission neutron branching fraction in the neutron source decay data distributed with the SOURCES code. The ${ }^{250} \mathrm{Cf}$ branching fraction was incorrectly assigned the value from ${ }^{252} \mathrm{Cf}$ of $3.092 \times 10^{-2}$. A corrected value of $7.700 \times 10^{-4}$ from ENDF/B-VII.1 is used.

Table 5.2.6. Neutron source data libraries

\begin{tabular}{ll}
\hline File name & \multicolumn{1}{c}{ Description } \\
\hline ALPHDEC & Neutron source decay data \\
STCOEFF & Stopping cross section expansion coefficients \\
ALPHYLD & Target $(\alpha, n)$ product level branching \\
ALPHAXS & Target $(\alpha, \mathrm{n})$ cross section \\
\hline
\end{tabular}

The neutron source decay contains spontaneous fission data for the 49 actinides listed in Table 5.2.7. These data include the spontaneous fission branching fraction, the number of neutrons per fission ( $v$ ), and the watt spectrum parameters for spontaneous fission. The spontaneous fission neutron energy spectrum is approximated using spectral parameters $\mathrm{A}$ and $\mathrm{B}$, such that 


$$
N(E) \cong C e^{-\frac{E}{A}} \sinh \sqrt{B E}
$$

where $E$ is the neutron energy and $C$ is a normalization constant.

Table 5.2.7. Nuclides with spontaneous fission data and spectral parameters

\begin{tabular}{lllll}
\hline${ }^{230} \mathrm{Th}$ & ${ }^{239} \mathrm{U}$ & ${ }^{240} \mathrm{Pu}$ & ${ }^{244} \mathrm{Am}$ & ${ }^{250} \mathrm{Cm}$ \\
${ }^{232} \mathrm{Th}$ & ${ }^{236} \mathrm{~Np}$ & ${ }^{241} \mathrm{Pu}$ & ${ }^{244} \mathrm{Am}$ & ${ }^{249} \mathrm{Bk}$ \\
${ }^{231} \mathrm{~Pa}$ & ${ }^{236 \mathrm{mp}}$ & ${ }^{242} \mathrm{Pu}$ & ${ }^{240} \mathrm{Cm}$ & ${ }^{248} \mathrm{Cf}$ \\
${ }^{232} \mathrm{U}$ & ${ }^{237} \mathrm{~Np}$ & ${ }^{243} \mathrm{Pu}$ & ${ }^{241} \mathrm{Cm}$ & ${ }^{250} \mathrm{Cf}$ \\
${ }^{233} \mathrm{U}$ & ${ }^{238} \mathrm{~Np}$ & ${ }^{244} \mathrm{Pu}$ & ${ }^{242} \mathrm{Cm}$ & ${ }^{252} \mathrm{Cf}$ \\
${ }^{234} \mathrm{U}$ & ${ }^{239} \mathrm{~Np}$ & ${ }^{240} \mathrm{Am}$ & ${ }^{243} \mathrm{Cm}$ & ${ }^{254} \mathrm{Cf}$ \\
${ }^{235} \mathrm{U}$ & ${ }^{236} \mathrm{Pu}$ & ${ }^{241} \mathrm{Am}$ & ${ }^{244} \mathrm{Cm}$ & ${ }^{253} \mathrm{Es}$ \\
${ }^{236} \mathrm{U}$ & ${ }^{237} \mathrm{Pu}$ & ${ }^{242} \mathrm{Am}$ & ${ }^{245} \mathrm{Cm}$ & ${ }^{254 m} \mathrm{Es}$ \\
${ }^{237} \mathrm{U}$ & ${ }^{238} \mathrm{Pu}$ & ${ }^{242 m} \mathrm{Am}$ & ${ }^{246} \mathrm{Cm}$ & ${ }^{255} \mathrm{Es}$ \\
${ }^{238} \mathrm{U}$ & ${ }^{239} \mathrm{Pu}$ & ${ }^{243} \mathrm{Am}$ & ${ }^{248} \mathrm{Cm}$ & \\
\hline
\end{tabular}

Delayed neutron branching fractions and neutron spectra for 105 fission products are listed in Table 5.2.8. The delayed neutron spectra are tabulated in discrete $10 \mathrm{keV}$ bins from $50 \mathrm{keV}$ to about $2 \mathrm{MeV}$.

Table 5.2.8. Nuclides with delayed neutron emission spectral data

\begin{tabular}{lllll}
\hline${ }^{79} \mathrm{Zn}$ & ${ }^{89} \mathrm{Br}$ & ${ }^{97} \mathrm{Y}$ & ${ }^{128} \mathrm{In}$ & ${ }^{41} \mathrm{I}$ \\
${ }^{79} \mathrm{Ga}$ & ${ }^{90} \mathrm{Br}$ & ${ }^{97 m} \mathrm{Y}$ & ${ }^{129} \mathrm{In}$ & ${ }^{42} \mathrm{I}$ \\
${ }^{80} \mathrm{Ga}$ & ${ }^{91} \mathrm{Br}$ & ${ }^{98} \mathrm{Y}$ & ${ }^{129} \mathrm{~m}$ & ${ }^{43} \mathrm{I}$ \\
${ }^{81} \mathrm{Ga}$ & ${ }^{92} \mathrm{Br}$ & ${ }^{98} \mathrm{Y}$ & ${ }^{130} \mathrm{In}$ & ${ }^{141} \mathrm{Xe}$ \\
${ }^{82} \mathrm{Ga}$ & ${ }^{93} \mathrm{Br}$ & ${ }^{99} \mathrm{Y}$ & ${ }^{131} \mathrm{In}$ & ${ }^{142} \mathrm{Xe}$ \\
${ }^{83} \mathrm{Ga}$ & ${ }^{92} \mathrm{Kr}$ & ${ }^{100} \mathrm{Y}$ & ${ }^{132} \mathrm{In}$ & ${ }^{143} \mathrm{Xe}$ \\
${ }^{83} \mathrm{Ge}$ & ${ }^{93} \mathrm{Kr}$ & ${ }^{104} \mathrm{Zr}$ & ${ }^{133} \mathrm{Sn}$ & ${ }^{144} \mathrm{Xe}$ \\
${ }^{84} \mathrm{Ge}$ & ${ }^{94} \mathrm{Kr}$ & ${ }^{105} \mathrm{Zr}$ & ${ }^{134} \mathrm{Sn}$ & ${ }^{141} \mathrm{Cs}$ \\
${ }^{85} \mathrm{Ge}$ & ${ }^{95} \mathrm{Kr}$ & ${ }^{103} \mathrm{Nb}$ & ${ }^{135} \mathrm{Sn}$ & ${ }^{142} \mathrm{Cs}$ \\
${ }^{86} \mathrm{Ge}$ & ${ }^{92} \mathrm{Rb}$ & ${ }^{104} \mathrm{Nb}$ & ${ }^{134} \mathrm{Sb}$ & ${ }^{143} \mathrm{Cs}$ \\
${ }^{84} \mathrm{As}$ & ${ }^{93} \mathrm{Rb}$ & ${ }^{105} \mathrm{Nb}$ & ${ }^{135} \mathrm{Sb}$ & ${ }^{144} \mathrm{Cs}$ \\
${ }^{85} \mathrm{As}$ & ${ }^{94} \mathrm{Rb}$ & ${ }^{106} \mathrm{Nb}$ & ${ }^{136} \mathrm{Sb}$ & ${ }^{145} \mathrm{Cs}$ \\
${ }^{86} \mathrm{As}$ & ${ }^{95} \mathrm{Rb}$ & ${ }^{109} \mathrm{Mo}$ & ${ }^{137} \mathrm{Sb}$ & ${ }^{146} \mathrm{Cs}$ \\
${ }^{87} \mathrm{As}$ & ${ }^{96} \mathrm{Rb}$ & ${ }^{110} \mathrm{Mo}$ & ${ }^{136} \mathrm{Te}$ & ${ }^{147} \mathrm{Cs}$ \\
${ }^{87} \mathrm{Se}$ & ${ }^{97} \mathrm{Rb}$ & ${ }^{109} \mathrm{Tc}$ & ${ }^{137} \mathrm{Te}$ & ${ }^{147} \mathrm{Ba}$ \\
${ }^{88} \mathrm{Se}$ & ${ }^{98} \mathrm{Rb}$ & ${ }^{110} \mathrm{Tc}$ & ${ }^{138} \mathrm{Te}$ & ${ }^{148} \mathrm{Ba}$ \\
${ }^{89} \mathrm{Se}$ & ${ }^{99} \mathrm{Rb}$ & ${ }^{139} \mathrm{Te}$ & ${ }^{149} \mathrm{Ba}$ \\
${ }^{90} \mathrm{Se}$ & ${ }^{122} \mathrm{Ag}$ & ${ }^{137} \mathrm{I}$ & ${ }^{150} \mathrm{Ba}$ \\
${ }^{91} \mathrm{Se}$ & ${ }^{138} \mathrm{Ag}$ & ${ }^{147} \mathrm{La}$ \\
${ }^{87} \mathrm{Br}$ & ${ }^{98} \mathrm{Sr}$ & ${ }^{128} \mathrm{Cd}$ & ${ }^{139} \mathrm{I}$ & ${ }^{149} \mathrm{La}$ \\
${ }^{88} \mathrm{Br}$ & ${ }^{99} \mathrm{Sr}$ & ${ }^{127} \mathrm{In}$ & ${ }^{127} \mathrm{La}$ \\
\hline
\end{tabular}

Neutron yields from $\alpha$-particle interaction are available for $19(\alpha, \mathrm{n})$ target nuclides: ${ }^{7} \mathrm{Li},{ }^{9} \mathrm{Be},{ }^{10} \mathrm{~B},{ }^{11} \mathrm{~B}$, ${ }^{13} \mathrm{C},{ }^{14} \mathrm{~N},{ }^{17} \mathrm{O},{ }^{18} \mathrm{O},{ }^{19} \mathrm{~F},{ }^{21} \mathrm{Ne},{ }^{22} \mathrm{Ne},{ }^{23} \mathrm{Na},{ }^{25} \mathrm{Mg},{ }^{26} \mathrm{Mg},{ }^{27} \mathrm{Al},{ }^{29} \mathrm{Si},{ }^{30} \mathrm{Si},{ }^{31} \mathrm{P}$, and ${ }^{37} \mathrm{Cl}$. The neutron decay data contain discrete $\alpha$-particle energies and branching fractions for 89 actinides and 7 fission products listed in Table 5.2.9. The sources of the level branching fraction data and the $(\alpha, n)$ cross section data are listed in Table 5.2.10. The stopping cross sections and $(\alpha, n)$ target cross section and product level branching 
libraries are used in calculating the neutron yield and spectra from Ziegler $1977^{18}$ for all elements with $\mathrm{Z}<93$, and from Wilson $1983{ }^{19}$ for all elements $\geq 93$.

\subsubsection{Beta Emission}

Beta emission rates and energy spectra are calculated using an analytic expression for the kinetic energy of the emitted $\beta$ - particles $^{16}$ :

$$
N(Z, W)=\frac{g^{2}}{2 \pi^{3}} F(Z, W) \rho W\left(W_{0}-W\right)^{2} S_{n}(W) d W
$$

where

$Z=$ atomic number of the daugher nucleus,

$g=$ weak interaction coupling constant,

$W=$ kinetic energy of beta particle (in $m_{e} c^{2}$ units),

$F(Z, W)=$ Fermi function,

$W_{0}=$ endpoint beta energy,

$\rho=\sqrt{W^{2}-1}=$ electron momentum,

$S_{n}(W)=$ spectral shape factor based on transition type, and

$n=$ classification of the transition type .

Internal conversion electron emission is not considered.

The calculation requires nuclear data on the fraction of the beta transition to each exicited state of the daughter nucleus, the maximum endpoint energy of the transition $\left(W_{0}\right)$, and a classification of the beta transition $(n)$ defined by the spin and parity change of the transition which defines the spectral shape factor. The transition classification uses $n=0$ for allowed and forbidden non-unique transitions, $n=1$ for first forbidden unique transitions, $n=2$ for second forbidden unique transitions, and $n=3$ for third forbidden unique transitions. These data are not stored in the decay data resource but are included in a separate beta decay resource used only for the beta calculation.

The beta decay data are stored in the formatted file origen. rev00.ensdf95beta. data. The data are derived from ENSDF as compiled in 1995. The file includes beta decay information for 715 beta decay nuclides and has 8486 beta transition branches.

\subsubsection{Alpha Emission}

Calculation of the alpha emission intensity and spectrum requires detailed information that is not available on the decay resource. The calculation requires the alpha particle energy and branching fraction for each transition branch. Unlike the beta spectrum, the alpha particles are emitted with discrete energies, and the source spectrum may be generated by straightforward binning into the user-defined group structure. Alpha particle emission data are also used in the $(\alpha, n)$ neutron source calculation. Therefore, the alpha emission spectra are calculated using the same alpha decay library in the neutron emission resource: origen.rev01.alphdec.data. 
Table 5.2.9. Nuclides with $\alpha$-particle emission data for neutron yield calculations

\begin{tabular}{|c|c|c|c|c|}
\hline${ }^{142} \mathrm{Ce}$ & ${ }^{216} \mathrm{Po}$ & ${ }^{226} \mathrm{Ac}$ & ${ }^{237} \mathrm{~Np}$ & ${ }^{245} \mathrm{Cm}$ \\
\hline${ }^{144} \mathrm{Nd}$ & ${ }^{218} \mathrm{Po}$ & ${ }^{227} \mathrm{Ac}$ & ${ }^{235} \mathrm{Pu}$ & ${ }^{246} \mathrm{Cm}$ \\
\hline${ }^{146} \mathrm{Sm}$ & ${ }^{215} \mathrm{At}$ & ${ }^{226} \mathrm{Th}$ & ${ }^{236} \mathrm{Pu}$ & ${ }^{247} \mathrm{Cm}$ \\
\hline${ }^{147} \mathrm{Sm}$ & ${ }^{217} \mathrm{At}$ & ${ }^{227} \mathrm{Th}$ & ${ }^{237} \mathrm{Pu}$ & ${ }^{248} \mathrm{Cm}$ \\
\hline${ }^{148} \mathrm{Sm}$ & ${ }^{218} \mathrm{At}$ & ${ }^{228} \mathrm{Th}$ & ${ }^{238} \mathrm{Pu}$ & ${ }^{249} \mathrm{Bk}$ \\
\hline${ }^{149} \mathrm{Sm}$ & ${ }^{219} \mathrm{At}$ & ${ }^{229} \mathrm{Th}$ & ${ }^{239} \mathrm{Pu}$ & ${ }^{248} \mathrm{Cf}$ \\
\hline${ }^{152} \mathrm{Gd}$ & ${ }^{217} \mathrm{Rn}$ & ${ }^{230} \mathrm{Th}$ & ${ }^{240} \mathrm{Pu}$ & ${ }^{249} \mathrm{Cf}$ \\
\hline${ }^{210} \mathrm{~Pb}$ & ${ }^{218} \mathrm{Rn}$ & ${ }^{232} \mathrm{Th}$ & ${ }^{241} \mathrm{Pu}$ & ${ }^{250} \mathrm{Cf}$ \\
\hline${ }^{210} \mathrm{Bi}$ & ${ }^{219} \mathrm{Rn}$ & ${ }^{230} \mathrm{~Pa}$ & ${ }^{242} \mathrm{Pu}$ & ${ }^{251} \mathrm{Cf}$ \\
\hline${ }^{211} \mathrm{Bi}$ & ${ }^{220} \mathrm{Rn}$ & ${ }^{231} \mathrm{~Pa}$ & ${ }^{244} \mathrm{Pu}$ & ${ }^{252} \mathrm{Cf}$ \\
\hline${ }^{212} \mathrm{Bi}$ & ${ }^{222} \mathrm{Rn}$ & ${ }^{230} \mathrm{U}$ & ${ }^{240} \mathrm{Am}$ & ${ }^{253} \mathrm{Cf}$ \\
\hline${ }^{213} \mathrm{Bi}$ & ${ }^{221} \mathrm{Fr}$ & ${ }^{231} \mathrm{U}$ & ${ }^{241} \mathrm{Am}$ & ${ }^{254} \mathrm{Cf}$ \\
\hline${ }^{214} \mathrm{Bi}$ & ${ }^{222} \mathrm{Fr}$ & ${ }^{232} \mathrm{U}$ & ${ }^{242 \mathrm{~m}} \mathrm{Am}$ & ${ }^{253} \mathrm{Es}$ \\
\hline${ }^{210} \mathrm{Po}$ & ${ }^{223} \mathrm{Fr}$ & ${ }^{233} \mathrm{U}$ & ${ }^{243} \mathrm{Am}$ & ${ }^{254} \mathrm{Es}$ \\
\hline${ }^{211} \mathrm{Po}$ & ${ }^{222} \mathrm{Ra}$ & ${ }^{234} \mathrm{U}$ & ${ }^{240} \mathrm{Cm}$ & ${ }^{254 \mathrm{~m}} \mathrm{Es}$ \\
\hline${ }^{212} \mathrm{Po}$ & ${ }^{223} \mathrm{Ra}$ & ${ }^{235} \mathrm{U}$ & ${ }^{241} \mathrm{Cm}$ & ${ }^{255} \mathrm{Es}$ \\
\hline${ }^{213} \mathrm{Po}$ & ${ }^{224} \mathrm{Ra}$ & ${ }^{236} \mathrm{U}$ & ${ }^{242} \mathrm{Cm}$ & ${ }^{254} \mathrm{Fm}$ \\
\hline${ }^{214} \mathrm{Po}$ & ${ }^{226} \mathrm{Ra}$ & ${ }^{238} \mathrm{U}$ & ${ }^{243} \mathrm{Cm}$ & ${ }^{255} \mathrm{Fm}$ \\
\hline \multirow[t]{2}{*}{${ }^{215} \mathrm{Po}$} & ${ }^{225} \mathrm{Ac}$ & ${ }^{235} \mathrm{~Np}$ & ${ }^{244} \mathrm{Cm}$ & ${ }^{256} \mathrm{Fm}$ \\
\hline & & & & ${ }^{257} \mathrm{Fm}$ \\
\hline
\end{tabular}

Table 5.2.10. Target $(\alpha, n)$ cross section and branching level isotopes and sources

\begin{tabular}{|c|c|c|c|}
\hline Isotope & ZAID & $\begin{array}{c}\text { Level branching fraction } \\
\text { source data }\end{array}$ & Cross section data \\
\hline${ }^{7} \mathrm{Li}$ & 30070 & GNASH & Gibbons and Macklin ${ }^{20}$ \\
\hline${ }^{9} \mathrm{Be}$ & 40090 & Geiger and Van der Zwain ${ }^{21}$ & Geiger and Van der Zwain \\
\hline${ }^{10} \mathrm{~B}$ & 50010 & GNASH & Bair et al. ${ }^{22}$ \\
\hline${ }^{11} \mathrm{~B}$ & 50110 & GNASH & Bair et al. \\
\hline${ }^{13} \mathrm{C}$ & 60130 & GNASH $^{a}$ & Bair and Haas ${ }^{23}$ \\
\hline${ }^{14} \mathrm{~N}$ & 70140 & $\mathrm{~N} / \mathrm{A}$ & GNASH \\
\hline${ }^{17} \mathrm{O}$ & 80170 & Lesser and Schenter ${ }^{24}$ & Perry and Wilson ${ }^{25}$ \\
\hline${ }^{18} \mathrm{O}$ & 80180 & Lesser and Schenter & Perry and Wilson \\
\hline${ }^{19} \mathrm{~F}$ & 90190 & Lesser and Schenter & Balakrishnan et al. $^{26}$ \\
\hline${ }^{21} \mathrm{Ne}$ & 100210 & N/A & GNASH \\
\hline${ }^{22} \mathrm{Ne}$ & 100220 & $\mathrm{~N} / \mathrm{A}$ & GNASH \\
\hline${ }^{23} \mathrm{Na}$ & 110230 & GNASH & $\mathrm{GNASH}^{a}$ \\
\hline${ }^{25} \mathrm{Mg}$ & 120250 & GNASH & GNASH \\
\hline${ }^{26} \mathrm{Mg}$ & 120260 & GNASH & GNASH \\
\hline${ }^{27} \mathrm{Al}$ & 130270 & GNASH & $\mathrm{GNASH}^{a}$ \\
\hline${ }^{29} \mathrm{Si}$ & 140290 & GNASH & $\mathrm{GNASH}^{a}$ \\
\hline${ }^{30} \mathrm{Si}$ & 140300 & GNASH & GNASH $^{a}$ \\
\hline${ }^{31} \mathrm{P}$ & 150310 & GNASH & GNASH \\
\hline${ }^{37} \mathrm{Cl}$ & 170370 & GNASH & Woosley et al. $^{27}$ \\
\hline
\end{tabular}

${ }^{a}$ GNASH calculated data and measured data are available for these nuclides in the library. By default, the GNASH values are used. To use the measured data, the user must reverse the order of the GNASH and measured data in the library since the code uses the first set encountered in the library (GNASH set). 


\subsubsection{References}

1. M. B. Chadwick et al., "ENDF/B-VII.1: Nuclear Data for Science and Technology: Cross Sections, Covariances, Fission Product Yields and Decay Data," Nucl. Data Sheets 112(2011)2887.

2. Code of Federal Regulations, Title 10, Part 20.

3. International Commission on Radiological Protection (ICRP). 1977. Age-Dependent Doses to Members of the Public from Intake of Radionuclides: Part 5, Compilation of Ingestion and Inhalation Dose Coefficients. ICRP Publication 72. International Commission on Radiological Protection, Pergamon Press, New York.

4. US Environmental Protection Agency (EPA). 1993. External Exposure to Radionuclides in Air, Water, and Soil. Federal Guidance Report 12, US Environmental Protection Agency, Washington, DC.

5. J-Ch. Sublet, A. J. Koning, R. A. Forrest and J. Kopecky, The JEFF-3.0/A Neutron Activation File-EAF-2003 into ENDF-6 Format, Commissariat à l'Energie Atomique, France, JEFDOC-982 (November 2003).

6. R. A. Forrest, J. Kopecky and J-Ch Sublet, The European Activation File: EAF-2003 Cross Section Library, UKAEA FUS-486, EURATOM/UKAEA Fusion (December 2002).

7. M. E. Dunn and N. M. Greene, "AMPX-2000: A Cross-Section Processing System for Generating Nuclear Data for Criticality Safety Applications,” Trans. Am. Nucl. Soc. 86, 118-119 (2002).

8. R. O. Gumprecht, "Current Status of the Isotopic Purity of Hanford Plutonium," Hanford Technical Record, HW-33874 (1954).

9. M. B. Chadwick, P. Oblozinsky, M. Herman et al., "ENDF/B-VII.0: Next Generation Evaluated Nuclear Data Library for Nuclear Science and Technology," Nuclear Data Sheets 107, 2931-3060 (2006).

10. M. T. Pigni, M. W. Francis, I. C. Gauld, Investigation of inconsistent ENDF/B-VII.1 independent and cumulative fission product yields with propossed revisions, Nuclear Data Sheets 123, 231 (2015).

11. M. W. Francis, C. F. Weber, M. T. Pigni, Reactor Fuel Isotopics and Code Validation for Nuclear Applications, ORNL TM-2014/464 (2015).

12. The JEF-2.2 Nuclear Data Library, Nuclear Energy Agency, Organisation For Economic CoOperation And Development, JEFF Report 17, April 2000.

13. A. G. Croft, R. L. Haese, and N. B. Grove, Updated Decay and Photon Libraries for the ORIGEN Code, ORNL/TM-6055, Union Carbide Corporation (Nuclear Division), Oak Ridge National Laboratory, Oak Ridge, TN, February 1979.

14. E. C. Fortune IV, I. C. Gauld, C-K C. Wang, "Gamma Dose Rate Near a New ${ }^{252} \mathrm{Cf}$ Brachytherapy Source," Nuc. Technol. (July 2011).

15. L. T. Dillman, W. S. Snyder, and M. R. Ford, "Nuclear Data Compilation of Utility in Medical and Biological Applications," in Proceedings of the Symposium on Application of Nuclear Data in Science and Technology, IAEA-FM-170/43, Vol. 2, pp. 529-539 (March 1973).

16. N. B. Gove and M. J. Martin, “Log-f Tables for Beta Decay,” Nucl. Data Tables 10, 206 (1971).

17. E. F. Shores, Data Updates for the SOURCES-4A Computer Code, LA-UR-00-5016, Los Alamos National Laboratory, Los Alamos, NM, October 2000. 
18. J. F. Ziegler, "Helium Stopping Power and Ranges in All Elemental Matter," Vol. 4 of The Stopping Power and Ranges of Ions in Matter Series, Pergamon Press, New York (1977).

19. W. B. Wilson, R. T. Pery, J. E. Stewart, T. R. England, D. G. Madland, and E. D. Arthur, Development of the SOURCES Code and Data Library for the Calculation of Neutron Sources and Spectra from $(\alpha, n)$ Reactions, Spontaneous Fission, and $\beta^{-}$Delayed Neutrons, LA-9841-PR, Los Alamos National Laboratory, Los Alamos, NM (1983).

20. J. H. Gibbons and R. L. Macklin, "Total Neutron Yields from Light Elements Under Proton and Alpha Bombardment," Phys. Rev. 114, 571 (1959).

21. K. W. Geiger and L. Van Der Zwain, "An Evaluation of the ${ }^{9} \mathrm{Be}(\alpha, n)$ Cross Section Data," Nucl. Instr. and Methods 131, 315 (1975).

22. J. K. Bair and J. Gomez Del Campo, "Neutron Yields from Alpha Particle Bombardment," Nucl. Sci. and Eng. 71, 18 (1979).

23. J. K. Bair and F. X. Hass, "Total Neutron Yield from the Reaction ${ }^{13} \mathrm{C}(\alpha, n){ }^{16} \mathrm{O}$ and ${ }^{17,18} \mathrm{O}(\alpha, \mathrm{n})$ ${ }^{20,21}$ Ne," Phys. Rev. C 7, 1356 (1973).

24. D. L. Lessor and R. E. Schenter, Neutron Spectra from $(\alpha, n)$ Reaction in Plutonium Compounds Calculated from Hauser-Feshbach Reaction Theory, BNWL-B-109, Brookhaven National Laboratory, Upton, NY (1971).

25. R. T. Perry and W. B. Wilson, Neutron Production from $(\alpha, n)$ Reactions and Spontaneous Fission in $\mathrm{ThO}_{2}, \mathrm{UO}_{2}$, and $(\mathrm{U}, \mathrm{Pu}) \mathrm{O}_{2}$ Fuels, LA-8869-MS, Los Alamos National Laboratory, Los Alamos, NM (1981).

26. M. Balakrishnan, S. Kailas, and M. K. Mehta, "A Study of the Reaction ${ }^{19} \mathrm{~F}(\alpha, n){ }^{22} \mathrm{Na}$ in the Bombarding Energy Range 2.6 to 5.1 MeV," Pramana 10, 329 (1978).

27. S. E. Woosley, W. A. Fowler, J. A. Holmes, and B. A. Zimmerman, Tables of Thermonuclear Reaction Rate Data for Intermediate Mass Nuclei, Preprint OAP-422, Kellogg Radiation Laboratory, California Institute of Technology, Pasadena, CA (1975). 


\section{APPENDIX 5.2.A - Decay Resource Format}

The decay resource is a simple text format file that can be processed by COUPLE to create a binary decay-only library that can be used directly by ORIGEN. In general, this is not necessary, as the decay resource distributed with SCALE has already been processed with COUPLE to produce the end7dec ORIGEN decay-only binary library file. Modifying the decay data or the set of nuclides ORIGEN tracks requires modification of the decay resource file. The format is described in Table 5.2.11. Note that as of the SCALE 6.2 release, ORIGEN now uses the SCALE Standard Composition resource for abundance data and the "ABUND" field shown below is ignored by COUPLE when reading the decay resource.

Table 5.2.11. Definitions of data in the decay resource

\begin{tabular}{ll}
\hline Data name & \\
\hline LIB & Nuclide sublib (used by COUPLE) \\
NUC1 & Nuclide identifier \\
IU & Units for the half life value (see Table 5.2.12) \\
HALFL & Value of the half life in IU units \\
FB1 & Beta decay transition leading to a daughter in the metastable state \\
FP & Positron emission decay fraction or orbital electron capture to the ground state \\
FP1 & Positron emission decay fraction or orbital electron capture to a metastable state \\
FA & Alpha particle emission decay fraction \\
FT & Isomeric transition decay fraction \\
LIB1 & Nuclide type in the library \\
FSF & Spontaneous fission decay fraction \\
FBN & Delayed neutron decay (beta particle and a neutron) fraction \\
Q & Recoverable energy per decay (MeV) \\
ABUND & Natural atom isotopic abundance in percent (no longer used) \\
AMPC & Maximum permissible concentration in air \\
WMPC & Maximum permissible concentration in water \\
LIB1 & Nuclide type in the library (used by COUPLE) \\
FG & Fraction of recoverable decay energy Q associated with gamma rays \\
FB & Beta decay transition leading to a daughter in the ground state \\
FBB & Double beta decay fraction \\
FN & Neutron decay fraction \\
FBA & Beta decay plus an alpha particle emission decay fraction \\
\hline &
\end{tabular}

The variable LIB (and LIB1) defines the nuclide sublibrary $(1 / 2 / 3=$ activation product/actinide/fission product). Variable LIBI is included for formatting purposes only. 
The nuclide identifier is read in variable NUC1 and is subsequently stored in array NUCL. The nuclide identifier is given by

$$
\mathrm{NUCL}=\mathrm{Z} * 10000+\mathrm{A} * 10+\mathrm{I}
$$

where $\mathrm{Z}$ is the atomic number, $\mathrm{A}$ is the atomic mass number, and $\mathrm{I}$ is the isomeric state, where $\mathrm{I}=0$ designates a ground state, and $\mathrm{I}=1$ is the first metastable state.

The variable HALFL is the physical half-life in units designated by the variable IU, as shown in Table 5.2.12. The definitions of 11 variables representing the different decay mode branching fractions are given in Table 5.2.11. The decay branching fractions are used in constructing the transition matrix.

Table 5.2.12. Units of half-life indicated by the variable IU

\begin{tabular}{cl}
\hline IU & Units of half-life \\
\hline 1 & seconds \\
2 & minutes \\
3 & hours \\
4 & days \\
5 & years \\
6 & stable \\
7 & $10^{3}$ years \\
8 & $10^{6}$ years \\
9 & $10^{9}$ years \\
\hline
\end{tabular}

The variable $Q$ is the total amount of recoverable energy $(\mathrm{MeV})$ per disintegration released by radioactive decay used for decay heat calculations. It does not include the energy of neutrinos emitted during beta decay transitions. The variable $\mathrm{FG}$ is the fraction of recoverable energy per disintegration that comes from gamma rays and $x$-rays. The value of $Q$ is obtained directly from ENDF/B-VII.1 as the sum of the average beta, gamma, and alpha decay energy values. The quantity includes the energy from all electron- related radiations such as $\beta^{-}, \beta+$, Auger electrons, etc., all gamma rays, x-rays, and annihilation radiations, and the average energy of all heavy charged particles and delayed neutrons. The contribution from alpha decay energy includes the energy of the recoil nucleus. A part of the recoverable energy per decay not included in the ENDF/B-VII.1 values is the additional contribution from spontaneous fission. This energy was calculated as the product of the spontaneous fission branching fraction and recoverable energy per fission using a value of $200 \mathrm{MeV}$ per fission and added to the ENDF/B-VII.1 recoverable Q energy. A value of $12.56 \mathrm{MeV}$ gamma energy per fission was used in computing the fraction of recoverable spontaneous fission energy from gamma rays.

External bremsstrahlung radiation is not included in the values of FG since the bremsstrahlung spectrum depends on electron interactions with the medium that contains the decay nuclide. The energy from capture gamma rays accompanying $(\alpha, n)$ reactions is also not included since it also depends on the medium. The variable ABUND is the atom percent abundance of naturally occurring isotopes. 


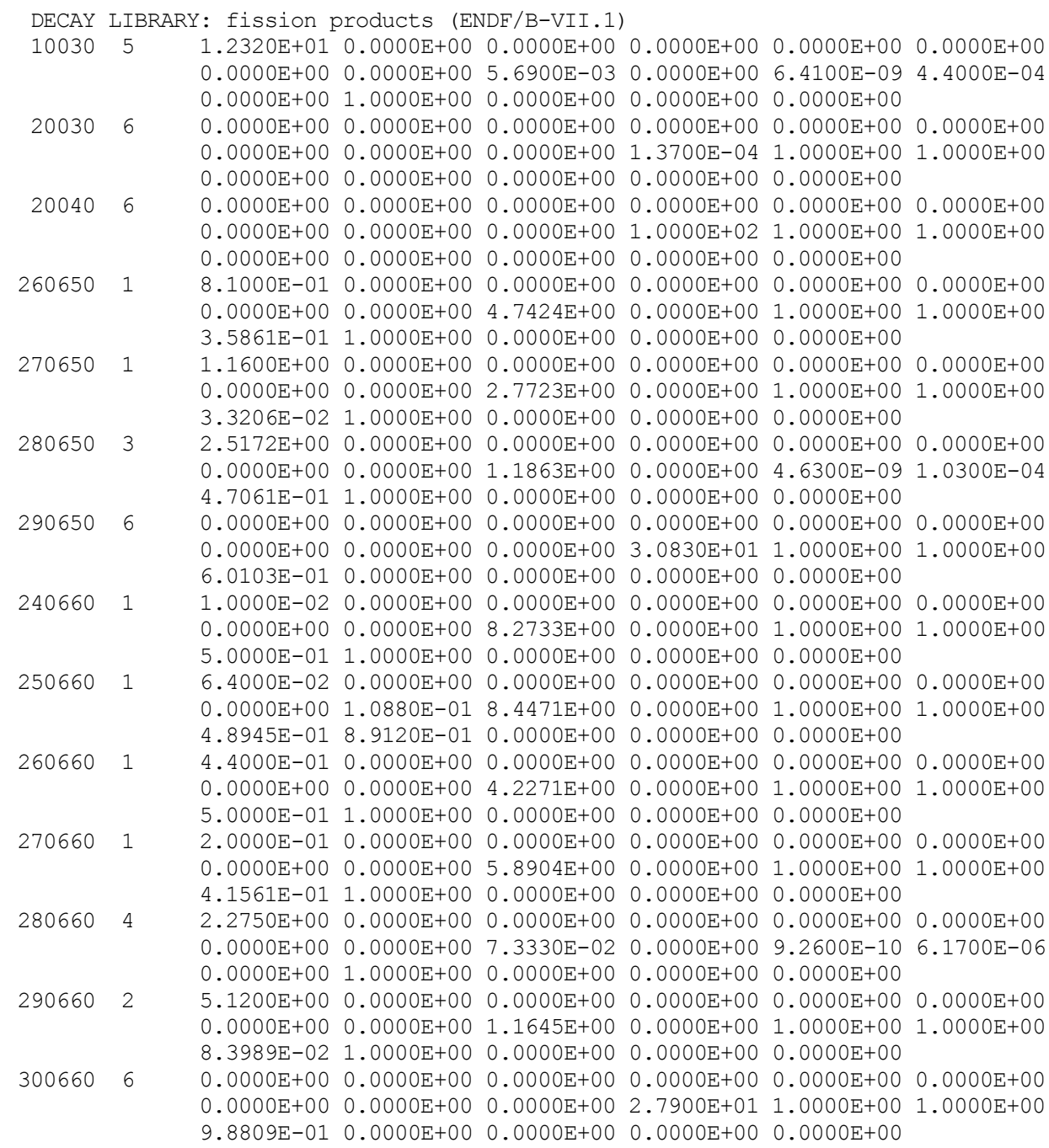

Figure 5.2.3. Example of the ENDF/B-VII.1 decay data resource entries for selected fission products. 


\section{APPENDIX 5.2.B -Fission Yield Resource Format}

The independent fission product yields are stored as a formatted text file. The header record for each set of fission product yields includes the fissionable nuclide ID and an unused entry (0.0), followed by the number of incident neutron energies included for this nuclide. The fission yields for each energy are preceeded by a single record containing the incident neutron energy $(\mathrm{eV})$, an unused entry $(0.0)$, an index for the incident energy, the number of data entries per fission product, the total number of entries for each incident energy, and the number of fission products. The fission product yields for each fissionable nuclide and incident neutron energy are then listed as pairs of entries for the fission product nuclide ID and the independent (direct) fission yield as atom percent per fission. An example of the format is shown below in Figure 5.2.4 for ${ }^{227} \mathrm{Th}$.

The number and order of the fission product yields must be the same for all fissionable nuclides and must correspond to the fission products in the nuclear decay data. The fission product yields for each fissionable nuclide, excluding the yields for the terniary fission products ${ }^{3} \mathrm{H},{ }^{3} \mathrm{He}$, and ${ }^{4} \mathrm{He}$, sum to 200 .

The fissionable nuclides and the tabulated incident neutron energies for which yields are available are listed in Table 5.2.2.

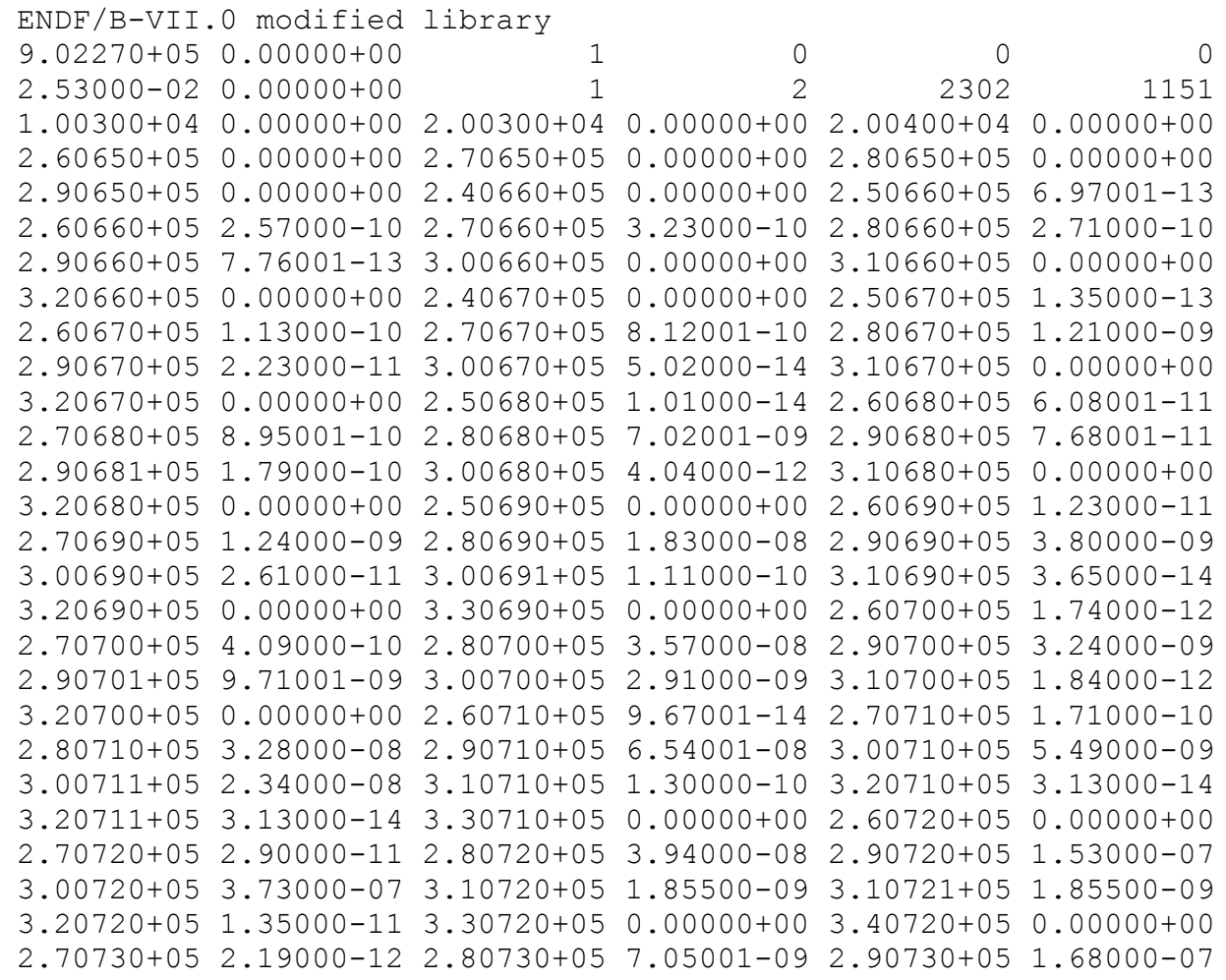

Figure 5.2.4. Fission yield format example showing a portion of ${ }^{227} \mathrm{Th}$. 


\section{APPENDIX 5.2.C - Gamma Resource Format}

An example of the photon data entries for the emissions from ${ }^{140}$ La decay is shown below in Figure 5.2.5. The header record for each nuclide contains the nuclide ID, the total number of emission lines in the evaluation, as well as the number of discrete x-ray lines, discrete gamma lines, and number of pseudo lines used to represent continuum data if present in an evaluation used to reconstruct continuous energy emission spectra from the discrete representation. The last entries in the header record include the total gamma energy $(\mathrm{MeV})$, and the character nuclide name. The emission spectrum is listed using pairs of entries for the photon energy $(\mathrm{MeV})$ and photon emission (photons per disintegration).

\begin{tabular}{|c|c|c|c|c|c|}
\hline 571400 & $\begin{array}{c}52 . \\
4.3847 \mathrm{E}-03 \\
6.0946 \mathrm{E}-03 \\
3.9196 \mathrm{E}-02 \\
3.9570 \mathrm{E}-02 \\
4.0340 \mathrm{E}-02 \\
6.4135 \mathrm{E}-02 \\
1.3112 \mathrm{E}-01 \\
2.6654 \mathrm{E}-01 \\
3.9752 \mathrm{E}-01 \\
4.4550 \mathrm{E}-01 \\
7.5164 \mathrm{E}-01 \\
9.1955 \mathrm{E}-01 \\
9.9290 \mathrm{E}-01 \\
1.3035 \mathrm{E}+00 \\
1.8773 \mathrm{E}+00 \\
2.3479 \mathrm{E}+00 \\
2.5473 \mathrm{E}+00 \\
3.3204 \mathrm{E}+00\end{array}$ & $\begin{array}{c}14 . \\
2.1017 \mathrm{E}-04 \\
2.3418 \mathrm{E}-04 \\
1.0523 \mathrm{E}-03 \\
1.6866 \mathrm{E}-05 \\
2.6312 \mathrm{E}-06 \\
1.4310 \mathrm{E}-04 \\
4.6746 \mathrm{E}-03 \\
4.6555 \mathrm{E}-03 \\
7.3458 \mathrm{E}-04 \\
2.8620 \mathrm{E}-05 \\
4.3312 \mathrm{E}-02 \\
2.6617 \mathrm{E}-02 \\
1.3356 \mathrm{E}-04 \\
4.1976 \mathrm{E}-04 \\
4.1022 \mathrm{E}-04 \\
8.4906 \mathrm{E}-03 \\
1.0112 \mathrm{E}-03 \\
3.8160 \mathrm{E}-05\end{array}$ & $\begin{array}{l}38 . \\
4.8247 \mathrm{E}-03 \\
3.4291 \mathrm{E}-02 \\
3.9285 \mathrm{E}-02 \\
4.0227 \mathrm{E}-02 \\
4.0344 \mathrm{E}-02 \\
6.8916 \mathrm{E}-02 \\
1.7354 \mathrm{E}-01 \\
3.0690 \mathrm{E}-01 \\
4.3249 \mathrm{E}-01 \\
4.8702 \mathrm{E}-01 \\
8.1577 \mathrm{E}-01 \\
9.2519 \mathrm{E}-01 \\
1.0451 \mathrm{E}+00 \\
1.4052 \mathrm{E}+00 \\
1.9246 \mathrm{E}+00 \\
2.4641 \mathrm{E}+00 \\
2.8996 \mathrm{E}+00\end{array}$ & $\begin{array}{c}0 . \\
1.7789 \mathrm{E}-03 \\
5.9015 \mathrm{E}-03 \\
2.0389 \mathrm{E}-03 \\
2.2404 \mathrm{E}-04 \\
3.5410 \mathrm{E}-06 \\
7.5366 \mathrm{E}-04 \\
1.2688 \mathrm{E}-03 \\
2.4804 \mathrm{E}-04 \\
2.9002 \mathrm{E}-02 \\
4.5506 \mathrm{E}-01 \\
2.3278 \mathrm{E}-01 \\
6.8974 \mathrm{E}-02 \\
2.4804 \mathrm{E}-04 \\
5.9148 \mathrm{E}-04 \\
1.3356 \mathrm{E}-04 \\
1.1448 \mathrm{E}-04 \\
6.6780 \mathrm{E}-04\end{array}$ & $\begin{array}{ll}0.2 .3083 \mathrm{E}+00 & 1 \mathrm{a} \\
5.3304 \mathrm{E}-03 & 1.5654 \mathrm{E}-03 \\
3.4743 \mathrm{E}-02 & 1.0817 \mathrm{E}-02 \\
3.9550 \mathrm{E}-02 & 1.2513 \mathrm{E}-05 \\
4.0247 \mathrm{E}-02 & 4.3591 \mathrm{E}-04 \\
2.4595 \mathrm{E}-02 & 1.4971 \mathrm{E}-05 \\
1.0942 \mathrm{E}-01 & 2.1942 \mathrm{E}-03 \\
2.4193 \mathrm{E}-01 & 4.1404 \mathrm{E}-03 \\
3.2876 \mathrm{E}-01 & 2.0320 \mathrm{E}-01 \\
4.3850 \mathrm{E}-01 & 3.9114 \mathrm{E}-04 \\
6.1812 \mathrm{E}-01 & 3.7206 \mathrm{E}-04 \\
8.6785 \mathrm{E}-01 & 5.5046 \mathrm{E}-02 \\
9.5099 \mathrm{E}-01 & 5.1898 \mathrm{E}-03 \\
1.0972 \mathrm{E}+00 & 2.2896 \mathrm{E}-04 \\
1.5962 \mathrm{E}+00 & 9.5400 \mathrm{E}-01 \\
2.0832 \mathrm{E}+00 & 1.1543 \mathrm{E}-04 \\
2.5214 \mathrm{E}+00 & 3.4630 \mathrm{E}-02 \\
3.1185 \mathrm{E}+00 & 2.4804 \mathrm{E}-04\end{array}$ \\
\hline
\end{tabular}

Figure 5.2.5. Gamma resource format example showing ${ }^{140} \mathrm{La} \mathrm{decay} \mathrm{photon} \mathrm{emission.}$ 


\section{APPENDIX 5.2.D -ORIGEN “end7dec" Nuclide Set}

Table 5.2.13 shows a list of the 2,237 nuclides on the origen. rev0 4 . end 7 dec ORIGEN binary decay-only library, and because this library provides the basis for all other libraries, effectively the set of nuclides tracked by ORIGEN in any decay or irradiation calculation. The "index" column is the index of that nuclide in the set (internally every ORIGEN isotopics vector has this order), the "sublib" column is the sublibrary ( $\mathrm{LT}=$ light nuclide, $\mathrm{AC}=$ actinide, $\mathrm{FP}=$ fission product) in which the nuclide resides, the "nuclide" column is the nuclide identifier, the "mass" column is the mass of the nuclide in grams per mole, the "abundance" column is the natural abundance in atom percent for the nuclide (note only light nuclides have abundances), and the "decay" column is the decay constant. Note that the mass and abundance data are embedded on the library with the values from the current SCALE Standard Composition Library.

Table 5.2.13. Nuclide listing for "end7dec" ORIGEN library.

\begin{tabular}{|c|c|c|c|c|c|}
\hline index & sublib & nuclide & $\begin{array}{c}\text { mass } \\
(\mathrm{g} / \mathrm{mol})\end{array}$ & $\begin{array}{c}\text { abundance } \\
\text { (atom\%) }\end{array}$ & $\begin{array}{l}\text { decay } \\
(1 / \mathrm{s})\end{array}$ \\
\hline 1 & LT & $1-\mathrm{H}-1$ & 1.0078 & $1.00 \mathrm{E}+02$ & $0.00 \mathrm{E}+00$ \\
\hline 2 & LT & $1-\mathrm{H}-2$ & 2.0141 & $1.15 \mathrm{E}-02$ & $0.00 \mathrm{E}+00$ \\
\hline 3 & LT & $1-\mathrm{H}-3$ & 3.0161 & $0.00 \mathrm{E}+00$ & $1.78 \mathrm{E}-09$ \\
\hline 4 & LT & 2-He-3 & 3.0160 & $1.00 \mathrm{E}-04$ & $0.00 \mathrm{E}+00$ \\
\hline 5 & LT & $2-\mathrm{He}-4$ & 4.0026 & $1.00 \mathrm{E}+02$ & $0.00 \mathrm{E}+00$ \\
\hline 6 & LT & 2-He-5 & 5.0122 & $0.00 \mathrm{E}+00$ & $6.93 \mathrm{E}+02$ \\
\hline 7 & LT & 2-He-6 & 6.0189 & $0.00 \mathrm{E}+00$ & $8.59 \mathrm{E}-01$ \\
\hline 8 & LT & 3-Li-6 & 6.0151 & $7.59 \mathrm{E}+00$ & $0.00 \mathrm{E}+00$ \\
\hline 9 & LT & 3-Li-7 & 7.0160 & $9.24 \mathrm{E}+01$ & $0.00 \mathrm{E}+00$ \\
\hline 10 & LT & 3-Li-8 & 8.0225 & $0.00 \mathrm{E}+00$ & $8.27 \mathrm{E}-01$ \\
\hline 11 & LT & 4-Be-7 & 7.0169 & $0.00 \mathrm{E}+00$ & $1.51 \mathrm{E}-07$ \\
\hline 12 & LT & 4-Be-8 & 8.0053 & $0.00 \mathrm{E}+00$ & $6.93 \mathrm{E}+02$ \\
\hline 13 & LT & 4-Be-9 & 9.0122 & $1.00 \mathrm{E}+02$ & $0.00 \mathrm{E}+00$ \\
\hline 14 & LT & 4-Be-10 & 10.0135 & $0.00 \mathrm{E}+00$ & $1.45 \mathrm{E}-14$ \\
\hline 15 & LT & 4-Be-11 & 11.0217 & $0.00 \mathrm{E}+00$ & $5.02 \mathrm{E}-02$ \\
\hline 16 & LT & 5-B-10 & 10.0129 & $1.99 \mathrm{E}+01$ & $0.00 \mathrm{E}+00$ \\
\hline 17 & LT & 5-B-11 & 11.0093 & $8.01 \mathrm{E}+01$ & $0.00 \mathrm{E}+00$ \\
\hline 18 & LT & $5-B-12$ & 12.0143 & $0.00 \mathrm{E}+00$ & $3.43 \mathrm{E}+01$ \\
\hline 19 & LT & 6-C-12 & 12.0000 & $9.89 \mathrm{E}+01$ & $0.00 \mathrm{E}+00$ \\
\hline 20 & LT & 6-C-13 & 13.0034 & $1.07 \mathrm{E}+00$ & $0.00 \mathrm{E}+00$ \\
\hline 21 & LT & 6-C-14 & 14.0032 & $0.00 \mathrm{E}+00$ & $3.85 \mathrm{E}-12$ \\
\hline 22 & LT & 6-C-15 & 15.0106 & $0.00 \mathrm{E}+00$ & $2.83 \mathrm{E}-01$ \\
\hline 23 & LT & $7-\mathrm{N}-13$ & 13.0057 & $0.00 \mathrm{E}+00$ & $1.16 \mathrm{E}-03$ \\
\hline 24 & LT & $7-\mathrm{N}-14$ & 14.0031 & $9.96 \mathrm{E}+01$ & $0.00 \mathrm{E}+00$ \\
\hline 25 & LT & $7-N-15$ & 15.0001 & 3.64E-01 & $0.00 \mathrm{E}+00$ \\
\hline 26 & LT & $7-\mathrm{N}-16$ & 16.0061 & $0.00 \mathrm{E}+00$ & $9.72 \mathrm{E}-02$ \\
\hline 27 & LT & $8-0-16$ & 15.9949 & $9.98 \mathrm{E}+01$ & $0.00 \mathrm{E}+00$ \\
\hline 28 & LT & $8-\mathrm{O}-17$ & 16.9991 & $3.80 \mathrm{E}-02$ & $0.00 \mathrm{E}+00$ \\
\hline 29 & LT & 8-O-18 & 17.9992 & $2.05 \mathrm{E}-01$ & $0.00 \mathrm{E}+00$ \\
\hline 30 & LT & 8-O-19 & 19.0036 & $0.00 \mathrm{E}+00$ & $2.58 \mathrm{E}-02$ \\
\hline 31 & LT & 9-F-19 & 18.9984 & $1.00 \mathrm{E}+02$ & $0.00 \mathrm{E}+00$ \\
\hline 32 & LT & 9-F-20 & 20.0000 & $0.00 \mathrm{E}+00$ & $6.21 \mathrm{E}-02$ \\
\hline 33 & LT & $10-\mathrm{Ne}-20$ & 19.9924 & $9.05 \mathrm{E}+01$ & $0.00 \mathrm{E}+00$ \\
\hline 34 & LT & $10-\mathrm{Ne}-21$ & 20.9939 & $2.70 \mathrm{E}-01$ & $0.00 \mathrm{E}+00$ \\
\hline 35 & LT & $10-\mathrm{Ne}-22$ & 21.9914 & $9.25 \mathrm{E}+00$ & $0.00 \mathrm{E}+00$ \\
\hline 36 & LT & $10-\mathrm{Ne}-23$ & 22.9945 & $0.00 \mathrm{E}+00$ & $1.86 \mathrm{E}-02$ \\
\hline 37 & LT & 11-Na-22 & 21.9944 & $0.00 \mathrm{E}+00$ & $8.44 \mathrm{E}-09$ \\
\hline 38 & LT & $11-\mathrm{Na}-23$ & 22.9898 & $1.00 \mathrm{E}+02$ & $0.00 \mathrm{E}+00$ \\
\hline 39 & LT & 11-Na-24 & 23.9910 & $0.00 \mathrm{E}+00$ & $1.28 \mathrm{E}-05$ \\
\hline 40 & LT & $11-\mathrm{Na}-24 \mathrm{~m}$ & 23.9910 & $0.00 \mathrm{E}+00$ & $3.43 \mathrm{E}+01$ \\
\hline 41 & LT & $11-\mathrm{Na}-25$ & 24.9900 & $0.00 \mathrm{E}+00$ & $1.17 \mathrm{E}-02$ \\
\hline 42 & LT & $12-\mathrm{Mg}-24$ & 23.9850 & $7.90 \mathrm{E}+01$ & $0.00 \mathrm{E}+00$ \\
\hline 43 & LT & $12-\mathrm{Mg}-25$ & 24.9858 & $1.00 \mathrm{E}+01$ & $0.00 \mathrm{E}+00$ \\
\hline 44 & LT & $12-\mathrm{Mg}-26$ & 25.9826 & $1.10 \mathrm{E}+01$ & $0.00 \mathrm{E}+00$ \\
\hline 45 & LT & $12-\mathrm{Mg}-27$ & 26.9843 & $0.00 \mathrm{E}+00$ & $1.22 \mathrm{E}-03$ \\
\hline
\end{tabular}




\begin{tabular}{|c|c|c|c|c|c|}
\hline 46 & $\mathrm{LT}$ & $12-\mathrm{Mg}-28$ & 27.9839 & $0.00 \mathrm{E}+00$ & $9.21 \mathrm{E}-06$ \\
\hline 47 & LT & $13-\mathrm{Al}-26$ & 25.9869 & $0.00 \mathrm{E}+00$ & $3.06 \mathrm{E}-14$ \\
\hline 48 & LT & $13-\mathrm{Al}-27$ & 26.9815 & $1.00 \mathrm{E}+02$ & $0.00 \mathrm{E}+00$ \\
\hline 49 & LT & $13-\mathrm{Al}-28$ & 27.9819 & $0.00 \mathrm{E}+00$ & $5.15 \mathrm{E}-03$ \\
\hline 50 & LT & $13-\mathrm{Al}-29$ & 28.9804 & $0.00 \mathrm{E}+00$ & $1.76 \mathrm{E}-03$ \\
\hline 51 & LT & $13-\mathrm{Al}-30$ & 29.9830 & $0.00 \mathrm{E}+00$ & $1.91 \mathrm{E}-01$ \\
\hline 52 & LT & 14-Si-28 & 27.9769 & $9.22 \mathrm{E}+01$ & $0.00 \mathrm{E}+00$ \\
\hline 53 & LT & 14-Si-29 & 28.9765 & $4.69 \mathrm{E}+00$ & $0.00 \mathrm{E}+00$ \\
\hline 54 & LT & 14-Si-30 & 29.9738 & $3.09 \mathrm{E}+00$ & $0.00 \mathrm{E}+00$ \\
\hline 55 & LT & 14-Si-31 & 30.9754 & $0.00 \mathrm{E}+00$ & $7.34 \mathrm{E}-05$ \\
\hline 56 & LT & 14-Si-32 & 31.9741 & $0.00 \mathrm{E}+00$ & $1.44 \mathrm{E}-10$ \\
\hline 57 & $\mathrm{LT}$ & $15-\mathrm{P}-31$ & 30.9738 & $1.00 \mathrm{E}+02$ & $0.00 \mathrm{E}+00$ \\
\hline 58 & LT & $15-\mathrm{P}-32$ & 31.9739 & $0.00 \mathrm{E}+00$ & $5.62 \mathrm{E}-07$ \\
\hline 59 & $\mathrm{LT}$ & 15-P-33 & 32.9717 & $0.00 \mathrm{E}+00$ & $3.17 \mathrm{E}-07$ \\
\hline 60 & LT & 15-P-34 & 33.9736 & $0.00 \mathrm{E}+00$ & $5.58 \mathrm{E}-02$ \\
\hline 61 & $\mathrm{LT}$ & $16-S-32$ & 31.9721 & $9.50 \mathrm{E}+01$ & $0.00 \mathrm{E}+00$ \\
\hline 62 & LT & $16-S-33$ & 32.9715 & $7.50 \mathrm{E}-01$ & $0.00 \mathrm{E}+00$ \\
\hline 63 & LT & $16-S-34$ & 33.9679 & $4.25 \mathrm{E}+00$ & $0.00 \mathrm{E}+00$ \\
\hline 64 & $\mathrm{LT}$ & $16-\mathrm{S}-35$ & 34.9690 & $0.00 \mathrm{E}+00$ & $9.17 \mathrm{E}-08$ \\
\hline 65 & LT & $16-S-36$ & 35.9671 & $1.00 \mathrm{E}-02$ & $0.00 \mathrm{E}+00$ \\
\hline 66 & $\mathrm{LT}$ & $16-S-37$ & 36.9711 & $0.00 \mathrm{E}+00$ & $2.29 \mathrm{E}-03$ \\
\hline 67 & LT & $17-\mathrm{Cl}-35$ & 34.9688 & $7.58 \mathrm{E}+01$ & $0.00 \mathrm{E}+00$ \\
\hline 68 & LT & $17-\mathrm{Cl}-36$ & 35.9683 & $0.00 \mathrm{E}+00$ & $7.30 \mathrm{E}-14$ \\
\hline 69 & LT & $17-\mathrm{Cl}-37$ & 36.9659 & $2.42 \mathrm{E}+01$ & $0.00 \mathrm{E}+00$ \\
\hline 70 & LT & $17-\mathrm{Cl}-38$ & 37.9680 & $0.00 \mathrm{E}+00$ & $3.10 \mathrm{E}-04$ \\
\hline 71 & LT & $17-\mathrm{Cl}-38 \mathrm{~m}$ & 37.9680 & $0.00 \mathrm{E}+00$ & $9.69 \mathrm{E}-01$ \\
\hline 72 & LT & $18-\mathrm{Ar}-36$ & 35.9675 & $3.37 \mathrm{E}-01$ & $0.00 \mathrm{E}+00$ \\
\hline 73 & LT & $18-\mathrm{Ar}-37$ & 36.9668 & $0.00 \mathrm{E}+00$ & $2.29 \mathrm{E}-07$ \\
\hline 74 & LT & $18-\mathrm{Ar}-38$ & 37.9627 & $6.32 \mathrm{E}-02$ & $0.00 \mathrm{E}+00$ \\
\hline 75 & $\mathrm{LT}$ & 18-Ar-39 & 38.9643 & $0.00 \mathrm{E}+00$ & $8.17 \mathrm{E}-11$ \\
\hline 76 & LT & $18-\mathrm{Ar}-40$ & 39.9624 & $9.96 \mathrm{E}+01$ & $0.00 \mathrm{E}+00$ \\
\hline 77 & $\mathrm{LT}$ & 18-Ar-41 & 40.9645 & $0.00 \mathrm{E}+00$ & $1.05 \mathrm{E}-04$ \\
\hline 78 & $\mathrm{LT}$ & 18-Ar-42 & 41.9631 & $0.00 \mathrm{E}+00$ & $6.68 \mathrm{E}-10$ \\
\hline 79 & $\mathrm{LT}$ & $19-K-39$ & 38.9637 & $9.33 \mathrm{E}+01$ & $0.00 \mathrm{E}+00$ \\
\hline 80 & $\mathrm{LT}$ & $19-K-40$ & 39.9640 & $1.17 \mathrm{E}-02$ & $1.76 \mathrm{E}-17$ \\
\hline 81 & $\mathrm{LT}$ & $19-K-41$ & 40.9618 & $6.73 \mathrm{E}+00$ & $0.00 \mathrm{E}+00$ \\
\hline 82 & LT & $19-K-42$ & 41.9624 & $0.00 \mathrm{E}+00$ & $1.56 \mathrm{E}-05$ \\
\hline 83 & $\mathrm{LT}$ & $19-\mathrm{K}-43$ & 42.9607 & $0.00 \mathrm{E}+00$ & $8.63 \mathrm{E}-06$ \\
\hline 84 & LT & $19-K-44$ & 43.9616 & $0.00 \mathrm{E}+00$ & $5.22 \mathrm{E}-04$ \\
\hline 85 & LT & $20-\mathrm{Ca}-40$ & 39.9626 & $9.69 \mathrm{E}+01$ & $0.00 \mathrm{E}+00$ \\
\hline 86 & LT & $20-\mathrm{Ca}-41$ & 40.9623 & $0.00 \mathrm{E}+00$ & $2.15 \mathrm{E}-13$ \\
\hline 87 & LT & $20-\mathrm{Ca}-42$ & 41.9586 & $6.47 \mathrm{E}-01$ & $0.00 \mathrm{E}+00$ \\
\hline 88 & $\mathrm{LT}$ & $20-\mathrm{Ca}-43$ & 42.9588 & $1.35 \mathrm{E}-01$ & $0.00 \mathrm{E}+00$ \\
\hline 89 & $\mathrm{LT}$ & 20-Ca-44 & 43.9555 & $2.09 \mathrm{E}+00$ & $0.00 \mathrm{E}+00$ \\
\hline 90 & LT & 20-Ca-45 & 44.9562 & $0.00 \mathrm{E}+00$ & 4.93E-08 \\
\hline 91 & LT & 20-Ca-46 & 45.9537 & $4.00 \mathrm{E}-03$ & $0.00 \mathrm{E}+00$ \\
\hline 92 & LT & $20-\mathrm{Ca}-47$ & 46.9546 & $0.00 \mathrm{E}+00$ & $1.77 \mathrm{E}-06$ \\
\hline 93 & LT & 20-Ca-48 & 47.9525 & $1.87 \mathrm{E}-01$ & $9.55 \mathrm{E}-28$ \\
\hline 94 & LT & 20-Ca-49 & 48.9557 & $0.00 \mathrm{E}+00$ & $1.33 \mathrm{E}-03$ \\
\hline 95 & $\mathrm{LT}$ & 21-Sc-44 & 43.9594 & $0.00 \mathrm{E}+00$ & $4.85 \mathrm{E}-05$ \\
\hline 96 & LT & $21-\mathrm{Sc}-44 \mathrm{~m}$ & 43.9594 & $0.00 \mathrm{E}+00$ & $3.29 \mathrm{E}-06$ \\
\hline 97 & LT & 21-Sc-45 & 44.9559 & $1.00 \mathrm{E}+02$ & $0.00 \mathrm{E}+00$ \\
\hline 98 & $\mathrm{LT}$ & $21-\mathrm{Sc}-45 \mathrm{~m}$ & 44.9559 & $0.00 \mathrm{E}+00$ & $2.18 \mathrm{E}+00$ \\
\hline 99 & LT & $21-\mathrm{Sc}-46$ & 45.9552 & $0.00 \mathrm{E}+00$ & $9.57 \mathrm{E}-08$ \\
\hline 100 & $\mathrm{LT}$ & $21-\mathrm{Sc}-46 \mathrm{~m}$ & 45.9552 & $0.00 \mathrm{E}+00$ & $3.70 \mathrm{E}-02$ \\
\hline 101 & LT & $21-\mathrm{Sc}-47$ & 46.9524 & $0.00 \mathrm{E}+00$ & $2.40 \mathrm{E}-06$ \\
\hline 102 & $\mathrm{LT}$ & $21-\mathrm{Sc}-48$ & 47.9522 & $0.00 \mathrm{E}+00$ & $4.41 \mathrm{E}-06$ \\
\hline 103 & $\mathrm{LT}$ & 21-Sc-49 & 48.9500 & $0.00 \mathrm{E}+00$ & 2.02E-04 \\
\hline 104 & $\mathrm{LT}$ & $21-S c-50$ & 49.9522 & $0.00 \mathrm{E}+00$ & $6.76 \mathrm{E}-03$ \\
\hline 105 & LT & $22-\mathrm{Ti}-44$ & 43.9597 & $0.00 \mathrm{E}+00$ & $3.66 \mathrm{E}-10$ \\
\hline 106 & LT & $22-\mathrm{Ti}-45$ & 44.9581 & $0.00 \mathrm{E}+00$ & $6.25 \mathrm{E}-05$ \\
\hline 107 & LT & $22-\mathrm{Ti}-46$ & 45.9526 & $8.25 \mathrm{E}+00$ & $0.00 \mathrm{E}+00$ \\
\hline 108 & LT & $22-\mathrm{Ti}-47$ & 46.9518 & $7.44 \mathrm{E}+00$ & $0.00 \mathrm{E}+00$ \\
\hline 109 & LT & 22-Ti-48 & 47.9479 & $7.37 \mathrm{E}+01$ & $0.00 \mathrm{E}+00$ \\
\hline 110 & LT & 22-Ti-49 & 48.9479 & $5.41 \mathrm{E}+00$ & $0.00 \mathrm{E}+00$ \\
\hline 111 & $\mathrm{LT}$ & $22-\mathrm{Ti}-50$ & 49.9448 & $5.18 \mathrm{E}+00$ & $0.00 \mathrm{E}+00$ \\
\hline
\end{tabular}




\begin{tabular}{|c|c|c|c|c|c|}
\hline 112 & $\mathrm{LT}$ & 22-Ti-51 & 50.9466 & $0.00 \mathrm{E}+00$ & $2.01 \mathrm{E}-03$ \\
\hline 113 & LT & $23-V-48$ & 47.9523 & $0.00 \mathrm{E}+00$ & $5.02 \mathrm{E}-07$ \\
\hline 114 & LT & $23-V-49$ & 48.9485 & $0.00 \mathrm{E}+00$ & $2.43 \mathrm{E}-08$ \\
\hline 115 & LT & $23-V-50$ & 49.9472 & $2.50 \mathrm{E}-01$ & $1.57 \mathrm{E}-25$ \\
\hline 116 & LT & $23-V-51$ & 50.9440 & $9.98 \mathrm{E}+01$ & $0.00 \mathrm{E}+00$ \\
\hline 117 & $\mathrm{LT}$ & $23-V-52$ & 51.9448 & $0.00 \mathrm{E}+00$ & $3.09 \mathrm{E}-03$ \\
\hline 118 & LT & $23-V-53$ & 52.9443 & $0.00 \mathrm{E}+00$ & $7.49 \mathrm{E}-03$ \\
\hline 119 & LT & $23-V-54$ & 53.9464 & $0.00 \mathrm{E}+00$ & $1.39 \mathrm{E}-02$ \\
\hline 120 & LT & $24-\mathrm{Cr}-48$ & 47.9540 & $0.00 \mathrm{E}+00$ & $8.93 \mathrm{E}-06$ \\
\hline 121 & LT & 24-Cr-49 & 48.9513 & $0.00 \mathrm{E}+00$ & $2.73 \mathrm{E}-04$ \\
\hline 122 & LT & $24-\mathrm{Cr}-50$ & 49.9460 & $4.35 \mathrm{E}+00$ & $0.00 \mathrm{E}+00$ \\
\hline 123 & LT & $24-\mathrm{Cr}-51$ & 50.9448 & $0.00 \mathrm{E}+00$ & $2.90 \mathrm{E}-07$ \\
\hline 124 & LT & $24-\mathrm{Cr}-52$ & 51.9405 & $8.38 \mathrm{E}+01$ & $0.00 \mathrm{E}+00$ \\
\hline 125 & LT & $24-\mathrm{Cr}-53$ & 52.9407 & $9.50 \mathrm{E}+00$ & $0.00 \mathrm{E}+00$ \\
\hline 126 & LT & $24-\mathrm{Cr}-54$ & 53.9389 & $2.37 \mathrm{E}+00$ & $0.00 \mathrm{E}+00$ \\
\hline 127 & $\mathrm{LT}$ & $24-\mathrm{Cr}-55$ & 54.9408 & $0.00 \mathrm{E}+00$ & $3.30 \mathrm{E}-03$ \\
\hline 128 & $\mathrm{LT}$ & $25-\mathrm{Mn}-52$ & 51.9456 & $0.00 \mathrm{E}+00$ & $1.43 \mathrm{E}-06$ \\
\hline 129 & LT & 25-Mn-53 & 52.9413 & $0.00 \mathrm{E}+00$ & $5.94 \mathrm{E}-15$ \\
\hline 130 & LT & $25-\mathrm{Mn}-54$ & 53.9404 & $0.00 \mathrm{E}+00$ & $2.57 \mathrm{E}-08$ \\
\hline 131 & LT & $25-\mathrm{Mn}-55$ & 54.9380 & $1.00 \mathrm{E}+02$ & $0.00 \mathrm{E}+00$ \\
\hline 132 & LT & $25-\mathrm{Mn}-56$ & 55.9389 & $0.00 \mathrm{E}+00$ & $7.47 \mathrm{E}-05$ \\
\hline 133 & LT & $25-\mathrm{Mn}-57$ & 56.9383 & $0.00 \mathrm{E}+00$ & $8.12 \mathrm{E}-03$ \\
\hline 134 & LT & $25-\mathrm{Mn}-58$ & 57.9400 & $0.00 \mathrm{E}+00$ & $2.31 \mathrm{E}-01$ \\
\hline 135 & LT & $26-\mathrm{Fe}-54$ & 53.9396 & $5.85 \mathrm{E}+00$ & $0.00 \mathrm{E}+00$ \\
\hline 136 & LT & $26-\mathrm{Fe}-55$ & 54.9383 & $0.00 \mathrm{E}+00$ & $8.00 \mathrm{E}-09$ \\
\hline 137 & $\mathrm{LT}$ & $26-\mathrm{Fe}-56$ & 55.9349 & $9.18 \mathrm{E}+01$ & $0.00 \mathrm{E}+00$ \\
\hline 138 & LT & $26-\mathrm{Fe}-57$ & 56.9354 & $2.12 \mathrm{E}+00$ & $0.00 \mathrm{E}+00$ \\
\hline 139 & LT & $26-\mathrm{Fe}-58$ & 57.9333 & $2.82 \mathrm{E}-01$ & $0.00 \mathrm{E}+00$ \\
\hline 140 & LT & $26-\mathrm{Fe}-59$ & 58.9349 & $0.00 \mathrm{E}+00$ & $1.80 \mathrm{E}-07$ \\
\hline 141 & LT & $26-\mathrm{Fe}-60$ & 59.9341 & $0.00 \mathrm{E}+00$ & $1.46 \mathrm{E}-14$ \\
\hline 142 & LT & $27-\mathrm{Co}-55$ & 54.9420 & $0.00 \mathrm{E}+00$ & $1.10 \mathrm{E}-05$ \\
\hline 143 & LT & $27-\mathrm{Co}-56$ & 55.9398 & $0.00 \mathrm{E}+00$ & $1.04 \mathrm{E}-07$ \\
\hline 144 & LT & 27-Co-57 & 56.9363 & $0.00 \mathrm{E}+00$ & $2.95 \mathrm{E}-08$ \\
\hline 145 & $\mathrm{LT}$ & 27-Co-58m & 57.9358 & $0.00 \mathrm{E}+00$ & $2.12 \mathrm{E}-05$ \\
\hline 146 & $\mathrm{LT}$ & $27-\mathrm{Co}-58$ & 57.9357 & $0.00 \mathrm{E}+00$ & $1.13 \mathrm{E}-07$ \\
\hline 147 & $\mathrm{LT}$ & 27-Co-59 & 58.9332 & $1.00 \mathrm{E}+02$ & $0.00 \mathrm{E}+00$ \\
\hline 148 & LT & 27-Co-60 & 59.9338 & $0.00 \mathrm{E}+00$ & 4.17E-09 \\
\hline 149 & LT & $27-\mathrm{Co}-60 \mathrm{~m}$ & 59.9338 & $0.00 \mathrm{E}+00$ & $1.10 \mathrm{E}-03$ \\
\hline 150 & $\mathrm{LT}$ & 27-Co-61 & 60.9325 & $0.00 \mathrm{E}+00$ & $1.17 \mathrm{E}-04$ \\
\hline 151 & LT & 27-Co-62 & 61.9341 & $0.00 \mathrm{E}+00$ & $7.70 \mathrm{E}-03$ \\
\hline 152 & LT & $28-\mathrm{Ni}-56$ & 55.9421 & $0.00 \mathrm{E}+00$ & $1.32 \mathrm{E}-06$ \\
\hline 153 & LT & $28-\mathrm{Ni}-57$ & 56.9398 & $0.00 \mathrm{E}+00$ & $5.41 \mathrm{E}-06$ \\
\hline 154 & LT & 28-Ni-58 & 57.9353 & $6.81 \mathrm{E}+01$ & $0.00 \mathrm{E}+00$ \\
\hline 155 & $\mathrm{LT}$ & 28-Ni-59 & 58.9343 & $0.00 \mathrm{E}+00$ & $2.89 \mathrm{E}-13$ \\
\hline 156 & LT & $28-\mathrm{Ni}-60$ & 59.9308 & $2.62 \mathrm{E}+01$ & $0.00 \mathrm{E}+00$ \\
\hline 157 & LT & 28-Ni-61 & 60.9311 & $1.14 \mathrm{E}+00$ & $0.00 \mathrm{E}+00$ \\
\hline 158 & $\mathrm{LT}$ & $28-\mathrm{Ni}-62$ & 61.9283 & $3.63 \mathrm{E}+00$ & $0.00 \mathrm{E}+00$ \\
\hline 159 & $\mathrm{LT}$ & $28-\mathrm{Ni}-63$ & 62.9297 & $0.00 \mathrm{E}+00$ & $2.17 \mathrm{E}-10$ \\
\hline 160 & $\mathrm{LT}$ & 28-Ni-64 & 63.9280 & $9.26 \mathrm{E}-01$ & $0.00 \mathrm{E}+00$ \\
\hline 161 & LT & $28-\mathrm{Ni}-65$ & 64.9301 & $0.00 \mathrm{E}+00$ & $7.65 \mathrm{E}-05$ \\
\hline 162 & LT & 28-Ni-66 & 65.9291 & $0.00 \mathrm{E}+00$ & $3.53 \mathrm{E}-06$ \\
\hline 163 & $\mathrm{LT}$ & 29-Cu-62 & 61.9326 & $0.00 \mathrm{E}+00$ & $1.19 \mathrm{E}-03$ \\
\hline 164 & LT & $29-\mathrm{Cu}-63$ & 62.9296 & $6.92 \mathrm{E}+01$ & $0.00 \mathrm{E}+00$ \\
\hline 165 & $\mathrm{LT}$ & $29-\mathrm{Cu}-64$ & 63.9298 & $0.00 \mathrm{E}+00$ & $1.52 \mathrm{E}-05$ \\
\hline 166 & LT & 29-Cu-65 & 64.9278 & $3.09 \mathrm{E}+01$ & $0.00 \mathrm{E}+00$ \\
\hline 167 & LT & 29-Cu-66 & 65.9289 & $0.00 \mathrm{E}+00$ & $2.26 \mathrm{E}-03$ \\
\hline 168 & $\mathrm{LT}$ & $29-\mathrm{Cu}-67$ & 66.9277 & $0.00 \mathrm{E}+00$ & $3.11 \mathrm{E}-06$ \\
\hline 169 & LT & $30-Z n-63$ & 62.9332 & $0.00 \mathrm{E}+00$ & $3.00 \mathrm{E}-04$ \\
\hline 170 & LT & $30-Z n-64$ & 63.9291 & $4.83 \mathrm{E}+01$ & $0.00 \mathrm{E}+00$ \\
\hline 171 & $\mathrm{LT}$ & $30-Z n-65$ & 64.9292 & $0.00 \mathrm{E}+00$ & $3.29 \mathrm{E}-08$ \\
\hline 172 & LT & $30-Z n-66$ & 65.9260 & $2.80 \mathrm{E}+01$ & $0.00 \mathrm{E}+00$ \\
\hline 173 & $\mathrm{LT}$ & $30-Z n-67$ & 66.9271 & $4.10 \mathrm{E}+00$ & $0.00 \mathrm{E}+00$ \\
\hline 174 & $\mathrm{LT}$ & $30-Z n-68$ & 67.9248 & $1.90 \mathrm{E}+01$ & $0.00 \mathrm{E}+00$ \\
\hline 175 & LT & $30-Z n-69$ & 68.9266 & $0.00 \mathrm{E}+00$ & $2.05 \mathrm{E}-04$ \\
\hline 176 & LT & $30-Z n-69 m$ & 68.9266 & $0.00 \mathrm{E}+00$ & $1.40 \mathrm{E}-05$ \\
\hline 177 & LT & $30-\mathrm{Zn}-70$ & 69.9253 & $6.31 \mathrm{E}-01$ & $0.00 \mathrm{E}+00$ \\
\hline
\end{tabular}




\begin{tabular}{|c|c|c|c|c|c|}
\hline 178 & $\mathrm{LT}$ & $30-Z n-71$ & 70.9277 & $0.00 \mathrm{E}+00$ & $4.72 \mathrm{E}-03$ \\
\hline 179 & LT & $30-\mathrm{Zn}-71 \mathrm{~m}$ & 70.9277 & $0.00 \mathrm{E}+00$ & $4.86 \mathrm{E}-05$ \\
\hline 180 & LT & $30-Z n-72$ & 71.9269 & $0.00 \mathrm{E}+00$ & $4.14 \mathrm{E}-06$ \\
\hline 181 & $\mathrm{LT}$ & 31-Ga-67 & 66.9282 & $0.00 \mathrm{E}+00$ & $2.46 \mathrm{E}-06$ \\
\hline 182 & $\mathrm{LT}$ & 31-Ga-68 & 67.9280 & $0.00 \mathrm{E}+00$ & $1.71 \mathrm{E}-04$ \\
\hline 183 & $\mathrm{LT}$ & 31-Ga-69 & 68.9256 & $6.01 \mathrm{E}+01$ & $0.00 \mathrm{E}+00$ \\
\hline 184 & LT & $31-\mathrm{Ga}-70$ & 69.9260 & $0.00 \mathrm{E}+00$ & $5.46 \mathrm{E}-04$ \\
\hline 185 & LT & 31-Ga-71 & 70.9247 & $3.99 \mathrm{E}+01$ & $0.00 \mathrm{E}+00$ \\
\hline 186 & $\mathrm{LT}$ & 31-Ga-72 & 71.9264 & $0.00 \mathrm{E}+00$ & $1.37 \mathrm{E}-05$ \\
\hline 187 & LT & $31-\mathrm{Ga}-72 \mathrm{~m}$ & 71.9264 & $0.00 \mathrm{E}+00$ & $1.75 \mathrm{E}+01$ \\
\hline 188 & LT & $32-\mathrm{Ge}-68$ & 67.9281 & $0.00 \mathrm{E}+00$ & $2.96 \mathrm{E}-08$ \\
\hline 189 & LT & $32-\mathrm{Ge}-69$ & 68.9280 & $0.00 \mathrm{E}+00$ & $4.93 \mathrm{E}-06$ \\
\hline 190 & $\mathrm{LT}$ & $32-\mathrm{Ge}-70$ & 69.9242 & $2.04 \mathrm{E}+01$ & $0.00 \mathrm{E}+00$ \\
\hline 191 & $\mathrm{LT}$ & $32-\mathrm{Ge}-71$ & 70.9249 & $0.00 \mathrm{E}+00$ & $7.02 \mathrm{E}-07$ \\
\hline 192 & $\mathrm{LT}$ & $32-\mathrm{Ge}-71 \mathrm{~m}$ & 70.9249 & $0.00 \mathrm{E}+00$ & $3.40 \mathrm{E}+01$ \\
\hline 193 & $\mathrm{LT}$ & $32-\mathrm{Ge}-72$ & 71.9221 & $2.73 \mathrm{E}+01$ & $0.00 \mathrm{E}+00$ \\
\hline 194 & $\mathrm{LT}$ & 32-Ge-73 & 72.9235 & $7.76 \mathrm{E}+00$ & $0.00 \mathrm{E}+00$ \\
\hline 195 & $\mathrm{LT}$ & $32-\mathrm{Ge}-73 \mathrm{~m}$ & 72.9235 & $0.00 \mathrm{E}+00$ & $1.39 \mathrm{E}+00$ \\
\hline 196 & LT & $32-\mathrm{Ge}-74$ & 73.9212 & $3.67 \mathrm{E}+01$ & $0.00 \mathrm{E}+00$ \\
\hline 197 & LT & $32-\mathrm{Ge}-75$ & 74.9229 & $0.00 \mathrm{E}+00$ & $1.40 \mathrm{E}-04$ \\
\hline 198 & LT & $32-\mathrm{Ge}-75 \mathrm{~m}$ & 74.9229 & $0.00 \mathrm{E}+00$ & $1.45 \mathrm{E}-02$ \\
\hline 199 & LT & $32-\mathrm{Ge}-76$ & 75.9214 & $7.83 \mathrm{E}+00$ & $0.00 \mathrm{E}+00$ \\
\hline 200 & LT & $32-\mathrm{Ge}-77$ & 76.9236 & $0.00 \mathrm{E}+00$ & $1.70 \mathrm{E}-05$ \\
\hline 201 & $\mathrm{LT}$ & $32-\mathrm{Ge}-77 \mathrm{~m}$ & 76.9236 & $0.00 \mathrm{E}+00$ & $1.31 \mathrm{E}-02$ \\
\hline 202 & LT & 33-As-71 & 70.9271 & $0.00 \mathrm{E}+00$ & $2.95 \mathrm{E}-06$ \\
\hline 203 & $\mathrm{LT}$ & 33-As-72 & 71.9268 & $0.00 \mathrm{E}+00$ & $7.41 \mathrm{E}-06$ \\
\hline 204 & LT & $33-A s-73$ & 72.9238 & $0.00 \mathrm{E}+00$ & 9.99E-08 \\
\hline 205 & LT & 33-As-74 & 73.9239 & $0.00 \mathrm{E}+00$ & $4.51 \mathrm{E}-07$ \\
\hline 206 & LT & $33-A s-75$ & 74.9216 & $1.00 \mathrm{E}+02$ & $0.00 \mathrm{E}+00$ \\
\hline 207 & LT & 33-As-75m & 74.9216 & $0.00 \mathrm{E}+00$ & $3.93 \mathrm{E}+01$ \\
\hline 208 & LT & 33-As-76 & 75.9224 & $0.00 \mathrm{E}+00$ & 7.34E-06 \\
\hline 209 & LT & 33-As-77 & 76.9206 & $0.00 \mathrm{E}+00$ & $4.96 \mathrm{E}-06$ \\
\hline 210 & LT & $34-\mathrm{Se}-72$ & 71.9271 & $0.00 \mathrm{E}+00$ & $9.55 \mathrm{E}-07$ \\
\hline 211 & $\mathrm{LT}$ & $34-\mathrm{Se}-73$ & 72.9268 & $0.00 \mathrm{E}+00$ & $2.69 \mathrm{E}-05$ \\
\hline 212 & $\mathrm{LT}$ & $34-\mathrm{Se}-74$ & 73.9225 & $8.90 \mathrm{E}-01$ & $0.00 \mathrm{E}+00$ \\
\hline 213 & $\mathrm{LT}$ & $34-\mathrm{Se}-75$ & 74.9225 & $0.00 \mathrm{E}+00$ & $6.70 \mathrm{E}-08$ \\
\hline 214 & LT & $34-\mathrm{Se}-76$ & 75.9192 & $9.37 \mathrm{E}+00$ & $0.00 \mathrm{E}+00$ \\
\hline 215 & LT & $34-\mathrm{Se}-77$ & 76.9199 & $7.63 \mathrm{E}+00$ & $0.00 \mathrm{E}+00$ \\
\hline 216 & $\mathrm{LT}$ & $34-\mathrm{Se}-77 \mathrm{~m}$ & 76.9199 & $0.00 \mathrm{E}+00$ & 3.99E-02 \\
\hline 217 & $\mathrm{LT}$ & 34-Se-78 & 77.9173 & $2.38 \mathrm{E}+01$ & $0.00 \mathrm{E}+00$ \\
\hline 218 & LT & $34-\mathrm{Se}-79$ & 78.9185 & $0.00 \mathrm{E}+00$ & $7.45 \mathrm{E}-14$ \\
\hline 219 & LT & 34-Se-79m & 78.9185 & $0.00 \mathrm{E}+00$ & $2.95 \mathrm{E}-03$ \\
\hline 220 & LT & $34-\mathrm{Se}-80$ & 79.9165 & $4.96 \mathrm{E}+01$ & $0.00 \mathrm{E}+00$ \\
\hline 221 & $\mathrm{LT}$ & $34-\mathrm{Se}-81$ & 80.9180 & $0.00 \mathrm{E}+00$ & $6.26 \mathrm{E}-04$ \\
\hline 222 & LT & $34-\mathrm{Se}-81 \mathrm{~m}$ & 80.9180 & $0.00 \mathrm{E}+00$ & $2.02 \mathrm{E}-04$ \\
\hline 223 & LT & $34-\mathrm{Se}-82$ & 81.9167 & $8.73 \mathrm{E}+00$ & $0.00 \mathrm{E}+00$ \\
\hline 224 & $\mathrm{LT}$ & $34-\mathrm{Se}-83$ & 82.9191 & $0.00 \mathrm{E}+00$ & $5.18 \mathrm{E}-04$ \\
\hline 225 & $\mathrm{LT}$ & $34-\mathrm{Se}-83 \mathrm{~m}$ & 82.9191 & $0.00 \mathrm{E}+00$ & $9.89 \mathrm{E}-03$ \\
\hline 226 & $\mathrm{LT}$ & $35-\mathrm{Br}-76$ & 75.9245 & $0.00 \mathrm{E}+00$ & $1.19 \mathrm{E}-05$ \\
\hline 227 & $\mathrm{LT}$ & $35-\mathrm{Br}-77$ & 76.9214 & $0.00 \mathrm{E}+00$ & $3.38 \mathrm{E}-06$ \\
\hline 228 & $\mathrm{LT}$ & $35-\mathrm{Br}-77 \mathrm{~m}$ & 76.9214 & $0.00 \mathrm{E}+00$ & $2.70 \mathrm{E}-03$ \\
\hline 229 & $\mathrm{LT}$ & $35-\mathrm{Br}-78$ & 77.9212 & $0.00 \mathrm{E}+00$ & $1.79 \mathrm{E}-03$ \\
\hline 230 & LT & $35-\mathrm{Br}-79$ & 78.9183 & $5.07 \mathrm{E}+01$ & $0.00 \mathrm{E}+00$ \\
\hline 231 & $\mathrm{LT}$ & $35-\mathrm{Br}-80$ & 79.9185 & $0.00 \mathrm{E}+00$ & $6.53 \mathrm{E}-04$ \\
\hline 232 & LT & $35-\mathrm{Br}-80 \mathrm{~m}$ & 79.9185 & $0.00 \mathrm{E}+00$ & 4.36E-05 \\
\hline 233 & LT & $35-\mathrm{Br}-81$ & 80.9163 & $4.93 \mathrm{E}+01$ & $0.00 \mathrm{E}+00$ \\
\hline 234 & $\mathrm{LT}$ & $35-\mathrm{Br}-82$ & 81.9168 & $0.00 \mathrm{E}+00$ & $5.46 \mathrm{E}-06$ \\
\hline 235 & LT & $35-\mathrm{Br}-82 \mathrm{~m}$ & 81.9168 & $0.00 \mathrm{E}+00$ & $1.88 \mathrm{E}-03$ \\
\hline 236 & LT & $35-\mathrm{Br}-83$ & 82.9152 & $0.00 \mathrm{E}+00$ & $8.02 \mathrm{E}-05$ \\
\hline 237 & $\mathrm{LT}$ & $36-\mathrm{Kr}-76$ & 75.9259 & $0.00 \mathrm{E}+00$ & $1.30 \mathrm{E}-05$ \\
\hline 238 & LT & $36-\mathrm{Kr}-77$ & 76.9247 & $0.00 \mathrm{E}+00$ & $1.55 \mathrm{E}-04$ \\
\hline 239 & $\mathrm{LT}$ & $36-\mathrm{Kr}-78$ & 77.9204 & $3.55 \mathrm{E}-01$ & $0.00 \mathrm{E}+00$ \\
\hline 240 & $\mathrm{LT}$ & $36-\mathrm{Kr}-79$ & 78.9201 & $0.00 \mathrm{E}+00$ & $5.49 \mathrm{E}-06$ \\
\hline 241 & LT & $36-\mathrm{Kr}-79 \mathrm{~m}$ & 78.9201 & $0.00 \mathrm{E}+00$ & $1.39 \mathrm{E}-02$ \\
\hline 242 & LT & $36-\mathrm{Kr}-80$ & 79.9164 & $2.29 \mathrm{E}+00$ & $0.00 \mathrm{E}+00$ \\
\hline 243 & LT & $36-\mathrm{Kr}-81$ & 80.9166 & $0.00 \mathrm{E}+00$ & $9.59 \mathrm{E}-14$ \\
\hline
\end{tabular}




\begin{tabular}{|c|c|c|c|c|c|}
\hline 244 & LT & $36-\mathrm{Kr}-81 \mathrm{~m}$ & 80.9166 & $0.00 \mathrm{E}+00$ & $5.29 \mathrm{E}-02$ \\
\hline 245 & LT & $36-\mathrm{Kr}-82$ & 81.9135 & $1.16 \mathrm{E}+01$ & $0.00 \mathrm{E}+00$ \\
\hline 246 & LT & $36-\mathrm{Kr}-83$ & 82.9141 & $1.15 \mathrm{E}+01$ & $0.00 \mathrm{E}+00$ \\
\hline 247 & LT & $36-\mathrm{Kr}-83 \mathrm{~m}$ & 82.9141 & $0.00 \mathrm{E}+00$ & $1.05 \mathrm{E}-04$ \\
\hline 248 & LT & $36-\mathrm{Kr}-84$ & 83.9115 & $5.70 \mathrm{E}+01$ & $0.00 \mathrm{E}+00$ \\
\hline 249 & $\mathrm{LT}$ & $36-\mathrm{Kr}-85$ & 84.9125 & $0.00 \mathrm{E}+00$ & $2.04 \mathrm{E}-09$ \\
\hline 250 & $\mathrm{LT}$ & $36-\mathrm{Kr}-85 \mathrm{~m}$ & 84.9125 & $0.00 \mathrm{E}+00$ & $4.30 \mathrm{E}-05$ \\
\hline 251 & $\mathrm{LT}$ & $36-\mathrm{Kr}-86$ & 85.9106 & $1.73 \mathrm{E}+01$ & $0.00 \mathrm{E}+00$ \\
\hline 252 & $\mathrm{LT}$ & $36-\mathrm{Kr}-87$ & 86.9134 & $0.00 \mathrm{E}+00$ & $1.51 \mathrm{E}-04$ \\
\hline 253 & $\mathrm{LT}$ & $36-\mathrm{Kr}-88$ & 87.9145 & $0.00 \mathrm{E}+00$ & $6.78 \mathrm{E}-05$ \\
\hline 254 & LT & $37-\mathrm{Rb}-82$ & 81.9182 & $0.00 \mathrm{E}+00$ & 9.19E-03 \\
\hline 255 & $\mathrm{LT}$ & $37-\mathrm{Rb}-83$ & 82.9151 & $0.00 \mathrm{E}+00$ & $9.31 \mathrm{E}-08$ \\
\hline 256 & LT & $37-\mathrm{Rb}-84$ & 83.9144 & $0.00 \mathrm{E}+00$ & $2.44 \mathrm{E}-07$ \\
\hline 257 & $\mathrm{LT}$ & $37-\mathrm{Rb}-85$ & 84.9118 & $7.22 \mathrm{E}+01$ & $0.00 \mathrm{E}+00$ \\
\hline 258 & LT & $37-\mathrm{Rb}-86$ & 85.9112 & $0.00 \mathrm{E}+00$ & $4.31 \mathrm{E}-07$ \\
\hline 259 & $\mathrm{LT}$ & $37-\mathrm{Rb}-86 \mathrm{~m}$ & 85.9112 & $0.00 \mathrm{E}+00$ & $1.14 \mathrm{E}-02$ \\
\hline 260 & $\mathrm{LT}$ & $37-\mathrm{Rb}-87$ & 86.9092 & $2.78 \mathrm{E}+01$ & $4.57 \mathrm{E}-19$ \\
\hline 261 & $\mathrm{LT}$ & $37-\mathrm{Rb}-88$ & 87.9113 & $0.00 \mathrm{E}+00$ & $6.50 \mathrm{E}-04$ \\
\hline 262 & $\mathrm{LT}$ & $37-\mathrm{Rb}-89$ & 88.9123 & $0.00 \mathrm{E}+00$ & $7.63 \mathrm{E}-04$ \\
\hline 263 & $\mathrm{LT}$ & $38-\mathrm{Sr}-82$ & 81.9184 & $0.00 \mathrm{E}+00$ & $3.16 \mathrm{E}-07$ \\
\hline 264 & $\mathrm{LT}$ & $38-\mathrm{Sr}-83$ & 82.9176 & $0.00 \mathrm{E}+00$ & $5.94 \mathrm{E}-06$ \\
\hline 265 & LT & $38-\mathrm{Sr}-84$ & 83.9134 & $5.60 \mathrm{E}-01$ & $0.00 \mathrm{E}+00$ \\
\hline 266 & LT & $38-\mathrm{Sr}-85$ & 84.9129 & $0.00 \mathrm{E}+00$ & $1.24 \mathrm{E}-07$ \\
\hline 267 & $\mathrm{LT}$ & $38-\mathrm{Sr}-85 \mathrm{~m}$ & 84.9129 & $0.00 \mathrm{E}+00$ & $1.71 \mathrm{E}-04$ \\
\hline 268 & LT & $38-\mathrm{Sr}-86$ & 85.9093 & $9.86 \mathrm{E}+00$ & $0.00 \mathrm{E}+00$ \\
\hline 269 & LT & $38-\mathrm{Sr}-87$ & 86.9089 & $7.00 \mathrm{E}+00$ & $0.00 \mathrm{E}+00$ \\
\hline 270 & LT & $38-\mathrm{Sr}-87 \mathrm{~m}$ & 86.9089 & $0.00 \mathrm{E}+00$ & $6.84 \mathrm{E}-05$ \\
\hline 271 & $\mathrm{LT}$ & $38-\mathrm{Sr}-88$ & 87.9056 & $8.26 \mathrm{E}+01$ & $0.00 \mathrm{E}+00$ \\
\hline 272 & $\mathrm{LT}$ & $38-\mathrm{Sr}-89$ & 88.9074 & $0.00 \mathrm{E}+00$ & $1.59 \mathrm{E}-07$ \\
\hline 273 & LT & $38-\mathrm{Sr}-90$ & 89.9077 & $0.00 \mathrm{E}+00$ & $7.63 \mathrm{E}-10$ \\
\hline 274 & LT & $38-\mathrm{Sr}-91$ & 90.9102 & $0.00 \mathrm{E}+00$ & $2.00 \mathrm{E}-05$ \\
\hline 275 & $\mathrm{LT}$ & $38-\mathrm{Sr}-93$ & 92.9140 & $0.00 \mathrm{E}+00$ & $1.56 \mathrm{E}-03$ \\
\hline 276 & LT & $39-Y-86$ & 85.9149 & $0.00 \mathrm{E}+00$ & $1.31 \mathrm{E}-05$ \\
\hline 277 & LT & $39-Y-87$ & 86.9109 & $0.00 \mathrm{E}+00$ & $2.41 \mathrm{E}-06$ \\
\hline 278 & LT & $39-Y-87 \mathrm{~m}$ & 86.9109 & $0.00 \mathrm{E}+00$ & $1.44 \mathrm{E}-05$ \\
\hline 279 & $\mathrm{LT}$ & $39-Y-88$ & 87.9095 & $0.00 \mathrm{E}+00$ & $7.52 \mathrm{E}-08$ \\
\hline 280 & LT & 39-Y-89 & 88.9059 & $1.00 \mathrm{E}+02$ & $0.00 \mathrm{E}+00$ \\
\hline 281 & $\mathrm{LT}$ & $39-Y-89 m$ & 88.9059 & $0.00 \mathrm{E}+00$ & $4.43 \mathrm{E}-02$ \\
\hline 282 & $\mathrm{LT}$ & $39-Y-90$ & 89.9072 & $0.00 \mathrm{E}+00$ & $3.01 \mathrm{E}-06$ \\
\hline 283 & LT & $39-Y-90 \mathrm{~m}$ & 89.9072 & $0.00 \mathrm{E}+00$ & $6.04 \mathrm{E}-05$ \\
\hline 284 & $\mathrm{LT}$ & $39-Y-91$ & 90.9073 & $0.00 \mathrm{E}+00$ & $1.37 \mathrm{E}-07$ \\
\hline 285 & LT & 39-Y-91m & 90.9073 & $0.00 \mathrm{E}+00$ & $2.32 \mathrm{E}-04$ \\
\hline 286 & $\mathrm{LT}$ & $39-Y-92$ & 91.9090 & $0.00 \mathrm{E}+00$ & $5.44 \mathrm{E}-05$ \\
\hline 287 & LT & $39-Y-93$ & 92.9096 & $0.00 \mathrm{E}+00$ & $1.89 \mathrm{E}-05$ \\
\hline 288 & LT & $39-Y-93 m$ & 92.9096 & $0.00 \mathrm{E}+00$ & $8.45 \mathrm{E}-01$ \\
\hline 289 & LT & $39-Y-94$ & 93.9116 & $0.00 \mathrm{E}+00$ & $6.18 \mathrm{E}-04$ \\
\hline 290 & LT & $39-Y-96$ & 95.9159 & $0.00 \mathrm{E}+00$ & $1.30 \mathrm{E}-01$ \\
\hline 291 & LT & $40-Z r-86$ & 85.9165 & $0.00 \mathrm{E}+00$ & $1.17 \mathrm{E}-05$ \\
\hline 292 & LT & $40-Z r-87$ & 86.9148 & $0.00 \mathrm{E}+00$ & $1.15 \mathrm{E}-04$ \\
\hline 293 & LT & $40-Z r-88$ & 87.9102 & $0.00 \mathrm{E}+00$ & $9.62 \mathrm{E}-08$ \\
\hline 294 & LT & $40-Z r-89$ & 88.9089 & $0.00 \mathrm{E}+00$ & $2.46 \mathrm{E}-06$ \\
\hline 295 & $\mathrm{LT}$ & $40-Z r-90$ & 89.9047 & $5.15 \mathrm{E}+01$ & $0.00 \mathrm{E}+00$ \\
\hline 296 & LT & $40-Z r-90 m$ & 89.9047 & $0.00 \mathrm{E}+00$ & $8.57 \mathrm{E}-01$ \\
\hline 297 & LT & $40-Z r-91$ & 90.9056 & $1.12 \mathrm{E}+01$ & $0.00 \mathrm{E}+00$ \\
\hline 298 & $\mathrm{LT}$ & $40-Z r-92$ & 91.9050 & $1.72 \mathrm{E}+01$ & $0.00 \mathrm{E}+00$ \\
\hline 299 & $\mathrm{LT}$ & $40-Z r-93$ & 92.9065 & $0.00 \mathrm{E}+00$ & $1.44 \mathrm{E}-14$ \\
\hline 300 & LT & $40-Z r-94$ & 93.9063 & $1.74 \mathrm{E}+01$ & $0.00 \mathrm{E}+00$ \\
\hline 301 & $\mathrm{LT}$ & $40-Z r-95$ & 94.9080 & $0.00 \mathrm{E}+00$ & $1.25 \mathrm{E}-07$ \\
\hline 302 & LT & $40-Z r-96$ & 95.9083 & $2.80 \mathrm{E}+00$ & $1.10 \mathrm{E}-27$ \\
\hline 303 & LT & $40-Z r-97$ & 96.9109 & $0.00 \mathrm{E}+00$ & $1.15 \mathrm{E}-05$ \\
\hline 304 & LT & $41-\mathrm{Nb}-90$ & 89.9113 & $0.00 \mathrm{E}+00$ & $1.32 \mathrm{E}-05$ \\
\hline 305 & $\mathrm{LT}$ & $41-\mathrm{Nb}-90 \mathrm{~m}$ & 89.9113 & $0.00 \mathrm{E}+00$ & $3.69 \mathrm{E}-02$ \\
\hline 306 & $\mathrm{LT}$ & $41-\mathrm{Nb}-91$ & 90.9070 & $0.00 \mathrm{E}+00$ & $3.23 \mathrm{E}-11$ \\
\hline 307 & $\mathrm{LT}$ & 41-Nb-91m & 90.9070 & $0.00 \mathrm{E}+00$ & $1.32 \mathrm{E}-07$ \\
\hline 308 & LT & $41-\mathrm{Nb}-92$ & 91.9072 & $0.00 \mathrm{E}+00$ & $6.33 \mathrm{E}-16$ \\
\hline 309 & LT & $41-\mathrm{Nb}-92 \mathrm{~m}$ & 91.9072 & $0.00 \mathrm{E}+00$ & $7.90 \mathrm{E}-07$ \\
\hline
\end{tabular}




\begin{tabular}{|c|c|c|c|c|c|}
\hline 310 & LT & 41-Nb-93 & 92.9064 & $1.00 \mathrm{E}+02$ & $0.00 \mathrm{E}+00$ \\
\hline 311 & LT & 41-Nb-93m & 92.9064 & $0.00 \mathrm{E}+00$ & $1.36 \mathrm{E}-09$ \\
\hline 312 & LT & $41-\mathrm{Nb}-94$ & 93.9073 & $0.00 \mathrm{E}+00$ & $1.08 \mathrm{E}-12$ \\
\hline 313 & LT & $41-\mathrm{Nb}-94 \mathrm{~m}$ & 93.9073 & $0.00 \mathrm{E}+00$ & $1.84 \mathrm{E}-03$ \\
\hline 314 & $\mathrm{LT}$ & 41-Nb-95 & 94.9068 & $0.00 \mathrm{E}+00$ & $2.29 \mathrm{E}-07$ \\
\hline 315 & LT & $41-\mathrm{Nb}-95 \mathrm{~m}$ & 94.9068 & $0.00 \mathrm{E}+00$ & $2.22 \mathrm{E}-06$ \\
\hline 316 & $\mathrm{LT}$ & $41-\mathrm{Nb}-96$ & 95.9081 & $0.00 \mathrm{E}+00$ & $8.25 \mathrm{E}-06$ \\
\hline 317 & LT & 41-Nb-97 & 96.9081 & $0.00 \mathrm{E}+00$ & $1.60 \mathrm{E}-04$ \\
\hline 318 & $\mathrm{LT}$ & $41-\mathrm{Nb}-97 \mathrm{~m}$ & 96.9081 & $0.00 \mathrm{E}+00$ & $1.18 \mathrm{E}-02$ \\
\hline 319 & LT & $41-\mathrm{Nb}-98$ & 97.9103 & $0.00 \mathrm{E}+00$ & $2.42 \mathrm{E}-01$ \\
\hline 320 & $\mathrm{LT}$ & $41-\mathrm{Nb}-100$ & 99.9142 & $0.00 \mathrm{E}+00$ & 4.62E-01 \\
\hline 321 & $\mathrm{LT}$ & 42-Мo-92 & 91.9068 & $1.48 \mathrm{E}+01$ & $0.00 \mathrm{E}+00$ \\
\hline 322 & LT & 42-Mo-93m & 92.9068 & $0.00 \mathrm{E}+00$ & $2.81 \mathrm{E}-05$ \\
\hline 323 & $\mathrm{LT}$ & 42-Mo-93 & 92.9068 & $0.00 \mathrm{E}+00$ & $5.49 \mathrm{E}-12$ \\
\hline 324 & LT & 42-Mo-94 & 93.9051 & $9.23 \mathrm{E}+00$ & $0.00 \mathrm{E}+00$ \\
\hline 325 & $\mathrm{LT}$ & 42-Mo-95 & 94.9058 & $1.59 \mathrm{E}+01$ & $0.00 \mathrm{E}+00$ \\
\hline 326 & $\mathrm{LT}$ & 42-Мo-96 & 95.9047 & $1.67 \mathrm{E}+01$ & $0.00 \mathrm{E}+00$ \\
\hline 327 & LT & 42-Мo-97 & 96.9060 & $9.56 \mathrm{E}+00$ & $0.00 \mathrm{E}+00$ \\
\hline 328 & LT & 42-Mo-98 & 97.9054 & $2.42 \mathrm{E}+01$ & $0.00 \mathrm{E}+00$ \\
\hline 329 & $\mathrm{LT}$ & 42-Мo-99 & 98.9077 & $0.00 \mathrm{E}+00$ & $2.92 \mathrm{E}-06$ \\
\hline 330 & LT & 42-Mo-100 & 99.9075 & $9.67 \mathrm{E}+00$ & $3.01 \mathrm{E}-27$ \\
\hline 331 & LT & 42-Mo-101 & 100.9103 & $0.00 \mathrm{E}+00$ & $7.91 \mathrm{E}-04$ \\
\hline 332 & LT & $43-\mathrm{Tc}-95$ & 94.9077 & $0.00 \mathrm{E}+00$ & $9.63 \mathrm{E}-06$ \\
\hline 333 & $\mathrm{LT}$ & 43-Tc-95m & 94.9077 & $0.00 \mathrm{E}+00$ & $1.32 \mathrm{E}-07$ \\
\hline 334 & $\mathrm{LT}$ & $43-\mathrm{Tc}-96$ & 95.9079 & $0.00 \mathrm{E}+00$ & $1.87 \mathrm{E}-06$ \\
\hline 335 & $\mathrm{LT}$ & 43-Tc-97 & 96.9064 & $0.00 \mathrm{E}+00$ & $5.22 \mathrm{E}-15$ \\
\hline 336 & LT & 43-Tc-97m & 96.9064 & $0.00 \mathrm{E}+00$ & $8.82 \mathrm{E}-08$ \\
\hline 337 & LT & 43-Tc-98 & 97.9072 & $0.00 \mathrm{E}+00$ & $5.23 \mathrm{E}-15$ \\
\hline 338 & LT & 43-Tc-99 & 98.9062 & $0.00 \mathrm{E}+00$ & $1.04 \mathrm{E}-13$ \\
\hline 339 & LT & 43-Tc-99m & 98.9062 & $0.00 \mathrm{E}+00$ & $3.21 \mathrm{E}-05$ \\
\hline 340 & LT & 43-Tc-100 & 99.9077 & $0.00 \mathrm{E}+00$ & $4.48 \mathrm{E}-02$ \\
\hline 341 & LT & 43-Tc-101 & 100.9073 & $0.00 \mathrm{E}+00$ & $8.14 \mathrm{E}-04$ \\
\hline 342 & LT & 44-Ru-96 & 95.9076 & $5.54 \mathrm{E}+00$ & $0.00 \mathrm{E}+00$ \\
\hline 343 & $\mathrm{LT}$ & 44-Ru-97 & 96.9076 & $0.00 \mathrm{E}+00$ & $2.83 \mathrm{E}-06$ \\
\hline 344 & LT & 44-Ru-98 & 97.9053 & $1.87 \mathrm{E}+00$ & $0.00 \mathrm{E}+00$ \\
\hline 345 & LT & 44-Ru-99 & 98.9059 & $1.28 \mathrm{E}+01$ & $0.00 \mathrm{E}+00$ \\
\hline 346 & LT & 44-Ru-100 & 99.9042 & $1.26 \mathrm{E}+01$ & $0.00 \mathrm{E}+00$ \\
\hline 347 & $\mathrm{LT}$ & 44-Ru-101 & 100.9056 & $1.71 \mathrm{E}+01$ & $0.00 \mathrm{E}+00$ \\
\hline 348 & $\mathrm{LT}$ & 44-Ru-102 & 101.9044 & $3.16 \mathrm{E}+01$ & $0.00 \mathrm{E}+00$ \\
\hline 349 & LT & 44-Ru-103 & 102.9063 & $0.00 \mathrm{E}+00$ & $2.04 \mathrm{E}-07$ \\
\hline 350 & LT & 44-Ru-104 & 103.9054 & $1.86 \mathrm{E}+01$ & $0.00 \mathrm{E}+00$ \\
\hline 351 & LT & 44-Ru-105 & 104.9078 & $0.00 \mathrm{E}+00$ & $4.34 \mathrm{E}-05$ \\
\hline 352 & LT & 44-Ru-106 & 105.9073 & $0.00 \mathrm{E}+00$ & $2.16 \mathrm{E}-08$ \\
\hline 353 & LT & 44-Ru-107 & 106.9099 & $0.00 \mathrm{E}+00$ & $3.08 \mathrm{E}-03$ \\
\hline 354 & $\mathrm{LT}$ & 45-Rh-99 & 98.9081 & $0.00 \mathrm{E}+00$ & 4.98E-07 \\
\hline 355 & LT & 45-Rh-99m & 98.9081 & $0.00 \mathrm{E}+00$ & $4.10 \mathrm{E}-05$ \\
\hline 356 & LT & 45-Rh-100 & 99.9081 & $0.00 \mathrm{E}+00$ & $9.26 \mathrm{E}-06$ \\
\hline 357 & $\mathrm{LT}$ & 45-Rh-101 & 100.9062 & $0.00 \mathrm{E}+00$ & $6.66 \mathrm{E}-09$ \\
\hline 358 & LT & 45-Rh-101m & 100.9062 & $0.00 \mathrm{E}+00$ & $1.85 \mathrm{E}-06$ \\
\hline 359 & LT & 45-Rh-102 & 101.9068 & $0.00 \mathrm{E}+00$ & $3.87 \mathrm{E}-08$ \\
\hline 360 & LT & $45-\mathrm{Rh}-102 \mathrm{~m}$ & 101.9068 & $0.00 \mathrm{E}+00$ & $5.87 \mathrm{E}-09$ \\
\hline 361 & $\mathrm{LT}$ & 45-Rh-103 & 102.9055 & $1.00 \mathrm{E}+02$ & $0.00 \mathrm{E}+00$ \\
\hline 362 & LT & 45-Rh-103m & 102.9055 & $0.00 \mathrm{E}+00$ & $2.06 \mathrm{E}-04$ \\
\hline 363 & LT & 45-Rh-104 & 103.9067 & $0.00 \mathrm{E}+00$ & $1.64 \mathrm{E}-02$ \\
\hline 364 & LT & 45-Rh-104m & 103.9067 & $0.00 \mathrm{E}+00$ & $2.66 \mathrm{E}-03$ \\
\hline 365 & LT & 45-Rh-105 & 104.9057 & $0.00 \mathrm{E}+00$ & $5.45 \mathrm{E}-06$ \\
\hline 366 & $\mathrm{LT}$ & $45-\mathrm{Rh}-105 \mathrm{~m}$ & 104.9057 & $0.00 \mathrm{E}+00$ & $1.73 \mathrm{E}-02$ \\
\hline 367 & LT & 45-Rh-106 & 105.9073 & $0.00 \mathrm{E}+00$ & $2.31 \mathrm{E}-02$ \\
\hline 368 & $\mathrm{LT}$ & 45-Rh-106m & 105.9073 & $0.00 \mathrm{E}+00$ & $8.82 \mathrm{E}-05$ \\
\hline 369 & $\mathrm{LT}$ & 45-Rh-107 & 106.9068 & $0.00 \mathrm{E}+00$ & $5.32 \mathrm{E}-04$ \\
\hline 370 & LT & 46-Pd-100 & 99.9085 & $0.00 \mathrm{E}+00$ & $2.21 \mathrm{E}-06$ \\
\hline 371 & LT & 46-Pd-101 & 100.9083 & $0.00 \mathrm{E}+00$ & $2.27 \mathrm{E}-05$ \\
\hline 372 & LT & 46-Pd-102 & 101.9056 & $1.02 \mathrm{E}+00$ & $0.00 \mathrm{E}+00$ \\
\hline 373 & LT & 46-Pd-103 & 102.9061 & $0.00 \mathrm{E}+00$ & $4.72 \mathrm{E}-07$ \\
\hline 374 & LT & 46-Pd-104 & 103.9040 & $1.11 \mathrm{E}+01$ & $0.00 \mathrm{E}+00$ \\
\hline 375 & LT & 46-Pd-105 & 104.9051 & $2.23 \mathrm{E}+01$ & $0.00 \mathrm{E}+00$ \\
\hline
\end{tabular}




\begin{tabular}{|c|c|c|c|c|c|}
\hline 376 & LT & 46-Pd-106 & 105.9035 & $2.73 \mathrm{E}+01$ & $0.00 \mathrm{E}+00$ \\
\hline 377 & LT & 46-Pd-107 & 106.9051 & $0.00 \mathrm{E}+00$ & $3.38 \mathrm{E}-15$ \\
\hline 378 & LT & 46-Pd-107m & 106.9051 & $0.00 \mathrm{E}+00$ & $3.25 \mathrm{E}-02$ \\
\hline 379 & LT & 46-Pd-108 & 107.9039 & $2.65 \mathrm{E}+01$ & $0.00 \mathrm{E}+00$ \\
\hline 380 & $\mathrm{LT}$ & 46-Pd-109 & 108.9060 & $0.00 \mathrm{E}+00$ & $1.41 \mathrm{E}-05$ \\
\hline 381 & LT & 46-Pd-109m & 108.9060 & $0.00 \mathrm{E}+00$ & $2.46 \mathrm{E}-03$ \\
\hline 382 & $\mathrm{LT}$ & $46-\mathrm{Pd}-110$ & 109.9052 & $1.17 \mathrm{E}+01$ & $0.00 \mathrm{E}+00$ \\
\hline 383 & LT & 46-Pd-111 & 110.9077 & $0.00 \mathrm{E}+00$ & $4.94 \mathrm{E}-04$ \\
\hline 384 & $\mathrm{LT}$ & 46-Pd-111m & 110.9077 & $0.00 \mathrm{E}+00$ & $3.50 \mathrm{E}-05$ \\
\hline 385 & LT & 46-Pd-112 & 111.9073 & $0.00 \mathrm{E}+00$ & $9.16 \mathrm{E}-06$ \\
\hline 386 & $\mathrm{LT}$ & 47-Ag-105 & 104.9065 & $0.00 \mathrm{E}+00$ & $1.94 \mathrm{E}-07$ \\
\hline 387 & $\mathrm{LT}$ & 47-Ag-106 & 105.9067 & $0.00 \mathrm{E}+00$ & $4.82 \mathrm{E}-04$ \\
\hline 388 & LT & 47-Ag-106m & 105.9067 & $0.00 \mathrm{E}+00$ & $9.69 \mathrm{E}-07$ \\
\hline 389 & LT & 47-Ag-107 & 106.9051 & $5.18 \mathrm{E}+01$ & $0.00 \mathrm{E}+00$ \\
\hline 390 & LT & $47-A g-107 m$ & 106.9051 & $0.00 \mathrm{E}+00$ & $1.56 \mathrm{E}-02$ \\
\hline 391 & $\mathrm{LT}$ & 47-Ag-108 & 107.9060 & $0.00 \mathrm{E}+00$ & $4.85 \mathrm{E}-03$ \\
\hline 392 & $\mathrm{LT}$ & 47-Ag-108m & 107.9060 & $0.00 \mathrm{E}+00$ & $5.01 \mathrm{E}-11$ \\
\hline 393 & LT & 47-Ag-109 & 108.9047 & $4.82 \mathrm{E}+01$ & $0.00 \mathrm{E}+00$ \\
\hline 394 & LT & 47-Ag-109m & 108.9047 & $0.00 \mathrm{E}+00$ & $1.75 \mathrm{E}-02$ \\
\hline 395 & $\mathrm{LT}$ & $47-\mathrm{Ag}-110$ & 109.9061 & $0.00 \mathrm{E}+00$ & $2.82 \mathrm{E}-02$ \\
\hline 396 & LT & $47-A g-110 \mathrm{~m}$ & 109.9062 & $0.00 \mathrm{E}+00$ & $3.21 \mathrm{E}-08$ \\
\hline 397 & LT & 47-Ag-111 & 110.9053 & $0.00 \mathrm{E}+00$ & $1.08 \mathrm{E}-06$ \\
\hline 398 & LT & 47-Ag-111m & 110.9053 & $0.00 \mathrm{E}+00$ & $1.07 \mathrm{E}-02$ \\
\hline 399 & LT & 47-Ag-112 & 111.9070 & $0.00 \mathrm{E}+00$ & $6.15 \mathrm{E}-05$ \\
\hline 400 & $\mathrm{LT}$ & $48-\mathrm{Cd}-106$ & 105.9065 & $1.25 \mathrm{E}+00$ & $0.00 \mathrm{E}+00$ \\
\hline 401 & $\mathrm{LT}$ & $48-\mathrm{Cd}-107$ & 106.9066 & $0.00 \mathrm{E}+00$ & $2.96 \mathrm{E}-05$ \\
\hline 402 & LT & $48-\mathrm{Cd}-108$ & 107.9042 & $8.90 \mathrm{E}-01$ & $0.00 \mathrm{E}+00$ \\
\hline 403 & LT & $48-\mathrm{Cd}-109$ & 108.9050 & $0.00 \mathrm{E}+00$ & $1.74 \mathrm{E}-08$ \\
\hline 404 & LT & $48-\mathrm{Cd}-110$ & 109.9030 & $1.25 \mathrm{E}+01$ & $0.00 \mathrm{E}+00$ \\
\hline 405 & LT & $48-\mathrm{Cd}-111$ & 110.9042 & $1.28 \mathrm{E}+01$ & $0.00 \mathrm{E}+00$ \\
\hline 406 & LT & $48-\mathrm{Cd}-111 \mathrm{~m}$ & 110.9042 & $0.00 \mathrm{E}+00$ & $2.38 \mathrm{E}-04$ \\
\hline 407 & LT & $48-\mathrm{Cd}-112$ & 111.9028 & $2.41 \mathrm{E}+01$ & $0.00 \mathrm{E}+00$ \\
\hline 408 & LT & $48-\mathrm{Cd}-113$ & 112.9044 & $1.22 \mathrm{E}+01$ & $2.73 \mathrm{E}-24$ \\
\hline 409 & $\mathrm{LT}$ & $48-\mathrm{Cd}-113 \mathrm{~m}$ & 112.9044 & $0.00 \mathrm{E}+00$ & $1.56 \mathrm{E}-09$ \\
\hline 410 & LT & $48-\mathrm{Cd}-114$ & 113.9034 & $2.87 \mathrm{E}+01$ & $0.00 \mathrm{E}+00$ \\
\hline 411 & LT & $48-\mathrm{Cd}-115$ & 114.9054 & $0.00 \mathrm{E}+00$ & $3.60 \mathrm{E}-06$ \\
\hline 412 & LT & $48-\mathrm{Cd}-115 \mathrm{~m}$ & 114.9051 & $0.00 \mathrm{E}+00$ & $1.80 \mathrm{E}-07$ \\
\hline 413 & $\mathrm{LT}$ & $48-\mathrm{Cd}-116$ & 115.9048 & $7.49 \mathrm{E}+00$ & $7.09 \mathrm{E}-28$ \\
\hline 414 & $\mathrm{LT}$ & $48-\mathrm{Cd}-117$ & 116.9072 & $0.00 \mathrm{E}+00$ & $7.73 \mathrm{E}-05$ \\
\hline 415 & LT & $48-\mathrm{Cd}-117 \mathrm{~m}$ & 116.9072 & $0.00 \mathrm{E}+00$ & $5.73 \mathrm{E}-05$ \\
\hline 416 & LT & $48-\mathrm{Cd}-119$ & 118.9099 & $0.00 \mathrm{E}+00$ & $4.29 \mathrm{E}-03$ \\
\hline 417 & LT & $48-\mathrm{Cd}-121$ & 120.9130 & $0.00 \mathrm{E}+00$ & $5.13 \mathrm{E}-02$ \\
\hline 418 & LT & 49-In-111 & 110.9051 & $0.00 \mathrm{E}+00$ & $2.86 \mathrm{E}-06$ \\
\hline 419 & LT & 49-In-112 & 111.9055 & $0.00 \mathrm{E}+00$ & $7.72 \mathrm{E}-04$ \\
\hline 420 & $\mathrm{LT}$ & 49-In-113 & 112.9041 & $4.29 \mathrm{E}+00$ & $0.00 \mathrm{E}+00$ \\
\hline 421 & LT & 49-In-113m & 112.9041 & $0.00 \mathrm{E}+00$ & $1.16 \mathrm{E}-04$ \\
\hline 422 & LT & 49-In-114 & 113.9049 & $0.00 \mathrm{E}+00$ & $9.64 \mathrm{E}-03$ \\
\hline 423 & $\mathrm{LT}$ & 49-In-114m & 113.9049 & $0.00 \mathrm{E}+00$ & $1.62 \mathrm{E}-07$ \\
\hline 424 & LT & 49-In-115 & 114.9039 & $9.57 \mathrm{E}+01$ & $4.98 \mathrm{E}-23$ \\
\hline 425 & LT & $49-\operatorname{In}-115 \mathrm{~m}$ & 114.9039 & $0.00 \mathrm{E}+00$ & $4.29 \mathrm{E}-05$ \\
\hline 426 & LT & 49-In-116 & 115.9053 & $0.00 \mathrm{E}+00$ & $4.92 \mathrm{E}-02$ \\
\hline 427 & $\mathrm{LT}$ & 49-In-116m & 115.9053 & $0.00 \mathrm{E}+00$ & $2.13 \mathrm{E}-04$ \\
\hline 428 & LT & 49-In-117 & 116.9045 & $0.00 \mathrm{E}+00$ & $2.67 \mathrm{E}-04$ \\
\hline 429 & LT & 49-In-117m & 116.9045 & $0.00 \mathrm{E}+00$ & $9.94 \mathrm{E}-05$ \\
\hline 430 & LT & 49-In-118 & 117.9063 & $0.00 \mathrm{E}+00$ & $1.39 \mathrm{E}-01$ \\
\hline 431 & LT & 49-In-119 & 118.9059 & $0.00 \mathrm{E}+00$ & $4.81 \mathrm{E}-03$ \\
\hline 432 & $\mathrm{LT}$ & 49-In-119m & 118.9059 & $0.00 \mathrm{E}+00$ & $6.42 \mathrm{E}-04$ \\
\hline 433 & LT & 49-In-120 & 119.9080 & $0.00 \mathrm{E}+00$ & $2.25 \mathrm{E}-01$ \\
\hline 434 & $\mathrm{LT}$ & 49-In-120m & 119.9080 & $0.00 \mathrm{E}+00$ & $1.50 \mathrm{E}-02$ \\
\hline 435 & $\mathrm{LT}$ & $49-\operatorname{In}-121$ & 120.9079 & $0.00 \mathrm{E}+00$ & $3.00 \mathrm{E}-02$ \\
\hline 436 & LT & $49-$ In- $121 \mathrm{~m}$ & 120.9079 & $0.00 \mathrm{E}+00$ & $2.98 \mathrm{E}-03$ \\
\hline 437 & LT & $50-$ Sn-112 & 111.9048 & $9.70 \mathrm{E}-01$ & $0.00 \mathrm{E}+00$ \\
\hline 438 & LT & $50-\mathrm{Sn}-113$ & 112.9052 & $0.00 \mathrm{E}+00$ & $6.97 \mathrm{E}-08$ \\
\hline 439 & LT & $50-\mathrm{Sn}-113 \mathrm{~m}$ & 112.9052 & $0.00 \mathrm{E}+00$ & $5.40 \mathrm{E}-04$ \\
\hline 440 & LT & $50-\mathrm{Sn}-114$ & 113.9028 & $6.60 \mathrm{E}-01$ & $0.00 \mathrm{E}+00$ \\
\hline 441 & LT & $50-\mathrm{Sn}-115$ & 114.9033 & $3.40 \mathrm{E}-01$ & $0.00 \mathrm{E}+00$ \\
\hline
\end{tabular}




\begin{tabular}{|c|c|c|c|c|c|}
\hline 442 & $\mathrm{LT}$ & $50-\mathrm{Sn}-116$ & 115.9017 & $1.45 \mathrm{E}+01$ & $0.00 \mathrm{E}+00$ \\
\hline 443 & LT & $50-\mathrm{Sn}-117$ & 116.9029 & $7.68 \mathrm{E}+00$ & $0.00 \mathrm{E}+00$ \\
\hline 444 & LT & $50-\mathrm{Sn}-117 \mathrm{~m}$ & 116.9029 & $0.00 \mathrm{E}+00$ & $5.90 \mathrm{E}-07$ \\
\hline 445 & $\mathrm{LT}$ & $50-$ Sn-118 & 117.9016 & $2.42 \mathrm{E}+01$ & $0.00 \mathrm{E}+00$ \\
\hline 446 & $\mathrm{LT}$ & 50-Sn-119 & 118.9033 & $8.59 \mathrm{E}+00$ & $0.00 \mathrm{E}+00$ \\
\hline 447 & $\mathrm{LT}$ & $50-\mathrm{Sn}-119 \mathrm{~m}$ & 118.9033 & $0.00 \mathrm{E}+00$ & $2.74 \mathrm{E}-08$ \\
\hline 448 & LT & $50-\mathrm{Sn}-120$ & 119.9022 & $3.26 \mathrm{E}+01$ & $0.00 \mathrm{E}+00$ \\
\hline 449 & LT & $50-\mathrm{Sn}-121$ & 120.9042 & $0.00 \mathrm{E}+00$ & $7.12 \mathrm{E}-06$ \\
\hline 450 & $\mathrm{LT}$ & $50-\mathrm{Sn}-121 \mathrm{~m}$ & 120.9042 & $0.00 \mathrm{E}+00$ & $5.00 \mathrm{E}-10$ \\
\hline 451 & LT & $50-\mathrm{Sn}-122$ & 121.9034 & $4.63 \mathrm{E}+00$ & $0.00 \mathrm{E}+00$ \\
\hline 452 & LT & $50-\mathrm{Sn}-123$ & 122.9057 & $0.00 \mathrm{E}+00$ & $6.21 \mathrm{E}-08$ \\
\hline 453 & LT & $50-\mathrm{Sn}-123 \mathrm{~m}$ & 122.9057 & $0.00 \mathrm{E}+00$ & $2.88 \mathrm{E}-04$ \\
\hline 454 & $\mathrm{LT}$ & $50-\mathrm{Sn}-124$ & 123.9053 & $5.79 \mathrm{E}+00$ & $0.00 \mathrm{E}+00$ \\
\hline 455 & LT & $50-\mathrm{Sn}-125$ & 124.9078 & $0.00 \mathrm{E}+00$ & $8.32 \mathrm{E}-07$ \\
\hline 456 & $\mathrm{LT}$ & $50-\mathrm{Sn}-125 \mathrm{~m}$ & 124.9078 & $0.00 \mathrm{E}+00$ & $1.21 \mathrm{E}-03$ \\
\hline 457 & $\mathrm{LT}$ & $50-\mathrm{Sn}-126$ & 125.9077 & $0.00 \mathrm{E}+00$ & $9.55 \mathrm{E}-14$ \\
\hline 458 & LT & 51-Sb-118 & 117.9055 & $0.00 \mathrm{E}+00$ & $3.21 \mathrm{E}-03$ \\
\hline 459 & $\mathrm{LT}$ & 51-Sb-119 & 118.9039 & $0.00 \mathrm{E}+00$ & $5.04 \mathrm{E}-06$ \\
\hline 460 & LT & $51-\mathrm{Sb}-120$ & 119.9051 & $0.00 \mathrm{E}+00$ & $7.27 \mathrm{E}-04$ \\
\hline 461 & LT & $51-\mathrm{Sb}-120 \mathrm{~m}$ & 119.9051 & $0.00 \mathrm{E}+00$ & $1.39 \mathrm{E}-06$ \\
\hline 462 & LT & 51-Sb-121 & 120.9038 & $5.72 \mathrm{E}+01$ & $0.00 \mathrm{E}+00$ \\
\hline 463 & LT & 51-Sb-122 & 121.9052 & $0.00 \mathrm{E}+00$ & $2.95 \mathrm{E}-06$ \\
\hline 464 & LT & $51-\mathrm{Sb}-122 \mathrm{~m}$ & 121.9052 & $0.00 \mathrm{E}+00$ & $2.76 \mathrm{E}-03$ \\
\hline 465 & $\mathrm{LT}$ & 51-Sb-123 & 122.9042 & $4.28 \mathrm{E}+01$ & $0.00 \mathrm{E}+00$ \\
\hline 466 & LT & 51-Sb-124 & 123.9059 & $0.00 \mathrm{E}+00$ & $1.33 \mathrm{E}-07$ \\
\hline 467 & $\mathrm{LT}$ & $51-\mathrm{Sb}-124 \mathrm{~m}$ & 123.9059 & $0.00 \mathrm{E}+00$ & $7.45 \mathrm{E}-03$ \\
\hline 468 & LT & 51-Sb-125 & 124.9053 & $0.00 \mathrm{E}+00$ & $7.96 \mathrm{E}-09$ \\
\hline 469 & LT & 51-Sb-126 & 125.9072 & $0.00 \mathrm{E}+00$ & $6.50 \mathrm{E}-07$ \\
\hline 470 & LT & $51-\mathrm{Sb}-126 \mathrm{~m}$ & 125.9072 & $0.00 \mathrm{E}+00$ & $6.03 \mathrm{E}-04$ \\
\hline 471 & LT & 51-Sb-127 & 126.9069 & $0.00 \mathrm{E}+00$ & $2.08 \mathrm{E}-06$ \\
\hline 472 & LT & 52-Te-118 & 117.9058 & $0.00 \mathrm{E}+00$ & $1.34 \mathrm{E}-06$ \\
\hline 473 & LT & 52-Te-119 & 118.9064 & $0.00 \mathrm{E}+00$ & $1.20 \mathrm{E}-05$ \\
\hline 474 & LT & 52-Te-119m & 118.9064 & $0.00 \mathrm{E}+00$ & $1.71 \mathrm{E}-06$ \\
\hline 475 & $\mathrm{LT}$ & $52-\mathrm{Te}-120$ & 119.9040 & $9.00 \mathrm{E}-02$ & $0.00 \mathrm{E}+00$ \\
\hline 476 & $\mathrm{LT}$ & $52-\mathrm{Te}-121$ & 120.9049 & $0.00 \mathrm{E}+00$ & $4.19 \mathrm{E}-07$ \\
\hline 477 & $\mathrm{LT}$ & $52-\mathrm{Te}-121 \mathrm{~m}$ & 120.9049 & $0.00 \mathrm{E}+00$ & $4.89 \mathrm{E}-08$ \\
\hline 478 & LT & 52-Te-122 & 121.9030 & $2.55 \mathrm{E}+00$ & $0.00 \mathrm{E}+00$ \\
\hline 479 & LT & $52-\mathrm{Te}-123$ & 122.9043 & $8.90 \mathrm{E}-01$ & $0.00 \mathrm{E}+00$ \\
\hline 480 & $\mathrm{LT}$ & $52-\mathrm{Te}-123 \mathrm{~m}$ & 122.9043 & $0.00 \mathrm{E}+00$ & $6.73 \mathrm{E}-08$ \\
\hline 481 & $\mathrm{LT}$ & $52-\mathrm{Te}-124$ & 123.9028 & $4.74 \mathrm{E}+00$ & $0.00 \mathrm{E}+00$ \\
\hline 482 & LT & $52-\mathrm{Te}-125$ & 124.9044 & $7.07 \mathrm{E}+00$ & $0.00 \mathrm{E}+00$ \\
\hline 483 & LT & $52-\mathrm{Te}-125 \mathrm{~m}$ & 124.9044 & $0.00 \mathrm{E}+00$ & $1.40 \mathrm{E}-07$ \\
\hline 484 & LT & $52-\mathrm{Te}-126$ & 125.9033 & $1.88 \mathrm{E}+01$ & $0.00 \mathrm{E}+00$ \\
\hline 485 & $\mathrm{LT}$ & 52-Te-127 & 126.9052 & $0.00 \mathrm{E}+00$ & $2.06 \mathrm{E}-05$ \\
\hline 486 & LT & $52-\mathrm{Te}-127 \mathrm{~m}$ & 126.9052 & $0.00 \mathrm{E}+00$ & 7.36E-08 \\
\hline 487 & LT & 52-Te-128 & 127.9045 & $3.17 \mathrm{E}+01$ & $2.50 \mathrm{E}-27$ \\
\hline 488 & $\mathrm{LT}$ & 52-Тe-129 & 128.9066 & $0.00 \mathrm{E}+00$ & $1.66 \mathrm{E}-04$ \\
\hline 489 & $\mathrm{LT}$ & $52-\mathrm{Te}-129 \mathrm{~m}$ & 128.9074 & $0.00 \mathrm{E}+00$ & $2.39 \mathrm{E}-07$ \\
\hline 490 & $\mathrm{LT}$ & 52-Te-130 & 129.9062 & $3.41 \mathrm{E}+01$ & $0.00 \mathrm{E}+00$ \\
\hline 491 & $\mathrm{LT}$ & 52-Te-131 & 130.9085 & $0.00 \mathrm{E}+00$ & $4.62 \mathrm{E}-04$ \\
\hline 492 & LT & $52-\mathrm{Te}-131 \mathrm{~m}$ & 130.9085 & $0.00 \mathrm{E}+00$ & $5.79 \mathrm{E}-06$ \\
\hline 493 & $\mathrm{LT}$ & 52-Te-132 & 131.9086 & $0.00 \mathrm{E}+00$ & $2.50 \mathrm{E}-06$ \\
\hline 494 & LT & $53-I-122$ & 121.9076 & $0.00 \mathrm{E}+00$ & $3.18 \mathrm{E}-03$ \\
\hline 495 & $\mathrm{LT}$ & $53-I-123$ & 122.9056 & $0.00 \mathrm{E}+00$ & $1.46 \mathrm{E}-05$ \\
\hline 496 & LT & $53-I-124$ & 123.9062 & $0.00 \mathrm{E}+00$ & $1.92 \mathrm{E}-06$ \\
\hline 497 & LT & $53-I-125$ & 124.9046 & $0.00 \mathrm{E}+00$ & $1.35 \mathrm{E}-07$ \\
\hline 498 & $\mathrm{LT}$ & $53-\mathrm{I}-126$ & 125.9056 & $0.00 \mathrm{E}+00$ & $6.20 \mathrm{E}-07$ \\
\hline 499 & LT & 53-I-127 & 126.9045 & $1.00 \mathrm{E}+02$ & $0.00 \mathrm{E}+00$ \\
\hline 500 & LT & $53-I-128$ & 127.9058 & $0.00 \mathrm{E}+00$ & $4.62 \mathrm{E}-04$ \\
\hline 501 & $\mathrm{LT}$ & $53-I-129$ & 128.9050 & $0.00 \mathrm{E}+00$ & $1.40 \mathrm{E}-15$ \\
\hline 502 & LT & $53-\mathrm{I}-130$ & 129.9067 & $0.00 \mathrm{E}+00$ & $1.56 \mathrm{E}-05$ \\
\hline 503 & $\mathrm{LT}$ & $53-\mathrm{I}-130 \mathrm{~m}$ & 129.9067 & $0.00 \mathrm{E}+00$ & $1.31 \mathrm{E}-03$ \\
\hline 504 & $\mathrm{LT}$ & $53-I-131$ & 130.9061 & $0.00 \mathrm{E}+00$ & $1.00 \mathrm{E}-06$ \\
\hline 505 & LT & $53-I-132$ & 131.9080 & $0.00 \mathrm{E}+00$ & $8.39 \mathrm{E}-05$ \\
\hline 506 & LT & $53-I-133$ & 132.9078 & $0.00 \mathrm{E}+00$ & $9.26 \mathrm{E}-06$ \\
\hline 507 & LT & $54-\mathrm{Xe}-122$ & 121.9084 & $0.00 \mathrm{E}+00$ & $9.58 \mathrm{E}-06$ \\
\hline
\end{tabular}




\begin{tabular}{|c|c|c|c|c|c|}
\hline 508 & $\mathrm{LT}$ & $54-\mathrm{Xe}-123$ & 122.9085 & $0.00 \mathrm{E}+00$ & $9.26 \mathrm{E}-05$ \\
\hline 509 & LT & $54-\mathrm{Xe}-124$ & 123.9059 & $9.52 \mathrm{E}-02$ & $0.00 \mathrm{E}+00$ \\
\hline 510 & LT & $54-\mathrm{Xe}-125$ & 124.9064 & $0.00 \mathrm{E}+00$ & $1.14 \mathrm{E}-05$ \\
\hline 511 & LT & $54-\mathrm{Xe}-125 \mathrm{~m}$ & 124.9064 & $0.00 \mathrm{E}+00$ & $1.22 \mathrm{E}-02$ \\
\hline 512 & $\mathrm{LT}$ & $54-\mathrm{Xe}-126$ & 125.9043 & $8.90 \mathrm{E}-02$ & $0.00 \mathrm{E}+00$ \\
\hline 513 & $\mathrm{LT}$ & $54-\mathrm{Xe}-127$ & 126.9052 & $0.00 \mathrm{E}+00$ & $2.20 \mathrm{E}-07$ \\
\hline 514 & LT & $54-\mathrm{Xe}-127 \mathrm{~m}$ & 126.9052 & $0.00 \mathrm{E}+00$ & $1.00 \mathrm{E}-02$ \\
\hline 515 & LT & $54-\mathrm{Xe}-128$ & 127.9035 & $1.91 \mathrm{E}+00$ & $0.00 \mathrm{E}+00$ \\
\hline 516 & LT & 54-Xe-129 & 128.9048 & $2.64 \mathrm{E}+01$ & $0.00 \mathrm{E}+00$ \\
\hline 517 & LT & $54-\mathrm{Xe}-129 \mathrm{~m}$ & 128.9048 & $0.00 \mathrm{E}+00$ & $9.03 \mathrm{E}-07$ \\
\hline 518 & LT & $54-\mathrm{Xe}-130$ & 129.9035 & $4.07 \mathrm{E}+00$ & $0.00 \mathrm{E}+00$ \\
\hline 519 & LT & $54-\mathrm{Xe}-131$ & 130.9051 & $2.12 \mathrm{E}+01$ & $0.00 \mathrm{E}+00$ \\
\hline 520 & $\mathrm{LT}$ & $54-\mathrm{Xe}-131 \mathrm{~m}$ & 130.9051 & $0.00 \mathrm{E}+00$ & $6.78 \mathrm{E}-07$ \\
\hline 521 & $\mathrm{LT}$ & $54-\mathrm{Xe}-132$ & 131.9041 & $2.69 \mathrm{E}+01$ & $0.00 \mathrm{E}+00$ \\
\hline 522 & $\mathrm{LT}$ & 54-Xe-133 & 132.9059 & $0.00 \mathrm{E}+00$ & $1.53 \mathrm{E}-06$ \\
\hline 523 & $\mathrm{LT}$ & $54-X e-133 m$ & 132.9059 & $0.00 \mathrm{E}+00$ & $3.66 \mathrm{E}-06$ \\
\hline 524 & $\mathrm{LT}$ & 54-Xe-134 & 133.9054 & $1.04 \mathrm{E}+01$ & $0.00 \mathrm{E}+00$ \\
\hline 525 & LT & $54-\mathrm{Xe}-135$ & 134.9072 & $0.00 \mathrm{E}+00$ & $2.11 \mathrm{E}-05$ \\
\hline 526 & LT & $54-\mathrm{Xe}-135 \mathrm{~m}$ & 134.9072 & $0.00 \mathrm{E}+00$ & $7.56 \mathrm{E}-04$ \\
\hline 527 & $\mathrm{LT}$ & $54-\mathrm{Xe}-136$ & 135.9072 & $8.86 \mathrm{E}+00$ & $0.00 \mathrm{E}+00$ \\
\hline 528 & LT & $54-\mathrm{Xe}-137$ & 136.9116 & $0.00 \mathrm{E}+00$ & $3.03 \mathrm{E}-03$ \\
\hline 529 & LT & 55-Cs-128 & 127.9078 & $0.00 \mathrm{E}+00$ & $3.19 \mathrm{E}-03$ \\
\hline 530 & LT & 55-Cs-129 & 128.9061 & $0.00 \mathrm{E}+00$ & $6.01 \mathrm{E}-06$ \\
\hline 531 & $\mathrm{LT}$ & 55-Cs-130 & 129.9067 & $0.00 \mathrm{E}+00$ & $3.95 \mathrm{E}-04$ \\
\hline 532 & LT & 55-Cs-131 & 130.9055 & $0.00 \mathrm{E}+00$ & $8.28 \mathrm{E}-07$ \\
\hline 533 & $\mathrm{LT}$ & 55-Cs-132 & 131.9064 & $0.00 \mathrm{E}+00$ & $1.24 \mathrm{E}-06$ \\
\hline 534 & LT & 55-Cs-133 & 132.9055 & $1.00 \mathrm{E}+02$ & $0.00 \mathrm{E}+00$ \\
\hline 535 & LT & 55-Cs-134 & 133.9067 & $0.00 \mathrm{E}+00$ & $1.06 \mathrm{E}-08$ \\
\hline 536 & LT & $55-\mathrm{Cs}-134 \mathrm{~m}$ & 133.9067 & $0.00 \mathrm{E}+00$ & $6.61 \mathrm{E}-05$ \\
\hline 537 & LT & 55-Cs-135 & 134.9060 & $0.00 \mathrm{E}+00$ & $9.55 \mathrm{E}-15$ \\
\hline 538 & LT & 55-Cs-136 & 135.9073 & $0.00 \mathrm{E}+00$ & $6.10 \mathrm{E}-07$ \\
\hline 539 & LT & 55-Cs-137 & 136.9071 & $0.00 \mathrm{E}+00$ & $7.30 \mathrm{E}-10$ \\
\hline 540 & LT & 55-Cs-138 & 137.9110 & $0.00 \mathrm{E}+00$ & $3.46 \mathrm{E}-04$ \\
\hline 541 & $\mathrm{LT}$ & 56-Ba-128 & 127.9083 & $0.00 \mathrm{E}+00$ & $3.30 \mathrm{E}-06$ \\
\hline 542 & $\mathrm{LT}$ & 56-Ba-129 & 128.9087 & $0.00 \mathrm{E}+00$ & $8.63 \mathrm{E}-05$ \\
\hline 543 & $\mathrm{LT}$ & 56-Ba-130 & 129.9063 & $1.06 \mathrm{E}-01$ & $0.00 \mathrm{E}+00$ \\
\hline 544 & LT & 56-Ba-131 & 130.9069 & $0.00 \mathrm{E}+00$ & $6.98 \mathrm{E}-07$ \\
\hline 545 & LT & 56-Ba-131m & 130.9069 & $0.00 \mathrm{E}+00$ & $7.91 \mathrm{E}-04$ \\
\hline 546 & $\mathrm{LT}$ & 56-Ba-132 & 131.9051 & $1.01 \mathrm{E}-01$ & $0.00 \mathrm{E}+00$ \\
\hline 547 & $\mathrm{LT}$ & 56-Ba-133 & 132.9060 & $0.00 \mathrm{E}+00$ & $2.09 \mathrm{E}-09$ \\
\hline 548 & LT & 56-Ba-133m & 132.9060 & $0.00 \mathrm{E}+00$ & $4.95 \mathrm{E}-06$ \\
\hline 549 & LT & 56-Ba-134 & 133.9045 & $2.42 \mathrm{E}+00$ & $0.00 \mathrm{E}+00$ \\
\hline 550 & LT & 56-Ba-135 & 134.9057 & $6.59 \mathrm{E}+00$ & $0.00 \mathrm{E}+00$ \\
\hline 551 & $\mathrm{LT}$ & 56-Ba-135m & 134.9057 & $0.00 \mathrm{E}+00$ & $6.71 \mathrm{E}-06$ \\
\hline 552 & LT & 56-Ba-136 & 135.9046 & $7.85 \mathrm{E}+00$ & $0.00 \mathrm{E}+00$ \\
\hline 553 & LT & 56-Ba-136m & 135.9046 & $0.00 \mathrm{E}+00$ & $2.25 \mathrm{E}+00$ \\
\hline 554 & $\mathrm{LT}$ & 56-Ba-137 & 136.9058 & $1.12 \mathrm{E}+01$ & $0.00 \mathrm{E}+00$ \\
\hline 555 & $\mathrm{LT}$ & 56-Ba-137m & 136.9058 & $0.00 \mathrm{E}+00$ & $4.53 \mathrm{E}-03$ \\
\hline 556 & $\mathrm{LT}$ & 56-Ba-138 & 137.9052 & $7.17 \mathrm{E}+01$ & $0.00 \mathrm{E}+00$ \\
\hline 557 & $\mathrm{LT}$ & 56-Ba-139 & 138.9088 & $0.00 \mathrm{E}+00$ & $1.39 \mathrm{E}-04$ \\
\hline 558 & LT & 56-Ba-140 & 139.9106 & $0.00 \mathrm{E}+00$ & $6.29 \mathrm{E}-07$ \\
\hline 559 & $\mathrm{LT}$ & 56-Ba-141 & 140.9144 & $0.00 \mathrm{E}+00$ & $6.32 \mathrm{E}-04$ \\
\hline 560 & LT & 57-La-134 & 133.9085 & $0.00 \mathrm{E}+00$ & $1.79 \mathrm{E}-03$ \\
\hline 561 & $\mathrm{LT}$ & 57-La-135 & 134.9070 & $0.00 \mathrm{E}+00$ & $9.87 \mathrm{E}-06$ \\
\hline 562 & $\mathrm{LT}$ & 57-La-136 & 135.9076 & $0.00 \mathrm{E}+00$ & $1.17 \mathrm{E}-03$ \\
\hline 563 & LT & 57-La-137 & 136.9065 & $0.00 \mathrm{E}+00$ & $3.66 \mathrm{E}-13$ \\
\hline 564 & $\mathrm{LT}$ & 57-La-138 & 137.9071 & $9.00 \mathrm{E}-02$ & $2.15 \mathrm{E}-19$ \\
\hline 565 & LT & 57-La-139 & 138.9064 & $9.99 \mathrm{E}+01$ & $0.00 \mathrm{E}+00$ \\
\hline 566 & LT & 57-La-140 & 139.9095 & $0.00 \mathrm{E}+00$ & $4.78 \mathrm{E}-06$ \\
\hline 567 & $\mathrm{LT}$ & 57-La-141 & 140.9110 & $0.00 \mathrm{E}+00$ & $4.91 \mathrm{E}-05$ \\
\hline 568 & LT & 58-Ce-134 & 133.9089 & $0.00 \mathrm{E}+00$ & $2.54 \mathrm{E}-06$ \\
\hline 569 & $\mathrm{LT}$ & 58-Ce-135 & 134.9091 & $0.00 \mathrm{E}+00$ & $1.09 \mathrm{E}-05$ \\
\hline 570 & $\mathrm{LT}$ & $58-\mathrm{Ce}-136$ & 135.9072 & $1.85 \mathrm{E}-01$ & $0.00 \mathrm{E}+00$ \\
\hline 571 & LT & 58-Ce-137 & 136.9078 & $0.00 \mathrm{E}+00$ & $2.14 \mathrm{E}-05$ \\
\hline 572 & LT & $58-\mathrm{Ce}-137 \mathrm{~m}$ & 136.9078 & $0.00 \mathrm{E}+00$ & $5.60 \mathrm{E}-06$ \\
\hline 573 & LT & $58-\mathrm{Ce}-138$ & 137.9060 & $2.51 \mathrm{E}-01$ & $0.00 \mathrm{E}+00$ \\
\hline
\end{tabular}




\begin{tabular}{|c|c|c|c|c|c|}
\hline 574 & $\mathrm{LT}$ & $58-\mathrm{Ce}-139$ & 138.9066 & $0.00 \mathrm{E}+00$ & $5.83 \mathrm{E}-08$ \\
\hline 575 & LT & $58-\mathrm{Ce}-139 \mathrm{~m}$ & 138.9066 & $0.00 \mathrm{E}+00$ & $1.26 \mathrm{E}-02$ \\
\hline 576 & LT & $58-\mathrm{Ce}-140$ & 139.9054 & $8.85 \mathrm{E}+01$ & $0.00 \mathrm{E}+00$ \\
\hline 577 & LT & 58-Ce-141 & 140.9083 & $0.00 \mathrm{E}+00$ & $2.47 \mathrm{E}-07$ \\
\hline 578 & $\mathrm{LT}$ & $58-\mathrm{Ce}-142$ & 141.9092 & $1.11 \mathrm{E}+01$ & $0.00 \mathrm{E}+00$ \\
\hline 579 & $\mathrm{LT}$ & 58-Ce-143 & 142.9124 & $0.00 \mathrm{E}+00$ & $5.83 \mathrm{E}-06$ \\
\hline 580 & LT & 58-Ce-144 & 143.9137 & $0.00 \mathrm{E}+00$ & $2.82 \mathrm{E}-08$ \\
\hline 581 & LT & 58-Ce-145 & 144.9172 & $0.00 \mathrm{E}+00$ & $3.84 \mathrm{E}-03$ \\
\hline 582 & LT & 59-Pr-140 & 139.9091 & $0.00 \mathrm{E}+00$ & $3.41 \mathrm{E}-03$ \\
\hline 583 & LT & 59-Pr-141 & 140.9077 & $1.00 \mathrm{E}+02$ & $0.00 \mathrm{E}+00$ \\
\hline 584 & LT & 59-Pr-142 & 141.9100 & $0.00 \mathrm{E}+00$ & $1.01 \mathrm{E}-05$ \\
\hline 585 & LT & 59-Pr-142m & 141.9100 & $0.00 \mathrm{E}+00$ & $7.91 \mathrm{E}-04$ \\
\hline 586 & $\mathrm{LT}$ & 59-Pr-143 & 142.9108 & $0.00 \mathrm{E}+00$ & $5.91 \mathrm{E}-07$ \\
\hline 587 & $\mathrm{LT}$ & 59-Pr-144 & 143.9133 & $0.00 \mathrm{E}+00$ & $6.69 \mathrm{E}-04$ \\
\hline 588 & $\mathrm{LT}$ & 59-Pr-144m & 143.9133 & $0.00 \mathrm{E}+00$ & $1.60 \mathrm{E}-03$ \\
\hline 589 & $\mathrm{LT}$ & 59-Pr-145 & 144.9145 & $0.00 \mathrm{E}+00$ & $3.22 \mathrm{E}-05$ \\
\hline 590 & LT & $60-\mathrm{Nd}-140$ & 139.9095 & $0.00 \mathrm{E}+00$ & $2.38 \mathrm{E}-06$ \\
\hline 591 & $\mathrm{LT}$ & $60-\mathrm{Nd}-141$ & 140.9096 & $0.00 \mathrm{E}+00$ & $7.73 \mathrm{E}-05$ \\
\hline 592 & LT & $60-\mathrm{Nd}-141 \mathrm{~m}$ & 140.9096 & $0.00 \mathrm{E}+00$ & $1.12 \mathrm{E}-02$ \\
\hline 593 & $\mathrm{LT}$ & $60-\mathrm{Nd}-142$ & 141.9077 & $2.72 \mathrm{E}+01$ & $0.00 \mathrm{E}+00$ \\
\hline 594 & LT & $60-\mathrm{Nd}-143$ & 142.9098 & $1.22 \mathrm{E}+01$ & $0.00 \mathrm{E}+00$ \\
\hline 595 & LT & $60-\mathrm{Nd}-144$ & 143.9101 & $2.38 \mathrm{E}+01$ & $9.59 \mathrm{E}-24$ \\
\hline 596 & LT & $60-\mathrm{Nd}-145$ & 144.9126 & $8.30 \mathrm{E}+00$ & $0.00 \mathrm{E}+00$ \\
\hline 597 & $\mathrm{LT}$ & $60-\mathrm{Nd}-146$ & 145.9131 & $1.72 \mathrm{E}+01$ & $0.00 \mathrm{E}+00$ \\
\hline 598 & LT & $60-\mathrm{Nd}-147$ & 146.9161 & $0.00 \mathrm{E}+00$ & $7.31 \mathrm{E}-07$ \\
\hline 599 & $\mathrm{LT}$ & 60-Nd-148 & 147.9169 & $5.70 \mathrm{E}+00$ & $0.00 \mathrm{E}+00$ \\
\hline 600 & LT & 60-Nd-149 & 148.9202 & $0.00 \mathrm{E}+00$ & $1.11 \mathrm{E}-04$ \\
\hline 601 & LT & $60-\mathrm{Nd}-150$ & 149.9209 & $5.60 \mathrm{E}+00$ & $2.78 \mathrm{E}-27$ \\
\hline 602 & $\mathrm{LT}$ & $60-\mathrm{Nd}-151$ & 150.9238 & $0.00 \mathrm{E}+00$ & $9.29 \mathrm{E}-04$ \\
\hline 603 & LT & 61-Pm-143 & 142.9109 & $0.00 \mathrm{E}+00$ & $3.03 \mathrm{E}-08$ \\
\hline 604 & LT & 61-Pm-144 & 143.9126 & $0.00 \mathrm{E}+00$ & $2.21 \mathrm{E}-08$ \\
\hline 605 & LT & 61-Pm-145 & 144.9128 & $0.00 \mathrm{E}+00$ & $1.24 \mathrm{E}-09$ \\
\hline 606 & $\mathrm{LT}$ & 61-Pm-146 & 145.9147 & $0.00 \mathrm{E}+00$ & $3.97 \mathrm{E}-09$ \\
\hline 607 & $\mathrm{LT}$ & 61-Pm-147 & 146.9151 & $0.00 \mathrm{E}+00$ & $8.37 \mathrm{E}-09$ \\
\hline 608 & $\mathrm{LT}$ & 61-Pm-148 & 147.9175 & $0.00 \mathrm{E}+00$ & $1.49 \mathrm{E}-06$ \\
\hline 609 & $\mathrm{LT}$ & 61-Pm-148m & 147.9207 & $0.00 \mathrm{E}+00$ & $1.94 \mathrm{E}-07$ \\
\hline 610 & LT & 61-Pm-149 & 148.9183 & $0.00 \mathrm{E}+00$ & $3.63 \mathrm{E}-06$ \\
\hline 611 & LT & 61-Pm-150 & 149.9210 & $0.00 \mathrm{E}+00$ & $7.18 \mathrm{E}-05$ \\
\hline 612 & $\mathrm{LT}$ & 61-Pm-151 & 150.9212 & $0.00 \mathrm{E}+00$ & $6.78 \mathrm{E}-06$ \\
\hline 613 & $\mathrm{LT}$ & 61-Pm-152 & 151.9235 & $0.00 \mathrm{E}+00$ & $2.80 \mathrm{E}-03$ \\
\hline 614 & LT & 62-Sm-144 & 143.9120 & $3.07 \mathrm{E}+00$ & $0.00 \mathrm{E}+00$ \\
\hline 615 & LT & 62-Sm-145 & 144.9134 & $0.00 \mathrm{E}+00$ & $2.36 \mathrm{E}-08$ \\
\hline 616 & LT & 62-Sm-146 & 145.9130 & $0.00 \mathrm{E}+00$ & $2.13 \mathrm{E}-16$ \\
\hline 617 & $\mathrm{LT}$ & 62-Sm-147 & 146.9149 & $1.50 \mathrm{E}+01$ & $2.07 \mathrm{E}-19$ \\
\hline 618 & LT & 62-Sm-148 & 147.9148 & $1.12 \mathrm{E}+01$ & $3.14 \mathrm{E}-24$ \\
\hline 619 & LT & 62-Sm-149 & 148.9172 & $1.38 \mathrm{E}+01$ & $0.00 \mathrm{E}+00$ \\
\hline 620 & $\mathrm{LT}$ & $62-\mathrm{Sm}-150$ & 149.9173 & $7.38 \mathrm{E}+00$ & $0.00 \mathrm{E}+00$ \\
\hline 621 & $\mathrm{LT}$ & 62-Sm-151 & 150.9199 & $0.00 \mathrm{E}+00$ & $2.44 \mathrm{E}-10$ \\
\hline 622 & $\mathrm{LT}$ & 62-Sm-152 & 151.9197 & $2.68 \mathrm{E}+01$ & $0.00 \mathrm{E}+00$ \\
\hline 623 & $\mathrm{LT}$ & 62-Sm-153 & 152.9221 & $0.00 \mathrm{E}+00$ & $4.14 \mathrm{E}-06$ \\
\hline 624 & LT & 62-Sm-154 & 153.9222 & $2.28 \mathrm{E}+01$ & $0.00 \mathrm{E}+00$ \\
\hline 625 & $\mathrm{LT}$ & $62-\mathrm{Sm}-155$ & 154.9246 & $0.00 \mathrm{E}+00$ & $5.18 \mathrm{E}-04$ \\
\hline 626 & LT & 63-Eu-145 & 144.9163 & $0.00 \mathrm{E}+00$ & $1.35 \mathrm{E}-06$ \\
\hline 627 & $\mathrm{LT}$ & 63-Eu-146 & 145.9172 & $0.00 \mathrm{E}+00$ & $1.75 \mathrm{E}-06$ \\
\hline 628 & LT & 63-Eu-147 & 146.9167 & $0.00 \mathrm{E}+00$ & $3.33 \mathrm{E}-07$ \\
\hline 629 & LT & 63-Eu-148 & 147.9181 & $0.00 \mathrm{E}+00$ & $1.47 \mathrm{E}-07$ \\
\hline 630 & $\mathrm{LT}$ & 63-Eu-149 & 148.9179 & $0.00 \mathrm{E}+00$ & $8.62 \mathrm{E}-08$ \\
\hline 631 & LT & 63-Eu-150 & 149.9197 & $0.00 \mathrm{E}+00$ & $5.95 \mathrm{E}-10$ \\
\hline 632 & LT & 63-Eu-150m & 149.9197 & $0.00 \mathrm{E}+00$ & $1.50 \mathrm{E}-05$ \\
\hline 633 & $\mathrm{LT}$ & 63-Eu-151 & 150.9198 & $4.78 \mathrm{E}+01$ & $0.00 \mathrm{E}+00$ \\
\hline 634 & LT & 63-Eu-152 & 151.9217 & $0.00 \mathrm{E}+00$ & $1.62 \mathrm{E}-09$ \\
\hline 635 & $\mathrm{LT}$ & 63-Eu-152m & 151.9217 & $0.00 \mathrm{E}+00$ & $2.07 \mathrm{E}-05$ \\
\hline 636 & $\mathrm{LT}$ & 63-Eu-153 & 152.9212 & $5.22 \mathrm{E}+01$ & $0.00 \mathrm{E}+00$ \\
\hline 637 & LT & 63-Eu-154 & 153.9230 & $0.00 \mathrm{E}+00$ & $2.55 \mathrm{E}-09$ \\
\hline 638 & LT & 63-Eu-155 & 154.9229 & $0.00 \mathrm{E}+00$ & $4.62 \mathrm{E}-09$ \\
\hline 639 & LT & 63-Eu-156 & 155.9247 & $0.00 \mathrm{E}+00$ & $5.28 \mathrm{E}-07$ \\
\hline
\end{tabular}




\begin{tabular}{|c|c|c|c|c|c|}
\hline 640 & LT & 63-Eu-157 & 156.9254 & $0.00 \mathrm{E}+00$ & $1.27 \mathrm{E}-05$ \\
\hline 641 & LT & 64-Gd-146 & 145.9183 & $0.00 \mathrm{E}+00$ & $1.66 \mathrm{E}-07$ \\
\hline 642 & LT & 64-Gd-147 & 146.9191 & $0.00 \mathrm{E}+00$ & $5.06 \mathrm{E}-06$ \\
\hline 643 & LT & 64-Gd-148 & 147.9181 & $0.00 \mathrm{E}+00$ & $2.94 \mathrm{E}-10$ \\
\hline 644 & LT & 64-Gd-149 & 148.9193 & $0.00 \mathrm{E}+00$ & $8.65 \mathrm{E}-07$ \\
\hline 645 & $\mathrm{LT}$ & 64-Gd-150 & 149.9187 & $0.00 \mathrm{E}+00$ & $1.23 \mathrm{E}-14$ \\
\hline 646 & LT & 64-Gd-151 & 150.9203 & $0.00 \mathrm{E}+00$ & $6.47 \mathrm{E}-08$ \\
\hline 647 & LT & 64-Gd-152 & 151.9198 & $2.00 \mathrm{E}-01$ & $2.03 \mathrm{E}-22$ \\
\hline 648 & LT & 64-Gd-153 & 152.9218 & $0.00 \mathrm{E}+00$ & $3.34 \mathrm{E}-08$ \\
\hline 649 & LT & 64-Gd-154 & 153.9209 & $2.18 \mathrm{E}+00$ & $0.00 \mathrm{E}+00$ \\
\hline 650 & LT & 64-Gd-155m & 154.9226 & $0.00 \mathrm{E}+00$ & $2.17 \mathrm{E}+01$ \\
\hline 651 & LT & 64-Gd-155 & 154.9226 & $1.48 \mathrm{E}+01$ & $0.00 \mathrm{E}+00$ \\
\hline 652 & LT & 64-Gd-156 & 155.9221 & $2.05 \mathrm{E}+01$ & $0.00 \mathrm{E}+00$ \\
\hline 653 & LT & 64-Gd-157 & 156.9240 & $1.57 \mathrm{E}+01$ & $0.00 \mathrm{E}+00$ \\
\hline 654 & LT & 64-Gd-158 & 157.9241 & $2.48 \mathrm{E}+01$ & $0.00 \mathrm{E}+00$ \\
\hline 655 & $\mathrm{LT}$ & 64-Gd-159 & 158.9264 & $0.00 \mathrm{E}+00$ & $1.04 \mathrm{E}-05$ \\
\hline 656 & LT & 64-Gd-160 & 159.9270 & $2.19 \mathrm{E}+01$ & $0.00 \mathrm{E}+00$ \\
\hline 657 & LT & 64-Gd-161 & 160.9297 & $0.00 \mathrm{E}+00$ & $3.16 \mathrm{E}-03$ \\
\hline 658 & LT & 64-Gd-162 & 161.9310 & $0.00 \mathrm{E}+00$ & $1.38 \mathrm{E}-03$ \\
\hline 659 & LT & $65-T b-152$ & 151.9241 & $0.00 \mathrm{E}+00$ & $1.10 \mathrm{E}-05$ \\
\hline 660 & LT & $65-\mathrm{Tb}-153$ & 152.9234 & $0.00 \mathrm{E}+00$ & $3.43 \mathrm{E}-06$ \\
\hline 661 & LT & $65-\mathrm{Tb}-154$ & 153.9247 & $0.00 \mathrm{E}+00$ & $8.96 \mathrm{E}-06$ \\
\hline 662 & LT & $65-\mathrm{Tb}-154 \mathrm{~m}$ & 153.9247 & $0.00 \mathrm{E}+00$ & $2.05 \mathrm{E}-05$ \\
\hline 663 & LT & $65-\mathrm{Tb}-155$ & 154.9235 & $0.00 \mathrm{E}+00$ & $1.51 \mathrm{E}-06$ \\
\hline 664 & LT & $65-\mathrm{Tb}-156$ & 155.9247 & $0.00 \mathrm{E}+00$ & $1.50 \mathrm{E}-06$ \\
\hline 665 & $\mathrm{LT}$ & $65-\mathrm{Tb}-156 \mathrm{~m}$ & 155.9247 & $0.00 \mathrm{E}+00$ & $7.89 \mathrm{E}-06$ \\
\hline 666 & LT & 65-Tb-157 & 156.9240 & $0.00 \mathrm{E}+00$ & $3.09 \mathrm{E}-10$ \\
\hline 667 & LT & $65-\mathrm{Tb}-158$ & 157.9254 & $0.00 \mathrm{E}+00$ & $1.22 \mathrm{E}-10$ \\
\hline 668 & LT & $65-\mathrm{Tb}-159$ & 158.9254 & $1.00 \mathrm{E}+02$ & $0.00 \mathrm{E}+00$ \\
\hline 669 & LT & $65-\mathrm{Tb}-160$ & 159.9272 & $0.00 \mathrm{E}+00$ & $1.11 \mathrm{E}-07$ \\
\hline 670 & LT & 65-Tb-161 & 160.9276 & $0.00 \mathrm{E}+00$ & $1.16 \mathrm{E}-06$ \\
\hline 671 & LT & $65-\mathrm{Tb}-162$ & 161.9295 & $0.00 \mathrm{E}+00$ & $1.52 \mathrm{E}-03$ \\
\hline 672 & LT & 66-Dy-154 & 153.9244 & $0.00 \mathrm{E}+00$ & $7.32 \mathrm{E}-15$ \\
\hline 673 & LT & 66-Dy-155 & 154.9258 & $0.00 \mathrm{E}+00$ & $1.94 \mathrm{E}-05$ \\
\hline 674 & $\mathrm{LT}$ & 66-Dy-156 & 155.9243 & $5.60 \mathrm{E}-02$ & $0.00 \mathrm{E}+00$ \\
\hline 675 & LT & 66-Dy-157 & 156.9255 & $0.00 \mathrm{E}+00$ & $2.37 \mathrm{E}-05$ \\
\hline 676 & LT & 66-Dy-158 & 157.9244 & $9.50 \mathrm{E}-02$ & $0.00 \mathrm{E}+00$ \\
\hline 677 & $\mathrm{LT}$ & 66-Dy-159 & 158.9257 & $0.00 \mathrm{E}+00$ & $5.56 \mathrm{E}-08$ \\
\hline 678 & LT & 66-Dy-160 & 159.9252 & $2.33 \mathrm{E}+00$ & $0.00 \mathrm{E}+00$ \\
\hline 679 & LT & 66-Dy-161 & 160.9269 & $1.89 \mathrm{E}+01$ & $0.00 \mathrm{E}+00$ \\
\hline 680 & LT & 66-Dy-162 & 161.9268 & $2.55 \mathrm{E}+01$ & $0.00 \mathrm{E}+00$ \\
\hline 681 & LT & 66-Dy-163 & 162.9287 & $2.49 \mathrm{E}+01$ & $0.00 \mathrm{E}+00$ \\
\hline 682 & LT & 66-Dy-164 & 163.9292 & $2.83 \mathrm{E}+01$ & $0.00 \mathrm{E}+00$ \\
\hline 683 & LT & 66-Dy-165 & 164.9317 & $0.00 \mathrm{E}+00$ & $8.25 \mathrm{E}-05$ \\
\hline 684 & LT & 66-Dy-165m & 164.9317 & $0.00 \mathrm{E}+00$ & 9.19E-03 \\
\hline 685 & LT & 66-Dy-166 & 165.9328 & $0.00 \mathrm{E}+00$ & $2.36 \mathrm{E}-06$ \\
\hline 686 & LT & 67-Ho-160 & 159.9287 & $0.00 \mathrm{E}+00$ & $4.51 \mathrm{E}-04$ \\
\hline 687 & LT & 67-Ho-160m & 159.9287 & $0.00 \mathrm{E}+00$ & $3.84 \mathrm{E}-05$ \\
\hline 688 & LT & 67-Нo-161 & 160.9279 & $0.00 \mathrm{E}+00$ & $7.76 \mathrm{E}-05$ \\
\hline 689 & $\mathrm{LT}$ & 67-Ho-163 & 162.9287 & $0.00 \mathrm{E}+00$ & $4.81 \mathrm{E}-12$ \\
\hline 690 & LT & 67-Ho-163m & 162.9287 & $0.00 \mathrm{E}+00$ & $6.36 \mathrm{E}-01$ \\
\hline 691 & LT & 67-Нo-164 & 163.9302 & $0.00 \mathrm{E}+00$ & $3.98 \mathrm{E}-04$ \\
\hline 692 & LT & 67-Ho-164m & 163.9302 & $0.00 \mathrm{E}+00$ & $3.08 \mathrm{E}-04$ \\
\hline 693 & LT & 67-Ho-165 & 164.9303 & $1.00 \mathrm{E}+02$ & $0.00 \mathrm{E}+00$ \\
\hline 694 & LT & 67-Ho-166 & 165.9323 & $0.00 \mathrm{E}+00$ & $7.18 \mathrm{E}-06$ \\
\hline 695 & LT & 67-Ho-166m & 165.9324 & $0.00 \mathrm{E}+00$ & $1.83 \mathrm{E}-11$ \\
\hline 696 & LT & $68-\mathrm{Er}-160$ & 159.9291 & $0.00 \mathrm{E}+00$ & $6.74 \mathrm{E}-06$ \\
\hline 697 & LT & 68-Er-161 & 160.9300 & $0.00 \mathrm{E}+00$ & $6.00 \mathrm{E}-05$ \\
\hline 698 & LT & $68-E r-162$ & 161.9288 & $1.39 \mathrm{E}-01$ & $0.00 \mathrm{E}+00$ \\
\hline 699 & LT & 68-Er-163 & 162.9300 & $0.00 \mathrm{E}+00$ & $1.54 \mathrm{E}-04$ \\
\hline 700 & LT & 68-Er-164 & 163.9292 & $1.60 \mathrm{E}+00$ & $0.00 \mathrm{E}+00$ \\
\hline 701 & LT & $68-\mathrm{Er}-165$ & 164.9307 & $0.00 \mathrm{E}+00$ & $1.86 \mathrm{E}-05$ \\
\hline 702 & LT & 68-Er-166 & 165.9303 & $3.35 \mathrm{E}+01$ & $0.00 \mathrm{E}+00$ \\
\hline 703 & LT & $68-E r-167$ & 166.9321 & $2.29 \mathrm{E}+01$ & $0.00 \mathrm{E}+00$ \\
\hline 704 & LT & 68 -Er-167m & 166.9321 & $0.00 \mathrm{E}+00$ & $3.05 \mathrm{E}-01$ \\
\hline 705 & LT & 68-Er-168 & 167.9324 & $2.70 \mathrm{E}+01$ & $0.00 \mathrm{E}+00$ \\
\hline
\end{tabular}




\begin{tabular}{|c|c|c|c|c|c|}
\hline 706 & LT & 68-Er-169 & 168.9346 & $0.00 \mathrm{E}+00$ & $8.54 \mathrm{E}-07$ \\
\hline 707 & LT & $68-E r-170$ & 169.9355 & $1.49 \mathrm{E}+01$ & $0.00 \mathrm{E}+00$ \\
\hline 708 & LT & 68-Er-171 & 170.9380 & $0.00 \mathrm{E}+00$ & $2.56 \mathrm{E}-05$ \\
\hline 709 & LT & 68-Er-172 & 171.9394 & $0.00 \mathrm{E}+00$ & $3.91 \mathrm{E}-06$ \\
\hline 710 & $\mathrm{LT}$ & 69-Tm-165 & 164.9324 & $0.00 \mathrm{E}+00$ & $6.41 \mathrm{E}-06$ \\
\hline 711 & LT & 69-Tm-166 & 165.9335 & $0.00 \mathrm{E}+00$ & $2.50 \mathrm{E}-05$ \\
\hline 712 & $\mathrm{LT}$ & 69-Tm-167 & 166.9328 & $0.00 \mathrm{E}+00$ & $8.67 \mathrm{E}-07$ \\
\hline 713 & LT & 69-Tm-168 & 167.9342 & $0.00 \mathrm{E}+00$ & $8.62 \mathrm{E}-08$ \\
\hline 714 & $\mathrm{LT}$ & 69-Tm-169 & 168.9342 & $1.00 \mathrm{E}+02$ & $0.00 \mathrm{E}+00$ \\
\hline 715 & LT & 69-Tm-170 & 169.9358 & $0.00 \mathrm{E}+00$ & $6.24 \mathrm{E}-08$ \\
\hline 716 & $\mathrm{LT}$ & 69-Tm-171 & 170.9364 & $0.00 \mathrm{E}+00$ & $1.14 \mathrm{E}-08$ \\
\hline 717 & $\mathrm{LT}$ & 69-Tm-172 & 171.9384 & $0.00 \mathrm{E}+00$ & $3.03 \mathrm{E}-06$ \\
\hline 718 & LT & 69-Tm-173 & 172.9396 & $0.00 \mathrm{E}+00$ & $2.34 \mathrm{E}-05$ \\
\hline 719 & $\mathrm{LT}$ & $70-Y b-166$ & 165.9339 & $0.00 \mathrm{E}+00$ & $3.40 \mathrm{E}-06$ \\
\hline 720 & LT & $70-Y b-167$ & 166.9350 & $0.00 \mathrm{E}+00$ & $6.60 \mathrm{E}-04$ \\
\hline 721 & $\mathrm{LT}$ & $70-Y b-168$ & 167.9339 & $1.30 \mathrm{E}-01$ & $0.00 \mathrm{E}+00$ \\
\hline 722 & $\mathrm{LT}$ & $70-Y b-169$ & 168.9352 & $0.00 \mathrm{E}+00$ & $2.51 \mathrm{E}-07$ \\
\hline 723 & LT & $70-Y b-170$ & 169.9348 & $3.04 \mathrm{E}+00$ & $0.00 \mathrm{E}+00$ \\
\hline 724 & LT & $70-Y b-171$ & 170.9363 & $1.43 \mathrm{E}+01$ & $0.00 \mathrm{E}+00$ \\
\hline 725 & $\mathrm{LT}$ & $70-Y b-172$ & 171.9364 & $2.18 \mathrm{E}+01$ & $0.00 \mathrm{E}+00$ \\
\hline 726 & LT & $70-Y b-173$ & 172.9382 & $1.61 \mathrm{E}+01$ & $0.00 \mathrm{E}+00$ \\
\hline 727 & LT & $70-Y b-174$ & 173.9389 & $3.18 \mathrm{E}+01$ & $0.00 \mathrm{E}+00$ \\
\hline 728 & LT & $70-Y b-175$ & 174.9413 & $0.00 \mathrm{E}+00$ & $1.92 \mathrm{E}-06$ \\
\hline 729 & LT & $70-Y b-175 \mathrm{~m}$ & 174.9413 & $0.00 \mathrm{E}+00$ & $1.02 \mathrm{E}+01$ \\
\hline 730 & LT & $70-Y b-176$ & 175.9426 & $1.28 \mathrm{E}+01$ & $0.00 \mathrm{E}+00$ \\
\hline 731 & $\mathrm{LT}$ & $70-Y b-177$ & 176.9453 & $0.00 \mathrm{E}+00$ & $1.01 \mathrm{E}-04$ \\
\hline 732 & LT & 71-Lu-169 & 168.9377 & $0.00 \mathrm{E}+00$ & $5.65 \mathrm{E}-06$ \\
\hline 733 & LT & 71-Lu-170 & 169.9385 & $0.00 \mathrm{E}+00$ & $3.99 \mathrm{E}-06$ \\
\hline 734 & LT & 71-Lu-171 & 170.9379 & $0.00 \mathrm{E}+00$ & $9.74 \mathrm{E}-07$ \\
\hline 735 & LT & 71-Lu-172 & 171.9391 & $0.00 \mathrm{E}+00$ & $1.20 \mathrm{E}-06$ \\
\hline 736 & LT & 71-Lu-172m & 171.9391 & $0.00 \mathrm{E}+00$ & $3.12 \mathrm{E}-03$ \\
\hline 737 & LT & 71-Lu-173 & 172.9389 & $0.00 \mathrm{E}+00$ & $1.60 \mathrm{E}-08$ \\
\hline 738 & LT & 71-Lu-174 & 173.9403 & $0.00 \mathrm{E}+00$ & $6.64 \mathrm{E}-09$ \\
\hline 739 & $\mathrm{LT}$ & 71-Lu-174m & 173.9403 & $0.00 \mathrm{E}+00$ & $5.65 \mathrm{E}-08$ \\
\hline 740 & LT & 71-Lu-175 & 174.9408 & $9.74 \mathrm{E}+01$ & $0.00 \mathrm{E}+00$ \\
\hline 741 & LT & 71-Lu-176 & 175.9427 & $2.59 \mathrm{E}+00$ & $5.84 \mathrm{E}-19$ \\
\hline 742 & LT & 71-Lu-176m & 175.9427 & $0.00 \mathrm{E}+00$ & $5.30 \mathrm{E}-05$ \\
\hline 743 & $\mathrm{LT}$ & 71-Lu-177 & 176.9438 & $0.00 \mathrm{E}+00$ & $1.21 \mathrm{E}-06$ \\
\hline 744 & $\mathrm{LT}$ & 71-Lu-177m & 176.9438 & $0.00 \mathrm{E}+00$ & $5.00 \mathrm{E}-08$ \\
\hline 745 & LT & 72-Hf-170 & 169.9396 & $0.00 \mathrm{E}+00$ & $1.20 \mathrm{E}-05$ \\
\hline 746 & LT & 72-Hf-171 & 170.9405 & $0.00 \mathrm{E}+00$ & $1.59 \mathrm{E}-05$ \\
\hline 747 & LT & 72-Hf-172 & 171.9395 & $0.00 \mathrm{E}+00$ & $1.17 \mathrm{E}-08$ \\
\hline 748 & LT & 72-Hf-173 & 172.9405 & $0.00 \mathrm{E}+00$ & $8.16 \mathrm{E}-06$ \\
\hline 749 & LT & 72-Hf-174 & 173.9400 & $1.60 \mathrm{E}-01$ & $1.10 \mathrm{E}-23$ \\
\hline 750 & $\mathrm{LT}$ & 72-Hf-175 & 174.9415 & $0.00 \mathrm{E}+00$ & $1.15 \mathrm{E}-07$ \\
\hline 751 & LT & 72-Hf-176 & 175.9414 & $5.26 \mathrm{E}+00$ & $0.00 \mathrm{E}+00$ \\
\hline 752 & LT & 72-Hf-177 & 176.9432 & $1.86 \mathrm{E}+01$ & $0.00 \mathrm{E}+00$ \\
\hline 753 & $\mathrm{LT}$ & 72-Hf-177m & 176.9432 & $0.00 \mathrm{E}+00$ & $6.36 \mathrm{E}-01$ \\
\hline 754 & LT & 72-Hf-178 & 177.9437 & $2.73 \mathrm{E}+01$ & $0.00 \mathrm{E}+00$ \\
\hline 755 & LT & 72-Hf-178m & 177.9437 & $0.00 \mathrm{E}+00$ & $1.73 \mathrm{E}-01$ \\
\hline 756 & LT & 72-Hf-179 & 178.9458 & $1.36 \mathrm{E}+01$ & $0.00 \mathrm{E}+00$ \\
\hline 757 & $\mathrm{LT}$ & 72-Hf-179m & 178.9458 & $0.00 \mathrm{E}+00$ & $3.71 \mathrm{E}-02$ \\
\hline 758 & LT & 72-Hf-180 & 179.9465 & $3.51 \mathrm{E}+01$ & $0.00 \mathrm{E}+00$ \\
\hline 759 & LT & 72-Hf-180m & 179.9465 & $0.00 \mathrm{E}+00$ & $3.50 \mathrm{E}-05$ \\
\hline 760 & LT & 72-Hf-181 & 180.9491 & $0.00 \mathrm{E}+00$ & 1.89E-07 \\
\hline 761 & LT & 72-Hf-182 & 181.9505 & $0.00 \mathrm{E}+00$ & $2.47 \mathrm{E}-15$ \\
\hline 762 & $\mathrm{LT}$ & 73-Тa-177 & 176.9445 & $0.00 \mathrm{E}+00$ & $3.40 \mathrm{E}-06$ \\
\hline 763 & LT & 73-Тa-178 & 177.9458 & $0.00 \mathrm{E}+00$ & $1.24 \mathrm{E}-03$ \\
\hline 764 & $\mathrm{LT}$ & 73-Тa-179 & 178.9459 & $0.00 \mathrm{E}+00$ & $1.21 \mathrm{E}-08$ \\
\hline 765 & $\mathrm{LT}$ & 73-Ta-180m & 179.9475 & $1.20 \mathrm{E}-02$ & $0.00 \mathrm{E}+00$ \\
\hline 766 & LT & 73-Тa-180 & 179.9475 & $0.00 \mathrm{E}+00$ & $2.36 \mathrm{E}-05$ \\
\hline 767 & LT & 73-Тa-181 & 180.9480 & $1.00 \mathrm{E}+02$ & $0.00 \mathrm{E}+00$ \\
\hline 768 & LT & 73-Ta-182 & 181.9501 & $0.00 \mathrm{E}+00$ & $6.99 \mathrm{E}-08$ \\
\hline 769 & LT & 73-Ta-182m & 181.9501 & $0.00 \mathrm{E}+00$ & $2.45 \mathrm{E}+00$ \\
\hline 770 & LT & 73-Тa-183 & 182.9514 & $0.00 \mathrm{E}+00$ & $1.57 \mathrm{E}-06$ \\
\hline 771 & LT & $74-W-178$ & 177.9459 & $0.00 \mathrm{E}+00$ & $3.71 \mathrm{E}-07$ \\
\hline
\end{tabular}




\begin{tabular}{|c|c|c|c|c|c|}
\hline 772 & LT & 74-W-180 & 179.9467 & $1.20 \mathrm{E}-01$ & $1.22 \mathrm{E}-26$ \\
\hline 773 & LT & 74-W-181 & 180.9482 & $0.00 \mathrm{E}+00$ & $6.62 \mathrm{E}-08$ \\
\hline 774 & LT & $74-W-182$ & 181.9482 & $2.65 \mathrm{E}+01$ & $0.00 \mathrm{E}+00$ \\
\hline 775 & LT & 74-W-183m & 182.9502 & $0.00 \mathrm{E}+00$ & $1.33 \mathrm{E}-01$ \\
\hline 776 & $\mathrm{LT}$ & 74-W-183 & 182.9502 & $1.43 \mathrm{E}+01$ & $0.00 \mathrm{E}+00$ \\
\hline 777 & LT & 74-W-184 & 183.9509 & $3.06 \mathrm{E}+01$ & $0.00 \mathrm{E}+00$ \\
\hline 778 & $\mathrm{LT}$ & $74-W-185$ & 184.9534 & $0.00 \mathrm{E}+00$ & $1.07 \mathrm{E}-07$ \\
\hline 779 & LT & 74-W-185m & 184.9534 & $0.00 \mathrm{E}+00$ & $6.92 \mathrm{E}-03$ \\
\hline 780 & $\mathrm{LT}$ & 74-W-186 & 185.9544 & $2.84 \mathrm{E}+01$ & $1.29 \mathrm{E}-28$ \\
\hline 781 & LT & 74-W-187 & 186.9572 & $0.00 \mathrm{E}+00$ & $8.02 \mathrm{E}-06$ \\
\hline 782 & $\mathrm{LT}$ & 74-W-188 & 187.9585 & $0.00 \mathrm{E}+00$ & $1.15 \mathrm{E}-07$ \\
\hline 783 & LT & 74-W-189 & 188.9619 & $0.00 \mathrm{E}+00$ & $1.08 \mathrm{E}-03$ \\
\hline 784 & LT & 75-Re-181 & 180.9501 & $0.00 \mathrm{E}+00$ & $9.68 \mathrm{E}-06$ \\
\hline 785 & $\mathrm{LT}$ & 75-Re-182 & 181.9512 & $0.00 \mathrm{E}+00$ & $3.01 \mathrm{E}-06$ \\
\hline 786 & LT & $75-\mathrm{Re}-182 \mathrm{~m}$ & 181.9512 & $0.00 \mathrm{E}+00$ & $1.52 \mathrm{E}-05$ \\
\hline 787 & $\mathrm{LT}$ & 75-Re-183 & 182.9508 & $0.00 \mathrm{E}+00$ & $1.15 \mathrm{E}-07$ \\
\hline 788 & $\mathrm{LT}$ & 75-Re-184 & 183.9525 & $0.00 \mathrm{E}+00$ & $2.27 \mathrm{E}-07$ \\
\hline 789 & LT & $75-\operatorname{Re}-184 \mathrm{~m}$ & 183.9525 & $0.00 \mathrm{E}+00$ & $4.75 \mathrm{E}-08$ \\
\hline 790 & LT & 75-Re-185 & 184.9530 & $3.74 \mathrm{E}+01$ & $0.00 \mathrm{E}+00$ \\
\hline 791 & $\mathrm{LT}$ & 75-Re-186 & 185.9550 & $0.00 \mathrm{E}+00$ & $2.16 \mathrm{E}-06$ \\
\hline 792 & LT & 75-Re-186m & 185.9550 & $0.00 \mathrm{E}+00$ & $1.10 \mathrm{E}-13$ \\
\hline 793 & LT & 75-Re-187 & 186.9557 & $6.26 \mathrm{E}+01$ & $5.07 \mathrm{E}-19$ \\
\hline 794 & LT & 75-Re-188 & 187.9581 & $0.00 \mathrm{E}+00$ & $1.13 \mathrm{E}-05$ \\
\hline 795 & $\mathrm{LT}$ & 75-Re-188m & 187.9581 & $0.00 \mathrm{E}+00$ & $6.21 \mathrm{E}-04$ \\
\hline 796 & LT & 75-Re-189 & 188.9592 & $0.00 \mathrm{E}+00$ & $7.92 \mathrm{E}-06$ \\
\hline 797 & $\mathrm{LT}$ & 76-Os-182 & 181.9521 & $0.00 \mathrm{E}+00$ & $8.82 \mathrm{E}-06$ \\
\hline 798 & LT & 76-Os-183 & 182.9531 & $0.00 \mathrm{E}+00$ & $1.48 \mathrm{E}-05$ \\
\hline 799 & LT & 76-Os-184 & 183.9525 & $2.00 \mathrm{E}-02$ & $0.00 \mathrm{E}+00$ \\
\hline 800 & LT & 76-Os-185 & 184.9540 & $0.00 \mathrm{E}+00$ & $8.57 \mathrm{E}-08$ \\
\hline 801 & LT & 76-Os-186 & 185.9538 & $1.59 \mathrm{E}+00$ & $1.10 \mathrm{E}-23$ \\
\hline 802 & LT & 76-Os-187 & 186.9557 & $1.96 \mathrm{E}+00$ & $0.00 \mathrm{E}+00$ \\
\hline 803 & LT & 76-Os-188 & 187.9558 & $1.32 \mathrm{E}+01$ & $0.00 \mathrm{E}+00$ \\
\hline 804 & LT & 76-Os-189 & 188.9581 & $1.62 \mathrm{E}+01$ & $0.00 \mathrm{E}+00$ \\
\hline 805 & $\mathrm{LT}$ & $76-$ Os- $189 \mathrm{~m}$ & 188.9581 & $0.00 \mathrm{E}+00$ & $3.31 \mathrm{E}-05$ \\
\hline 806 & LT & 76-Os-190 & 189.9585 & $2.63 \mathrm{E}+01$ & $0.00 \mathrm{E}+00$ \\
\hline 807 & LT & 76-Os-190m & 189.9585 & $0.00 \mathrm{E}+00$ & $1.17 \mathrm{E}-03$ \\
\hline 808 & LT & 76-Os-191 & 190.9609 & $0.00 \mathrm{E}+00$ & $5.21 \mathrm{E}-07$ \\
\hline 809 & $\mathrm{LT}$ & 76-Os-191m & 190.9609 & $0.00 \mathrm{E}+00$ & $1.47 \mathrm{E}-05$ \\
\hline 810 & $\mathrm{LT}$ & 76-Os-192 & 191.9615 & $4.08 \mathrm{E}+01$ & $0.00 \mathrm{E}+00$ \\
\hline 811 & LT & 76-Os-193 & 192.9642 & $0.00 \mathrm{E}+00$ & $6.39 \mathrm{E}-06$ \\
\hline 812 & LT & 76-Os-194 & 193.9652 & $0.00 \mathrm{E}+00$ & $3.66 \mathrm{E}-09$ \\
\hline 813 & LT & $77-I r-185$ & 184.9567 & $0.00 \mathrm{E}+00$ & $1.34 \mathrm{E}-05$ \\
\hline 814 & LT & 77-Ir-186 & 185.9579 & $0.00 \mathrm{E}+00$ & $1.16 \mathrm{E}-05$ \\
\hline 815 & LT & $77-I r-188$ & 187.9588 & $0.00 \mathrm{E}+00$ & $4.64 \mathrm{E}-06$ \\
\hline 816 & $\mathrm{LT}$ & 77-Ir-189 & 188.9587 & $0.00 \mathrm{E}+00$ & $6.08 \mathrm{E}-07$ \\
\hline 817 & LT & $77-\mathrm{Ir}-189 \mathrm{~m}$ & 188.9587 & $0.00 \mathrm{E}+00$ & $5.21 \mathrm{E}+01$ \\
\hline 818 & LT & $77-I r-190$ & 189.9606 & $0.00 \mathrm{E}+00$ & $6.81 \mathrm{E}-07$ \\
\hline 819 & $\mathrm{LT}$ & 77-Ir-191 & 190.9606 & $3.73 \mathrm{E}+01$ & $0.00 \mathrm{E}+00$ \\
\hline 820 & LT & 77-Ir-191m & 190.9606 & $0.00 \mathrm{E}+00$ & $1.41 \mathrm{E}-01$ \\
\hline 821 & LT & $77-I r-192$ & 191.9626 & $0.00 \mathrm{E}+00$ & $1.09 \mathrm{E}-07$ \\
\hline 822 & LT & 77-Ir-192m & 191.9626 & $0.00 \mathrm{E}+00$ & 7.97E-03 \\
\hline 823 & $\mathrm{LT}$ & 77-Ir-193 & 192.9629 & $6.27 \mathrm{E}+01$ & $0.00 \mathrm{E}+00$ \\
\hline 824 & LT & 77-Ir-193m & 192.9629 & $0.00 \mathrm{E}+00$ & $7.62 \mathrm{E}-07$ \\
\hline 825 & LT & 77-Ir-194 & 193.9651 & $0.00 \mathrm{E}+00$ & $9.99 \mathrm{E}-06$ \\
\hline 826 & LT & 77-Ir-194m & 193.9651 & $0.00 \mathrm{E}+00$ & $2.18 \mathrm{E}+01$ \\
\hline 827 & LT & $77-I r-196$ & 195.9684 & $0.00 \mathrm{E}+00$ & $1.33 \mathrm{E}-02$ \\
\hline 828 & $\mathrm{LT}$ & 77-Ir-196m & 195.9684 & $0.00 \mathrm{E}+00$ & $1.38 \mathrm{E}-04$ \\
\hline 829 & LT & 78-Pt-188 & 187.9594 & $0.00 \mathrm{E}+00$ & 7.87E-07 \\
\hline 830 & $\mathrm{LT}$ & 78-Pt-189 & 188.9608 & $0.00 \mathrm{E}+00$ & $1.77 \mathrm{E}-05$ \\
\hline 831 & $\mathrm{LT}$ & 78-Pt-190 & 189.9599 & $1.40 \mathrm{E}-02$ & $3.38 \mathrm{E}-20$ \\
\hline 832 & LT & 78-Pt-191 & 190.9617 & $0.00 \mathrm{E}+00$ & $2.86 \mathrm{E}-06$ \\
\hline 833 & LT & 78-Pt-192 & 191.9610 & $7.82 \mathrm{E}-01$ & $0.00 \mathrm{E}+00$ \\
\hline 834 & LT & 78-Pt-193 & 192.9630 & $0.00 \mathrm{E}+00$ & $4.39 \mathrm{E}-10$ \\
\hline 835 & LT & 78-Pt-193m & 192.9630 & $0.00 \mathrm{E}+00$ & $1.85 \mathrm{E}-06$ \\
\hline 836 & LT & 78-Pt-194 & 193.9627 & $3.30 \mathrm{E}+01$ & $0.00 \mathrm{E}+00$ \\
\hline 837 & LT & 78-Pt-195 & 194.9648 & $3.38 \mathrm{E}+01$ & $0.00 \mathrm{E}+00$ \\
\hline
\end{tabular}




\begin{tabular}{|c|c|c|c|c|c|}
\hline 838 & LT & 78-Pt-195m & 194.9648 & $0.00 \mathrm{E}+00$ & $2.00 \mathrm{E}-06$ \\
\hline 839 & LT & 78-Pt-196 & 195.9650 & $2.52 \mathrm{E}+01$ & $0.00 \mathrm{E}+00$ \\
\hline 840 & LT & 78-Pt-197 & 196.9673 & $0.00 \mathrm{E}+00$ & $9.68 \mathrm{E}-06$ \\
\hline 841 & LT & 78-Pt-197m & 196.9673 & $0.00 \mathrm{E}+00$ & $1.21 \mathrm{E}-04$ \\
\hline 842 & LT & 78-Pt-198 & 197.9679 & $7.16 \mathrm{E}+00$ & $0.00 \mathrm{E}+00$ \\
\hline 843 & LT & 78-Pt-199 & 198.9706 & $0.00 \mathrm{E}+00$ & $3.75 \mathrm{E}-04$ \\
\hline 844 & LT & 78-Pt-199m & 198.9706 & $0.00 \mathrm{E}+00$ & $5.10 \mathrm{E}-02$ \\
\hline 845 & LT & 78-Pt-200 & 199.9714 & $0.00 \mathrm{E}+00$ & $1.53 \mathrm{E}-05$ \\
\hline 846 & LT & 79-Au-193 & 192.9642 & $0.00 \mathrm{E}+00$ & $1.09 \mathrm{E}-05$ \\
\hline 847 & LT & 79-Au-194 & 193.9654 & $0.00 \mathrm{E}+00$ & $5.06 \mathrm{E}-06$ \\
\hline 848 & LT & 79-Au-195 & 194.9650 & $0.00 \mathrm{E}+00$ & $4.31 \mathrm{E}-08$ \\
\hline 849 & $\mathrm{LT}$ & 79-Au-195m & 194.9650 & $0.00 \mathrm{E}+00$ & $2.27 \mathrm{E}-02$ \\
\hline 850 & LT & 79-Au-196 & 195.9666 & $0.00 \mathrm{E}+00$ & $1.30 \mathrm{E}-06$ \\
\hline 851 & LT & 79-Au-197 & 196.9666 & $1.00 \mathrm{E}+02$ & $0.00 \mathrm{E}+00$ \\
\hline 852 & LT & 79-Au-197m & 196.9666 & $0.00 \mathrm{E}+00$ & 8.97E-02 \\
\hline 853 & $\mathrm{LT}$ & 79-Au-198 & 197.9682 & $0.00 \mathrm{E}+00$ & $2.98 \mathrm{E}-06$ \\
\hline 854 & LT & 79-Au-198m & 197.9682 & $0.00 \mathrm{E}+00$ & $3.53 \mathrm{E}-06$ \\
\hline 855 & LT & 79-Au-199 & 198.9688 & $0.00 \mathrm{E}+00$ & $2.56 \mathrm{E}-06$ \\
\hline 856 & LT & 79-Au-200 & 199.9707 & $0.00 \mathrm{E}+00$ & $2.39 \mathrm{E}-04$ \\
\hline 857 & LT & 79-Au-200m & 199.9707 & $0.00 \mathrm{E}+00$ & $1.03 \mathrm{E}-05$ \\
\hline 858 & LT & 80-Hg-193 & 192.9667 & $0.00 \mathrm{E}+00$ & $5.07 \mathrm{E}-05$ \\
\hline 859 & LT & $80-\mathrm{Hg}-193 \mathrm{~m}$ & 192.9667 & $0.00 \mathrm{E}+00$ & $1.63 \mathrm{E}-05$ \\
\hline 860 & LT & 80-Hg-194 & 193.9654 & $0.00 \mathrm{E}+00$ & $4.95 \mathrm{E}-11$ \\
\hline 861 & LT & 80-Hg-195 & 194.9667 & $0.00 \mathrm{E}+00$ & $1.83 \mathrm{E}-05$ \\
\hline 862 & LT & $80-\mathrm{Hg}-195 \mathrm{~m}$ & 194.9667 & $0.00 \mathrm{E}+00$ & $4.63 \mathrm{E}-06$ \\
\hline 863 & $\mathrm{LT}$ & 80-Hg-196 & 195.9658 & $1.50 \mathrm{E}-01$ & $0.00 \mathrm{E}+00$ \\
\hline 864 & LT & $80-\mathrm{Hg}-197$ & 196.9672 & $0.00 \mathrm{E}+00$ & $3.00 \mathrm{E}-06$ \\
\hline 865 & LT & $80-\mathrm{Hg}-197 \mathrm{~m}$ & 196.9672 & $0.00 \mathrm{E}+00$ & $8.09 \mathrm{E}-06$ \\
\hline 866 & LT & 80-Hg-198 & 197.9668 & $9.97 \mathrm{E}+00$ & $0.00 \mathrm{E}+00$ \\
\hline 867 & LT & 80-Hg-199 & 198.9683 & $1.69 \mathrm{E}+01$ & $0.00 \mathrm{E}+00$ \\
\hline 868 & LT & $80-\mathrm{Hg}-199 \mathrm{~m}$ & 198.9683 & $0.00 \mathrm{E}+00$ & $2.71 \mathrm{E}-04$ \\
\hline 869 & LT & $80-\mathrm{Hg}-200$ & 199.9683 & $2.31 \mathrm{E}+01$ & $0.00 \mathrm{E}+00$ \\
\hline 870 & LT & 80-Hg-201 & 200.9703 & $1.32 \mathrm{E}+01$ & $0.00 \mathrm{E}+00$ \\
\hline 871 & LT & $80-\mathrm{Hg}-202$ & 201.9706 & $2.99 \mathrm{E}+01$ & $0.00 \mathrm{E}+00$ \\
\hline 872 & LT & 80-Hg-203 & 202.9729 & $0.00 \mathrm{E}+00$ & $1.72 \mathrm{E}-07$ \\
\hline 873 & LT & 80-Hg-204 & 203.9735 & $6.87 \mathrm{E}+00$ & $0.00 \mathrm{E}+00$ \\
\hline 874 & LT & $80-\mathrm{Hg}-205$ & 204.9761 & $0.00 \mathrm{E}+00$ & $2.25 \mathrm{E}-03$ \\
\hline 875 & $\mathrm{LT}$ & $80-\mathrm{Hg}-206$ & 205.9775 & $0.00 \mathrm{E}+00$ & $1.39 \mathrm{E}-03$ \\
\hline 876 & LT & $81-\mathrm{Tl}-200$ & 199.9710 & $0.00 \mathrm{E}+00$ & $7.38 \mathrm{E}-06$ \\
\hline 877 & LT & 81-T1-201 & 200.9708 & $0.00 \mathrm{E}+00$ & $2.64 \mathrm{E}-06$ \\
\hline 878 & LT & 81-T1-202 & 201.9721 & $0.00 \mathrm{E}+00$ & $6.52 \mathrm{E}-07$ \\
\hline 879 & LT & 81-Tl-203 & 202.9723 & $2.95 \mathrm{E}+01$ & $0.00 \mathrm{E}+00$ \\
\hline 880 & LT & 81-T1-204 & 203.9739 & $0.00 \mathrm{E}+00$ & $5.81 \mathrm{E}-09$ \\
\hline 881 & LT & 81-T1-205 & 204.9744 & $7.05 \mathrm{E}+01$ & $0.00 \mathrm{E}+00$ \\
\hline 882 & LT & 81-T1-206 & 205.9761 & $0.00 \mathrm{E}+00$ & $2.75 \mathrm{E}-03$ \\
\hline 883 & LT & 81-T1-207 & 206.9774 & $0.00 \mathrm{E}+00$ & $2.42 \mathrm{E}-03$ \\
\hline 884 & LT & $82-\mathrm{Pb}-200$ & 199.9718 & $0.00 \mathrm{E}+00$ & $8.96 \mathrm{E}-06$ \\
\hline 885 & LT & $82-\mathrm{Pb}-202$ & 201.9722 & $0.00 \mathrm{E}+00$ & $4.18 \mathrm{E}-13$ \\
\hline 886 & LT & $82-\mathrm{Pb}-203$ & 202.9734 & $0.00 \mathrm{E}+00$ & $3.71 \mathrm{E}-06$ \\
\hline 887 & $\mathrm{LT}$ & $82-\mathrm{Pb}-204$ & 203.9730 & $1.40 \mathrm{E}+00$ & $1.58 \mathrm{E}-25$ \\
\hline 888 & LT & $82-\mathrm{Pb}-205$ & 204.9745 & $0.00 \mathrm{E}+00$ & $1.27 \mathrm{E}-15$ \\
\hline 889 & LT & $82-\mathrm{Pb}-205 \mathrm{~m}$ & 204.9745 & $0.00 \mathrm{E}+00$ & $1.25 \mathrm{E}+02$ \\
\hline 890 & LT & $82-\mathrm{Pb}-206$ & 205.9745 & $2.41 \mathrm{E}+01$ & $0.00 \mathrm{E}+00$ \\
\hline 891 & LT & $82-\mathrm{Pb}-207$ & 206.9759 & $2.21 \mathrm{E}+01$ & $0.00 \mathrm{E}+00$ \\
\hline 892 & LT & $82-\mathrm{Pb}-207 \mathrm{~m}$ & 206.9759 & $0.00 \mathrm{E}+00$ & $8.60 \mathrm{E}-01$ \\
\hline 893 & LT & $82-\mathrm{Pb}-208$ & 207.9767 & $5.24 \mathrm{E}+01$ & $0.00 \mathrm{E}+00$ \\
\hline 894 & LT & $82-\mathrm{Pb}-209$ & 208.9811 & $0.00 \mathrm{E}+00$ & 5.92E-05 \\
\hline 895 & LT & $82-\mathrm{Pb}-210$ & 209.9842 & $0.00 \mathrm{E}+00$ & $9.89 \mathrm{E}-10$ \\
\hline 896 & LT & $83-\mathrm{Bi}-205$ & 204.9774 & $0.00 \mathrm{E}+00$ & $5.24 \mathrm{E}-07$ \\
\hline 897 & LT & $83-\mathrm{Bi}-206$ & 205.9785 & $0.00 \mathrm{E}+00$ & $1.29 \mathrm{E}-06$ \\
\hline 898 & LT & $83-\mathrm{Bi}-207$ & 206.9785 & $0.00 \mathrm{E}+00$ & $6.96 \mathrm{E}-10$ \\
\hline 899 & LT & $83-\mathrm{Bi}-208$ & 207.9797 & $0.00 \mathrm{E}+00$ & $5.97 \mathrm{E}-14$ \\
\hline 900 & LT & $83-\mathrm{Bi}-209$ & 208.9804 & $1.00 \mathrm{E}+02$ & $1.16 \mathrm{E}-27$ \\
\hline 901 & LT & $83-\mathrm{Bi}-210$ & 209.9841 & $0.00 \mathrm{E}+00$ & $1.60 \mathrm{E}-06$ \\
\hline 902 & LT & $83-\mathrm{Bi}-210 \mathrm{~m}$ & 209.9841 & $0.00 \mathrm{E}+00$ & $7.23 \mathrm{E}-15$ \\
\hline 903 & LT & $83-\mathrm{Bi}-211$ & 210.9873 & $0.00 \mathrm{E}+00$ & $5.40 \mathrm{E}-03$ \\
\hline
\end{tabular}




\begin{tabular}{|c|c|c|c|c|c|}
\hline 904 & LT & 84-Po-206 & 205.9805 & $0.00 \mathrm{E}+00$ & $9.12 \mathrm{E}-07$ \\
\hline 905 & LT & 84-Po-207 & 206.9816 & $0.00 \mathrm{E}+00$ & $3.32 \mathrm{E}-05$ \\
\hline 906 & LT & 84-Po-208 & 207.9812 & $0.00 \mathrm{E}+00$ & $7.58 \mathrm{E}-09$ \\
\hline 907 & $\mathrm{LT}$ & 84-Po-209 & 208.9824 & $0.00 \mathrm{E}+00$ & $2.15 \mathrm{E}-10$ \\
\hline 908 & LT & 84-Po-210 & 209.9829 & $0.00 \mathrm{E}+00$ & 5.80E-08 \\
\hline 909 & LT & 84-Po-211 & 210.9866 & $0.00 \mathrm{E}+00$ & $1.34 \mathrm{E}+00$ \\
\hline 910 & $\mathrm{LT}$ & 84-Po-211m & 210.9866 & $0.00 \mathrm{E}+00$ & $2.75 \mathrm{E}-02$ \\
\hline 911 & $\mathrm{AC}$ & $2-\mathrm{He}-3$ & 3.0160 & $0.00 \mathrm{E}+00$ & $0.00 \mathrm{E}+00$ \\
\hline 912 & $\mathrm{AC}$ & $2-\mathrm{He}-4$ & 4.0026 & $0.00 \mathrm{E}+00$ & $0.00 \mathrm{E}+00$ \\
\hline 913 & $\mathrm{AC}$ & 3-Li-6 & 6.0151 & $0.00 \mathrm{E}+00$ & $0.00 \mathrm{E}+00$ \\
\hline 914 & $\mathrm{AC}$ & 3-Li-7 & 7.0160 & $0.00 \mathrm{E}+00$ & $0.00 \mathrm{E}+00$ \\
\hline 915 & $\mathrm{AC}$ & 4-Be-7 & 7.0169 & $0.00 \mathrm{E}+00$ & $1.51 \mathrm{E}-07$ \\
\hline 916 & $\mathrm{AC}$ & $6-C-12$ & 12.0000 & $0.00 \mathrm{E}+00$ & $0.00 \mathrm{E}+00$ \\
\hline 917 & $\mathrm{AC}$ & $80-\mathrm{Hg}-206$ & 205.9775 & $0.00 \mathrm{E}+00$ & $1.39 \mathrm{E}-03$ \\
\hline 918 & $\mathrm{AC}$ & 81-T1-203 & 202.9723 & $0.00 \mathrm{E}+00$ & $0.00 \mathrm{E}+00$ \\
\hline 919 & $\mathrm{AC}$ & 81-T1-205 & 204.9744 & $0.00 \mathrm{E}+00$ & $0.00 \mathrm{E}+00$ \\
\hline 920 & $\mathrm{AC}$ & 81-T1-206 & 205.9761 & $0.00 \mathrm{E}+00$ & $2.75 \mathrm{E}-03$ \\
\hline 921 & $\mathrm{AC}$ & 81-T1-207 & 206.9774 & $0.00 \mathrm{E}+00$ & $2.42 \mathrm{E}-03$ \\
\hline 922 & $\mathrm{AC}$ & 81-T1-208 & 207.9820 & $0.00 \mathrm{E}+00$ & $3.78 \mathrm{E}-03$ \\
\hline 923 & $\mathrm{AC}$ & 81-T1-209 & 208.9854 & $0.00 \mathrm{E}+00$ & $5.25 \mathrm{E}-03$ \\
\hline 924 & $\mathrm{AC}$ & 81-Tl-210 & 209.9901 & $0.00 \mathrm{E}+00$ & 8.89E-03 \\
\hline 925 & $\mathrm{AC}$ & $82-\mathrm{Pb}-203$ & 202.9734 & $0.00 \mathrm{E}+00$ & $3.71 \mathrm{E}-06$ \\
\hline 926 & $\mathrm{AC}$ & $82-\mathrm{Pb}-204$ & 203.9730 & $0.00 \mathrm{E}+00$ & $1.58 \mathrm{E}-25$ \\
\hline 927 & $\mathrm{AC}$ & $82-\mathrm{Pb}-205$ & 204.9745 & $0.00 \mathrm{E}+00$ & $1.27 \mathrm{E}-15$ \\
\hline 928 & $\mathrm{AC}$ & $82-\mathrm{Pb}-206$ & 205.9745 & $0.00 \mathrm{E}+00$ & $0.00 \mathrm{E}+00$ \\
\hline 929 & $\mathrm{AC}$ & $82-\mathrm{Pb}-207$ & 206.9759 & $0.00 \mathrm{E}+00$ & $0.00 \mathrm{E}+00$ \\
\hline 930 & $\mathrm{AC}$ & $82-\mathrm{Pb}-207 \mathrm{~m}$ & 206.9759 & $0.00 \mathrm{E}+00$ & $8.60 \mathrm{E}-01$ \\
\hline 931 & $\mathrm{AC}$ & $82-\mathrm{Pb}-208$ & 207.9767 & $0.00 \mathrm{E}+00$ & $0.00 \mathrm{E}+00$ \\
\hline 932 & $\mathrm{AC}$ & $82-\mathrm{Pb}-209$ & 208.9811 & $0.00 \mathrm{E}+00$ & $5.92 \mathrm{E}-05$ \\
\hline 933 & $\mathrm{AC}$ & $82-\mathrm{Pb}-210$ & 209.9842 & $0.00 \mathrm{E}+00$ & $9.89 \mathrm{E}-10$ \\
\hline 934 & $\mathrm{AC}$ & $82-\mathrm{Pb}-211$ & 210.9887 & $0.00 \mathrm{E}+00$ & $3.20 \mathrm{E}-04$ \\
\hline 935 & $\mathrm{AC}$ & $82-\mathrm{Pb}-212$ & 211.9919 & $0.00 \mathrm{E}+00$ & $1.81 \mathrm{E}-05$ \\
\hline 936 & $\mathrm{AC}$ & $82-\mathrm{Pb}-214$ & 213.9998 & $0.00 \mathrm{E}+00$ & $4.31 \mathrm{E}-04$ \\
\hline 937 & $\mathrm{AC}$ & $83-\mathrm{Bi}-206$ & 205.9785 & $0.00 \mathrm{E}+00$ & $1.29 \mathrm{E}-06$ \\
\hline 938 & $\mathrm{AC}$ & $83-\mathrm{Bi}-207$ & 206.9785 & $0.00 \mathrm{E}+00$ & $6.96 \mathrm{E}-10$ \\
\hline 939 & $\mathrm{AC}$ & $83-\mathrm{Bi}-208$ & 207.9797 & $0.00 \mathrm{E}+00$ & $5.97 \mathrm{E}-14$ \\
\hline 940 & $\mathrm{AC}$ & 83-Bi-209 & 208.9804 & $0.00 \mathrm{E}+00$ & $1.16 \mathrm{E}-27$ \\
\hline 941 & $\mathrm{AC}$ & $83-\mathrm{Bi}-210 \mathrm{~m}$ & 209.9841 & $0.00 \mathrm{E}+00$ & $7.23 \mathrm{E}-15$ \\
\hline 942 & $\mathrm{AC}$ & $83-\mathrm{Bi}-210$ & 209.9841 & $0.00 \mathrm{E}+00$ & $1.60 \mathrm{E}-06$ \\
\hline 943 & $\mathrm{AC}$ & $83-\mathrm{Bi}-211$ & 210.9873 & $0.00 \mathrm{E}+00$ & $5.40 \mathrm{E}-03$ \\
\hline 944 & $\mathrm{AC}$ & $83-\mathrm{Bi}-212$ & 211.9913 & $0.00 \mathrm{E}+00$ & $1.91 \mathrm{E}-04$ \\
\hline 945 & $\mathrm{AC}$ & $83-B i-212 m$ & 211.9913 & $0.00 \mathrm{E}+00$ & $4.62 \mathrm{E}-04$ \\
\hline 946 & $\mathrm{AC}$ & $83-\mathrm{Bi}-213$ & 212.9944 & $0.00 \mathrm{E}+00$ & $2.53 \mathrm{E}-04$ \\
\hline 947 & $\mathrm{AC}$ & $83-\mathrm{Bi}-214$ & 213.9987 & $0.00 \mathrm{E}+00$ & $5.81 \mathrm{E}-04$ \\
\hline 948 & $\mathrm{AC}$ & 84-Po-207 & 206.9816 & $0.00 \mathrm{E}+00$ & $3.32 \mathrm{E}-05$ \\
\hline 949 & $\mathrm{AC}$ & 84-Po-208 & 207.9812 & $0.00 \mathrm{E}+00$ & $7.58 \mathrm{E}-09$ \\
\hline 950 & $\mathrm{AC}$ & 84-Рo-209 & 208.9824 & $0.00 \mathrm{E}+00$ & $2.15 \mathrm{E}-10$ \\
\hline 951 & $\mathrm{AC}$ & 84-Po-210 & 209.9829 & $0.00 \mathrm{E}+00$ & $5.80 \mathrm{E}-08$ \\
\hline 952 & $\mathrm{AC}$ & 84-Po-211m & 210.9866 & $0.00 \mathrm{E}+00$ & $2.75 \mathrm{E}-02$ \\
\hline 953 & $\mathrm{AC}$ & 84-Po-211 & 210.9866 & $0.00 \mathrm{E}+00$ & $1.34 \mathrm{E}+00$ \\
\hline 954 & $\mathrm{AC}$ & 84-Po-212 & 211.9889 & $0.00 \mathrm{E}+00$ & $6.93 \mathrm{E}+02$ \\
\hline 955 & $\mathrm{AC}$ & 84-Po-213 & 212.9929 & $0.00 \mathrm{E}+00$ & $6.93 \mathrm{E}+02$ \\
\hline 956 & $\mathrm{AC}$ & 84-Po-214 & 213.9952 & $0.00 \mathrm{E}+00$ & $6.93 \mathrm{E}+02$ \\
\hline 957 & $\mathrm{AC}$ & 84-Po-215 & 214.9994 & $0.00 \mathrm{E}+00$ & $3.89 \mathrm{E}+02$ \\
\hline 958 & $\mathrm{AC}$ & 84-Po-216 & 216.0019 & $0.00 \mathrm{E}+00$ & $4.78 \mathrm{E}+00$ \\
\hline 959 & $\mathrm{AC}$ & 84-Po-218 & 218.0090 & $0.00 \mathrm{E}+00$ & $3.73 \mathrm{E}-03$ \\
\hline 960 & $\mathrm{AC}$ & $85-A t-216$ & 216.0024 & $0.00 \mathrm{E}+00$ & $6.93 \mathrm{E}+02$ \\
\hline 961 & $\mathrm{AC}$ & $85-A t-217$ & 217.0047 & $0.00 \mathrm{E}+00$ & $2.15 \mathrm{E}+01$ \\
\hline 962 & $\mathrm{AC}$ & $85-A t-218$ & 218.0087 & $0.00 \mathrm{E}+00$ & $4.62 \mathrm{E}-01$ \\
\hline 963 & $\mathrm{AC}$ & $86-\mathrm{Rn}-216$ & 216.0003 & $0.00 \mathrm{E}+00$ & $6.93 \mathrm{E}+02$ \\
\hline 964 & $\mathrm{AC}$ & 86-Rn-217 & 217.0039 & $0.00 \mathrm{E}+00$ & $6.93 \mathrm{E}+02$ \\
\hline 965 & $\mathrm{AC}$ & $86-\mathrm{Rn}-218$ & 218.0056 & $0.00 \mathrm{E}+00$ & $1.98 \mathrm{E}+01$ \\
\hline 966 & $\mathrm{AC}$ & $86-\mathrm{Rn}-219$ & 219.0095 & $0.00 \mathrm{E}+00$ & $1.75 \mathrm{E}-01$ \\
\hline 967 & $\mathrm{AC}$ & $86-\mathrm{Rn}-220$ & 220.0114 & $0.00 \mathrm{E}+00$ & $1.25 \mathrm{E}-02$ \\
\hline 968 & $\mathrm{AC}$ & $86-\mathrm{Rn}-222$ & 222.0176 & $0.00 \mathrm{E}+00$ & $2.10 \mathrm{E}-06$ \\
\hline 969 & $\mathrm{AC}$ & $87-F r-220$ & 220.0123 & $0.00 \mathrm{E}+00$ & $2.53 \mathrm{E}-02$ \\
\hline
\end{tabular}




\begin{tabular}{|c|c|c|c|c|c|}
\hline 970 & $\mathrm{AC}$ & $87-F r-221$ & 221.0143 & $0.00 \mathrm{E}+00$ & $2.36 \mathrm{E}-03$ \\
\hline 971 & $\mathrm{AC}$ & $87-F r-222$ & 222.0175 & $0.00 \mathrm{E}+00$ & $8.14 \mathrm{E}-04$ \\
\hline 972 & $\mathrm{AC}$ & $87-F r-223$ & 223.0197 & $0.00 \mathrm{E}+00$ & $5.25 \mathrm{E}-04$ \\
\hline 973 & $\mathrm{AC}$ & $88-\mathrm{Ra}-220$ & 220.0110 & $0.00 \mathrm{E}+00$ & $3.85 \mathrm{E}+01$ \\
\hline 974 & $\mathrm{AC}$ & 88-Ra-222 & 222.0154 & $0.00 \mathrm{E}+00$ & $1.92 \mathrm{E}-02$ \\
\hline 975 & $\mathrm{AC}$ & 88-Ra-223 & 223.0185 & $0.00 \mathrm{E}+00$ & $7.02 \mathrm{E}-07$ \\
\hline 976 & $\mathrm{AC}$ & $88-\mathrm{Ra}-224$ & 224.0202 & $0.00 \mathrm{E}+00$ & $2.19 \mathrm{E}-06$ \\
\hline 977 & $\mathrm{AC}$ & 88-Ra-225 & 225.0236 & $0.00 \mathrm{E}+00$ & $5.38 \mathrm{E}-07$ \\
\hline 978 & $\mathrm{AC}$ & 88-Ra-226 & 226.0254 & $0.00 \mathrm{E}+00$ & $1.37 \mathrm{E}-11$ \\
\hline 979 & $\mathrm{AC}$ & $88-\mathrm{Ra}-227$ & 227.0292 & $0.00 \mathrm{E}+00$ & $2.74 \mathrm{E}-04$ \\
\hline 980 & $\mathrm{AC}$ & 88-Ra-228 & 228.0311 & $0.00 \mathrm{E}+00$ & $3.82 \mathrm{E}-09$ \\
\hline 981 & $\mathrm{AC}$ & 89-Ac-224 & 224.0217 & $0.00 \mathrm{E}+00$ & $6.93 \mathrm{E}-05$ \\
\hline 982 & $\mathrm{AC}$ & $89-A c-225$ & 225.0232 & $0.00 \mathrm{E}+00$ & $8.02 \mathrm{E}-07$ \\
\hline 983 & $\mathrm{AC}$ & $89-A c-226$ & 226.0261 & $0.00 \mathrm{E}+00$ & $6.56 \mathrm{E}-06$ \\
\hline 984 & $\mathrm{AC}$ & $89-A c-227$ & 227.0278 & $0.00 \mathrm{E}+00$ & $1.01 \mathrm{E}-09$ \\
\hline 985 & $\mathrm{AC}$ & 89-Ac-228 & 228.0310 & $0.00 \mathrm{E}+00$ & $3.13 \mathrm{E}-05$ \\
\hline 986 & $\mathrm{AC}$ & 90-Th-226 & 226.0249 & $0.00 \mathrm{E}+00$ & $3.78 \mathrm{E}-04$ \\
\hline 987 & $\mathrm{AC}$ & 90-Th-227 & 227.0277 & $0.00 \mathrm{E}+00$ & $4.29 \mathrm{E}-07$ \\
\hline 988 & $\mathrm{AC}$ & 90-Th-228 & 228.0287 & $0.00 \mathrm{E}+00$ & $1.15 \mathrm{E}-08$ \\
\hline 989 & $\mathrm{AC}$ & 90-Th-229 & 229.0318 & $0.00 \mathrm{E}+00$ & $2.99 \mathrm{E}-12$ \\
\hline 990 & $\mathrm{AC}$ & 90-Th-230 & 230.0331 & $0.00 \mathrm{E}+00$ & $2.91 \mathrm{E}-13$ \\
\hline 991 & $\mathrm{AC}$ & 90-Th-231 & 231.0363 & $0.00 \mathrm{E}+00$ & $7.55 \mathrm{E}-06$ \\
\hline 992 & $\mathrm{AC}$ & 90-Th-232 & 232.0381 & $0.00 \mathrm{E}+00$ & $1.56 \mathrm{E}-18$ \\
\hline 993 & $\mathrm{AC}$ & 90-Th-233 & 233.0416 & $0.00 \mathrm{E}+00$ & $5.18 \mathrm{E}-04$ \\
\hline 994 & $\mathrm{AC}$ & 90-Th-234 & 234.0436 & $0.00 \mathrm{E}+00$ & $3.33 \mathrm{E}-07$ \\
\hline 995 & $\mathrm{AC}$ & 91-Pa-228 & 228.0311 & $0.00 \mathrm{E}+00$ & $8.75 \mathrm{E}-06$ \\
\hline 996 & $\mathrm{AC}$ & 91-Pa-229 & 229.0321 & $0.00 \mathrm{E}+00$ & $5.35 \mathrm{E}-06$ \\
\hline 997 & $\mathrm{AC}$ & 91-Pa-230 & 230.0345 & $0.00 \mathrm{E}+00$ & $4.61 \mathrm{E}-07$ \\
\hline 998 & $\mathrm{AC}$ & 91-Pa-231 & 231.0359 & $0.00 \mathrm{E}+00$ & $6.70 \mathrm{E}-13$ \\
\hline 999 & $\mathrm{AC}$ & 91-Pa-232 & 232.0386 & $0.00 \mathrm{E}+00$ & $6.08 \mathrm{E}-06$ \\
\hline 1000 & $\mathrm{AC}$ & 91-Pa-233 & 233.0403 & $0.00 \mathrm{E}+00$ & $2.97 \mathrm{E}-07$ \\
\hline 1001 & $\mathrm{AC}$ & 91-Pa-234m & 234.0433 & $0.00 \mathrm{E}+00$ & $9.97 \mathrm{E}-03$ \\
\hline 1002 & $\mathrm{AC}$ & 91-Pa-234 & 234.0433 & $0.00 \mathrm{E}+00$ & $2.87 \mathrm{E}-05$ \\
\hline 1003 & $\mathrm{AC}$ & 91-Pa-235 & 235.0454 & $0.00 \mathrm{E}+00$ & $4.73 \mathrm{E}-04$ \\
\hline 1004 & $\mathrm{AC}$ & $92-U-230$ & 230.0339 & $0.00 \mathrm{E}+00$ & $3.86 \mathrm{E}-07$ \\
\hline 1005 & $\mathrm{AC}$ & $92-U-231$ & 231.0363 & $0.00 \mathrm{E}+00$ & $1.91 \mathrm{E}-06$ \\
\hline 1006 & $\mathrm{AC}$ & $92-U-232$ & 232.0372 & $0.00 \mathrm{E}+00$ & $3.19 \mathrm{E}-10$ \\
\hline 1007 & $\mathrm{AC}$ & $92-U-233$ & 233.0396 & $0.00 \mathrm{E}+00$ & $1.38 \mathrm{E}-13$ \\
\hline 1008 & $\mathrm{AC}$ & $92-U-234$ & 234.0410 & $0.00 \mathrm{E}+00$ & $8.95 \mathrm{E}-14$ \\
\hline 1009 & $\mathrm{AC}$ & $92-U-235$ & 235.0439 & $0.00 \mathrm{E}+00$ & $3.12 \mathrm{E}-17$ \\
\hline 1010 & $\mathrm{AC}$ & 92-U-235m & 235.0439 & $0.00 \mathrm{E}+00$ & $4.44 \mathrm{E}-04$ \\
\hline 1011 & $\mathrm{AC}$ & 92-U-236 & 236.0456 & $0.00 \mathrm{E}+00$ & $9.38 \mathrm{E}-16$ \\
\hline 1012 & $\mathrm{AC}$ & $92-U-237$ & 237.0487 & $0.00 \mathrm{E}+00$ & $1.19 \mathrm{E}-06$ \\
\hline 1013 & $\mathrm{AC}$ & $92-U-238$ & 238.0508 & $0.00 \mathrm{E}+00$ & $4.92 \mathrm{E}-18$ \\
\hline 1014 & $\mathrm{AC}$ & 92-U-239 & 239.0543 & $0.00 \mathrm{E}+00$ & 4.93E-04 \\
\hline 1015 & $\mathrm{AC}$ & $92-U-240$ & 240.0566 & $0.00 \mathrm{E}+00$ & $1.37 \mathrm{E}-05$ \\
\hline 1016 & $\mathrm{AC}$ & $92-U-241$ & 241.0603 & $0.00 \mathrm{E}+00$ & $2.31 \mathrm{E}-03$ \\
\hline 1017 & $\mathrm{AC}$ & 93-Np-234 & 234.0429 & $0.00 \mathrm{E}+00$ & $1.82 \mathrm{E}-06$ \\
\hline 1018 & $\mathrm{AC}$ & 93-Np-235 & 235.0441 & $0.00 \mathrm{E}+00$ & $2.03 \mathrm{E}-08$ \\
\hline 1019 & $\mathrm{AC}$ & 93-Np-236m & 236.0466 & $0.00 \mathrm{E}+00$ & $8.56 \mathrm{E}-06$ \\
\hline 1020 & $\mathrm{AC}$ & 93-Np-236 & 236.0466 & $0.00 \mathrm{E}+00$ & $1.44 \mathrm{E}-13$ \\
\hline 1021 & $\mathrm{AC}$ & 93-Np-237 & 237.0482 & $0.00 \mathrm{E}+00$ & $1.02 \mathrm{E}-14$ \\
\hline 1022 & $\mathrm{AC}$ & 93-Np-238 & 238.0509 & $0.00 \mathrm{E}+00$ & $3.79 \mathrm{E}-06$ \\
\hline 1023 & $\mathrm{AC}$ & 93-Np-239 & 239.0529 & $0.00 \mathrm{E}+00$ & $3.41 \mathrm{E}-06$ \\
\hline 1024 & $\mathrm{AC}$ & 93-Np-240m & 240.0562 & $0.00 \mathrm{E}+00$ & $1.60 \mathrm{E}-03$ \\
\hline 1025 & $\mathrm{AC}$ & 93-Np-240 & 240.0562 & $0.00 \mathrm{E}+00$ & $1.87 \mathrm{E}-04$ \\
\hline 1026 & $\mathrm{AC}$ & 93-Np-241 & 241.0582 & $0.00 \mathrm{E}+00$ & $8.31 \mathrm{E}-04$ \\
\hline 1027 & $\mathrm{AC}$ & 94-Pu-236 & 236.0461 & $0.00 \mathrm{E}+00$ & 7.69E-09 \\
\hline 1028 & $\mathrm{AC}$ & 94-Pu-237m & 237.0484 & $0.00 \mathrm{E}+00$ & $3.85 \mathrm{E}+00$ \\
\hline 1029 & $\mathrm{AC}$ & 94-Pu-237 & 237.0484 & $0.00 \mathrm{E}+00$ & $1.76 \mathrm{E}-07$ \\
\hline 1030 & $\mathrm{AC}$ & 94-Pu-238 & 238.0496 & $0.00 \mathrm{E}+00$ & $2.50 \mathrm{E}-10$ \\
\hline 1031 & $\mathrm{AC}$ & 94-Pu-239 & 239.0522 & $0.00 \mathrm{E}+00$ & $9.11 \mathrm{E}-13$ \\
\hline 1032 & $\mathrm{AC}$ & 94-Pu-240 & 240.0538 & $0.00 \mathrm{E}+00$ & $3.35 \mathrm{E}-12$ \\
\hline 1033 & $\mathrm{AC}$ & 94-Pu-241 & 241.0569 & $0.00 \mathrm{E}+00$ & $1.54 \mathrm{E}-09$ \\
\hline 1034 & $\mathrm{AC}$ & 94-Pu-242 & 242.0587 & $0.00 \mathrm{E}+00$ & $5.88 \mathrm{E}-14$ \\
\hline 1035 & $\mathrm{AC}$ & 94-Pu-243 & 243.0620 & $0.00 \mathrm{E}+00$ & $3.89 \mathrm{E}-05$ \\
\hline
\end{tabular}




\begin{tabular}{|c|c|c|c|c|c|}
\hline 1036 & $\mathrm{AC}$ & 94-Pu-244 & 244.0642 & $0.00 \mathrm{E}+00$ & $2.71 \mathrm{E}-16$ \\
\hline 1037 & $\mathrm{AC}$ & 94-Pu-245 & 245.0677 & $0.00 \mathrm{E}+00$ & $1.83 \mathrm{E}-05$ \\
\hline 1038 & $\mathrm{AC}$ & 94-Pu-246 & 246.0702 & $0.00 \mathrm{E}+00$ & $7.40 \mathrm{E}-07$ \\
\hline 1039 & $\mathrm{AC}$ & 94-Pu-247 & 247.0741 & $0.00 \mathrm{E}+00$ & $3.53 \mathrm{E}-06$ \\
\hline 1040 & $\mathrm{AC}$ & 95-Am-239 & 239.0530 & $0.00 \mathrm{E}+00$ & $1.62 \mathrm{E}-05$ \\
\hline 1041 & $\mathrm{AC}$ & 95-Am-240 & 240.0553 & $0.00 \mathrm{E}+00$ & $3.79 \mathrm{E}-06$ \\
\hline 1042 & $\mathrm{AC}$ & 95-Am-241 & 241.0568 & $0.00 \mathrm{E}+00$ & $5.08 \mathrm{E}-11$ \\
\hline 1043 & $\mathrm{AC}$ & 95-Am-242m & 242.0595 & $0.00 \mathrm{E}+00$ & $1.56 \mathrm{E}-10$ \\
\hline 1044 & $\mathrm{AC}$ & 95-Am-242 & 242.0596 & $0.00 \mathrm{E}+00$ & $1.20 \mathrm{E}-05$ \\
\hline 1045 & $\mathrm{AC}$ & 95-Am-243 & 243.0614 & $0.00 \mathrm{E}+00$ & $2.98 \mathrm{E}-12$ \\
\hline 1046 & $\mathrm{AC}$ & 95-Am-244m & 244.0646 & $0.00 \mathrm{E}+00$ & 4.44E-04 \\
\hline 1047 & $\mathrm{AC}$ & 95-Am-244 & 244.0643 & $0.00 \mathrm{E}+00$ & $1.91 \mathrm{E}-05$ \\
\hline 1048 & $\mathrm{AC}$ & 95-Am-245 & 245.0665 & $0.00 \mathrm{E}+00$ & 9.39E-05 \\
\hline 1049 & $\mathrm{AC}$ & 95-Am-246 & 246.0698 & $0.00 \mathrm{E}+00$ & $2.96 \mathrm{E}-04$ \\
\hline 1050 & $\mathrm{AC}$ & 95-Am-246m & 246.0698 & $0.00 \mathrm{E}+00$ & $4.62 \mathrm{E}-04$ \\
\hline 1051 & $\mathrm{AC}$ & 95-Am-247 & 247.0721 & $0.00 \mathrm{E}+00$ & $5.02 \mathrm{E}-04$ \\
\hline 1052 & $\mathrm{AC}$ & 96-Cm-240 & 240.0555 & $0.00 \mathrm{E}+00$ & $2.97 \mathrm{E}-07$ \\
\hline 1053 & $\mathrm{AC}$ & 96-Cm-241 & 241.0576 & $0.00 \mathrm{E}+00$ & $2.45 \mathrm{E}-07$ \\
\hline 1054 & $\mathrm{AC}$ & 96-Cm-242 & 242.0588 & $0.00 \mathrm{E}+00$ & $4.92 \mathrm{E}-08$ \\
\hline 1055 & $\mathrm{AC}$ & 96-Cm-243 & 243.0614 & $0.00 \mathrm{E}+00$ & $7.55 \mathrm{E}-10$ \\
\hline 1056 & $\mathrm{AC}$ & 96-Cm-244 & 244.0627 & $0.00 \mathrm{E}+00$ & $1.21 \mathrm{E}-09$ \\
\hline 1057 & $\mathrm{AC}$ & 96-Cm-245 & 245.0655 & $0.00 \mathrm{E}+00$ & $2.58 \mathrm{E}-12$ \\
\hline 1058 & $\mathrm{AC}$ & 96-Cm-246 & 246.0672 & $0.00 \mathrm{E}+00$ & $4.61 \mathrm{E}-12$ \\
\hline 1059 & $\mathrm{AC}$ & 96-Cm-247 & 247.0703 & $0.00 \mathrm{E}+00$ & $1.41 \mathrm{E}-15$ \\
\hline 1060 & $\mathrm{AC}$ & 96-Cm-248 & 248.0724 & $0.00 \mathrm{E}+00$ & $6.31 \mathrm{E}-14$ \\
\hline 1061 & $\mathrm{AC}$ & 96-Cm-249 & 249.0759 & $0.00 \mathrm{E}+00$ & $1.80 \mathrm{E}-04$ \\
\hline 1062 & $\mathrm{AC}$ & 96-Cm-250 & 250.0784 & $0.00 \mathrm{E}+00$ & $2.65 \mathrm{E}-12$ \\
\hline 1063 & $\mathrm{AC}$ & 96-Cm-251 & 251.0823 & $0.00 \mathrm{E}+00$ & $6.88 \mathrm{E}-04$ \\
\hline 1064 & $\mathrm{AC}$ & 97-Bk-245 & 245.0664 & $0.00 \mathrm{E}+00$ & $1.62 \mathrm{E}-06$ \\
\hline 1065 & $\mathrm{AC}$ & 97-Bk-246 & 246.0687 & $0.00 \mathrm{E}+00$ & $4.46 \mathrm{E}-06$ \\
\hline 1066 & $\mathrm{AC}$ & 97-Bk-247 & 247.0703 & $0.00 \mathrm{E}+00$ & $1.59 \mathrm{E}-11$ \\
\hline 1067 & $\mathrm{AC}$ & 97-Bk-248 & 248.0731 & $0.00 \mathrm{E}+00$ & $2.44 \mathrm{E}-09$ \\
\hline 1068 & $\mathrm{AC}$ & 97-Bk-248m & 248.0731 & $0.00 \mathrm{E}+00$ & $8.12 \mathrm{E}-06$ \\
\hline 1069 & $\mathrm{AC}$ & 97-Bk-249 & 249.0750 & $0.00 \mathrm{E}+00$ & $2.51 \mathrm{E}-08$ \\
\hline 1070 & $\mathrm{AC}$ & 97-Bk-250 & 250.0783 & $0.00 \mathrm{E}+00$ & 5.99E-05 \\
\hline 1071 & $\mathrm{AC}$ & 97-Bk-251 & 251.0808 & $0.00 \mathrm{E}+00$ & $2.08 \mathrm{E}-04$ \\
\hline 1072 & $\mathrm{AC}$ & 98-Cf-246 & 246.0688 & $0.00 \mathrm{E}+00$ & 5.39E-06 \\
\hline 1073 & $\mathrm{AC}$ & 98-Cf-248 & 248.0722 & $0.00 \mathrm{E}+00$ & $2.41 \mathrm{E}-08$ \\
\hline 1074 & $\mathrm{AC}$ & 98-Cf-249 & 249.0748 & $0.00 \mathrm{E}+00$ & $6.26 \mathrm{E}-11$ \\
\hline 1075 & $\mathrm{AC}$ & $98-\mathrm{Cf}-250$ & 250.0764 & $0.00 \mathrm{E}+00$ & $1.68 \mathrm{E}-09$ \\
\hline 1076 & $\mathrm{AC}$ & 98-Cf-251 & 251.0796 & $0.00 \mathrm{E}+00$ & $2.45 \mathrm{E}-11$ \\
\hline 1077 & $\mathrm{AC}$ & 98-Cf-252 & 252.0816 & $0.00 \mathrm{E}+00$ & $8.30 \mathrm{E}-09$ \\
\hline 1078 & $\mathrm{AC}$ & 98-Cf-253 & 253.0851 & $0.00 \mathrm{E}+00$ & $4.50 \mathrm{E}-07$ \\
\hline 1079 & $\mathrm{AC}$ & 98-Cf-254 & 254.0873 & $0.00 \mathrm{E}+00$ & $1.33 \mathrm{E}-07$ \\
\hline 1080 & $\mathrm{AC}$ & 98-Cf-255 & 255.0910 & $0.00 \mathrm{E}+00$ & $1.36 \mathrm{E}-04$ \\
\hline 1081 & $\mathrm{AC}$ & 99-Es-251 & 251.0800 & $0.00 \mathrm{E}+00$ & $5.83 \mathrm{E}-06$ \\
\hline 1082 & $\mathrm{AC}$ & 99-Es-252 & 252.0830 & $0.00 \mathrm{E}+00$ & $1.70 \mathrm{E}-08$ \\
\hline 1083 & $\mathrm{AC}$ & 99-Es-253 & 253.0848 & $0.00 \mathrm{E}+00$ & $3.92 \mathrm{E}-07$ \\
\hline 1084 & $\mathrm{AC}$ & 99-Es-254m & 254.0880 & $0.00 \mathrm{E}+00$ & $4.90 \mathrm{E}-06$ \\
\hline 1085 & $\mathrm{AC}$ & 99-Es-254 & 254.0880 & $0.00 \mathrm{E}+00$ & $2.91 \mathrm{E}-08$ \\
\hline 1086 & $\mathrm{AC}$ & 99-Es-255 & 255.0903 & $0.00 \mathrm{E}+00$ & $2.02 \mathrm{E}-07$ \\
\hline 1087 & $\mathrm{FP}$ & $1-\mathrm{H}-3$ & 3.0161 & $0.00 \mathrm{E}+00$ & $1.78 \mathrm{E}-09$ \\
\hline 1088 & $\mathrm{FP}$ & $2-\mathrm{He}-3$ & 3.0160 & $0.00 \mathrm{E}+00$ & $0.00 \mathrm{E}+00$ \\
\hline 1089 & $\mathrm{FP}$ & $2-\mathrm{He}-4$ & 4.0026 & $0.00 \mathrm{E}+00$ & $0.00 \mathrm{E}+00$ \\
\hline 1090 & $\mathrm{FP}$ & $26-\mathrm{Fe}-65$ & 64.9454 & $0.00 \mathrm{E}+00$ & $8.56 \mathrm{E}-01$ \\
\hline 1091 & $\mathrm{FP}$ & $27-\mathrm{Co}-65$ & 64.9365 & $0.00 \mathrm{E}+00$ & $5.98 \mathrm{E}-01$ \\
\hline 1092 & $\mathrm{FP}$ & $28-\mathrm{Ni}-65$ & 64.9301 & $0.00 \mathrm{E}+00$ & $7.65 \mathrm{E}-05$ \\
\hline 1093 & $\mathrm{FP}$ & $29-\mathrm{Cu}-65$ & 64.9278 & $0.00 \mathrm{E}+00$ & $0.00 \mathrm{E}+00$ \\
\hline 1094 & FP & $24-\mathrm{Cr}-66$ & 65.9734 & $0.00 \mathrm{E}+00$ & $6.93 \mathrm{E}+01$ \\
\hline 1095 & FP & $25-\mathrm{Mn}-66$ & 65.9611 & $0.00 \mathrm{E}+00$ & $1.08 \mathrm{E}+01$ \\
\hline 1096 & FP & $26-\mathrm{Fe}-66$ & 65.9468 & $0.00 \mathrm{E}+00$ & $1.58 \mathrm{E}+00$ \\
\hline 1097 & FP & 27-Co-66 & 65.9398 & $0.00 \mathrm{E}+00$ & $3.47 \mathrm{E}+00$ \\
\hline 1098 & $\mathrm{FP}$ & $28-\mathrm{Ni}-66$ & 65.9291 & $0.00 \mathrm{E}+00$ & $3.53 \mathrm{E}-06$ \\
\hline 1099 & FP & 29-Cu-66 & 65.9289 & $0.00 \mathrm{E}+00$ & $2.26 \mathrm{E}-03$ \\
\hline 1100 & $\mathrm{FP}$ & $30-Z n-66$ & 65.9260 & $0.00 \mathrm{E}+00$ & $0.00 \mathrm{E}+00$ \\
\hline 1101 & $\mathrm{FP}$ & 31-Ga-66 & 65.9316 & $0.00 \mathrm{E}+00$ & $2.03 \mathrm{E}-05$ \\
\hline
\end{tabular}




\begin{tabular}{|c|c|c|c|c|c|}
\hline 1102 & FP & $32-\mathrm{Ge}-66$ & 65.9338 & $0.00 \mathrm{E}+00$ & $8.52 \mathrm{E}-05$ \\
\hline 1103 & FP & $24-\mathrm{Cr}-67$ & 66.9796 & $0.00 \mathrm{E}+00$ & $1.39 \mathrm{E}+01$ \\
\hline 1104 & FP & $25-\mathrm{Mn}-67$ & 66.9641 & $0.00 \mathrm{E}+00$ & $1.47 \mathrm{E}+01$ \\
\hline 1105 & FP & $26-\mathrm{Fe}-67$ & 66.9510 & $0.00 \mathrm{E}+00$ & $1.67 \mathrm{E}+00$ \\
\hline 1106 & FP & 27-Co-67 & 66.9409 & $0.00 \mathrm{E}+00$ & $1.63 \mathrm{E}+00$ \\
\hline 1107 & FP & $28-\mathrm{Ni}-67$ & 66.9316 & $0.00 \mathrm{E}+00$ & $3.30 \mathrm{E}-02$ \\
\hline 1108 & FP & $29-\mathrm{Cu}-67$ & 66.9277 & $0.00 \mathrm{E}+00$ & $3.11 \mathrm{E}-06$ \\
\hline 1109 & FP & $30-Z n-67$ & 66.9271 & $0.00 \mathrm{E}+00$ & $0.00 \mathrm{E}+00$ \\
\hline 1110 & FP & 31-Ga-67 & 66.9282 & $0.00 \mathrm{E}+00$ & $2.46 \mathrm{E}-06$ \\
\hline 1111 & FP & $32-\mathrm{Ge}-67$ & 66.9327 & $0.00 \mathrm{E}+00$ & $6.11 \mathrm{E}-04$ \\
\hline 1112 & FP & $25-\mathrm{Mn}-68$ & 67.9693 & $0.00 \mathrm{E}+00$ & $2.48 \mathrm{E}+01$ \\
\hline 1113 & FP & $26-\mathrm{Fe}-68$ & 67.9537 & $0.00 \mathrm{E}+00$ & $3.71 \mathrm{E}+00$ \\
\hline 1114 & FP & 27-Co-68 & 67.9449 & $0.00 \mathrm{E}+00$ & $3.48 \mathrm{E}+00$ \\
\hline 1115 & FP & $28-\mathrm{Ni}-68$ & 67.9319 & $0.00 \mathrm{E}+00$ & $2.39 \mathrm{E}-02$ \\
\hline 1116 & FP & 29-Cu-68 & 67.9296 & $0.00 \mathrm{E}+00$ & $2.23 \mathrm{E}-02$ \\
\hline 1117 & FP & $29-\mathrm{Cu}-68 \mathrm{~m}$ & 67.9296 & $0.00 \mathrm{E}+00$ & $3.08 \mathrm{E}-03$ \\
\hline 1118 & FP & $30-Z n-68$ & 67.9248 & $0.00 \mathrm{E}+00$ & $0.00 \mathrm{E}+00$ \\
\hline 1119 & FP & 31-Ga-68 & 67.9280 & $0.00 \mathrm{E}+00$ & $1.71 \mathrm{E}-04$ \\
\hline 1120 & $\mathrm{FP}$ & $32-\mathrm{Ge}-68$ & 67.9281 & $0.00 \mathrm{E}+00$ & $2.96 \mathrm{E}-08$ \\
\hline 1121 & FP & $25-\mathrm{Mn}-69$ & 68.9728 & $0.00 \mathrm{E}+00$ & $4.95 \mathrm{E}+01$ \\
\hline 1122 & FP & $26-\mathrm{Fe}-69$ & 68.9588 & $0.00 \mathrm{E}+00$ & $6.36 \mathrm{E}+00$ \\
\hline 1123 & FP & 27-Co-69 & 68.9463 & $0.00 \mathrm{E}+00$ & $3.15 \mathrm{E}+00$ \\
\hline 1124 & FP & 28-Ni-69 & 68.9356 & $0.00 \mathrm{E}+00$ & $6.08 \mathrm{E}-02$ \\
\hline 1125 & FP & 29-Cu-69 & 68.9294 & $0.00 \mathrm{E}+00$ & $4.05 \mathrm{E}-03$ \\
\hline 1126 & FP & $30-Z n-69$ & 68.9266 & $0.00 \mathrm{E}+00$ & $2.05 \mathrm{E}-04$ \\
\hline 1127 & FP & $30-Z n-69 m$ & 68.9266 & $0.00 \mathrm{E}+00$ & $1.40 \mathrm{E}-05$ \\
\hline 1128 & FP & 31-Ga-69 & 68.9256 & $0.00 \mathrm{E}+00$ & $0.00 \mathrm{E}+00$ \\
\hline 1129 & $\mathrm{FP}$ & $32-\mathrm{Ge}-69$ & 68.9280 & $0.00 \mathrm{E}+00$ & $4.93 \mathrm{E}-06$ \\
\hline 1130 & FP & $33-A s-69$ & 68.9323 & $0.00 \mathrm{E}+00$ & $7.59 \mathrm{E}-04$ \\
\hline 1131 & FP & $26-\mathrm{Fe}-70$ & 69.9615 & $0.00 \mathrm{E}+00$ & $7.37 \mathrm{E}+00$ \\
\hline 1132 & FP & $27-\mathrm{Co}-70$ & 69.9510 & $0.00 \mathrm{E}+00$ & $5.82 \mathrm{E}+00$ \\
\hline 1133 & FP & $28-\mathrm{Ni}-70$ & 69.9365 & $0.00 \mathrm{E}+00$ & $1.16 \mathrm{E}-01$ \\
\hline 1134 & FP & 29-Cu-70 & 69.9324 & $0.00 \mathrm{E}+00$ & $1.56 \mathrm{E}-02$ \\
\hline 1135 & FP & 29-Cu-70m & 69.9324 & $0.00 \mathrm{E}+00$ & $2.10 \mathrm{E}-02$ \\
\hline 1136 & FP & $30-\mathrm{Zn}-70$ & 69.9253 & $0.00 \mathrm{E}+00$ & $0.00 \mathrm{E}+00$ \\
\hline 1137 & FP & $31-\mathrm{Ga}-70$ & 69.9260 & $0.00 \mathrm{E}+00$ & $5.46 \mathrm{E}-04$ \\
\hline 1138 & FP & $32-\mathrm{Ge}-70$ & 69.9242 & $0.00 \mathrm{E}+00$ & $0.00 \mathrm{E}+00$ \\
\hline 1139 & FP & $26-\mathrm{Fe}-71$ & 70.9667 & $0.00 \mathrm{E}+00$ & $2.48 \mathrm{E}+01$ \\
\hline 1140 & FP & 27-Co-71 & 70.9529 & $0.00 \mathrm{E}+00$ & $8.77 \mathrm{E}+00$ \\
\hline 1141 & FP & $28-\mathrm{Ni}-71$ & 70.9407 & $0.00 \mathrm{E}+00$ & $2.71 \mathrm{E}-01$ \\
\hline 1142 & FP & 29-Cu-71 & 70.9327 & $0.00 \mathrm{E}+00$ & $3.55 \mathrm{E}-02$ \\
\hline 1143 & FP & $30-Z n-71$ & 70.9277 & $0.00 \mathrm{E}+00$ & $4.72 \mathrm{E}-03$ \\
\hline 1144 & FP & $30-Z n-71 m$ & 70.9277 & $0.00 \mathrm{E}+00$ & $4.86 \mathrm{E}-05$ \\
\hline 1145 & FP & 31-Ga-71 & 70.9247 & $0.00 \mathrm{E}+00$ & $0.00 \mathrm{E}+00$ \\
\hline 1146 & FP & $32-\mathrm{Ge}-71$ & 70.9249 & $0.00 \mathrm{E}+00$ & $7.02 \mathrm{E}-07$ \\
\hline 1147 & FP & $32-\mathrm{Ge}-71 \mathrm{~m}$ & 70.9249 & $0.00 \mathrm{E}+00$ & $3.40 \mathrm{E}+01$ \\
\hline 1148 & FP & 33-As-71 & 70.9271 & $0.00 \mathrm{E}+00$ & $2.95 \mathrm{E}-06$ \\
\hline 1149 & FP & $26-\mathrm{Fe}-72$ & 71.9696 & $0.00 \mathrm{E}+00$ & $6.93 \mathrm{E}+02$ \\
\hline 1150 & FP & 27-Co-72 & 71.9578 & $0.00 \mathrm{E}+00$ & $1.16 \mathrm{E}+01$ \\
\hline 1151 & FP & $28-\mathrm{Ni}-72$ & 71.9421 & $0.00 \mathrm{E}+00$ & $4.42 \mathrm{E}-01$ \\
\hline 1152 & FP & 29-Cu-72 & 71.9358 & $0.00 \mathrm{E}+00$ & $1.05 \mathrm{E}-01$ \\
\hline 1153 & FP & $30-Z n-72$ & 71.9269 & $0.00 \mathrm{E}+00$ & $4.14 \mathrm{E}-06$ \\
\hline 1154 & FP & 31-Ga-72 & 71.9264 & $0.00 \mathrm{E}+00$ & $1.37 \mathrm{E}-05$ \\
\hline 1155 & FP & 31-Ga-72m & 71.9264 & $0.00 \mathrm{E}+00$ & $1.75 \mathrm{E}+01$ \\
\hline 1156 & FP & $32-\mathrm{Ge}-72$ & 71.9221 & $0.00 \mathrm{E}+00$ & $0.00 \mathrm{E}+00$ \\
\hline 1157 & FP & $33-A s-72$ & 71.9268 & $0.00 \mathrm{E}+00$ & $7.41 \mathrm{E}-06$ \\
\hline 1158 & FP & $34-\mathrm{Se}-72$ & 71.9271 & $0.00 \mathrm{E}+00$ & $9.55 \mathrm{E}-07$ \\
\hline 1159 & FP & 27-Co-73 & 72.9602 & $0.00 \mathrm{E}+00$ & $1.69 \mathrm{E}+01$ \\
\hline 1160 & FP & $28-\mathrm{Ni}-73$ & 72.9465 & $0.00 \mathrm{E}+00$ & $8.25 \mathrm{E}-01$ \\
\hline 1161 & FP & $29-\mathrm{Cu}-73$ & 72.9367 & $0.00 \mathrm{E}+00$ & $1.65 \mathrm{E}-01$ \\
\hline 1162 & FP & $30-Z n-73$ & 72.9298 & $0.00 \mathrm{E}+00$ & $2.95 \mathrm{E}-02$ \\
\hline 1163 & FP & 31-Ga-73 & 72.9252 & $0.00 \mathrm{E}+00$ & $3.96 \mathrm{E}-05$ \\
\hline 1164 & FP & $32-\mathrm{Ge}-73$ & 72.9235 & $0.00 \mathrm{E}+00$ & $0.00 \mathrm{E}+00$ \\
\hline 1165 & FP & $32-\mathrm{Ge}-73 \mathrm{~m}$ & 72.9235 & $0.00 \mathrm{E}+00$ & $1.39 \mathrm{E}+00$ \\
\hline 1166 & FP & 33-As-73 & 72.9238 & $0.00 \mathrm{E}+00$ & 9.99E-08 \\
\hline 1167 & FP & $34-\mathrm{Se}-73$ & 72.9268 & $0.00 \mathrm{E}+00$ & $2.69 \mathrm{E}-05$ \\
\hline
\end{tabular}




\begin{tabular}{|c|c|c|c|c|c|}
\hline 1168 & FP & $34-\mathrm{Se}-73 \mathrm{~m}$ & 72.9268 & $0.00 \mathrm{E}+00$ & $2.90 \mathrm{E}-04$ \\
\hline 1169 & FP & 27-Co-74 & 73.9654 & $0.00 \mathrm{E}+00$ & $2.31 \mathrm{E}+01$ \\
\hline 1170 & FP & $28-\mathrm{Ni}-74$ & 73.9481 & $0.00 \mathrm{E}+00$ & $1.02 \mathrm{E}+00$ \\
\hline 1171 & FP & 29-Cu-74 & 73.9399 & $0.00 \mathrm{E}+00$ & $3.96 \mathrm{E}-01$ \\
\hline 1172 & FP & $30-Z n-74$ & 73.9295 & $0.00 \mathrm{E}+00$ & $7.25 \mathrm{E}-03$ \\
\hline 1173 & FP & 31-Ga-74 & 73.9269 & $0.00 \mathrm{E}+00$ & $1.42 \mathrm{E}-03$ \\
\hline 1174 & FP & 31-Ga-74m & 73.9269 & $0.00 \mathrm{E}+00$ & $7.30 \mathrm{E}-02$ \\
\hline 1175 & FP & $32-\mathrm{Ge}-74$ & 73.9212 & $0.00 \mathrm{E}+00$ & $0.00 \mathrm{E}+00$ \\
\hline 1176 & FP & 33-As-74 & 73.9239 & $0.00 \mathrm{E}+00$ & $4.51 \mathrm{E}-07$ \\
\hline 1177 & FP & 34-Se-74 & 73.9225 & $0.00 \mathrm{E}+00$ & $0.00 \mathrm{E}+00$ \\
\hline 1178 & FP & 27-Co-75 & 74.9683 & $0.00 \mathrm{E}+00$ & $2.04 \mathrm{E}+01$ \\
\hline 1179 & FP & $28-\mathrm{Ni}-75$ & 74.9529 & $0.00 \mathrm{E}+00$ & $1.16 \mathrm{E}+00$ \\
\hline 1180 & FP & 29-Cu-75 & 74.9419 & $0.00 \mathrm{E}+00$ & $5.66 \mathrm{E}-01$ \\
\hline 1181 & FP & $30-Z n-75$ & 74.9329 & $0.00 \mathrm{E}+00$ & $6.80 \mathrm{E}-02$ \\
\hline 1182 & FP & 31-Ga-75 & 74.9265 & $0.00 \mathrm{E}+00$ & $5.50 \mathrm{E}-03$ \\
\hline 1183 & FP & $32-\mathrm{Ge}-75$ & 74.9229 & $0.00 \mathrm{E}+00$ & $1.40 \mathrm{E}-04$ \\
\hline 1184 & FP & $32-\mathrm{Ge}-75 \mathrm{~m}$ & 74.9229 & $0.00 \mathrm{E}+00$ & $1.45 \mathrm{E}-02$ \\
\hline 1185 & FP & $33-A s-75$ & 74.9216 & $0.00 \mathrm{E}+00$ & $0.00 \mathrm{E}+00$ \\
\hline 1186 & FP & $33-A s-75 m$ & 74.9216 & $0.00 \mathrm{E}+00$ & $3.93 \mathrm{E}+01$ \\
\hline 1187 & FP & $34-\mathrm{Se}-75$ & 74.9225 & $0.00 \mathrm{E}+00$ & $6.70 \mathrm{E}-08$ \\
\hline 1188 & FP & $35-\mathrm{Br}-75$ & 74.9258 & $0.00 \mathrm{E}+00$ & $1.19 \mathrm{E}-04$ \\
\hline 1189 & FP & $28-\mathrm{Ni}-76$ & 75.9553 & $0.00 \mathrm{E}+00$ & $2.91 \mathrm{E}+00$ \\
\hline 1190 & FP & 29-Cu-76 & 75.9453 & $0.00 \mathrm{E}+00$ & $1.06 \mathrm{E}+00$ \\
\hline 1191 & FP & $30-Z n-76$ & 75.9333 & $0.00 \mathrm{E}+00$ & $1.22 \mathrm{E}-01$ \\
\hline 1192 & FP & 31-Ga-76 & 75.9288 & $0.00 \mathrm{E}+00$ & $2.13 \mathrm{E}-02$ \\
\hline 1193 & FP & $32-\mathrm{Ge}-76$ & 75.9214 & $0.00 \mathrm{E}+00$ & $0.00 \mathrm{E}+00$ \\
\hline 1194 & FP & 33-As-76 & 75.9224 & $0.00 \mathrm{E}+00$ & $7.34 \mathrm{E}-06$ \\
\hline 1195 & FP & $34-\mathrm{Se}-76$ & 75.9192 & $0.00 \mathrm{E}+00$ & $0.00 \mathrm{E}+00$ \\
\hline 1196 & FP & $28-\mathrm{Ni}-77$ & 76.9605 & $0.00 \mathrm{E}+00$ & $1.14 \mathrm{E}+01$ \\
\hline 1197 & FP & 29-Cu-77 & 76.9479 & $0.00 \mathrm{E}+00$ & $1.48 \mathrm{E}+00$ \\
\hline 1198 & FP & $30-Z n-77$ & 76.9370 & $0.00 \mathrm{E}+00$ & $3.33 \mathrm{E}-01$ \\
\hline 1199 & FP & 31-Ga-77 & 76.9292 & $0.00 \mathrm{E}+00$ & $5.25 \mathrm{E}-02$ \\
\hline 1200 & FP & 32-Ge-77 & 76.9236 & $0.00 \mathrm{E}+00$ & $1.70 \mathrm{E}-05$ \\
\hline 1201 & FP & $32-\mathrm{Ge}-77 \mathrm{~m}$ & 76.9236 & $0.00 \mathrm{E}+00$ & $1.31 \mathrm{E}-02$ \\
\hline 1202 & FP & 33-As-77 & 76.9206 & $0.00 \mathrm{E}+00$ & $4.96 \mathrm{E}-06$ \\
\hline 1203 & FP & 34-Se-77 & 76.9199 & $0.00 \mathrm{E}+00$ & $0.00 \mathrm{E}+00$ \\
\hline 1204 & FP & $34-\mathrm{Se}-77 \mathrm{~m}$ & 76.9199 & $0.00 \mathrm{E}+00$ & 3.99E-02 \\
\hline 1205 & FP & $35-\mathrm{Br}-77$ & 76.9214 & $0.00 \mathrm{E}+00$ & $3.38 \mathrm{E}-06$ \\
\hline 1206 & FP & $35-\mathrm{Br}-77 \mathrm{~m}$ & 76.9214 & $0.00 \mathrm{E}+00$ & $2.70 \mathrm{E}-03$ \\
\hline 1207 & FP & $36-\mathrm{Kr}-77$ & 76.9247 & $0.00 \mathrm{E}+00$ & $1.55 \mathrm{E}-04$ \\
\hline 1208 & FP & $28-\mathrm{Ni}-78$ & 77.9632 & $0.00 \mathrm{E}+00$ & $6.30 \mathrm{E}+00$ \\
\hline 1209 & FP & 29-Cu-78 & 77.9520 & $0.00 \mathrm{E}+00$ & $2.07 \mathrm{E}+00$ \\
\hline 1210 & FP & $30-Z n-78$ & 77.9384 & $0.00 \mathrm{E}+00$ & $4.72 \mathrm{E}-01$ \\
\hline 1211 & FP & 31-Ga-78 & 77.9316 & $0.00 \mathrm{E}+00$ & $1.36 \mathrm{E}-01$ \\
\hline 1212 & FP & $32-\mathrm{Ge}-78$ & 77.9229 & $0.00 \mathrm{E}+00$ & $1.31 \mathrm{E}-04$ \\
\hline 1213 & FP & 33-As-78 & 77.9218 & $0.00 \mathrm{E}+00$ & $1.27 \mathrm{E}-04$ \\
\hline 1214 & FP & 34-Se-78 & 77.9173 & $0.00 \mathrm{E}+00$ & $0.00 \mathrm{E}+00$ \\
\hline 1215 & FP & $35-\mathrm{Br}-78$ & 77.9212 & $0.00 \mathrm{E}+00$ & $1.79 \mathrm{E}-03$ \\
\hline 1216 & FP & $36-\mathrm{Kr}-78$ & 77.9204 & $0.00 \mathrm{E}+00$ & $0.00 \mathrm{E}+00$ \\
\hline 1217 & FP & 29-Cu-79 & 78.9546 & $0.00 \mathrm{E}+00$ & $3.69 \mathrm{E}+00$ \\
\hline 1218 & FP & $30-Z n-79$ & 78.9426 & $0.00 \mathrm{E}+00$ & $6.97 \mathrm{E}-01$ \\
\hline 1219 & FP & 31-Ga-79 & 78.9329 & $0.00 \mathrm{E}+00$ & $2.43 \mathrm{E}-01$ \\
\hline 1220 & FP & $32-\mathrm{Ge}-79$ & 78.9254 & $0.00 \mathrm{E}+00$ & $3.65 \mathrm{E}-02$ \\
\hline 1221 & FP & $32-\mathrm{Ge}-79 \mathrm{~m}$ & 78.9254 & $0.00 \mathrm{E}+00$ & $1.78 \mathrm{E}-02$ \\
\hline 1222 & FP & 33-As-79 & 78.9210 & $0.00 \mathrm{E}+00$ & $1.28 \mathrm{E}-03$ \\
\hline 1223 & FP & $34-\mathrm{Se}-79$ & 78.9185 & $0.00 \mathrm{E}+00$ & $7.45 \mathrm{E}-14$ \\
\hline 1224 & FP & $34-\mathrm{Se}-79 \mathrm{~m}$ & 78.9185 & $0.00 \mathrm{E}+00$ & $2.95 \mathrm{E}-03$ \\
\hline 1225 & FP & $35-\mathrm{Br}-79$ & 78.9183 & $0.00 \mathrm{E}+00$ & $0.00 \mathrm{E}+00$ \\
\hline 1226 & FP & $35-\mathrm{Br}-79 \mathrm{~m}$ & 78.9183 & $0.00 \mathrm{E}+00$ & $1.43 \mathrm{E}-01$ \\
\hline 1227 & FP & $36-\mathrm{Kr}-79$ & 78.9201 & $0.00 \mathrm{E}+00$ & $5.49 \mathrm{E}-06$ \\
\hline 1228 & FP & $36-\mathrm{Kr}-79 \mathrm{~m}$ & 78.9201 & $0.00 \mathrm{E}+00$ & $1.39 \mathrm{E}-02$ \\
\hline 1229 & FP & $37-\mathrm{Rb}-79$ & 78.9240 & $0.00 \mathrm{E}+00$ & $5.04 \mathrm{E}-04$ \\
\hline 1230 & FP & $29-\mathrm{Cu}-80$ & 79.9609 & $0.00 \mathrm{E}+00$ & $4.08 \mathrm{E}+00$ \\
\hline 1231 & FP & $30-Z n-80$ & 79.9443 & $0.00 \mathrm{E}+00$ & $1.28 \mathrm{E}+00$ \\
\hline 1232 & FP & $31-\mathrm{Ga}-80$ & 79.9365 & $0.00 \mathrm{E}+00$ & $4.14 \mathrm{E}-01$ \\
\hline 1233 & FP & $32-\mathrm{Ge}-80$ & 79.9254 & $0.00 \mathrm{E}+00$ & $2.35 \mathrm{E}-02$ \\
\hline
\end{tabular}




\begin{tabular}{|c|c|c|c|c|c|}
\hline 1234 & FP & 33-As-80 & 79.9225 & $0.00 \mathrm{E}+00$ & $4.56 \mathrm{E}-02$ \\
\hline 1235 & $\mathrm{FP}$ & $34-\mathrm{Se}-80$ & 79.9165 & $0.00 \mathrm{E}+00$ & $0.00 \mathrm{E}+00$ \\
\hline 1236 & FP & $35-\mathrm{Br}-80$ & 79.9185 & $0.00 \mathrm{E}+00$ & $6.53 \mathrm{E}-04$ \\
\hline 1237 & FP & $35-\mathrm{Br}-80 \mathrm{~m}$ & 79.9185 & $0.00 \mathrm{E}+00$ & $4.36 \mathrm{E}-05$ \\
\hline 1238 & $\mathrm{FP}$ & $36-\mathrm{Kr}-80$ & 79.9164 & $0.00 \mathrm{E}+00$ & $0.00 \mathrm{E}+00$ \\
\hline 1239 & $\mathrm{FP}$ & $30-Z n-81$ & 80.9505 & $0.00 \mathrm{E}+00$ & $2.17 \mathrm{E}+00$ \\
\hline 1240 & FP & 31-Ga-81 & 80.9378 & $0.00 \mathrm{E}+00$ & $5.70 \mathrm{E}-01$ \\
\hline 1241 & FP & $32-\mathrm{Ge}-81$ & 80.9288 & $0.00 \mathrm{E}+00$ & $9.12 \mathrm{E}-02$ \\
\hline 1242 & FP & 32-Ge-81m & 80.9288 & $0.00 \mathrm{E}+00$ & $9.12 \mathrm{E}-02$ \\
\hline 1243 & FP & 33-As-81 & 80.9221 & $0.00 \mathrm{E}+00$ & $2.08 \mathrm{E}-02$ \\
\hline 1244 & FP & 34-Se-81 & 80.9180 & $0.00 \mathrm{E}+00$ & $6.26 \mathrm{E}-04$ \\
\hline 1245 & FP & $34-\mathrm{Se}-81 \mathrm{~m}$ & 80.9180 & $0.00 \mathrm{E}+00$ & $2.02 \mathrm{E}-04$ \\
\hline 1246 & FP & $35-\mathrm{Br}-81$ & 80.9163 & $0.00 \mathrm{E}+00$ & $0.00 \mathrm{E}+00$ \\
\hline 1247 & FP & $36-\mathrm{Kr}-81$ & 80.9166 & $0.00 \mathrm{E}+00$ & $9.59 \mathrm{E}-14$ \\
\hline 1248 & FP & $36-\mathrm{Kr}-81 \mathrm{~m}$ & 80.9166 & $0.00 \mathrm{E}+00$ & $5.29 \mathrm{E}-02$ \\
\hline 1249 & $\mathrm{FP}$ & $37-R b-81$ & 80.9190 & $0.00 \mathrm{E}+00$ & $4.21 \mathrm{E}-05$ \\
\hline 1250 & FP & $30-Z n-82$ & 81.9544 & $0.00 \mathrm{E}+00$ & $1.33 \mathrm{E}+01$ \\
\hline 1251 & FP & 31-Ga-82 & 81.9430 & $0.00 \mathrm{E}+00$ & $1.16 \mathrm{E}+00$ \\
\hline 1252 & FP & $32-\mathrm{Ge}-82$ & 81.9296 & $0.00 \mathrm{E}+00$ & $1.52 \mathrm{E}-01$ \\
\hline 1253 & FP & 33-As-82 & 81.9245 & $0.00 \mathrm{E}+00$ & $3.63 \mathrm{E}-02$ \\
\hline 1254 & FP & 33-As-82m & 81.9245 & $0.00 \mathrm{E}+00$ & $5.10 \mathrm{E}-02$ \\
\hline 1255 & FP & $34-\mathrm{Se}-82$ & 81.9167 & $0.00 \mathrm{E}+00$ & $0.00 \mathrm{E}+00$ \\
\hline 1256 & FP & $35-\mathrm{Br}-82$ & 81.9168 & $0.00 \mathrm{E}+00$ & $5.46 \mathrm{E}-06$ \\
\hline 1257 & FP & $35-\mathrm{Br}-82 \mathrm{~m}$ & 81.9168 & $0.00 \mathrm{E}+00$ & $1.88 \mathrm{E}-03$ \\
\hline 1258 & FP & $36-\mathrm{Kr}-82$ & 81.9135 & $0.00 \mathrm{E}+00$ & $0.00 \mathrm{E}+00$ \\
\hline 1259 & FP & $30-Z n-83$ & 82.9610 & $0.00 \mathrm{E}+00$ & $1.61 \mathrm{E}+01$ \\
\hline 1260 & $\mathrm{FP}$ & $31-\mathrm{Ga}-83$ & 82.9470 & $0.00 \mathrm{E}+00$ & $2.25 \mathrm{E}+00$ \\
\hline 1261 & FP & $32-\mathrm{Ge}-83$ & 82.9346 & $0.00 \mathrm{E}+00$ & $3.75 \mathrm{E}-01$ \\
\hline 1262 & FP & $33-A s-83$ & 82.9250 & $0.00 \mathrm{E}+00$ & $5.17 \mathrm{E}-02$ \\
\hline 1263 & FP & $34-\mathrm{Se}-83$ & 82.9191 & $0.00 \mathrm{E}+00$ & $5.18 \mathrm{E}-04$ \\
\hline 1264 & FP & $34-\mathrm{Se}-83 \mathrm{~m}$ & 82.9191 & $0.00 \mathrm{E}+00$ & $9.89 \mathrm{E}-03$ \\
\hline 1265 & FP & $35-\mathrm{Br}-83$ & 82.9152 & $0.00 \mathrm{E}+00$ & $8.02 \mathrm{E}-05$ \\
\hline 1266 & FP & $36-\mathrm{Kr}-83$ & 82.9141 & $0.00 \mathrm{E}+00$ & $0.00 \mathrm{E}+00$ \\
\hline 1267 & FP & $36-\mathrm{Kr}-83 \mathrm{~m}$ & 82.9141 & $0.00 \mathrm{E}+00$ & $1.05 \mathrm{E}-04$ \\
\hline 1268 & FP & $37-R b-83$ & 82.9151 & $0.00 \mathrm{E}+00$ & $9.31 \mathrm{E}-08$ \\
\hline 1269 & FP & $38-\mathrm{Sr}-83$ & 82.9176 & $0.00 \mathrm{E}+00$ & $5.94 \mathrm{E}-06$ \\
\hline 1270 & $\mathrm{FP}$ & 31-Ga-84 & 83.9527 & $0.00 \mathrm{E}+00$ & $8.15 \mathrm{E}+00$ \\
\hline 1271 & FP & $32-\mathrm{Ge}-84$ & 83.9375 & $0.00 \mathrm{E}+00$ & $7.27 \mathrm{E}-01$ \\
\hline 1272 & FP & 33-As-84 & 83.9291 & $0.00 \mathrm{E}+00$ & $1.65 \mathrm{E}-01$ \\
\hline 1273 & FP & $34-\mathrm{Se}-84$ & 83.9185 & $0.00 \mathrm{E}+00$ & $3.54 \mathrm{E}-03$ \\
\hline 1274 & FP & $35-\mathrm{Br}-84$ & 83.9165 & $0.00 \mathrm{E}+00$ & $3.64 \mathrm{E}-04$ \\
\hline 1275 & FP & $35-\mathrm{Br}-84 \mathrm{~m}$ & 83.9165 & $0.00 \mathrm{E}+00$ & $1.93 \mathrm{E}-03$ \\
\hline 1276 & FP & $36-\mathrm{Kr}-84$ & 83.9115 & $0.00 \mathrm{E}+00$ & $0.00 \mathrm{E}+00$ \\
\hline 1277 & FP & $37-\mathrm{Rb}-84$ & 83.9144 & $0.00 \mathrm{E}+00$ & $2.44 \mathrm{E}-07$ \\
\hline 1278 & $\mathrm{FP}$ & $38-\mathrm{Sr}-84$ & 83.9134 & $0.00 \mathrm{E}+00$ & $0.00 \mathrm{E}+00$ \\
\hline 1279 & FP & 31-Ga-85 & 84.9570 & $0.00 \mathrm{E}+00$ & $1.44 \mathrm{E}+01$ \\
\hline 1280 & $\mathrm{FP}$ & $32-\mathrm{Ge}-85$ & 84.9430 & $0.00 \mathrm{E}+00$ & $1.30 \mathrm{E}+00$ \\
\hline 1281 & FP & 33-As-85 & 84.9320 & $0.00 \mathrm{E}+00$ & $3.43 \mathrm{E}-01$ \\
\hline 1282 & FP & $34-\mathrm{Se}-85$ & 84.9222 & $0.00 \mathrm{E}+00$ & $2.19 \mathrm{E}-02$ \\
\hline 1283 & $\mathrm{FP}$ & $35-\mathrm{Br}-85$ & 84.9156 & $0.00 \mathrm{E}+00$ & $3.98 \mathrm{E}-03$ \\
\hline 1284 & FP & $36-\mathrm{Kr}-85$ & 84.9125 & $0.00 \mathrm{E}+00$ & $2.04 \mathrm{E}-09$ \\
\hline 1285 & FP & $36-\mathrm{Kr}-85 \mathrm{~m}$ & 84.9125 & $0.00 \mathrm{E}+00$ & $4.30 \mathrm{E}-05$ \\
\hline 1286 & FP & $37-\mathrm{Rb}-85$ & 84.9118 & $0.00 \mathrm{E}+00$ & $0.00 \mathrm{E}+00$ \\
\hline 1287 & FP & $38-\mathrm{Sr}-85$ & 84.9129 & $0.00 \mathrm{E}+00$ & $1.24 \mathrm{E}-07$ \\
\hline 1288 & FP & $38-\mathrm{Sr}-85 \mathrm{~m}$ & 84.9129 & $0.00 \mathrm{E}+00$ & $1.71 \mathrm{E}-04$ \\
\hline 1289 & $\mathrm{FP}$ & $39-Y-85$ & 84.9164 & $0.00 \mathrm{E}+00$ & $7.18 \mathrm{E}-05$ \\
\hline 1290 & $\mathrm{FP}$ & 31-Ga-86 & 85.9631 & $0.00 \mathrm{E}+00$ & $2.39 \mathrm{E}+01$ \\
\hline 1291 & FP & $32-\mathrm{Ge}-86$ & 85.9465 & $0.00 \mathrm{E}+00$ & $7.30 \mathrm{E}+00$ \\
\hline 1292 & $\mathrm{FP}$ & 33-As-86 & 85.9365 & $0.00 \mathrm{E}+00$ & $7.33 \mathrm{E}-01$ \\
\hline 1293 & FP & $34-\mathrm{Se}-86$ & 85.9243 & $0.00 \mathrm{E}+00$ & $4.85 \mathrm{E}-02$ \\
\hline 1294 & FP & $35-\mathrm{Br}-86$ & 85.9188 & $0.00 \mathrm{E}+00$ & $1.26 \mathrm{E}-02$ \\
\hline 1295 & FP & $36-\mathrm{Kr}-86$ & 85.9106 & $0.00 \mathrm{E}+00$ & $0.00 \mathrm{E}+00$ \\
\hline 1296 & FP & $37-\mathrm{Rb}-86$ & 85.9112 & $0.00 \mathrm{E}+00$ & $4.31 \mathrm{E}-07$ \\
\hline 1297 & FP & $37-\mathrm{Rb}-86 \mathrm{~m}$ & 85.9112 & $0.00 \mathrm{E}+00$ & $1.14 \mathrm{E}-02$ \\
\hline 1298 & FP & $38-\mathrm{Sr}-86$ & 85.9093 & $0.00 \mathrm{E}+00$ & $0.00 \mathrm{E}+00$ \\
\hline 1299 & $\mathrm{FP}$ & $32-\mathrm{Ge}-87$ & 86.9525 & $0.00 \mathrm{E}+00$ & $4.95 \mathrm{E}+00$ \\
\hline
\end{tabular}




\begin{tabular}{|c|c|c|c|c|c|}
\hline 1300 & FP & $33-A s-87$ & 86.9399 & $0.00 \mathrm{E}+00$ & $1.24 \mathrm{E}+00$ \\
\hline 1301 & FP & $34-\mathrm{Se}-87$ & 86.9285 & $0.00 \mathrm{E}+00$ & $1.26 \mathrm{E}-01$ \\
\hline 1302 & FP & $35-\mathrm{Br}-87$ & 86.9207 & $0.00 \mathrm{E}+00$ & $1.25 \mathrm{E}-02$ \\
\hline 1303 & FP & $36-\mathrm{Kr}-87$ & 86.9134 & $0.00 \mathrm{E}+00$ & $1.51 \mathrm{E}-04$ \\
\hline 1304 & FP & $37-\mathrm{Rb}-87$ & 86.9092 & $0.00 \mathrm{E}+00$ & $4.57 \mathrm{E}-19$ \\
\hline 1305 & FP & $38-\mathrm{Sr}-87$ & 86.9089 & $0.00 \mathrm{E}+00$ & $0.00 \mathrm{E}+00$ \\
\hline 1306 & FP & $38-\mathrm{Sr}-87 \mathrm{~m}$ & 86.9089 & $0.00 \mathrm{E}+00$ & $6.84 \mathrm{E}-05$ \\
\hline 1307 & FP & $39-Y-87$ & 86.9109 & $0.00 \mathrm{E}+00$ & $2.41 \mathrm{E}-06$ \\
\hline 1308 & FP & $39-Y-87 \mathrm{~m}$ & 86.9109 & $0.00 \mathrm{E}+00$ & $1.44 \mathrm{E}-05$ \\
\hline 1309 & FP & $40-Z r-87$ & 86.9148 & $0.00 \mathrm{E}+00$ & $1.15 \mathrm{E}-04$ \\
\hline 1310 & FP & $32-\mathrm{Ge}-88$ & 87.9569 & $0.00 \mathrm{E}+00$ & $1.05 \mathrm{E}+01$ \\
\hline 1311 & FP & $33-A s-88$ & 87.9449 & $0.00 \mathrm{E}+00$ & $6.19 \mathrm{E}+00$ \\
\hline 1312 & FP & $34-\mathrm{Se}-88$ & 87.9314 & $0.00 \mathrm{E}+00$ & $4.53 \mathrm{E}-01$ \\
\hline 1313 & FP & $35-\mathrm{Br}-88$ & 87.9241 & $0.00 \mathrm{E}+00$ & $4.26 \mathrm{E}-02$ \\
\hline 1314 & FP & $36-\mathrm{Kr}-88$ & 87.9145 & $0.00 \mathrm{E}+00$ & $6.78 \mathrm{E}-05$ \\
\hline 1315 & FP & $37-\mathrm{Rb}-88$ & 87.9113 & $0.00 \mathrm{E}+00$ & $6.50 \mathrm{E}-04$ \\
\hline 1316 & FP & $38-\mathrm{Sr}-88$ & 87.9056 & $0.00 \mathrm{E}+00$ & $0.00 \mathrm{E}+00$ \\
\hline 1317 & FP & $39-Y-88$ & 87.9095 & $0.00 \mathrm{E}+00$ & $7.52 \mathrm{E}-08$ \\
\hline 1318 & FP & $40-Z r-88$ & 87.9102 & $0.00 \mathrm{E}+00$ & $9.62 \mathrm{E}-08$ \\
\hline 1319 & FP & $32-\mathrm{Ge}-89$ & 88.9638 & $0.00 \mathrm{E}+00$ & $1.78 \mathrm{E}+01$ \\
\hline 1320 & FP & $33-A s-89$ & 88.9494 & $0.00 \mathrm{E}+00$ & $1.17 \mathrm{E}+01$ \\
\hline 1321 & FP & 34-Se-89 & 88.9364 & $0.00 \mathrm{E}+00$ & $1.69 \mathrm{E}+00$ \\
\hline 1322 & FP & $35-\mathrm{Br}-89$ & 88.9264 & $0.00 \mathrm{E}+00$ & $1.58 \mathrm{E}-01$ \\
\hline 1323 & $\mathrm{FP}$ & $36-\mathrm{Kr}-89$ & 88.9176 & $0.00 \mathrm{E}+00$ & $3.67 \mathrm{E}-03$ \\
\hline 1324 & FP & $37-\mathrm{Rb}-89$ & 88.9123 & $0.00 \mathrm{E}+00$ & $7.63 \mathrm{E}-04$ \\
\hline 1325 & FP & $38-\mathrm{Sr}-89$ & 88.9074 & $0.00 \mathrm{E}+00$ & $1.59 \mathrm{E}-07$ \\
\hline 1326 & FP & $39-Y-89$ & 88.9059 & $0.00 \mathrm{E}+00$ & $0.00 \mathrm{E}+00$ \\
\hline 1327 & FP & $39-Y-89 m$ & 88.9059 & $0.00 \mathrm{E}+00$ & $4.43 \mathrm{E}-02$ \\
\hline 1328 & FP & $40-Z r-89$ & 88.9089 & $0.00 \mathrm{E}+00$ & $2.46 \mathrm{E}-06$ \\
\hline 1329 & FP & $40-Z r-89 m$ & 88.9089 & $0.00 \mathrm{E}+00$ & $2.78 \mathrm{E}-03$ \\
\hline 1330 & FP & $41-\mathrm{Nb}-89$ & 88.9134 & $0.00 \mathrm{E}+00$ & $9.48 \mathrm{E}-05$ \\
\hline 1331 & FP & $33-A s-90$ & 89.9555 & $0.00 \mathrm{E}+00$ & $1.61 \mathrm{E}+01$ \\
\hline 1332 & FP & $34-\mathrm{Se}-90$ & 89.9400 & $0.00 \mathrm{E}+00$ & $4.31 \mathrm{E}+00$ \\
\hline 1333 & FP & $35-\mathrm{Br}-90$ & 89.9306 & $0.00 \mathrm{E}+00$ & $3.61 \mathrm{E}-01$ \\
\hline 1334 & FP & $36-\mathrm{Kr}-90$ & 89.9195 & $0.00 \mathrm{E}+00$ & $2.14 \mathrm{E}-02$ \\
\hline 1335 & FP & $37-\mathrm{Rb}-90$ & 89.9148 & $0.00 \mathrm{E}+00$ & $4.39 \mathrm{E}-03$ \\
\hline 1336 & FP & 37-Rb-90m & 89.9148 & $0.00 \mathrm{E}+00$ & $2.69 \mathrm{E}-03$ \\
\hline 1337 & FP & $38-\mathrm{Sr}-90$ & 89.9077 & $0.00 \mathrm{E}+00$ & $7.63 \mathrm{E}-10$ \\
\hline 1338 & FP & $39-Y-90$ & 89.9072 & $0.00 \mathrm{E}+00$ & $3.01 \mathrm{E}-06$ \\
\hline 1339 & FP & $39-Y-90 \mathrm{~m}$ & 89.9072 & $0.00 \mathrm{E}+00$ & $6.04 \mathrm{E}-05$ \\
\hline 1340 & FP & $40-Z r-90$ & 89.9047 & $0.00 \mathrm{E}+00$ & $0.00 \mathrm{E}+00$ \\
\hline 1341 & FP & $40-Z r-90 m$ & 89.9047 & $0.00 \mathrm{E}+00$ & $8.57 \mathrm{E}-01$ \\
\hline 1342 & FP & $41-\mathrm{Nb}-90$ & 89.9113 & $0.00 \mathrm{E}+00$ & $1.32 \mathrm{E}-05$ \\
\hline 1343 & FP & $41-\mathrm{Nb}-90 \mathrm{~m}$ & 89.9113 & $0.00 \mathrm{E}+00$ & $3.69 \mathrm{E}-02$ \\
\hline 1344 & FP & 42-Мo-90 & 89.9139 & $0.00 \mathrm{E}+00$ & $3.40 \mathrm{E}-05$ \\
\hline 1345 & FP & 33-As-91 & 90.9604 & $0.00 \mathrm{E}+00$ & $1.58 \mathrm{E}+01$ \\
\hline 1346 & FP & $34-S e-91$ & 90.9460 & $0.00 \mathrm{E}+00$ & $2.57 \mathrm{E}+00$ \\
\hline 1347 & FP & $35-\mathrm{Br}-91$ & 90.9340 & $0.00 \mathrm{E}+00$ & $1.28 \mathrm{E}+00$ \\
\hline 1348 & FP & $36-\mathrm{Kr}-91$ & 90.9234 & $0.00 \mathrm{E}+00$ & $8.09 \mathrm{E}-02$ \\
\hline 1349 & FP & 37-Rb-91 & 90.9165 & $0.00 \mathrm{E}+00$ & $1.19 \mathrm{E}-02$ \\
\hline 1350 & FP & 38-Sr-91 & 90.9102 & $0.00 \mathrm{E}+00$ & $2.00 \mathrm{E}-05$ \\
\hline 1351 & FP & $39-Y-91$ & 90.9073 & $0.00 \mathrm{E}+00$ & $1.37 \mathrm{E}-07$ \\
\hline 1352 & FP & $39-\mathrm{Y}-91 \mathrm{~m}$ & 90.9073 & $0.00 \mathrm{E}+00$ & $2.32 \mathrm{E}-04$ \\
\hline 1353 & FP & $40-Z r-91$ & 90.9056 & $0.00 \mathrm{E}+00$ & $0.00 \mathrm{E}+00$ \\
\hline 1354 & FP & 41-Nb-91 & 90.9070 & $0.00 \mathrm{E}+00$ & $3.23 \mathrm{E}-11$ \\
\hline 1355 & FP & $41-\mathrm{Nb}-91 \mathrm{~m}$ & 90.9070 & $0.00 \mathrm{E}+00$ & $1.32 \mathrm{E}-07$ \\
\hline 1356 & FP & 42-Мo-91 & 90.9118 & $0.00 \mathrm{E}+00$ & $7.46 \mathrm{E}-04$ \\
\hline 1357 & FP & 33-As-92 & 91.9668 & $0.00 \mathrm{E}+00$ & $2.57 \mathrm{E}+01$ \\
\hline 1358 & FP & $34-\mathrm{Se}-92$ & 91.9499 & $0.00 \mathrm{E}+00$ & $7.45 \mathrm{E}+00$ \\
\hline 1359 & FP & $35-\mathrm{Br}-92$ & 91.9393 & $0.00 \mathrm{E}+00$ & $2.02 \mathrm{E}+00$ \\
\hline 1360 & FP & $36-\mathrm{Kr}-92$ & 91.9262 & $0.00 \mathrm{E}+00$ & $3.77 \mathrm{E}-01$ \\
\hline 1361 & FP & $37-\mathrm{Rb}-92$ & 91.9197 & $0.00 \mathrm{E}+00$ & $1.54 \mathrm{E}-01$ \\
\hline 1362 & FP & $38-\mathrm{Sr}-92$ & 91.9110 & $0.00 \mathrm{E}+00$ & $7.10 \mathrm{E}-05$ \\
\hline 1363 & FP & $39-Y-92$ & 91.9090 & $0.00 \mathrm{E}+00$ & $5.44 \mathrm{E}-05$ \\
\hline 1364 & FP & $40-Z r-92$ & 91.9050 & $0.00 \mathrm{E}+00$ & $0.00 \mathrm{E}+00$ \\
\hline 1365 & FP & 41-Nb-92 & 91.9072 & $0.00 \mathrm{E}+00$ & $6.33 \mathrm{E}-16$ \\
\hline
\end{tabular}




\begin{tabular}{|c|c|c|c|c|c|}
\hline 1366 & FP & $41-\mathrm{Nb}-92 \mathrm{~m}$ & 91.9072 & $0.00 \mathrm{E}+00$ & $7.90 \mathrm{E}-07$ \\
\hline 1367 & FP & 42-Mo-92 & 91.9068 & $0.00 \mathrm{E}+00$ & $0.00 \mathrm{E}+00$ \\
\hline 1368 & FP & 34-Se-93 & 92.9563 & $0.00 \mathrm{E}+00$ & $1.12 \mathrm{E}+01$ \\
\hline 1369 & FP & $35-\mathrm{Br}-93$ & 92.9430 & $0.00 \mathrm{E}+00$ & $6.80 \mathrm{E}+00$ \\
\hline 1370 & FP & $36-\mathrm{Kr}-93$ & 92.9313 & $0.00 \mathrm{E}+00$ & 5.39E-01 \\
\hline 1371 & FP & $37-\mathrm{Rb}-93$ & 92.9220 & $0.00 \mathrm{E}+00$ & $1.19 \mathrm{E}-01$ \\
\hline 1372 & FP & $38-\mathrm{Sr}-93$ & 92.9140 & $0.00 \mathrm{E}+00$ & $1.56 \mathrm{E}-03$ \\
\hline 1373 & FP & $39-Y-93$ & 92.9096 & $0.00 \mathrm{E}+00$ & $1.89 \mathrm{E}-05$ \\
\hline 1374 & FP & 39-Y-93m & 92.9096 & $0.00 \mathrm{E}+00$ & $8.45 \mathrm{E}-01$ \\
\hline 1375 & FP & $40-Z r-93$ & 92.9065 & $0.00 \mathrm{E}+00$ & $1.44 \mathrm{E}-14$ \\
\hline 1376 & FP & $41-\mathrm{Nb}-93$ & 92.9064 & $0.00 \mathrm{E}+00$ & $0.00 \mathrm{E}+00$ \\
\hline 1377 & FP & $41-\mathrm{Nb}-93 \mathrm{~m}$ & 92.9064 & $0.00 \mathrm{E}+00$ & $1.36 \mathrm{E}-09$ \\
\hline 1378 & FP & 42-Mo-93 & 92.9068 & $0.00 \mathrm{E}+00$ & $5.49 \mathrm{E}-12$ \\
\hline 1379 & FP & 42-Mo-93m & 92.9068 & $0.00 \mathrm{E}+00$ & $2.81 \mathrm{E}-05$ \\
\hline 1380 & FP & 43-Tc-93 & 92.9102 & $0.00 \mathrm{E}+00$ & $7.00 \mathrm{E}-05$ \\
\hline 1381 & FP & 34-Se-94 & 93.9605 & $0.00 \mathrm{E}+00$ & $1.17 \mathrm{E}+01$ \\
\hline 1382 & FP & $35-\mathrm{Br}-94$ & 93.9487 & $0.00 \mathrm{E}+00$ & $9.90 \mathrm{E}+00$ \\
\hline 1383 & FP & $36-\mathrm{Kr}-94$ & 93.9344 & $0.00 \mathrm{E}+00$ & $3.27 \mathrm{E}+00$ \\
\hline 1384 & FP & 37-Rb-94 & 93.9264 & $0.00 \mathrm{E}+00$ & $2.57 \mathrm{E}-01$ \\
\hline 1385 & FP & $38-\mathrm{Sr}-94$ & 93.9154 & $0.00 \mathrm{E}+00$ & $9.20 \mathrm{E}-03$ \\
\hline 1386 & FP & 39-Y-94 & 93.9116 & $0.00 \mathrm{E}+00$ & $6.18 \mathrm{E}-04$ \\
\hline 1387 & FP & $40-Z r-94$ & 93.9063 & $0.00 \mathrm{E}+00$ & $0.00 \mathrm{E}+00$ \\
\hline 1388 & FP & $41-\mathrm{Nb}-94$ & 93.9073 & $0.00 \mathrm{E}+00$ & $1.08 \mathrm{E}-12$ \\
\hline 1389 & FP & $41-\mathrm{Nb}-94 \mathrm{~m}$ & 93.9073 & $0.00 \mathrm{E}+00$ & $1.84 \mathrm{E}-03$ \\
\hline 1390 & FP & 42-Mo-94 & 93.9051 & $0.00 \mathrm{E}+00$ & $0.00 \mathrm{E}+00$ \\
\hline 1391 & FP & $35-\mathrm{Br}-95$ & 94.9529 & $0.00 \mathrm{E}+00$ & $1.05 \mathrm{E}+01$ \\
\hline 1392 & FP & $36-\mathrm{Kr}-95$ & 94.9398 & $0.00 \mathrm{E}+00$ & $6.08 \mathrm{E}+00$ \\
\hline 1393 & FP & $37-\mathrm{Rb}-95$ & 94.9293 & $0.00 \mathrm{E}+00$ & $1.84 \mathrm{E}+00$ \\
\hline 1394 & FP & $38-\mathrm{Sr}-95$ & 94.9194 & $0.00 \mathrm{E}+00$ & $2.90 \mathrm{E}-02$ \\
\hline 1395 & FP & $39-Y-95$ & 94.9128 & $0.00 \mathrm{E}+00$ & $1.12 \mathrm{E}-03$ \\
\hline 1396 & FP & $40-Z r-95$ & 94.9080 & $0.00 \mathrm{E}+00$ & $1.25 \mathrm{E}-07$ \\
\hline 1397 & FP & $41-\mathrm{Nb}-95$ & 94.9068 & $0.00 \mathrm{E}+00$ & $2.29 \mathrm{E}-07$ \\
\hline 1398 & FP & $41-\mathrm{Nb}-95 \mathrm{~m}$ & 94.9068 & $0.00 \mathrm{E}+00$ & $2.22 \mathrm{E}-06$ \\
\hline 1399 & FP & 42-Мo-95 & 94.9058 & $0.00 \mathrm{E}+00$ & $0.00 \mathrm{E}+00$ \\
\hline 1400 & FP & 43-Tc-95 & 94.9077 & $0.00 \mathrm{E}+00$ & $9.63 \mathrm{E}-06$ \\
\hline 1401 & FP & 43-Tc-95m & 94.9077 & $0.00 \mathrm{E}+00$ & $1.32 \mathrm{E}-07$ \\
\hline 1402 & FP & 44-Ru-95 & 94.9104 & $0.00 \mathrm{E}+00$ & $1.17 \mathrm{E}-04$ \\
\hline 1403 & FP & $35-\mathrm{Br}-96$ & 95.9585 & $0.00 \mathrm{E}+00$ & $1.65 \mathrm{E}+01$ \\
\hline 1404 & FP & $36-\mathrm{Kr}-96$ & 95.9431 & $0.00 \mathrm{E}+00$ & $8.66 \mathrm{E}+00$ \\
\hline 1405 & FP & $37-\mathrm{Rb}-96$ & 95.9343 & $0.00 \mathrm{E}+00$ & $3.41 \mathrm{E}+00$ \\
\hline 1406 & FP & $38-\mathrm{Sr}-96$ & 95.9217 & $0.00 \mathrm{E}+00$ & $6.48 \mathrm{E}-01$ \\
\hline 1407 & FP & $39-Y-96$ & 95.9159 & $0.00 \mathrm{E}+00$ & $1.30 \mathrm{E}-01$ \\
\hline 1408 & FP & 39-Y-96m & 95.9159 & $0.00 \mathrm{E}+00$ & $7.22 \mathrm{E}-02$ \\
\hline 1409 & FP & $40-Z r-96$ & 95.9083 & $0.00 \mathrm{E}+00$ & $1.10 \mathrm{E}-27$ \\
\hline 1410 & FP & $41-\mathrm{Nb}-96$ & 95.9081 & $0.00 \mathrm{E}+00$ & $8.25 \mathrm{E}-06$ \\
\hline 1411 & FP & 42-Mo-96 & 95.9047 & $0.00 \mathrm{E}+00$ & $0.00 \mathrm{E}+00$ \\
\hline 1412 & FP & 43-Tc-96 & 95.9079 & $0.00 \mathrm{E}+00$ & $1.87 \mathrm{E}-06$ \\
\hline 1413 & FP & 44-Ru-96 & 95.9076 & $0.00 \mathrm{E}+00$ & $0.00 \mathrm{E}+00$ \\
\hline 1414 & FP & $35-\mathrm{Br}-97$ & 96.9628 & $0.00 \mathrm{E}+00$ & $1.73 \mathrm{E}+01$ \\
\hline 1415 & FP & $36-\mathrm{Kr}-97$ & 96.9486 & $0.00 \mathrm{E}+00$ & $1.10 \mathrm{E}+01$ \\
\hline 1416 & FP & 37-Rb-97 & 96.9373 & $0.00 \mathrm{E}+00$ & $4.10 \mathrm{E}+00$ \\
\hline 1417 & FP & 38-Sr-97 & 96.9261 & $0.00 \mathrm{E}+00$ & $1.62 \mathrm{E}+00$ \\
\hline 1418 & $\mathrm{FP}$ & $39-Y-97$ & 96.9181 & $0.00 \mathrm{E}+00$ & $1.85 \mathrm{E}-01$ \\
\hline 1419 & FP & $39-Y-97 m$ & 96.9181 & $0.00 \mathrm{E}+00$ & $5.92 \mathrm{E}-01$ \\
\hline 1420 & FP & $40-Z r-97$ & 96.9109 & $0.00 \mathrm{E}+00$ & $1.15 \mathrm{E}-05$ \\
\hline 1421 & $\mathrm{FP}$ & $41-\mathrm{Nb}-97$ & 96.9081 & $0.00 \mathrm{E}+00$ & $1.60 \mathrm{E}-04$ \\
\hline 1422 & FP & $41-\mathrm{Nb}-97 \mathrm{~m}$ & 96.9081 & $0.00 \mathrm{E}+00$ & $1.18 \mathrm{E}-02$ \\
\hline 1423 & FP & 42-Mo-97 & 96.9060 & $0.00 \mathrm{E}+00$ & $0.00 \mathrm{E}+00$ \\
\hline 1424 & FP & 43-Tc-97 & 96.9064 & $0.00 \mathrm{E}+00$ & $5.22 \mathrm{E}-15$ \\
\hline 1425 & FP & $43-T c-97 m$ & 96.9064 & $0.00 \mathrm{E}+00$ & $8.82 \mathrm{E}-08$ \\
\hline 1426 & FP & 44-Ru-97 & 96.9076 & $0.00 \mathrm{E}+00$ & $2.83 \mathrm{E}-06$ \\
\hline 1427 & FP & $36-\mathrm{Kr}-98$ & 97.9519 & $0.00 \mathrm{E}+00$ & $1.51 \mathrm{E}+01$ \\
\hline 1428 & FP & 37-Rb-98 & 97.9418 & $0.00 \mathrm{E}+00$ & $6.08 \mathrm{E}+00$ \\
\hline 1429 & $\mathrm{FP}$ & 38-Sr-98 & 97.9285 & $0.00 \mathrm{E}+00$ & $1.06 \mathrm{E}+00$ \\
\hline 1430 & $\mathrm{FP}$ & 39-Y-98 & 97.9222 & $0.00 \mathrm{E}+00$ & $1.26 \mathrm{E}+00$ \\
\hline 1431 & FP & $39-Y-98 m$ & 97.9222 & $0.00 \mathrm{E}+00$ & $3.47 \mathrm{E}-01$ \\
\hline
\end{tabular}




\begin{tabular}{|c|c|c|c|c|c|}
\hline 1432 & FP & $40-Z r-98$ & 97.9127 & $0.00 \mathrm{E}+00$ & $2.26 \mathrm{E}-02$ \\
\hline 1433 & FP & 41-Nb-98 & 97.9103 & $0.00 \mathrm{E}+00$ & $2.42 \mathrm{E}-01$ \\
\hline 1434 & FP & $41-\mathrm{Nb}-98 \mathrm{~m}$ & 97.9103 & $0.00 \mathrm{E}+00$ & $2.25 \mathrm{E}-04$ \\
\hline 1435 & $\mathrm{FP}$ & 42-Mo-98 & 97.9054 & $0.00 \mathrm{E}+00$ & $0.00 \mathrm{E}+00$ \\
\hline 1436 & FP & 43-Tc-98 & 97.9072 & $0.00 \mathrm{E}+00$ & $5.23 \mathrm{E}-15$ \\
\hline 1437 & FP & 44-Ru-98 & 97.9053 & $0.00 \mathrm{E}+00$ & $0.00 \mathrm{E}+00$ \\
\hline 1438 & FP & $36-\mathrm{Kr}-99$ & 98.9576 & $0.00 \mathrm{E}+00$ & $2.57 \mathrm{E}+01$ \\
\hline 1439 & FP & 37-Rb-99 & 98.9454 & $0.00 \mathrm{E}+00$ & $1.28 \mathrm{E}+01$ \\
\hline 1440 & FP & 38-Sr-99 & 98.9332 & $0.00 \mathrm{E}+00$ & $2.57 \mathrm{E}+00$ \\
\hline 1441 & FP & $39-Y-99$ & 98.9246 & $0.00 \mathrm{E}+00$ & $4.72 \mathrm{E}-01$ \\
\hline 1442 & FP & $40-Z r-99$ & 98.9165 & $0.00 \mathrm{E}+00$ & $3.30 \mathrm{E}-01$ \\
\hline 1443 & FP & $41-\mathrm{Nb}-99$ & 98.9116 & $0.00 \mathrm{E}+00$ & $4.62 \mathrm{E}-02$ \\
\hline 1444 & FP & 41-Nb-99m & 98.9116 & $0.00 \mathrm{E}+00$ & $4.62 \mathrm{E}-03$ \\
\hline 1445 & FP & 42-Мo-99 & 98.9077 & $0.00 \mathrm{E}+00$ & $2.92 \mathrm{E}-06$ \\
\hline 1446 & FP & 43-Tc-99 & 98.9062 & $0.00 \mathrm{E}+00$ & $1.04 \mathrm{E}-13$ \\
\hline 1447 & FP & 43-Tc-99m & 98.9062 & $0.00 \mathrm{E}+00$ & $3.21 \mathrm{E}-05$ \\
\hline 1448 & FP & 44-Ru-99 & 98.9059 & $0.00 \mathrm{E}+00$ & $0.00 \mathrm{E}+00$ \\
\hline 1449 & FP & 45-Rh-99 & 98.9081 & $0.00 \mathrm{E}+00$ & $4.98 \mathrm{E}-07$ \\
\hline 1450 & FP & 45-Rh-99m & 98.9081 & $0.00 \mathrm{E}+00$ & $4.10 \mathrm{E}-05$ \\
\hline 1451 & FP & 46-Pd-99 & 98.9118 & $0.00 \mathrm{E}+00$ & $5.40 \mathrm{E}-04$ \\
\hline 1452 & FP & $36-\mathrm{Kr}-100$ & 99.9611 & $0.00 \mathrm{E}+00$ & $9.90 \mathrm{E}+01$ \\
\hline 1453 & FP & $37-\mathrm{Rb}-100$ & 99.9499 & $0.00 \mathrm{E}+00$ & $1.36 \mathrm{E}+01$ \\
\hline 1454 & FP & $38-\mathrm{Sr}-100$ & 99.9353 & $0.00 \mathrm{E}+00$ & $3.43 \mathrm{E}+00$ \\
\hline 1455 & FP & $39-Y-100$ & 99.9278 & $0.00 \mathrm{E}+00$ & $9.43 \mathrm{E}-01$ \\
\hline 1456 & FP & $40-Z r-100$ & 99.9178 & $0.00 \mathrm{E}+00$ & $9.76 \mathrm{E}-02$ \\
\hline 1457 & FP & $41-\mathrm{Nb}-100$ & 99.9142 & $0.00 \mathrm{E}+00$ & $4.62 \mathrm{E}-01$ \\
\hline 1458 & FP & $41-\mathrm{Nb}-100 \mathrm{~m}$ & 99.9142 & $0.00 \mathrm{E}+00$ & $2.32 \mathrm{E}-01$ \\
\hline 1459 & FP & 42-Mo-100 & 99.9075 & $0.00 \mathrm{E}+00$ & $3.01 \mathrm{E}-27$ \\
\hline 1460 & FP & $43-T c-100$ & 99.9077 & $0.00 \mathrm{E}+00$ & $4.48 \mathrm{E}-02$ \\
\hline 1461 & FP & 44-Ru-100 & 99.9042 & $0.00 \mathrm{E}+00$ & $0.00 \mathrm{E}+00$ \\
\hline 1462 & FP & 37-Rb-101 & 100.9532 & $0.00 \mathrm{E}+00$ & $2.17 \mathrm{E}+01$ \\
\hline 1463 & FP & $38-\mathrm{Sr}-101$ & 100.9405 & $0.00 \mathrm{E}+00$ & $5.87 \mathrm{E}+00$ \\
\hline 1464 & FP & $39-Y-101$ & 100.9303 & $0.00 \mathrm{E}+00$ & $1.54 \mathrm{E}+00$ \\
\hline 1465 & FP & $40-Z r-101$ & 100.9211 & $0.00 \mathrm{E}+00$ & $3.01 \mathrm{E}-01$ \\
\hline 1466 & FP & 41-Nb-101 & 100.9153 & $0.00 \mathrm{E}+00$ & $9.76 \mathrm{E}-02$ \\
\hline 1467 & FP & 42-Mo-101 & 100.9103 & $0.00 \mathrm{E}+00$ & $7.91 \mathrm{E}-04$ \\
\hline 1468 & FP & 43-Tc-101 & 100.9073 & $0.00 \mathrm{E}+00$ & $8.14 \mathrm{E}-04$ \\
\hline 1469 & FP & 44-Ru-101 & 100.9056 & $0.00 \mathrm{E}+00$ & $0.00 \mathrm{E}+00$ \\
\hline 1470 & FP & 45-Rh-101 & 100.9062 & $0.00 \mathrm{E}+00$ & $6.66 \mathrm{E}-09$ \\
\hline 1471 & FP & $45-\mathrm{Rh}-101 \mathrm{~m}$ & 100.9062 & $0.00 \mathrm{E}+00$ & $1.85 \mathrm{E}-06$ \\
\hline 1472 & FP & $46-P d-101$ & 100.9083 & $0.00 \mathrm{E}+00$ & $2.27 \mathrm{E}-05$ \\
\hline 1473 & FP & 37-Rb-102 & 101.9589 & $0.00 \mathrm{E}+00$ & $1.87 \mathrm{E}+01$ \\
\hline 1474 & FP & $38-\mathrm{Sr}-102$ & 101.9430 & $0.00 \mathrm{E}+00$ & $1.00 \mathrm{E}+01$ \\
\hline 1475 & FP & $39-Y-102$ & 101.9336 & $0.00 \mathrm{E}+00$ & $1.93 \mathrm{E}+00$ \\
\hline 1476 & FP & $40-Z r-102$ & 101.9230 & $0.00 \mathrm{E}+00$ & $2.39 \mathrm{E}-01$ \\
\hline 1477 & FP & $41-\mathrm{Nb}-102$ & 101.9180 & $0.00 \mathrm{E}+00$ & $1.61 \mathrm{E}-01$ \\
\hline 1478 & FP & 41-Nb-102m & 101.9180 & $0.00 \mathrm{E}+00$ & $5.33 \mathrm{E}-01$ \\
\hline 1479 & FP & 42-Mo-102 & 101.9103 & $0.00 \mathrm{E}+00$ & $1.02 \mathrm{E}-03$ \\
\hline 1480 & FP & 43-Tc-102 & 101.9092 & $0.00 \mathrm{E}+00$ & $1.31 \mathrm{E}-01$ \\
\hline 1481 & FP & $43-\mathrm{Tc}-102 \mathrm{~m}$ & 101.9092 & $0.00 \mathrm{E}+00$ & $2.66 \mathrm{E}-03$ \\
\hline 1482 & FP & 44-Ru-102 & 101.9044 & $0.00 \mathrm{E}+00$ & $0.00 \mathrm{E}+00$ \\
\hline 1483 & FP & 45-Rh-102 & 101.9068 & $0.00 \mathrm{E}+00$ & $3.87 \mathrm{E}-08$ \\
\hline 1484 & FP & 45-Rh-102m & 101.9068 & $0.00 \mathrm{E}+00$ & $5.87 \mathrm{E}-09$ \\
\hline 1485 & FP & 46-Pd-102 & 101.9056 & $0.00 \mathrm{E}+00$ & $0.00 \mathrm{E}+00$ \\
\hline 1486 & FP & 38-Sr-103 & 102.9490 & $0.00 \mathrm{E}+00$ & $1.02 \mathrm{E}+01$ \\
\hline 1487 & FP & $39-Y-103$ & 102.9367 & $0.00 \mathrm{E}+00$ & $3.01 \mathrm{E}+00$ \\
\hline 1488 & FP & $40-Z r-103$ & 102.9266 & $0.00 \mathrm{E}+00$ & $5.33 \mathrm{E}-01$ \\
\hline 1489 & FP & $41-\mathrm{Nb}-103$ & 102.9191 & $0.00 \mathrm{E}+00$ & 4.62E-01 \\
\hline 1490 & FP & 42-Mo-103 & 102.9132 & $0.00 \mathrm{E}+00$ & $1.03 \mathrm{E}-02$ \\
\hline 1491 & FP & 43-Tc-103 & 102.9092 & $0.00 \mathrm{E}+00$ & $1.28 \mathrm{E}-02$ \\
\hline 1492 & FP & 44-Ru-103 & 102.9063 & $0.00 \mathrm{E}+00$ & $2.04 \mathrm{E}-07$ \\
\hline 1493 & FP & 45-Rh-103 & 102.9055 & $0.00 \mathrm{E}+00$ & $0.00 \mathrm{E}+00$ \\
\hline 1494 & FP & 45-Rh-103m & 102.9055 & $0.00 \mathrm{E}+00$ & $2.06 \mathrm{E}-04$ \\
\hline 1495 & FP & 46-Pd-103 & 102.9061 & $0.00 \mathrm{E}+00$ & 4.72E-07 \\
\hline 1496 & FP & 47-Ag-103 & 102.9090 & $0.00 \mathrm{E}+00$ & $1.76 \mathrm{E}-04$ \\
\hline 1497 & FP & $38-\mathrm{Sr}-104$ & 103.9523 & $0.00 \mathrm{E}+00$ & $1.61 \mathrm{E}+01$ \\
\hline
\end{tabular}




\begin{tabular}{|c|c|c|c|c|c|}
\hline 1498 & FP & $39-Y-104$ & 103.9410 & $0.00 \mathrm{E}+00$ & $3.85 \mathrm{E}+00$ \\
\hline 1499 & FP & $40-Z r-104$ & 103.9288 & $0.00 \mathrm{E}+00$ & $5.78 \mathrm{E}-01$ \\
\hline 1500 & FP & $41-\mathrm{Nb}-104$ & 103.9225 & $0.00 \mathrm{E}+00$ & $1.41 \mathrm{E}-01$ \\
\hline 1501 & FP & $41-\mathrm{Nb}-104 \mathrm{~m}$ & 103.9225 & $0.00 \mathrm{E}+00$ & $7.37 \mathrm{E}-01$ \\
\hline 1502 & FP & 42-Mo-104 & 103.9138 & $0.00 \mathrm{E}+00$ & $1.16 \mathrm{E}-02$ \\
\hline 1503 & FP & 43-Tc-104 & 103.9115 & $0.00 \mathrm{E}+00$ & $6.31 \mathrm{E}-04$ \\
\hline 1504 & FP & 44-Ru-104 & 103.9054 & $0.00 \mathrm{E}+00$ & $0.00 \mathrm{E}+00$ \\
\hline 1505 & FP & 45-Rh-104 & 103.9067 & $0.00 \mathrm{E}+00$ & $1.64 \mathrm{E}-02$ \\
\hline 1506 & FP & $45-\mathrm{Rh}-104 \mathrm{~m}$ & 103.9067 & $0.00 \mathrm{E}+00$ & $2.66 \mathrm{E}-03$ \\
\hline 1507 & FP & 46-Pd-104 & 103.9040 & $0.00 \mathrm{E}+00$ & $0.00 \mathrm{E}+00$ \\
\hline 1508 & FP & $38-\mathrm{Sr}-105$ & 104.9586 & $0.00 \mathrm{E}+00$ & $1.25 \mathrm{E}+01$ \\
\hline 1509 & FP & $39-Y-105$ & 104.9449 & $0.00 \mathrm{E}+00$ & $7.88 \mathrm{E}+00$ \\
\hline 1510 & FP & $40-Z r-105$ & 104.9331 & $0.00 \mathrm{E}+00$ & $1.16 \mathrm{E}+00$ \\
\hline 1511 & FP & $41-\mathrm{Nb}-105$ & 104.9239 & $0.00 \mathrm{E}+00$ & $2.35 \mathrm{E}-01$ \\
\hline 1512 & FP & 42-Mo-105 & 104.9170 & $0.00 \mathrm{E}+00$ & $1.95 \mathrm{E}-02$ \\
\hline 1513 & FP & 43-Tc-105 & 104.9117 & $0.00 \mathrm{E}+00$ & $1.52 \mathrm{E}-03$ \\
\hline 1514 & FP & 44-Ru-105 & 104.9078 & $0.00 \mathrm{E}+00$ & $4.34 \mathrm{E}-05$ \\
\hline 1515 & FP & 45-Rh-105 & 104.9057 & $0.00 \mathrm{E}+00$ & $5.45 \mathrm{E}-06$ \\
\hline 1516 & $\mathrm{FP}$ & $45-\mathrm{Rh}-105 \mathrm{~m}$ & 104.9057 & $0.00 \mathrm{E}+00$ & $1.73 \mathrm{E}-02$ \\
\hline 1517 & FP & 46-Pd-105 & 104.9051 & $0.00 \mathrm{E}+00$ & $0.00 \mathrm{E}+00$ \\
\hline 1518 & FP & 47-Ag-105 & 104.9065 & $0.00 \mathrm{E}+00$ & $1.94 \mathrm{E}-07$ \\
\hline 1519 & FP & $47-A g-105 m$ & 104.9065 & $0.00 \mathrm{E}+00$ & $1.60 \mathrm{E}-03$ \\
\hline 1520 & FP & $48-\mathrm{Cd}-105$ & 104.9095 & $0.00 \mathrm{E}+00$ & $2.08 \mathrm{E}-04$ \\
\hline 1521 & FP & $39-Y-106$ & 105.9498 & $0.00 \mathrm{E}+00$ & $1.05 \mathrm{E}+01$ \\
\hline 1522 & FP & $40-Z r-106$ & 105.9359 & $0.00 \mathrm{E}+00$ & $2.57 \mathrm{E}+00$ \\
\hline 1523 & FP & 41-Nb-106 & 105.9280 & $0.00 \mathrm{E}+00$ & $7.45 \mathrm{E}-01$ \\
\hline 1524 & FP & 42-Mo-106 & 105.9181 & $0.00 \mathrm{E}+00$ & $7.94 \mathrm{E}-02$ \\
\hline 1525 & FP & $43-\mathrm{Tc}-106$ & 105.9144 & $0.00 \mathrm{E}+00$ & $1.95 \mathrm{E}-02$ \\
\hline 1526 & FP & 44-Ru-106 & 105.9073 & $0.00 \mathrm{E}+00$ & $2.16 \mathrm{E}-08$ \\
\hline 1527 & FP & 45-Rh-106 & 105.9073 & $0.00 \mathrm{E}+00$ & $2.31 \mathrm{E}-02$ \\
\hline 1528 & FP & 45-Rh-106m & 105.9073 & $0.00 \mathrm{E}+00$ & $8.82 \mathrm{E}-05$ \\
\hline 1529 & FP & 46-Pd-106 & 105.9035 & $0.00 \mathrm{E}+00$ & $0.00 \mathrm{E}+00$ \\
\hline 1530 & FP & 47-Ag-106 & 105.9067 & $0.00 \mathrm{E}+00$ & $4.82 \mathrm{E}-04$ \\
\hline 1531 & FP & 47-Ag-106m & 105.9067 & $0.00 \mathrm{E}+00$ & $9.69 \mathrm{E}-07$ \\
\hline 1532 & FP & 48-Cd-106 & 105.9065 & $0.00 \mathrm{E}+00$ & $0.00 \mathrm{E}+00$ \\
\hline 1533 & FP & $39-Y-107$ & 106.9541 & $0.00 \mathrm{E}+00$ & $2.31 \mathrm{E}+01$ \\
\hline 1534 & FP & $40-Z r-107$ & 106.9408 & $0.00 \mathrm{E}+00$ & $4.62 \mathrm{E}+00$ \\
\hline 1535 & FP & 41-Nb-107 & 106.9303 & $0.00 \mathrm{E}+00$ & $2.31 \mathrm{E}+00$ \\
\hline 1536 & FP & 42-Mo-107 & 106.9217 & $0.00 \mathrm{E}+00$ & $1.98 \mathrm{E}-01$ \\
\hline 1537 & FP & 43-Tc-107 & 106.9151 & $0.00 \mathrm{E}+00$ & $3.27 \mathrm{E}-02$ \\
\hline 1538 & FP & 44-Ru-107 & 106.9099 & $0.00 \mathrm{E}+00$ & $3.08 \mathrm{E}-03$ \\
\hline 1539 & FP & 45-Rh-107 & 106.9068 & $0.00 \mathrm{E}+00$ & $5.32 \mathrm{E}-04$ \\
\hline 1540 & FP & 46-Pd-107 & 106.9051 & $0.00 \mathrm{E}+00$ & $3.38 \mathrm{E}-15$ \\
\hline 1541 & FP & 46-Pd-107m & 106.9051 & $0.00 \mathrm{E}+00$ & $3.25 \mathrm{E}-02$ \\
\hline 1542 & FP & 47-Ag-107 & 106.9051 & $0.00 \mathrm{E}+00$ & $0.00 \mathrm{E}+00$ \\
\hline 1543 & FP & $47-A g-107 m$ & 106.9051 & $0.00 \mathrm{E}+00$ & $1.56 \mathrm{E}-02$ \\
\hline 1544 & FP & $48-\mathrm{Cd}-107$ & 106.9066 & $0.00 \mathrm{E}+00$ & $2.96 \mathrm{E}-05$ \\
\hline 1545 & FP & 49-In-107 & 106.9103 & $0.00 \mathrm{E}+00$ & $3.57 \mathrm{E}-04$ \\
\hline 1546 & FP & $39-Y-108$ & 107.9595 & $0.00 \mathrm{E}+00$ & $1.44 \mathrm{E}+01$ \\
\hline 1547 & FP & $40-Z r-108$ & 107.9440 & $0.00 \mathrm{E}+00$ & $8.66 \mathrm{E}+00$ \\
\hline 1548 & FP & $41-\mathrm{Nb}-108$ & 107.9348 & $0.00 \mathrm{E}+00$ & $3.59 \mathrm{E}+00$ \\
\hline 1549 & FP & 42-Mo-108 & 107.9234 & $0.00 \mathrm{E}+00$ & $6.36 \mathrm{E}-01$ \\
\hline 1550 & FP & 43-Tc-108 & 107.9185 & $0.00 \mathrm{E}+00$ & $1.34 \mathrm{E}-01$ \\
\hline 1551 & FP & 44-Ru-108 & 107.9102 & $0.00 \mathrm{E}+00$ & $2.54 \mathrm{E}-03$ \\
\hline 1552 & FP & 45-Rh-108 & 107.9087 & $0.00 \mathrm{E}+00$ & $4.13 \mathrm{E}-02$ \\
\hline 1553 & FP & $45-\mathrm{Rh}-108 \mathrm{~m}$ & 107.9087 & $0.00 \mathrm{E}+00$ & $1.93 \mathrm{E}-03$ \\
\hline 1554 & FP & 46-Pd-108 & 107.9039 & $0.00 \mathrm{E}+00$ & $0.00 \mathrm{E}+00$ \\
\hline 1555 & FP & 47-Ag-108 & 107.9060 & $0.00 \mathrm{E}+00$ & $4.85 \mathrm{E}-03$ \\
\hline 1556 & FP & $47-A g-108 m$ & 107.9060 & $0.00 \mathrm{E}+00$ & $5.01 \mathrm{E}-11$ \\
\hline 1557 & FP & $48-\mathrm{Cd}-108$ & 107.9042 & $0.00 \mathrm{E}+00$ & $0.00 \mathrm{E}+00$ \\
\hline 1558 & FP & $40-Z r-109$ & 108.9492 & $0.00 \mathrm{E}+00$ & $5.92 \mathrm{E}+00$ \\
\hline 1559 & FP & $41-\mathrm{Nb}-109$ & 108.9376 & $0.00 \mathrm{E}+00$ & $3.65 \mathrm{E}+00$ \\
\hline 1560 & FP & 42-Mo-109 & 108.9278 & $0.00 \mathrm{E}+00$ & $1.31 \mathrm{E}+00$ \\
\hline 1561 & FP & 43-Tc-109 & 108.9200 & $0.00 \mathrm{E}+00$ & $8.06 \mathrm{E}-01$ \\
\hline 1562 & FP & 44-Ru-109 & 108.9132 & $0.00 \mathrm{E}+00$ & $2.01 \mathrm{E}-02$ \\
\hline 1563 & FP & 45-Rh-109 & 108.9087 & $0.00 \mathrm{E}+00$ & $8.66 \mathrm{E}-03$ \\
\hline
\end{tabular}




\begin{tabular}{|c|c|c|c|c|c|}
\hline 1564 & FP & 46-Pd-109 & 108.9060 & $0.00 \mathrm{E}+00$ & $1.41 \mathrm{E}-05$ \\
\hline 1565 & FP & 46-Pd-109m & 108.9060 & $0.00 \mathrm{E}+00$ & $2.46 \mathrm{E}-03$ \\
\hline 1566 & FP & 47-Ag-109 & 108.9047 & $0.00 \mathrm{E}+00$ & $0.00 \mathrm{E}+00$ \\
\hline 1567 & FP & 47-Ag-109m & 108.9047 & $0.00 \mathrm{E}+00$ & $1.75 \mathrm{E}-02$ \\
\hline 1568 & $\mathrm{FP}$ & 48-Cd-109 & 108.9050 & $0.00 \mathrm{E}+00$ & $1.74 \mathrm{E}-08$ \\
\hline 1569 & FP & 49-In-109 & 108.9072 & $0.00 \mathrm{E}+00$ & $4.62 \mathrm{E}-05$ \\
\hline 1570 & $\mathrm{FP}$ & $40-Z r-110$ & 109.9529 & $0.00 \mathrm{E}+00$ & $7.07 \mathrm{E}+00$ \\
\hline 1571 & FP & $41-\mathrm{Nb}-110$ & 109.9424 & $0.00 \mathrm{E}+00$ & $4.08 \mathrm{E}+00$ \\
\hline 1572 & $\mathrm{FP}$ & 42-Mo-110 & 109.9297 & $0.00 \mathrm{E}+00$ & $2.31 \mathrm{E}+00$ \\
\hline 1573 & FP & $43-\mathrm{Tc}-110$ & 109.9238 & $0.00 \mathrm{E}+00$ & $7.53 \mathrm{E}-01$ \\
\hline 1574 & $\mathrm{FP}$ & 44-Ru-110 & 109.9141 & $0.00 \mathrm{E}+00$ & $5.98 \mathrm{E}-02$ \\
\hline 1575 & $\mathrm{FP}$ & $45-\mathrm{Rh}-110$ & 109.9111 & $0.00 \mathrm{E}+00$ & $2.17 \mathrm{E}-01$ \\
\hline 1576 & FP & $45-\mathrm{Rh}-110 \mathrm{~m}$ & 109.9111 & $0.00 \mathrm{E}+00$ & $2.43 \mathrm{E}-02$ \\
\hline 1577 & FP & 46-Pd-110 & 109.9052 & $0.00 \mathrm{E}+00$ & $0.00 \mathrm{E}+00$ \\
\hline 1578 & FP & 47-Ag-110 & 109.9061 & $0.00 \mathrm{E}+00$ & $2.82 \mathrm{E}-02$ \\
\hline 1579 & FP & $47-\mathrm{Ag}-110 \mathrm{~m}$ & 109.9062 & $0.00 \mathrm{E}+00$ & $3.21 \mathrm{E}-08$ \\
\hline 1580 & FP & $48-\mathrm{Cd}-110$ & 109.9030 & $0.00 \mathrm{E}+00$ & $0.00 \mathrm{E}+00$ \\
\hline 1581 & $\mathrm{FP}$ & $41-\mathrm{Nb}-111$ & 110.9456 & $0.00 \mathrm{E}+00$ & $8.66 \mathrm{E}+00$ \\
\hline 1582 & $\mathrm{FP}$ & 42-Mo-111 & 110.9344 & $0.00 \mathrm{E}+00$ & $3.47 \mathrm{E}+00$ \\
\hline 1583 & FP & 43-Tc-111 & 110.9257 & $0.00 \mathrm{E}+00$ & $2.39 \mathrm{E}+00$ \\
\hline 1584 & FP & 44-Ru-111 & 110.9177 & $0.00 \mathrm{E}+00$ & $3.27 \mathrm{E}-01$ \\
\hline 1585 & $\mathrm{FP}$ & 45-Rh-111 & 110.9116 & $0.00 \mathrm{E}+00$ & $6.30 \mathrm{E}-02$ \\
\hline 1586 & $\mathrm{FP}$ & 46-Pd-111 & 110.9077 & $0.00 \mathrm{E}+00$ & $4.94 \mathrm{E}-04$ \\
\hline 1587 & FP & 46-Pd-111m & 110.9077 & $0.00 \mathrm{E}+00$ & $3.50 \mathrm{E}-05$ \\
\hline 1588 & FP & 47-Ag-111 & 110.9053 & $0.00 \mathrm{E}+00$ & $1.08 \mathrm{E}-06$ \\
\hline 1589 & $\mathrm{FP}$ & $47-\mathrm{Ag}-111 \mathrm{~m}$ & 110.9053 & $0.00 \mathrm{E}+00$ & $1.07 \mathrm{E}-02$ \\
\hline 1590 & FP & $48-\mathrm{Cd}-111$ & 110.9042 & $0.00 \mathrm{E}+00$ & $0.00 \mathrm{E}+00$ \\
\hline 1591 & FP & $48-\mathrm{Cd}-111 \mathrm{~m}$ & 110.9042 & $0.00 \mathrm{E}+00$ & $2.38 \mathrm{E}-04$ \\
\hline 1592 & FP & $49-\operatorname{In}-111$ & 110.9051 & $0.00 \mathrm{E}+00$ & $2.86 \mathrm{E}-06$ \\
\hline 1593 & $\mathrm{FP}$ & 49-In-111m & 110.9051 & $0.00 \mathrm{E}+00$ & $1.50 \mathrm{E}-03$ \\
\hline 1594 & $\mathrm{FP}$ & $50-\mathrm{Sn}-111$ & 110.9077 & $0.00 \mathrm{E}+00$ & $3.27 \mathrm{E}-04$ \\
\hline 1595 & FP & $41-\mathrm{Nb}-112$ & 111.9508 & $0.00 \mathrm{E}+00$ & $1.00 \mathrm{E}+01$ \\
\hline 1596 & $\mathrm{FP}$ & 42-Mo-112 & 111.9368 & $0.00 \mathrm{E}+00$ & $2.42 \mathrm{E}+00$ \\
\hline 1597 & FP & 43-Tc-112 & 111.9292 & $0.00 \mathrm{E}+00$ & $2.48 \mathrm{E}+00$ \\
\hline 1598 & FP & 44-Ru-112 & 111.9190 & $0.00 \mathrm{E}+00$ & $3.96 \mathrm{E}-01$ \\
\hline 1599 & FP & 45-Rh-112 & 111.9144 & $0.00 \mathrm{E}+00$ & $3.30 \mathrm{E}-01$ \\
\hline 1600 & FP & 46-Pd-112 & 111.9073 & $0.00 \mathrm{E}+00$ & $9.16 \mathrm{E}-06$ \\
\hline 1601 & FP & 47-Ag-112 & 111.9070 & $0.00 \mathrm{E}+00$ & $6.15 \mathrm{E}-05$ \\
\hline 1602 & $\mathrm{FP}$ & 48-Cd-112 & 111.9028 & $0.00 \mathrm{E}+00$ & $0.00 \mathrm{E}+00$ \\
\hline 1603 & $\mathrm{FP}$ & $49-\operatorname{In}-112$ & 111.9055 & $0.00 \mathrm{E}+00$ & $7.72 \mathrm{E}-04$ \\
\hline 1604 & FP & $49-\operatorname{In}-112 \mathrm{~m}$ & 111.9055 & $0.00 \mathrm{E}+00$ & $5.62 \mathrm{E}-04$ \\
\hline 1605 & FP & $50-\mathrm{Sn}-112$ & 111.9048 & $0.00 \mathrm{E}+00$ & $0.00 \mathrm{E}+00$ \\
\hline 1606 & FP & $41-\mathrm{Nb}-113$ & 112.9547 & $0.00 \mathrm{E}+00$ & $2.31 \mathrm{E}+01$ \\
\hline 1607 & FP & 42-Mo-113 & 112.9419 & $0.00 \mathrm{E}+00$ & $6.93 \mathrm{E}+00$ \\
\hline 1608 & FP & 43-Tc-113 & 112.9316 & $0.00 \mathrm{E}+00$ & $4.33 \mathrm{E}+00$ \\
\hline 1609 & FP & 44-Ru-113 & 112.9225 & $0.00 \mathrm{E}+00$ & $8.66 \mathrm{E}-01$ \\
\hline 1610 & $\mathrm{FP}$ & 45-Rh-113 & 112.9155 & $0.00 \mathrm{E}+00$ & $2.48 \mathrm{E}-01$ \\
\hline 1611 & FP & 46-Pd-113 & 112.9101 & $0.00 \mathrm{E}+00$ & $7.45 \mathrm{E}-03$ \\
\hline 1612 & FP & 47-Ag-113 & 112.9066 & $0.00 \mathrm{E}+00$ & $3.59 \mathrm{E}-05$ \\
\hline 1613 & FP & $47-A g-113 m$ & 112.9066 & $0.00 \mathrm{E}+00$ & $1.01 \mathrm{E}-02$ \\
\hline 1614 & $\mathrm{FP}$ & $48-\mathrm{Cd}-113$ & 112.9044 & $0.00 \mathrm{E}+00$ & $2.73 \mathrm{E}-24$ \\
\hline 1615 & FP & $48-\mathrm{Cd}-113 \mathrm{~m}$ & 112.9044 & $0.00 \mathrm{E}+00$ & $1.56 \mathrm{E}-09$ \\
\hline 1616 & $\mathrm{FP}$ & 49-In-113 & 112.9041 & $0.00 \mathrm{E}+00$ & $0.00 \mathrm{E}+00$ \\
\hline 1617 & FP & 49-In-113m & 112.9041 & $0.00 \mathrm{E}+00$ & $1.16 \mathrm{E}-04$ \\
\hline 1618 & $\mathrm{FP}$ & $50-\mathrm{Sn}-113$ & 112.9052 & $0.00 \mathrm{E}+00$ & $6.97 \mathrm{E}-08$ \\
\hline 1619 & FP & $50-\mathrm{Sn}-113 \mathrm{~m}$ & 112.9052 & $0.00 \mathrm{E}+00$ & $5.40 \mathrm{E}-04$ \\
\hline 1620 & FP & $51-S b-113$ & 112.9094 & $0.00 \mathrm{E}+00$ & $1.73 \mathrm{E}-03$ \\
\hline 1621 & FP & 42-Mo-114 & 113.9449 & $0.00 \mathrm{E}+00$ & $8.66 \mathrm{E}+00$ \\
\hline 1622 & $\mathrm{FP}$ & 43-Tc-114 & 113.9359 & $0.00 \mathrm{E}+00$ & $4.62 \mathrm{E}+00$ \\
\hline 1623 & $\mathrm{FP}$ & 44-Ru-114 & 113.9243 & $0.00 \mathrm{E}+00$ & $1.33 \mathrm{E}+00$ \\
\hline 1624 & $\mathrm{FP}$ & 45-Rh-114 & 113.9188 & $0.00 \mathrm{E}+00$ & $3.75 \mathrm{E}-01$ \\
\hline 1625 & FP & 46-Pd-114 & 113.9104 & $0.00 \mathrm{E}+00$ & $4.77 \mathrm{E}-03$ \\
\hline 1626 & FP & 47-Ag-114 & 113.9088 & $0.00 \mathrm{E}+00$ & $1.51 \mathrm{E}-01$ \\
\hline 1627 & $\mathrm{FP}$ & $48-\mathrm{Cd}-114$ & 113.9034 & $0.00 \mathrm{E}+00$ & $0.00 \mathrm{E}+00$ \\
\hline 1628 & $\mathrm{FP}$ & 49-In-114 & 113.9049 & $0.00 \mathrm{E}+00$ & $9.64 \mathrm{E}-03$ \\
\hline 1629 & $\mathrm{FP}$ & $49-\operatorname{In}-114 \mathrm{~m}$ & 113.9049 & $0.00 \mathrm{E}+00$ & $1.62 \mathrm{E}-07$ \\
\hline
\end{tabular}




\begin{tabular}{|c|c|c|c|c|c|}
\hline 1630 & FP & $50-\mathrm{Sn}-114$ & 113.9028 & $0.00 \mathrm{E}+00$ & $0.00 \mathrm{E}+00$ \\
\hline 1631 & FP & 42-Mo-115 & 114.9503 & $0.00 \mathrm{E}+00$ & $7.53 \mathrm{E}+00$ \\
\hline 1632 & FP & 43-Tc-115 & 114.9387 & $0.00 \mathrm{E}+00$ & $9.50 \mathrm{E}+00$ \\
\hline 1633 & FP & 44-Ru-115 & 114.9287 & $0.00 \mathrm{E}+00$ & $9.37 \mathrm{E}-01$ \\
\hline 1634 & $\mathrm{FP}$ & 45-Rh-115 & 114.9203 & $0.00 \mathrm{E}+00$ & $7.00 \mathrm{E}-01$ \\
\hline 1635 & FP & 46-Pd-115 & 114.9137 & $0.00 \mathrm{E}+00$ & $2.77 \mathrm{E}-02$ \\
\hline 1636 & $\mathrm{FP}$ & 47-Ag-115 & 114.9088 & $0.00 \mathrm{E}+00$ & $5.78 \mathrm{E}-04$ \\
\hline 1637 & FP & $47-A g-115 \mathrm{~m}$ & 114.9088 & $0.00 \mathrm{E}+00$ & $3.85 \mathrm{E}-02$ \\
\hline 1638 & $\mathrm{FP}$ & $48-\mathrm{Cd}-115$ & 114.9054 & $0.00 \mathrm{E}+00$ & $3.60 \mathrm{E}-06$ \\
\hline 1639 & FP & $48-\mathrm{Cd}-115 \mathrm{~m}$ & 114.9051 & $0.00 \mathrm{E}+00$ & $1.80 \mathrm{E}-07$ \\
\hline 1640 & FP & 49-In-115 & 114.9039 & $0.00 \mathrm{E}+00$ & $4.98 \mathrm{E}-23$ \\
\hline 1641 & $\mathrm{FP}$ & $49-\operatorname{In}-115 \mathrm{~m}$ & 114.9039 & $0.00 \mathrm{E}+00$ & $4.29 \mathrm{E}-05$ \\
\hline 1642 & FP & $50-\mathrm{Sn}-115$ & 114.9033 & $0.00 \mathrm{E}+00$ & $0.00 \mathrm{E}+00$ \\
\hline 1643 & FP & 51-Sb-115 & 114.9066 & $0.00 \mathrm{E}+00$ & $3.60 \mathrm{E}-04$ \\
\hline 1644 & FP & 52-Te-115 & 114.9119 & $0.00 \mathrm{E}+00$ & 1.99E-03 \\
\hline 1645 & FP & 43-Tc-116 & 115.9434 & $0.00 \mathrm{E}+00$ & $7.70 \mathrm{E}+00$ \\
\hline 1646 & FP & 44-Ru-116 & 115.9308 & $0.00 \mathrm{E}+00$ & $3.40 \mathrm{E}+00$ \\
\hline 1647 & $\mathrm{FP}$ & 45-Rh-116 & 115.9241 & $0.00 \mathrm{E}+00$ & $1.02 \mathrm{E}+00$ \\
\hline 1648 & $\mathrm{FP}$ & 46-Pd-116 & 115.9142 & $0.00 \mathrm{E}+00$ & $5.87 \mathrm{E}-02$ \\
\hline 1649 & FP & 47-Ag-116 & 115.9114 & $0.00 \mathrm{E}+00$ & $2.92 \mathrm{E}-03$ \\
\hline 1650 & FP & $47-A g-116 \mathrm{~m}$ & 115.9114 & $0.00 \mathrm{E}+00$ & $3.47 \mathrm{E}-02$ \\
\hline 1651 & $\mathrm{FP}$ & $48-\mathrm{Cd}-116$ & 115.9048 & $0.00 \mathrm{E}+00$ & $7.09 \mathrm{E}-28$ \\
\hline 1652 & $\mathrm{FP}$ & 49-In-116 & 115.9053 & $0.00 \mathrm{E}+00$ & $4.92 \mathrm{E}-02$ \\
\hline 1653 & FP & 49-In-116m & 115.9053 & $0.00 \mathrm{E}+00$ & $2.13 \mathrm{E}-04$ \\
\hline 1654 & FP & $50-\mathrm{Sn}-116$ & 115.9017 & $0.00 \mathrm{E}+00$ & $0.00 \mathrm{E}+00$ \\
\hline 1655 & $\mathrm{FP}$ & 43-Tc-117 & 116.9465 & $0.00 \mathrm{E}+00$ & $1.73 \mathrm{E}+01$ \\
\hline 1656 & FP & 44-Ru-117 & 116.9356 & $0.00 \mathrm{E}+00$ & $4.88 \mathrm{E}+00$ \\
\hline 1657 & FP & 45-Rh-117 & 116.9260 & $0.00 \mathrm{E}+00$ & $1.58 \mathrm{E}+00$ \\
\hline 1658 & FP & 46-Pd-117 & 116.9178 & $0.00 \mathrm{E}+00$ & $1.61 \mathrm{E}-01$ \\
\hline 1659 & $\mathrm{FP}$ & 47-Ag-117 & 116.9117 & $0.00 \mathrm{E}+00$ & $9.52 \mathrm{E}-03$ \\
\hline 1660 & $\mathrm{FP}$ & $47-A g-117 \mathrm{~m}$ & 116.9117 & $0.00 \mathrm{E}+00$ & $1.30 \mathrm{E}-01$ \\
\hline 1661 & FP & $48-\mathrm{Cd}-117$ & 116.9072 & $0.00 \mathrm{E}+00$ & $7.73 \mathrm{E}-05$ \\
\hline 1662 & $\mathrm{FP}$ & $48-\mathrm{Cd}-117 \mathrm{~m}$ & 116.9072 & $0.00 \mathrm{E}+00$ & 5.73E-05 \\
\hline 1663 & FP & 49-In-117 & 116.9045 & $0.00 \mathrm{E}+00$ & $2.67 \mathrm{E}-04$ \\
\hline 1664 & FP & 49-In-117m & 116.9045 & $0.00 \mathrm{E}+00$ & $9.94 \mathrm{E}-05$ \\
\hline 1665 & FP & $50-\mathrm{Sn}-117$ & 116.9029 & $0.00 \mathrm{E}+00$ & $0.00 \mathrm{E}+00$ \\
\hline 1666 & FP & $50-\mathrm{Sn}-117 \mathrm{~m}$ & 116.9029 & $0.00 \mathrm{E}+00$ & $5.90 \mathrm{E}-07$ \\
\hline 1667 & FP & 51-Sb-117 & 116.9048 & $0.00 \mathrm{E}+00$ & $6.88 \mathrm{E}-05$ \\
\hline 1668 & $\mathrm{FP}$ & 52-Te-117 & 116.9087 & $0.00 \mathrm{E}+00$ & $1.86 \mathrm{E}-04$ \\
\hline 1669 & $\mathrm{FP}$ & 43-Tc-118 & 117.9515 & $0.00 \mathrm{E}+00$ & $1.05 \mathrm{E}+01$ \\
\hline 1670 & FP & 44-Ru-118 & 117.9378 & $0.00 \mathrm{E}+00$ & $5.64 \mathrm{E}+00$ \\
\hline 1671 & FP & 45-Rh-118 & 117.9301 & $0.00 \mathrm{E}+00$ & $2.61 \mathrm{E}+00$ \\
\hline 1672 & $\mathrm{FP}$ & 46-Pd-118 & 117.9190 & $0.00 \mathrm{E}+00$ & $3.65 \mathrm{E}-01$ \\
\hline 1673 & FP & 47-Ag-118 & 117.9146 & $0.00 \mathrm{E}+00$ & $1.84 \mathrm{E}-01$ \\
\hline 1674 & $\mathrm{FP}$ & 47-Ag-118m & 117.9146 & $0.00 \mathrm{E}+00$ & $3.47 \mathrm{E}-01$ \\
\hline 1675 & FP & $48-\mathrm{Cd}-118$ & 117.9069 & $0.00 \mathrm{E}+00$ & $2.30 \mathrm{E}-04$ \\
\hline 1676 & $\mathrm{FP}$ & 49-In-118 & 117.9063 & $0.00 \mathrm{E}+00$ & $1.39 \mathrm{E}-01$ \\
\hline 1677 & FP & $49-\operatorname{In}-118 \mathrm{~m}$ & 117.9063 & $0.00 \mathrm{E}+00$ & $2.60 \mathrm{E}-03$ \\
\hline 1678 & FP & $50-\mathrm{Sn}-118$ & 117.9016 & $0.00 \mathrm{E}+00$ & $0.00 \mathrm{E}+00$ \\
\hline 1679 & FP & 51-Sb-118 & 117.9055 & $0.00 \mathrm{E}+00$ & $3.21 \mathrm{E}-03$ \\
\hline 1680 & $\mathrm{FP}$ & 51-Sb-118m & 117.9055 & $0.00 \mathrm{E}+00$ & $3.85 \mathrm{E}-05$ \\
\hline 1681 & FP & 52-Te-118 & 117.9058 & $0.00 \mathrm{E}+00$ & $1.34 \mathrm{E}-06$ \\
\hline 1682 & $\mathrm{FP}$ & 44-Ru-119 & 118.9428 & $0.00 \mathrm{E}+00$ & $4.28 \mathrm{E}+00$ \\
\hline 1683 & FP & 45-Rh-119 & 118.9321 & $0.00 \mathrm{E}+00$ & $4.05 \mathrm{E}+00$ \\
\hline 1684 & $\mathrm{FP}$ & 46-Pd-119 & 118.9231 & $0.00 \mathrm{E}+00$ & 7.53E-01 \\
\hline 1685 & $\mathrm{FP}$ & 47-Ag-119 & 118.9157 & $0.00 \mathrm{E}+00$ & $3.30 \mathrm{E}-01$ \\
\hline 1686 & FP & $48-\mathrm{Cd}-119$ & 118.9099 & $0.00 \mathrm{E}+00$ & $4.29 \mathrm{E}-03$ \\
\hline 1687 & FP & $48-\mathrm{Cd}-119 \mathrm{~m}$ & 118.9099 & $0.00 \mathrm{E}+00$ & $5.25 \mathrm{E}-03$ \\
\hline 1688 & $\mathrm{FP}$ & 49-In-119 & 118.9059 & $0.00 \mathrm{E}+00$ & $4.81 \mathrm{E}-03$ \\
\hline 1689 & $\mathrm{FP}$ & 49-In-119m & 118.9059 & $0.00 \mathrm{E}+00$ & $6.42 \mathrm{E}-04$ \\
\hline 1690 & $\mathrm{FP}$ & $50-\mathrm{Sn}-119$ & 118.9033 & $0.00 \mathrm{E}+00$ & $0.00 \mathrm{E}+00$ \\
\hline 1691 & FP & $50-\mathrm{Sn}-119 \mathrm{~m}$ & 118.9033 & $0.00 \mathrm{E}+00$ & $2.74 \mathrm{E}-08$ \\
\hline 1692 & FP & 51-Sb-119 & 118.9039 & $0.00 \mathrm{E}+00$ & $5.04 \mathrm{E}-06$ \\
\hline 1693 & $\mathrm{FP}$ & 52-Te-119 & 118.9064 & $0.00 \mathrm{E}+00$ & $1.20 \mathrm{E}-05$ \\
\hline 1694 & $\mathrm{FP}$ & 44-Ru-120 & 119.9453 & $0.00 \mathrm{E}+00$ & $4.65 \mathrm{E}+00$ \\
\hline 1695 & $\mathrm{FP}$ & $45-\mathrm{Rh}-120$ & 119.9364 & $0.00 \mathrm{E}+00$ & $5.10 \mathrm{E}+00$ \\
\hline
\end{tabular}




\begin{tabular}{|c|c|c|c|c|c|}
\hline 1696 & FP & 46-Pd-120 & 119.9247 & $0.00 \mathrm{E}+00$ & $1.39 \mathrm{E}+00$ \\
\hline 1697 & FP & 47-Ag-120 & 119.9188 & $0.00 \mathrm{E}+00$ & $5.64 \mathrm{E}-01$ \\
\hline 1698 & FP & $47-A g-120 \mathrm{~m}$ & 119.9188 & $0.00 \mathrm{E}+00$ & $2.17 \mathrm{E}+00$ \\
\hline 1699 & FP & $48-\mathrm{Cd}-120$ & 119.9099 & $0.00 \mathrm{E}+00$ & $1.36 \mathrm{E}-02$ \\
\hline 1700 & FP & $49-\operatorname{In}-120$ & 119.9080 & $0.00 \mathrm{E}+00$ & $2.25 \mathrm{E}-01$ \\
\hline 1701 & FP & 49-In-120m & 119.9080 & $0.00 \mathrm{E}+00$ & $1.50 \mathrm{E}-02$ \\
\hline 1702 & FP & $50-\mathrm{Sn}-120$ & 119.9022 & $0.00 \mathrm{E}+00$ & $0.00 \mathrm{E}+00$ \\
\hline 1703 & FP & 51-Sb-120 & 119.9051 & $0.00 \mathrm{E}+00$ & $7.27 \mathrm{E}-04$ \\
\hline 1704 & FP & $51-\mathrm{Sb}-120 \mathrm{~m}$ & 119.9051 & $0.00 \mathrm{E}+00$ & $1.39 \mathrm{E}-06$ \\
\hline 1705 & FP & $52-\mathrm{Te}-120$ & 119.9040 & $0.00 \mathrm{E}+00$ & $0.00 \mathrm{E}+00$ \\
\hline 1706 & FP & 45-Rh-121 & 120.9387 & $0.00 \mathrm{E}+00$ & $4.59 \mathrm{E}+00$ \\
\hline 1707 & FP & 46-Pd-121 & 120.9289 & $0.00 \mathrm{E}+00$ & $2.43 \mathrm{E}+00$ \\
\hline 1708 & FP & 47-Ag-121 & 120.9199 & $0.00 \mathrm{E}+00$ & $8.89 \mathrm{E}-01$ \\
\hline 1709 & FP & $48-\mathrm{Cd}-121$ & 120.9130 & $0.00 \mathrm{E}+00$ & $5.13 \mathrm{E}-02$ \\
\hline 1710 & FP & $48-\mathrm{Cd}-121 \mathrm{~m}$ & 120.9130 & $0.00 \mathrm{E}+00$ & $8.35 \mathrm{E}-02$ \\
\hline 1711 & FP & 49-In-121 & 120.9079 & $0.00 \mathrm{E}+00$ & $3.00 \mathrm{E}-02$ \\
\hline 1712 & FP & 49-In-121m & 120.9079 & $0.00 \mathrm{E}+00$ & $2.98 \mathrm{E}-03$ \\
\hline 1713 & FP & $50-\mathrm{Sn}-121$ & 120.9042 & $0.00 \mathrm{E}+00$ & $7.12 \mathrm{E}-06$ \\
\hline 1714 & FP & $50-\mathrm{Sn}-121 \mathrm{~m}$ & 120.9042 & $0.00 \mathrm{E}+00$ & $5.00 \mathrm{E}-10$ \\
\hline 1715 & FP & 51-Sb-121 & 120.9038 & $0.00 \mathrm{E}+00$ & $0.00 \mathrm{E}+00$ \\
\hline 1716 & FP & $52-\mathrm{Te}-121$ & 120.9049 & $0.00 \mathrm{E}+00$ & 4.19E-07 \\
\hline 1717 & FP & $52-\mathrm{Te}-121 \mathrm{~m}$ & 120.9049 & $0.00 \mathrm{E}+00$ & $4.89 \mathrm{E}-08$ \\
\hline 1718 & FP & $53-I-121$ & 120.9074 & $0.00 \mathrm{E}+00$ & $9.08 \mathrm{E}-05$ \\
\hline 1719 & FP & 45-Rh-122 & 121.9432 & $0.00 \mathrm{E}+00$ & $6.42 \mathrm{E}+00$ \\
\hline 1720 & FP & 46-Pd-122 & 121.9305 & $0.00 \mathrm{E}+00$ & $3.96 \mathrm{E}+00$ \\
\hline 1721 & FP & $47-A g-122$ & 121.9235 & $0.00 \mathrm{E}+00$ & $1.31 \mathrm{E}+00$ \\
\hline 1722 & FP & $47-\mathrm{Ag}-122 \mathrm{~m}$ & 121.9235 & $0.00 \mathrm{E}+00$ & $3.47 \mathrm{E}+00$ \\
\hline 1723 & FP & $48-\mathrm{Cd}-122$ & 121.9133 & $0.00 \mathrm{E}+00$ & $1.32 \mathrm{E}-01$ \\
\hline 1724 & FP & 49-In-122 & 121.9103 & $0.00 \mathrm{E}+00$ & $4.62 \mathrm{E}-01$ \\
\hline 1725 & FP & 49-In-122m & 121.9103 & $0.00 \mathrm{E}+00$ & $6.73 \mathrm{E}-02$ \\
\hline 1726 & FP & $50-\mathrm{Sn}-122$ & 121.9034 & $0.00 \mathrm{E}+00$ & $0.00 \mathrm{E}+00$ \\
\hline 1727 & FP & 51-Sb-122 & 121.9052 & $0.00 \mathrm{E}+00$ & $2.95 \mathrm{E}-06$ \\
\hline 1728 & FP & $51-\mathrm{Sb}-122 \mathrm{~m}$ & 121.9052 & $0.00 \mathrm{E}+00$ & $2.76 \mathrm{E}-03$ \\
\hline 1729 & FP & $52-\mathrm{Te}-122$ & 121.9030 & $0.00 \mathrm{E}+00$ & $0.00 \mathrm{E}+00$ \\
\hline 1730 & FP & 46-Pd-123 & 122.9349 & $0.00 \mathrm{E}+00$ & $2.84 \mathrm{E}+00$ \\
\hline 1731 & FP & 47-Ag-123 & 122.9249 & $0.00 \mathrm{E}+00$ & $2.31 \mathrm{E}+00$ \\
\hline 1732 & FP & $48-\mathrm{Cd}-123$ & 122.9170 & $0.00 \mathrm{E}+00$ & $3.30 \mathrm{E}-01$ \\
\hline 1733 & FP & $48-\mathrm{Cd}-123 \mathrm{~m}$ & 122.9170 & $0.00 \mathrm{E}+00$ & $3.81 \mathrm{E}-01$ \\
\hline 1734 & FP & 49-In-123 & 122.9104 & $0.00 \mathrm{E}+00$ & $1.12 \mathrm{E}-01$ \\
\hline 1735 & FP & 49-In-123m & 122.9104 & $0.00 \mathrm{E}+00$ & $1.46 \mathrm{E}-02$ \\
\hline 1736 & FP & $50-\mathrm{Sn}-123$ & 122.9057 & $0.00 \mathrm{E}+00$ & $6.21 \mathrm{E}-08$ \\
\hline 1737 & FP & $50-\mathrm{Sn}-123 \mathrm{~m}$ & 122.9057 & $0.00 \mathrm{E}+00$ & $2.88 \mathrm{E}-04$ \\
\hline 1738 & FP & $51-\mathrm{Sb}-123$ & 122.9042 & $0.00 \mathrm{E}+00$ & $0.00 \mathrm{E}+00$ \\
\hline 1739 & FP & $52-\mathrm{Te}-123$ & 122.9043 & $0.00 \mathrm{E}+00$ & $0.00 \mathrm{E}+00$ \\
\hline 1740 & FP & $52-\mathrm{Te}-123 \mathrm{~m}$ & 122.9043 & $0.00 \mathrm{E}+00$ & $6.73 \mathrm{E}-08$ \\
\hline 1741 & FP & $53-I-123$ & 122.9056 & $0.00 \mathrm{E}+00$ & $1.46 \mathrm{E}-05$ \\
\hline 1742 & FP & 46-Pd-124 & 123.9369 & $0.00 \mathrm{E}+00$ & $1.82 \mathrm{E}+01$ \\
\hline 1743 & FP & $47-\mathrm{Ag}-124$ & 123.9286 & $0.00 \mathrm{E}+00$ & $4.03 \mathrm{E}+00$ \\
\hline 1744 & FP & $48-\mathrm{Cd}-124$ & 123.9176 & $0.00 \mathrm{E}+00$ & $5.55 \mathrm{E}-01$ \\
\hline 1745 & FP & 49-In-124 & 123.9132 & $0.00 \mathrm{E}+00$ & $2.22 \mathrm{E}-01$ \\
\hline 1746 & FP & 49-In-124m & 123.9132 & $0.00 \mathrm{E}+00$ & $1.87 \mathrm{E}-01$ \\
\hline 1747 & FP & $50-\mathrm{Sn}-124$ & 123.9053 & $0.00 \mathrm{E}+00$ & $0.00 \mathrm{E}+00$ \\
\hline 1748 & $\mathrm{FP}$ & $51-\mathrm{Sb}-124$ & 123.9059 & $0.00 \mathrm{E}+00$ & $1.33 \mathrm{E}-07$ \\
\hline 1749 & FP & $51-S b-124 m$ & 123.9059 & $0.00 \mathrm{E}+00$ & $7.45 \mathrm{E}-03$ \\
\hline 1750 & FP & $52-\mathrm{Te}-124$ & 123.9028 & $0.00 \mathrm{E}+00$ & $0.00 \mathrm{E}+00$ \\
\hline 1751 & $\mathrm{FP}$ & 53-I-124 & 123.9062 & $0.00 \mathrm{E}+00$ & $1.92 \mathrm{E}-06$ \\
\hline 1752 & FP & $54-\mathrm{Xe}-124$ & 123.9059 & $0.00 \mathrm{E}+00$ & $0.00 \mathrm{E}+00$ \\
\hline 1753 & FP & $47-A g-125$ & 124.9304 & $0.00 \mathrm{E}+00$ & $4.18 \mathrm{E}+00$ \\
\hline 1754 & FP & $48-\mathrm{Cd}-125$ & 124.9212 & $0.00 \mathrm{E}+00$ & $1.02 \mathrm{E}+00$ \\
\hline 1755 & FP & 49-In-125 & 124.9136 & $0.00 \mathrm{E}+00$ & $2.94 \mathrm{E}-01$ \\
\hline 1756 & FP & 49-In-125m & 124.9136 & $0.00 \mathrm{E}+00$ & $5.68 \mathrm{E}-02$ \\
\hline 1757 & FP & $50-\mathrm{Sn}-125$ & 124.9078 & $0.00 \mathrm{E}+00$ & $8.32 \mathrm{E}-07$ \\
\hline 1758 & FP & $50-\mathrm{Sn}-125 \mathrm{~m}$ & 124.9078 & $0.00 \mathrm{E}+00$ & $1.21 \mathrm{E}-03$ \\
\hline 1759 & $\mathrm{FP}$ & 51-Sb-125 & 124.9053 & $0.00 \mathrm{E}+00$ & $7.96 \mathrm{E}-09$ \\
\hline 1760 & $\mathrm{FP}$ & $52-\mathrm{Te}-125$ & 124.9044 & $0.00 \mathrm{E}+00$ & $0.00 \mathrm{E}+00$ \\
\hline 1761 & FP & $52-\mathrm{Te}-125 \mathrm{~m}$ & 124.9044 & $0.00 \mathrm{E}+00$ & $1.40 \mathrm{E}-07$ \\
\hline
\end{tabular}




\begin{tabular}{|c|c|c|c|c|c|}
\hline 1762 & FP & $53-\mathrm{I}-125$ & 124.9046 & $0.00 \mathrm{E}+00$ & $1.35 \mathrm{E}-07$ \\
\hline 1763 & FP & $54-\mathrm{Xe}-125$ & 124.9064 & $0.00 \mathrm{E}+00$ & $1.14 \mathrm{E}-05$ \\
\hline 1764 & FP & $54-\mathrm{Xe}-125 \mathrm{~m}$ & 124.9064 & $0.00 \mathrm{E}+00$ & $1.22 \mathrm{E}-02$ \\
\hline 1765 & FP & $47-A g-126$ & 125.9345 & $0.00 \mathrm{E}+00$ & $6.48 \mathrm{E}+00$ \\
\hline 1766 & FP & $48-\mathrm{Cd}-126$ & 125.9223 & $0.00 \mathrm{E}+00$ & $1.35 \mathrm{E}+00$ \\
\hline 1767 & FP & 49-In-126 & 125.9165 & $0.00 \mathrm{E}+00$ & $4.53 \mathrm{E}-01$ \\
\hline 1768 & FP & $49-\mathrm{In}-126 \mathrm{~m}$ & 125.9165 & $0.00 \mathrm{E}+00$ & $4.23 \mathrm{E}-01$ \\
\hline 1769 & FP & $50-\mathrm{Sn}-126$ & 125.9077 & $0.00 \mathrm{E}+00$ & $9.55 \mathrm{E}-14$ \\
\hline 1770 & FP & 51-Sb-126 & 125.9072 & $0.00 \mathrm{E}+00$ & $6.50 \mathrm{E}-07$ \\
\hline 1771 & FP & 51-Sb-126m & 125.9072 & $0.00 \mathrm{E}+00$ & $6.03 \mathrm{E}-04$ \\
\hline 1772 & FP & $52-\mathrm{Te}-126$ & 125.9033 & $0.00 \mathrm{E}+00$ & $0.00 \mathrm{E}+00$ \\
\hline 1773 & FP & $53-I-126$ & 125.9056 & $0.00 \mathrm{E}+00$ & $6.20 \mathrm{E}-07$ \\
\hline 1774 & FP & $54-\mathrm{Xe}-126$ & 125.9043 & $0.00 \mathrm{E}+00$ & $0.00 \mathrm{E}+00$ \\
\hline 1775 & FP & $47-A g-127$ & 126.9368 & $0.00 \mathrm{E}+00$ & $6.36 \mathrm{E}+00$ \\
\hline 1776 & FP & $48-\mathrm{Cd}-127$ & 126.9264 & $0.00 \mathrm{E}+00$ & $1.87 \mathrm{E}+00$ \\
\hline 1777 & FP & 49-In-127 & 126.9174 & $0.00 \mathrm{E}+00$ & $6.36 \mathrm{E}-01$ \\
\hline 1778 & FP & 49-In-127m & 126.9174 & $0.00 \mathrm{E}+00$ & $1.89 \mathrm{E}-01$ \\
\hline 1779 & FP & $50-\mathrm{Sn}-127$ & 126.9104 & $0.00 \mathrm{E}+00$ & $9.17 \mathrm{E}-05$ \\
\hline 1780 & $\mathrm{FP}$ & $50-\mathrm{Sn}-127 \mathrm{~m}$ & 126.9104 & $0.00 \mathrm{E}+00$ & $2.80 \mathrm{E}-03$ \\
\hline 1781 & FP & 51-Sb-127 & 126.9069 & $0.00 \mathrm{E}+00$ & $2.08 \mathrm{E}-06$ \\
\hline 1782 & FP & 52-Te-127 & 126.9052 & $0.00 \mathrm{E}+00$ & $2.06 \mathrm{E}-05$ \\
\hline 1783 & FP & $52-\mathrm{Te}-127 \mathrm{~m}$ & 126.9052 & $0.00 \mathrm{E}+00$ & $7.36 \mathrm{E}-08$ \\
\hline 1784 & FP & $53-I-127$ & 126.9045 & $0.00 \mathrm{E}+00$ & $0.00 \mathrm{E}+00$ \\
\hline 1785 & $\mathrm{FP}$ & $54-\mathrm{Xe}-127$ & 126.9052 & $0.00 \mathrm{E}+00$ & $2.20 \mathrm{E}-07$ \\
\hline 1786 & FP & $54-\mathrm{Xe}-127 \mathrm{~m}$ & 126.9052 & $0.00 \mathrm{E}+00$ & $1.00 \mathrm{E}-02$ \\
\hline 1787 & FP & 55-Cs-127 & 126.9074 & $0.00 \mathrm{E}+00$ & $3.08 \mathrm{E}-05$ \\
\hline 1788 & FP & $47-A g-128$ & 127.9412 & $0.00 \mathrm{E}+00$ & $1.20 \mathrm{E}+01$ \\
\hline 1789 & FP & $48-\mathrm{Cd}-128$ & 127.9278 & $0.00 \mathrm{E}+00$ & $2.48 \mathrm{E}+00$ \\
\hline 1790 & FP & 49-In-128 & 127.9202 & $0.00 \mathrm{E}+00$ & $8.25 \mathrm{E}-01$ \\
\hline 1791 & FP & 49-In-128m & 127.9202 & $0.00 \mathrm{E}+00$ & 9.63E-01 \\
\hline 1792 & FP & $50-\mathrm{Sn}-128$ & 127.9105 & $0.00 \mathrm{E}+00$ & $1.96 \mathrm{E}-04$ \\
\hline 1793 & FP & $50-\mathrm{Sn}-128 \mathrm{~m}$ & 127.9105 & $0.00 \mathrm{E}+00$ & $1.07 \mathrm{E}-01$ \\
\hline 1794 & FP & 51-Sb-128 & 127.9092 & $0.00 \mathrm{E}+00$ & $2.14 \mathrm{E}-05$ \\
\hline 1795 & FP & 51-Sb-128m & 127.9092 & $0.00 \mathrm{E}+00$ & $1.11 \mathrm{E}-03$ \\
\hline 1796 & FP & 52-Te-128 & 127.9045 & $0.00 \mathrm{E}+00$ & $2.50 \mathrm{E}-27$ \\
\hline 1797 & FP & $53-\mathrm{I}-128$ & 127.9058 & $0.00 \mathrm{E}+00$ & $4.62 \mathrm{E}-04$ \\
\hline 1798 & FP & $54-\mathrm{Xe}-128$ & 127.9035 & $0.00 \mathrm{E}+00$ & $0.00 \mathrm{E}+00$ \\
\hline 1799 & FP & $47-A g-129$ & 128.9437 & $0.00 \mathrm{E}+00$ & $1.51 \mathrm{E}+01$ \\
\hline 1800 & FP & $48-\mathrm{Cd}-129$ & 128.9321 & $0.00 \mathrm{E}+00$ & $2.57 \mathrm{E}+00$ \\
\hline 1801 & FP & 49-In-129 & 128.9217 & $0.00 \mathrm{E}+00$ & $1.14 \mathrm{E}+00$ \\
\hline 1802 & FP & $49-\operatorname{In}-129 \mathrm{~m}$ & 128.9217 & $0.00 \mathrm{E}+00$ & $5.64 \mathrm{E}-01$ \\
\hline 1803 & FP & $50-\mathrm{Sn}-129$ & 128.9135 & $0.00 \mathrm{E}+00$ & $5.18 \mathrm{E}-03$ \\
\hline 1804 & FP & $50-\mathrm{Sn}-129 \mathrm{~m}$ & 128.9135 & $0.00 \mathrm{E}+00$ & $1.67 \mathrm{E}-03$ \\
\hline 1805 & FP & 51-Sb-129 & 128.9091 & $0.00 \mathrm{E}+00$ & $4.38 \mathrm{E}-05$ \\
\hline 1806 & FP & 51-Sb-129m & 128.9091 & $0.00 \mathrm{E}+00$ & $6.53 \mathrm{E}-04$ \\
\hline 1807 & FP & 52-Te-129 & 128.9066 & $0.00 \mathrm{E}+00$ & $1.66 \mathrm{E}-04$ \\
\hline 1808 & FP & $52-\mathrm{Te}-129 \mathrm{~m}$ & 128.9074 & $0.00 \mathrm{E}+00$ & $2.39 \mathrm{E}-07$ \\
\hline 1809 & FP & $53-I-129$ & 128.9050 & $0.00 \mathrm{E}+00$ & $1.40 \mathrm{E}-15$ \\
\hline 1810 & FP & $54-\mathrm{Xe}-129$ & 128.9048 & $0.00 \mathrm{E}+00$ & $0.00 \mathrm{E}+00$ \\
\hline 1811 & FP & $54-\mathrm{Xe}-129 \mathrm{~m}$ & 128.9048 & $0.00 \mathrm{E}+00$ & $9.03 \mathrm{E}-07$ \\
\hline 1812 & FP & 55-Cs-129 & 128.9061 & $0.00 \mathrm{E}+00$ & $6.01 \mathrm{E}-06$ \\
\hline 1813 & FP & 56-Вa-129 & 128.9087 & $0.00 \mathrm{E}+00$ & $8.63 \mathrm{E}-05$ \\
\hline 1814 & FP & 47-Ag-130 & 129.9505 & $0.00 \mathrm{E}+00$ & $1.39 \mathrm{E}+01$ \\
\hline 1815 & FP & $48-\mathrm{Cd}-130$ & 129.9339 & $0.00 \mathrm{E}+00$ & $4.28 \mathrm{E}+00$ \\
\hline 1816 & FP & 49-In-130 & 129.9250 & $0.00 \mathrm{E}+00$ & $2.39 \mathrm{E}+00$ \\
\hline 1817 & FP & 49-In-130m & 129.9250 & $0.00 \mathrm{E}+00$ & $1.28 \mathrm{E}+00$ \\
\hline 1818 & FP & $50-\mathrm{Sn}-130$ & 129.9140 & $0.00 \mathrm{E}+00$ & $3.11 \mathrm{E}-03$ \\
\hline 1819 & FP & $50-\mathrm{Sn}-130 \mathrm{~m}$ & 129.9140 & $0.00 \mathrm{E}+00$ & $6.80 \mathrm{E}-03$ \\
\hline 1820 & FP & $51-\mathrm{Sb}-130$ & 129.9117 & $0.00 \mathrm{E}+00$ & $2.92 \mathrm{E}-04$ \\
\hline 1821 & FP & 51-Sb-130m & 129.9117 & $0.00 \mathrm{E}+00$ & $1.83 \mathrm{E}-03$ \\
\hline 1822 & FP & $52-\mathrm{Te}-130$ & 129.9062 & $0.00 \mathrm{E}+00$ & $0.00 \mathrm{E}+00$ \\
\hline 1823 & FP & $53-\mathrm{I}-130$ & 129.9067 & $0.00 \mathrm{E}+00$ & $1.56 \mathrm{E}-05$ \\
\hline 1824 & FP & $53-\mathrm{I}-130 \mathrm{~m}$ & 129.9067 & $0.00 \mathrm{E}+00$ & $1.31 \mathrm{E}-03$ \\
\hline 1825 & FP & $54-\mathrm{Xe}-130$ & 129.9035 & $0.00 \mathrm{E}+00$ & $0.00 \mathrm{E}+00$ \\
\hline 1826 & FP & $48-\mathrm{Cd}-131$ & 130.9407 & $0.00 \mathrm{E}+00$ & $1.02 \mathrm{E}+01$ \\
\hline 1827 & FP & 49-In-131 & 130.9268 & $0.00 \mathrm{E}+00$ & $2.48 \mathrm{E}+00$ \\
\hline
\end{tabular}




\begin{tabular}{|c|c|c|c|c|c|}
\hline 1828 & FP & 49-In-131m & 130.9268 & $0.00 \mathrm{E}+00$ & $1.98 \mathrm{E}+00$ \\
\hline 1829 & FP & 50-Sn-131 & 130.9170 & $0.00 \mathrm{E}+00$ & $1.24 \mathrm{E}-02$ \\
\hline 1830 & FP & $50-\mathrm{Sn}-131 \mathrm{~m}$ & 130.9170 & $0.00 \mathrm{E}+00$ & $1.19 \mathrm{E}-02$ \\
\hline 1831 & FP & 51-Sb-131 & 130.9120 & $0.00 \mathrm{E}+00$ & $5.02 \mathrm{E}-04$ \\
\hline 1832 & FP & $52-\mathrm{Te}-131$ & 130.9085 & $0.00 \mathrm{E}+00$ & $4.62 \mathrm{E}-04$ \\
\hline 1833 & FP & $52-\mathrm{Te}-131 \mathrm{~m}$ & 130.9085 & $0.00 \mathrm{E}+00$ & $5.79 \mathrm{E}-06$ \\
\hline 1834 & FP & $53-\mathrm{I}-131$ & 130.9061 & $0.00 \mathrm{E}+00$ & $1.00 \mathrm{E}-06$ \\
\hline 1835 & FP & 54-Xe-131 & 130.9051 & $0.00 \mathrm{E}+00$ & $0.00 \mathrm{E}+00$ \\
\hline 1836 & FP & $54-\mathrm{Xe}-131 \mathrm{~m}$ & 130.9051 & $0.00 \mathrm{E}+00$ & $6.78 \mathrm{E}-07$ \\
\hline 1837 & FP & 55-Cs-131 & 130.9055 & $0.00 \mathrm{E}+00$ & $8.28 \mathrm{E}-07$ \\
\hline 1838 & FP & 56-Ba-131 & 130.9069 & $0.00 \mathrm{E}+00$ & $6.98 \mathrm{E}-07$ \\
\hline 1839 & FP & $48-\mathrm{Cd}-132$ & 131.9456 & $0.00 \mathrm{E}+00$ & $7.15 \mathrm{E}+00$ \\
\hline 1840 & FP & 49-In-132 & 131.9330 & $0.00 \mathrm{E}+00$ & $3.35 \mathrm{E}+00$ \\
\hline 1841 & FP & $50-\mathrm{Sn}-132$ & 131.9178 & $0.00 \mathrm{E}+00$ & $1.75 \mathrm{E}-02$ \\
\hline 1842 & FP & 51-Sb-132 & 131.9145 & $0.00 \mathrm{E}+00$ & $4.14 \mathrm{E}-03$ \\
\hline 1843 & FP & $51-S b-132 m$ & 131.9145 & $0.00 \mathrm{E}+00$ & $2.82 \mathrm{E}-03$ \\
\hline 1844 & FP & $52-\mathrm{Te}-132$ & 131.9086 & $0.00 \mathrm{E}+00$ & $2.50 \mathrm{E}-06$ \\
\hline 1845 & FP & 53-I-132 & 131.9080 & $0.00 \mathrm{E}+00$ & $8.39 \mathrm{E}-05$ \\
\hline 1846 & FP & $53-\mathrm{I}-132 \mathrm{~m}$ & 131.9080 & $0.00 \mathrm{E}+00$ & $1.39 \mathrm{E}-04$ \\
\hline 1847 & FP & $54-\mathrm{Xe}-132$ & 131.9041 & $0.00 \mathrm{E}+00$ & $0.00 \mathrm{E}+00$ \\
\hline 1848 & FP & 55-Cs-132 & 131.9064 & $0.00 \mathrm{E}+00$ & $1.24 \mathrm{E}-06$ \\
\hline 1849 & FP & 56-Ba-132 & 131.9051 & $0.00 \mathrm{E}+00$ & $0.00 \mathrm{E}+00$ \\
\hline 1850 & FP & 49-In-133 & 132.9378 & $0.00 \mathrm{E}+00$ & $4.20 \mathrm{E}+00$ \\
\hline 1851 & FP & $50-\mathrm{Sn}-133$ & 132.9238 & $0.00 \mathrm{E}+00$ & $4.75 \mathrm{E}-01$ \\
\hline 1852 & FP & $51-\mathrm{Sb}-133$ & 132.9153 & $0.00 \mathrm{E}+00$ & $4.62 \mathrm{E}-03$ \\
\hline 1853 & FP & $52-\mathrm{Te}-133$ & 132.9110 & $0.00 \mathrm{E}+00$ & $9.24 \mathrm{E}-04$ \\
\hline 1854 & FP & 52-Te-133m & 132.9110 & $0.00 \mathrm{E}+00$ & $2.09 \mathrm{E}-04$ \\
\hline 1855 & FP & $53-I-133$ & 132.9078 & $0.00 \mathrm{E}+00$ & $9.26 \mathrm{E}-06$ \\
\hline 1856 & FP & 53-I-133m & 132.9078 & $0.00 \mathrm{E}+00$ & $7.70 \mathrm{E}-02$ \\
\hline 1857 & FP & 54-Xe-133 & 132.9059 & $0.00 \mathrm{E}+00$ & $1.53 \mathrm{E}-06$ \\
\hline 1858 & FP & $54-\mathrm{Xe}-133 \mathrm{~m}$ & 132.9059 & $0.00 \mathrm{E}+00$ & $3.66 \mathrm{E}-06$ \\
\hline 1859 & FP & 55-Cs-133 & 132.9055 & $0.00 \mathrm{E}+00$ & $0.00 \mathrm{E}+00$ \\
\hline 1860 & FP & 56-Ba-133 & 132.9060 & $0.00 \mathrm{E}+00$ & $2.09 \mathrm{E}-09$ \\
\hline 1861 & FP & 57-La-133 & 132.9082 & $0.00 \mathrm{E}+00$ & $4.92 \mathrm{E}-05$ \\
\hline 1862 & FP & 49-In-134 & 133.9442 & $0.00 \mathrm{E}+00$ & $4.95 \mathrm{E}+00$ \\
\hline 1863 & FP & $50-\mathrm{Sn}-134$ & 133.9283 & $0.00 \mathrm{E}+00$ & $6.60 \mathrm{E}-01$ \\
\hline 1864 & FP & 51-Sb-134 & 133.9204 & $0.00 \mathrm{E}+00$ & 8.89E-01 \\
\hline 1865 & FP & $51-S b-134 m$ & 133.9204 & $0.00 \mathrm{E}+00$ & $6.88 \mathrm{E}-02$ \\
\hline 1866 & FP & $52-\mathrm{Te}-134$ & 133.9114 & $0.00 \mathrm{E}+00$ & $2.76 \mathrm{E}-04$ \\
\hline 1867 & FP & 53-I-134 & 133.9097 & $0.00 \mathrm{E}+00$ & $2.20 \mathrm{E}-04$ \\
\hline 1868 & FP & $53-\mathrm{I}-134 \mathrm{~m}$ & 133.9097 & $0.00 \mathrm{E}+00$ & $3.28 \mathrm{E}-03$ \\
\hline 1869 & FP & 54-Xe-134 & 133.9054 & $0.00 \mathrm{E}+00$ & $0.00 \mathrm{E}+00$ \\
\hline 1870 & FP & 54-Xe-134m & 133.9054 & $0.00 \mathrm{E}+00$ & $2.39 \mathrm{E}+00$ \\
\hline 1871 & FP & 55-Cs-134 & 133.9067 & $0.00 \mathrm{E}+00$ & $1.06 \mathrm{E}-08$ \\
\hline 1872 & FP & 55-Cs- $134 \mathrm{~m}$ & 133.9067 & $0.00 \mathrm{E}+00$ & $6.61 \mathrm{E}-05$ \\
\hline 1873 & FP & 56-Ba-134 & 133.9045 & $0.00 \mathrm{E}+00$ & $0.00 \mathrm{E}+00$ \\
\hline 1874 & FP & 49-In-135 & 134.9493 & $0.00 \mathrm{E}+00$ & $7.53 \mathrm{E}+00$ \\
\hline 1875 & FP & $50-\mathrm{Sn}-135$ & 134.9347 & $0.00 \mathrm{E}+00$ & $1.31 \mathrm{E}+00$ \\
\hline 1876 & FP & 51-Sb-135 & 134.9252 & $0.00 \mathrm{E}+00$ & 4.13E-01 \\
\hline 1877 & FP & 52-Te-135 & 134.9164 & $0.00 \mathrm{E}+00$ & $3.65 \mathrm{E}-02$ \\
\hline 1878 & FP & $53-\mathrm{I}-135$ & 134.9100 & $0.00 \mathrm{E}+00$ & $2.93 \mathrm{E}-05$ \\
\hline 1879 & FP & $54-\mathrm{Xe}-135$ & 134.9072 & $0.00 \mathrm{E}+00$ & $2.11 \mathrm{E}-05$ \\
\hline 1880 & $\mathrm{FP}$ & $54-\mathrm{Xe}-135 \mathrm{~m}$ & 134.9072 & $0.00 \mathrm{E}+00$ & $7.56 \mathrm{E}-04$ \\
\hline 1881 & FP & $55-\mathrm{Cs}-135$ & 134.9060 & $0.00 \mathrm{E}+00$ & $9.55 \mathrm{E}-15$ \\
\hline 1882 & FP & $55-\mathrm{Cs}-135 \mathrm{~m}$ & 134.9060 & $0.00 \mathrm{E}+00$ & $2.18 \mathrm{E}-04$ \\
\hline 1883 & $\mathrm{FP}$ & 56-Ba-135 & 134.9057 & $0.00 \mathrm{E}+00$ & $0.00 \mathrm{E}+00$ \\
\hline 1884 & FP & 56-Ba-135m & 134.9057 & $0.00 \mathrm{E}+00$ & $6.71 \mathrm{E}-06$ \\
\hline 1885 & FP & 57-La-135 & 134.9070 & $0.00 \mathrm{E}+00$ & $9.87 \mathrm{E}-06$ \\
\hline 1886 & FP & $58-\mathrm{Ce}-135$ & 134.9091 & $0.00 \mathrm{E}+00$ & $1.09 \mathrm{E}-05$ \\
\hline 1887 & FP & $50-\mathrm{Sn}-136$ & 135.9393 & $0.00 \mathrm{E}+00$ & $2.77 \mathrm{E}+00$ \\
\hline 1888 & FP & 51-Sb-136 & 135.9303 & $0.00 \mathrm{E}+00$ & $7.51 \mathrm{E}-01$ \\
\hline 1889 & FP & $52-\mathrm{Te}-136$ & 135.9201 & $0.00 \mathrm{E}+00$ & $3.96 \mathrm{E}-02$ \\
\hline 1890 & FP & $53-I-136$ & 135.9147 & $0.00 \mathrm{E}+00$ & $8.31 \mathrm{E}-03$ \\
\hline 1891 & $\mathrm{FP}$ & 53-I-136m & 135.9147 & $0.00 \mathrm{E}+00$ & $1.48 \mathrm{E}-02$ \\
\hline 1892 & $\mathrm{FP}$ & 54-Xe-136 & 135.9072 & $0.00 \mathrm{E}+00$ & $0.00 \mathrm{E}+00$ \\
\hline 1893 & FP & 55-Cs-136 & 135.9073 & $0.00 \mathrm{E}+00$ & $6.10 \mathrm{E}-07$ \\
\hline
\end{tabular}




\begin{tabular}{|c|c|c|c|c|c|}
\hline 1894 & FP & 55-Cs-136m & 135.9073 & $0.00 \mathrm{E}+00$ & $3.65 \mathrm{E}-02$ \\
\hline 1895 & FP & 56-Ba-136 & 135.9046 & $0.00 \mathrm{E}+00$ & $0.00 \mathrm{E}+00$ \\
\hline 1896 & FP & 56-Ba-136m & 135.9046 & $0.00 \mathrm{E}+00$ & $2.25 \mathrm{E}+00$ \\
\hline 1897 & FP & $50-\mathrm{Sn}-137$ & 136.9460 & $0.00 \mathrm{E}+00$ & $3.65 \mathrm{E}+00$ \\
\hline 1898 & FP & 51-Sb-137 & 136.9353 & $0.00 \mathrm{E}+00$ & $1.54 \mathrm{E}+00$ \\
\hline 1899 & FP & 52-Te-137 & 136.9253 & $0.00 \mathrm{E}+00$ & $2.78 \mathrm{E}-01$ \\
\hline 1900 & FP & $53-\mathrm{I}-137$ & 136.9179 & $0.00 \mathrm{E}+00$ & $2.83 \mathrm{E}-02$ \\
\hline 1901 & FP & 54-Xe-137 & 136.9116 & $0.00 \mathrm{E}+00$ & $3.03 \mathrm{E}-03$ \\
\hline 1902 & FP & 55-Cs-137 & 136.9071 & $0.00 \mathrm{E}+00$ & $7.30 \mathrm{E}-10$ \\
\hline 1903 & FP & 56-Ba-137 & 136.9058 & $0.00 \mathrm{E}+00$ & $0.00 \mathrm{E}+00$ \\
\hline 1904 & FP & 56-Ba-137m & 136.9058 & $0.00 \mathrm{E}+00$ & $4.53 \mathrm{E}-03$ \\
\hline 1905 & FP & 57-La-137 & 136.9065 & $0.00 \mathrm{E}+00$ & $3.66 \mathrm{E}-13$ \\
\hline 1906 & FP & 58-Ce-137 & 136.9078 & $0.00 \mathrm{E}+00$ & $2.14 \mathrm{E}-05$ \\
\hline 1907 & FP & 51-Sb-138 & 137.9408 & $0.00 \mathrm{E}+00$ & $4.13 \mathrm{E}+00$ \\
\hline 1908 & FP & 52-Te-138 & 137.9292 & $0.00 \mathrm{E}+00$ & 4.95E-01 \\
\hline 1909 & FP & $53-I-138$ & 137.9223 & $0.00 \mathrm{E}+00$ & $1.11 \mathrm{E}-01$ \\
\hline 1910 & FP & $54-\mathrm{Xe}-138$ & 137.9140 & $0.00 \mathrm{E}+00$ & $8.20 \mathrm{E}-04$ \\
\hline 1911 & FP & $55-\mathrm{Cs}-138$ & 137.9110 & $0.00 \mathrm{E}+00$ & $3.46 \mathrm{E}-04$ \\
\hline 1912 & $\mathrm{FP}$ & $55-\mathrm{Cs}-138 \mathrm{~m}$ & 137.9110 & $0.00 \mathrm{E}+00$ & $3.97 \mathrm{E}-03$ \\
\hline 1913 & FP & 56-Ba-138 & 137.9052 & $0.00 \mathrm{E}+00$ & $0.00 \mathrm{E}+00$ \\
\hline 1914 & FP & 57-La-138 & 137.9071 & $0.00 \mathrm{E}+00$ & $2.15 \mathrm{E}-19$ \\
\hline 1915 & FP & $58-\mathrm{Ce}-138$ & 137.9060 & $0.00 \mathrm{E}+00$ & $0.00 \mathrm{E}+00$ \\
\hline 1916 & FP & 51-Sb-139 & 138.9460 & $0.00 \mathrm{E}+00$ & $5.46 \mathrm{E}+00$ \\
\hline 1917 & $\mathrm{FP}$ & 52-Te-139 & 138.9347 & $0.00 \mathrm{E}+00$ & $2.00 \mathrm{E}+00$ \\
\hline 1918 & FP & $53-I-139$ & 138.9261 & $0.00 \mathrm{E}+00$ & $3.04 \mathrm{E}-01$ \\
\hline 1919 & FP & $54-\mathrm{Xe}-139$ & 138.9188 & $0.00 \mathrm{E}+00$ & $1.75 \mathrm{E}-02$ \\
\hline 1920 & FP & 55-Cs-139 & 138.9134 & $0.00 \mathrm{E}+00$ & $1.25 \mathrm{E}-03$ \\
\hline 1921 & FP & 56-Ba-139 & 138.9088 & $0.00 \mathrm{E}+00$ & $1.39 \mathrm{E}-04$ \\
\hline 1922 & FP & 57-La-139 & 138.9064 & $0.00 \mathrm{E}+00$ & $0.00 \mathrm{E}+00$ \\
\hline 1923 & FP & 58-Ce-139 & 138.9066 & $0.00 \mathrm{E}+00$ & 5.83E-08 \\
\hline 1924 & FP & $58-\mathrm{Ce}-139 \mathrm{~m}$ & 138.9066 & $0.00 \mathrm{E}+00$ & $1.26 \mathrm{E}-02$ \\
\hline 1925 & FP & 59-Pr-139 & 138.9089 & $0.00 \mathrm{E}+00$ & $4.37 \mathrm{E}-05$ \\
\hline 1926 & FP & $52-\mathrm{Te}-140$ & 139.9388 & $0.00 \mathrm{E}+00$ & $2.28 \mathrm{E}+00$ \\
\hline 1927 & FP & $53-\mathrm{I}-140$ & 139.9310 & $0.00 \mathrm{E}+00$ & $8.06 \mathrm{E}-01$ \\
\hline 1928 & FP & $54-\mathrm{Xe}-140$ & 139.9216 & $0.00 \mathrm{E}+00$ & $5.10 \mathrm{E}-02$ \\
\hline 1929 & FP & $55-\mathrm{Cs}-140$ & 139.9173 & $0.00 \mathrm{E}+00$ & $1.09 \mathrm{E}-02$ \\
\hline 1930 & FP & 56-Ba-140 & 139.9106 & $0.00 \mathrm{E}+00$ & $6.29 \mathrm{E}-07$ \\
\hline 1931 & FP & 57-La-140 & 139.9095 & $0.00 \mathrm{E}+00$ & $4.78 \mathrm{E}-06$ \\
\hline 1932 & FP & 58-Ce-140 & 139.9054 & $0.00 \mathrm{E}+00$ & $0.00 \mathrm{E}+00$ \\
\hline 1933 & FP & 59-Pr-140 & 139.9091 & $0.00 \mathrm{E}+00$ & $3.41 \mathrm{E}-03$ \\
\hline 1934 & FP & $60-\mathrm{Nd}-140$ & 139.9095 & $0.00 \mathrm{E}+00$ & $2.38 \mathrm{E}-06$ \\
\hline 1935 & FP & $52-\mathrm{Te}-141$ & 140.9447 & $0.00 \mathrm{E}+00$ & $3.25 \mathrm{E}+00$ \\
\hline 1936 & FP & 53-I-141 & 140.9350 & $0.00 \mathrm{E}+00$ & $1.61 \mathrm{E}+00$ \\
\hline 1937 & FP & $54-\mathrm{Xe}-141$ & 140.9267 & $0.00 \mathrm{E}+00$ & $4.01 \mathrm{E}-01$ \\
\hline 1938 & FP & 55-Cs-141 & 140.9200 & $0.00 \mathrm{E}+00$ & $2.79 \mathrm{E}-02$ \\
\hline 1939 & FP & 56-Ba-141 & 140.9144 & $0.00 \mathrm{E}+00$ & $6.32 \mathrm{E}-04$ \\
\hline 1940 & FP & 57-La-141 & 140.9110 & $0.00 \mathrm{E}+00$ & $4.91 \mathrm{E}-05$ \\
\hline 1941 & FP & 58-Ce-141 & 140.9083 & $0.00 \mathrm{E}+00$ & $2.47 \mathrm{E}-07$ \\
\hline 1942 & FP & 59-Pr-141 & 140.9077 & $0.00 \mathrm{E}+00$ & $0.00 \mathrm{E}+00$ \\
\hline 1943 & FP & $60-\mathrm{Nd}-141$ & 140.9096 & $0.00 \mathrm{E}+00$ & $7.73 \mathrm{E}-05$ \\
\hline 1944 & FP & $60-\mathrm{Nd}-141 \mathrm{~m}$ & 140.9096 & $0.00 \mathrm{E}+00$ & $1.12 \mathrm{E}-02$ \\
\hline 1945 & FP & 61-Pm-141 & 140.9136 & $0.00 \mathrm{E}+00$ & $5.53 \mathrm{E}-04$ \\
\hline 1946 & FP & $52-\mathrm{Te}-142$ & 141.9491 & $0.00 \mathrm{E}+00$ & $3.47 \mathrm{E}+00$ \\
\hline 1947 & FP & $53-I-142$ & 141.9402 & $0.00 \mathrm{E}+00$ & $3.12 \mathrm{E}+00$ \\
\hline 1948 & FP & $54-\mathrm{Xe}-142$ & 141.9297 & $0.00 \mathrm{E}+00$ & $5.64 \mathrm{E}-01$ \\
\hline 1949 & FP & 55-Cs-142 & 141.9243 & $0.00 \mathrm{E}+00$ & $4.12 \mathrm{E}-01$ \\
\hline 1950 & FP & 56-Ba-142 & 141.9164 & $0.00 \mathrm{E}+00$ & $1.09 \mathrm{E}-03$ \\
\hline 1951 & FP & 57-La-142 & 141.9141 & $0.00 \mathrm{E}+00$ & $1.27 \mathrm{E}-04$ \\
\hline 1952 & FP & $58-\mathrm{Ce}-142$ & 141.9092 & $0.00 \mathrm{E}+00$ & $0.00 \mathrm{E}+00$ \\
\hline 1953 & FP & 59-Pr-142 & 141.9100 & $0.00 \mathrm{E}+00$ & $1.01 \mathrm{E}-05$ \\
\hline 1954 & FP & 59-Pr-142m & 141.9100 & $0.00 \mathrm{E}+00$ & $7.91 \mathrm{E}-04$ \\
\hline 1955 & FP & $60-N d-142$ & 141.9077 & $0.00 \mathrm{E}+00$ & $0.00 \mathrm{E}+00$ \\
\hline 1956 & FP & $53-I-143$ & 142.9446 & $0.00 \mathrm{E}+00$ & $2.34 \mathrm{E}+00$ \\
\hline 1957 & FP & $54-\mathrm{Xe}-143$ & 142.9351 & $0.00 \mathrm{E}+00$ & $2.31 \mathrm{E}+00$ \\
\hline 1958 & FP & $55-\mathrm{Cs}-143$ & 142.9274 & $0.00 \mathrm{E}+00$ & $3.87 \mathrm{E}-01$ \\
\hline 1959 & FP & 56-Ba-143 & 142.9206 & $0.00 \mathrm{E}+00$ & $4.78 \mathrm{E}-02$ \\
\hline
\end{tabular}




\begin{tabular}{|c|c|c|c|c|c|}
\hline 1960 & FP & 57-La-143 & 142.9161 & $0.00 \mathrm{E}+00$ & $8.14 \mathrm{E}-04$ \\
\hline 1961 & FP & $58-\mathrm{Ce}-143$ & 142.9124 & $0.00 \mathrm{E}+00$ & $5.83 \mathrm{E}-06$ \\
\hline 1962 & $\mathrm{FP}$ & 59-Pr-143 & 142.9108 & $0.00 \mathrm{E}+00$ & 5.91E-07 \\
\hline 1963 & FP & $60-\mathrm{Nd}-143$ & 142.9098 & $0.00 \mathrm{E}+00$ & $0.00 \mathrm{E}+00$ \\
\hline 1964 & FP & 61-Pm-143 & 142.9109 & $0.00 \mathrm{E}+00$ & 3.03E-08 \\
\hline 1965 & $\mathrm{FP}$ & 62-Sm-143 & 142.9146 & $0.00 \mathrm{E}+00$ & $1.32 \mathrm{E}-03$ \\
\hline 1966 & $\mathrm{FP}$ & 62-Sm-143m & 142.9146 & $0.00 \mathrm{E}+00$ & $1.05 \mathrm{E}-02$ \\
\hline 1967 & $\mathrm{FP}$ & $53-I-144$ & 143.9500 & $0.00 \mathrm{E}+00$ & $3.57 \mathrm{E}+00$ \\
\hline 1968 & $\mathrm{FP}$ & 54-Xe-144 & 143.9385 & $0.00 \mathrm{E}+00$ & $6.03 \mathrm{E}-01$ \\
\hline 1969 & $\mathrm{FP}$ & 55-Cs-144 & 143.9321 & $0.00 \mathrm{E}+00$ & $6.97 \mathrm{E}-01$ \\
\hline 1970 & FP & 56-Ba-144 & 143.9229 & $0.00 \mathrm{E}+00$ & $6.03 \mathrm{E}-02$ \\
\hline 1971 & $\mathrm{FP}$ & 57-La-144 & 143.9196 & $0.00 \mathrm{E}+00$ & $1.70 \mathrm{E}-02$ \\
\hline 1972 & FP & 58-Ce-144 & 143.9137 & $0.00 \mathrm{E}+00$ & $2.82 \mathrm{E}-08$ \\
\hline 1973 & $\mathrm{FP}$ & 59-Pr-144 & 143.9133 & $0.00 \mathrm{E}+00$ & $6.69 \mathrm{E}-04$ \\
\hline 1974 & FP & 59-Pr-144m & 143.9133 & $0.00 \mathrm{E}+00$ & $1.60 \mathrm{E}-03$ \\
\hline 1975 & $\mathrm{FP}$ & $60-\mathrm{Nd}-144$ & 143.9101 & $0.00 \mathrm{E}+00$ & $9.59 \mathrm{E}-24$ \\
\hline 1976 & $\mathrm{FP}$ & 61-Pm-144 & 143.9126 & $0.00 \mathrm{E}+00$ & $2.21 \mathrm{E}-08$ \\
\hline 1977 & $\mathrm{FP}$ & 62-Sm-144 & 143.9120 & $0.00 \mathrm{E}+00$ & $0.00 \mathrm{E}+00$ \\
\hline 1978 & $\mathrm{FP}$ & $54-\mathrm{Xe}-145$ & 144.9441 & $0.00 \mathrm{E}+00$ & $3.69 \mathrm{E}+00$ \\
\hline 1979 & $\mathrm{FP}$ & 55-Cs-145 & 144.9355 & $0.00 \mathrm{E}+00$ & $1.18 \mathrm{E}+00$ \\
\hline 1980 & $\mathrm{FP}$ & 56-Ba-145 & 144.9276 & $0.00 \mathrm{E}+00$ & $1.61 \mathrm{E}-01$ \\
\hline 1981 & $\mathrm{FP}$ & 57-La-145 & 144.9216 & $0.00 \mathrm{E}+00$ & $2.80 \mathrm{E}-02$ \\
\hline 1982 & $\mathrm{FP}$ & 58-Ce-145 & 144.9172 & $0.00 \mathrm{E}+00$ & $3.84 \mathrm{E}-03$ \\
\hline 1983 & FP & 59-Pr-145 & 144.9145 & $0.00 \mathrm{E}+00$ & $3.22 \mathrm{E}-05$ \\
\hline 1984 & $\mathrm{FP}$ & $60-\mathrm{Nd}-145$ & 144.9126 & $0.00 \mathrm{E}+00$ & $0.00 \mathrm{E}+00$ \\
\hline 1985 & FP & 61-Pm-145 & 144.9128 & $0.00 \mathrm{E}+00$ & $1.24 \mathrm{E}-09$ \\
\hline 1986 & $\mathrm{FP}$ & 62-Sm-145 & 144.9134 & $0.00 \mathrm{E}+00$ & $2.36 \mathrm{E}-08$ \\
\hline 1987 & $\mathrm{FP}$ & $54-\mathrm{Xe}-146$ & 145.9478 & $0.00 \mathrm{E}+00$ & $1.88 \mathrm{E}+00$ \\
\hline 1988 & $\mathrm{FP}$ & $55-\mathrm{Cs}-146$ & 145.9403 & $0.00 \mathrm{E}+00$ & $2.16 \mathrm{E}+00$ \\
\hline 1989 & FP & 56-Ba-146 & 145.9302 & $0.00 \mathrm{E}+00$ & $3.12 \mathrm{E}-01$ \\
\hline 1990 & FP & 57-La-146 & 145.9258 & $0.00 \mathrm{E}+00$ & $1.11 \mathrm{E}-01$ \\
\hline 1991 & $\mathrm{FP}$ & 57-La-146m & 145.9258 & $0.00 \mathrm{E}+00$ & $6.93 \mathrm{E}-02$ \\
\hline 1992 & $\mathrm{FP}$ & $58-\mathrm{Ce}-146$ & 145.9188 & $0.00 \mathrm{E}+00$ & $8.54 \mathrm{E}-04$ \\
\hline 1993 & FP & 59-Pr-146 & 145.9176 & $0.00 \mathrm{E}+00$ & $4.78 \mathrm{E}-04$ \\
\hline 1994 & FP & $60-\mathrm{Nd}-146$ & 145.9131 & $0.00 \mathrm{E}+00$ & $0.00 \mathrm{E}+00$ \\
\hline 1995 & $\mathrm{FP}$ & 61-Pm-146 & 145.9147 & $0.00 \mathrm{E}+00$ & $3.97 \mathrm{E}-09$ \\
\hline 1996 & $\mathrm{FP}$ & 62-Sm-146 & 145.9130 & $0.00 \mathrm{E}+00$ & $2.13 \mathrm{E}-16$ \\
\hline 1997 & $\mathrm{FP}$ & $54-\mathrm{Xe}-147$ & 146.9536 & $0.00 \mathrm{E}+00$ & $6.93 \mathrm{E}+00$ \\
\hline 1998 & FP & 55-Cs-147 & 146.9442 & $0.00 \mathrm{E}+00$ & $3.01 \mathrm{E}+00$ \\
\hline 1999 & $\mathrm{FP}$ & 56-Ba-147 & 146.9350 & $0.00 \mathrm{E}+00$ & $7.75 \mathrm{E}-01$ \\
\hline 2000 & $\mathrm{FP}$ & 57-La-147 & 146.9282 & $0.00 \mathrm{E}+00$ & $1.71 \mathrm{E}-01$ \\
\hline 2001 & $\mathrm{FP}$ & $58-\mathrm{Ce}-147$ & 146.9227 & $0.00 \mathrm{E}+00$ & $1.23 \mathrm{E}-02$ \\
\hline 2002 & $\mathrm{FP}$ & 59-Pr-147 & 146.9190 & $0.00 \mathrm{E}+00$ & $8.62 \mathrm{E}-04$ \\
\hline 2003 & FP & $60-\mathrm{Nd}-147$ & 146.9161 & $0.00 \mathrm{E}+00$ & $7.31 \mathrm{E}-07$ \\
\hline 2004 & FP & 61-Pm-147 & 146.9151 & $0.00 \mathrm{E}+00$ & 8.37E-09 \\
\hline 2005 & $\mathrm{FP}$ & 62-Sm-147 & 146.9149 & $0.00 \mathrm{E}+00$ & $2.07 \mathrm{E}-19$ \\
\hline 2006 & $\mathrm{FP}$ & 63-Eu-147 & 146.9167 & $0.00 \mathrm{E}+00$ & $3.33 \mathrm{E}-07$ \\
\hline 2007 & FP & 64-Gd-147 & 146.9191 & $0.00 \mathrm{E}+00$ & $5.06 \mathrm{E}-06$ \\
\hline 2008 & FP & 55-Cs-148 & 147.9492 & $0.00 \mathrm{E}+00$ & $4.75 \mathrm{E}+00$ \\
\hline 2009 & FP & 56-Ba-148 & 147.9377 & $0.00 \mathrm{E}+00$ & $1.13 \mathrm{E}+00$ \\
\hline 2010 & $\mathrm{FP}$ & 57-La-148 & 147.9322 & $0.00 \mathrm{E}+00$ & $5.50 \mathrm{E}-01$ \\
\hline 2011 & $\mathrm{FP}$ & 58-Ce-148 & 147.9244 & $0.00 \mathrm{E}+00$ & $1.24 \mathrm{E}-02$ \\
\hline 2012 & $\mathrm{FP}$ & 59-Pr-148 & 147.9221 & $0.00 \mathrm{E}+00$ & $5.04 \mathrm{E}-03$ \\
\hline 2013 & $\mathrm{FP}$ & 59-Pr-148m & 147.9221 & $0.00 \mathrm{E}+00$ & $5.75 \mathrm{E}-03$ \\
\hline 2014 & $\mathrm{FP}$ & $60-\mathrm{Nd}-148$ & 147.9169 & $0.00 \mathrm{E}+00$ & $0.00 \mathrm{E}+00$ \\
\hline 2015 & $\mathrm{FP}$ & 61-Pm-148 & 147.9175 & $0.00 \mathrm{E}+00$ & $1.49 \mathrm{E}-06$ \\
\hline 2016 & $\mathrm{FP}$ & 61-Pm-148m & 147.9207 & $0.00 \mathrm{E}+00$ & $1.94 \mathrm{E}-07$ \\
\hline 2017 & $\mathrm{FP}$ & 62-Sm-148 & 147.9148 & $0.00 \mathrm{E}+00$ & $3.14 \mathrm{E}-24$ \\
\hline 2018 & FP & 55-Cs-149 & 148.9529 & $0.00 \mathrm{E}+00$ & $1.39 \mathrm{E}+01$ \\
\hline 2019 & FP & 56-Ba-149 & 148.9426 & $0.00 \mathrm{E}+00$ & $2.02 \mathrm{E}+00$ \\
\hline 2020 & FP & 57-La-149 & 148.9347 & $0.00 \mathrm{E}+00$ & $6.60 \mathrm{E}-01$ \\
\hline 2021 & $\mathrm{FP}$ & 58-Ce-149 & 148.9284 & $0.00 \mathrm{E}+00$ & $1.31 \mathrm{E}-01$ \\
\hline 2022 & $\mathrm{FP}$ & 59-Pr-149 & 148.9237 & $0.00 \mathrm{E}+00$ & $5.11 \mathrm{E}-03$ \\
\hline 2023 & FP & $60-\mathrm{Nd}-149$ & 148.9202 & $0.00 \mathrm{E}+00$ & $1.11 \mathrm{E}-04$ \\
\hline 2024 & $\mathrm{FP}$ & 61-Pm-149 & 148.9183 & $0.00 \mathrm{E}+00$ & $3.63 \mathrm{E}-06$ \\
\hline 2025 & $\mathrm{FP}$ & 62-Sm-149 & 148.9172 & $0.00 \mathrm{E}+00$ & $0.00 \mathrm{E}+00$ \\
\hline
\end{tabular}




\begin{tabular}{|c|c|c|c|c|c|}
\hline 2026 & FP & 63-Eu-149 & 148.9179 & $0.00 \mathrm{E}+00$ & $8.62 \mathrm{E}-08$ \\
\hline 2027 & FP & 64-Gd-149 & 148.9193 & $0.00 \mathrm{E}+00$ & $8.65 \mathrm{E}-07$ \\
\hline 2028 & FP & $55-C s-150$ & 149.9582 & $0.00 \mathrm{E}+00$ & $1.39 \mathrm{E}+01$ \\
\hline 2029 & FP & $56-\mathrm{Ba}-150$ & 149.9457 & $0.00 \mathrm{E}+00$ & $2.31 \mathrm{E}+00$ \\
\hline 2030 & $\mathrm{FP}$ & 57-La-150 & 149.9388 & $0.00 \mathrm{E}+00$ & $8.06 \mathrm{E}-01$ \\
\hline 2031 & FP & $58-\mathrm{Ce}-150$ & 149.9304 & $0.00 \mathrm{E}+00$ & $1.73 \mathrm{E}-01$ \\
\hline 2032 & $\mathrm{FP}$ & 59-Pr-150 & 149.9267 & $0.00 \mathrm{E}+00$ & $1.12 \mathrm{E}-01$ \\
\hline 2033 & FP & $60-\mathrm{Nd}-150$ & 149.9209 & $0.00 \mathrm{E}+00$ & $2.78 \mathrm{E}-27$ \\
\hline 2034 & $\mathrm{FP}$ & 61-Pm-150 & 149.9210 & $0.00 \mathrm{E}+00$ & $7.18 \mathrm{E}-05$ \\
\hline 2035 & FP & $62-\mathrm{Sm}-150$ & 149.9173 & $0.00 \mathrm{E}+00$ & $0.00 \mathrm{E}+00$ \\
\hline 2036 & $\mathrm{FP}$ & 55-Cs-151 & 150.9622 & $0.00 \mathrm{E}+00$ & $1.39 \mathrm{E}+01$ \\
\hline 2037 & FP & 56-Ba-151 & 150.9508 & $0.00 \mathrm{E}+00$ & $2.68 \mathrm{E}+00$ \\
\hline 2038 & FP & 57-La-151 & 150.9417 & $0.00 \mathrm{E}+00$ & $8.91 \mathrm{E}-01$ \\
\hline 2039 & FP & $58-\mathrm{Ce}-151$ & 150.9340 & $0.00 \mathrm{E}+00$ & $3.94 \mathrm{E}-01$ \\
\hline 2040 & FP & 59-Pr-151 & 150.9283 & $0.00 \mathrm{E}+00$ & $3.67 \mathrm{E}-02$ \\
\hline 2041 & FP & $60-\mathrm{Nd}-151$ & 150.9238 & $0.00 \mathrm{E}+00$ & $9.29 \mathrm{E}-04$ \\
\hline 2042 & FP & 61-Pm-151 & 150.9212 & $0.00 \mathrm{E}+00$ & $6.78 \mathrm{E}-06$ \\
\hline 2043 & $\mathrm{FP}$ & 62-Sm-151 & 150.9199 & $0.00 \mathrm{E}+00$ & $2.44 \mathrm{E}-10$ \\
\hline 2044 & $\mathrm{FP}$ & 63-Eu-151 & 150.9198 & $0.00 \mathrm{E}+00$ & $0.00 \mathrm{E}+00$ \\
\hline 2045 & FP & 64-Gd-151 & 150.9203 & $0.00 \mathrm{E}+00$ & $6.47 \mathrm{E}-08$ \\
\hline 2046 & FP & $65-\mathrm{Tb}-151$ & 150.9231 & $0.00 \mathrm{E}+00$ & $1.09 \mathrm{E}-05$ \\
\hline 2047 & $\mathrm{FP}$ & 56-Ba-152 & 151.9543 & $0.00 \mathrm{E}+00$ & $3.04 \mathrm{E}+00$ \\
\hline 2048 & $\mathrm{FP}$ & 57-La-152 & 151.9462 & $0.00 \mathrm{E}+00$ & $1.54 \mathrm{E}+00$ \\
\hline 2049 & FP & 58-Ce-152 & 151.9365 & $0.00 \mathrm{E}+00$ & $4.95 \mathrm{E}-01$ \\
\hline 2050 & FP & 59-Pr-152 & 151.9315 & $0.00 \mathrm{E}+00$ & $1.91 \mathrm{E}-01$ \\
\hline 2051 & $\mathrm{FP}$ & $60-\mathrm{Nd}-152$ & 151.9247 & $0.00 \mathrm{E}+00$ & $1.01 \mathrm{E}-03$ \\
\hline 2052 & FP & 61-Pm-152 & 151.9235 & $0.00 \mathrm{E}+00$ & $2.80 \mathrm{E}-03$ \\
\hline 2053 & FP & 61-Pm-152m & 151.9235 & $0.00 \mathrm{E}+00$ & $1.54 \mathrm{E}-03$ \\
\hline 2054 & FP & 62-Sm-152 & 151.9197 & $0.00 \mathrm{E}+00$ & $0.00 \mathrm{E}+00$ \\
\hline 2055 & $\mathrm{FP}$ & 63-Eu-152 & 151.9217 & $0.00 \mathrm{E}+00$ & $1.62 \mathrm{E}-09$ \\
\hline 2056 & $\mathrm{FP}$ & 63-Eu-152m & 151.9217 & $0.00 \mathrm{E}+00$ & $2.07 \mathrm{E}-05$ \\
\hline 2057 & FP & 64-Gd-152 & 151.9198 & $0.00 \mathrm{E}+00$ & $2.03 \mathrm{E}-22$ \\
\hline 2058 & $\mathrm{FP}$ & 56-Ba-153 & 152.9596 & $0.00 \mathrm{E}+00$ & $4.39 \mathrm{E}+00$ \\
\hline 2059 & FP & 57-La-153 & 152.9496 & $0.00 \mathrm{E}+00$ & $2.03 \mathrm{E}+00$ \\
\hline 2060 & FP & $58-\mathrm{Ce}-153$ & 152.9406 & $0.00 \mathrm{E}+00$ & $7.08 \mathrm{E}-01$ \\
\hline 2061 & FP & 59-Pr-153 & 152.9338 & $0.00 \mathrm{E}+00$ & $1.62 \mathrm{E}-01$ \\
\hline 2062 & FP & $60-\mathrm{Nd}-153$ & 152.9277 & $0.00 \mathrm{E}+00$ & $2.19 \mathrm{E}-02$ \\
\hline 2063 & FP & 61-Pm-153 & 152.9241 & $0.00 \mathrm{E}+00$ & $2.20 \mathrm{E}-03$ \\
\hline 2064 & $\mathrm{FP}$ & 62-Sm-153 & 152.9221 & $0.00 \mathrm{E}+00$ & $4.14 \mathrm{E}-06$ \\
\hline 2065 & $\mathrm{FP}$ & 63-Eu-153 & 152.9212 & $0.00 \mathrm{E}+00$ & $0.00 \mathrm{E}+00$ \\
\hline 2066 & FP & 64-Gd-153 & 152.9218 & $0.00 \mathrm{E}+00$ & $3.34 \mathrm{E}-08$ \\
\hline 2067 & FP & $65-T b-153$ & 152.9234 & $0.00 \mathrm{E}+00$ & $3.43 \mathrm{E}-06$ \\
\hline 2068 & FP & 57-La-154 & 153.9545 & $0.00 \mathrm{E}+00$ & $3.04 \mathrm{E}+00$ \\
\hline 2069 & FP & $58-\mathrm{Ce}-154$ & 153.9434 & $0.00 \mathrm{E}+00$ & $8.94 \mathrm{E}-01$ \\
\hline 2070 & FP & 59-Pr-154 & 153.9375 & $0.00 \mathrm{E}+00$ & $3.01 \mathrm{E}-01$ \\
\hline 2071 & FP & $60-\mathrm{Nd}-154$ & 153.9295 & $0.00 \mathrm{E}+00$ & $2.68 \mathrm{E}-02$ \\
\hline 2072 & $\mathrm{FP}$ & 61-Pm-154 & 153.9265 & $0.00 \mathrm{E}+00$ & $6.68 \mathrm{E}-03$ \\
\hline 2073 & FP & 61-Pm-154m & 153.9265 & $0.00 \mathrm{E}+00$ & $4.31 \mathrm{E}-03$ \\
\hline 2074 & FP & 62-Sm-154 & 153.9222 & $0.00 \mathrm{E}+00$ & $0.00 \mathrm{E}+00$ \\
\hline 2075 & FP & 63-Eu-154 & 153.9230 & $0.00 \mathrm{E}+00$ & $2.55 \mathrm{E}-09$ \\
\hline 2076 & $\mathrm{FP}$ & 63-Eu-154m & 153.9230 & $0.00 \mathrm{E}+00$ & $2.51 \mathrm{E}-04$ \\
\hline 2077 & FP & 64-Gd-154 & 153.9209 & $0.00 \mathrm{E}+00$ & $0.00 \mathrm{E}+00$ \\
\hline 2078 & $\mathrm{FP}$ & 57-La-155 & 154.9583 & $0.00 \mathrm{E}+00$ & $3.77 \mathrm{E}+00$ \\
\hline 2079 & FP & $58-\mathrm{Ce}-155$ & 154.9480 & $0.00 \mathrm{E}+00$ & $1.47 \mathrm{E}+00$ \\
\hline 2080 & $\mathrm{FP}$ & 59-Pr-155 & 154.9401 & $0.00 \mathrm{E}+00$ & $8.14 \mathrm{E}-01$ \\
\hline 2081 & FP & $60-\mathrm{Nd}-155$ & 154.9329 & $0.00 \mathrm{E}+00$ & $7.79 \mathrm{E}-02$ \\
\hline 2082 & FP & 61-Pm-155 & 154.9281 & $0.00 \mathrm{E}+00$ & $1.67 \mathrm{E}-02$ \\
\hline 2083 & FP & 62-Sm-155 & 154.9246 & $0.00 \mathrm{E}+00$ & $5.18 \mathrm{E}-04$ \\
\hline 2084 & FP & 63-Eu-155 & 154.9229 & $0.00 \mathrm{E}+00$ & $4.62 \mathrm{E}-09$ \\
\hline 2085 & $\mathrm{FP}$ & 64-Gd-155 & 154.9226 & $0.00 \mathrm{E}+00$ & $0.00 \mathrm{E}+00$ \\
\hline 2086 & $\mathrm{FP}$ & 64-Gd-155m & 154.9226 & $0.00 \mathrm{E}+00$ & $2.17 \mathrm{E}+01$ \\
\hline 2087 & FP & 65-Tb-155 & 154.9235 & $0.00 \mathrm{E}+00$ & $1.51 \mathrm{E}-06$ \\
\hline 2088 & FP & 66-Dy-155 & 154.9258 & $0.00 \mathrm{E}+00$ & $1.94 \mathrm{E}-05$ \\
\hline 2089 & $\mathrm{FP}$ & $58-\mathrm{Ce}-156$ & 155.9513 & $0.00 \mathrm{E}+00$ & $1.88 \mathrm{E}+00$ \\
\hline 2090 & $\mathrm{FP}$ & 59-Pr-156 & 155.9443 & $0.00 \mathrm{E}+00$ & $9.46 \mathrm{E}-01$ \\
\hline 2091 & $\mathrm{FP}$ & $60-\mathrm{Nd}-156$ & 155.9350 & $0.00 \mathrm{E}+00$ & $1.26 \mathrm{E}-01$ \\
\hline
\end{tabular}




\begin{tabular}{|c|c|c|c|c|c|}
\hline 2092 & FP & 61-Pm-156 & 155.9311 & $0.00 \mathrm{E}+00$ & $2.60 \mathrm{E}-02$ \\
\hline 2093 & FP & 62-Sm-156 & 155.9255 & $0.00 \mathrm{E}+00$ & $2.05 \mathrm{E}-05$ \\
\hline 2094 & FP & 63-Eu-156 & 155.9247 & $0.00 \mathrm{E}+00$ & $5.28 \mathrm{E}-07$ \\
\hline 2095 & FP & 64-Gd-156 & 155.9221 & $0.00 \mathrm{E}+00$ & $0.00 \mathrm{E}+00$ \\
\hline 2096 & FP & $65-\mathrm{Tb}-156$ & 155.9247 & $0.00 \mathrm{E}+00$ & $1.50 \mathrm{E}-06$ \\
\hline 2097 & FP & $65-\mathrm{Tb}-156 \mathrm{~m}$ & 155.9247 & $0.00 \mathrm{E}+00$ & $7.89 \mathrm{E}-06$ \\
\hline 2098 & FP & 66-Dy-156 & 155.9243 & $0.00 \mathrm{E}+00$ & $0.00 \mathrm{E}+00$ \\
\hline 2099 & FP & 58-Ce-157 & 156.9563 & $0.00 \mathrm{E}+00$ & $2.85 \mathrm{E}+00$ \\
\hline 2100 & FP & 59-Pr-157 & 156.9474 & $0.00 \mathrm{E}+00$ & $1.16 \mathrm{E}+00$ \\
\hline 2101 & FP & $60-\mathrm{Nd}-157$ & 156.9390 & $0.00 \mathrm{E}+00$ & $3.64 \mathrm{E}-01$ \\
\hline 2102 & FP & 61-Pm-157 & 156.9330 & $0.00 \mathrm{E}+00$ & $6.56 \mathrm{E}-02$ \\
\hline 2103 & FP & 62-Sm-157 & 156.9284 & $0.00 \mathrm{E}+00$ & $1.44 \mathrm{E}-03$ \\
\hline 2104 & FP & 63-Eu-157 & 156.9254 & $0.00 \mathrm{E}+00$ & $1.27 \mathrm{E}-05$ \\
\hline 2105 & FP & 64-Gd-157 & 156.9240 & $0.00 \mathrm{E}+00$ & $0.00 \mathrm{E}+00$ \\
\hline 2106 & FP & $65-\mathrm{Tb}-157$ & 156.9240 & $0.00 \mathrm{E}+00$ & $3.09 \mathrm{E}-10$ \\
\hline 2107 & FP & 66-Dy-157 & 156.9255 & $0.00 \mathrm{E}+00$ & $2.37 \mathrm{E}-05$ \\
\hline 2108 & FP & 59-Pr-158 & 157.9520 & $0.00 \mathrm{E}+00$ & $5.17 \mathrm{E}+00$ \\
\hline 2109 & FP & $60-\mathrm{Nd}-158$ & 157.9416 & $0.00 \mathrm{E}+00$ & $5.21 \mathrm{E}-01$ \\
\hline 2110 & FP & 61-Pm-158 & 157.9366 & $0.00 \mathrm{E}+00$ & $1.44 \mathrm{E}-01$ \\
\hline 2111 & FP & 62-Sm-158 & 157.9300 & $0.00 \mathrm{E}+00$ & $2.18 \mathrm{E}-03$ \\
\hline 2112 & FP & 63-Eu-158 & 157.9279 & $0.00 \mathrm{E}+00$ & $2.52 \mathrm{E}-04$ \\
\hline 2113 & FP & 64-Gd-158 & 157.9241 & $0.00 \mathrm{E}+00$ & $0.00 \mathrm{E}+00$ \\
\hline 2114 & FP & $65-\mathrm{Tb}-158$ & 157.9254 & $0.00 \mathrm{E}+00$ & $1.22 \mathrm{E}-10$ \\
\hline 2115 & FP & $65-\mathrm{Tb}-158 \mathrm{~m}$ & 157.9254 & $0.00 \mathrm{E}+00$ & $6.48 \mathrm{E}-02$ \\
\hline 2116 & FP & 66-Dy-158 & 157.9244 & $0.00 \mathrm{E}+00$ & $0.00 \mathrm{E}+00$ \\
\hline 2117 & FP & 59-Pr-159 & 158.9555 & $0.00 \mathrm{E}+00$ & $6.57 \mathrm{E}+00$ \\
\hline 2118 & FP & $60-\mathrm{Nd}-159$ & 158.9461 & $0.00 \mathrm{E}+00$ & $8.97 \mathrm{E}-01$ \\
\hline 2119 & FP & 61-Pm-159 & 158.9390 & $0.00 \mathrm{E}+00$ & $4.72 \mathrm{E}-01$ \\
\hline 2120 & FP & 62-Sm-159 & 158.9332 & $0.00 \mathrm{E}+00$ & $6.10 \mathrm{E}-02$ \\
\hline 2121 & FP & 63-Eu-159 & 158.9291 & $0.00 \mathrm{E}+00$ & $6.38 \mathrm{E}-04$ \\
\hline 2122 & FP & 64-Gd-159 & 158.9264 & $0.00 \mathrm{E}+00$ & $1.04 \mathrm{E}-05$ \\
\hline 2123 & FP & $65-\mathrm{Tb}-159$ & 158.9254 & $0.00 \mathrm{E}+00$ & $0.00 \mathrm{E}+00$ \\
\hline 2124 & FP & 66-Dy-159 & 158.9257 & $0.00 \mathrm{E}+00$ & $5.56 \mathrm{E}-08$ \\
\hline 2125 & FP & 67-Ho-159 & 158.9277 & $0.00 \mathrm{E}+00$ & $3.50 \mathrm{E}-04$ \\
\hline 2126 & FP & 67-Ho-159m & 158.9277 & $0.00 \mathrm{E}+00$ & $8.35 \mathrm{E}-02$ \\
\hline 2127 & FP & $60-\mathrm{Nd}-160$ & 159.9491 & $0.00 \mathrm{E}+00$ & $1.18 \mathrm{E}+00$ \\
\hline 2128 & FP & 61-Pm-160 & 159.9430 & $0.00 \mathrm{E}+00$ & $4.44 \mathrm{E}-01$ \\
\hline 2129 & FP & $62-\mathrm{Sm}-160$ & 159.9351 & $0.00 \mathrm{E}+00$ & $7.22 \mathrm{E}-02$ \\
\hline 2130 & FP & 63-Eu-160 & 159.9320 & $0.00 \mathrm{E}+00$ & $1.82 \mathrm{E}-02$ \\
\hline 2131 & FP & 64-Gd-160 & 159.9270 & $0.00 \mathrm{E}+00$ & $0.00 \mathrm{E}+00$ \\
\hline 2132 & FP & $65-\mathrm{Tb}-160$ & 159.9272 & $0.00 \mathrm{E}+00$ & $1.11 \mathrm{E}-07$ \\
\hline 2133 & FP & 66-Dy-160 & 159.9252 & $0.00 \mathrm{E}+00$ & $0.00 \mathrm{E}+00$ \\
\hline 2134 & FP & $60-\mathrm{Nd}-161$ & 160.9539 & $0.00 \mathrm{E}+00$ & $1.42 \mathrm{E}+00$ \\
\hline 2135 & FP & 61-Pm-161 & 160.9459 & $0.00 \mathrm{E}+00$ & $6.51 \mathrm{E}-01$ \\
\hline 2136 & FP & 62-Sm-161 & 160.9388 & $0.00 \mathrm{E}+00$ & $1.44 \mathrm{E}-01$ \\
\hline 2137 & FP & 63-Eu-161 & 160.9337 & $0.00 \mathrm{E}+00$ & $2.67 \mathrm{E}-02$ \\
\hline 2138 & FP & 64-Gd-161 & 160.9297 & $0.00 \mathrm{E}+00$ & $3.16 \mathrm{E}-03$ \\
\hline 2139 & FP & $65-\mathrm{Tb}-161$ & 160.9276 & $0.00 \mathrm{E}+00$ & $1.16 \mathrm{E}-06$ \\
\hline 2140 & FP & 66-Dy-161 & 160.9269 & $0.00 \mathrm{E}+00$ & $0.00 \mathrm{E}+00$ \\
\hline 2141 & FP & 67-Ho-161 & 160.9279 & $0.00 \mathrm{E}+00$ & $7.76 \mathrm{E}-05$ \\
\hline 2142 & FP & 67-Ho-161m & 160.9279 & $0.00 \mathrm{E}+00$ & $1.03 \mathrm{E}-01$ \\
\hline 2143 & FP & 68-Er-161 & 160.9300 & $0.00 \mathrm{E}+00$ & $6.00 \mathrm{E}-05$ \\
\hline 2144 & $\mathrm{FP}$ & 61-Pm-162 & 161.9503 & $0.00 \mathrm{E}+00$ & $2.59 \mathrm{E}+00$ \\
\hline 2145 & FP & 62-Sm-162 & 161.9412 & $0.00 \mathrm{E}+00$ & $2.89 \mathrm{E}-01$ \\
\hline 2146 & FP & 63-Eu-162 & 161.9370 & $0.00 \mathrm{E}+00$ & $6.54 \mathrm{E}-02$ \\
\hline 2147 & $\mathrm{FP}$ & 64-Gd-162 & 161.9310 & $0.00 \mathrm{E}+00$ & $1.38 \mathrm{E}-03$ \\
\hline 2148 & FP & $65-T b-162$ & 161.9295 & $0.00 \mathrm{E}+00$ & $1.52 \mathrm{E}-03$ \\
\hline 2149 & FP & 66-Dy-162 & 161.9268 & $0.00 \mathrm{E}+00$ & $0.00 \mathrm{E}+00$ \\
\hline 2150 & FP & 67-Ho-162 & 161.9291 & $0.00 \mathrm{E}+00$ & $7.70 \mathrm{E}-04$ \\
\hline 2151 & FP & 67-Ho-162m & 161.9291 & $0.00 \mathrm{E}+00$ & $1.72 \mathrm{E}-04$ \\
\hline 2152 & FP & 68-Er-162 & 161.9288 & $0.00 \mathrm{E}+00$ & $0.00 \mathrm{E}+00$ \\
\hline 2153 & FP & 61-Pm-163 & 162.9537 & $0.00 \mathrm{E}+00$ & $3.47 \mathrm{E}+00$ \\
\hline 2154 & FP & 62-Sm-163 & 162.9454 & $0.00 \mathrm{E}+00$ & $3.97 \mathrm{E}-01$ \\
\hline 2155 & $\mathrm{FP}$ & 63-Eu-163 & 162.9392 & $0.00 \mathrm{E}+00$ & $9.00 \mathrm{E}-02$ \\
\hline 2156 & $\mathrm{FP}$ & 64-Gd-163 & 162.9340 & $0.00 \mathrm{E}+00$ & $1.02 \mathrm{E}-02$ \\
\hline 2157 & FP & $65-T b-163$ & 162.9306 & $0.00 \mathrm{E}+00$ & $5.92 \mathrm{E}-04$ \\
\hline
\end{tabular}




\begin{tabular}{|c|c|c|c|c|c|}
\hline 2158 & FP & 66-Dy-163 & 162.9287 & $0.00 \mathrm{E}+00$ & $0.00 \mathrm{E}+00$ \\
\hline 2159 & FP & 67-Нo-163 & 162.9287 & $0.00 \mathrm{E}+00$ & $4.81 \mathrm{E}-12$ \\
\hline 2160 & FP & 67-Ho-163m & 162.9287 & $0.00 \mathrm{E}+00$ & $6.36 \mathrm{E}-01$ \\
\hline 2161 & FP & 68-Er-163 & 162.9300 & $0.00 \mathrm{E}+00$ & $1.54 \mathrm{E}-04$ \\
\hline 2162 & FP & 62-Sm-164 & 163.9483 & $0.00 \mathrm{E}+00$ & $5.65 \mathrm{E}-01$ \\
\hline 2163 & FP & 63-Eu-164 & 163.9430 & $0.00 \mathrm{E}+00$ & $2.44 \mathrm{E}-01$ \\
\hline 2164 & FP & 64-Gd-164 & 163.9359 & $0.00 \mathrm{E}+00$ & $1.54 \mathrm{E}-02$ \\
\hline 2165 & FP & $65-T b-164$ & 163.9333 & $0.00 \mathrm{E}+00$ & $3.85 \mathrm{E}-03$ \\
\hline 2166 & FP & 66-Dy-164 & 163.9292 & $0.00 \mathrm{E}+00$ & $0.00 \mathrm{E}+00$ \\
\hline 2167 & FP & 67-Ho-164 & 163.9302 & $0.00 \mathrm{E}+00$ & $3.98 \mathrm{E}-04$ \\
\hline 2168 & FP & 67-Ho-164m & 163.9302 & $0.00 \mathrm{E}+00$ & $3.08 \mathrm{E}-04$ \\
\hline 2169 & FP & 68-Er-164 & 163.9292 & $0.00 \mathrm{E}+00$ & $0.00 \mathrm{E}+00$ \\
\hline 2170 & FP & $62-\mathrm{Sm}-165$ & 164.9530 & $0.00 \mathrm{E}+00$ & $9.07 \mathrm{E}-01$ \\
\hline 2171 & FP & 63-Eu-165 & 164.9457 & $0.00 \mathrm{E}+00$ & $3.01 \mathrm{E}-01$ \\
\hline 2172 & FP & 64-Gd-165 & 164.9394 & $0.00 \mathrm{E}+00$ & $6.73 \mathrm{E}-02$ \\
\hline 2173 & FP & $65-\mathrm{Tb}-165$ & 164.9349 & $0.00 \mathrm{E}+00$ & $5.47 \mathrm{E}-03$ \\
\hline 2174 & FP & 66-Dy-165 & 164.9317 & $0.00 \mathrm{E}+00$ & $8.25 \mathrm{E}-05$ \\
\hline 2175 & FP & 66-Dy-165m & 164.9317 & $0.00 \mathrm{E}+00$ & $9.19 \mathrm{E}-03$ \\
\hline 2176 & FP & 67-Ho-165 & 164.9303 & $0.00 \mathrm{E}+00$ & $0.00 \mathrm{E}+00$ \\
\hline 2177 & FP & $68-E r-165$ & 164.9307 & $0.00 \mathrm{E}+00$ & $1.86 \mathrm{E}-05$ \\
\hline 2178 & FP & 69-Tm-165 & 164.9324 & $0.00 \mathrm{E}+00$ & $6.41 \mathrm{E}-06$ \\
\hline 2179 & FP & 63-Eu-166 & 165.9500 & $0.00 \mathrm{E}+00$ & $1.73 E+00$ \\
\hline 2180 & FP & 64-Gd-166 & 165.9416 & $0.00 \mathrm{E}+00$ & $1.44 \mathrm{E}-01$ \\
\hline 2181 & FP & $65-\mathrm{Tb}-166$ & 165.9380 & $0.00 \mathrm{E}+00$ & $2.76 \mathrm{E}-02$ \\
\hline 2182 & FP & 66-Dy-166 & 165.9328 & $0.00 \mathrm{E}+00$ & $2.36 \mathrm{E}-06$ \\
\hline 2183 & FP & 67-Ho-166 & 165.9323 & $0.00 \mathrm{E}+00$ & $7.18 \mathrm{E}-06$ \\
\hline 2184 & FP & 67-Ho-166m & 165.9324 & $0.00 \mathrm{E}+00$ & $1.83 \mathrm{E}-11$ \\
\hline 2185 & FP & $68-E r-166$ & 165.9303 & $0.00 \mathrm{E}+00$ & $0.00 \mathrm{E}+00$ \\
\hline 2186 & FP & 69-Tm-166 & 165.9335 & $0.00 \mathrm{E}+00$ & $2.50 \mathrm{E}-05$ \\
\hline 2187 & FP & $70-Y b-166$ & 165.9339 & $0.00 \mathrm{E}+00$ & $3.40 \mathrm{E}-06$ \\
\hline 2188 & FP & 63-Eu-167 & 166.9532 & $0.00 \mathrm{E}+00$ & $3.47 \mathrm{E}+00$ \\
\hline 2189 & FP & 64-Gd-167 & 166.9456 & $0.00 \mathrm{E}+00$ & $2.31 \mathrm{E}-01$ \\
\hline 2190 & FP & $65-T b-167$ & 166.9400 & $0.00 \mathrm{E}+00$ & $3.57 \mathrm{E}-02$ \\
\hline 2191 & FP & 66-Dy-167 & 166.9357 & $0.00 \mathrm{E}+00$ & $1.86 \mathrm{E}-03$ \\
\hline 2192 & FP & 67-Ho-167 & 166.9331 & $0.00 \mathrm{E}+00$ & $6.21 \mathrm{E}-05$ \\
\hline 2193 & FP & 68-Er-167 & 166.9321 & $0.00 \mathrm{E}+00$ & $0.00 \mathrm{E}+00$ \\
\hline 2194 & FP & $68-E r-167 \mathrm{~m}$ & 166.9321 & $0.00 \mathrm{E}+00$ & $3.05 \mathrm{E}-01$ \\
\hline 2195 & FP & 69-Tm-167 & 166.9328 & $0.00 \mathrm{E}+00$ & $8.67 \mathrm{E}-07$ \\
\hline 2196 & FP & $70-Y b-167$ & 166.9350 & $0.00 \mathrm{E}+00$ & $6.60 \mathrm{E}-04$ \\
\hline 2197 & FP & 64-Gd-168 & 167.9484 & $0.00 \mathrm{E}+00$ & $2.31 \mathrm{E}+00$ \\
\hline 2198 & FP & $65-T b-168$ & 167.9436 & $0.00 \mathrm{E}+00$ & $8.45 \mathrm{E}-02$ \\
\hline 2199 & FP & 66-Dy-168 & 167.9371 & $0.00 \mathrm{E}+00$ & $1.33 \mathrm{E}-03$ \\
\hline 2200 & FP & 67-Ho-168 & 167.9355 & $0.00 \mathrm{E}+00$ & $3.86 \mathrm{E}-03$ \\
\hline 2201 & FP & $68-E r-168$ & 167.9324 & $0.00 \mathrm{E}+00$ & $0.00 \mathrm{E}+00$ \\
\hline 2202 & FP & 69-Tm-168 & 167.9342 & $0.00 \mathrm{E}+00$ & $8.62 \mathrm{E}-08$ \\
\hline 2203 & FP & $70-Y b-168$ & 167.9339 & $0.00 \mathrm{E}+00$ & $0.00 \mathrm{E}+00$ \\
\hline 2204 & FP & 64-Gd-169 & 168.9529 & $0.00 \mathrm{E}+00$ & $6.93 \mathrm{E}-01$ \\
\hline 2205 & FP & $65-\mathrm{Tb}-169$ & 168.9462 & $0.00 \mathrm{E}+00$ & $3.47 \mathrm{E}-01$ \\
\hline 2206 & FP & 66-Dy-169 & 168.9403 & $0.00 \mathrm{E}+00$ & $1.78 \mathrm{E}-02$ \\
\hline 2207 & FP & 67-Ho-169 & 168.9369 & $0.00 \mathrm{E}+00$ & $2.45 \mathrm{E}-03$ \\
\hline 2208 & FP & 68-Er-169 & 168.9346 & $0.00 \mathrm{E}+00$ & $8.54 \mathrm{E}-07$ \\
\hline 2209 & FP & 69-Tm-169 & 168.9342 & $0.00 \mathrm{E}+00$ & $0.00 \mathrm{E}+00$ \\
\hline 2210 & FP & $70-Y b-169$ & 168.9352 & $0.00 \mathrm{E}+00$ & $2.51 \mathrm{E}-07$ \\
\hline 2211 & FP & $70-Y b-169 m$ & 168.9352 & $0.00 \mathrm{E}+00$ & $1.51 \mathrm{E}-02$ \\
\hline 2212 & FP & 71-Lu-169 & 168.9377 & $0.00 \mathrm{E}+00$ & $5.65 \mathrm{E}-06$ \\
\hline 2213 & FP & 71-Lu-169m & 168.9377 & $0.00 \mathrm{E}+00$ & $4.33 \mathrm{E}-03$ \\
\hline 2214 & FP & $65-\mathrm{Tb}-170$ & 169.9503 & $0.00 \mathrm{E}+00$ & $2.31 \mathrm{E}-01$ \\
\hline 2215 & FP & 66-Dy-170 & 169.9424 & $0.00 \mathrm{E}+00$ & $2.31 \mathrm{E}-02$ \\
\hline 2216 & FP & 67-Ho-170 & 169.9396 & $0.00 \mathrm{E}+00$ & $4.19 \mathrm{E}-03$ \\
\hline 2217 & FP & 67-Ho-170m & 169.9396 & $0.00 \mathrm{E}+00$ & $1.61 \mathrm{E}-02$ \\
\hline 2218 & $\mathrm{FP}$ & $68-E r-170$ & 169.9355 & $0.00 \mathrm{E}+00$ & $0.00 \mathrm{E}+00$ \\
\hline 2219 & FP & 69-Tm-170 & 169.9358 & $0.00 \mathrm{E}+00$ & $6.24 \mathrm{E}-08$ \\
\hline 2220 & FP & $70-Y b-170$ & 169.9348 & $0.00 \mathrm{E}+00$ & $0.00 \mathrm{E}+00$ \\
\hline 2221 & FP & $65-\mathrm{Tb}-171$ & 170.9533 & $0.00 \mathrm{E}+00$ & $1.39 \mathrm{E}+00$ \\
\hline 2222 & FP & 66-Dy-171 & 170.9462 & $0.00 \mathrm{E}+00$ & $1.16 \mathrm{E}-01$ \\
\hline 2223 & FP & 67-Ho-171 & 170.9415 & $0.00 \mathrm{E}+00$ & $1.31 \mathrm{E}-02$ \\
\hline
\end{tabular}




\begin{tabular}{|rllllr|}
\hline 2224 & FP & $68-\mathrm{Er}-171$ & 170.9380 & $0.00 \mathrm{E}+00$ & $2.56 \mathrm{E}-05$ \\
\hline 2225 & FP & $69-T m-171$ & 170.9364 & $0.00 \mathrm{E}+00$ & $1.14 \mathrm{E}-08$ \\
\hline 2226 & FP & $70-\mathrm{Yb}-171$ & 170.9363 & $0.00 \mathrm{E}+00$ & $0.00 \mathrm{E}+00$ \\
\hline 2227 & FP & $71-\mathrm{Lu}-171$ & 170.9379 & $0.00 \mathrm{E}+00$ & $9.74 \mathrm{E}-07$ \\
\hline 2228 & FP & $71-\mathrm{Lu}-171 \mathrm{~m}$ & 170.9379 & $0.00 \mathrm{E}+00$ & $8.77 \mathrm{E}-03$ \\
\hline 2229 & FP & $72-\mathrm{Hf}-171$ & 170.9405 & $0.00 \mathrm{E}+00$ & $1.59 \mathrm{E}-05$ \\
\hline 2230 & FP & $66-\mathrm{Dy}-172$ & 171.9488 & $0.00 \mathrm{E}+00$ & $2.31 \mathrm{E}-01$ \\
\hline 2231 & FP & $67-\mathrm{Ho}-172$ & 171.9448 & $0.00 \mathrm{E}+00$ & $2.77 \mathrm{E}-02$ \\
\hline 2232 & FP & $68-\mathrm{Er}-172$ & 171.9394 & $0.00 \mathrm{E}+00$ & $3.91 \mathrm{E}-06$ \\
\hline 2233 & FP & $69-\mathrm{Tm}-172$ & 171.9384 & $0.00 \mathrm{E}+00$ & $3.03 \mathrm{E}-06$ \\
\hline 2234 & FP & $70-\mathrm{Yb}-172$ & 171.9364 & $0.00 \mathrm{E}+00$ & $0.00 \mathrm{E}+00$ \\
\hline 2235 & FP & $71-\mathrm{Lu}-172$ & 171.9391 & $0.00 \mathrm{E}+00$ & $1.20 \mathrm{E}-06$ \\
\hline 2236 & FP & $71-\mathrm{Lu}-172 \mathrm{~m}$ & 171.9391 & $0.00 \mathrm{E}+00$ & $3.12 \mathrm{E}-03$ \\
\hline 2237 & FP & $72-\mathrm{Hf}-172$ & 171.9395 & $0.00 \mathrm{E}+00$ & $1.17 \mathrm{E}-08$ \\
\hline
\end{tabular}




\section{APPENDIX 5.2.E -Decay Resource Contents}

A concise representation of the data contained in the decay resource is shown below in Figure 5.2.6. Note that the actual decay resource file currently also defines the complete ORIGEN nuclide set, including the multiple versions of some nuclides by sublibrary, e.g., ${ }^{155} \mathrm{Gd}-155$, as both a light nuclide/activation product and a fission product. The content list below contains no such duplication.

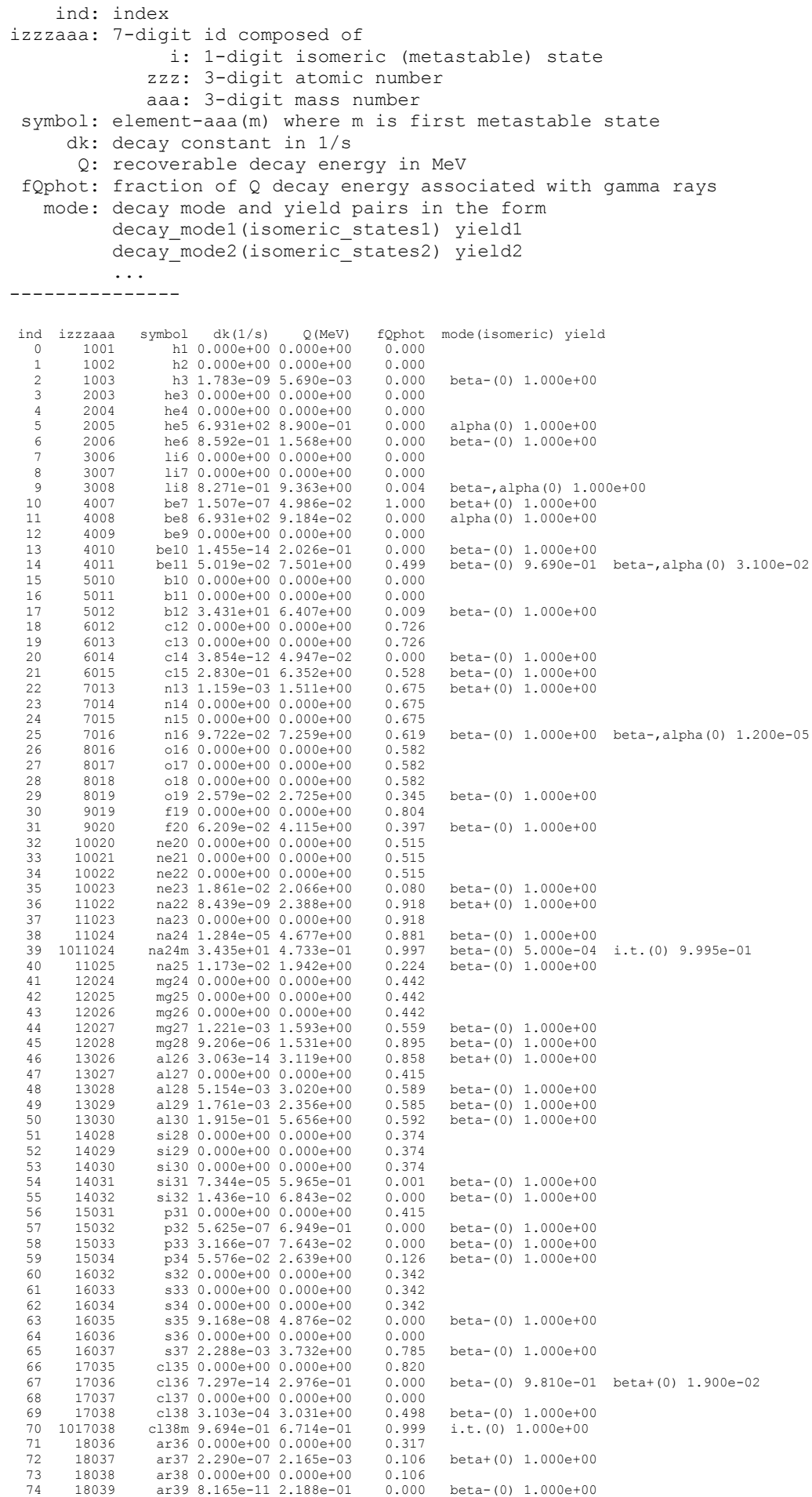




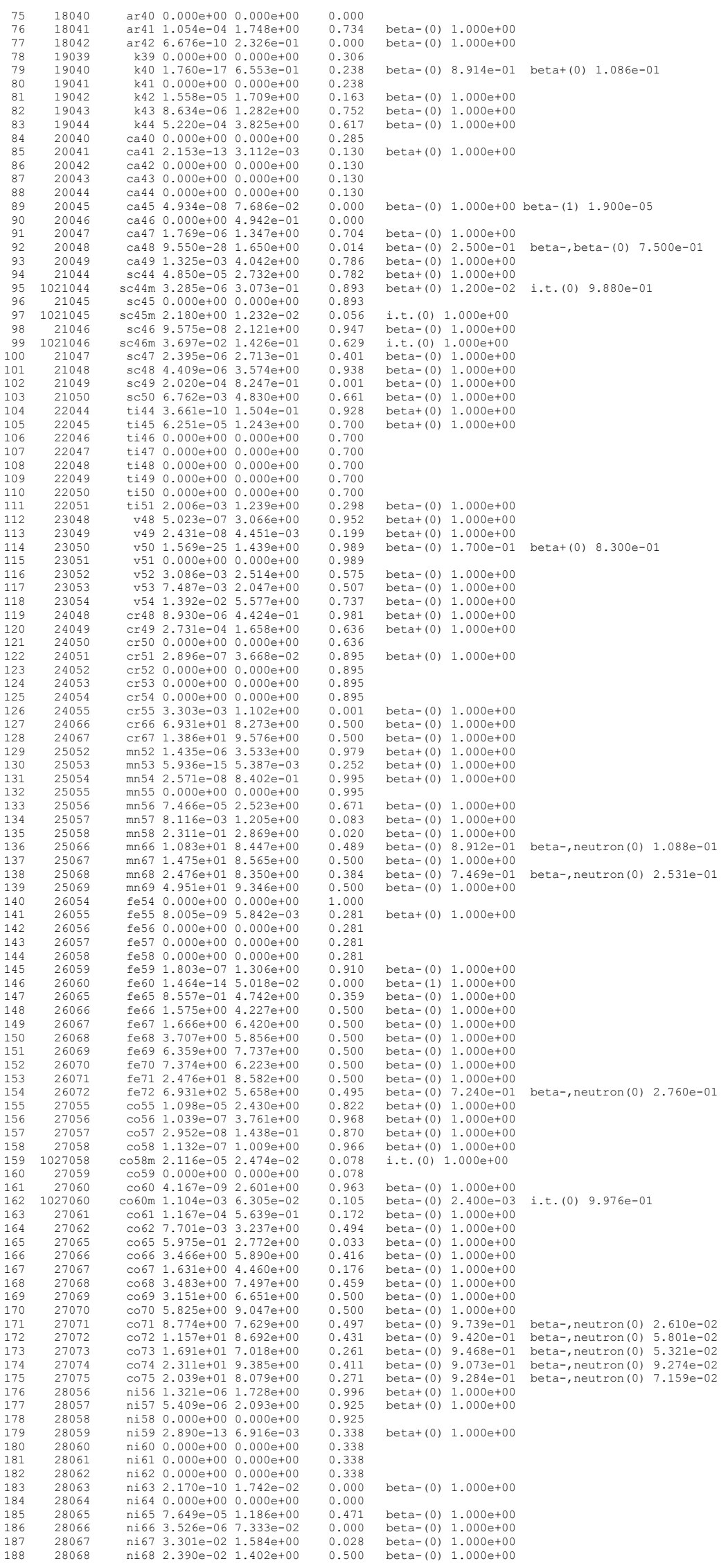




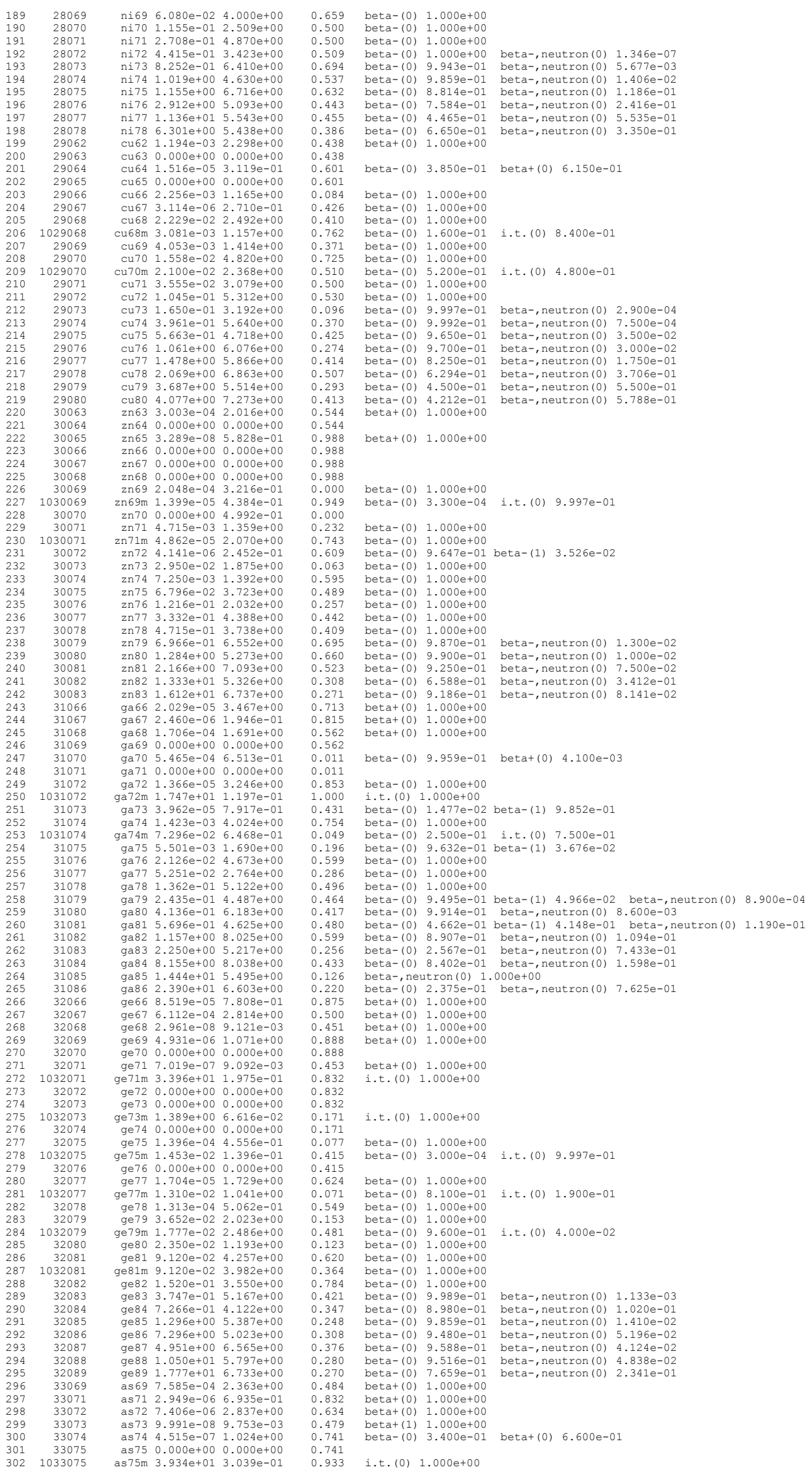




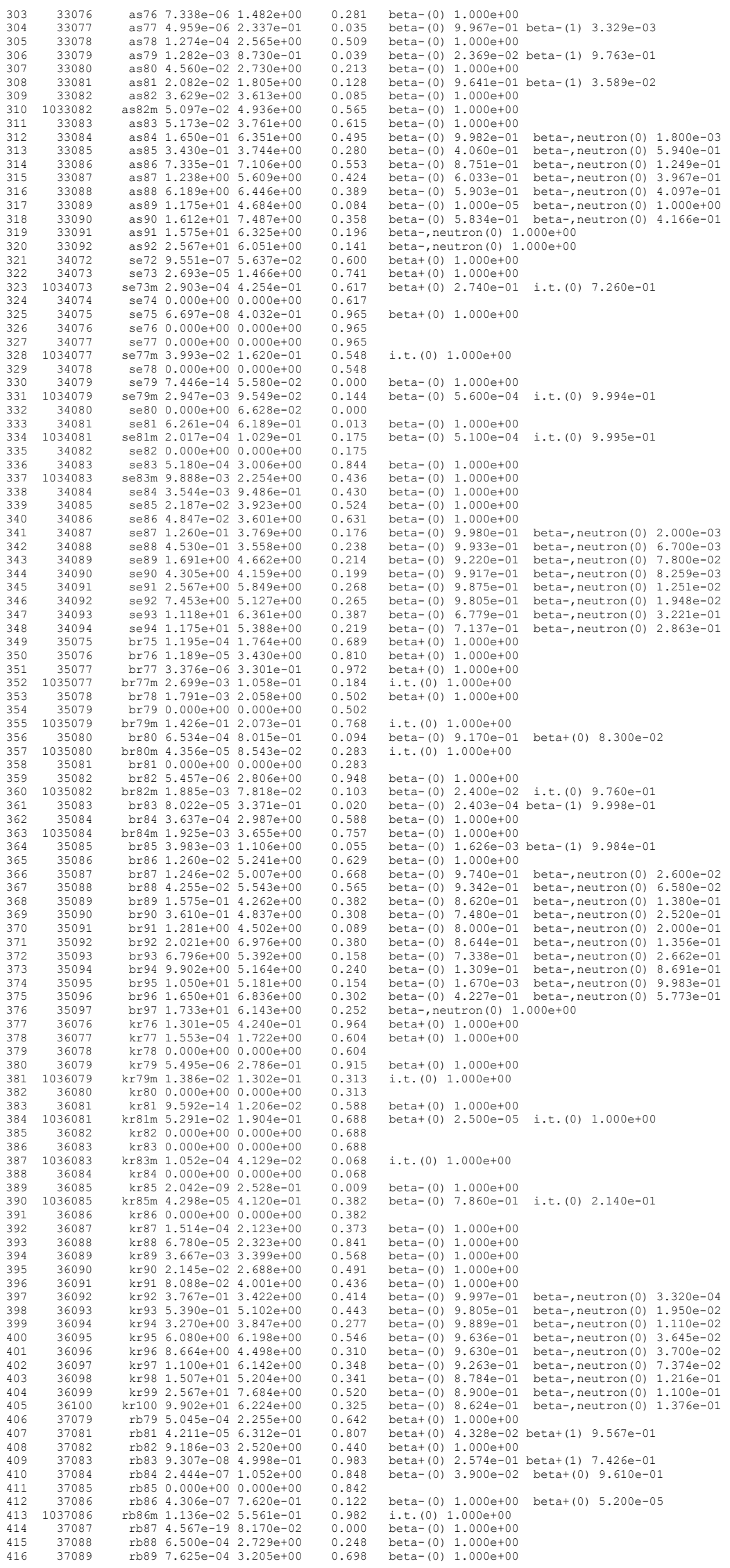




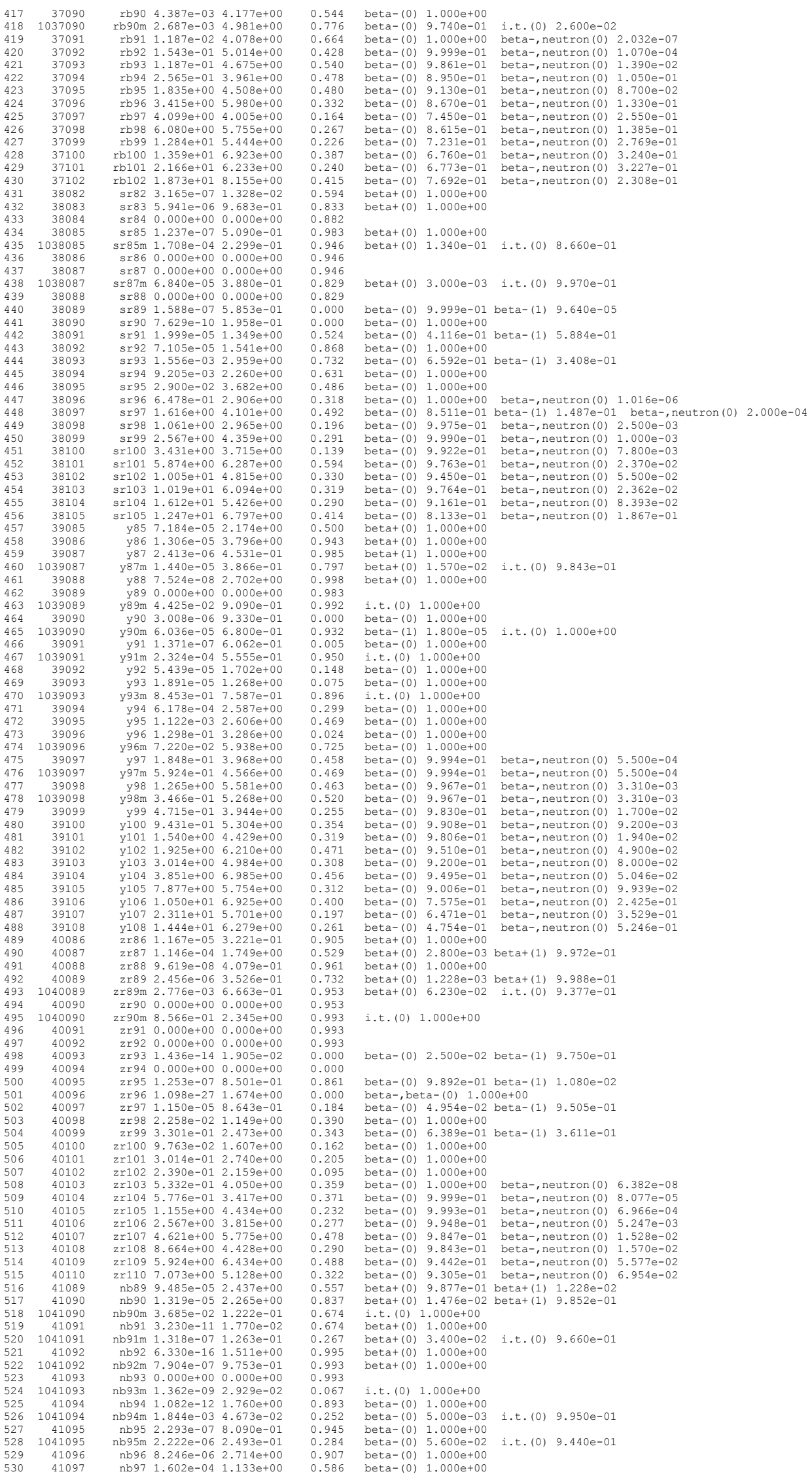




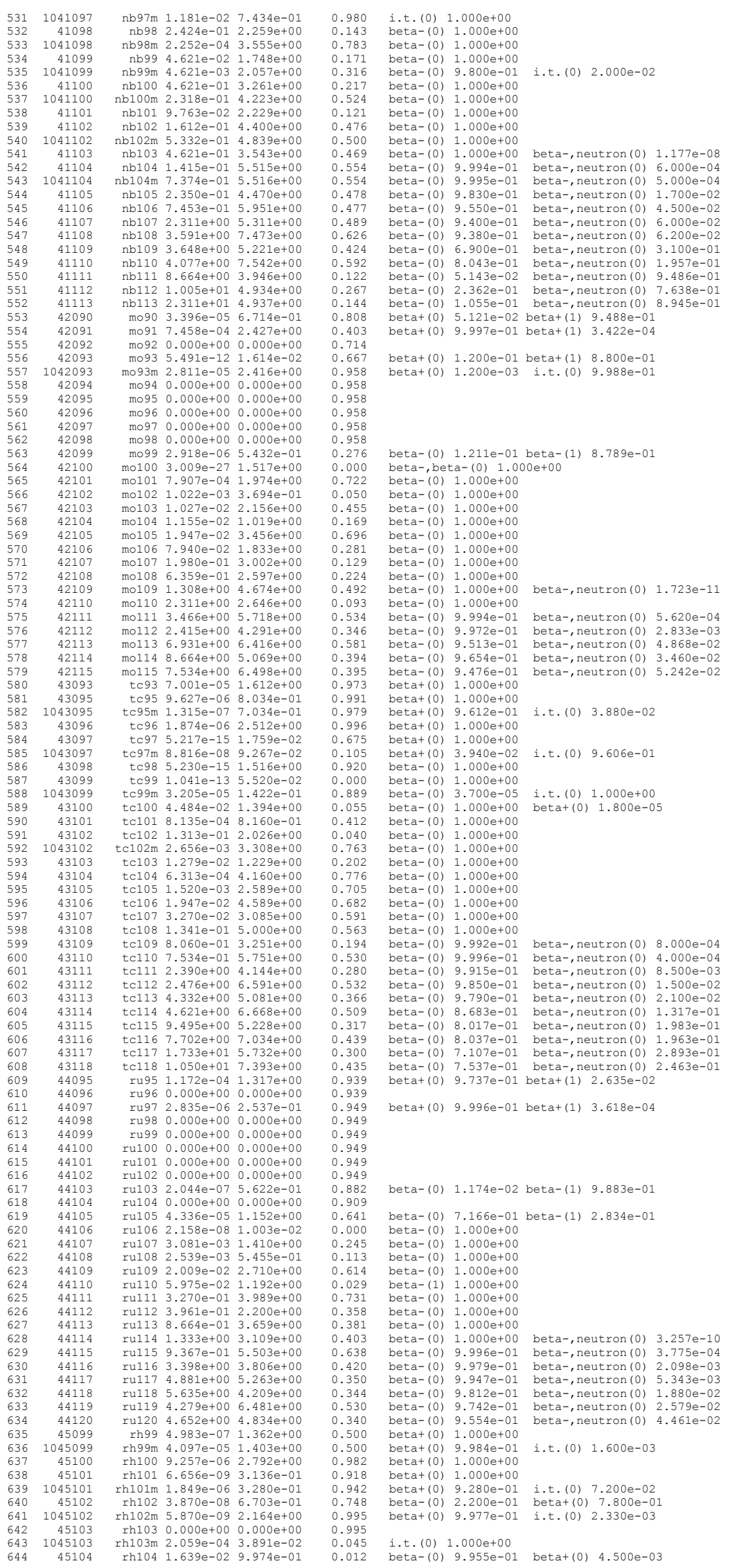




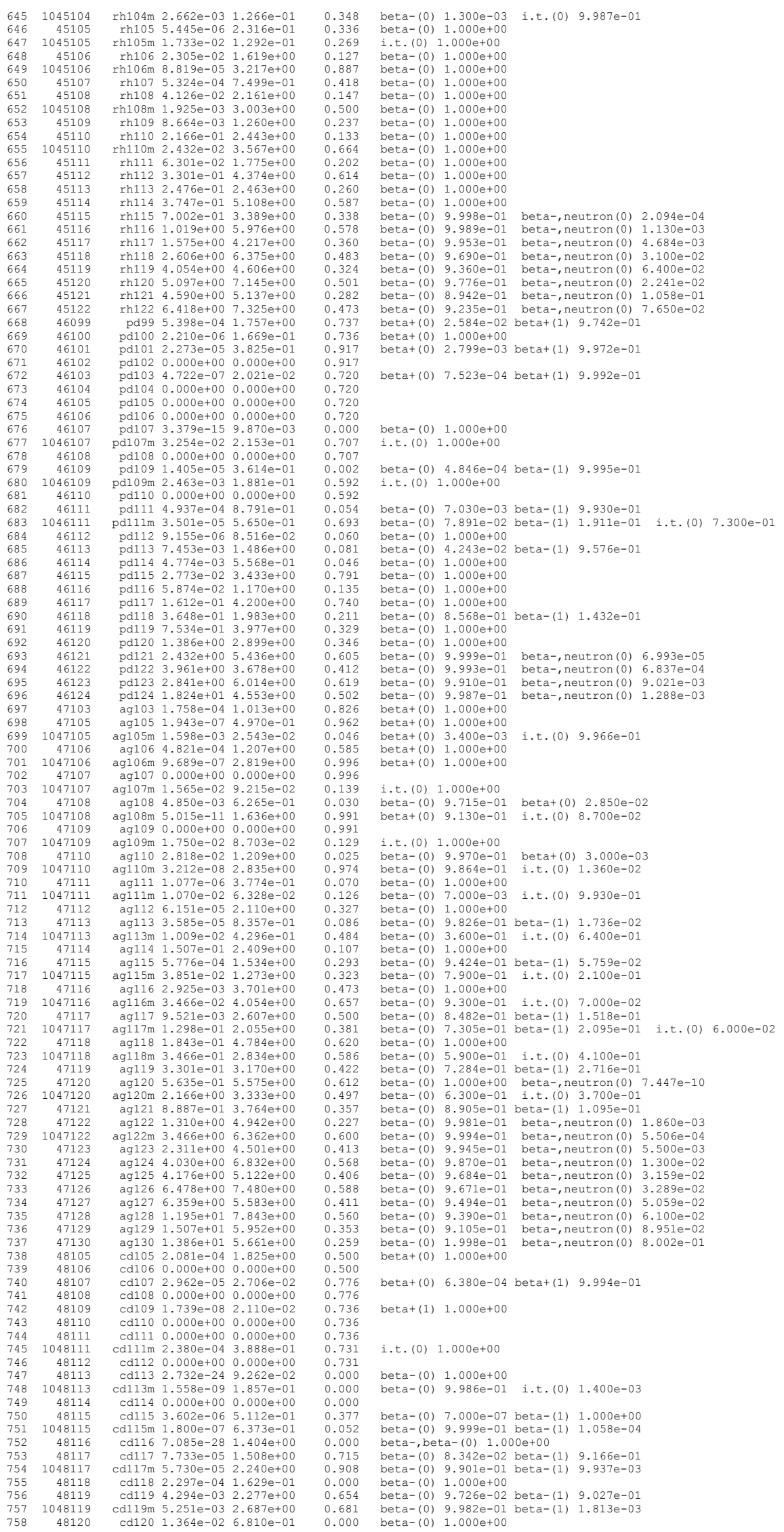




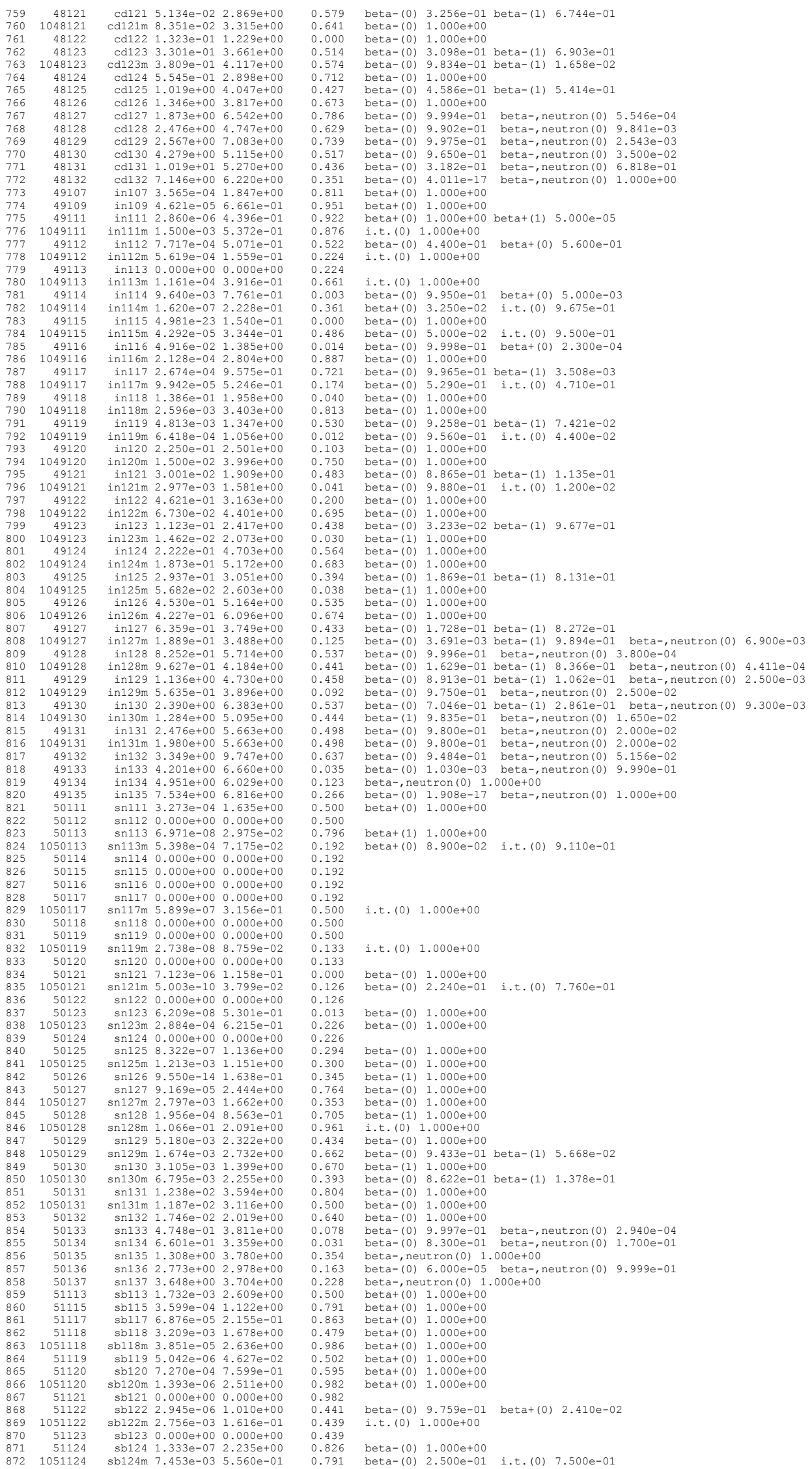




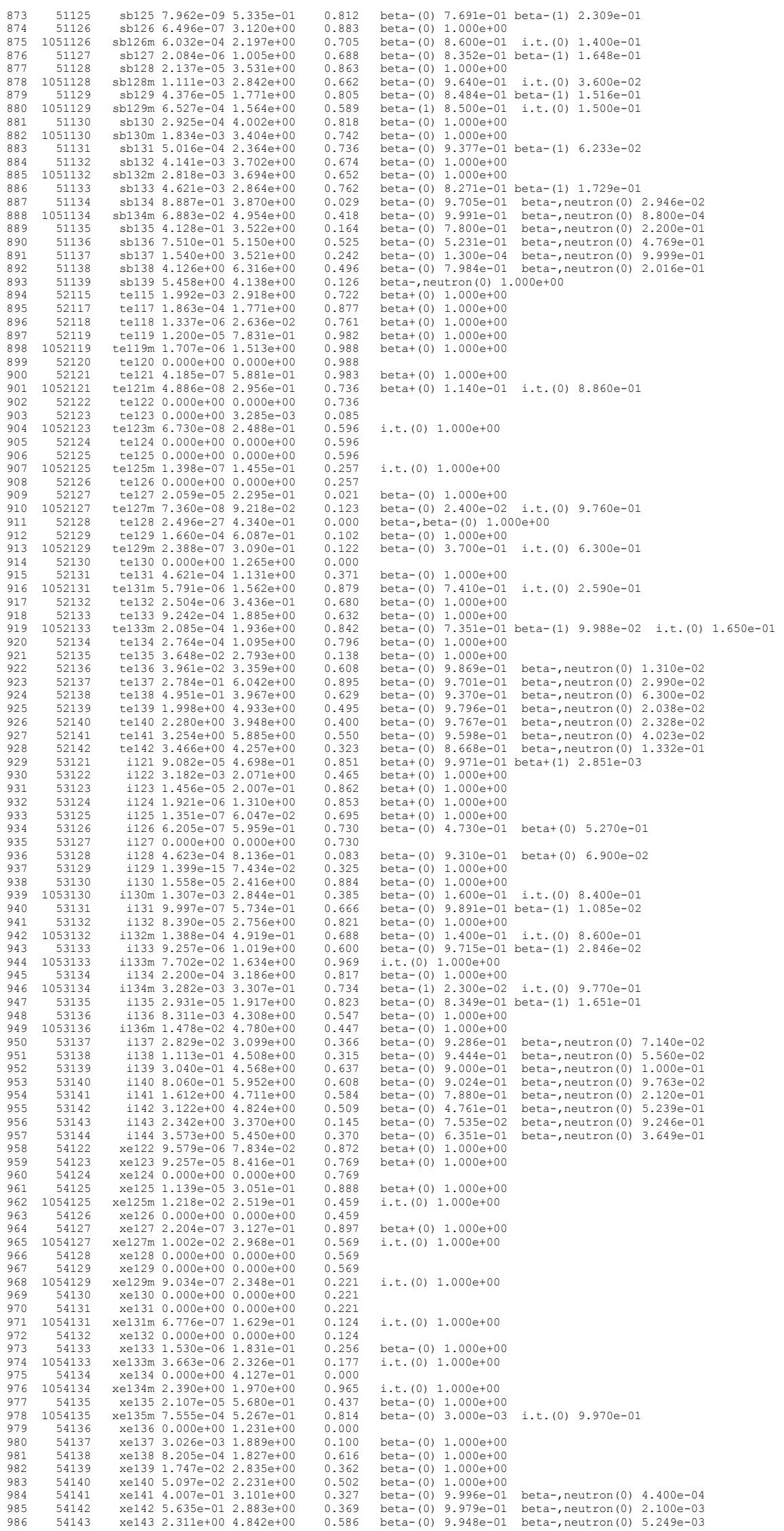




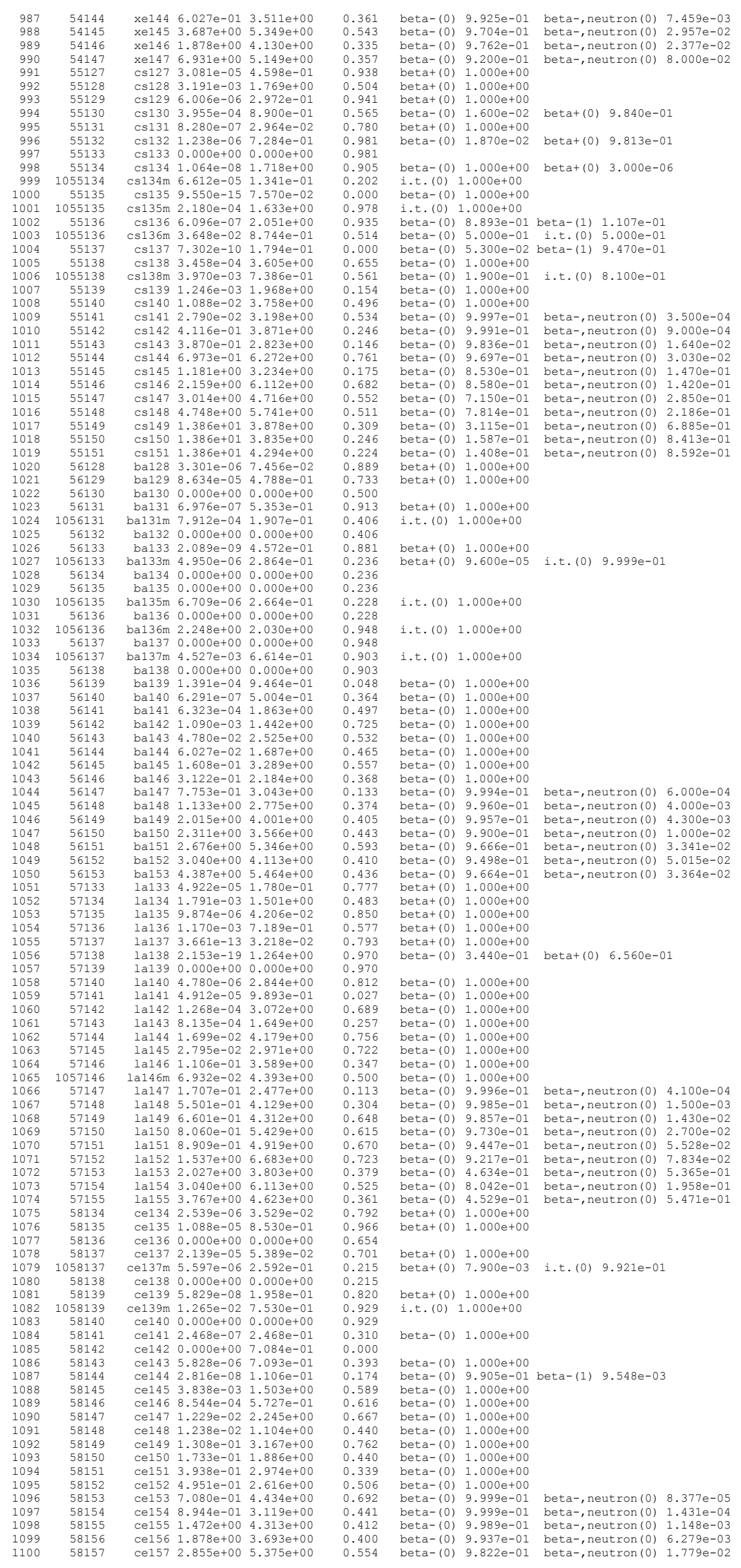




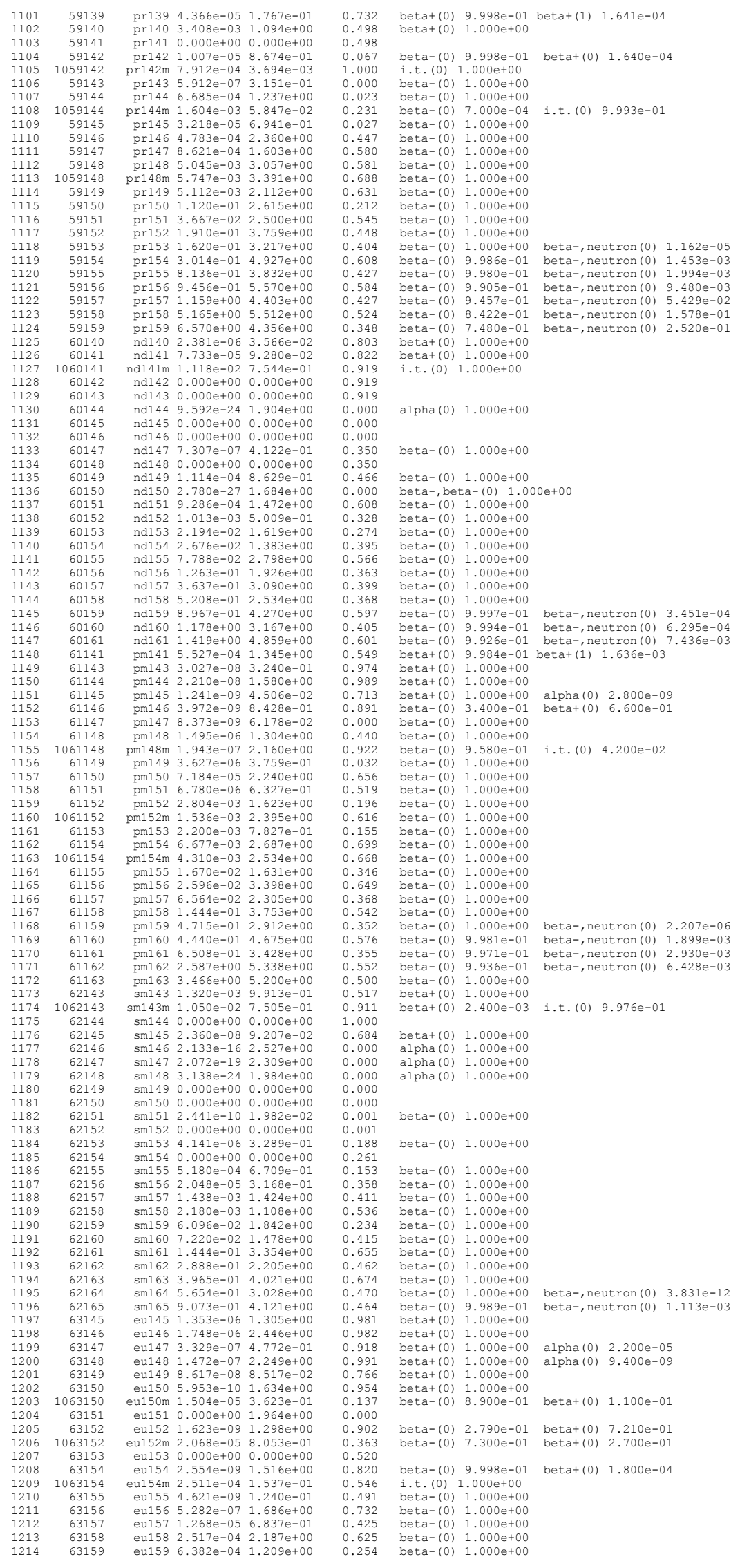




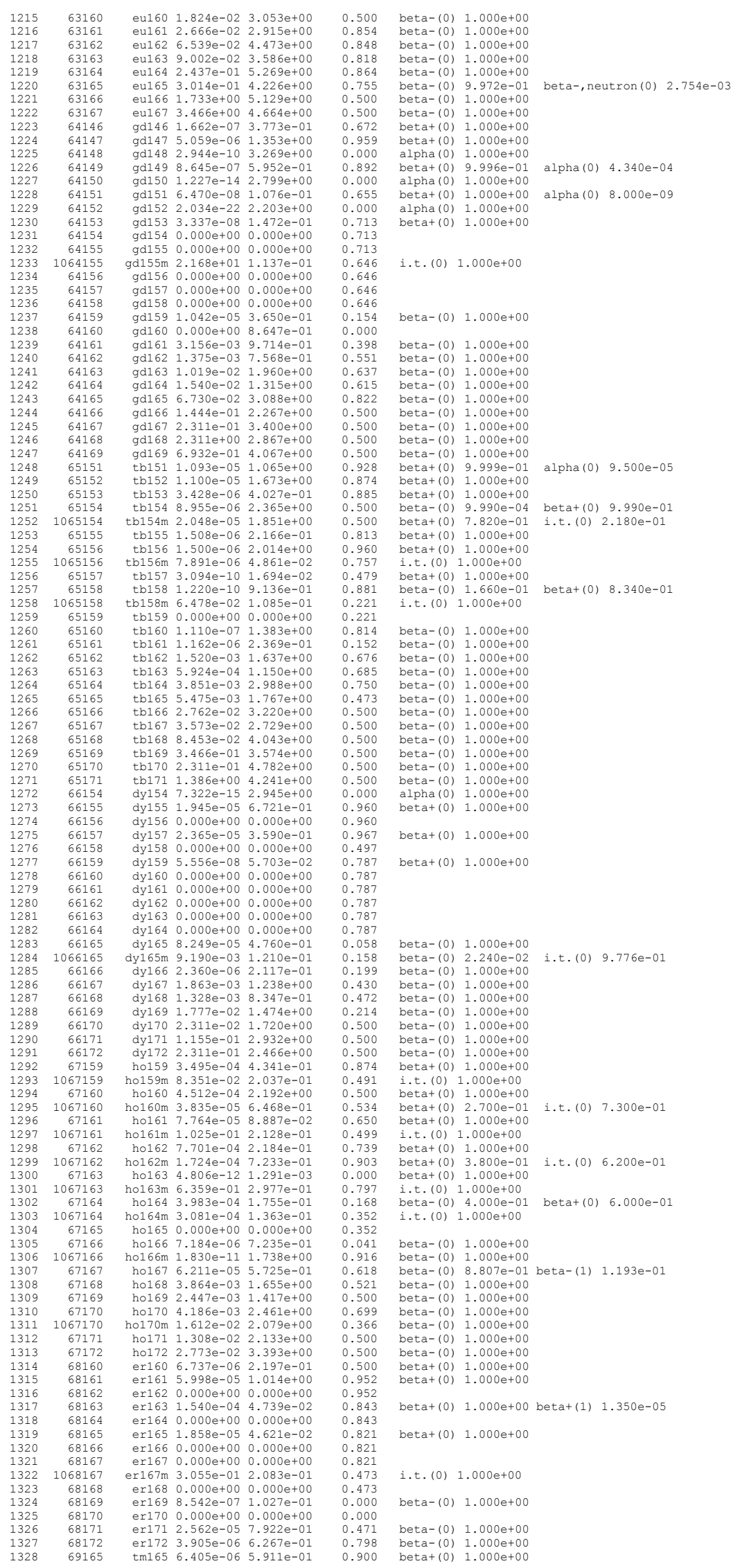




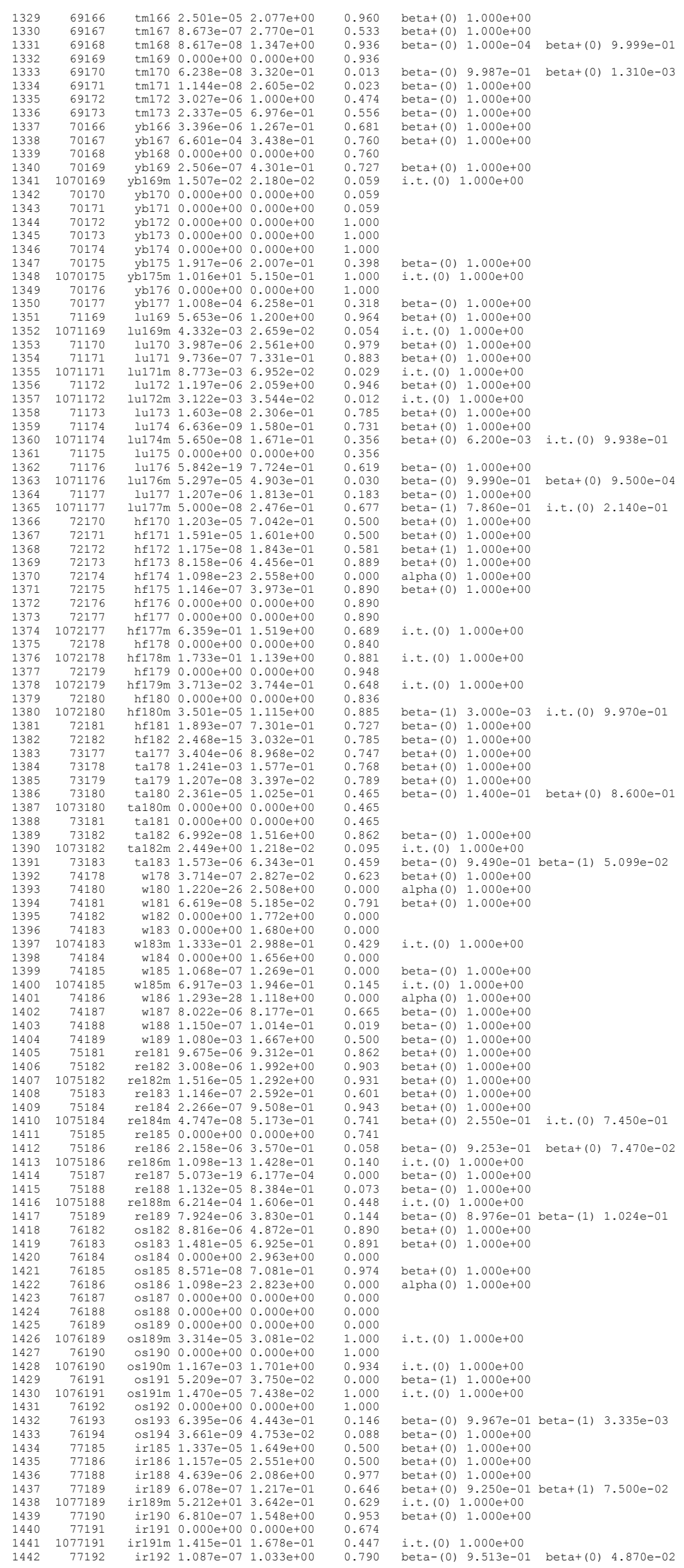




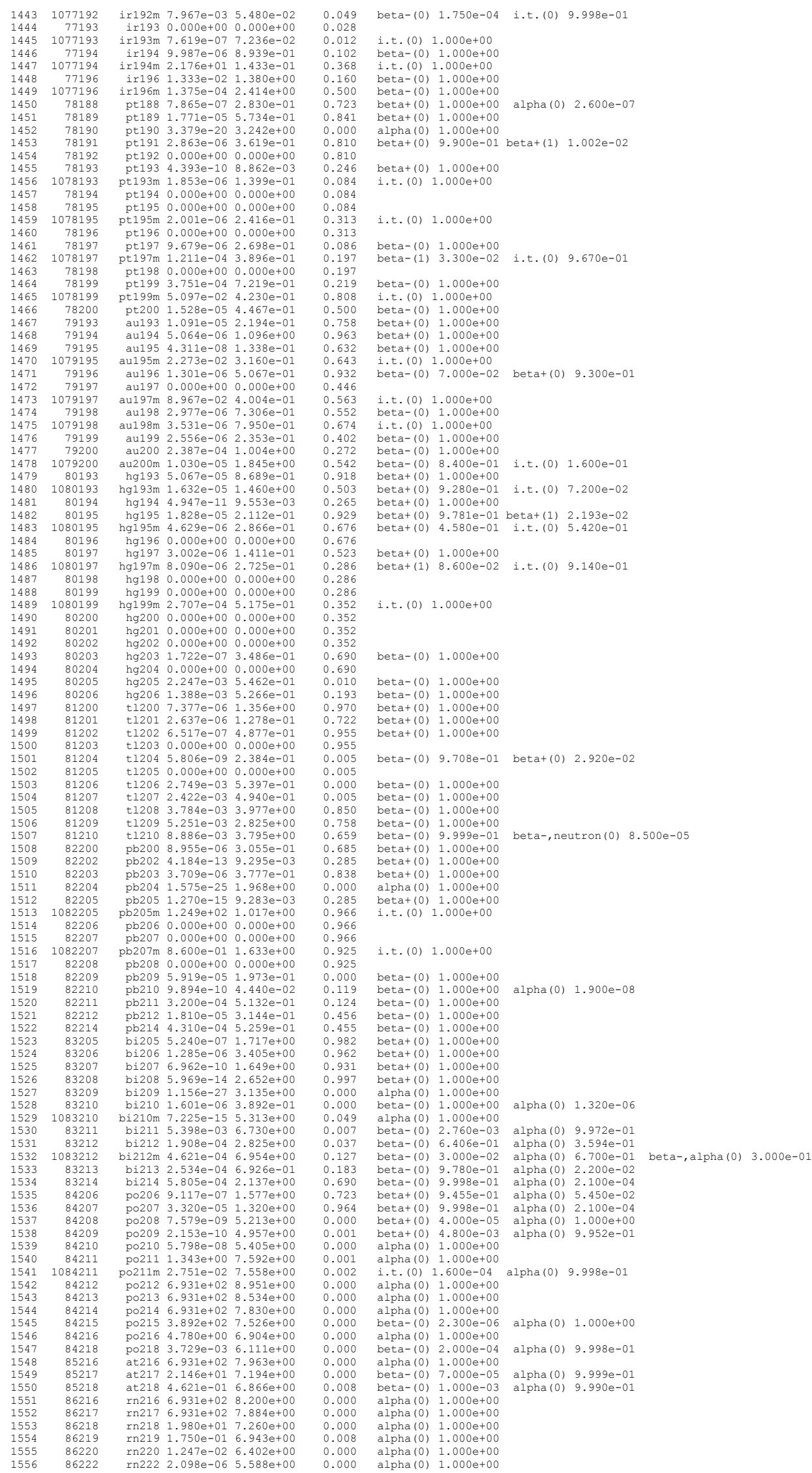




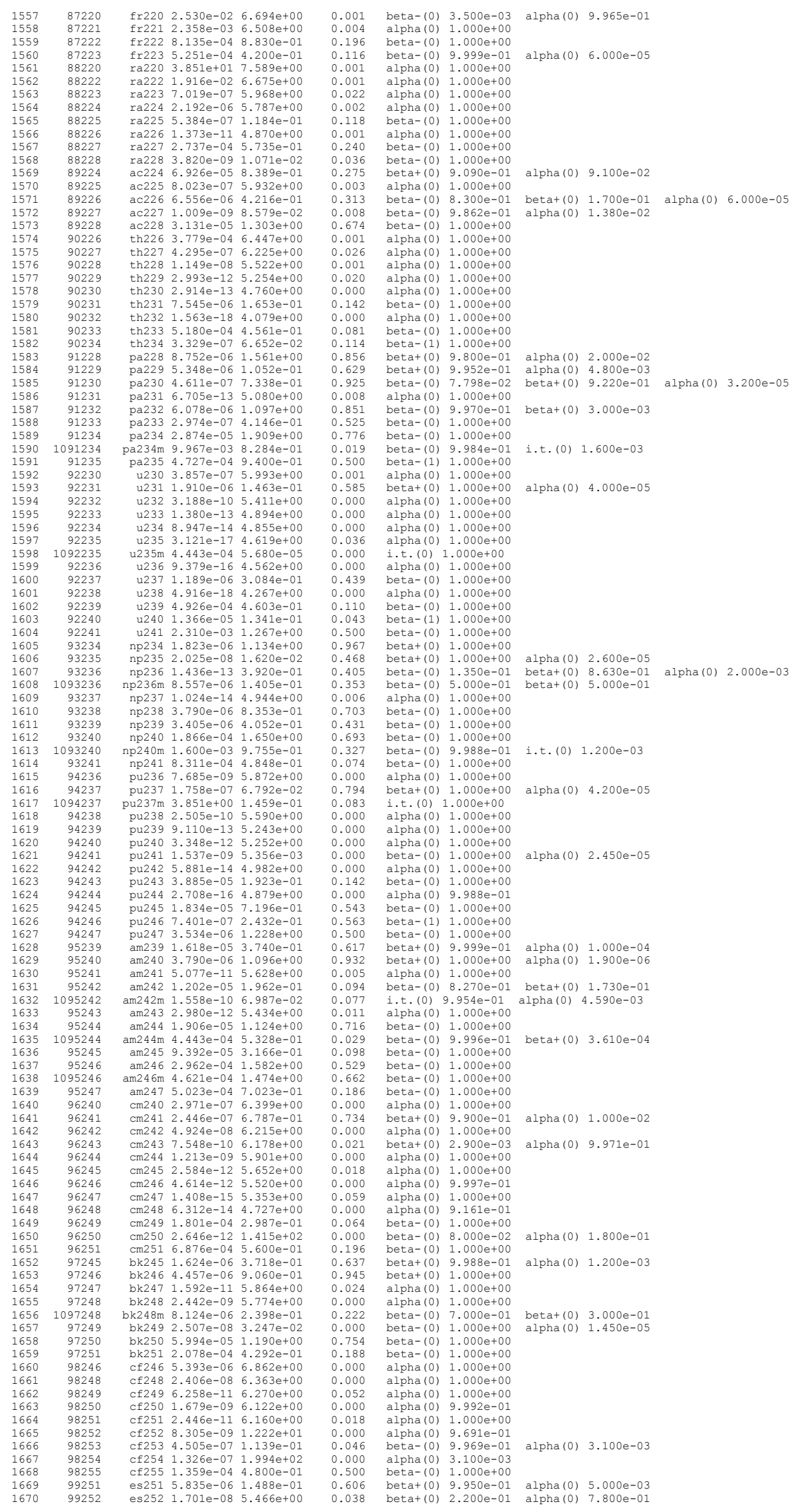




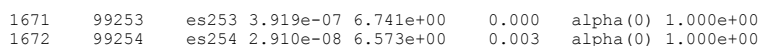

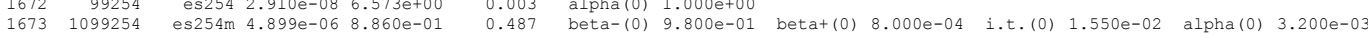

1674.99255 es $254 \mathrm{~m}$. $4.899 \mathrm{e}-06 \quad 8.860 \mathrm{e}-01$

Figure 5.2.6. Contents of decay resource. 


\section{APPENDIX 5.2.F - Reaction Resource Contents}

A concise representation of the data contained in the reaction resource is shown below in Figure 5.2.7. This type of output may be generated from any AMPX multigroup library using the PALEALE utility.

JEFF-3.0/A Neutron Activation File

J-Ch Sublet, A J Koning, R A Forrest and J Kopecky March 2003

238-group Library developed by I C Gauld and D Wiarda

Updated: $01 / 11 / 11$

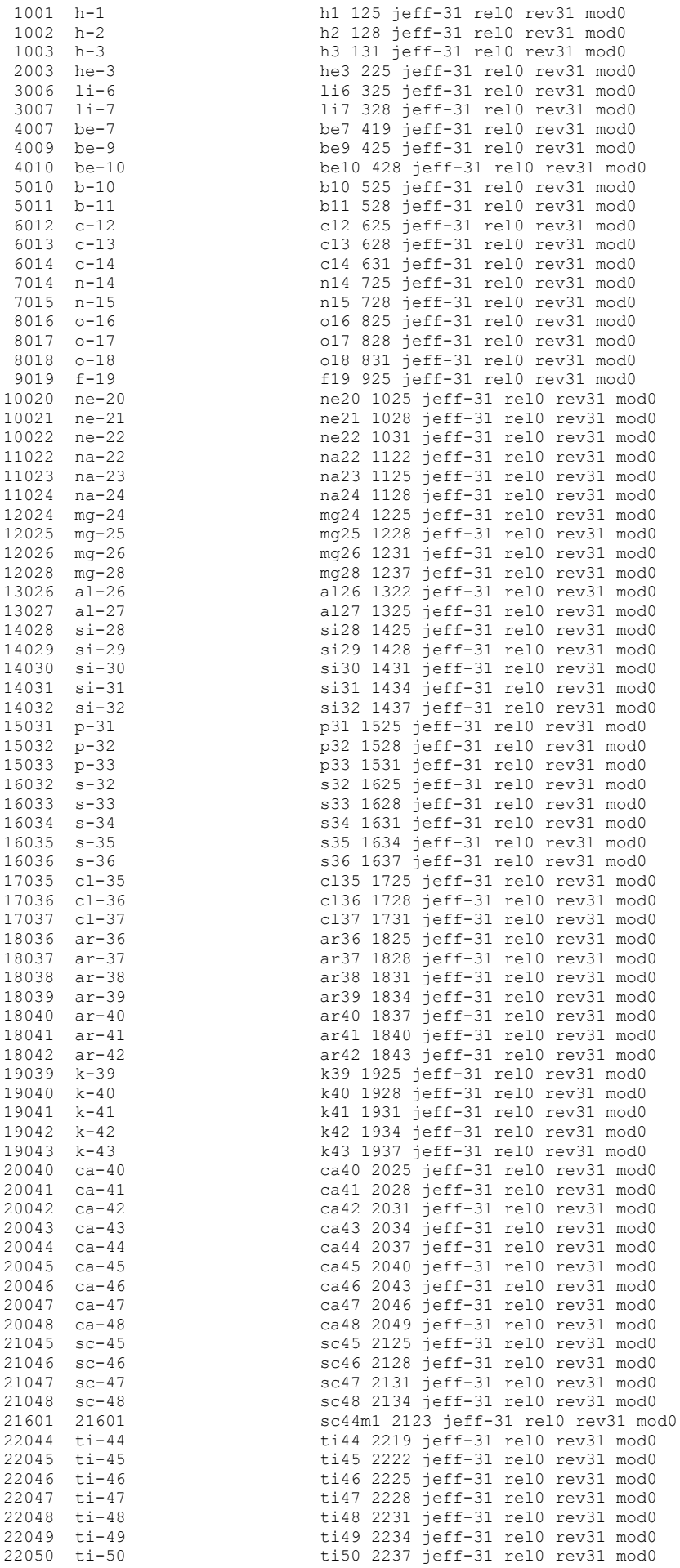




\begin{tabular}{|c|c|}
\hline 48 & \\
\hline 049 & $v-49$ \\
\hline & $v-50$ \\
\hline J & $v-51$ \\
\hline & $\mathrm{Cr}-48$ \\
\hline & cr -50 \\
\hline 051 & cr -51 \\
\hline & cr -52 \\
\hline 053 & cr -53 \\
\hline & cr -54 \\
\hline & $m n-52$ \\
\hline 053 & $m n-53$ \\
\hline 054 & $m n-54$ \\
\hline 055 & $m n-55$ \\
\hline 54 & $f e-54$ \\
\hline 55 & $\mathrm{fe}-55$ \\
\hline & fe-56 \\
\hline 57 & $\mathrm{fe}-57$ \\
\hline & $\mathrm{fe}-58$ \\
\hline 059 & fe-5 \\
\hline 60 & $f e-60$ \\
\hline 055 & co- 55 \\
\hline 056 & co- 56 \\
\hline 057 & $\mathrm{co}-57$ \\
\hline 058 & $\mathrm{CO}-5$ \\
\hline 059 & co- 59 \\
\hline ace & $\mathrm{co}-60$ \\
\hline 01 & $\mathrm{Co}-58$ \\
\hline 0 & ni-56 \\
\hline & ni-5 \\
\hline 58 & ni-58 \\
\hline 059 & ni-59 \\
\hline 0 & ni-60 \\
\hline 01 & ni-61 \\
\hline $06 ?$ & ni- 62 \\
\hline 063 & ni-63 \\
\hline 4 & ni-6 \\
\hline & ni- 66 \\
\hline 163 & $\mathrm{cu}-63$ \\
\hline 64 & $\mathrm{cu}-64$ \\
\hline 5 & $\mathrm{cu}-65$ \\
\hline 67 & $\mathrm{cu}-67$ \\
\hline V & $\mathrm{zn}-6$ \\
\hline 65 & $\mathrm{zn}-65$ \\
\hline 066 & $\mathrm{zn}-66$ \\
\hline 7 & $\mathrm{zn}-67$ \\
\hline 68 & $\mathrm{zn}-68$ \\
\hline 0 & $\mathrm{zn}-70$ \\
\hline 2 & $\mathrm{zn}-72$ \\
\hline 1 & 30601 \\
\hline 7 & ga -67 \\
\hline 9 & $9 a-69$ \\
\hline 1 & ga-71 \\
\hline ? & ga -72 \\
\hline & ge -68 \\
\hline 69 & ge- 69 \\
\hline 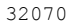 & ge -70 \\
\hline 1 & ge-71 \\
\hline 2 & ge -72 \\
\hline 3 & ge -73 \\
\hline 4 & ge -7 \\
\hline 6 & ge -76 \\
\hline 7 & ge -77 \\
\hline 1 & as -71 \\
\hline 2 & as -72 \\
\hline 073 & as -73 \\
\hline 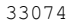 & as -74 \\
\hline 5 & as -75 \\
\hline & as -76 \\
\hline 7 & as -77 \\
\hline 2 & $\mathrm{se}-72$ \\
\hline & se -73 \\
\hline 4 & se -74 \\
\hline 5 & se -75 \\
\hline & se -76 \\
\hline 7 & se -77 \\
\hline & se -78 \\
\hline 79 & se -79 \\
\hline 80 & se -80 \\
\hline & se -82 \\
\hline 076 & br -76 \\
\hline & br -77 \\
\hline 79 & br -79 \\
\hline 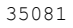 & br -81 \\
\hline & $\mathrm{br}-8$ \\
\hline & $k r-76$ \\
\hline 078 & $\mathrm{kr}-78$ \\
\hline & $\mathrm{kr}-79$ \\
\hline 88 & $\mathrm{kr}-80$ \\
\hline & $\mathrm{kr}-81$ \\
\hline & $\mathrm{kr}-82$ \\
\hline & $\mathrm{kr}-$ \\
\hline & $\mathrm{kr}-84$ \\
\hline & $k r-85$ \\
\hline & \\
\hline
\end{tabular}

v48 2319 jeff-31 rel0 rev31 modo v49 2322 jeff-31 rel0 rev31 modo v50 2325 jeff-31 rel0 rev31 modo v51 2328 jeff-31 relo rev31 modo cr48 2419 jeff-31 rel0 rev31 modo cr50 2425 jeff-31 rel0 rev31 modo cr51 2428 jeff-31 relo rev31 modo cr52 2431 jeff-31 relo rev31 modo cr52 2431 jeff-31 relo rev31 modo cr53 2434 jeft-31 relo rev31 modo cr54 2437 jeff-31 relo rev31 modo mn52 2516 jeff-31 relo rev31 modo mn53 2519 jeff-31 rel0 rev31 modo mn54 2522 jeff-31 rel0 rev31 modo mn55 2525 jeff-31 rel0 rev31 mod0 fe54 2625 jeff-31 rel0 rev31 modo fe55 2628 jeff-31 rel0 rev31 modo fe56 2631 jeff-31 relo rev31 modo fe57 2634 jeff-31 relo rev31 modo fe58 2637 jeff-31 rel0 rev31 modo fes8 2637 jeff-31 relo rev31 modo fe 60 2643 jeff-31 relo rev31 modo fe60 2643 jeff-31 relo rev31 modo co55 2713 jeff-31 relo rev31 modo co56 2716 jeff-31 rel0 rev31 modo co57 2719 jeff-31 rel0 rev31 modo co58 2722 jeff-31 relo rev31 modo co59 2725 jeff-31 rel0 rev31 modo co60 2728 jeff-31 rel0 rev31 modo co58m1 2723 jeff-31 rel0 rev31 modo ni56 2819 jeff-31 relo rev31 modo ni57 2822 jeff -31 rel0 rev31 modo ni57 2822 jeff 31 relo rev31 modo ni5 2825 jeff-31 relo rev31 modo ni59 2828 jeff-31 relo rev31 modo ni60 2831 jeff-31 relo rev31 modo ni61 2834 jeff-31 rel0 rev31 modo ni62 2837 jeff-31 rel0 rev31 modo ni63 2840 jeff-31 rel0 rev31 modo ni64 2843 jeff-31 rel0 rev31 modo ni66 2849 jeff-31 relo rev31 modo cu63 2925 jeff-31 rel0 rev31 modo cu64 2928 jeff-31 relo rev31 modo cu65 2931 jeff-31 rel0 rev31 modo cu6 672937 jeff-31 relo rev31 modo cu67 2937 jeft-31 relo rev31 modo 2n64 3025 jeft -31 relo rev31 modo zn65 3028 jeff-31 relo rev31 modo zn66 3031 jeff-31 rel0 rev31 modo zn67 3034 jeff-31 rel0 rev31 modo zn68 3037 jeff-31 relo rev31 modo zn70 3043 jeff-31 rel0 rev31 modo zn72 3049 jeff-31 rel0 rev31 modo zn69m1 3041 jeff-31 rel0 rev31 modo ga67 3119 jeff-31 rel0 rev31 modo ga69 3125 jeff-31 rel0 rev31 modo ga69 3125 jeff-31 relo rev31 modo ga71 3131 jeff-31 relo rev31 modo ga72 3134 jeff-31 relo rev31 modo ge68 3219 jeff-31 relo rev31 modo ge69 3222 jeft-31 relo rev31 modo ge70 3225 jeff-31 rel0 rev31 modo ge71 3228 jeff-31 relo rev31 modo ge72 3231 jeff-31 rel0 rev31 mod0 ge73 3234 jeff-31 rel0 rev31 modo ge74 3237 jeff-31 rel0 rev31 modo ge76 3243 jeff-31 relo rev31 modo ge77 3246 jeff-31 relo rev31 modo as 31313 jeff -31 rel0 rev31 modo as 1 3313 jeff 31 relo rev31 modo 373319 jef $f-31$ relo rev31 modo as 73 3319 je as74 3322 jeft-31 relo rev31 modo as75 3325 jeff-31 relo rev31 modo as76 3328 jeff-31 rel0 rev31 modo as77 3331 jeff-31 rel0 rev31 modo se72 3419 jeff-31 rel0 rev31 modo se73 3422 jeff-31 rel0 rev31 modo se74 3425 jeff-31 rel0 rev31 modo se75 3428 jeff-31 relo rev31 modo se76 3431 jeff-31 rel0 rev31 modo se77 3434 jeff-31 relo rev31 modo se 783437 jeff-31 rel0 rev31 modo se78 3437 jeff-31 relo rev31 modo se79 3440 jeff -31 relo rev31 modo se80 3443 jeff-31 relo rev31 modo se82 3449 jeff-31 rel0 rev31 modo br76 3516 jeff-31 rel0 rev31 modo br77 3519 jeff-31 rel0 rev31 mod0 br79 3525 jeff-31 relo rev31 modo br81 3531 jeff-31 rel0 rev31 modo br82 3534 jeff-31 rel0 rev31 modo kr76 3619 jeff-31 rel0 rev31 modo kr78 3625 jeff-31 relo rev31 modo kr78 3625 jeff-31 relo rev31 modo kr79 3628 jeff -31 rel0 rev31 nodo kr80 3631 jeff-31 relo rev31 modo kr81 3634 jeff-31 relo rev31 modo kr82 3637 jeff-31 rel0 rev31 modo kr83 3640 jeff-31 rel0 rev31 modo kr84 3643 jeff-31 relo rev31 modo kr85 3646 jeff-31 rel0 rev31 modo kr86 3649 jeff-31 rel0 rev31 modo 
rb83 3719 jeff-31 rel0 rev31 modo rb84 3722 jeff-31 rel0 rev31 modo rb85 3725 jeff-31 relo rev31 modo rb86 3728 jeff-31 rel0 rev31 modo rb87 3731 jeff-31 rel0 rev31 modo sr82 3819 jeff-31 rel0 rev31 modo sr83 3822 jeff-31 rel0 rev31 modo sr83 3822 jeff 31 relo rev31 modo sr85 3828 jeff-31 relo rev31 modo sr86 3831 jeff-31 relo rev31 modo sr87 3834 jeff-31 relo rev31 modo sr88 3837 jeff-31 relo rev31 modo sr89 3840 jeft-31 relo rev31 modo sr90 3843 jeff-31 relo rev31 modo y86 3916 jeff-31 rel0 rev31 modo y87 3919 jeff-31 rel0 rev31 modo y88 3922 jeff-31 rel0 rev31 modo y89 3925 jeff-31 rel0 rev31 modo y 3028 jeff-31 relo rev31 modo y90 3928 jeff-31 relo rev31 modo y87m1 3920 jeff-31 relo rev31 modo y $87 \mathrm{ml} 3920$ jeff -31 relo rev31 modo zr86 4013 jeff-31 relo rev31 modo zr88 4019 jeff-31 rel0 rev31 modo zr89 4022 jeff-31 relo rev31 modo zr90 4025 jeff-31 rel0 rev31 modo zr91 4028 jeff-31 rel0 rev31 modo zr92 4031 jeff-31 rel0 rev31 modo zr93 4034 jeff-31 relo rev31 modo zr94 4037 jeff-31 rel0 rev31 modo $2 \times 954040$ jeff -31 rel0 rev31 modo zrg5 4040 jeff-31 relo rev31 modo 2964043 jeff-31 relo rev31 modo zr97 4046 jeff-31 relo rev31 modo nb90 4116 jeff-31 relo rev31 modo nb91 4119 jeff-31 rel0 rev31 modo nb92 4122 jeff-31 rel0 rev31 modo nb93 4125 jeff-31 rel0 rev31 modo nb94 4128 jeff-31 relo rev31 modo nb95 4131 jeff-31 relo rev31 modo nb96 4134 jeff-31 rel0 rev31 modo nb91m1 4120 jeff-31 relo rev31 modo nb92m1 4123 jeff-31 relo rev31 modo nag2m1 4123 jeff 31 relo rev31 modo nb95m1 4132 jeff-31 rel0 rev31 modo mo92 4225 jeff-31 relo rev31 modo mo93 4228 jeff-31 relo rev31 modo mo94 4231 jeff-31 rel0 rev31 modo mo95 4234 jeff-31 rel0 rev31 modo mo96 4237 jeff-31 rel0 rev31 mod0 mo97 4240 jeff-31 rel0 rev31 modo mo98 4243 jeff-31 relo rev31 modo mo99 4246 jeff-31 relo rev31 modo mo100 4249 jeff-31 relo rev31 modo tc95 4313 jeff-31 relo rev31 modo tc96 4316 jef $f-31$ relo revi modo tc97 4319 jeff-31 relo rev31 modo tc97 4319 jeff-31 relo rev31 modo tc98 4322 jeff-31 rel0 rev31 modo tc99 4325 jeff-31 relo rev31 modo tc95m1 4314 jeff-31 rel0 rev31 modo tc97m1 4320 jeff-31 rel0 rev31 modo ru96 4425 jeff-31 rel0 rev31 modo ru97 4428 jeff-31 relo rev31 modo ru98 4431 jeff-31 rel0 rev31 modo rug9 4434 jeff-31 relo rev31 modo rul0 4437 jeff -31 rel0 rev31 modo ruloo 4437 jeff-31 relo rev31 modo rul 44402 jeff -31 relo rev31 modo rulo2 4443 jeft 31 relo rev31 modo ru103 4446 jeff-31 relo rev31 modo ru104 4449 jeff-31 rel0 rev31 modo ru105 4452 jeff-31 relo rev31 modo ru106 4455 jeff-31 relo rev31 modo rh99 4513 jeff-31 rel0 rev31 modo rh100 4516 jeff-31 relo rev31 modo rh101 4519 jeff-31 rel0 rev31 modo rh102 4522 jeff-31 rel0 rev31 modo rh103 4525 jeff-31 rel0 rev31 modo h105 4531 jeff -31 relo rev31 modo th105 4531 jeff 31 relo rev31 modo rh9m1 4514 jeft-31 relo rev31 modo rh101m1 4520 jeff-31 relo rev31 modo rh102m1 4523 jeff-31 rel0 rev31 modo pd100 4619 jeff-31 relo rev31 modo pd101 4622 jeff-31 rel0 rev31 modo pd102 4625 jeff-31 rel0 rev31 modo pd103 4628 jeff-31 relo rev31 modo pd104 4631 jeff-31 rel0 rev31 modo pd105 4634 jeff-31 rel0 rev31 modo pd106 4637 jeff-31 rel0 rev31 modo 1074640 jeff -31 rel 0 rev31 modo palo 4603 jeff 31 relo rev31 modo pdice 4643 jeff-31 relo rev31 nodo pd109 4646 jeff-31 relo rev31 modo pd110 4649 jeff-31 relo rev31 modo pd112 4655 jeff-31 rel0 rev31 modo ag105 4719 jeff-31 rel0 rev31 modo ag107 4725 jeff-31 rel0 rev31 modo ag109 4731 jeff-31 relo rev31 mod0 ag111 4737 jeff-31 relo rev31 modo 
ag106m1 4723 jeff-31 relo rev31 modo ag108m1 4729 jeff-31 relo rev31 modo ag110m1 4735 jeff-31 rel0 rev31 modo cd106 4825 jeff-31 rel0 rev31 modo cd108 4831 jeff-31 rel0 rev31 modo cd109 4834 jeff-31 rel0 rev31 modo cd110 4837 jeff-31 rel0 rev31 modo cd110 4837 jeff 31 relo rev31 modo cd111 4830 jeff 31 relo rev31 modo cd112 4843 jeff-31 relo rev31 modo cd113 4846 jeff-31 relo rev31 modo cd114 4849 jeft-31 relo rev31 modo cd115 4852 jeff-31 rel0 rev31 modo cd116 4855 jeff-31 rel0 rev31 modo cd113m1 4847 jeff-31 relo rev31 modo cd115m1 4853 jeff-31 rel0 rev31 modo in111 4919 jeff-31 rel0 rev31 modo in113 4925 jeff-31 relo rev31 modo in115 4931 jeff-31 relo rev31 modo in114m1 4929 jeff-31 relo rev31 modo in114m1 4929 jeff-31 relo rev31 modo sn112 5025 jeff-31 relo rev31 modo sn113 5028 jeff 31 relo rev31 modo sn114 5031 jeff-31 relo rev31 modo sn115 5034 jeff-31 relo rev31 modo sn116 5037 jeff-31 rel0 rev31 modo sn117 5040 jeff-31 relo rev31 modo sn118 5043 jeff-31 relo rev31 modo sn119 5046 jeff-31 relo rev31 modo sn120 5049 jeff-31 relo rev31 modo sn121 5052 jeff-31 relo rev31 modo nn122 5055 jeff -31 rel0 rev31 nodo sn122 5055 jeft-31 relo rev31 modo sn123 5058 jefe -31 relo rev31 modo sn124 5061 jeff 31 relo rev31 modo sn125 5064 jelf-31 relo rev31 modo sn126 5067 jeff-31 rel0 rev31 modo sn117m1 5041 jeff-31 relo rev31 modo sn119m1 5047 jeff-31 rel0 rev31 modo sn121m1 5053 jeff-31 rel0 rev31 modo sb119 5119 jeff-31 rel0 rev31 modo sb121 5125 jeff-31 rel0 rev31 modo sb122 5128 jeff-31 rel0 rev31 modo sb123 5131 jeff-31 rel0 rev31 modo bit 5131 jeff-31 relo rev31 modo sb124 5134 jeff -31 relo rev31 modo sb125 513 sb126 5140 jeff-31 relo rev31 modo sb127 5143 jeff-31 relo rev31 modo sb120m1 5123 jeff-31 relo rev31 modo
te118 5219 jeff-31 rel0 rev31 modo te119 5222 jeff-31 relo rev31 modo te120 5225 jeff-31 relo rev31 modo te121 5228 jeff-31 relo rev31 modo te122 5231 jeff-31 relo rev31 modo te123 5234 jeff-31 rel0 rev31 modo te124 $5237 \mathrm{jeff}-31$ rel0 rev31 modo te $1255240 \mathrm{jeff}-31$ rel 0 rev 31 modo tello $5240 \mathrm{j}$ tel26 5243 jeff-31 relo rev31 modo te127 5246 jeft-31 relo rev31 modo te128 5249 jeff-31 rel0 rev31 modo te129 5252 jeff-31 rel0 rev31 modo te130 5255 jeff-31 relo rev31 modo te132 5261 jeff-31 relo rev31 modo tel19m1 5223 jeff-31 relo rev31 modo te121m1 5229 jeff-31 relo rev31 modo te123m1 5235 jeff-31 rel0 rev31 modo te $125 \mathrm{~m} 15241$ jeff -31 rel0 rev31 n tel25m1 5241 jeff 31 relo rev31 modo te129m1 5253 jeff -31 rel0 rev31 modo tel29m1 5253 jefr-31 relo rev31 mod0 te131mi 5259 jeff-31 relo rev31 modo i123 5313 jeff-31 rel0 rev31 modo 11245316 jeff-31 relo rev31 modo i125 5319 jeff-31 rel0 rev31 modo i126 5322 jeff-31 rel0 rev31 modo i127 5325 jeff-31 rel0 rev31 modo i128 5328 jeff-31 rel0 rev31 modo i129 5331 jeff-31 rel0 rev31 modo 11305334 jeff-31 rel0 rev31 modo i131 5337 jeff-31 relo rev31 modo i133 5343 jeff-31 relo rev31 modo 11335343 jeffesi relo rev31 modo xel22 5419 jeff-31 relo rev31 modo xe124 5425 jeff-31 relo rev31 modo xe125 5428 jeff-31 relo rev31 modo xe126 5431 jeff-31 relo rev31 modo xe127 5434 jeff-31 relo rev31 modo xe128 5437 jeff-31 rel0 rev31 modo xe129 5440 jeff-31 relo rev31 modo xe130 5443 jeff-31 rel0 rev31 modo xe131 5446 jeff-31 relo rev31 modo xe132 5449 jeff 31 relo rev31 modo xe132 5449 jeff 31 relo rev31 modo xe134 5455 jeff-31 relo rev31 modo xe135 5458 jeff-31 relo rev31 modo xe136 5461 jeff-31 rel0 rev31 modo xe129m1 5441 jeff-31 relo rev31 modo xe131m1 5447 jeff-31 relo rev31 modo xe133m1 5453 jeff-31 relo rev31 modo cs129 5513 jeff-31 relo rev31 modo 


\begin{tabular}{|c|c|c|c|}
\hline 55131 & Cs -131 & $\operatorname{cs} 131$ & 5519 \\
\hline 55132 & Cs -132 & $\operatorname{cs} 132$ & $5522 j$ \\
\hline 55133 & CS -133 & $\operatorname{cs} 133$ & $5525 j$ \\
\hline 55134 & cs -134 & $\operatorname{cs} 134$ & $5528 j$ \\
\hline 55135 & CS -135 & $\operatorname{cs} 135$ & $5531 \mathrm{j}$ \\
\hline 55136 & cs -136 & $\operatorname{cs} 136$ & $5534 j$ \\
\hline 55137 & CS -137 & $\operatorname{cs} 137$ & $5537 \mathrm{j}$ \\
\hline 56128 & ba -128 & ba128 & $5619 j$ \\
\hline 56129 & ba -129 & ba129 & $5622 j$ \\
\hline 56130 & ba -130 & ba130 & $5625 j$ \\
\hline 56131 & ba-131 & ba131 & 5628 \\
\hline 56132 & ba-132 & ba132 & $5631 j$ \\
\hline 56133 & $b a-133$ & ba133 & $5634 j$ \\
\hline 56134 & ba-134 & ba134 & $5637 \mathrm{j}$ \\
\hline 56135 & ba-135 & ba135 & $5640 j$ \\
\hline 56136 & ba-136 & ba136 & $5643 j$ \\
\hline 56137 & ba -137 & ba137 & $5646 j$ \\
\hline 56138 & ba -138 & ba138 & $5649 j$ \\
\hline 56139 & ba-139 & ba139 & 5652 \\
\hline 56140 & ba -140 & ba140 & $5655 \mathrm{j}$ \\
\hline 56601 & 56601 & ba133m & n1 5635 \\
\hline 56611 & 56611 & ba135m & n1 5641 \\
\hline 57135 & $1 a-135$ & la135 & $5716 j$ \\
\hline 57137 & $1 a-137$ & $\operatorname{la} 137$ & 5722 \\
\hline 57138 & $1 a-138$ & la138 & $5725 j$ \\
\hline 57139 & $1 a-139$ & $\operatorname{la} 139$ & 5728 \\
\hline 57140 & $1 a-140$ & la140 & $5731 j$ \\
\hline 57141 & $1 a-141$ & la141 & $5734 j$ \\
\hline 58134 & $\mathrm{ce}-134$ & ce134 & $5819 j$ \\
\hline 58135 & $\mathrm{ce}-135$ & ce135 & $5822 j$ \\
\hline 58136 & $\mathrm{ce}-136$ & ce136 & $5825 j$ \\
\hline 58138 & $\mathrm{ce}-138$ & ce138 & $5831 j$ \\
\hline 58139 & $\mathrm{ce}-139$ & ce139 & $5834 j$ \\
\hline 58140 & $\mathrm{ce}-140$ & ce140 & $5837 \mathrm{j}$ \\
\hline 58141 & $\mathrm{ce}-141$ & $\mathrm{ce} 141$ & $5840 j$ \\
\hline 58142 & $\mathrm{ce}-142$ & ce142 & $5843 j$ \\
\hline 58143 & $\mathrm{ce}-143$ & $\mathrm{ce} 143$ & 5846 \\
\hline 58144 & $\mathrm{ce}-144$ & ce144 & $5849 j$ \\
\hline 58601 & 58601 & $\mathrm{ce} 137 \mathrm{~m}$ & n1 5829 \\
\hline 59141 & pr -141 & pr141 & $5925 j$ \\
\hline 59142 & pr -142 & pr142 & $5928 j$ \\
\hline 59143 & pr-143 & pr143 & $5931 \mathrm{j}$ \\
\hline 60140 & nd -140 & nd140 & $6019 j$ \\
\hline 60141 & nd-141 & nd141 & $6022 j$ \\
\hline 60142 & nd -142 & nd1 42 & $6025 j$ \\
\hline 60143 & nd -143 & nd143 & $6028 j$ \\
\hline 60144 & nd -144 & nd144 & $6031 j$ \\
\hline 60145 & nd-145 & nd145 & $6034 j$ \\
\hline 60146 & nd-146 & nd146 & $6037 j$ \\
\hline 60147 & nd-147 & nd147 & $6040 j$ \\
\hline 60148 & nd -148 & nd148 & $6043 j$ \\
\hline 60149 & nd -149 & nd149 & $6046 j$ \\
\hline 60150 & nd -150 & nd150 & $6049 j$ \\
\hline 61143 & $\mathrm{pm}-143$ & pm143 & $6137 \mathrm{j}$ \\
\hline 61144 & $\mathrm{pm}-144$ & pm144 & $6140 j$ \\
\hline 61145 & $\mathrm{pm}-145$ & pm145 & $6143 j$ \\
\hline 61146 & $\mathrm{pm}-146$ & pm146 & 6146 \\
\hline 61147 & $\mathrm{pm}-147$ & pm147 & $6149 j$ \\
\hline 61148 & $\mathrm{pm}-148$ & pm148 & $6152 j$ \\
\hline 61149 & $\mathrm{pm}-149$ & pm149 & $6155 j$ \\
\hline 61150 & $\mathrm{pm}-150$ & pm150 & $6158 j$ \\
\hline 61151 & $\mathrm{pm}-151$ & pm151 & $6161 \mathrm{j}$ \\
\hline 61601 & $\mathrm{pm}-148 \mathrm{~m}$ & $\mathrm{pm} 148 \mathrm{~m}$ & n1 6153 \\
\hline 62144 & $\mathrm{sm}-144$ & $\operatorname{sm1} 44$ & 6225 \\
\hline 62145 & $\mathrm{sm}-145$ & $\operatorname{sm} 145$ & 6228 \\
\hline 62146 & $\mathrm{sm}-146$ & $\operatorname{sm} 146$ & $6231 j$ \\
\hline 62147 & $\mathrm{sm}-147$ & $\operatorname{sm1} 47$ & $6234 j$ \\
\hline 62148 & $\mathrm{sm}-148$ & $\operatorname{sm1} 48$ & $6237 j$ \\
\hline 62149 & $\mathrm{sm}-149$ & $\operatorname{sm1} 49$ & $6240 j$ \\
\hline 62150 & $\mathrm{sm}-150$ & sm150 & 6243 \\
\hline 62151 & $\mathrm{sm}-151$ & sm151 & $6246 j$ \\
\hline 62152 & $\mathrm{sm}-152$ & sm152 & $6249 j$ \\
\hline 62153 & $\mathrm{sm}-153$ & $\operatorname{sm1} 153$ & $6252 j$ \\
\hline 62154 & $\mathrm{sm}-154$ & sm154 & 6255 \\
\hline 63145 & eu-145 & eu145 & 6307 \\
\hline 63146 & eu-146 & eu146 & 6310 \\
\hline 63147 & eu-147 & eu147 & 6313 \\
\hline 63148 & eu-148 & eu148 & 6316 \\
\hline 63149 & eu-149 & eu149 & 6319 \\
\hline 63150 & eu-150 & eu150 & 6322 \\
\hline 63151 & eu-151 & eu151 & 6325 \\
\hline 63152 & $\mathrm{eu}-152$ & eu152 & 6328 \\
\hline 63153 & eu-153 & eu153 & 6331 \\
\hline 63154 & eu-154 & eu154 & 6334 \\
\hline 63155 & eu-155 & eu155 & 6337 \\
\hline 63156 & eu-156 & eu156 & 6340 \\
\hline 63157 & eu-157 & eu157 & 6343 \\
\hline 63601 & 63601 & eu $150 \mathrm{~m}$ & n1 632 \\
\hline 63611 & 63611 & eu $152 \mathrm{~m}$ & n1 6329 \\
\hline 64146 & $g d-146$ & gd146 & 6407 \\
\hline 64147 & gd-147 & gd147 & 6410 \\
\hline 64148 & $g d-148$ & $\operatorname{gd} 148$ & 6413 \\
\hline 64149 & gd-149 & gd149 & 6416 \\
\hline 64150 & $g d-150$ & gd150 & 6419 \\
\hline 64151 & $\mathrm{gd}-15$ & 501 & 6422 \\
\hline
\end{tabular}


gd152 6425 jeff-31 rel0 rev31 modo gd153 6428 jeff-31 rel0 rev31 modo gd154 6431 jeff-31 relo rev31 modo gd155 6434 jeff-31 rel0 rev31 modo gd156 6437 jeff-31 relo rev31 modo gd157 6440 jeff-31 rel0 rev31 modo gd158 6443 jeff-31 rel0 rev31 mod0 gd159 6446 jeff-31 relo gd159 6446 jeff 31 relo rev31 modo ga160 6449 tb151 6501 jeff-31 relo rev31 modo tb152 6504 jeff-31 relo rev31 modo tb153 6507 jeff-31 rel0 rev31 modo tb154 6510 jeff-31 rel0 rev31 mod0 tb155 6513 jeff-31 relo rev31 modo tb156 6516 jeff-31 rel0 rev31 modo tb157 6519 jeff-31 rel0 rev31 modo tb158 6522 jeff-31 rel0 rev31 modo tb159 6525 jeff-31 relo rev31 modo tb160 6528 jeff -31 rel0 rev31 modo tb160 6528 jeff-31 relo rev31 modo tb161 6531 jeff 31 relo rev31 modo tb156m1 6517 jeff-31 rel0 rev31 modo tb154m2 6512 jeff-31 relo rev31 mod0 tb156m2 6518 jeff-31 rel0 rev31 modo dy154 6619 jeff-31 relo rev31 modo dy155 6622 jeff-31 relo rev31 modo dy156 6625 jeff-31 relo rev31 modo dy157 6628 jeff-31 relo rev31 modo dy158 6631 jeff-31 rel0 rev31 modo dy159 6634 jeff-31 relo rev31 modo dy160 6637 jeff -31 rel0 rev31 modo dy160 6637 jeff-31 relo rev31 modo dy161 6640 jeff -31 relo rev31 modo dy162 6643 jeff 31 relo rev31 modo dy163 6646 jeff-31 relo rev31 modo dy164 6649 jeff-31 relo rev31 modo dy165 6652 jeff-31 rel0 rev31 modo dy166 6655 jeff-31 relo rev31 modo ho163 6719 jeff-31 rel0 rev31 modo ho164 6722 jeff-31 relo rev31 modo ho165 6725 jeff-31 rel0 rev31 modo ho166 6728 jeff-31 rel0 rev31 modo ho164m1 6723 jeff-31 relo rev31 modo hol66m1 6729 jeff-31 relo rev31 modo er160 6819 jeff-31 relo rev31 modo er161 6822 jeff-31 relo rev31 modo er162 6825 jeff-31 relo rev31 modo er164 6831 jeff-31 rel0 rev31 modo er165 6834 jeff-31 rel0 rev31 modo er166 6837 jeff-31 rel0 rev31 modo er167 6840 jeff-31 relo rev31 modo er168 6843 jeff-31 relo rev31 modo er169 6846 jeff-31 rel0 rev31 modo er170 6849 jeff-31 rel0 rev31 mod0 er171 6852 jeff-31 rel0 rev31 modo er172 6855 jeff -31 relo rev31 modo (9.172 6855 tm165 6913 jetr-31 relo rev31 modo tm166 6916 jeft-31 relo rev31 modo tm167 6919 jeff-31 rel0 rev31 modo tm168 6922 jeff-31 relo rev31 modo tm169 6925 jeff-31 relo rev31 modo tm170 6928 jeff-31 relo rev31 modo tm171 6931 jeff-31 relo rev31 modo tm172 6934 jeff-31 rel0 rev31 modo vb166 7019 jeff-31 rel0 rev31 modo yb168 7025 jeff-31 rel0 rev31 Yb168 7025 jefe-31 relo rev31 mod0 yb171 7034 jeff-31 relo rev31 modo yb172 7037 jeff-31 rel0 rev31 modo yb173 7040 jeff-31 rel0 rev31 modo yb174 7043 jeff-31 relo rev31 modo yb175 7046 jeff-31 relo rev31 modo yb176 7049 jeff-31 relo rev31 modo lu169 7107 jeff-31 relo rev31 modo 1 u170 7110 jeff-31 relo rev31 modo 11717113 jeff -31 relo rev31 modo lu172 7116 jeff-31 rel0 rev31 modo lu173 7119 jeff-31 relo rev31 modo lu174 7122 jeff-31 relo rev31 lu175 7125 jeff-31 rel0 rev31 modo lu176 7128 jeff-31 rel0 rev31 modo lu177 7131 jeff-31 rel0 rev31 modo lu174m1 7123 jeff-31 relo rev31 modo lu177m1 7132 jeff-31 rel0 rev31 modo hf170 7213 jeff-31 rel0 rev31 modo hf171 7216 jeff-31 relo rev31 hf172 7219 jeff-31 rel0 rev31 modo he173 7222 jeff 31 relo rev31 nodo he173 7222 jeff 31 relo rev31 modo hfl75 7228 jeff-31 relo rev31 modo hf176 7231 jeff-31 relo rev31 modo hf177 7234 jeff-31 relo rev31 modo hf178 7237 jeff-31 relo rev31 modo hf179 7240 jeff-31 relo rev31 modo hf180 7243 jeff-31 relo rev31 modo hf181 7246 jeff-31 relo rev31 modo 


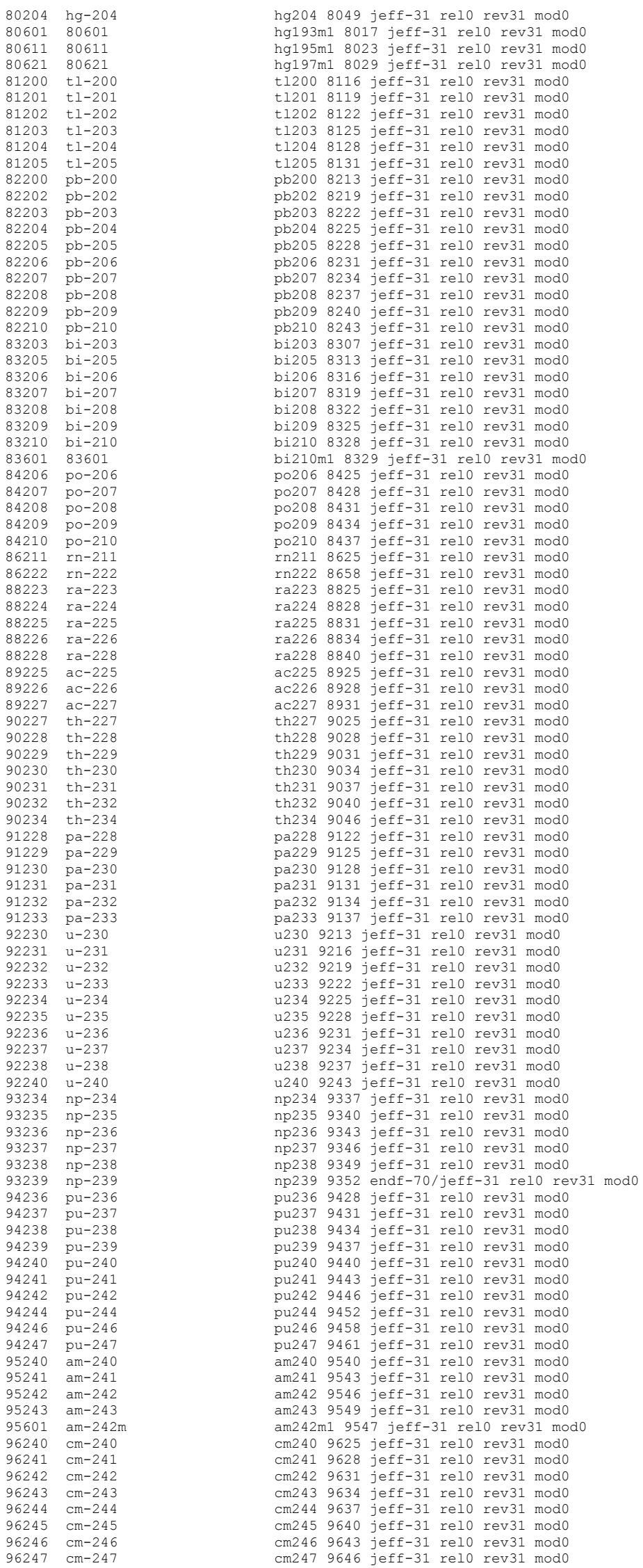




$\begin{array}{rl}96248 & \mathrm{~cm}-248 \\ 96249 & \mathrm{~cm}-249 \\ 96250 & \mathrm{~cm}-250 \\ 97245 & \mathrm{bk}-245 \\ 97246 & \mathrm{bk}-246 \\ 97247 & \mathrm{bk}-247 \\ 97248 & \mathrm{bk}-248 \\ 97249 & \mathrm{bk}-249 \\ 97250 & \mathrm{bk}-250 \\ 97601 & 97601 \\ 98246 & \mathrm{cf}-246 \\ 98248 & \mathrm{cf}-248 \\ 98249 & \mathrm{cf}-249 \\ 98250 & \mathrm{cf}-250 \\ 98251 & \mathrm{cf}-251 \\ 98252 & \mathrm{cf}-252 \\ 98253 & \mathrm{cf}-253 \\ 98254 & \mathrm{cf}-254 \\ 99251 & \mathrm{es}-251 \\ 99252 & \mathrm{es}-252 \\ 99253 & \mathrm{es}-253 \\ 99254 & \mathrm{es}-254 \\ 99255 & \mathrm{es}-255 \\ 99601 & 99601 \\ 100252 & \mathrm{fm}-252 \\ 100253 & \mathrm{fm}-253 \\ 100255 & \mathrm{fm}-255 \\ 100257 & \mathrm{fm}-257\end{array}$

cm248 9649 jeff-31 rel0 rev31 modo cm249 9652 jeff-31 relo rev31 modo cm250 9655 jeff-31 relo rev31 modo bk245 9740 jeff-31 relo rev31 modo bk246 9743 jeff-31 rel0 rev31 modo bk247 9746 jeff-31 rel0 rev31 modo bk248 9749 jeff-31 rel0 rev31 mod0 bk249 9752 jeff-31 relo rev31 modo kk250 9755 jeff-31 relo rev31 modo bk248m1 9750 jeff-31 relo rev31 modo bk248m1 9750 jeff-31 rel0 rev31 modo cf246 9843 jeff-31 rel0 rev31 modo cf249 9852 jeff-31 relo rev31 modo cf250 9855 jeff-31 rel0 rev31 modo cf251 9858 jeff-31 relo rev31 modo cf252 9861 jeff-31 rel0 rev31 modo cf253 9864 jeff-31 rel0 rev31 modo cf254 9867 jeff-31 rel0 rev31 modo e 2519911 jeff-31 relo rev31 modo es251 9911 jeff-31 relo rev31 modo es 2529912 jeff 31 relo rev31 modo es253 9913 jefr-31 relo rev31 modo es254 9914 jeft-31 relo rev31 modo es255 9916 jeff-31 rel0 rev31 modo es254m1 9915 jeff-31 relo rev31 modo
fm252 9933 jeff-31 relo rev31 modo fm253 9934 jeff-31 relo rev31 modo fm255 9936 jeff-31 rel0 rev31 modo

Figure 5.2.7. Contents of reaction resource. 
5.3 ORIGEN REACTOR LIBRARIES

B. R. Betzler, B. J. Ade 


\section{TABLE OF CONTENTS}

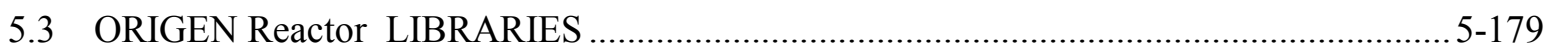

5.3.1 Description of ORIGEN Reactor Libraries in SCALE .......................................5-182

5.3.2 How to Generate ORIGEN Cross-Section Libraries ..................................................... 5-185 


\section{LIST OF TABLES}

$\underline{\text { Page }}$

Table 5.3.1. Summary of ORIGEN libraries (1470 total libraries) ................................................. 5-183

Table 5.3.2. Additional information on ORIGEN libraries. ........................................................ 5-184 


\subsubsection{Description of ORIGEN Reactor Libraries in SCALE}

The ARP code creates burnup-dependent ORIGEN cross-section libraries by interpolating over reactor cross-section libraries generated in advance using reactor physics transport methods. The reactor cross-section libraries distributed in SCALE include many classes of commercial power reactor designs and a range of fuel assembly designs.

Cross-section libraries suitable for use with ORIGEN are available in SCALE for the following reactor and fuel assembly designs:

- $\quad$ BWR 7×7, 8×8-1, 8×8-2, 9×9-2, 9×9-9, 10×10-9, 10×10-8, SVEA-64, SVEA-96, and SVEA-100;

- PWR $14 \times 14,15 \times 15,16 \times 16,17 \times 17,18 \times 18$;

- CANDU reactor (19-, 28-, and 37-element bundle designs);

- Magnox graphite reactor;

- Advanced Gas-Cooled Reactor (AGR);

- VVER 440 and VVER 1000;

- RBMK;

- IRT;

- MOX BWR 7×7, 8×8-1, 8×8-2, 9×9-2, 9×9-9, 10×10-9, 10×10-8, SVEA-64, SVEA-96, and SVEA-100;

- MOX PWR $14 \times 14,15 \times 15,16 \times 16,17 \times 17,18 \times 18$.

All of the libraries distributed with SCALE were developed using ENDF/B-VII.1-based 252group cross section libraries and the SCALE 6.2 TRITON module. The assembly design and the identifier for each library are summarized in Table 1. All TRITON templates used to generate these libraries are provided with the release; these templates have been developed to reflect the most recent publicly available data and to adhere to current SCALE best practices. MOX templates use the same set of BWR and PWR lattices, with MOX compositions replacing the $\mathrm{UO}_{2}$ fuel.

Additionally, a Python script (slig.py) has been developed to semi-automate the process of generating sets of libraries from information in these templates. This script calls another Python script to automatically generate a PDF documentation file with $\mathrm{L}^{\mathrm{A}} \mathrm{T}_{\mathrm{E}} \mathrm{X}$. This SLIG script is made available to the user; more details are available in the SLIG manual. 
Table 5.3.1. Summary of ORIGEN libraries (1470 total libraries).

\begin{tabular}{|c|c|c|}
\hline Reactor type & Assembly design description & Library Name(s) \\
\hline \multirow{9}{*}{$\begin{array}{l}\text { PWR LEU* } \\
\text { and } \mathrm{MOX}^{\dagger}\end{array}$} & Babcock \& Wilcox $15 \times 15$ & bw15x15, mox_bw15x15 \\
\hline & Siemens $14 \times 14$ & s14x14, mox_s14x14 \\
\hline & Siemens $18 \times 18$ & s18x18, mox_s18x18 \\
\hline & Westinghouse $14 \times 14$ & w14x14, mox_w14x14 \\
\hline & Westinghouse $15 \times 15$ & w15x15, mox_w15x15 \\
\hline & Westinghouse $17 \times 17$ & w17x17, mox_w17x17 \\
\hline & Westinghouse $17 \times 17$-OFA & w17x17_ofa, mox_w17x17_ofa \\
\hline & Westinghouse CE $14 \times 14$ & ce14x14, mox_ce14x14 \\
\hline & Westinghouse CE $16 \times 16$ & ce16x16, mox_ce16x16 \\
\hline \multirow{11}{*}{$\begin{array}{l}\text { BWR LEU } \\
\text { and MOX }\end{array}$} & ABB $8 \times 8-1$ & abb8x8-1, mox_abb8x8-1 \\
\hline & ATRIUM $9 \times 9-9$ & atrium9x9-9, mox_atrium9x9-9 \\
\hline & ATRIUM 10×10-9 & atrium10x10-9, mox_atrium10x10-9 \\
\hline & General Electric $7 \times 7-0$ & ge7 $7 \times 7-0$, mox_ge $7 \times 7-0$ \\
\hline & General Electric $8 \times 8-1$ & ge8x8-1, mox_ge8x8-1 \\
\hline & General Electric $8 \times 8-2$ & ge8x8-2, mox_ge8x8-2 \\
\hline & General Electric $9 \times 9-2$ & ge9x9-2, mox_ge9x9-2 \\
\hline & General Electric $10 \times 10-8$ & ge10x10-8, mox_ge10x10-8 \\
\hline & SVEA $64(8 \times 8-1)$ & svea64-1, mox_svea64-1 \\
\hline & SVEA $96(10 \times 10-4)$ & svea96-0, mox_svea96-0 \\
\hline & SVEA $100(10 \times 10-0)$ & svea100-0, mox_svea100-0 \\
\hline \multirow[t]{5}{*}{ VVER } & VVER-440 flat enrichment & vver440 \\
\hline & $\begin{array}{l}\text { VVER-440 radial enr. profile, } \\
\text { avg. } 3.82 \%\end{array}$ & vver440_3.82 \\
\hline & $\begin{array}{l}\text { VVER-440 radial enr. profile, } \\
\text { avg. } 4.25 \%\end{array}$ & vver440_4.25 \\
\hline & $\begin{array}{l}\text { VVER-440 radial enr. profile, } \\
\text { avg. } 4.38 \%\end{array}$ & vver440_4.38 \\
\hline & VVER-1000 & vver1000 \\
\hline RBMK & RBMK 1000 , flat enrichment & rbmk1000 \\
\hline \multirow{3}{*}{ CANDU } & 19-element bundle design & candu19 \\
\hline & 28-element bundle design & candu28 \\
\hline & 37-element bundle design & candu37 \\
\hline Magnox & Magnox graphite reactor (Calder Hall) & magnox \\
\hline AGR & Advanced gas-cooled reactor & agr \\
\hline \multirow[t]{6}{*}{ IRT } & IRT-2M (3 tube) & irt2m3tube36enrich, irt2m3tube \\
\hline & IRT-2M (4 tube) & irt2m4tube36enrich, irt2m4tube \\
\hline & IRT-3M (6 tube) & irt3m6tube36enrich, irt3m6tube90enrich \\
\hline & IRT-3M (8 tube) & irt3m8tube36enrich, irt3m8tube90enrich \\
\hline & IRT-4M (6 tube) & irt4m6tube \\
\hline & IRT-4M (8 tube) & irt4m8tube \\
\hline
\end{tabular}

\footnotetext{
${ }^{*}$ LEU $=$ Low-enriched uranium

$\dagger$ MOX $=$ Mixed-oxide
} 
The libraries for BWR and RBMK assembly designs all include variable coolant density. Table 2 lists the number of cross-section files associated with each individual assembly design; this number is often a product of the number of enrichments (or Pu contents for MOX assemblies) and the number of coolant density values. Unless otherwise noted in Table 2, the PWR and BWR LEU libraries were generated for seven enrichment values: $0.5,1.5,2.0,3.0,4.0,5.0$, and 6.0 $\mathrm{wt} \%{ }^{235} \mathrm{U}$. The cross sections are represented at several burnup values in the range from 0 to the maximum burnup listed. The number of burnup values on the library has been optimized with a post-processing script to minimize the size of the data libraries without losing significant fidelity in the cross section data. Note that these optimized burnup steps are not the same burnup steps used in the TRITON calculations; more burnup steps are used to calculate the depletion of the fuel. The fuel assembly designations used for many of the BWR designs include the basic lattice type, followed optionally by the number of empty (non fuel) lattice positions. For example, the $9 \times 9-9$ designation refers to the Atrium design with a $9 \times 9$ assembly lattice with 9 non-fuel (water) locations. Additional data on the reactor libraries is available in the library information PDF file released with SCALE. This document includes details on the fuel lattice type, the fuel vendor (where design information is specific to a vendor design), the assembly model, basic fuel design and reactor operating data, and the range of the variable parameters associated with the libraries (e.g., enrichment range, burnup range, etc.). This document references separate reports from which a more detailed description of the data for these libraries can be found.

Table 5.3.2. Additional information on ORIGEN libraries.

\begin{tabular}{|l|l|l|c|c|}
\hline $\begin{array}{l}\text { Assembly } \\
\text { type }\end{array}$ & $\begin{array}{l}\text { Enrichments } \\
{[\%]}\end{array}$ & $\begin{array}{l}\text { Coolant/moderator } \\
\text { densities }\left[\mathrm{g} / \mathrm{cm}^{3}\right]\end{array}$ & $\begin{array}{c}\text { Maximum burnup } \\
{[\mathrm{GWd} / \mathrm{MTU}]}\end{array}$ & $\begin{array}{c}\text { Number of } \\
\text { Libraries }\end{array}$ \\
\hline PWR LEU & $0.5,1.5,2,3,4,5,6$ & $\sim 0.73$ & 70.5 & 7 \\
\hline BWR LEU & $0.5,1.5,2,3,4,5,6$ & $0.1,0.3,0.5,0.7,0.9$ & 70.5 & 35 \\
\hline PWR MOX & $*$ & $\sim 0.73$ & 70.5 & 15 \\
\hline BWR MOX & $*$ & $0.1,0.3,0.5,0.7,0.9$ & 70.5 & 75 \\
\hline AGR & $0.5,1.5,2,3,4,5$ & 1.65 & 48.7 & 6 \\
\hline CANDU & 0.711 & 0.8445 & 13.7 & 3 \\
\hline IRT & $19.75,36,80,90$ & 0.989 & 159 & 12 \\
\hline Magnox & $0.7,0.8,0.9,1$ & 1.628 & 13.7 & 4 \\
\hline RBMK & $1.8,2.2,2.6,3$ & $0.15,0.28,0.41,0.54,0.67$, & 24.3 & 24 \\
\hline VVER-440 & $1.6,2.4,3.6$, profiled & 0.73 & 70.5 & 6 \\
\hline VVER-1000 & $0.5,1.5,2,3,4,5,6$ & 0.7145 & 70.5 & 7 \\
\hline
\end{tabular}

${ }^{*} \mathrm{Pu}$ contents [\%]: 4, 7, 10; ${ }^{239} \mathrm{Pu}$ contents [\%]: 50, 55, 60, 65, 70 


\subsubsection{How to Generate ORIGEN Cross-Section Libraries}

This section describes the basic procedures one needs to follow to generate ORIGEN crosssection libraries for fuel types not included in SCALE. Note that extension of the methods and data beyond $5 \mathrm{wt} \%$ enrichment has not been widely investigated at ORNL because of a lack of accessible validation data for non-commercial reactor fuels. Such applications of the methodology should be tested carefully and validated. The following discussion and examples relate primarily to generating commercial LWR fuel libraries. Note that the parameter ranges used as examples are not necessarily appropriate to non-LWR applications, and will need to be modified for different reactor types and fuel designs.

The first step is to construct a physics model of the fuel lattice with the descriptions of the reactor assembly under consideration. For a given initial fuel enrichment, a TRITON depletion calculation is performed using one of the depletion analysis sequences of SCALE. TRITON can use either an explicit 2-D representation of the fuel assembly using the NEWT discrete ordinates transport code, or a 3-D representation of the assembly using the KENO Monte Carlo transport code. TRITON allows multiple fuel types to be defined and depleted independently.

The depletion analysis sequences are used to simulate irradiation and depletion of the fuel over the required irradiation history. A burnup analysis is typically modeled using a series of time intervals, or cycles. During the simulation, cross-sections that are representative of the mid-point of each cycle are created and saved in the library by the depletion sequence. In addition, cross sections that are representative of fresh fuel conditions (zero burnup) are now automatically saved in the library, without the need for the user to add a small burnup step to represent initial cross sections, as required in earlier versions of SCALE.

Past experience in creating LWR fuel libraries has indicated that depletion simulations with burnup steps of 3,000 MWd/MTU are generally adequate to represent the cross section variations with burnup. Each set of burnup-dependent cross sections is stored within the single library, and each set is accessed sequentially by its position in the library. The position 1 set contains freshfuel cross sections and the remaining cross-section sets (positions) correspond to burnup levels characterizing the midpoint of each burnup step in the depletion sequence calculation.

For fuels with multiple enrichment values, the above procedure is repeated for each enrichment. For fuels with a single enrichment value (e.g., natural uranium), only one depletion case is required. Cross-section changes with enrichment are generally represented using approximately $1.0 \mathrm{wt} \%$ increments, e.g., $0.5,1.5,2.0,3.0,4.0,5.0$, and $6.0 \mathrm{wt} \%$ of ${ }^{235} \mathrm{U}$. Because the crosssection variation is generally well behaved and smooth with changes in enrichment, it may be possible to maintain accuracy using fewer enrichment points. For BWR designs, or reactor designs involving considerable moderator density variations, one has also to consider the effect of water density variation on the cross sections. The recommended water density values for BWRs are typically 0.1 to $0.9 \mathrm{~g} / \mathrm{cm}^{3}$, with increments of about $0.2 \mathrm{~g} / \mathrm{cm}^{3}$. Again, fewer points may be adequate for many applications. Water density variation has typically not been included for PWR libraries developed at ORNL because of the relatively small variation in pressurized water reactors. When the variation in moderator density is considered, the libraries must be 
calculated for each combination of enrichment and moderator density. This may involve a considerable number of depletion analysis simulations to prepare all of the required cross-section libraries.

The SLIG utility has been created to help automate the creation of input files that represent each of the discrete parameter combinations. Instead of having to manually create input files for each parameter value, a single template input file is constructed with generic flags substituted for the parameters that vary from case-to-case (i.e., enrichment and water density). The specific values required for each parameter are specified in the header of the template file; SLIG reads the template and substitutes the values for the generic flags in the input file. SLIG generates a unique subdirectory and input file for each parameter combination. A description of SLIG and examples are provided in the SLIG manual.

Another utility provides an optional procedure that can be used to reduce the size of the crosssection libraries by eliminating some of the burnup-dependent cross-section sets in the libraries in regimes where the cross sections do not vary appreciably with burnup. In general, the change in cross-section values is more pronounced early in irradiation, and tends to approach asymptotic values at higher burnup. Therefore, interpolation of the cross sections using fewer data points may yield acceptable accuracy in this range. This utility is currently unavailable in SCALE 6.2.

As an example of this procedure, the basic steps in creating a set of cross-section libraries for a typical PWR assembly are illustrated. In this example the burnup is calculated up to 72 $\mathrm{GWd} / \mathrm{MTU}$. To generate these basic cross-section libraries, the following steps are performed for each fuel enrichment value:

1. Create a 2-D or 3-D reactor physics model using TRITON for the fuel assembly design and fuel type being considered.

2. Run a depletion analysis calculation to the maximum burnup under consideration. For example, set a maximum burnup at $72 \mathrm{GWd} / \mathrm{MTU}$, and simulate using 24 intervals (cycles) with one library per cycle. Each of these cycles is $3 \mathrm{GWd} / \mathrm{MTU}$ in size. The library generated by this analysis will contain 25 sets of cross sections: initial fresh fuel cross sections plus 24 burnup-dependent cross sections.

3. Include as many extra nuclides in the fresh fuel composition specification as is practical in trace concentrations (e.g., 1E-20 atoms/barn-cm). This procedure ensures that updated cross sections for each nuclide added will be used in creating the ORIGEN library. The depletion analysis sequences automatically add many of the most important actinides and fission products. However, the addition of more nuclides by the user will result in more complete updating of the cross-section data. The addition of trace nuclides is handled automatically in TRITON using the ADDNUX= parameter entry.

4. The above procedure is repeated for each enrichment value. If moderator density variation is to be included, a separate calculation is required for each enrichment-moderator density value combination.

5. Finally, the ARPDATA.TXT file must be updated to include the data for the new crosssection libraries created. This file contains information on the library, including the number of values for each variable parameter, the parameter values, the burnup values for each 
library position, and the actual library file names. The burnup values for each set of cross sections in the library are printed in the TRITON output file to assist the user in determining the appropriate library entries. Once the library information is registered in the ARPDATA.TXT file, it can be used directly by ORIGEN. 


\title{
5.4 ORIGAMI: A CODE FOR COMPUTING ASSEMBLY ISOTOPICS WITH ORIGEN
}

M. L. Williams, S. E. Skutnik ${ }^{1}$, I. C. Gauld, W. A. Wieselquist, and R. A. Lefebvre

\begin{abstract}
ORIGAMI computes detailed isotopic compositions for light water reactor assemblies containing $\mathrm{UO}_{2}$ fuel by using the ORIGEN transmutation code with pre-generated ORIGEN libraries, for a specified assembly power distribution. The fuel may be modeled using either lumped or pinwise representations with the option of including axial zones. In either case, ORIGAMI performs ORIGEN burnup calculations for each of the specified power regions to obtain the spatial distribution of isotopes in the burned fuel. Multiple cycles with varying burn-times and downtimes may be used. ORIGAMI produces several types of output files, including one containing stacked ORIGEN binary output data ("ft71 file") for each depletion zone; files with nuclide concentrations at the last time-step for each axial depletion region, in the format of SCALE standard composition input data or as MCNP material cards; a file containing the axial decay heat at the final time-step; and gamma and neutron radiation source spectra.
\end{abstract}

\footnotetext{
${ }^{1}$ University of Tennessee, Knoxville
} 


\section{TABLE OF CONTENTS}

Page

5.4 ORIGAMI: A Code for Computing Assembly Isotopics with ORIGEN.............................5-188

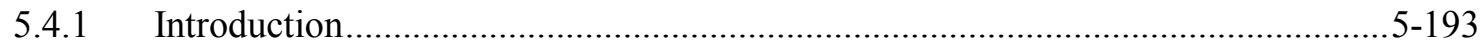

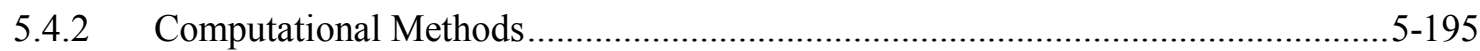

5.4.3 ORIGAMI Input Description ............................................................................... 5-200

5.4.4 ORIGAMI Input/Output Files.......................................................................... 5-211

5.4.5 Parallel Execution on Linux Clusters................................................................5-218

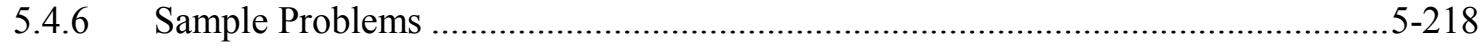

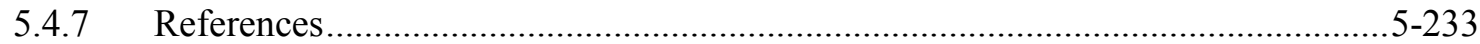




\section{LIST OF FIGURES}

Page

Figure 5.4.1. Fulcrum UO2 express form for creating ORIGAMI input

Figure 5.4.2. MeshView plots of total plutonium content in the 3D depletion regions (left: XZ plane; right: XY plane).

Figure 5.4.3. MeshView Plot of Axial Burnup Map (MWd/MTU) for Sample Problem 5. 


\section{LIST OF TABLES}

Table 5.4.1. Template for ORIGAMI Input Data.........................................................................5-201

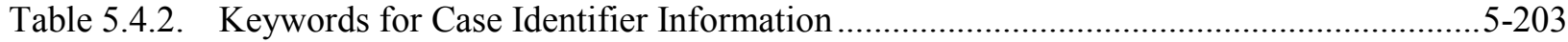

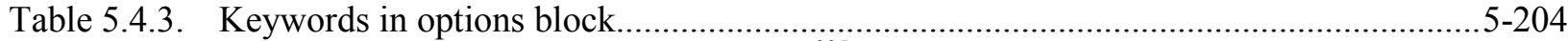

Table 5.4.4. Uranium isotope dependence on $\mathrm{X} \mathrm{wt} \%{ }^{235} \mathrm{U}$ enrichment .......................................5-206

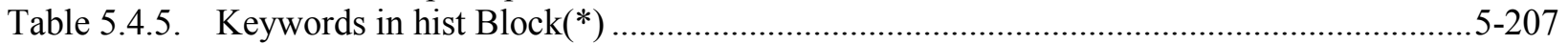

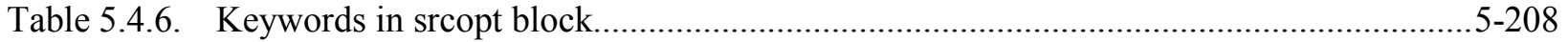

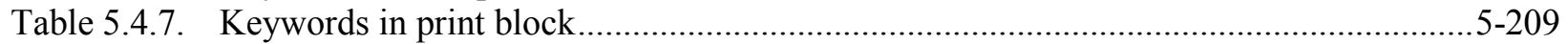

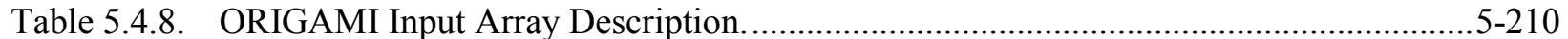

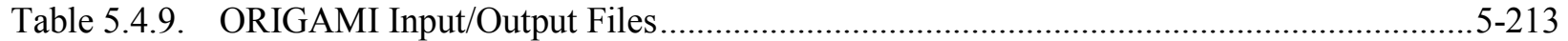

Table 5.4.10. Default Burnup Credit Nuclides in Standard Comp File ............................................5-215

Table 5.4.11. Input for ORIGAMI Sample Problem 1 ....................................................................... 5-219

Table 5.4.12. Calculated Actinide Isotopics for Sample Problem 1 ...................................................5-219

Table 5.4.13. Input for ORIGAMI Sample Problem 2 ............................................................... 5-220

Table 5.4.14. Calculated Actinide Isotopics by Axial Zone for Sample Problem 2 ...........................5-221

Table 5.4.15. Sample Problem 2: Standard Composition File (Default Burnup Credit Nuclides) .......5-222

Table 5.4.16. Sample Problem 2: Decay Heat File (Watts) by Axial Interval ....................................5-226

Table 5.4.17. Input for ORIGAMI Sample Problem 3 ..................................................................... 5-227

Table 5.4.18. Calculated Actinide Isotopics by Axial Zone for Sample Problem 3 ............................5-228

Table 5.4.19. Input for ORIGAMI Sample Problem 4 .........................................................................5-229

Table 5.4.20. Actinide Isotopics by Axial Zone for Pins $(1,1)$ and $(1,2)$ in Sample Problem 4 ...........5-229

Table 5.4.21. Actinide Isotopics Blended over All Pins, by Axial Zone in Sample Problem 4 ............5-230

Table 5.4.22. Sample Problem 4: Decay Heat (Watts) by Axial Interval ............................................5-230

Table 5.4.23. Input for ORIGAMI Sample Problem 5 ........................................................................5-231 


\section{ACKNOWLEDGMENTS}

ORIGAMI is based on the PinDeplete code developed by Steve Skutnik of the University of Tennessee, and it also includes techniques taken from the Orella code written by Ian Gauld. Support for development of ORIGAMI was provided by the U.S. Department of Energy, Office of Nuclear Energy, Nuclear Fuels Storage and Transportation Planning Project. 


\subsubsection{Introduction}

ORIGAMI ( $\underline{\boldsymbol{O R I G}} \mathrm{EN} \underline{\boldsymbol{A}}$ ssembly $\underline{\boldsymbol{I}} \mathrm{sotopics})$ provides the capability to perform isotopic depletion and decay calculations for a light water reactor fuel assembly model using one or more ORIGEN calculations. The assembly may be modeled using either lumped or pinwise representations with the option of including axial zones. ORIGAMI automates the performance of ORIGEN depletion calculations for each region and thus simulates zero-, one-, two-, and three -dimensional (0D, 1D, 2D, and 3D) modeling of a fuel assembly. Multiple cycles with different specific powers and exposure and decay times may be treated, and the power distribution is described in terms of fractional pin powers in the XY plane and axial distributions along the $\mathrm{Z}$ axis, which define the burnup regions for the ORIGEN computations. ORIGAMI allows for easy and flexible material composition specification through the standard SCALE mixture processor for composition input, the same as in TRITON (see XSPROC chapter). While ORIGAMI cannot presently treat axially non-uniform lattice features (e.g. axially varying enrichment or the partial-length rods found in many boiling water reactor designs) within a single input, these problems can still be easily modeled by splitting the problem across sequential ORIGAMI input cases residing on the same file.

The ORIGEN calculations performed by ORIGAMI use the methodology originally established for the SCALE sequence ORIGEN-ARP (see ARP in section 5.1). This approach provides an efficient mechanism to perform stand-alone reactor depletion calculations using pre-generated ORIGEN libraries which contain self-shielded, collapsed one-group cross sections as a function of selected independent variables, such as burnup, enrichment, and moderator density, for different reactor systems. Typically the data in these libraries are obtained from 2D, multigroup lattice transport calculations (e.g., TRITON) coupled with depletion calculations for burnup. The library cross sections may be flux-weighted over the lattice to obtain data representative of the entire homogenized assembly for lumped depletion; or alternatively, it is also possible to generate multiple ORIGEN libraries corresponding to individual or groups of pins within the lattice for multi-pin depletion. The burnup-dependent ORIGEN libraries are analogous to the parameterized cross section data produced by lattice physics codes for reactor core simulators, except that data for many more nuclides and reactions are included to allow ORIGEN to compute detailed isotopics for more than 2200 nuclides.

ORIGAMI extends the capabilities previously provided by ORIGEN-ARP to perform a suite of ORIGEN calculations in order to represent the isotopic distribution of fuel within an assembly in more detail. The pre-generated ORIGEN libraries provided with SCALE tabulate the assembly-average one-group cross sections, in order to accurately reproduce assembly-average isotopics. When performing pin-by-pin calculations in ORIGAMI, users can increase the fidelity with respect to proximity to features such as assembly edges, water holes, burnable poison rods, etc. by creating and employing zone-specific libraries for different pins. By specifying the individual library assignments for each pin, users can capture these local spectrum changes in the ORIGAMI calculation through the use of one-group libraries based on these local conditions. Currently, the specification of individual libraries is limited to pin-level specification only (i.e., the same library is used for all axial zones corresponding to a pin for 3D cases) with an allowed axial moderator density distribution and radial and axial power distributions.

ORIGAMI can produce the following output files in addition to the standard ORIGEN output for each depletion zone:

- $\quad$ isotopics in ORIGEN binary concentration (ft71) files including

$\circ$ in each depletion zone at times specified by the options block, $f t 71$ key and

$\circ$ in each axial zone (summed over all pins at a particular axial level) at the final time;

- nuclide concentrations by axial zone, written as a SCALE "standard composition block" that can be used directly as input for SCALE transport codes such as the KENO Monte Carlo criticality code; 
- axially-dependent decay heat source for input to a thermal analysis code such as COBRA, so that the temperature distribution within a storage cask can be computed;

- nuclide concentrations for each axial zone, given in the format of MCNP material cards; and

- $\quad$ space-dependent radiation source energy spectra and magnitudes in a simple text file.

ORIGAMI is tightly integrated with the SCALE Graphical User Interface, Fulcrum. Using Fulcrum and the "UO2 express form (configurable)", one can create a simple $\mathrm{UO}_{2}$ assembly depletion case in seconds (see Figure 5.4.1). Finally, ORIGAMI has the ability to perform the depletion/decay calculations for each zone in parallel using the MPI (Message Passing Interface), however this requires a special SCALE installation built with MPI in order to do so. ${ }^{1}$

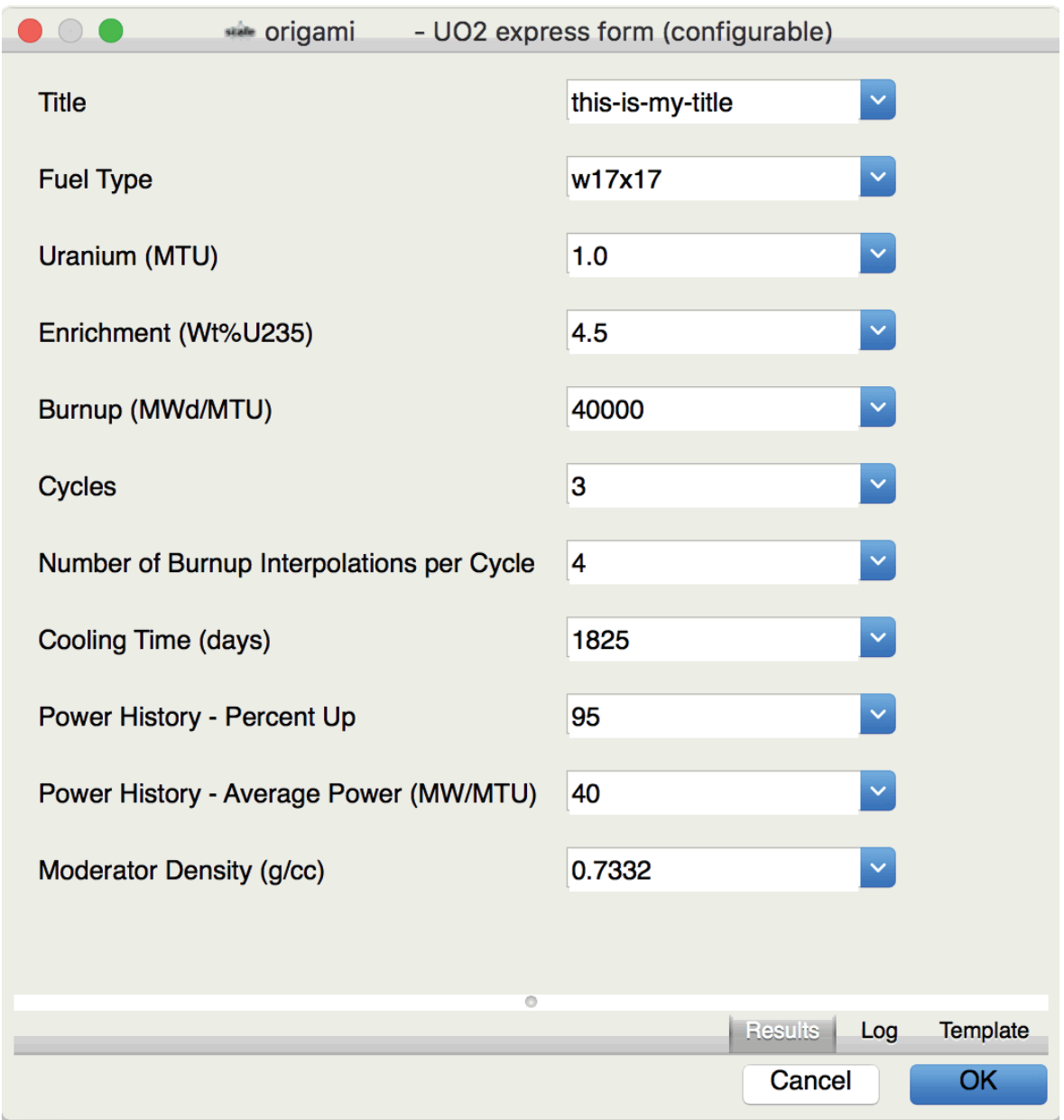

Figure 5.4.1. Fulcrum UO2 express form for creating ORIGAMI input. 


\subsubsection{Computational Methods}

\subsubsection{ORIGAMI assembly model}

The basic model for ORIGAMI is a fuel assembly, which may be modeled in several ways with varying degrees of complexity. The most primitive model represents the assembly materials as a single mass lump that is depleted using the value of the specific power input in the power-history block. In this case, a single ORIGEN calculation is performed to obtain isotopics representing the entire assembly. This 0D model is equivalent to the current ORIGEN-ARP procedure. A more detailed model applies an input axial power profile to the (radially) lumped assembly materials. This lumped axial depletion model produces a 1D axially varying burnup distribution, but no allowance is made for variations in the relative pin powers within the assembly. Thus, if the axial power distribution is defined by $\mathrm{N}_{Z}$ axial zones, ORIGEN calculations are performed for $\mathrm{N}_{Z}$ different depletion regions. The 1D axial depletion model has been found to be adequate for most criticality and decay heat analysis of spent fuel assemblies. ${ }^{2,3}$ Note that both the $0 \mathrm{D}$ and 1D modes are fully consistent with the 2D TRITON calculations used to generate ORIGEN reactor libraries distributed with SCALE, in that these modes employ spatially-homogenized cross-sections to represent assembly-averaged flux and cross-sections. For 2D and 3D depletion models (wherein individual pin-specific libraries may optionally be specified), the user is advised that the ORIGEN reactor data libraries distributed with SCALE are representative of an assembly axial plane as a whole; in as much, the user is advised to generate their own zone-specific libraries (i.e., based on individual material zones) within TRITON if they wish to capture regional neutronic effects within the assembly (such as proximity to water holes, burnable absorbers, etc.)

By specifying a radial pin-power map, a 2D or 3D calculation may be performed. Currently the axial and radial power shapes are fixed for the entire calculation but do still result in a fully 3D isotopic distribution. ${ }^{1,2}$ If there are $\mathrm{N}_{\mathrm{P}}$ pins in the assembly and each has $\mathrm{N}_{\mathrm{Z}}$ axial zones, ORIGAMI will perform ORIGEN calculations for $\mathrm{N}_{P} \times \mathrm{N}_{Z}$ depletion regions. For example, an assembly with a $17 \times 17$ array with 264 fuel pins and $\mathrm{N}_{Z}=24$ axial zones requires 6336 independent ORIGEN calculations. For these types of simulations, the parallel mode with MPI is highly recommended.

\subsubsection{Definition of initial composition}

The initial mass in metric tons of heavy metal is $M_{m t u}$, set by the input parameter $m t u$. The default value of $m t u$ is equal to 1.0 , so that by default the ORIGEN calculations are performed on the basis of "per metric ton of heavy metal". Given that the sum over all zones must have the total heavy metal content $\left(M_{m t u}\right)$, one arrives at zone-wise heavy metal masses of:

$$
\mathrm{M}_{\mathrm{XY}, \mathrm{Z}}=\frac{\mathrm{M}_{\mathrm{mtu}}}{\sum_{\mathrm{Z}=1}^{\mathrm{N}_{\mathrm{Z}}} \mathrm{f}_{\mathrm{Z}} \sum_{\mathrm{XY}=1}^{\mathrm{N}_{\mathrm{P}}} \mathrm{m}_{\mathrm{XY}}} \mathrm{f}_{\mathrm{Z}} \mathrm{m}_{\mathrm{XY}}
$$

where the relative amount of heavy metal in each radial position, $m_{X Y}$, is calculated from the mixture specification; fractional axial height, $f_{Z}$, from the zone specification; and $N_{P}$ and $N_{Z}$ are the total number of fuel pins and axial zones, respectively. Note that some pin locations in the assembly may not contain fuel, and these are not included in the value of $\mathrm{N}_{\mathrm{P}}$. The fractional axial height is given as

$$
\begin{aligned}
& \mathrm{f}_{\mathrm{Z}}=\frac{\Delta \mathrm{Z}}{\mathrm{Z}_{\mathrm{tot}}} \text { is the fraction of the active fuel height occupied by axial zone } \mathrm{Z} \\
& \Delta \mathrm{Z} \text { is the length of axial zone } \mathrm{Z} \\
& \mathrm{Z}_{\text {tot }} \text { is the total length of the active fuel. }
\end{aligned}
$$

Whenever an axial zone mesh is input (with array meshz), the value of $f_{Z}$ is computed from the values of the zone boundaries (see input description in Sect. 5.4.3.6). If an axial mesh array is not input, the axial 
zones are assumed to be uniformly distributed. In this case, the axial zones all have the same height, so that $\mathrm{f}_{Z}=1 / \mathrm{N}_{Z}$, where $\mathrm{N}_{Z}$ is the number of uniform axial zones in the assembly.

The uranium mass in a single axial zone for all $N_{P}$ fuel pins in the assembly $\left(M_{z}\right)$ is thus:

$$
\mathrm{M}_{\mathrm{Z}}=\mathrm{N}_{\mathrm{P}} \times \mathrm{M}_{\mathrm{XY}, \mathrm{Z}}=\mathrm{M}_{\mathrm{mtu}} \times \mathrm{f}_{\mathrm{Z}}
$$

In addition to the fuel mixture in an assembly, non-fuel materials (e.g., structural materials) may also be present. These materials contribute to the overall power production due to the energy produced by neutron capture reactions.. For a given value of the total assembly power, this reduces the power from the fuel mass and thus may slightly alter the fuel burnup and isotopics. In addition, activation of non-fuel materials produces additional radiation source terms in the spent fuel, which contribute to the decay heat and activity. Therefore ORIGAMI provides an option for including the non-fuel elements in the input array, nonfuel. The units of the non-fuel element masses are $\mathrm{kg}$ per MTU, and the materials are distributed uniformly within all fuel depletion zones. Note that the input non-fuel materials should not include oxygen in $\mathrm{UO}_{2}$ if $\mathrm{UO}_{2}$ is specified as the fuel material, as oxygen is already included in proportion to the uranium mass basis. Finally, because ORIGAMI accesses the StdComp library, any SCALE StdComp composition, e.g. "zirc4" for reactor cladding material Zircaloy-4, may be used in either structural or fuel materials.

\subsubsection{Restart cases}

ORIGAMI also allows the initial nuclide concentrations to be obtained from a previously produced ORIGEN binary output file. A restart case is indicated by setting restart=yes in the parameter array. The restart file has the name "assembly_restart.f71" and must be copied (or linked) to the SCALE temporary directory used for calculations. The restart file is normally obtained from an earlier ORIGAMI calculation, which always produces an ORIGEN restart file named "\$\{OUTBASENAME\}.assm.f71", where $\$\{$ OUTBASENAME\} is an output prefix defined by the name of the input file and any userspecified prefix with the prefix $=$ key. Generally the restart file from ORIGAMI contains stacked concentrations, corresponding to each axial zone and then a final entry for the lumped assembly concentrations; hence, the initial composition for a restart case varies with axial zone, unlike the case for fresh fuel. ORIGAMI does not currently allow pin-dependent restart calculations. A restart case may be useful for performing decay-only calculations of spent fuel inventory, using the burned fuel composition previously computed for the assembly exposure during reactor operation. For decay-only cases, a value for the input parameter $n z$ must be input in order to indicate the number of axial depletion regions in the previous burnup calculation.

\subsubsection{Definition of power distribution}

The radial power distribution is defined by the XY fractional pin powers in the input array $p x y$, and the axial fractional powers in the input array $p z$. The input values in arrays $p x y$ and $p z$ are normalized to unity by the code. The fractional power for a fuel pin "XY" is designated here to be $r_{X Y}$, with the normalization $\sum_{\mathrm{XY}=1}^{\mathrm{N}_{\mathrm{P}}} \mathrm{r}_{\mathrm{XY}}$. Similarly the fractional axial power for an axial zone $\mathrm{Z}$ is $\mathrm{a}_{\mathrm{Z}}$, which is normalized to $\sum_{\mathrm{Z}=1}^{\mathrm{N}_{\mathrm{Z}}} \mathrm{a}_{\mathrm{Z}}$. The shapes of both the radial $\mathrm{XY}$ and axial $\mathrm{Z}$ distributions must be obtained prior to the ORIGAMI calculation, either from neutron transport calculations or experimental measurements. The input distributions remain constant during the ORIGEN burn calculations for all cycles; but in reality, the power distributions may vary with time - for example, the initial axial power distribution tends to flatten after a period of burnup since the higher power zones deplete the fuel faster. For this reason it is strongly recommended to use the relative burnup distribution (at final discharge) rather than the relative power 
density distribution for the input values. The burnup shape corresponds to the shape of the time-averaged flux distribution during the exposure period. This ensures that the final burnup distribution matches the desired shape.

For a given cycle, the assembly-specific power $\mathrm{P}^{(\mathrm{SP})}$ is equal to the value of input variable power, read in the power-history block (see Sect. 5.4.3.3). The assembly-specific power has units of megawatts per MTU (MW/MTU). Therefore, the total power produced by the fuel assembly is

$$
\mathrm{P}_{\text {tot }}=\mathrm{P}_{\mathrm{A}}^{(\mathrm{SP})} \cdot \mathrm{M}_{\mathrm{mtu}}
$$

where $\mathrm{P}_{\text {tot }}$ is the assembly total power, and $\mathrm{P}_{\mathrm{A}}^{(\mathrm{SP})}$ is the specific power for the assembly, read from input. The absolute power (MW) in fuel pin " $\mathrm{XY}$ " is:

$$
\mathrm{P}_{\mathrm{P}}=\mathrm{P}_{\text {tot }} \cdot \mathrm{r}_{\mathrm{xy}}=\mathrm{P}_{\mathrm{A}}^{(\mathrm{SP})} \cdot \mathrm{M}_{\mathrm{mtu}} \cdot \mathrm{r}_{\mathrm{xy}}
$$

and the power produced in axial zone $\mathrm{Z}$ of this fuel pin $\mathrm{XY}$ is:

$$
\mathrm{P}_{\mathrm{XY}, \mathrm{Z}}=\mathrm{P}_{\mathrm{tot}} \cdot \mathrm{r}_{\mathrm{xy}} \cdot \mathrm{a}_{\mathrm{Z}}=\mathrm{P}_{\mathrm{A}}^{(\mathrm{SP})} \cdot \mathrm{M}_{\mathrm{mtu}} \cdot \mathrm{r}_{\mathrm{xy}} \cdot \mathrm{a}_{\mathrm{Z}}
$$

The absolute power produced in a single axial zone $\mathrm{Z}$ for all pins is:

$$
\mathrm{P}_{\mathrm{Z}}=\sum_{\mathrm{XY}=1}^{\mathrm{N}_{\mathrm{P}}} \mathrm{P}_{\mathrm{XY}, \mathrm{Z}}=\mathrm{P}_{\mathrm{TOT}} \times \mathrm{a}_{\mathrm{Z}}=\mathrm{P}_{\mathrm{A}}^{(\mathrm{SP})} \times \mathrm{M}_{\mathrm{mtu}} \times \mathrm{a}_{\mathrm{Z}}
$$

The ORIGEN depletion calculations are performed with the absolute powers defined in Eqs. (5.4.4) and (5.4.6) for each depletion region in the $2 \mathrm{D} / 3 \mathrm{D}$ pin-wise or $0 \mathrm{D} / 1 \mathrm{D}$ axial depletion models, respectively. However, cross sections in the ORIGEN libraries are parameterized as a function of burnup, which depends on the specific power rather than absolute power for a given depletion region. The specific power (MW/MTU) in axial zone $\mathrm{Z}$ of pin $\mathrm{XY}$ is equal to:

$$
\mathrm{P}_{\mathrm{XY}, \mathrm{Z}}^{(\mathrm{SP})}=\frac{\mathrm{P}_{\mathrm{XY}, \mathrm{Z}}}{\mathrm{M}_{\mathrm{XY}, \mathrm{Z}}}
$$

Substituting Eqs. (5.4.1) and (5.4.5) into Eq. (5.4.7) gives:

$$
\mathrm{P}_{\mathrm{XY}, \mathrm{Z}}^{(\mathrm{SP})}=\frac{\mathrm{P}_{\mathrm{A}}^{(\mathrm{SP})} \cdot \mathrm{r}_{\mathrm{xy}} \cdot \mathrm{a}_{\mathrm{Z}} \cdot \mathrm{N}_{\mathrm{P}}}{\mathrm{f}_{\mathrm{Z}}}
$$

In a similar manner, it can be shown that the specific power for all fuel pins in axial plane $\mathrm{Z}$ is:

$$
\mathrm{P}_{\mathrm{Z}}^{(\mathrm{SP})}=\frac{\mathrm{P}_{\mathrm{A}}^{(\mathrm{SP})} \cdot \mathrm{a}_{\mathrm{Z}}}{\mathrm{f}_{\mathrm{Z}}}
$$

ORIGAMI permits two modes for user-specified power distributions along the axial and radial meshes: absolute fractions (i.e., where powers along the axial mesh points are expressed as fractions of the total assembly power in $\mathrm{MW}$ ) and relative normalization (i.e., in which specific powers - in MW/MTU - of 
axial zones are expressed as a relative modifiers of the assembly specific powers input in the power history block). Relative power shape modifiers assume that the specific powers expressed in the power history block represent the average assembly specific power(s) $\mathrm{P}_{\mathbf{A}}^{(S P)}$; thus, ORIGAMI will convert these factors into axial \& pin power fractions - i.e., the factors $\mathbf{r}_{\mathrm{XY}}$ and $\mathbf{a}_{\mathrm{Z}}$ found in Eqs. (5.4.4) and (5.4.6) used to calculate the absolute pin power and axial zone power, respectively. The conversion from relative specific power modifiers to absolute power fractions is accomplished through the following normalization procedure:

$$
\left(\mathrm{a}_{\mathrm{Z}}\right)_{\mathrm{i}}=\frac{\left(\mathrm{R}_{\mathrm{Z}}\right)_{\mathrm{i}} \cdot \mathrm{M}_{\mathrm{MTU}} \cdot\left(\frac{\Delta \mathrm{Z}}{\mathrm{Z}_{\mathrm{tot}}}\right)_{\mathrm{i}}}{\sum\left(\mathrm{R}_{\mathrm{Z}}\right)_{\mathrm{i}} \cdot \mathrm{M}_{\mathrm{MTU}} \cdot\left(\frac{\Delta \mathrm{Z}}{\mathrm{Z}_{\mathrm{tot}}}\right)_{\mathrm{i}}}=\frac{\left(\mathrm{R}_{\mathrm{Z}}\right)_{\mathrm{i}} \cdot\left(\frac{\Delta \mathrm{Z}}{\mathrm{Z}_{\mathrm{tot}}}\right)_{\mathrm{i}}}{\sum\left(\mathrm{R}_{\mathrm{Z}}\right)_{\mathrm{i}} \cdot\left(\frac{\Delta \mathrm{Z}}{\mathrm{Z}_{\mathrm{tot}}}\right)_{\mathrm{i}}}
$$

where $\left(\mathrm{a}_{\mathrm{Z}}\right)_{\mathrm{i}}$ is the axial power fraction for axial zone $i$ and $\left(\mathrm{R}_{\mathrm{Z}}\right)_{\mathrm{i}}$ is the relative axial zone specific power modifier for axial zone $i$. Obviously, for a uniformly-spaced axial mesh, the conversion from relative specific powers (using relative power modifiers) is precisely the same as that for absolute fractional axial zone powers; i.e., the relative power modifiers simply become axial power fractions by virtue of the fact that the term $\left(\frac{\Delta \mathrm{Z}}{\mathrm{Z}_{\mathrm{tot}}}\right)_{\mathrm{i}}$ becomes a constant, thereby reducing Eq. (5.4.10) back to a direct calculation of the fractional axial power based on a relative power modifier following normalization.

Because it is assumed that the assembly mass is uniformly distributed across the pins, it can similarly be shown that the use of relative power modifiers for the XY pin map $\left(r_{X Y}\right)_{i}$ will always produce the same result as using pre-normalized absolute fractional powers in the pin map, i.e.:

$$
\left(r_{X Y}\right)_{i}=\frac{\left(R_{X Y}\right)_{i} \cdot \frac{M_{M T U}}{N_{P}}}{\sum\left(R_{X Y}\right)_{i} \cdot \frac{M_{M T U}}{N_{P}}}=\frac{\left(R_{X Y}\right)_{i}}{\sum\left(R_{X Y}\right)_{i}}
$$

This option is provided as the relnorm option in the parameters block (discussed further in Sect. 5.4.3.2). The motivation for providing an alternative normalization for axial power shape factors is twofold. First, it is generally assumed that information on the axial power shape is obtained from axial measurements relative to an assembly-average value (i.e., axial gamma scans to determine the burnup profile based upon the gross gamma intensity or isotopic ratios of burnup indicators such as ${ }^{134} \mathrm{Cs} /{ }^{137} \mathrm{Cs}$, etc.). Therefore, by using the relative normalization option (i.e., treating axial power shape factors as relative modifiers of the assembly specific power), users can directly input shape factors obtained from techniques such as nondestructive analysis (NDA) fuel measurements into ORIGAMI to model assembly isotopic distributions. 
The second motivation for the relative normalization option comes from potential problems that can arise if treating axial power shape factors as absolute fractional powers $($ relnorm $=n o$ ) in conjunction with nonuniform axial mesh spacing defined by the user in the $z$ array (see Sect. 5.4.3.7 for details).

Important: If using the relnorm=no option, the fractional axial powers must be consistent with the axial mesh sizes defined or else incorrect zone specific powers will result from Eq. (5.4.9), therefore leading to incorrect results and likely causing the ARP sequence to fail (and therefore the ORIGAMI calculation to halt) due to calculated burnup values for the depletion zone being out of the library range. Users are thus strongly cautioned when using absolute fractional axial powers $($ relnorm $=$ no $)$ to ensure proper consistency between the axial power fractions and the axial mesh sizes.

For this reason, relative power shape factor normalization is turned on by default (relnorm $=y e s$ ).

\subsubsection{Computation of neutron and gamma energy spectra}

ORIGAMI includes an option to generate multi-group neutron and gamma source spectra due to radioactive decay, for each depletion zone. Multi-group values are calculated by binning the discrete line and continuum spectra produced by radioactive decay and nuclear reactions into arbitrary energy group structures defined by user input. Whenever neutron energy group boundaries are input in array ngrp, neutron source spectra due to spontaneous fission, delayed neutron emission, and $(\alpha, n)$ reactions are calculated. Similarly, gamma source spectra are computed if gamma energy group bounds are input in array ggrp. The gamma source includes photons produced by all types of radioactive decays, and also may include bremsstrahlung radiation produced by beta interactions. Input options can specify the type of nuclides included in the source term (i.e., light elements, actinides, fission products, or all nuclides), and the materials used for $(\alpha, n)$ reactions and bremsstrahlung production. If source spectra are calculated, the values are always included in the ORIGEN output $\mathrm{ft} 71$ binary file; and optionally the source spectra may also be output in a text file. The source text file only includes the average over all pins for each axial zone, while the $\mathrm{ft} 71$ file includes sources for all pins and axial zones.

The source spectra output by ORIGAMI are calculated in ORIGEN using the following expression:

$$
\mathrm{S}_{\mathrm{Z} . \mathrm{g}}^{(\mathrm{p})}=\sum_{\mathrm{i}=1}^{\mathrm{itot}} \mathrm{Y}_{\mathrm{i}, \mathrm{g}}^{(\mathrm{p})} \lambda_{\mathrm{i}} \frac{\mathrm{M}_{\mathrm{Z}}^{(\mathrm{i})}}{\mathrm{A}^{(\mathrm{i})}} \cdot \mathrm{N}_{\mathrm{A}}
$$

where

$\mathrm{S}_{\mathrm{Z} \cdot \mathrm{g}}^{(\mathrm{p})} \quad=$ source spectrum $(\mathrm{p} / \mathrm{s})$ in energy group $g$ for particles of type $p$ and axial zone $Z$;

$\mathrm{Y}_{\mathrm{i}, \mathrm{g}}^{(\mathrm{p})} \quad=$ number of particles of type $p$ emitted per decay of nuclide $i$; with energy in group $g$;

$\mathrm{M}_{\mathrm{Z}}^{(\mathrm{i})} \quad=$ mass $(\mathrm{g})$ of nuclide $i$ in axial zone $Z$, obtained from ORIGEN calculation;

$\mathrm{N}_{\mathrm{A}} \quad=$ Avogadro's number (number atoms of nuclide $i$ per mole);

$\mathrm{A}^{(\mathrm{i})} \quad=$ mass $(\mathrm{g})$ of 1 mole of nuclide $i$;

$\lambda_{\mathrm{i}} \quad=$ decay constant $\left(\mathrm{s}^{-1}\right)$ for nuclide $i$, and

itot $=$ total number of nuclides in burned fuel.

More details on the ORIGEN calculation of the source spectra can be found in the ORIGEN section of SCALE documentation. 


\subsubsection{ORIGAMI Input Description}

ORIGAMI uses free-form, keyword-driven input with the SCALE Object Notation (SON) syntax also used for ORIGEN input, and is described in more detail there. The general outline of ORIGAMI input is as follows.

(a) Case Identifier Information

(b) Options Block

(c) Fuel Composition Block

(d) Power-History Block

(e) Source-Options Block

(f) Output-Print Options Block

(g) Input Data Arrays

The above input data may be entered in any order. Data blocks and parameters which are not needed, or for which default values are desired, can be omitted. Table 5.4.1 provides a template containing all of the ORIGAMI input data blocks and arrays, with example values assigned. Note that much of the information shown in the template is optional, and typically is not needed for many cases. The following subsections provide a more detailed description of the input. 
Table 5.4.1. Template for ORIGAMI Input Data

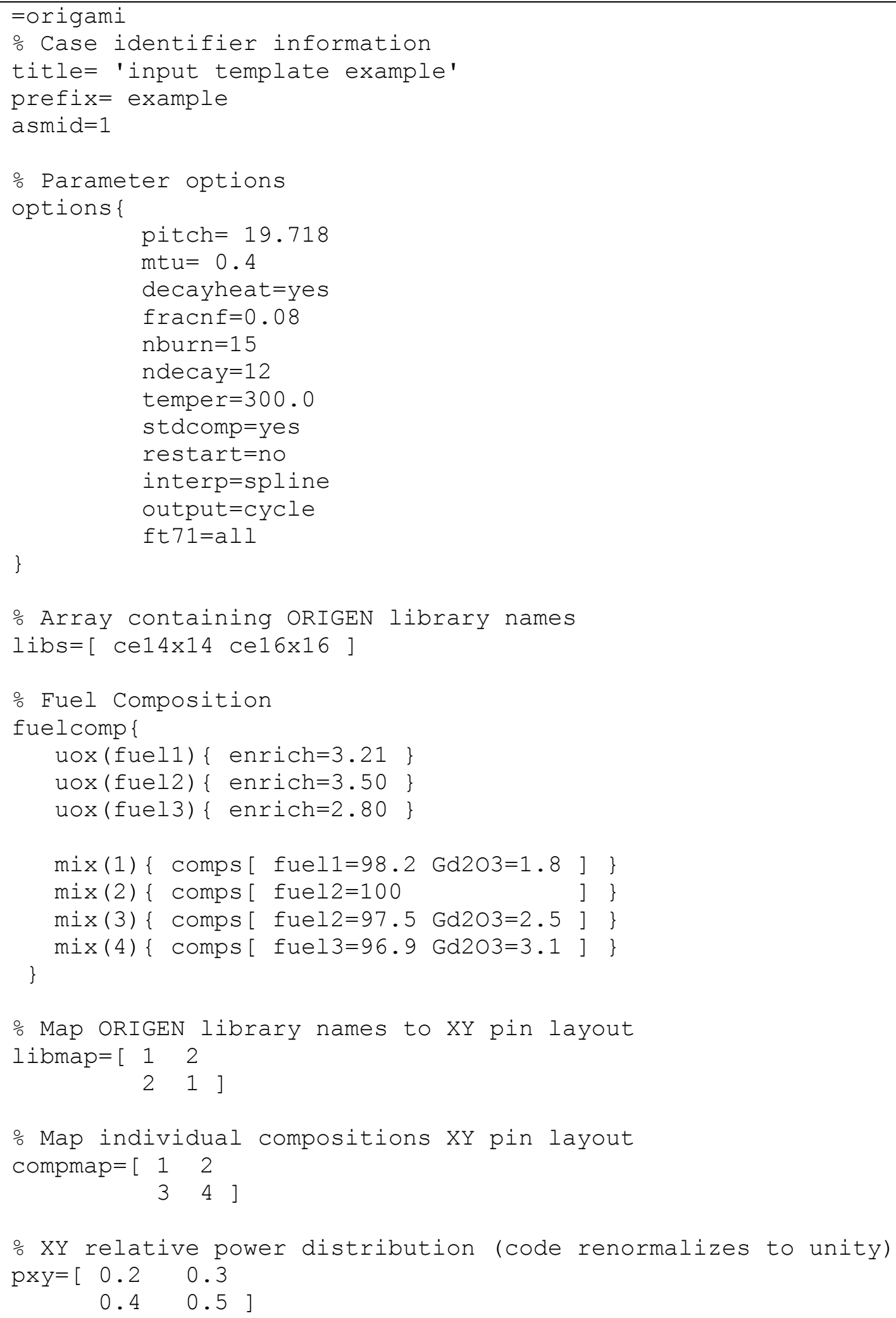




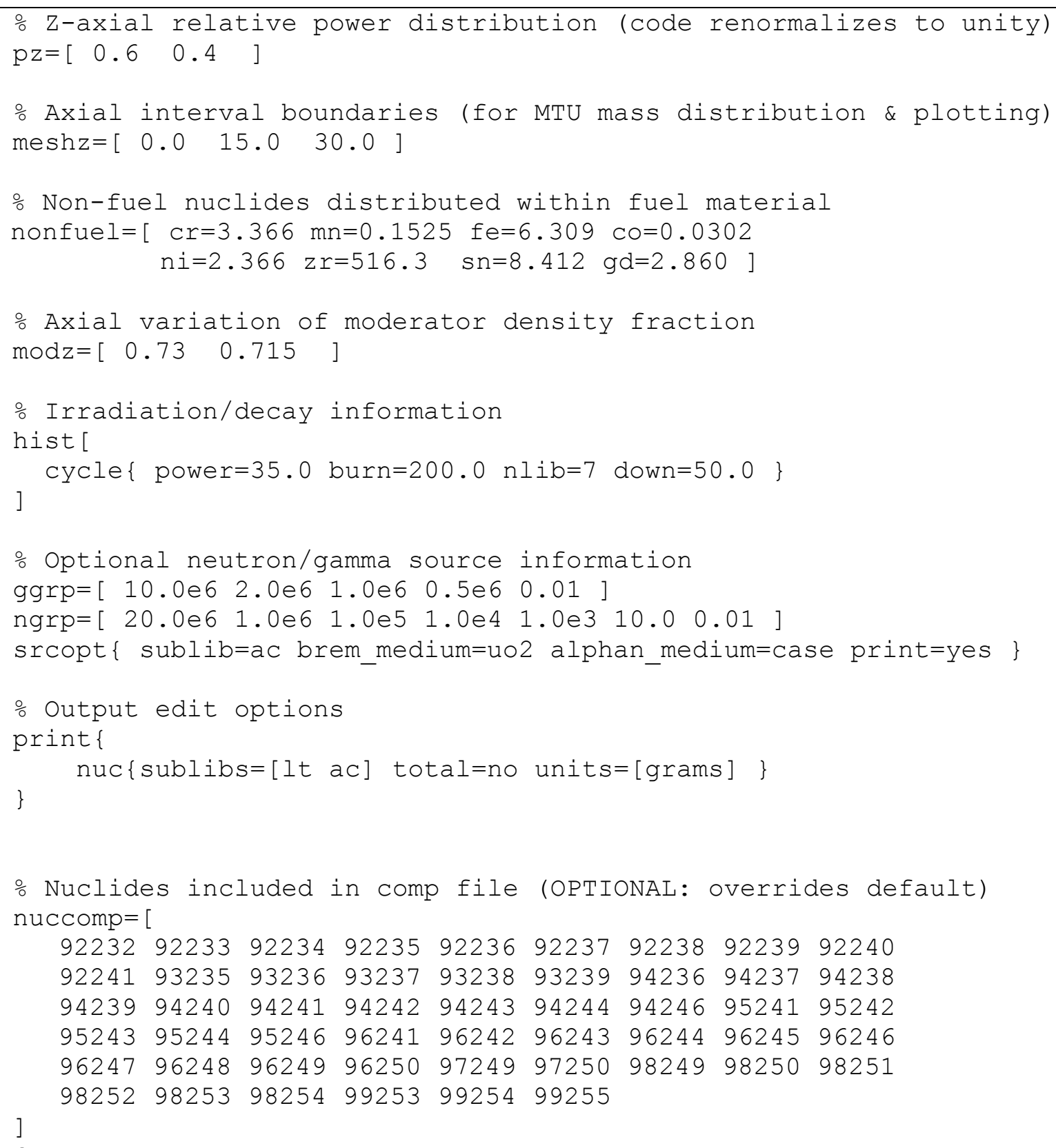

\subsubsection{Case and identifier information}

ORIGAMI has three optional identifiers for the case. These are summarized in Table 5.4.2. The title is included as a descriptor in the printed output file. The character string prefix is added to the front of the output file names described in Section 5.4.4 and in Table 5.4.9. Finally, the integer variable asmid is an arbitrary assembly identifier used in defining mixture numbers in the SCALE standard composition output file. Equation (5.4.14) in Section 5.4.4.1 describes how the mixture ID is determined. 
Table 5.4.2. Keywords for Case Identifier Information

\begin{tabular}{|r|l|c|}
\hline \multicolumn{1}{|c|}{ Keyword } & \multicolumn{1}{|c|}{ Description } & Default \\
\hline title $=$ & up to 50 characters describing the case title, quoted if embedded blanks & blank \\
\hline prefix $=$ & up to 16 characters (no embedded blanks) appended to output file names & blank \\
\hline asmid $=$ & $\begin{array}{l}\text { integer used to identify mixture ID in generated SCALE standard } \\
\text { composition block [see Eq. (5.4.14)] }\end{array}$ & 1 \\
\hline
\end{tabular}




\subsubsection{Options block}

The options block has the following form: options $\{. .$. keywords ...\}

Table 5.4.3 shows the Keywords for allowable parameters. An example parameter block is

options $\{$ stdcomp=yes decayheat=yes $\}$

Table 5.4.3. Keywords in options block

\begin{tabular}{|c|c|c|}
\hline Keyword & Description & Default \\
\hline mtu= & Metric tons of heavy metal in the assembly & 1.0 \\
\hline fracnf $=$ & $\begin{array}{l}\text { Total non-fuel mass in assembly, given as fraction of heavy metal mass } \\
\text { defined by input } m t u=. \text { See description of input array nonfuel }=\end{array}$ & none \\
\hline $\mathrm{nz}=$ & $\begin{array}{l}\text { Number of axial intervals. If not input, } n z \text { is equal to the number of } \\
\text { entries in the input axial power array } p z=\text {. Required for decay-only } \\
\text { restarts. }\end{array}$ & $\begin{array}{l}\text { Determined } \\
\text { by code }\end{array}$ \\
\hline nburn= & Number of substeps used in ORIGEN burn calculations & 10 \\
\hline ndecay $=$ & Number of substeps used in ORIGEN decay calculations & 10 \\
\hline pitch $=$ & $\begin{array}{l}\text { Assembly pitch }(\mathrm{cm}), \text { if }>0.0 \text {. Only used to define } X Y \text { mesh in viewing } \\
\text { results. If this parameter is input, array } p x y=\text { must also be entered. }\end{array}$ & 0.0 \\
\hline temper $=$ & Temperature (Kelvin) assigned to materials in standard composition file & 293.0 \\
\hline offsetz= & $\begin{array}{l}\text { Axial numbering offset; used for sequential ORIGAMI cases to uniquely } \\
\text { identify axial zones (i.e., such as when using sequential cases to modify } \\
\text { changing axial geometry). [integer] }\end{array}$ & 0 \\
\hline relnorm= & $\begin{array}{l}\text { Normalization of axial power shaping factors }(p z=) \text { to be used } \\
\text { no: axial power shape factors treated as absolute fractions (does not } \\
\text { normalize all axial burnups to } 1.00 \text { ) } \\
\text { yes: axial power shape factors treated as relative modifiers of assembly } \\
\text { specific power (i.e., power= entries in the power history block) [yes/no] }\end{array}$ & Yes \\
\hline $\mathrm{mcnp}=$ & no/yes $\rightarrow$ do not /do generate an MCNP material and gamma/neutron file & Yes \\
\hline stdcomp $=$ & $\begin{array}{l}\text { no/yes } \rightarrow \text { do not /do generate a standard composition file containing } \\
\text { burnup-credit nuclide number densities for each axial zone. }\end{array}$ & No \\
\hline decayheat $=$ & $\begin{array}{l}\text { no/yes } \rightarrow \text { do not /do produce a decay heat file containing heat sources for } \\
\text { each axial zone. }\end{array}$ & No \\
\hline restart $=$ & $\begin{array}{l}\text { no/yes } \rightarrow \text { do not } / \text { do restart using initial composition from a previously } \\
\text { generated ORIGEN ft } 71 \text { file. }\end{array}$ & No \\
\hline solver $=$ & $\begin{array}{l}\text { matrex/cram } \rightarrow \text { use the standard ("MATREX") solver or the Chebyshev } \\
\text { Rational Approximation Method (CRAM) solver. }\end{array}$ & Matrex \\
\hline small= & $\begin{array}{l}\text { no/yes } \rightarrow \text { keep .out file small by suppressing all spectra and } \\
\text { concentrations output except for lumped, assembly-averaged } \\
\text { concentrations and spectra (note: all results are still written to relevant } \\
\text { files). }\end{array}$ & No \\
\hline interp= & lagrange/spline $\rightarrow$ method for interpolating cross sections in ARP & Lagrange \\
\hline output $=$ & last/cycle/all $\rightarrow$ time steps for output print edits & Last \\
\hline ft71= & last/cycle/all $\rightarrow$ time steps included in output ft71 file & Last \\
\hline
\end{tabular}

(*) Required input value 
Additional notes on input parameters

(a) pitch = is only used for visualization of the results, and may be omitted if this is not of interest;

(b) $m t u=$ is discussed in Sect. 5.4.2.2;

(c) $n z=$ is not required except decay-only restart cases; it must equal the number of entries in the array $p z=$

(d) $n b u r n=$ and $n d e c a y=$ are discussed in Sect. 5.4.3.3;

(e) fracnf= is discussed in Sect. 5.4.3.7, where the input array of non-fuel materials is described;

(f) relnorm = is discussed in Sect. 5.4.2.4, in the definition of the assembly power distribution;

(g) $s t d c o m p=$,fdens $=$, and temper $=$ are discussed in Sect.5.4.4;

(h) offsetz = is an optional feature designed to allow for ORIGAMI cases to be split across multiple inputs to capture axially-dependent features (such as partial-length rods); its use is discussed in further detail in the context of output generation in Sect. 5.4.4;

(i) decayheat = is discussed in Sect. 5.4.4.3;

(j) restart $=$ is discussed in Sect. 5.4.2.3.

(k) output=, $f t 71=$, are discussed in Sect. 5.4.3.6.

\subsubsection{Fuel composition block}

The purpose of the fuelcomp block is to create a set of mixtures (via the mix blocks inside) to specify the pin-wise distribution of initial isotopics. The example below, defines three mixtures (with IDs 1, 2, and 3 ); these are referenced in the compmap array for this $2 \times 2$ array of fuel pins.

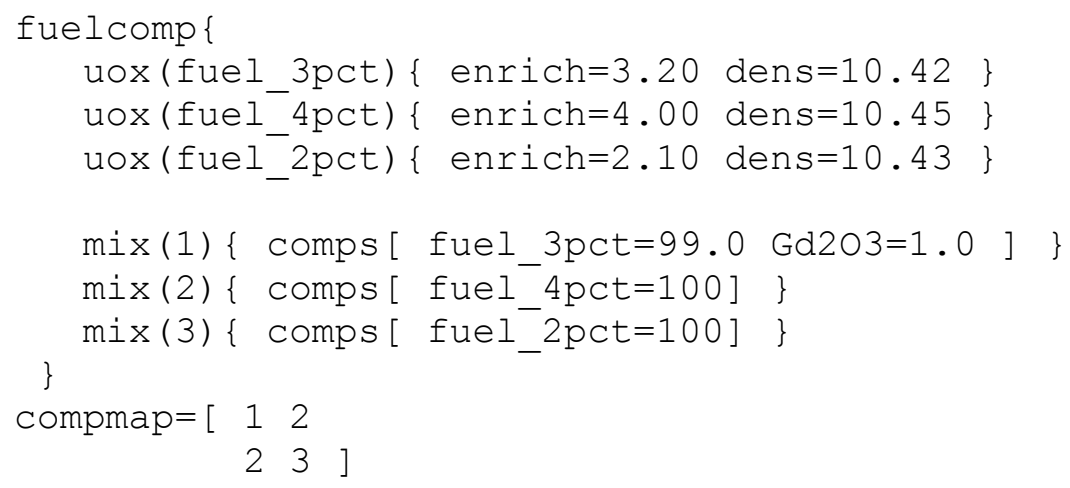

The mix block defines an array of compositions by their weight \%. E.g., in the case of mix 2 and 3, it is $100 \%$ the "fuel_4pct" and "fuel_2pct" compositions defined on the uox blocks above. In the case of mix 1 , it is $99 \%$ by weight "fuel_3pct" and $1 \%$ by weight the SCALE StdComp Gd2O3 (gadolinia). Each mixture number (defined by numbered mix objects) is then referenced in the compmap array to define an individual pin composition. For $\mathrm{UO}_{\mathrm{x}}$-based fuels, ORIGAMI automatically calculates the pin enrichment for cross-section library interpolation via ARP. (Interpolation for MOX-based fuels is not supported by ORIGAMI at this time.)

The uox keyword is an ORIGAM-specific shortcut to allow for easy specification of $\mathrm{UO}_{2}$-based fuels along with their enrichment; ORIGAMI automatically expands the uox keyword into a SCALE StdComp block with a $\mathrm{UO}_{2}$ base and explicitly-calculated uranium isotopics per Table 5.4.4. For example, the uox block "fuel_3pct" expands to the following: 


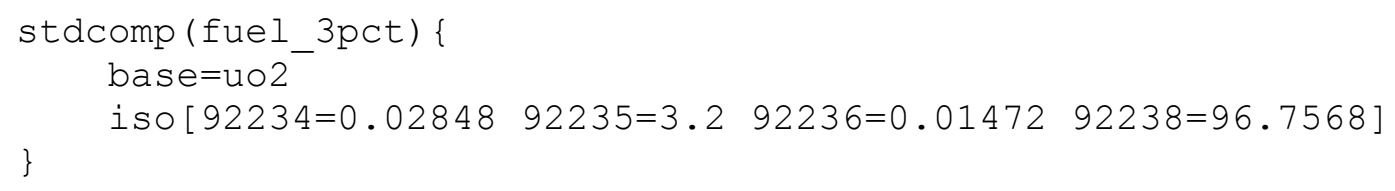

For $u o x$-based entries, the uranium isotopic distribution is calculated from the user-specified enrichment per the following formula ${ }^{4,5}$ :

Table 5.4.4. Uranium isotope dependence on $\mathrm{X} \mathrm{wt} \%{ }^{235} \mathrm{U}$ enrichment

\begin{tabular}{ll}
\hline Isotope & Isotope wt\% \\
\hline${ }^{234} \mathrm{U}$ & $0.0089 \mathrm{X}$ \\
${ }^{235} \mathrm{U}$ & $1.0000 \mathrm{X}$ \\
${ }^{236} \mathrm{U}$ & $0.0046 \mathrm{X}$ \\
${ }^{238} \mathrm{U}$ & $100-1.0135 \mathrm{X}$ \\
\hline
\end{tabular}

Users may also specify materials directly using SCALE mixture processor conventions; for example, the user could simply enter fuel mixture 2 directly as a StdComp as follows:

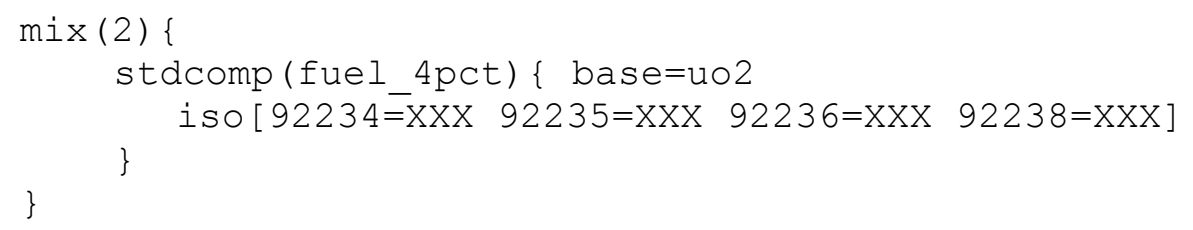

Or similarly:

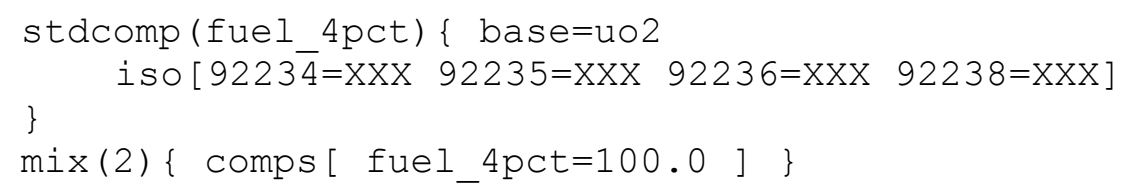

The uox keyword is thus useful when a user wishes to quickly specify a $\mathrm{UO}_{2}$-based fuel; however, in cases where the user wishes to specify the isotopic fractions of each uranium isotope, the use of a StdComp object is recommended.

CAVEAT: The mixture composition system in ORIGAMI is very flexible but it should be cautioned ORIGAMI does not rigorously check that the specified composition is neutronically similar to that used to generate the ORIGEN library used in the calculation. For example, use of gadolinia burnable absorbers in the ORIGAMI input will yield incorrect results if the ORIGEN library was generated without gadolinia, due to the extreme thermal flux depression that gadolinia creates. It is therefore up to the user to verify that the libraries specified for the depletion zone are matched neutronically to the compositions specified.

\subsubsection{Power history block}

The data contained in the power history block is the same as in the BURNDATA block of the TRITON lattice physics depletion sequence in SCALE (see TRITON chapter, section BURNDATA block). The power-history block describes the burnup and decay of the assembly and has the following general form: 


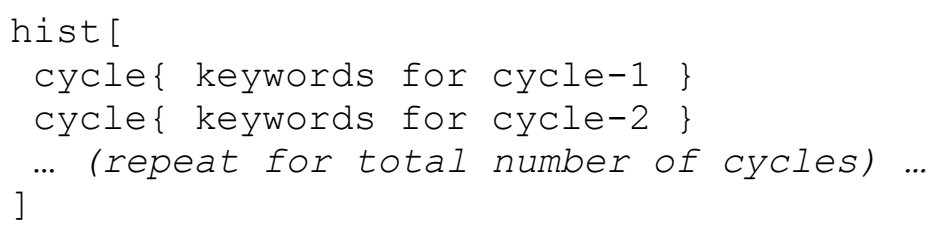

Because the cycles must be processed in order, the array syntax with "[]" is used for the "hist" block. (The block syntax " \{\} " implies no order for its contents.) The "hist" array consists of one or more "cycle" blocks, each describing the assembly irradiation and/or decay for some period of time. Each cycle is defined by (a) the assembly total specific power; (b) number of exposure days at this power; (c) the number of ORIGEN library burnup interpolations during the exposure period; and (d) number of days of decay following the exposure period. The keywords defining this information are given in Table 5.4.5.

Table 5.4.5. Keywords in hist Block(*)

\begin{tabular}{|r|l|c|}
\hline \multicolumn{1}{|c|}{ Keyword } & \multicolumn{1}{|c|}{ Description } & Default \\
\hline power $=$ & assembly specific power (MW/MTU) for the cycle & 0.0 \\
\hline burn $=$ & length of the cycle exposure period in days & 0.0 \\
\hline nlib $=$ & number of ORIGEN library burnup-interpolations during the cycle & 1 \\
\hline down $=$ & downtime in days following the exposure & 0.0 \\
\hline
\end{tabular}

(*) Keywords are repeated for each cycle.

The following is an example for the power-history block for four cycles:

$\begin{array}{llllll}\text { hist }[ & & & & \\ \text { cycle }\{\text { power=35.6 } & \text { burn=400 } & \text { nlib=6 } & \text { down=30 } & \} \\ \text { cycle }\{\text { power=38.2 } & \text { burn=350 } & \text { nlib=6 } & \text { down=30 } & \} \\ \text { cycle }\{\text { power=30.0 } & \text { burn=200 } & \text { nlib=4 } & \text { down=30 } & \} \\ \text { cycle }\{\text { down=10000 } & \} & & & & \\ \text { ] } & & & & \end{array}$

ORIGAMI discretizes time intervals first by cycles (composed of a fixed power over a set burn time interval and / or decay time), where each cycle is composed of a number of substeps. The power-history block, along with values of $n b u r n=$ and $n d e c a y=$ from the input parameter block, define various types of nested time intervals (substeps) for the ORIGEN calculations. The entire time period for an ORIGAMI case is first of all divided into the cycles defined within the power-history block. Each cycle is divided into an exposure interval $($ burn $=)$ and a decay $($ down $=)$ interval. The exposure interval has a constant specific power, but it is further subdivided into a number of equally spaced burnup steps defined by $n l i b=$ in the power-history block. This parameter specifies the number of burnup-dependent ORIGEN libraries to use during the exposure interval. Cross section values for each burnup step are interpolated using the burnup at the midpoint of the step and remain constant throughout the burnup step. The burnup period associated with a single ORIGEN library, or a decay period, is called a time "step." Finally, each burnup step, as well as the entire decay step, is divided into a number of computational "substeps"- the actual time steps used in the ORIGEN solver kernel. The number of substeps in each burnup step is given by the value of $n b u r n=$, while the number of decay substeps is equal to the value $n$ decay $=$. The default number of substeps for both burnup and decay is equal to 10. The substeps for irradiation are equally spaced but for decay follow the rule of threes, i.e. each substep increases in duration by a factor of three over the previous substep.

For the example given above, there are four cycles. The first three cycles include both exposure and decay intervals, while the last cycle is decay only. In the first cycle, the assembly-specific power is 
35.6 MW/MTU, which remains constant over the 400-day exposure interval; therefore, the total burnup for the exposure period is $400 * 35.6=14240 \mathrm{MWD} / \mathrm{MTU}$. This exposure period is divided into six burnup steps of 66.67 days, each with a cross-section library based on the midpoint burnup of that step. Thus, ORIGEN libraries are interpolated at 1186.7, 3560.0, 5933.3, 8306.7, 10680.0, and 13053.3 MWD/MTU. Each of the six burnup steps is further subdivided into 10 computational substeps. Likewise, the decay interval of 30 days is divided into 10 computational substeps.

\subsubsection{Source options block}

This block defines options used in computing neutron and gamma sources. The block is only used if the input energy group boundary arrays ggr $=$ or $n g r p=$ is given, which indicates that radiation decay source spectra are to be computed. The general form of this block is:

$$
\text { srcopt }\{\quad . . \text { keyword-value pairs ... }\}
$$

Keywords are defined in Table 5.4.6. The following is an example of the srcopt input block:

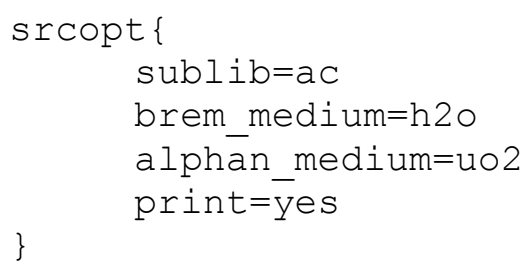

If "print=yes" then text files with axial neutron and gamma sources are created.

Table 5.4.6. Keywords in srcopt block

\begin{tabular}{|c|c|c|}
\hline Keyword & Description & Default \\
\hline sublib= & $\begin{array}{l}l t / f p / a c / \text { all } \rightarrow \text { gamma sources from: } \\
\text { light elements / fission products / actinides / all } \\
\text { nuclides }\end{array}$ & all \\
\hline brem_medium= & $\begin{array}{l}\text { none / } \mathrm{H} 2 \mathrm{O} / \mathrm{UO} 2 / \rightarrow \text { bremsstrahlung production } \\
\text { based on: } \\
\text { no bremsstrahlung / water } / \mathrm{UO}_{2}\end{array}$ & uo2 \\
\hline alphan_medium= & $\begin{array}{l}\text { UO2 / borosilicate/ case } \rightarrow \text { (alpha,n) source } \\
\text { computed for: } \\
\mathrm{UO}_{2} / \text { borosilicate glass / case-specific mixture }\end{array}$ & case \\
\hline print $=$ & $\begin{array}{l}\text { yes / no } \rightarrow \text { write output text file containing sources / } \\
\text { only write sources in binary output } \mathrm{ft} 71 \text { file }\end{array}$ & no \\
\hline
\end{tabular}

\subsubsection{Output print-options block}

This block defines the desired ORIGEN output response edits to be printed by ORIGAMI.

The following is an example input which edits response values for the mass in grams, activities in Curies, and concentrations in atoms/barn-cm, for all nuclides (isotopes) broken down by actinides or fission products as well as curies by element, totaled over all nuclide sub-libraries (sublibs). 


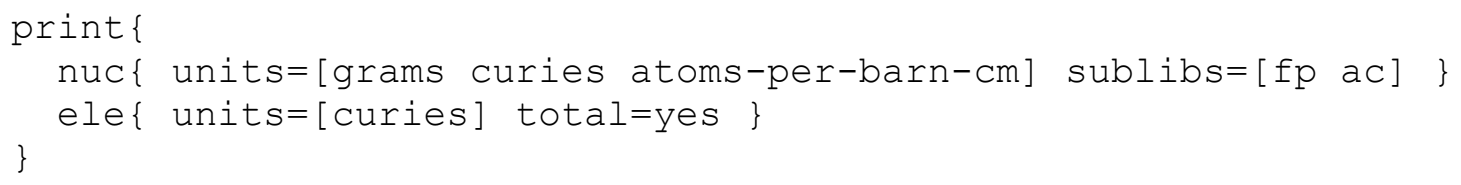

Table 5.4.7. Keywords in print block

\begin{tabular}{|c|l|c|}
\hline Keyword & \multicolumn{1}{|c|}{ Description } & Default \\
\hline nuc / ele & Specify print options for output by individual nuclides / elements & N/A \\
\hline units $=$ & $\begin{array}{l}\text { moles / gram-atoms / grams / curies / becquerels / watts / g-watts / } \\
\text { m3_air / m3_water /weight_ppm / atoms_ppm / } \\
\text { atoms-per-barn-cm }\end{array}$ & $\begin{array}{l}\text { Output concentrations in units of gram-atoms (moles), grams, } \\
\text { curies, becquerels, total thermal power (alpha, beta, and gamma), } \\
\text { thermal power from gammas only, radiotoxicity / dilution factors } \\
\text { for air and water, mass fraction (in ppm), atom fraction (in ppm), } \\
\text { atoms / barn-cm, respectively. }\end{array}$ \\
\hline sublibs $=$ & $\begin{array}{l}\text { le / fp / ac / all } \rightarrow \text { output concentration units for light element / } \\
\text { fission product / actinide sub-libraries. }\end{array}$ & all \\
\hline total $=$ & $\begin{array}{l}\text { yes / no } \rightarrow \text { print out total concentration for nuclides / elements for } \\
\text { each output unit type }\end{array}$ & yes \\
\hline
\end{tabular}

\subsubsection{Input data arrays}

Table 5.4.8 shows the remaining input arrays for ORIGAMI. The input values for all arrays are entered in either of the general forms (with or without "=")

$$
\begin{aligned}
& \operatorname{array}\left[\begin{array}{l}
\ldots \\
\text { values }
\end{array}\right] \\
& \operatorname{array}=\left[\begin{array}{lll}
\text {... values } & . .
\end{array}\right]
\end{aligned}
$$

The array libs=, which defines the ORIGEN library files, is the only one that is strictly required for all cases. Cases that simulate $0 \mathrm{D}$ or $1 \mathrm{D}$ lumped-assembly models typically only require one entry for a single ORIGEN library (assuming uniform axial enrichment), while the simulated 3D depletion model may utilize multiple libraries if specific ORIGEN libraries are pre-generated for different pin locations (e.g., adjacent to a water hole, Gd rods, etc.). If multiple libraries are used, the array libmap = is required to identify the pin locations associated with the input libraries. The numbering of these libraries in the libmap array corresponds to the ordering of libraries in the libs= array; i.e., a "1" corresponds to the first library specified, " 2 " to the second, and so on. A zero-value entry in the array indicates that the location is not to be depleted (i.e., a non-fuel region, such as a water hole or guide tube).

For single array values, the array bracket syntax is not required. For example, each of the following is equivalent:

$$
\begin{gathered}
\text { compmap }=[1] \\
\text { compmap }[1] \\
\text { compmap }=1
\end{gathered}
$$

Note that the assignment operator $\left(_{(}\right)$is likewise optional for arrays when using the square-bracket syntax. 
Unless the 0D lumped-assembly model (i.e., lumped mass with no axial power variation) is used, at least one of the arrays $(p z, p x y)$ describing the power variations must also be entered. The 1D axial depletion model requires that the $p z=$ array be entered, while the pin-wise depletion model additionally requires the array $p x y=$. The data in arrays $p x y=$ and $p z=$ correspond to the variables $r_{X Y}$ and $a_{\mathrm{Z}}$, respectively, described in Sect. 5.4.2.3. The axial and XY power distributions are normalized to unity inside the code, so that only the ratios of the input array values are significant. As discussed in Sect. 5.4.2.3, it is generally recommended to use the final burnup distributions rather than the relative power distributions for the values in the pxy and $p z$ arrays.

The array nuccomp defines the nuclides to be included in the output compBlock file, described in more detail in Sect.5.4.4. The nuclides in the array are identified by their seven digit IZZZAAA identifier defined as $\mathrm{ID}=\mathrm{I} * 1000000+\mathrm{Z} * 1000+\mathrm{A}$, where $\mathrm{Z}$ is the atomic number; $\mathrm{A}$ is the mass number, and $\mathrm{I}$ is the isomeric state ( $\mathrm{I}=0$ for ground; $\mathrm{I}=1$ for first metastable; etc.). For example, identifiers for ${ }^{16} \mathrm{O}$ and ${ }^{242 \mathrm{~m}} \mathrm{Am}$ are 8016 and 1095242 , respectively. If this array is omitted, the nuclides in Table 5.4.10 are used. This is described in more detail in Sect.5.4.4.1.

The optional array describing the non-fuel elements in the assembly contains pairs of values (element, mass), where "element" is the chemical symbol for a particular element, and "mass" is the mass of the element in kilograms per MTU. For example, the array

$$
\text { nonfuel }=\left[\begin{array}{lll}
\operatorname{zr}=520.3 & \mathrm{sn}=8.4
\end{array}\right]
$$

indicates that the assembly contains 520.3 kilograms of zirconium and 8.4 kilograms of tin for each metric ton of uranium (MTU) in the assembly. Note that elemental masses are specified - the isotopic masses are computed internally by the code using natural abundances in the data library. It is also possible to normalize the total mass of non-fuel elements to a specified fraction of the MTU mass using the parameter fracnf $=$ in the parameter block. In this case, only the relative amounts of each non-fuel element are needed for the nonfuel array. Non-fuel masses are distributed uniformly among all the fuel depletion regions.

Table 5.4.8. ORIGAMI Input Array Description.

\begin{tabular}{|c|c|c|}
\hline Array Name & Description & Default \\
\hline$(*)$ libs & List of ORIGEN library file names for fuel in assembly. [characters] & None \\
\hline libmap & $\begin{array}{l}\text { XY map of library identifiers associated with each pin in assembly. } \\
\text { Library identifiers correspond to the order of the ORIGEN libraries } \\
\text { entered in the libs block. [integers] }\end{array}$ & 1 \\
\hline compmap & $\begin{array}{l}\text { XY map of mixture identifiers that correspond to the mixture ID in the } \\
\text { fuelcomp block. [integers] }\end{array}$ & 1 \\
\hline $\mathrm{pxy}$ & $\begin{array}{l}\text { XY map of pin power shaping factors / fractional powers. Must be a } \\
\text { square array (e.g., } 15 \times 15) \text {. Defaults to lumped assembly model (no } \\
\text { individual pins). [real numbers] }\end{array}$ & 1.0 \\
\hline $\mathrm{pz}$ & $\begin{array}{l}\text { Axial }(Z) \text { power shaping factors / fractional power distribution for the } \\
\text { assembly. [real numbers] }\end{array}$ & 1.0 \\
\hline
\end{tabular}




\begin{tabular}{|c|c|c|}
\hline meshz & $\begin{array}{l}\text { Axial mesh boundaries (cm) for the axial relative power zones. Only } \\
\text { required to define axial mesh for viewing results; but if entered, it must } \\
\text { be consistent with axial power shape. The number of entries should be } \\
\text { one greater than number of entries in } p z=\text { array. } \\
\text { [real numbers] }\end{array}$ & None \\
\hline $\bmod z$ & $\begin{array}{l}\text { Axial variation in water density }(\mathrm{g} / \mathrm{cc}) \text { corresponding to the axial } \\
\text { power zones. [real numbers] }\end{array}$ & 0.723 \\
\hline nonfuel & $\begin{array}{l}\text { Non-fuel materials contained in assembly. Values are entered in pairs } \\
\text { of (element-symbol=mass }(\mathrm{kg}) \text { per mtu of } \mathrm{HM}) \text {. If parameter fracnf is } \\
\text { input, mass of non-fuel materials is normalized to this fraction of fuel } \\
\text { mtu. } \\
\text { NOTE: Oxygen mass in } \mathrm{UO}_{2} \text { should not be entered here (i.e., this is } \\
\text { pre-supplied by ORIGAMI). [character / real number pairs] }\end{array}$ & None \\
\hline ggrp & $\begin{array}{l}\text { Energy boundaries (eV) for defining decay gamma source spectrum. } \\
\text { [real numbers] }\end{array}$ & None \\
\hline ngrp & $\begin{array}{l}\text { Energy boundaries }(\mathrm{eV}) \text { for defining }(\alpha, \mathrm{n}) \text { and spontaneous fission } \\
\text { neutron source spectrum. [real numbers] }\end{array}$ & None \\
\hline nuccomp & List of nuclide IZZZAAAs to be included in output compBlock file. & $\begin{array}{l}\text { Table 5.4.10 } \\
\text { Nuclides }\end{array}$ \\
\hline
\end{tabular}

$\left(^{*}\right)$ indicates required input

\subsubsection{ORIGAMI Input/Output Files}

Table 5.4.9 gives the input and output files for ORIGAMI. ORIGAMI produces printed output results as well as several optional output files described in this section. In order to reduce the potentially voluminous amount of printout, by default ORIGAMI only prints the concentrations in grams for selected actinides in each axial zone of every pin, and only for the last time step (e.g., decay step) of the last cycle in the power-history block. Time-dependent results are given for all substeps in the last step (i.e, there are nburn and ndecay substeps within a burn step or decay step, respectively) In addition, the blended actinide concentrations over all pins are printed for each axial zone, and for the entire lumped assembly. Additional types of printed output can be specified in the print block. The concentrations, as well as optional neutron and gamma source spectra information, for all nuclides, in all pins and axial zones are also stored in the ORIGEN binary output file, often called an " $\mathrm{ft} 71$ " file. The contents and format of the binary file are described in the ORIGEN documentation of the SCALE manual. The binary file information can be edited by the OPUS module in SCALE. Like the printed output, the $\mathrm{ft} 71$ file is written by default only for the last step of the last cycle. However, both the printed output and binary file results can be obtained at additional time steps by specifying the input variables output and $\mathrm{ft} 71$, respectively, in the OPTIONS input block. These input parameters can have the keywords:

$\mathrm{ft} 71=$ last, cycle, all ; and/or, output=last, cycle, all ;

where:

last $=>$ save results only for the substeps in last step of the last cycle (default)

cycle $=>$ save results for substeps in the last irradiation and decay steps in every cycle

all $\quad=>$ save results for all substeps of all irradiation and decay steps in every cycle

The output files are written in the user output directory for the calculation (i.e., the same directory where the printed output file is written - the default is the directory from where the case was submitted). File names are prefixed by an extension consisting of the input file base-name appended to an optional 
character string given by the input keyword prefix $=$. For example, if the ORIGAMI input file is named ORIGAMICase.inp, the base-name is ORIGAMICase. Thus, if the keyword prefix= is not included in the input, the file containing the axial decay heat results is named ORIGAMICase_AxialDecayHeat. On the other hand, if the input contains the keyword prefix=CE16X16, the file is named ORIGAMICase_CE16X16_AxialDecayHeat.

In order to capture axially dependent features of an assembly (such as partial-length rods), users may elect to construct sequential ORIGAMI cases that modify the XY pin map features (e.g., library and enrichment maps) between cases. In order to allow for these types of "continuation" cases (in which the sequential case represents an adjacent axial span of the assembly), the offsetz= feature is provided, which adjusts the axial numbering for ORIGAMI outputs (such as for MCNP materials \& spectra cards, axial decay heat, etc.). The offsetz= parameter offsets the axial numbering for these output files, where the (integer) value provided corresponds to the last axial zone number calculated by ORIGAMI (default: 0 ). For more details on the syntax of the options block, see Sect. 5.4.3.2. 
Table 5.4.9. ORIGAMI Input/Output Files

\begin{tabular}{|c|c|c|c|}
\hline File Name (*) & Description & Type & Format \\
\hline compBlock & $\begin{array}{l}\text { Mixture compositions in standard composition } \\
\text { format for input to SCALE codes such as KENO }\end{array}$ & out & text \\
\hline MCNP_matls.inp & $\begin{array}{l}\text { Nuclide identifiers and weight fractions in format } \\
\text { for MCNP material cards }\end{array}$ & out & text \\
\hline MCNP_gamma.inp & $\begin{array}{l}\text { Total gamma source intensity in MCNP source } \\
\text { format. Only output if gamma energy group } \\
\text { boundaries are entered in input array ggrp= }\end{array}$ & out & text \\
\hline MCNP_neutron.inp & $\begin{array}{l}\text { Total neutron source intensity in MCNP source } \\
\text { format. Only output if neutron energy group } \\
\text { boundaries are entered in input array ngrp }=\end{array}$ & out & text \\
\hline AxialGammaSpec & $\begin{array}{l}\text { Gamma spectrum (photons/sec) by axial zone, } \\
\text { enabled by "srcopt }\{\text { print=yes }\} " \text {. }\end{array}$ & out & text \\
\hline AxialNeutSpec & $\begin{array}{l}\text { Neutron spectrum (neutron/sec) by axial zone, } \\
\text { enabled by "srcopt }\{\text { print=yes }\} " \text {. }\end{array}$ & out & text \\
\hline AxialDecayHeat & $\begin{array}{l}\text { Decay heat source (watts) by axial zone, enabled } \\
\text { by "options }\{\text { decayheat=yes }\} \text { " }\end{array}$ & out & text \\
\hline assm.f71 & $\begin{array}{l}\text { Output stacked ORIGEN ft71 files for each axial } \\
\text { zone }\end{array}$ & out & binary \\
\hline assembly_restart.f71 & $\begin{array}{l}\text { Input stacked ORIGEN ft71 files for each axial } \\
\text { zone }\end{array}$ & in & binary \\
\hline. $\mathrm{f71}$ & $\begin{array}{l}\text { Output of stacked ORIGEN ft71 files for each } \\
\text { pin and axial zone }\end{array}$ & out & binary \\
\hline actinideMesh.3dmap & $\begin{array}{l}\text { Binary MeshView file of selected actinide } \\
\text { masses by depletion cell }\end{array}$ & out & binary \\
\hline actinideMesh.ASCII.txt & $\begin{array}{l}\text { Plaintext MeshView file of selected actinide } \\
\text { masses by depletion cell }\end{array}$ & out & text \\
\hline fpMesh. 3dmap & $\begin{array}{l}\text { Binary MeshView file of selected fission product } \\
\text { masses by depletion cell }\end{array}$ & out & binary \\
\hline fpMesh.ASCII.txt & $\begin{array}{l}\text { Plaintext MeshView file of selected fission } \\
\text { product masses by depletion cell }\end{array}$ & out & text \\
\hline burnupMesh.3dmap & Binary MeshView file of depletion node burnups & out & binary \\
\hline
\end{tabular}

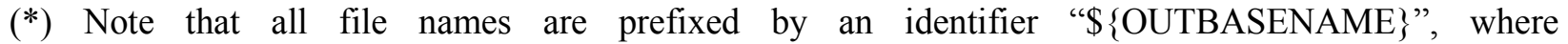
$\$\{$ OUTBASENAME $\}$ is a prefix constructed from the input file base name followed by the character string given by input keyword prefix=. For example, the input file named "my.inp" with prefix=sample would give an output prefix "my_sample"; e.g., my_sample.f71, my_sample.assm.f71,my_sample_MCNP_matls.inp, etc.

\subsubsection{Generation of SCALE standard composition data file}

If input parameter $s t d c o m p=y e s$ is specified, ORIGAMI produces a text file containing a SCALE standard composition description for each axial interval. The file is written in the form of a stdcomp block that can 
be directly used as input to any SCALE module that requires a composition block. If a 1D axial depletion model is used for the assembly, the composition for each axial zone is given a unique mixture number defined for an axial node ' $Z$ ' as:

(1D axial model)

$$
\operatorname{mix}=1000+(\text { asmid }-1) \times \mathrm{N}_{Z}+\mathrm{Z}
$$

where $\mathrm{N}_{\mathrm{Z}}$ is the number of axial zones and asmid is the input identifier. For example, if there are 12 axial zones and the input for asmid is 20, then the mixture number associated with axial zone number 1 is mix $=1229$, and the mixture for zone 12 is $\operatorname{mix}=1240$. If an assembly is represented by a 3D multiple-pin model, the mixture number is defined,

$(3 \mathrm{D}$ model $) \quad \operatorname{mix}=1000+($ asmid -1$) \times \mathrm{N}_{\mathrm{Z}}+\mathrm{Z}+\mathrm{X} \times 100000+\mathrm{Y} \times 10000000$

where $\mathrm{X}$ and $\mathrm{Y}$ correspond to the row and column numbers of the pin.

The nuclides components of the mixtures may be specified in the input array compnuc=, or by default the mixture may consist of the nuclides given in Table 5.4.10, which are the nuclides recommended in Ref. 3 for burnup credit analysis, plus ${ }^{16} \mathrm{O}$. The temperatures of the mixtures are set by the value of parameter temper $=$, which defaults to a value of 293 Kelvin. The number densities of the nuclides in the mixtures are calculated using the following expressions:

$$
\begin{aligned}
\mathrm{N}_{\mathrm{Z}}^{(\mathrm{i})} & =\rho \frac{\mathrm{M}_{\mathrm{Z}}^{(\mathrm{i})}}{\mathrm{M}_{\mathrm{Z}} \cdot 10^{6}} \cdot \frac{\mathrm{N}_{\mathrm{A}}}{\mathrm{A}^{(\mathrm{i})}} \cdot 0.8814 \cdot 10^{-24} \\
& =\rho \frac{\mathrm{M}_{\mathrm{Z}}^{(\mathrm{i})}}{\mathrm{M}_{\mathrm{Z}} \cdot \mathrm{A}^{(\mathrm{i})}} \cdot 5.309 \cdot 10^{-7}
\end{aligned}
$$

Where:

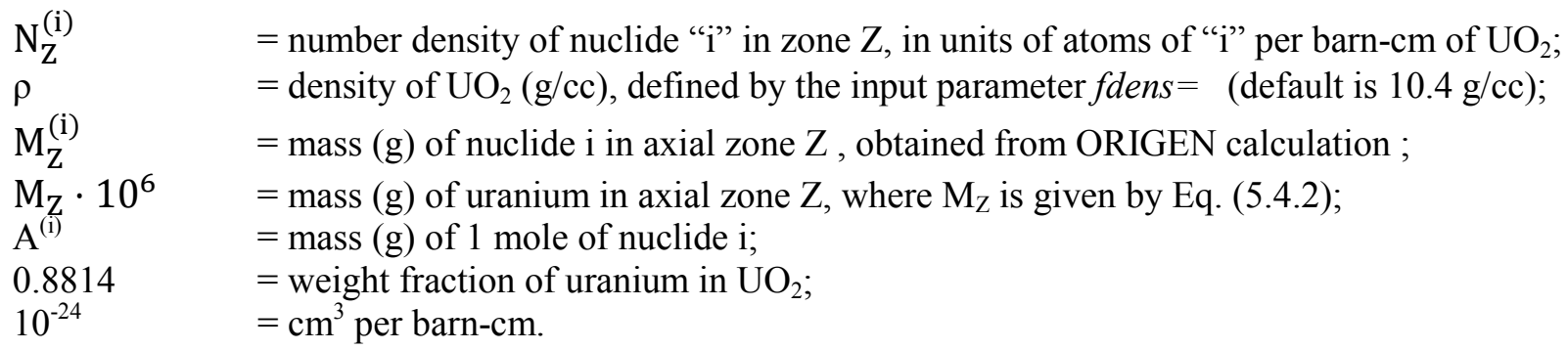

The definitions of other parameters appearing in this equation are given in Sect. 5.4.2.5. An example of the standard composition file produced by ORIGAMI is given in Sect. 5.4.6, Table 5.4.15 (illustrated in sample problem 2) 
Table 5.4.10. Default Burnup Credit Nuclides in Standard Comp File

\begin{tabular}{|c|c|c|}
\hline Nuclide & ZAID & Nuclide type \\
\hline${ }^{16} \mathrm{O}$ & 8016 & light element \\
\hline${ }^{234} \mathrm{U}$ & 92234 & actinide \\
\hline${ }^{235} \mathrm{U}$ & 92235 & actinide \\
\hline${ }^{236} \mathrm{U}$ & 92236 & actinide \\
\hline${ }^{238} \mathrm{U}$ & 92238 & actinide \\
\hline${ }^{237} \mathrm{~Np}$ & 93237 & actinide \\
\hline${ }^{238} \mathrm{Pu}$ & 94238 & actinide \\
\hline${ }^{239} \mathrm{Pu}$ & 94239 & actinide \\
\hline${ }^{240} \mathrm{Pu}$ & 94240 & actinide \\
\hline${ }^{241} \mathrm{Pu}$ & 94241 & actinide \\
\hline${ }^{242} \mathrm{Pu}$ & 94242 & actinide \\
\hline${ }^{241} \mathrm{Am}$ & 95241 & actinide \\
\hline${ }^{243} \mathrm{Am}$ & 95243 & actinide \\
\hline${ }^{95} \mathrm{Mo}$ & 42095 & fission product \\
\hline${ }^{99} \mathrm{Tc}$ & 43099 & fission product \\
\hline${ }^{101} \mathrm{Ru}$ & 44101 & fission product \\
\hline${ }^{103} \mathrm{Rh}$ & 45103 & fission product \\
\hline${ }^{109} \mathrm{Ag}$ & 47109 & fission product \\
\hline${ }^{133} \mathrm{Cs}$ & 55133 & fission product \\
\hline${ }^{143} \mathrm{Nd}$ & 60143 & fission product \\
\hline${ }^{145} \mathrm{Nd}$ & 60145 & fission product \\
\hline${ }^{147} \mathrm{Sm}$ & 62147 & fission product \\
\hline${ }^{149} \mathrm{Sm}$ & 62149 & fission product \\
\hline${ }^{150} \mathrm{Sm}$ & 62150 & fission product \\
\hline${ }^{151} \mathrm{Sm}$ & 62151 & fission product \\
\hline${ }^{152} \mathrm{Sm}$ & 62152 & fission product \\
\hline${ }^{151} \mathrm{Eu}$ & 63151 & fission product \\
\hline${ }^{153} \mathrm{Eu}$ & 63153 & fission product \\
\hline & 64155 & fission product \\
\hline
\end{tabular}

\subsubsection{MCNP data files}

If the input parameter mcnp=yes is set in the options block, the computed weight fractions for the materials in each axial zone also are output in a file in the format of MCNP material cards. These material cards are designed to be coupled to a corresponding MCNP assembly geometry using the same numbering convention for the depletion zones. Sect. 5.4.6 shows an example of the MCNP material information produced by ORIGAMI. The numbering convention of the MCNP materials cards works by combining the axial and pin numbers into a material card, where pins are counted sequentially by row, starting with the bottom-left row of input, counting from left to right across each row to the top-right pin (i.e., the bottom-left pin is pin \#1, etc.). The pin numbers reset with each axial zone, starting from the bottom zone, counting up from 1 . The naming convention for materials cards is thus the pin number (1999) followed by the zone number (1-99); for example, pin \#15 of axial zone \#12 would be m1512. Accompanying each material card is a list of ZAID numbers and final concentrations (following depletion/decay) for the cell expressed in weight fractions. The weight fractions are given as negative values in accordance with MCNP convention. The fuel density, which may be used in the MCNP cell card, is equal to the value of the input parameter $f d e n s=$. 
When parameter $m c n p=y e s$ is set, ORIGAMI also produces output files containing the fuel assembly radiation source magnitude by depletion zone, to support modeling with MCNP. The gamma/neutron source term cards correspond to the total gamma or neutron intensity (particles/s) from each respective depletion region, using the same numbering convention as that for the MCNP material cards. The source magnitude is computed by summing over the MG source spectra defined in Eq. (5.4.12).

$$
\mathrm{S}_{\mathrm{Z}}^{(\mathrm{p})}=\sum_{\mathrm{g}} \mathrm{S}_{\mathrm{Z}, \mathrm{g}}^{(\mathrm{p})}
$$

Where:

$\mathrm{S}_{\mathrm{Z}}^{(\mathrm{p})} \quad=$ total source magnitude $(\mathrm{p} / \mathrm{s})$ for particles of type $p$;

$\mathrm{S}_{\mathrm{Z}, \mathrm{g}}^{(\mathrm{p})}=$ multigroup source magnitude $(\mathrm{p} / \mathrm{s})$ for energy group $g$, and particles of type $p$

More details on the ORIGEN calculation of the source terms can be found in the ORIGEN section of SCALE documentation.

\subsubsection{Decay heat calculation}

When input parameter decayheat $=y e s$ is specified in the input, a text file containing the decay heat source by axial zone, summed over all pins, is generated as output. The decay heat in zone $Z$ is given in watts and is computed from the expression

$$
\mathrm{H}_{\mathrm{Z}}=\sum_{\mathrm{i}=1}^{\mathrm{itot}} \mathrm{Q}_{\mathrm{i}} \lambda_{\mathrm{i}} \frac{\mathrm{M}_{\mathrm{Z}}^{(\mathrm{i})}}{\mathrm{A}^{(\mathrm{i})}} \cdot 1.602 \cdot 10^{-13} \cdot \mathrm{N}_{\mathrm{A}}=9.65 \cdot 10^{10} \sum_{\mathrm{i}=1}^{\mathrm{itot}} \mathrm{Q}_{\mathrm{i}} \lambda_{\mathrm{i}} \frac{\mathrm{M}_{\mathrm{Z}}^{(\mathrm{i})}}{\mathrm{A}^{(\mathrm{i})}}
$$

where

$\mathrm{Q}_{\mathrm{i}} \quad=$ decay energy in $\mathrm{MeV}$ for nuclide $i$;

$\lambda_{\mathrm{i}} \quad=$ decay constant $\left(\mathrm{s}^{-1}\right)$ for nuclide $i$;

$\mathrm{M}_{\mathrm{Z}}^{(\mathrm{i})} \quad=$ mass $(\mathrm{g})$ of nuclide $i$ in axial zone $Z$, obtained from ORIGEN calculation;

$\mathrm{A}^{(\mathrm{i})} \quad=$ mass $(\mathrm{g})$ of 1 mole of nuclide $i$;

itot $\quad=$ total number of nuclides in burned fuel, and

$1.602 \times 10^{-19}=$ number of joules per MeV.

An example output decay heat file produced by ORIGAMI is shown in Sect. 5.4.6, Table 5.4.16 (from sample problem 2).

\subsubsection{ORIGEN results files}

The ORIGEN computation for each depletion region produces an ORIGEN binary concentrations output file, historically called an "ft71" because it was written on "Fortran tape" number 71. The file named "\$\{OUTBASENAME\}.f71" contains the concentrations for all depletion regions, stacked within a single binary file, where "\$\{OUTBASENAME\}" is the base of the output file name, e.g. the "my" in "my.out". 
The order of stored cases on the $\mathrm{f} 71$ file corresponds to the order in which ORGAMI processes individual depletion cases, starting with the bottom-left row in the user-supplied power map (pin \#1) and looping left to right, progressively up through the series of rows. This process repeats for each axial zone, starting from the bottom of the assembly and working upward (i.e., starting with pin \#1, axial zone \#1, looping through each pin on axial zone $\# 1$, and then proceeding to pin $\# 1$ on axial zone $\# 2$, etc.). This convention is the same as that used for TRITON arrays.

In addition, the compositions are blended over all pins for each axial zone to obtain the axially-dependent compositions for the lumped assembly, stored in a file named "\$\{OUTBASENAME $\}$.assm.f71". If saved, this file may be input as a restart file, as discussed in Sect. 5.4.2.3.

\subsubsection{Plotting features}

ORIGAMI creates three separate mesh summaries of material inventories for individual depletion regions, useful for 3D visualization and inspection. These include maps of (1) depletion region burnups, (2) selected actinide concentrations (including isotopes of $\mathrm{U}, \mathrm{Pu}, \mathrm{A} \mathrm{m}$, and $\mathrm{Cm}$ ), and (3) selected fission products typically used for burnup evaluation, including isotopes of $\mathrm{Cs}, \mathrm{Y}, \mathrm{Ag}, \mathrm{Rh}, \mathrm{Ru}, \mathrm{Eu}, \mathrm{Sm}, \mathrm{Nd}, \mathrm{Gd}$, and others). Additionally, ORIGAMI outputs a separate mesh tally of individual node burnups. These outputs are described in Table 5.4.9. Note: The mesh files are only created if the user specifies the (optional) input arguments for assembly pitch (pitch $=$ ) and axial zone locations (meshz array).

These output mesh-dependent maps can be visualized using the Java-based Mesh File Viewer program included with SCALE. An example MeshView visualization of one of these outputs is shown in Figure 5.4.2. MeshView is installed in " $\$\{$ SCALE $\} /$ Meshview", where " $\$\{$ SCALE $\}$ " is the installation directory. A script to run MeshView is located at " $\$\{\mathrm{SCALE}\} / \mathrm{cmds} / \mathrm{meshview".}$
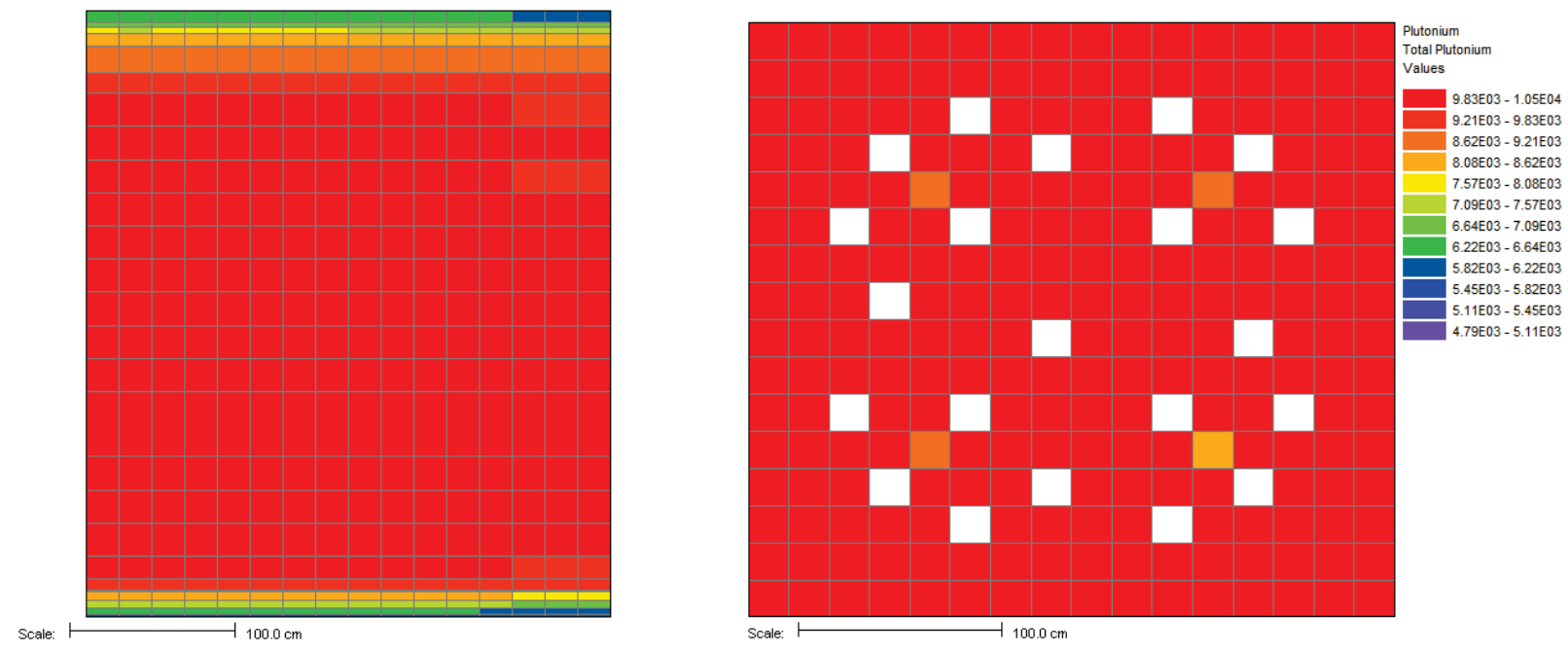

Figure 5.4.2. MeshView plots of total plutonium content in the 3D depletion regions (left: XZ plane; right: XY plane). 


\subsubsection{Parallel Execution on Linux Clusters}

For large 3D depletion problems it is advantageous to execute the ORIGEN calculations for different depletion regions in parallel. This can be done on Linux clusters using MPI. When parallel execution mode is enabled, ORIGAMI distributes the individual depletion cases across the pin rows, columns, and axial zones across several processors; the depletion calculation is thus split across several processors. ORIGAMI then collects the inventories from each calculation node and concatenates the output.

To execute ORIGAMI in parallel mode, a parallel-enabled MPI build of SCALE must be used and ORIGAMI should be invoked with the percent (\%) prefix:

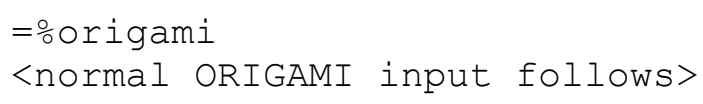

Additionally, for parallel jobs spanning multiple computational nodes (as opposed to those just using multiple processors on the same node, it is recommended to use the $-\mathrm{T}$ option to specify a common temporary directory (such as a network-mounted directory accessible to all nodes). This is due to the way ORIGAMI divides the problem space in parallel mode; each computational node stores its respective binary dump file of the individual pin/zone concentrations. Upon completion of execution, the master node must be able to locate these individual problem node-generated binary dump files; thus, by using a common temporary directory, ORIGAMI can correctly re-assembly the individual pinwise dumpfiles into a single consolidated "master" dump file.

The following is a typical execution command line to execute ORIGAMI in parallel.

$$
\begin{aligned}
& \text { scalerte }-\mathrm{N} \text { [number of nodes] }-\mathrm{M} \text { [machine file] -T [tmpdir] } \\
& \text { [input_file.inp] }
\end{aligned}
$$

For more information on executing SCALE in parallel, see the SCALE Readme file.

\subsubsection{Sample Problems}

This section shows sample problems for each of the three types of simulated assembly models: 0D fully lumped, 2D lumped axial depletion, and 3D pinwise depletion, and also demonstrates a restart case.

\subsubsection{Sample problem 1: fully lumped assembly model}

The first example corresponds to a fully lumped assembly model in which the materials are depleted with a space-independent (i.e., assembly average) flux distribution. The assembly contains $0.38 \mathrm{MTU}$, and the fuel is $2.8 \mathrm{wt} \%$ enriched. The assembly also includes several non-fuel materials corresponding to cladding and other structural materials. Note that the non-fuel concentrations are specified in $\mathrm{kg} / \mathrm{MTU}$, and thus are not the actual total non-fuel masses in the $0.38 \mathrm{MTU}$ assembly. The assembly is depleted for three cycles with specific powers of 40.0, 38.6, and 25.2 MW/MTU, respectively. The ORIGEN library data are interpolated for eight different burnup steps during the irradiation periods of the first two cycles, and for six burnup steps in the last cycle. Table 5.4.11 shows the ORIGAMI input for this case, and Table 5.4.12 gives the calculated actinide concentrations at the end of the third cycle. 
Table 5.4.11. Input for ORIGAMI Sample Problem 1

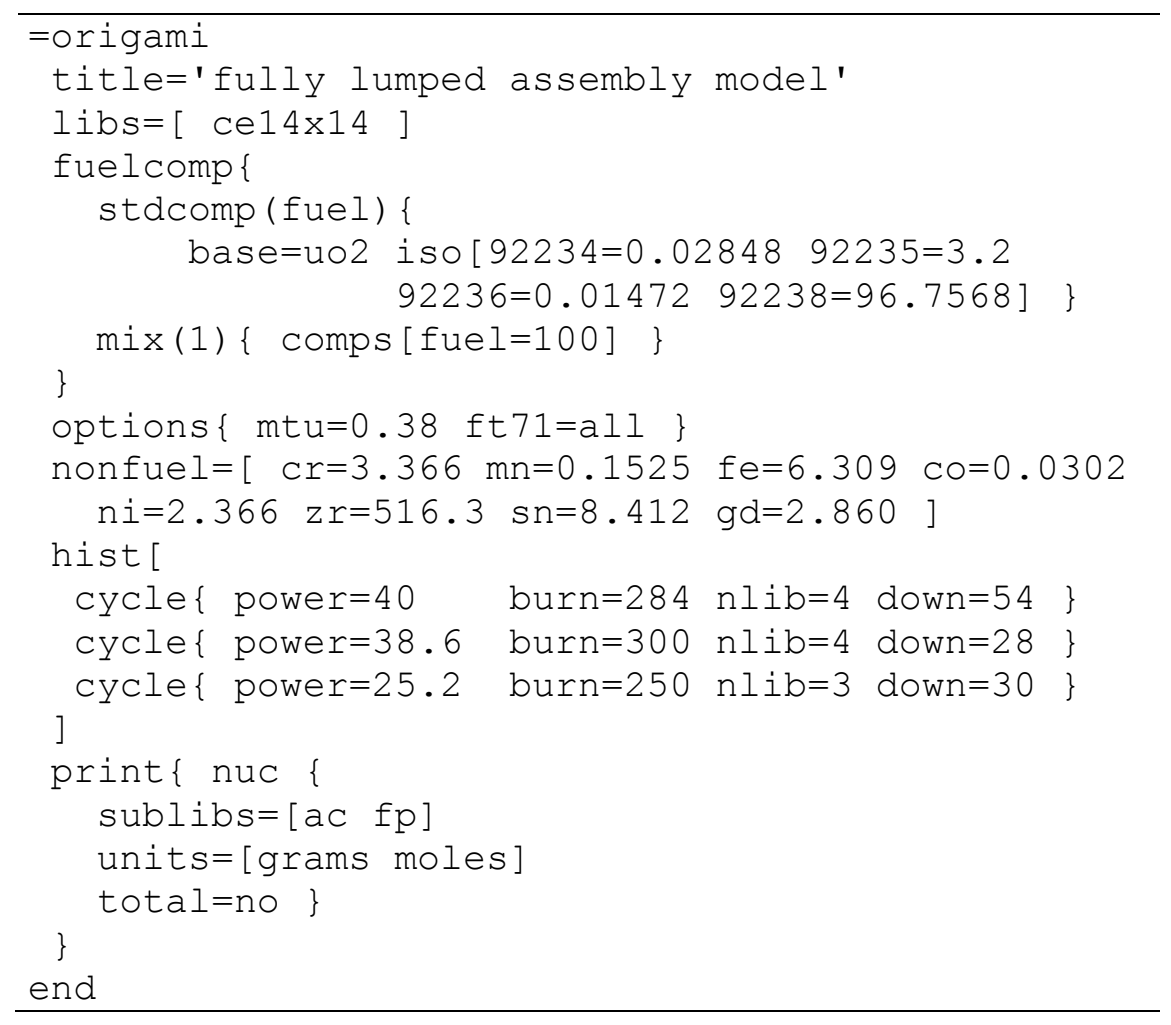

Table 5.4.12. Calculated Actinide Isotopics for Sample Problem 1

\begin{tabular}{|c|c|}
\hline Nuclide $(*)$ & Mass $(\mathrm{g})$ \\
\hline${ }^{234} \mathrm{U}$ & $6.820 \mathrm{E}+01$ \\
\hline${ }^{235} \mathrm{U}$ & $3.621 \mathrm{E}+03$ \\
\hline${ }^{236} \mathrm{U}$ & $1.487 \mathrm{E}+03$ \\
\hline${ }^{238} \mathrm{U}$ & $3.598 \mathrm{E}+05$ \\
\hline${ }^{237} \mathrm{~Np}$ & $1.348 \mathrm{E}+02$ \\
\hline${ }^{238} \mathrm{Pu}$ & $3.862 \mathrm{E}+01$ \\
\hline${ }^{239} \mathrm{Pu}$ & $1.919 \mathrm{E}+03$ \\
\hline${ }^{240} \mathrm{Pu}$ & $7.820 \mathrm{E}+02$ \\
\hline${ }^{241} \mathrm{Pu}$ & $3.960 \mathrm{E}+02$ \\
\hline${ }^{242} \mathrm{Pu}$ & $1.394 \mathrm{E}+02$ \\
\hline${ }^{241} \mathrm{Am}$ & $1.474 \mathrm{E}+01$ \\
\hline${ }^{243} \mathrm{Am}$ & $2.491 \mathrm{E}+01$ \\
\hline${ }^{242} \mathrm{Cm}$ & $2.663 \mathrm{E}+00$ \\
\hline${ }^{244} \mathrm{Cm}$ & $5.698 \mathrm{E}+00$ \\
\hline TOTAL & $6.820 \mathrm{E}+01$ \\
\hline
\end{tabular}

$\left.{ }^{*}\right)$ Actinides with concentrations less than 0.0001 are not shown. 


\subsubsection{Sample problem 2: lumped axial depletion assembly model}

The second example has the same lumped assembly and power history as sample problem 1, except in this case an axial power distribution is provided for eight zones, so that the fuel burnup will vary axially. Table 5.4.13 shows the ORIGAMI input for this case. Also, the options to generate standard composition and decay output files are requested.

Table 5.4.14 gives the computed actinide concentrations in grams for the first four of the eight axial zones. Since the input axial power distribution is symmetrical about the assembly midplane, the last four zones have identical concentrations as the first four. The last column in the table shows actinide masses for the entire assembly.

Table 5.4.15 is a listing of the contents of the compBlock file, which contains standard composition input for the eight axial zones in the assembly at the end of cycle 3. A complete description of the SCALE standard composition input format is given in the MIPLIB chapter. The first entry on each line in Table 5.4.15 corresponds to the SCALE nuclide identifier. Only the default burnup credit analysis are included. The second entry is the mixture number associated with a particular axial zone. The mixture number for an axial zone is obtained using Eq. (5.4.14). The third entry is always zero in this file, and the fourth entry corresponds to the number density in atoms per barn-cm. The next entry on the line is the temperature, which has the default value of 293.0 since the input parameter temper $=$ was not specified. The final entry is an "end" statement. The information in this file can be used as the read comp input block for any SCALE module.

Table 5.4.16 shows a listing of the file AxialDecayHeat, which contains the heat source at the end of the third cycle. The entries in the file correspond to the decay power in watts for the eight axial zones, which are computed using Eq. (5.4.18).

Table 5.4.13. Input for ORIGAMI Sample Problem 2

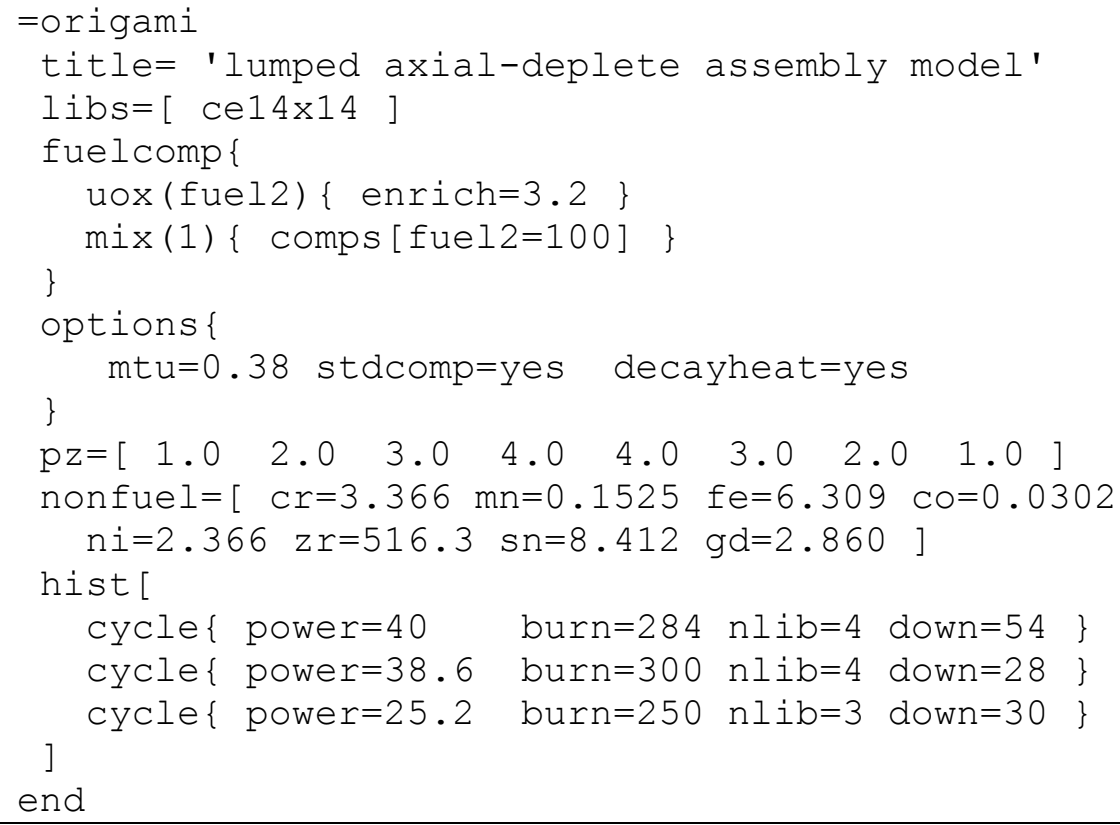


Table 5.4.14. Calculated Actinide Isotopics by Axial Zone for Sample Problem 2

\begin{tabular}{|c|c|c|c|c|c|}
\hline Nuclide & $\begin{array}{c}\text { Axial Zone 1 } \\
\text { Mass (g) }\end{array}$ & $\begin{array}{c}\text { Axial Zone 2 } \\
\text { Mass (g) }\end{array}$ & $\begin{array}{c}\text { Axial Zone 3 } \\
\text { Mass (g) }\end{array}$ & $\begin{array}{c}\text { Axial Zone 4 } \\
\text { Mass (g) }\end{array}$ & $\begin{array}{c}\text { TOTAL } \\
\text { Mass (g) }\end{array}$ \\
\hline${ }^{234} \mathrm{U}$ & $1.1384 \mathrm{E}+01$ & $9.4326 \mathrm{E}+00$ & $7.6666 \mathrm{E}+00$ & $6.11 \mathrm{E}+00$ & $6.92 \mathrm{E}+01$ \\
\hline${ }^{235} \mathrm{U}$ & $9.7324 \mathrm{E}+02$ & $5.9453 \mathrm{E}+02$ & $3.3795 \mathrm{E}+02$ & $1.78 \mathrm{E}+02$ & $4.17 \mathrm{E}+03$ \\
\hline${ }^{236} \mathrm{U}$ & $1.0392 \mathrm{E}+02$ & $1.6544 \mathrm{E}+02$ & $2.0053 \mathrm{E}+02$ & $2.15 \mathrm{E}+02$ & $1.37 \mathrm{E}+03$ \\
\hline${ }^{238} \mathrm{U}$ & $4.5604 \mathrm{E}+04$ & $4.5202 \mathrm{E}+04$ & $4.4752 \mathrm{E}+04$ & $4.43 \mathrm{E}+04$ & $3.60 \mathrm{E}+05$ \\
\hline${ }^{237} \mathrm{~Np}$ & $4.7874 \mathrm{E}+00$ & $1.2622 \mathrm{E}+01$ & $2.0984 \mathrm{E}+01$ & $2.83 \mathrm{E}+01$ & $1.33 \mathrm{E}+02$ \\
\hline${ }^{238} \mathrm{Pu}$ & $5.5445 \mathrm{E}-01$ & $2.9024 \mathrm{E}+00$ & $7.1722 \mathrm{E}+00$ & $1.26 \mathrm{E}+01$ & $4.65 \mathrm{E}+01$ \\
\hline${ }^{239} \mathrm{Pu}$ & $1.7378 \mathrm{E}+02$ & $2.2968 \mathrm{E}+02$ & $2.4438 \mathrm{E}+02$ & $2.46 \mathrm{E}+02$ & $1.79 \mathrm{E}+03$ \\
\hline${ }^{240} \mathrm{Pu}$ & $3.3124 \mathrm{E}+01$ & $7.7894 \mathrm{E}+01$ & $1.1457 \mathrm{E}+02$ & $1.40 \mathrm{E}+02$ & $7.30 \mathrm{E}+02$ \\
\hline${ }^{241} \mathrm{Pu}$ & $1.2974 \mathrm{E}+01$ & $3.8401 \mathrm{E}+01$ & $5.8714 \mathrm{E}+01$ & $7.09 \mathrm{E}+01$ & $3.62 \mathrm{E}+02$ \\
\hline${ }^{242} \mathrm{Pu}$ & $1.4543 \mathrm{E}+00$ & $1.0115 \mathrm{E}+01$ & $2.6393 \mathrm{E}+01$ & $4.75 \mathrm{E}+01$ & $1.71 \mathrm{E}+02$ \\
\hline${ }^{241} \mathrm{Am}$ & $5.3938 \mathrm{E}-01$ & $1.5121 \mathrm{E}+00$ & $2.0433 \mathrm{E}+00$ & $2.12 \mathrm{E}+00$ & $1.24 \mathrm{E}+01$ \\
\hline${ }^{243} \mathrm{Am}$ & $9.5029 \mathrm{E}-02$ & $1.4341 \mathrm{E}+00$ & $5.6093 \mathrm{E}+00$ & $1.29 \mathrm{E}+01$ & $4.00 \mathrm{E}+01$ \\
\hline${ }^{242} \mathrm{Cm}$ & $(*)$ & $2.0213 \mathrm{E}-01$ & $4.7744 \mathrm{E}-01$ & $7.56 \mathrm{E}-01$ & $2.93 \mathrm{E}+00$ \\
\hline${ }^{244} \mathrm{Cm}$ & $(*)$ & $2.4685 \mathrm{E}-01$ & $1.6315 \mathrm{E}+00$ & $5.54 \mathrm{E}+00$ & $1.49 \mathrm{E}+01$ \\
\hline${ }^{245} \mathrm{Cm}$ & $(*)$ & $(*)$ & $5.8836 \mathrm{E}-02$ & $2.40 \mathrm{E}-01$ & $6.11 \mathrm{E}-01$ \\
\hline TOTAL & $4.6920 \mathrm{E}+04$ & $4.6346 \mathrm{E}+04$ & $4.5780 \mathrm{E}+04$ & $4.5221 \mathrm{E}+04$ & $3.6854 \mathrm{E}+05$ \\
\hline
\end{tabular}

$\left(^{*}\right)$ Values $<0.0001$ are not shown 
Table 5.4.15. Sample Problem 2: Standard Composition File (Default Burnup Credit Nuclides)

\begin{tabular}{|c|c|c|c|c|c|}
\hline $0-16$ & 1001 & 0 & $4.6395 \mathrm{E}-02$ & 293.0 & end \\
\hline$u-234$ & 1001 & 0 & $5.6529 \mathrm{E}-06$ & 293.0 & end \\
\hline$u-235$ & 1001 & 0 & $4.8120 \mathrm{E}-04$ & 293.0 & end \\
\hline$u-236$ & 1001 & 0 & $5.1165 \mathrm{E}-05$ & 293.0 & end \\
\hline$u-238$ & 1001 & 0 & $2.2263 E-02$ & 293.0 & end \\
\hline$n p-237$ & 1001 & 0 & $2.3470 E-06$ & 293.0 & end \\
\hline$p u-238$ & 1001 & 0 & $2.7067 \mathrm{E}-07$ & 293.0 & end \\
\hline$p u-239$ & 1001 & 0 & $8.4481 E-05$ & 293.0 & end \\
\hline pu-240 & 1001 & 0 & $1.6036 \mathrm{E}-05$ & 293.0 & end \\
\hline pu-241 & 1001 & 0 & $6.2547 \mathrm{E}-06$ & 293.0 & end \\
\hline$p u-242$ & 1001 & 0 & $6.9823 E-07$ & 293.0 & end \\
\hline$a m-241$ & 1001 & 0 & $2.6003 \mathrm{E}-07$ & 293.0 & end \\
\hline$a m-243$ & 1001 & 0 & $4.5435 E-08$ & 293.0 & end \\
\hline mo-95 & 1001 & 0 & $1.5700 \mathrm{E}-05$ & 293.0 & end \\
\hline$t c-99$ & 1001 & 0 & $1.7475 E-05$ & 293.0 & end \\
\hline$r u-101$ & 1001 & 0 & $1.5272 \mathrm{E}-05$ & 293.0 & end \\
\hline$r h-103$ & 1001 & 0 & $9.4877 \mathrm{E}-06$ & 293.0 & end \\
\hline ag-109 & 1001 & 0 & $7.6473 E-07$ & 293.0 & end \\
\hline$c s-133$ & 1001 & 0 & $1.8563 \mathrm{E}-05$ & 293.0 & end \\
\hline$n d-143$ & 1001 & 0 & $1.4504 \mathrm{E}-05$ & 293.0 & end \\
\hline$n d-145$ & 1001 & 0 & $1.0445 E-05$ & 293.0 & end \\
\hline $\mathrm{sm}-147$ & 1001 & 0 & $1.5451 \mathrm{E}-06$ & 293.0 & end \\
\hline $\mathrm{sm}-149$ & 1001 & 0 & $8.0469 \mathrm{E}-08$ & 293.0 & end \\
\hline$s m-150$ & 1001 & 0 & $3.3359 \mathrm{E}-06$ & 293.0 & end \\
\hline$s m-151$ & 1001 & 0 & $3.5230 \mathrm{E}-07$ & 293.0 & end \\
\hline$s m-152$ & 1001 & 0 & $1.7469 \mathrm{E}-06$ & 293.0 & end \\
\hline eu-151 & 1001 & 0 & $1.5582 \mathrm{E}-09$ & 293.0 & end \\
\hline eu-153 & 1001 & 0 & $9.1077 \mathrm{E}-07$ & 293.0 & end \\
\hline$g d-155$ & 1001 & 0 & $1.3966 \mathrm{E}-09$ & 293.0 & end \\
\hline $0-16$ & 1002 & 0 & $4.6394 \mathrm{E}-02$ & 293.0 & end \\
\hline$u-234$ & 1002 & 0 & $4.6837 \mathrm{E}-06$ & 293.0 & end \\
\hline$u-235$ & 1002 & 0 & $2.9395 E-04$ & 293.0 & end \\
\hline$u-236$ & 1002 & 0 & $8.1452 \mathrm{E}-05$ & 293.0 & end \\
\hline$u-238$ & 1002 & 0 & $2.2067 \mathrm{E}-02$ & 293.0 & end \\
\hline$n p-237$ & 1002 & 0 & $6.1878 \mathrm{E}-06$ & 293.0 & end \\
\hline$p u-238$ & 1002 & 0 & $1.4169 \mathrm{E}-06$ & 293.0 & end \\
\hline pu-239 & 1002 & 0 & $1.1165 \mathrm{E}-04$ & 293.0 & end \\
\hline$p u-240$ & 1002 & 0 & $3.7710 \mathrm{E}-05$ & 293.0 & end \\
\hline$p u-241$ & 1002 & 0 & $1.8513 \mathrm{E}-05$ & 293.0 & end \\
\hline$p u-242$ & 1002 & 0 & $4.8561 \mathrm{E}-06$ & 293.0 & end \\
\hline$a m-241$ & 1002 & 0 & $7.2896 \mathrm{E}-07$ & 293.0 & end \\
\hline$a m-243$ & 1002 & 0 & $6.8566 \mathrm{E}-07$ & 293.0 & end \\
\hline mo-95 & 1002 & 0 & $2.9706 \mathrm{E}-05$ & 293.0 & end \\
\hline tc-99 & 1002 & 0 & $3.3424 E-05$ & 293.0 & end \\
\hline$r u-101$ & 1002 & 0 & $3.0467 \mathrm{E}-05$ & 293.0 & end \\
\hline$r h-103$ & 1002 & 0 & $1.8486 \mathrm{E}-05$ & 293.0 & end \\
\hline ag-109 & 1002 & 0 & $2.2784 \mathrm{E}-06$ & 293.0 & end \\
\hline$c s-133$ & 1002 & 0 & $3.5311 \mathrm{E}-05$ & 293.0 & end \\
\hline$n d-143$ & 1002 & 0 & $2.4869 \mathrm{E}-05$ & 293.0 & end \\
\hline
\end{tabular}




\begin{tabular}{|c|c|c|c|c|c|}
\hline$n d-145$ & 1002 & 0 & $1.9393 E-05$ & 293.0 & end \\
\hline $\mathrm{sm}-147$ & 1002 & 0 & $2.4415 \mathrm{E}-06$ & 293.0 & end \\
\hline $\mathrm{sm}-149$ & 1002 & 0 & $1.0154 \mathrm{E}-07$ & 293.0 & end \\
\hline $\mathrm{sm}-150$ & 1002 & 0 & $7.3182 \mathrm{E}-06$ & 293.0 & end \\
\hline $\mathrm{sm}-151$ & 1002 & 0 & $4.4543 E-07$ & 293.0 & end \\
\hline $\mathrm{sm}-152$ & 1002 & 0 & $3.4915 E-06$ & 293.0 & end \\
\hline eu-151 & 1002 & 0 & $1.1064 \mathrm{E}-09$ & 293.0 & end \\
\hline eu-153 & 1002 & 0 & $2.5199 \mathrm{E}-06$ & 293.0 & end \\
\hline$g d-155$ & 1002 & 0 & $2.5157 \mathrm{E}-09$ & 293.0 & end \\
\hline $0-16$ & 1003 & 0 & $4.6392 \mathrm{E}-02$ & 293.0 & end \\
\hline$u-234$ & 1003 & 0 & $3.8068 E-06$ & 293.0 & end \\
\hline$u-235$ & 1003 & 0 & $1.6709 \mathrm{E}-04$ & 293.0 & end \\
\hline$u-236$ & 1003 & 0 & $9.8728 \mathrm{E}-05$ & 293.0 & end \\
\hline$u-238$ & 1003 & 0 & $2.1847 \mathrm{E}-02$ & 293.0 & end \\
\hline$n p-237$ & 1003 & 0 & $1.0287 \mathrm{E}-05$ & 293.0 & end \\
\hline$p u-238$ & 1003 & 0 & $3.5014 \mathrm{E}-06$ & 293.0 & end \\
\hline$p u-239$ & 1003 & 0 & $1.1880 \mathrm{E}-04$ & 293.0 & end \\
\hline$p u-240$ & 1003 & 0 & $5.5465 \mathrm{E}-05$ & 293.0 & end \\
\hline$p u-241$ & 1003 & 0 & $2.8306 \mathrm{E}-05$ & 293.0 & end \\
\hline$p u-242$ & 1003 & 0 & $1.2671 \mathrm{E}-05$ & 293.0 & end \\
\hline$a m-241$ & 1003 & 0 & $9.8507 \mathrm{E}-07$ & 293.0 & end \\
\hline$a m-243$ & 1003 & 0 & $2.6819 E-06$ & 293.0 & end \\
\hline mo-95 & 1003 & 0 & $4.2205 E-05$ & 293.0 & end \\
\hline$t c-99$ & 1003 & 0 & $4.7742 E-05$ & 293.0 & end \\
\hline ru-101 & 1003 & 0 & $4.5361 E-05$ & 293.0 & end \\
\hline rh-103 & 1003 & 0 & $2.6134 \mathrm{E}-05$ & 293.0 & end \\
\hline ag-109 & 1003 & 0 & $4.1770 \mathrm{E}-06$ & 293.0 & end \\
\hline$c s-133$ & 1003 & 0 & $5.0069 \mathrm{E}-05$ & 293.0 & end \\
\hline$n d-143$ & 1003 & 0 & $3.1519 \mathrm{E}-05$ & 293.0 & end \\
\hline$n d-145$ & 1003 & 0 & $2.6993 E-05$ & 293.0 & end \\
\hline $\mathrm{sm}-147$ & 1003 & 0 & $2.8511 \mathrm{E}-06$ & 293.0 & end \\
\hline$s m-149$ & 1003 & 0 & $1.2277 \mathrm{E}-07$ & 293.0 & end \\
\hline $\mathrm{sm}-150$ & 1003 & 0 & $1.1691 \mathrm{E}-05$ & 293.0 & end \\
\hline $\mathrm{sm}-151$ & 1003 & 0 & $5.2472 E-07$ & 293.0 & end \\
\hline$s m-152$ & 1003 & 0 & $5.0112 E-06$ & 293.0 & end \\
\hline eu-151 & 1003 & 0 & $9.0563 \mathrm{E}-10$ & 293.0 & end \\
\hline eu-153 & 1003 & 0 & $4.4446 E-06$ & 293.0 & end \\
\hline$g d-155$ & 1003 & 0 & $4.0054 E-09$ & 293.0 & end \\
\hline $0-16$ & 1004 & 0 & $4.6390 \mathrm{E}-02$ & 293.0 & end \\
\hline$u-234$ & 1004 & 0 & $3.0343 E-06$ & 293.0 & end \\
\hline$u-235$ & 1004 & 0 & $8.7951 E-05$ & 293.0 & end \\
\hline$u-236$ & 1004 & 0 & $1.0588 \mathrm{E}-04$ & 293.0 & end \\
\hline$u-238$ & 1004 & 0 & $2.1605 E-02$ & 293.0 & end \\
\hline$n p-237$ & 1004 & 0 & $1.3864 \mathrm{E}-05$ & 293.0 & end \\
\hline$p u-238$ & 1004 & 0 & $6.1605 E-06$ & 293.0 & end \\
\hline$p u-239$ & 1004 & 0 & $1.1946 \mathrm{E}-04$ & 293.0 & end \\
\hline$p u-240$ & 1004 & 0 & $6.7542 E-05$ & 293.0 & end \\
\hline$p u-241$ & 1004 & 0 & $3.4172 E-05$ & 293.0 & end \\
\hline$p u-242$ & 1004 & 0 & $2.2786 \mathrm{E}-05$ & 293.0 & end \\
\hline$a m-241$ & 1004 & 0 & $1.0210 \mathrm{E}-06$ & 293.0 & end \\
\hline$a m-243$ & 1004 & 0 & $6.1463 E-06$ & 293.0 & end \\
\hline
\end{tabular}




\begin{tabular}{|c|c|c|c|c|c|}
\hline mo-95 & 1004 & 0 & $5.3319 \mathrm{E}-05$ & 293.0 & end \\
\hline$t c-99$ & 1004 & 0 & $6.0395 E-05$ & 293.0 & end \\
\hline ru-101 & 1004 & 0 & $5.9831 \mathrm{E}-05$ & 293.0 & end \\
\hline$r h-103$ & 1004 & 0 & $3.2151 E-05$ & 293.0 & end \\
\hline ag-109 & 1004 & 0 & $6.2338 E-06$ & 293.0 & end \\
\hline$c s-133$ & 1004 & 0 & $6.2779 \mathrm{E}-05$ & 293.0 & end \\
\hline$n d-143$ & 1004 & 0 & $3.4995 E-05$ & 293.0 & end \\
\hline$n d-145$ & 1004 & 0 & $3.3350 \mathrm{E}-05$ & 293.0 & end \\
\hline $\mathrm{sm}-147$ & 1004 & 0 & $2.9202 E-06$ & 293.0 & end \\
\hline $\mathrm{sm}-149$ & 1004 & 0 & $1.4469 \mathrm{E}-07$ & 293.0 & end \\
\hline $\mathrm{sm}-150$ & 1004 & 0 & $1.6101 \mathrm{E}-05$ & 293.0 & end \\
\hline $\mathrm{sm}-151$ & 1004 & 0 & $6.0099 \mathrm{E}-07$ & 293.0 & end \\
\hline$s m-152$ & 1004 & 0 & $6.3684 E-06$ & 293.0 & end \\
\hline eu-151 & 1004 & 0 & $8.1899 \mathrm{E}-10$ & 293.0 & end \\
\hline eu-153 & 1004 & 0 & $6.4006 \mathrm{E}-06$ & 293.0 & end \\
\hline$g d-155$ & 1004 & 0 & $5.5315 E-09$ & 293.0 & end \\
\hline $0-16$ & 1005 & 0 & $4.6390 \mathrm{E}-02$ & 293.0 & end \\
\hline$u-234$ & 1005 & 0 & $3.0343 E-06$ & 293.0 & end \\
\hline$u-235$ & 1005 & 0 & $8.7951 \mathrm{E}-05$ & 293.0 & end \\
\hline$u-236$ & 1005 & 0 & $1.0588 \mathrm{E}-04$ & 293.0 & end \\
\hline$u-238$ & 1005 & 0 & $2.1605 \mathrm{E}-02$ & 293.0 & end \\
\hline$n p-237$ & 1005 & 0 & $1.3864 \mathrm{E}-05$ & 293.0 & end \\
\hline$p u-238$ & 1005 & 0 & $6.1605 \mathrm{E}-06$ & 293.0 & end \\
\hline$p u-239$ & 1005 & 0 & $1.1946 \mathrm{E}-04$ & 293.0 & end \\
\hline$p u-240$ & 1005 & 0 & $6.7542 \mathrm{E}-05$ & 293.0 & end \\
\hline pu-241 & 1005 & 0 & $3.4172 \mathrm{E}-05$ & 293.0 & end \\
\hline$p u-242$ & 1005 & 0 & $2.2786 \mathrm{E}-05$ & 293.0 & end \\
\hline$a m-241$ & 1005 & 0 & $1.0210 \mathrm{E}-06$ & 293.0 & end \\
\hline$a m-243$ & 1005 & 0 & $6.1463 E-06$ & 293.0 & end \\
\hline mo-95 & 1005 & 0 & $5.3319 \mathrm{E}-05$ & 293.0 & end \\
\hline tc-99 & 1005 & 0 & $6.0395 E-05$ & 293.0 & end \\
\hline$r u-101$ & 1005 & 0 & $5.9831 E-05$ & 293.0 & end \\
\hline$r h-103$ & 1005 & 0 & $3.2151 \mathrm{E}-05$ & 293.0 & end \\
\hline ag-109 & 1005 & 0 & $6.2338 E-06$ & 293.0 & end \\
\hline$C S-133$ & 1005 & 0 & $6.2779 \mathrm{E}-05$ & 293.0 & end \\
\hline$n d-143$ & 1005 & 0 & $3.4995 E-05$ & 293.0 & end \\
\hline$n d-145$ & 1005 & 0 & $3.3350 \mathrm{E}-05$ & 293.0 & end \\
\hline $\mathrm{sm}-147$ & 1005 & 0 & $2.9202 E-06$ & 293.0 & end \\
\hline $\mathrm{sm}-149$ & 1005 & 0 & $1.4469 \mathrm{E}-07$ & 293.0 & end \\
\hline$s m-150$ & 1005 & 0 & $1.6101 \mathrm{E}-05$ & 293.0 & end \\
\hline $\mathrm{sm}-151$ & 1005 & 0 & $6.0099 \mathrm{E}-07$ & 293.0 & end \\
\hline $\mathrm{sm}-152$ & 1005 & 0 & $6.3684 \mathrm{E}-06$ & 293.0 & end \\
\hline eu-151 & 1005 & 0 & $8.1899 \mathrm{E}-10$ & 293.0 & end \\
\hline eu-153 & 1005 & 0 & $6.4006 \mathrm{E}-06$ & 293.0 & end \\
\hline$g d-155$ & 1005 & 0 & $5.5315 E-09$ & 293.0 & end \\
\hline $0-16$ & 1006 & 0 & $4.6392 \mathrm{E}-02$ & 293.0 & end \\
\hline$u-234$ & 1006 & 0 & $3.8068 E-06$ & 293.0 & end \\
\hline$u-235$ & 1006 & 0 & $1.6709 \mathrm{E}-04$ & 293.0 & end \\
\hline$u-236$ & 1006 & 0 & $9.8728 \mathrm{E}-05$ & 293.0 & end \\
\hline$u-238$ & 1006 & 0 & $2.1847 \mathrm{E}-02$ & 293.0 & end \\
\hline$n p-237$ & 1006 & 0 & $1.0287 \mathrm{E}-05$ & 293.0 & end \\
\hline
\end{tabular}




\begin{tabular}{|c|c|c|c|c|c|}
\hline$p u-238$ & 1006 & 0 & $3.5014 \mathrm{E}-06$ & 293.0 & end \\
\hline pu-239 & 1006 & 0 & $1.1880 \mathrm{E}-04$ & 293.0 & end \\
\hline pu-2 40 & 1006 & 0 & $5.5465 \mathrm{E}-05$ & 293.0 & end \\
\hline pu-241 & 1006 & 0 & $2.8306 \mathrm{E}-05$ & 293.0 & end \\
\hline$p u-242$ & 1006 & 0 & $1.2671 \mathrm{E}-05$ & 293.0 & end \\
\hline$a m-241$ & 1006 & 0 & $9.8507 \mathrm{E}-07$ & 293.0 & end \\
\hline$a m-243$ & 1006 & 0 & $2.6819 E-06$ & 293.0 & end \\
\hline mo-95 & 1006 & 0 & $4.2205 E-05$ & 293.0 & end \\
\hline tc-99 & 1006 & 0 & $4.7742 E-05$ & 293.0 & end \\
\hline$r u-101$ & 1006 & 0 & $4.5361 \mathrm{E}-05$ & 293.0 & end \\
\hline$r h-103$ & 1006 & 0 & $2.6134 \mathrm{E}-05$ & 293.0 & end \\
\hline ag-109 & 1006 & 0 & $4.1770 \mathrm{E}-06$ & 293.0 & end \\
\hline$c s-133$ & 1006 & 0 & $5.0069 \mathrm{E}-05$ & 293.0 & end \\
\hline$n d-143$ & 1006 & 0 & $3.1519 \mathrm{E}-05$ & 293.0 & end \\
\hline$n d-145$ & 1006 & 0 & $2.6993 E-05$ & 293.0 & end \\
\hline sm-147 & 1006 & 0 & $2.8511 \mathrm{E}-06$ & 293.0 & end \\
\hline $\mathrm{sm}-149$ & 1006 & 0 & $1.2277 \mathrm{E}-07$ & 293.0 & end \\
\hline$s m-150$ & 1006 & 0 & $1.1691 \mathrm{E}-05$ & 293.0 & end \\
\hline $\mathrm{sm}-151$ & 1006 & 0 & $5.2472 \mathrm{E}-07$ & 293.0 & end \\
\hline $\mathrm{sm}-152$ & 1006 & 0 & $5.0112 \mathrm{E}-06$ & 293.0 & end \\
\hline eu-151 & 1006 & 0 & $9.0563 \mathrm{E}-10$ & 293.0 & end \\
\hline eu-153 & 1006 & 0 & $4.4446 \mathrm{E}-06$ & 293.0 & end \\
\hline$g d-155$ & 1006 & 0 & $4.0054 \mathrm{E}-09$ & 293.0 & end \\
\hline $0-16$ & 1007 & 0 & $4.6394 E-02$ & 293.0 & end \\
\hline$u-234$ & 1007 & 0 & $4.6837 \mathrm{E}-06$ & 293.0 & end \\
\hline$u-235$ & 1007 & 0 & $2.9395 E-04$ & 293.0 & end \\
\hline$u-236$ & 1007 & 0 & $8.1452 \mathrm{E}-05$ & 293.0 & end \\
\hline$u-238$ & 1007 & 0 & $2.2067 \mathrm{E}-02$ & 293.0 & end \\
\hline$n p-237$ & 1007 & 0 & $6.1878 \mathrm{E}-06$ & 293.0 & end \\
\hline $\mathrm{pu}-238$ & 1007 & 0 & $1.4169 \mathrm{E}-06$ & 293.0 & end \\
\hline$p u-239$ & 1007 & 0 & $1.1165 \mathrm{E}-04$ & 293.0 & end \\
\hline$p u-240$ & 1007 & 0 & $3.7710 \mathrm{E}-05$ & 293.0 & end \\
\hline $\mathrm{pu}-241$ & 1007 & 0 & $1.8513 E-05$ & 293.0 & end \\
\hline pu-242 & 1007 & 0 & $4.8561 \mathrm{E}-06$ & 293.0 & end \\
\hline$a m-241$ & 1007 & 0 & $7.2896 \mathrm{E}-07$ & 293.0 & end \\
\hline$a m-243$ & 1007 & 0 & $6.8566 \mathrm{E}-07$ & 293.0 & end \\
\hline mo-95 & 1007 & 0 & $2.9706 \mathrm{E}-05$ & 293.0 & end \\
\hline tc-99 & 1007 & 0 & $3.3424 E-05$ & 293.0 & end \\
\hline ru-101 & 1007 & 0 & $3.0467 \mathrm{E}-05$ & 293.0 & end \\
\hline$r h-103$ & 1007 & 0 & $1.8486 \mathrm{E}-05$ & 293.0 & end \\
\hline ag-109 & 1007 & 0 & $2.2784 E-06$ & 293.0 & end \\
\hline$c s-133$ & 1007 & 0 & $3.5311 \mathrm{E}-05$ & 293.0 & end \\
\hline$n d-143$ & 1007 & 0 & $2.4869 \mathrm{E}-05$ & 293.0 & end \\
\hline$n d-145$ & 1007 & 0 & $1.9393 E-05$ & 293.0 & end \\
\hline $\mathrm{sm}-147$ & 1007 & 0 & $2.4415 E-06$ & 293.0 & end \\
\hline$s m-149$ & 1007 & 0 & $1.0154 \mathrm{E}-07$ & 293.0 & end \\
\hline $\mathrm{sm}-150$ & 1007 & 0 & $7.3182 E-06$ & 293.0 & end \\
\hline $\mathrm{sm}-151$ & 1007 & 0 & $4.4543 E-07$ & 293.0 & end \\
\hline $\mathrm{sm}-152$ & 1007 & 0 & $3.4915 E-06$ & 293.0 & end \\
\hline eu-151 & 1007 & 0 & $1.1064 \mathrm{E}-09$ & 293.0 & end \\
\hline eu-153 & 1007 & 0 & $2.5199 \mathrm{E}-06$ & 293.0 & end \\
\hline
\end{tabular}




\begin{tabular}{|c|c|c|c|c|c|}
\hline$g d-155$ & 1007 & 0 & $2.5157 \mathrm{E}-09$ & 293.0 & end \\
\hline $0-16$ & 1008 & 0 & $4.6395 E-02$ & 293.0 & end \\
\hline$u-234$ & 1008 & 0 & $5.6529 \mathrm{E}-06$ & 293.0 & end \\
\hline$u-235$ & 1008 & 0 & $4.8120 E-04$ & 293.0 & end \\
\hline$u-236$ & 1008 & 0 & $5.1165 \mathrm{E}-05$ & 293.0 & end \\
\hline$u-238$ & 1008 & 0 & $2.2263 E-02$ & 293.0 & end \\
\hline$n p-237$ & 1008 & 0 & $2.3470 \mathrm{E}-06$ & 293.0 & end \\
\hline pu -238 & 1008 & 0 & $2.7067 \mathrm{E}-07$ & 293.0 & end \\
\hline$p u-239$ & 1008 & 0 & $8.4481 E-05$ & 293.0 & end \\
\hline pu-240 & 1008 & 0 & $1.6036 \mathrm{E}-05$ & 293.0 & end \\
\hline$p u-241$ & 1008 & 0 & $6.2547 \mathrm{E}-06$ & 293.0 & end \\
\hline$p u-242$ & 1008 & 0 & $6.9823 E-07$ & 293.0 & end \\
\hline$a m-241$ & 1008 & 0 & $2.6003 E-07$ & 293.0 & end \\
\hline$a m-243$ & 1008 & 0 & $4.5435 \mathrm{E}-08$ & 293.0 & end \\
\hline$m o-95$ & 1008 & 0 & $1.5700 \mathrm{E}-05$ & 293.0 & end \\
\hline$t c-99$ & 1008 & 0 & $1.7475 \mathrm{E}-05$ & 293.0 & end \\
\hline$r u-101$ & 1008 & 0 & $1.5272 \mathrm{E}-05$ & 293.0 & end \\
\hline$r h-103$ & 1008 & 0 & $9.4877 \mathrm{E}-06$ & 293.0 & end \\
\hline $\mathrm{ag}-109$ & 1008 & 0 & $7.6473 E-07$ & 293.0 & end \\
\hline$c s-133$ & 1008 & 0 & $1.8563 \mathrm{E}-05$ & 293.0 & end \\
\hline$n d-143$ & 1008 & 0 & $1.4504 \mathrm{E}-05$ & 293.0 & end \\
\hline$n d-145$ & 1008 & 0 & $1.0445 \mathrm{E}-05$ & 293.0 & end \\
\hline$s m-147$ & 1008 & 0 & $1.5451 \mathrm{E}-06$ & 293.0 & end \\
\hline$s m-149$ & 1008 & 0 & $8.0469 \mathrm{E}-08$ & 293.0 & end \\
\hline $\mathrm{sm}-150$ & 1008 & 0 & $3.3359 \mathrm{E}-06$ & 293.0 & end \\
\hline $\mathrm{sm}-151$ & 1008 & 0 & $3.5230 \mathrm{E}-07$ & 293.0 & end \\
\hline$s m-152$ & 1008 & 0 & $1.7469 \mathrm{E}-06$ & 293.0 & end \\
\hline eu-151 & 1008 & 0 & $1.5582 \mathrm{E}-09$ & 293.0 & end \\
\hline eu-153 & 1008 & 0 & $9.1077 \mathrm{E}-07$ & 293.0 & end \\
\hline$g d-155$ & 1008 & 0 & $1.3966 \mathrm{E}-09$ & 293.0 & end \\
\hline
\end{tabular}

Table 5.4.16. Sample Problem 2: Decay Heat File (Watts) by Axial Interval

\begin{tabular}{l}
$7.11397 \mathrm{E}+02$ \\
$1.43786 \mathrm{E}+03$ \\
$2.18631 \mathrm{E}+03$ \\
$2.95428 \mathrm{E}+03$ \\
$2.95428 \mathrm{E}+03$ \\
$2.18631 \mathrm{E}+03$ \\
$1.43786 \mathrm{E}+03$ \\
$7.11397 \mathrm{E}+02$ \\
\hline
\end{tabular}

\subsubsection{Sample problem 3: restart decay calculation for lumped axial depletion assembly model}

The third example shows a restart decay-only calculation, using the ORIGEN ft71 binary file obtained from sample problem 2. This case calculates the composition of the burned fuel produced in sample problem 2 after 100,000 additional days of decay. The input this problem is given in Table 5.4.17. Because the input parameter restart $=y e s$ is specified, the initial composition of the assembly is obtained 
from a file named "assembly_restart.f71". The shell input that precedes the ORIGAMI input in Table 5.4.17 copies the output $\mathrm{ft} 71$ file produced in sample problem 2, which was named "assembly_dump.f71", into a file named "assembly_restart.f71" in the temporary directory for SCALE calculations. The restart file contains the complete inventory of nuclide compositions for eight axial zones. Because this restart case is decay only (i.e., power= value is not given in the power-history block), it is necessary to provide the input parameter $n z=8$ because this value is used to determine how many axial zones were used in the previous burnup calculations.

Table 5.4.18 shows the actinide composition of the first four axial of the (symmetrical) eight zones after 100,000 days of decay. The initial masses of these nuclides before decay are the values given in Table 5.4.15. The last column in Table 5.4.18 shows actinide masses for the entire assembly after the decay period.

Table 5.4.17. Input for ORIGAMI Sample Problem 3

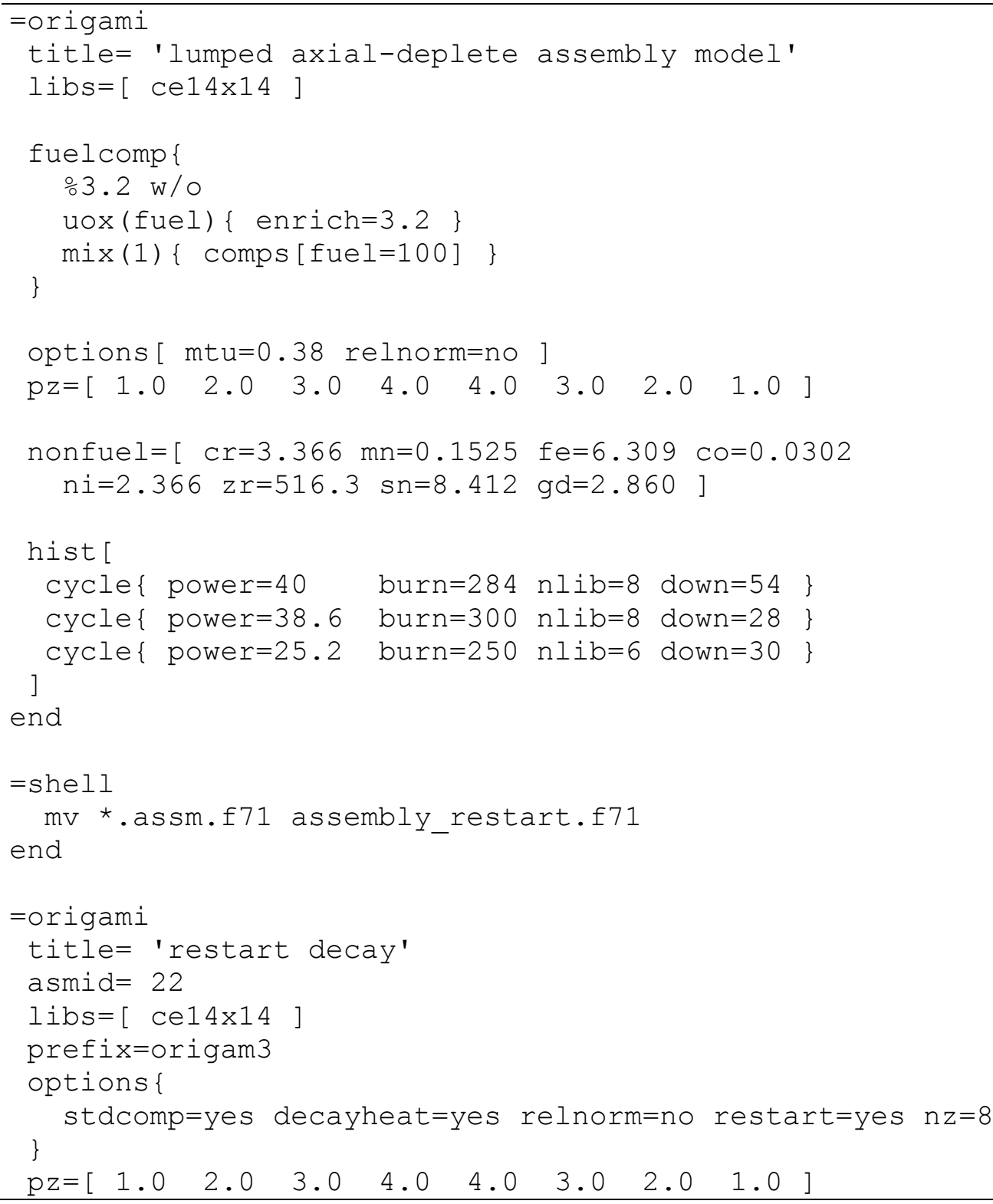




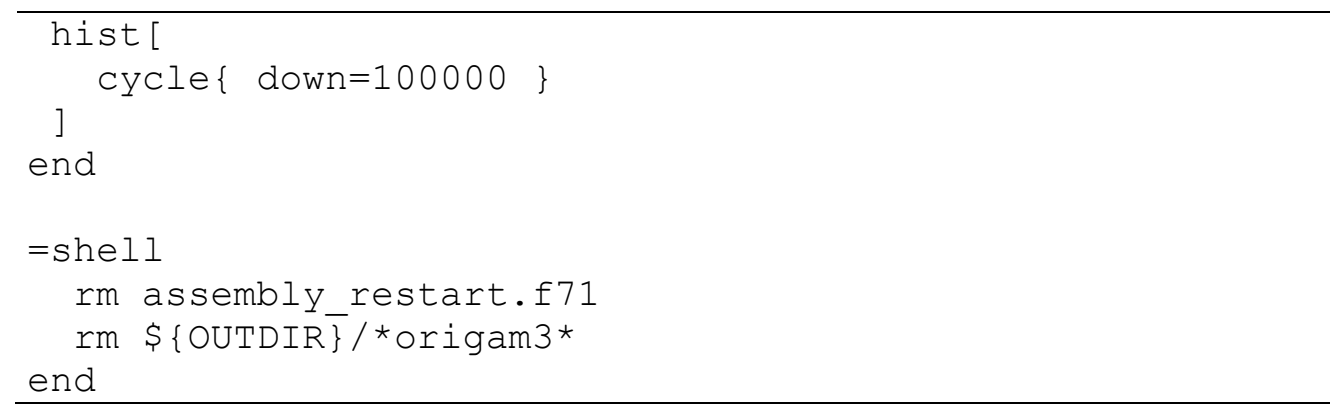

Table 5.4.18. Calculated Actinide Isotopics by Axial Zone for Sample Problem 3

\begin{tabular}{|c|c|c|c|c|c|}
\hline Nuclide & $\begin{array}{c}\text { Axial Zone 1 } \\
\text { Mass }(\mathrm{g})\end{array}$ & $\begin{array}{c}\text { Axial Zone 2 } \\
\text { Mass }(\mathrm{g})\end{array}$ & $\begin{array}{c}\text { Axial Zone 3 } \\
\text { Mass }(\mathrm{g})\end{array}$ & $\begin{array}{c}\text { Axial Zone 4 } \\
\text { Mass }(\mathrm{g})\end{array}$ & $\begin{array}{c}\text { TOTAL } \\
\text { Mass }(\mathrm{g})\end{array}$ \\
\hline${ }^{234} \mathrm{U}$ & $1.1889 \mathrm{E}+01$ & $1.2133 \mathrm{E}+01$ & $1.4319 \mathrm{E}+01$ & $1.7738 \mathrm{E}+01$ & $1.1216 \mathrm{E}+02$ \\
\hline${ }^{235} \mathrm{U}$ & $9.7454 \mathrm{E}+02$ & $5.9616 \mathrm{E}+02$ & $3.3963 \mathrm{E}+02$ & $1.7955 \mathrm{E}+02$ & $4.1798 \mathrm{E}+03$ \\
\hline${ }^{236} \mathrm{U}$ & $1.0486 \mathrm{E}+02$ & $1.6764 \mathrm{E}+02$ & $2.0378 \mathrm{E}+02$ & $2.1907 \mathrm{E}+02$ & $1.3907 \mathrm{E}+03$ \\
\hline${ }^{238} \mathrm{U}$ & $4.5604 \mathrm{E}+04$ & $4.5202 \mathrm{E}+04$ & $4.4752 \mathrm{E}+04$ & $4.4256 \mathrm{E}+04$ & $3.5963 \mathrm{E}+05$ \\
\hline${ }^{237} \mathrm{~Np}$ & $9.2242 \mathrm{E}+00$ & $2.5723 \mathrm{E}+01$ & $4.0929 \mathrm{E}+01$ & $5.2246 \mathrm{E}+01$ & $2.5625 \mathrm{E}+02$ \\
\hline${ }^{238} \mathrm{Pu}$ & $6.8535 \mathrm{E}-02$ & $3.6104 \mathrm{E}-01$ & $8.8478 \mathrm{E}-01$ & $1.5422 \mathrm{E}+00$ & $5.7132 \mathrm{E}+00$ \\
\hline${ }^{239} \mathrm{Pu}$ & $1.7243 \mathrm{E}+02$ & $2.2794 \mathrm{E}+02$ & $2.4262 \mathrm{E}+02$ & $2.4413 \mathrm{E}+02$ & $1.7742 \mathrm{E}+03$ \\
\hline${ }^{240} \mathrm{Pu}$ & $3.2192 \mathrm{E}+01$ & $7.5957 \mathrm{E}+01$ & $1.1297 \mathrm{E}+02$ & $1.4095 \mathrm{E}+02$ & $7.2412 \mathrm{E}+02$ \\
\hline${ }^{241} \mathrm{Pu}$ & $(*)$ & $(*)$ & $(*)$ & $(*)$ & $(*)$ \\
\hline${ }^{242} \mathrm{Pu}$ & $1.4538 \mathrm{E}+00$ & $1.0107 \mathrm{E}+01$ & $2.6379 \mathrm{E}+01$ & $4.7456 \mathrm{E}+01$ & $1.7079 \mathrm{E}+02$ \\
\hline${ }^{241} \mathrm{Am}$ & $8.9945 \mathrm{E}+00$ & $2.6564 \mathrm{E}+01$ & $4.0451 \mathrm{E}+01$ & $4.8623 \mathrm{E}+01$ & $2.4927 \mathrm{E}+02$ \\
\hline${ }^{243} \mathrm{Am}$ & $9.2577 \mathrm{E}-02$ & $1.3965 \mathrm{E}+00$ & $5.4616 \mathrm{E}+00$ & $1.2516 \mathrm{E}+01$ & $3.8934 \mathrm{E}+01$ \\
\hline${ }^{242} \mathrm{Cm}$ & $(*)$ & $(*)$ & $(*)$ & $(*)$ & $(*)$ \\
\hline${ }^{244} \mathrm{Cm}$ & $(*)$ & $(*)$ & $(*)$ & $(*)$ & $(*)$ \\
\hline${ }^{245} \mathrm{Cm}$ & $(*)$ & $(*)$ & $5.7288 \mathrm{E}-02$ & $2.3382 \mathrm{E}-01$ & $5.9479 \mathrm{E}-01$ \\
\hline TOTAL & $4.6920 \mathrm{E}+04$ & $4.6346 \mathrm{E}+04$ & $4.5780 \mathrm{E}+04$ & $4.5220 \mathrm{E}+04$ & $3.6853 \mathrm{E}+05$ \\
\hline
\end{tabular}

$(*)$ Values $<0.0001$ are not shown

\subsubsection{Sample problem 4: Simplified 3D multi-pin model}

The fourth example is a simulation of a simplified 3D depletion model. The ORIGAMI 3D model normally includes all fuel pins within the assembly, such as a $14 \times 14$ array. However to keep this example case simple and the execution time low, only a $2 \times 2$ array of four individual pins is considered for illustrative purposes. An axial fractional power distribution is also specified for two axial zones. Therefore the total number of depletion regions will be eight - two axial for each of the four pins. Two different ORIGEN libraries are used to obtain cross sections for the four pins. This is done whenever fuel pins in different locations in the assembly have significantly different neutron spectra, such as if some pins are adjacent to a control rod. In this sample problem, ORIGEN libraries for two different types of assembly designs CE (Combustion Engineering) $14 \times 14$ and $16 \times 16$ assembly designs, respectively are used to demonstrate the use of pin-dependent libraries, although in reality the ORIGEN libraries normally would be pre-generated for different pin locations within a single type of assembly configuration. The values specified in libmap = indicate which library is to be used for each pin location. Table 5.4.19 shows the input for this sample problem. 
Table 5.4.19. Input for ORIGAMI Sample Problem 4

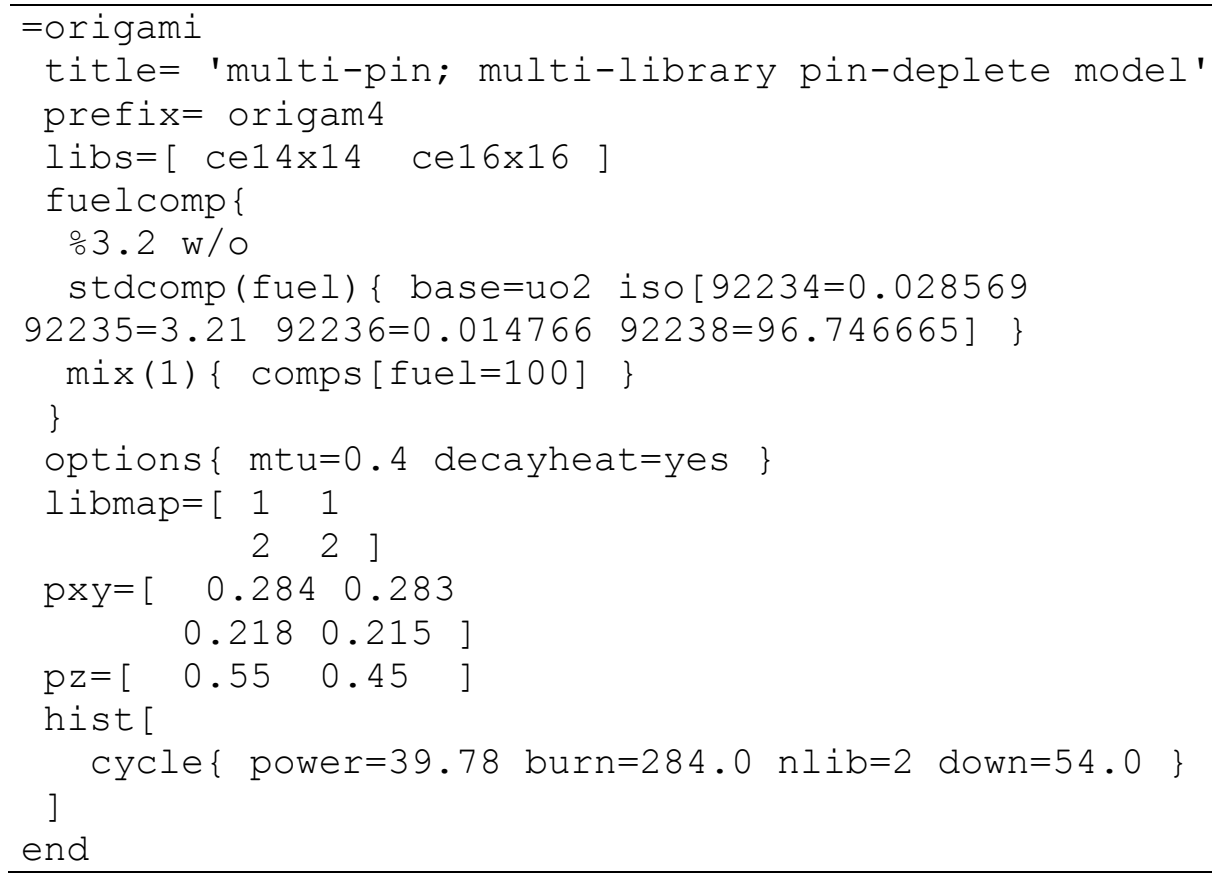

Table 5.4.20 shows selected actinide compositions for the first row of two pins, that is, locations $(1,1)$ and $(1,2)$, for each of the two axial zones. The blended compositions over all fuel pins, for the two axial zones, are given in Table 5.4.21. The output decay heat file for the assembly is shown in Table 5.4.22, as a function of axial zone, summed over all pins. Note that this file has the prefix "sample4_" appended to the standard file name, since prefix=sample 4 is specified in the input.

Table 5.4.20. Actinide Isotopics by Axial Zone for Pins $(1,1)$ and $(1,2)$ in Sample Problem 4

\begin{tabular}{|c|c|c|c|c|}
\hline Nuclide & $\begin{array}{c}\text { pin }(1,1) \\
\text { Axial Zone 1 } \\
\text { Mass (g) }\end{array}$ & $\begin{array}{c}\text { pin (1,1) } \\
\text { Axial Zone 2 } \\
\text { Mass (g) }\end{array}$ & $\begin{array}{c}\text { pin (1,2) } \\
\text { Axial Zone 1 } \\
\text { Mass (g) }\end{array}$ & $\begin{array}{c}\text { pin }(1,2) \\
\text { Axial Zone 2 } \\
\text { Mass (g) }\end{array}$ \\
\hline${ }^{234} \mathrm{U}$ & $1.2115 \mathrm{E}+01$ & $1.2493 \mathrm{E}+01$ & $1.2143 \mathrm{E}+01$ & $1.2516 \mathrm{E}+01$ \\
\hline${ }^{235} \mathrm{U}$ & $1.0701 \mathrm{E}+03$ & $1.1528 \mathrm{E}+03$ & $1.0762 \mathrm{E}+03$ & $1.1581 \mathrm{E}+03$ \\
\hline${ }^{236} \mathrm{U}$ & $1.0383 \mathrm{E}+02$ & $8.9286 \mathrm{E}+01$ & $1.0277 \mathrm{E}+02$ & $8.8348 \mathrm{E}+01$ \\
\hline${ }^{237} \mathrm{U}$ & $1.0664 \mathrm{E}-03$ & $(*)$ & $1.0428 \mathrm{E}-03$ & $(*)$ \\
\hline${ }^{238} \mathrm{U}$ & $4.8003 \mathrm{E}+04$ & $4.8074 \mathrm{E}+04$ & $4.8009 \mathrm{E}+04$ & $4.8078 \mathrm{E}+04$ \\
\hline${ }^{237} \mathrm{~Np}$ & $4.8000 \mathrm{E}+00$ & $3.6009 \mathrm{E}+00$ & $4.7055 \mathrm{E}+00$ & $3.5303 \mathrm{E}+00$ \\
\hline${ }^{239} \mathrm{~Np}$ & $5.8742 \mathrm{E}-07$ & $4.4485 \mathrm{E}-07$ & $5.7555 \mathrm{E}-07$ & $4.3692 \mathrm{E}-07$ \\
\hline${ }^{238} \mathrm{Pu}$ & $4.7198 \mathrm{E}-01$ & $2.9453 \mathrm{E}-01$ & $4.5684 \mathrm{E}-01$ & $2.8512 \mathrm{E}-01$ \\
\hline${ }^{239} \mathrm{Pu}$ & $1.8855 \mathrm{E}+02$ & $1.6706 \mathrm{E}+02$ & $1.8705 \mathrm{E}+02$ & $1.6560 \mathrm{E}+02$ \\
\hline${ }^{240} \mathrm{Pu}$ & $3.3235 \mathrm{E}+01$ & $2.5225 \mathrm{E}+01$ & $3.2625 \mathrm{E}+01$ & $2.4736 \mathrm{E}+01$ \\
\hline${ }^{241} \mathrm{Pu}$ & $1.3906 \mathrm{E}+01$ & $9.2953 \mathrm{E}+00$ & $1.3537 \mathrm{E}+01$ & $9.0304 \mathrm{E}+00$ \\
\hline${ }^{242} \mathrm{Pu}$ & $1.3733 \mathrm{E}+00$ & $7.3260 \mathrm{E}-01$ & $1.3161 \mathrm{E}+00$ & $7.0081 \mathrm{E}-01$ \\
\hline${ }^{241} \mathrm{Am}$ & $2.3603 \mathrm{E}-01$ & $1.5773 \mathrm{E}-01$ & $2.2978 \mathrm{E}-01$ & $1.5322 \mathrm{E}-01$ \\
\hline${ }^{243} \mathrm{Am}$ & $8.4143 \mathrm{E}-02$ & $(*)$ & $7.9365 \mathrm{E}-02$ & $(*)$ \\
\hline TOTAL & $4.9432 \mathrm{E}+04$ & $4.9535 \mathrm{E}+04$ & $4.9440 \mathrm{E}+04$ & $4.9541 \mathrm{E}+04$ \\
\hline
\end{tabular}

$\left(^{*}\right)$ Values $<0.0001$ are not shown 
Table 5.4.21. Actinide Isotopics Blended over All Pins, by Axial Zone in Sample Problem 4

\begin{tabular}{|c|c|c|c|}
\hline Nuclide & $\begin{array}{c}\text { Axial Zone 1 } \\
\text { Mass (g) }\end{array}$ & $\begin{array}{c}\text { Axial Zone 2 } \\
\text { Mass (g) }\end{array}$ & $\begin{array}{c}\text { TOTAL } \\
\text { Mass (g) }\end{array}$ \\
\hline${ }^{234} \mathrm{U}$ & $4.7388 \mathrm{E}+01$ & $4.9082 \mathrm{E}+01$ & $9.6469 \mathrm{E}+01$ \\
\hline${ }^{235} \mathrm{U}$ & $4.0115 \mathrm{E}+03$ & $4.3778 \mathrm{E}+03$ & $8.3894 \mathrm{E}+03$ \\
\hline${ }^{236} \mathrm{U}$ & $4.5851 \mathrm{E}+02$ & $3.9537 \mathrm{E}+02$ & $8.5387 \mathrm{E}+02$ \\
\hline${ }^{238} \mathrm{U}$ & $1.9184 \mathrm{E}+05$ & $1.9216 \mathrm{E}+05$ & $3.8399 \mathrm{E}+05$ \\
\hline${ }^{237} \mathrm{~Np}$ & $2.2821 \mathrm{E}+01$ & $1.7132 \mathrm{E}+01$ & $3.9953 \mathrm{E}+01$ \\
\hline${ }^{238} \mathrm{Pu}$ & $2.5739 \mathrm{E}+00$ & $1.6049 \mathrm{E}+00$ & $4.1789 \mathrm{E}+00$ \\
\hline${ }^{239} \mathrm{Pu}$ & $7.8246 \mathrm{E}+02$ & $6.9963 \mathrm{E}+02$ & $1.4821 \mathrm{E}+03$ \\
\hline${ }^{240} \mathrm{Pu}$ & $1.5436 \mathrm{E}+02$ & $1.1801 \mathrm{E}+02$ & $2.7238 \mathrm{E}+02$ \\
\hline${ }^{241} \mathrm{Pu}$ & $6.7918 \mathrm{E}+01$ & $4.6455 \mathrm{E}+01$ & $1.1437 \mathrm{E}+02$ \\
\hline${ }^{242} \mathrm{Pu}$ & $8.1693 \mathrm{E}+00$ & $4.4411 \mathrm{E}+00$ & $1.2610 \mathrm{E}+01$ \\
\hline${ }^{241} \mathrm{Am}$ & $1.1476 \mathrm{E}+00$ & $7.8696 \mathrm{E}-01$ & $1.9346 \mathrm{E}+00$ \\
\hline${ }^{243} \mathrm{Am}$ & $6.0150 \mathrm{E}-01$ & $2.5995 \mathrm{E}-01$ & $8.6145 \mathrm{E}-01$ \\
\hline TOTAL & $1.9740 \mathrm{E}+05$ & $1.9787 \mathrm{E}+05$ & $3.9526 \mathrm{E}+05$ \\
\hline \multicolumn{3}{|c}{}
\end{tabular}

Table 5.4.22. Sample Problem 4:

Decay Heat (Watts) by Axial Interval

$6.96472 \mathrm{E}+03$

$5.72539 \mathrm{E}+03$

\subsubsection{Sample problem 5: PWR 3D assembly model}

This sample problem shows the input for a simulated full 3D pressurized water reactor (PWR) assemblydepletion model, which corresponds to a $16 \times 16$ lattice with 26 axial zones. Table 5.4.23 shows the ORIGAMI input for this case. The arrays $p x y=$ and $p z=$ define the $3 \mathrm{D} X Y-Z$ fractional power distribution. Four different pre-processed ORIGEN libraries are used to describe the pin-averaged ORIGEN cross-sections for the assembly. The array libmap= assigns these libraries to the appropriate pin locations. It can be seen that the libmap = array contains values of zero at 21 locations. These correspond to non-depleting (i.e., zero power) locations. The input includes the information (parameter pitch=, and array $z=$ ) necessary to generate 3D mesh summary maps for subsequent visualization. In this case, the axial mesh is not uniform. Since a total of 6110 ORIGEN cases are executed for the depletable pins, this ORIGAMI calculation was performed in parallel using MPI. Figure 5.4.3 shows a plot of the axial burnup distribution, summed over all pins. 
Table 5.4.23. Input for ORIGAMI Sample Problem 5

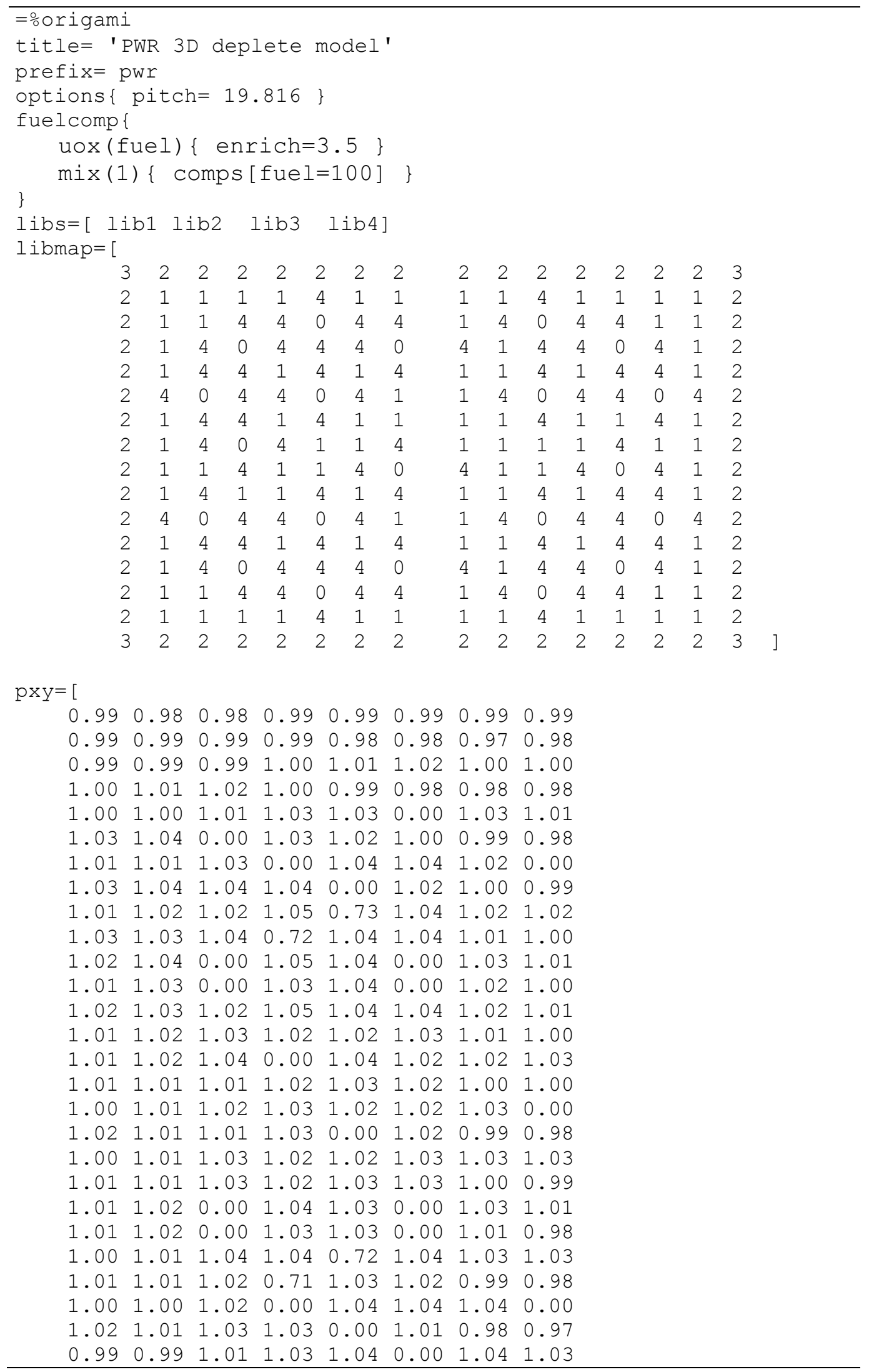




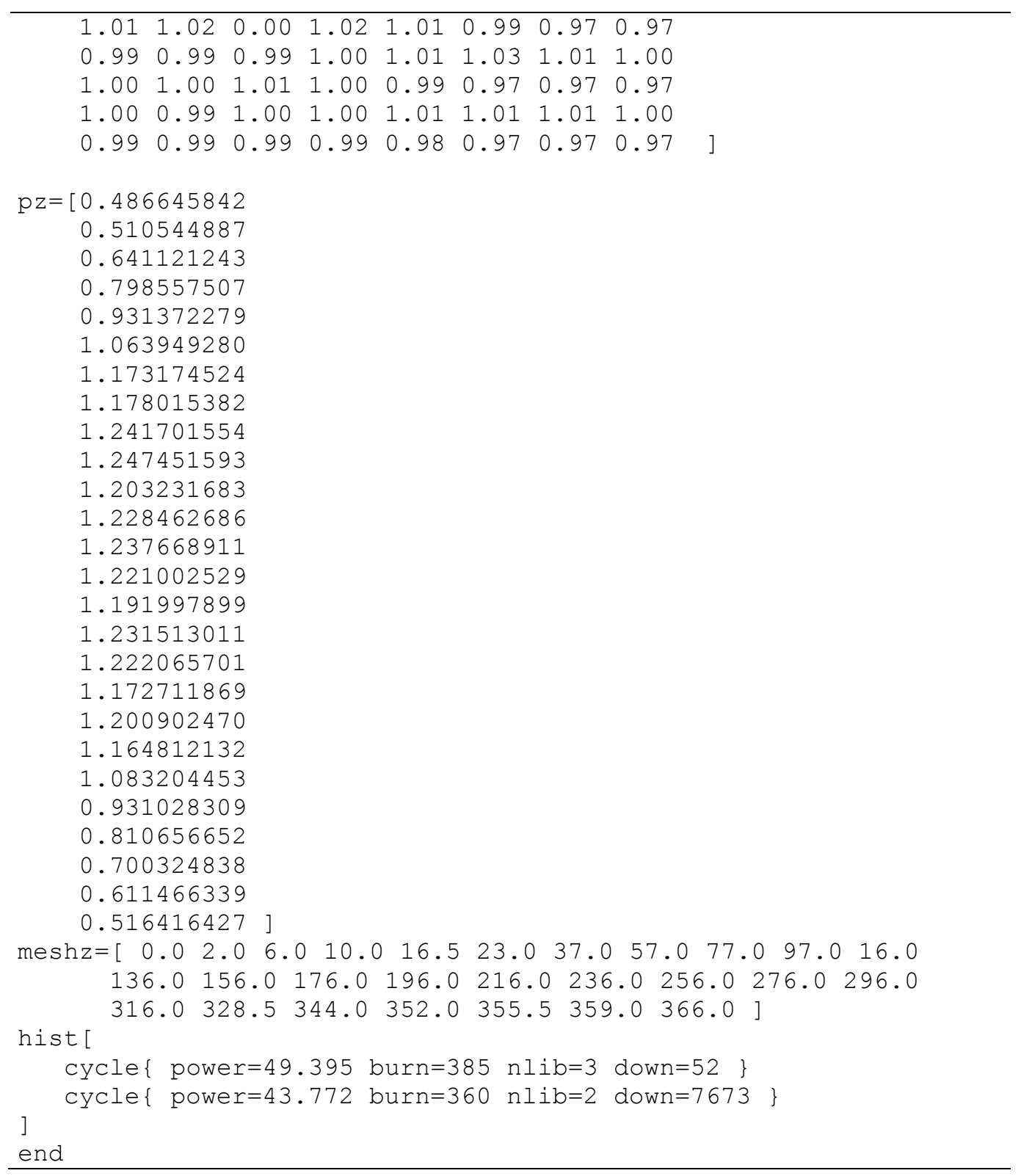




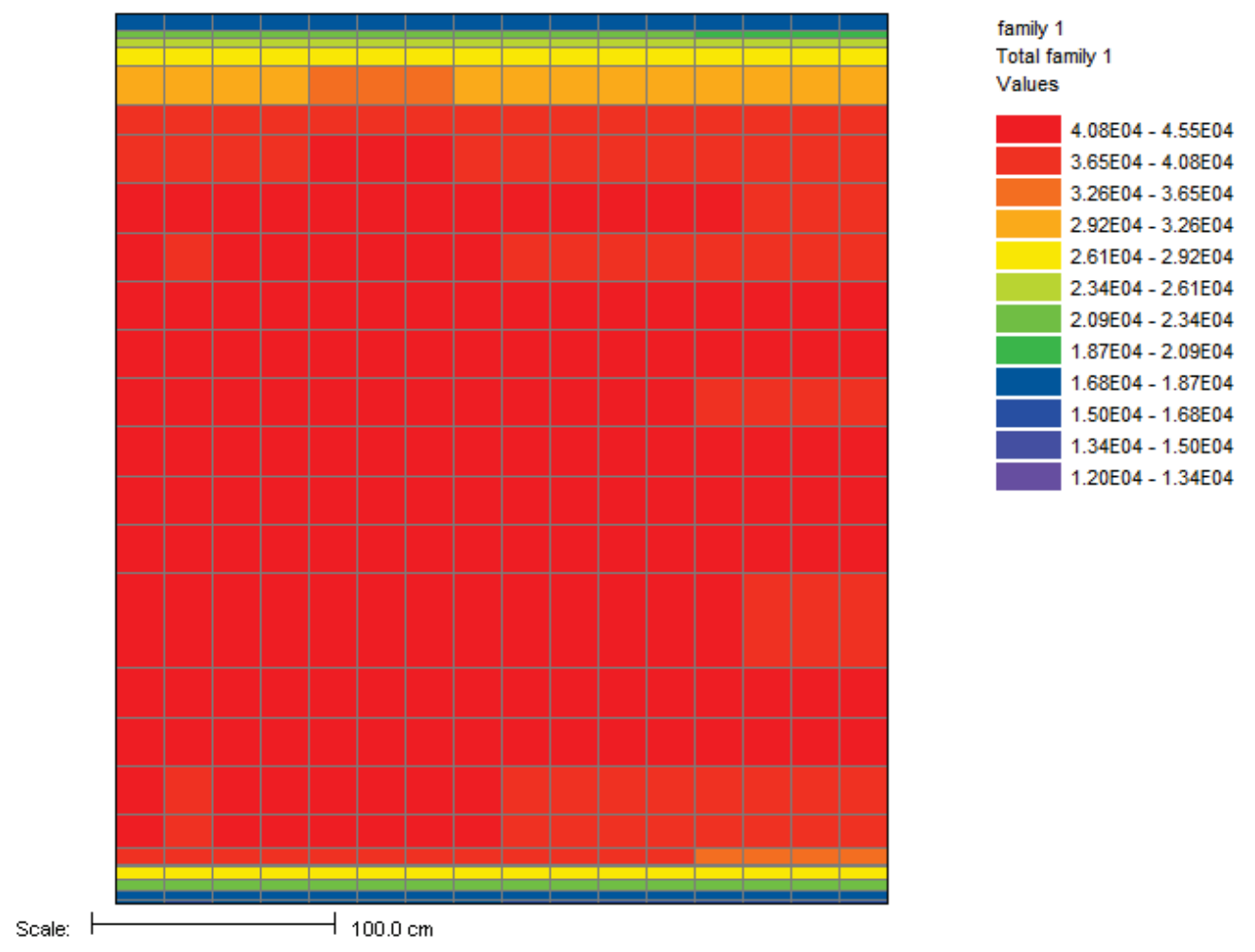

Figure 5.4.3. MeshView Plot of Axial Burnup Map (MWd/MTU) for Sample Problem 5.

\subsubsection{References}

1. S. Skutnik, F. Havlůj, D. Lago, and I. Gauld, "Development of an Object-Oriented ORIGEN for Advanced Nuclear Fuel Modeling Applications," International Conference on Mathematics and Computational Methods Applied to Nuclear Science \& Engineering (M\&C 2013), Sun Valley, ID, May 2013.

2. S. Skutnik, I.C. Gauld, C.E. Romano, and H.R. Trellue, "Creating NDA 'Working Standards' through High-Fidelity Spent Fuel Modeling," Proceedings of the Institute of Nuclear Materials Management, 53rd Annual Meeting, July, 2012.

3. G. Radulescu, I. C. Gauld, G. llas, and J. C. Wagner, An Approach for Validating Actinide and Fission Product Burnup Credit Criticality Safety Analyses - Isotopic Composition Predictions, NUREG/CR7108, ORNL/TM-2011/509, April 2012.

4. O.W. Hermann et al., Technical Support for a Proposed Decay Heat Guide Using SAS2H/ORIGEN Data, NUREG/CR-5625 (ORNL-6698), Martin Marietta Energy Systems, Inc., Oak Ridge National Laboratory, September 1994.

5. G. Radulescu, I. C. Gauld, G. Ilas, "SCALE 5.1 Predictions of PWR Spent Nuclear Fuel Isotopic Compositions," ORNL/TM-2010/44, Oak Ridge National Laboratory, March 2010. 


\title{
5.A SCALE/ORIGEN LIBRARY GENERATOR (SLIG)
}

B. R. Betzler

\begin{abstract}
This Python script (slig.py) semi-automates the process of generating sets of cross-section libraries for ORIGEN calculations for spent fuel depletion, decay, and source term analysis. SLIG performs several tasks:

1. reads standard-format template files, obtaining information (e.g, enrichments, burnups, moderator densities, etc.) from the file header;

2. generates a set of input files according to this header information;

3. builds a directory tree to house these input files;

4. writes the addition to the arpdata.txt file;

5. moves the final libraries to a new directory; and

6. reads the burnup list for each set of libraries and writes them in the addition to the arpdata.txt file.
\end{abstract}

This manual is divided into five main sections based on the desired application:

(5.A.1) for running SCALE on a local machine or a computing cluster without a queue system, see Quick Start Directions;

(5.A.2) for running SCALE on a cluster with a queue system, see Advanced Options;

(5.A.3) for editing and writing template files, see Template File Rules and Examples;

(5.A.4) for issues running SLIG, see Troubleshooting; and

(5.A.5) for making changes to the code, see Code Information. 


\section{TABLE OF CONTENTS}

$\underline{\text { Page }}$

5.A SCALE/ORIGEN LIBRARY GENERATOR (SLIG) …................................................ $5-234$

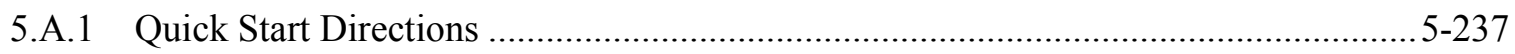

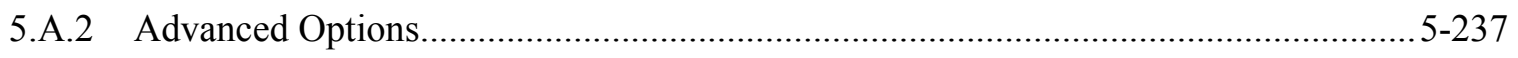

5.A.3 Template File Rules and Examples.................................................................... 5-238

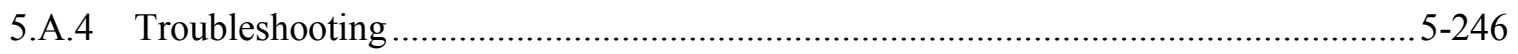

5.A.5 Code Information ............................................................................................ 5-246

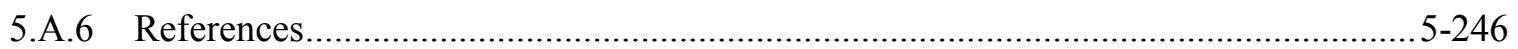




\section{LIST OF FIGURES}

Page

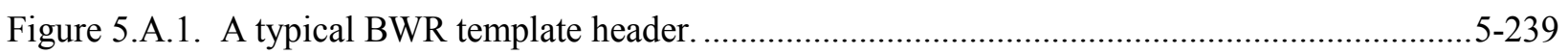

Figure 5.A.2. A typical BWR MOX template header.................................................................5-240

Figure 5.A.3. MOX zoning layout for a Westinghouse $14 \times 14$ PWR assembly showing the corner (green), edge (salmon), inside edge (magenta), and inner (red) pin zones. .........5-242

Figure 5.A.4. A typical BWR MOX documentation header.............................................................5-245 


\section{A.1 Quick Start Directions}

The SLIG package is located in the SCALE source directory in ./packages/etc/slig/. Because it is necessary to run SCALE to generate the appropriate cross-section libraries, SLIG runs in a disjointed, three-step process:

1. Copy the contents of the ./slig/src/ directory into ./slig/testing/. Move to the ./slig/testing/ directory and use ./slig.py -g to perform tasks i through iv. If SLIG returns an error, follow the directions in the error message (see §5.A.4). After a successful run, three new items will appear in the current directory:

runSpace / - the directory tree containing the input files,

arpLiblist.txt - a list of the locations of the libraries in the directory tree, and

addToArpData.txt — additional lines for the arpdata.txt file.

2. Run SCALE with each input within the directory tree (runSpace/). Each input is located within the directory tree in the location ./runSpace/templateName/inputID/, where templateName is the prefix of the template file (i.e., templateName template.inp) and inputID is a string identifying the enrichment (eNN), moderator density (wMM, if applicable), and plutonium vector ( $V P P$, if applicable) for the input.

3. After all SCALE calculations are complete, use ./slig.py $-\mathrm{f}$ to perform task $\mathrm{v}$ and vi. SLIG will identify any missing libraries. After a successful run, two changes will be made in the current directory:

newLibraries / - this directory containing the new libraries will appear, and addToArpData.txt - the burnup lists will be written on the this file.

The final result is an addition to the arpdata.txt file (addToArpData.txt) and a directory containing all generated libraries (newLibraries/). Do not delete any files associated with slig.py unless it directs you to do so. The following options are available to the user (see §5.A.2 for more options):

-p PATH or - path=PATH

This specifies the location of the template files. SLIG will search PATH and all its subdirectories for template files. The default path is the current directory.

$-x$ XSLIB or - xsections=XSLIB

This specifies the cross section library used in all calculations. The default is ' $\mathrm{V} 7-252$ '.

\section{A.2 Advanced Options}

The following are more advanced options available to the user:

-e EXT or -extension=EXT 
This specifies the file extension that identifies a template file. By default, SLIG searches for files ending in '_template.inp').

$-a$ or $-a d d$

This flag is used with the $-g$ option (./slig.py -ga) to add input files to the current directory tree (runSpace/). It also adds library information to addToArpData.txt and adds the location of the libraries in the directory tree to arpLiblist.txt.

This flag is used with the $-\mathrm{f}$ option $(. / \mathrm{slig} \cdot \mathrm{py}-\mathrm{fa})$ to add newly generated libraries to the current library directory (newLibraries/).

$-\mathrm{d}$ or -document

This flag turns on documentation routines that automatically generate a $\mathrm{L}^{A} \mathrm{~T}_{\mathrm{E}} \mathrm{X}$ file (libraryInformation.tex) with data about each template file using information on the file header. This also generates a .pdf file (libraryInformation.pdf) using pdflatex (required for this to function properly). Additionally, any figures referenced within the templates must be located within the current directory or any of its subdirectories.

$-s$ or -submit

This flag turns on routines that generate a PBS submit script for each input file for running on clusters with a queue system. It also generates a shell script to submit these jobs (with qsub). The PBS submit script template (run1proc.pbs) must be placed in the current directory. The first two steps of the three-step process for running SLIG (see §5.A.1) change:

1. Use ./slig.py -gs to perform tasks i through iv. After a successful run, four new items will appear in the current directory:

runSpace/ - the directory tree containing the input files, arpLiblist.txt - a list of the locations of the libraries in the directory tree, addToArpData.txt — additional lines for the arpdata.txt file, and submitSLIGjobs - a shell script for submitting jobs to the queue.

2. Run the shell script (./submitSLIGjobs) to submit jobs to the queue. Note that this may submit a very large number of jobs to the queue.

When using ./slig.py -ga, SLIG will check for documentation and submit scripts and will proceed with the same settings that were used for the initial run of SLIG. For example, if the user initially runs . / slig.py - gds to generate documentation and submit scripts then later uses . /slig.py -ga, SLIG will proceed with adding documentation for the additional templates and generating submit scripts. When SLIG generates additional submit scripts, a separate numbered file is generated for each new template (e.g., submitSLIGjobs1).

\section{A.3 Template File Rules and Examples}

Example templates are provided with the SCALE distribution. For creating new templates, it is best to use these templates as starter files and make incremental changes as necessary for the new 
application. The header at the top of each template file contains the information that will be used to generate input files and documentation. Each line of this header must start with an apostrophe; each line is a comment line according to the SCALE standard input. There are two main sections in this header:

1. The template header (see Figure 5.A.1 and Figure 5.A.2). This header has a parameter list and an option list:

(a) The parameter list identifies the strings within the template file that SLIG will replace as it generates input files. These parameters are found throughout the template file; SLIG will replace these with values as it generates each input files. SLIG identifies the parameter list starting from the first line containing the 'parameter' string and each line thereafter that is indented more than five blank spaces (not including the apostrophe). The following is a list of rules for entering parameters:

- Each parameter is entered on a separate line in the form "parameter description”.

- Each parameter must be a unique string of characters.

- Each parameter is a single string without spaces.

- SLIG uses the "description" to characterize the parameter. If these descriptions are changed, SLIG may not be able to properly characterize each parameter. Thus, it is suggested that the descriptions in the following list are not changed.

- There is some flexibility in changing the parameter strings.

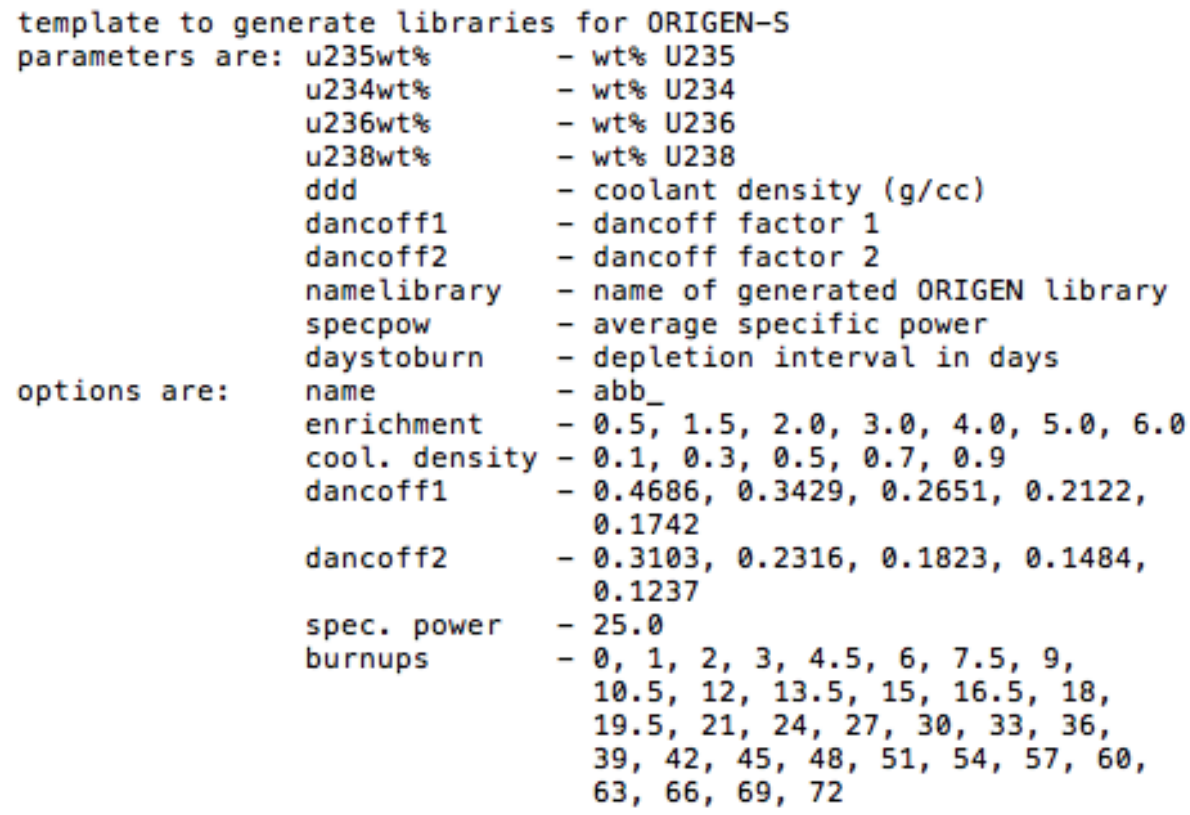

Figure 5.A.1. A typical BWR template header. 


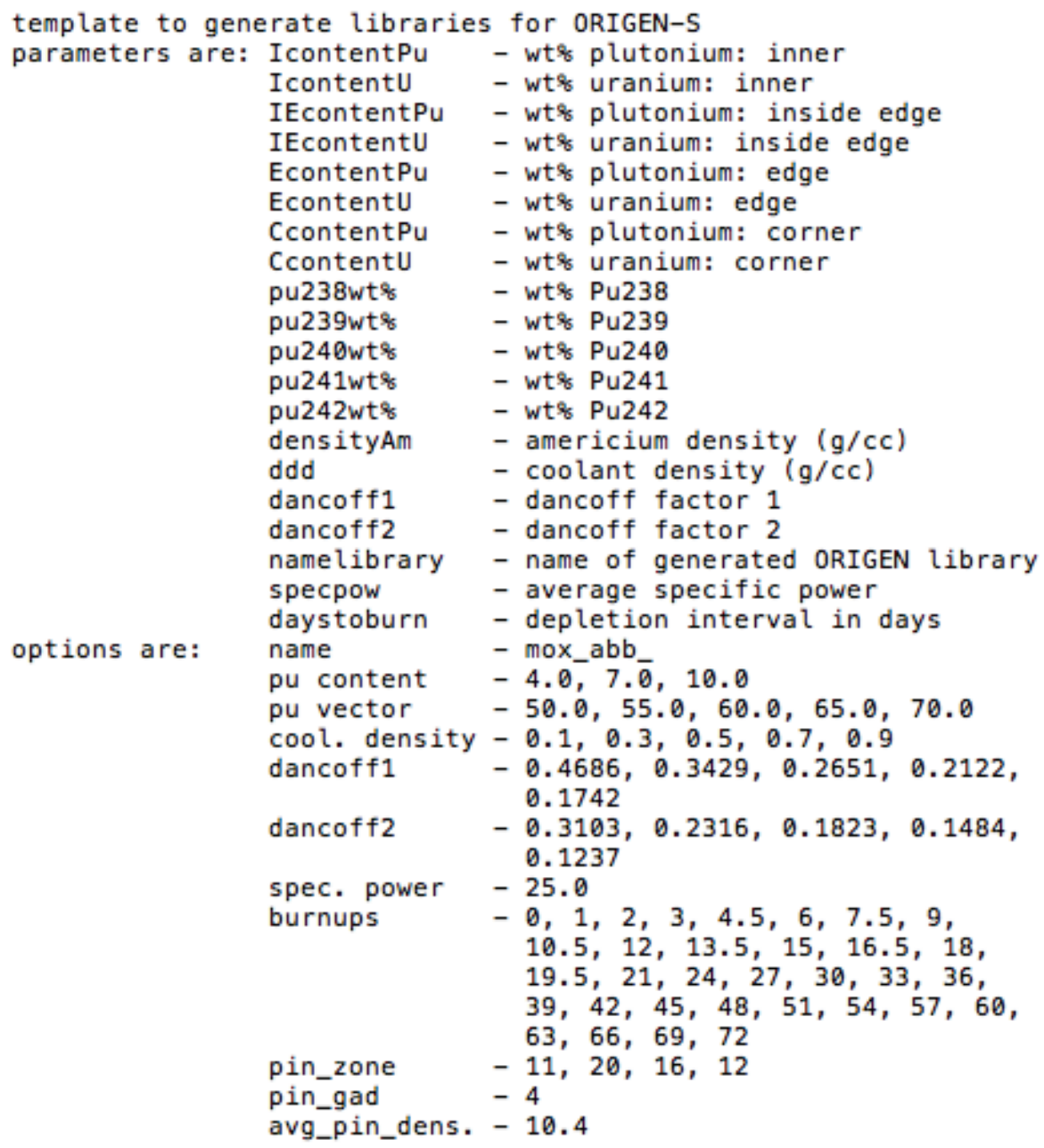

Figure 5.A.2. A typical BWR MOX template header.

The following is a list of some of the parameters available to the user for working with most PWR and BWR lattices:

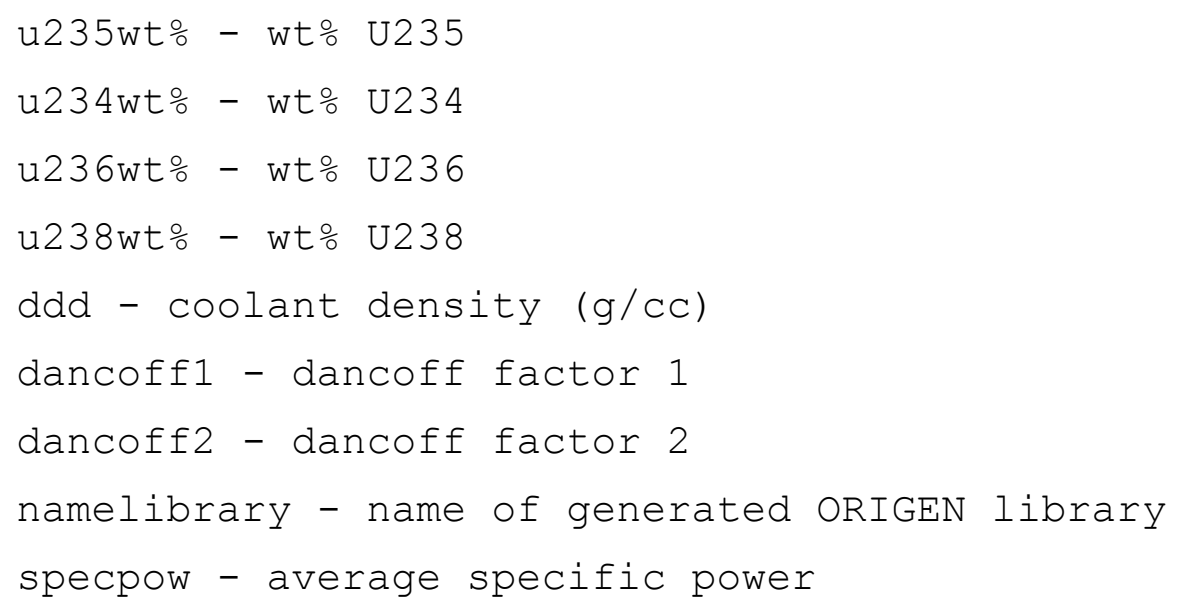




$$
\text { daystoburn - depletion interval in days }
$$

For mixed oxide (MOX) PWR and BWR assemblies, there is a different set of parameters available to the user. SLIG will recognize a file as a MOX template by searching for the Pu-239 parameter. SLIG automatically generates a MOX zoning pattern (see Figure 3) for BWR and PWR lattices.

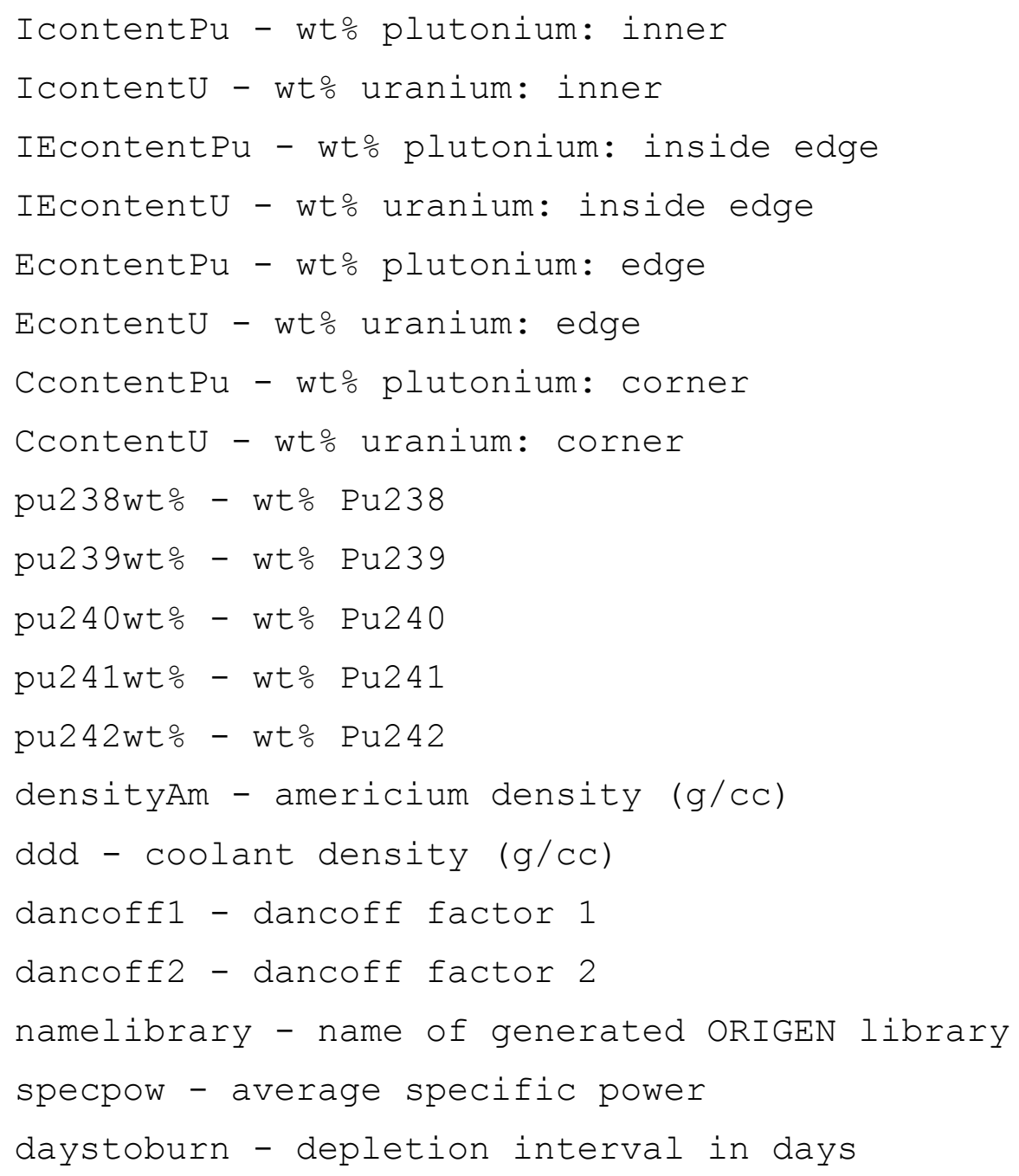




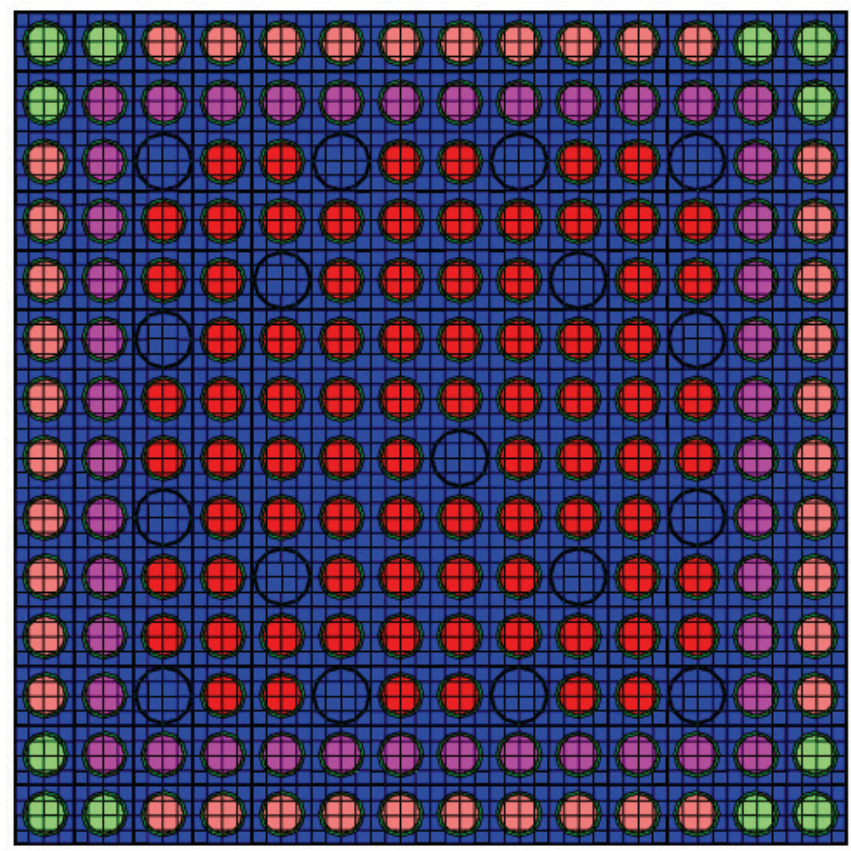

Figure 5.A.3. MOX zoning layout for a Westinghouse $14 \times 14$ PWR assembly showing the corner (green), edge (salmon), inside edge (magenta), and inner (red) pin zones.

(b) The option list identifies the quantities or values that SLIG uses to determine the values that will replace the parameters in each input file. SLIG identifies the option list starting from the first line containing the 'option' string and each line thereafter that is indented more than five blank spaces (not including the apostrophe). The following is a list of rules for entering options:

- Each option is entered on a separate line in the form "option - values".

- SLIG uses the "option" to recognize what to do with the values. If this is changed, SLIG may not be able to properly characterize each option. Thus, it is suggested that the options (to the left of the dash) in the following list are not changed.

- For options with multiple values, each value must be separated with a comma.

- For values to continue onto the next line, the last value in a line must end with a comma and the next line must be indented and must begin with the next value.

- The values may be changed.

The following is a list of the options available to the user for working with most PWR and BWR lattices:

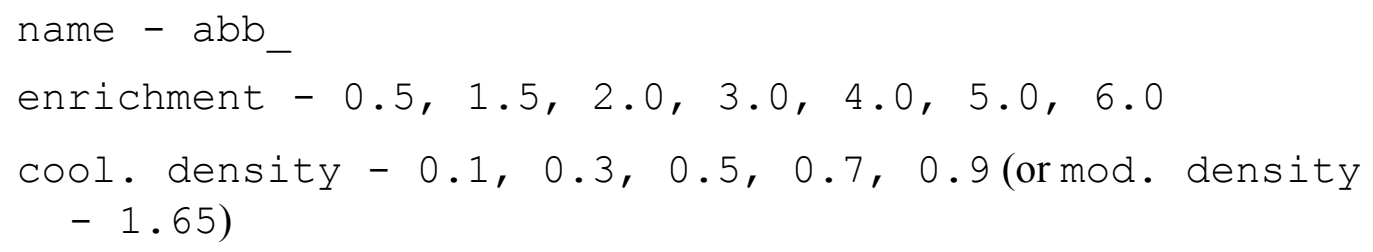




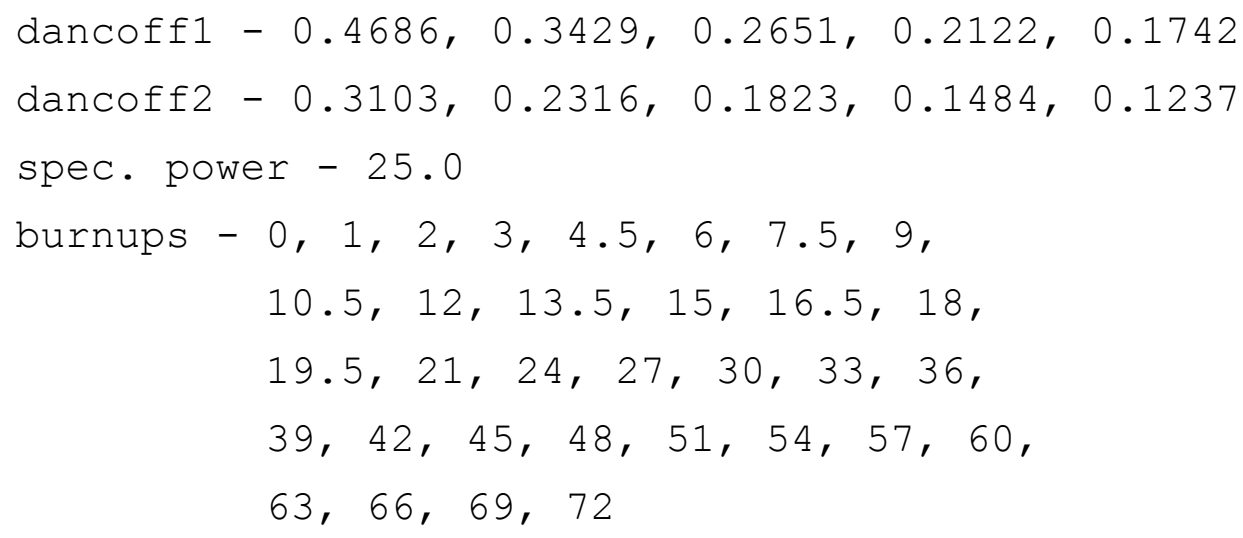

For mixed oxide (MOX) PWR and BWR assemblies, there is a different set of options available to the user. SLIG automatically generates the MOX zoning pattern according to the values of "pin_zone", "pin_gad", and "avg_pin_dens.".

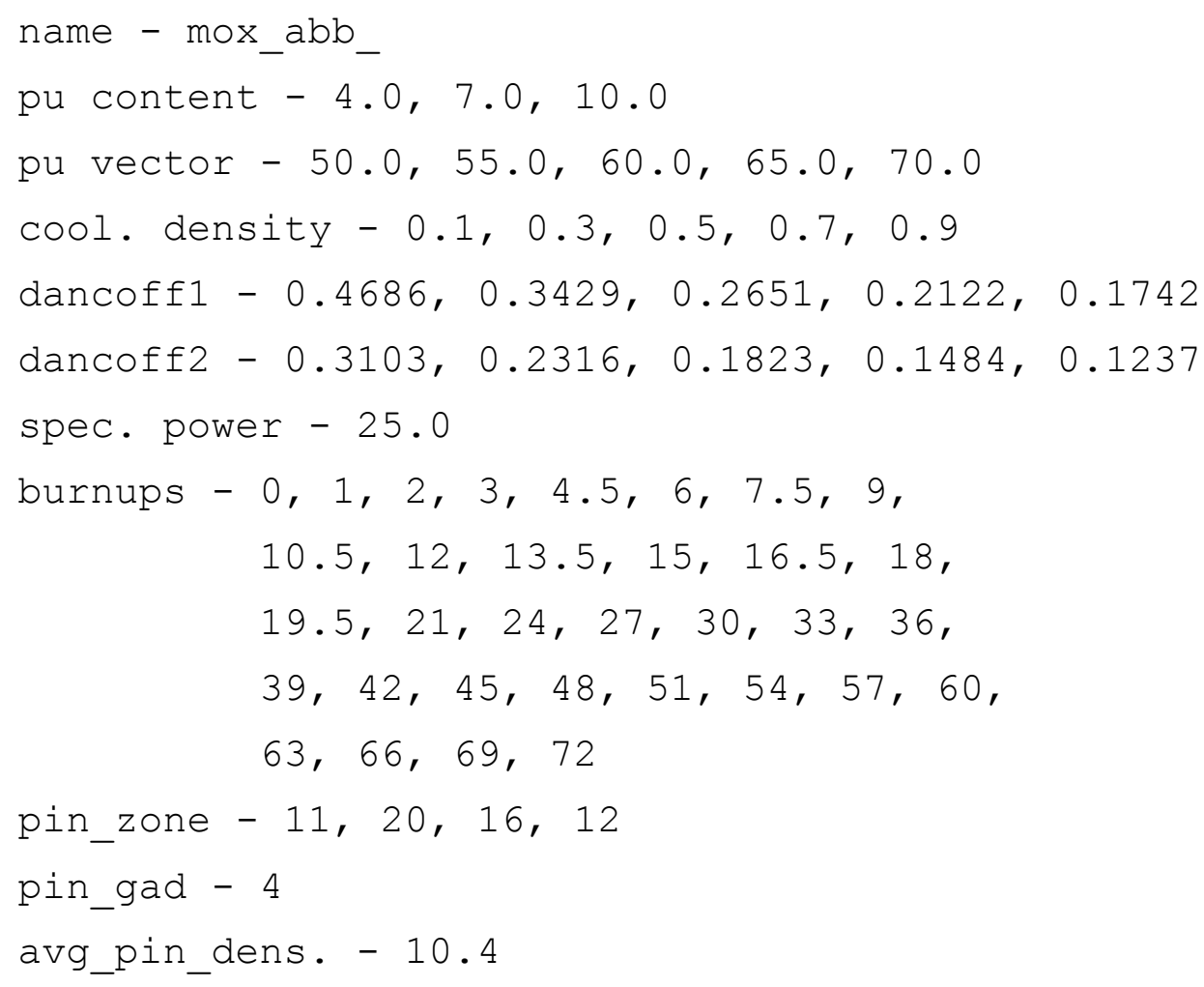

The following is an explanation of each option:

name

This is the prefix for the name of the final generated libraries. It should be unique to the template (i.e., other templates should have a different name).

enrichment

This is a list of the ${ }^{235} \mathrm{U}$ enrichments [\%] for which SLIG will create separate inputs.

cool. density or mod. density 
This is a list of the coolant or moderator densities $\left[\mathrm{g} / \mathrm{cm}^{3}\right]$ for which SLIG will create separate inputs.

\section{dancoffN}

This is a list of dancoff factors that correspond to pin N. The length of this list must match the length of the coolant/moderator density list (the dancoff factor varies significantly with this density).

spec. power

This is the specific power [MW/MTU] of the assembly.

burnups

This is a list of cumulative burnup steps [GWd/MTU] that will be used to create the depletion steps. If a small step is not included, SLIG will print a warning and automatically insert one at the beginning of the calculation (for Xe equilibrium).

pu content

This is a MOX-specific parameter that is a list of the average content of plutonium [\%] in the assembly for which SLIG will create separate inputs. These quantities are calculated as a percentage of the total heavy metal loading in the assembly.

pu vector

This is a MOX-specific parameter that is a list of the enrichments of ${ }^{239} \mathrm{Pu}[\%]$ for which SLIG will create separate inputs. These quantities are calculated as a percentage of the total plutonium in the assembly and are used to calculate the entire plutonium vector ${ }^{1}$.

pin_zone

This is a MOX-specific parameter that lists of the number of pins in the inner, inside edge, edge, and corner zones (see Figure 5.A.3). This varies for each assembly.

pin_gad

This is a MOX-specific parameter that specifies the number of Gd-bearing pins in the assembly. SLIG uses this quantity to calculate ensure that the specified $\mathrm{Pu}$ content of the assembly is correctly represented. ${ }^{2}$ avg_pin_dens.

This is a MOX-specific parameter that specifies the average density $\left[\mathrm{g} / \mathrm{cm}^{3}\right]$ of the pins in the assembly. SLIG uses this quantity to calculate ensure that the specified $\mathrm{Pu}$ content of the assembly is correctly represented.

2. The documentation header (see Figure 5.A.4). The documentation header has two purposes. First, it is used to track information about the template (e.g., author, date created, and methodology) and the source documents that contributed to the template's creation. This simplifies updating input files for new versions of SCALE. Second, SLIG uses this 
header information to generate documentation files. SLIG identifies the documentation header starting from the first line containing 'Documentation' to the line starting with an apostrophe followed by a space and five dashes (' -----).

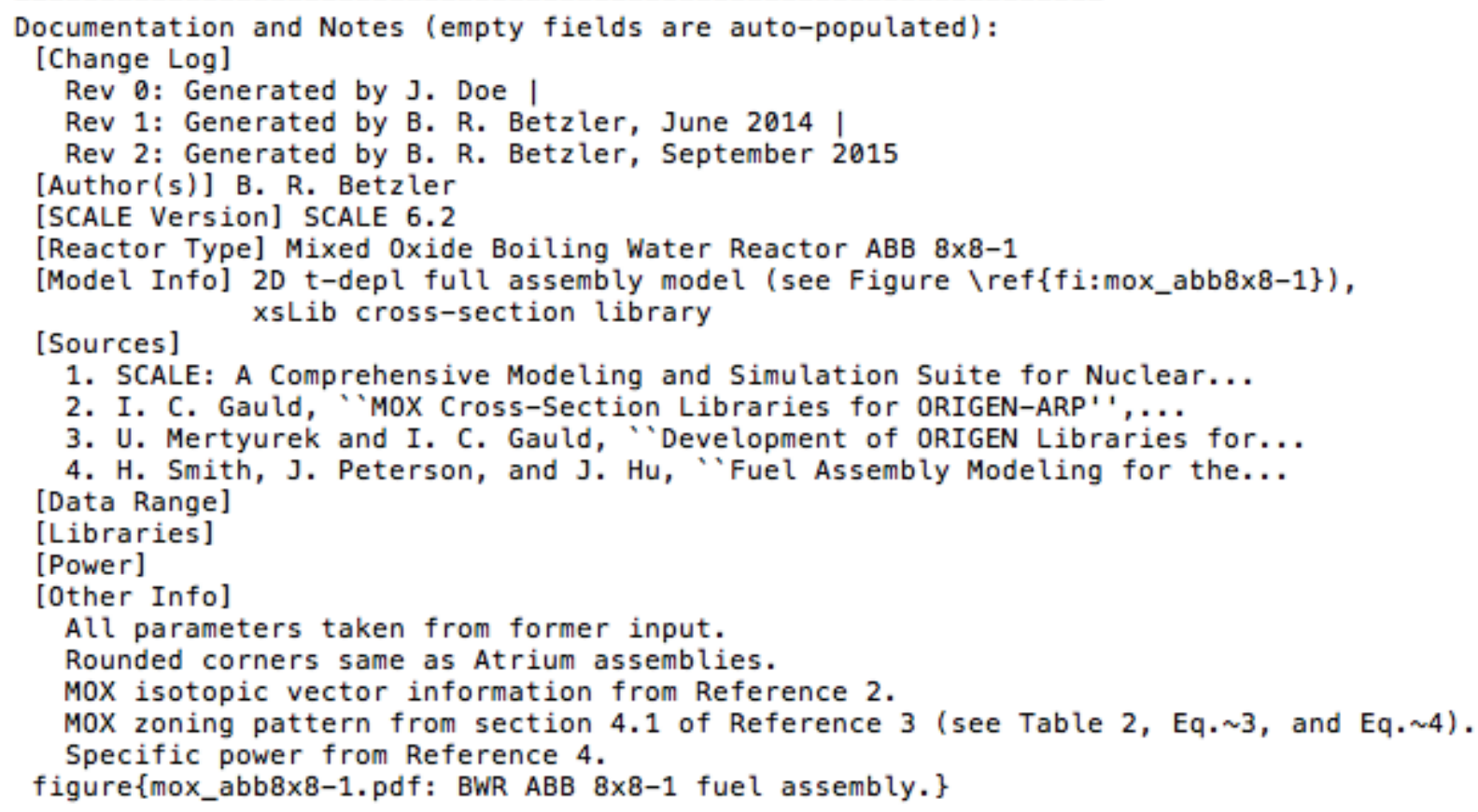

Figure 5.A.4. A typical BWR MOX documentation header.

The documentation header has a free form-style entry, where sections are specified by "[Section Name]" and the text for each section follows afterward. Figures are referenced as "figure ffigureName.pdf: Figure caption.\}". Any reference to the figure within the header must be in $\mathrm{L}^{\mathrm{A}} \mathrm{T}_{\mathrm{E}} \mathrm{X}$-like form; for this, the figure is labeled as "fi:figureName". The section name keywords "[Data Range]", "[Libraries]", and "[Power]" are reserved to be auto-populated by SLIG according to information provided within the options list. All information within this header is transferred to the documentation file.

The parameters in the file headers are located within the template file in the appropriate locations. See the example templates for the proper usage. The burndata card in the input file should only be three lines:

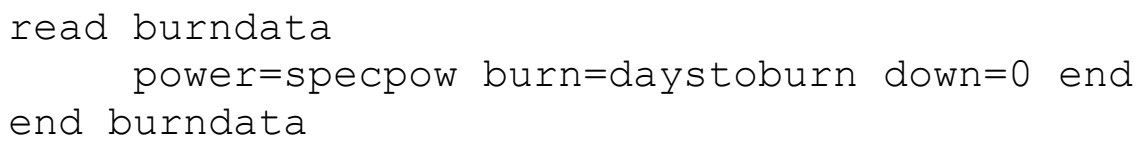

because it will be populated by the burnup points listed in the file header. There should also be a shell command at the end of the input file to save the cross-section library:

$$
\begin{aligned}
& \text { shell } \\
& \quad \mathrm{cp} \text { ft33f001. cmbined \$RTNDIR/namelibrary }
\end{aligned}
$$


end

\section{A.4 Troubleshooting}

If at any time an error occurs, SLIG will exit after printing the reason for the error and offer a possible solution. Following these instructions should resolve most issues with SLIG. The following is a list of factors to consider when having trouble running SLIG.

- SLIG uses the module argparse.py https://docs.python.org/3/library/argparse.html) to handle command line arguments; SLIG will crash if it does not have access to it. For versions of Python that do not inherently support this module, download the argparse.py file and include it in the directory with slig.py.

- If SLIG is crashing, there may be an issue with the version of Python. SLIG was developed to be functional with Python 2.7.6.

- After running ./slig.py -g, the addToArpData.txt file will have dummy placeholders instead of the burnup lists. These placeholders are replaced with the burnup lists that are read off of the SCALE output files. The burnup lists in the documentation file are the burnup lists specified in the template files.

\section{A.5 Code Information}

SLIG consists of a main, one SCALE-specific class (manageTemplate), and three generic classes (messenger, manageDirectory, and manageFile):

- main reads and validates arguments, and contains a loop to make inputs and collect libraries

- manageTemplate manages templates (reads/sorts options, calculates concentrations)

- messenger manages all prints to the screen

- manageDirectory manages external directories (searching, making, etc.)

- managefile manages external files (reading, writing, searching, copying, etc.)

SLIG calls a separate Python script (collectinfov04.py) to perform documentation functions. This script uses a template (basedoc. v04.tex) to generate the documentation file.

\section{A.6 References}

1. I. C. Gauld, "MOX Cross-Section Libraries for ORIGEN-ARP," ORNL/TM-2003/2, Oak Ridge National Laboratory, Oak Ridge, Tennessee, 2003. 
2. U. Mertyurek and I. C. Gauld, "Development of ORIGEN Libraries for Mixed Oxide (MOX) Fuel Assembly Designs," to be published, 2015. 


\title{
5.B UTILITY PROGRAMS
}

I. C. Gauld, S. M. Bowman, and W. A. Wieselquist

\begin{abstract}
There are three utility programs that may be of interest to ORIGEN users: PRISM, ARPLIB, and XSECLIST. The traditional use of the PRISM module has been to produce the set of TRITON input files necessary to compile an ORIGEN reactor library, e.g. with varied enrichment and moderator density. For the most part, this capability has been replaced by the SLIG capability described in Appendix 5.A. The ARPLIB utility's main purpose is to remove burnup points from existing ORIGEN libraries, e.g. to reduce the size on disk. The final utility, XSECLIST, provides a simple way to print the burnupdependent cross sections contained in the ORIGEN library.
\end{abstract}




\section{TABLE OF CONTENTS}

$\underline{\text { PAGE }}$

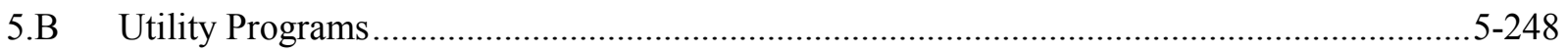

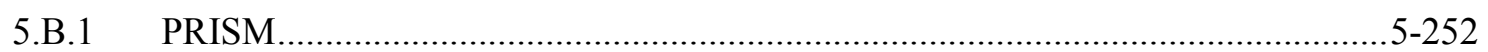

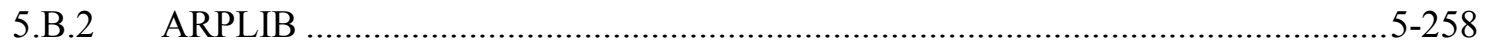

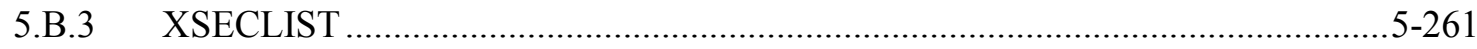




\section{TABLE OF FIGURES}

$\underline{\text { PAGE }}$

Figure 5.B.1. PRISM input example to generate TRITON input files.............................................5-254

Figure 5.B.2. Generic TRITON input template for PRISM........................................................5-254

Figure 5.B.3. ARPLIB example input to reduce size of ORIGEN-ARP cross-section libraries. .........5-260

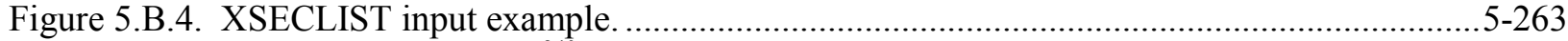

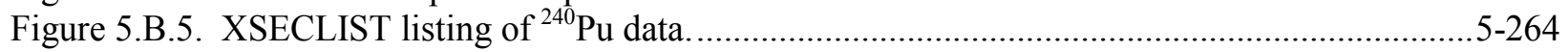




\section{LIST OF TABLES}

$\underline{\text { PAGE }}$

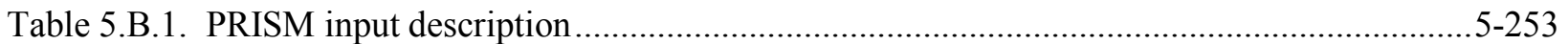

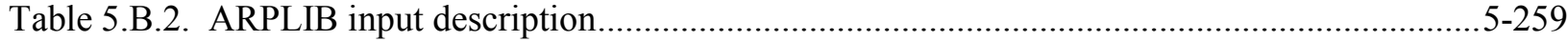

Table 5.B.3. XSECLIST input description 


\section{B.1 PRISM}

PRISM is a utility that reads a single input template file containing generic parameter flags and replaces them with specific values designated by the user to generate any number of files containing desired combinations of specific parameter values. PRISM provides a procedure to convert a generic input file for a particular fuel assembly design into a large number of input files containing combinations of specific fuel enrichment and moderator densities and/or other parameters for generating basic cross-section libraries. The program was designed in a general manner so PRISM can be used to generate multiple files from any generic file.

The input description for PRISM is presented in Table 5.B.1. The input format is free form. The user input includes the name of the template file to be read; the pattern for the name of the output files to be generated, using the generic parameter flags; the number of generic parameter flags; the number of files to be generated; each generic parameter flag and the specific values to be substituted in each output file.

The template file contains generic parameter flags. PRISM creates copies of the template file and substitutes specific values for the generic flags. Note that the character length of each specific value must be the same as that of the associated generic flag.

An example using PRISM to generate input files for six fuel enrichments is presented in and Figure 5.B.2. In this example a TRITON input file for a Westinghouse $17 \times 17$ fuel assembly (Figure 5.B.2) is processed by PRISM using the input file for PRISM listed in Figure 5.B.1. The case generates 6 specific input files from the template file. 
Table 5.B.1. PRISM input description

\begin{tabular}{|c|c|c|c|}
\hline Line No. & Parameter & Description & Comments \\
\hline 1 & TEMPLATE & Template file name & 80 characters maximum \\
\hline 2 & OUT_TEMPLATE & Pattern for output file names & $\begin{array}{l}80 \text { characters maximum } \\
\text { Must contain enough generic parameter } \\
\text { names to create unique filename for } \\
\text { each output file }\end{array}$ \\
\hline \multirow[t]{3}{*}{3} & NUMPARMS & $\begin{array}{l}\text { Number of generic parameter } \\
\text { types }\end{array}$ & \\
\hline & NUMFILES & $\begin{array}{l}\text { Number of output files to be } \\
\text { generated }\end{array}$ & \\
\hline & \multicolumn{3}{|l|}{ NOTE: } \\
\hline 4 & PARAM_NAME & $\begin{array}{l}\text { Generic parameter name as it } \\
\text { appears in template file }\end{array}$ & 80 characters maximum \\
\hline $5^{a}$ & PARAMETERS & $\begin{array}{l}\text { Specific values of generic } \\
\text { parameter for each output } \\
\text { file }\end{array}$ & $\begin{array}{l}\text { NUMFILES entries required. Length of } \\
\text { value must be same as length of } \\
\text { PARAM_NAME }\end{array}$ \\
\hline
\end{tabular}

${ }^{a}$ May be continued on subsequent lines as needed. 


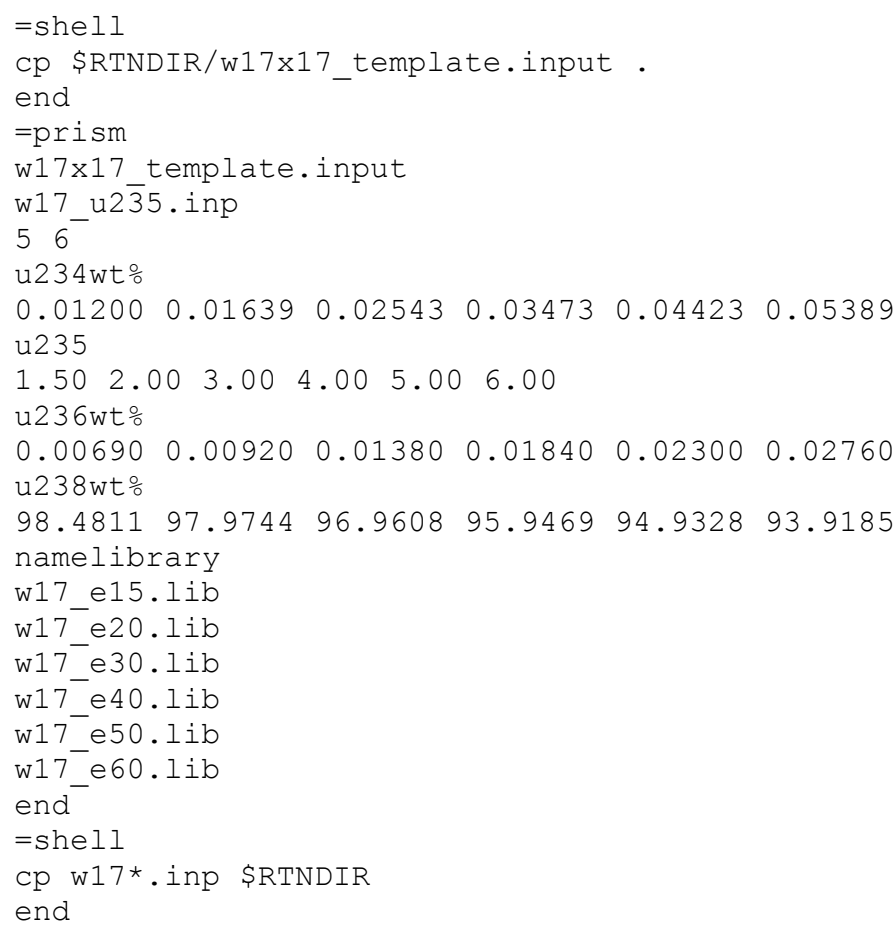

Figure 5.B.1. PRISM input example to generate TRITON input files.

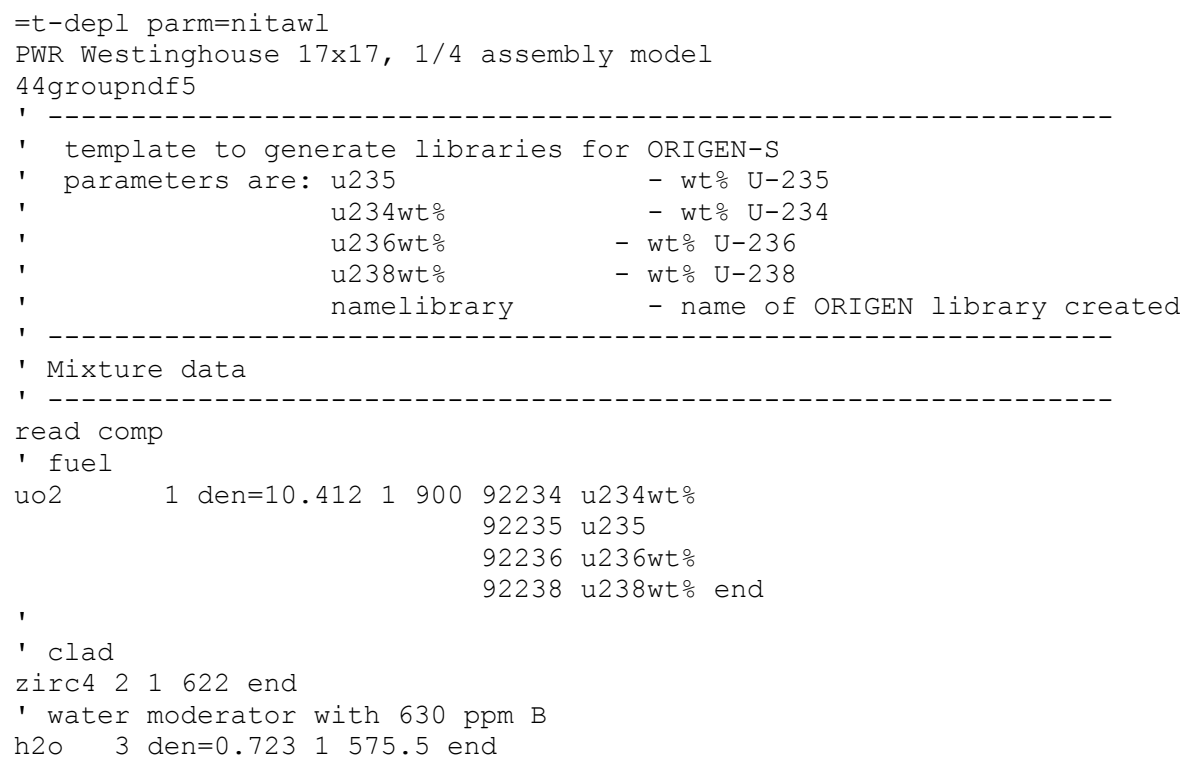

Figure 5.B.2. Generic TRITON input template for PRISM. 


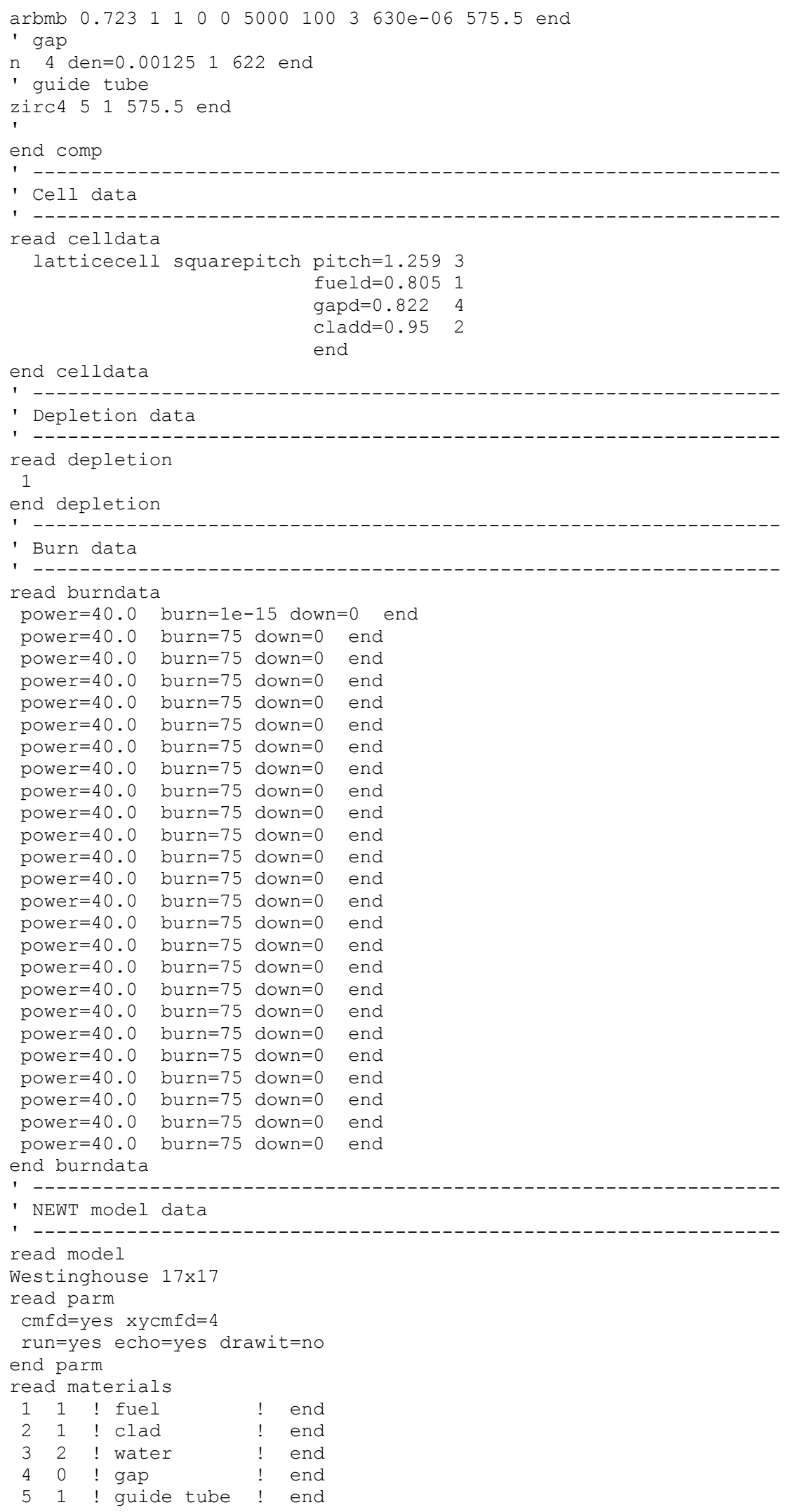

Figure 5.B.2. Generic TRITON input template for PRISM. (continued) 


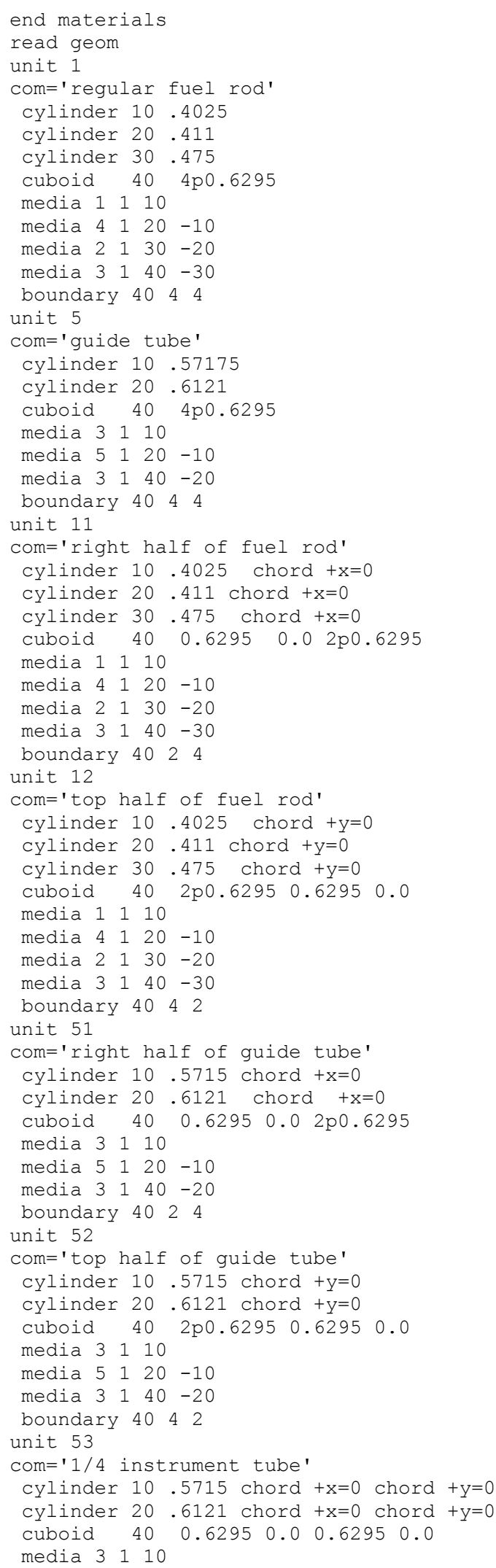

Figure 5.B.2. Generic TRITON input template for PRISM. (continued) 


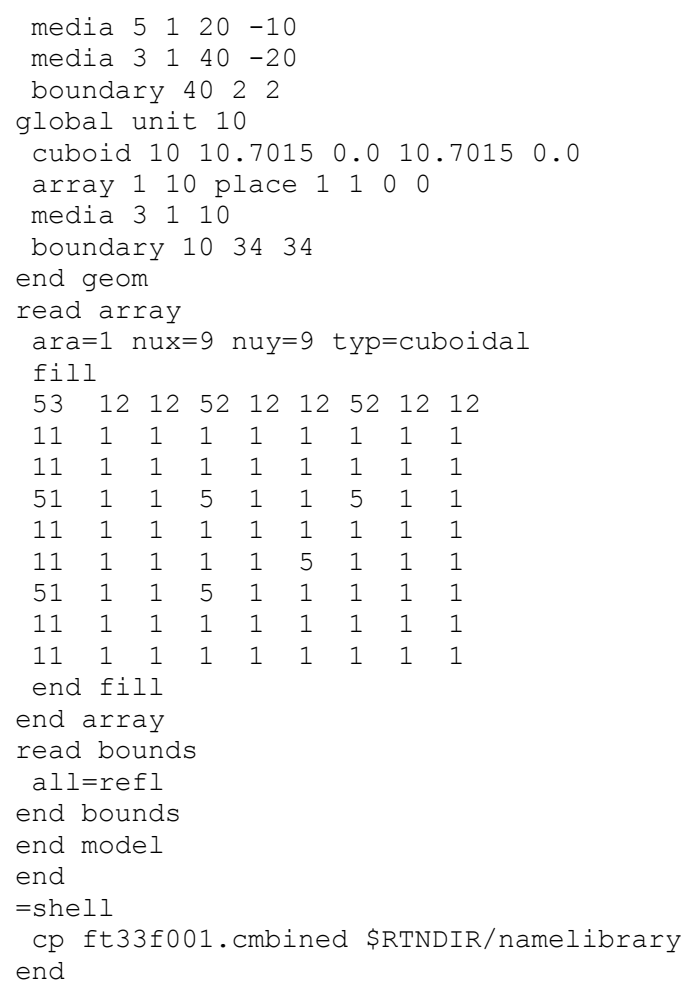

Figure 5.B.2. Generic TRITON input template for PRISM. (continued) 


\section{B.2 ARPLIB}

ARPLIB is a utility program designed to read a burnup-dependent binary ORIGEN-ARP cross-section library and copy the cross-section data from only the desired burnup positions to create a new ORIGENARP cross-section library. The default behavior of ARPLIB is to read a library with 21 burnupdependent cross-section sets and reduce it to a 10-burnup-step cross-section library. By default, the ten burnup positions in the library are $1,2,3,4,5,6,7,12,17$, and 21 (see Table 5.4.A.2). However, any position in the original library may be omitted or retained.

The input for ARPLIB is described in Table 5.B.2. The input is free format. The sign (+/-) of the first input parameter depends upon whether the user wants to select the ten default burnup positions from the multiburnup library, or specify different positions. If the default positions are desired, the first parameter is the total number of multiburnup library files to be converted (NUMLIBS). If the user wants to override the defaults, the NUMLIBS parameter should be any negative integer. If a negative integer is used, it should be followed by the number of burnup positions in each multiburnup library (NL), flags for each burnup position (IN(I), I=1, NL), and the total number of multiburnup libraries to be converted (NUMLIBS). The final portion of the input file in all cases is the filename of each library, followed by the filename of the reduced cross-section library to be created from it.

An example of the input to ARPLIB is given in Figure 5.B.3. Note that the first burnup position is always saved, regardless of the input value for IN(1). The cross sections in the first burnup position are always assumed to be the fresh fuel cross sections. 
Table 5.B.2. ARPLIB input description

\begin{tabular}{|c|c|c|}
\hline Parameter & Description & Comments \\
\hline \multirow[t]{2}{*}{ NUMLIBS } & $\begin{array}{l}\text { Number of multi-burnup library files }(>0) \\
\text { or flag to read following optional data } \\
(<0)\end{array}$ & $\begin{array}{l}\text { NUMLIBS }<0 \text { if number of burnups } \\
\text { and/or burnup positions to keep differ } \\
\text { from default }{ }^{a}\end{array}$ \\
\hline & \multicolumn{2}{|c|}{ NOTE: The following data are read only if NUMLIBS $<0$. } \\
\hline NL & $\begin{array}{l}\text { Number of burnup positions on each } \\
\text { multiburnup library }\end{array}$ & $\begin{array}{l}\text { Input if NUMLIBS }<0 \\
\text { Default }=21 .\end{array}$ \\
\hline $\mathrm{IN}$ & $\begin{array}{l}\text { Flag for each burnup position to keep or } \\
\text { remove cross sections }{ }^{a}\end{array}$ & $\begin{array}{l}\text { Input if NUMLIBS }<0 \\
\text { NL entries required } \\
=0 \text { Remove cross sections } \\
>0 \text { Keep cross sections }\end{array}$ \\
\hline NUMLIBS & Number of multiburnup library files & Input if NUMLIBS $<0$ \\
\hline
\end{tabular}

NOTE: End of optional input for NUMLIBS $<0$.

NOTE: The following entries are entered in pairs NUMLIB times.

FILENAME Input library filename 80 characters maximum

FILENAME2 Output library filename 80 characters maximum

${ }^{a}$ Default is to keep cross sections from the following ten burnup positions of a 21-burnup cross-section library: $1,2,3,4,5,6,7,12,17,21$. The first burnup position (i.e., fresh fuel) is always kept, regardless of user input. 


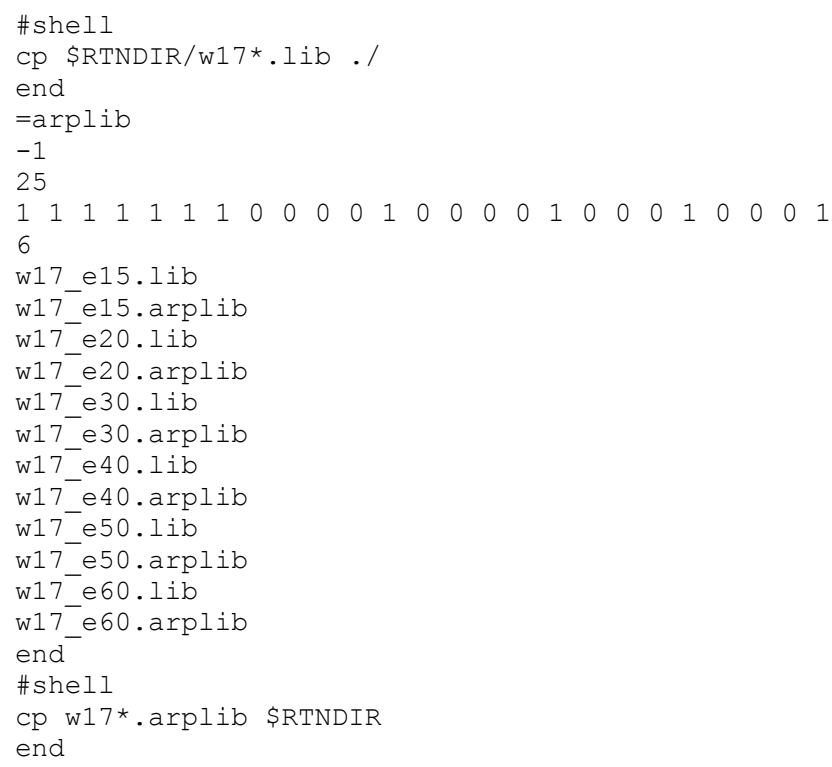

Figure 5.B.3. ARPLIB example input to reduce size of ORIGEN-ARP cross-section libraries. 


\section{B.3 XSECLIST}

The XSECLIST program is intended to provide an interpreted listing of any ORIGEN-ARP cross-section library. This utility program allows users to list the absorption and/or fission cross sections of any or all nuclides in the library as a function of burnup. The absorption cross sections are given for light elements, actinides, and fission products. Some of the light-element isotopes in the library may appear also as fission products; therefore, some isotopes may be listed twice. Fission cross sections may be listed for any or all actinides for which nonzero values of the cross sections exist. ORIGEN-S cross sections are typically normalized to thermal flux, rather than the total flux.

The nuclide ID numbers used in the library listings have the form IZ*10000+IA*10+IS,

where

$$
\begin{aligned}
& I Z=\text { the atomic number; } \\
& I A=\text { the atomic weight; } \\
& I S=0, \text { for ground state; } \\
& \text { IS }=1, \text { for metastable state }
\end{aligned}
$$

The XSECLIST input is described in Table 5.B.3. The input is free format. The user specifies the library filename, the total number of burnup positions in the library, and the burnup values (GWd/MTU) corresponding to each burnup position. The user then indicates whether the cross-section data listings are for absorption, fission, or both; and whether the listings are for all nuclides or only certain specified ones.

An example input file for XSECLIST is shown in Figure 5.B.4. This example contains two cases. The first case lists both absorption and fission cross sections for ${ }^{240} \mathrm{Pu}$ in the ORIGEN-ARP $5 \mathrm{wt} \%$ enriched PWR $14 \times 14$ basic cross-section library. The output listing for this case is displayed in Figure 5.B.5. These are microscopic cross sections listed in units of barns. The second case lists the fission cross sections for all nuclides in the ORIGEN-ARP $1.5 \mathrm{wt} \%$ enriched PWR $14 \times 14$ basic cross-section library. The output from this case is not presented here because of its size. 
Table 5.B.3. XSECLIST input description

\begin{tabular}{|c|c|c|c|}
\hline Line No. & Parameter & Description & Comments \\
\hline 1 & FILENAME & Library filename & 30-character maximum \\
\hline 2 & NL & $\begin{array}{l}\text { Number of burnup positions in } \\
\text { library }\end{array}$ & \\
\hline $3^{a}$ & BURN & $\begin{array}{l}\text { Burnup (GWd/MTU) of each } \\
\text { burnup position in library }\end{array}$ & NL entries required \\
\hline 4 & CHARD & Cross-section data to be printed & $\begin{array}{l}\mathrm{a}=\text { absorption } \\
\mathrm{f}=\text { fission } \\
\mathrm{b}=\text { both }\end{array}$ \\
\hline \multirow[t]{2}{*}{5} & CHARL & List entire library (all nuclides) & $\begin{array}{l}\mathrm{y}=\text { yes } \\
\mathrm{n}=\text { no }\end{array}$ \\
\hline & \multicolumn{3}{|c|}{ NOTE: The following optional data are entered only if CHARL $=n$. } \\
\hline 6 & MT & $\begin{array}{l}\text { Number of nuclides for which } \\
\text { cross-section listings are desired }\end{array}$ & \\
\hline $7^{a}$ & MTRD & Nuclide IDs ${ }^{b}$ & MT entries required \\
\hline
\end{tabular}

${ }^{a}$ Input may be continued on subsequent lines as needed.

${ }^{b}$ Nuclide ID $=$ Atomic No. ${ }^{*} 10000+$ Atomic wt $* 10+$ IS, where IS $=0$ for ground state and 1 for metastable state. 


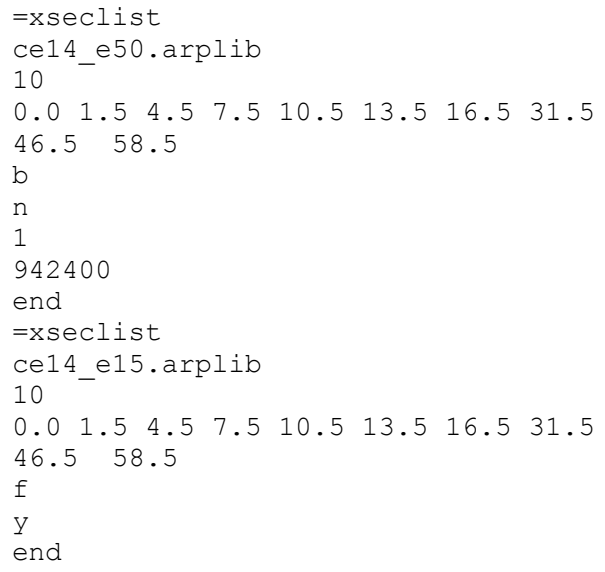

Figure 5.B.4. XSECLIST input example. 


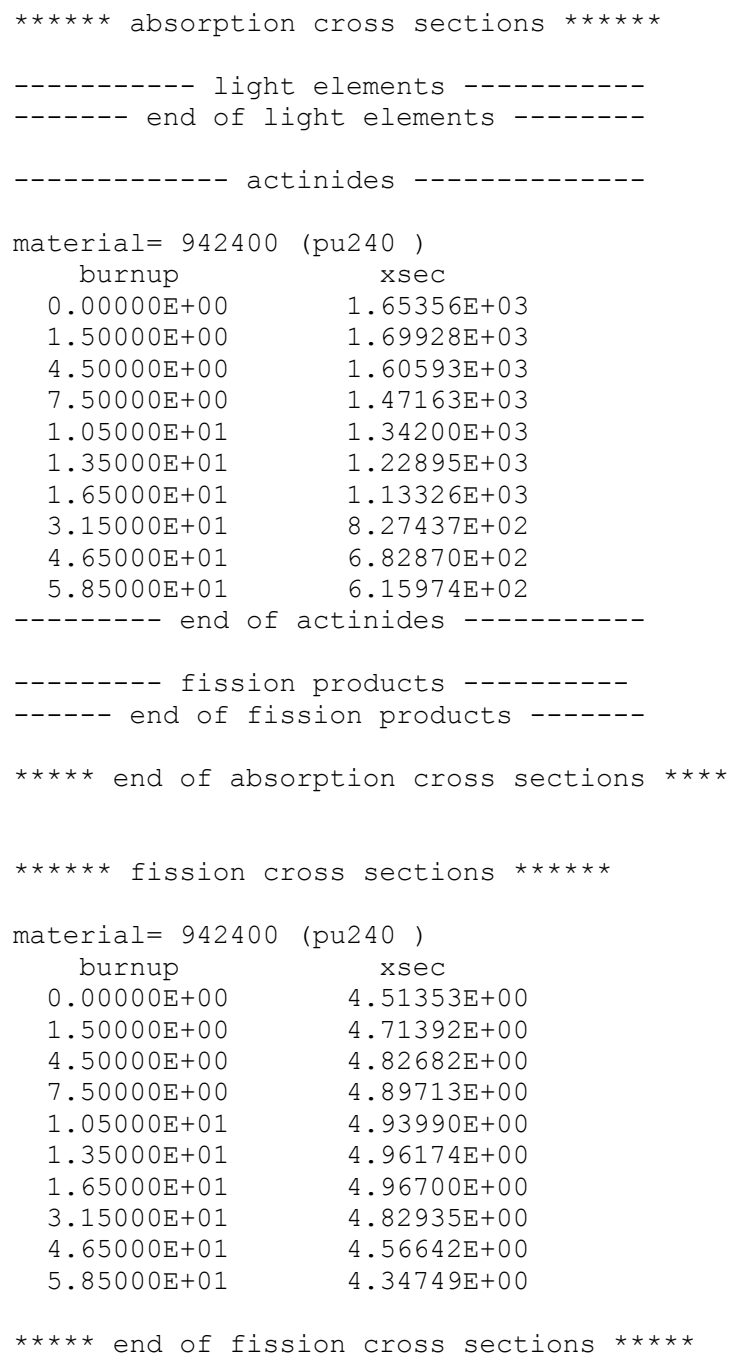

Figure 5.B.5. XSECLIST listing of ${ }^{240} \mathrm{Pu}$ data. 


\subsection{SENSITIVITY AND UNCERTAINTY ANALYSIS}

\section{Introduction by B. T. Rearden}

SCALE provides a suite of computational tools for sensitivity and uncertainty analysis to (1) identify important processes in safety analysis and design, (2) provide a quantifiable basis for neutronics validation for criticality safety and reactor physics analysis based on similarity assessment, and (3) quantify the effects of uncertainties in nuclear data and physical parameters for safety analysis. ${ }^{1,2}$

\section{Sensitivity Analysis and Uncertainty Quantification}

Sensitivity analysis provides a unique insight into system performance in that the predicted response of the system to a change in some input process is quantified. Important processes can be identified as those that cause the largest changes in the response per unit change in the input. In neutron transport numerical simulations, calculating important responses such as $k_{\text {eff, }}$, reaction rates, and reactivity coefficients requires many input parameters, including material compositions, system geometry, temperatures, and neutron cross section data. Because of the complexity of nuclear data and its evaluation process, the response of neutron transport models to the cross section data can provide valuable information to analysts. The SCALE sensitivity and uncertainty ( $\mathrm{S} / \mathrm{U})$ analysis sequences - known as the Tools for Sensitivity and Uncertainty Analysis Methodology Implementation (TSUNAMI) - quantify the predicted change in $k_{\text {eff }}$, reaction rates, or reactivity differences due to changes in the energy-dependent, nuclide-reaction-specific cross section data, whether continuous-energy or multigroup.

Uncertainty quantification is useful for identifying potential sources of computational biases and highlighting parameters important to code validation. When applying uncertainties in the neutron cross section data, the sensitivity of the system to the cross section data can be applied to propagate the uncertainties in the cross section data to an uncertainty in the system response. Additionally, SCALE provides the ability to stochastically sample uncertainties in nuclear data or any other model input parameter (e.g., dimensions, densities, temperatures) and propagate these input uncertainties to uncertainties not only as traditional TSUNAMI responses of $k_{\text {eff }}$, reaction rates, and reactivity, but also in any general output quantity such as burnup isotopics, dose rates, etc. Additionally, where the same input quantities are used in multiple models, the sampling techniques can be applied to quantify the correlation in uncertainties of multiple systems due to the use of the same uncertain parameters across these systems.

\section{Validation of Codes and Data}

Modern neutron transport codes such as the KENO Monte Carlo codes in the SCALE code system can predict $k_{\text {eff }}$ with a high degree of precision. Still, computational biases of one percent or more are often found when using these codes to model critical benchmark experiments. The primary source of this computational bias is believed to be errors in the cross section data as bounded by their uncertainties. These errors can be tabulated in cross section covariance data. To predict or bound the computational bias for a design system of interest, the American National Standards for Nuclear Criticality Safety in Operations with Fissionable Material Outside Reactors (ANSI/ANS-8.1-1998) ${ }^{3}$ and the American National Standard for Validation of Neutron Transport Methods for Nuclear Criticality Safety Calculations (ANSI/ANS-8.24-2007) ${ }^{4}$ allow calculations to be used to determine subcritical limits for the design of fissionable material systems. The standards require validation of the analytical methods and data used in nuclear criticality safety calculations to quantify any computational bias and the uncertainty in the bias. The validation procedure must be conducted through comparison of computed results with experimental data, and the design system for which the subcritical limit is established must fall within the area of applicability of the experiments chosen for validation. The ANS-8.1 standard defines the area(s) of applicability as "the limiting ranges of material compositions, geometric arrangements, neutron-energy 
spectra, and other relevant parameters (e.g., heterogeneity, leakage, interaction, absorption, etc.) within which the bias of a computational method is established."

\section{TSUNAMI Techniques for Code Validation}

The TSUNAMI software provides a unique means to determine the similarity of nuclear criticality experiments to safety applications. ${ }^{5}$ The TSUNAMI validation techniques are based on the assumption that computational biases are primarily caused by errors in cross section data, the potential for which are quantified in cross section covariance data. TSUNAMI provides two methods to establish the computational bias introduced through cross section data.

For the first method, instead of using one or more average physical parameters to characterize a system, TSUNAMI determines the uncertainties in the computed response that are shared between two systems due to cross section uncertainties. These shared uncertainties directly relate to the bias shared by the two systems. To accomplish this, the sensitivity to each group-wise nuclide-reaction-specific cross section is computed for all systems considered in the analysis. Correlation coefficients are developed by propagating the uncertainties in neutron cross section data to uncertainties in the computed response for experiments and safety applications through sensitivity coefficients. The bias in the experiments, as a function of correlated uncertainty with the intended application, is extrapolated to predict the bias and bias uncertainty in the target application. This correlation coefficient extrapolation method is useful where many experiments with uncertainties that are highly correlated to the target application are available.

For the second method, data adjustment or data assimilation techniques are applied to predict computational biases, and more general responses, including but not limited to $k_{\text {eff, }}$ can be addressed simultaneously. ${ }^{5}$ This technique uses $\mathrm{S} / \mathrm{U}$ data to identify a single set of adjustments to nuclear data and experimental responses, taking into account their correlated uncertainties, which would improve the agreement between the response values from the experimental results and computational simulations. The same data adjustments are then used to predict an unbiased response (e.g., $k_{\text {eff }}$ ) value for the application and an uncertainty on the adjusted response value. The difference between the originally calculated response value and the new post-adjustment response value represents the bias in the original calculation, and the uncertainty in the adjusted value represents the uncertainty in this bias. If experiments are available to validate the use of a particular nuclide in the application, the uncertainty of the bias for this nuclide may be reduced. If similar experiments are not available, the uncertainty in the bias for the given nuclide is high. Thus, with a complete set of experiments to validate important components in the application, a precise bias with a small uncertainty can be predicted. Where the experimental coverage is lacking, a bias can be predicted with an appropriately large uncertainty. The data assimilation method presents many advantages over other techniques in that biases can be projected from an agglomeration of benchmark experiments, each of which may represent only a small component of the bias of the target application. Also, contributors to the computational bias can be analyzed on an energy-dependent nuclidereaction-specific basis. However, this technique requires additional data that are not generally available and must be quantified or approximated by the analyst, specifically the correlation coefficients that quantify the relative independence of experimental measurements that use the same equipment, whether nuclear fuel, reactivity devices, or measurement tools.

\section{Sensitivity and Uncertainty Analysis Tools in SCALE}

The TSUNAMI-1D, TSUNAMI-2D and TSUNAMI-3D analysis sequences compute the sensitivity of $k_{\text {eff }}$ and reaction rates to energy-dependent cross section data for each reaction of each nuclide in a system model. The one-dimensional (1D) transport calculations are performed with XSDRNPM, twodimensional (2D) transport calculations are preformed using NEWT, and the three-dimensional (3D) calculations are performed with KENO V.a or KENO-VI. The Monte Carlo capabilities of TSUNAMI-3D provide for $\mathrm{S} / \mathrm{U}$ analysis from either continuous-energy or multigroup neutron transport, where the 
deterministic capabilities of TSUNAMI-1D and TSUNAMI-2D only operate in multigroup mode. SAMS (Sensitivity Analysis Module for SCALE) is applied within each analysis sequence to provide the requested $\mathrm{S} / \mathrm{U}$ data. Whether performing a continuous-energy or multigroup calculation, energydependent sensitivity data are stored in multigroup-binned form in a sensitivity data file (SDF) for subsequent analysis. Additionally, these sequences use the energy-dependent cross section-covariance data to compute the uncertainty in the response value due to the cross section-covariance data. As TSUNAMI-2D operates as an extension of the TRITON sequence, it is documented in the "Reactor Physics" section of this document.

TSAR (Tool for Sensitivity Analysis of Reactivity Responses) computes the sensitivity of the reactivity change between two $k_{\text {eff }}$ calculations, using SDFs from TSUNAMI-1D, TSUNAMI-2D, and/or TSUNAMI-3D. TSAR also computes the uncertainty in the reactivity difference due to the cross section covariance data.

TSUNAMI-IP (TSUNAMI Indices and Parameters) uses the SDFs generated from TSUNAMI-1D, TSUNAMI-2D, TSUNAMI-3D, or TSAR for a series of systems to compute correlation coefficients that determine the amount of shared uncertainty between each target application and each benchmark experiment considered in the analysis. TSUNAMI-IP offers a wide range of options for more detailed assessment of system-to-system similarity. Additionally, TSUNAMI-IP can generate input for the USLSTATS (Upper Subcritical Limit Statistical Software) ${ }^{6}$ trending analysis and compute a penalty, or additional margin, needed for the gap analysis. USLSTATS is distributed as a graphical user interface with SCALE, but its use is documented in the TSUNAMI Primer, ${ }^{7}$ not in this documentation chapter.

TSURFER (Tool for S/U Analysis of Response Functions Using Experimental Results) is a bias and bias uncertainty prediction tool that implements the generalized linear least-squares (GLLS) approach to data assimilation and cross section data adjustment that also uses the SDFs generated from TSUNAMI-1D, TSUNAMI-2D, TSUNAMI-3D, or TSAR. The data adjustments produced by TSURFER are not used to produce adjusted cross section data libraries for subsequent use; rather, they are used only to predict biases in application systems.

The TSUNAMI Primer also documents the use of the graphical user interfaces for TSUNAMI, specifically ExSITE (Extensible SCALE Intelligent Text Editor) that facilitates analysis with TSUNAMIIP, TSURFER, TSAR, and USLSTATS as well as VIBE (Validation, Interpretation and Bias Estimation) for examining SDF files, creating sets of benchmark experiments for subsequent analysis, and gathering additional information about each benchmark experiment.

Sampler is a "super-sequence" that performs general uncertainty analysis by stochastically sampling uncertain parameters that can be applied to any type of SCALE calculation, propagating uncertainties throughout a computational sequence. Sampler treats uncertainties from two sources: (1) nuclear data and (2) input parameters. Sampler generates the uncertainty in any result generated by any computational sequence through stochastic means by repeating numerous passes through the computational sequence, each with a randomly perturbed sample of the requested uncertain quantities. The mean value and uncertainty in each parameter is reported, along with the correlation in uncertain parameters where multiple systems are simultaneously sampled with correlated uncertainties.

Used in conjunction with nuclear data covariances available in SCALE, Sampler is a general technique to obtain uncertainties for many types of applications. SCALE includes covariances for multigroup neutron cross section data, as well as for fission product yields, and radioactive decay data, which allow uncertainty calculations to be performed for most MG computational sequences in SCALE. At the present time, nuclear data sampling cannot be applied to SCALE CE Monte Carlo calculations, although the fundamental approach is still valid.

Used in conjunction with uncertainties in input data, Sampler can determine the uncertainties and correlations in computed results due to uncertainties in dimensions, densities, distributions of material 
compositions, temperatures, or any quantities that are defined in the user input for any SCALE computational sequence. This methodology was developed to produce uncertainties and correlations in criticality safety benchmark experiments, ${ }^{8}$ but it has a wide range of applications in numerous scenarios in nuclear safety analysis and design. The input sampling capabilities of Sampler also include a parametric capability to determine the response of a system to a systematic variation of an input parameter.

\section{References}

1. B. T. Rearden, M. L. Williams, M. A. Jessee, D. E. Mueller, and D. A. Wiarda, "Sensitivity and Uncertainty Analysis Capabilities and Data in SCALE," Nucl. Technol. 174(2), 236-288 (2011).

2. M. L. Williams, G. Ilas, M. A. Jessee, B. T. Rearden, D. Wiarda, W. Zwermann, L. Gallner, M. Klein, B. Krzykacz-Hausmann, and A. Pautz, "A Statistical Sampling Method for Uncertainty Analysis with SCALE and XSUSA," Nucl. Tech. 183, 515-526 (2013).

3. American National Standard for Nuclear Criticality Safety in Operations with Fissionable Materials outside Reactors, ANSI/ANS-8.1-1998, American Nuclear Society (1998).

4. American National Standard for Validation of Neutron Transport Methods for Nuclear Criticality Safety Calculations, ANSI/ANS-8.24-2007, American Nuclear Society (2007).

5. B. L. Broadhead et al., "Sensitivity- and Uncertainty-Based Criticality Safety Validation Techniques," Nucl. Sci. Eng. 146, 340-366 (2004).

6. J. J. Lichtenwalter et al., Criticality Benchmark Guide for Light-Water-Reactor Fuel in Transportation and Storage Packages, NUREG/CR-6361 (ORNL/TM-13211), Oak Ridge National Laboratory (1997).

7. B. T. Rearden, D. E. Mueller, S. M. Bowman, R. D. Busch, and S. J. Emerson, TSUNAMI Primer: A Primer for Sensitivity/Uncertainty Calculations with SCALE, ORNL/TM-2009/027, Oak Ridge National Laboratory (2009).

8. W. J. Marshall and B. T. Rearden, "Determination of Experimental Correlations Using the Sampler Sequence within SCALE 6.2," ICNC 2015, Charlotte, NC (2015). 


\title{
6.1 TSUNAMI-1D: CONTROL MODULE FOR ONE-DIMENSIONAL CROSS-SECTION SENSITIVITY AND UNCERTAINTY
}

\author{
B. T. Rearden, M. A. Jessee, L. M. Petrie, and M. L. Williams
}

\begin{abstract}
TSUNAMI-1D (Tools for Sensitivity and Uncertainty Analysis Methodology Implementation in One Dimension) is a SCALE control module that facilitates the application of sensitivity and uncertainty analysis theory to nuclear systems analyses. TSUNAMI-1D was originally developed to provide sensitivity and uncertainty analysis of $k_{\text {eff }}$ for criticality safety applications, and subsequent updates provide for analysis of system responses other than $k_{\text {eff, }}$ using generalized perturbation theory. TSUNAMI-1D provides for automated processing of material input, processing of cross-section data, calculation of neutron transport solutions, calculation of sensitivity coefficients, and the calculation of uncertainties in system responses due to cross-section-covariance data. The XSDRNPM module is used for the transport solver. XSDRNPM uses the method of discrete ordinates to calculate $k_{\text {eff }}$ for applications that are appropriate for 1D modeling. The SAMS module is used to determine the sensitivities of the calculated value of $k_{\text {eff }}$ and other system responses to the nuclear data used in the calculation as a function of nuclide, reaction type, and energy. The uncertainties in the calculated value of $k_{\text {eff }}$ and other system responses, resulting from uncertainties in the basic nuclear data used in the calculation, are estimated using energy-dependent cross-section-covariance matrices. The implicit effects of the resonance selfshielding calculations are predicted using BONAMIST.
\end{abstract}




\section{TABLE OF CONTENTS}

$\underline{\text { Page }}$

6.1 TSUNAMI-1D: Control Module for One-Dimensional Cross-Section Sensitivity and

Uncertainty.... $6-5$

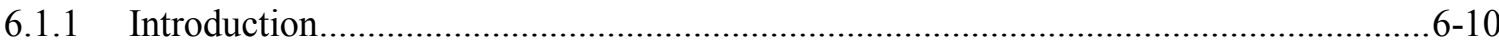

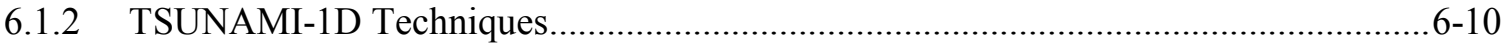

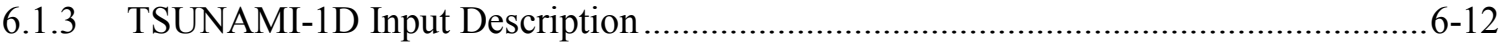

6.1.3.1 Analytical sequence specification record ............................................... 6-13

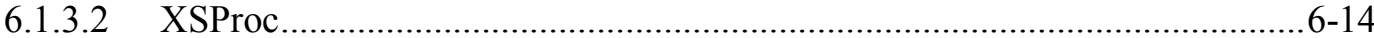

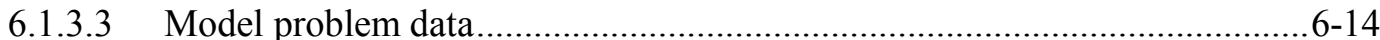

6.1.3.4 Sensitivity and uncertainty calculation data ...........................................6-17

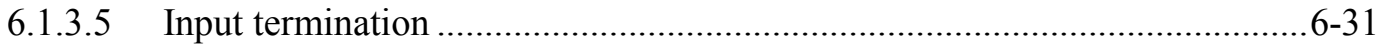

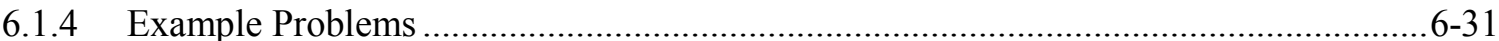

6.1.4.1 INFHOMMEDIUM sample problem .................................................. 6-32

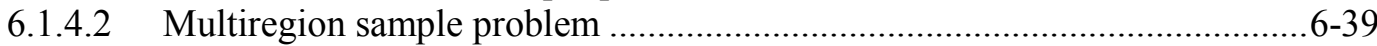

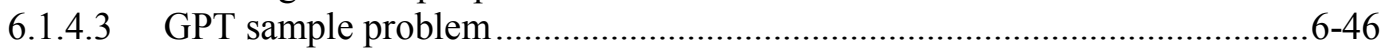

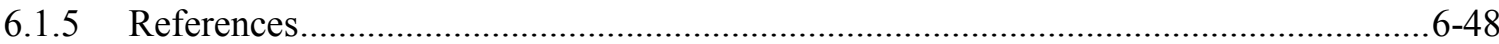

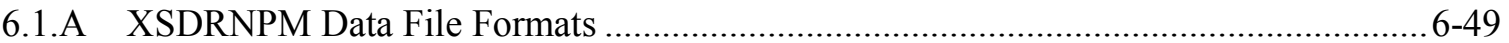

6.1.A.1 XSDRNPM Forward Output File ................................................................ 6-49

6.1.A.2 XSDRNPM Adjoint Output File ….............................................................. 6-50 


\section{LIST OF FIGURES}

$\underline{\text { Page }}$

Figure 6.1.1. General flow diagram of TSUNAMI-1D.

$6-12$

Figure 6.1.2. TSUNAMI-1D INFHOMMEDIUM sample problem input...........................................6-33

Figure 6.1.3. Uncertainty information from INFHOMMEDIUM sample problem...........................6-36

Figure 6.1.4. Energy-dependent sensitivity profiles from TSUNAMI-1D for INFHOMMEDIUM sample problem.

Figure 6.1.5. Comparison of 238U capture sensitivities from TSUNAMI-1D and TSUNAMI-1D with PARM=CENTRM for INFHOMMEDIUM sample problem..................................6-38

Figure 6.1.6. TSUNAMI-1D MULTIREGION sample problem input............................................... 6-40

Figure 6.1.7. Uncertainty information in HTML output from MULTIREGION sample problem.........6-43

Figure 6.1.8. Sensitivity profiles from TSUNAMI-1D for ${ }^{235} U$ fission in zone 1 and zone 2 of MULTIREGION sample problem.

Figure 6.1.9. Sensitivity profiles from TSUNAMI-1D for ${ }^{238} U$ capture in zone 1 and zone 2 of MULTIREGION sample problem.

Figure 6.1.10. Input for TSUNAMI-1D9 sample problem. .6-47

Figure 6.1.11. Response Sensitivities to ${ }^{238} \mathrm{U}$ n, gamma cross section for the TSUNAMI-1D9 sample problem 6-48 


\section{LIST OF TABLES}

$\underline{\text { Page }}$

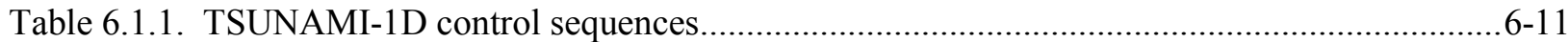

Table 6.1.2. Optional parameter input for the criticality problem data...............................................6-16

Table 6.1.3. Response function keywords in DEFINITIONS block ...................................................6-18

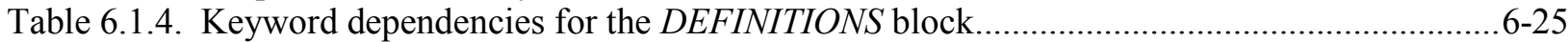

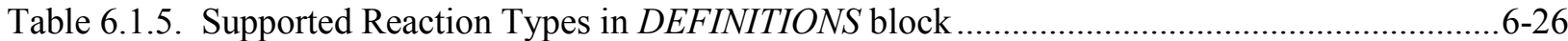

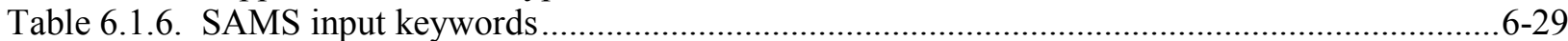

Table 6.1.7. SAMS input keywords for default covariance data ..........................................................6-30

Table 6.1.8. Energy- and region-integrated sensitivity coefficients from TSUNAMI-1D

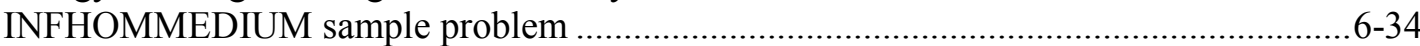

Table 6.1.9. Energy- and region-integrated sensitivity coefficients from TSUNAMI-1D

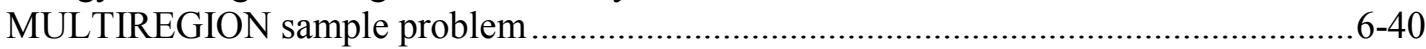




\section{ACKNOWLEDGMENTS}

The authors acknowledge Bryan Broadhead of Oak Ridge National Laboratory, and R. L. Childs, formerly of the Oak Ridge National Laboratory, for their assistance with this work. The support and encouragement of Calvin Hopper, Cecil Parks, and Don Mueller of Oak Ridge National Laboratory is also appreciated. Additionally, the authors wish to acknowledge Debbie Weaver and Sheila Walker for their assistance in preparing this document. 


\subsubsection{Introduction}

TSUNAMI-1D (Tools for Sensitivity and Uncertainty Analysis Methodology Implementation in One Dimension) is a SCALE control module that facilitates the application of sensitivity and uncertainty theory to nuclear system analyses. The data computed with TSUNAMI-1D are the sensitivity of a system response, such as $k_{\text {eff, }}$, to each constituent cross-section data component used in the calculation. The sensitivity data are coupled with cross-section uncertainty data, in the form of multigroup covariance matrices, to produce an uncertainty in the system response due to uncertainties in the underlying nuclear data. The group-wise sensitivity data computed with TSUNAMI-1D are stored in a sensitivity data file (.sdf file) that is suitable for use in assessing system similarity for code validation purposes using TSUNAMI-IP, (see TSUNAMI-IP chapter), and for advanced bias assessment using TSURFER, see the TSURFER chapter.

This manual is intended to provide the user with a detailed reference on code input options and provide some examples of the application of TSUNAMI-1D to generate sensitivity and uncertainty data. A detailed description of code input is provided in Section 6.1.3, three sample problems are given in Section 6.1.4, the techniques used in each computational sequence are described in Section 6.1.2, and additional information is provided in the appendices. A new user may wish to begin by reviewing the sample problems, and then refer to the input details in Section 6.1.3 to customize an input for his specific needs.

TSUNAMI-1D provides automated, problem-dependent cross sections using the same methods and input as the Criticality Safety Analysis Sequences (CSAS). The BONAMIST code computes the sensitivity of resonance self-shielded cross to the input data, the so-called "implicit sensitivities."

After the cross sections are processed, the TSUNAMI-1D sequence performs two XSDRNPM criticality calculations, one forward and one adjoint. Finally, the sequence calls the SAMS module to calculate the sensitivity coefficients that indicate the sensitivity of the calculated values to changes in the cross sections and the uncertainty in the calculated value due to uncertainties in the basic nuclear data. SAMS prints energy-integrated sensitivity coefficients and their statistical uncertainties to the SCALE output file and generates a separate data file containing the energy-dependent sensitivity coefficients.

In addition to the sensitivity and uncertainty analysis sequence, the TSUNAMI-1DC sequence can be used to verify the accuracy of the TSUNAMI-1D calculations with direct perturbation criticality calculations. The verification of computed sensitivity coefficients is imported for systems where the cellweighted material is not the only material used in the model. By default, TSUNAMI-1DC performs the same functions as the TSUNAMI-1D sequence with PARM=CENTRM, except that it does not perform the adjoint XSDRNPM calculation and does not call the SAMS module.

\subsubsection{TSUNAMI-1D Techniques}

TSUNAMI-1D is a SCALE control module. As such, its primary function is to control a sequence of calculations that are performed by other codes. The input for each of the TSUNAMI-1D sequences is very similar to that used for CSAS1, with the addition of the system model description and optional sensitivity calculation data. TSUNAMI-1D uses the same material and cell data input as all other SCALE sequences. The control sequences available in TSUNAMI-1D are summarized in Table 6.1.1, where the functional modules executed by each control sequence are also shown. A general flow diagram of TSUNAMI-1D is shown in Figure 6.1.1. 
Table 6.1.1. TSUNAMI-1D control sequences

\begin{tabular}{|c|c|c|c|c|c|}
\hline $\begin{array}{c}\text { Control } \\
\text { sequence }\end{array}$ & \multicolumn{4}{|c|}{ Functional modules executed by the control module } \\
\hline TSUNAMI-1D & XSProc & $\begin{array}{c}\text { XSDRNPM } \\
\text { forward }\end{array}$ & $\begin{array}{c}\text { XSDRNPM } \\
\text { adjoint* }\end{array}$ & BONAMIST & SAMS* \\
\hline TSUNAMI-1DC & XSProc & $\begin{array}{c}\text { XSDRNPM } \\
\text { forward }\end{array}$ & & \\
\hline *The XSDRNPM adjoint calculation and SAMS calculation are repeated for each system response defined by the user. \\
\hline
\end{tabular}

TSUNAMI-1D and many other SCALE sequences apply a standardized procedure to provide appropriate cross sections for the calculation. This procedure is carried out by routines of the XSProc that generate number densities and related information, prepare geometry data for resonance self-shielding and fluxweighting cell calculations, and create data input files for the cross-section processing codes.

By default, the TSUNAMI-1D sequence performs cross-section processing with XSProc, exercising all available options there, performs the forward and adjoint XSDRNPM calculations, calls BONAMIST to produce implicit sensitivity coefficients, then calls SAMS to produce sensitivity and uncertainty output and $s d f$ files. Optional sequence level parameters can be used to change methods applied in resonance self-shielding and exclude the implicit sensitivity calculation, which detailed later in this document. If additional system responses are requested in the input, TSUNAMI-1D executes additional generalized adjoint XSDRNPM and SAMS calculations for each system response.

The input requirements for the model description are very similar to those used for multiregion cell descriptions in the cell data section of input. The definition of system responses other than $k_{\text {eff }}$ requires both the DEFINITIONS and SYSTEMRESPONSE block of input data. These blocks of data are described in Section 6.1.3.4. TSUNAMI-1D also reads and prepares inputs for the SAMS calculation. The additional input blocks for the SAMS module are optional. The input format of the SAMS blocks of data are described in the SAMS chapter. 


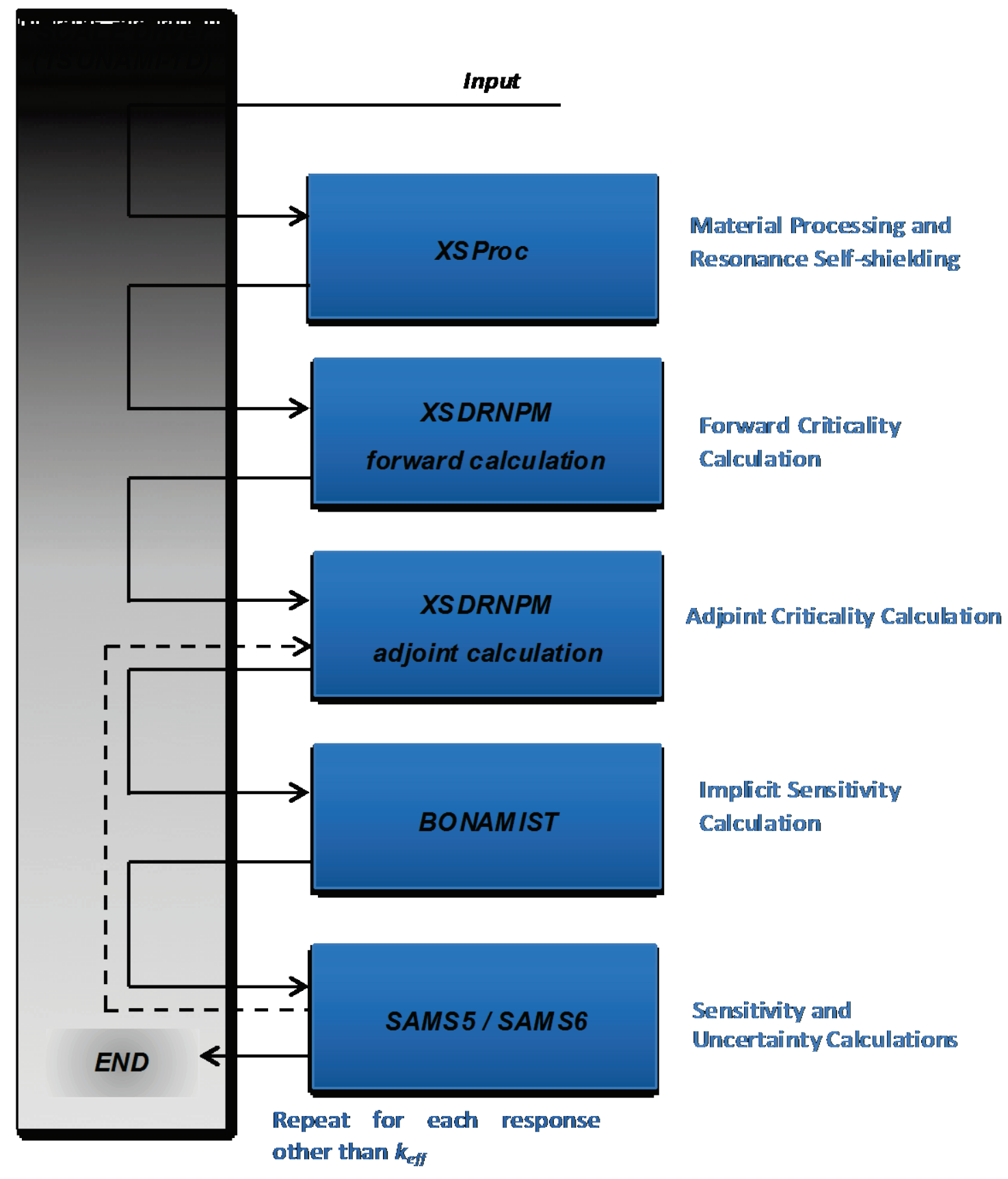

Figure 6.1.1. General flow diagram of TSUNAMI-1D.

\subsubsection{TSUNAMI-1D Input Description}

The input to TSUNAMI-1D consists of a SCALE Analytical Sequence Specification Record, SCALE XSProc data, model problem data, optional sensitivity and uncertainty calculation data, and optional 
system response characterization data. The data for each of these segments are entered using the SCALE free-form format, allowing alphanumeric data, floating-point data, and integer data to be entered in an unstructured manner. The input is not case sensitive, so either upper- or lowercase letters may be used. A maximum of 252 columns per line may be used for input. Data can usually start or end in any column with a few exceptions. As an example, the word END beginning in column 1 and followed by two blank spaces will end the problem, any data following will be ignored. Each data entry must be followed by one or more blanks to terminate the data entry. For numeric data, either a comma or a blank can be used to terminate each data entry. Integers may be entered for floating values. For example, 10 will be interpreted as 10.0. Imbedded blanks are not allowed within a data entry unless an E precedes a single blank as in an unsigned exponent in a floating-point number. For example, 1.0E 4 would be correctly interpreted as $1.0 \times 10^{4}$. A comment is initiated with a single quote, ', and continues until the end of the input line.

\subsubsection{Analytical sequence specification record}

The analytical sequence specification begins in column 1 of the first line of the input file and must contain one of the following:

$=$ TSUNAMI-1D This sequence is used for sensitivity and uncertainty calculations.

$=$ TSUNAMI-1DC This sequence allows more flexibility than CSAS1 and is used for criticality calculations where the criticality problem description contains more detail than that specified in a single unit cell description.

Optional keyword input may be entered, starting after column 10 of the analytical sequence specification record. These keywords are

PARM=CHECK This option allows the input data to be read and checked without executing any $\mathrm{PARM}=\mathrm{CHK}$ functional modules.

$\mathrm{PARM}=\mathrm{SIZE}=\mathrm{n} \quad$ The amount of memory requested in four-byte words may be set with this entry. The default value for $\mathrm{n}$ is 20000000 . This value only affects calculations in BONAMIST, where this value of the SIZE parameter is used for allocation of storage for the derivatives. Please see the documentation on BONAMIST in the Sensitivity Utility Modules chapter for more details. All other codes use dynamic memory allocation and this value has no effect.

PARM=BONAMIST This is the default configuration for MG TSUNAMI-1D calculations. XSProc with BONAMI and CENTRM is used for cross-section processing, and implicit sensitivities are produced with BONAMIST. 
PARM $=$ CENTRM XSProc with BONAMI and CENTRM is used for cross-section processing, but BONAMIST is not run. TSUNAMI-1D sequence with PARM=CENTRM does not produce the implicit portions of the sensitivity coefficients, and should be used with caution.

PARM=BONAMI XSProc with BONAMI is used for cross-section processing, but BONAMIST is not run. TSUNAMI-1D sequence with PARM=BONAMI does not produce the implicit portions of the sensitivity coefficients, and should be used with caution.

PARM=2REGION XSProc with BONAMI and CENTRM are run where Dancoff factors are to compute the escape probabilities for an accelerated, yet more approximate, CENTRM calculation. Implicit sensitivities are computed with BONAMIST.

Multiple parameters can be used simultaneously by enclosing them in parentheses and separating them with commas such as $\mathrm{PARM}=(\mathrm{SIZE}=2000000, \mathrm{CHECK})$.

Multiple parameters can be used simultaneously by enclosing them in parentheses and separating them with commas such as $\mathrm{PARM}=(\mathrm{SIZE}=2000000$, $\mathrm{CHECK})$.

\subsubsection{XSProc}

XSProc reads the standard composition specification data and the unit cell geometry specifications. It then produces the mixing table and unit cell information necessary for processing the cross sections. The XSProc chapter provides a detailed description of the input data for the Material Information Processor.

\subsubsection{Model problem data}

The model problem data are used by the TSUNAMI-1D sequences to prepare input for the XSDRNPM transport calculation. This input section consists of two data blocks, one block contains a geometry description and one contains optional parameters.

\subsection{Geometry data}

The TSUNAMI-1D geometry data block begins with the keywords READ GEOM and ends with the keywords END GEOM. This data block is always required. The following data is contained within this data block:

1. A line containing the geometry and boundary conditions for the XSDRNPM criticality case. The first entry on this line describes the geometry and must be SLAB, CYLINDRICAL, or SPHERICAL. The second entry is optional and describes the right-boundary condition. The default value for the right-boundary condition is VACUUM. The third entry on this line is optional and describes the left-boundary condition. The default value for the left boundary condition is REFLECTED. The last entry on this line is END. Valid entries for the boundary conditions are the following:

\begin{tabular}{|c|c|}
\hline VACUUM & No return at boundary \\
\hline REFLECTED & - Specular (mirror-like) return at boundary \\
\hline PERIODIC & Infinite array of cells in slab geometry \\
\hline WHITE & - Isotropic return at boundary \\
\hline
\end{tabular}


2. A line containing the following two entries for each zone of the XSDRNPM case:

a. mixture number in the zone and

b. zone outer dimension (in $\mathrm{cm}$ ).

Mixture numbers and zone dimensions are entered in pairs until the entire geometry is defined. The mixture numbers must be defined in the material input processor input. Mixture 0 is used for voids, and a mixture number defined with CELLMIX = in the MIP section of the input may be used here. It should be noted that, due to a restriction in XSDRNPM, the mixture number identified with CELLMIX= may not appear in the output file, even though it is input in this section. TSUNAMI-1D automatically renumbers the cell mixed mixture to the next available mixture number for use in XSDRNPM. A message is printed in the output identifying this change. TSUNAMI-1D uses the same techniques as CSAS1X to automatically prepare a spatial mesh appropriate for the input materials and dimensions.

\subsection{Parameter data}

An optional data block may be entered to change parameters of the XSDRNPM forward and adjoint calculations. This data block begins with the keywords READ PARA or READ PARM and must end with either END PARA or END PARM, corresponding to the read keyword. In this data block, the user may enter optional lines that contain entries for selected XSDRNPM input parameters. A list of the parameters and their default values are found in Table 6.1.2. 
Table 6.1.2. Optional parameter input for the criticality problem data

\begin{tabular}{|c|c|c|}
\hline Name & Default & Meaning \\
\hline $\mathrm{ISN}=$ & 16 & Order of angular quadrature \\
\hline $\mathrm{IIM}=$ & 20 & Inner-iteration maximum \\
\hline $\mathrm{ICM}=$ & 100 & Outer-iteration maximum \\
\hline \multirow[t]{4}{*}{$\mathrm{ID} 1=$} & -1 & Flux-edit option: \\
\hline & & -1 no flux print \\
\hline & & $0 \quad$ scalar flux print \\
\hline & & 1 scalar and angular flux print \\
\hline $\mathrm{SCT}=$ & 5 & Order of Legendre expansion for cross sections \\
\hline \multirow[t]{4}{*}{$\mathrm{PRT}=$} & -2 & Cross-section print option: \\
\hline & & -2 no cross-section print \\
\hline & & -1 print $1-\mathrm{D}$ cross sections \\
\hline & & $0 / \mathrm{N}$ print 2 -D cross sections through order $\mathrm{N}$ \\
\hline \multirow[t]{3}{*}{$\mathrm{PBT}=$} & 0 & Balance table print option: \\
\hline & & -1 no balance table print \\
\hline & & 0 fine group balance table print \\
\hline $\mathrm{EPS}=$ & $1 . \mathrm{E}-6$ & Outer-iteration convergence criteria \\
\hline $\mathrm{PTC}=$ & $1 . \mathrm{E}-6$ & Inner-iteration convergence criteria \\
\hline $\mathrm{DY}=$ & 0 & $\begin{array}{l}\text { First-transverse dimension }(\mathrm{cm}) \text { for buckling correction } \\
\text { (i.e., height of cylinder or slab) }\end{array}$ \\
\hline $\mathrm{DZ}=$ & 0 & $\begin{array}{l}\text { Second-transverse dimension }(\mathrm{cm}) \text { for buckling correction } \\
\text { (i.e., depth of slab) }\end{array}$ \\
\hline $\mathrm{SZF}=$ & 1.5 & $\begin{array}{l}\text { Size factor of spatial computational mesh intervals. Increasing } \\
\text { this number will cause the forward and adjoint XSDRNPM } \\
\text { calculations to be conducted with larger mesh intervals and fewer } \\
\text { mesh points. } 0.0<\mathrm{SZF}<1.5 \text { gives a finer mesh, } \mathrm{SZF}>1.5 \text { gives a } \\
\text { coarser mesh. }\end{array}$ \\
\hline
\end{tabular}




\subsubsection{Sensitivity and uncertainty calculation data}

The data blocks for controlling the sensitivity and uncertainty calculation are optional. The optional data blocks include the SAMS block, the HTML block, the COVARIANCE block, the DEFINITIONS block, and the SYSTEMRESPONSES block. These data blocks begin with the keywords READ BLOCKNAME and end with the keywords END BLOCKNAME, where BLOCKNAME is one of SAMS, HTML, COVARIANCE, DEFINITONS, or SYSTEMRESPONSES. These data blocks can be input in any order with the following two exceptions. First, all five data blocks must appear in the input file after the composition and cell data blocks of data. Second, if a SAMS block is specified, the $H T M L$ and COVARIANCE data blocks must come after the SAMS block, if they are to be specified. In addition, both the DEFINITONS and SYSTEMRESPONSES data blocks must be present for additional analysis of system responses other than $k_{\text {eff. }}$. If only one or both of the data blocks are omitted, then analysis is only performed for $k_{\text {eff. }}$ The following sub-sections describe these blocks of data in detail.

\subsection{Response definition data}

The DEFINITIONS and SYSTEMRESPONSES blocks are used to define system responses for additional sensitivities and uncertainty analysis in SAMS. For criticality calculations, the sensitivities of system responses other than $k_{\text {eff }}$ are calculated in TSUNAMI-1D using generalized perturbation theory (GPT). The details of the GPT methodology are provided in General Perturbation Theory section of the SAMS chapter. Using GPT, a system response, denoted $\mathrm{R}$, is defined as a ratio such as:

$$
R=\frac{\sum_{g} \int d \bar{r} H_{N, g}(\bar{r}) \phi_{g}(\bar{r})}{\sum_{g} \int d \bar{r} H_{D, g}(\bar{r}) \phi_{g}(\bar{r})}
$$

In this equation, $\phi_{g}(\bar{r})$ is the space-dependent multi-group scalar flux and $H_{N, g}(\bar{r}), H_{D, g}(\bar{r})$ are referred to as the space-dependent, multi-group response functions. In TSUNAMI-1D, the response functions are specified in the DEFINITIONS data block and the system responses are defined in the SYSTEMRESPONSES data block. Responses (other $k_{\text {eff) }}$ treated in TSUNAMI-1D MUST be ratios.

The DEFINITIONS data block is used by TSUNAMI-1D similarly to that of the MAVRIC and MONACO modules in SCALE. The format of the DEFINITIONS block is as follows:

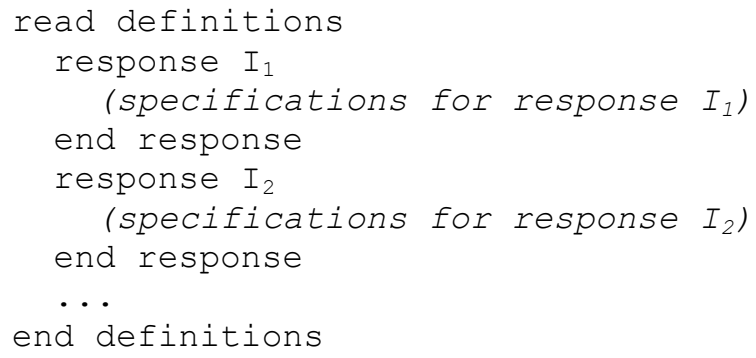

The DEFINITIONS block of data begins with READ DEFINITIONS and terminates with END DEFINITIONS. Likewise, each response function definition begins with RESPONSE - followed by a unique, positive integer identifier - and terminates with END RESPONSE. The keyword entries summarized in Table 6.1.3 are allowed for each response specification. Keywords ending with '=' must be followed by the value to be assigned to the corresponding variable. All keywords are optional and can 
be entered in any order. However certain keywords are required depending one of the seven basic types of response functions described in the following subsections. The required keywords are summarized for each of the seven basic response function types in Table 6.1.4 at the end of this section.

Table 6.1.3. Response function keywords in DEFINITIONS block

\begin{tabular}{|c|c|c|c|}
\hline Keyword & Type & $\begin{array}{c}\text { Default } \\
\text { value }\end{array}$ & Description \\
\hline title $=$ & String & “" “ & $\begin{array}{l}\text { Response function title. The title must begin and end with } \\
\text { quotes and have a maximum of } 256 \text { characters. }\end{array}$ \\
\hline macro & Logical & $\mathrm{F}$ & $\begin{array}{l}\text { Macroscopic cross-section flag. If specified, macroscopic } \\
\text { cross-sections are used to define the response function. }\end{array}$ \\
\hline micro & Logical & $\mathrm{T}$ & $\begin{array}{l}\text { Microscopic cross-section flag. If specified, microscopic } \\
\text { cross-sections are used to define the response function. }\end{array}$ \\
\hline $\begin{array}{l}\text { nuclide }= \\
\text { or } \\
\text { zaid }=\end{array}$ & $\begin{array}{l}\text { Integer } \\
\text { or string }\end{array}$ & Undefined & $\begin{array}{l}\text { Nuclide identifier for which cross-sections are used to } \\
\text { define the response function. The nuclide can be specified } \\
\text { in integer format (92235) or in character string format (u- } \\
\text { 235). }\end{array}$ \\
\hline $\begin{array}{c}\text { reaction }= \\
\text { or } \\
\mathrm{mt}=\end{array}$ & $\begin{array}{l}\text { Integer } \\
\text { or string }\end{array}$ & Undefined & $\begin{array}{l}\text { Reaction identifier for which cross-sections are used to } \\
\text { define the response function. The reaction can be specified } \\
\text { as an MT number (18) or as a character string (fission). } \\
\text { Supported reaction types are listed below. }\end{array}$ \\
\hline $\begin{array}{l}\text { material }= \\
\text { or } \\
\text { mixture }=\end{array}$ & Integer & Undefined & $\begin{array}{l}\text { Mixture identifier for which cross-sections are used to } \\
\text { define the response function. }\end{array}$ \\
\hline $\begin{array}{l}\text { multimix ... end } \\
\text { or } \\
\text { multimat } \ldots \text { end }\end{array}$ & $\begin{array}{l}\text { Integer } \\
\text { array }\end{array}$ & Undefined & $\begin{array}{l}\text { Array of mixture identifiers for which cross-sections are } \\
\text { used to define the response function. }\end{array}$ \\
\hline unity & Logical & $\mathrm{F}$ & $\begin{array}{l}\text { Flux response function flag. If specified, cross-sections are } \\
\text { not used to define the response function. }\end{array}$ \\
\hline multiplier & Real & 1.0 & Response function multiplier. \\
\hline ehigh $=$ & Real & $10^{25}$ & Upper energy $(\mathrm{eV})$ boundary of the response function. \\
\hline elow $=$ & Real & 0.0 & Lower energy $(\mathrm{eV})$ boundary of the response function. \\
\hline ehightransfer $=$ & Real & $10^{25}$ & $\begin{array}{l}\text { Upper energy }(\mathrm{eV}) \text { boundary used for cross-sections with } \\
\text { secondary particle distributions (elastic, inelastic, scatter, } \\
\text { and } n, 2 n) \text {. }\end{array}$ \\
\hline elowtransfer $=$ & Real & 0.0 & $\begin{array}{l}\text { Lower energy }(\mathrm{eV}) \text { boundary used for cross-sections with } \\
\text { secondary particle distributions (elastic, inelastic, scatter, } \\
\text { and } n, 2 n) \text {. }\end{array}$ \\
\hline
\end{tabular}




\subsection{Single-mixture flux response function}

A single-mixture flux response is simply the integration of the neutron flux wherever a specified mixture is defined in the problem geometry. Therefore, the response function $H_{g}(\bar{r})$ for a single mixture-j is defined as:

$$
H_{g}(\bar{r})=c^{*} \delta_{g} * \delta_{j}(\bar{r})
$$

where

$$
\begin{gathered}
\delta_{g}=\left\{\begin{array}{cc}
1.0 & E_{\text {High }}>E_{g}^{\text {Lower }}, E_{\text {Low }}<E_{g}^{\text {Upper }} \\
0 & \text { otherwise }
\end{array}\right. \\
\text { and } \\
\delta_{j}(\bar{r})=\left\{\begin{array}{cc}
1.0 & \text { mixture jis used at } \bar{r} \\
0 & \text { otherwise }
\end{array}\right.
\end{gathered}
$$

In this expression, the constant $c$ is the scalar multiplier defined by the multiplier $=$ keyword. For example, the "fast" and "thermal" flux responses for mixture 5 would be:

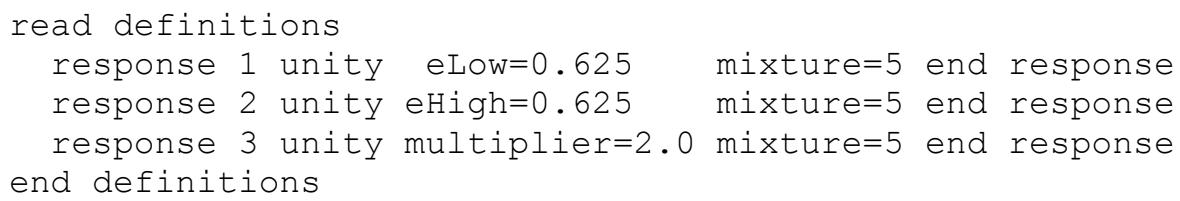

In this example, the energy cutoff between the fast group and the thermal group is defined as $0.625 \mathrm{eV}$. Response 3 reflects the total energy-integrated flux - scaled by a factor of 2.0 - because the default values of eHigh and eLow are used.

For single-mixture flux responses, keywords unity and mixture are required; multiplier, eHigh, and eLow are optional; title, nuclide, reaction, micro, macro, eHighTransfer, and eLowTransfer are optional but are not used; and multimix is not allowed. These keyword dependencies are outlined for each response type in Table 6.1.4.

\subsection{Multiple-mixture flux response}

A multiple-mixture flux response is the integration of the neutron flux wherever a set of mixtures are defined in the problem geometry. Therefore, the response function $H_{g}(\bar{r})$ for a set of mixtures " $S$ " is defined as:

$$
H_{g}(\bar{r})=c^{*} \delta_{g} * \sum_{j \in S} \delta_{j}(\bar{r})
$$


For example, the following definition is for the energy-integrated flux response, and spatially-integrated wherever mixtures 5, 7, and 9 are used:

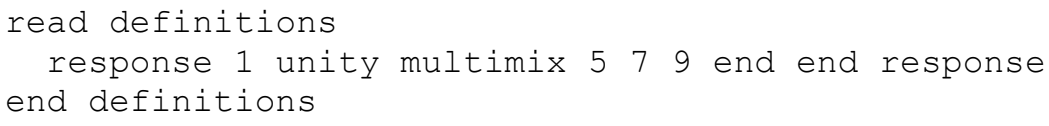

or alternatively,

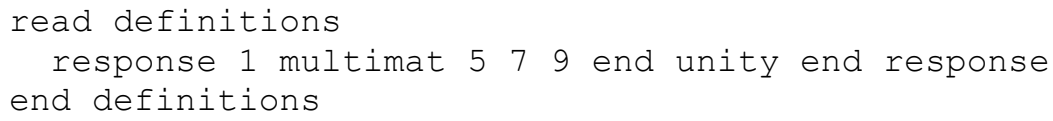

For multiple-mixture flux responses, keywords unity and multimix are required; multiplier, eHigh, and eLow are optional; title, nuclide, reaction, micro, macro, eHighTransfer, and eLowTransfer are optional but are not used; and mixture is not allowed.

\subsection{Single-mixture, single-nuclide, microscopic cross-section response}

A single-mixture, single-nuclide, microscopic cross-section response is the integration of the neutron flux multiplied by a microscopic cross-section. The microscopic cross-section used in the integral is defined by a specific mixture, nuclide, and reaction type. Therefore, the response function $H_{g}(\bar{r})$ is defined as:

$$
H_{g}(\bar{r})=c^{*} \delta_{g} * \delta_{j}(\bar{r}) * \sigma_{x, g}^{j, n}
$$

In this expression, $\sigma_{x, g}^{j, n}$ is the microscopic cross-section for mixture- $j$, nuclide- $n$, reaction type- $x$, and energy group- $g$. For transfer reaction types - scatter, elastic, inelastic, and $n, 2 n$ - the expression above is slightly modified so the user can define the energy range of the secondary particles, i.e.,

$$
H_{g}(\bar{r})=c^{*} \delta_{g} * \delta_{j}(\bar{r})^{*} \sum_{g^{\prime}} \delta_{g^{\prime}}^{\text {Transfer }} \sigma_{x, g \rightarrow g^{\prime}}^{j, n}
$$

where

$$
\delta_{g}^{\text {Transfer }}=\left\{\begin{array}{cc}
1.0 & E_{\text {HighTransfer }}>E_{g}^{\text {Lower }}, E_{\text {LowTransfer }}<E_{g}^{\text {Upper }} \\
0 & \text { otherwise }
\end{array}\right.
$$

Likewise, the expression for $H_{g}(\bar{r})$ is also modified for fission distribution responses (chi), which are usually integrated by the energy-integrated neutron production rate rather than the neutron flux:

$$
H_{g}(\bar{r})=c^{*} \delta_{j}(\bar{r}) * \bar{v}_{f, g}^{j, n} * \sigma_{f, g}^{j, n} * \sum_{g^{\prime}} \delta_{g^{\prime}} * \chi_{g^{\prime}}^{j, n}
$$

For examples of this response type, the following DEFINITION block has response definitions for

- total nu-fission rate of U-235 in mixture 1, 
- "fast" n,gamma capture rate of U-238 in mixture 1 (energy cutoff is $0.625 \mathrm{eV}$ ),

- downscatter rate of H-1 in mixture 2, and

- number fission neutrons born from Pu-239 fissions in mixture 1 in the intermediate energy range $(\mathrm{E}>0.625 \mathrm{eV}$ and $\mathrm{E}<25 \mathrm{keV})$

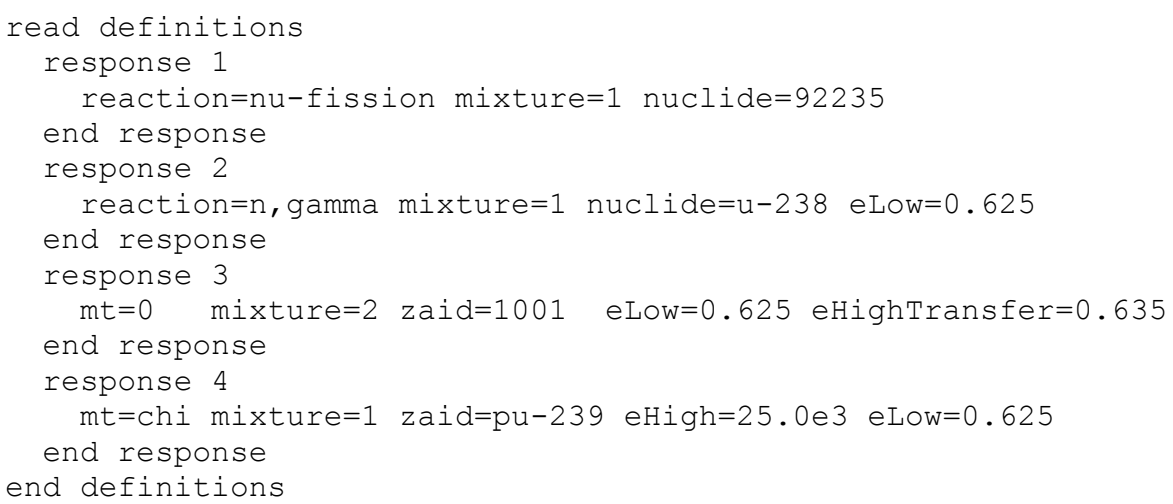

For single-mixture, single-nuclide microscopic cross-section responses, keywords mixture, nuclide, and reaction are required; multiplier, eHigh, eLow, eHighTransfer, eLowTransfer, and micro, are optional; title is optional but not used; and multimix, macro, and unity are not allowed. A list of supported crosssection reaction types is provided at the end of this section in Table 6.1.5.

\subsection{Single-mixture, single-nuclide, macroscopic cross-section response}

A single-mixture, single-nuclide, macroscopic cross-section response is the integration of the neutron flux multiplied by a macroscopic cross-section. The macroscopic cross-section used in the integral is defined by a specific mixture, nuclide, and reaction type. The response function $H_{g}(\bar{r})$ is defined as:

$$
H_{g}(\bar{r})=c * \delta_{g} * \delta_{j}(\bar{r}) * \Sigma_{x, g}^{j, n}
$$

In this expression, $\Sigma_{x, g}^{j, n}$ is the macroscopic cross-section $\left(N^{j, n} * \sigma_{x, g}^{j, n}\right)$ for mixture- $j$, nuclide- $n$, reaction type- $x$, and energy group- $g$. The modifications to this expression for transfer reactions and chi are similar to that of single-mixture, single-nuclide, microscopic cross-section responses. Using the same example as above, the single-mixture, single-nuclide, macroscopic cross-section responses are given as:

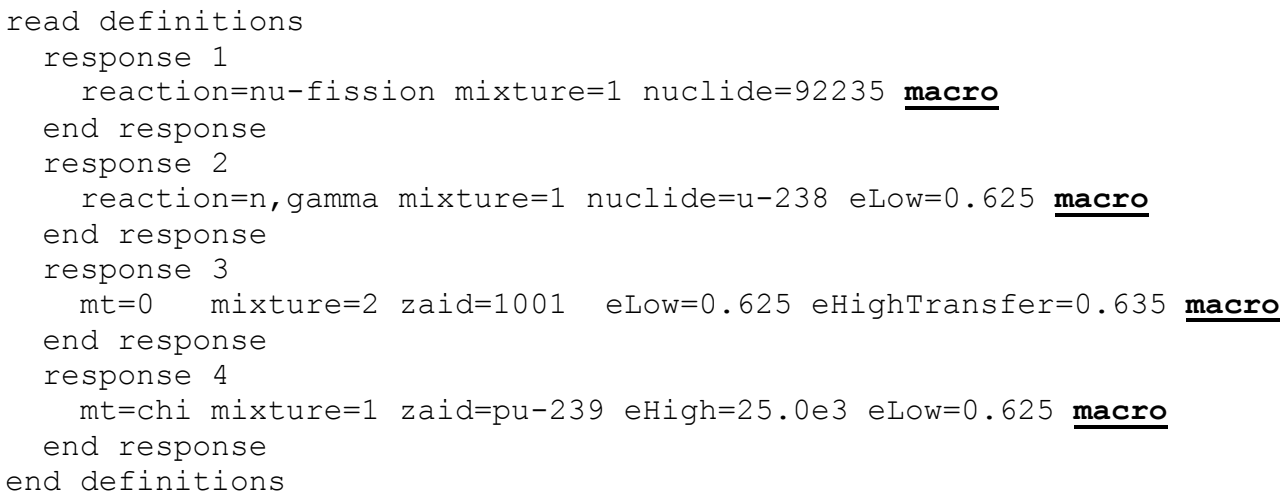


For single-mixture, single-nuclide macroscopic cross-section responses, keywords mixture, nuclide, macro, and reaction are required; multiplier, eHigh, eLow, eHighTransfer, and eLowTransfer, are optional; title is optional but not used; and multimix, micro, and unity are not allowed.

\subsection{Single-mixture, multiple-nuclide, macroscopic cross-section response}

A single-mixture, multiple-nuclide, macroscopic cross-section response is the integration of the neutron flux multiplied by a macroscopic cross-section. The macroscopic cross-section used in the integral is defined by a specific mixture, and reaction type. The response function $H_{g}(\bar{r})$ is defined as:

$$
H_{g}(\bar{r})=c^{*} \delta_{g} * \delta_{j}(\bar{r}) * \Sigma_{x, g}^{j}
$$

In this expression, $\Sigma_{x, g}^{j}$ is the mixture macroscopic cross-section defined as $\sum_{n} N^{j, n} * \sigma_{x, g}^{j, n}$ for mixture-j, reaction type- $x$, and energy group- $g$. The modifications to this expression for transfer reactions is similar to that defined in previous subsections. For mixture chi responses, $H_{g}(\bar{r})$ is rewritten as

$$
H_{g}(\bar{r})=c^{*} \delta_{j}(\bar{r}) * \sum_{n} \bar{\nu}_{f, g}^{j, n} * \sum_{f, g}^{j, n} * \sum_{g^{\prime}} \delta_{g^{\prime}} * \chi_{g^{\prime}}^{j, n}
$$

For examples of this response type, the following DEFINITIONS block has response definitions for

- total nu-fission rate in mixture 1 ,

- "fast" n,gamma capture rate in mixture 1 (energy cutoff is $0.625 \mathrm{eV}$ ),

- downscatter rate in mixture 2 , and

- number fission neutrons born in mixture 1 in the intermediate energy range $(\mathrm{E}>0.625 \mathrm{eV}$ and $\mathrm{E}<25 \mathrm{keV}$ )

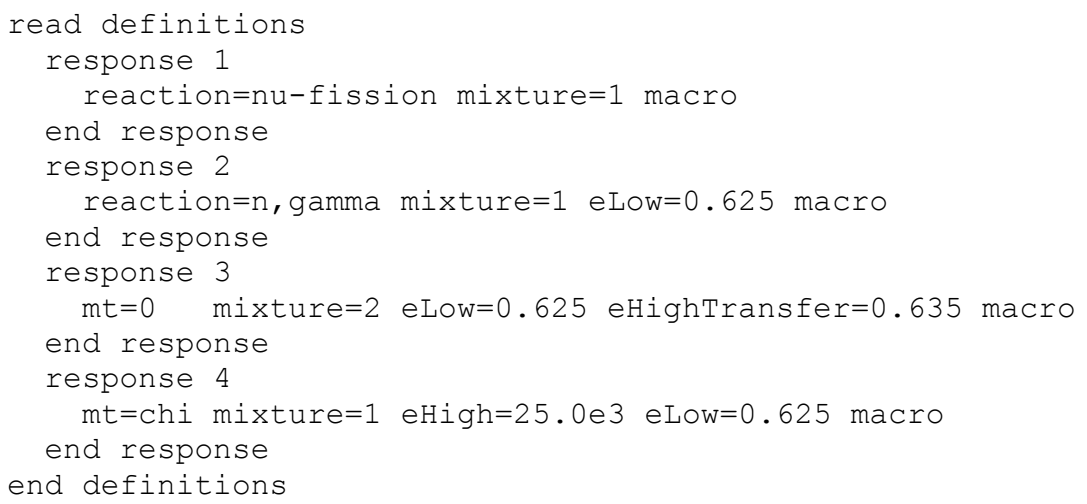

For single-mixture, multiple-nuclide macroscopic cross-section responses, keywords mixture, macro, and reaction are required; multiplier, eHigh, eLow, eHighTransfer, and eLowTransfer, are optional; title is optional but not used; and multimix, micro, nuclide, and unity are not allowed. 


\subsection{Multiple-mixture, single-nuclide, macroscopic cross-section response}

A multiple-mixture, single-nuclide, macroscopic cross-section response is the integration of the neutron flux multiplied by a macroscopic cross-section over a set of mixtures defined in the problem geometry. The macroscopic cross-section used in the integral is defined by a specific mixture, nuclide, and reaction type. The response function $H_{g}(\bar{r})$ is defined as:

$$
H_{g}(\bar{r})=c^{*} \delta_{g} * \sum_{j \in S} \delta_{j}(\bar{r}) * \sum_{x, g}^{j, n}
$$

In this expression, $\Sigma_{x, g}^{j, n}$ is the macroscopic cross-section $\left(N^{j, n} * \sigma_{x, g}^{j, n}\right)$ for mixture-j, nuclide- $n$, reaction type- $x$, and energy group- $g$. The summation of mixtures in this expression is for a set of user-defined mixtures, denoted $S$. The modifications to this expression for transfer reactions and chi are applied similarly to previously defined response types above.

For examples of this response type, the following DEFINITIONS block has response definitions for

- total nu-fission rate of U-235 in the fuel mixtures (mixtures $1,3,5$ )

- "fast" n,gamma capture rate of U-238 in the fuel mixtures

- downscatter rate of H-1 in the moderator mixtures (mixtures 2,4)

- number fission neutrons born in the intermediate energy range $(\mathrm{E}>0.625 \mathrm{eV}$ and $\mathrm{E}<25 \mathrm{keV}$ ) in $\mathrm{Pu}-239$ in the fuel mixtures

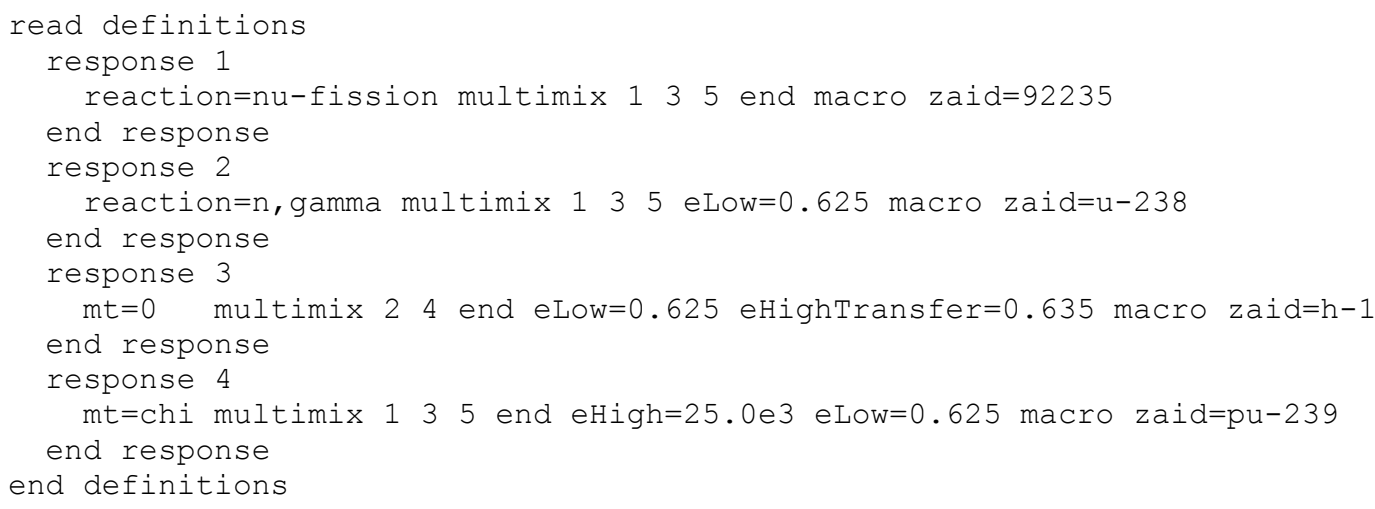

For multiple-mixture, single-nuclide macroscopic cross-section responses, keywords multimix, nuclide, macro, and reaction are required; multiplier, eHigh, eLow, eHighTransfer, and eLowTransfer, are optional; title is optional but not used; and mixture, micro, and unity are not allowed.

\subsection{Multiple-mixture, multiple-nuclide, macroscopic cross-section response}

A multiple-mixture, multiple-nuclide, macroscopic cross-section response is the integration of the neutron flux multiplied by a macroscopic cross-section over a set of mixtures defined in the problem geometry. The macroscopic cross-section used in the integral is defined by a specific mixture, and reaction type. The response function $H_{g}(\bar{r})$ is defined as: 


$$
H_{g}(\bar{r})=c^{*} \delta_{g} * \sum_{j \in S} \delta_{j}(\bar{r})^{*} \sum_{x, g}^{j}
$$

In this expression, $\Sigma_{x, g}^{j}$ is the mixture macroscopic cross-section for mixture- $j$ and reaction type- $x$, and energy group-g. The summation of mixtures in this expression is for a set of user-defined mixtures, denoted $S$. The modifications to this expression for transfer reactions and chi are applied similarly to the previously defined response types above.

For examples of this response type, the following DEFINITIONS block has response definitions for

- total nu-fission rate in the fuel mixtures (mixtures $1,3,5$ )

- "fast" n,gamma capture rate in the fuel mixtures

- downscatter rate in the moderator mixtures (mixtures 2,4)

- number fission neutrons born in the intermediate energy range $(\mathrm{E}>0.625 \mathrm{eV}$ and $\mathrm{E}<25$ $\mathrm{keV}$ ) in the fuel mixtures

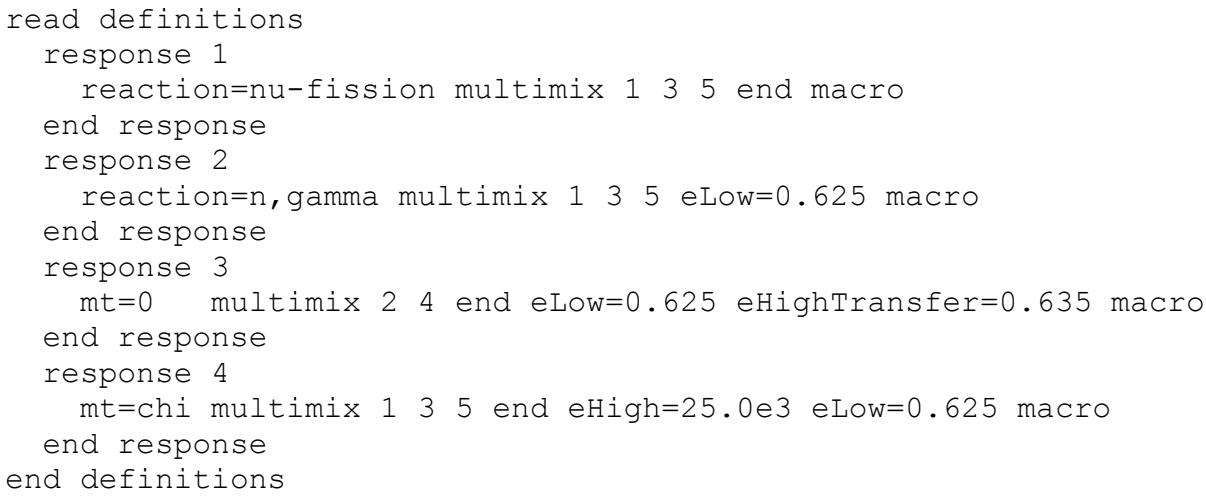

For multiple-mixture, multiple-nuclide macroscopic cross-section responses, keywords multimix, macro, and reaction are required; multiplier, eHigh, eLow, eHighTransfer, and eLowTransfer, are optional; title is optional but not used; and mixture, micro, nuclide, and unity are not allowed. 
Table 6.1.4. Keyword dependencies for the DEFINITIONS block

\begin{tabular}{|c|c|c|c|c|}
\hline Response type & $\begin{array}{l}\text { Required } \\
\text { keywords }\end{array}$ & $\begin{array}{l}\text { Unallowed } \\
\text { keywords }\end{array}$ & Optional keywords & $\begin{array}{l}\text { Optional, but not } \\
\text { used keywords }\end{array}$ \\
\hline Single-mixture flux & unity, mixture & multimix & $\begin{array}{l}\text { multiplier, eHigh, } \\
\text { eLow }\end{array}$ & $\begin{array}{l}\text { title, } \\
\text { reaction, muclide, } \\
\text { macro, } \\
\text { eHighTransfer, } \\
\text { eLowTransfer }\end{array}$ \\
\hline $\begin{array}{l}\text { Multiple-mixture } \\
\text { flux }\end{array}$ & unity, multimix & mixture & $\begin{array}{l}\text { multiplier, } \\
\text { eLow }\end{array}$ & $\begin{array}{l}\text { title, nuclide, } \\
\text { reaction, micro, } \\
\text { macro, } \\
\text { eHighTransfer, } \\
\text { eLowTransfer }\end{array}$ \\
\hline $\begin{array}{l}\text { Single-mixture, } \\
\text { single-nuclide, } \\
\text { microscopic cross- } \\
\text { section }\end{array}$ & $\begin{array}{l}\text { mixture, nuclide, } \\
\text { reaction }\end{array}$ & $\begin{array}{l}\text { unity, } \\
\text { multimix }\end{array}$ & $\begin{array}{l}\text { multiplier, eHigh, } \\
\text { eLow, micro, } \\
\text { eHighTransfer }^{\mathrm{a}} \text {, } \\
\text { eLowTransfer }^{\mathrm{a}}\end{array}$ & title \\
\hline $\begin{array}{l}\text { Single-mixture, } \\
\text { single-nuclide, } \\
\text { macroscopic cross- } \\
\text { section }\end{array}$ & $\begin{array}{l}\text { mixture, nuclide, } \\
\text { reaction,macro }\end{array}$ & $\begin{array}{l}\text { unity, } \\
\text { multimix }\end{array}$ & $\begin{array}{l}\text { multiplier, eHigh, } \\
\text { eLow, } \\
\text { eHighTransfer }^{\mathrm{a}} \text {, } \\
\text { eLowTransfer }^{\mathrm{a}}\end{array}$ & title \\
\hline $\begin{array}{l}\text { Single-mixture, } \\
\text { multiple-nuclide, } \\
\text { macroscopic cross- } \\
\text { section }\end{array}$ & $\begin{array}{l}\text { mixture, } \\
\text { reaction,macro }\end{array}$ & $\begin{array}{l}\text { unity, micro, } \\
\text { multimix, nuclide }\end{array}$ & $\begin{array}{l}\text { multiplier, eHigh, } \\
\text { eLow, } \\
\text { eHighTransfer }^{\mathrm{a}} \text {, } \\
\text { eLowTransfer }^{\mathrm{a}}\end{array}$ & title \\
\hline $\begin{array}{l}\text { Multiple-mixture, } \\
\text { single-nuclide, } \\
\text { macroscopic cross- } \\
\text { section }\end{array}$ & $\begin{array}{l}\text { multimix, } \\
\text { nuclide } \\
\text { reaction,macro }\end{array}$ & $\begin{array}{l}\text { unity, } \\
\text { mixture }\end{array}$ & $\begin{array}{l}\text { multiplier, eHigh, } \\
\text { eLow, } \\
\text { eHighTransfer }^{\mathrm{a}} \text {, } \\
\text { eLowTransfer }^{\mathrm{a}}\end{array}$ & title \\
\hline $\begin{array}{l}\text { Multiple-mixture, } \\
\text { multiple-nuclide, } \\
\text { macroscopic cross- } \\
\text { section }\end{array}$ & $\begin{array}{l}\text { multimix, } \\
\text { reaction,macro }\end{array}$ & $\begin{array}{l}\text { unity, micro, } \\
\text { mixture, nuclide }\end{array}$ & $\begin{array}{l}\text { multiplier, eHigh, } \\
\text { eLow, } \\
\text { eHighTransfer }{ }^{\mathrm{a}} \text {, } \\
\text { eLowTransfer }^{\mathrm{a}}\end{array}$ & title \\
\hline
\end{tabular}

${ }^{a}$ Keywords eHighTransfer and eLowTransfer are only used for the following reaction types: scatter $(\mathrm{mt}=0)$, elastic $(\mathrm{mt}=2)$, inelastic $(\mathrm{mt}=4), \mathrm{n}, 2 \mathrm{n}(\mathrm{mt}=16)$. For all other reaction types, these keywords are optional, but not used. 
Table 6.1.5. Supported Reaction Types in DEFINITIONS block

\begin{tabular}{ccc}
\hline MT & Reaction & String Identifier \\
\hline 1 & total & Total \\
2 & elastic scattering & Elastic \\
4 & inelastic scattering & Inelastic \\
$16^{\mathrm{a}}$ & effective $\mathrm{n}, 2 \mathrm{n}$ & $\mathrm{n}, 2 \mathrm{n}$ \\
0 & sum of scattering $(2+4+16)$ & Scatter \\
18 & fission & Fission \\
102 & $\mathrm{n}, \gamma$ & $\mathrm{n}, \mathrm{gamma}$ \\
103 & $\mathrm{n}, \mathrm{p}$ & $\mathrm{n}, \mathrm{p}$ \\
104 & $\mathrm{n}, \mathrm{d}$ & $\mathrm{n}, \mathrm{d}$ \\
105 & $\mathrm{n}, \mathrm{t}$ & $\mathrm{n}, \mathrm{t}$ \\
106 & $\mathrm{n},{ }^{\mathrm{h}} \mathrm{he}$ & $\mathrm{n}, \mathrm{he}-3$ \\
107 & $\mathrm{n}, \alpha$ & $\mathrm{n}, \mathrm{alpha}$ \\
101 & Neutron disappearance & capture \\
452 & $(102+103+104+105+106+107)$ & nubar \\
1452 & $\bar{v}$ & nu-fission \\
1018 & $\bar{v}$ times fission & chi \\
\hline${ }^{\mathrm{a}}$ The effective $\mathrm{n}, 2 \mathrm{n}$ is defined by the summation of transfer matrices of the \\
following reaction types: $(\mathrm{n}, 2 \mathrm{n}),(\mathrm{n}, 2 \mathrm{n}+\alpha),(\mathrm{n}, 2 \mathrm{n}+2 \alpha),(\mathrm{n}, 3 \mathrm{n}),(\mathrm{n}, 3 \mathrm{n}+\alpha)$, and $(\mathrm{n}, 4 \mathrm{n})$. \\
The individual transfer matrices are scaled by the number exit channel neutrons, i.e., \\
2,3 , or 4.
\end{tabular}

\subsection{System response definition data}

The SYSTEMRESPONSES block is used to define the set of system responses for which TSUNAMI-1D will perform sensitivity and uncertainty analysis additional to $k_{\text {eff. }}$ For SCALE 6.1, only system response ratios are supported in TSUNAMI-1D. The system response ratios are defined from the response function definitions created in the DEFINITIONS block. The format of the SYSTEMRESPONSES block is as follows:

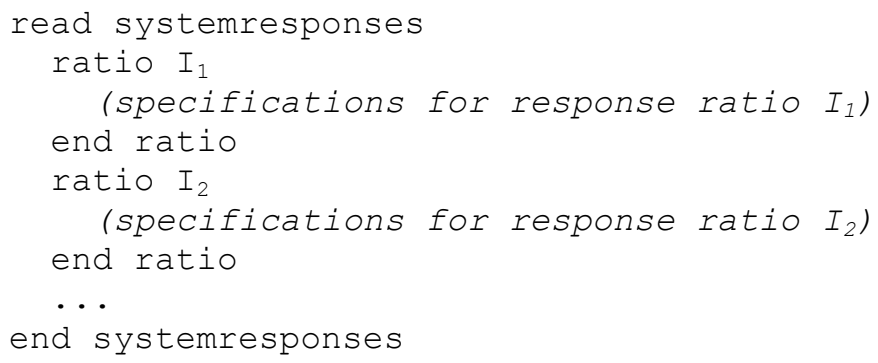

The SYSTEMRESPONSES block of data begins with READ SYSTEMRESPONSES and terminates with END SYSTEMRESPONSES. Likewise, each system response ratio definition begins with RATIO followed by a unique, positive integer identifier - and terminates with END RATIO. For each response ratio definition, the keywords title $=$, numer, and denom are allowed in any order. The title $=$ specification is optional. However, if specified, the title must be begin and end with quotes and have a maximum of 20 characters. If omitted, the title of the ratio is "rsp ratio NNNNNNNNNN" where NNNNNNNNNN is a zero-padded 10-digit integer that is equal to the ratio identifier. The title is used as labels in both the TSUNAMI-1D text and html output. The title is also used by SAMS to generate the filename for the sensitivity data file for the ratio system response discussed further below. 
The numer array is a list of integers that correlate to response function identifiers defined in the DEFINITIONS block. These response functions are added together to form the composite response function used in the numerator of the ratio. Likewise, the denom array is a list of integers that correlate to response function identifiers defined in the DEFINITIONS block. These response functions are added together to form the composite response function used in the denominator of the response ratio. Multiple response function ratios can be defined in a single input file.

For a simple example of the SYSTEMRESPONSES block, suppose the ratio system response of interest is the resonance escape probability for a given system. Using 2-group theory, this is equivalent to the following expression:

$$
p=\frac{\left\langle\Sigma_{s, 1 \rightarrow 2}\right\rangle}{\left\langle\Sigma_{r, 1}\right\rangle}=\frac{\int d \bar{r} \sum_{g \in 1} \phi_{g}(\bar{r}) \sum_{g^{\prime} \in 2} \Sigma_{s, g \rightarrow g^{\prime}}(\bar{r})}{\int d \bar{r} \sum_{g \in 1} \phi_{g}(\bar{r}) \Sigma_{r, g}(\bar{r})}
$$

where $\Sigma_{r, g}(\bar{r})$ is the removal cross-section defined as the total cross-section minus the within group cross-section $\left(\Sigma_{t, g}(\bar{r})-\Sigma_{s, g \rightarrow g}(\bar{r})\right)$. The TSUNAMI-1D model uses three mixtures whose ids are 6, 7, and 10 . The thermal energy cutoff is $0.625 \mathrm{eV}$.

This ratio can be defined in multiple ways. First, the ratio can be defined with three response function definitions:

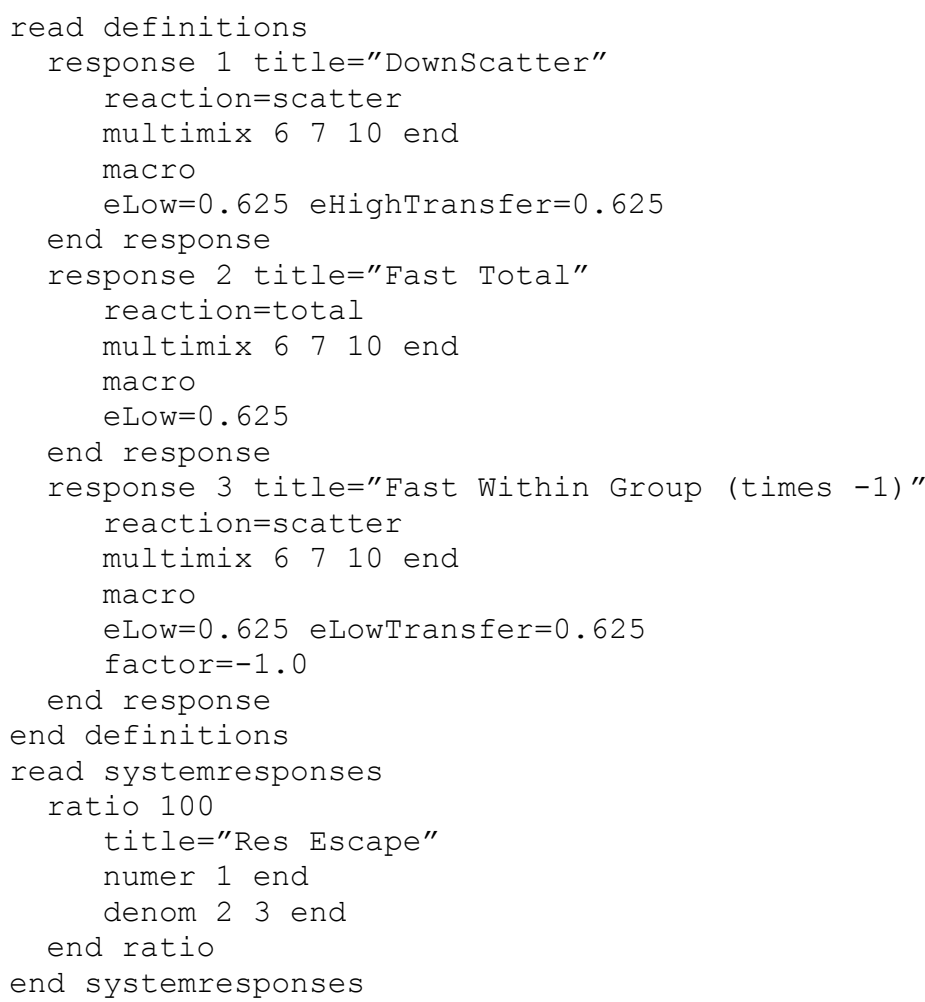


In the above input, the numerator of the response ratio is defined by a single response function (id=1), which represents the rate at which neutrons slow down from fast energies to slow energies. The denominator of the response ratio is defined by two response functions ( $\mathrm{id}=2$ and $\mathrm{id}=3$ ). The addition of these two response functions represents the "total minus within group scattering" calculation to formulate the fast neutron removal rate. In this input, the title of the response ratio is set to "Res Escape". Because only one response ratio is defined, TRITON will invoke SAMS twice, first for the $k_{\text {eff }}$ sensitivity and uncertainty analysis and second for the analysis of the resonance escape probability. SAMS will generate two .sdf files, the first will be jobname.sdf for $k_{\text {eff }}$ sensitivities and the second will be jobname.Res_Escape.sdf. jobname is the name of the input file. An underscore is used to replace blanks and special characters in the response ratio title in the sdf filename.

Similarly, the resonance escape probability can be defined in a variety of different ways. For example, the numerator response function can be expressed as the sum of individual mixture downscattering rates:

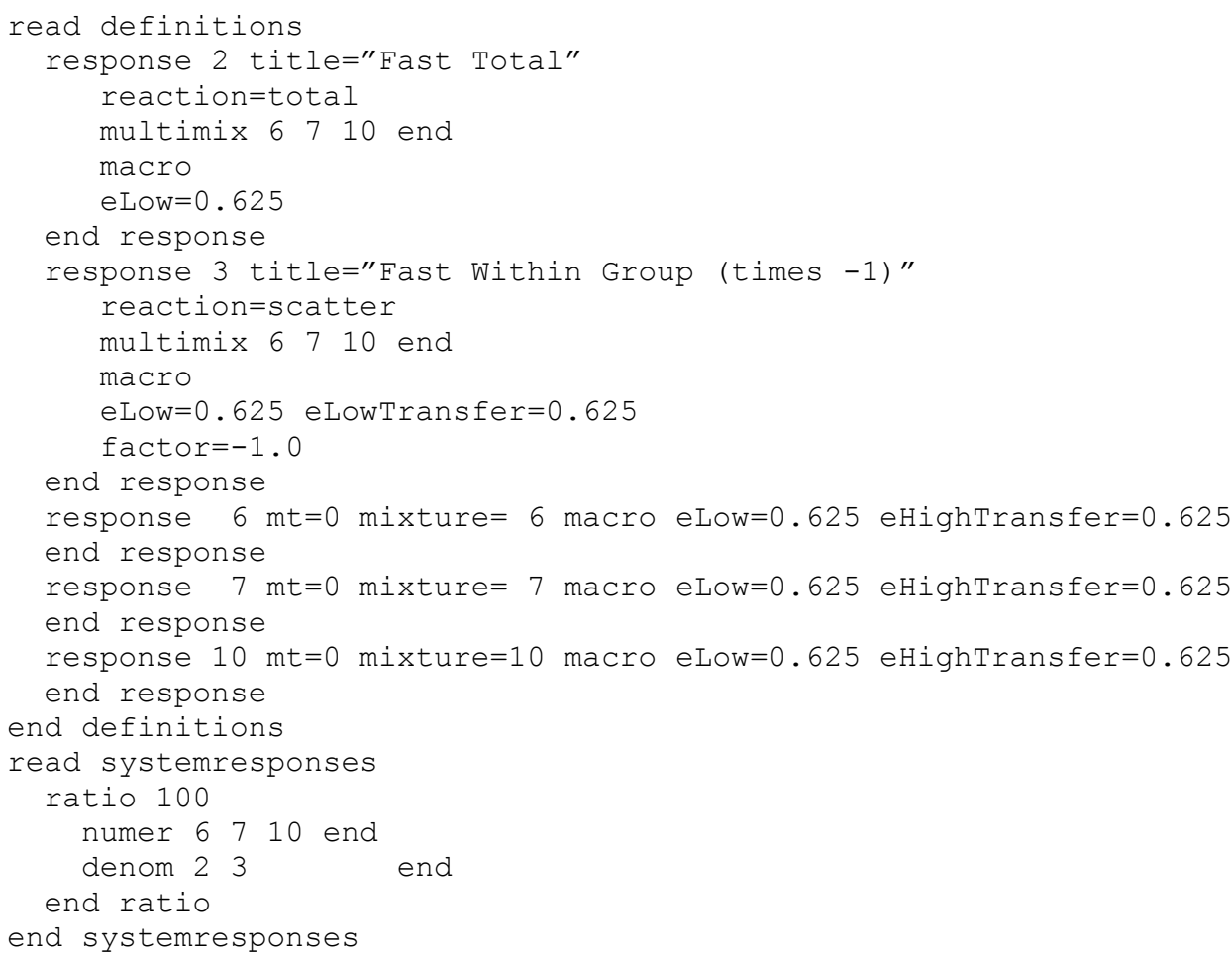

In this input, the numerator of the response ratio is defined by adding the individual mixture downscattering rates together. Because a title was not given for the response ratio, SAMS will generate the filename of the response ratio sdf file as jobname.rsp_ratio_0000000100.sdf. 


\subsection{SAMS data}

The $S A M S$ block is used for controlling certain aspects of the sensitivity and uncertainty calculation. This data block begins with the keywords $R E A D S A M S$ and ends with the keywords $E N D S A M S$. Any of the optional SAMS input data may be entered in free form format between the READ $S A M S$ and $E N D S A M S$ keywords. This optional SAMS input data is shown in

Table 6.1.6, with the default values specific to TSUNAMI-1D. Parameters used to specify default covariance data to supplement or correct values on the files specified by coverx= are shown in Table 6.1.7. A more detailed explanation of the SAMS parameters may be found in the SAMS chapter.

Table 6.1.6. SAMS input keywords

\begin{tabular}{|c|c|c|}
\hline Keyword & Default value & Description \\
\hline binsen & $\mathrm{F}$ & Produces SENPRO formatted binary sensitivity data file on unit 40 \\
\hline coverx $=$ & 56groupcov7.1 & Name of covariance data file to use for uncertainty analysis \\
\hline largeimp $=$ & 100.0 & $\begin{array}{l}\text { Value for the absolute value of implicit sensitivities, which if } \\
\text { exceeded, will be reset to } 0.0 \text { and print a warning message. }\end{array}$ \\
\hline nocovar & $\mathrm{T}$ & Flag to cause uncertainty edit to be turned off (sets print_covar to F) \\
\hline nohtml & $\mathrm{F}$ & Flag to cause HTML output to not be produced. \\
\hline nomix & $\mathrm{F}$ & Flag to cause the sensitivities by mixture to be turned off \\
\hline $\mathrm{pn}=$ & 3 & Legendre order for moment calculations \\
\hline prtgeom & $\mathrm{F}$ & Flag to cause the sensitivities to be output for each geometry region \\
\hline prtimp & $\mathrm{F}$ & $\begin{array}{l}\text { Prints explicit sensitivities coefficients, implicit sensitivity coefficients } \\
\text { and complete sensitivity coefficients }\end{array}$ \\
\hline prtvols & $\mathrm{F}$ & Flag to cause the volumes of the regions to be printed by SAMS \\
\hline unconstrainedchi & $\mathrm{F}$ & $\begin{array}{l}\text { Flag to generate pre-SCALE } 6 \text { unconstrained chi (fission spectrum) } \\
\text { sensitivities }\end{array}$ \\
\hline
\end{tabular}


Table 6.1.7. SAMS input keywords for default covariance data

\begin{tabular}{|c|c|c|}
\hline Keyword & Default value & Description \\
\hline use_dcov & $\mathrm{F}$ & Use default covariance data \\
\hline use_icov & $\mathrm{F}$ & Use user-input covariance data \\
\hline cov_fix & $\mathrm{F}$ & $\begin{array}{l}\text { Correct covariance data if the uncertainty is large }>1000 \% \text { or } \\
\text { zero }\end{array}$ \\
\hline large_cov & 10.0 & Relative Standard deviation to apply cov_fix \\
\hline return_work_cov & $\mathrm{F}$ & $\begin{array}{l}\text { Create a new covariance data file with only the cross-section } \\
\text { covariance data used in the analysis. }\end{array}$ \\
\hline udcov $=$ & 0.05 & $\begin{array}{l}\text { User-defined default value of standard deviation in cross- } \\
\text { section data to use for all groups for nuclide-reaction pairs for } \\
\text { which cross-section-covariance data are too large or not } \\
\text { available on input covariance data library. }\end{array}$ \\
\hline udcov_corr $=$ & 1.0 & $\begin{array}{l}\text { User-defined default correlation value to use for nuclide- } \\
\text { reaction pairs for which cross-section-covariance data are not } \\
\text { available on the input covariance library. }\end{array}$ \\
\hline udcov_corr_type $=$ & zone & $\begin{array}{l}\text { User-defined default correlation to use for nuclide-reaction } \\
\text { pairs for which cross-section-covariance data are not available } \\
\text { on the input covariance library. Allowed values are long, zone, } \\
\text { and short. See the table Input Data for Covariance Block of } \\
\text { TSAR Input in the TSAR chapter for details on long, zone, and } \\
\text { short. }\end{array}$ \\
\hline udcov_therm $=$ & 0.0 & $\begin{array}{l}\text { User-defined default value of standard deviation in cross- } \\
\text { section data to use for thermal data for nuclide-reaction pairs } \\
\text { for which cross-section-covariance data are too large or not } \\
\text { available on input covariance data library. If input, the } u d c o v- \\
\text { therm overrides the } u d c o v \text { value in the thermal range (i.e. } \\
\text { neutron energies below } 0.625 \mathrm{eV} \text { ). }\end{array}$ \\
\hline udcov_inter $=$ & 0.0 & $\begin{array}{l}\text { User-defined default value of standard deviation in cross- } \\
\text { section data to use for intermediate data for nuclide-reaction } \\
\text { pairs for which cross-section-covariance data are too large or } \\
\text { not available on input covariance data library. If input, the } \\
u d c o v \text { inter overrides the udcov value in the intermediate } \\
\text { range (i.e. neutron energies above } 0.625 \mathrm{eV} \text { and below } 25 \\
\mathrm{keV} \text { ). }\end{array}$ \\
\hline udcov_fast $=$ & 0.0 & $\begin{array}{l}\text { User-defined default value of standard deviation in cross- } \\
\text { section data to use for fast data for nuclide-reaction pairs for } \\
\text { which cross-section-covariance data are too large or not } \\
\text { available on input covariance data library. If input, the } u d c o v- \\
\text { fast overrides the } u d c o v \text { value in the fast range (i.e. neutron } \\
\text { energies above } 25 \mathrm{keV} \text { ). }\end{array}$ \\
\hline
\end{tabular}




\subsection{HTML and user-input covariance data}

User-defined covariance data can be specified for individual nuclides and reactions using the COVARIANCE data block. This data begins with the keywords READ COVARIANCE and ends with the keywords END COVARIANCE. Any of the optional COVARIANCE input data may be entered in free form format between the READ COVARIANCE and END COVARIANCE keywords. The specifications for the COVARIANCE data block are described in User Input Covariance Data of the TSUNAMI Utility Modules chapter.

As the SAMS module generates HTML output, the optional HTML data block will provides user control over some formats of the output. This data begins with the keywords READ HTML and ends with the keywords END HTML. Any of the optional HTML input data may be entered in free form format between the READ HTML and END HTML keywords. The specifications for the HTML data block are described in Sect. 18.1.6.6 of the TSUNAMI Utility Modules manual.

\subsubsection{Input termination}

The input specification for all TSUNAMI-1D sequences must terminate with a line containing $E N D$ in column 1. This END terminates the control sequence.

\subsubsection{Example Problems}

Nine TSUNAMI-1D sample problems are included in the SCALE package to verify successful installation and to provide examples for users. They are provided in the smplprbs directory of the software distribution. Three example problems are presented in this section and comparisons among the different methods for cross-section processing are discussed. The first problem presented is a variant of the TSUNAMI-1D1 $k_{\text {eff }}$ sensitivity sample problem with some addition input parameters in the READ SAMS data block and using INFHOMMEDIUM unit cell type. The second example problem presented in this section generates $k_{\text {eff }}$ sensitivities using the MULTIREGION unit cell type. The third example problem is similar to the TSUNAMI-1D5 sample problem that demonstrates the GPT capabilities. The five sample problems in the software package are designed to run quickly and test most code features. The three examples presented here are designed to produce accurate results, but may require more computational resources.

For all problems the validity of the sensitivity coefficients should be confirmed through the use of direct perturbation sensitivity calculations. For each sensitivity coefficient examined by direct perturbation, the $k_{\text {eff }}$ of the system is computed first with the nominal values of the input quantities, then with a selected input value increased by a certain percentage, and then with the value decreased by the same percentage. The direct perturbation sensitivity coefficient of $k_{\text {eff }}$ to some input value $\alpha$ is computed as

$$
S_{k, \alpha}=\frac{\alpha}{k} \times \frac{d k}{d \alpha}=\frac{\alpha}{k} \times \frac{k_{\alpha^{+}}-k_{\alpha^{-}}}{\alpha^{+}-\alpha^{-}}
$$

where $\alpha^{+}$and $\alpha^{-}$represent the increased and decreased values, respectively, of the input quantity $\alpha$ and $k_{\alpha^{+}}$and $k_{\alpha^{-}}$represent the corresponding values of $k_{e f f}$. 
The use of direct perturbation calculations to confirm the validity of sensitivity coefficients is strongly encouraged. Inconsistent modeling between the resonance-self shielding input and the criticality problem description can lead to erroneous sensitivity results. These erroneous results can be revealed through the use of direct perturbation confirmation of the energy-integrated sensitivity results for the total cross section. The total cross-section sensitivities are equivalent to number density sensitivities on an energy-integrated basis.

The results shown here were generated with a previous version of SCALE, so current data libraries and code implementations may product different results. However, the techniques demonstrated are applicable to the current version of TSUNAMI-1D.

\subsubsection{INFHOMMEDIUM sample problem}

The selected sample problem with INFHOMMEDIUM cross-section processing is based on an unreflected rectangular parallelepiped consisting of a homogeneous mixture of $\mathrm{UF}_{4}$ and paraffin with an enrichment of $2 \%$ in ${ }^{235} \mathrm{U}$. The $\mathrm{H} /{ }^{235} \mathrm{U}$ atomic ratio is $294: 1$. The dimensions of the experiment were $56.22 \mathrm{~cm} \times 56.22 \mathrm{~cm} \times 122.47 \mathrm{~cm}^{1}{ }^{1}$ For the purposes of this exercise, this experiment was modeled as a sphere with a critical radius of $38.50 \mathrm{~cm}$. This model is consistent with SCALE sample problem TSUNAMI-1D1, which utilizes the 238-group ENDF/B-VII cross-section library, and the default crosssection processing with BONAMIST and CENTRM/PMC/WORKER.

An annotated TSUNAMI-1D1 input for this experiment is shown in Figure 6.1.2. The composition data is input as number densities for each nuclide. Because the material is treated as INFHOMMEDIUM, no explicit unit cell model is necessary, and the READ CELL block is omitted. The criticality description contains optional parameter data to change the default $\mathrm{S}_{16}$ angular quadrature set to $\mathrm{S}_{8}$. The change in angular quadrature is made only to demonstrate the input capabilities of TSUNAMI-1D and has little effect on this calculation. The criticality problem geometry uses a spherical coordinate system with the default boundary conditions (reflected left, vacuum right). The system consists of a single material zone containing mixture 1 with a radius of $38.50 \mathrm{~cm}$. The optional sensitivity calculation data block was entered to request the extended edit of sensitivity by material zone (prtgeom), the extended edits of the explicit, implicit and complete sensitivity coefficients (prtimp), and corrections in the cross-section covariance data (use_dcov, covffix).

Prior to producing the output of the functional modules, TSUNAMI-1D produces output from the XSProc routines as it is processing the user input and creating internal inputs for the resonance processing codes. TSUNAMI-1D also prints information regarding the criticality description. 


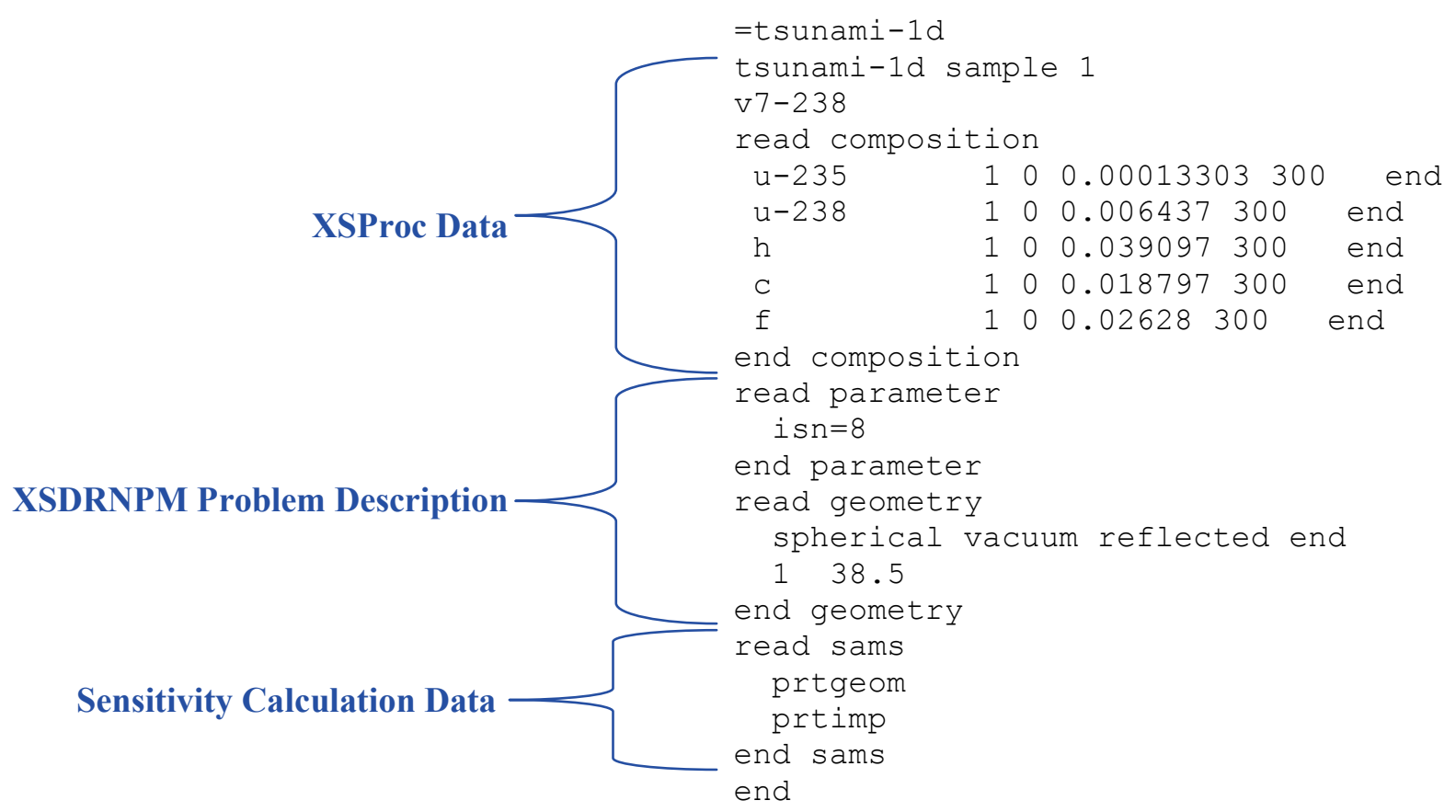

Figure 6.1.2. TSUNAMI-1D INFHOMMEDIUM sample problem input. 
For this problem, direct perturbation results were obtained for the number densities of each nuclide. In these calculations, the number density of each nuclide was perturbed by $\pm 2 \%$ and the calculation was repeated using the TSUNAMI-1DC sequence. The sensitivity of $k_{\text {eff }}$ to the number density is equivalent to the sensitivity of $k_{\text {eff }}$ to the total cross section, integrated over energy. The direct perturbation sensitivity coefficients were computed by using the $k_{\text {eff }}$ values from the unperturbed and perturbed cases in Eq. (6.1.13). To demonstrate the importance of the sensitivity to the resonance processing implicit sensitivity computed by BONAMIST, the same model shown in Figure 6.1.2 was run with TSUNAMI-1D with PARM=CENTRM. The results from the INFHOMMEDIUM sample problem are given in Table 6.1.8. The TSUNAMI-1D results using the default codes for resonance processing show good agreement with the direct perturbation results for all nuclides. Due to omission of the implicit terms, the TSUNAMI-1D results with PARM=CENTRM do not show good agreement with the direct perturbation for this thermal system. The maximum difference between the direct perturbation results and the TSUNAMI-1D results occurs for ${ }^{238} \mathrm{U}$ with a magnitude of $1.5 \%$. The maximum difference between the direct perturbation results and the TSUNAMI-1D with PARM $=C E N T R M$ results occurs for ${ }^{238} U$ with a magnitude of $19 \%$. Thus, the use of the default PARM=BONAMIST is recommended.

Table 6.1.8. Energy- and region-integrated sensitivity coefficients from TSUNAMI-1D INFHOMMEDIUM sample problem

\begin{tabular}{cllcc}
\hline Isotope & Reaction & $\begin{array}{c}\text { Direct } \\
\text { perturbation }\end{array}$ & TSUNAMI-1D & $\begin{array}{c}\text { TSUNAMI-1D } \\
\text { PARM=CENTRM }\end{array}$ \\
\hline${ }^{1} \mathrm{H}$ & total & $2.20 \mathrm{E}-01$ & $2.18 \mathrm{E}-01$ & $2.52 \mathrm{E}-01$ \\
${ }^{1} \mathrm{H}$ & scatter & & $3.19 \mathrm{E}-01$ & $3.53 \mathrm{E}-01$ \\
${ }^{1} \mathrm{H}$ & elastic & & $3.19 \mathrm{E}-01$ & $3.53 \mathrm{E}-01$ \\
${ }^{1} \mathrm{H}$ & capture & & $-1.01 \mathrm{E}-01$ & $-1.01 \mathrm{E}-01$ \\
${ }^{1} \mathrm{H}$ & $\mathrm{n}, \gamma$ & $-1.01 \mathrm{E}-01$ & $-1.01 \mathrm{E}-01$ \\
\hline${ }^{12} \mathrm{C}$ & total & $2.41 \mathrm{E}-02$ & $2.38 \mathrm{E}-02$ & $2.76 \mathrm{E}-02$ \\
${ }^{12} \mathrm{C}$ & scatter & & $2.45 \mathrm{E}-02$ & $2.83 \mathrm{E}-02$ \\
${ }^{12} \mathrm{C}$ & elastic & & $2.43 \mathrm{E}-02$ & $2.80 \mathrm{E}-02$ \\
${ }^{12} \mathrm{C}$ & $n, n '$ & $2.20 \mathrm{E}-04$ & $2.20 \mathrm{E}-04$ \\
${ }^{12} \mathrm{C}$ & capture & & $-6.83 \mathrm{E}-04$ & $-6.83 \mathrm{E}-04$ \\
${ }^{12} \mathrm{C}$ & n, $\gamma$ & $-4.98 \mathrm{E}-04$ & $-4.98 \mathrm{E}-04$ \\
${ }^{12} \mathrm{C}$ & $n, p$ & $-3.53 \mathrm{E}-08$ & $-3.53 \mathrm{E}-08$ \\
${ }^{12} \mathrm{C}$ & $n, \mathrm{~d}$ & & $-7.33 \mathrm{E}-08$ & $-7.33 \mathrm{E}-08$ \\
${ }^{12} \mathrm{C}$ & $n, \alpha$ & $-1.85 \mathrm{E}-04$ & $-1.85 \mathrm{E}-04$ \\
\hline${ }^{19} \mathrm{~F}$ & total & $4.10 \mathrm{E}-02$ & $4.06 \mathrm{E}-02$ & $4.47 \mathrm{E}-02$ \\
${ }^{19} \mathrm{~F}$ & scatter & & $4.62 \mathrm{E}-02$ & $5.03 \mathrm{E}-02$ \\
${ }^{19} \mathrm{~F}$ & elastic & & $2.94 \mathrm{E}-02$ & $3.34 \mathrm{E}-02$ \\
${ }^{19} \mathrm{~F}$ & $n, n '$ & $1.58 \mathrm{E}-02$ & $1.58 \mathrm{E}-02$ \\
${ }^{19} \mathrm{~F}$ & $n, 2 \mathrm{n}$ & $2.89 \mathrm{E}-06$ & $2.89 \mathrm{E}-06$ \\
${ }^{19} \mathrm{~F}$ & capture & $-5.59 \mathrm{E}-03$ & $-5.59 \mathrm{E}-03$ \\
${ }^{19} \mathrm{~F}$ & $n, \gamma$ & $-2.39 \mathrm{E}-03$ & $-2.39 \mathrm{E}-03$ \\
& & &
\end{tabular}


Table 6.1.8. Energy- and region-integrated sensitivity coefficients from TSUNAMI-1D INFHOMMEDIUM sample problem (continued)

\begin{tabular}{|c|c|c|c|c|}
\hline Isotope & Reaction & $\begin{array}{c}\text { Direct } \\
\text { perturbation }\end{array}$ & TSUNAMI-1D & $\begin{array}{c}\text { TSUNAMI-1D } \\
\text { PARM=CENTRM }\end{array}$ \\
\hline${ }^{19} \mathrm{~F}$ & $\mathrm{n}, \mathrm{p}$ & & $-2.37 \mathrm{E}-04$ & $-2.37 \mathrm{E}-04$ \\
\hline${ }^{19} \mathrm{~F}$ & $\mathrm{n}, \mathrm{d}$ & & $-1.27 \mathrm{E}-05$ & $-1.27 \mathrm{E}-05$ \\
\hline${ }^{19} \mathrm{~F}$ & $\mathrm{n}, \mathrm{t}$ & & $-2.72 \mathrm{E}-06$ & $-2.72 \mathrm{E}-06$ \\
\hline${ }^{19} \mathrm{~F}$ & $\mathrm{n}, \alpha$ & & $-2.96 \mathrm{E}-03$ & $-2.96 \mathrm{E}-03$ \\
\hline${ }^{235} \mathrm{U}$ & total & $2.52 \mathrm{E}-01$ & $2.52 \mathrm{E}-01$ & $2.53 \mathrm{E}-01$ \\
\hline${ }^{235} \mathrm{U}$ & scatter & & 4.32E-04 & $5.03 \mathrm{E}-04$ \\
\hline${ }^{235} \mathrm{U}$ & elastic & & 2.02E-04 & 2.73E-04 \\
\hline${ }^{235} \mathrm{U}$ & $\mathrm{n}, \mathrm{n}^{\prime}$ & & $2.13 \mathrm{E}-04$ & $2.13 \mathrm{E}-04$ \\
\hline${ }^{235} \mathrm{U}$ & $\mathrm{n}, 2 \mathrm{n}$ & & $1.70 \mathrm{E}-05$ & $1.70 \mathrm{E}-05$ \\
\hline${ }^{235} \mathrm{U}$ & fission & & $3.64 \mathrm{E}-01$ & 3.65E-01 \\
\hline${ }^{235} \mathrm{U}$ & capture & & $-1.13 \mathrm{E}-01$ & $-1.12 \mathrm{E}-01$ \\
\hline${ }^{235} \mathrm{U}$ & $\mathrm{n}, \gamma$ & & $-1.13 \mathrm{E}-01$ & $-1.12 \mathrm{E}-01$ \\
\hline${ }^{235} \mathrm{U}$ & nubar & & $9.50 \mathrm{E}-01$ & $9.50 \mathrm{E}-01$ \\
\hline${ }^{235} \mathrm{U}$ & chi & & 8.52E-08 & $8.52 \mathrm{E}-08$ \\
\hline${ }^{238} \mathrm{U}$ & total & $-2.08 \mathrm{E}-01$ & $-2.05 \mathrm{E}-01$ & $-2.47 \mathrm{E}-01$ \\
\hline${ }^{238} \mathrm{U}$ & scatter & & $4.81 \mathrm{E}-02$ & $2.46 \mathrm{E}-02$ \\
\hline${ }^{238} \mathrm{U}$ & elastic & & $3.46 \mathrm{E}-02$ & $1.10 \mathrm{E}-02$ \\
\hline${ }^{238} \mathrm{U}$ & $\mathrm{n}, \mathrm{n}^{\prime}$ & & $1.25 \mathrm{E}-02$ & $1.25 \mathrm{E}-02$ \\
\hline${ }^{238} \mathrm{U}$ & $n, 2 n$ & & $1.02 \mathrm{E}-03$ & $1.02 \mathrm{E}-03$ \\
\hline${ }^{238} \mathrm{U}$ & fission & & $3.35 \mathrm{E}-02$ & $3.35 \mathrm{E}-02$ \\
\hline${ }^{238} \mathrm{U}$ & capture & & $-2.86 \mathrm{E}-01$ & $-3.05 \mathrm{E}-01$ \\
\hline${ }^{238} \mathrm{U}$ & $\mathrm{n}, \gamma$ & & $-2.86 \mathrm{E}-01$ & $-3.05 \mathrm{E}-01$ \\
\hline${ }^{238} \mathrm{U}$ & nubar & & $5.02 \mathrm{E}-02$ & $5.02 \mathrm{E}-02$ \\
\hline${ }^{238} \mathrm{U}$ & chi & & 4.54E-09 & 4.54E-09 \\
\hline
\end{tabular}

The uncertainty information from SAMS for the INFHOMMEDIUM sample problem is shown in Figure 6.1.3. Based on the 44GROUPCOV covariance data library, documented in the COVLIB chapter, the uncertainty in $k_{\text {eff }}$ due to these covariance data is $0.6064 \% \Delta \mathrm{k} / \mathrm{k}$. A more detailed description of the uncertainty information is given in Chapter 6.3. Some plots of the energy-dependent sensitivity data were generated with Fulcrum. The energy-dependent data is available in the sensitivity data file, which is returned to the same directory as the input file and given the same name as the user's input file with the extension .sdf. Energy-dependent sensitivity profiles for ${ }^{235} \mathrm{U}$ fission and ${ }^{1} \mathrm{H}$ elastic scattering are shown in Figure 6.1.4. The ${ }^{238} \mathrm{U}$ capture sensitivity profiles from TSUNAMI-1D and TSUNAMI-1D with PARM=CENTRM are shown in Figure 6.1.5. The effect of the implicit component of the sensitivity coefficients can be seen in the resonance region in the difference between the TSUNAMI-1D and TSUNAMI-1D PARM=CENTRM profiles. 
Uncertainty Information

the relative standard deviation of k-eff ( $\frac{\circ}{\circ}$ delta-k/k) due to cross-section covariance data is:

$0.6064 \div \mathrm{delta-k/k}$

contributions to uncertainty in k-eff (\% delta-k/k) by individual energy covariance matrices:

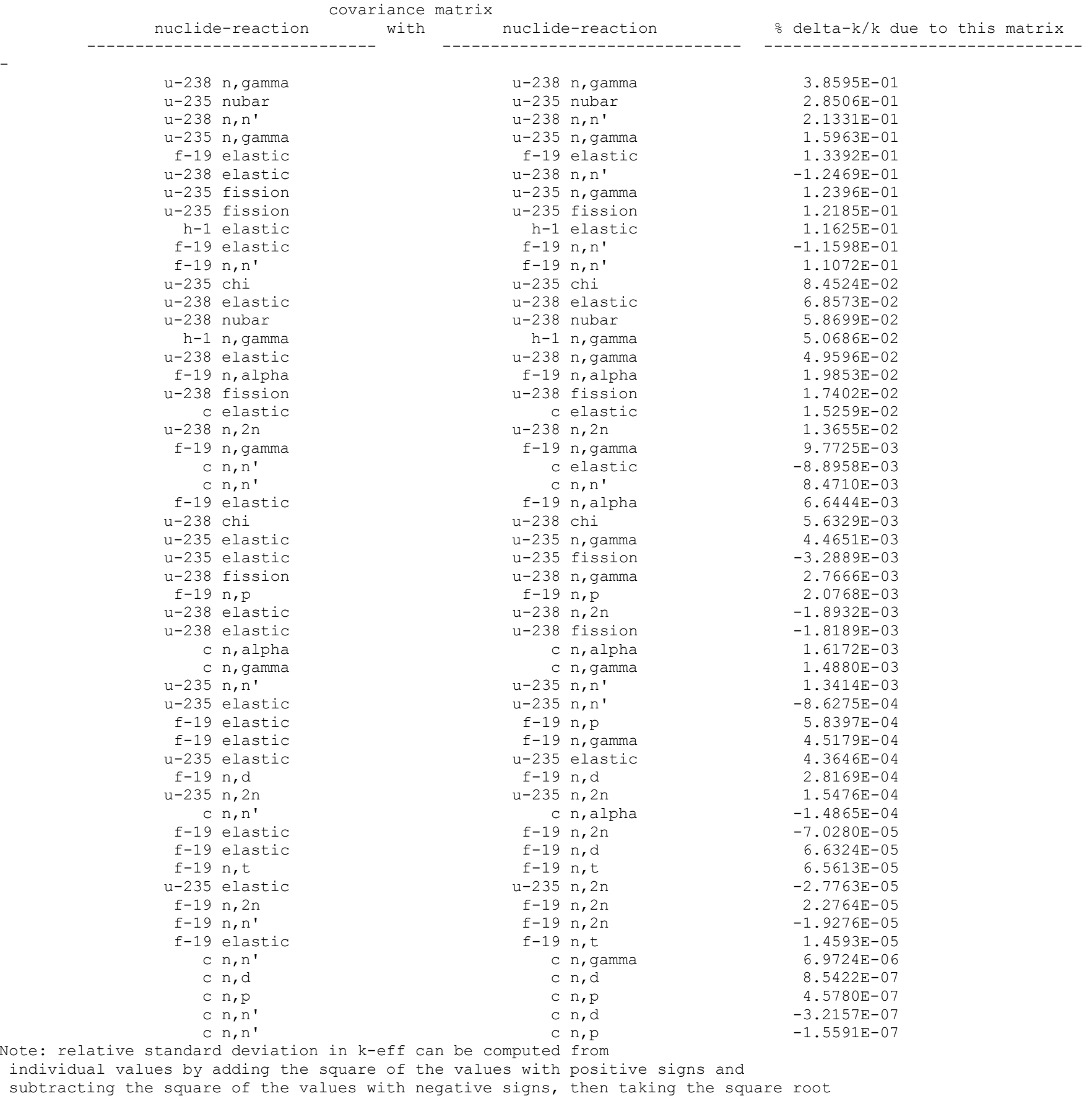

Figure 6.1.3. Uncertainty information from INFHOMMEDIUM sample problem. 


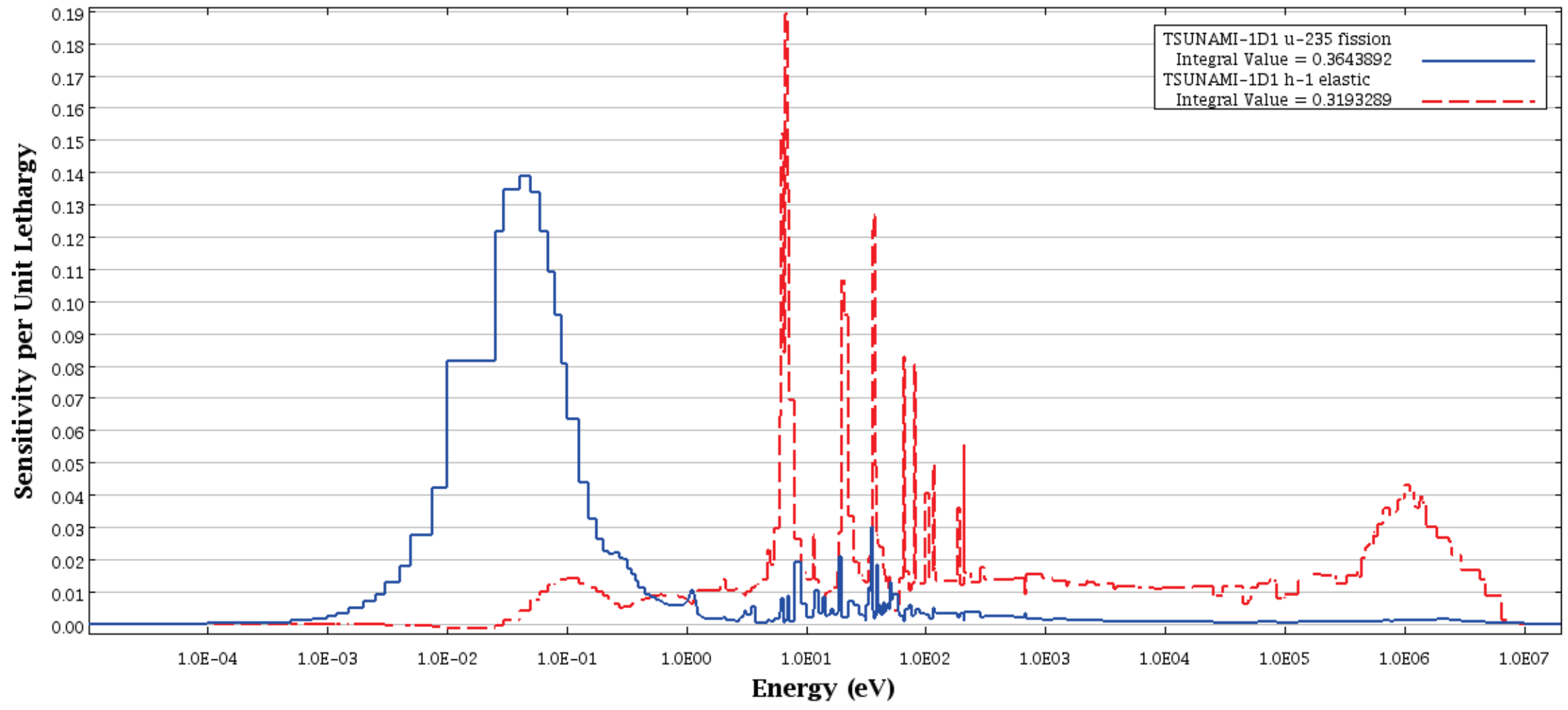

Figure 6.1.4. Energy-dependent sensitivity profiles from TSUNAMI-1D for INFHOMMEDIUM sample problem. 


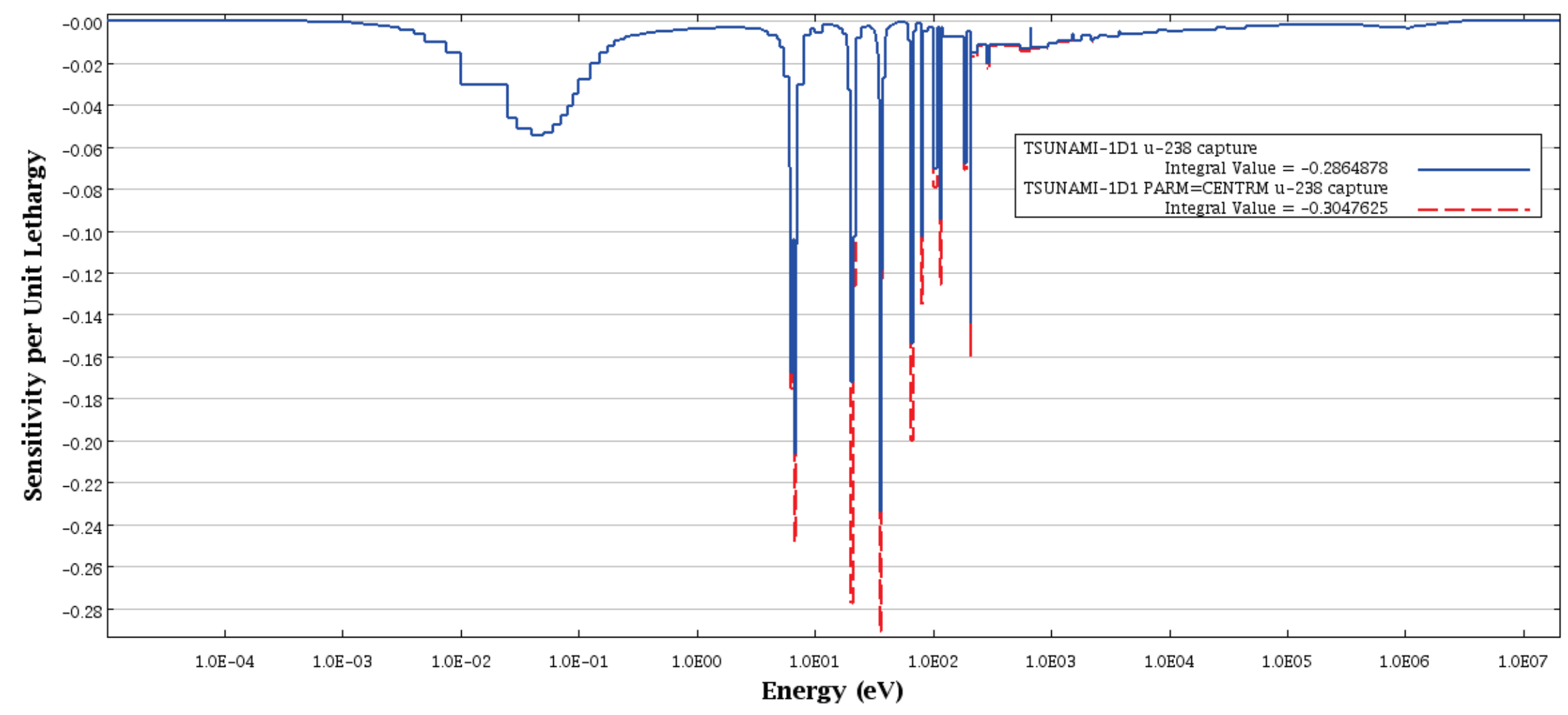

Figure 6.1.5. Comparison of 238U capture sensitivities from TSUNAMI-1D and TSUNAMI-1D with PARM=CENTRM for INFHOMMEDIUM sample problem. 


\subsubsection{Multiregion sample problem}

The sample problem selected to demonstrate the use of TSUNAMI-1D with MULTIREGION crosssection processing is the FLATTOP-25 metal system from the Cross-Section Evaluation Working Group benchmark specifications. $^{2}$ The system consists of a $6.116-\mathrm{cm}$ sphere of $93 \%$-enriched uranium with a natural uranium reflector. The outer radius of the reflector is $24.13 \mathrm{~cm}$. The system is used for sample problems TSUNAMI-1D4 - TSUNAMI-1D7. For this example, input for TSUNAMI-1D4 was modified to use the SCALE 238-group ENDF/B-VII library with multiregion cell data as shown in Figure 6.1.6. The multiregion cell data processes the cross sections in the same geometry as the criticality model. Therefore, the dimensions of the system are input twice in this model: once in the unit cell specification portion of the input and once in the criticality portion of the input. The unit cell specification geometry is used to generate input for BONAMIST and CENTRM/PMC/WORKER, and the criticality model is used to generate input for the forward and adjoint XSDRNPM calculations. The optional sensitivity calculation data block was entered to request the extended edit of sensitivity by material zone (prtgeom), the extended edits of the explicit, implicit and complete sensitivity coefficients (prtimp), and to allow larger implicit sensitivity values to be computed without producing warning messages (largeimp $=1000)$.

This model was executed with TSUNAMI-1D and also with TSUNAMI-1D with PARM=CENTRM. Direct perturbation sensitivity results were obtained for the number densities of all nuclides, which correspond to the sensitivity of $k_{\text {eff }}$ to the total cross section, integrated over energy. The energyintegrated sensitivity results are shown in Table 6.1.9. The TSUNAMI-1D results agree well with the direct perturbation results for this system. The maximum difference occurs for ${ }^{238} \mathrm{U}$ in the reflector region with a magnitude of $0.9 \%$. Because this is a fast system, the effect of the resonance processing on the sensitivity coefficients is minimal. Thus, the TSUNAMI-1D PARM=CENTRM results are almost identical to the default TSUNAMI-1D results with BONAMIST. 


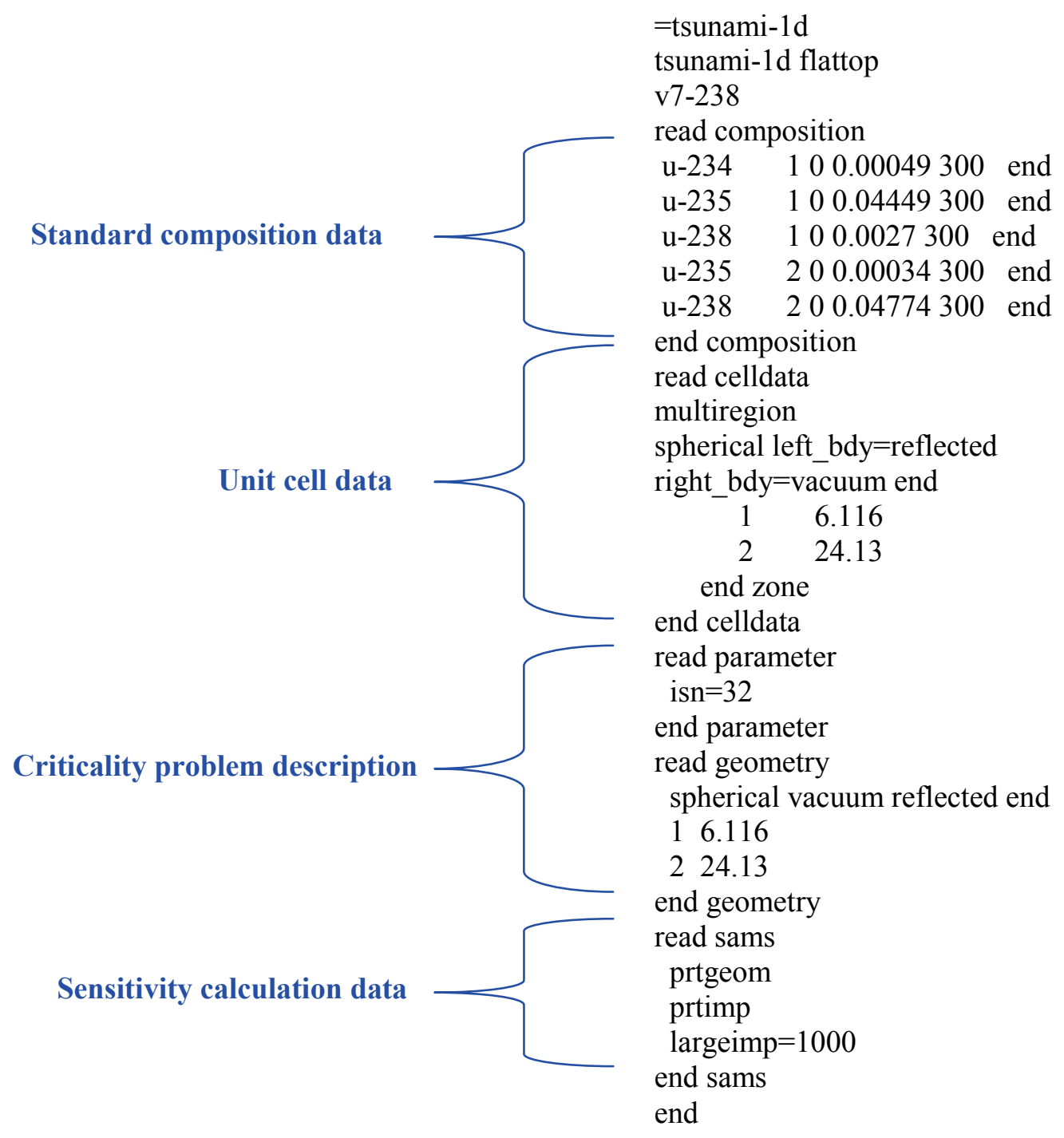

Figure 6.1.6. TSUNAMI-1D MULTIREGION sample problem input. 
Table 6.1.9. Energy- and region-integrated sensitivity coefficients from TSUNAMI-1D MULTIREGION sample problem

\begin{tabular}{|c|c|c|c|c|}
\hline Isotope/Location & Reaction & $\begin{array}{c}\text { Direct } \\
\text { perturbation }\end{array}$ & TSUNAMI-1D & $\begin{array}{c}\text { TSUNAMI-1D } \\
\text { PARM }=\text { CENTRM }\end{array}$ \\
\hline${ }^{234} \mathrm{U}$ in core & total & $4.53 \mathrm{E}-03$ & $4.51 \mathrm{E}-03$ & $4.51 \mathrm{E}-03$ \\
\hline${ }^{234} \mathrm{U}$ in core & scatter & & $6.64 \mathrm{E}-04$ & $6.64 \mathrm{E}-04$ \\
\hline${ }^{234} \mathrm{U}$ in core & elastic & & $4.01 \mathrm{E}-04$ & $4.01 \mathrm{E}-04$ \\
\hline${ }^{234} \mathrm{U}$ in core & $\mathrm{n}, \mathrm{n}^{\prime}$ & & $2.62 \mathrm{E}-04$ & $2.62 \mathrm{E}-04$ \\
\hline${ }^{234} \mathrm{U}$ in core & $\mathrm{n}, 2 \mathrm{n}$ & & $1.44 \mathrm{E}-06$ & $1.44 \mathrm{E}-06$ \\
\hline${ }^{234} \mathrm{U}$ in core & fission & & $4.30 \mathrm{E}-03$ & 4.30E-03 \\
\hline${ }^{234} \mathrm{U}$ in core & capture & & $-4.56 \mathrm{E}-04$ & $-4.56 \mathrm{E}-04$ \\
\hline${ }^{234} \mathrm{U}$ in core & $\mathrm{n}, \gamma$ & & $-4.56 \mathrm{E}-04$ & $-4.56 \mathrm{E}-04$ \\
\hline${ }^{234} \mathrm{U}$ in core & nubar & & $6.56 \mathrm{E}-03$ & $6.56 \mathrm{E}-03$ \\
\hline${ }^{234} \mathrm{U}$ in core & chi & & $1.72 \mathrm{E}-10$ & $1.72 \mathrm{E}-10$ \\
\hline${ }^{235} \mathrm{U}$ in core & total & $5.84 \mathrm{E}-01$ & $5.84 \mathrm{E}-01$ & $5.84 \mathrm{E}-01$ \\
\hline${ }^{235} \mathrm{U}$ in core & scatter & & $6.58 \mathrm{E}-02$ & $6.58 \mathrm{E}-02$ \\
\hline${ }^{235} \mathrm{U}$ in core & elastic & & $3.16 \mathrm{E}-02$ & $3.16 \mathrm{E}-02$ \\
\hline${ }^{235} \mathrm{U}$ in core & $\mathrm{n}, \mathrm{n}^{\prime}$ & & $3.31 \mathrm{E}-02$ & $3.31 \mathrm{E}-02$ \\
\hline${ }^{235} \mathrm{U}$ in core & $\mathrm{n}, 2 \mathrm{n}$ & & $1.08 \mathrm{E}-03$ & $1.08 \mathrm{E}-03$ \\
\hline${ }^{235} \mathrm{U}$ in core & fission & & $5.66 \mathrm{E}-01$ & $5.66 \mathrm{E}-01$ \\
\hline${ }^{235} \mathrm{U}$ in core & capture & & $-4.79 \mathrm{E}-02$ & $-4.79 \mathrm{E}-02$ \\
\hline${ }^{235} \mathrm{U}$ in core & $\mathrm{n}, \gamma$ & & $-4.79 \mathrm{E}-02$ & $-4.79 \mathrm{E}-02$ \\
\hline${ }^{235} \mathrm{U}$ in core & nubar & & $9.04 \mathrm{E}-01$ & $9.04 \mathrm{E}-01$ \\
\hline${ }^{235} \mathrm{U}$ in core & chi & & $-2.35 \mathrm{E}-08$ & $-2.35 \mathrm{E}-08$ \\
\hline${ }^{238} \mathrm{U}$ in core & total & $8.01 \mathrm{E}-03$ & $8.01 \mathrm{E}-03$ & $8.01 \mathrm{E}-03$ \\
\hline${ }^{238} \mathrm{U}$ in core & scatter & & $4.66 \mathrm{E}-03$ & $4.66 \mathrm{E}-03$ \\
\hline${ }^{238} \mathrm{U}$ in core & elastic & & $2.37 \mathrm{E}-03$ & $2.37 \mathrm{E}-03$ \\
\hline${ }^{238} \mathrm{U}$ in core & $\mathrm{n}, \mathrm{n}^{\prime}$ & & $2.21 \mathrm{E}-03$ & $2.21 \mathrm{E}-03$ \\
\hline${ }^{238} \mathrm{U}$ in core & $\mathrm{n}, 2 \mathrm{n}$ & & $7.25 \mathrm{E}-05$ & $7.25 \mathrm{E}-05$ \\
\hline${ }^{238} \mathrm{U}$ in core & fission & & $5.03 \mathrm{E}-03$ & $5.03 \mathrm{E}-03$ \\
\hline${ }^{238} \mathrm{U}$ in core & capture & & $-1.69 \mathrm{E}-03$ & $-1.69 \mathrm{E}-03$ \\
\hline${ }^{238} \mathrm{U}$ in core & $\mathrm{n}, \gamma$ & & $-1.69 \mathrm{E}-03$ & $-1.69 \mathrm{E}-03$ \\
\hline${ }^{238} \mathrm{U}$ in core & nubar & & $7.66 \mathrm{E}-03$ & $7.66 \mathrm{E}-03$ \\
\hline${ }^{238} \mathrm{U}$ in core & chi & & $-4.85 \mathrm{E}-10$ & $-4.85 \mathrm{E}-10$ \\
\hline${ }^{235} \mathrm{U}$ in reflector & total & $8.31 \mathrm{E}-03$ & 8.24E-03 & $8.25 \mathrm{E}-03$ \\
\hline${ }^{235} \mathrm{U}$ in reflector & scatter & & $1.15 \mathrm{E}-03$ & $1.16 \mathrm{E}-03$ \\
\hline${ }^{235} \mathrm{U}$ in reflector & elastic & & $7.98 \mathrm{E}-04$ & $8.03 \mathrm{E}-04$ \\
\hline${ }^{235} \mathrm{U}$ in reflector & $\mathrm{n}, \mathrm{n}^{\prime}$ & & $3.53 \mathrm{E}-04$ & $3.53 \mathrm{E}-04$ \\
\hline${ }^{235} \mathrm{U}$ in reflector & $\mathrm{n}, 2 \mathrm{n}$ & & $4.43 \mathrm{E}-06$ & $4.43 \mathrm{E}-06$ \\
\hline${ }^{235} \mathrm{U}$ in reflector & fission & & $7.73 \mathrm{E}-03$ & $7.74 \mathrm{E}-03$ \\
\hline${ }^{235} \mathrm{U}$ in reflector & capture & & $-6.45 \mathrm{E}-04$ & $-6.44 \mathrm{E}-04$ \\
\hline${ }^{235} \mathrm{U}$ in reflector & $\mathrm{n}, \gamma$ & & $-6.45 \mathrm{E}-04$ & $-6.44 \mathrm{E}-04$ \\
\hline
\end{tabular}


Table 6.1.9. Energy- and region-integrated sensitivity coefficients from TSUNAMI-1D MULTIREGION sample problem (continued)

\begin{tabular}{lllcc}
\hline Isotope/Location & Reaction & $\begin{array}{c}\text { Direct } \\
\text { perturbation }\end{array}$ & TSUNAMI-1D & $\begin{array}{c}\text { TSUNAMI-1D } \\
\text { PARM }=\text { CENTRM }\end{array}$ \\
\hline${ }^{235} \mathrm{U}$ in reflector & nubar & & $1.10 \mathrm{E}-02$ & $1.10 \mathrm{E}-02$ \\
${ }^{235} \mathrm{U}$ in reflector & chi & & $4.65 \mathrm{E}-10$ & $4.65 \mathrm{E}-10$ \\
\hline${ }^{238} \mathrm{U}$ in reflector & total & $2.07 \mathrm{E}-01$ & $2.09 \mathrm{E}-01$ & $2.09 \mathrm{E}-01$ \\
${ }^{238} \mathrm{U}$ in reflector & scatter & & $2.06 \mathrm{E}-01$ & $2.06 \mathrm{E}-01$ \\
${ }^{238} \mathrm{U}$ in reflector & elastic & & $1.41 \mathrm{E}-01$ & $1.41 \mathrm{E}-01$ \\
${ }^{238} \mathrm{U}$ in reflector & n,n' & & $6.38 \mathrm{E}-02$ & $6.38 \mathrm{E}-02$ \\
${ }^{238} \mathrm{U}$ in reflector & $n, 2 \mathrm{n}$ & & $6.93 \mathrm{E}-04$ & $6.93 \mathrm{E}-04$ \\
${ }^{238} \mathrm{U}$ in reflector & fission & & $5.06 \mathrm{E}-02$ & $5.06 \mathrm{E}-02$ \\
${ }^{238} \mathrm{U}$ in reflector & capture & $-4.70 \mathrm{E}-02$ & $-4.71 \mathrm{E}-02$ \\
${ }^{238} \mathrm{U}$ in reflector & n, $\gamma$ & $-4.70 \mathrm{E}-02$ & $-4.71 \mathrm{E}-02$ \\
${ }^{238} \mathrm{U}$ in reflector & nubar & $7.08 \mathrm{E}-02$ & $7.08 \mathrm{E}-02$ \\
${ }^{238} \mathrm{U}$ in reflector & chi & $1.24 \mathrm{E}-09$ & $1.24 \mathrm{E}-09$ \\
\hline
\end{tabular}

The uncertainty information from SAMS HTML output for the multiregion sample problem is shown in Figure 6.1.7. Based on the 44GROUPCOV covariance data file, the uncertainty in $k_{\text {eff }}$ due to these covariance data is $1.2743 \% \Delta \mathrm{k} / \mathrm{k}$. The contributions to this uncertainty are listed by nuclide. These data are explained in more detail in the SAMS chapter.

Sensitivity profiles from TSUNAMI-1D for ${ }^{235} \mathrm{U}$ fission in zone 1 (core) and zone 2 (reflector) were generated with Fulcrum and are shown in Figure 6.1.8. Additionally, sensitivity profiles for ${ }^{238} \mathrm{U}$ capture in zone 1 and zone 2 are shown in Figure 6.1.9. Note that the capture sensitivities are negative, such that the lower curve has the greater magnitude. In ${ }^{235} \mathrm{U}$ and ${ }^{238} \mathrm{U}$ sensitivity profiles, the effect of the differing enrichments in the core and the reflector of this system are demonstrated with the much greater sensitivity to ${ }^{235} \mathrm{U}$ fission in the core and to ${ }^{238} \mathrm{U}$ capture in the reflector. 


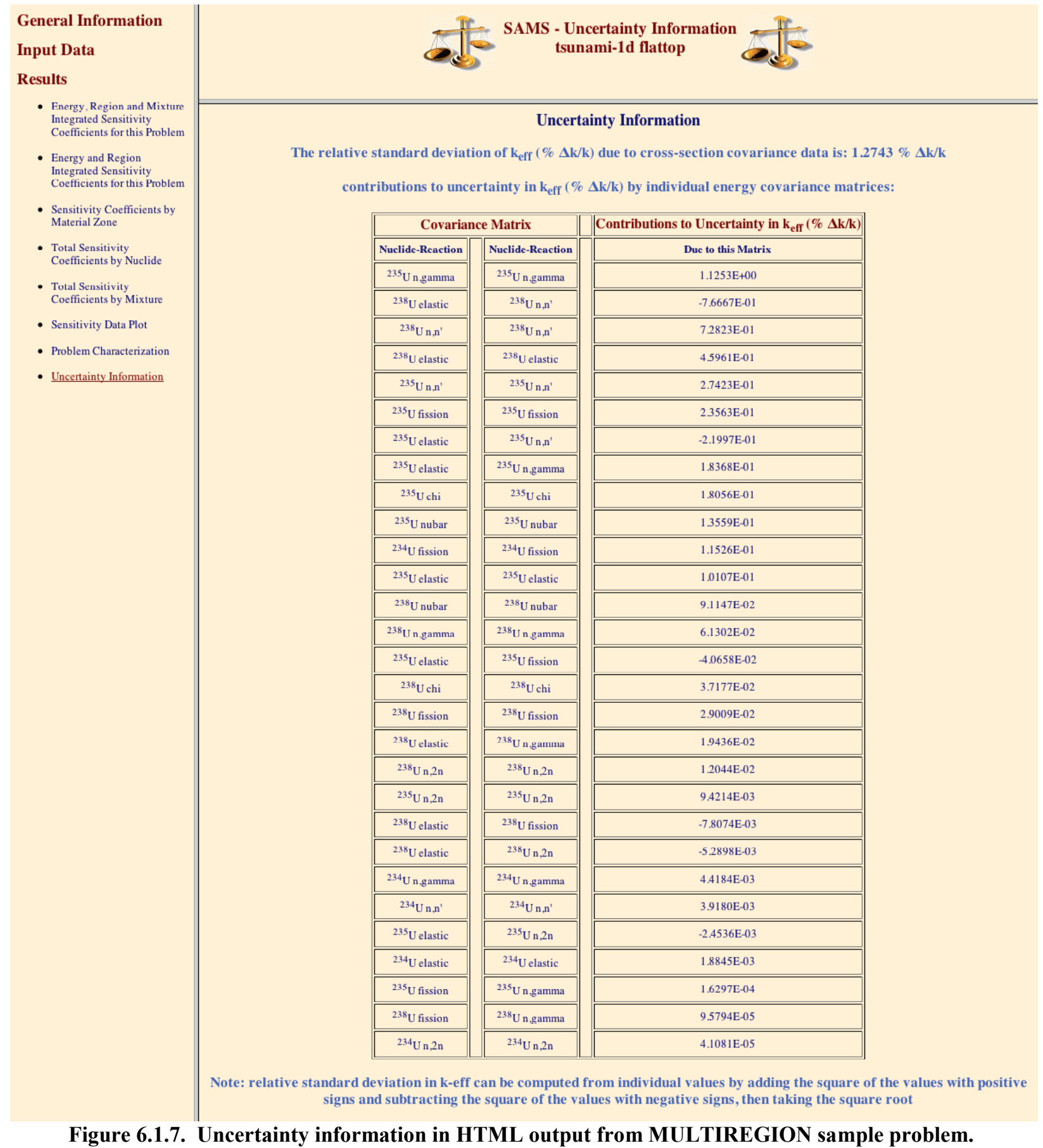

Figure 6.1.7. Uncertainty information in HTML output from MULTIREGION sample problem. 


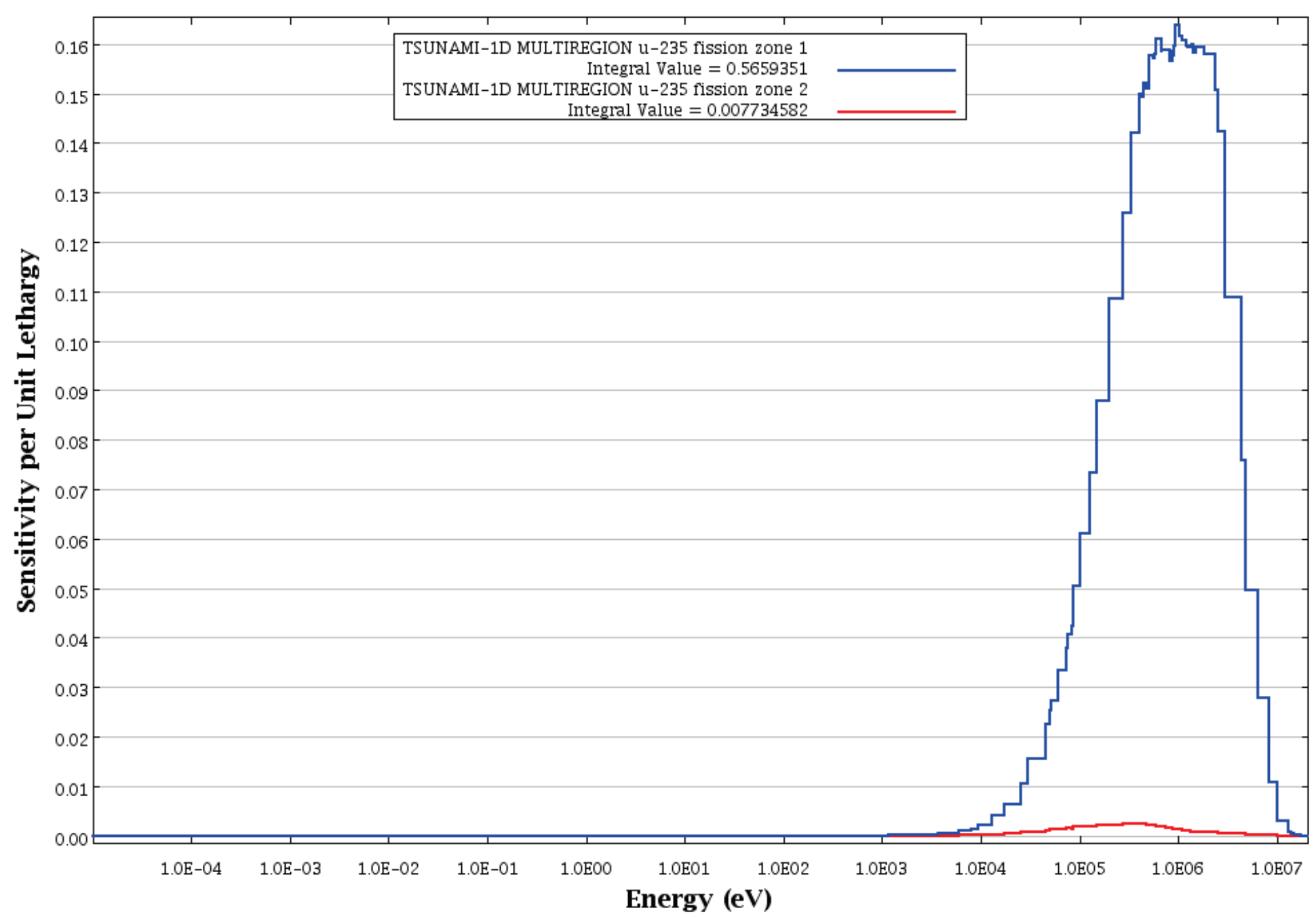

Figure 6.1.8. Sensitivity profiles from TSUNAMI-1D for ${ }^{235} U$ fission in zone 1 and zone 2 of MULTIREGION sample problem. 


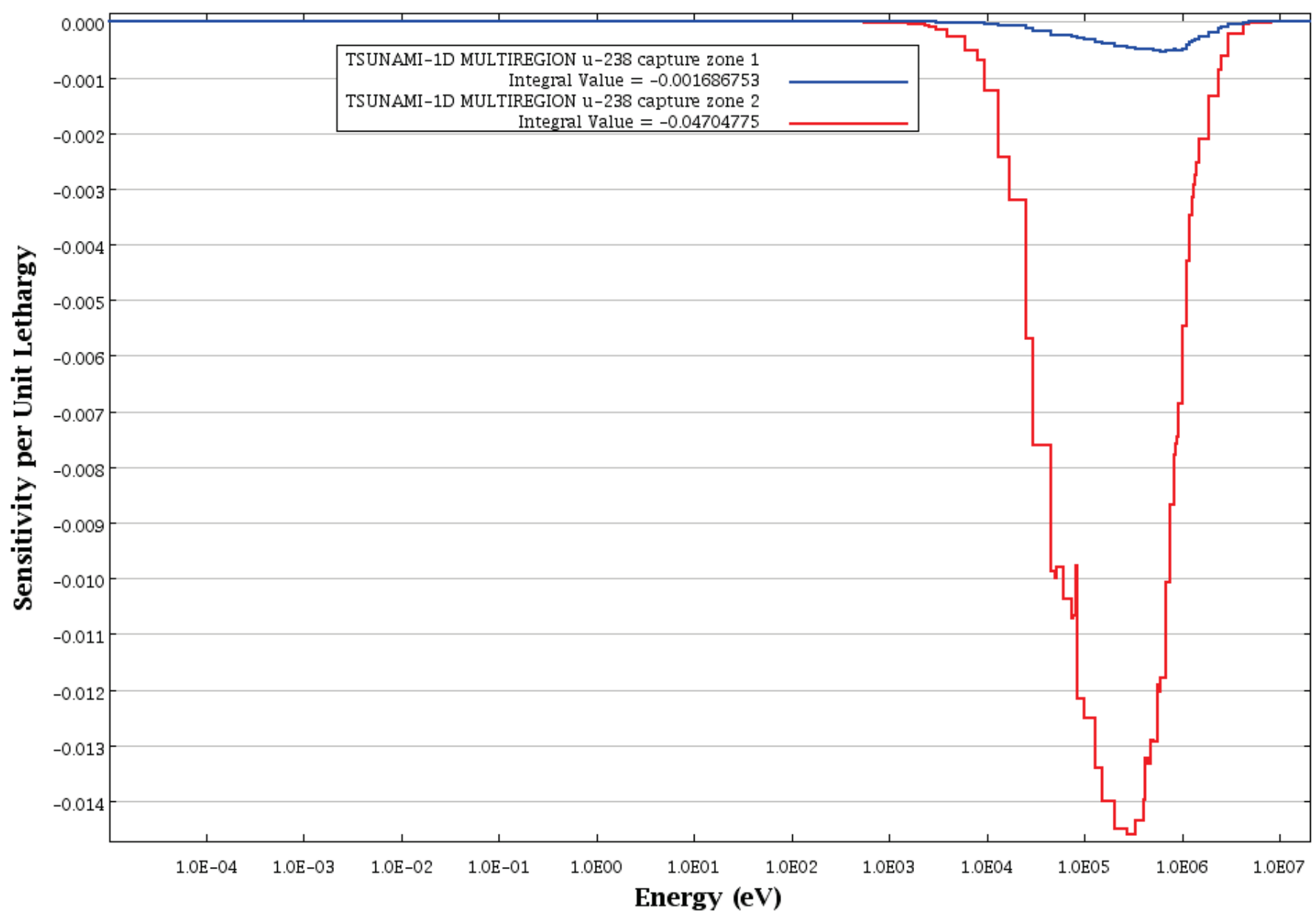

Figure 6.1.9. Sensitivity profiles from TSUNAMI-1D for ${ }^{238} \mathrm{U}$ capture in zone 1 and zone 2 of MULTIREGION sample problem. 


\subsubsection{GPT sample problem}

The sample problem selected to demonstrate the use of TSUNAMI-1D with Generalized Perturbation theory is from the OECD LWR Uncertainty Analysis in Modeling benchmark specification. ${ }^{3}$ The system consists of a $4.85 \%$ enriched uranium PWR fuel pin modeled at $551 \mathrm{~K}$. This system is used for sample problem TSUNAMI-1D9. For this example, the DEFINITIONS and SYSTEMRESPONSES blocks are used to define six additional response ratios for sensitivity and uncertainty analysis. The requested responses in the benchmark were for the energy-integrated fission and absorption microscopic crosssections for ${ }^{234} \mathrm{U},{ }^{235} \mathrm{U}$, and ${ }^{238} \mathrm{U}$. The input for this sample problem is provided in Figure 6.1.10. For this sample, seven separate sensitivity data files are generated, one for each of the six defined responses in addition to $k_{\text {eff. }}$ Selected sensitivity profiles are shown in Figure 6.1.11 for the ${ }^{238} \mathrm{U}(\mathrm{n}, \gamma)$ cross-section. This figure shows the negative sensitivity of $k_{\text {eff }}$ due to ${ }^{238} \mathrm{U}$ resonance absorption in the blue profile. The red profile shows the positive sensitivity of the energy-integrated ${ }^{238} \mathrm{U}$ absorption cross-section due to the multigroup ${ }^{238} \mathrm{U}(\mathrm{n}, \gamma)$ cross-section. The large positive magnitude of this sensitivity is predominantly due to the presence of the ${ }^{238} \mathrm{U}(\mathrm{n}, \gamma)$ cross-section directly in the definition of the response ratio. In contrast, the black sensitivity profile shows the negative sensitivity of the energy-integrated ${ }^{235} \mathrm{U}$ fission crosssection due to the multigroup ${ }^{238} \mathrm{U}(\mathrm{n}, \gamma)$ cross-section. In this response, positive perturbations to the ${ }^{238} \mathrm{U}$ $(\mathrm{n}, \gamma)$ multigroup cross-sections induce changes in the flux spectra that lead to a decrease in the energyintegrated ${ }^{235} \mathrm{U}$ fission cross-section. These indirect sensitivity effects are determined by the solution of the generalized adjoint calculations. 


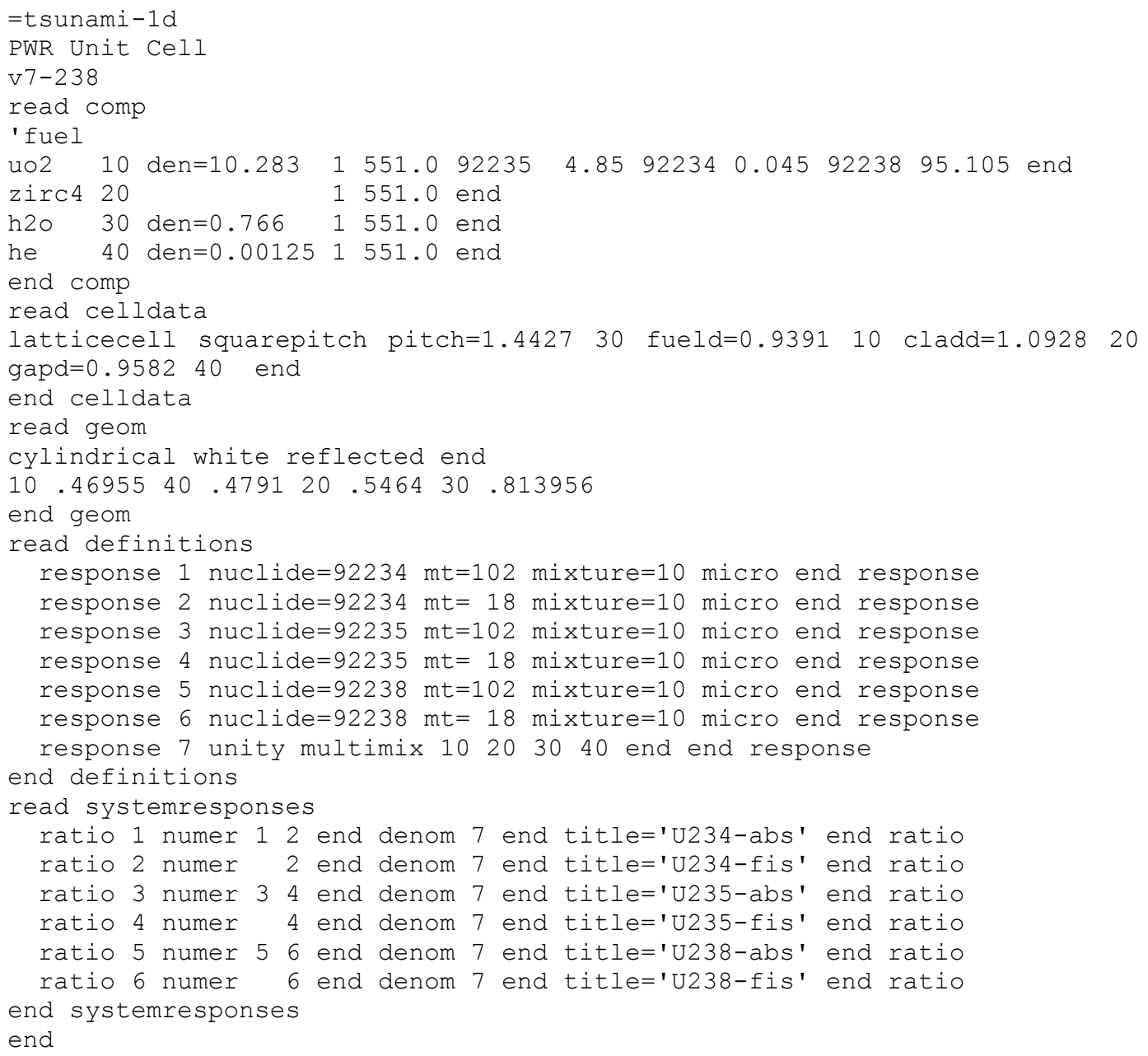

Figure 6.1.10. Input for TSUNAMI-1D9 sample problem. 


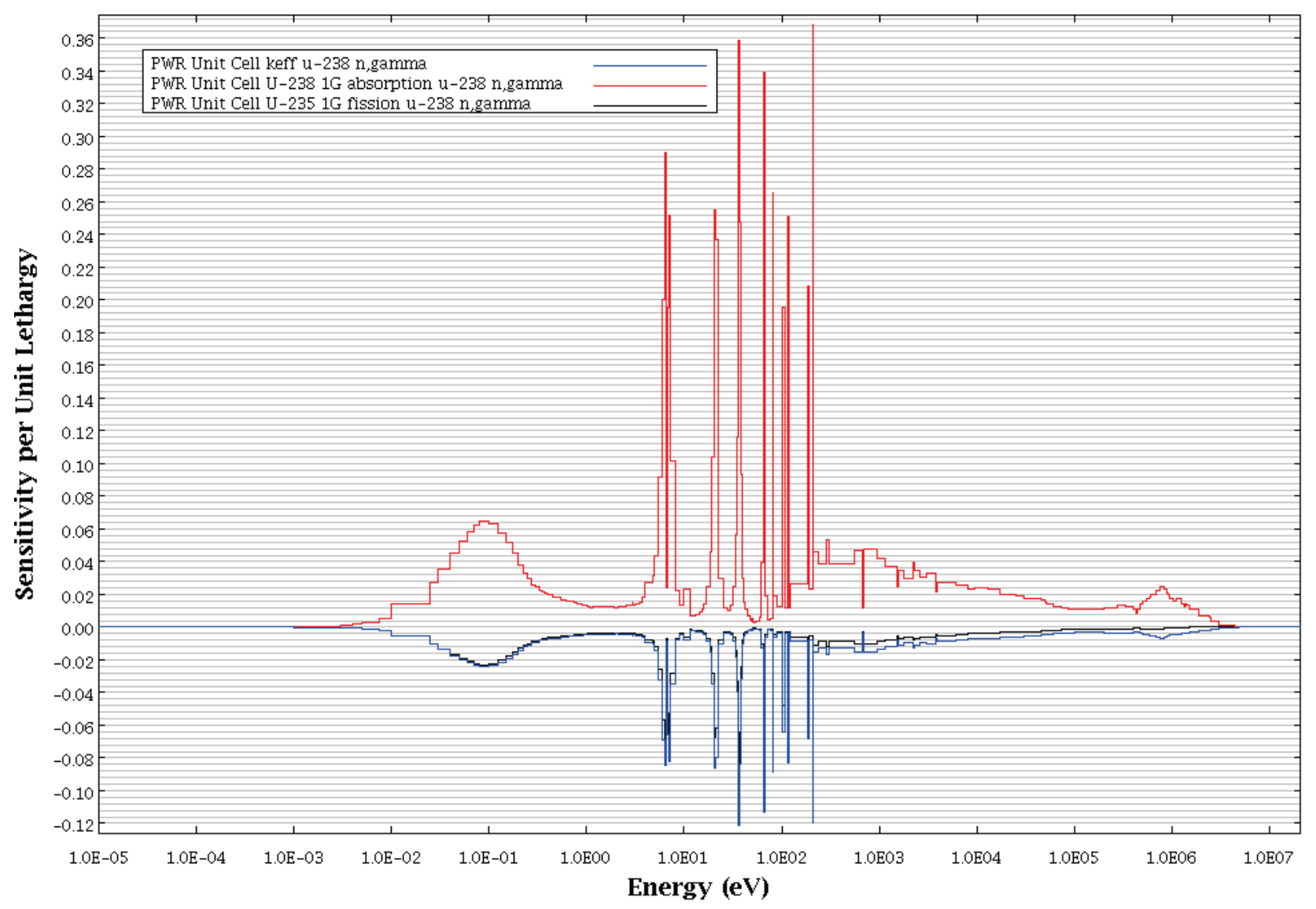

Figure 6.1.11. Response Sensitivities to ${ }^{238} \mathrm{U}$ n,gamma cross section for the TSUNAMI-1D9 sample problem.

\subsubsection{References}

1. W. C. Jordan, N. F. Landers, and L. M. Petrie, Validation of KENO V.a Comparison with Critical Experiments, ORNL/CSD/TM-238, Martin Marietta Energy Systems, Inc., Oak Ridge National Laboratory (1986).

2. Cross Section Evaluation Working Group Benchmark Specification, BNL 19302 (ENDF-202), Brookhaven National Laboratory, November 1974.

3. Benchmark for Uncertainty Analysis in Modeling (UAM) for Design, Operation and Safety Analysis of LWRs, OECD Nuclear Energy Agency, December 2007. 


\subsection{A XSDRNPM Data File Formats}

As part of the development of the sensitivity capabilities in SCALE, the XSDRNPM module was modified to allow the writing of a new interface file containing information needed for sensitivity calculations. This same data file is used by TSUNAMI-1D. This information is written on unit NTD in the XSDRNPM input, when NTD is positive. When TSUNAMI-1D creates the inputs for the forward and adjoint XSDRNPM criticality calculations, NTD is set to 31 for the forward case and 32 for the adjoint case. The files written are $\mathrm{ft} 31 \mathrm{f001}$ and $\mathrm{ft} 32 \mathrm{f} 001$, respectively.

\subsection{A.1 XSDRNPM forward output file}

For a forward case, XSDRNPM writes the following unformatted records on unit NTD:

\section{RECORD 1: IZM,IM,MXX,MS,ISCT,MM,JT,IGM}

$\begin{array}{ll}\text { IZM } & \text { Number of zones } \\ \text { IM } & \text { Number of spatial intervals } \\ \text { MXX } & \text { Number of compositions (mixtures) } \\ \text { MS } & \text { Length of the XSDRNPM mixing table } \\ \text { ISCT } & \text { Order of the Legendre scattering expansion } \\ \text { MM } & \text { Number of angles in the angular quadrature } \\ \text { JT } & \text { Number of flux moments } \\ \text { IGM } & \text { Number of energy groups }\end{array}$

\section{RECORD 2: IGE,IBL,IBR,ISN,IFTG,MMT,NT1,T}

$\begin{array}{ll}\text { IGE } & \text { Geometry: } 1 / 2 / 3=\text { plane/cylinder/sphere } \\ \text { IBL } & \text { Left-boundary condition: } 0 / 1 / 2 / 3=\text { vacuum/reflected/periodic/white } \\ \text { IBR } & \text { Right-boundary condition } \\ \text { ISN } & \text { S }_{\mathrm{n}} \text { quadrature order } \\ \text { IFTG } & \text { First thermal group } \\ \text { MMT } & \text { Number of neutron groups } \\ \text { NT1 } & \text { Unit number of working cross-section library } \\ \text { T } & \text { Problem title containing } 80 \text { characters }\end{array}$

RECORD 3: V,R

$\mathrm{V}(\mathrm{IM})$

$\mathrm{R}(\mathrm{IM}+1)$

\section{RECORD 4: W,PNC}

$\mathrm{W}(\mathrm{MM})$

PNC(MM,JT)
Volumes of the spatial mesh cells (single-precision)

Boundaries of the spatial mesh cells (single-precision)
Weights in the angular quadrature (single-precision)

Scattering constants used to obtain flux moments from angular fluxes (singleprecision) 
RECORD 5: MA,MZ

MA(IM) Zone number by interval

MZ(IZM) Mixture number by zone

\section{RECORD 6: MB,MC,XMD}

MB(MS) Mixture number in the cross-section mixing table

MC(MS) Component (nuclide) in the cross-section mixing table

$\mathrm{XMD}(\mathrm{MS}) \quad$ Atom density in the cross-section mixing table (single-precision)

\section{RECORD 7: CHI,FISNU}

CHI(IGM,MXX) $\quad \chi$ for each mixture (single-precision)

FISNU(IGM,MXX) $\quad \bar{v}$ times the fission cross section for each mixture (single-precision)

\section{RECORD 8: EIGEN}

EIGEN $\quad k_{\text {eff }}$ (single-precision)

\section{NEXT IGM RECORDS: XNDC}

XNDC(IM,MM) Mesh cell centered angular flux for one group (double-precision)

\section{LAST RECORD: TLEAKAGE}

TLEAKAGE(IGM) Total leakage from the system (single-precision)

\subsection{A.2 XSDRNPM adjoint output file}

For an adjoint case, XSDRNPM writes the following unformatted records on unit NTD, containing the following information:

\section{RECORD 1: EIGEN}

EIGEN $\quad k_{\text {eff }}$ value (single-precision)

\section{NEXT IGM RECORDS: XNDC}

XNDC(IM,MM) Mesh cell centered angular flux for one group (double-precision)

The adjoint angular fluxes are reversed in direction such that each angular flux is the importance for that direction in the forward case. This reversal is done by using the reflected angle. Also, the records are written in forward order such that the first record corresponds to the highest-energy group. 


\title{
6.2 TSUNAMI-3D: CONTROL MODULE FOR THREE-DIMENSIONAL CROSS SECTION SENSITIVITY AND UNCERTAINTY ANALYSIS FOR CRITICALITY
}

B. T. Rearden, C. M. Perfetti, and L. M. Petrie

\begin{abstract}
TSUNAMI-3D (Tools for Sensitivity and Uncertainty Analysis Methodology Implementation in Three Dimensions) is a SCALE control module that facilitates the application of sensitivity and uncertainty analysis theory to criticality safety analysis. In multigroup (MG) mode, TSUNAMI-3D provides for resonance self-shielding of cross section data, calculation of the implicit effects of resonance selfshielding calculations, calculation of forward and adjoint Monte Carlo neutron transport solutions, and calculation of eigenvalue sensitivity coefficients. In continuous-energy (CE) mode, sensitivity coefficients are computed in a single forward Monte Carlo neutron transport calculation for either eigenvalue or generalized reaction rate ratio responses. In both MG and CE modes, the KENO V.a or KENO-VI module is used for transport solvers, and the SAMS module is used to compute and/or create edits of the sensitivity of the calculated responses to the nuclear data used in the calculation as a function of nuclide, reaction type, and energy. SAMS also computes the uncertainty in the calculated responses resulting from uncertainties in the basic nuclear data used in the calculation through energy-dependent cross section covariance matrices. In both MG and CE calculations, a sensitivity data file is produced for use in subsequent analysis.
\end{abstract}




\section{TABLE OF CONTENTS}

$\underline{\text { Page }}$

6.2 TSUNAMI-3D: Control Module for Three-Dimensional Cross Section Sensitivity and

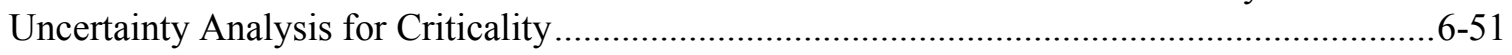

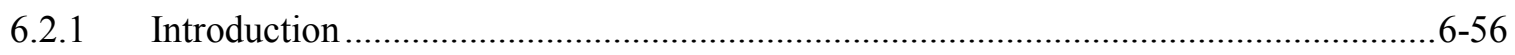

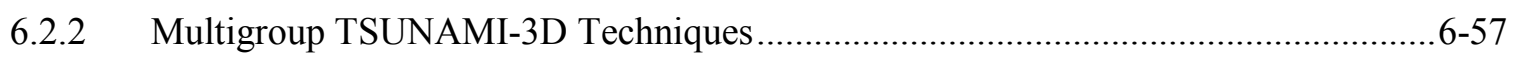

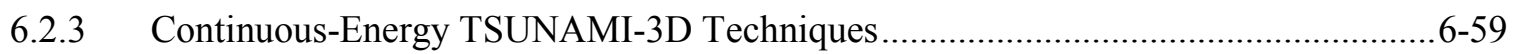

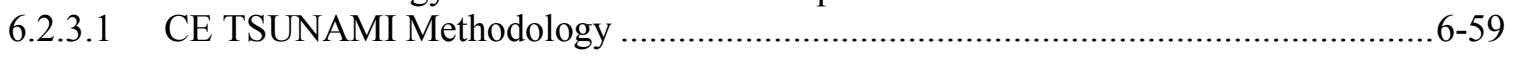

6.2.3.2 CE TSUNAMI Generalized Perturbation Theory Capability ...................................6-63

6.2.3.3 CE TSUNAMI Sequence Description ....................................................................6-66

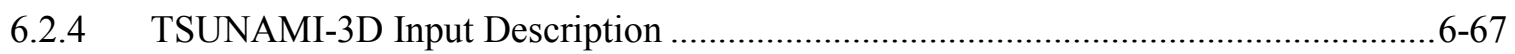

6.2.4.1 Analytical Sequence Specification Record..........................................................6-68

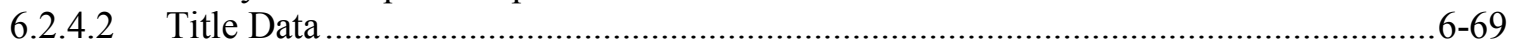

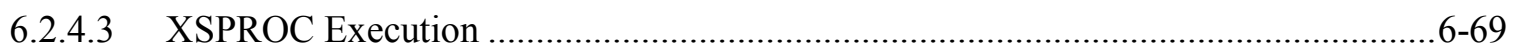

6.2.4.4 KENO V.A or KENO-VI Problem Description ..................................................6-69

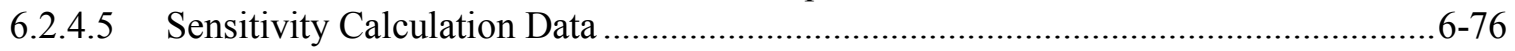

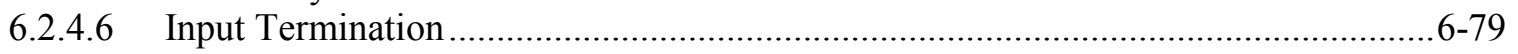

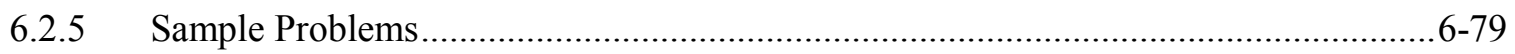

6.2.5.1 Generating Reference Direct Perturbation Sensitivity Coefficients ..........................6-79

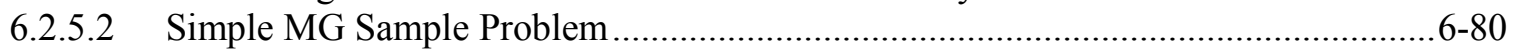

6.2.5.3 Multigroup Sample Problem with Spatial Flux Mesh .............................................6-88

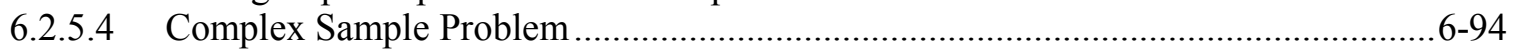

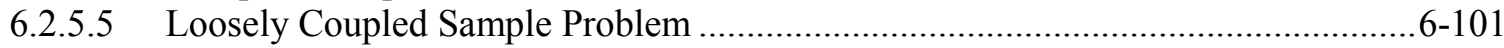

6.2.5.6 CE TSUNAMI Sample Problem ...................................................................... $6-105$

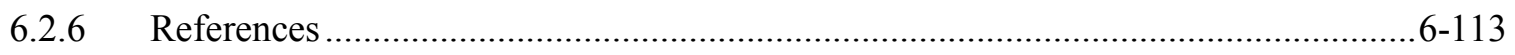




\section{LIST OF FIGURES}

$\underline{\text { Page }}$

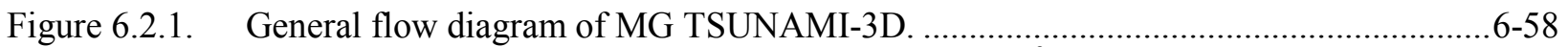

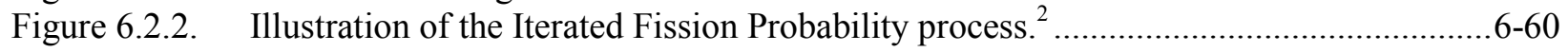

Figure 6.2.3. General flow diagram of CE TSUNAMI-3D .....................................................6-67

Figure 6.2.4 $F^{*}(r)$ mesh from a sample CLUTCH eigenvalue sensitivity calculation. .....................6-73

Figure 6.2.5. $\quad F^{*}(r)$ meshes from a sample CLUTCH GPT sensitivity calculation.............................6-74

Figure 6.2.6. TSUNAMI-3D-K5 simple sample problem input. ...................................................6-81

Figure 6.2.7. Uncertainty information from $\mathrm{UF}_{4}$ sample problem..............................................6-86

Figure 6.2.8. Energy-dependent sensitivity profiles from TSUNAMI-3D-K5 for simple sample

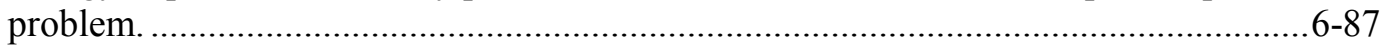

Figure 6.2.9. TSUNAMI-3D-K5 input for LEU-COMP-THERM-033 case 45 .............................6-89

Figure 6.2.10. TSUNAMI-3D-K5 input LEU-COMP-THERM-033 case 45 with manual

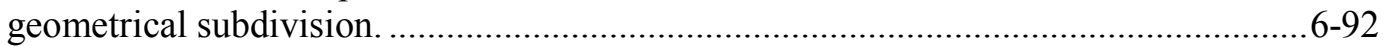

Figure 6.2.11. Cutaway view of LEU-COMP-THERM-033 case 45 with manual subdivision. ..........6-92

Figure 6.2.12. TSUNAMI-3D-K5 input for LEU-COMP-THERM-033 case 45 with $8 \mathrm{~cm}$

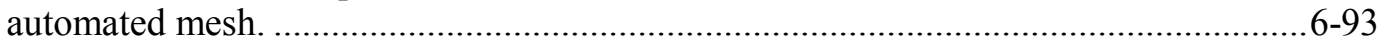

Figure 6.2.13. Cutaway view of LEU-COMP-THERM-033 case 45 with $15 \mathrm{~cm}$ automated mesh. ...6-93

Figure 6.2.14. TSUNAMI-3D LEU-COMP-THERM-009 case 10 sample problem input................6-95

Figure 6.2.15. Graphical representation of KENO V.a geometry for LEU-COMP-THERM-009

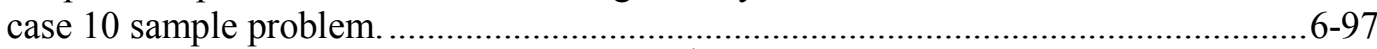

Figure 6.2.16. Sensitivity profiles from TSUNAMI-3D for ${ }^{1} \mathrm{H}$ elastic scattering in mixture 2 of LEU-COMP-THERM-009 case 10 sample problem.............................................6-100

Figure 6.2.17. TSUNAMI-3D-K5 input for sample problem PU-SOL-THERM-014 case 19..........6-102

Figure 6.2.18. Cutaway view of PU-SOL-THERM-014 case 19 sample problem..........................6-102

Figure 6.2.19. Cutaway view of single solution tank from PU-SOL-THERM-014 case 19

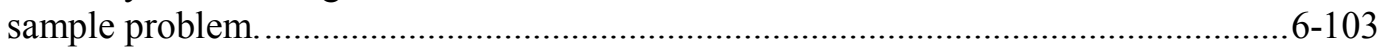

Figure 6.2.20. TSUNAMI-3D-K5 input with GRID input for sample problem PU-SOL-THERM-014 case 19........................................................................6-105

Figure 6.2.21. CE TSUNAMI-3D-K6 input for the Godiva system using the IFP method...............6-106

Figure 6.2.22. CE TSUNAMI-3D-K6 input for the Godiva system using the CLUTCH method......6-107

Figure 6.2.23. CE TSUNAMI-3D-K6 input for the Godiva system using the CLUTCH method

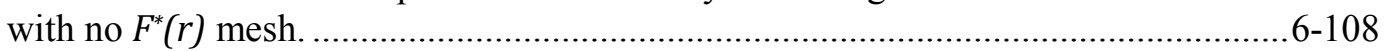

Figure 6.2.24. CE TSUNAMI-3D-K6 input for the Godiva system using GEAR-MC with only the CLUTCH method....................................................................................... $6-110$

Figure 6.2.25. CE TSUNAMI-3D-K6 input for the Godiva system using GEAR-MC with the CLUTCH and IFP methods. 6-111 


\section{LIST OF TABLES}

$\underline{\text { Page }}$

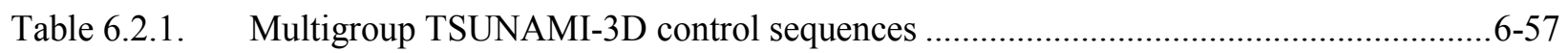

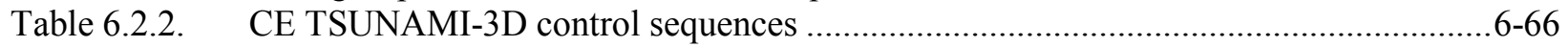

Table 6.2.3. Default values KENO parameters in MG TSUNAMI-3D ........................................6-70

Table 6.2.4. Default values of TSUNAMI-3D parameters for KENO MG adjoint calculation ........6-70

Table 6.2.5. CE TSUNAMI-3D parameters and default values ..................................................6-73

Table 6.2.6. CE TSUNAMI-3D GPT sensitivity parameters and default values .............................6-76

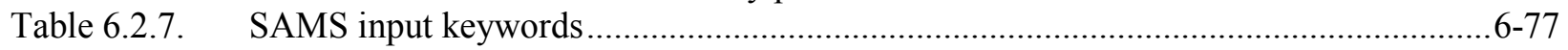

Table 6.2.8. SAMS input keywords for default covariance data ................................................... 6-78

Table 6.2.9. Energy- and region-integrated sensitivity coefficients from TSUNAMI-3D UF 4

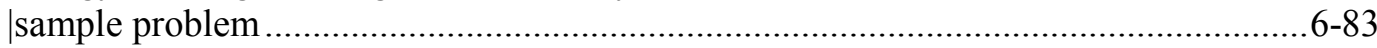

Table 6.2.10. Energy- and region-integrated sensitivity coefficients from LEU-COMP-THERM-033

case 45 sample problem

Table 6.2.11. Energy- and region-integrated total sensitivity coefficients for LEU-COMP-THERM-009 case 10.

Table 6.2.12. Energy- and region-integrated total sensitivity coefficients for PU-SOL-THERM-014 case 19 $6-104$

Table 6.2.13. CE TSUNAMI Godiva sensitivity coefficient comparison 6-109 


\section{ACKNOWLEDGMENTS}

The author wishes to acknowledge M. L. Williams, B. L. Broadhead, and K. B. Bekar, of Oak Ridge National Laboratory (ORNL), and R. L. Childs, formerly of ORNL, for their assistance with this work. The support and encouragement of C. M. Hopper, formerly of ORNL, and C. V. Parks and D. E Mueller of ORNL is also appreciated. The continued support of the U.S. Department of Energy Nuclear Criticality Safety Program and the ORNL Laboratory-Directed Research and Development Program is gratefully acknowledged. 


\subsubsection{Introduction}

TSUNAMI-3D (Tools for Sensitivity and Uncertainty Analysis Methodology Implementation in Three Dimensions) is a SCALE control module that facilitates the application of sensitivity and uncertainty theory to criticality safety analysis. The data computed with TSUNAMI-3D are the sensitivity of computed responses (e.g. $k_{\text {eff }}$ and ratios of reaction rates) to each constituent nuclear data component used in the calculation. The sensitivity data are coupled with cross section uncertainty data, in the form of multigroup (MG) covariance matrices, to produce an uncertainty in the computed responses due to uncertainties in the underlying nuclear data. The group-wise sensitivity data computed with TSUNAMI-3D are stored in a sensitivity data file format (.sdf file) that is suitable for use in data visualization with Fulcrum, similarity assessment with TSUNAMI-IP, and bias assessment with TSURFER.

This manual is intended to provide the user with a detailed reference on code input options and some examples of the application of TSUNAMI-3D to generate sensitivity and uncertainty data. The techniques used in the MG and continuous-energy (CE) versions of TSUNAMI-3D are described in Sects. 6.2.2 and 6.2.3, respectively. The input for TSUNAMI-3D is presented in Sect. 6.2.4, and the use of TSUNAMI-3D to obtain accurate sensitivity coefficients for several sample problems is given in Sect. 6.2.5. Additional information is provided in the appendix. A new user may wish to review the sample problems and then refer to the input description in Sect. 6.2.4 to obtain specific guidance on preparing input for specific models.

TSUNAMI-3D provides automated, problem-dependent cross sections using the same methods and input as the Criticality Safety Analysis Sequences (CSASs). Although CE calculations with TSUNAMI do not require resonance self-shielding calculations because of its use of CE cross sections, MG TSUNAMI-3D uses self-shielding codes that are similar to those used in MG CSAS calculations. The BONAMIST code computes the sensitivity of resonance self-shielded cross to the input data, the so-called "implicit sensitivities" for MG calculations.

After the cross sections are processed, the MG TSUNAMI-3D-K5 sequence performs two KENO V.a criticality calculations, one forward and one adjoint; the MG TSUNAMI-3D-K6 sequence performs two KENO-VI calculations. Finally, the sequence calls the SAMS module to calculate the sensitivity coefficients that indicate the sensitivity of the computed responses to changes in the cross sections and the uncertainty in the computed responses that is due to uncertainties in the basic nuclear data. SAMS prints energy-integrated sensitivity coefficients and their statistical uncertainties to the SCALE output file and generates a separate data file containing the energy-dependent sensitivity coefficients. CE calculations in TSUNAMI do not require a separate adjoint criticality calculation; instead, they calculate sensitivity coefficients and produce a sensitivity data file during a single forward simulation. The SAMS module is used to print user output for CE calculations.

Choosing poor values for any of several adjustable parameters in TSUNAMI inputs may result in inaccurate sensitivity coefficient estimates; thus, users are advised to always compare their calculated sensitivity coefficients to reference values to verify the suitability of their TSUNAMI input parameters. The Sample problems section describes the direct perturbation approach for generating reference sensitivity coefficients for the total cross section of nuclides in a system. It is difficult to calculate high fidelity, energy-dependent, reference sensitivity coefficients using direct perturbation (although possible using stochastic sampling of cross section data), but at the minimum users are advised to verify the accuracy of TSUNAMI-produced total nuclide sensitivity coefficients for all key nuclides in their application. 


\subsubsection{Multigroup TSUNAMI-3D Techniques}

TSUNAMI-3D is a SCALE control module. As such, its primary function is to control a sequence of calculations that are performed by other codes. A thorough theoretical development of MG eigenvalue sensitivity theory is described in the SAMS section of the SCALE documentation. Currently, two computational sequences are available with TSUNAMI-3D: TSUNAMI-3D-K5 and TSUNAMI-3D-K6. The input for TSUNAMI-3D-K5 is very similar to that used for CSAS5 and the input for TSUNAMI-3D-K6 is very similar to that of CSAS6. TSUNAMI-3D uses the same material and cell data input as all other SCALE sequences. TSUNAMI-3D can calculate eigenvalue sensitivity coefficients using either MG or CE Monte Carlo simulations, but the theoretical approaches for each calculation mode differ greatly. MG TSUNAMI-3D techniques will be discussed in this section, and CE TSUNAMI-3D calculations will be discussed in Sect. 6.2.3. The control sequences available in MG TSUNAMI-3D are summarized in Table 6.2.1, where the functional modules that are executed are also shown. A general flow diagram of MG TSUNAMI-3D is shown in Figure 6.2.1.

Table 6.2.1. Multigroup TSUNAMI-3D control sequences

\begin{tabular}{|c|c|c|c|c|c|}
\hline \multirow{2}{*}{$\begin{array}{c}\begin{array}{c}\text { Control } \\
\text { sequence }\end{array} \\
\text { TSUNAMI-3D-K5 }\end{array}$} & \multicolumn{5}{|c|}{ Functional modules executed by the control module } \\
\hline & XSProc & $\begin{array}{l}\text { KENO V.a } \\
\text { forward }\end{array}$ & $\begin{array}{l}\text { KENO V.a } \\
\text { adjoint }\end{array}$ & BONAMIST & SAMS5 \\
\hline TSUNAMI-3D-K6 & XSProc & $\begin{array}{l}\text { KENO-VI } \\
\text { forward }\end{array}$ & $\begin{array}{c}\text { KENO-VI } \\
\text { adjoint }\end{array}$ & BONAMIST & SAMS6 \\
\hline
\end{tabular}

TSUNAMI-3D and many other SCALE sequences apply a standardized procedure to provide appropriate cross sections for the calculation. This procedure is carried out by routines of the XSProc module, which generate number densities and related information, prepare geometry data for resonance self-shielding and flux-weighting cell calculations, and create data input files for the cross section processing codes.

By default, the MG TSUNAMI-3D sequence performs cross-section processing with XSProc, exercising all available options there, performs the forward and adjoint KENO calculations, calls BONAMIST to produce implicit sensitivity coefficients, then calls SAMS to produce sensitivity and uncertainty output and $s d f$ files. Optional sequence level parameters can be used to change methods applied in resonance self-shielding and exclude the implicit sensitivity calculation, which are detailed later in this document. 


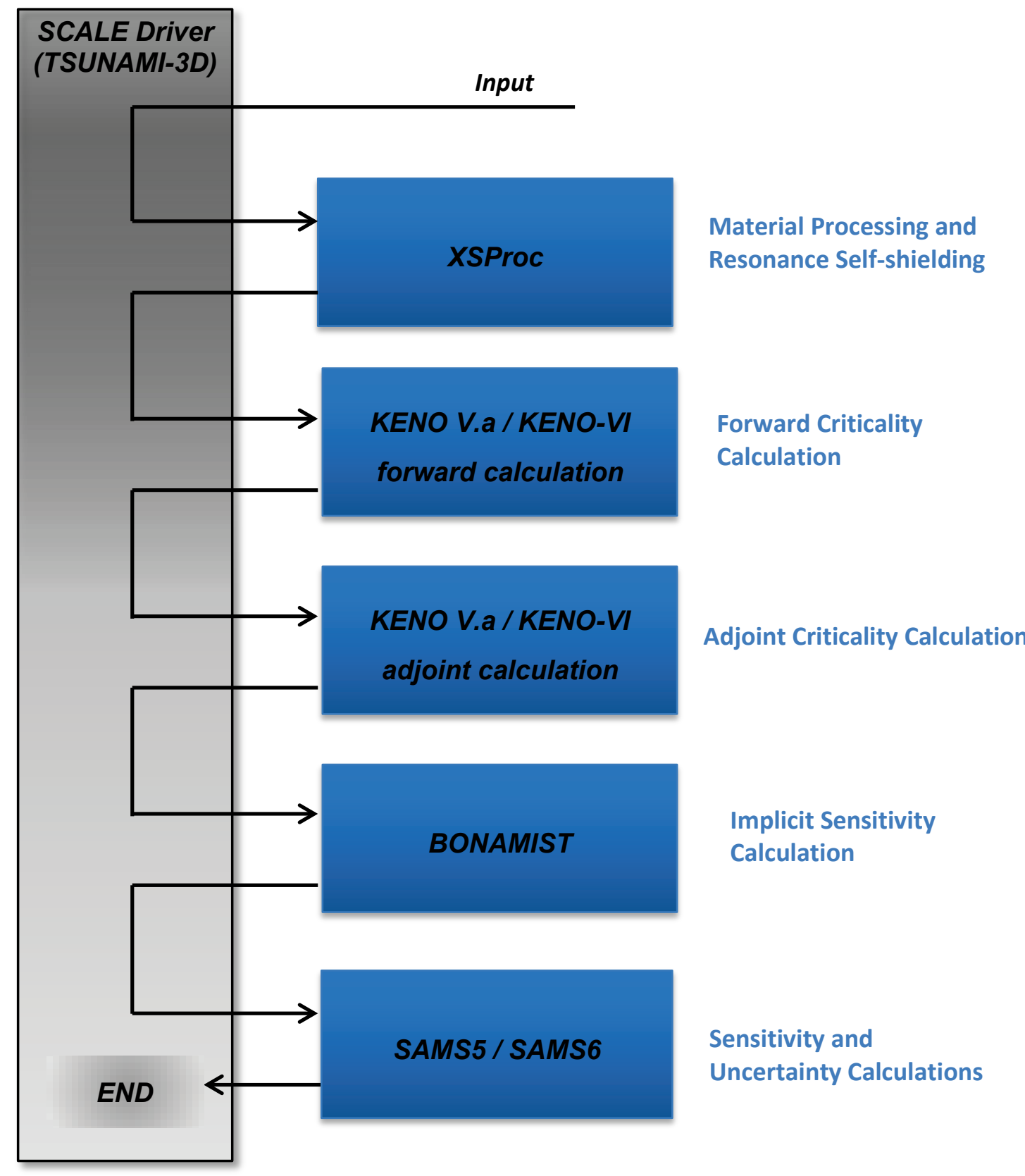

Figure 6.2.1. General flow diagram of MG TSUNAMI-3D. 
Once the appropriate AMPX libraries are prepared, TSUNAMI-3D prepares KENO V.a or KENO-VI inputs for forward and adjoint calculations from the criticality model provided by the user. The input requirements for the KENO V.a input sections are identical to those for the CSAS5 sequence, with some optional additional data. Also, the input requirements for the KENO-VI are identical to those for CSAS6, with some optional additional data. Additional input is prepared for the SAMS module using an optional user-defined input block for SAMS. TSUNAMI-3D executes forward and adjoint KENO calculations, generates implicit sensitivity data with BONAMIST, and then executes the SAMS module to compute the sensitivity and uncertainty data using the data accumulated from the codes previously executed in the sequence. Details concerning calculation of sensitivity and uncertainty data using MG forward and adjoint calculations are provided in the SAMS chapter of the SCALE manual. Of particular interest, the filename.sdf file, where filename is the name of the input file less any extensions, contains energydependent sensitivity coefficients. SCALE returns this file to the same directory as the input file.

The XSProc module is responsible for reading the standard composition data and other engineering-type specifications, including volume fraction or percent theoretical density, temperature, and isotopic distribution as well as the unit cell data. The techniques used in the XSProc module and their applications and limitations are discussed in the XSProc chapter. The input data for XSProc is the same for all analytical sequences available through TSUNAMI-3D, TSUNAMI-1D, CSAS, and many other SCALE sequences.

\subsubsection{Continuous-Energy TSUNAMI-3D Techniques}

Like MG TSUNAMI-3D, the CE TSUNAMI-3D capability is a control module that uses codes within the SCALE code package to calculate eigenvalue sensitivity coefficients and other information for models of eigenvalue problems. The CE and MG TSUNAMI-3D calculations differ dramatically in their approach for calculating sensitivity coefficients and as a result have different user interfaces. CE TSUNAMI calculations are automatically enabled when the user selects a CE cross-section library.

\subsubsection{CE TSUNAMI methodology}

CE TSUNAMI currently contains two separate approaches for performing eigenvalue sensitivity coefficient calculations: Iterated Fission Probability (IFP) approach and Contributon-Linked eigenvalue sensitivity/Uncertainty estimation via Tracklength importance Characterization (CLUTCH) approach. Both IFP and CLUTCH calculate sensitivity coefficients during a single forward Monte Carlo (KENO) simulation, and, unlike MG TSUNAMI-3D, do not require the simulation of adjoint histories, calculation of angular flux moments using a flux mesh, volume calculations, or treatment of implicit sensitivity effects. The theoretical background of each method is discussed in detail in the following sections, but in general IFP is easier to use than $\mathrm{CLUTCH}$, but $\mathrm{CLUTCH}$ has greater computational efficiency and a lower memory footprint.

\subsection{IFP methodology}

The IFP methodology, developed by Hurwitz in the 1940s and 1950s, determines the importance of events during a particle history using the notion that an event's importance is proportional to the number of neutrons present in the system during some future generation that are descendants, or progeny, of the original event. ${ }^{1,2}$ In practice, the IFP method requires storing reaction rate tallies for particles for some number of generations until the average population of their descendants in the system, or "asymptotic population," is obtained. This process is illustrated in Figure 6.2.2. Once obtained, the asymptotic population of the original neutron is used to weight reaction rate tallies for that neutron and to produce sensitivity coefficient estimates via the first-order perturbation equation. ${ }^{1,2}$ A number of generations, referred to as the "latent generations," must be skipped before calculating the asymptotic population for an event to guarantee that the progeny of the event have had sufficient time to impact all regions in the 
system and to converge to a true estimate of the asymptotic population. The number of latent generations required to calculate accurate sensitivity coefficients varies based on the complexity of the system and the desired sensitivity coefficient fidelity, but in general 20 generations is a conservative number of latent generations to ensure convergence to the asymptotic population. The IFP method is useful for benchmarking the accuracy of other sensitivity coefficient methods and is very easy to use because the only assumption of the IFP method (besides the standard CE Monte Carlo and first order perturbation theory assumptions) is that the asymptotic population that is reached after the chosen number of latent generations is representative of the importance of the original event. Thus a user who is new to sensitivity methods can assume a conservative number of latent generations and can use the IFP method to accurately calculate sensitivity coefficients for a system so long as the user's computer has sufficient computational memory for the simulation.

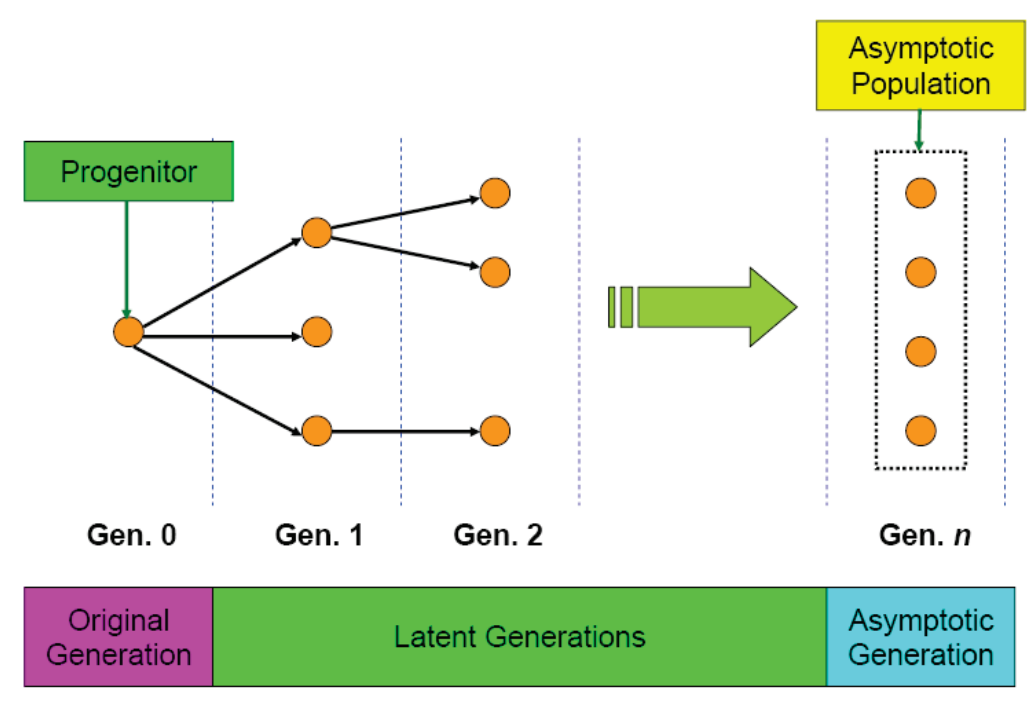

Figure 6.2.2. Illustration of the Iterated Fission Probability process. ${ }^{2}$

The IFP method requires storage of region-, isotope-, reaction-, and energy-dependent reaction rates for every particle for some number of latent generations. Complex problems can require simulating tens of thousands or hundreds of thousands of particles during each generation, and IFP simulations for these systems can easily require many gigabytes of computational memory storage. The IFP implementation in TSUNAMI-3D makes use of dynamic memory allocation to minimize memory requirements, but the method frequently produces large memory footprints despite these optimizations. The IFP memory requirements are proportional to the number of latent generations used in the calculations, and perhaps the best approach for minimizing the memory requirements of the IFP method is to minimize the number of latent generations used in a calculation. Twenty is typically a conservative number of latent generations, and it is recommended that the user always use as few latent generations as possible for IFP simulations. The 10 latent generations that IFP assumes by default is a reasonable starting guess, but users should compare IFP nuclide sensitivity coefficients with direct perturbation sensitivities to determine if they need to use more or fewer latent generations. Determining the adequate number of latent generations can be done by starting with a small number of latent generations and slowly increasing the number until the IFP-calculated sensitivity coefficients agree well with reference sensitivities. The memory requirements of the IFP method are also proportional to the number of particles used in each generation (NPG), and high-memory simulations can decrease NPG to decrease the memory requirements of the IFP method. This should be done with caution because making NPG too small can affect the fission source convergence and thus can produce poor tally Monte Carlo variance estimates. 


\subsection{CLUTCH methodology}

The CLUTCH method was developed by Perfetti in 2012, based in part on the Contributon theory explored by M. L. Williams of ORNL, to produce an accurate and efficient means for calculating eigenvalue sensitivity coefficients with a small computational memory footprint. ${ }^{3}$ Like the IFP method, CLUTCH calculates sensitivities during a single forward Monte Carlo calculation and does not require tallying angular flux moments. Instead, the CLUTCH method calculates the importance of events during a particle's lifetime by examining how many fission neutrons are created by that particle after those events occur. Consider a neutron source $Q$ that is equal to the fission source of a system:

$$
Q=\lambda F \phi
$$

Multiplying this source by the adjoint flux and integrating over phase space gives

$$
\left\langle\phi^{*} Q\right\rangle=\lambda\left\langle\phi^{*} F \phi\right\rangle .
$$

Consider now a neutron emitted in phase space $\tau_{s}$ such that $Q\left(\tau_{s}\right)=Q_{S} \delta\left(\tau-\tau_{s}\right)$. This source definition reduces Eq. (6.2.2) and allows the importance of the neutron in phase space $\tau_{s}$ to be calculated by

$$
\phi^{*}\left(\tau_{s}\right)=\frac{\lambda}{Q_{s}} \int_{V} G\left(\tau_{s} \rightarrow r\right) F^{*}(r) d r,
$$

where the transfer function $G\left(\tau_{s} \rightarrow r\right)$ is equal to the expected number of fission neutrons generated in all energies and directions at $r$ due to a neutron emitted at phase space $\tau_{s}$ and is given by

$$
G\left(\tau_{s} \rightarrow r\right)=\frac{1}{Q_{s}} \int_{E} \int_{\Omega} v \Sigma_{f}(r, E) \phi\left(r, E, \Omega \mid Q\left(\tau_{s}\right)\right) d \Omega d E,
$$

where $\phi\left(r, E, \Omega \mid Q\left(\tau_{s}\right)\right)$ is the flux created in phase space $(r, E, \Omega)$ given the source $Q\left(\tau_{s}\right)$. The weighting function $F^{*}(r)$ is defined to be equal to the expected importance generated by a fission neutron emitted at $r$ and is given by

$$
F^{*}(r)=\int_{E} \int_{\Omega} \frac{\chi(r, E)}{4 \pi} \phi^{*}(r, E, \Omega) d \Omega d E .
$$

In practice, the CLUTCH method calculates the integral of $G\left(\tau_{s} \rightarrow r\right)$, weighted by $F^{*}(r)$, to calculate the importance of every event in a particle's lifetime. For example, the importance of a scattering event would be determined by tallying how many fission neutrons, weighted by the value of $F^{*}(r)$ at the sites where they are born, are created by the neutron that emerges from the scattering collision. The CLUTCH method cannot calculate the importance of events until after the particle that caused these events dies, which requires that CLUTCH store reaction rate information for every collision that a particle undergoes during its lifetime. This is a manageable amount of information because these tallies are not energy dependent (a particle's energy is constant between any two collisions) and because this information can be deleted once the particle dies. In contrast, the IFP method requires much more memory because it stores energy-dependent reaction rate tallies for every particle for some number of generations.

The only assumption made by the CLUTCH method (besides the standard CE Monte Carlo assumptions) is that $F^{*}(r)$ provides an accurate estimate of the average importance of fission neutrons at $r$. The current approach for calculating $F^{*}(r)$ takes advantage of the definition of the unconstrained fission spectrum sensitivity coefficient, which is given by 


$$
S_{k, \chi}(r)=\frac{1}{D} \frac{1}{k} \int_{0}^{\infty} \int_{4 \pi} \bar{v} \Sigma_{f}(r, E) \phi(r, E, \Omega) d \Omega d E \int_{0}^{\infty} \int_{4 \pi} \frac{\chi\left(r, E^{\prime}\right)}{4 \pi} \phi^{*}\left(r, E^{\prime}, \Omega^{\prime}\right) d \Omega^{\prime} d E^{\prime}
$$

where $D$ is the adjoint-weighted fission source for the system. The right-most integral of Eq. (6.2.6) is recognized as the definition of $F^{*}(r)$ in Eq. (6.2.5) and the terms in Eq. (6.2.6) can be rearranged to define $F^{*}(r)$ as

$$
F^{*}(r)=\frac{D \times S_{k, \chi}(r)}{\int_{0}^{\infty} \int_{4 \pi} \frac{\bar{v} \Sigma_{f}(r, E) \phi(r, E, \Omega)}{k} d \Omega d E}
$$

This approach assumes that the energy spectrum of neutrons emitted from a fission event is not strongly dependent on the parent isotope or the energy of the neutron causing the fission. The current CLUTCH implementation tallies the unconstrained chi sensitivity in the numerator of Eq. (6.2.7) during the inactive generations to estimate $F^{*}(r)$ for the active generation sensitivity coefficient calculations. The spatial dependence of $F^{*}(r)$ is currently accounted for by calculating and storing $F^{*}(r)$ on a spatial mesh, although kernel density estimators might be used in the future to store $F^{*}(r)$ using spatially continuous functional representations. ${ }^{4}$ Because $F^{*}(r)$ is only nonzero for regions containing fissionable material, the $F^{*}(r)$ mesh used in a CE TSUNAMI calculation must only encompass all fissionable material in the system, rather than the entire system as required by MG TSUNAMI. The $D$ term in Eq. (6.2.7) can be ignored because it is constant for all regions in a problem and is cancelled out by the presence of $F^{*}(r)$ terms in both the numerator and denominator of the first-order perturbation equation. The denominator in Eq. (6.2.7) is simply the total weight of fission neutrons born in each $F^{*}(r)$ mesh region, which must also be tallied during the inactive generations.

Because $F^{*}(r)$ describes the contribution to the chi sensitivity that is created per fission neutron born at a point, a converged fission source is not required to begin $F^{*}(r)$ calculations; thus, $F^{*}(r)$ tallies begin during the inactive generations of Monte Carlo simulations to obtain useful information from the typically discarded inactive particle histories. The fission source must converge well enough during the inactive generations so that $F^{*}(r)$ is tallied in all fissile regions to a desired statistical uncertainty, and it is sometimes necessary to simulate extra inactive generations to allow $F^{*}(r)$ tallies to fully converge; however, the ability to begin $F^{*}(r)$ tallies before the fission source is converged essentially provides "free" tallies while the fission source is converging.

Although several approaches exist for calculating the unconstrained chi sensitivity coefficients needed to calculate $F^{*}(r)$, an IFP-based approach has been determined to be the best approach. ${ }^{3}$ Although the IFP method can produce large memory footprints for full sensitivity coefficient calculations, the amount of memory required by the method to calculate unconstrained chi sensitivity coefficients and $F^{*}(r)$ is generally negligible. The spatial dependence of $F^{*}(r)$ is currently described using a spatial mesh, and an interval of 1 to $2 \mathrm{~cm}$ mesh is typically sufficiently refined to obtain accurate $F^{*}(r)$ estimates. Users should simulate at least on the order of 50 to 100 inactive histories per mesh interval to allow for sufficient $F^{*}(r)$ convergence; sometimes this necessitates simulating a large number of additional inactive histories/generations. 


\subsubsection{CE TSUNAMI Generalized Perturbation Theory capability}

The CLUTCH and IFP methods were combined in 2013 by Perfetti to enable sensitivity calculations for generalized neutronic response ratios. ${ }^{5}$ This Generalized Perturbation Theory (GPT) capability is known as the GEneralized Adjoint Responses in Monte Carlo (GEAR-MC) method. Applications for GPT sensitivity coefficients differ from the traditional criticality safety applications of TSUNAMI, and may include $\mathrm{S} / \mathrm{U}$ analyses for multigroup cross sections that are produced by a continuous-energy Monte Carlo code, the relative power of pins in a LWR, or ratios of foil activities in an irradiation experiment.

Two approaches for performing the GEAR-MC method have been included in this release as demonstration capabilities. One implementation relies heavily on the IFP method for sensitivity calculations and is therefore subject to the long runtimes and large memory footprint that may be encountered when using the IFP method. The other implementation does not use the IFP method when calculating sensitivities. Instead, it uses only the CLUTCH method with an $F^{*}(r)$ mesh that has been modified for performing generalized response sensitivity calculations. Because it does not use the IFP method except for calculating the $F^{*}(r)$ function, this approach typically produces a significantly lower memory footprint than the first GEAR-MC implementation and can be performed in a parallel environment. ${ }^{6}$ Both approaches are experimental capabilities and have been included in this release to demonstrate the expanding SCALE S/U capabilities. The current format for inputting response information allows for the sensitivity calculations for a single response in each sensitivity calculation; future implementations will allow for the calculation of multiple response sensitivities in a single calculation.

The theory behind the GEAR-MC method will now be described. Rather than calculating eigenvalue sensitivities, the GEAR-MC method calculates the sensitivity of a response, $R$, to perturbations or uncertainties in the system parameters. The generalized response sensitivity coefficient for the system parameter $\Sigma_{x}$ is defined as

$$
S_{R, \Sigma_{x}}=\frac{\delta R / R}{\delta \Sigma_{x} / \Sigma_{x}}
$$

The GEAR-MC method calculates sensitivities of responses that are defined as ratios of neutron reaction rates integrated over some region of phase space such that

$$
R=\frac{\left\langle\Sigma_{1} \phi\right\rangle}{\left\langle\Sigma_{2} \phi\right\rangle}
$$

where $\Sigma_{1}$ and $\Sigma_{2}$ are nuclear cross sections. The reaction rates in Eq. (6.2.9) can be isotope- or materialdependent reaction rates and can also represent neutron flux responses if $\Sigma=1$. The fractional change in $R$ due to a perturbation $\delta \Sigma_{x}$ to the system parameter $\Sigma_{x}$ is given by

$$
\frac{\delta R}{R}=\left\langle\frac{1}{R} \frac{\partial R}{\partial \Sigma_{x}} \delta \Sigma_{x}+\frac{1}{R} \frac{\partial R}{\partial \phi} \frac{\partial \phi}{\partial \Sigma_{x}} \delta \Sigma_{x}\right\rangle
$$

The first term in Eq. (6.2.10) is known as the "direct effect term" and describes how perturbations in $\Sigma_{x}$ affect the response function of the response reaction rates. The second term, known as the "indirect effect term," describes how perturbations affect the neutron flux spectrum in the response region. ${ }^{7}$ Calculating the sensitivity of the response to the direct effect term is relatively simple and involves tallying the fraction of the total numerator and denominator responses that is generated for each energy, region, isotope, and material in the response region(s). For example, consider a response that is defined as the ratio of the energy-integrated fission rate to the energy-integrated capture rate in a uranium fuel pin. The 
direct effect sensitivity of this response to the thermal fission cross section is simply the fraction of the fission reaction rate in the pin that is caused by neutrons with thermal energies.

The indirect effect term in Eq. (6.2.10) cannot be calculated as simply as the direct effect term and has historically posed a greater challenge. The GEAR-MC method offers an approach for calculating the indirect effect term during a single, unperturbed Monte Carlo transport calculation. The neutron balance equation for an eigenvalue problem is given by

$$
L \phi-\lambda P \phi=0
$$

where $L$ is the neutron loss operator and $P$ is the fission neutron production operator. The change induced in the neutron balance equation in response to a first-order perturbation is given by

$$
(L-\lambda P) \delta \phi=\delta \lambda P \phi+(\lambda \delta P-\delta L) \phi
$$

Consider now the generalized adjoint balance equation

$$
\left(L^{*}-\lambda P^{*}\right) \Gamma^{*}=S^{*}
$$

where $L^{*}$ is the adjoint loss operator, $P^{*}$ is the adjoint fission neutron production operator, $S^{*}$ is a source of importance for the response that is defined such that $\left\langle\phi S^{*}\right\rangle=0$, and $\Gamma^{*}$ is the generalized importance function that provides the solution to this equation. ${ }^{7}$ Multiplying Eq. (6.2.12) and Eq. (6.2.13) by $\Gamma^{*}$ and $\delta \phi$, respectively, and taking the inner product gives, respectively,

$$
\left\langle\Gamma^{*}(L-\lambda P) \delta \phi\right\rangle=\delta \lambda\left\langle\Gamma^{*} P \phi\right\rangle+\left\langle\Gamma^{*}(\lambda \delta P-\delta L) \phi\right\rangle
$$

and

$$
\left\langle\delta \phi\left(L^{*}-\lambda P^{*}\right) \Gamma^{*}\right\rangle=\left\langle\delta \phi S^{*}\right\rangle
$$

The source of adjoint importance in Eq. (6.2.15) is defined to conveniently provide an expression for the indirect effect term. ${ }^{7}$ Defining $S^{*}$ as

$$
S^{*} \equiv \frac{1}{R} \frac{\delta R}{\delta \phi}=\frac{\Sigma_{1}}{\left\langle\Sigma_{1} \phi\right\rangle}-\frac{\Sigma_{2}}{\left\langle\Sigma_{2} \phi\right\rangle}
$$

and applying the adjoint property allows Eqs. (6.2.14) and (6.2.15) to be combined to express the indirect effect term as

$$
\left\langle\frac{1}{R} \frac{\delta R}{\delta \phi} \delta \phi\right\rangle=\left\langle\delta \lambda \Gamma^{*} P \phi\right\rangle+\left\langle\Gamma^{*}(\lambda \delta P-\delta L) \phi\right\rangle
$$

The $\left\langle\delta \lambda \Gamma^{*} P \phi\right\rangle$ term in Eq. (6.2.17) is usually equal to zero because $\Gamma^{*}$ is typically orthogonal to $P \phi$. The effect of this orthogonality can be interpreted in a more physical manner by realizing that perturbations to the eigenvalue of a system do not alter the steady-state neutron flux shape or spectrum of the system. As a result, perturbations affect the response numerator and denominator terms equally.

The GEAR-MC methodology uses Eqs. (6.2.13) and (6.2.17) to calculate the generalized importance function $\Gamma^{*}$ for neutrons during a single forward Monte Carlo simulation, thus enabling the calculation of 
the indirect effect term in Eq. (6.2.10) and thus sensitivity coefficients for generalized responses using GPT. The approach developed for calculating $\Gamma^{*}$ is similar to the approach used by the CLUTCH method for calculating eigenvalue sensitivity coefficients.

Assuming that the fission production term, $\lambda P \phi$, in Eq. (6.2.11) is the sole source of neutron production in a system, $Q$, multiplying Eqs. (6.2.11) and (6.2.13) by $\Gamma^{*}$ and $\phi$, respectively, and integrating over all phase space gives

$$
\left\langle\Gamma^{*} L \phi\right\rangle=\left\langle\Gamma^{*} Q\right\rangle
$$

and

$$
\left\langle\phi L^{*} \Gamma^{*}\right\rangle=\lambda\left\langle\phi P^{*} \Gamma^{*}\right\rangle+\left\langle\phi S^{*}\right\rangle
$$

Nonfission neutron production reactions, such as $(n, X n)$ reactions, are included in the $L^{*}$ adjoint loss term in Eq. (6.2.19). Combining Eqs. (6.2.18) and (6.2.19) using the adjoint property gives

$$
\left\langle\Gamma^{*} Q\right\rangle=\lambda\left\langle\Gamma^{*} P \phi\right\rangle+\left\langle\phi S^{*}\right\rangle
$$

The terms in Eq. (6.2.20) are all equal to zero in inner product space, but Eq. (6.2.20) can be used to extract information about the importance of events by considering the neutron source to be a single neutron traveling through the phase space $\tau_{s}$, such as a neutron entering or leaving a collision at some point. This concept is used similarly in Williams' Contributon theory for calculating eigenvalue sensitivity coefficients and assumes that

$$
Q=Q_{s} \delta\left(\tau-\tau_{s}\right)
$$

where $Q_{S}$ is the source strength for this neutron. ${ }^{8,9}$ Substituting Eq. (6.2.21) into Eq. (6.2.20) produces an expression for the generalized importance function at $\tau_{s} .{ }^{5}$

$$
\begin{aligned}
\Gamma^{*}\left(\tau_{s}\right) & =\frac{1}{Q_{s}}\left\langle S^{*}(r) \phi\left(\tau_{s} \rightarrow r\right)\right\rangle+\frac{\lambda}{Q_{s}}\left\langle\Gamma^{*}(r) P \phi\left(\tau_{s} \rightarrow r\right)\right\rangle \\
& =\frac{1}{Q_{s}}\left\langle\frac{1}{R} \frac{\delta R}{\delta \phi}(r) \phi\left(\tau_{s} \rightarrow r\right)\right\rangle+\frac{\lambda}{Q_{s}}\left\langle\Gamma^{*}(r) P \phi\left(\tau_{s} \rightarrow r\right)\right\rangle
\end{aligned}
$$

where $\phi\left(\tau_{s} \rightarrow r\right)$ is the neutron flux created at $r$ by the neutron originating at $\tau_{s}$. The two terms on the right-hand side of Eqs. (6.2.20) and (6.2.22) represent the intragenerational and intergenerational effects of an event on the importance of a particle, respectively. The intragenerational effect term describes how much importance the neutron in phase space $\tau_{s}$ generates in the response region(s) during its lifetime; the intergenerational effect term describes how many fission neutrons this neutron creates and how much importance these fission neutrons will generate in future generations. The intragenerational term can be determined by tallying the amount of flux generated in the response region(s) and weighted by $S^{*}(r)$ from Eq. (6.2.16) from the time the particle enters phase space $\tau_{s}$ until its death. Thus the intragenerational term is given by

$$
\left\langle S^{*}(r) \phi\left(\tau_{s} \rightarrow r\right)\right\rangle=\frac{\Sigma_{1} \phi\left(\tau_{s} \rightarrow r\right)}{\left\langle\Sigma_{1} \phi\right\rangle}-\frac{\Sigma_{2} \phi\left(\tau_{s} \rightarrow r\right)}{\left\langle\Sigma_{2} \phi\right\rangle}
$$


The approach for calculating the intragenerational importance term in Eq. (6.2.23) is similar to the approach used by the CLUTCH method during eigenvalue sensitivity coefficient calculations and requires storing tracklength information for each collision that a particle enters and determining the importance of that collision after the particle dies. ${ }^{3,6}$ The presence of both positive and negative terms in Eq. (6.2.23) allows a single event to generate either a positive or negative importance. The intergenerational contribution to the importance function can be calculated by tallying the cumulative score of $S^{*}(r) \phi\left(\tau_{s} \rightarrow r\right)$ that is generated by the particle's daughter fission neutrons, or "progeny," over some number of generations. The GEAR-MC method estimates the intergenerational importance by summing the intragenerational importance, $\Gamma_{i}^{*}$, generated by the fission production, $F_{i}$, of neutrons in the $i$ th generation of a fission chain over some number of generations:

$$
\lambda\left\langle\Gamma^{*}(r) P \phi\left(\tau_{s} \rightarrow r\right)\right\rangle=\Gamma_{1}^{*} F_{1}+\Gamma_{2}^{*} F_{2}+\Gamma_{3}^{*} F_{3}+\ldots+0
$$

This approach is used similarly by the IFP approach for calculating the importance of events during eigenvalue sensitivity calculations, except that the IFP method tallies the importance only one time after the daughter neutrons have established an asymptotic population in the system. ${ }^{2,3}$ The $\delta \lambda$ term in Eq. (6.2.17) demands that the $\left\langle\Gamma^{*} P \phi\right\rangle$ term be equal to zero, which causes the $\Gamma_{i}^{*} F_{i}$ terms in Eq. (6.2.24) to approach zero as $i$ approaches infinity; therefore, the intergenerational importance term is obtained by taking the sum of the $\Gamma_{i}^{*} F_{i}$ terms as they asymptotically approach zero.

\subsubsection{CE TSUNAMI sequence description}

The code flow of CE calculations with TSUNAMI is significantly simpler than the flow of MG TSUNAMI because CE Monte Carlo does not require resonance self-shielding of MG cross sections or the calculation of implicit sensitivity coefficients and calculates sensitivity coefficients during a single forward calculation. The CE control sequences available in TSUNAMI are summarized in Table 6.2.2, where the functional modules executed are also shown. A general flow diagram of a CE calculation with TSUNAMI is shown in Figure 6.2.3.

Table 6.2.2. CE TSUNAMI-3D control sequences

\begin{tabular}{ccc}
\hline $\begin{array}{c}\text { Control } \\
\text { sequence }\end{array}$ & $\begin{array}{c}\text { Functional modules executed by the } \\
\text { control module }\end{array}$ \\
\hline TSUNAMI-3D-K5 & KENO V.a & SAMS5 \\
& & \\
TSUNAMI-3D-K6 & KENO-VI & SAMS6 \\
& & \\
\hline
\end{tabular}




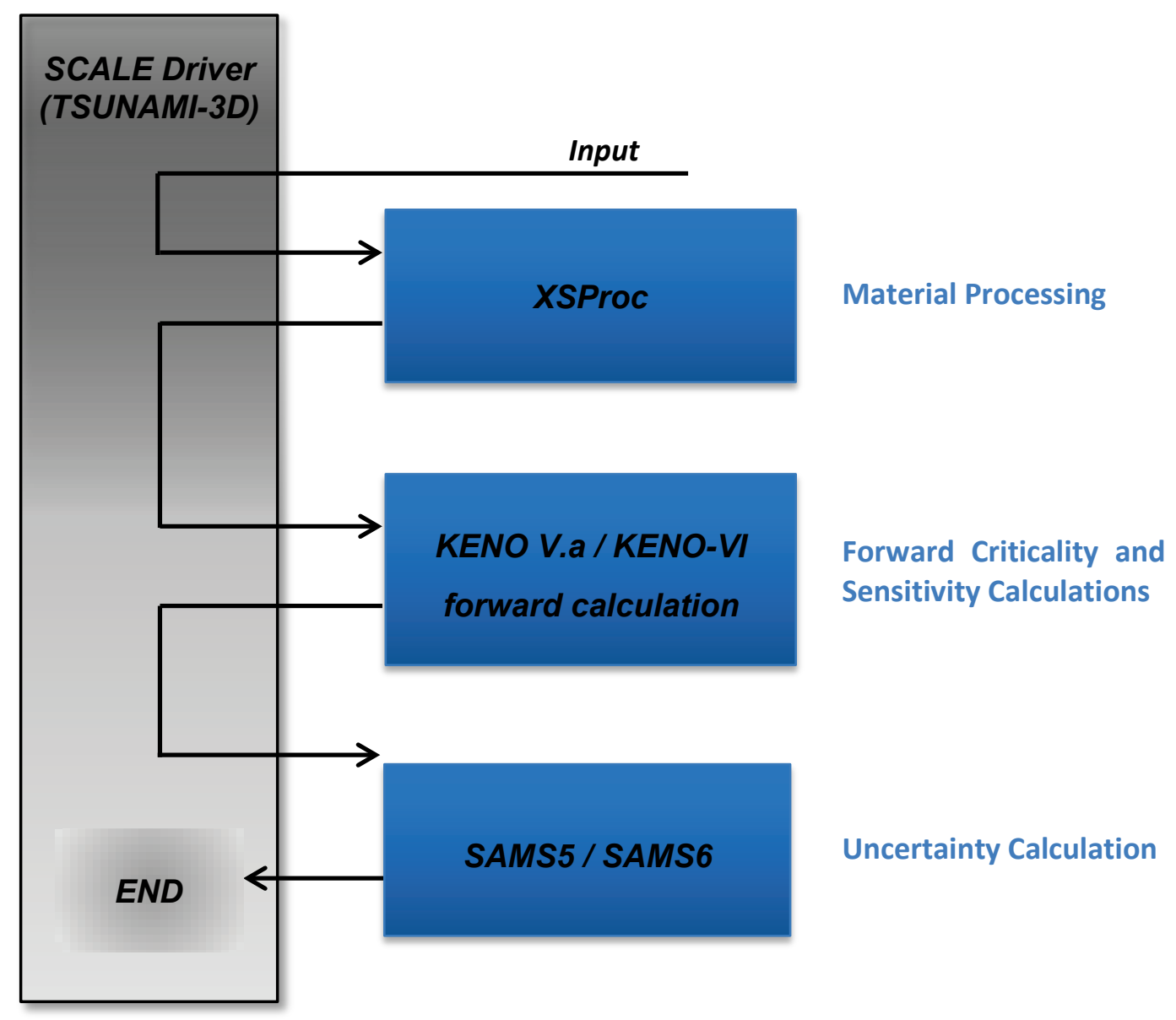

Figure 6.2.3. General flow diagram of CE TSUNAMI-3D.

Eigenvalue sensitivity coefficients are calculated during the KENO Monte Carlo transport calculation, and the energy-dependent sensitivity coefficients are summarized by KENO in a sensitivity data file (sdf). The SAMS module then uses the .sdf file produced by KENO and cross section covariance data to complete the eigenvalue uncertainty analysis for the problem and estimate the data-induced eigenvalue uncertainty.

\subsubsection{TSUNAMI-3D Input Description}

The input for TSUNAMI-3D is designed to be very compatible with those used for the CSAS criticality safety analysis sequences. Given a CSAS input, MG TSUNAMI-3D calculations only require several input modifications to obtain adequate flux solutions, and CE TSUNAMI calculations require as little as one additional parameter. Additional optional input may be added to control the sensitivity calculations.

The input to TSUNAMI-3D consists of an input title, SCALE analytical sequence specification record, SCALE XSProc data, KENO V.a or KENO-VI input descriptions with some additional optional parameter data, and optional sensitivity and uncertainty data. These data are processed using the SCALE free-form reading routines, which allow alphanumeric data, floating-point data, and integer data to be entered in an unstructured manner. The input is not case sensitive, so either upper- or lowercase letters may be used. A maximum of 252 columns per line may be used for input, although some exceptions for this rule exist, such as the 80 character title data. Data can usually start or end in any column with a few exceptions. As an example, the word END beginning in column 1 and followed by two blank spaces will 
end the problem, any data following will be ignored. Each data entry must be followed by one or more blanks to terminate the data entry. For numeric data, either a comma or a blank can be used to terminate each data entry. Integers may be entered for floating values. For example, 10 will be interpreted as 10.0. Imbedded blanks are not allowed within a data entry unless an E precedes a single blank as in an unsigned exponent in a floating-point number. For example, 1.0E 4 would be correctly interpreted as $1.0 \times 10^{4}$.

\subsubsection{Analytical sequence specification record}

The analytical sequence specification begins in column 1 of the first line of the input file and must contain the following:

$=$ TSUNAMI-3D-K5 This sequence is used for sensitivity and uncertainty calculations with KENO V.a.

$=$ TSUNAMI-3D-K6 This sequence is used for sensitivity and uncertainty calculations with KENO-VI.

Optional keyword input may be entered after the analytical sequence specification record. These keywords are

PARM=CHECK This option allows the input data to be read and checked without executing any $\mathrm{PARM}=\mathrm{CHK} \quad$ functional modules.

The following PARM setting only apply to MG calculations and are ignored for CE calculations:

$\mathrm{PARM}=\mathrm{SIZE}=\mathrm{n} \quad$ The amount of memory requested in four-byte words may be set with this entry. The default value for $\mathrm{n}$ is 20000000 . This value only affects calculations in BONAMIST, where this value of the SIZE parameter is used for allocation of storage for the derivatives. Please see the documentation on BONAMIST in the Sensitivity Utility Modules chapter for more details. All other codes use dynamic memory allocation and this value has no effect.

PARM=BONAMIST This is the default configuration for MG TSUNAMI-3D calculations. XSProc is used with BONAMI and CENTRM for cross section processing. Implicit sensitivities are produced with BONAMIST.

PARM $=$ CENTRM $\quad$ XSProc is used with BONAMI and CENTRM for cross section processing, but BONAMIST is not run. MG TSUNAMI-3D calculations with PARM=CENTRM do not account for contributions by implicit sensitivity effects, and should be used with caution.

PARM=BONAMI $\quad$ XSProc is used with BONAMI for cross section processing, but BONAMIST is not run. MG TSUNAMI-3D calculations with PARM=BONAMI do not account for contributions by implicit sensitivity effects, and should be used with caution.

PARM=2REGION XSProc (with BONAMI and CENTRM) use Dancoff factors to compute neutron escape probabilities for an accelerated, yet more approximate, CENTRM calculation. Implicit sensitivities are computed with BONAMIST.

Multiple parameters can be used simultaneously by enclosing them in parentheses and separating them with commas such as PARM=(SIZE=2000000, CHECK). 


\subsubsection{Title data}

A title, a character string, must be entered as the second line of the input file. The syntax for the title is a string of characters with a length of up to 80 characters, including blanks.

\subsubsection{XSProc Execution}

XSProc reads the standard composition specification data for MG and $\mathrm{CE}$ and the unit cell geometry specifications for MG resonance self-shielding calculations. CE TSUNAMI calculations do not require the specification of unit cells. Please see chapters on the Material Information Processor for input specifications. The cross section data library that is to be used by TSUNAMI must also be entered as the third line of the TSUNAMI input; a list of the currently available libraries are listed in the table Standard $S C A L E$ cross section libraries of the XSLIB chapter.

\subsubsection{KENO V.A or KENO-VI problem description}

The KENO V.a or KENO-VI problem description follows the Material Input Processor section in the TSUNAMI-3D input. The input for KENO V.a and KENO-VI in TSUNAMI-3D is very similar to that described in section KENO V.a Data Guide in chapter KENO V.a or section KENO-VI Data Guide in the KENO-VI chapter, with only a few differences in the default values for the parameter data and a few additional parameters to control the adjoint criticality calculation. Otherwise, geometry, array, biasing, boundary, start, and plot data are entered exactly as described for KENO. Default parameter values for MG TSUNAMI-3D that are different from those used for other MG KENO calculations are shown in Table 6.2.3. Parameters that are unique to TSUNAMI-3D and are used to control to the MG adjoint calculation are shown in Table 6.2.4. These adjoint parameters are optional and can be entered with other parameters in the standard READ PARAMETER input block in KENO problem description. Parameters that are unique to CE TSUNAMI-3D are shown in Table 6.2.5; these parameters can also be entered in the KENO READ PARAMETER input block.

Several features were added to KENO V.a and KENO-VI to allow the calculation of sensitivity coefficients from the MG Monte Carlo analysis. One significant addition is the calculation of neutron flux moments and/or angular fluxes, both of which give directional components to the neutron flux solution required to compute the sensitivity of $k_{\text {eff }}$ to scattering cross sections. Another significant addition is the ability to compute fluxes that are subdivided over a cubic mesh or Cartesian spatial grid. These mesh fluxes simplify the accurate computation of the product of the forward and adjoint flux solutions, sometimes called the "inner product." Both of the flux moments and the spatial flux mesh options must be correctly applied to obtain accurate sensitivity coefficients. Generally, the refinement of the flux mesh to sufficiently small intervals to capture relevant spatial effects while maintaining a manageable memory footprint is the most challenging aspect of performing MG calculations with TSUNAMI-3D. It is important to note that TSUNAMI-3D provides no default mesh for these flux tallies, and the user must input a mesh using either the MSH parameter or a GridGeometry input to access this feature.

New input data that aid in the accurate calculation of sensitivity coefficients are the parameter inputs: NQD, PNM, MFX, MSH, TFM, APG, AGN, ABK, ASG, CET, CFP, CGD, FST, NNC, NMA, NMT, DNC, DMA, DMT, NMX, NMN, DMX, and DMN. Thus, these parameters may require modification when using TSUNAMI-3D. The READ GRID block of KENO input is used with MSH to input the planar grid for MG TSUNAMI-3D calculations and also to input the spatial grid for the calculation and storage of $F^{*}(r)$ for CE TSUNAMI calculations. Also, if using the coordinate transform for angular flux or flux moment calculations, use of the optional CENTER modifier in the KENO geometry input may be required. Users should note that when using the xlinear, ylinear, or zlinear options in the READ GRID block that KENO checks to determine whether the boundaries of each grid coincide with the global unit 
boundaries. If any planes in the grid boundaries are identical to the planes used in the global boundary, then KENO will extend the outermost plane in that grid by a distance equal to one-tenth of the grid mesh interval to ensure that the grid mesh covers the entire geometry.

Some KENO parameters (e.g., SCD, MFX, CDS, GFX, and CGD) may be used to assign user provided grid definitions for use with specific tallies. These are described in Section 9.1.2.3 of the SCALE KENO chapter. Grid IDs in the READ GRID blocks should be limited to no more than 4 integer characters (i.e., $1 \leq \mathrm{ID} \leq 9999$ ).

Table 6.2.3. Default values KENO parameters in MG TSUNAMI-3D

\begin{tabular}{cccl}
\hline Parameter & $\begin{array}{c}\text { Default value for } \\
\text { TSUNAMI-3D }\end{array}$ & $\begin{array}{c}\text { Default value for } \\
\text { KENO in CSAS } \\
\text { Sequences or as } \\
\text { stand-alone code }\end{array}$ & Description \\
\hline CFX & YES & NO & Collect fluxes \\
GEN & 550 & 203 & $\begin{array}{c}\text { Number of generations to be run } \\
\text { Number of generations to be omitted when } \\
\text { collecting results } \\
\text { NSK }\end{array}$ \\
PNM & 30 & 3 & $\begin{array}{c}\text { Highest order of flux moments tallies } \\
\text { Perform coordinate transform for flux moment and } \\
\text { angular flux calculations }\end{array}$ \\
TFM & YES & 0 & NO \\
\hline
\end{tabular}

Table 6.2.4. Default values of TSUNAMI-3D parameters for KENO MG adjoint calculation

\begin{tabular}{|c|c|c|c|}
\hline Parameter & $\begin{array}{l}\text { Default value for } \\
\text { TSUNAMI-3D }\end{array}$ & $\begin{array}{c}\text { Corresponding } \\
\text { KENO } \\
\text { parameter }\end{array}$ & Description \\
\hline $\mathrm{ABK}$ & $\mathrm{APG} \times 2$ & NBK & $\begin{array}{l}\text { Number of positions in the neutron bank for the } \\
\text { adjoint calculation }\end{array}$ \\
\hline AGN & GEN - NSK + ASK & GEN & $\begin{array}{l}\text { Number of generations to be run for the adjoint } \\
\text { calculation - default value produces the } \\
\text { same number of active generations as the } \\
\text { forward calculation }\end{array}$ \\
\hline APG & $\mathrm{NPG} \times 3$ & NPG & Number of particles per generation \\
\hline ASG & SIG (default SIG=0) & SIG & $\begin{array}{l}\text { if }>0.0 \text {, this is the standard deviation at which } \\
\text { the adjoint problem will terminate }\end{array}$ \\
\hline ASK & $\mathrm{NSK} \times 3$ & NSK & $\begin{array}{l}\text { Number of generations to be omitted when } \\
\text { collecting results for the adjoint calculation }\end{array}$ \\
\hline
\end{tabular}

MG TSUNAMI sensitivity coefficient estimates can be very sensitive to the values of a problem's input parameters, and users should always check the accuracy of their sensitivity coefficients by comparing them with reference direct perturbation sensitivities, discussed in Sect. 6.2.5.1. The default values for parameters in Table 6.2.3 and Table 6.2.4 generally serve as good starting values for a MG TSUNAMI calculation, but users may need to use a higher order of flux moments to better capture the angular 
dependence of the flux (typically $\mathrm{PNM}=5$ is sufficient), and may need to simulate more histories/generations to reduce the uncertainty in sensitivity coefficient estimates. The dimensions of the flux mesh are another important MG TSUNAMI parameter, and a reasonable starting guess for the width of the flux mesh intervals is $1 / 10^{\text {th }}$ of the diameter of the fuel-containing region. Users should take care when increasing the order of the flux moment tallies and the number of intervals in the spatial flux mesh as the memory footprint of a MG TSUNAMI calculation increases quickly as these parameters increase.

CE TSUNAMI calculations are in many respects simpler than MG TSUNAMI calculations because they use state-of-the-art sensitivity methodologies that typically require less user input to perform sensitivity calculations. CE TSUNAMI calculations do not use any of the input parameters in Table 6.2.3 or Table 6.2.4 and do not require flux moment tallies, flux mesh tallies (except for calculating $F^{*}(r)$ ), or the simulation of adjoint histories. CET, CFP, and CDG are the three parameters that control CE TSUNAMI eigenvalue sensitivity calculations. CET specifies which CE sensitivity method (either CLUTCH, IFP, or GEAR-MC) will be used in the CE TSUNAMI calculation. CFP specifies how many latent generations will be used by the IFP method for either calculating sensitivity coefficients $(\mathrm{CET}=2 / 5)$ or for calculating $F^{*}(r)$ during the inactive generations $(\mathrm{CET}=1 / 4)$; if $\mathrm{CET}=1$ or 4 and $\mathrm{CFP}=-1$, then CE TSUNAMI will perform a CLUTCH calculation assuming that $F^{*}(r)$ is equal to one everywhere for CET=1 and zero everywhere for $\mathrm{CET}=4$. The number of latent generations (CFP) and the number of generations skipped for fission source convergence (NSK) control very different things.

The typical workflow for generating an IFP-based CE TSUNAMI $k_{\text {eff }}$ sensitivity input is given below:

1) Set $\mathrm{CET}=2$.

2) Set your number of latent generations using CFP=\# (usually between 5 and 10).

The typical workflow for generating a CLUTCH-based CE TSUNAMI $k_{\text {eff }}$ sensitivity input is given below:

1) Set CET $=1$.

2) Set your number of latent generations using CFP=\# (usually between 5 and 10).

3) Create a GridGeometry for the $F^{*}(r)$ mesh; this mesh must cover all fissionable regions of the problem and the width of the mesh voxels is usually between 1 and $2 \mathrm{~cm}$ in the $\mathrm{X}-, \mathrm{Y}-$, and Zdimensions.

4) Tell CE TSUNAMI the ID of this GridGeometry using the CGD=\# parameter.

5) Consider simulating extra inactive generations to allow the $F^{*}(r)$ mesh to converge - most $F^{*}(r)$ mesh calculations require between 10 and 100 inactive histories per mesh voxel to sufficiently converge.

Ex: A problem using a $20 \times 30 \times 40 F^{*}(r)$ mesh contains 24,000 voxels. Assuming at least 10 inactive histories per mesh interval means this problem will require 240,000 inactive histories for the $F^{*}(r)$ mesh to converge. If the problem uses 1,000 particles per generation $(\mathrm{NPG}=1000)$, then the user should use at least 240 skipped generations $(\mathrm{NSK}=240)$ to allow $F^{*}(r)$ mesh tallies to converge.

When performing CLUTCH calculations using $F^{*}(r)$ (i.e., when CET=1 or 4 and CFP is not -1 ) a spatial grid for $F^{*}(r)$ must be specified in the READ GRIDGEOMETRY block of KENO input. CGD specifies the ID of this $F^{*}(r)$ grid. Failure to specify a grid or using the ID of a nonexistent grid results in an error message. The entries in the $F^{*}(r)$ grid can be printed to a 3dmap file by setting the parameter FST=YES. This information is printed to a file with the same name as the input but with a _FStar_map.3dmap extension (i.e., problem.inp prints information to problem_FStar_map.3dmap). This .3dmap file can be viewed using the Fulcrum interface, as shown for a CLUTCH $k_{e f f}$ sensitivity test problem in Figure 6.2.4. 
Values for $F^{*}(r)$ are set by default to one/zero in CLUTCH $k_{\text {eff }} / \mathrm{GPT}$ sensitivity calculations, respectively, for regions that do not contain fissionable material and/or did not generate any $F^{*}(r)$ tallies. When doing GPT sensitivity calculations using $F^{*}(r)$ CE TSUNAMI will produce two $F^{*}(r)$ meshes: one for the numerator term in the response of interest and another for the denominator term; therefore, 3dmaps that are produced from CE TSUNAMI GPT $F^{*}(r)$ calculations will contain two meshes, as shown in Figure 6.2.5. 
Table 6.2.5. CE TSUNAMI-3D parameters and default values

\begin{tabular}{|c|c|c|}
\hline Parameter & $\begin{array}{l}\text { Default value for } \\
\text { TSUNAMI-3D }\end{array}$ & Description \\
\hline CET & 1 & $\begin{array}{l}\text { Mode for CE TSUNAMI } \\
0=\text { No sensitivity calculations } \\
1=\text { CLUTCH sensitivity calculation } \\
2=\text { IFP sensitivity calculation } \\
4=\text { GEAR-MC calculation (with CLUTCH only) } \\
5=\text { GEAR-MC calculation (with CLUTCH+IFP) } \\
7=\text { Undersampling metric calculation }\end{array}$ \\
\hline CFP & 5 & $\begin{array}{l}\text { Number of latent generations used for IFP sensitivity } \\
\text { or } F^{*}(r) \text { calculations. Note: } \\
\text { - If CET=1 and CFP }=-1 \text { then } F^{*}(r) \text { is } \\
\text { assumed to equal one everywhere. } \\
\text { - If CET }=4 \text { and CFP }=-1 \text { then } F^{*}(r) \text { is } \\
\text { assumed to equal zero everywhere. }\end{array}$ \\
\hline CGD & NONE & $\begin{array}{l}\text { ID of the gridgeom mesh used for CLUTCH } F^{*}(r) \\
\text { calculations. }\end{array}$ \\
\hline FST & NO & Print the $F^{*}(r)$ grid values to a $.3 \mathrm{dmap}$ file. \\
\hline
\end{tabular}

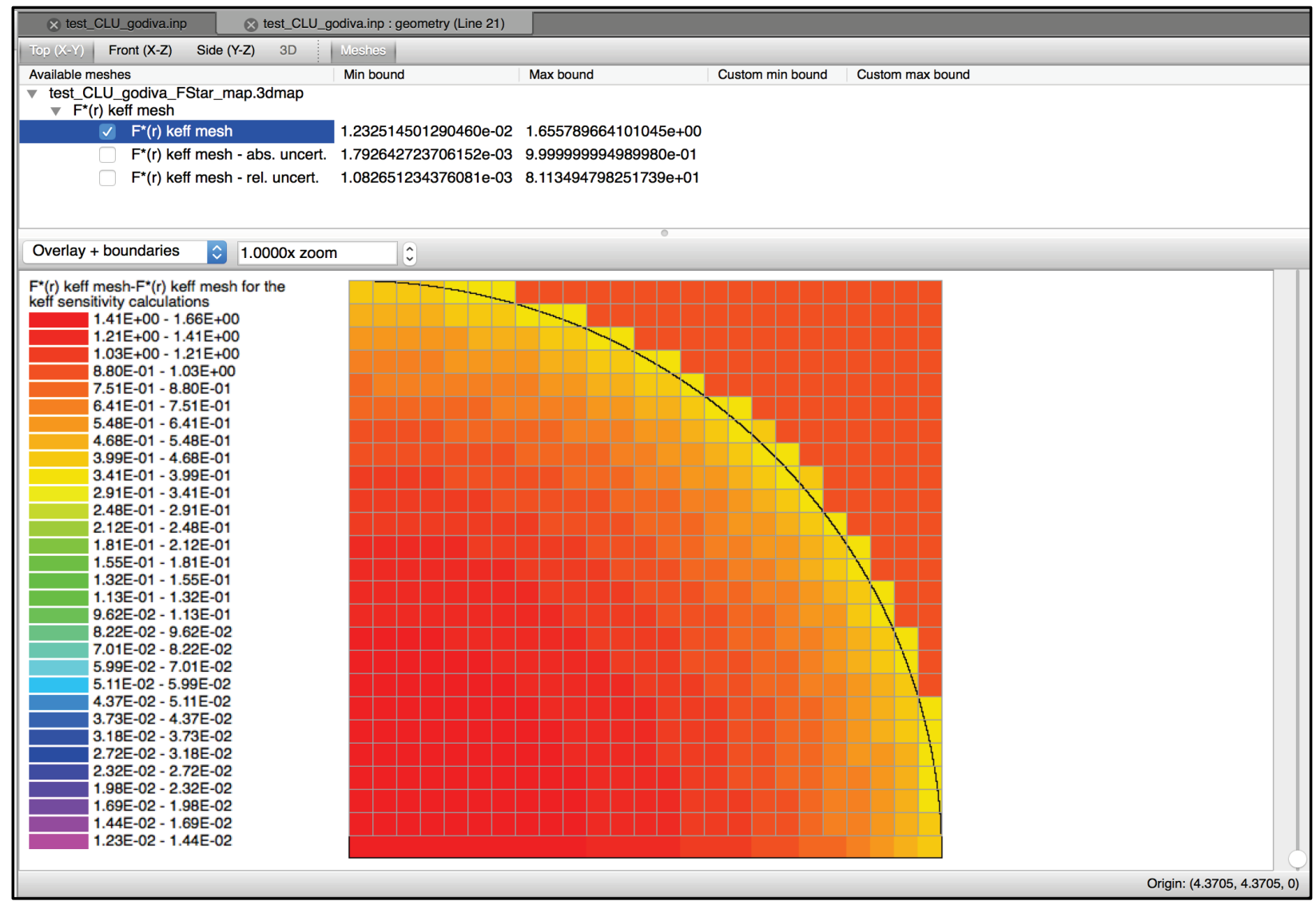

Figure 6.2.4 $F^{*}(r)$ mesh from a sample CLUTCH eigenvalue sensitivity calculation. 


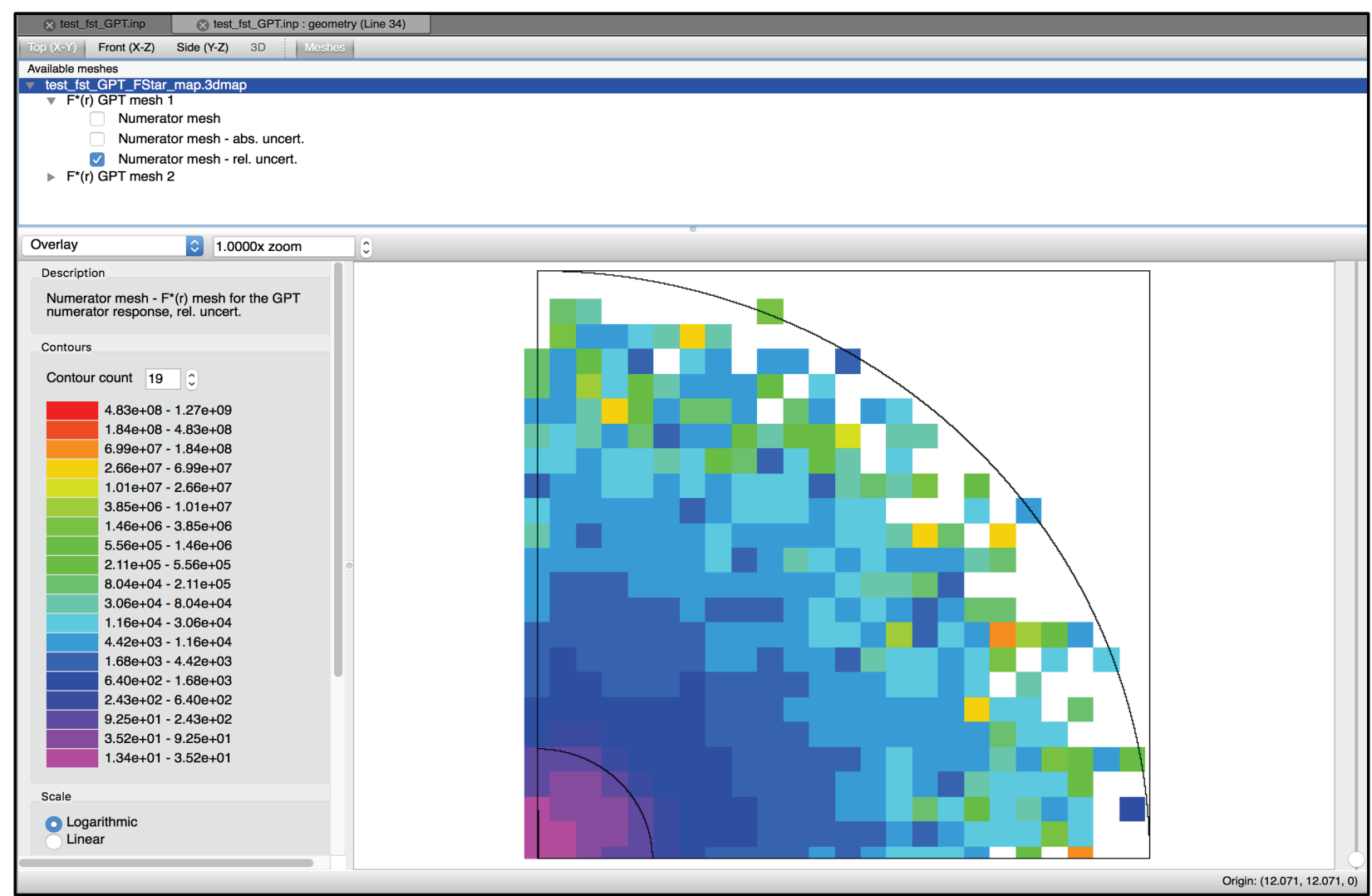

Figure 6.2.5. $F^{*}(r)$ meshes from a sample CLUTCH GPT sensitivity calculation.

Setting CET=7 will activate an experimental capability for detecting computational biases due to the undersampling of fission sites and particle histories during a Monte Carlo simulation. This capability does not calculate sensitivity coefficients and does not require selecting a number of latent generations or building an $F^{*}(r)$ mesh. Instead, this approach scores flux and reaction rate tallies for various materials and nuclides in a system and reports the tallies to a.$s d f$ file after the simulation ends, along with scores for various statistical metrics that may predict undersampling biases in the reported tallies. An undersampling metric calculation will produce four .sdf files, as described below:

$\begin{array}{lll}\text { input_name_metric1.sdf } & = & \text { Reaction rate and flux tallies } \\ \text { input_name_metric2.sdf } & = & \text { Number of nonzero scores per generation for each tally } \\ \text { input_name_metric3.sdf } & = & \text { Tally Entropy scores for each tally } \\ \text { input_name_metric4.sdf } & = & \text { Heidelberger-Welch RHW scores for each tally }\end{array}$

Each of these undersampling metrics is described in detail in Reference 10. The values reported in the metric2/3/4.sdf files correspond to each of the tallies scored in the metric1.sdf file. As in typical sensitivity coefficient .sdf files, the undersampling metric .sdfs report energy-dependent and energyintegrated information for various reaction rates in every material and nuclide in a system; however, the undersampling metric calculations also report information for flux tallies within each material/nuclide under the name of the fictitious isotope $\mathrm{H}-111$. This undersampling metric capability is only peripherally related to the scope and application of sensitivity coefficient calculations and was included with CE TSUNAMI-3D predominantly to take advantage of the established TSUNAMI tally scoring framework and sensitivity visualization/postprocessing tools (i.e., Fulcrum). 
Performing GPT sensitivity calculations with CE TSUNAMI requires the user to enter some additional input to specify the GPT response being examined in the calculation. For the IFP+CLUTCH GPT implementation $(\mathrm{CET}=5)$ users can define GPT reaction rate ratios using the "Definitions" and "SystemResponses" blocks (please see Sensitivity and uncertainty calculation data in the TSUNAMI-1D chapter), which are used similarly in TSUNAMI-1D and TSUNAMI-2D. Both TSUNAMI-3D GPT capabilities can currently only accept total cross section (MT=1), fission (MT=18), n,gamma (MT=102), nu-fission (MT=452), or flux reaction response definitions. The CLUTCH-only GPT implementation $(\mathrm{CET}=4)$ currently cannot accept GPT response input from the Definitions and SystemResponses blocks, and may only calculate GPT sensitivity coefficients for one response per TSUNAMI-3D simulation. The input parameters for defining the GPT response in these $(\mathrm{CET}=4)$ calculations are described in Table 6.2.6. The two GEAR-MC implementations $(\mathrm{CET}=4$ or $\mathrm{CET}=5)$ both require the user to specify a value for CFP; the CLUTCH-only GEAR-MC implementation requires the user to specify an $F^{*}(r)$ mesh using the gridGeom block and the CGD parameter. 
Table 6.2.6. CE TSUNAMI-3D GPT sensitivity parameters and default values

\begin{tabular}{|c|c|c|}
\hline Parameter & $\begin{array}{l}\text { Default value for } \\
\text { TSUNAMI-3D }\end{array}$ & Description \\
\hline $\mathrm{NNC}$ & 92235 & $\begin{array}{l}\text { ZAID for the nuclide used in the GPT Response } \\
\text { Numerator term. Setting NNC=-1 tallies the response } \\
\text { reaction (NMT) for all nuclides in the response } \\
\text { material (NMA). }\end{array}$ \\
\hline NMA & -1 & $\begin{array}{l}\text { Composition ID for the material used in the GPT } \\
\text { Response Numerator term. Setting NMA=-1 tallies } \\
\text { the response reaction (NMT) for the response nuclide } \\
\text { (NNC) in all materials of the problem. }\end{array}$ \\
\hline \multirow[t]{2}{*}{ NMT } & 18 & $\begin{array}{l}\text { Reaction MT number for the GPT Response } \\
\text { Numerator term. Only several of the MT numbers in } \\
\text { ENDF are currently supported, including }\end{array}$ \\
\hline & & $\begin{array}{l}0=\text { flux response }(\text { i.e. }, \Sigma=1) \\
1=\text { total cross section response } \\
18=\text { fission cross section response } \\
102=(n, \gamma) \text { cross section response }\end{array}$ \\
\hline NMX & $10^{8} \mathrm{eV}$ & $\begin{array}{l}\text { Upper energy threshold (units of } \mathrm{eV} \text { ) for the GPT } \\
\text { Response Numerator term. }\end{array}$ \\
\hline $\mathrm{NMN}$ & $10^{-6} \mathrm{eV}$ & $\begin{array}{l}\text { Lower energy threshold (units of } \mathrm{eV} \text { ) for the GPT } \\
\text { Response Numerator term. }\end{array}$ \\
\hline $\mathrm{DNC}$ & -1 & $\begin{array}{l}\text { ZAID for the nuclide used in the GPT Response } \\
\text { Denominator term. Setting DNC=-1 tallies the } \\
\text { response reaction (DMT) for all nuclides in the } \\
\text { response material (DMA). }\end{array}$ \\
\hline DMA & -1 & $\begin{array}{l}\text { Composition ID for the material used in the GPT } \\
\text { Response Denominator term. Setting DMA=-1 tallies } \\
\text { the response reaction (DMT) for the response nuclide } \\
\text { (DNC) in all materials of the problem. }\end{array}$ \\
\hline \multirow[t]{2}{*}{ DMT } & 18 & $\begin{array}{l}\text { Reaction MT number for the GPT Response } \\
\text { Denominator term. Only several of the MT numbers } \\
\text { in ENDF are currently supported, including: }\end{array}$ \\
\hline & & $\begin{array}{l}0=\text { flux response }(\text { i.e., } \Sigma=1) \\
1=\text { total cross section response } \\
18=\text { fission cross section response } \\
102=(n, \gamma) \text { cross section response }\end{array}$ \\
\hline DMX & $10^{8} \mathrm{eV}$ & $\begin{array}{l}\text { Upper energy threshold (units of eV) for the GPT } \\
\text { Response Denominator term. }\end{array}$ \\
\hline $\mathrm{DMN}$ & $10^{-6} \mathrm{eV}$ & $\begin{array}{l}\text { Lower energy threshold (units of } \mathrm{eV} \text { ) for the GPT } \\
\text { Response Denominator term. }\end{array}$ \\
\hline
\end{tabular}

\subsubsection{Sensitivity calculation data}

A data block for controlling the sensitivity calculation is optional. If included, this data block begins with the keywords READ SAMS and ends with the keywords END SAMS. Any of the optional SAMS input data may be 
entered in free-form format between the READ SAMS and END SAMS keywords. The optional SAMS input data are shown with the default values specific to TSUNAMI-3D in Table 6.2.7. Certain options in Table 6.2.8 are not available for $\mathrm{CE}$ calculations. Parameters used to specify default covariance data to supplement or correct values on the files specified by coverx = are shown in Table 6.2.8. A more detailed explanation of the SAMS parameters is provided in the SAMS chapter.

Table 6.2.7. SAMS input keywords

\begin{tabular}{|c|c|c|}
\hline KEYWORD & DEFAULT VALUE & DESCRIPTION \\
\hline binsen & $\mathrm{F}$ & $\begin{array}{l}\text { Produces SENPRO formatted binary sensitivity data file on } \\
\text { unit } 40\end{array}$ \\
\hline cover $x=$ & 56groupcov7.1 & Name of covariance data file to use for uncertainty analysis \\
\hline largeimp $=$ & 100 & $\begin{array}{l}\text { Value for the absolute value of implicit sensitivities, which if } \\
\text { exceeded, will be reset to } 0.0 \text { and print a warning message. }\end{array}$ \\
\hline makeimp $^{*}$ & $\mathrm{~T}$ & Flag to cause implicit sensitivity coefficients to be generated. \\
\hline nocovar & $\mathrm{T}$ & $\begin{array}{l}\text { Flag to cause uncertainty edit to be turned off (sets print_covar } \\
\text { to F) }\end{array}$ \\
\hline nohtml & $\mathrm{F}$ & Flag to cause HTML output to not be produced. \\
\hline nomix & $\mathrm{F}$ & Flag to cause the sensitivities by mixture to be turned off \\
\hline $\mathrm{pn}={ }^{*}$ & 3 & Legendre order for moment calculations \\
\hline prtgeom $^{*}$ & $\mathrm{~F}$ & $\begin{array}{l}\text { Flag to cause the sensitivities to be output for each geometry } \\
\text { region }\end{array}$ \\
\hline prtimp $^{*}$ & $\mathrm{~F}$ & $\begin{array}{l}\text { Prints explicit sensitivities coefficients, implicit sensitivity } \\
\text { coefficients and complete sensitivity coefficients }\end{array}$ \\
\hline prtvols $^{*}$ & $\mathrm{~F}$ & Flag to cause the volumes of the regions to be printed by SAMS \\
\hline unconstrainedchi & $\mathrm{F}$ & $\begin{array}{l}\text { Flag to generate pre-SCALE } 6 \text { unconstrained chi (fission } \\
\text { spectrum) sensitivities }\end{array}$ \\
\hline useang $^{*}$ & $\mathrm{~F}$ & $\begin{array}{l}\text { Flag to cause the angular flux calculated in KENO V.a to be } \\
\text { used to calculate flux moments for sensitivity calculations. If } \\
\text { angular fluxes were not computed by KENO V.a, useang is set } \\
\text { to the false internally. }\end{array}$ \\
\hline usemom $^{*}$ & $\mathrm{~T}$ & $\begin{array}{l}\text { Flag to cause the flux moments calculated with KENO V.a to be } \\
\text { used to in sensitivity coefficient generation. If flux moments } \\
\text { were not computed by KENO V.a, usemom is set to false } \\
\text { internally. }\end{array}$ \\
\hline
\end{tabular}

\footnotetext{
"Not available in CE mode
} 
Table 6.2.8. SAMS input keywords for default covariance data

\begin{tabular}{|c|c|c|}
\hline KEYWORD & DEFAULT VALUE & DESCRIPTION \\
\hline use_dcov & $\mathrm{F}$ & Use default values for any covariance updates \\
\hline use_icov & $\mathrm{F}$ & Use user-input values for specified covariance updates \\
\hline cov_fix & $\mathrm{F}$ & $\begin{array}{l}\text { Correct covariance data if the uncertainty is large } \\
>1000 \% \text { or zero with user-input and/or default values }\end{array}$ \\
\hline large_cov & 10.0 & Relative standard deviation to apply cov_fix \\
\hline return_work_cov & $\mathrm{F}$ & $\begin{array}{l}\text { Create a new covariance data file with only the cross } \\
\text { section covariance data used in the analysis. }\end{array}$ \\
\hline $\mathrm{udcov}=$ & 0.05 & $\begin{array}{l}\text { User-defined default value of relative standard deviation in } \\
\text { cross section data to use for all groups for nuclide-reaction } \\
\text { pairs for which cross section covariance data are too large } \\
\text { or not available on input covariance data library. }\end{array}$ \\
\hline udcov_corr $=$ & 1.0 & $\begin{array}{l}\text { User-defined default correlation value to use for nuclide- } \\
\text { reaction pairs for which cross section covariance data are } \\
\text { not available on the input covariance library. }\end{array}$ \\
\hline \multirow[t]{2}{*}{ udcov_corr_type $=$} & zone & $\begin{array}{l}\text { Type of correlation applied from group-to-group } \\
\text { covariance values. Allowable options are: }\end{array}$ \\
\hline & & $\begin{array}{l}\text { - long - correlation is applied between all groups } \\
\text { - short - correlation is applied only between adjacent } \\
\text { groups } \\
\text { - zone - correlation is applied for groups within fast, } \\
\text { intermediate, and thermal energy regimes, but no } \\
\text { correlation is applied between energy regimes }\end{array}$ \\
\hline udcov_therm $=$ & 0.0 & $\begin{array}{l}\text { User-defined default value of relative standard deviation in } \\
\text { cross section data to use for thermal data for nuclide- } \\
\text { reaction pairs for which cross section covariance data are } \\
\text { too large or not available on input covariance data library. } \\
\text { If input, the } u d c o v \text { therm overrides the } u d c o v \text { value in the } \\
\text { thermal range (typically neutron energies below } 0.625 \mathrm{eV} \text { ). }\end{array}$ \\
\hline udcov_inter $=$ & 0.0 & $\begin{array}{l}\text { User-defined default value of relative standard deviation in } \\
\text { cross section data to use for intermediate data for nuclide- } \\
\text { reaction pairs for which cross section covariance data are } \\
\text { too large or not available on input covariance data library. } \\
\text { If input, the } u d c o v \text { inter overrides the } u d c o v \text { value in the } \\
\text { intermediate range (typically neutron energies above } 0.625 \\
\text { eV and below } 25 \mathrm{keV}) \text {. }\end{array}$ \\
\hline udcov_fast $=$ & 0.0 & $\begin{array}{l}\text { User-defined default value of relative standard deviation in } \\
\text { cross section data to use for fast data for nuclide-reaction } \\
\text { pairs for which cross section covariance data are too large } \\
\text { or not available on input covariance data library. If input, } \\
\text { the } u d c o v \text { fast overrides the } u d c o v \text { value in the fast range } \\
\text { (typically neutron energies above } 25 \mathrm{keV} \text { ). }\end{array}$ \\
\hline
\end{tabular}


Additionally, user-defined covariance data can be specified for individual nuclides and reactions using the COVARIANCE data block. This data block begins with the keywords READ COVARIANCE and ends with the keywords END COVARIANCE. Any of the optional COVARIANCE input data may be entered in free-form format between the READ COVARIANCE and END COVARIANCE keywords. The specifications for the COVARIANCE data block are described in the "User Input Covariance Data" section of the TSUNAMI_IP chapter of the TSUNAMI Utility Modules manual.

As the SAMS module generates HTML output, the optional HTML data block provides user control over some formats of the output. This data block begins with the keywords READ HTML and ends with the keywords END HTML. Any of the optional HTML input data may be entered in free-form format between the READ HTML and END HTML keywords. The specifications for the HTML data block are described in the "HTML Data" section or the TSUNAMI_IP chapter of the TSUNAMI Utility Modules manual.

\subsubsection{Input termination}

The input for TSUNAMI-3D must terminate with a line containing END in column 1. This END terminates the control sequence.

\subsubsection{Sample problems}

Five sample problems are given in this section. In each example, the use of a new feature is explained to guide the user in the proper definition of input models so that reliable sensitivity coefficients can be obtained. The user must ensure that the necessary options are employed for each model to obtain accurate results. Please note, the results shown here were generated with a previous version of SCALE, so current data libraries and code implementations may product different results. However, the techniques demonstrated are applicable to the current version of TSUNAMI-3D.

\subsubsection{Generating reference direct perturbation sensitivity coefficients}

The accuracy of the energy-integrated sensitivity coefficients can be confirmed through the use of central difference direct perturbation sensitivity calculations. Through this technique, the sensitivity of $k_{\text {eff }}$ to the number density of particular nuclide can be obtained. This sensitivity of $k_{\text {eff }}$ to the number density is equivalent to the sensitivity of $k_{\text {eff }}$ to the total cross section integrated over energy. Because the total cross section sensitivity coefficient tests much of the data used to compute all other sensitivity coefficients, it is considered an adequate test for verification. For each sensitivity coefficient examined by direct perturbation, the $k_{\text {eff }}$ of the system is computed first with the nominal values of the input quantities, then with a selected nominal input value increased by a certain percentage, and then with the nominal value decreased by the same percentage. The direct perturbation sensitivity coefficient of $k_{\text {eff }}$ to some input value $\alpha$ is computed as

$$
S_{k, \alpha}=\frac{\alpha}{k} \times \frac{d k}{d \alpha}=\frac{\alpha}{k} \times \frac{k_{\alpha^{+}}-k_{\alpha^{-}}}{\alpha^{+}-\alpha^{-}}
$$

where $\alpha^{+}$and $\alpha^{-}$represent the increased and decreased values, respectively, of the input quantity $\alpha$ and $k_{\alpha^{+}}$and $k_{\alpha^{-}}$represent the corresponding values of $k_{\text {eff. }}$ In general, perturbations used for calculating direct perturbation sensitivities should be large enough to induce a statistically significant $\left(\begin{array}{lll}10 & \sigma_{k e f f}\end{array}\right)$ change in the eigenvalue of the system but not large enough to induce second-order effects in the 
perturbed eigenvalue. Statistical uncertainties in the computed values of $k_{\text {eff }}$ are propagated to uncertainties in direct perturbation sensitivity coefficients by standard error propagation techniques as

$$
\sigma_{S}=\left(\left(\frac{\left(\sigma_{k^{+}}^{2}+\sigma_{k^{-}}^{2}\right)}{\left(k^{+}-k^{-}\right)^{2}}+\frac{\sigma_{k}^{2}}{k^{2}}\right) \times\left(\frac{k^{+}-k^{-}}{k}\right)^{2}\right)^{1 / 2} \times \frac{\alpha}{\alpha^{+}-\alpha^{-}} .
$$

In MG TSUNAMI sensitivity calculations it is important to ensure that the $k_{\text {eff }}$ value of the forward and adjoint solutions closely agree. If the $k_{\text {eff }}$ values do not agree, then the quality of at least one of the transport calculations may be in question. Typically, the transport calculation of concern is the adjoint calculation. By default, TSUNAMI-3D triples the number of histories per generation requested for the forward case to produce the adjoint solution. Experience has shown that agreement to less than $0.5 \%$ difference in $k_{\text {eff }}$ between the forward and adjoint calculations is adequate to obtain accurate sensitivity coefficients.

\subsubsection{Simple MG Sample Problem}

A simple sample problem with INFHOMMEDIUM MG cross section processing is based on an unreflected rectangular parallelepiped consisting of a homogeneous mixture $\mathrm{UF}_{4}$ and paraffin with an enrichment of $2 \mathrm{wt} \%$ in ${ }^{235} \mathrm{U}$. The $\mathrm{H} /{ }^{235} \mathrm{U}$ atomic ratio is $294: 1$. The dimensions of the experiment are $56.22 \times 56.22 \times 122.47 \mathrm{~cm} .{ }^{11}$ For consistency with a TSUNAMI-1D model of the same sample problem, this experiment was modeled as a sphere with a critical radius of $38.50 \mathrm{~cm}$. This configuration is used in the TSUNAMI-3D_K5-1 and TSUNAMI-3D_K6-1 sample problems distributed with SCALE. An annotated TSUNAMI-3D-K5 input for this experiment is shown in Figure 6.2.6. The composition data are input as number densities for each nuclide. Because the material is treated as INFHOMMEDIUM, no explicit unit cell model is necessary, and the READ CELL block is omitted. The KENO V.a problem description contains parameter data to request 10,000 generations $(g e n=10000)$ with 3000 neutrons per generation $(n p g=3000)$, deactivate the HTML output for KENO V.a $(\mathrm{htm}=n o)$, stop the forward calculation when $k_{\text {eff }}$ has converged to one standard deviation of 0.005 (sig=0.005), and to stop the adjoint calculation when $k_{\text {eff }}$ has converged to one standard deviation of 0.010 (asg=0.010). The KENO V.a geometry consists of nine concentric spheres, up to an outer radius of $38.5 \mathrm{~cm}$, with each sphere containing the material defined as mixture 1 . The geometry subdivision is necessary to adequately resolve the spatial dependence of the angular moments of the forward and adjoint flux solutions. The optional sensitivity calculation data block is used to request edits of the sensitivity coefficients for each region (prtgeom) and edits of the explicit, implicit, and complete sensitivity coefficients (prtimp). The code output from each functional module is not given here, but is described in the manual section for each functional module. 


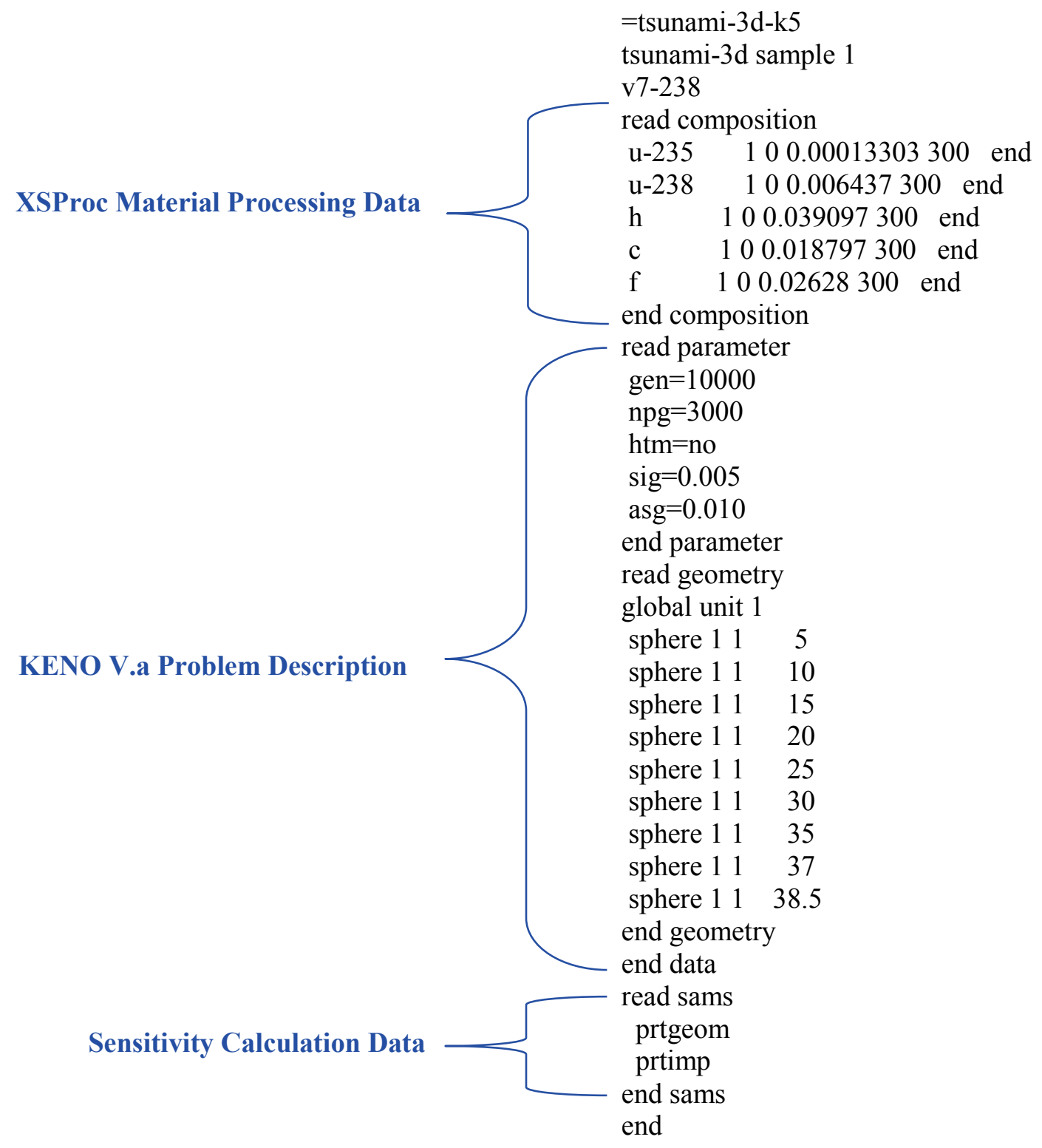

Figure 6.2.6. TSUNAMI-3D-K5 simple sample problem input.

For this problem, direct perturbation results were obtained for the number densities of each nuclide using an equivalent $1 \mathrm{D}$ model. In these calculations, the number density of each nuclide was perturbed by $\pm 2 \%$ and the calculation was repeated using the TSUNAMI-1DC sequence. The sensitivity of $k_{\text {eff }}$ to the number density is equivalent to the sensitivity of $k_{\text {eff }}$ to the total cross section, integrated over energy. The direct perturbation sensitivity coefficients were computed by using the $k_{\text {eff }}$ values from the unperturbed and perturbed cases in Eq. (6.2.26).

This experiment was modeled with the nine-region model shown in Figure 6.2.6, and as a single computational region (not shown). Flux moments were expanded to third order in both cases, which is the default configuration. TSUNAMI-3D-K5 automatically increases the number of particles per generation by a factor of three for the adjoint analysis. For the single region case, the $k_{\text {eff }}$ values for the forward and 
adjoint cases are in good agreement at $1.00682+/-0.00094$, and $1.0013+/-0.0049$, respectively. The sensitivity results shown in Table 6.2.9 were extracted from the output file from the edit titled "Energy, Region, and Mixture Integrated Sensitivity Coefficients for this Problem." The uncertainty in the sensitivity coefficients represents one standard deviation and is present due to the use of Monte Carlo methods to compute the fluxes and $k_{\text {eff }}$. These results indicate similarity with the direct perturbation results for some nuclides but not for others. Differences between the TSUNAMI-3D-K5 results and the direct perturbation results vary $1 \%$ for ${ }^{238} \mathrm{U}$ up to $16 \%$ for ${ }^{1} \mathrm{H}$. The results from the TSUNAMI-3D-K5 analysis with the model divided into nine spherical shells, with all other parameters held constant, are also shown in Table 6.2.9. These results compare much more favorably with the direct perturbation results. For this model, all TSUNAMI-3D-K5 sensitivities agree with the direct perturbation values within $0.1 \%$ for ${ }^{1} \mathrm{H}$ up to a maximum difference of $1.5 \%$ for ${ }^{238} \mathrm{U}$.

The differences in the results from the two TSUNAMI-3D models, one region and nine regions, are due to the summation of the product of the forward and adjoint fluxes over the regions in the problem. For a region in which the flux moments vary greatly by position, subdividing the geometry will provide better resolution of the variation of the flux across the system and will produce more accurate results. The number of regions necessary for accurate computation of the sensitivity coefficients was determined through an iterative process. Models divided into more regions produce the equivalent results to those produced by the nine-region model. Increasing the number of computational regions increases the run time for this problem by about $10 \%$.

The sensitivity results from the nine-region model using TSUNAMI-3D-K5 with PARM=CENTRM, which does not include the contributions from the implicit sensitivity coefficients, are also shown in Table 6.2.9. The differences between the TSUNAMI-3D-K5 PARM=CENTRM and the direct perturbation results are $16 \%$ for ${ }^{1} \mathrm{H}$ and $19 \%$ for ${ }^{238} \mathrm{U}$. The use of the default cross section processing with the sensitivity versions of the resonance processing codes is strongly recommended. However, TSUNAMI-3D-K5 with PARM=CENTRM should produce accurate results for fast systems where resonance self-shielding is not important. This is illustrated with the second sample problem for TSUNAMI-1D and will not be repeated here. 


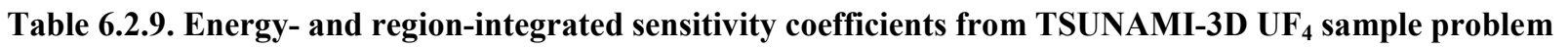

\begin{tabular}{|c|c|c|c|c|c|}
\hline Isotope & Reaction & $\begin{array}{c}\text { Direct } \\
\text { perturbation }\end{array}$ & $\begin{array}{c}\text { TSUNAMI-3D } \\
\text { one computational } \\
\text { region }\end{array}$ & $\begin{array}{c}\text { TSUNAMI-3D } \\
\text { nine computational } \\
\text { regions }\end{array}$ & $\begin{array}{c}\text { TSUNAMI-3D } \\
\text { PARM=CENTRM } \\
\text { nine computational } \\
\text { regions }\end{array}$ \\
\hline${ }^{1} \mathrm{H}$ & total & $2.20 \mathrm{E}-01$ & $2.55 \mathrm{E}-01 \pm 6.61 \mathrm{E}-05$ & $2.20 \mathrm{E}-01 \pm 1.99 \mathrm{E}-04$ & $2.54 \mathrm{E}-01 \pm 1.99 \mathrm{E}-04$ \\
\hline${ }^{1} \mathrm{H}$ & scatter & & $3.60 \mathrm{E}-01 \pm 6.06 \mathrm{E}-05$ & $3.22 \mathrm{E}-01 \pm 1.98 \mathrm{E}-04$ & $3.56 \mathrm{E}-01 \pm 1.98 \mathrm{E}-04$ \\
\hline${ }^{1} \mathrm{H}$ & elastic & & $3.60 \mathrm{E}-01 \pm 6.06 \mathrm{E}-05$ & $3.22 \mathrm{E}-01 \pm 1.98 \mathrm{E}-04$ & $3.56 \mathrm{E}-01 \pm 1.98 \mathrm{E}-04$ \\
\hline${ }^{1} \mathrm{H}$ & capture & & $-1.05 \mathrm{E}-01 \pm 2.82 \mathrm{E}-05$ & $-1.02 \mathrm{E}-01 \pm 1.25 \mathrm{E}-05$ & $-1.02 \mathrm{E}-01 \pm 1.25 \mathrm{E}-05$ \\
\hline${ }^{1} \mathrm{H}$ & $\mathrm{n}, \gamma$ & & $-1.05 \mathrm{E}-01 \pm 2.82 \mathrm{E}-05$ & $-1.02 \mathrm{E}-01 \pm 1.25 \mathrm{E}-05$ & $-1.02 \mathrm{E}-01 \pm 1.25 \mathrm{E}-05$ \\
\hline${ }^{12} \mathrm{C}$ & total & $2.41 \mathrm{E}-02$ & $2.78 \mathrm{E}-02 \pm 6.54 \mathrm{E}-06$ & $2.42 \mathrm{E}-02 \pm 1.43 \mathrm{E}-05$ & $2.79 \mathrm{E}-02 \pm 1.43 \mathrm{E}-05$ \\
\hline${ }^{12} \mathrm{C}$ & scatter & & $2.85 \mathrm{E}-02 \pm 6.54 \mathrm{E}-06$ & $2.48 \mathrm{E}-02 \pm 1.43 \mathrm{E}-05$ & $2.86 \mathrm{E}-02 \pm 1.43 \mathrm{E}-05$ \\
\hline${ }^{12} \mathrm{C}$ & elastic & & $2.82 \mathrm{E}-02 \pm 6.52 \mathrm{E}-06$ & $2.46 \mathrm{E}-02 \pm 1.43 \mathrm{E}-05$ & $2.84 \mathrm{E}-02 \pm 1.43 \mathrm{E}-05$ \\
\hline${ }^{12} \mathrm{C}$ & $\mathrm{n}, \mathrm{n}^{\prime}$ & & $2.46 \mathrm{E}-04 \pm 1.90 \mathrm{E}-07$ & $2.25 \mathrm{E}-04 \pm 1.54 \mathrm{E}-07$ & $2.25 \mathrm{E}-04 \pm 1.54 \mathrm{E}-07$ \\
\hline${ }^{12} \mathrm{C}$ & capture & & $-6.82 \mathrm{E}-04 \pm 1.70 \mathrm{E}-07$ & $-6.85 \mathrm{E}-04 \pm 8.42 \mathrm{E}-08$ & $-6.85 \mathrm{E}-04 \pm 8.42 \mathrm{E}-08$ \\
\hline${ }^{12} \mathrm{C}$ & $\mathrm{n}, \gamma$ & & $-5.15 \mathrm{E}-04 \pm 1.38 \mathrm{E}-07$ & $-5.00 \mathrm{E}-04 \pm 6.12 \mathrm{E}-08$ & $-5.00 \mathrm{E}-04 \pm 6.12 \mathrm{E}-08$ \\
\hline${ }^{12} \mathrm{C}$ & $\mathrm{n}, \mathrm{p}$ & & $-1.40 \mathrm{E}-08 \pm 1.40 \mathrm{E}-11$ & $-2.97 \mathrm{E}-08 \pm 2.46 \mathrm{E}-10$ & $-2.97 \mathrm{E}-08 \pm 2.46 \mathrm{E}-10$ \\
\hline${ }^{12} \mathrm{C}$ & $\mathrm{n}, \mathrm{d}$ & & $-1.55 \mathrm{E}-08 \pm 1.79 \mathrm{E}-11$ & $-5.93 \mathrm{E}-08 \pm 6.94 \mathrm{E}-10$ & $-5.93 \mathrm{E}-08 \pm 6.94 \mathrm{E}-10$ \\
\hline${ }^{12} \mathrm{C}$ & $\mathrm{n}, \alpha$ & & $-1.68 \mathrm{E}-04 \pm 9.95 \mathrm{E}-08$ & $-1.86 \mathrm{E}-04 \pm 5.77 \mathrm{E}-08$ & $-1.86 \mathrm{E}-04 \pm 5.77 \mathrm{E}-08$ \\
\hline${ }^{19} \mathrm{~F}$ & total & $4.10 \mathrm{E}-02$ & $4.75 \mathrm{E}-02 \pm 1.01 \mathrm{E}-05$ & $4.14 \mathrm{E}-02 \pm 2.00 \mathrm{E}-05$ & $4.54 \mathrm{E}-02 \pm 2.00 \mathrm{E}-05$ \\
\hline${ }^{19} \mathrm{~F}$ & scatter & & $5.29 \mathrm{E}-02 \pm 1.03 \mathrm{E}-05$ & $4.70 \mathrm{E}-02 \pm 2.00 \mathrm{E}-05$ & $5.10 \mathrm{E}-02 \pm 2.00 \mathrm{E}-05$ \\
\hline${ }^{19} \mathrm{~F}$ & elastic & & $3.46 \mathrm{E}-02 \pm 7.50 \mathrm{E}-06$ & $2.98 \mathrm{E}-02 \pm 1.73 \mathrm{E}-05$ & $3.38 \mathrm{E}-02 \pm 1.73 \mathrm{E}-05$ \\
\hline${ }^{19} \mathrm{~F}$ & $\mathrm{n}, \mathrm{n}^{\prime}$ & & $1.74 \mathrm{E}-02 \pm 3.91 \mathrm{E}-06$ & $1.61 \mathrm{E}-02 \pm 5.46 \mathrm{E}-06$ & $1.61 \mathrm{E}-02 \pm 5.46 \mathrm{E}-06$ \\
\hline${ }^{19} \mathrm{~F}$ & $\mathrm{n}, 2 \mathrm{n}$ & & $2.54 \mathrm{E}-06 \pm 1.55 \mathrm{E}-09$ & $2.78 \mathrm{E}-06 \pm 3.60 \mathrm{E}-09$ & $2.78 \mathrm{E}-06 \pm 3.60 \mathrm{E}-09$ \\
\hline${ }^{19} \mathrm{~F}$ & capture & & $-5.45 \mathrm{E}-03 \pm 1.58 \mathrm{E}-06$ & $-5.59 \mathrm{E}-03 \pm 7.86 \mathrm{E}-07$ & $-5.59 \mathrm{E}-03 \pm 7.86 \mathrm{E}-07$ \\
\hline${ }^{19} \mathrm{~F}$ & $\mathrm{n}, \gamma$ & & $-2.46 \mathrm{E}-03 \pm 6.05 \mathrm{E}-07$ & $-2.39 \mathrm{E}-03 \pm 2.69 \mathrm{E}-07$ & $-2.39 \mathrm{E}-03 \pm 2.69 \mathrm{E}-07$ \\
\hline${ }^{19} \mathrm{~F}$ & $\mathrm{n}, \mathrm{p}$ & & $-2.16 \mathrm{E}-04 \pm 1.17 \mathrm{E}-07$ & $-2.38 \mathrm{E}-04 \pm 6.25 \mathrm{E}-08$ & $-2.38 \mathrm{E}-04 \pm 6.25 \mathrm{E}-08$ \\
\hline${ }^{19} \mathrm{~F}$ & $\mathrm{n}, \mathrm{d}$ & & $-1.12 \mathrm{E}-05 \pm 6.31 \mathrm{E}-09$ & $-1.26 \mathrm{E}-05 \pm 4.43 \mathrm{E}-09$ & $-1.26 \mathrm{E}-05 \pm 4.43 \mathrm{E}-09$ \\
\hline${ }^{19} \mathrm{~F}$ & $\mathrm{n}, \mathrm{t}$ & & $-2.24 \mathrm{E}-06 \pm 1.65 \mathrm{E}-09$ & $-2.63 \mathrm{E}-06 \pm 1.52 \mathrm{E}-09$ & $-2.63 \mathrm{E}-06 \pm 1.52 \mathrm{E}-09$ \\
\hline${ }^{19} \mathrm{~F}$ & $\mathrm{n}, \alpha$ & & $-2.76 \mathrm{E}-03 \pm 1.38 \mathrm{E}-06$ & $-2.95 \mathrm{E}-03 \pm 6.93 \mathrm{E}-07$ & $-2.95 \mathrm{E}-03 \pm 6.93 \mathrm{E}-07$ \\
\hline
\end{tabular}


Table 6.2.9 Energy- and region-integrated sensitivity coefficients from TSUNAMI-3D UF 4 sample problem (continued)

\begin{tabular}{|c|c|c|c|c|c|}
\hline Isotope & Reaction & $\begin{array}{c}\text { Direct } \\
\text { perturbation }\end{array}$ & $\begin{array}{c}\text { TSUNAMI-3D } \\
\text { one computational } \\
\text { region }\end{array}$ & $\begin{array}{c}\text { TSUNAMI-3D } \\
\text { nine computational } \\
\text { regions }\end{array}$ & $\begin{array}{c}\text { TSUNAMI-3D } \\
\text { PARM=CENTRM } \\
\text { nine computational } \\
\text { regions }\end{array}$ \\
\hline${ }^{235} \mathrm{U}$ & total & $2.52 \mathrm{E}-01$ & $2.31 \mathrm{E}-01 \pm 8.79 \mathrm{E}-05$ & $2.50 \mathrm{E}-01 \pm 4.36 \mathrm{E}-05$ & $5.13 \mathrm{E}-04 \pm 1.22 \mathrm{E}-07$ \\
\hline${ }^{235} \mathrm{U}$ & scatter & & $5.10 \mathrm{E}-04 \pm 9.11 \mathrm{E}-08$ & $4.42 \mathrm{E}-04 \pm 1.22 \mathrm{E}-07$ & $2.76 \mathrm{E}-04 \pm 1.08 \mathrm{E}-07$ \\
\hline${ }^{235} \mathrm{U}$ & elastic & & $2.57 \mathrm{E}-04 \pm 4.65 \mathrm{E}-08$ & $2.05 \mathrm{E}-04 \pm 1.08 \mathrm{E}-07$ & $2.20 \mathrm{E}-04 \pm 4.62 \mathrm{E}-08$ \\
\hline${ }^{235} \mathrm{U}$ & $\mathrm{n}, \mathrm{n}^{\prime}$ & & $2.36 \mathrm{E}-04 \pm 6.35 \mathrm{E}-08$ & $2.20 \mathrm{E}-04 \pm 4.62 \mathrm{E}-08$ & $1.73 \mathrm{E}-05 \pm 4.49 \mathrm{E}-09$ \\
\hline${ }^{235} \mathrm{U}$ & $\mathrm{n}, 2 \mathrm{n}$ & & $1.65 \mathrm{E}-05 \pm 8.98 \mathrm{E}-09$ & $1.73 \mathrm{E}-05 \pm 4.49 \mathrm{E}-09$ & $3.63 \mathrm{E}-01 \pm 5.30 \mathrm{E}-05$ \\
\hline${ }^{235} \mathrm{U}$ & fission & & $3.47 \mathrm{E}-01 \pm 1.10 \mathrm{E}-04$ & $3.63 \mathrm{E}-01 \pm 5.30 \mathrm{E}-05$ & $-1.13 \mathrm{E}-01 \pm 1.27 \mathrm{E}-05$ \\
\hline${ }^{235} \mathrm{U}$ & capture & & $-1.16 \mathrm{E}-01 \pm 2.86 \mathrm{E}-05$ & $-1.13 \mathrm{E}-01 \pm 1.27 \mathrm{E}-05$ & $-1.13 \mathrm{E}-01 \pm 1.27 \mathrm{E}-05$ \\
\hline${ }^{235} \mathrm{U}$ & $\mathrm{n}, \gamma$ & & $-1.16 \mathrm{E}-01 \pm 2.86 \mathrm{E}-05$ & $-1.13 \mathrm{E}-01 \pm 1.27 \mathrm{E}-05$ & $9.50 \mathrm{E}-01 \pm 1.65 \mathrm{E}-04$ \\
\hline${ }^{235} \mathrm{U}$ & nubar & & $9.51 \mathrm{E}-01 \pm 3.64 \mathrm{E}-04$ & $9.50 \mathrm{E}-01 \pm 1.65 \mathrm{E}-04$ & $8.52 \mathrm{E}-08 \pm 9.89 \mathrm{E}-05$ \\
\hline${ }^{235} \mathrm{U}$ & chi & & $8.54 \mathrm{E}-08 \pm 2.18 \mathrm{E}-04$ & $8.52 \mathrm{E}-08 \pm 9.89 \mathrm{E}-05$ & $-2.47 \mathrm{E}-01 \pm 2.33 \mathrm{E}-05$ \\
\hline${ }^{238} \mathrm{U}$ & total & $-2.08 \mathrm{E}-01$ & $-2.11 \mathrm{E}-01 \pm 5.14 \mathrm{E}-05$ & $-2.05 \mathrm{E}-01 \pm 2.39 \mathrm{E}-05$ & $2.52 \mathrm{E}-02 \pm 5.17 \mathrm{E}-06$ \\
\hline${ }^{238} \mathrm{U}$ & scatter & & $5.29 \mathrm{E}-02 \pm 7.93 \mathrm{E}-06$ & $4.88 \mathrm{E}-02 \pm 5.85 \mathrm{E}-06$ & $1.13 \mathrm{E}-02 \pm 3.86 \mathrm{E}-06$ \\
\hline${ }^{238} \mathrm{U}$ & elastic & & $3.82 \mathrm{E}-02 \pm 6.53 \mathrm{E}-06$ & $3.49 \mathrm{E}-02 \pm 4.73 \mathrm{E}-06$ & $1.29 \mathrm{E}-02 \pm 2.95 \mathrm{E}-06$ \\
\hline${ }^{238} \mathrm{U}$ & $\mathrm{n}, \mathrm{n}^{\prime}$ & & $1.37 \mathrm{E}-02 \pm 3.83 \mathrm{E}-06$ & $1.29 \mathrm{E}-02 \pm 2.95 \mathrm{E}-06$ & $1.03 \mathrm{E}-03 \pm 3.01 \mathrm{E}-07$ \\
\hline${ }^{238} \mathrm{U}$ & $\mathrm{n}, 2 \mathrm{n}$ & & $9.85 \mathrm{E}-04 \pm 5.90 \mathrm{E}-07$ & $1.03 \mathrm{E}-03 \pm 3.01 \mathrm{E}-07$ & $3.35 \mathrm{E}-02 \pm 5.49 \mathrm{E}-06$ \\
\hline${ }^{238} \mathrm{U}$ & fission & & $3.24 \mathrm{E}-02 \pm 1.18 \mathrm{E}-05$ & $3.35 \mathrm{E}-02 \pm 5.49 \mathrm{E}-06$ & $-3.06 \mathrm{E}-01 \pm 2.14 \mathrm{E}-05$ \\
\hline${ }^{238} \mathrm{U}$ & capture & & $-2.96 \mathrm{E}-01 \pm 4.82 \mathrm{E}-05$ & $-2.87 \mathrm{E}-01 \pm 2.16 \mathrm{E}-05$ & $-3.06 \mathrm{E}-01 \pm 2.14 \mathrm{E}-05$ \\
\hline${ }^{238} \mathrm{U}$ & $\mathrm{n}, \gamma$ & & $-2.96 \mathrm{E}-01 \pm 4.82 \mathrm{E}-05$ & $-2.87 \mathrm{E}-01 \pm 2.16 \mathrm{E}-05$ & $5.02 \mathrm{E}-02 \pm 1.11 \mathrm{E}-05$ \\
\hline${ }^{238} \mathrm{U}$ & nubar & & $4.85 \mathrm{E}-02 \pm 2.37 \mathrm{E}-05$ & $5.02 \mathrm{E}-02 \pm 1.11 \mathrm{E}-05$ & $4.53 \mathrm{E}-09 \pm 5.18 \mathrm{E}-06$ \\
\hline${ }^{238} \mathrm{U}$ & chi & & $4.38 \mathrm{E}-09 \pm 1.10 \mathrm{E}-05$ & $4.53 \mathrm{E}-09 \pm 5.18 \mathrm{E}-06$ & $5.13 \mathrm{E}-04 \pm 1.22 \mathrm{E}-07$ \\
\hline
\end{tabular}


The uncertainty information from SAMS for the $\mathrm{UF}_{4}$ sample problem is shown in Figure 6.2.7. Based on the 44GROUPCOV covariance data file, the uncertainty in $k_{\text {eff }}$ due to these covariance data is $0.6110 \%$ $\Delta \mathrm{k} / \mathrm{k}$. A more detailed description of the uncertainty information is given in the SAMS chapter.

The energy-dependent sensitivity data are available in the sensitivity data file, which is returned to the same directory as the input file and given the same name as the users input file with the extension .sdf. In the case of the nine-region model, the sensitivity data file contains 495 individual sensitivity profiles with varying reaction types, each in the 238-group energy structure. There are 45 profiles that are integrated over all regions, one for each reaction of each nuclide in the system. The sum of the sensitivity coefficients for the same nuclide in all mixtures is printed unless nomix is entered in the SAMS data block, so there are an additional 45 profiles, one for each reaction of each nuclide in mixture 1. Additionally, because prtgeom was entered in the SAMS data block, each reaction of each nuclide for each region in the system model is represented with a sensitivity profile. There are nine regions in the model, each with 45 sensitivity profiles, making for a total of 495 sensitivity profiles on the data file and a total of 117,810 energy-dependent sensitivity coefficients.

Some plots of the energy-dependent sensitivity data from the nine-region model of the sample problem were generated with the plotting capabilities of Fulcrum. Energy-dependent sensitivity profiles for ${ }^{235} \mathrm{U}$ fission and ${ }^{1} \mathrm{H}$ elastic scattering are shown in Figure 6.2.8. The error bars represent one standard deviation for the statistical uncertainty due to the use of Monte Carlo methods to compute the fluxes and $k_{\text {eff. }}$ 
Uncertainty Information

the relative standard deviation of k-eff (\% delta-k/k) due to cross-section covariance data is:

$0.6110+/-0.0000 \% \mathrm{delta-k/k}$

contributions to uncertainty in k-eff (\% delta-k/k) by individual energy covariance matrices:

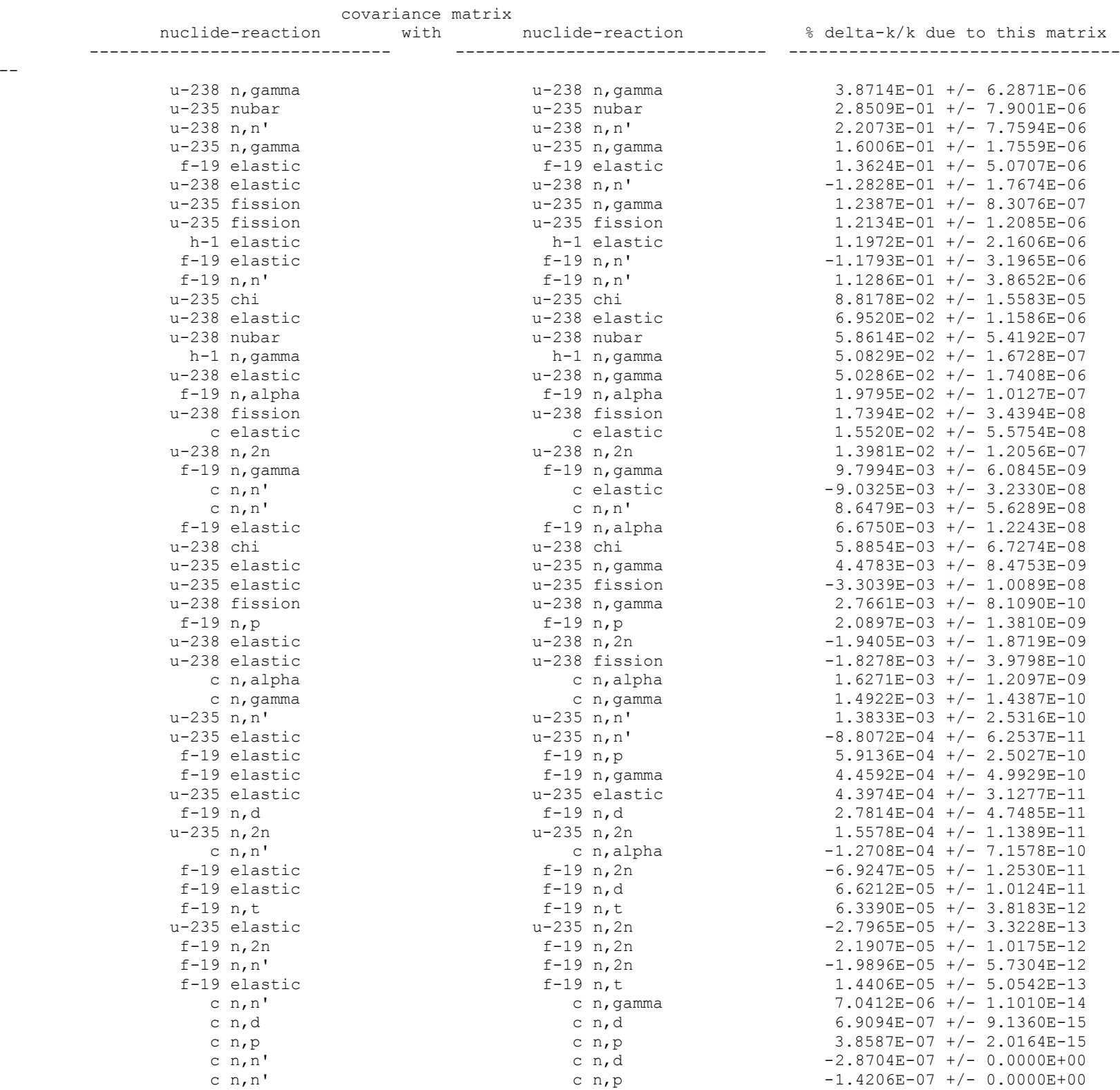

Note: relative standard deviation in k-eff can be computed from

individual values by adding the square of the values with positive signs and

subtracting the square of the values with negative signs, then taking the square root

Figure 6.2.7. Uncertainty information from $\mathrm{UF}_{4}$ sample problem. 


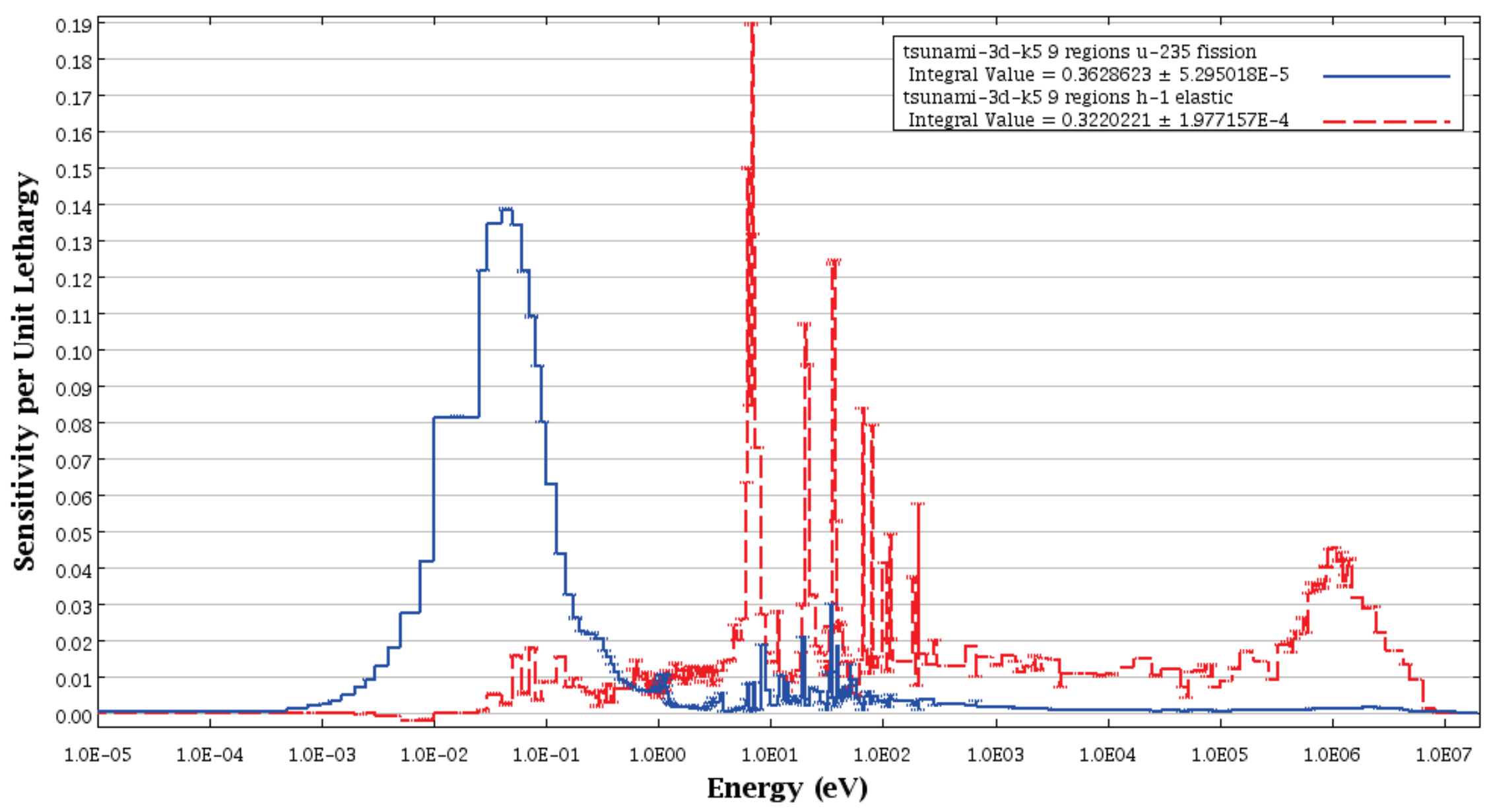

Figure 6.2.8. Energy-dependent sensitivity profiles from TSUNAMI-3D-K5 for simple sample problem. 


\subsubsection{Multigroup sample problem with spatial flux mesh}

In the previous example, subdivision of the system geometry was necessary to obtain adequate resolution of the flux solution to obtain the appropriate product of the forward and adjoint fluxes necessary for the sensitivity calculations. To simplify the geometry refinement procedure, the meshing of KENO V.a or KENO-VI is used where fluxes are tallied in a cubic mesh that is superimposed over each region of the system geometry. If mesh fluxes are generated in the KENO solution, they are automatically used by the SAMS module in the calculation of the sensitivity coefficients.

To demonstrate the use of the mesh flux option in TSUNAMI-3D-K5, another simple system has been selected. This system is an unreflected rectangular parallelepiped consisting of a homogeneous mixture of $\mathrm{UF}_{4}$ and paraffin with an enrichment of $2 \mathrm{wt} \%$ in ${ }^{235} \mathrm{U}$. The $\mathrm{H} /{ }^{235} \mathrm{U}$ atomic ratio is $972: 1$. The dimensions of the experiment are $81.45 \times 86.70 \times 88.22 \mathrm{~cm}$. This system is identified as LEU-COMP-THERM- 033 case 45 from the International Handbook of Evaluated Criticality Safety Benchmark Experiments (IHECSBE). ${ }^{11}$ The model provided in the IHECSBE was converted to a TSUNAMI-3D-K5 input and is shown in Figure 6.2.9. Here the experiment is modeled as a single cuboid. This model is included as an example and is not distributed as a TSUNAMI-3D sample problem.

Direct perturbation sensitivity results were obtained for ${ }^{235} \mathrm{U},{ }^{238} \mathrm{U}$, and ${ }^{1} \mathrm{H}$ with a $\pm 4 \%$ change in the number density. Each direct perturbation calculation was conducted with 15,000 particles per generation and 1400 active generations. All TSUNAMI-3D-K5 results presented in this section were obtained with 10,000 particles per generation for the forward case and 30,000 particles per generation for the adjoint case. Both the forward and adjoint cases requested up to 10,000 active generations, but the calculation was requested to stop when the $k_{\text {eff }}$ value converged to a standard deviation of $0.0001(\mathrm{sig}=0.0001)$. For the model shown in Figure 6.2.9, the forward and adjoint $k_{\text {eff }}$ values agreed well at $0.99250 \pm 0.00056$ and $0.9905 \pm 0.0049$, respectively. The direct perturbation results and the TSUNAMI-3D-K5 results are shown in Table 6.2.10. When modeled as a single region, the TSUNAMI-3D-K5 results differed from the direct perturbation results by $2.6 \%$ for ${ }^{238} \mathrm{U}, 3.1 \%$ for ${ }^{235} \mathrm{U}$, and $15 \%$ for ${ }^{1} \mathrm{H}$.

To improve the agreement in the values, the model was divided into 6 cuboids nested inside of each other. The input for this model is given in Figure 6.2.10 and is illustrated in Figure 6.2.11. The results for this model are also given in Table 6.2.10. Here the TSUNAMI-3D-K5 sensitivity coefficients agree with the direct perturbation within $0.3 \%$ for ${ }^{238} \mathrm{U}, 1.7 \%$ for ${ }^{235} \mathrm{U}$, and $0.8 \%$ for ${ }^{1} \mathrm{H}$.

To simplify the generation of a refined geometrical representation of this system, the same geometry as the initial model was used with an $8 \mathrm{~cm}$ mesh for the flux tallies. The input for this model is shown in Figure 6.2.12. The mesh is illustrated in Figure 6.2.13, using a larger $(15 \mathrm{~cm})$ mesh interval for illustrative purposes. The mesh flux option is activated by entering $m f x=y e s$ in the input, and the size of the mesh is defined with the $m s h=8$ entry. The 8 following $m s h=$ indicates that a cubic grid with a length of $8 \mathrm{~cm}$ on the side of each cube will be superimposed on each geometry region. For this system, the flux will be tallied in 1728 mesh intervals. When the forward and adjoint mesh flux solutions are processed by the SAMS module, the product of the forward and adjoint solutions are produced for each mesh interval, then summed for each region. This technique provides a simple input parameter to produce accurate sensitivity coefficients. The results from TSUNAMI-3D-K5 with an $8 \mathrm{~cm}$ mesh flux are given in Table 6.2.10. Similar to the results with the manual subdivision, sensitivity coefficients agree with the direct perturbation within $0.7 \%$ for ${ }^{238} \mathrm{U}, 2.1 \%$ for ${ }^{235} \mathrm{U}$, and $0.03 \%$ for ${ }^{1} \mathrm{H}$. 
The sensitivity results for ${ }^{235} \mathrm{U}$ and ${ }^{238} \mathrm{U}$ show little variation with modifications in the geometry subdivision. The products of the forward and adjoint flux moments, which are derived from the angular flux solution, are most impacted by the mesh flux. The flux moments are used to compute the scattering terms of the sensitivity coefficients. For isotopes with limited scattering cross sections, such as ${ }^{235} \mathrm{U}$ and ${ }^{238} \mathrm{U}$, the impact of refinement of the flux solution is reduced.

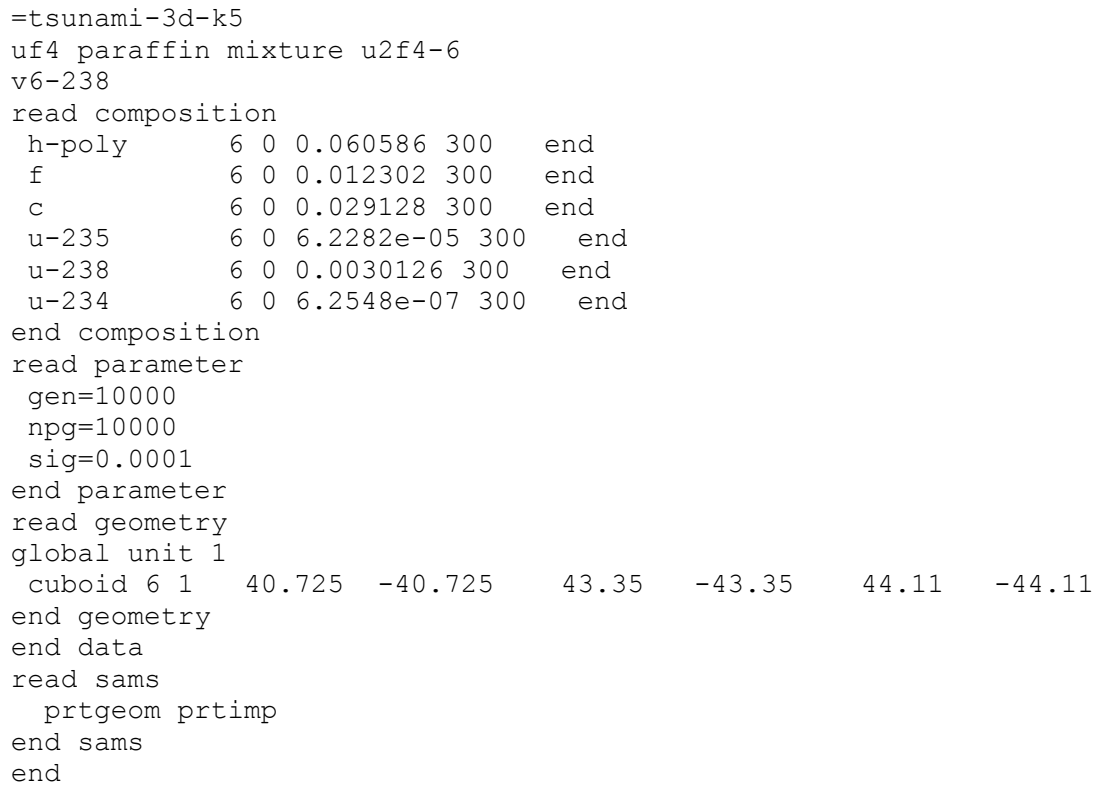

Figure 6.2.9. TSUNAMI-3D-K5 input for LEU-COMP-THERM-033 case 45. 
Table 6.2.10. Energy- and region-integrated sensitivity coefficients from LEU-COMP-THERM-033 case 45 sample problem

\begin{tabular}{|c|c|c|c|c|c|c|c|c|c|c|c|c|c|}
\hline \multirow{2}{*}{$\frac{\text { Isotope }}{{ }^{1} \mathrm{H}}$} & \multirow{2}{*}{$\frac{\text { Reaction }}{\text { total }}$} & \multicolumn{3}{|c|}{$\begin{array}{c}\text { Direct } \\
\text { perturbation }\end{array}$} & \multicolumn{3}{|c|}{$\begin{array}{c}\text { TSUNAMI-3D } \\
\text { one computational } \\
\text { region }\end{array}$} & \multicolumn{3}{|c|}{$\begin{array}{c}\text { TSUNAMI-3D } \\
\text { six computational } \\
\text { regions } \\
\end{array}$} & \multicolumn{3}{|c|}{$\begin{array}{c}\text { TSUNAMI-3D } \\
8 \mathrm{~cm} \text { mesh } \\
\text { (1728 mesh regions) } \\
\end{array}$} \\
\hline & & $-1.00 \mathrm{E}-01$ & \pm & $4.74 \mathrm{E}-03$ & $-8.46 \mathrm{E}-02$ & \pm & $7.44 \mathrm{E}-05$ & $-1.01 \mathrm{E}-01$ & \pm & $2.70 \mathrm{E}-04$ & $-1.00 \mathrm{E}-01$ & \pm & $1.51 \mathrm{E}-03$ \\
\hline${ }^{1} \mathrm{H}$ & elastic & & & & $1.93 \mathrm{E}-01$ & \pm & $5.26 \mathrm{E}-05$ & $1.79 \mathrm{E}-01$ & \pm & $2.65 \mathrm{E}-04$ & $1.79 \mathrm{E}-01$ & \pm & $1.50 \mathrm{E}-03$ \\
\hline${ }^{1} \mathrm{H}$ & capture & & & & $-2.77 \mathrm{E}-01$ & \pm & $5.12 \mathrm{E}-05$ & $-2.80 \mathrm{E}-01$ & \pm & $3.20 \mathrm{E}-05$ & $-2.79 \mathrm{E}-01$ & \pm & $5.44 \mathrm{E}-05$ \\
\hline${ }^{1} \mathrm{H}$ & $\mathrm{n}, \gamma$ & & & & $-2.77 \mathrm{E}-01$ & \pm & $5.12 \mathrm{E}-05$ & $-2.80 \mathrm{E}-01$ & \pm & $3.20 \mathrm{E}-05$ & $-2.79 \mathrm{E}-01$ & \pm & 5.44E-05 \\
\hline${ }^{12} \mathrm{C}$ & elastic & & & & $2.46 \mathrm{E}-02$ & \pm & $4.84 \mathrm{E}-06$ & $1.65 \mathrm{E}-02$ & \pm & $1.82 \mathrm{E}-05$ & $1.78 \mathrm{E}-02$ & \pm & $1.04 \mathrm{E}-04$ \\
\hline${ }^{12} \mathrm{C}$ & $\mathrm{n}, \mathrm{n}^{\prime}$ & & & & $3.60 \mathrm{E}-04$ & \pm & $1.35 \mathrm{E}-07$ & $3.36 \mathrm{E}-04$ & \pm & $1.15 \mathrm{E}-07$ & $3.47 \mathrm{E}-04$ & \pm & $6.72 \mathrm{E}-07$ \\
\hline${ }^{12} \mathrm{C}$ & capture & & & & $-1.62 \mathrm{E}-03$ & \pm & $2.67 \mathrm{E}-07$ & $-1.64 \mathrm{E}-03$ & \pm & $1.69 \mathrm{E}-07$ & $-1.63 \mathrm{E}-03$ & \pm & $3.17 \mathrm{E}-07$ \\
\hline${ }^{12} \mathrm{C}$ & $\mathrm{n}, \gamma$ & & & & $-1.36 \mathrm{E}-03$ & \pm & $2.50 \mathrm{E}-07$ & $-1.37 \mathrm{E}-03$ & \pm & $1.56 \mathrm{E}-07$ & $-1.37 \mathrm{E}-03$ & \pm & $2.66 \mathrm{E}-07$ \\
\hline${ }^{12} \mathrm{C}$ & $\mathrm{n}, \mathrm{p}$ & & & & $-4.87 \mathrm{E}-08$ & \pm & $4.71 \mathrm{E}-11$ & $-2.75 \mathrm{E}-08$ & \pm & $3.05 \mathrm{E}-11$ & $-4.52 \mathrm{E}-08$ & \pm & $8.03 \mathrm{E}-10$ \\
\hline${ }^{12} \mathrm{C}$ & $\mathrm{n}, \mathrm{d}$ & & & & $-1.32 \mathrm{E}-07$ & \pm & $1.54 \mathrm{E}-10$ & $-5.57 \mathrm{E}-08$ & \pm & $8.13 \mathrm{E}-11$ & $-1.17 \mathrm{E}-07$ & \pm & $2.67 \mathrm{E}-09$ \\
\hline${ }^{19} \mathrm{~F}$ & $\mathrm{n}, \mathrm{n}^{\prime}$ & & & & $4.66 \mathrm{E}-03$ & \pm & $6.67 \mathrm{E}-07$ & $3.71 \mathrm{E}-03$ & \pm & $1.07 \mathrm{E}-06$ & $3.90 \mathrm{E}-03$ & \pm & $6.34 \mathrm{E}-06$ \\
\hline${ }^{19} \mathrm{~F}$ & $\mathrm{n}, 2 \mathrm{n}$ & & & & $1.69 \mathrm{E}-06$ & \pm & $5.84 \mathrm{E}-10$ & $1.53 \mathrm{E}-06$ & \pm & $5.20 \mathrm{E}-10$ & $1.70 \mathrm{E}-06$ & \pm & $4.82 \mathrm{E}-09$ \\
\hline${ }^{19} \mathrm{~F}$ & capture & & & & $-3.24 \mathrm{E}-03$ & \pm & $5.40 \mathrm{E}-07$ & $-3.26 \mathrm{E}-03$ & \pm & $3.39 \mathrm{E}-07$ & $-3.23 \mathrm{E}-03$ & \pm & $6.03 \mathrm{E}-07$ \\
\hline${ }^{19} \mathrm{~F}$ & $\mathrm{n}, \gamma$ & & & & $-1.76 \mathrm{E}-03$ & \pm & $3.00 \mathrm{E}-07$ & $-1.78 \mathrm{E}-03$ & \pm & $1.88 \mathrm{E}-07$ & $-1.77 \mathrm{E}-03$ & \pm & $3.19 \mathrm{E}-07$ \\
\hline${ }^{19} \mathrm{~F}$ & $\mathrm{n}, \mathrm{p}$ & & & & $-1.03 \mathrm{E}-04$ & \pm & $3.50 \mathrm{E}-08$ & $-1.04 \mathrm{E}-04$ & \pm & $2.23 \mathrm{E}-08$ & $-1.01 \mathrm{E}-04$ & \pm & $4.57 \mathrm{E}-08$ \\
\hline${ }^{19} \mathrm{~F}$ & $\mathrm{n}, \mathrm{d}$ & & & & $-4.97 \mathrm{E}-06$ & \pm & $1.63 \mathrm{E}-09$ & $-4.99 \mathrm{E}-06$ & \pm & $1.10 \mathrm{E}-09$ & $-4.85 \mathrm{E}-06$ & \pm & $3.69 \mathrm{E}-09$ \\
\hline${ }^{19} \mathrm{~F}$ & $\mathrm{n}, \mathrm{t}$ & & & & $-1.13 \mathrm{E}-06$ & \pm & 4.63E-10 & $-1.10 \mathrm{E}-06$ & \pm & $3.33 \mathrm{E}-10$ & $-1.10 \mathrm{E}-06$ & \pm & $1.55 \mathrm{E}-09$ \\
\hline${ }^{19} \mathrm{~F}$ & $\mathrm{n}, \alpha$ & & & & $-1.36 \mathrm{E}-03$ & \pm & 4.23E-07 & $-1.37 \mathrm{E}-03$ & \pm & $2.66 \mathrm{E}-07$ & $-1.35 \mathrm{E}-03$ & \pm & $4.79 \mathrm{E}-07$ \\
\hline${ }^{234} \mathrm{U}$ & total & $1.51 \mathrm{E}-03$ & \pm & $5.79 \mathrm{E}-03$ & $-1.13 \mathrm{E}-03$ & \pm & $2.10 \mathrm{E}-07$ & $-1.15 \mathrm{E}-03$ & \pm & $1.32 \mathrm{E}-07$ & $-1.14 \mathrm{E}-03$ & \pm & $2.31 \mathrm{E}-07$ \\
\hline${ }^{234} \mathrm{U}$ & scatter & & & & $1.68 \mathrm{E}-06$ & \pm & $1.95 \mathrm{E}-10$ & $1.25 \mathrm{E}-06$ & \pm & $1.70 \mathrm{E}-10$ & $1.34 \mathrm{E}-06$ & 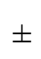 & $2.19 \mathrm{E}-09$ \\
\hline${ }^{234} \mathrm{U}$ & elastic & & & & $1.02 \mathrm{E}-06$ & \pm & $1.14 \mathrm{E}-10$ & $6.53 \mathrm{E}-07$ & \pm & $1.21 \mathrm{E}-10$ & $7.19 \mathrm{E}-07$ & \pm & $1.84 \mathrm{E}-09$ \\
\hline${ }^{234} \mathrm{U}$ & $\mathrm{n}, \mathrm{n}^{\prime}$ & & & & $6.38 \mathrm{E}-07$ & \pm & $1.14 \mathrm{E}-10$ & $5.79 \mathrm{E}-07$ & \pm & $8.78 \mathrm{E}-11$ & $5.95 \mathrm{E}-07$ & \pm & $5.33 \mathrm{E}-10$ \\
\hline
\end{tabular}


Table 6.2.10. Energy- and region-integrated sensitivity coefficients from LEU-COMP-THERM-033 case 45 sample problem (continued)

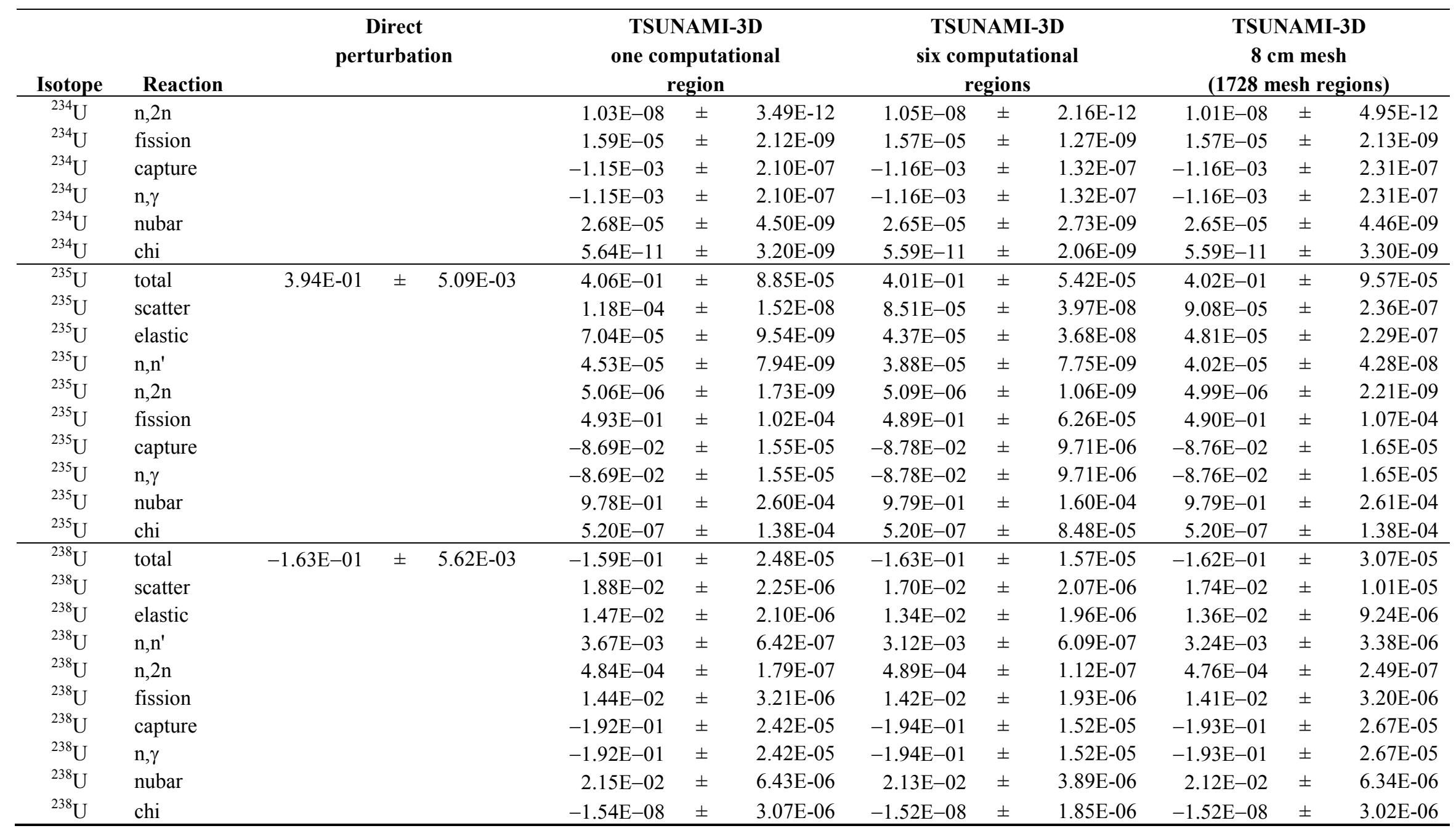




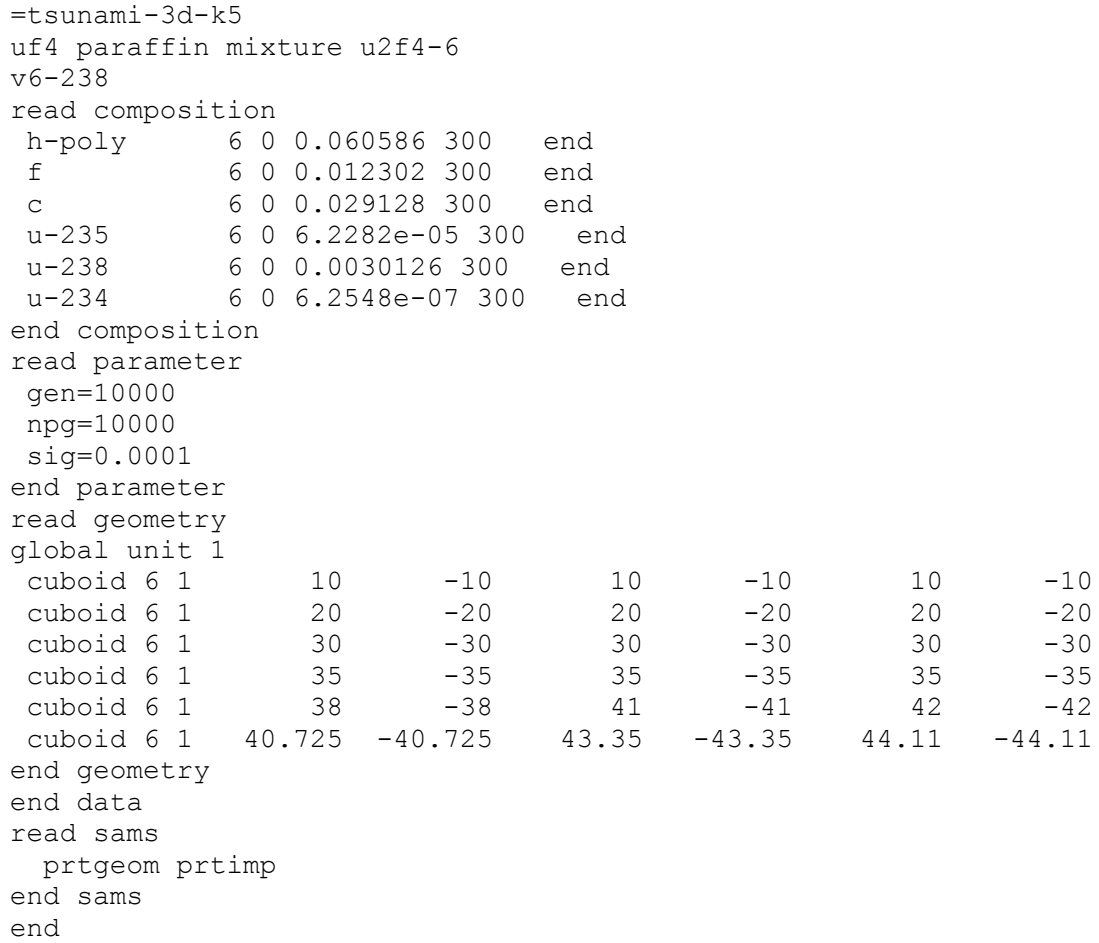

$\begin{array}{rrrrrr}10 & -10 & 10 & -10 & 10 & -10 \\ 20 & -20 & 20 & -20 & 20 & -20 \\ 30 & -30 & 30 & -30 & 30 & -30 \\ 35 & -35 & 35 & -35 & 35 & -35 \\ 38 & -38 & 41 & -41 & 42 & -42 \\ 40.725 & -40.725 & 43.35 & -43.35 & 44.11 & -44.11\end{array}$

Figure 6.2.10. TSUNAMI-3D-K5 input LEU-COMP-THERM-033 case 45 with manual geometrical subdivision.

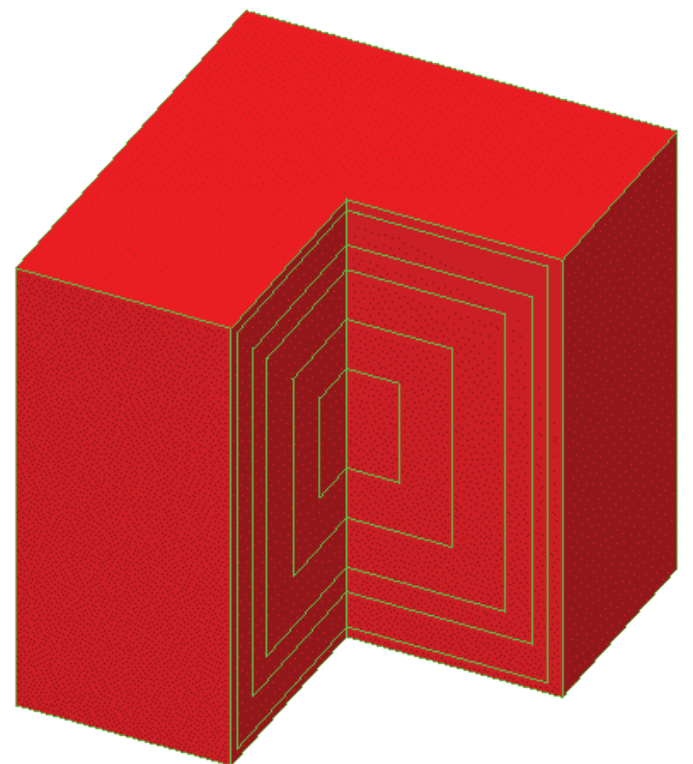

Figure 6.2.11. Cutaway view of LEU-COMP-THERM-033 case 45 with manual subdivision. 


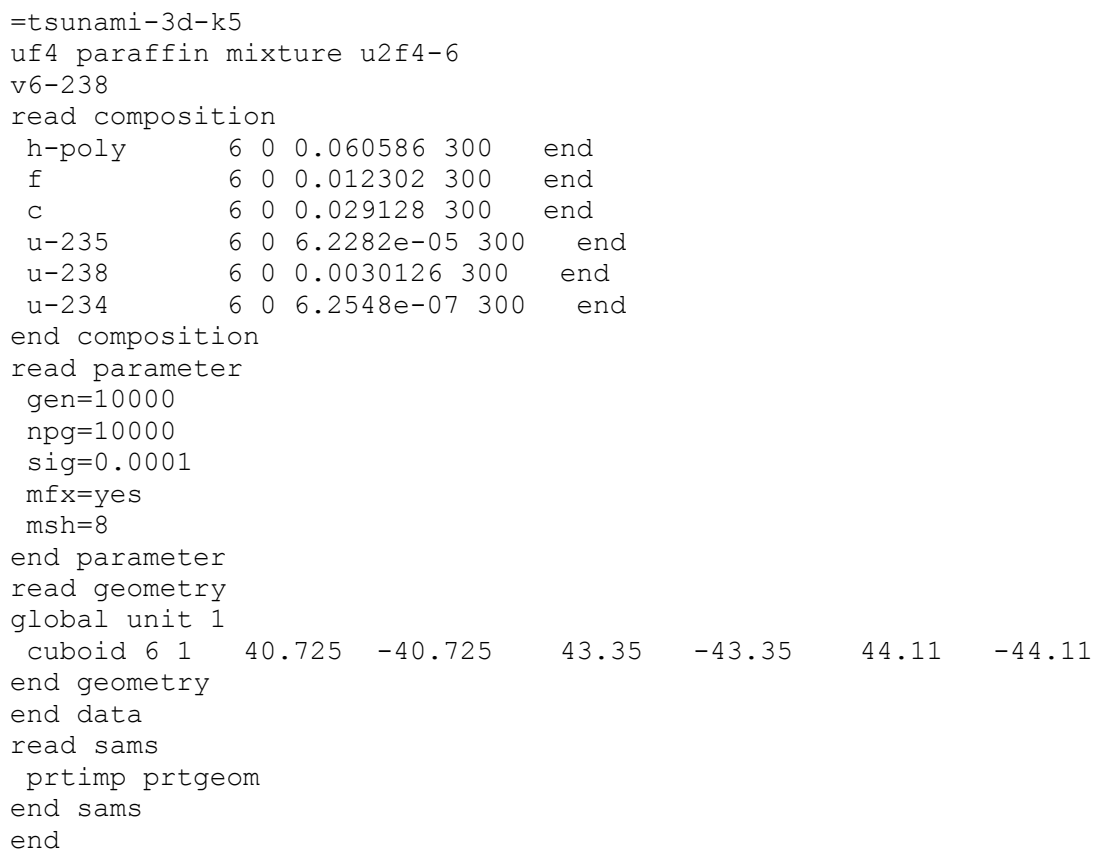

Figure 6.2.12. TSUNAMI-3D-K5 input for LEU-COMP-THERM-033 case 45 with $8 \mathrm{~cm}$ automated mesh.

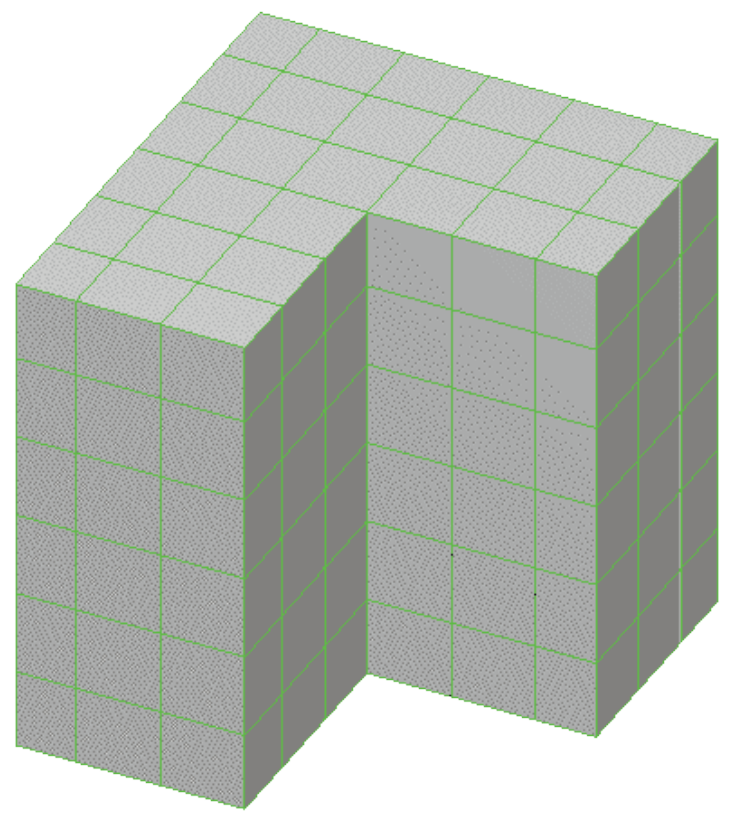

Figure 6.2.13. Cutaway view of LEU-COMP-THERM-033 case 45 with $15 \mathrm{~cm}$ automated mesh. 


\subsubsection{Complex sample problem}

A more complex sample problem is a critical assembly of $4.31 \mathrm{wt} \%$-enriched $\mathrm{UO}_{2}$ fuel rods with a pitch of $2.54 \mathrm{~cm}$ in clusters that are separated by copper plates. This system is identified as LEU-COMPTHERM-009 case 10 from the IHECSBE ${ }^{11}$. This system is used for sample problem TSUNAMI-3D_K52 that is distributed with SCALE. The TSUNAMI-3D_K5-2 sample problem input is shown in Figure 6.2.14, and the geometry is illustrated in Figure 6.2.15. The KENO V.a input section for this model is essentially the same as that presented in the IHECSBE except that the parameter data have been modified for the TSUNAMI calculation. Also, in the IHECSBE model, all water was assigned to the same mixture. For this model, the water in the reflector region is entered as a separate mixture from the water in the pin cell to generate separate sensitivity coefficients in these regions.

For this system, direct perturbation results were obtained for ${ }^{235} \mathrm{U},{ }^{238} \mathrm{U}$, and ${ }^{1} \mathrm{H}$ in the pin cell model (mixture 2) and for ${ }^{1} \mathrm{H}$ in the reflector (mixture 7). Direct perturbation sensitivity coefficients can be difficult to obtain for large systems using Monte Carlo techniques. For a small change in a number density ( $1 \%$ or $2 \%$ ), the effect of $k_{\text {eff }}$ may not be significant enough to be observed outside of statistical uncertainties. If a larger perturbation $(5 \%$ or $10 \%)$ is used, the effect on $k_{\text {eff }}$ may not be linear and may produce misleading results. Also, if the magnitude of the difference in $k_{\text {eff }}$ between the baseline value and the increased density perturbed model is different from the magnitude of the difference in $k_{\text {eff }}$ between the baseline model and the decreased density perturbed model, the response may be nonlinear. In these calculations, the amount of the perturbation was carefully chosen to produce approximately 10 standard deviations of change in $k_{\text {eff }}$ between the perturbed and unperturbed case. The amount of perturbation for each nuclide is shown in Table 6.2.11.

TSUNAMI-3D-K5 was executed with no flux mesh, a $15 \mathrm{~cm}$ flux mesh, and a $5 \mathrm{~cm}$ flux mesh using the default coordinate transform setting $(\mathrm{tfm}=y e s)$. Also, the $15 \mathrm{~cm}$ and $5 \mathrm{~cm}$ mesh cases were run with the coordinate transform turned off $(t f m=n o)$.

Where a region is repeatedly used in KENO, the flux data are averaged over all occurrences of a region. In this model, only one fuel pin is explicitly modeled. This single pin is used repeatedly in arrays to create the system model. The flux data for this single pin are averaged over all occurrences throughout the system to create the sensitivity data. Using the mesh flux option, the flux is accumulated for the fuel pin in each mesh interval. For the model with no mesh, the flux in the $\mathrm{UO}_{2}$ portion of the fuel pin is averaged over a single region. With the $15 \mathrm{~cm}$ mesh, the flux in $\mathrm{UO}_{2}$ is accumulated in 126 separate mesh intervals distributed across the core. For the $5 \mathrm{~cm}$ mesh, the flux in $\mathrm{UO}_{2}$ is accumulated in 2280 mesh intervals. For all regions of the model with no mesh, the flux is only stored for 17 unique locations, one for each region. For the $15 \mathrm{~cm}$ mesh, the flux is stored in 1968 unique mesh intervals. For the $5 \mathrm{~cm}$ mesh mode, the flux is stored in 34,298 unique mesh intervals. 


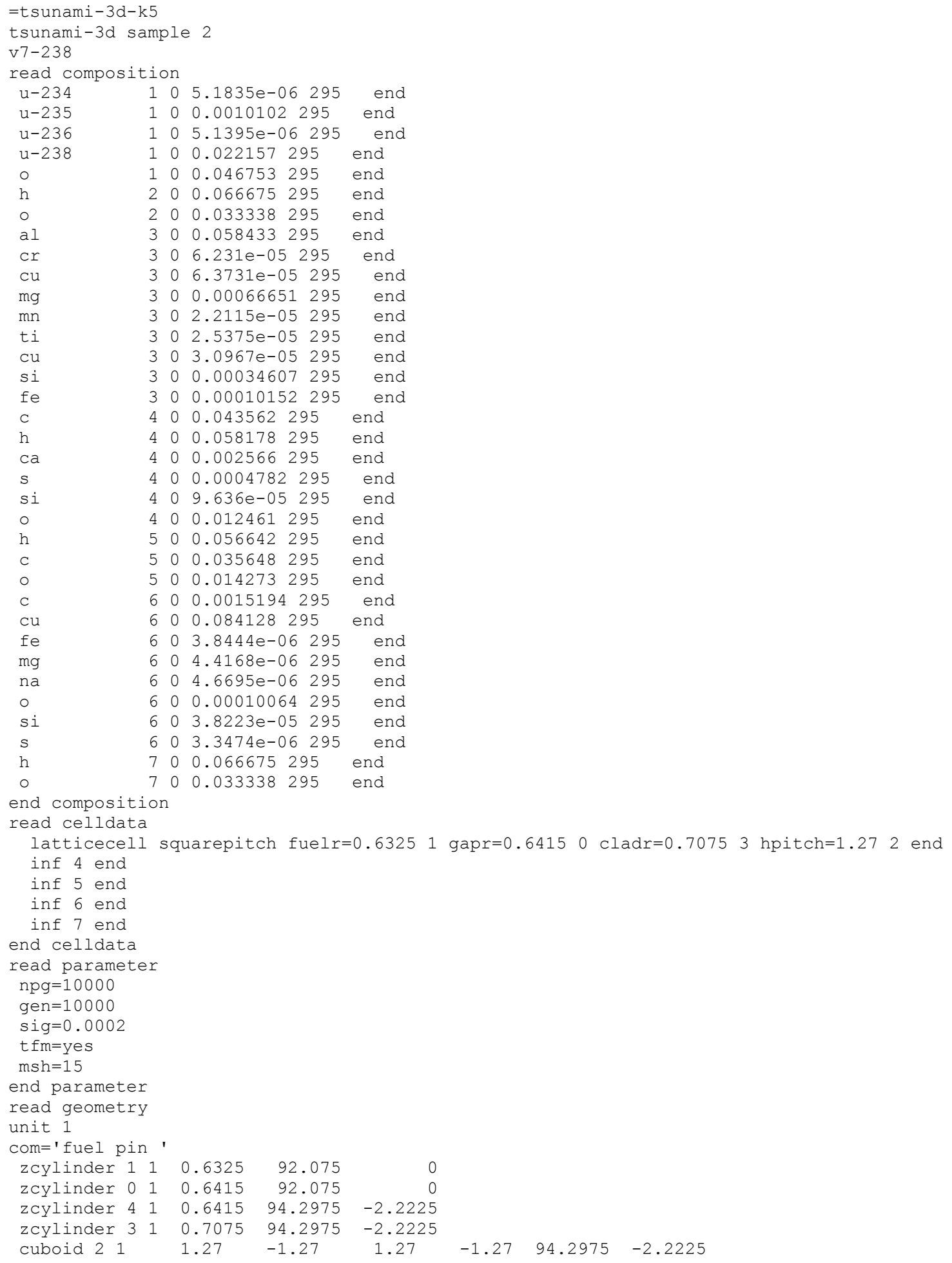

Figure 6.2.14. TSUNAMI-3D LEU-COMP-THERM-009 case 10 sample problem input. 


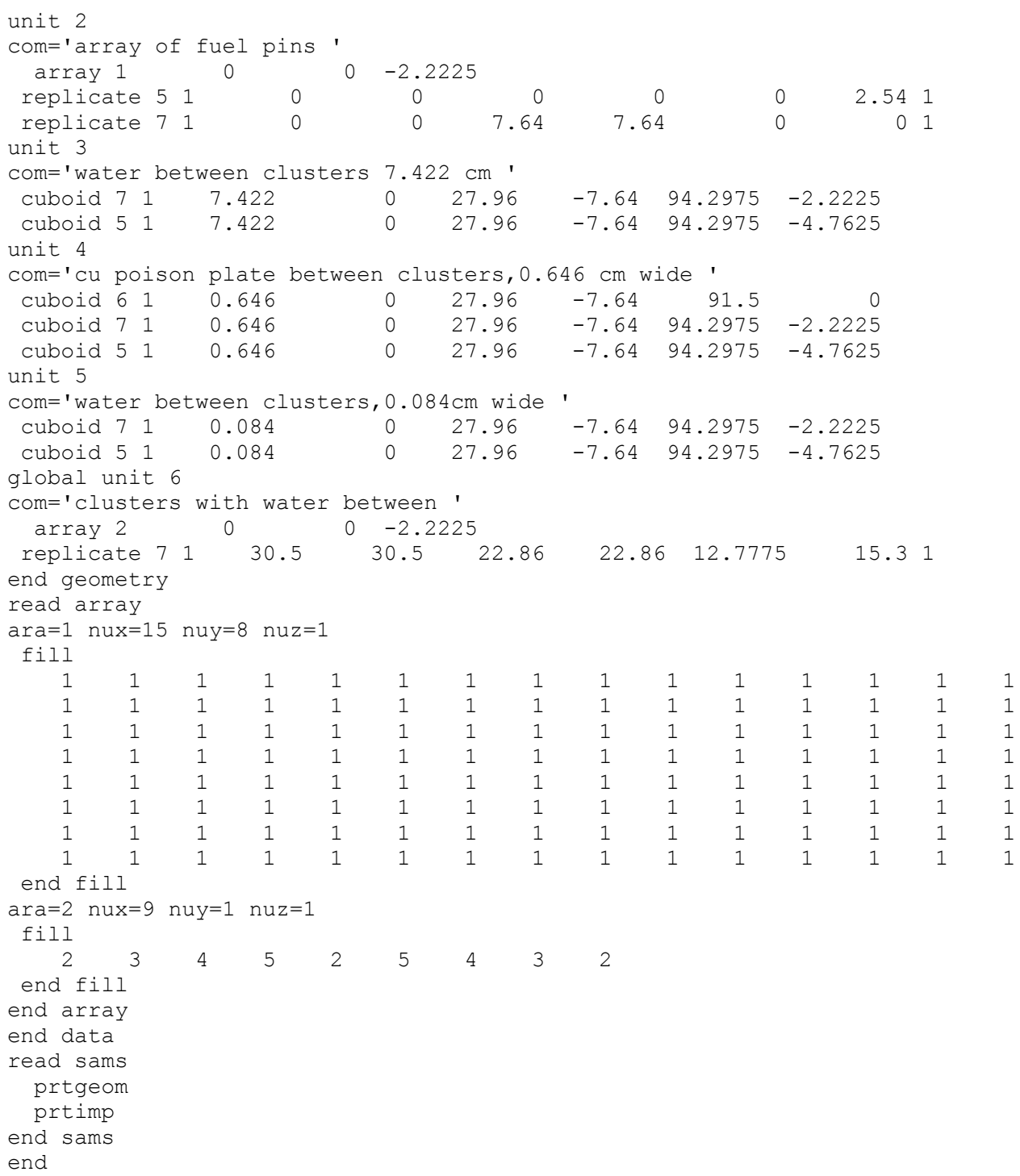

Figure 6.2.14. TSUNAMI-3D LEU-COMP-THERM-009 case 10 sample problem input.. (continued) 


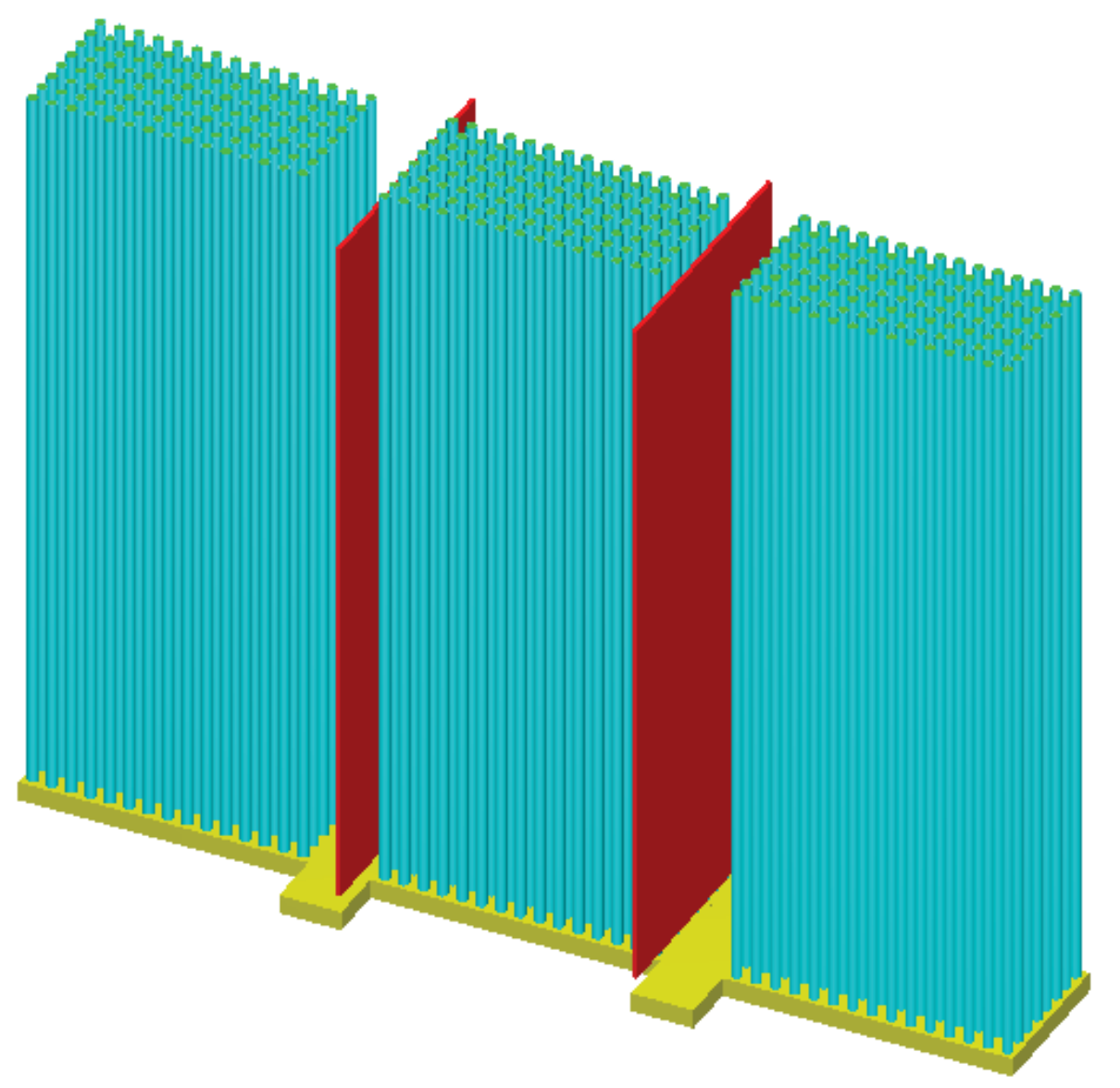

Figure 6.2.15. Graphical representation of KENO V.a geometry for LEU-COMP-THERM-009 case 10 sample problem. 
Sensitivity coefficients and their statistical uncertainties are shown in Table 6.2.11. For ${ }^{235} \mathrm{U}$, the TSUNAMI-3D and direct perturbation results agree within one standard deviation for all cases. For ${ }^{238} \mathrm{U}$, TSUNAMI-3D agrees within two standard deviations for all cases except the no-mesh case, which disagrees by more than four standard deviations. Similarly for ${ }^{1} \mathrm{H}$ in the pin cell, all meshed cases agree with the direct perturbation results to less than one standard deviation, but the case with no geometrical subdivision disagrees by five standard deviations. For ${ }^{1} \mathrm{H}$ in the reflector, again, the meshed cases all agree well with the direct perturbation results, but the non-mesh case shows large discrepancy of 21 standard deviations.

Also, only small differences are observed with and without the transform where an adequate flux mesh is used. Because of the computational resources required for computing the transform while performing neutron tracking, setting $t f m=n o$ can decrease runtime by $\sim 50 \%$. However, in cases where an adequate computational mesh is not used, setting $t f m=n o$ can lead to erroneous results.

The energy-dependent sensitivity profiles for ${ }^{1} \mathrm{H}$ elastic scattering for the three TSUNAMI-3D models are shown in Figure 6.2.16. The differences in the groupwise sensitivity coefficients are most pronounced in the intermediate- to high-energy regions. 
Table 6.2.11. Energy- and region-integrated total sensitivity coefficients for LEU-COMP-THERM-009 case 10

\begin{tabular}{|c|c|c|c|c|c|c|c|c|c|c|c|c|c|c|c|c|c|c|c|}
\hline \multirow{2}{*}{$\begin{array}{r}\text { Nuclide } \\
{ }^{235} \mathrm{U}\end{array}$} & \multirow{2}{*}{$\begin{array}{c}\begin{array}{c}\text { Perturb. } \\
\text { size }\end{array} \\
0.97 \%\end{array}$} & \multicolumn{3}{|c|}{ Direct perturbation } & \multicolumn{3}{|c|}{$\begin{array}{l}\text { TSUNAMI-3D } \\
\text { no mesh } \\
\text { tfm=yes }\end{array}$} & \multicolumn{3}{|c|}{$\begin{array}{l}\text { TSUNAMI-3D } \\
15 \mathrm{~cm} \text { mesh } \\
\text { tfm=yes }\end{array}$} & \multicolumn{3}{|c|}{$\begin{array}{l}\text { TSUNAMI-3D } \\
5 \mathrm{~cm} \text { mesh } \\
\text { tfm=yes }\end{array}$} & \multicolumn{3}{|c|}{$\begin{array}{c}\text { TSUNAMI-3D } \\
15 \mathrm{~cm} \text { mesh } \\
\text { tfm=no }\end{array}$} & \multicolumn{3}{|c|}{$\begin{array}{l}\text { TSUNAMI-3D } \\
5 \mathrm{~cm} \text { mesh } \\
\text { tfm=no }\end{array}$} \\
\hline & & $2.00 \mathrm{E}-01$ & \pm & $1.39 \mathrm{E}-02$ & $2.07 \mathrm{E}-01$ & \pm & $1.77 \mathrm{E}-05$ & 2.07E-01 & \pm & $2.74 \mathrm{E}-05$ & $2.06 \mathrm{E}-01$ & \pm & $7.04 \mathrm{E}-05$ & 2.07E-01 & \pm & $2.75 \mathrm{E}-05$ & $2.06 \mathrm{E}-01$ & \pm & $7.05 \mathrm{E}-05$ \\
\hline${ }^{238} \mathrm{U}$ & $3.36 \%$ & $-5.51 \mathrm{E}-02$ & \pm & $4.01 \mathrm{E}-03$ & $-7.27 \mathrm{E}-02$ & \pm & $4.90 \mathrm{E}-06$ & $-6.18 \mathrm{E}-02$ & \pm & $8.21 \mathrm{E}-06$ & $-5.96 \mathrm{E}-02$ & \pm & $2.68 \mathrm{E}-05$ & $-6.18 \mathrm{E}-02$ & \pm & $8.22 \mathrm{E}-06$ & $-5.96 \mathrm{E}-02$ & \pm & 2.69E-05 \\
\hline${ }^{1} \mathrm{H}$ in pin cell & $0.99 \%$ & $1.92 \mathrm{E}-01$ & \pm & $1.36 \mathrm{E}-02$ & $1.25 \mathrm{E}-01$ & \pm & $1.79 \mathrm{E}-05$ & $1.84 \mathrm{E}-01$ & \pm & $1.87 \mathrm{E}-04$ & $2.02 \mathrm{E}-01$ & \pm & 4.65E-04 & $1.85 \mathrm{E}-01$ & \pm & $1.87 \mathrm{E}-04$ & $2.02 \mathrm{E}-01$ & \pm & 4.65E-04 \\
\hline${ }^{1} \mathrm{H}$ in reflector & $6.09 \%$ & $-3.30 \mathrm{E}-02$ & \pm & $2.21 \mathrm{E}-03$ & $-7.98 \mathrm{E}-02$ & \pm & $3.22 \mathrm{E}-05$ & $-3.24 \mathrm{E}-02$ & \pm & $2.85 \mathrm{E}-04$ & $-3.28 \mathrm{E}-02$ & \pm & $6.01 \mathrm{E}-04$ & $-3.24 \mathrm{E}-02$ & \pm & $2.85 \mathrm{E}-04$ & $-3.28 \mathrm{E}-02$ & \pm & $6.01 \mathrm{E}-04$ \\
\hline
\end{tabular}




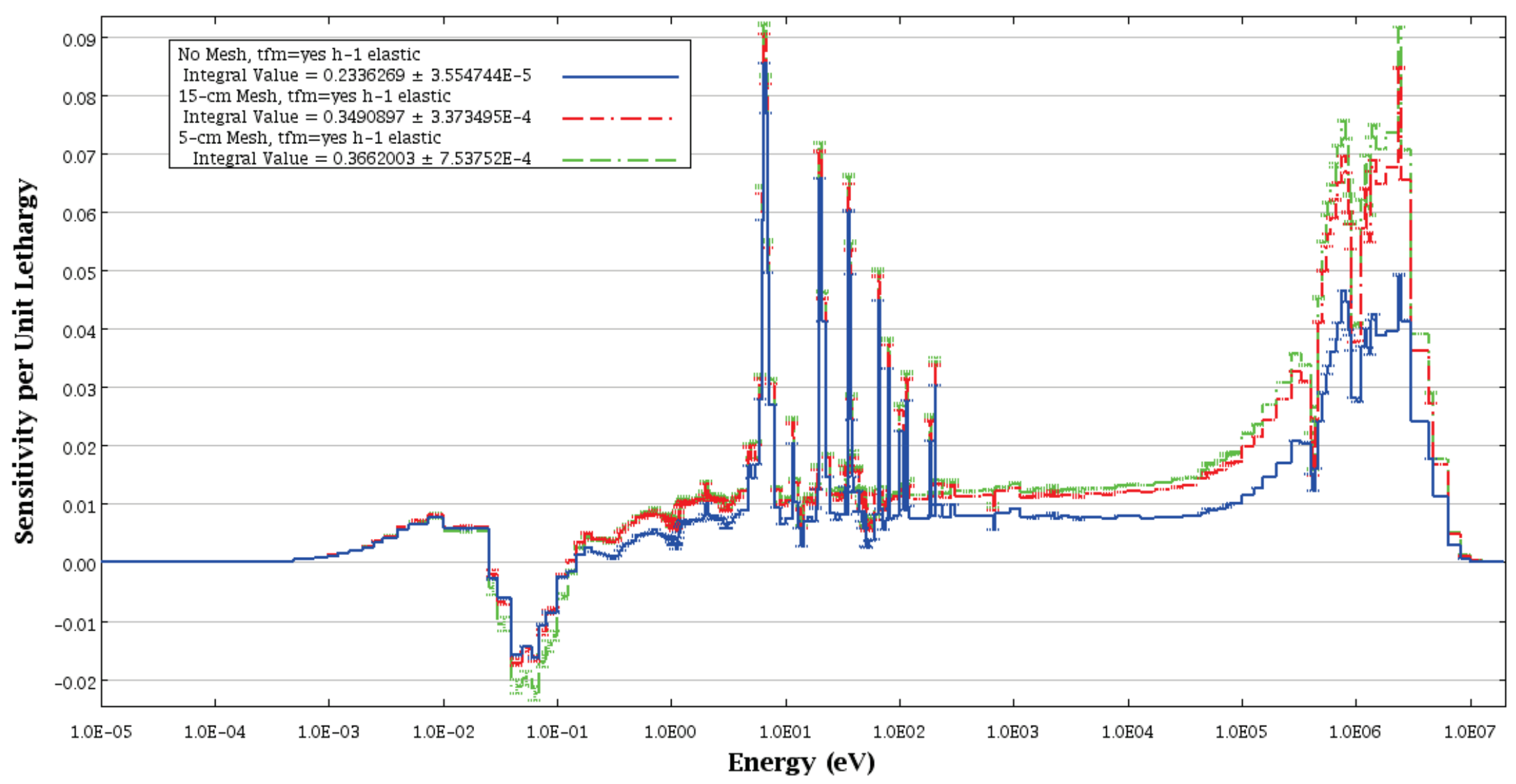

Figure 6.2.16. Sensitivity profiles from TSUNAMI-3D for ${ }^{1} \mathrm{H}$ elastic scattering in mixture 2 of LEU-COMP-THERM-009 case 10 sample problem. 


\subsubsection{Loosely coupled sample problem}

Another sample problem demonstrates the use of the GRID input block to input a nonuniform mesh. This sample problem consists of three tanks containing plutonium nitrate solution that are suspended in air inside of a large concrete room. This system is identified as PU-SOL-THERM-014 case 19 in the IHECSBE. ${ }^{11}$ A TSUNAMI-3D-K5 input for this system is shown in Figure 6.2.17. The system geometry is illustrated in Figure 6.2.18, where the concrete room is shown in light blue and the solution tanks are shown in dark blue. A single tank is shown in Figure 6.2.19, where the tank wall is shown in dark blue and the plutonium nitrate solution is shown in gray.

The KENO V.a option to compute the matrix $k_{\text {eff }}$ by hole was used ( $m k h=y e s$ ) to give the selfmultiplication of each tank. This analysis revealed a system $k_{\text {eff }}$ of $1.0049 \pm 0.0003$ for the forward case and $1.002 \pm 0.002$ for the adjoint case. The $k_{\text {eff }}$ of a single tank for the forward solution was found to be $1.0044 \pm 0.0017$. Thus the interaction between the tanks has a limited effect on the system multiplication factor.

Direct perturbation sensitivity coefficients were generated for ${ }^{239} \mathrm{Pu},{ }^{240} \mathrm{Pu}$, and ${ }^{1} \mathrm{H}$ in the solution using number density perturbations to produce a 20 standard deviation change in $k_{\text {efff }}$, where the requested $k_{\text {eff }}$ convergence for the CSAS direct perturbation calculation was $10^{-4}$. These values are shown in Table 6.2.12 along with the corresponding TSUNAMI-3D results. The results generated by the model shown in Figure 6.2.17, which has no geometric subdivision, agree well with the direct perturbation results for ${ }^{239} \mathrm{Pu}$, but the ${ }^{1} \mathrm{H}$ values differ by more than six standard deviations, indicating poor agreement.

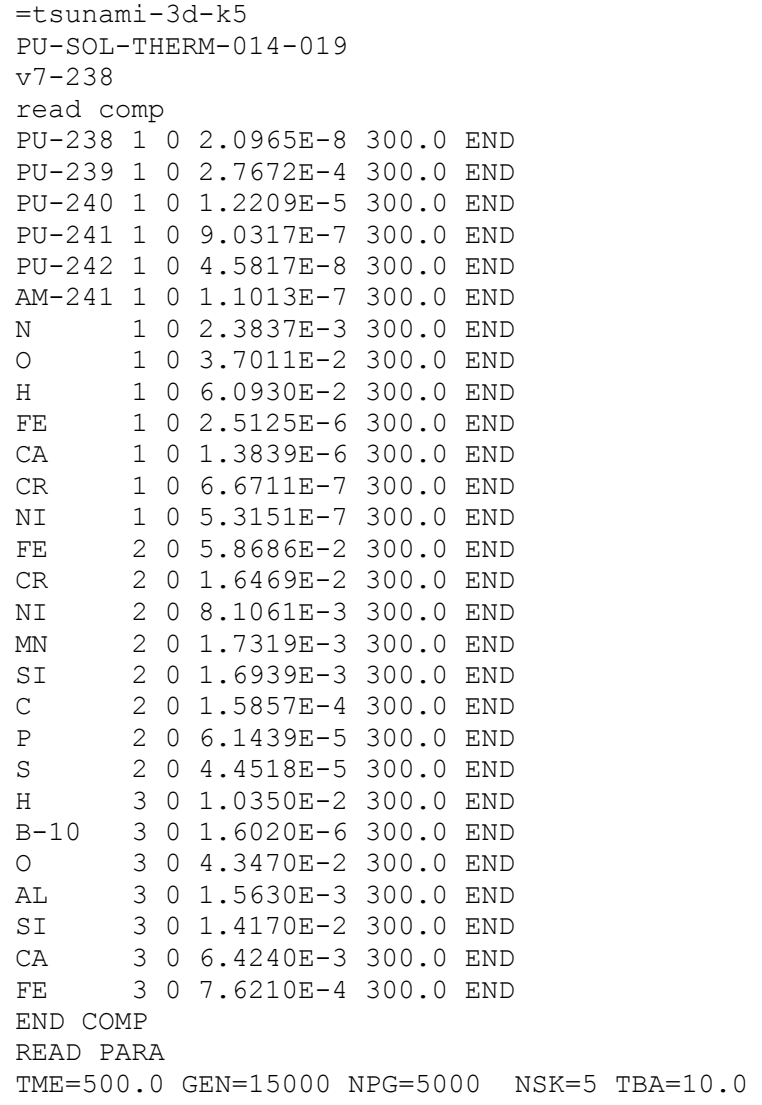


$\mathrm{RUN}=\mathrm{YES} \quad \mathrm{AMX}=\mathrm{NO} \quad \mathrm{FLX}=\mathrm{NO} \quad \mathrm{FDN}=\mathrm{NO} \quad \mathrm{FAR}=\mathrm{NO} \quad \mathrm{PLT}=\mathrm{NO} \quad \mathrm{sig}=0.0001$

END PARA

READ GEOM

UNIT 1

COM='CYLINDER + SOLUTION'

CYLINDER $1114.7-10.7555-50.5855$

$\begin{array}{lllllll}\text { CYLINDER } & 0 & 1 & 14.7 & 50.5855 & -50.5855\end{array}$

CYLINDER $2 \quad 1 \quad 15.0 \quad 51.7855 \quad-51.9145$

GLOBAL

UNIT 2

$\mathrm{COM}=$ 'CONCRETE BUILDING + ARRAY'

CUBOID 0 1 2P605.0 2P440.0 2P500.0

$\begin{array}{llrl}\text { HOLE } 1 & -310.0 & 55.0 & -344.0855\end{array}$

HOLE $1-160.0 \quad-95.0 \quad-344.0855$

HOLE $1-310.0 \quad-95.0 \quad-344.0855$

CUBOID 312 P $750.0 \quad 2$ P585.0 $570.0 \quad-540.0$

END GEOM

READ STAR

$\mathrm{NST}=1$

END STAR

END DATA

end

Figure 6.2.17. TSUNAMI-3D-K5 input for sample problem PU-SOL-THERM-014 case 19.

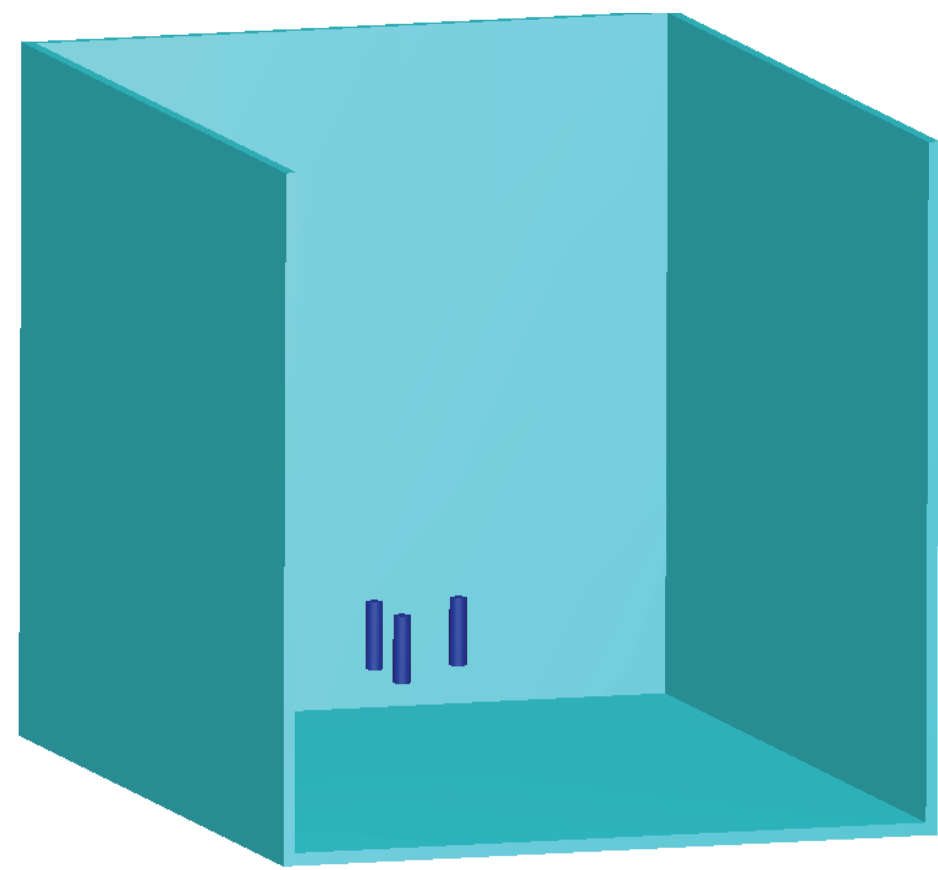

Figure 6.2.18. Cutaway view of PU-SOL-THERM-014 case 19 sample problem. 


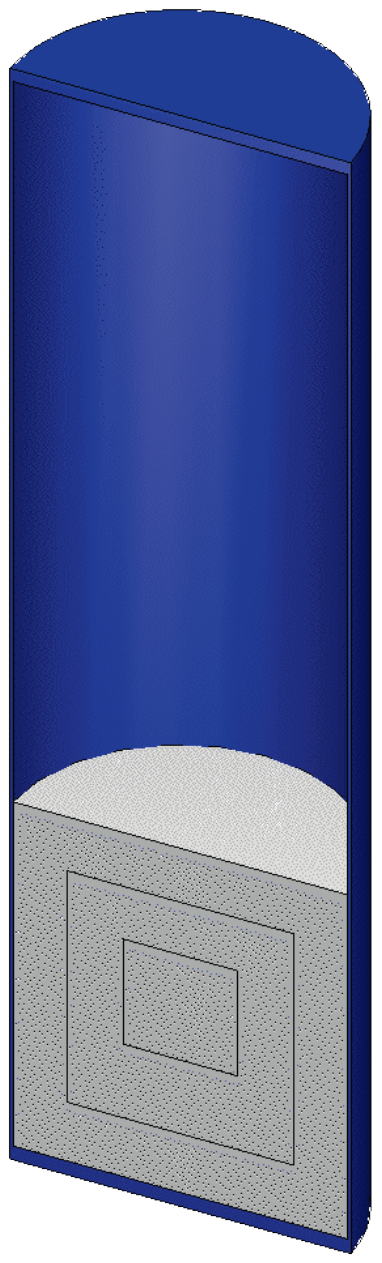

Figure 6.2.19. Cutaway view of single solution tank from PU-SOL-THERM-014 case 19 sample problem. 
Table 6.2.12. Energy- and region-integrated total sensitivity coefficients for PU-SOL-THERM-014 case 19

\begin{tabular}{lll}
\hline & \multicolumn{1}{c}{${ }^{\mathbf{1}} \mathbf{H}$} & \multicolumn{1}{c}{${ }^{\mathbf{2 3 9}} \mathbf{P u}$} \\
\hline Percent change in number density for direct perturbation & 0.32 & 2.39 \\
Direct perturbation & $5.99 \mathrm{E}-01 \pm 2.56 \mathrm{E}-02$ & $7.57 \mathrm{E}-02 \pm 8.29 \mathrm{E}-03$ \\
TSUNAMI-3D-K5 base model & $4.34 \mathrm{E}-01 \pm 1.82 \mathrm{E}-05$ & $7.58 \mathrm{E}-02 \pm 4.42 \mathrm{E}-06$ \\
TSUNAMI-3D-K5 grid geometry model & $6.05 \mathrm{E}-01 \pm 3.42 \mathrm{E}-04$ & $7.84 \mathrm{E}-02 \pm 3.80 \mathrm{E}-05$ \\
\hline
\end{tabular}

Because of the large size of the room included in this model relative to the size of the fuel tanks, use of the uniform mesh flux proved to be impractical due to excessive memory requirements. The directional dependence of the flux needs to be computed separately for each tank through the use of adequate spatial refinement with the flux mesh through the use of the grid geometry input.

The GRID block in KENO V.a and KENO-VI enables the use of a nonuniform mesh for this problem that is coarse throughout the room to save memory, yet refined within the fueled regions to obtain accurate results. As with previous examples, the use of a sufficiently fine mesh provides accurate results without the use of the transform $(t \mathrm{fm}=n o$ ). The TSUNAMI-3D-K5 input listing for this model using the userdefined GRID is shown in Figure 6.2.20, where the transform is disabled with $t f m=n o$, the mesh fluxes are requested with $m f x=y e s$, and the grid is defined in the READ GRID block. The msh parameter that defines a uniform mesh is not used. The input requirements for the grid are described in the READ GRID section of the KENO documentation and will not be repeated here. It is required that the user-defined mesh completely encloses the geometry of the system. In the model in Figure 6.2.23, the grid extends past the geometry by $1 \mathrm{~cm}$ in each direction to ensure that potential round-off errors do not cause the grid to appear to terminate inside the geometry at any point.

When the user-defined grid is used without the transform, the TSUNAMI-3D-K5 and direct perturbation sensitivities agree within one standard deviation for both ${ }^{1} \mathrm{H}$ and ${ }^{239} \mathrm{Pu}$, as shown in Table 6.2.12.

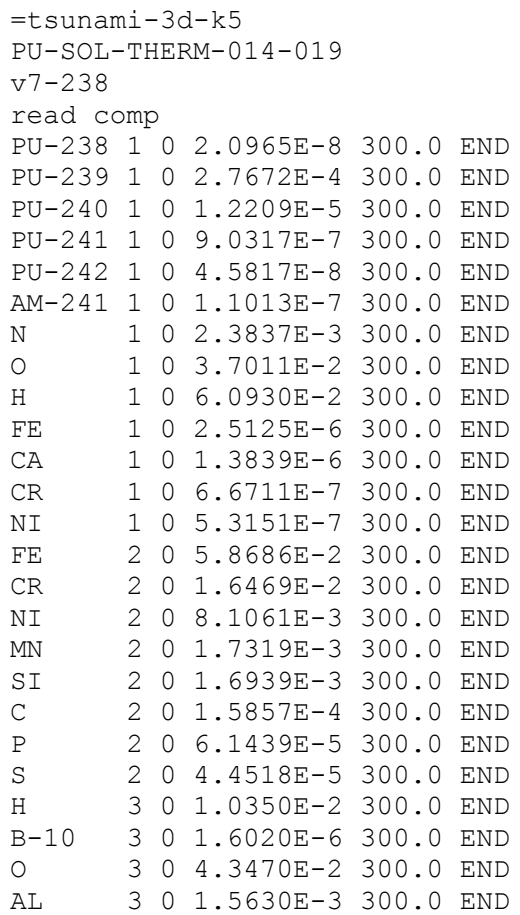




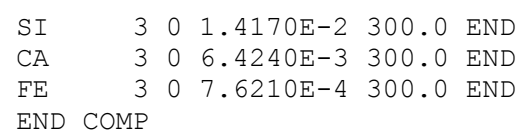

Figure 6.2.20. TSUNAMI-3D-K5 input with GRID input for sample problem PU-SOL-THERM-014 case 19.

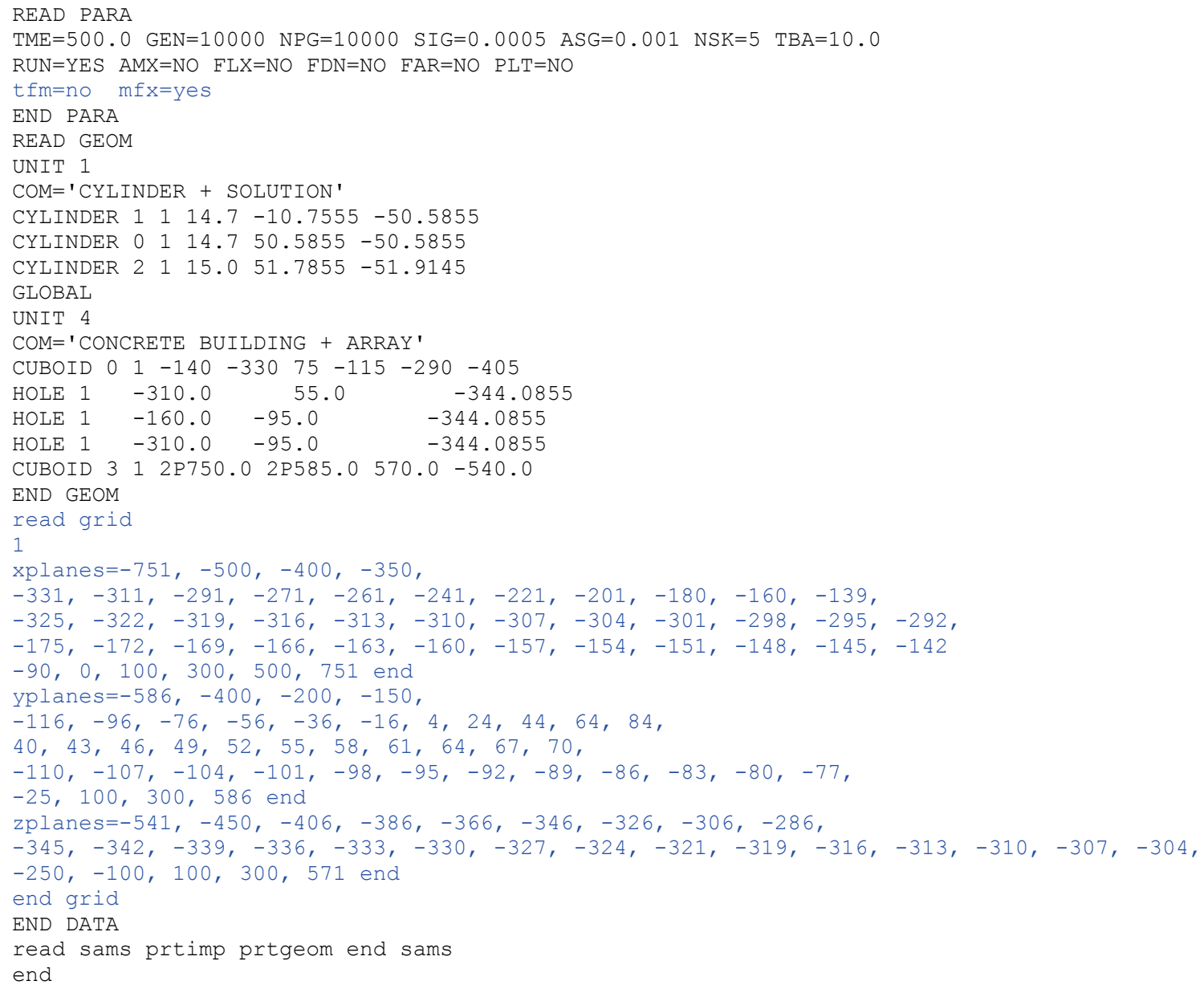

Figure 6.2.20. TSUNAMI-3D-K5 input with GRID input for sample problem $P U-S O L-T H E R M-014$ case 19 . (continued)

\subsubsection{CE TSUNAMI Sample Problem}

The last sample problem focuses on performing CE TSUNAMI sensitivity calculations starting from a CE KENO-VI model. The model used in this example is a highly enriched uranium sphere (Godiva) using one-eighth symmetry; this case is identified as HEU-MET-FAST-001 in IHECSBE. ${ }^{11}$ CE TSUNAMI inputs are in many ways easier to prepare than MG TSUNAMI inputs because CE TSUNAMI does not require flux moment calculations, a flux mesh (except for CLUTCH $F^{*}(r)$ calculations), implicit sensitivity calculations, or an adjoint transport calculation, and thus require much less user input. Figure 6.2.21 shows the CE TSUNAMI input for this system. The input lines that differ from those in the CE KENO-VI input are highlighted in blue. This simulation uses the IFP sensitivity method (cet=2) and 
assumes that daughter fission neutrons diffuse through the system and produce an asymptotic population after five latent generations $(\mathrm{cfp}=5)$. In general, IFP simulations require a $\mathrm{cfp}$ between 5 and 10 generations to produce accurate sensitivity estimates, although IFP sensitivity calculations can produce accurate sensitivities for this system using as few as 2 latent generations. The runtime and memory requirements of the IFP method are proportional to $\mathrm{cfp}$, and users should minimize $\mathrm{cfp}$ when performing IFP sensitivity calculations to maximize calculation efficiency and minimize calculation memory footprint.

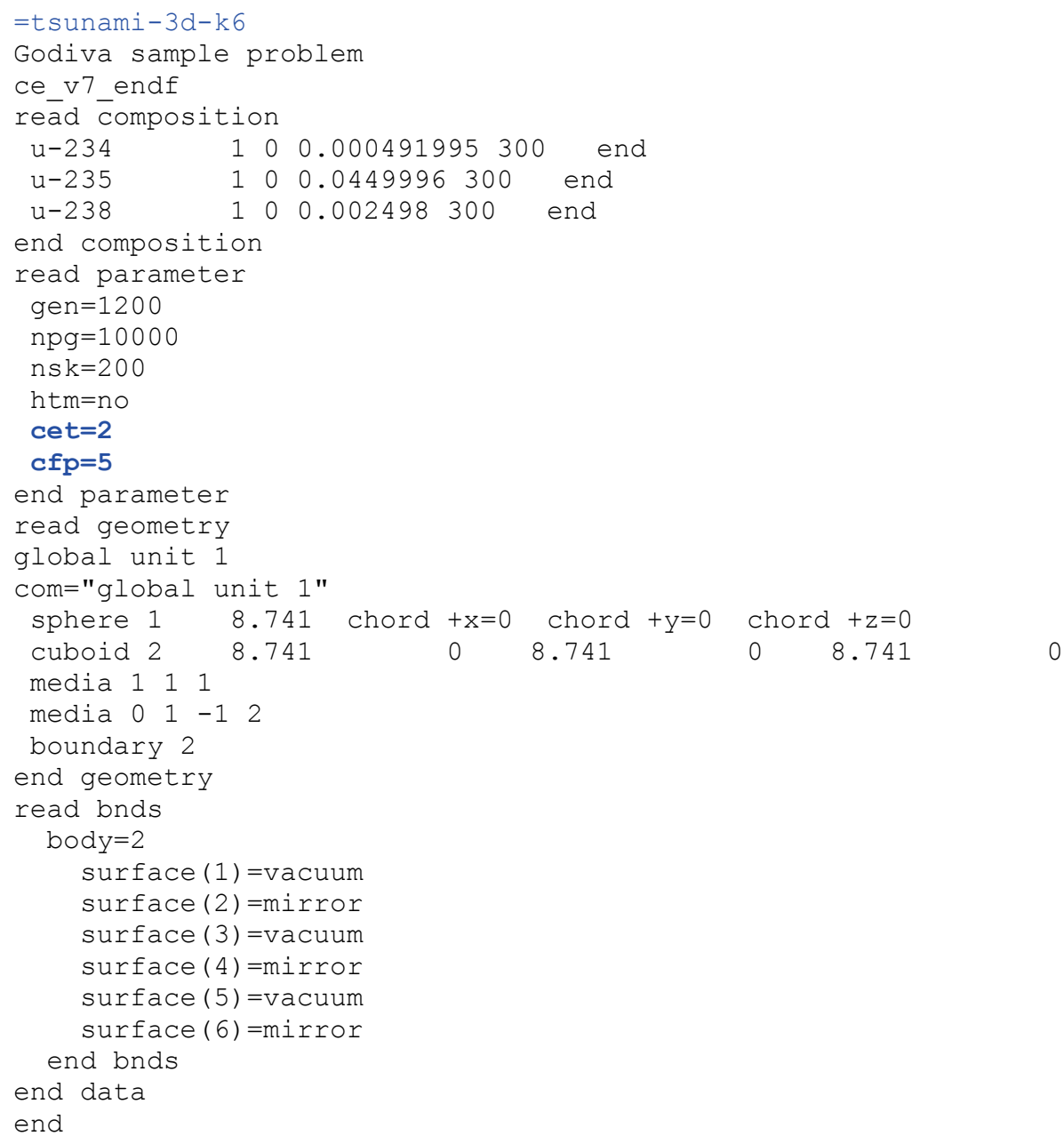

Figure 6.2.21. CE TSUNAMI-3D-K6 input for the Godiva system using the IFP method.

Figure 6.2.22 shows the CE TSUNAMI input for the Godiva system using the CLUTCH sensitivity method (cet=1). This input is different from the IFP input in that the CLUTCH method requires a spatial grid for tallying the CLUTCH $F^{*}(r)$ function. This grid is specified in the "read gridGeometry" block and the ID of the grid specified in this block is passed to CLUTCH in the parameter block $(\mathrm{cgd}=11)$. In general, $\mathrm{CLUTCH}$ calculations are much faster than IFP calculations and produce a significantly smaller memory footprint, but CLUTCH calculations must have an accurate $F^{*}(r)$ mesh to obtain accurate sensitivity coefficients. The $F^{*}(r)$ mesh is currently calculated during inactive generations using the IFP method, and the $\mathrm{c} f \mathrm{p}=5$ card specifies how many latent generations are used in 
this calculation. Using more latent generations will increase the accuracy of the $F^{*}(r)$ mesh and will not significantly affect the memory footprint of the CLUTCH method, but it will increase the variance of the $F^{*}(r)$ mesh values. In general an $F^{*}(r)$ mesh with $1 \mathrm{~cm}$ to $2 \mathrm{~cm}$ mesh intervals is sufficiently refined to obtain accurate sensitivity coefficients. $F^{*}(r)$ is calculated during the inactive generations, and in general users should simulate at least on the order of 10 to 100 inactive histories per mesh interval to allow for sufficient $F^{*}(r)$ convergence. This sometimes necessitates simulating a large number of additional inactive histories/generations. Fission source convergence is not necessary to begin accurate $F^{*}(r)$ estimates. Therefore, $F^{*}(r)$ can be tallied during the inactive generations while the fission source is still converging.

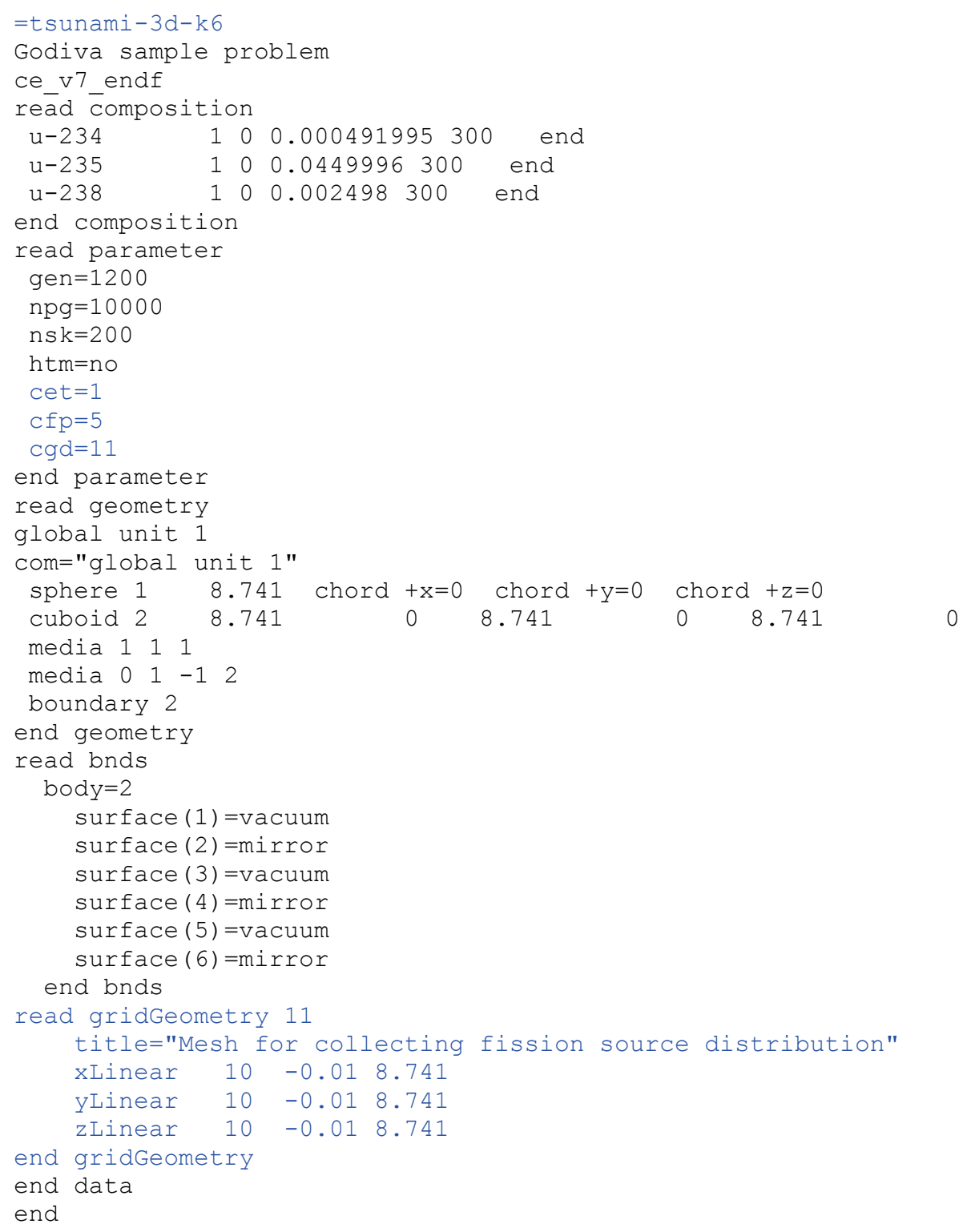

Figure 6.2.22. CE TSUNAMI-3D-K6 input for the Godiva system using the CLUTCH method. 
It is possible to perform a CE TSUNAMI CLUTCH sensitivity calculation using the assumption that $F^{*}(r)$ equals one everywhere. This will not produce accurate sensitivity coefficient estimates for systems where the importance of fission neutrons varies significantly as a function of space, but it will produce accurate sensitivity coefficients for systems with relatively flat importance functions, such as an infinitely reflected model of a single fuel pin or an infinite media problem. This calculation mode requires no additional inactive generations for the calculation of $F^{*}(r)$ and is useful for estimating the runtime or memory requirements of a CLUTCH calculation and for obtaining rough estimates of the sensitivity coefficients of complicated systems. Figure 6.2.23 shows the CE TSUNAMI input for the Godiva system using the CLUTCH sensitivity method $(\mathrm{cet}=1)$. The $F^{*}(r)$ mesh calculation is disabled using $\mathrm{cfp}=-1$, making the "read gridGeometry" and "cgd=\#\#\#" inputs not required.

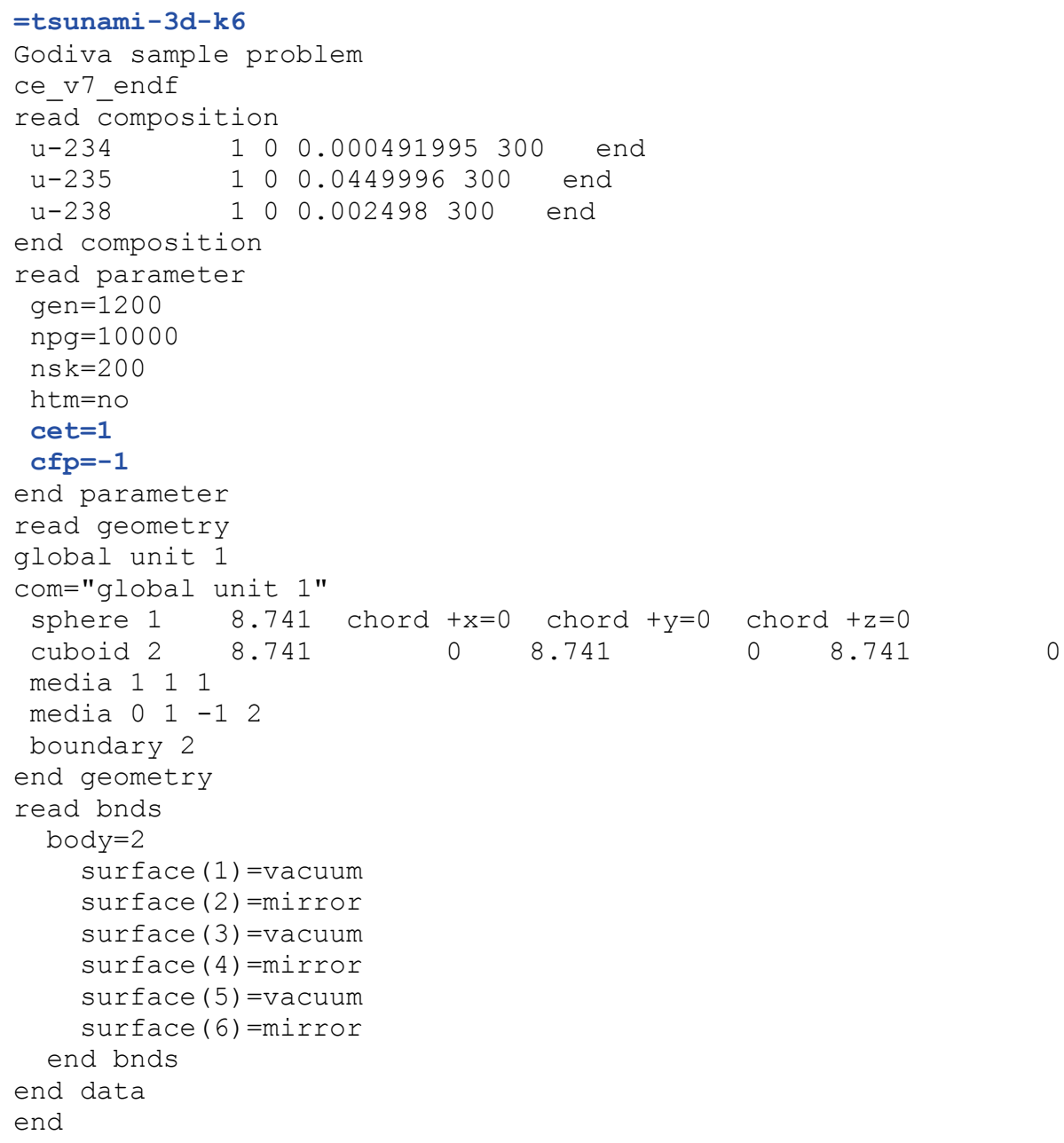

Figure 6.2.23. CE TSUNAMI-3D-K6 input for the Godiva system using the CLUTCH method with no $F^{*}(r)$ mesh.

The three previously described CE TSUNAMI inputs were simulated. The total nuclide sensitivity coefficients from these runs are compared with reference direct perturbation and MG TSUNAMI-3D sensitivities in Table 6.2.13. The difference between the calculated and reference sensitivities, in terms of the effective number of standard deviations, is given in the table in parentheses below each calculated sensitivity coefficient. Although the reference direct perturbation sensitivity estimates had uncertainties 
that were a bit large (it is recommended that their relative uncertainties are less than $5 \%$ ), the four TSUNAMI calculations all produced sensitivity coefficients that seem to agree well with the reference sensitivities.

Table 6.2.13. CE TSUNAMI Godiva sensitivity coefficient comparison

\begin{tabular}{|c|c|c|c|c|c|}
\hline Nuclide & $\begin{array}{c}\text { Direct } \\
\text { Perturbation }\end{array}$ & MG TSUNAMI-3D & IFP & CLUTCH & $\begin{array}{c}\text { CLUTCH } \\
\text { (no } F^{*}(\boldsymbol{r}) \text { mesh) }\end{array}$ \\
\hline${ }^{234} \mathbf{U}$ & $\begin{array}{c}0.00646 \pm \\
0.00050\end{array}$ & $\begin{array}{c}0.00727 \pm 0.000005 \\
\left(1.62 \sigma_{e f f}\right)\end{array}$ & $\begin{array}{c}0.00725 \pm 0.00030 \\
\left(1.35 \sigma_{e f f}\right)\end{array}$ & $\begin{array}{c}0.00738 \pm 0.00006 \\
\left(1.83 \sigma_{e f f}\right)\end{array}$ & $\begin{array}{c}0.00708 \pm 0.00006 \\
\left(1.23 \sigma_{e f f}\right)\end{array}$ \\
\hline${ }^{235} \mathbf{U}$ & $\begin{array}{c}0.80959 \pm \\
0.06937\end{array}$ & $\begin{array}{c}0.79648 \pm 0.00048 \\
\left(-0.19 \sigma_{e f f}\right)\end{array}$ & $\begin{array}{c}0.80378 \pm 0.00289 \\
\left(-0.08 \sigma_{e f f}\right)\end{array}$ & $\begin{array}{l}0.80359 \pm 0.00062 \\
\quad\left(-0.09 \sigma_{\text {eff }}\right)\end{array}$ & $\begin{array}{c}0.77705 \pm 0.00059 \\
\left(-0.47 \sigma_{e f f}\right)\end{array}$ \\
\hline${ }^{238} \mathbf{U}$ & $\begin{array}{c}0.01896 \pm \\
0.00178\end{array}$ & $\begin{array}{c}0.01768 \pm 0.00002 \\
\left(-0.72 \sigma_{\text {eff }}\right)\end{array}$ & $\begin{array}{c}0.01816 \pm 0.00064 \\
\left(-0.42 \sigma_{e f f}\right)\end{array}$ & $\begin{array}{c}0.01792 \pm 0.00013 \\
\quad\left(-0.58 \sigma_{\text {eff }}\right)\end{array}$ & $\begin{array}{c}0.01633 \pm 0.00012 \\
\left(-1.47 \sigma_{\text {eff }}\right)\end{array}$ \\
\hline
\end{tabular}

Two sample inputs have been included to illustrate how a user can run GEAR-MC sensitivity calculations starting from the CE KENO-VI Godiva model. The first, CLUTCH-only GPT input requires specifying a number of latent generations for the GPT $F^{*}(r)$ calculation $(\mathrm{cfp}=5)$, creating a gridGeom for the $F^{*}(r)$ mesh, and specifying reaction rates, materials, and energy ranges for the numerator and denominator response terms. The generalized response examined in this problem is the ${ }^{235} \mathrm{U}$ fast $(>0.6$ $\mathrm{MeV}$ and $<20 \mathrm{MeV}$ ) fission cross section; in other words:

$$
R=\frac{\left\langle\Sigma_{f}^{U-235} \phi\right\rangle^{\text {fast }}}{\langle\phi\rangle^{\text {fast }}}
$$

Therefore, the reaction rate in the numerator term of this response is the ${ }^{235} \mathrm{U}$ fast fission reaction rate in material $1(n n c=92235, n m a=1, n m t=18, n m x=20 e 7$, and $n m n=600000)$ and the denominator term is the fast flux in material $1(d n c=-1, d m a=1, d m t=0, d m x=20 e 7$, and $d m n=600000)$. 


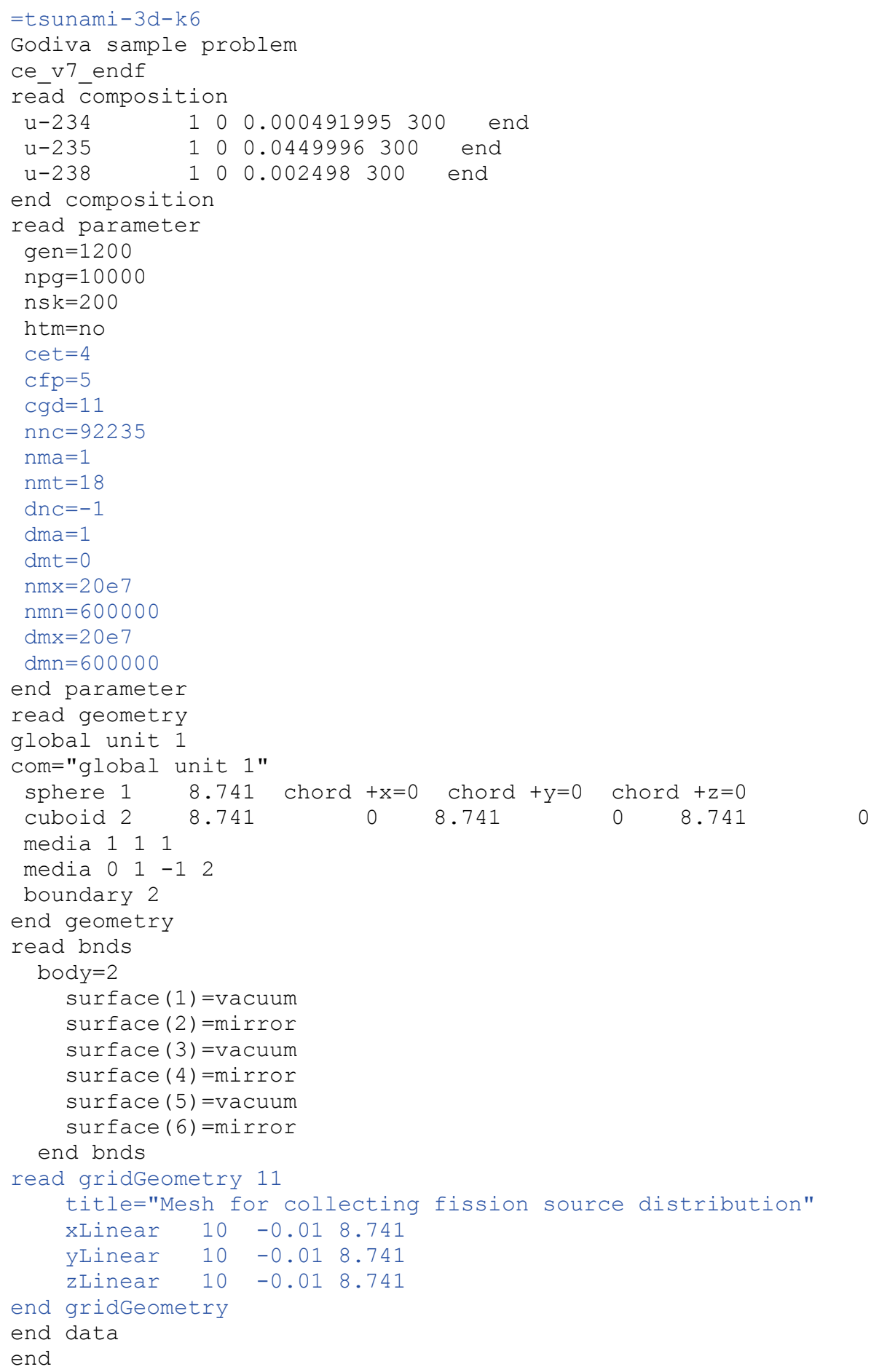

Figure 6.2.24. CE TSUNAMI-3D-K6 input for the Godiva system using GEAR-MC with only the CLUTCH method.

This problem can be specified using the input option cet $=5$, which uses the IFP method to estimate the intergenerational effect term on the fly rather than tallying the effect in an $F^{*}(r)$ mesh. For this cet=5 case the Definitions and SystemResponses blocks (found in the SystemResponses block sections in the 
TSUNAMI-1D chapter of the SCALE manual) are used to specify the GPT responses rather than using KENO input parameters; eventually, both $c e t=4$ and cet $=5$ will use exclusively the Definitions and SystemResponses blocks for specifying GPT responses.

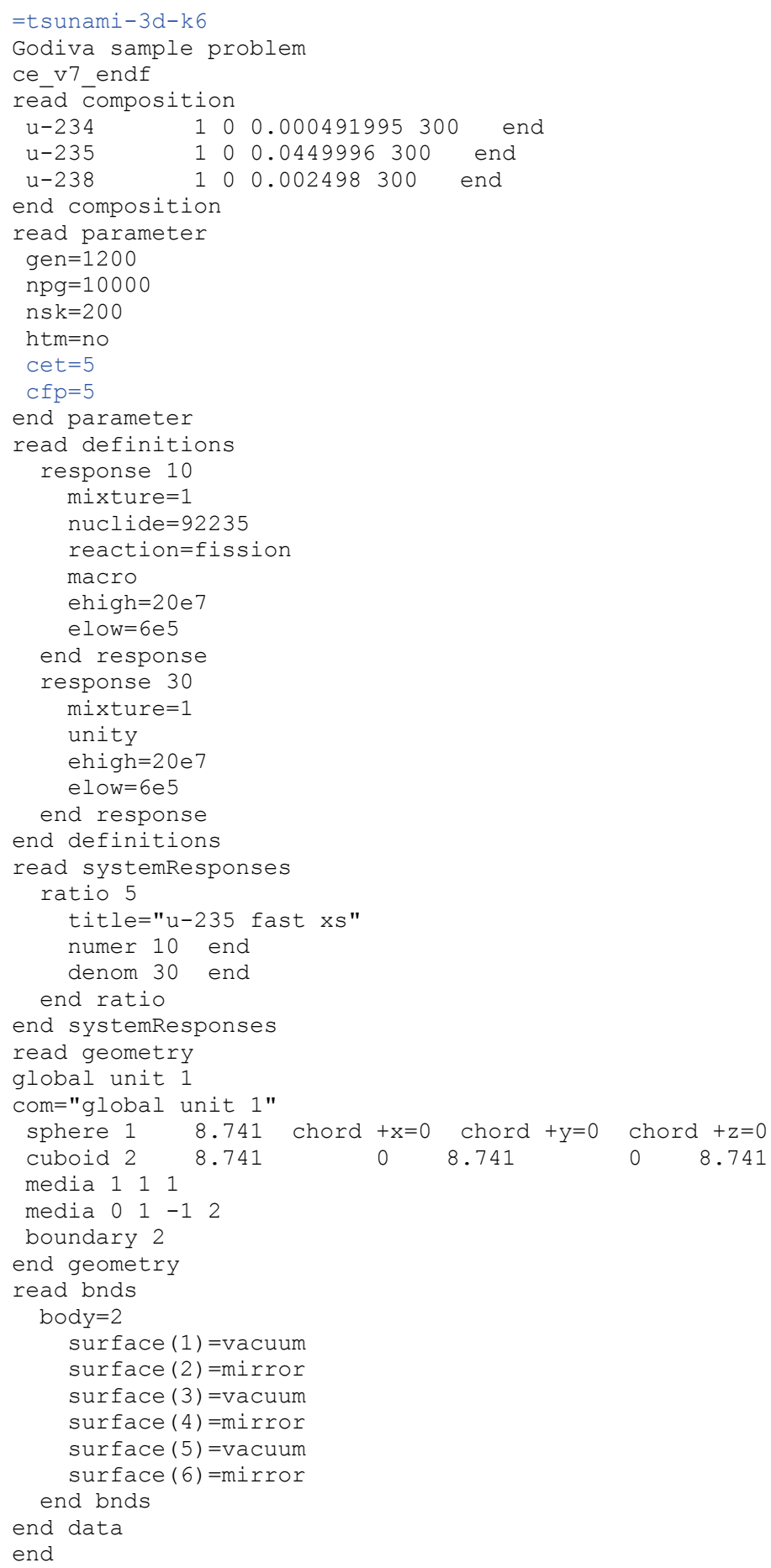

Figure 6.2.25. CE TSUNAMI-3D-K6 input for the Godiva system using GEAR-MC with the CLUTCH and IFP methods. 
The CE TSUNAMI GPT sensitivity calculations do not output the sensitivity coefficients in the usual filename.sdf output file; instead, the cet $=4$ option will produce a GPT sdf with the name filename_GPT.sdf, and the cet $=5$ option will produce an sdf with the name filename_responseXX.sdf, where XX is the ID of this response in the SystemResponses block. The cet $=5$ GPT mode will also print two additional sdf's: one for the intragenerational (i.e., CLUTCH-only) contribution to the response, and one for the intergenerational (i.e., IFP-only) contribution. 


\subsubsection{References}

1. H. Hurwitz, Jr., "A Note on the Theory of Danger Coefficients, Tech. Rep. KAPL-98, Knolls Atomic Power Laboratory, 1948.

2. B. C. Kiedrowski, “Adjoint Weighting for Continuous-Energy Monte Carlo Radiation Transport,”, doctoral dissertation, University of Wisconsin (2009).

3. C. M. Perfetti, “Advanced Monte Carlo Methods for Eigenvalue Sensitivity Coefficient Calculation,”, doctoral dissertation, University of Michigan (2012).

4. K. Banerjee, W. M. Martin, "Kernel Density Estimate Monte Carlo Global Flux Tallies," Proceedings of the International Conference on Mathematics, Computational Methods \& Reactor Physics (M\&C 2009), Saratoga Springs, New York, May 3-7, 2009.

5. C. M. Perfetti, B. T. Rearden, "Continuous-Energy Monte Carlo Methods for Calculating Generalized Response Sensitivities using TSUNAMI-3D," Proceedings of the 2014 International Conference on the Physics of Reactors (PHYSOR 2014), Kyoto, Japan, September 28-October 3, 2014.

6. C. M. Perfetti, B. T. Rearden, "Performance Enhancements to the SCALE TSUNAMI-3D Generalized Response Sensitivity Capability," Trans. Am. Nucl. Soc. 112 (2014).

7. M. L. Williams, "Perturbation Theory for Nuclear Reactor Analysis," in Y. Ronen, CRC Handbook of Nuclear Reactor Calculations: Volume III, Boca Raton, Florida: CRC Press, Inc. (1986).

8. M. L. Williams, "Generalized Contributon Response Theory,” Nucl. Sci. \& Engr., 108, pp. 355-383 (1991).

9. M. L. Williams, Equations for Contributon Eigenvalue Solution. Unpublished document (2007).

10. C. M. Perfetti, B. T. Rearden, "Metrics for Diagnosing Undersampling in Monte Carlo Tally Estimates," Proceedings of $M \& C$ 2015, Nashville, Tennessee, April 19-23, 2015

11. International Handbook of Evaluated Criticality Safety Benchmark Experiments, Nuclear Energy Agency Nuclear Science Committee of the Organization for Economic Co-operation and Development, NEA/NSC/DOC(95)03 (2010). Available at: http://icsbep.inl.gov/handbook.shtml 


\title{
6.3 SAMS: SENSITIVITY ANALYSIS MODULE FOR SCALE
}

B. T. Rearden, L. M. Petrie, M. A. Jessee, M. L Williams

\begin{abstract}
The Sensitivity Analysis Module for SCALE (SAMS) calculates sensitivity coefficients that predict the expected changes of the calculated value of system responses such and $k_{\text {eff }}$ and reaction rate ratios due to perturbations in constituent cross-section data. Additionally, the uncertainty in each response due to cross-section-covariance data can be predicted. SAMS performs sensitivity calculations using linear perturbation theory and requires the calculation of the forward and adjoint flux moments. Multigroup sensitivity coefficients computed by SAMS also include the implicit effect due to resonance self-shielding calculations. In multigroup mode SAMS works in conjunction with XSDRNPM, NEWT, KENO V.a or KENO-VI, which are capable of calculating the desired responses as well as associated fluxes, flux moments and/or mesh fluxes. The implicit calculations are performed with data generated by a combination of the BONAMIST and SENLIB codes specially developed to produce resonance selfshielded cross sections and their sensitivities to input data. SAMS is also used to edit the sensitivity coefficients generated by KENO continuous energy sensitivity calculations.
\end{abstract}

SAMS automatically selects all of the sensitivity parameters that can be calculated or edited for each nuclide in each region of the system based on available cross-section data. Sensitivity parameters for a given nuclide may be generated for a number of parameters, including total, scatter, capture, and fission cross sections, as well as $\bar{v}$ and $\chi$. The uncertainty information is produced for all processes available on the cross-section-covariance data file.

SAMS has been designed for automated operation with the TSUNAMI-1D, TSUNAMI-2D, TSUNAMI-3D-K5 and TSUNAMI-3D-K6 SCALE control modules and produces data suitable for use with the TSUNAMI-IP, TSURFER, and TSAR modules. 


\section{TABLE OF CONTENTS}

Page

6.3 SAMS: Sensitivity Analysis Module for SCALE ............................................................... $6-114$

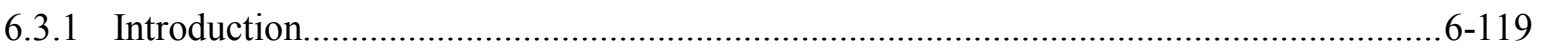

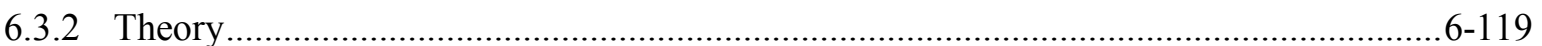

6.3.2.1 Explicit Sensitivity Coefficient Generation....................................................... 6-120

6.3.2.2 Implicit Sensitivity Coefficient Generation.................................................... 6-125

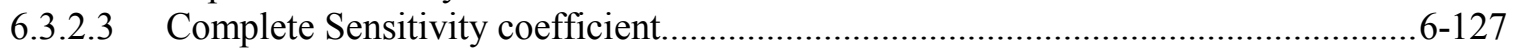

6.3.2.4 Summary of Sensitivity Coefficients Calculated by SAMS ..................................6-127

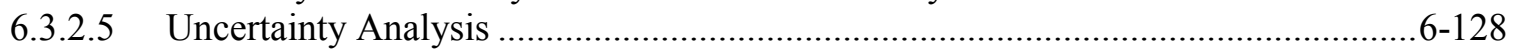

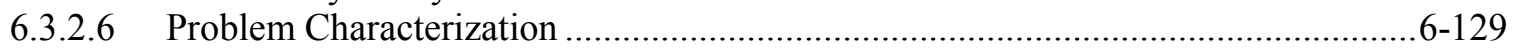

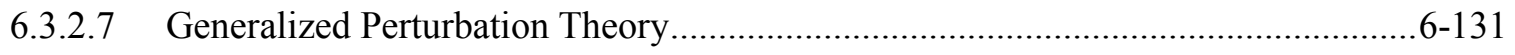

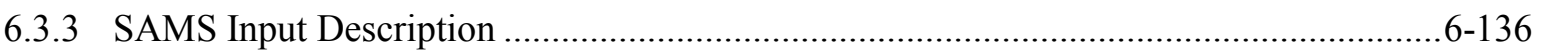

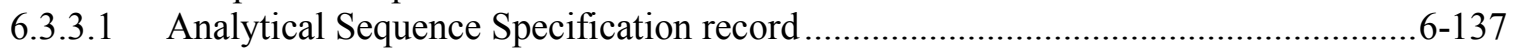

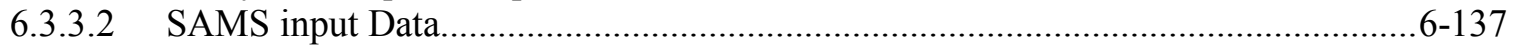

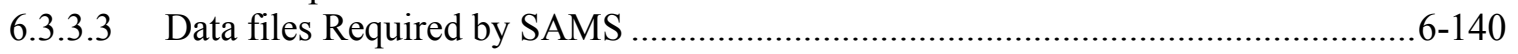

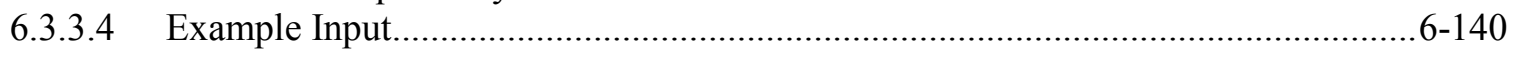

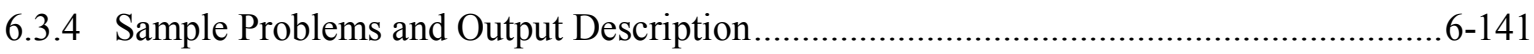

6.3.4.1 Example Problem Using XSDRNPM.................................................................. $6-141$

6.3.4.2 Example Problem Using KENO V.A ................................................................ $6-164$

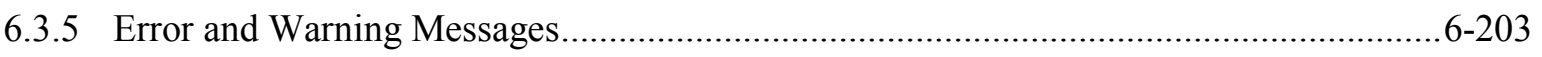

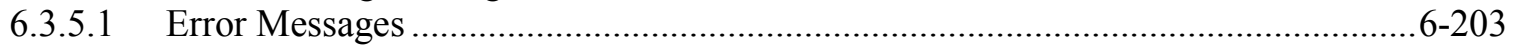

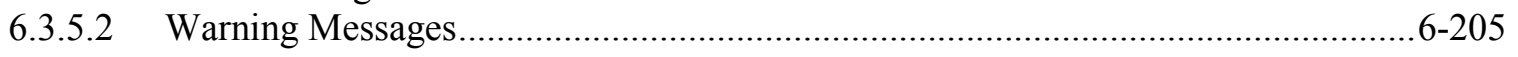

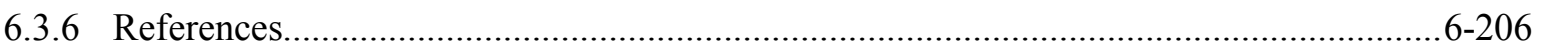

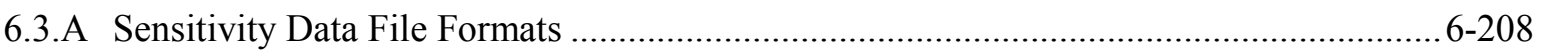

6.3.A.1 Format of TSUNAMI-A sensitivity data file .............................................. 6-208

6.3.A.2 Format of TSUNAMI-B sensitivity data file............................................ 6-213 


\section{LIST OF FIGURES}

Page

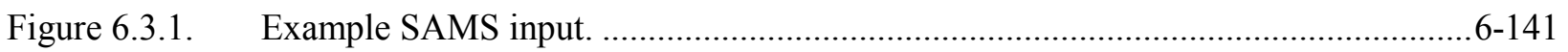

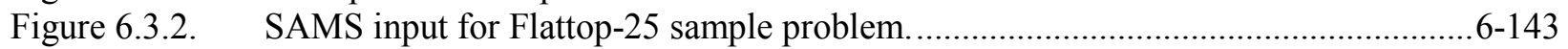

Figure 6.3.3. SAMS output for Flattop-25 sample problem.......................................................6-144

Figure 6.3.4. Sample of sensitivity data file for Flattop-25 sample problem. ...............................6-151

Figure 6.3.5. Region-dependent fission sensitivity profiles for Flattop-25 sample problem. .........6-154

Figure 6.3.6. Region-dependent capture sensitivity profiles for Flattop-25 sample problem. ........6-155

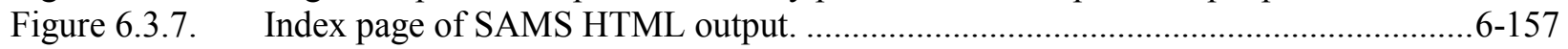

Figure 6.3.8. Program verification page of SAMS HTML output. ............................................6-158

Figure 6.3.9. Input parameters from SAMS HTML output..................................................6-159

Figure 6.3.10. Summary of transport calculations from SAMS HTML output..............................6-160

Figure 6.3.11. Energy- region- and mixture-integrated sensitivity coefficients from SAMS

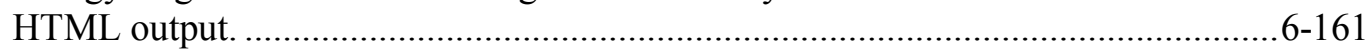

Figure 6.3.12. Energy- region- and mixture-integrated sensitivity coefficients from SAMS

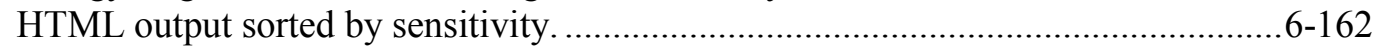

Figure 6.3.13. Sensitivity data plot from SAMS HTML output................................................6-163

Figure 6.3.14. KENO3D rendering of KENO V.a model of LEU-COMP-THERM-009 case 10...6-164

Figure 6.3.15. SAMS input LEU-COMP-THERM-009 case 10 sample problem. .........................6-166

Figure 6.3.16. SAMS output for LEU-COMP-THERM-009 case 10 sample problem....................6-167

Figure 6.3.17. Region-integrated sensitivity profiles from test case LEU-COMP-THERM-009

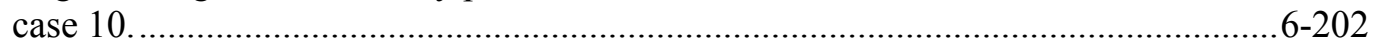

Figure 6.3.A.1. Truncated sensitivity data file for Flattop-25 sample problem. ..............................6-210

Figure 6.3.A.2. Truncated sensitivity data file for LEU-COMP-THERM-009 sample problem........ 6-216 


\section{LIST OF TABLES}

Page

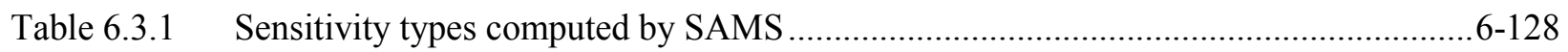

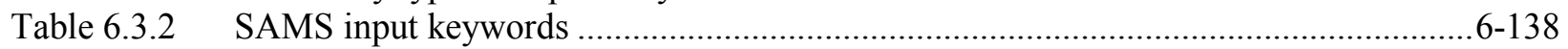

Table 6.3.3. SAMS input keywords for default covariance data .................................................. 6-139

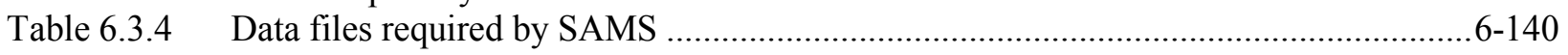

Table 6.3.A.1. Format specification for TSUNAMI-A sensitivity data file ....................................... 6-209

Table 6.3.A.2. Format specification for TSUNAMI-B sensitivity data file....................................... 6-214 


\section{ACKNOWLEDGMENTS}

The author wishes to acknowledge B. L. Broadhead of the Oak Ridge National Laboratory and R. L. Childs, formerly of the Oak Ridge National Laboratory, for their assistance with this work. The support and encouragement of C. V. Parks of the Oak Ridge National Laboratory and C. M. Hopper, formerly of the Oak Ridge National Laboratory is also appreciated. The support of U.S. Department of Energy Nuclear Criticality Safety Program is greatly appreciated. 


\subsubsection{Introduction}

SAMS is the Sensitivity Analysis Module for SCALE, which has been designed for automated operation with the TSUNAMI-1D, TSUNAMI-2D, TSUNAMI-3D-K5 and TSUNAMI-3D-K6 SCALE control modules. In multigroup mode, SAMS utilizes data from forward and adjoint neutron transport analyses as well as the sensitivity of resonance self-shielded cross-section data to input data to compute sensitivity coefficients that represent the percentage effect on a system response, such as multiplication factor $\left(k_{e f f}\right)$ or reaction rate ratio, to a percentage change in the nuclear reaction probabilities or cross sections. Sensitivity coefficients computed with SAMS can be presented as an energy-integrated value, which represents a uniform change in a given cross section across all energies, or as a "profile" in which a value is calculated for each discrete energy group. When combined with the uncertainties in the cross-section data, the cross-section-covariance data, the uncertainty in $k_{\text {eff }}$ due to uncertainties in the cross-section data can be assessed. The sensitivity data produced by SAMS are appropriate for use with the TSUNAMI-IP, TSURFER and TSAR functional modules of SCALE and may be plotted in the Fulcrum SCALE user interface.

The methodology for calculating sensitivity and uncertainty parameters in SAMS is an extension of that used in the Fantastic Oak Ridge Sensitivity System (FORSS). ${ }^{1-3}$ The FORSS system was capable of calculating the sensitivity of the system $k_{\text {eff }}$ to changes in group-wise cross-section data for any given isotope for a number of reaction types. This data could also be used in conjunction with cross-section covariance data to determine the uncertainty in the calculated $k_{\text {eff }}$ due to uncertainties in the nuclear data library. The FORSS methodology requires the calculation of the forward and adjoint angular and scalar fluxes and flux moments. Once these flux solutions are determined, the relative change in the system response due to a change in a component cross section can be determined. These sensitivities are calculated for each material region, nuclide, reaction type, and energy group in the system model.

In multigroup transport solution mode, the generation of sensitivity coefficients with SAMS requires forward and adjoint criticality calculations to compute the system responses, required fluxes as well as and the volume of each flux region. Additionally, the neutron cross-section data used in the transport solution and the sensitivity of the resonance self-shielded cross-section data to the input material number densities must be available. SAMS currently functions with neutron transport solutions from XSDRNPM, NEWT, KENO V.a and KENO-VI. The sensitivities of the resonance self-shielded crosssection data to the input material number densities is computed by routines from SENLIB and BONAMIST. SAMS reads the required data files and produces sensitivity coefficients for many reactions for all nuclides for all regions in the problem. SAMS offers several output options to provide convenient transfer of the sensitivity data for plotting and additional analysis with other modules, such as TSUNAMI-IP, TSURFER, TSAR and Fulcrum.

As is described in the SCALE TSUNAMI-3D manual section, SAMS is also used in continuous energy mode transport solution sensitivity calculations. In these calculations, sensitivities are generated by TSUNAMI-3D and SAMS is used only to provide edits of sensitivity and uncertainty results. Sensitivity coefficients generated in continuous energy mode are stored in a multigroup sensitivity data file (SDF) format that is consistent with the various SCALE tools that use SDFs. The text provided in the rest of the SAMS manual chapter is oriented toward SAMS use with multigroup transport solutions.

\subsubsection{Theory}

The methodology used by SAMS to produce sensitivity coefficients is presented in this section. The explicit portion of the sensitivity coefficient represents the sensitivity of the system responses to the problem-dependent resonance self-shielded multigroup cross-section data used in the analysis. The implicit portion of the complete sensitivity coefficient is the sensitivity of the resonance self-shielded 
multigroup cross-section data to the data input to the resonance self-shielding calculation. Summing the implicit and explicit contributions from a particular cross-section data component produces the complete sensitivity coefficient. Uncertainty analysis involves propagating the uncertainty information from the cross-section-covariance data file to the computed value of $k_{\text {eff }}$ via the sensitivity coefficients. Problem characterization data are computed system average quantities such as the energy of average lethargy causing fission. Each of these quantities is computed by SAMS and is explained in this section. When executed as part of one of the TSUNAMI sequences, the SAMS input is generated automatically by the control module for that sequence.

\subsubsection{Explicit sensitivity coefficient generation}

This methodology used to generate the explicit portion of the sensitivity coefficients is identical to that used in the FORSS ${ }^{3}$ code system for fast reactor applications, with the addition of the sensitivity of $k_{\text {eff }}$ to the fission energy spectrum $(\chi)$. The sensitivity coefficients produced with these techniques give the sensitivity of the computed $k_{\text {eff }}$ to a particular component of the group-wise cross-section data.

The explicit sensitivity coefficients are calculated using the well-established adjoint-based perturbation theory approach. ${ }^{4-7}$ The full derivation of the general procedure is not given here; however, the specific theory for the generation of $k_{\text {eff }}$ sensitivities is presented below. For the full derivation of the general sensitivity equations the reader is referred to Ref. 3 .

The Boltzmann transport equation can be written in the form

$$
[A-\lambda B] \phi=0
$$

where

$$
\begin{aligned}
& \phi=\text { neutron flux, } \\
& \lambda=\text { represents the eigenvalues where the largest eigenvalue is } 1 / k_{\text {eff }}, \\
& A=\text { operator that represents all of the transport equation except for the fission term, } \\
& B=\text { operator that represents the fission term of the transport equation. }
\end{aligned}
$$

Defining perturbed transport operators and the perturbed eigenvalues as

$$
\begin{aligned}
& A^{\prime}=A+\delta A, \\
& B^{\prime}=B+\delta B, \text { and } \\
& \lambda^{\prime}=\lambda+\delta \lambda,
\end{aligned}
$$

where $\delta A$ and $\delta B$ represent small linear perturbations in their corresponding transport operators and $\delta \lambda$ represents the resulting change in the eigenvalues. The perturbed transport equation can be written in the form

$$
\left[A^{\prime}-\lambda^{\prime} B^{\prime}\right] \phi^{\prime}=0
$$

The equation adjoint to Eq. (6.3.1) is

$$
\left[A^{\dagger}-\lambda B^{\dagger}\right] \phi^{\dagger}=0,
$$


where $\phi^{\dagger}$ is the adjoint flux and has a special physical significance as the "importance" of the particles within the system, and $A^{\dagger}$ and $B^{\dagger}$ are the adjoint operators corresponding to $A$ and $B$.

Multiplying Eq. (6.3.3) by $\phi \hbar$, and integrating over all phase space yields

$$
\left\langle\phi^{\dagger}\left(A^{\prime}-\lambda^{\prime} B^{\prime}\right) \phi^{\prime}\right\rangle=0
$$

where $<>$ represents integration over all phase space (volume, energy, and direction).

Expanding Eq. (6.3.5) in terms of Eq. (6.3.2) yields

$$
\left\langle\phi^{\dagger}(A-\lambda B+\delta A-\lambda \delta B-B \delta \lambda-\delta \lambda \delta B) \phi^{\prime}\right\rangle=0 .
$$

Using the property of adjointness (i.e., $\left.\left\langle\phi^{\dagger}(A-\lambda B) \phi^{\prime}\right\rangle=\left\langle\phi^{\prime}\left(A^{\dagger}-\lambda^{\dagger} B^{\dagger}\right) \phi^{\dagger}\right\rangle\right)$ and Eq. (6.3.4) to reduce the number of terms yields

$$
\left\langle\phi^{\dagger}(\delta A-\lambda \delta B-B \delta \lambda-\delta \lambda \delta B) \phi^{\prime}\right\rangle=0
$$

Eq. (6.3.7) is further simplified by ignoring the second-order perturbation term $(\delta \lambda \delta B)$ and substituting $\phi^{\prime}$ with $\phi$, indicating that the perturbations in the transport operators do not cause significant perturbations in the flux solution. The eigenvalue perturbation becomes

$$
\frac{\delta \lambda}{\lambda}=\frac{\left\langle\phi^{\dagger}(\delta A-\lambda \delta B) \phi\right\rangle}{\left\langle\phi^{\dagger}(\lambda B) \phi\right\rangle}
$$

Substituting the perturbation terms with partial derivatives with respect to a macroscopic cross section, $\Sigma$, of the transport operator at some point in phase space $\vec{r}$, the relative sensitivity of $\lambda$, becomes

$$
\frac{\delta \lambda}{\lambda}=\frac{\left\langle\phi^{\dagger}(\vec{\xi})\left(\frac{\partial A[\Sigma(\vec{\xi})]}{\partial \Sigma(\vec{r})}-\lambda \frac{\partial B[\Sigma(\vec{\xi})]}{\partial \Sigma(\vec{r})}\right) \phi(\vec{\xi})\right\rangle}{\left\langle\phi^{\dagger}(\vec{\xi}) \lambda B[\Sigma(\vec{\xi})] \phi(\vec{\xi})\right\rangle} .
$$

where $\vec{\xi}$ is the phase space vector and the brackets indicate integration over space, direction and energy variables. 


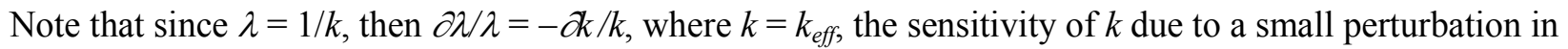
a macroscopic cross section, $\Sigma$, of the transport operator at some point in phase space $\vec{r}$ can be expressed as

$$
S_{k, \Sigma(\vec{r})} \equiv \frac{\Sigma(\vec{r})}{k} \frac{\partial k}{\partial \Sigma(\vec{r})}=-\frac{\Sigma(\vec{r})}{k} \frac{\left\langle\phi^{\dagger}(\vec{\xi})\left(\frac{\partial A[\Sigma(\vec{\xi})]}{\partial \Sigma(\vec{r})}-\frac{1}{k} \frac{\partial B[\Sigma(\vec{\xi})]}{\partial \Sigma(\vec{r})}\right) \phi(\vec{\xi})\right\rangle}{\left\langle\phi^{\dagger}(\vec{\xi}) \frac{1}{k^{2}} B[\Sigma(\vec{\xi})] \phi(\vec{\xi})\right\rangle},
$$

The $k$ sensitivity for individual cross sections can be obtained from Eq. (6.3.10) using the discrete ordinates form of the transport equation. In doing so, the phase space vector, $\bar{\xi}$, has been replaced by indices representing discretization in space, energy and angle. It has been demonstrated in Ref. 3 that sensitivity coefficients for reaction $x$, isotope $i$, energy group $g$, and computational region $z$ can be represented as

$$
S_{k, \Sigma_{x, g, z}^{i}}=\frac{T_{1, x, g, z}^{i}+T_{2, g, z}^{i}+T_{3, x, g, z}^{i}}{D} .
$$

where the denominator, $D$, is expressed as

$$
D=\frac{1}{k} \sum_{i=1}^{I} \sum_{z=1}^{R} V_{z} \sum_{g=1}^{G}\left(\bar{v}_{g, z}^{i} \Sigma_{f, g, z}^{i} \phi_{g, z}\right) \sum_{g^{\prime}=1}^{G}\left(\chi_{g^{\prime}, z}^{i} \phi_{g^{\prime}, z}^{\dagger}\right)
$$

where

$$
\begin{aligned}
\chi_{g^{\prime}, z}^{i}= & \begin{array}{l}
\text { average fraction of fission neutrons emitted into energy group } g^{\prime} \text { from fission of } \\
\end{array} \\
\bar{v}_{g, z}^{i}= & \begin{array}{l}
\text { average number of fission neutrons emitted from fission of isotope } i \text { in region } z \text { in } \\
\\
\text { energy group } g,
\end{array} \\
\Sigma_{f, g, z}^{i}= & \text { macroscopic cross section for fission of isotope } i \text { in region } z \text { and energy group } g, \\
I= & \text { number of isotopes in the system model, } \\
R= & \text { number of computational regions in the system model, } \\
G= & \text { number of neutron energy groups in the system model. }
\end{aligned}
$$

Energy-integrated coefficients are obtained by summing the group-wise coefficients over all energy groups. The $T$ terms of Eq. (6.3.11) represent the transport processes for neutron loss, fission production and scattering to the group of interest in $T_{1}, T_{2}$ and $T_{3}$, respectively. The first term is expressed as

$$
T_{1, x, g, z}^{i}=-\sum_{x, g, z}^{i} V_{z} \sum_{j=0}^{N M O M}(2 \ell+1) \tilde{\phi}_{g, z}^{\dagger j} \tilde{\phi}_{g, z}^{j}
$$

where

$$
\begin{aligned}
\Sigma_{x, g, z}^{i}= & \text { macroscopic cross section for some reaction } x \text {, of isotope } i \text {, energy group } g \text {, in region } \\
& z,
\end{aligned}
$$


$\left[=\right.$ Legendre order that corresponds to the $j^{\text {th }}$ real valued flux moment,

$\tilde{\phi}_{g, z}^{\dagger j}=j^{\text {th }}$ component real valued adjoint flux moment for energy group $g$, and region $z$,

$N M O M=$ the total number of real valued flux moments corresponding to the desired Legendre order of expansion.

$V_{z} \quad=$ the volume of region $z$

The second and third terms can be expressed as

$$
\begin{gathered}
T_{2, g, z}^{i}=\frac{1}{k} V_{z} \bar{v}_{g, z}^{i} \Sigma_{f, g, z}^{i} \phi_{g, z} \sum_{g^{\prime}=1}^{G} \phi_{g^{\prime}, z}^{\dagger} \chi_{g^{\prime}, z}, \\
T_{3, x, g, z}^{i}=\sum_{j=0}^{N M O M} V_{z} \sum_{g^{\prime}=1}^{G} \tilde{\phi}_{g^{\prime}, z}^{\dagger j} \tilde{\phi}_{g, z}^{j} \Sigma_{x, g^{\prime} \rightarrow g, z}^{\ell, i},
\end{gathered}
$$

where

$$
\begin{aligned}
\sum_{x, g \rightarrow g^{\prime}, z}^{\ell, i}= & l^{\text {th }} \text { moment of the transfer cross section for reaction } x \text { of isotope } i, \text { from energy } \\
& \text { group } g^{\prime} \text { to energy group } g \text { in region } z .
\end{aligned}
$$

For specific reactions, not all of the $T$ terms defined above are needed to calculate the sensitivity coefficient. The application of Eq. (6.3.11) for each type of reaction is outlined below. The computational form of each equation is expressed with the volume integrated product of the forward and adjoint flux moments as

$$
P_{g, g^{\prime}, z}^{\ell}=V_{z} \sum_{j}^{\tilde{\phi}_{g^{\prime}, z}^{\dagger j}} \tilde{\phi}_{g, z}^{j}
$$

For KENO calculations without mesh fluxes, the flux product is computed with Eq. (6.3.16), where $z$ simply represents each material region. For KENO calculations using the mesh flux generation option, the flux product for each material region is computed as

$$
P_{g, g^{\prime}, z}^{\ell}=\sum_{j} \sum_{m} \tilde{\phi}_{g^{\prime}, z_{m}}^{\dagger j} \tilde{\phi}_{g, z_{m}}^{j} V_{z_{m}}
$$

where $m$ represents flux meshes that occur in region $z$, and the subscript $z_{m}$ denotes fluxes computed in mesh $m$ of region $z$. Also, $V_{z_{m}}$ is the volume of mesh $m$ in region $z$.

Similarly, for transport calculations using XSDRNPM and NEWT, the flux product is computed over the computational mesh using Eq. (6.3.17) where $m$ represents the computational mesh used in the transport calculation defined for each material region, $z$.

Once the flux products are computed for each material region or zone, the sensitivity coefficients for each reaction type can be computed as follows.

\section{Capture Reaction Sensitivity (non-fission, non-scattering)}

Only the $T_{1, x, g, z}^{i}$ term is used for this class of reactions where $\Sigma_{x, g, z}^{i}$ is the absorption cross section of interest $((n, \gamma),(n, \alpha),(n, p)$, etc.) and can be expressed as 


$$
S_{x, g, z}^{i}=\frac{-\sum_{x, g, z}^{i} \sum_{\ell=0}^{I S C T}(2 \ell+1) P_{g, g, z}^{\ell}}{D},
$$

where $\quad I S C T=$ the highest Legendre order of scattering used in the sensitivity calculations.

2. Fission Reaction Sensitivity

The fission reaction requires $T_{1, x, g, z}^{i}$ and $T_{2, g, z}^{i}$, where $\Sigma_{x, g, z}^{i}$ in the definition of $T_{1, x, g, z}^{i}$ is the fission cross section and can be expressed as

$$
\begin{gathered}
S_{f, g, z}^{i}=\frac{1}{D}\left[\left(\frac{1}{k} \bar{v}_{g, z}^{i} \Sigma_{f, g, z}^{i} \chi_{g, z}^{i}-\sum_{f, g, z}^{i}\right) P_{g, g, z}^{0}+\sum_{\substack{g^{\prime}=1 \\
g \neq g^{\prime}}}^{G} \frac{1}{k} \bar{v}_{g, z}^{i} \Sigma_{f, g, z}^{i} \chi_{g^{\prime}, z}^{i} P_{g, g^{\prime}, z}^{0}\right. \\
\left.-\sum_{f, g, z}^{i} \sum_{\ell=1}^{I S C T}(2 \ell+1) P_{g, g, z}^{\ell}\right]
\end{gathered}
$$

3. $\quad \bar{v}$ Sensitivity

The $\bar{v}$ reaction only requires $T_{2, g, z}^{i}$ and can be expressed as

$$
S_{\bar{v}, g, z}^{i}=\frac{\frac{1}{k} \sum_{g^{\prime}=1}^{G} \bar{v}_{g, z}^{i} \Sigma_{f, g, z}^{i} \chi_{g^{\prime}, z}^{i} P_{g, g^{\prime}, z}^{0}}{D} .
$$

4. $\chi$ Sensitivity

The computation of the unconstrained $\chi$ reaction sensitivity only requires $T_{2, g, z}^{i}$, with the $\chi$ and $v \Sigma_{f}$ terms interchanged and is expressed as

$$
S_{\chi, g, z}^{i}=\frac{\frac{1}{k} \sum_{g^{\prime}=1}^{G} \bar{\nu}_{g^{\prime}, z}^{i} \sum_{f, g^{\prime}, z}^{i} \chi_{g, z}^{i} P_{g^{\prime}, g, z}^{0}}{D}
$$

In Eq. (6.3.21), the sensitivities coefficients sum to 1.0 when added over all energy groups and nuclides. However, since the fission spectrum probability distribution for any nuclide must, by definition, sum to 1.0 over all energy groups, the sensitivity of $k_{\text {eff }}$ to the fission spectrum should sum to 0.0 , as any change in fission spectrum in any group must be compensated by changes in other groups to maintain the constraint that all values sum to 1.0. This methodology was first developed for the SAGEP code ${ }^{8}$ and is implemented in SAMS as

$$
\tilde{S}_{\chi, g, z}^{i}=S_{\chi, g, z}^{i}-\chi_{g, z}^{i} \sum_{g^{\prime}=1}^{G} S_{\chi, g^{\prime}, z}^{i}
$$

The constrained $\chi$ calculation from Eq. (6.3.22) is the default for SAMS. 


\section{Scattering Reaction Sensitivity}

All scattering reactions (elastic, inelastic, and (n, 2n) reactions) require $T_{1, x, g, z}^{i}$ and $T_{3, x, g, z}^{i}$ where $\Sigma_{x, g, z}^{i}$ in the definition of $T_{1, x, g, z}^{i}$ is the scattering cross section and $\sum_{x, g^{\prime} \rightarrow g, k}^{\ell, i}$ in the definition of $T_{3, x, g, z}^{i}$ is a component of the group-to-group scattering matrix for the $\ell^{\text {th }}$ scattering moment of reaction $x$.

$$
S_{x, g, z}^{i}=\frac{1}{D}\left(\sum_{\ell=0}^{I S C T}\left(\sum_{x, g \rightarrow g, z}^{\ell, i}-(2 \ell+1) \sum_{x, g, z}^{i}\right) P_{g, g, z}^{\ell}+\sum_{\substack{g^{\prime}=1 \\ g^{\prime} \neq g}}^{G} \sum_{x, g \rightarrow g^{\prime}, z}^{\ell, i} P_{g, g^{\prime}, z}^{\ell}\right)
$$

\section{6. $\quad$ Total Reaction Sensitivity}

The total reaction requires $T_{1, x, g, z}^{i}, T_{2, g, z}^{i}$, and $T_{3, x, g, z}^{i}$. Here, $\Sigma_{x, g, z}^{i}$ in the definition of $T_{1, x, g, z}^{i}$ is the total cross section and $\sum_{x, g^{\prime} \rightarrow g, k}^{\ell, i}$ in the definition of $T_{3, x, g, z}^{i}$ is a component of the group-to-group scattering matrix for the $\ell^{\text {th }}$ scattering moment. For non-fissionable isotopes, $T_{2, g, z}^{i}$ will be zero. The total reaction sensitivity coefficient can be expressed as

$$
\begin{aligned}
& S_{t, g, z}^{i}=\frac{1}{D}\left[\left(\Sigma_{s, g \rightarrow g, z}^{0, i}+\frac{1}{k} \bar{v}_{g, z}^{i} \Sigma_{f, g, z}^{i} \chi_{g, z}^{i}-\Sigma_{t, g, z}^{i}\right) P_{g, g, z}^{0}\right. \\
&+\sum_{\substack{g^{\prime}=1 \\
g \neq g^{\prime}}}^{G}\left(\Sigma_{s, g \rightarrow g^{\prime}, z}^{0, i}+\frac{1}{k} \bar{v}_{g, z}^{i} \Sigma_{f, g, z}^{i} \chi_{g^{\prime}, z}^{i}\right) P_{g, g^{\prime}, z}^{0} \\
&\left.+\sum_{\ell=1}^{I S C T}\left(\Sigma_{s, g \rightarrow g, z}^{\ell, i}-(2 \ell+1) \Sigma_{t, g, z}^{i}\right) P_{g, g, z}^{l}+\sum_{\substack{g^{\prime}=1 \\
g^{\prime} \neq g}}^{G} \Sigma_{s, g \rightarrow g^{\prime}, z}^{\ell, i} P_{g, g^{\prime}, z}^{l}\right] .
\end{aligned}
$$

\subsubsection{Implicit sensitivity coefficient generation}

The methodology to calculate the sensitivity coefficients, as presented in the previous section, was developed for fast reactor applications in which the effect of resonance self-shielding in the multigroup cross-section data is minimal. To provide an accurate estimation of the sensitivity coefficients for systems in which resonance self-shielding is important, the sensitivity coefficients as computed in Eq. (6.3.11) require additional terms to account for the first-order implicit effect of perturbations in the material number densities or nuclear data upon the shielded group-wise macroscopic cross-section data. ${ }^{9}$

The sensitivity of the cross-section data to the input data in turn affects the $k_{\text {eff }}$ sensitivities. The implicit portion of the sensitivity coefficient, the sensitivity of the group-wise data to the input quantities, is defined as

$$
S_{\Sigma_{x, g}, \omega_{i}}=\frac{\omega_{i}}{\Sigma_{x, g}} \frac{\partial \Sigma_{x, g}}{\partial \omega_{i}}
$$

where $\omega_{i}$ is some input quantity. The $\omega_{i}$ term could represent the number density of a particular material, a certain nuclear data component or a physical dimension of a system. For the sensitivity coefficients 
produced by SAMS, which are the sensitivities of $k_{\text {eff }}$ to the group-wise cross-section data, the effect on $k_{\text {eff }}$ of perturbing one cross section that affects the resonance-shielded values of other cross sections must be computed. If $\omega_{i}$ is a certain cross-section data component for process $y$ of nuclide $j$ in energy group $h$ expressed as $\sum_{y, h}^{j}$, which is sensitive to perturbations in process $x$ in energy group $g$ for nuclide $i$ expressed as $\Sigma_{x, g}^{i}$, the complete sensitivity of $k_{\text {eff }}$ due to perturbations of $\Sigma_{x, g}^{i}$ can be defined using the chain rule for derivatives as

$$
\begin{gathered}
\left(S_{k, \Sigma_{x, g}^{i}}\right)_{\text {complete }}=\frac{\sum_{x, g}^{i}}{k} \frac{d k}{d \Sigma_{x, g}^{i}}=\frac{\sum_{x, g}^{i}}{k} \frac{\partial k}{\partial \sum_{x, g}^{i}}+\sum_{j} \sum_{h} \frac{\Sigma_{y, h}^{j}}{k} \frac{\partial k}{\partial \Sigma_{y, h}^{j}} \times \frac{\sum_{x, g}^{i}}{\sum_{y, h}^{j}} \frac{\partial \Sigma_{y, h}^{j}}{\partial \Sigma_{x, g}^{i}} \\
=S_{k, \Sigma_{x, g}^{i}}+\sum_{j} \sum_{h} S_{k, \Sigma_{y, h}^{j}} S_{\Sigma_{y, h}^{j}, \Sigma_{x, g}^{i}},
\end{gathered}
$$

where the sensitivity coefficients with respect to $k_{\text {eff }}$ are the explicit components as computed in Eq. (6.3.11), with the region subscript, $z$, omitted, and $j$ and $h$ are varied to include all processes that are influenced by the value of $\Sigma_{x, g}^{i}$.

Subsequent to the computation of the explicit portion of the sensitivity coefficients, data from SENLIB and BONAMIST are used to compute the implicit portion of the sensitivity coefficients. This implementation of the implicit sensitivity coefficients is more general than that presented in the example calculation of Ref. 9, as it allows for the assessment of the implicit components for all reactions due to interaction with all nuclides. Because the sensitivity of a response to a material number density is equivalent to the sensitivity of the same response to the corresponding total macroscopic cross section, the computation of the implicit sensitivity coefficients can be based on the sensitivity to the input material number densities. The implicit effect of the number densities on $k_{\text {eff }}$ must be accounted for from several sources. One source is the effect of the number densities input to BONAMIST on the shielded cross sections in the unresolved resonance region. For this case, the implicit sensitivity of $k_{\text {eff }}$ to the total cross section of nuclide $i$ is

$$
\begin{aligned}
& \left(S_{k, \Sigma_{T, g}^{i}}\right)_{\text {implicit }}=\sum_{j} \sum_{y} \sum_{h} \frac{\Sigma_{y, h}^{j}}{k} \frac{\partial k}{\partial \Sigma_{y, h}^{j}} \times \frac{\Sigma_{T}^{i}}{\sum_{y, h}^{j}} \frac{\partial \Sigma_{y, h}^{j}}{\partial \Sigma_{T}^{i}} \times \frac{\Sigma_{T, g}^{i}}{\Sigma_{T}^{i}} \frac{\partial \Sigma_{T}^{i}}{\partial \Sigma_{T, g}^{i}} \\
& =\sum_{j} \sum_{y} \sum_{h} S_{k, \Sigma_{y, h}^{j}} S_{\Sigma_{y, h}^{j}, \Sigma_{T}^{i}} S_{\Sigma_{T}^{i}, \Sigma_{T, g}^{i}}=\sum_{j} \sum_{y} \sum_{h} S_{k, \Sigma_{y, h}^{j}} S_{\Sigma_{y, h}^{j}, N^{i}} S_{\Sigma_{T}^{i}, \Sigma_{T, g}^{i}},
\end{aligned}
$$

where $j$ and $y$ are varied to include all processes that are sensitive to $N^{i}$, the number density of the $i^{\text {th }}$ nuclide. Additionally, the energy group for the implicit sensitivity, $g$, is varied over all energies. The sensitivity of the total macroscopic cross section to the group-wise macroscopic total cross section, $S_{\Sigma_{T}^{i}, \Sigma_{T, g}^{i}}$, is simply 1.0. For data computed by SENLIB and input to BONAMIST, an additional term is necessary to account for the sensitivity of the SENLIB parameter, denoted $\omega_{m}$. The chain rule for derivatives can again be used to propagate this sensitivity to a $k_{\text {eff }}$ sensitivity. The implicit sensitivity of $k_{\text {eff }}$ to the input number densities, in this case, is

$$
\left(S_{k, \Sigma_{T, g}^{i}}\right)_{i m p l i c i t}=\sum_{m} \sum_{j} \sum_{y} \sum_{h} \frac{\Sigma_{y, h}^{j}}{k} \frac{\partial k}{\partial \Sigma_{y, h}^{j}} \times \frac{\omega_{m}}{\Sigma_{y, h}^{j}} \frac{\partial \Sigma_{y, h}^{j}}{\partial \omega_{m}} \times \frac{\Sigma_{T}^{i}}{\omega_{m}} \frac{\partial \omega_{m}}{\partial \Sigma_{T}^{i}} \times \frac{\Sigma_{T, g}^{i}}{\Sigma_{T}^{i}} \frac{\partial \Sigma_{T}^{i}}{\partial \Sigma_{T, g}^{i}}
$$




$$
=\sum_{m} \sum_{j} \sum_{y} \sum_{h} S_{k, \Sigma_{y, h}^{j}} S_{\Sigma_{y, h}^{j}, \omega_{m}} S_{\omega_{m}, \Sigma_{T}^{i}} S_{\Sigma_{T}^{i}, \Sigma_{T, g}^{i}}=\sum_{m} \sum_{j} \sum_{y} \sum_{h} S_{k, \Sigma_{y, h}^{j}} S_{\Sigma_{y, h}^{j}, \omega_{m}} S_{\omega_{m}, N^{i}} S_{\Sigma_{T}^{i}, \Sigma_{T, g}^{i}},
$$

where $m$ is varied to include all SENLIB computed parameters that use the material number densities in their calculation and are input to BONAMIST.

The calculation of the implicit sensitivity of a total cross-section component in the unresolved resonance region requires the sum of the implicit quantities computed in Eq. (6.3.27) and Eq. (6.3.28) with $\omega_{m}$ varied to include the Dancoff factor for each zone used in the BONAMIST calculation.

To compute the implicit portion of sensitivity coefficients for reactions, $x$, other than total, an additional term must be employed. With the implicit sensitivity of $k_{\text {eff }}$ to the total cross section computed, the chain rule for derivatives is again applied to propagate the sensitivity of $k_{\text {eff }}$ to the total cross section to the sensitivity of $k_{\text {eff }}$ to some other process. This is accomplished using the sensitivity of the total cross section to the particular processes, computed from the unshielded cross-section data as

$$
\left(S_{k, \Sigma_{x, g}^{i}}\right)_{\text {implicit }}=\left(\frac{\Sigma_{T, g}^{i}}{k} \frac{\partial k}{\partial \Sigma_{T, g}^{i}}\right)_{\text {implicit }} \times\left(\frac{\Sigma_{x, g}^{i}}{\Sigma_{T, g}^{i}} \frac{\partial \Sigma_{T, g}^{i}}{\partial \Sigma_{x, g}^{i}}\right) .
$$

The second term on the right hand side of Eq. (6.3.29) is computed analytically using data from the short master cross-section data library.

\subsubsection{Complete sensitivity coefficient}

With the implicit sensitivities properly computed, the complete sensitivity coefficient by group can be computed as the sum of the explicit and implicit terms as

$$
\left(S_{k, \Sigma_{x, g}^{i}}\right)_{\text {complete }}=\left(S_{k, \Sigma_{x, g}^{i}}\right)_{\text {explicit }}+\left(S_{k, \Sigma_{x, g}^{i}}\right)_{\text {implicit }} .
$$

When a Monte Carlo transport solution is used to produce sensitivity coefficients, uncertainties in the forward and adjoint flux solutions and the value of $k_{\text {eff }}$ are propagated to the final sensitivity results using standard error propagation techniques. ${ }^{10}$ The forward and adjoint fluxes are treated as uncorrelated to each other. Also, the group-wise values of each flux solution are treated as uncorrelated. The flux moments within each group are treated as fully correlated. Although this method provides an adequate assessment of the statistical uncertainty in the sensitivity coefficients, a more robust technique may be implemented in subsequent revisions.

\subsubsection{Summary of sensitivity coefficients calculated by SAMS}

Sensitivity coefficients are calculated for the sensitivity of $k_{\text {eff }}$ to the reactions listed in Table 6.3.1, if appropriate cross-section data is available. The identifier used in the SAMS output for each of these sensitivity types is also given. The MT of 0 assigned to scattering is arbitrary, as a sum of scattering reaction does not exist in the AMPX format. 
Table 6.3.1 Sensitivity types computed by SAMS

\begin{tabular}{|c|c|c|}
\hline MT & Reaction & SAMS identifier \\
\hline 0 & Sum of scattering & scatter \\
\hline 1 & Total & total \\
\hline 2 & Elastic scattering & elastic \\
\hline 4 & Inelastic scattering & $\mathrm{n}, \mathrm{n}^{\prime}$ \\
\hline 16 & $\mathrm{n}, 2 \mathrm{n}$ & $n, 2 n$ \\
\hline 18 & Fission & fission \\
\hline 101 & Neutron disappearance & capture \\
\hline 102 & $\mathrm{n}, \gamma$ & n,gamma \\
\hline 103 & $\mathrm{n}, \mathrm{p}$ & $\mathrm{n}, \mathrm{p}$ \\
\hline 104 & $\mathrm{n}, \mathrm{d}$ & $\mathrm{n}, \mathrm{d}$ \\
\hline 105 & $\mathrm{n}, \mathrm{t}$ & $\mathrm{n}, \mathrm{t}$ \\
\hline 106 & $\mathrm{n},{ }^{3} \mathrm{He}$ & n,he-3 \\
\hline 107 & $\mathrm{n}, \alpha$ & n,alpha \\
\hline 452 & $\bar{v}$ & nubar \\
\hline 1018 & $\chi$ & chi \\
\hline
\end{tabular}

\subsubsection{Uncertainty analysis}

Given uncertainty information for the cross sections for all nuclides and reaction processes that are important to the system of interest, it is possible to estimate the uncertainty in the calculated system multiplication factor due to these data uncertainties.

The nuclear data parameters are represented by the vector $\boldsymbol{\alpha}$, the elements of which are $\left(\alpha_{x, g}^{i}\right)$, where $i$ is varied over all isotopes, $x$ is varied over all reactions for each isotope and $g$ is varied over all energy groups. If $M$ is the number of nuclide-reaction pairs $\times$ the number of energy groups (i.e., the number of elements in $\boldsymbol{\alpha}$ ), the symmetric $M \times M$ matrix containing the relative variances (diagonal elements) and relative covariances (off-diagonal elements) in the nuclear data is $\mathbf{C}_{\alpha \alpha}$. The elements of $\mathbf{C}_{\alpha \alpha}$ are

$$
\left(C_{\alpha_{x, g}^{i} \alpha_{y, g^{\prime}}^{j}}\right)=\frac{\operatorname{COV}\left(\alpha_{x, g}^{i}, \alpha_{y, g^{\prime}}^{j}\right)}{\alpha_{x, g}^{i} \alpha_{y, g^{\prime}}^{j}},
$$

where $i$ and $j$ are varied over all isotopes, $x$ and $y$ are varied over all reactions for each isotope and $g$ and $g$ 'are varied over all energy groups. Additionally,

$$
\operatorname{COV}\left(\alpha_{x, g}^{i}, \alpha_{y, g^{\prime}}^{j}\right)=\left\langle\delta \alpha_{x, g}^{i} \delta \alpha_{y, g^{\prime}}^{j}\right\rangle
$$

where $\delta \alpha_{x, g}^{i}$ and $\delta \alpha_{y, g^{\prime}}^{j}$ represent the difference between the values and expectation values of the nuclear data parameters and \langle\rangle represents integration over the ranges of $\alpha_{x, g}^{i}$ and $\alpha_{y, g^{\prime}}^{j}$ weighted with a 
probability density function. A rigorous definition of the cross-section-covariance data is given in Ref. 11. SAMS simply reads the covariance data from a standard COVERX data file.

The vector of length $M$ containing sensitivities of the calculated $k_{\text {eff }}$ to the $\alpha$ parameters is represented by $\mathbf{S}_{\mathbf{k}}$, where each element is

$$
\left(S_{k, \alpha_{x, g}^{i}}\right)=\frac{\alpha_{x, g}^{i}}{k} \frac{\partial k}{\partial \alpha_{x, g}^{i}}
$$

For the purposes of SAMS uncertainty calculations, the $\alpha_{x, g}^{i}$ parameters are simply the group-wise cross-section data. If a particular material is present in more than one material region, the sensitivity coefficients for all regions are summed prior to creating the $\mathbf{S}_{\mathbf{k}}$ vector.

The variance for the system $k_{\text {eff }}$ value is given as

$$
\sigma_{k}^{2}=\mathbf{S}_{\mathbf{k}} \mathbf{C}_{\alpha \alpha} \mathbf{S}_{\mathbf{k}}^{T}
$$

where $T$ indicates a transpose.

The covariance in $k_{\text {eff }}$ due to the energy correlations of two particular processes can be assessed by examining a subset of the elements of $\mathbf{C}_{\alpha \alpha}$, where $i, j, x$ and $y$ are held constant. If $G$ is the number of energy groups, the covariance data for a particular process is represented as the $G \times G$ matrix $\mathbf{C}_{\boldsymbol{\alpha}_{x}^{\mathrm{i}} \alpha_{y}^{j}}$ and the group-wise sensitivity vectors, of length $G$, for the processes are represented as $\mathbf{S}_{\mathbf{k}, \boldsymbol{\alpha}_{\mathbf{x}}^{\mathrm{i}}}$ and $\mathbf{S}_{\mathbf{k}, \boldsymbol{\alpha}_{\mathbf{y}}^{\mathrm{j}}}$. The relative covariance in $k_{\text {eff }}$ due to the particular process or processes is given as

$$
\sigma_{k_{x, y}^{i, j}}^{2}=\mathbf{S}_{\mathbf{k}, \alpha_{x}^{\mathbf{i}}} \mathbf{C}_{\alpha_{\mathbf{x}}^{\mathrm{i}} \mathbf{\alpha}_{y}^{\mathbf{j}}} \mathbf{S}_{\mathbf{k}, \alpha_{y}^{\mathrm{j}}}^{T} \cdot
$$

In actuality, the COVERX data file represents the covariance data in the form of multiple $\mathbf{C}_{\boldsymbol{\alpha}_{x}^{i} \alpha_{y}^{j}}$ matrices. Thus, although commonly used for its mathematical convenience, $\mathbf{C}_{\alpha \alpha}$ does not exist as a continuous matrix. In the COVERX format, if $\mathbf{C}_{\alpha_{x}^{i} \alpha_{y}^{j}}$ is present on the data file with $i \neq j$ and/or $x \neq y$, then the transpose matrix $\mathbf{C}_{\alpha_{y}^{j} \alpha_{x}^{i}}$ is not present. Thus, using each matrix on the COVERX file only once, an upper (or lower) triangular $\mathbf{C}_{\alpha \alpha}$ matrix could be constructed, but not a full matrix.

In SAMS, the value of $\sigma_{k}^{2}$ is calculated by first determining the values of the variances or covariance as in Eq. (6.3.59) for all processes in the system under consideration, excluding the total reaction. The total reaction is excluded because it is the sum of the other processes and its inclusion would increase the variance from its actual value. The value of $\sigma_{k}^{2}$ is then computed as the sum of the variances (diagonal elements of $\mathbf{C}_{\alpha \alpha}$ plus twice the sum of the covariances (off-diagonal elements of $\mathbf{C}_{\alpha \alpha}$ ). The standard deviation of $k_{\text {eff }}$ is simply the square root of $\sigma_{k}^{2}$.

\subsubsection{Problem characterization}

SAMS computes a number of characteristic parameters of fissile systems for the convenience of the user for multigroup sensitivity calculations. These characterization parameters are not calculated in continuous-energy mode. These include the median fission group, average fission group, average energy 
causing fission, and energy of average lethargy causing fission. Corresponding parameters are also computed for capture and scattering.

The median fission group is computed as the first group $M$ that satisfies the inequality

where

$$
\frac{\sum_{z=1}^{Z} \sum_{g=1}^{M} \phi_{g, z} \Sigma_{f, g, z}}{\sum_{z=1}^{Z} \sum_{g=1}^{G} \phi_{g, z} \Sigma_{f, g, z}} \geq \frac{1}{2},
$$

$$
\begin{aligned}
& z=\text { index for material regions or zones, } \\
& Z=\text { total number of regions or zones in the system model, } \\
& G=\text { total number of energy groups. }
\end{aligned}
$$

The average fission group (AFG) is computed as

$$
\mathrm{AFG}=\frac{\sum_{z=1}^{Z} \sum_{g=1}^{G} g \phi_{g, z} \Sigma_{f, g, z}}{\sum_{z=1}^{Z} \sum_{g=1}^{G} \phi_{g, z} \Sigma_{f, g, z}} .
$$

The average energy causing fission (AECF) is computed as

$$
\mathrm{AECF}=\frac{\sum_{z=1}^{Z} \sum_{g=1}^{G} \bar{E}_{g} \phi_{g, z} \Sigma_{f, g, z}}{\sum_{z=1}^{Z} \sum_{g=1}^{G} \phi_{g, z} \Sigma_{f, g, z}},
$$

where

$$
\bar{E}_{g}=\text { the average energy of group } g \text {, computed as }\left(E_{g}+E_{g+1}\right) / 2 \text {. }
$$

Lethargy is defined as $u=\ln \left(E_{0} / E\right)$, where $E_{0}$ is the maximum energy considered, which is assumed for the lethargy calculation to be $10 \mathrm{MeV}$. The average lethargy causing fission is computed as

$$
\bar{u}=\frac{\sum_{z=1}^{Z} \sum_{g=1}^{G} \bar{u}_{g} \phi_{g, z} \Sigma_{f, g, z}}{\sum_{z=1}^{Z} \sum_{g=1}^{G} \phi_{g, z} \Sigma_{f, g, z}},
$$

where

$\bar{u}_{g}=$ the average lethargy of group $g$, computed as $\left(u_{g}+u_{g+1}\right) / 2$. 
The energy of the average lethargy causing fission (EALF) is

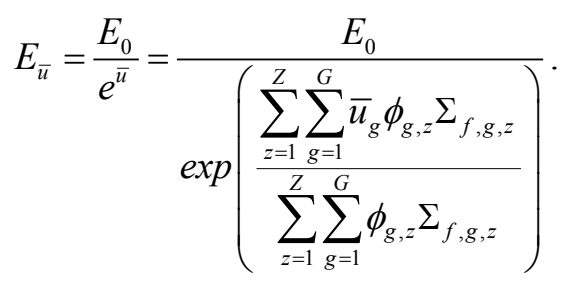

The above definitions are applicable to determining corresponding parameters for capture and scattering reactions where the capture and 1-D scattering cross-section data are substituted for the fission cross-section data.

\subsubsection{Generalized perturbation theory}

\section{General Responses for Critical Systems}

The previous sections have presented perturbation theory expressions for the explicit and implicit sensitivity coefficients relating changes in cross section data to changes in the fundamental eigenvalue of the neutron transport equation in Eq. (6.3.1). Generalized perturbation theory (GPT) can be used to extend the deterministic S/U methods in TSUNAMI-1D and TSUNAMI-2D to address more general responses that depend on the solution of the neutron transport equation. There is currently no TSUNAMI-3D GPT capability based on MG KENO Monte Carlo calculations, but a CE capability exists, as described in the TSUNAMI-3D section of the SCALE manual. Here the basic expressions used in GPT computations are described. More details and background material on GPT can be found in Ref. 12 .

The neutron transport equation is homogeneous because the fundamental eigenvalue effectively forces the multiplying medium to satisfy a pseudo-critical condition by scaling the fission production term to exactly balance the neutron losses. Since the solution of a homogeneous equation can only be found within an arbitrary normalization factor, the responses of interest in a critical system are generally ratios of linear functionals of the neutron flux, such as

$$
R=\frac{\left\langle H_{N} \phi\right\rangle}{\left\langle H_{D} \phi\right\rangle},
$$

where $\mathrm{H}_{\mathrm{N}}$ and $\mathrm{H}_{\mathrm{D}}$ are response functions defining the response of interest. The value for a ratio response is independent of the flux normalization since it cancels from the numerator and denominator; thus the response is uniquely defined. The following are some examples of ratio responses:

(a) reaction rate ratios such as the conversion factor:

$$
\begin{aligned}
& R=\frac{\left\langle\Sigma_{c}^{(\text {fertile })} \phi\right\rangle}{\left\langle\sum_{a}^{(\text {fissile })} \phi\right\rangle}, \\
& \Rightarrow H_{N}=\Sigma_{c}^{(\text {fertile })} ; H_{D}=\Sigma_{a}^{(\text {fissile })} ;
\end{aligned}
$$

(b) relative power density in fuel region $V_{z}$ (e.g., fuel pin): 


$$
\begin{array}{r}
R=\frac{\left\langle\frac{h\left(\mathrm{~V}_{z}\right)}{\mathrm{V}_{z}} \Sigma_{f} \phi\right\rangle}{\left\langle\frac{1}{\mathrm{~V}_{t o t}} \Sigma_{f} \phi\right\rangle}, \\
\Rightarrow H_{N}=\frac{h\left(\mathrm{~V}_{z}\right)}{\mathrm{V}_{z}} \Sigma_{f} ; H_{D}=\frac{1}{\mathrm{~V}_{t o t}} \Sigma_{f} ;
\end{array}
$$

where

$$
h\left(V_{z}\right)=1, r \in V_{z} \quad ; \quad h\left(V_{z}\right)=0, r \notin V_{z} ;
$$

(c) fast-to-thermal flux ratio:

$$
\begin{gathered}
R=\frac{\left\langle\left[1-h\left(E<E_{t h}\right)\right] \phi\right\rangle}{\left\langle\left(h\left(E<E_{t h}\right)\right) \phi\right\rangle}, \\
\Rightarrow H_{N}=\left[1-h\left(E>E_{t h}\right)\right] ; H_{D}=h\left(E<E_{t h}\right) ; E_{t h} \equiv \text { thermal energy boundary. }
\end{gathered}
$$

where

$$
h\left(E<E_{t h}\right)=1, E<E_{t h} \quad ; \quad h\left(E<E_{t h}\right)=0, E>E_{t h} ;
$$

(d) collapsed/homogenized thermal absorption cross section:

$$
\begin{array}{r}
R=\frac{\left\langle h\left(E<E_{t h}\right) \Sigma_{a} \phi\right\rangle}{\left\langle h\left(E<E_{t h}\right) \phi\right\rangle}, \\
\Rightarrow H_{N}=\Sigma_{a}(E) h\left(E<E_{t h}\right) ; H_{N}=h\left(E<E_{t h}\right) .
\end{array}
$$

Generalized Perturbation Theory for Response Ratios

GPT is used to relate variations in input data to changes in the calculated ratio-response. Let $\alpha$ be an arbitrary input parameter (number density, multigroup cross section, nubar, chi, etc.) that affects the response ratio because the response functions $\mathrm{H}_{\mathrm{N}}$ and/or $\mathrm{H}_{\mathrm{D}}$ depend on $\alpha$, or because the parameter appears in the transport equation, so that the flux is a function of $\alpha$; i.e.,

$$
R=R\left[H_{N}(\alpha), H_{D}(\alpha), \phi(\alpha)\right] .
$$

The response perturbation due to a sufficiently small variation in $\alpha$ can be approximated by the first-order term in a functional Taylor series expansion, 


$$
\delta R \cong\left\langle\left(\frac{\partial R}{\partial H_{N}} \frac{\partial H_{N}}{\partial \alpha}+\frac{\partial R}{\partial H_{D}} \frac{\partial H_{D}}{\partial \alpha}+\frac{\partial R}{\partial \phi} \frac{d \phi}{d \alpha}\right) \delta \alpha(\vec{\xi})\right\rangle
$$

In GPT it is common to express the response perturbation as the sum of a "direct" and an "indirect" component. Eq. (6.3.37) is rearranged slightly to obtain:

$$
\delta R=\delta R^{(\text {direct })}+\delta R^{(\text {indirect })}
$$

where

$$
\begin{gathered}
\delta R^{(\text {direct })}=\left\langle\left(\frac{\partial R}{\partial H_{N}} \frac{\partial H_{N}}{\partial \alpha}+\frac{\partial R}{\partial H_{D}} \frac{\partial H_{D}}{\partial \alpha}\right) \delta \alpha\right\rangle \\
\delta R^{(\text {indirect })}=\left\langle\left(\frac{\partial R}{\partial \phi} \frac{d \phi}{d \alpha}\right) \delta \alpha\right\rangle .
\end{gathered}
$$

The direct component accounts for response changes due to perturbations in the response functions $\mathrm{H}_{\mathrm{N}}$ and/or $\mathrm{H}_{\mathrm{D}}$ that depend explicitly (or implicitly through self-shielding) on data parameter $\alpha$. The second term, called the indirect component, accounts for the response change due to a flux perturbation in the numerator or denominator of Eq. (6.3.31), which is caused by varying $\alpha$ in the transport equation.

If the response functions depend explicitly on $\alpha$, then the functional derivatives appearing in the direct effect component can be evaluated to give

$$
\delta R^{(\text {direct })} \equiv \frac{R}{\left\langle H_{N} \phi\right\rangle}\left\langle\frac{\partial H_{N}}{\partial \alpha} \delta \alpha\right\rangle-\frac{R}{\left\langle H_{D} \phi\right\rangle}\left\langle\frac{\partial H_{D}}{\partial \alpha} \delta \alpha\right\rangle .
$$

In order to evaluate the indirect effect it is necessary to determine the impact of the data perturbation $\delta \alpha$ on the neutron balance equation. The operators $A$ and $B$, as well as the flux $\phi$ and eigenvalue $\lambda$ may all be functions of $\alpha$. Taking the first-order expansion for each of these functions and neglecting higher order terms gives

$$
[A-\lambda B]\left(\frac{d \phi}{d \alpha} \delta \alpha\right)=-\left[\left(\frac{\partial A}{\partial \alpha}-\lambda \frac{\partial B}{\partial \alpha}\right) \delta \alpha\right] \phi+\delta \lambda B \phi .
$$

An equation called the generalized adjoint equation is introduced as follows

$$
\left[A^{\dagger}-\lambda B^{\dagger}\right] \Gamma^{*}=\frac{1}{R} \frac{\partial R}{\partial \phi}=Q^{*}
$$


where $\Gamma^{*}$ is known as the generalized adjoint, or generalized importance, function. The adjoint source term in Eq. (6.3.43) is equal to the functional derivative of the response ratio with respect to the neutron flux, which corresponds to

$$
\frac{1}{R} \frac{\partial R}{\partial \phi}=\frac{H_{N}}{\left\langle H_{N} \phi\right\rangle}-\frac{H_{D}}{\left\langle H_{D} \phi\right\rangle}
$$

Taking the inner product of $\Gamma^{*}$ with Eq. (6.3.42), and the inner product of $\left(\frac{d \phi}{d \alpha} \delta \alpha\right)$ with Eq. (6.3.43), and then applying the property of adjoint operators and rearranging, gives:

$$
\left\langle\left(\frac{\partial R}{\partial \phi} \frac{d \phi}{d \alpha}\right) \delta \alpha\right\rangle=-\left\langle\Gamma^{*}\left(\frac{\partial A}{\partial \alpha}-\lambda \frac{\partial B}{\partial \alpha}\right) \phi \delta \alpha\right\rangle+\delta \lambda\left\langle\Gamma^{*} B \phi\right\rangle .
$$

The left side of Eq. (6.3.45) is identical to the indirect component defined in Eq. (6.3.40). As shown in the next section, the generalized adjoint function is calculated such that the term $\left\langle\Gamma^{*} B \phi\right\rangle$ is equal to zero; thus

$$
\delta R^{(\text {indirect })}=-\left\langle\Gamma^{*}\left(\frac{\partial A}{\partial \alpha}-\lambda \frac{\partial B}{\partial \alpha}\right) \phi \delta \alpha\right\rangle
$$

Combining Eqs. (6.3.41) and (6.3.46) gives the response perturbation due to both direct and indirect components:

$$
\delta R \equiv\left\{\frac{R}{\left\langle H_{N} \phi\right\rangle}\left\langle\frac{\partial H_{N}}{\partial \alpha} \delta \alpha\right\rangle-\frac{R}{\left\langle H_{D} \phi\right\rangle}\left\langle\frac{\partial H_{D}}{\partial \alpha} \delta \alpha\right\rangle\right\}-\left\langle\Gamma^{*}\left(\frac{\partial A}{\partial \alpha}-\lambda \frac{\partial B}{\partial \alpha}\right) \phi \delta \alpha\right\rangle .
$$

\section{Calculation of the Generalized Adjoint Function}

The forward flux $\phi$ and adjoint function $\phi^{*}$ are fundamental eigenfunctions of the homogeneous equations in Eqs. (6.3.1) and (6.3.3), respectively. In contrast, the generalized adjoint function $\Gamma^{*}$ obeys an inhomogeneous equation. Furthermore, the operator $\left(A^{\dagger}-\lambda B^{\dagger}\right)$ in Eq. (6.3.43) is singular, since by definition $\lambda$ is an eigenvalue. Taking the inner production of $\phi$ with Eq. (6.3.43) and applying the property of adjoint operators gives

$$
\left\langle\phi Q^{*}\right\rangle=\left\langle\phi \frac{1}{R} \frac{\partial R}{\partial \phi}\right\rangle=0
$$

The above relation is necessary for the singular inhomogeneous equation to have a solution; i.e., the adjoint source must be orthogonal to the forward flux. It is easily shown that the terms in Eq. (6.3.44) satisfy this condition. However a solution to Eq. (6.3.43) is not unique-the general solution for $\Gamma^{*}$ is the 
sum of a particular and a homogeneous solution. If $\Gamma_{p}^{*}$ is any particular solution, then the function $\left(\Gamma_{\mathrm{p}}^{*}+a \phi^{\dagger}\right)$ is also a solution, where " $\mathrm{a}$ " is any constant. This can be proved by direct substitution into Eq. (6.3.43). For GPT calculations it is convenient to define an auxiliary condition that "normalizes" the generalized fission source $B^{\dagger} \Gamma^{*}$ to contain no fundamental mode; i.e.,

$$
\left\langle\phi B^{\dagger} \Gamma^{*}\right\rangle=\left\langle\Gamma^{*} B \phi\right\rangle=0
$$

The above relation is satisfied by defining the constant to be $a=\frac{\left\langle\phi B^{\dagger} \Gamma_{\mathrm{p}}^{*}\right\rangle}{\left\langle\phi B^{\dagger} \phi^{\dagger}\right\rangle}$, so that:

$$
\Gamma^{*} \rightarrow \Gamma_{\mathrm{p}}^{*}-\frac{\left\langle\phi B^{\dagger} \Gamma_{\mathrm{p}}^{*}\right\rangle}{\left\langle\phi B^{\dagger} \phi^{\dagger}\right\rangle} \phi^{\dagger}
$$

The normalization in Eq.(6.3.49) is done for two reasons. First, the $2^{\text {nd }}$ term on the right side of Eq. (6.3.45) vanishes, so that the indirect effect is stationary with respect to eigenvalue perturbations introduced by $\delta \alpha$. The second reason is due to numerical difficulties in converging the generalized fission source. During outer iterations the fundamental mode component multiplies like a resonance frequency compared to other harmonics. This can lead to loss of numerical significance in the general solution. Theoretically, if the initial outer iteration has no fundamental mode in $B^{\dagger} \Gamma^{*}$, then subsequent outer iterations also will not. However in practice numerical approximations and incomplete convergence of inner iterations tend to re-introduce the fundamental mode. Therefore the operation in Eq. (6.3.50) is applied after each outer iteration to remove fundamental mode "contamination."

\section{$\underline{\text { Sensitivity Coefficients for General Responses }}$}

Analogous to the eigenvalue sensitivities, the response sensitivity coefficient at phase space coordinate $\vec{\xi}$ is defined as the relative change in $\mathrm{R}$ due to a relative change in $\alpha(\vec{\xi})$ :

$$
S_{R, \alpha}(\vec{\xi}) \equiv \frac{\delta R / R}{\delta \alpha / \alpha}
$$

The total response perturbation is obtained by summing over the response perturbations due to arbitrary $\alpha$-variations throughout phase space

$$
\frac{\delta R}{R} \cong\left\langle S_{R, \alpha}(\vec{\xi}) \frac{\delta \alpha}{\alpha}(\vec{\xi})\right\rangle
$$


Comparing Eqs. (6.3.37) and (6.3.47) shows that the sensitivity coefficient is equal to

$$
S_{R, \alpha}(\vec{\xi})=\underbrace{\left\{\frac{1}{\left\langle H_{N} \phi\right\rangle} \frac{\partial H_{N}}{\partial \alpha}-\frac{1}{\left\langle H_{D} \phi\right\rangle} \frac{\partial H_{D}}{\partial \alpha}\right\}}_{S^{(\text {direct })}}-\underbrace{\frac{1}{R} \Gamma^{*}\left(\frac{\partial A}{\partial \alpha}-\lambda \frac{\partial B}{\partial \alpha}\right)}_{S^{(\text {indirect })}} \phi .
$$

As in the case for eigenvalue sensitivity theory, the response perturbations introduced by $\delta \alpha$ may include explicit as well as implicit effects caused by changes in self-shielded cross sections $\tilde{\alpha}$ that are impacted by the value of parameter $\alpha$. This can be treated by extending the partial derivative operator with respect to ato be a total derivative, so that

$$
\frac{\partial}{\partial \alpha} \rightarrow \frac{d}{d \alpha}=\frac{\partial}{\partial \alpha}+\sum_{\tilde{\alpha}}\left(\frac{\partial \tilde{\alpha}}{\partial \alpha}\right) \frac{\partial}{\partial \tilde{\alpha}}
$$

When the above substitution is made into Eq. (6.3.53), the complete sensitivity coefficient is obtained

$$
\begin{aligned}
S_{R, \alpha}^{(\text {complete })} & =\overbrace{\left\{\frac{1}{\left\langle H_{N} \phi\right\rangle} \frac{\partial H_{N}}{\partial \alpha}-\frac{1}{\left\langle H_{D} \phi\right\rangle} \frac{\partial H_{D}}{\partial \alpha}\right\}}^{S_{\text {explicit }}^{(\text {direct }}}+\overbrace{\left\{\sum_{\tilde{\alpha}}\left(\frac{1}{\left\langle H_{N} \phi\right\rangle} \frac{\partial H_{N}}{\partial \tilde{\alpha}}-\frac{1}{\left\langle H_{D} \phi\right\rangle} \frac{\partial H_{D}}{\partial \tilde{\alpha}}\right)\left(\frac{\partial \tilde{\alpha}}{\partial \alpha}\right)\right\}}^{S_{\text {implicit }}^{(\text {direct })}} \\
& +\overbrace{\left\{\frac{1}{R} \Gamma^{*}\left(\frac{\partial A}{\partial \alpha}-\lambda \frac{\partial B}{\partial \alpha}\right) \phi\right\}}^{S_{\text {explicit }}^{(\text {indirect })}}+\overbrace{\left\{\sum_{\tilde{\alpha}}\left(\left\{\frac{1}{R} \Gamma^{*}\left(\frac{\partial A}{\partial \tilde{\alpha}}-\lambda \frac{\partial B}{\partial \tilde{\alpha}}\right) \phi\right\}\right)\left(\frac{\partial \tilde{\alpha}}{\partial \alpha}\right)\right\}}^{S_{\text {implicit }}^{(\text {indirect })}} .
\end{aligned}
$$

It can be seen that for GPT there are four distinct contributions to the complete sensitivity coefficient:

$$
S_{R, \alpha}^{(\text {complete })}=S_{\text {explicit }}^{(\text {diret })}+S_{\text {implicit }}^{(\text {direct })}+S_{\text {explicit }}^{(\text {indiret })}+S_{\text {implicit }}^{(\text {indirect }} .
$$

The SAMS code evaluates sensitivity coefficients for GPT as well as eigenvalue perturbation theory. Direct sensitivity coefficients for GPT are easily determined since they depend only on the response functions and do not require an adjoint calculation. On the other hand, the expressions for indirect explicit sensitivities are very similar to those presented in Section 6.3.2.1 for eigenvalue responses. The detailed expressions in Eqs. (6.3.11)-(6.3.24) for eigenvalue sensitivity coefficients with respect to each type of nuclear data are also valid for general responses, by replacing the fundamental mode adjoint function $\phi^{\dagger}$ with the generalized adjoint $\Gamma^{*}$, and setting the value of the denominator $\mathrm{D}$ equal to the response R. The treatment of implicit effects described in Section 6.3.2.2 for eigenvalue responses is also the same for general responses.

\subsubsection{SAMS Input Description}

The input to SAMS consists of a SCALE Analytical Sequence Specification Record, a single block of data that contains keyword input that controls the code execution, and a SCALE input termination END record. Very little input is required to obtain sensitivity and uncertainty results, given the availability of appropriate data files, as the default values are suitable for a basic analysis. The input data for SAMS are 
entered using the SCALE free-form reading routines. The input is not case sensitive, so either upper- or lowercase letters may be used. A maximum of 252 columns per line may be used for input.

\subsubsection{Analytical sequence specification record}

The analytical sequence specification begins in column 1 of the first line of the input file and must contain the following line.

=SAMS5 (for XSDRN or KENO V.a calculations)

=SAMS6 (for KENO-VI calculations)

This signals the SCALE driver to execute the appropriate SAMS functional module. Input data following the Analytical Sequence Specification Record and preceding the END record are passed to SAMS as input.

\subsubsection{SAMS input data}

SAMS input data consists of a single SCALE input data block, the INITIAL block. The input must begin with the keywords READ INITIAL and end with the keywords END INITIAL. The keyword entries summarized in Table 6.3.2 and Table 6.3.3 are allowed between the READ INITIAL and END INITIAL keywords. Keywords ending with ' $=$ ' must be followed by the value to be assigned to the corresponding variable. Default values listed in Tables 6.3.2 and 6.3.3 may be set or altered if SAMS is executed as part of a sequence, such as TSUNAMI-3D-K5 or TSUNAMI-3D-K6; in these cases, the sequence manual for that sequence should also be consulted for default variable values. 
Table 6.3.2 SAMS input keywords

\begin{tabular}{|c|c|c|}
\hline Keyword & Default value & Description \\
\hline binsen & $\bar{F}$ & $\begin{array}{l}\text { Produces SENPRO formatted binary sensitivity data file on } \\
\text { unit } 40\end{array}$ \\
\hline coverx $=$ & 56groupcov7.1 & Name of covariance data file to use for uncertainty analysis \\
\hline kenova & Kenova & $\begin{array}{l}\text { Indicates whether KENO V.a or KENO-VI data files are } \\
\text { present on logical unit number kunit and kunita }\end{array}$ \\
\hline kenovi & kenova & $\begin{array}{l}\text { Indicates whether KENO V.a or KENO-VI data files are } \\
\text { present on logical unit number kunit and kunita }\end{array}$ \\
\hline kunit $=$ & 35 & $\begin{array}{l}\text { Logical unit number of the binary data file from the forward } \\
\text { calculation }\end{array}$ \\
\hline kunita $=$ & 36 & $\begin{array}{l}\text { Logical unit number of the binary data file from the adjoint } \\
\text { case }\end{array}$ \\
\hline largeimp $=$ & 100.0 & $\begin{array}{l}\text { Value for the absolute value of implicit sensitivities, which if } \\
\text { exceeded, will be reset to } 0.0 \text { and print a warning message. }\end{array}$ \\
\hline makeimp & $\mathrm{F}$ & Flag to cause implicit sensitivity coefficients to be generated. \\
\hline newt & Kenova & $\begin{array}{l}\text { Indicates that NEWT data files are present on logical unit } \\
\text { number kunit and kunita }\end{array}$ \\
\hline nocovar & $\mathrm{T}$ & $\begin{array}{l}\text { Flag to cause uncertainty edit to be turned off (sets print_covar } \\
\text { to F) }\end{array}$ \\
\hline nohtml & $\mathrm{F}$ & Flag to cause HTML output to not be produced. \\
\hline nomix & $\mathrm{F}$ & Flag to cause the sensitivities by mixture to be turned off \\
\hline pmeshvol & $\mathrm{F}$ & Flag to print mesh volumes \\
\hline $\mathrm{pn}=$ & 3 & Legendre order for moment calculations \\
\hline pltp & $\mathrm{F}$ & $\begin{array}{l}\text { Flag to cause a Javapeño 3D file containing } \phi \phi^{*} \text { matrices to be } \\
\text { written inside the .htmd directory of the output. }\end{array}$ \\
\hline prtgeom & $\mathrm{F}$ & $\begin{array}{l}\text { Flag to cause the sensitivities to be output for each geometry } \\
\text { region }\end{array}$ \\
\hline prtimp & $\mathrm{F}$ & $\begin{array}{l}\text { Prints explicit sensitivities coefficients, implicit sensitivity } \\
\text { coefficients and complete sensitivity coefficients }\end{array}$ \\
\hline prtp & $\mathrm{F}$ & Flag to cause $\phi \phi^{*}$ matrices to be written to the output file. \\
\hline prtvols & $\mathrm{F}$ & $\begin{array}{l}\text { Flag to cause the volumes of the regions to be printed by } \\
\text { SAMS }\end{array}$ \\
\hline useang & $\mathrm{F}$ & $\begin{array}{l}\text { Flag to cause the angular flux calculated in KENO to be used } \\
\text { to calculate flux moments for sensitivity calculations. If } \\
\text { angular fluxes were not computed by KENO, useang is set to } \\
\text { false internally. }\end{array}$ \\
\hline
\end{tabular}


Table 6.3.2. SAMS input keywords (continued)

\begin{tabular}{ccl}
\hline Keyword & Default value & \multicolumn{1}{c}{ Description } \\
\hline usemom & $\mathrm{T}$ & $\begin{array}{l}\text { Flag to cause the flux moments calculated with KENO to be } \\
\text { used to in sensitivity coefficient generation. If flux moments } \\
\text { were not computed by KENO, usemom is set to false internally. }\end{array}$ \\
xsdrn & kenova & $\begin{array}{l}\text { Indicates that XSDRN data files are present on kunit and kunita } \\
\text { logical unit numbers }\end{array}$ \\
xunit= & 4 & $\begin{array}{l}\text { Logical unit number of the AMPX working formatted cross- } \\
\text { section library }\end{array}$ \\
xunitm= & 42 & $\begin{array}{l}\text { Logical unit number for master sensitivity library } \\
\text { shortx= }\end{array}$ \\
unconstrainedchi & $\mathrm{F}$ & $\begin{array}{l}\text { Logical unit number of the AMPX short master formatted } \\
\text { cross-section library. }\end{array}$ \\
\hline
\end{tabular}

Due to differences in the continuous-energy and multigroup sensitivity coefficient calculation methods, SAMS input files that use continuous-energy TSUNAMI do not use most of the input parameters that are described in Table 6.3.2. In fact, continuous-energy TSUNAMI calculations will only use the nomix and unconstrainedchi input parameters; all other parameters that are input will be ignored.

Table 6.3.3. SAMS input keywords for default covariance data

\begin{tabular}{lcl}
\hline \multicolumn{1}{c}{ Keyword } & Default value & \multicolumn{1}{c}{ Description } \\
\hline use_dcov & F & Use default covariance data \\
use_icov & F & Use user-input covariance data \\
cov_fix & F & $\begin{array}{l}\text { Correct covariance data if the uncertainty is large } \\
>1000 \% \text { or zero }\end{array}$ \\
large_cov & 10.0 & $\begin{array}{l}\text { Relative Standard deviation to apply cov_fix } \\
\text { return_work_cov }\end{array}$ \\
udcov= & F & $\begin{array}{l}\text { Create a new covariance data file with only the } \\
\text { cross-section covariance data used in the analysis }\end{array}$ \\
udcov_corr= & 1.0 & $\begin{array}{l}\text { User-defined default value of standard deviation for } \\
\text { all energy groups } \\
\text { User-defined default correlation value }\end{array}$ \\
udcov_corr_type $=$ & zone & $\begin{array}{l}\text { User-defined default correlation type; allowed values } \\
\text { are long, zone, and short } \\
\text { Udcov_therm= }\end{array}$ \\
udcov_inter= & 0.0 & $\begin{array}{l}\text { User-defined default value of standard deviation for } \\
\text { thermal energy groups } \\
\text { User-defined default value of standard deviation for } \\
\text { intermediate energy groups }\end{array}$
\end{tabular}


Additionally, user-defined covariance data can be specified for individual nuclides and reactions using the COVARIANCE data block. This data begins with the keywords READ COVARIANCE and ends with the keywords END COVARIANCE. Any of the optional COVARIANCE input data may be entered in free form format between the READ COVARIANCE and END COVARIANCE keywords. The specifications for the COVARIANCE data block are described in the "User Input Covariance Data" section of the TSUNAMI Utility Modules chapter of the SCALE manual.

As the SAMS module generates HTML output, the optional HTML data block will provide user control over some formats of the output. This data begins with the keywords READ HTML and ends with the keywords END HTML. Any of the optional HTML input data may be entered in free form format between the READ HTML and END HTML keywords. The specifications for the HTML data block are described in the HTML Data section of the TSUNAMI Utility Modules chapter of the SCALE manual.

\subsubsection{Data files required by SAMS}

SAMS requires a number of data files to be available in the directory in which in the program executes to calculate MG sensitivities. These files are summarized in Table 6.3.4, but are not needed for CE sensitivity calculations.

Table 6.3.4 Data files required by SAMS

\begin{tabular}{|c|c|c|}
\hline Filename & When required & Description \\
\hline ftkunitf001 & Always & Data from forward transport solution \\
\hline ftkunitaf001 & Always & Data from adjoint transport solution \\
\hline ftxunitf001 & Always & $\begin{array}{l}\text { AMPX working formatted cross-section data library containing } \\
\text { resonance self-shielded cross-section data used in transport } \\
\text { calculations }\end{array}$ \\
\hline ftxunitmf001 & Always & $\begin{array}{l}\text { Master sensitivity cross-section data library; this library contains } \\
\text { resonance self-shielded data for more reactions than are available } \\
\text { on the working formatted library }\end{array}$ \\
\hline ftxunitsxf001 & Implicit Sensitivities & $\begin{array}{l}\text { Short master cross-section data library containing infinitely dilute } \\
\text { cross-section data }\end{array}$ \\
\hline senlib.sen & Implicit Sensitivities & $\begin{array}{l}\text { Sensitivity data file produced by SENLIB; contains sensitivity of } \\
\text { values input to resonance self-shielding codes to input material } \\
\text { number densities }\end{array}$ \\
\hline bonamist.sen & Implicit Sensitivities & $\begin{array}{l}\text { Sensitivity data file produced by BONAMIST; contains the } \\
\text { sensitivity of shielded group-wise cross sections to data input to } \\
\text { BONAMIST }\end{array}$ \\
\hline coverx & Uncertainty Analysis & COVERX formatted cross-section-covariance data file \\
\hline
\end{tabular}

\subsubsection{Example input}

An example input file is given in Figure 6.3.1. In this example, XSDRNPM was used as the transport solver ( $x s d r n$ ), the data file from the forward transport solution is read from file ft33f001 (kunit=33), and the data file from the adjoint transport solution is read from file $\mathrm{ft} 32 \mathrm{f001}$ (kunita=32). SAMS will 
generate implicit sensitivity coefficients (makeimp) and print them in detail (prtimp). SAMS will compute and print sensitivity coefficients by region (prtgeom) and print the fluxes (prtflx).

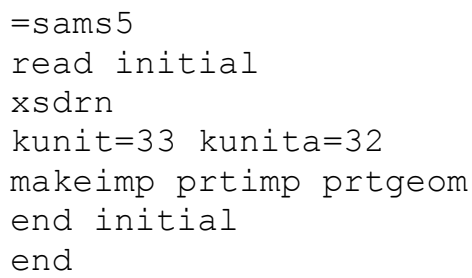

Figure 6.3.1. Example SAMS input.

\subsubsection{Sample Problems And Output Description}

\subsubsection{Example problem using XSDRNPM}

This sample problem is the Flattop-25 metal system from the Cross-Section Evaluation Working Group benchmark specifications. ${ }^{13}$ The system consists of a $6.116-\mathrm{cm}$ sphere of $93 \%$-enriched uranium with a natural uranium reflector. The outer radius of the reflector is $24.13 \mathrm{~cm}$.

Prior to running SAMS, the data files listed in Table 6.3.4 were generated for this system. It is recommended that users perform sensitivity analyses on 1-D systems using the TSUNAMI-1D control module, which executes the appropriate codes to generate the required data files in an automated manner from a single input. SAMS is executed automatically as a part of the TSUNAMI-1D control sequence. However, given appropriate data files SAMS can be run as stand-alone program.

Input and text output

The SAMS input is shown in Figure 6.3.2 and the output is shown in Figure 6.3.3. Each section of the output is described below. Note that output shown in this section is intended only to represent the format of output that the user will encounter. The actual computed results may vary.

\section{Parameter Table and Summary of Transport Calculation}

In the output listing, the SAMS Parameter Table gives the values of the SAMS input data. Next, the title from the XSDRNPM input file is given and a brief summary of the transport solution is given.

2. Energy-, Region-, and Mixture Integrated Sensitivity Coefficients

Next the energy-, region-, and mixture integrated sensitivity coefficients are given for each reaction of each nuclide. The nuclide symbol and reaction name are given. The data given in the Sensitivity column represents the expected change in $k_{\text {eff }}$ of this system due to a uniform fractional change in the cross section of the given nuclide and reaction in all energy groups for all regions and mixtures that contain this nuclide. For example, a uniform fractional change of 0.01 (or $1 \%$ ) in the fission cross section of ${ }^{235} \mathrm{U}$ would produce a fractional change in $k_{\text {eff }}$ of $5.7367 \times 10^{-1} \times 0.01$ or $5.7367 \times 10^{-3}(0.57367 \% \Delta \mathrm{k} / \mathrm{k})$. Because the keyword PRTIMP was entered in the SAMS input, the explicit, implicit and complete sensitivity coefficients are listed in separate columns. If PRTIMP were not entered, only the complete sensitivity would be given in the Sensitivity column. 


\section{Energy- and Region-Integrated Sensitivity Coefficients}

Next the energy- and region-integrated sensitivity coefficients are given for each reaction of each nuclide. The mixture number, nuclide symbol and reaction name are given. The data given in the Sensitivity column represents the expected change in $k_{\text {eff }}$ of this system due to a uniform fractional change in the cross section of the given nuclide and reaction in all energy groups for all regions that contain the particular mixture. For example, a uniform fractional change of 0.01 (or 1\%) in the fission cross section of ${ }^{235} \mathrm{U}$ in mixture 1 would produce a fractional change in $k_{\text {eff }}$ of $5.6594 \times 10^{-1} \times 0.01$ or $5.6594 \times 10^{-3}(0.56594 \% \Delta \mathrm{k} / \mathrm{k})$. Because the keyword PRTIMP was entered in the SAMS input, the explicit, implicit and complete sensitivity coefficients are listed in separate columns. If PRTIMP were not entered, only the complete sensitivity would be given in the Sensitivity column.

\section{Total Sensitivity Coefficients by Nuclide}

The next section of the output summarizes the total sensitivity by nuclide and mixture. The sensitivity values given here are the same as the values given in the previous section for the sensitivity of $k_{\text {eff }}$ to the total cross section. Also included in this edit are the atom densities for each nuclide in each mixture.

\section{Total Sensitivity Coefficients by Mixture}

The next output edit gives the total sensitivity of $k_{\text {eff }}$ to the mixture. Here, the sensitivity of $k_{\text {eff }}$ to the total cross section is summed over each nuclide in a given mixture.

6. Problem Characterization (MG Calculations Only)

The next output edit is for the problem characterization data. This section includes the median group of neutrons causing fission, capture and scattering; average group for fission, capture and scattering; the average energy causing fission, capture and scattering; and the energy of the average lethargy causing fission, capture and scattering.

\section{Sensitivity Coefficients by Material Zone}

Because the keyword PRTGEOM was entered in the input, the next output edit gives the energyintegrated sensitivity coefficients for each material zone defined in the XSDRNPM criticality model. First, the zone number, material number and volume of the zone are given. Next, the sensitivity coefficients are given in the same format as for the region-integrated data. The edit is repeated for each material zone in the XSDRNPM criticality model.

\section{Uncertainty Information}

The next output edit contains the uncertainty information. First, the percent relative standard deviation in $k_{\text {eff }}\left(\Delta k_{\text {eff }} k_{\text {eff }} \times 100 \%\right)$ due to cross-section-covariance data is given. Next, a listing of the uncertainty terms as produced by Eq. (6.3.59) are given. The nuclide-reaction to nuclide-reaction covariance matrix responsible for the uncertainty contribution is given followed by the contribution to the uncertainty in terms of $\% \Delta \mathrm{k} / \mathrm{k}$. The total uncertainty can be computed from individual values by adding the square of the values with positive signs and subtracting the square of the values with negative signs, then taking the square root. The negative values are the result of anti-correlations in the cross-section-covariance data. 
9. Execution Complete

The final edit produced simply states that the execution is complete and gives the elapsed time.

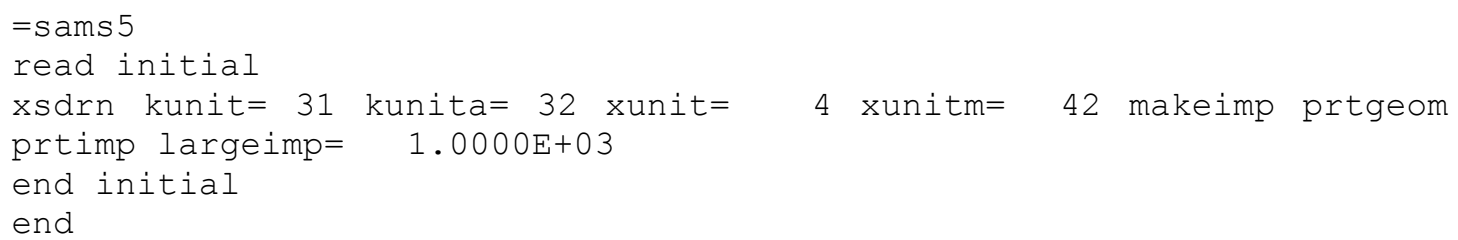

Figure 6.3.2. SAMS input for Flattop-25 sample problem. 
SAMS: Sensitivity Analysis Module for SCALE

SAMS Parameter Table

$$
\begin{aligned}
& \text { transport solution from: XSDRNPM } \\
& \text { forward transport solution on unit: } 31 \\
& \text { adjoint transport solution on unit: } 32 \\
& \text { working cross-section library on unit: } 4 \\
& \text { master sensitivity cross-section library on unit: } 42 \\
& \text { short master cross-section library on unit: } 11 \\
& \text { covariance data library read from file: 56groupcov7.1 } \\
& \begin{array}{ll}
\text { print mesh volumes: false } \\
\text { ry SENPRO data file: false }
\end{array}
\end{aligned}
$$

HTML Format Options

Background color: PapayaWhip

Major Headings color: Maroon

Sub-headings color: Navy

Plain text color: Black

Hyperlink color: Navy

Hypelink style: none

Default Covariance color: Blue

User Input Covariance color: Red

Corrected with default covariance data color: RoyalBlue

corrected with user input covariance data color: Green

Figure 6.3.3. SAMS output for Flattop-25 sample problem. 
Energy, Region and Mixture Integrated Sensitivity Coefficients for this Problem

\begin{tabular}{|c|c|c|c|c|}
\hline Nuclide & Reaction & Explicit & Implicit & Sensitivity \\
\hline$u-234$ & $\begin{array}{l}----- \\
\text { total }\end{array}$ & $4.5108 \mathrm{E}-03$ & $1.4614 \mathrm{E}-07$ & \\
\hline$u-234$ & scatter & $6.6431 \mathrm{E}-04$ & 1. $2145 \mathrm{E}-07$ & $6.6443 E-04$ \\
\hline$u-234$ & elastic & $4.0086 \mathrm{E}-04$ & $1.1594 \mathrm{E}-07$ & $4.0097 E-04$ \\
\hline$u-234$ & $n, n^{\prime}$ & $2.6201 \mathrm{E}-04$ & $5.5685 \mathrm{E}-09$ & $2.6202 \mathrm{E}-04$ \\
\hline$u-234$ & $n, 2 n$ & $1.4354 \mathrm{E}-06$ & $-5.7151 \mathrm{E}-11$ & $1.4354 \mathrm{E}-06$ \\
\hline$u-234$ & fission & $4.3022 \mathrm{E}-03$ & $1.6639 \mathrm{E}-08$ & $4.3022 \mathrm{E}-03$ \\
\hline$u-234$ & capture & $-4.5566 \mathrm{E}-04$ & $8.0468 \mathrm{E}-09$ & $-4.5565 E-04$ \\
\hline$u-234$ & $\mathrm{n}$, gamma & $-4.5566 \mathrm{E}-04$ & $8.0468 E-09$ & $-4.5565 E-04$ \\
\hline$u-234$ & nubar & $6.5581 \mathrm{E}-03$ & $0.0000 \mathrm{E}+00$ & $6.5581 \mathrm{E}-03$ \\
\hline$u-234$ & $\operatorname{chi}$ & $1.7178 \mathrm{E}-10$ & $0.0000 \mathrm{E}+00$ & $1.7178 \mathrm{E}-10$ \\
\hline$u-235$ & total & $5.9204 \mathrm{E}-01$ & $-4.7099 \mathrm{E}-06$ & $5.9204 \mathrm{E}-01$ \\
\hline$u-235$ & scatter & $6.6931 \mathrm{E}-02$ & $-3.6751 E-06$ & $6.6927 \mathrm{E}-02$ \\
\hline$u-235$ & elastic & $3.2362 \mathrm{E}-02$ & $-3.6651 \mathrm{E}-06$ & $3.2358 \mathrm{E}-02$ \\
\hline$u-235$ & $n, n^{\prime}$ & $3.3483 E-02$ & $-8.6100 \mathrm{E}-09$ & $3.3483 \mathrm{E}-02$ \\
\hline$u-235$ & $n, 2 n$ & $1.0842 \mathrm{E}-03$ & $-1.4191 \mathrm{E}-09$ & $1.0842 \mathrm{E}-03$ \\
\hline$u-235$ & fission & $5.7367 \mathrm{E}-01$ & $-7.7390 \mathrm{E}-07$ & $5.7367 \mathrm{E}-01$ \\
\hline$u-235$ & capture & $-4.8559 \mathrm{E}-02$ & $-2.6087 \mathrm{E}-07$ & $-4.8559 \mathrm{E}-02$ \\
\hline$u-235$ & $\mathrm{n}$, gamma & $-4.8559 \mathrm{E}-02$ & $-2.6087 \mathrm{E}-07$ & $-4.8559 \mathrm{E}-02$ \\
\hline$u-235$ & nubar & $9.1500 \mathrm{E}-01$ & $0.0000 \mathrm{E}+00$ & $9.1500 \mathrm{E}-01$ \\
\hline$u-235$ & chi & $-2.3075 E-08$ & $0.0000 \mathrm{E}+00$ & $-2.3075 E-08$ \\
\hline$u-238$ & total & $2.1733 \mathrm{E}-01$ & $6.2352 \mathrm{E}-05$ & $2.1740 \mathrm{E}-01$ \\
\hline$u-238$ & scatter & $2.1045 \mathrm{E}-01$ & $6.0318 \mathrm{E}-05$ & $2.1051 \mathrm{E}-01$ \\
\hline$u-238$ & elastic & $1.4370 \mathrm{E}-01$ & $5.9909 \mathrm{E}-05$ & $1.4376 \mathrm{E}-01$ \\
\hline$u-238$ & $n, n^{\prime}$ & $6.5971 \mathrm{E}-02$ & $3.8252 \mathrm{E}-07$ & $6.5971 \mathrm{E}-02$ \\
\hline$u-238$ & $n, 2 n$ & $7.6541 \mathrm{E}-04$ & $2.6330 \mathrm{E}-08$ & $7.6544 \mathrm{E}-04$ \\
\hline
\end{tabular}

Figure 6.3.3. SAMS output for Flattop-25 sample problem. (continued) 


$\begin{array}{llrrr}\text { u-238 } & \text { fission } & \mathbf{5 . 5 6 2 4 \mathrm { E } - 0 2} & \mathbf{4 . 9 5 1 2 \mathrm { E } - 0 8} & \mathbf{5 . 5 6 2 4 \mathrm { E } - 0 2} \\ \mathrm{u}-238 & \text { capture } & \mathbf{- 4 . 8 7 3 6 \mathrm { E } - 0 2} & \mathbf{1 . 9 8 4 7 \mathrm { E } - 0 6} & \mathbf{- 4 . 8 7 3 4 \mathrm { E } - 0 2} \\ \mathrm{u}-238 & \text { n, gamma } & -4.8736 \mathrm{E}-02 & 1.9847 \mathrm{E}-06 & -4.8734 \mathrm{E}-02 \\ \mathrm{u}-238 & \text { nubar } & 7.8442 \mathrm{E}-02 & 0.0000 \mathrm{E}+00 & 7.8442 \mathrm{E}-02 \\ \mathrm{u}-238 & \text { chi } & 7.5020 \mathrm{E}-10 & 0.0000 \mathrm{E}+00 & 7.5020 \mathrm{E}-10\end{array}$

Energy and Region Integrated Sensitivity Coefficients for this Problem

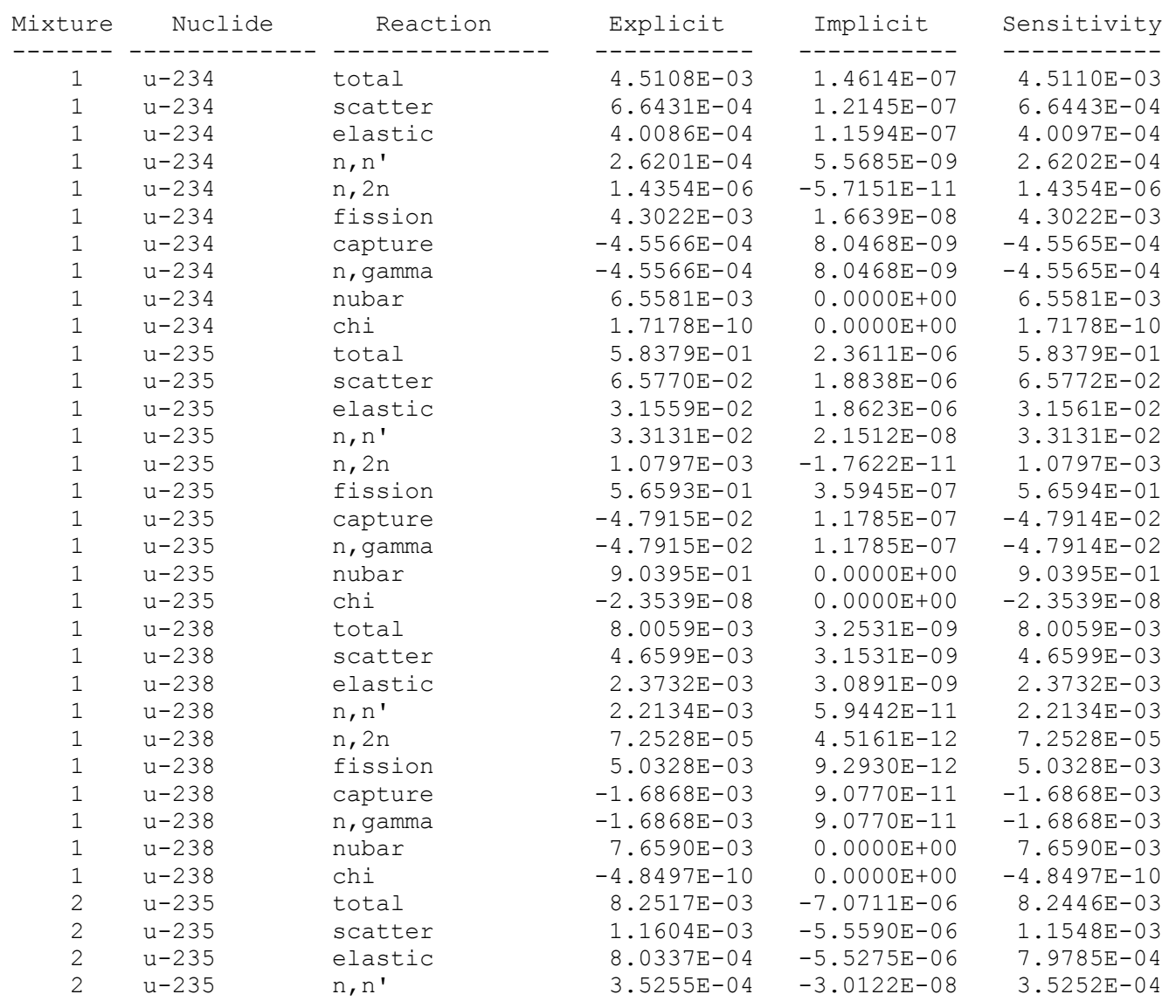

Figure 6.3.3. SAMS output for Flattop-25 sample problem. (continued) 


$\begin{array}{rrlrrr}2 & \mathrm{u}-235 & \text { n,2n } & 4.4336 \mathrm{E}-06 & -1.4015 \mathrm{E}-09 & 4.4322 \mathrm{E}-06 \\ 2 & \mathrm{u}-235 & \text { fission } & 7.7357 \mathrm{E}-03 & -1.1333 \mathrm{E}-06 & 7.7346 \mathrm{E}-03 \\ 2 & \mathrm{u}-235 & \text { capture } & -6.4439 \mathrm{E}-04 & -3.7872 \mathrm{E}-07 & -6.4477 \mathrm{E}-04 \\ 2 & \mathrm{u}-235 & \text { n,gamma } & -6.4439 \mathrm{E}-04 & -3.7872 \mathrm{E}-07 & -6.4477 \mathrm{E}-04 \\ 2 & \mathrm{u}-235 & \text { nubar } & 1.1046 \mathrm{E}-02 & 0.0000 \mathrm{E}+00 & 1.1046 \mathrm{E}-02 \\ 2 & \mathrm{u}-235 & \text { chi } & 4.6475 \mathrm{E}-10 & 0.0000 \mathrm{E}+00 & 4.6475 \mathrm{E}-10 \\ 2 & \mathrm{u}-238 & \text { total } & 2.0933 \mathrm{E}-01 & 6.2348 \mathrm{E}-05 & 2.0939 \mathrm{E}-01 \\ 2 & \mathrm{u}-238 & \text { scatter } & 2.0579 \mathrm{E}-01 & 6.0314 \mathrm{E}-05 & 2.0585 \mathrm{E}-01 \\ 2 & \mathrm{u}-238 & \text { elastic } & 1.4133 \mathrm{E}-01 & 5.9906 \mathrm{E}-05 & 1.4139 \mathrm{E}-01 \\ 2 & \mathrm{u}-238 & \text { n, n' } & 6.3758 \mathrm{E}-02 & 3.8246 \mathrm{E}-07 & 6.3758 \mathrm{E}-02 \\ 2 & \mathrm{u}-238 & \text { n, 2n } & 6.9288 \mathrm{E}-04 & 2.6325 \mathrm{E}-08 & 6.9291 \mathrm{E}-04 \\ 2 & \mathrm{u}-238 & \text { fission } & 5.0591 \mathrm{E}-02 & 4.9503 \mathrm{E}-08 & 5.0591 \mathrm{E}-02 \\ 2 & \mathrm{u}-238 & \text { capture } & -4.7050 \mathrm{E}-02 & 1.9846 \mathrm{E}-06 & -4.7048 \mathrm{E}-02 \\ 2 & \mathrm{u}-238 & \text { n, gamma } & -4.7050 \mathrm{E}-02 & 1.9846 \mathrm{E}-06 & -4.7048 \mathrm{E}-02 \\ 2 & \mathrm{u}-238 & \text { nubar } & 7.0783 \mathrm{E}-02 & 0.0000 \mathrm{E}+00 & 7.0783 \mathrm{E}-02 \\ 2 & \mathrm{u}-238 & \text { chi } & 1.2352 \mathrm{E}-09 & 0.0000 \mathrm{E}+00 & 1.2352 \mathrm{E}-09\end{array}$

Total Sensitivity Coefficients by Nuclide

\begin{tabular}{|c|c|c|c|}
\hline Mixture & Nuclide & Atom Density & Sensitivity \\
\hline---- & ---------- & ----------- & --------- \\
\hline 1 & $u-234$ & $4.9000 \mathrm{E}-04$ & $4.5110 \mathrm{E}-03$ \\
\hline 1 & $u-235$ & $4.4490 \mathrm{E}-02$ & $5.8379 \mathrm{E}-01$ \\
\hline 2 & $u-235$ & $3.4000 \mathrm{E}-04$ & $8.2446 \mathrm{E}-03$ \\
\hline 1 & $u-238$ & $2.7000 \mathrm{E}-03$ & $8.0059 \mathrm{E}-03$ \\
\hline 2 & $u-238$ & $4.7740 \mathrm{E}-02$ & $2.0939 \mathrm{E}-01$ \\
\hline
\end{tabular}

Total Sensitivity Coefficients by Mixture

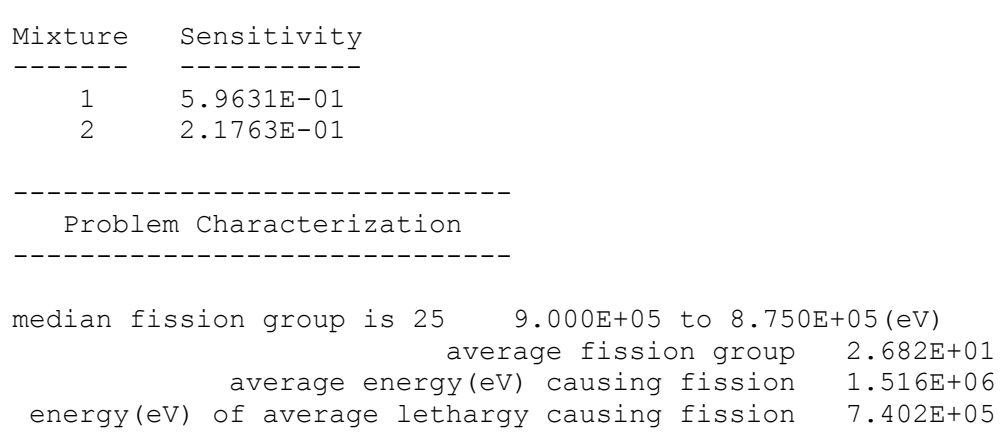

Figure 6.3.3. SAMS output for Flattop-25 sample problem. (continued) 
median capture group is $42 \quad 2.000 \mathrm{E}+05$ to $1.500 \mathrm{E}+05(\mathrm{eV})$ average capture group $4.216 \mathrm{E}+01$ average energy (eV) causing capture $3.014 \mathrm{E}+05$ energy (eV) of average lethargy causing capture $1.498 \mathrm{E}+05$ median scatter group is $412.700 \mathrm{E}+05$ to $2.000 \mathrm{E}+05(\mathrm{eV})$ average scatter group $3.915 \mathrm{E}+01$ average energy(eV) causing scatter $4.743 \mathrm{E}+05$ energy(eV) of average lethargy causing scatter $2.231 \mathrm{E}+05$

Sensitivity Coefficients by Material Zone

Zone 1 Material 1 Volume of zone $=9.58277 \mathrm{E}+02 \mathrm{~cm}^{\wedge} 3$

\begin{tabular}{rrrrrr} 
Mixture & Nuclide & Reaction & \multicolumn{1}{c}{ Explicit } & \multicolumn{1}{c}{ Implicit } & Sensitivity \\
\hline 1 & u-234 & total & $4.5108 \mathrm{E}-03$ & $1.4614 \mathrm{E}-07$ & $-1.5110 \mathrm{E}-03$ \\
1 & $\mathrm{u}-234$ & scatter & $6.6431 \mathrm{E}-04$ & $1.2145 \mathrm{E}-07$ & $6.6443 \mathrm{E}-04$ \\
1 & $\mathrm{u}-234$ & elastic & $4.0086 \mathrm{E}-04$ & $1.1594 \mathrm{E}-07$ & $4.0097 \mathrm{E}-04$ \\
1 & $\mathrm{u}-234$ & n,n' & $2.6201 \mathrm{E}-04$ & $5.5685 \mathrm{E}-09$ & $2.6202 \mathrm{E}-04$ \\
1 & $\mathrm{u}-234$ & n,2n & $1.4354 \mathrm{E}-06$ & $-5.7151 \mathrm{E}-11$ & $1.4354 \mathrm{E}-06$ \\
1 & $\mathrm{u}-234$ & fission & $4.3022 \mathrm{E}-03$ & $1.6639 \mathrm{E}-08$ & $4.3022 \mathrm{E}-03$ \\
1 & $\mathrm{u}-234$ & capture & $-4.5566 \mathrm{E}-04$ & $8.0468 \mathrm{E}-09$ & $-4.5565 \mathrm{E}-04$ \\
1 & $\mathrm{u}-234$ & n, gamma & $-4.5566 \mathrm{E}-04$ & $8.0468 \mathrm{E}-09$ & $-4.5565 \mathrm{E}-04$ \\
1 & $\mathrm{u}-234$ & nubar & $6.5581 \mathrm{E}-03$ & $0.0000 \mathrm{E}+00$ & $6.5581 \mathrm{E}-03$ \\
1 & $\mathrm{u}-234$ & chi & $1.7178 \mathrm{E}-10$ & $0.0000 \mathrm{E}+00$ & $1.7178 \mathrm{E}-10$ \\
1 & $\mathrm{u}-235$ & total & $5.8379 \mathrm{E}-01$ & $2.3611 \mathrm{E}-06$ & $5.8379 \mathrm{E}-01$ \\
1 & $\mathrm{u}-235$ & scatter & $6.5770 \mathrm{E}-02$ & $1.8838 \mathrm{E}-06$ & $6.5772 \mathrm{E}-02$ \\
1 & $\mathrm{u}-235$ & elastic & $3.1559 \mathrm{E}-02$ & $1.8623 \mathrm{E}-06$ & $3.1561 \mathrm{E}-02$ \\
1 & $\mathrm{u}-235$ & n, n' & $3.3131 \mathrm{E}-02$ & $2.1512 \mathrm{E}-08$ & $3.3131 \mathrm{E}-02$ \\
1 & $\mathrm{u}-235$ & n,2n & $1.0797 \mathrm{E}-03$ & $-1.7622 \mathrm{E}-11$ & $1.0797 \mathrm{E}-03$ \\
1 & $\mathrm{u}-235$ & fission & $5.6593 \mathrm{E}-01$ & $3.5945 \mathrm{E}-07$ & $5.6594 \mathrm{E}-01$ \\
1 & $\mathrm{u}-235$ & capture & $-4.7915 \mathrm{E}-02$ & $1.1785 \mathrm{E}-07$ & $-4.7914 \mathrm{E}-02$ \\
1 & $\mathrm{u}-235$ & n,gamma & $-4.7915 \mathrm{E}-02$ & $1.1785 \mathrm{E}-07$ & $-4.7914 \mathrm{E}-02$ \\
1 & $\mathrm{u}-235$ & nubar & $9.0395 \mathrm{E}-01$ & $0.0000 \mathrm{E}+00$ & $9.0395 \mathrm{E}-01$ \\
1 & $\mathrm{u}-235$ & chi & $-2.3539 \mathrm{E}-08$ & $0.0000 \mathrm{E}+00$ & $-2.3539 \mathrm{E}-08$ \\
1 & $\mathrm{u}-238$ & total & $8.0059 \mathrm{E}-03$ & $3.2531 \mathrm{E}-09$ & $8.0059 \mathrm{E}-03$ \\
1 & $\mathrm{u}-238$ & scatter & $4.6599 \mathrm{E}-03$ & $3.1531 \mathrm{E}-09$ & $4.6599 \mathrm{E}-03$ \\
1 & $\mathrm{u}-238$ & elastic & $2.3732 \mathrm{E}-03$ & $3.0891 \mathrm{E}-09$ & $2.3732 \mathrm{E}-03$ \\
1 & $\mathrm{u}-238$ & n,n' & $2.2134 \mathrm{E}-03$ & $5.9442 \mathrm{E}-11$ & $2.2134 \mathrm{E}-03$ \\
1 & $\mathrm{u}-238$ & n,2n & $7.2528 \mathrm{E}-05$ & $4.5161 \mathrm{E}-12$ & $7.2528 \mathrm{E}-05$ \\
1 & $\mathrm{u}-238$ & fission & $5.0328 \mathrm{E}-03$ & $9.2930 \mathrm{E}-12$ & $5.0328 \mathrm{E}-03$ \\
1 & $\mathrm{u}-238$ & capture & $-1.6868 \mathrm{E}-03$ & $9.0770 \mathrm{E}-11$ & $-1.6868 \mathrm{E}-03$
\end{tabular}

Figure 6.3.3. SAMS output for Flattop-25 sample problem. (continued 


$\begin{array}{rrlrrr}1 & \mathrm{u}-238 & \mathrm{n}, \text { gamma } & -1.6868 \mathrm{E}-03 & 9.0770 \mathrm{E}-11 & -1.6868 \mathrm{E}-03 \\ 1 & \mathrm{u}-238 & \text { nubar } & 7.6590 \mathrm{E}-03 & 0.0000 \mathrm{E}+00 & 7.6590 \mathrm{E}-03 \\ 1 & \mathrm{u}-238 & \text { chi } & -4.8497 \mathrm{E}-10 & 0.0000 \mathrm{E}+00 & -4.8497 \mathrm{E}-10\end{array}$

\begin{tabular}{|c|c|c|c|c|c|}
\hline Mixture & Nuclide & Reaction & Explicit & Implicit & Sensitivity \\
\hline 2 & -------1 & total & ----------- & $\begin{array}{l}------1--- \\
-7-0711 \mathrm{~F}-06\end{array}$ & \\
\hline & $u-235$ & total & $8.2517 \mathrm{E}-03$ & & $\begin{array}{l}8.2446 \mathrm{E}-03 \\
\end{array}$ \\
\hline $\begin{array}{l}2 \\
2\end{array}$ & $\begin{array}{l}u-235 \\
u-235\end{array}$ & $\begin{array}{l}\text { scatter } \\
\text { elastic }\end{array}$ & $\begin{array}{l}1.1604 \mathrm{E}-03 \\
8.0337 \mathrm{E}-04\end{array}$ & $\begin{array}{l}-5.5590 \mathrm{E}-06 \\
-5.5275 \mathrm{E}-06\end{array}$ & $\begin{array}{l}1.1548 \mathrm{E}-03 \\
7.9785 \mathrm{E}-04\end{array}$ \\
\hline $\begin{array}{l}2 \\
2\end{array}$ & $u-235$ & $n, n^{\prime}$ & $3.5255 \mathrm{E}-04$ & $-3.0122 \mathrm{E}-08$ & $3.5252 \mathrm{E}-04$ \\
\hline 2 & $u-235$ & $n, 2 n$ & $4.4336 \mathrm{E}-06$ & $-1.4015 E-09$ & $4.4322 E-06$ \\
\hline 2 & $u-235$ & fission & $7.7357 \mathrm{E}-03$ & $-1.1333 E-06$ & $7.7346 \mathrm{E}-03$ \\
\hline 2 & $u-235$ & capture & $-6.4439 E-04$ & $-3.7872 \mathrm{E}-07$ & $-6.4477 \mathrm{E}-04$ \\
\hline 2 & $u-235$ & $n$, gamma & $-6.4439 E-04$ & $-3.7872 \mathrm{E}-07$ & $-6.4477 E-04$ \\
\hline 2 & $u-235$ & nubar & $1.1046 \mathrm{E}-02$ & $0.0000 \mathrm{E}+00$ & $1.1046 \mathrm{E}-02$ \\
\hline 2 & $u-235$ & chi & $4.6475 \mathrm{E}-10$ & $0.0000 \mathrm{E}+00$ & $4.6475 \mathrm{E}-10$ \\
\hline 2 & $u-238$ & total & $2.0933 \mathrm{E}-01$ & $6.2348 E-05$ & $2.0939 \mathrm{E}-01$ \\
\hline 2 & $u-238$ & scatter & $2.0579 \mathrm{E}-01$ & $6.0314 \mathrm{E}-05$ & $2.0585 \mathrm{E}-01$ \\
\hline 2 & $u-238$ & elastic & $1.4133 \mathrm{E}-01$ & $5.9906 \mathrm{E}-05$ & $1.4139 \mathrm{E}-01$ \\
\hline 2 & $u-238$ & $n, n^{\prime}$ & $6.3758 \mathrm{E}-02$ & $3.8246 \mathrm{E}-07$ & $6.3758 \mathrm{E}-02$ \\
\hline 2 & $u-238$ & $n, 2 n$ & $6.9288 \mathrm{E}-04$ & $2.6325 \mathrm{E}-08$ & $6.9291 \mathrm{E}-04$ \\
\hline 2 & $u-238$ & fission & $5.0591 \mathrm{E}-02$ & $4.9503 E-08$ & $5.0591 \mathrm{E}-02$ \\
\hline 2 & $u-238$ & capture & $-4.7050 \mathrm{E}-02$ & $1.9846 \mathrm{E}-06$ & $-4.7048 E-02$ \\
\hline 2 & $u-238$ & $n$, gamma & $-4.7050 \mathrm{E}-02$ & $1.9846 \mathrm{E}-06$ & $-4.7048 \mathrm{E}-02$ \\
\hline 2 & $u-238$ & nubar & $7.0783 \mathrm{E}-02$ & $0.0000 \mathrm{E}+00$ & $7.0783 \mathrm{E}-02$ \\
\hline 2 & $u-238$ & chi & $1.2352 \mathrm{E}-09$ & $0.0000 \mathrm{E}+00$ & $1.2352 \mathrm{E}-09$ \\
\hline
\end{tabular}

Generating working covariance matrix ...

Working covariance matrix created for future processing.

Uncertainty Information

the relative standard deviation of k-eff $\left(\frac{\circ}{0}\right.$ delta-k/k) due to cross-section covariance data is:

$1.2743 \div \mathrm{delta-k/k}$

Figure 6.3.3. SAMS output for Flattop-25 sample problem. (continued) 
contributions to uncertainty in k-eff ( $\frac{0}{0}$ delta-k/k) by individual energy covariance matrices:

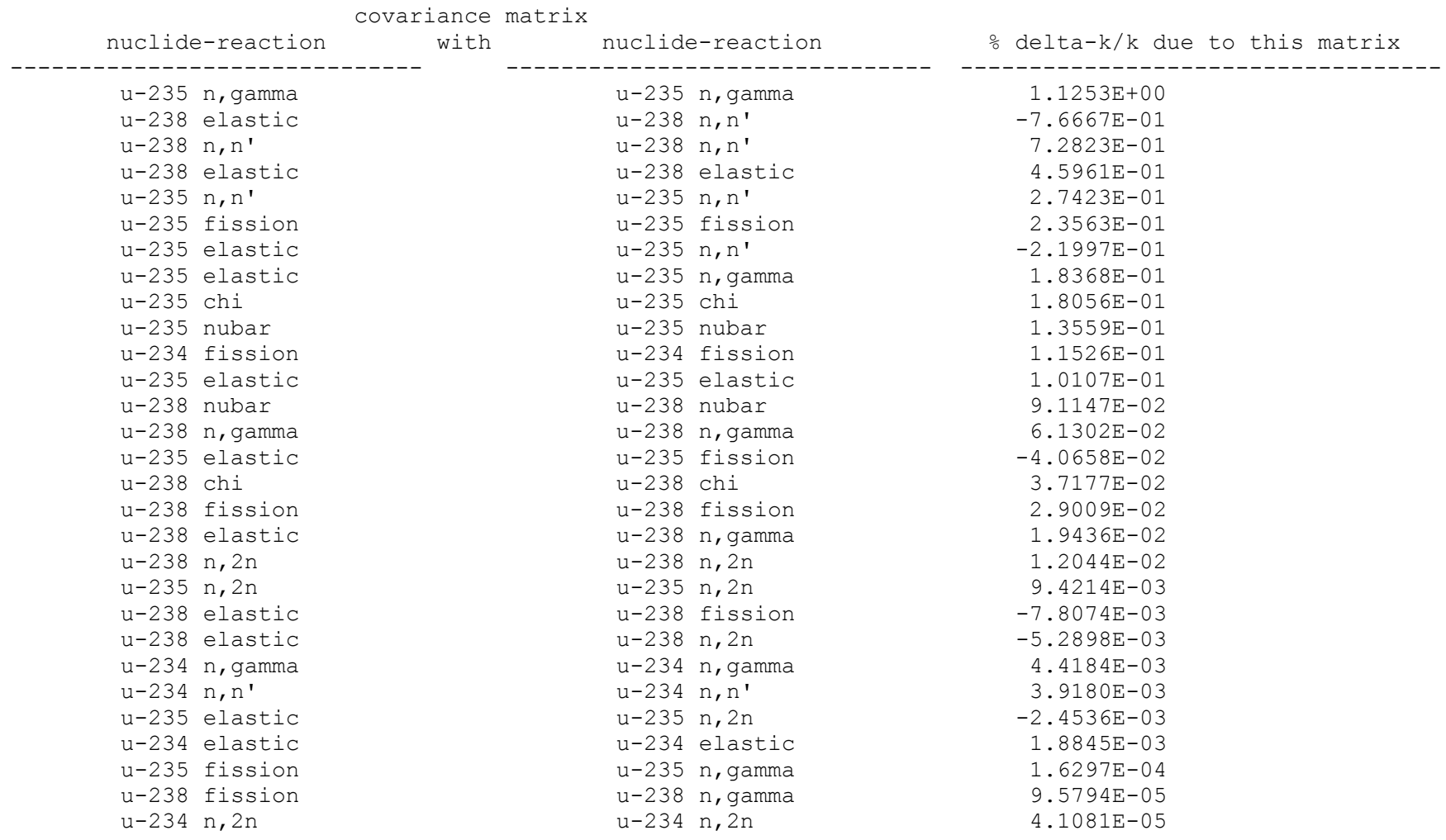

Note: relative standard deviation in k-eff can be computed from

individual values by adding the square of the values with positive signs and

subtracting the square of the values with negative signs, then taking the square root

SAMS execution complete

Elapsed time: 0.02717 minutes

Figure 6.3.3. SAMS output for Flattop-25 sample problem. (continued) 


\section{Sensitivity data file}

The energy-dependent sensitivity data computed by SAMS is written to a sensitivity data file (SDF), which SCALE returns to the same directory as the output file with the extension ".sdf". The SDF is generated directly in KENO in CE TSUNAMI-3D calculations. When XSDRNPM is used for the transport solution, the data file is presented in the TSUNAMI-A format described in Appendix A. This data file is suitable for visualization with the Fulcrum SCALE user interface or for system comparison with the TSUNAMI-IP code, which is described in section 6.5. A sample section of the TSUNAMI-A formatted sensitivity data file is show in Figure 6.3.4.

\begin{tabular}{|c|c|c|c|c|}
\hline \multicolumn{5}{|c|}{ tsunami-1d flattop } \\
\hline $238 \mathrm{nu}$ & r of neutron & coups & & \\
\hline 130 & mber of sensi & ivity profiles & 30 & are region integrated \\
\hline 1.005337 & -eff from the & forward case & & \\
\hline nergy boun & $S:$ & & & \\
\hline $2.000000 \mathrm{E}+07$ & $1.733300 \mathrm{E}+07$ & $1.568300 \mathrm{E}+07$ & $1.455000 \mathrm{E}+07$ & $1.384000 \mathrm{E}+07$ \\
\hline 1.284000E+07 & $1.000000 \mathrm{E}+07$ & $8.187300 \mathrm{E}+06$ & $6.434000 E+06$ & $4.800000 \mathrm{E}+06$ \\
\hline $4.304000 E+06$ & $3.000000 \mathrm{E}+06$ & $2.479000 \mathrm{E}+06$ & $2.354000 \mathrm{E}+06$ & $1.850000 \mathrm{E}+06$ \\
\hline $1.500000 \mathrm{E}+06$ & $1.400000 \mathrm{E}+06$ & $1.356000 \mathrm{E}+06$ & $1.317000 \mathrm{E}+06$ & $1.250000 \mathrm{E}+06$ \\
\hline $1.200000 \mathrm{E}+06$ & $1.100000 \mathrm{E}+06$ & $1.010000 \mathrm{E}+06$ & $9.200000 \mathrm{E}+05$ & $9.000000 \mathrm{E}+05$ \\
\hline $8.750000 \mathrm{E}+05$ & $8.611000 \mathrm{E}+05$ & $8.200000 \mathrm{E}+05$ & $7.500000 \mathrm{E}+05$ & $6.790000 \mathrm{E}+05$ \\
\hline $6.700000 \mathrm{E}+05$ & $6.000000 \mathrm{E}+05$ & $5.730000 \mathrm{E}+05$ & $5.500000 \mathrm{E}+05$ & $4.995200 \mathrm{E}+05$ \\
\hline $4.700000 E+05$ & $4.400000 \mathrm{E}+05$ & $4.200000 \mathrm{E}+05$ & $4.000000 E+05$ & $3.300000 \mathrm{E}+05$ \\
\hline $2.700000 E+05$ & $2.000000 \mathrm{E}+05$ & $1.500000 \mathrm{E}+05$ & $1.283000 \mathrm{E}+05$ & $1.000000 \mathrm{E}+05$ \\
\hline $8.500000 E+04$ & $8.200000 \mathrm{E}+04$ & $7.500000 \mathrm{E}+04$ & $7.300000 \mathrm{E}+04$ & $6.000000 \mathrm{E}+04$ \\
\hline $5.200000 \mathrm{E}+04$ & $5.000000 \mathrm{E}+04$ & $4.500000 \mathrm{E}+04$ & $3.000000 \mathrm{E}+04$ & $2.500000 \mathrm{E}+04$ \\
\hline $1.700000 \mathrm{E}+04$ & $1.300000 \mathrm{E}+04$ & $9.500000 \mathrm{E}+03$ & $8.030000 \mathrm{E}+03$ & $6.000000 \mathrm{E}+03$ \\
\hline $3.900000 \mathrm{E}+03$ & $3.740000 \mathrm{E}+03$ & $3.000000 \mathrm{E}+03$ & $2.580000 \mathrm{E}+03$ & $2.290000 \mathrm{E}+03$ \\
\hline $2.200000 \mathrm{E}+03$ & $1.800000 \mathrm{E}+03$ & $1.550000 \mathrm{E}+03$ & $1.500000 \mathrm{E}+03$ & $1.150000 \mathrm{E}+03$ \\
\hline $9.500000 \mathrm{E}+02$ & $6.830000 \mathrm{E}+02$ & $6.700000 \mathrm{E}+02$ & $5.500000 \mathrm{E}+02$ & $3.050000 \mathrm{E}+02$ \\
\hline $2.850000 \mathrm{E}+02$ & $2.400000 \mathrm{E}+02$ & $2.100000 \mathrm{E}+02$ & $2.075000 \mathrm{E}+02$ & 1.925000E+02 \\
\hline $1.860000 \mathrm{E}+02$ & $1.220000 \mathrm{E}+02$ & $1.190000 \mathrm{E}+02$ & $1.150000 \mathrm{E}+02$ & $1.080000 \mathrm{E}+02$ \\
\hline $1.000000 \mathrm{E}+02$ & $9.000000 \mathrm{E}+01$ & $8.200000 \mathrm{E}+01$ & $8.000000 \mathrm{E}+01$ & $7.600000 \mathrm{E}+01$ \\
\hline $7.200000 \mathrm{E}+01$ & $6.750000 \mathrm{E}+01$ & $6.500000 \mathrm{E}+01$ & $6.100000 \mathrm{E}+01$ & $5.900000 \mathrm{E}+01$ \\
\hline $5.340000 \mathrm{E}+01$ & $5.200000 \mathrm{E}+01$ & $5.060000 \mathrm{E}+01$ & $4.920000 \mathrm{E}+01$ & $4.830000 \mathrm{E}+01$ \\
\hline $4.700000 \mathrm{E}+01$ & $4.520000 \mathrm{E}+01$ & $4.400000 \mathrm{E}+01$ & $4.240000 \mathrm{E}+01$ & $4.100000 \mathrm{E}+01$ \\
\hline $3.960000 \mathrm{E}+01$ & $3.910000 \mathrm{E}+01$ & $3.800000 \mathrm{E}+01$ & $3.700000 \mathrm{E}+01$ & $3.550000 \mathrm{E}+01$ \\
\hline $3.460000 \mathrm{E}+01$ & $3.375000 \mathrm{E}+01$ & $3.325000 \mathrm{E}+01$ & $3.175000 \mathrm{E}+01$ & $3.125000 \mathrm{E}+01$ \\
\hline $3.000000 \mathrm{E}+01$ & $2.750000 \mathrm{E}+01$ & $2.500000 \mathrm{E}+01$ & $2.250000 \mathrm{E}+01$ & $2.100000 \mathrm{E}+01$ \\
\hline 2.000000E+01 & $1.900000 \mathrm{E}+01$ & $1.850000 \mathrm{E}+01$ & $1.700000 \mathrm{E}+01$ & $1.600000 \mathrm{E}+01$ \\
\hline $1.509990 \mathrm{E}+01$ & $1.440000 \mathrm{E}+01$ & $1.375000 \mathrm{E}+01$ & $1.290000 \mathrm{E}+01$ & $1.190000 \mathrm{E}+01$ \\
\hline $1.150000 \mathrm{E}+01$ & $1.000000 \mathrm{E}+01$ & $9.099990 \mathrm{E}+00$ & $8.099990 \mathrm{E}+00$ & $7.150000 \mathrm{E}+00$ \\
\hline $7.000000 \mathrm{E}+00$ & $6.750000 \mathrm{E}+00$ & $6.500000 \mathrm{E}+00$ & $6.250000 \mathrm{E}+00$ & $6.000000 \mathrm{E}+00$ \\
\hline $5.400000 \mathrm{E}+00$ & $5.000000 \mathrm{E}+00$ & $4.750000 \mathrm{E}+00$ & $4.000000 \mathrm{E}+00$ & $3.730000 \mathrm{E}+00$ \\
\hline $3.500000 \mathrm{E}+00$ & $3.150000 \mathrm{E}+00$ & $3.049990 \mathrm{E}+00$ & $3.000000 \mathrm{E}+00$ & $2.969990 \mathrm{E}+00$ \\
\hline $2.870000 \mathrm{E}+00$ & $2.770000 \mathrm{E}+00$ & $2.669990 \mathrm{E}+00$ & $2.570000 \mathrm{E}+00$ & $2.469990 \mathrm{E}+00$ \\
\hline $2.379990 \mathrm{E}+00$ & $2.299990 \mathrm{E}+00$ & $2.209990 \mathrm{E}+00$ & $2.120000 \mathrm{E}+00$ & $2.000000 \mathrm{E}+00$ \\
\hline $1.940000 \mathrm{E}+00$ & $1.860000 \mathrm{E}+00$ & $1.770000 \mathrm{E}+00$ & $1.679990 \mathrm{E}+00$ & $1.589990 \mathrm{E}+00$ \\
\hline $1.500000 \mathrm{E}+00$ & $1.450000 \mathrm{E}+00$ & 1. $400000 \mathrm{E}+00$ & $1.349990 \mathrm{E}+00$ & $1.299990 \mathrm{E}+00$ \\
\hline $1.250000 \mathrm{E}+00$ & $1.224990 \mathrm{E}+00$ & $1.200000 \mathrm{E}+00$ & $1.174990 \mathrm{E}+00$ & $1.150000 \mathrm{E}+00$ \\
\hline $1.139990 \mathrm{E}+00$ & $1.129990 \mathrm{E}+00$ & $1.120000 \mathrm{E}+00$ & $1.110000 \mathrm{E}+00$ & $1.099990 \mathrm{E}+00$ \\
\hline $1.089990 \mathrm{E}+00$ & $1.080000 \mathrm{E}+00$ & $1.070000 \mathrm{E}+00$ & $1.059990 \mathrm{E}+00$ & $1.049990 \mathrm{E}+00$ \\
\hline $1.040000 \mathrm{E}+00$ & $1.030000 \mathrm{E}+00$ & $1.020000 \mathrm{E}+00$ & $1.009990 \mathrm{E}+00$ & $1.000000 \mathrm{E}+00$ \\
\hline $9.750000 \mathrm{E}-01$ & $9.500000 \mathrm{E}-01$ & $9.250000 \mathrm{E}-01$ & $9.000000 \mathrm{E}-01$ & $8.500000 \mathrm{E}-01$ \\
\hline $8.000000 E-01$ & $7.500000 \mathrm{E}-01$ & $7.000000 \mathrm{E}-01$ & $6.500000 \mathrm{E}-01$ & $6.250000 \mathrm{E}-01$ \\
\hline $6.000000 \mathrm{E}-01$ & $5.500000 \mathrm{E}-01$ & $5.000000 \mathrm{E}-01$ & $4.500000 \mathrm{E}-01$ & $4.000000 \mathrm{E}-01$ \\
\hline $3.750000 \mathrm{E}-01$ & $3.500000 \mathrm{E}-01$ & $3.250000 \mathrm{E}-01$ & $3.000000 \mathrm{E}-01$ & $2.750000 \mathrm{E}-01$ \\
\hline $2.500000 \mathrm{E}-01$ & $2.250000 \mathrm{E}-01$ & $2.000000 \mathrm{E}-01$ & $1.750000 \mathrm{E}-01$ & $1.500000 \mathrm{E}-01$ \\
\hline 1.250000E-01 & $1.000000 \mathrm{E}-01$ & $9.000000 \mathrm{E}-02$ & $8.000000 \mathrm{E}-02$ & 7. $000000 \mathrm{E}-02$ \\
\hline $6.000000 \mathrm{E}-02$ & $5.000000 \mathrm{E}-02$ & $4.000000 \mathrm{E}-02$ & $3.000000 \mathrm{E}-02$ & $2.530000 \mathrm{E}-02$ \\
\hline $1.000000 \mathrm{E}-02$ & $7.500000 \mathrm{E}-03$ & $5.000000 \mathrm{E}-03$ & $4.000000 \mathrm{E}-03$ & $3.000000 \mathrm{E}-03$ \\
\hline $2.500000 \mathrm{E}-03$ & $2.000000 \mathrm{E}-03$ & $1.500000 \mathrm{E}-03$ & $1.200000 \mathrm{E}-03$ & $1.000000 \mathrm{E}-03$ \\
\hline $7.500000 \mathrm{E}-04$ & $5.000000 \mathrm{E}-04$ & $1.000000 \mathrm{E}-04$ & $1.000000 \mathrm{E}-05$ & \\
\hline$u-234$ & tal & 92234 & 0.000 & \\
\hline
\end{tabular}

Figure 6.3.4. Sample of sensitivity data file for Flattop-25 sample problem. 


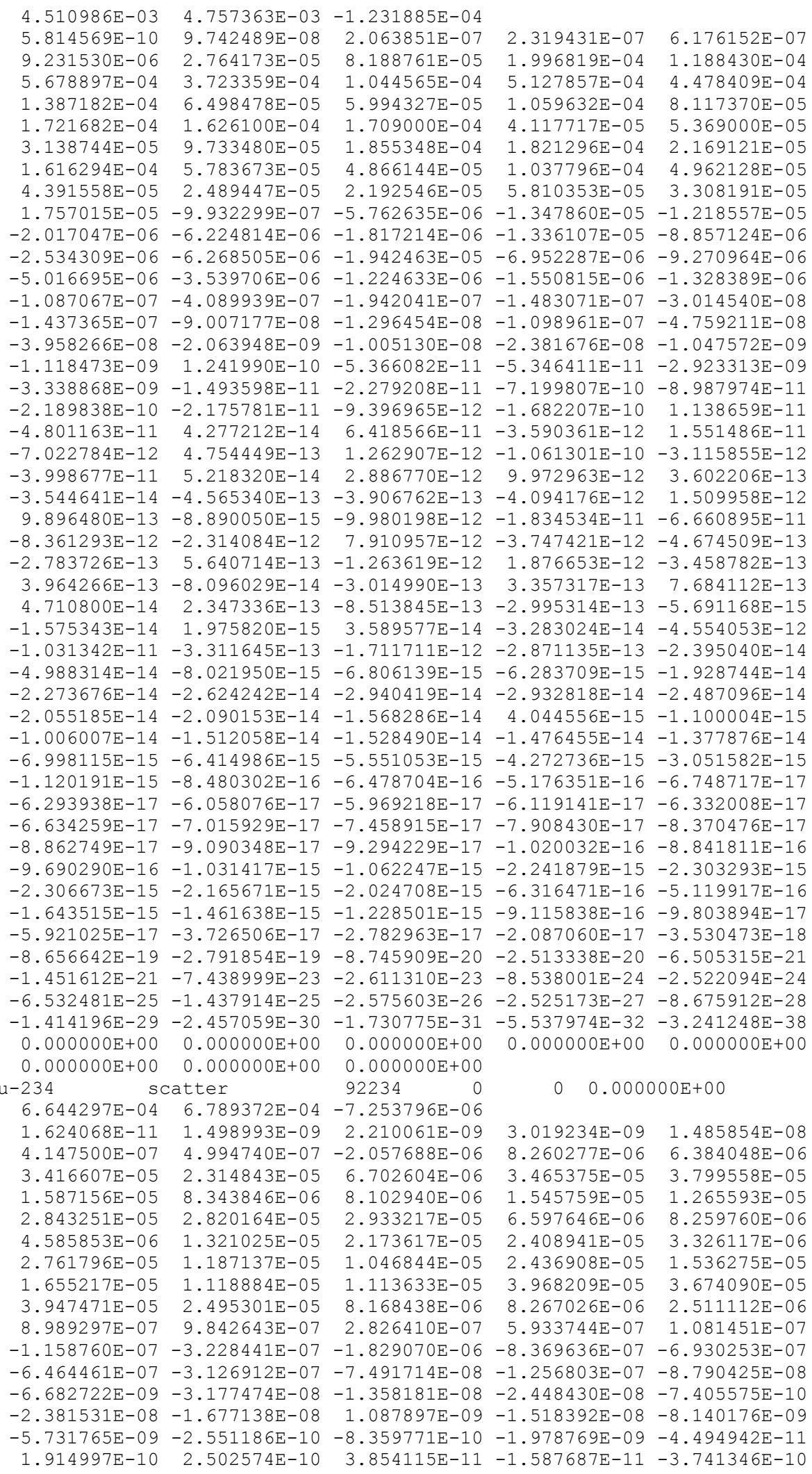

Figure 6.3.4. Sample of sensitivity data file for Flattop-25 sample problem. (continued) 


\begin{tabular}{|c|c|c|c|c|}
\hline & & & & \\
\hline & & & & \\
\hline$-4.719829 E-11$ & 1. $913950 \mathrm{E}-13$ & $6.404752 \mathrm{E}-11$ & $486064 \mathrm{E}-12$ & 1. \\
\hline$-6.699422 E-12$ & $604126 \mathrm{E}-13$ & $181300 \mathrm{E}-12$ & $-2.223242 E-12$ & 4. \\
\hline $5680 E-13$ & $69277 \mathrm{E}-13$ & $106 E-12$ & $39 \mathrm{E}$ & 4. \\
\hline $746 \mathrm{E}-14$ & $-4.034699 E-13$ & $-3.260798 E-13$ & $-3.978481 E-12$ & 1.4 \\
\hline $468 E-13$ & $34317 \mathrm{E}$ & $-7.658717 \mathrm{E}$ & & 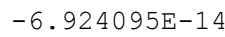 \\
\hline $5669 \mathrm{E}-12$ & $119092 \mathrm{E}-12$ & $89 E-12$ & $189 \mathrm{E}-12$ & -4 \\
\hline$E-13$ & תים & -1.20 & 2 & -3 \\
\hline $594 E-13$ & $50363 E-14$ & $-2.712372 E-13$ & $388504 \mathrm{E}-13$ & 7.4 \\
\hline-13 & 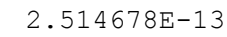 & -7 & -6 & -1 \\
\hline $4347 E-15$ & $90198 E-15$ & $2.890316 \mathrm{E}-14$ & -15 & -2.3 \\
\hline-14 & 9 & 1. & & -9 \\
\hline $\mathrm{E}-16$ & $94 E-16$ & -2.43 & $E-16$ & $-1 \cdot 1$ \\
\hline-1 & 3. & -7 & -5 & -7 \\
\hline$E-16$ & $9 E-15$ & -2.4 & 15 & 1 . \\
\hline$E-17$ & -5 & -5 & -5 & -5 \\
\hline-2 . & $0 E-16$ & -2 & -1 & -4 \\
\hline$E-17$ & -2 . & -2 & & -4 \\
\hline$E-18$ & $E-18$ & -4 & - & -5.2 \\
\hline$E-18$ & -5 & -6 & - & -7 \\
\hline$E-18$ & $3 E-18$ & -7 & $-\varepsilon$ & -2 \\
\hline$E-17$ & $E-17$ & $E-17$ & - & -5 . \\
\hline $2 E-17$ & $3 E-17$ & $21 E-17$ & -2 & -2 \\
\hline $1 E-17$ & $5 E-17$ & $E-17$ & -8 & -3 \\
\hline$E-18$ & $6 E-19$ & $1 E-19$ & -3 & -1 \\
\hline $3 E-20$ & $6 \mathrm{E}-20$ & $2 E-21$ & -1 & -2 \\
\hline$E-23$ & $12 E-24$ & $2 E-25$ & -2 & -6.3 \\
\hline-1 & -2 & $5 E-28$ & -3 & -2.3 \\
\hline-6 & $8 E-33$ & -8 & -3 & $E+00$ \\
\hline $\mathrm{E}+00$ & $\mathrm{OE}+\mathrm{OC}$ & $\mathrm{E}+00$ & $0 E+00$ & $E+00$ \\
\hline $0 E+00$ & $00 E+0$ & $\mathrm{OE}+0$ & & \\
\hline
\end{tabular}

Figure 6.3.4. Sample of sensitivity data file for Flattop-25 sample problem. (continued)

Sample plots of the energy-dependent sensitivity profiles from the SDF are shown below. Figures 6.3.5 and 6.3.6 depict the region-dependent sensitivity profiles for ${ }^{235} U$ fission and ${ }^{238} U$ capture, respectively, in zones 1 and 2 of the Flattop-25 sample problem, which are the core and the reflector regions, respectively. 


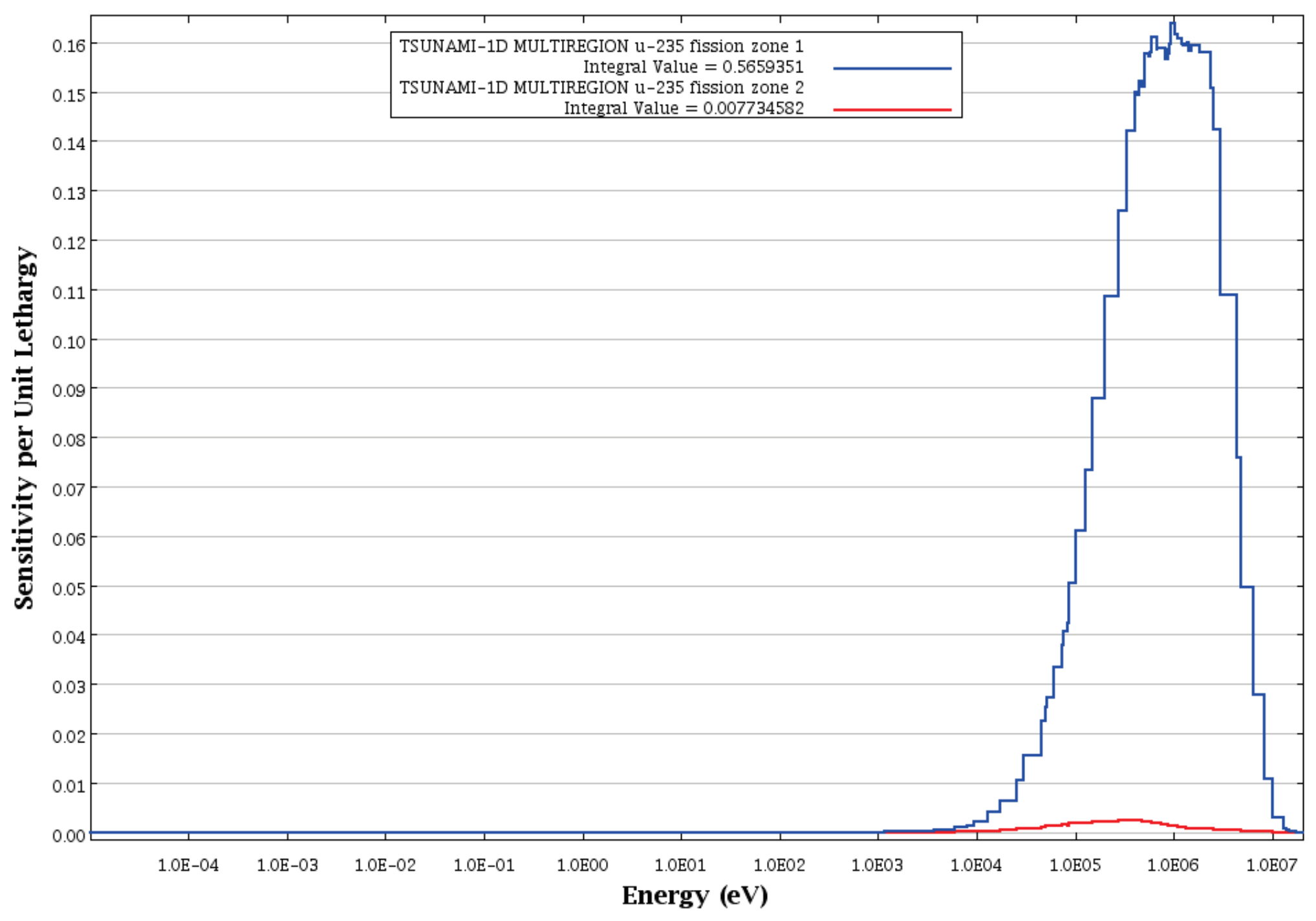

Figure 6.3.5. Region-dependent fission sensitivity profiles for Flattop-25 sample problem. 


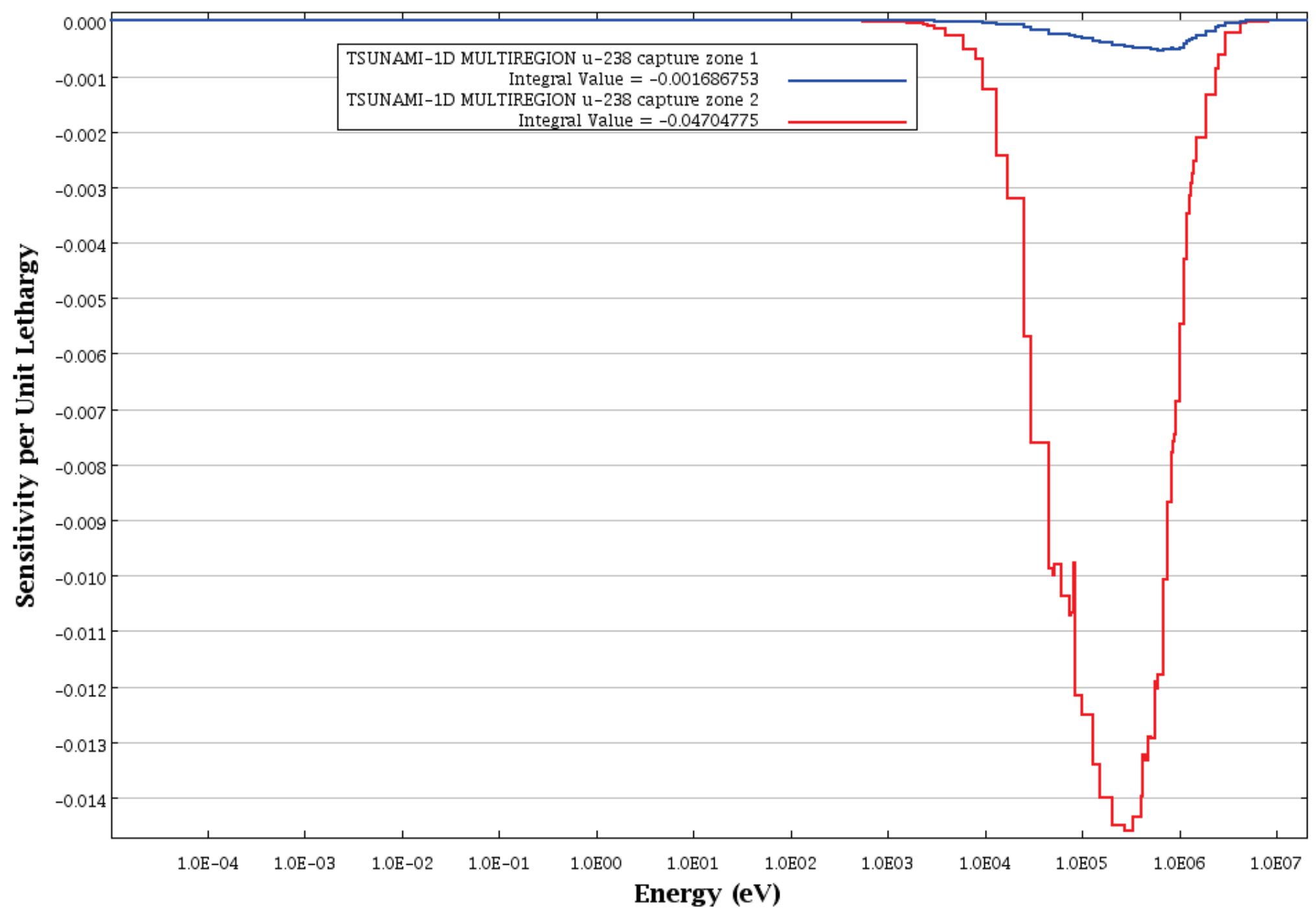

Figure 6.3.6. Region-dependent capture sensitivity profiles for Flattop-25 sample problem. 


\section{HTML formatted output}

SAMS will, by default, present the sensitivity results in HTML formatted output for interactive viewing in a web browser. SCALE will return an HTML file to the users' directory with the same name as the output file, but with the extension ".html". Opening this file in a web browser will allow the user to begin viewing the output. Other files necessary for the HTML output are stored in the ".htmd" directory associated with the output file and some utilities for data visualization are stored in the applet resources directory, which is created in the same directory as the output file.

When the .html file is opened, an index page like the one in Figure 6.3 .7 will be shown. In this example, only one SCALE module with HTML output, SAMS, was executed from the input file. If more than one set of HTML output was available, additional modules would be listed on this page. To begin viewing the HTML output, click on the word SAMS in the middle of the page. Once SAMS is selected, the program verification information page is displayed, as shown in Figure 6.3.8. The output data is sorted into four sections, three of which are shown on the left side of the window in this example. General Information consists of the Program Verification Information and the Elapsed Time for program execution. Input Data lists data input to the calculation including user input and data read from the required forward and adjoint transport calculations. Results contains the computed results, and an additional Messages section, not shown, would contain any error or warning messages generated during the SAMS calculation.

Clicking on the Input Data menu item reveals the menu of pages containing input data. Clicking on the Input Parameters item reveals the page shown in Figure 6.3.9. Clicking on the Summary of Transport Calculations item reveals the page shown in Figure 6.3.10.

Clicking on the Results menu item reveals the results available for viewing. Clicking on Energy, Region and Mixture Integrated Sensitivity Coefficients for this Problem under the results menu reveals the energy-, region- and mixture integrated sensitivity coefficients for this problem, as shown in Figure 6.3.11. As with most of the tables in the HTML output, the table of sensitivity coefficients on this page can be sorted in ascending and descending order by clicking on the heading of the column for which the sorting is desired. When clicking twice on the sensitivity column, the sensitivity results are sorted in descending order as shown in Figure 6.3.12.

Skipping several output edits for brevity and selecting the Sensitivity Data Plot item will open a page with an applet version of Javapeño with the sensitivity data file preloaded as shown in Figure 6.3.13. To create a plot of sensitivity data, double-click on the desired information on the right side of the window. Multiple items can be simultaneously selected in the list using standard platform specific modifier keys such as shift or control. Right-clicking on an item in the list will present a menu with various options for plotting. For more information on the use of Javapeño, please select Help from the Javapeño help menu. 


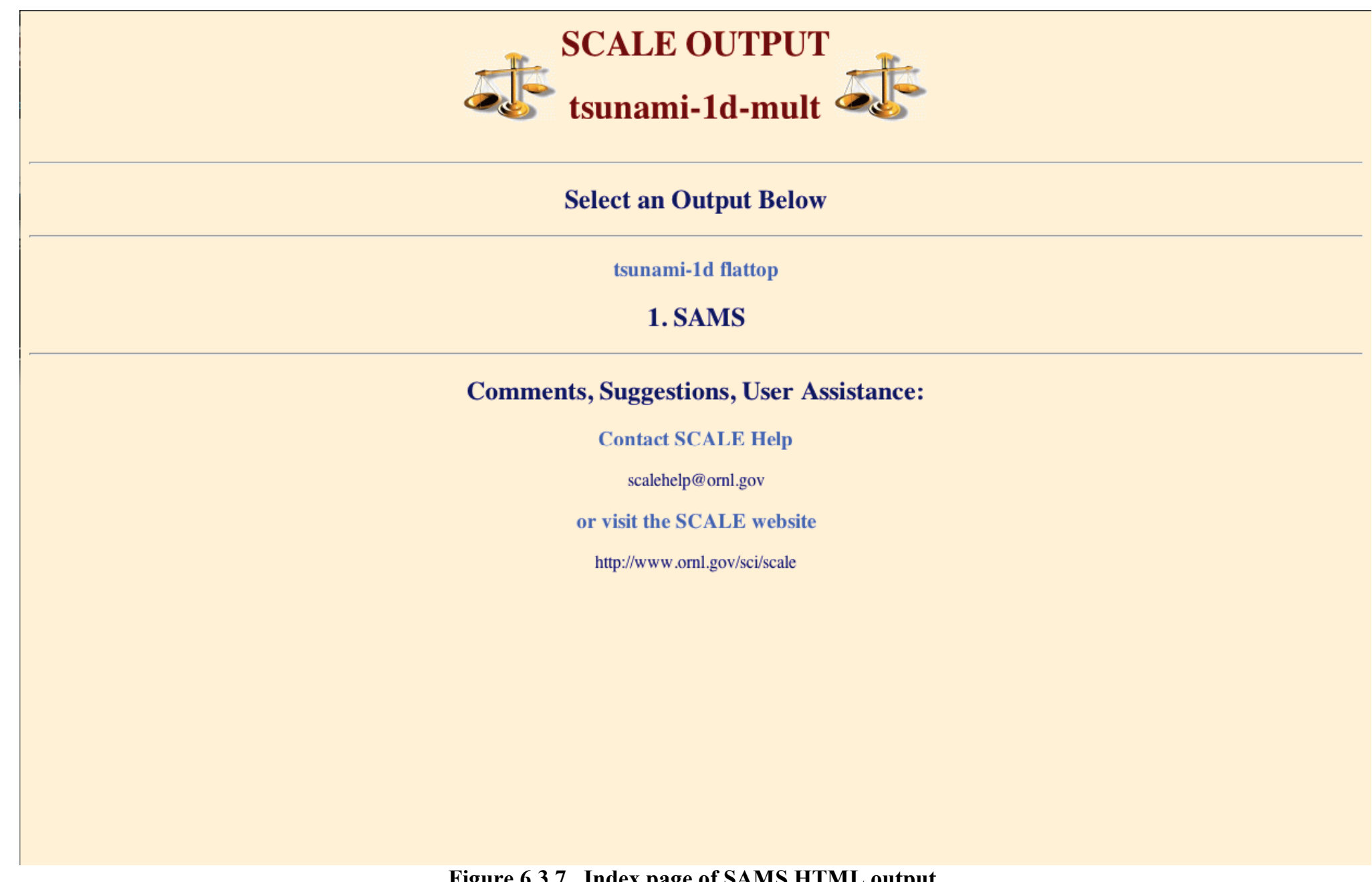

Figure 6.3.7. Index page of SAMS HTML output. 


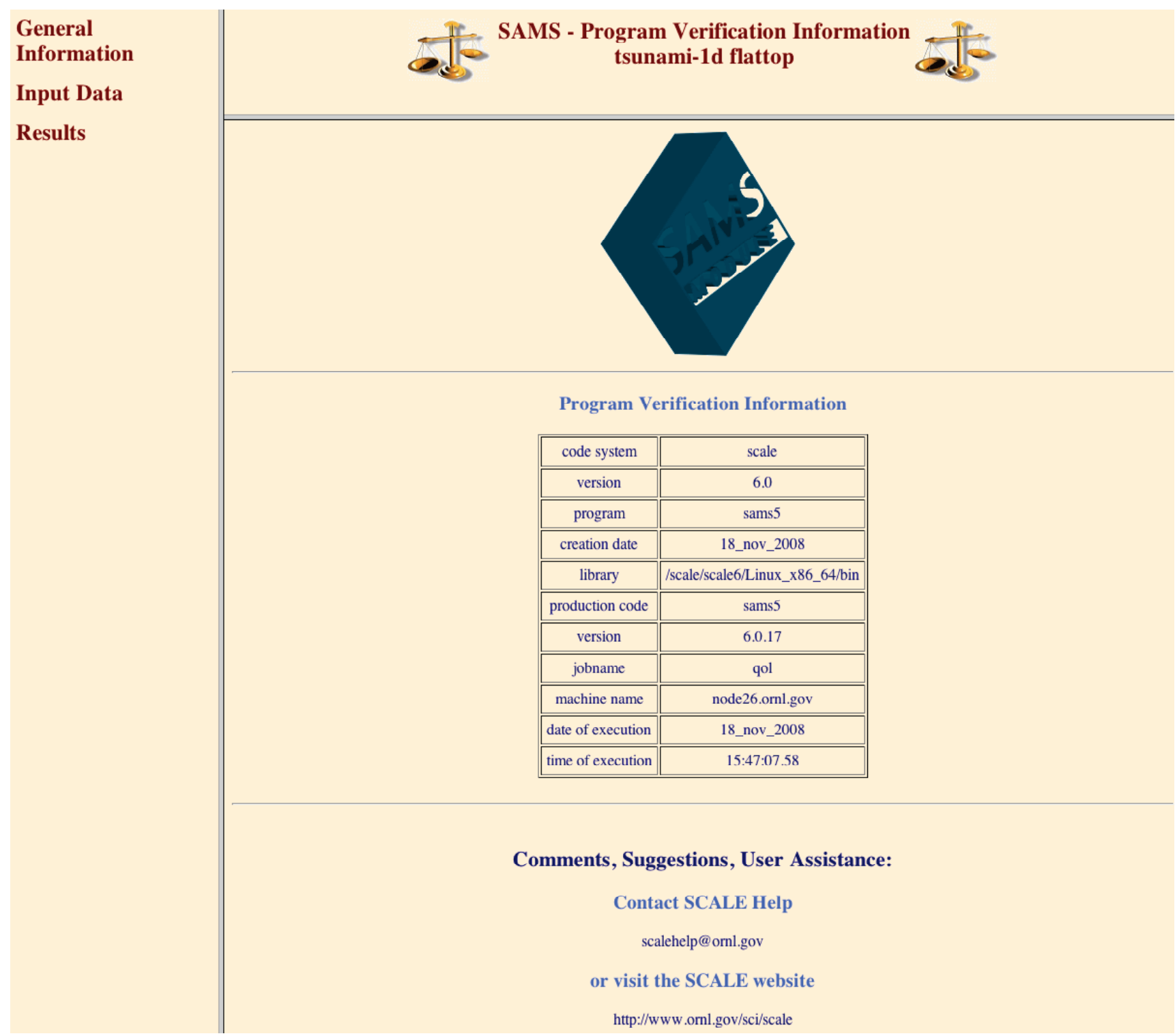

Figure 6.3.8. Program verification page of SAMS HTML output. 


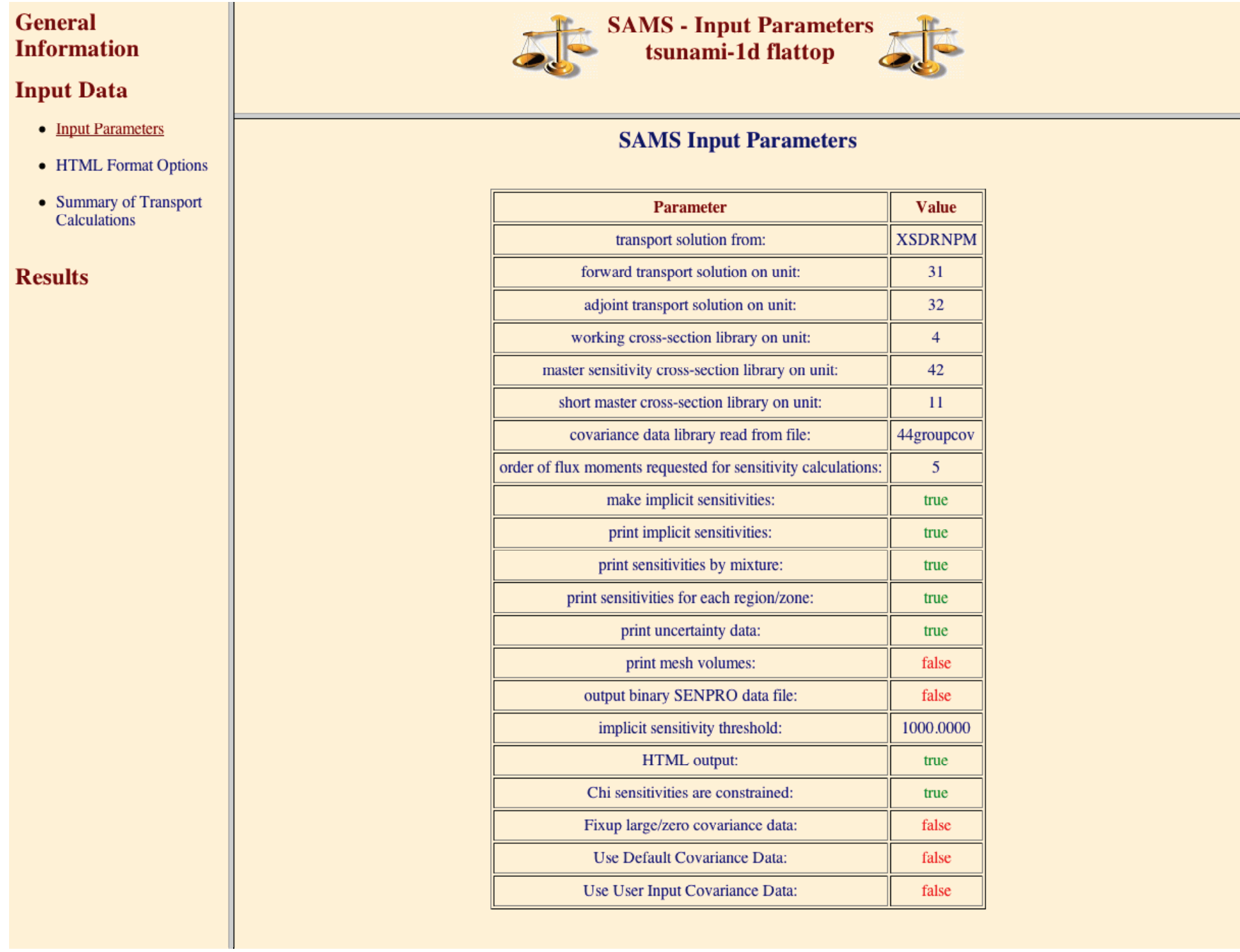

Figure 6.3.9. Input parameters from SAMS HTML output. 

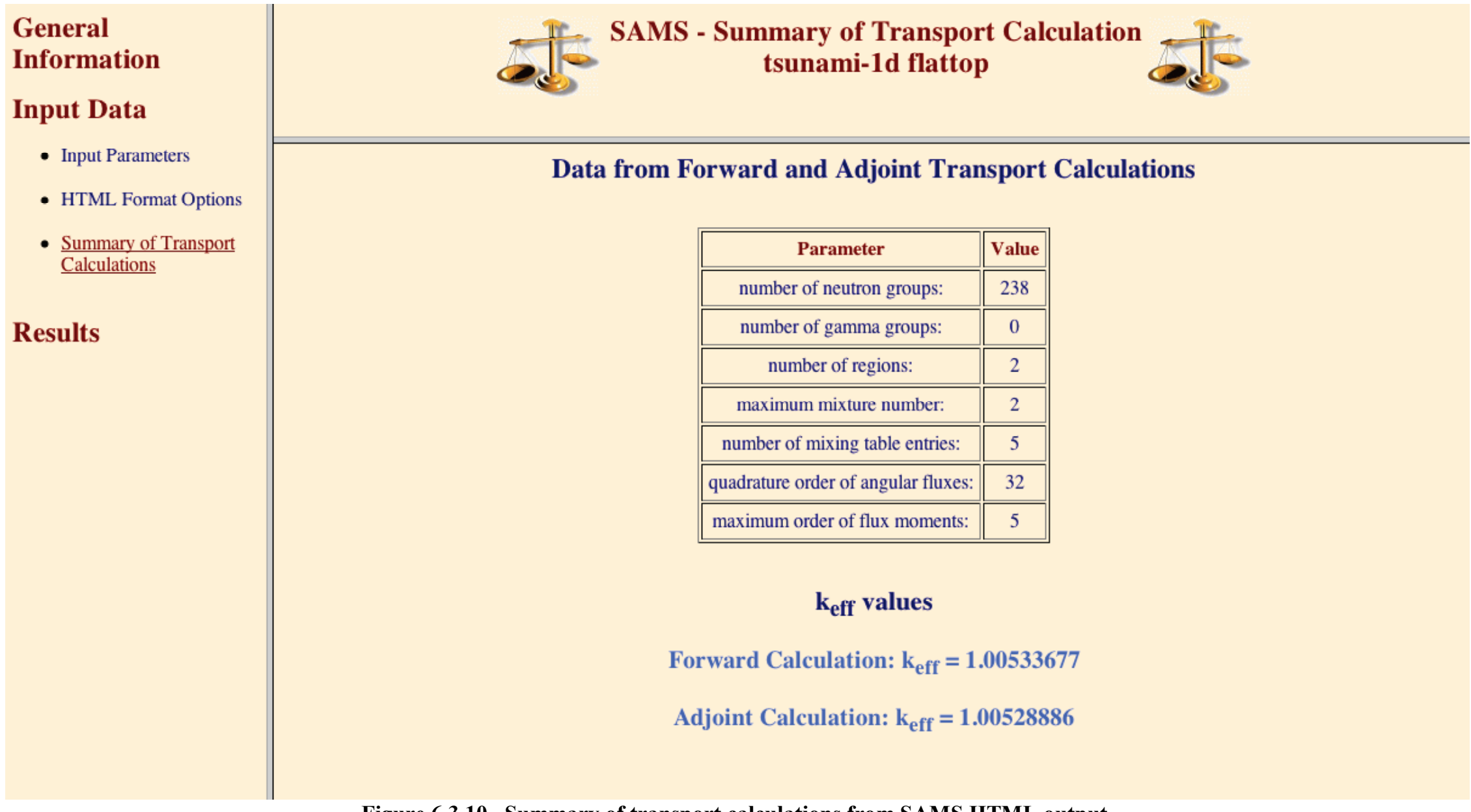

Figure 6.3.10. Summary of transport calculations from SAMS HTML output. 


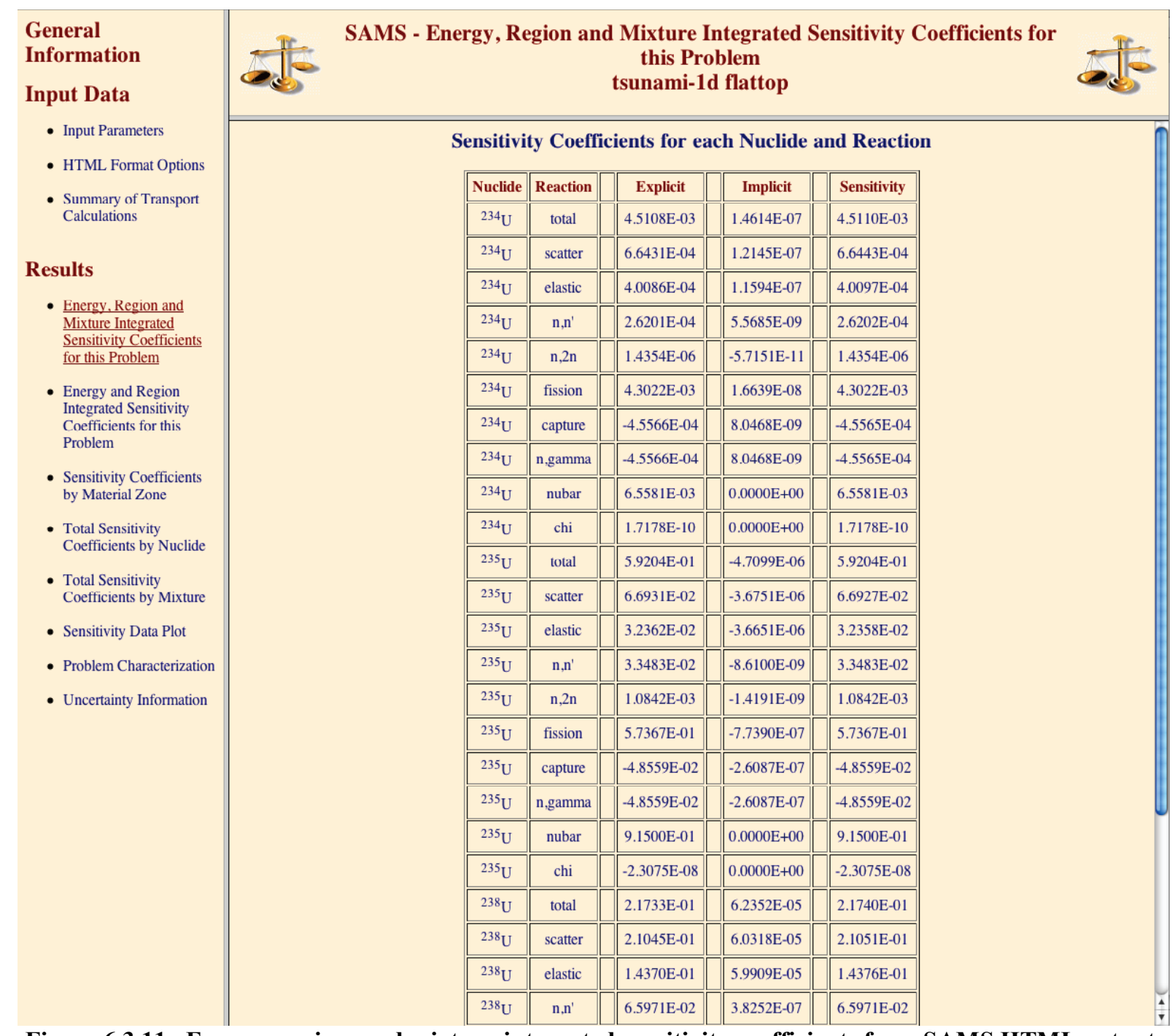

Figure 6.3.11. Energy- region- and mixture-integrated sensitivity coefficients from SAMS HTML output. 


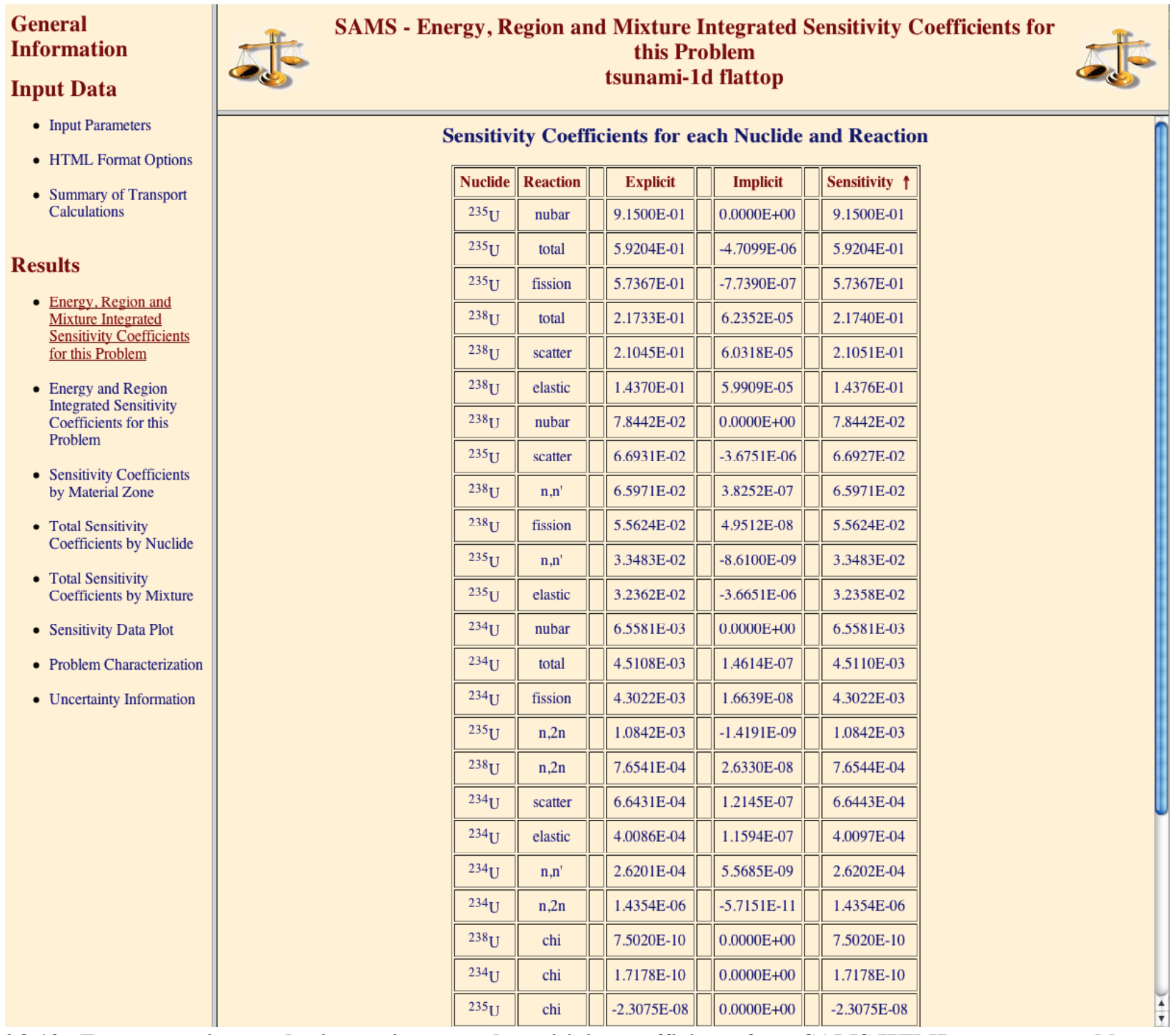

Figure 6.3.12. Energy- region- and mixture-integrated sensitivity coefficients from SAMS HTML output sorted by sensitivity. 


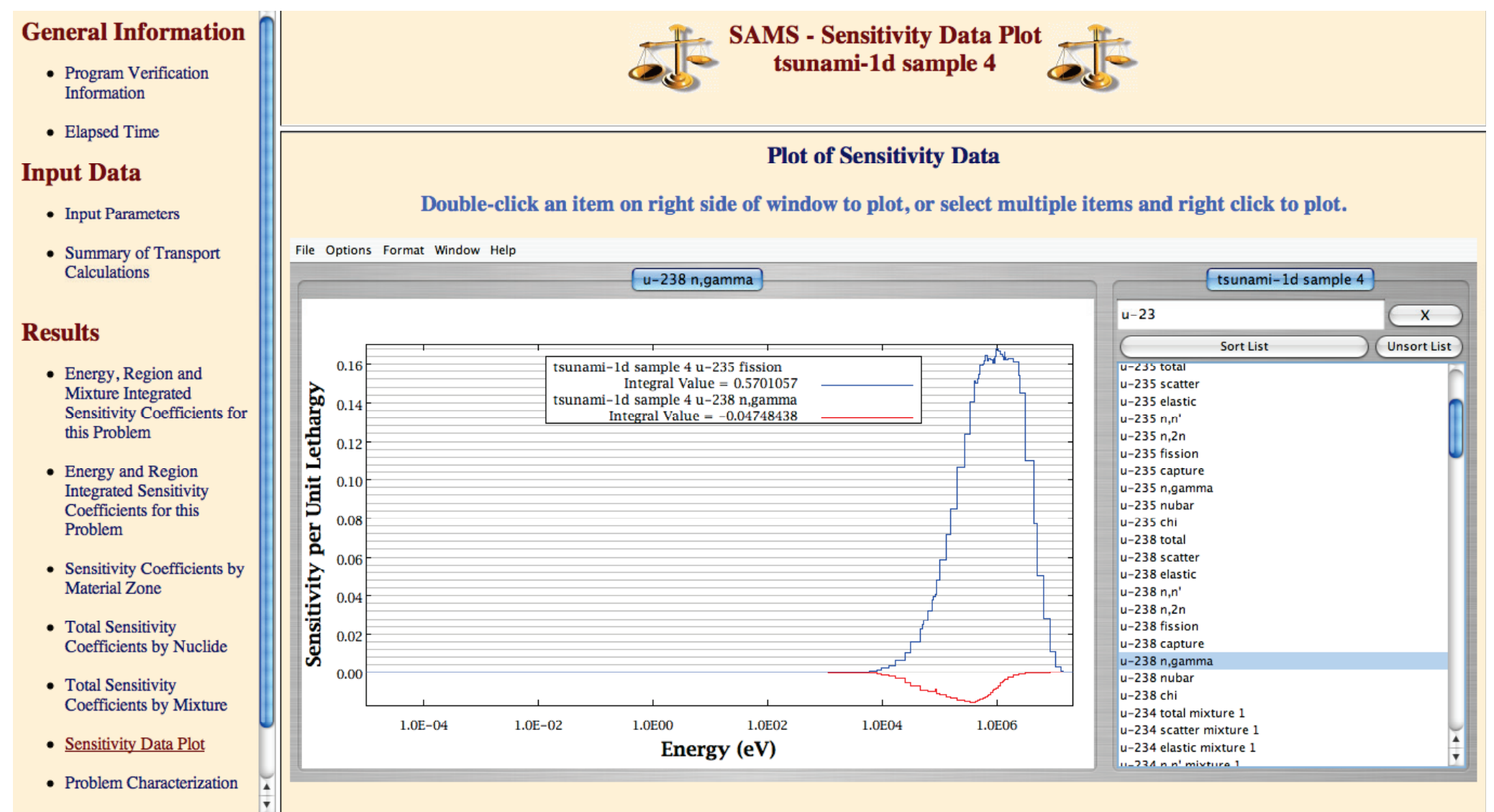

Figure 6.3.13. Sensitivity data plot from SAMS HTML output. 


\subsubsection{Example problem using KENO V.a}

This sample problem is critical experiment number 10 from evaluation LEU-COMP-THERM-009 of the ICSBEP Handbook. ${ }^{14}$ The critical assembly consists of three $8 \times 15$ fuel-rod arrays separated by 6.46-mm thick copper plates. The $\mathrm{U}(4.31) \mathrm{O}_{2}$ fuel rods are $92.075-\mathrm{cm}$ long. A KENO3D rendering of the KENO V.a geometry model with the water moderator removed is shown in Figure 6.3.14.

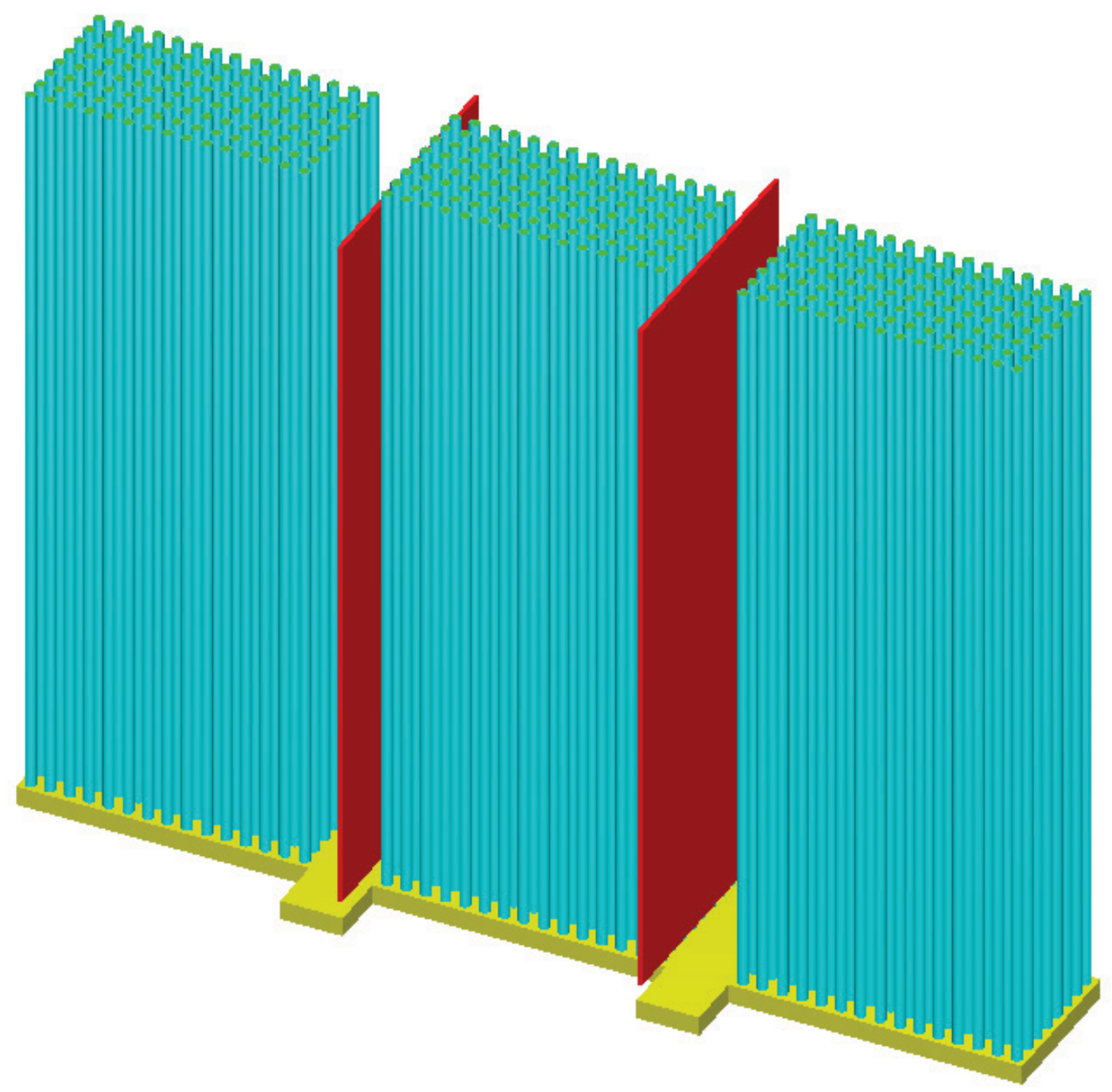

Figure 6.3.14. KENO3D rendering of KENO V.a model of LEU-COMP-THERM-009 case 10.

Prior to executing SAMS, the data files listed in Table 6.3.4 were generated for this system. It is recommended that users perform sensitivity analyses on 3-D systems using the TSUNAMI-3D control module, which executes the appropriate codes to generate the required data files in an automated manner from a single input. SAMS is executed as a part of the TSUNAMI-3D control sequence. However, given appropriate data files SAMS can be run as a stand-alone program. 
Input data and text output

The SAMS input is shown in Figure 6.3.15 and the output is shown in Figure 6.3.16. Each section of the output is described below. Note that output shown in this section is intended only to represent the format of output that the user will encounter. The actual computed results may vary.

\section{Parameter Table and Summary of Transport Calculation}

In the output listing, the SAMS Parameter Table gives the values of the SAMS input data. Next, the title from the KENO V.a input file is given and a brief summary of the transport solution is given.

2. Energy-, Region-, and Mixture Integrated Sensitivity Coefficients

Next the energy-, region-, and mixture integrated sensitivity coefficients are given for each reaction of each nuclide. The nuclide symbol and reaction name are given. The data given in the Sensitivity column represents the expected change in $k_{\text {eff }}$ of this system due to a uniform fractional change in the cross section of the given nuclide and reaction in all energy groups for all regions and mixtures that contain this nuclide. The standard deviations represent stochastic uncertainties from the Monte Carlo calculation. For example, a uniform fractional change of 0.01 (or $1 \%$ ) in the fission cross section of ${ }^{235} \mathrm{U}$ would produce a fractional change in $k_{\text {eff }}$ of $3.2433 \times 10^{-1} \times 0.01$ or $3.2433 \times 10^{-3}(0.32433 \% \Delta \mathrm{k} / \mathrm{k})$. Because the keyword PRTIMP was not entered in the SAMS input, only the complete sensitivity would be given in the Sensitivity column. If PRTIMP was entered, the explicit, implicit and complete sensitivity coefficients are listed in separate columns. Region-integrated sensitivity profiles for ${ }^{1} \mathrm{H}$ elastic scattering, ${ }^{238} \mathrm{U}$ capture and ${ }^{235} \mathrm{U}$ fission are shown in Figure 6.3.17.

3. Energy- and Region-Integrated Sensitivity Coefficients

Next the energy- and region-integrated sensitivity coefficients are given for each reaction of each nuclide. The mixture number, nuclide symbol and reaction name are given. The data given in the Sensitivity column represents the expected change in $k_{\text {eff }}$ of this system due to a uniform fractional change in the cross section of the given nuclide and reaction in all energy groups for all regions that contain the particular mixture. The standard deviations represent stochastic uncertainties from the Monte Carlo calculation. Because the keyword PRTIMP was not entered in the SAMS input, only the complete sensitivity would be given in the Sensitivity column. If PRTIMP was entered the explicit, implicit and complete sensitivity coefficients are listed in separate columns.

\section{Total Sensitivity Coefficients by Nuclide}

The next section of the output summarizes the total sensitivity by nuclide. The sensitivity values given here are the same as the values given in the previous section for the sensitivity of $k_{\text {eff }}$ to the total cross section. Also included in this edit are the atom densities for each nuclide in each mixture.

\section{Total Sensitivity Coefficients by Mixture}

The next output edit gives the total sensitivity of $k_{\text {eff }}$ to the mixture. Here, the sensitivity of $k_{\text {eff }}$ to the total cross section is summed over each nuclide in a given mixture. 


\section{Problem Characterization (MG Calculations Only)}

The next output edit is for the problem characterization data. This section includes the median neutron energy group causing fission, capture and scattering, average group for fission, capture and scattering, the average energy causing fission, capture and scattering, and the energy of the average lethargy causing fission, capture and scattering.

\section{Sensitivity Coefficients by Region}

If the keyword, PRTGEOM was entered in the SAMS input, the next output edit would give the energy-integrated sensitivity coefficients for each region of each unit defined in the KENO V.a geometry input. Note that for brevity, this output edit was not requested.

\section{Uncertainty Information}

The next output edit contains the uncertainty information. First, the percent relative standard deviation in $k_{\text {eff }}\left(\Delta k_{\text {eff }} k_{\text {eff }} \times 100 \%\right)$ due to cross-section-covariance data is given. Next a listing of the uncertainty terms as produced by Eq. (6.3.59) are given. The nuclide-reaction to nuclidereaction covariance matrix responsible for the uncertainty contribution is given followed by the contribution to the uncertainty in terms of $\% \Delta \mathrm{k} / \mathrm{k}$. The total uncertainty can be computed from individual values by adding the square of the values with positive signs and subtracting the square of the values with negative signs, then taking the square root. The negative values are the result of anti-correlations in the cross-section-covariance data.

\section{Execution Complete}

The final edit produced simply states that the execution is complete and gives the elapsed time.

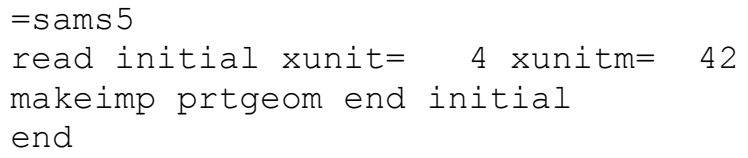

Figure 6.3.15. SAMS input LEU-COMP-THERM-009 case 10 sample problem. 


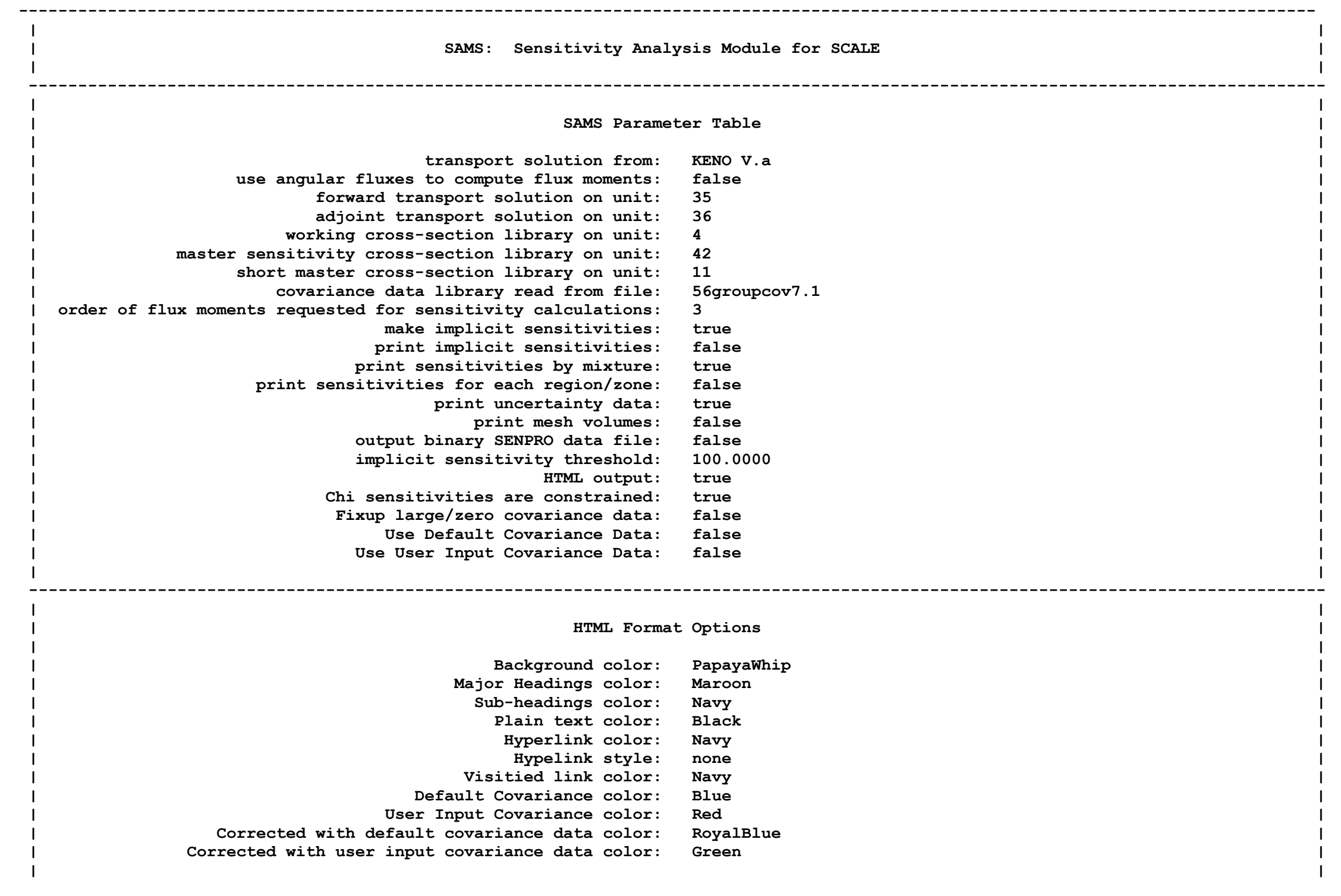

Figure 6.3.16. SAMS output for LEU-COMP-THERM-009 case 10 sample problem. 


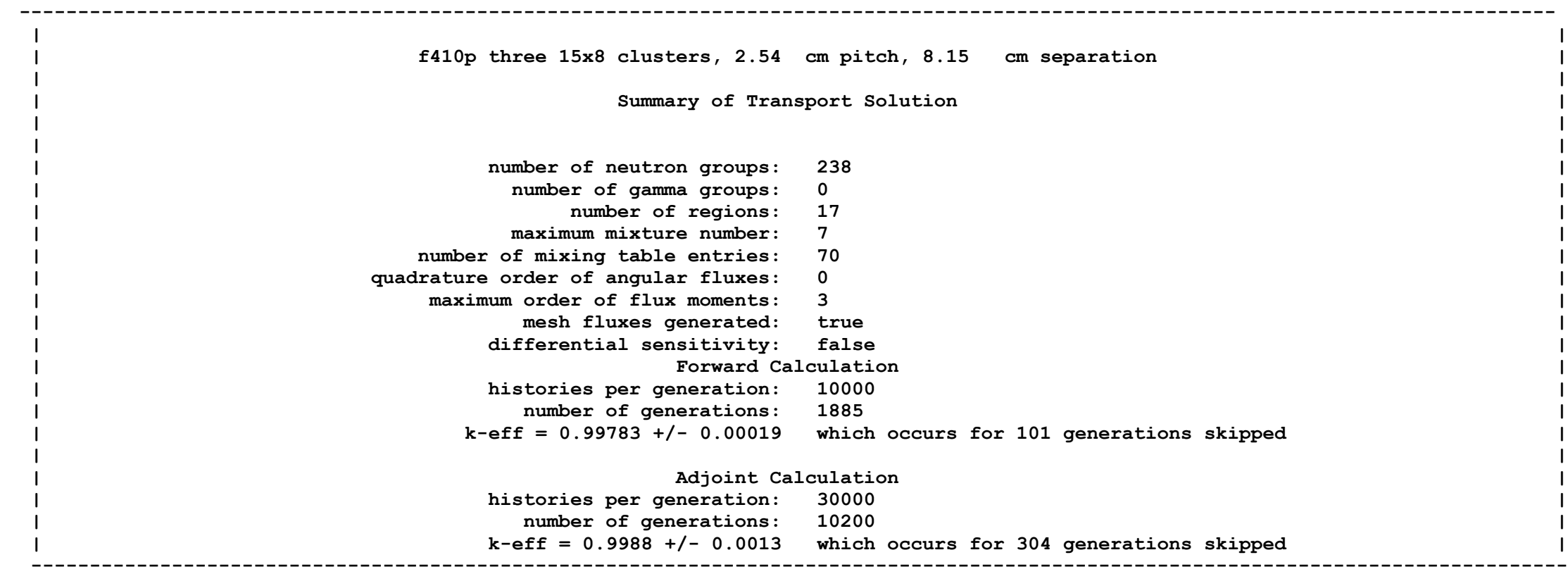

Energy, Region and Mixture Integrated Sensitivity Coefficients for this Problem

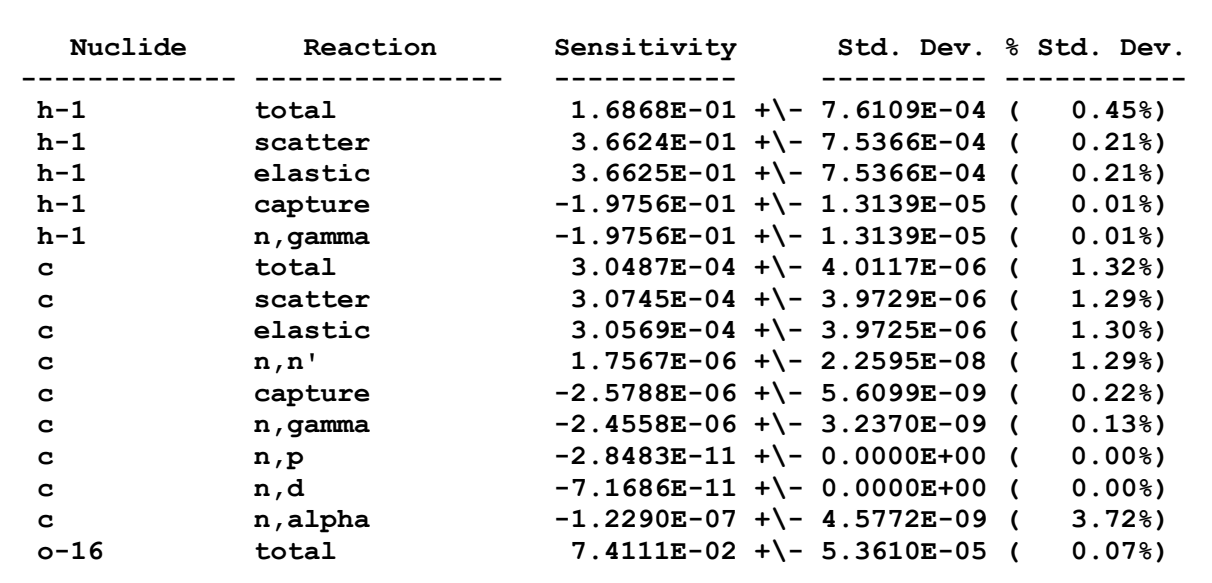

Figure 6.3.16. SAMS output for LEU-COMP-THERM-009 case 10 sample problem. (continued) 


\begin{tabular}{|c|c|c|c|c|}
\hline $0-16$ & scatter & $7.5947 \mathrm{E}-02$ & $+\backslash-5.3599 \mathrm{E}-05$ & $0.07 \%)$ \\
\hline $0-16$ & elastic & $7.5552 \mathrm{E}-02$ & $+\backslash-5.3596 \mathrm{E}-05$ & $0.07 \%)$ \\
\hline $0-16$ & $\mathrm{n}, \mathrm{n}^{\prime}$ & $3.8299 \mathrm{E}-04$ & $+1-2.5943 \mathrm{E}-07$ & $0.07 \%)$ \\
\hline $0-16$ & $n, 2 n$ & $3.7303 E-11$ & $+\backslash-1.5211 \mathrm{E}-17$ & $0.00 \div)$ \\
\hline $0-16$ & capture & $-1.8367 \mathrm{E}-03$ & $+1-2.2526 \mathrm{E}-07$ & $0.01 \%)$ \\
\hline $0-16$ & $\mathrm{n}$, gamma & $-6.6536 \mathrm{E}-05$ & $+\backslash-3.9010 \mathrm{E}-09$ & $0.01 \%)$ \\
\hline $0-16$ & $\mathrm{n}, \mathrm{p}$ & $-8.9997 E-06$ & $+1-8.7659 \mathrm{E}-09$ & $0.10 \%)$ \\
\hline $0-16$ & $\mathrm{n}, \mathrm{d}$ & $-9.2619 \mathrm{E}-07$ & $+1-1.7089 \mathrm{E}-09$ & $0.18 \%)$ \\
\hline $0-16$ & $n, t$ & $-3.3277 \mathrm{E}-12$ & $+1-2.7563 \mathrm{E}-18$ & $0.00 \%)$ \\
\hline $0-16$ & n, alpha & $-1.7602 \mathrm{E}-03$ & $+\backslash-2.2246 \mathrm{E}-07$ & $0.01 \%)$ \\
\hline na-23 & total & $2.3175 \mathrm{E}-08$ & $+\backslash-1.4447 \mathrm{E}-09$ & $6.23 \%)$ \\
\hline na-23 & scatter & $5.7809 \mathrm{E}-08$ & $+\backslash-1.4371 \mathrm{E}-09$ & $2.49 \%)$ \\
\hline na-23 & elastic & $5.2606 \mathrm{E}-08$ & $+\backslash-1.4371 \mathrm{E}-09$ & $2.73 \%)$ \\
\hline na-23 & $\mathrm{n}, \mathrm{n}^{\prime}$ & $5.2023 E-09$ & $+\backslash-7.8251 \mathrm{E}-15$ & $0.00 \%)$ \\
\hline na-23 & $\mathrm{n}, 2 \mathrm{n}$ & $4.5318 E-13$ & $+1-0.0000 \mathrm{E}+00$ & $0.00 \%)$ \\
\hline na-23 & capture & $-3.4634 \mathrm{E}-08$ & $+\backslash-2.0188 \mathrm{E}-11$ & $0.06 \%)$ \\
\hline na-23 & $\mathrm{n}$, gamma & $-3.4409 E-08$ & $+\backslash-2.0188 \mathrm{E}-11$ & $0.06 \%)$ \\
\hline na-23 & $\mathrm{n}, \mathrm{p}$ & $-1.5160 \mathrm{E}-10$ & $+\backslash-3.2974 \mathrm{E}-18$ & $0.00 \%)$ \\
\hline na-23 & n,alpha & $-7.2671 \mathrm{E}-11$ & $+\backslash-5.0907 \mathrm{E}-18$ & $0.00 \%)$ \\
\hline $\mathrm{mg}-24$ & total & $4.5199 \mathrm{E}-05$ & $+1-4.5339 \mathrm{E}-07$ & $1.00 \%)$ \\
\hline $\mathrm{mg}-24$ & scatter & $5.5375 \mathrm{E}-05$ & $+\backslash-4.5356 \mathrm{E}-07$ & $0.82 \%)$ \\
\hline $\mathrm{mg}-24$ & elastic & $4.3580 \mathrm{E}-05$ & $+\backslash-4.5024 \mathrm{E}-07$ & $1.03 \%)$ \\
\hline $\mathrm{mg}-24$ & $\mathrm{n}, \mathrm{n}^{\prime}$ & $1.1783 \mathrm{E}-05$ & $+1-3.3434 \mathrm{E}-08$ & $0.28 \%)$ \\
\hline $\mathrm{mg}-24$ & $n, 2 n$ & $5.8604 \mathrm{E}-13$ & $+\backslash-1.0107 \mathrm{E}-22$ & $0.00 \%)$ \\
\hline $\mathrm{mg}-24$ & capture & $-1.0176 \mathrm{E}-05$ & $+1-3.6669 \mathrm{E}-09$ & $0.04 \%)$ \\
\hline $\mathrm{mg}-24$ & $\mathrm{n}$, gamma & $-9.1064 \mathrm{E}-06$ & $+1-3.1240 \mathrm{E}-09$ & $0.03 \%)$ \\
\hline $\mathrm{mg}-24$ & $\mathrm{n}, \mathrm{p}$ & $-2.5472 E-07$ & $+\backslash-5.7541 \mathrm{E}-10$ & $0.23 \%)$ \\
\hline $\mathrm{mg}-24$ & n, alpha & $-8.1482 E-07$ & $+\backslash-1.3622 \mathrm{E}-09$ & $0.17 \%)$ \\
\hline $\mathrm{mg}-25$ & total & $1.2162 \mathrm{E}-06$ & $+1-3.2733 \mathrm{E}-08$ & $2.69 \%)$ \\
\hline$m g-25$ & scatter & $5.8494 \mathrm{E}-06$ & $+1-3.1369 \mathrm{E}-08$ & $0.54 \%)$ \\
\hline$m g-25$ & elastic & $4.2267 \mathrm{E}-06$ & $+1-3.0490 \mathrm{E}-08$ & $0.72 \%)$ \\
\hline $\mathrm{mg}-25$ & $\mathrm{n}, \mathrm{n}^{\prime}$ & $1.5857 \mathrm{E}-06$ & $+\backslash-3.7904 \mathrm{E}-09$ & $0.24 \%)$ \\
\hline $\mathrm{mg}-25$ & $n, 2 n$ & $3.6950 \mathrm{E}-08$ & $+\backslash-9.6404 \mathrm{E}-11$ & $0.26 \%)$ \\
\hline $\mathrm{mg}-25$ & capture & $-4.6331 \mathrm{E}-06$ & $+\backslash-1.5204 \mathrm{E}-09$ & $0.03 \%)$ \\
\hline $\mathrm{mg}-25$ & $\mathrm{n}$, gamma & $-4.2713 E-06$ & $+\backslash-1.4848 \mathrm{E}-09$ & $0.03 \%)$ \\
\hline$m g-25$ & $\mathrm{n}, \mathrm{p}$ & $-3.2385 E-08$ & $+\backslash-3.3458 \mathrm{E}-11$ & $0.10 \%)$ \\
\hline $\mathrm{mg}-25$ & n,alpha & $-3.2950 E-07$ & $+1-2.8796 \mathrm{E}-10$ & $0.09 \%)$ \\
\hline$m g-26$ & total & $5.3028 \mathrm{E}-06$ & $+1-3.2698 \mathrm{E}-08$ & $0.62 \%)$ \\
\hline$m g-26$ & scatter & $6.2567 \mathrm{E}-06$ & $+1-3.2364 \mathrm{E}-08$ & $0.52 \%)$ \\
\hline$m g-26$ & elastic & $4.8131 \mathrm{E}-06$ & $+\backslash-3.1809 \mathrm{E}-08$ & $0.66 \%)$ \\
\hline$m g-26$ & $\mathrm{n}, \mathrm{n}^{\prime}$ & $1.4401 \mathrm{E}-06$ & $+1-3.4023 \mathrm{E}-09$ & $0.24 \%)$ \\
\hline$m g-26$ & $n, 2 n$ & $3.6129 \mathrm{E}-09$ & $+1-2.0677 \mathrm{E}-18$ & $0.00 \%)$ \\
\hline$m g-26$ & capture & $-9.5390 \mathrm{E}-07$ & $+1-3.2481 \mathrm{E}-10$ & $0.03 \%)$ \\
\hline $\mathrm{mg}-26$ & $\mathrm{n}$, gamma & $-9.4936 \mathrm{E}-07$ & $+1-3.2481 \mathrm{E}-10$ & $0.03 \%)$ \\
\hline$m g-26$ & $\mathrm{n}, \mathrm{p}$ & $-5.2412 \mathrm{E}-10$ & $+\backslash-1.6179 \mathrm{E}-19$ & $0.00 \%)$ \\
\hline$m g-26$ & n, alpha & $-4.0217 \mathrm{E}-09$ & $+\backslash-5.7744 \mathrm{E}-19$ & $0.00 \%)$ \\
\hline al-27 & total & $-6.7582 E-05$ & $+1-2.3201 \mathrm{E}-05$ & $34.33 \%)$ \\
\hline al-27 & scatter & $4.6080 \mathrm{E}-03$ & $-2.2663 E-05$ & $0.49 \%)$ \\
\hline
\end{tabular}

Figure 6.3.16. SAMS output for LEU-COMP-THERM-009 case 10 sample problem. (continued) 


\begin{tabular}{|c|c|c|c|c|}
\hline al-27 & elastic & $3.2847 \mathrm{E}-03$ & $+\backslash-2.1856 \mathrm{E}-05$ & $0.67 \%)$ \\
\hline al-27 & $\mathrm{n}, \mathrm{n}^{\prime}$ & $1.3189 \mathrm{E}-03$ & $+1-3.0167 \mathrm{E}-06$ & $0.23 \%)$ \\
\hline al-27 & $\mathrm{n}, 2 \mathrm{n}$ & $5.9944 \mathrm{E}-08$ & $+\backslash-1.4100 \mathrm{E}-09$ & $2.35 \%)$ \\
\hline al-27 & capture & $-4.6756 E-03$ & $+1-1.6107 \mathrm{E}-06$ & $0.03 \%)$ \\
\hline al-27 & $\mathrm{n}$, gamma & $-4.5949 E-03$ & $+\backslash-1.6079 \mathrm{E}-06$ & $0.03 \%)$ \\
\hline al-27 & $\mathrm{n}, \mathrm{p}$ & $-6.7911 \mathrm{E}-05$ & $+1-6.9477 \mathrm{E}-08$ & $0.10 \%)$ \\
\hline al-27 & $\mathrm{n}, \mathrm{d}$ & $-1.9733 E-07$ & $+\backslash-1.4218 \mathrm{E}-09$ & $0.72 \%)$ \\
\hline al-27 & $n, t$ & $-6.6132 E-09$ & $+\backslash-5.0053 E-16$ & $0.00 \%)$ \\
\hline al-27 & n, alpha & $-1.2531 \mathrm{E}-05$ & $+1-3.2986 \mathrm{E}-08$ & $0.26 \%)$ \\
\hline si-28 & total & $4.6433 E-06$ & $+1-1.2249 \mathrm{E}-07$ & $2.64 \%)$ \\
\hline si-28 & scatter & $2.3947 E-05$ & $+\backslash-1.2025 \mathrm{E}-07$ & $0.50 \%)$ \\
\hline si-28 & elastic & $1.9104 \mathrm{E}-05$ & $+\backslash-1.1760 \mathrm{E}-07$ & $0.62 \%)$ \\
\hline si-28 & $\mathrm{n}, \mathrm{n}^{\prime}$ & $4.8407 E-06$ & $+\backslash-1.3004 \mathrm{E}-08$ & $0.27 \%)$ \\
\hline si-28 & capture & $-1.9303 E-05$ & $+\backslash-6.5597 \mathrm{E}-09$ & $0.03 \%)$ \\
\hline si-28 & $\mathrm{n}$, gamma & $-1.8340 E-05$ & $+\backslash-6.3655 \mathrm{E}-09$ & $0.03 \%)$ \\
\hline si-28 & $\mathrm{n}, \mathrm{p}$ & $-6.6369 E-07$ & $+1-1.0533 \mathrm{E}-09$ & $0.16 \%)$ \\
\hline si-28 & $n, d$ & $-8.9840 \mathrm{E}-10$ & $+\backslash-1.1828 \mathrm{E}-18$ & $0.00 \%)$ \\
\hline si-28 & n, alpha & $-2.9909 E-07$ & $+1-5.3824 \mathrm{E}-10$ & $0.18 \%)$ \\
\hline si-29 & total & $5.5254 \mathrm{E}-07$ & $+\backslash-5.6554 \mathrm{E}-09$ & $1.02 \%)$ \\
\hline si-29 & scatter & $1.2730 \mathrm{E}-06$ & $+1-4.0360 \mathrm{E}-09$ & $0.32 \%)$ \\
\hline si-29 & elastic & $8.2855 \mathrm{E}-07$ & $+1-3.6454 \mathrm{E}-09$ & $0.44 \%)$ \\
\hline si-29 & $\mathrm{n}, \mathrm{n}^{\prime}$ & $4.4317 \mathrm{E}-07$ & $+\backslash-9.9759 \mathrm{E}-10$ & $0.23 \%)$ \\
\hline si-29 & $\mathrm{n}, 2 \mathrm{n}$ & $1.2964 \mathrm{E}-09$ & $+1-8.4820 \mathrm{E}-19$ & $0.00 \%)$ \\
\hline si-29 & capture & $-7.2047 \mathrm{E}-07$ & $+1-2.3029 \mathrm{E}-10$ & $0.03 \%)$ \\
\hline si-29 & $\mathrm{n}$, gamma & $-6.7259 E-07$ & $+1-2.2493 \mathrm{E}-10$ & $0.03 \%)$ \\
\hline si-29 & $\mathrm{n}, \mathrm{p}$ & $-1.7997 \mathrm{E}-08$ & $+\backslash-1.7962 \mathrm{E}-11$ & $0.10 \%)$ \\
\hline si-29 & n, alpha & $-2.9884 \mathrm{E}-08$ & $+1-2.2339 \mathrm{E}-11$ & $0.07 \%)$ \\
\hline si-30 & total & $1.4343 \mathrm{E}-07$ & $+\backslash-4.4925 \mathrm{E}-09$ & $3.13 \%)$ \\
\hline si-30 & scatter & $7.5256 \mathrm{E}-07$ & $+1-3.2216 \mathrm{E}-09$ & $0.43 \%)$ \\
\hline si-30 & elastic & $5.6016 \mathrm{E}-07$ & $+\backslash-3.1391 \mathrm{E}-09$ & $0.56 \%)$ \\
\hline si-30 & $\mathrm{n}, \mathrm{n}^{\prime}$ & $1.9152 \mathrm{E}-07$ & $+1-4.2667 \mathrm{E}-10$ & $0.22 \%)$ \\
\hline si-30 & $n, 2 n$ & $8.8318 E-10$ & $+\backslash-5.2234 \mathrm{E}-19$ & $0.00 \%)$ \\
\hline si-30 & capture & $-6.0913 E-07$ & $+\backslash-8.1712 \mathrm{E}-10$ & $0.13 \div)$ \\
\hline si-30 & $\mathrm{n}$, gamma & $-6.0862 E-07$ & $+\backslash-8.1712 \mathrm{E}-10$ & $0.13 \%)$ \\
\hline si-30 & $\mathrm{n}, \mathrm{p}$ & $-9.3872 E-11$ & $+\backslash-1.2377 \mathrm{E}-19$ & $0.00 \%)$ \\
\hline si-30 & n,alpha & $-4.1605 E-10$ & $+1-3.2227 \mathrm{E}-19$ & $0.00 \%)$ \\
\hline$s-32$ & total & $-9.4450 E-07$ & $+1-1.5023 \mathrm{E}-08$ & $1.59 \%)$ \\
\hline$s-32$ & scatter & $1.1163 \mathrm{E}-06$ & $+\backslash-1.1617 \mathrm{E}-08$ & $1.04 \%)$ \\
\hline$s-32$ & elastic & $1.0496 \mathrm{E}-06$ & $+1-1.1393 \mathrm{E}-08$ & $1.09 \%)$ \\
\hline$s-32$ & $\mathrm{n}, \mathrm{n}^{\prime}$ & $6.6511 \mathrm{E}-08$ & $+1-8.6196 \mathrm{E}-10$ & $1.30 \%)$ \\
\hline$s-32$ & $n, 2 n$ & $1.0953 \mathrm{E}-14$ & $+1-0.0000 \mathrm{E}+00$ & $0.00 \%)$ \\
\hline$s-32$ & capture & $-2.0608 \mathrm{E}-06$ & $+1-4.4706 \mathrm{E}-09$ & $0.22 \%)$ \\
\hline$s-32$ & $\mathrm{n}$, gamma & $-1.9066 \mathrm{E}-06$ & $+\backslash-4.2718 \mathrm{E}-09$ & $0.22 \%)$ \\
\hline$s-32$ & $\mathrm{n}, \mathrm{p}$ & $-4.8964 \mathrm{E}-08$ & $+1-5.3423 \mathrm{E}-10$ & $1.09 \%)$ \\
\hline$s-32$ & n,alpha & $-1.0530 \mathrm{E}-07$ & $+1-5.3326 \mathrm{E}-10$ & $0.51 \%)$ \\
\hline$s-33$ & total & $-1.2276 \mathrm{E}-08$ & $+1-2.3332 \mathrm{E}-14$ & $0.00 \%)$ \\
\hline$s-33$ & scatter & $8.0190 \mathrm{E}-09$ & $+\backslash-2.1686 \mathrm{E}-14$ & $0.00 \%)$ \\
\hline$s-33$ & elastic & $7.2275 E-09$ & $+\backslash-2.1662 \mathrm{E}-14$ & $0.00 \%)$ \\
\hline
\end{tabular}

Figure 6.3.16. SAMS output for LEU-COMP-THERM-009 case 10 sample problem. (continued) 


\begin{tabular}{|c|c|c|c|c|}
\hline$s-33$ & $\mathrm{n}, \mathrm{n}^{\prime}$ & $7.8504 \mathrm{E}-10$ & $+\backslash-1.7509 \mathrm{E}-16$ & $0.00 \%)$ \\
\hline$s-33$ & $\mathrm{n}, 2 \mathrm{n}$ & $5.0222 \mathrm{E}-12$ & $+1-2.0474 \mathrm{E}-20$ & $0.00 \%)$ \\
\hline$s-33$ & capture & $-2.0295 E-08$ & $+\backslash-1.6612 \mathrm{E}-15$ & $0.00 \%)$ \\
\hline$s-33$ & $\mathrm{n}$, gamma & $-9.9685 E-09$ & $+1-3.3428 \mathrm{E}-17$ & $0.00 \%)$ \\
\hline$s-33$ & $\mathrm{n}, \mathrm{p}$ & $-4.7345 E-10$ & $+\backslash-1.0045 \mathrm{E}-16$ & $0.00 \%)$ \\
\hline$s-33$ & n, alpha & $-9.8528 \mathrm{E}-09$ & $+\backslash-1.6143 \mathrm{E}-15$ & $0.00 \%)$ \\
\hline$s-34$ & total & $1.2906 \mathrm{E}-08$ & $+1-3.9241 \mathrm{E}-10$ & $3.04 \%)$ \\
\hline$s-34$ & scatter & $4.8732 \mathrm{E}-08$ & $+\backslash-1.6902 \mathrm{E}-10$ & $0.35 \%)$ \\
\hline$s-34$ & elastic & $4.4628 \mathrm{E}-08$ & $+\backslash-1.1281 \mathrm{E}-10$ & $0.25 \%)$ \\
\hline$s-34$ & $\mathrm{n}, \mathrm{n}^{\prime}$ & $4.0885 E-09$ & $+1-7.8533 \mathrm{E}-16$ & $0.00 \%)$ \\
\hline$s-34$ & $n, 2 n$ & $6.7127 \mathrm{E}-12$ & $+\backslash-1.8198 \mathrm{E}-20$ & $0.00 \%)$ \\
\hline$s-34$ & capture & $-3.5826 E-08$ & $+1-6.1670 \mathrm{E}-11$ & $0.17 \%)$ \\
\hline$s-34$ & $\mathrm{n}$, gamma & $-3.5723 E-08$ & $+\backslash-6.1670 \mathrm{E}-11$ & $0.17 \%)$ \\
\hline$s-34$ & $\mathrm{n}, \mathrm{p}$ & $-1.9406 \mathrm{E}-11$ & $+\backslash-1.4282 \mathrm{E}-18$ & $0.00 \%)$ \\
\hline$s-34$ & n, alpha & $-8.3072 E-11$ & $+\backslash-9.4595 \mathrm{E}-18$ & $0.00 \%)$ \\
\hline$s-36$ & total & $1.3056 \mathrm{E}-10$ & $+\backslash-5.1210 \mathrm{E}-16$ & $0.00 \%)$ \\
\hline$s-36$ & scatter & $2.4907 \mathrm{E}-10$ & $+\backslash-5.1183 \mathrm{E}-16$ & $0.00 \%)$ \\
\hline$s-36$ & elastic & $2.3590 \mathrm{E}-10$ & $+\backslash-5.1180 \mathrm{E}-16$ & $0.00 \%)$ \\
\hline$s-36$ & $\mathrm{n}, \mathrm{n}^{\prime}$ & $1.2962 \mathrm{E}-11$ & $+\backslash-1.1331 \mathrm{E}-18$ & $0.00 \%)$ \\
\hline$s-36$ & $\mathrm{n}, 2 \mathrm{n}$ & $2.0465 \mathrm{E}-13$ & $+1-8.5709 \mathrm{E}-22$ & $0.00 \%)$ \\
\hline$s-36$ & capture & $-1.1851 \mathrm{E}-10$ & $+\backslash-3.2691 \mathrm{E}-19$ & $0.00 \%)$ \\
\hline$s-36$ & $\mathrm{n}$, gamma & $-1.1850 \mathrm{E}-10$ & $+1-3.2691 \mathrm{E}-19$ & $0.00 \%)$ \\
\hline$s-36$ & $\mathrm{n}, \mathrm{p}$ & $-7.0565 E-17$ & $+\backslash-1.4329 \mathrm{E}-24$ & $0.00 \%)$ \\
\hline$s-36$ & n, alpha & $-5.9656 \mathrm{E}-15$ & $+1-2.0535 \mathrm{E}-22$ & $0.00 \%)$ \\
\hline $\mathrm{ca}-40$ & total & $-1.7949 E-06$ & $+\backslash-1.1894 \mathrm{E}-07$ & $6.63 \%)$ \\
\hline $\mathrm{ca}-40$ & scatter & $6.7330 \mathrm{E}-06$ & $+\backslash-9.7909 \mathrm{E}-08$ & $1.45 \%)$ \\
\hline $\mathrm{ca}-40$ & elastic & $6.5226 \mathrm{E}-06$ & $+1-9.7589 \mathrm{E}-08$ & $1.50 \%)$ \\
\hline $\mathrm{ca}-40$ & $\mathrm{n}, \mathrm{n}^{\prime}$ & $2.0785 \mathrm{E}-07$ & $+1-3.3003 \mathrm{E}-09$ & $1.59 \%)$ \\
\hline $\mathrm{ca}-40$ & $n, 2 n$ & $4.5607 \mathrm{E}-14$ & $+1-0.0000 \mathrm{E}+00$ & $0.00 \%)$ \\
\hline $\mathrm{ca}-40$ & capture & $-8.5279 E-06$ & $+\backslash-1.9716 \mathrm{E}-08$ & $0.23 \%)$ \\
\hline $\mathrm{ca}-40$ & $\mathrm{n}$, gamma & $-7.9727 \mathrm{E}-06$ & $+\backslash-1.8069 \mathrm{E}-08$ & $0.23 \%)$ \\
\hline$c a-40$ & $\mathrm{n}, \mathrm{p}$ & $-3.7411 E-07$ & $+\backslash-5.5225 \mathrm{E}-09$ & $1.48 \%)$ \\
\hline $\mathrm{ca}-40$ & $n, d$ & $-5.9766 \mathrm{E}-11$ & $+1-6.5478 \mathrm{E}-19$ & $0.00 \%)$ \\
\hline $\mathrm{ca}-40$ & $n, t$ & $-4.2787 E-15$ & $+1-0.0000 \mathrm{E}+00$ & $0.00 \%)$ \\
\hline$c a-40$ & $\mathrm{n}, \mathrm{he}-3$ & $-5.7096 E-14$ & $+1-0.0000 \mathrm{E}+00$ & $0.00 \%)$ \\
\hline $\mathrm{ca}-40$ & n,alpha & $-1.8104 \mathrm{E}-07$ & $+1-2.0743 \mathrm{E}-09$ & $1.15 \%)$ \\
\hline $\mathrm{ca}-42$ & total & $-3.3798 E-08$ & $+1-4.2370 \mathrm{E}-10$ & $1.25 \%)$ \\
\hline $\mathrm{ca}-42$ & scatter & $5.5595 \mathrm{E}-08$ & $+\backslash-1.8840 \mathrm{E}-10$ & $0.34 \%)$ \\
\hline $\mathrm{ca}-42$ & elastic & $4.4749 \mathrm{E}-08$ & $+1-7.7226 \mathrm{E}-14$ & $0.00 \%)$ \\
\hline $\mathrm{ca}-42$ & $\mathrm{n}, \mathrm{n}^{\prime}$ & $1.0831 \mathrm{E}-08$ & $+1-3.5480 \mathrm{E}-15$ & $0.00 \%)$ \\
\hline $\mathrm{ca}-42$ & $\mathrm{n}, 2 \mathrm{n}$ & $4.2807 \mathrm{E}-12$ & $+1-0.0000 \mathrm{E}+00$ & $0.00 \%)$ \\
\hline $\mathrm{ca}-42$ & capture & $-8.9393 E-08$ & $+\backslash-1.9154 \mathrm{E}-10$ & $0.21 \%)$ \\
\hline $\mathrm{ca}-42$ & $\mathrm{n}$, gamma & $-8.9285 E-08$ & $+\backslash-1.9154 \mathrm{E}-10$ & $0.21 \%)$ \\
\hline $\mathrm{ca}-42$ & $\mathrm{n}, \mathrm{p}$ & $-6.3001 E-11$ & $+\backslash-6.1936 \mathrm{E}-18$ & $0.00 \%)$ \\
\hline$c a-42$ & $\mathrm{n}, \mathrm{d}$ & $-3.9571 E-14$ & $+1-0.0000 \mathrm{E}+00$ & $0.00 \%)$ \\
\hline $\mathrm{ca}-42$ & $n, t$ & $-1.1401 \mathrm{E}-16$ & $+1-0.0000 \mathrm{E}+00$ & $0.00 \%)$ \\
\hline $\mathrm{ca}-42$ & $\mathrm{n}, \mathrm{he}-3$ & $-2.8628 \mathrm{E}-18$ & $+1-0.0000 \mathrm{E}+00$ & $0.00 \%)$ \\
\hline$c a-42$ & n,alpha & $-4.4886 \mathrm{E}-11$ & $+1-4.9613 \mathrm{E}-18$ & $0.00 \%)$ \\
\hline
\end{tabular}

Figure 6.3.16. SAMS output for LEU-COMP-THERM-009 case 10 sample problem. (continued) 


\begin{tabular}{|c|c|c|c|c|}
\hline$c a-43$ & total & $-2.9992 E-07$ & $+\backslash-8.5309 \mathrm{E}-10$ & $0.28 \%)$ \\
\hline $\mathrm{ca}-43$ & scatter & $1.7308 \mathrm{E}-08$ & $+\backslash-9.3864 \mathrm{E}-15$ & $0.00 \%)$ \\
\hline $\mathrm{ca}-43$ & elastic & $1.4035 \mathrm{E}-08$ & $+\backslash-8.9886 \mathrm{E}-15$ & $0.00 \%)$ \\
\hline $\mathrm{ca}-43$ & $\mathrm{n}, \mathrm{n}^{\prime}$ & $3.2593 E-09$ & $+\backslash-1.0415 \mathrm{E}-15$ & $0.00 \%)$ \\
\hline $\mathrm{ca}-43$ & $\mathrm{n}, 2 \mathrm{n}$ & $1.3992 \mathrm{E}-11$ & $+\backslash-1.0619 \mathrm{E}-22$ & $0.00 \div)$ \\
\hline $\mathrm{ca}-43$ & capture & $-3.1723 E-07$ & $+\backslash-7.1329 \mathrm{E}-10$ & $0.22 \%)$ \\
\hline $\mathrm{ca}-43$ & $\mathrm{n}$, gamma & $-3.1720 E-07$ & $+\backslash-7.1328 \mathrm{E}-10$ & $0.22 \%)$ \\
\hline $\mathrm{ca}-43$ & $\mathrm{n}, \mathrm{p}$ & $-1.2736 \mathrm{E}-11$ & $+\backslash-1.4953 \mathrm{E}-18$ & $0.00 \%)$ \\
\hline $\mathrm{ca}-43$ & $\mathrm{n}, \mathrm{d}$ & $-3.9211 E-15$ & $+1-0.0000 \mathrm{E}+00$ & $0.00 \div)$ \\
\hline $\mathrm{ca}-43$ & $n, t$ & $-2.0903 E-16$ & $+1-0.0000 \mathrm{E}+00$ & $0.00 \div)$ \\
\hline $\mathrm{ca}-43$ & $\mathrm{n}, \mathrm{he}-3$ & $-8.8381 E-21$ & $+1-0.0000 \mathrm{E}+00$ & $0.00 \%)$ \\
\hline $\mathrm{ca}-43$ & n, alpha & $-1.8078 E-11$ & $+\backslash-1.6658 \mathrm{E}-18$ & $0.00 \%)$ \\
\hline $\mathrm{ca}-44$ & total & $-1.9121 \mathrm{E}-07$ & $+\backslash-2.9350 \mathrm{E}-09$ & $1.53 \%)$ \\
\hline $\mathrm{ca}-44$ & scatter & $1.8201 \mathrm{E}-07$ & $+\backslash-9.8994 \mathrm{E}-10$ & $0.54 \%)$ \\
\hline $\mathrm{ca}-44$ & elastic & $1.4003 \mathrm{E}-07$ & $+\backslash-6.5307 \mathrm{E}-10$ & $0.47 \%$ \\
\hline $\mathrm{ca}-44$ & $\mathrm{n}, \mathrm{n}^{\prime}$ & $4.1934 \mathrm{E}-08$ & $+1-3.9319 \mathrm{E}-10$ & $0.94 \%)$ \\
\hline $\mathrm{ca}-44$ & $n, 2 n$ & $3.7701 \mathrm{E}-11$ & $+\backslash-0.0000 \mathrm{E}+00$ & $0.00 \%)$ \\
\hline $\mathrm{ca}-44$ & capture & $-3.7322 \mathrm{E}-07$ & $+1-8.4317 \mathrm{E}-10$ & $0.23 \%)$ \\
\hline $\mathrm{ca}-44$ & $\mathrm{n}$, gamma & $-3.7321 E-07$ & $+1-8.4317 \mathrm{E}-10$ & $0.23 \%)$ \\
\hline $\mathrm{ca}-44$ & $\mathrm{n}, \mathrm{p}$ & $-4.3462 E-12$ & $+\backslash-1.1952 \mathrm{E}-19$ & $0.00 \%)$ \\
\hline $\mathrm{ca}-44$ & $\mathrm{n}, \mathrm{d}$ & $-1.9015 E-14$ & $+1-0.0000 \mathrm{E}+00$ & $0.00 \%)$ \\
\hline $\mathrm{ca}-44$ & $n, t$ & $-3.6521 \mathrm{E}-17$ & $+1-0.0000 \mathrm{E}+00$ & $0.00 \%)$ \\
\hline $\mathrm{ca}-44$ & $\mathrm{n}, \mathrm{he}-3$ & $-5.7133 E-22$ & $+1-0.0000 \mathrm{E}+00$ & $0.00 \%)$ \\
\hline $\mathrm{ca}-44$ & n, alpha & $-2.1833 E-12$ & $+\backslash-5.2524 \mathrm{E}-20$ & $0.00 \%)$ \\
\hline $\mathrm{ca}-46$ & total & $-9.1789 E-11$ & $+\backslash-2.9314 \mathrm{E}-16$ & $0.00 \%)$ \\
\hline $\mathrm{ca}-46$ & scatter & $5.0404 \mathrm{E}-10$ & $+\backslash-2.9308 \mathrm{E}-16$ & $0.00 \%)$ \\
\hline$c a-46$ & elastic & $4.3313 E-10$ & $+1-2.8501 \mathrm{E}-16$ & $0.00 \%$ \\
\hline$c a-46$ & $\mathrm{n}, \mathrm{n}^{\prime}$ & $7.0696 \mathrm{E}-11$ & $+\backslash-2.2304 \mathrm{E}-17$ & $0.00 \%)$ \\
\hline $\mathrm{ca}-46$ & $n, 2 n$ & $2.0718 \mathrm{E}-13$ & $+1-0.0000 \mathrm{E}+00$ & $0.00 \%)$ \\
\hline $\mathrm{ca}-46$ & capture & $-5.9583 E-10$ & $+\backslash-5.9929 \mathrm{E}-20$ & $0.00 \%)$ \\
\hline $\mathrm{ca}-46$ & $\mathrm{n}$, gamma & $-5.9583 E-10$ & $+\backslash-5.9929 \mathrm{E}-20$ & $0.00 \%)$ \\
\hline $\mathrm{ca}-46$ & $\mathrm{n}, \mathrm{p}$ & $-3.2267 \mathrm{E}-16$ & $+1-3.6714 \mathrm{E}-26$ & $0.00 \%)$ \\
\hline $\mathrm{ca}-46$ & $\mathrm{n}, \mathrm{d}$ & $-6.8722 E-18$ & $+1-0.0000 \mathrm{E}+00$ & $0.00 \%)$ \\
\hline $\mathrm{ca}-46$ & $n, t$ & $-3.3633 E-21$ & $+1-0.0000 \mathrm{E}+00$ & $0.00 \%)$ \\
\hline $\mathrm{ca}-46$ & n, alpha & $-2.2633 \mathrm{E}-17$ & $+1-0.0000 \mathrm{E}+00$ & $0.00 \%)$ \\
\hline $\mathrm{ca}-48$ & total & $-2.4743 E-08$ & $+\backslash-1.8724 \mathrm{E}-10$ & $0.76 \%$ \\
\hline $\mathrm{ca}-48$ & scatter & $1.6341 \mathrm{E}-08$ & $+\backslash-8.2057 \mathrm{E}-15$ & $0.00 \%)$ \\
\hline $\mathrm{ca}-48$ & elastic & $1.5512 \mathrm{E}-08$ & $+\backslash-8.2022 \mathrm{E}-15$ & $0.00 \%)$ \\
\hline $\mathrm{ca}-48$ & $\mathrm{n}, \mathrm{n}^{\prime}$ & $8.1356 \mathrm{E}-10$ & $+\backslash-6.9284 \mathrm{E}-17$ & $0.00 \%)$ \\
\hline $\mathrm{ca}-48$ & $n, 2 n$ & $1.5225 \mathrm{E}-11$ & $+\backslash-0.0000 \mathrm{E}+00$ & $0.00 \%)$ \\
\hline $\mathrm{ca}-48$ & capture & $-4.1084 E-08$ & $+\backslash-7.9141 \mathrm{E}-11$ & $0.19 \%)$ \\
\hline $\mathrm{ca}-48$ & $\mathrm{n}$, gamma & $-4.1084 \mathrm{E}-08$ & $+\backslash-7.9141 \mathrm{E}-11$ & $0.19 \%)$ \\
\hline $\mathrm{ca}-48$ & $\mathrm{n}, \mathrm{p}$ & $-1.0552 \mathrm{E}-16$ & $+1-0.0000 \mathrm{E}+00$ & $0.00 \%)$ \\
\hline $\mathrm{ca}-48$ & $\mathrm{n}, \mathrm{d}$ & $-2.1494 E-17$ & $+1-0.0000 \mathrm{E}+00$ & $0.00 \%)$ \\
\hline $\mathrm{ca}-48$ & $n, t$ & $-5.0004 E-22$ & $+1-0.0000 \mathrm{E}+00$ & $0.00 \%)$ \\
\hline $\mathrm{ca}-48$ & n,alpha & $-1.9945 \mathrm{E}-17$ & $+\backslash-0.0000 \mathrm{E}+00$ & $0.00 \%)$ \\
\hline ti-46 & total & $-1.8856 \mathrm{E}-07$ & $-8.6800 \mathrm{E}-10$ & $0.46 \%)$ \\
\hline
\end{tabular}

Figure 6.3.16. SAMS output for LEU-COMP-THERM-009 case 10 sample problem. (continued) 


\begin{tabular}{|c|c|c|c|c|}
\hline$t i-46$ & scatter & $2.1933 E-07$ & $+\backslash-4.7014 \mathrm{E}-10$ & $0.21 \%)$ \\
\hline ti-46 & elastic & $1.2896 \mathrm{E}-07$ & $+1-3.2795 \mathrm{E}-10$ & $0.25 \%)$ \\
\hline ti-46 & $\mathrm{n}, \mathrm{n}^{\prime}$ & $9.0312 \mathrm{E}-08$ & $+\backslash-1.9591 \mathrm{E}-10$ & $0.22 \%)$ \\
\hline ti-46 & $\mathrm{n}, 2 \mathrm{n}$ & $4.8498 \mathrm{E}-12$ & $+\backslash-2.8827 \mathrm{E}-21$ & $0.00 \%)$ \\
\hline ti-46 & capture & $-4.0789 E-07$ & $+\backslash-1.3319 \mathrm{E}-10$ & $0.03 \%)$ \\
\hline ti-46 & $\mathrm{n}$, gamma & $-3.9961 E-07$ & $+\backslash-1.3319 \mathrm{E}-10$ & $0.03 \%)$ \\
\hline ti-46 & $\mathrm{n}, \mathrm{p}$ & $-7.7485 E-09$ & $+\backslash-3.8514 \mathrm{E}-18$ & $0.00 \%)$ \\
\hline ti-46 & $\mathrm{n}, \mathrm{d}$ & $-3.1806 E-13$ & $+1-2.3627 \mathrm{E}-22$ & $0.00 \%)$ \\
\hline ti-46 & $n, t$ & $-9.9763 \mathrm{E}-17$ & $+1-3.2655 \mathrm{E}-26$ & $0.00 \%)$ \\
\hline ti-46 & $\mathrm{n}, \mathrm{he}-3$ & $-4.2439 E-16$ & $+\backslash-1.9547 \mathrm{E}-25$ & $0.00 \%)$ \\
\hline ti-46 & n, alpha & $-5.2794 \mathrm{E}-10$ & $+\backslash-2.5965 \mathrm{E}-19$ & $0.00 \%)$ \\
\hline ti-47 & total & $-8.7007 E-07$ & $+1-2.4463 \mathrm{E}-09$ & $0.28 \%)$ \\
\hline ti-47 & scatter & $2.4268 \mathrm{E}-07$ & $+\backslash-2.2853 \mathrm{E}-09$ & $0.94 \%)$ \\
\hline ti-47 & elastic & $1.3531 \mathrm{E}-07$ & $+1-2.2669 \mathrm{E}-09$ & $1.68 \%)$ \\
\hline ti-47 & $\mathrm{n}, \mathrm{n}^{\prime}$ & $1.0717 \mathrm{E}-07$ & $+\backslash-1.8872 \mathrm{E}-10$ & $0.18 \%)$ \\
\hline ti-47 & $\mathrm{n}, 2 \mathrm{n}$ & $1.9211 \mathrm{E}-10$ & $+1-4.0015 \mathrm{E}-20$ & $0.00 \%)$ \\
\hline ti-47 & capture & $-1.1128 \mathrm{E}-06$ & $+1-3.6552 \mathrm{E}-10$ & $0.03 \%)$ \\
\hline ti-47 & $\mathrm{n}$, gamma & $-1.1003 E-06$ & $+1-3.6552 \mathrm{E}-10$ & $0.03 \%)$ \\
\hline ti-47 & $\mathrm{n}, \mathrm{p}$ & $-1.0646 E-08$ & $+\backslash-1.4197 \mathrm{E}-16$ & $0.00 \%)$ \\
\hline ti-47 & $\mathrm{n}, \mathrm{d}$ & $-2.0197 E-13$ & $+\backslash-1.5135 \mathrm{E}-22$ & $0.00 \%)$ \\
\hline ti-47 & $n, t$ & $-4.3601 E-15$ & $+1-3.0922 \mathrm{E}-24$ & $0.00 \%)$ \\
\hline ti-47 & $\mathrm{n}, \mathrm{he}-3$ & $-2.3300 \mathrm{E}-17$ & $+1-7.4079 \mathrm{E}-27$ & $0.00 \%)$ \\
\hline ti-47 & n,alpha & $-1.8482 E-09$ & $+\backslash-1.3222 \mathrm{E}-17$ & $0.00 \%)$ \\
\hline ti-48 & total & $-4.5957 \mathrm{E}-05$ & $+1-3.3263 \mathrm{E}-08$ & $0.07 \%)$ \\
\hline ti-48 & scatter & $3.0030 \mathrm{E}-06$ & $+1-2.3419 \mathrm{E}-08$ & $0.78 \%)$ \\
\hline ti-48 & elastic & $2.1566 \mathrm{E}-06$ & $+1-2.3249 \mathrm{E}-08$ & $1.08 \%)$ \\
\hline ti-48 & $\mathrm{n}, \mathrm{n}^{\prime}$ & $8.4526 \mathrm{E}-07$ & $+\backslash-1.8984 \mathrm{E}-09$ & $0.22 \%)$ \\
\hline ti-48 & $n, 2 n$ & $1.0593 E-09$ & $+\backslash-5.3938 E-19$ & $0.00 \%)$ \\
\hline ti-48 & capture & $-4.8960 \mathrm{E}-05$ & $+\backslash-1.7297 \mathrm{E}-08$ & $0.04 \%)$ \\
\hline ti-48 & $\mathrm{n}$, gamma & $-4.8958 \mathrm{E}-05$ & $+\backslash-1.7297 \mathrm{E}-08$ & $0.04 \%)$ \\
\hline ti-48 & $\mathrm{n}, \mathrm{p}$ & $-1.6058 \mathrm{E}-09$ & $+\backslash-9.4652 \mathrm{E}-19$ & $0.00 \%)$ \\
\hline$t i-48$ & $n, d$ & $-1.6876 \mathrm{E}-12$ & $+1-1.6673 \mathrm{E}-21$ & $0.00 \%)$ \\
\hline ti-48 & $n, t$ & $-8.0952 E-16$ & $+1-2.7332 \mathrm{E}-25$ & $0.00 \%)$ \\
\hline ti-48 & $\mathrm{n}, \mathrm{he}-3$ & $-1.9723 E-17$ & $+\backslash-6.7861 \mathrm{E}-27$ & $0.00 \div)$ \\
\hline$t i-48$ & n, alpha & $-1.5654 \mathrm{E}-10$ & $+\backslash-4.0991 \mathrm{E}-20$ & $0.00 \%)$ \\
\hline ti-49 & total & $-6.8733 E-07$ & $+1-6.2660 \mathrm{E}-10$ & $0.09 \%)$ \\
\hline ti-49 & scatter & $1.6784 \mathrm{E}-07$ & $+1-2.2439 \mathrm{E}-09$ & $1.34 \%)$ \\
\hline ti-49 & elastic & $1.0265 \mathrm{E}-07$ & $+1-2.2349 \mathrm{E}-09$ & $2.18 \%)$ \\
\hline ti-49 & $n, n^{\prime}$ & $6.4463 \mathrm{E}-08$ & $+\backslash-1.2032 \mathrm{E}-10$ & $0.19 \%)$ \\
\hline ti-49 & $n, 2 n$ & $7.2472 \mathrm{E}-10$ & $+1-7.6807 \mathrm{E}-20$ & $0.00 \%)$ \\
\hline ti-49 & capture & $-8.5517 \mathrm{E}-07$ & $+1-2.9502 \mathrm{E}-10$ & $0.03 \%)$ \\
\hline ti-49 & $\mathrm{n}$, gamma & $-8.5476 E-07$ & $+1-2.9502 \mathrm{E}-10$ & $0.03 \%)$ \\
\hline ti-49 & $\mathrm{n}, \mathrm{p}$ & $-3.3313 E-10$ & $+1-3.2208 \mathrm{E}-19$ & $0.00 \%)$ \\
\hline ti-49 & $\mathrm{n}, \mathrm{d}$ & $-7.4647 E-14$ & $+1-6.7611 \mathrm{E}-23$ & $0.00 \%)$ \\
\hline ti-49 & $n, t$ & $-2.1162 E-15$ & $+\backslash-9.3763 E-25$ & $0.00 \%)$ \\
\hline ti-49 & $\mathrm{n}, \mathrm{he}-3$ & $-9.3750 \mathrm{E}-20$ & $+1-0.0000 \mathrm{E}+00$ & $0.00 \%)$ \\
\hline ti-49 & n,alpha & $-7.1068 \mathrm{E}-11$ & $+1-4.3628 \mathrm{E}-20$ & $0.00 \%)$ \\
\hline ti-50 & total & $5.1758 \mathrm{E}-08$ & $+\backslash-4.1741 \mathrm{E}-10$ & $0.81 \%)$ \\
\hline
\end{tabular}

Figure 6.3.16. SAMS output for LEU-COMP-THERM-009 case 10 sample problem. (continued) 


\begin{tabular}{|c|c|c|c|c|}
\hline ti-50 & scatter & $1.3378 \mathrm{E}-07$ & $+\backslash-1.9577 \mathrm{E}-10$ & $0.15 \%)$ \\
\hline ti-50 & elastic & $8.8832 E-08$ & $+\backslash-1.1755 \mathrm{E}-10$ & $0.13 \%)$ \\
\hline ti-50 & $\mathrm{n}, \mathrm{n}^{\prime}$ & $4.4731 \mathrm{E}-08$ & $+1-8.8290 \mathrm{E}-11$ & $0.20 \%)$ \\
\hline ti-50 & $\mathrm{n}, 2 \mathrm{n}$ & $2.1770 \mathrm{E}-10$ & $+1-7.0963 \mathrm{E}-20$ & $0.00 \%)$ \\
\hline ti-50 & capture & $-8.2023 E-08$ & $+\backslash-2.5593 \mathrm{E}-11$ & $0.03 \%)$ \\
\hline ti-50 & $\mathrm{n}$, gamma & $-8.2018 \mathrm{E}-08$ & $+\backslash-2.5593 \mathrm{E}-11$ & $0.03 \%)$ \\
\hline ti-50 & $\mathrm{n}, \mathrm{p}$ & $-2.9356 E-12$ & $+1-9.4805 \mathrm{E}-22$ & $0.00 \%)$ \\
\hline ti-50 & $\mathrm{n}, \mathrm{d}$ & $-1.7101 E-14$ & $+\backslash-1.1908 \mathrm{E}-23$ & $0.00 \%)$ \\
\hline ti-50 & $n, t$ & $-1.0953 \mathrm{E}-17$ & $+\backslash-2.8155 \mathrm{E}-27$ & $0.00 \%)$ \\
\hline ti-50 & $\mathrm{n}, \mathrm{he}-3$ & $-4.0903 E-23$ & $+1-0.0000 \mathrm{E}+00$ & $0.00 \%)$ \\
\hline ti-50 & n, alpha & $-1.7272 \mathrm{E}-12$ & $+\backslash-6.5310 \mathrm{E}-22$ & $0.00 \%)$ \\
\hline cr -50 & total & $-1.3040 E-05$ & $+\backslash-9.1891 \mathrm{E}-09$ & $0.07 \%)$ \\
\hline cr -50 & scatter & $1.3376 \mathrm{E}-06$ & $+\backslash-7.2895 \mathrm{E}-09$ & $0.54 \%)$ \\
\hline cr -50 & elastic & $1.2307 \mathrm{E}-06$ & $+\backslash-7.2762 \mathrm{E}-09$ & $0.59 \%)$ \\
\hline cr -50 & $\mathrm{n}, \mathrm{n}^{\prime}$ & $1.0681 \mathrm{E}-07$ & $+\backslash-2.3841 \mathrm{E}-10$ & $0.22 \%)$ \\
\hline cr -50 & $\mathrm{n}, 2 \mathrm{n}$ & $3.1922 \mathrm{E}-12$ & $+1-2.3793 \mathrm{E}-21$ & $0.00 \%)$ \\
\hline cr -50 & capture & $-1.4377 \mathrm{E}-05$ & $+1-5.0788 \mathrm{E}-09$ & $0.04 \%)$ \\
\hline cr -50 & $\mathrm{n}$, gamma & $-1.4333 E-05$ & $+\backslash-5.0787 \mathrm{E}-09$ & $0.04 \%)$ \\
\hline cr -50 & $\mathrm{n}, \mathrm{p}$ & $-4.1672 E-08$ & $+1-3.2114 \mathrm{E}-11$ & $0.08 \%)$ \\
\hline cr -50 & $\mathrm{n}, \mathrm{d}$ & $-7.5849 \mathrm{E}-12$ & $+1-5.4355 \mathrm{E}-19$ & $0.00 \%)$ \\
\hline cr-50 & n,alpha & $-2.7989 E-09$ & $+\backslash-1.7847 \mathrm{E}-15$ & $0.00 \%)$ \\
\hline$c r-52$ & total & $-8.7501 E-06$ & $+1-2.6630 \mathrm{E}-08$ & $0.30 \%)$ \\
\hline$c r-52$ & scatter & $4.4850 \mathrm{E}-06$ & $+1-2.1763 \mathrm{E}-08$ & $0.49 \%)$ \\
\hline$c r-52$ & elastic & $2.3489 \mathrm{E}-06$ & $+1-2.0602 \mathrm{E}-08$ & $0.88 \%)$ \\
\hline$c r-52$ & $\mathrm{n}, \mathrm{n}^{\prime}$ & $2.1346 \mathrm{E}-06$ & $+1-4.5774 \mathrm{E}-09$ & $0.21 \%)$ \\
\hline$c r-52$ & $n, 2 n$ & $1.3180 \mathrm{E}-09$ & $+\backslash-9.1937 \mathrm{E}-19$ & $0.00 \%)$ \\
\hline$c r-52$ & capture & $-1.3235 E-05$ & $+\backslash-4.5982 \mathrm{E}-09$ & $0.03 \%)$ \\
\hline$c r-52$ & $\mathrm{n}$, gamma & $-1.3217 \mathrm{E}-05$ & $+\backslash-4.5982 \mathrm{E}-09$ & $0.03 \%)$ \\
\hline$c r-52$ & $\mathrm{n}, \mathrm{p}$ & $-1.5832 E-08$ & $+\backslash-1.8313 \mathrm{E}-11$ & $0.12 \%)$ \\
\hline$c r-52$ & n, alpha & $-1.9304 \mathrm{E}-09$ & $+\backslash-3.8775 \mathrm{E}-17$ & $0.00 \%)$ \\
\hline cr -53 & total & $-3.4245 E-05$ & $+1-2.1670 \mathrm{E}-08$ & $0.06 \%)$ \\
\hline cr -53 & scatter & $1.3865 \mathrm{E}-06$ & $+\backslash-1.4475 E-08$ & $1.04 \%)$ \\
\hline cr -53 & elastic & $1.0474 \mathrm{E}-06$ & $+1-1.4432 \mathrm{E}-08$ & $1.38 \%)$ \\
\hline cr -53 & $\mathrm{n}, \mathrm{n}^{\prime}$ & $3.3527 \mathrm{E}-07$ & $+\backslash-7.1251 \mathrm{E}-10$ & $0.21 \%)$ \\
\hline$c r-53$ & $n, 2 n$ & $3.8152 \mathrm{E}-09$ & $+\backslash-3.6614 \mathrm{E}-17$ & $0.00 \%)$ \\
\hline cr -53 & capture & $-3.5632 E-05$ & $+\backslash-1.2596 \mathrm{E}-08$ & $0.04 \%)$ \\
\hline cr -53 & $\mathrm{n}$, gamma & $-3.5630 E-05$ & $+1-1.2596 \mathrm{E}-08$ & $0.04 \%)$ \\
\hline cr -53 & $\mathrm{n}, \mathrm{p}$ & $-6.9509 E-10$ & $+\backslash-5.2410 \mathrm{E}-17$ & $0.00 \%)$ \\
\hline cr -53 & n, alpha & $-1.5386 \mathrm{E}-09$ & $+\backslash-1.4981 \mathrm{E}-16$ & $0.00 \%)$ \\
\hline cr -54 & total & $-1.6695 \mathrm{E}-08$ & $+\backslash-4.1104 \mathrm{E}-10$ & $2.46 \%)$ \\
\hline cr -54 & scatter & $1.6318 \mathrm{E}-07$ & $+1-2.3409 \mathrm{E}-10$ & $0.14 \%)$ \\
\hline cr-54 & elastic & $9.3303 E-08$ & $+\backslash-7.9919 \mathrm{E}-11$ & $0.09 \%)$ \\
\hline cr-54 & $\mathrm{n}, \mathrm{n}^{\prime}$ & $6.9373 E-08$ & $+\backslash-1.3815 \mathrm{E}-10$ & $0.20 \%)$ \\
\hline$c r-54$ & $n, 2 n$ & $5.0486 \mathrm{E}-10$ & $+1-8.3567 \mathrm{E}-20$ & $0.00 \%)$ \\
\hline$c r-54$ & capture & $-1.7988 E-07$ & $+\backslash-5.9710 \mathrm{E}-11$ & $0.03 \%)$ \\
\hline cr-54 & $\mathrm{n}$, gamma & $-1.7985 E-07$ & $+\backslash-5.9710 \mathrm{E}-11$ & $0.03 \%)$ \\
\hline $\operatorname{cr}-54$ & $\mathrm{n}, \mathrm{p}$ & $-4.2582 \mathrm{E}-12$ & $+\backslash-1.1391 \mathrm{E}-21$ & $0.00 \%)$ \\
\hline cr -54 & n,alpha & $-2.1413 E-11$ & $+\backslash-1.0788 \mathrm{E}-20$ & $0.00 \%)$ \\
\hline
\end{tabular}

Figure 6.3.16. SAMS output for LEU-COMP-THERM-009 case 10 sample problem. (continued) 


\begin{tabular}{|c|c|c|c|c|}
\hline$m n-55$ & total & $-9.6360 \mathrm{E}-05$ & $+\backslash-1.4079 \mathrm{E}-07$ & $0.15 \%)$ \\
\hline$m n-55$ & scatter & $6.8704 \mathrm{E}-06$ & $+\backslash-1.3262 \mathrm{E}-07$ & $1.93 \%)$ \\
\hline$m n-55$ & elastic & $5.7292 E-06$ & $+\backslash-1.3254 \mathrm{E}-07$ & $2.31 \%)$ \\
\hline$m n-55$ & $\mathrm{n}, \mathrm{n}^{\prime}$ & $1.1366 \mathrm{E}-06$ & $+\backslash-2.5436 \mathrm{E}-09$ & $0.22 \%)$ \\
\hline$m n-55$ & $n, 2 n$ & $4.4184 \mathrm{E}-09$ & $+\backslash-1.0180 \mathrm{E}-18$ & $0.00 \%)$ \\
\hline$m n-55$ & capture & $-1.0323 E-04$ & $+1-3.5222 \mathrm{E}-08$ & $0.03 \%)$ \\
\hline$m n-55$ & $\mathrm{n}$, gamma & $-1.0323 E-04$ & $+1-3.5222 \mathrm{E}-08$ & $0.03 \%)$ \\
\hline$m n-55$ & $\mathrm{n}, \mathrm{p}$ & $-3.7300 E-09$ & $+\backslash-9.1810 \mathrm{E}-18$ & $0.00 \%)$ \\
\hline$m n-55$ & $\mathrm{n}, \mathrm{d}$ & $-3.5791 \mathrm{E}-11$ & $+\backslash-6.7992 \mathrm{E}-21$ & $0.00 \%)$ \\
\hline$m n-55$ & $n, t$ & $-1.3299 \mathrm{E}-12$ & $+\backslash-1.2646 \mathrm{E}-21$ & $0.00 \%)$ \\
\hline$m n-55$ & $\mathrm{n}, \mathrm{he}-3$ & $-4.6031 E-14$ & $+\backslash-4.8445 \mathrm{E}-23$ & $0.00 \%)$ \\
\hline$m n-55$ & n,alpha & $-8.3737 E-10$ & $+\backslash-6.0947 \mathrm{E}-19$ & $0.00 \%)$ \\
\hline $\mathrm{fe}-54$ & total & $-3.9443 E-06$ & $+\backslash-8.5217 \mathrm{E}-09$ & $0.22 \%)$ \\
\hline $\mathrm{fe}-54$ & scatter & $7.5526 \mathrm{E}-07$ & $+1-8.1028 \mathrm{E}-09$ & $1.07 \%)$ \\
\hline$f e-54$ & elastic & $5.5856 \mathrm{E}-07$ & $+1-8.0723 \mathrm{E}-09$ & $1.45 \%)$ \\
\hline $\mathrm{fe}-54$ & $\mathrm{n}, \mathrm{n}^{\prime}$ & $1.9618 \mathrm{E}-07$ & $+1-4.3649 \mathrm{E}-10$ & $0.22 \%)$ \\
\hline $\mathrm{fe}-54$ & $n, 2 n$ & $3.8024 \mathrm{E}-12$ & $+\backslash-1.3165 \mathrm{E}-21$ & $0.00 \%)$ \\
\hline $\mathrm{fe}-54$ & capture & $-4.6996 \mathrm{E}-06$ & $+\backslash-1.5839 \mathrm{E}-09$ & $0.03 \%)$ \\
\hline $\mathrm{fe}-54$ & $\mathrm{n}$, gamma & $-4.5471 E-06$ & $+\backslash-1.5807 \mathrm{E}-09$ & $0.03 \%)$ \\
\hline $\mathrm{fe}-54$ & $\mathrm{n}, \mathrm{p}$ & $-1.5084 \mathrm{E}-07$ & $+\backslash-9.9241 \mathrm{E}-11$ & $0.07 \%)$ \\
\hline $\mathrm{fe}-54$ & $\mathrm{n}, \mathrm{d}$ & $-2.5872 \mathrm{E}-11$ & $+\backslash-1.0217 \mathrm{E}-20$ & $0.00 \%)$ \\
\hline$f e-54$ & n,alpha & $-1.5885 E-09$ & $+1-6.8285 \mathrm{E}-19$ & $0.00 \%)$ \\
\hline $\mathrm{fe}-56$ & total & $-6.9531 E-05$ & $+\backslash-1.3507 \mathrm{E}-07$ & $0.19 \%)$ \\
\hline$f e-56$ & scatter & $1.1558 \mathrm{E}-05$ & $+\backslash-1.1066 \mathrm{E}-07$ & $0.96 \%)$ \\
\hline $\mathrm{fe}-56$ & elastic & $7.5772 \mathrm{E}-06$ & $+\backslash-1.0976 \mathrm{E}-07$ & $1.45 \%)$ \\
\hline $\mathrm{fe}-56$ & $\mathrm{n}, \mathrm{n}^{\prime}$ & $3.9750 \mathrm{E}-06$ & $+1-9.5385 \mathrm{E}-09$ & $0.24 \%)$ \\
\hline $\mathrm{fe}-56$ & $\mathrm{n}, 2 \mathrm{n}$ & $5.5701 \mathrm{E}-09$ & $+\backslash-3.2182 \mathrm{E}-18$ & $0.00 \%)$ \\
\hline $\mathrm{fe}-56$ & capture & $-8.1090 \mathrm{E}-05$ & $+\backslash-2.8413 \mathrm{E}-08$ & $0.04 \%)$ \\
\hline $\mathrm{fe}-56$ & $\mathrm{n}$, gamma & $-8.1049 E-05$ & $+\backslash-2.8413 \mathrm{E}-08$ & $0.04 \%)$ \\
\hline $\mathrm{fe}-56$ & $\mathrm{n}, \mathrm{p}$ & $-2.8313 E-08$ & $+\backslash-4.3821 \mathrm{E}-11$ & $0.15 \%)$ \\
\hline $\mathrm{fe}-56$ & $\mathrm{n}, \mathrm{d}$ & $-6.3279 \mathrm{E}-11$ & $+1-5.4153 \mathrm{E}-20$ & $0.00 \%)$ \\
\hline $\mathrm{fe}-56$ & $n, t$ & $-1.5517 \mathrm{E}-13$ & $+\backslash-1.2163 \mathrm{E}-22$ & $0.00 \%)$ \\
\hline $\mathrm{fe}-56$ & $\mathrm{n}, \mathrm{he}-3$ & $-1.7491 \mathrm{E}-14$ & $+\backslash-1.2250 \mathrm{E}-23$ & $0.00 \%)$ \\
\hline $\mathrm{fe}-56$ & n, alpha & $-1.1979 \mathrm{E}-08$ & $+1-1.2530 \mathrm{E}-11$ & $0.10 \%)$ \\
\hline fe- 57 & total & $-1.4596 \mathrm{E}-06$ & $+1-2.4578 \mathrm{E}-09$ & $0.17 \%)$ \\
\hline fe- 57 & scatter & $2.9573 \mathrm{E}-07$ & $+1-2.2278 \mathrm{E}-09$ & $0.75 \%)$ \\
\hline fe- 57 & elastic & $1.6174 \mathrm{E}-07$ & $+1-2.1139 \mathrm{E}-09$ & $1.31 \%)$ \\
\hline fe- 57 & $n, n^{\prime}$ & $1.3200 \mathrm{E}-07$ & $+1-2.4140 \mathrm{E}-10$ & $0.18 \%)$ \\
\hline fe- 57 & $n, 2 n$ & $1.9892 \mathrm{E}-09$ & $+1-3.7761 \mathrm{E}-19$ & $0.00 \%)$ \\
\hline fe- 57 & capture & $-1.7553 \mathrm{E}-06$ & $+1-6.0368 \mathrm{E}-10$ & $0.03 \%)$ \\
\hline fe- -57 & $\mathrm{n}$, gamma & $-1.7542 E-06$ & $+1-6.0368 \mathrm{E}-10$ & $0.03 \%)$ \\
\hline fe- 57 & $n, p$ & $-4.5820 \mathrm{E}-10$ & $+\backslash-1.9581 \mathrm{E}-19$ & $0.00 \%)$ \\
\hline fe- 57 & n, alpha & $-6.5747 \mathrm{E}-10$ & $+1-3.5652 \mathrm{E}-19$ & $0.00 \%)$ \\
\hline $\mathrm{fe}-58$ & total & $-8.6319 \mathrm{E}-08$ & $+1-9.2863 \mathrm{E}-11$ & $0.11 \%)$ \\
\hline $\mathrm{fe}-58$ & scatter & $3.2999 \mathrm{E}-08$ & $+\backslash-2.1157 \mathrm{E}-13$ & $0.00 \%)$ \\
\hline $\mathrm{fe}-58$ & elastic & $1.9560 \mathrm{E}-08$ & $+\backslash-2.1157 \mathrm{E}-13$ & $0.00 \%)$ \\
\hline $\mathrm{fe}-58$ & $\mathrm{n}, \mathrm{n}^{\prime}$ & 1. $3357 \mathrm{E}-08$ & $+\backslash-1.7645 \mathrm{E}-16$ & $0.00 \%)$ \\
\hline $\mathrm{fe}-58$ & $n, 2 n$ & $8.1863 \mathrm{E}-11$ & $1-2.4070 \mathrm{E}-20$ & $0.00 \%)$ \\
\hline
\end{tabular}

Figure 6.3.16. SAMS output for LEU-COMP-THERM-009 case 10 sample problem. (continued) 


\begin{tabular}{|c|c|c|c|c|}
\hline$f e-58$ & capture & $-1.1932 E-07$ & $+\backslash-3.5967 \mathrm{E}-11$ & $0.03 \%)$ \\
\hline$f e-58$ & $\mathrm{n}$, gamma & $-1.1931 \mathrm{E}-07$ & $+\backslash-3.5967 \mathrm{E}-11$ & $0.03 \%)$ \\
\hline $\mathrm{fe}-58$ & $\mathrm{n}, \mathrm{p}$ & $-8.9843 E-13$ & $+1-3.3131 \mathrm{E}-22$ & $0.00 \%)$ \\
\hline $\mathrm{fe}-58$ & n, alpha & $-5.5353 E-12$ & $+\backslash-1.7256 \mathrm{E}-21$ & $0.00 \%)$ \\
\hline $\mathrm{cu}-63$ & total & $-3.2291 \mathrm{E}-03$ & $+\backslash-6.7644 \mathrm{E}-06$ & $0.21 \%)$ \\
\hline $\mathrm{cu}-63$ & scatter & $5.2401 \mathrm{E}-04$ & $+\backslash-4.5049 \mathrm{E}-06$ & $0.86 \%)$ \\
\hline $\mathrm{cu}-63$ & elastic & $4.4000 \mathrm{E}-04$ & $+\backslash-4.1846 \mathrm{E}-06$ & $0.95 \%)$ \\
\hline $\mathrm{cu}-63$ & $\mathrm{n}, \mathrm{n}^{\prime}$ & $8.2502 \mathrm{E}-05$ & $+\backslash-1.1401 \mathrm{E}-06$ & $1.38 \%)$ \\
\hline $\mathrm{cu}-63$ & $\mathrm{n}, 2 \mathrm{n}$ & $2.1792 \mathrm{E}-07$ & $+\backslash-7.0126 \mathrm{E}-09$ & $3.22 \%)$ \\
\hline $\mathrm{cu}-63$ & capture & $-3.7531 E-03$ & $+\backslash-3.1714 \mathrm{E}-06$ & $0.08 \%)$ \\
\hline $\mathrm{cu}-63$ & $\mathrm{n}$, gamma & $-3.7267 E-03$ & $+\backslash-3.1712 \mathrm{E}-06$ & $0.09 \%)$ \\
\hline $\mathrm{cu}-63$ & $\mathrm{n}, \mathrm{p}$ & $-2.5132 E-05$ & $+\backslash-3.5516 \mathrm{E}-08$ & $0.14 \%)$ \\
\hline $\mathrm{cu}-63$ & $n, d$ & $-5.3278 E-07$ & $+\backslash-1.5798 \mathrm{E}-09$ & $0.30 \%)$ \\
\hline $\mathrm{cu}-63$ & $\mathrm{n}, \mathrm{he}-3$ & $-3.1134 \mathrm{E}-12$ & $+\backslash-3.9260 \mathrm{E}-19$ & $0.00 \%)$ \\
\hline $\mathrm{cu}-63$ & n, alpha & $-6.8776 E-07$ & $+\backslash-2.7471 \mathrm{E}-09$ & $0.40 \%)$ \\
\hline $\mathrm{cu}-65$ & total & $-6.2532 E-04$ & $+\backslash-4.7383 E-06$ & $0.76 \%)$ \\
\hline $\mathrm{cu}-65$ & scatter & $1.8352 \mathrm{E}-04$ & $+1-4.2466 \mathrm{E}-06$ & $2.31 \%)$ \\
\hline$c u-65$ & elastic & $1.4901 \mathrm{E}-04$ & $+\backslash-4.1861 \mathrm{E}-06$ & $2.81 \%)$ \\
\hline $\mathrm{cu}-65$ & $\mathrm{n}, \mathrm{n}^{\prime}$ & $3.4114 \mathrm{E}-05$ & $+\backslash-4.9055 \mathrm{E}-07$ & $1.44 \%)$ \\
\hline $\mathrm{cu}-65$ & $\mathrm{n}, 2 \mathrm{n}$ & $3.8621 \mathrm{E}-07$ & $+1-7.8368 \mathrm{E}-09$ & $2.03 \%)$ \\
\hline $\mathrm{cu}-65$ & capture & $-8.0884 \mathrm{E}-04$ & $+\backslash-6.8255 \mathrm{E}-07$ & $0.08 \%)$ \\
\hline $\mathrm{cu}-65$ & $\mathrm{n}$, gamma & $-8.0848 E-04$ & $+\backslash-6.8255 \mathrm{E}-07$ & $0.08 \%)$ \\
\hline $\mathrm{cu}-65$ & $\mathrm{n}, \mathrm{p}$ & $-3.1527 E-07$ & $+1-7.8886 \mathrm{E}-10$ & $0.25 \%)$ \\
\hline $\mathrm{cu}-65$ & $n, d$ & $-4.1498 \mathrm{E}-08$ & $+\backslash-1.8559 \mathrm{E}-10$ & $0.45 \%)$ \\
\hline $\mathrm{cu}-65$ & $n, t$ & $-1.0755 E-11$ & $+1-2.7949 \mathrm{E}-18$ & $0.00 \%)$ \\
\hline $\mathrm{cu}-65$ & $\mathrm{n}, \mathrm{he}-3$ & $-3.9455 E-13$ & $+\backslash-1.3313 \mathrm{E}-20$ & $0.00 \%)$ \\
\hline $\mathrm{cu}-65$ & n, alpha & $-9.0375 E-09$ & $+\backslash-1.3569 \mathrm{E}-15$ & $0.00 \%)$ \\
\hline $\mathrm{u}-234$ & total & $-9.0979 E-04$ & $+1-3.1204 \mathrm{E}-07$ & $0.03 \%)$ \\
\hline$u-234$ & scatter & $6.2441 \mathrm{E}-06$ & $+\backslash-1.2041 \mathrm{E}-08$ & $0.19 \%)$ \\
\hline$u-234$ & elastic & $4.0083 E-06$ & $+\backslash-1.1854 \mathrm{E}-08$ & $0.30 \%)$ \\
\hline$u-234$ & $\mathrm{n}, \mathrm{n}^{\prime}$ & $2.2050 \mathrm{E}-06$ & $+\backslash-1.4630 \mathrm{E}-09$ & $0.07 \%)$ \\
\hline$u-234$ & $n, 2 n$ & $3.0794 \mathrm{E}-08$ & $+\backslash-1.7472 \mathrm{E}-11$ & $0.06 \%)$ \\
\hline$u-234$ & fission & $2.2909 \mathrm{E}-05$ & $+\backslash-1.7241 \mathrm{E}-09$ & $0.01 \%)$ \\
\hline$u-234$ & capture & $-9.3894 E-04$ & $+\backslash-3.0291 \mathrm{E}-07$ & $0.03 \div)$ \\
\hline$u-234$ & $\mathrm{n}$, gamma & $-9.3894 \mathrm{E}-04$ & $+\backslash-3.0291 \mathrm{E}-07$ & $0.03 \%)$ \\
\hline$u-234$ & nubar & $3.6021 \mathrm{E}-05$ & $+1-2.6348 \mathrm{E}-09$ & $0.01 \%)$ \\
\hline$u-234$ & chi & $8.7265 E-13$ & $+\backslash-1.5382 \mathrm{E}-09$ & $(-999.99 \%)$ \\
\hline$u-235$ & total & $2.0616 \mathrm{E}-01$ & $+1-7.0501 \mathrm{E}-05$ & $0.03 \%)$ \\
\hline$u-235$ & scatter & $8.8279 E-04$ & $+1-1.0661 \mathrm{E}-06$ & $0.12 \%)$ \\
\hline$u-235$ & elastic & $3.1799 \mathrm{E}-04$ & $+\backslash-9.6547 \mathrm{E}-07$ & $0.30 \%)$ \\
\hline$u-235$ & $\mathrm{n}, \mathrm{n}^{\prime}$ & $5.3198 \mathrm{E}-04$ & $+1-3.3888 \mathrm{E}-07$ & $0.06 \%)$ \\
\hline$u-235$ & $n, 2 n$ & $3.2795 \mathrm{E}-05$ & $+\backslash-1.0524 \mathrm{E}-08$ & $0.03 \%)$ \\
\hline$u-235$ & fission & $3.2433 E-01$ & $+1-6.2460 \mathrm{E}-05$ & $0.02 \%)$ \\
\hline$u-235$ & capture & $-1.1906 \mathrm{E}-01$ & $+\backslash-1.1810 \mathrm{E}-05$ & $0.01 \%)$ \\
\hline$u-235$ & $\mathrm{n}$, gamma & $-1.1906 \mathrm{E}-01$ & $+\backslash-1.1810 \mathrm{E}-05$ & $0.01 \%)$ \\
\hline$u-235$ & nubar & $9.6382 \mathrm{E}-01$ & $+1-8.0129 \mathrm{E}-05$ & $0.01 \%)$ \\
\hline$u-235$ & chi & $-6.6884 \mathrm{E}-08$ & $+1-4.6474 \mathrm{E}-05$ & $(-999.99 \%)$ \\
\hline$u-236$ & total & $-2.1805 E-04$ & $+\backslash-1.2725 \mathrm{E}-07$ & $0.06 \%)$ \\
\hline
\end{tabular}

Figure 6.3.16. SAMS output for LEU-COMP-THERM-009 case 10 sample problem. (continued) 


\begin{tabular}{|c|c|c|c|c|}
\hline$u-236$ & scatter & $5.6471 \mathrm{E}-06$ & $+1-9.6565 \mathrm{E}-09$ & $0.17 \%)$ \\
\hline$u-236$ & elastic & $2.3929 \mathrm{E}-06$ & $+1-9.2543 \mathrm{E}-09$ & $0.39 \%)$ \\
\hline$u-236$ & $\mathrm{n}, \mathrm{n}^{\prime}$ & $3.1626 \mathrm{E}-06$ & $+1-2.0863 \mathrm{E}-09$ & $0.07 \%)$ \\
\hline$u-236$ & $\mathrm{n}, 2 \mathrm{n}$ & $9.1002 \mathrm{E}-08$ & $+\backslash-4.1257 \mathrm{E}-11$ & $0.05 \%)$ \\
\hline$u-236$ & fission & $1.2829 \mathrm{E}-05$ & $+1-1.7760 \mathrm{E}-09$ & $0.01 \%)$ \\
\hline$u-236$ & capture & $-2.3653 E-04$ & $+\backslash-1.2003 \mathrm{E}-07$ & $0.05 \%)$ \\
\hline$u-236$ & $\mathrm{n}$, gamma & $-2.3653 E-04$ & $+\backslash-1.2003 \mathrm{E}-07$ & $0.05 \%)$ \\
\hline$u-236$ & nubar & $2.1111 \mathrm{E}-05$ & $+\backslash-1.6677 \mathrm{E}-09$ & $0.01 \%)$ \\
\hline$u-236$ & chi & $-4.2095 E-13$ & $+1-1.0077 \mathrm{E}-09$ & $(-999.99 \%)$ \\
\hline$u-238$ & total & $-5.9609 \mathrm{E}-02$ & $+1-2.6857 \mathrm{E}-05$ & $0.05 \%)$ \\
\hline$u-238$ & scatter & $2.9406 \mathrm{E}-02$ & $+1-2.0373 \mathrm{E}-05$ & $0.07 \%)$ \\
\hline$u-238$ & elastic & 1. $3217 \mathrm{E}-02$ & $+\backslash-1.6047 \mathrm{E}-05$ & $0.12 \%)$ \\
\hline$u-238$ & $\mathrm{n}, \mathrm{n}^{\prime}$ & $1.5251 \mathrm{E}-02$ & $+1-9.7098 \mathrm{E}-06$ & $0.06 \%)$ \\
\hline$u-238$ & $n, 2 n$ & $9.2840 \mathrm{E}-04$ & $+1-3.7033 \mathrm{E}-07$ & $0.04 \%)$ \\
\hline$u-238$ & fission & $2.4727 \mathrm{E}-02$ & $+1-2.4196 \mathrm{E}-06$ & $0.01 \%)$ \\
\hline$u-238$ & capture & $-1.1374 \mathrm{E}-01$ & $+1-9.9579 \mathrm{E}-06$ & $0.01 \%)$ \\
\hline$u-238$ & $\mathrm{n}$, gamma & $-1.1374 E-01$ & $+\backslash-9.9579 \mathrm{E}-06$ & $0.01 \%)$ \\
\hline$u-238$ & nubar & $3.6122 \mathrm{E}-02$ & $+1-3.8010 \mathrm{E}-06$ & $0.01 \%)$ \\
\hline$u-238$ & chi & $-2.9925 E-09$ & $+\backslash-1.7124 \mathrm{E}-06$ & $(-999.99 \%)$ \\
\hline
\end{tabular}

Energy and Region Integrated Sensitivity Coefficients for this Problem

\begin{tabular}{|c|c|c|c|c|c|c|}
\hline Mixture & Nuclide & Reaction & Sensitivity & & Std. Dev. & \% Std. Dev. \\
\hline 1 & $0-16$ & total & $1.1444 \mathrm{E}-02$ & $+/-$ & $3.3431 E-05$ & $0.29 \%)$ \\
\hline 1 & $0-16$ & scatter & $1.2013 \mathrm{E}-02$ & $+/-$ & $3.3421 \mathrm{E}-05$ & $0.28 \%$ ) \\
\hline 1 & $0-16$ & elastic & $1.1922 \mathrm{E}-02$ & $+/-$ & $3.3419 \mathrm{E}-05$ & $0.28 \%$ ) \\
\hline 1 & $0-16$ & $\mathrm{n}, \mathrm{n}^{\prime}$ & $8.7378 \mathrm{E}-05$ & $+/-$ & $1.9449 \mathrm{E}-07$ & $0.22 \%)$ \\
\hline 1 & $0-16$ & $n, 2 n$ & $1.0127 \mathrm{E}-11$ & $+/-$ & $1.6420 \mathrm{E}-20$ & $0.00 \%$ ) \\
\hline 1 & $0-16$ & capture & $-5.6947 \mathrm{E}-04$ & $+1-$ & $1.5760 \mathrm{E}-07$ & $0.03 \%)$ \\
\hline 1 & $0-16$ & $\mathrm{n}$, gamma & $-1.0052 \mathrm{E}-05$ & $+/-$ & $1.0479 \mathrm{E}-09$ & $0.01 \%$ ) \\
\hline 1 & $0-16$ & $\mathrm{n}, \mathrm{p}$ & $-2.8703 E-06$ & $+/-$ & $6.5195 \mathrm{E}-09$ & $0.23 \%)$ \\
\hline 1 & $0-16$ & $\mathrm{n}, \mathrm{d}$ & $-2.9590 \mathrm{E}-07$ & $+1-$ & $1.2700 \mathrm{E}-09$ & $0.43 \%)$ \\
\hline 1 & $0-16$ & $n, t$ & $-1.0698 \mathrm{E}-12$ & $+/-$ & $2.9755 E-21$ & $0.00 \%)$ \\
\hline 1 & $0-16$ & n, alpha & $-5.5625 E-04$ & $+1-$ & $1.5540 \mathrm{E}-07$ & $0.03 \%)$ \\
\hline 1 & $u-234$ & total & $-9.0979 E-04$ & $+/-$ & $3.1204 \mathrm{E}-07$ & $0.03 \%)$ \\
\hline 1 & $u-234$ & scatter & $6.2441 \mathrm{E}-06$ & $+/-$ & $1.2041 E-08$ & $0.19 \%)$ \\
\hline 1 & $u-234$ & elastic & $4.0083 E-06$ & $+1-$ & $1.1854 \mathrm{E}-08$ & $0.30 \%)$ \\
\hline 1 & $u-234$ & $\mathrm{n}, \mathrm{n}^{\prime}$ & $2.2050 \mathrm{E}-06$ & $+/-$ & $1.4630 \mathrm{E}-09$ & $0.07 \%$ ) \\
\hline 1 & $u-234$ & $n, 2 n$ & $3.0794 \mathrm{E}-08$ & $+/-$ & $1.7472 \mathrm{E}-11$ & $0.06 \%)$ \\
\hline 1 & $u-234$ & fission & $2.2909 \mathrm{E}-05$ & $+/-$ & $1.7241 \mathrm{E}-09$ & $0.01 \%)$ \\
\hline 1 & $u-234$ & capture & $-9.3894 \mathrm{E}-04$ & $+/-$ & $3.0291 \mathrm{E}-07$ & $0.03 \%)$ \\
\hline 1 & $u-234$ & $\mathrm{n}$, gamma & $-9.3894 \mathrm{E}-04$ & $+1-$ & $3.0291 \mathrm{E}-07$ & $0.03 \%)$ \\
\hline 1 & $u-234$ & nubar & $3.6021 \mathrm{E}-05$ & $+/-$ & $2.6348 \mathrm{E}-09$ & $0.01 \%)$ \\
\hline 1 & $u-234$ & chi & $8.7265 \mathrm{E}-13$ & $+/-$ & $1.5382 \mathrm{E}-09$ & $(-999.99 \%)$ \\
\hline 1 & $u-235$ & total & $2.0616 \mathrm{E}-01$ & $+1-$ & $7.0501 E-05$ & $0.03 \%$ ) \\
\hline
\end{tabular}

Figure 6.3.16. SAMS output for LEU-COMP-THERM-009 case 10 sample problem. (continued) 


\begin{tabular}{|c|c|c|c|c|}
\hline$u-235$ & scatter & $8.8279 E-04$ & $+/-1.0661 \mathrm{E}-06$ & $0.12 \%)$ \\
\hline$u-235$ & elastic & $3.1799 \mathrm{E}-04$ & $+/-9.6547 \mathrm{E}-07$ & $0.30 \%)$ \\
\hline$u-235$ & $\mathrm{n}, \mathrm{n}^{\prime}$ & $5.3198 \mathrm{E}-04$ & $+/-3.3888 \mathrm{E}-07$ & $0.06 \%)$ \\
\hline$u-235$ & $n, 2 n$ & $3.2795 E-05$ & $+/-1.0524 \mathrm{E}-08$ & $0.03 \%)$ \\
\hline$u-235$ & fission & $3.2433 \mathrm{E}-01$ & $+/-6.2460 \mathrm{E}-05$ & $0.02 \%)$ \\
\hline$u-235$ & capture & $-1.1906 \mathrm{E}-01$ & $+/-1.1810 \mathrm{E}-05$ & $0.01 \%)$ \\
\hline$u-235$ & $\mathrm{n}$, gamma & $-1.1906 \mathrm{E}-01$ & $+/-1.1810 \mathrm{E}-05$ & $0.01 \%)$ \\
\hline$u-235$ & nubar & $9.6382 \mathrm{E}-01$ & $+/-8.0129 \mathrm{E}-05$ & $0.01 \%)$ \\
\hline$u-235$ & chi & $-6.6884 \mathrm{E}-08$ & $+/-4.6474 \mathrm{E}-05$ & $(-999.99 \%)$ \\
\hline$u-236$ & total & $-2.1805 E-04$ & $+/-1.2725 \mathrm{E}-07$ & $0.06 \%)$ \\
\hline$u-236$ & scatter & $5.6471 \mathrm{E}-06$ & $+/-9.6565 \mathrm{E}-09$ & $0.17 \%)$ \\
\hline$u-236$ & elastic & $2.3929 \mathrm{E}-06$ & $+/-9.2543 \mathrm{E}-09$ & $0.39 \%$ \\
\hline$u-236$ & $\mathrm{n}, \mathrm{n}^{\prime}$ & $3.1626 \mathrm{E}-06$ & $+/-2.0863 \mathrm{E}-09$ & $0.07 \%)$ \\
\hline$u-236$ & $n, 2 n$ & $9.1002 \mathrm{E}-08$ & $+/-4.1257 \mathrm{E}-11$ & $0.05 \%)$ \\
\hline$u-236$ & fission & $1.2829 \mathrm{E}-05$ & $+/-1.7760 \mathrm{E}-09$ & $0.01 \%)$ \\
\hline$u-236$ & capture & $-2.3653 \mathrm{E}-04$ & $+/-1.2003 \mathrm{E}-07$ & $0.05 \%)$ \\
\hline$u-236$ & $\mathrm{n}$, gamma & $-2.3653 E-04$ & $+/-1.2003 \mathrm{E}-07$ & $0.05 \%)$ \\
\hline$u-236$ & nubar & $2.1111 \mathrm{E}-05$ & $+/-1.6677 \mathrm{E}-09$ & $0.01 \%)$ \\
\hline$u-236$ & chi & $-4.2095 E-13$ & $+/-1.0077 \mathrm{E}-09$ & $(-999.99 \%)$ \\
\hline$u-238$ & total & $-5.9609 \mathrm{E}-02$ & $+/-2.6857 \mathrm{E}-05$ & $0.05 \%)$ \\
\hline$u-238$ & scatter & $2.9406 \mathrm{E}-02$ & $+/-2.0373 \mathrm{E}-05$ & $0.07 \%)$ \\
\hline$u-238$ & elastic & $1.3217 \mathrm{E}-02$ & $+/-1.6047 \mathrm{E}-05$ & $0.12 \%)$ \\
\hline$u-238$ & $\mathrm{n}, \mathrm{n}^{\prime}$ & $1.5251 \mathrm{E}-02$ & $+/-9.7098 \mathrm{E}-06$ & $0.06 \%)$ \\
\hline$u-238$ & $n, 2 n$ & $9.2840 \mathrm{E}-04$ & $+/-3.7033 E-07$ & $0.04 \%)$ \\
\hline$u-238$ & fission & $2.4727 E-02$ & $+/-2.4196 \mathrm{E}-06$ & $0.01 \%)$ \\
\hline$u-238$ & capture & $-1.1374 \mathrm{E}-01$ & $+/-9.9579 \mathrm{E}-06$ & $0.01 \%)$ \\
\hline$u-238$ & $\mathrm{n}$, gamma & $-1.1374 \mathrm{E}-01$ & $+/-9.9579 \mathrm{E}-06$ & $0.01 \%)$ \\
\hline$u-238$ & nubar & $3.6122 \mathrm{E}-02$ & $+/-3.8010 \mathrm{E}-06$ & $0.01 \%)$ \\
\hline$u-238$ & chi & $-2.9925 \mathrm{E}-09$ & $+/-1.7124 \mathrm{E}-06$ & $(-999.99 \%)$ \\
\hline $\mathrm{h}-1$ & total & $2.0159 \mathrm{E}-01$ & $+/-4.6480 \mathrm{E}-04$ & $0.23 \%)$ \\
\hline $\mathrm{h}-1$ & scatter & 3. $4592 \mathrm{E}-01$ & $+/-4.6062 \mathrm{E}-04$ & $0.13 \%)$ \\
\hline $\mathrm{h}-1$ & elastic & 3. $4592 \mathrm{E}-01$ & $+/-4.6062 \mathrm{E}-04$ & $0.13 \%)$ \\
\hline $\mathrm{h}-1$ & capture & $-1.4433 \mathrm{E}-01$ & $+/-1.0386 \mathrm{E}-05$ & $0.01 \%)$ \\
\hline $\mathrm{h}-1$ & $\mathrm{n}$, gamma & $-1.4433 \mathrm{E}-01$ & $+/-1.0386 \mathrm{E}-05$ & $0.01 \%)$ \\
\hline $0-16$ & total & $3.6005 \mathrm{E}-02$ & $+/-2.9143 \mathrm{E}-05$ & $0.08 \%)$ \\
\hline $0-16$ & scatter & $3.7111 \mathrm{E}-02$ & $+/-2.9137 \mathrm{E}-05$ & $0.08 \%)$ \\
\hline $0-16$ & elastic & 3. $6871 \mathrm{E}-02$ & $+/-2.9136 \mathrm{E}-05$ & $0.08 \%)$ \\
\hline $0-16$ & $\mathrm{n}, \mathrm{n}^{\prime}$ & $2.3245 \mathrm{E}-04$ & $+/-1.5061 \mathrm{E}-07$ & $0.06 \%)$ \\
\hline $0-16$ & $\mathrm{n}, 2 \mathrm{n}$ & 2. $3322 \mathrm{E}-11$ & $+/-1.2879 \mathrm{E}-17$ & $0.00 \%)$ \\
\hline $0-16$ & capture & $-1.1055 E-03$ & $+/-1.4843 \mathrm{E}-07$ & $0.01 \%)$ \\
\hline $0-16$ & $\mathrm{n}$, gamma & $-4.1305 E-05$ & $+/-2.9719 \mathrm{E}-09$ & $0.01 \%)$ \\
\hline $0-16$ & $\mathrm{n}, \mathrm{p}$ & $-5.3552 \mathrm{E}-06$ & $+/-5.0619 \mathrm{E}-09$ & $0.09 \%)$ \\
\hline $0-16$ & $\mathrm{n}, \mathrm{d}$ & $-5.5135 \mathrm{E}-07$ & $+/-9.9279 \mathrm{E}-10$ & $0.18 \%)$ \\
\hline $0-16$ & $n, t$ & $-2.0353 \mathrm{E}-12$ & $+/-2.3337 \mathrm{E}-18$ & $0.00 \%)$ \\
\hline $0-16$ & n, alpha & $-1.0583 E-03$ & $+/-1.4700 \mathrm{E}-07$ & $0.01 \%)$ \\
\hline$m g-24$ & total & $4.5166 \mathrm{E}-05$ & $+/-4.5339 \mathrm{E}-07$ & $1.00 \%)$ \\
\hline$m g-24$ & scatter & $5.5339 \mathrm{E}-05$ & $+/-4.5356 \mathrm{E}-07$ & $0.82 \%)$ \\
\hline$m g-24$ & elastic & 4. $3547 \mathrm{E}-05$ & $+/-4.5024 \mathrm{E}-07$ & $1.03 \%$ \\
\hline
\end{tabular}

Figure 6.3.16. SAMS output for LEU-COMP-THERM-009 case 10 sample problem. (continued) 


\begin{tabular}{|c|c|c|c|c|}
\hline$m g-24$ & $\mathrm{n}, \mathrm{n}^{\prime}$ & $1.1781 \mathrm{E}-05$ & $+/-3.3434 \mathrm{E}-08$ & $0.28 \%)$ \\
\hline$m g-24$ & $n, 2 n$ & $5.8600 \mathrm{E}-13$ & $+/-1.0107 \mathrm{E}-22$ & $0.00 \%)$ \\
\hline$m g-24$ & capture & $-1.0173 E-05$ & $+/-3.6669 \mathrm{E}-09$ & $0.04 \%)$ \\
\hline $\mathrm{mg}-24$ & $\mathrm{n}$, gamma & $-9.1039 E-06$ & $+/-3.1240 \mathrm{E}-09$ & $0.03 \%)$ \\
\hline$m g-24$ & $\mathrm{n}, \mathrm{p}$ & $-2.5459 \mathrm{E}-07$ & $+/-5.7541 \mathrm{E}-10$ & $0.23 \%)$ \\
\hline$m g-24$ & n,alpha & $-8.1441 \mathrm{E}-07$ & $+/-1.3622 \mathrm{E}-09$ & $0.17 \%)$ \\
\hline $\mathrm{mg}-25$ & total & $1.2140 \mathrm{E}-06$ & $+/-3.2733 E-08$ & $2.70 \%)$ \\
\hline $\mathrm{mg}-25$ & scatter & $5.8458 \mathrm{E}-06$ & $+/-3.1369 \mathrm{E}-08$ & $0.54 \%)$ \\
\hline$m g-25$ & elastic & $4.2233 \mathrm{E}-06$ & $+/-3.0490 \mathrm{E}-08$ & $0.72 \%)$ \\
\hline$m g-25$ & $\mathrm{n}, \mathrm{n}^{\prime}$ & $1.5854 \mathrm{E}-06$ & $+/-3.7904 \mathrm{E}-09$ & $0.24 \%)$ \\
\hline $\mathrm{mg}-25$ & $n, 2 n$ & $3.6936 \mathrm{E}-08$ & $+/-9.6404 \mathrm{E}-11$ & $0.26 \%)$ \\
\hline $\mathrm{mg}-25$ & capture & $-4.6318 E-06$ & $+/-1.5204 \mathrm{E}-09$ & $0.03 \%)$ \\
\hline $\mathrm{mg}-25$ & $\mathrm{n}$, gamma & $-4.2701 \mathrm{E}-06$ & $+/-1.4848 \mathrm{E}-09$ & $0.03 \%)$ \\
\hline$m g-25$ & $\mathrm{n}, \mathrm{p}$ & $-3.2369 \mathrm{E}-08$ & $+/-3.3458 \mathrm{E}-11$ & $0.10 \%)$ \\
\hline$m g-25$ & n,alpha & $-3.2935 E-07$ & $+/-2.8796 \mathrm{E}-10$ & $0.09 \%)$ \\
\hline$m g-26$ & total & $5.2992 \mathrm{E}-06$ & $+/-3.2698 \mathrm{E}-08$ & $0.62 \%$ \\
\hline$m g-26$ & scatter & $6.2529 \mathrm{E}-06$ & $+/-3.2364 \mathrm{E}-08$ & $0.52 \%)$ \\
\hline$m g-26$ & elastic & $4.8094 \mathrm{E}-06$ & $+/-3.1809 \mathrm{E}-08$ & $0.66 \%)$ \\
\hline$m g-26$ & $\mathrm{n}, \mathrm{n}^{\prime}$ & $1.4399 \mathrm{E}-06$ & $+/-3.4023 E-09$ & $0.24 \%)$ \\
\hline $\mathrm{mg}-26$ & $n, 2 n$ & $3.6117 \mathrm{E}-09$ & $+/-2.0668 \mathrm{E}-18$ & $0.00 \%)$ \\
\hline $\mathrm{mg}-26$ & capture & $-9.5364 \mathrm{E}-07$ & $+/-3.2481 \mathrm{E}-10$ & $0.03 \%)$ \\
\hline$m g-26$ & $\mathrm{n}$, gamma & $-9.4910 \mathrm{E}-07$ & $+/-3.2481 \mathrm{E}-10$ & $0.03 \%)$ \\
\hline$m g-26$ & $\mathrm{n}, \mathrm{p}$ & $-5.2381 E-10$ & $+/-1.5687 \mathrm{E}-19$ & $0.00 \%)$ \\
\hline $\mathrm{mg}-26$ & n,alpha & $-4.0194 \mathrm{E}-09$ & $+/-5.3317 \mathrm{E}-19$ & $0.00 \%$ \\
\hline al-27 & total & $-6.7582 E-05$ & $+/-2.3201 \mathrm{E}-05$ & ( $34.33 \%)$ \\
\hline al-27 & scatter & $4.6080 \mathrm{E}-03$ & $+/-2.2663 \mathrm{E}-05$ & $0.49 \%$ \\
\hline al-27 & elastic & $3.2847 E-03$ & $+/-2.1856 \mathrm{E}-05$ & $0.67 \%$ \\
\hline al-27 & $\mathrm{n}, \mathrm{n}^{\prime}$ & $1.3189 \mathrm{E}-03$ & $+/-3.0167 \mathrm{E}-06$ & $0.23 \%)$ \\
\hline al-27 & $n, 2 n$ & $5.9944 \mathrm{E}-08$ & $+/-1.4100 \mathrm{E}-09$ & $2.35 \%$ \\
\hline al-27 & capture & $-4.6756 \mathrm{E}-03$ & $+/-1.6107 \mathrm{E}-06$ & $0.03 \%)$ \\
\hline al-27 & $\mathrm{n}$, gamma & $-4.5949 E-03$ & $+/-1.6079 \mathrm{E}-06$ & $0.03 \%)$ \\
\hline al-27 & $\mathrm{n}, \mathrm{p}$ & $-6.7911 \mathrm{E}-05$ & $+/-6.9477 \mathrm{E}-08$ & $0.10 \%)$ \\
\hline al-27 & $\mathrm{n}, \mathrm{d}$ & $-1.9733 E-07$ & $+/-1.4218 \mathrm{E}-09$ & $0.72 \%)$ \\
\hline al-27 & $n, t$ & $-6.6132 \mathrm{E}-09$ & $+/-5.0053 \mathrm{E}-16$ & $0.00 \%)$ \\
\hline al-27 & n, alpha & $-1.2531 \mathrm{E}-05$ & $+/-3.2986 \mathrm{E}-08$ & $0.26 \%)$ \\
\hline si-28 & total & 4. $3107 \mathrm{E}-06$ & $+/-1.2244 \mathrm{E}-07$ & $2.84 \%$ \\
\hline si-28 & scatter & $2.3404 \mathrm{E}-05$ & $+/-1.2022 \mathrm{E}-07$ & $0.51 \%)$ \\
\hline si-28 & elastic & $1.8601 \mathrm{E}-05$ & $+/-1.1757 \mathrm{E}-07$ & $0.63 \%)$ \\
\hline si-28 & $n, n^{\prime}$ & $4.8005 \mathrm{E}-06$ & $+/-1.3001 \mathrm{E}-08$ & $0.27 \%)$ \\
\hline si-28 & capture & $-1.9094 \mathrm{E}-05$ & $+/-6.5542 \mathrm{E}-09$ & $0.03 \%)$ \\
\hline si-28 & $\mathrm{n}$, gamma & $-1.8139 \mathrm{E}-05$ & $+/-6.3599 \mathrm{E}-09$ & $0.04 \%)$ \\
\hline si-28 & $\mathrm{n}, \mathrm{p}$ & $-6.5750 \mathrm{E}-07$ & $+/-1.0533 \mathrm{E}-09$ & $0.16 \%)$ \\
\hline si-28 & $\mathrm{n}, \mathrm{d}$ & $-8.8870 \mathrm{E}-10$ & $+/-3.5446 \mathrm{E}-19$ & $0.00 \%)$ \\
\hline si-28 & n, alpha & $-2.9630 \mathrm{E}-07$ & $+/-5.3824 \mathrm{E}-10$ & $0.18 \%)$ \\
\hline si-29 & total & $5.3687 \mathrm{E}-07$ & $+/-5.6554 \mathrm{E}-09$ & $1.05 \%$ \\
\hline si-29 & scatter & $1.2496 \mathrm{E}-06$ & $+/-4.0360 \mathrm{E}-09$ & $0.32 \%)$ \\
\hline si-29 & elastic & $8.0857 \mathrm{E}-07$ & $+/-3.6454 \mathrm{E}-09$ & $0.45 \%)$ \\
\hline si-29 & $n, n^{\prime}$ & 4. $3972 \mathrm{E}-07$ & $+/-9.9759 \mathrm{E}-10$ & $0.23 \%$ \\
\hline
\end{tabular}

Figure 6.3.16. SAMS output for LEU-COMP-THERM-009 case 10 sample problem. (continued) 


\begin{tabular}{|c|c|c|c|c|}
\hline si-29 & $\mathrm{n}, 2 \mathrm{n}$ & $1.2847 E-09$ & $+/-2.7509 \mathrm{E}-19$ & $0.00 \%)$ \\
\hline si-29 & capture & $-7.1270 \mathrm{E}-07$ & $+/-2.3029 \mathrm{E}-10$ & $0.03 \%)$ \\
\hline si-29 & $\mathrm{n}$, gamma & $-6.6526 \mathrm{E}-07$ & $+/-2.2493 \mathrm{E}-10$ & $0.03 \%)$ \\
\hline si-29 & $\mathrm{n}, \mathrm{p}$ & $-1.7830 \mathrm{E}-08$ & $+/-1.7962 \mathrm{E}-11$ & $0.10 \%)$ \\
\hline si-29 & n, alpha & $-2.9617 \mathrm{E}-08$ & $+/-2.2339 \mathrm{E}-11$ & $0.08 \%)$ \\
\hline si-30 & total & $1.3408 \mathrm{E}-07$ & $+/-4.4925 \mathrm{E}-09$ & $3.35 \%$ \\
\hline si-30 & scatter & $7.3741 \mathrm{E}-07$ & $+/-3.2216 \mathrm{E}-09$ & $0.44 \%)$ \\
\hline si-30 & elastic & $5.4630 \mathrm{E}-07$ & $+/-3.1391 E-09$ & $0.57 \%)$ \\
\hline si-30 & $\mathrm{n}, \mathrm{n}^{\prime}$ & $1.9023 \mathrm{E}-07$ & $+/-4.2667 \mathrm{E}-10$ & $0.22 \%)$ \\
\hline si-30 & $\mathrm{n}, 2 \mathrm{n}$ & $8.7604 \mathrm{E}-10$ & $+/-2.6538 \mathrm{E}-19$ & $0.00 \%)$ \\
\hline si-30 & capture & $-6.0334 \mathrm{E}-07$ & $+/-8.1712 \mathrm{E}-10$ & $0.14 \%)$ \\
\hline si-30 & $\mathrm{n}$, gamma & $-6.0283 E-07$ & $+/-8.1712 \mathrm{E}-10$ & $0.14 \%)$ \\
\hline si-30 & $\mathrm{n}, \mathrm{p}$ & $-9.2891 \mathrm{E}-11$ & $+/-2.3442 \mathrm{E}-20$ & $0.00 \%)$ \\
\hline si-30 & n, alpha & $-4.1193 \mathrm{E}-10$ & $+/-7.5633 \mathrm{E}-20$ & $0.00 \%)$ \\
\hline ti-46 & total & $-1.8856 \mathrm{E}-07$ & $+/-8.6800 \mathrm{E}-10$ & $0.46 \%)$ \\
\hline ti-46 & scatter & $2.1933 \mathrm{E}-07$ & $+/-4.7014 \mathrm{E}-10$ & $0.21 \%)$ \\
\hline ti-46 & elastic & $1.2896 \mathrm{E}-07$ & $+/-3.2795 \mathrm{E}-10$ & $0.25 \%)$ \\
\hline ti-46 & $\mathrm{n}, \mathrm{n}^{\prime}$ & $9.0312 \mathrm{E}-08$ & $+/-1.9591 \mathrm{E}-10$ & $0.22 \%)$ \\
\hline ti-46 & $\mathrm{n}, 2 \mathrm{n}$ & 4. $8498 \mathrm{E}-12$ & $+/-2.8827 \mathrm{E}-21$ & $0.00 \%)$ \\
\hline ti-46 & capture & $-4.0789 E-07$ & $+/-1.3319 \mathrm{E}-10$ & $0.03 \%)$ \\
\hline ti-46 & $\mathrm{n}$, gamma & $-3.9961 \mathrm{E}-07$ & $+/-1.3319 \mathrm{E}-10$ & $0.03 \%)$ \\
\hline ti-46 & $n, p$ & $-7.7485 E-09$ & $+/-3.8514 \mathrm{E}-18$ & $0.00 \%)$ \\
\hline ti-46 & $\mathrm{n}, \mathrm{d}$ & $-3.1806 E-13$ & $+/-2.3627 \mathrm{E}-22$ & $0.00 \%)$ \\
\hline ti-46 & $n, t$ & $-9.9763 E-17$ & $+/-3.2655 \mathrm{E}-26$ & $0.00 \%)$ \\
\hline ti-46 & $\mathrm{n}, \mathrm{he}-3$ & $-4.2439 E-16$ & $+/-1.9547 \mathrm{E}-25$ & $0.00 \%)$ \\
\hline ti-46 & n, alpha & $-5.2794 \mathrm{E}-10$ & $+/-2.5965 \mathrm{E}-19$ & $0.00 \%)$ \\
\hline ti-47 & total & $-8.7007 \mathrm{E}-07$ & $+/-2.4463 \mathrm{E}-09$ & $0.28 \%)$ \\
\hline ti-47 & scatter & $2.4268 \mathrm{E}-07$ & $+/-2.2853 \mathrm{E}-09$ & $0.94 \%)$ \\
\hline ti-47 & elastic & $1.3531 \mathrm{E}-07$ & $+/-2.2669 \mathrm{E}-09$ & $1.68 \%)$ \\
\hline ti-47 & $\mathrm{n}, \mathrm{n}^{\prime}$ & $1.0717 \mathrm{E}-07$ & $+/-1.8872 \mathrm{E}-10$ & $0.18 \%)$ \\
\hline ti-47 & $\mathrm{n}, 2 \mathrm{n}$ & $1.9211 \mathrm{E}-10$ & $+/-4.0015 \mathrm{E}-20$ & $0.00 \% 1$ \\
\hline ti-47 & capture & $-1.1128 \mathrm{E}-06$ & $+/-3.6552 \mathrm{E}-10$ & $0.03 \%)$ \\
\hline ti-47 & $\mathrm{n}$, gamma & $-1.1003 E-06$ & $+/-3.6552 \mathrm{E}-10$ & $0.03 \%)$ \\
\hline ti-47 & $n, p$ & $-1.0646 \mathrm{E}-08$ & $+/-1.4197 \mathrm{E}-16$ & $0.00 \%)$ \\
\hline ti-47 & $\mathrm{n}, \mathrm{d}$ & $-2.0197 \mathrm{E}-13$ & $+/-1.5135 \mathrm{E}-22$ & $0.00 \%)$ \\
\hline ti-47 & $n, t$ & $-4.3601 \mathrm{E}-15$ & $+/-3.0922 \mathrm{E}-24$ & $0.00 \%)$ \\
\hline$t i-47$ & $n$, he-3 & $-2.3300 E-17$ & $+/-7.4079 \mathrm{E}-27$ & $0.00 \%$ ) \\
\hline ti-47 & n, alpha & $-1.8482 \mathrm{E}-09$ & $+/-1.3222 \mathrm{E}-17$ & $0.00 \%)$ \\
\hline ti-48 & total & $-4.5957 \mathrm{E}-05$ & $+/-3.3263 \mathrm{E}-08$ & $0.07 \%)$ \\
\hline$t i-48$ & scatter & $3.0030 \mathrm{E}-06$ & $+/-2.3419 \mathrm{E}-08$ & $0.78 \%)$ \\
\hline$t i-48$ & elastic & $2.1566 \mathrm{E}-06$ & $+/-2.3249 \mathrm{E}-08$ & $1.08 \%)$ \\
\hline$t i-48$ & $\mathrm{n}, \mathrm{n}^{\prime}$ & $8.4526 \mathrm{E}-07$ & $+/-1.8984 \mathrm{E}-09$ & $0.22 \%$ \\
\hline$t i-48$ & $\mathrm{n}, 2 \mathrm{n}$ & $1.0593 \mathrm{E}-09$ & $+/-5.3938 \mathrm{E}-19$ & $0.00 \%)$ \\
\hline$t i-48$ & capture & $-4.8960 \mathrm{E}-05$ & $+/-1.7297 \mathrm{E}-08$ & $0.04 \%)$ \\
\hline ti-48 & $\mathrm{n}$, gamma & $-4.8958 E-05$ & $+/-1.7297 \mathrm{E}-08$ & $0.04 \%)$ \\
\hline$t i-48$ & $\mathrm{n}, \mathrm{p}$ & $-1.6058 \mathrm{E}-09$ & $+/-9.4652 \mathrm{E}-19$ & $0.00 \%)$ \\
\hline$t i-48$ & $\mathrm{n}, \mathrm{d}$ & $-1.6876 \mathrm{E}-12$ & $+/-1.6673 \mathrm{E}-21$ & $0.00 \%)$ \\
\hline$t i-48$ & $n, t$ & $-8.0952 \mathrm{E}-16$ & $+/-2.7332 E-25$ & $0.00 \%$ ) \\
\hline
\end{tabular}

Figure 6.3.16. SAMS output for LEU-COMP-THERM-009 case 10 sample problem. (continued) 


\begin{tabular}{|c|c|c|c|c|}
\hline ti-48 & $\mathrm{n}, \mathrm{he}-3$ & $-1.9723 E-17$ & $+/-6.7861 E-27$ & $0.00 \%)$ \\
\hline$t i-48$ & n, alpha & $-1.5654 \mathrm{E}-10$ & $+/-4.0991 \mathrm{E}-20$ & $0.00 \%)$ \\
\hline ti-49 & total & $-6.8733 E-07$ & $+/-6.2660 \mathrm{E}-10$ & $0.09 \%)$ \\
\hline ti-49 & scatter & $1.6784 \mathrm{E}-07$ & $+/-2.2439 E-09$ & $1.34 \%)$ \\
\hline ti-49 & elastic & $1.0265 \mathrm{E}-07$ & $+/-2.2349 \mathrm{E}-09$ & $2.18 \%)$ \\
\hline ti-49 & $\mathrm{n}, \mathrm{n}^{\prime}$ & $6.4463 E-08$ & $+/-1.2032 \mathrm{E}-10$ & $0.19 \%)$ \\
\hline ti-49 & $n, 2 n$ & $7.2472 \mathrm{E}-10$ & $+/-7.6807 \mathrm{E}-20$ & $0.00 \%)$ \\
\hline ti-49 & capture & $-8.5517 E-07$ & $+/-2.9502 E-10$ & $0.03 \%)$ \\
\hline ti-49 & $\mathrm{n}$, gamma & $-8.5476 \mathrm{E}-07$ & $+/-2.9502 E-10$ & $0.03 \%)$ \\
\hline ti-49 & $\mathrm{n}, \mathrm{p}$ & $-3.3313 E-10$ & $+/-3.2208 E-19$ & $0.00 \%)$ \\
\hline ti-49 & $n, d$ & $-7.4647 \mathrm{E}-14$ & $+/-6.7611 \mathrm{E}-23$ & $0.00 \%)$ \\
\hline ti-49 & $n, t$ & $-2.1162 E-15$ & $+/-9.3763 E-25$ & $0.00 \%)$ \\
\hline ti-49 & $\mathrm{n}, \mathrm{he}-3$ & $-9.3750 \mathrm{E}-20$ & $+/-0.0000 \mathrm{E}+00$ & $0.00 \%)$ \\
\hline ti-49 & n, alpha & $-7.1068 E-11$ & $+/-4.3628 \mathrm{E}-20$ & $0.00 \%)$ \\
\hline ti-50 & total & $5.1758 \mathrm{E}-08$ & $+/-4.1741 \mathrm{E}-10$ & $0.81 \%)$ \\
\hline ti-50 & scatter & 1. $3378 \mathrm{E}-07$ & $+/-1.9577 \mathrm{E}-10$ & $0.15 \%)$ \\
\hline ti-50 & elastic & $8.8832 \mathrm{E}-08$ & $+/-1.1755 \mathrm{E}-10$ & $0.13 \%)$ \\
\hline$t i-50$ & $\mathrm{n}, \mathrm{n}^{\prime}$ & $4.4731 E-08$ & $+/-8.8290 \mathrm{E}-11$ & $0.20 \%)$ \\
\hline ti-50 & $n, 2 n$ & $2.1770 \mathrm{E}-10$ & $+/-7.0963 E-20$ & $0.00 \%)$ \\
\hline ti-50 & capture & $-8.2023 E-08$ & $+/-2.5593 E-11$ & $0.03 \%)$ \\
\hline ti-50 & $\mathrm{n}$, gamma & $-8.2018 E-08$ & $+/-2.5593 E-11$ & $0.03 \%)$ \\
\hline ti-50 & $\mathrm{n}, \mathrm{p}$ & $-2.9356 E-12$ & $+/-9.4805 \mathrm{E}-22$ & $0.00 \%)$ \\
\hline ti-50 & $n, d$ & $-1.7101 E-14$ & $+/-1.1908 \mathrm{E}-23$ & $0.00 \%)$ \\
\hline ti-50 & $n, t$ & $-1.0953 E-17$ & $+/-2.8155 E-27$ & $0.00 \%)$ \\
\hline ti-50 & $\mathrm{n}, \mathrm{he}-3$ & $-4.0903 E-23$ & $+/-0.0000 \mathrm{E}+00$ & $0.00 \%)$ \\
\hline ti-50 & n, alpha & $-1.7272 \mathrm{E}-12$ & $+/-6.5310 \mathrm{E}-22$ & $0.00 \%)$ \\
\hline cr -50 & total & $-1.3040 \mathrm{E}-05$ & $+/-9.1891 \mathrm{E}-09$ & $0.07 \%)$ \\
\hline cr -50 & scatter & $1.3376 \mathrm{E}-06$ & $+/-7.2895 \mathrm{E}-09$ & $0.54 \%)$ \\
\hline cr-50 & elastic & $1.2307 \mathrm{E}-06$ & $+/-7.2762 E-09$ & $0.59 \%)$ \\
\hline $\operatorname{cr}-50$ & $\mathrm{n}, \mathrm{n}^{\prime}$ & $1.0681 \mathrm{E}-07$ & $+/-2.3841 \mathrm{E}-10$ & $0.22 \%)$ \\
\hline $\operatorname{cr}-50$ & $\mathrm{n}, 2 \mathrm{n}$ & $3.1922 \mathrm{E}-12$ & $+/-2.3793 E-21$ & $0.00 \%)$ \\
\hline cr-50 & capture & $-1.4377 \mathrm{E}-05$ & $+/-5.0788 \mathrm{E}-09$ & $0.04 \%)$ \\
\hline cr-50 & $\mathrm{n}$, gamma & $-1.4333 E-05$ & $+/-5.0787 \mathrm{E}-09$ & $0.04 \%)$ \\
\hline cr -50 & $\mathrm{n}, \mathrm{p}$ & $-4.1672 E-08$ & $+/-3.2114 \mathrm{E}-11$ & $0.08 \%)$ \\
\hline cr-50 & $\mathrm{n}, \mathrm{d}$ & $-7.5849 \mathrm{E}-12$ & $+/-5.4355 \mathrm{E}-19$ & $0.00 \%)$ \\
\hline cr-50 & n, alpha & $-2.7989 E-09$ & $+/-1.7847 \mathrm{E}-15$ & $0.00 \%)$ \\
\hline$c r-52$ & total & $-8.7501 E-06$ & $+/-2.6630 \mathrm{E}-08$ & $0.30 \%)$ \\
\hline$c r-52$ & scatter & $4.4850 \mathrm{E}-06$ & $+/-2.1763 E-08$ & $0.49 \%)$ \\
\hline$c r-52$ & elastic & $2.3489 \mathrm{E}-06$ & $+/-2.0602 \mathrm{E}-08$ & $0.88 \%)$ \\
\hline$c r-52$ & $\mathrm{n}, \mathrm{n}^{\prime}$ & $2.1346 \mathrm{E}-06$ & $+/-4.5774 \mathrm{E}-09$ & $0.21 \%)$ \\
\hline$c r-52$ & $n, 2 n$ & 1. $3180 \mathrm{E}-09$ & $+/-9.1937 \mathrm{E}-19$ & $0.00 \%)$ \\
\hline cr -52 & capture & $-1.3235 \mathrm{E}-05$ & $+/-4.5982 E-09$ & $0.03 \%)$ \\
\hline$c r-52$ & n, gamma & $-1.3217 \mathrm{E}-05$ & $+/-4.5982 \mathrm{E}-09$ & $0.03 \%)$ \\
\hline$c r-52$ & $n, p$ & $-1.5832 E-08$ & $+/-1.8313 E-11$ & $0.12 \%)$ \\
\hline$c r-52$ & n, alpha & $-1.9304 \mathrm{E}-09$ & $+/-3.8775 E-17$ & $0.00 \%)$ \\
\hline cr -53 & total & $-3.4245 E-05$ & $+/-2.1670 \mathrm{E}-08$ & $0.06 \%)$ \\
\hline cr -53 & scatter & $1.3865 E-06$ & $+/-1.4475 \mathrm{E}-08$ & $1.04 \%)$ \\
\hline cr -53 & elastic & $1.0474 \mathrm{E}-06$ & $+/-1.4432 \mathrm{E}-08$ & $1.38 \%)$ \\
\hline
\end{tabular}

Figure 6.3.16. SAMS output for LEU-COMP-THERM-009 case 10 sample problem. (continued) 


\begin{tabular}{|c|c|c|c|c|}
\hline cr -53 & $\mathrm{n}, \mathrm{n}^{\prime}$ & $3.3527 E-07$ & $+/-7.1251 \mathrm{E}-10$ & $0.21 \%)$ \\
\hline cr-53 & $\mathrm{n}, 2 \mathrm{n}$ & $3.8152 \mathrm{E}-09$ & $+/-3.6614 \mathrm{E}-17$ & $0.00 \%)$ \\
\hline cr -53 & capture & $-3.5632 \mathrm{E}-05$ & $+/-1.2596 \mathrm{E}-08$ & $0.04 \%)$ \\
\hline cr-53 & $\mathrm{n}$, gamma & $-3.5630 \mathrm{E}-05$ & $+/-1.2596 \mathrm{E}-08$ & $0.04 \%)$ \\
\hline cr-53 & $\mathrm{n}, \mathrm{p}$ & $-6.9509 E-10$ & $+/-5.2410 \mathrm{E}-17$ & $0.00 \%)$ \\
\hline cr-53 & n,alpha & $-1.5386 \mathrm{E}-09$ & $+/-1.4981 \mathrm{E}-16$ & $0.00 \%)$ \\
\hline cr-54 & total & $-1.6695 \mathrm{E}-08$ & $+/-4.1104 \mathrm{E}-10$ & $2.46 \%)$ \\
\hline cr-54 & scatter & $1.6318 \mathrm{E}-07$ & $+/-2.3409 \mathrm{E}-10$ & $0.14 \%)$ \\
\hline cr-54 & elastic & $9.3303 E-08$ & $+/-7.9919 \mathrm{E}-11$ & $0.09 \%)$ \\
\hline cr-54 & $\mathrm{n}, \mathrm{n}^{\prime}$ & $6.9373 E-08$ & $+/-1.3815 \mathrm{E}-10$ & $0.20 \%)$ \\
\hline cr-54 & $\mathrm{n}, 2 \mathrm{n}$ & $5.0486 \mathrm{E}-10$ & $+/-8.3567 \mathrm{E}-20$ & $0.00 \%)$ \\
\hline cr-54 & capture & $-1.7988 \mathrm{E}-07$ & $+/-5.9710 \mathrm{E}-11$ & $0.03 \%)$ \\
\hline cr-54 & $\mathrm{n}$, gamma & $-1.7985 E-07$ & $+/-5.9710 \mathrm{E}-11$ & $0.03 \%)$ \\
\hline cr-54 & $\mathrm{n}, \mathrm{p}$ & $-4.2582 \mathrm{E}-12$ & $+/-1.1391 \mathrm{E}-21$ & $0.00 \%)$ \\
\hline cr-54 & n, alpha & $-2.1413 E-11$ & $+/-1.0788 \mathrm{E}-20$ & $0.00 \%)$ \\
\hline$m n-55$ & total & $-9.6360 \mathrm{E}-05$ & $+/-1.4079 \mathrm{E}-07$ & $0.15 \%)$ \\
\hline$m n-55$ & scatter & $6.8704 \mathrm{E}-06$ & $+/-1.3262 \mathrm{E}-07$ & $1.93 \%)$ \\
\hline$m n-55$ & elastic & $5.7292 \mathrm{E}-06$ & $+/-1.3254 \mathrm{E}-07$ & $2.31 \%)$ \\
\hline$m n-55$ & $\mathrm{n}, \mathrm{n}^{\prime}$ & $1.1366 \mathrm{E}-06$ & $+/-2.5436 \mathrm{E}-09$ & $0.22 \%)$ \\
\hline$m n-55$ & $n, 2 n$ & $4.4184 \mathrm{E}-09$ & $+/-1.0180 \mathrm{E}-18$ & $0.00 \div)$ \\
\hline$m n-55$ & capture & $-1.0323 E-04$ & $+/-3.5222 \mathrm{E}-08$ & $0.03 \%)$ \\
\hline$m n-55$ & $\mathrm{n}$, gamma & $-1.0323 E-04$ & $+/-3.5222 \mathrm{E}-08$ & $0.03 \%)$ \\
\hline$m n-55$ & $\mathrm{n}, \mathrm{p}$ & $-3.7300 \mathrm{E}-09$ & $+/-9.1810 \mathrm{E}-18$ & $0.00 \%)$ \\
\hline$m n-55$ & $\mathrm{n}, \mathrm{d}$ & $-3.5791 E-11$ & $+/-6.7992 \mathrm{E}-21$ & $0.00 \div)$ \\
\hline$m n-55$ & $n, t$ & $-1.3299 \mathrm{E}-12$ & $+/-1.2646 \mathrm{E}-21$ & $0.00 \div)$ \\
\hline$m n-55$ & $\mathrm{n}, \mathrm{he}-3$ & $-4.6031 E-14$ & $+/-4.8445 \mathrm{E}-23$ & $0.00 \%)$ \\
\hline$m n-55$ & n,alpha & $-8.3737 \mathrm{E}-10$ & $+/-6.0947 \mathrm{E}-19$ & $0.00 \%)$ \\
\hline$f e-54$ & total & $-3.9407 \mathrm{E}-06$ & $+/-8.5216 \mathrm{E}-09$ & $0.22 \%)$ \\
\hline$f e-54$ & scatter & $7.5145 \mathrm{E}-07$ & $+/-8.1027 \mathrm{E}-09$ & $1.08 \%)$ \\
\hline$f e-54$ & elastic & $5.5492 \mathrm{E}-07$ & $+/-8.0723 E-09$ & $1.45 \%)$ \\
\hline$f e-54$ & $\mathrm{n}, \mathrm{n}^{\prime}$ & $1.9601 \mathrm{E}-07$ & $+/-4.3649 \mathrm{E}-10$ & $0.22 \%)$ \\
\hline$f e-54$ & $\mathrm{n}, 2 \mathrm{n}$ & $3.7980 \mathrm{E}-12$ & $+/-1.3165 \mathrm{E}-21$ & $0.00 \%)$ \\
\hline$f e-54$ & capture & $-4.6921 \mathrm{E}-06$ & $+/-1.5839 \mathrm{E}-09$ & $0.03 \%)$ \\
\hline$f e-54$ & $\mathrm{n}$, gamma & $-4.5400 \mathrm{E}-06$ & $+/-1.5807 \mathrm{E}-09$ & $0.03 \%)$ \\
\hline$f e-54$ & $\mathrm{n}, \mathrm{p}$ & $-1.5046 \mathrm{E}-07$ & $+/-9.9241 \mathrm{E}-11$ & $0.07 \%)$ \\
\hline$f e-54$ & $\mathrm{n}, \mathrm{d}$ & $-2.5789 E-11$ & $+/-3.7341 \mathrm{E}-21$ & $0.00 \%)$ \\
\hline$f e-54$ & n, alpha & $-1.5839 E-09$ & $+/-6.7397 \mathrm{E}-19$ & $0.00 \%)$ \\
\hline$f e-56$ & total & $-6.9426 \mathrm{E}-05$ & $+/-1.3507 \mathrm{E}-07$ & $0.19 \%)$ \\
\hline$f e-56$ & scatter & $1.1537 \mathrm{E}-05$ & $+/-1.1066 \mathrm{E}-07$ & $0.96 \%)$ \\
\hline$f e-56$ & elastic & $7.5606 \mathrm{E}-06$ & $+/-1.0976 \mathrm{E}-07$ & $1.45 \%)$ \\
\hline$f e-56$ & $\mathrm{n}, \mathrm{n}^{\prime}$ & $3.9702 \mathrm{E}-06$ & $+/-9.5385 \mathrm{E}-09$ & $0.24 \%)$ \\
\hline$f e-56$ & $n, 2 n$ & $5.5596 \mathrm{E}-09$ & $+/-3.1334 \mathrm{E}-18$ & $0.00 \%)$ \\
\hline$f e-56$ & capture & $-8.0963 E-05$ & $+/-2.8413 \mathrm{E}-08$ & $0.04 \%)$ \\
\hline$f e-56$ & $\mathrm{n}$, gamma & $-8.0923 E-05$ & $+/-2.8413 \mathrm{E}-08$ & $0.04 \%)$ \\
\hline$f e-56$ & $\mathrm{n}, \mathrm{p}$ & $-2.8231 E-08$ & $+/-4.3821 \mathrm{E}-11$ & $0.16 \%)$ \\
\hline$f e-56$ & $n, d$ & $-6.3061 E-11$ & $+/-4.9191 \mathrm{E}-20$ & $0.00 \%)$ \\
\hline$f e-56$ & $n, t$ & $-1.5472 \mathrm{E}-13$ & $+/-1.2160 \mathrm{E}-22$ & $0.00 \%)$ \\
\hline$f e-56$ & $\mathrm{n}, \mathrm{he}-3$ & $-1.7440 \mathrm{E}-14$ & $+/-1.2169 \mathrm{E}-23$ & $0.00 \%)$ \\
\hline
\end{tabular}

Figure 6.3.16. SAMS output for LEU-COMP-THERM-009 case 10 sample problem. (continued) 


\begin{tabular}{|c|c|c|c|c|}
\hline$f e-56$ & n, alpha & $-1.1945 E-08$ & $+/-1.2530 \mathrm{E}-11$ & $0.10 \%)$ \\
\hline$f e-57$ & total & $-1.4580 \mathrm{E}-06$ & $+/-2.4578 \mathrm{E}-09$ & $0.17 \%)$ \\
\hline$f e-57$ & scatter & $2.9453 E-07$ & $+/-2.2278 \mathrm{E}-09$ & $0.76 \%$ \\
\hline fe-57 & elastic & $1.6082 \mathrm{E}-07$ & $+/-2.1139 \mathrm{E}-09$ & $1.31 \%)$ \\
\hline$f e-57$ & $\mathrm{n}, \mathrm{n}^{\prime}$ & 1. $3172 \mathrm{E}-07$ & $+/-2.4140 \mathrm{E}-10$ & $0.18 \%)$ \\
\hline$f e-57$ & $n, 2 n$ & $1.9852 \mathrm{E}-09$ & $+/-1.4642 \mathrm{E}-19$ & $0.00 \%)$ \\
\hline fe-57 & capture & $-1.7526 E-06$ & $+/-6.0368 \mathrm{E}-10$ & $0.03 \%)$ \\
\hline fe-57 & $\mathrm{n}$, gamma & $-1.7515 E-06$ & $+/-6.0368 \mathrm{E}-10$ & $0.03 \%)$ \\
\hline$f e-57$ & $\mathrm{n}, \mathrm{p}$ & $-4.5690 \mathrm{E}-10$ & $+/-1.9448 \mathrm{E}-19$ & $0.00 \%)$ \\
\hline fe-57 & n,alpha & $-6.5570 \mathrm{E}-10$ & $+/-3.5601 \mathrm{E}-19$ & $0.00 \%)$ \\
\hline$f e-58$ & total & $-8.6249 \mathrm{E}-08$ & $+/-9.2863 \mathrm{E}-11$ & $0.11 \%)$ \\
\hline$f e-58$ & scatter & $3.2882 E-08$ & $+/-2.1096 \mathrm{E}-13$ & $0.00 \%)$ \\
\hline$f e-58$ & elastic & $1.9457 \mathrm{E}-08$ & $+/-2.1096 \mathrm{E}-13$ & $0.00 \%)$ \\
\hline$f e-58$ & $\mathrm{n}, \mathrm{n}^{\prime}$ & $1.3343 E-08$ & $+/-1.7621 \mathrm{E}-16$ & $0.00 \%)$ \\
\hline$f e-58$ & $n, 2 n$ & $8.1702 \mathrm{E}-11$ & $+/-1.5798 \mathrm{E}-20$ & $0.00 \%$ \\
\hline$f e-58$ & capture & $-1.1913 E-07$ & $+/-3.5967 \mathrm{E}-11$ & $0.03 \%)$ \\
\hline$f e-58$ & $\mathrm{n}$, gamma & $-1.1912 \mathrm{E}-07$ & $+/-3.5967 \mathrm{E}-11$ & $0.03 \%)$ \\
\hline$f e-58$ & $\mathrm{n}, \mathrm{p}$ & $-8.9556 \mathrm{E}-13$ & $+/-2.0142 \mathrm{E}-22$ & $0.00 \%)$ \\
\hline$f e-58$ & n,alpha & $-5.5186 \mathrm{E}-12$ & $+/-1.4040 \mathrm{E}-21$ & $0.00 \%$ \\
\hline $\mathrm{cu}-63$ & total & $-9.5973 E-05$ & $+/-7.0063 \mathrm{E}-08$ & $0.07 \%$ \\
\hline $\mathrm{cu}-63$ & scatter & $8.9795 E-06$ & $+/-3.9903 E-08$ & $0.44 \%$ \\
\hline $\mathrm{cu}-63$ & elastic & $5.1356 \mathrm{E}-06$ & $+/-3.8382 \mathrm{E}-08$ & $0.75 \%$ \\
\hline $\mathrm{cu}-63$ & $\mathrm{n}, \mathrm{n}^{\prime}$ & $3.8154 \mathrm{E}-06$ & $+/-7.8316 \mathrm{E}-09$ & $0.21 \%)$ \\
\hline $\mathrm{cu}-63$ & $n, 2 n$ & $4.8437 E-09$ & $+/-1.9022 \mathrm{E}-18$ & $0.00 \%$ \\
\hline $\mathrm{cu}-63$ & capture & $-1.0495 E-04$ & $+/-3.5784 \mathrm{E}-08$ & $0.03 \%)$ \\
\hline $\mathrm{cu}-63$ & $\mathrm{n}$, gamma & $-1.0450 \mathrm{E}-04$ & $+/-3.5783 E-08$ & $0.03 \%)$ \\
\hline $\mathrm{cu}-63$ & $\mathrm{n}, \mathrm{p}$ & $-4.3880 E-07$ & $+/-2.3679 \mathrm{E}-10$ & $0.05 \%)$ \\
\hline $\mathrm{cu}-63$ & $\mathrm{n}, \mathrm{d}$ & $-7.9573 E-09$ & $+/-9.1280 \mathrm{E}-17$ & $0.00 \%$ \\
\hline $\mathrm{cu}-63$ & $\mathrm{n}, \mathrm{he}-3$ & $-3.8432 E-14$ & $+/-1.6090 \mathrm{E}-23$ & $0.00 \%)$ \\
\hline $\mathrm{cu}-63$ & n, alpha & $-1.0048 E-08$ & $+/-7.9401 \mathrm{E}-17$ & $0.00 \%$ \\
\hline $\mathrm{cu}-65$ & total & $-1.7689 E-05$ & $+/-4.4209 \mathrm{E}-08$ & $0.25 \%)$ \\
\hline $\mathrm{cu}-65$ & scatter & $4.5920 \mathrm{E}-06$ & $+/-3.3439 E-08$ & $0.73 \%)$ \\
\hline $\mathrm{cu}-65$ & elastic & $2.9304 \mathrm{E}-06$ & $+/-3.3117 \mathrm{E}-08$ & $1.13 \%)$ \\
\hline $\mathrm{cu}-65$ & $\mathrm{n}, \mathrm{n}^{\prime}$ & $1.6531 \mathrm{E}-06$ & $+/-3.3430 \mathrm{E}-09$ & $0.20 \%)$ \\
\hline $\mathrm{cu}-65$ & $n, 2 n$ & $8.2988 \mathrm{E}-09$ & $+/-2.5459 \mathrm{E}-11$ & $0.31 \%)$ \\
\hline $\mathrm{cu}-65$ & capture & $-2.2281 E-05$ & $+/-7.6516 \mathrm{E}-09$ & $0.03 \%)$ \\
\hline $\mathrm{cu}-65$ & $\mathrm{n}$, gamma & $-2.2276 \mathrm{E}-05$ & $+/-7.6516 \mathrm{E}-09$ & $0.03 \%)$ \\
\hline$c u-65$ & $n, p$ & $-4.8468 E-09$ & $+/-2.3482 \mathrm{E}-16$ & $0.00 \%$ \\
\hline $\mathrm{cu}-65$ & $\mathrm{n}, \mathrm{d}$ & $-5.9497 \mathrm{E}-10$ & $+/-1.8412 \mathrm{E}-17$ & $0.00 \%)$ \\
\hline $\mathrm{cu}-65$ & $n, t$ & $-1.3215 E-13$ & $+/-7.8888 \mathrm{E}-23$ & $0.00 \%$ \\
\hline $\mathrm{cu}-65$ & $\mathrm{n}, \mathrm{he}-3$ & $-4.7968 \mathrm{E}-15$ & $+/-6.6710 \mathrm{E}-24$ & $0.00 \%)$ \\
\hline $\mathrm{cu}-65$ & n,alpha & $-1.2252 \mathrm{E}-10$ & $+/-1.7122 \mathrm{E}-18$ & $0.00 \%$ \\
\hline$h-1$ & total & $-3.3487 E-05$ & $+/-3.3383 \mathrm{E}-05$ & $99.69 \%)$ \\
\hline$h-1$ & scatter & $1.1785 \mathrm{E}-04$ & $+/-3.3082 \mathrm{E}-05$ & $28.07 \%)$ \\
\hline $\mathrm{h}-1$ & elastic & $1.1785 \mathrm{E}-04$ & $+/-3.3082 \mathrm{E}-05$ & $28.07 \%)$ \\
\hline$h-1$ & capture & $-1.5134 \mathrm{E}-04$ & $+/-3.4431 \mathrm{E}-07$ & $0.23 \%)$ \\
\hline$h-1$ & $\mathrm{n}$, gamma & $-1.5134 \mathrm{E}-04$ & $+/-3.4431 \mathrm{E}-07$ & $0.23 \%)$ \\
\hline c & total & $1.4324 \mathrm{E}-04$ & $+/-3.4393 E-06$ & $2.40 \%$ \\
\hline
\end{tabular}

Figure 6.3.16. SAMS output for LEU-COMP-THERM-009 case 10 sample problem. (continued) 


\begin{tabular}{|c|c|c|c|}
\hline er & $1.4444 \mathrm{E}-04$ & $+/-3.4379 \mathrm{E}-06$ & $2.38 \%)$ \\
\hline elastic & $1.4356 \mathrm{E}-04$ & $+/-3.4374 \mathrm{E}-06$ & $2.39 \%)$ \\
\hline $\mathrm{n}, \mathrm{n}^{\prime}$ & $8.7941 E-07$ & $+/-2.1229 \mathrm{E}-08$ & $2.41 \%)$ \\
\hline capture & $-1.2004 \mathrm{E}-06$ & $+/-5.0249 \mathrm{E}-09$ & $0.42 \%)$ \\
\hline $\mathrm{n}$, gamma & $-1.1534 \mathrm{E}-06$ & $+/-2.6118 \mathrm{E}-09$ & $0.23 \%)$ \\
\hline $\mathrm{n}, \mathrm{p}$ & $-1.2812 \mathrm{E}-11$ & $+/-0.0000 \mathrm{E}+00$ & $0.00 \%)$ \\
\hline $\mathrm{n}, \mathrm{d}$ & $-3.3016 \mathrm{E}-11$ & $+/-0.0000 \mathrm{E}+00$ & $0.00 \%)$ \\
\hline n, alpha & $-4.6915 E-08$ & $+/-4.2884 \mathrm{E}-09$ & $9.14 \%)$ \\
\hline total & $3.9480 \mathrm{E}-05$ & $+/-8.0523 \mathrm{E}-07$ & $2.04 \%)$ \\
\hline scatter & $3.9628 \mathrm{E}-05$ & $+/-8.0503 \mathrm{E}-07$ & $2.03 \%)$ \\
\hline elastic & $3.9526 \mathrm{E}-05$ & $+/-8.0498 \mathrm{E}-07$ & $2.04 \%)$ \\
\hline $\mathrm{n}, \mathrm{n}^{\prime}$ & $1.0040 \mathrm{E}-07$ & $+/-3.9571 \mathrm{E}-09$ & $3.94 \%)$ \\
\hline$n, 2 n$ & $5.9840 \mathrm{E}-15$ & $+/-0.0000 \mathrm{E}+00$ & $0.00 \%)$ \\
\hline capture & $-1.4730 \mathrm{E}-07$ & $+/-2.8085 \mathrm{E}-09$ & $1.91 \%)$ \\
\hline $\mathrm{n}$, gamma & $-1.8551 \mathrm{E}-08$ & $+/-3.2427 \mathrm{E}-11$ & $0.17 \%$ \\
\hline $\mathrm{n}, \mathrm{p}$ & $-4.6597 \mathrm{E}-10$ & $+/-2.6874 \mathrm{E}-13$ & $0.06 \%)$ \\
\hline$n, d$ & $-4.2225 \mathrm{E}-11$ & $+/-1.3904 \mathrm{E}-14$ & $0.03 \%)$ \\
\hline$n, t$ & $-3.5750 \mathrm{E}-16$ & $+/-0.0000 \mathrm{E}+00$ & $0.00 \%)$ \\
\hline n, alpha & $-1.2824 E-07$ & $+/-2.8083 \mathrm{E}-09$ & $2.19 \%)$ \\
\hline total & $1.5659 \mathrm{E}-07$ & $+/-3.1168 E-09$ & $1.99 \%$ \\
\hline scatter & $2.7569 \mathrm{E}-07$ & $+/-2.1958 \mathrm{E}-09$ & $0.80 \%)$ \\
\hline elastic & $2.4930 \mathrm{E}-07$ & $+/-2.0614 \mathrm{E}-09$ & $0.83 \%)$ \\
\hline $\mathrm{n}, \mathrm{n}^{\prime}$ & $2.6386 \mathrm{E}-08$ & $+/-2.5243 \mathrm{E}-10$ & $0.96 \%)$ \\
\hline capture & $-1.1911 \mathrm{E}-07$ & $+/-2.5877 \mathrm{E}-10$ & $0.22 \%)$ \\
\hline $\mathrm{n}$, gamma & $-1.1814 E-07$ & $+/-2.5877 \mathrm{E}-10$ & $0.22 \%)$ \\
\hline $\mathrm{n}, \mathrm{p}$ & $-6.7032 E-10$ & $+/-6.8669 \mathrm{E}-17$ & $0.00 \%)$ \\
\hline$n, d$ & $-7.0401 \mathrm{E}-13$ & $+/-0.0000 \mathrm{E}+00$ & $0.00 \%)$ \\
\hline n, alpha & $-2.9677 E-10$ & $+/-2.4154 \mathrm{E}-17$ & $0.00 \%)$ \\
\hline total & $8.3353 E-09$ & $+/-7.5001 \mathrm{E}-15$ & $0.00 \%$ \\
\hline scatter & $1.2660 \mathrm{E}-08$ & $+/-7.4989 \mathrm{E}-15$ & $0.00 \%)$ \\
\hline elastic & $1.0360 \mathrm{E}-08$ & $+/-7.1408 \mathrm{E}-15$ & $0.00 \%)$ \\
\hline $\mathrm{n}, \mathrm{n}^{\prime}$ & $2.2970 \mathrm{E}-09$ & $+/-7.6907 \mathrm{E}-16$ & $0.00 \%)$ \\
\hline$n, 2 n$ & 3. $6953 \mathrm{E}-12$ & $+/-0.0000 \mathrm{E}+00$ & $0.00 \%)$ \\
\hline capture & $-4.3250 E-09$ & $+/-5.9139 \mathrm{E}-18$ & $0.00 \%)$ \\
\hline $\mathrm{n}$, gamma & $-4.2731 E-09$ & $+/-1.0615 \mathrm{E}-18$ & $0.00 \%)$ \\
\hline $\mathrm{n}, \mathrm{p}$ & $-1.8703 E-11$ & $+/-2.1885 \mathrm{E}-18$ & $0.00 \%)$ \\
\hline n,alpha & $-3.3206 \mathrm{E}-11$ & $+/-3.9362 E-18$ & $0.00 \%)$ \\
\hline total & $5.0684 \mathrm{E}-09$ & $+/-6.4263 \mathrm{E}-15$ & $0.00 \%)$ \\
\hline scatter & $8.0334 \mathrm{E}-09$ & $+/-6.4252 \mathrm{E}-15$ & $0.00 \%)$ \\
\hline elastic & $7.1395 \mathrm{E}-09$ & $+/-6.3753 \mathrm{E}-15$ & $0.00 \%)$ \\
\hline $\mathrm{n}, \mathrm{n}^{\prime}$ & $8.9160 \mathrm{E}-10$ & $+/-2.1621 \mathrm{E}-16$ & $0.00 \%)$ \\
\hline$n, 2 n$ & $2.2872 \mathrm{E}-12$ & $+/-0.0000 \mathrm{E}+00$ & $0.00 \%)$ \\
\hline capture & $-2.9651 E-09$ & $+/-1.4537 \mathrm{E}-18$ & $0.00 \%)$ \\
\hline $\mathrm{n}$, gamma & $-2.9646 E-09$ & $+/-1.4536 \mathrm{E}-18$ & $0.00 \%)$ \\
\hline $\mathrm{n}, \mathrm{p}$ & $-7.7936 E-14$ & $+/-3.6748 \mathrm{E}-26$ & $0.00 \%)$ \\
\hline n,alpha & $-3.6427 \mathrm{E}-13$ & $+/-5.9486 \mathrm{E}-21$ & $0.00 \%)$ \\
\hline total & $-9.3027 \mathrm{E}-07$ & $+/-1.5023 E-08$ & $1.61 \%)$ \\
\hline scatter & $1.0965 \mathrm{E}-06$ & $+/-1.1617 \mathrm{E}-08$ & $1.06 \%)$ \\
\hline
\end{tabular}

Figure 6.3.16. SAMS output for LEU-COMP-THERM-009 case 10 sample problem. (continued) 


\begin{tabular}{|c|c|c|c|c|}
\hline$s-32$ & elastic & $1.0303 E-06$ & $+/-1.1393 E-08$ & $1.11 \%)$ \\
\hline$s-32$ & $\mathrm{n}, \mathrm{n}^{\prime}$ & $6.5967 \mathrm{E}-08$ & $+/-8.6196 \mathrm{E}-10$ & $1.31 \%)$ \\
\hline$s-32$ & $n, 2 n$ & $1.0859 \mathrm{E}-14$ & $+/-0.0000 \mathrm{E}+00$ & $0.00 \%)$ \\
\hline$s-32$ & capture & $-2.0268 \mathrm{E}-06$ & $+/-4.4706 \mathrm{E}-09$ & $0.22 \%)$ \\
\hline$s-32$ & $\mathrm{n}$, gamma & $-1.8835 \mathrm{E}-06$ & $+/-4.2718 \mathrm{E}-09$ & $0.23 \%)$ \\
\hline$s-32$ & $\mathrm{n}, \mathrm{p}$ & $-4.4415 \mathrm{E}-08$ & $+/-5.3423 \mathrm{E}-10$ & $1.20 \%)$ \\
\hline$s-32$ & n,alpha & $-9.8944 \mathrm{E}-08$ & $+/-5.3326 \mathrm{E}-10$ & $0.54 \%)$ \\
\hline$s-33$ & total & $-1.1925 E-08$ & $+/-1.0473 \mathrm{E}-14$ & $0.00 \%)$ \\
\hline$s-33$ & scatter & $7.9053 \mathrm{E}-09$ & $+/-9.6281 \mathrm{E}-15$ & $0.00 \%)$ \\
\hline$s-33$ & elastic & $7.1213 \mathrm{E}-09$ & $+/-9.5751 \mathrm{E}-15$ & $0.00 \%)$ \\
\hline$s-33$ & $\mathrm{n}, \mathrm{n}^{\prime}$ & $7.7770 \mathrm{E}-10$ & $+/-1.7507 \mathrm{E}-16$ & $0.00 \%)$ \\
\hline$s-33$ & $n, 2 n$ & $4.8458 \mathrm{E}-12$ & $+/-0.0000 E+00$ & $0.00 \%)$ \\
\hline$s-33$ & capture & $-1.9830 \mathrm{E}-08$ & $+/-8.4932 \mathrm{E}-16$ & $0.00 \%)$ \\
\hline$s-33$ & $\mathrm{n}$, gamma & $-9.8480 \mathrm{E}-09$ & $+/-1.5761 \mathrm{E}-18$ & $0.00 \%)$ \\
\hline$s-33$ & $\mathrm{n}, \mathrm{p}$ & $-4.3988 \mathrm{E}-10$ & $+/-9.9510 \mathrm{E}-17$ & $0.00 \%)$ \\
\hline$s-33$ & n, alpha & $-9.5421 \mathrm{E}-09$ & $+/-8.0138 \mathrm{E}-16$ & $0.00 \%)$ \\
\hline$s-34$ & total & $1.2596 \mathrm{E}-08$ & $+/-3.9241 \mathrm{E}-10$ & $3.12 \%)$ \\
\hline$s-34$ & scatter & $4.7978 \mathrm{E}-08$ & $+/-1.6902 \mathrm{E}-10$ & $0.35 \%)$ \\
\hline$s-34$ & elastic & $4.3911 \mathrm{E}-08$ & $+/-1.1281 \mathrm{E}-10$ & $0.26 \%)$ \\
\hline$s-34$ & $\mathrm{n}, \mathrm{n}^{\prime}$ & $4.0515 E-09$ & $+/-7.8532 \mathrm{E}-16$ & $0.00 \%)$ \\
\hline$s-34$ & $\mathrm{n}, 2 \mathrm{n}$ & $6.4633 \mathrm{E}-12$ & $+/-0.0000 \mathrm{E}+00$ & $0.00 \%)$ \\
\hline$s-34$ & capture & $-3.5381 \mathrm{E}-08$ & $+/-6.1670 \mathrm{E}-11$ & $0.17 \%)$ \\
\hline$s-34$ & $\mathrm{n}$, gamma & $-3.5292 E-08$ & $+/-6.1670 \mathrm{E}-11$ & $0.17 \%)$ \\
\hline$s-34$ & $\mathrm{n}, \mathrm{p}$ & $-1.6841 \mathrm{E}-11$ & $+/-1.4263 \mathrm{E}-18$ & $0.00 \%$ \\
\hline$s-34$ & n,alpha & $-7.2939 \mathrm{E}-11$ & $+/-9.4585 \mathrm{E}-18$ & $0.00 \%)$ \\
\hline$s-36$ & total & $1.2812 \mathrm{E}-10$ & $+/-1.6526 \mathrm{E}-16$ & $0.00 \%)$ \\
\hline$s-36$ & scatter & 2. $4510 \mathrm{E}-10$ & $+/-1.6524 \mathrm{E}-16$ & $0.00 \%)$ \\
\hline$s-36$ & elastic & 2. $3204 \mathrm{E}-10$ & $+/-1.6517 \mathrm{E}-16$ & $0.00 \%)$ \\
\hline$s-36$ & $\mathrm{n}, \mathrm{n}^{\prime}$ & $1.2864 \mathrm{E}-11$ & $+/-1.1331 \mathrm{E}-18$ & $0.00 \%)$ \\
\hline$s-36$ & $n, 2 n$ & $1.9756 \mathrm{E}-13$ & $+/-0.0000 \mathrm{E}+00$ & $0.00 \%)$ \\
\hline$s-36$ & capture & $-1.1697 \mathrm{E}-10$ & $+/-2.0921 \mathrm{E}-20$ & $0.00 \%)$ \\
\hline$s-36$ & $\mathrm{n}$, gamma & $-1.1697 \mathrm{E}-10$ & $+/-2.0919 \mathrm{E}-20$ & $0.00 \%)$ \\
\hline$s-36$ & $\mathrm{n}, \mathrm{p}$ & $-5.8200 \mathrm{E}-17$ & $+/-0.0000 E+00$ & $0.00 \%)$ \\
\hline$s-36$ & n, alpha & $-5.1249 \mathrm{E}-15$ & $+/-2.0171 \mathrm{E}-22$ & $0.00 \%)$ \\
\hline $\mathrm{ca}-40$ & total & $-1.7949 \mathrm{E}-06$ & $+/-1.1894 \mathrm{E}-07$ & $6.63 \%)$ \\
\hline$c a-40$ & scatter & $6.7330 \mathrm{E}-06$ & $+/-9.7909 \mathrm{E}-08$ & $1.45 \%)$ \\
\hline$c a-40$ & elastic & $6.5226 \mathrm{E}-06$ & $+/-9.7589 \mathrm{E}-08$ & $1.50 \%)$ \\
\hline$c a-40$ & $\mathrm{n}, \mathrm{n}^{\prime}$ & $2.0785 \mathrm{E}-07$ & $+/-3.3003 \mathrm{E}-09$ & $1.59 \%$ \\
\hline $\mathrm{ca}-40$ & $\mathrm{n}, 2 \mathrm{n}$ & $4.5607 \mathrm{E}-14$ & $+/-0.0000 \mathrm{E}+00$ & $0.00 \%)$ \\
\hline$c a-40$ & capture & $-8.5279 \mathrm{E}-06$ & $+/-1.9716 \mathrm{E}-08$ & $0.23 \%)$ \\
\hline$c a-40$ & $\mathrm{n}$, gamma & $-7.9727 \mathrm{E}-06$ & $+/-1.8069 \mathrm{E}-08$ & $0.23 \%)$ \\
\hline$c a-40$ & $\mathrm{n}, \mathrm{p}$ & $-3.7411 \mathrm{E}-07$ & $+/-5.5225 \mathrm{E}-09$ & $1.48 \%)$ \\
\hline $\mathrm{ca}-40$ & $\mathrm{n}, \mathrm{d}$ & $-5.9766 \mathrm{E}-11$ & $+/-6.5478 \mathrm{E}-19$ & $0.00 \%)$ \\
\hline$c a-40$ & $n, t$ & $-4.2787 E-15$ & $+/-0.0000 \mathrm{E}+00$ & $0.00 \%)$ \\
\hline$c a-40$ & $\mathrm{n}, \mathrm{he}-3$ & $-5.7096 \mathrm{E}-14$ & $+/-0.0000 \mathrm{E}+00$ & $0.00 \%)$ \\
\hline$c a-40$ & n, alpha & $-1.8104 \mathrm{E}-07$ & $+/-2.0743 \mathrm{E}-09$ & $1.15 \%$ \\
\hline $\mathrm{ca}-42$ & total & $-3.3798 \mathrm{E}-08$ & $+/-4.2370 \mathrm{E}-10$ & $1.25 \%$ \\
\hline $\mathrm{ca}-42$ & scatter & $5.5595 \mathrm{E}-08$ & $+/-1.8840 \mathrm{E}-10$ & $0.34 \%$ \\
\hline
\end{tabular}

Figure 6.3.16. SAMS output for LEU-COMP-THERM-009 case 10 sample problem. (continued) 


\begin{tabular}{|c|c|c|c|c|}
\hline $\mathrm{ca}-42$ & elastic & $4.4749 E-08$ & $+/-7.7226 \mathrm{E}-14$ & $0.00 \%)$ \\
\hline $\mathrm{ca}-42$ & $\mathrm{n}, \mathrm{n}^{\prime}$ & $1.0831 \mathrm{E}-08$ & $+/-3.5480 \mathrm{E}-15$ & $0.00 \%)$ \\
\hline$c a-42$ & $\mathrm{n}, 2 \mathrm{n}$ & 4. $2807 \mathrm{E}-12$ & $+/-0.0000 \mathrm{E}+00$ & $0.00 \%)$ \\
\hline$c a-42$ & capture & $-8.9393 E-08$ & $+/-1.9154 \mathrm{E}-10$ & $0.21 \%)$ \\
\hline $\mathrm{ca}-42$ & $\mathrm{n}$, gamma & $-8.9285 E-08$ & $+/-1.9154 \mathrm{E}-10$ & $0.21 \%)$ \\
\hline $\mathrm{ca}-42$ & $n, p$ & $-6.3001 \mathrm{E}-11$ & $+/-6.1936 \mathrm{E}-18$ & $0.00 \%)$ \\
\hline$c a-42$ & $\mathrm{n}, \mathrm{d}$ & $-3.9571 E-14$ & $+/-0.0000 \mathrm{E}+00$ & $0.00 \%)$ \\
\hline$c a-42$ & $n, t$ & $-1.1401 \mathrm{E}-16$ & $+/-0.0000 \mathrm{E}+00$ & $0.00 \%)$ \\
\hline $\mathrm{ca}-42$ & $\mathrm{n}, \mathrm{he}-3$ & $-2.8628 \mathrm{E}-18$ & $+/-0.0000 \mathrm{E}+00$ & $0.00 \%)$ \\
\hline $\mathrm{ca}-42$ & n, alpha & $-4.4886 \mathrm{E}-11$ & $+/-4.9613 \mathrm{E}-18$ & $0.00 \%)$ \\
\hline$c a-43$ & total & $-2.9992 E-07$ & $+/-8.5309 \mathrm{E}-10$ & $0.28 \%)$ \\
\hline$c a-43$ & scatter & $1.7308 \mathrm{E}-08$ & $+/-9.3864 \mathrm{E}-15$ & $0.00 \%)$ \\
\hline$c a-43$ & elastic & $1.4035 \mathrm{E}-08$ & $+/-8.9886 \mathrm{E}-15$ & $0.00 \%)$ \\
\hline$c a-43$ & $n, n^{\prime}$ & 3. 2593E-09 & $+/-1.0415 \mathrm{E}-15$ & $0.00 \%)$ \\
\hline$c a-43$ & $n, 2 n$ & 1. 3992E-11 & $+/-1.0619 \mathrm{E}-22$ & $0.00 \% 1$ \\
\hline$c a-43$ & capture & $-3.1723 E-07$ & $+/-7.1329 \mathrm{E}-10$ & $0.22 \%)$ \\
\hline$c a-43$ & $\mathrm{n}$, gamma & $-3.1720 \mathrm{E}-07$ & $+/-7.1328 \mathrm{E}-10$ & $0.22 \%)$ \\
\hline $\mathrm{ca}-43$ & $n, p$ & $-1.2736 \mathrm{E}-11$ & $+/-1.4953 \mathrm{E}-18$ & $0.00 \%)$ \\
\hline$c a-43$ & $\mathrm{n}, \mathrm{d}$ & $-3.9211 E-15$ & $+/-0.0000 \mathrm{E}+00$ & $0.00 \%)$ \\
\hline$c a-43$ & $n, t$ & $-2.0903 E-16$ & $+/-0.0000 \mathrm{E}+00$ & $0.00 \%)$ \\
\hline$c a-43$ & $\mathrm{n}, \mathrm{he}-3$ & $-8.8381 \mathrm{E}-21$ & $+/-0.0000 \mathrm{E}+00$ & $0.00 \%)$ \\
\hline$c a-43$ & $\mathrm{n}$, alpha & $-1.8078 \mathrm{E}-11$ & $+/-1.6658 \mathrm{E}-18$ & $0.00 \%)$ \\
\hline$c a-44$ & total & $-1.9121 E-07$ & $+/-2.9350 \mathrm{E}-09$ & $1.53 \%)$ \\
\hline$c a-44$ & scatter & $1.8201 \mathrm{E}-07$ & $+/-9.8994 \mathrm{E}-10$ & $0.54 \%)$ \\
\hline$c a-44$ & elastic & $1.4003 \mathrm{E}-07$ & $+/-6.5307 \mathrm{E}-10$ & $0.47 \%)$ \\
\hline $\mathrm{ca}-44$ & $\mathrm{n}, \mathrm{n}^{\prime}$ & $4.1934 \mathrm{E}-08$ & $+/-3.9319 \mathrm{E}-10$ & $0.94 \%)$ \\
\hline $\mathrm{ca}-44$ & $\mathrm{n}, 2 \mathrm{n}$ & $3.7701 \mathrm{E}-11$ & $+/-0.0000 \mathrm{E}+00$ & $0.00 \%)$ \\
\hline$c a-44$ & capture & $-3.7322 E-07$ & $+/-8.4317 \mathrm{E}-10$ & $0.23 \%)$ \\
\hline$c a-44$ & $\mathrm{n}$, gamma & $-3.7321 E-07$ & $+/-8.4317 \mathrm{E}-10$ & $0.23 \%)$ \\
\hline$c a-44$ & $\mathrm{n}, \mathrm{p}$ & $-4.3462 \mathrm{E}-12$ & $+/-1.1952 \mathrm{E}-19$ & $0.00 \%)$ \\
\hline $\mathrm{ca}-44$ & $\mathrm{n}, \mathrm{d}$ & $-1.9015 \mathrm{E}-14$ & $+/-0.0000 \mathrm{E}+00$ & $0.00 \%)$ \\
\hline $\mathrm{ca}-44$ & $n, t$ & $-3.6521 \mathrm{E}-17$ & $+/-0.0000 \mathrm{E}+00$ & $0.00 \%)$ \\
\hline$c a-44$ & $\mathrm{n}, \mathrm{he}-3$ & $-5.7133 E-22$ & $+/-0.0000 \mathrm{E}+00$ & $0.00 \%)$ \\
\hline $\mathrm{ca}-44$ & n, alpha & $-2.1833 E-12$ & $+/-5.2524 \mathrm{E}-20$ & $0.00 \%)$ \\
\hline $\mathrm{ca}-46$ & total & $-9.1789 \mathrm{E}-11$ & $+/-2.9314 \mathrm{E}-16$ & $0.00 \%)$ \\
\hline $\mathrm{ca}-46$ & scatter & $5.0404 \mathrm{E}-10$ & $+/-2.9308 \mathrm{E}-16$ & $0.00 \%)$ \\
\hline$c a-46$ & elastic & 4. $3313 \mathrm{E}-10$ & $+/-2.8501 \mathrm{E}-16$ & $0.00 \%$ ) \\
\hline $\mathrm{ca}-46$ & $\mathrm{n}, \mathrm{n}^{\prime}$ & $7.0696 \mathrm{E}-11$ & $+/-2.2304 \mathrm{E}-17$ & $0.00 \%)$ \\
\hline $\mathrm{ca}-46$ & $\mathrm{n}, 2 \mathrm{n}$ & $2.0718 \mathrm{E}-13$ & $+/-0.0000 \mathrm{E}+00$ & $0.00 \%)$ \\
\hline$c a-46$ & capture & $-5.9583 E-10$ & $+/-5.9929 \mathrm{E}-20$ & $0.00 \%)$ \\
\hline$c a-46$ & $\mathrm{n}$, gamma & $-5.9583 E-10$ & $+/-5.9929 \mathrm{E}-20$ & $0.00 \%)$ \\
\hline$c a-46$ & $n, p$ & $-3.2267 \mathrm{E}-16$ & $+/-3.6714 \mathrm{E}-26$ & $0.00 \%)$ \\
\hline $\mathrm{ca}-46$ & $\mathrm{n}, \mathrm{d}$ & $-6.8722 \mathrm{E}-18$ & $+/-0.0000 \mathrm{E}+00$ & $0.00 \%)$ \\
\hline$c a-46$ & $n, t$ & $-3.3633 E-21$ & $+/-0.0000 \mathrm{E}+00$ & $0.00 \%)$ \\
\hline$c a-46$ & n, alpha & $-2.2633 E-17$ & $+/-0.0000 \mathrm{E}+00$ & $0.00 \%)$ \\
\hline $\mathrm{ca}-48$ & total & $-2.4743 E-08$ & $+/-1.8724 \mathrm{E}-10$ & $0.76 \%)$ \\
\hline $\mathrm{ca}-48$ & scatter & $1.6341 \mathrm{E}-08$ & $+/-8.2057 \mathrm{E}-15$ & $0.00 \%)$ \\
\hline $\mathrm{ca}-48$ & elastic & $1.5512 \mathrm{E}-08$ & $+/-8.2022 \mathrm{E}-15$ & $0.00 \%$ ) \\
\hline
\end{tabular}

Figure 6.3.16. SAMS output for LEU-COMP-THERM-009 case 10 sample problem. (continued) 


\begin{tabular}{|c|c|c|c|c|}
\hline$c a-48$ & $\mathrm{n}, \mathrm{n}^{\prime}$ & $8.1356 \mathrm{E}-10$ & $+/-6.9284 \mathrm{E}-17$ & $0.00 \%)$ \\
\hline$c a-48$ & $n, 2 n$ & $1.5225 \mathrm{E}-11$ & $+/-0.0000 \mathrm{E}+00$ & $0.00 \%)$ \\
\hline$c a-48$ & capture & $-4.1084 E-08$ & $+/-7.9141 \mathrm{E}-11$ & $0.19 \%)$ \\
\hline$c a-48$ & $\mathrm{n}$, gamma & $-4.1084 E-08$ & $+/-7.9141 \mathrm{E}-11$ & $0.19 \%)$ \\
\hline$c a-48$ & $\mathrm{n}, \mathrm{p}$ & $-1.0552 \mathrm{E}-16$ & $+/-0.0000 \mathrm{E}+00$ & $0.00 \%)$ \\
\hline$c a-48$ & $\mathrm{n}, \mathrm{d}$ & $-2.1494 \mathrm{E}-17$ & $+/-0.0000 \mathrm{E}+00$ & $0.00 \%)$ \\
\hline$c a-48$ & $n, t$ & $-5.0004 E-22$ & $+/-0.0000 \mathrm{E}+00$ & $0.00 \%)$ \\
\hline$c a-48$ & n, alpha & $-1.9945 E-17$ & $+/-0.0000 \mathrm{E}+00$ & $0.00 \%)$ \\
\hline $\mathrm{h}-1$ & total & $-4.6343 E-05$ & $+/-2.5632 \mathrm{E}-05$ & $55.31 \%)$ \\
\hline $\mathrm{h}-1$ & scatter & $1.4599 \mathrm{E}-04$ & $+/-2.5374 \mathrm{E}-05$ & $17.38 \%)$ \\
\hline $\mathrm{h}-1$ & elastic & $1.4599 \mathrm{E}-04$ & $+/-2.5374 \mathrm{E}-05$ & $17.38 \%)$ \\
\hline $\mathrm{h}-1$ & capture & $-1.9233 E-04$ & $+/-2.9929 \mathrm{E}-07$ & $0.16 \%)$ \\
\hline h-1 & $\mathrm{n}$, gamma & $-1.9233 E-04$ & $+/-2.9929 \mathrm{E}-07$ & $0.16 \%)$ \\
\hline c & total & $1.5186 \mathrm{E}-04$ & $+/-2.0605 \mathrm{E}-06$ & $1.36 \%)$ \\
\hline c & scatter & $1.5313 \mathrm{E}-04$ & $+/-1.9864 \mathrm{E}-06$ & $1.30 \%)$ \\
\hline c & elastic & $1.5229 \mathrm{E}-04$ & $+/-1.9863 \mathrm{E}-06$ & $1.30 \%)$ \\
\hline c & $\mathrm{n}, \mathrm{n}^{\prime}$ & 8. $3833 \mathrm{E}-07$ & $+/-7.6391 \mathrm{E}-09$ & $0.91 \%)$ \\
\hline c & capture & $-1.2702 E-06$ & $+/-2.4828 \mathrm{E}-09$ & $0.20 \%)$ \\
\hline c & $\mathrm{n}$, gamma & $-1.2318 \mathrm{E}-06$ & $+/-1.9115 \mathrm{E}-09$ & $0.16 \%)$ \\
\hline c & $\mathrm{n}, \mathrm{p}$ & $-9.4682 \mathrm{E}-12$ & $+/-0.0000 \mathrm{E}+00$ & $0.00 \%)$ \\
\hline c & $n, d$ & $-2.8081 E-11$ & $+/-0.0000 \mathrm{E}+00$ & $0.00 \%)$ \\
\hline c & n, alpha & $-3.8362 E-08$ & $+/-1.5828 \mathrm{E}-09$ & $4.13 \%)$ \\
\hline $0-16$ & total & $5.7694 \mathrm{E}-05$ & $+/-6.3654 \mathrm{E}-07$ & $1.10 \%)$ \\
\hline $0-16$ & scatter & $5.7871 \mathrm{E}-05$ & $+/-6.3645 \mathrm{E}-07$ & $1.10 \%)$ \\
\hline $0-16$ & elastic & $5.7729 \mathrm{E}-05$ & $+/-6.3644 \mathrm{E}-07$ & $1.10 \%)$ \\
\hline $0-16$ & $\mathrm{n}, \mathrm{n}^{\prime}$ & 1. $3911 \mathrm{E}-07$ & $+/-2.0477 \mathrm{E}-09$ & $1.47 \%$ \\
\hline $0-16$ & $n, 2 n$ & $1.1747 \mathrm{E}-14$ & $+/-0.0000 \mathrm{E}+00$ & $0.00 \%)$ \\
\hline $0-16$ & capture & $-1.7668 \mathrm{E}-07$ & $+/-1.5046 \mathrm{E}-09$ & $0.85 \%)$ \\
\hline $0-16$ & $\mathrm{n}$, gamma & $-2.7736 \mathrm{E}-08$ & $+/-3.4230 \mathrm{E}-11$ & $0.12 \%)$ \\
\hline $0-16$ & $\mathrm{n}, \mathrm{p}$ & $-6.3458 \mathrm{E}-10$ & $+/-1.2415 \mathrm{E}-13$ & $0.02 \%)$ \\
\hline $0-16$ & $n, d$ & $-5.2519 \mathrm{E}-11$ & $+/-6.4231 \mathrm{E}-15$ & $0.01 \%)$ \\
\hline $0-16$ & $n, t$ & $-4.4058 E-16$ & $+/-0.0000 \mathrm{E}+00$ & $0.00 \%)$ \\
\hline $0-16$ & n, alpha & $-1.4826 \mathrm{E}-07$ & $+/-1.4006 \mathrm{E}-09$ & $0.94 \%)$ \\
\hline c & total & $9.7668 \mathrm{E}-06$ & $+/-1.3853 \mathrm{E}-07$ & $1.42 \%)$ \\
\hline c & scatter & $9.8750 \mathrm{E}-06$ & $+/-1.3850 \mathrm{E}-07$ & $1.40 \%)$ \\
\hline c & elastic & $9.8360 \mathrm{E}-06$ & $+/-1.3846 \mathrm{E}-07$ & $1.41 \%)$ \\
\hline c & $\mathrm{n}, \mathrm{n}^{\prime}$ & $3.8987 \mathrm{E}-08$ & $+/-1.2258 \mathrm{E}-09$ & $3.14 \%)$ \\
\hline c & capture & $-1.0818 \mathrm{E}-07$ & $+/-2.4021 \mathrm{E}-10$ & $0.22 \%)$ \\
\hline c & $\mathrm{n}$, gamma & $-7.0538 \mathrm{E}-08$ & $+/-5.6578 \mathrm{E}-11$ & $0.08 \%$ \\
\hline c & $\mathrm{n}, \mathrm{p}$ & $-6.2023 E-12$ & $+/-0.0000 \mathrm{E}+00$ & $0.00 \%)$ \\
\hline c & $n, d$ & $-1.0588 \mathrm{E}-11$ & $+/-0.0000 \mathrm{E}+00$ & $0.00 \%)$ \\
\hline c & n, alpha & $-3.7621 \mathrm{E}-08$ & $+/-2.3320 \mathrm{E}-10$ & $0.62 \%)$ \\
\hline $0-16$ & total & $6.9207 \mathrm{E}-07$ & $+/-7.5312 \mathrm{E}-09$ & $1.09 \%)$ \\
\hline $0-16$ & scatter & $7.0981 \mathrm{E}-07$ & $+/-7.5282 \mathrm{E}-09$ & $1.06 \%)$ \\
\hline $0-16$ & elastic & $7.0868 \mathrm{E}-07$ & $+/-7.5282 \mathrm{E}-09$ & $1.06 \%)$ \\
\hline $0-16$ & $n, n^{\prime}$ & $1.0108 \mathrm{E}-09$ & $+/-2.8433 \mathrm{E}-16$ & $0.00 \%)$ \\
\hline $0-16$ & $n, 2 n$ & $6.5082 \mathrm{E}-17$ & $+/-0.0000 \mathrm{E}+00$ & $0.00 \%)$ \\
\hline $0-16$ & capture & $-1.7739 E-08$ & $+/-2.1801 \mathrm{E}-11$ & $0.12 \%)$ \\
\hline
\end{tabular}

Figure 6.3.16. SAMS output for LEU-COMP-THERM-009 case 10 sample problem. (continued) 


$\begin{array}{ll}\text { o-16 } & \text { n,gamma } \\ \text { o-16 } & \text { n,p } \\ \text { o-16 } & \text { n,d } \\ \text { o-16 } & \text { n,t } \\ \text { o-16 } & \text { n,alpha } \\ \text { na-23 } & \text { total } \\ \text { na-23 } & \text { scatter } \\ \text { na-23 } & \text { elastic } \\ \text { na-23 } & \text { n,n' } \\ \text { na-23 } & \text { n,2n } \\ \text { na-23 } & \text { capture } \\ \text { na-23 } & \text { n,gamma } \\ \text { na-23 } & \text { n,p } \\ \text { na-23 } & \text { n,alpha } \\ \text { mg-24 } & \text { total } \\ \text { mg-24 } & \text { scatter } \\ \text { mg-24 } & \text { elastic } \\ \text { mg-24 } & \text { n,n' } \\ \text { mg-24 } & \text { n,2n } \\ \text { mg-24 } & \text { capture } \\ \text { mg-24 } & \text { n,gamma } \\ \text { mg-24 } & \text { n,p } \\ \text { mg-24 } & \text { n,alpha } \\ \text { mg-25 } & \text { total } \\ \text { mg-25 } & \text { scatter } \\ \text { mg-25 } & \text { elastic } \\ \text { mg-25 } & \text { n,n' } \\ \text { mg-25 } & \text { n,2n } \\ \text { mg-25 } & \text { capture } \\ \text { mg-25 } & \text { n,gamma } \\ \text { mg-25 } & \text { n,p } \\ \text { mg-25 } & \text { n,alpha } \\ \text { mg-26 } & \text { total } \\ \text { mg-26 } & \text { scatter } \\ \text { mg-26 } & \text { elastic } \\ \text { mg-26 } & \text { n, n' } \\ \text { mg-26 } & \text { n,2n } \\ \text { mg-26 } & \text { capture } \\ \text { mg-26 } & \text { n,gamma } \\ \text { mg-26 } & \text { n,p } \\ \text { mg-26 } & \text { n,alpha } \\ \text { si-28 } & \text { total } \\ \text { si-28 } & \text { scatter } \\ \text { si-28 } & \text { elastic } \\ \text { si-28 } & \text { n, n' } \\ \text { si-28 } & \text { capture } \\ \text { si-28 } & \text { n,gamma } \\ \text { si-28 } & \text { n,p } \\ & \\ & \end{array}$

\begin{tabular}{|c|c|c|}
\hline-10 & $+/-$ & \\
\hline$E-10$ & $+1-$ & \\
\hline-11 & $3008 \mathrm{E}-19$ & $00 \%)$ \\
\hline $.2926 \mathrm{E}-17$ & $0.0000 \mathrm{E}+00$ & $00 \div)$ \\
\hline $356 \mathrm{E}-08$ & $1 \mathrm{E}-11$ & \\
\hline $2.3175 \mathrm{E}-08$ & $+/-1.4447 \mathrm{E}-09$ & $3 \%)$ \\
\hline $5.7809 \mathrm{E}-08$ & $+/-1.4371 \mathrm{E}-09$ & \\
\hline & & \\
\hline $5.2023 E-09$ & $7.8251 \mathrm{E}-15$ & \\
\hline $4.5318 \mathrm{E}-13$ & $+/-0.0000 \mathrm{E}+00$ & $0 \%)$ \\
\hline 3.4 & $+1-2.0$ & \\
\hline 88 & $8 E-11$ & \\
\hline 1.5 & $3.2974 \mathrm{E}-18$ & \\
\hline-7.26 & $5.0907 \mathrm{E}-18$ & \\
\hline 3. & $2 E-12$ & \\
\hline 3.5 & $E-12$ & \\
\hline 3.3 & $/-5.83$ & \\
\hline 2.2 & 16 & \\
\hline 4.24 & 0.00 & \\
\hline 3. & 2.0 & \\
\hline 2.5 & $+1-$ & \\
\hline 1.3 & 4.39 & \\
\hline 0 & & \\
\hline 2.2 & -12 & \\
\hline 3.6 & $\mathrm{E}-12$ & \\
\hline 3.3 & L. 0 & \\
\hline 2. & 1.2 & \\
\hline 1.3 & $+/-1.1$ & \\
\hline-1.3 & 7.29 & \\
\hline & & \\
\hline-1.5 & 1.84 & \\
\hline-1.5 & .45 & \\
\hline & L3 & \\
\hline 3.8 & .4 & \\
\hline 3.6 & -13 & \\
\hline 2.0 & +1 & \\
\hline 1.2 & & \\
\hline-2.6 & -17 & \\
\hline-2.5 & E-17 & \\
\hline & & \\
\hline-2.2 & 2.21 & \\
\hline 1 & .66 & \\
\hline 2.6 & & \\
\hline 2.5 & 1.52 & \\
\hline 1. $3815 E-08$ & $1.7317 \mathrm{E}-15$ & 0 \\
\hline-9.06 & OE-11 & \\
\hline & 11 & \\
\hline 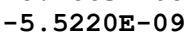 & & 0 . \\
\hline
\end{tabular}

Figure 6.3.16. SAMS output for LEU-COMP-THERM-009 case 10 sample problem. (continued) 


\begin{tabular}{|c|c|c|c|c|}
\hline si-28 & $\mathrm{n}, \mathrm{d}$ & $-8.9972 \mathrm{E}-12$ & $+/-1.1284 \mathrm{E}-18$ & $0.00 \%)$ \\
\hline si-28 & n, alpha & $-2.4934 \mathrm{E}-09$ & $+/-5.7790 \mathrm{E}-17$ & $0.00 \%)$ \\
\hline si-29 & total & $7.3370 \mathrm{E}-09$ & $+/-1.5194 \mathrm{E}-12$ & $0.02 \%)$ \\
\hline si-29 & scatter & $1.0779 \mathrm{E}-08$ & $+/-1.5176 \mathrm{E}-12$ & $0.01 \%)$ \\
\hline si-29 & elastic & $9.6163 \mathrm{E}-09$ & $+/-1.5176 \mathrm{E}-12$ & $0.02 \%)$ \\
\hline si-29 & $\mathrm{n}, \mathrm{n}^{\prime}$ & $1.1542 \mathrm{E}-09$ & $+/-1.7759 \mathrm{E}-16$ & $0.00 \%)$ \\
\hline si-29 & $\mathrm{n}, 2 \mathrm{n}$ & $8.0511 \mathrm{E}-12$ & $+/-8.0235 E-19$ & $0.00 \%)$ \\
\hline si-29 & capture & $-3.4416 \mathrm{E}-09$ & $+/-7.1336 \mathrm{E}-15$ & $0.00 \%)$ \\
\hline si-29 & $\mathrm{n}$, gamma & $-3.0602 E-09$ & $+/-7.1336 \mathrm{E}-15$ & $0.00 \%)$ \\
\hline si-29 & $n, p$ & $-1.4767 \mathrm{E}-10$ & $+/-2.5051 \mathrm{E}-18$ & $0.00 \%)$ \\
\hline si-29 & n, alpha & $-2.3373 E-10$ & $+/-3.3600 \mathrm{E}-18$ & $0.00 \%)$ \\
\hline si-30 & total & $4.2864 \mathrm{E}-09$ & $+/-9.7142 \mathrm{E}-13$ & $0.02 \%)$ \\
\hline si-30 & scatter & $7.1185 \mathrm{E}-09$ & $+/-9.6378 \mathrm{E}-13$ & $0.01 \%)$ \\
\hline si-30 & elastic & $6.7125 \mathrm{E}-09$ & $+/-9.6378 \mathrm{E}-13$ & $0.01 \%)$ \\
\hline si-30 & $\mathrm{n}, \mathrm{n}^{\prime}$ & $4.0116 \mathrm{E}-10$ & $+/-5.5897 \mathrm{E}-17$ & $0.00 \% 1$ \\
\hline si-30 & $n, 2 n$ & $4.8546 \mathrm{E}-12$ & $+/-4.4990 \mathrm{E}-19$ & $0.00 \%)$ \\
\hline si-30 & capture & $-2.8321 E-09$ & $+/-1.8920 \mathrm{E}-14$ & $0.00 \%)$ \\
\hline si-30 & $\mathrm{n}$, gamma & $-2.8275 E-09$ & $+/-1.8920 \mathrm{E}-14$ & $0.00 \%)$ \\
\hline si-30 & $\mathrm{n}, \mathrm{p}$ & $-9.0348 \mathrm{E}-13$ & $+/-1.2153 \mathrm{E}-19$ & $0.00 \%)$ \\
\hline si-30 & n,alpha & $-3.7528 \mathrm{E}-12$ & $+/-3.1321 \mathrm{E}-19$ & $0.00 \%)$ \\
\hline$s-32$ & total & $-1.4228 \mathrm{E}-08$ & $+/-1.7286 \mathrm{E}-12$ & $0.01 \%)$ \\
\hline$s-32$ & scatter & $1.9787 \mathrm{E}-08$ & $+/-1.7227 \mathrm{E}-12$ & $0.01 \%)$ \\
\hline$s-32$ & elastic & $1.9229 \mathrm{E}-08$ & $+/-1.7227 \mathrm{E}-12$ & $0.01 \%)$ \\
\hline$s-32$ & $\mathrm{n}, \mathrm{n}^{\prime}$ & $5.4483 \mathrm{E}-10$ & $+/-8.3488 \mathrm{E}-17$ & $0.00 \%)$ \\
\hline$s-32$ & $\mathrm{n}, 2 \mathrm{n}$ & $9.4026 \mathrm{E}-17$ & $+/-0.0000 \mathrm{E}+00$ & $0.00 \%)$ \\
\hline$s-32$ & capture & $-3.4015 E-08$ & $+/-9.3425 \mathrm{E}-15$ & $0.00 \%)$ \\
\hline$s-32$ & $\mathrm{n}$, gamma & $-2.3112 \mathrm{E}-08$ & $+/-3.3672 \mathrm{E}-15$ & $0.00 \%)$ \\
\hline$s-32$ & $\mathrm{n}, \mathrm{p}$ & $-4.5487 E-09$ & $+/-5.5174 \mathrm{E}-17$ & $0.00 \%)$ \\
\hline$s-32$ & n, alpha & $-6.3539 E-09$ & $+/-7.3703 E-15$ & $0.00 \% 1$ \\
\hline$s-33$ & total & $-3.5098 \mathrm{E}-10$ & $+/-2.0849 \mathrm{E}-14$ & $0.01 \%)$ \\
\hline$s-33$ & scatter & $1.1379 \mathrm{E}-10$ & $+/-1.9431 \mathrm{E}-14$ & $0.02 \%)$ \\
\hline$s-33$ & elastic & $1.0619 \mathrm{E}-10$ & $+/-1.9431 \mathrm{E}-14$ & $0.02 \%)$ \\
\hline$s-33$ & $\mathrm{n}, \mathrm{n}^{\prime}$ & 7. $3395 \mathrm{E}-12$ & $+/-2.0279 \mathrm{E}-18$ & $0.00 \%)$ \\
\hline$s-33$ & $\mathrm{n}, 2 \mathrm{n}$ & $1.7646 \mathrm{E}-13$ & $+/-2.0474 \mathrm{E}-20$ & $0.00 \%)$ \\
\hline$s-33$ & capture & $-4.6477 \mathrm{E}-10$ & $+/-1.4277 \mathrm{E}-15$ & $0.00 \%)$ \\
\hline$s-33$ & $\mathrm{n}$, gamma & $-1.2051 \mathrm{E}-10$ & $+/-3.3390 \mathrm{E}-17$ & $0.00 \%)$ \\
\hline$s-33$ & $n, p$ & $-3.3567 \mathrm{E}-11$ & $+/-1.3744 \mathrm{E}-17$ & $0.00 \%$ ) \\
\hline$s-33$ & n, alpha & $-3.1070 \mathrm{E}-10$ & $+/-1.4014 \mathrm{E}-15$ & $0.00 \%)$ \\
\hline$s-34$ & total & $3.0984 \mathrm{E}-10$ & $+/-8.4943 \mathrm{E}-14$ & $0.03 \%)$ \\
\hline$s-34$ & scatter & $7.5435 \mathrm{E}-10$ & $+/-8.4929 \mathrm{E}-14$ & $0.01 \%$ ) \\
\hline$s-34$ & elastic & $7.1661 \mathrm{E}-10$ & $+/-8.4929 \mathrm{E}-14$ & $0.01 \%)$ \\
\hline$s-34$ & $\mathrm{n}, \mathrm{n}^{\prime}$ & $3.6985 \mathrm{E}-11$ & $+/-4.3271 \mathrm{E}-18$ & $0.00 \%)$ \\
\hline$s-34$ & $\mathrm{n}, 2 \mathrm{n}$ & $2.4941 \mathrm{E}-13$ & $+/-1.8198 \mathrm{E}-20$ & $0.00 \%)$ \\
\hline$s-34$ & capture & $-4.4451 \mathrm{E}-10$ & $+/-3.1938 \mathrm{E}-17$ & $0.00 \%)$ \\
\hline$s-34$ & n, gamma & $-4.3181 \mathrm{E}-10$ & $+/-3.1937 \mathrm{E}-17$ & $0.00 \%)$ \\
\hline$s-34$ & $\mathrm{n}, \mathrm{p}$ & $-2.5649 \mathrm{E}-12$ & $+/-7.4283 \mathrm{E}-20$ & $0.00 \%)$ \\
\hline$s-34$ & n, alpha & $-1.0132 \mathrm{E}-11$ & $+/-1.3880 \mathrm{E}-19$ & $0.00 \%)$ \\
\hline$s-36$ & total & $2.4367 \mathrm{E}-12$ & $+/-4.8470 \mathrm{E}-16$ & $0.02 \%$ ) \\
\hline
\end{tabular}

Figure 6.3.16. SAMS output for LEU-COMP-THERM-009 case 10 sample problem. (continued) 


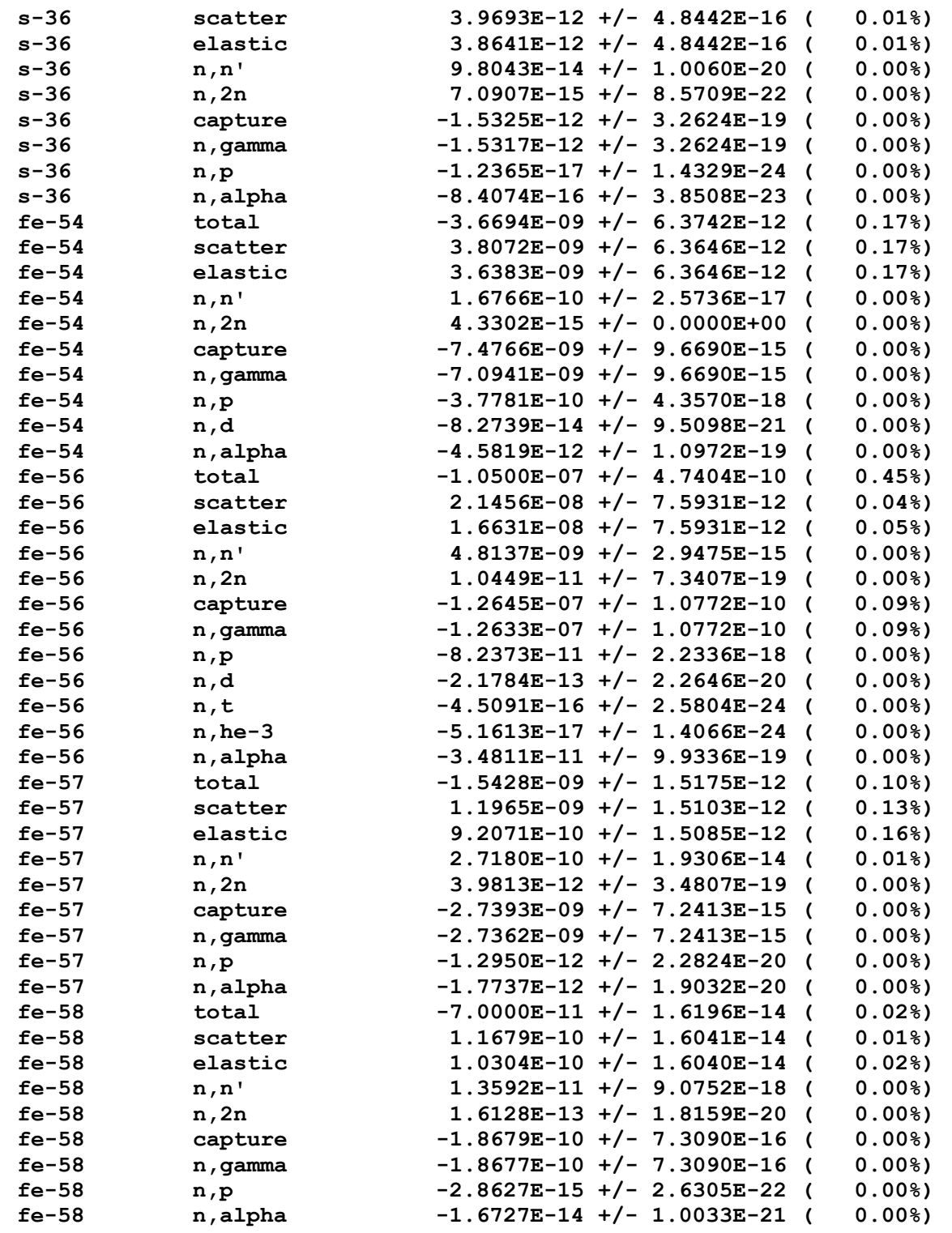

Figure 6.3.16. SAMS output for LEU-COMP-THERM-009 case 10 sample problem. (continued) 


\begin{tabular}{|c|c|c|c|c|}
\hline $\mathrm{cu}-63$ & total & $-3.1331 E-03$ & $+/-6.7640 \mathrm{E}-06$ & $0.22 \%)$ \\
\hline $\mathrm{cu}-63$ & scatter & $5.1503 E-04$ & $+/-4.5047 \mathrm{E}-06$ & $0.87 \%)$ \\
\hline $\mathrm{cu}-63$ & elastic & $4.3487 \mathrm{E}-04$ & $+/-4.1844 \mathrm{E}-06$ & $0.96 \%)$ \\
\hline $\mathrm{cu}-63$ & $\mathrm{n}, \mathrm{n}^{\prime}$ & $7.8687 \mathrm{E}-05$ & $+/-1.1401 \mathrm{E}-06$ & $1.45 \%)$ \\
\hline $\mathrm{cu}-63$ & $n, 2 n$ & $2.1308 \mathrm{E}-07$ & $+/-7.0126 \mathrm{E}-09$ & $3.29 \%$ \\
\hline $\mathrm{cu}-63$ & capture & $-3.6481 E-03$ & $+/-3.1712 \mathrm{E}-06$ & $0.09 \%)$ \\
\hline $\mathrm{cu}-63$ & $\mathrm{n}$, gamma & $-3.6222 E-03$ & $+/-3.1710 \mathrm{E}-06$ & $0.09 \%)$ \\
\hline $\mathrm{cu}-63$ & $\mathrm{n}, \mathrm{p}$ & $-2.4693 E-05$ & $+/-3.5515 \mathrm{E}-08$ & $0.14 \%)$ \\
\hline $\mathrm{cu}-63$ & $\mathrm{n}, \mathrm{d}$ & $-5.2482 \mathrm{E}-07$ & $+/-1.5798 \mathrm{E}-09$ & $0.30 \%)$ \\
\hline $\mathrm{cu}-63$ & $\mathrm{n}, \mathrm{he}-3$ & $-3.0750 \mathrm{E}-12$ & $+/-3.9260 \mathrm{E}-19$ & $0.00 \%)$ \\
\hline $\mathrm{cu}-63$ & n,alpha & $-6.7771 E-07$ & $+/-2.7471 \mathrm{E}-09$ & $0.41 \%)$ \\
\hline $\mathrm{cu}-65$ & total & $-6.0763 E-04$ & $+/-4.7381 E-06$ & $0.78 \%)$ \\
\hline $\mathrm{cu}-65$ & scatter & $1.7893 \mathrm{E}-04$ & $+/-4.2465 \mathrm{E}-06$ & $2.37 \%$ \\
\hline$c u-65$ & elastic & $1.4608 \mathrm{E}-04$ & $+/-4.1860 \mathrm{E}-06$ & $2.87 \%$ \\
\hline $\mathrm{cu}-65$ & $\mathrm{n}, \mathrm{n}^{\prime}$ & $3.2461 \mathrm{E}-05$ & $+/-4.9054 \mathrm{E}-07$ & $1.51 \%)$ \\
\hline$c u-65$ & $n, 2 n$ & $3.7791 \mathrm{E}-07$ & $+/-7.8368 \mathrm{E}-09$ & $2.07 \%)$ \\
\hline $\mathrm{cu}-65$ & capture & $-7.8656 \mathrm{E}-04$ & $+/-6.8251 \mathrm{E}-07$ & $0.09 \%)$ \\
\hline$c u-65$ & $\mathrm{n}$, gamma & $-7.8620 \mathrm{E}-04$ & $+/-6.8251 \mathrm{E}-07$ & $0.09 \%)$ \\
\hline $\mathrm{cu}-65$ & $\mathrm{n}, \mathrm{p}$ & $-3.1043 E-07$ & $+/-7.8886 \mathrm{E}-10$ & $0.25 \%)$ \\
\hline $\mathrm{cu}-65$ & $\mathrm{n}, \mathrm{d}$ & $-4.0903 E-08$ & $+/-1.8559 \mathrm{E}-10$ & $0.45 \%$ \\
\hline $\mathrm{cu}-65$ & $n, t$ & $-1.0623 E-11$ & $+/-2.7949 \mathrm{E}-18$ & $0.00 \%)$ \\
\hline $\mathrm{cu}-65$ & $n, h e-3$ & $-3.8976 \mathrm{E}-13$ & $+/-1.3313 \mathrm{E}-20$ & $0.00 \%)$ \\
\hline$c u-65$ & n, alpha & $-8.9150 E-09$ & $+/-1.3569 \mathrm{E}-15$ & $0.00 \%)$ \\
\hline $\mathrm{h}-1$ & total & $-3.2827 E-02$ & $+/-6.0121 \mathrm{E}-04$ & $1.83 \%)$ \\
\hline $\mathrm{h}-1$ & scatter & $2.0058 \mathrm{E}-02$ & $+/-5.9506 \mathrm{E}-04$ & $2.97 \%)$ \\
\hline $\mathrm{h}-1$ & elastic & $2.0058 \mathrm{E}-02$ & $+/-5.9506 \mathrm{E}-04$ & $2.97 \%)$ \\
\hline $\mathrm{h}-1$ & capture & $-5.2884 \mathrm{E}-02$ & $+/-8.0351 \mathrm{E}-06$ & $0.02 \%$ \\
\hline $\mathrm{h}-1$ & $\mathrm{n}$, gamma & $-5.2884 E-02$ & $+/-8.0351 \mathrm{E}-06$ & $0.02 \%$ \\
\hline $0-16$ & total & $2.6563 \mathrm{E}-02$ & $+/-3.0100 \mathrm{E}-05$ & $0.11 \%)$ \\
\hline $0-16$ & scatter & $2.6725 \mathrm{E}-02$ & $+/-3.0097 \mathrm{E}-05$ & $0.11 \%)$ \\
\hline $0-16$ & elastic & $2.6660 \mathrm{E}-02$ & $+/-3.0097 \mathrm{E}-05$ & $0.11 \%)$ \\
\hline $0-16$ & $\mathrm{n}, \mathrm{n}^{\prime}$ & $6.2924 \mathrm{E}-05$ & $+/-8.2297 \mathrm{E}-08$ & $0.13 \%)$ \\
\hline $0-16$ & $n, 2 n$ & $3.8366 \mathrm{E}-12$ & $+/-8.0943 E-18$ & $0.00 \%)$ \\
\hline $0-16$ & capture & $-1.6136 \mathrm{E}-04$ & $+/-6.2177 \mathrm{E}-08$ & $0.04 \%)$ \\
\hline $0-16$ & $\mathrm{n}$, gamma & $-1.5133 \mathrm{E}-05$ & $+/-2.2991 \mathrm{E}-09$ & $0.02 \%)$ \\
\hline $0-16$ & $\mathrm{n}, \mathrm{p}$ & $-7.7305 \mathrm{E}-07$ & $+/-2.9519 \mathrm{E}-09$ & $0.38 \%)$ \\
\hline $0-16$ & $\mathrm{n}, \mathrm{d}$ & $-7.8828 E-08$ & $+/-5.6744 \mathrm{E}-10$ & $0.72 \%)$ \\
\hline $0-16$ & $n, t$ & $-2.2174 E-13$ & $+/-1.4667 \mathrm{E}-18$ & $0.00 \%)$ \\
\hline $0-16$ & n, alpha & $-1.4538 \mathrm{E}-04$ & $+/-6.1000 \mathrm{E}-08$ & $0.04 \%)$ \\
\hline
\end{tabular}

Total Sensitivity Coefficients by Nuclide

\begin{tabular}{|c|c|c|c|c|c|c|}
\hline$x t$ & de & Atom Density & ity & & v. & td. Dev. \\
\hline 1 & $\mathrm{u}$ & & & $+/-$ & 07 & \\
\hline 1 & $u-235$ & $102 \mathrm{E}-03$ & $2.0616 \mathrm{E}-01$ & $-1-$ & $7.0501 \mathrm{E}-05$ & $0.03 \%)$ \\
\hline
\end{tabular}

Figure 6.3.16. SAMS output for LEU-COMP-THERM-009 case 10 sample problem. (continued) 


\begin{tabular}{|c|c|c|c|c|c|}
\hline$u-236$ & $5.1395 \mathrm{E}-06$ & $-2.1805 E-04$ & $+/-$ & $1.2725 \mathrm{E}-07$ & $0.06 \%)$ \\
\hline$u-238$ & $2.2157 \mathrm{E}-02$ & $-5.9609 \mathrm{E}-02$ & $+1-$ & $2.6857 \mathrm{E}-05$ & $0.05 \%)$ \\
\hline $0-16$ & $4.6753 \mathrm{E}-02$ & $1.1444 \mathrm{E}-02$ & $+/-$ & $3.3431 \mathrm{E}-05$ & $0.29 \%)$ \\
\hline $0-16$ & $3.3338 \mathrm{E}-02$ & $3.6005 \mathrm{E}-02$ & $+/-$ & $2.9143 E-05$ & $0.08 \%)$ \\
\hline $0-16$ & $1.2461 \mathrm{E}-02$ & $3.9480 \mathrm{E}-05$ & $+/-$ & $8.0523 E-07$ & $2.04 \%)$ \\
\hline $0-16$ & $1.4273 \mathrm{E}-02$ & $5.7694 \mathrm{E}-05$ & $+/-$ & $6.3654 \mathrm{E}-07$ & $1.10 \%)$ \\
\hline $0-16$ & $1.0064 \mathrm{E}-04$ & $6.9207 \mathrm{E}-07$ & $+1-$ & $7.5312 \mathrm{E}-09$ & $1.09 \%)$ \\
\hline $0-16$ & $3.3338 \mathrm{E}-02$ & $2.6563 \mathrm{E}-02$ & $+1-$ & $3.0100 \mathrm{E}-05$ & $0.11 \%)$ \\
\hline$h-1$ & $6.6675 \mathrm{E}-02$ & $2.0159 \mathrm{E}-01$ & $+1-$ & $4.6480 \mathrm{E}-04$ & $0.23 \%)$ \\
\hline $\mathrm{h}-1$ & $5.8178 \mathrm{E}-02$ & $-3.3487 \mathrm{E}-05$ & $+/-$ & $3.3383 E-05$ & $99.69 \%)$ \\
\hline$h-1$ & $5.6642 \mathrm{E}-02$ & $-4.6343 \mathrm{E}-05$ & $+/-$ & $2.5632 \mathrm{E}-05$ & $55.31 \%$ \\
\hline $\mathrm{h}-1$ & $6.6675 \mathrm{E}-02$ & $-3.2827 \mathrm{E}-02$ & $+/-$ & $6.0121 \mathrm{E}-04$ & $1.83 \%)$ \\
\hline al-27 & $5.8433 \mathrm{E}-02$ & $-6.7582 E-05$ & $+1-$ & $2.3201 \mathrm{E}-05$ & $34.33 \%)$ \\
\hline cr -50 & $2.7074 \mathrm{E}-06$ & $-1.3040 \mathrm{E}-05$ & $+/-$ & $9.1891 \mathrm{E}-09$ & $0.07 \%)$ \\
\hline$c r-52$ & $5.2210 \mathrm{E}-05$ & $-8.7501 \mathrm{E}-06$ & $+/-$ & $2.6630 \mathrm{E}-08$ & $0.30 \%)$ \\
\hline cr -53 & $5.9194 \mathrm{E}-06$ & $-3.4245 \mathrm{E}-05$ & $+/-$ & $2.1670 \mathrm{E}-08$ & $0.06 \%)$ \\
\hline cr -54 & $1.4736 \mathrm{E}-06$ & $-1.6695 E-08$ & $+/-$ & $4.1104 \mathrm{E}-10$ & $2.46 \%)$ \\
\hline $\mathrm{cu}-63$ & $6.5503 \mathrm{E}-05$ & $-9.5973 E-05$ & $+/-$ & $7.0063 \mathrm{E}-08$ & $0.07 \%)$ \\
\hline $\mathrm{cu}-63$ & $5.8191 \mathrm{E}-02$ & $-3.1331 \mathrm{E}-03$ & $+/-$ & $6.7640 \mathrm{E}-06$ & $0.22 \%)$ \\
\hline $\mathrm{cu}-65$ & $2.9195 \mathrm{E}-05$ & $-1.7689 \mathrm{E}-05$ & $+/-$ & $4.4209 \mathrm{E}-08$ & $0.25 \%)$ \\
\hline$c u-65$ & $2.5937 \mathrm{E}-02$ & $-6.0763 E-04$ & $+/-$ & $4.7381 E-06$ & $0.78 \%)$ \\
\hline$m g-24$ & $5.2648 \mathrm{E}-04$ & $4.5166 \mathrm{E}-05$ & $+/-$ & $4.5339 \mathrm{E}-07$ & $1.00 \%)$ \\
\hline$m g-24$ & $3.4888 \mathrm{E}-06$ & $3.2809 \mathrm{E}-08$ & $+/-$ & $5.8412 \mathrm{E}-12$ & $0.02 \%)$ \\
\hline$m g-25$ & $6.6651 \mathrm{E}-05$ & $1.2140 \mathrm{E}-06$ & $+/-$ & $3.2733 \mathrm{E}-08$ & $2.70 \%)$ \\
\hline$m g-25$ & $4.4168 \mathrm{E}-07$ & $2.2861 \mathrm{E}-09$ & $+/-$ & 1. $0962 \mathrm{E}-12$ & $0.05 \%)$ \\
\hline$m g-26$ & $7.3383 \mathrm{E}-05$ & $5.2992 \mathrm{E}-06$ & $+1-$ & $3.2698 \mathrm{E}-08$ & $0.62 \%)$ \\
\hline$m g-26$ & $4.8629 \mathrm{E}-07$ & $3.6092 \mathrm{E}-09$ & $+1-$ & $4.4230 \mathrm{E}-13$ & $0.01 \%)$ \\
\hline$m n-55$ & $2.2115 \mathrm{E}-05$ & $-9.6360 \mathrm{E}-05$ & $+/-$ & $1.4079 \mathrm{E}-07$ & $0.15 \%)$ \\
\hline$t i-46$ & $2.0300 \mathrm{E}-06$ & $-1.8856 \mathrm{E}-07$ & $+/-$ & $8.6800 \mathrm{E}-10$ & $0.46 \%)$ \\
\hline ti-47 & $1.8524 \mathrm{E}-06$ & $-8.7007 \mathrm{E}-07$ & $+1-$ & $2.4463 E-09$ & $0.28 \%)$ \\
\hline$t i-48$ & $1.8727 \mathrm{E}-05$ & $-4.5957 \mathrm{E}-05$ & $+1-$ & $3.3263 E-08$ & $0.07 \%)$ \\
\hline$t i-49$ & $1.3956 \mathrm{E}-06$ & $-6.8733 E-07$ & $+/-$ & $6.2660 \mathrm{E}-10$ & $0.09 \%)$ \\
\hline$t i-50$ & 1. $3702 \mathrm{E}-06$ & $5.1758 \mathrm{E}-08$ & $+/-$ & $4.1741 \mathrm{E}-10$ & $0.81 \%)$ \\
\hline$s i-28$ & $3.1918 \mathrm{E}-04$ & 4. $3107 \mathrm{E}-06$ & $+/-$ & $1.2244 \mathrm{E}-07$ & $2.84 \%)$ \\
\hline si-28 & $8.8873 E-05$ & $1.5659 \mathrm{E}-07$ & $+/-$ & $3.1168 \mathrm{E}-09$ & $1.99 \%)$ \\
\hline si-28 & $3.5253 \mathrm{E}-05$ & $1.7607 \mathrm{E}-07$ & $+/-$ & $1.6638 \mathrm{E}-09$ & $0.94 \%)$ \\
\hline si-29 & $1.6161 \mathrm{E}-05$ & $5.3687 \mathrm{E}-07$ & $+/-$ & $5.6554 \mathrm{E}-09$ & $1.05 \%)$ \\
\hline si-29 & $4.5000 \mathrm{E}-06$ & $8.3353 \mathrm{E}-09$ & $+1-$ & $7.5001 \mathrm{E}-15$ & $0.00 \%)$ \\
\hline si-29 & $1.7850 \mathrm{E}-06$ & $7.3370 \mathrm{E}-09$ & $+1-$ & 1. $5194 \mathrm{E}-12$ & $0.02 \%)$ \\
\hline si-30 & $1.0728 \mathrm{E}-05$ & $1.3408 \mathrm{E}-07$ & $+/-$ & $4.4925 \mathrm{E}-09$ & $3.35 \%)$ \\
\hline si-30 & $2.9872 \mathrm{E}-06$ & $5.0684 \mathrm{E}-09$ & $+/-$ & $6.4263 \mathrm{E}-15$ & $0.00 \%)$ \\
\hline si-30 & $1.1849 \mathrm{E}-06$ & $4.2864 \mathrm{E}-09$ & $+/-$ & $9.7142 \mathrm{E}-13$ & $0.02 \%)$ \\
\hline $\mathrm{fe}-54$ & $5.9897 \mathrm{E}-06$ & $-3.9407 E-06$ & $+/-$ & $8.5216 \mathrm{E}-09$ & $0.22 \%)$ \\
\hline $\mathrm{fe}-54$ & $2.2682 \mathrm{E}-07$ & $-3.6694 \mathrm{E}-09$ & $+/-$ & $6.3742 \mathrm{E}-12$ & $0.17 \%)$ \\
\hline $\mathrm{fe}-56$ & $9.3114 \mathrm{E}-05$ & $-6.9426 \mathrm{E}-05$ & $+1-$ & 1. $3507 \mathrm{E}-07$ & $0.19 \%)$ \\
\hline fe-56 & $3.5261 \mathrm{E}-06$ & $-1.0500 \mathrm{E}-07$ & $+/-$ & $4.7404 \mathrm{E}-10$ & $0.45 \%)$ \\
\hline$f e-57$ & $2.1319 \mathrm{E}-06$ & $-1.4580 \mathrm{E}-06$ & $+/-$ & $2.4578 E-09$ & $0.17 \%)$ \\
\hline$f e-57$ & $8.0732 \mathrm{E}-08$ & $-1.5428 \mathrm{E}-09$ & $+1-$ & 1. $5175 \mathrm{E}-12$ & $0.10 \%)$ \\
\hline
\end{tabular}

Figure 6.3.16. SAMS output for LEU-COMP-THERM-009 case 10 sample problem. (continued) 


\begin{tabular}{|c|c|c|c|c|c|c|c|}
\hline 3 & $f e-58$ & $2.8426 \mathrm{E}-07$ & $-8.6249 \mathrm{E}-08$ & $+/-$ & $9.2863 E-11$ & ( & $0.11 \%)$ \\
\hline 6 & $f e-58$ & $1.0764 \mathrm{E}-08$ & $-7.0000 \mathrm{E}-11$ & $+/-$ & 1. $6196 \mathrm{E}-14$ & . & $0.02 \% 1$ \\
\hline 4 & c & $4.3562 \mathrm{E}-02$ & $1.4324 \mathrm{E}-04$ & $+/-$ & 3. $4393 E-06$ & $i$ & $2.40 \%)$ \\
\hline 5 & c & $3.5648 \mathrm{E}-02$ & $1.5186 \mathrm{E}-04$ & $+/-$ & $2.0605 E-06$ & ( & $1.36 \%)$ \\
\hline 6 & c & $1.5194 \mathrm{E}-03$ & $9.7668 \mathrm{E}-06$ & $+1-$ & 1. $3853 \mathrm{E}-07$ & 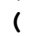 & $1.42 \%$ \\
\hline 4 & $\mathrm{Ca}-40$ & $2.4875 \mathrm{E}-03$ & $-1.7949 \mathrm{E}-06$ & $+/-$ & $1.1894 \mathrm{E}-07$ & ( & $6.63 \% 1$ \\
\hline 4 & $c a-42$ & $1.6602 \mathrm{E}-05$ & $-3.3798 E-08$ & $+/-$ & $4.2370 \mathrm{E}-10$ & i & $1.25 \%)$ \\
\hline 4 & $c a-43$ & $3.4641 \mathrm{E}-06$ & $-2.9992 \mathrm{E}-07$ & $+/-$ & $8.5309 E-10$ & ( & $0.28 \%)$ \\
\hline 4 & $\mathrm{ca}-44$ & $5.3527 \mathrm{E}-05$ & $-1.9121 E-07$ & $+1-$ & $2.9350 \mathrm{E}-09$ & i & $1.53 \circ)$ \\
\hline 4 & $c a-46$ & $1.0264 \mathrm{E}-07$ & $-9.1789 \mathrm{E}-11$ & $+1-$ & $2.9314 \mathrm{E}-16$ & i & $0.00 \% 1$ \\
\hline 4 & $\mathrm{ca}-48$ & $4.7984 \mathrm{E}-06$ & $-2.4743 E-08$ & $+/-$ & $1.8724 \mathrm{E}-10$ & i & $0.76 \%)$ \\
\hline 4 & $s-32$ & $4.5439 \mathrm{E}-04$ & $-9.3027 \mathrm{E}-07$ & $+/-$ & $1.5023 E-08$ & ( & $1.61 \%)$ \\
\hline 6 & $s-32$ & $3.1807 E-06$ & $-1.4228 \mathrm{E}-08$ & $+/-$ & $1.7286 \mathrm{E}-12$ & ( & $0.01 \div)$ \\
\hline 4 & $s-33$ & $3.5865 \mathrm{E}-06$ & $-1.1925 \mathrm{E}-08$ & $+/-$ & $1.0473 E-14$ & ( & $0.00 \div)$ \\
\hline 6 & $s-33$ & $2.5106 \mathrm{E}-08$ & $-3.5098 E-10$ & $+/-$ & $2.0849 \mathrm{E}-14$ & ( & $0.01 \%)$ \\
\hline 4 & $s-34$ & $2.0132 \mathrm{E}-05$ & $1.2596 \mathrm{E}-08$ & $+/-$ & $3.9241 \mathrm{E}-10$ & $i$ & $3.12 \%$ \\
\hline 6 & $s-34$ & $1.4093 \mathrm{E}-07$ & $3.0984 \mathrm{E}-10$ & $+/-$ & $8.4943 E-14$ & ( & $0.03 \%)$ \\
\hline 4 & $s-36$ & $9.5640 \mathrm{E}-08$ & $1.2812 \mathrm{E}-10$ & $+/-$ & $1.6526 \mathrm{E}-16$ & i & $0.00 \% 1$ \\
\hline 6 & $s-36$ & $6.6948 \mathrm{E}-10$ & $2.4367 E-12$ & $+/-$ & $4.8470 \mathrm{E}-16$ & $i$ & $0.02 \%)$ \\
\hline 6 & na-23 & $4.6695 \mathrm{E}-06$ & $2.3175 \mathrm{E}-08$ & $+/-$ & $1.4447 \mathrm{E}-09$ & i & $6.23 \% 1$ \\
\hline
\end{tabular}

Total Sensitivity Coefficients by Mixture

\begin{tabular}{|c|c|c|c|c|}
\hline Mixture & Sensitivity & & Std. Dev. & \% Std. Dev. \\
\hline 1 & $1.5686 \mathrm{E}-01$ & $+1-$ & $8.2519 \mathrm{E}-05$ & $0.05 \%)$ \\
\hline 2 & $2.3760 \mathrm{E}-01$ & $+/-$ & $4.6571 \mathrm{E}-04$ & $0.20 \%)$ \\
\hline 3 & $-3.9956 \mathrm{E}-04$ & $+/-$ & $2.3207 \mathrm{E}-05$ & $5.81 \%)$ \\
\hline 4 & 1. $4613 \mathrm{E}-04$ & $+/-$ & $3.3569 \mathrm{E}-05$ & ( $22.97 \%)$ \\
\hline 5 & $1.6321 \mathrm{E}-04$ & $+1-$ & $2.5723 \mathrm{E}-05$ & ( $15.76 \%)$ \\
\hline 6 & $-3.7302 \mathrm{E}-03$ & $+/-$ & $8.2596 \mathrm{E}-06$ & $0.22 \%)$ \\
\hline 7 & $-6.2632 \mathrm{E}-03$ & $+/-$ & $6.0196 \mathrm{E}-04$ & $9.61 \%)$ \\
\hline
\end{tabular}

Problem Characterization

median fission group is $222 \quad 5.000 \mathrm{E}-02$ to $4.000 \mathrm{E}-02(\mathrm{eV})$

average fission group $2.077 \mathrm{E}+02+/-3.212 \mathrm{E}-01$

average energy $(\mathrm{eV})$ causing fission $1.115 \mathrm{E}+05+/-2.059 \mathrm{E}+02$

energy (eV) of average lethargy causing fission $1.128 \mathrm{E}-01+/-1.749 \mathrm{E}-04$

median capture group is $2234.000 \mathrm{E}-02$ to $3.000 \mathrm{E}-02(\mathrm{eV})$

average capture group $2.072 \mathrm{E}+02+/-1.110 \mathrm{E}-01$

average energy $(\mathrm{eV})$ causing capture $3.771 \mathrm{E}+04+/-4.509 \mathrm{E}+01$

energy $(\mathrm{eV})$ of average lethargy causing capture $8.751 \mathrm{E}-02+/-4.773 \mathrm{E}-05$

Figure 6.3.16. SAMS output for LEU-COMP-THERM-009 case 10 sample problem. (continued) 
median scatter group is $221 \quad 6.000 \mathrm{E}-02$ to $5.000 \mathrm{E}-02(\mathrm{eV})$

average scatter group $1.903 \mathrm{E}+02+/-5.331 \mathrm{E}-02$

average energy $(\mathrm{eV})$ causing scatter $6.721 \mathrm{E}+04+/-2.875 \mathrm{E}+01$

energy $(\mathrm{eV})$ of average lethargy causing scatter $3.839 \mathrm{E}-01+/-1.069 \mathrm{E}-04$

Generating working covariance matrix ...

Working covariance matrix created for future processing.

Uncertainty Information

the relative standard deviation of $k$-eff $(\%$ delta-k/k) due to cross-section covariance data is:

$0.6109+/-0.0000 \div \operatorname{delta-k/k}$

contributions to uncertainty in k-eff ( $\% \operatorname{delta-k/k})$ by individual energy covariance matrices:

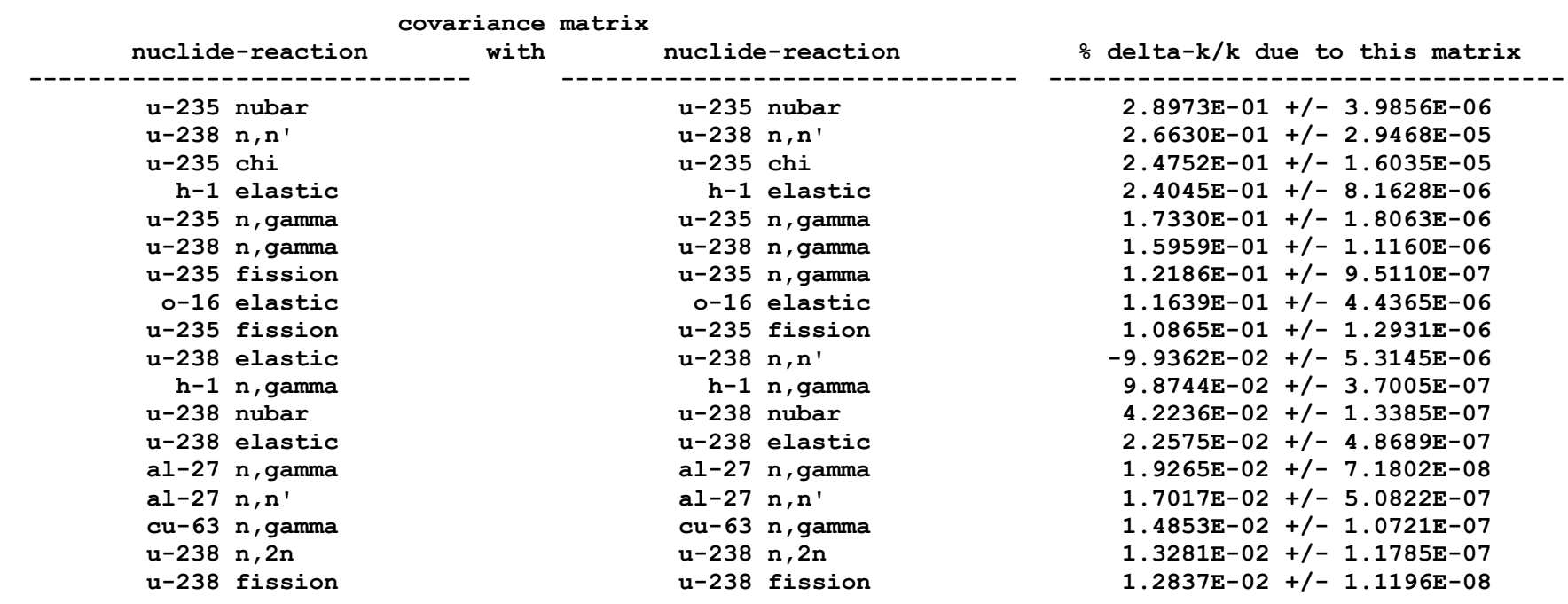

Figure 6.3.16. SAMS output for LEU-COMP-THERM-009 case 10 sample problem. (continued) 


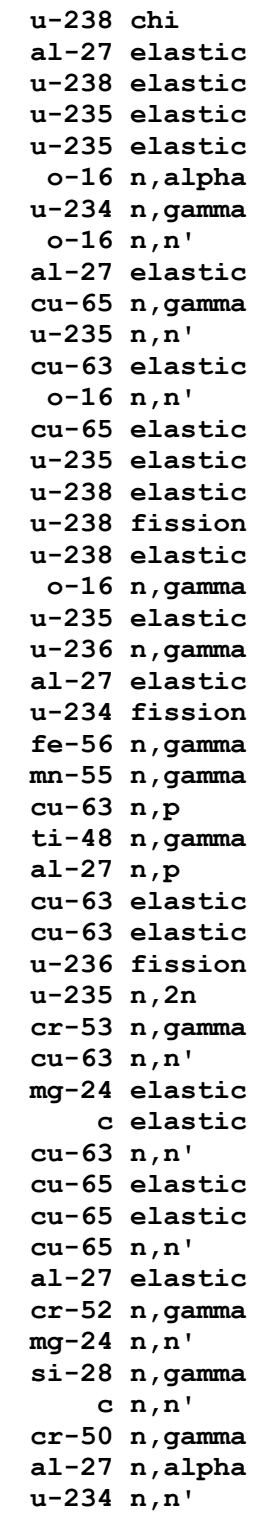

u-238 chi

al-27 n, n

$\mathrm{u}-238 \mathrm{n}$, gamma

$\mathrm{u}-235 \mathrm{n}$, gamma

u-235 fission

o-16 n, alpha

u-234 n, gamma

0-16 $n, n^{\prime}$

al-27 elastic

cu-65 n, gamma

$\mathrm{u}-235 \mathrm{n}, \mathrm{n}^{\prime}$

cu-63 elastic

-16 elastic

cu-65 elastic

$\mathrm{u}-235 \mathrm{n}, \mathrm{n}^{\prime}$

u-238 fission

u-238 n, gamma

$\mathrm{u}-238 \mathrm{n}, 2 \mathrm{n}$

0-16 n, gamma

u-235 elastic

u-236 n, gamma

al-27 n, gamma

u-234 fission

fe-56 $n$, gamma

mn-55 n, gamma

$\mathrm{cu}-63 \mathrm{n}, \mathrm{p}$

ti-48 n, gamma

al-27 n,p

cu-63 n, gamma

cu-63 n, n'

$\mathrm{u}-236$ fission

u-235 n, 2n

cr-53 n, gamma

cu-63 n, n'

mg-24 elastic

c elastic

$\mathrm{cu}-63 \mathrm{n}, \mathrm{p}$

$\mathrm{cu}-65 \mathrm{n}$, gamma

$\mathrm{cu}-65 \mathrm{n}, \mathrm{n}$

cu-65 $n, n$

al-27 $n, p$

cr-52 n, gamma

$\mathrm{mg}-24 \mathrm{n}, \mathrm{n}$

si-28 n, gamma

c elastic

cr-50 n, gamma

al-27 $n$, alpha

$\mathrm{u}-234 \mathrm{n}, \mathrm{n}$
$1.1469 \mathrm{E}-02+/-3.3598 \mathrm{E}-08$

$-1.0157 \mathrm{E}-02+/-2.1624 \mathrm{E}-07$

$8.5476 \mathrm{E}-03+/-7.8644 \mathrm{E}-07$

$6.0964 \mathrm{E}-03+/-7.9059 \mathrm{E}-08$

$-4.4946 \mathrm{E}-03+/-7.3563 \mathrm{E}-08$

$4.0401 \mathrm{E}-03+/-2.6708 \mathrm{E}-09$

$3.8813 \mathrm{E}-03+/-2.0326 \mathrm{E}-08$

3. $8417 \mathrm{E}-03+/-1.1648 \mathrm{E}-08$

$3.6255 \mathrm{E}-03+/-4.5685 \mathrm{E}-08$

3. $4344 \mathrm{E}-03+/-5.5487 \mathrm{E}-09$

3. $3168 \mathrm{E}-03+1-4.5674 \mathrm{E}-09$

$3.3168 \mathrm{E}-03+/-4.5674 \mathrm{E}-09$

3. $2014 \mathrm{E}-03+/-4.7334 \mathrm{E}-08$

$-2.1989 \mathrm{E}-03+/-3.3250 \mathrm{E}-09$
$1.6081 \mathrm{E}-03+/-1.1779 \mathrm{E}-08$

$1.6081 \mathrm{E}-03+/-1.1779 \mathrm{E}-08$
$-1.5190 \mathrm{E}-03+/-1.0084 \mathrm{E}-09$

$-1.5190 \mathrm{E}-03+/-1.0084 \mathrm{E}-09$
$-1.2258 \mathrm{E}-03+/-8.6756 \mathrm{E}-10$

$1.1071 \mathrm{E}-03+/-1.6581 \mathrm{E}-10$

$-1.0603 \mathrm{E}-03+/-8.3265 \mathrm{E}-09$

$6.6497 \mathrm{E}-04+1-1.4657 \mathrm{E}-11$

$6.4491 \mathrm{E}-04+1-5.5253 \mathrm{E}-10$

$6.4491 \mathrm{E}-04+/-5.5253 \mathrm{E}-10$

$6.4328 \mathrm{E}-04+/-4.8310 \mathrm{E}-10$

$6.3680 \mathrm{E}-04+/-4.9874 \mathrm{E}-08$

$5.5055 \mathrm{E}-04+/-1.4300 \mathrm{E}-11$

$4.6510 \mathrm{E}-04+/-4.5069 \mathrm{E}-11$

$4.2641 \mathrm{E}-04+/-3.3567 \mathrm{E}-11$

$4.1549 \mathrm{E}-04+/-2.0900 \mathrm{E}-10$

$4.1164 \mathrm{E}-04+/-5.1774 \mathrm{E}-11$

$4.1164 \mathrm{E}-04+/-5.1774 \mathrm{E}-11$

$4.0075 \mathrm{E}-04+/-1.5438 \mathrm{E}-10$

$3.5387 \mathrm{E}-04+/-6.2656 \mathrm{E}-10$

$-3.5353 \mathrm{E}-04+/-8.2827 \mathrm{E}-10$

3. $0090 \mathrm{E}-04+/-5.0084 \mathrm{E}-12$

$2.9426 \mathrm{E}-04+/-7.2192 \mathrm{E}-11$

$2.8295 \mathrm{E}-04+/-1.8411 \mathrm{E}-11$

$2.7900 \mathrm{E}-04+/-9.5920 \mathrm{E}-10$

2. $2982 \mathrm{E}-04+/-3.3498 \mathrm{E}-10$

$1.8308 \mathrm{E}=04+1-1.3865 \mathrm{E}-10$

$1.8308 \mathrm{E}-04+/-1.3865 \mathrm{E}-10$

$1.8186 \mathrm{E}-04+/-2.1087 \mathrm{E}-10$

$1.7182 \mathrm{E}-04+/-1.7540 \mathrm{E}-10$

$-1.4020 \mathrm{E}-04+/-1.3870 \mathrm{E}-10$

$1.1168 \mathrm{E}-04+/-1.6558 \mathrm{E}-10$

$1.0924 \mathrm{E}-04+/-5.0806 \mathrm{E}-11$

$9.4888 \mathrm{E}-05+/-2.0408 \mathrm{E}-12$

8. $9307 \mathrm{E}-05+/-1.6296 \mathrm{E}-11$

8. $3099 \mathrm{E}-05+/-1.6055 \mathrm{E}-12$

$8.30998-05+1-1.60558-12$

$6.0959 \mathrm{E}-05+/-3.4172 \mathrm{E}-11$

$6.1797 \mathrm{E}-05+/-7.7139 \mathrm{E}-13$

$5.1627 \mathrm{E}-05+/-6.3487 \mathrm{E}-12$

4.9075E-05 +/- 1.3555E-12

Figure 6.3.16. SAMS output for LEU-COMP-THERM-009 case 10 sample problem. (continued) 
c $\mathrm{n}, \mathrm{n}^{\prime}$

$0-16 n, p$

mn-55 elastic

cu-63 n, n'

$\mathrm{u}-236 \mathrm{n}, \mathrm{n}^{\prime}$

ca-40 n, gamma

al-27 $n, n^{\prime}$

fe-54 n, gamma

mg-24 n, gamma

mg-25 elastic

u-235 elastic

ca-40 elastic

si-28 elastic

al-27 $n, n^{\prime}$

si-28 $n, n^{\prime}$

fe-57 n, gamma

$\mathrm{cu}-63 \mathrm{n}, \mathrm{d}$

u-234 elastic

o-16 n, d

fe-56 elastic

al-27 elastic

mn-55 elastic

ti-46 n, gamma

u-236 elastic

cr-52 elastic

si-28 elastic

fe-56 n, n'

al-27 n, n'

mg-25 n, gamma

si-28 n,p

cu-65 $n, n^{\prime}$

mg-25 n, n'

ca-43 n, gamma

cr-50 elastic

cu-63 n, n'

$m g-26 n, n^{\prime}$

c $n$, gamma

cu-65 n, p

s-32 elastic

si-28 n, n'

fe-56 elastic

cr-52 $n, n^{\prime}$

cr-53 elastic

s-32 n, gamma

$\mathrm{s}-32 \mathrm{n}$, gam

$\mathrm{mn}-55 \mathrm{n}, \mathrm{n}$
$\mathrm{si}-28 \mathrm{n}$, alpha c $n, n^{\prime}$

0-16 n, p

$\mathrm{mn}-55$ elastic

cu-63 n, gamma

$\mathrm{u}-236 \mathrm{n}, \mathrm{n}^{\prime}$

ca-40 n, gamma

al-27 $n, p$

fe-54 $n$, gamma

mg-26 elastic

mg-24 $\mathrm{n}$, gamma

m-16 n,

mg-25 elastic

$\mathrm{u}-235 \mathrm{n}, 2 \mathrm{n}$

ca-40 elastic

si-28 elastic

al-27 n, alpha

si-28 n, n'

fe-57 n, gamma

cu-63 n, d

u-234 elastic

o-16 n, d

fe-56 elastic

al-27 n, alpha

mn-55 n, gamma

ti-46 n, gamma

u-236 elastic

cr-52 elastic

si-28 n, n'

fe-56 n, n

al-27 n, gamm

mg-25 n, gamma

si-28 n, p

cu-65 n, gamma

mg-25 $n, n^{\prime}$

ca-43 n, gamma

cr-50 elastic

cu-63 n, d

mg-26 n, n

c $n$, gamma

$\mathrm{cu}-65 \mathrm{n}, \mathrm{p}$

s-32 elastic

si-28 n,p

fe-56 $n, n^{\prime}$

cr-52 $n, n$

cr-53 elastic

s-32 n, gamma

mn-55 n, n

si-28 n, alpha
$4.8570 \mathrm{E}-05+/-3.6800 \mathrm{E}-11$

$4.7167 \mathrm{E}-05+/-3.5470 \mathrm{E}-12$

$4.3763 \mathrm{E}-05+/-6.4296 \mathrm{E}-11$

$4.1035 \mathrm{E}-05+/-1.8996 \mathrm{E}-11$

3. $8927 \mathrm{E}-05+/-1.4655 \mathrm{E}-12$

3. $8300 \mathrm{E}-05+/-2.0407 \mathrm{E}-12$

3. $8185 \mathrm{E}-05+/-1.9522 \mathrm{E}-11$

$3.2900 \mathrm{E}-05+/-2.4380 \mathrm{E}-13$

$3.0966 \mathrm{E}-05+/-4.0780 \mathrm{E}-12$

2. $9109 \mathrm{E}-05+/-1.6944 \mathrm{E}-13$

2. $9062 \mathrm{E}-05+/-6.2232 \mathrm{E}-12$

2.5701 $05+/ /-3.0122 \mathrm{E}-12$

$2.5701 \mathrm{E}-05+/-3.0122 \mathrm{E}-12$

$2.5517 \mathrm{E}-05+/-6.3158 \mathrm{E}-12$

$2.4956 \mathrm{E}-05+/-1.5456 \mathrm{E}-11$

2.3690E-05 +/- 1.8751E-12

$1.8266 \mathrm{E}-05+/-3.6345 \mathrm{E}-12$

$1.8068 \mathrm{E}-05+/-7.2723 \mathrm{E}-13$

$1.7622 \mathrm{E}-05+/-8.0437 \mathrm{E}-14$

$1.7414 \mathrm{E}-05+/-9.7220 \mathrm{E}-13$

1. $6232 \mathrm{E}-05+/ /-1.9361 \mathrm{E}-12$

1. $3977 \mathrm{E}-05+1-5.9002 \mathrm{E}-13$

1.3490E-05 +/- $1.2632 \mathrm{E}-12$

$1.1830 \mathrm{E}-05+/-1.6930 \mathrm{E}-12$

$1.1605 \mathrm{E}-05+/-1.0979 \mathrm{E}-12$

$1.1500 \mathrm{E}-05+/-2.6908 \mathrm{E}-14$

$1.1425 \mathrm{E}-05+/-8.1487 \mathrm{E}-13$

$1.1243 \mathrm{E}-05+/-7.7884 \mathrm{E}-13$

$1.0955 \mathrm{E}-05+/ 1-3.0843 \mathrm{E}-13$

$1.095 \mathrm{n}-05+/-3.0843 \mathrm{E}-13$

$1.0721 \mathrm{E}-05+/-2.4123 \mathrm{E}-13$

$1.0698 \mathrm{E}-05+/-7.9823 \mathrm{E}-13$

$1.0650 \mathrm{E}-05+/-1.6936 \mathrm{E}-14$

$1.0124 \mathrm{E}-05+/-1.7907 \mathrm{E}-13$

$9.9993 \mathrm{E}-06+/-9.1006 \mathrm{E}-13$

$9.7352 \mathrm{E}-06+/-1.3961 \mathrm{E}-13$

$9.5474 \mathrm{E}-06+/-1.2570 \mathrm{E}-13$

$9.4119 \mathrm{E}-06+1-7.7575 \mathrm{E}-13$

$9.4119 \mathrm{E}-06+1-7.7575 \mathrm{E}-13$

$9.0483 \mathrm{E}-06+1-9.7927 \mathrm{E}-13$

$8.6371 \mathrm{E}-06+/-1.2850 \mathrm{E}-13$

7. 3604E-06 +/- 4.2545E-14

$7.2791 \mathrm{E}-06+/-1.2170 \mathrm{E}-13$

$6.8662 \mathrm{E}-06+/-8.4130 \mathrm{E}-13$

$6.6795 \mathrm{E}-06+/-1.9459 \mathrm{E}-13$

$-6.2701 \mathrm{E}-06+/-7.5901 \mathrm{E}-14$

$6.0843 \mathrm{E}-06+1-8.2734 \mathrm{E}-14$

$5.4910 \mathrm{n}-06+1-9.0167 \mathrm{E}-13$

$5.4910 \mathrm{E}-06+/-9.0167 \mathrm{E}-13$

$5.0179 \mathrm{E}-06+/-3.4498 \mathrm{E}-1$

$5.0115 \mathrm{E}-06+/-4.1826 \mathrm{E}-14$

4. 8192E-06 +/- 5.1984E-14

Figure 6.3.16. SAMS output for LEU-COMP-THERM-009 case 10 sample problem. (continued) 
si-30 n, gamma

ti $-48 \mathrm{n}, \mathrm{n}$

$\mathrm{u}-236 \mathrm{n}, 2 \mathrm{n}$

cu- $65 \mathrm{n}, \mathrm{p}$

cr-53 n, gamma

cr-52 $n, n$

si-28 n, alpha

si-29 n, gamma

ti-47 elastic

fe-56 n, gamma

ti-47 n, gamma

si-29 elastic

fe-54 elastic

ti-49 elastic

si-29 n, n'

ca-44 n, gamma

cu-63 n, alpha

c n, alpha

ti-46 elastic

ti-46 elastic

cr-54 n, gamma

cr-50 $\mathrm{n}$, gamma

al-27 n, 2n

mg-26 n, gamma

ca-40 n, n'

si-29 $n, n$

cu-65 n, d

si-30 elastic

cr-52 n, gamm

cu-63 n, 2n

c n, alpha
50 elastic

cu-63 n, alpha

cr-53 n, n'

ca-44 elastic

ti-46 n, n'

fe-57 elastic

ti-47 n, n

ca-42 n, gamma

si-30 $n, n$

al-27 n, 2n

$\mathrm{cu}-65 \mathrm{n}, \mathrm{d}$

fe-54 $n, n$

u-234 n

$u-234 n, 2 n$

$\mathrm{cu}-63 \mathrm{n}, 2 \mathrm{n}$
$\mathrm{cr}-53 \mathrm{n}, \mathrm{n}$

fe-58 n, gamm

si-30 $\mathrm{n}$, gamma
$4.7027 \mathrm{E}-06+/-1.0526 \mathrm{E}-13$

3. $9882 \mathrm{E}-06+/-2.8137 \mathrm{E}-14$

3. $8665 \mathrm{E}-06+/-6.9405 \mathrm{E}-15$

3. $6181 \mathrm{E}-06+/-1.2145 \mathrm{E}-13$

3. $2232 \mathrm{E}-06+/-4.5743 \mathrm{E}-14$

$-3.1662 \mathrm{E}-06+/-2.3274 \mathrm{E}-14$

$3.1124 \mathrm{E}-06+/-5.5246 \mathrm{E}-14$

$2.9509 \mathrm{E}-06+/-0.0000 \mathrm{E}+00$

$2.9274 \mathrm{E}-06+/-2.6958 \mathrm{E}-13$

2. $7043 \mathrm{E}-06+/-3.8368 \mathrm{E}-14$

2. $6204 \mathrm{E}-06+1-0.0000 \mathrm{E}+00$

2. $5109 \mathrm{E}-06+/ /-2.3146 \mathrm{E}-14$

$2.5109 \mathrm{E}-06+/-2.3146 \mathrm{E}-14$

$2.4053 \mathrm{E}-06+/-1.4319 \mathrm{E}-13$

$2.2186 \mathrm{E}-06+/-1.6191 \mathrm{E}-13$

$2.1944 \mathrm{E}-06+/-9.7431 \mathrm{E}-15$

$2.0769 \mathrm{E}-06+/-5.0792 \mathrm{E}-15$

$1.9167 \mathrm{E}-06+/-1.6316 \mathrm{E}-14$

$1.9077 \mathrm{E}-06+/-1.1726 \mathrm{E}-13$

$1.8792 \mathrm{E}-06+/-0.0000 \mathrm{E}+00$

$1.8174 \mathrm{E}-06+1-1.9222 \mathrm{E}-14$

$1.8174 \mathrm{E}-06+/-1.9222 \mathrm{E}-1$

$1.8052 \mathrm{E}-06+/ /-0.0000 \mathrm{E}+00$

$-1.6885 \mathrm{E}-06+/-2.2389 \mathrm{E}-15$

$1.6378 \mathrm{E}-06+/-1.0328 \mathrm{E}-13$

$1.4435 \mathrm{E}-06+/-0.0000 \mathrm{E}+00$

$1.3555 \mathrm{E}-06+/-3.3924 \mathrm{E}-14$

$-1.3141 \mathrm{E}-06+/-2.9905 \mathrm{E}-15$

$1.2882 \mathrm{E}-06+/-8.1066 \mathrm{E}-15$

$1.2448 \mathrm{E}-06+/ /-3.8759 \mathrm{E}-15$

$1.2448 \mathrm{E}-06+1-3.8759 \mathrm{E}-15$

$-1.2364 \mathrm{E}-06+/-2.5560 \mathrm{E}-15$

$1.2318 \mathrm{E}-06+/-7.9923 \mathrm{E}-14$

$1.0668 \mathrm{E}-06+/-6.1427 \mathrm{E}-14$

$1.0218 \mathrm{E}-06+/-0.0000 \mathrm{E}+00$

$1.0189 \mathrm{E}-06+/-1.5895 \mathrm{E}-14$

$9.9320 \mathrm{E}-07+/-1.7846 \mathrm{E}-15$

$9.9005 \mathrm{E}-07+/-9.0779 \mathrm{E}-15$

$9.6859=07+1-0.0000 E+00$

$9.6859 \mathrm{E}-07+/-0.0000 \mathrm{E}+00$

$9.5215 \mathrm{E}-07+/-0.6326 \mathrm{E}-15$

$9.0613 \mathrm{E}-07+/-0.0000 \mathrm{E}+00$

8. $9913 \mathrm{E}-07+/-0.0000 \mathrm{E}+00$

$8.6223 \mathrm{E}-07+/-0.0000 \mathrm{E}+00$

$-8.2603 \mathrm{E}-07+/-1.6896 \mathrm{E}-14$

$6.9595 \mathrm{E}-07+/-8.0999 \mathrm{E}-15$

$6.7558 \mathrm{E}-07+/-0.0000 \mathrm{E}+00$

$6.5672=07+1-0.0000=+00$

$6.5672 \mathrm{E}-07+1-0.0000$

$-5.9352 \mathrm{E}-07+1-2.0173 \mathrm{E}-14$

$-5.8452 \mathrm{E}-07+/-0.0000 \mathrm{E}+00$

$5.8445 \mathrm{E}-07+/-0.0000 \mathrm{E}+00$

$5.6922 \mathrm{E}-07+/-2.2346 \mathrm{E}-14$

Figure 6.3.16. SAMS output for LEU-COMP-THERM-009 case 10 sample problem. (continued) 
fe-56 $n, n^{\prime}$

ca-48 n, gamma

cu-65 n, 2n

ca-44 n, n'

si-30 elastic

ti-49 n, n

mg-25 n, 2n

cr-52 $n, n$

fe-57 n, n'

si-29 n, alpha

cr-54 elastic

si-28 elastic

cr-50 $n, n^{\prime}$

fe-54 elastic

$\mathrm{fe}-54 \mathrm{n}, \mathrm{p}$

cr-50 $n, p$

$\mathrm{s}-32 \mathrm{n}, \mathrm{n}^{\prime}$

ti-50 $n, n^{\prime}$

fe-57 elastic

si-28 $n, n^{\prime}$

si-29 n, n'

-34 elastic

s-34 n, gamma

ca-42 elastic

fe-54 $n, n^{\prime}$

cr-50 $n, n^{\prime}$

cu-65 n, n'

si-29 $n$

si-29 n, p

ca-43 elastic

cr-50 elastic

na-23 elastic

cu-65 n, alpha

cr-54 n, n'

fe-56 n, alpha

$\mathrm{cr}-52 \mathrm{n}, \mathrm{p}$

fe-54 elastic

si-29 n, n

cr-52 $n, n$

fe-56 $n, n^{\prime}$

$\mathrm{fe}-56 \mathrm{n}, 2 \mathrm{n}$

ca-48 elastic

ti-50 n, gamma

s-33 n, gamma

fe-58 elastic

cr-54 elastic

cu-65 n, n'

ca-42 $n, n^{\prime}$

fe-57 elastic fe-56 n, gamma

ca-48 n, gamma

cu-65 n, 2n

ca-44 n, n

si-30 $\mathrm{n}, \mathrm{n}$

ti-49n, n

mg-25 n, 2n

cr-52 $\mathrm{n}$, gamma

fe-57 $n, n^{\prime}$

si-29 n,alpha

cr-54 elastic

si-28 n, gamma

cr-50 n, $n$

fe-54 $n, n$

$\mathrm{fe}-54 \mathrm{n}, \mathrm{p}$

cr-50 $n, p$

$s-32 n, n^{\prime}$

ti-50 n, n

fe-57 $n, n$

si-28 n, gamma

si-29 n, alpha

s-34 elastic

s-34 n, gamma

ca-42 elastic

fe-54 $\mathrm{n}, \mathrm{p}$

cr-50 $n, p$

cu-65 n, 2n

si-29 n,p

ca-43 elastic

cr-50 n, n'

na-23 elastic

cu-65 n,alpha

cr-54 n, n'

fe-56 n, alpha

cr-52 $n, p$

fe-54 n, gamma

si-29 $n, p$

cr-52 n, p

fe-56 n,alpha

fe-56 n, $2 \mathrm{n}$

ca-48 elastic

ti-50 $\mathrm{n}$, gamma

s-33 n, gamma

fe-58 elastic

cr-54 $n, n^{\prime}$

$\mathrm{cu}-65 \mathrm{n}$, alpha

ca-42 n, n

fe-57 $n$, gamma
$5.2558 \mathrm{E}-07+/-0.0000 \mathrm{E}+00$

$5.1948 \mathrm{E}-07+1-0.0000 \mathrm{E}+00$

$4.8462 \mathrm{E}-07+/-7.8007 \mathrm{E}-15$

$4.5030=07+1-0.0000=+00$

$-4.4830 \mathrm{E}-07+/-0.0000 \mathrm{E}+00$

$4.4467 \mathrm{E}-07+/-0.0000 \mathrm{E}+00$

$4.2964 \mathrm{E}-07+/-0.0000 \mathrm{E}+00$

$4.1052 \mathrm{E}-07+/-0.0000 \mathrm{E}+00$

$3.9365 \mathrm{E}-07+/-0.0000 \mathrm{E}+00$

3. $8432 \mathrm{E}-07+/-0.0000 \mathrm{E}+00$

$3.6988 \mathrm{E}-07+1-0.0000 \mathrm{E}+00$

$3.6988 \mathrm{E}-07+1-0.0000 \mathrm{E}+00$

$3.6913 \mathrm{E}-07+/-0.0000 \mathrm{E}+00$

$3.5176 \mathrm{E}-07+/-0.00000 \mathrm{E}+00$

$-3.4272 \mathrm{E}-07+/-0.0000 \mathrm{E}+00$

$3.2528 \mathrm{E}-07+/-0.0000 \mathrm{E}+00$

$3.2089 \mathrm{E}-07+/-0.0000 \mathrm{E}+00$

$3.1845 \mathrm{E}-07+/-0.0000 \mathrm{E}+00$

$3.1488 \mathrm{E}-07+/-0.0000 \mathrm{E}+00$

$2.9570 \mathrm{E}-07+/-0.0000 \mathrm{E}+00$

$2.8067 \mathrm{E}-07+1-0.0000 \mathrm{E}+00$

$2.8067 \mathrm{E}-07+/-0.0000 \mathrm{E}+00$

$2.6995 \mathrm{E}-07+/-0.0000 \mathrm{E}+00$

2.5320E-07 +/- $0.0000 \mathrm{E}+00$

2. $3668 \mathrm{E}-07+/-0.0000 \mathrm{E}+00$

2.3320E-07 +/- $0.0000 \mathrm{E}+00$

$2.3313 \mathrm{E}-07+/-0.0000 \mathrm{E}+00$

$2.3306 \mathrm{E}-07+/-0.0000 \mathrm{E}+00$

$2.3141 \mathrm{E}-07+/-2.7300 \mathrm{E}-15$

2. $2789 \mathrm{E}-07+1-0.0000 \mathrm{E}+00$

$2.2169=07+1-0.0000=+00$

$2.2169 \mathrm{E}-07+/-0.0000 \mathrm{E}+00$

. $1587 \mathrm{E}-07+/-0.0000 \mathrm{E}+00$

$2.0908 \mathrm{E}-07+/-2.2505 \mathrm{E}-15$

$2.0897 \mathrm{E}-07+/-0.0000 \mathrm{E}+00$

$2.0240 \mathrm{E}-07+/-0.0000 \mathrm{E}+00$

$1.8941 \mathrm{E}-07+/-0.0000 \mathrm{E}+00$

$1.8760 \mathrm{E}-07+/-0.0000 \mathrm{E}+00$

$1.8132 \mathrm{E}-07+1-0.0000 \mathrm{E}+00$

$1.8132 \mathrm{E}-07+/-0.0000 \mathrm{E}+00$

$1.6557 \mathrm{E}-07+/-0.0000 \mathrm{E}+00$

$1.5092 \mathrm{E}-07+/-0.0000 \mathrm{E}+00$

$1.4803 \mathrm{E}-07+/-0.0000 \mathrm{E}+00$

$1.4651 \mathrm{E}-07+/-0.0000 \mathrm{E}+00$

$1.4271 \mathrm{E}-07+/-0.0000 \mathrm{E}+00$

$1.3989 \mathrm{E}-07+/-0.0000 \mathrm{E}+00$

$1.2657 \mathrm{E}-07+/-0.0000 \mathrm{E}+00$

$1.2168 \mathrm{E}-07+/-0.0000 \mathrm{E}+00$

$1.2057 \mathrm{E}-07+1-0.0000 \mathrm{E}+0$

$1.2057 \mathrm{E}-07+/-0.0000 \mathrm{E}+00$

$1.1181 \mathrm{E}-07+/-0.0000 \mathrm{E}+00$

$1.0111 \mathrm{E}-07+/-0.0000 \mathrm{E}+00$

$9.3777 \mathrm{E}-08+/-0.0000 \mathrm{E}+00$

Figure 6.3.16. SAMS output for LEU-COMP-THERM-009 case 10 sample problem. (continued) 


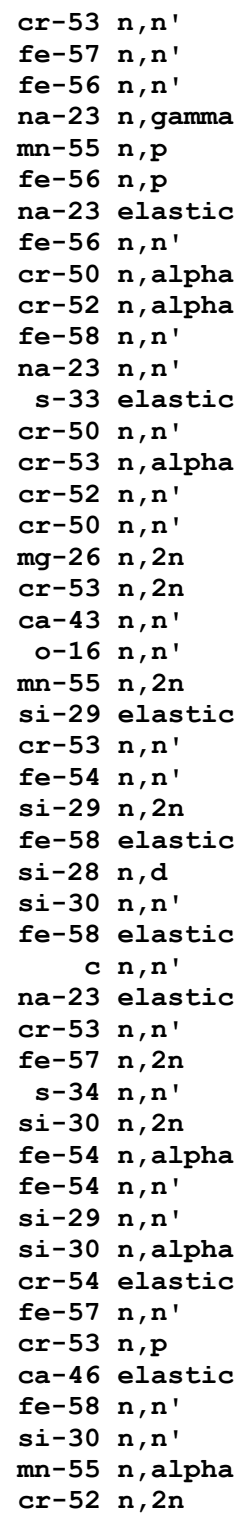

cr-53 n, gamma

fe-57 $n$, gamma

fe-56 n, 2n

na-23 n, gamma

$\mathrm{mn}-55 \mathrm{n}, \mathrm{p}$

fe-56 $n, p$
$n a-23 n, n$

fe-56 n,p

cr-50 n, alpha

cr-52 n, alpha

fe-58 $n, n^{\prime}$

$n a-23 n, n^{\prime}$

s-33 elastic

cr-50 n, alpha

cr-53 n, alpha

cr-52 n, alpha

cr-50 n, gamma

mg-26 n, 2n

$c r-53 n, 2 n$

ca-43 n, n'

o-16 n, gamma

$\mathrm{mn}-55 \mathrm{n}, 2 \mathrm{n}$

si-29 n, gamma

cr-53 n, alpha

fe-54 n, gamma

si-29 n, 2n

fe-58 $n, n^{\prime}$

si-28 n, d

si-30 n, gamma

e-58 $n$, gamma

c n, gamma

cr-53 n, 2n

fe-57 $n, 2 n$

$s-34 n, n^{\prime}$

si-30 n,

fe-54 n, alph

fe-54 n,alpha

si-29 n, 2n

si-30 n, alpha

cr-54 n, gamma

fe-57 n, 2n

cr-53 n, p

ca-46 elastic

fe-58 $n$, gamm

si-30 n, 2n

$\mathrm{mn}-55 \mathrm{n}$, alpha
$\mathrm{cr}-52 \mathrm{n}, 2 \mathrm{n}$
$8.4457 \mathrm{E}-08+/-0.0000 \mathrm{E}+00$

$7.6615 \mathrm{E}-08+/-0.0000 \mathrm{E}+00$

$-7.4856 \mathrm{E}-08+/-0.0000 \mathrm{E}+00$

$6.9611 \mathrm{E}-08+/-0.0000 \mathrm{E}+00$

$5.8834 \mathrm{E}-08+/-0.0000 \mathrm{E}+00$

$5.6198 \mathrm{E}-08+/-0.0000 \mathrm{E}+00$

$-4.8025 \mathrm{E}-08+/-0.0000 \mathrm{E}+00$

$4.5081 \mathrm{E}-08+/-0.0000 \mathrm{E}+00$

$4.4847 \mathrm{E}-08+/-0.0000 \mathrm{E}+00$

$4.3919 \mathrm{E}-08+/-0.0000 \mathrm{E}+00$

$4.2403 E-08+1-0.0000 E+00$

$4.2403 \mathrm{E}-08+1-0.0000 \mathrm{E}+00$

$3.9418 \mathrm{E}-08+/-0.0000 \mathrm{E}+00$

$3.7973 \mathrm{E}-08+/-0.0000 \mathrm{E}+00$

$3.6143 \mathrm{E}-08+/-0.0000 \mathrm{E}+00$

$3.5247 \mathrm{E}-08+/-0.0000 \mathrm{E}+00$

$3.4289 \mathrm{E}-08+/-0.0000 \mathrm{E}+00$

$3.3993 \mathrm{E}-08+/-0.0000 \mathrm{E}+00$

$3.2987 \mathrm{E}-08+/-0.0000 \mathrm{E}+00$

$3.0637 \mathrm{E}-08+/-0.0000 \mathrm{E}+00$

2. $9945 \mathrm{E}-08+1-0.0000 \mathrm{E}+00$

$2.9945 \mathrm{E}-08+1-0.0000 \mathrm{E}+00$

$2.8202 \mathrm{E}-08+/-0.0000 \mathrm{E}+00$

$2.8181 \mathrm{E}-08+/-0.0000 \mathrm{E}+00$

$2.7325 \mathrm{E}-08+/-0.0000 \mathrm{E}+00$

2. $6753 \mathrm{E}-08+/-0.0000 \mathrm{E}+00$

$2.5858 \mathrm{E}-08+/-0.0000 \mathrm{E}+00$

$2.4668 \mathrm{E}-08+/-0.0000 \mathrm{E}+00$

$-2.3267 \mathrm{E}-08+/-0.0000 \mathrm{E}+00$

$2.2815 \mathrm{E}-08+1-0.0000 \mathrm{E}+00$

$2.2815 \mathrm{E}-08+/-0.0000 \mathrm{E}+00$

$2.1466 \mathrm{E}-08+/-0.0000 \mathrm{E}+00$

$1.9178 \mathrm{E}-08+/-0.0000 \mathrm{E}+00$

$1.7660 \mathrm{E}-08+/-0.0000 \mathrm{E}+00$

$-1.6839 \mathrm{E}-08+/-0.0000 \mathrm{E}+00$

$-1.6046 \mathrm{E}-08+/-0.0000 \mathrm{E}+00$

$1.5101 \mathrm{E}-08+/-0.0000 \mathrm{E}+00$

$1.4729 \mathrm{E}-08+/-0.0000 \mathrm{E}+00$

$1.3545 \mathrm{E}-08+1-0.0000 \mathrm{E}+00$

$1.3545 \mathrm{E}-08+1-0.0000 \mathrm{E}+00$

$1.3005 \mathrm{E}-08+/-0.0000 \mathrm{E}+00$

$1.0111 \mathrm{E}-08+/-0.000 \mathrm{E}+00$

$-1.0081 \mathrm{E}-08+/-0.0000 \mathrm{E}+00$

$8.4209 \mathrm{E}-09+/-0.0000 \mathrm{E}+00$

$8.3271 \mathrm{E}-09+/-0.0000 \mathrm{E}+00$

$-7.9525 \mathrm{E}-09+/-0.0000 \mathrm{E}+00$

$7.9053 \mathrm{E}-09+/-0.0000 \mathrm{E}+00$

$7.64775-09+1-0.0000 E+00$

$7.2381 \mathrm{E}-09+1-0.0000 \mathrm{E}+00$

$7.2381 \mathrm{E}-09+1-0.0000 \mathrm{E}+00$

$7.0930 \mathrm{E}-09+/-0.0000 \mathrm{E}+00$

$7.0079 \mathrm{E}-09+/-0.0000 \mathrm{E}+00$

$6.5167 \mathrm{E}-09+/-0.0000 \mathrm{E}+00$

Figure 6.3.16. SAMS output for LEU-COMP-THERM-009 case 10 sample problem. (continued) 


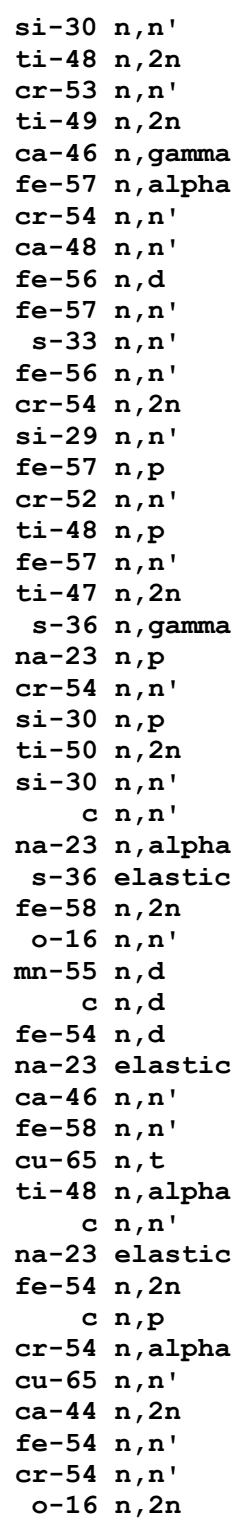

si-30 n, alpha

ti-48 n, 2n

cr-53 $\mathrm{n}, \mathrm{p}$

ti-49 n, 2n

ca-46 n, gamma

fe-57 $n$, alpha

cr-54 n, gamma

ca-48 $n, n^{\prime}$

fe-56 n, d

fe-57 $n$, alpha

$\mathrm{s}-33 \mathrm{n}, \mathrm{n}$ '

fe-56 $n, d$

cr-54 n, 2n

si-29 n, gamma

fe-57 $n, p$

cr-52 n, 2n

ti-48 $\mathrm{n}, \mathrm{p}$

$\mathrm{fe}-57 \mathrm{n}, \mathrm{p}$

ti-47n, $\mathrm{n}$

s-36 n, gamma

na-23 $\mathrm{n}, \mathrm{p}$

cr-54 n, 2n

si-30 n, p

ti-50 n, 2n

$\mathrm{si}-30 \mathrm{n}, \mathrm{p}$$$
\text { c } n, d
$$

na-23 n, alpha

fe-58 $n, 2 n$

$0-16 n, 2 n$

$\mathrm{mn}-55 \mathrm{n}, \mathrm{d}$

$c n, d$

fe-54 $n, d$

na-23 $n, p$

ca-46 n, n'

fe-58 n, $2 n$

$\mathrm{cu}-65 \mathrm{n}, \mathrm{t}$

ti-48 n, alpha c $n, p$

na-23 n,alpha

$\mathrm{fe}-54 \mathrm{n}, 2 \mathrm{n}$

$$
\text { c } n, p
$$

cr-54 n, alpha

cu-65 $n, t$

ca-44 n, 2n

fe-54 n, 2n

$\begin{aligned} & \text { cr-54 } n, \text { alpha } \\ & 0-16 n, 2 n\end{aligned}$
$6.4283 \mathrm{E}-09+/-0.0000 \mathrm{E}+00$

$6.2875 \mathrm{E}-09+/-0.0000 \mathrm{E}+00$

$6.2628 \mathrm{E}-09+1-0.0000 \mathrm{E}+00$

$5.8476 \mathrm{E}-09+1-0.0000 \mathrm{E}+00$

$5.5651 \mathrm{E}-09+/-0.0000 \mathrm{E}+00$

$5.3090 \mathrm{E}-09+/-0.0000 \mathrm{E}+00$

$4.9562 \mathrm{E}-09+/-0.0000 \mathrm{E}+00$

$4.9102 \mathrm{E}-09+/-0.0000 \mathrm{E}+00$

$4.7681 \mathrm{E}-09+/-0.0000 \mathrm{E}+00$

3. $9611 \mathrm{E}-09+/-0.0000 \mathrm{E}+00$

3. $7151 \mathrm{E}-09+/-0.0000 \mathrm{E}+00$

3.

$3.6804 \mathrm{E}-09+/-0.0000 \mathrm{E}+00$

$3.6592 \mathrm{E}-09+/-0.0000 \mathrm{E}+00$

$3.4777 \mathrm{E}-09+/-0.0000 \mathrm{E}+00$

$3.4511 \mathrm{E}-09+/-0.0000 \mathrm{E}+00$

$-3.4308 \mathrm{E}-09+/-0.0000 \mathrm{E}+00$

$2.9116 \mathrm{E}-09+/-0.0000 \mathrm{E}+00$

$2.6805 \mathrm{E}-09+/-0.0000 \mathrm{E}+00$

2. $6563 \mathrm{E}-09+/-0.0000 \mathrm{E}+00$

$2.5689=09+1-0.0000 E+00$

$2.5689 \mathrm{E}-09+1-0.0000 \mathrm{E}+00$

$2.1365 \mathrm{E}-09+/-0.0000 \mathrm{E}+00$

$2.0358 \mathrm{E}-09+/-0.0000 \mathrm{E}+00$

$1.9130 \mathrm{E}-09+/-0.0000 \mathrm{E}+00$

$1.4840 \mathrm{E}-09+/-0.0000 \mathrm{E}+00$

$1.4354 \mathrm{E}-09+/-0.0000 \mathrm{E}+00$

$1.3648 \mathrm{E}-09+/-0.0000 \mathrm{E}+00$

1. $3262 \mathrm{E}-09+/-0.0000 \mathrm{E}+00$

$1.3262 \mathrm{E}-09+1-0.0000 \mathrm{t}$

$1.2017 \mathrm{E}-09+/-0.0000 \mathrm{E}+00$

$-9.4097 \mathrm{E}-10+/-0.0000 \mathrm{E}+00$

$8.7967 \mathrm{E}-10+/-0.0000 \mathrm{E}+00$

$8.3498 \mathrm{E}-10+/-0.0000 \mathrm{E}+00$

$7.9457 \mathrm{E}-10+/-0.0000 \mathrm{E}+00$

$7.7621 \mathrm{E}-10+/-0.0000 \mathrm{E}+00$

$7.5538 \mathrm{E}-10+/-0.0000 \mathrm{E}+00$

$-6.7419 \mathrm{E}-10+/-0.0000 \mathrm{E}+\mathrm{C}$

$6.7419 \mathrm{E}-10+1-0.0000 \mathrm{E}+00$

$6.555 \mathrm{E}-10+/ /-0.0000 \mathrm{E}+00$

$6.2508 \mathrm{E}-10+/-0.0000 \mathrm{E}+00$

$6.0127 \mathrm{E}-10+/-0.0000 \mathrm{E}+00$

$4.5436 \mathrm{E}-10+/-0.0000 \mathrm{E}+00$

$3.8024 \mathrm{E}-10+/-0.0000 \mathrm{E}+00$

$3.6950 \mathrm{E}-10+/-0.0000 \mathrm{E}+00$

3. $3662 \mathrm{E}-10+/-0.0000 \mathrm{E}+00$

3. $3605 E-10+1-0.0000 E+00$

3. $3085 \mathrm{E}-10+/ 1-0.0000 \mathrm{E}+00$

$3.3085 \mathrm{E}-10+/-0.000 \mathrm{E}+00$

$-3.2000 \mathrm{E}-10+/-0.0000 \mathrm{E}+00$

$2.7557 \mathrm{E}-10+/-0.0000 \mathrm{E}+00$
$2.4315 \mathrm{E}-10+/-0.0000 \mathrm{E}+00$

Figure 6.3.16. SAMS output for LEU-COMP-THERM-009 case 10 sample problem. (continued) 


$$
\begin{aligned}
& \text { ca-43 n, 2n } \\
& \text { fe-56 n, n' } \\
& \begin{array}{ll}
\text { fe-56 } n, n \\
\text { cr-50 } n, d
\end{array} \\
& \begin{array}{l}
c r-50 n, d \\
\text { ca-48 } n, 2 n
\end{array} \\
& \text { cr-50 } \mathrm{n}, \mathrm{n}^{\prime} \\
& \text { cu- } 63 \mathrm{n}, \mathrm{he}-3 \\
& \text { s-33n, 2n } \\
& \text { ti-46 n, 2n } \\
& \text { fe-58 n,alpha } \\
& \text { cr-50 } n, 2 n \\
& \mathrm{~s}-36 \mathrm{n}, \mathrm{n}^{\prime} \\
& \text { ca-42 n, 2n } \\
& \text { fe-58 } n, n^{\prime} \\
& \mathrm{cu}-63 \mathrm{n}, \mathrm{n}^{\prime} \\
& \mathrm{s}-34 \mathrm{n}, 2 \mathrm{n} \\
& \text { cr-50 } \mathrm{n}, \mathrm{n}^{\prime} \\
& \text { cr-54 } n, p \\
& \text { fe-56 } n, n^{\prime} \\
& \mathrm{mn}-55 \mathrm{n}, \mathrm{t} \\
& 0-16 n, t \\
& c r-54 n, n \\
& \text { fe-56 } n, t \\
& \text { fe-58 n,p } \\
& \text { fe-58 } n, n^{\prime} \\
& \text { cu-65 } n, \text { he-3 } \\
& \text { mg-24 n, 2n } \\
& \text { cu-65 } n, n^{\prime} \\
& \begin{array}{ll}
\mathrm{cu}-65 & \mathrm{n}, \mathrm{n} \\
\mathrm{na}-23 \mathrm{n}, 2 \mathrm{n}
\end{array} \\
& \begin{array}{r}
n a-23 n, 2 n \\
s-36 n, 2 n
\end{array} \\
& \text { fe-56 } n, h e-3 \\
& \text { ca-46 n, 2n } \\
& \text { na-23 elastic } \\
& \text { mn-55 } \mathrm{n}, \mathrm{he}-3 \\
& \text { ca- } 40 \mathrm{n}, 2 \mathrm{n} \\
& \mathrm{s}-32 \mathrm{n}, 2 \mathrm{n}
\end{aligned}
$$

$1.7648 \mathrm{E}-10+/-0.0000 \mathrm{E}+00$

$1.5852 \mathrm{E}-10+/-0.0000 \mathrm{E}+00$ $1.5158 \mathrm{E}-10+/-0.0000 \mathrm{E}+00$

$1.5158 \mathrm{E}-10+/ 10.0 .000 \mathrm{E}+00$

$1.3661 \mathrm{E}-10+/-0.0000 \mathrm{E}+00$

$1.2412 \mathrm{E}-10+/-0.0000 \mathrm{E}+0$

$1.1688 \mathrm{E}-10+/-0.0000 \mathrm{E}+00$

$1.1455 \mathrm{E}-10+/-0.0000 \mathrm{E}+00$

$8.3916 \mathrm{E}-11+/-0.0000 \mathrm{E}+00$

$8.3616 \mathrm{E}-11+/-0.0000 \mathrm{E}+00$

$7.9460 \mathrm{E}-11+/-0.0000 \mathrm{E}+00$

$7.3825 \mathrm{E}-11+1-0.0000 \mathrm{E}+00$

$7.3825 \mathrm{E}-11+1-0.0000 \mathrm{E}+00$

$7.0527 \mathrm{E}-11+/-0.0000 \mathrm{E}+00$

$6.8070 \mathrm{E}-11+/-0.0000 \mathrm{E}+00$

$5.9819 \mathrm{E}-11+/-0.0000 \mathrm{E}+00$

$5.5409 \mathrm{E}-11+/-0.0000 \mathrm{E}+00$

$-4.5867 \mathrm{E}-11+/-0.0000 \mathrm{E}+00$

$3.4617 \mathrm{E}-11+/-0.0000 \mathrm{E}+00$

$2.9868 \mathrm{E}-11+/-0.0000 \mathrm{E}+00$

$2.9356 \mathrm{E}-11+/-0.0000 \mathrm{E}+00$

$2.9008 \mathrm{E}-11+1-0.0000 \mathrm{E}+00$

$2.9008 \mathrm{E}-11+/-0.0000 \mathrm{E}+00$

$2.7365 \mathrm{E}-11+/ /-0.0000 \mathrm{E}+00$

$1.5517 \mathrm{E}-11+/-0.0000 \mathrm{E}+00$

1. 3800E-11 +/- $0.0000 \mathrm{E}+00$

$1.1055 \mathrm{E}-11+/-0.0000 \mathrm{E}+00$

$6.2232 \mathrm{E}-12+/-0.0000 \mathrm{E}+00$

$3.6825 \mathrm{E}-12+/-0.0000 \mathrm{E}+00$

3. $1211 \mathrm{E}-12+/-0.0000 \mathrm{E}+00$

$2.9396 \mathrm{E}-12+1-0.0000 \mathrm{E}+00$

$2.9396 \mathrm{E}-12+/ /-0.0000 \mathrm{E}+00$

$1.8272 \mathrm{E}-12+/-0.0000 \mathrm{E}+00$

$1.7491 \mathrm{E}-12+/-0.0000 \mathrm{E}+00$

$1.7030 \mathrm{E}-12+/-0.0000 \mathrm{E}+00$

$-1.0631 \mathrm{E}-12+/-0.0000 \mathrm{E}+00$

$8.9376 \mathrm{E}-13+/-0.0000 \mathrm{E}+00$

$4.9330 \mathrm{E}-13+/-0.0000 \mathrm{E}+00$

$1.1554 \mathrm{E}-13+/-0.0000 \mathrm{E}+00$

Note: relative standard deviation in $\mathrm{k}$-eff can be computed from

individual values by adding the square of the values with positive signs and

subtracting the square of the values with negative signs, then taking the square root

SAMS execution complete

Elapsed time: 8.10467 minutes

Figure 6.3.16. SAMS output for LEU-COMP-THERM-009 case 10 sample problem. (continued) 


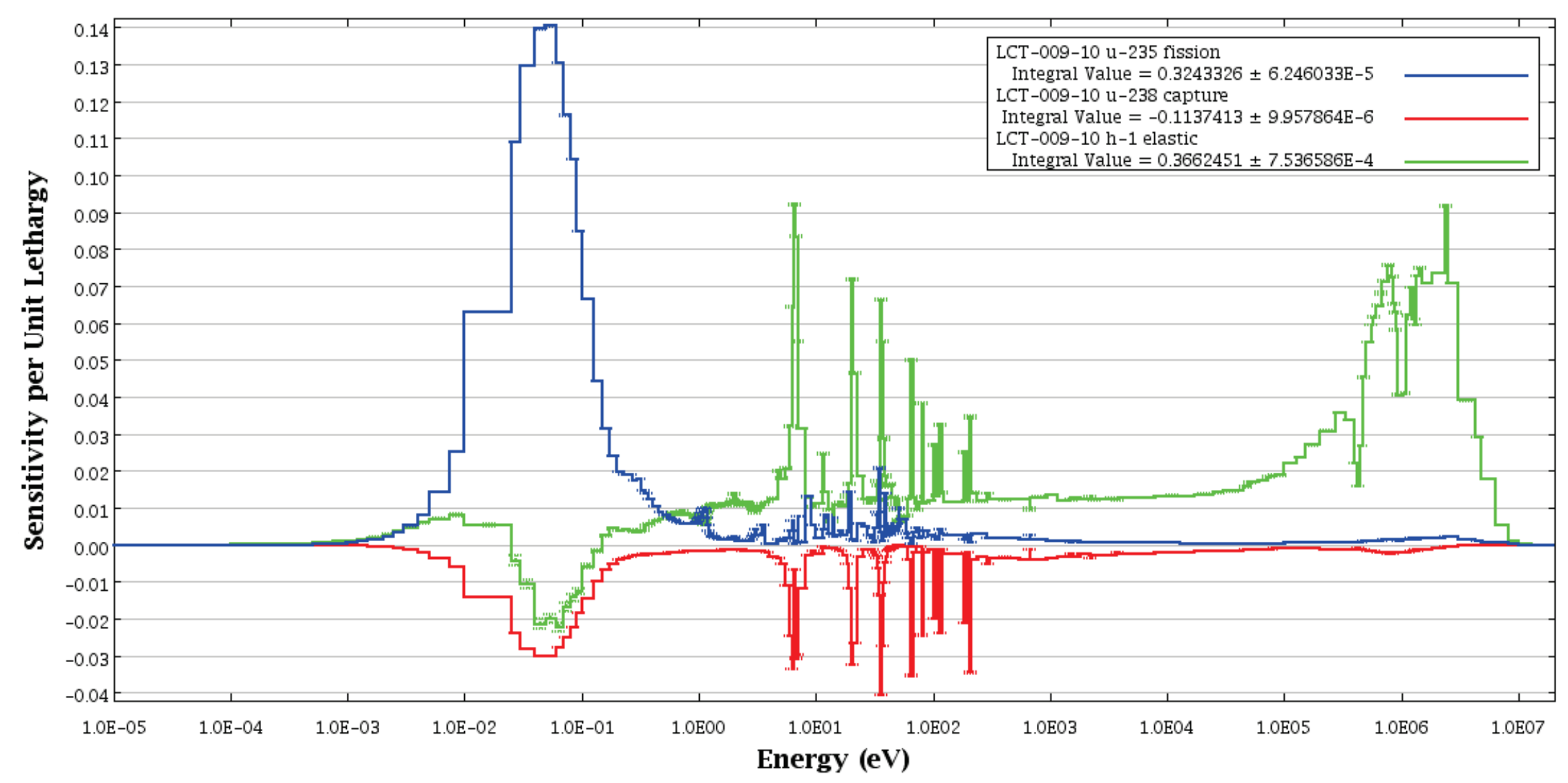

Figure 6.3.17. Region-integrated sensitivity profiles from test case LEU-COMP-THERM-009 case 10. 


\subsubsection{Error And Warning Messages}

SAMS will display a message when a predetermined condition is encountered. Error messages are displayed prior to code termination. Warning messages are displayed when a potentially abnormal condition is encountered, but code execution continues.

\subsubsection{Error Messages}

SAMS Error 001: Selected calculation type is not valid.

This message is displayed by VIP when the user enters a calculation type other than kenova or xsdrn.

\section{SAMS Error 002: There was a zero response value.}

This message is displayed by SENSITIVITY_SUMMATION when there was no fissile material in the problem or the forward or adjoint flux in all regions containing fissile material was zero. This message can be encountered when the adjoint solution fails to proceed far enough to accumulate active generations for flux accumulation in TSUNAMI-3D models.

SAMS Error 003: Error reading covariance data io= $\mathbf{m t 2}=$ nblok $=$ ngroup=

$\operatorname{mat} 1=$

mt1 $=$ $\operatorname{mat} 2=$

This error is displayed by SAMCAVA when a non-zero return code is obtained when reading from the covariance data library.

\section{SAMS Error 004: Adjoint flux file from KENO was not found on unit}

This error is displayed by GET_FLUXES when the adjoint flux unit cannot be opened.

\section{SAMS Error 005: Forward flux file from KENO was not found on unit}

This error is displayed by GET_FLUXES when the forward flux unit cannot be opened.

\section{SAMS Error 006: Adjoint fluxes were not calculated in the source case}

This error is displayed by GET_FLUXES when an adjoint data file is found, but it does not contain flux data. The adjoint case should be rerun with flux accumulation turned on.

\section{SAMS Error 007: Only the scalar fluxes were calculated. Execution is stopping.}

This message is displayed by GET_FLUXES when the KENO calculation was run with both $\mathrm{PNM}=0$ and $\mathrm{NQD}=0$. The KENO case does not contain sufficient information to produce sensitivity coefficients. 
SAMS Error 008: Error reading the forward restart file, record type

This message is displayed by GET_FLUXES when an unknown record type from the KENO forward solution is encountered.

SAMS Error 009: Error reading the adjoint restart file, record type

This message is displayed by GET_FLUXES if an unknown record type from the KENO adjoint solution is encountered.

SAMS Error 010: Invalid read key

This message is displayed by GET_INPUT when an invalid word is encountered in the input before a "read" card is encountered.

SAMS Error 011: Invalid block key . The first block must be initial.

This message is displayed by GET_INPUT when the first word after the first "read" is not "initial".

SAMS Error 012: End of input data reached. Expecting more data.

This message is displayed by GET_INPUT when the end of the input stream is unexpectedly encountered.

SAMS Error 013: Ending block keyword does not equal the beginning block keyword

This message is displayed by GET_INPUT when the word following "END" does not match the word after "READ" for the current input block.

SAMS Error 014: Unknown read return flag

This message is displayed by GET_INPUT when a reading error occurs. This is likely due to an input error.

\section{Error allocating}

This message is displayed by GET_FLUXES when insufficient computational resources exist to allocate memory for processing the problem. If this message is encountered, try reducing the size of the calculation by reducing the number of mesh intervals (e.g., increase the mesh size, MSH in KENO), reducing the order of flux moments or angular fluxes accumulated (PNM and NQD in KENO), or using a cross-section data library with fewer energy groups. 


\subsubsection{Warning Messages}

SAMS Warning 001: Use of mesh flux was requested $(\mathrm{mfx}=\mathrm{yes})$, but a mesh size of was requested. Please enter a positive value for $\mathrm{msh}=$ in the KENO parameter data. S $\overline{\mathrm{AMS}}$ will continue its calculation with the region fluxes, equivalent to $\mathrm{mfx}=\mathrm{no}$.

This message is displayed by GET_FLUXES when MFX=YES is entered in KENO parameter data, but the mesh size MSH is not entered or is non-positive. However, if the user specifies another mesh in a different section of a TSUNAMI-3D input (i.e. in the read GridGeometry block), then TSUNAMI will default to use that mesh.

SAMS Warning 002: The k-eff values for the forward and adjoint calculations differ by more than one percent.

This message is displayed by PRINT_TRANS when the k-eff values from the forward and adjoint calculations differ by more than one percent. This typically indicates poor convergence of the adjoint Monte Carlo calculation. The calculation should be rerun with more histories to improve agreement between the forward and adjoint k-eff values.

SAMS Warning 003: Implicit sensitivities were requested but no implicit datafiles were found. Proceeding with explicit sensitivity coefficients only.

This message is displayed by IMPLICIT_SENSITIVITIES when MAKEIMP is present in the SAMS input, but no datafiles from SENLIB or BONAMIST are found. The calculation will continue with explicit sensitivity coefficients only.

SAMS Warning 004: Implicit sensitivity of and has been reset to 0.0 . to group from had a value of

This message is displayed by IMPLICIT_SENSITIVITIES when an implicit sensitivity value greater than that set by LARGEIMP is encountered. The large implicit value is likely due to an inconsistency between the CELLDATA specification for the material identified in the message and the use of that material in the geometry of the model.

SAMS Warning 005: Implicit sensitivity for mixture exceeds the explicit sensitivity by more than an order of magnitude. Implicit sensitivity: Explicit sensitivity: Please check for appropriate resonance self-shielding models in celldata.

This message is displayed by IMPLICIT_SENSITIVITIES when the implicit sensitivity value exceeds the explicit sensitivity value by more than an order of magnitude. The large implicit value is possibly due to an inconsistency between the CELLDATA specification for the material identified in the message and the use of that material in the geometry of the model. 
SAMS Warning 006: An implicit sensitivity was generated by the resonance self-shielding calculation in for mixture with mixture which was not found in the model. This potentially important information will be skipped. Please check the celldata input.

This message is displayed by IMPLICIT_SENSITIVITIES when implicit data is generated by the resonance self-shielding codes, but a material needed for the calculation is not present in the geometry model. Please check for consistent use of materials in the CELLDATA input and in the geometry model.

SAMS Warning 007: USEANG was requested in the input, but angular fluxes were not computed. USEMOM has been set to true, and flux moments will be used to compute sensitivity coefficients.

This message is displayed by GET_FLUXES when USEANG is true but angular fluxes were not computed in the transport solution. In this case the flux moments computed in the transport solution are used to compute sensitivity coefficients. If neither angular fluxes nor flux moments are found, SAMS Error 007 will stop code execution prior to reaching this warning.

SAMS Warning 008: USEMOM was requested in the input, but flux moments were not computed. USEANG has been set to true, and angular fluxes will be used to compute sensitivity coefficients.

This message is displayed by GET_FLUXES when USEMOM is true but flux moments were not computed in the transport solution. In this case the angular fluxes computed in the transport solution are used to compute sensitivity coefficients. If neither angular fluxes nor flux moments are found, SAMS Error 007 will stop code execution prior to reaching this warning.

\subsubsection{References}

1. J. L. Lucius et al., A Users Manual for the FORSS Sensitivity and Uncertainty Analysis Code System, ORNL-5316, Union Carbide Corp., Oak Ridge National Laboratory (1981).

2. C. R. Weisbin, E. M. Oblow, J. H. Harable, R. W. Peele, and J. L. Lucius, "Application of Sensitivity and Uncertainty Methodology to Fast Reactor Integral Experiment Analysis," Nucl. Sci. Eng. 66, 307 (1978).

3. C. R. Weisbin et al., Application of FORSS Sensitivity and Uncertainty Methodology to Fast Reactor Benchmark Analysis, ORNL/TM-5563, Union Carbide Corp., Oak Ridge National Laboratory (1976).

4. E. M. Oblow, "Sensitivity Theory from a Differential Viewpoint," Nucl. Sci. Eng. 59, 187 (1976).

5. W. M. Stacey, Jr., "Variational Estimates and Generalized Perturbation Theory for the Ratios of Linear and Bilinear Functionals," J. Math. Phys. 13, 1119 (1972); see also W. M. Stacey, Jr., "Variational Estimates of Reactivity Worths and Reaction Rate Ratios in Critical Nuclear Reactors," Nucl. Sci. Eng. 48, 444 (1972).

6. L. N. Usachev, "Perturbation Theory for the Breeding Ratio and for Other Number Ratios Pertaining to Various Reactor Processes,” J. Nucl. Energy Pts. A/B, 18, 571 (1964).

7. A. Gandini, "A Generalized Perturbation Method for Bilinear Functionals of the Real and Adjoint Neutron Fluxes," J. Nucl. Energy 21, 755 (1967).

8. A. Hara, T. Takeda, Y. Kikuchi, "SAGEP: Two-Dimensional Sensitivity Analysis Code Based on Generalized Perturbation Theory,”JAERI-M 84-027 (Japan) (1984). 
9. M. L. Williams, B. L. Broadhead, and C. V. Parks, "Eigenvalue Sensitivity Theory for ResonanceShielded Cross Sections," Nucl. Sci. Eng. 138, 17-191 (2001).

10. P. R. Bevington, Data Reduction and Error Analysis for the Physical Sciences, McGraw-Hill Book Company, New York, New York (1969).

11. M. E. Dunn, PUFF-III: A Code for Processing ENDF Uncertainty Data Into Multigroup Covariance Matrices, ORNL/TM-1999/235 (NUREG/CR-6650), U.S. Nuclear Regulatory Commission, Oak Ridge National Laboratory (2000).

12. M. L. Williams, "Perturbation Theory for Reactor Analysis," CRC Handbook of Nuclear Reactors Calculations, Vol. 3, pp. 63-188, CRC Press, 1986.

13. Cross Section Evaluation Working Group Benchmark Specification, BNL 19302 (ENDF-202), Brookhaven National Laboratory, November 1974.

14. LEU-COMP-THERM-009 in International Handbook of Evaluated Criticality Safety Benchmark Experiments, Nuclear Energy Agency Nuclear Science Committee of the Organization for Economic Co-operation and Development, NEA/NSC/DOC(95)03, Paris (1999). 


\subsection{A Sensitivity Data File Formats}

\subsection{A.1 Format of TSUNAMI-A sensitivity data file}

The format of the TSUNAMI-A sensitivity data file produced by SAMS for cases with deterministic transport solutions is given in Table 6.3.A.1. The occurrence of each entry in the data file is followed by an identification of the data contained on each line of the file and the FORTRAN edit descriptor denoting the format of each line. A brief description of each line is also presented.

A sample of the TSUNAMI-A data file for the Flattop-25 sample problem is provided in Figure 6.3.A.1. Here, only two profiles out of the 130 computed are shown. 
Table 6.3.A.1. Format specification for TSUNAMI-A sensitivity data file

\begin{tabular}{|c|c|c|c|}
\hline Occurrence & Data & Format & Description \\
\hline \multirow{6}{*}{$\begin{array}{l}\text { Once at beginning } \\
\text { of file. }\end{array}$} & title & $\mathrm{a} 80$ & $\begin{array}{l}\text { Title extracted from transport } \\
\text { calculation }\end{array}$ \\
\hline & number of neutron groups & $\mathrm{i} 10$ & $\begin{array}{l}\text { Number of neutron groups in } \\
\text { calculation }\end{array}$ \\
\hline & $\begin{array}{l}\text { total number of profiles, } \\
\text { text descriptor, } \\
\text { number of total profiles that are } \\
\text { region integrated }\end{array}$ & $\mathrm{i} 10, \mathrm{a} 35, \mathrm{i} 10$ & $\begin{array}{l}\text { Total number of sensitivity } \\
\text { profiles in data file, separated by a } \\
\text { text descriptor, then the number of } \\
\text { profiles that contain region- } \\
\text { integrated data }\end{array}$ \\
\hline & $k_{\text {eff }}$ & f10.6 & $\begin{array}{l}\text { Value of } k_{\text {eff }} \text { from the forward } \\
\text { transport calculation }\end{array}$ \\
\hline & 'energy boundaries:' & $\mathrm{a}$ & Text \\
\hline & energy boundary data & $\begin{array}{l}5 \text { es } 14.6 \\
\text { (repeats until } \\
\text { all data is } \\
\text { printed) }\end{array}$ & $\begin{array}{l}\text { Values of boundaries of energy } \\
\text { groups. Begins with upper value } \\
\text { for highest energy group and ends } \\
\text { with lower value of lowest energy } \\
\text { group. }\end{array}$ \\
\hline \multirow{3}{*}{$\begin{array}{l}\text { Repeats for each } \\
\text { profile. }\end{array}$} & $\begin{array}{l}\text { isotope name, } \\
\text { sensitivity reaction name, } \\
\text { nuclide ID, } \\
\text { MT number, } \\
\text { Zone number or negative of material } \\
\text { number, } \\
\text { zone volume }\end{array}$ & $\begin{array}{l}\text { a12, } 1 x, \text { a15, } \\
3 \mathrm{i} 12, \text { es } 14.6\end{array}$ & $\begin{array}{l}\text { Provides data identifying the } \\
\text { sensitivity data that follows. } \\
\text { Note, if sensitivity data is region- } \\
\text { integrated, zone number and zone } \\
\text { volume are both } 0 \text {. If data is } \\
\text { integrated over all zones } \\
\text { containing the same material, the } \\
\text { material number is given in place } \\
\text { of the zone number as a negative } \\
\text { number. }\end{array}$ \\
\hline & $\begin{array}{l}\text { Energy integrated sensitivity } \\
\text { coefficient, sum of absolute value of } \\
\text { group-wise sensitivities, sum of the } \\
\text { group-wise sensitivities with } \\
\text { opposite sign as energy integrated } \\
\text { value (osc) }\end{array}$ & 3 es 14.6 & $\begin{array}{l}\text { Energy-integrated sensitivity } \\
\text { coefficients for this profile. }\end{array}$ \\
\hline & group-wise sensitivity coefficients & $\begin{array}{l}5 \text { es14.6 } \\
\text { (repeats until } \\
\text { all data is } \\
\text { printed) }\end{array}$ & $\begin{array}{l}\text { Energy-dependent sensitivity } \\
\text { coefficients. Begins with highest } \\
\text { energy group. }\end{array}$ \\
\hline Once at end of file. & & & $\begin{array}{l}\text { Block of file verification } \\
\text { information. }\end{array}$ \\
\hline
\end{tabular}




\begin{tabular}{|c|c|c|c|c|}
\hline \multicolumn{5}{|l|}{ flattop-25 } \\
\hline 238 & \multicolumn{4}{|c|}{ number of neutron groups } \\
\hline 130 & mber of sensi & vity profiles & \multirow[t]{2}{*}{30} & are region integrated \\
\hline 1.002969 & -eff from the & forward case & & \\
\hline \multicolumn{5}{|c|}{ energy boundaries: } \\
\hline 2.000000E+07 & $1.733300 \mathrm{E}+07$ & $1.568300 \mathrm{E}+07$ & $1.455000 \mathrm{E}+07$ & $1.384000 \mathrm{E}+07$ \\
\hline $1.284000 \mathrm{E}+07$ & $1.000000 \mathrm{E}+07$ & $8.187300 \mathrm{E}+06$ & $6.434000 \mathrm{E}+06$ & $4.800000 \mathrm{E}+06$ \\
\hline $4.304000 \mathrm{E}+06$ & $3.000000 \mathrm{E}+06$ & $2.479000 \mathrm{E}+06$ & $2.354000 \mathrm{E}+06$ & $1.850000 \mathrm{E}+06$ \\
\hline $1.500000 \mathrm{E}+06$ & $1.400000 \mathrm{E}+06$ & $1.356000 \mathrm{E}+06$ & $1.317000 \mathrm{E}+06$ & $1.250000 \mathrm{E}+06$ \\
\hline $1.200000 \mathrm{E}+06$ & $1.100000 \mathrm{E}+06$ & $1.010000 \mathrm{E}+06$ & $9.200000 \mathrm{E}+05$ & $9.000000 \mathrm{E}+05$ \\
\hline $8.750000 \mathrm{E}+05$ & $8.611000 \mathrm{E}+05$ & $8.200000 \mathrm{E}+05$ & $7.500000 \mathrm{E}+05$ & $6.790000 \mathrm{E}+05$ \\
\hline $6.700000 \mathrm{E}+05$ & $6.000000 \mathrm{E}+05$ & $5.730000 \mathrm{E}+05$ & $5.500000 \mathrm{E}+05$ & $4.995200 \mathrm{E}+05$ \\
\hline $4.700000 \mathrm{E}+05$ & $4.400000 E+05$ & $4.200000 \mathrm{E}+05$ & $4.000000 \mathrm{E}+05$ & $3.300000 \mathrm{E}+05$ \\
\hline $2.700000 \mathrm{E}+05$ & $2.000000 E+05$ & $1.500000 \mathrm{E}+05$ & $1.283000 \mathrm{E}+05$ & $1.000000 \mathrm{E}+05$ \\
\hline $8.500000 \mathrm{E}+04$ & $8.200000 E+04$ & $7.500000 \mathrm{E}+04$ & $7.300000 \mathrm{E}+04$ & $6.000000 \mathrm{E}+04$ \\
\hline $5.200000 \mathrm{E}+04$ & $5.000000 \mathrm{E}+04$ & $4.500000 \mathrm{E}+04$ & $3.000000 \mathrm{E}+04$ & $2.500000 \mathrm{E}+04$ \\
\hline $1.700000 \mathrm{E}+04$ & $1.300000 \mathrm{E}+04$ & $9.500000 \mathrm{E}+03$ & $8.030000 \mathrm{E}+03$ & $6.000000 \mathrm{E}+03$ \\
\hline $3.900000 \mathrm{E}+03$ & $3.740000 \mathrm{E}+03$ & $3.000000 \mathrm{E}+03$ & $2.580000 \mathrm{E}+03$ & $2.290000 \mathrm{E}+03$ \\
\hline $2.200000 \mathrm{E}+03$ & $1.800000 \mathrm{E}+03$ & $1.550000 \mathrm{E}+03$ & $1.500000 \mathrm{E}+03$ & $1.150000 \mathrm{E}+03$ \\
\hline $9.500000 \mathrm{E}+02$ & $6.830000 \mathrm{E}+02$ & $6.700000 \mathrm{E}+02$ & $5.500000 \mathrm{E}+02$ & $3.050000 \mathrm{E}+02$ \\
\hline $2.850000 \mathrm{E}+02$ & $2.400000 \mathrm{E}+02$ & $2.100000 \mathrm{E}+02$ & $2.075000 \mathrm{E}+02$ & 1. $925000 \mathrm{E}+02$ \\
\hline $1.860000 \mathrm{E}+02$ & $1.220000 \mathrm{E}+02$ & $1.190000 \mathrm{E}+02$ & $1.150000 \mathrm{E}+02$ & $1.080000 \mathrm{E}+02$ \\
\hline $1.000000 \mathrm{E}+02$ & $9.000000 \mathrm{E}+01$ & $8.200000 \mathrm{E}+01$ & $8.000000 \mathrm{E}+01$ & $7.600000 \mathrm{E}+01$ \\
\hline $7.200000 \mathrm{E}+01$ & $6.750000 \mathrm{E}+01$ & $6.500000 \mathrm{E}+01$ & $6.100000 \mathrm{E}+01$ & $5.900000 \mathrm{E}+01$ \\
\hline $5.340000 \mathrm{E}+01$ & $5.200000 \mathrm{E}+01$ & $5.060000 \mathrm{E}+01$ & $4.920000 \mathrm{E}+01$ & $4.830000 \mathrm{E}+01$ \\
\hline $4.700000 \mathrm{E}+01$ & $4.520000 \mathrm{E}+01$ & $4.400000 \mathrm{E}+01$ & $4.240000 \mathrm{E}+01$ & $4.100000 \mathrm{E}+01$ \\
\hline $3.960000 \mathrm{E}+01$ & $3.910000 \mathrm{E}+01$ & $3.800000 \mathrm{E}+01$ & $3.700000 \mathrm{E}+01$ & $3.550000 \mathrm{E}+01$ \\
\hline $3.460000 \mathrm{E}+01$ & $3.375000 \mathrm{E}+01$ & $3.325000 \mathrm{E}+01$ & $3.175000 \mathrm{E}+01$ & $3.125000 \mathrm{E}+01$ \\
\hline $3.000000 \mathrm{E}+01$ & $2.750000 \mathrm{E}+01$ & $2.500000 \mathrm{E}+01$ & $2.250000 \mathrm{E}+01$ & $2.100000 \mathrm{E}+01$ \\
\hline $2.000000 \mathrm{E}+01$ & $1.900000 \mathrm{E}+01$ & $1.850000 \mathrm{E}+01$ & $1.700000 \mathrm{E}+01$ & $1.600000 \mathrm{E}+01$ \\
\hline $1.509990 \mathrm{E}+01$ & $1.440000 \mathrm{E}+01$ & $1.375000 \mathrm{E}+01$ & $1.290000 \mathrm{E}+01$ & $1.190000 \mathrm{E}+01$ \\
\hline $1.150000 \mathrm{E}+01$ & $1.000000 \mathrm{E}+01$ & $9.099990 \mathrm{E}+00$ & $8.099990 \mathrm{E}+00$ & $7.150000 \mathrm{E}+00$ \\
\hline $7.000000 \mathrm{E}+00$ & $6.750000 \mathrm{E}+00$ & $6.500000 \mathrm{E}+00$ & $6.250000 \mathrm{E}+00$ & $6.000000 \mathrm{E}+00$ \\
\hline $5.400000 \mathrm{E}+00$ & $5.000000 \mathrm{E}+00$ & $4.750000 \mathrm{E}+00$ & $4.000000 \mathrm{E}+00$ & $3.730000 \mathrm{E}+00$ \\
\hline $3.500000 \mathrm{E}+00$ & $3.150000 \mathrm{E}+00$ & $3.049990 \mathrm{E}+00$ & $3.000000 \mathrm{E}+00$ & $2.969990 \mathrm{E}+00$ \\
\hline $2.870000 \mathrm{E}+00$ & $2.770000 \mathrm{E}+00$ & $2.669990 \mathrm{E}+00$ & $2.570000 \mathrm{E}+00$ & $2.469990 \mathrm{E}+00$ \\
\hline $2.379990 \mathrm{E}+00$ & $2.299990 \mathrm{E}+00$ & 2.209990E+00 & $2.120000 \mathrm{E}+00$ & $2.000000 \mathrm{E}+00$ \\
\hline $1.940000 \mathrm{E}+00$ & $1.860000 \mathrm{E}+00$ & $1.770000 \mathrm{E}+00$ & $1.679990 \mathrm{E}+00$ & $1.589990 \mathrm{E}+00$ \\
\hline $1.500000 \mathrm{E}+00$ & $1.450000 \mathrm{E}+00$ & $1.400000 \mathrm{E}+00$ & $1.349990 \mathrm{E}+00$ & $1.299990 \mathrm{E}+00$ \\
\hline $1.250000 \mathrm{E}+00$ & $1.224990 \mathrm{E}+00$ & $1.200000 \mathrm{E}+00$ & $1.174990 \mathrm{E}+00$ & $1.150000 \mathrm{E}+00$ \\
\hline $1.139990 \mathrm{E}+00$ & $1.129990 \mathrm{E}+00$ & $1.120000 \mathrm{E}+00$ & $1.110000 \mathrm{E}+00$ & $1.099990 \mathrm{E}+00$ \\
\hline $1.089990 \mathrm{E}+00$ & $1.080000 \mathrm{E}+00$ & $1.070000 \mathrm{E}+00$ & $1.059990 \mathrm{E}+00$ & $1.049990 \mathrm{E}+00$ \\
\hline $1.040000 \mathrm{E}+00$ & $1.030000 \mathrm{E}+00$ & $1.020000 \mathrm{E}+00$ & $1.009990 \mathrm{E}+00$ & $1.000000 \mathrm{E}+00$ \\
\hline $9.750000 \mathrm{E}-01$ & $9.500000 \mathrm{E}-01$ & $9.250000 \mathrm{E}-01$ & $9.000000 \mathrm{E}-01$ & $8.500000 \mathrm{E}-01$ \\
\hline $8.000000 \mathrm{E}-01$ & $7.500000 \mathrm{E}-01$ & $7.000000 \mathrm{E}-01$ & $6.500000 \mathrm{E}-01$ & $6.250000 \mathrm{E}-01$ \\
\hline $6.000000 \mathrm{E}-01$ & $5.500000 \mathrm{E}-01$ & $5.000000 \mathrm{E}-01$ & $4.500000 \mathrm{E}-01$ & $4.000000 \mathrm{E}-01$ \\
\hline $3.750000 \mathrm{E}-01$ & $3.500000 \mathrm{E}-01$ & $3.250000 \mathrm{E}-01$ & $3.000000 \mathrm{E}-01$ & $2.750000 \mathrm{E}-01$ \\
\hline $2.500000 \mathrm{E}-01$ & $2.250000 \mathrm{E}-01$ & $2.000000 \mathrm{E}-01$ & $1.750000 \mathrm{E}-01$ & $1.500000 \mathrm{E}-01$ \\
\hline
\end{tabular}

Figure 6.3.A.1. Truncated sensitivity data file for Flattop-25 sample problem. 


\begin{tabular}{|c|c|c|c|c|}
\hline $50000 \mathrm{E}-01$ & $.000000 \mathrm{E}-01$ & $9.000000 \mathrm{E}-02$ & $8.000000 \mathrm{E}-02$ & $7.000000 \mathrm{E}-02$ \\
\hline $6.000000 \mathrm{E}-02$ & $5.000000 \mathrm{E}-02$ & $4.000000 \mathrm{E}-02$ & $3.000000 \mathrm{E}-02$ & $2.530000 \mathrm{E}-02$ \\
\hline $000000 \mathrm{E}-02$ & $7.500000 \mathrm{E}-03$ & $5.000000 \mathrm{E}-03$ & $4.000000 \mathrm{E}-03$ & $3.000000 \mathrm{E}-03$ \\
\hline $0000 E-03$ & $0000 \mathrm{E}$ & $00000 \mathrm{E}$ & $1.200000 \mathrm{E}$ & 1.00 \\
\hline $7.500000 \mathrm{E}-04$ & $5.000000 \mathrm{E}-04$ & $1.000000 \mathrm{E}-04$ & $1.000000 \mathrm{E}-05$ & \\
\hline-234 & & 92234 & 1 & $0.000000 \mathrm{E}+$ \\
\hline $4.717915 \mathrm{E}-03$ & $.975886 \mathrm{E}-03$ & $-1.289843 \mathrm{E}-04$ & & \\
\hline & & 07 & $3.212515 \mathrm{E}-07$ & 8.004773 \\
\hline .06 & 77621 & $8.2-2$ & -04 & \\
\hline 6.0 & 4.0446421 & 1.1350 & 5.6 & 4. \\
\hline 1.4 & 6. & 6.2892 & $966 E-04$ & \\
\hline 1.7 & 1.6 & 1.761 & -05 & \\
\hline 3.2 & 1.0 & 1.8 & -04 & \\
\hline 1. & & 4.8 & 1 . & \\
\hline & & & & \\
\hline 1.4 & -3.8 & -8.1 & -1.5 & -1 \\
\hline-1.8 & -5 . & -1 & $-1 \cdot 32$ & -9.32 \\
\hline & -6 & -1.968 & $-7.035957 \mathrm{E}-06$ & -9.44630 \\
\hline-4 & -3. & -1.2 & -1.4 & -1.2 \\
\hline 8 & -3 & -1 & -1 . & -2 . \\
\hline & & & & \\
\hline-3 & -1.7 & -9.1 & -1.9 & -7 \\
\hline-1 & -2 & -7.6 & & -2 \\
\hline-2 & -8 . & -1 & & -1 \\
\hline-8 . & -8 . & -1 & -2 & -1 \\
\hline-2 . & 3. & 3. & -1 . & \\
\hline & -2 & -1 & & \\
\hline-2 . & -2 . & 4. & 12 & -2 \\
\hline 5 & -1 & 2.3 & & -6 \\
\hline & & & -1 & -2 . \\
\hline & -1.3 & -6. & 4. & \\
\hline-7. & -3 & -1 & -1 & \\
\hline & & -5 & & \\
\hline 5.7 & -2.6 & -5.2 & -1.3 & -9 \\
\hline 13 & -2.3 & -1.6311191 & 8.36 & -2.8967 \\
\hline & -1.0 & -1 . & & -3 \\
\hline-2 & 5 . & -5 & 5 & -2 \\
\hline-4 . & -2 & -2 . & -2 . & -1 . \\
\hline & -1 & -1 & & \\
\hline 5 & -1 & -1.03 & -9.93 & -9.776219 \\
\hline 5 & -4 . & -3 & & -6.1 \\
\hline-1 . & -5 . & & & \\
\hline & -2 & & & \\
\hline & -1 & -2 . & & \\
\hline & & & & -3 \\
\hline & -7 . & -7 & -1 & -1 \\
\hline-1 & -1 . & -1 & -2.95 & $00 E-16$ \\
\hline & -1 & -3.1 & -2. & 1.0 \\
\hline & 4.7 & 5.1 & $05 E-18$ & -7.3 \\
\hline 19 & 1.8 & 1.2 & $-1.217866 \mathrm{E}-22$ & $6.410866 \mathrm{E}-2$ \\
\hline
\end{tabular}

Figure 6.3.A.1. Truncated sensitivity data file for Flattop-25 sample problem. (continued) 


\begin{tabular}{|c|c|c|c|c|}
\hline & & & & \\
\hline-1.4 & & & & \\
\hline-32 & $0.000000 \mathrm{E}+00$ & $0.000000 \mathrm{E}+00$ & $0.000000 \mathrm{E}+00$ & $0.000000 \mathrm{E}+$ \\
\hline $0.000000 \mathrm{E}+00$ & $0.000000 \mathrm{E}+00$ & $0.000000 \mathrm{E}+00$ & $0.000000 \mathrm{E}+00$ & $0.000000 \mathrm{E}+$ \\
\hline $000 \mathrm{E}+00$ & $0.000000 \mathrm{E}+00$ & $0.000000 \mathrm{E}+00$ & & \\
\hline 234 & tter & & 1 & $0.000000 \mathrm{E}-$ \\
\hline $8.032846 \mathrm{E}-04$ & $8.241044 \mathrm{E}-04$ & $-1.041006 \mathrm{E}-05$ & & \\
\hline$-3.2-2 \cdot x \cdot x$ & & & 4.894583 & 8.37 \\
\hline & & & & \\
\hline 3. & & & & 4.5 \\
\hline 2 & & & & \\
\hline & & & & \\
\hline & 1. & & & \\
\hline & & & & \\
\hline & & & & \\
\hline 4. & 2 . & & & \\
\hline 1 . & & & & \\
\hline & & & & \\
\hline 7 & -1 & -2 & & \\
\hline-3 & -2 & & -1 & \\
\hline & -1 & & & -5 \\
\hline-2 . & -1 & -3 & & \\
\hline & -5.4 & & & \\
\hline 0 & -7 & -1 & & \\
\hline & -4 & -9 & & \\
\hline-1 . & 3. & & -1 & \\
\hline & -1 & -4 & & \\
\hline & -6.3 & & & \\
\hline & -7 & & & -3 \\
\hline & & & & \\
\hline & -8 & & & \\
\hline & -2 & -7 & -1 & \\
\hline-5 & & -3.4 & & -1.3 \\
\hline 4. & 5. & -3.6 & & -4 \\
\hline & -1 & -3 & & \\
\hline & -1 & & & \\
\hline & & & -4 & -4 \\
\hline-1 . & -2 . & -1 & & \\
\hline & -8 & -2 . & & \\
\hline 16 & -8 & & & -2 \\
\hline & -8.5 & & & -1 \\
\hline & & & & \\
\hline & -9 & -7 & & -2 \\
\hline & -4 & & & -6 \\
\hline-1 & 1 . & & & \\
\hline 6 & -8 & -1.3 & 2.67 & 9.881 \\
\hline & 1.0 & -1 . & & -1.0 \\
\hline & -2 . & -7 & & \\
\hline & & & & -2. \\
\hline
\end{tabular}

Figure 6.3.A.1. Truncated sensitivity data file for Flattop- 25 sample problem. (continued) 


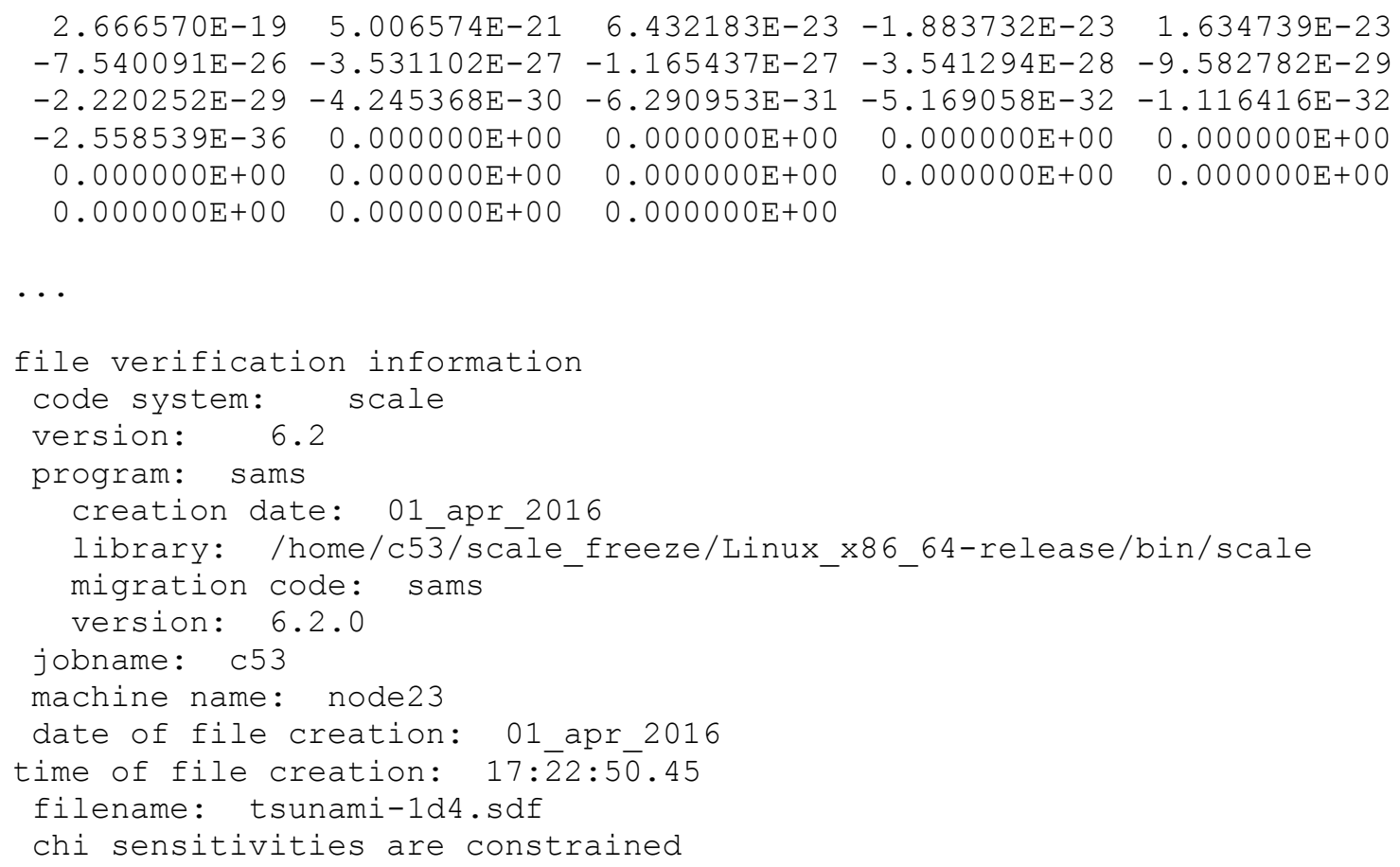

Figure 6.3.A.1. Truncated sensitivity data file for Flattop-25 sample problem. (continued)

\subsection{A.2 Format of TSUNAMI-B sensitivity data file}

The format of the TSUNAMI-B sensitivity data file produced by SAMS for cases with Monte Carlo transport solutions is given in Table 6.3.A.2. The occurrence of each entry in the data file is followed by an identification of the data contained on each line of the file and the FORTRAN edit descriptor denoting the format of each line. A brief description of each line is also presented.

A sample of the TSUNAMI-B data file for the LEU-COMP-THERM-009 case 10 sample problem is provided in Figure 6.3.A.2. Here, only two profiles out of the 3389 computed are shown. 
Table 6.3.A.2. Format specification for TSUNAMI-B sensitivity data file

\begin{tabular}{|c|c|c|c|}
\hline Occurrence & Data & Format & Description \\
\hline \multirow{6}{*}{$\begin{array}{l}\text { Once at beginning of } \\
\text { file. }\end{array}$} & title & $\mathrm{a} 80$ & $\begin{array}{l}\text { Title extracted from transport } \\
\text { calculation }\end{array}$ \\
\hline & number of neutron groups & i10 & $\begin{array}{l}\text { Number of neutron groups in } \\
\text { calculation }\end{array}$ \\
\hline & $\begin{array}{l}\text { total number of profiles, } \\
\text { text descriptor, } \\
\text { number of total profiles that are region } \\
\text { integrated }\end{array}$ & $\mathrm{i} 10, \mathrm{a} 35, \mathrm{i} 10$ & $\begin{array}{l}\text { Total number of sensitivity } \\
\text { profiles in data file, separated } \\
\text { by a text descriptor, then the } \\
\text { number of profiles that contain } \\
\text { region-integrated data }\end{array}$ \\
\hline & $k_{e f f}{ }^{'}+/-^{\prime} \sigma$ & $\begin{array}{l}\mathrm{f} 10.6,1 \mathrm{x}, \mathrm{a} \\
1 \mathrm{x}, \mathrm{f} 10.6\end{array}$ & $\begin{array}{l}\text { Value of } k_{\text {eff }} \text { from the forward } \\
\text { transport calculation and its } \\
\text { standard deviation }\end{array}$ \\
\hline & 'energy boundaries:' & $\mathrm{a}$ & Text \\
\hline & energy boundary data & $\begin{array}{l}\text { 5es } 14.6 \text { (repeats } \\
\text { until all data is } \\
\text { printed) }\end{array}$ & $\begin{array}{l}\text { Values of boundaries of } \\
\text { energy groups. Begins with } \\
\text { upper value for highest energy } \\
\text { group and ends with lower } \\
\text { value of lowest energy group. }\end{array}$ \\
\hline \multirow{3}{*}{$\begin{array}{l}\text { Repeats for each } \\
\text { profile. }\end{array}$} & $\begin{array}{l}\text { isotope name, } \\
\text { sensitivity reaction name, } \\
\text { nuclide ID, } \\
\text { MT number }\end{array}$ & $\begin{array}{l}\text { a12, } 1 \mathrm{x}, \quad \mathrm{a} 15, \\
2 \mathrm{i} 12\end{array}$ & $\begin{array}{l}\text { Provides data identifying the } \\
\text { sensitivity data that follows. } \\
\text { Note, if sensitivity data is } \\
\text { region-integrated, zone } \\
\text { number and zone volume are } \\
\text { both 0. If data is integrated } \\
\text { over all zones containing the } \\
\text { same material, the material } \\
\text { number is given in place of the } \\
\text { zone number as a negative } \\
\text { number. }\end{array}$ \\
\hline & $\begin{array}{l}\text { Unit number, } \\
\text { number of the region within the unit, } \\
\text { unit comments }\end{array}$ & $2 \mathrm{i} 7, \mathrm{a} 50$ & \\
\hline & $\begin{array}{l}\text { Zone number or negative of material } \\
\text { number, } \\
\text { zone volume, } \\
\text { number of times unit is referenced in } \\
\text { the problem, } \\
\text { MatId }\end{array}$ & 2es14.6, $2 \mathrm{i} 7$ & $\begin{array}{l}\text { Note, if sensitivity data is } \\
\text { region-integrated, zone } \\
\text { number and zone volume are } \\
\text { both } 0 \text {. If data is integrated } \\
\text { over all zones containing the } \\
\text { same material, the material } \\
\text { number is given in place of the } \\
\text { zone number as a negative } \\
\text { number. }\end{array}$ \\
\hline
\end{tabular}


Table 6.3.A.2. Format specification for TSUNAMI-B sensitivity data file (continued)

\begin{tabular}{|c|c|c|c|}
\hline Occurrence & Data & Format & Description \\
\hline & $\begin{array}{l}\text { energy integrated sensitivity } \\
\text { coefficient, } \\
\text { standard deviation for energy } \\
\text { integrated sensitivity coefficient, } \\
\text { sum of absolute value of group-wise } \\
\text { sensitivities, } \\
\text { sum of the group-wise } \\
\text { sensitivities with opposite sign as } \\
\text { energy integrated value (osc), } \\
\text { standard deviation for osc. }\end{array}$ & 5 es 14.6 & $\begin{array}{l}\text { Energy-integrated sensitivity } \\
\text { coefficients and their } \\
\text { standard deviations for this } \\
\text { profile. }\end{array}$ \\
\hline & group-wise sensitivity coefficients & $\begin{array}{l}\text { 5es14.6 (repeats } \\
\text { until all data is } \\
\text { printed) }\end{array}$ & $\begin{array}{l}\text { Energy-dependent sensitivity } \\
\text { coefficients. Begins with } \\
\text { highest energy group. }\end{array}$ \\
\hline & $\begin{array}{l}\text { standard deviation in group-wise } \\
\text { sensitivity coefficients }\end{array}$ & $\begin{array}{l}\text { 5es14.6 (repeats } \\
\text { until all data is } \\
\text { printed) }\end{array}$ & $\begin{array}{l}\text { Standard deviation for energy- } \\
\text { dependent } \\
\text { coefficients. Begins witivity } \\
\text { highest energy group. }\end{array}$ \\
\hline Once at end of file. & & & $\begin{array}{l}\text { Block of file verification } \\
\text { information. }\end{array}$ \\
\hline
\end{tabular}




\begin{tabular}{|c|c|c|c|c|}
\hline \multicolumn{5}{|c|}{$\begin{array}{l}\text { sample } 3-\text { tsunami-3d } \\
238 \text { number of neutron groups }\end{array}$} \\
\hline $\begin{array}{r}3389 \\
1.004674+/-\end{array}$ & $\begin{array}{l}\text { nber of sensit } \\
0.000543 \mathrm{k}-\epsilon\end{array}$ & $\begin{array}{l}\text { lvity protiles } \\
\text { ff from the fo }\end{array}$ & ward case & are region integrated \\
\hline \multicolumn{5}{|c|}{ energy boundaries: } \\
\hline $2.000000 \mathrm{E}+07$ & $1.733300 \mathrm{E}+07$ & $1.568300 \mathrm{E}+07$ & $1.455000 \mathrm{E}+07$ & $1.384000 \mathrm{E}+07$ \\
\hline $1.284000 \mathrm{E}+07$ & $1.000000 \mathrm{E}+07$ & $8.187300 \mathrm{E}+06$ & $6.434000 \mathrm{E}+06$ & $4.800000 \mathrm{E}+06$ \\
\hline $4.304000 \mathrm{E}+06$ & $3.000000 \mathrm{E}+06$ & $2.479000 \mathrm{E}+06$ & $2.354000 \mathrm{E}+06$ & $1.850000 \mathrm{E}+06$ \\
\hline $1.500000 \mathrm{E}+06$ & $1.400000 \mathrm{E}+06$ & $1.356000 \mathrm{E}+06$ & $1.317000 \mathrm{E}+06$ & $1.250000 \mathrm{E}+06$ \\
\hline $1.200000 \mathrm{E}+06$ & $1.100000 \mathrm{E}+06$ & $1.010000 \mathrm{E}+06$ & $9.200000 \mathrm{E}+05$ & $9.000000 \mathrm{E}+05$ \\
\hline $8.750000 \mathrm{E}+05$ & $8.611000 \mathrm{E}+05$ & $8.200000 \mathrm{E}+05$ & $7.500000 \mathrm{E}+05$ & $6.790000 \mathrm{E}+05$ \\
\hline $6.700000 \mathrm{E}+05$ & $6.000000 \mathrm{E}+05$ & $5.730000 \mathrm{E}+05$ & $5.500000 \mathrm{E}+05$ & $4.995200 \mathrm{E}+05$ \\
\hline $4.700000 \mathrm{E}+05$ & $4.400000 \mathrm{E}+05$ & $4.200000 \mathrm{E}+05$ & $4.000000 \mathrm{E}+05$ & $3.300000 \mathrm{E}+05$ \\
\hline $2.700000 \mathrm{E}+05$ & $2.000000 \mathrm{E}+05$ & $1.500000 \mathrm{E}+05$ & $1.283000 \mathrm{E}+05$ & $1.000000 \mathrm{E}+05$ \\
\hline $8.500000 \mathrm{E}+04$ & $8.200000 \mathrm{E}+04$ & $7.500000 \mathrm{E}+04$ & $7.300000 \mathrm{E}+04$ & $6.000000 \mathrm{E}+04$ \\
\hline $5.200000 \mathrm{E}+04$ & $5.000000 \mathrm{E}+04$ & $4.500000 \mathrm{E}+04$ & $3.000000 \mathrm{E}+04$ & $2.500000 \mathrm{E}+04$ \\
\hline $1.700000 \mathrm{E}+04$ & $1.300000 \mathrm{E}+04$ & $9.500000 \mathrm{E}+03$ & $8.030000 \mathrm{E}+03$ & $6.000000 \mathrm{E}+03$ \\
\hline $3.900000 \mathrm{E}+03$ & $3.740000 \mathrm{E}+03$ & $3.000000 \mathrm{E}+03$ & $2.580000 \mathrm{E}+03$ & $2.290000 \mathrm{E}+03$ \\
\hline $2.200000 \mathrm{E}+03$ & $1.800000 \mathrm{E}+03$ & $1.550000 \mathrm{E}+03$ & $1.500000 \mathrm{E}+03$ & $1.150000 \mathrm{E}+03$ \\
\hline $9.500000 \mathrm{E}+02$ & $6.830000 \mathrm{E}+02$ & $6.700000 \mathrm{E}+02$ & $5.500000 \mathrm{E}+02$ & $3.050000 \mathrm{E}+02$ \\
\hline $2.850000 \mathrm{E}+02$ & $2.400000 \mathrm{E}+02$ & $2.100000 \mathrm{E}+02$ & $2.075000 \mathrm{E}+02$ & 1. $925000 \mathrm{E}+02$ \\
\hline $1.860000 \mathrm{E}+02$ & $1.220000 \mathrm{E}+02$ & $1.190000 \mathrm{E}+02$ & $1.150000 \mathrm{E}+02$ & $1.080000 \mathrm{E}+02$ \\
\hline $1.000000 \mathrm{E}+02$ & $9.000000 \mathrm{E}+01$ & $8.200000 \mathrm{E}+01$ & $8.000000 \mathrm{E}+01$ & $7.600000 \mathrm{E}+01$ \\
\hline $7.200000 \mathrm{E}+01$ & $6.750000 \mathrm{E}+01$ & $6.500000 \mathrm{E}+01$ & $6.100000 \mathrm{E}+01$ & $5.900000 \mathrm{E}+01$ \\
\hline $5.340000 \mathrm{E}+01$ & $5.200000 \mathrm{E}+01$ & $5.060000 \mathrm{E}+01$ & $4.920000 \mathrm{E}+01$ & $4.830000 \mathrm{E}+01$ \\
\hline $4.700000 \mathrm{E}+01$ & $4.520000 \mathrm{E}+01$ & $4.400000 \mathrm{E}+01$ & $4.240000 \mathrm{E}+01$ & $4.100000 \mathrm{E}+01$ \\
\hline $3.960000 \mathrm{E}+01$ & $3.910000 \mathrm{E}+01$ & $3.800000 \mathrm{E}+01$ & $3.700000 \mathrm{E}+01$ & $3.550000 \mathrm{E}+01$ \\
\hline $3.460000 \mathrm{E}+01$ & $3.375000 \mathrm{E}+01$ & $3.325000 \mathrm{E}+01$ & $3.175000 \mathrm{E}+01$ & $3.125000 \mathrm{E}+01$ \\
\hline $3.000000 \mathrm{E}+01$ & $2.750000 \mathrm{E}+01$ & $2.500000 \mathrm{E}+01$ & $2.250000 \mathrm{E}+01$ & $2.100000 \mathrm{E}+01$ \\
\hline $2.000000 \mathrm{E}+01$ & $1.900000 \mathrm{E}+01$ & $1.850000 \mathrm{E}+01$ & $1.700000 \mathrm{E}+01$ & $1.600000 \mathrm{E}+01$ \\
\hline $1.509990 \mathrm{E}+01$ & $1.440000 \mathrm{E}+01$ & $1.375000 \mathrm{E}+01$ & $1.290000 \mathrm{E}+01$ & $1.190000 \mathrm{E}+01$ \\
\hline $1.150000 \mathrm{E}+01$ & $1.000000 \mathrm{E}+01$ & $9.099990 \mathrm{E}+00$ & $8.099990 \mathrm{E}+00$ & $7.150000 \mathrm{E}+00$ \\
\hline $7.000000 \mathrm{E}+00$ & $6.750000 \mathrm{E}+00$ & $6.500000 \mathrm{E}+00$ & $6.250000 \mathrm{E}+00$ & $6.000000 \mathrm{E}+00$ \\
\hline $5.400000 \mathrm{E}+00$ & $5.000000 \mathrm{E}+00$ & $4.750000 \mathrm{E}+00$ & $4.000000 \mathrm{E}+00$ & $3.730000 \mathrm{E}+00$ \\
\hline $3.500000 \mathrm{E}+00$ & $3.150000 \mathrm{E}+00$ & $3.049990 \mathrm{E}+00$ & $3.000000 \mathrm{E}+00$ & $2.969990 \mathrm{E}+00$ \\
\hline $2.870000 \mathrm{E}+00$ & $2.770000 \mathrm{E}+00$ & $2.669990 \mathrm{E}+00$ & $2.570000 \mathrm{E}+00$ & $2.469990 \mathrm{E}+00$ \\
\hline $2.379990 \mathrm{E}+00$ & $2.299990 \mathrm{E}+00$ & $2.209990 E+00$ & $2.120000 \mathrm{E}+00$ & $2.000000 \mathrm{E}+00$ \\
\hline $1.940000 \mathrm{E}+00$ & $1.860000 \mathrm{E}+00$ & $1.770000 \mathrm{E}+00$ & $1.679990 \mathrm{E}+00$ & $1.589990 \mathrm{E}+00$ \\
\hline $1.500000 \mathrm{E}+00$ & $1.450000 \mathrm{E}+00$ & $1.400000 \mathrm{E}+00$ & $1.349990 \mathrm{E}+00$ & $1.299990 \mathrm{E}+00$ \\
\hline $1.250000 \mathrm{E}+00$ & $1.224990 \mathrm{E}+00$ & $1.200000 \mathrm{E}+00$ & $1.174990 \mathrm{E}+00$ & $1.150000 \mathrm{E}+00$ \\
\hline $1.139990 \mathrm{E}+00$ & $1.129990 \mathrm{E}+00$ & $1.120000 \mathrm{E}+00$ & $1.110000 \mathrm{E}+00$ & $1.099990 \mathrm{E}+00$ \\
\hline $1.089990 \mathrm{E}+00$ & $1.080000 \mathrm{E}+00$ & $1.070000 \mathrm{E}+00$ & $1.059990 \mathrm{E}+00$ & $1.049990 \mathrm{E}+00$ \\
\hline $1.040000 \mathrm{E}+00$ & $1.030000 \mathrm{E}+00$ & $1.020000 \mathrm{E}+00$ & $1.009990 \mathrm{E}+00$ & $1.000000 \mathrm{E}+00$ \\
\hline $9.750000 \mathrm{E}-01$ & $9.500000 \mathrm{E}-01$ & $9.250000 \mathrm{E}-01$ & $9.000000 \mathrm{E}-01$ & $8.500000 \mathrm{E}-01$ \\
\hline $8.000000 E-01$ & $7.500000 \mathrm{E}-01$ & $7.000000 \mathrm{E}-01$ & $6.500000 \mathrm{E}-01$ & $6.250000 \mathrm{E}-01$ \\
\hline $6.000000 \mathrm{E}-01$ & $5.500000 \mathrm{E}-01$ & $5.000000 \mathrm{E}-01$ & $4.500000 \mathrm{E}-01$ & $4.000000 \mathrm{E}-01$ \\
\hline $3.750000 \mathrm{E}-01$ & $3.500000 \mathrm{E}-01$ & $3.250000 \mathrm{E}-01$ & $3.000000 \mathrm{E}-01$ & $2.750000 \mathrm{E}-01$ \\
\hline $2.500000 \mathrm{E}-01$ & $2.250000 \mathrm{E}-01$ & $2.000000 \mathrm{E}-01$ & $1.750000 \mathrm{E}-01$ & $1.500000 \mathrm{E}-01$ \\
\hline $1.250000 \mathrm{E}-01$ & $1.000000 \mathrm{E}-01$ & $9.000000 \mathrm{E}-02$ & $8.000000 \mathrm{E}-02$ & $7.000000 \mathrm{E}-02$ \\
\hline 22 & $5.000000 \mathrm{E}-02$ & $4.000000 \mathrm{E}-02$ & $3.000000 \mathrm{E}-02$ & $\$ 30000$ \\
\hline
\end{tabular}

Figure 6.3.A.2. Truncated sensitivity data file for LEU-COMP-THERM-009 sample problem. 


\begin{tabular}{|c|c|c|c|c|}
\hline $1.000000 \mathrm{E}-02$ & $7.500000 \mathrm{E}-03$ & $5.000000 \mathrm{E}-03$ & $4.000000 \mathrm{E}-03$ & \\
\hline $2.500000 \mathrm{E}-03$ & $2.000000 \mathrm{E}-03$ & $1.500000 \mathrm{E}-03$ & $1.200000 \mathrm{E}-03$ & $1.000000 \mathrm{E}-03$ \\
\hline $0 \mathrm{E}-04$ & $5.000000 \mathrm{E}-04$ & $000000 \mathrm{E}-04$ & $1.000000 \mathrm{E}-05$ & \\
\hline & & 1100 & & \\
\hline 0 & & & & \\
\hline$E+00$ & $0 \mathrm{E}+00$ & S & & \\
\hline $5.878199 \mathrm{E}-01$ & $1.080812 \mathrm{E}-02$ & $5.393673 \mathrm{E}-01$ & $-2.577372 \mathrm{E}-02$ & $6.213759 \mathrm{E}-03$ \\
\hline $0.000000 \mathrm{E}+00$ & $5.495367 \mathrm{E}-07$ & $2.331587 \mathrm{E}-06$ & $3.300773 \mathrm{E}-06$ & \\
\hline $1.664451 \mathrm{E}-04$ & $6.565088 \mathrm{E}-04$ & $2.936479 \mathrm{E}-03$ & $1.086949 \mathrm{E}-02$ & $535-03$ \\
\hline $3.153059 \mathrm{E}-02$ & $94 E-02$ & $866 \mathrm{E}-03$ & $85 E-02$ & \\
\hline $9.882265 \mathrm{E}-03$ & $4542 E-03$ & $62922 \mathrm{E}-03$ & & \\
\hline $9.352559 \mathrm{E}-03$ & $6.455556 \mathrm{E}-03$ & $6.329793 \mathrm{E}-03$ & $2.219300 \mathrm{E}-03$ & \\
\hline $1.846754 \mathrm{E}-03$ & $5.699152 \mathrm{E}-03$ & $1.105599 \mathrm{E}-02$ & $1.187824 \mathrm{E}-02$ & $E-03$ \\
\hline $1.163906 \mathrm{E}-02$ & & $4.090504 \mathrm{E}-03$ & & \\
\hline 3.1 & 1 & -03 & & \\
\hline 1.6 & 02 & -03 & & \\
\hline 1.183 & $2 . \varepsilon$ & 4 & & \\
\hline 1.1 & 3. & 03 & & \\
\hline $6.009586 \mathrm{E}$ & $502 E-03$ & $5383 E-03$ & $21 E-03$ & \\
\hline & & & & \\
\hline & & & & \\
\hline 5.2 & .04 & 03 & & \\
\hline 2.2 & 3 & 4 & & \\
\hline $7.128428 \mathrm{E}$ & $4.2 x-2.2$ & 4 & & \\
\hline $1.660348 \mathrm{E}-03$ & $3 E-04$ & 3081871 & & \\
\hline 1.0 & 3. & & & \\
\hline & & & & \\
\hline & & & & \\
\hline 2.3 & 6.3 & 04 & & \\
\hline $3.620459 \mathrm{E}-04$ & 2.29 & -04 & & \\
\hline & & & & \\
\hline 4 & 4 & & & \\
\hline 1 & 4.2 & 3 & & \\
\hline & & & & \\
\hline- & 4.7 & 04 & & -03 \\
\hline $1.341676 \mathrm{E}-03$ & $54 \mathrm{E}-04$ & $2.380048 \mathrm{E}-03$ & & $6 E-04$ \\
\hline 3 & 4.6 & -04 & 1. & \\
\hline & & & & \\
\hline & & & & \\
\hline $3920 E^{3}$ & 6.92 & $2597 E-04$ & 9. & -03 \\
\hline 4 & 7.5 & 92 & & \\
\hline & & & & \\
\hline & 8.2 & & & \\
\hline & & & & \\
\hline & & & & \\
\hline t广 & 1.1 & 8. & & -03 \\
\hline $18 \mathrm{E}-1-3$ & 1.1 & $5521 \mathrm{E}$ & 4. & $24 \mathrm{E}-04$ \\
\hline & & & & \\
\hline & & 7 . & & \\
\hline $46 \mathrm{E}-$ & $1.298630 \mathrm{E}-03$ & $1.487261 \mathrm{E}-03$ & $1.447523 \mathrm{E}-03$ & $2.061059 \mathrm{E}-\mathrm{C}$ \\
\hline
\end{tabular}

Figure 6.3.A.2. Truncated sensitivity data file for LEU-COMP-THERM-009 sample problem. (continued) 


\begin{tabular}{|c|c|c|c|c|}
\hline $3669 \mathrm{E}-03$ & 04 & $D E-04$ & $E-05$ & $3 E-03$ \\
\hline & & $86 \mathrm{E}-03$ & $-2.081466 \mathrm{E}-03$ & $-9.899241 \mathrm{E}-03$ \\
\hline & & -04 & $5 E-04$ & \\
\hline 04 & -04 & $43808 \mathrm{E}-05$ & $-6.213754 E-05$ & -6.412 \\
\hline & -6 & $-5.596935 \mathrm{E}-06$ & & \\
\hline & $669 E-07$ & $4.563308 \mathrm{E}-07$ & $5.797961 E-07$ & 1.0525891 \\
\hline $409 E-06$ & $07 E-05$ & $83163 E-05$ & $1.976880 \mathrm{E}-04$ & $1.499190 \mathrm{E}-$ \\
\hline $8 \mathrm{E}-04$ & $4 \mathrm{E}-04$ & & & \\
\hline & 04 & -04 & ע1 & \\
\hline $1421 \mathrm{E}-04$ & $6 \mathrm{E}-04$ & $91 \mathrm{E}-04$ & $26265 E-04$ & \\
\hline $89384 \mathrm{E}-04$ & $3.598922 \mathrm{E}-04$ & $6.532525 \mathrm{E}-04$ & $6.485064 \mathrm{E}-04$ & 1.182 \\
\hline $41 E-04$ & $550 \mathrm{E}-04$ & $876 \mathrm{E}$ & 6 . & \\
\hline $352 \mathrm{E}-04$ & $5750 E-04$ & $68595 \mathrm{E}-04$ & $3 E-03$ & \\
\hline $0829 \mathrm{E}-03$ & $332 E-03$ & & & \\
\hline $3224 \mathrm{E}-04$ & 5 . & -04 & & \\
\hline $4300 \mathrm{E}$ & 6.2 & 1. & 9. & \\
\hline $38510 \mathrm{E}$ & $1.511362 \mathrm{E}$ & $9.038528 \mathrm{E}$ & 57087 & 1.91 \\
\hline $1742 E$ & 1 . & 7 . & & \\
\hline 3 & & & & \\
\hline 1.6 & 1. & -03 & & \\
\hline $31 \mathrm{E}-04$ & 7.0 & -04 & 4 . & 2 . \\
\hline $1.745300 \mathrm{E}-03$ & 1. & 2 . & 3. & 4. \\
\hline $5.449888 \mathrm{E}-04$ & $4.941223 \mathrm{E}-04$ & $91 / 61$ & 3. & 3. \\
\hline 4 & 2.3 & 3. & & \\
\hline 4 & & & & \\
\hline $9 E-04$ & 1. & 04 & & \\
\hline $949 E-04$ & -04 & -04 & 2 . & 1. \\
\hline $1.802619 \mathrm{E}-04$ & $1.265510 \mathrm{E}-04$ & $2.860560 \mathrm{E}-04$ & 1.303 & 2.6 \\
\hline $4.822369 \mathrm{E}$ & $5.053777 \mathrm{E}$ & 5.572759 & 3.7 & 2.5 \\
\hline & & & & \\
\hline 2 . & 04 & 04 & & 04 \\
\hline $6 \mathrm{E}-04$ & 4 . & 5. & & \\
\hline $2270 E-04$ & $59 \mathrm{E}-04$ & $0 E-04$ & 2 . & 5 . \\
\hline $4.148698 \mathrm{E}-04$ & $2.984723 \mathrm{E}-04$ & $9708 \mathrm{E}-04$ & 3.7827 & 3.5 \\
\hline $5.266942 \mathrm{E}-04$ & $1.997956 \mathrm{E}-04$ & 4097 & 9. & 2 . \\
\hline & & & & \\
\hline 2.2 & & 04 & & 04 \\
\hline 2. & 2 . & & & \\
\hline 04 & 2. & 2 . & 2. & 2. \\
\hline $1.491903 \mathrm{E}-04$ & $1.504862 \mathrm{E}-04$ & $1.526581 \mathrm{E}-04$ & $1.494485 \mathrm{E}-04$ & 7.853 \\
\hline $7.694254 \mathrm{E}-05$ & $7.521152 \mathrm{E}-05$ & $7.139824 \mathrm{E}-05$ & 6.321 & 5.3 \\
\hline $7381 E$ & $44781 \mathrm{E}$ & 2 . & 2.42 & 2.6 \\
\hline 5 & $4.518938 \mathrm{E}-05$ & 5. & $1 E-05$ & $1.491623 \mathrm{E}-04$ \\
\hline $1.711490 \mathrm{E}-04$ & $1.797697 \mathrm{E}-04$ & $1.924294 \mathrm{E}-04$ & $E-04$ & 3.6 \\
\hline & $4.127060 \mathrm{E}-04$ & $4.406396 \mathrm{E}-$ & $2.723181 \mathrm{E}-$ & 2.8 \\
\hline $5 \pi$ & $5.409453 \mathrm{E}$ & $0452 \mathrm{E}$ & 5.9332191 & 3.3468861 \\
\hline $73575 \mathrm{~F}$ & $2.671577 \mathrm{E}$ & $2.257729 \mathrm{E}-04$ & $2.317883 \mathrm{E}$ & $2.815263 \mathrm{E}$ \\
\hline $24861 \mathrm{~F}$ & 84778 & $6.219380 \mathrm{E}-$ & $232623 \mathrm{E}$ & $\perp$ \\
\hline $840 E-03$ & $1433 E-03$ & $1.278081 \mathrm{E}-03$ & $1.529288 \mathrm{E}-03$ & $1.839648 \mathrm{E}-03$ \\
\hline
\end{tabular}

Figure 6.3.A.2. Truncated sensitivity data file for LEU-COMP-THERM-009 sample problem. (continued) 


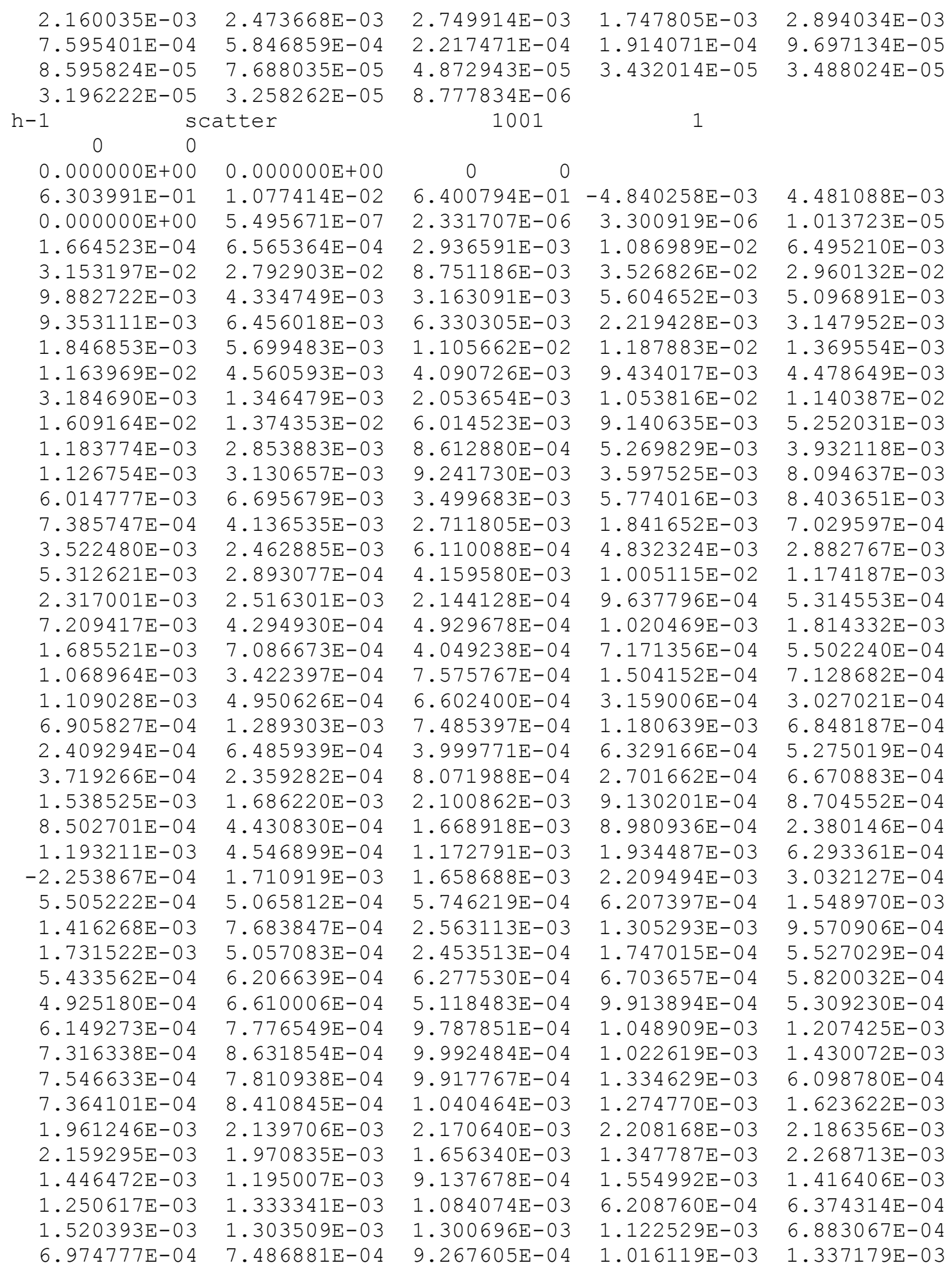

Figure 6.3.A.2. Truncated sensitivity data file for LEU-COMP-THERM-009 sample problem. (continued) 


\begin{tabular}{|c|c|c|c|c|}
\hline $1901 E-03$ & $4987 E-03$ & $2.065219 \mathrm{E}-03$ & $2.269599 \mathrm{E}-03$ & $2 E-03$ \\
\hline $4.496744 \mathrm{E}-03$ & $1.651763 \mathrm{E}-03$ & $1.099488 \mathrm{E}-03$ & $1.646667 \mathrm{E}-03$ & $8.793735 \mathrm{E}-04$ \\
\hline $13625 \mathrm{E}-03$ & $1.724868 \mathrm{E}-03$ & $55957 \mathrm{E}-04$ & $3.661996 \mathrm{E}-05$ & -2 \\
\hline $224 \mathrm{E}-04$ & $-5.189590 \mathrm{E}-04$ & $-1.363285 E-04$ & $-1.360189 \mathrm{E}-04$ & \\
\hline & $-6.221425 E-05$ & $75 E-05$ & $-2.253157 E-05$ & $-2.094053 E-$ \\
\hline & $-2.462690 \mathrm{E}-05$ & $-2.170317 \mathrm{E}-06$ & & \\
\hline $00 \mathrm{E}+00$ & $2.166573 \mathrm{E}-07$ & $4.563122 \mathrm{E}-07$ & $5.797737 \mathrm{E}-07$ & $1.052550 \mathrm{E}-06$ \\
\hline $227220 \mathrm{E}-06$ & $358 \mathrm{E}-05$ & $3009 \mathrm{E}-05$ & & \\
\hline 4 & 4 & $30 E-04$ & & \\
\hline $23 E-04$ & $374 E-04$ & $01 E-04$ & $2.896345 \mathrm{E}-04$ & \\
\hline $911378 E-04$ & $4.089002 \mathrm{E}-04$ & $4.619455 \mathrm{E}-04$ & $1.526254 \mathrm{E}-04$ & 1.96468 \\
\hline $39375 \mathrm{E}-04$ & $8896 \mathrm{E}-04$ & $6.532479 \mathrm{E}-04$ & 6.4850191 & $1.108+3$ \\
\hline $2796 \mathrm{E}-04$ & $3.167530 \mathrm{E}-04$ & $80858 E-04$ & $E-04$ & 4 \\
\hline $3.285334 \mathrm{E}-04$ & 2.205 & $579 E-04$ & & \\
\hline $818 \mathrm{E}-03$ & $21 E-03$ & -04 & 03 & 4 \\
\hline 4 & 5.3 & 2 . & 03 & 4 \\
\hline $2.744358 \mathrm{E}-04$ & $6.274046 \mathrm{E}-04$ & $1.948488 \mathrm{E}$ & 9.86557 & 1.823805 \\
\hline & $1.511324 \mathrm{E}$ & & & \\
\hline & & & & \\
\hline 3 & & & & \\
\hline $12093 E-03$ & $6 E-04$ & -03 & 03 & 3. \\
\hline $8.649064 \mathrm{E}-04$ & $7.028914 \mathrm{E}-04$ & -04 & 04 & 2. \\
\hline $1.744864 \mathrm{E}-03$ & $1.777447 \mathrm{E}-04$ & $00 \mathrm{E}-04$ & 3.594 & 4.17 \\
\hline $5.44 \xi>2>$ & 4. & 04 & & \\
\hline & & & & \\
\hline 1. & 1. & & & \\
\hline $2.471996 \mathrm{E}-04$ & -04 & $57 E-04$ & -04 & 2.2 \\
\hline $1.131494 \mathrm{E}-04$ & $1.943583 \mathrm{E}-04$ & $1.879164 \mathrm{E}-04$ & 2.617 & 1.8 \\
\hline $1.801841 \mathrm{E}-04$ & $1.264961 \mathrm{E}-04$ & 2.8 & 1.3 & 2.61 \\
\hline & & & & \\
\hline 4 & 04 & 4 & & \\
\hline 2. & 2 . & & & \\
\hline $581 \mathrm{E}$ & $2 E-04$ & -04 & -04 & 1 . \\
\hline $2.230120 \mathrm{E}-04$ & $2.313091 \mathrm{E}-04$ & $2.422020 \mathrm{E}-04$ & $2.482344 \mathrm{E}-04$ & 5.4185 \\
\hline $4.144033 E-04$ & $2.981307 \mathrm{E}-04$ & -04 & 3.7 & 3. \\
\hline & & & & \\
\hline 2.2 & & 04 & 04 & 04 \\
\hline 2. & & & & \\
\hline & 2 . & 3. & 3. & \\
\hline $2.213007 \mathrm{E}-04$ & $2.267429 \mathrm{E}-04$ & $2.309969 \mathrm{E}-04$ & $2.360546 \mathrm{E}-04$ & $2.429477 \mathrm{E}-$ \\
\hline $38554 \mathrm{E}-04$ & $1.501431 \mathrm{E}-04$ & $1.523043 \mathrm{E}-04$ & $1.490992 \mathrm{E}-04$ & 7.83 \\
\hline & 7 & 7.7 r & 6.3 & 5 \\
\hline $4.217917 \mathrm{E}-05$ & $3.237631 \mathrm{E}-05$ & $2.594829 \mathrm{E}-05$ & $2.435751 \mathrm{E}-05$ & $4 E-05$ \\
\hline $3.477155 \mathrm{E}$ & $4.508479 \mathrm{E}-05$ & $404 \mathrm{E}-05$ & $748 E-05$ & 1.48 \\
\hline & $1.793168 \mathrm{E}-$ & $1.919402 \mathrm{E}$ & & 3.615 \\
\hline 50 & $4.114530 \mathrm{E}$ & $4.392739 \mathrm{E}$ & $2.714958 \mathrm{E}$ & 2.8010941 \\
\hline $011157 \mathrm{~F}$ & $390380 \mathrm{E}$ & $5.729596 \mathrm{E}-04$ & $5.910309 \mathrm{E}$ & 3.3346161 \\
\hline $61873 F$ & $61426 \mathrm{E}$ & $248651 \mathrm{E}-$ & $2.0000 / 0 \mathrm{E}$ & 年 \\
\hline 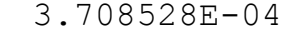 & $9 E-04$ & $2 E-04$ & $8.189344 \mathrm{E}-04$ & $1.110189 \mathrm{E}-03$ \\
\hline
\end{tabular}

Figure 6.3.A.2. Truncated sensitivity data file for LEU-COMP-THERM-009 sample problem. (continued) 


$\begin{array}{llllll}1.577516 \mathrm{E}-03 & 1.075698 \mathrm{E}-03 & 1.270736 \mathrm{E}-03 & 1.520277 \mathrm{E}-03 & 1.828479 \mathrm{E}-03 \\ 2.146210 \mathrm{E}-03 & 2.457027 \mathrm{E}-03 & 2.729725 \mathrm{E}-03 & 1.737714 \mathrm{E}-03 & 2.854629 \mathrm{E}-03 \\ 7.541633 \mathrm{E}-04 & 5.796042 \mathrm{E}-04 & 2.197686 \mathrm{E}-04 & 1.895209 \mathrm{E}-04 & 9.598149 \mathrm{E}-05 \\ 8.502876 \mathrm{E}-05 & 7.599381 \mathrm{E}-05 & 4.814730 \mathrm{E}-05 & 3.389918 \mathrm{E}-05 & 3.443490 \mathrm{E}-05 \\ 3.153711 \mathrm{E}-05 & 3.212018 \mathrm{E}-05 & 8.655023 \mathrm{E}-06 & & \end{array}$

$\cdots$

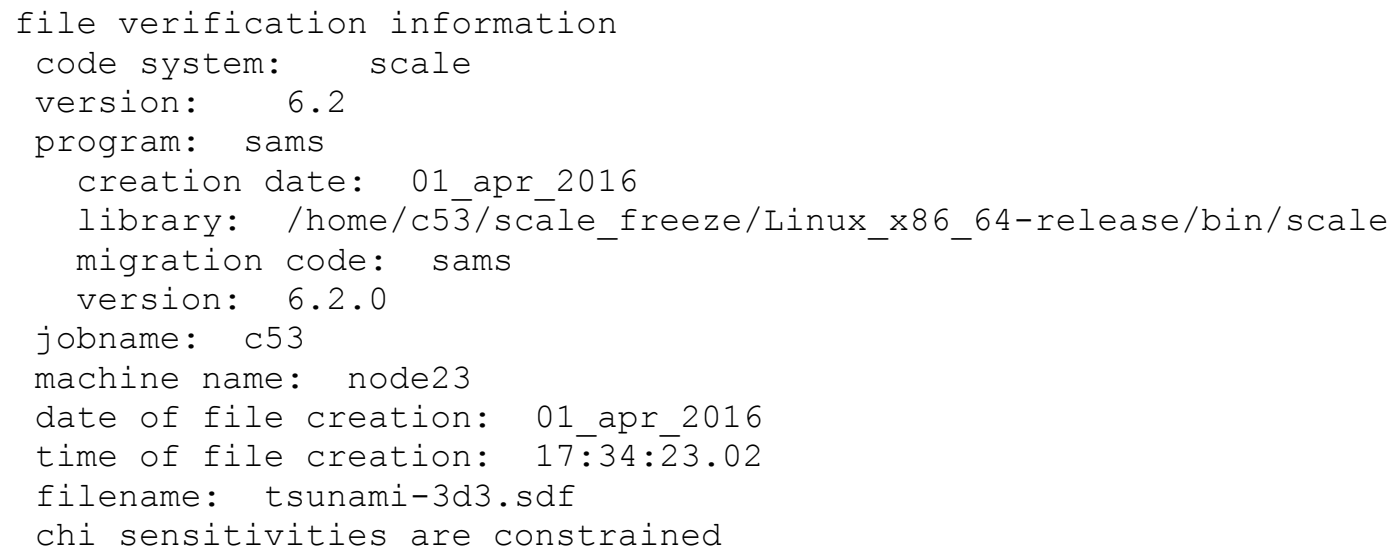

Figure 6.3.A.2. Truncated sensitivity data file for LEU-COMP-THERM-009 sample problem. (continued) 


\title{
6.4 TSAR: TOOL FOR SENSITIVITY ANALYSIS OF REACTIVITY RESPONSES
}

M. L. Williams and M. A. Jessee

\begin{abstract}
TSAR (Tool for Sensitivity Analysis of Reactivity Responses) is a SCALE functional module that computes nuclear data sensitivity coefficients for eigenvalue-difference responses such as reactor reactivity and worth coefficients. Examples include void reactivity, Doppler coefficients, and control rod worths. TSAR reads previously computed sensitivity coefficients for the k-eigenvalues at two states of a reactor system (or for two different systems) and combines them to obtain sensitivity coefficients for the difference. The k-eigenvalue sensitivities are typically obtained using the TSUNAMI-3D or -1D control sequences in SCALE. The reactivity sensitivity coefficients are combined with nuclear data covariance information to determine the uncertainty in the reactivity response.
\end{abstract}




\section{TABLE OF CONTENTS}

$\underline{\text { Page }}$

6.4 TSAR: Tool for Sensitivity Analysis of Reactivity Responses …........................................ 6-222

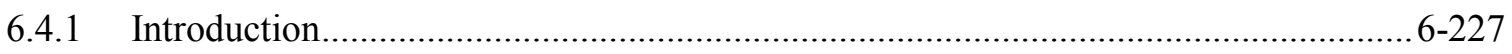

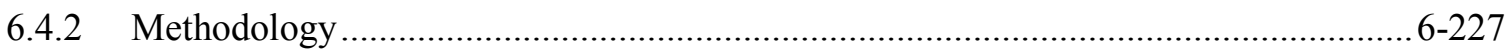

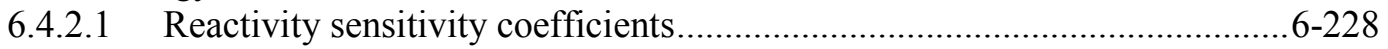

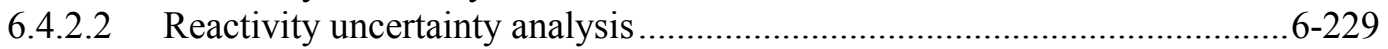

6.4.2.3 Cross-section-covariance data ............................................................ 6-230

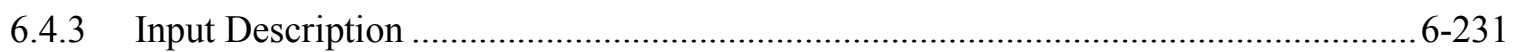

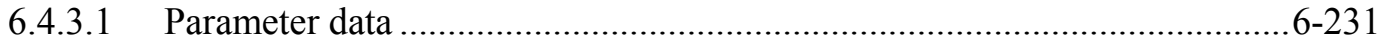

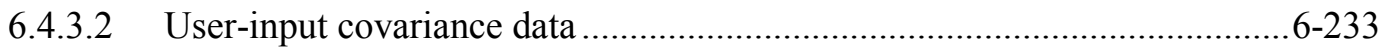

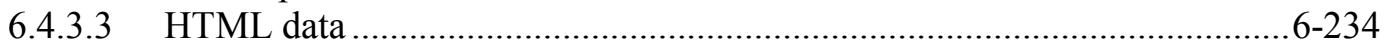

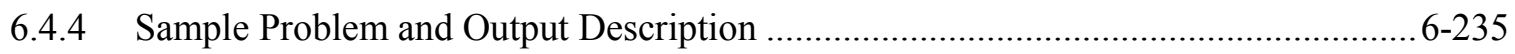

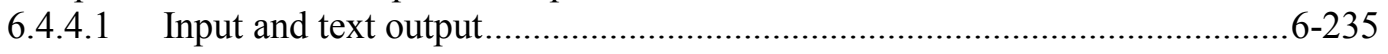

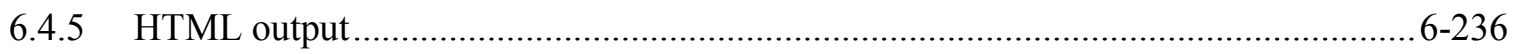

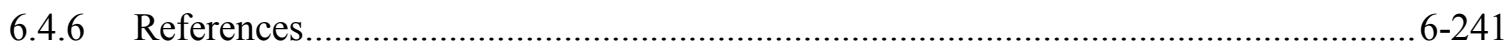




\section{LIST OF FIGURES}

Page

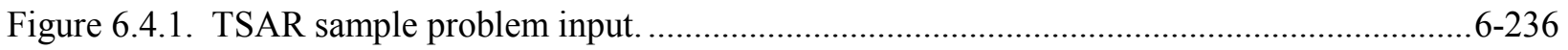

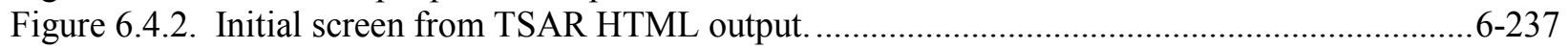

Figure 6.4.3. Program verification screen from TSAR HTML output. .............................................6-238

Figure 6.4.4. Input parameters from TSAR HTML output............................................................. $6-239$

Figure 6.4.5. Global integral indices from TSAR HTML output. ................................................. $6-240$

Figure 6.4.6. Covariance data from TSAR HTML output............................................................... 6-241 


\section{LIST OF TABLES}

$\underline{\text { Page }}$

Table 6.4.1. Input data for parameter block of TSAR input ....................................................... 6-231

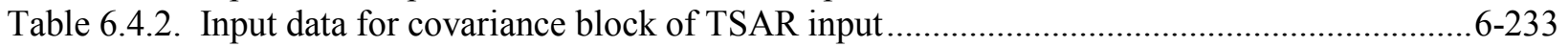

Table 6.4.3. Input data for $\mathrm{html}$ block of TSAR input............................................................... $6-235$ 


\section{ACKNOWLEDGMENT}

Development of the TSAR code was funded by the U. S. Nuclear Regulatory Commission Office of Research. 


\subsubsection{Introduction}

The TSUNAMI-1D, $-2 \mathrm{D}$, or $-3 \mathrm{D}$ control sequences in SCALE compute multigroup sensitivity coefficients and uncertainties for the critical multiplication factor $k$, the reciprocal of the $\lambda$-eigenvalue of the neutron transport equation for a multiplying medium. The TSAR module in SCALE performs sensitivity/uncertainty $(\mathrm{S} / \mathrm{U})$ calculations for responses represented by the difference of two eigenvalues. These types of responses are often of interest in reactor physics applications. For example, TSAR can compute data sensitivities and uncertainties of reactivity responses such as control rod worths, fuel and moderator temperature coefficients, and void coefficients for two defined states of a power reactor. ${ }^{1}$ Another potential application is in the analysis of benchmark critical experiments for nuclear data testing and validation studies. Data and methods deficiencies can introduce a computational bias manifested as a trend in calculated critical eigenvalues versus experiment parameters. TSAR can be applied to the difference in the computed eigenvalues of two benchmarks to establish the sensitivity of the bias trend to various nuclear data used in the calculations.

TSAR builds upon capabilities of other SCALE modules. TSUNAMI is first used to calculate sensitivities for the multiplication factors of the reference and altered states of the reactor, respectively. TSAR reads the sensitivity data files (.sdf file) produced by TSUNAMI $k_{\text {eff }}$ calculations and uses them to compute relative or absolute sensitivities of an eigenvalue-difference response. The reactivity sensitivities are written to an output file for subsequent applications or visualization. TSAR also combines the calculated reactivity sensitivity coefficients with input nuclear data covariance matrices included in SCALE to determine the uncertainty of the reactivity response.

\subsubsection{Methodology}

A detailed description of the $\mathrm{S} / \mathrm{U}$ methodology for reactivity responses is given in Reference 2 ; thus, only a brief overview is presented here. The lambda-eigenvalue form of the neutron transport equation for a multiplying medium is given by

$$
(\mathbf{L}-\lambda \mathbf{P}) \Phi=0
$$

where $\mathrm{L}$ and $\mathrm{P}$ are the loss and production operators, respectively, for the Boltzmann equation describing a multiplying medium and $\lambda=\frac{1}{\mathrm{k}}$ is the fundamental lambda-eigenvalue. It is assumed that the system is initially in a well-defined state 1 having a lambda- eigenvalue of $\lambda_{1}$. The reactivity for state 1 is defined as $\rho_{1}=1-\lambda_{1}$. Suppose that changes in $L$ and/or $P$ transformed the original system into a new distinct configuration designated as state 2 , with the lambda eigenvalue of $\lambda_{2}$ and static reactivity of $\rho_{2}=1-\lambda_{2}$. For example, the configuration change could be caused by moving a control rod or by voiding of the coolant. The reactivity insertion/withdrawal associated with the designated change in conditions is defined as

$$
\rho_{1 \rightarrow 2}=\rho_{2}-\rho_{1}=\lambda_{1}-\lambda_{2}
$$

Equation (6.4.2) defines the eigenvalue-difference (i.e., reactivity) response addressed by TSAR. The code edits the eigenvalues for the two reactor states and the value of the reactivity obtained from Eq. (6.4.2). 


\subsubsection{Reactivity sensitivity coefficients}

The relative k-sensitivity coefficient for an arbitrary data parameter $\alpha$ appearing in the transport equation is equal to

$$
\mathrm{S}_{\mathrm{k}, \alpha}=\frac{\alpha \partial \mathrm{k}}{\mathrm{k} \partial \alpha}=-\frac{\alpha \partial \lambda}{\lambda \partial \alpha}
$$

An analogous expression defines the relative sensitivity coefficient of the reactivity response:

$$
\mathrm{S}_{\rho, \alpha}=\frac{\alpha \partial \rho_{1 \rightarrow 2}}{\rho_{1 \rightarrow 2} \partial \alpha}
$$

Unlike the multiplication factor, the reactivity response can be negative. This can be source of confusion when interpreting the relative sensitivity coefficient; hence, by convention TSAR defines sensitivities relative to the absolute value of the reactivity; thus,

$$
\mathrm{S}_{\rho, \alpha} \rightarrow \frac{\alpha}{\left|\rho_{1 \rightarrow 2}\right|} \frac{\partial \rho_{1 \rightarrow 2}}{\partial \alpha} .
$$

In this way, a positive value for the relative sensitivity coefficient means that increasing the value of $\alpha$ always increases the value of the reactivity (i.e., a positive $\rho$ becomes more positive, and a negative $\rho$ becomes less negative). Conversely, a negative relative sensitivity coefficient means that increasing $\alpha$ always decreases the reactivity (i.e., a positive $\rho$ becomes less positive, and a negative $\rho$ becomes more negative). This convention is used in TSAR for all relative quantities involving the reactivity.

From the definitions in Eqs. (6.4.2) and (6.4.3), Eq. (6.4.4) is simplified to the following expression used in TSAR:

$$
\mathrm{S}_{\rho, \alpha}=\frac{\lambda_{2} \mathrm{~S}_{\mathrm{k} 2, \alpha}-\lambda_{1} \mathrm{~S}_{\mathrm{k} 1, \alpha}}{\left|\rho_{1 \rightarrow 2}\right|}
$$

where $\mathrm{S}_{\mathrm{k} 1, \alpha}$ and $\mathrm{S}_{\mathrm{k} 2, \alpha}$ are the k-sensitivities for the two states. The relative change in the reactivity response due to an arbitrary relative variation (or uncertainty) in parameter $\alpha$ can be found very easily once the $\rho$-sensitivities are determined that is,

$$
\frac{\Delta \rho_{1 \rightarrow 2}}{\left|\rho_{1 \rightarrow 2}\right|} \sim \mathrm{S}_{\rho, \alpha} \frac{\Delta \alpha}{\alpha} .
$$

In cases where the net reactivity change is very small, the denominator of Eq. (6.4.6) approaches zero; thus, the relative sensitivity coefficient can increase without bound. For this reason TSAR provides an input option to compute absolute rather than relative sensitivity coefficients. Absolute quantities are indicated here by the presence of a tilde $(\sim)$, while relative quantities have no tilde. The absolute sensitivity coefficient is defined in TSAR as the absolute change in the reactivity, expressed in pcm (percent-milli), due to a fractional change in data: 


$$
\tilde{\mathrm{S}}_{\rho, \alpha}=\left(\lambda_{2} \mathrm{~S}_{\mathrm{k} 2, \alpha}-\lambda_{1} \mathrm{~S}_{\mathrm{k} 1, \alpha}\right) \times 10^{5},
$$

so that

$$
\Delta \rho_{1 \rightarrow 2}(p c m) \sim \tilde{\mathrm{S}}_{\rho, \alpha} \frac{\Delta \alpha}{\alpha} .
$$

Prior to executing TSAR, it is necessary to perform TSUNAMI calculations for each reactor state, in order to evaluate the relative k-sensitivity coefficients in Eq. (6.4.3). These are written out in the SDF sensitivity file format and saved for input to TSAR. TSAR reads the two previously prepared files and uses them to evaluate Eq. (6.4.6) or (6.4.8) for the reactivity sensitivities. The $\rho$-sensitivities are then output to another SDF file. As discussed in Chapter 6.3 of the SCALE documentation, the complete sensitivities calculated by TSUNAMI include implicit effects associated with perturbations in resonance self-shielding; hence, the reactivity sensitivities also account for these effects, which can be significant.

TSAR prints the number of different sensitivity profiles that are computed and optionally can edit the input $\mathrm{k}$-sensitivities corresponding to each reactor state, as well the calculated $\rho$-sensitivities. Sensitivities may be edited by a sum over group for each nuclide-reaction pair. In addition, the TSAR output SDF file containing multigroup $\rho$-sensitivities for each nuclide-reaction pair can be read by Fulcrum to produce plots of the energy-dependent sensitivities. The filename of the reactivity sensitivity data file is given as job_name.react.sdf, where job_name is the name of the TSAR input file (i.e., job_name.inp,job_name.input, or simply job_name). Additionally, if the user requests edits of the input $\mathrm{k}$-sensitivities using the print=all option, the k-sensitivity data files are copied to the return directory as job_name.kstate1.sdf and job_name.kstate2.sdf. The print keyword is further described in the TSAR input section.

\subsubsection{Reactivity uncertainty analysis}

TSAR performs uncertainty analysis for eigenvalue-difference responses in much the same manner as the TSUNAMI sequences for the multiplication factor, except that either absolute or relative uncertainties may be computed. Assume that the transport calculations for the eigenvalues of the two reactor states use "N" input parameters, consisting of nuclear data for all groups, reaction types, and nuclides. The relative sensitivity coefficients for these data can be expressed as components of the N-dimension column vector $\mathbf{S}_{\boldsymbol{\rho}}$, and similarly for the absolute sensitivities, $\tilde{\mathbf{S}}_{\boldsymbol{\rho}}$. The relative and absolute reactivity variancesindicated as $\sigma_{\rho}^{2}$ and $\tilde{\sigma}_{\rho}^{2}$, respectively - are calculated in TSAR as

$$
\sigma_{\rho}^{2}=\frac{\tilde{\sigma}_{\rho}^{2}}{\rho^{2}}=\mathbf{S}_{\rho}^{\mathbf{T}} \mathbf{C}_{\boldsymbol{\alpha} \alpha} \mathbf{S}_{\boldsymbol{\rho}} \quad \text { and } \quad \tilde{\sigma}_{\rho}^{2}=\tilde{\mathbf{S}}_{\mathbf{p}}^{\mathbf{T}} \mathbf{C}_{\boldsymbol{\alpha} \alpha \boldsymbol{S}} \tilde{\mathbf{S}}_{\boldsymbol{\rho}}
$$

where $\mathbf{C}_{\alpha \alpha}$ is the relative covariance matrix describing nuclear data uncertainties and correlations, which are read from the SCALE covariance libraries. Due to the manner in which the absolute sensitivity coefficient is defined in Eq. (6.4.8), the absolute variance of the reactivity still uses the relative covariance matrix of the nuclear data.

The reactivity variance is related to the uncertainties and correlations in the calculated eigenvalues of the two reactor states. It can be shown that expression in Eq. (6.4.10) for the relative variance in an eigenvalue-difference response is equivalent to2 


$$
\sigma_{\rho}^{2}=\frac{\lambda_{1}^{2}}{\left(\lambda_{1}-\lambda_{2}\right)^{2}} \sigma_{\mathrm{k} 1}^{2}+\frac{\lambda_{2}^{2}}{\left(\lambda_{1}-\lambda_{2}\right)^{2}} \sigma_{\mathrm{k} 2}^{2}-2 \mathrm{c}_{1 \rightarrow 2} \frac{2 \lambda_{1} \lambda_{2}}{\left(\lambda_{1}-\lambda_{2}\right)^{2}} \sigma_{\mathrm{k} 1} \sigma_{\mathrm{k} 2}
$$

In the above equations, $\sigma_{\mathrm{k} 1}$ and $\sigma_{\mathrm{k} 2}$ are relative standard deviations of the multiplication factors and the correlation coefficient between the two reactor states is designated as $c_{1 \rightarrow 2} \in[-1,1]$. The eigenvalue calculations of the two states are correlated because they both use the same nuclear data libraries; therefore, the variance in the eigenvalue-difference is not simply the sum of the variances of the eigenvalues. A positive correlation (i.e., $\mathrm{c}_{1 \rightarrow 2}>0$ ) reduces the uncertainty in the reactivity because common uncertainties tend to cancel from the eigenvalue difference. On the other hand, negative correlations increase the reactivity uncertainty. It also can be seen from Eq. (6.4.11) that whenever the difference in the eigenvalues of the two states is small, the relative variance of the reactivity is substantially greater than the individual eigenvalue variances because the coefficients of $\sigma_{\mathrm{k} 1}^{2}$ and $\sigma_{\mathrm{k} 2}^{2}$ are large. Since this is usually the case for reactivity changes in a reactor, relative uncertainties in reactivity responses tend to be much larger than those for eigenvalues. If the reactivity response is close to zero, it is usually preferable to consider absolute rather than relative uncertainties. Whenever the eigenvalue difference is less than $10^{-10}$, TSAR will abort the calculation if a relative uncertainty is specified.

TSAR calculates the variance in the reactivity response using the expressions in Eq. (6.4.10). The square root of the variance corresponds to the standard deviation, which indicates the reactivity uncertainty. TSAR edits this value, as well the individual contributions of each nuclide-reaction pair (including cross correlations) to the overall uncertainty.

\subsubsection{Cross-section-covariance data}

The cross-section-covariance data are read from the COVERX-formatted covariance library identified by coverx $=$ in the PARAMETER data block on the TSAR input file. Cross-section-covariance data files distributed with SCALE are discussed in Chapter 11.3, and the format of the COVERX data file is presented in Appendix C.6.5.A.. The recommended SCALE covariance libraries include uncertainty data for all materials in ENDF/B-VII. These data were obtained from a variety of sources, including ENDF/BVI and VII, JENDL, and approximate values based on uncertainties in measured integral parameters. ${ }^{3}$ The COVARIANCE data block can be used to override and/or supplement the library uncertainty values for specified nuclide-reaction pairs. The keyword use icov is entered in the PARAMETER data block to utilize the covariance data defined in the COVARIANCE data block. Additionally, a default uniform standard deviation value can be assigned for any missing covariance data. This default value is defined by the $u d c o v=$ keyword in the PARAMETER data block, and the keyword use_dcov is entered to activate the option. Warning messages are printed to identify substituted covariance matrices.

When use_dcov and/or use_icov and cov_fix are specified in the PARAMETER data block, and a reaction has zero or large (standard deviation $>1000 \%$ ) values on the diagonal of the covariance matrix, the diagonal elements and off-diagonal terms are replaced according to the user-input criteria. Warning messages are printed to identify the replaced values. Additional options for user-specified covariance data are given in Sect. 6.4.3.2.

In the reactivity-uncertainty-analysis edit, a single asterisk (“*”) identifies uncertainty contributions from nuclide-reaction pairs for which the default cross-section-covariance data is applied. Likewise, the markers ("**”), (“***”), (“****”), denote (a) user-input covariance data, (b) covariance library data replaced by default values, and (c) covariance library data replaced by user-input values, respectively. In the HTML output, these uncertainty contributions are distinguished by using unique HTML colors. 
The different HTML colors are specified by the HTML block keywords $u d \_c l r=, u i_{-} c l r=, u d f i x \_c l r=$, and uifix_clr=. The HTML block keyword options are discussed in more detail in Sect. $\overline{6}$.4.3.3.

TSAR computes a problem-specific covariance library that contains cross-section covariances only for the nuclide-reaction pairs (including cross correlations) listed on the $\mathrm{k}_{1}$-and $\mathrm{k}_{2}$-sensitivity data files. This covariance library, referred to as the working covariance library, is written in COVERX format like the SCALE covariance library. The working covariance library contains any default or user-input covariance data for nuclide-reaction pairs that were not on the input covariance library as well as any modified crosssection-covariance data. The working covariance library can be read by the Javapeño plotting tool to visualize the cross-section-covariance data used in the reactivity uncertainty analysis.

\subsubsection{Input Description}

The user input for TSAR consists of a SCALE Analytic Sequence Specification Record (i.e., =tsar), an optional title followed by three blocks of data in free-form keyword format, and a SCALE input termination END record. The data blocks begin with READ KEYNAME and end with END $\boldsymbol{K E Y N A M E}$, where $\boldsymbol{K E Y N A M E}$ is the name of an individual data block. The PARAMETER data block is the only required block of data, while the HTML and COVARIANCE data blocks are optional. The data blocks can be entered in any order.

\subsubsection{Parameter data}

The PARAMETER block of data is used to specify the name of the previously prepared k-sensitivity data files, the name of the $\rho$-sensitivity data file, and other keyword options that control the code execution. The keyword options are listed in Table 6.4.1 along with their default values and description. A keyword that ends with "=" must be followed by additional data. Keywords that do not end with "=" are Boolean flags that are used to turn on certain features of the code, such as the computation of certain data or certain output edits. If the keyword is present for a Boolean entry, the value is set to true. Otherwise, the value is set to false.

Table 6.4.1. Input data for parameter block of TSAR input

\begin{tabular}{|c|c|c|}
\hline Keyword & Default value & Description \\
\hline $\begin{array}{l}\operatorname{cov} f i x \\
\text { (optional) }\end{array}$ & False & $\begin{array}{l}\text { Replace zero and large (standard deviation > large_cov) values on } \\
\text { diagonal of cross-section-covariance data with user-input values or } \\
\text { default values. }\end{array}$ \\
\hline $\begin{array}{l}\text { coverx }= \\
\text { (optional) }\end{array}$ & 44groupcov & Name of cross-section-covariance data file to use in analysis. \\
\hline $\begin{array}{l}\text { large_cov }= \\
\text { (optional) }\end{array}$ & 10.0 & $\begin{array}{l}\text { Cutoff fractional standard deviation value for } \operatorname{cov} \text { fix. Covariance data } \\
\text { with uncertainties larger than large_cov are replaced with user- } \\
\text { defined or default values. Default }=10 \text {, which is } 1000 \% \text { uncertainty. }\end{array}$ \\
\hline $\begin{array}{c}\text { nocovar } \\
\text { (optional) }\end{array}$ & False & If nocovar is present, the reactivity uncertainty calculation is bypassed. \\
\hline $\begin{array}{c}\text { nohtml } \\
\text { (optional) }\end{array}$ & False & If nohtml is present, HTML-formatted output is not generated. \\
\hline $\begin{array}{l}\text { print }= \\
\text { (optional) }\end{array}$ & rho & $\begin{array}{l}\text { Available options are } r h o, \text { all, or none. If print }=\text { rho, } \rho \text {-sensitivities edits } \\
\text { are generated. If print }=\text { all, } \rho \text {-, } \mathrm{k}_{1^{-}}, \text {and } \mathrm{k}_{2} \text {-sensitivities edits are } \\
\text { generated. The } \text { print }=\text { none option turns off all sensitivity edits. }\end{array}$ \\
\hline
\end{tabular}




\begin{tabular}{|c|c|c|}
\hline $\begin{array}{l}\text { return_work_cov } \\
\quad \text { (optional) }\end{array}$ & False & $\begin{array}{l}\text { If return_work_cov is present, the working covariance library is copied to } \\
\text { the return directory with the file name job_name.wrk.cov, where } \\
\text { job_name is the name of the input file. If return_work_cov is not } \\
\text { present, the working covariance library remains in the temporary } \\
\text { working directory with the file name job_name.wrk. }\end{array}$ \\
\hline $\begin{array}{l}\text { sdf file_1 }= \\
\text { (required) }\end{array}$ & $\mathrm{n} / \mathrm{a}$ & The file name of the initial state $\mathrm{k}_{1}$-sensitivity data file. \\
\hline $\begin{array}{l}\text { sdf_file_2 }= \\
\text { (required) }\end{array}$ & $\mathrm{n} / \mathrm{a}$ & The file name of the final state $\mathrm{k}_{2}$-sensitivity data file. \\
\hline $\begin{array}{c}\text { type }= \\
\text { (optional) }\end{array}$ & relative & $\begin{array}{l}\text { Available options are relative (or } r e l \text { ) and absolute (or } a b s \text { ). If } \\
\text { type }=\text { relative, the output reactivity sensitivity data file contains } \\
\text { relative } \rho-\text { sensitivities. If type }=\text { absolute, the output reactivity } \\
\text { sensitivity data file contains absolute } \rho-\text {-sensitivities (i.e., pcm units). }\end{array}$ \\
\hline $\begin{array}{c}u d c o v= \\
\text { (optional) }\end{array}$ & 0.05 & $\begin{array}{l}\text { User-defined default value of standard deviation in cross-section data to } \\
\text { use for all groups for nuclide-reaction pairs for which cross-section- } \\
\text { covariance data are too large or not available on the input covariance } \\
\text { library. }\end{array}$ \\
\hline $\begin{array}{l}\text { udcov_corr }= \\
\quad(\text { optional) }\end{array}$ & 1.0 & $\begin{array}{l}\text { User-defined default correlation value to use for nuclide-reaction pairs for } \\
\text { which cross-section-covariance data are not available on the input } \\
\text { covariance library. }\end{array}$ \\
\hline $\begin{array}{l}\text { udcov_corr_type }= \\
\text { (optional) }\end{array}$ & zone & $\begin{array}{l}\text { User-defined default correlation in cross-section data to use for nuclide- } \\
\text { reaction pairs for which cross-section-covariance data are not } \\
\text { available on the input SCALE covariance library. Allowed values } \\
\text { are long, zone, and short. See Table } 6.4 .2 \text { for details on long, zone, } \\
\text { and short. }\end{array}$ \\
\hline $\begin{array}{l}\text { udcov_therm }= \\
\text { (optional) }\end{array}$ & 0.0 & $\begin{array}{l}\text { User-defined default value of standard deviation in cross-section data to } \\
\text { use for thermal data for nuclide-reaction pairs for which cross- } \\
\text { section-covariance data are too large or not available on the input } \\
\text { covariance library. If input, the } u d c o v \text { therm value overrides the } \\
\text { udcov value in the thermal range (i.e., neutron energies below } 0.625 \\
\text { eV). }\end{array}$ \\
\hline $\begin{array}{l}\text { udcov_inter }= \\
\quad(\text { optional) }\end{array}$ & 0.0 & $\begin{array}{l}\text { User-defined default value of standard deviation in cross-section data to } \\
\text { use for intermediate data for nuclide-reaction pairs for which cross- } \\
\text { section-covariance data are too large or not available on the input } \\
\text { covariance library. If input, the } u d c o v \text { inter value overrides the } \\
\text { udcov value in the intermediate range (i.e., neutron energies above } \\
0.625 \mathrm{eV} \text { and below } 25 \mathrm{keV}) \text {. }\end{array}$ \\
\hline $\begin{array}{l}\text { udcov_fast }= \\
\text { (optional) }\end{array}$ & 0.0 & $\begin{array}{l}\text { User-defined default value of standard deviation in cross-section data to } \\
\text { use for fast data for nuclide-reaction pairs for which cross-section- } \\
\text { covariance data are too large or not available on the input covariance } \\
\text { library. If input, the } u d c o v \text { fast value overrides the } u d c o v \text { value in } \\
\text { the fast range (i.e., neutron energies above } 25 \mathrm{keV} \text { ). }\end{array}$ \\
\hline $\begin{array}{l}\text { use_dcov } \\
\text { (optional) }\end{array}$ & False & $\begin{array}{l}\text { Use user-defined default cross-section-covariance data for nuclide- } \\
\text { reaction pairs not included on the input covariance library. }\end{array}$ \\
\hline $\begin{array}{l}\text { use_icov } \\
\text { (optional) }\end{array}$ & False & $\begin{array}{l}\text { Use user-defined cross-section-covariance data input in the } \\
C O V A R I A N C E \text { input data block in place of the default values for } \\
\text { user-input nuclide-reaction pairs that are not on the input covariance } \\
\text { library. }\end{array}$ \\
\hline
\end{tabular}




\subsubsection{User-input covariance data}

The COVARIANCE data block described in Table 6.4.2 allows the user to specify a covariance matrix for specific nuclide-reaction pairs for which covariance data are not present on the input covariance library or that have zero or large values on the diagonal. The COVARIANCE data block must begin with READ COVARIANCE and end with END COVARIANCE.

Table 6.4.2. Input data for covariance block of TSAR input

\begin{tabular}{|c|c|c|c|c|}
\hline $\begin{array}{c}\text { Input } \\
\text { parameter }\end{array}$ & Requirement & $\begin{array}{c}\text { Default } \\
\text { value }\end{array}$ & Allowed values & Description \\
\hline Nuclide & Required & none & $\begin{array}{l}\text { Nuclide name } \\
\text { or ZA number }\end{array}$ & $\begin{array}{l}\text { Nuclide for which covariance data are to } \\
\text { be entered }\end{array}$ \\
\hline Reaction & Required & none & $\begin{array}{l}\text { Reaction name } \\
\text { or ZA number }\end{array}$ & $\begin{array}{l}\text { Reaction for which covariance data are to } \\
\text { be entered. See the TSUNAMI-IP manual } \\
\text { for available reaction types. }\end{array}$ \\
\hline all $=$ & Optional & 0.0 & any number & $\begin{array}{l}\text { Fractional standard deviation value to be } \\
\text { applied to all groups }\end{array}$ \\
\hline fast $=$ & Optional & 0.0 & any number & $\begin{array}{l}\text { Fractional standard deviation value to be } \\
\text { applied to fast groups. If input, the fast } \\
\text { value overrides the all value in the fast } \\
\text { range (i.e., neutron energies above } 25 \\
\text { keV). }\end{array}$ \\
\hline therm $=$ & Optional & 0.0 & any number & $\begin{array}{l}\text { Fractional standard deviation value to be } \\
\text { applied to thermal groups. If input, the } \\
\text { therm value overrides the all value in the } \\
\text { thermal range (i.e., neutron energies below } \\
0.625 \mathrm{eV} \text { ). }\end{array}$ \\
\hline inter $=$ & Optional & 0.0 & any number & $\begin{array}{l}\text { Fractional standard deviation value to be } \\
\text { applied to intermediate groups. If input, } \\
\text { the inter value overrides the all value in } \\
\text { the intermediate range (i.e., neutron } \\
\text { energies above } 0.625 \mathrm{eV} \text { and below } 25 \\
\mathrm{keV} \text { ). }\end{array}$ \\
\hline corr $=$ & Optional & 1.0 & $\begin{array}{l}\text { any number } \\
\text { from }-1.0 \text { to } 1.0\end{array}$ & Correlation between groups \\
\hline & & & & $\begin{array}{l}\text { Type of correlation applied from group-to- } \\
\text { group covariance values }\end{array}$ \\
\hline & & & & $\begin{array}{l}\text { long - correlation is applied between all } \\
\text { groups }\end{array}$ \\
\hline corr_type $=$ & Optional & zone & $\begin{array}{l}\text { long, short, } \\
\text { zone }\end{array}$ & $\begin{array}{l}\text { short - correlation is applied only between } \\
\text { adjacent groups }\end{array}$ \\
\hline & & & & $\begin{array}{l}\text { zone - correlation is applied within fast, } \\
\text { intermediate, and thermal groups, but no } \\
\text { correlation is applied between zones }\end{array}$ \\
\hline end & Required & & & $\begin{array}{l}\text { Denotes end of input for current } \\
\text { nuclide/reaction }\end{array}$ \\
\hline
\end{tabular}

Any MT number or reaction name will be treated as a valid input, but only those present on the $\mathrm{k}_{1}-\mathrm{or}_{\mathrm{k}_{2}}$ sensitivity data files will impact the results. The reaction sensitivity types computed by SAMS from TSUNAMI-1D and TSUNAMI-3D are described in the TSUNAMI-IP manual. An energy-covariance matrix is created for the specified nuclide-reaction pair with the square of the entered standard deviation 
for the diagonal terms for all groups using the all= value. Groups in the fast, intermediate, and thermal energies are then set to the square of the standard deviation value entered for fast $=$, inter $=$, and therm $=$, respectively. The off-diagonal terms of the energy matrix are generated according to the input for corr $=$, and corr type $=$, with default settings of 1.0 and zone. Data entered in this block are used only for missing data and do not override values on the input covariance data file. The SCALE 5.1 input format is supported where data are entered in triplets with the nuclide name (e.g., u-235), then the reaction MT number or name (e.g., 18 or fission), and then a standard deviation value. In this case, the end keyword must not be entered. The standard deviation value is applied to all groups with default setting for correlations. These data are only used if use icov is specified in the PARAMETER data block. When both use icov and cov fix are specified in the PARAMETER data block, and a reaction has zero or large (standard deviation $>1000 \%$ ) values on the diagonal of the covariance matrix, these values are replaced with the square of the user input standard deviation value, and the corresponding off-diagonal terms are substituted according to the values of corr and corr_type.

\subsubsection{HTML data}

The optional HTML data block is used to customize HTML-formatted output. The HTML data block must begin with READ HTML and end with END HTML. The data input to the HTML data block consists of several keywords that are shown, along with their default values and descriptions, in Table 6.4.3. A keyword that ends with "=" must be followed by text data. For color entries, any valid HTML color name can be entered or the hexadecimal representation can be used if preceded by a \# sign. For example, to change the background color of the HTML output to white, $b g_{-} c l r=w h i t e$ and $b g_{-}$clr=\#fffff have the same effect, because ffffff is the hexadecimal representation of white. An extensive list of available colors for customized output is shown in Appendix 6.5.B. Please note that not all features are supported by all browsers. 
Table 6.4.3. Input data for html block of TSAR input

\begin{tabular}{|c|c|c|}
\hline Keyword & Default value & Description \\
\hline$b g \_c l r=$ & papayawhip & Background color \\
\hline$h 1 \_c l r=$ & maroon & Color used for major headings \\
\hline$h 2_{-} c l r=$ & navy & Color used for sub-headings \\
\hline$t x t \_c l r=$ & black & Color for plain text \\
\hline $\operatorname{lnk} \_l r=$ & navy & Color for hyperlinks \\
\hline $\operatorname{lnk}{ }_{-} d e c=$ & none & Decoration for hyperlinks. (none, underline, overline, line-through, blink) \\
\hline$v l n k \_c l r$ & navy & Color for visited hyperlinks \\
\hline$u d \_l r=$ & blue & Color for values in tables that use default covariance data \\
\hline$u i \_c l r=$ & red & Color for values in tables that use user-input covariance data \\
\hline udfix_clr= & royalblue & Color for values in tables that use default corrected covariance data \\
\hline uifix_clr= & green & Color for values in tables that use user-input corrected covariance data \\
\hline
\end{tabular}

\subsubsection{Sample Problem and Output Description}

\subsubsection{Input and text output}

An example of TSAR input is given in Figure 6.4.1. Each section of the text output, not shown, is described in order below.

1. Input Listing and Summary of Calculations - The TSAR input data is printed for each data block. Both user-specified and default values for the various keywords are edited.

2. $\underline{k}_{1}$-Sensitivity Data File Summary and Sensitivity Coefficients - The header information for the $\mathrm{k}_{1}$-sensitivity data files follows the input data. This includes the title on the sensitivity data file, the number of energy groups, the number of sensitivity profiles, and the values of $\mathrm{k}_{1}$ and $\lambda_{1}$. If print $=$ all is specified in the PARAMETER data block, the energy, region, and mixture-integrated $\mathrm{k}_{1}$-sensitivity coefficients are edited for each nuclide-reaction pair.

3. $\underline{\mathrm{k}}_{2}$-Sensitivity Data File Summary and Sensitivity Coefficients - The header information for the $\mathrm{k}_{2}$-sensitivity data files follows the $\mathrm{k}_{1}$-sensitivity data file edit. This includes the title on the sensitivity data file, the number of energy groups, the number of sensitivity profiles, and the 
values of $\mathrm{k}_{2}$ and $\lambda_{1}$. If print=all is specified in the PARAMETER data block, the energy, region, and mixture-integrated $\mathrm{k}_{2}$-sensitivity coefficients are edited for each nuclide-reaction pair.

4. Reactivity Value and Optional $\rho$-Sensitivity Coefficients - After the $\mathrm{k}_{1}$ and $\mathrm{k}_{2}$ sensitivity edits, the reactivity between the two states is edited in pcm units. If print=all or print $=$ rho is specified in the PARAMETER data block, the energy, region, and mixture-integrated $\rho$-sensitivity coefficients are edited for each nuclide-reaction pair.

5. Reactivity Uncertainty Analysis - Following the edit of the reactivity value and the optional $\rho$-sensitivity edit, the reactivity uncertainty analysis is printed on the text output. First, a message is printed that states that the working covariance library is being generated. If PARAMETER block keywords use dcov, use icov, and/or cov fix are specified, covariance warnings are printed whenever user-input covariance data are included in the reactivity uncertainty analysis. Following the covariance warnings, the total reactivity uncertainty is printed along with the reactivity uncertainty contributions from individual energy covariance matrices. The reactivity uncertainty contributions are sorted in descending order.

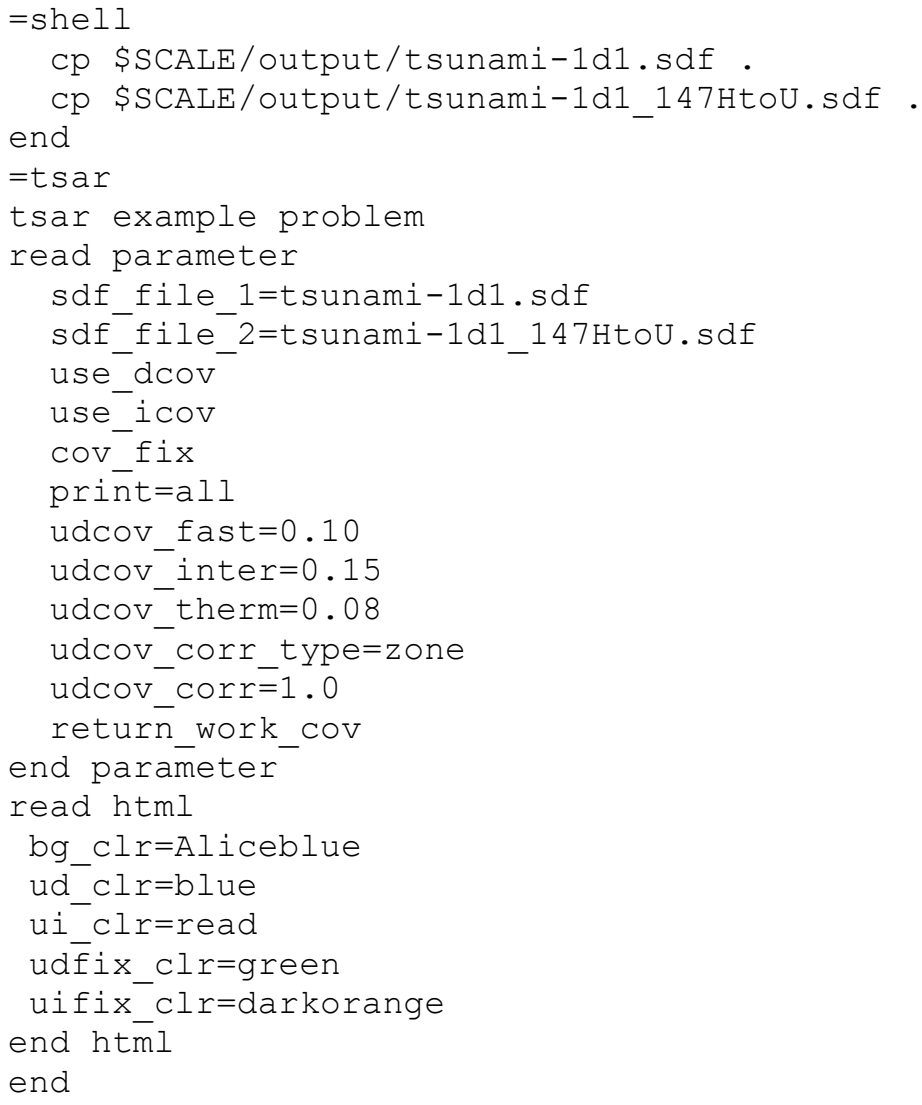

Figure 6.4.1. TSAR sample problem input.

\subsubsection{HTML output}

The input file for the TSAR sample problem shown in Figure 6.4.1 is named tsar1.input. In this case, the HTML-formatted output is stored in a file called tsarl.html and additional resources are stored in 
directories called tsar1.htmd and applet_resources. This section contains example TSAR HTMLformatted output only for demonstration of the interface. The problem does not correspond to the sample problem distributed with SCALE and is included for illustrative purposes only. When tsar1.html is opened in a web browser, the information shown in Figure 6.4.2 is displayed.

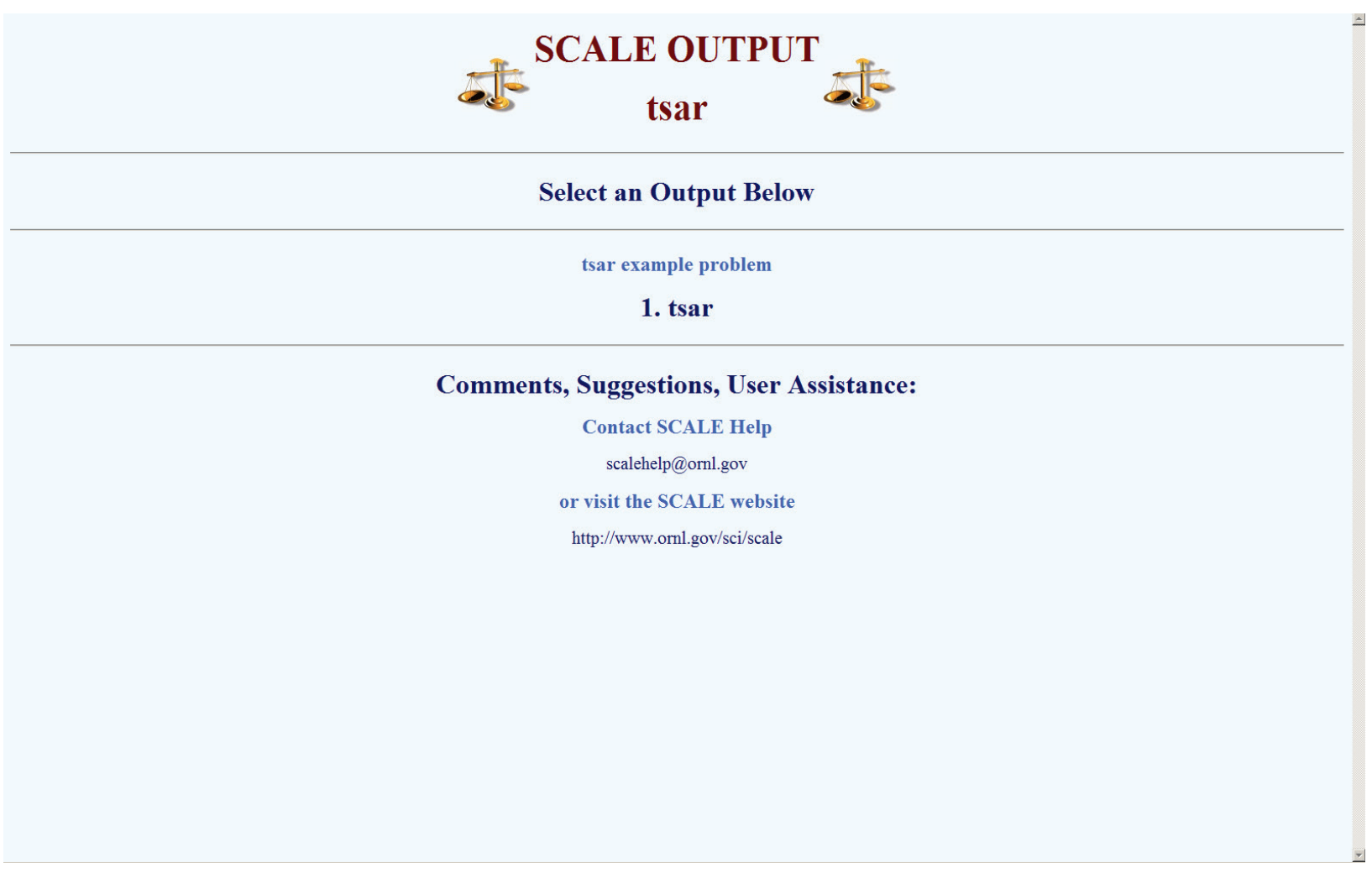

Figure 6.4.2. Initial screen from TSAR HTML output. 
The title of the input file is displayed between the two SCALE logos. Clicking on the SCALE logo will link the user directly to the SCALE website, if Internet access is available. Because this SCALE input file only executed TSAR, only a single output listing is available. The text " 1 . TSAR" is a hyperlink to view the output from TSAR. Clicking on the "1. TSAR" hyperlink will bring up the information shown in Figure 6.4.3.

The initial page of output from TSAR is shown in Figure 6.4.3. Program verification information is shown in the table under the TSAR logo. This table includes information about the code that was executed and the date and time it was run. The menu on the left side of the screen contains hyperlinks to specific portions of the code output. Echoes of the input data are available in the Input Data section. Any errors or warning messages are available in the Messages sections. Results from the code execution are shown in the results section.

General
Information
Input Data
Results

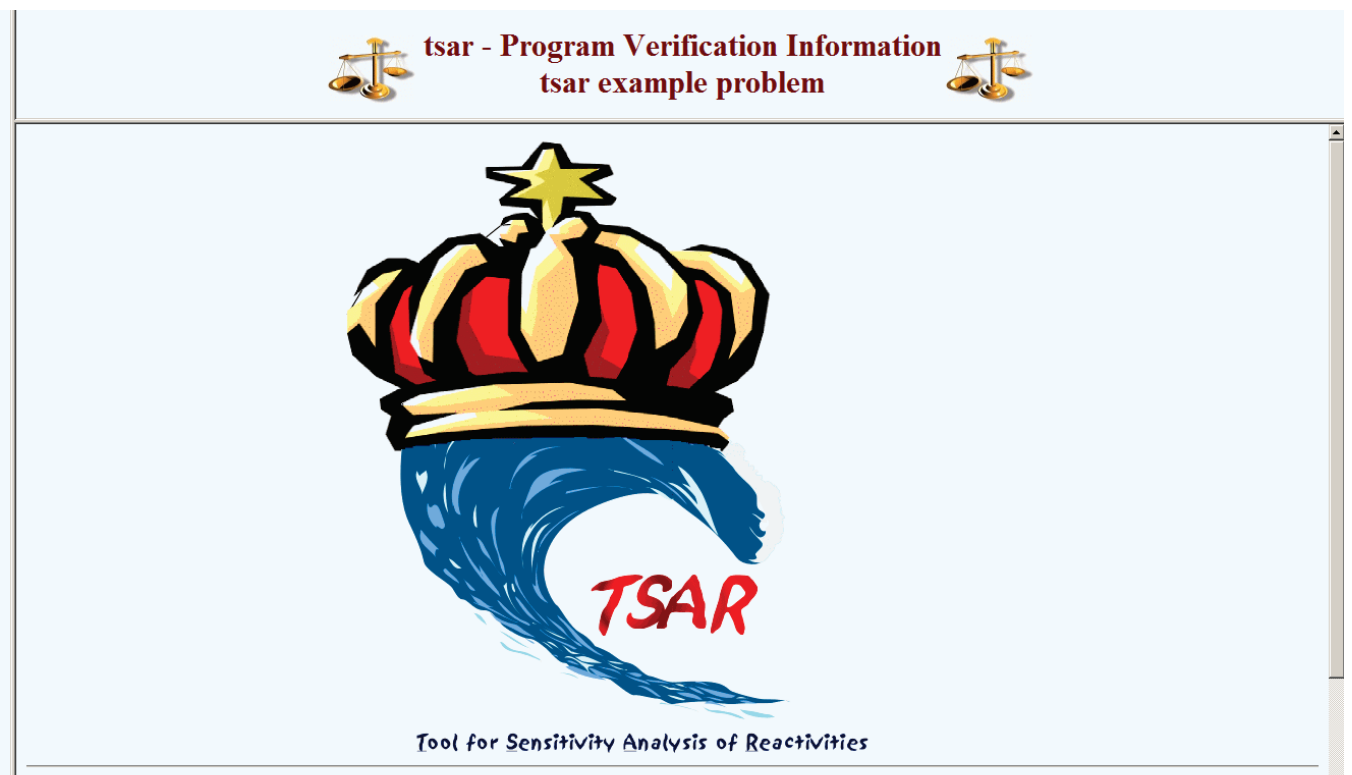

Program Verification Information

\begin{tabular}{|c|c|}
\hline code system & scale \\
\hline version & 6.0 \\
\hline program & tsar \\
\hline creation date & 11_nov_2008 \\
\hline lihrarv & /scale/scale $6 /$ / inix $\times 86$ 64/hin \\
\hline
\end{tabular}

Figure 6.4.3. Program verification screen from TSAR HTML output. 
Selecting Input Parameters will reveal the menu of available input data. Selecting Input Parameters causes the table shown in Figure 6.4.4 to be displayed. Other input data can also be displayed by selecting the desired data from the menu.

\begin{tabular}{|c|c|c|c|}
\hline \multirow{6}{*}{$\begin{array}{l}\text { General } \\
\text { Information } \\
\text { - Program Verification } \\
\text { Information } \\
\text { Input Data } \\
\text { - Input Parameters } \\
\text { - Filenames } \\
\text { - HTML Format Options }\end{array}$} & \multicolumn{3}{|r|}{$\begin{array}{l}\text { tsar - Input Parameters } \\
\text { tsar example problem }\end{array}$} \\
\hline & \\
\hline & PARAMETER & VALUE & DESCRIPTION \\
\hline & cov_fix & true & Replace zero and large values on diagonal of cross-section covariance data with user input values and dcov value. \\
\hline & large_cov= & 10.0000 & Cutoff fractional standard deviation value for cov_fix. \\
\hline & nocovar & false & Flag to cause the reactivity uncertainty edit to be turned off. \\
\hline \multirow{14}{*}{ Results } & nohtml & false & Flag to cause HTML output to not be produced. \\
\hline & print $=$ & all & $\begin{array}{l}\text { If print=all (or nprt=2), Sensitivity edits are generated forthe initial state, final state, and reactivity. If print=rho (or } \\
\text { nprt=1), reactivity sensitivities are editted. If print=none (or nprt=0), Sensitivity edits are not produced. }\end{array}$ \\
\hline & return_work_cov & true & Option to copy the working covariance data file back to the return directory. \\
\hline & type $=$ & Relative & Format of the reactivity sensitivity data file. \\
\hline & udcov $=$ & 0.0500 & $\begin{array}{l}\text { User-defined default value of standard deviation in cross-section data to use for nuclide-reaction pairs for which } \\
\text { covariance data are not available on the selected data file. }\end{array}$ \\
\hline & udcov_corr= & 1.0000 & $\begin{array}{l}\text { User-defined default correlation value to use for nuclide-reaction pairs for which covariance data are not available on } \\
\text { the selected data file. }\end{array}$ \\
\hline & udcov_corr_type $=$ & zone & $\begin{array}{l}\text { User-defined default correlation in cross-section data to use for nuclide-reaction pairs for which covariance data are not } \\
\text { available on the selected data file. (long, zone, short) }\end{array}$ \\
\hline & udcov_fast $=$ & 0.1000 & $\begin{array}{l}\text { User-defined default value of standard deviation in cross-section data to use for fast data for nuclide-reaction pairs for } \\
\text { which covariance data are not available on the selected data file. }\end{array}$ \\
\hline & udcov_inter= & 0.1500 & $\begin{array}{l}\text { User-defined default value of standard deviation in cross-section data to use for intermediate data for nuclide-reaction } \\
\text { pairs for which covariance data are not available on the selected data file. }\end{array}$ \\
\hline & udcov_therm $=$ & 0.0800 & $\begin{array}{l}\text { User-defined default value of standard deviation in cross-section data to use for thermal data for nuclide-reaction pairs } \\
\text { for which covariance data are not available on the selected data file. }\end{array}$ \\
\hline & use_dcov & true & Use user-defined default covariance data, udcov, for nuclide reaction pairs not included on the covariance data file. \\
\hline & use_icov & true & $\begin{array}{l}\text { Use user-defined data input in COVARIANCE input data block in place of udcov value for user input nuclide-reaction } \\
\text { pairs that are not on the covariance data file. }\end{array}$ \\
\hline & sdf1 $=$ & 41 & Logical unit number for the initial state SDF file (lun1). \\
\hline & $\operatorname{sdf} 2=$ & 42 & Togical unit number for the final state SDF file (lun2). \\
\hline
\end{tabular}

Figure 6.4.4. Input parameters from TSAR HTML output. 
Selecting Results causes a menu of available results to be revealed. From this menu, selecting Reactivity Sensitivities causes a submenu to be revealed. From the submenu, the Reactivity Sensitivities can be visualized in tabular format or plot format. Selecting Energy, Region, and Mixture Integrated Sensitivity Coefficients from this submenu causes the information shown in Figure 6.4.5 to appear.

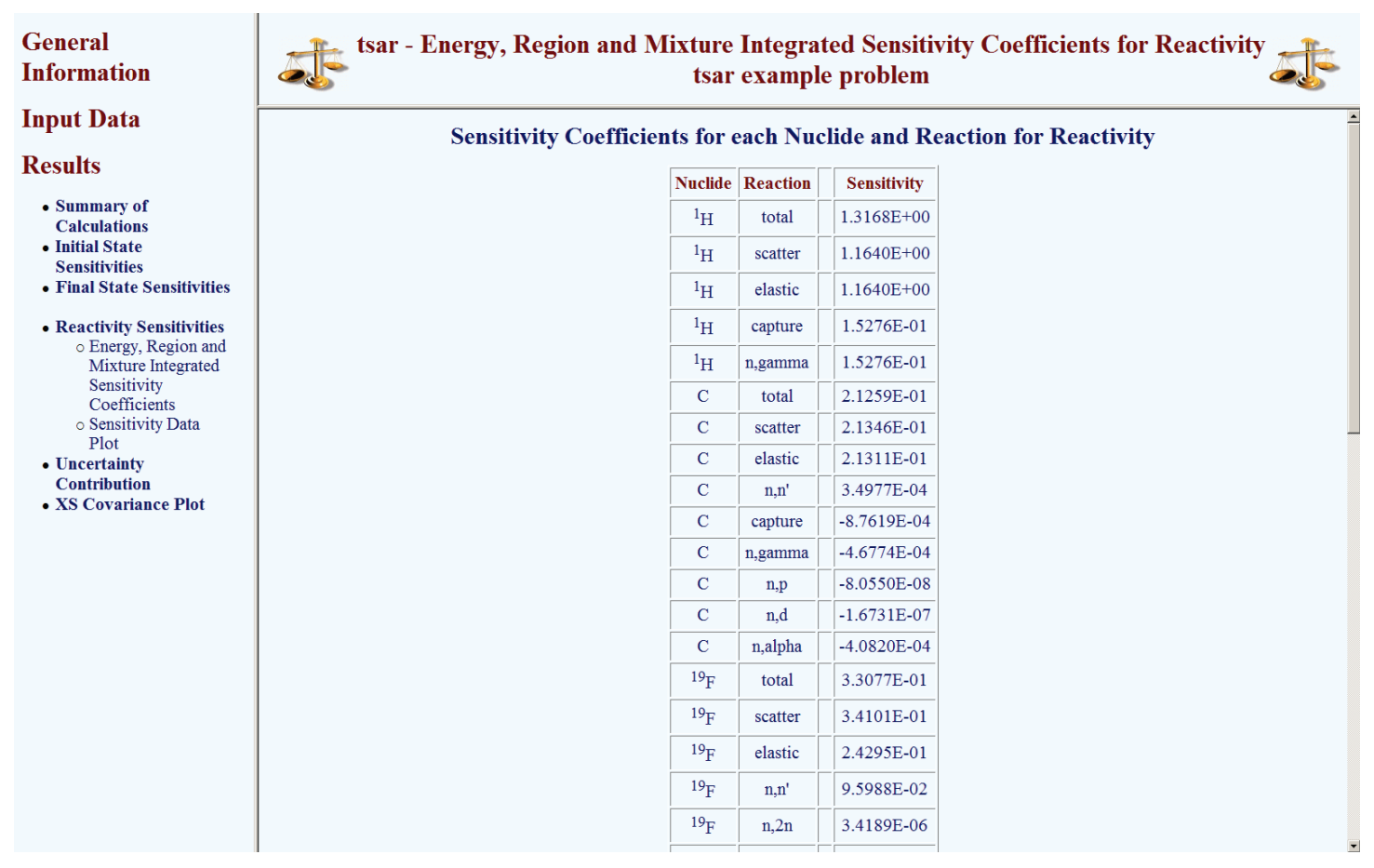

Figure 6.4.5. Global integral indices from TSAR HTML output. 
Plots of sensitivity data from the initial and final states as well as the reactivity sensitivities are available for viewing in the TSAR HTML output. If the return_work_cov keyword option is included in the PARAMETER block on the TSAR input file, then the covariance data can be viewed by selecting "XS Covariance Plot" in the results submenu. A Java applet version of Javapeño will appear in the browser window with the working covariance library preloaded. Data can be added to the plot by double-clicking on the list of available data on the right side of Javapeño. The plot shown in Figure 6.4.6 was produced with this procedure.

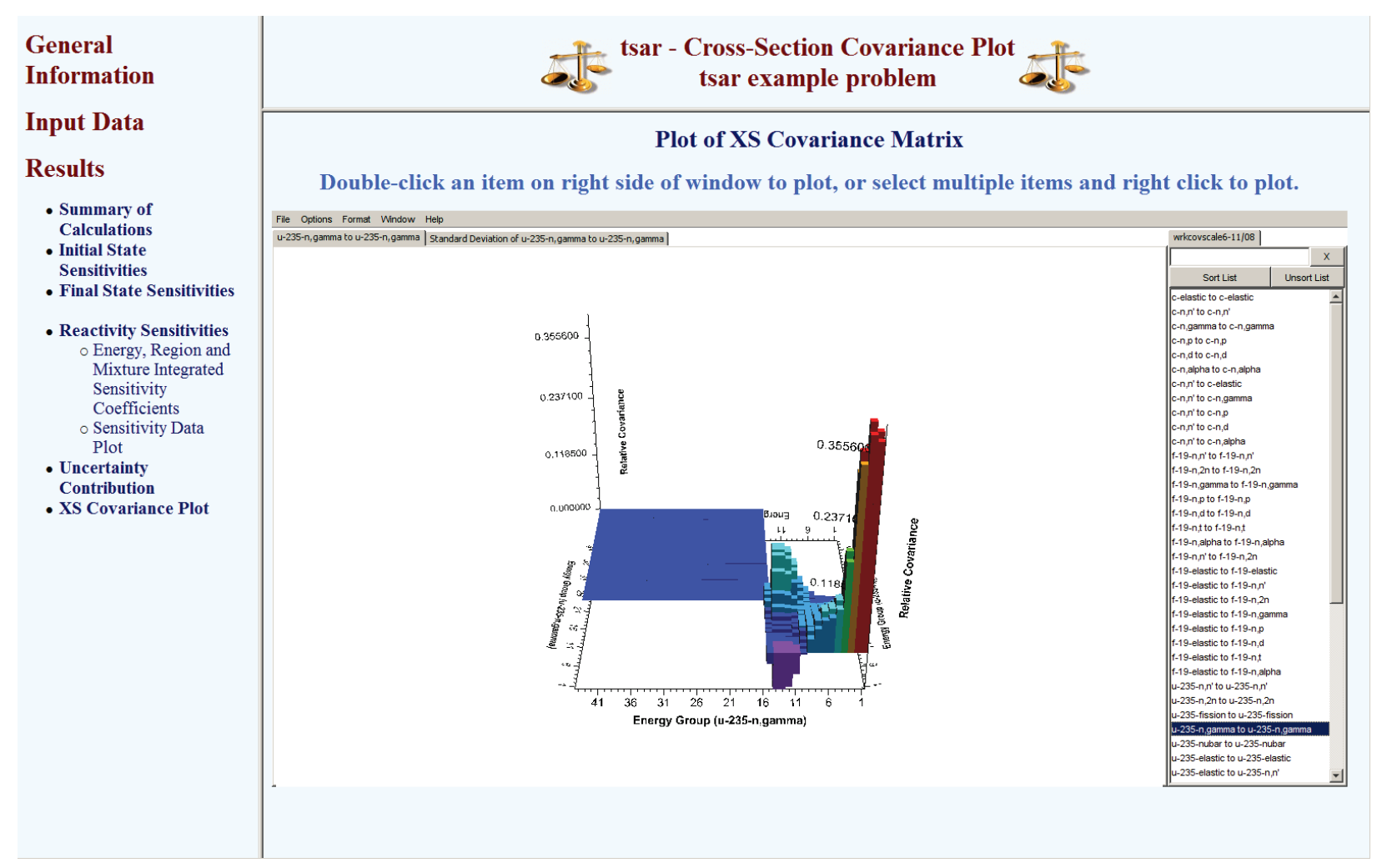

Figure 6.4.6. Covariance data from TSAR HTML output.

\subsubsection{References}

1 M. L. Williams, J. C. Gehin, and K. T. Clarno, "Sensitivity Analysis of Reactivity Responses Using One-Dimensional Discrete Ordinates and Three-Dimensional Monte Carlo Methods," C135.pdf in Proc. of PHYSOR-2006, American Nuclear Society Topical Meeting on Reactor Physics: Advances in Nuclear Analysis and Simulation, September 10-14, 2006, Vancouver, British Columbia, Canada.

2 M. L. Williams, "Sensitivity and Uncertainty Analysis for Eigenvalue-Difference Responses," Nucl. Sci. \& Engr., 155, p.18-36 (2007).

3 M. L. Williams and B. T. Rearden, "SCALE-6 Sensitivity/Uncertainty Methods and Covariance Data," Nuclear Data Sheets, Vol. 109, Issue 12, December 2008, pp. 2796-2800. 


\title{
6.5 TSUNAMI UTILITY MODULES
}

B. T. Rearden and M. A. Jessee

\begin{abstract}
Several modules have been developed to assist with the sensitivity and uncertainty analysis techniques included in SCALE. TSUNAMI-IP (Tools for Sensitivity and Uncertainty Analysis Methodology Implementation - Indices and Parameters) uses sensitivity data generated by TSUNAMI-1D, -2D, or -3D to propagate the effect of uncertainty in nuclear data to a response of interest, and to generate several relational parameters and indices that predict the degree of similarity between two systems. Additionally, the formats of files used by SCALE for sensitivity analyses are described herein.
\end{abstract}




\section{TABLE OF CONTENTS}

$\underline{\text { Page }}$

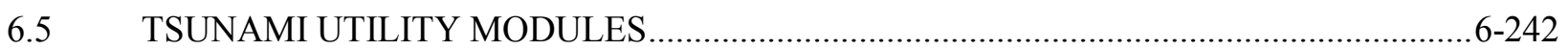

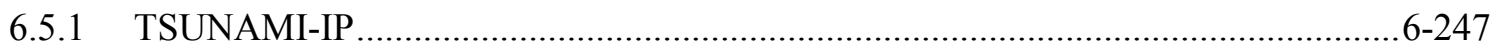

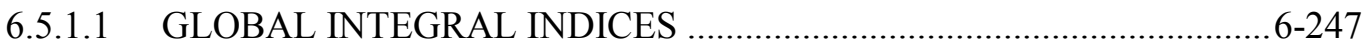

6.5.1.2 NUCLIDE-REACTION SPECIFIC INTEGRAL INDICES .....................6-253

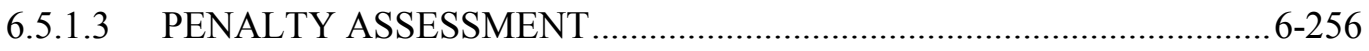

6.5.1.4 OTHER PARAMETERS.............................................................. 6-259

6.5.1.5 MISCELLANEOUS OPTIONS ........................................................ 6-263

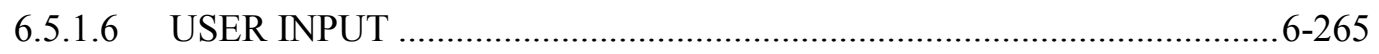

6.5.1.7 EXAMPLE PROBLEM............................................................... $6-279$

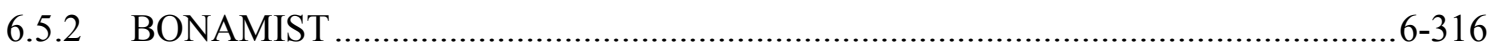

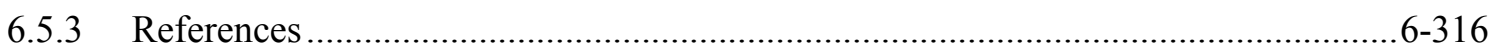

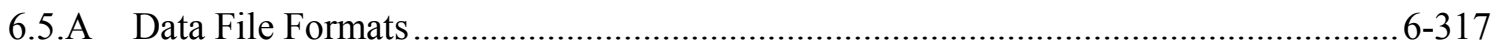

6.5.A.1 Format of TSUNAMI-A sensitivity data file ......................................... 6-317

6.5.A.2 Format of TSUNAMI-B sensitivity data file ....................................... 6-318

6.5.A.3 Format of SENLIB.SEN data file ................................................. 6-320

6.5.A.4 Format of BONAMIST.SEN data file ................................................. 6-320

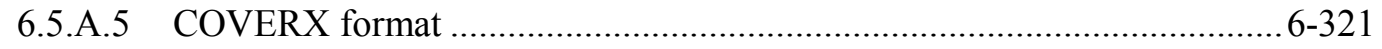

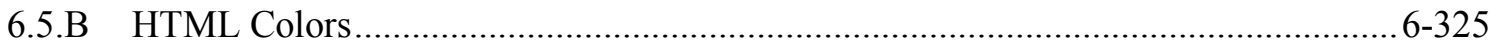




\section{LIST OF FIGURES}

$\underline{\text { Page }}$

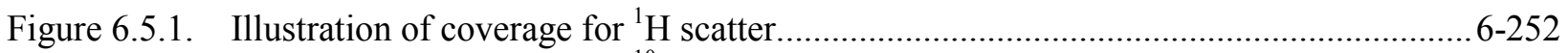

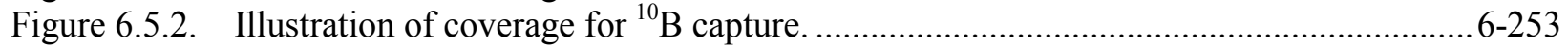

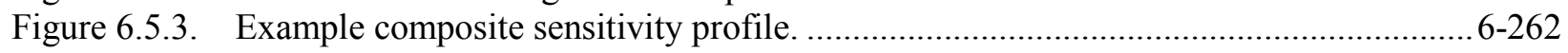

Figure 6.5.4. Example TSUNAMI-IP input listing.................................................................. $6-278$

Figure 6.5.5. Example TSUNAMI-IP input listing with Response Block....................................6-279

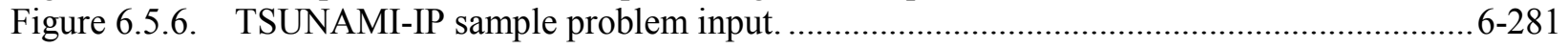

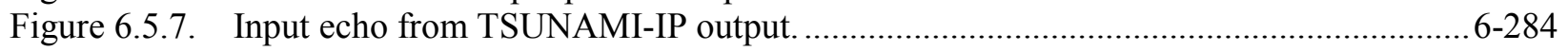

Figure 6.5.8. Warning messages from TSUNAMI-IP output ...................................................... $6-291$

Figure 6.5.9. Global integral indices values table from TSUNAMI-IP output............................... 6-292

Figure 6.5.10. Extended uncertainty edit from TSUNAMI-IP output. ............................................. 6-293

Figure 6.5.11. Extended $c_{k}$ edit from TSUNAMI-IP output........................................................ 6-294

Figure 6.5.12. Nuclide-reaction specific integral indices from TSUNAMI-IP output........................ 6-295

Figure 6.5.13. Extended penalty assessment from TSUNAMI-IP output....................................... 6-297

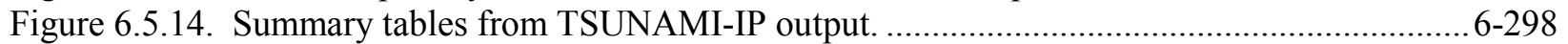

Figure 6.5.15. Composite sensitivity data from TSUNAMI-IP output........................................... 6-302

Figure 6.5.16. Initial screen from TSUNAMI-IP HTML output................................................ 6-310

Figure 6.5.17. Program verification screen from TSUNAMI-IP HTML output................................6-311

Figure 6.5.18. Input parameters from TSUNAMI-IP HTML output................................................ 6-312

Figure 6.5.19. Warning messages from TSUNAMI-IP HTML output............................................. 6-313

Figure 6.5.20. Global integral indices from TSUNAMI-IP HTML output........................................ 6-314

Figure 6.5.21. Composite sensitivity data plot from TSUNAMI-IP HTML output. .......................... 6-315 


\section{LIST OF TABLES}

$\underline{\text { Page }}$

Table 6.5.1. Keynames and descriptions for TSUNAMI-IP input data blocks..............................6-265

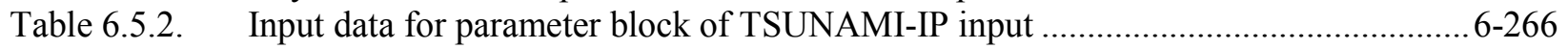

Table 6.5.3. Input options for user-defined covariance data................................................... 6-272

Table 6.5.4. Reaction sensitivity types computed by SAMS ...................................................... $6-273$

Table 6.5.5. Input data for HTML block of TSUNAMI-IP input ...............................................6-277

Table 6.5.A.1. Format specification for TSUNAMI-A sensitivity data file ....................................... 6-317

Table 6.5.A.2. Format specification for TSUNAMI-B sensitivity data file .......................................6-319

Table 6.5.A.3. Parameter identifiers for SENLIB.SEN data file ..................................................... 6-320

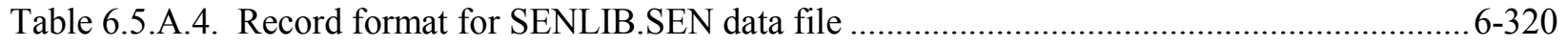

Table 6.5.A.5. Record format for BONAMIST.SEN data file ..................................................... 6-321

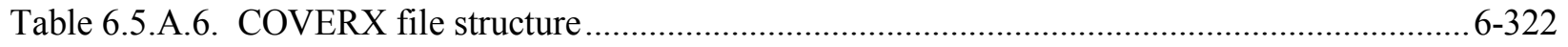

Table 6.5.A.7. Description of records on COVERX formatted file................................................. 6-323

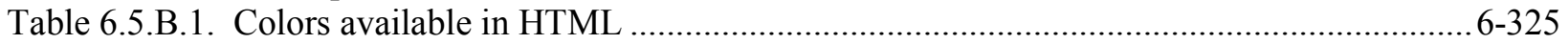




\section{ACKNOWLEDGMENTS}

The author wishes to acknowledge C. M. Hopper, B. L. Broadhead of the Oak Ridge National Laboratory (ORNL), S. Goluoglu, and R. L. Childs, formerly of the ORNL, and J. J. Wagschal of The Hebrew University of Jerusalem, for their assistance with the development of TSUNAMI-IP and L. M. Petrie, I. C. Gauld, and J. E. Horwedel of the Oak Ridge National Laboratory for their assistance in the development of SENLIB and BONAMIST. 


\subsubsection{TSUNAMI-IP}

TSUNAMI-IP (Tools for Sensitivity and Uncertainty Analysis Methodology Implementation-Indices and Parameters) uses sensitivity data generated by TSUNAMI-1D, $-2 \mathrm{D}$, or $-3 \mathrm{D}$ and cross-sectioncovariance data described in the COVLIB chapter to generate several relational parameters and indices that can be used to determine the degree of similarity between two systems. TSUNAMI-IP combines many techniques developed over several years into a comprehensive package with simplified input. Most of the techniques developed for TSUNAMI-IP are documented in Ref. 1, other techniques are documented in Refs. 2, 3 and 4, and others are originally presented in this document.

Depending on the user selected options, voluminous output can be generated by TSUNAMI-IP. To assist with review of these data, TSUNAMI-IP was the first SCALE module to offer HTML formatted output. This document first describes the indices and parameters that are computed, presents a description of the user input, then finally a sample problem is presented where the standard text output and the HTML formatted output are described.

TSUNAMI-IP was introduced in SCALE 5.0 and improved in SCALE 5.1. In SCALE 6.0, TSUNAMIIP now includes an individual $c_{k}$ to better quantify uncertainty-based similarity on a nuclide-reaction specific level. The $E_{\text {sum }}$ parameter has since been replaced with a sensitivity-only parameter, simply called $E$, which is based on more rigorous mathematical definition. The input format has been expanded with additional keywords to identify sensitivity data and a RESPONSE data block that allows users to define applications and experiments within the same input block. Uncertainty and penalty edits can also be presented with relative or absolute values, where previously only relative values were available. The input of user-defined covariance data now allows much greater flexibility, and the covariance data used in the problem can now be exported to a COVERX formatted data file for subsequent plotting in Javapeño.

\subsubsection{Global integral indices}

Three global integral indices that assess the similarity of a particular application and a single experiment on a system-wide basis for all nuclides and reactions are defined in this section. The integral indices are $c_{k}, E$, and $G$. Each of these indices is defined in subsequent subsections, and TSUNAMI-IP input options used to generate these indices and produce specific output edits are explained. Each integral index is normalized such that a value of 1.0 indicates complete similarity between the application and the experiment and a value of 0.0 indicates no similarity.

\subsection{Integral index $c k$}

A rigorous approach to assessing the similarity of two systems for purposes of criticality code validation is the use of uncertainty analysis, which propagates the tabulated cross-section-uncertainty information to the calculated $k_{\text {eff }}$ value of a given system via the energy-dependent sensitivity coefficients.1 Mathematically, the system uncertainty is computed with a quadratic product of the group-wise sensitivity profile vectors by nuclide and reaction type with the group-wise cross-section uncertainty matrices by nuclide and reaction type. The result of this procedure is not only an estimate of the uncertainty in the system $k_{\text {eff }}$ due to cross sections, but also an estimate of the correlated uncertainty between systems. These correlated uncertainties can be represented by correlation coefficients, which represent the degree of correlation in the uncertainties between the two systems. This correlation coefficient index, denoted as $c_{k}$, not only has the desirability of a single quantity relating the two systems, but also measures the similarity of the systems in terms of related uncertainty. These correlation coefficients are particularly useful when used in traditional trending analyses for criticality safety validation in that the correlation coefficient relates the degree in which the uncertainties in the critical benchmarks are coupled with the uncertainties in the application of interest. This coupling with the common uncertainties in the various 
systems is expected to closely mimic the coupling in predicted biases between the various systems, since they should both be related to the cross-section uncertainties.

The cross-section-covariance data are read from a COVERX formatted data file identified by coverx $=$ in the PARAMETER input. The cross-section-covariance data file 44GROUPCOV distributed with SCALE is discussed in the COVLIB chapter, and the format of the COVERX data file is presented in Appendix A, COVERX Format. A prerequisite in the uncertainty analysis approach is that cross-sectionuncertainty data for all nuclides and reactions of interest have been evaluated and processed for use by these procedures. However, evaluated cross-section-uncertainty data are not available for all nuclidereaction pairs. Nuclide-reaction pairs without data are omitted from this analysis, but it is assumed that either the cross-section data values from these pairs are well known (i.e., small uncertainties), or that the sensitivity of the system $k_{\text {eff }}$ to these nuclide-reaction pairs is small. Where these assumptions hold, the nuclide-reaction pairs without cross-section-uncertainty data present a negligible contribution to the uncertainty-based analysis. For situations where this negligible contribution assumption is judged to be invalid, the use of uncertainty analysis is not appropriate. However, the COVARIANCE data block can be used to input uncertainty values for the cross-section data for particular nuclide-reaction pairs to assess the impact of additional covariance data. To utilize the covariance data generated by user input in the COVARIANCE data block, the keyword use_icov must be entered in the PARAMETER data block. Additionally, default uncertainty values can be assigned for all unknown covariance data. This default uncertainty data is input in the PARAMETER data block and the keyword use_dcov must be entered to activate its use. Warning messages are printed to identify substituted covariance matrices.

When use dcov and/or use icov and cov fix are specified in the PARAMETER data block, and a reaction has zero or large (standard deviation $>1000 \%$ ) values on the diagonal of the covariance matrix, these values are replaced with the square of the user input or default standard deviation values, and the corresponding off-diagonal terms are substituted according to the user input or default correlation values. Warning messages are printed to identify which values were replaced and which standard deviation value was used in the replacement.

The mathematical development of the integral index $c_{k}$ is presented here based on the development given in Ref. 1. The nuclear data parameters (i.e., group wise nuclide-reaction specific cross sections) are represented by the vector $\boldsymbol{\alpha} \equiv\left(\alpha_{m}\right), m=1,2, \ldots, M$, where $M$ is the number of nuclide-reaction pairs $\times$ the number of energy groups. The corresponding symmetric $M \times M$ matrix containing the relative variances (diagonal elements) and covariances (off-diagonal elements) in the nuclear data are

$$
\mathbf{C}_{\alpha \alpha} \equiv\left\lfloor\frac{\operatorname{COV}\left(\alpha_{m}, \alpha_{p}\right)}{\alpha_{m} \alpha_{p}}\right\rfloor, m=1,2, \ldots, M ; p=1,2, \ldots, M,
$$

where

$$
\operatorname{COV}\left(\alpha_{m}, \alpha_{p}\right)=\left\langle\delta \alpha_{m} \delta \alpha_{p}\right\rangle
$$

where $\delta \alpha_{m}$ and $\delta \alpha_{p}$ represent the difference between the values and expectation values of the nuclear data parameters and \langle\rangle represents integration over the ranges of $\alpha_{m}$ and $\alpha_{p}$ weighted with a probability density function. A rigorous definition of the cross-section-covariance data are given in Ref. 5 .

The matrix containing sensitivities of the calculated $k_{\text {eff }}$ to the $\boldsymbol{\alpha}$ parameters is given as 


$$
\mathbf{S}_{\mathbf{k}} \equiv\left[\frac{\alpha_{m}}{k_{i}} \frac{\partial k_{i}}{\partial \alpha_{m}}\right], i=1,2, \ldots, I ; m=1,2, \ldots, M
$$

where $I$ is the number of critical systems being considered. In TSUNAMI-IP, the elements of $\mathbf{S}_{\mathbf{k}}$ are the sensitivity coefficients read from the sensitivity data files identified in the APPLICATIONS and EXPERIMENTS data blocks. The uncertainty matrix for the system $k_{\text {eff }}$ values, $\mathbf{C}_{\mathbf{k k}}$, is given as

$$
\mathrm{C}_{\mathrm{kk}}=\mathrm{S}_{\mathrm{k}} \mathrm{C}_{\alpha \alpha} \mathbf{S}_{\mathrm{k}}^{\dagger}
$$

where $\%$ indicates a transpose.

$\mathbf{S}_{\mathbf{k}}$ is an $I \times M$ matrix; $\mathbf{C}_{\alpha \alpha}$ is an $M \times M$ matrix; and the resulting $\mathbf{C}_{\mathbf{k k}}$ matrix is of dimension $I \times I$. The $\mathbf{C}_{\mathbf{k k}}$ matrix consists of relative variance values, $\sigma_{i}^{2}$, for each of the systems under consideration (the diagonal elements), as well as the relative covariance between systems, $\sigma_{i j}^{2}$ (the off-diagonal elements). These off-diagonal elements represent the shared or common variance between two systems. The off-diagonal elements are typically divided by the square root of the corresponding diagonal elements (i.e., the respective standard deviations) to generate a correlation coefficient matrix. Thus, the correlation coefficient is defined as

$$
c_{k}=\frac{\sigma_{i j}^{2}}{\left(\sigma_{i} \sigma_{j}\right)},
$$

such that the single $c_{k}$ value represents the correlation coefficient between uncertainties in system $i$ and system $j$.

These correlations are primarily due to the fact that the uncertainties in the calculated $k_{\text {eff }}$ values for two different systems are related, since they contain the same materials. Cross-section uncertainties propagate to all systems containing these materials. Systems with the same materials and similar spectra would be correlated, while systems with different materials or spectra would not be correlated. The interpretation of the correlation coefficient is the following: a value of 0.0 represents no correlation between the systems, a value of 1.0 represents full correlation between the systems, and a value of -1.0 represents a full anti-correlation.

To request the computation of $c_{k}$ for each application identified in the APPLICATIONS section of the input in relation to each experiment in the EXPERIMENTS portion of the input, simply enter the input $c$ in the PARAMETER section of the input. Additionally, the values table must be requested to output the full listing of $c_{k}$ values. The csummary edit prints a listing of all experiments that have a $c_{k}$ value exceeding the criteria value set by cvalue . 


\subsection{Integral index $c_{r}$}

The integral index $c_{r}$ is intended for investigative analysis and is defined as $c_{k}$ with user-defined reactions removed from consideration. The EXCLUSIONS data block is used to identify which reactions will be excluded from consideration in the calculation of $c_{r}$. If the user identifies no reactions, then $c_{r}$ will compute the same value as $c_{k}$. Using $c_{r}$ is equivalent to removing all sensitivities for a given reaction or series of reactions for all nuclides from the sensitivity data file for all applications and all experiments considered in the analysis.

To request the computation of $c_{r}$ for each application identified in the APPLICATIONS section of the input in relation to each experiment in the EXPERIMENTS portion of the input, simply enter the input $\mathrm{cr}$ in the PARAMETER section of the input. Additionally, the values table must be requested to output the full listing of $c_{r}$ values. The crsummary edit prints a listing of all experiments that have a $c_{r}$ value exceeding the criteria value set by crvalue $=$.

\subsection{Integral index $E$}

The $E$ index is a replacement for the previous $E_{\text {sum }}$ index, which was present in TSUNAMI-IP for SCALE 5.0 and 5.1.1 If the group-wise sensitivity data for all nuclides and reactions for each system is thought of as a vector, then the integral index $E$ is the cosine of the angle between the two sensitivity vectors for the analyzed systems. If the two sensitivity vectors are parallel, i.e., proportional, the systems are similar. $E$ does not require cross-section covariance data and is normalized such that an $E$ value of 0.0 indicates the systems are totally dissimilar, and an $E$ value of 1.0 indicates the two systems are the same. Mathematically, an $E$ value as low as -1.0 could be generated, but this would be the result of a rare combination of system sensitivity coefficients such that the sensitivity of the respective system responses would have to be exactly proportional in magnitude and opposite in sign, which seems not to be physically feasible. The $E$ parameter is considered global in nature because its single quantity assesses similarity between two systems based on the magnitude and shape of all sensitivity profiles. The vector $\mathbf{S}_{\mathbf{i}}$ is defined as the sensitivity vector (not matrix) for a particular application or experiment "i." The magnitude of the sensitivity vector corresponds to the L2 norm: $\left|\mathbf{S}_{\mathbf{i}}\right|=\sqrt{\mathbf{S}_{\mathbf{i}}^{\mathbf{T}} \mathbf{S}_{\mathbf{i}}}$. The $E$ value for a given application $a$ with experiment $e$ is then

$$
E \equiv \frac{\mathbf{S}_{\mathrm{a}}^{\mathbf{T}} \mathbf{S}_{\mathbf{e}}}{\left|\mathbf{S}_{\mathrm{a}}\right|\left|\mathbf{S}_{\mathrm{e}}\right|}
$$

The similarity of systems in terms of their sensitivities to only the fission, capture or scatter reactions can also be evaluated as

$$
E_{x} \equiv \frac{\mathbf{S}_{\mathbf{x}, \mathbf{a}}^{\mathbf{T}} \mathbf{S}_{\mathbf{x}, \mathbf{e}}}{\left|\mathbf{S}_{\mathbf{x}, \mathbf{a}}\right| \mathbf{S}_{\mathbf{x}, \mathbf{e}} \mid},
$$

where the vectors $\mathbf{S}_{\mathrm{x}, \mathrm{a}}$ and $\mathbf{S}_{\mathrm{x}, \mathrm{e}}$ represent the sensitivity of application and experiment to fission, capture, or scatter reaction $x$.

To request the computation of $E$ for each application identified in the APPLICATIONS section of the input in relation to each experiment in the EXPERIMENTS portion of the input, simply enter the input $e$ in the PARAMETER section of the input. Additionally, the values table must be requested to output the full 
listing of $E$ values. The reaction components of $E$ (i.e., $E_{f}, E_{c}$ and $E_{s}$ ) are included in the values table if prtparts is entered in the input. The reaction-specific components of $E$ are each normalized between -1 and 1, the same as $E$ itself. The esummary edit prints a listing of all experiments that have an $E$ value exceeding the criteria value set by evalue $=$. Note the warning provided in Table 6.5.2 that $E$ values cannot be calculated without also specifying an option that exercises the covariance library.

\subsection{Integral index $G$}

The $G$ index assesses the similarity of two systems based on normalized differences in the energydependent sensitivity data for fission, capture, and scatter. ${ }^{2}$ The similarity measure used for $G$ is based on the concept of coverage of the application by an experiment. A physical interpretation of the $G$ index is the ratio of the sum of the sensitivity coefficients of the application that are covered by the experiment to the sum of the sensitivity coefficients for the application. The $G$ index, sometimes referred to as "big G" is defined as:

$$
G=1-\frac{\sum_{n} \sum_{x} \sum_{j}\left(S_{x, j}^{a, n}-S_{x, j}^{e^{\prime}, n}\right)}{\sum_{n} \sum_{x} \sum_{j} S_{x, j}^{a, n}}
$$

where,

$$
S_{x, j}^{e^{\prime}, n}=\left\{\begin{array}{ll}
S_{x, j}^{e, n}, \text { where } & \left|S_{x, j}^{a, n}\right| \geq\left|S_{x, j}^{e, n}\right| \text { and } \frac{S_{x, j}^{a, n}}{\left|S_{x, j}^{a, n}\right|}=\frac{S_{x, j}^{e, n}}{\left|S_{x, j}^{e, n}\right|} \\
S_{x, j}^{a, n}, \text { where } & \left|S_{x, j}^{a, n}\right|<\left|S_{x, j}^{e, n}\right|
\end{array} \text { and } \frac{S_{x, j}^{a, n}}{\left|S_{x, j}^{a, n}\right|}=\frac{S_{x, j}^{e, n}}{\left|S_{x, j}^{e, n}\right|},\right.
$$

the $n$ summation is performed over all nuclides present in the application system,

the $x$ summation is performed over fission, capture and scatter reactions $(f, c$, and $s)$ as appropriate for each nuclide, and

the $j$ summation is performed over all energy groups.

The use of 1 minus the normalized difference makes the range of this index consistent with $c_{k}$ and $E_{\text {sum }}$. Hence, a $G$ of 1 indicates complete similarity and a $G$ value of 0 indicates no similarity.

The definition of $S_{x, j}^{e^{\prime}, n}$ restricts the coverage of the application by the experiment to the portion of the experiment's sensitivity coefficient that does not exceed that of the application in magnitude. Additionally, the application's sensitivity coefficient and that of the experiment must have the same sign. The coverage for ${ }^{1} \mathrm{H}$ scatter for an example application and experiment is illustrated in Figure 6.5.1 where the energy-dependent sensitivity profiles for the application, the experiment and the coverage of the application by the experiment are shown in green, red and blue, respectively. Because the sensitivity coefficients for the application and the experiment have opposite signs at energies just above $1 \times 10^{-2} \mathrm{eV}$, the application provides no coverage for the experiment for these groups. Also, for several groups, the 
sensitivity of the application exceeds that of the experiment and only partial coverage is provided. Partial coverage is illustrated where the application data (green) exceeds the coverage (blue). In other groups, the sensitivity of the experiment exceeds that of the application. In these groups, full coverage is provided, but the coverage is not allowed to exceed the sensitivity of the application. This is illustrated in groups where the experiment data (red) exceeds the coverage data (blue). With the limitation of the coverage as the value of the applications sensitivity coefficient, the experiment cannot provide "extra credit" in coverage for sensitivity coefficients that exceed those of the application.

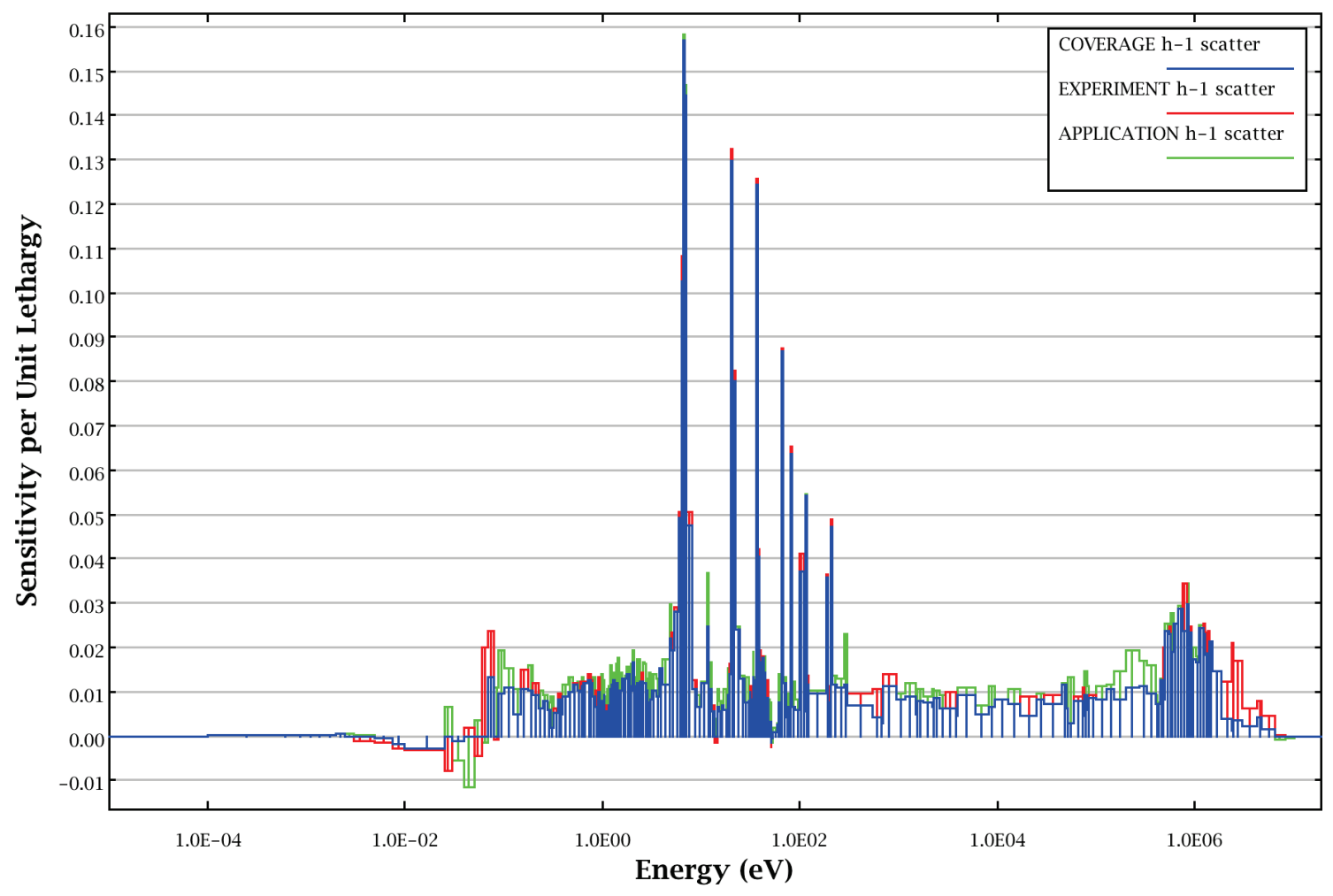

Figure 6.5.1. Illustration of coverage for ${ }^{1} \mathrm{H}$ scatter.

The coverage for ${ }^{10} \mathrm{~B}$ capture for an example application and experiment is illustrated inFigure 6.5.2. In this figure, the sensitivity coefficients are all negative. The magnitudes of the sensitivity coefficients for the experiment far exceed those of the application at thermal energies. However, coverage is only provided to the magnitudes of the sensitivity coefficients of the application. 


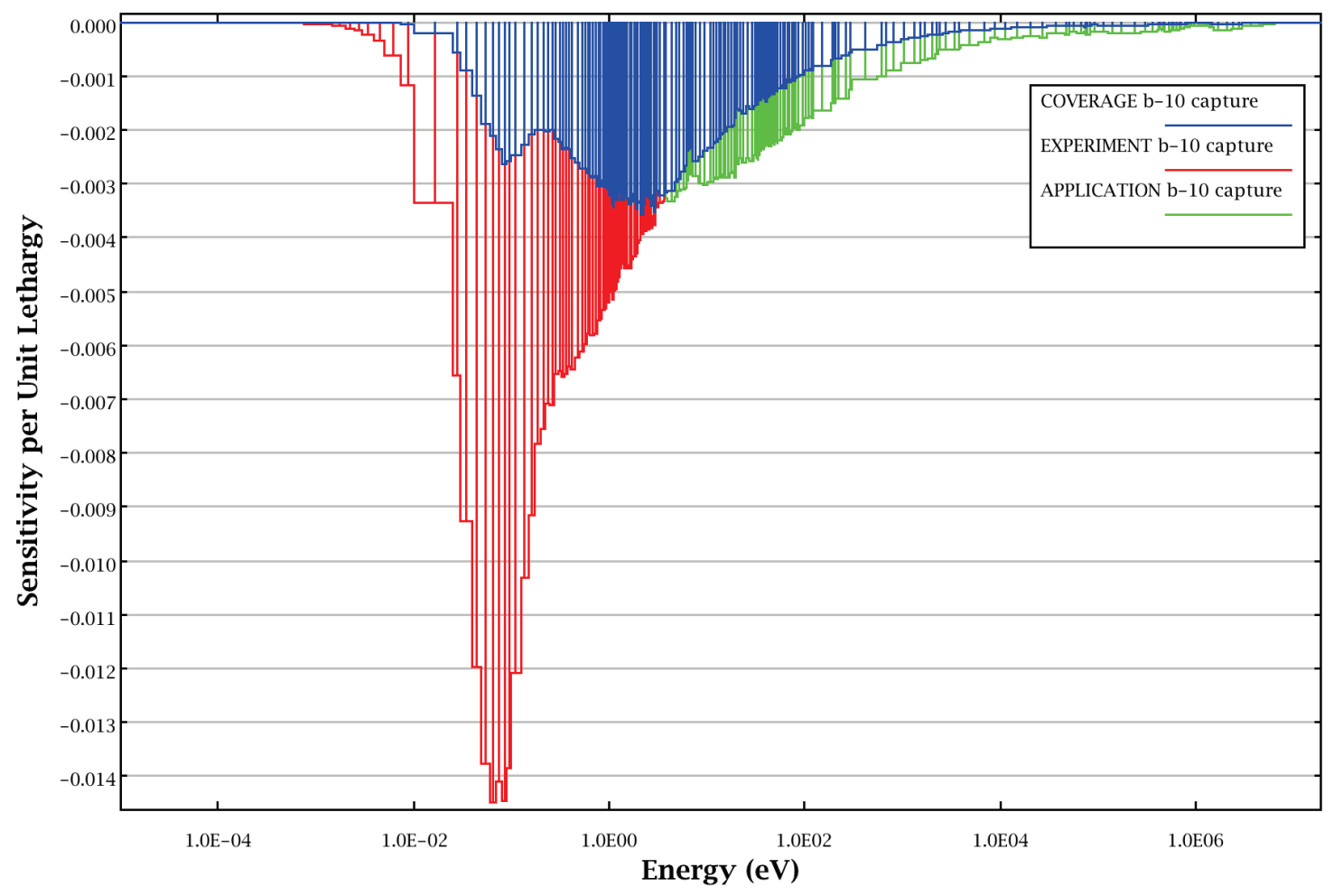

Figure 6.5.2. Illustration of coverage for ${ }^{10} \mathrm{~B}$ capture.

Assessment of similarity over a particular reaction type (fission, capture or scatter) can be made with a partial $G$ value. A $G_{x}$ value can be computed by eliminating the sum over reactions, $x$ in Eq. (6.5.10), as

$$
G_{x}=1-\frac{\sum_{n} \sum_{j}\left(S_{x, j}^{a, n}-S_{x, j}^{e^{\prime}, n}\right)}{\sum_{n} \sum_{j} S_{x, j}^{a, n}},
$$

where $x$ is $f, c$, or $s$.

To request the computation of $G$ for each application identified in the APPLICATIONS section of the input in relation to each experiment in the EXPERIMENTS portion of the input, simply enter the input $g$ in the PARAMETER section of the input. Additionally, the values table must be requested to output the full listing of $G$ values. The reaction components of $G$ (i.e., $G_{f}, G_{c}$ and $G_{s}$ ) are included in the values table if prtparts is entered in the input. The $G_{x}$ indices are normalized such they also have a range of 0 to 1 , where a $G_{x}$ of 1 indicates complete similarity of sensitivity coefficients for that particular reaction type $x$ between the application and experiment. The gsummary edit prints a listing of all experiments that have a $G$ value exceeding the criteria value set by gvalue $=$.

\subsubsection{Nuclide-reaction specific integral indices}

The global integral indices described in Sect. 6.5.1.10 assess system similarity for all nuclides and reactions in the application system. It is also possible and sometimes desirable to produce values 
analogous to $G, E$, and $c_{k}$ for each nuclide-reaction pair, such that similarity can be assessed on a nuclidereaction-specific level.

\subsection{Nuclide-reaction specific integral index $g$}

The nuclide-reaction specific integral index based on the same coverage criteria as $\mathrm{G}$ is denoted $\mathrm{g}$, and sometimes referred to as "little g." 2 It is defined in terms of the normalized differences of the group wise sensitivity coefficients for a particular nuclide, $n$, and reaction, $\mathrm{x}$, summed over all energy groups, $\mathrm{j}$, as

$$
g_{x}^{n}=1-\frac{\sum_{j}\left(S_{x, j}^{a, n}-S_{x, j}^{e^{\prime}, n}\right)}{\sum_{j} S_{x, j}^{a, n}},
$$

where,

$$
S_{x, j}^{e^{\prime}, n}= \begin{cases}S_{x, j}^{e, n}, \text { where } & \left|S_{x, j}^{a, n}\right| \geq\left|S_{x, j}^{e, n}\right| \text { and } \frac{S_{x, j}^{a, n}}{\left|S_{x, j}^{a, n}\right|}=\frac{S_{x, j}^{e, n}}{\left|S_{x, j}^{e, n}\right|} \\ S_{x, j}^{a, n}, \text { where } & \left|S_{x, j}^{a, n}\right|<\left|S_{x, j}^{e, n}\right| \text { and } \frac{S_{x, j}^{a, n}}{\left|S_{x, j}^{a, n}\right|}=\frac{S_{x, j}^{e, n}}{\left|S_{x, j}^{e, n}\right|} \\ 0, & \text { otherwise }\end{cases}
$$

and the $j$ summation is performed over all energy groups.

The criteria for $g$ are the same as those explained in Sect. 0 . The only difference in $g$ and $G$ is that the summations over nuclide and reaction have been removed. As with other integral indices, the $g$ index is normalized such that a $g$ value of 1 indicates complete coverage of the application by the experiment for the particular nuclide-reaction pair. A g value of 0 indicates no coverage of the application by the experiment for the particular nuclide-reaction pair.

To request the computation of $g$ ("little g") for each application identified in the APPLICATIONS section of the input in relation to each experiment in the EXPERIMENTS portion of the input for fission, capture and scatter reactions for each nuclide in the application, simply enter the input $l g$ in the PARAMETER section of the input. The $l g$ data appears in the nuclide-reaction specific edit for each application and only includes nuclide-reaction pairs with energy integrated sensitivity coefficients with at least the magnitude of sencut. Additionally, $g$ values are printed for each nuclide-reaction pair specified in the REACTIONS data block. The lgall edit prints all the $\mathrm{g}$ values for fission, capture and scatter for all nuclides in the application in relation to all experiments regardless of the magnitude of the energy-integrated sensitivity value. The lgsum edit prints a listing of all $\mathrm{g}$ values exceeding the criteria value set by lgvalue $=$ for fission, capture and scatter reactions for each nuclide. The lggroups edit prints a long table listing the numbers of experiments that are at least as sensitive as the application for each group for each nuclidereaction pair requested in the REACTIONS data block.

\subsection{Extended $c_{k}$}

The extended $c_{k}$ edit lists the contribution of each nuclide-reaction-to-nuclide-reaction energy covariance matrix to the global integral index $c_{k}$. A single entry in this edit is computed by utilizing a subset of the 
$\mathbf{C}_{\alpha \alpha}$ matrix and the $\mathbf{S}_{\mathbf{k}}$ sensitivity vectors for the application and the experiment. Thus, the extended $c_{k}$ for a nuclide-reaction pair in the application in relation to a nuclide-reaction pair in the experiment is defined as

$$
c_{k,(n-j)_{a},(m-k)_{e}}^{\text {contribution }}=\frac{\sigma_{(n-j)_{a},(m-k)_{e}}^{2}}{\left(\sigma_{a} \sigma_{e}\right)}
$$

where

$$
\begin{array}{ll}
(n-j)_{a} & \text { represents the nuclide } n \text { and reaction } j \text { of the application } a, \\
(m-k)_{e} & \text { represents the nuclide } m \text { and reaction } k \text { of the experiment } e, \\
\sigma_{(n-j)_{a},(m-k)_{e}}^{2} & \text { represents the covariance between application } a \text { and experiment } e \text { due to this } \\
& \text { nuclide-reaction pairs, } \\
\sigma_{a} & \text { is the standard deviation in } k_{e f f} \text { for the application due to all cross-section } \\
& \text { covariance data, and } \\
\sigma_{e} & \text { is the standard deviation in } k_{e f f} \text { for the experiment due to all cross-section } \\
& \text { covariance data. }
\end{array}
$$

The global integral index $c_{k}$ can be reconstructed from the extended $c_{k}$ components by summing the extended $c_{k}$ over all nuclide-reaction-to-nuclide-reaction values. Thus, the extended $c_{k}$ allows the user to determine the contribution of nuclide-reaction-to-nuclide-reaction covariances to $c_{k}$. All covariance data, default covariance data and user-input covariance data used in the calculation of $c_{k}$, as outlined in Sect. 0 , are also applied to the extended $c_{k}$ data. Nuclide-reaction-to-nuclide-reaction covariances using default and user-input values are identified with one and two asterisks, respectively, in the text output, and are identified with unique colors in the HTML output. Nuclide-reaction-to-nuclide-reaction covariances using default and user-input values for cov fix adjustments are identified with three and four asterisks, respectively, in the text output, and are identified with unique colors in the HTML output. Warning messages are printed to identify which zero values were replaced and which standard deviation value was used in the substitution.

The extended $c_{k}$ edit also includes an individual $c_{k}$ to examine the similarity of an application and experiment based only on a single nuclide-reaction pair. The individual $c_{k}$ is similar to the $c_{k}$ contribution from Eq.(6.5.11), except that it is normalized between -1 and 1 for each nuclide-reaction pair as

$$
c_{k,(n-j)_{a},(m-k)_{e}}^{\text {individual }}=\frac{\sigma_{(n-j)_{a},(m-k)_{e}}^{2}}{\left(\sigma_{(n-j)_{a}} \sigma_{(m-k)_{e}}\right)} .
$$

Note that individual $c_{k}$ values computed for a nuclide-reaction pair in an application to the same nuclidereaction pair in the experiment. Although cross-reaction and cross-nuclide covariance data are available, the cross-relationship has no physical interpretation for assessing for assessing the similarity of systems for a specific nuclide-reaction pair.

To request the computation of extended $c_{k}$ for each application identified in the APPLICATIONS section of the input in relation to each experiment in the EXPERIMENTS portion of the input, simply enter the input $c_{-}$long in the PARAMETER section of the input. 


\subsection{Extended $c_{r}$}

Similar to the extended $c_{k}$ edit, the extended $c_{r}$ edit lists the contribution of each nuclide-reaction-tonuclide-reaction energy covariance matrix to the global integral index $c_{r}$. An individual $c_{r}$ evaluating the similarity of each application to each experiment in terms of only one nuclide-reaction pair, normalized from -1 to 1 , is also available. The computation of the extended $c_{r}$ is the same as that of extended $c_{k}$, but with user-defined reactions removed from consideration. The EXCLUSIONS data block is used to identify which reactions will be excluded from consideration in the calculation of $c_{r}$ and its components as presented in extended $c_{r}$. If the user identifies no reactions, then $c_{r}$ and extended $c_{r}$ will be computed the same as $c_{k}$ and extended $c_{k}$. The $c_{r}$ parameter and extended $c_{r}$ edit are equivalent to removing all sensitivities for a given reaction or series of reactions from the sensitivity data file for all applications and all experiments considered in the analysis.

As with extended $c_{k}$, nuclide-reaction-to-nuclide-reaction covariances using default and user-input values are identified with one and two asterisks, respectively, in the text output, and are identified with unique colors in the HTML output. Nuclide-reaction-to-nuclide-reaction covariances using default and user-input values for cov fix adjustments are identified with three and four asterisks, respectively, in the text output, and are identified with unique colors in the HTML output. Warning messages are printed to identify which values were replaced and which standard deviation value was used in the substitution.

To request the computation of extended $c_{k}$ for each application identified in the APPLICATIONS section of the input in relation to each experiment in the EXPERIMENTS portion of the input, simply enter the input $\mathrm{cr}$ _long in the PARAMETER section of the input.

\subsubsection{Penalty assessment}

A method is available to assess an additional margin to subcriticality, or penalty, where sufficient experiments are not available to provide complete coverage for a particular application.3 This penalty is intended as an additional uncertainty component that can be added to the calculated value of $k_{\text {eff }}$ to provide an added measure of safety for application systems where validation coverage is lacking. The penalty calculation is based on the criteria for coverage explained in Sect. 0 . The criteria for coverage in this implementation of the penalty assessment is that if a single experiment that passes qualification tests for the particular application exhibits a sensitivity coefficient for a particular energy group for a particular nuclide-reaction pair that is at least as great in magnitude and has the same sign as the corresponding sensitivity coefficient for the application, then adequate coverage exists for the code validation of the application. For group-wise nuclide-reaction specific sensitivity coefficients for the application that are not fully covered by the experiments, the uncovered portion of the sensitivity coefficient is used to compute an uncertainty in $k_{\text {eff }}$ though the cross-section-covariance data.

Any experiment used in the penalty assessment calculation must pass a qualification test to determine global similarity of the experiment, based on a global integral index $\left(c_{k}, E\right.$, or $\left.G\right)$, and may also have to pass a nuclide-reaction specific qualification test based on the $g$ integral index. Thus, only experiments that exhibit a certain degree of similarity to the application can be considered in this calculation, unless the tests are deactivated at the user's discretion. Additionally, a number of similar experiments may be required before any penalty assessment is produced.

To compute the penalty, a vector of the minimum differences in the sensitivity coefficients, $\mathbf{Z}_{\mathbf{a}}$, for the application with respect to all experiments can be obtained as

$$
\mathbf{Z}_{\mathbf{a}} \equiv\left[Z_{x, j}^{a, n}\right], n=1, \ldots, N, x=1, \ldots, X, j=1, . ., J
$$


where,

$Z_{x, j}^{a, n}=S_{x, j}^{a, n}-C_{x, j}^{a, n}$,

$C_{x, j}^{a, n}$ is a composite of the best available sensitivity data from all experiments and is defined as

$C_{x, j}^{a, n}=S_{x, j}^{e^{\prime}, n}$ for the experiment that satisfies $\min \left|S_{x, j}^{a, n}-S_{x, j}^{e^{\prime}, n}\right|, e^{\prime}=1, \ldots, E$,

$S_{x, j}^{e^{\prime}, n}= \begin{cases}S_{x, j}^{e, n}, \text { where } & \left|S_{x, j}^{a, n}\right| \geq\left|S_{x, j}^{e, n}\right| \text { and } \frac{S_{x, j}^{a, n}}{\left|S_{x, j}^{a, n}\right|}=\frac{S_{x, j}^{e, n}}{\left|S_{x, j}^{e, n}\right|} \\ S_{x, j}^{a, n}, \text { where } & \left|S_{x, j}^{a, n}\right|<\left|S_{x, j}^{e, n}\right| \text { and } \frac{S_{x, j}^{a, n}}{\left|S_{x, j}^{a, n}\right|}=\frac{S_{x, j}^{e, n}}{\left|S_{x, j}^{e, n}\right|} \\ 0, & \text { otherwise }\end{cases}$

a represents a particular application,

e represents a particular experiment,

$n$ represents a particular nuclide,

$x \quad$ represents a particular reaction,

j represents a particular energy group,

$N=$ number of nuclides in the application system,

$X=$ number of reactions for each nuclide,

$J=$ number of energy groups, and

$E=$ number of experiments meeting the qualification tests.

Once $\mathbf{Z}_{\mathbf{a}}$ is computed, the portion of the sensitivity of the application that is not covered by the experiments can be used to propagate the uncertainty in the cross-section data to a relative uncertainty in $k_{\text {eff }}$ as

$$
\Delta k_{e f f} / k_{e f f}=\sqrt{\mathbf{Z}_{\mathbf{a}} \mathbf{C}_{\alpha \alpha} \mathbf{Z}_{\mathbf{a}}^{\dagger}}
$$

where $\digamma$ indicates a transpose and $\mathbf{C}_{\alpha \alpha}$ is the matrix of the cross-section-covariance data defined in Eq. (6.5.1). In the above equation, the elements of $\mathbf{Z}_{\mathbf{a}}$ are each expressed in terms of $\left(\Delta k_{\text {eff }} / k_{\text {eff }}\right) /(\Delta \Sigma / \Sigma)$, and the elements of $\mathbf{C}_{\alpha \alpha}$ are expressed in terms of relative variances or 
covariances as $(\Delta \Sigma / \Sigma)^{2}$, so that the final penalty is expressed as a relative uncertainty in $k_{\text {eff }}, \Delta k_{\text {eff }} / k_{\text {eff }}$. This relative uncertainty in $k_{\text {eff }}$ is written to the output file in the penalty calculations.

To request the penalty computation for each application identified in the APPLICATIONS section of the input in relation to all experiments in the EXPERIMENTS section of the input, simply enter the input penalty in the PARAMETER section of the input. Additionally, a list of the contribution each nuclidereaction-to-nuclide-reaction covariance matrix to the total penalty can be viewed by entering penlong in the PARAMETER input. This creates an edit similar to the Uncertainty Information edit of the SAMS module. Each value shown in this output edit represents the relative penalty in percent $(\% \Delta k / k)$ due to the specified nuclide-reaction-to-nuclide-reaction covariance matrix. The values are sorted in order of descending magnitude. The cumulative penalty can be constructed by squaring the individual values, then adding those that had positive signs and subtracting those that had negative signs, then taking the square root. Negative values in the table result from covariance matrices that have anticorrelated values.

The qualification test for including or excluding experiments from the development of the penalty for a particular application is set by several input parameters. The purpose of the qualification test is to ensure that some relevant data are used in the calculation. Otherwise, in the limit that no relevant data are used in the penalty calculation, the $\mathbf{Z}_{\mathbf{a}}$ minimum difference sensitivity vector becomes $\mathbf{S}_{\mathbf{a}}$, the sensitivity vector for the application. In this case, the maximum penalty assessed is simply the uncertainty in the application's criticality calculation due to cross-section-covariance data.

A global qualification test, to test the similarity of a particular experiment to the given application based on a global integral index, is configured with the keywords: penusec, penusee and penuseg. These keywords are used to produce a penalty calculation that only includes experiments with $c_{k}, E$, or $G$ values at least as great as cvalue, evalue or gvalue, respectively. The default value is penusec. If more than one of the keywords penusec, penusee or penuseg are included in the input, only the last one entered will be used. The penalty is only computed for a given application if the number of experiments passing the global integral index qualification test is at least as great as penminx (default $=10$ ). If no global test is desired, enter penmin $x=0$.

The pencut $=$ keyword allows the user to set a discriminator for excluding nuclide-reaction pairs from the application with small sensitivity coefficients from the penalty calculation. If the sum of the absolute values of the group-wise sensitivity coefficients is below pencut, the nuclide reaction pair is excluded from the penalty calculation. The default value is 0.0 , which includes all nuclide-reaction pairs in the penalty calculation. Additionally, penlgv= sets a discriminator that only includes experiments with a $g$ value relative to the application for a given nuclide-reaction pair that meets or exceeds penlgv. Thus, using penlgv may allow some nuclide-reaction pairs from a given experiment to be included in the penalty calculation, but exclude others from the same experiment that do not meet the criteria. The default value is 0.0 , which means include all nuclide-reaction pairs that pass the other qualification tests.

The keyword penwarn activates a penalty warning edit that details which experiments were excluded from the penalty calculation based on failing the global qualification test, and which nuclide-reaction pairs were excluded from the penalty calculation based on failing the nuclide-reaction specific tests.

The composite of the best available sensitivity data from all experiments that meet the requested criteria, as used in the calculation of the penalty, can be viewed with the composite sensitivity data for nuclides and reactions requested in the REACTIONS input data block with the input keyword prtcomp described in Sect. 6.5.1.4.3. 
The $\mathbf{C}_{\alpha \alpha}$ matrix in Eq. (6.5.14) is the cross-section covariance data read in the COVERX formatted data file identified by cover $=$. As in the calculation of $c_{k}$, nuclide-reaction pairs without available covariance data are omitted from this analysis, but it is assumed that either the cross-section data values from these pairs are well known (i.e., small uncertainties), or that the sensitivity of the system $k_{\text {eff }}$ to these nuclidereaction pairs is small. Where these assumptions hold, the nuclide-reaction pairs without cross-sectionuncertainty data present a negligible contribution to the penalty calculation. For situations where this negligible contribution assumption is judged not to be valid, the use of uncertainty analysis for the computation of a penalty is not appropriate. However, the COVARIANCE data block can be used to input uncertainty values for the cross-section data for particular nuclide-reaction pairs to assess the impact of additional covariance data. To utilize the covariance data generated by user input in the COVARIANCE data block, the keyword use icov must be entered in the PARAMETER data block. Additionally, default uncertainty values can be assigned for all unknown covariance data. The default uncertainty data input in the PARAMETER data block and the keyword use_dcov must be entered to activate its use. In the extended penalty edit, nuclide-reaction-to-nuclide-reaction covariances using default and user-input values are identified with one and two asterisks, respectively, in the text output, and are identified with unique colors in the HTML output. Warning messages are also printed to identify substituted covariance matrices.

When use dcov and/or use icov and cov fix are specified in the PARAMETER data block, and a reaction has zero or large (standard deviation $>1000 \%$ ) values on the diagonal of the covariance matrix, these values are replaced with the square of the user input or default standard deviation values, and the corresponding off-diagonal terms are substituted according to the user input or default correlation values. In the extended penalty edit, nuclide-reaction-to-nuclide-reaction covariances using default and user-input values for cov fix adjustments are identified with three and four asterisks, respectively, in the text output, and are identified with unique colors in the HTML output. Warning messages are printed to identify which values were replaced and which standard deviation value was used in the substitution.

\subsubsection{Other parameters}

TSUNAMI-IP can produce a number of other parameters that are useful for analysis of systems. These are briefly explained in this section.

\subsection{Uncertainty information}

The keyword uncert activates the calculation of the uncertainty in $k_{\text {eff }}$ due to the cross-section-covariance data read from the COVERX formatted data file identified by coverx $=$. The uncertainty is computed for each application and each experiment and is printed in the values table as a standard deviation value with its statistical uncertainty, if appropriate. The uncertainty value printed is a relative uncertainty in percent (i.e., $\Delta k_{\text {eff }} / k_{\text {eff }} \times 100 \%$ ).

Nuclide-reaction pairs without available data are omitted from this analysis, but it is assumed that either the cross-section data values from these pairs are well known (i.e., small uncertainties), or that the sensitivity of the system $k_{\text {eff }}$ to these nuclide-reaction pairs is small. Where these assumptions hold, the nuclide-reaction pairs without cross-section-uncertainty data present a negligible contribution to the uncertainty-based analysis. For situations where this negligible contribution assumption is judged not to be valid, the use of uncertainty analysis is not appropriate. However, the COVARIANCE data block can be used to input uncertainty values for the cross-section data for particular nuclide-reaction pairs to assess the impact of additional covariance data. To utilize the covariance data generated by user input in the COVARIANCE data block, the keyword use icov must be entered in the PARAMETER data block. Additionally, default uncertainty data can be assigned for all unknown covariance data. This default data is input in the PARAMETER data block and the keyword use_dcov must be entered to activate its use. 
To request a listing of the contributions nuclide-reaction-to-nuclide-reaction covariance matrix to the uncertainty in the $k_{\text {eff }}$ value for each application identified in the APPLICATIONS section of the input enter the input keyword uncert_long in the PARAMETER section of the input. This creates an edit similar to the Uncertainty Information edit of the SAMS module. Each value shown in this output edit represents the relative uncertainty in percent $(\% \Delta k / k)$ due to the specified nuclide-reaction-to-nuclide-reaction covariance matrix. The values are sorted in order of descending magnitude. The cumulative uncertainty can be constructed by squaring the individual values, then adding those that had positive signs and subtracting those that had negative signs, then taking the square root. Negative values in the table result from covariance matrices that have anticorrelated values.

In the extended uncertainty edit, nuclide-reaction-to-nuclide-reaction covariances using default and userinput values are identified with one and two asterisks, respectively, in the text output, and are identified with unique colors in the HTML output. Nuclide-reaction-to-nuclide-reaction covariances using default and user-input values for cov fix adjustments are identified with three and four asterisks, respectively, in the text output, and are identified with unique colors in the HTML output. Warning messages are printed to identify which values were replaced and which standard deviation value was used in the substitution.

\subsection{Completeness parameter}

A parameter has been developed to assess the completeness of a set of experiments for the code validation of a given application. 4 The set of experiments is "complete" in the sense that it completely tests all the important cross-section elements in the particular application of interest. The availability of sensitivity coefficients provides a key element in the definition of this completeness parameter.

The completeness parameter, $R$, is defined as follows:

where

$$
R=S_{a} / S_{t}
$$

$$
\begin{gathered}
\mathrm{S}_{\mathrm{t}}=\sum_{\mathrm{n}} \sum_{\mathrm{x}} \sum_{\mathrm{j}}\left|\mathrm{S}_{\mathrm{x}, \mathrm{j}}^{\mathrm{a}, \mathrm{n}}\right| \\
S_{a}=\sum_{n} \sum_{x} \sum_{j}\left|d S_{x, j}^{a, n}\right|
\end{gathered}
$$

and

$$
\begin{aligned}
& \qquad d=\left\{\begin{array}{l}
1, \text { if } N_{x, j}^{n} \geq \text { nix } \lim \\
0, \text { if } N_{x, j}^{n}<\text { nix } \lim
\end{array}\right. \\
& N_{x, j}^{n}=\text { number of systems for which } S_{x, j}^{e, n}>\left|\operatorname{senfac} \times S_{x, j}^{a, n}\right|, \\
& e \quad=\quad \text { experiment, } \\
& a \quad=\quad \text { application, } \\
& S_{x, j}^{e, n}=\quad \text { the sensitivity of } k_{\text {eff }} \text { of an experiment to the cross sections of the constituent material } \\
& \quad \text { nuclide } n, \text { reaction } x, \text { and energy group } j,
\end{aligned}
$$




$$
\begin{aligned}
S_{x, j}^{a, n}= & \text { the sensitivity of } k_{\text {eff }} \text { of the application to the cross sections of the constituent material } \\
& \text { nuclide } n, \text { reaction } x, \text { and energy group } j, \\
\text { nixlim }= & \text { an integer, and } \\
\text { senfac }= & \text { a real number such that } 0.0 \leq \text { senfac } \leq 1.0 .
\end{aligned}
$$

The completeness parameter is designed to give the effective fraction of the total sensitivity for each application system that is "covered" by the benchmark set. This coverage is defined by comparing the magnitude of each group-wise sensitivity coefficient for the application with respect to each of the corresponding sensitivities of the benchmark systems. The completeness parameter is computed for each application for each experiment if the keyword $c p$ is entered in the PARAMETER data. The minimum coverage of the sensitivity coefficients for the experiment systems is defined as senfac $\times 100 \%$ of the application sensitivity in the definition of $N_{x, j}^{n}$. The value of senfac is set with the keyword input senfac $=$, and the default value is 0.9 . Thus, the experiment must have a sensitivity coefficient at least $90 \%$ as great as that of the application for the particular nuclide, reaction and energy group to count in $N_{x, j}^{n}$. The number of experiments counted in $N_{x, j}^{n}$ must be at least nixlim to change $d$ from 0 to 1 , which indicates coverage for the particular nuclide, reaction and group of the application. The value of nixlim is set by the nixlim= keyword, and it has a default value of 10

\subsection{Composite sensitivity data}

A composite of the best available sensitivity data from all experiments included in the analysis, based on the coverage criteria used for the $G$ integral index in Sect. 0 is produced for each application for each nuclide-reaction pair in the REACTIONS input if the keyword prtcomp is entered. The composite profile for a particular application $a$ for nuclide $n$ and reaction $x$ is defined as the vector

where,

$$
\mathbf{C}_{\mathbf{x}}^{\mathbf{a}, \mathbf{n}} \equiv\left[C_{x, j}^{a, n}\right], j=1, \ldots, J
$$

$C_{x, j}^{a, n}$ is $S_{x, j}^{e^{\prime}, n}$ for the experiment that satisfies $\min \left|S_{x, j}^{a, n}-S_{x, j}^{e^{\prime}, n}\right|, e^{\prime}=1, \ldots, E$,

$$
S_{x, j}^{e^{\prime}, n}= \begin{cases}S_{x, j}^{e, n}, \text { where } & \left|S_{x, j}^{a, n}\right| \geq\left|S_{x, j}^{e, n}\right| \text { and } \frac{S_{x, j}^{a, n}}{\left|S_{x, j}^{a, n}\right|}=\frac{S_{x, j}^{e, n}}{\left|S_{x, j}^{e, n}\right|} \\ S_{x, j}^{a, n}, \text { where } & \left|S_{x, j}^{a, n}\right|<\left|S_{x, j}^{e, n}\right| \text { and } \frac{S_{x, j}^{a, n}}{\left|S_{x, j}^{a, n}\right|}=\frac{S_{x, j}^{e, n}}{\left|S_{x, j}^{e, n}\right|}, \\ 0, & \text { otherwise }\end{cases}
$$

a represents a particular application,

e represents a particular experiment,

$n$ represents a particular nuclide, 
j represents a particular energy group,

$J=$ number of energy groups, and

$E=$ number of experiments.

An example composite sensitivity profile for ${ }^{1} \mathrm{H}$ total is shown in Figure 6.5.3. Here, the composite sensitivity profile comprised of the best available data from unidentified experiments $1-5$ is shown in black. The sensitivity of the unidentified application is shown in red. The areas where the red application curve exceeds the black composite curve are considered uncovered. Areas where the experiment data exceed the application data are considered fully covered. Note that the composite data does not exceed the application data.

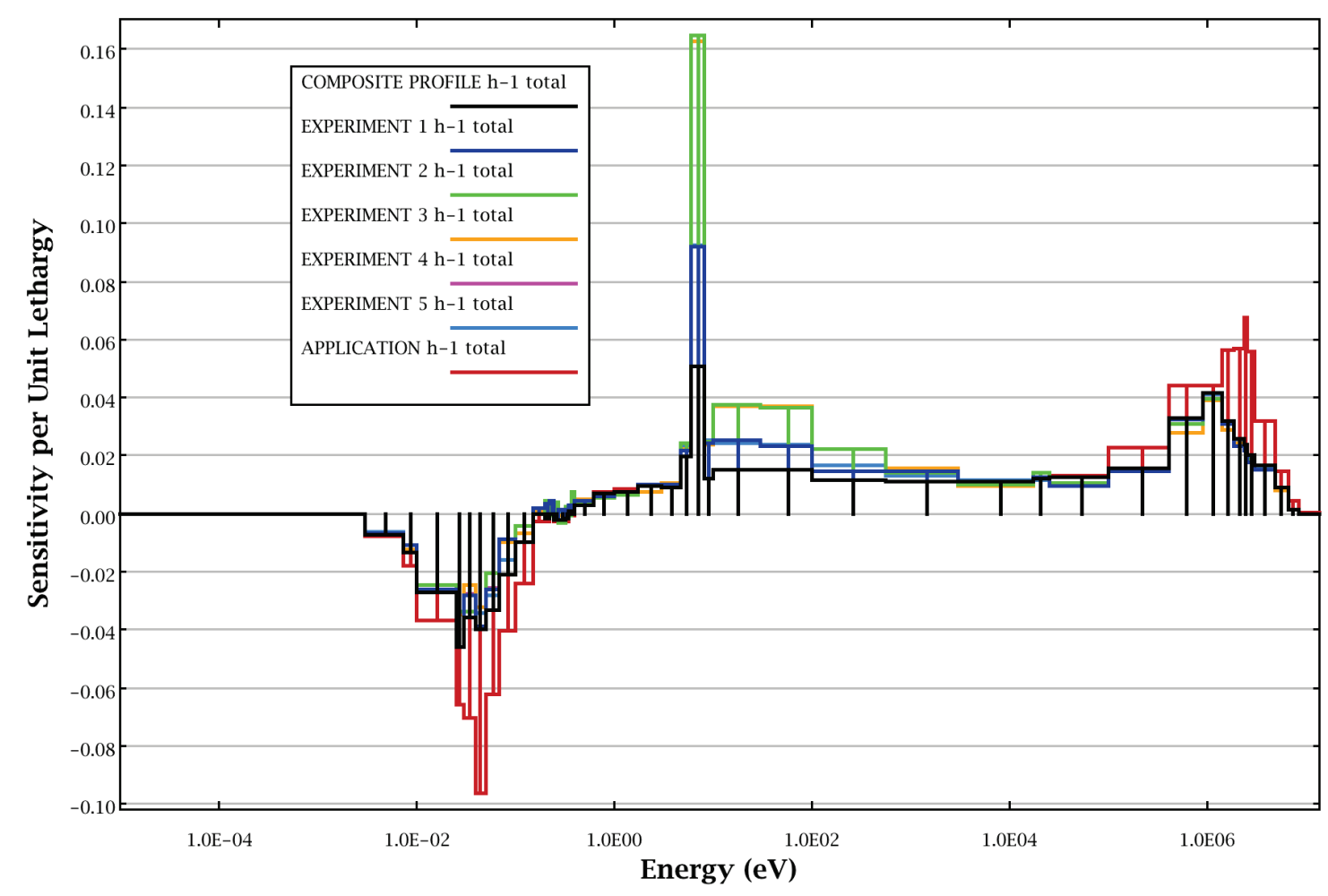

Figure 6.5.3. Example composite sensitivity profile.

If the composite data for a particular nuclide-reaction pair for a particular energy group are added to the minimum difference data as defined in Eq. (6.5.13), the sensitivity of the application would result as

$$
C_{x, j}^{a, n}+Z_{x, j}^{a, n}=S_{x, j}^{a, n}
$$

A sensitivity data file containing the composite sensitivity data is generated to permit further analysis. Only data for those nuclides and reaction identified in the REACTIONS input block are included in the composite sensitivity data file. The data file is identified with the TSUNAMI-IP input file name with the extension ".sdf" and is suitable for use with Javapeño. This data file is presented as an interactive plot in the HTML output. The following data are included on the composite sensitivity data file for each 
nuclide-reaction pair identified in the REACTIONS input block for each application: the sensitivity of the application identified with "Application" and the application number, the composite sensitivity data as defined in Eq. (6.5.16), and the composite profile used in the definition of the minimum difference profile from the penalty calculation (Sect. 6.5.1.3), which may be reduced from the full composite by excluding experiments not meeting the cutoff criteria for the penalty calculation. The composite data as used in the penalty calculation are identified as "Cut Composite."

\subsection{Non-coverage}

A summary of non-coverage for the nuclide-reaction pairs entered in the REACTIONS section of the input can be produced if the keyword prtnotcv is entered in the PARAMETER data. The non-coverage edit gives the number of groups that are not fully covered according to the $G$ criteria and gives the sum of the non-covered portion of the sensitivity coefficients. It also lists the group with largest sensitivity, regardless of whether or not it is covered, and gives the sensitivity value for this group. The experiment that best covers the group with the maximum sensitivity is given and the sensitivity of this group for the best matching experiment is given.

\subsubsection{Miscellaneous options}

Several input parameters that provide a wide range of options in TSUNAMI-IP are explained here. These keywords are entered in the PARAMETER data block.

\subsection{USLSTATS input files}

USLSTATS is a data regression tool described in Ref. 6. It is included in the Windows version of SCALE and is available for download from the SCALE website. The uslstats keyword requests input files for USLSTATS to be generated from all integral indices computed by TSUNAMI-IP. The uslsummary keyword requests USLSTATS input files to be generated from all integral indices exceeding their particular acceptance criteria (cvalue, evalue, and gvalue). The uncertainties in the $k_{\text {eff }}$ values for USLSTATS can be adjusted with two options. The default setting is that the uncertainty for each $k_{\text {eff }}$ value included in the USLSTATS input file consists of the square root of the sum of the squares of the Monte Carlo uncertainty and a uniform experimental uncertainty of $0.3 \%$. To quadratically add the uncertainty in $k_{\text {eff }}$ due to cross-section-covariance data to this uncertainty, enter the keyword usl_uncert. To modify the uniform experimental uncertainty, use the $u s l$ sigs $=$ keyword. When a positive value is entered for usl_sigs, this value is treated as the total uncertainty for each experiment, and no other contributions, Monte Carlo or cross section, are considered. When a negative value is entered for usl_sigs, this value is treated as the experimental uncertainty for each experiment and Monte Carlo and cross section uncertainties are added as appropriate. Currently, no option exists to input a unique experimental uncertainty for each experiment.

The USLSTATS input files contain the extension .usl and the filenames are presented in a descriptive format as title_xxxx_p_yyyy.usl, where title is the filename of the TSUNAMI-IP input case, $x x x x$ is the application number in the TSUNAMI-IP input, $p$ is the name of the integral index ( $c k$ for $c_{k}, e$ for $E$, and $g$ for $G$ ), and yyyyy is the TSUNAMI-IP execution number (typically 0001). When the summary inputs are requested with uslsummary, the filename is of the format title_xxxx_p_sum_yyyy.usl, where sum denotes that this USLSTATS input file contains only the summary of experiments that exceed the requested criteria for the specific integral index.

\subsection{Covariance data directory listing}

The prtmtrix keyword causes a listing of the energy covariances of the COVERX formatted cross-section data file, identified by cover $x=$, to be printed. 


\subsection{HTML output}

The keyword html causes TSUNAMI-IP to create an HTML formatted output. This output edit is accessed by opening the root.html file, where root is the name of the user's input file without extensions. Additional resources for the html output are placed in new directories called root.htmd and applet_resources. The root.html directory contains files needed to display data produced by the current case and the applet_resources directory contains Java applets for data plotting within the HTML interface and can be shared by multiple output files within the same directory. The root.html file and associated directories are placed in the same location as the user's input file. The HTML formatted output is color coded and more easily navigated than the standard plain text formatted output file. The HTML output can be customized with the HTML data block.

\subsection{Case sensitive input}

The inptcase keyword sets a flag in the SCALE free form input reader to prevent SCALE from translating all input to lower case. The purpose of this keyword is the identification of file and directory names that have upper case characters on a case-sensitive operating system. By default, the SCALE input reader translates all text to lower case. However, any input entered after inptcase is treated as case sensitive. All subsequent keyword entries and data block names must be entered in lower case, or errors will result.

\subsection{Print filenames}

The usename keyword causes TSUNAMI-IP to identify files with their file names in the code output. By default, TSUNAMI-IP identifies files according to the title on the TSUNAMI-A or TSUNAMI-B sensitivity data files. For files that have the same titles, or have long or non-descriptive titles, usename can provide for simplified interpretation of the output.

\subsubsection{6 $\quad c_{k}$ and $E$ differences}

The keywords $\operatorname{cechk}=$ and $c e d i f f=$ allow the user to input discriminators that govern the output of warning messages when $c_{k}$ and $E$ values for a particular application and experiment differ by more than cediff and $c_{k}$ and $E$ are both at least as great as cechk.

\subsection{Plots}

The keyword plot causes Javapeño formatted plot (.plt) files of the global integral indices to be generated. A plot file containing all computed integral index values for each application as a function of experiment number is titled rootxxxx.plt, where root is the base name of the input file and xxxx is the TSUNAMI-IP execution number (typically 0001). Plot file containing the number of integral index values that exceeded the acceptance criteria for each application is titled rootsummaryxxxx.plt, where summary indicated it is a summary plot. Both plots are displayed in the HTML output or can be view with Javapeño. If the .plt files are opened in Javapeño with "Open Dataset..." each set of data can be manually added to the plot as desired by the user. If the .plt files are opened with "Open Plot...," all data are immediately displayed.

\subsection{Absolute sensitivity option}

All global indices and parameters can also be calculated using absolute sensitivities. This capability is useful when working with TSAR-generated reactivity sensitivity data files. TSAR creates a sensitivity data file with reactivity sensitivities tabulated in either relative format or absolute format $(\Delta \rho /|\rho|) / \Delta \sigma / \sigma)$. If keyword relative is specified in the PARAMETER block, any absolute reactivity sensitivities are internally converted to $(\Delta \rho) / \Delta \sigma / \sigma$ ). (Note that the reactivity sensitivity is normalized by $\rho$ rather than $|\rho|$. Normalizing by $|\rho|$ can potentially lead to $c_{k}$ and $E$ indices with the wrong sign.) Likewise, if the keyword absolute is specified, any relative reactivity sensitivities are internally converted to $(\Delta \rho / \rho) / \Delta \sigma / \sigma)$, and the SAMS-generated relative $k_{\text {eff }}$ sensitivities are internally converted to 
$\left(\Delta k_{\text {eff }} / k_{\text {eff }} / \Delta \sigma / \sigma\right)$. If both the relative and absolute keywords are specified, the last keyword entered in the PARAMETER block is used to determine the sensitivity format. If neither keyword is entered, the default relative format is applied.

It is important to note that the absolute sensitivity option has been added for user control over the format of the uncertainty edit and the extended uncertainty edit. Using absolute sensitivities, the absolute standard deviation in $k_{\text {eff }}$ or $\rho$ is edited. Using relative sensitivities, the relative standard deviation in $k_{\text {eff }}$ or $\rho$ is edited. The global indices $c_{k}$ and $E$ are the same regardless of the sensitivity format option. For other indices and parameters (i.e., $G$, penalty assessment, and completeness parameter), their respective values can change depending on sensitivity format option. These indices and parameters play an important role in nuclear criticality safety validation, and the default relative format option is recommended.

\subsubsection{User input}

The user input for TSUNAMI-IP is described in this section. The input consists of an optional title on a single line followed by one required and six optional blocks of data which are identified in Table 6.5.1 and individually described in subsequent subsections. These data blocks must begin with READ KEYNAME and end with END KEYNAME, where $K E Y N A M E$ is the name of an individual data block. The required PARAMETER data block should be entered first, followed by the remaining blocks of data in any order. Note that this is different than the SCALE 5 and SCALE 5.1 versions of TSUNAMI-IP, which allowed the blocks of data to be in any order. The SCALE 6 version will continue if the PARAMETER data block is not entered first, but the keywords absolute and use_diff_groups (explained below) will have no effect.

Table 6.5.1. Keynames and descriptions for TSUNAMI-IP input data blocks

\begin{tabular}{|c|c|c|}
\hline Keyname & Description & Required/Optional \\
\hline PARAMETER & $\begin{array}{l}\text { Indices and parameters to be computed are input in this } \\
\text { section. Output edits are requested, and user-input criteria } \\
\text { values for numeric data are entered. }\end{array}$ & Required \\
\hline APPLICATIONS & $\begin{array}{l}\text { File paths to sensitivity data files representing application } \\
\text { systems for which validation by the experiments are assessed } \\
\text { are input in this section. }\end{array}$ & Optional $^{*}$ \\
\hline EXPERIMENTS & $\begin{array}{l}\text { File paths to sensitivity data files representing experiments to } \\
\text { be used in the analysis are input in this section. }\end{array}$ & Optional $^{*}$ \\
\hline RESPONSE & $\begin{array}{l}\text { File paths to sensitivity data files representing experiments or } \\
\text { applications to be used in the analysis are input in this section. }\end{array}$ & Optional $^{*}$ \\
\hline REACTIONS & $\begin{array}{l}\text { Specific nuclide-reaction pairs for which analysis with certain } \\
\text { indices and parameters are desired can be entered in this } \\
\text { section. }\end{array}$ & Optional \\
\hline COVARIANCE & $\begin{array}{l}\text { User input standard deviation for nuclide-reaction pairs for } \\
\text { which cross-section-covariance data are not available can be } \\
\text { entered in this section. }\end{array}$ & Optional \\
\hline
\end{tabular}


Table 6.5.1. Keynames and descriptions for TSUNAMI-IP input data blocks (continued)

\begin{tabular}{lll}
\hline \multicolumn{1}{c}{ Keyname } & Description & Required/Optional \\
\hline EXCLUSIONS & $\begin{array}{l}\text { User input reactions to exclude from } c_{r} \text { and Extended } c_{r} \\
\text { calculations. }\end{array}$ & Optional \\
HTML & $\begin{array}{l}\text { Parameters to customize the HTML formatted output can be } \\
\text { entered in this section. }\end{array}$ & Optional \\
\hline
\end{tabular}

*Although the EXPERIMENTS, APPLICATIONS, and RESPONSE data blocks are optional, at least one application and one experiment must be specified on the TSUNAMI-IP input file. This can be done in a variety of ways explained below.

\subsection{Parameter data}

The PARAMETER data block is used to request the calculation of the various indices and parameters available in TSUNAMI-IP, request output edits and set criteria values. The parameter block must begin with READ PARAMETER and end with END PARAMETER. The data input to the parameter data block consist of numerous keywords that are shown, along with their default values and descriptions, in Table 6.5.2. A keyword that ends with $=$ must be followed by numeric data. Keywords that do not end with $=$ are Boolean flags that are used to turn on certain features of the code, such as the computation of certain data or certain output edits. If the keyword is present for a Boolean entry, the value is set to true. Otherwise, the Boolean flag is set to its default value. If no data are requested in the PARAMETER section using the Boolean flags, then no data will be produced by the code. The input is designed to maximize user control over the operation of the code. A more detailed description of the indices and parameters are given in Sects. 247-6.5.1.4

Table 6.5.2. Input data for parameter block of TSUNAMI-IP input

\begin{tabular}{|c|c|c|}
\hline Keyword & Default value & Description \\
\hline absolute & False & $\begin{array}{l}\left.\text { Use absolute sensitivities [e.g. }\left(\Delta k_{e f f} / \Delta \sigma / \sigma\right)\right] \text { for all applications } \\
\text { and experiments in the analysis. If absolute is entered, the } \\
\text { keyword relative is set to False. }\end{array}$ \\
\hline $\mathrm{c}$ & False & $\begin{array}{l}\text { Compute } c_{k} \text { values for each application compared to each } \\
\text { experiment. }\end{array}$ \\
\hline c_long & False & $\begin{array}{l}\text { Produces extended } c_{k} \text { output edit for each application compared } \\
\text { to each experiment. }\end{array}$ \\
\hline cechck $=$ & 0.5 & $\begin{array}{l}\text { Level of } E \text { and } c_{k} \text { values that trigger the cediff warning. If } E \text { or } \\
c_{k} \text { are below this value, no warning is printed. }\end{array}$ \\
\hline cediff $=$ & 0.1 & $\begin{array}{l}\text { If the } E \text { and } c_{k} \text { values for a given application and experiment } \\
\text { differ by more than cediff, a warning message is printed. }\end{array}$ \\
\hline cov_fix & False & $\begin{array}{l}\text { Replace zero and large (standard deviation }>1000 \% \text { ) values on } \\
\text { diagonal of cross-section-covariance data with user input values } \\
\text { and default values. }\end{array}$ \\
\hline
\end{tabular}


Table 6.5.2. Input data for parameter block of TSUNAMI-IP input (continued)

\begin{tabular}{|c|c|c|}
\hline Keyword & Default value & Description \\
\hline coverx $=$ & 44groupcov & Name of cross-section covariance data file to use in analysis. \\
\hline $\mathrm{cp}$ & False & $\begin{array}{l}\text { Compute and print completeness parameter for each } \\
\text { application. }\end{array}$ \\
\hline $\mathrm{cr}$ & False & $\begin{array}{l}\text { Compute } c_{r} \text { values for each application compared to each } \\
\text { experiment. }\end{array}$ \\
\hline cr_long & False & $\begin{array}{l}\text { Produces extended } c_{r} \text { output edit for each application compared } \\
\text { to each experiment. }\end{array}$ \\
\hline crsummary & False & $\begin{array}{l}\text { Print summary table of } c_{r} \text { values that meet or exceed the crvalue } \\
\text { limit. Sets cr to true. }\end{array}$ \\
\hline crvalue $=$ & .9000 & Threshold value of $c_{r}$ for inclusion in the summary table. \\
\hline $\mathrm{e}$ & False & $\begin{array}{l}\text { Compute } \mathrm{E}(\mathrm{sum}) \text { values for each application compared to each } \\
\text { experiment. }\end{array}$ \\
\hline \multicolumn{3}{|c|}{$\begin{array}{l}\text { WARNING: E values cannot be calculated in TSUNAMI-IP in any SCALE version up to and including SCALE } \\
6.2 .2 \text { without also specifying an option that uses the covariance library. These options include, but are not limited } \\
\text { to, c, cr, and uncert. }\end{array}$} \\
\hline esummary & False & $\begin{array}{l}\text { Print summary table of } \mathrm{E}(\mathrm{sum}) \text { values that meet or exceed the } \\
\text { evalue limit. Sets e to true. }\end{array}$ \\
\hline evalue $=$ & .9000 & Threshold value of E(sum) for inclusion in the summary table. \\
\hline g & False & Compute G values. \\
\hline gsummary & False & $\begin{array}{l}\text { Print summary table of } \mathrm{G} \text { values that meet or exceed the gvalue } \\
\text { limit. Sets } g \text { to true. }\end{array}$ \\
\hline gvalue $=$ & .9000 & Threshold value of $\mathrm{G}$ for inclusion in summary table. \\
\hline html & False & $\begin{array}{l}\text { Generate output in html format in addition to the standard text } \\
\text { output. }\end{array}$ \\
\hline inptcase & False & $\begin{array}{l}\text { This sets the SCALE free form reader to leave the case of the } \\
\text { input data as read. This is useful when sensitivity data file } \\
\text { names have upper case letters. If this option is set, all other } \\
\text { input keywords MUST be in lower case to be correctly } \\
\text { interpreted by SCALE. This keyword must be entered in the } \\
\text { input prior to the reading of any titles of the sensitivity data } \\
\text { files with capital letters. }\end{array}$ \\
\hline large_cov $=$ & 10 & $\begin{array}{l}\text { Cutoff fractional standard deviation value for cov_fix. } \\
\text { Covariance data with uncertainties larger than large_cov are } \\
\text { replaced with user-defined or default values. Default }=10 \text {, } \\
\text { which is } 1000 \% \text { uncertainty. }\end{array}$ \\
\hline
\end{tabular}




\begin{tabular}{|c|c|c|}
\hline $\lg$ & False & $\begin{array}{l}\text { Compute } g \text { values for fission, capture and scatter for each } \\
\text { nuclide for each experiment compared to each application and } \\
\text { print them in a table if the application's sensitivity for the } \\
\text { corresponding nuclide-reaction pair is greater than or equal to } \\
\text { sencut. Also compute } g \text { values for reactions specified in the } \\
\text { REACTIONS data block. }\end{array}$ \\
\hline lgall & False & $\begin{array}{l}\text { Print } g \text { values for fission, capture and scatter for all nuclides for } \\
\text { all experiments for each application. }\end{array}$ \\
\hline lggroups & False & $\begin{array}{l}\text { Print a table listing the numbers of experiments that are at least } \\
\text { as sensitive as the application for each group for each reaction } \\
\text { requested in the REACTIONS data block. }\end{array}$ \\
\hline $\operatorname{lgsum}$ & False & $\begin{array}{l}\text { Print a summary table of } g \text { for each application for each } \\
\text { experiment that exceeds lgvalue for each nuclide's capture, } \\
\text { fission and scatter reactions }\end{array}$ \\
\hline lgvalue $=$ & 0.9 & Threshold value of $g$ for inclusion in summary table. \\
\hline nixlim= & 10 & $\begin{array}{l}\text { Minimum number of experiments with group-wise values } \\
\text { exceeding senfac times the group-wise value for the application } \\
\text { for the group-wise value to be added to the completeness } \\
\text { parameter. }\end{array}$ \\
\hline penalty & False & $\begin{array}{l}\text { Create penalty assessment based on differences in the } \\
\text { application's sensitivity profile for a particular nuclide-reaction } \\
\text { pair, and the corresponding composite profile for all qualifying } \\
\text { experiments. }\end{array}$ \\
\hline
\end{tabular}


Table 6.5.2. Input data for parameter block of TSUNAMI-IP input (continued)

\begin{tabular}{|c|c|c|}
\hline Keyword & Default value & Description \\
\hline pencut $=$ & 0.0 & $\begin{array}{l}\text { Cutoff value for excluding sensitivities from the penalty } \\
\text { calculation. If the sum of the absolute values of the energy- } \\
\text { dependent sensitivity data for a particular nuclide-reaction pair } \\
\text { is below this number, the nuclide-reaction pair will not be } \\
\text { included in the penalty calculation. }\end{array}$ \\
\hline penlgv $=$ & 0.0 & $\begin{array}{l}\text { Use only nuclide-reaction pairs with } g \text { values exceeding penlgv } \\
\text { in the penalty assessment. }\end{array}$ \\
\hline penlong & False & $\begin{array}{l}\text { Print detailed edits of components of penalty assessments } \\
\text { showing each component of the penalty. }\end{array}$ \\
\hline penmin $x=$ & 10 & $\begin{array}{l}\text { Minimum number of qualifying experiments for each } \\
\text { application for penalty calculation. }\end{array}$ \\
\hline pensusec & True & $\begin{array}{l}\text { Use only experiments with } c_{k} \text { values exceeding cvalue in the } \\
\text { penalty assessment. If penusec is entered, the keywords } \\
\text { penusee and penuseg are set to False. }\end{array}$ \\
\hline penusee & False & $\begin{array}{l}\text { Use only experiments with } E \text { values exceeding evalue in the } \\
\text { penalty assessment. If penusee is entered, the keywords } \\
\text { penusec and penuseg are set to False. }\end{array}$ \\
\hline penuseg & False & $\begin{array}{l}\text { Use only experiments with } G \text { values exceeding gvalue in the } \\
\text { penalty assessment. If penuseg is entered, the keywords } \\
\text { penusec and penusee are set to False. }\end{array}$ \\
\hline penwarn & False & $\begin{array}{l}\text { Print list of warning messages noting the experiments and } \\
\text { nuclide-reaction pairs that were excluded from the penalty } \\
\text { calculation. }\end{array}$ \\
\hline plot & False & $\begin{array}{l}\text { Produces Javapeño formatted plot (.plt) files for integral values } \\
\text { and composite sensitivity data. }\end{array}$ \\
\hline prtcomp & False & $\begin{array}{l}\text { Print "composite" of experiment sensitivity profiles for } \\
\text { reactions selected in REACTIONS data block. Also write data } \\
\text { to sensitivity data file. }\end{array}$ \\
\hline prtmtrix & False & $\begin{array}{l}\text { Print directory of data available on the cross-section-covariance } \\
\text { data library. }\end{array}$ \\
\hline prtnotcv & False & $\begin{array}{l}\text { Print a table summarizing the non-coverage for the nuclide- } \\
\text { reaction pairs entered in the } R E A C T I O N S \text { data block. }\end{array}$ \\
\hline prtparts & False & $\begin{array}{l}\text { Print the components of } E \text { and } G \text { for fission capture in scatter in } \\
\text { the values table. }\end{array}$ \\
\hline relative & True & $\begin{array}{l}\left.\text { Use relative sensitivities [e.g., }\left(\Delta k_{\text {eff }} / k_{\text {eff }} / \Delta \sigma / \sigma\right)\right] \text { for all } \\
\text { applications and experiments in the analysis. If relative is } \\
\text { entered, the keyword absolute is set to False. }\end{array}$ \\
\hline return_work_cov & False & $\begin{array}{l}\text { Copy the working covariance library to the return directory } \\
\text { with the file name job_name.wrk.cov, where job_name is the } \\
\text { name of the input file. If return_work_cov is not present, the } \\
\text { working covariance library remains in the temporary working } \\
\text { directory with the file namejob_name.wrk. }\end{array}$ \\
\hline
\end{tabular}


Table 6.5.2. Input data for parameter block of TSUNAMI-IP input (continued)

\begin{tabular}{|c|c|c|}
\hline Keyword & Default value & Description \\
\hline sencut $=$ & 0.01 & $\begin{array}{l}\text { Cutoff value for ignoring low valued sensitivities in nuclide- } \\
\text { reaction specific edit tables. If the absolute value of the sum of } \\
\text { the energy-dependent sensitivity data for a particular nuclide- } \\
\text { reaction pair is below this number, the nuclide-reaction pair } \\
\text { will not be included in the edit. }\end{array}$ \\
\hline senfac $=$ & 0.9 & $\begin{array}{l}\text { Value used in calculation of completeness parameter. Group- } \\
\text { wise sensitivity for the application is counted as validated by } \\
\text { the experiment if the sensitivity from the experiment is greater } \\
\text { than the application sensitivity times senfac. }\end{array}$ \\
\hline udcov $=$ & 0.05 & $\begin{array}{l}\text { User-defined default value of standard deviation in cross- } \\
\text { section data to use for all groups for nuclide-reaction pairs for } \\
\text { which covariance data are not available on the selected data } \\
\text { file. }\end{array}$ \\
\hline udcov_corr= & 1.0 & $\begin{array}{l}\text { User-defined default correlation value to use for nuclide- } \\
\text { reaction pairs for which covariance data are not available on the } \\
\text { selected data file. }\end{array}$ \\
\hline udcov_corr_type $=$ & zone & $\begin{array}{l}\text { User-defined default correlation in cross-section data to use for } \\
\text { nuclide-reaction pairs for which covariance data are not } \\
\text { available on the selected data file. Allowed values are long, } \\
\text { zone, and short. (See Sect. 6.5.1.6.3 for details on long, zone, } \\
\text { and short.) }\end{array}$ \\
\hline udcov_fast $=$ & 0.0 & $\begin{array}{l}\text { User-defined default value of standard deviation in cross- } \\
\text { section data to use for fast data for nuclide-reaction pairs for } \\
\text { which covariance data are too large or not available on the } \\
\text { selected data file. }\end{array}$ \\
\hline udcov_inter $=$ & 0.0 & $\begin{array}{l}\text { User-defined default value of standard deviation in cross- } \\
\text { section data to use for intermediate data for nuclide-reaction } \\
\text { pairs for which covariance data are too large or not available on } \\
\text { the selected data file. }\end{array}$ \\
\hline udcov_therm= & 0.0 & $\begin{array}{l}\text { User-defined default value of standard deviation in cross- } \\
\text { section data to use for thermal data for nuclide-reaction pairs } \\
\text { for which covariance data are too large or not available on the } \\
\text { selected data file. }\end{array}$ \\
\hline uncert & False & Computes uncertainty in $k_{\text {eff }}$ due to covariance data \\
\hline uncert_long & False & $\begin{array}{l}\text { Prints extended table of uncertainty in } k_{\text {eff }} \text { due to covariance } \\
\text { data. }\end{array}$ \\
\hline use_dcov & False & $\begin{array}{l}\text { Use user-defined default covariance data for nuclide reaction } \\
\text { pairs not included on the covariance data file. The user-defined } \\
\text { data will be used in the penalty assessment as well as the } \\
\text { computation of } c_{k} \text { and uncertainty calculations. }\end{array}$ \\
\hline use_diff_groups & False & $\begin{array}{l}\text { Allow sensitivity data files to have different energy groups. } \\
\text { Data files with different energy groups will be internally } \\
\text { converted to the energy group structure of the covariance data } \\
\text { file. }\end{array}$ \\
\hline
\end{tabular}


Table 6.5.2. Input data for parameter block of TSUNAMI-IP input (continued)

\begin{tabular}{|c|c|c|}
\hline Keyword & Default value & Description \\
\hline use_icov & False & $\begin{array}{l}\text { Use user-defined data input in COVARIANCE input data block } \\
\text { in place of default values for user input nuclide-reaction pairs } \\
\text { that are not on the covariance data file. The user-defined data } \\
\text { will be used in the penalty assessment as well as the } \\
\text { computation of } c_{k} \text { and uncertainty calculations. }\end{array}$ \\
\hline usename & False & $\begin{array}{l}\text { Use the name of the sensitivity data file in place of its title in all } \\
\text { output. }\end{array}$ \\
\hline uslstats & False & $\begin{array}{l}\text { Produces USLSTATS input files for trending analysis with all } \\
\text { experiments for each global integral index }\left(c_{k}, E \text {, and } G\right) \\
\text { requested for each application }\end{array}$ \\
\hline uslsummary & False & $\begin{array}{l}\text { Produces USLSTATS input files for trending analysis with } \\
\text { experiments exceeding cutoff value (cvalue, evalue or gvalue) } \\
\text { for each global integral index }\left(c_{k}, E \text {, and } G\right) \text { requested for each } \\
\text { application }\end{array}$ \\
\hline usl $\_p=$ & 0.9990 & $\begin{array}{l}\text { Value of P in USLSTATS input files. } P \text { is the portion of the } \\
\text { population falling above the lower tolerance level }\end{array}$ \\
\hline usl_1-g= & 0.9500 & $\begin{array}{l}\text { Value of } 1-\gamma \text { in USLSTATS input files. } 1-\gamma \text { is the confidence on } \\
\text { the fit. }\end{array}$ \\
\hline usl_alpha= & 0.9990 & $\begin{array}{l}\text { Value of } \alpha \text { in USLSTATS input files. } \alpha \text { is the confidence on } \\
\text { the proportion of P. }\end{array}$ \\
\hline usl_xmin= & 0.0000 & $\begin{array}{l}\text { Value of } x(\min ) \text { in USLSTATS input files. } x(\min ) \text { is the } \\
\text { minimum value of the parameter } x \text {. }\end{array}$ \\
\hline usl_xmax= & 1.0000 & $\begin{array}{l}\text { Value of } x(\max ) \text { in USLSTATS input files. } x(\max ) \text { is the } \\
\text { maximum value of the parameter } x \text {. }\end{array}$ \\
\hline usl_sigs= & -0.0030 & $\begin{array}{l}\text { Value of } \sigma_{\mathrm{s}} \text { in USLSTATS input files. If a positive value is } \\
\text { entered, it is applied as the total uncertainty for each } \\
\text { experiment. If a negative or zero value is entered, it is used as } \\
\text { the experimental measurement uncertainty and any } \\
\text { Monte Carlo and cross section uncertainties are added } \\
\text { quadratically to the absolute value of usl_sigs for each } \\
\text { experiment. }\end{array}$ \\
\hline usl_dkm= & 0.0500 & $\begin{array}{l}\text { Value of } \Delta k_{\mathrm{m}} \text { in USLSTATS input files. } \Delta k_{\mathrm{m}} \text { is the } \\
\text { administrative margin used to ensure subcriticality. }\end{array}$ \\
\hline usl_uncert & False & $\begin{array}{l}\text { Includes uncertainty in } k_{\text {eff }} \text { due to cross section covariance data } \\
\text { from } k_{\text {eff }} \text { uncertainty written to USLSTATS input files. }\end{array}$ \\
\hline values & False & $\begin{array}{l}\text { Print all computed "global" indices }\left(E, c_{k} \text {, and } G\right) \text { in a table for } \\
\text { each application. If prtparts is input, also include the partial } \\
\text { values. }\end{array}$ \\
\hline
\end{tabular}

\subsection{Reaction Data}

The REACTIONS data block is used to specify nuclide-reaction pairs for the $\lg$ and lggroups edits. The reactions block must begin with READ REACTIONS and end with END REACTIONS. Data are entered 
in pairs with the nuclide number (e.g., 92235) followed by the reaction MT number (e.g., 18). Alphanumeric input is also accepted (e.g., u-235 fission) for the nuclide-reaction pairs. Mixed input is also acceptable (e.g., 92235 fission). Available reaction types are given in Table 6.5.4.

\subsection{User input covariance data}

The COVARIANCE data block allows the user to specify a covariance matrix for specific nuclide-reaction pairs for which covariance data are not present on the covariance data file or that have zero or large values on the diagonal. The COVARIANCE data block must begin with READ COVARIANCE and end with END COVARIANCE.

Table 6.5.3. Input options for user-defined covariance data

\begin{tabular}{|c|c|c|c|c|}
\hline $\begin{array}{c}\text { Input } \\
\text { Parameter }\end{array}$ & Requirement & $\begin{array}{c}\text { Default } \\
\text { Value }\end{array}$ & $\begin{array}{c}\text { Allowed } \\
\text { Values }\end{array}$ & Description \\
\hline Nuclide & Required & none & $\begin{array}{l}\text { Nuclide name } \\
\text { or ZA number }\end{array}$ & $\begin{array}{l}\text { Nuclide for which covariance data are to } \\
\text { be entered }\end{array}$ \\
\hline Reaction & Required & none & $\begin{array}{l}\text { Reaction name } \\
\text { or MT number }\end{array}$ & $\begin{array}{l}\text { Reaction for which covariance data are to } \\
\text { be entered. See Table } 6.5 .4 \text { for available } \\
\text { reaction types. }\end{array}$ \\
\hline all $=$ & Optional & 0.0 & any number & $\begin{array}{l}\text { Fractional standard deviation value to be } \\
\text { applied to all groups. }\end{array}$ \\
\hline fast $=$ & Optional & 0.0 & any number & $\begin{array}{l}\text { Fractional standard deviation value to be } \\
\text { applied to fast groups. The fast value } \\
\text { overrides the all value in the fast groups. }\end{array}$ \\
\hline therm $=$ & Optional & 0.0 & any number & $\begin{array}{l}\text { Fractional standard deviation value to be } \\
\text { applied to thermal groups. The therm } \\
\text { value overrides the all value in the thermal } \\
\text { groups. }\end{array}$ \\
\hline inter $=$ & Optional & 0.0 & any number & $\begin{array}{l}\text { Fractional standard deviation value to be } \\
\text { applied to intermediate groups. The inter } \\
\text { value overrides the all value in the } \\
\text { intermediate groups. }\end{array}$ \\
\hline corr $=$ & Optional & 1.0 & $\begin{array}{l}\text { any number } \\
\text { from }-1.0 \text { to } 1.0\end{array}$ & $\begin{array}{l}\text { Correlation between groups (see corr_type } \\
\text { for use) }\end{array}$ \\
\hline & & & & $\begin{array}{l}\text { Type of correlation applied form group-to- } \\
\text { group covariance values. }\end{array}$ \\
\hline & & & & $\begin{array}{l}\text { long - correlation is applied between all } \\
\text { groups }\end{array}$ \\
\hline corr_type & Optional & zone & $\begin{array}{l}\text { long, short, } \\
\text { zone }\end{array}$ & $\begin{array}{l}\text { short - correlation is applied only between } \\
\text { adjacent groups }\end{array}$ \\
\hline & & & & $\begin{array}{l}\text { zone - correlation is applied within fast, } \\
\text { intermediate and thermal groups, but no } \\
\text { correlation is applied between zones }\end{array}$ \\
\hline end & Required & & & $\begin{array}{l}\text { Denotes end of input for current } \\
\text { nuclide/reaction }\end{array}$ \\
\hline
\end{tabular}

Any MT number or reaction name will be treated as a valid input, but only those present on the sensitivity data files will produce useful information. The reaction sensitivity types computed by SAMS from TSUNAMI-1D and TSUNAMI-3D are shown in Table 6.5.4. An energy-covariance matrix is created for the specified nuclide-reaction pair with the square of the entered standard deviation for the diagonal terms for all groups using the all= value. Groups in the fast, intermediate and thermal energies are then set to the square of the standard deviation value entered for fast=, inter=, and therm $=$, respectively. The off- 
diagonal terms of the energy matrix are generated according to the input for corr=, and corr_type=, with default settings of 1.0 and zone. Data entered in this block do not override data present on the covariance data file. The SCALE 5.1 input format is supported where data are entered in triplets with the nuclide name (e.g., u-235), then the reaction MT number or name (e.g., 18 or fission), and then a standard deviation value. In this case, the end card must not be entered. The standard deviation value is applied to all groups with default setting for correlations. These data are only used if use_icov is specified in the PARAMETER data block.

Table 6.5.4. Reaction sensitivity types computed by SAMS

\begin{tabular}{rll}
\hline MT & \multicolumn{1}{c}{ Reaction } & \multicolumn{1}{c}{ SCALE identifier } \\
\hline 0 & Sum of scattering & scatter \\
1 & Total & total \\
2 & Elastic scattering & elastic \\
4 & Inelastic scattering & n,n' \\
16 & $\mathrm{n}, 2 \mathrm{n}$ & $\mathrm{n}, 2 \mathrm{n}$ \\
18 & Fission & fission \\
101 & Neutron disappearance & capture \\
102 & $\mathrm{n}, \gamma$ & $\mathrm{n}, \mathrm{gamma}$ \\
103 & $\mathrm{n}, \mathrm{p}$ & $\mathrm{n}, \mathrm{p}$ \\
104 & $\mathrm{n}, \mathrm{d}$ & $\mathrm{n}, \mathrm{d}$ \\
105 & $\mathrm{n}, \mathrm{t}$ & $\mathrm{n}, \mathrm{t}$ \\
106 & $\mathrm{n},{ }^{3} \mathrm{He}$ & $\mathrm{n}, \mathrm{he}-3$ \\
107 & $\mathrm{n}, \alpha$ & $\mathrm{n}, \mathrm{alpha}$ \\
452 & $\bar{v}$ & nubar \\
1018 & $\chi$ & Chi \\
\hline
\end{tabular}

If use icov is specified, these data are used for the calculation of the uncertainty in $k_{\text {eff }}$ (uncert and uncert_long edits), calculation of $c_{k}$, Extended $c_{k}$, and the penalty assessment for each application. These user input values are only applied where an application has a sensitivity profile for which there is no corresponding covariance matrix on the covariance data file. When both use icov and cov fix are specified in the PARAMETER data block, and a reaction has zero or large (standard deviation $>1000 \%$ ) values on the diagonal of the covariance matrix, these values are replaced with the square of the user input standard deviation value, and the corresponding off-diagonal terms are substituted according to the values of corr and corr_type.

Specifying user input covariance data for the summative reactions total, scatter and capture have no affect on the results of TSUNAMI-IP, as the summative reactions are not used in uncertainty analysis calculations. 


\subsection{Application, experiment, and response data}

Sensitivity data files are designated as either application systems or experiment systems by the APPLICATIONS, EXPERIMENTS, or RESPONSE data blocks. For each application system, TSUNAMI-IP will calculate integral parameters against each experiment system. Each data block must begin with READ KEYNAME and end with END KEYNAME where KEYNAME can be APPLICATIONS, EXPERIMENTS, or RESPONSE.

Inside each data block, sensitivity data files are listed using response definition records. A response definition record is a single line of input that contains the sensitivity data filename and nine optional keyword specifications. The sensitivity data filename and optional keywords can be entered in any order, with the following format:

$$
\text { filename }(\text { name }=N)(u s e=U)(\text { type }=T)(\text { omit })(e v=E)(u v=U)(n u=P) \quad \text { (absolute or abs) (relative or rel) }
$$

where

$$
\begin{aligned}
& \text { filename }=\text { sensitivity data filename. The filename can include the file path. } \\
& \mathrm{N} \quad=\text { A descriptive identifier for the sensitivity data file in TSUNAMI-IP output edits. } \\
& \text { The identifier is limited to } 20 \text { alphanumeric characters (spaces are not allowed). } \\
& \mathrm{U}=\text { The file usage. Allowed values are appl, expt, and omit, signifying the use of the file } \\
& \text { as an application or an experiment or to exclude the file from the analysis, } \\
& \text { respectively. The default values for the use keyword are described below. } \\
& \mathrm{T}=8 \text {-character alphanumeric identifier for the response type (e.g. 'keff'). The response } \\
& \text { type is used in various output edits along with the name }=N \text { identifier. } \\
& \text { omit - Optional keyword used to omit filename from the analysis, can be used independent } \\
& \text { of } u s e=\text {. } \\
& \left.\mathrm{E}=\text { experimental value of the response (e.g. } k_{\text {eff }}\right) \text {. } \\
& \mathrm{U} \quad=\text { uncertainty value of the response. } \\
& \mathrm{P}=\text { number of uncertainty components to characterize the experiment uncertainty for } \\
& \text { this response. }
\end{aligned}
$$

By default, filenames listed in the APPLICATIONS data block are designated as application systems. Likewise, filenames listed in the EXPERIMENTS or RESPONSE data blocks are designated as experiment systems. The sensitivity data files must be in either the TSUNAMI-A or TSUNAMI-B file format. The formats of these data files are detailed in Appendix A. Only the region-integrated sensitivity coefficients from the sensitivity data files are used by TSUNAMI-IP. These data are energy-dependent, but any mixture-dependent or region-dependent data present on the data files are not used. Case-sensitive filenames and their file paths are allowed. However, spaces are not allowed in the filenames or file paths.

The nine optional keywords provide more user control over how each sensitivity data file is used in the analysis, and how each sensitivity data file is identified in the TSUNAMI-IP output. By default, TSUNAMI-IP identifies files in the output according to the title on the sensitivity data files. For files that have the same titles, or have long or non-descriptive titles, the usename keyword in the PARAMETER data block can be used to identify sensitivity data files by their filename. Although file names are unique, they can also be non-descriptive. For this reason, the name keyword on the response definition record can 
be used to create a new identifier for the sensitivity data file in the TSUNAMI-IP output. Similarly, the type keyword can be used to identify the response type in the output. Because TSUNAMI-IP was initially intended for similarity assessment of critical systems, the default response type identifier is ' $k e f f$ '; the type identifier for Generalized Perturbation Theory reaction rate sensitivity responses is ' $g p t$ '. For reactivity sensitivity data files produced by the TSAR module (Chapter 6.4), the default response type identifier is 'rho'. The type keyword can be used to specify a descriptive response type identifier, such as type $=k \_s d m$ or type $=$ alpha_t.

The use keyword specifies how the sensitivity data file is used in the analysis. Allowed values are expt, appl, or omit. All sensitivity data files on response definition records with $u s e=\operatorname{expt}$ are designated as experiment systems, regardless of what data block it is in. Likewise, all sensitivity data files on response definition records with $u s e=a p p l$ are designated as application systems. In addition, the user can omit the sensitivity data file from the analysis by entering either use=omit or simply omit on the response definition record.

The experiment value of the response $(e v=)$ and the experiment uncertainty of the response $(u v=)$ are used in generating USLSTATS input files for trending analysis. If the experiment value is not provided, the default value used in USLSTATS is 1.0. If the experiment uncertainty value is not provided, the default used in USLSTATS is the absolute value of the usl_sigs value in the PARAMETER data block.

The $e v=$ and $u v=$ keywords do not impact the computed results in TSUNAMI-IP and are only used if the uslstats keyword is included in the PARAMETER data block. Experiment values of $k_{\text {eff }}$ or reactivity only need to be provided for experiment systems. TSUNAMI-IP skips over $e v=$ and $u v=$ keyword specifications given for application or omitted systems.

In addition to the $u v=$ keyword specification, TSUNAMI-IP allows for the experiment uncertainty value to be given in terms of uncertainty components. The $n u=$ keyword defines the number of uncertainty components that characterize the experiment uncertainty. If the experiment response uncertainty is given in terms of uncertainty components, the $u v=$ keyword specification is optional. An uncertainty component definition record follows the response definition record if the $n u=$ keyword specification is given. The uncertainty component definition record has the following format:

$$
\text { uncmp1 val1 uncmp2 val2 ....... uncmpP valP }
$$

where

$$
\begin{array}{ll}
\begin{array}{ll}
\text { uncmp1 } & =4 \text {-character alphanumeric identifier for the } 1^{\text {st }} \text { uncertainty component. } \\
\text { val1 } & =\text { experiment uncertainty for component } \text { uncmp } 1 . \\
\text { uncmp } 2 & =4 \text {-character alphanumeric identifier for the } 2^{\text {nd }} \text { uncertainty component. } \\
\text { val2 } & =\text { experiment uncertainty for component } \text { uncmp } 2 . \\
\text {...... } & \\
\text { uncmpP } & =4 \text {-character alphanumeric identifier for the } \mathrm{P}^{\text {th }} \text { uncertainty component. } \\
\text { valP } & =\text { experiment uncertainty for component } \text { uncmpP. }
\end{array}
\end{array}
$$

The uncertainty component definition record contains $n u=P$ pairs of alphanumeric identifiers and numeric values. The experiment uncertainty value is determined by taking the square root of the sum of the squares of each uncertainty component value. 
The final optional keywords $a b s$ and $r e l$ are used to determine the format of sensitivity and uncertainty data on the response definition record and the uncertainty component definition record. For a $k_{\text {eff }}$ response, the following four input definitions produce equivalent uncertainties:

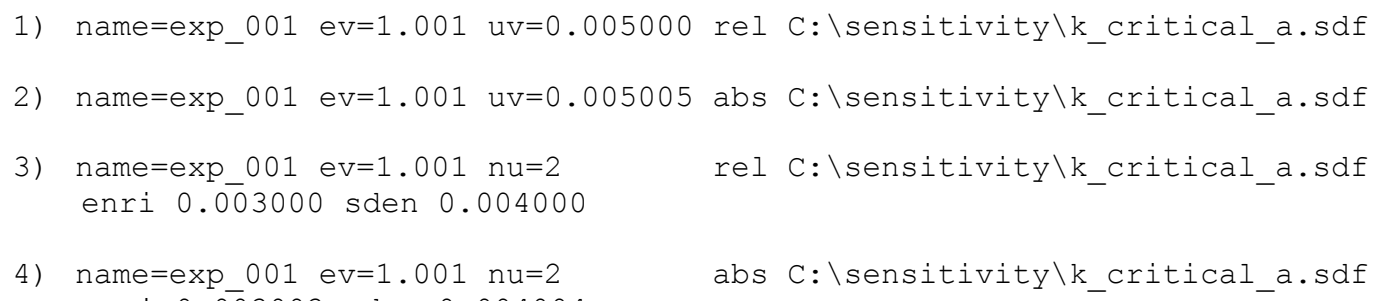

In the example above, the measured $k_{\text {eff }}$ is $1.001 \pm 0.5 \%$ or $1.001 \pm 0.005005$. The sensitivity data filename is given as C: $\mid$ sensitivity $\mid k \_c r i t i c a l \_a . s d f$, and the experiment response is referred to as exp_001 in the TSUNAMI-IP output. In 1), the relative format is used to specify the relative standard deviation of the measured response as 0.005 . In 2), the absolute format is used to specify the absolute standard deviation of the measured response as 0.005005 . Because the TSUNAMI-generated sensitivity data file is in relative format, TSUNAMI-IP internally renormalizes the relative sensitivities to absolute sensitivities. In 3), the relative format is used to specify the relative standard deviation of $k_{\text {eff }}$ due to two components (enri and sden) as 0.003 and 0.004 respectively. Using the square root of the sum of the squares approach, the relative standard deviation of $k_{\text {eff }}$ is computed to be 0.005 . In 4), the absolute format is used to specify the absolute standard deviation of $k_{\text {eff }}$ due to two components (enri and sden) as 0.003003 and 0.004004 respectively. The absolute standard deviation of $k_{\text {eff }}$ is computed to be 0.005005 . Like 2), the sensitivity data file is internally converted to contain absolute sensitivities.

Because of the high-degree of flexibility in designating application systems and experiment systems in the TSUNAMI-IP input, the following two methods are recommended. The first method is consistent with previous versions of TSUNAMI-IP. That is, application systems are listed in the APPLICATIONS data block and experiment systems are listed in the EXPERIMENTS data block. The second method would be to list both set of systems in a single RESPONSE data block and use the $u s e=U$ keyword specification to designate how each file is to be used. Sample input files for both methods are given for the TSUNAMIIP example problem in Sect. 6.5.1.6.7.

\subsection{Reactions excluded from $c_{r}$}

The EXCLUSIONS data block contains lists of reaction types that will be removed from consideration in the calculation of the $c_{r}$ integral index and in the extended $c_{r}$ edit. Valid input for this block are reaction MT numbers or names (e.g., 18 or fission). Values must be separated by a space of a line break. Any MT number or reaction name will be treated as a valid input, but only those present on the sensitivity data files will produce useful information. The reaction sensitivity types computed by SAMS from TSUNAMI-1D and TSUNAMI-3D are shown in Table 6.5.4.

\subsection{HTML data}

The optional HTML data block is used to customize HTML formatted output. The HTML data block must begin with READ HTML and end with END HTML. The data input to the HTML data block consist of several keywords that are shown, along with their default values and descriptions, in Table 6.5.5. A keyword that ends with = must be followed by text data. For color entries, any valid HTML color name can be entered or the hexadecimal representation can be used if preceded by a \# sign. For example, to change the background color of the HTML output to white, $b g_{-}$clr=white and bg_clr=\#ffffff have the same effect, because ffffff is the hexadecimal representation of white. An extensive list of available 
colors for customized output is shown in Appendix B. Please note that not all features are supported by all browsers.

Table 6.5.5. Input data for HTML block of TSUNAMI-IP input

\begin{tabular}{ccl}
\hline Keyword & Default value & \multicolumn{1}{c}{ Description } \\
\hline bg_clr $=$ & papayawhip & Background color. \\
h1_clr $=$ & maroon & Color used for major headings. \\
h2_clr $=$ & navy & Color used for sub-headings. \\
txt_clr $=$ & black & Color for plain text. \\
lnk_clr $=$ & maroon & Color for hyperlinks. \\
lnk_dec $=$ & none & Decoration for hyperlinks. (none, underline, overline, line-through, blink). \\
vlnk_clr & navy & Color for visited hyperlinks. \\
max_clr $=$ & maroon & Color for maximum values in tables. \\
cut_clr $=$ & navy & Color for values in tables that exceed cutoff values. \\
ud_clr $=$ & blue & Color for values in tables that use default covariance data. \\
ui_clr $=$ & Red & Color for values in tables that use user-input covariance data. \\
udfix_clr $=$ & royalblue & Color for values in tables that use default corrected covariance data. \\
uifix_clr $=$ & green & Color for values in tables that use user-input corrected covariance data. \\
\hline
\end{tabular}

\subsection{Example TSUNAMI-IP input}

An example TSUNAMI-IP input listing is given in Figure 6.5.4. In this example, the optional title for this analysis is entered as "tsunami-ip example". The parameter data are used to request that $E, c_{k}, G$, and $g$ be computed (e,c,g, and $l g$, respectively). The integral indices $E, c_{k}$, and $G$ will be listed in a table for each application containing the values of each index for each experiment (values). The cutoff values for $E$ and $c_{k}$ for inclusion in the summary tables are set to 0.8 (evalue $=0.8$ and cvalue $=0.8$ ). Summary tables listing experiments with $E$ values exceeding evalue, $c_{k}$ values exceeding cvalue will be produced (esummary, and csummary, respectively). The REACTIONS data block requests that the $n, \gamma$ reaction for ${ }^{235} \mathrm{U}$ and the elastic scattering reaction for ${ }^{1} \mathrm{H}$ be assessed for coverage with the $g$ index. 


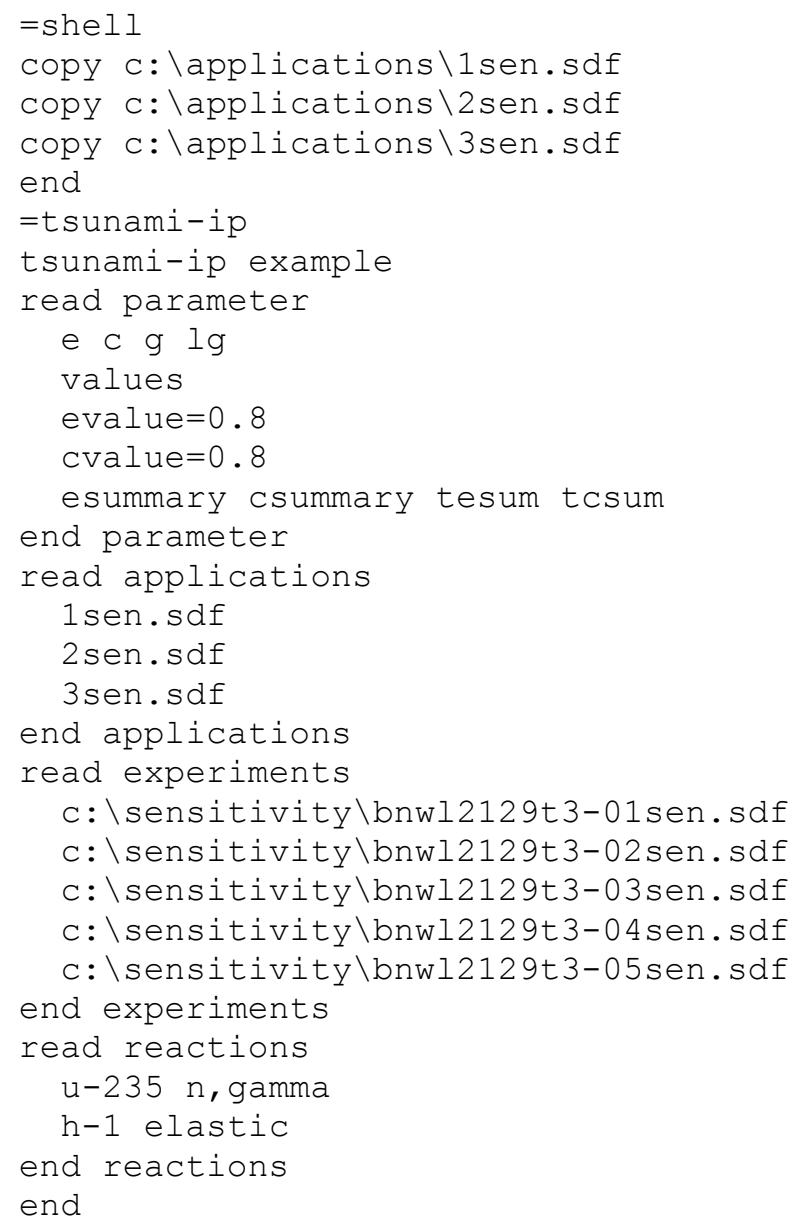

Figure 6.5.4. Example TSUNAMI-IP input listing.

The application systems are represented by the sensitivity data files named 1 sen.sdf, 2 sen.sdf and 3 sen.sdf. In this example input, these data files are copied (or they could be linked in UNIX) to the temporary directory where SCALE executes and writes its scratch files via system commands that are input to the SHELL utility at the top of the input file. In a typical SCALE installation on a PC, this directory is called c: $\mid$ scale6\tmpdir when running SCALE6 from the command line. On a UNIX system, this directory is defined by the environment variable \$TMPDIR when executing scale6 or batch6 with the $-t$ option. When executing batch 6 without the $-t$ option, the temporary directory will change each time batch6 is executed. The experiments for which the indices will be computed for each application are listed in the EXPERIMENTS data block. Here, an explicit file path is specified in the input. This could also have been done in the APPLICATIONS data block. In this case, the 5 data files would have to exist in a directory called c: Isensitivity. An equivalent input file using a single RESPONSE data block is given in Figure 6.5.5. 


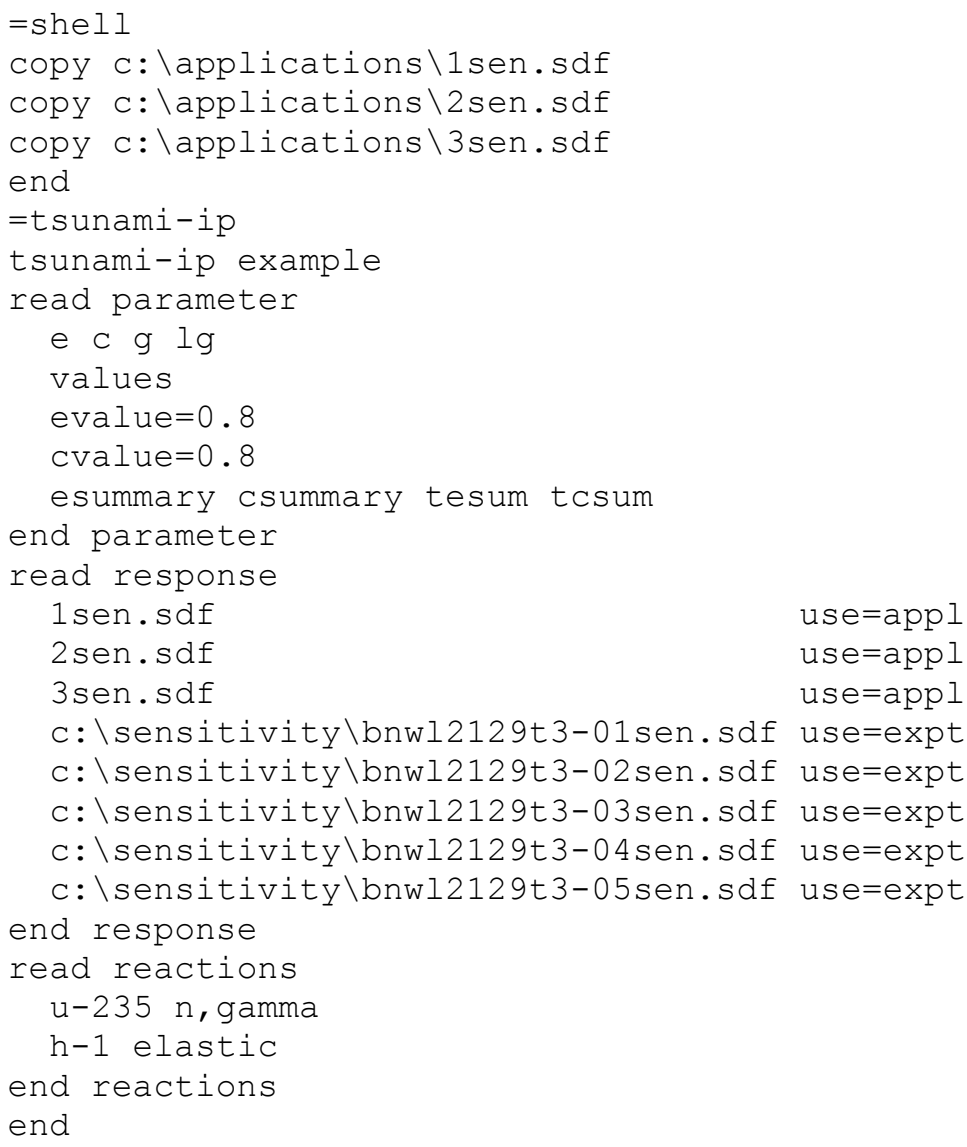

Figure 6.5.5. Example TSUNAMI-IP input listing with Response Block.

\subsubsection{Example PROBLEM}

An example problem to demonstrate the use of TSUNAMI-IP is based on the TSUNAMI-1D and TSUNAMI-3D sample problems available with the SCALE distribution. The systems included in the sample problem are not intended to provide a rigorous demonstration of the methodologies of TSUNAMI-IP, but merely to demonstrate how to use the software and read the output. The problem does not correspond to the sample problem distributed with SCALE and is included for illustrative purposes only.

\subsection{Input description}

The example problem input listing is shown in Figure 6.5.6. The input begins with the SCALE shell module, which executes a series of copy commands to move the sensitivity data files required for code execution from the user's directory (\$root_dir\} on Unix, \%RTNDIR\% on Windows) to the current working directory. Next TSUNAMI-IP is called with =tsunami-ip. The optional title card is entered as "tsunami-ip example 2." In the PARAMETER data block, the values table is requested and it will contain $c_{k}, E$ and $G$, since $c$ e $g$ are entered on the next line of input. The cutoff value for inclusion of $c_{k}$ values in the summary table is set at 0.8 with cvalue $=0.8$ and the $c_{k}$ summary table is requested with csummary. The nuclide-reaction specific integral indices extended $c_{k}$ and $g$ are requested with the input $c$ long and $l g$. The summary table of $g$ values exceeding the cutoff is requested with lgsum, and the cutoff value for this table is set to 0.75 with lgvalue $=0.75$. The composite sensitivity profiles for the reactions entered in the REACTIONS input section are requested with prtcomp, and the summary of non-coverage edit is 
requested with prtnotcv. The uncertainty in the $k_{\text {eff }}$ values for the experiments and applications due to cross section uncertainties is requested with uncert and the extended uncertainty edits for the applications are requested with uncert_long. The penalty assessment is requested with penalty, the minimum number of experiments exceeding the global integral index; the cutoff value is set to 0 with penmin $x=0$. In this way, the limited number of experiments used in this sample problem will still produce a penalty edit. The detailed edit of the components of the penalty are requested with penlong. The user-defined default value for covariance data are set to $100 \%$ uncertainty for cross sections without covariance data with $u d c o v=1.0$. The use_dcov input keyword activates the use of the $u d c o v$ value in the computation of $c_{k}$ and the penalty assessment. The use of filenames in place of the titles on the data files is requested with usename.

This input defines two systems as applications in the APPLICATIONS data block. The application systems are defined as the system with sensitivity data in the data files tsunami-3d_k5-1.sdf and tsunami3d_k5-2.sdf. Three experiments are defined in the EXPERIMENTS data block. These systems have sensitivity data in the data files tsunami-1d1.sdf, tsunami-1d4.sdf, and tsunami-3d_k5-3.sdf. 


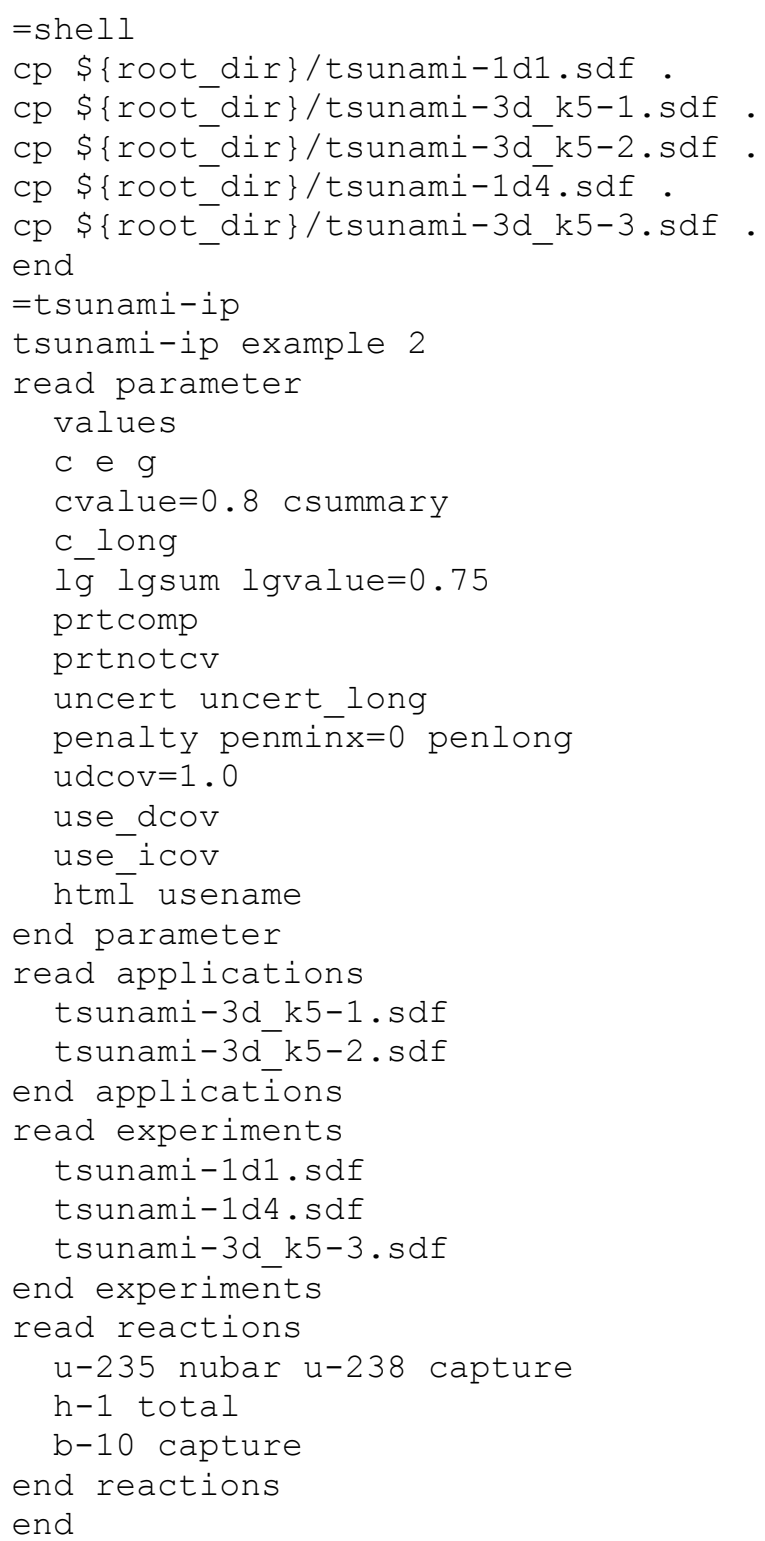

Figure 6.5.6. TSUNAMI-IP sample problem input. 


\subsection{Output Description}

The output listings shown here are for descriptive purposes only. The data generated from an actual execution of this problem may differ from that shown below. The Applications List and the Experiments List provide some details from the sensitivity data files. These include the title on the data files, the filename, the number of energy groups and the number of sensitivity profiles. After the Experiments List, Covariance Warnings are printed during the creation of the working (i.e., problem-dependent) covariance library. The nuclide-reactions pairs present on the sensitivity data files for which covariance data are not available on the coverx file are detailed. This edit is shown in Figure 6.5.8.

The next output edit, shown in Figure 6.5.9 details the global integral indices for the current application compared to all experiments. The edit is only present if values is entered in the PARAMETER data block. The first row of data in the table displays the application compared to itself. This is useful to confirm the normalization of the integral indices. The data detailing the application compared to itself is not displayed in the HTML output. The next rows display the values of the integral indices requested by the user for each experiment as compared to the current application. The values of the response (i.e., $k_{e f f}$ or reactivity) for the application and each experiment are also shown in this table. If the sensitivity data were computed with Monte Carlo methods, the statistical uncertainties are shown. In Figure 6.5.9, the $k_{\text {eff }}$ value for tsunami-1d1 does not show a statistical uncertainty because these sensitivity data were computed with TSUNAMI-1D. The integral values of the application compared to tsunami-1d1 do show statistical uncertainties because the application system was computed with TSUNAMI-3D. The column titles "xsec unc \%" gives the relative uncertainty in the response due to cross section data uncertainties, in percent.

The next output edit is the extended uncertainty edit for the application and is shown in Figure 6.5.10. This edit details the uncertainty in the response due to each nuclide-reaction to nuclide-reaction covariance matrix. Where default or user input values for covariance data are used, they are indicated in the output edit. The total uncertainty can be computed from the individual values by squaring each term, adding the values that had positive signs, subtracting the values that had negative signs, then taking the square root. The edit is present because uncert_long was included in the parameter input.

The next output edit is the extended $c_{k}$ edit, and an example edit for Application 1 with Experiment 1 is shown in Figure 6.5.11. This edit details the contribution to $c_{k}$ due to each nuclide-reaction to nuclidereaction covariance matrix. Where default or user input values for covariance data are used, they are indicated in the output edit. The edit is present because $c_{-}$long was included in the parameter input. The $c_{k}$ value can be computed by summing the individual values. Similar tables present the output for each application compared to each experiment.

The next output edit includes the nuclide-reaction specific integral indices and is shown in Figure 6.5.12. In this sample problem, $g$ indices for fission capture and scatter reactions for each nuclide-reaction pair with a sensitivity coefficient exceeding sencut in magnitude are printed for each experiment compared to the application. The first table of values is for the application compared to itself. This initial edit is useful to test the normalization of the integral indices for the application. This initial table of the application compared with itself is not available in the HTML output. The energy-integrated sensitivity coefficient for the application is also shown for each reaction. Statistical uncertainties in these integral values are not available in this version of TSUNAMI-IP. This table is repeated for each experiment compared to the application. Because some reactions were requested in the REACTIONS section of the input, and the $g$ integral index was computed, a table of the user requested $g$ values for the application compared to each experiment is given. In this sample problem, ${ }^{10} \mathrm{~B}$ was not present in the application, even though it was requested in the input. TSUNAMI-IP lists all requested reactions in this table, but 
leaves the values blank when the nuclide or reaction is not available on the sensitivity data file for the application.

The next output edit, shown in Figure 6.5.13, gives the extended penalty assessment for application 1. This edit is present because penlong was entered in the parameter data block. The penalty edit utilizes the user defined default uncertainties defined by udcov because use_dcov was entered in the PARAMETER data block. Nuclide reaction pairs that utilized default uncertainty data are indicated with *. In this case, when the sensitivity coefficients of the application that are not covered by the experiments are propagated to an uncertainty in the application response with the cross-section-covariance data value of $0.0097 \% \pm$ $0.00007 \%$ results. Next a listing of the contributions to the penalty from each nuclide-reaction to nuclidereaction covariance matrix is given. As with the extended uncertainty edit, the cumulative penalty can be computed from the individual values by squaring each term, adding the values that had positive signs, subtracting the values that had negative signs, then taking the square root.

Each of the previous edits would repeat in the full output listing for each application. The next series of output edits, shown in Figure 6.5.14, are the summary edits requested by the user. The $c_{k}$ summary table, requested with the keyword csummary, indicates that one experiment has a $c_{k}$ value at least as great as 0.8 compared to each application. The $g$ summary table lists the $g$ values that exceed lgvalue for the fission, capture and scatter reactions for each nuclide in the application with an energy-integrated sensitivity coefficient exceeding sencut in magnitude. The value of the sensitivity coefficient for the application is also given. The summary of non-coverage edit is present because prtnotcv was entered in the PARAMETER input data block. This edit lists a number of properties of each reaction input in the REACTIONS input data block. The final summary table is the penalty summary table, which is activated when penalty is listed in the PARAMETER data block. The penalties for all applications identified in the APPLICATIONS input data block are listed in the penalty summary table.

The composite sensitivity data are listed next in the TSUNAMI-IP output as shown in Figure 6.5.15. The group-wise values for the composite profiles for each nuclide-reaction pair identified in the REACTIONS data block are listed here for each application identified in the APPLICATIONS data block. If a reaction is requested that is not present in the application, the reaction is left blank in the table, as is the case in this sample problem for ${ }^{10} \mathrm{~B}$ capture. The composite sensitivity profiles are also written to a TSUNAMI-B formatted sensitivity data file. In this data file, the composite sensitivity profiles are written for each application in the order they were requested in the REACTIONS data block. If a requested reaction is not available in the application, it is not written to the data file. The application number is listed on the data file in the position of the material number but with a value that is the negative of the application number. All unit and region information are entered as zeros as is the $k_{\text {eff }}$ value. This data file can be correctly read by Javapeño such that the composite data can be plotted. In Javapeño, the application number appears in the list of available sensitivity profiles identified as "composite." The application sensitivity data and the composite data as used in the penalty calculation are also included on the composite sensitivity data file. These new data are identified as "application" and "cut composite" respectively. 


\begin{tabular}{|c|c|c|}
\hline TSUI & IP: Tools for & $\begin{array}{c}\text { Sensitivity and UNcertainty Analysis Methodology Implementation - Indices and Parameters } \\
\text { tsunami-ip example } 2\end{array}$ \\
\hline \multicolumn{3}{|r|}{ TSUNAMI-IP Parameter Table } \\
\hline PARAMETER & VALUE & DESCRIPTION \\
\hline absolute & false & $\begin{array}{l}\text { Print uncertainty values and penalty assessments in absolute format. This is the default } \\
\text { format. Relative format can be specified using the "rel" keyword in the APPLICATIONS, } \\
\text { EXPERIMENTS, or RESPONSE input blocks. }\end{array}$ \\
\hline c & true & Compute $c(k)$ values for each application compared to each experiment. \\
\hline c_long & true & $\begin{array}{l}\text { Produces extended } c(k) \text { output edit for each application compared to each experiment. } \\
\text { Sets } c \text { to true. }\end{array}$ \\
\hline cechck = & 0.5000 & $\begin{array}{l}\text { Level of } E \text { and } c(k) \text { values that trigger the cediff warning. If } E \text { or } c(k) \text { are below this } \\
\text { value, no warning is printed. }\end{array}$ \\
\hline cediff= & 0.1000 & $\begin{array}{l}\text { If the } E \text { and } c(k) \text { values for a given application and experiment differ by more than } \\
\text { cediff, a warning message is printed. }\end{array}$ \\
\hline coverx= & 44 groupcov & Name of cross section covariance data file. \\
\hline cov_fix & false & $\begin{array}{l}\text { Replace zero and large values on diagonal of cross-section covariance data with user } \\
\text { input values and dcov value. }\end{array}$ \\
\hline $\mathrm{cp}$ & false & Compute and print completeness parameter for each application. \\
\hline csummary & true & Print summary table of $c(k)$ values that meet or exceed the cvalue limit. Sets $c$ to true. \\
\hline cvalue= & 0.8000 & Threshold value of $c(k)$ for inclusion in the summary table. \\
\hline cr & false & Compute $c(r)$ values for each application compared to each experiment. \\
\hline cr_long & false & $\begin{array}{l}\text { Produces extended } c(r) \text { output edit for each application compared to each experiment. } \\
\text { Sets cr to true. }\end{array}$ \\
\hline crsummary & false & $\begin{array}{l}\text { Print summary table of } c(r) \text { values that meet or exceed the crvalue limit. Sets cr to } \\
\text { true. }\end{array}$ \\
\hline
\end{tabular}

Figure 6.5.7. Input echo from TSUNAMI-IP output. 


$\begin{array}{ll}\text { crvalue }= & 0.9000 \\ \text { esummary } & \text { true } \\ \text { false } & \\ \text { evalue= } & 0.9000 \\ \text { g } & \text { true } \\ \text { gsummary } & \text { false } \\ \text { gvalue= } & 0.9000 \\ \text { html } & \text { true } \\ \text { inptcase } & \text { false } \\ \text { large_cov= } & 10.0000 \\ \text { lg } & \text { true } \\ \text { penalty } & \text { true } \\ \text { lgall } & 0.7500 \\ \text { lggroups } & \text { false } \\ & \text { false } \\ & \end{array}$

Threshold value of $c(r)$ for inclusion in the summary table.

Compute $\mathbf{E}$ (sum) values for each application compared to each experiment.

Print summary table of $\mathrm{E}$ (sum) values that meet or exceed the evalue limit. Sets e to true.

Threshold value of $\mathrm{E}$ (sum) for inclusion in the summary table.

Compute $\mathrm{G}$ values.

Print summary table of $G$ values that meet or exceed the gvalue limit. Sets $g$ to true.

Threshold value of $G$ for inclusion in summary table.

Generate output in html format in addition to the standard text output.

Set the SCALE free form reader to leave the case of the input data as read.

Cutoff fractional standard deviation value for cov fix. Covariance data with uncertainties larger than large_cov are replaced with user-defined or default values.

Compute $g$ values for fission, capture and scatter for each nuclide for each experiment compared to each application and print them in a table if the application's sensitivity for the corresponding nuclide-reaction pair is greater than or equal to sencut.

Print $g$ values for fission, capture and scatter for all nuclides for all experiments for each application.

Print a table listing the number of experiments that are at least as sensitive as the application for each group for each reaction requested in the REACTIONS input block.

Prints a summary table of $\mathrm{g}$ for each application for each experiment that exceeds lgvalue for each nuclide's capture, fission and scatter reactions.

Threshold value of $\mathrm{g}$ for inclusion in summary table.

Minimum number of experiments with group-wise values exceeding senfac times the group-wise value for the application for the group-wise value to be added to the completeness parameter.

Creates penalty assessment based on differences in the application's sensitivity profile for a particular nuclide-reaction pair, and the corresponding composite profile for all qualifying experiments.

Figure 6.5.7. Input echo from TSUNAMI-IP output.(continued) 


\begin{tabular}{|c|c|}
\hline pencut= & $0.0000 \mathrm{E}$ \\
\hline penlgv= & $0.0000 \mathrm{E}$ \\
\hline penlong & true \\
\hline penmin $x=$ & 0 \\
\hline penusec & true \\
\hline penusee & false \\
\hline penuseg & false \\
\hline penwarn & false \\
\hline plot & false \\
\hline prtcomp & true \\
\hline prtmtrix & false \\
\hline prtnotcv & true \\
\hline prtparts & false \\
\hline relative & true \\
\hline return_work_cov & false \\
\hline sencut= & 0.0100 \\
\hline senfac $=$ & 0.9000 \\
\hline
\end{tabular}

Cutoff value for excluding sensitivities from the penalty calculation. If the sum of the absolute values of the energy-dependent sensitivity data for a particular nuclide-reaction pair is below this number, the nuclide-reaction pair will not be included in the penalty calculation.

Use only nuclide-reaction pairs with $g$ values exceeding penlgv in the penalty assessment. Print detailed edits of components of penalty assessments.

Minimum number of qualifying experiments for each application for penalty calculation.

Use only experiments with $c(k)$ values exceeding cvalue in the penalty assessment. Sets $c$ to true.

Use only experiments with $\mathrm{E}$ (sum) values exceeding evalue in the penalty assessment. Sets e to true.

Use only experiments with $G$ values exceeding gvalue in the penalty assessment. Sets $g$ to true.

Print list of warning messages noting the experiments and nuclide-reaction pairs that were excluded from the penalty calculation.

Produces Javapeno formatted plot (.plt) files for integral values and composite sensitivity data.

Print "composite" of experiment sensitivity profiles for reactions selected in REACTIONS input block and write data to sensitivity data file. Sets g to true.

Prints directory of data available on the cross section covariance data library.

Print a table summarizing the non-coverage for the nuclide-reaction pairs entered in the REACTIONS input block. Sets $g$ to true.

Print the components of $\mathbf{E}$ and $\mathrm{G}$ for fission capture in scatter in the values table.

Print uncertainty values and penalty assessments in relative format. This is the default format. Absolute format can be specified using the "abs" keyword in the APPLICATIONS, EXPERIMENTS, or RESPONSE input blocks.

Option to copy the working covariance data file back to the return directory.

Cutoff value for ignoring low valued sensitivities in nuclide-reaction specific edit tables.

Value used in calculation of completeness parameter. Group-wise sensitivity for the application is counted as validated by the experiment if the sensitivity from the experiment is greater than the application sensitivity times senfac.

Figure 6.5.7. Input echo from TSUNAMI-IP output. (continued) 


\begin{tabular}{|c|c|}
\hline uncert & true \\
\hline uncert_long & true \\
\hline use_dcov & true \\
\hline udcove & 1.0000 \\
\hline udcov_corr= & 1.0000 \\
\hline udcov_corr_type $=$ & zone \\
\hline udcov_fast= & 0.0000 \\
\hline udcov_inter= & 0.0000 \\
\hline udcov_therm= & 0.0000 \\
\hline use_diff_groups & false \\
\hline use_icov & true \\
\hline usename & true \\
\hline uslstats & false \\
\hline uslsummary & false \\
\hline
\end{tabular}

Computes uncertainty in response due to covariance data.

Prints extended table of uncertainty in response due to covariance data. sets uncert to true.

Use user-defined default covariance data, udcov, for nuclide reaction pairs not included on the covariance data file. The user-defined data will be used in the fourth penalty assessment as well as the computation of $\mathrm{c}(\mathrm{k})$ and uncertainty calculations.

User-defined default value of standard deviation in cross-section data to use for nuclide-reaction pairs for which covariance data are not available on the selected data file.

User-defined default correlation value to use for nuclide-reaction pairs for which covariance data are not available on the selected data file.

User-defined default correlation in cross-section data to use for nuclide-reaction pairs for which covariance data are not available on the selected data file. (long, zone, short)

User-defined default value of standard deviation in cross-section data to use for fast data for nuclide-reaction pairs for which covariance data are not available on the selected data file.

User-defined default value of standard deviation in cross-section data to use for intermediate data for nuclide-reaction pairs for which covariance data are not available on the selected data file.

User-defined default value of standard deviation in cross-section data to use for thermal data for nuclide-reaction pairs for which covariance data are not available on the selected data file.

Allow sensitivity data files to have different energy group structures.

Use user-defined data input in COVARIANCE input data block in place of udcov value for user input nuclide-reaction pairs that are not on the covariance data file. The user-defined data will be used in the fourth penalty assessment as well as the computation of $c(k)$ and uncertainty calculations.

Use the name of the sensitivity data file in place of its title in all output.

Produces USLSTATS input files for trending analysis with all experiments for each global integral index (c(k), E, and $G$ ) requested for each application

Produces USLSTATS input files for trending analysis with experiments exceeding cutoff value (cvalue, crvalue, evalue or gralue) for each global integral index (c(k), E, and G) requested for each application

Figure 6.5.7. Input echo from TSUNAMI-IP output. (continued) 


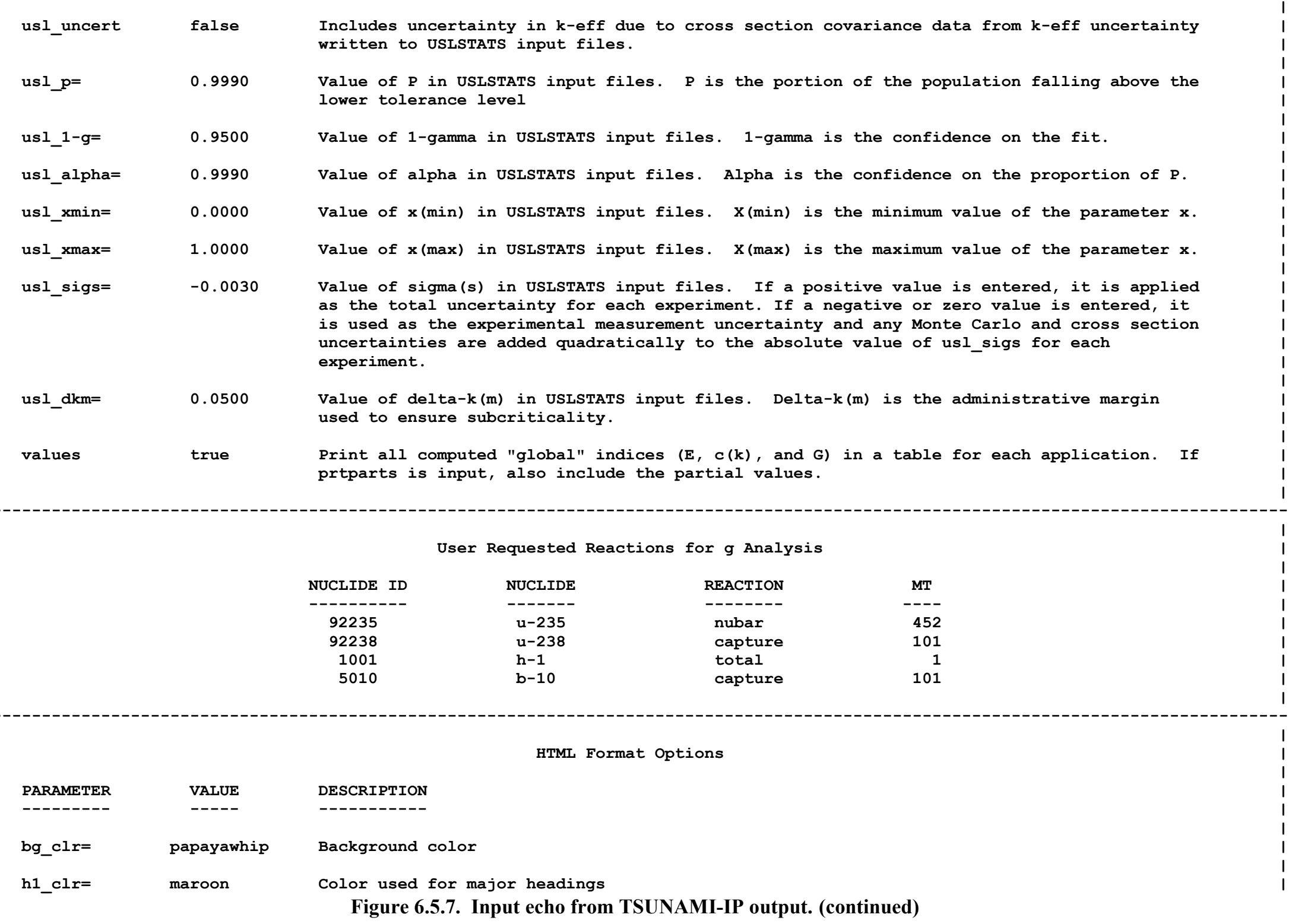




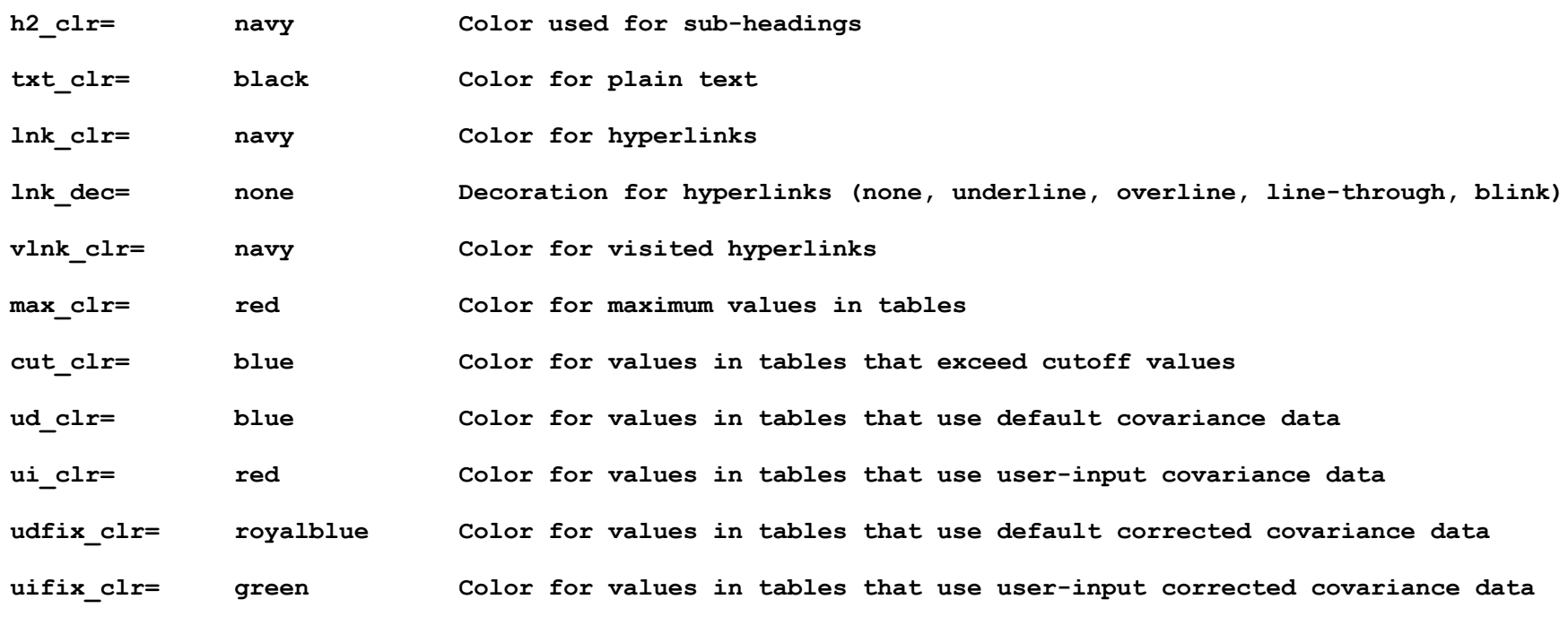


Title: tsunami-1d sample

Name: tsunami-1d4, Type: keff, Format: Relative

Name: tsunami-1d4, Type: kef

File

238 groups, 30 Sensitivity profiles

Title: tsunami-3d sample 3

Name: tsunami-3d k5-3, Type: keff, Format: Relative

Filename: tsunami-3d k5-3.sdf

238 groups, 182 Sensitivity profiles

Figure 6.5.7. Input echo from TSUNAMI-IP output. (continued) 

all $=1.0000$ therm $=0 \begin{aligned} & \text { Default data will be used for } \mathrm{mg} \mathrm{n}_{1} \mathrm{n}^{\prime} \\ & \text { Default data will be used for } \mathrm{mg} \mathrm{n}, 2 \mathrm{n}\end{aligned}$

all $=1.0000$ therm $=0.0000$ inter $=0.0000$ fast $=0.0000$ corr $=1.0000$ corr_type $=$ zone Default data will be used for $\mathrm{mg} n, p$

all $=1.0000$ therm $=0.0000$ inter $=0.0000$ fast $=0.0000$ corr $=1.0000$ corr type $=$ zone Default data will be used for $\mathrm{mg} n$, alpha

all $=1.0000$ therm $=0.0000$ inter $=0.0000$ fast $=0.0000$ corr $=1.0000$ corr_type $=$ zone Default data will be used for al-27 $\mathrm{n}, \mathrm{d}$

all $=1.0000$ therm $=0.0000$ inter $=0.0000$ fast $=0.0000$ corr $=1.0000$ corr_type $=$ zone Default data will be used for al-27 $\mathrm{n}, \mathrm{t}$

all $=1.0000$ therm $=0.0000$ inter $=0.0000$ fast $=0.0000$ corr $=1.0000$ corr_type $=$ zone Default data will be used for si $n, 2 n$

all $=1.0000$ therm $=0.0000$ inter $=0.0000$ fast $=0.0000$ corr $=1.0000$ corr type $=$ zone Default data will be used for si $n, d$

all $=1.0000$ therm $=0.0000$ inter $=0.0000$ fast $=0.0000$ corr $=1.0000$ corr_type $=$ zone

all $=1.0000$ therm $=0.0000$ inter $=0.0000$ fast $=0.0000$ corr $=1.0000$ corr_type= zone Default data will be used for $s \mathrm{n}, 2 \mathrm{n}$

all $=1.0000$ therm $=0.0000$ inter $=0.0000$ fast $=0.0000$ corr $=1.0000$ corr_type $=$ zone Default data will be used for $s$ fission

$\mathrm{all}=1.0000$ therm $=0.0000$ inter $=0.0000$ fast $=0.0000$ corr $=1.0000$ corr type $=$ zone Default data will be used for $s \mathrm{n}, \mathrm{p}$

all $=1.0000$ therm $=0.0000$ inter $=0.0000$ fast $=0.0000$ corr $=1.0000$ corr type $=$ zone

all $=1.0000$ therm $=0.0000$ inter $=0.0000$ fast $=0.0000$ corr $=1.0000$ corr_type $=0$ zone Default data will be used for $s \mathrm{n}, \mathrm{t}$

all $=1.0000$ therm $=0.0000$ inter $=0.0000$ fast $=0.0000$ corr $=1.0000$ corr_type $=$ zone Default data will be used for $s \mathrm{n}$, alpha

all $=1.0000$ therm $=0.0000$ inter $=0.0000$ fast $=0.0000$ corr $=1.0000$ corr_type $=$ zone Default data will be used for ca $n, n^{\prime}$

all $=1.0000$ therm $=0.0000$ inter $=0.0000$ fast $=0.0000$ corr $=1.0000$ corr type $=$ zone

all $=1.0000$ therm $=0.0000$ inter $=0.0000$ fast $=0.0000$ corr $=1.0000$ corr_type= zone Default data will be used for ca $n, p$

all $=1.0000$ therm $=0.0000$ inter $=0.0000$ fast $=0.0000$ corr $=1.0000$ corr_type $=$ zone Default data will be used for ca $n$, d

all $=1.0000$ therm $=0.0000$ inter $=0.0000$ fast $=0.0000$ corr $=1.0000$ corr_type $=$ zone Default data will be used for ca $n, t$

all $=1.0000$ therm $=0.0000$ inter $=0.0000$ fast $=0.0000$ corr $=1.0000$ corr_type $=$ zone

Figure 6.5.8. Warning messages from TSUNAMI-IP output. 
Integral Values for Application \#1

\begin{tabular}{|c|c|c|c|c|c|c|c|c|c|c|c|c|}
\hline & Experiment & Type & Value & s.d. & xsec unc $\%$ & s.d. & $c(k)$ & s.d. & $\mathrm{E}$ & s.d. & G & s.d. \\
\hline 0 & tsunami-3d k5-1 & $\begin{array}{l}----- \\
\operatorname{keff}\end{array}$ & $\begin{array}{r}-0078 \mathrm{E}+0 \\
1.007\end{array}$ & $\begin{array}{l}-------- \\
1.0000 \mathrm{E}-3\end{array}$ & $\begin{array}{l}-------- \\
6.10990 \mathrm{E}-1\end{array}$ & $\begin{array}{l}-------- \\
2.1762 \mathrm{E}-5\end{array}$ & $\begin{array}{l}------ \\
1.0000\end{array}$ & $\begin{array}{l}------ \\
0.0001\end{array}$ & $\begin{array}{l}------ \\
1.0000\end{array}$ & $\begin{array}{l}------ \\
0.0005\end{array}$ & 1.0000 & \\
\hline 1 & tsunami-1d $\overline{1}$ & keff & $1.0073 \mathrm{E}+0$ & & $6.06419 \mathrm{E}-1$ & & 0.9999 & 0.0000 & 0.9999 & 0.0003 & 0.9801 & 0.000 \\
\hline 2 & tsunami-1d4 & keff & $1.0040 \mathrm{E}+0$ & & $1.42001 \mathrm{E}+0$ & & 0.0988 & 0.0000 & 0.0164 & 0.0000 & 0.0542 & 0.0016 \\
\hline 3 & tsunami-3d k5-3 & keff & $9.9575 \mathrm{E}-1$ & $9.9300 \mathrm{E}-4$ & $7.48243 \mathrm{E}-1$ & $1.2500 \mathrm{E}-4$ & 0.6442 & 0.0001 & 0.9647 & 0.0008 & 0.6754 & 0.0026 \\
\hline
\end{tabular}

Figure 6.5.9. Global integral indices values table from TSUNAMI-IP output. 
Extended Uncertainty Edit for Application 1 tsunami-3d1

Input covariance file: $44 \mathrm{groupcov}$

Working covariance file: tsunami-ip.wrk

The standard deviation of keff $(\div \mathrm{dk} / \mathrm{k})$ is:

$6.110 \mathrm{E}-01+/-2.176 \mathrm{E}-05$ percent

* indicates default covariance data

contributions to uncertainty in keff ( $\left.\frac{\circ}{\mathrm{d}} \mathrm{k} / \mathrm{k}\right)$ by individual energy covariance matrices:

covariance matrix

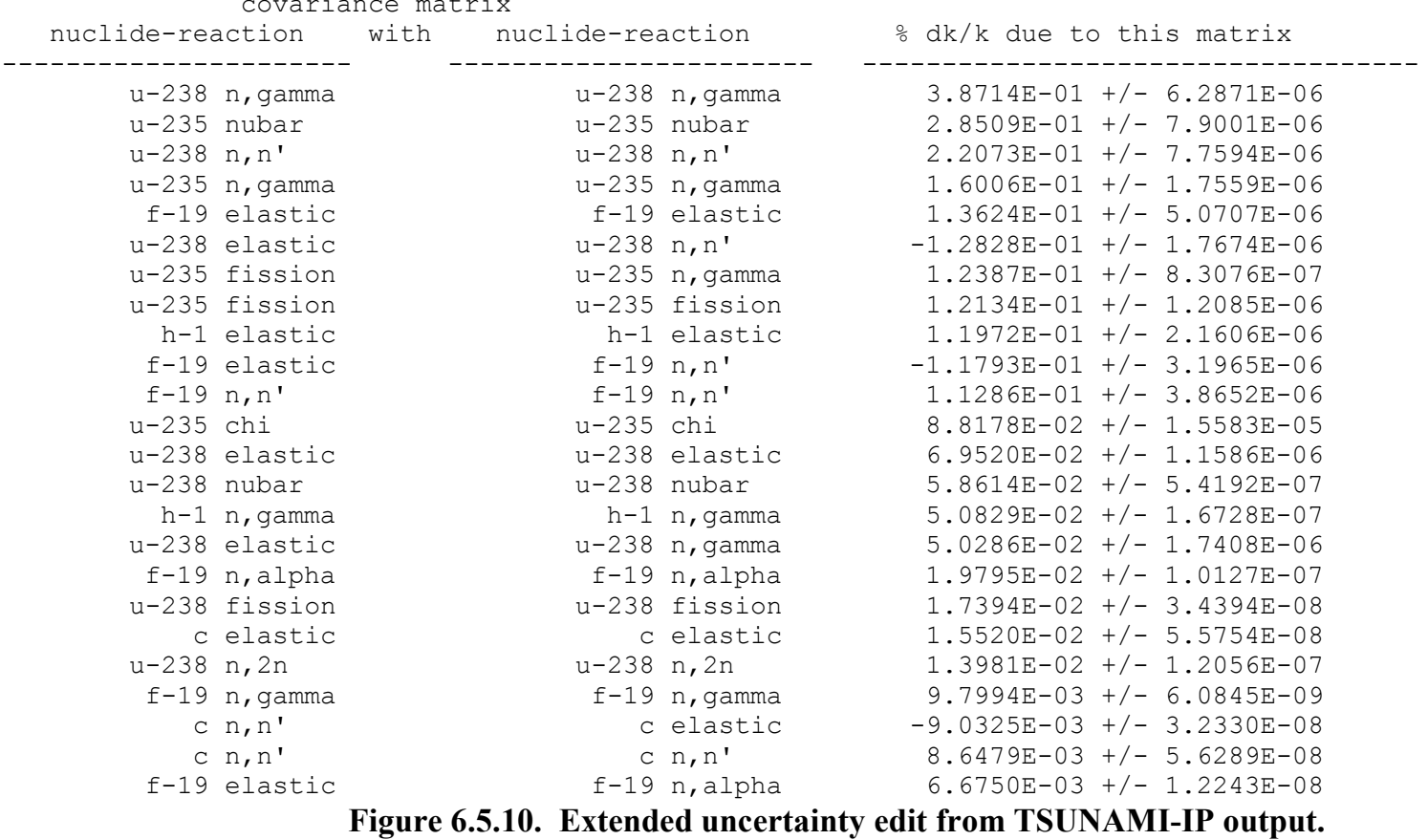


Extended $\mathrm{c}(\mathrm{k})$ Edit for Application 1 tsunami-3dl with Experiment 1 tsunami-1d1

the $c(k)$ value is:

$1.0000+/-0.0001$

Input covariance file: 44 groupcov

Working covariance file: tsunami-ip.wrk

* indicates default covariance data

contributions to $\mathrm{c}(\mathrm{k})$ by individual energy covariance matrices

the $c(k)$ value is the sum of the individual contributions

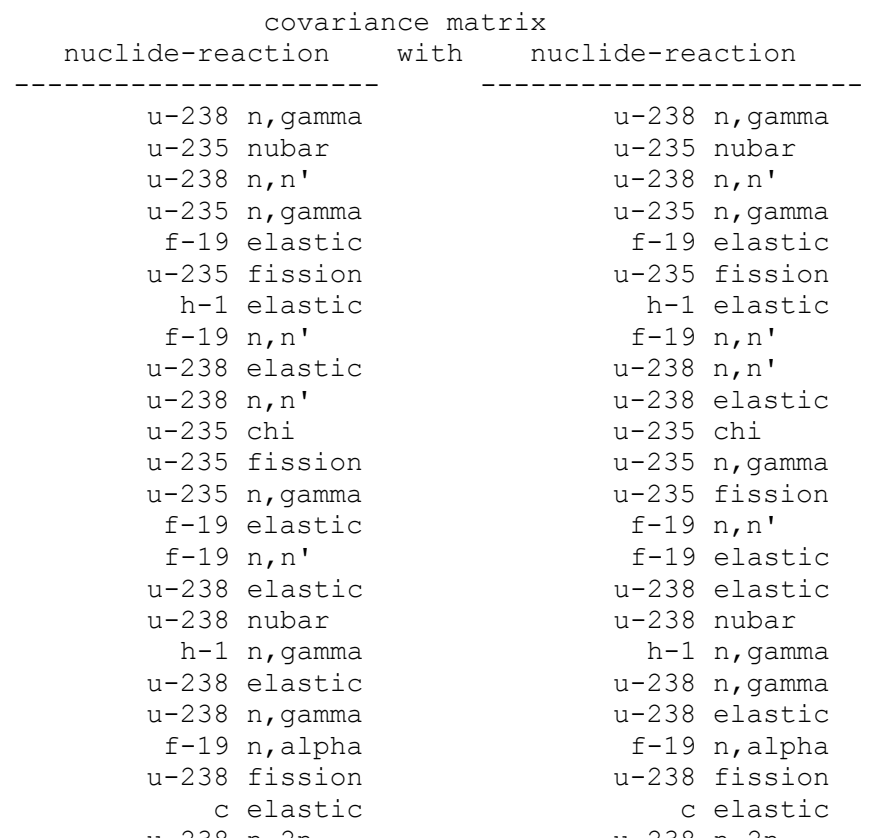

$\mathrm{u}-238 \mathrm{n}, 2 \mathrm{n}$

$\begin{aligned} & C(\mathrm{k}) \text { contribution from this matrix } \\ &- .0149 \mathrm{E}-01+/-1.4552 \mathrm{E}-05 \\ & 2.1772 \mathrm{E}-01+/-1.8286 \mathrm{E}-05 \\ & 1.3051 \mathrm{E}-01+/-1.7960 \mathrm{E}-05 \\ & 6.8631 \mathrm{E}-02+/-4.0642 \mathrm{E}-06 \\ & 4.9723 \mathrm{E}-02+/-1.1737 \mathrm{E}-05 \\ & 3.9440 \mathrm{E}-02+/-2.7972 \mathrm{E}-06 \\ & 3.8391 \mathrm{E}-02+/-5.0010 \mathrm{E}-06 \\ & 3.4122 \mathrm{E}-02+/-8.9464 \mathrm{E}-06 \\ &-2.2039 \mathrm{E}-02+/-2.8927 \mathrm{E}-06 \\ &-2.2039 \mathrm{E}-02+/-2.8927 \mathrm{E}-06 \\ & 2.0828 \mathrm{E}-02+/-3.6069 \mathrm{E}-05 \\ & 2.0550 \mathrm{E}-02+/-1.3597 \mathrm{E}-06 \\ & 2.0550 \mathrm{E}-02+/-1.3597 \mathrm{E}-06 \\ &-1.8628 \mathrm{E}-02+/-5.2316 \mathrm{E}-06 \\ &-1.8628 \mathrm{E}-02+/-5.2316 \mathrm{E}-06 \\ & 1.2947 \mathrm{E}-02+/-2.6818 \mathrm{E}-06 \\ & 9.2031 \mathrm{E}-03+/-1.2543 \mathrm{E}-06 \\ & 6.9207 \mathrm{E}-03+/-3.8719 \mathrm{E}-07 \\ & 3.3869 \mathrm{E}-03+/-2.8491 \mathrm{E}-06 \\ & 3.3869 \mathrm{E}-03+/-2.8491 \mathrm{E}-06 \\ & 1.0496 \mathrm{E}-03+/-2.3439 \mathrm{E}-07 \\ & 8.1042 \mathrm{E}-04+/-7.9610 \mathrm{E}-08 \\ & 6.4522 \mathrm{E}-04+/-1.2905 \mathrm{E}-07 \\ & 5.2362 \mathrm{E}-04+/-2.7904 \mathrm{E}-07\end{aligned}$

Figure 6.5.11. Extended $c_{k}$ edit from TSUNAMI-IP output. individual $\mathrm{c}(\mathrm{k})$

$1.0000 \mathrm{E}+00+/-3.6246 \mathrm{E}-05$

$1.0000 \mathrm{E}+00+/-8.3989 \mathrm{E}-05$

$1.0000 \mathrm{E}+00+/-1.3761 \mathrm{E}-04$

$1.0000 \mathrm{E}+00+/-5.9217 \mathrm{E}-05$

$1.0000 \mathrm{E}+00+/-2.3604 \mathrm{E}-04$

$1.0000 \mathrm{E}+00+/-7.0924 \mathrm{E}-05$

$1.0000 \mathrm{E}+00+/-1.3026 \mathrm{E}-04$

$1.0000 \mathrm{E}+00+/-2.6219 \mathrm{E}-04$

$1.0000 \mathrm{E}+00+/-1.7317 \mathrm{E}-03$

$1.0000 \mathrm{E}+00+/-2.0714 \mathrm{E}-04$ $1.0000 \mathrm{E}+00+/-1.3629 \mathrm{E}-04$

$1.0000 \mathrm{E}+00+/-5.5946 \mathrm{E}-05$

$1.0000 \mathrm{E}+00+/-2.2331 \mathrm{E}-04$ $1.0000 \mathrm{E}+00+/-9.8233 \mathrm{E}-05$

$1.0000 \mathrm{E}+00+/-2.0001 \mathrm{E}-04$

$1.0000 \mathrm{E}+00+/-5.3290 \mathrm{E}-04$ 


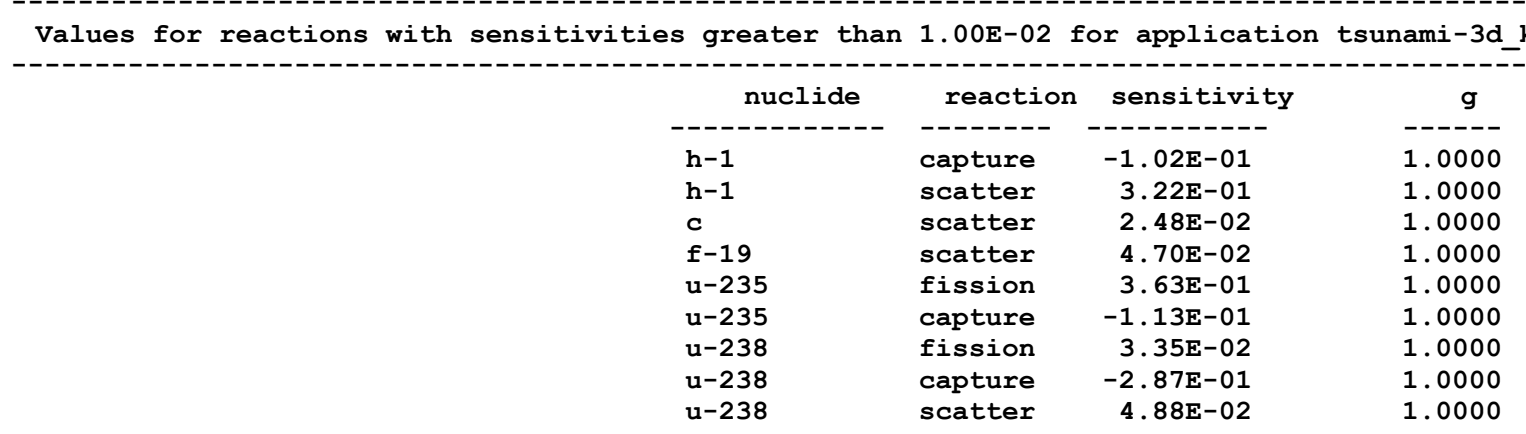

$\begin{array}{rrrr}\text { Values for reactions with sensitivities greater than } 1.00 \mathrm{E}-02 & \text { for application tsunami-3d_k5-1 with experiment tsunami-1d1 } \\ \text { nuclide } & \text { reaction sensitivity } & \text { g } \\ \text { h-1 } & \text { capture } & -1.02 \mathrm{E}-01 & 0.9971 \\ \mathrm{~h}-1 & \text { scatter } & 3.22 \mathrm{E}-01 & 0.9413 \\ \mathrm{c} & \text { scatter } & 2.48 \mathrm{E}-02 & 0.9491 \\ \mathrm{f}-19 & \text { scatter } & 4.70 \mathrm{E}-02 & 0.9511 \\ \mathrm{u}-235 & \text { fission } & 3.63 \mathrm{E}-01 & 0.9998 \\ \mathrm{u}-235 & \text { capture } & -1.13 \mathrm{E}-01 & 0.9967 \\ \mathrm{u}-238 & \text { fission } & 3.35 \mathrm{E}-02 & 0.9978 \\ \mathrm{u}-238 & \text { capture } & -2.87 \mathrm{E}-01 & 0.9960\end{array}$

Figure 6.5.12. Nuclide-reaction specific integral indices from TSUNAMI-IP output. 


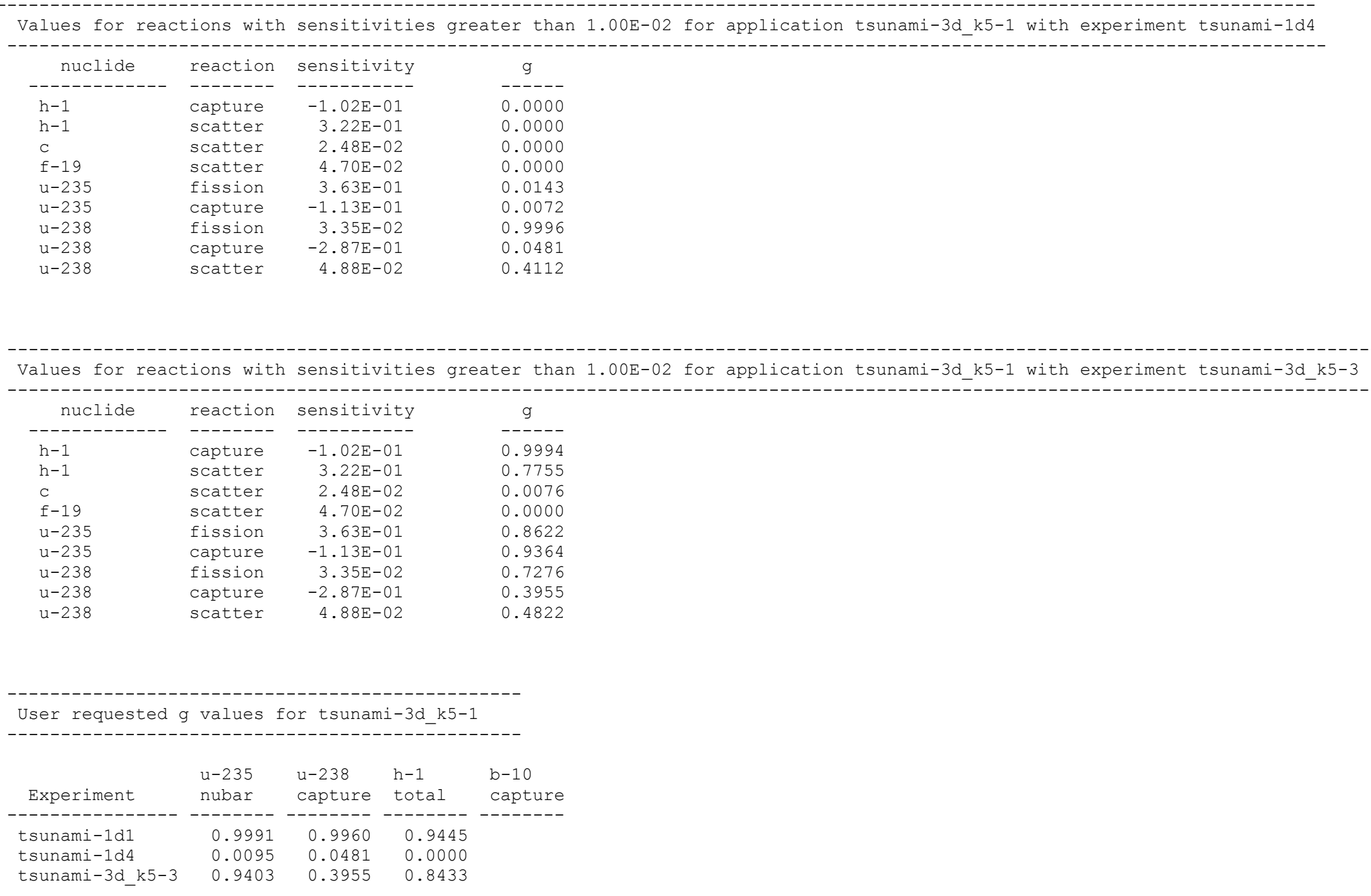

Figure 6.5.12. Nuclide-reaction specific integral indices from TSUNAMI-IP output. (continued) 
Penalty Assessment for Application 1 tsunami-3dl

the standard deviation of keff due to uncovered sensitivity data is:

$9.747 \mathrm{E}-03+/-6.584 \mathrm{E}-05(\div \mathrm{dk} / \mathrm{k})$

Input covariance file: 44 groupcov

Working covariance file: tsunami-ip.wrk

* indicates default covariance data

contributions to uncertainty in keff $\left(\frac{\circ}{\circ} \mathrm{k} / \mathrm{k}\right)$ by individual energy covariance matrices:

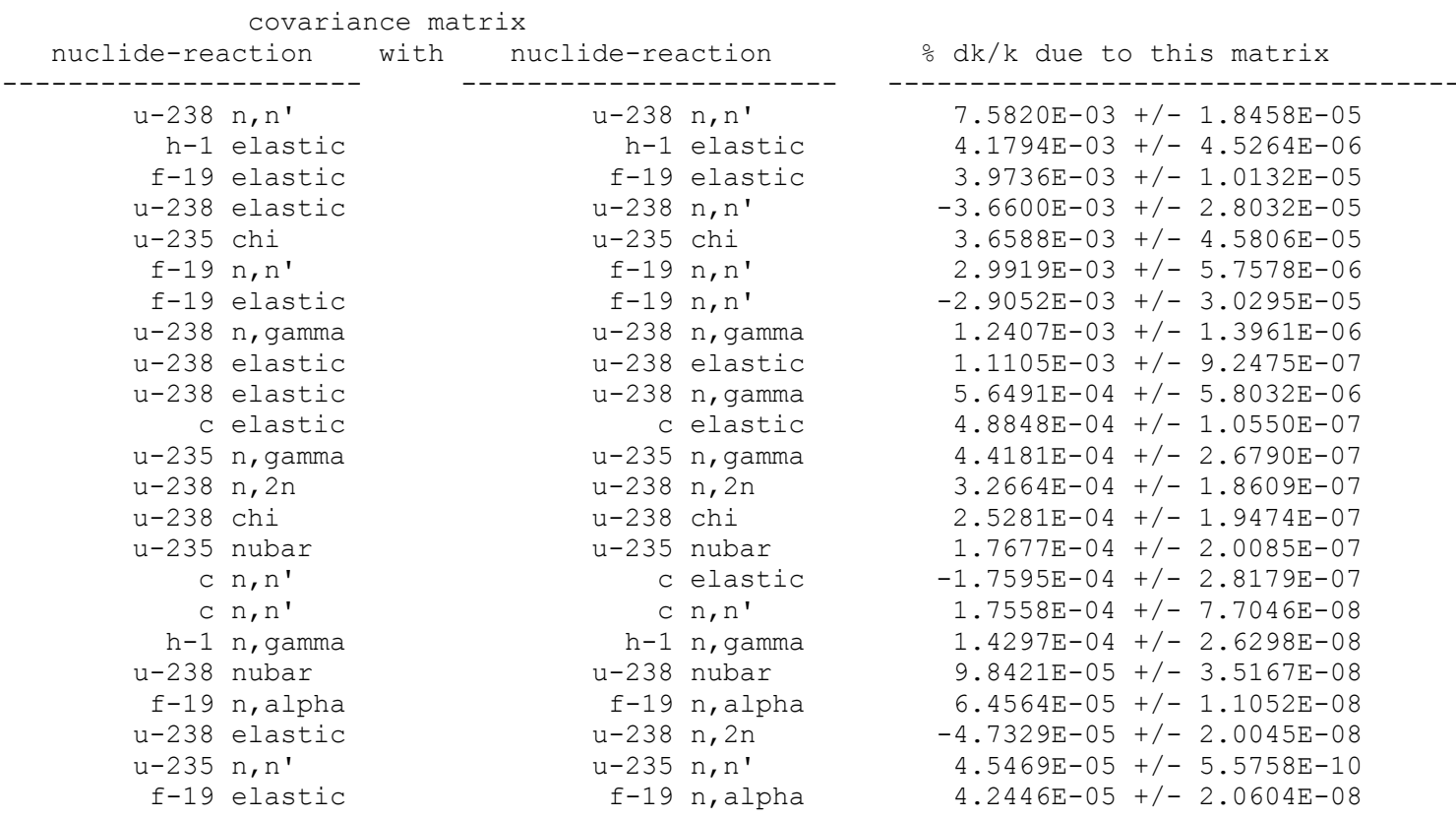

Figure 6.5.13. Extended penalty assessment from TSUNAMI-IP output. 
application \# 1 tsunami-3d_k5-1 has $1 \mathrm{c}(\mathrm{k})$ values $>=0.8000$
1 tsunami-1d1
$0.9999+/-0.0000$

application \#2 tsunami-3d_k5-2 has $1 \mathrm{c}(\mathrm{k})$ values $>=0.8000$
1 tsunami-1d 1
$0.8175+/-0.0001$

Summary of Integral Values Meeting Acceptance Criteria

$\begin{array}{lcc}\text { Application } & \mathrm{C}(\mathrm{k})>= & 0.8000 \\ ---------1 & ----1 \\ \text { tsunami-3d_k5-1 } & 1 \\ \text { tsunami-3d_k5-2 } & \end{array}$

Figure 6.5.14. Summary tables from TSUNAMI-IP output. 


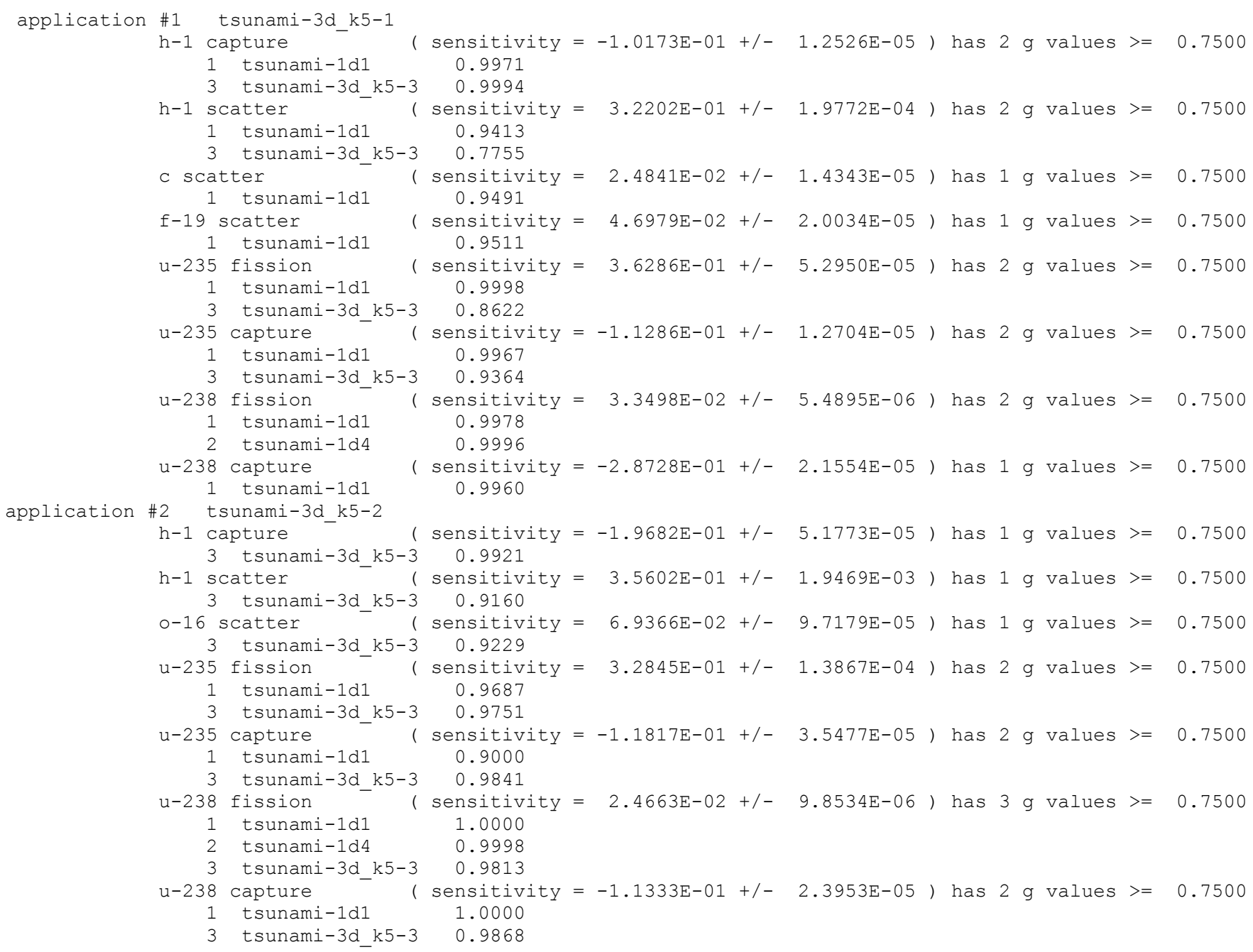

\section{Figure 6.5.14. Summary tables from TSUNAMI-IP output. (continued)}


Non-coverage for user-requested reactions for tsunami-3d k5-1

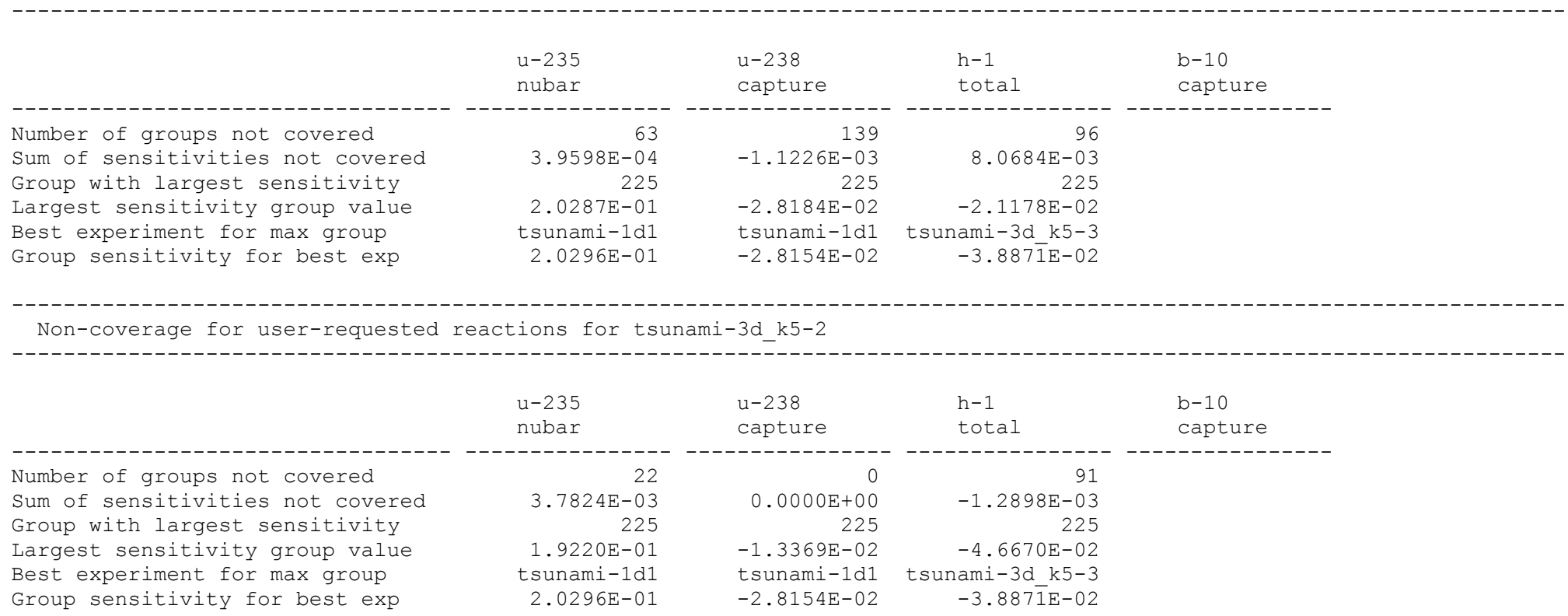

Figure 6.5.14. Summary tables from TSUNAMI-IP output. (continued) 
Standard Deviation in Application Response Due to Uncovered Sensitivity Coefficients

\begin{tabular}{|c|c|c|c|}
\hline Application & Type & Penalty & SD Units \\
\hline nami-3d_k5-1 & keff & $9.7475 \mathrm{E}-3+/-6.5845 \mathrm{E}-5$ & o $\mathrm{dk} / \mathrm{k}$ \\
\hline anami-3d_k5-2 & keff & $2.3500 \mathrm{E}-1+/-1.2902 \mathrm{E}-4$ & $\div \mathrm{dk} / \mathrm{k}$ \\
\hline
\end{tabular}

Figure 6.5.14. Summary tables from TSUNAMI-IP output. (continued) 


\begin{tabular}{|c|c|c|c|c|}
\hline Group & $\begin{array}{l}u-235 \\
\text { nubar }\end{array}$ & $\begin{array}{l}u-238 \\
\text { capture }\end{array}$ & $\begin{array}{l}h-1 \\
\text { total }\end{array}$ & $\begin{array}{l}\mathrm{b}-10 \\
\text { capture }\end{array}$ \\
\hline-------- & ---------------- & ---------------- & ---------------- & ----------------- \\
\hline 1 & $0.0000 \mathrm{E}+00$ & $0.0000 \mathrm{E}+00$ & $0.0000 \mathrm{E}+00$ & \\
\hline 2 & $8.3121 E-08$ & $-2.1269 \mathrm{E}-10$ & $-4.8103 E-07$ & \\
\hline 3 & $2.8391 E-07$ & $-1.1742 \mathrm{E}-09$ & $-1.2188 E-06$ & \\
\hline 4 & $5.1635 \mathrm{E}-07$ & $-2.9305 E-09$ & $-1.4983 E-06$ & \\
\hline 5 & $7.6669 \mathrm{E}-07$ & $-6.5763 E-09$ & $-3.0912 E-06$ & \\
\hline 6 & $1.2087 \mathrm{E}-05$ & $-1.3860 E-07$ & $-4.2146 E-05$ & \\
\hline 7 & $3.2436 \mathrm{E}-05$ & $-2.5827 E-07$ & $-7.0385 E-05$ & \\
\hline 8 & $9.4203 E-05$ & $-1.5178 E-06$ & $2.2338 E-04$ & \\
\hline 9 & $1.7111 \mathrm{E}-04$ & $-9.8460 E-06$ & $2.6160 \mathrm{E}-03$ & \\
\hline 10 & $9.5881 \mathrm{E}-05$ & $-9.6314 \mathrm{E}-06$ & $1.4498 \mathrm{E}-03$ & \\
\hline 11 & $4.6498 E-04$ & $-8.6324 E-05$ & $6.0348 E-03$ & \\
\hline 12 & $3.3668 E-04$ & $-1.0881 E-04$ & $4.1605 E-03$ & \\
\hline 13 & $1.0308 \mathrm{E}-04$ & $-4.2404 E-05$ & $1.5022 \mathrm{E}-03$ & \\
\hline 14 & $4.9039 E-04$ & $-2.8588 E-04$ & $6.9219 E-03$ & \\
\hline 15 & $4.1834 \mathrm{E}-04$ & $-3.6981 E-04$ & $6.6199 \mathrm{E}-03$ & \\
\hline 16 & $1.3739 \mathrm{E}-04$ & $-1.5075 E-04$ & $2.9036 \mathrm{E}-03$ & \\
\hline 17 & $6.1269 \mathrm{E}-05$ & $-7.3358 E-05$ & $1.2879 E-03$ & \\
\hline 18 & $5.3656 \mathrm{E}-05$ & $-6.8243 E-05$ & $1.1029 \mathrm{E}-03$ & \\
\hline 19 & $9.3382 \mathrm{E}-05$ & $-1.2782 \mathrm{E}-04$ & $1.8069 \mathrm{E}-03$ & \\
\hline 20 & $7.2274 E-05$ & $-1.0611 E-04$ & $1.7937 \mathrm{E}-03$ & \\
\hline 21 & $1.6121 \mathrm{E}-04$ & $-2.6852 \mathrm{E}-04$ & $3.6259 \mathrm{E}-03$ & \\
\hline 22 & $1.5722 \mathrm{E}-04$ & $-2.9405 E-04$ & $3.6598 E-03$ & \\
\hline 23 & $1.6032 \mathrm{E}-04$ & $-3.2036 E-04$ & $3.6221 \mathrm{E}-03$ & \\
\hline 24 & $3.6667 \mathrm{E}-05$ & $-7.5670 E-05$ & $8.7492 \mathrm{E}-04$ & \\
\hline 25 & $4.6036 E-05$ & $-9.5868 E-05$ & $1.1263 E-03$ & \\
\hline 26 & $2.4917 \mathrm{E}-05$ & $-5.2550 E-05$ & $5.7950 \mathrm{E}-04$ & \\
\hline 27 & 7. $3525 E-05$ & $-1.5597 E-04$ & $1.6697 \mathrm{E}-03$ & \\
\hline 28 & $1.3207 E-04$ & $-2.7919 E-04$ & $3.1426 \mathrm{E}-03$ & \\
\hline 29 & $1.4917 \mathrm{E}-04$ & $-3.1499 E-04$ & $3.3212 E-03$ & \\
\hline 30 & $1.9524 \mathrm{E}-05$ & $-4.0694 \mathrm{E}-05$ & $4.7128 E-04$ & \\
\hline 31 & $1.5260 \mathrm{E}-04$ & $-3.1113 E-04$ & $3.5947 E-03$ & \\
\hline 32 & $5.9914 \mathrm{E}-05$ & $-1.2004 \mathrm{E}-04$ & $1.0086 \mathrm{E}-03$ & \\
\hline 33 & $5.4703 E-05$ & $-1.0849 \mathrm{E}-04$ & $1.0609 \mathrm{E}-03$ & \\
\hline 34 & $1.2067 \mathrm{E}-04$ & $-2.3813 E-04$ & $2.0801 \mathrm{E}-03$ & \\
\hline 35 & $7.4939 E-05$ & $-1.4661 E-04$ & $1.3644 \mathrm{E}-03$ & \\
\hline 36 & $7.5277 E-05$ & $-1.4645 E-04$ & 1. $3422 \mathrm{E}-03$ & \\
\hline 37 & $4.5884 \mathrm{E}-05$ & $-8.8788 E-05$ & $6.8414 \mathrm{E}-04$ & \\
\hline 38 & $5.1306 E-05$ & $-9.8727 E-05$ & $9.3662 \mathrm{E}-04$ & \\
\hline 39 & $1.9597 \mathrm{E}-04$ & $-3.7831 E-04$ & $3.5664 \mathrm{E}-03$ & \\
\hline 40 & $1.8431 E-04$ & $-3.6387 E-04$ & $1.8026 \mathrm{E}-03$ & \\
\hline 41 & $2.8555 E-04$ & $-5.5723 E-04$ & $4.2242 E-03$ & \\
\hline 42 & $2.6119 \mathrm{E}-04$ & $-5.3510 E-04$ & $4.7957 E-03$ & \\
\hline 43 & $1.3711 \mathrm{E}-04$ & $-2.9893 E-04$ & $1.8811 \mathrm{E}-03$ & \\
\hline 44 & 1. $9055 \mathrm{E}-04$ & $-4.3852 E-04$ & $2.1220 \mathrm{E}-03$ & \\
\hline 45 & $1.2409 \mathrm{E}-04$ & $-3.1979 E-04$ & $1.1164 \mathrm{E}-03$ & \\
\hline 46 & $3.6535 \mathrm{E}-05$ & $-9.7941 E-05$ & $4.6072 \mathrm{E}-04$ & \\
\hline 47 & $8.7249 E-05$ & $-2.3882 E-04$ & $8.8505 E-04$ & \\
\hline 48 & $2.5653 E-05$ & $-6.9180 E-05$ & $3.5130 \mathrm{E}-04$ & \\
\hline 49 & $1.6739 \mathrm{E}-04$ & $-5.0475 E-04$ & $2.2125 E-03$ & \\
\hline 50 & $1.1716 \mathrm{E}-04$ & $-3.9760 E-04$ & $1.5983 E-03$ & \\
\hline 51 & $2.4526 \mathrm{E}-05$ & $-9.6985 E-05$ & 1. 5119E-04 & \\
\hline 52 & $6.4964 E-05$ & $-2.6803 E-04$ & $7.1621 \mathrm{E}-04$ & \\
\hline 53 & $3.3839 \mathrm{E}-04$ & $-1.4814 \mathrm{E}-03$ & $4.7531 \mathrm{E}-03$ & \\
\hline 54 & $1.3114 \mathrm{E}-04$ & $-6.1020 E-04$ & $1.8245 \mathrm{E}-03$ & \\
\hline 55 & $3.5173 E-04$ & $-1.7047 E-03$ & $5.1134 \mathrm{E}-03$ & \\
\hline
\end{tabular}

Figure 6.5.15. Composite sensitivity data from TSUNAMI-IP output. 


\begin{tabular}{|c|c|c|c|}
\hline 56 & $2.4929 \mathrm{E}-04$ & $-1.2514 \mathrm{E}-03$ & $3.3044 \mathrm{E}-03$ \\
\hline 57 & $3.1251 \mathrm{E}-04$ & $-1.5616 \mathrm{E}-03$ & $3.2685 \mathrm{E}-03$ \\
\hline 58 & $1.7934 \mathrm{E}-04$ & $-7.8059 \mathrm{E}-04$ & $1.6846 \mathrm{E}-03$ \\
\hline 59 & $3.3768 \mathrm{E}-04$ & $-1.5847 \mathrm{E}-03$ & $3.0949 \mathrm{E}-03$ \\
\hline 60 & $5.7218 \mathrm{E}-04$ & $-2.6453 \mathrm{E}-03$ & $4.6208 \mathrm{E}-03$ \\
\hline 61 & $6.2408 \mathrm{E}-05$ & $-2.1987 \mathrm{E}-04$ & $2.8987 \mathrm{E}-04$ \\
\hline 62 & $3.4221 \mathrm{E}-04$ & $-1.6321 \mathrm{E}-03$ & $2.6850 \mathrm{E}-03$ \\
\hline 63 & $2.5176 \mathrm{E}-04$ & $-1.0549 \mathrm{E}-03$ & $1.7729 \mathrm{E}-03$ \\
\hline 64 & $2.1465 \mathrm{E}-04$ & $-9.5589 \mathrm{E}-04$ & $1.3821 \mathrm{E}-03$ \\
\hline 65 & $7.3062 \mathrm{E}-05$ & $-3.8956 \mathrm{E}-04$ & $5.2412 \mathrm{E}-04$ \\
\hline 66 & $3.7161 \mathrm{E}-04$ & $-1.4107 \mathrm{E}-03$ & $2.3877 \mathrm{E}-03$ \\
\hline 67 & $3.2093 \mathrm{E}-04$ & $-1.3730 \mathrm{E}-03$ & $1.6616 \mathrm{E}-03$ \\
\hline 68 & $5.8195 \mathrm{E}-05$ & $-2.1647 \mathrm{E}-04$ & $3.7317 \mathrm{E}-04$ \\
\hline 69 & $6.6370 \mathrm{E}-04$ & $-2.5516 \mathrm{E}-03$ & $3.5190 \mathrm{E}-03$ \\
\hline 70 & $4.5913 \mathrm{E}-04$ & $-2.0723 E-03$ & $2.4579 \mathrm{E}-03$ \\
\hline 71 & $9.7426 \mathrm{E}-04$ & $-4.1869 \mathrm{E}-03$ & $4.9772 \mathrm{E}-03$ \\
\hline 72 & $1.0966 \mathrm{E}-04$ & $-5.8274 \mathrm{E}-05$ & $1.8589 \mathrm{E}-04$ \\
\hline 73 & $7.1828 \mathrm{E}-04$ & $-2.6476 \mathrm{E}-03$ & $2.7657 \mathrm{E}-03$ \\
\hline 74 & $2.4703 \mathrm{E}-03$ & $-6.6484 \mathrm{E}-03$ & $7.4726 \mathrm{E}-03$ \\
\hline 75 & $2.6381 \mathrm{E}-04$ & $-1.3566 \mathrm{E}-03$ & $1.1842 \mathrm{E}-03$ \\
\hline 76 & $1.1644 \mathrm{E}-03$ & $-1.9283 E-03$ & $2.1884 \mathrm{E}-03$ \\
\hline 77 & $9.0059 \mathrm{E}-04$ & $-1.9983 E-03$ & $2.0712 \mathrm{E}-03$ \\
\hline 78 & $1.3932 \mathrm{E}-05$ & $-1.7300 \mathrm{E}-03$ & $6.6140 \mathrm{E}-04$ \\
\hline 79 & $4.7174 \mathrm{E}-04$ & $-3.7028 E-04$ & $5.4439 E-04$ \\
\hline 80 & $1.2166 \mathrm{E}-04$ & $-2.3242 \mathrm{E}-03$ & $1.2190 \mathrm{E}-03$ \\
\hline 81 & $2.6867 \mathrm{E}-03$ & $-3.1503 E-03$ & $5.5591 \mathrm{E}-03$ \\
\hline 82 & $1.7934 \mathrm{E}-04$ & $-7.5046 \mathrm{E}-05$ & $4.2063 E-04$ \\
\hline 83 & $2.9797 \mathrm{E}-04$ & $-3.1650 \mathrm{E}-03$ & $1.6682 \mathrm{E}-03$ \\
\hline 84 & $1.8506 \mathrm{E}-04$ & $-1.7962 \mathrm{E}-04$ & $6.9532 \mathrm{E}-04$ \\
\hline 85 & $3.8625 \mathrm{E}-04$ & $-5.4319 \mathrm{E}-03$ & $3.0824 \mathrm{E}-03$ \\
\hline 86 & $6.6282 \mathrm{E}-04$ & $-2.9944 \mathrm{E}-04$ & $1.2789 \mathrm{E}-03$ \\
\hline 87 & $8.2978 \mathrm{E}-04$ & $-4.5155 \mathrm{E}-04$ & $1.1380 \mathrm{E}-03$ \\
\hline 88 & $1.1337 \mathrm{E}-04$ & $-2.5098 \mathrm{E}-03$ & $1.9443 \mathrm{E}-03$ \\
\hline 89 & $2.1511 \mathrm{E}-04$ & $-6.1408 \mathrm{E}-05$ & $6.1627 \mathrm{E}-04$ \\
\hline 90 & $5.9065 \mathrm{E}-04$ & $-6.5874 \mathrm{E}-05$ & $5.6950 \mathrm{E}-04$ \\
\hline 91 & $5.4779 \mathrm{E}-04$ & $-3.0607 \mathrm{E}-04$ & $1.0246 \mathrm{E}-03$ \\
\hline 92 & $3.7293 \mathrm{E}-05$ & $-5.7959 E-03$ & $3.1081 \mathrm{E}-03$ \\
\hline 93 & $2.7815 \mathrm{E}-04$ & $-6.1499 E-04$ & $8.7462 \mathrm{E}-04$ \\
\hline 94 & $2.5372 \mathrm{E}-04$ & $-4.8609 E-05$ & $1.2161 \mathrm{E}-04$ \\
\hline 95 & $1.8329 \mathrm{E}-03$ & $-8.5639 \mathrm{E}-05$ & $4.8164 \mathrm{E}-04$ \\
\hline 96 & $3.9099 \mathrm{E}-04$ & $-1.8614 \mathrm{E}-05$ & $1.2128 \mathrm{E}-04$ \\
\hline 97 & $7.1230 \mathrm{E}-04$ & $-1.9738 \mathrm{E}-05$ & $1.5539 \mathrm{E}-04$ \\
\hline 98 & $2.4608 \mathrm{E}-04$ & $-3.5551 \mathrm{E}-05$ & $3.8414 \mathrm{E}-04$ \\
\hline 99 & $2.2019 \mathrm{E}-04$ & $-1.5836 \mathrm{E}-05$ & $1.7509 \mathrm{E}-04$ \\
\hline 100 & $3.1350 \mathrm{E}-04$ & $-2.7103 E-05$ & $3.2313 \mathrm{E}-04$ \\
\hline 101 & $2.9855 \mathrm{E}-04$ & $-6.8308 E-05$ & $5.6510 \mathrm{E}-04$ \\
\hline 102 & $3.5827 \mathrm{E}-04$ & $-5.9559 \mathrm{E}-05$ & $4.5259 \mathrm{E}-04$ \\
\hline 103 & $2.1822 \mathrm{E}-04$ & $-1.0055 \mathrm{E}-04$ & $8.6367 \mathrm{E}-04$ \\
\hline 104 & $3.8254 \mathrm{E}-04$ & $-1.3022 \mathrm{E}-04$ & $7.8610 \mathrm{E}-04$ \\
\hline 105 & $2.6271 \mathrm{E}-04$ & $-2.6041 \mathrm{E}-04$ & $7.6712 \mathrm{E}-04$ \\
\hline 106 & $4.5665 \mathrm{E}-04$ & $-1.6304 \mathrm{E}-04$ & $3.4290 \mathrm{E}-04$ \\
\hline 107 & $1.3250 \mathrm{E}-04$ & $-7.8605 E-04$ & $1.3860 \mathrm{E}-03$ \\
\hline 108 & $5.8291 \mathrm{E}-05$ & $-3.1200 \mathrm{E}-03$ & $3.3141 \mathrm{E}-03$ \\
\hline 109 & $2.5917 \mathrm{E}-04$ & $-9.6788 \mathrm{E}-03$ & $5.0386 \mathrm{E}-03$ \\
\hline 110 & $1.5727 \mathrm{E}-03$ & $-9.5678 \mathrm{E}-04$ & $5.9505 E-04$ \\
\hline 111 & $5.1745 \mathrm{E}-04$ & $-3.9567 \mathrm{E}-04$ & $5.1849 \mathrm{E}-04$ \\
\hline 112 & $3.3689 \mathrm{E}-04$ & $-1.4825 E-04$ & $2.9832 \mathrm{E}-04$ \\
\hline 113 & $5.5476 \mathrm{E}-04$ & $-2.9394 \mathrm{E}-04$ & $5.5464 \mathrm{E}-04$ \\
\hline 114 & $2.2143 \mathrm{E}-05$ & $-6.9191 E-05$ & $1.6138 \mathrm{E}-04$ \\
\hline 115 & $1.9064 \mathrm{E}-04$ & $-1.4094 \mathrm{E}-04$ & $5.3270 \mathrm{E}-04$ \\
\hline 116 & $5.0989 \mathrm{E}-04$ & $-2.2832 \mathrm{E}-04$ & $1.2496 \mathrm{E}-03$ \\
\hline 117 & $1.1009 \mathrm{E}-03$ & $-3.0055 E-04$ & $1.6754 \mathrm{E}-03$ \\
\hline 118 & $1.4392 \mathrm{E}-03$ & $-8.5266 \mathrm{E}-04$ & $3.3368 \mathrm{E}-03$ \\
\hline 119 & $2.4313 \mathrm{E}-04$ & $-7.0828 \mathrm{E}-03$ & $6.5606 \mathrm{E}-03$ \\
\hline 120 & $1.7047 \mathrm{E}-04$ & $-8.4025 E-03$ & $5.1957 \mathrm{E}-03$ \\
\hline 121 & $2.2684 \mathrm{E}-03$ & $-1.6808 \mathrm{E}-03$ & $1.4346 \mathrm{E}-03$ \\
\hline 122 & $2.0681 \mathrm{E}-04$ & $-3.2469 \mathrm{E}-04$ & $4.1713 \mathrm{E}-04$ \\
\hline 123 & $5.3283 \mathrm{E}-04$ & $-5.4572 \mathrm{E}-04$ & $9.2483 \mathrm{E}-04$ \\
\hline
\end{tabular}

Figure 6.5.15. Composite sensitivity data from TSUNAMI-IP output.. (continued) 


\begin{tabular}{|c|c|c|c|}
\hline 124 & $5.6983 E-04$ & $-2.1805 E-04$ & $5.6677 \mathrm{E}-04$ \\
\hline 125 & $3.4396 \mathrm{E}-04$ & $-1.5890 \mathrm{E}-04$ & $4.4437 \mathrm{E}-04$ \\
\hline 126 & $2.2707 \mathrm{E}-04$ & $-1.1013 E-04$ & $4.5908 E-04$ \\
\hline 127 & $8.3158 E-04$ & $-9.6956 \mathrm{E}-05$ & $1.8533 \mathrm{E}-04$ \\
\hline 128 & $4.5995 \mathrm{E}-04$ & $-1.2467 E-04$ & $4.7548 E-04$ \\
\hline 129 & $1.7971 \mathrm{E}-03$ & $-1.4955 \mathrm{E}-04$ & $8.4077 \mathrm{E}-04$ \\
\hline 130 & $3.2110 \mathrm{E}-04$ & $-6.5461 E-05$ & $9.1569 \mathrm{E}-04$ \\
\hline 131 & $6.1328 \mathrm{E}-04$ & $-8.3373 E-04$ & $1.7860 \mathrm{E}-03$ \\
\hline 132 & $1.2347 \mathrm{E}-03$ & $-3.2726 \mathrm{E}-04$ & $1.4198 \mathrm{E}-03$ \\
\hline 133 & $4.8042 E-03$ & $-7.7205 E-04$ & $2.9930 \mathrm{E}-03$ \\
\hline 134 & $1.9295 \mathrm{E}-04$ & $-3.7846 \mathrm{E}-03$ & $8.5681 \mathrm{E}-03$ \\
\hline 135 & $3.2715 E-04$ & $-2.2262 E-03$ & $2.7745 \mathrm{E}-03$ \\
\hline 136 & $6.6065 \mathrm{E}-05$ & $-7.5091 E-03$ & $6.8661 \mathrm{E}-03$ \\
\hline 137 & $1.3387 \mathrm{E}-05$ & $-3.9274 E-03$ & $3.1875 \mathrm{E}-03$ \\
\hline 138 & $5.5488 \mathrm{E}-04$ & $-6.5404 E-03$ & $5.8490 \mathrm{E}-03$ \\
\hline 139 & $3.2411 E-04$ & $-2.8026 E-03$ & $2.5508 E-03$ \\
\hline 140 & $3.8712 E-04$ & $-2.8312 E-03$ & $2.6262 \mathrm{E}-03$ \\
\hline 141 & $1.4925 E-04$ & $-9.6337 E-04$ & $1.3378 \mathrm{E}-03$ \\
\hline 142 & $1.4422 \mathrm{E}-04$ & $-4.5295 E-04$ & $1.1235 \mathrm{E}-03$ \\
\hline 143 & $1.5490 \mathrm{E}-04$ & $-1.2351 E-03$ & $2.1938 \mathrm{E}-03$ \\
\hline 144 & $6.4744 \mathrm{E}-05$ & $-3.3445 E-04$ & $7.5336 \mathrm{E}-04$ \\
\hline 145 & $8.2229 E-04$ & $-2.7165 E-04$ & $6.6269 \mathrm{E}-04$ \\
\hline 146 & $6.7978 \mathrm{E}-04$ & $-4.1042 E-04$ & $7.9458 \mathrm{E}-04$ \\
\hline 147 & $3.2938 E-04$ & $-1.1761 E-04$ & $1.9318 \mathrm{E}-04$ \\
\hline 148 & $1.0859 \mathrm{E}-04$ & $-5.9175 E-05$ & 1. $3243 E-04$ \\
\hline 149 & $5.9627 \mathrm{E}-05$ & $-3.7701 E-05$ & $9.7112 \mathrm{E}-05$ \\
\hline 150 & $1.7015 \mathrm{E}-04$ & $-1.2409 \mathrm{E}-04$ & $2.8871 \mathrm{E}-04$ \\
\hline 151 & $1.3042 \mathrm{E}-04$ & $-1.2721 E-04$ & $3.4573 E-04$ \\
\hline 152 & $7.9303 E-05$ & $-1.3007 E-04$ & $3.5622 \mathrm{E}-04$ \\
\hline 153 & $8.0252 \mathrm{E}-05$ & $-1.3289 \mathrm{E}-04$ & $3.9765 E-04$ \\
\hline 154 & $9.3218 \mathrm{E}-05$ & $-1.3512 E-04$ & $3.3495 \mathrm{E}-04$ \\
\hline 155 & $9.3312 \mathrm{E}-05$ & $-1.2361 E-04$ & $2.5897 \mathrm{E}-04$ \\
\hline 156 & $9.2711 \mathrm{E}-05$ & $-1.1448 \mathrm{E}-04$ & $3.3308 \mathrm{E}-04$ \\
\hline 157 & $1.1417 \mathrm{E}-04$ & $-1.3297 E-04$ & $3.5854 \mathrm{E}-04$ \\
\hline 158 & $1.2864 \mathrm{E}-04$ & $-1.3720 \mathrm{E}-04$ & $3.7838 \mathrm{E}-04$ \\
\hline 159 & $3.0061 \mathrm{E}-04$ & $-1.9035 E-04$ & $6.5148 \mathrm{E}-04$ \\
\hline 160 & $1.2085 E-04$ & $-9.9337 E-05$ & $3.2785 \mathrm{E}-04$ \\
\hline 161 & $1.4351 \mathrm{E}-04$ & $-1.3909 \mathrm{E}-04$ & $3.1532 \mathrm{E}-04$ \\
\hline 162 & $1.6682 E-04$ & $-1.6273 E-04$ & $4.2556 \mathrm{E}-04$ \\
\hline 163 & $1.8495 \mathrm{E}-04$ & $-1.7393 E-04$ & $3.0967 \mathrm{E}-04$ \\
\hline 164 & $2.0345 E-04$ & $-1.8509 \mathrm{E}-04$ & $4.6764 \mathrm{E}-04$ \\
\hline 165 & $2.2132 \mathrm{E}-04$ & $-1.9666 \mathrm{E}-04$ & $4.2683 E-04$ \\
\hline 166 & 1. $3542 \mathrm{E}-04$ & $-1.1483 E-04$ & $2.8152 \mathrm{E}-04$ \\
\hline 167 & $1.5182 \mathrm{E}-04$ & $-1.2110 \mathrm{E}-04$ & $3.3331 \mathrm{E}-04$ \\
\hline 168 & $1.7660 \mathrm{E}-04$ & $-1.2685 E-04$ & $2.7533 E-04$ \\
\hline 169 & $2.1245 \mathrm{E}-04$ & $-1.3208 E-04$ & $3.0394 \mathrm{E}-04$ \\
\hline 170 & $2.6320 \mathrm{E}-04$ & $-1.3841 E-04$ & $2.7320 E-04$ \\
\hline 171 & $1.9419 \mathrm{E}-04$ & $-7.1841 E-05$ & $1.6409 \mathrm{E}-04$ \\
\hline 172 & $2.7519 E-04$ & $-7.3711 E-05$ & $1.4999 \mathrm{E}-04$ \\
\hline 173 & $3.8550 \mathrm{E}-04$ & $-7.5674 E-05$ & $1.4648 \mathrm{E}-04$ \\
\hline 174 & $5.0044 \mathrm{E}-04$ & $-7.7661 \mathrm{E}-05$ & $8.4438 \mathrm{E}-05$ \\
\hline 175 & $2.2717 \mathrm{E}-04$ & $-3.1699 \mathrm{E}-05$ & $3.8770 \mathrm{E}-05$ \\
\hline 176 & $2.2913 E-04$ & $-3.1410 \mathrm{E}-05$ & $2.9601 \mathrm{E}-05$ \\
\hline 177 & $2.3709 E-04$ & $-3.2293 E-05$ & $4.2854 \mathrm{E}-05$ \\
\hline 178 & $2.4257 \mathrm{E}-04$ & $-3.2709 E-05$ & $3.4193 E-05$ \\
\hline 179 & $2.3003 E-04$ & $-3.2775 E-05$ & $2.9616 \mathrm{E}-05$ \\
\hline 180 & $2.2349 E-04$ & $-3.2936 E-05$ & $3.7056 \mathrm{E}-05$ \\
\hline 181 & $2.2113 E-04$ & $-3.3917 E-05$ & $3.6280 \mathrm{E}-05$ \\
\hline 182 & $2.1354 \mathrm{E}-04$ & $-3.4383 E-05$ & $3.6401 \mathrm{E}-05$ \\
\hline 183 & $2.0719 \mathrm{E}-04$ & $-3.4859 \mathrm{E}-05$ & $5.0084 \mathrm{E}-05$ \\
\hline 184 & $2.0168 \mathrm{E}-04$ & $-3.5283 E-05$ & $4.0429 \mathrm{E}-05$ \\
\hline 185 & $1.9414 \mathrm{E}-04$ & $-3.5733 E-05$ & $5.6482 \mathrm{E}-05$ \\
\hline 186 & $1.8530 \mathrm{E}-04$ & $-3.6287 E-05$ & $5.3350 \mathrm{E}-05$ \\
\hline 187 & $1.8060 \mathrm{E}-04$ & $-3.6876 \mathrm{E}-05$ & $4.6883 E-05$ \\
\hline 188 & $1.7542 \mathrm{E}-04$ & $-3.7408 E-05$ & $4.8868 \mathrm{E}-05$ \\
\hline 189 & $1.7875 \mathrm{E}-04$ & $-3.8136 \mathrm{E}-05$ & $1.7370 \mathrm{E}-05$ \\
\hline 190 & $4.1786 \mathrm{E}-04$ & $-9.7698 E-05$ & $1.3305 \mathrm{E}-04$ \\
\hline 191 & $4.0193 E-04$ & $-1.0043 E-04$ & $1.4039 \mathrm{E}-04$ \\
\hline
\end{tabular}

Figure 6.5.15. Composite sensitivity data from TSUNAMI-IP output.. (continued) 


\begin{tabular}{|c|c|c|c|}
\hline 192 & $4.0255 E-04$ & $-1.0398 E-04$ & 1. $5921 \mathrm{E}-0$ \\
\hline 193 & $3.9780 \mathrm{E}-04$ & $-1.0814 E-04$ & $1.2778 \mathrm{E}-0$ \\
\hline 194 & $8.0388 E-04$ & $-2.3098 E-04$ & $2.8322 \mathrm{E}-0$ \\
\hline 195 & $8.5863 E-04$ & $-2.5171 E-04$ & $1.3920 \mathrm{E}-0$ \\
\hline 196 & $9.2365 E-04$ & $-2.7401 E-04$ & $3.7939 \mathrm{E}-0$ \\
\hline 197 & $1.0456 E-03$ & $-3.0662 E-04$ & $4.0857 \mathrm{E}-0$ \\
\hline 198 & $1.1698 \mathrm{E}-03$ & $-3.3550 E-04$ & $4.2186 \mathrm{E}-\mathrm{C}$ \\
\hline 199 & $6.4963 E-04$ & $-1.8310 E-04$ & $2.6318 \mathrm{E}-\mathrm{C}$ \\
\hline 200 & $7.1981 \mathrm{E}-04$ & $-1.9880 \mathrm{E}-04$ & $2.2883 E-c$ \\
\hline 201 & $1.6158 \mathrm{E}-03$ & $-4.2613 E-04$ & $3.6152 \mathrm{E}-\mathrm{C}$ \\
\hline 202 & $2.0254 \mathrm{E}-03$ & $-4.9966 E-04$ & $2.8211 \mathrm{E}-\mathrm{C}$ \\
\hline 203 & $2.4707 E-03$ & $-5.6340 E-04$ & $2.8843 E-c$ \\
\hline 204 & $3.3624 E-03$ & $-6.7630 E-04$ & $2.8969 \mathrm{E}-\mathrm{C}$ \\
\hline 205 & $2.1844 E-03$ & $-3.8558 E-04$ & $-8.4664 E-C$ \\
\hline 206 & $2.7103 E-03$ & $-4.3373 E-04$ & $1.1227 \mathrm{E}-\mathrm{C}$ \\
\hline 207 & $3.2507 E-03$ & $-4.6733 E-04$ & $0.0000 \mathrm{E}+\mathrm{C}$ \\
\hline 208 & $4.1765 E-03$ & $-5.5377 E-04$ & $1.9915 \mathrm{E}-\mathrm{C}$ \\
\hline 209 & $4.6469 \mathrm{E}-03$ & $-6.0428 E-04$ & $-6.5711 E-0$ \\
\hline 210 & $5.4292 E-03$ & $-7.4815 E-04$ & $-5.8270 \mathrm{E}-\mathrm{C}$ \\
\hline 211 & $5.9319 E-03$ & $-8.9850 E-04$ & $-4.1482 E-C$ \\
\hline 212 & $6.8645 E-03$ & $-1.1352 E-03$ & $3.6827 \mathrm{E}-0$ \\
\hline 213 & $9.0697 E-03$ & $-1.5740 E-03$ & $9.5084 \mathrm{E}-\mathrm{C}$ \\
\hline 214 & $1.3105 E-02$ & $-2.3116 E-03$ & $-6.2132 E-C$ \\
\hline 215 & $2.0944 \mathrm{E}-02$ & $-3.6390 E-03$ & $0.0000 \mathrm{E}+\mathrm{C}$ \\
\hline 216 & $3.7206 \mathrm{E}-02$ & $-6.2563 E-03$ & $-2.7238 E-0$ \\
\hline 217 & $2.2374 E-02$ & $-3.6629 E-03$ & $-1.8224 \mathrm{E}-\mathrm{C}$ \\
\hline 218 & $2.9838 E-02$ & $-4.7756 E-03$ & $-3.1442 E-C$ \\
\hline 219 & $3.8551 E-02$ & $-6.0551 E-03$ & $-2.1233 E-C$ \\
\hline 220 & $4.9816 E-02$ & $-7.6542 E-03$ & $-4.8489 E-C$ \\
\hline 221 & $6.4973 E-02$ & $-9.7526 \mathrm{E}-03$ & $-4.3945 E-C$ \\
\hline 222 & $8.2484 E-02$ & $-1 \cdot 2128 E-02$ & $-8.4682 \mathrm{E}-\mathrm{C}$ \\
\hline 223 & $1.0354 \mathrm{E}-01$ & $-1.4855 E-02$ & $-9.5965 E-c$ \\
\hline 224 & $5.5503 E-02$ & $-7.8609 E-03$ & $-5.7398 E-c$ \\
\hline 225 & $2.0287 E-01$ & $-2.8154 E-02$ & $-2.1178 \mathrm{E}-\mathrm{C}$ \\
\hline 226 & $3.2324 E-02$ & $-4.4137 E-03$ & $-3.9011 E-C$ \\
\hline 227 & $2.9803 E-02$ & $-4.0284 E-03$ & $-3.8972 \mathrm{E}-\mathrm{C}$ \\
\hline 228 & $1.0735 E-02$ & $-1.4428 E-03$ & $-1.2831 E-C$ \\
\hline 229 & $9.7841 \mathrm{E}-03$ & $-1.3106 \mathrm{E}-03$ & $-1.2401 \mathrm{E}-\mathrm{C}$ \\
\hline 230 & $4.4405 E-03$ & $-5.9294 E-04$ & $-4.6629 E-C$ \\
\hline 231 & $4.0564 \mathrm{E}-03$ & $-5.4338 E-04$ & $-4.6124 \mathrm{E}-\mathrm{C}$ \\
\hline 232 & $3.6568 E-03$ & $-4.8680 E-04$ & $-4.1461 \mathrm{E}-\mathrm{C}$ \\
\hline 233 & $1.9509 \mathrm{E}-03$ & $-2.5916 E-04$ & $-2.2200 E-C$ \\
\hline 234 & $1.1574 \mathrm{E}-03$ & $-1.5422 \mathrm{E}-04$ & $-1.3472 \mathrm{E}-\mathrm{C}$ \\
\hline 235 & $1.3127 E-03$ & $-1.7411 E-04$ & $-1.4945 E-C$ \\
\hline 236 & $1.1070 \mathrm{E}-03$ & $-1.4705 E-04$ & $-1.1858 \mathrm{E}-\mathrm{C}$ \\
\hline 237 & $1.2172 \mathrm{E}-03$ & $-1.6145 E-04$ & $-1.3854 \mathrm{E}-\mathrm{C}$ \\
\hline 238 & $1.0399 \mathrm{E}-04$ & $-1.3798 E-05$ & $-1.1822 \mathrm{E}-\mathrm{C}$ \\
\hline
\end{tabular}

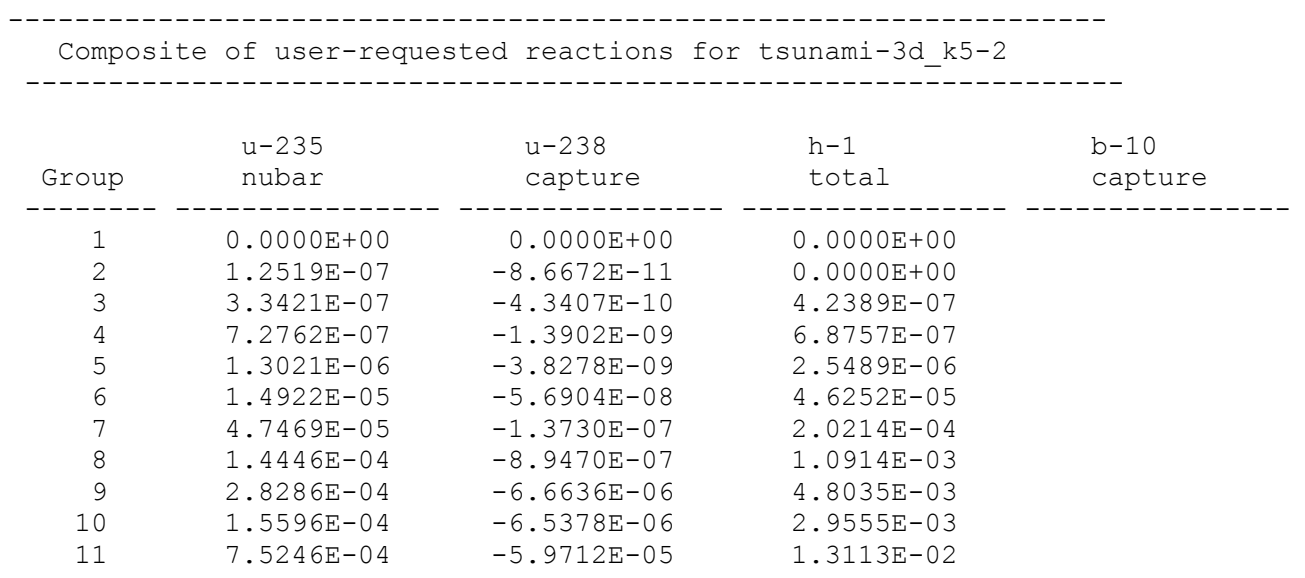

Figure 6.5.15. Composite sensitivity data from TSUNAMI-IP output.. (continued) 


\begin{tabular}{|c|c|c|c|}
\hline 12 & $5.8627 \mathrm{E}-04$ & $-7.9447 E-05$ & $1.2557 \mathrm{E}-02$ \\
\hline 13 & $1.7542 \mathrm{E}-04$ & $-3.0813 E-05$ & $4.0300 \mathrm{E}-03$ \\
\hline 14 & $7.3736 \mathrm{E}-04$ & $-1.8899 E-04$ & $1.5889 \mathrm{E}-02$ \\
\hline 15 & $6.2601 \mathrm{E}-04$ & $-2.4990 E-04$ & $1.3073 E-02$ \\
\hline 16 & $2.0457 \mathrm{E}-04$ & $-1.0331 E-04$ & $4.7828 \mathrm{E}-03$ \\
\hline 17 & $9.0359 \mathrm{E}-05$ & $-4.9631 E-05$ & $2.1468 \mathrm{E}-03$ \\
\hline 18 & $7.3377 \mathrm{E}-05$ & $-4.3188 E-05$ & $1.6032 \mathrm{E}-03$ \\
\hline 19 & $1.3573 E-04$ & $-8.6428 E-05$ & $2.6278 E-03$ \\
\hline 20 & $1.1161 \mathrm{E}-04$ & $-7.6638 E-05$ & $2.4900 \mathrm{E}-03$ \\
\hline 21 & $2.2033 E-04$ & $-1.7115 E-04$ & $4.9231 \mathrm{E}-03$ \\
\hline 22 & $1.8239 \mathrm{E}-04$ & $-1.6205 E-04$ & $3.4382 \mathrm{E}-03$ \\
\hline 23 & $1.9578 \mathrm{E}-04$ & $-1.8691 E-04$ & $3.6221 \mathrm{E}-03$ \\
\hline 24 & $4.7147 \mathrm{E}-05$ & $-4.5670 E-05$ & $1.1450 \mathrm{E}-03$ \\
\hline 25 & $6.3898 \mathrm{E}-05$ & $-6.1821 E-05$ & $1.6513 \mathrm{E}-03$ \\
\hline 26 & $3.7065 \mathrm{E}-05$ & $-3.6333 E-05$ & $9.5190 \mathrm{E}-04$ \\
\hline 27 & $1.1856 \mathrm{E}-04$ & $-1.1649 \mathrm{E}-04$ & $3.1639 \mathrm{E}-03$ \\
\hline 28 & $2.2510 \mathrm{E}-04$ & $-2.2146 E-04$ & $5.6953 E-03$ \\
\hline 29 & $2.1545 E-04$ & $-2.1077 E-04$ & $6.2475 E-03$ \\
\hline 30 & $2.7193 E-05$ & $-2.6428 E-05$ & $7.8781 \mathrm{E}-04$ \\
\hline 31 & $2.1388 \mathrm{E}-04$ & $-2.0401 E-04$ & $6.4215 \mathrm{E}-03$ \\
\hline 32 & $8.3295 E-05$ & $-7.7147 E-05$ & $2.3947 E-03$ \\
\hline 33 & $7.1583 E-05$ & $-6.6441 E-05$ & $2.0713 E-03$ \\
\hline 34 & $1.5905 E-04$ & $-1.4732 E-04$ & $4.5168 E-03$ \\
\hline 35 & $9.2541 \mathrm{E}-05$ & $-8.5375 E-05$ & $2.5578 \mathrm{E}-03$ \\
\hline 36 & $8.1119 \mathrm{E}-05$ & $-7.5870 E-05$ & $1.5017 E-03$ \\
\hline 37 & $4.9873 E-05$ & $-4.6393 E-05$ & $6.8414 \mathrm{E}-04$ \\
\hline 38 & $6.3817 E-05$ & $-5.9216 E-05$ & $9.7337 \mathrm{E}-04$ \\
\hline 39 & $2.7366 \mathrm{E}-04$ & $-2.5218 E-04$ & $6.3032 \mathrm{E}-03$ \\
\hline 40 & $2.7217 E-04$ & $-2.5472 E-04$ & $6.6936 \mathrm{E}-03$ \\
\hline 41 & $3.4925 E-04$ & $-3.2612 E-04$ & $8.1596 \mathrm{E}-03$ \\
\hline 42 & $2.8678 \mathrm{E}-04$ & $-2.8720 E-04$ & $6.4855 E-03$ \\
\hline 43 & $1.4163 \mathrm{E}-04$ & $-1.4926 \mathrm{E}-04$ & $3.3112 \mathrm{E}-03$ \\
\hline 44 & $2.1400 \mathrm{E}-04$ & $-2.3936 E-04$ & $4.3949 \mathrm{E}-03$ \\
\hline 45 & $1.2424 \mathrm{E}-04$ & $-1.5558 \mathrm{E}-04$ & $3.0408 \mathrm{E}-03$ \\
\hline 46 & $2.7340 \mathrm{E}-05$ & $-3.5790 E-05$ & $5.9417 \mathrm{E}-04$ \\
\hline 47 & $7.2215 \mathrm{E}-05$ & $-9.6257 E-05$ & $1.5233 \mathrm{E}-03$ \\
\hline 48 & $2.2974 \mathrm{E}-05$ & $-3.0302 E-05$ & $4.7674 \mathrm{E}-04$ \\
\hline 49 & $1.5246 \mathrm{E}-04$ & $-2.2541 E-04$ & $2.9677 \mathrm{E}-03$ \\
\hline 50 & $1.1097 \mathrm{E}-04$ & $-1.8360 E-04$ & $1.7649 \mathrm{E}-03$ \\
\hline 51 & $2.8048 E-05$ & $-5.3701 E-05$ & $5.0807 \mathrm{E}-04$ \\
\hline 52 & $7.6335 E-05$ & $-1.5167 \mathrm{E}-04$ & $1.3984 \mathrm{E}-03$ \\
\hline 53 & $2.9500 \mathrm{E}-04$ & $-6.2994 E-04$ & $5.4989 \mathrm{E}-03$ \\
\hline 54 & $1.3718 \mathrm{E}-04$ & $-3.1099 E-04$ & $1.9844 \mathrm{E}-03$ \\
\hline 55 & $3.0486 \mathrm{E}-04$ & $-7.2088 E-04$ & $5.0718 \mathrm{E}-03$ \\
\hline 56 & $2.2166 \mathrm{E}-04$ & $-5.3923 E-04$ & $2.8143 E-03$ \\
\hline 57 & $2.8217 \mathrm{E}-04$ & $-6.7714 \mathrm{E}-04$ & $3.5159 \mathrm{E}-03$ \\
\hline 58 & $1.6164 \mathrm{E}-04$ & $-3.3247 E-04$ & $2.1026 \mathrm{E}-03$ \\
\hline 59 & $3.0000 E-04$ & $-6.5640 E-04$ & $3.1621 \mathrm{E}-03$ \\
\hline 60 & $5.0678 \mathrm{E}-04$ & $-1.0792 E-03$ & $4.8527 \mathrm{E}-03$ \\
\hline 61 & $5.7681 E-05$ & $-8.7342 E-05$ & $4.7504 \mathrm{E}-04$ \\
\hline 62 & $3.0594 \mathrm{E}-04$ & $-6.2686 E-04$ & $2.0945 E-03$ \\
\hline 63 & $2.2535 E-04$ & $-4.0760 E-04$ & $1.7729 \mathrm{E}-03$ \\
\hline 64 & $1.9245 \mathrm{E}-04$ & $-3.5872 E-04$ & $1.4543 \mathrm{E}-03$ \\
\hline 65 & $6.6674 E-05$ & $-1.3797 E-04$ & $4.3374 E-04$ \\
\hline 66 & $3.3064 \mathrm{E}-04$ & $-5.1874 \mathrm{E}-04$ & $2.2962 \mathrm{E}-03$ \\
\hline 67 & $2.9095 E-04$ & $-4.5419 E-04$ & $1.8807 E-03$ \\
\hline 68 & $5.1987 \mathrm{E}-05$ & $-7.5088 E-05$ & $3.1526 \mathrm{E}-04$ \\
\hline 69 & $5.8854 \mathrm{E}-04$ & $-8.5478 E-04$ & $3.0208 E-03$ \\
\hline 70 & $4.1099 \mathrm{E}-04$ & $-7.1338 E-04$ & $1.7490 \mathrm{E}-03$ \\
\hline 71 & $8.5819 E-04$ & $-1.3405 E-03$ & $4.3646 E-03$ \\
\hline 72 & $9.7106 \mathrm{E}-05$ & $-2.5341 E-05$ & $2.1843 E-04$ \\
\hline 73 & $6.3698 \mathrm{E}-04$ & $-7.7299 E-04$ & $2.5855 E-03$ \\
\hline 74 & $2.1654 \mathrm{E}-03$ & $-2.0489 \mathrm{E}-03$ & $6.4043 E-03$ \\
\hline 75 & $2.4547 E-04$ & $-3.7235 E-04$ & $6.9292 E-04$ \\
\hline 76 & $1.0286 \mathrm{E}-03$ & $-5.5024 E-04$ & $1.9443 E-03$ \\
\hline 77 & $7.9496 \mathrm{E}-04$ & $-5.2036 \mathrm{E}-04$ & $2.0518 \mathrm{E}-03$ \\
\hline 78 & $1.1633 \mathrm{E}-05$ & $-4.1199 \mathrm{E}-04$ & $4.3688 E-04$ \\
\hline 79 & $4.1893 E-04$ & $-1.6219 E-04$ & $8.4874 \mathrm{E}-04$ \\
\hline
\end{tabular}

Figure 6.5.15. Composite sensitivity data from TSUNAMI-IP output.. (continued) 


\begin{tabular}{|c|c|c|c|}
\hline 80 & $1.0671 \mathrm{E}-04$ & $-6.8021 E-04$ & $7.8182 \mathrm{E}-04$ \\
\hline 81 & $2.3203 E-03$ & $-9.9401 E-04$ & $4.6543 E-03$ \\
\hline 82 & $1.5328 \mathrm{E}-04$ & $-3.1823 E-05$ & $3.2999 \mathrm{E}-04$ \\
\hline 83 & $2.7539 E-04$ & $-8.2324 E-04$ & $1.0520 \mathrm{E}-03$ \\
\hline 84 & $1.6432 \mathrm{E}-04$ & $-7.7786 \mathrm{E}-05$ & $6.4772 \mathrm{E}-04$ \\
\hline 85 & $3.3128 E-04$ & $-1.4793 E-03$ & $1.9299 \mathrm{E}-03$ \\
\hline 86 & $5.8071 E-04$ & $-1.2966 \mathrm{E}-04$ & $9.6470 \mathrm{E}-04$ \\
\hline 87 & $7.2897 E-04$ & $-1.8707 E-04$ & $1.0648 E-03$ \\
\hline 88 & $1.0165 E-04$ & $-6.0134 E-04$ & $9.5099 \mathrm{E}-04$ \\
\hline 89 & $1.9705 \mathrm{E}-04$ & $-2.7280 E-05$ & $5.4711 \mathrm{E}-04$ \\
\hline 90 & $5.1470 \mathrm{E}-04$ & $-2.8447 E-05$ & $4.2588 \mathrm{E}-04$ \\
\hline 91 & $4.7360 \mathrm{E}-04$ & $-1.2936 \mathrm{E}-04$ & $8.4947 E-04$ \\
\hline 92 & $2.6906 E-05$ & $-1.3329 E-03$ & $1.8984 \mathrm{E}-03$ \\
\hline 93 & $2.5613 E-04$ & $-2.6531 E-04$ & $6.5075 E-04$ \\
\hline 94 & $2.3538 E-04$ & $-2.1992 E-05$ & $2.0144 \mathrm{E}-04$ \\
\hline 95 & $1.5775 \mathrm{E}-03$ & $-3.7759 \mathrm{E}-05$ & $5.4441 \mathrm{E}-04$ \\
\hline 96 & $3.3280 \mathrm{E}-04$ & $-7.9568 E-06$ & $1.7036 \mathrm{E}-04$ \\
\hline 97 & $5.9346 \mathrm{E}-04$ & $-8.3159 E-06$ & $6.5965 E-05$ \\
\hline 98 & $2.2511 \mathrm{E}-04$ & $-1.5893 \mathrm{E}-05$ & $3.1849 \mathrm{E}-04$ \\
\hline 99 & $2.0225 E-04$ & $-7.0445 E-06$ & $1.9922 \mathrm{E}-04$ \\
\hline 100 & $2.7342 \mathrm{E}-04$ & $-1.1609 \mathrm{E}-05$ & $3.2313 E-04$ \\
\hline 101 & $2.6957 \mathrm{E}-04$ & $-3.0399 E-05$ & $4.6923 E-04$ \\
\hline 102 & $3.3244 \mathrm{E}-04$ & $-2.7060 \mathrm{E}-05$ & $3.2659 \mathrm{E}-04$ \\
\hline 103 & $1.9461 \mathrm{E}-04$ & $-4.2923 E-05$ & $5.9301 \mathrm{E}-04$ \\
\hline 104 & $3.2556 \mathrm{E}-04$ & $-5.2598 E-05$ & $5.4021 E-04$ \\
\hline 105 & $2.3730 E-04$ & $-1.1291 E-04$ & $5.1509 \mathrm{E}-04$ \\
\hline 106 & $3.4607 \mathrm{E}-04$ & $-5.8981 E-05$ & $2.2766 \mathrm{E}-04$ \\
\hline 107 & $1.1425 \mathrm{E}-04$ & $-2.9850 \mathrm{E}-04$ & $7.9441 \mathrm{E}-04$ \\
\hline 108 & $4.1191 E-05$ & $-6.7330 E-04$ & $1.4840 E-03$ \\
\hline 109 & $1.9773 \mathrm{E}-04$ & $-1.6031 \mathrm{E}-03$ & $2.7789 \mathrm{E}-03$ \\
\hline 110 & $1.1533 \mathrm{E}-03$ & $-3.4513 E-04$ & $2.8717 \mathrm{E}-04$ \\
\hline 111 & $4.0019 \mathrm{E}-04$ & $-1.5092 \mathrm{E}-04$ & $4.0941 \mathrm{E}-04$ \\
\hline 112 & $2.7434 \mathrm{E}-04$ & $-5.7168 E-05$ & $2.7772 \mathrm{E}-04$ \\
\hline 113 & $4.5490 \mathrm{E}-04$ & $-1.2471 E-04$ & $4.5389 \mathrm{E}-04$ \\
\hline 114 & $2.0751 E-05$ & $-3.1118 \mathrm{E}-05$ & $1.8411 \mathrm{E}-04$ \\
\hline 115 & $1.7034 \mathrm{E}-04$ & $-5.9649 E-05$ & $5.3270 E-04$ \\
\hline 116 & $4.5462 E-04$ & $-9.8437 E-05$ & $8.1464 \mathrm{E}-04$ \\
\hline 117 & $1.0112 \mathrm{E}-03$ & $-1.3308 E-04$ & $9.1834 \mathrm{E}-04$ \\
\hline 118 & $1.2441 E-03$ & $-3.4617 E-04$ & $1.8797 \mathrm{E}-03$ \\
\hline 119 & $1.4613 \mathrm{E}-04$ & $-1.7858 E-03$ & $3.0512 \mathrm{E}-03$ \\
\hline 120 & $1.1927 \mathrm{E}-04$ & $-1.4793 E-03$ & $3.4795 E-03$ \\
\hline 121 & $1.6146 \mathrm{E}-03$ & $-6.3939 E-04$ & $8.1885 E-04$ \\
\hline 122 & $2.0288 E-04$ & $-1.4691 E-04$ & $2.7061 \mathrm{E}-04$ \\
\hline 123 & $4.9382 E-04$ & $-2.3581 E-04$ & $9.1055 E-04$ \\
\hline 124 & $5.0753 E-04$ & $-9.0473 E-05$ & $4.6140 \mathrm{E}-04$ \\
\hline 125 & $3.2433 E-04$ & $-7.0258 E-05$ & $5.3142 E-04$ \\
\hline 126 & $2.1587 \mathrm{E}-04$ & $-4.8428 E-05$ & $4.5908 \mathrm{E}-04$ \\
\hline 127 & $7.7506 \mathrm{E}-04$ & $-4.2735 E-05$ & $1.8533 \mathrm{E}-04$ \\
\hline 128 & $4.4479 E-04$ & $-5.6181 E-05$ & $4.7548 E-04$ \\
\hline 129 & $1.3698 \mathrm{E}-03$ & $-5.9501 E-05$ & $1.0153 \mathrm{E}-03$ \\
\hline 130 & $2.7637 \mathrm{E}-04$ & $-2.5432 E-05$ & $7.1524 \mathrm{E}-04$ \\
\hline 131 & $5.7207 \mathrm{E}-04$ & $-3.5591 E-04$ & $1.2909 \mathrm{E}-03$ \\
\hline 132 & $1.1350 \mathrm{E}-03$ & $-1.4174 E-04$ & $7.8511 E-04$ \\
\hline 133 & $3.6109 \mathrm{E}-03$ & $-3.1864 E-04$ & $1.2579 \mathrm{E}-03$ \\
\hline 134 & $1.7268 \mathrm{E}-04$ & $-1.4722 E-03$ & $3.9073 E-03$ \\
\hline 135 & $2.5746 \mathrm{E}-04$ & $-6.2532 E-04$ & $1.1511 \mathrm{E}-03$ \\
\hline 136 & $4.3943 E-05$ & $-1.0605 E-03$ & $2.9541 \mathrm{E}-03$ \\
\hline 137 & $5.7508 E-06$ & $-2.4973 E-04$ & $3.3501 E-03$ \\
\hline 138 & $3.7473 E-04$ & $-1.2848 E-03$ & $2.3995 E-03$ \\
\hline 139 & $2.8865 E-04$ & $-9.6386 E-04$ & $1.2706 \mathrm{E}-03$ \\
\hline 140 & $3.6819 \mathrm{E}-04$ & $-1.1046 \mathrm{E}-03$ & $2.0471 \mathrm{E}-03$ \\
\hline 141 & $1.4780 \mathrm{E}-04$ & $-3.9461 E-04$ & $1.3134 \mathrm{E}-03$ \\
\hline 142 & $1.3985 \mathrm{E}-04$ & $-1.8380 \mathrm{E}-04$ & $1.0559 \mathrm{E}-03$ \\
\hline 143 & $1.5490 \mathrm{E}-04$ & $-5.5020 \mathrm{E}-04$ & $1.4747 \mathrm{E}-03$ \\
\hline 144 & $6.4744 \mathrm{E}-05$ & $-1.5547 \mathrm{E}-04$ & $7.5336 \mathrm{E}-04$ \\
\hline 145 & $8.1785 \mathrm{E}-04$ & $-1.1830 \mathrm{E}-04$ & $6.1400 \mathrm{E}-04$ \\
\hline 146 & $6.7829 \mathrm{E}-04$ & $-1.7972 \mathrm{E}-04$ & $9.0948 \mathrm{E}-04$ \\
\hline 147 & $3.2822 \mathrm{E}-04$ & $-5.0617 \mathrm{E}-05$ & $2.4536 \mathrm{E}-04$ \\
\hline
\end{tabular}

Figure 6.5.15. Composite sensitivity data from TSUNAMI-IP output.. (continued) 


\begin{tabular}{|c|c|c|c|}
\hline 148 & $1.0819 \mathrm{E}-04$ & $-2.5691 \mathrm{E}-05$ & $1.1098 \mathrm{E}-04$ \\
\hline 149 & $6.4077 E-05$ & $-1.7786 \mathrm{E}-05$ & $9.2894 \mathrm{E}-05$ \\
\hline 150 & 1.7080E-04 & $-5.5107 \mathrm{E}-05$ & $2.8871 E-04$ \\
\hline 151 & $1.3528 E-04$ & $-5.7736 E-05$ & $3.6300 \mathrm{E}-04$ \\
\hline 152 & $7.9303 E-05$ & $-5.8377 \mathrm{E}-05$ & $3.3702 E-04$ \\
\hline 153 & $8.0053 E-05$ & $-5.7765 \mathrm{E}-05$ & $2.9098 E-04$ \\
\hline 154 & $9.4301 E-05$ & $-6.1205 E-05$ & $3.5845 \mathrm{E}-04$ \\
\hline 155 & $9.5100 \mathrm{E}-05$ & $-5.5160 \mathrm{E}-05$ & $3.1008 \mathrm{E}-04$ \\
\hline 156 & $9.2093 E-05$ & $-4.9272 E-05$ & $3.3308 \mathrm{E}-04$ \\
\hline 157 & $1.1417 \mathrm{E}-04$ & $-6.0554 \mathrm{E}-05$ & $3.5854 \mathrm{E}-04$ \\
\hline 158 & $1.2913 E-04$ & $-6.2998 E-05$ & $3.6015 \mathrm{E}-0$ \\
\hline 159 & $3.0061 E-04$ & $-8.2329 E-05$ & $7.1490 \mathrm{E}-0$ \\
\hline 160 & $1.2367 E-04$ & $-4.5006 E-05$ & $2.9688 \mathrm{E}-0$ \\
\hline 161 & $1.4840 \mathrm{E}-04$ & $-6.7230 E-05$ & $3.8590 \mathrm{E}-0$ \\
\hline 162 & $1.7126 \mathrm{E}-04$ & $-7.3391 E-05$ & $4.2556 \mathrm{E}-0$ \\
\hline 163 & $1.8465 \mathrm{E}-04$ & $-7.6909 \mathrm{E}-05$ & $4.4451 \mathrm{E}-0$ \\
\hline 164 & $2.0831 E-04$ & $-8.3782 E-05$ & $4.5114 \mathrm{E}-0$ \\
\hline 165 & $2.2843 E-04$ & $-8.8648 E-05$ & 4. $6432 E-0$ \\
\hline 166 & $1.4190 \mathrm{E}-04$ & $-5.2854 E-05$ & $2.4605 \mathrm{E}-0$ \\
\hline 167 & $1.5012 E-04$ & $-5.2102 E-05$ & $3.1876 \mathrm{E}-0$ \\
\hline 168 & $1.8061 \mathrm{E}-04$ & $-5.6757 E-05$ & $3.1259 \mathrm{E}-0$ \\
\hline 169 & $2.1245 E-04$ & $-6.0593 E-05$ & $3.0542 \mathrm{E}-0$ \\
\hline 170 & $2.6343 E-04$ & $-6.3934 E-05$ & $3.4215 \mathrm{E}-0$ \\
\hline 171 & $1.9448 E-04$ & $-3.1752 E-05$ & $1.3489 \mathrm{E}-0$ \\
\hline 172 & $2.6013 E-04$ & $-3.0902 E-05$ & $1.1947 \mathrm{E}-0$ \\
\hline 173 & $3.7054 E-04$ & $-3.2340 E-05$ & $1.2321 E-0$ \\
\hline 174 & $4.7482 \mathrm{E}-04$ & $-3.2632 E-05$ & $1.1158 \mathrm{E}-0$ \\
\hline 175 & $2.1975 E-04$ & $-1.3672 E-05$ & $3.2459 \mathrm{E}-0$ \\
\hline 176 & $2.1632 \mathrm{E}-04$ & $-1.3213 E-05$ & $4.0032 E-0$ \\
\hline 177 & $2.2152 E-04$ & $-1.3437 E-05$ & $4.5984 \mathrm{E}-0$ \\
\hline 178 & $2.3849 E-04$ & $-1.4859 \mathrm{E}-05$ & $1.8672 \mathrm{E}-0$ \\
\hline 179 & $2.1745 E-04$ & $-1.4007 \mathrm{E}-05$ & $2.7954 \mathrm{E}-0$ \\
\hline 180 & $2.2646 E-04$ & $-1.4890 \mathrm{E}-05$ & $3.7056 \mathrm{E}-0$ \\
\hline 181 & $2.1143 E-04$ & $-1.4534 \mathrm{E}-05$ & $3.4536 \mathrm{E}-0$ \\
\hline 182 & $2.0606 \mathrm{E}-04$ & $-1.4947 \mathrm{E}-05$ & $6.3531 \mathrm{E}-0$ \\
\hline 183 & $1.9077 E-04$ & $-1.4574 \mathrm{E}-05$ & $5.0864 \mathrm{E}-0$ \\
\hline 184 & $1.9528 \mathrm{E}-04$ & $-1.5566 \mathrm{E}-05$ & $4.4139 \mathrm{E}-0$ \\
\hline 185 & $1.8839 E-04$ & $-1.5884 \mathrm{E}-05$ & $4.6885 E-0$ \\
\hline 186 & $1.8455 E-04$ & $-1.6210 \mathrm{E}-05$ & $4.8693 \mathrm{E}-0$ \\
\hline 187 & $1.8045 E-04$ & $-1.6486 E-05$ & $1.6260 \mathrm{E}-0$ \\
\hline 188 & $1.7154 \mathrm{E}-04$ & $-1.6166 \mathrm{E}-05$ & $4.8868 \mathrm{E}-0$ \\
\hline 189 & $1.7634 \mathrm{E}-04$ & $-1.7504 E-05$ & $2.2074 \mathrm{E}-0$ \\
\hline 190 & $4.1297 \mathrm{E}-04$ & $-4.3034 \mathrm{E}-05$ & $1.3305 \mathrm{E}-0$ \\
\hline 191 & $4.0310 E-04$ & $-4.5888 E-05$ & $1.4039 \mathrm{E}-0$ \\
\hline 192 & $3.9172 E-04$ & $-4.5833 E-05$ & $1.5921 \mathrm{E}-0$ \\
\hline 193 & $3.8144 \mathrm{E}-04$ & $-4.6490 \mathrm{E}-05$ & $1.0588 \mathrm{E}-0$ \\
\hline 194 & $8.3313 E-04$ & $-1.0632 E-04$ & $3.3357 \mathrm{E}-0$ \\
\hline 195 & $8.7545 E-04$ & $-1.1477 \mathrm{E}-04$ & $2.7002 \mathrm{E}-0$ \\
\hline 196 & $9.0294 \mathrm{E}-04$ & $-1.1976 E-04$ & $3.7939 \mathrm{E}-0$ \\
\hline 197 & $1.0334 \mathrm{E}-03$ & $-1.3672 \mathrm{E}-04$ & $3.6811 \mathrm{E}-0$ \\
\hline 198 & $1.1556 \mathrm{E}-03$ & $-1.4920 \mathrm{E}-04$ & $4.2186 \mathrm{E}-0$ \\
\hline 199 & $6.6467 \mathrm{E}-04$ & $-8.4875 E-05$ & 1. $4783 \mathrm{E}-0$ \\
\hline 200 & $7.0922 E-04$ & $-8.7084 E-05$ & $2.2883 E-0$ \\
\hline 201 & $1.6056 \mathrm{E}-03$ & $-1.9185 \mathrm{E}-04$ & $4.5221 \mathrm{E}-0$ \\
\hline 202 & $1.9989 \mathrm{E}-03$ & $-2.2347 E-04$ & $4.2581 E-0$ \\
\hline 203 & $2.4690 \mathrm{E}-03$ & $-2.5230 \mathrm{E}-04$ & 4.1739E-0 \\
\hline 204 & $3.3101 E-03$ & $-3.0014 E-04$ & $0.0000 \mathrm{E}+0$ \\
\hline 205 & $2.0584 \mathrm{E}-03$ & $-1.6521 \mathrm{E}-04$ & $1.0675 \mathrm{E}-0$ \\
\hline 206 & $2.5065 E-03$ & $-1.8312 \mathrm{E}-04$ & $0.0000 \mathrm{E}+0$ \\
\hline 207 & $2.9577 E-03$ & $-1.9366 \mathrm{E}-04$ & $0.0000 \mathrm{E}+0$ \\
\hline 208 & $3.8129 \mathrm{E}-03$ & $-2.3258 E-04$ & $-1.4373 \mathrm{E}-0$ \\
\hline 209 & $4.2586 \mathrm{E}-03$ & $-2.5331 E-04$ & $9.6694 \mathrm{E}-0$ \\
\hline 210 & $4.8357 E-03$ & $-3.0345 E-04$ & $-1.5320 \mathrm{E}-0$ \\
\hline 211 & $5.4913 E-03$ & $-3.8129 E-04$ & $-2.2865 \mathrm{E}-0$ \\
\hline 212 & $6.4549 E-03$ & $-4.8716 \mathrm{E}-04$ & $-3.0036 \mathrm{E}-0$ \\
\hline 213 & $8.8549 E-03$ & $-7.0184 \mathrm{E}-04$ & $0.0000 \mathrm{E}+0$ \\
\hline 214 & 1. 3538E-02 & $-1.0984 \mathrm{E}-03$ & $-2.0716 \mathrm{E}-0$ \\
\hline 215 & $2.2933 E-02$ & $-1.8610 \mathrm{E}-03$ & $-4.7201 \mathrm{E}-0$ \\
\hline
\end{tabular}

Figure 6.5.15. Composite sensitivity data from TSUNAMI-IP output.. (continued) 


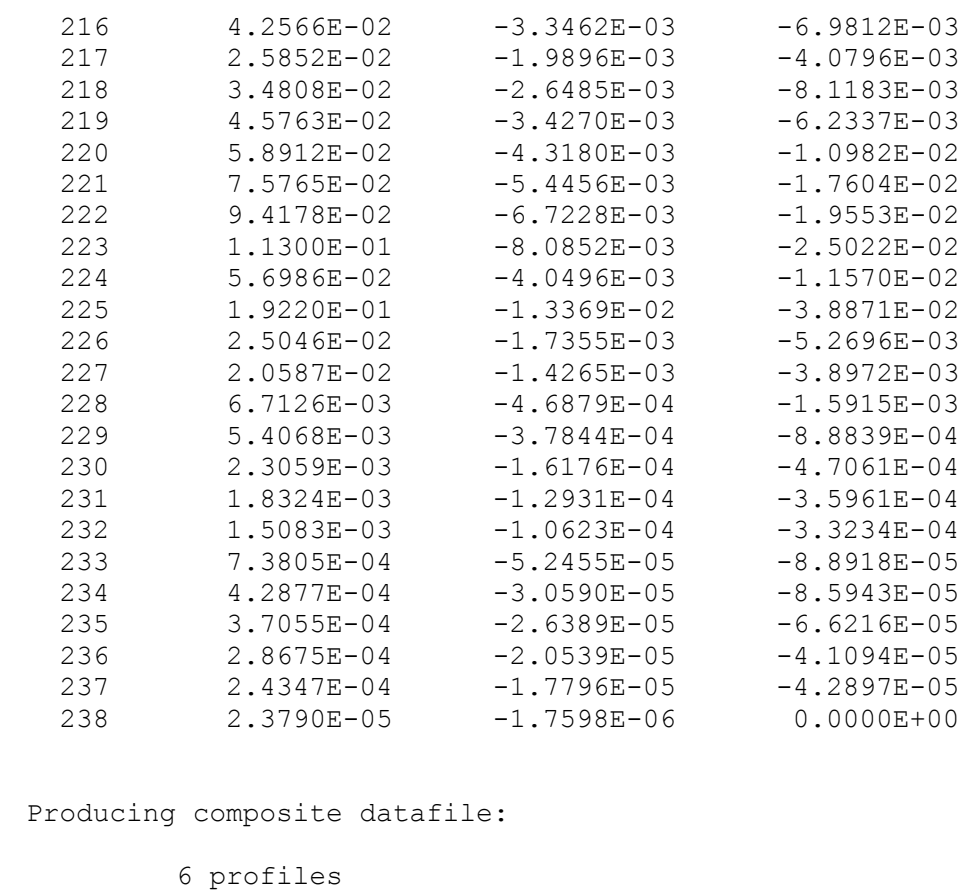

Figure 6.5.15. Composite sensitivity data from TSUNAMI-IP output.. (continued)

\subsection{HTML output description}

The HTML formatted output from the TSUNAMI-IP sample problem is described in this section. The HTML output is generated when the html keyword is included in the PARAMETER block keyword The input for this sample problem is named tsunami-ip.input. In this case, the HTML formatted output is stored in a file called tsunami-ip.html and additional resources are stored in directories called tsunami-ip.htmd and applet_resources. These sections contain example TSUNAMI-IP HTML formatted output only for demonstration of the interface. When tsunami-ip.html is opened in a web browser, the information shown in Figure 6.5.16 is displayed. The title of the input file is displayed between the two SCALE logos. Because this SCALE input file only executed tsunami-ip, only a single output listing is available. The text "1. TSUNAMI-IP" is a hyperlink to view the output from TSUNAMI-IP. Clicking on the "1. TSUNAMI-IP" hyperlink will present the information shown in Figure 6.5.17. 


\section{SCALE OUTPUT tsunami-ip}

Select an Output Below

tsunami-ip example 2

\section{TSUNAMI-IP}

Comments, Suggestions, User Assistance:

Contact SCALE Help

scalehelp@ornl.gov

or visit the SCALE website

http://www.ornl.gov/sci/scale

Figure 6.5.16. Initial screen from TSUNAMI-IP HTML output. 
The initial page of output from TSUNAMI-IP is shown in Figure 6.5.17. Program verification information is shown in the table under the TSUNAMI logo. This table includes information about the code that was executed and the date and time it was run. The menu on the left side of the screen contains hyperlinks to specific portions of the code output. Echoes of the input data are available in the Input Data section. Any errors or warning messages are available in the Messages sections. Results from the code execution are shown in the results section.

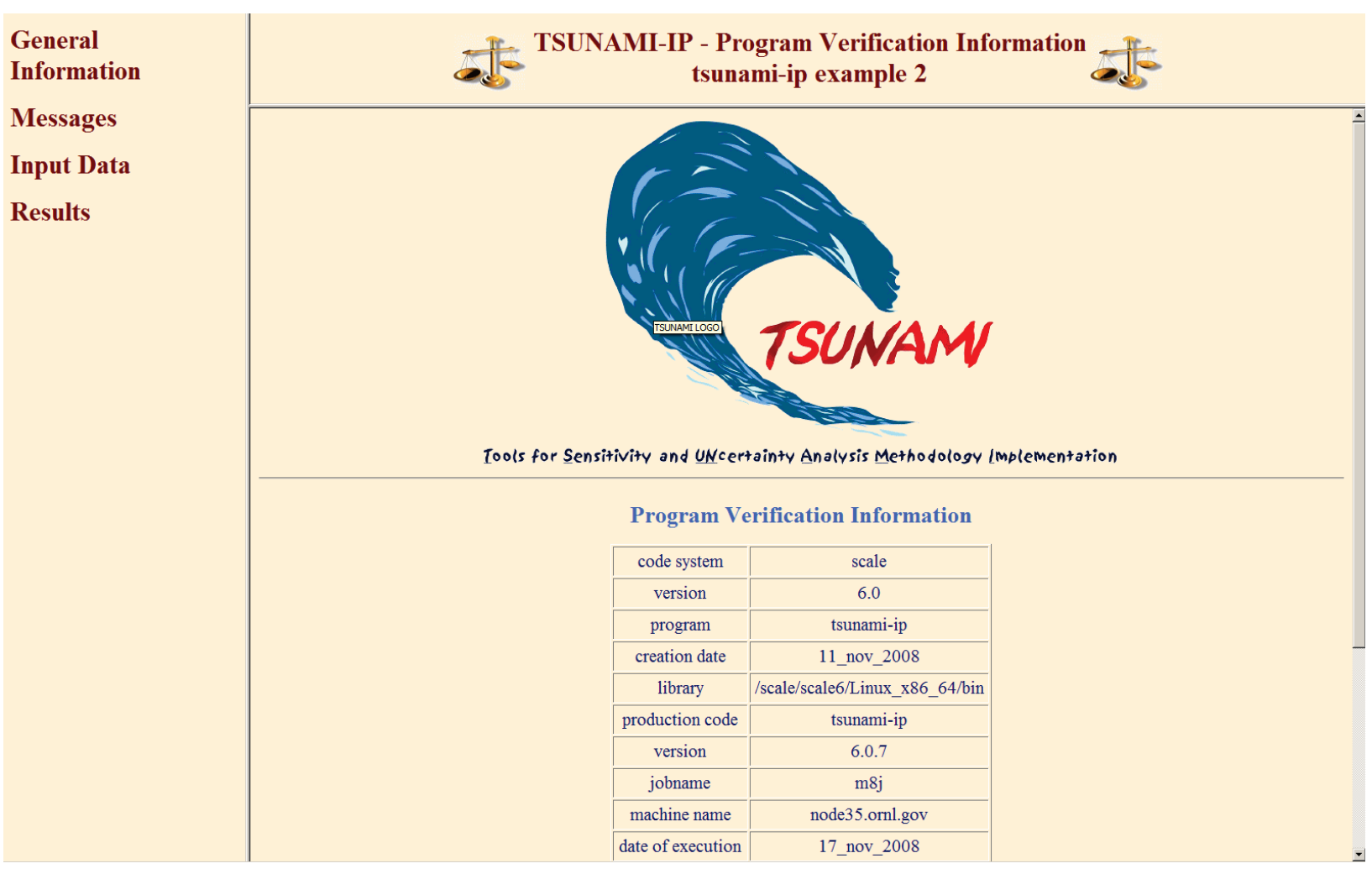

Figure 6.5.17. Program verification screen from TSUNAMI-IP HTML output. 
Selecting Input Parameters will reveal the menu of available input data. Selecting Input Parameters causes the table shown in Figure 6.5.18 to be displayed. Other input data can also be displayed by selecting the desired data from the menu.

\begin{tabular}{|c|c|c|c|}
\hline \multirow{7}{*}{$\begin{array}{l}\text { General } \\
\text { Information } \\
\text { Messages } \\
\text { Input Data } \\
\text { - Input Parameters } \\
\text { - Applications List } \\
\text { - Experiments List } \\
\text { - User Requested } \\
\text { Reactions } \\
\text { - HTML Format Options }\end{array}$} & \multicolumn{3}{|r|}{$\begin{array}{c}\text { TSUNAMI-IP - Input Parameters } \\
\text { tsunami-ip example } 2\end{array}$} \\
\hline & \multirow[b]{2}{*}{ PARAMETER } & \multirow[b]{2}{*}{ VALUE } & \multirow[b]{2}{*}{ DESCRIPTION } \\
\hline & & & \\
\hline & absolute & false & $\begin{array}{l}\text { Print uncertainty values and penalty assessments in absolute format. This is the default format. Relative format can } \\
\text { be specified using the "rel" keyword in the APPLICATIONS, EXPERIMENTS, or RESPONSE input blocks. }\end{array}$ \\
\hline & c & true & Compute $\mathrm{c}(\mathrm{k})$ values for each application compared to each experiment. \\
\hline & c_long & true & Produces extended c(k) output edit for each application compared to each experiment. Sets $\mathrm{c}$ to true. \\
\hline & cechck $=$ & 0.5000 & Level of $\mathrm{E}$ and $\mathrm{c}(\mathrm{k})$ values that trigger the cediff warning. If $\mathrm{E}$ or $\mathrm{c}(\mathrm{k})$ are below this value, no warning is printed. \\
\hline \multirow[t]{16}{*}{ Results } & cediff= & 0.1000 & $\begin{array}{l}\text { If the } \mathrm{E} \text { and } \mathrm{c}(\mathrm{k}) \text { values for a given application and experiment differ by more than cediff, a warning message is } \\
\text { printed. }\end{array}$ \\
\hline & coverx $=$ & 44groupcov & Name of cross section covariance data file. \\
\hline & cov_fix & false & Replace zero and large values on diagonal of cross-section covariance data with user input values and dcov value. \\
\hline & cp & false & Compute and print completeness parameter for each application. \\
\hline & csummary & true & Print summary table of $\mathrm{c}(\mathrm{k})$ values that meet or exceed the cvalue limit. Sets $\mathrm{c}$ to true. \\
\hline & cvalue $=$ & 0.8000 & Threshold value of $\mathrm{c}(\mathrm{k})$ for inclusion in the summary table. \\
\hline & $\mathrm{cr}$ & false & Compute $\mathrm{c}(\mathrm{r})$ values for each application compared to each experiment. \\
\hline & cr_long & false & Produces extended c(r) output edit for each application compared to each experiment. Sets cr to true. \\
\hline & crsummary & false & Print summary table of $\mathrm{c}(\mathrm{r})$ values that meet or exceed the crvalue limit. Sets $\mathrm{cr}$ to true. \\
\hline & crvalue $=$ & 0.9000 & Threshold value of $\mathrm{c}(\mathrm{r})$ for inclusion in the summary table. \\
\hline & e & true & Compute $\mathrm{E}$ (sum) values for each application compared to each experiment. \\
\hline & esummary & false & Print summary table of $\mathrm{E}(\mathrm{sum})$ values that meet or exceed the evalue limit. Sets e to true. \\
\hline & evalue $=$ & 0.9000 & Threshold value of $\mathrm{E}$ (sum) for inclusion in the summary table. \\
\hline & $\mathrm{g}$ & true & Compute $\mathrm{G}$ values. \\
\hline & gsummary & false & Print summary table of $\mathrm{G}$ values that meet or exceed the gvalue limit. Sets $\mathrm{g}$ to true. \\
\hline & gvalue $=$ & 0.9000 & Threshold value of $\mathrm{G}$ for inclusion in summary table. \\
\hline
\end{tabular}

Figure 6.5.18. Input parameters from TSUNAMI-IP HTML output. 
Selecting Messages will reveal a menu of available messages. Selecting Warning Messages from the Messages section of the menu causes the information shown in Figure 6.5.19 to appear. The Warning Messages edit contains all warning messages that were generated during the execution of the code. If errors were encountered in the code execution, an Error Messages item would have also been available in the menu under Messages.

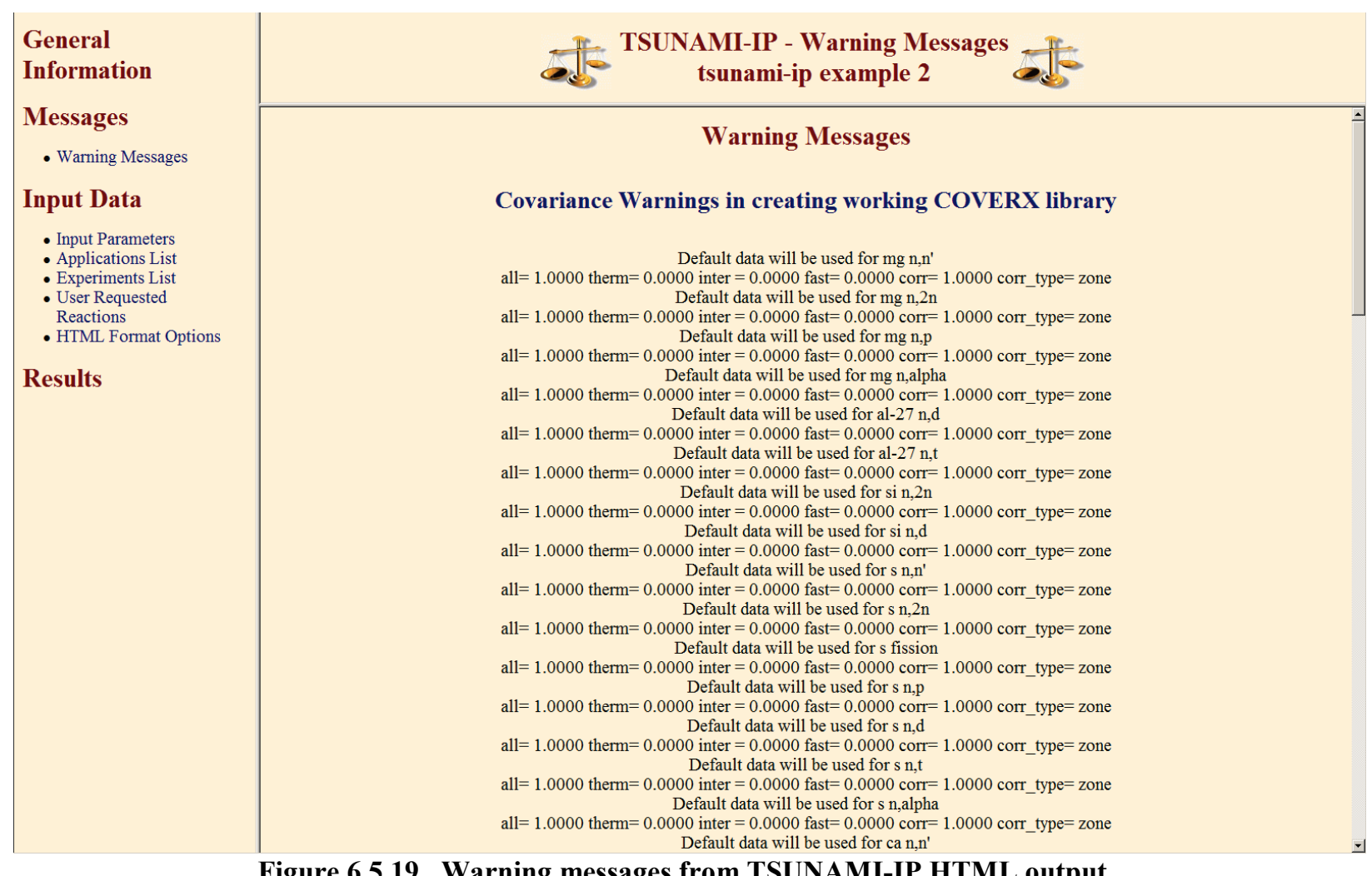

Figure 6.5.19. Warning messages from TSUNAMI-IP HTML output. 
Selecting Results causes a menu of available results to be revealed. From this menu, selecting Global Integral Indices causes a submenu of available global integral indices to be revealed. Selecting Integral Index Values from this submenu causes the information shown in Figure 6.5.20 to appear. In certain results edits, a second menu appears on the right such that the information for a particular application can be quickly reached. The table shown in Figure 6.5.20 corresponds to the values table from the standard output file. One advantage of the HTML output is the use of color coding. Values exceeding the cutoff value for a particular integral index are printed in the color that is set by the HMTL data block input cut$c l r=$. The maximum value for each index for each application is printed in the color that is set by the $\bar{H} M T L$ data block input max_clr=. In this case, the values that exceed the cutoff values are also the maximum values and are colored as such. Edits for each type of data requested in the PARAMETER data block are available in the results section by selecting the appropriate submenu.

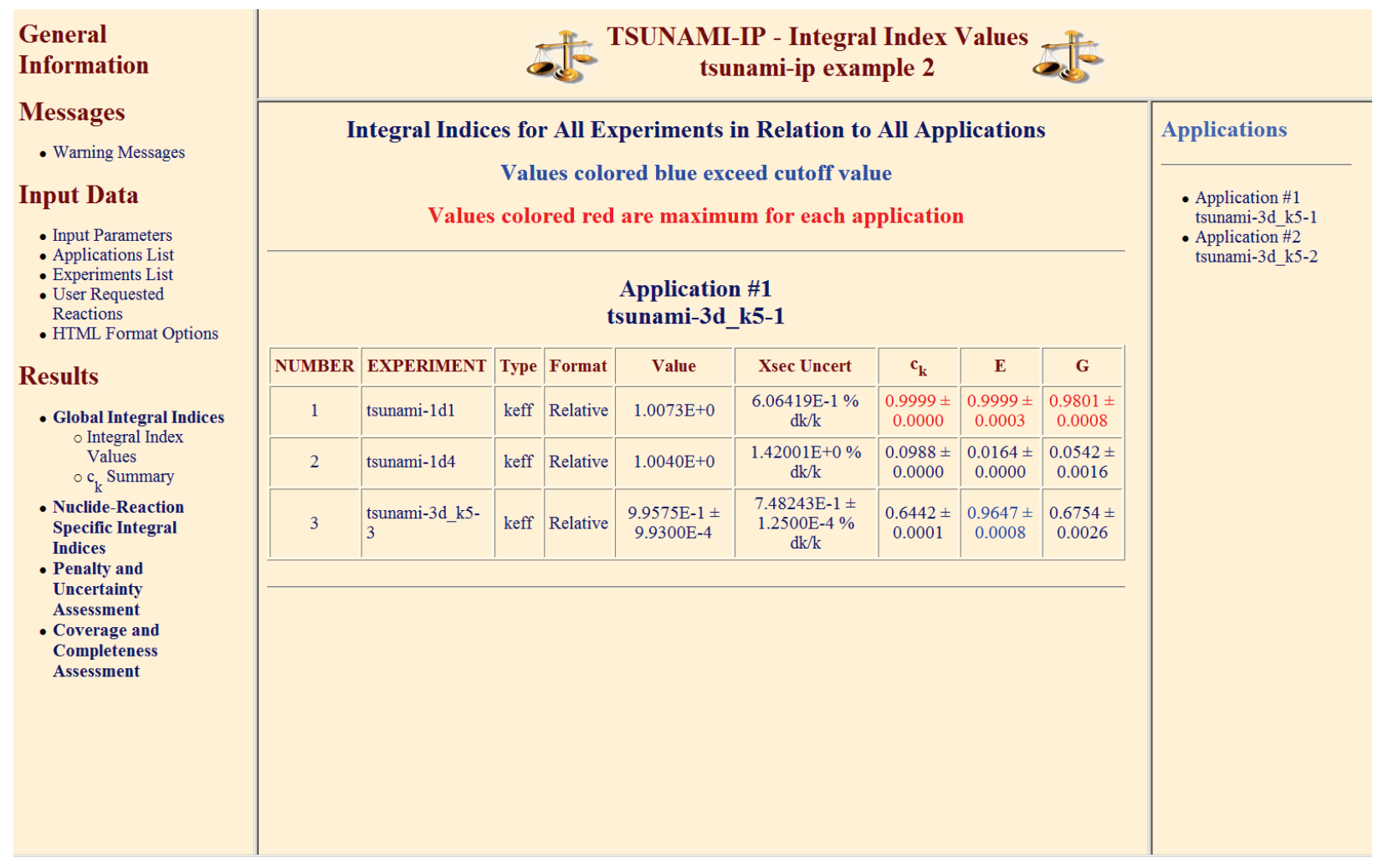

Figure 6.5.20. Global integral indices from TSUNAMI-IP HTML output. 
Data plots can also be directly viewed in the HTML output. The composite data can be viewed by selecting Coverage and Completeness Assessment then Composite Sensitivity Data Plot. A Java applet version of Javapeño will appear in the browser window with the appropriate datafile preloaded. Data can be added to the plot by double-clicking on the list of available data on the right side of Javapeño. The plot shown in Figure 6.5.21 was produced with this procedure.

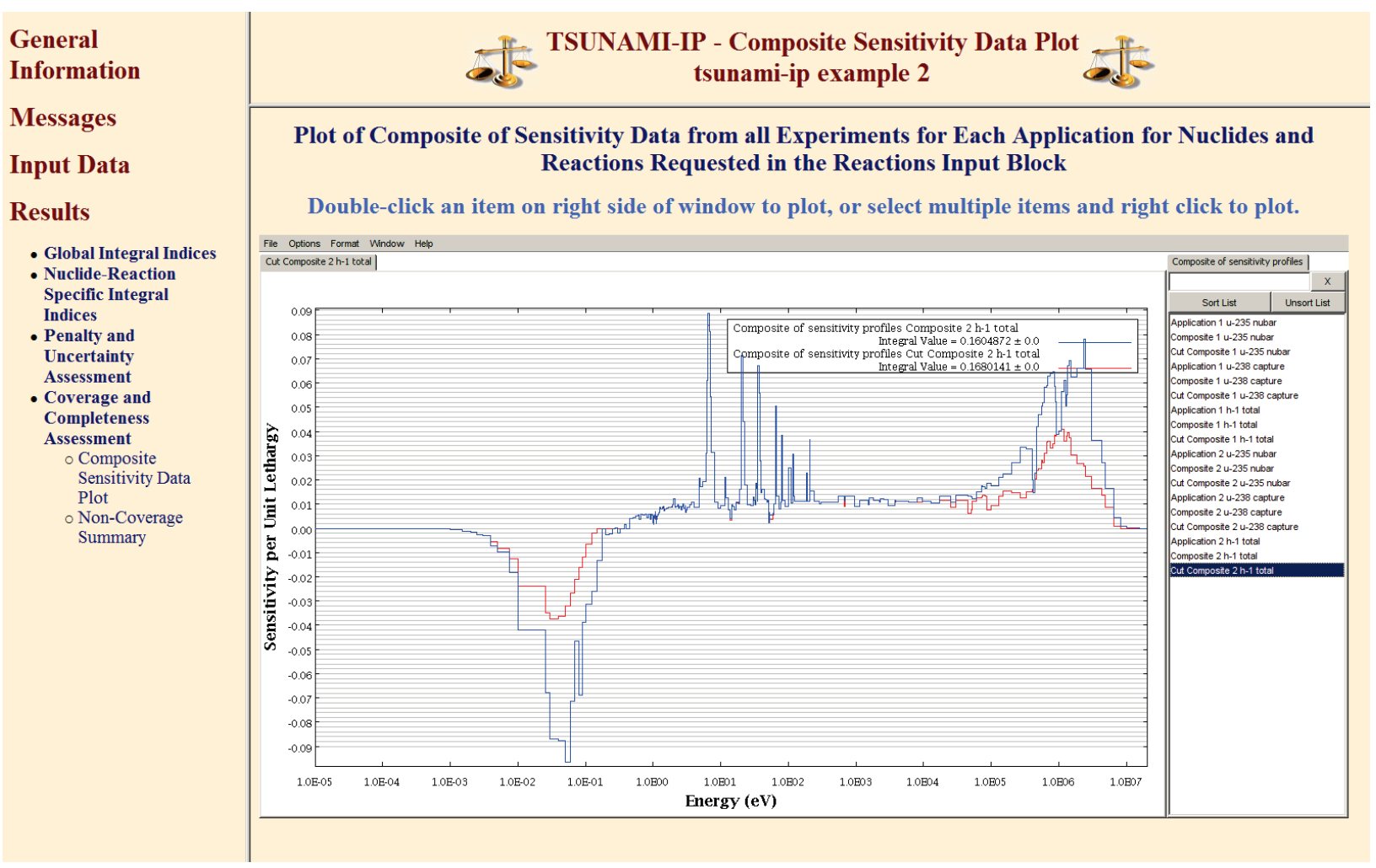

Figure 6.5.21. Composite sensitivity data plot from TSUNAMI-IP HTML output. 


\subsubsection{BONAMIST}

BONAMIST is a sensitivity version of the SCALE 4.4a BONAMI module. BONAMI performs Bondarenko calculations for resonance self-shielding. The entire FORTRAN 77 source for BONAMI was processed with the GRESS pre-compiler so that the sensitivities of the self-shielded cross sections to the data input to the code are computed. BONAMIST utilizes many of the routines from SCALELIB. BONAMIST writes the resonance self-shielded cross sections to an AMPX formatted data file, just as BONAMI does. However, an additional data file, bonamist.sen, is also written. The bonamist.sen file contains the sensitivities of resonance self-shielded cross sections generated by BONAMIST to the quantities input to BONAMIST.

The methodologies for computing the resonance self-shielded cross sections in BONAMIST are identical to those used in BONAMI, and equivalent cross-section results are obtained. The bonamist.sen data file contains the sensitivity of the self-shielded cross sections to the number density of each nuclide and the extra cross-section input to BONAMIST. The extra cross section is used by TSUNAMI-1D, TSUNAMI-3D, CSAS and other SCALE sequences to pass the Dancoff factor computed in MIPLIB or SENLIB to BONAMI. The format of the bonamist.sen data file is given in Appendix A.

The input, output, methodologies and program flow of BONAMIST are not described here. The user is referred to the BONAMI chapter. The input of BONAMIST is identical to that required for BONAMI. The sensitivities are automatically computed and the bonamist.sen data file is automatically generated without user intervention.

\subsubsection{References}

1. B. L. Broadhead, B. T. Rearden, C. M. Hopper, J. J. Wagschal, and C. V. Parks, "Sensitivity- and Uncertainty-Based Criticality Safety Validation Techniques," Nucl. Sci. Eng., 146, 340-366 (2004).

2. S. Golouglu, C. M. Hopper, and B. T. Rearden, "Extended Interpretation of Sensitivity Data for Benchmark Areas of Applicability," Trans. Am. Nuc. Soc., 88, 77-79 (2003).

3. S. Goluoglu, K. R. Elam, B. T. Rearden, B. L. Broadhead, and C. M. Hopper, Sensitivity Analysis Applied to the Validation of the ${ }^{10} B$ Capture Reaction in Nuclear Fuel Casks, NUREG/CR-6845 (ORNL/TM-2004/48) U.S. Nuclear Regulatory Commission, Oak Ridge National Laboratory, August 2004.

4. B. L. Broadhead, C. M. Hopper, K. R. Elam, B. T. Rearden, and R. L. Childs, "Criticality Safety Applications of S/U Validation Methods," Trans. Am. Nucl. Soc. 83, 107-113 (2000).

5. M. E. Dunn, PUFF-III: A Code for Processing ENDF Uncertainty Data Into Multigroup Covariance Matrices, ORNL/TM-1999/235 (NUREG/CR-6650), U.S. Nuclear Regulatory Commission, Oak Ridge National Laboratory, June 2000.

6. J. J. Lichtenwalter, S. M. Bowman, M. D. DeHart, and C. M. Hopper, Criticality Benchmark Guide for Light-Water-Reactor Fuel in Transportation and Storage Packages, NUREG/CR-6361 (ORNL/TM-13211), U.S. Nuclear Regulatory Commission, Oak Ridge National Laboratory, March 1997. 


\subsection{A Data File Formats}

Several data file formats that are used by the sensitivity and uncertainty analysis codes are presented in this appendix.

\subsection{A.1 Format of TSUNAMI-A sensitivity data file}

The format of the TSUNAMI-A sensitivity data file produced by SAMS, which is executed as part of TSUNAMI-1D/2D/3D, is shown in Table 6.5.A.1. The occurrence of each entry in the data file is followed by an identification of the data contained on each line of the file and the FORTRAN edit descriptor denoting the format of each line. A brief description of each line is also presented.

Table 6.5.A.1. Format specification for TSUNAMI-A sensitivity data file

\begin{tabular}{|c|c|c|c|}
\hline Occurrence & Data & Format & Description \\
\hline \multirow{6}{*}{$\begin{array}{l}\text { Once at beginning } \\
\text { of file. }\end{array}$} & title & $\mathrm{a} 80$ & $\begin{array}{l}\text { Title extracted from transport } \\
\text { calculation }\end{array}$ \\
\hline & number of neutron groups & i10 & $\begin{array}{l}\text { Number of neutron groups in } \\
\text { calculation }\end{array}$ \\
\hline & $\begin{array}{l}\text { total number of profiles, } \\
\text { text descriptor, } \\
\text { number of total profiles that are } \\
\text { region integrated }\end{array}$ & $\mathrm{i} 10, \mathrm{a} 35, \mathrm{i} 10$ & $\begin{array}{l}\text { Total number of sensitivity } \\
\text { profiles in data file, separated by a } \\
\text { text descriptor, then the number of } \\
\text { profiles that contain region- } \\
\text { integrated data }\end{array}$ \\
\hline & $k_{\text {eff }}$ & f10.6 & $\begin{array}{l}\text { Value of } k_{\text {eff }} \text { from the forward } \\
\text { transport calculation }\end{array}$ \\
\hline & 'energy boundaries:' & $\mathrm{a}$ & Text \\
\hline & energy boundary data & $\begin{array}{l}\text { 5es14.6 } \\
\text { (repeats until } \\
\text { all data is } \\
\text { printed) }\end{array}$ & $\begin{array}{l}\text { Values of boundaries of energy } \\
\text { groups. Begins with upper value } \\
\text { for highest energy group and ends } \\
\text { with lower value of lowest energy } \\
\text { group. }\end{array}$ \\
\hline \multirow{3}{*}{$\begin{array}{l}\text { Repeats for each } \\
\text { profile. }\end{array}$} & $\begin{array}{l}\text { isotope name, } \\
\text { sensitivity reaction name, } \\
\text { nuclide ID, } \\
\text { MT number, } \\
\text { Zone number or negative of material } \\
\text { number, } \\
\text { zone volume }\end{array}$ & $\begin{array}{l}\text { a12, } 1 \mathrm{x}, \text { a15, } \\
3 \mathrm{i} 12, \text { es } 14.6\end{array}$ & $\begin{array}{l}\text { Provides data identifying the } \\
\text { sensitivity data that follows. } \\
\text { Note, if sensitivity data is region- } \\
\text { integrated, zone number and zone } \\
\text { volume are both } 0 \text {. If data is } \\
\text { integrated over all zones } \\
\text { containing the same material, the } \\
\text { material number is given in place } \\
\text { of the zone number as a negative } \\
\text { number. }\end{array}$ \\
\hline & $\begin{array}{l}\text { Energy integrated sensitivity } \\
\text { coefficient, sum of absolute value of } \\
\text { group-wise sensitivities, sum of the } \\
\text { group-wise sensitivities with } \\
\text { opposite sign as energy integrated } \\
\text { value (osc) }\end{array}$ & $3 \mathrm{es} 14.6$ & $\begin{array}{l}\text { Energy-integrated sensitivity } \\
\text { coefficients for this profile. }\end{array}$ \\
\hline & group-wise sensitivity coefficients & $\begin{array}{l}\text { 5es14.6 } \\
\text { (repeats until } \\
\text { all data is } \\
\text { printed) }\end{array}$ & $\begin{array}{l}\text { Energy-dependent sensitivity } \\
\text { coefficients. Begins with highest } \\
\text { energy group. }\end{array}$ \\
\hline Once at end of file. & & & $\begin{array}{l}\text { Block of file verification } \\
\text { information. }\end{array}$ \\
\hline
\end{tabular}




\subsection{A.2 Format of TSUNAMI-B sensitivity data file}

The format of the TSUNAMI-B sensitivity data file produced by SAMS, which is executed as part of TSUNAMI-3D-K5, is shown in Table 6.5.A.2. The occurrence of each entry in the data file is followed by an identification of the data contained on each line of the file and the FORTRAN edit descriptor denoting the format of each line. A brief description of each line is also presented. 
Table 6.5.A.2. Format specification for TSUNAMI-B sensitivity data file

\begin{tabular}{|c|c|c|c|}
\hline Occurrence & Data & Format & Description \\
\hline \multirow{6}{*}{$\begin{array}{l}\text { Once at beginning } \\
\text { of file. }\end{array}$} & title & $\mathrm{a} 80$ & $\begin{array}{l}\text { Title extracted from transport } \\
\text { calculation }\end{array}$ \\
\hline & number of neutron groups & $\mathrm{i} 10$ & $\begin{array}{l}\text { Number of neutron groups in } \\
\text { calculation }\end{array}$ \\
\hline & $\begin{array}{l}\text { total number of profiles, } \\
\text { text descriptor, } \\
\text { number of total profiles that are } \\
\text { region integrated }\end{array}$ & $\mathrm{i} 10, \mathrm{a} 35, \mathrm{i} 10$ & $\begin{array}{l}\text { Total number of sensitivity } \\
\text { profiles in data file, separated by a } \\
\text { text descriptor, then the number of } \\
\text { profiles that contain region- } \\
\text { integrated data }\end{array}$ \\
\hline & $k_{\text {eff }}$ & f10.6 & $\begin{array}{l}\text { Value of } k_{\text {eff }} \text { from the forward } \\
\text { transport calculation }\end{array}$ \\
\hline & 'energy boundaries:' & $\mathrm{a}$ & Text \\
\hline & energy boundary data & $\begin{array}{l}5 \text { es } 14.6 \\
\text { (repeats until } \\
\text { all data is } \\
\text { printed) }\end{array}$ & $\begin{array}{l}\text { Values of boundaries of energy } \\
\text { groups. Begins with upper value } \\
\text { for highest energy group and ends } \\
\text { with lower value of lowest energy } \\
\text { group. }\end{array}$ \\
\hline \multirow{3}{*}{$\begin{array}{l}\text { Repeats for each } \\
\text { profile. }\end{array}$} & $\begin{array}{l}\text { isotope name, } \\
\text { sensitivity reaction name, } \\
\text { nuclide ID, } \\
\text { MT number, } \\
\text { Zone number or negative of material } \\
\text { number, } \\
\text { zone volume }\end{array}$ & $\begin{array}{l}\text { a12, } 1 x, \text { a15, } \\
3 \mathrm{i} 12, \text { es } 14.6\end{array}$ & $\begin{array}{l}\text { Provides data identifying the } \\
\text { sensitivity data that follows. } \\
\text { Note, if sensitivity data is region- } \\
\text { integrated, zone number and zone } \\
\text { volume are both } 0 \text {. If data is } \\
\text { integrated over all zones } \\
\text { containing the same material, the } \\
\text { material number is given in place } \\
\text { of the zone number as a negative } \\
\text { number. }\end{array}$ \\
\hline & $\begin{array}{l}\text { Energy integrated sensitivity } \\
\text { coefficient, sum of absolute value of } \\
\text { group-wise sensitivities, sum of the } \\
\text { group-wise sensitivities with } \\
\text { opposite sign as energy integrated } \\
\text { value (osc) }\end{array}$ & 3 es 14.6 & $\begin{array}{l}\text { Energy-integrated sensitivity } \\
\text { coefficients for this profile. }\end{array}$ \\
\hline & group-wise sensitivity coefficients & $\begin{array}{l}5 \text { es14.6 } \\
\text { (repeats until } \\
\text { all data is } \\
\text { printed) }\end{array}$ & $\begin{array}{l}\text { Energy-dependent sensitivity } \\
\text { coefficients. Begins with highest } \\
\text { energy group. }\end{array}$ \\
\hline Once at end of file. & & & $\begin{array}{l}\text { Block of file verification } \\
\text { information. }\end{array}$ \\
\hline
\end{tabular}




\subsection{A.3 Format of SENLIB.SEN data file}

The SENLIB.SEN data file is generated by the SENLIB routines used in the TSUNAMI modules to compute implicit sensitivity effects. The data file contains the sensitivity of data computed in SENLIB and input to the resonance processing code BONAMIST. This file also contains the sensitivities of the extra cross sections input to BONAMIST to the number densities of each nuclide for each region of the BONAMIST input. In BONAMIST, the extra cross section contains data for the Dancoff factor. Each of the parameters for which sensitivity coefficients are computed has a unique identification number. The parameter identification numbers, corresponding parameters, and their locations in the resonance processing code input are shown in Table 6.5.A.3. The format of the records of the SENLIB.SEN data file is given in Table 6.5.A.4. Each sensitivity coefficient is identified by three records. These records are repeated until all sensitivities are listed on the data file.

Table 6.5.A.3. Parameter identifiers for SENLIB.SEN data file

\begin{tabular}{|c|c|c|}
\hline $\begin{array}{c}\text { Identifier } \\
\text { number }\end{array}$ & Parameter & $\begin{array}{l}\text { Location of parameter } \\
\text { in resonance processing code input }\end{array}$ \\
\hline-1004 & $\begin{array}{l}\text { Extra cross section }\left(\mathrm{cm}^{-1}\right) \text { by zone, } \\
\text { account for Dancoff factor in } \\
\text { BONAMIST }\end{array}$ & $9^{*}$ array in data block 2 of BONAMIST input \\
\hline
\end{tabular}

Table 6.5.A.4. Record format for SENLIB.SEN data file

\begin{tabular}{clll}
\hline Record number & \multicolumn{1}{c}{ Data } & Format & \multicolumn{1}{c}{ Description } \\
\hline 1 & $\begin{array}{l}\text { Region for BONAMIST data } \\
\text { and parameter }\end{array}$ & $\begin{array}{l}\text { BONAMIST region and parameter for which } \\
\text { sensitivity coefficient is computed. }\end{array}$ \\
2 & $\begin{array}{l}\text { Nuclide and reaction MT 2i12 } \\
\text { number to which sensitivity } \\
\text { coefficient is computed }\end{array}$ & $\begin{array}{l}\text { Nuclide and reaction MT number to which the } \\
\text { sensitivity of the parameter is computed. For data } \\
\text { currently computed by SENLIB, only the } \\
\text { sensitivities of the parameters to the number } \\
\text { densities of the nuclides are computed. Thus, the } \\
\text { reaction MT number is always 1. }\end{array}$ \\
& & & \\
3 & Sensitivity coefficient & es14.6 & $\begin{array}{l}\text { Relative sensitivity of parameter identified in first } \\
\text { record to nuclide and reaction identified in second } \\
\text { record. }\end{array}$ \\
\hline
\end{tabular}

\subsection{A.4 Format of BONAMIST.SEN data file}

The BONAMIST.SEN data file is generated by the BONAMIST code, which is executed as part of the TSUNAMI-1D sequence. This data file contains the sensitivities of the groupwise cross sections that are resonance shielded in BONAMIST to the certain data input to BONAMIST. The data to which the 
sensitivity coefficients are computed are the number densities of the nuclides and the extra cross section by region, which contains data for the Dancoff factor. These data are listed in the data file according to the identifiers listed in Table 6.5.A.3. Additionally, the sensitivity of group cross sections to the number density of a particular nuclide is identified with the nuclide number from the AMPX cross-section data library.

The format of the records in the data file is identified in Table 6.5.A.5 Blocks of these records are printed for the non-zero sensitivities for all nuclides and reactions for which resolved resonances are processed.

Table 6.5.A.5. Record format for BONAMIST.SEN data file

\begin{tabular}{|c|c|c|c|}
\hline Record number & Data & Format & Description \\
\hline 1 & $\begin{array}{l}\text { Nuclide and MT number for } \\
\text { cross sections for which } \\
\text { sensitivity coefficients are } \\
\text { computed }\end{array}$ & $2 \mathrm{i} 12$ & $\begin{array}{l}\text { Nuclide identifier and MT number of reaction for } \\
\text { which sensitivity data are computed. }\end{array}$ \\
\hline 2 & $\begin{array}{l}\text { Parameter or nuclide and the } \\
\text { MT number to which the } \\
\text { sensitivity data are } \\
\text { computed }\end{array}$ & $2 \mathrm{i} 12$ & $\begin{array}{l}\text { Parameter identifier for scattering cross sections } \\
\text { and Dancoff factor or nuclide identifier and } \\
\text { MT number to which the sensitivity } \\
\text { coefficients are computed. If a parameter } \\
\text { identifier is used, the MT number is set to } 1 . \\
\text { Currently only the sensitivities to number } \\
\text { densities are computed in BONAMIST, so the } \\
\text { only MT number printed is } 1 \text {. }\end{array}$ \\
\hline 3 & $\begin{array}{l}\text { First and last group for which } \\
\text { sensitivity coefficients are } \\
\text { printed }\end{array}$ & $2 \mathrm{i} 12$ & $\begin{array}{l}\text { First and last group for the sensitivity coefficients } \\
\text { that follow. }\end{array}$ \\
\hline 4 & $\begin{array}{l}\text { Sensitivity coefficients, record } \\
\text { repeats until all groups } \\
\text { identified by record } 3 \text { are } \\
\text { printed }\end{array}$ & $5 \mathrm{es} 14.6$ & $\begin{array}{l}\text { Relative sensitivities of cross sections for nuclide } \\
\text { and process identified by record } 1 \text { to } \\
\text { parameter or nuclide identified in record } 2 \text { for } \\
\text { groups corresponding to those identified by } \\
\text { record 3. }\end{array}$ \\
\hline
\end{tabular}

\subsection{A.5 COVERX format}

The COVERX data file format for storing multigroup cross-section uncertainty information is presented in this section. The COVERX format was used with the FORSS code system at ORNL. The covariance data processing code PUFF-III outputs multigroup data in the COVERX format. This the only covariance format available for use with the sensitivity and uncertainty analysis codes in SCALE.

The overall structure of a COVERX file is given in Table 6.5.A.6. The occurrence of the particular record type, the name of the record type and when a given record type is present on the file are identified. 
Table 6.5.A.6. COVERX file structure

\begin{tabular}{clc}
\hline Occurrence & \multicolumn{1}{c}{ Record type } & Present if \\
\hline & File Identification & Always \\
& File Control & Always \\
& File Description & NHOLL $>0$ \\
Once at beginning of file & Neutron Group Boundaries & NNGRUP $>0$ \\
& Gamma Group Boundaries & NGGRUP $>0$ \\
& Material-Reaction Control & Always \\
Repeat for all material-reaction & Material-Reaction Type Cross $\quad$ Sections and & Always \\
type pairs & Error Files & Always \\
Repeat for all matrices & Matrix Control & Always \\
Repeat for all blocks & Block Control & Always \\
\hline
\end{tabular}

The details of each record type identified in Table 6.5.A.6 are presented in Table 6.5.A.7. Here, the name of the record type, the variables present on the record, the length of the record, the format of the record in FORTRAN context and a description of the content of the record are given. 
Table 6.5.A.7. Description of records on COVERX formatted file

\begin{tabular}{|c|c|c|c|c|c|}
\hline Record type & Variables & Length & Format & \multicolumn{2}{|r|}{ Description } \\
\hline \multirow{3}{*}{ File Identification } & \multirow{3}{*}{$\begin{array}{l}\text { HNAME, (HUSE(I), } \\
\text { I=1,2), IVERS }\end{array}$} & \multirow{3}{*}{$1+3 *$ MULT } & \multirow{3}{*}{$\begin{array}{c}(\text { OV COVERX ‘, A6, ‘*’, } \\
\text { 2A6, '*', I6) }\end{array}$} & HUSE & User identification (A6) \\
\hline & & & & IVERS & File version number \\
\hline & & & & MULT & $\begin{array}{l}1-\mathrm{A} 6 \text { is a single precision word } \\
2-\mathrm{A} 6 \text { is double precision word }\end{array}$ \\
\hline \multirow{7}{*}{ File Control } & \multirow{7}{*}{$\begin{array}{l}\text { NGROUP, NNGRUP, } \\
\text { NGGRUP, NTYPE, } \\
\text { NMMP, NMTRIX, } \\
\text { NHOLL }\end{array}$} & \multirow{7}{*}{7} & \multirow{7}{*}{$(' 1 \mathrm{D},, 7 \mathrm{I} 6)$} & NGROUP & Number of energy groups \\
\hline & & & & NNGRUP & Number of neutron groups \\
\hline & & & & NGGRUP & Number of gamma groups \\
\hline & & & & NTYPE & $\begin{array}{l}1-\text { Covariance matrix, standard } \\
\text { deviation } \\
2-\text { Relative covariance matrix, } \\
\text { relative standard deviation } \\
3-\text { Correlation matrix, standard } \\
\text { deviation }\end{array}$ \\
\hline & & & & NMMP & Number of MAT-MT pairs \\
\hline & & & & NMTRIX & Number of matrices \\
\hline & & & & NHOLL & $\begin{array}{l}\text { Number of Hollerith words in } \\
\text { description }\end{array}$ \\
\hline File Description & $\begin{array}{l}\text { (WORDS(J), } \\
\mathrm{J}=1, \mathrm{NHOLL})\end{array}$ & MULT*NHOLL & $\left({ }^{*} 2 \mathrm{D}{ }^{\prime},{ }^{*} ’, 11 \mathrm{~A} 6 /(11 \mathrm{~A} 6)\right)$ & WORDS(J) & Hollerith description of file \\
\hline \multirow{2}{*}{$\begin{array}{l}\text { Neutron Group } \\
\text { Boundaries }\end{array}$} & \multirow{2}{*}{$\begin{array}{c}(\mathrm{GPBN}(\mathrm{J}), \\
\mathrm{J}=1, \text { NNGRUP }) \\
\text { ENMIN }\end{array}$} & \multirow{2}{*}{ NNGRUP+1 } & \multirow{2}{*}{$\left({ }^{\prime} 3 \mathrm{D} ‘, 5 \mathrm{E} 12.4 /(6 \mathrm{E} 12.4)\right)$} & $\operatorname{GPBN}(\mathrm{J})$ & $\begin{array}{l}\text { Maximum energy bound of neutron } \\
\text { group }(\mathrm{J})(\mathrm{eV})\end{array}$ \\
\hline & & & & ENMIN & $\begin{array}{l}\text { Minimum energy of neutron in } \\
\text { energy range }\end{array}$ \\
\hline \multirow{2}{*}{$\begin{array}{l}\text { Gamma Group } \\
\text { Boundaries }\end{array}$} & \multirow{2}{*}{$\begin{array}{c}(\mathrm{GPBG}(\mathrm{J}), \\
\mathrm{J}=1, \text { NGGRUP }), \\
\text { EGMIN }\end{array}$} & \multirow{2}{*}{ NGGRUP+1 } & \multirow{2}{*}{$\left({ }^{\prime} 4 \mathrm{D}\right.$ ‘,5E12.4/(6E12.4)) } & GPBG(J) & $\begin{array}{l}\text { Maximum energy bound of gamma } \\
\text { group }(J)(\mathrm{eV})\end{array}$ \\
\hline & & & & EGMIN & $\begin{array}{l}\text { Minimum energy of neutron in } \\
\text { energy range }\end{array}$ \\
\hline
\end{tabular}


Table 6.5.A.7. Description of records on COVERX formatted file (continued)

\begin{tabular}{|c|c|c|c|c|c|}
\hline Record type & Variables & Length & Format & & Description \\
\hline & & \multirow{7}{*}{$3 * \mathrm{NMMP}$} & \multirow{7}{*}{ ( ‘ 5D ‘,11I6,(12I6)) } & MATID(I) & Material identification number \\
\hline & & & & $\operatorname{MTID}(\mathrm{I})$ & Reaction type identification number \\
\hline Material- & (MATID(I), & & & & Cross section weighting option \\
\hline Reaction & MTID(I), MWGT(I), & & & & $1-$ Constant \\
\hline Control & $\mathrm{I}=1, \mathrm{NMMP})$ & & & MWGT(I) & $2-1 / \mathrm{E}$ \\
\hline & & & & & $\begin{array}{l}3-\text { Thermal }+1 / E+\text { Fission } \\
4-\text { Arbitrary }\end{array}$ \\
\hline \multirow{4}{*}{$\begin{array}{c}\text { Material- } \\
\text { Reaction Type } \\
\text { Cross Sections } \\
\text { and Error Files }\end{array}$} & & & & & 5-Combined CTR CRBR \\
\hline & $(\mathrm{CRS}(\mathrm{J})$ & \multirow[b]{2}{*}{$2 *$ NGROUP } & \multirow[b]{2}{*}{$(‘ 6 \mathrm{D}$ ‘,5E12.4/(6E12.4)) } & CRS & Cross section \\
\hline & $\begin{array}{c}\mathrm{J}=1, \mathrm{NGROUP}), \\
(\mathrm{ERROR}(\mathrm{J}), \\
\mathrm{J}=1, \mathrm{NGROUP})\end{array}$ & & & ERROR & Standard deviation \\
\hline & \multirow{5}{*}{$\begin{array}{c}\text { MAT1, MT1, MAT2, } \\
\text { MT2, NBLOK }\end{array}$} & \multirow{5}{*}{5} & \multirow{5}{*}{$\left({ }^{\prime} 7 \mathrm{D} \cdot, 5 \mathrm{I} 6\right)$} & MAT1 & Material 1 identification number \\
\hline \multirow{4}{*}{ Matrix Control } & & & & MT1 & $\begin{array}{l}\text { Reaction type } 1 \text { identification } \\
\text { number }\end{array}$ \\
\hline & & & & MAT2 & Material 2 identification number \\
\hline & & & & MT2 & $\begin{array}{l}\text { Reaction type } 2 \text { identification } \\
\text { number }\end{array}$ \\
\hline & & & & NBLOK & $\begin{array}{l}\text { Number of blocks into which matrix } \\
\text { is divided }\end{array}$ \\
\hline \multirow{3}{*}{ Block Control } & $(\mathrm{JBAND}(\mathrm{J}), \mathrm{IJJ}(\mathrm{J})$, & \multirow{3}{*}{$2 * \mathrm{NGROUP} * \mathrm{NBLOK}$} & \multirow{3}{*}{$\left({ }^{\circ} 8 \mathrm{D} `, 11 \mathrm{I} 6 /(12 \mathrm{I} 6)\right)$} & $\mathrm{JBAND}(\mathrm{J})$ & Bandwidth for group $\mathrm{J}$ \\
\hline & $\begin{array}{c}\mathrm{J}=1, \mathrm{NGROUP}) \\
(\mathrm{LGRP}(\mathrm{N})\end{array}$ & & & $\operatorname{IJJ}(J)$ & $\begin{array}{l}\text { Position of diagonal element for } \\
\text { group J }\end{array}$ \\
\hline & $\mathrm{N}=1, \mathrm{NBLOK})$ & & & $\operatorname{LGRP}(\mathrm{N})$ & Number of groups in block(N) \\
\hline Matrix Data & $\begin{array}{c}(\operatorname{COV}(\mathrm{K}) \\
\mathrm{K}=1, \mathrm{KMAX})\end{array}$ & KMAX & $\left({ }^{\prime} 9 \mathrm{D}\right.$ ‘,5E12.4/(6E12.4)) & $\mathrm{COV}$ & NTYPE matrix data \\
\hline
\end{tabular}




\subsection{B HTML Colors}

Colors available for use in HTML formatted output are shown in Table 6.5.B.1. This table was created from data available at http://www.w3schools.com/css/css_colornames.asp. Color names are not case sensitive.

Table 6.5.B.1. Colors available in HTML

\begin{tabular}{|c|c|c|}
\hline Color name & Color HEX & Color \\
\hline AliceBlue & \#F0F8FF & \\
\hline AntiqueWhite & \#FAEBD7 & \\
\hline Aqua & \#00FFFF & \\
\hline Aquamarine & \#7FFFD4 & \\
\hline Azure & \#F0FFFF & \\
\hline Beige & \#F5F5DC & \\
\hline Bisque & \#FFE4C4 & \\
\hline Black & $\# 000000$ & \\
\hline BlanchedAlmond & \#FFEBCD & \\
\hline Blue & $\# 0000 \mathrm{FF}$ & \\
\hline BlueViolet & \#8A2BE2 & \\
\hline Brown & \#A52A2A & \\
\hline BurlyWood & \#DEB887 & \\
\hline CadetBlue & \#5F9EA0 & \\
\hline Chartreuse & \#7FFF00 & \\
\hline Chocolate & \#D2691E & \\
\hline Coral & \#FF7F50 & \\
\hline CornflowerBlue & \#6495ED & \\
\hline Cornsilk & \#FFF8DC & \\
\hline Crimson & \#DC143C & \\
\hline Cyan & \#00FFFF & \\
\hline DarkBlue & $\# 00008 B$ & \\
\hline DarkCyan & \#008B8B & \\
\hline DarkGoldenRod & \#B8860B & \\
\hline DarkGray & \#A9A9A9 & \\
\hline DarkGreen & $\# 006400$ & \\
\hline DarkKhaki & \#BDB76B & \\
\hline DarkMagenta & \#8B008B & \\
\hline DarkOliveGreen & \#556B2F & \\
\hline Darkorange & \#FF8C00 & \\
\hline DarkOrchid & \#9932CC & \\
\hline DarkRed & \#8B0000 & \\
\hline
\end{tabular}


Table 6.5.B.1. Colors available in HTML (continued)

\begin{tabular}{|c|c|c|}
\hline Color name & Color HEX & Color \\
\hline DarkSalmon & \#E9967A & \\
\hline DarkSeaGreen & \#8FBC8F & \\
\hline DarkSlateBlue & \#483D8B & \\
\hline DarkSlateGray & $\# 2 \mathrm{~F} 4 \mathrm{~F} 4 \mathrm{~F}$ & \\
\hline DarkTurquoise & \#00CED1 & \\
\hline DarkViolet & \#9400D3 & \\
\hline DeepPink & \#FF1493 & \\
\hline DeepSkyBlue & \#00BFFF & \\
\hline DimGray & \#696969 & \\
\hline DodgerBlue & \#1E90FF & \\
\hline Feldspar & \#D19275 & \\
\hline FireBrick & \#B22222 & \\
\hline FloralWhite & \#FFFAF0 & \\
\hline ForestGreen & \#228B22 & \\
\hline Fuchsia & \#FF00FF & \\
\hline Gainsboro & \#DCDCDC & \\
\hline GhostWhite & \#F8F8FF & \\
\hline Gold & \#FFD700 & \\
\hline GoldenRod & \#DAA520 & \\
\hline Gray & \#808080 & \\
\hline Green & $\# 008000$ & \\
\hline GreenYellow & \#ADFF2F & \\
\hline HoneyDew & \#F0FFF0 & \\
\hline HotPink & \#FF69B4 & \\
\hline IndianRed & \#CD5C5C & \\
\hline Indigo & \#4B0082 & \\
\hline Ivory & \#FFFFF0 & \\
\hline Khaki & \#F0E68C & \\
\hline Lavender & \#E6E6FA & \\
\hline LavenderBlush & \#FFF0F5 & \\
\hline LawnGreen & \#7CFC00 & \\
\hline LemonChiffon & \#FFFACD & \\
\hline LightBlue & \#ADD8E6 & \\
\hline LightCoral & \#F08080 & \\
\hline LightCyan & \#E0FFFF & \\
\hline LightGoldenRodYellow & \#FAFAD2 & \\
\hline LightGrey & \#D3D3D3 & \\
\hline LightGreen & \#90EE90 & \\
\hline
\end{tabular}


Table 6.5.B.1. Colors available in HTML (continued)

\begin{tabular}{|c|c|c|}
\hline Color name & Color HEX & Color \\
\hline LightPink & \#FFB6C1 & \\
\hline LightSalmon & \#FFA07A & \\
\hline LightSeaGreen & \#20B2AA & \\
\hline LightSkyBlue & \#87CEFA & \\
\hline LightSlateBlue & \#8470FF & \\
\hline LightSlateGray & \#778899 & \\
\hline LightSteelBlue & \#B0C4DE & \\
\hline LightYellow & \#FFFFE0 & \\
\hline Lime & \#00FF00 & \\
\hline LimeGreen & \#32CD32 & \\
\hline Linen & \#FAF0E6 & \\
\hline Magenta & \#FF00FF & \\
\hline Maroon & $\# 800000$ & \\
\hline MediumAquaMarine & \#66CDAA & \\
\hline MediumBlue & $\# 0000 \mathrm{CD}$ & \\
\hline MediumOrchid & \#BA55D3 & \\
\hline MediumPurple & \#9370D8 & \\
\hline MediumSeaGreen & \#3CB371 & \\
\hline MediumSlateBlue & \#7B68EE & \\
\hline MediumSpringGreen & \#00FA9A & \\
\hline MediumTurquoise & \#48D1CC & \\
\hline MediumVioletRed & \#C71585 & \\
\hline MidnightBlue & \#191970 & \\
\hline MintCream & \#F5FFFA & \\
\hline MistyRose & \#FFE4E1 & \\
\hline Moccasin & \#FFE4B5 & \\
\hline NavajoWhite & \#FFDEAD & \\
\hline Navy & $\# 000080$ & \\
\hline OldLace & \#FDF5E6 & \\
\hline Olive & \#808000 & \\
\hline OliveDrab & \#6B8E23 & \\
\hline Orange & \#FFA500 & \\
\hline OrangeRed & \#FF4500 & \\
\hline Orchid & \#DA70D6 & \\
\hline PaleGoldenRod & \#EEE8AA & \\
\hline PaleGreen & \#98FB98 & \\
\hline PaleTurquoise & \#AFEEEE & \\
\hline PaleVioletRed & \#D87093 & \\
\hline
\end{tabular}


Table 6.5.B.1. Colors available in HTML (continued)

\begin{tabular}{|c|c|c|}
\hline Color name & Color HEX & Color \\
\hline PapayaWhip & \#FFEFD5 & \\
\hline PeachPuff & \#FFDAB9 & \\
\hline Peru & \#CD853F & \\
\hline Pink & \#FFC0CB & \\
\hline Plum & \#DDA0DD & \\
\hline PowderBlue & \#B0E0E6 & \\
\hline Purple & $\# 800080$ & \\
\hline Red & \#FF0000 & \\
\hline RosyBrown & \#BC8F8F & \\
\hline RoyalBlue & \#4169E1 & \\
\hline SaddleBrown & \#8B4513 & \\
\hline Salmon & \#FA8072 & \\
\hline SandyBrown & \#F4A460 & \\
\hline SeaGreen & \#2E8B57 & \\
\hline SeaShell & \#FFF5EE & \\
\hline Sienna & \#A0522D & \\
\hline Silver & $\# \mathrm{C} 0 \mathrm{C} 0 \mathrm{C} 0$ & \\
\hline SkyBlue & \#87CEEB & \\
\hline SlateBlue & \#6A5ACD & \\
\hline SlateGray & \#708090 & \\
\hline Snow & \#FFFAFA & \\
\hline SpringGreen & $\# 00$ FF7F & \\
\hline SteelBlue & \#4682B4 & \\
\hline Tan & \#D2B48C & \\
\hline Teal & $\# 008080$ & \\
\hline Thistle & \#D8BFD8 & \\
\hline Tomato & \#FF6347 & \\
\hline Turquoise & \#40E0D0 & \\
\hline Violet & \#EE82EE & \\
\hline VioletRed & \#D02090 & \\
\hline Wheat & \#F5DEB3 & \\
\hline White & \#FFFFFF & \\
\hline WhiteSmoke & \#F5F5F5 & \\
\hline Yellow & \#FFFF00 & \\
\hline YellowGreen & \#9ACD32 & \\
\hline
\end{tabular}




\title{
6.6 TSURFER: AN ADJUSTMENT CODE TO DETERMINE BIASES AND UNCERTAINTIES IN NUCLEAR SYSTEM RESPONSES BY CONSOLIDATING DIFFERENTIAL DATA AND BENCHMARK INTEGRAL EXPERIMENTS
}

M. L. Williams, B. L. Broadhead, M. A. Jessee, J. J. Wagschal ${ }^{\text {a }}$, and R.A. Lefebvre

\begin{abstract}
The TSURFER code uses the generalized linear least-squares method to consolidate a prior set of measured integral responses (such as $k_{\text {eff }}$ ) and corresponding calculated values obtained using the SCALE nuclear analysis code system. The initial estimates for the computed and measured responses are improved by adjusting the experimental values and the nuclear data used in the transport calculationstaking into account their correlated uncertainties - so that the most self-consistent set of data is obtained. This procedure makes the refined estimates of the calculated and measured responses agree within first-order accuracy, while constraining the data variations to minimize a generalized chi-square parameter. Consolidation of the original integral experiment data and calculated results reduces the prior uncertainty in the response estimates because additional information has been incorporated. The method can also address one or more "application responses" for which no experimental measurements are available. TSURFER computes an updated estimate for the application responses and provides an estimate for the computational bias and application uncertainty. The methodology is useful in validation studies for criticality safety and reactor analysis.
\end{abstract}

a Racah Institute of Physics, The Hebrew University of Jerusalem, 91904, Jerusalem, Israel 


\section{TABLE OF CONTENTS}

$\underline{\text { Page }}$

6.6 TSURFER: An Adjustment Code to Determine Biases and Uncertainties in Nuclear

System Responses by Consolidating Differential Data and Benchmark Integral

Experiments

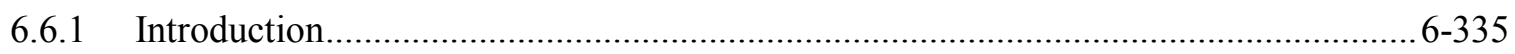

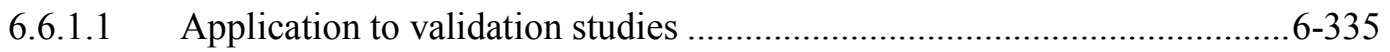

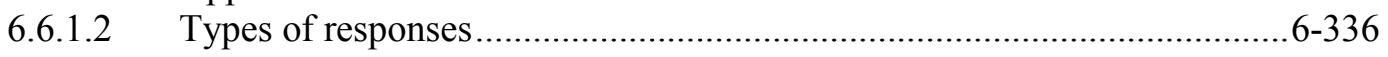

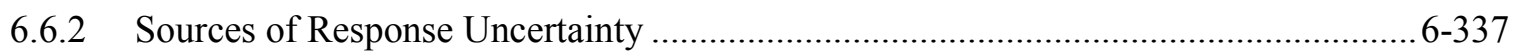

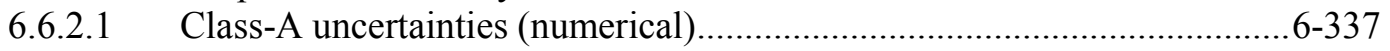

6.6.2.2 Class-B uncertainties (modeling/experimental) .....................................6-337

6.6.2.3 Class-C uncertainties (nuclear data) ....................................................6-338

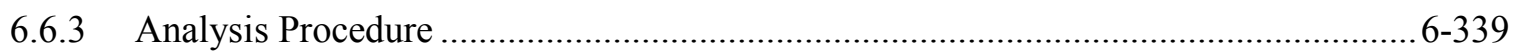

6.6.3.1 Functional relation to other SCALE modules........................................6-339

6.6.3.2 Guidelines for TSURFER analysis .....................................................6-339

6.6.3.3 Required data for TSURFER ........................................................ $6-341$

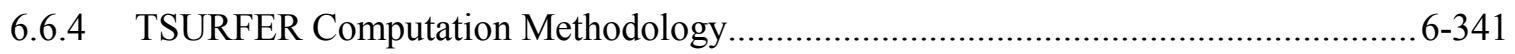

6.6.4.1 Representation of experimental uncertainty components .........................6-344

6.6.4.2 Generalized linear least-squares equations ..........................................6-345

6.6.4.3 Expressions for response similarity parameters....................................6-352

6.6.4.4 Convergence of reference application response........................................6-354

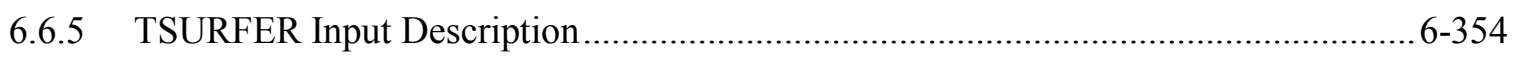

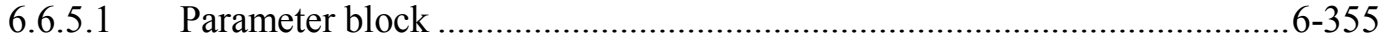

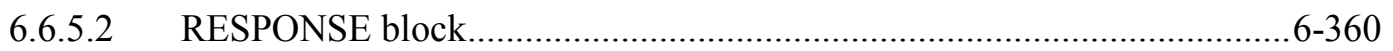

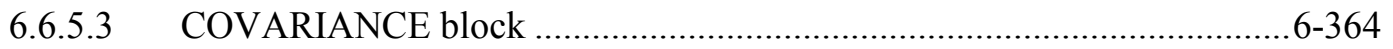

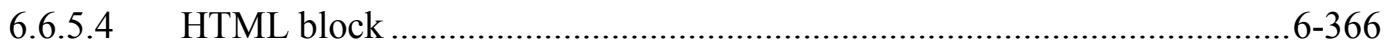

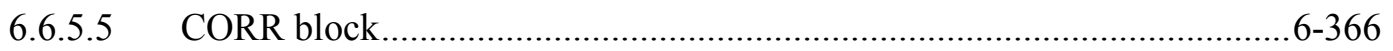

6.6.6 Sample Problem Input and Output Description ..........................................................6-369

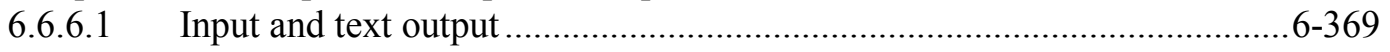

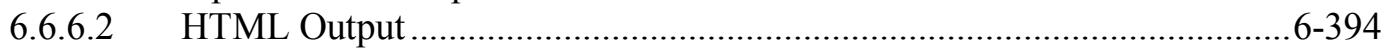

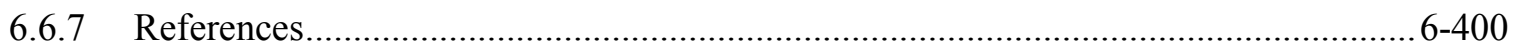




\section{TABLE OF CONTENTS (continued)}

$\underline{\text { Page }}$

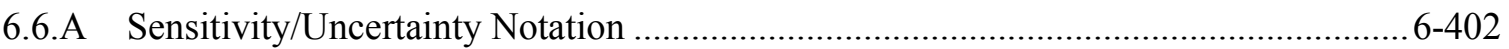

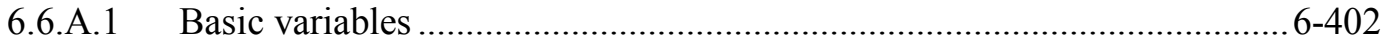

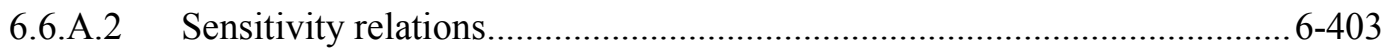

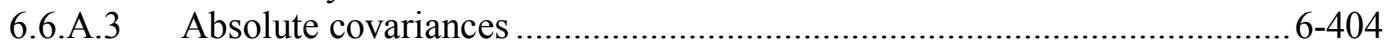

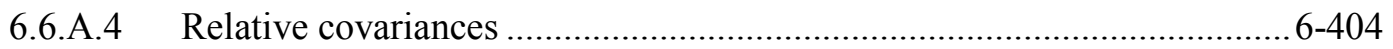

6.6.A.5 Mixed absolute-relative covariances...................................................... 6-405

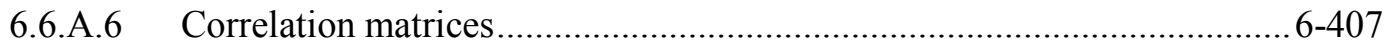




\section{LIST OF FIGURES}

$\underline{\text { Page }}$

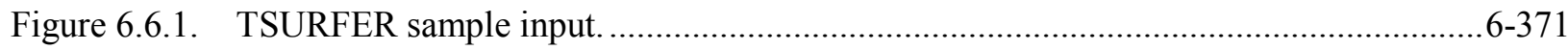

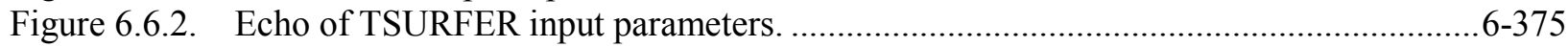

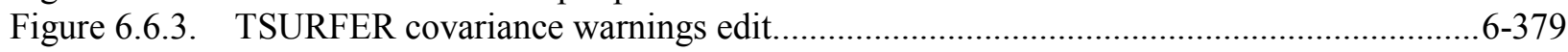

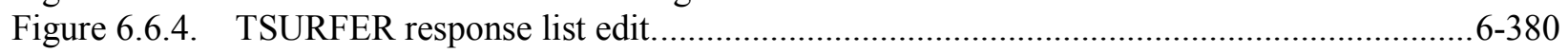

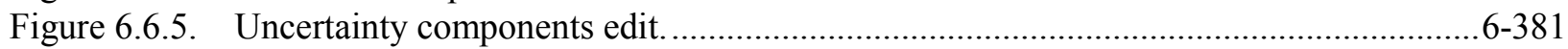

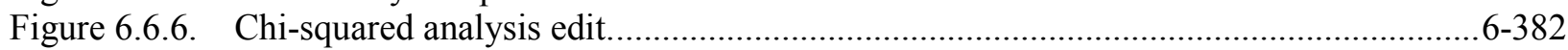

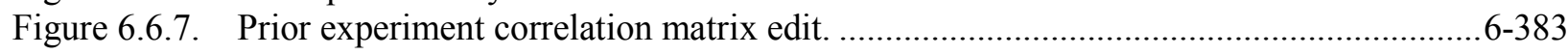

Figure 6.6.8. Prior calculated response correlation matrix edit. ................................................... $6-384$

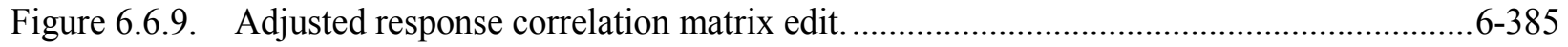

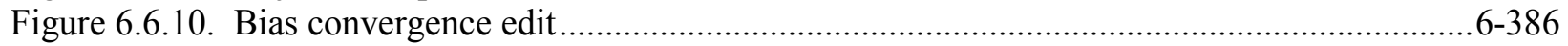

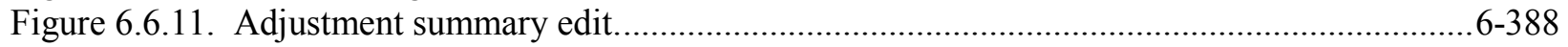

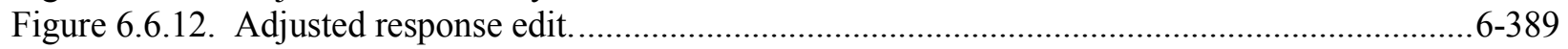

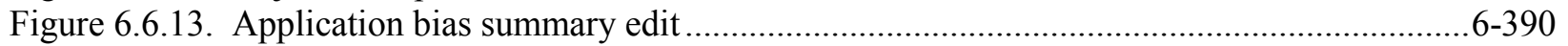

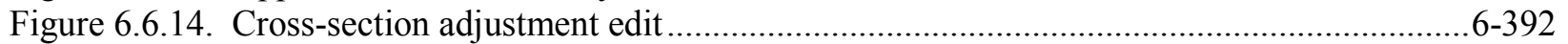

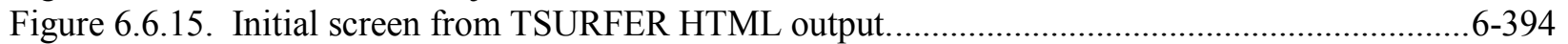

Figure 6.6.16. Program verification screen from TSURFER HTML output. .....................................6-395

Figure 6.6.17. Input parameters from TSURFER HTML output. ................................................6-396

Figure 6.6.18. Warning messages from TSURFER HTML output. ..................................................6-397

Figure 6.6.19. Cross-section adjustments from TSURFER HTML output......................................6-398

Figure 6.6.20. Three-dimensional plot of correlation matrix in TSURFER HTML output.................6-399 


\section{LIST OF TABLES}

Page

Table 6.6.1. Table keynames and descriptions for TSURFER input data blocks ...............................6-355

Table 6.6.2. Input data for the Parameter block of TSURFER .....................................................6-356

Table 6.6.3. Input data for the Covariance block of TSURFER ........................................................ 6-365

Table 6.6.4. Input data for HTML block of TSURFER ….................................................................. $6-366$ 


\section{ACKNOWLEDGMENTS}

The authors would like to acknowledge R. L. Childs, formerly of ORNL, for his contributions. Development of the TSURFER code was funded by the U.S. Department of Energy Nuclear Criticality Safety Program. 


\subsubsection{Introduction}

This report describes the TSURFER code (Tool for Sensitivity/Uncertainty analysis of Response Functionals using Experimental Results - pronounced "surfer," with silent "T" like TSUNAMI), which is a functional module in the SCALE sensitivity and uncertainty $(\mathrm{S} / \mathrm{U}$ ) analysis methodology (see TSUNAMI-1D chapter). The main functions of the code are to (a) compute uncertainties in calculated integral responses such as $k_{\text {eff }}$, due to uncertainties in the input nuclear data; (b) reduce discrepancies between the measured and calculated responses by adjusting the nuclear data and experimental values such that the overall consistency is maximized; (c) analyze measured responses from benchmark experiments to establish the bias and associated uncertainty in some application response that has been calculated.

TSURFER utilizes the generalized linear least-squares (GLLS) methodology based on S/U techniques originally developed in the 1970's and 1980's for a variety of applications, including nuclear data evaluation, ${ }^{1}$ fast reactor design studies, ${ }^{2,3}$ and reactor pressure vessel damage predictions. ${ }^{4} \mathrm{~A}$ recent GLLS application has been in the area of criticality safety analysis, in which critical benchmarks are used to validate the computational methodology for predicting subcritical quantities and configurations of fissile materials. ${ }^{5}$ Similar validation procedures also could be performed for other integral responses of interest for nuclear reactor analysis. These include responses such as reactivity coefficients associated with coolant voiding or Doppler broadening, peak power values, or in-core instrumentation readings. Although not limited to this area, the application of TSURFER to criticality safety validation studies is emphasized here.

\subsubsection{Application to validation studies}

Historically the validity of a calculation performed for some application has been established by considering how well the same calculational methods and nuclear data perform for a set of representative benchmark experiments. While a simple comparison of the computed and experimental results is very informative, it does not fully take advantage of the valuable information provided by the measured integral responses. If the original sets of calculated and experimental responses are consolidated in a consistent manner (i.e., correctly accounting for uncertainties), then the "adjusted" results should be a better estimate for the true responses, since the revised response values are based upon more information than was available in either the original calculations or measurements alone. This is essentially a statement of Bayes Theorem from probability theory, which indicates how prior information (calculated responses) can be evolved by incorporating additional information (integral measurements) into more reliable posterior results (the adjusted responses). The equations used for the GLLS methodology are equivalent to those obtained from Bayes Theorem. ${ }^{6}$

Cross-section libraries for neutron transport calculations are processed from fundamental evaluated nuclear data files such as ENDF/B. Because the "true" values of the nuclear data are not known precisely, it is reasonable to view the ENDF data as being selected from a probability distribution of allowable values. Nuclear data uncertainties are described by covariance matrices that contain variances in the group cross sections for a given nuclide and reaction type, as well as covariances arising from correlations between energy groups, and possibly between reactions and materials. Discrepancies in the ENDF nuclear data caused by uncertainties in the evaluation process propagate to errors in group cross sections, which in general cause computed responses to disagree with the corresponding measured values. The GLLS approach considers potential variations in data parameters and measured integral responses that minimize the differences in measured and calculated integral responses (such as $k_{\text {eff) for a }}$ set of benchmark experiments, taking into account uncertainties and correlations in the ENDF data and in the integral measurements. Since there are generally many more cross-section values than measured integral responses, the determination of the data modifications is an under-determined problem. If the 
data variations are not performed in a reasonable manner, non-physical changes may be obtained. Data adjustments in the GLLS methodology are constrained by the magnitude of the nuclear data uncertainties and their correlations. TSURFER also accounts for uncertainties and correlations in the integral response measurements, arising from experimental uncertainties in parameters such as enrichment, density, impurities, etc. As long as realistic data covariances are used, the resulting data adjustments may be considered the "best estimates"-within the limitations of the GLLS linearity approximation-for realistic data alterations that improve the computed integral responses. It can be shown that the GLLS equations provide the maximum likelihood estimator for the correct nuclear data if the evaluated nuclear data and system parameters obey a multivariate normal probability distribution. ${ }^{6}$

Some previous studies have applied the GLLS methodology to produce an adjusted nuclear data library in order to improve calculations of nuclear reactors with similar characteristics as the experiments used in the adjustment. ${ }^{7}$ In criticality safety analysis this procedure runs the risk of applying the adjusted library to systems beyond the limits for which the data modifications are appropriate. The usual function of TSURFER is not to output an adjusted nuclear data library but rather to obtain an adjusted application response $\left(k_{\text {eff }}\right)$ and to provide a quantitative estimate for its accuracy. Hence, it is more appropriate to view TSURFER as a tool to establish biases and uncertainties in calculated responses. Nuclear data adjustments are a by-product of this procedure.

Traditional validation of criticality safety calculations estimates the computational bias based on trends in the calculated $k_{\text {eff }}$ values versus system parameters such as hydrogen-to-fissile ratios $(\mathrm{H} / \mathrm{X})$ or energy of average lethargy causing fission (EALF). These trending parameters are frequently used as measures of "similarity" between critical systems, hence their use as bias-predictors. Recent studies have shown that data sensitivity coefficients, either alone or in combination with cross-section uncertainty information, are good indicators of system similarity. Thus S/U-based indices have also been used in trending analyses, analogously to the commonly used physical parameters. ${ }^{5}$ The input data for S/U trending analysis (i.e., calculated and measured responses, sensitivity coefficients, cross-section and experimental uncertainties) are almost identical to those needed for GLLS analysis; therefore, it is not surprising that some aspects of the TSURFER calculations are also used for trending results. However, TSURFER provides an alternative to the trending approach to determine the bias and can address other validation issues. For example, TSURFER is useful when there are few or no existing experiments that are similar to a particular application area, since the GLLS technique can include individual experiments that separately validate portions of the application area, even though none can be considered entirely similar to the application. $^{8}$

\subsubsection{Types of responses}

A response corresponds to a particular integral response type (e.g., $k_{\text {eff, }}$, reaction rate ratio, material worth, radiation dose, etc.) in a particular nuclear system (e.g., a benchmark experiment or a proposed storage arrangement of reactor fuel assemblies or a reactor core). In the TSURFER input, responses may be classified either as "experiments" or "applications" or "omitted."

An experiment response has both calculated and measured values input to TSURFER, and these play an active role in the GLLS procedure, which minimizes the difference between the two results. A value for the uncertainty in the measured response and any correlations with other experiments are also input for experimental responses. Examples of experimental integral responses are the multiplication factor for the GODIVA critical benchmark experiment, the measured $\rho^{28}$ (ratio of epithermal to thermal capture rate for

${ }^{238} \mathrm{U}$ ) in the TRX-1 critical benchmark lattice, or the coolant voiding reactivity in a power reactor.

Applications are responses for which a calculated value is known but no measured value is available. Applications often correspond to hypothetical systems being considered within the context of a design 
study or a criticality safety analysis for which the computational bias and uncertainty are desired. Examples of application responses are the multiplication factor (subcritical) for a proposed fuel assembly storage rack or for a shipping cask. An application response plays a passive role in the GLLS procedure. Since the application has no experimental results, it does not impact the active responses included in the consolidation procedure; conversely, the GLLS procedure may modify the calculated application value if it is "similar" to some of the experimental responses. In this case the application response shares similar data sensitivity characteristics with one or more of the active responses and hence will be indirectly affected by the same data adjustments that impact the similar experimental responses. This provides a systematic, well-defined method for utilizing experimental benchmark measurements to establish a bias and uncertainty in the calculation of application response.

A response designated as omitted in the TSURFER input neither affects other responses nor is affected by them. These responses are completely isolated from the GLLS procedure. This capability is sometimes useful to easily "turn off" an active system to observe its impact on the application results or on the consistency (chi-square) of the set of remaining experimental responses.

\subsubsection{Sources of Response Uncertainty}

Transport calculations of responses such as the neutron multiplication factor inherently have biases and uncertainties due to several factors that can be grouped into three classes:

(A) numerical approximations in the transport code;

(B) system modeling approximations; and

(C) input data uncertainties.

\subsubsection{Class-A uncertainties (numerical)}

Class-A uncertainties are sometimes referred to as "methods uncertainties." In Monte Carlo calculations these may be caused by imperfections in random number generation routines, approximations in techniques for scoring neutron multiplication (e.g., incomplete convergence of fission source distribution, neglect of correlations between generations, etc.), and biases from algorithms used to represent nuclear data and to sample probability distributions, as well as the basic statistical uncertainty that is fundamental to the Monte Carlo method. Deterministic methods have uncertainties from using finite space-energydirection meshes, truncated (rather than infinite) expansions of functions, incomplete convergence of iterations, and especially self-shielding approximations for the multigroup cross sections. Computational benchmark studies often can establish a reasonable upper limit for these effects, which may be judged either as negligible or as requiring some conservative bias to be applied to the application calculations. Here it is assumed that class-A uncertainties in the calculated response can be made acceptably small (e.g., by running more histories or refining mesh sizes) or at least have been previous quantified and can be bounded by a margin applied to the computation. Hence class (A) uncertainties are considered as systematic "tolerance," and are not addressed in the present GLLS methodology used by TSURFER.

\subsubsection{Class-B uncertainties (modeling/experimental)}

Class-B uncertainties occur because the mathematical model used in the transport computations of an application or an experimental response does not correspond exactly to the "true" system. The response uncertainty caused by modeling effects may either be associated with (i) direct computational simplifications such as omitting or homogenizing some components in the calculation model or (ii) fundamental uncertainties in the material compositions, densities, and dimensions of the experiment. The former are systematic uncertainties similar in effect to Class-A numerical uncertainties and may be 
addressed in the same manner; that is, by bounding the magnitude of the uncertainty through the applied safety margins. However, the latter are true random uncertainties that in theory have probability distributions, and these may be addressed with the TSURFER code.

Even "clean" critical benchmark experiments have uncertainties in the nominal system parameters - such as fuel enrichment, impurities, densities, critical dimensions, and numerous other components - that may lead to discrepancies in the measured and calculated responses for the system. In TSURFER the impact of these uncertainties is designated as the "experimental uncertainty" in the response, since this uncertainty will be present even if no simplifications or approximations are made in the model used for the transport computation. The terminology is sometimes a source of confusion. For example the measured $k_{\text {eff }}$ in a critical experiment usually is known to be unity with a very small uncertainty associated with the long, but finite, stable period. While there is little doubt about $k_{\text {eff }}$ for a critical experiment, there may be considerable uncertainty in the system parameter values describing the benchmark configuration. This contribution to the modeling uncertainty could be justifiably considered either "experimental" (because system parameters such as material compositions and dimensions are specified by the experimentalists) or "computational" (because uncertainties in the system parameters affect the calculation model), but in TSURFER they are designated as experimental uncertainties. In any case the uncertainty in each system parameter must be propagated to an uncertainty in the measured response. For a $k_{\text {eff }}$ response this may be done experimentally by physically varying the system parameter and measuring the reactivity effect or, more commonly, by performing auxiliary transport calculations to determine the eigenvalue variation. This is discussed in a somewhat more quantitative manner in Sect. 6.6.4.1.

The response uncertainty components associated with the respective modeling uncertainties in system parameters determine the overall experimental uncertainty. Many benchmark experiment descriptions in the International Handbook of Evaluated Criticality Safety Benchmark Experiments ${ }^{9}$ include information about uncertainties in the system parameters and their estimated impact on the multiplication factor. The standard deviations in $k_{\text {eff }}$ due to uncertainties in various system parameters are assigned by the benchmark evaluators based on published or archived experiment descriptions, and sometimes on other considerations.

A complication in specifying experimental uncertainties is how to treat correlations among the experiments. Response correlations in two benchmark experiments may be caused by factors such as use of the same fuel pins and container tank, and common instrumentation (same detectors, hydrometers, etc.). For example, if two different experiments use the same fuel material, then it is not reasonable to conclude that the enrichment in one is too high while the other is too low, even if both differences fall within the specified standard deviation. Reference 10 has shown that these correlations may not be negligible when applying the GLLS technique to a set of benchmark experiments. Only a limited amount of experiment correlation data has been published, but more is expected in future revisions to the International Handbook of Evaluated Criticality Safety Benchmark Experiments. TSURFER allows experimental uncertainties caused by uncertainties in system modeling parameters to be input for individual components and correlation coefficients can be specified for the shared system parameters of each response. This approach provides the capability for users to describe the actual sources of benchmark experiment correlations without having to know the overall correlation between two different experiments. See Sects. 6.6.4.1 and 6.6.4.2.

\subsubsection{Class- $\mathrm{C}$ uncertainties (nuclear data)}

In many applications, the major source of uncertainty in the calculated response is due to uncertainties in evaluated nuclear data such as microscopic cross sections, fission spectra, neutron yield (nu-bar), and scattering distributions that are contained in ENDF/B. These arise from uncertainties in experimental 
nuclear data measurements, as well as uncertainties in the evaluation process itself, which in general combine differential experimental information with nuclear physics theory to generate the basic data in compilations like ENDF/B. Class-C uncertainties are governed by probability distributions. The actual probabilities are unknown, but the evaluated data values are assumed to represent the mean of the distribution, and the evaluated variance represents a measure of the distribution width. Correlations as well as uncertainties in nuclear data can have a significant impact on the overall uncertainty in the calculated response; thus, it is important to include covariances as well as variances in the TSURFER calculations. The uncertainties in fundamental nuclear data also impact resonance self-shielding of multigroup cross-section values, further contributing to the response uncertainty. ${ }^{11}$ In the SCALE S/U methodology the effects of implicit changes in self-shielded cross sections are included in the overall response sensitivity coefficients, rather than in the covariance data, so that the fundamental data uncertainties are isolated from problem-specific effects. ${ }^{12}$

Covariance information is currently quite limited in all evaluated nuclear data compilations such as ENDF/B. A more complete library of multigroup uncertainties has been created for SCALE using data from a variety of sources, including ENDF/B-VI and VII, JENDL3.1, and approximate covariances based on uncertainties in measured integral data and nuclear model calculations. ${ }^{12,13}$ A detailed description of the SCALE covariance libraries is found in the COVLIB chapter.

The GLLS methodology in TSURFER is mainly concerned with treating Class-C uncertainties due to nuclear data, along with Class-B experimental uncertainties.

\subsubsection{Analysis Procedure}

\subsubsection{Functional relation to other SCALE modules}

TSURFER is a functional module within the overall SCALE S/U methodology. Other modules in SCALE and outside SCALE perform complementary calculations and provide data files used by TSURFER, as described below.

- PUFF-III: ${ }^{14}$ AMPX code that processes ENDF/B nuclear data covariances and generates multigroup covariance data; creates nuclear data uncertainty files for input to TSURFER.

- TSUNAMI-1D/2D/3D: SCALE control sequence that computes sensitivity coefficients for $k_{e f f}$ or other responses in a 1D/2D/3D model of the experiment or application system; creates sensitivity files used by TSURFER.

- TSAR: SCALE functional module that computes sensitivity coefficients for eigenvaluedifference responses such as reactor reactivity parameters, using $k_{\text {eff }}$ sensitivities from a TSUNAMI sequence; creates sensitivity files used by TSURFER.

- TSUNAMI-IP: SCALE functional module that computes similarity and completeness indices for a set of responses with sensitivity coefficients. Prior to running TSURFER, it may be advantageous to perform scoping studies with TSUNAMI-IP to determine if the selected set of benchmark experiments provides adequate "coverage" for data uncertainties that have a significant impact on the application response.

\subsubsection{Guidelines for TSURFER analysis}

Both active and passive responses may be included in the TSURFER calculation. For example, in criticality safety validation procedures, responses of interest typically correspond to the system 
multiplication factor, $k_{\text {eff. }}$ The desired sub-critical design configuration would be a passive application system, while the critical benchmarks used for validation are active experiment systems. TSURFER determines the application bias and uncertainty by propagating data variations obtained from GLLS analysis of the active systems to the calculated multiplication factor of the passive system. The bias represents the change of the application's original $k_{\text {eff }}$ as a result of the consolidation of all the active critical benchmark experiments and the adjusted nuclear data parameters. An increase in $k_{\text {eff }}$ computed for the applications system response indicates that the calculated value was initially too low (a negative bias), and a decrease that the application's multiplication factor was too high (a positive bias).

TSURFER also computes uncertainties in the initial and adjusted estimates for the system responses (e.g., multiplication factors). These response uncertainties include effects of experimental uncertainties (class-B) and nuclear data uncertainties (class-C) but not the impact of simplifications made to the experiment specifications and numerical approximations (class-A) - which should be included in the safety margin.

Several quantities can be examined to give confidence to the predicted results. ${ }^{15}$ The first is the completeness parameter, R, given in the output of the TSUNAMI-IP code. It has been suggested that values of $\mathrm{R}$ greater than about $0.7-0.8$ for a set of active experiments indicate adequate cross-section coverage for an application response in the GLLS procedure; however, this is a preliminary conclusion that may change as more experience is gained. ${ }^{8}$

The chi-square $\left(\chi^{2}\right)$ statistic indicates the overall consistency of the suite of benchmarks and is key to proper interpretation of the TSURFER results. The value of $\chi^{2}$ per degree of freedom represents the average discrepancy between the calculated and measured responses, expressed in units of the combined variance of the calculation plus experiment. Values of chi-square per degree of freedom ideally should be within about $\pm 20 \%$ of unity, indicating that the calculations and measurements on average agree within about one standard deviation. Results in which this test is not strictly met may still be valid, but in general these should be viewed with skepticism unless the reasons for the test failure are understood. An excessively large $\chi^{2}$ can lead to unreliable results in the GLLS adjustment. TSURFER provides the total $\chi^{2}$ value, as well as estimated contributions from each experiment (see Sect. 6.6.4.2.1). Individual $\chi^{2}$ values suggest which experiments may contain inconsistencies (i.e., the difference between the measured and calculated $k_{\text {eff }}$ is larger than their combined uncertainties).

Several methods can be used to improve the initial value of chi-square. One approach is to reevaluate the measurement uncertainties and their correlations for identified discrepant experiments. If the experimental or data uncertainties are underestimated, the data adjustments will correspond to an excessive number of standard deviations, as reflected in high $\chi^{2}$ values. Values of $\chi^{2}$ that are too low suggest that the input uncertainty estimates may be too high, and again a reevaluation should be considered. Thus it is quite important to utilize realistic (not conservative) estimates for the uncertainties in nuclear data and experimental measurements.

Even when best estimates are used for all input uncertainties, it is not uncommon to encounter a few active responses that are inconsistent with the others, especially when dealing with a large number of benchmark experiments. In this case the best alternative to improve $\chi^{2}$ is to remove the outliers, either by transforming those experiments into passive responses or by omitting them entirely from the GLLS adjustment. TSURFER provides a "chi-square filtering" procedure that automatically omits inconsistent experiments until a specified target value of chi-square is achieved. Several options are provided to select the experiments to be omitted, as discussed in Sect. 6.6.4.2.1. Omitted experiments should be examined to ensure that simple errors in the problem description are not present. 
An internal consistency test such as described in Ref. 15 also may be useful. The consistency test is performed by changing one of the benchmark experiments that is similar (see Sect. 6.6.4.3) to the application response into a passive, pseudo-application response. The predicted bias for this passive response should be close to that of the original application; furthermore, in this case the bias prediction can be checked because this passive response actually has a measured experimental value.

\subsubsection{Required data for TSURFER}

Active and passive responses considered in the GLLS analysis should have sensitivity data provided for each nuclide-reaction pair that significantly impacts the response. The sensitivity coefficients are precalculated using other SCALE modules as described in Sect. 6.6.3.1 and are stored in individual files for each response included in the TSURFER analysis. The sensitivity data files must be in either TSUNAMIA format (described in Appendix A, Format of TSUNAMI-A Sensitivity Data File of TSUNAMI-IP) or in TSUNAMI-B format (described in Appendix A, Format of TSUNAMI-B Sensitivity Data File of TSUNAMI-IP). The locations of the sensitivity files are specified in the TSURFER input data. It is not required for all sensitivity files to have the same group structures; for example, the sensitivity coefficients for one response may have been computed using a 238-group cross-section library, while sensitivities for another response could have a 44-group structure. Whatever the group structure of the sensitivity data, it is mapped into the same group structure as the covariance file. At present the SCALE covariance files use the SCALE 44-group structure.

A file of nuclear data covariances also must be input to the TSURFER calculation. The covariance data file must be in COVERX format described in the TSUNAMI_IP Appendix A section COVERX Format. SCALE includes a comprehensive applications-oriented covariance library that includes evaluated covariances taken from ENDF/B-VII, ENDF/B-VI, and JENDL nuclear data files ${ }^{12}$ described in the COVLIB chapter. Ideally, the covariance files should contain data for all nuclide-reaction pairs on the response sensitivity data files. However, cross-section covariance data are not available for all nuclidereaction pairs. Nuclide-reaction pairs without available covariance data are omitted from the GLLS analysis, but it is assumed that either the cross-section covariance data values for these pairs are well known (i.e., small uncertainties) or that the sensitivity to these nuclide-reaction pairs is small. Where these assumptions hold, the cross sections for these nuclide-reaction pairs should not be adjusted and can be omitted from the GLLS analysis. For situations where these assumptions are judged to be invalid, the use of GLLS analysis is not appropriate. However, TSURFER provides several input options to define uncertainty values for nuclide-reaction pairs with missing covariance data to assess the impact of the additional covariance data on the GLLS analysis. These input options are discussed in more detail in Sections 6.6.5.1 and 6.6.5.3.

\subsubsection{TSURFER Computation Methodology}

A recent detailed derivation of the GLLS formalism is given in Ref. 15. The general formalism allows cross correlations between the initial integral experiment measurements and the original nuclear data, such as would be present if the calculations used a previously "adjusted" library of nuclear data. Since this is not normally done in SCALE, correlations between the benchmark experiment measurements and the cross-section data in the multigroup libraries are not considered in the TSURFER code; therefore, the GLLS equations presented here are somewhat simplified compared to the more general expressions in Ref. 15.

At present, the SCALE cross-section-covariance data files characterize nuclear data uncertainties in terms of relative covariances. Therefore, response sensitivity data in TSURFER are defined in terms of relative 
changes in the nuclear data. An absolute response sensitivity is defined as an absolute change in response due to a relative change in the nuclear data, that is,

$$
\tilde{\mathrm{S}}_{\alpha}=\alpha \frac{\partial \mathrm{R}}{\partial \alpha}
$$

In this equation, $\mathrm{R}$ represents the response, $\alpha$ represents the nuclear data, and the tilde will be used to represent absolute sensitivity and uncertainty data. Likewise, a relative response sensitivity is defined as a relative change in response due to a relative change in the nuclear data, that is,

$$
\mathrm{S}_{\alpha}=\frac{\alpha}{\mathrm{R}} \frac{\partial \mathrm{R}}{\partial \alpha}
$$

The initial development that follows is for relative, rather than absolute, response sensitivity and uncertainty parameters. It is then shown how to express the quantities in absolute form for reactivity analysis and mixed relative-absolute form for combined $k_{\text {eff }}$ and reactivity analysis. A summary of the notation and definitions used in this section can be found in Appendix A.

The methodology consists of calculating values for a set of I integral responses ( $k_{\text {eff }}$, reaction rates, etc.), some of which have been measured in selected benchmark experiments. Responses with no measured values are the selected "applications," described previously. The set of measured response values $\left\{\mathrm{m}_{\mathrm{i}}\right.$; $\mathrm{i}=1,2, \ldots ., \mathrm{I}\}$ can be arranged into an I-dimension column vector designated as $\mathbf{m}$. By convention the (unknown) experimental values corresponding to applications are represented by the corresponding calculated values. As discussed in Sect. 6.6.2.2, the measured integral responses have uncertaintiespossibly correlated - due to uncertainties in the system parameter specifications. The I $\times$ I covariance matrix describing the relative experimental uncertainties is defined to be $\mathbf{C}_{\mathbf{m m}}$.

The calculated integral values for each experiment and application are obtained by neutron transport calculations, producing a set of calculated responses $\left\{\mathrm{k}_{\mathrm{i}} ; \mathrm{i}=1,2, \ldots, \mathrm{I}\right\}$ arranged in a I-dimension vector $\mathbf{k}$. The multigroup cross-section data for all nuclide-reaction pairs used in the transport calculations of all responses comprise a set $\left\{\alpha_{n} ; n=1,2, \ldots, M\right\}$, where $M$ is the number of unique nuclide-reaction pairs multiplied by the number of energy groups. It is convenient to arrange these data into a M-dimensional column vector $\boldsymbol{\alpha}$, so that the dependence of the initial calculated responses upon the input nuclear data values can be indicated as $\mathbf{k}=\mathbf{k}(\boldsymbol{\alpha})$. The prior covariance matrix for the nuclear data is equal to the $\mathrm{M} \times \mathrm{M}$ matrix $\mathbf{C}_{\boldsymbol{\alpha} \alpha}$, which contains relative variances along the diagonal and relative covariances in the off-diagonal positions. These data describe uncertainties in the infinitely dilute multigroup cross sections.

Nuclear data uncertainties cause uncertainties in the calculated response values. In general, these uncertainties are correlated because the same nuclear data library is used for all the transport calculations. The covariance matrix describing uncertainties in the calculated responses due to class- $\mathrm{C}$ uncertainties is designated as $\mathbf{C}_{\mathbf{k k} .}$. Using expressions for propagation of error (the so called sandwich rule), the following relationship is obtained for the relative uncertainty in the calculated responses:

$$
\mathrm{C}_{\mathrm{kk}}=\mathrm{S}_{\mathrm{k} \alpha} \mathrm{C}_{\alpha \alpha} \mathrm{S}_{\mathrm{k} \alpha}^{\mathrm{T}}
$$


where $\mathbf{S}_{\mathbf{k} \boldsymbol{a}}$ is the relative sensitivity matrix, whose (i,n) element is equal to the relative sensitivity of the $i_{\text {th }}$ response with respect to the $n_{\text {th }}$ data value, that is, $\frac{1}{R_{i}} \frac{\partial R_{i}}{\partial \alpha_{n}} \alpha_{n}$. Sensitivity coefficients appearing in the sensitivity matrix are computed using first-order perturbation theory. A description of the equations used to compute sensitivity coefficients using first order perturbation theory can be found in Ref. 16 or in the SAMS chapter. In the SCALE methodology, the sensitivity coefficients consist of an "explicit" component that accounts for the direct impact of the data on the neutron transport calculation, as well as an "implicit" component that accounts for its impact on other self-shielded multigroup data. ${ }^{11}$ For example, a variation in the hydrogen multigroup elastic cross section has an explicit effect on $k_{\text {eff }}$ through its impact on neutron moderation and leakage in the transport solution and has an implicit effect on the self-shielded ${ }^{238} U$ multigroup cross sections, which causes additional change in $k_{\text {eff. }}$ Because selfshielding effects are addressed through the sensitivity coefficients, the nuclear data uncertainties in the covariance matrix correspond to the infinitely dilute values.

Each row $\mathrm{i}$ of the sensitivity matrix contains sensitivity coefficients for all nuclear data used in the transport calculation of response i. These data also can be arranged into an M-component sensitivity vector $\mathbf{S}_{\mathrm{i}}$ for a particular response "i", which may be either an experiment or application. For example, the sensitivity vector $\mathbf{S}_{\mathrm{i}}$ is an $\mathbf{M}$ dimensional vector whose $\mathrm{n}_{\mathrm{th}}$ element is equal to the sensitivity coefficient of response "i" to data element $\alpha_{n}$ as specified previously.

It is often convenient to express covariance matrices in terms of standard deviations [represented as $\sigma_{\mathrm{i}}$ for variable $i$ ] and correlation coefficients [represented by $\rho_{\mathrm{i}, \mathrm{j}}$ for the variable pair $\left.(i, j)\right]$. The correlation coefficient is related to the corresponding covariance value by the equation

$$
\rho_{i, j}=\frac{\operatorname{Cov}(i, j)}{\sigma_{i} \sigma_{j}}
$$

Correlation coefficients, which have values between -1 and 1 , indicate the degree of correlation between the pair of variables, where a value of 1.0 indicates full correlation, 0.0 no correlation, and -1.0 full anticorrelation. Using matrix notation, relative standard deviations are arranged in a diagonal matrix $\boldsymbol{\sigma}$ and the correlation coefficients in a square matrix $\mathbf{R}$ (symmetrical, but generally non-diagonal). The previously defined covariance matrices can be expressed as follows:

$$
\begin{gathered}
C_{\mathrm{mm}}=\sigma_{\mathrm{m}} \mathbf{R}_{\mathrm{mm}} \sigma_{\mathrm{m}} \\
\mathrm{C}_{\alpha \alpha}=\sigma_{\alpha} \mathbf{R}_{\alpha \alpha} \sigma_{\alpha} ; \\
\mathrm{C}_{\mathrm{kk}}=\sigma_{\mathrm{k}} \mathbf{R}_{\mathrm{kk}} \sigma_{\mathrm{k}}
\end{gathered}
$$

Equations (6.6.3) and (6.6.4) can be substituted into Eq. (6.6.1) and rearranged to relate the nuclear data correlations to the correlations in the computed responses:

$$
\mathbf{R}_{\mathrm{kk}}=\left[\sigma_{\mathrm{k}}^{-1} \mathbf{S}_{\mathrm{k} \alpha} \boldsymbol{\sigma}_{\alpha}\right] \mathbf{R}_{\alpha \alpha}\left[\boldsymbol{\sigma}_{\mathrm{k}}^{-1} \mathbf{S}_{\mathrm{k} \alpha} \boldsymbol{\sigma}_{\alpha}\right]^{\mathrm{T}}
$$

The bracketed term on the right side of the above equation is an $\mathrm{I} \times \mathrm{M}$ matrix whose elements equal the number of relative standard deviations $\left(\boldsymbol{\sigma}_{\mathbf{k}}\right)$ that the response changes, due to a one relative standard 
deviation change in the nuclear data $\left(\boldsymbol{\sigma}_{\boldsymbol{\alpha}}\right)$. Even if the nuclear data are not correlated, - - that is, $\mathbf{R}_{\boldsymbol{\alpha} \alpha}$ is an identity matrix and $\mathbf{R}_{\mathbf{k k}}$ is generally not diagonal.

The expressions thus far describe relative response sensitivities and covariances (i.e., uncertainties). Similar expressions can also be derived for absolute quantities. In this report, absolute response sensitivities and covariances are denoted by a tilde, such as $\widetilde{\mathbf{C}}_{\mathbf{m m}}, \widetilde{\mathbf{C}}_{\mathbf{k k}}$, and $\widetilde{\mathbf{S}}_{\mathbf{k} \boldsymbol{\alpha}}$, which are explicitly defined in Appendix A.

TSURFER allows for a mixed relative and absolute response sensitivities and covariances to be used in the analysis. In the TSURFER input (described in Sect. 6.6.5), each response is designated as an absolute-formatted response or a relative-formatted response using the input keywords "absolute" or "relative". This flexibility allows for the simultaneous use of both relative-formatted $k_{\text {eff }}$ sensitivity data (generated by TSUNAMI modules) and absolute-formatted eigenvalue-difference sensitivity data (generated by TSAR) in the same analysis. In this report, mixed relative-absolute response sensitivities and covariances are denoted by a caret, such as $\hat{\mathbf{C}}_{\mathbf{m m}}, \hat{\mathbf{C}}_{\mathbf{k k}}$, and $\hat{\mathbf{S}}_{\mathbf{k} \boldsymbol{\alpha}}$.

\subsubsection{Representation of experimental uncertainty components}

Experimental uncertainties (i.e., type-B uncertainties as described in Sect. 6.6.2.2) may be entered directly in the TSURFER input, or alternatively, it may be specified by defining individual "uncertainty components." The latter approach is useful in defining experimental correlations between measured responses. In this case, an index " $\ell$ " is introduced to identify the response uncertainty components associated with a particular system parameter, $\mathrm{p}_{\ell}$. For example, the measured $k_{\text {eff }}$ uncertainty components for a particular critical experiment consisting of uranyl nitrate dissolved in water might correspond to the following eight $p_{\ell}$ contributors, as identified by the value of $(\ell)$ : (1) isotopic composition; (2) fuel concentration in the solution; (3) solution density; (4) excess acid concentration in the solution; (5) fuel impurities; (6) dimension of the solution tank; (7) thickness of the solution tank; (8) composition of the tank. ${ }^{10}$

The relative standard deviation of a measured response $m_{i}$ due to an uncertainty in the system parameter $\mathrm{p}_{\ell}$ is designated as the uncertainty component $\sigma_{\mathrm{m}, \mathrm{i}}^{(\ell)}$. Assuming that uncertainties in system parameters are uncorrelated, the response uncertainty component is related to the uncertainty in system parameter $\mathrm{p}_{\ell}$ by the expression

$$
\sigma_{\mathrm{m}, \mathrm{i}}^{(\ell)}=\frac{1}{\mathrm{~m}_{\mathrm{i}}}\left(\frac{\partial \mathrm{k}_{\mathrm{i}}}{\partial \mathrm{p}_{\ell}} \mathrm{p}_{\ell}\right) \sigma_{\mathrm{p} \ell}=\mathrm{S}_{\mathrm{m}_{\mathrm{p} \ell},} \sigma_{\mathrm{p} \ell}
$$

where $\sigma_{p_{\ell}}$ is the relative standard deviation of system parameter $\mathrm{p}_{\ell}$ and $\mathrm{S}_{\mathrm{m}_{\mathrm{i}} \mathrm{p}_{\ell}}$ is the relative sensitivity coefficient relating $\mathrm{p}_{\ell}$ to the measured response $\mathrm{m}_{\mathrm{i}}$. In principle, the system parameter values and uncertainties could be treated directly in the TSURFER calculation by providing the sensitivity coefficients $\mathrm{S}_{\mathrm{m}_{\mathrm{i}} \mathrm{p}_{\ell}}$, thus allowing the experiment parameters to be included in the GLLS adjustment. However, at the present time the response uncertainty components $\boldsymbol{\sigma}_{m, i}^{(\ell)}$ must be determined prior to the TSURFER calculation and are read into TSURFER. Values for the response uncertainty components sometimes can be found in the benchmark experiment specifications, ${ }^{9}$ or auxiliary sensitivity analysis 
may be necessary. The relative experimental standard deviation of the $\mathrm{i}_{\mathrm{th}}$ measured response is calculated from

$$
\sigma_{\mathrm{m}, \mathrm{i}}=\sqrt{\sum_{\mathrm{i}}\left(\sigma_{\mathrm{m}, \mathrm{i}}^{(\mathrm{I})}\right)^{2}}
$$

The $(\mathrm{i}, \mathrm{i})$ diagonal element of the relative covariance matrix corresponds to the relative experimental variance in response $\mathrm{i}$, which is equal the square of $\sigma_{m, i}$ above. Note that similar expressions can be derived for uncertainty components using absolute sensitivities and uncertainties. For absolute-formatted responses, the uncertainty components on the TSURFER input must be entered in terms of absolute standard deviation. This is discussed in more detail in Sect. 6.6.5.2.

If a different response $\mathrm{j}$ is measured in a benchmark system that shares some or all of the same uncertainty components as response $\mathrm{i}$, then the two experiment responses have correlated uncertainties. In such a case the $(i, j)$ element of the relative covariance matrix $\mathbf{C}_{\mathbf{m m}}$ is equal to

$$
\mathrm{C}_{\mathrm{mm}}(\mathrm{i}, \mathrm{j})=\sum_{\ell}\left(\sigma_{\mathrm{m}, \mathrm{i}}^{(\ell)} \rho_{\mathrm{i}, \mathrm{j}}^{(\ell)} \sigma_{\mathrm{m}, \mathrm{j}}^{(\ell)}\right)
$$

and the total correlation coefficient for response pair $(i, j)$ is

$$
R_{m m}(i, j)=\frac{C_{m m}(i, j)}{\sigma_{m, i} \sigma_{m, j}}=\frac{\sum_{\ell}\left(\sigma_{m, i}^{(\ell)} \rho_{i, j}^{(\ell)} \sigma_{m, j}^{(\ell)}\right)}{\sigma_{m, i} \sigma_{m, j}}
$$

where $\rho_{\mathrm{i}, \mathrm{j}}^{(\ell)}$ is the correlation coefficient for responses $\mathrm{i}$ and $\mathrm{j}$ due uncertainty component $\ell$.

TSURFER allows the user to input text-identifiers for the various experiment uncertainty components, along with the associated values for relative standard deviations, $\sigma_{\mathrm{m}, \mathrm{i}}^{(\ell)}$. Response correlation coefficients $\rho_{\mathrm{i}, \mathrm{j}}^{(\ell)}$ can be input for each type of uncertainty component, by response pair $(\mathrm{i}, \mathrm{j})$.

The previous discussion applies only to experiment responses for which measurements have been performed. In the case of an application response for which no experimental measurement is known, the uncertainty is set internally by TSURFER to the large value of $10^{10}$, to approximate the "infinite" uncertainty in the unknown measurement, and correlations to other responses are set to zero. The large uncertainty for an application response has the effect of letting the response "float" in a passive manner; that is, the application response has a negligible effect on the adjustment of active responses, but the GLLS consolidation of the active responses with finite uncertainties can impact the adjusted value for the application.

\subsubsection{Generalized linear least-squares equations}

Discrepancies in the calculated and measured responses are defined by the I dimensional column vector 


$$
\mathbf{d}=\left\{\mathrm{d}_{i}=\frac{\mathrm{k}_{\mathrm{i}}(\alpha)-\mathrm{m}_{i}}{\mathrm{k}_{\mathrm{i}}(\alpha)}, i=1, \ldots, I\right\} .
$$

In TSURFER the components of $\mathbf{d}$ corresponding to application responses are set to zero because applications have no measured values. Using the standard formula for propagation of error and assuming no correlations between $\mathrm{k}$ and $\mathrm{m}$, the relative uncertainty matrix for the discrepancy vector $\mathbf{d}$ can be expressed as the I by I matrix:

$$
C_{d d}=C_{k k}+F_{m / k} C_{m m} F_{m / k}=S_{k \alpha} C_{\alpha \alpha} S_{k \alpha}^{T}+F_{m / k} C_{m m} F_{m / k}
$$

where the expression in Eq. (6.6.1) was substituted for $\mathbf{C}_{\mathbf{k} \mathbf{k}}$, and $\mathbf{F}_{\mathbf{m} / \mathbf{k}}$ is an $\mathrm{I} \times$ I diagonal matrix containing $\mathrm{m} / \mathrm{k}$ factors, that is, $\frac{\mathrm{E}}{\mathrm{C}}$ factors (ratio of experimental to calculated response values). The inverse of the matrix $\mathbf{C}_{\mathbf{d d}}$ appears in several expressions presented later in this section. In TSURFER the inversion is performed using routines from the LINPAC software package.

The goal of the GLLS method is to vary the nuclear data $\left(\alpha \rightarrow \alpha^{\prime}\right)$ and the measured integral responses $\left(\mathrm{m} \rightarrow \mathrm{m}^{\prime}\right)$, such that they are most consistent with their respective uncertainty matrices, $\mathbf{C}_{\boldsymbol{\alpha} \alpha}$ and $\mathbf{C}_{\mathbf{m m}}$. This is done by minimizing chi-square, expressed as

$$
\begin{aligned}
\chi^{2} & =\left[\frac{\alpha^{\prime}-\alpha}{\alpha}\right]^{\mathrm{T}} \mathbf{C}_{\alpha \alpha}^{-1}\left[\frac{\alpha^{\prime}-\alpha}{\alpha}\right]+\left[\frac{\mathbf{m}^{\prime}-\mathbf{m}}{\mathbf{m}}\right]^{\mathrm{T}} \mathbf{C}_{\mathbf{m m}}^{-1}\left[\frac{\mathbf{m}^{\prime}-\mathbf{m}}{\mathbf{m}}\right], \\
& =[\Delta \alpha]^{\mathrm{T}} \mathbf{C}_{\alpha \cdot \alpha}^{-1}[\Delta \alpha]+[\Delta \mathbf{m}]^{\mathrm{T}} \mathbf{C}_{\mathbf{m m}}^{-1}[\Delta \mathbf{m}]
\end{aligned}
$$

where $\Delta \alpha_{i}=\frac{\alpha_{i}^{\prime}-\alpha_{i}}{\alpha_{i}}$ and $\Delta \mathrm{m}_{i}=\frac{\mathrm{m}_{i}^{\prime}-\mathrm{m}_{i}}{\mathrm{~m}_{i}}$. Equation (6.6.11) is rearranged to give

$$
\chi^{2}=\left[\sigma_{\alpha}^{-1} \Delta \alpha\right]^{\mathrm{T}} \mathbf{R}_{\alpha \alpha}^{-1}\left[\sigma_{\alpha}^{-1} \Delta \alpha\right]+\left[\sigma_{\mathrm{m}}^{-1} \Delta \mathrm{m}\right]^{\mathrm{T}} \mathbf{R}_{\mathrm{mm}}^{-1}\left[\sigma_{\mathrm{m}}^{-1} \Delta \mathrm{m}\right]
$$

Eq. (6.6.12) expresses the variations in the nuclear data and measured responses in units of their respective standard deviations; that is, $\left[\boldsymbol{\sigma}_{\alpha}^{-1} \Delta \boldsymbol{\alpha}\right]$ and $\left[\sigma_{\mathrm{m}}^{-1} \Delta \mathbf{m}\right]$.

Chi-square is a quadratic form indicating the squared magnitude of the combined data variations with respect to their uncertainties. This is easily seen for the simple case in which $\left[\mathbf{R}_{\boldsymbol{\alpha} \alpha}\right]^{-1}$ and $\left[\mathbf{R}_{\mathbf{m m}}\right]^{-1}$ in Eq. (6.6.12) are identity matrices, so that Eq. (6.6.12) reduces to just the diagonal contributions:

$$
\chi^{2} \rightarrow \sum_{\mathrm{n}=1}^{\mathrm{M}}\left(\frac{\alpha_{n}^{\prime}-\alpha_{n}}{\tilde{\sigma}_{\alpha_{n}}}\right)^{2}+\sum_{\mathrm{i}=1}^{\mathrm{I}}\left(\frac{\mathrm{m}_{\mathrm{i}}^{\prime}-\mathrm{m}_{\mathrm{i}}}{\tilde{\sigma}_{\mathrm{m}_{i}}}\right)^{2}
$$

The first term on the on the right side of Eq. (6.6.13) is equal to the sum of the squares of the individual nuclear data variations expressed in units of their standard deviations while the second term represents a similar quantity for the measured integral responses. In the general case where correlations exist, the 
inverse matrices in Eq. (6.6.12) are not diagonal, and the value of chi-square must be evaluated using the indicated matrix multiplication.

Thus it can be seen that the GLLS method determines adjustments in the nuclear data and experimental measurements that (a) make the calculated and measured responses agree [i.e., $\mathbf{k}^{\prime}=\mathbf{k}^{\prime}\left(\boldsymbol{\alpha}^{\prime}\right)=\mathbf{m}^{\prime}$, within the limitations of first-order sensitivity theory], and (b) minimize Eq. (6.6.11) so that the adjustments are most consistent with the data uncertainties. Although many possible combinations of data variations may make $\mathrm{k}^{\prime}=\mathrm{m}^{\prime}$, there is a unique set that also minimizes $\chi^{2}$.

In TSURFER the term "chi-square" normally is meant to signify the minimum value of the quadratic form in Eq. (6.6.11). The significance of this minimum value is discussed in Sect. 6.6.4.2.1.

The following variations minimize Eq. (6.6.11), subject to the constraint $\mathbf{k}^{\prime}\left(\boldsymbol{\alpha}^{\prime}\right)=\mathbf{m}^{\mathbf{\prime}^{15}}$ and the linearity condition $[\Delta \mathbf{k}]=\mathbf{S}_{\mathbf{k} \alpha}[\Delta \boldsymbol{\alpha}]$ where $\Delta \mathrm{k}_{i}=\frac{\mathrm{k}_{i}^{\prime}-\mathrm{k}_{i}}{\mathrm{k}_{i}}$ :

$$
\begin{gathered}
\Delta \alpha=-\left[C_{a \alpha} S_{k \alpha}^{T} C_{d d}^{-1}\right] d \\
\Delta m=\left[C_{m m} F_{m / k} C_{d d}^{-1}\right] d
\end{gathered}
$$

In the above equations the initial response discrepancy vector $\mathbf{d}$ is operated on by the transformation matrix in square brackets to obtain the desired variations in nuclear data and integral measurements; thus, it is the discrepancy components that drive the adjustments. If the linearity assumption is valid, then the changes in the calculated responses are found to be

$$
\Delta \mathrm{k}=\mathrm{F}_{\mathrm{m} / \mathrm{k}} \Delta \mathrm{m}-\mathrm{d}=\mathrm{S}_{\mathrm{k} \alpha} \Delta \alpha
$$

Eq. (6.6.16) relates the adjustments in calculated responses, measured responses, and nuclear data.

As previously discussed, consolidation of the calculated and measured responses reduces the prior uncertainties for $\boldsymbol{\alpha}, \mathbf{m}$, and $\mathbf{k}$ because additional knowledge has been incorporated. This is indicated by their modified covariance matrices $\mathbf{C}_{\mathbf{a}^{\prime} \mathbf{a}^{\prime}}, \mathbf{C}_{\mathbf{m}^{\prime} \mathbf{m}^{\prime}}, \mathbf{C}_{\mathbf{k}^{\prime} \mathbf{k}^{\prime}}$, respectively, given by ${ }^{15}$

$$
\begin{gathered}
C_{\alpha^{\prime} \alpha^{\prime}}=C_{\alpha \alpha}-\left[C_{\alpha \alpha} S_{k \alpha}^{T} C_{d d}^{-1} S_{k \alpha} C_{\alpha \alpha}\right] \\
C_{m^{\prime} m^{\prime}}=C_{m m}-\left[C_{m m} F_{m / k} C_{d d}^{-1} F_{m / k} C_{m m}\right] \\
C_{k^{\prime} k^{\prime}}=C_{k k}-\left[C_{k k} C_{d d}^{-1} C_{k k}\right] .
\end{gathered}
$$


If all the responses on the TSURFER input are relative formatted, then the adjusted data and response values edited by TSURFER are obtained from Eqs. (6.6.14)-(6.6.16), while the square roots of diagonal elements in Eqs. (6.6.17)-(6.6.19) correspond to the edited values for adjusted uncertainties in the nuclear data and in the experiment responses, respectively.

The adjustment formulas must be modified slightly to be consistent with the absolute-formatted responses. In the following expressions, absolute response covariance and response sensitivity data are denoted by a tilde [see Appendix A.]:

$$
\begin{gathered}
\tilde{\mathbf{d}}=\mathbf{k}(\boldsymbol{\alpha})-\mathbf{m} \\
\tilde{\mathbf{C}}_{\mathrm{dd}}=\tilde{\mathbf{C}}_{\mathbf{k k}}+\tilde{\mathbf{C}}_{\mathbf{m m}}=\tilde{\mathbf{S}}_{\mathrm{k} \alpha} \mathbf{C}_{\alpha \alpha} \tilde{\mathbf{S}}_{\mathrm{k} \alpha}^{\mathrm{T}}+\tilde{\mathbf{C}}_{\mathbf{m m}} \\
\Delta \tilde{\boldsymbol{\alpha}}=\boldsymbol{\alpha}^{\prime}-\boldsymbol{\alpha}=-\left[\mathbf{C}_{\alpha \alpha} \tilde{\mathbf{S}}_{\mathrm{k} \alpha}^{\mathrm{T}} \tilde{\mathbf{C}}_{\mathrm{dd}}^{-1}\right] \tilde{\mathbf{d}} \\
\Delta \tilde{\mathbf{m}}=\mathbf{m}^{\prime}-\mathbf{m}=\left[\tilde{\mathbf{C}}_{\mathrm{mm}} \tilde{\mathbf{C}}_{\mathrm{dd}}^{-1}\right] \tilde{\mathbf{d}} \\
\Delta \tilde{\mathbf{k}}=\mathbf{k}^{\prime}-\mathbf{k}=\left(\mathbf{m}^{\prime}-\mathbf{m}\right)-\tilde{\mathbf{d}}=\mathbf{S}_{\mathbf{k} \alpha}\left(\alpha^{\prime}-\alpha\right) .
\end{gathered}
$$

Relative covariances for the posterior values of the nuclear data and measured responses are given as

$$
\begin{gathered}
\mathbf{C}_{\alpha^{\prime} \alpha^{\prime}}=\mathbf{C}_{\alpha \alpha}-\left[\mathbf{C}_{\alpha \alpha} \tilde{\mathbf{S}}_{\mathrm{k} \alpha}^{\mathrm{T}}\right] \tilde{\mathbf{C}}_{\mathrm{dd}}^{-1}\left[\tilde{\mathbf{S}}_{\mathrm{k} \alpha} \mathbf{C}_{\alpha \alpha}\right] \\
\tilde{\mathbf{C}}_{\mathrm{m}^{\prime} \mathrm{m}^{\prime}}=\tilde{\mathbf{C}}_{\mathrm{mm}}-\left[\tilde{\mathbf{C}}_{\mathrm{mm}} \tilde{\mathbf{C}}_{\mathrm{dd}}^{-1} \tilde{\mathbf{C}}_{\mathrm{mm}}\right] .
\end{gathered}
$$

If all the responses on the TSURFER input are absolute formatted, the adjusted data and response values edited by TSURFER are obtained from Eqs. (6.6.22)-(6.6.24), while the square roots of diagonal elements in Eqs. (6.6.25)-(6.6.26) correspond to the edited values for adjusted uncertainties in the nuclear data and in the experiment responses, respectively. 
The adjustment formulas again must be modified slightly given a set of mixed relative/absolute-formatted responses. In the following expressions, mixed response covariance and response sensitivity data are denoted by a caret (see Appendix A.), and $\hat{\mathbf{F}}_{\mathbf{m} / \mathbf{k}}$ is an $\mathrm{I} \times \mathrm{I}$ diagonal matrix containing $\mathrm{m} / \mathrm{k}$ factors for relative-formatted responses or a value of one for absolute-formatted responses:

$$
\begin{aligned}
& \hat{\mathbf{d}}_{i}= \begin{cases}\frac{\mathbf{k}(\boldsymbol{\alpha})_{i}-\mathbf{m}_{i}}{\mathbf{k}(\boldsymbol{\alpha})_{i}} & i_{\text {th }} \text { response is relative-formatted } \\
\mathbf{k}(\boldsymbol{\alpha})_{i}-\mathbf{m}_{i} & i_{\text {th }} \text { response is absolute }- \text { formatted }\end{cases} \\
& \Delta \hat{\mathbf{m}}_{\mathbf{i}}=\left\{\begin{array}{cc}
\frac{\mathbf{m}_{\mathbf{i}}^{\prime}-\mathbf{m}_{\mathbf{i}}}{\mathbf{m}_{\mathbf{i}}} & \text { relative } \\
\mathbf{m}_{\mathbf{i}}^{\prime}-\mathbf{m}_{\mathbf{i}} & \text { absolute }
\end{array}\right. \\
& \Delta \hat{\mathbf{k}}_{\mathbf{i}} \quad= \begin{cases}\frac{\mathbf{k}^{\prime}\left(\boldsymbol{\alpha}^{\prime}\right)_{i}-\mathbf{k}(\boldsymbol{\alpha})_{i}}{\mathbf{k}(\boldsymbol{\alpha})_{i}} & \text { relative } \\
\mathbf{k}(\boldsymbol{\alpha})_{i}-\mathbf{k}(\boldsymbol{\alpha})_{i} & \text { absolute }\end{cases} \\
& \hat{\mathbf{C}}_{\mathrm{dd}}^{-1}=\hat{\mathbf{C}}_{\mathrm{kk}}+\hat{\mathbf{F}}_{\mathrm{m} / \mathrm{k}} \hat{\mathbf{C}}_{\mathrm{mm}} \hat{\mathbf{F}}_{\mathrm{m} / \mathrm{k}}=\hat{\mathbf{S}}_{\mathrm{k} \alpha} \mathbf{C}_{\mathrm{dd}} \hat{\mathbf{S}}_{\mathrm{k} \alpha}^{\mathrm{T}}+\hat{\mathbf{F}}_{\mathrm{m} / \mathrm{k}} \hat{\mathbf{C}}_{\mathrm{mm}} \hat{\mathbf{F}}_{\mathrm{m} / \mathrm{k}} \\
& \Delta \hat{\boldsymbol{\alpha}} \quad=-\left[\mathbf{C}_{\mathrm{dd}} \hat{\mathbf{S}}_{\mathrm{k} \alpha}^{\mathrm{T}} \hat{\mathbf{C}}_{\mathrm{dd}}^{-1}\right] \hat{\mathbf{d}} \\
& \Delta \hat{\mathbf{m}} \quad=\left[\hat{\mathbf{C}}_{\mathrm{mm}} \hat{\mathbf{F}}_{\mathrm{m} / \mathrm{k}} \hat{\mathbf{C}}_{\mathrm{dd}}^{-1}\right] \hat{\mathbf{d}} \\
& \Delta \hat{\mathbf{k}} \quad=\hat{\mathbf{S}}_{\mathbf{k} \alpha} \Delta \hat{\boldsymbol{\alpha}} .
\end{aligned}
$$

Covariances for the posterior values of the nuclear data and measured responses are given as

$$
\begin{gathered}
\mathbf{C}_{\alpha^{\prime} \alpha^{\prime}}=\mathbf{C}_{\alpha \alpha}-\left[\mathbf{C}_{\alpha \alpha} \hat{\mathbf{S}}_{\mathrm{k} \alpha}^{\mathrm{T}}\right] \hat{\mathbf{C}}_{\mathrm{dd}}^{-1}\left[\hat{\mathbf{S}}_{\mathrm{k} \alpha} \mathbf{C}_{\alpha \alpha}\right] \\
\hat{\mathbf{C}}_{\mathrm{m}^{\prime} \mathrm{m}^{\prime}}=\hat{\mathbf{C}}_{\mathrm{mm}}-\left[\hat{\mathbf{C}}_{\mathrm{mm}} \hat{\mathbf{F}}_{\mathrm{m} / \mathrm{k}} \hat{\mathbf{C}}_{\mathrm{dd}}^{-1} \hat{\mathbf{F}}_{\mathrm{m} / \mathbf{k}} \hat{\mathbf{C}}_{\mathrm{mm}}\right] .
\end{gathered}
$$

If responses on the TSURFER input are both relative formatted and formatted, the adjusted data and response values edited by TSURFER are obtained from Eqs. (6.6.31)-(6.6.33), while the square roots of diagonal elements in Eqs. (6.6.34)-(6.6.35) correspond to the edited values for adjusted uncertainties in the nuclear data and in the experiment responses, respectively. 


\subsection{Consistency relations and chi-square filtering}

The variations for $\Delta \mathbf{m}$ and $\Delta \boldsymbol{\alpha}$ defined by Eq. (6.6.14) and Eq. (6.6.15)() are those that give the smallest value of the quadratic form $\chi^{2}$. This minimum $\chi^{2}$ value is found by substituting these equations into Eq. (6.6.11):

$$
\chi_{\min }^{2}=\mathbf{d}^{\mathrm{T}} \mathbf{C}_{\mathbf{d d}}{ }^{-1} \mathbf{d}=\mathbf{d}^{\mathrm{T}}\left[\mathbf{C}_{\mathbf{k k}}+\mathbf{F}_{\mathbf{m} / \mathbf{k}} \mathbf{C}_{\mathbf{m m}} \mathbf{F}_{\mathbf{m} / \mathbf{k}}\right]^{-1} \mathbf{d}
$$

It is interesting to observe that Eq. (6.6.36) does not depend upon adjustments in nuclear data or integral experiments and physically expresses a measure of the initial discrepancies (d) in all responses, compared to their combined calculation and experiment uncertainties $\left(\mathbf{C}_{\mathbf{k k}}+\mathbf{F}_{\mathbf{m} / \mathbf{k}} \mathbf{C}_{\mathbf{m m}} \mathbf{F}_{\mathbf{m} / \mathbf{k}}\right)$. In fact, the parameter

$\chi_{\min }^{2}$ is identical to the chi-square statistic discussed in Sect. 6.6.3.2 that denotes consistency between the calculations and measurements. Equation (6.6.36) can be viewed as an inherent limit on the consistency of the GLLS adjustment procedure. If the initial calculated and measured responses are not consistent with their stated uncertainties, then adjustments in nuclear data and experiment values obtained by TSURFER cannot be consistent either.

TSURFER provides an option for "chi-square filtering" to ensure that a given set of benchmark experiments is consistent; that is, that the input responses have an acceptable $\chi_{\min }^{2}$ defined by Eq.(6.6.36). The code progressively removes individual experiments until the calculated $\chi_{\min }^{2}$ is less than the input target value "target_chi". Each iteration removes one experiment estimated to have the greatest impact on chi-square per degree of freedom. The method used to assess individual contributions to $\chi_{\min }^{2}$ is specified by input parameter "chi_sq_filter", which refers to the different approaches described below.

Independent Chi-Square Option (chi_sq_filter=independent).

The consistency of the i-th measured and calculated response values, disregarding any other integral response, is equal to the discrepancy in the measured and calculated value squared divided by the variance of the discrepancy of the $i$-th response:

$$
\chi_{\text {ind }, \mathbf{i}}^{2}=\frac{\left(\mathrm{k}_{\mathbf{i}}-\mathbf{m}_{\mathbf{i}}\right)^{2}}{\sigma_{\mathbf{k}_{\mathrm{i}}}^{2}+\sigma_{\mathbf{m}_{\mathrm{i}}}^{2}} .
$$

Equation (6.6.37) is strictly valid only when no correlations exist, but it may be a useful approximation to estimate the experiment having the greatest impact on chi-square per degree of freedom. Hence, this expression is called the "independent chi-square" approximation in TSURFER. This approximation executes fast since no matrix inversions are required.

Diagonal Chi-Square Option (chi_sq_filter=diagonal)

The "diagonal chi-square" approach uses diagonal values of the original inverse $\mathbf{C}_{\mathbf{d d}}$ matrix to estimate the experiment having the greatest impact on chi-square per degree of freedom:

$$
\chi_{\text {dia,i }}^{2} \equiv\left(k_{i}-m_{i}\right)^{2} C_{d d}^{-1}(i, i)
$$


In this method the correlations in all responses are taken into account to some extent. The original $\mathbf{C}_{\mathrm{dd}}^{-1}$ is used in each iteration; therefore, the diagonal chi-square method requires only a single matrix inversion.

Iterative-Diagonal Chi-Square Option (chi_sq_filter=iter_diag).

This approach is identical to the diagonal chi-square method, except that a updated value of $\mathbf{C}_{\mathrm{dd}}^{-1}$ is computed each iteration to reevaluate the total chi-square from Eq. (6.6.36). Thus one matrix inversion is performed per iteration.

\section{Delta Chi-Square Option $($ chi_sq_filter=delta_chi).}

The most rigorous method to determine the impact of an individual response on the overall consistency is called the "delta chi-square" method in TSURFER. This method ${ }^{17}$ calculates the change in chi-square whenever a particular response is omitted for the analysis; that is, omitting the ith response results in

$$
\Delta \chi_{\mathrm{i}}^{2}=\left[\mathbf{d}^{\mathrm{T}} \mathbf{C}_{\mathbf{d d}}^{-1} \mathbf{d}\right]-\left[\mathbf{d}_{\neq \mathrm{i}}^{\mathrm{T}}\left(\mathbf{C}_{\mathbf{d d}}^{\neq \mathrm{i}}\right)^{-1} \mathbf{d}_{\neq \mathrm{i}}\right]
$$

where $\mathbf{d}_{\neq \mathrm{i}}$ and $\mathbf{C}_{\mathbf{d d}}^{\neq \mathrm{i}}$ are, respectively, the discrepancy vector and discrepancy covariance with response $\mathrm{i}$ omitted. While Eq. (6.6.39) is the most rigorous method, it also requires the most computation effort. A matrix inversion must be performed for every omitted response, in each iteration.

It has been observed that independent chi-square and diagonal chi-square options execute fast but often eliminate more experiments than necessary to obtain the target chi-square value. The diagonal chi-square option is somewhat faster than the iterative-diagonal chi-square option but also sometimes omits more than the minimum number of experiments. The delta chi-square option is currently default in TSURFER.

\subsection{Expressions for computational bias}

The computational "bias" is defined here as the observed difference between a calculated and measured response. In conventional validation studies the expected bias in an application response (for which there is no measurement, by definition) often is estimated as the sample mean of the biases for a set of benchmark experiments and the uncertainty in the application bias is estimated by the sample standard deviation of the experimental biases.

The GLLS technique provides another method to compute the bias of an application response. The application response bias $\beta_{a}$ is defined as the expected deviation of the original calculated response $k_{a}$ from the best estimate of the measured response, which is unknown but has some probability distribution. Note that if the application response actually did have a prior measured value $m_{a}$, then the best estimate for the experiment value would be the final adjusted value $\mathrm{m}_{\mathrm{a}}$ ' obtained from the GLLS procedure. For this reason the notation $\mathrm{m}_{\mathrm{a}}{ }^{\prime}$ is used here to represent the (unknown) best estimate for the application's projected measured response, so that

$$
\beta_{\mathrm{a}}=\mathrm{E}\left[\mathrm{k}_{\mathrm{a}}-\mathrm{m}_{\mathrm{a}}{ }^{\prime}\right],
$$

where $\mathrm{E}$ is the expectation operator. The application's projected experiment value can be expressed as $\mathrm{m}_{\mathrm{a}}{ }^{\prime}=\mathrm{k}_{\mathrm{a}}\left(\alpha^{\prime}\right)-\delta \mathrm{m}_{\mathrm{a}}$, where $\delta \mathrm{m}_{\mathrm{a}}$ represents the difference between the best computed response obtained with the adjusted data $\alpha^{\prime}$ and the expected value of the experimental measurement. Therefore Eq. (6.6.40) can be expressed 


$$
\beta_{\mathrm{a}}=\mathrm{E}\left[\mathrm{k}_{\mathrm{a}}-\mathrm{k}_{\mathrm{a}}\left(\alpha^{\prime}\right)+\delta \mathrm{m}_{\mathrm{a}}\right]=\mathrm{k}_{\mathrm{a}}-\mathrm{k}_{\mathrm{a}}\left(\alpha^{\prime}\right)+\mathrm{E}\left[\delta \mathrm{m}_{\mathrm{a}}\right]
$$

Recall that all experiment responses are sure to have $\delta \mathrm{m}_{\mathrm{i}}=0$, because the GLLS procedure forces $\mathrm{k}^{\prime}=\mathrm{m}^{\prime}$ within the approximation of first order theory. However, $\delta m_{a}\left(=k_{a}{ }^{\prime}-m_{a}{ }^{\prime}\right)$ for the application is not guaranteed to be zero, since there is no known measured value. Nevertheless the application response calculated using the best cross sections $\alpha^{\prime}$ should approach the desired (unknown) experiment value if a "sufficient" number of experiments similar to the application of interest are considered ${ }^{15}$ so that under these conditions $E\left[\delta \mathrm{m}_{\mathrm{a}}\right] \rightarrow 0$ for the application as well. More details concerning the suitable degree of similarity and the sufficient number of experiments necessary for convergence of the GLLS methodology are discussed in other publications. ${ }^{5,8,15}$ TSURFER also provides an automated procedure to examine the convergence of the bias, which is described in Sect. 6.6.4.4.

Assuming an adequate benchmark data base such that $\mathrm{E}\left[\delta \mathrm{m}_{\mathrm{a}}\right] \sim 0$, Eq. (6.6.41) simplifies to

$$
\beta_{\mathrm{a}}=\mathrm{k}_{\mathrm{a}}-\mathrm{k}_{\mathrm{a}}{ }^{\prime}\left(\alpha^{\prime}\right) \sim-\left(k_{a}\right) \mathbf{S}_{\mathrm{a}}^{\mathbf{T}} \Delta \boldsymbol{\alpha}
$$

or, stated in absolute terms,

$$
\beta_{\mathrm{a}} \sim-\tilde{\mathbf{S}}_{\mathbf{a}}^{\mathbf{T}} \Delta \boldsymbol{\alpha}
$$

In the above equations $\mathbf{S}_{\mathbf{a}}$ is the column vector of relative sensitivities for the application response. A negative bias indicates that the original computed value was too low; therefore, the adjusted application result will be higher than the original $\left(\mathrm{k}_{\mathrm{a}}{ }^{\prime}>\mathrm{k}_{\mathrm{a}}\right)$. Similarly, a positive bias means that the original response was calculated too high, and therefore $\mathrm{k}_{\mathrm{a}}{ }^{\prime}<\mathrm{k}_{\mathrm{a}}$.

\subsubsection{Expressions for response similarity parameters}

TSURFER estimates the similarity in a pair of responses using one of three internally computed similarity coefficients - respectively designated as E, G, and C_-specified by the input parameter "sim_type". These are essentially equivalent to the corresponding similarity coefficients described in Ref. 5, although there are slight differences in the definitions of E and G. Similarity coefficients are defined so that a value of zero indicates no similarity between the systems, and unity is maximum similarity. It is also theoretically possible, but unusual, to have a negative similarity in the range $[-1,0]$, indicating systems that are "anti-correlated" in some sense - in which case they are treated as completely dissimilar. Input parameter "sim_min" specifies the minimum similarity coefficient (compared to a specified reference application) of systems to be included in the GLLS procedure. TSURFER also optionally edits the I by I similarity matrix whose elements are the similarity coefficients for every response-pair combination (including both experimental and application responses).

The three types of similarity coefficients used in TSURFER are described below. In these expressions $\mathbf{S}_{\mathbf{i}}$ is defined as the sensitivity vector (not matrix) for a particular response " $i$ " which may be an experiment or application. The magnitude of the sensitivity vector corresponds to the L2 norm: $\left|\mathbf{S}_{\mathbf{i}}\right|=\sqrt{\mathbf{S}_{\mathbf{i}}^{\mathbf{T}} \mathbf{S}_{\mathbf{i}}}$. 


\subsection{The $E$ similarity parameter (sim_type $=E)$}

The E-similarity coefficient relating two responses $i$ and $j$ is defined analogously to the cosine of the angle between two direction vectors:

$$
E_{i, j} \equiv \frac{\mathbf{S}_{\mathbf{i}}^{\mathbf{T}} \mathbf{S}_{\mathbf{j}}}{\left|\mathbf{S}_{\mathbf{i}}\right|\left|\mathbf{S}_{\mathbf{j}}\right|}
$$

A value of E=1.0 corresponds to the case when $\mathbf{S}_{\mathbf{i}}$ and $\mathbf{S}_{\mathbf{j}}$ are "parallel", such as would occur when the two sensitivity vectors are proportional. A value of $E=0.0$ corresponds to the case when $\mathbf{S}_{\mathbf{i}}$ and $\mathbf{S}_{\mathbf{j}}$ are "perpendicular", such as occurs when the two sensitivity vectors have no common components (i.e., for every non-zero component in one, the corresponding component is zero in the other). Thus, E indicates the "relative direction" of the two sensitivity vectors in an N-dimensional vector space, with the assumption that the larger the parallel component, the greater the similarity. In theory, E could also take on the negative values in the interval $[-1,0]$ if the two responses are anti-parallel. In addition, the $\mathrm{E}$ coefficient is the same for absolute-formatted sensitivities (i.e., $\tilde{\mathbf{S}}_{\mathbf{i}}$ and $\tilde{\mathbf{S}}_{\mathbf{j}}$ ) or mixed relative-absolute sensitivities (e.g., $\tilde{\mathbf{S}}_{\mathbf{i}}$ and $\mathbf{S}_{\mathbf{j}}$ ).

\subsection{The $G$ similarity parameter $($ sim_type $=G)$}

The G-similarity coefficient for responses $\mathrm{i}$ and $\mathrm{j}$ is defined as,

$$
\mathrm{G}_{\mathrm{ij}} \equiv 1-\frac{\left|\mathbf{S}_{\mathbf{i}}-\mathbf{S}_{\mathbf{j}}\right|^{2}}{\left|\mathbf{S}_{\mathbf{i}}\right|^{2}+\left|\mathbf{S}_{\mathbf{j}}\right|^{2}}=\frac{\mathbf{S}_{\mathbf{i}}^{\mathbf{T}} \mathbf{S}_{\mathbf{j}}}{\overline{|\mathbf{S}|^{2}}}
$$

where $\overline{|\mathbf{S}|^{2}} \equiv \frac{\left|\mathbf{S}_{\mathbf{i}}\right|^{2}+\left|\mathbf{S}_{\mathbf{j}}\right|^{2}}{2}$.

As seen in the last term, the G parameter is similar to the E parameter, except the denominators are different. The effect of the different normalization is that $\mathrm{G}$ will be unity only if $\mathrm{S}_{\mathrm{i}}$ and $\mathrm{S}_{\mathrm{j}}$ are identical, while $\mathrm{E}$ indicates maximum similarity if they are proportional. It is important to note that the expression for the G parameter in the TSUNAMI-IP manual is different from Eq. (6.6.45). In both the TSURFER and TSUNAMI-IP formulations of G, the calculated parameter depends on the sensitivity format. It is recommended that Eq. (6.6.45) be used with relative-formatted sensitivities to calculate $\mathrm{G}$ for $k_{\text {eff }}$ responses.

\subsection{The $C$ similarity parameter $\left(\operatorname{sim} \_t y p e=C\right)$}

The C similarity coefficient represents the correlation in two calculated responses due to the shared uncertainty from common nuclear data. While $\mathrm{E}$ and $\mathrm{G}$ similarity coefficients only depend on the sensitivity vectors of two responses, the $\mathrm{C}$ parameter also involves cross-section covariance data. The $\mathrm{C}$-similarity parameter for responses $\mathrm{i}$ and $\mathrm{j}$ is the value of the correlation coefficient $\left(\rho_{\mathrm{ij}}\right)$ in position $(\mathrm{i}, \mathrm{j})$ of the $\mathrm{R}_{\mathrm{kk}}$ correlation matrix; thus, 


$$
\mathrm{C}_{\mathrm{ij}} \equiv \rho_{\mathrm{ij}}=\mathrm{R}_{\mathrm{kk}}(\mathrm{i}, \mathrm{j})=\frac{\mathbf{S}_{\mathbf{i}}^{\mathbf{T}} \mathbf{C}_{\boldsymbol{\alpha} \boldsymbol{\alpha}} \mathbf{S}_{\mathbf{j}}}{\boldsymbol{\sigma}_{\mathbf{i}} \boldsymbol{\sigma}_{\mathbf{j}}}
$$

The $\mathrm{C}$ coefficient has the usual interpretation of a correlation coefficient: 1.0 implies that the two responses are completely correlated by their nuclear data; 0.0 means no correlation; and -1.0 means full

anti-correlation. The $\mathrm{C}$ coefficient is the same for absolute-formatted sensitivities (i.e., $\tilde{\mathbf{S}}_{\mathbf{i}}$ and $\tilde{\mathbf{S}}_{\mathbf{j}}$ ) or mixed relative-absolute sensitivities (e.g., $\tilde{\mathbf{S}}_{\mathbf{i}}$ and $\mathbf{S}_{\mathbf{j}}$ ).

\subsubsection{Convergence of reference application response}

It is sometimes useful to consider how the GLLS procedure "converges" the estimated bias in an application response, as the number and similarity of integral experiment responses included in the analysis is increased. ${ }^{15}$ In TSURFER, the bias-convergence can be edited for any one of the application responses, called the "reference application," which is defined by the value of "ref_app" in the TSURFER input. Inserting the keyword "calc_cumul_effect" on the TSURFER input activates the option to edit the cumulative impact of increasing the number of benchmark experiments in the GLLS calculation. In this case, the range of similarity coefficients $[0.0 \rightarrow 1.0]$ is subdivided in bins of constant width set by the TSURFER input parameter "bin_width," and the experiment responses are sorted into the bins according their similarity to the reference application response. Any experiments with negative similarity coefficients are included in the first bin. Each bin of benchmark experiments is successively added to the GLLS calculation, going from low to high response similarity, until the whole suite of benchmarks is included. Ideally, the calculated reference application bias $\left(\beta_{\mathrm{a}}\right)$ should converge and stabilize at some value as the number and similarity of the experiment responses increases. Under these conditions the value $\mathrm{E}\left[\delta \mathrm{m}_{\mathrm{a}}\right]$ in Eq. (6.6.41) is approximately zero.

\subsubsection{TSURFER Input Description}

The user input for TSURFER is described in this section. The input consists of an optional title on a single line followed by one required and four optional blocks of data which are identified in Table 6.6.1 and individually described in subsequent subsections. These data blocks must begin with READ KEYNAME and end with END KEYNAME, where KEYNAME is the name of an individual data block. The PARAMETER data block, if requested, should be entered first after the optional title. The $H T M L$, COVARIANCE, and RESPONSE data blocks may follow in any order. If the CORR data block is necessary to specify experiment correlations, it should be the last block of data on the input. All keyword inputs are internally translated to lowercase with the exception of sensitivity data filenames and their file paths. 
Table 6.6.1. Table keynames and descriptions for TSURFER input data blocks

\begin{tabular}{|c|c|c|}
\hline Keyname & Description & Required/Optional \\
\hline PARAMETER & $\begin{array}{l}\text { Parameters that specify the covariance data file, chi- } \\
\text { square filtering options, similarity filtering options, } \\
\text { output edit options, and approximate cross-section } \\
\text { covariance data options can be entered in this } \\
\text { section. }\end{array}$ & Optional \\
\hline $\begin{array}{l}\text { RESPONSE } \\
\text { EXPERIMENTS }^{a} \\
\text { APPLICATIONS }^{a}\end{array}$ & $\begin{array}{l}\text { File paths to sensitivity data files representing } \\
\text { experiments or applications are input in this } \\
\text { section. Measured response values and measured } \\
\text { response uncertainties are also input in this section. }\end{array}$ & Required \\
\hline COVARIANCE & $\begin{array}{l}\text { User-input standard deviation for nuclide-reaction pairs } \\
\text { for which cross-section-covariance data are not } \\
\text { available can be entered in this section. }\end{array}$ & Optional \\
\hline$H T M L$ & $\begin{array}{l}\text { Parameters to customize the HTML-formatted output } \\
\text { can be entered in this section. }\end{array}$ & Optional \\
\hline CORR & $\begin{array}{l}\text { Correlations between measured responses and measured } \\
\text { response uncertainty components can be entered in } \\
\text { this section. }\end{array}$ & Optional \\
\hline
\end{tabular}

\subsubsection{Parameter block}

The PARAMETER data block is used to specify various keyword options used to control the execution of the code. These options include the name of the cross-section covariance data file, output edits, default covariance data, and chi-square or similarity filtering options. The parameter block must begin with READ PARAMETER and end with END PARAMETER. The data input to the parameter data block consist of numerous keywords that are shown, along with their default values and descriptions, in

Table 6.6.2. A keyword that ends with " $=$ " must be followed by numeric data or a character string. Keywords that do not end with "=" are Boolean flags that are used to turn on certain features of the code, such as the computation of certain data or certain output edits. If the keyword is present for a Boolean entry, the value is set to true. Otherwise, the value is set to false. All PARAMETER block keywords are optional. 
Table 6.6.2. Input data for the Parameter block of TSURFER

\begin{tabular}{|c|c|c|}
\hline Keyword & Default value & Description \\
\hline absolute or $a b s$ & False & $\begin{array}{l}\text { Use absolute sensitivities and uncertainties for all } \\
\text { applications and experiments in the analysis unless } \\
\text { specifically overridden by experiment or application } \\
\text { input. }\end{array}$ \\
\hline adj_cov_cut & 0.000001 & $\begin{array}{l}\text { Cutoff value for including an adjusted cross-section- } \\
\text { covariance matrix in the post adjustment analysis } \\
\text { and data file. If a nuclide-reaction to nuclide- } \\
\text { reaction covariance contains no values exceeding } \\
\text { adj_cov_cut,the matrix is excluded from further } \\
\text { analysis. Note that adj_cov_cut represents a } \\
\text { variance, not a standard deviation. }\end{array}$ \\
\hline adjcut & 0.00001 & $\begin{array}{l}\text { Cutoff value for the cross-section adjustment edit. If the } \\
\text { maximum (absolute value) multigroup cross-section } \\
\text { adjustment for a given nuclide-reaction pair is less } \\
\text { than adjcut, then the nuclide-reaction pair is not } \\
\text { included in the cross-section adjustment edit. }\end{array}$ \\
\hline bin_width ${ }^{a}$ & 0.01 & $\begin{array}{l}\text { Size of the similarity bins for the cumulative iteration } \\
\text { edits. }\end{array}$ \\
\hline $\operatorname{cov}$ fix & False & $\begin{array}{l}\text { Replace zero and large (standard deviation }>1000 \% \text { ) } \\
\text { values on diagonal of cross-section-covariance data } \\
\text { with user-input values and default values. }\end{array}$ \\
\hline cover $x=$ & 44groupcov & $\begin{array}{l}\text { Name of cross-section covariance data file to use in } \\
\text { analysis. See the COVLIB chapter of SCALE } \\
\text { documentation for detailed description of the } \\
\text { available covariance library. }\end{array}$ \\
\hline calc_cumul_effect ${ }^{a}$ & False & Perform cumulative iteration edit. \\
\hline chi_sq_filter ${ }^{b}$ & delta_chi & $\begin{array}{l}\text { Method used for chi-square filtering. Allowed values are } \\
\text { independent, diagonal, iter_diag, and delta_chi. }\end{array}$ \\
\hline def_min $=$ & 0.001 & $\begin{array}{l}\text { Minimum sensitivity criteria to adjust nuclear data. The } \\
\text { minimum sensitivity criteria is only applied to } \\
\text { nuclide-reaction pairs with missing covariance data } \\
\text { and if use_dcov or use_icov is entered. }\end{array}$ \\
\hline large_cov $=$ & 10.0 & $\begin{array}{l}\text { Cutoff fractional standard deviation value for cov fix. } \\
\text { Cross-section-covariance data with uncertainties } \\
\text { larger than large_cov are replaced with user-input or } \\
\text { default values. Default }=10 \text {, which is } 1000 \% \\
\text { uncertainty. }\end{array}$ \\
\hline
\end{tabular}

nohtml

False

If nohtml is present, HTML-formatted output is not 


\begin{tabular}{|c|c|c|}
\hline Keyword & Default value & Description \\
\hline & & generated. \\
\hline print $=$ & regular & $\begin{array}{l}\text { Level of ouput edits discussed below. Options are } \\
\text { minimum and regular. }\end{array}$ \\
\hline print_adjustments & False & Option to print cross-section adjustment edit. \\
\hline print_adj_corr & False & Option to print the adjusted response correlation matrix. \\
\hline print_init_corr & False & Option to print the initial response correlation matrix. \\
\hline print_sim_matrix & False & Option to print the initial response similarity matrix. \\
\hline$r e f \_a p p={ }^{a}$ & $\begin{array}{l}\text { First Application on } \\
\text { Input }\end{array}$ & $\begin{array}{l}\text { If application systems are included, ref_app is the index } \\
\text { to the reference application response. Additional } \\
\text { output edits are given for the reference application } \\
\text { described in Sect 6.6.6. }\end{array}$ \\
\hline relative or rel & True & $\begin{array}{l}\text { Use relative sensitivities and uncertainties for all } \\
\text { applications and experiments in the analysis. This is } \\
\text { the default and keyword relative is not required. }\end{array}$ \\
\hline return_adj_cov & False & $\begin{array}{l}\text { Option to create a COVERX-formatted covariance data } \\
\text { file of the adjusted cross-section-covariance matrix. } \\
\text { If return_adj_cov is present, the adjusted covariance } \\
\text { data file is returned to the working directory with the } \\
\text { file name job_name.adj.cov where job_name is the } \\
\text { name of the input file. }\end{array}$ \\
\hline return_work_cov & False & $\begin{array}{l}\text { If return_work_cov is present, the working covariance } \\
\text { library is copied to the return directory with the file } \\
\text { name job_name.wrk.cov where job_name is the } \\
\text { name of the input file. If return_work_cov is not } \\
\text { present, the working covariance library remains in } \\
\text { the temporary working directory with the file name } \\
\text { job_name.wrk. }\end{array}$ \\
\hline sim_min $=^{c}$ & -1 & $\begin{array}{l}\text { Minimum similarity coefficient of experimental responses } \\
\text { with the reference application response to be } \\
\text { included in the adjustment. }\end{array}$ \\
\hline sim_type $=^{c}$ & None & $\begin{array}{l}\text { Criteria to calculate initial response similarity matrix. } \\
\text { Allowed values are none, } E, C \text {, and } G \text {. }\end{array}$ \\
\hline target_chi $=^{b}$ & 1.2 & $\begin{array}{l}\text { Target chi-square per degree of freedom for consistency } \\
\text { acceptance. If target_chi=0.0, chi-square filtering is } \\
\text { not performed. }\end{array}$ \\
\hline
\end{tabular}




\begin{tabular}{|c|c|c|}
\hline Keyword & Default value & Description \\
\hline $\begin{array}{c}u d c o v= \\
\text { (optional) }\end{array}$ & 0.05 & $\begin{array}{l}\text { User-defined default value of standard deviation in cross- } \\
\text { section data to use for all groups for nuclide-reaction } \\
\text { pairs for which cross-section-covariance data are not } \\
\text { available on the input covariance library. }\end{array}$ \\
\hline $\begin{array}{c}u d c o v \_c o r r= \\
\text { (optional) }\end{array}$ & 1.0 & $\begin{array}{l}\text { User-defined default correlation value to use for nuclide- } \\
\text { reaction pairs for which cross-section-covariance data } \\
\text { are not available on the input covariance library. }\end{array}$ \\
\hline $\begin{array}{c}\text { udcov_corr_type }= \\
\text { (optional) }\end{array}$ & zone & $\begin{array}{l}\text { User-defined default correlation in cross-section data to } \\
\text { use for nuclide-reaction pairs for which cross-section- } \\
\text { covariance data are not available on the input SCALE } \\
\text { covariance library. Allowed values are long, zone, } \\
\text { and short. }\end{array}$ \\
\hline $\begin{array}{l}\text { udcov_therm }= \\
\text { (optional) }\end{array}$ & 0.0 & $\begin{array}{l}\text { User-defined default value of standard deviation in cross- } \\
\text { section data to use for thermal data for nuclide- } \\
\text { reaction pairs for which cross-section-covariance data } \\
\text { are not available on the input covariance library. }\end{array}$ \\
\hline $\begin{array}{l}\text { udcov_inter }= \\
\quad(\text { optional) }\end{array}$ & 0.0 & $\begin{array}{l}\text { User-defined default value of standard deviation in cross- } \\
\text { section data to use for intermediate data for nuclide- } \\
\text { reaction pairs for which cross-section-covariance data } \\
\text { are not available on the input covariance library. }\end{array}$ \\
\hline $\begin{array}{c}\text { udcov_fast }= \\
\text { (optional) }\end{array}$ & 0.0 & $\begin{array}{l}\text { User-defined default value of standard deviation in cross- } \\
\text { section data to use for fast data for nuclide-reaction } \\
\text { pairs for which cross-section-covariance data are not } \\
\text { available on the input covariance library. }\end{array}$ \\
\hline uncert_long & False & $\begin{array}{l}\text { Prints extended table of uncertainty in response due to } \\
\text { covariance data. }\end{array}$ \\
\hline use_dcov & False & $\begin{array}{l}\text { Use default cross-section-covariance data for nuclide- } \\
\text { reaction pairs not included on the input covariance } \\
\text { data file. }\end{array}$ \\
\hline use_diff_groups $=$ & true & $\begin{array}{l}\text { Permit sensitivity data files to have different energy group } \\
\text { structures. Allowed values are true and false. }\end{array}$ \\
\hline usename & False & $\begin{array}{l}\text { Use the name of the sensitivity data file as the default } \\
\text { response identifier in the TSURFER output. }\end{array}$ \\
\hline use_icov & False & $\begin{array}{l}\text { Use user-defined cross-section-covariance data input in } \\
\text { the COVARIANCE input data block in place of the } \\
\text { default values for user-input nuclide-reaction pairs } \\
\text { that are not on the input covariance data file. }\end{array}$ \\
\hline
\end{tabular}




Keyword Default value Description

${ }^{a}$ See Sect. 6.6.4.4 for description of Bias Convergence Analysis.

${ }^{b}$ See Sect. 6.6.4.2.1 for definitions of chi-square filtering methods.

${ }^{c}$ See Sect. 6.6.4.3 for description of similarity coefficients.

The PARAMETER block keyword print controls the general level of the TSURFER output print. The minimum print level "print=minimum" summarizes the input values for experimental responses and uncertainties, edits chi-square values, and prints GLLS results for the application responses. The regular print option "print=regular" additionally shows GLLS results computed for all adjusted experimental responses.

The PARAMETER block keywords—use_dcov, udcov, udcov_therm, udcov_inter, udcov_fast, udcov_corr, and udcov_corr type - are used to specify the default covariance data for nuclide-reaction pairs that do not have covariance data available on the SCALE covariance data file. The Boolean flag keyword use_dcov activates the use of default covariance data for nuclide-reaction pairs with missing covariance data. The udcov keyword specifies a default relative standard deviation for all energy groups. The keywords udcov_therm, udcov_inter, and udcov_fast can be used to specify the default relative standard deviation for the thermal energy groups, intermediate energy groups, and fast energy groups, respectively. If either udcov_therm, udcov_inter, or udcov_fast are omitted from the input, the default uncertainty applied for their respective energy groups is the udcov value. The keyword udcov_corr specifies the correlation coefficient for the default covariance data, and udcov_corr_type specifies the correlation type. The correlation type options are (a) long - apply correlation coefficient in all energy groups, (b) short - apply correlation coefficient in adjacent groups, and (c) zone - apply correlation within fast, intermediate, and thermal groups, but no correlation is applied between different group ranges.

For additional user control over the approximate cross-section covariance data, the COVARIANCE data block can be used to input uncertainty values for particular nuclide-reaction pairs. To utilize the covariance data generated by user-input in the COVARIANCE data block, the keyword use icov must be entered in the PARAMETER data block. Approximate covariance data specified in the COVARIANCE data block are referred to as user-input data. The input for the COVARIANCE data block is described in more detail in Sect. 6.6.5.3.

When use_dcov and/or use_icov and cov_fix are specified in the PARAMETER data block, and a reaction has zero or large (standard deviation $>1000 \%$ ) values on the diagonal of the covariance matrix, these values are replaced with the square of the user-input or default standard deviation value, and the corresponding off-diagonal terms are substituted according to the user-input or default correlation values. Warning messages are printed to identify which values were replaced and which standard deviation value was used in the replacement. The maximum relative standard deviation in which to apply the covariance correction can be specified by the user with the large_cov keyword.

The def_min keyword is used to determine if the default or user-input covariance data is applied for nuclide-reaction pairs with missing covariance data. For each nuclide-reaction pair with missing covariance data, TSURFER calculates the maximum, absolute-value, groupwise response sensitivity over all active (i.e., experiment) and passive (i.e., application) responses on the input. If the maximum sensitivity value for a given nuclide-reaction pair is greater than def_min, the default or user-input covariance data is applied and the cross-section data for the nuclide-reaction pair is adjusted in the analysis. If the relative keyword is entered in the PARAMETER data block, the value of def_min is interpreted as a relative-formatted sensitivity. Likewise, if the absolute keyword is entered in the PARAMETER data block, the value of def_min is interpreted as an absolute-formatted sensitivity. If both 
relative and absolute are entered, the last keyword in the PARAMETER data block sets the format for both def_min and the response sensitivity data files. If both relative and absolute are omitted, the default format for def_min is relative. The minimum sensitivity criterion is slightly different if both relativeformatted $k_{\text {eff }}$ responses and absolute-formatted eigenvalue-difference (reactivity) responses are included in the analysis. In this case, the minimum sensitivity criteria can be entered for each response in the RESPONSE block described in the next section.

During the GLLS analysis, TSURFER computes a new covariance data file that contains cross-sectioncovariance data only for the nuclide-reaction pairs that are listed in the response sensitivity data files. The new covariance data file, referred to as the working covariance data file, is written in COVERX format like the input SCALE covariance data file. The working covariance data file contains any default or userinput cross-section-covariance data for nuclide-reaction pairs that were not in the input SCALE covariance data file as well as any corrected cross-section-covariance data if the cov fix keyword is entered on the input. The working covariance data file can be read by the data plotting tool in Fulcrum to visualize the cross section covariance data used in the analysis.

\subsubsection{RESPONSE block}

In the RESPONSE data block, sensitivity data files are designated as either application responses or experiment responses. The RESPONSE data block is also used to specify experimental response values, experimental response uncertainties, and uncertainties of experimental response components. The TSUNAMI-IP block keynames EXPERIMENTS and APPLICATIONS are also allowed. Each data block must begin with READ KEYNAME and end with END KEYNAME where KEYNAME can be APPLICATIONS, EXPERIMENTS, or RESPONSE.

By default, sensitivity data files listed in RESPONSE or EXPERIMENTS data blocks are designated as experiment responses, while files listed in the APPLICATIONS data block are designated as application responses. Multiple RESPONSE, EXPERIMENTS, and APPLICATIONS data blocks are allowed, and they can be entered in any order. However, the order of the sensitivity data files in the TSURFER input is important when defining experiment correlations. Two recommended input methods are (a) define all experiment and application responses in a single RESPONSE data block using the use keyword and specify the role of each response in the analysis or (b) define all experiment responses in a single EXPERIMENTS block, and define all application responses in a single APPLICATIONS block.

Inside each data block, sensitivity data files are listed using response definition records. A response definition record is a single line of input that contains the sensitivity data filename, two required keywords and eight optional keywords shown in parentheses. The sensitivity data filename and keywords can be entered in any order, with the following format:

$$
\text { filename }(\text { use }=R)(\text { name }=N)(\text { type }=T) \text { ev }=E u v=U(c v=C)(\text { nu=P) (omit) (abs) (rel) }(\text { msen }=M)
$$

where

$$
\begin{aligned}
\text { filename }= & \text { sensitivity data filename. The filename can include the file path. } \\
R & =\text { adjustment role. Allowed values are appl, expt, and omit. The default value is expt } \\
& \text { in the RESPONSE or EXPERIMENT block and appl in the APPLICATION block. } \\
\mathrm{N}= & \text { 20-character maximum alphanumeric response identifier in TSURFER output. } \\
\mathrm{T}= & \text { 8-character maximum alphanumeric identifier for the response type (e.g., "keff", } \\
& \text { "gpt", or "rho"). The response-type identifier is used in various output edits along } \\
& \text { with the response name identifier. }
\end{aligned}
$$




\begin{tabular}{|c|c|}
\hline U & uncertainty value of the response. \\
\hline$C$ & calculated value of the response. \\
\hline$P$ & $\begin{array}{l}=\text { number of uncertainty components to characterize the experiment uncertainty for } \\
\text { this response. }\end{array}$ \\
\hline omit & $\begin{array}{l}\text { - Optional keyword used to omit the response from the analysis. This can also be } \\
\text { done by the } u s e=\text { omit keyword specification. }\end{array}$ \\
\hline$a b s$ & $\begin{array}{l}\text { - Optional keyword that specifies absolute sensitivities, absolute experiment } \\
\text { uncertainties, and absolute components of uncertainty that are used for this } \\
\text { response. The keyword absolute is also valid. }\end{array}$ \\
\hline I & $\begin{array}{l}\text { - Optional keyword that specifies relative sensitivities, relative experiment } \\
\text { uncertainties, and relative components of uncertainty that are used for this } \\
\text { response. The keyword relative is also valid. }\end{array}$ \\
\hline$M$ & $\begin{array}{l}=\text { minimum sensitivity criteria for this response. This value will replace the def_min } \\
\text { value in the PARAMETER block to determine if nuclide-reaction pairs with } \\
\text { missing covariance data are included in the adjustment. }\end{array}$ \\
\hline
\end{tabular}

Case-sensitive filenames and file paths are allowed for sensitivity data filename. However, spaces are not allowed in the filenames or file paths. The sensitivity data filename is limited to 80 characters, and the total length of the response definition must not exceed 255 characters. The use keyword specifies the role of the response in the GLLS analysis. "use=expt" designates the corresponding sensitivity data file as an experiment response. Likewise, "use=appl" designates the corresponding data file as an application response. In addition, the user can omit the sensitivity data file from the analysis by entering either "use=omit" or simply omit on the response definition record. If the use keyword is not included, the role of the response is determined by the data block name; that is, "use=appl" is implied for the APPLICATIONS block and " $u s e=e x p t$ " is implied for the EXPERIMENTS and RESPONSE blocks.

By default, TSURFER identifies responses in the output according to the title on the sensitivity data files. For files that have the same titles, or have long or non-descriptive titles, the usename keyword in the PARAMETER data block can be used to identify the response by their sensitivity data filename. Although filenames are unique, they can also be non-descriptive. For this reason, the name keyword on the response definition record can be used to create a new identifier for the response in the TSURFER output. Similarly, the type keyword can be used to identify the response type in the output. The default response type is "keff" for $k_{\text {eff }}$ responses and "rho" for eigenvalue-difference responses. It may be useful to include a sequence number in the response name, in order to more easily associate the response number to the input response data. For example, the response names for the first three responses entered could be name $=1 \_G O D I V A$, name $=2 \_J E Z E B E L$, and name $=3 \_Z P R 4$. In the CORR data block and in the printed output, responses are identified by their sequence number (i.e., the order read in), so it is convenient to show this number in the response Name, especially when dealing with a large number of responses.

The measured value of the response $(e v=)$ and the measured uncertainty $(u v=)$ are required for experiment responses. For application or omitted responses, the $e v$ and $u v$ keywords are permitted but are not required. The calculated response value is read from the sensitivity data file, but can be overridden by the $c v$ keyword. The $n u=$ keyword defines the number of uncertainty components that characterize the experiment response uncertainty. If the experiment response uncertainty is given in terms of uncertainty components, the $u v=$ keyword specification is optional. An uncertainty component definition record follows the response definition record if the $n u=$ keyword specification is given. The uncertainty component definition record has the following format:

$$
\text { ucmp1 val1 ucmp2 val2 …... ucmpP valP, }
$$


where
uncmpl $1=4$-character alphanumeric identifier for the $1^{\text {st }}$ uncertainty component, vall $=$ experiment uncertainty for component uncmpl,
Uncmp2 = 4-character alphanumeric identifier for the $2^{\text {nd }}$ uncertainty component,
val2 $=$ experiment uncertainty for component uncmp2,
uncmpP $=$ 4-character alphanumeric identifier for the $\mathrm{P}^{\text {th }}$ uncertainty component, and
valP $=$ experiment uncertainty for component uncmpP.

The uncertainty component definition record contains $n u=P$ pairs of alphanumeric identifiers and numeric values. The measured uncertainty value is determined by Eq. (6.6.6).

The keywords $a b s$ and $r e l$ are used to determine the format of sensitivity and uncertainty data on the response definition record and the uncertainty component definition record. For a $k_{\text {eff }}$ response, the following four input definitions are equivalent:

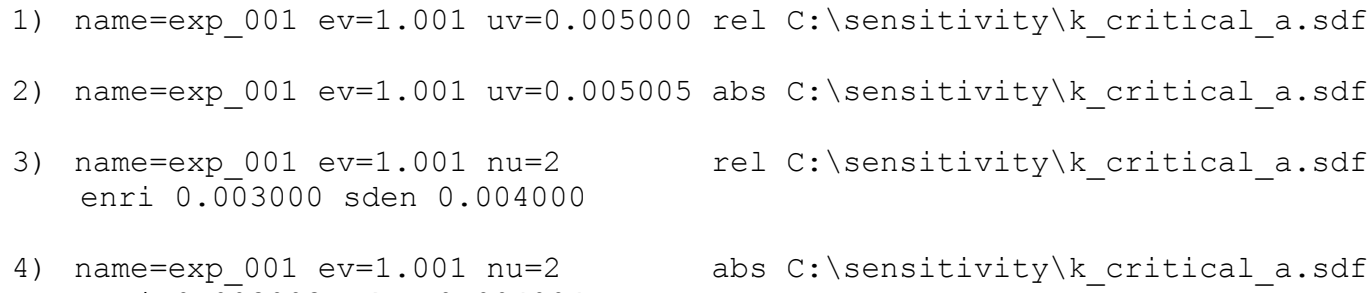
enri $0.0 \overline{0} 3003$ sden 0.004004

In the example above, the measured $k_{\text {eff }}$ is $1.001 \pm 0.5 \%$ or $1.001 \pm 0.005005$. (Although most critical experiments have measured $k_{\text {eff }}=1$, this contrived example reveals the difference between the absolute format and the response format.) The sensitivity data filename is given as C:|sensitivity|k_critical_a.sdf, and the experiment response is referred to as exp_001 in the TSURFER output. In 1), the relative format is used to specify the relative standard deviation of the measured response as 0.005 . In 2), the absolute format is used to specify the absolute standard deviation of the measured response as 0.005005 . Because the TSUNAMI-generated sensitivity data file is in relative format, TSURFER internally renormalizes the relative sensitivities to absolute sensitivities. In 3 ), the relative format is used to specify the relative standard deviation of $k_{\text {eff }}$ due to two components (enri and sden) as 0.003 and 0.004 , respectively. Using Eq. (6.6.6), the relative standard deviation of $k_{\text {eff }}$ is computed to be 0.005 . In 4), the absolute format is used to specify the absolute standard deviation of $k_{\text {eff }}$ due to two components (enri and sden) as 0.003003 and 0.004004 , respectively. The absolute standard deviation of $k_{\text {eff }}$ is computed to be 0.005005 . Like 2), the sensitivity data file is internally converted to contain absolute sensitivities.

For a second example, the following input definitions are equivalent for an eigenvalue-difference, or reactivity, response:

1) $\mathrm{C}: \backslash$ sensitivity \reactivity.sdf ev=15.0000 uv=3.0 abs

2) $\mathrm{C}: \backslash$ sensitivity \reactivity.sdf ev=0.00015 uv=0.2 rel

In this example, the measured reactivity is $15 \mathrm{pcm}$ (percent-mille) $\pm 3 \mathrm{pcm}$ or $0.00015 \pm 20 \%$. TSAR creates reactivity sensitivity files in either (a) absolute format where the calculated reactivity response and sensitivities are in pcm units or (b) relative format with relative sensitivities and the calculated reactivity response are not in pcm units. The TSURFER response definition records are designed to be consistent with the TSAR formats. In 1), the absolute format is used to specify the absolute standard deviation of the measured response as $3 \mathrm{pcm}$. In 2), the relative format is used to specify the measured reactivity response as 0.00015 with a relative standard deviation of 0.20 (or 20\%). Because TSAR-generated 
reactivity sensitivity data files may be in absolute format or relative format, TSURFER internally renormalizes the reactivity sensitivity data file to the user-requested format. On occasion, it is desired to adjust a set of nuclear data with zero-valued reactivity responses (i.e., $e v=0.0$ ). For this case, the absolute format should be used because the relative standard deviation of the measured response approaches infinity.

If the abs or rel keywords are not included on the response definition record, the default format is determined by the abs or rel keywords in the PARAMETER data block. If more than one formatting keyword is entered in either the PARAMETER data block or response definition record, the last keyword entered sets the format. As an example, the following inputs are equivalent:

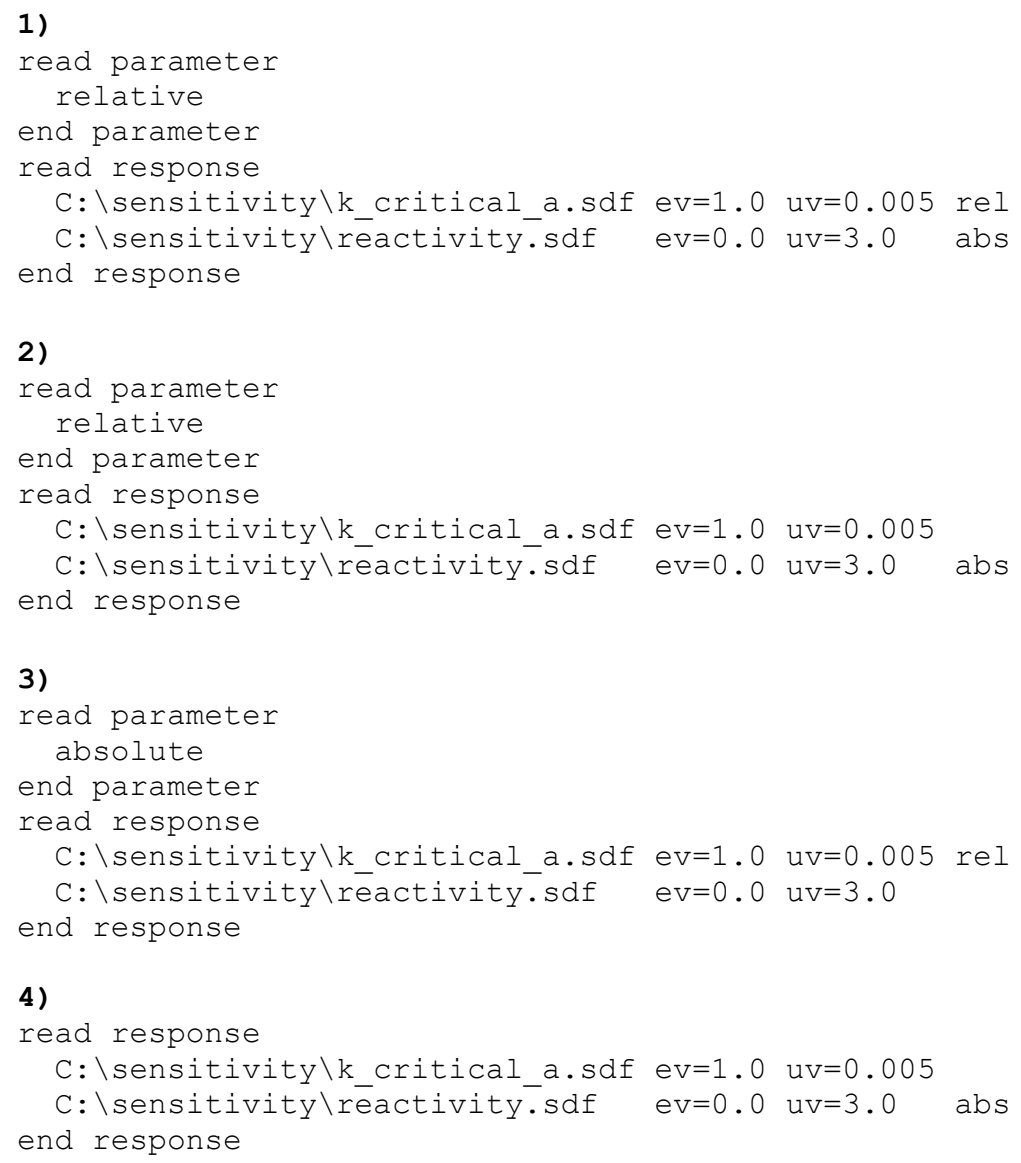

In this example, two experiment responses are given. The first response is a relative-formatted $k_{\text {eff }}$ response. The second response is an absolute-formatted reactivity response. In 1), the format is determined by the formatting keyword on the response definition record. In 2), the relative-format is set as the default format by the PARAMETER block and the absolute format for the reactivity response is specified on its response definition record. In 3), the absolute-format is set as the default format by the PARAMETER block and the relative format for the $k_{\text {eff }}$ is set by its response definition record. Case 4) is the same as case 2) where the default relative format is applied if no PARAMETER block is included.

The final optional keyword for the response definition record is msen=M. This record sets the minimum sensitivity criteria for nuclide-reaction pairs with missing covariance data. The keyword is useful when dealing with mixed formatted responses. For example, the following input contains three relative formatted $k_{\text {eff }}$ responses and two absolute-formatted reactivity responses. 
1)

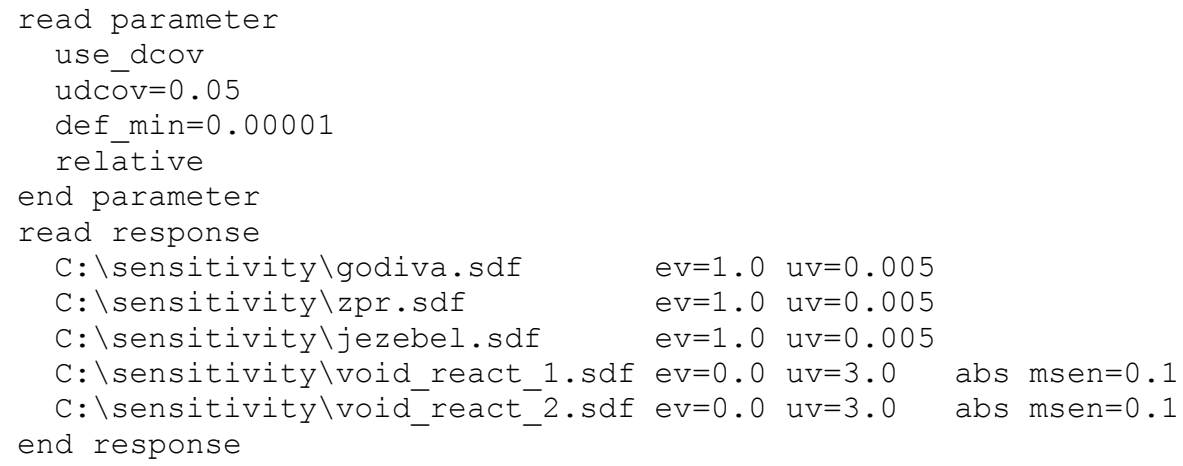

In this example, the PARAMETER block keywords initialize all the responses that follow as relativeformatted responses and the minimum sensitivity criteria for applying default covariance data is 0.00001 or $0.001 \%$. This criteria is used for the three $k_{\text {eff }}$ responses. In the response definition records for the two reactivity responses, the absolute-format is specified and the minimum sensitivity criteria is $0.1 \mathrm{pcm}$. Therefore, default covariance data is used for a nuclide-reaction pair with missing cross-section covariance data if at least one $k_{\text {eff }}$ sensitivity for one of the three $k_{\text {eff }}$ responses is greater than $0.001 \%$ or at least one reactivity sensitivity for one of the two reactivity responses is greater than $0.1 \mathrm{pcm}$. Similar to the example above, this example has the following equivalent input:

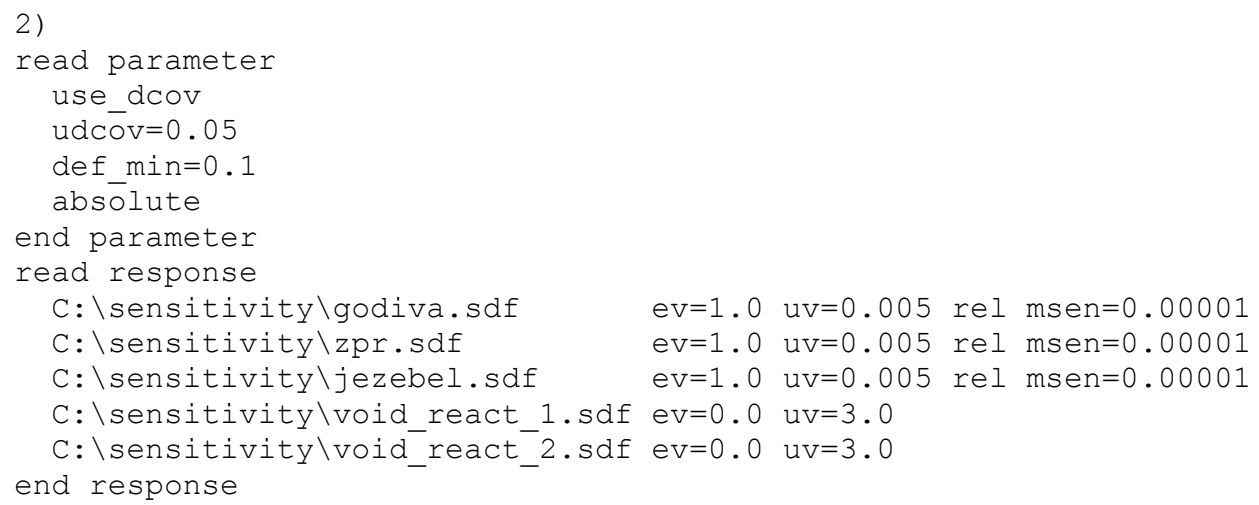

\subsubsection{COVARIANCE block}

The COVARIANCE data block allows the user to specify a covariance matrix for specific nuclide-reaction pairs for which covariance data are not present on the input SCALE covariance library or that have zero or large values on the diagonal. The COVARIANCE data block must begin with READ COVARIANCE and end with END COVARIANCE. The available COVARIANCE data block keywords and their default values are given in Table 6.6.3. 
Table 6.6.3. Input data for the Covariance block of TSURFER

\begin{tabular}{|c|c|c|c|c|}
\hline $\begin{array}{c}\text { Input } \\
\text { parameter }\end{array}$ & Requirement & $\begin{array}{c}\text { Default } \\
\text { value }\end{array}$ & Allowed values & Description \\
\hline Nuclide & Required & none & $\begin{array}{l}\text { Nuclide name or } \\
\text { ZA number }\end{array}$ & $\begin{array}{l}\text { Nuclide for which covariance data are } \\
\text { to be entered }\end{array}$ \\
\hline Reaction & Required & none & $\begin{array}{l}\text { Reaction name or } \\
\text { ZA number }\end{array}$ & $\begin{array}{l}\text { Reaction for which covariance data are } \\
\text { to be entered }\end{array}$ \\
\hline all $=$ & Optional & 0.0 & any number & $\begin{array}{l}\text { Fractional standard deviation value to } \\
\text { be applied to all groups }\end{array}$ \\
\hline fast $=$ & Optional & 0.0 & any number & $\begin{array}{l}\text { Fractional standard deviation value to } \\
\text { be applied to fast groups }\end{array}$ \\
\hline therm $=$ & Optional & 0.0 & any number & $\begin{array}{l}\text { Fractional standard deviation value to } \\
\text { be applied to thermal groups }\end{array}$ \\
\hline inter $=$ & Optional & 0.0 & any number & $\begin{array}{l}\text { Fractional standard deviation value to } \\
\text { be applied to intermediate groups }\end{array}$ \\
\hline \multirow[t]{3}{*}{$\operatorname{corr}=$} & \multirow[t]{3}{*}{ Optional } & \multirow[t]{3}{*}{1.0} & \multirow[t]{3}{*}{$\begin{array}{l}\text { any number from } \\
-1.0 \text { to } 1.0\end{array}$} & Correlation between groups \\
\hline & & & & $\begin{array}{l}\text { Type of correlation applied from } \\
\text { group-to-group covariance values }\end{array}$ \\
\hline & & & & $\begin{array}{l}\text { long - correlation is applied between } \\
\text { all groups }\end{array}$ \\
\hline \multirow[t]{2}{*}{ corr_type $=$} & \multirow[t]{2}{*}{ Optional } & \multirow[t]{2}{*}{ zone } & \multirow[t]{3}{*}{ long, short, zone } & $\begin{array}{l}\text { short - correlation is applied only } \\
\text { between adjacent groups }\end{array}$ \\
\hline & & & & $\begin{array}{l}\text { zone - correlation is applied within } \\
\text { fast, intermediate, and thermal groups, } \\
\text { but no correlation is applied between } \\
\text { zones }\end{array}$ \\
\hline end & Required & & & $\begin{array}{l}\text { Denotes end of input for current } \\
\text { nuclide/reaction (must not start in } \\
\text { column 1) }\end{array}$ \\
\hline
\end{tabular}

Any MT number or reaction name will be treated as a valid input, but only those present on the response sensitivity data files will produce useful information. The available reaction sensitivity types are shown in table Reaction Sensitivity Types Computed by SAMS in the TSUNAMI-IP manual. An energycovariance matrix is created for the specified nuclide-reaction pair with the square of the entered standard deviation for the diagonal terms for all groups using the all= value. Groups in the fast, intermediate, and thermal energies are then set to the square of the standard deviation value entered for fast $=$, inter $=$, and therm $=$, respectively. The off-diagonal terms of the energy matrix are generated according to the input for corr=, and corr_type=, with default settings of 1.0 and zone. Data entered in this block do not override data present on the covariance data file. The SCALE 5.1 input format is supported where data are entered in triplets with the nuclide name or ZA identifier (e.g., u-235 or 92235), then the reaction MT name or number (e.g., 18 or fission), and then a standard deviation value. In this case, the end keyword must not be entered. These data are only used if use_icov is specified in the PARAMETER data block. When both use_icov and cov fix are specified in the PARAMETER data block, and a reaction has zero or large (standard deviation $>1000 \%$ ) values on the diagonal of the covariance matrix, these values are 
replaced with the square of the user input standard deviation value, and the corresponding off-diagonal terms are substituted according to the values of corr and corr_type.

\subsubsection{HTML block}

The optional HTML data block is used to customize HTML-formatted output. The HTML data block must begin with READ HTML and end with END HTML. The data input in the HTML data block consist of several keywords that are shown, along with their default values and descriptions, in

Table 6.6.4. A keyword that ends with "=" must be followed by text data. For color entries, any valid HTML color name can be entered or the hexadecimal representation can be used if preceded by a \# sign. For example, to change the background color of the HTML output to white, $b g_{-} c l r=w h i t e$ and $b g_{\text {c }}$ lr=\#ffffff have the same effect, because ffffff is the hexadecimal representation of white. An extensive list of available colors for customized output is shown in Appendix B of the TSUNAMI-IP chapter. Please note that not all features are supported by all browsers.

Table 6.6.4. Input data for HTML block of TSURFER

\begin{tabular}{|c|c|c|}
\hline Keyword & Default value & Description \\
\hline$b g \_c l r=$ & papayawhip & Background color. \\
\hline$h 1 \_c l r=$ & maroon & Color used for major headings. \\
\hline$h 2 \_c l r=$ & navy & Color used for sub-headings. \\
\hline$t x t \_c l r=$ & black & Color for plain text. \\
\hline $\ln k_{-} c l r=$ & navy & Color for hyperlinks. \\
\hline $\operatorname{lnk}{ }_{-} d e c=$ & none & Decoration for hyperlinks. (none, underline, overline, line-through, blink). \\
\hline$v l n k \_c l r$ & navy & Color for visited hyperlinks. \\
\hline ud_clr= & blue & Color for values in tables that use default covariance data. \\
\hline$u i \_c l r=$ & red & Color for values in tables that use user-input covariance data. \\
\hline udfix_clr= & royalblue & Color for values in tables that use default corrected covariance data. \\
\hline uifix_clr= & green & Color for values in tables that use user-input corrected covariance data. \\
\hline
\end{tabular}

\subsubsection{CORR block}

The CORR block specifies correlation coefficients between different experiment responses. When present, this block must be the last data block in the input file. The correlation block must begin with READ CORR and end with END PARAMETER. Correlation coefficients for experimental response 
uncertainties may be entered either as the total correlation coefficient for a pair of responses; or for a particular uncertainty-component shared by two responses. Values for correlation coefficients are input in the form:

$$
\text { corr_typ } \quad(i, j)=\rho \quad \ldots \ldots . . \text { repeat for } \mathrm{I}=1, \mathrm{~N}\} \quad \text { end, }
$$

where

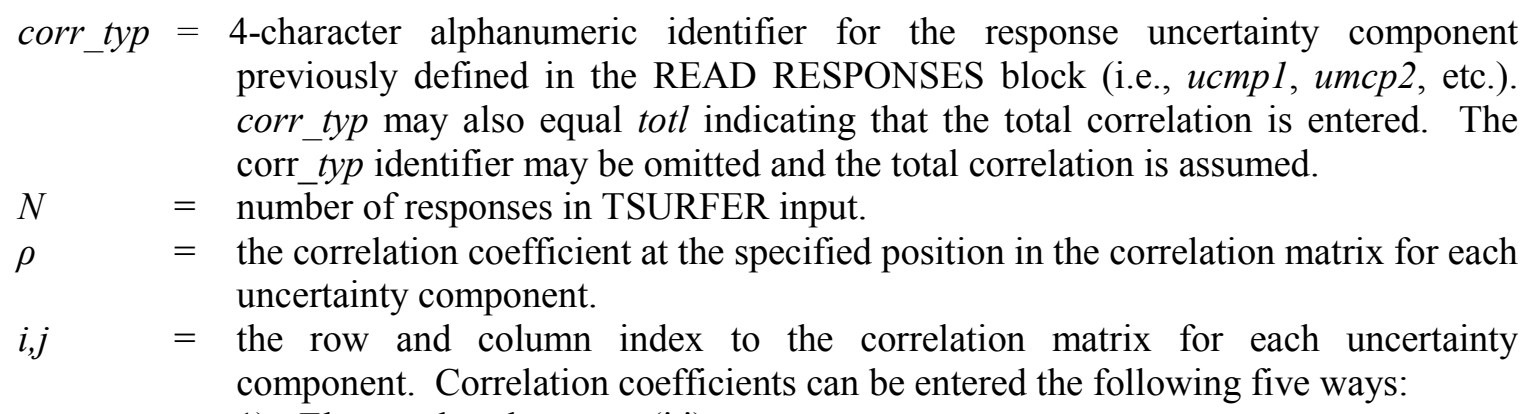

1) Element-by-element $-(\mathrm{i}, \mathrm{j})=\rho$

2) By row $-(\mathrm{i}, \mathrm{j} 1: \mathrm{j} 2)=\rho$

3) $\underline{\text { By column }}-(\mathrm{i} 1: \mathrm{j} 2, \mathrm{j})=\rho$

4) By block - (i1:i2,j1:j2) $=\rho$. This option can be used to set a large block of the correlation matrix to one number. All diagonal elements in the block are reset to 1.0 .

5) By shorthand block $-(\mathrm{i} 1: \mathrm{i} 2)=\rho$. This is identical to $(\mathrm{i} 1: \mathrm{i} 2, \mathrm{i} 1: \mathrm{i} 2)=\rho$. All diagonal elements in the block are reset to 1.0 .

TSURFER initializes each correlation matrix as an $\mathrm{N}$ by $\mathrm{N}$ identity matrix. Therefore, all uncorrelated elements (i.e., values equal to 0 ) do not have to be entered. The correlation matrix can be specified using multiple lines of input with each line having a maximum of 255 characters. As each correlation coefficient is processed, the symmetric element of the correlation matrix is assigned to the same value. Therefore only the upper or lower triangular portion of each correlation matrix must be specified. For example, given the following RESPONSE block:

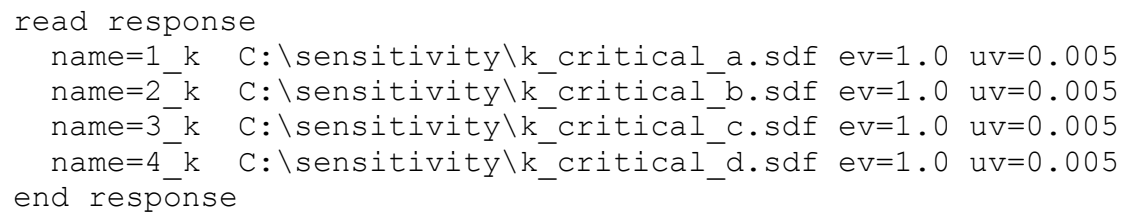

then the following forms of the CORR block are equivalent in specifying the $4 \times 4$ total correlation matrix as:

$$
\left[\begin{array}{cccc}
1 & .2 & .3 & .2 \\
.2 & 1 & .2 & .2 \\
.3 & .2 & 1 & .1 \\
.2 & .2 & .1 & 1
\end{array}\right]
$$

1) Specify the upper triangular portion of the matrix element by element: 


$$
\begin{aligned}
& \text { read } \operatorname{corr} \\
& \quad \operatorname{tot}(1,2)=.2 \quad(1,3)=.3 \quad(1,4)=.2 \quad(2,3)=.2 \quad(2,4)=.2 \quad(3,4)=.1 \text { end } \\
& \text { end } \operatorname{corr}
\end{aligned}
$$

2) Specify the lower triangular portion of the matrix element by element:

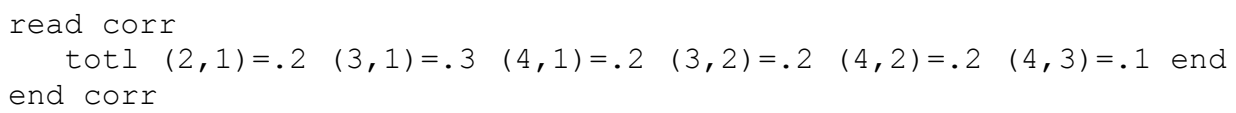

3) Use the colon character to specify multiple elements at one time in the upper triangular portion of the matrix:

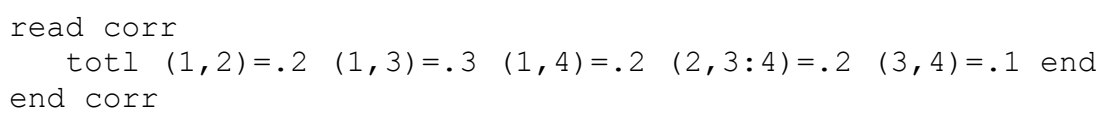

Values entered for the total correlation matrix will override any component correlations entered. Correlation coefficients may be specified in the CORR data block for responses that share one or more of the same uncertainty components. The value of corr_typ must correspond to one of the 4-character alphanumeric identifiers given to an uncertainty component. Only those uncertainty components that appear in more than one response description should be entered, since these are the only ones with correlations. An END keyword is required to terminate the data of an individual uncertainty component, and the input is repeated for each type of correlated uncertainty component.

**NOTE** The experiment covariance matrix should be positive definite to ensure a physical result for all possible sensitivities. If the input correlation values do not satisfy this constraint, a warning message is printed. Use of several fully correlated uncertainties can lead to an over-constrained system, which may result in a non-positive-definite covariance matrix. In order to help avoid this problem, correlation values usually should be limited to a maximum of 0.95, suggesting that a small random component is always present.

As an example of correlation matrices for uncertainty components, consider the following input:

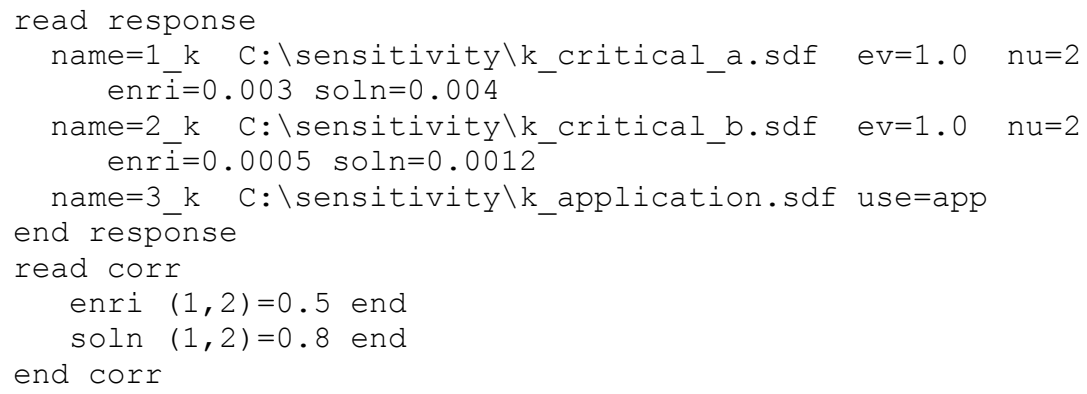

In this example, two uncertainty components are used, identified as enri and soln. Using the propagation of error formula Eq. (6.6.6), the relative standard deviation of the experiment responses $1 \_k$ and $2 \_k$ are determined as $\sqrt{ }\left(0.003^{2}+0.004^{2}\right)=0.005$ and $\sqrt{ }\left(0.0005^{2}+0.0012^{2}\right)=0.0013$, respectively. The correlation matrix enri and soln are given as $\left[\begin{array}{cc}1 & .5 \\ .5 & 1\end{array}\right]$ and $\left[\begin{array}{ll}1 & .8 \\ .8 & 1\end{array}\right]$. Using Eq. (6.6.7), the relative covariance 
between response $1 \_k$ and $2 \_k$ is calculated as: $0.003 * 0.5 * 0.0005$ (enri component) $+0.0005 * 0.8 * 0.0012$ $($ soln component $)=1.23 \mathrm{E}-6$.

\subsubsection{Sample Problem Input and Output Description}

\subsubsection{Input and text output}

An example TSURFER input is given in Figure 6.6.1 and the text output is shown in Figure 6.6.2Figure 6.6.14. In this sample problem, $40 k_{\text {eff }}$ responses are specified in the RESPONSE block, 37 experiments and 3 applications. All calculation options are turned on including the use of default and user-input covariance data, similarity filtering to the reference application, chi-square filtering for consistency, and bias convergence analysis. Each section of the text output is described in order below. Some of the figures of the text output have been truncated from their original length. The examples of output are for illustrative purposes and only demonstrate the format of the TSURFER results.

1. Echo of Input (Figure 6.6.2) - The TSURFER input data are printed for the PARAMETER, $H T M L$, and COVARIANCE data blocks. Both user-specified and default values for the various keywords are edited.

2. Covariance Warnings (Figure 6.6.3) - If the PARAMETER block keywords use_dcov and/or use_icov and/or cov_fix are entered, covariance warnings are listed that specify the nuclidereaction pairs for which approximate covariance data is applied.

3. Listing of Input Responses (Figure 6.6.4) - Various information is listed for each response. This includes the response index, name, title, adjustment role (e.g., expt), type, calculated response value, measured response value, and similarity coefficient to the reference application. The similarity coefficient column is only edited if a reference application is listed on the input.

4. Experiment Uncertainties (Figure 6.6.5) - The experiment standard deviations, as well as any input uncertainty components, are edited for each measured response. When uncertainty components are given, the total standard deviation is computed from Eq. (6.6.6).

5. Chi-square summary (Figure 6.6.6) - Different chi-squared values are edited based on the GLLS analysis. This includes the initial value of chi-squared, the target value of chi-squared based on the target_chi keyword, and the final value of chi-squared. The independent and diagonal chisquares are also edited.

6. Correlation Matrices (Figure 6.6.7-Figure 6.6.9) - Correlation matrices are printed after the chisquared edit in the following order: (1) response similarity matrix if print_sim_matrix is entered in the input, (2) the prior calculated response correlation matrix and prior measured response correlation matrix if print_init_corr is entered in the input, and (3) the adjusted response correlation matrix if print_adj_corr is entered in the input. The value of the keyword sim_type designates the type of similarity coefficient appearing in the similarity matrix. See Sect 6.6.4.3 for description of the types of similarity coefficients. The response correlation matrices are defined in Appendix A.

7. Cumulative Convergence Edit (Figure 6.6.10) - The cumulative convergence edit follows the printout of the requested correlation matrices. TSURFER only performs the cumulative convergence calculation if the keyword calc_cumul_effect is entered in the input. Four columns of data are printed that specify the cumulative range number, the maximum similarity coefficient allowed for each adjustment, the number of experiments with similarity coefficients within the 
specified range, and the computed application bias for each range, shown as o/v(A-C)/C, where A represents the adjusted $k_{\text {eff }}$ value and $\mathrm{C}$ represents the original calculated $k_{\text {eff }}$ value.

8. Summary of Adjustments (Figure 6.6.11) - The adjustment summary table is an 11-column table that summarizes the prior and posterior values of each response. The 11 columns include the response adjustment role (i.e., expt, appl, or omit), the name and type identifiers, the prior and posterior uncertainties of each response, the independent and diagonal chi-squared values, and the change in the response between the prior and posterior values.

9. Summary of Adjusted Responses (Figure 6.6.12) - Following the adjustment summary table, the adjusted values of each response are listed in tabular format. The adjusted uncertainty values of the response are also included.

10. Application and Bias Summary (Figure 6.6.13) - The application and bias summary table follows the adjusted response table. This edit is only printed if applications are specified on the TSURFER input. For each application, the following values are tabulated: the name and type of the response, the prior and posterior values of the application response, the prior and posterior values of the application uncertainty, and the application bias as determined by Eq. (6.6.33). If the application is a relative-formatted response, the fractional bias is also included in the table. Following this table, a second table is printed that lists the contribution to the reference application bias for each nuclide-reaction pair used in the analysis. The nuclide-reaction pairs are listed in descending order based on the fraction of bias L1-norm, defined as

$$
f_{x}=\frac{\sum_{g}\left|S_{x, g} \Delta \alpha_{x, g}\right|}{\sum_{x^{\prime}} \sum_{g}\left|S_{x^{\prime}, g} \Delta \alpha_{x^{\prime}, g}\right|} .
$$

11. Multigroup Cross-Section Adjustment Table (Figure 6.6.14) - The Multigroup cross-section adjustment tables are printed if the print_adjustments keyword is included in the TSURFER input. For each nuclide-reaction pair, a table is printed that includes the relative adjustment of each multigroup cross-section, and the prior and posterior values of the cross-section uncertainty. If an application is included in the TSURFER input, the bias contribution and fraction of bias L1norm are also edited. The order of the nuclide-reaction pairs is determined by the fraction of bias L1-norm. 


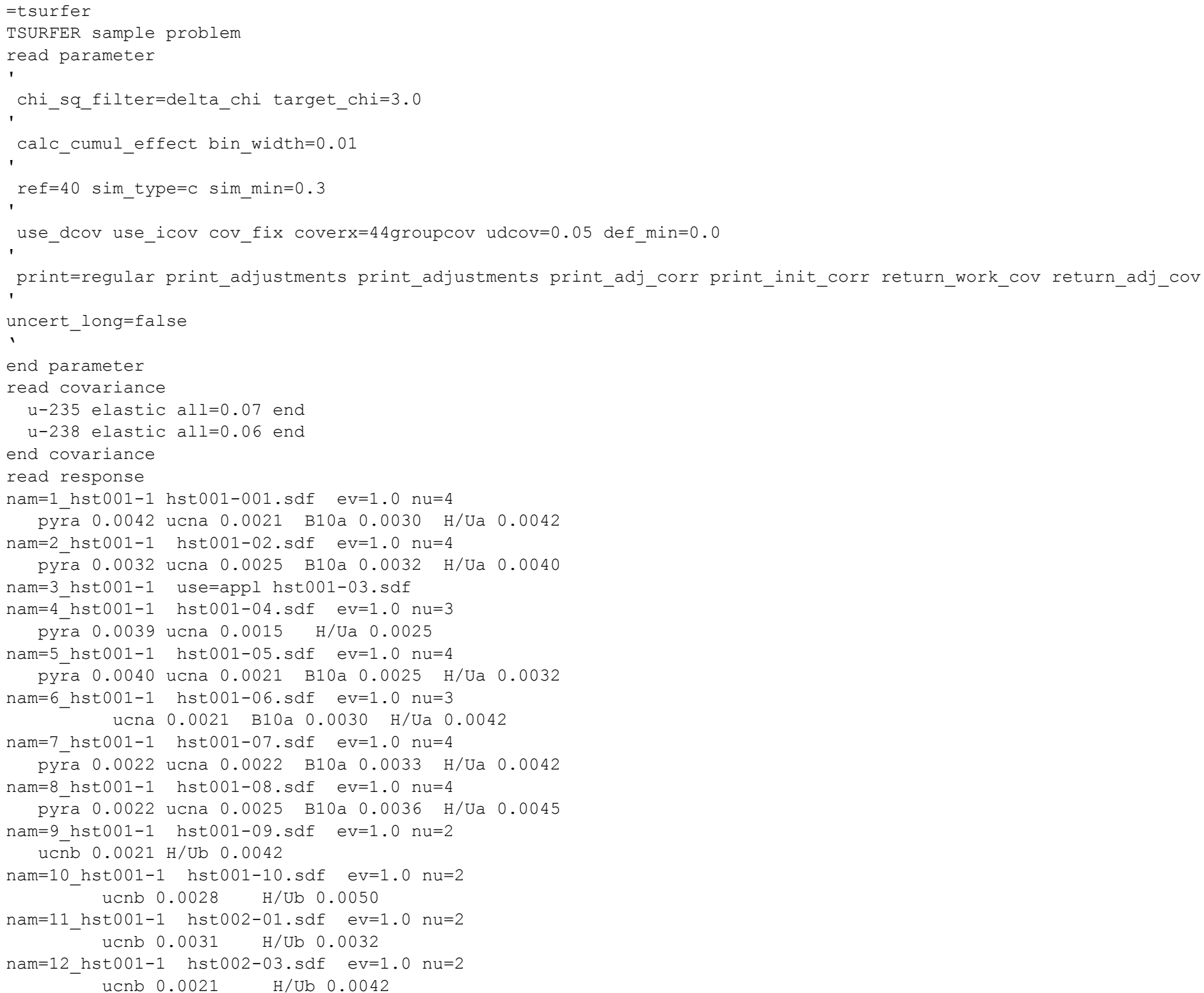

Figure 6.6.1. TSURFER sample input. 
nam=13 hst001-1 hst002-09.sdf ev=1.0 nu=2 ucnb $0.0021 \mathrm{H} / \mathrm{Ub} 0.0042$

nam=14 hst001-1 hst003-03.sdf ev=1.0 nu=2 ucnb $0.0011 \mathrm{H} / \mathrm{Ub} 0.0022$

nam=15 hst001-1 hst003-08.sdf ev=1.0 nu=2 ucnb $0.0021 \quad \mathrm{H} / \mathrm{Ub} \quad 0.0042$

nam=16 hst001-1 hst003-18.sdf ev=1.0 nu=2 ucnb $0.0021 \quad \mathrm{H} / \mathrm{Ub} 0.0042$

nam=17 hst001-1 hst004-003.sdf ev=1.0 nu=2 ucnb $0.0021 \quad \mathrm{H} / \mathrm{Ub} 0.0042$

nam=18_hst001-1 hst021-030.sdf ev=1.0 nu=2 ucnb $0.0021 \mathrm{H} / \mathrm{Ub} 0.0042$

nam=19_hst001-1 hst025-02.sdf ev=1.0 nu=2 ucnb $0.0021 \mathrm{H} / \mathrm{Ub} 0.0042$

nam $=20$ hst001-1 hst025-04.sdf ev=1.0 nu=2 ucnb 0.0021 H/Ub 0.0042

nam=21_hst001-1 hst025-05.sdf ev=1.0 nu=4

pyrc 0.0042 ucnc $0.0021 \mathrm{~B} 10 \mathrm{C} 0.0030 \mathrm{H} / \mathrm{UC}_{\mathrm{C}} 0.0042$

nam=22 hst001-1 hst027-01.sdf ev=1.0 nu=4

pyrc 0.0045 ucnc 0.0025 B10C 0.0033 H/UC 0.0040 nam=23_hst001-1 hst29i-01.sdf ev=1.0 nu=4

pyrc 0.0039 ucnc 0.0021 B10c $0.0030 \mathrm{H} / \mathrm{Uc} 0.0045$

nam=24 hst001-1 hst29i-02.sdf ev=1.0 nu=4

pyrc 0.0042 ucnc 0.0030 B10C 0.0020 H/UC 0.0022 nam=25_hst001-1 hst29i-03.sdf ev=1.0 nu=4

pyrc 0.0042 ucnc 0.0021 B10c 0.0030 H/Uc 0.0042

nam=26 hst001-1 hst29i-04.sdf ev=1.0 nu=4

pyrc 0.0022 ucnc 0.0031 B10C 0.0040 H/UC 0.0036

nam=27_hst001-1 hst29i-05.sdf ev=1.0 nu=4

pyrc 0.0042 ucnc $0.0021 \mathrm{~B} 10 \mathrm{C} 0.0030 \mathrm{H} / \mathrm{UC} 0.0042$

nam=28 hst001-1 hst29i-06.sdf ev=1.0 nu=4

pyrc 0.0032 ucnc 0.0021 B10c 0.0045 H/Uc 0.0032

nam=29 hst001-1 hst29i-07.sdf ev=1.0 nu=4

pyrc 0.0042 ucnc $0.0025 \mathrm{~B} 10 \mathrm{C} 0.0030 \mathrm{H} / \mathrm{UC} 0.0036$

nam=30 hst001-1 hst30i-01.sdf ev=1.0 nu=3

ucnc 0.0021 B10C $0.0030 \mathrm{H} / \mathrm{Uc} 0.0042$

nam=31_hst001-1 hst30i-02.sdf ev=1.0 nu=3

ucnc 0.0031 B10C $0.0030 \mathrm{H} / \mathrm{UC} \quad 0.0042$

nam=32 hst001-1 use=appl hst30i-03.sdf ev=1.0 uv=0.003

nam=33 hst001-1 hst30i-04.sdf ev=1.0 nu=3

ucnc 0.0031 B10C 0.0040 H/UC 0.0038

nam=34_hst001-1 hst30i-05.sdf ev=1.0 nu=3

ucnc $0.0021 \mathrm{~B} 10 \mathrm{C} 0.0030 \mathrm{H} / \mathrm{Uc} 0.0042$

nam=35 hst001-1 hst30i-06.sdf ev=1.0 nu=3

ucnc 0.0025 B10 C $0.0040 \mathrm{H} / \mathrm{UC} 0.00436$

Figure 6.6.1. TSURFER sample input (continued). 


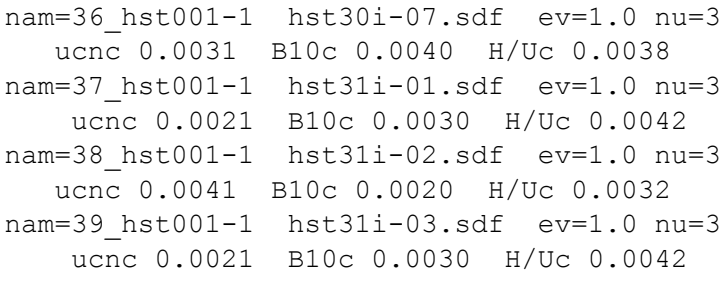

pyra $\quad(1,2)=0.2 \quad(1,4)=0.6 \quad(1,5)=0.5 \quad(1,7)=0.2 \quad(1,8)=0.6$ $(2,4)=0.6 \quad(2,5)=0.5 \quad(2,7)=0.2 \quad(2,8)=0.6 \quad(4,5)=0.6$ $(4,7)=0.5 \quad(4,8)=0.5 \quad(5,7)=0.2 \quad(5,8)=0.6 \quad(7,8)=0.6 \quad$ end

ucna $(1,2)=0.2 \quad(1,4)=0.2 \quad(1,5)=0.2 \quad(1,6)=0.2 \quad(1,7)=0.2$ $(1,8)=0.2 \quad(2,4)=0.2 \quad(2,5)=0.2 \quad(2,6)=0.2 \quad(2,7)=0.2$ $(2,8)=0.2 \quad(4,5)=0.6 \quad(4,6)=0.6 \quad(4,7)=0.5 \quad(4,8)=0.5$ $(5,6)=0.8 \quad(5,7)=0.2 \quad(5,8)=0.6 \quad(6,7)=0.8 \quad(6,8)=0.6$ $(7,8)=0.6 \quad(5,7)=0.2(5,8)=0.6 \quad(6,7)=0.8 \quad(6,8)=0.6$

$\mathrm{H} / \mathrm{Ua} \quad(1,2)=0.2 \quad(1,4)=0.2 \quad(1,5)=0.2 \quad(1,6)=0.2 \quad(1,7)=0.2$ $(1,8)=0.2 \quad(2,4)=0.2 \quad(2,5)=0.2 \quad(2,6)=0.2 \quad(2,7)=0.2$ $(2,8)=0.2 \quad(4,5)=0.6 \quad(4,6)=0.6 \quad(4,7)=0.5 \quad(4,8)=0.5$ $(5,6)=0.8 \quad(5,7)=0.2 \quad(5,8)=0.6 \quad(6,7)=0.8 \quad(6,8)=0.6$ $(7,8)=0.6$ $(9,15)=0.2 \quad(9,16)=0.2 \quad(9,17)=0.2 \quad(9,18)=0.2 \quad(9,19)=0.2$ $(9,20)=0.2 \quad(10,11)=0.2 \quad(10,12)=0.2 \quad(10,13)=0.2$ $(10,14)=0.2 \quad(10,15)=0.2 \quad(10,16)=0.2 \quad(10,17)=0.2$ $(10,18)=0.2 \quad(10,19)=0.2 \quad(10,20)=0.2 \quad(11,12)=0.2$ $(11,13)=0.2 \quad(11,14)=0.2 \quad(11,15)=0.2 \quad(11,16)=0.2$ $(11,17)=0.2 \quad(11,18)=0.2 \quad(11,19)=0.2 \quad(11,20)=0.2$ $(12,13)=0.2 \quad(12,14)=0.2 \quad(12,15)=0.2 \quad(12,16)=0.2$ $(12,17)=0.2 \quad(12,18)=0.2 \quad(12,19)=0.2 \quad(12,20)=0.2$ $(13,14)=0.2 \quad(13,15)=0.2 \quad(13,16)=0.2 \quad(13,17)=0.2$ $(13,18)=0.2 \quad(13,19)=0.2 \quad(13,20)=0.2 \quad(14,15)=0.2$ $(14,16)=0.2 \quad(14,17)=0.2 \quad(14,18)=0.2 \quad(14,19)=0.2$ $(14,20)=0.2 \quad(15,16)=0.2 \quad(15,17)=0.2 \quad(15,18)=0.2$ $(15,19)=0.2 \quad(15,20)=0.2 \quad(16,17)=0.2 \quad(16,18)=0.2$ $(16,19)=0.2 \quad(16,20)=0.2 \quad(17,18)=0.2 \quad(17,19)=0.2$

$\mathrm{H} / \mathrm{Ub} \quad(9,10)=0.2 \quad(9,11)=0.2 \quad(9,12)=0.2 \quad(9,13)=0.2 \quad(9,14)=0.2$ $(9,15)=0.2 \quad(9,16)=0.2 \quad(9,17)=0.2 \quad(9,18)=0.2 \quad(9,19)=0.2$ $(9,20)=0.2 \quad(10,11)=0.2 \quad(10,12)=0.2 \quad(10,13)=0.2$ $(10,14)=0.2 \quad(10,15)=0.2 \quad(10,16)=0.2$

Figure 6.6.1. TSURFER sample input (continued). 
$(10,18)=0.2 \quad(10,19)=0.2 \quad(10,20)=0.2 \quad(11,12)=0.2$

$(11,13)=0.2 \quad(11,14)=0.2 \quad(11,15)=0.2 \quad(11,16)=0.2$

$(11,17)=0.2 \quad(11,18)=0.2 \quad(11,19)=0.2 \quad(11,20)=0.2$

$(12,13)=0.2 \quad(12,14)=0.2 \quad(12,15)=0.2 \quad(12,16)=0.2$

$(12,17)=0.2 \quad(12,18)=0.2 \quad(12,19)=0.2 \quad(12,20)=0.2$

$(13,14)=0.2 \quad(13,15)=0.2 \quad(13,16)=0.2 \quad(13,17)=0.2$

$(13,18)=0.2 \quad(13,19)=0.2 \quad(13,20)=0.2 \quad(14,15)=0.2$

$(14,16)=0.2 \quad(14,17)=0.2 \quad(14,18)=0.2 \quad(14,19)=0.2$

$(14,20)=0.2 \quad(15,16)=0.2 \quad(15,17)=0.2 \quad(15,18)=0.2$

$(15,19)=0.2 \quad(15,20)=0.2 \quad(16,17)=0.2 \quad(16,18)=0.2$

$(16,19)=0.2 \quad(16,20)=0.2 \quad(17,18)=0.2 \quad(17,19)=0.2$

$(17,20)=0.2 \quad(18,19)=0.2 \quad(19,20)=0.2$

end

ucnc $(21: 31,21: 31)=0.2 \quad(21: 31,33: 39)=0.2 \quad(33: 39,33: 39)=0.2$ end

end corr

end

Figure 6.6.1. TSURFER sample input (continued). 


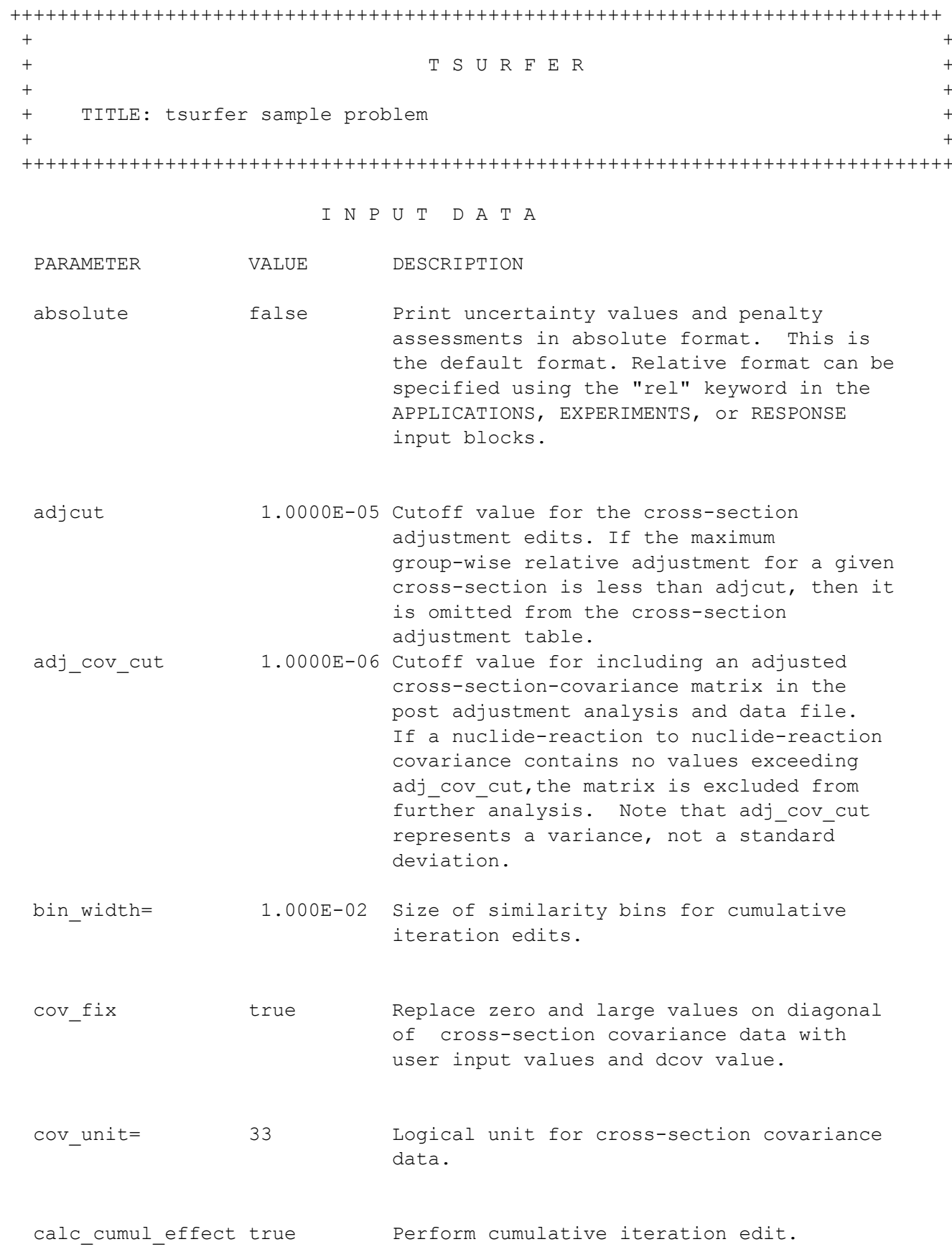

Figure 6.6.2. Echo of TSURFER input parameters. 


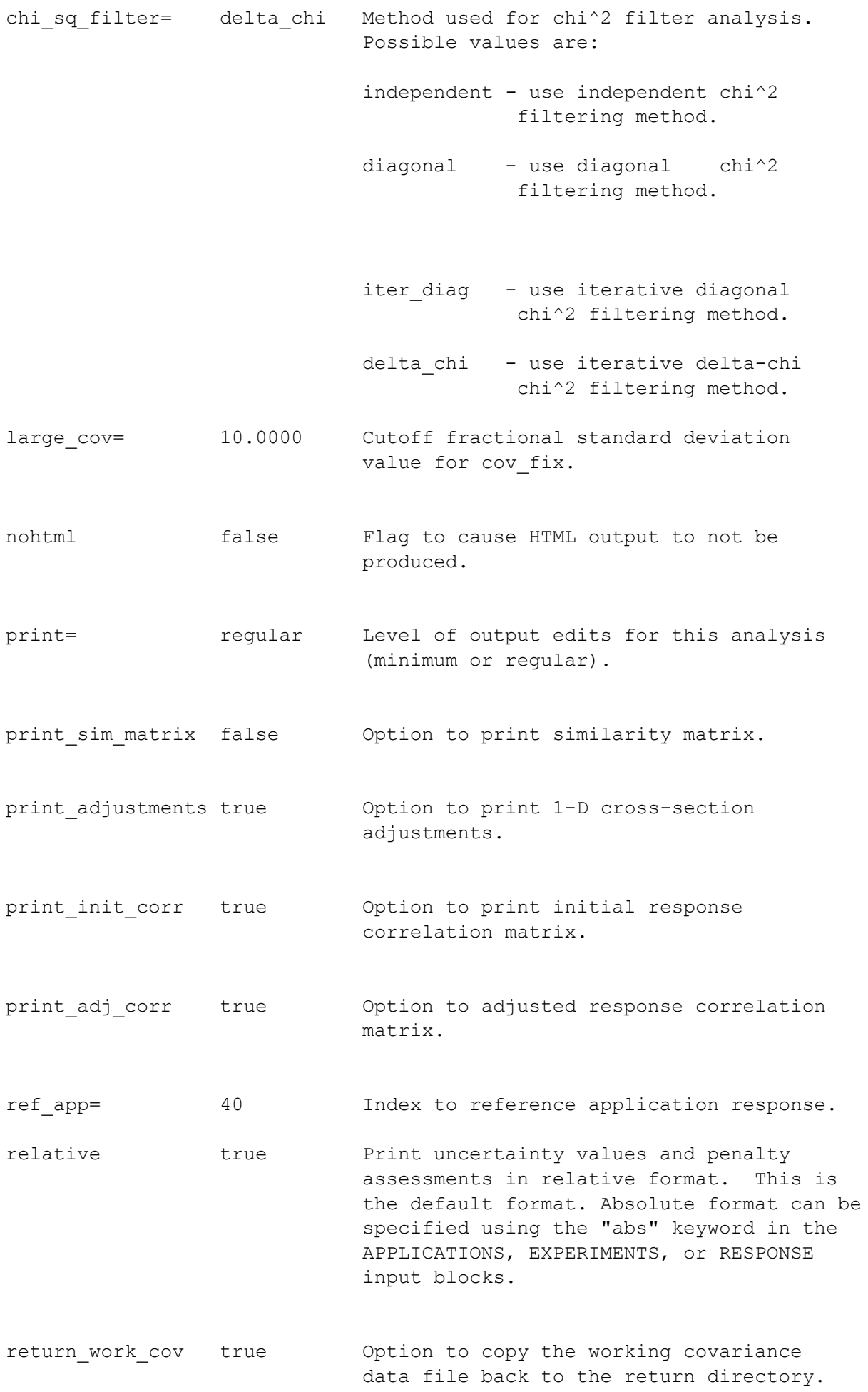

Figure 6.6.2. Echo of TSURFER input parameters (continued). 


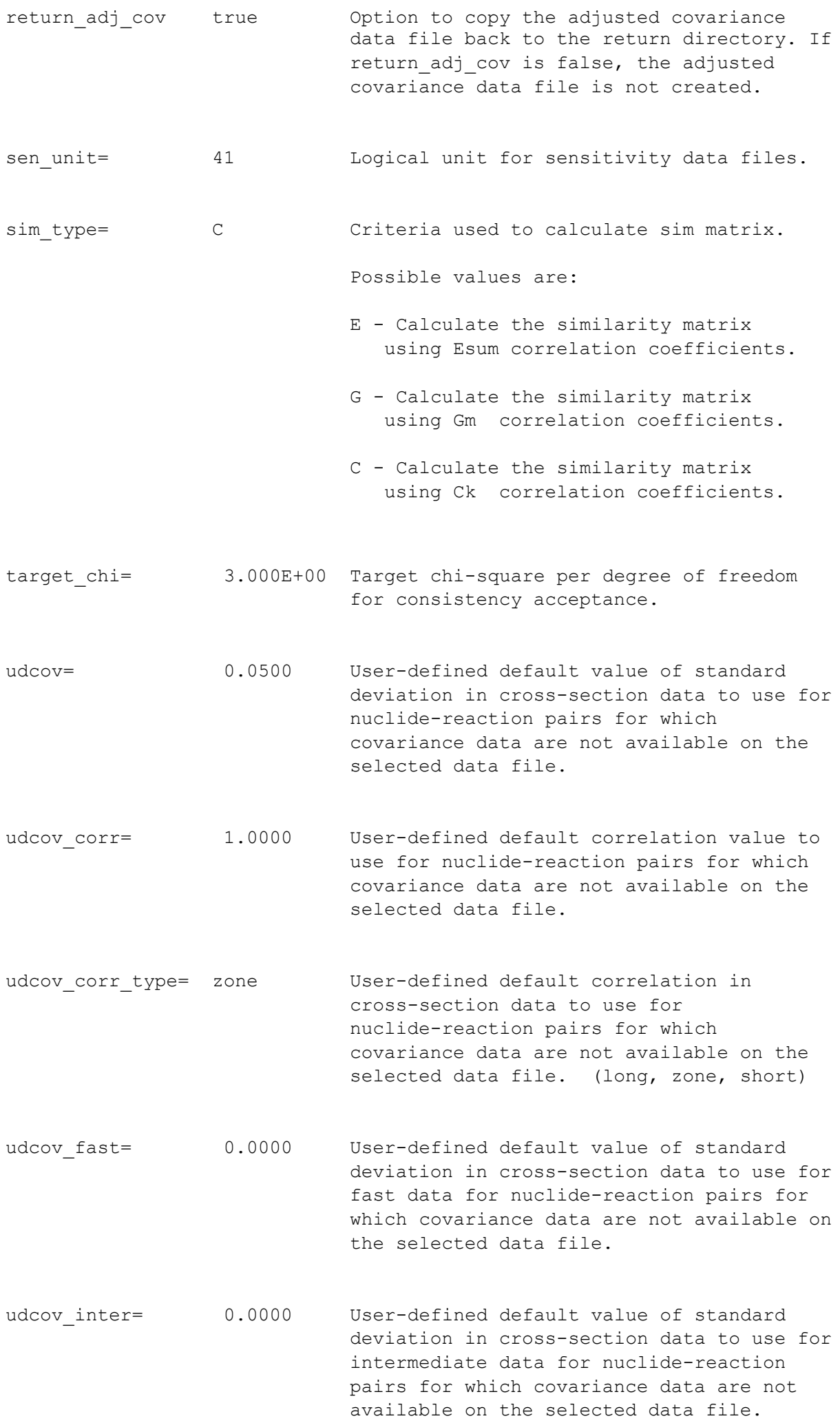

Figure 6.6.2. Echo of TSURFER input parameters (continued). 


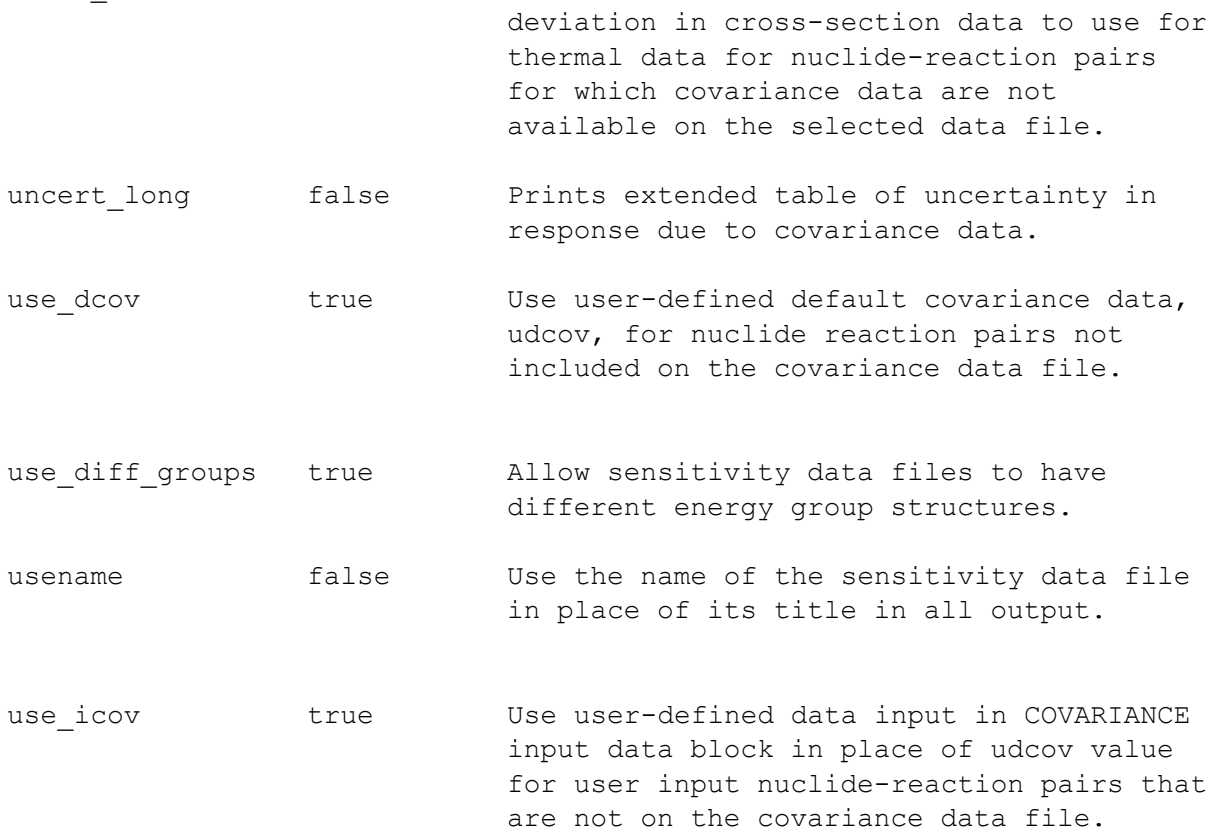

USER COVARIANCE DATA

\begin{tabular}{|c|c|c|c|c|c|c|c|c|c|}
\hline $\mathrm{ZA}$ & NUCLIDE & REACTION & $\mathrm{MT}$ & ALL & THERMAL & INTER & FAST & CORREL & TYPE \\
\hline------ & ------- & -------- & ---- & ---------- & ---------- & ---------- & ---------- & -------- & ------ \\
\hline 92235 & $u-235$ & elastic & 2 & 7. $00 \mathrm{E}-02$ & $0.00 \mathrm{E}+00$ & $0.00 \mathrm{E}+00$ & $0.00 \mathrm{E}+00$ & 1.00 & zone \\
\hline 92238 & $u-238$ & elastic & 2 & $6.00 E-02$ & $0.00 \mathrm{E}+00$ & $0.00 \mathrm{E}+00$ & $0.00 \mathrm{E}+00$ & 1.00 & zone \\
\hline
\end{tabular}

HTML Format Options

$\begin{array}{cll}\text { PARAMETER } & \text { VALUE } & \text { DESCRIPTION } \\ \text { bg_clr= } & \text { papayawhip } & \text { Background color } \\ \text { hl_clr= } & \text { maroon } & \text { Color used for major headings } \\ \text { txt_clr= } & \text { navy } & \text { Color used for sub-headings } \\ \text { lnk_clr= } & \text { black } & \text { Color for plain text } \\ \text { lnk_dec }= & \text { navy } & \text { Color for hyperlinks } \\ \text { through, blink) } & \text { none } & \text { Decoration for hyperlinks (none, underline, overline, line- } \\ \text { vlnk_clr= } & \text { navy } & \text { Color for visited hyperlinks }\end{array}$

Figure 6.6.2. Echo of TSURFER input parameters (continued). 


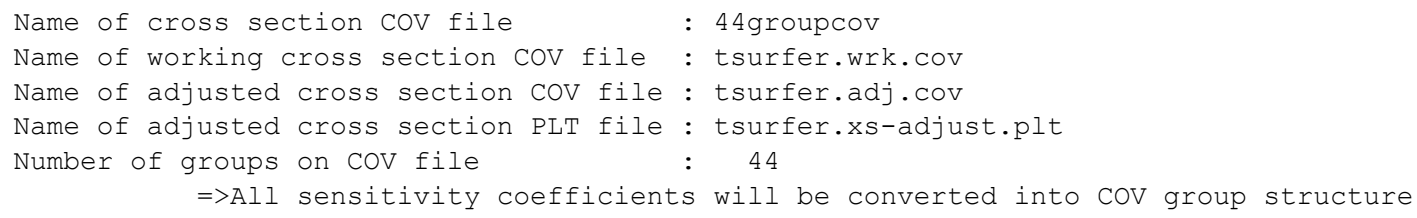

Figure 6.6.2. Echo of TSURFER input parameters (continued).

WARNING: cov_fix applied for b-10 n, p

Default standard deviation data value 0.0500 will replace 0.0000 for group 15 Default standard deviation data value 0.0500 will replace 0.0000 for group 16 Default standard deviation data value 0.0500 will replace 0.0000 for group 17 Default standard deviation data value 0.0500 will replace 0.0000 for group 18 Default standard deviation data value 0.0500 will replace 0.0000 for group 19 Default standard deviation data value 0.0500 will replace 0.0000 for group 20 Default standard deviation data value 0.0500 will replace 0.0000 for group 21 Default standard deviation data value 0.0500 will replace 0.0000 for group 22 Default standard deviation data value 0.0500 will replace 0.0000 for group 23 Default standard deviation data value 0.0500 will replace 0.0000 for group 24 Default standard deviation data value 0.0500 will replace 0.0000 for group 25 Default standard deviation data value 0.0500 will replace 0.0000 for group 26 Default standard deviation data value 0.0500 will replace 0.0000 for group 27 Default standard deviation data value 0.0500 will replace 0.0000 for group 28 Default standard deviation data value 0.0500 will replace 0.0000 for group 29 Default standard deviation data value 0.0500 will replace 0.0000 for group 30 Default standard deviation data value 0.0500 will replace 0.0000 for group 31 Default standard deviation data value 0.0500 will replace 0.0000 for group 32 Default standard deviation data value 0.0500 will replace 0.0000 for group 33 Default standard deviation data value 0.0500 will replace 0.0000 for group 34 Default standard deviation data value 0.0500 will replace 0.0000 for group 35 Default standard deviation data value 0.0500 will replace 0.0000 for group 36 Default standard deviation data value 0.0500 will replace 0.0000 for group 37 Default standard deviation data value 0.0500 will replace 0.0000 for group 38 Default standard deviation data value 0.0500 will replace 0.0000 for group 39 Default standard deviation data value 0.0500 will replace 0.0000 for group 40 Default standard deviation data value 0.0500 will replace 0.0000 for group 41 Default standard deviation data value 0.0500 will replace 0.0000 for group 42 Default standard deviation data value 0.0500 will replace 0.0000 for group 43 Default standard deviation data value 0.0500 will replace 0.0000 for group 44

$\cdots$

Working covariance matrix created for future processing.

Figure 6.6.3. TSURFER covariance warnings edit. 
Number of Input Sensitivity Files $=40$

$=>$ Number of Applications (passive) Included in GLLSM: 3

$=>$ Number of Benchmarks (active) Included in GLLSM : 37

=>Number of Responses (active+passive) used in GLLSM: 40

=>Number of Input Systems Omitted from GLLSM(*) : 0

** Description of Prior Responses **

RESP.\# EXPERIMENT NAME SENS. TITLE USE

REFERENCE APPLICATION

\begin{tabular}{|c|c|c|c|c|c|c|c|}
\hline 1 & 1 hst001-1 & $r 1$ & expt & keff & $1.0015 \mathrm{E}+00$ & $1.0000 \mathrm{E}+00$ & $9.597 \mathrm{E}-01$ \\
\hline 2 & 2 hst001-1 & $r 2$ & expt & $\operatorname{keff}$ & $9.9852 \mathrm{E}-01$ & $1.0000 \mathrm{E}+00$ & $9.569 \mathrm{E}-01$ \\
\hline 3 & 3-hst001-1 & r3 & appl & keff & $1.0024 \mathrm{E}+00$ & $<(N A)\rangle$ & $9.589 \mathrm{E}-01$ \\
\hline 4 & 4 hst $001-1$ & r4 & expt & keff & $1.0008 \mathrm{E}+00$ & $1.0000 \mathrm{E}+00$ & $9.558 \mathrm{E}-01$ \\
\hline 5 & 5 hst $001-1$ & $r 5$ & expt & keff & $1.0011 \mathrm{E}+00$ & $1.0000 \mathrm{E}+00$ & $9.640 \mathrm{E}-01$ \\
\hline 6 & 6_hst $001-1$ & $r 6$ & expt & keff & $1.0046 \mathrm{E}+00$ & $1.0000 \mathrm{E}+00$ & $9.640 \mathrm{E}-01$ \\
\hline 7 & 7_hst001-1 & $r 7$ & expt & $\operatorname{keff}$ & $9.9994 \mathrm{E}-01$ & $1.0000 \mathrm{E}+00$ & $9.593 \mathrm{E}-01$ \\
\hline 8 & 8 hst $001-1$ & r8 & expt & keff & $9.9975 E-01$ & $1.0000 \mathrm{E}+00$ & $9.589 \mathrm{E}-01$ \\
\hline 9 & 9-hst001-1 & r9 & expt & keff & $9.9634 \mathrm{E}-01$ & $1.0000 \mathrm{E}+00$ & $9.561 \mathrm{E}-01$ \\
\hline 10 & $1 \overline{0}$ hst $001-1$ & r10 & expt & $\operatorname{keff}$ & $9.9521 E-01$ & $1.0000 \mathrm{E}+00$ & $9.660 \mathrm{E}-01$ \\
\hline 11 & 11 hst001-1 & rot2 tank in cen & expt & keff & $1.0046 \mathrm{E}+00$ & $1.0000 \mathrm{E}+00$ & $9.723 E-01$ \\
\hline 12 & 12_hst001-1 & rot 7 tank in cen & expt & $\operatorname{keff}$ & $1.0010 \mathrm{E}+00$ & $1.0000 \mathrm{E}+00$ & $9.609 \mathrm{E}-01$ \\
\hline 13 & 13_hst001-1 & rot 38 tank in ce & expt & $\operatorname{keff}$ & $1.0009 \mathrm{E}+00$ & $1.0000 \mathrm{E}+00$ & $9.661 \mathrm{E}-01$ \\
\hline 14 & 14 hst $001-1$ & rot 4 tank in cen & expt & keff & $1.0019 \mathrm{E}+00$ & $1.0000 \mathrm{E}+00$ & $9.638 \mathrm{E}-01$ \\
\hline 15 & 15-hst $001-1$ & rot 14 tank in ce & expt & keff & $1.0049 \mathrm{E}+00$ & $1.0000 \mathrm{E}+00$ & $9.621 \mathrm{E}-01$ \\
\hline 16 & 16_hst001-1 & rot29 tank in ce & expt & $\operatorname{keff}$ & $9.9888 \mathrm{E}-01$ & $1.0000 \mathrm{E}+00$ & $9.641 \mathrm{E}-01$ \\
\hline 17 & 17 hst001-1 & ol3ne 15.5 in. s & expt & keff & $1.0031 \mathrm{E}+00$ & $1.0000 \mathrm{E}+00$ & $3.882 E-01$ \\
\hline 18 & 18_hst001-1 & case 30 experime & expt & $\operatorname{keff}$ & $9.9962 \mathrm{E}-01$ & $1.0000 \mathrm{E}+00$ & $9.858 \mathrm{E}-01$ \\
\hline 19 & 19_hst001-1 & heu-sol-therm-02 & expt & $\operatorname{keff}$ & $1.0023 E+00$ & $1.0000 \mathrm{E}+00$ & $9.720 \mathrm{E}-01$ \\
\hline 20 & 20_hst001-1 & heu-sol-therm-02 & expt & $\operatorname{keff}$ & $1.0031 E+00$ & $1.0000 \mathrm{E}+00$ & $9.744 \mathrm{E}-01$ \\
\hline 21 & 21_hst001-1 & heu-sol-therm-02 & expt & $\operatorname{keff}$ & $1.0060 \mathrm{E}+00$ & $1.0000 \mathrm{E}+00$ & $9.767 \mathrm{E}-01$ \\
\hline 22 & 22_hst001-1 & heu-sol-therm-02 & expt & $\operatorname{keff}$ & $9.9845 \mathrm{E}-01$ & $1.0000 \mathrm{E}+00$ & $9.660 \mathrm{E}-01$ \\
\hline 23 & 23-hst001-1 & heu-sol-therm-02 & expt & $\operatorname{keff}$ & $1.0050 \mathrm{E}+00$ & $1.0000 \mathrm{E}+00$ & $9.828 \mathrm{E}-01$ \\
\hline 24 & 24_hst001-1 & heu-sol-therm-02 & expt & $\operatorname{keff}$ & $1.0086 \mathrm{E}+00$ & $1.0000 \mathrm{E}+00$ & $9.937 \mathrm{E}-01$ \\
\hline 25 & 25_hst001-1 & heu-sol-therm-02 & expt & $\operatorname{keff}$ & $1.0014 \mathrm{E}+00$ & $1.0000 \mathrm{E}+00$ & $9.952 E-01$ \\
\hline 26 & 26_hst001-1 & heu-sol-therm-02 & expt & $\operatorname{keff}$ & $9.9857 E-01$ & $1.0000 \mathrm{E}+00$ & $9.988 \mathrm{E}-01$ \\
\hline 27 & 27_hst001-1 & heu-sol-therm-02 & expt & $\operatorname{keff}$ & $1.0045 \mathrm{E}+00$ & $1.0000 \mathrm{E}+00$ & $9.989 \mathrm{E}-01$ \\
\hline 28 & 28_hst001-1 & heu-sol-therm-02 & expt & $\operatorname{keff}$ & $1.0051 \mathrm{E}+00$ & $1.0000 \mathrm{E}+00$ & $9.971 \mathrm{E}-01$ \\
\hline 29 & 29_hst001-1 & heu-sol-therm-02 & expt & $\operatorname{keff}$ & $1.0057 \mathrm{E}+00$ & $1.0000 \mathrm{E}+00$ & $9.931 \mathrm{E}-01$ \\
\hline 30 & 30_hst001-1 & heu-sol-therm-03 & expt & $\operatorname{keff}$ & $9.9998 \mathrm{E}-01$ & $1.0000 \mathrm{E}+00$ & $9.798 \mathrm{E}-01$ \\
\hline 31 & 31_hst001-1 & heu-sol-therm-03 & expt & $\operatorname{keff}$ & $1.0014 \mathrm{E}+00$ & $1.0000 \mathrm{E}+00$ & $9.862 \mathrm{E}-01$ \\
\hline 32 & 32_hst001-1 & heu-sol-therm-03 & appl & $\operatorname{keff}$ & $9.9990 E-01$ & $<(N A)>$ & $9.852 \mathrm{E}-01$ \\
\hline 33 & 33_hst001-1 & heu-sol-therm-03 & expt & $\operatorname{keff}$ & $1.0083 \mathrm{E}+00$ & $1.0000 \mathrm{E}+00$ & $9.820 \mathrm{E}-01$ \\
\hline 34 & 34_hst001-1 & heu-sol-therm-03 & expt & keff & $1.0036 \mathrm{E}+00$ & $1.0000 \mathrm{E}+00$ & $9.910 \mathrm{E}-01$ \\
\hline 35 & 35_hst001-1 & heu-sol-therm-03 & expt & $\operatorname{keff}$ & $1.0048 \mathrm{E}+00$ & $1.0000 \mathrm{E}+00$ & $9.936 \mathrm{E}-01$ \\
\hline 36 & 36_hst001-1 & heu-sol-therm-03 & expt & $\operatorname{keff}$ & $1.0029 \mathrm{E}+00$ & $1.0000 \mathrm{E}+00$ & $9.979 \mathrm{E}-01$ \\
\hline 37 & 37_hst001-1 & heu-sol-therm-03 & expt & $\operatorname{keff}$ & $1.0045 \mathrm{E}+00$ & $1.0000 \mathrm{E}+00$ & $9.944 \mathrm{E}-01$ \\
\hline 38 & 38_hst001-1 & heu-sol-therm-03 & expt & $\operatorname{keff}$ & $1.0051 E+00$ & $1.0000 \mathrm{E}+00$ & $9.978 \mathrm{E}-01$ \\
\hline 39 & 39_hst001-1 & heu-sol-therm-03 & expt & $\operatorname{keff}$ & $1.0043 \mathrm{E}+00$ & $1.0000 \mathrm{E}+00$ & $9.967 \mathrm{E}-01$ \\
\hline 40 & 40_hst001-1 & heu-sol-therm-03 & appl & keff & $1.0017 \mathrm{E}+00$ & $<(\mathrm{NA})>$ & $1.000 \mathrm{E}+00$ \\
\hline 31 & 31_hst001-1 & heu-sol-therm-03 & expt & $\operatorname{keff}$ & $9.9987 \mathrm{E}-01$ & 1.0000E+00 & $9.565 \mathrm{E}-01$ \\
\hline 32 & 32_hst001-1 & heu-sol-therm-03 & appl & $\operatorname{keff}$ & $9.9894 \mathrm{E}-01$ & $<(\mathrm{NA})\rangle$ & $9.527 \mathrm{E}-01$ \\
\hline 33 & 33_hst001-1 & heu-sol-therm-03 & expt & $\operatorname{keff}$ & $1.0069 \mathrm{E}+00$ & $1.0000 \mathrm{E}+00$ & $9.782 \mathrm{E}-01$ \\
\hline 34 & 34_hst001-1 & heu-sol-therm-03 & expt & $\operatorname{keff}$ & $1.0033 E+00$ & $1.0000 \mathrm{E}+00$ & $9.820 \mathrm{E}-01$ \\
\hline 35 & 35_hst001-1 & heu-sol-therm-03 & expt & $\operatorname{keff}$ & $1.0042 \mathrm{E}+00$ & $1.0000 \mathrm{E}+00$ & $9.831 \mathrm{E}-01$ \\
\hline 36 & 36_hst001-1 & heu-sol-therm-03 & expt & $\operatorname{keff}$ & $1.0026 \mathrm{E}+00$ & $1.0000 \mathrm{E}+00$ & $9.878 \mathrm{E}-01$ \\
\hline 37 & 37_hst001-1 & heu-sol-therm-03 & expt & $\operatorname{keff}$ & $1.0039 \mathrm{E}+00$ & $1.0000 \mathrm{E}+00$ & $9.842 \mathrm{E}-01$ \\
\hline 38 & 38_hst001-1 & heu-sol-therm-03 & expt & $\operatorname{keff}$ & $1.0040 \mathrm{E}+00$ & $1.0000 \mathrm{E}+00$ & $9.800 \mathrm{E}-01$ \\
\hline 39 & 39_hst001-1 & heu-sol-therm-03 & expt & $\operatorname{keff}$ & $1.0028 \mathrm{E}+00$ & $1.0000 \mathrm{E}+00$ & $9.868 \mathrm{E}-01$ \\
\hline 40 & 40_hst001-1 & $\begin{array}{r}\text { heu-sol-therm-03 } \\
\text { ave. calc } \\
\text { sample star } \\
\text { Chi-fissior }\end{array}$ & $\begin{array}{l}\text { appl } \\
\text { exp }\end{array}$ & $\begin{array}{l}\text { keff } \\
\text { alue }\end{array}$ & $\begin{array}{l}1.0007 E+00 \\
-E \mid=3.122 \\
-E \mid=2.2236\end{array}$ & $\begin{aligned} &<(N A)> \\
& E-03 \\
& E-03\end{aligned}$ & $1.000 \mathrm{E}+00$ \\
\hline
\end{tabular}

Figure 6.6.4. TSURFER response list edit. 
** Input Experiment Response Uncertainties **

RESP.\# TYPE UNC. UNITS

\begin{tabular}{|c|c|c|}
\hline ff & $\because 0$ & \\
\hline keff & $\frac{\circ}{0}$ & $\mathrm{dk} / \mathrm{k}$ \\
\hline reff & $\frac{\circ}{0}$ & $\mathrm{dk} / \mathrm{k}$ \\
\hline keff & $\frac{\circ}{0}$ & $\mathrm{dk} / \mathrm{k}$ \\
\hline keff & $\div$ & $\mathrm{dk} / \mathrm{k}$ \\
\hline keff & $\%$ & $\mathrm{dk} / \mathrm{k}$ \\
\hline keff & $\frac{\circ}{0}$ & $\mathrm{dk} / \mathrm{k}$ \\
\hline keff & $\frac{\circ}{0}$ & $\mathrm{dk} / \mathrm{k}$ \\
\hline keff & $\frac{\circ}{0}$ & $\mathrm{dk} / \mathrm{k}$ \\
\hline keff & $\frac{\circ}{0}$ & $\mathrm{dk} / \mathrm{k}$ \\
\hline keff & $\frac{\circ}{0}$ & $\mathrm{dk} / \mathrm{k}$ \\
\hline keff & $\frac{\circ}{0}$ & $\mathrm{dk} / \mathrm{k}$ \\
\hline keff & $\frac{\circ}{0}$ & $\mathrm{dk} / \mathrm{k}$ \\
\hline keff & $\frac{\circ}{0}$ & $\mathrm{dk} / \mathrm{k}$ \\
\hline keff & $\%$ & $\mathrm{dk} / \mathrm{k}$ \\
\hline keff & 응 & $\mathrm{dk} / \mathrm{k}$ \\
\hline keff & $\frac{\circ}{0}$ & $\mathrm{dk} / \mathrm{k}$ \\
\hline keff & $\frac{\circ}{0}$ & $\mathrm{dk} / \mathrm{k}$ \\
\hline keff & $\div$ & $\mathrm{dk} / \mathrm{k}$ \\
\hline keff & $\%$ & $\mathrm{dk} / \mathrm{k}$ \\
\hline keff & $\frac{\circ}{0}$ & $\mathrm{dk} / \mathrm{k}$ \\
\hline keff & $\frac{\circ}{0}$ & $\mathrm{dk} / \mathrm{k}$ \\
\hline keff & $\frac{\circ}{0}$ & $\mathrm{dk} / \mathrm{k}$ \\
\hline keff & $\frac{\circ}{0}$ & $\mathrm{dk} / \mathrm{k}$ \\
\hline keff & $\frac{\circ}{0}$ & $\mathrm{dk} / \mathrm{k}$ \\
\hline keff & $\frac{\circ}{0}$ & $\mathrm{dk} / \mathrm{k}$ \\
\hline keff & $\frac{\circ}{0}$ & $\mathrm{dk} / \mathrm{k}$ \\
\hline keff & $\frac{\circ}{0}$ & $\mathrm{dk} / \mathrm{k}$ \\
\hline keff & $\frac{\circ}{0}$ & $\mathrm{dk} / \mathrm{k}$ \\
\hline keff & $\frac{\circ}{0}$ & $\mathrm{dk} / \mathrm{k}$ \\
\hline keff & $\frac{\circ}{0}$ & $\mathrm{dk} / \mathrm{k}$ \\
\hline keff & $\%$ & $\mathrm{dk} / \mathrm{k}$ \\
\hline keff & $\%$ & $\mathrm{dk} / \mathrm{k}$ \\
\hline keff & $\%$ & $\mathrm{dk} / \mathrm{k}$ \\
\hline keff & 0 & $\mathrm{dk} / \mathrm{k}$ \\
\hline keff & $\frac{\circ}{0}$ & $\mathrm{dk} / \mathrm{k}$ \\
\hline keff & 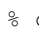 & $\mathrm{dk} / \mathrm{k}$ \\
\hline keff & $\%$ & $\mathrm{dk} / \mathrm{k}$ \\
\hline keff & & $\mathrm{dk} / \mathrm{k}$ \\
\hline & & \\
\hline
\end{tabular}

UNCERT. COMPONENTS (std. dev.):

STD D

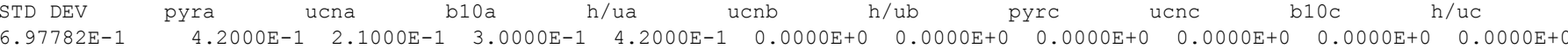
ucna b10a $\begin{array}{lcccccccccc}\text { STD DEV } & \text { pyra } & \text { ucna } & \text { bloa } & \text { h/ua } & \text { ucnb } & \mathrm{h} / \mathrm{ub} & \text { pyrc } & u c h c & \mathrm{~b} 10 \mathrm{C} & \mathrm{h} / \mathrm{uc} \\ 6.97782 \mathrm{E}-1 & 4.2000 \mathrm{E}-1 & 2.1000 \mathrm{E}-1 & 3.0000 \mathrm{E}-1 & 4.2000 \mathrm{E}-1 & 0.0000 \mathrm{E}+0 & 0.0000 \mathrm{E}+0 & 0.0000 \mathrm{E}+0 & 0.0000 \mathrm{E}+0 & 0.0000 \mathrm{E}+0 & 0.0000 \mathrm{E}+0\end{array}$ ucnb

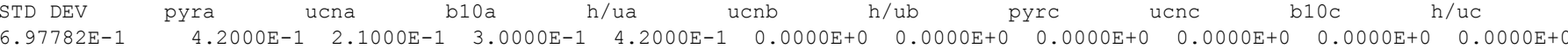

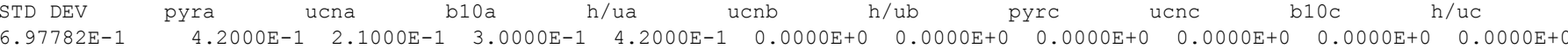
ucne

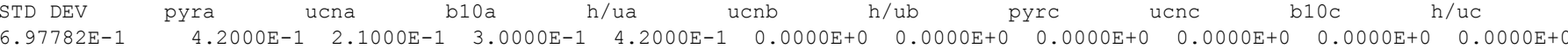
$\begin{array}{lcccccccccc}\text { STD DEV } & \text { pyra } & \text { ucna } & \text { bloa } & \text { h/ua } & \text { ucnb } & \mathrm{h} / \mathrm{ub} & \text { pyrc } & u c h c & \mathrm{~b} 10 \mathrm{C} & \mathrm{h} / \mathrm{uc} \\ 6.97782 \mathrm{E}-1 & 4.2000 \mathrm{E}-1 & 2.1000 \mathrm{E}-1 & 3.0000 \mathrm{E}-1 & 4.2000 \mathrm{E}-1 & 0.0000 \mathrm{E}+0 & 0.0000 \mathrm{E}+0 & 0.0000 \mathrm{E}+0 & 0.0000 \mathrm{E}+0 & 0.0000 \mathrm{E}+0 & 0.0000 \mathrm{E}+0\end{array}$ $\begin{array}{llllllllllll}6.53682 \mathrm{E}-1 & 3.2000 \mathrm{E}-1 & 2.5000 \mathrm{E}-1 & 3.2000 \mathrm{E}-1 & 4.0000 \mathrm{E}-1 & 0.0000 \mathrm{E}+0 & 0.0000 \mathrm{E}+0 & 0.0000 \mathrm{E}+0 & 0.0000 \mathrm{E}+0 & 0.0000 \mathrm{E}+0 & 0.0000 \mathrm{E}+0\end{array}$ $\begin{array}{lllllllllll}0.00000 \mathrm{E}+0 & 0.0000 \mathrm{E}+0 & 0.0000 \mathrm{E}+0 & 0.0000 \mathrm{E}+0 & 0.0000 \mathrm{E}+0 & 0.0000 \mathrm{E}+0 & 0.0000 \mathrm{E}+0 & 0.0000 \mathrm{E}+0 & 0.0000 \mathrm{E}+0 & 0.0000 \mathrm{E}+0 & 0.0000 \mathrm{E}+0\end{array}$ $\begin{array}{lllllllllll}4.86929 \mathrm{E}-1 & 3.9000 \mathrm{E}-1 & 1.5000 \mathrm{E}-1 & 0.0000 \mathrm{E}+0 & 2.5000 \mathrm{E}-1 & 0.0000 \mathrm{E}+0 & 0.0000 \mathrm{E}+0 & 0.0000 \mathrm{E}+0 & 0.0000 \mathrm{E}+0 & 0.0000 \mathrm{E}+0 & 0.0000 \mathrm{E}+0\end{array}$ $\begin{array}{lllllllllll}6.07454 \mathrm{E}-1 & 4.0000 \mathrm{E}-1 & 2.1000 \mathrm{E}-1 & 2.5000 \mathrm{E}-1 & 3.2000 \mathrm{E}-1 & 0.0000 \mathrm{E}+0 & 0.0000 \mathrm{E}+0 & 0.0000 \mathrm{E}+0 & 0.0000 \mathrm{E}+0 & 0.0000 \mathrm{E}+0 & 0.0000 \mathrm{E}+0\end{array}$ $\begin{array}{lllllllllll}5.57225 \mathrm{E}-1 & 0.0000 \mathrm{E}+0 & 2.1000 \mathrm{E}-1 & 3.0000 \mathrm{E}-1 & 4.2000 \mathrm{E}-1 & 0.0000 \mathrm{E}+0 & 0.0000 \mathrm{E}+0 & 0.0000 \mathrm{E}+0 & 0.0000 \mathrm{E}+0 & 0.0000 \mathrm{E}+0 & 0.0000 \mathrm{E}+0\end{array}$ $\begin{array}{lllllllllll}6.18142 \mathrm{E}-1 & 2.2000 \mathrm{E}-1 & 2.2000 \mathrm{E}-1 & 3.3000 \mathrm{E}-1 & 4.2000 \mathrm{E}-1 & 0.0000 \mathrm{E}+0 & 0.0000 \mathrm{E}+0 & 0.0000 \mathrm{E}+0 & 0.0000 \mathrm{E}+0 & 0.0000 \mathrm{E}+0 & 0.0000 \mathrm{E}+0\end{array}$ $\begin{array}{lllllllllll}6.65582 \mathrm{E}-1 & 2.2000 \mathrm{E}-1 & 2.5000 \mathrm{E}-1 & 3.6000 \mathrm{E}-1 & 4.5000 \mathrm{E}-1 & 0.0000 \mathrm{E}+0 & 0.0000 \mathrm{E}+0 & 0.0000 \mathrm{E}+0 & 0.0000 \mathrm{E}+0 & 0.0000 \mathrm{E}+0 & 0.0000 \mathrm{E}+0\end{array}$ $\begin{array}{lllllllllll}4.69574 \mathrm{E}-1 & 0.0000 \mathrm{E}+0 & 0.0000 \mathrm{E}+0 & 0.0000 \mathrm{E}+0 & 0.0000 \mathrm{E}+0 & 2.1000 \mathrm{E}-1 & 4.2000 \mathrm{E}-1 & 0.0000 \mathrm{E}+0 & 0.0000 \mathrm{E}+0 & 0.0000 \mathrm{E}+0 & 0.0000 \mathrm{E}+0\end{array}$ $\begin{array}{lllllllllll}5.73062 \mathrm{E}-1 & 0.0000 \mathrm{E}+0 & 0.0000 \mathrm{E}+0 & 0.0000 \mathrm{E}+0 & 0.0000 \mathrm{E}+0 & 2.8000 \mathrm{E}-1 & 5.0000 \mathrm{E}-1 & 0.0000 \mathrm{E}+0 & 0.0000 \mathrm{E}+0 & 0.0000 \mathrm{E}+0 & 0.0000 \mathrm{E}+0\end{array}$ $\begin{array}{lllllllllll}4.45533 \mathrm{E}-1 & 0.0000 \mathrm{E}+0 & 0.0000 \mathrm{E}+0 & 0.0000 \mathrm{E}+0 & 0.0000 \mathrm{E}+0 & 3.1000 \mathrm{E}-1 & 3.2000 \mathrm{E}-1 & 0.0000 \mathrm{E}+0 & 0.0000 \mathrm{E}+0 & 0.0000 \mathrm{E}+0 & 0.0000 \mathrm{E}+0\end{array}$ $\begin{array}{lllllllllll}4.69574 \mathrm{E}-1 & 0.0000 \mathrm{E}+0 & 0.0000 \mathrm{E}+0 & 0.0000 \mathrm{E}+0 & 0.0000 \mathrm{E}+0 & 2.1000 \mathrm{E}-1 & 4.2000 \mathrm{E}-1 & 0.0000 \mathrm{E}+0 & 0.0000 \mathrm{E}+0 & 0.0000 \mathrm{E}+0 & 0.0000 \mathrm{E}+0\end{array}$ $\begin{array}{lllllllllll}4.69574 \mathrm{E}-1 & 0.0000 \mathrm{E}+0 & 0.0000 \mathrm{E}+0 & 0.0000 \mathrm{E}+0 & 0.0000 \mathrm{E}+0 & 2.1000 \mathrm{E}-1 & 4.2000 \mathrm{E}-1 & 0.0000 \mathrm{E}+0 & 0.0000 \mathrm{E}+0 & 0.0000 \mathrm{E}+0 & 0.0000 \mathrm{E}+0\end{array}$

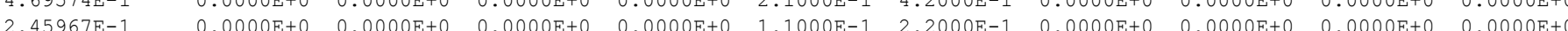

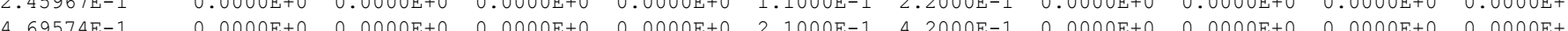

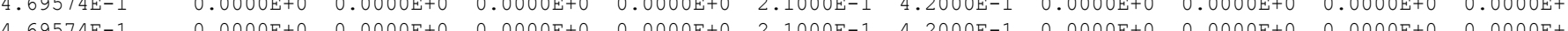
$\begin{array}{lllllllllll}4.69574 \mathrm{E}-1 & 0.0000 \mathrm{E}+0 & 0.0000 \mathrm{E}+0 & 0.0000 \mathrm{E}+0 & 0.0000 \mathrm{E}+0 & 2.1000 \mathrm{E}-1 & 4.2000 \mathrm{E}-1 & 0.0000 \mathrm{E}+0 & 0.0000 \mathrm{E}+0 & 0.0000 \mathrm{E}+0 & 0.0000 \mathrm{E}+0\end{array}$ $\begin{array}{llllllllllll}4.69574 \mathrm{E}-1 & 0.0000 \mathrm{E}+0 & 0.0000 \mathrm{E}+0 & 0.0000 \mathrm{E}+0 & 0.0000 \mathrm{E}+0 & 2.1000 \mathrm{E}-1 & 4.2000 \mathrm{E}-1 & 0.0000 \mathrm{E}+0 & 0.0000 \mathrm{E}+0 & 0.0000 \mathrm{E}+0 & 0.0000 \mathrm{E}+0\end{array}$ $\begin{array}{llllllllllll}4.69574 \mathrm{E}-1 & 0.0000 \mathrm{E}+0 & 0.0000 \mathrm{E}+0 & 0.0000 \mathrm{E}+0 & 0.0000 \mathrm{E}+0 & 2.1000 \mathrm{E}-1 & 4.2000 \mathrm{E}-1 & 0.0000 \mathrm{E}+0 & 0.0000 \mathrm{E}+0 & 0.0000 \mathrm{E}+0 & 0.0000 \mathrm{E}+0\end{array}$ $\begin{array}{lllllllllll}4.69574 \mathrm{E}-1 & 0.0000 \mathrm{E}+0 & 0.0000 \mathrm{E}+0 & 0.0000 \mathrm{E}+0 & 0.0000 \mathrm{E}+0 & 2.1000 \mathrm{E}-1 & 4.2000 \mathrm{E}-1 & 0.0000 \mathrm{E}+0 & 0.0000 \mathrm{E}+0 & 0.0000 \mathrm{E}+0 & 0.0000 \mathrm{E}+0\end{array}$ $\begin{array}{lllllllllll}4.69574 \mathrm{E}-1 & 0.0000 \mathrm{E}+0 & 0.0000 \mathrm{E}+0 & 0.0000 \mathrm{E}+0 & 0.0000 \mathrm{E}+0 & 2.1000 \mathrm{E}-1 & 4.2000 \mathrm{E}-1 & 0.0000 \mathrm{E}+0 & 0.0000 \mathrm{E}+0 & 0.0000 \mathrm{E}+0 & 0.0000 \mathrm{E}+0\end{array}$ $\begin{array}{llllllllllll}6.97782 \mathrm{E}-1 & 0.0000 \mathrm{E}+0 & 0.0000 \mathrm{E}+0 & 0.0000 \mathrm{E}+0 & 0.0000 \mathrm{E}+0 & 0.0000 \mathrm{E}+0 & 0.0000 \mathrm{E}+0 & 4.2000 \mathrm{E}-1 & 2.1000 \mathrm{E}-1 & 3.0000 \mathrm{E}-1 & 4.2000 \mathrm{E}-1\end{array}$ $\begin{array}{llllllllllll}7.30685 \mathrm{E}-1 & 0.0000 \mathrm{E}+0 & 0.0000 \mathrm{E}+0 & 0.0000 \mathrm{E}+0 & 0.0000 \mathrm{E}+0 & 0.0000 \mathrm{E}+0 & 0.0000 \mathrm{E}+0 & 4.5000 \mathrm{E}-1 & 2.5000 \mathrm{E}-1 & 3.3000 \mathrm{E}-1 & 4.0000 \mathrm{E}-1\end{array}$ $\begin{array}{llllllllllll}6.99071 \mathrm{E}-1 & 0.0000 \mathrm{E}+0 & 0.0000 \mathrm{E}+0 & 0.0000 \mathrm{E}+0 & 0.0000 \mathrm{E}+0 & 0.0000 \mathrm{E}+0 & 0.0000 \mathrm{E}+0 & 3.9000 \mathrm{E}-1 & 2.1000 \mathrm{E}-1 & 3.0000 \mathrm{E}-1 & 4.5000 \mathrm{E}-1\end{array}$ $\begin{array}{llllllllllll}5.95651 \mathrm{E}-1 & 0.0000 \mathrm{E}+0 & 0.0000 \mathrm{E}+0 & 0.0000 \mathrm{E}+0 & 0.0000 \mathrm{E}+0 & 0.0000 \mathrm{E}+0 & 0.0000 \mathrm{E}+0 & 4.2000 \mathrm{E}-1 & 3.0000 \mathrm{E}-1 & 2.0000 \mathrm{E}-1 & 2.2000 \mathrm{E}-1\end{array}$ $\begin{array}{llllllllllll}6.97782 \mathrm{E}-1 & 0.0000 \mathrm{E}+0 & 0.0000 \mathrm{E}+0 & 0.0000 \mathrm{E}+0 & 0.0000 \mathrm{E}+0 & 0.0000 \mathrm{E}+0 & 0.0000 \mathrm{E}+0 & 4.2000 \mathrm{E}-1 & 2.1000 \mathrm{E}-1 & 3.0000 \mathrm{E}-1 & 4.2000 \mathrm{E}-1\end{array}$ $\begin{array}{lllllllllll}6.58863 \mathrm{E}-1 & 0.0000 \mathrm{E}+0 & 0.0000 \mathrm{E}+0 & 0.0000 \mathrm{E}+0 & 0.0000 \mathrm{E}+0 & 0.0000 \mathrm{E}+0 & 0.0000 \mathrm{E}+0 & 2.2000 \mathrm{E}-1 & 3.1000 \mathrm{E}-1 & 4.0000 \mathrm{E}-1 & 3.6000 \mathrm{E}-1\end{array}$ $6.97782 \mathrm{E}-1 \quad 0.0000 \mathrm{E}+0 \quad 0.0000 \mathrm{E}+0 \quad 0.0000 \mathrm{E}+0 \quad 0.0000 \mathrm{E}+0 \quad 0.0000 \mathrm{E}+0 \quad 0.0000 \mathrm{E}+0=4.2000 \mathrm{E}-1 \quad 2.1000 \mathrm{E}-1 \quad 3.0000 \mathrm{E}-1 \quad 4.2000 \mathrm{E}-1$

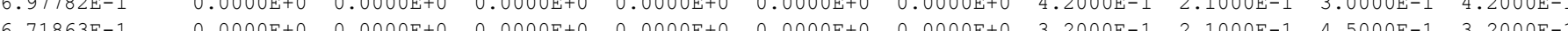

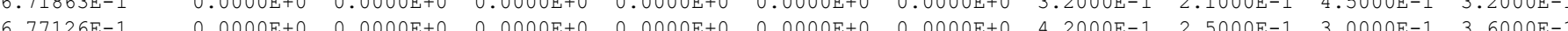
$\begin{array}{llllllllllll}6.77126 \mathrm{E}-1 & 0.0000 \mathrm{E}+0 & 0.0000 \mathrm{E}+0 & 0.0000 \mathrm{E}+0 & 0.0000 \mathrm{E}+0 & 0.0000 \mathrm{E}+0 & 0.0000 \mathrm{E}+0 & 4.2000 \mathrm{E}-1 & 2.5000 \mathrm{E}-1 & 3.0000 \mathrm{E}-1 & 3.6000 \mathrm{E}-1\end{array}$ $\begin{array}{lllllllllll}5.57225 \mathrm{E}-1 & 0.0000 \mathrm{E}+0 & 0.0000 \mathrm{E}+0 & 0.0000 \mathrm{E}+0 & 0.0000 \mathrm{E}+0 & 0.0000 \mathrm{E}+0 & 0.0000 \mathrm{E}+0 & 0.0000 \mathrm{E}+0 & 2.1000 \mathrm{E}-1 & 3.0000 \mathrm{E}-1 & 4.2000 \mathrm{E}-1\end{array}$ $\begin{array}{llllllllllll}6.02080 \mathrm{E}-1 & 0.0000 \mathrm{E}+0 & 0.0000 \mathrm{E}+0 & 0.0000 \mathrm{E}+0 & 0.0000 \mathrm{E}+0 & 0.0000 \mathrm{E}+0 & 0.0000 \mathrm{E}+0 & 0.0000 \mathrm{E}+0 & 3.1000 \mathrm{E}-1 & 3.0000 \mathrm{E}-1 & 4.2000 \mathrm{E}-1\end{array}$ $\begin{array}{llllllllllll}0.00000 \mathrm{E}+0 & 0.0000 \mathrm{E}+0 & 0.0000 \mathrm{E}+0 & 0.0000 \mathrm{E}+0 & 0.0000 \mathrm{E}+0 & 0.0000 \mathrm{E}+0 & 0.0000 \mathrm{E}+0 & 0.0000 \mathrm{E}+0 & 0.0000 \mathrm{E}+0 & 0.0000 \mathrm{E}+0 & 0.0000 \mathrm{E}+0\end{array}$ $\begin{array}{llllllllllll}6.32851 \mathrm{E}-1 & 0.0000 \mathrm{E}+0 & 0.0000 \mathrm{E}+0 & 0.0000 \mathrm{E}+0 & 0.0000 \mathrm{E}+0 & 0.0000 \mathrm{E}+0 & 0.0000 \mathrm{E}+0 & 0.0000 \mathrm{E}+0 & 3.1000 \mathrm{E}-1 & 4.0000 \mathrm{E}-1 & 3.8000 \mathrm{E}-1\end{array}$ $\begin{array}{llllllllllll}5.57225 \mathrm{E}-1 & 0.0000 \mathrm{E}+0 & 0.0000 \mathrm{E}+0 & 0.0000 \mathrm{E}+0 & 0.0000 \mathrm{E}+0 & 0.0000 \mathrm{E}+0 & 0.0000 \mathrm{E}+0 & 0.0000 \mathrm{E}+0 & 2.1000 \mathrm{E}-1 & 3.0000 \mathrm{E}-1 & 4.2000 \mathrm{E}-1\end{array}$ $\begin{array}{lllllllllll}6.42336 \mathrm{E}-1 & 0.0000 \mathrm{E}+0 & 0.0000 \mathrm{E}+0 & 0.0000 \mathrm{E}+0 & 0.0000 \mathrm{E}+0 & 0.0000 \mathrm{E}+0 & 0.0000 \mathrm{E}+0 & 0.0000 \mathrm{E}+0 & 2.5000 \mathrm{E}-1 & 4.0000 \mathrm{E}-1 & 4.3600 \mathrm{E}-1\end{array}$ $\begin{array}{lllllllllll}6.32851 \mathrm{E}-1 & 0.0000 \mathrm{E}+0 & 0.0000 \mathrm{E}+0 & 0.0000 \mathrm{E}+0 & 0.0000 \mathrm{E}+0 & 0.0000 \mathrm{E}+0 & 0.0000 \mathrm{E}+0 & 0.0000 \mathrm{E}+0 & 3.1000 \mathrm{E}-1 & 4.0000 \mathrm{E}-1 & 3.8000 \mathrm{E}-1\end{array}$ $\begin{array}{llllllllllll}5.57225 \mathrm{E}-1 & 0.0000 \mathrm{E}+0 & 0.0000 \mathrm{E}+0 & 0.0000 \mathrm{E}+0 & 0.0000 \mathrm{E}+0 & 0.0000 \mathrm{E}+0 & 0.0000 \mathrm{E}+0 & 0.0000 \mathrm{E}+0 & 2.1000 \mathrm{E}-1 & 3.0000 \mathrm{E}-1 & 4.2000 \mathrm{E}-1\end{array}$ $\begin{array}{llllllllllll}5.57225 \mathrm{E}-1 & 0.0000 \mathrm{E}+0 & 0.0000 \mathrm{E}+0 & 0.0000 \mathrm{E}+0 & 0.0000 \mathrm{E}+0 & 0.0000 \mathrm{E}+0 & 0.0000 \mathrm{E}+0 & 0.0000 \mathrm{E}+0 & 4.1000 \mathrm{E}-1 & 2.0000 \mathrm{E}-1 & 3.2000 \mathrm{E}-1\end{array}$

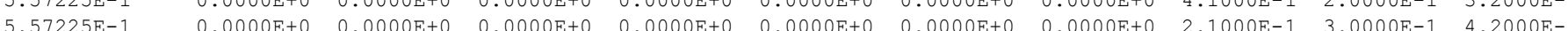
$\begin{array}{llllllllllll}0.00000 \mathrm{E}+0 & 0.0000 \mathrm{E}+0 & 0.0000 \mathrm{E}+0 & 0.0000 \mathrm{E}+0 & 0.0000 \mathrm{E}+0 & 0.0000 \mathrm{E}+0 & 0.0000 \mathrm{E}+0 & 0.0000 \mathrm{E}+0 & 0.0000 \mathrm{E}+0 & 0.0000 \mathrm{E}+0 & 0.0000 \mathrm{E}+0\end{array}$

Figure 6.6.5. Uncertainty components edit. 


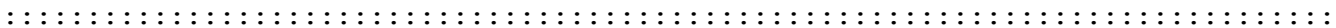

CHI-SQUARED ANALYSIS FOR INCLUDED EXPERIMENTS

INITIAL CHI-SQUARE PER DEGR. OF FREEDOM =

$2.781 \mathrm{E}-01$

$3.000 \mathrm{E}+00$

FINAL CHI-SQUARE PER DEGR. OF FREEDOM

$2.781 \mathrm{E}-01$

* final number of degrees of freedom

* final chi-square per degr. of freedom.... diagonal contribution

* final chi-square per degr. of freedom... without correlations

$1.561 \mathrm{E}-01$

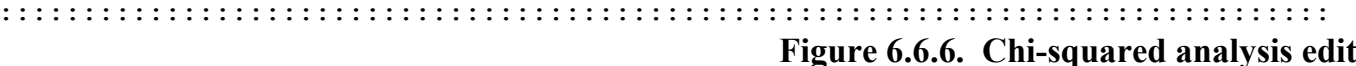


Prior Experimental-Response Correlation Matrix

\begin{tabular}{|c|c|c|c|c|c|c|c|c|}
\hline resp & resp 1 & resp 2 & resp 3 & resp 4 & resp 5 & resp 6 & resp 7 & resp \\
\hline 1 & $1.000 \mathrm{E}+00$ & $1.556 \mathrm{E}-01$ & $0.000 \mathrm{E}+00$ & $3.696 \mathrm{E}-01$ & $2.824 \mathrm{E}-01$ & $1.134 \mathrm{E}-01$ & $1.461 \mathrm{E}-01$ & $2.234 \mathrm{E}-01$ \\
\hline 2 & $1.556 \mathrm{E}-01$ & $1.000 \mathrm{E}+00$ & $0.000 \mathrm{E}+00$ & $3.216 \mathrm{E}-01$ & $2.521 \mathrm{E}-01$ & $1.211 \mathrm{E}-01$ & $1.452 \mathrm{E}-01$ & $2.086 \mathrm{E}-01$ \\
\hline 3 & $0.000 \mathrm{E}+00$ & $0.000 \mathrm{E}+00$ & $1.000 \mathrm{E}+00$ & $0.000 \mathrm{E}+00$ & $0.000 \mathrm{E}+00$ & $0.000 \mathrm{E}+00$ & $0.000 \mathrm{E}+00$ & $0.000 \mathrm{E}+00$ \\
\hline 4 & $3.696 \mathrm{E}-01$ & $3.216 \mathrm{E}-01$ & $0.000 \mathrm{E}+00$ & $1.000 \mathrm{E}+00$ & $5.426 \mathrm{E}-01$ & $3.018 \mathrm{E}-01$ & $3.718 \mathrm{E}-01$ & $3.638 \mathrm{E}-01$ \\
\hline 5 & $2.824 \mathrm{E}-01$ & $2.521 \mathrm{E}-01$ & $0.000 \mathrm{E}+00$ & $5.426 \mathrm{E}-01$ & $1.000 \mathrm{E}+00$ & $4.219 \mathrm{E}-01$ & $1.431 \mathrm{E}-01$ & $4.222 \mathrm{E}-01$ \\
\hline 6 & $1.134 \mathrm{E}-01$ & $1.211 \mathrm{E}-01$ & $0.000 \mathrm{E}+00$ & $3.018 \mathrm{E}-01$ & $4.219 \mathrm{E}-01$ & $1.000 \mathrm{E}+00$ & $5.170 \mathrm{E}-01$ & $3.907 \mathrm{E}-01$ \\
\hline 7 & $1.461 \mathrm{E}-01$ & $1.452 \mathrm{E}-01$ & $0.000 \mathrm{E}+00$ & $3.718 \mathrm{E}-01$ & $1.431 \mathrm{E}-01$ & $5.170 \mathrm{E}-01$ & $1.000 \mathrm{E}+00$ & $4.264 \mathrm{E}-01$ \\
\hline 8 & $2.234 \mathrm{E}-01$ & $2.086 \mathrm{E}-01$ & $0.000 \mathrm{E}+00$ & $3.638 \mathrm{E}-01$ & $4.222 \mathrm{E}-01$ & $3.907 \mathrm{E}-01$ & $4.264 \mathrm{E}-01$ & $1.000 \mathrm{E}+00$ \\
\hline \multirow[t]{2}{*}{9} & $0.000 \mathrm{E}+00$ & $0.000 \mathrm{E}+00$ & $0.000 \mathrm{E}+00$ & $0.000 \mathrm{E}+00$ & $0.000 \mathrm{E}+00$ & $0.000 \mathrm{E}+00$ & $0.000 \mathrm{E}+00$ & $0.000 \mathrm{E}+00$ \\
\hline & 10 thru & resp & same as above & & & & & \\
\hline resp & & \multirow{2}{*}{ resp 10} & resp 11 & resp 12 & resp 13 & resp 14 & resp 15 & resp 16 \\
\hline \multirow[t]{2}{*}{1} & $0.000 \mathrm{E}+00$ & & $0.000 \mathrm{E}+00$ & $0.000 \mathrm{E}+00$ & $0.000 \mathrm{E}+00$ & $0.000 \mathrm{E}+00$ & $0.000 \mathrm{E}+00$ & $0.000 \mathrm{E}+00$ \\
\hline & resp & resp & same as above & & & & & \\
\hline 9 & $1.000 \mathrm{E}+00$ & $1.998 \mathrm{E}-01$ & $1.907 \mathrm{E}-01$ & $2.000 \mathrm{E}-01$ & $2.000 \mathrm{E}-01$ & $2.000 \mathrm{E}-01$ & $2.000 \mathrm{E}-01$ & $2.000 \mathrm{E}-01$ \\
\hline 10 & $1.998 \mathrm{E}-01$ & $1.000 \mathrm{E}+00$ & $1.933 \mathrm{E}-01$ & $1.998 \mathrm{E}-01$ & $1.998 \mathrm{E}-01$ & $1.998 \mathrm{E}-01$ & $1.998 \mathrm{E}-01$ & $1.998 \mathrm{E}-01$ \\
\hline 11 & $1.907 \mathrm{E}-01$ & $1.933 \mathrm{E}-01$ & $1.000 \mathrm{E}+00$ & $1.907 \mathrm{E}-01$ & $1.907 \mathrm{E}-01$ & $1.907 \mathrm{E}-01$ & $1.907 \mathrm{E}-01$ & $1.907 \mathrm{E}-01$ \\
\hline 12 & $2.000 \mathrm{E}-01$ & $1.998 \mathrm{E}-01$ & $1.907 \mathrm{E}-01$ & $1.000 \mathrm{E}+00$ & $2.000 \mathrm{E}-01$ & $2.000 \mathrm{E}-01$ & $2.000 \mathrm{E}-01$ & $2.000 \mathrm{E}-01$ \\
\hline 13 & $2.000 \mathrm{E}-01$ & $1.998 \mathrm{E}-01$ & $1.907 \mathrm{E}-01$ & $2.000 \mathrm{E}-01$ & $1.000 \mathrm{E}+00$ & $2.000 \mathrm{E}-01$ & $2.000 \mathrm{E}-01$ & $2.000 \mathrm{E}-01$ \\
\hline 14 & $2.000 \mathrm{E}-01$ & $1.998 \mathrm{E}-01$ & $1.907 \mathrm{E}-01$ & $2.000 \mathrm{E}-01$ & $2.000 \mathrm{E}-01$ & $1.000 \mathrm{E}+00$ & $2.000 \mathrm{E}-01$ & $2.000 \mathrm{E}-01$ \\
\hline 15 & $2.000 \mathrm{E}-01$ & $1.998 \mathrm{E}-01$ & $1.907 \mathrm{E}-01$ & $2.000 \mathrm{E}-01$ & $2.000 \mathrm{E}-01$ & $2.000 \mathrm{E}-01$ & $1.000 \mathrm{E}+00$ & $2.000 \mathrm{E}-01$ \\
\hline 16 & \multirow{2}{*}{$\begin{array}{l}2.000 \mathrm{E}-01 \\
2.000 \mathrm{E}-01\end{array}$} & $1.998 \mathrm{E}-01$ & $1.907 \mathrm{E}-01$ & $2.000 \mathrm{E}-01$ & $2.000 \mathrm{E}-01$ & $2.000 \mathrm{E}-01$ & $2.000 \mathrm{E}-01$ & $1.000 \mathrm{E}+00$ \\
\hline 17 & & $1.998 \mathrm{E}-01$ & $1.907 \mathrm{E}-01$ & $2.000 \mathrm{E}-01$ & $2.000 \mathrm{E}-01$ & $2.000 \mathrm{E}-01$ & $2.000 \mathrm{E}-01$ & $2.000 \mathrm{E}-01$ \\
\hline & resp 18 thru & resp & same as above & & & & & \\
\hline \multirow[t]{2}{*}{21} & $0.000 \mathrm{E}+00$ & $0.000 \mathrm{E}+00$ & $0.000 \mathrm{E}+00$ & $0.000 \mathrm{E}+00$ & $0.000 \mathrm{E}+00$ & $0.000 \mathrm{E}+00$ & $0.000 \mathrm{E}+00$ & $0.000 \mathrm{E}+00$ \\
\hline & resp 22 thru & resp & same as above & & & & & \\
\hline resp & \multirow{2}{*}{$\begin{array}{l}\text { resp } 17 \\
0.000 \text { +00 }\end{array}$} & resp 18 & resp 19 & resp 20 & resp 21 & resp 22 & resp 23 & resp 24 \\
\hline \multirow[t]{2}{*}{1} & & $0.000 \mathrm{E}+00$ & $0.000 \mathrm{E}+00$ & $0.000 \mathrm{E}+00$ & $0.000 \mathrm{E}+00$ & $0.000 \mathrm{E}+00$ & $0.000 \mathrm{E}+00$ & $0.000 \mathrm{E}+00$ \\
\hline & 2 thru & resp & same as above & & & & & \\
\hline 9 & $2.000 \mathrm{E}-01$ & $2.000 \mathrm{E}-01$ & $2.000 \mathrm{E}-01$ & $2.000 \mathrm{E}-01$ & $0.000 \mathrm{E}+00$ & $0.000 \mathrm{E}+00$ & $0.000 \mathrm{E}+00$ & $0.000 \mathrm{E}+00$ \\
\hline 10 & $1.998 \mathrm{E}-01$ & $1.998 \mathrm{E}-01$ & $1.998 \mathrm{E}-01$ & $1.998 \mathrm{E}-01$ & $0.000 \mathrm{E}+00$ & $0.000 \mathrm{E}+00$ & $0.000 \mathrm{E}+00$ & $0.000 \mathrm{E}+00$ \\
\hline 11 & $1.907 \mathrm{E}-01$ & $1.907 \mathrm{E}-01$ & $1.907 \mathrm{E}-01$ & $1.907 \mathrm{E}-01$ & $0.000 \mathrm{E}+00$ & $0.000 \mathrm{E}+00$ & $0.000 \mathrm{E}+00$ & $0.000 \mathrm{E}+00$ \\
\hline 12 & $2.000 \mathrm{E}-01$ & $2.000 \mathrm{E}-01$ & $2.000 \mathrm{E}-01$ & $2.000 \mathrm{E}-01$ & $0.000 \mathrm{E}+00$ & $0.000 \mathrm{E}+00$ & $0.000 \mathrm{E}+00$ & $0.000 \mathrm{E}+00$ \\
\hline & 13 thru & resp & same as above & & & & & \\
\hline 17 & $1.000 \mathrm{E}+00$ & $2.000 \mathrm{E}-01$ & $2.000 \mathrm{E}-01$ & $2.000 \mathrm{E}-01$ & $0.000 \mathrm{E}+00$ & $0.000 \mathrm{E}+00$ & $0.000 \mathrm{E}+00$ & $0.000 \mathrm{E}+00$ \\
\hline 18 & $2.000 \mathrm{E}-01$ & $1.000 \mathrm{E}+00$ & $2.000 \mathrm{E}-01$ & $0.000 \mathrm{E}+00$ & $0.000 \mathrm{E}+00$ & $0.000 \mathrm{E}+00$ & $0.000 \mathrm{E}+00$ & $0.000 \mathrm{E}+00$ \\
\hline 19 & $2.000 \mathrm{E}-01$ & $2.000 \mathrm{E}-01$ & $1.000 \mathrm{E}+00$ & $2.000 \mathrm{E}-01$ & $0.000 \mathrm{E}+00$ & $0.000 \mathrm{E}+00$ & $0.000 \mathrm{E}+00$ & $0.000 \mathrm{E}+00$ \\
\hline 20 & $2.000 \mathrm{E}-01$ & $0.000 \mathrm{E}+00$ & $2.000 \mathrm{E}-01$ & $1.000 \mathrm{E}+00$ & $0.000 \mathrm{E}+00$ & $0.000 \mathrm{E}+00$ & $0.000 \mathrm{E}+00$ & $0.000 \mathrm{E}+00$ \\
\hline 21 & $0.000 \mathrm{E}+00$ & $0.000 \mathrm{E}+00$ & $0.000 \mathrm{E}+00$ & $0.000 \mathrm{E}+00$ & $1.000 \mathrm{E}+00$ & $2.059 \mathrm{E}-02$ & $1.808 \mathrm{E}-02$ & $3.032 \mathrm{E}-02$ \\
\hline
\end{tabular}

Figure 6.6.7. Prior experiment correlation matrix edit. 
Prior Calculated-Response Correlation Matrix (omitted responses are 0 )

\begin{tabular}{|c|c|c|c|c|c|c|c|c|}
\hline resp & resp & resp & resp & resp & resp & resp & resp & resp \\
\hline 1 & $1.000 \mathrm{E}+00$ & $9.941 \mathrm{E}-01$ & $9.988 \mathrm{E}-01$ & $9.922 \mathrm{E}-01$ & $9.918 \mathrm{E}-01$ & $9.932 \mathrm{E}-01$ & $9.988 \mathrm{E}-01$ & $9.988 \mathrm{E}-01$ \\
\hline 2 & $9.941 \mathrm{E}-01$ & $1.000 \mathrm{E}+00$ & $9.925 \mathrm{E}-01$ & $9.987 \mathrm{E}-01$ & $9.768 \mathrm{E}-01$ & $9.790 \mathrm{E}-01$ & $9.921 \mathrm{E}-01$ & $9.928 \mathrm{E}-01$ \\
\hline 3 & $9.988 \mathrm{E}-01$ & $9.925 \mathrm{E}-01$ & $1.000 \mathrm{E}+00$ & $9.931 \mathrm{E}-01$ & $9.931 \mathrm{E}-01$ & $9.945 \mathrm{E}-01$ & $1.000 \mathrm{E}+00$ & $1.000 \mathrm{E}+00$ \\
\hline 4 & $9.922 \mathrm{E}-01$ & $9.987 \mathrm{E}-01$ & $9.931 \mathrm{E}-01$ & $1.000 \mathrm{E}+00$ & $9.769 \mathrm{E}-01$ & $9.791 \mathrm{E}-01$ & $9.926 \mathrm{E}-01$ & $9.933 \mathrm{E}-01$ \\
\hline 5 & $9.918 \mathrm{E}-01$ & $9.768 \mathrm{E}-01$ & $9.931 \mathrm{E}-01$ & $9.769 \mathrm{E}-01$ & $1.000 \mathrm{E}+00$ & $9.999 \mathrm{E}-01$ & $9.936 \mathrm{E}-01$ & $9.928 \mathrm{E}-01$ \\
\hline 6 & $9.932 \mathrm{E}-01$ & $9.790 \mathrm{E}-01$ & $9.945 \mathrm{E}-01$ & $9.791 \mathrm{E}-01$ & $9.999 \mathrm{E}-01$ & $1.000 \mathrm{E}+00$ & $9.950 \mathrm{E}-01$ & $9.943 \mathrm{E}-01$ \\
\hline 7 & $9.988 \mathrm{E}-01$ & $9.921 \mathrm{E}-01$ & $1.000 \mathrm{E}+00$ & $9.926 \mathrm{E}-01$ & $9.936 \mathrm{E}-01$ & $9.950 \mathrm{E}-01$ & $1.000 \mathrm{E}+00$ & $1.000 \mathrm{E}+00$ \\
\hline 8 & $9.988 \mathrm{E}-01$ & $9.928 \mathrm{E}-01$ & $1.000 \mathrm{E}+00$ & $9.933 \mathrm{E}-01$ & $9.928 \mathrm{E}-01$ & $9.943 \mathrm{E}-01$ & $1.000 \mathrm{E}+00$ & $1.000 \mathrm{E}+00$ \\
\hline 9 & $9.922 \mathrm{E}-01$ & $9.986 \mathrm{E}-01$ & $9.931 \mathrm{E}-01$ & $1.000 \mathrm{E}+00$ & $9.770 \mathrm{E}-01$ & $9.792 \mathrm{E}-01$ & $9.927 \mathrm{E}-01$ & $9.934 \mathrm{E}-01$ \\
\hline 10 & $9.934 \mathrm{E}-01$ & $9.793 \mathrm{E}-01$ & $9.947 \mathrm{E}-01$ & $9.794 \mathrm{E}-01$ & $9.998 \mathrm{E}-01$ & $1.000 \mathrm{E}+00$ & $9.951 \mathrm{E}-01$ & $9.944 \mathrm{E}-01$ \\
\hline 11 & $9.923 \mathrm{E}-01$ & $9.847 \mathrm{E}-01$ & $9.927 \mathrm{E}-01$ & $9.836 \mathrm{E}-01$ & $9.883 \mathrm{E}-01$ & $9.896 \mathrm{E}-01$ & $9.926 \mathrm{E}-01$ & $9.927 \mathrm{E}-01$ \\
\hline 12 & $9.943 \mathrm{E}-01$ & $9.992 \mathrm{E}-01$ & $9.923 \mathrm{E}-01$ & $9.977 \mathrm{E}-01$ & $9.786 \mathrm{E}-01$ & $9.805 \mathrm{E}-01$ & $9.920 \mathrm{E}-01$ & $9.925 \mathrm{E}-01$ \\
\hline 13 & $9.901 \mathrm{E}-01$ & $9.748 \mathrm{E}-01$ & $9.913 \mathrm{E}-01$ & $9.750 \mathrm{E}-01$ & $9.995 \mathrm{E}-01$ & $9.992 \mathrm{E}-01$ & $9.919 \mathrm{E}-01$ & $9.909 \mathrm{E}-01$ \\
\hline 14 & $9.995 \mathrm{E}-01$ & $9.929 \mathrm{E}-01$ & $9.983 \mathrm{E}-01$ & $9.909 \mathrm{E}-01$ & $9.935 \mathrm{E}-01$ & $9.946 \mathrm{E}-01$ & $9.984 \mathrm{E}-01$ & $9.982 \mathrm{E}-01$ \\
\hline 15 & $9.985 \mathrm{E}-01$ & $9.918 \mathrm{E}-01$ & $9.997 \mathrm{E}-01$ & $9.924 \mathrm{E}-01$ & $9.945 \mathrm{E}-01$ & $9.957 \mathrm{E}-01$ & $9.998 \mathrm{E}-01$ & $9.996 \mathrm{E}-01$ \\
\hline 16 & $9.932 \mathrm{E}-01$ & $9.983 \mathrm{E}-01$ & $9.942 \mathrm{E}-01$ & $9.994 \mathrm{E}-01$ & $9.805 \mathrm{E}-01$ & $9.825 \mathrm{E}-01$ & $9.938 \mathrm{E}-01$ & $9.944 \mathrm{E}-01$ \\
\hline 17 & $3.190 \mathrm{E}-01$ & $3.558 \mathrm{E}-01$ & $3.167 \mathrm{E}-01$ & $3.588 \mathrm{E}-01$ & $3.060 \mathrm{E}-01$ & $3.064 \mathrm{E}-01$ & $3.164 \mathrm{E}-01$ & $3.175 \mathrm{E}-01$ \\
\hline 18 & $9.745 \mathrm{E}-01$ & $9.771 \mathrm{E}-01$ & $9.756 \mathrm{E}-01$ & $9.771 \mathrm{E}-01$ & $9.692 \mathrm{E}-01$ & $9.705 \mathrm{E}-01$ & $9.754 \mathrm{E}-01$ & $9.757 \mathrm{E}-01$ \\
\hline 19 & $9.782 \mathrm{E}-01$ & $9.598 \mathrm{E}-01$ & $9.774 \mathrm{E}-01$ & $9.579 \mathrm{E}-01$ & $9.925 \mathrm{E}-01$ & $9.915 \mathrm{E}-01$ & $9.783 \mathrm{E}-01$ & $9.771 \mathrm{E}-01$ \\
\hline 20 & $9.804 \mathrm{E}-01$ & $9.626 \mathrm{E}-01$ & $9.785 \mathrm{E}-01$ & $9.592 \mathrm{E}-01$ & $9.923 \mathrm{E}-01$ & $9.915 \mathrm{E}-01$ & $9.793 \mathrm{E}-01$ & $9.781 \mathrm{E}-01$ \\
\hline 21 & $9.860 \mathrm{E}-01$ & $9.715 \mathrm{E}-01$ & $9.831 \mathrm{E}-01$ & $9.670 \mathrm{E}-01$ & $9.909 \mathrm{E}-01$ & $9.907 \mathrm{E}-01$ & $9.836 \mathrm{E}-01$ & $9.829 \mathrm{E}-01$ \\
\hline 22 & $9.994 \mathrm{E}-01$ & $9.918 \mathrm{E}-01$ & $9.979 \mathrm{E}-01$ & $9.893 \mathrm{E}-01$ & $9.939 \mathrm{E}-01$ & $9.950 \mathrm{E}-01$ & $9.980 \mathrm{E}-01$ & $9.978 \mathrm{E}-01$ \\
\hline 23 & $9.715 \mathrm{E}-01$ & $9.696 \mathrm{E}-01$ & $9.715 \mathrm{E}-01$ & $9.684 \mathrm{E}-01$ & $9.659 \mathrm{E}-01$ & $9.673 \mathrm{E}-01$ & $9.713 \mathrm{E}-01$ & $9.717 \mathrm{E}-01$ \\
\hline 24 & $9.738 \mathrm{E}-01$ & $9.709 \mathrm{E}-01$ & $9.735 \mathrm{E}-01$ & $9.697 \mathrm{E}-01$ & $9.723 \mathrm{E}-01$ & $9.731 \mathrm{E}-01$ & $9.735 \mathrm{E}-01$ & $9.736 \mathrm{E}-01$ \\
\hline 25 & $9.697 \mathrm{E}-01$ & $9.666 \mathrm{E}-01$ & $9.694 \mathrm{E}-01$ & $9.654 \mathrm{E}-01$ & $9.696 \mathrm{E}-01$ & $9.702 \mathrm{E}-01$ & $9.695 \mathrm{E}-01$ & $9.695 \mathrm{E}-01$ \\
\hline 26 & $9.581 \mathrm{E}-01$ & $9.543 \mathrm{E}-01$ & $9.576 \mathrm{E}-01$ & $9.531 \mathrm{E}-01$ & $9.621 \mathrm{E}-01$ & $9.622 \mathrm{E}-01$ & $9.579 \mathrm{E}-01$ & $9.576 \mathrm{E}-01$ \\
\hline 27 & $9.653 \mathrm{E}-01$ & $9.621 \mathrm{E}-01$ & $9.647 \mathrm{E}-01$ & $9.609 \mathrm{E}-01$ & $9.675 \mathrm{E}-01$ & $9.678 \mathrm{E}-01$ & $9.650 \mathrm{E}-01$ & $9.647 \mathrm{E}-01$ \\
\hline 28 & $9.756 \mathrm{E}-01$ & $9.732 \mathrm{E}-01$ & $9.748 \mathrm{E}-01$ & $9.719 \mathrm{E}-01$ & $9.750 \mathrm{E}-01$ & $9.756 \mathrm{E}-01$ & $9.750 \mathrm{E}-01$ & $9.749 \mathrm{E}-01$ \\
\hline 29 & $9.833 \mathrm{E}-01$ & $9.815 \mathrm{E}-01$ & $9.826 \mathrm{E}-01$ & $9.803 \mathrm{E}-01$ & $9.798 \mathrm{E}-01$ & $9.807 \mathrm{E}-01$ & $9.826 \mathrm{E}-01$ & $9.826 \mathrm{E}-01$ \\
\hline 30 & $9.828 \mathrm{E}-01$ & $9.674 \mathrm{E}-01$ & $9.828 \mathrm{E}-01$ & $9.659 \mathrm{E}-01$ & $9.914 \mathrm{E}-01$ & $9.912 \mathrm{E}-01$ & $9.833 \mathrm{E}-01$ & $9.826 \mathrm{E}-01$ \\
\hline 31 & $9.663 \mathrm{E}-01$ & $9.478 \mathrm{E}-01$ & $9.663 \mathrm{E}-01$ & $9.461 \mathrm{E}-01$ & $9.828 \mathrm{E}-01$ & $9.817 \mathrm{E}-01$ & $9.671 \mathrm{E}-01$ & $9.659 \mathrm{E}-01$ \\
\hline 32 & $9.511 \mathrm{E}-01$ & $9.310 \mathrm{E}-01$ & $9.511 \mathrm{E}-01$ & $9.293 \mathrm{E}-01$ & $9.717 \mathrm{E}-01$ & $9.702 \mathrm{E}-01$ & $9.521 \mathrm{E}-01$ & $9.507 \mathrm{E}-01$ \\
\hline 33 & $9.875 \mathrm{E}-01$ & $9.864 \mathrm{E}-01$ & $9.870 \mathrm{E}-01$ & $9.854 \mathrm{E}-01$ & $9.796 \mathrm{E}-01$ & $9.810 \mathrm{E}-01$ & $9.868 \mathrm{E}-01$ & $9.871 \mathrm{E}-01$ \\
\hline 34 & $9.830 \mathrm{E}-01$ & $9.809 \mathrm{E}-01$ & $9.825 \mathrm{E}-01$ & $9.798 \mathrm{E}-01$ & $9.788 \mathrm{E}-01$ & $9.799 \mathrm{E}-01$ & $9.825 \mathrm{E}-01$ & $9.826 \mathrm{E}-01$ \\
\hline 35 & $9.773 \mathrm{E}-01$ & $9.749 \mathrm{E}-01$ & $9.770 \mathrm{E}-01$ & $9.738 \mathrm{E}-01$ & $9.750 \mathrm{E}-01$ & $9.759 \mathrm{E}-01$ & $9.770 \mathrm{E}-01$ & $9.771 \mathrm{E}-01$ \\
\hline 36 & $9.680 \mathrm{E}-01$ & $9.649 \mathrm{E}-01$ & $9.676 \mathrm{E}-01$ & $9.637 \mathrm{E}-01$ & $9.692 \mathrm{E}-01$ & $9.697 \mathrm{E}-01$ & $9.678 \mathrm{E}-01$ & $9.676 \mathrm{E}-01$ \\
\hline 37 & $9.738 \mathrm{E}-01$ & $9.711 \mathrm{E}-01$ & $9.736 \mathrm{E}-01$ & $9.701 \mathrm{E}-01$ & $9.725 \mathrm{E}-01$ & $9.733 \mathrm{E}-01$ & $9.736 \mathrm{E}-01$ & $9.737 \mathrm{E}-01$ \\
\hline
\end{tabular}

Figure 6.6.8. Prior calculated response correlation matrix edit. 
Adjusted-Response Correlation Matrix

\begin{tabular}{|c|c|c|c|c|c|c|c|c|}
\hline resp & resp 1 & resp & resp & resp & resp & resp & resp & resp \\
\hline 1 & $1.000 \mathrm{E}+00$ & $8.916 \mathrm{E}-01$ & $9.629 \mathrm{E}-01$ & $8.530 \mathrm{E}-01$ & $8.277 \mathrm{E}-01$ & $8.526 \mathrm{E}-01$ & $9.625 \mathrm{E}-01$ & $9.634 \mathrm{E}-01$ \\
\hline 2 & $8.916 \mathrm{E}-01$ & $1.000 \mathrm{E}+00$ & $8.490 \mathrm{E}-01$ & $9.648 \mathrm{E}-01$ & $5.905 \mathrm{E}-01$ & $6.214 \mathrm{E}-01$ & $8.413 \mathrm{E}-01$ & $8.529 \mathrm{E}-01$ \\
\hline 3 & $9.629 \mathrm{E}-01$ & $8.490 \mathrm{E}-01$ & $1.000 \mathrm{E}+00$ & $8.796 \mathrm{E}-01$ & $8.663 \mathrm{E}-01$ & $8.917 \mathrm{E}-01$ & $9.997 \mathrm{E}-01$ & $9.998 \mathrm{E}-01$ \\
\hline 4 & $8.530 \mathrm{E}-01$ & $9.648 \mathrm{E}-01$ & $8.796 \mathrm{E}-01$ & $1.000 \mathrm{E}+00$ & $6.189 \mathrm{E}-01$ & $6.501 \mathrm{E}-01$ & $8.721 \mathrm{E}-01$ & $8.838 \mathrm{E}-01$ \\
\hline 5 & $8.277 \mathrm{E}-01$ & $5.905 \mathrm{E}-01$ & $8.663 \mathrm{E}-01$ & $6.189 \mathrm{E}-01$ & $1.000 \mathrm{E}+00$ & $9.985 \mathrm{E}-01$ & $8.765 \mathrm{E}-01$ & $8.606 \mathrm{E}-01$ \\
\hline 6 & $8.526 \mathrm{E}-01$ & $6.214 \mathrm{E}-01$ & $8.917 \mathrm{E}-01$ & $6.501 \mathrm{E}-01$ & $9.985 \mathrm{E}-01$ & $1.000 \mathrm{E}+00$ & $9.009 \mathrm{E}-01$ & $8.865 \mathrm{E}-01$ \\
\hline 7 & $9.625 \mathrm{E}-01$ & $8.413 \mathrm{E}-01$ & $9.997 \mathrm{E}-01$ & $8.721 \mathrm{E}-01$ & $8.765 \mathrm{E}-01$ & $9.009 \mathrm{E}-01$ & $1.000 \mathrm{E}+00$ & $9.992 \mathrm{E}-01$ \\
\hline 8 & $9.634 \mathrm{E}-01$ & $8.529 \mathrm{E}-01$ & $9.998 \mathrm{E}-01$ & $8.838 \mathrm{E}-01$ & $8.606 \mathrm{E}-01$ & $8.865 \mathrm{E}-01$ & $9.992 \mathrm{E}-01$ & $1.000 \mathrm{E}+00$ \\
\hline 9 & $8.534 \mathrm{E}-01$ & $9.646 \mathrm{E}-01$ & $8.799 \mathrm{E}-01$ & $9.999 \mathrm{E}-01$ & $6.208 \mathrm{E}-01$ & $6.518 \mathrm{E}-01$ & $8.726 \mathrm{E}-01$ & $8.842 \mathrm{E}-01$ \\
\hline 10 & $8.541 \mathrm{E}-01$ & $6.235 \mathrm{E}-01$ & $8.941 \mathrm{E}-01$ & $6.520 \mathrm{E}-01$ & $9.968 \mathrm{E}-01$ & $9.990 \mathrm{E}-01$ & $9.033 \mathrm{E}-01$ & $8.888 \mathrm{E}-01$ \\
\hline 11 & $8.150 \mathrm{E}-01$ & $6.935 \mathrm{E}-01$ & $8.277 \mathrm{E}-01$ & $6.829 \mathrm{E}-01$ & $7.351 \mathrm{E}-01$ & $7.573 \mathrm{E}-01$ & $8.238 \mathrm{E}-01$ & $8.291 \mathrm{E}-01$ \\
\hline 12 & $8.904 \mathrm{E}-01$ & $9.808 \mathrm{E}-01$ & $8.354 \mathrm{E}-01$ & $9.423 \mathrm{E}-01$ & $6.150 \mathrm{E}-01$ & $6.419 \mathrm{E}-01$ & $8.309 \mathrm{E}-01$ & $8.392 \mathrm{E}-01$ \\
\hline 13 & $7.950 \mathrm{E}-01$ & $5.588 \mathrm{E}-01$ & $8.308 \mathrm{E}-01$ & $5.896 \mathrm{E}-01$ & $9.877 \mathrm{E}-01$ & $9.833 \mathrm{E}-01$ & $8.433 \mathrm{E}-01$ & $8.244 \mathrm{E}-01$ \\
\hline 14 & $9.869 \mathrm{E}-01$ & $8.703 \mathrm{E}-01$ & $9.488 \mathrm{E}-01$ & $8.280 \mathrm{E}-01$ & $8.583 \mathrm{E}-01$ & $8.786 \mathrm{E}-01$ & $9.509 \mathrm{E}-01$ & $9.466 \mathrm{E}-01$ \\
\hline 15 & $9.537 \mathrm{E}-01$ & $8.341 \mathrm{E}-01$ & $9.916 \mathrm{E}-01$ & $8.669 \mathrm{E}-01$ & $8.944 \mathrm{E}-01$ & $9.158 \mathrm{E}-01$ & $9.936 \mathrm{E}-01$ & $9.896 \mathrm{E}-01$ \\
\hline 16 & $8.567 \mathrm{E}-01$ & $9.549 \mathrm{E}-01$ & $8.878 \mathrm{E}-01$ & $9.898 \mathrm{E}-01$ & $6.606 \mathrm{E}-01$ & $6.891 \mathrm{E}-01$ & $8.817 \mathrm{E}-01$ & $8.902 \mathrm{E}-01$ \\
\hline 17 & $1.856 \mathrm{E}-01$ & $2.321 \mathrm{E}-01$ & $1.808 \mathrm{E}-01$ & $2.363 \mathrm{E}-01$ & $1.675 \mathrm{E}-01$ & $1.688 \mathrm{E}-01$ & $1.812 \mathrm{E}-01$ & $1.819 \mathrm{E}-01$ \\
\hline 18 & $5.222 \mathrm{E}-01$ & $5.967 \mathrm{E}-01$ & $5.551 \mathrm{E}-01$ & $6.082 \mathrm{E}-01$ & $4.698 \mathrm{E}-01$ & $4.846 \mathrm{E}-01$ & $5.491 \mathrm{E}-01$ & $5.553 \mathrm{E}-01$ \\
\hline 19 & $6.311 \mathrm{E}-01$ & $3.866 \mathrm{E}-01$ & $6.173 \mathrm{E}-01$ & $3.713 \mathrm{E}-01$ & $8.690 \mathrm{E}-01$ & $8.530 \mathrm{E}-01$ & $6.318 \mathrm{E}-01$ & $6.111 \mathrm{E}-01$ \\
\hline 20 & $6.622 \mathrm{E}-01$ & $4.169 \mathrm{E}-01$ & $6.154 \mathrm{E}-01$ & $3.653 \mathrm{E}-01$ & $8.540 \mathrm{E}-01$ & $8.398 \mathrm{E}-01$ & $6.286 \mathrm{E}-01$ & $6.100 \mathrm{E}-01$ \\
\hline 21 & $7.295 \mathrm{E}-01$ & $5.167 \mathrm{E}-01$ & $6.495 \mathrm{E}-01$ & $4.285 \mathrm{E}-01$ & $7.865 \mathrm{E}-01$ & $7.834 \mathrm{E}-01$ & $6.582 \mathrm{E}-01$ & $6.456 \mathrm{E}-01$ \\
\hline 22 & $9.864 \mathrm{E}-01$ & $8.495 \mathrm{E}-01$ & $9.378 \mathrm{E}-01$ & $7.951 \mathrm{E}-01$ & $8.603 \mathrm{E}-01$ & $8.801 \mathrm{E}-01$ & $9.396 \mathrm{E}-01$ & $9.365 \mathrm{E}-01$ \\
\hline 23 & $4.880 \mathrm{E}-01$ & $4.903 \mathrm{E}-01$ & $4.928 \mathrm{E}-01$ & $4.794 \mathrm{E}-01$ & $3.958 \mathrm{E}-01$ & $4.132 \mathrm{E}-01$ & $4.863 \mathrm{E}-01$ & $4.972 \mathrm{E}-01$ \\
\hline 24 & $5.166 \mathrm{E}-01$ & $5.003 \mathrm{E}-01$ & $5.130 \mathrm{E}-01$ & $4.885 \mathrm{E}-01$ & $5.022 \mathrm{E}-01$ & $5.113 \mathrm{E}-01$ & $5.123 \mathrm{E}-01$ & $5.148 \mathrm{E}-01$ \\
\hline 25 & $4.678 \mathrm{E}-01$ & $4.510 \mathrm{E}-01$ & $4.650 \mathrm{E}-01$ & $4.406 \mathrm{E}-01$ & $4.786 \mathrm{E}-01$ & $4.847 \mathrm{E}-01$ & $4.654 \mathrm{E}-01$ & $4.662 \mathrm{E}-01$ \\
\hline 26 & $3.542 \mathrm{E}-01$ & $3.359 \mathrm{E}-01$ & $3.504 \mathrm{E}-01$ & $3.294 \mathrm{E}-01$ & $4.347 \mathrm{E}-01$ & $4.321 \mathrm{E}-01$ & $3.551 \mathrm{E}-01$ & $3.493 \mathrm{E}-01$ \\
\hline 27 & $4.191 \mathrm{E}-01$ & $4.060 \mathrm{E}-01$ & $4.111 \mathrm{E}-01$ & $3.969 \mathrm{E}-01$ & $4.747 \mathrm{E}-01$ & $4.745 \mathrm{E}-01$ & $4.154 \mathrm{E}-01$ & $4.104 \mathrm{E}-01$ \\
\hline 28 & $5.372 \mathrm{E}-01$ & $5.292 \mathrm{E}-01$ & $5.228 \mathrm{E}-01$ & $5.150 \mathrm{E}-01$ & $5.446 \mathrm{E}-01$ & $5.493 \mathrm{E}-01$ & $5.259 \mathrm{E}-01$ & $5.229 \mathrm{E}-01$ \\
\hline 29 & $6.543 \mathrm{E}-01$ & $6.511 \mathrm{E}-01$ & $6.374 \mathrm{E}-01$ & $6.342 \mathrm{E}-01$ & $6.022 \mathrm{E}-01$ & $6.136 \mathrm{E}-01$ & $6.379 \mathrm{E}-01$ & $6.391 \mathrm{E}-01$ \\
\hline 30 & $6.667 \mathrm{E}-01$ & $4.381 \mathrm{E}-01$ & $6.730 \mathrm{E}-01$ & $4.322 \mathrm{E}-01$ & $8.187 \mathrm{E}-01$ & $8.159 \mathrm{E}-01$ & $6.816 \mathrm{E}-01$ & $6.694 \mathrm{E}-01$ \\
\hline 31 & $4.770 \mathrm{E}-01$ & $2.395 \mathrm{E}-01$ & $4.821 \mathrm{E}-01$ & $2.341 \mathrm{E}-01$ & $7.442 \mathrm{E}-01$ & $7.284 \mathrm{E}-01$ & $4.955 \mathrm{E}-01$ & $4.767 \mathrm{E}-01$ \\
\hline 32 & $3.553 \mathrm{E}-01$ & $1.294 \mathrm{E}-01$ & $3.622 \mathrm{E}-01$ & $1.263 \mathrm{E}-01$ & $6.524 \mathrm{E}-01$ & $6.326 \mathrm{E}-01$ & $3.763 \mathrm{E}-01$ & $3.564 \mathrm{E}-01$ \\
\hline 33 & $7.158 \mathrm{E}-01$ & $7.218 \mathrm{E}-01$ & $7.060 \mathrm{E}-01$ & $7.095 \mathrm{E}-01$ & $5.640 \mathrm{E}-01$ & $5.864 \mathrm{E}-01$ & $7.012 \mathrm{E}-01$ & $7.099 \mathrm{E}-01$ \\
\hline 34 & $6.492 \mathrm{E}-01$ & $6.403 \mathrm{E}-01$ & $6.387 \mathrm{E}-01$ & $6.257 \mathrm{E}-01$ & $5.781 \mathrm{E}-01$ & $5.927 \mathrm{E}-01$ & $6.370 \mathrm{E}-01$ & $6.413 \mathrm{E}-01$ \\
\hline 35 & $5.624 \mathrm{E}-01$ & $5.510 \mathrm{E}-01$ & $5.579 \mathrm{E}-01$ & $5.404 \mathrm{E}-01$ & $5.327 \mathrm{E}-01$ & $5.433 \mathrm{E}-01$ & $5.572 \mathrm{E}-01$ & $5.598 \mathrm{E}-01$ \\
\hline 36 & $4.467 \mathrm{E}-01$ & $4.327 \mathrm{E}-01$ & $4.414 \mathrm{E}-01$ & $4.238 \mathrm{E}-01$ & $4.848 \mathrm{E}-01$ & $4.872 \mathrm{E}-01$ & $4.443 \mathrm{E}-01$ & $4.412 \mathrm{E}-01$ \\
\hline
\end{tabular}

Figure 6.6.9. Adjusted response correlation matrix edit. 
$\star \star \star$ Cumulative Conv. of $(\mathrm{A}-\mathrm{C}) / \mathrm{C}$ For Application $* \star \star$

$=>$ Edited for reference response: 40

=>Based on similarity parameter : $\mathrm{Ck}$

$\Rightarrow$ Minimum similarity included : 0.300

=>Similarity bin width for edit : 0.010

\begin{tabular}{|c|c|c|c|}
\hline CUM RANGE & MAX. SIM & NO. EXP & $\div(A-C) / C$ \\
\hline NO. & INCLUDED & INCLUDED & \\
\hline 1 & 0.390 & 1 & -0.057 \\
\hline 2 & 0.400 & 1 & -0.057 \\
\hline 3 & 0.410 & 1 & -0.057 \\
\hline 4 & 0.420 & 1 & -0.057 \\
\hline 5 & 0.430 & 1 & -0.057 \\
\hline 6 & 0.440 & 1 & -0.057 \\
\hline 7 & 0.450 & 1 & -0.057 \\
\hline 8 & 0.460 & 1 & -0.057 \\
\hline 9 & 0.470 & 1 & -0.057 \\
\hline 10 & 0.480 & 1 & -0.057 \\
\hline 11 & 0.490 & 1 & -0.057 \\
\hline 12 & 0.500 & 1 & -0.057 \\
\hline 13 & 0.510 & 1 & -0.057 \\
\hline 14 & 0.520 & 1 & -0.057 \\
\hline 15 & 0.530 & 1 & -0.057 \\
\hline 16 & 0.540 & 1 & -0.057 \\
\hline 17 & 0.550 & 1 & -0.057 \\
\hline 18 & 0.560 & 1 & -0.057 \\
\hline 19 & 0.570 & 1 & -0.057 \\
\hline 20 & 0.580 & 1 & -0.057 \\
\hline 21 & 0.590 & 1 & -0.057 \\
\hline 22 & 0.600 & 1 & -0.057 \\
\hline 23 & 0.610 & 1 & -0.057 \\
\hline 24 & 0.620 & 1 & -0.057 \\
\hline 25 & 0.630 & 1 & -0.057 \\
\hline 26 & 0.640 & 1 & -0.057 \\
\hline 27 & 0.650 & 1 & -0.057 \\
\hline 28 & 0.660 & 1 & -0.057 \\
\hline 29 & 0.670 & 1 & -0.057 \\
\hline 30 & 0.680 & 1 & -0.057 \\
\hline 31 & 0.690 & 1 & -0.057 \\
\hline 32 & 0.700 & 1 & -0.057 \\
\hline 33 & 0.710 & 1 & -0.057 \\
\hline
\end{tabular}

Figure 6.6.10. Bias convergence edit 


$\begin{array}{llll}34 & 0.720 & 1 & -0.057 \\ 35 & 0.730 & 1 & -0.057 \\ 36 & 0.740 & 1 & -0.057 \\ 37 & 0.750 & 1 & -0.057 \\ 38 & 0.760 & 1 & -0.057 \\ 39 & 0.770 & 1 & -0.057 \\ 40 & 0.780 & 1 & -0.057 \\ 41 & 0.790 & 1 & -0.057 \\ 42 & 0.800 & 1 & -0.057 \\ 43 & 0.810 & 1 & -0.057 \\ 44 & 0.820 & 1 & -0.057 \\ 45 & 0.830 & 1 & -0.057 \\ 46 & 0.840 & 1 & -0.057 \\ 47 & 0.850 & 1 & -0.057 \\ 48 & 0.860 & 1 & -0.057 \\ 49 & 0.870 & 1 & -0.057 \\ 50 & 0.880 & 1 & -0.057 \\ 51 & 0.890 & 1 & -0.057 \\ 52 & 0.900 & 1 & -0.057 \\ 53 & 0.910 & 1 & -0.057 \\ 54 & 0.920 & 1 & -0.057 \\ 55 & 0.930 & 1 & -0.057 \\ 56 & 0.940 & 1 & -0.057 \\ 57 & 0.950 & 1 & -0.057 \\ 58 & 0.960 & 7 & 0.063 \\ 59 & 0.970 & 16 & -0.076 \\ 60 & 0.980 & 21 & -0.133 \\ 61 & 0.990 & 25 & -0.170 \\ 62 & 1.000 & 37 & -0.246\end{array}$

Figure 6.6.10. Bias convergence edit (continued). 
*** Summary of Adjustments by Response ***

$\begin{aligned} \text { NOTATION: calc } & =\text { prior calculated value } \\ \text { exp } & =\text { prior experimental value } \\ \text { adj } & =\text { adjusted calculated value } \\ & =\text { adjusted experimental value }\end{aligned}$

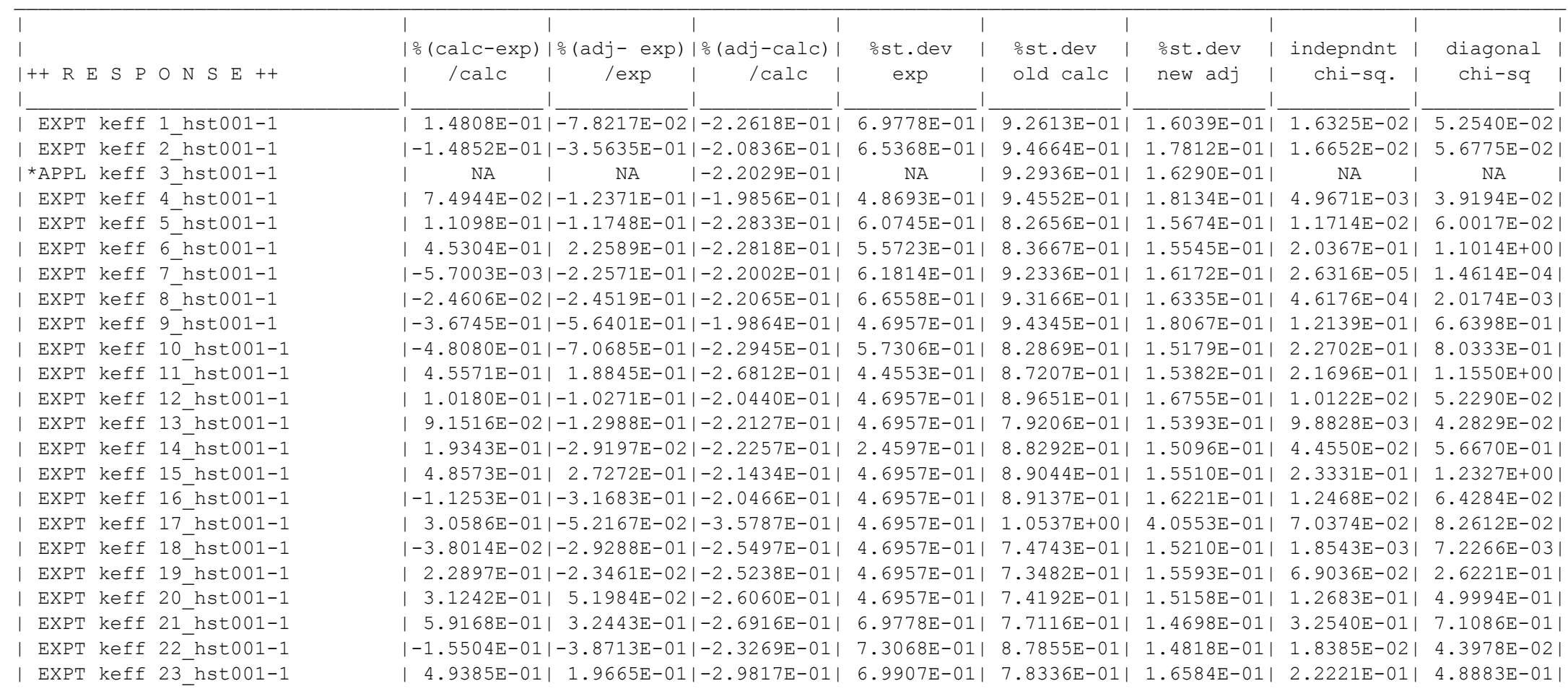

Figure 6.6.11. Adjustment summary edit. 
** Description of Adjusted Responses **

$\begin{array}{cl}\text { RESP.\# } & \text { EXPERIMENT NAME } \\ 1 & \text { 1_hst001-1 } \\ 2 & \text { 2_hst001-1 } \\ 3 & \text { 3_hst001-1 } \\ 4 & \text { 4_hst001-1 } \\ 5 & 5 \text { hst001-1 } \\ 6 & 6 \text { hst001-1 } \\ 7 & 7 \text { hst001-1 } \\ 8 & 8 \text { hst001-1 } \\ 9 & 9 \text { hst001-1 } \\ 10 & 10 \text { hst001-1 } \\ 11 & 11 \text { hst001-1 } \\ 12 & 12 \text { hst001-1 } \\ 13 & 13 \text { hst001-1 } \\ 14 & 14 \text { hst001-1 } \\ 15 & 15 \text { hst001-1 } \\ 16 & 16 \text { hst001-1 } \\ 17 & 17 \text { hst001-1 } \\ 18 & 18 \text { hst001-1 } \\ 19 & 19 \text { hst001-1 } \\ 20 & 20 \text { hst001-1 } \\ 21 & 21 \text { hst001-1 } \\ 22 & 22 \text { hst001-1 } \\ 23 & 23 \text { hst001-1 } \\ 24 & 24 \text { hst001-1 } \\ 25 & 25 \text { hst001-1 } \\ 26 & 26 \text { hst001-1 } \\ 27 & 27 \text { hst001-1 } \\ & \\ & \end{array}$

$\begin{array}{lll}\text { SENS. TITLE } & \text { USE } & \text { TYPE } \\ \text { r1 } & \text { expt } & \text { keff } \\ \text { r2 } & \text { expt } & \text { keff } \\ \text { r3 } & \text { appl } & \text { keff } \\ \text { r4 } & \text { expt } & \text { keff } \\ \text { r5 } & \text { expt } & \text { keff } \\ r 6 & \text { expt } & \text { keff } \\ \text { r7 } & \text { expt } & \text { keff } \\ \text { r8 } & \text { expt } & \text { keff } \\ \text { r9 } & \text { expt } & \text { keff } \\ \text { r10 } & \text { expt } & \text { keff } \\ \text { rot2 tank in cen } & \text { expt } & \text { keff } \\ \text { rot7 tank in cen } & \text { expt } & \text { keff } \\ \text { rot38 tank in ce } & \text { expt } & \text { keff } \\ \text { rot4 tank in cen } & \text { expt } & \text { keff } \\ \text { rot14 tank in ce } & \text { expt } & \text { keff } \\ \text { rot29 tank in ce } & \text { expt } & \text { keff } \\ \text { ol3ne 15.5 in. S } & \text { expt } & \text { keff } \\ \text { case 30 experime } & \text { expt } & \text { keff } \\ \text { heu-sol-therm-02 } & \text { expt } & \text { keff } \\ \text { heu-sol-therm-02 } & \text { expt } & \text { keff } \\ \text { heu-sol-therm-02 } & \text { expt } & \text { keff } \\ \text { heu-sol-therm-02 } & \text { expt } & \text { keff } \\ \text { heu-sol-therm-02 } & \text { expt } & \text { keff } \\ \text { heu-sol-therm-02 } & \text { expt } & \text { keff } \\ \text { heu-sol-therm-02 } & \text { expt } & \text { keff } \\ \text { heu-sol-therm-02 } & \text { expt } & \text { keff } \\ \text { heu-sol-therm-02 } & \text { expt } & \text { keff }\end{array}$

ADJUSTED RESPONSE

$9.9922 \mathrm{E}-01+/-\quad 1.6063 \mathrm{E}-03$

$9.9644 \mathrm{E}-01+/-1.7785 \mathrm{E}-03$

$1.0002 \mathrm{E}+00+/-1.6330 \mathrm{E}-03$

$9.9876 \mathrm{E}-01+/-\quad 1.8147 \mathrm{E}-03$

$9.9883 \mathrm{E}-01+/-\quad 1.5691 \mathrm{E}-03$

$1.0023 \mathrm{E}+00+/-1.5616 \mathrm{E}-03$

$9.9774 \mathrm{E}-01+/-\quad 1.6171 \mathrm{E}-03$

$9.9755 \mathrm{E}-01+/-\quad 1.6331 \mathrm{E}-03$

$9.9436 \mathrm{E}-01+/-\quad 1.8001 \mathrm{E}-03$

$9.9293 \mathrm{E}-01+/-1.5106 \mathrm{E}-03$

$1.0019 \mathrm{E}+00+/-1.5453 \mathrm{E}-03$

$9.9897 \mathrm{E}-01+/-\quad 1.6772 \mathrm{E}-03$

$9.9870 \mathrm{E}-01+/-\quad 1.5407 \mathrm{E}-03$

$9.9971 \mathrm{E}-01+/-1.5125 \mathrm{E}-03$

$1.0027 \mathrm{E}+00+/-1.5586 \mathrm{E}-03$

$9.9683 \mathrm{E}-01+/-1.6202 \mathrm{E}-03$

$9.9948 \mathrm{E}-01+/-\quad 4.0678 \mathrm{E}-03$

$9.9707 \mathrm{E}-01+/-\quad 1.5204 \mathrm{E}-03$

$9.9977 \mathrm{E}-01+/-1.5629 \mathrm{E}-03$

$1.0005 \mathrm{E}+00+/-1.5205 \mathrm{E}-03$

$1.0032 \mathrm{E}+00+/-\quad 1.4786 \mathrm{E}-03$

$9.9613 \mathrm{E}-01+/-\quad 1.4795 \mathrm{E}-03$

$1.0020 \mathrm{E}+00+/-1.6666 \mathrm{E}-03$

$1.0059 \mathrm{E}+00+/-1.3850 \mathrm{E}-03$

$9.9870 \mathrm{E}-01+/-1.3849 \mathrm{E}-03$

$\begin{array}{ll}9.9870 \mathrm{E}-01+/- & 1.3849 \mathrm{E}-03 \\ 9.9596 \mathrm{E}-01+/- & 1.4012 \mathrm{E}-03\end{array}$

heu-sol-therm-02 expt keff

$1.0019 \mathrm{E}+00+/-1.3609 \mathrm{E}-03$

Figure 6.6.12. Adjusted response edit. 
APPLICATION AND BIAS SUMMARY

$\begin{array}{lllrlrrr}\text { APPLICATION } & \text { TYPE CALC VALUE } & \text { PRIOR UNC } & \text { REL. BIAS } & \text { BIAS } & \text { ADJ VALUE } & \text { ADJ UNC } \\ - & \text { keff } & 1.0024 \mathrm{E}+00 & 9.2936 \mathrm{E}-01 & 2.2029 \mathrm{E}-01 & 2.2083 \mathrm{E}-03 & 1.0002 \mathrm{E}+00 & 1.6290 \mathrm{E}-01 \\ \text { 3_hst001-1 } & \text { keff } & 9.9990 \mathrm{E}-01 & 6.2270 \mathrm{E}-01 & 2.7119 \mathrm{E}-01 & 2.7116 \mathrm{E}-03 & 9.9719 \mathrm{E}-01 & 1.5301 \mathrm{E}-01 \\ \text { 32_hst001-1 } & \text { keff } & 1.0017 \mathrm{E}+00 & 6.0802 \mathrm{E}-01 & 2.4635 \mathrm{E}-01 & 2.4677 \mathrm{E}-03 & 9.9922 \mathrm{E}-01 & 1.3718 \mathrm{E}-01\end{array}$

NOTE: The relative bias and uncertainty values are normalized to the calculated response value.

CONTRIBUTION TO THE REFERENCE APPLICATION BIAS FROM NUCLIDE-REACTION PAIRS

\begin{tabular}{|c|c|c|c|}
\hline NUCLIDE & REACTION & $\begin{array}{l}\text { CONTRIB. TO BIAS } \\
\therefore \mathrm{dk} / \mathrm{k}\end{array}$ & $\begin{array}{l}\text { FRACTION OF BIAS } \\
\text { LI-NORM }\end{array}$ \\
\hline$u-235$ & nubar & $1.2213 \mathrm{E}-01$ & $3.1407 E-01$ \\
\hline$u-235$ & chi & $1.0203 \mathrm{E}-01$ & $2.6237 \mathrm{E}-01$ \\
\hline$u-235$ & $\mathrm{n}$, gamma & $5.6672 \mathrm{E}-02$ & $1.4647 \mathrm{E}-01$ \\
\hline$h-1$ & elastic & $-4.9761 \mathrm{E}-02$ & $1.2798 \mathrm{E}-01$ \\
\hline$u-235$ & fission & $2.3867 \mathrm{E}-02$ & $6.1390 \mathrm{E}-02$ \\
\hline $0-16$ & elastic & $-2.0793 E-02$ & $5.3522 \mathrm{E}-02$ \\
\hline $\mathrm{fe}$ & $\mathrm{n}$, gamma & $6.2393 \mathrm{E}-03$ & $1.6044 \mathrm{E}-02$ \\
\hline $\mathrm{fe}$ & elastic & $2.1543 E-03$ & $5.5536 \mathrm{E}-03$ \\
\hline$h-1$ & $\mathrm{n}$, gamma & $1.0344 \mathrm{E}-03$ & $2.6600 \mathrm{E}-03$ \\
\hline $\mathrm{fe}$ & $n, n^{\prime}$ & $9.3138 \mathrm{E}-04$ & $2.3950 \mathrm{E}-03$ \\
\hline $\mathrm{Cr}$ & n, gamma & $7.2797 \mathrm{E}-04$ & $1.8720 \mathrm{E}-03$ \\
\hline ni & n, gamma & $3.7890 \mathrm{E}-04$ & $9.7433 \mathrm{E}-04$ \\
\hline$u-234$ & n, gamma & $-3.0719 E-04$ & $8.0394 \mathrm{E}-04$ \\
\hline
\end{tabular}

Figure 6.6.13. Application bias summary edit 


\begin{tabular}{|c|c|c|c|}
\hline $\mathrm{Cr}$ & elastic & $2.4825 E-04$ & $6.3853 E-04$ \\
\hline$u-238$ & $\mathrm{n}$, gamma & $2.4072 E-04$ & $6.1902 \mathrm{E}-04$ \\
\hline$b-10$ & $\mathrm{n}, \mathrm{alpha}$ & $2.1488 \mathrm{E}-04$ & $5.5255 \mathrm{E}-04$ \\
\hline$n-14$ & $\mathrm{n}, \mathrm{p}$ & $2.1113 E-04$ & $5.4291 \mathrm{E}-04$ \\
\hline$u-235$ & $n, n^{\prime}$ & $-1.5322 \mathrm{E}-04$ & $3.9401 \mathrm{E}-04$ \\
\hline $\mathrm{ni}$ & elastic & $1.0020 \mathrm{E}-04$ & $2.5830 \mathrm{E}-04$ \\
\hline$m n-55$ & $\mathrm{n}$, gamma & $8.5311 \mathrm{E}-05$ & $2.1938 \mathrm{E}-04$ \\
\hline $\mathrm{Cr}$ & $\mathrm{n}, \mathrm{n}^{\prime}$ & $4.9517 \mathrm{E}-05$ & $1.2733 E-04$ \\
\hline$n-14$ & elastic & $-4.0198 E-05$ & $1.0883 \mathrm{E}-04$ \\
\hline$u-235$ & elastic & $4.1221 E-06$ & $9.1845 \mathrm{E}-05$ \\
\hline $0-16$ & n, alpha & $3.5175 E-05$ & $9.0453 \mathrm{E}-05$ \\
\hline$u-238$ & elastic & $8.6693 E-06$ & $4.1429 \mathrm{E}-05$ \\
\hline$b-11$ & elastic & $1.3516 \mathrm{E}-05$ & $3.6278 \mathrm{E}-05$ \\
\hline ni & $\mathrm{n}, \mathrm{n}^{\prime}$ & $1.0318 \mathrm{E}-05$ & $2.6532 \mathrm{E}-05$ \\
\hline$m n-55$ & elastic & $1.0216 \mathrm{E}-05$ & $2.6270 \mathrm{E}-05$ \\
\hline$u-234$ & fission & $-7.9124 \mathrm{E}-06$ & $2.0356 \mathrm{E}-05$ \\
\hline$u-236$ & $\mathrm{n}$, gamma & $-5.3796 \mathrm{E}-06$ & $1.4106 \mathrm{E}-05$ \\
\hline$u-238$ & $n, n^{\prime}$ & $4.7750 \mathrm{E}-06$ & $1.2292 \mathrm{E}-05$ \\
\hline$n-14$ & $\mathrm{n}$, gamma & $4.2428 \mathrm{E}-06$ & $1.0911 \mathrm{E}-05$ \\
\hline$b-10$ & $n, n^{\prime}$ & $3.5966 \mathrm{E}-06$ & $9.5464 \mathrm{E}-06$ \\
\hline$n-14$ & n, alpha & $3.5931 \mathrm{E}-06$ & $9.2396 \mathrm{E}-06$ \\
\hline$n-14$ & $n, n^{\prime}$ & $5.9234 \mathrm{E}-07$ & $7.7528 \mathrm{E}-06$ \\
\hline $\mathrm{c}$ & elastic & $2.2321 \mathrm{E}-06$ & $6.1840 \mathrm{E}-06$ \\
\hline$b-10$ & elastic & $-8.9644 \mathrm{E}-07$ & $6.1541 \mathrm{E}-06$ \\
\hline$b-10$ & $n, p$ & $1.5897 \mathrm{E}-06$ & $4.0880 \mathrm{E}-06$ \\
\hline ni & $n, p$ & $1.3670 \mathrm{E}-06$ & $3.5153 \mathrm{E}-06$ \\
\hline $\mathrm{c}$ & $n, n^{\prime}$ & $-1.0409 \mathrm{E}-06$ & $2.6767 \mathrm{E}-06$ \\
\hline$u-234$ & $n, n^{\prime}$ & $-8.8825 E-07$ & $2.4901 \mathrm{E}-06$ \\
\hline $0-16$ & $n, n^{\prime}$ & $-7.8209 \mathrm{E}-07$ & $2.3129 \mathrm{E}-06$ \\
\hline$u-236$ & fission & $-7.4671 \mathrm{E}-07$ & $1.9550 \mathrm{E}-06$ \\
\hline si & elastic & $6.9545 \mathrm{E}-07$ & $1.8014 \mathrm{E}-06$ \\
\hline$u-234$ & nubar & $-6.3015 E-07$ & $1.6282 \mathrm{E}-06$ \\
\hline$u-235$ & $n, 2 n$ & $-4.4596 \mathrm{E}-07$ & $1.1468 \mathrm{E}-06$ \\
\hline$m n-55$ & $n, n^{\prime}$ & $4.2551 \mathrm{E}-07$ & $1.0942 \mathrm{E}-06$ \\
\hline$u-238$ & nubar & $3.9169 \mathrm{E}-07$ & $1.0072 \mathrm{E}-06$ \\
\hline $\mathrm{fe}$ & $\mathrm{n}, \mathrm{p}$ & $3.7711 \mathrm{E}-07$ & $9.6973 \mathrm{E}-07$ \\
\hline ti & elastic & $2.3945 E-07$ & $6.1575 \mathrm{E}-07$ \\
\hline ti & n, gamma & $1.6831 \mathrm{E}-07$ & $4.3281 \mathrm{E}-07$ \\
\hline$b-11$ & $n, n^{\prime}$ & $1.0274 \mathrm{E}-07$ & $2.6419 \mathrm{E}-07$ \\
\hline $0-16$ & n, gamma & $1.0048 \mathrm{E}-07$ & $2.5839 \mathrm{E}-07$ \\
\hline ti & $\mathrm{n}, \mathrm{n}^{\prime}$ & $7.4571 \mathrm{E}-08$ & $1.9176 \mathrm{E}-07$ \\
\hline si & $n, n^{\prime}$ & $5.2998 \mathrm{E}-08$ & $1.3628 \mathrm{E}-07$ \\
\hline$u-236$ & nubar & $-5.0705 E-08$ & $1.3039 \mathrm{E}-07$ \\
\hline
\end{tabular}

Figure 6.6.13. Application bias summary edit (continued). 
$:::::::::::::::::::::::::::::::::::::::::::::::::::::::::::::::::::::::::::::::::::::::$

Multigroup Cross Section Changes Inferred from GLSS Adjustment

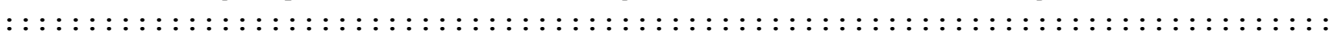

\begin{tabular}{|c|c|c|c|c|c|c|}
\hline $\begin{array}{l}\text { group } \\
\text { number }\end{array}$ & $\begin{array}{l}\text { delta-XS } \\
\left(\frac{\circ}{0}\right)\end{array}$ & $\begin{array}{l}\text { st. dev. } \\
\left.\text { old ( } \frac{0}{0}\right)\end{array}$ & $\begin{array}{l}\text { st. dev. } \\
\text { new }\left(\frac{\circ}{0}\right)\end{array}$ & $\begin{array}{c}\text { rel sen } \\
\text { coeff. }\end{array}$ & $\begin{array}{l}\text { contribution to } \\
\text { appl. bias (\%) }\end{array}$ & $\begin{array}{l}\text { fraction of } \\
\text { bias - L1-norm }\end{array}$ \\
\hline 1 & $8.798 \mathrm{E}-06$ & $8.125 \mathrm{E}-01$ & $8.125 \mathrm{E}-01$ & $1.585 \mathrm{E}-04$ & $-1.395 \mathrm{E}-09$ & $3.586 \mathrm{E}-09$ \\
\hline 2 & $-1.194 \mathrm{E}-04$ & $2.310 \mathrm{E}-01$ & $2.309 \mathrm{E}-01$ & $3.486 \mathrm{E}-04$ & $4.160 \mathrm{E}-08$ & $1.070 \mathrm{E}-07$ \\
\hline 3 & $-1.044 \mathrm{E}-04$ & $2.811 \mathrm{E}-01$ & $2.811 \mathrm{E}-01$ & $6.741 \mathrm{E}-04$ & $7.035 \mathrm{E}-08$ & $1.809 \mathrm{E}-07$ \\
\hline 4 & $-1.079 \mathrm{E}-04$ & $2.298 \mathrm{E}-01$ & $2.298 \mathrm{E}-01$ & $2.170 \mathrm{E}-03$ & $2.342 \mathrm{E}-07$ & $6.023 \mathrm{E}-07$ \\
\hline 5 & $-9.178 \mathrm{E}-05$ & $3.071 \mathrm{E}-01$ & $3.071 \mathrm{E}-01$ & $1.384 \mathrm{E}-03$ & $1.270 \mathrm{E}-07$ & $3.265 \mathrm{E}-07$ \\
\hline 6 & $-5.405 \mathrm{E}-05$ & $2.888 \mathrm{E}-01$ & $2.887 E-01$ & $4.064 \mathrm{E}-04$ & $2.197 \mathrm{E}-08$ & $5.648 \mathrm{E}-08$ \\
\hline 7 & $-5.449 \mathrm{E}-05$ & $2.887 \mathrm{E}-01$ & $2.886 \mathrm{E}-01$ & $1.693 \mathrm{E}-03$ & $9.228 \mathrm{E}-08$ & $2.373 E-07$ \\
\hline 8 & $-8.681 \mathrm{E}-05$ & $2.444 \mathrm{E}-01$ & $2.443 \mathrm{E}-01$ & $1.896 \mathrm{E}-03$ & $1.646 \mathrm{E}-07$ & $4.232 \mathrm{E}-07$ \\
\hline 9 & $-1.706 \mathrm{E}-04$ & $2.544 \mathrm{E}-01$ & $2.543 \mathrm{E}-01$ & $2.392 \mathrm{E}-03$ & $4.082 \mathrm{E}-07$ & $1.050 \mathrm{E}-06$ \\
\hline 10 & $-2.719 \mathrm{E}-04$ & $1.946 \mathrm{E}-01$ & $1.945 \mathrm{E}-01$ & $3.378 \mathrm{E}-03$ & $9.187 \mathrm{E}-07$ & $2.362 \mathrm{E}-06$ \\
\hline 11 & $-3.341 \mathrm{E}-04$ & $1.828 \mathrm{E}-01$ & $1.827 \mathrm{E}-01$ & $3.793 \mathrm{E}-03$ & $1.267 \mathrm{E}-06$ & $3.259 \mathrm{E}-06$ \\
\hline 12 & $-4.506 \mathrm{E}-04$ & $2.503 \mathrm{E}-01$ & $2.502 \mathrm{E}-01$ & $2.920 \mathrm{E}-03$ & $1.316 \mathrm{E}-06$ & $3.384 \mathrm{E}-06$ \\
\hline 13 & $-1.078 \mathrm{E}-03$ & $3.416 \mathrm{E}-01$ & $3.415 \mathrm{E}-01$ & $8.418 \mathrm{E}-04$ & $9.071 \mathrm{E}-07$ & $2.333 \mathrm{E}-06$ \\
\hline 14 & $-1.230 \mathrm{E}-03$ & $2.896 \mathrm{E}-01$ & $2.894 \mathrm{E}-01$ & $5.316 \mathrm{E}-03$ & $6.538 \mathrm{E}-06$ & $1.681 \mathrm{E}-05$ \\
\hline 15 & $-1.187 \mathrm{E}-03$ & $2.036 \mathrm{E}-01$ & $2.034 \mathrm{E}-01$ & $1.087 \mathrm{E}-02$ & $1.289 \mathrm{E}-05$ & $3.316 \mathrm{E}-05$ \\
\hline 16 & $-3.654 \mathrm{E}-03$ & $4.411 \mathrm{E}-01$ & $4.393 \mathrm{E}-01$ & $2.317 \mathrm{E}-02$ & $8.467 \mathrm{E}-05$ & $2.177 \mathrm{E}-04$ \\
\hline 17 & $-4.655 E-02$ & $3.057 \mathrm{E}-01$ & $2.995 \mathrm{E}-01$ & $3.238 \mathrm{E}-02$ & $1.507 \mathrm{E}-03$ & $3.875 \mathrm{E}-03$ \\
\hline 18 & $-1.238 \mathrm{E}-01$ & $3.167 \mathrm{E}-01$ & $2.829 \mathrm{E}-01$ & $2.907 \mathrm{E}-02$ & $3.598 \mathrm{E}-03$ & $9.251 \mathrm{E}-03$ \\
\hline 19 & $-1.238 \mathrm{E}-01$ & $3.167 \mathrm{E}-01$ & $2.829 \mathrm{E}-01$ & $1.322 \mathrm{E}-02$ & $1.636 \mathrm{E}-03$ & $4.208 \mathrm{E}-03$ \\
\hline 20 & $-1.238 \mathrm{E}-01$ & $3.167 \mathrm{E}-01$ & $2.829 \mathrm{E}-01$ & $4.597 \mathrm{E}-03$ & $5.689 \mathrm{E}-04$ & $1.463 \mathrm{E}-03$ \\
\hline 21 & $-1.257 \mathrm{E}-01$ & $3.109 \mathrm{E}-01$ & $2.752 \mathrm{E}-01$ & $1.735 \mathrm{E}-03$ & $2.182 \mathrm{E}-04$ & $5.610 \mathrm{E}-04$ \\
\hline 22 & $-1.337 E-01$ & $3.108 \mathrm{E}-01$ & $2.703 E-01$ & $5.343 E-03$ & $7.141 \mathrm{E}-04$ & $1.836 \mathrm{E}-03$ \\
\hline 23 & $-1.337 \mathrm{E}-01$ & $3.108 \mathrm{E}-01$ & $2.703 E-01$ & $4.306 \mathrm{E}-03$ & $5.755 \mathrm{E}-04$ & $1.480 \mathrm{E}-03$ \\
\hline 24 & $-1.337 E-01$ & $3.108 \mathrm{E}-01$ & $2.703 E-01$ & $1.447 \mathrm{E}-02$ & $1.934 \mathrm{E}-03$ & $4.973 E-03$ \\
\hline 25 & $-1.337 \mathrm{E}-01$ & $3.108 \mathrm{E}-01$ & $2.703 \mathrm{E}-01$ & $1.678 \mathrm{E}-02$ & $2.242 \mathrm{E}-03$ & $5.766 \mathrm{E}-03$ \\
\hline 26 & $-1.337 \mathrm{E}-01$ & $3.108 \mathrm{E}-01$ & $2.703 \mathrm{E}-01$ & $2.346 \mathrm{E}-02$ & $3.136 \mathrm{E}-03$ & $8.065 \mathrm{E}-03$ \\
\hline 27 & $-1.337 \mathrm{E}-01$ & $3.108 \mathrm{E}-01$ & $2.703 \mathrm{E}-01$ & $4.807 \mathrm{E}-03$ & $6.424 \mathrm{E}-04$ & $1.652 \mathrm{E}-03$ \\
\hline 28 & $-1.337 E-01$ & $3.108 \mathrm{E}-01$ & $2.703 E-01$ & $5.858 \mathrm{E}-03$ & $7.830 \mathrm{E}-04$ & $2.013 \mathrm{E}-03$ \\
\hline 29 & $-1.337 \mathrm{E}-01$ & $3.108 \mathrm{E}-01$ & $2.703 E-01$ & $7.113 \mathrm{E}-03$ & $9.507 \mathrm{E}-04$ & $2.445 \mathrm{E}-03$ \\
\hline 30 & $-1.337 \mathrm{E}-01$ & $3.108 \mathrm{E}-01$ & $2.703 \mathrm{E}-01$ & $1.844 \mathrm{E}-02$ & $2.464 \mathrm{E}-03$ & $6.337 \mathrm{E}-03$ \\
\hline 31 & $-1.337 \mathrm{E}-01$ & $3.108 \mathrm{E}-01$ & $2.703 \mathrm{E}-01$ & $1.076 \mathrm{E}-02$ & $1.438 \mathrm{E}-03$ & $3.699 \mathrm{E}-03$ \\
\hline 32 & $-1.337 \mathrm{E}-01$ & $3.108 \mathrm{E}-01$ & $2.703 \mathrm{E}-01$ & $1.164 \mathrm{E}-02$ & $1.555 \mathrm{E}-03$ & $3.999 \mathrm{E}-03$ \\
\hline
\end{tabular}

Figure 6.6.14. Cross-section adjustment edit 


$\begin{array}{llllll}-1.337 \mathrm{E}-01 & 3.108 \mathrm{E}-01 & 2.703 \mathrm{E}-01 & 1.316 \mathrm{E}-02 & 1.759 \mathrm{E}-03 & 4.524 \mathrm{E}-03 \\ -1.337 \mathrm{E}-01 & 3.108 \mathrm{E}-01 & 2.703 \mathrm{E}-01 & 3.669 \mathrm{E}-02 & 4.904 \mathrm{E}-03 & 1.261 \mathrm{E}-02 \\ -1.337 \mathrm{E}-01 & 3.108 \mathrm{E}-01 & 2.703 \mathrm{E}-01 & 7.544 \mathrm{E}-02 & 1.008 \mathrm{E}-02 & 2.593 \mathrm{E}-02 \\ -1.337 \mathrm{E}-01 & 3.108 \mathrm{E}-01 & 2.703 \mathrm{E}-01 & 9.663 \mathrm{E}-02 & 1.292 \mathrm{E}-02 & 3.321 \mathrm{E}-02 \\ -1.337 \mathrm{E}-01 & 3.108 \mathrm{E}-01 & 2.703 \mathrm{E}-01 & 1.070 \mathrm{E}-01 & 1.430 \mathrm{E}-02 & 3.678 \mathrm{E}-02 \\ -1.337 \mathrm{E}-01 & 3.108 \mathrm{E}-01 & 2.703 \mathrm{E}-01 & 7.069 \mathrm{E}-02 & 9.448 \mathrm{E}-03 & 2.430 \mathrm{E}-02 \\ -1.337 \mathrm{E}-01 & 3.108 \mathrm{E}-01 & 2.703 \mathrm{E}-01 & 8.255 \mathrm{E}-02 & 1.103 \mathrm{E}-02 & 2.837 \mathrm{E}-02 \\ -1.337 \mathrm{E}-01 & 3.108 \mathrm{E}-01 & 2.703 \mathrm{E}-01 & 4.197 \mathrm{E}-02 & 5.609 \mathrm{E}-03 & 1.442 \mathrm{E}-02 \\ -1.336 \mathrm{E}-01 & 3.106 \mathrm{E}-01 & 2.700 \mathrm{E}-01 & 1.461 \mathrm{E}-01 & 1.951 \mathrm{E}-02 & 5.017 \mathrm{E}-02 \\ -1.331 \mathrm{E}-01 & 3.108 \mathrm{E}-01 & 2.706 \mathrm{E}-01 & 2.156 \mathrm{E}-02 & 2.871 \mathrm{E}-03 & 7.383 \mathrm{E}-03 \\ -1.331 \mathrm{E}-01 & 3.108 \mathrm{E}-01 & 2.706 \mathrm{E}-01 & 3.154 \mathrm{E}-02 & 4.199 \mathrm{E}-03 & 1.080 \mathrm{E}-02 \\ -1.331 \mathrm{E}-01 & 3.108 \mathrm{E}-01 & 2.706 \mathrm{E}-01 & 1.072 \mathrm{E}-02 & 1.427 \mathrm{E}-03 & 3.670 \mathrm{E}-03 \\ -3.625 \mathrm{E}+00 & ----------------- & 9.994 \mathrm{E}-01 & 1.221 \mathrm{E}-01 & 3.141 \mathrm{E}-01\end{array}$

NOTE: The contribution to the application bias is tabulated in units of $\% \mathrm{dk} / \mathrm{k}$.

The contribution to the application bias is normalized to the calculated response value.

NOTE: The fraction of the bias L1-norm is equal to the absolute value of the bias contribution divided by the sum of the absolute value of all groupwise bias contributions.

\begin{tabular}{|c|c|c|c|c|c|c|}
\hline $\begin{array}{l}\text { group } \\
\text { number }\end{array}$ & $\begin{array}{c}\text { delta-XS } \\
\left(\frac{\circ}{0}\right)\end{array}$ & $\begin{array}{l}\text { st. dev. } \\
\text { old (\%) }\end{array}$ & $\begin{array}{l}\text { st. dev. } \\
\text { new }\left(\frac{\circ}{0}\right)\end{array}$ & $\begin{array}{l}\text { rel sen } \\
\text { coeff. }\end{array}$ & $\begin{array}{l}\text { contribution to } \\
\text { appl. bias ( }\left(\frac{\circ}{0}\right)\end{array}$ & $\begin{array}{l}\text { fraction of } \\
\text { bias - L1-norm }\end{array}$ \\
\hline 1 & $3.011 \mathrm{E}+00$ & $1.165 \mathrm{E}+01$ & $8.267 \mathrm{E}+00$ & $-2.143 E-03$ & $6.453 \mathrm{E}-03$ & $1.659 \mathrm{E}-02$ \\
\hline 2 & $2.165 \mathrm{E}+00$ & $8.336 \mathrm{E}+00$ & $5.877 \mathrm{E}+00$ & $-5.079 E-03$ & $1.100 \mathrm{E}-02$ & $2.828 \mathrm{E}-02$ \\
\hline 3 & $1.528 \mathrm{E}+00$ & $5.869 \mathrm{E}+00$ & $4.122 \mathrm{E}+00$ & $-1.255 \mathrm{E}-02$ & $1.917 \mathrm{E}-02$ & $4.930 \mathrm{E}-02$ \\
\hline 4 & $8.165 \mathrm{E}-01$ & $3.138 \mathrm{E}+00$ & $2.200 \mathrm{E}+00$ & $-2.584 \mathrm{E}-02$ & $2.110 \mathrm{E}-02$ & $5.426 \mathrm{E}-02$ \\
\hline 5 & $3.741 \mathrm{E}-01$ & $1.451 \mathrm{E}+00$ & $1.024 \mathrm{E}+00$ & $-8.856 \mathrm{E}-03$ & $3.313 \mathrm{E}-03$ & $8.518 \mathrm{E}-03$ \\
\hline 6 & $2.299 \mathrm{E}-01$ & $9.050 \mathrm{E}-01$ & $6.464 \mathrm{E}-01$ & $-1.880 \mathrm{E}-03$ & $4.321 \mathrm{E}-04$ & $1.111 \mathrm{E}-03$ \\
\hline 7 & $7.697 \mathrm{E}-02$ & $3.463 \mathrm{E}-01$ & $2.717 \mathrm{E}-01$ & $-3.955 E-03$ & $3.044 \mathrm{E}-04$ & $7.827 \mathrm{E}-04$ \\
\hline
\end{tabular}

$<<<<<$ NORMAL END OF CALCULATION $>>>>>$

Figure 6.6.14. Cross-section adjustment edit (continued). 


\subsubsection{HTML Output}

The input file for the TSURFER sample problem is named tsurfer.inp. In this case, the HTML-formatted output is stored in a file called tsurfer.html and additional resources are stored in directories called tsurfer.htmd and applet_resources. This section contains example TSURFER HTML-formatted output only for demonstration of the interface. When tsurfer.html is opened in a web browser, the information shown in Figure 6.6.15 is displayed. The title of the input file is displayed between the two SCALE logos. Because this SCALE input file only executed TSURFER, only a single-output listing is available. The text "1. TSURFER" is a hyperlink to view the output from TSURFER. Clicking on the "1. TSURFER" hyperlink will bring up the information shown in Figure 6.6.16. Clicking on the SCALE logos will link the user to the SCALE website, if external internet access is available.

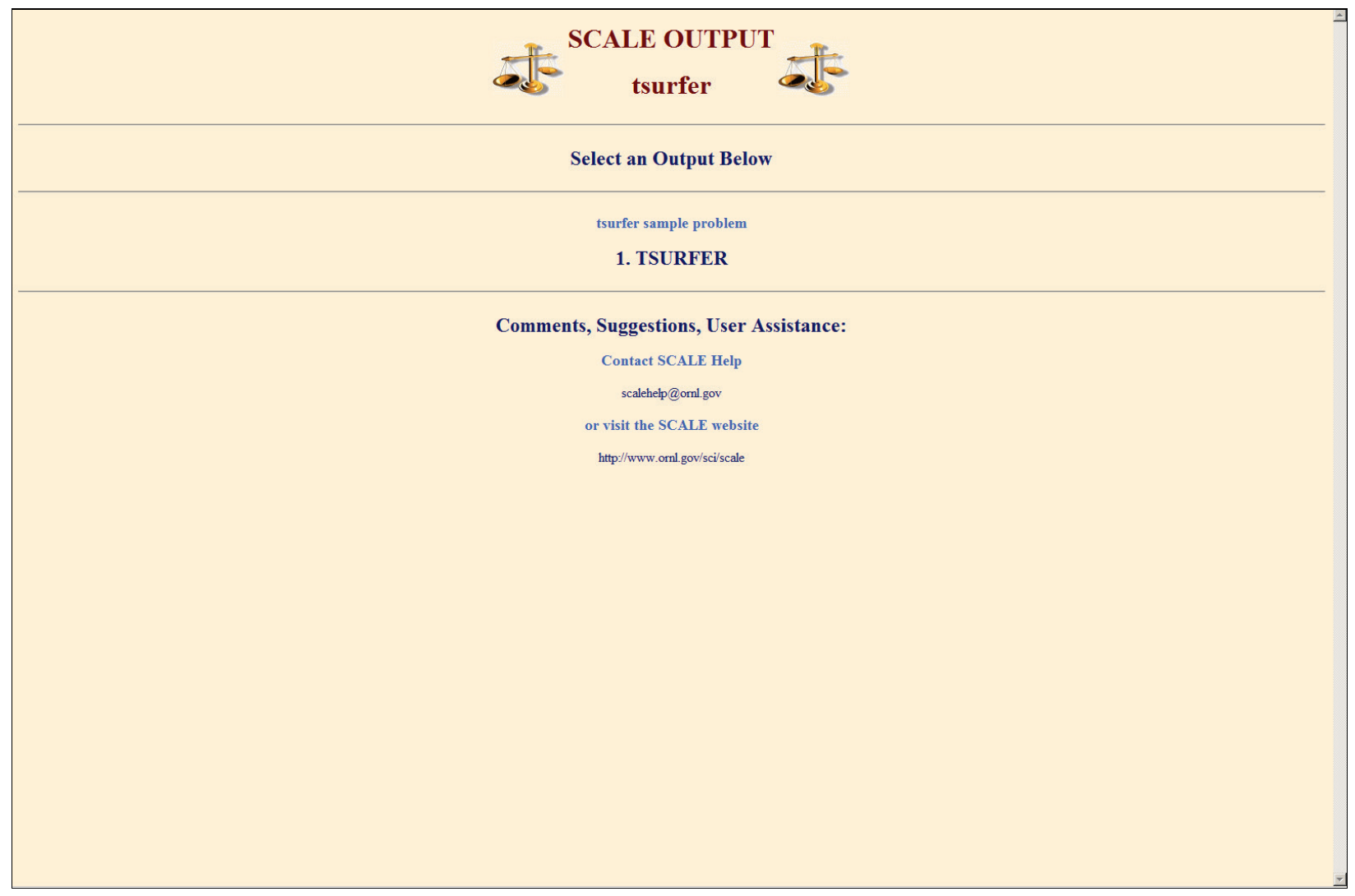

Figure 6.6.15. Initial screen from TSURFER HTML output.

The initial page of output from TSURFER is shown in Figure 6.6.16. Program verification information is shown in the table under the TSURFER logo. This table includes information about the code that was executed and the date and time it was run. The menu on the left side of the screen contains hyperlinks to specific portions of the code output. Echoes of the input data are available in the Input Data section. Any errors or warning messages are available in the Messages sections. Results from the code execution are shown in the results section. 


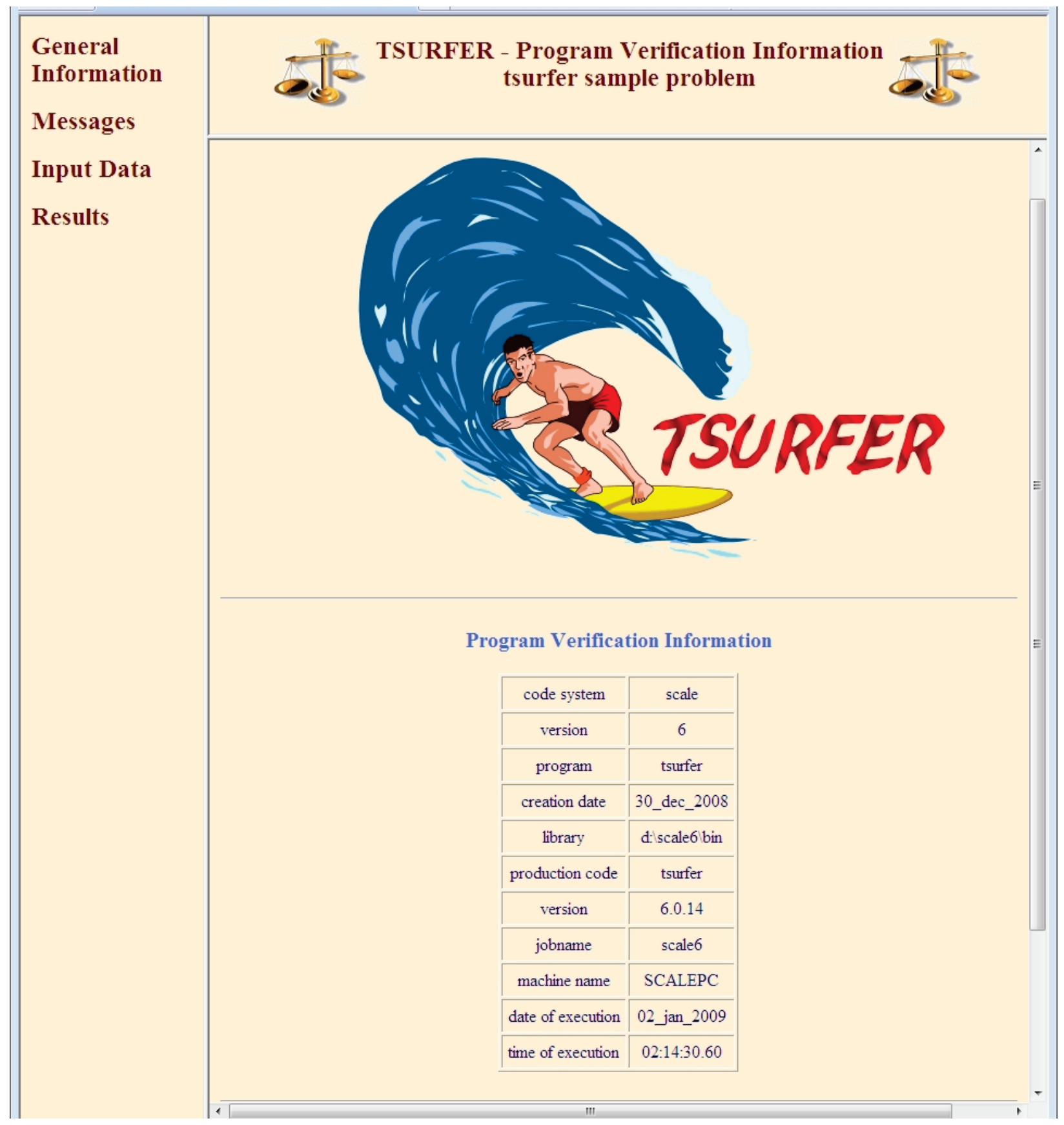

Figure 6.6.16. Program verification screen from TSURFER HTML output. 
Selecting Input Parameters will reveal the menu of available input data. Selecting Input Parameters causes the table shown in Figure 6.6.17 to be displayed. Other input data can also be displayed by selecting the desired data from the menu.

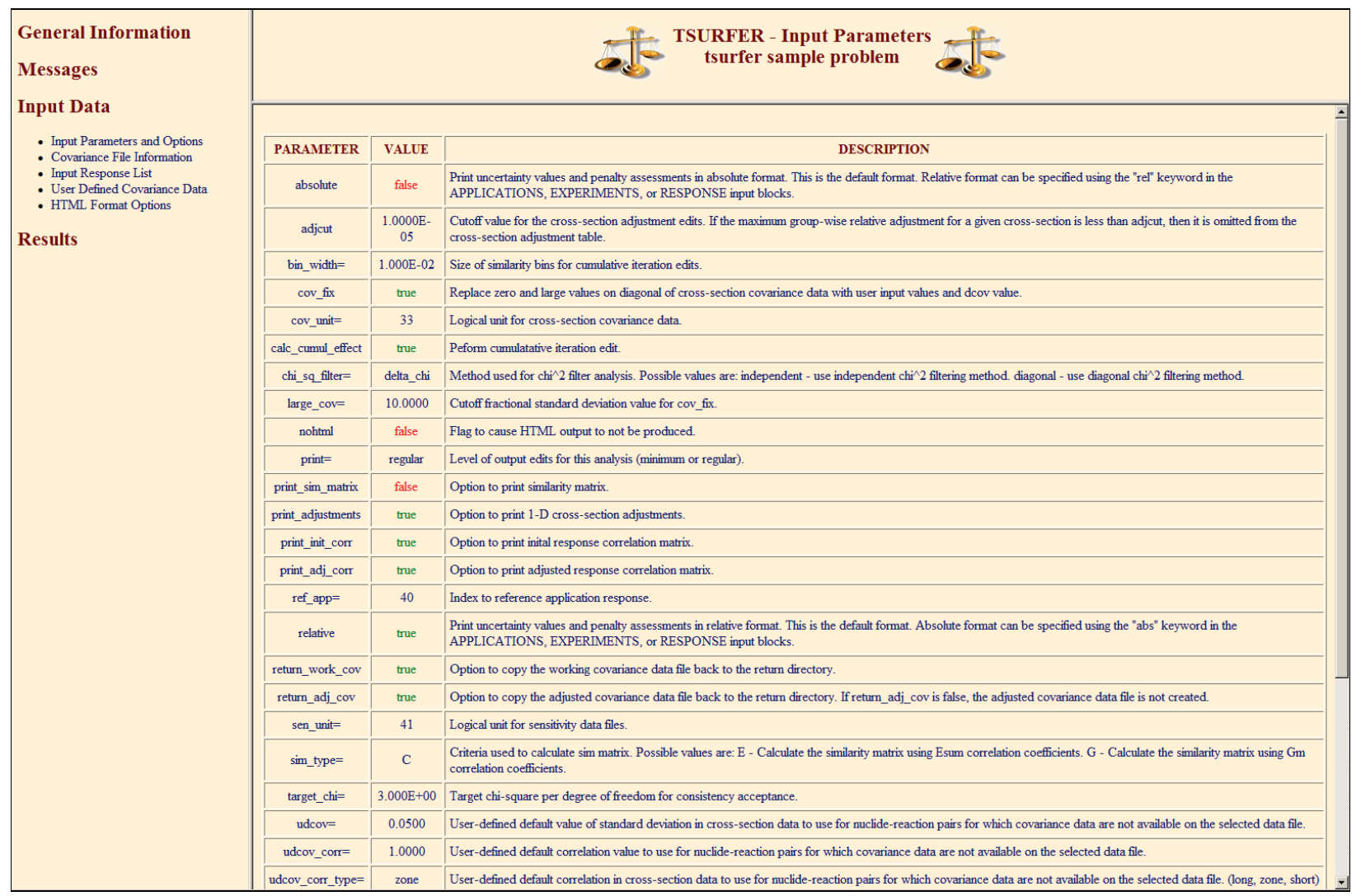

Figure 6.6.17. Input parameters from TSURFER HTML output. 
Selecting Messages will reveal a menu of available messages. Selecting Warning Messages from the Messages section of the menu causes the information shown in Figure 6.6.18 to appear. The Warning Messages edit contains all warning messages that were generated during the execution of the code. If errors were encountered in the code execution, an Error Messages item would have also been available in the menu under Messages.

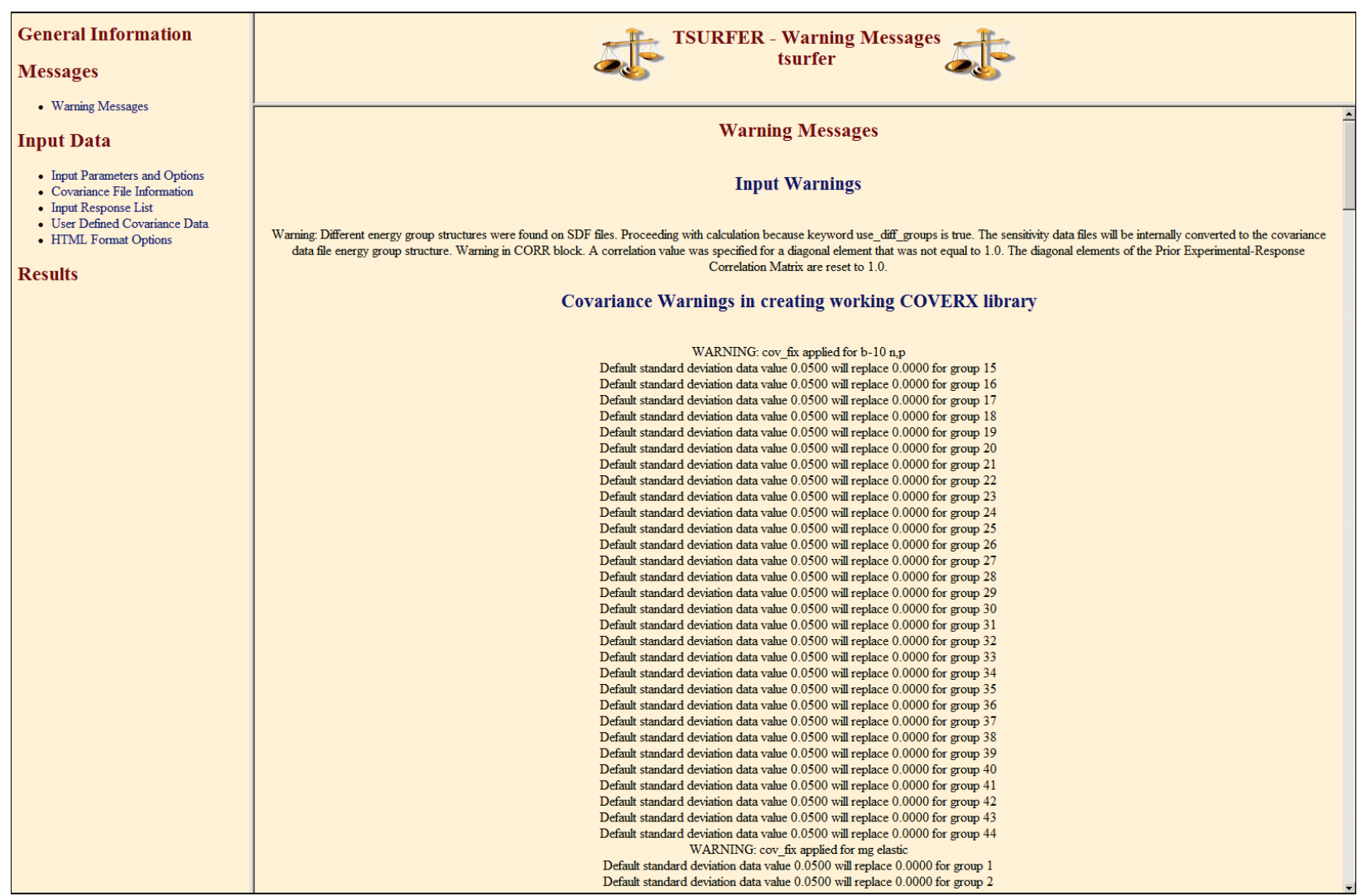

Figure 6.6.18. Warning messages from TSURFER HTML output. 
Selecting Results causes a menu of available results to be revealed. From this menu, selecting CrossSection Adjustments causes a menu on the right to be revealed. From this menu, nuclide-reaction pairs can be selected to visualize their cross-section adjustments in tabular format. The U-235 nubar adjustments are shown in Figure 6.6.19.

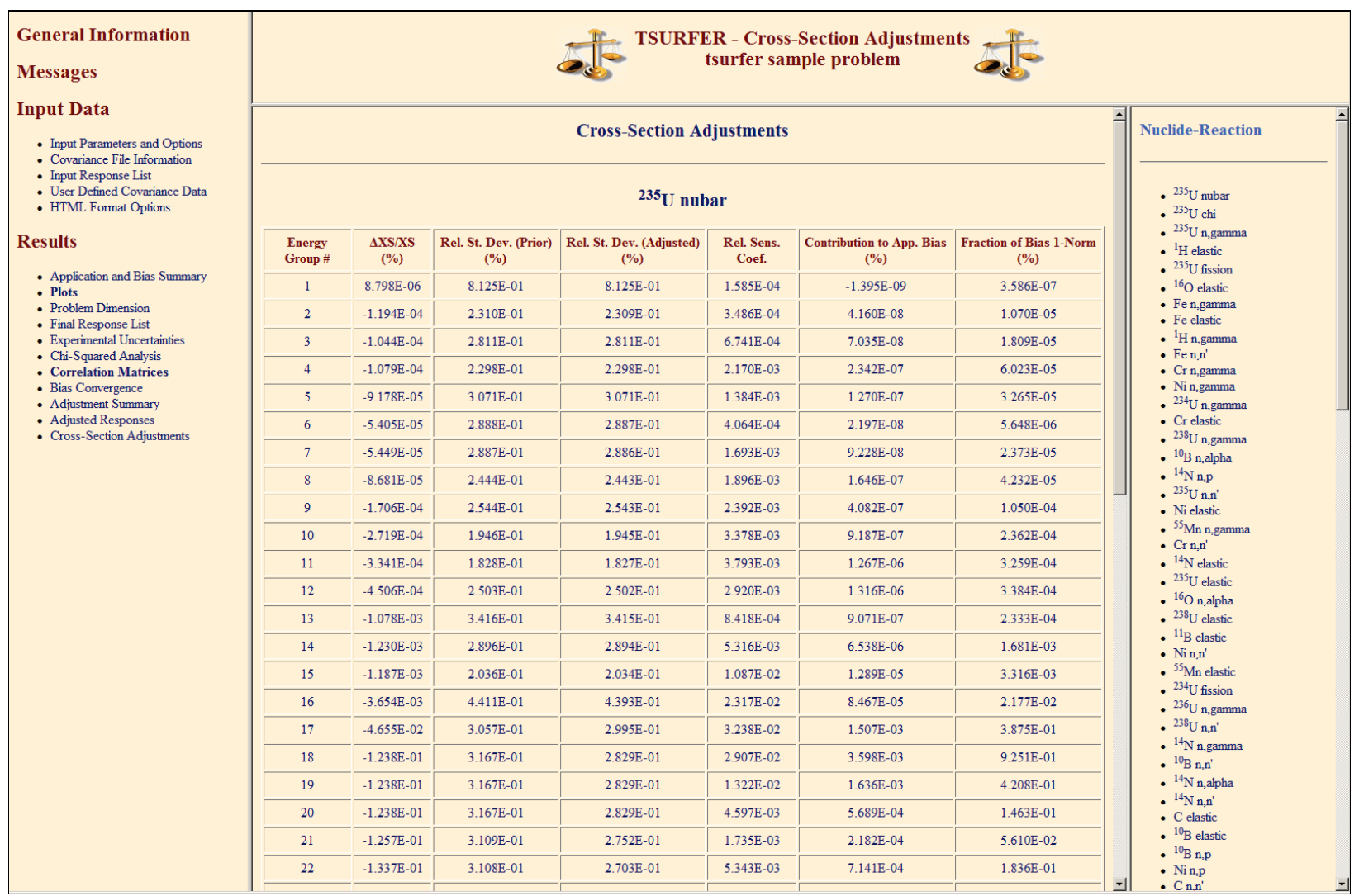

Figure 6.6.19. Cross-section adjustments from TSURFER HTML output. 
Various plots can also be viewed in the TSURFER HTML output. Selecting "Plots" in the Results menu brings up a submenu of various TSURFER plots. The correlation matrices may be viewed by selecting "Correlation Matrices" in the Plots submenu. A Java applet version of Javapeño will appear in the browser window with the correlation matrices preloaded. Data can be added to the plot by doubleclicking on the list of available data on the right side of Javapeño. The plot shown in Figure 6.6.20 was produced with this procedure.

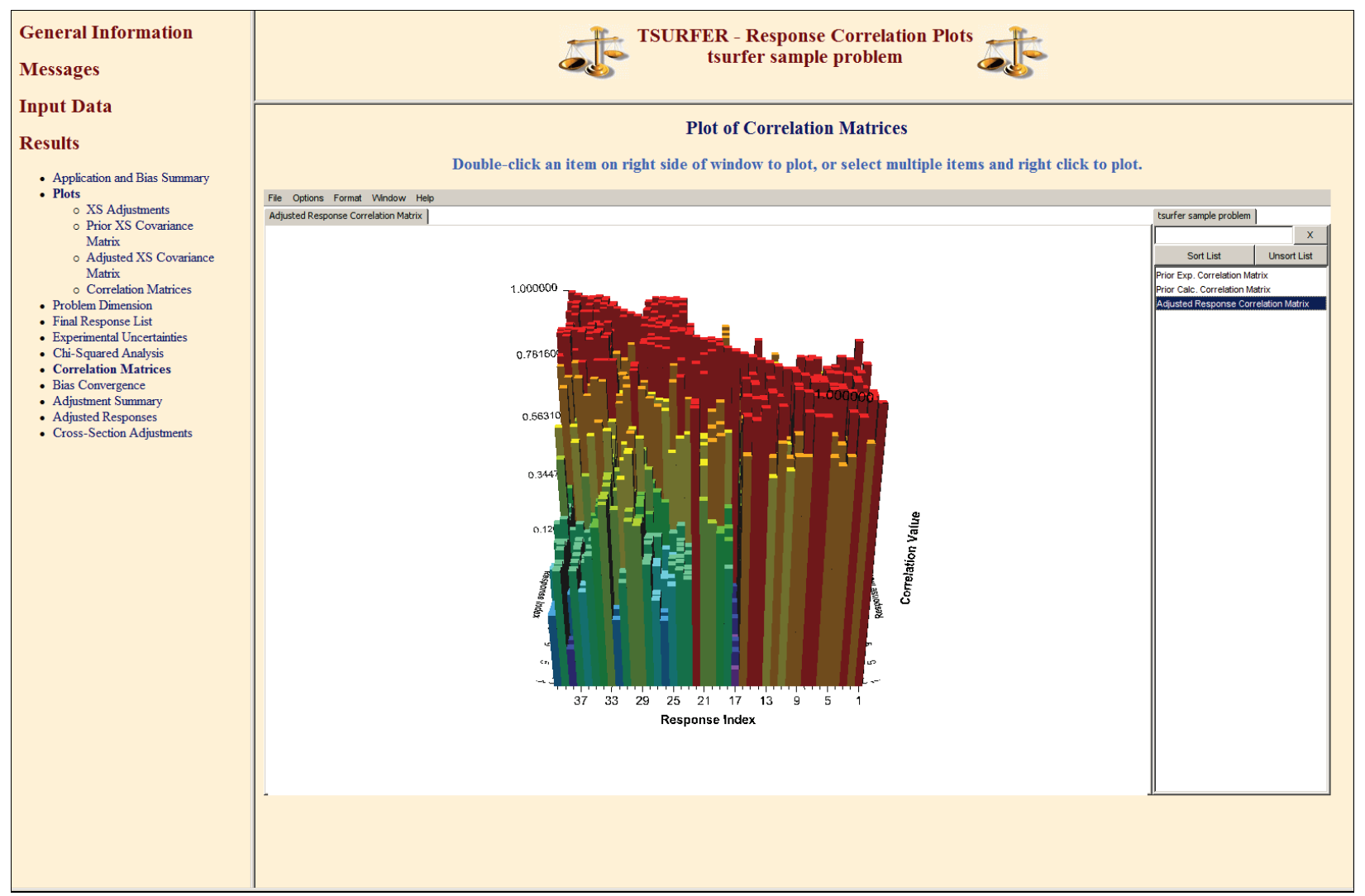

Figure 6.6.20. Three-dimensional plot of correlation matrix in TSURFER HTML output. 


\subsubsection{References}

1. A. Pazy, G. Rakavy, I. Reiss, J. J. Wagschal, A. Ya'ari, and Y. Yeivin, "The Role of Integral Data in Neutron Cross Section Evaluation,” Nucl. Sci. Eng. 55, 280-295 (1974).

2. C. R. Weisbin, J. H. Marable, J. L. Lucius, E. M. Oblow, F. R. Mynatt, R. W. Peelle, and F. G. Perey, Application of FORSS Sensitivity and Uncertainty Methodology to Fast Reactor Benchmark Analysis, ORNL/TM-5563, Union Carbide Corporation, Oak Ridge National Laboratory, December 1976.

3. W. P. Poenitz and P. J. Collins, "Utilization of Experimental Integral Data for the Adjustment and Uncertainty Evaluation of Reactor Design Quantities," Applications of Critical Experiments and Operating Data to Core Design via Formal Methods of Cross Section Data Adjustment, NEACRPL-307, Jackson Hole, WY (September 1988).

4. R. E. Maerker, J. J. Wagschal, and B. L. Broadhead, "Development and Demonstration of an Advanced Methodology for LWR Dosimetry Applications," EPRI NP-2188, Electric Power Research Institute, December 1981.

5. B. L. Broadhead, B. T. Rearden, C. M. Hopper, J. J. Wagschal, and C. V. Parks, "Sensitivity- and Uncertainty-Based Criticality Safety Validation," Nucl. Sci. Eng. 146(3), 340-366 (2004).

6. R. N. Hwang, "Topics in Data Adjustment Theory and Applications," Applications of Critical Experiments and Operating Data to Core Design via Formal Methods of Cross Section Data Adjustment, NEACRP-L-307, Jackson Hole, WY (September 1988).

7. T. Takeda, M. Takamoto, A. Yoshimura, and K. Shirakata, "Adjustment of JENDL-2 Cross Sections and Prediction Accuracy for FBR Core Parameters Using Jupiter Integral Data," Applications of Critical Experiments and Operating Data to Core Design via Formal Methods of Cross Section Data Adjustment, NEACRP-L-307, Jackson Hole, WY (September 1988).

8. S. Goluoglu, K. R. Elam, B. T. Rearden, B. L. Broadhead, and C. M. Hopper, Sensitivity Analysis Applied to the Validation of the ${ }^{10} B$ Capture Reaction in Nuclear Fuel Casks, NUREG/CR-6845 (ORNL/TM-2004/48), U.S. Nuclear Regulatory Commission, Oak Ridge National Laboratory, August 2004.

9. International Handbook of Evaluated Criticality Safety Benchmark Experiments, Organization for Economic Cooperation and Development-Nuclear Energy Agency, NEA/NSC/DOC(95)03 (2006 Edition).

10. T. T. Ivanova, M. N. Nikolaev, K. F. Raskach, E. V. Rozhikhin, and A. M. Tsiboulia, "Influence of the Correlations of Experimental Uncertainties on Criticality Prediction," Nucl. Sci. Eng. 145(1), 97-104 (September 2003).

11. M. L. Williams, B. L. Broadhead, and C. V. Parks, "Eigenvalue Sensitivity Theory for ResonanceShielded Cross Sections," Nucl. Sci. Eng. 138(2), 177-191 (2001).

12. M. L. Williams and B. T. Rearden, "SCALE-6 Sensitivity/Uncertainty Methods and Covariance Data," Nuclear Data Sheets, Vol. 109, Issue 12, December 2008, pp. 2796-2800.

13. R. C. Little, T. Kawano, G. D. Hale, M. T. Pigni, M. Herman, P. Obložinský, M. L. Williams, M. E. Dunn, G. Arbanas, D. Wiarda, R. D. McKnight, J. N. McKamy, and J. R. Felty, "Lowfidelity Covariance Project," Nuclear Data Sheets, Vol. 109, Issue 12, December 2008, pp. 28282833. 
14. D. Wiarda and M. E. Dunn, PUFF-IV: A Code for Processing ENDF Uncertainty Data into Multigroup Covariance Matrices, ORNL/TM-2006/147, UT-Battelle, LLC, Oak Ridge National Laboratory, October 2006.

15. B. L. Broadhead, C. M. Hopper, R. L. Childs, and C. V. Parks, Sensitivity and Uncertainty Analyses Applied to Criticality Safety Validation, NUREG/CR-6655, Vols. 1 and 2 (ORNL/TM-13692/V1 and V2), U.S. Nuclear Regulatory Commission, Oak Ridge National Laboratory, November 1999.

16. M. L. Williams, "Perturbation Theory for Reactor Analysis," CRC Handbook of Nuclear Reactor Calculations, pp. 63-188, CRC Press, 1986.

17. Y. Yeivin, J. J. Wagschal, J. H. Marable, and C. R. Weisbin, "Relative Consistency of ENDF/B-IV and $-\mathrm{V}$ with Fast Reactor Benchmarks," Proc. Int. Conf. on Nuclear Cross Sections for Technology, J. L. Fowler, C. H. Johnson, and C. D. Bowman, eds., NBS SP 594 (1980). 


\subsection{A Sensitivity/Uncertainty Notation}

In the following expressions, the notation $\mathrm{E}[\mathrm{X}]$ represents the expected value of random variable $\mathrm{X}$, which is equal to the integral of $\mathrm{X}$ weighted by its probability density function over the range of allowable values.

\subsection{A.1 Basic variables}

I $=$ number of integral response (experiment and applications) used in GLLS analysis

$\mathrm{M}=$ number of nuclear data parameters used in transport calculations (i.e., number of unique nuclide-reaction pairs multiplied by the number of energy groups)

$\boldsymbol{\alpha}=$ M dimensional vector of prior nuclear data parameters, where component $-i=\alpha_{\mathrm{i}}$

$\mathbf{A}=\mathrm{M}$ by $\mathrm{M}$ diagonal matrix of prior nuclear data parameters, where diagonal element $\mathbf{A}(\mathrm{i}, \mathrm{i})=\alpha_{\mathrm{i}}$

$\mathbf{m}=$ I dimensional vector of prior measured responses, where component- $i=\mathrm{m}_{\mathrm{i}}$

$\mathbf{M}=$ I by I diagonal matrix of prior measured responses, where diagonal element $\mathbf{M}(\mathrm{i}, \mathrm{i})=\mathrm{m}_{\mathrm{i}}$

$\mathbf{k}(\boldsymbol{\alpha})=$ I dimensional vector of prior calculated responses obtained with nuclear data $\alpha$, where component $\mathrm{I}=\mathrm{k}_{\mathrm{i}}$

$\mathbf{K}=$ I by I diagonal matrix of prior calculated responses, where diagonal element $\mathbf{K}(\mathrm{i}, \mathrm{i})=\mathrm{k}_{\mathrm{i}}$

$\mathbf{F}_{\mathbf{m} / \mathbf{k}}=$ I by I diagonal matrix of "E/C" values $=\mathbf{M} \mathbf{K}^{-1}=\mathbf{K}^{-1} \mathbf{M}$, where diagonal element $\mathrm{F}_{\mathrm{m} / \mathrm{k}}(\mathrm{i}, \mathrm{i})=\frac{\mathrm{m}_{\mathrm{i}}}{\mathrm{k}_{\mathrm{i}}}$

$\hat{\mathbf{F}}_{\mathbf{m} / \mathbf{k}}=$ I by I diagonal matrix, where diagonal element $F_{m / k}(i, i)=\frac{m_{i}}{k_{i}}$ for a relative-formatted response and $\mathrm{F}_{\mathrm{m} / \mathrm{k}}(\mathrm{i}, \mathrm{i})=1$ for an absolute-formatted response

$\boldsymbol{\alpha}^{\prime}=$ M dimensional vector of adjusted nuclear data parameters produced by GLLS procedure

$\mathbf{m}^{\prime} \quad=$ I dimensional vector of adjusted measured responses produced by GLLS procedure

$\mathbf{k}^{\prime}\left(\boldsymbol{\alpha}^{\prime}\right)=$ I dimensional vector of adjusted calculated responses obtained with modified nuclear data $\boldsymbol{\alpha}^{\prime}$

>>> N NOTE: $\boldsymbol{k}^{\prime}\left(\alpha^{\prime}\right)=\boldsymbol{m}^{\prime}$, due to GLLS adjustment procedure. $\quad<<<<$

$\widetilde{\mathbf{d}}=$ original absolute discrepancy vector $=\mathbf{k}-\mathbf{m}$, where component-i $=k_{i}-m_{i}$ 
d $=$ original relative discrepancy vector $=\mathbf{K}^{-1}(\mathbf{k}-\mathbf{m})$, where component-i $=\left(\mathrm{k}_{\mathrm{i}}-\mathrm{m}_{\mathrm{i}}\right) / \mathrm{k}_{\mathrm{i}}$

$\hat{\mathbf{d}}=$ original mixed absolute-relative discrepancy vector, where component- $\mathrm{i}=\left(\mathrm{k}_{\mathrm{i}}-\mathrm{m}_{\mathrm{i}}\right) / \mathrm{k}_{\mathrm{i}}$ for a relative-formatted response and $\left(\mathrm{k}_{\mathrm{i}}-\mathrm{m}_{\mathrm{i}}\right)$ for an absolute-formatted response

$[\boldsymbol{\Delta \alpha}]=\mathrm{M}$ dimensional vector of relative variations in nuclear data $=\mathbf{A}^{-1}\left(\boldsymbol{\alpha}^{\prime}-\boldsymbol{\alpha}\right)$, where component-i $=\frac{\alpha_{i}^{\prime}-\alpha_{i}}{\alpha_{i}}$

$[\Delta \mathbf{m}]=$ I dimensional vector of relative variations in measured responses $=\mathbf{M}^{-\mathbf{1}}\left(\mathbf{m}^{\prime}-\mathbf{m}\right)$, where component- $\mathrm{i}=\frac{\mathrm{m}_{\mathrm{i}}^{\prime}-\mathrm{m}_{\mathrm{i}}}{\mathrm{m}_{\mathrm{i}}} \rightarrow \frac{\mathrm{k}_{\mathrm{i}}^{\prime}-\mathrm{m}_{\mathrm{i}}}{\mathrm{m}_{\mathrm{i}}}$

$[\Delta \tilde{\mathbf{m}}]=$ I dimensional vector of absolute variations in measured responses $=\mathbf{m}^{\prime}-\mathbf{m}$, where component- $\mathrm{i}=\mathrm{m}_{\mathrm{i}}^{\prime}-\mathrm{m}_{\mathrm{i}} \rightarrow \mathrm{k}_{\mathrm{i}}^{\prime}-\mathrm{m}_{\mathrm{i}}$

$[\boldsymbol{\Delta} \hat{\mathbf{m}}]=$ I dimensional vector of mixed absolute-relative variations in measured responses, where component- $\mathrm{i}=\frac{\mathrm{m}_{\mathrm{i}}^{\prime}-\mathrm{m}_{\mathrm{i}}}{\mathrm{m}_{\mathrm{i}}}$ for a relative-formatted response and $\mathrm{m}_{\mathrm{i}}^{\prime}-\mathrm{m}_{\mathrm{i}}$ for an absoluteformatted response

$[\Delta \mathbf{k}]=$ I dimensional vector of relative variations in calculated responses $=\mathbf{K}^{-1}\left(\mathbf{k}^{\mathbf{\prime}}-\mathbf{k}\right)$, where component-i $=\frac{\mathrm{k}_{\mathrm{i}}^{\prime}-\mathrm{k}_{\mathrm{i}}}{\mathrm{k}_{\mathrm{i}}}$

$[\mathbf{\Delta} \tilde{\mathbf{k}}]=\mathrm{I}$ dimensional vector of absolute variations in calculated responses $=\mathbf{k}^{\mathbf{\prime}}-\mathbf{k}$, where component-i $=\mathrm{k}_{\mathrm{i}}^{\prime}-\mathrm{k}_{\mathrm{i}}$

$[\mathbf{\Delta} \hat{\mathbf{k}}]=$ I dimensional vector of mixed absolute-relative variations in calculated responses, where component-i $=\frac{\mathrm{k}_{\mathrm{i}}^{\prime}-\mathrm{k}_{\mathrm{i}}}{\mathrm{k}_{\mathrm{i}}}$ for a relative-formatted response and $\mathrm{k}_{\mathrm{i}}^{\prime}-\mathrm{k}_{\mathrm{i}}$ for an absolute formatted response

\subsection{A.2 Sensitivity relations}

$\widetilde{\mathbf{S}}_{\mathbf{k} \boldsymbol{}}=$ I by M absolute sensitivity matrix; where element $\widetilde{\mathbf{S}}_{\mathbf{k} \boldsymbol{\alpha}}(\mathrm{i}, \mathrm{n})=\alpha_{\mathrm{n}} \frac{\partial \mathbf{k}_{\mathrm{i}}}{\partial \alpha_{\mathrm{n}}}$

$\mathbf{S}_{\mathbf{k} \boldsymbol{\alpha}}=$ I by M relative sensitivity matrix $=\mathbf{K}^{-1} \mathbf{S}_{\mathbf{k} \alpha}$, where element $\mathbf{S}_{k \alpha}(i, n)=\frac{\alpha_{\mathrm{n}}}{\mathrm{k}_{\mathrm{i}}} \frac{\partial \mathrm{k}_{\mathrm{i}}}{\partial \alpha_{\mathrm{n}}}$ 
$\hat{\mathbf{S}}_{\mathbf{k} \boldsymbol{\alpha}}=\mathrm{I}$ by $\mathrm{M}$ mixed absolute-relative sensitivity matrix, where element $\hat{\mathbf{S}}_{\mathbf{k} \alpha}(\mathrm{i}, \mathrm{n})=\frac{\alpha_{\mathrm{n}}}{\mathrm{k}_{\mathrm{i}}} \frac{\partial \mathrm{k}_{\mathrm{i}}}{\partial \alpha_{\mathrm{n}}}$ if response-i is relative-formatted and $\hat{\mathbf{S}}_{\mathbf{k} \boldsymbol{\alpha}}(\mathrm{i}, \mathrm{n})=\alpha_{\mathrm{n}} \frac{\partial \mathbf{k}_{\mathrm{i}}}{\partial \alpha_{\mathrm{n}}}$ if response-i is absolute-formatted

$$
\begin{aligned}
& {[\Delta \tilde{\mathbf{k}}]=\tilde{\mathbf{S}}_{\mathbf{k} \alpha}[\Delta \alpha]} \\
& {[\Delta \mathbf{k}]=\mathbf{S}_{\mathbf{k} \alpha}[\Delta \alpha]} \\
& {[\Delta \hat{\mathbf{k}}]=\hat{\mathbf{S}}_{\mathbf{k} \alpha}[\Delta \alpha]}
\end{aligned}
$$

\subsection{A.3 Absolute covariances}

$\tilde{\mathbf{C}}_{\mathbf{m m}}=$ I by I covariance matrix for prior measured experiment responses where element $\tilde{\mathbf{C}}_{\mathbf{m m}}(\mathrm{i}, \mathrm{j})=$ $\mathrm{E}\left(\delta \mathrm{m}_{\mathrm{i}} \delta \mathrm{m}_{\mathrm{j}}\right)$

$\tilde{\mathbf{C}}_{\mathbf{k k}}=$ I by I covariance matrix for prior calculated responses, where element $\tilde{\mathbf{C}}_{\mathbf{k k}}(\mathrm{i}, \mathrm{j})=\mathrm{E}\left(\delta \mathrm{k}_{\mathrm{i}} \delta \mathrm{k}_{\mathrm{j}}\right)$

$\tilde{\mathbf{C}}_{\mathbf{d d}}=$ I by I covariance matrix for the discrepancies $(\mathrm{k}-\mathrm{m})$, where element $\tilde{\mathbf{C}}_{\mathbf{d d}}(\mathrm{i}, \mathrm{j})=\mathrm{E}\left(\delta \mathrm{d}_{\mathrm{i}} \delta \mathrm{d}_{\mathrm{j}}\right)=$ $\mathrm{E}\left(\delta\left(\mathrm{k}_{\mathrm{i}}-\mathrm{m}_{\mathrm{i}}\right) \delta\left(\mathrm{k}_{\mathrm{j}}-\mathrm{m}_{\mathrm{j}}\right)\right)$

$\tilde{\mathbf{C}}_{\mathbf{k}^{\prime} \mathbf{k}^{\prime}}=$ I by I covariance matrix for adjusted responses, where element $\tilde{\mathbf{C}}_{\mathbf{k}^{\prime} \mathbf{k}^{\prime}}(\mathrm{i}, \mathrm{j})=\mathrm{E}\left(\delta \mathrm{k}_{\mathrm{i}}^{\prime} \delta \mathrm{k}_{\mathrm{j}}{ }_{\mathrm{j}}\right)$

$\tilde{\boldsymbol{\sigma}}_{\mathbf{m}}=\mathrm{I}$ by I diagonal matrix containing standard deviations in prior measured responses, where diagonal element $\widetilde{\sigma}_{\mathrm{m}}(\mathrm{i}, \mathrm{i})=\sqrt{\widetilde{\mathrm{C}}_{\mathrm{mm}}(\mathrm{i}, \mathrm{i})}$

$\tilde{\boldsymbol{\sigma}}_{\mathbf{k}}=\mathrm{I}$ by I diagonal matrix containing standard deviations in prior calculated responses, where diagonal element $\widetilde{\sigma}_{\mathrm{k}}(\mathrm{i}, \mathrm{i})=\sqrt{\widetilde{\mathrm{C}}_{\mathrm{kk}}(\mathrm{i}, \mathrm{i})}$

$\tilde{\boldsymbol{\sigma}}_{\mathbf{k}^{\prime}}=$ I by I diagonal matrix containing standard deviations in adjusted calculated responses, where diagonal element $\tilde{\sigma}_{k^{\prime}}(\mathrm{i}, \mathrm{i})=\sqrt{\tilde{\mathrm{C}}_{\mathrm{k}^{\prime} \mathrm{k}^{\prime}}(\mathrm{i}, \mathrm{i})}$

\subsection{A.4 Relative covariances}

$\mathbf{C}_{\mathbf{m m}}=\mathrm{I}$ by I relative covariance matrix for prior measured responses, $=\mathbf{M}^{-1}\left[\tilde{\mathbf{C}}_{\mathbf{m m}}\right] \mathbf{M}^{-1}$; where element $C_{m m}(i, j)=\frac{\tilde{\mathrm{C}}_{\mathrm{mm}}(\mathrm{i}, \mathrm{j})}{\mathrm{m}_{\mathrm{i}} \mathrm{m}_{\mathrm{j}}}$ 
$\mathbf{C}_{\alpha \boldsymbol{\alpha}}=\mathrm{M}$ by $\mathrm{M}$ relative covariance matrix for prior nuclear data, where element $\widetilde{\mathrm{C}}_{\alpha \alpha}(\mathrm{i}, \mathrm{j})=$ $\frac{E\left(\delta \alpha_{i} \delta \alpha_{j}\right)}{\alpha_{i} \alpha_{j}}$

$\mathbf{C}_{\mathbf{k} \mathbf{k}}=\mathrm{I}$ by I relative covariance matrix for prior calculated responses $=\mathbf{K}^{-1}\left[\tilde{\mathbf{C}}_{\mathbf{k}}\right] \mathbf{K}^{-1}$, where element $C_{k k}(i, j)=\frac{\tilde{\mathrm{C}}_{\mathrm{kk}}(\mathrm{i}, \mathrm{j})}{\mathrm{k}_{\mathrm{i}} \mathrm{k}_{\mathrm{j}}}$

$\mathbf{C}_{\mathbf{d d}}=$ I by I relative covariance matrix for response discrepancies; $=\mathbf{K}^{-1}\left[\tilde{\mathbf{C}}_{\mathbf{d d}}\right] \mathbf{K}^{-1}$, where element $C_{d d}(i, j)=\frac{\tilde{\mathrm{C}}_{\mathrm{dd}}(\mathrm{i}, \mathrm{j})}{\mathrm{k}_{\mathrm{i}} \mathrm{k}_{\mathrm{j}}}$

$\boldsymbol{\sigma}_{\mathbf{m}}=\mathrm{I}$ by I diagonal matrix containing relative standard deviations in measured responses, where diagonal element $\sigma_{\mathrm{m}}(\mathrm{i}, \mathrm{i})=\sqrt{\mathrm{C}_{\mathrm{mm}}(\mathrm{i}, \mathrm{i})}$

$\boldsymbol{\sigma}_{\mathbf{k}}=$ I by I diagonal matrix containing relative standard deviations in calculated responses, where diagonal element $\sigma_{\mathrm{k}^{\prime}}(\mathrm{i}, \mathrm{i})=\sqrt{\mathrm{C}_{\mathrm{k}^{\prime} \mathrm{k}^{\prime}}(\mathrm{i}, \mathrm{i})}$

$\boldsymbol{\sigma}_{\boldsymbol{\alpha}}=\mathrm{M}$ by $\mathrm{M}$ diagonal matrix containing standard deviations in nuclear data, where diagonal element $\boldsymbol{\sigma}_{\boldsymbol{\alpha}}(\mathrm{i}, \mathrm{i})=\sqrt{C_{\alpha \alpha}(i, i)}$

\subsection{A.5 Mixed absolute-relative covariances}

If response-i and response- $j$ are both absolute formatted, then

$$
\begin{gathered}
\hat{\mathrm{C}}_{\mathrm{kk}}(\mathrm{i}, \mathrm{j})=\tilde{\mathrm{C}}_{\mathrm{kk}}(\mathrm{i}, \mathrm{j}) \\
\hat{\mathrm{C}}_{\mathrm{mm}}(\mathrm{i}, \mathrm{j})=\tilde{\mathrm{C}}_{\mathrm{mm}}(\mathrm{i}, \mathrm{j}) \\
\hat{\mathrm{C}}_{\mathrm{dd}}(\mathrm{i}, \mathrm{j})=\tilde{\mathrm{C}}_{\mathrm{dd}}(\mathrm{i}, \mathrm{j}) \\
\hat{\mathrm{C}}_{\mathrm{k}^{\prime} \mathrm{k}^{\prime}}(\mathrm{i}, \mathrm{j})=\tilde{\mathrm{C}}_{\mathrm{k}^{\prime} \mathrm{k}^{\prime}}(\mathrm{i}, \mathrm{j}) .
\end{gathered}
$$

Likewise, if both response-i and response-j are relative-formatted, then

$$
\begin{gathered}
\hat{\mathrm{C}}_{\mathrm{kk}}(\mathrm{i}, \mathrm{j})=\mathrm{C}_{\mathrm{kk}}(\mathrm{i}, \mathrm{j})=\frac{\tilde{\mathrm{C}}_{\mathrm{kk}}(\mathrm{i}, \mathrm{j})}{\mathrm{k}_{\mathrm{i}} \mathrm{k}_{\mathrm{j}}} \\
\hat{\mathrm{C}}_{\mathrm{mm}}(\mathrm{i}, \mathrm{j})=\mathrm{C}_{\mathrm{mm}}(\mathrm{i}, \mathrm{j})=\frac{\tilde{\mathrm{C}}_{\mathrm{mm}}(\mathrm{i}, \mathrm{j})}{\mathrm{m}_{\mathrm{i}} \mathrm{m}_{\mathrm{j}}}
\end{gathered}
$$




$$
\begin{gathered}
\hat{\mathrm{C}}_{\mathrm{dd}}(\mathrm{i}, \mathrm{j})=\mathrm{C}_{\mathrm{dd}}(\mathrm{i}, \mathrm{j})=\frac{\tilde{\mathrm{C}}_{\mathrm{dd}}(\mathrm{i}, \mathrm{j})}{\mathrm{d}_{\mathrm{i}} \mathrm{d}_{\mathrm{j}}} \\
\hat{\mathrm{C}}_{\mathrm{k}^{\prime} \mathrm{k}^{\prime}}(\mathrm{i}, \mathrm{j})=\mathrm{C}_{\mathrm{k}^{\prime} \mathrm{k}^{\prime}}(\mathrm{i}, \mathrm{j})=\frac{\tilde{\mathrm{C}}_{\mathrm{k}^{\prime} \mathrm{k}^{\prime}}(\mathrm{i}, \mathrm{j})}{\mathrm{k}_{\mathrm{i}}^{\prime} \mathrm{k}_{\mathrm{j}}^{\prime}} .
\end{gathered}
$$

If response-i is absolute-formatted and response-j is relative-formatted, then

$$
\begin{gathered}
\hat{\mathrm{C}}_{\mathrm{kk}}(\mathrm{i}, \mathrm{j})=\frac{\tilde{\mathrm{C}}_{\mathrm{kk}}(\mathrm{i}, \mathrm{j})}{\mathrm{k}_{\mathrm{j}}} \\
\hat{\mathrm{C}}_{\mathrm{mm}}(\mathrm{i}, \mathrm{j})=\frac{\tilde{\mathrm{C}}_{\mathrm{mm}}(\mathrm{i}, \mathrm{j})}{\mathrm{m}_{\mathrm{j}}} \\
\hat{\mathrm{C}}_{\mathrm{dd}}(\mathrm{i}, \mathrm{j})=\frac{\tilde{\mathrm{C}}_{\mathrm{dd}}(\mathrm{i}, \mathrm{j})}{\mathrm{d}_{\mathrm{j}}} \\
\hat{\mathrm{C}}_{\mathrm{k}^{\prime} \mathrm{k}^{\prime}}(\mathrm{i}, \mathrm{j})=\frac{\tilde{\mathrm{C}}_{\mathrm{k}^{\prime} \mathrm{k}^{\prime}}(\mathrm{i}, \mathrm{j})}{\mathrm{k}_{\mathrm{j}}^{\prime}} .
\end{gathered}
$$

Similar expressions can be derived if response- $i$ is relative-formatted, and response- $j$ is absoluteformatted. The I by I diagonal matrices of standard deviation values are the following:

$$
\begin{aligned}
& \hat{\sigma}_{\mathrm{k}}(\mathrm{i}, \mathrm{i})= \begin{cases}\tilde{\sigma}_{\mathrm{k}}(\mathrm{i}, \mathrm{i}) & \text { absolute-formatted } \\
\sigma_{\mathrm{k}}(\mathrm{i}, \mathrm{i}) & \text { relative-formatted }\end{cases} \\
& \hat{\sigma}_{\mathrm{m}}(\mathrm{i}, \mathrm{i})= \begin{cases}\tilde{\sigma}_{\mathrm{m}}(\mathrm{i}, \mathrm{i}) & \text { absolute-formatted } \\
\sigma_{\mathrm{m}}(\mathrm{i}, \mathrm{i}) & \text { relative-formatted }\end{cases} \\
& \hat{\sigma}_{\mathrm{d}}(\mathrm{i}, \mathrm{i})= \begin{cases}\tilde{\sigma}_{\mathrm{d}}(\mathrm{i}, \mathrm{i}) & \text { absolute-formatted } \\
\sigma_{\mathrm{d}}(\mathrm{i}, \mathrm{i}) & \text { relative-formatted }\end{cases} \\
& \hat{\sigma}_{\mathrm{k}^{\prime}}(\mathrm{i}, \mathrm{i})= \begin{cases}\tilde{\sigma}_{\mathrm{k}^{\prime}}(\mathrm{i}, \mathrm{i}) & \text { absolute-formatted } \\
\sigma_{\mathrm{k}^{\prime}}(\mathrm{i}, \mathrm{i}) & \text { relative-formatted }\end{cases}
\end{aligned}
$$




\subsection{A.6 Correlation matrices}

$\mathbf{R}_{\mathbf{k k}}=\mathrm{I}$ by $\mathrm{I}$ correlation matrix for prior calculated responses, where element $\mathrm{R}_{\mathrm{kk}}(\mathrm{i}, \mathrm{j})=$ $\frac{C_{k k}(i, j)}{\sigma_{k}(i, i) \sigma_{k}(j, j)}=\frac{\tilde{C}_{k k}(i, j)}{\tilde{\sigma}_{k}(i, i) \tilde{\sigma}_{k}(j, j)}=\frac{\hat{C}_{k k}(i, j)}{\hat{\sigma}_{k}(i, i) \hat{\sigma}_{k}(j, j)}$

$\mathbf{R}_{\mathbf{m m}}=\mathrm{I}$ by $\mathrm{I}$ correlation matrix for prior measured responses, where element $\mathrm{R}_{\mathrm{mm}}(\mathrm{i}, \mathrm{j})=$ $\frac{C_{m m}(i, j)}{\sigma_{m}(i, i) \sigma_{m}(j, j)}=\frac{\tilde{C}_{m m}(i, j)}{\tilde{\sigma}_{m}(i, i) \tilde{\sigma}_{m}(j, j)}=\frac{\hat{C}_{m m}(i, j)}{\hat{\sigma}_{m}(i, i) \hat{\sigma}_{m}(j, j)}$.

$\mathbf{R}_{\mathbf{\alpha} \alpha}=$ M by M correlation matrix for prior nuclear data, where element $R_{\alpha \alpha}(i, j)=\frac{C_{\alpha \alpha}(i, j)}{\sigma_{\alpha}(i, i) \sigma_{\alpha}(j, j)}$

$\mathbf{R}_{\mathbf{k}^{\prime} \mathbf{k}^{\prime}}=\mathrm{I}$ by $\mathrm{I}$ correlation matrix for adjusted responses, where element $\mathrm{R}_{\mathbf{k}^{\prime} \mathrm{k}^{\prime}}(\mathrm{i}, \mathrm{j})=$ $\frac{\mathrm{C}_{k^{\prime} k^{\prime}}(i, j)}{\sigma_{k^{\prime}}(i, i) \sigma_{k^{\prime}}(j, j)}=\frac{\tilde{C}_{k^{\prime} k^{\prime}}(i, j)}{\tilde{\sigma}_{k^{\prime}}(i, i) \tilde{\sigma}_{k^{\prime}}(j, j)}=\frac{\hat{C}_{k^{\prime} k^{\prime}}(i, j)}{\hat{\sigma}_{k^{\prime}}(i, i) \hat{\sigma}_{k^{\prime}}(j, j)}$. 


\title{
6.7 SAMPLER: A MODULE FOR STATISTICAL UNCERTAINTY ANALYSIS WITH SCALE SEQUENCES
}

W. A. Wieselquist, M. L. Williams, F. Havlůj ${ }^{1}$, R. A. Lefebvre, W. Zwermann ${ }^{2}$, M. Klein ${ }^{2}$, D. Wiarda, M. T. Pigni, I. C. Gauld, M. A. Jessee, J. P. Lefebvre, K. J. Dugan ${ }^{3}$, and B. T. Rearden

\begin{abstract}
Sampler is a "super-sequence" that performs general uncertainty analysis for SCALE sequences by statistically sampling the input data and analyzing the output distributions for specified responses. Among the input parameters that can be sampled are multigroup nuclear data, resonance self-shielding data (shielding factors and CENTRM pointwise cross sections), depletion data such as fission product yields and decay data, and model parameters such as nuclide concentrations, temperatures, and simple dimension specifications. Random perturbation factors for nuclear cross sections and depletion data are pre-computed with the XSUSA module Medusa by sampling covariance information and are stored in libraries read during the Sampler execution, while model parameters are sampled "on the fly". A wide variety of output response types for virtually all SCALE sequences can be specified for the uncertainty analysis, and correlations in uncertain parameters between multiple systems are also generated.
\end{abstract}

\footnotetext{
${ }^{1}$ ÚJV Řež, a. s., Czech Republic

${ }^{2}$ Gesellschaft für Anlagen- und Reaktorsicherheit (GRS)

${ }^{3}$ Texas A\&M University
} 


\section{CONTENTS}

$\underline{\text { Page }}$

6.7 SAMPLER: A Modular for Statistical Uncertainty Analysis with SCALE Sequences............... 6-408

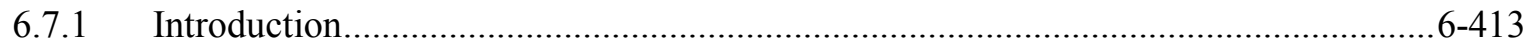

6.7.1.1 Uncertainty analysis with stochastic versus perturbation methods............6-413

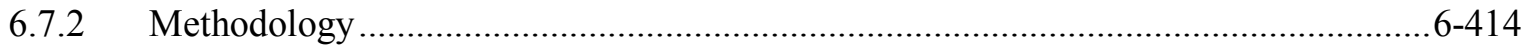

6.7.2.1 Definition of input data perturbations.....................................................6-414

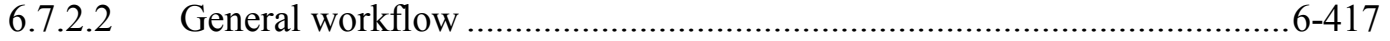

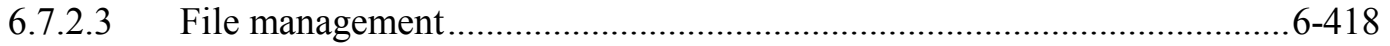

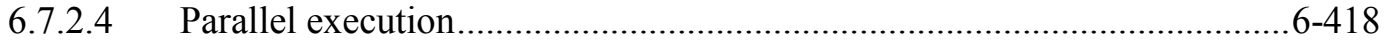

6.7.2.5 Behavior when encountering errors ..................................................6-418

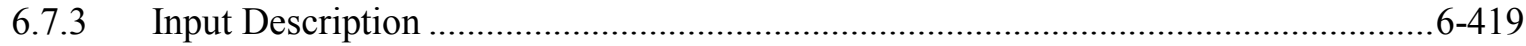

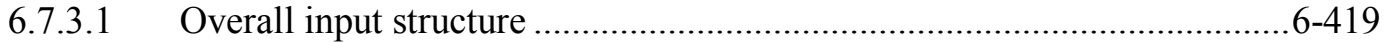

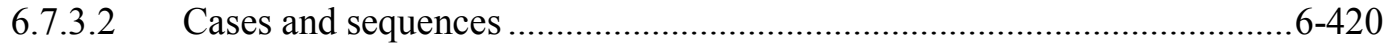

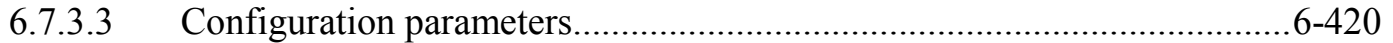

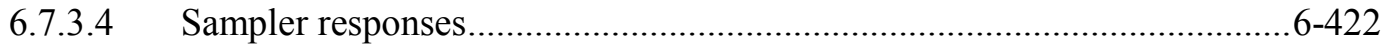

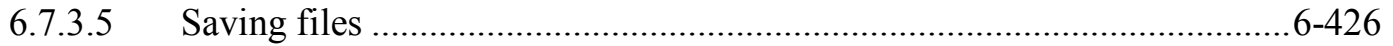

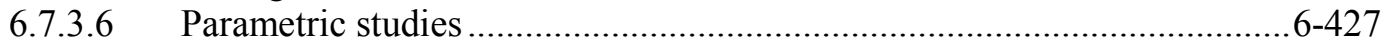

6.7.3.7 Geometry and material perturbations.................................................6-427

6.7.3.8 Converting a standard SCALE input deck to a Sampler input deck..........6-432

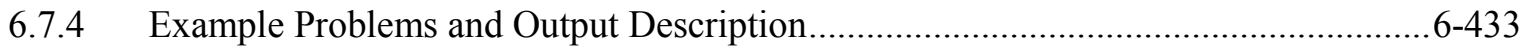

6.7.4.1 Output description...............................................................................6-433

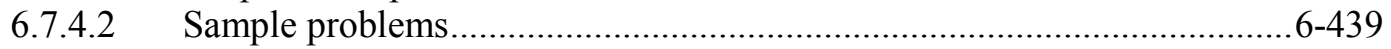

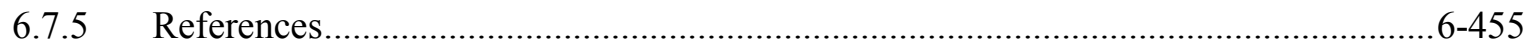

6.7.A APPENDIX A - SCALE Input Retrieval ENgine (SIREN) .....................................6-456

6.7.B APPENDIX B - Expression Operators and Functions for Sampler ............................ 6-461

6.7.C APPENDIX C - Guidelines for Running Sampler in Parallel.................................... 6-463 


\section{TABLE OF FIGURES}

$\underline{\text { Page }}$

Figure 6.7.1. Example histogram viewed in Fulcrum ..................................................................6-437

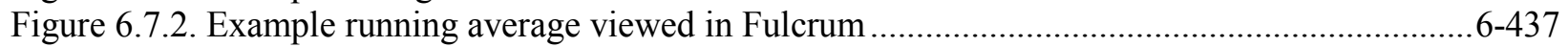

Figure 6.7.3. Time-dependent average plus standard deviation plot ............................................... 6-438

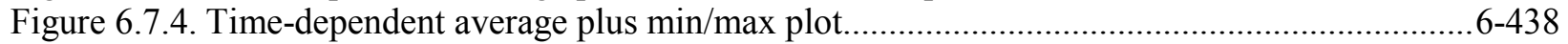

Figure 6.7.5. Distribution of lambda (k-eff) obtained from sample problem 1 .................................6-440

Figure 6.7.6. Dependency of lambda (k-eff) on coolant density and fuel temperature for sample

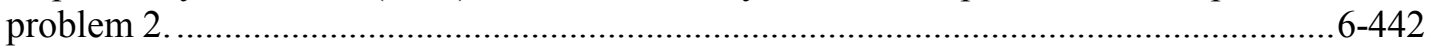

Figure 6.7.7. Distribution of multiplication factor with sampled enrichment distribution ...................6-443

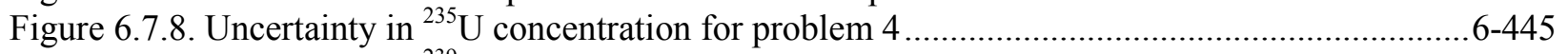

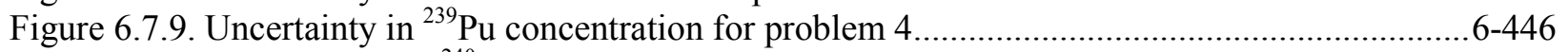

Figure 6.7.10. Uncertainty in ${ }^{240} \mathrm{Pu}$ concentration for problem 4 ....................................................6-646

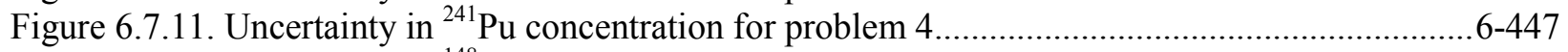

Figure 6.7.12. Uncertainty in ${ }^{148} \mathrm{Nd}$ concentration for problem 4 .................................................6-447

Figure 6.7.13. Uncertainty in ${ }^{150} \mathrm{Sm}$ concentration for problem 4 ...............................................6-448

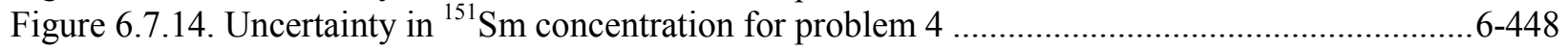

Figure 6.7.15. Correlation matrix for concentrations in sample problem 5 ..................................6-450

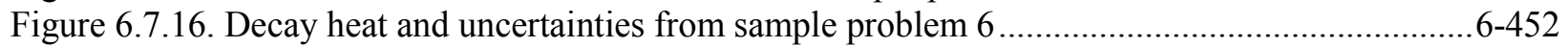

Figure 6.7.17. Flux as a function of shielding thickness for sample problem 7 ................................ 6-455 


\section{LIST OF TABLES}

$\underline{\text { Page }}$

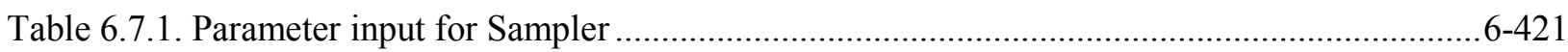

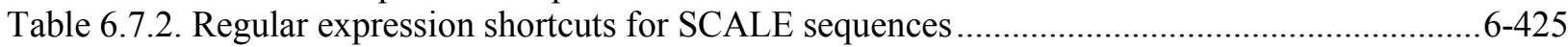

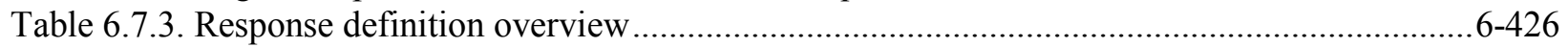

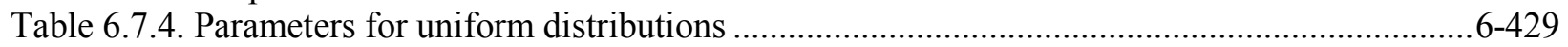

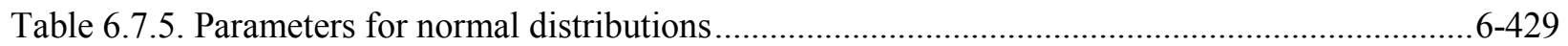

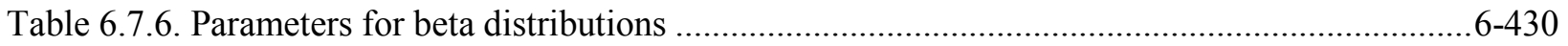

Table 6.7.7. Decay heat and uncertainties from sample problem 6 .............................................6-451 


\section{ACKNOWLEDGMENTS}

Contributions from the Gesellschaft für Anlagen- und Reaktorsicherheit (GRS) in Germany are gratefully acknowledged. The development of the SCALE Sampler module is based on GRS's suggestion that their XSUSA code could be used in conjunction with SCALE for stochastic uncertainty calculations. The original Sampler sequence was developed based on the XSUSA sampling sequence as well as collaboration and knowledge exchange with GRS staff members. The GRS module Medusa is used to generate perturbations of the MG cross sections, fission yields, and decay data.

The U.S. Nuclear Regulatory Commission Office of Nuclear Regulatory Research, the U.S. DOE Nuclear Fuel Storage and Transportation Planning Project, and the U.S. DOE Nuclear Criticality Safety Program supported the development of Sampler. 


\subsubsection{Introduction}

The SCALE nuclear analysis code system provides a unified set of computational tools and data libraries to address a wide range of applications, including criticality safety, reactor physics, spent fuel characterization, burnup credit, national security, and neutron/photon radiation shielding. ${ }^{1}$ In addition to determining the problem solutions, SCALE also provides tools to compute uncertainties in the results, arising from uncertainties in the data used for the calculations. Due to the diverse types of computational methods in SCALE, robust sensitivity/uncertainty $(\mathrm{S} / \mathrm{U})$ methods are necessary. Sampler implements stochastic sampling of uncertain parameters that can be applied to any type of SCALE calculation, propagating uncertainties throughout a computational sequence. Sampler treats uncertainties from two sources: 1) nuclear data and 2) input parameters. Sampler generates the uncertainty in any result generated by the computational sequence through stochastic means by repeating numerous passes through the computational sequence, each with a randomly perturbed sample of the requested uncertain quantities. The mean value and uncertainty in each parameter is reported along with the correlation in uncertain parameters where multiple systems are simultaneously sampled with correlated uncertainties.

Used in conjunction with nuclear data covariances available in SCALE, Sampler is a general, overarching sequence for obtaining uncertainties for many types of applications. SCALE includes covariances for multigroup neutron cross-section data, as well as for fission product yields and radioactive decay data, which allows uncertainty calculations to be performed for most multigroup (MG) computational sequences in SCALE. At the present time, nuclear data sampling cannot be applied to SCALE continuous energy (CE) Monte Carlo calculations (i.e., CE-KENO and CE-Monaco), although the fundamental approach is still valid.

Used in conjunction with uncertainties in input data, Sampler can determine the uncertainties and correlations in computed results due to uncertainties in dimensions, densities, distributions of material compositions, temperatures, or many other quantities that are defined in the user input for any SCALE computational sequence. This methodology was especially developed to produce uncertainties and correlations in criticality safety benchmark experiments, but it has a wide range of applications in numerous scenarios in nuclear safety analysis and design. The input sampling capabilities of Sampler also include a parametric capability to determine the response of a system to a systematic variation of an arbitrary number of input parameters.

\subsubsection{Uncertainty analysis with stochastic versus perturbation methods}

Two quite different approaches may be used for uncertainty analysis. One method uses first order perturbation theory expressions to compute sensitivity coefficients for a given response. This requires performing a forward transport calculation for the specified system and (sometimes) adjoint calculations for each response of interest. After the forward and adjoint transport solutions are obtained, sensitivity coefficients for all nuclear cross sections and material concentrations can be computed very efficiently with perturbation theory. ${ }^{1}$ The sensitivities may be folded with covariance matrices to obtain response uncertainties due to nuclear data. The TSUNAMI modules and sequences in SCALE use perturbation theory for S/U analysis in this manner (see TSUNAMI-1D, TSUNAMI-2D, and TSUNAMI-3D).

For some types of applications, the adjoint-based perturbation methodology is not adequate or is inefficient. These include:

(a) Cases requiring codes with no adjoint functionality. SCALE has capability for critical eigenvalue adjoint solutions and generalized adjoint calculations using XSDRN, NEWT or KENO, but adjoint methods are not currently available for coupled neutronics-depletion calculations. 
(b) Cases for which first order perturbation theory is not valid (i.e., problems with significant second order effects).

The Sampler module described in this section provides an alternative method for uncertainty analysis based on stochastic sampling (Monte Carlo) and does not require adjoint calculations. This approach samples joint probability density functions (PDFs) - such as given in the SCALE nuclear data covariance library-- to produce a random sample for the nuclear cross sections used a transport calculation. If PDFs are available for other parameters such as depletion data or model parameters, etc., then these too can be sampled and included in the perturbed input vector. The perturbed data vector can be input to any SCALE sequence or functional module to obtain a single forward solution for all desired perturbed responses. The process is repeated for the desired number of samples-typically a few hundred; and the output distributions of results are analyzed to obtain standard deviations and correlation coefficients for all responses. The stochastic sampling method is not restricted to current SCALE modules; any new sequences or codes can be used for the forward calculations, without having to develop the capability for adjoint calculations.

Output distributions from the SCALE sampling also may be propagated to downstream codes for followon uncertainty analysis. For example, input for the TRITON lattice physics sequence can be sampled to produce a random set of output assembly-averaged, two-group cross section libraries. The two-group libraries can be input to a 3D core simulator that performs steady-state or transient calculations, and statistical analysis of the simulator output provides response uncertainties (possibly time-dependent) due to the SCALE input data uncertainties. Response uncertainties computed with this approach are not limited to first order accuracy; i.e., they account for all non-linearities and discontinuities with the same accuracy as the original codes.

Thus there are several advantages to the statistical sampling method because it requires only forward calculations. The typical Sampler computational procedure perturbs the entire input data vector simultaneously, so that the total uncertainty in all responses, due to all data uncertainties, is obtained. This standard approach does not provide individual data sensitivity coefficients, unlike the perturbation theory method. In this sense, the statistical sampling method is complementary to the adjoint-based sensitivity method in the TSUNAMI modules. Computation of sensitivities using only forward calculations requires that each input parameter be varied individually, rather than collectively; and this may require a large number of simulations to obtain a full set of sensitivity coefficients.

\subsubsection{Methodology}

The main components of a Sampler calculation are the procedures for perturbing input data, obtaining the desired responses, and performing statistical analysis of the output distributions.

\subsubsection{Definition of input data perturbations}

The input data for a SCALE computation will generally be one of three types:

(a) Nuclear data for transport calculations. This includes multigroup (MG) and continuous energy (CE) cross sections, multiplicities, secondary particle distributions, and data used for resonance self-shielding of MG cross sections.

(b) Nuclide transmutation data for depletion and burnup calculations. This includes fission product yield data, decay constants, branching ratios to excited states, decay energies and distributions.

(c) Modeling parameters for the system. This includes information for defining nuclide number densities (e.g., density, weight fractions, enrichment, void fraction, etc.), temperature, and dimensions. 
In principle Sampler can perform uncertainty analysis for all the above types of input data if uncertainties and correlations are known. The main restriction at this time is that CE cross sections for Monte Carlo calculations are not sampled (although the continuous data used for self-shielding are treated), so data perturbation applications are limited to $\mathrm{MG}$ calculations. Perturbations to input number densities and model dimensions are not impacted by this data limitation.

\subsection{Nuclear data perturbations for multigroup calculations}

Input MG nuclear data for SCALE sequences are obtained from an AMPX Master formatted library, which contains infinitely-dilute one-dimensional (1D) cross sections, two-dimensional (2D) scattering distributions, and Bondarenko self-shielding factors for various types of reactions. Only the 1D data and Bondarenko factors are varied in Sampler because no covariance data are available for the 2D scattering distributions; however, the 2D data are renormalized to be consistent the perturbed 1D scattering cross sections.

The Medusa module of the XSUSA program ${ }^{2}$ is used to generate perturbation factors for the $1 \mathrm{D}$ cross sections on the MG library, assuming that the probability density functions are multivariate normal distributions with covariances given in the SCALE nuclear data covariance library. The library covariance data are given as infinitely-dilute, relative values; therefore a random sample for cross section $\sigma_{\mathrm{x}, \mathrm{g}}$

corresponds to $\frac{\Delta \sigma_{x, g}}{\sigma_{x, g}}$, where subscript $x$ defines the nuclide/reaction type and $g$ is the group number. The relative variations are transformed to multiplicative perturbation factors, defined by

$$
\mathrm{Q}_{\mathrm{x}, \mathrm{g}}=1+\frac{\Delta \sigma_{\mathrm{x}, \mathrm{g}}}{\sigma_{\mathrm{x}, \mathrm{g}}}
$$

that can be applied to the reference data to obtain the altered infinitely-dilute values. A master sample file containing perturbation factors for 1000 samples $^{4}$ of the infinitely-dilute $1 \mathrm{D}$ data has been pre-computed and stored in the SCALE data directory. Each sample in the file contains perturbation factors for all groups and reactions in all materials. The master sample file is used for all cases, which avoids having to perform the data sampling during SCALE execution.

Because the 1D data in the MG library are infinitely-dilute (i.e., problem-independent), SCALE sequences include modules that compute resonance shielding corrections for the MG data. The selfshielding calculations generally require two additional types of input data: (a) Bondarenko self-shielding factors for the BONAMI module, which typically performs self-shielding calculations outside of the resolved resonance range; and (b) CE cross sections for the CENTRM/PMC modules, which compute pointwise (PW) flux spectra and process self-shielded cross sections for the resolved resonance range. Perturbations in the Bondarenko factors and CE cross sections used in self-shielding calculations must be consistent with perturbations made to the infinitely dilute $1 \mathrm{D}$ cross sections since all these data are based on the same fundamental ENDF/B information. It was shown in reference [3] that consistent perturbations can be obtained by using the same perturbation factors $Q_{x, g}$ in following expressions:

\footnotetext{
${ }^{4}$ The tradeoff of size on disk of the pre-calculated samples distributed with SCALE versus the maximum number of perturbations required in practice has lead to the current maximum of 1000 samples. Based on limited experience, correlation coefficients of near zero require the most samples to converge and typically about 1000 samples has been sufficient.
} 
(a) infinitely-dilute MG cross sections $\sigma_{x, g}$ :

$$
\sigma_{x, g}^{\prime}=Q_{x, g} \sigma_{x, g}
$$

(b) Bondarenko factors $f\left(\sigma_{0}, T\right)$, at background cross section $\sigma_{0}$ and temperature T:

$$
\mathrm{f}_{\mathrm{x}, \mathrm{g}}^{\prime}\left(\sigma_{0}, \mathrm{~T}\right)=\mathrm{f}_{\mathrm{x}, \mathrm{g}}\left(\sigma_{0}^{\prime}, \mathrm{T}\right) ; \text { where } \sigma_{0}^{\prime}=\sigma_{0} / \mathrm{Q}_{\mathrm{x}, \mathrm{g}}
$$

(c) CE data $\sigma_{\mathrm{x}}(\mathrm{E})$ :

$$
\sigma_{x}^{\prime}(E)=Q_{x, g} \sigma_{x}(E), \text { for } E \in g
$$

In the above expressions, subscript $\mathrm{x}$ defines the nuclide/reaction type and $\mathrm{g}$ is the group number.

During Sampler execution the module ClarolPlus reads perturbation factors $\left(Q_{x, g}\right)$ for a specified sample number from the master sample file, and evaluates Equations (6.7.2) and (6.7.3). ClarolPlus also writes a file containing perturbation factors only for the particular sample number used by the CrawdadPlus module, as described below.

\subsection{Depletion data perturbations}

Multiplicative perturbation factors for fission product yields have been generated with XSUSA by sampling the covariances for the independent yield uncertainties. The yield uncertainties are taken from ENDF/B VII.1, which in general are given by fissionable nuclide and for up to three energies: $0.025 \mathrm{eV}$, $0.5 \mathrm{MeV}$, and $14 \mathrm{MeV}$. The ENDF/B yield uncertainties do not include correlations between fission products, which may arise due to constraints such as (a) the sum of all yields must always be two (i.e., the uncertainty in the yield sum is zero), and (b) the uncertainties in independent yields should be consistent with uncertainties given for cumulative yields. The constraints generally introduce positive and negative correlations into the yields covariance matrix. A method developed by Pigni ${ }^{4}$ was used to determine the correlations in ${ }^{235} \mathrm{U}$ yields. Correlations in yields from other fissionable nuclides are not available in SCALE at this time.

During Sampler execution the perturbation factors are read for a given data sample, compute a complete set of perturbed independent yields for all fissionable nuclides and energies, and renormalize the yields to ensure that they sum to two. An output file containing the perturbed yield data is written to an external file in the format read by ORIGEN. The perturbation factors are read once each time a sequence executed (i.e., for each data sample).

A set of 1,000 decay data perturbations has also been generated with XSUSA and stored in decay-only ORIGEN library files. Sampler automatically aliases the appropriate sample to the file "end7dec". Note: In order for decay data perturbations to be performed, the "end7dec" decay library must be used directly. Typical TRITON and Polaris calculations do not use "end7dec" directly, due to using the unperturbed decay data embedded in a special ORIGEN reaction library aliased to "transition.def" as the basis for all coupled transport/depletion calculations. Experience has been that decay data contributes very little additional uncertainty compared to yield data and cross section data. 


\subsection{Model data perturbations}

An approach presented by Areva NP GmbH utilizes statistical sampling on uncertain parameters to assess the uncertainty in individual system as well as correlations between multiple systems. ${ }^{5}$ In this approach, values for individual parameters in the input model are randomly modified within the reported uncertainty and distribution function and a series of perturbed values are obtained. Where sufficient samples are made, the distribution of the perturbed values is used to determine the uncertainty in the computed quantity due to uncertainties in the input parameters. In cases where the same uncertain parameters influence multiple experiments the simultaneous perturbation of the parameter for multiple cases will provide the correlation in uncertainties between the different configurations.

To obtain the uncertainty and correlation due to all uncertain parameters, all parameters are randomly perturbed for each calculation and the uncertainties and correlations are determined. Mathematically, the uncertainty in an individual output parameter $k$ is determined as shown in Eq. (6.7.4).

$$
\Delta k^{\exp }(i)=\hat{\mu}_{i}=\sqrt{\frac{1}{n-1} \sum_{a=1}^{n}\left(\left(k_{\text {calc }}^{M C}(i)\right)_{a}-\overline{k_{\text {calc }}^{M C}(i)}\right)^{2}}
$$

where $\Delta k^{\exp }(i)$ is the uncertainty (in terms of standard deviation) in system $i$ due to uncertainties in the

input parameters. $\left(k_{\text {calc }}^{M C}(i)\right)_{a}$ is the $a^{\text {th }}$ Monte Carlo (MC) sample of system $i$, where all uncertain input parameters have been randomly varied within the specified distribution.

The covariance between two systems, $i$ and $j$, is determined as shown in Eq. (6.7.4).

$$
\hat{\Sigma}_{i j}=\sqrt{\frac{1}{n-1} \sum_{a=1}^{n}\left(\left(k_{\text {calc }}^{M C}(i)\right)_{a}-\overline{k_{\text {calc }}^{M C}(i)}\right)\left(\left(k_{\text {calc }}^{M C}(j)\right)_{a}-\overline{k_{\text {calc }}^{M C}(j)}\right)} .
$$

The correlation coefficient between systems $i$ and $j$ can be determined from Eqs. (6.7.4) and (6.7.4) as shown in Eq. (6.7.4).

$$
c_{i j}=\frac{\hat{\Sigma}_{i j}}{\hat{\mu}_{i} \hat{\mu}_{j}} .
$$

The correlation coefficients determined with Eq. (6.7.4) are the values needed to perform the Generalized Least Linear Square (GLLS) analysis using TSURFER, which solves for a set of cross section data perturbations that would improve agreement between the computational simulations and experimental benchmark results.

\subsubsection{General workflow}

The overall workflow for Sampler is as follows:

- for each sample, pick the perturbation factors and generate geometry perturbations

$\circ$ for each case, build SCALE input decks, which include: 
- calls to the perturbation modules, which generate the perturbed data libraries (based on the perturbation factors for this sample)

- user sequence inputs

- output data retrieval

○ insert each of the constructed input decks into the processing queue

- run all SCALE cases from the queue (serial or parallel, as available)

- perform data extraction (using the response mechanism) and statistical analysis

- $\quad$ print output and generate data files

The advantage of this workflow is that the individual SCALE runs are completely identified by the sample number (so they are reproducible) and they are independent. Each of the runs is executed within its own environment (with SCALE runtime as a subprocess), with its own decay data, fission yield and cross section library. This arrangement is very robust and, as there is no coupling between the runs, can be effectively parallelized.

\subsubsection{File management}

For every SCALE run, Sampler creates a subdirectory within its own temporary directory. Each subdirectory has a name in the form (case name)_pert_(sample number). Within this directory all the useful data for the particular run are stored: the input file, the output file, the message file, the terminal $\log$ file (which is a joint capture of SCALE both standard output and standard error stream) along with the saved data files (ft71, xfile016, PTP files etc.)

By retaining the temporary directory, the user can then examine and possibly reuse saved files for the individual SCALE runs.

\subsubsection{Parallel execution}

Since the Sampler calculations usually consist of several hundred mutually completely independent calculations, it is desirable to run the subcases in parallel.

Sampler supports both threading and MPI for parallel computing. No special settings are needed in the input deck; Sampler fully relies on the parallel capabilities of the SCALE runtime environment (ScaleRTE).

In order to run Sampler in parallel, use $=\frac{\circ}{\circ}$ ampler as the sequence declarator and specify either the $-\mathrm{N}$ (for MPI) or -I (for multi-threads) command line arguments to ScaleRTE. For serial and multi-threaded runs, the declaratory =sampler may be used to remove the warning about a parallel version not being available.

Note: Where MPI is used, the Sampler case temporary directory has to be accessible by all of the computing nodes. Therefore, use $-\mathrm{T}$ to place the temporary directory into a shared storage space.

\subsubsection{Behavior when encountering errors}

Any time a parameter within a SCALE input is perturbed, there is the possibility that the perturbation will cause unrealistic behavior (fuel pellet passing through cladding, etc.) that will cause SCALE to fail. The default behavior of Sampler is to finish all perturbed cases and check whether there are errors present for each case once all cases have been run. This behavior can be changed, so that the output of each case is checked as soon as the case has finished and exit immediately if there are errors present, by specifying the parameter "continue_if_errors=no". 
It is advisable to use this parameter during the development of a Sampler input when a small number of samples are being made. Once there is confidence that the perturbed parameters will not cause errors in the SCALE sequences, this parameter can be returned to its default value and a larger number of samples can be taken.

Note: This parameter only has an effect in serial and multi-threaded mode. At the present time, if this parameter is declared as "no" in MPI mode there will be no change in behavior (Sampler will finish all cases and check for errors afterwards).

\subsubsection{Input Description}

This section describes the Sampler input file format.

\subsubsection{Overall input structure}

The order of the blocks is arbitrary, with the exception of dependent variables (see Sect. 6.7.3.7.3). Below is the layout of a typical sampler input.

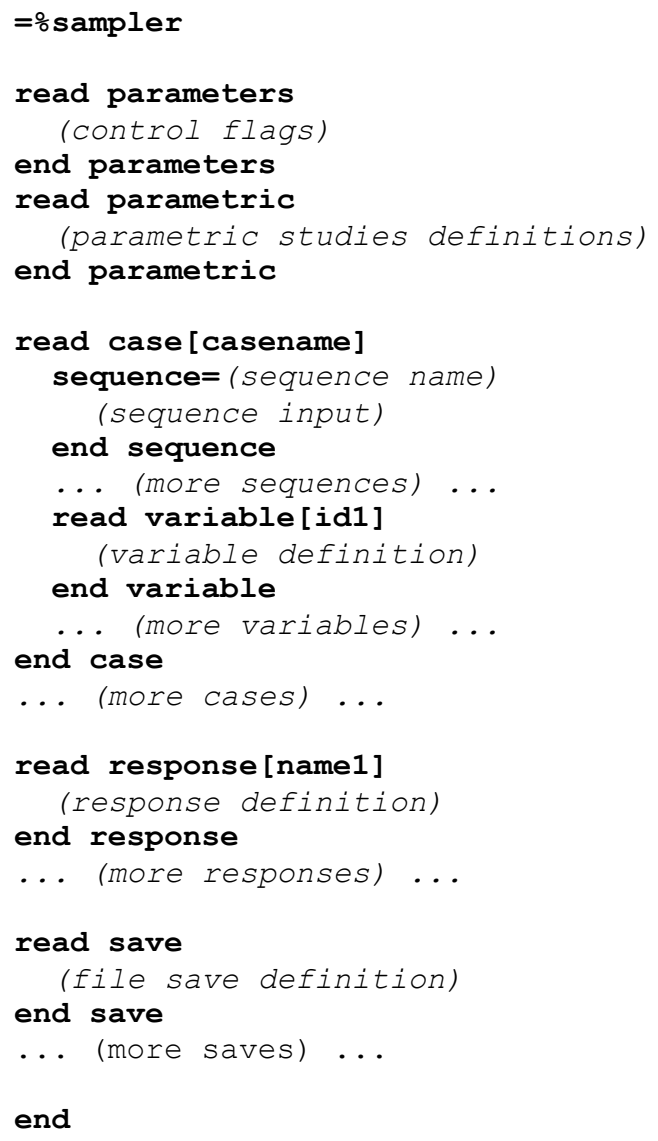

Every Sampler input file has to contain the parameters block and at least one case block. Other input blocks are optional. 


\subsubsection{Cases and sequences}

Within Sampler, multiple independent SCALE calculations, or cases, can be included. Since the same set of responses is extracted from each of the cases, these should have the same structure (i.e. produce the same kind of output files); the benefit of having multiple cases within one Sampler input deck is that it is possible to generate cross-correlations between cases as an output.

Every case contains one or more stacked sequences. The whole case is always run together.

Each case has an unique identifier. The identifier is a single word beginning with a letter followed by letters, numbers and underscores. Note that the dash "-" cannot be used in a case identifier.

Within the case block, the user can enter any number of sequences, which contain the actual user input. The format of each sequence block is:

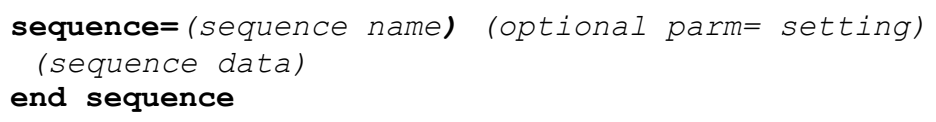

The sequence data is a SCALE input that is not processed by Sampler, except to substitute sampled values for variable placeholders (see Sect. 6.7.3.7.4 for more information) . For parm= settings, no limit on column number is enforced.

\subsection{Importing input data from external files}

Instead of directly specifying the SCALE sequence input within the Sampler input file, the user can specify the path to a previously generated input file which can be imported for use within Sampler as:

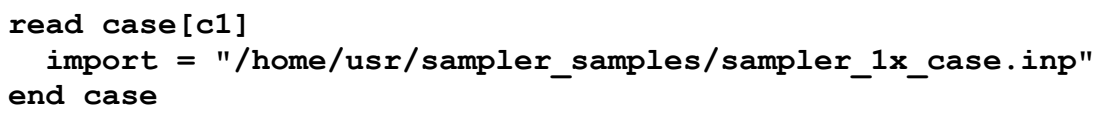

In this case, absolute paths should be used (or a shell sequence before invoking Sampler, to copy the appropriate files into the temporary directory). This approach provides concise input files and is advantageous for quality assurance controlled input data.

\subsubsection{Configuration parameters}

In the parameters block the user can control the main workflow and output parameters for sampler. Valid keywords are shown in Table 6.7.1. Note that of the major perturbation modes, only "perturb_geometry=yes" is on by default. (Bondarenko factor and pointwise data perturbation for CENTRM controls how "perturb xs=yes" is performed.) 
Table 6.7.1. Parameter input for Sampler

\begin{tabular}{|c|c|c|}
\hline KEYWORD & DESCRIPTION & DEFAULT \\
\hline $\mathrm{n}_{\text {_samples }}=\mathrm{N}$ & $\begin{array}{l}\text { Number of samples }(1-1000 \text { for nuclear data } \\
\text { perturbations, unlimited for input file } \\
\text { perturbations) }\end{array}$ & none \\
\hline first_sample $=\mathrm{N}$ & Number of the first sample & 1 \\
\hline perturb_geometry $=($ yes $/$ no $)$ & $\begin{array}{l}\text { Perform input file/model data/geometry } \\
\text { perturbations }\end{array}$ & yes \\
\hline perturb_xs=(yes/no) & Perform cross-section (XS) perturbation & no \\
\hline perturb_yields=(yes/no $)$ & Perform fission yield perturbation & no \\
\hline perturb_decay=(yes/no) & Perform decay data perturbation & no \\
\hline perturb_bondarenko=(yes/no) & When perturbing XS, perturb Bondarenko factors & yes \\
\hline perturb_pointwise $=($ yes $/$ no $)$ & When perturbing XS, perturb pointwise data & yes \\
\hline library=".." & $\begin{array}{l}\text { Name of the master XS library (in quotes), it is } \\
\text { possible to use the filename or an alias (e.g. "“ } \\
\text { v7.1-252n") }\end{array}$ & none \\
\hline perturbed_library=".." & $\begin{array}{l}\text { Name of the perturbed library (in quotes); this is } \\
\text { the perturbed XS library used by the actual } \\
\text { computational sequences }\end{array}$ & $\begin{array}{l}\text { (same as } \\
\text { library) }\end{array}$ \\
\hline mg_factors_library=" .." & $\begin{array}{l}\text { Name of the multigroup XS perturbation factors } \\
\text { library (in quotes); if not given, built-in library is } \\
\text { used }\end{array}$ & (blank) \\
\hline run_cases $=($ yes $/$ no $)$ & $\begin{array}{l}\text { Actually run inputs through SCALERTE or just } \\
\text { generates them. }\end{array}$ & yes \\
\hline force_run $=($ yes/no $)$ & $\begin{array}{l}\text { Enforce running SCALE even when the output } \\
\text { files are present }\end{array}$ & no \\
\hline $\mathrm{plt}=($ yes $/ \mathrm{no})$ & $\begin{array}{l}\text { Produce plot file histograms (PTP format) with } \\
\text { response distributions that can be viewed with } \\
\text { Fulcrum }\end{array}$ & yes \\
\hline $\mathrm{csv}=(\mathrm{yes} / \mathrm{no})$ & Produce CSV files with individual tables & yes \\
\hline print_data $=($ yes/no) & Print per-sample values in the main output & no \\
\hline print_corr $=($ yes/no $)$ & Print correlation matrices in the main output & no \\
\hline print_cov $=($ yes/no $)$ & Print covariance matrices in the main output & no \\
\hline print_chi2=(yes/no $)$ & Print chi-square normality test in the main output & no \\
\hline continue_if_errors=(yes/no) & $\begin{array}{l}\text { Complete all perturbed cases even if errors are } \\
\text { discovered in the output of perturbed case }\end{array}$ & yes \\
\hline
\end{tabular}

\section{Notes on sample numbers:}

The samples are selected from the perturbation factor libraries (except for geometry perturbation); it is up to the user to fit inside the range of samples available (i.e. $n$ _samples+first_sample-1 must be less or equal to the number of samples). The built-in perturbation libraries based nuclear data covariances contain 1000 samples.

\section{Note on perturbed library name:}

The default behavior for Sampler is to set perturbed_library to the same name as library. Since Sampler creates a local file in the temporary directory, which is used by SCALE instead of the library in the lookup table, it in general results in the desired behavior. SCALE sequences only provide pre-defined 
resonance self-shielding options for known libraries, so where perturbed_library differs from the name of a standard SCALE library, the type of resonance self-shielding calculation desired must be specified (via the $P A R M=$ setting). Please review the documentation of the specified sequence for available options, such as $P A R M=C E N T R M$.

Beware: The library name must result in a valid filename. In some cases the use of "xn252v7.1" instead of "v7.1-252" is recommended because the dash might result in improper links to the perturbed library. This guidance applies only to the cases when perturb_xs=yes is used; otherwise, Sampler does not generate a perturbed library.

See Sect. 6.7.3.8 for an example of properly referencing the perturbed library.

\subsubsection{Sampler responses}

For every case run within Sampler any number of responses can be extracted. A response can be a single number or a time-dependent series, which is assigned a name and optionally several parameters. The responses are entered once and shared across all the cases, i.e. every case returns the same set of responses. This allows Sampler to provide the user with inter-case comparisons and statistics (i.e. covariance and correlation matrices).

Sampler recognizes these kinds of responses:

- opus_plt - data from an OPUS-generated PLT file

- triton - TRITON homogenized cross-sections (xfile016)

- stdcmp - standard composition files

- $\quad f 71$ - concentrations from the F71 ORIGEN dump

- grep - general expression from the text output file

- variables - the geometry perturbation sampled values

.

\subsection{General response block format}

The general format of the response block is:

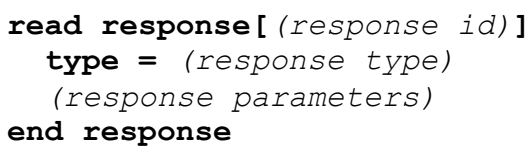

The (response id) is an arbitrary identifier (a single word) by which the the response is denoted in the results. The (response type) is one of the keywords opus_plt, triton, stdcmp, f71, variables and grep. Response parameters are different for each response type and are explained below.

\subsection{OPUS PLT file responses}

This response extracts any data from a PLT file generated by OPUS. The user specifies which PLT file should be used and which elements/nuclides should be used. 
Parameter ndataset provides the number of the selected PLT file, i.e. ndataset $=1$ will read data from the file ending with .00000000000000001.plt (which is the second generated PLT file in the given case).

Parameter nuclides=...end specifies the list of nuclides (or elements) which are read from the PLT file; nuclides can be specified as alphanumeric identifiers (U-235, ba137m) or six-digit ZAI identifiers (922350). In addition to that, any other PLT file response identifiers (i.e. the character strings in the first column of the plot table) may be used, which allows for example the usage of total and subtotal keywords.

\section{Example:}

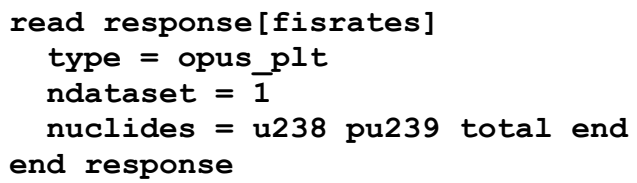

\subsection{TRITON homogenized cross-section responses}

This response extracts the homogenized cross-section data saved by TRITON on the xfile016.

Responses are retrieved for a selected homogenized mixture and all branches (which are then denoted by response name suffixes).

Using a data $=\ldots$ end assignment specifies which data types are to be saved.

The available options for data entries are:

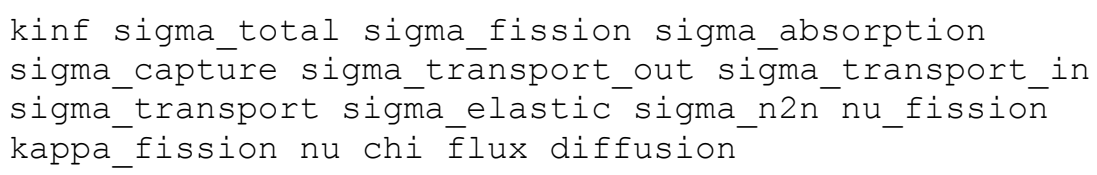

\section{Example:}

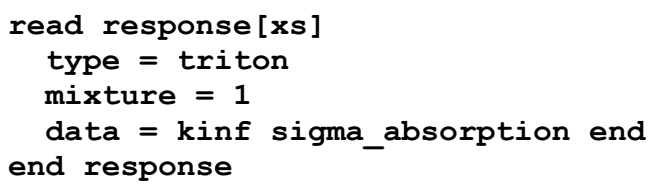

\subsection{Standard composition file responses}

This response retrieves isotopic concentrations (in atoms/barn-cm) from the standard composition file. Parameter nuclides=...end specifies which nuclides should be retrieved. The parameter mixture specifies the number of the StdCmpMix file, so mixture=10 would load concentrations from the file StdCmpMix00010_* (for all time steps).

\section{Example:}

read response [ $\mathrm{mix} 10]$

nuclides $=\mathrm{u}-235 \mathrm{pu}-239$ end

mixture $=10$ 
end response

\subsection{ORIGEN concentration (F71) responses}

This response retrieves the isotopic concentrations (in gram-atoms) from the ORIGEN concentration edit in the $f t 71 f 001$ file.

Parameter nuclides=...end specifies the list of nuclides (or elements) which are read from the F71 file; nuclides can be specified as alphanumeric identifiers (U-235, ba137m) or six-digit ZAI identifiers (922350).

Two options are available to choose the positions on the file from which data should be retrieved. Either step_from=start and step_to=end can be used to select a range of positions, or mixture $=N$ can be used to choose either an ORIGEN case or a TRITON case mixture. This is convenient for TRITON cases where step numbers are usually not known in advance.

\section{Example:}

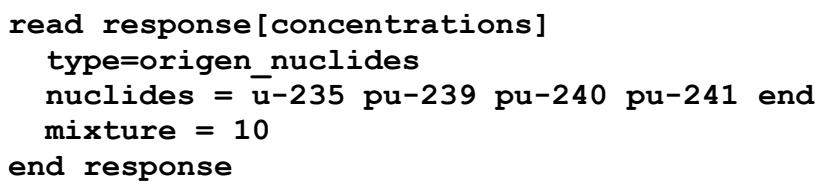

\subsection{Generic regular expression (GREP) responses}

In order to allow the user to collect other responses from the SCALE output, a generic regular expression (regexp) mechanism is provided by Sampler. For every response the user can enter one (or more) regular expressions, which are applied (using the "grep" system tool) to the main output file. "grep" is executed with the "-o" option, which returns only the matched portion of the line (and not the whole line). Usually it is necessary to use two expressions, one to find the line of interest and another to extract only the desired value. The POSIX character classes are supported in the grep used - the most commonly used are "[[:digit:]]" to match a single digit 0-9 and [[:space:]] to match a single space or tab. The "+" and "*" are used to match one or more and zero or more repeats, respectively. Note that as per standard regexp rules, "." matches any character and an escape is necessary, i.e. "।.", in order to match a period.

Each regular expression is defined by the keyword regexp="...". The result of the last regular expressions should be a single number (and is treated as such by Sampler).

The following example defines regular expression for extraction of k-effective from a CSAS5/6 output file:

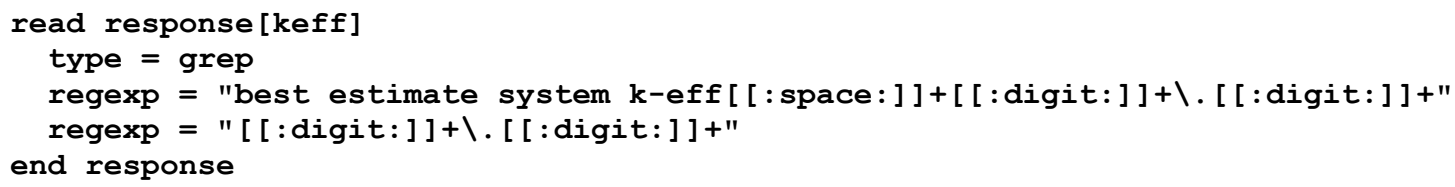

With the first regexp statement, a line containing "best estimate system k-eff" followed by a number is found, and then just the number part is extracted with the second regexp statement.

For ease of use, Sampler provides several regular expression shortcuts shown in Table 6.7.2. 
Table 6.7.2. Regular expression shortcuts for SCALE sequences

\begin{tabular}{|l|l|}
\hline regexp $=$ ": kenova.keff:" & k-eff from KENO5 / CSAS5 sequence \\
\hline regexp $=":$ kenova.ealf:" & EALF from KENO5 / CSAS5 sequence \\
\hline regexp $=":$ kenova.nubar:" & nu-bar from KENO5 / CSAS5 sequence \\
\hline regexp $=":$ kenovi.keff $: "$ & keff from KENO6 / CSAS6 sequence \\
\hline regexp $=":$ xsdrn.lambda:" & lambda from XSDRN / CSAS1 sequence \\
\hline regexp $=":$ scale.number:" & matches any number (e.g. "1", "1.0", "1.23e-7", "-0.3") \\
\hline
\end{tabular}

Thus, the previous example may be alternately rephrased as such:

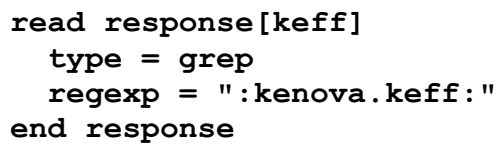

In addition to this, the grep response also supports extraction of data with uncertainties. In order to get the response uncertainty, use the eregexp= keyword, which follows the same rules as regexp=. The same shortcuts as for regexp= may be used as well (for KENO V.a/VI multiplication coefficient). Therefore, to get KENO multiplication factor including the uncertainty, one might define the response like this:

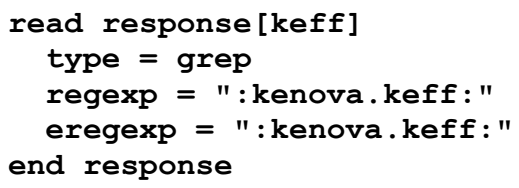

\subsection{Sampled variable values}

Using the variables response, the user can extract information from the sampled values of the geometry/material perturbation variables. The data $=$ key contains the list of variable identifiers of interest.

This option is useful to generate the correlations between geometry/material perturbations and the responses of interest.

\section{Example:}

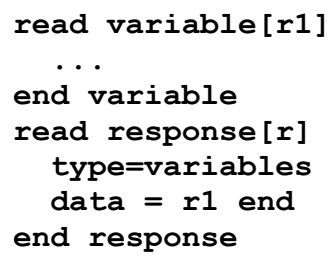




\subsection{Quick response definition overview}

Table 6.7.3 summarizes the available options for the different response types. The nuclides specification can either be in terms of the standard alphanumeric identifier, e.g. "u235m" for ${ }^{235 \mathrm{~m}} \mathrm{U}$, or the IZZZAAA integer identifier, e.g. " 1092235 " for ${ }^{235 \mathrm{~m}} \mathrm{U}$.

Table 6.7.3. Response definition overview

\begin{tabular}{|c|c|}
\hline Key & Description \\
\hline \multicolumn{2}{|c|}{ response type opus plt } \\
\hline Ndataset & Number of the PTP file \\
\hline Nuclides & List of nuclides (alphanumeric/IZZZAAA, terminated by end) \\
\hline \multicolumn{2}{|c|}{ response type stdemp } \\
\hline Mixture & Number of the StdCmpMix file \\
\hline Nuclides & List of nuclides (alphanumeric/IZZZAAA, terminated by end) \\
\hline \multicolumn{2}{|c|}{ response type triton } \\
\hline Mixture & Homogenized mixture index \\
\hline Nuclides & List of nuclides (alphanumeric/IZZZAAA, terminated by end) \\
\hline Data & List of homogenized data types (terminated by end) \\
\hline \multicolumn{2}{|l|}{ response type $f 71$} \\
\hline Nuclides & List of nuclides (alphanumeric/IZZZAAA, terminated by end) \\
\hline Mixture & ORIGEN case number / TRITON mixture number \\
\hline step_from & Lower bound of position range \\
\hline step_to & Upper bound \\
\hline \multicolumn{2}{|l|}{ response type grep } \\
\hline regexp & Regular expression for the response value (quoted) \\
\hline eregexp & Regular expression for the response uncertainty (quoted) \\
\hline \multicolumn{2}{|c|}{ response type variables } \\
\hline data & List of variable names (terminated by end) \\
\hline
\end{tabular}

\subsubsection{Saving files}

By default, Sampler saves from each run the input, output, message and terminal log files. In addition to that, if respective responses are requested, it saves the ft71f001 as basename.f71, xfile016 as basename. $\times 16$ and the StaCmpMix* files.

The user might specify additional files to be saved into the sample subdirectory; this is achieved by defining one or more save blocks.

Each save block contains a file="..." parameter, which specifies the filename in the sample run temporary directory. Optionally, the user can specify name $=" . . . "$ to let Sampler rename the file to basename.extension, where extension is the value of the name parameter. If name is not specified, the file is not renamed and is just copied to the sample subdirectory. The name parameter cannot be used if wildcards are used in the file parameter.

The quotes for both name and file parameter values are mandatory. 


\section{Example 1:}

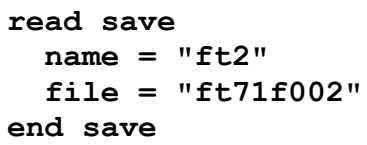

Example 2:

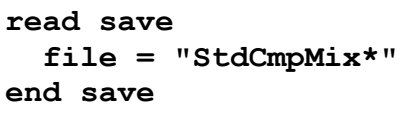

\subsubsection{Parametric studies}

The Sampler infrastructure allows an efficient implementation of studies of parameter variation effects on various responses. This mode is activated by entering the read parametric ... end parametric block.

This block contains two arrays: variables $=\ldots$ end and $n$ samples $=\ldots$ end. The variables array lists the variables of the parametric study. The variables must have "distribution=uniform", and the minimum and maximum becomes the range for that variable in the parametric study. For each variable, the corresponding value in the $n_{-}$samples $=\ldots$ end array indicates the number of evenly spaced values to assume in that dimension. The total number of calculations is therefore the multiplication of all the n_samples values. Note that for a single sample with n_samples=1, only the minimum value is used. Below is as an example of the parametric block.

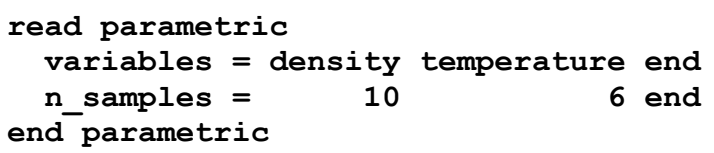

The two variables are density and temperature, and there will be 10 evenly spaced density values (including the minimum and maximum) and 6 evenly spaced temperature values, for a total of $10 \times 6=$ 60 calculations. To perform the same number of samples in each dimension, the keyword n_samples in the parameters block may also be used.

Sampler generates a summary table of the parametric study, including values for which the minimum and maximum of each response occurs. Sampler also generates PTP plot files showing the dependency of each response on each variable.

\subsubsection{Geometry and material perturbations}

In addition to data perturbations, Sampler also allows the user to include geometry and material uncertainties in the calculation. This is achieved by defining variable blocks. Each variable may be linked to a particular value in the input and is associated either with a random variable distribution or with an arithmetic expression. The expression capability allows for dependent or derived parameters, such as ${ }^{238} \mathrm{U}$ content depending on enrichment or outer clad radius depending on the inner radius. 
For each sample, Sampler creates a perturbed input by generating a set of variable values and substituting them into the input. For every variable, the user has to define the variable and specify its distribution (using one of the predefined random variable distributions described in Sect. 0) or its dependence on other variables (using an arithmetic expression). If desired, the user can also specify which part of the input will be replaced by the variable. This can be achieved either by specifying a SCALE Input Retrieval ENgine (SIREN) expression or by putting placeholders directly inside the input deck (see Appendix A for details on SIREN).

\subsection{Variable definition}

Variables are defined by a read variable..end variable block. The general format of the block is:

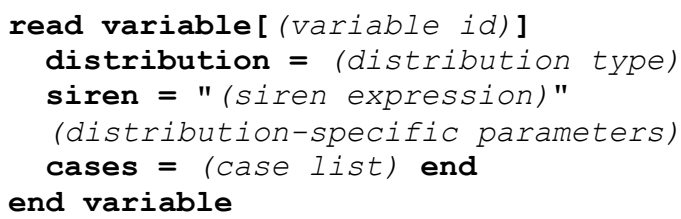

The variable id is an arbitrary, single word consisting of letters, numbers and underscores (with number not being the first character). The variable id has to be unique and is case dependent. distribution is one of the distribution-type keywords (see Sect. 0) or expression for the dependent variable definition. The cases $=$ specification applies only to the variables defined at the global scope (see below). The siren= specification is optional, see below.

The block can be placed either:

a) inside a case block. This variable applies only to this particular case.

In the following example, variable $\mathrm{x}$ applies to the case $\mathrm{c} 1$ and the variable $\mathrm{y}$ applies to the case $\mathrm{c}$.

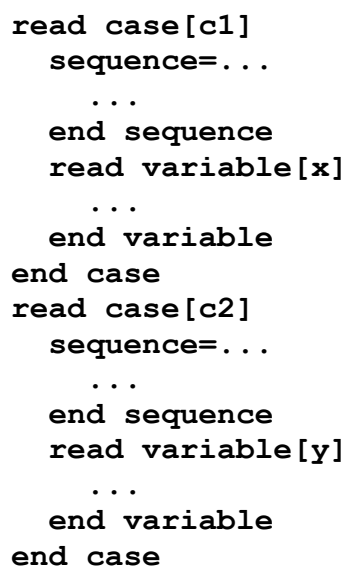

b) at the global scope. The cases=... end has to be used to specify the cases to which this variable applies.

In this example, variable $\mathrm{x}$ applies to the both cases $\mathrm{c} 1$ and $\mathrm{c} 2$ and the variable $\mathrm{y}$ applies only to the case $\mathrm{c} 1$. 


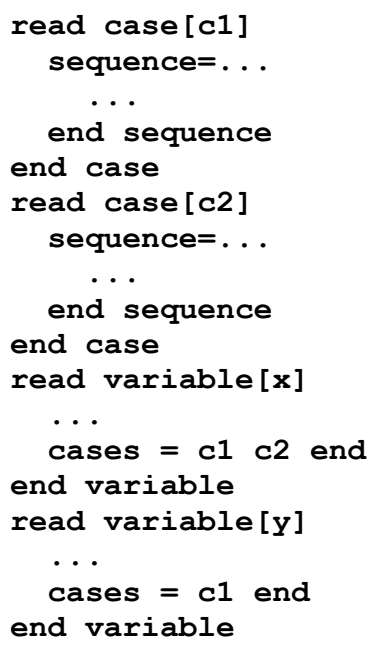

\subsection{Distribution types}

Sampler supports three random distribution types, selected using the distribution= keyword.

1) uniform: uniform distribution over a (closed) interval.

Additional parameters for a uniform distribution are shown in Table 6.7.4.

Table 6.7.4. Parameters for uniform distributions

\begin{tabular}{|l|l|l|}
\hline KEYWORD & DESCRIPTION & NOTE \\
\hline value $=$ & Base (unperturbed) value & required \\
\hline minimum $=$ & Lower bound value & required \\
\hline maximum $=$ & Upper bound value & required \\
\hline
\end{tabular}

Example:

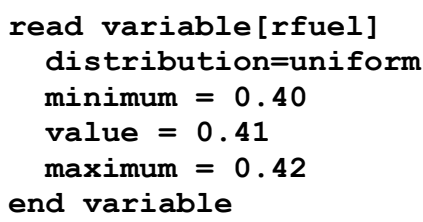

2) normal: normal (optionally truncated) distribution.

Additional parameters for normal distribution are shown in Table 6.7.5.

Table 6.7.5. Parameters for normal distributions

\begin{tabular}{|l|l|l|}
\hline KEYWORD & DESCRIPTION & NOTE \\
\hline value $=$ & Mean value & required \\
\hline stddev $=$ & Standard deviation & required \\
\hline minimum $=$ & Lower cutoff value & optional \\
\hline maximum $=$ & Upper cutoff value & optional \\
\hline
\end{tabular}


The user can specify both minimum and maximum, one of them, or neither. If a cutoff is not specified, the distribution is not truncated on that side.

\section{Example:}

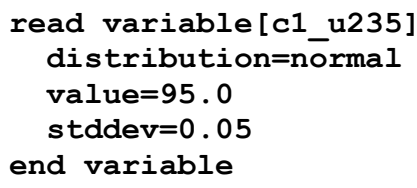

3) beta: beta distribution.

Additional parameters for the beta distribution are shown in Table 6.7.5.

Table 6.7.6. Parameters for beta distributions

\begin{tabular}{|l|l|l|}
\hline KEYWORD & DESCRIPTION & NOTE \\
\hline value $=$ & Mean value & required \\
\hline minimum $=$ & Lower cutoff value & required \\
\hline maximum $=$ & Upper cutoff value & required \\
\hline beta_a $=$ & First parameter for distribution & required \\
\hline beta_b $=$ & Second parameter for distribution & required \\
\hline
\end{tabular}

The Beta distribution is defined in the standard way given by Eq. (6.7.8). The parameters for the distribution determine where the peak is located in the interval [minimum, maximum] and the variance of the distribution; the parameters $\alpha$ and $\beta$ are required to be integer values. Note that value does not need to be the midpoint between minimum and maximum.

$$
f(x ; \alpha, \beta)=\frac{\Gamma(\alpha+\beta)}{\Gamma(\alpha) \Gamma(\beta)} x^{\alpha-1}(1-x)^{\beta-1},
$$

\section{Example:}

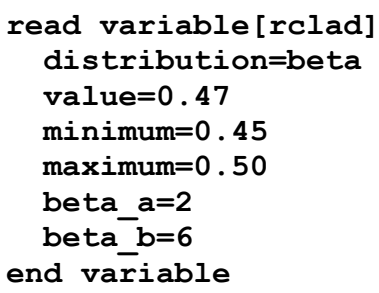

\subsection{Dependent variables (expressions)}

Using distribution=expression allows the user to specify a variable using the values of other variables. Setting expression="(expression)" then specifies how to evaluate the variable. Sampler supports basic arithmetic operators and other variables can be used as well. However, Sampler currently provides no variable dependency resolution and therefore only variables that were defined (using the variable block) previously in the input deck can be referenced in an expression. 


\section{Example:}

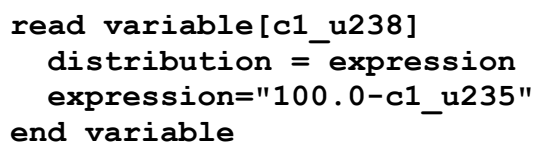

\subsection{Using placeholders}

Inside the sequence $=$ blocks, a \# $\{$ variable $i d\}$ placeholder can be used. This will be replaced by a variable value when Sampler builds the particular input deck. Only a simple variable reference can be used; no expressions are allowed here.

\section{Example:}

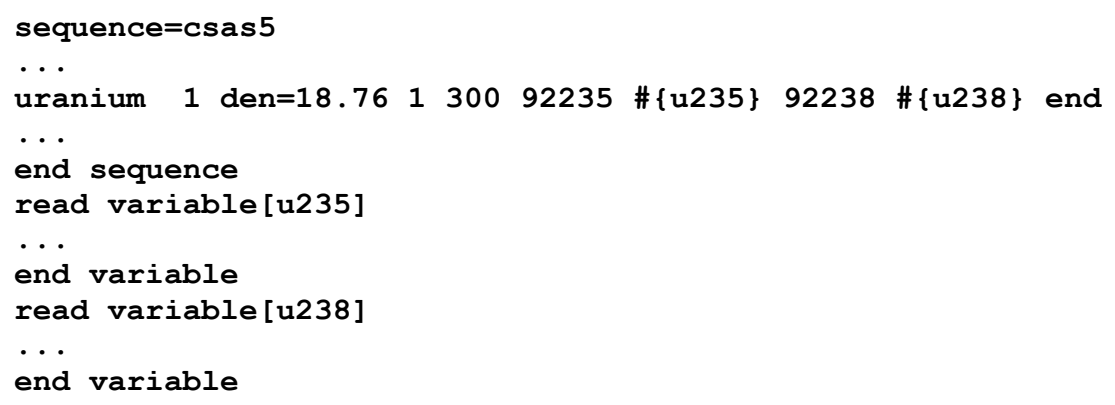

In the input deck snippet the ${ }^{235} \mathrm{U}$ and ${ }^{238} \mathrm{U}$ content are inserted directly to the respective places (defined by variables $\mathrm{u} 235$ and $\mathrm{u} 238$ ).

Using placeholders is straightforward and simple; however, if for input deck quality assurance or other reasons it is not desirable to modify the input deck directly, SIREN expressions can be used.

\subsection{Using SIREN expressions}

SIREN is a package which provides an XPath-like interface to the SCALE input deck represented by a Document Object Model (DOM). In Sampler, the user can specify siren="path" to have the respective token(s) replaced by a variable value.

Please refer to Appendix A for more details on specifying the SIREN path expressions.

\section{Example:}

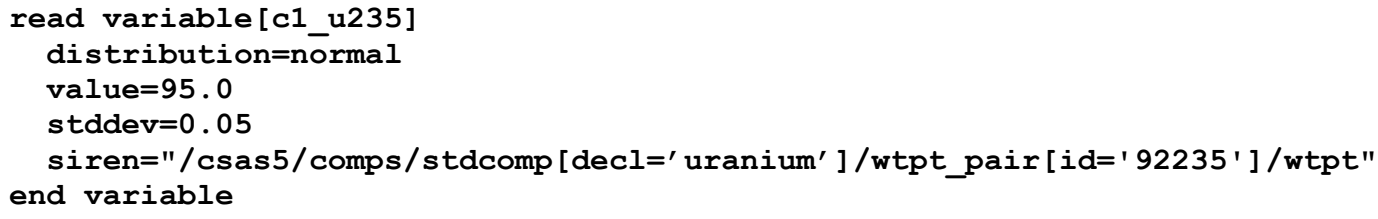

The variable c1_u235 value is inserted as the weight percent of ${ }^{235} \mathrm{U}$ in the basic standard composition declared as "uranium" in the CSAS5 sequence. 


\subsubsection{Converting a standard SCALE input deck to a Sampler input deck}

In this section, a short walkthrough is provided on how to convert a "normal" SCALE input into a Sampler input for cross section uncertainty propagation.

Beginning with a simple CSAS5 input deck:

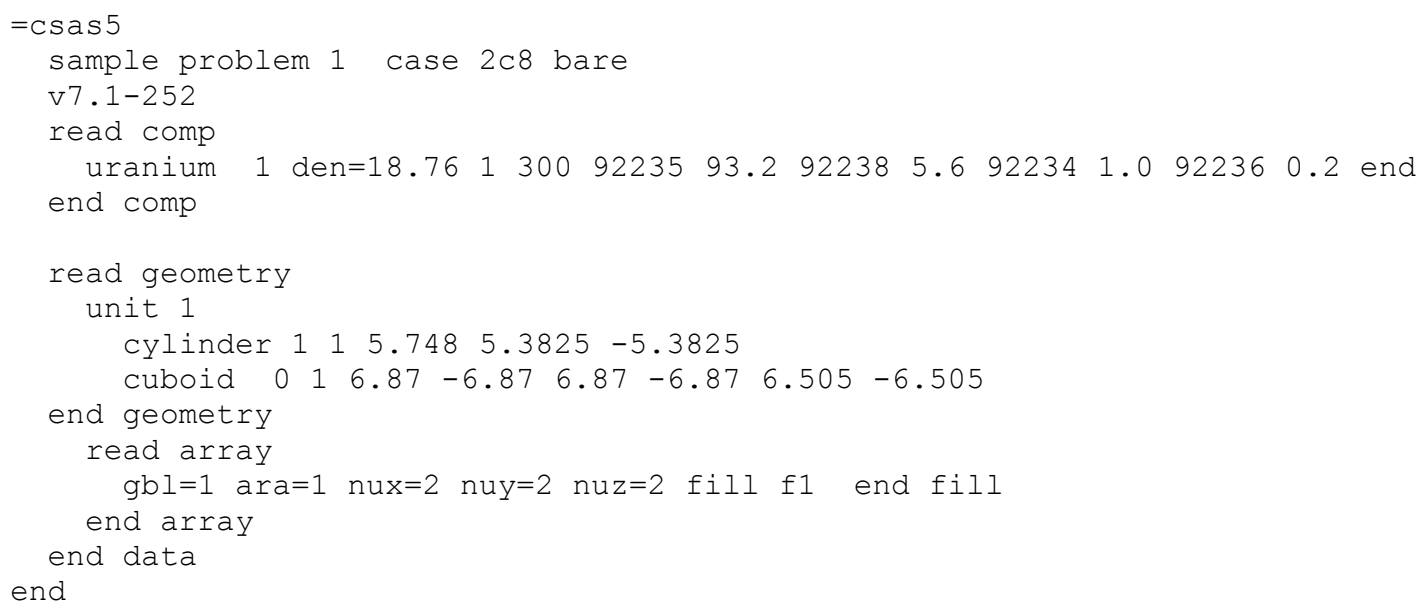

First, wrap the given input in sequence and case blocks and assign the case an arbitrary identifier (c1). It is also recommended to change the library alias to the actual filename.

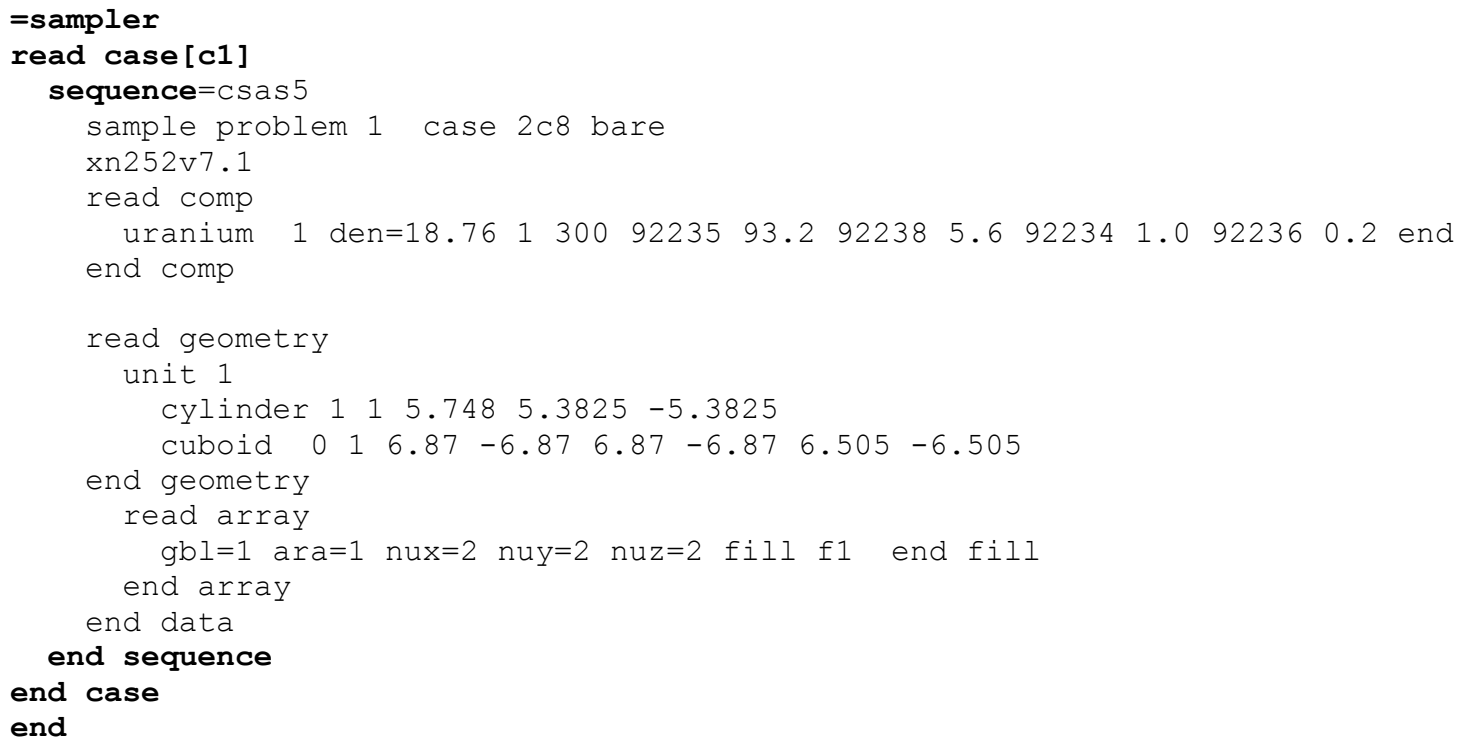

Second, add the parameters block to identify the base cross-section library used for building the perturbed ones. Note that this is the only SCALE module that uses the plural form of parameters and that the reference to the library matches exactly the one inside the CSAS5 input.

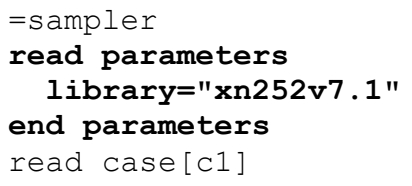




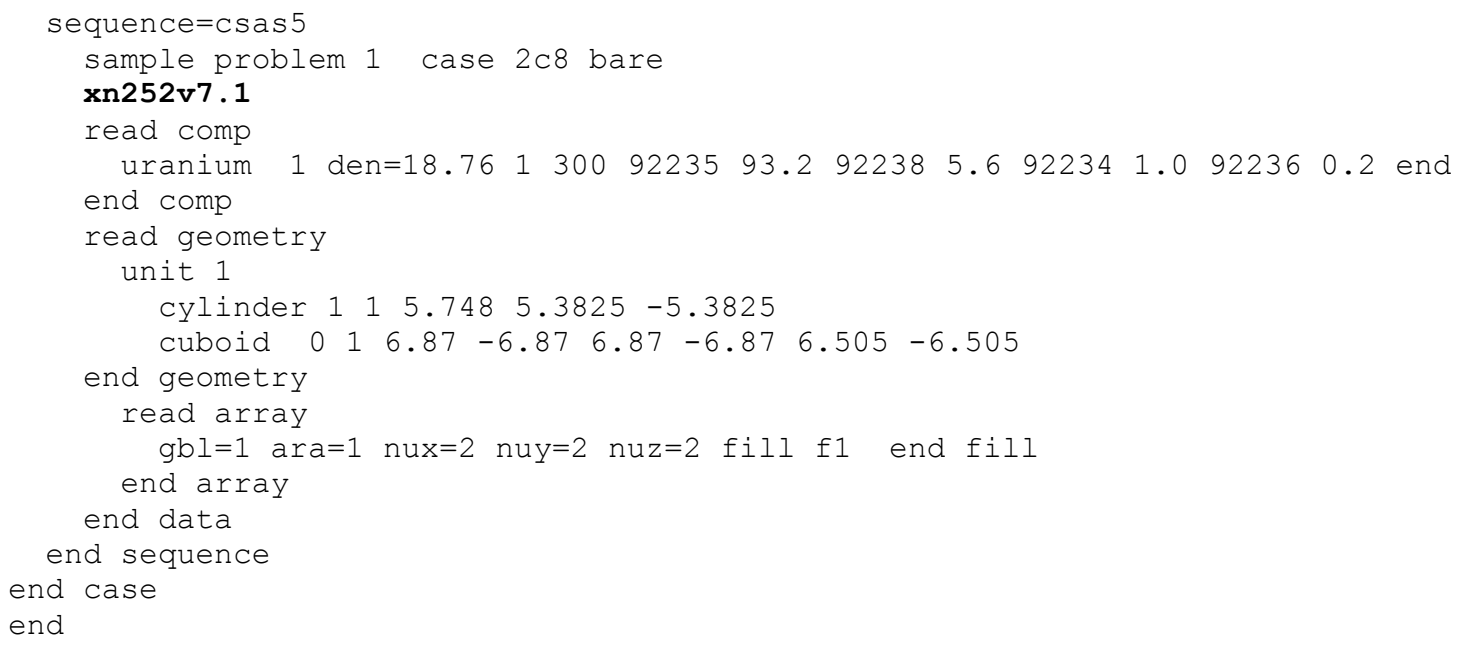

Finally, set up the perturbations (number of samples and what to perturb):

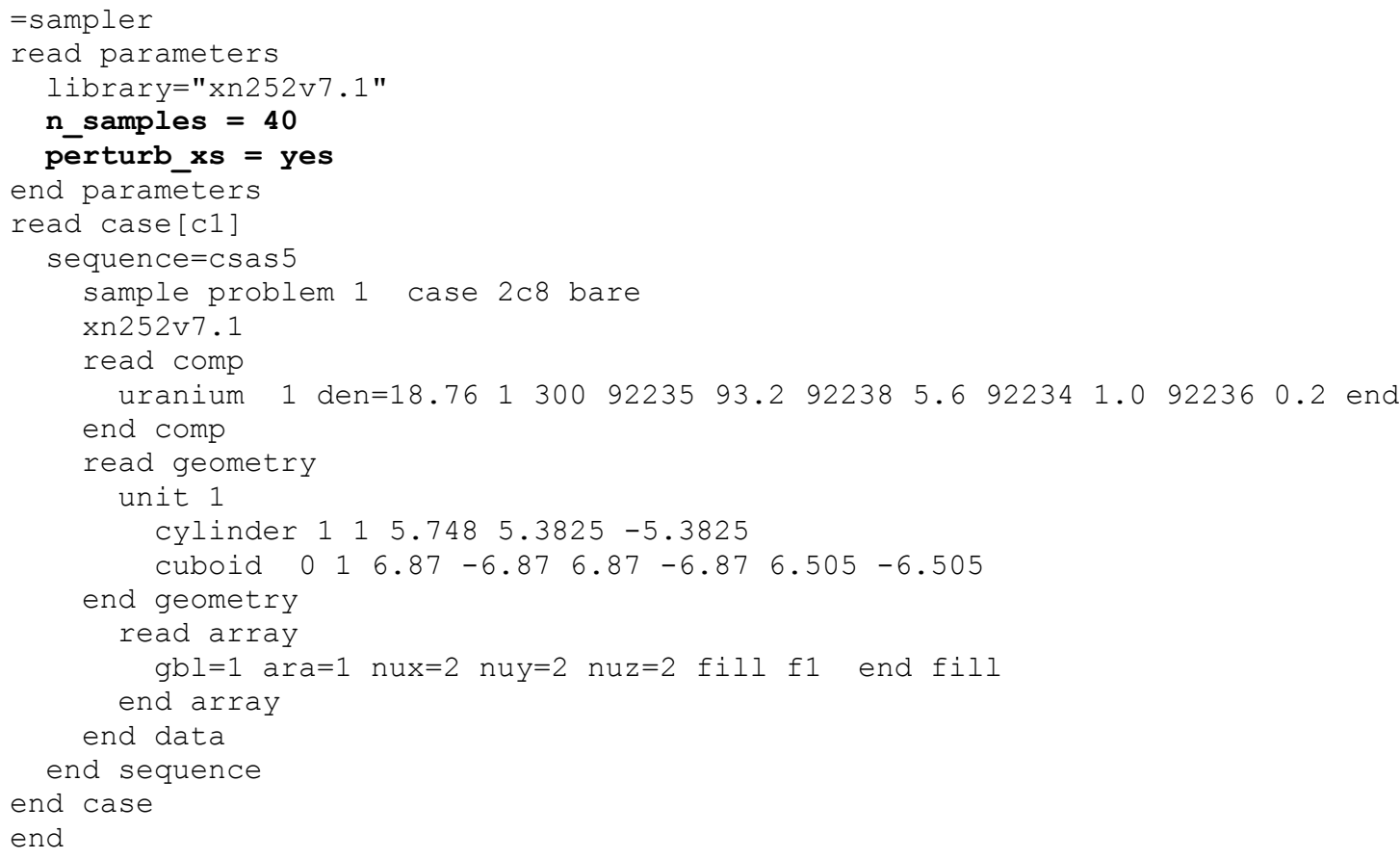

\subsubsection{Example Problems and Output Description}

This section describes output files created by Sampler and provides several sample cases.

\subsubsection{Output description}

This section describes the contents of the main Sampler output file, as well as the other files generated by Sampler.

All of the CSV, PTP and SDF files are, for convenience, copied into a separate directory called $\$\{O U T B A S E N A M E\}$. samplerfiles, where $\$\{O U T B A S E N A M E\}$ is the base name of the main SCALE output file, e.g. "my" in "my.out". 


\subsection{Main text output}

The main text output summarizes the Sampler run progress and presents the most important results.

\subsection{Sampler banner}

The program verification information banner shows the program version and the main execution information (date and time, user name, computer name).

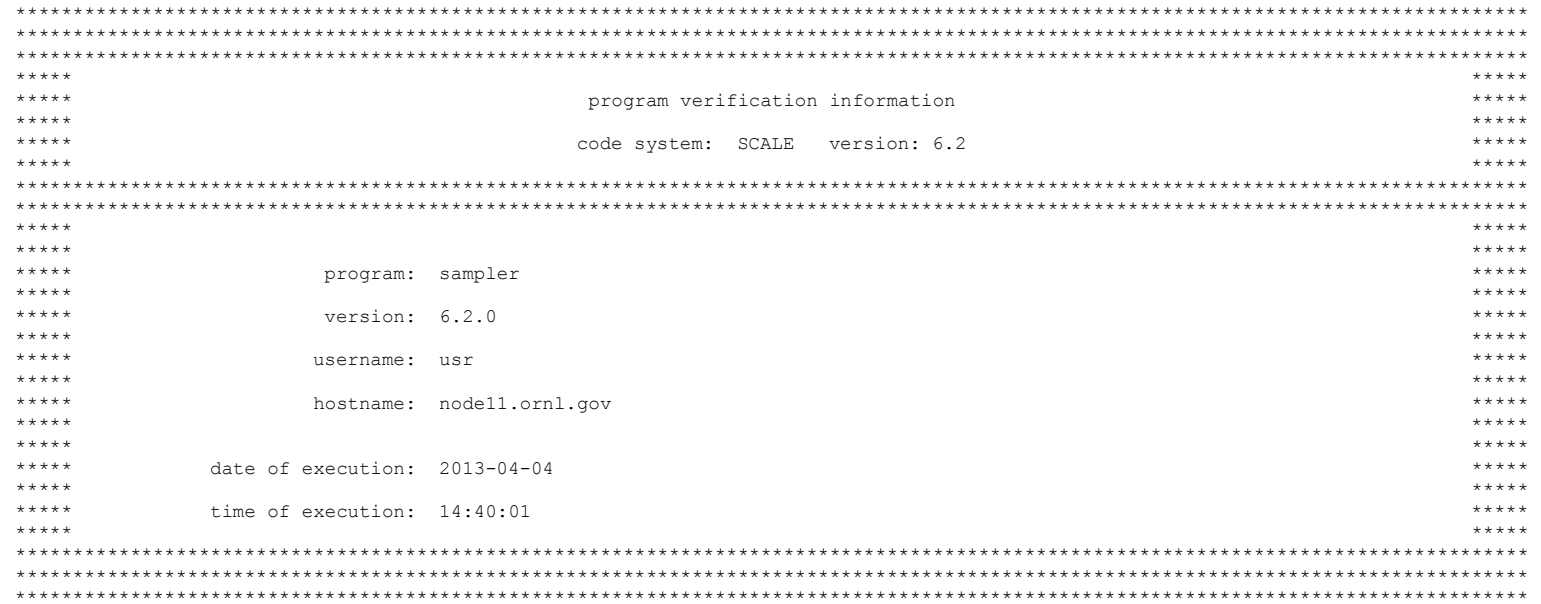

\subsection{Input parameters echo}

Input echo table summarizes the user selected parameters and options.

\begin{tabular}{|c|c|}
\hline & Input parameters echo \\
\hline $\begin{array}{l}\text { Number of cases } \\
\text { Number of samples } \\
\text { First sample index } \\
\text { Number of MAT-MT pairs }\end{array}$ & 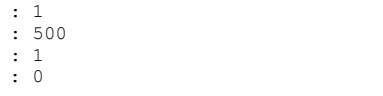 \\
\hline $\begin{array}{l}\text { Perturb cross-sections } \\
\text { Perturb decay data } \\
\text { Perturb fission yields }\end{array}$ & $\begin{array}{l}: \text { yes } \\
: \text { no } \\
: \text { no }\end{array}$ \\
\hline $\begin{array}{l}\text { Perturb pointwise XS } \\
\text { Perturb Bondarenko factors }\end{array}$ & $\begin{array}{l}: \text { yes } \\
: \text { yes }\end{array}$ \\
\hline $\begin{array}{l}\text { Master XS library } \\
\text { Perturbed XS library } \\
\text { Multigroup factors library } \\
\text { Sensitivity factors library } \\
\text { Covariance library }\end{array}$ & $\begin{array}{l}: \text { xn238v7 } \\
: \text { xn238v7 } \\
\vdots \\
: \text { sensitivity_factors } \\
: 44 \text { groupcov }\end{array}$ \\
\hline $\begin{array}{l}\text { Print CSV tables } \\
\text { Print PTP histograms/histories } \\
\text { Print per-sample data } \\
\text { Print covariances } \\
\text { Print correlations } \\
\text { Print chi-square test }\end{array}$ & $\begin{array}{l}: \text { yes } \\
: \text { yes } \\
: \text { no } \\
: \text { no } \\
: \text { no } \\
: \text { no }\end{array}$ \\
\hline
\end{tabular}

\subsection{SCALE run overview}

The run overview table displays the list of SCALE calculations processed by Sampler, i.e. for each case the baseline calculation (sample 0) and the requested number of samples. 


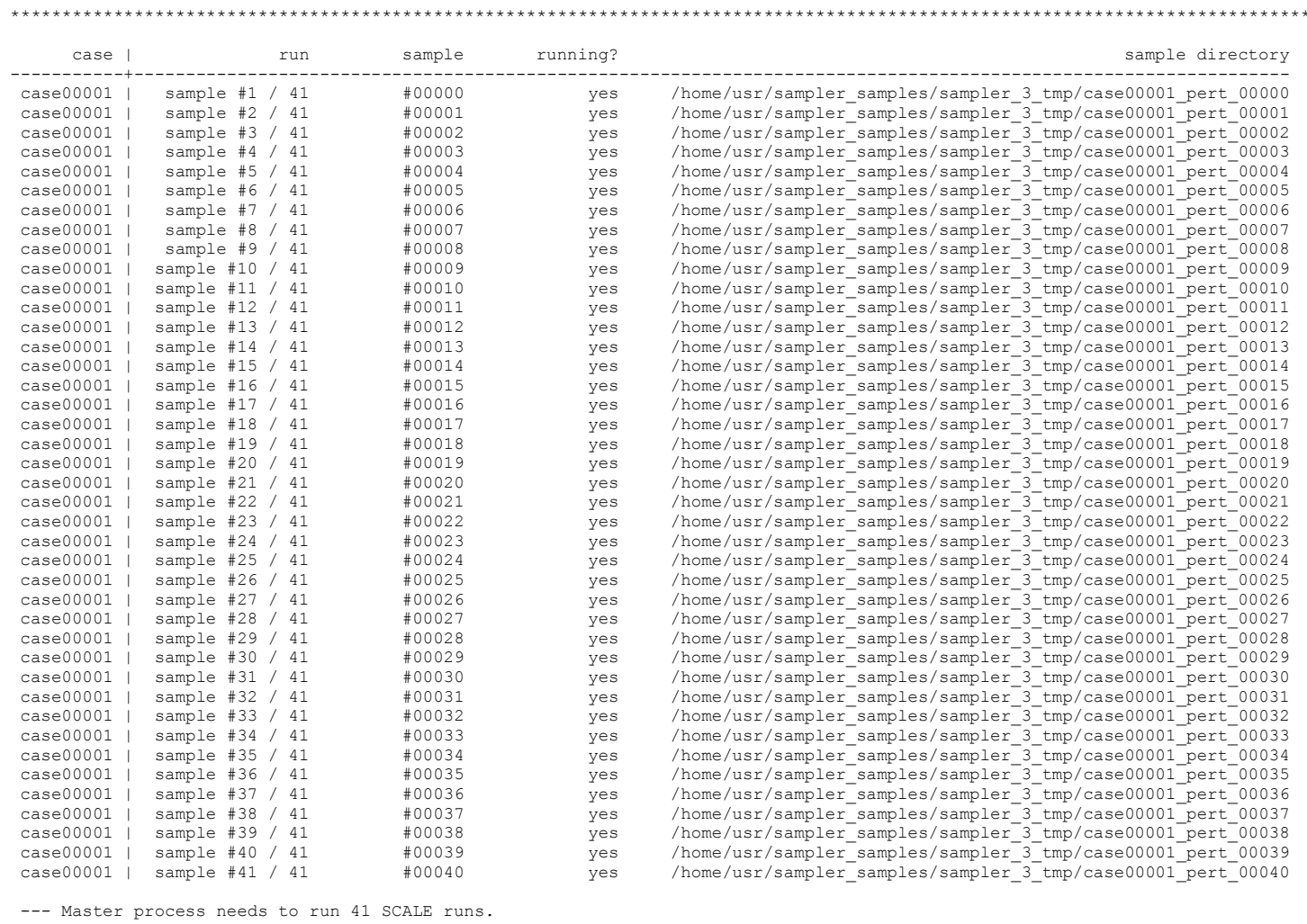

The table shows case name, sample index, sample number (i.e. the number in the perturbation factor library), whether the case has to be executed (if not it means that the results were already available in the samplerfiles directory) and the full path to the run subdirectory.

\subsection{Response tables}

According to the print flags set by the user in the parameters block, Sampler prints the following tables:

- values of all responses for all samples (printed if print_data=yes)

- average values and standard deviation over the samples population (always printed)

- comparison of average and baseline value (always printed)

- chi-square normality test (printed if print_chi2=yes)

- covariance matrices (printed if print_cov=yes)

- correlation matrices (printed if print_corr=yes)

- case- and response- specific tables

The case-specific tables contain only responses for a given case (so it is possible to explore correlations only within a given case). The response-specific tables are, on the other hand, contain responses across all cases, so they are useful for case cross-correlation analysis.

All of the tables are, regardless of the print flags, saved in the CSV files (see the following section). 


\subsection{CSV tables}

Every table produced by Sampler is (regardless of whether it has been selected for the main text output) saved also in the CSV (comma separated values) format, which makes it convenient to process Sampler results with a spreadsheet program, plotting package, or any scripting workflow.

These types of tables are created:

- values for every sample for time-independent responses

(response_table.static.val.all.csv)

- correlation matrix for time-independent responses

(response_table.static.corr.all.csv)

- covariance matrix for time-independent responses

(response_table.static.cov.all.csv)

- values for every sample for time-independent responses for each case

(response_table.static.val.case-*.csv)

- correlation matrix for time-independent responses for each case (response_table.static.corr.case-*.csv)

- covariance matrix for time-independent responses for each case (response_table.static.cov.case-*.csv)

- values for every sample for time-independent responses for each response (response_table.static.val.response-*.csv)

- correlation matrix for time-independent responses for each response (response_table.static.corr.response-*.csv)

- covariance matrix for time-independent responses for each response (response_table.static.cov.response-*.csv)

- values for every sample for time-dependent responses (response_table.*.csv)

- average values for time-dependent responses (response_table.*.avg.csv)

- standard deviations for time-dependent responses (response_table.*.stddev.csv)

- correlation matrices for every time step for time-dependent responses (response_table.*.corr.step*.csv)

- covariance matrices for every time step for time-dependent responses (response_table.*.cov.step*.csv)

\subsection{Sampling histograms and running averages}

In order to provide information on sampling convergence, Sampler provides two plots for each response at every time step:

- $\quad$ histogram plot - distribution of the response values in directory \$\{OUTBASENAME\}.samplerfiles/histogram

- running average plot - average and standard deviation for first $\mathrm{N}$ samples of the population in directory $\$\{O U T B A S E N A M E\}$.samplerfiles/running_averages

Both plots are in the PTP format and can be plotted by Fulcrum, as shown in Figure 6.7.1 and Figure 6.7.2. 


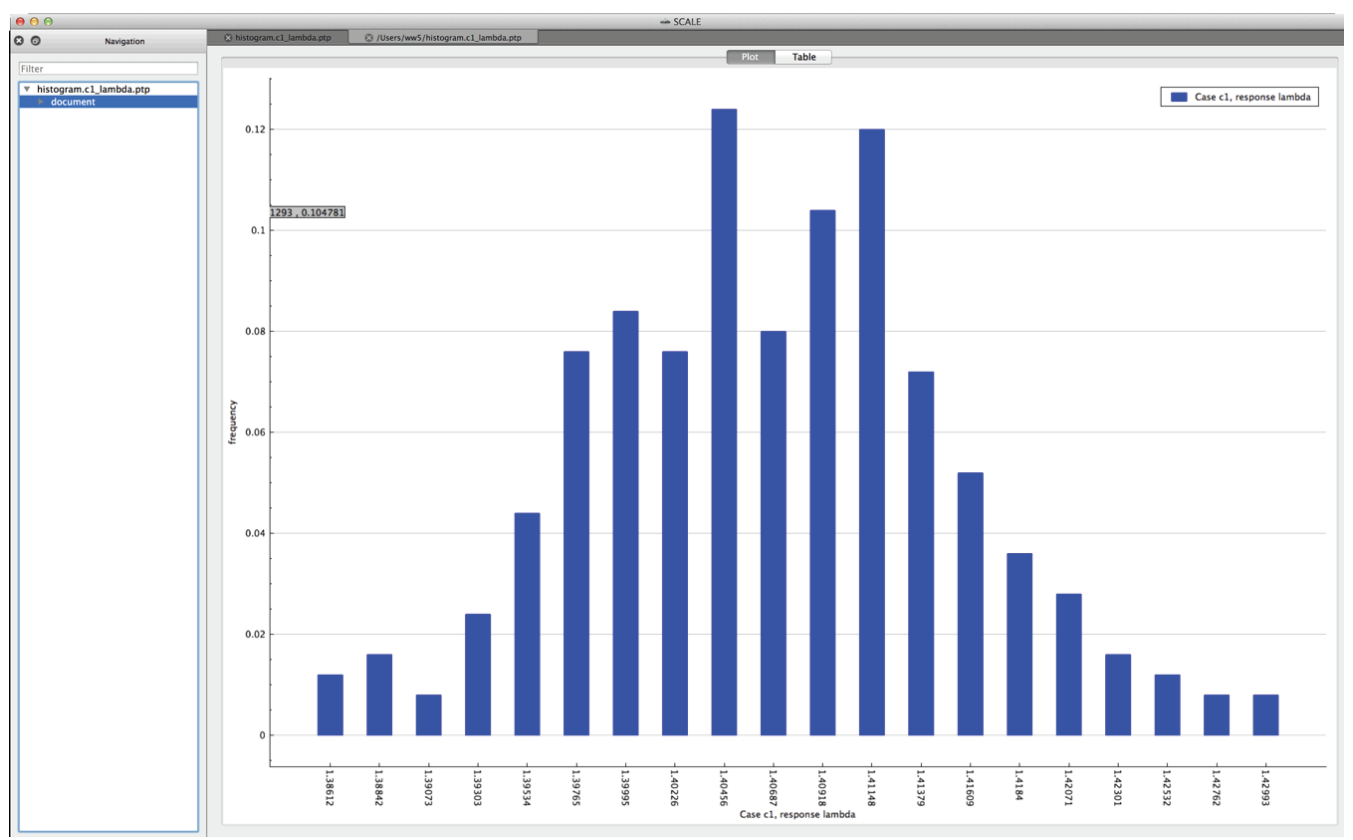

Figure 6.7.1. Example histogram viewed in Fulcrum

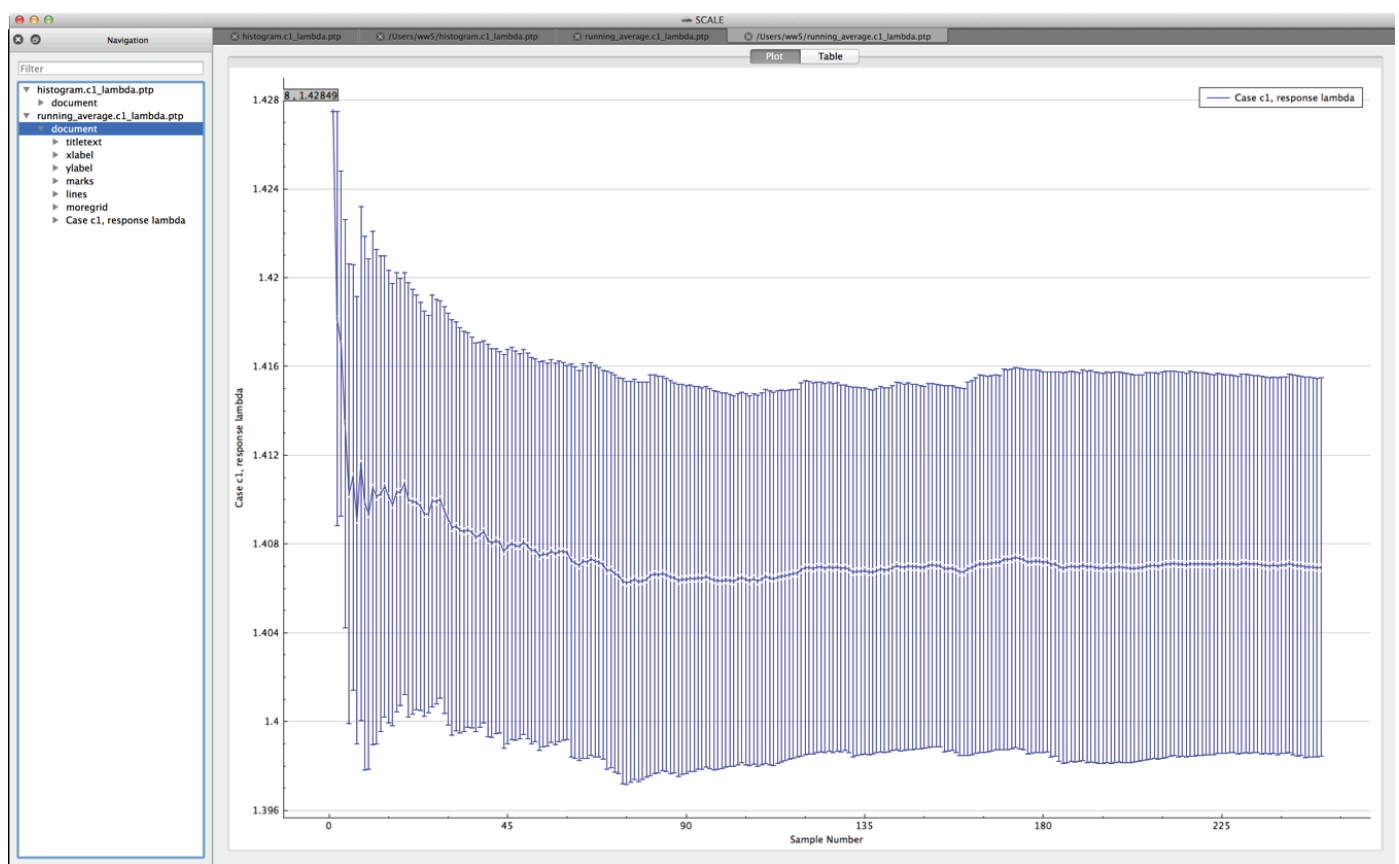

Figure 6.7.2. Example running average viewed in Fulcrum

\subsection{Response histories}

For time-dependent responses, Sampler produces two plots with time-dependent summary data.

- standard deviation plot - time-dependent average response with 1-sigma uncertainty bars in directory $\$\{O U T B A S E N A M E\}$.samplerfiles/histories/history.avg.* 
- $\min / \max$ plot - time-dependent average response with $\min / \max$ error bars in directory $\$\{O U T B A S E N A M E\}$.samplerfiles/ histories/history.min_max.*

An example of the standard deviation plot is shown Figure 6.7.3 and the min/max plot in Figure 6.7.4.

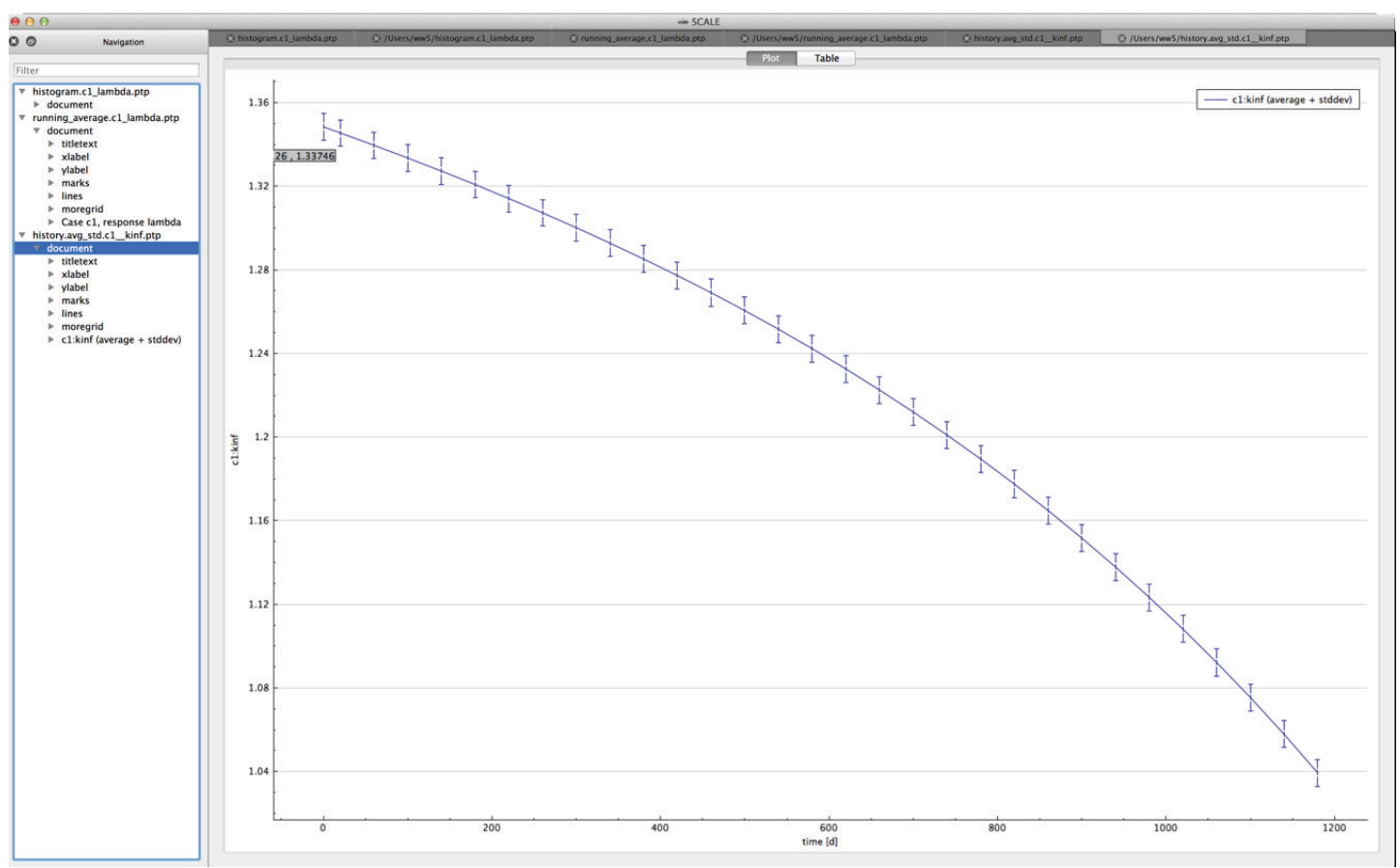

Figure 6.7.3. Time-dependent average plus standard deviation plot

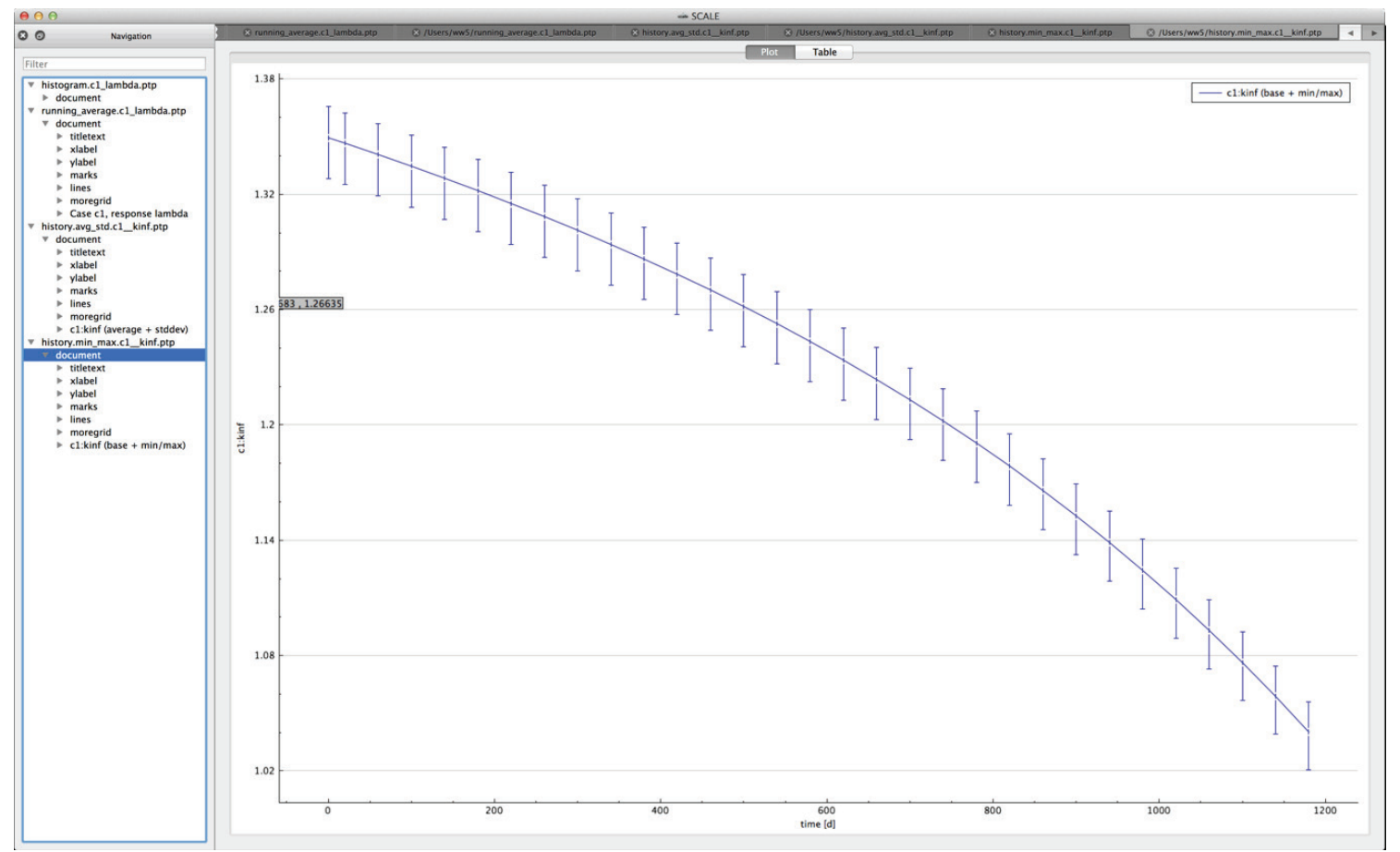

Figure 6.7.4. Time-dependent average plus $\min / \max$ plot 


\subsubsection{Sample problems}

The following sample problems demonstrate various computational and output capabilities of Sampler in various situations, for both uncertainty and parametric calculations.

Input files for those sample problems can be found in the samples/input directory of the SCALE installation. The naming convention for the inputs is sampler_N.inp, where $N$ is the sample problem number. The number of samples (n_samples) shown here may vary from the number included in the sample inputs.

\subsection{Sample problem 1}

This simple, single-case problem, evaluates uncertainty in eigenvalue for a T-XSDRN calculation of a MOX pincell. Only the cross-sections are perturbed.

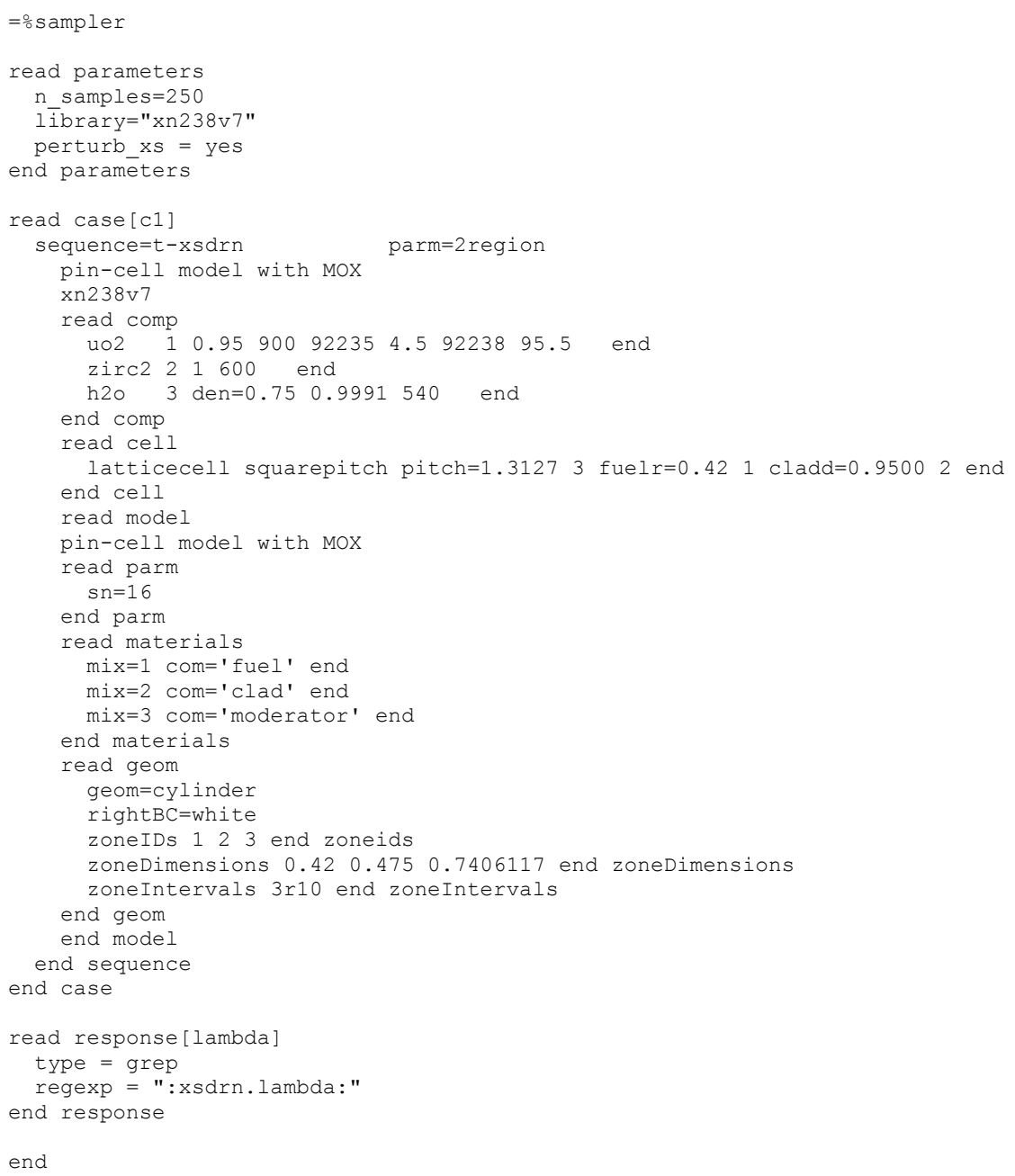

The distribution of lambda (k-eff) from sample problem 1 is shown in Figure 6.7.5. 


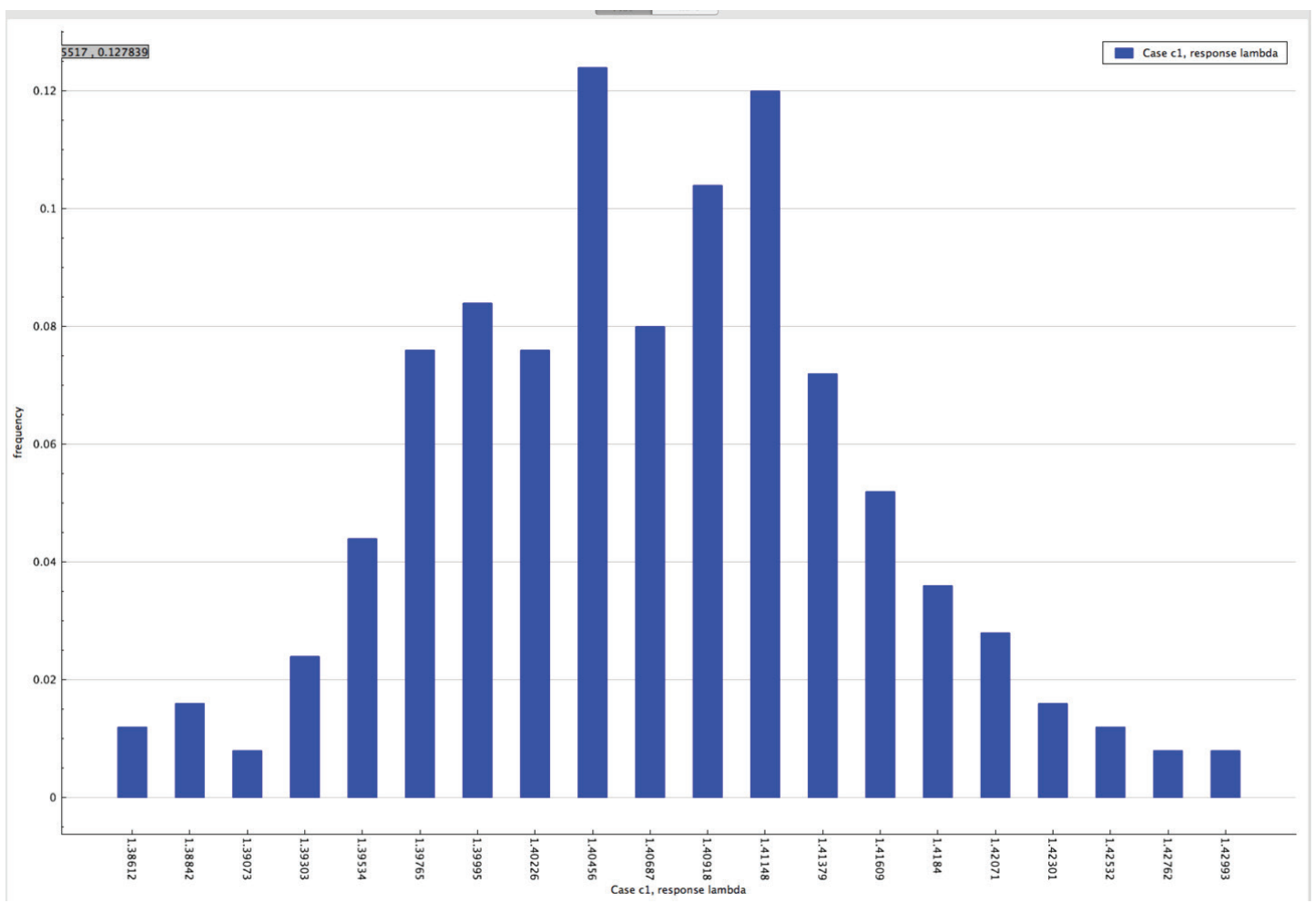

Figure 6.7.5. Distribution of lambda (k-eff) obtained from sample problem 1

\subsection{Sample problem 2}

This problem demonstrates a two-dimensional parametric study (using inline placeholders) for two pincell systems.

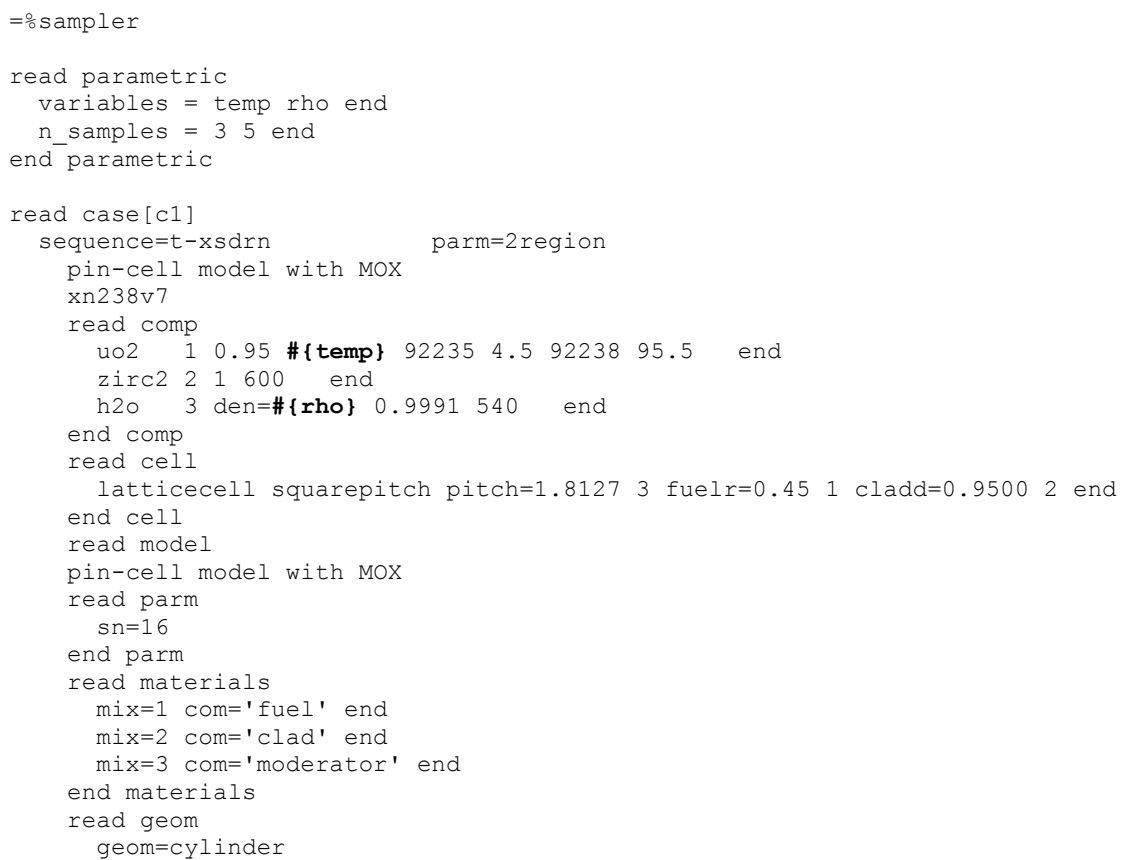




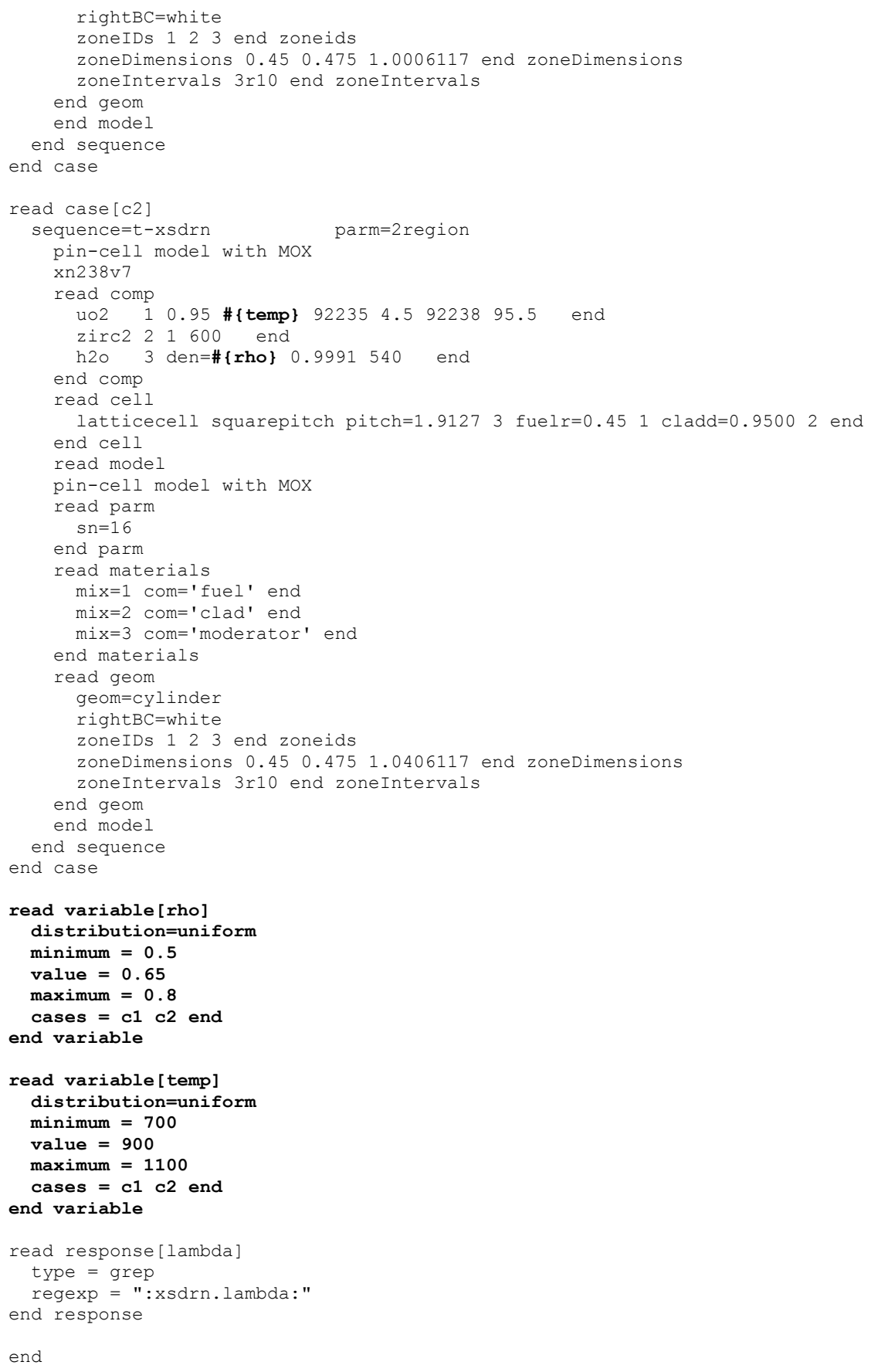

The dependency of eigenvalue on coolant density for the two systems is printed to the output file, as shown in Figure 6.7.6. 


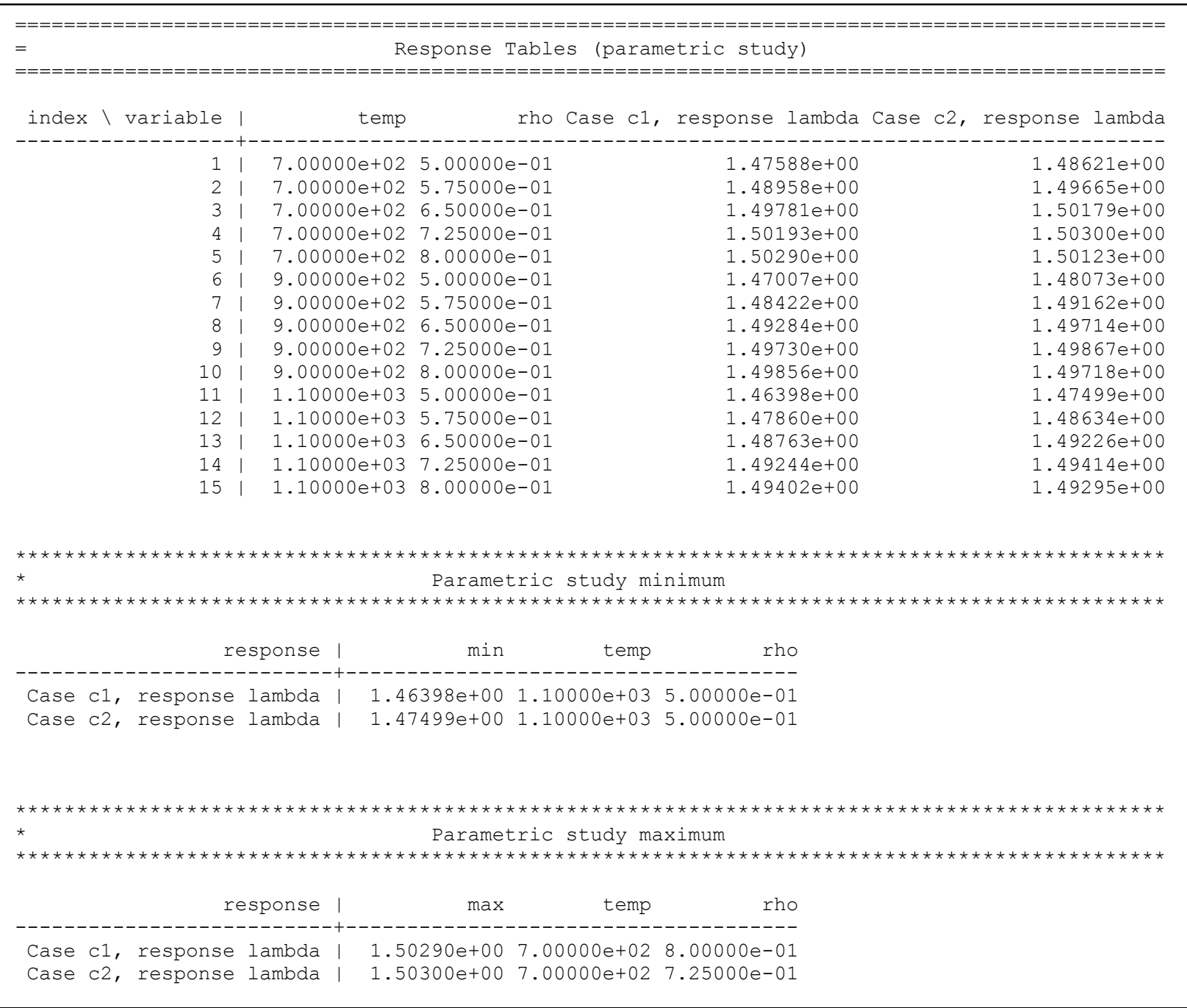

Figure 6.7.6. Dependency of lambda (k-eff) on coolant density and fuel temperature for sample problem 2.

\subsection{Sample problem 3}

This sample problem demonstrates enrichment variation using SIREN expressions and dependent variables.

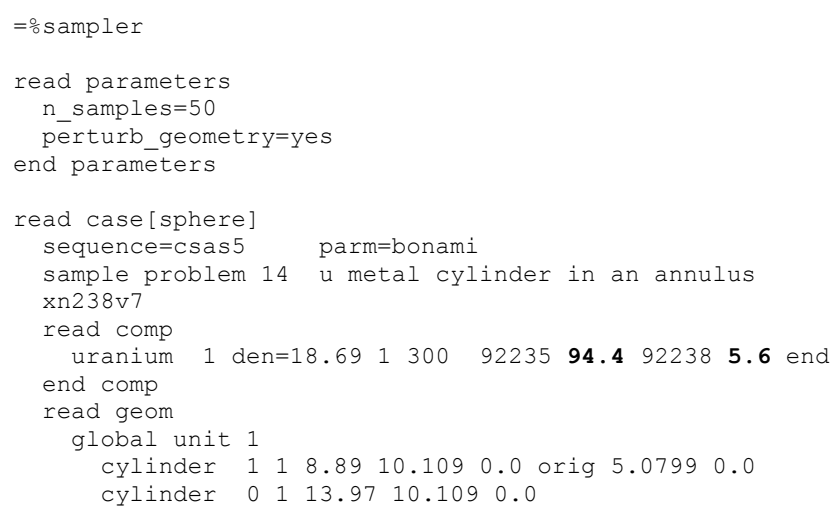




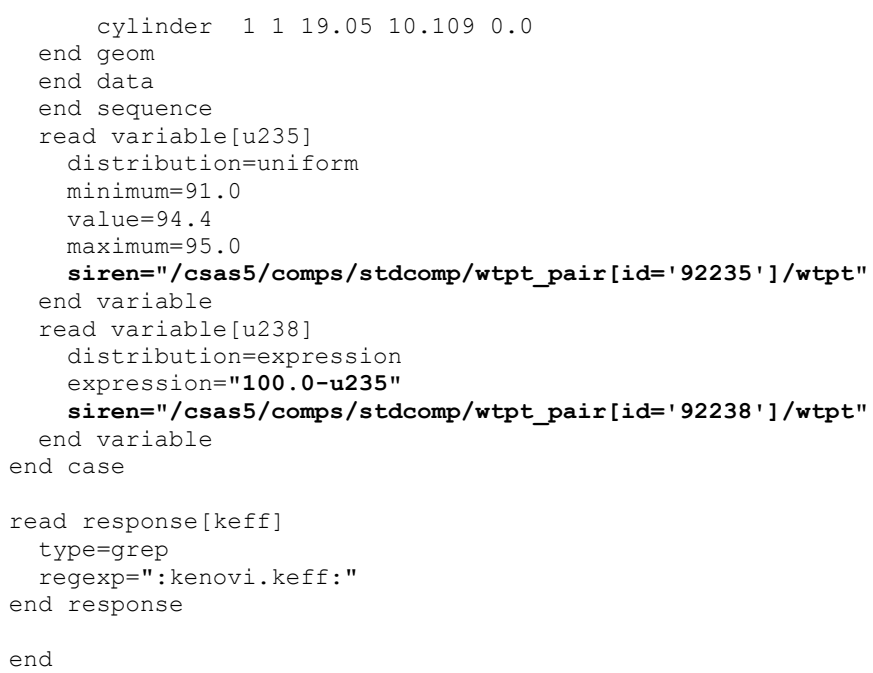

The distribution of the multiplication factor with the specified enrichment distribution is shown in Figure 6.7.7.

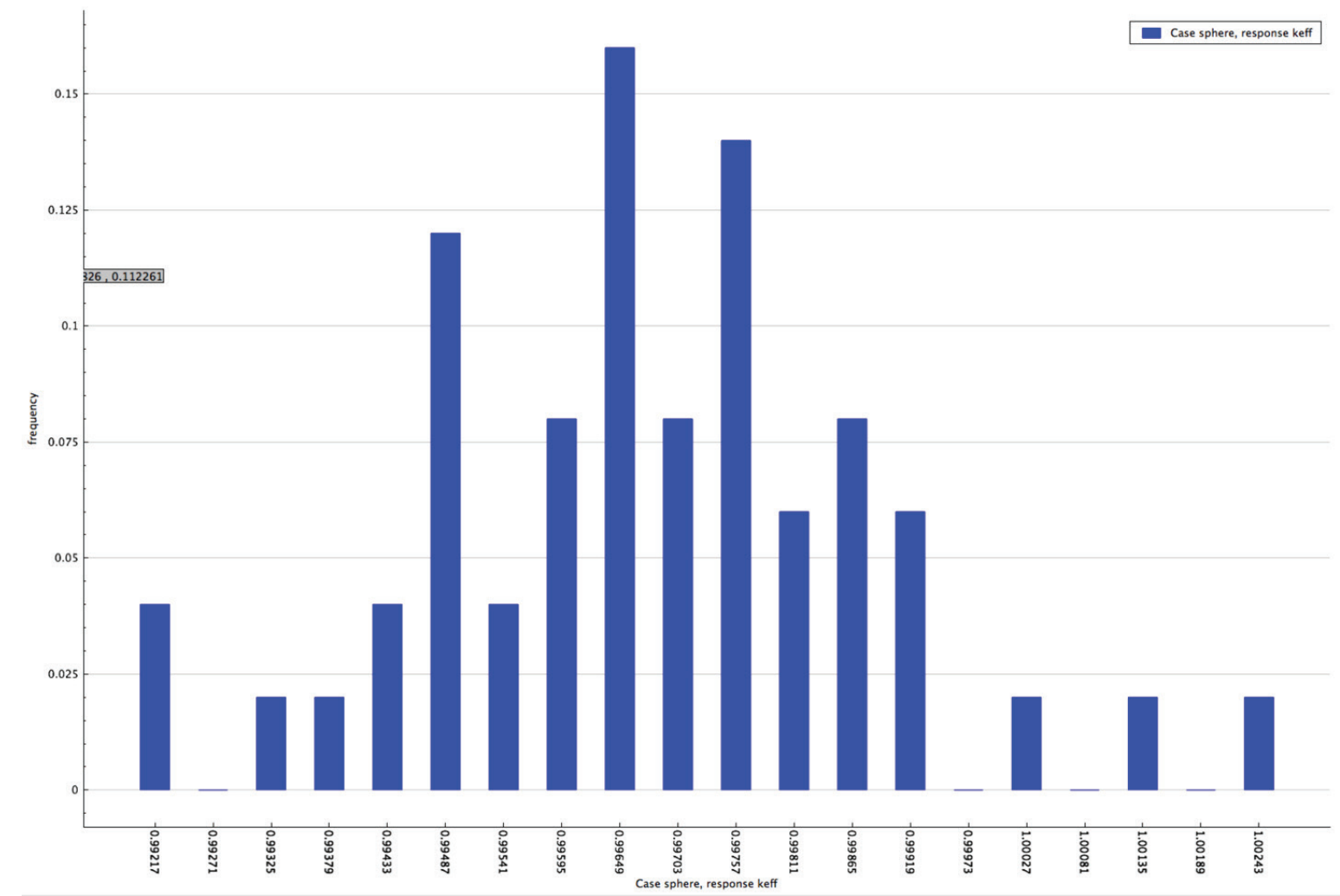

Figure 6.7.7. Distribution of multiplication factor with sampled enrichment distribution for sample problem 3

\subsection{Sample problem 4}

Sample problem 4 demonstrates sampling with covariance data for neutron cross sections and fission product yields. Note that decay sampling does not work with TRITON at this time due to not using the 
perturbed ORIGEN decay libraries directly. Additionally, it demonstrates how to extract reaction rates from a TRITON case, combining an additional OPUS run with the opus_plt response.

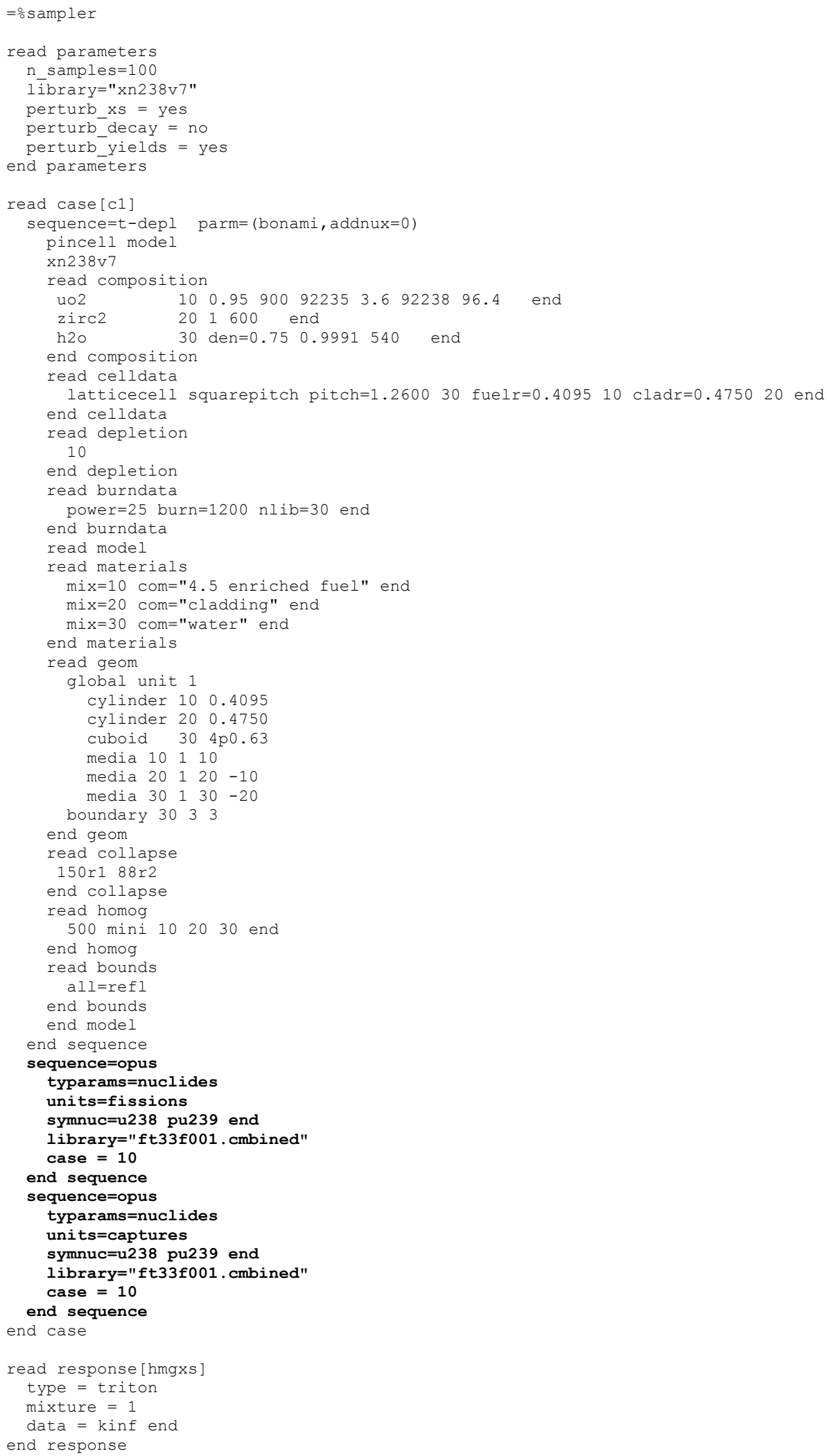




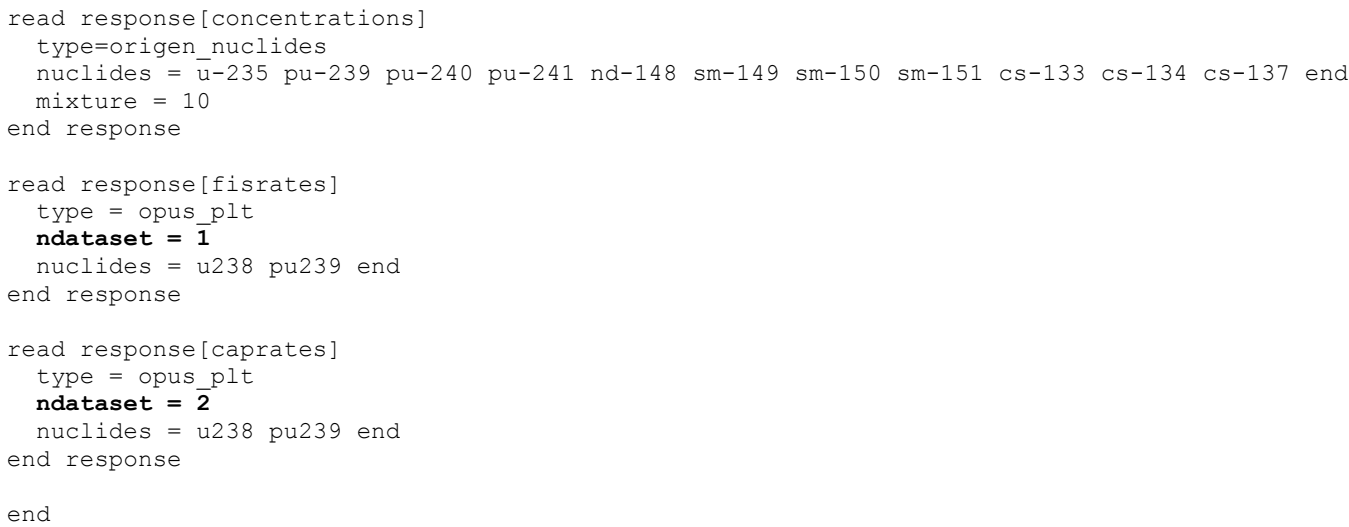

Plots of various isotopic uncertainties as a function of time are shown in Figure 6.7 .8 for ${ }^{235} \mathrm{U}$, Figure 6.7.9 for ${ }^{239} \mathrm{Pu}$, Figure 6.7.10 for ${ }^{240} \mathrm{Pu}$, Figure 6.7.11 for ${ }^{241} \mathrm{Pu}$, Figure 6.7 .12 for ${ }^{148} \mathrm{Nd}$, Figure 6.7.13 for ${ }^{150} \mathrm{Sm}$, and Figure 6.7.14 for ${ }^{151} \mathrm{Sm}$ in terms of the unperturbed with error bars for the minimum and maximum, that is over the 100 samples performed, the minimum value at that time and the maximum value at that time. Note that most isotopes accumulate uncertainty over time, but this is not always the case, e.g. for ${ }^{151} \mathrm{Sm}$. Two common burnup indicators (measured in spent fuel to infer/confirm burnup) ${ }^{148} \mathrm{Nd}$ and ${ }^{150} \mathrm{Sm}$ show linear increase with time (as expected for a burnup indicator).

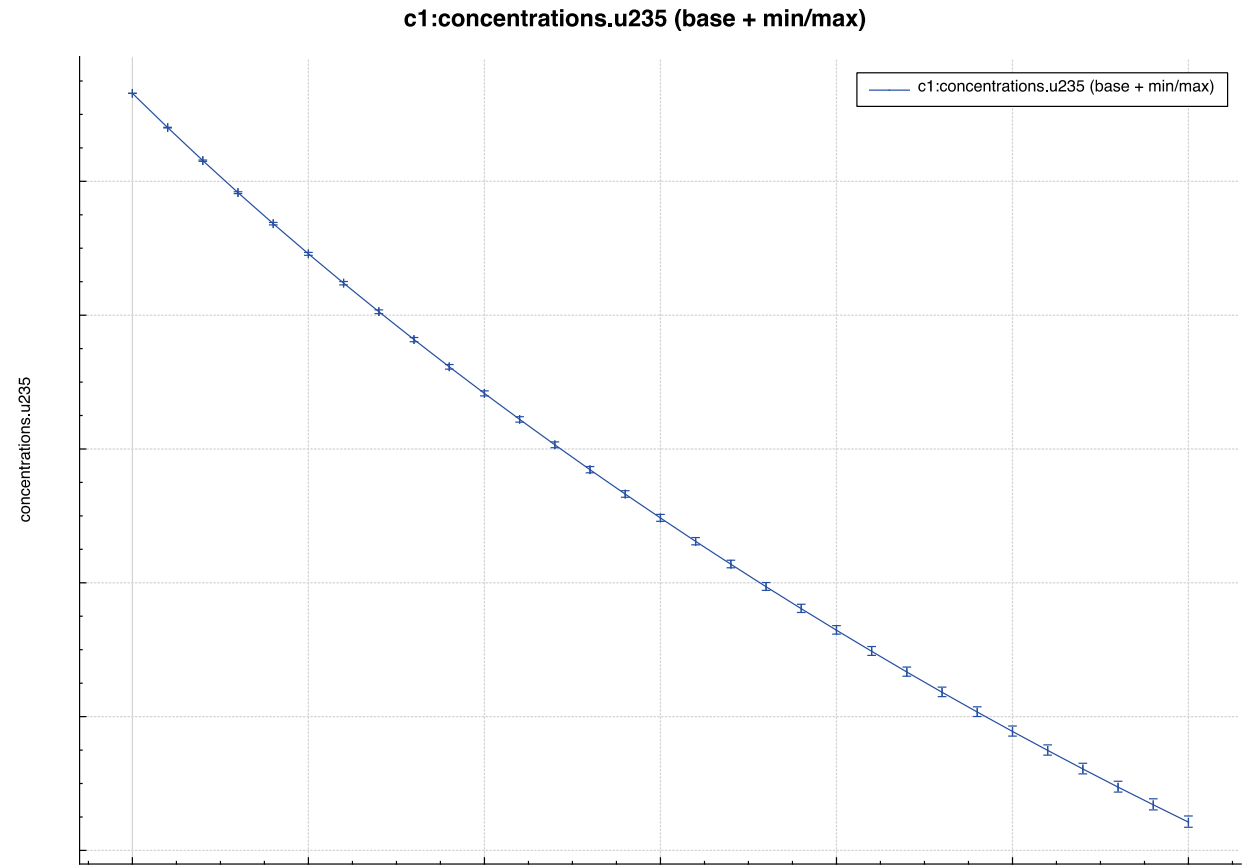

Figure 6.7.8. Uncertainty in ${ }^{235} U$ concentration for problem 4 


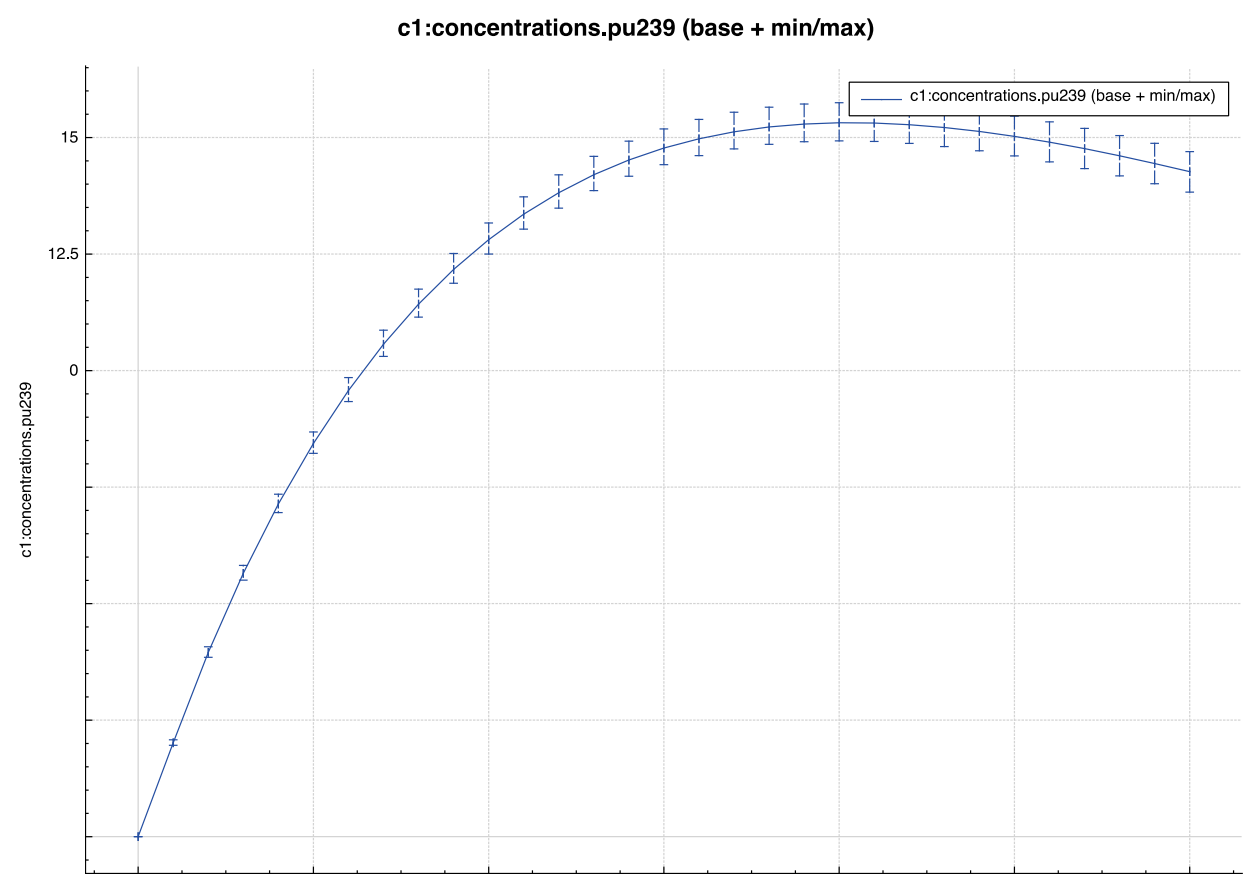

Figure 6.7.9. Uncertainty in ${ }^{239} \mathrm{Pu}$ concentration for problem 4

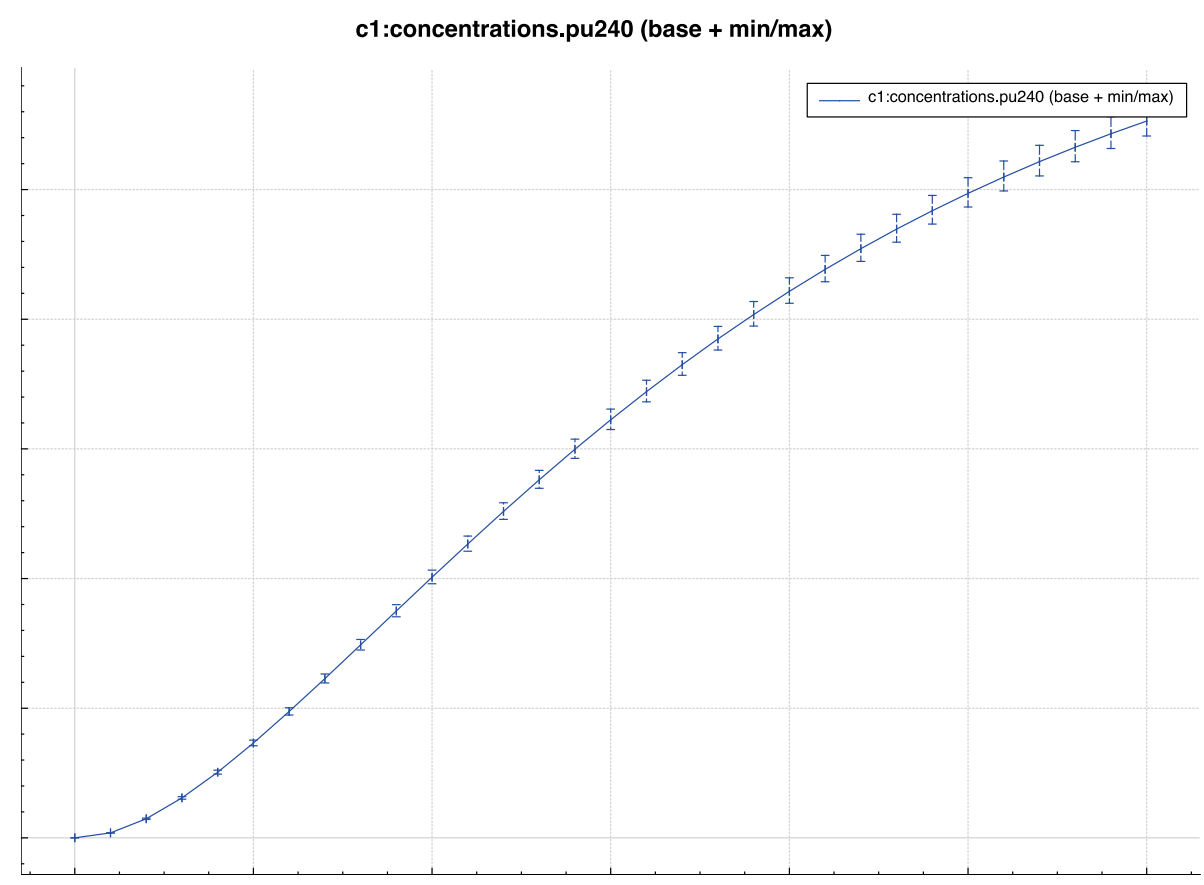

Figure 6.7.10. Uncertainty in ${ }^{240} \mathrm{Pu}$ concentration for problem 4 
c1:concentrations.pu241 (base $+\min / \max$ )

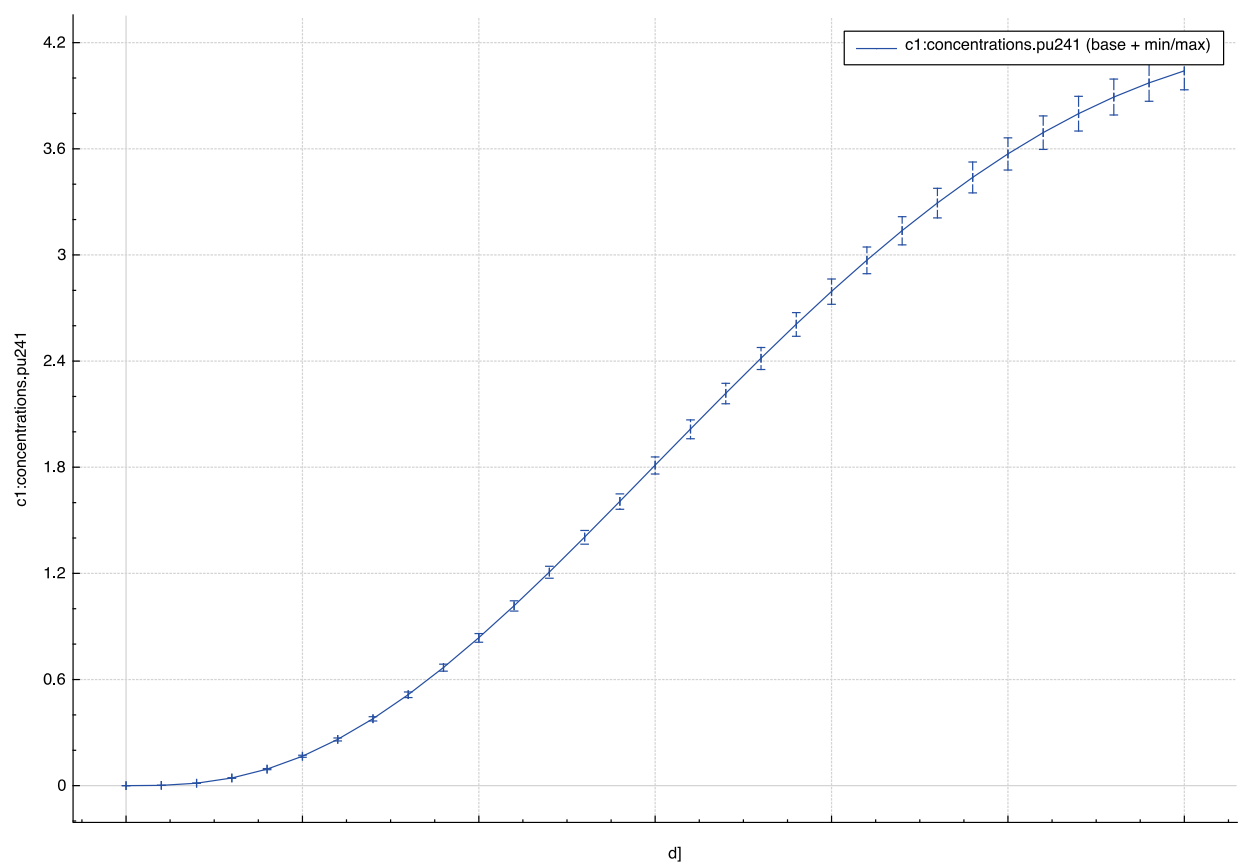

Figure 6.7.11. Uncertainty in ${ }^{241} \mathrm{Pu}$ concentration for problem 4

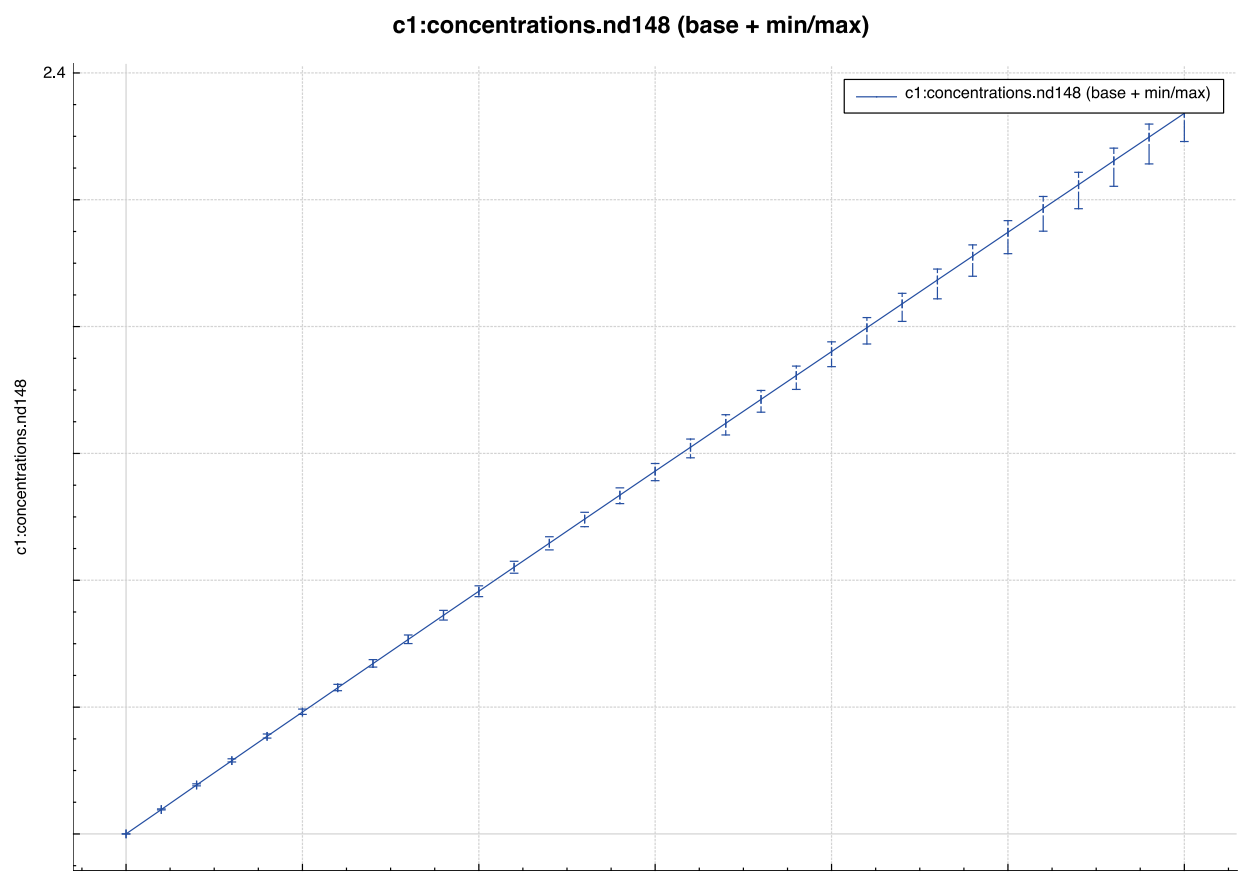

Figure 6.7.12. Uncertainty in ${ }^{148} \mathrm{Nd}$ concentration for problem 4 


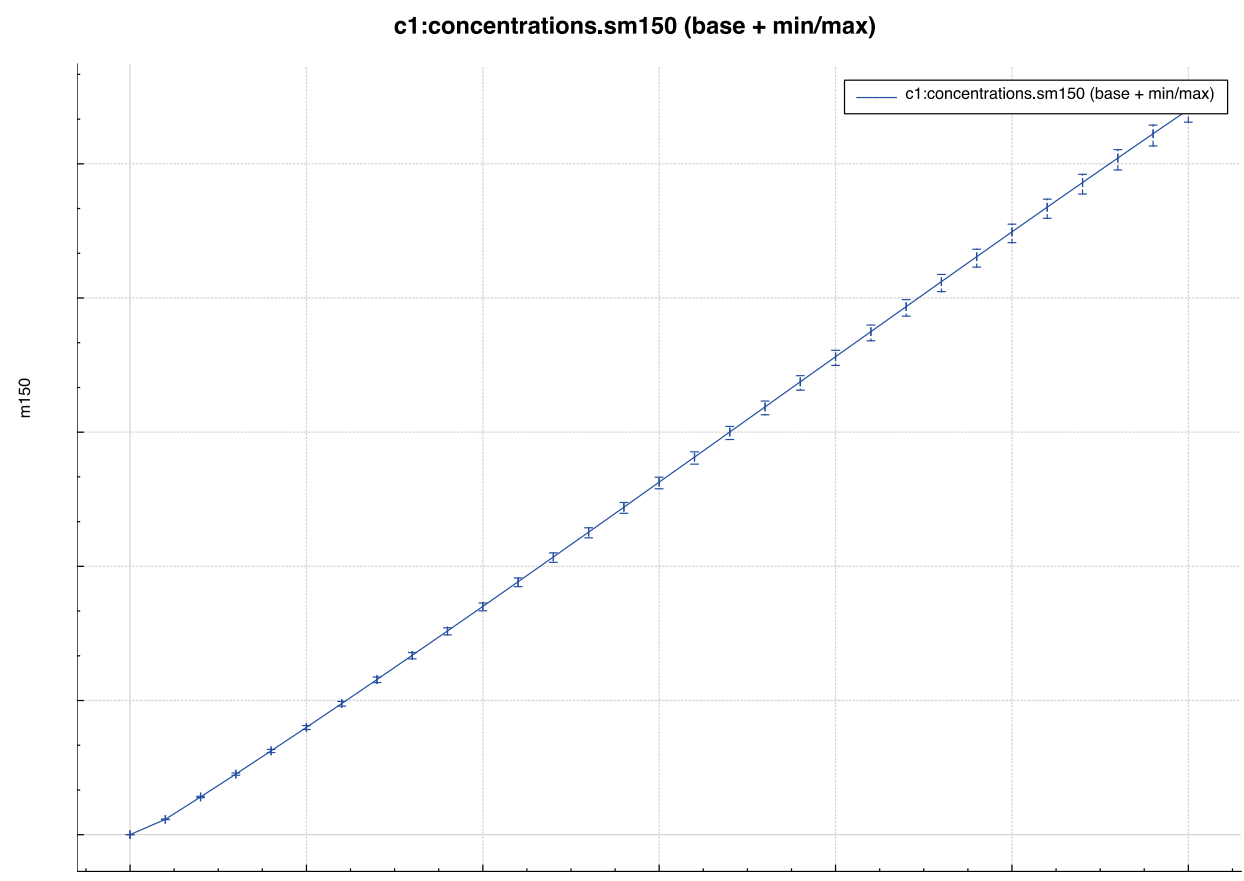

Figure 6.7.13. Uncertainty in ${ }^{150} \mathrm{Sm}$ concentration for problem 4

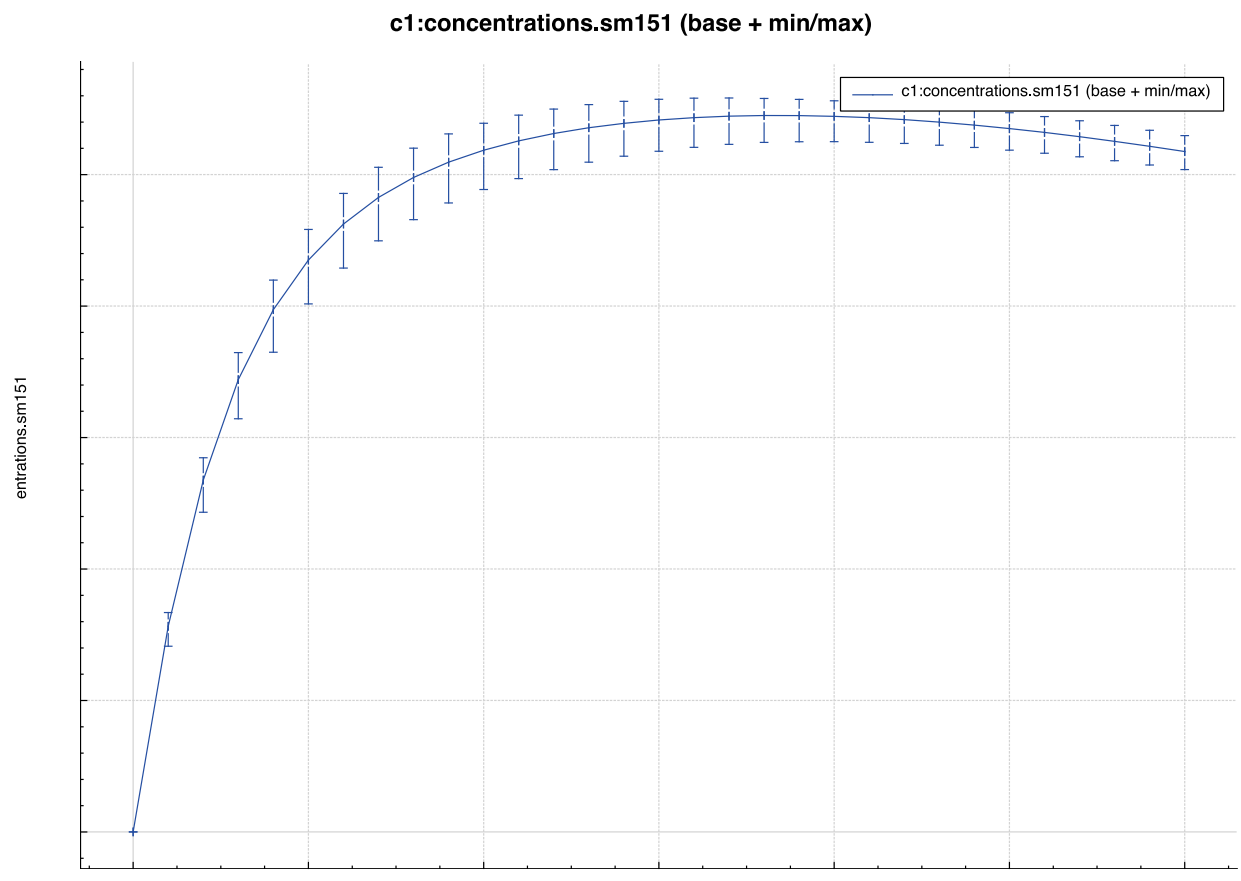

Figure 6.7.14. Uncertainty in ${ }^{151} \mathrm{Sm}$ concentration for problem 4 


\subsection{Sample problem 5}

This problem provides an example of two correlated depletion cases. The second "c2" case has a larger pitch and lower enrichment fuel. All data libraries are perturbed.

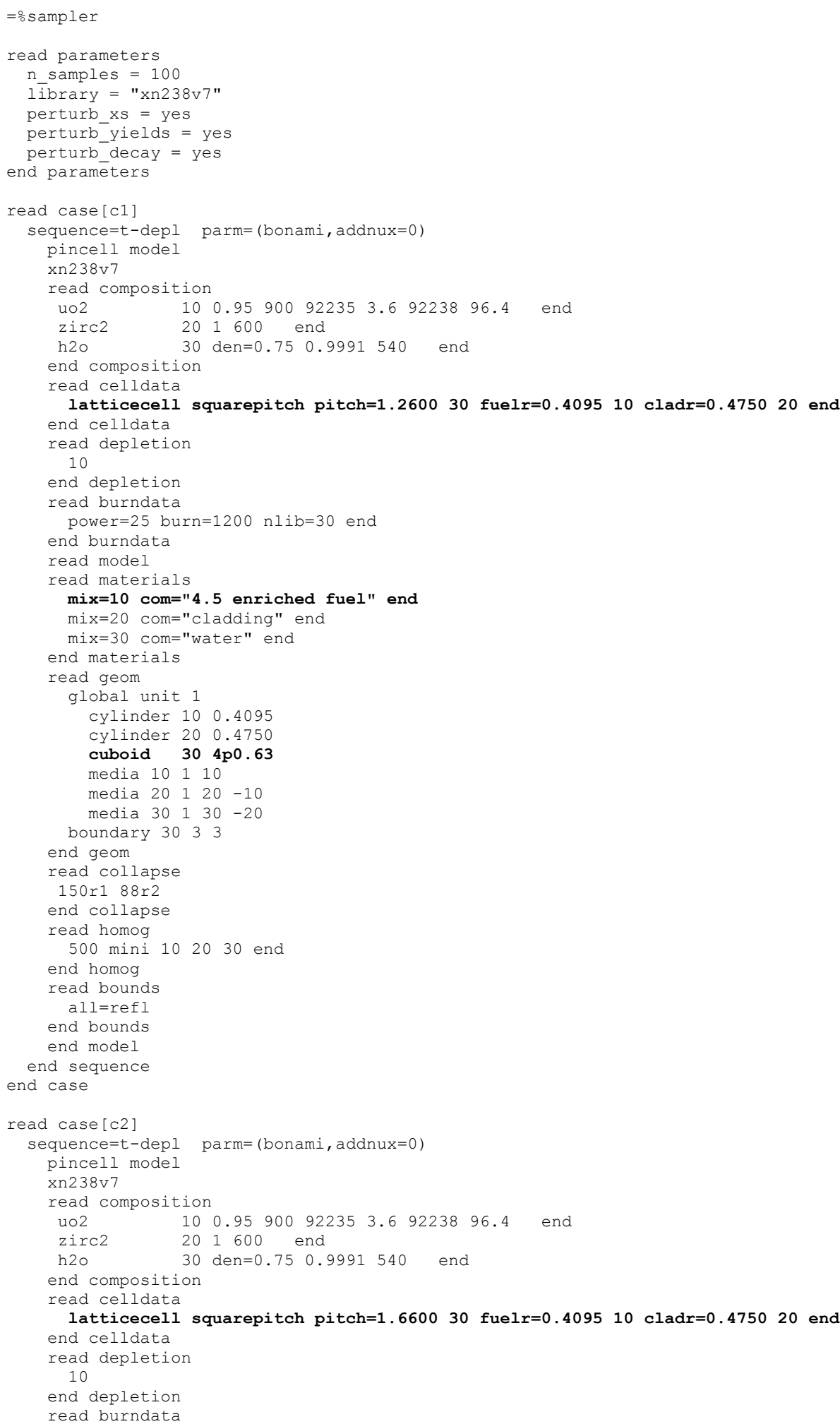




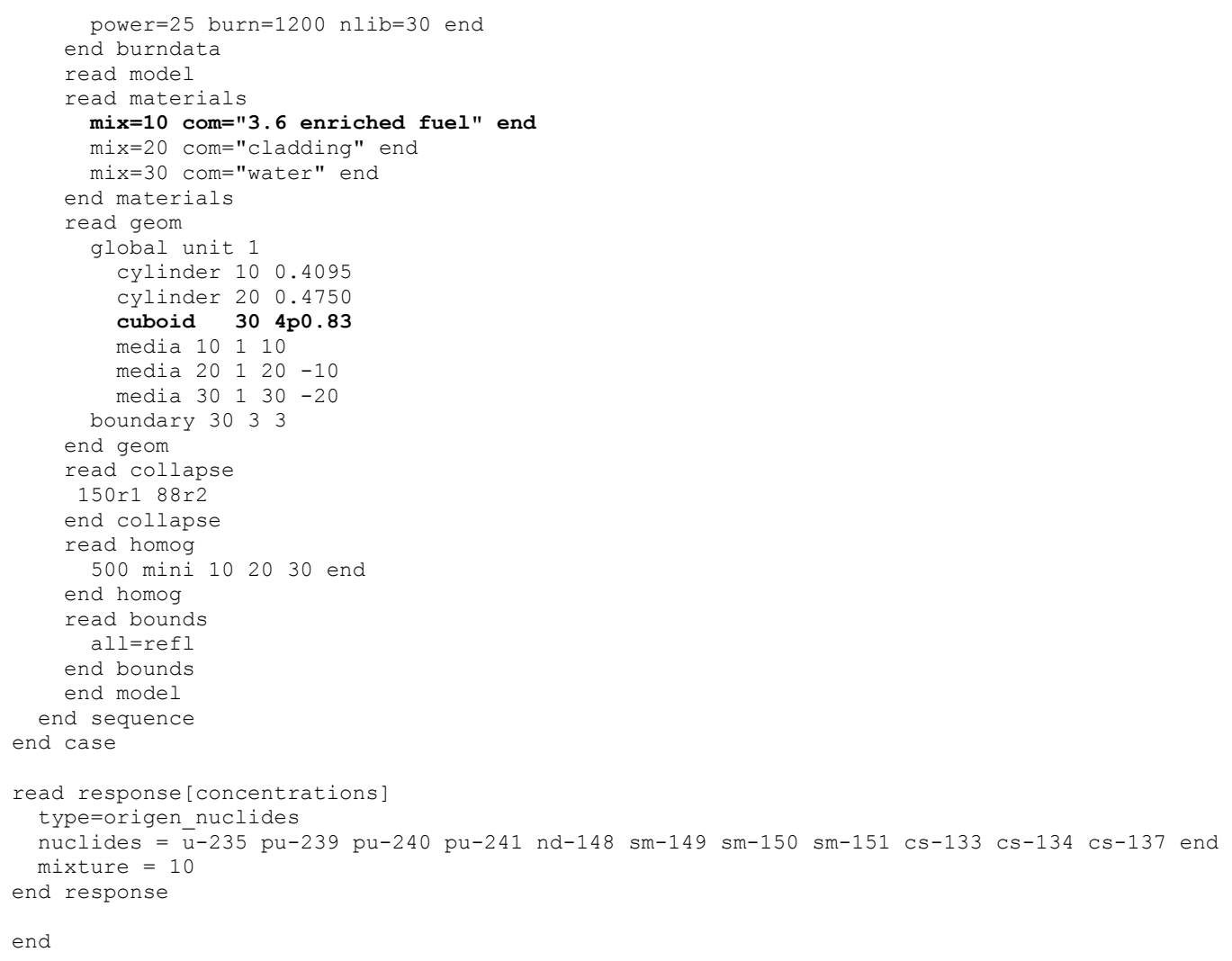

The correlation matrix for concentrations at 1200 days of irradiation is shown in Figure 6.7.15, postprocessed with Excel to show high and low correlation coefficients. The bold and underline entries indicate that the each extracted isotope shows a correlation coefficient of 1.0 between the two cases. This means that the two cases are the same in terms of their isotopic response to data perturbations.

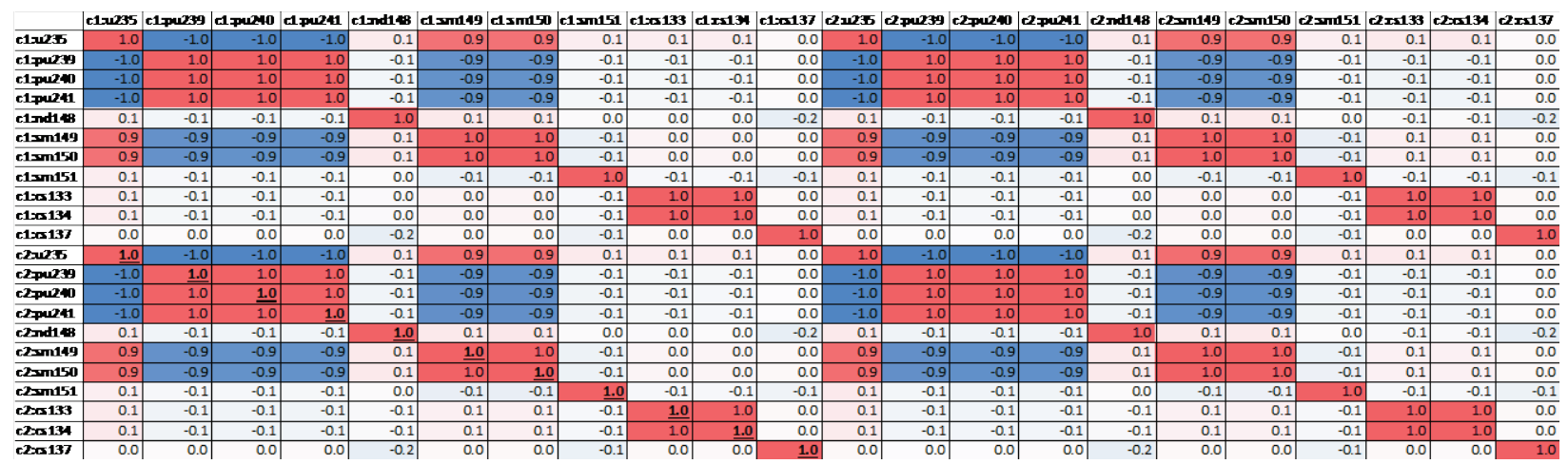

Figure 6.7.15. Correlation matrix for concentrations in sample problem 5

\subsection{Sample problem 6}

Sample problem 6 provides an example of fission yield and decay data sampling. First, COUPLE is run to assemble an ORIGEN library (on file ft33f001) from the perturbed yields and decay data libraries. Burst fission with long decay is then performed by ORIGEN and OPUS is used to extract the fission product decay heat. 


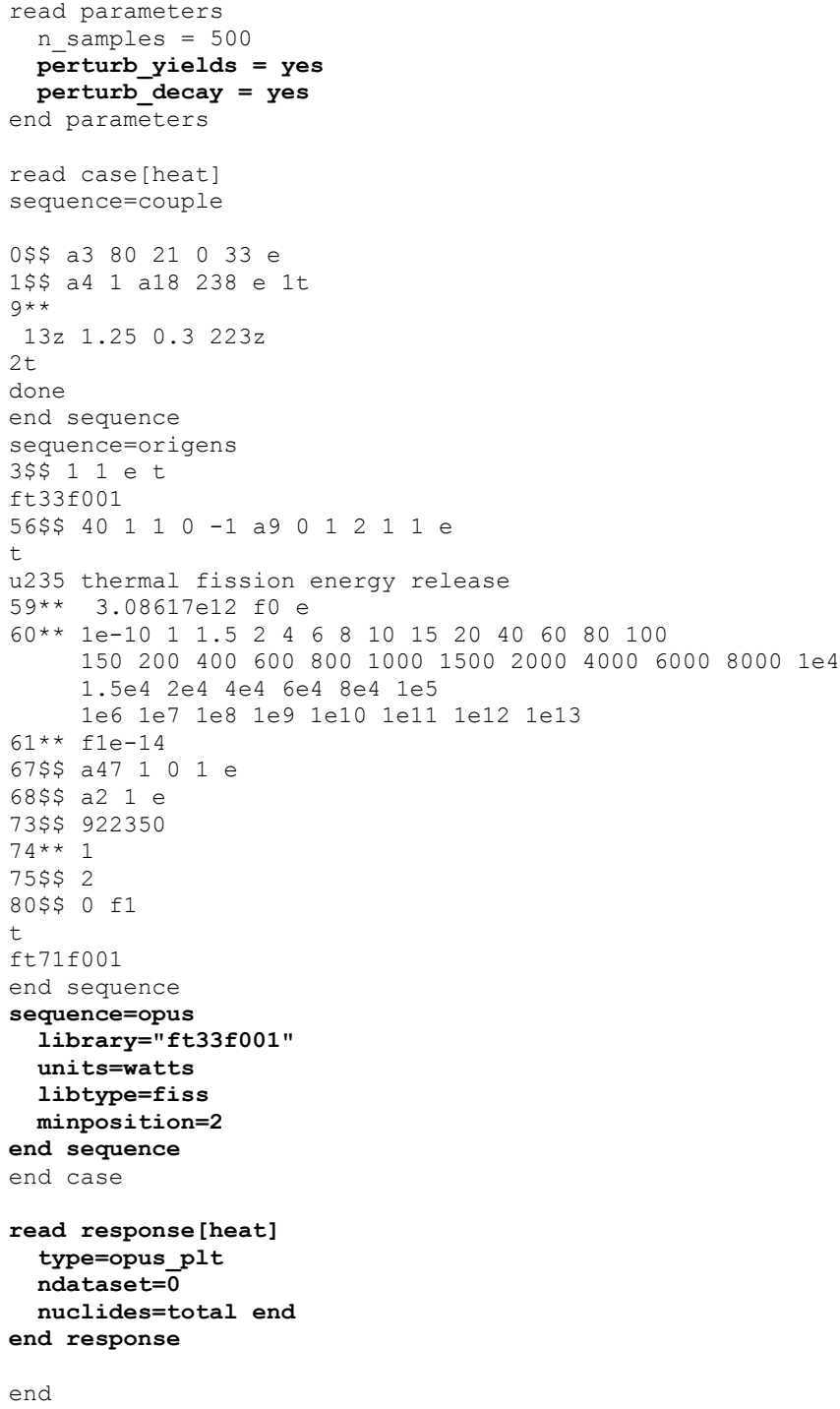

The total decay heat with the uncertainty is shown in Table 6.7.7 and Figure 6.7.16.

Table 6.7.7. Decay heat and uncertainties from sample problem 6

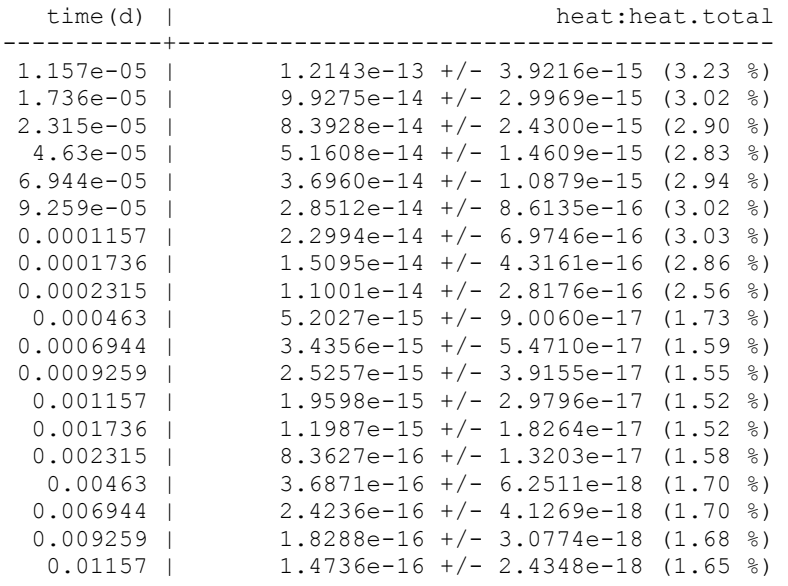



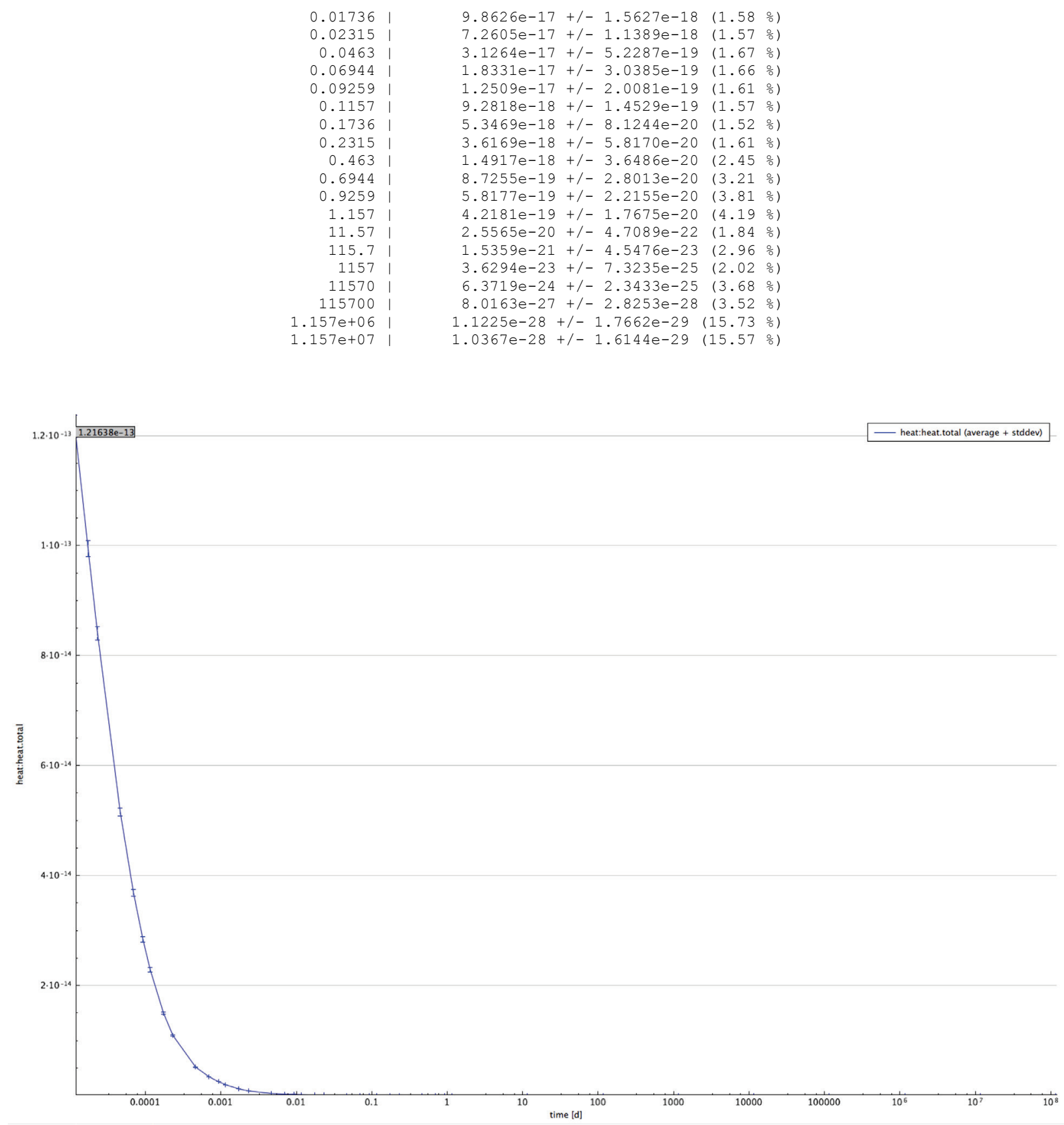

Figure 6.7.16. Decay heat and uncertainties from sample problem 6

\subsection{Sample problem 7}

Sample problem 7 illustrates an example of a shielding parametric study with MAVRIC. It also demonstrates usage of the grep response for both response value and uncertainty.

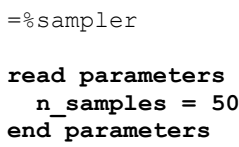




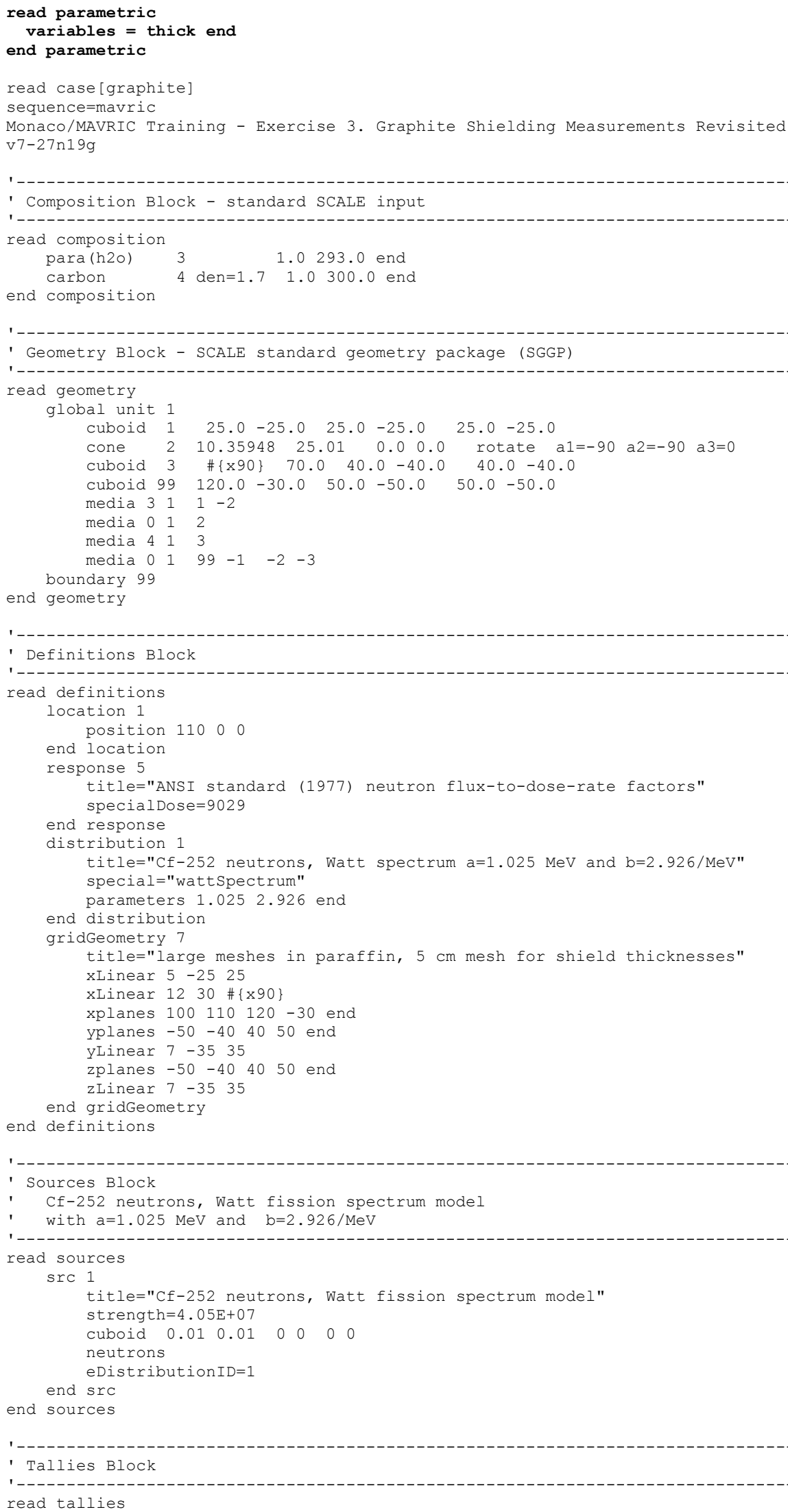




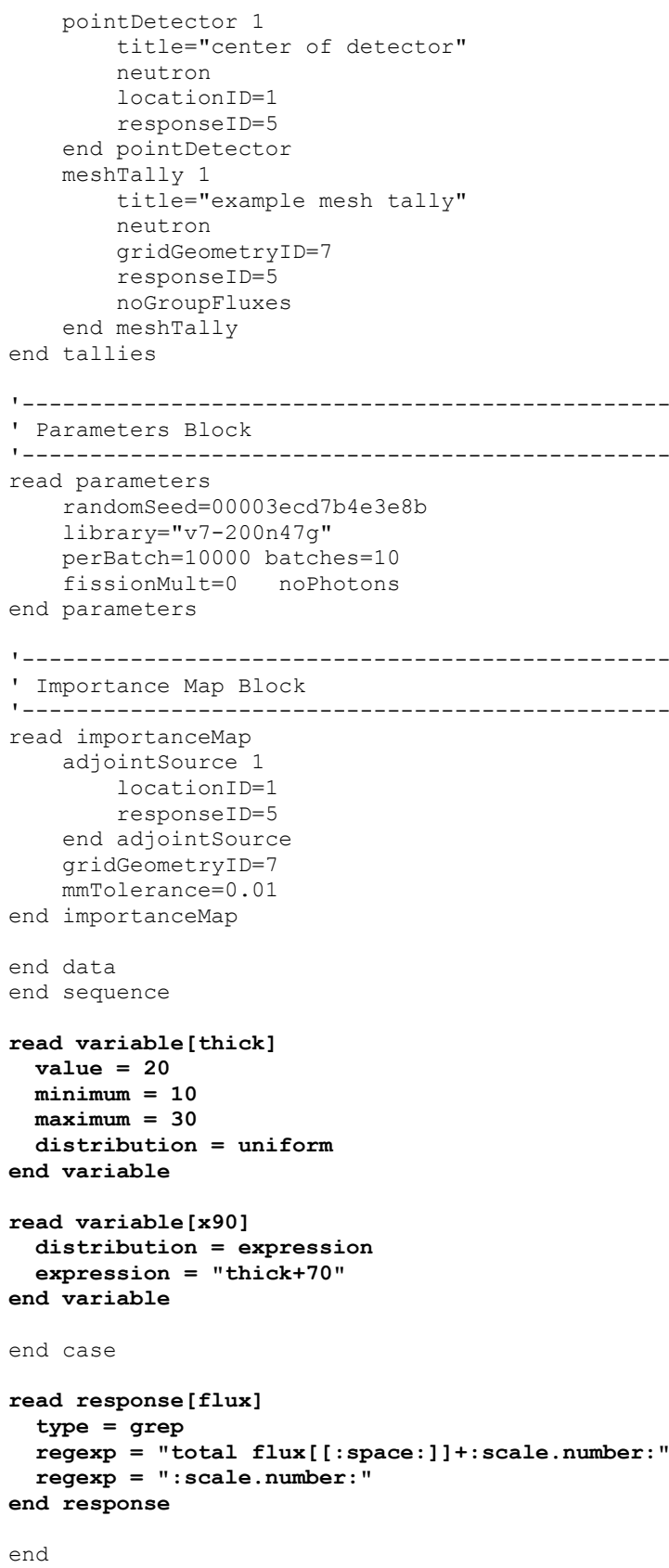

Flux dependence on the shielding thickness is shown in Figure 6.7.17. 


\section{Case graphite, response flux vs thick}

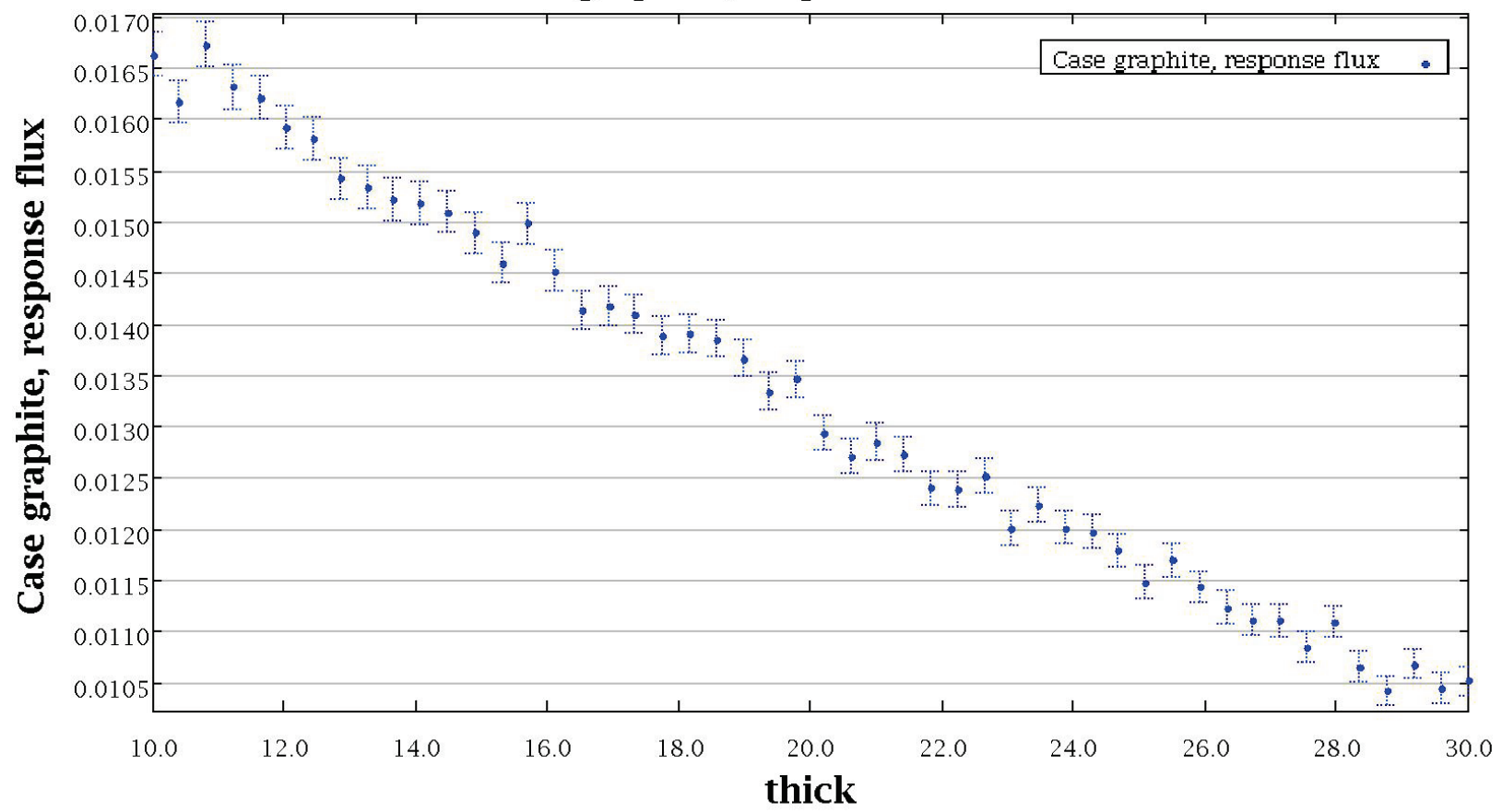

Figure 6.7.17. Flux as a function of shielding thickness for sample problem 7

\subsubsection{References}

1. B. T. Rearden, M. L. Williams, M. A. Jessee, D. E. Mueller, D. A. Wiarda. "Sensitivity and Uncertainty Analysis Capabilities in SCALE," Nuclear Technology, 174, 236-288 (2011).

2. B. Krzykacz, E. Hofer, M. Kloos, "A Software System for Probabilistic Uncertainty and Sensitivity Analysis of Results from Computer Models," Proc. International Conference on Probabilistic Safety Assessment and Management (PSAM-II), San Diego, CA (1994).

3. M. L. Williams, G. Ilas, M. A. Jessee, B. T. Rearden, D. Wiarda, W. Zwermann, L. Gallner, M. Klein, B. Krzykacz-Hausmann, and A. Pautz, "A Statistical Sampling Method for Uncertainty Analysis with SCALE and XSUSA,” Nuclear Technology, 183, 515-526 (2013).

4. M.T. Pigni, M.W. Francis, and I.C. Gauld, "Investigation of Inconsistent ENDF/B-VII.1 Independent and Cumulative Fission Product Yields with Proposed Revisions", Nuclear Data Sheets, 123, 231-236 (2015).

5. O. Buss, A. Hoefer, J. C. Neuber, and M. Schmid, "Hierarchical Monte-Carlo Approach to Bias Estimation for Criticality Safety Calculations," Proceedings of PHYSOR 2010, Advances in Reactor Physics to Power the Nuclear Renaissance, Pittsburgh, PA, May 9-14, 2010. 


\section{APPENDIX 6.7 A - SCALE Input Retrieval ENgine (SIREN)}

SIREN (SCALE Input Retrieval ENgine) provides a hierarchical representation of SCALE input and enables access to individual input components or sets of related input parameters. SIREN sorts the contents of supported SCALE input sequences into a tree structure so that every component in a SCALE input can be accessed using an XPath designation. The SIREN package is used by the SCALE supersequence Sampler to select individual components within an input file, perturb the parameter in memory, and write the perturbed input to a new file. In this way, components within an input file can be perturbed without modification to the original input file, which can be maintained as a read-only file under configuration control for quality assurance purposes.

To explore the use of SIREN expressions, two utilities are available in the SCALE bin directory. The utilities are the executables "InputViewer" and "InputSelector." The InputViewer utility accepts a SCALE input file and outputs the XPath designation for each component of that input file. The InputSelector utility accepts a SCALE input file and a SIREN select statement and then outputs the part of the input file specified by the SIREN select statement. The remainder of this section provides practical examples in the use of SIREN select statements to designate specific components of a SCALE input.

A simple CSAS6 input file, which models a sphere of HEU, is used to show how to use SIREN select statements to access specific portions of a SCALE input. The contents of the example CSAS6 input file are shown below.

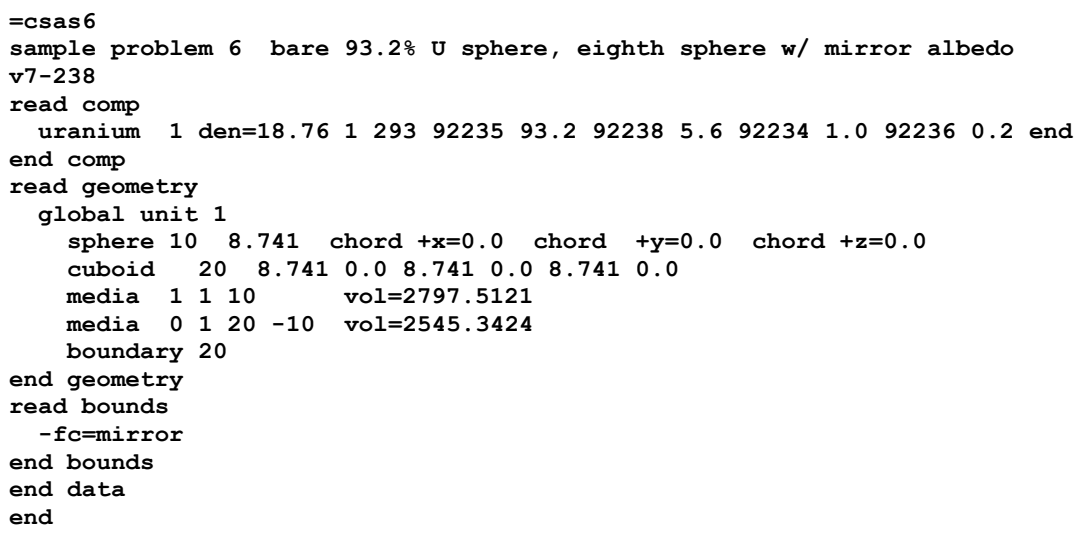

The InputViewer utility is used to view the contents of the example input file with their respective XPath designations. The utility accepts the path to the input file as an argument so that the usage is:

\$ InputViewer inputfile

A portion of the output from InputViewer is shown below; this portion corresponds to the material definition in the example input file. Notice that each component of the composition has a specific XPath designation. For instance, the "wtptPair" has several pieces of information (ids for each component and the weight percent for that component). To gain access to a specific component, the InputSelector utility is introduced below.

/csas6/comps

/csas6/comps/decl (read comp)

/csas6/comps/stdcomp

/csas6/comps/stdcomp/name (uranium)

/csas6/comps/stdcomp/mixture (1) 


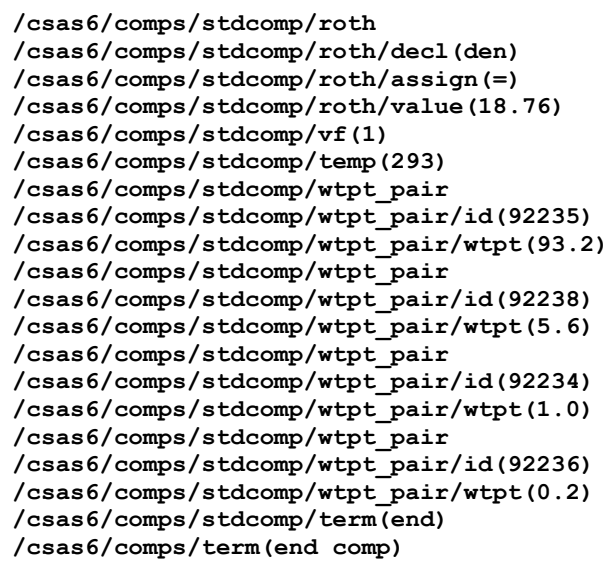

To explore how SIREN select statements are used, the InputSelector utility is used for a few cases with the example CSAS6 input file. Suppose the ${ }^{235} \mathrm{U}$ weight percent needed to be accessed for perturbation; the SIREN expression to access the ${ }^{235} \mathrm{U}$ weight percent is as follows:

Long form:

'/csas6/comps/stdcomp/wtpt_pair[id="92235"]/wtpt'

Short form:

'///wtpt_pair[id="92235"]/wtpt'

This statement tells SIREN to look for the weight percent with an ID of 92235 in the weight percent pair. A short form of the select statement (designated by the triple slash) can be used when there are unique qualities about a component; for instance, there is only a single material with an ID of 92235 , so the weight percent pair must belong to that material. There are many combinations of select statements that can point to a specific input component, and the developers suggest using the InputSelector utility to familiarize oneself with this capability. The usage of InputSelector is:

\$ InputSelector inputfile quoted_Select_Statement

Below is the screen output from the InputSelector with the example CSAS6 input file and the select statement for the ${ }^{235} \mathrm{U}$ weight percent.

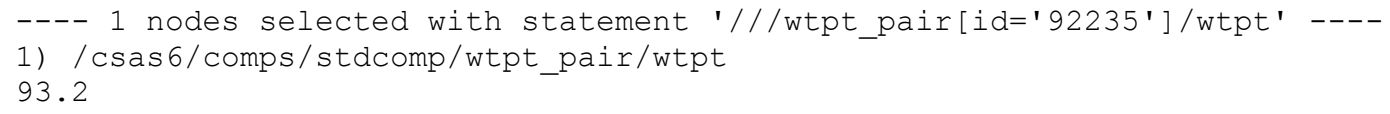

The output from InputSelector shows that the select statement points to a single component in the SCALE input, which is indeed the value for the ${ }^{235} \mathrm{U}$ weight percent. There are cases where a select statement points to a portion of the input that has multiple components and the select statement may need to be refined to point to a specific component. For instance, the cuboid defined in the geometry section of the input has multiple dimensions $(+\mathrm{x},-\mathrm{x},+\mathrm{y},-\mathrm{y}$, etc.). By using the select statement below, the dimensions of the cuboid are shown

'//cuboid/[id="20"]/dimensions' 
The output from using InputSelector with this select statement is shown below.

---- 1 nodes selected with statement '//cuboid/[id="20"]/dimensions' -.--

1) /csas6/geometry/global_unit/cuboid/dimensions

8.7410 .08 .7410 .08 .7410 .0

The output from InputSelector shows six values, which correspond to the dimensions of the cuboid $(+x,-x,+y,-y,+z,-z$ faces$)$. To select a single dimension, the select statement should include which component is desired. For instance, to select the height of the cuboid the select statement becomes:

'//cuboid/[id="20"]/dimensions/plus_z'

In the same way, the radius of the sphere can be selected using the SIREN expression that identifies the radius of a sphere with an ID of 10:

'//sphere/[id="10"]/dimensions/r'

The output from InputSelector is shown below.

---- 1 nodes selected with statement '//sphere/[id="10"]/dimensions/r' ----

1) /csas6/geometry/global_unit/sphere/dimensions/r

8.741

There are scenarios where there is no unique or obvious identifier for a component. As an example, the media volume can utilize an array index to retrieve the volume value.

'//media/volume/value'

The output from InputSelector illustrates both media's volume value are selected below.

---- 2 nodes selected with statement '//media/volume/value' ----

1) /csas6/geometry/global_unit/media/volume/value

2797.5121

2) /csas6/geometry/global_unit/media/volume/value

2545.3424

The printed index (1) can be utilized to narrow the selection.

'//media[1]/volume/value'

Here the first media is selected and subsequently, the media's volume value, removing the selection of the second media entirely. The output from InputSelector is shown below.

---- 1 nodes selected with statement '//media[1]/volume/value' ----

1) /csas6/geometry/global_unit/media/volume/value

2797.5121

For the sake of completeness, the following example illustrates selection of the media's volume value via the mixture's value comparator.

'//media [mixture="1"]/volume/value' 
This illustrates the first media's unique mixture component can be used to select the volume for the first media. The output from InputSelector is shown below.

1) /csas6/geometry/global_unit/media/volume/value

2797.5121

The above examples were intended to provide users with an idea of how to use SIREN select statements to access specific components within a SCALE input. The select statements are primarily used in cases where the input is to be perturbed by the Sampler super-sequence, but modification to the original input is not desirable. The recommended way to ensure that the select statements are correct is to use the InputSelector utility to check that a select statement points to the correct input component.

For viewing the input paths, an alternative to the InputViewer command line utility is available within the Fulcrum graphical user interface (GUI). By clicking on the Run Button drop-down and selecting "Input Listing" as shown in Figure 6.7.18, the full listing of the current input file is displayed in the "Messages" box. Also, notice the path is displayed in the bottom left corner for the input component located at the cursor.

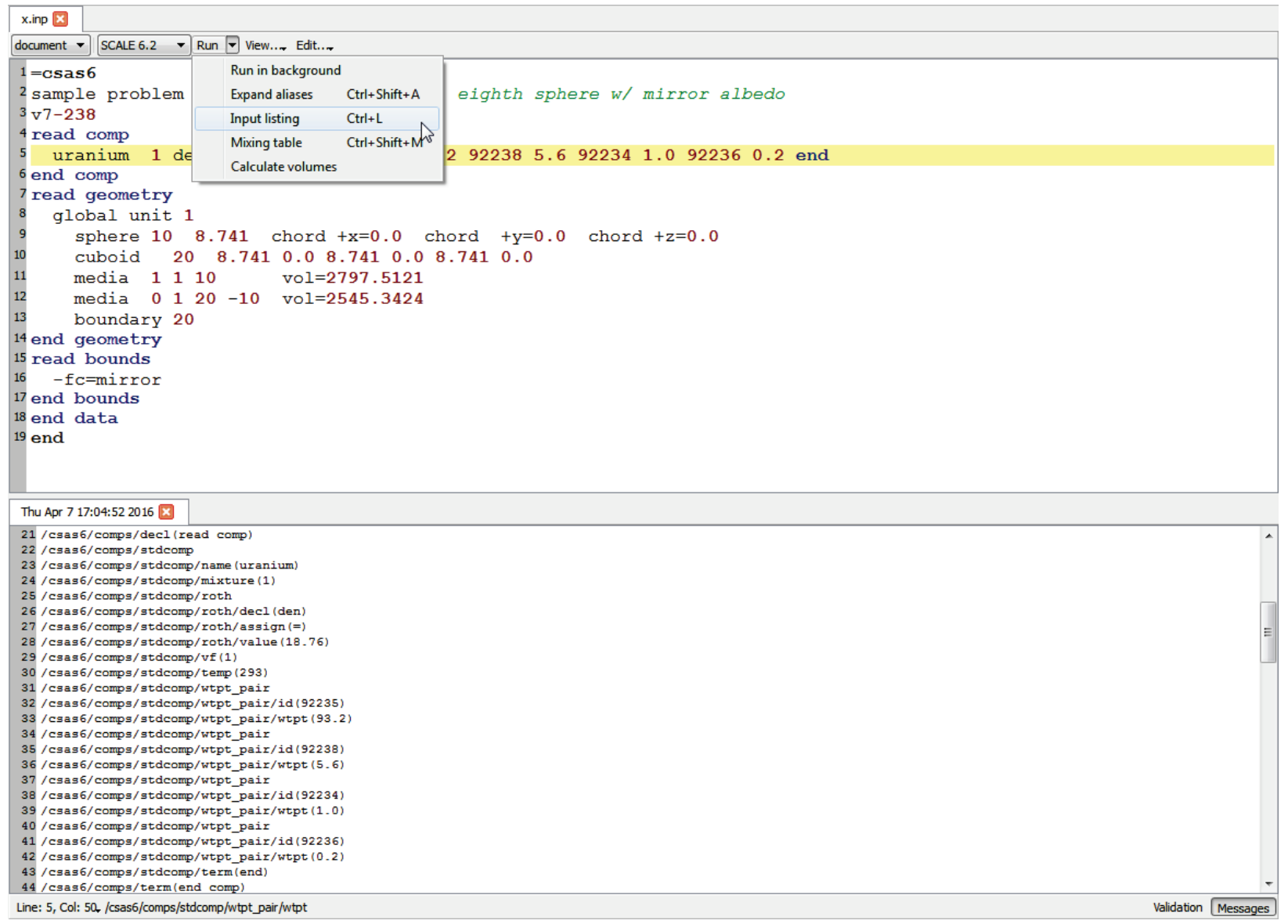

Figure 6.7.18. Input Listing in Fulcrum 
A table of SIREN expressions are as follows.

Table 6.7.2. Expressions for SIREN

\begin{tabular}{|c|c|c|}
\hline Expressions & Description & Examples \\
\hline component & Component match. & csas6 \\
\hline 1 & $\begin{array}{l}\text { Component separator. } \\
\text { When the expression begins } \\
\text { with this, it selects the root of } \\
\text { the document. }\end{array}$ & $\begin{array}{l}\text { csas6/xslib } \\
\text { / }\end{array}$ \\
\hline$/ /$ & $\begin{array}{l}\text { This expression is the 'Any } \\
\text { Child' select. }\end{array}$ & $\begin{array}{l}\text { csas6//uranium } \\
\text { // }\end{array}$ \\
\hline [index] & $\begin{array}{l}\text { Array index selector. When } \\
\text { an array of objects has been } \\
\text { selected, this expression } \\
\text { selects the object at the given } \\
\text { index. }\end{array}$ & csas6/geometry/global_unit/media[1] \\
\hline [sindex:eindex] & $\begin{array}{l}\text { Subarray index selector. } \\
\text { When an array of objects has } \\
\text { been selected, this expression } \\
\text { selects a subarray. }\end{array}$ & csas6/ comps/stdcomp/uranium/wtpt_pair[2:3] \\
\hline [sindex:eindex:stride] & $\begin{array}{l}\text { Subarray index selector. } \\
\text { When an array of objects has } \\
\text { been selected, this expression } \\
\text { selects a subarray and } \\
\text { subsequently components } \\
\text { stride apart. }\end{array}$ & csas6/comps/stdcomp/uranium/wtptPair[1:4:2] \\
\hline$*$ & $\begin{array}{l}\text { Component wild card. This } \\
\text { can only be used in place of a } \\
\text { component or to wild card the } \\
\text { remainder of a component. }\end{array}$ & $\begin{array}{l}\text { csas6/read comp/* } \\
\text { csas6/read comp/ur* }\end{array}$ \\
\hline
\end{tabular}




\section{APPENDIX 6.7 B - Expression Operators and Functions for Sampler}

The expression engine utilized by Sampler supports all basic mathematical and logical operators, as well as a number of functions. Logical expressions and functions evaluate to the number 1 for true, and the number 0 for false.

Table 6.7.2. Expression operators and functions for Sampler

\begin{tabular}{|c|c|c|}
\hline Operator & Description & Example \\
\hline+ & Addition & $3+3, x+y$ \\
\hline+ & Unary Plus & $+3,+(-3),+x$ \\
\hline- & Subtraction & $3-3, x-y$ \\
\hline- & Unary Minus & $-3,-(3+3),-x$ \\
\hline$*$ & Multiplication & $3 * 3, x * y$ \\
\hline l & Division & $3 / 3, x / y$ \\
\hline$\%$ & Modulo & $3 \% 3, x \% y$ \\
\hline$\wedge$ & Power & $3^{\wedge} 3, x^{\wedge} y$ \\
\hline$==$ & Equal to & $3==3, x==y$ \\
\hline$!=$ & Not equal to & $3 !=3, x !=y$ \\
\hline$<$ & Less than & $3<3, x<y$ \\
\hline$>$ & Greater than & $3>3, x>y$ \\
\hline$<=$ & Less than or equal to & $3<=3, x<=y$ \\
\hline$>=$ & Greater than or equal to & $3>=3, x>=y$ \\
\hline and & Left is true and right is true & 1 and $1, x==y$ and $y==z$ \\
\hline or & Left is true or right is true & 1 or $1, x==y$ or $y==z$ \\
\hline nand & Not and & 1 nand 1,1 nand $0, x$ nand $y$ \\
\hline xor & Exclusive or & 1 xor 1,1 xor $0, \mathrm{x}$ xor $\mathrm{y}$ \\
\hline nor & Not or & 1 nor 1,1 nor $0, x$ nor $y$ \\
\hline $\operatorname{abs}(x)$ & Absolute value & $\operatorname{abs}(1), \operatorname{abs}(-1), \operatorname{abs}(x), \operatorname{abs}(y)$ \\
\hline $\min (x, \ldots n)$ & Minimum of values & $\min (1), \min (1,2,3,4,5, \mathrm{x}, \mathrm{y})$, etc \\
\hline $\max (\mathrm{x}, \ldots \mathrm{n})$ & Maximum of values & $\max (1), \max (1,2,3,4,5, \mathrm{x}, \mathrm{y})$, etc \\
\hline $\operatorname{avg}(x, \ldots n)$ & Average of values & $\operatorname{avg}(1), \operatorname{avg}(1,2,3,4,5, x, y)$, etc \\
\hline $\operatorname{Sum}(\mathrm{x}, \ldots \mathrm{n})$ & Summation of values & $\operatorname{sum}(1), \operatorname{sum}(1,2,3,4,5, x, y)$, etc \\
\hline $\operatorname{mul}(\mathrm{x}, \ldots \mathrm{n})$ & Multiply values & $\operatorname{mul}(1), \operatorname{mul}(1,2,3,4,5, \mathrm{x}, \mathrm{y})$, etc \\
\hline floor $(\mathrm{x})$ & Floor of the value & $\begin{array}{l}\text { floor }(1)=1, \quad \text { floor }(-1.0)=-1.0, \quad \text { floor }(- \\
1.1)=-2.0\end{array}$ \\
\hline $\operatorname{ceil}(\mathrm{x})$ & Ceiling of the value & $\operatorname{ceil}(1)=1, \operatorname{ceil}(-1.1)=-1$ \\
\hline $\operatorname{round}(\mathrm{x})$ & Round the value to nearest integer & $\operatorname{round}(1.1)=1, \operatorname{round}(1.5)=2, \operatorname{round}(1.9)=2$ \\
\hline roundn $(\mathrm{x}, \mathrm{n})$ & $\begin{array}{l}\text { Round the value to the nth } \\
\text { decimal position }\end{array}$ & $\begin{array}{l}\operatorname{roundn}(1.15,1)=1.2, \text { roundn }(1.519,2)= \\
1.52\end{array}$ \\
\hline $\cos (\mathrm{x})$ & Cosine of the value & $\cos (0.0)=1$ \\
\hline $\sin (\mathrm{x})$ & Sine of the value & $\sin (0.0)=0$ \\
\hline $\tan (\mathrm{x})$ & Tangent of the value & $\tan (0.0)=0$ \\
\hline $\sec (x)$ & Secant of the value & $\sec (0.0)=1$ \\
\hline $\csc (\mathrm{x})$ & Cosecant of the value & $\csc (1.0)=1.1884$ \\
\hline $\cot (\mathrm{x})$ & Cotangent of the value & $\cot (1.0)=0.642093$ \\
\hline $\sinh (\mathrm{x})$ & Hyperbolic sine of the value & $\sinh (1.0)=1.1752$ \\
\hline $\cosh (\mathrm{x})$ & Hyperbolic cosine of the value & $\cosh (1.0)=1.54308$ \\
\hline
\end{tabular}




\begin{tabular}{|c|c|c|}
\hline $\tanh (\mathrm{x})$ & Hyperbolic tangent of the value & $\tanh (1.0)=0.761594$ \\
\hline $\log (\mathrm{x})$ & Natural $\log$ of the value & $\log (2.7182818)=1$ \\
\hline $\log 10(\mathrm{x})$ & Common log of the value & $\log 10(10.0)=1$ \\
\hline $\exp (x)$ & Exponential of the value & $\exp (1)=2.7182818$ \\
\hline $\operatorname{clamp}(1, \mathrm{x}, \mathrm{u})$ & $\begin{array}{l}\text { Clamp the value } \mathrm{x} \text { between } \\
\text { lowerbound } 1 \text { and upperbound } \mathrm{u}\end{array}$ & $\operatorname{clamp}(-1,1,+1)=1, \operatorname{clamp}(-1,-5,+1)=-1$ \\
\hline inrange $(1, \mathrm{x}, \mathrm{u})$ & $\begin{array}{l}\text { Determine if value } \mathrm{x} \text { is greater } \\
\text { than or equal to lowerbound } 1 \text {, and } \\
\text { less than or equal to upperbound } \mathrm{u}\end{array}$ & inrange $(-1,1,+1)=1$, inrange $(-1,-5,+1)=0$ \\
\hline $\operatorname{sgn}(x)$ & Determine the sign of $\mathrm{x}$. & $\begin{array}{l}\operatorname{sgn}(+1)=1, \operatorname{sgn}(-1)=- \\
1, \operatorname{sgn}(0)=0, \operatorname{sgn}(5)=1\end{array}$ \\
\hline sqrt(x) & Square Root of the value & $\operatorname{sqrt}(1)=1, \operatorname{sqrt}(4)=2$ \\
\hline $\operatorname{root}(\mathrm{x}, \mathrm{y})$ & Root of $x$ given $y$ & $\operatorname{root}(4,2)=2, \operatorname{root}(8,3)=2$ \\
\hline true & Constant true value & true \\
\hline false & Constant false value & False \\
\hline if(condition,true,false) & $\begin{array}{l}\text { If function that evaluates the } \\
\text { condition. If true, the true value is } \\
\text { substituted, else the false value is } \\
\text { substituted }\end{array}$ & $\operatorname{if}(x>y, x, y)=x$ \\
\hline
\end{tabular}




\section{APPENDIX 6.7 C - Guidelines for Running Sampler in Parallel}

Sampler calculations consist of three basic steps:

1. generate $N$ perturbed input files and libraries,

2. run $N$ independent SCALE jobs, and

3. analyze $N$ results sets.

At this time, the preferred way of performing Sampler calculations is to use run_cases=no (in the parameters block) and perform step 2 above outside of Sampler, i.e. using scripting or your system's queuing system to launch the $N$ SCALE jobs. The "\$\{OUTBASENAME\}.samplerfiles" directory that Sampler creates in step 1 will contain all the perturbed input files. Each of these input files should be run as a separate SCALE job. When all jobs are complete, Sampler should be run again (still with run_cases $=n o$ ). Sampler will see that all jobs have completed, and perform the analysis in step 3 . The Sampler sample problem 8 is a quick-running calculation that can be used to test and, if necessary adjust, the manual 3-step scheme described above on your system.

The internal frameworks for launching SCALE jobs in parallel are experimental in this release. Users must accept all responsibility for using these parallel capabilities on their systems. The following information is provided for different platforms on which the parallel capability has been tested.

\section{OpenMP Thread-based Parallelism}

The recommended parallel capability is based on parallel threads with OpenMP. This capability is available on all platforms SCALE supports (e.g. Windows, Mac, and Linux) and allows scaling up to the number of threads on a personal computer/workstation (typically 4 or more on current machines) or a single node of a Linux cluster (typically 8 to 16 on current machines). To launch a Sampler calculation with 5 parallel threads, one would issue the following from the command line:

scalerte -r - I 5 -m sample_8.inp

This will perform all 3 steps of the Sampler process in sequence, first generating all the inputs, then using 5 parallel threads to launch jobs ( 1 for the main "master" process that organizes the workers, and 4 worker processes that actually perform the perturbed calculations).

\section{MPI-based Parallelism}

The main limitation of OpenMP's thread-based parallelism is that it is limited to a single node in a Linux cluster environment, a common platform for modern scientific computing. The MPI-based parallelism requires building SCALE from source with MPI enabled. There is one workaround necessary with the MPI-enabled Sampler. MPI-enabled Sampler cannot call another MPI-enabled SCALE module, such as kenovi or $x k b a$. For this reason, if kenovi or $x k b a$ (or any other MPI-parallel sequence) is required in the Sampler calculation, the user must make the serial versions of these executables available to Sampler during calculations, e.g. by copying them into the temporary directories via $a=$ shell command in the input file.

To launch a Sampler calculation with 32 parallel calculations on two nodes with 16 cores each, one would issue the following from the command line:

scalerte $-\mathrm{T}$ temp $-\mathrm{M}$ machine.txt $-\mathrm{N} 32-\mathrm{m}$ sample_8.inp

where the machine.txt file contains: 
nodeA slots $=16$

nodeB slots $=16$

where "nodeA" and "nodeB" are the names of nodes recognized by the system. Note that the temporary directory "temp" must be available to all nodes, such that the saved results can be copied back after execution. This requires the temp directory be on a "shared filesystem", which in turn means that some input/output (I/O) will be performed over this shared filesystem. If too many parallel jobs are requested, the system I/O may be swamped by the Sampler file transfer requests, impacting all users on the system. For modest sized Linux clusters, a maximum number of parallel jobs of 50 (i.e. $-\mathrm{N} 50$ ) is recommended, although for I/O intensive sequences such as TRITON, this may still be too much. The maximum number of jobs per node should take into account not only the number of cores on that node, but the memory demands of the SCALE sequences to be called by Sampler. Ensuring sufficient memory is available may limit the number of cores per node ("slots") that should be requested.

Finally, some experience suggests that using the utility "nohup" to detach the main Sampler calculation, e.g. to logout of ssh and have the job continue to run, can cause runs to fail. It is recommended to use alternatives to "nohup" such as "screen" or "disown" to detach the main Sampler calculation from the user's login session. 


\title{
7.0 MATERIAL SPECIFICATION AND CROSS SECTION PROCESSING
}

\author{
Introduction by M. L. Williams and B. T. Rearden
}

XSProc (Cross Section Processing) provides material input and multigroup (MG) cross section preparation for most SCALE sequences. XSProc allows users to specify problem materials using easily remembered and easily recognizable keywords associated with mixtures, elements, nuclides, and fissile solutions provided in the SCALE Standard Composition Library. For MG calculations, XSProc provides cross section temperature correction and resonance self-shielding as well as energy group collapse and spatial homogenization for systems that can be represented in celldata input as infinite media, finite 1D systems, or repeating structures of 1D systems, such as uniform arrays of fuel units. Improved resonance self-shielding treatment for nonuniform lattices can be achieved through the use of the MCDancoff (Monte Carlo Dancoff) code that generates Dancoff factors for generalized 3D geometries for subsequent use in XSProc. Cross sections are generated on a microscopic and/or macroscopic basis as needed. Although XSProc is most often used as part of an integrated sequence, it can be run without subsequent calculations to generate problem-dependent MG data for use in other tools.

This chapter provides detailed descriptions of the methods and modules used for self-shielding. Selfshielding calculations are effectively a problem-specific extension of the processing procedures used to create the SCALE cross section libraries. SCALE includes continuous energy (CE) and several MG (MG) cross section libraries described in the chapter on SCALE Cross Section Libraries. The AMPX nuclear data processing system ${ }^{1}$ was used to convert evaluated data from ENDF/B into CE cross sections, which were then averaged into problem-independent MG data at a reference temperature of $300 \mathrm{~K}$, weighted with a generic energy spectrum (see the SCALE Cross Section Libraries chapter). After being transformed in probability distributions by AMPX, the CE data require no further modifications for application to a specific problem except for possible interpolation to the required temperatures. However, in $M G$ calculations, reaction rates depend strongly on the problem-specific energy distribution of the flux, which implies that the problem-independent MG data on the library should be modified into problemdependent values representative of the actual flux spectrum rather than the library generic spectrum. The neutron energy spectrum is especially sensitive to the concentrations and heterogeneous arrangement of resonance absorbers, which may dramatically reduce the flux at the resonance peaks of a nuclide, thus reducing its own reaction rate -a phenomenon known as self-shielding. In general, the higher the concentration of a resonance nuclide and the more the interaction between heterogeneous lumps (e.g. fuel pins), the greater the degree of self-shielding for the nuclide.

Reference 2 gives a general description of the SCALE self-shielding methods. The individual computational modules perform distinct functions within the overall all self-shielding methodology of XSProc. More theoretical details about individual computational modules are given in Chapters 7.2-7.7. XSProc provides capabilities for two different types of self-shielding methods, which are summarized below.

\section{Bondarenko Method}

The Bondarenko approach ${ }^{3}$ uses MG cross sections pre-computed over a range of self-shielding conditions, varying from negligibly (infinitely dilute) to highly self-shielded. Based on the following approximations $^{4}$ it can be shown that the degree of self-shielding in both homogeneous and heterogeneous systems depends only on a single parameter called the background cross section, "sigma0," and on the Doppler broadening temperature:

(a) neglect of resonance interference effects,

(b) intermediate resonance approximation, and 
(c) equivalence theory.

During the SCALE MG library processing with AMPX, self-shielded cross sections are computed using a CE flux calculated at several background cross section values and temperatures. These are used to calculate ratios of the shielded to unshielded cross sections, called "Bondarenko factors" (a.k.a. shielding factors or f-factors). As described in the SCALE Cross Section Libraries chapter, Bondarenko factors are tabulated on the SCALE libraries as a function of sigma0 values and Doppler temperatures for all energy groups of each nuclide.

Bondarenko factors are multiplicative correction factors that convert the generic unshielded data into problem-dependent self-shielded values. The BONAMI computational module performs self-shielding calculations with the Bondarenko method by using the input concentrations and unit cell geometry to calculate a sigma0 value for each nuclide and then interpolating the appropriate MG shielding factors from the tabulated library values.

\section{CENTRM/PMC Method}

Self-shielding calculations with BONAMI are fast and are always performed for all SCALE MG sequences. However, due to the approximations (a)-(c) listed in the previous section, a more rigorous method is also provided which can replace the BONAMI results over a specified energy range, usually encompassing the resolved resonance ranges of important absorber nuclides. This approach is designated as the CENTRM/PMC method, named after the two main computational modules, although several additional modules are also used. CENTRM/PMC eliminates the main approximations of the BONAMI approach by performing detailed neutron transport calculations with a combination of MG and CE cross sections for the actual problem-dependent compositions and unit cell descriptions. ${ }^{5}$ This provides a problem-dependent pointwise (PW) flux spectrum for averaging MG cross sections, which reflects resonance cross-interference effects, an accurate slowing down treatment, and geometry-specific transport calculations using several available options. Shielded MG cross sections processed with CENTRM/PMC are usually more accurate than BONAMI, so it is the default for most SCALE MG sequences. However, depending on the selected transport option, CENTRM/PMC may run considerably longer than BONAMI alone.

The CENTRM/PMC methodology first executes BONAMI, which provides shielded cross sections outside the specified range of the PW flux calculation. Then the computational module CRAWDAD reads CE cross section files and bound thermal scatter kernels and interpolates the data to the desired temperatures for CENTRM. Using a combination of shielded MG data from BONAMI and CE data from CRAWDAD, CENTRM calculates PW flux spectra by solving the deterministic neutron transport equation for all unit cells described in the input. CENTRM calculations cover the energy interval $10^{-5} \mathrm{eV}$ to $2 \times 10^{7} \mathrm{eV}$ spanned by the SCALE MG libraries. This energy range is subdivided into three sections: (a) upper MG range: $\mathrm{E}>\operatorname{demax}$, (b) $\mathrm{PW}$ range: demin $<\mathrm{E}<$ demax, and (c) lower $\mathrm{MG}$ range: $\mathrm{E}<$ demin, where demin and demax are the boundaries of the PW range, which can be defined by user input. The default values are demin $=10^{-3} \mathrm{eV}$ and demax $=2 \times 10^{4}$. The values encompass the resolved resonance ranges of essentially all actinide and fission product nuclides. MG transport calculations are performed in the upper and lower ranges, which are coupled to the PW transport calculation by the scattering sources.

Several methods are available for the CENTRM transport solutions within each energy range, and the default methods can be changed through parameters in the XSProc input. The discrete $S_{n}$ method is default for homogeneous media and for arbitrary one dimensional (1D) slab, spherical, and cylindrical geometries with general boundary conditions. A unit cell model is used for self-shielding arrays of spherical or cylindrical fuel regions. For the common case of a square-pitch lattice with cylindrical fuel pins, the default transport solver is the 2D method of characteristics (MoC). The CENTRM MoC solution 
exactly models the outer rectangular cell surface using a reflected boundary condition. CENTRM also has an option for discrete $S_{n}$ calculations using a 1D Wigner-Seitz cell with a white outer boundary condition. The 1D cell model is always used for spherical fuel arrays (e.g., pebbles), and can also be selected as a faster alternative than MoC for cylindrical fuel lattices. Finally, a two-region collision probability method can be used for any type of array. The two-region solver executes very fast but is usually more approximate than the $\mathrm{MoC}$ and $\mathrm{S}_{\mathrm{n}}$ methods.

After CENTRM computes the average PW flux for each material zone, PMC uses the spectra to process the $\mathrm{CE}$ cross sections into problem-specific $\mathrm{MG}$ values for each material zone. A typical energy grid for the flux solution consists of 50,000-90,000 points, providing good resolution of the spectral fine-structure caused by resonance self-shielding. PMC has several options for processing the MG data, such as correcting for resonance absorption effects on the elastic removal. Shielded cross sections from PMC may also be used to perform an optional MG eigenvalue calculation with the XSDRNPM $\mathrm{S}_{\mathrm{n}}$ module for cellaveraging and/or group collapsing of the MG values.

A variation of the standard CENTRM/PMC method is used to perform self-shielding for doubly heterogeneous cells in which cylindrical or spherical fuel elements, composed of small spherical fuel particles dispersed in a moderator material, are distributed in an array configuration. Self-shielding of this type of system requires multiple CENTRM/PMC passes, effectively representing the two levels of heterogeneity. ${ }^{6}$ First-level CENTRM calculations are performed for each type of fuel particle using a spherical unit cell to represent the array of multi-layered fuel particles distributed in the moderator matrix. Space-dependent CE fluxes from these calculations are used in the CHOPS module to compute CE disadvantage factors (fuel-average flux divided by cell-average flux) for generating cell-averaged, CE cross sections representative of the homogenized fuel compact. The spatially averaged CE cross sections are used in a second-level CENTRM transport calculation corresponding to a 1D unit cell model for the array of fuel elements, with homogenized number densities for the fuel compact. The CE flux spectrum from this calculation is used in PMC to process the final MG, problem-dependent cross sections. This entire procedure is transparent to the user and has been automated in XSProc. Reference 2 provides more details about the SCALE treatment for doubly heterogeneous fuel.

\section{Treatment of Non-Uniform Lattice Effects}

For self-shielding of lattice configurations, both the BONAMI and CENTRM/PMC approaches assume that the fuel is arranged in an infinite, uniform array of identical cells. For most pins in an actual lattice, the uniform-array approximation is satisfactory; however, self-shielding of some cells may be affected by boundary effects along the edge of the array or by the presence of water holes or control rods. These effects can be treated by incorporating a nonuniform Dancoff factor into the self-shielding calculations for the affected cells. The SCALE module MCDancoff performs a simplified one-group Monte Carlo calculation to compute Dancoff factors for arbitrary absorber mixtures within a complex (nonuniform) 3D array. The input for MCDancoff is described in Sect. 7.7. This module must be run as a standalone executable prior to the self-shielding calculations for a given sequence, and the computed Dancoff factors must be entered as XSProc input. The input Dancoff factor is used directly in defining the background cross section for BONAMI calculations. In the CENTRM/PMC methodology, the input Dancoff factor is used in CENTRM to calculate a Dancoff-equivalent unit cell, which defines a uniform lattice pitch that produces the same Dancoff value as the nonuniform lattice. The CENTRM transport calculation then proceeds as usual using $2 \mathrm{D} \mathrm{MoC}$ or $1 \mathrm{D} \mathrm{S}_{\mathrm{n}}$ for the unit cell. 


\section{References}

1. D. Wiarda, M. L. Williams, C. Celik, and M. E. Dunn, "AMPX: A Modern Cross Section Processing System For Generating Nuclear Data Libraries," Proceedings of International Conference on Nuclear Criticality Safety, Charlotte, NC, Sept 13-17, 2015.

2. M. L. Williams, "Resonance Self-Shielding Methodologies in SCALE," Nuclear Technology 174, 149-168 (May 2011).

3. I. I. Bondarenko, Ed., Group Constants for Nuclear Reactor Calculations, Consultants Bureau, New York, 1964.

4. R. J. J. Stamm'ler and M. J. Abbate, Methods of Steady-State Reactor Physics in Nuclear Design, Academic Press, London, 1983.

5. M. L. Williams and M. Asgari 1995. "Computation of Continuous-Energy Neutron Spectra with Discrete Ordinates Transport Theory," Nucl. Sci. \& Engr. 121, 173-201, 1995.

6. S. Goluoglu and M. L. Williams, "Modeling Doubly Heterogeneous Systems in SCALE," Tran. Am. Nucl. Soc. 92, Washington, D.C., November 2005. 


\title{
7.1 XSPROC: THE MATERIAL AND CROSS SECTION PROCESSING MODULE FOR SCALE
}

M. L. Williams, L. M. Petrie, R. A. Lefebvre, K. T. Clarno, J. P. Lefebvre, U. Merturyek, D. Wiarda, and B. T. Rearden

\begin{abstract}
The modern material and cross section processing module of SCALE (XSProc) was developed for the 6.2 release to prepare data for continuous-energy and multigroup calculations. XSProc expands material input from Standard Composition Library definitions into atom number densities and, for multigroup calculations, performs cross section resonance self-shielding, energy group collapse, and spatial homogenization. XSProc implements capabilities for problem-dependent temperature interpolation, calculation of Dancoff factors, resonance self-shielding using Bondarenko factors with full-range intermediate resonance treatment, as well as use of continuous energy resonance self-shielding in the resolved resonance region. XSProc integrates and enhances the capabilities previously implemented independently in BONAMI, CENTRM, PMC, WORKER, ICE, and XSDRNPM, along with some additional capabilities that were provided by MIPLIB and SCALELIB. The use of the modern XSProc sequence instead of the legacy codes of previous versions of SCALE generally results in the preparation of cross sections in less time, with substantial speedups for more I/O bound problems. Additionally, the memory requirements of XSProc are improved by generating only the data needed for a particular calculation instead of generating a general-purpose library that contains substantial amounts of data that are not needed for a particular calculation.
\end{abstract}




\section{TABLE OF CONTENTS}

Page

7.1 XSPROC: The MATERIAL AND CROSS SECTION PROCESSING MODULE

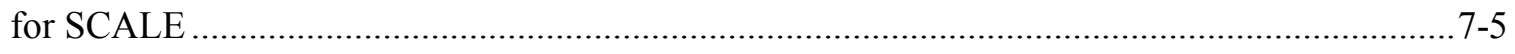

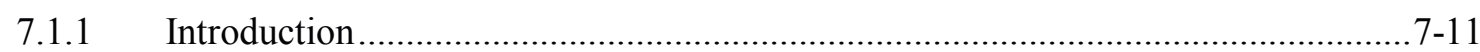

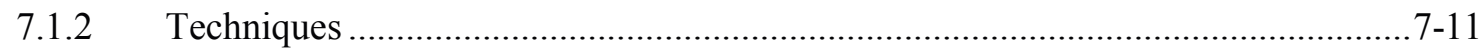

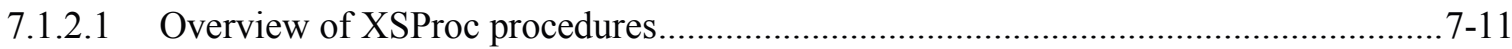

7.1.2.2 Standard composition material processing..............................................................

7.1.2.3 Unit cells for MG resonance self-shielding ........................................................... 7-12

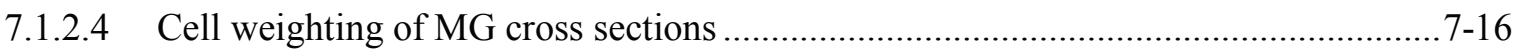

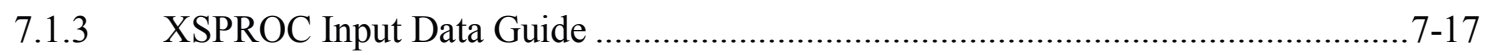

7.1.3.1 XSProc data checking and resonance processing options......................................... $7-17$

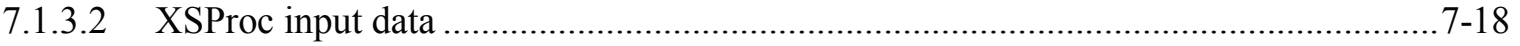

7.1.3.3 Standard composition specification data................................................................

7.1.3.4 Unit cell specification for infinite homogeneous problems ...................................... 7-36

7.1.3.5 Unit cell specification for LATTICECELL problems ................................................

7.1.3.6 Unit cell specification for MULTIREGION cells..................................................... 7-46

7.1.3.7 Unit cell specification for doubly heterogeneous (DOUBLEHET) cells....................7-51

7.1.3.8 Optional MORE DATA parameter data .................................................................... 7-60

7.1.3.9 Optional CENTRM DATA parameter data ................................................................

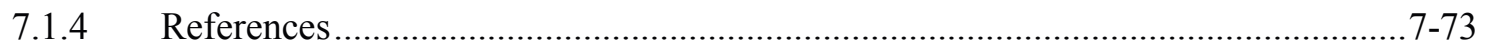

Appendix 7.1.A Standard Composition Examples .............................................................. 7-74

7.1.A.1. Standard composition fundamentals ....................................... 7-74

7.1.A.2. Basic standard composition specifications................................ 7-76

7.1.A.3. User-defined (arbitrary) chemical compound

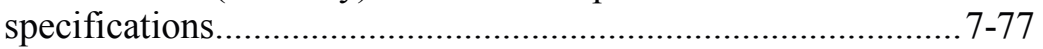

7.1.A.4. User-defined (arbitrary) mixture/alloy specifications ............... 7-78

7.1.A.5. Fissile solution specifications ............................................... 7-78

7.1.A.6. Combinations of standard composition materials to define a

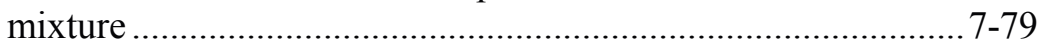

7.1.A.7. Combinations of user-defined compound and userdefined mixture/alloy to define a mixture.................................... 7-81

7.1.A.8. Combinations of solutions to define a mixture ........................ 7-82

7.1.A.9. Combinations of basic and user-defined standard compositions to define a mixture ............................................ 7-82

7.1.A.10. Combinations of basic and solution standard compositions to define a mixture ............................................. 7-85

7.1.A.11. Combinations of user-defined compound and solution to define a mixture 
Appendix 7.1.B Examples of Unit Cell Input Data ............................................................

7.1.B.1. Infinite homogeneous medium unit cell data ........................... 7-88

7.1.B.2. LATTICECELL unit cell data ................................................ 7-88

7.1.B.3. MULTIREGION unit cell data ................................................

7.1.B.4. DOUBLEHET unit cell data ..................................................

7.1.B.5. Optional parameter data ..................................................... 7-97

7.1.B.6. CENTRM DATA examples................................................ 7-97

Appendix 7.1.C Examples of Complete XSProc Input Data ............................................. 7-99

7.1.C.1. Infinite homogeneous medium input data............................... 7-99

7.1.C.2. LATTICECELL input data .................................................. 7-100

7.1.C.3. MULTIREGION input data ............................................ 7-102

7.1.C.4. DOUBLEHET input data................................................. 7-103

7.1.C.5. Two methods of specifying a fissile solution.......................... 7-104

7.1.C.6. Multiple unit cells in a single problem................................... 7-107

7.1.C.7. Multiple fissile mixtures in a single unit cell .......................... 7-108

7.1.C.8. Cell weighting an infinite homogeneous problem .................. 7-109

7.1.C.9. Cell weighting a LATTICECELL problem ............................7-109

7.1.C.10. Cell weighting a MULTIREGION problem ............................ 7-110 


\section{LIST OF FIGURES}

Page

Figure 7.1.1. Arrangement of materials in a SQUAREPITCH and SPHSQUAREP unit cell................7-43

Figure 7.1.2. Arrangement of materials in a TRIANGPITCH and SPHTRIANGP unit cell. ................7-43

Figure 7.1.3. Arrangement of materials in a SYMMSLABCELL unit cell having reflected left

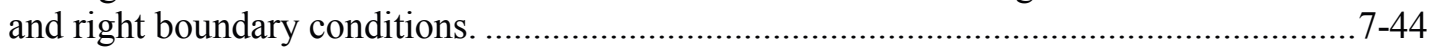

Figure 7.1.4. Arrangement of materials in an ASQUAREPITCH and ASPHSQUAREP unit cell.........7-45

Figure 7.1.5. Arrangement of materials in an ATRIANGPITCH and ASPHTRIANGP unit cell...........7-45

Figure 7.1.6. Arrangement of materials in an ASYMSLABCELL unit cell having reflected left

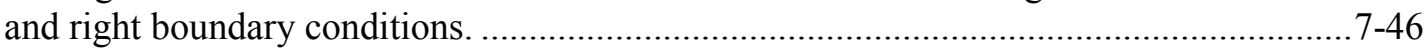

Figure 7.1.7. Arrangement of materials in a grain (first level cell) in a DOUBLEHET unit cell............7-55 


\section{LIST OF TABLES}

Page

Table 7.1.1. Outline of XSProc input data ................................................................................ 7-20

Table 7.1.2. Outline of standard composition specification options .............................................. 7-22

Table 7.1.2.a. Standard composition specification for basic mixtures ................................................ 7-24

Table 7.1.2.b. Standard composition specification for solutions.................................................. 7-25

Table 7.1.2.c. Standard composition specification for chemical compounds .................................... 7-26

Table 7.1.2.d. Input specification for user-specified mixture/alloy data ............................................ 7-27

Table 7.1.3. Unit cell specifications for INFHOMMEDIUM problems ......................................... 7-37

Table 7.1.4. Unit cell specification for LATTICECELL problems .................................................... 7-41

Table 7.1.5. Unit cell specification for MULTIREGION problems................................................. 7-49

Table 7.1.6. Unit cell specification for DOUBLEHET problems …................................................ 7-56 


\section{ACKNOWLEDGMENTS}

XSProc has evolved from the concept of a Material Information Processor library (MIPLIB) that used alphanumeric material specifications, which was initially proposed and developed by R. M. Westfall. J. R. Knight and J. A. Bucholz expanded and refined MIPLIB in early SCALE releases. Through SCALE 6.1, many enhancements were made by S. Goluoglu, D. F. Hollenbach, N. F. Landers, J. A. Bucholz, C. F. Weber, and C. M. Hopper, with L. M. Petrie taking the lead responsibility. With the SCALE modernization initiative beginning in SCALE 6.2, MIPLIB is no longer part of the XSProc analysis, but the original concepts and input formatting were preserved in the new implementation. The authors wish to thank Dan Ilas for helping convert the original MIPLIB documentation and Sheila Walker for editing and formatting this document. Special thanks to Don Mueller for his detailed review and checking of the document. 


\subsubsection{Introduction}

Self-shielding of multigroup cross sections is required in SCALE sequences for criticality safety, reactor physics, radiation shielding, and sensitivity analysis. In all previous versions of SCALE, resonance selfshielding calculations were done by executing a series of stand-alone executable codes, each dedicated to a specific aspect of the self-shielding operations. Each sequence had its own unique internal coding to launch the executable codes. Multigroup (MG) and continuous-energy (CE) cross sections and other data were passed between the individual executable codes by external $\mathrm{I} / \mathrm{O}$, which could require a substantial amount of clock time. In the modern version of SCALE, all self-shielding operations are consolidated into a single driver module named XSProc, and the stand-alone executable codes have been transformed into callable "computational modules." 1,2 The functions of XSProc are to (a) read input data, (b) generate inmemory data structures (objects) containing problem-definition information (compositions, cell geometries, computation options), as well as self-shielding information (MG and CE cross sections and fluxes), and (c) execute appropriate computational modules for the requested self-shielding option. Calculated results produced by one module may be stored in the internal data objects and passed to other modules through application program interfaces (APIs). At the completion of XSProc the self-shielded MG cross sections on the data objects can be passed along to transport solvers for continued execution of the control sequence or can be written to an external AMPX library file.

In the future, XSProc will be extended to parallel computations in which self-shielding calculations are done simultaneously for multiple types of unit cells. At the present time, however, XSProc is limited to serial computations; but even in serial mode it typically requires less time than older versions of SCALE to process shielded cross sections, and significant speedups have been observed for heavily I/O bound problems. Integrating the self-shielding capabilities into a single module has a number of additional benefits as well, including maintainability, extensibility, and the ability to easily replace an entire computational module with a future implementation containing new features. Additionally, the size of the problem-dependent MG library generated by XSProc may be greatly reduced compared to previous versions of SCALE because macroscopic cross sections are stored rather than a general-purpose library of microscopic data.

\subsubsection{Techniques}

XSProc integrates and enhances the capabilities previously implemented independently in BONAMI, CENTRM, PMC, WORKER, ICE, and XSDRNPM, as well as other capabilities formerly provided by MIPLIB and SCALELIB. It provides capabilities for problem-dependent temperature interpolation of both CE and MG nuclear data, calculation of Dancoff factors, and resonance self-shielding of MG cross sections using several available options. XSProc produces shielded microscopic data for each nuclide or macroscopic data for each material. Additionally, a flux-weighting spectrum can be applied to collapse cross sections to a coarser group structure and/or to integrate over volumes for homogenized cross sections. The flux-weighting spectrum can be input by the user or calculated using one-dimensional (1-D) coupled neutron/gamma transport model. These operations are performed by the sequences CSAS-MG, CSAS1, CSASI, and T-XSEC described in section 7.1.3.2.

\subsubsection{Overview of XSProc procedures}

XSProc reads the COMPOSITION and CELL DATA blocks of the SCALE input, which are described in the following sections. After reading the user input data, XSProc loads the specified MG library to be self-shielded and, depending on the selected self-shielding method, additional CE data files for nuclides appearing in the problem specification. Finally XSProc performs MG self-shielding calculations for all compositions by calling APIs to computational modules such as BONAMI (BONdarenko AMPX 


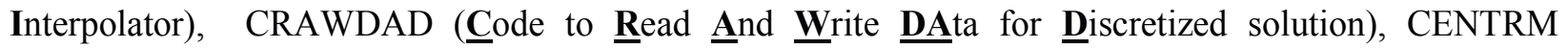
(Continuous ENergy TRansport Module), PMC (Produce Multigroup Cross sections), CHOPS (Compute HOmogenized Pointwise Stuff), CAJUN (CE AJAX UNiter), WAX (Working AJAX), XSDRNPM (X-Section Development for Reactor Nucleonics with Petrie Modifications), and/or MIXMACRO to provide a problem-dependent cross section library. Many computational modules have been modernized compared to earlier executable codes distributed in previous versions of SCALE.

Like earlier versions of SCALE, XSProc provides several options for self-shielding an input MG library. ${ }^{3}$ The first, based on the Bondarenko method, ${ }^{4}$ uses the computational module BONAMI. BONAMI is always used to compute self-shielded cross sections for all energy groups. If parm=bonami is specified, the shielded cross sections provided by BONAMI are the final values output from XSProc. However the Bondarenko method has several limitations, especially in the resolved resonance range. Therefore XSProc provides another self-shielding method, with several computation options, which often produces more accurate MG data in the resolved resonance and thermal energy ranges. If parm=centrm or parm=2region is specified on the sequence line, XSProc calls APIs for the modules CRAWDAD, CENTRM, and PMC to compute CE flux spectra for processing problem-specific, self-shielded cross sections "on the fly. ${ }^{5}$ CENTRM performs MG transport calculations in the fast and lower energy ranges, coupled to pointwise (PW) transport calculations that use CE cross sections in the resonance range. PMC uses the PW flux spectra from CENTRM to compute MG values, which replace the previous values obtained from BONAMI over the specified range of the CE calculation. The original BONAMI shielded cross sections are retained for all other groups.

The CENTRM/PMC approach is the default for criticality and lattice physics calculations, while the BONAMI-only method is default for radiation shielding calculations. The end results of an XSProc calculation are self-shielded macroscopic and/or microscopic MG cross sections stored in memory for subsequent transport calculations; or alternatively a shielded MG AMPX library can be written to an external file and saved for future use.

\subsubsection{Standard composition material processing}

A primary function of the XSProc module is to expand user input in the COMPOSITION block into nuclear number densities (nuclei/b-cm) for every nuclide in each defined mixture. Mixtures can be specified through the direct use of materials presented in the Standard Composition Library, which includes individual nuclides, elements with natural abundances, numerous compounds, alloys and mixtures found in engineering practice, as well several variations of fissile solutions. Additionally, users may define their own materials as atom percent or weight percent combinations. Nuclear masses and theoretical densities are provided in the Standard Composition Library, and methods are available to determine equilibrium states for fissile solutions. Input options for composition data are described in Section 7.1.3.3 with several examples provided in Appendix A.

\subsubsection{Unit cells for MG resonance self-shielding}

XSProc utilizes a unit cell description to provide information for resonance self-shielding calculations of the input mixtures. As many unit cells as needed to describe the problem may be specified; however, each mixture (other than 0 for a void mixture) can appear only in one unit cell in the CELLDATA block. If a nuclide appears in more than one mixture, multiple sets of self-shielded cross sections are calculated for the nuclide - one for each mixture in each unit cell. Four types of cells are available for self-shielding 
calculations: INFHOMMEDIUM, LATTICECELL, MULTIREGION, and DOUBLEHET. The default calculation type is CENTRM/PMC for CSAS (see chapter 2), TRITON, (see chapter 3) and TSUNAMI (see chapter 6) sequences and BONAMI for MAVRIC. All materials not specified in a unit cell are treated as infinite homogeneous media and shielded with BONAMI only, unless the mixture contains a fissionable nuclide, in which case an infinite medium CENTRM/PMC model is used. Note that previous versions of SCALE used infinite medium CENTRM/PMC calculations for all unassigned mixtures. The default type of self-shielding calculation can be overridden, as described in Section 7.1.3.2. The following is a brief description of the types of unit cells that can be input in CELLDATA and the computation procedures used.

\section{$\underline{\text { INFHOMMEDIUM (infinite homogeneous medium) Treatment }}$}

The INFHOMMEDIUM treatment is best suited for large masses of materials where the size of each material is large compared with the average mean-free path of the material or where the fraction of the material that is a mean-free path from the surface of the material is very small. When INFHOMMEDIUM cell is specified, the material in the unit cell is treated as an infinite homogeneous lump. Systems composed of small fuel lumps or resonance nuclides sandwiched between moderating regions should not be treated as infinite homogeneous media. In these cases a MULTIREGION or LATTICECELL geometry should be used.

\section{$\underline{\text { LATTICECELL Treatment }}$}

The LATTICECELL model is appropriate for arrays of resonance absorber mixtures-with or without clad - arranged in a square or a triangular pitch configuration within a moderator. Annular fuel (e.g., with an internal moderator in the center) can also be addressed. Input data for the LATTICECELL treatment are described in Section 7.1.3.5. Self-shielded cross sections are generated for each material zone in a unit cell of the lattice. If a nuclide appears in more than one zone, self-shielded cross sections are produced for each zone where the nuclide is present. Limitations of the LATTICECELL treatment are listed below.

1. The cell description is limited to unit cells for arrays of spherical, plate (slab), or cylindrical fuel bodies. In the case of cylindrical pins in a square-pitch lattice, the default (parm=centrm) selfshielding calculation uses the CENTRM method of characteristics (MoC) option to represent the 2D rectangular unit cell with reflected boundary conditions. By default, self-shielding for all other arrays uses a CENTRM 1D $\mathrm{S}_{\mathrm{N}}$ calculation for the unit cell (spherical and cylindrical geometries use Wigner-Seitz cells). If parm=bonami is specified, heterogeneous self-shielding effects are treated by equivalence theory. ${ }^{3}$ The computation option parm=2region, described in Section 7.1.3.1, can also be used for self-shielding lattice cells.

2. Only predefined choices of cell configurations are available. The available options are described in detail in Section 7.1.3.5.

3. The basic treatment for LATTICECELL assumes an infinite, uniform array of unit cells. This assumption is a good approximation for interior fuel regions within a large, uniform array. The approximation becomes less rigorous for fuel regions on the periphery of the array or adjacent to a nonuniformity (e.g., control rod, water hole, etc.) in the lattice. For some cases it may be desirable to address this issue by specifying a different lattice cell for this type of fuel pin and using a modified procedure to define an effective unit cell, as described below. 
***** LATTICECELL treatment for nonuniform arrays.

Nonuniform lattice effects may be treated in CENTRM calculation by specifying the keyword DAN2PITCH=dancoff in the optional CENTRM DATA (see section 7.1.3.9). In this approach, the SCALE standalone code MCDancoff must be run prior to the self-shielding calculation in order to compute Dancoff factors for the fuel regions of interest in the nonuniform lattice configuration. MCDancoff performs a simplified one-group Monte Carlo calculation to compute Dancoff factors for complex geometries (see section 7.8). The Dancoff value for the fuel region of interest is assigned to the DAN2PITCH keyword in the input for the corresponding cell. Using an iterative procedure, CENTRM computes the pitch of a uniform lattice that has the same Dancoff value as the nonuniform lattice.

\section{MULTIREGION Treatment}

The MULTIREGION treatment is appropriate for 1-D geometric regions where the geometry effects may be important, but the limited number of zones and boundary conditions in the LATTICECELL treatment are not applicable. The MULTIREGION unit cell allows more flexibility in the placement of the mixtures but requires all regions of the cell to have the same geometric shape (i.e., slab, cylinder, sphere, buckled slab, or buckled cylinder). Lattice arrangements can be approximated by specifying a non-vacuum boundary condition on the outer boundary. See section 7.1.3.6 for more details. Limitations of the MULTIREGION cell treatment are listed below.

1. A MULTIREGION cell is limited to a 1-D approximation of the system being represented. An exact 1D model can be defined for the following multizone geometries with vacuum boundary conditions: spheres, infinitely long cylinders, and slabs; and for an infinite array of slabs with reflected or periodic boundaries.

2. The shape of the outer boundary of the MULTIREGION cell is the same as the shape of the inner regions. Cells with curved outer surfaces cannot be stacked physically to create arrays; however, arrays can be approximated by a Wigner-Sietz cell with a white outer boundary condition, where the outer radius is defined to preserve the area of the true rectangular or hexagonal unit cell.

3. Boundary conditions available in a MULTIREGION problem include vacuum (eliminated at the boundary), reflected (reflected about the normal to the surface at the point of impact), periodic (a particle exiting the surface effectively enters an identical cell having the same orientation and continues traveling in the same direction), and white (isotropic return about the point of impact). Reflected and periodic boundary conditions on a slab can represent real physical situations but are not valid on a curved outer surface. A single, non-interacting cell has a vacuum outer boundary condition. If the cell outer boundary condition is not a vacuum boundary, the unit cell approximates some type of array.

4. When using the CENTRM/PMC self-shielding method, the MULTIREGION cell model must include fissionable material. This can be accomplished by adding a trace amount of a fissionable material to one or more mixtures, or by modeling a region of homogenized fuel and water, or by adding a thin (e.g., 1e-6 cm-thick) layer containing at least a trace of a fissionable nuclide on the periphery of the problem. 


\section{DOUBLEHET Treatment}

DOUBLEHET cells use a specialized CENTRM/PMC calculational approach to treat resonance selfshielding in "doubly heterogeneous" systems. The fuel for these systems typically consists of small, heterogeneous, spherical fuel particles (grains) embedded in a moderator matrix to form the fuel compact. The fuel-grain/matrix compact constitutes the first level of heterogeneity. Cylindrical(rod). spherical (pebble), or slab fuel elements composed of the compact material are arranged in a moderating medium to form a regular or irregular lattice, producing the second level of heterogeneity. The fuel elements are also referred to as "macro cells." Advanced reactor fuel designs that use TRISO (tri-material, isotopic) or fully ceramic microencapsulated (FCM) fuel require the DOUBLEHET treatment to account for both levels of heterogeneities in the self-shielding calculations. Simply ignoring the double-heterogeneity by volumeweighting the fuel grains and matrix material into a homogenized compact mixture can result in a large reactivity bias.

In the DOUBLEHET cell input, keywords and the geometry description for grains are similar to those of the MULTIREGION treatment, while keywords and the geometry for the fuel element (macro-cell) are similar to those of the LATTICECELL treatment. The following rules apply to the DOUBLEHET cell treatment and must be followed. Violation of any rules may cause a fatal error.

1. As many grain types as needed may be specified for each unique fuel element. Note that grain type is different from the number of grains of a certain type. For example, a fuel element that contains both $\mathrm{UO}_{2}$ and $\mathrm{PuO}_{2}$ grains has two grain types. The same fuel element may contain $10000 \mathrm{UO}_{2}$ grains and $5000 \mathrm{PuO}_{2}$ grains. In this case, the number of grains of type $\mathrm{UO}_{2}$ is 10000 , and the number of grains of type $\mathrm{PuO}_{2}$ is 5000 .

2. As many fuel elements as needed may be specified, each requiring its own DOUBLEHET cell. This may be the case for systems with many fuel elements at different fuel enrichments, burnable poisons, etc. Each fuel element may have one or more grain types.

3. Since the grains are homogenized into a new mixture to be used in the fuel element (macro-cell) cell calculation, a unique fuel mixture number must be entered. XSProc creates a new material with the new mixture number designated by the keyword fuelmix=, containing all the nuclides that are homogenized. The user must assign the new mixture number in the transport solver geometry (e.g., KENO) input unless a cell-weighted mixture is created.

4. The type of lattice or array configuration for the fuel-element may be spheres on a triangular pitch (SPHTRIANGP), spheres on a square pitch (SPHSQUAREP), annular spheres on a triangular pitch (ASPHTRIANGP), annular spheres on a square pitch (ASPHSQUAREP), cylindrical rods on a triangular pitch (TRIANGPITCH), cylindrical rods on a square pitch (SQUAREPITCH), annular cylinderical rods on a triangular pitch (ATRIANGPITCH), annular cylindrical rods on a square pitch (ASQUAREPITCH), a symmetric slab (SYMMSLABCELL), or an asymmetric slab (ASYMSLABCELL).

5. If there is only one grain type for a fuel element, the user must enter either the pitch, the aggregate number of particles in the element, or the volume fraction for the grains. The code needs the pitch and will directly use it if entered. If pitch is not given, then the volume fraction (if given) is used to calculate the pitch. If neither the pitch nor the volume fraction is given, then the number of particles is used to calculate the pitch and the volume fraction. The user should only enter one of these items.

If the fuel matrix contains more than one grain type, all types are homogenized into a single mixture for the compact. As for the one grain type case, the pitch is needed for the spherical cell 
calculations. However, the pitch by itself is not sufficient to perform the homogenization. Since each grain's volume is known (grain dimensions must always be entered), entering the number of particles for each grain type essentially provides the total volume of each grain type and therefore enables the calculation of the volume fraction and the pitch. Likewise, entering the volume fraction for each grain type essentially provides the total volume of each grain type and therefore enables the calculation of the number of particles and the pitch. Therefore, one of these two quantities must be entered for multiple grain types. In these cases, since pitch is not given, the available matrix material is distributed around the grains of each grain type proportional to the grain volume and is used to calculate the corresponding pitch. Over-specification is allowed as long as the values are not inconsistent to greater than $0.01 \%$.

6. For cylindrical rods and for slabs, fuel height must also be specified. For slabs the slab width must also be specified.

7. The CENTRM calculation option must be $S_{n}$.

\subsubsection{Cell weighting of MG cross sections}

Cell-weighted self-shielded cross sections are created when CELLMIX $=$ is specified in a LATTICECELL or MULTIREGION cell input. In this case, after finishing the self-shielding calculations for all mixtures in the cell, XSProc calls the computational module XSDRNPM, which solves the 1-D MG transport equation to obtain $\mathrm{k}_{\infty}$ and space-dependent MG fluxes for the cell. The resultant fluxes are used to compute $\mathrm{MG}$ flux disadvantage factors for processing cell-weighted cross sections of all nuclides in the cell. When the cell-weighted cross sections are used with homogenized number densities of the cell nuclides, the reaction rates of the homogenized mixture preserve the spatially averaged reactions rates of the heterogeneous configuration. The user must input a new mixture ID to identify the homogenized mixture associated with the cell-weighted cross sections.

This homogenized mixture should not be used in the heterogeneous geometry data for other transport codes such as KENO, NEWT, etc. Instead, the cell-homogenized mixture that is created should be used at the location of the original cell. Also, cell weighted homogenized cross sections should not be used in MG sensitivity data calculations performed using the TSUNAMI sequences. 


\subsubsection{XSPROC Input Data Guide}

XSProc input data are entered in free form, allowing alphanumeric data, floating-point data, and integer data to be entered in an unstructured manner. Up to 252 characters per line are allowed. Data can usually start or end in any column. Each data entry must be followed by one or more blanks to terminate the data entry. For numeric data, either a comma or a blank can be used to terminate each data entry. Integers may be entered for floating values. For example, 10 will be interpreted as 10.0 if a floating point value is required. Imbedded blanks are not allowed within a data entry unless an E precedes a single blank as in an unsigned exponent in a floating-point number. For example, 1.0E 4 would be correctly interpreted as $1.0 \times 10^{4}$. A number with a negative exponent must include an "E". For example 1.0-4 cannot be used for $1.0 \mathrm{E}-4$.

The word "END" is a special data item. An END may have a name or label associated with it. The name or label associated with an END is separated from the END by a single blank and is a maximum of 12 characters long. At least two blanks or a new line MUST follow every labeled and unlabeled END. WARNING: It is the user's responsibility to ensure compliance with this restriction. Failure to observe this restriction can result in the use of incorrect or incomplete data without the benefit of warning or error messages.

Multiple entries of the same data value can be achieved by specifying the number of times the data value is to be entered, followed by either $\mathrm{R}, *$, or $\$$, followed by the data value to be repeated. Imbedded blanks are not allowed between the number of repeats and the repeat flag. For example, $5 \mathrm{R} 12,5 * 12,5 \$ 12$, or 5R 12, etc., will enter five successive 12's in the input data. Multiple zeros can be specified as $\mathrm{nZ}$ where $\mathrm{n}$ is the number of zeroes to be entered.

\subsubsection{XSProc data checking and resonance processing options}

To check the XSProc input data, run CSAS-MG and specify PARM=CHECK or PARM=CHK after the sequence specification as shown below.

\section{$=\mathrm{CSAS}-\mathrm{MG} \quad \mathrm{PARM}=\mathrm{CHK}$}

In this case the actual XSProc cross section processing calculations are not performed. The input data are checked, the problem description is printed, appropriate error and warning messages are printed, and a table of additional data is printed.

Resonance processing will automatically be performed by the default method for the sequence selected. The default methods are CENTRM/PMC for CSAS, TRITON, and TSUNAMI sequences and BONAMI for the MAVRIC sequences. Alternatively, a resonance processing procedure may be chosen by entering PARM=option, where option CENTRM selects the recommended CENTRM/PMC transport method for each cell type, option 2REGION selects the CENTRM/PMC two-region calculation, and option BONAMI applies full range Bondarenko factors to all energy groups without utilizing CENTRM/PMC. For example, to run CSAS1X sequence using only BONAMI for self-shielding, rather than the default CENTRM/PMC method, enter the computational sequence specification shown below.

$$
=\text { CSAS1X } \quad \text { PARM=BONAMI }
$$

Multiple PARM options are specified by enclosing parameters in parenthesis, such as

$$
=\mathrm{CSAS} 1 \mathrm{X} \quad \mathrm{PARM}=(\mathrm{CHK}, \mathrm{BONAMI})
$$

XSProc resonance self-shielding options are summarized below. 


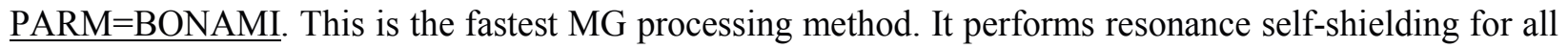
energy groups using the Bondarenko method. BONAMI computes the appropriate background cross section of a given unit cell and then interpolates the corresponding shielding factor from Bondarenko factors on the MG library. Dancoff factors needed to evaluate the background cross section for lattices are computed internally, but these can be overridden by input values in the MORE DATA block. More details on this method are given in the BONAMI section of the manual.

$\underline{\text { PARM }=\text { CENTRM. }}$. This executes the CENTRM/PMC modules to process shielded MG cross sections using CE flux spectra calculated with the recommended type of CE transport solver for the designated type of cell. The CENTRM-recommended CE transport solvers are (a) infinite homogeneous medium calculation for INFHOMMEDIUM cells; (b) 2D MoC transport calculation for a LATTICECELL consisting of cylindrical fuel pins in a square lattice; and (c) 1-D discrete $S_{n}$ transport for all other LATTICECELLs and for all MULTIREGION cells. The recommended type of transport solver can be overridden for individual cells, as well as for selected energy ranges, by using the CENTRM DATA block described in Section 7.1.3.9.

\section{PARM=2REGION.}

The CENTRM two-region (2R) option computes the PW flux using a simplified collision probability method for an absorber (e.g., fuel) region surrounded by an external moderator region which has an asymptotic energy spectrum. To account for the heterogeneous effects of a lattice, a correction known as the Dancoff factor is applied to the escape probabilities in the $2 \mathrm{R}$ calculation (see the CENTRM chapter of the SCALE manual). These Dancoff factors are calculated internally by XSProc for a uniform array of mixtures in slab, spherical, or cylindrical geometries. These mixture-dependent Dancoff factors can be modified by user input using the DAN parameters contained in the MORE DATA block, as defined in Section 7.1.3.8.

Note on CENTRM/PMC self-shielding options:

The energy range of the CENTRM flux calculation is subdivided into three sections: fast, PW, and low energy. PMC only computes self-shielded cross sections for groups within the PW range defined by parameters demax and demin, which, respectively, define the upper and lower energies of the CENTRM PW flux calculation. Problem-dependent cross sections for groups in the fast and low energy ranges are obtained with the more approximate BONAMI method. Default values for parameters demax and demin are defined appropriately for self-shielding of important resonance materials in thermal reactor systems. The PW self-shielding range can be extended or decreased for individual cells by modifying these parameters using CENTRM DATA.

\subsubsection{XSProc input data}

The types of input data required for XSProc are given in Table 7.1.1, and individual entries are explained in the text following the table. The title, cross section library name (either CE or MG), and standard composition specification data (READ COMP input block) are required for all sequences that use XSProc. The name of the cross section library is used to determine if the transport solver is executed using CE or MG data (e.g., CE or MG KENO calculations). The unit cell descriptions (READ CELL input block) are only used for MG self-shielding calculations. If the specified sequence executes in CE mode, the cell data input can be omitted, or it will be skipped if present. If the cell data information is omitted for MG calculations, all mixtures are self-shielded using the infinite medium approximation.

There are seven standard SCALE sequences that run just XSProc, and produce a MG cross section library or libraries. 
$=$ XSPROC produces three libraries with an optional fourth library.

- sysin.microLib is a self-shielded library of the individual nuclides in the problem for use in a later transport calculation,

- sysin.macroLib is a self-shielded library of the mixture cross sections in the problem for use in a later transport calculation,

- sysin.smallMicroLib is a self-shielded library of specific reaction rate cross sections and the elastic and total inelastic scattering transfer matrices for later use in calculating reaction rates and sensitivity values, and

- sysin.xsdrnWeightedLib is an optional library produced if the input specifies having XSDRN do a weighting calculation. This can be a cell weighted and/or a group collapse calculation. The library can be either individual nuclides or mixtures, depending on input.

$=\boldsymbol{C S} \boldsymbol{A S}-\boldsymbol{M G}$ produces an ft04f001 library that is equivalent to the sysin.microLib. With appropriate input it can also produce an ft03f001 which is equivalent to sysin.xsdrnWeightedLib above.

$=\boldsymbol{C S A S I}$ or $=\boldsymbol{C S A S I X}$ produce an ft04f001 library that is equivalent to sysin.microLib, and an ft02f001 library that is equivalent to sysin.macroLib. CSASIX will run an XSDRN on the first cell without any MOREDATA input. With appropriate input they both can produce an ft03f001 that is the equivalent of sysin.xsdrnWeightedLib.

$=C S A S 1$ or $=C S A S 1 X$ produce an ft04f001 library that is the equivalent of sysin.microLib. Both sequences will run an XSDRN on the first cell. With appropriate input, they both can produce an ft03f001 that is the equivalent of sysin.xsdrnWeightLib.

$=\boldsymbol{T}-X S E C$ produces an $\mathbf{f t 0 4 f 0 0 1 ~ l i b r a r y ~ t h a t ~ i s ~ e q u i v a l e n t ~ t o ~ s y s i n . m i c r o L i b . ~}$ 
Table 7.1.1. Outline of XSProc input data

\begin{tabular}{|c|c|c|c|}
\hline $\begin{array}{c}\text { Data } \\
\text { Position }\end{array}$ & Type of Data & Data Entry & Comments \\
\hline 1 & Title & Enter title & Limit to 80 characters \\
\hline 2 & Cross section library name & & $\begin{array}{l}\text { The currently available libraries are listed in } \\
\text { the table Standard SCALE cross-section } \\
\text { libraries of the XSLib chapter. }\end{array}$ \\
\hline 3 & $\begin{array}{l}\text { Standard composition } \\
\text { specification data }\end{array}$ & Enter the appropriate data & $\begin{array}{l}\text { Begin this data block with } \\
\text { READ COMP } \\
\text { and terminate with } \\
\text { END COMP. } \\
\text { See Section 7.1.3.3. }\end{array}$ \\
\hline \multirow[t]{6}{*}{4} & $\begin{array}{l}\text { Unit cell(s) description } \\
\text { for } \mathrm{MG} \text { calculations } \\
\text { only }\end{array}$ & & $\begin{array}{l}\text { Begin this data block with } \\
\text { READ CELL (or CELLDATA) }\end{array}$ \\
\hline & $\begin{array}{l}\text { a. Type of self shielding } \\
\text { calculation }\end{array}$ & $\begin{array}{l}\text { INFHOMMEDIUM } \\
\text { LATTICECELL } \\
\text { MULTIREGION } \\
\text { DOUBLEHET }\end{array}$ & $\begin{array}{l}\text { These are the available options. } \\
\text { See the explanation in Section 7.1.3.2. }\end{array}$ \\
\hline & $\begin{array}{l}\text { b. Unit cell geometry } \\
\text { specification }\end{array}$ & Enter the appropriate data & $\begin{array}{l}\text { See Section 7.1.3.4 for INFHOMMEDIUM. } \\
\text { See Section 7.1.3.5 for LATTICECELL. } \\
\text { See Section 7.1.3.6 for MULTIREGION. } \\
\text { See Section 7.1.3.7 for DOUBLEHET. }\end{array}$ \\
\hline & $\begin{array}{l}\text { Optional MORE } \\
\text { parameter data }\end{array}$ & Enter the desired data & $\begin{array}{l}\text { Begin this data block with } \\
\text { MORE DATA (or MOREDATA) } \\
\text { and terminate with } \\
\text { END MORE (or END MOREDATA). } \\
\text { Use only if MORE parameter data are to be } \\
\text { entered; otherwise, omit these data entirely. } \\
\text { See Section 7.1.3.8. }\end{array}$ \\
\hline & $\begin{array}{l}\text { d. Optional CENTRM } \\
\text { parameter data }\end{array}$ & Enter the desired data & $\begin{array}{l}\text { Begin this data block with } \\
\text { CENTRM DATA (or CENTRMDATA) } \\
\text { and terminate with } \\
\text { END CENTRM (or END CENTRMDATA). } \\
\text { Use only if CENTRM parameter data are to be } \\
\text { entered; otherwise, omit these data entirely. }\end{array}$ \\
\hline & e. End of unit cell data & & $\begin{array}{l}\text { Terminate with END CELL (or END } \\
\text { CELLDATA) }\end{array}$ \\
\hline
\end{tabular}

Repeat positions $4 \mathrm{a}-4 \mathrm{~d}$ as needed to specify all unit cells. Position 4 data are applicable to the MG calculations only. 
1. TITLE. An 80-character maximum title is required. The title is the first 80 characters of the XSPROC data.

2. CROSS SECTION LIBRARY NAME. This item specifies the cross section library that is to be used in the calculation. See Table Standard SCALE cross-section libraries in the XSLIB chapter of the SCALE manual for a discussion of the available libraries.

3. The keywords READ COMP followed by the standard compositions specifications. These data are used to define mixtures used in the problem. See Section 7.1.3.3 and Table 7.1.2 for a description of the standard composition specification data. These data are required for every problem. After all mixtures have been entered, the keywords END COMP must be entered.

4. The keywords READ CELLDATA followed by the input describing each unit cell as defined below. After all unit cells are described, the keywords END CELLDATA terminate this input block.

a. TYPE OF CALCULATION. The options are INFHOMMEDIUM, LATTICECELL, MULTIREGION, DOUBLEHET, or nothing. A description of these cell types and the associated computational methods are provided in Section 7.1.2.3. If all input mixtures are to be treated as infinite homogeneous media, the CELLDATA block can be omitted. In this case the self-shielding calculations will not account for any geometrical effects, so users should be careful in applying this approach. Similarly, mixtures not explicitly assigned to a cell are treated as infinite homogeneous media in the manner discussed in Section 7.1.2.3.

b. CELL GEOMETRY SPECIFICATION. See Section 7.1.3.4 and Table 7.1.3 for an explanation of the optional unit cell data associated with an INFHOMMEDIUM problem. See Section 7.1.3.5 for an explanation of the data associated with LATTICECELL problems. Section 7.1.3.6 explains the data required for a MULTIREGION problem. Section 7.1.3.7 explains the required data for a DOUBLEHET problem. The DOUBLEHET input may be thought of as a combination of MULTIREGION input for the fuel grains and LATTICECELL input for the fuel element.

c. OPTIONAL MORE PARAMETER DATA. This option allows certain defaulted parameters to be re-specified by the user. This block begins with MORE DATA and is used by XSDRN. These data apply only to the unit cell immediately preceding them. Data placed prior to all unit cell data apply to all materials not listed in any unit cell and are treated as infinite homogeneous media. Omit these data unless they are needed. This block ends with END MORE. See Section 7.1.3.8.

d. OPTIONAL CENTRM PARAMETER DATA. This optional data block begins with CENTRM DATA and ends with END CENTRM. These data allow the user to override default data for CENTRM and PMC. These data apply only to the unit cell immediately preceding them. Data placed prior to all unit cell data apply to all materials not listed in any unit cell and are treated as infinite homogeneous media.

\subsubsection{Standard composition specification data}

Mixtures utilized in the problem are defined using standard composition specification data. The standard composition input begins with the keywords READ COMP, followed by standard composition specifications for all mixtures in the problem. When all mixtures have been described, enter the words END COMP to signal the completion of this block of data. XSProc computes macroscopic cross sections for all mixtures defined in the COMP block. 
The required input for the standard composition specification data varies, depending on the type of standard composition material. However, every standard composition specification must include the following:

1. a standard composition material name.

2. a mixture number (MX) that contains this material, and

3. a terminator for the standard composition specification data (enter the word END).

The types of standard compositions in SCALE are (a) basic mixtures, (b) fissile solutions, (c) chemical compounds, and (d) alloys. The four general options for inputting these types of data are shown in Table 7.1.2. For some cases, more than one option could possibly be used to specify the mixture. The user may select whichever options are most convenient to define a particular mixture, and these may be entered in any order.

Table 7.1.2. Outline of standard composition specification options

(Mixtures can be defined using one or more of these options in any order)

\begin{tabular}{|c|c|}
\hline $\begin{array}{l}\text { Input data } \\
\text { name }\end{array}$ & Comments \\
\hline READ COMP & $\begin{array}{l}\text { Enter once for a problem. Enter the words READ COMP prior to entering any standard } \\
\text { composition data. }\end{array}$ \\
\hline sc & $\begin{array}{l}\text { This option is used for defining basic mixtures. Enter one of the alphanumeric identifiers, } \\
\text { symbols, or names from Standard Composition Library tables Isotopes in standard composition } \\
\text { library, Isotopes and their natural abundances, Elements and special nuclide symbols, } \\
\text { Compounds, or Alloys and mixtures in place of SC. This indicates the isotope, nuclide, } \\
\text { compound, or alloy that will make up this standard composition. See Table } 7.1 .2 \text { a for additional } \\
\text { required and optional data for each standard composition. }\end{array}$ \\
\hline SOLUTION & $\begin{array}{l}\text { This option is used to specify a fissile solution mixture. See Table } 7.1 .2 \mathrm{~b} \text { for additional required } \\
\text { and optional data for each solution. End the data with an END. }\end{array}$ \\
\hline АТОМ & $\begin{array}{l}\text { This option creates a chemical compound mixture composed of the specified nuclide in } \\
\text { the compound. Each nuclide is entered followed by the relative number of atoms of the nuclide } \\
\text { in the compound. All compounds must begin with the four letters ATOM followed by up to } \\
\text { eight additional alphanumeric characters. See Table } 7.1 .2 \mathrm{c} \text { for additional required and optional } \\
\text { data for each compound. }\end{array}$ \\
\hline WTPT & $\begin{array}{l}\text { This option creates a mixture/alloy composed of the specified nuclides in the mixture/alloy. Each } \\
\text { nuclide is entered followed by the weight percent of the nuclide in the mixture/alloy. All } \\
\text { mixture/alloys must begin with the four letters WTPT followed by up to eight additional } \\
\text { alphanumeric characters. See Table } 7.1 .2 \mathrm{~d} \text { for additional required and optional data for each } \\
\text { arbitrary physical mixture or alloy. }\end{array}$ \\
\hline END COMP & $\begin{array}{l}\text { Enter once for a problem. Enter the exact words END COMP when all the standard composition } \\
\text { components have been described. At least two blanks or a new line must follow the words END } \\
\text { COMP prior to continuing data entry. }\end{array}$ \\
\hline
\end{tabular}


Names of the standard composition materials (the alphanumeric identifiers) appearing in the COMP block input must be selected from the tables of elements, compounds, solutions, and alloys given in the SCALE manual section describing the Standard Composition Library. An error message will be printed if the user enters an invalid standard composition material name or if any isotopes in the compound do not exist in the specified library

Input data to define each of the standard composition types in Table 7.1.2 are summarized in Tables 7.1.2a - 7.1.2d. Optional input is indicated by curly brackets \{\} . Since some of the input is not keyword based, the order of entries is important in the standard composition specification. The temperature specification is used for Doppler broadening and/or determination of the proper thermal scattering data. Input material densities are not modified for temperature effects. Additional description of the standard composition input for each type of material is given following all the tables. As in the tables, input parameters enclosed by curly brackets \{\} indicate that these are optional. 
Table 7.1.2.a. Standard composition specification for basic mixtures

\begin{tabular}{|c|c|c|c|c|}
\hline $\begin{array}{l}\text { Entry } \\
\text { no. }\end{array}$ & $\begin{array}{l}\text { Data } \\
\text { name }\end{array}$ & Data type & $\begin{array}{l}\text { Entry } \\
\text { requirement }\end{array}$ & Comment \\
\hline 1 & sc & $\begin{array}{l}\text { Standard } \\
\text { composition } \\
\text { name }\end{array}$ & Always & $\begin{array}{l}\text { Enter one of the alphanumeric identifiers, symbols, or names from } \\
\text { Tables Isotopes in standard composition library, Isotopes and } \\
\text { their natural abundances, Elements and special nuclide symbols, } \\
\text { Compounds, or Alloys and mixtures in the STDCMP chapter in } \\
\text { place of sc. This indicates the isotope, nuclide, compound, or alloy } \\
\text { that will make up this standard composition. This entry is required. }\end{array}$ \\
\hline 2 & $m x$ & $\begin{array}{l}\text { Mixture ID } \\
\text { number }\end{array}$ & Always & $\begin{array}{l}\text { Skip at least one blank after SC prior to entering the mixture } \\
\text { number. This must be an integer greater than zero. This entry is } \\
\text { required. }\end{array}$ \\
\hline 3 & $\mathbf{D E N}=$ roth & Density & Optional & $\begin{array}{l}\text { If the density of a basic standard composition (roth) is to be } \\
\text { entered, add DEN = roth, where roth is the density in } \mathrm{g} / \mathrm{cm}^{3} \text {, } \\
\text { following the mixture ID number with at least one space between } \\
m x \text { and the keyword DEN. }\end{array}$ \\
\hline 4 & $\{\mathbf{V F}=\} v f$ & $\begin{array}{l}\text { Density } \\
\text { multiplier }\end{array}$ & $\begin{array}{l}\text { See } \\
\text { comment }\end{array}$ & $\begin{array}{l}\text { Default value is } 1 \text {. Enter the density multiplier (density fraction, } \\
\text { volume fraction, or a combination). VF }=0 \text { indicates that syntax } 2 \\
\text { is to be used and that the next number entered will be ADEN. In } \\
\text { this case entries } 7-8 \text { are omitted. }\end{array}$ \\
\hline 5 & aden & $\begin{array}{l}\text { Number } \\
\text { density }\end{array}$ & $\mathbf{V F}=0$ & $\begin{array}{l}\text { Atom density of the nuclide in atoms / barn-cm. This can only be } \\
\text { entered for elements and isotopes. See chapter STDCMP tables } \\
\text { Isotopes in standard composition library, Isotopes and their } \\
\text { natural abundances, and Elements and special nuclide symbols. }\end{array}$ \\
\hline 6 & temp & $\begin{array}{l}\text { Temperature } \\
\qquad(\mathrm{K})\end{array}$ & $\begin{array}{l}\text { See } \\
\text { comment }\end{array}$ & $\begin{array}{l}\text { Default value is } 300 \mathrm{~K} \text {. This entry may be omitted if the default } \\
\text { temperature is acceptable and iza / wpt data are not entered. }\end{array}$ \\
\hline 7 & $i z a$ & $\begin{array}{l}\text { Isotope's ZA } \\
\text { number }\end{array}$ & $\mathbf{V F} \neq 0$ & $\begin{array}{l}\text { Enter for each isotope in a multiple isotope nuclide. Omit if VF = } \\
0 \text { or the default values are acceptable. Entries } 7 \text { and } 8 \text { are entered } \\
\text { in pairs until each isotope in the nuclide is defined. See STDCMP } \\
\text { chapter tables Elements and special nuclide symbols, Compounds, } \\
\text { and Alloys and mixtures for isotope IDs contained in nuclides, } \\
\text { compounds, and alloys. }\end{array}$ \\
\hline 8 & $w t p$ & $\begin{array}{l}\text { weight } \\
\text { percent of } \\
\text { isotope }\end{array}$ & $\mathbf{V F} \neq 0$ & $\begin{array}{l}\text { Enter the weight percent of the isotope in the nuclide. Omit if VF } \\
=0 \text { or the default values are acceptable. For each multiple isotope } \\
\text { nuclide, the weight percents of the isotopes must sum to } 100 . \\
\text { Entries } 7 \text { and } 8 \text { are entered in pairs until each isotope in the nuclide } \\
\text { is defined. }\end{array}$ \\
\hline 9 & END & $\begin{array}{l}\text { Terminate } \\
\text { the standard } \\
\text { composition }\end{array}$ & Always & $\begin{array}{l}\text { This terminates the data for a standard composition. Enter the } \\
\text { keyword END to terminate standard composition. A tag, up to } \\
12 \text { characters long, may follow the keyword END preceded by a } \\
\text { single blank. At least two blanks or an end of line must separate } \\
\text { this entry from the next entry. }\end{array}$ \\
\hline
\end{tabular}


Table 7.1.2.b. Standard composition specification for solutions

\begin{tabular}{|c|c|c|c|c|}
\hline $\begin{array}{l}\text { Entry } \\
\text { no. }\end{array}$ & Data name & Data type & $\begin{array}{l}\text { Entry } \\
\text { requirement }\end{array}$ & Comment \\
\hline 1 & SOLUTION & keyword & Always & $\begin{array}{l}\text { Identifies a new fissile solution. } \\
\text { See the Standard Composition } \\
\text { Library documentation. }\end{array}$ \\
\hline 2 & $\{\mathbf{M I X}=\} m x$ & mixture number & Always & $\begin{array}{l}\text { If the mixture number is not } \\
\text { keyworded, it must immediately } \\
\text { follow the SOLUTION } \\
\text { keyword. }\end{array}$ \\
\hline$* 3 a$ & $\mathbf{R H O}[$ name $]=r h o$ & $\begin{array}{c}\text { metal } \\
\text { density }\end{array}$ & Optional & $\begin{array}{l}\text { The density of the metal in } \\
\text { name in grams/liter }\end{array}$ \\
\hline$* 3 b$ & MOLAR $[$ name $]=$ molar & molarity & Optional & $\begin{array}{l}\text { moles of name per liter of } \\
\text { solution }\end{array}$ \\
\hline$* 3 c$ & $\begin{array}{l}\text { MASSFRAC }[\text { name }]= \\
\text { massfrac }\end{array}$ & relative density & Optional & $\begin{array}{l}\text { grams of metal in name/gram of } \\
\text { solution }\end{array}$ \\
\hline$* 3 \mathrm{~d}$ & $\begin{array}{l}\text { MOLEFRAC }[\text { name }]= \\
\text { molefrac }\end{array}$ & $\begin{array}{l}\text { fractional } \\
\text { molarity }\end{array}$ & Optional & $\begin{array}{l}\text { moles of name per mole of } \\
\text { solution }\end{array}$ \\
\hline$* 3 \mathrm{e}$ & $\begin{array}{l}\text { MOLALITY }[\text { name }]= \\
\text { molality }\end{array}$ & molality & Optional & $\begin{array}{l}\text { moles of name per kilogram of } \\
\text { water }\end{array}$ \\
\hline 4 & DENSITY $=$ density & solution density & Optional & $\begin{array}{l}\text { density of the solution in } \\
\text { grams/milliliter }\end{array}$ \\
\hline 5 & $\begin{array}{l}\text { TEMPERATURE }= \\
\text { temperature }\end{array}$ & temperature & Optional & $\begin{array}{l}\text { temperature of the solution in } \\
\text { Kelvin }\end{array}$ \\
\hline 6 & VOL_FRAC $=v f$ & $\begin{array}{l}\text { density } \\
\text { multiplier }\end{array}$ & Optional & $\begin{array}{l}\text { an overall, after the fact } \\
\text { multiplier of the solution density }\end{array}$ \\
\hline 7 & END SOLUTION & keyword & Always & ends the solution input \\
\hline
\end{tabular}

"Nuclides that occur in an item 3 will, by default, have naturally occurring isotopics. If this is not appropriate, the desired isotope and weight percent of each isotope making up the nuclide can be input in pairs following the value associated with the specified item 3. 
Table 7.1.2.c. Standard composition specification for chemical compounds

\begin{tabular}{|c|c|c|c|c|}
\hline $\begin{array}{l}\text { Entry } \\
\text { no. }\end{array}$ & $\begin{array}{l}\text { Data } \\
\text { name }\end{array}$ & Data type & $\begin{array}{l}\text { Entry } \\
\text { requirement }\end{array}$ & Comment \\
\hline 1 & АТОМ & $\begin{array}{l}\text { Arbitrary } \\
\text { compound } \\
\text { name }\end{array}$ & Always & $\begin{array}{l}\text { Enter the four characters ATOM followed by up to } \\
12 \text { additional alphanumeric characters in place of ATOM. As } \\
\text { many compounds as required may be entered, but each must } \\
\text { have a unique name. This entry is required. }\end{array}$ \\
\hline 2 & $m x$ & $\begin{array}{l}\text { Mixture ID } \\
\text { number }\end{array}$ & Always & $\begin{array}{l}\text { Skip at least one blank after the compound name prior to } \\
\text { entering the mixture number. This must be an integer greater } \\
\text { than zero. This entry is required. }\end{array}$ \\
\hline 3 & roth & Density & Always & This is the mixture density in $\mathrm{g} / \mathrm{cm}^{3}$. \\
\hline 4 & nel & $\begin{array}{l}\text { Number of } n c z a \\
\quad \text { entries }\end{array}$ & Always & $\begin{array}{l}\text { This is the number of elements or nuclides that make up the } \\
\text { compound. }\end{array}$ \\
\hline 5 & $n c z a$ & $\begin{array}{l}\text { Nuclide ID } \\
\text { number }\end{array}$ & Always & $\begin{array}{l}\text { Repeat entries } 6 \text { and } 7 \text { for each element in the arbitrary } \\
\text { compound before entering temp. Enter the ID number from } \\
\text { the far right column of tables Isotopes in Standard } \\
\text { composition library or Isotopes and their natural } \\
\text { abundances. (Premixed standard compositions cannot be } \\
\text { used in a chemical compound definition.) }\end{array}$ \\
\hline 6 & atpm & $\begin{array}{l}\text { Atoms per } \\
\text { molecule }\end{array}$ & Always & $\begin{array}{l}\text { Enter the number of atoms of this element per molecule of } \\
\text { compound following each ncza. Repeat entries } 6 \text { and } 7 \text { for } \\
\text { each element in the arbitrary compound before entering } \\
\text { temp. }\end{array}$ \\
\hline 7 & $v f$ & $\begin{array}{l}\text { Density } \\
\text { multiplier }\end{array}$ & Always & $\begin{array}{l}\text { Enter the density multiplier (density fraction, volume } \\
\text { fraction, or a combination). A value of } 1 \text { means the material } \\
\text { density is roth. }\end{array}$ \\
\hline 8 & temp & $\begin{array}{l}\text { Temperature } \\
\qquad(\mathrm{K})\end{array}$ & $\begin{array}{l}\text { See } \\
\text { comment }\end{array}$ & $\begin{array}{l}\text { Default value is } 300 \mathrm{~K} \text {. This entry may be omitted if the } \\
\text { default temperature is acceptable and iza / wpt data are not } \\
\text { entered. }\end{array}$ \\
\hline 9 & $i z a$ & $\begin{array}{l}\text { Isotope's ZA } \\
\text { number }\end{array}$ & Optional & $\begin{array}{l}\text { Enter for each isotope in a multiple isotope nuclide. } \\
\text { Entries } 9 \text { and } 10 \text { are entered in pairs until each isotope in the } \\
\text { nuclide is defined. See table Isotopes and their natural } \\
\text { abundances for multiple isotope nuclide IDs and table } \\
\text { Elements and special nuclide symbols in the Standard } \\
\text { Composition Library for a list of isotopes in a multiple } \\
\text { isotope nuclide. }\end{array}$ \\
\hline 10 & $w t p$ & $\begin{array}{l}\text { weight percent } \\
\text { of isotope }\end{array}$ & Optional & $\begin{array}{l}\text { Enter the weight percent of the isotope in the nuclide. For } \\
\text { each nuclide the weight percents must sum to } 100 \text {. Entries } 9 \\
\text { and } 10 \text { are entered in pairs until each isotope in the nuclide is } \\
\text { defined. }\end{array}$ \\
\hline 11 & END & $\begin{array}{l}\text { Terminate the } \\
\text { standard } \\
\text { composition }\end{array}$ & Always & $\begin{array}{l}\text { This terminates the data for an compound. Enter the keyword } \\
\text { END to terminate compound. A tag, up to } 12 \text { characters } \\
\text { long, may follow the keyword END preceded by a single } \\
\text { blank. At least two blanks or a new line must separate this } \\
\text { entry from the next entry. }\end{array}$ \\
\hline
\end{tabular}


Table 7.1.2.d. Input specification for user-specified mixture/alloy data

\begin{tabular}{|c|c|c|c|c|}
\hline $\begin{array}{c}\text { Entry } \\
\text { no. }\end{array}$ & $\begin{array}{l}\text { Data } \\
\text { name }\end{array}$ & Data type & $\begin{array}{c}\text { Entry } \\
\text { requirement }\end{array}$ & Comment \\
\hline 1 & WTPT & $\begin{array}{l}\text { Mixture/alloy } \\
\text { name }\end{array}$ & Always & $\begin{array}{l}\text { Enter the four characters WTPT followed by up to } \\
12 \text { additional alphanumeric characters in place of WTPT. As } \\
\text { many physical mixtures or alloys as required may be entered } \\
\text { but each must have a unique name. This entry is required. }\end{array}$ \\
\hline 2 & $m x$ & $\begin{array}{l}\text { Mixture ID } \\
\text { number }\end{array}$ & Always & $\begin{array}{l}\text { Skip at least one blank after the alloy name prior to entering } \\
\text { the mixture number. This must be an integer greater than } \\
\text { zero. This entry is required. }\end{array}$ \\
\hline 3 & roth & Density & Always & This is the mixture density in $\mathrm{g} / \mathrm{cm}^{3}$. \\
\hline 4 & nel & $\begin{array}{c}\text { Number of } n c z a \\
\text { entries }\end{array}$ & Always & $\begin{array}{l}\text { This is the number of elements or nuclides that make up the } \\
\text { mixture/alloy. }\end{array}$ \\
\hline 5 & $n c z a$ & $\begin{array}{l}\text { Nuclide or } \\
\text { element ID } \\
\text { number }\end{array}$ & Always & $\begin{array}{l}\text { Repeat the sequences } 6 \text { and } 7 \text { for each element in the } \\
\text { mixture/alloy before entering temp. Enter the ID number } \\
\text { from the far right column of table Isotopes in Standard } \\
\text { composition library or Isotopes and their natural } \\
\text { abundances in the Standard Composition Library section. } \\
\text { (Premixed standard compositions cannot be used in an } \\
\text { arbitrary mixture/alloy definition.) }\end{array}$ \\
\hline 6 & wpct & $\begin{array}{l}\text { Weight percent } \\
\text { of nuclide or } \\
\text { element }\end{array}$ & Always & $\begin{array}{l}\text { Enter the weight percent of this element in the mixture/alloy } \\
\text { following each ncza. Weight percents must sum to } 100 \text {. } \\
\text { Repeat the sequence } 6 \text { and } 7 \text { for each element in the mixture } \\
\text { before entering temp. }\end{array}$ \\
\hline 7 & $v f$ & $\begin{array}{l}\text { Density } \\
\text { multiplier }\end{array}$ & Always & $\begin{array}{l}\text { Enter the density multiplier (density fraction, volume } \\
\text { fraction, or a combination). A value of } 1 \text { means the material } \\
\text { density is roth. }\end{array}$ \\
\hline 8 & temp & $\begin{array}{l}\text { Temperature } \\
\quad(\mathrm{K})\end{array}$ & $\begin{array}{l}\text { See } \\
\text { comment }\end{array}$ & $\begin{array}{l}\text { Default value is } 300 \mathrm{~K} \text {. This entry may be omitted if the } \\
\text { default temperature is acceptable and iza / wpt data is not } \\
\text { entered. }\end{array}$ \\
\hline 9 & $i z a$ & $\begin{array}{l}\text { Isotope's ZA } \\
\text { number }\end{array}$ & Optional & $\begin{array}{l}\text { Enter for each isotope in a multiple isotope nuclide. } \\
\text { Entries } 9 \text { and } 10 \text { are entered in pairs until each isotope in the } \\
\text { nuclide is defined. See the Standard Composition Library } \\
\text { tables Isotopes and their natural abundances in for multiple } \\
\text { isotope nuclide IDs and Elements and special nuclide } \\
\text { symbols for a list of isotopes in a multiple isotope nuclide. }\end{array}$ \\
\hline 10 & $w t p$ & $\begin{array}{l}\text { Weight percent } \\
\text { of isotope }\end{array}$ & Optional & $\begin{array}{l}\text { Enter the weight percent of the isotope in the nuclide. } \\
\text { For each nuclide the weight percents must sum to } 100 \text { for } \\
\text { each isotope in a multiple isotope nuclide. Entries } 9 \text { and } 10 \\
\text { are entered in pairs until each isotope in the nuclide is } \\
\text { defined. }\end{array}$ \\
\hline 11 & END & $\begin{array}{l}\text { Terminate the } \\
\text { standard } \\
\text { composition }\end{array}$ & Always & $\begin{array}{l}\text { This terminates the data for an arbitrary mixture. Enter the } \\
\text { keyword END to terminate arbitrary mixture/alloy. Repeat } \\
\text { entries } 1 \text { through } 11 \text { until all the mixtures have been defined. } \\
\text { A tag, up to } 12 \text { characters long, may follow the keyword } \\
\text { END preceded by a single blank. At least two blanks or a } \\
\text { new line must separate this entry from the next entry. }\end{array}$ \\
\hline
\end{tabular}




\section{STANDARD COMPOSITION INPUT FOR BASIC MIXTURES (see Table 7.1.2.a)}

Two input syntaxes are available for standard composition specifications of basic mixtures in the COMP block. The first uses information (e.g., densities, atomic weights, physical constants, etc.) contained in the Standard Composition Library, along with user specified input, to automatically compute the number densities for mixture components. In the second option, the user computes the nuclide number densities, and inputs these directly for each component of the mixture. XSProc recognizes syntax 2 if the third entry of the composition specification is zero, as shown below. It is allowable to use syntax 1 for some standard composition specifications and syntax 2 for others. The two syntaxes to define basic mixtures with the standard composition specifications are shown below.

\section{syntax 1: Standard Composition Library data used to compute number densities.}

sc $m x \quad \mathbf{D E N}=$ roth $\{\mathbf{V F}=\}$ of $\quad$ temp $i z a_{1} w t p_{1} \ldots i z a_{N} w t p_{N} \quad$ END

\section{syntax 2: User input number densities}

sc $m x \quad 0$ aden temp END

The definitions for these input parameters are given below.

A1. sc STANDARD COMPOSITION MATERIAL NAME. This corresponds to one of the material names given in the Standard Composition Library for isotopes, elements, thermal moderators and activity materials, chemical compounds, and alloys/mixtures. Some types of these materials require entering certain data such as the volume fraction or theoretical density and other engineering-type data. For standard compositions containing more than one isotope of an element (such as $\mathrm{UO}_{2}$ ), the user is free to specify the weight percent for each isotope, such that they total $100 \%$. See the Basic standard composition specifications section for examples of basic standard compositions.

A2. $m x \quad$ MIXTURE ID NUMBER. An arbitrary mixture number is required on every standard composition specification for both syntaxes. It defines the mixture that contains the material defined by the standard composition specification data. The mixture numbers are utilized in the CELLDATA block Cell Block for INFHOMMEDIUM, LATTICECELL, MULTIREGION, or DOUBLEHET problems and the geometry data.

A3. DEN=roth MIXTURE DENSITY. The keyword DEN is assigned a value of roth, where roth is the specified density of the mixture component in $\mathrm{g} / \mathrm{cm}^{3}$. It should always be entered for materials that contain enriched multi-isotopic nuclides. The effective density of the material component is equal to the product of roth and $v f$. An example of this is demonstrated in Appendix A. 
A4. $\{\mathbf{V F}=\}$ vf VOLUME FRACTION. The keyword VF is assigned a value of $v f$. It is also allowable to omit the keyword $\mathbf{V F}=$ and just enter the value $v f$. The default value of the volume fraction is 1.0. The volume fraction can be interpreted as

a. the volume fraction of this standard composition component in the mixture,

b. the density of the standard composition component in this application divided by the theoretical or default density given in the Standard Composition Library, or

c. the product of (a) and (b).

Appendix A discusses the interaction between roth and $v f$. For example, assume a homogenized mixture representing the water moderator and Zircaloy cladding around a fuel pin is to be described. If the volume of the clad is $5.32 \mathrm{cc}$ and the volume of the water moderator is $44.68 \mathrm{cc}$, the mixture can be described using $\mathrm{H}_{2} \mathrm{O}$ with a volume fraction of 0.8936 [i.e., 44.68/(44.68+5.32)] and ZIRCALOY with a volume fraction of 0.1064 [i.e., $5.32 /(44.68+5.32)]$.

A5. aden NUMBER DENSITY (not used for syntax 1, but required for 2). The number density is entered ONLY if $3^{\text {rd }}$ entry on the standard composition specification is entered as zero. The number density is entered in units of atoms per barn-cm.

A6. temp TEMPERATURE. The default value of the temperature is $300 \mathrm{~K}$. The temperature can be omitted if entries A7 and A8 are also omitted.

A7. iza

ISOTOPE ZA NUMBER. Enter a value for each isotope in the standard composition component, entry 1. Do not enter a value if the volume fraction, VF, is zero (A4 above).

The ZA number of the isotope is entered if the user wishes to specify the isotopic distribution. This is done by entering $i z a$ and $w t p$ for each isotope until all the desired isotopes have been described. In most cases the "ZA" ID number is $(\mathrm{A}+1000 * \mathrm{Z})$, where $\mathrm{A}$ is the atomic mass or weight of the isotope, and $\mathrm{Z}$ is the atomic number. For example, the ZA number for ${ }^{235} \mathrm{U}$ is 92235 .

Entries A7 and A8 can be skipped if the default values listed in Table 7.1.2 of section 7.1 are acceptable.

A8. wtp WEIGHT PERCENT OF THE ISOTOPE. If entry A7 is entered, a value must also be entered for A8. The weight percent of the isotope is the percent of this isotope in the element. The weight percent of all specified isotopes of the element must sum to $100( \pm$ $0.01)$.

A9. END The word END is entered to terminate the input data for a standard composition component. This END can have a label associated with it that can be as long as 12 characters. The label is optional, and if entered must be preceded from the END by a single blank. At least two blanks or a new line must separate this item from the next data entry. 
Syntax:

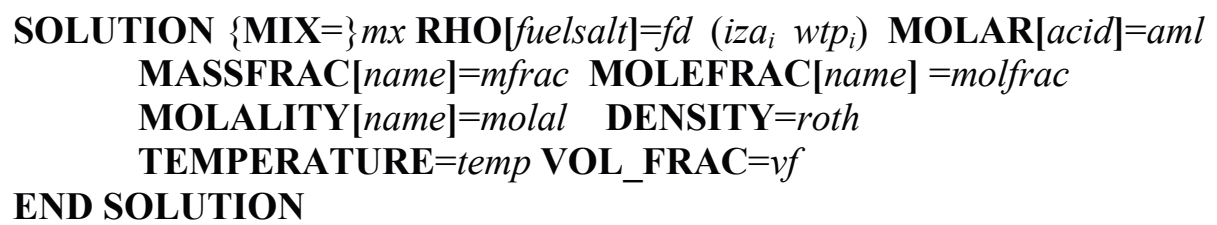

where
$m x \quad$ is the mixture number,
fuelsalt is the Standard Composition Library component name of one of the fissile compounds
$f d \quad$ is the fuel density in grams of uranium or plutonium per liter of solution
acid is one of the Standard Composition Library acid compounds (e.g., HNO3 or HFACID)
name is one of the Standard Composition Library solution components
aml is the acid molarity of the acid component (moles of acid/liter of solution)
mfrac is the mass fraction of name in the solution (grams of metal in name/gram solution)
molfrac is the mole fraction of name in the solution (moles of name/mole solution)
molal is the mass fraction of name in the solution (moles of name $/ \mathrm{kg}$ water)
roth is the density of the solution,
$v f \quad$ is the density multiplier (ratio of actual to theoretical density of the solution),
temp is the temperature in Kelvin,
iza is the isotope ID number from table Available fissile solution components, and
wtp is the weight percent of the isotope in the material.

Below are the input data for fissile solutions.

1. SOLUTION

2. $m x$
Keyword starting a solution specification. Solutions require the specification of the mixture and at least one component. Current possible components are given in the Standard Composition Library table, Available fissile solution components. Only the mixture number and one component are required. Appendix A contains examples of the input data for solutions.

MIXTURE ID NUMBER. A mixture number is required on every standard composition specification. It defines the mixture that contains the material defined by the standard composition specification data. The mixture numbers are utilized in the Unit Cell Specification for INFHOMMEDIUM, LATTICECELL, or MULTIREGION.

KEYWORD PARAMETERS TO DEFINE CONCENTRATIONS OF SOLUTION COMPONENTS. Each keyword specifies the unit, the component name from the Standard Composition Library and the component value, as shown Table 7.1.2.b. Up to three components can be specified for a solution if one is an acid. After the value, the 
4. DENSITY=roth

5. TEMPERATURE $=$ temp

6. VOLFRAC $=v f$ isotopic enrichments of the nuclides can be given as pairs of isotope IDs and weight percent. NOTE: the square brackets [ ] containing the component name are required.

Keyword specifying the overall solution density as grams per cubic centimeter or as a "?", meaning it is to be solved for. Solving for the density is the default behavior, but the density can be given, and a component value can be solved for instead.

Keyword defining temperature of the solution. The default value is $300 \mathrm{~K}$.

Keyword defining volume fraction - the default volume fraction is 1.0. This value must be greater than 0.0 . The volume fraction can be interpreted as:

a. the volume fraction of this solution specification in the mixture,

b. the density of the solution in this application divided by the calculated density of the solution, or

c. the product of (a) and (b).

\section{END SOLUTION}


STANDARD COMPOSITION INPUT FOR CHEMICAL COMPOUNDS (see Table 7.1.2.c) Syntax:

ATOMnn $m x$ roth nel $n c z a_{1}$ atpm $_{1} \ldots n c z a_{\text {nel }} \operatorname{atpm}_{\text {nel }}\left\{v f\left\{\right.\right.$ temp $\left.\left.\left\{i z a_{1} w t p_{1} \ldots\right\}\right\}\right\}$ END

Below are the data for user-defined chemical compounds.

C1. ATOMnn COMPOUND NAME. User-specified compounds (also defined as "arbitrary" in older versions of SCALE) require the user to provide all the information normally found in the Standard Composition Library. This option allows specifying a compound not available in the Standard Composition Library by utilizing nuclides and elements available in the library. An user-specified compound name must start with the four characters "ATOM." A maximum of twelve characters is allowed for the compound name, and imbedded blanks are not allowed.

C2. $m x \quad$ MIXTURE ID NUMBER. A mixture number is required on every standard composition specification. It defines the mixture that contains the material defined by the compound specification data. The mixture numbers are utilized in the Unit Cell Specification for INFHOMMEDIUM, LATTICECELL, or MULTIREGION problems and the KENO V.a or KENO-VI geometry data.

C3. roth MIXTURE DENSITY. The density of the arbitrary material is entered in units of $\mathrm{g} / \mathrm{cm}^{3}$. roth and $v f$ interact to produce the density of the mixture used in the problem. Note that this is a required entry and does not use "DEN=" keyword.

C4. nel NUMBER OF ELEMENTS IN THE MATERIAL. Enter the number of components from the Standard Composition Library that are to be used to define this material.

C5. ncza ID NUMBER. This is the "ZA" ID number for the element or isotope. Usually, $n c z a=\mathrm{A}+1000^{*} \mathrm{Z}$, where $\mathrm{A}$ is the atomic mass or weight of the nuclide, and $\mathrm{Z}$ is the atomic number.

C6. atpm ATOMIC or ELEMENT ABUNDANCE. Enter the number of atoms of this element per molecule of compound. Repeat the sequence ncza and atpm (C5 and C6) for every element in the compound before going to entry $\mathrm{C} 7$.

C7. vf VOLUME FRACTION. The default value of the volume fraction is 1.0. This value must be greater than 0.0 . The volume fraction can be interpreted as

a. the volume fraction of this compound in the mixture,

b. the density of the compound in this application divided by the input density of the compound, or

c. the product of (a) and (b).

C8. temp TEMPERATURE. The default value of the temperature is $300 \mathrm{~K}$. The temperature can be omitted if entries C9 and C10 are also omitted. 
C9. iza ISOTOPE ZA NUMBER. Enter a value for each isotope in the element in the compound. The ZA number of the isotope is entered if the user wishes to specify the isotopic distribution. This is done by entering $i z a$ and $w t p$ for each isotope until all the desired isotopes have been described. In most cases the "ZA" ID number is $\left(A+1000^{*} \mathrm{Z}\right)$, where $\mathrm{A}$ is the atomic mass or weight of the isotope, and $\mathrm{Z}$ is the atomic number.

Entries C9 and C10 can be skipped if the default values listed in Table 7.1.2 of section 7.1 are acceptable.

C10. wtp WEIGHT PERCENT OF THE ISOTOPE. If entry C9 is entered, a value must also be entered for $\mathrm{C} 10$. The weight percent of the isotope is the percent of this isotope in the element. The weight percents of all specified isotopes of the element must sum to 100 $( \pm 0.01)$.

Repeat the sequence iza wtp until the sum of the $w t p$ s sum to 100. The sequence $i z a w t p$ is repeated until all of the desired isotopes have been specified.

C11. END The word END is entered to terminate the input data for compound. This END can have a label associated with it that can be as long as 12 characters. The label is optional, and if entered must be preceded from the END by a single blank. At least two blanks or a new line must separate item $\mathrm{C} 11$ from the next data entry. 
STANDARD COMPOSITION INPUT FOR MIXTURES AND ALLOYS (see Table 7.1.2.d)

Syntax:
WTPTnn $m x$ roth nel $n c z a_{1}$ wpct $_{1} \ldots n c z a_{\text {nel }}$ wpct $_{\text {nel }}\left\{v f\left\{\right.\right.$ temp $\left.\left.\left\{i z a_{1} w t p_{1} \ldots\right\}\right\}\right\}$ END

Below are the input data for arbitrary (i.e., user-defined) physical mixture or alloy.

D1. WTPTnn ARBITRARY MIXTURE/ALLOY NAME. The arbitrary user-specified mixture/alloy option allows specifying a mixture or an alloy not available in the Standard Composition Library by utilizing the nuclides and elements available in the library. An arbitrary mixture/alloy name must start with the four characters "WTPT." A maximum of 12 characters is allowed for the arbitrary mixture/alloy name. Imbedded blanks are not allowed in an arbitrary mixture/alloy name. Appendix A contains input data for arbitrary mixture/alloys.

D2. $m x \quad$ MIXTURE ID NUMBER. A mixture number is required on every standard composition specification. It defines the mixture that contains the material defined by the arbitrary compound specification data. The mixture numbers are utilized in the Unit Cell Specification for INFHOMMEDIUM, LATTICECELL, MULTIREGION, or DOUBLEHET problems and the KENO V.a or KENO-VI geometry data.

D3. roth MIXTURE DENSITY. The density of the arbitrary material is entered in units of $\mathrm{g} / \mathrm{cm}^{3}$. roth and $v f$ interact to produce the density of the mixture used in the problem. Note that this is a required entry and does not use "DEN=" keyword.

D4. nel NUMBER OF ELEMENTS IN THE MATERIAL. Enter the number of components from the Standard Composition Library that are to be used to define this arbitrary material.

D5. $n c z a$ ID NUMBER. This is the "ZA" ID number for the element or isotope. Usually, $n c z a=\mathrm{A}+1000 * \mathrm{Z}$, where $\mathrm{A}$ is the atomic mass or weight of the nuclide, and $\mathrm{Z}$ is the atomic number.

D6. wpet ATOMIC or ELEMENT ABUNDANCE. Enter the weight percent of this element in the arbitrary alloy. The sum of all the weight percents for each specified element in the arbitrary alloy MUST be 100.0. Repeat the sequence $n c z a$ and wpct (D5 and D6) for every element in the arbitrary mixture/alloy before going to entry D7.

D7. $v f \quad$ VOLUME FRACTION. The default value of the volume fraction is 1.0. This value must be greater than 0.0 . The volume fraction can be interpreted as:

a. the volume fraction of this mixture or alloy in the mixture,

b. the density of the mixture or alloy in this application divided by the input density (roth) of the mixture or alloy, or

c. the product of (a) and (b). 
D8. temp TEMPERATURE. The default value of the temperature is $300 \mathrm{~K}$. The temperature can be omitted if entries D9 and D10 are also omitted.

D9. iza ISOTOPE ZA NUMBER. Enter a value for each isotope in the element in the arbitrary alloy. The ZA number of the isotope is entered if the user wishes to specify the isotopic distribution. This is done by entering iza and $w t p$ for each isotope until all the desired isotopes have been described. In most cases the "ZA" ID number is $(A+1000 * Z)$, where $\mathrm{A}$ is the atomic mass or weight of the isotope, and $\mathrm{Z}$ is the atomic number.

Entries D9 and D10 can be skipped if the default values listed in Table 7.1.2 of section 7.1 are acceptable.

D10. wtp WEIGHT PERCENT OF THE ISOTOPE. If entry D9 is entered, a value must also be entered for D10. The weight percent of the isotope is the percent of this isotope in the element. Weight percents of all specified isotopes of the element must sum to 100 $( \pm 0.01)$.

D11. END The word END is entered to terminate the input data for an arbitrary compound. This END can have a label associated with it that can be as long as 12 characters. The label is optional and if entered must be preceded from the END by a single blank. At least two blanks or a new line must separate this item from the next data entry. 


\subsubsection{Unit cell specification for infinite homogeneous problems}

This section describes the unit cell data that can be entered for an INFHOMMEDIUM problem. Additional information is available in Appendix B.

Syntax:

INFHOMMEDIUM $m x\{$ CELLMIX $\{=\} m i x\}$ END

The data required to specify the unit cell for an INFHOMMEDIUM unit cell are given in Table 7.1.3. The individual entries are explained in the following text.

1. celltype INFHOMMEDIUM. The keyword INFHOMMEDIUM is entered to indicate this unit cell contains one mixture with no geometry corrections. This data must be entered. The keyword may be truncated to any number of characters as long as the characters present are identical from the beginning of the keyword (i.e., INF is acceptable). All mixtures not in a defined unit cell are by default processed as infhommedium.

2. $m x \quad$ MIXTURE NUMBER. The mixture number defines the mixture to be used in the cell. This data must be entered. Be sure the mixture number entered is defined in the standard composition data.

3. CELLMIX=mix CELL-WEIGHTED MIXTURE NUMBER. (the $=$ sign can be replaced by a space if desired). Enter ONLY if a cell-weighted mixture is to be generated. Enter a unique mixture number to be used by XSDRN to create the cell-weighted mixture (Section 7.1.2.4). For INFHOMMEDIUM cells, cross sections for the cell mixture are equal to the shielded values of the original mixture.

4. END The word END is entered to terminate the INFHOMMEDIUM data. An optional label can be associated with this END. The label can be as many as 12 characters long and is separated from the END by a single blank. At least two blanks must follow this entry. 
Table 7.1.3. Unit cell specifications for INFHOMMEDIUM problems

\begin{tabular}{|c|c|c|c|}
\hline $\begin{array}{l}\text { Entry } \\
\text { no. }\end{array}$ & $\begin{array}{c}\text { Input } \\
\text { data }\end{array}$ & $\begin{array}{l}\text { Data } \\
\text { type }\end{array}$ & Comments \\
\hline 1 & INFHOMMEDIUM & Keyword & $\begin{array}{l}\text { Keyword to begin infhommedium unit cell. Enter the } \\
\text { keyword INFHOMMEDIUM. This word may be truncated to } \\
\text { any number of letters as long as they exactly replicate the } \\
\text { beginning part of the keyword (e.g., INF is acceptable). }\end{array}$ \\
\hline 2 & $m x$ & $\begin{array}{c}\text { Cell } \\
\text { mixture } \\
\text { number }\end{array}$ & Specifies the mixture number to be used in the cell. \\
\hline 3 & CELLMIX $=m i x$ & $\begin{array}{l}\text { Keyword }+ \\
\text { new } \\
\text { mixture } \\
\text { number }\end{array}$ & $\begin{array}{l}\text { Enter the keyword CELLMIX= followed immediately by a } \\
\text { unique positive integer (mix). The integer will be a new } \\
\text { mixture number that has the neutronic properties of the self- } \\
\text { shielded unit cell. }{ }^{a}\end{array}$ \\
\hline 4 & END & & Terminate INFHOMMEDIUM data \\
\hline \multicolumn{4}{|c|}{$\begin{array}{l}{ }^{a} \text { Note: If CELLMIX is entered for a INFHOMMEDIUM cell, XSDRNPM is executed to compute } \mathrm{k}_{\infty} \text {, but cross } \\
\text { sections for the "homogenized" mixture are identical to the shielded values for the original cell. }\end{array}$} \\
\hline 7.1.3.5 & \multicolumn{3}{|c|}{ Unit cell specification for LATTICECELL problems } \\
\hline \multicolumn{4}{|c|}{$\begin{array}{l}\text { This section describes the unit cell input data for a LATTICECELL problem. The LATTICECELL } \\
\text { description is especially suited to self-shield arrays of repeated cells such as a fuel assembly lattice. The } \\
\text { unit cell specification plays a major role in providing accurate problem-dependent cross sections using the } \\
\text { computational procedures described in Section 7.1.2.3. Unit cells are limited to (a) infinitely long } \\
\text { cylindrical rods in a square or triangular lattice, (b) spheres in a cubic or triangular lattice, (c) a symmetric } \\
\text { array of slabs, or (d) an asymmetric array of slabs. Both "regular" and "annular" fuel geometries can be } \\
\text { used in LATTICECELL problems. "Regular" cells allow a concentric spherical, cylindrical, or } \\
\text { symmetric slab configuration, where the central region is fuel, surrounded by an optional gap, an optional } \\
\text { clad, and an external moderator. "Annular" cells also allow concentric spherical, cylindrical, or } \\
\text { asymmetric slab configurations, but the central region corresponds to an inner moderator region which is } \\
\text { surrounded by a fuel region having an optional gap and optional clad on each side of the fuel. An inner } \\
\text { gap may be specified inside the fuel region, and an outer gap may be specified outside the fuel region } \\
\text { Similarly an inner clad may be specified inside the fuel region, and an outer clad may be specified outside } \\
\text { the fuel region. For both regular and annular fuel cells, the outer boundary of the unit cell is determined } \\
\text { from the square or triangular pitch of the array. }\end{array}$} \\
\hline
\end{tabular}

Regular cells are SQUAREPITCH, TRIANGPITCH, SPHSQUAREP, SPHTRIANGP, and SYMMSLABCELL.

Annular cells are ASQUAREPITCH (or ASQP), ATRIANGPITCH (or ATRP), ASPHSQUAREP (or ASSP), ASPHTRIANGP (or ASTP), and ASYMSLABCELL

Syntax: 


\section{celltype ctp PITCH (or HPITCH) pitch $\mathrm{mm}$ FUELD (or FUELR) fuel $\mathrm{mf}$ GAPD (or GAPR) gap mg CLADD (or CLADR) clad mc IMODD (or IMODR) imod mim IGAPD (or IGAPR) igap mig ICLADD (or ICLADR) iclad mic $\quad\{$ CELLMIX $=m i x\} \quad$ END}

The unit cell geometry data required to specify a LATTICECELL problem are given in Table 7.1.4. The individual entries are explained in the text below.

LATTICECELL. The keyword LATTICECELL is entered to indicate this unit cell contains mixtures that are positioned in a regular array. This data must be entered. The keyword may be truncated to any number of characters as long as the characters present are identical from the beginning of the keyword (e.g., LAT is acceptable). This unit cell is normally used for regular arrays of materials such as fuel pins in an assembly.

TYPE OF LATTICE. This defines the type of lattice or array configuration. Any one of the following alphanumeric descriptions may be used. Note that the alphanumeric description must be separated from subsequent data entries by one or more blanks. Figure 7.1.1 Mixture and position data are entered using keywords. Mixture number 0 may be entered for void and may be used multiple times in each and all unit cells. For regular cells, the minimum requirement is that a fuel region and a moderator region are specified and no other inner components are specified. For annular cells, the minimum requirement is the fuel and outer moderator and inner moderator regions are specified. Regular and annular cell configurations are specified as shown below.

\section{Regular Cells}

SQUAREPITCH is used for an array of cylinders arranged in a square lattice, as shown in Figure 7.1.1. The clad and/or gap can be omitted.

TRIANGPITCH is used for an array of cylinders arranged in a triangular-pitch lattice as shown in Figure 7.1.2. The clad and/or gap can be omitted.

SPHSQUAREP is used for an array of spheres arranged in a square-pitch lattice. A cross section view through a cell is represented by Figure 7.1.1. The clad and/or gap can be omitted.

SPHTRIANGP is used for an array of spheres arranged in a triangular-pitch (dodecahedral) lattice. A cross section view through a cell is represented by Figure 7.1.2. The clad and/or gap can be omitted.

SYMMSLABCELL is used for an infinite array of symmetric slab cells, as shown in Figure 7.1.3. The clad and/or gap can be omitted. 


\section{Annular Cells}

ASQUAREPITCH or ASQP is used for annular cylindrical rods in a square-pitch lattice as shown in Figure 7.1.4. The inner and outer clad and gap are independently entered so they must be different materials and dimensions. Note that each mixture in the problem can be used only once and in only one zone of a cell.

ATRIANGPITCH or ATRP is used for annular cylindrical rods in a triangularpitch lattice as shown in Figure 7.1.5. The inner and outer clad and gap are independently entered, so they must be different materials and dimensions.

ASPHSQUAREP or ASSP is used for spherical shells in a square-pitch lattice as shown in Figure 7.1.4. The inner and outer clad and gap are independently entered, so they must be different materials and dimensions.

ASPHTRIANGP

or ASTP is used for spherical shells in a triangular-pitch (dodecahedral) lattice as shown in Figure 7.1.5. The inner and outer clad and gap are independently entered, so they must be different materials and dimensions.

ASYMSLABCELL is used for a periodic, but asymmetric, array of slabs as shown in Figure 7.1.6. The inner and outer clad and gap are independently entered, so they may be different materials and dimensions.

3. PITCH or HPITCH

4. FUELD or FUELR

5. GAPD or GAPR

6. CLADD or CLADR
ARRAY PITCH. This is the center-to-center spacing or half-spacing between the fuel lumps (rods, pellets, or slabs), pitch, in $\mathrm{cm}$ followed by the outer moderator material number, $\mathrm{mm}$, as shown in Figure 7.1.1 through Figure 7.1.6.

OUTSIDE DIMENSION OF FUEL. This is the outside diameter or radius of the fuel, fuel, in $\mathrm{cm}$ followed by the fuel mixture number, $m f$, as shown in Figure 7.1.1 through Figure 7.1.6.

OUTSIDE DIMENSION OF OUTER GAP. Enter only if outer gap is present. This is the outside diameter or radius of the outer gap, gap, in $\mathrm{cm}$ followed by the gap mixture number, $m g$, as shown in Figure 7.1.1 through Figure 7.1.6.

OUTSIDE DIMENSION OF OUTER CLAD. Enter ONLY if a clad is present. This is the outside diameter or radius of the outer clad, clad, in $\mathrm{cm}$ followed by the clad mixture number, $m c$, as shown in Figure 7.1.1 through Figure 7.1.6. 
7. IMODD or IMODR

8. IGAPD or IGAPR

9. ICLADD or ICLADR
DIMENSION OF INNER MODERATOR. Enter ONLY if an annular cell is specified. This is the outside diameter or radius of the inner moderator, imod, in $\mathrm{cm}$ followed by the inner moderator mixture number, mim, as shown in Figure 7.1.4 through Figure 7.1.6.

OUTSIDE DIMENSION OF INNER GAP. Enter ONLY if an annular cell is specified and inner gap is present. This is the outside diameter or radius of the inner gap, igap, in cm followed by the inner gap mixture number, mig, as shown in Figure 7.1.4 through Figure 7.1.6.

OUTSIDE DIMENSION OF INNER CLAD. Enter ONLY if an annular cell is specified and inner clad is present. This is the outside diameter or radius of the inner clad, iclad, in $\mathrm{cm}$ followed by the inner clad mixture number, mic, as shown in Figure 7.1.4 through Figure 7.1.6.

10. $\{$ CELLMIX $=\} \boldsymbol{m i x}$ CELL-WEIGHTED MIXTURE NUMBER. [the $=$ sign can be replaced by a space if desired). Enter ONLY if a cell-weighted mixture is to be generated. Enter a unique mixture number to be used by XSDRN to create the cell-weighted mixture (Section 7.1.2.4).

11. END

The word END is entered to terminate the LATTICECELL data. An optional label can be associated with this END. The label can be as many as 12 characters long and is separated from the END by a single blank. At least two blanks must follow this entry. Must not start in column 1. 
Table 7.1.4. Unit cell specification for LATTICECELL problems

\begin{tabular}{|c|c|c|c|c|c|}
\hline \multirow{2}{*}{$\begin{array}{c}\text { Entry } \\
\text { no. }\end{array}$} & \multicolumn{2}{|c|}{$\begin{array}{c}\text { Input } \\
\text { keyword }\end{array}$} & \multicolumn{3}{|r|}{ Comments } \\
\hline & \multicolumn{2}{|c|}{ LATTICECELL } & \multicolumn{3}{|c|}{$\begin{array}{l}\text { Keyword to begin LATTICECELL unit cell. Enter the keyword } \\
\text { LATTICECELL. This word may be truncated to any number of letters as long } \\
\text { as they exactly replicate the beginning part of the keyword (e.g., LAT is } \\
\text { acceptable). }\end{array}$} \\
\hline \multirow[t]{15}{*}{2} & & & \multicolumn{3}{|c|}{$\begin{array}{l}\text { One of the following keywords is specified. This keyword determines the type } \\
\text { of lattice or array configuration and which subsequent data need to be } \\
\text { specified. }\end{array}$} \\
\hline & \multicolumn{2}{|c|}{ SQUAREPITCH } & \multicolumn{3}{|c|}{ Used for cylindrical rods in a square pitch. } \\
\hline & \multicolumn{2}{|c|}{ ASQUAREPITCH } & \multicolumn{3}{|c|}{ Used for annular cylindrical rods in a square pitch. } \\
\hline & \multicolumn{2}{|l|}{ ASQP } & \multicolumn{3}{|c|}{ Used for annular cylindrical rods in a square pitch. } \\
\hline & \multicolumn{2}{|c|}{ TRIANGPITCH } & \multicolumn{3}{|c|}{ Used for cylindrical rods in a triangular pitch. } \\
\hline & \multicolumn{2}{|c|}{ ATRIANGPITCH } & \multicolumn{3}{|c|}{ Used for annular cylindrical rods in a triangular pitch. } \\
\hline & \multicolumn{2}{|l|}{ ATRP } & \multicolumn{3}{|c|}{ Used for annular cylindrical rods in a triangular pitch. } \\
\hline & \multicolumn{2}{|c|}{ SPHSQUAREP } & \multicolumn{3}{|c|}{ Used for spherical pellets in a cubic lattice. } \\
\hline & \multicolumn{2}{|c|}{ ASPHSQUAREP } & \multicolumn{3}{|c|}{ Used for annular spherical pellets in a cubic lattice. } \\
\hline & \multicolumn{2}{|l|}{ ASSP } & \multicolumn{3}{|c|}{ Used for annular spherical pellets in a cubic lattice. } \\
\hline & \multicolumn{2}{|c|}{ SPHTRIANGP } & \multicolumn{3}{|c|}{$\begin{array}{l}\text { Used for spherical pellets in a bi-centered or face-centered hexagonal close- } \\
\text { packed lattice. }\end{array}$} \\
\hline & \multicolumn{2}{|c|}{$\begin{array}{l}\text { ASPHTRIANGP } \\
\quad \text { or }\end{array}$} & \multicolumn{3}{|c|}{$\begin{array}{l}\text { Used for annular spherical pellets in a bi-centered or face-centered hexagonal } \\
\text { close-packed lattice. }\end{array}$} \\
\hline & \multicolumn{5}{|l|}{ ASTP } \\
\hline & \multicolumn{2}{|c|}{ SYMMSLABCELL } & \multicolumn{3}{|c|}{ Used for a symmetric array of slabs. } \\
\hline & \multicolumn{2}{|c|}{ ASYMSLABCELL } & \multicolumn{3}{|c|}{ Used for a periodic but asymmetric array of slabs. } \\
\hline \multicolumn{6}{|c|}{$\begin{array}{l}\text { Enter the following keywords and subordinate data as required to specify the unit cell. Each dimension can be entered as } \\
\text { a diameter or radius using the appropriate keyword. The following keywords may be entered in any order. }\end{array}$} \\
\hline 3 & PITCH & HPITCH & $\begin{array}{l}\text { Cell pitch or } \\
\text { half-pitch }(\mathrm{cm}) \\
+ \text { moderator } \\
\text { mixture } \\
\end{array}$ & Always & $\begin{array}{l}\text { The pitch is the center-to-center spacing }(\mathrm{cm}) \\
\text { between fuel lumps. For asymmetric slab cell, } \\
\text { PITCH is the center-to-center distance between } \\
\text { inner moderators }(\mathrm{cm}) \text {. }\end{array}$ \\
\hline 4 & FUELD & FUELR & $\begin{array}{l}\text { Outside diameter or } \\
\text { radius of fuel } \\
(\mathrm{cm})+\text { fuel } \\
\text { mixture }\end{array}$ & Always & $\begin{array}{l}\text { Outside diameter (radius) of fuel }(\mathrm{cm}) \text { or the } \\
\text { thickness (half-thickness) of the fuel in a slab. For } \\
\text { asymmetric slab cells, FUELR is measured from } \\
\text { the centerline of the inner moderator. }\end{array}$ \\
\hline 5 & GAPD & GAPR & $\begin{array}{l}\text { Outside diameter or } \\
\text { radius of outer } \\
\text { gap }(\mathrm{cm}) \\
+ \text { gap mixture }\end{array}$ & $\begin{array}{l}\text { Outer gap } \\
\text { Present }\end{array}$ & $\begin{array}{l}\text { OMIT IF NO GAP between the fuel and clad } \\
\text { outer clad. A mixture number of zero is often } \\
\text { used. For asymmetric slab cells, GAPR is } \\
\text { measured from the centerline of the inner } \\
\text { moderator. }\end{array}$ \\
\hline
\end{tabular}




\begin{tabular}{|c|c|c|c|c|c|}
\hline 6 & CLADD & CLADR & $\begin{array}{l}\text { Outside diameter or } \\
\text { radius of outer } \\
\text { clad }(\mathrm{cm})+\text { clad } \\
\text { mixture }\end{array}$ & $\begin{array}{l}\text { Outer clad } \\
\text { Present }\end{array}$ & $\begin{array}{l}\text { OMIT IF NO CLAD between fuel and outer } \\
\text { moderator. For asymmetric slab cells, CLADR is } \\
\text { measured from the centerline of the inner } \\
\text { moderator. }\end{array}$ \\
\hline 7 & IMODD & IMODR & $\begin{array}{l}\text { Outside diameter } \\
\text { or radius of inner } \\
\text { moderator }(\mathrm{cm}) \\
+ \text { moderator } \\
\text { mixture }\end{array}$ & Annular cell & $\begin{array}{l}\text { Dimension of inner moderator mixture inside the } \\
\text { fuel. For a slab, this is the moderator on the other } \\
\text { side of the slab. Enter for annular cells only. }\end{array}$ \\
\hline 8 & IGAPD & IGAPR & $\begin{array}{l}\text { Outside diameter } \\
\text { or radius of inner } \\
\text { gap }(\mathrm{cm}) \\
+ \text { gap mixture } \\
\end{array}$ & $\begin{array}{l}\text { Annular cell }+ \\
\text { Inner Gap } \\
\text { Present }\end{array}$ & $\begin{array}{l}\text { OMIT IF NO GAP between the inner clad and } \\
\text { fuel. For an asymmetric slab, IGAPR is the } \\
\text { distance from the center of the inner moderator to } \\
\text { the outside of the inner gap }(\mathrm{cm}) \text {. }\end{array}$ \\
\hline 9 & ICLADD & ICLADR & $\begin{array}{l}\text { Outside diameter } \\
\text { or radius of inner } \\
\text { clad }(\mathrm{cm})+ \\
\text { clad mixture }\end{array}$ & $\begin{array}{l}\text { Annular cell + } \\
\text { Inner Clad } \\
\text { Present }\end{array}$ & $\begin{array}{l}\text { OMIT IF NO CLAD between fuel and inner } \\
\text { moderator. For an asymmetric slab, ICLADR is } \\
\text { the distance from the center of the inner } \\
\text { moderator to the outside of the inner clad }(\mathrm{cm}) \text {. }\end{array}$ \\
\hline 10 & CELLMIX & & $\begin{array}{l}\text { Unique mixture } \\
\text { number }\end{array}$ & $\begin{array}{l}\text { Cell-weighted } \\
\text { mixture }\end{array}$ & $\begin{array}{l}\text { Used if a cell-weighted mixture is specified. } \\
\text { Calls XSDRN to produce a cell-weighted mixture } \\
\text { (Section } 7.1 .2 .4 \text { ). }\end{array}$ \\
\hline 11 & END & & $\begin{array}{l}\text { Terminate } \\
\text { LATTICECELL } \\
\text { data }\end{array}$ & Always & $\begin{array}{l}\text { Terminate the LATTICECELL input data by } \\
\text { entering the word END. Do not start in column } 1 \text {. } \\
\text { At least two blanks or a new line must follow } \\
\text { END. }\end{array}$ \\
\hline
\end{tabular}




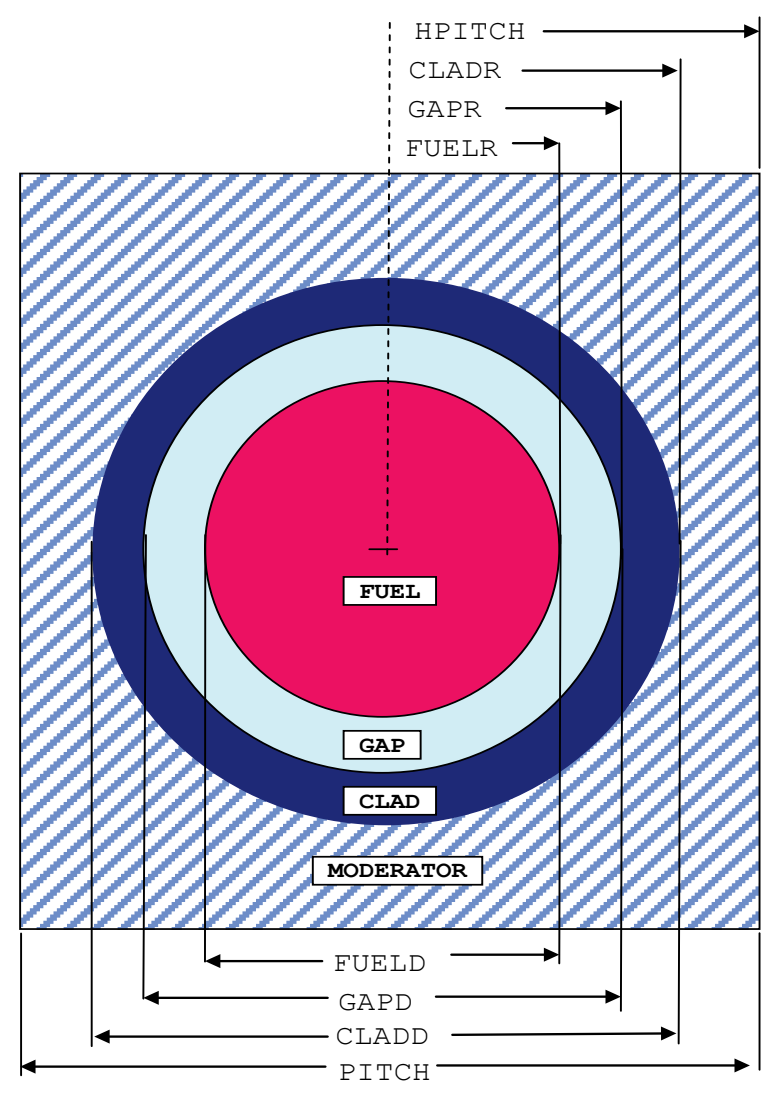

Figure 7.1.1. Arrangement of materials in a SQUAREPITCH and SPHSQUAREP unit cell.

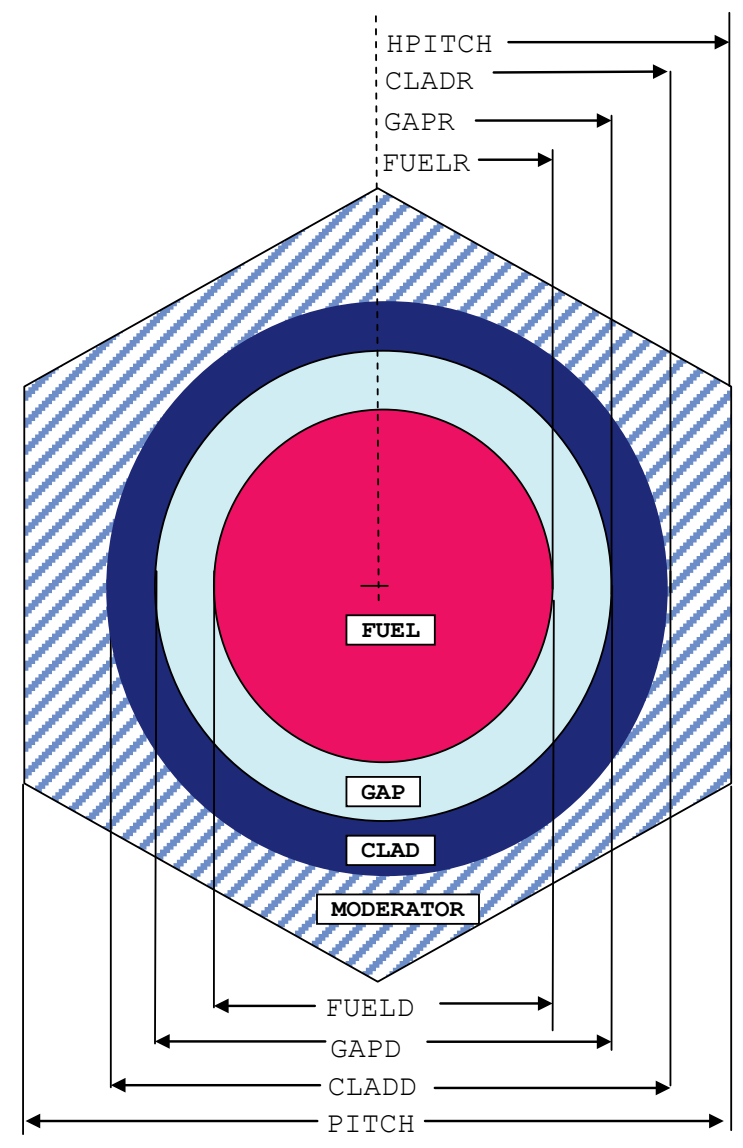

Figure 7.1.2. Arrangement of materials in a TRIANGPITCH and SPHTRIANGP unit cell. 


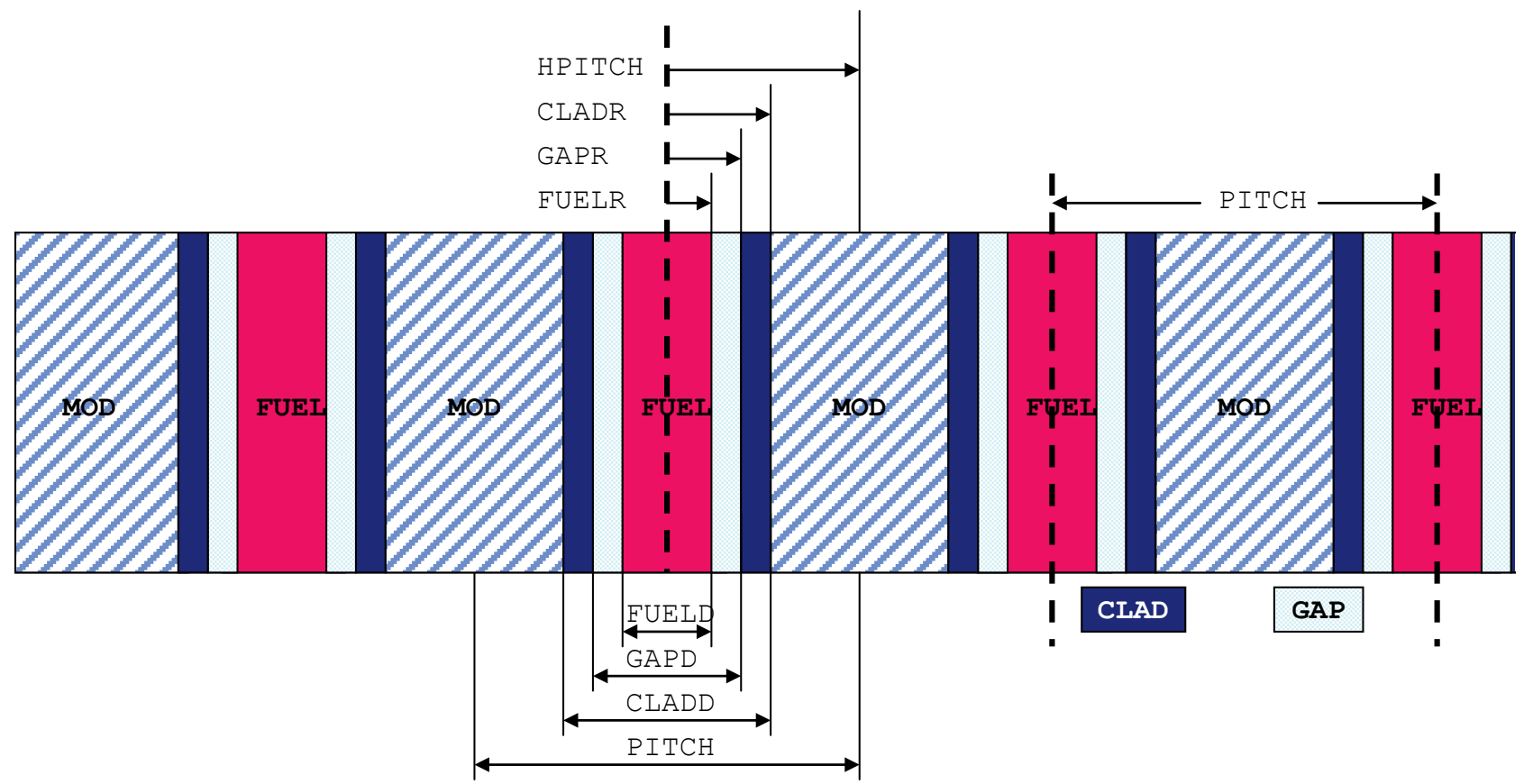

Figure 7.1.3. Arrangement of materials in a SYMMSLABCELL unit cell having reflected left and right boundary conditions. 


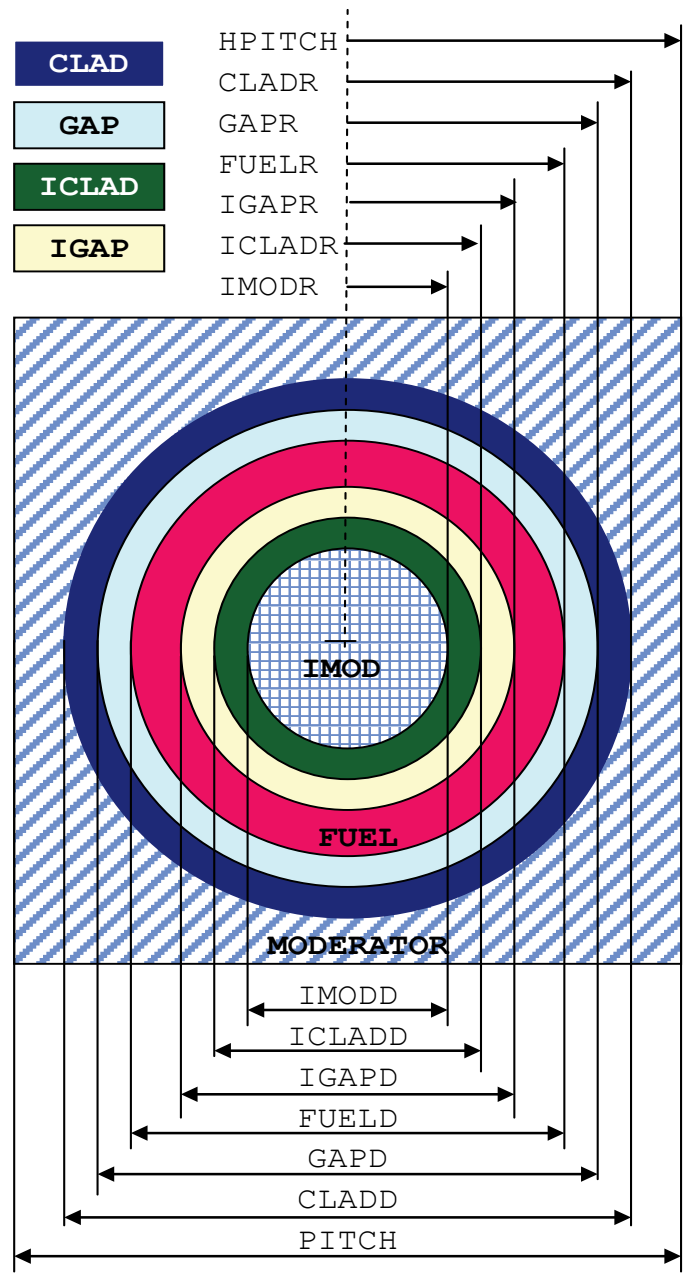

Figure 7.1.4. Arrangement of materials in an ASQUAREPITCH and ASPHSQUAREP unit cell.

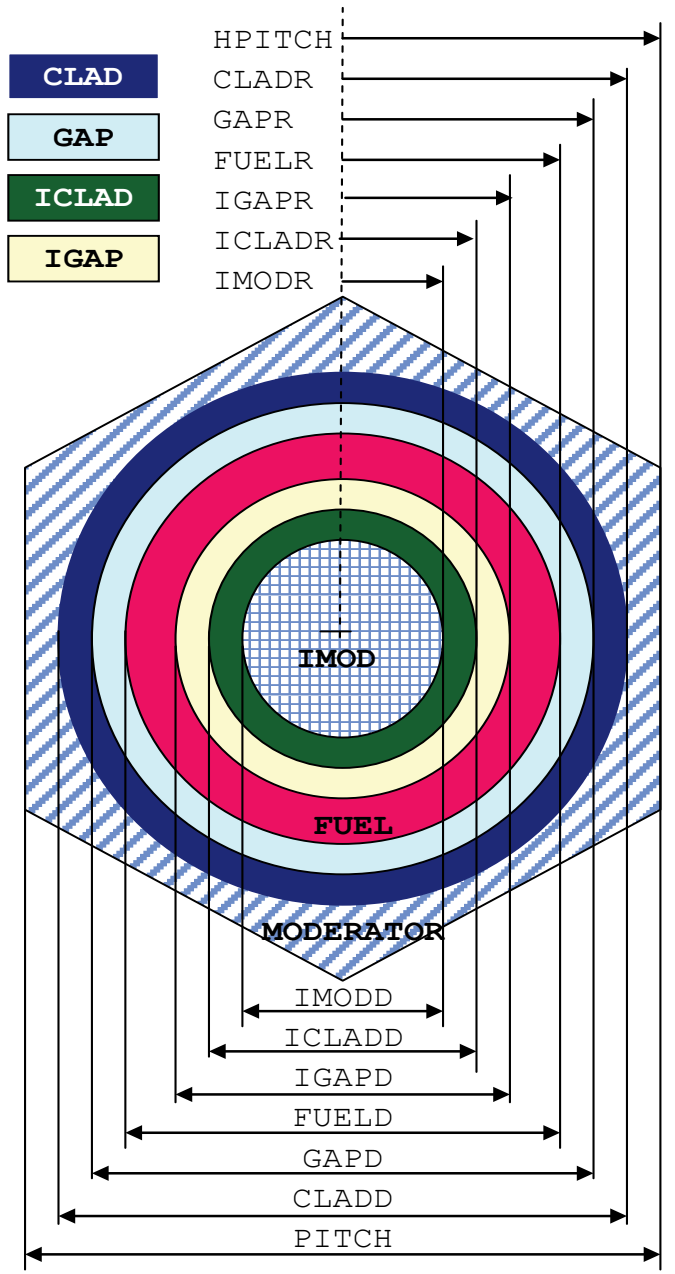

Figure 7.1.5. Arrangement of materials in an ATRIANGPITCH and ASPHTRIANGP unit cell. 


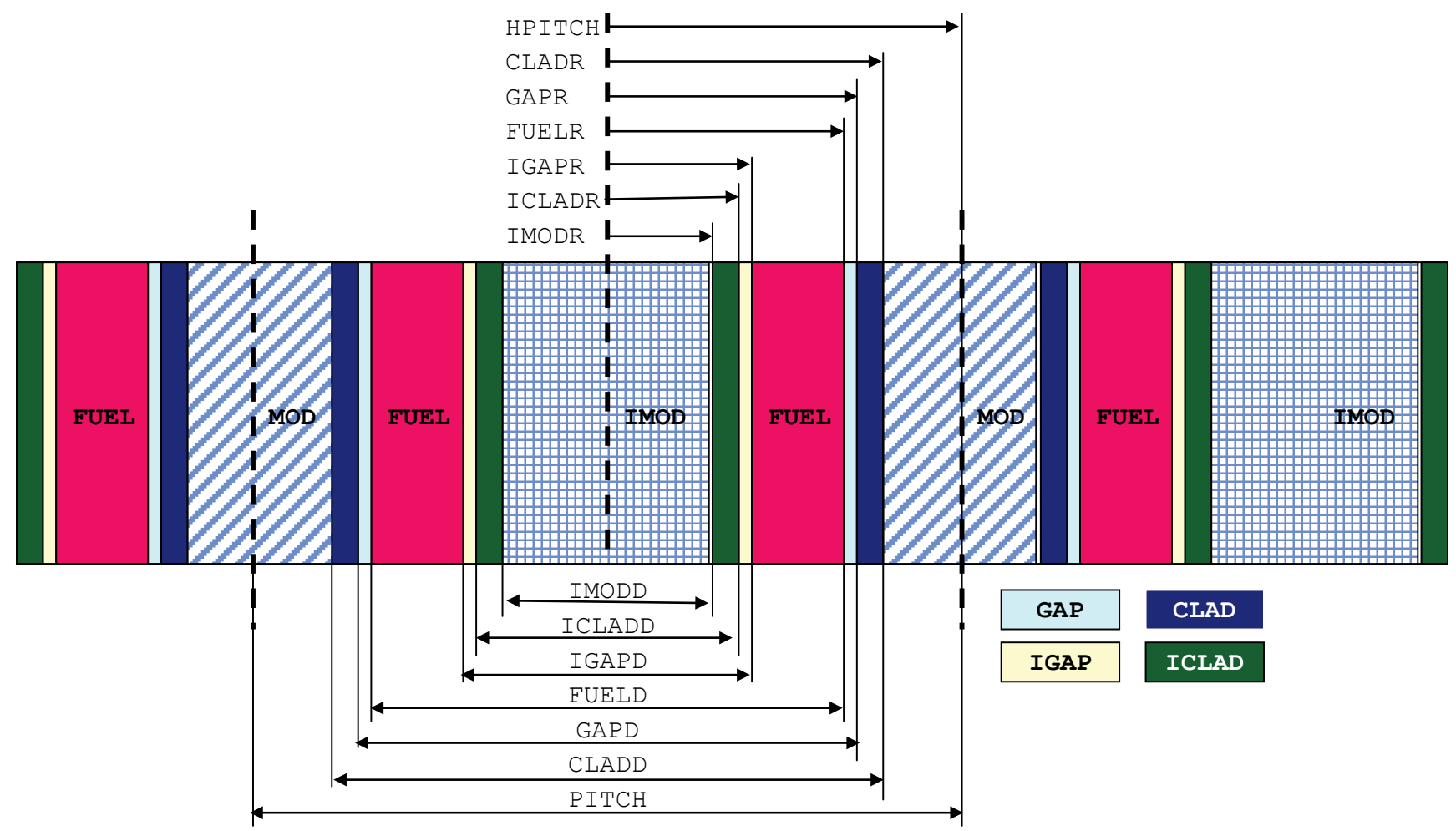

Figure 7.1.6. Arrangement of materials in an ASYMSLABCELL unit cell having reflected left and right boundary conditions.

\subsubsection{Unit cell specification for MULTIREGION cells}

A MULTIREGION cell can be used to define a 1-D geometric arrangement that is more general than allowed by a LATTICECELL. It can also be used for large geometric regions where the geometry effects for the cross sections are small. For CENTRM/PMC self-shielding, lattice effects can be approximated by applying reflected, periodic, or white external boundary conditions to a MULTIREGION cell. HOWEVER, MULTIREGION CELLS SHOULD NOT BE USED FOR BONAMI-ONLY SELF-SHIELDING OF AN ARRAY UNIT CELL. In this case a LATTICECELL should always be used for BONAMI self-shielding in order to incorporate the proper Dancoff effects.

The data required for a MULTIREGION cell are given in Table 7.1.5 and explained in the following text.

1. celltype

MULTIREGION. The keyword MULTIREGION is used to represent arbitrary 1-D geometries, with no restrictions the on number or placement of mixtures in the cell. The keyword may be truncated to any number of characters as long as the characters presented are identical from the beginning of the keyword (i.e., $\mathrm{M}$ is acceptable). 
TYPE OF GEOMETRY. The type of geometry must always be specified for a MULTIREGION cell. The available geometry options are listed below.

SLAB. This is used to describe a slab geometry.

CYLINDRICAL. This is used to describe cylindrical geometry.

SPHERICAL. This is used to describe spherical geometry.

BUCKLEDSLAB. This is used for slab geometry with a buckling correction for the two transverse directions.

BUCKLEDCYL. This is used for cylindrical geometry with a buckling correction in the axial direction.

3. RIGHT_BDY RIGHT BOUNDARY CONDITION. This is defaulted to VACUUM. The available options and their qualifications are listed below.

VACUUM. This imposes a vacuum at the boundary of the system.

REFLECTED. This imposes mirror image reflection at the boundary. Do not use for CYLINDRICAL or SPHERICAL.

PERIODIC. This imposes periodic reflection at the boundary. Do not use for CYLINDRICAL or SPHERICAL.

WHITE. This imposes isotropic return at the boundary.

4. LEFT_BDY LEFT BOUNDARY CONDITION. This is defaulted to REFLECTED. The available options and their qualifications are listed below.

VACUUM. This imposes a vacuum at the boundary of the system.

REFLECTED. This imposes mirror image reflection at the boundary. For CYLINDRICAL or SPHERICAL, this is the only valid boundary condition because the left boundary corresponds to the centerline of the cylinder or the center of the sphere.

PERIODIC. This imposes periodic reflection at the boundary.

WHITE. This imposes isotropic return at the boundary.

5. ORIGIN LOCATION OF LEFT BOUNDARY ON THE ORIGIN. The default value of ORIGIN is 0.0. This is the only value allowed for CYLINDRICAL or SPHERICAL geometry. For SLABs, enter the location of the left boundary on the $\mathrm{X}$-axis perpendicular to the slab (in $\mathrm{cm})$.

6. DY

BUCKLING HEIGHT. This is the buckling height in $\mathrm{cm}$. It corresponds to one of the transverse dimensions of an actual 3-D slab assembly or the length of a finite cylinder. 
7. DZ

8. CELLMIX

9. END

10. $\mathbf{m x z}$

11. rz

12. END ZONE
BUCKLING DEPTH. This is the buckling width in $\mathrm{cm}$. It corresponds to the second transverse dimension of an actual 3-D slab assembly.

CELL-WEIGHTED MIXTURE NUMBER. Enter ONLY if a cell-weighted mixture is required. Enter a unique mixture number used to create a cell-weighted homogeneous mixture (Section 7.1.2.4).

The word END is entered to terminate these data before entering the zone description data. It must not be entered in columns 1 through 3, and at least two blanks must separate it from the zone description. A label can be associated with this END. The label can be a maximum of 12 characters and is separated from the END by a single blank. At least two blanks must follow this entry.

The zone description data are entered at this point. Entries 10 and 11 are entered for each zone, and the sequence is repeated until all the desired zones have been described. To terminate the data, enter the words END ZONE. Zone dimensions must be in increasing order.

MIXTURE NUMBER IN THE ZONE. Enter the mixture number of the material that is present in the zone. Enter a zero for a void. Repeat the sequence of entries 10 and 11 for each zone. Mixtures other than zero must not be used more than once in a cell and may be used in no more than one cell.

OUTSIDE RADIUS OF THE ZONE. Enter the outside dimension of the zone in $\mathrm{cm}$. In SLAB geometry, $\mathbf{r z}$ is the location of the zone's right boundary on the X-axis. Repeat the sequence of entries 10 and 11 for each zone.

Is used to terminate the MULTIREGION zone data. Enter the words END ZONE when all the zones have been described. Note that ZONE is a label associated with this END. This label can be as long as 12 characters, but the first four characters must be ZONE. At least two blanks must follow this entry. 
Table 7.1.5. Unit cell specification for MULTIREGION problems

\begin{tabular}{|c|c|c|c|c|}
\hline $\begin{array}{c}\text { Entry } \\
\text { no. }\end{array}$ & $\begin{array}{c}\text { Input } \\
\text { keyword }\end{array}$ & $\begin{array}{l}\text { Data following } \\
\text { keyword }\end{array}$ & $\begin{array}{l}\text { Entry } \\
\text { requirement }\end{array}$ & Comments \\
\hline 1 & MULTIREGION & & Always & $\begin{array}{l}\text { Keyword to begin multiregion unit cell. Enter } \\
\text { the keyword MULTIREGION. This word } \\
\text { may be truncated to any number of letters as } \\
\text { long as they exactly replicate the beginning } \\
\text { part of the keyword (e.g., M is acceptable). }\end{array}$ \\
\hline 2 & & & Always & $\begin{array}{l}\text { One of the following keywords is specified. } \\
\text { This keyword determines the type of unit cell } \\
\text { geometry. }\end{array}$ \\
\hline & SLAB & & & Used for slab geometry. \\
\hline & CYLINDRICAL & & & Used for cylindrical geometry. \\
\hline & SPHERICAL & & & Used for spherical geometry. \\
\hline & BUCKLEDSLAB & & & $\begin{array}{l}\text { Used for slab geometry with a buckling } \\
\text { correction for the two transverse directions. }\end{array}$ \\
\hline & BUCKLEDCYL & & & $\begin{array}{l}\text { Used for cylindrical geometry with a } \\
\text { buckling correction in the axial direction. }\end{array}$ \\
\hline 3 & RIGHT_BDY & $\begin{array}{l}\text { VACUUM } \\
\text { REFLECTED } \\
\text { PERIODIC } \\
\text { WHITE }\end{array}$ & $\begin{array}{l}\text { Optional for all } \\
\text { geometries }\end{array}$ & $\begin{array}{l}\text { Default is VACUUM. Describes the } \\
\text { right/outer boundary condition. This provides } \\
\text { a non-return condition at the boundary. } \\
\text { REFLECTED or PERIODIC not allowed } \\
\text { for cylindrical or spherical. }\end{array}$ \\
\hline 4 & LEFT_BDY & $\begin{array}{l}\text { VACUUM } \\
\text { REFLECTED } \\
\text { PERIODIC } \\
\text { WHITE }\end{array}$ & $\begin{array}{l}\text { Optional for slab } \\
\text { type geometries }\end{array}$ & $\begin{array}{l}\text { Default is REFLECTED. Describes the } \\
\text { left/inner boundary condition. Do not change } \\
\text { for cylindrical or spherical. } \\
\text { VACUUM provides a non-return condition } \\
\text { at the boundary. WHITE provides isotropic } \\
\text { return at the boundary. }\end{array}$ \\
\hline 5 & ORIGIN & $\begin{array}{l}\text { Left boundary } \\
\text { location }(\mathrm{cm})\end{array}$ & $\begin{array}{l}\text { Optional for slab } \\
\text { type geometries }\end{array}$ & $\begin{array}{l}\text { Default is } 0.0 \text {. Should not be changed for } \\
\text { cylindrical or spherical geometry. }\end{array}$ \\
\hline 6 & DY & $\begin{array}{l}\text { Buckling height } \\
\qquad(\mathrm{cm})\end{array}$ & & $\begin{array}{l}\text { OMIT FOR SLAB, CYLINDRICAL, and } \\
\text { SPHERICAL. This corresponds to one of } \\
\text { the transverse dimensions of an actual } 3-D \\
\text { slab assembly or to the length of a finite } \\
\text { cylinder. }\end{array}$ \\
\hline 7 & DZ & $\begin{array}{l}\text { Bucking depth } \\
(\mathrm{cm})\end{array}$ & & $\begin{array}{l}\text { OMIT UNLESS BUCKLEDSLAB IS } \\
\text { SPECIFIED. This is the buckling depth } \\
\text { corresponding to the second transverse } \\
\text { dimension of a 3-D slab assembly. }\end{array}$ \\
\hline
\end{tabular}


Table 7.1.5. Unit cell specification for MULTIREGION problems (continued)

\begin{tabular}{|c|c|c|c|c|}
\hline $\begin{array}{c}\text { Entry } \\
\text { no. }\end{array}$ & $\begin{array}{c}\text { Input } \\
\text { keyword }\end{array}$ & $\begin{array}{l}\text { Data following } \\
\text { keyword }\end{array}$ & $\begin{array}{l}\text { Entry } \\
\text { requirement }\end{array}$ & Comments \\
\hline 8 & CELLMIX & $\begin{array}{l}\text { Unique } \\
\text { mixture no. }\end{array}$ & $\begin{array}{l}\text { Cell-weighted } \\
\text { mixture required }\end{array}$ & $\begin{array}{l}\text { Used if a cell-weighted mixture is specified. } \\
\text { Enter a unique mixture number in the } \\
\text { problem (Section 7.1.2.4). }\end{array}$ \\
\hline 9 & END & & Always & $\begin{array}{l}\text { Enter the word END. Do not start in } \\
\text { column 1. At least two blanks or a new line } \\
\text { must separate END from the next entry. }\end{array}$ \\
\hline 10 & & $\begin{array}{l}\text { Zone mixture } \\
\text { number }\end{array}$ & Always & $\begin{array}{l}\text { Enter mixture number in zone. Repeat } 10 \text { and } \\
11 \text { until all zones are specified. }\end{array}$ \\
\hline 11 & & $\begin{array}{l}\text { Zone outside } \\
\text { radius }(\mathrm{cm})\end{array}$ & Always & $\begin{array}{l}\text { Enter outside radius of zone. Repeat } 10 \text { and } \\
11 \text { until all zones are specified. }\end{array}$ \\
\hline 12 & END ZONE & & Always & $\begin{array}{l}\text { Terminates MULTIREGION data. At least } \\
\text { two blanks must follow. }\end{array}$ \\
\hline
\end{tabular}




\subsubsection{Unit cell specification for doubly heterogeneous (DOUBLEHET) cells}

The data required for a DOUBLEHET cell are given in Table 7.1.6 and explained in the following text.

Details about the computation procedures for DOUBLEHET cells can be found in Section 7.1.2.3.

"Grain" refers to a spherical fuel particle surrounded by one or more coating zones and does not include the matrix material the grains are in. "Grain type" refers to a grain that has specified dimensions and mixtures such as a $0.025-\mathrm{cm}$-radius $\mathrm{UO}_{2}$ fuel kernel with a $0.01-\mathrm{cm}$-thick carbon coating. Another grain type could be a $0.012-\mathrm{cm}$-radius $\mathrm{PuO}_{2}$ fuel kernel with a $0.01-\mathrm{cm}$-thick carbon coating. The user must first define all grain types in a fuel element. Next, all fuel element-related data must be entered.

Since all grains and the matrix material are homogenized into a single uniform mixture for the fuel element, there are restrictions on how each grain type must be defined so that the volume fraction of each grain type within the homogenized fuel mixture can be determined. Related entries are PITCH, NUMPAR (number of particles), and VF (volume fraction). If there is only one grain type for a fuel element, the code needs the pitch and will directly use the input value if entered. If PITCH is not given, then the VF (if given) is used to calculate the pitch. If neither PITCH nor VF is given, then NUMPAR is used to calculate the pitch and the volume fraction. The user should only enter one of these items.

If more than one grain type is present, additional information is needed since all grain types are homogenized into a single mixture. Similar to the one grain type case, the pitch is needed to perform the CENTRM spherical cell calculations. However, the pitch by itself is not sufficient to perform the homogenization. Therefore, the user needs to input VF or NUMPAR for each grain type. Since each grain's volume is known (grain dimensions must always be entered), entering NUMPAR or VF for each grain type essentially provides the total volume of each grain type and therefore enables the calculation of the other unknowns (VF or NUMPAR, and PITCH). In this case, since pitch is not given, the available matrix material is distributed around the grains of each grain type proportional to the grain volume to calculate the corresponding pitch.

Syntax:

DOUBLEHET fuelmix END

$\mathbf{G F}(\mathbf{D} \mid \mathbf{R})=$ fuel $m g \quad(\mathbf{C O A T}(\mathbf{D} \mid \mathbf{R})=$ coat $\quad m c) \mid(\mathbf{C O A T T}=$ coat $\quad m c) \quad\{\mathbf{H}\} \mathbf{P I T C H}=$ mod $\quad$ MATRIX $=m m$ NUMPAR $=n p a r$ VF $=v f$ END GRAIN

mct ctp $\quad$ FUEL $(\mathbf{D} \mid \mathbf{R})=m f u e l \quad\{$ FUELH $=h f u e l\} \quad\{$ FUELW $=$ wfuel $\} \quad\{\mathbf{G A P}(\mathbf{D} \mid \mathbf{R})=m g a p \quad m m g\}$ $\{\mathbf{C L A D}(\mathbf{D} \mid \mathbf{R})=$ mclad $m m c\} \quad\{\mathbf{H}\}$ PITCH $=$ mpitch $m m m \quad\{\mathbf{C E L L M I X}=m c m x\}$ END

1. celltype DOUBLEHET. The keyword DOUBLEHET is used to represent a doubly heterogeneous problem such as fuel units that are composed of grains of fuel..

2. fuelmix HOMOGENIZED MIXTURE NUMBER. Enter a unique mixture number to be used for the homogenized grains and matrix material.

3. END The word END is entered to terminate these data before entering the grain and fuel element description data. It must not be entered in columns 1 through 3 , and at least two blanks must separate it from the zone description. A label can be associated with this END. The label can be a maximum of 12 characters and is separated from the END by a single blank. At least two blanks must follow this entry. 
The grain description data are entered at this point. Entries 5 through 12 are entered for each grain, and the sequence is repeated until all the grains have been described. To terminate the data, enter the words END GRAIN. Data may be entered in any order.

4. PITCH EQUIVALENT CELL DIMENSION. This is the equivalent spherical diameter (or or HPITCH radius), in $\mathrm{cm}$, of the "average" unit cell for this grain, as shown in Figure 7.1.7. Physically, the volume of the average unit cell is equal to the volume of the fuel element divided by the total number of all grain types.

5. GFD OUTSIDE DIMENSION OF FUEL. This is the outside diameter or radius of the fuel or GFR zone in a grain, fuel, in $\mathrm{cm}$ followed by the fuel mixture number, $m g$, as shown in Figure 7.1.7.

6. COATD OUTSIDE DIMENSION OF COATING. This is the outside diameter or radius of a or COATR coating zone, coat, in $\mathrm{cm}$ followed by the coating mixture number, $m c$, as shown in Figure 7.1.7. As many coating-mixture pairs as desired may be entered. If the coating dimensions are entered using COATD or COATR, then the COATT keyword should not be used.

7. COATT THICKNESS OF COATING. This is the thickness of a coating zone, coat, in $\mathrm{cm}$ followed by the coating mixture number, $m c$, as shown in Figure 7.1.7. As many coating-mixture pairs as desired may be entered. If the coating dimensions are entered using COATT, then the COATD or COATR keyword should not be used.

8. MATRIX MIXTURE NUMBER OF THE MATRIX MATERIAL. This is the mixture number, $m m$, of the matrix material that encloses the grains.

9. NUMPAR NUMBER OF PARTICLES. This is the number of grains, npar, of this type in each fuel element.

10. VF

VOLUME FRACTION. This is the volume fraction, $v f$, of grains of this type in each fuel element's fuel zone. A fuel element's fuel zone is entered using the entry number 16-FUELD (or FUELR).

11. END GRAIN This is used to terminate the grain zone data for this grain type. At least two blanks must follow this entry.

REPEAT ENTRIES 4-11 FOR EACH GRAIN TYPE IN A FUEL ELEMENT. 
12. met

13. ctp
TYPE OF FUEL ELEMENT (macro cell type). One of the keywords PEBBLE or ROD is entered to indicate the type of the fuel element, i.e., the second layer of heterogeneity. This data must be entered. The keyword may NOT be truncated. PEBBLE is used for spherical fuel elements; ROD is used for cylindrical fuel elements.

TYPE OF LATTICE. This defines the type of lattice or array configuration. Any one of the following alphanumeric descriptions may be used. Note that the alphanumeric description must be separated from subsequent data entries by one or more blanks. Figure 7.1.1 Mixture and position data are entered using keywords. Mixture number 0 may be entered for void and may be used multiple times in each and all unit cells. For regular cells, the minimum requirement is that a fuel region and a moderator region are specified and no inner components are specified. For annular cells, the minimum requirement is the fuel and outer moderator and inner moderator regions are specified. Regular and annular cell configurations are specified as shown below.

\section{Regular Cells}

SQUAREPITCH is used for an array of cylinders arranged in a square lattice, as shown in Figure 7.1.1. The clad and/or gap can be omitted.

TRIANGPITCH is used for an array of cylinders arranged in a triangular-pitch lattice as shown in Figure 7.1.2. The clad and/or gap can be omitted.

SPHSQUAREP is used for an array of spheres arranged in a square-pitch lattice. A cross section view through a cell is represented by Figure 7.1.1. The clad and/or gap can be omitted.

SPHTRIANGP is used for an array of spheres arranged in a triangular-pitch (dodecahedral) lattice. A cross section view through a cell is represented by Figure 7.1.2. The clad and/or gap can be omitted.

SYMMSLABCELL is used for an infinite array of symmetric slab cells, as shown in Figure 7.1.3. The clad and/or gap can be omitted.

Annular Cells

ASQUAREPITCH or ASQP is used for annular cylindrical rods in a square-pitch lattice as shown in Figure 7.1.4. The inner and outer clad and gap are independently entered so they may be different materials and dimensions.

ATRIANGPITCH or ATRP is used for annular cylindrical rods in a triangularpitch lattice as shown in Figure 7.1.5. The inner and outer clad and gap are independently entered, so they may be different materials and dimensions. 
ASPHSQUAREP

ASPHTRIANGP or ASSP is used for spherical shells in a square-pitch lattice as shown in Figure 7.1.4. The inner and outer clad and gap are independently entered, so they may be different materials and dimensions.

or ASTP is used for spherical shells in a triangular-pitch (dodecahedral) lattice as shown in Figure 7.1.5. The inner and outer clad and gap are independently entered, so they may be different materials and dimensions.

ASYMSLABCELL is used for a periodic, but asymmetric, array of slabs as shown in Figure 7.1.6. The inner and outer clad and gap are independently entered, so they may be different materials and dimensions.

14. PITCH or HPITCH

15. FUELD or FUELR

16. FUELH

17. FUELW

18. GAPD or GAPR

19. CLADD or CLADR

20. CELLMIX

21. IMODD or IMODR

22. IGAPD or IGAPR

23. ICLADD or ICLADR
ARRAY PITCH. This is the center-to-center spacing or half-spacing between the fuel lumps (pebbles or rods), mpitch, in $\mathrm{cm}$ followed by the outer moderator material number, $\mathrm{mmm}$, as shown in Figure 7.1.1 and Figure 7.1.2.

OUTSIDE DIMENSION OF FUEL. This is the outside diameter or radius of the fuel, mfuel, in cm, as shown in Figure 7.1.1 and Figure 7.1.2.

HEIGHT OF FUEL ROD OR SLAB. This is the height of the fuel, hfuel, in $\mathrm{cm}$.

WIDTH OF FUEL ROD. This is the width of the fuel, wfuel, in $\mathrm{cm}$.

OUTSIDE DIMENSION OF GAP. Enter only if outer gap is present. This is the outside diameter or radius of the outer gap, mgap, in $\mathrm{cm}$ followed by the gap mixture number, $m m g$, as shown in Figure 7.1.1 and Figure 7.1.2.

OUTSIDE DIMENSION OF CLAD. Enter ONLY if a clad is present. This is the outside diameter or radius of the outer clad, mclad, in $\mathrm{cm}$ followed by the clad mixture number, $m m c$, as shown in Figure 7.1.1 and Figure 7.1.2.

CELL-WEIGHTED MIXTURE NUMBER. Enter ONLY if cell-weighted mixture, $m c m x$, is to be created.

DIMENSION OF INNER MODERATOR. Enter ONLY if an annular cell is specified. This is the outside diameter or radius of the inner moderator, imod, in $\mathrm{cm}$ followed by the inner moderator mixture number, mim, as shown in Figure 7.1.4 through Figure 7.1.6.

OUTSIDE DIMENSION OF INNER GAP. Enter ONLY if an annular cell is specified and inner gap is present. This is the outside diameter or radius of the inner gap, igap, in $\mathrm{cm}$ followed by the inner gap mixture number, $\mathrm{mig}$, as shown in Figure 7.1.4 through Figure 7.1.6.

OUTSIDE DIMENSION OF INNER CLAD. Enter ONLY if an annular cell is specified and inner clad is present. This is the outside diameter or radius of the inner clad, iclad, in $\mathrm{cm}$ followed by the inner clad mixture number, mic, as shown in Figure 7.1.4 through Figure 7.1.6. 
The word END is entered to terminate the DOUBLEHET data. An optional label can be associated with this END. The label can be as many as 12 characters long and is separated from the END by a single blank. At least two blanks must follow this entry.

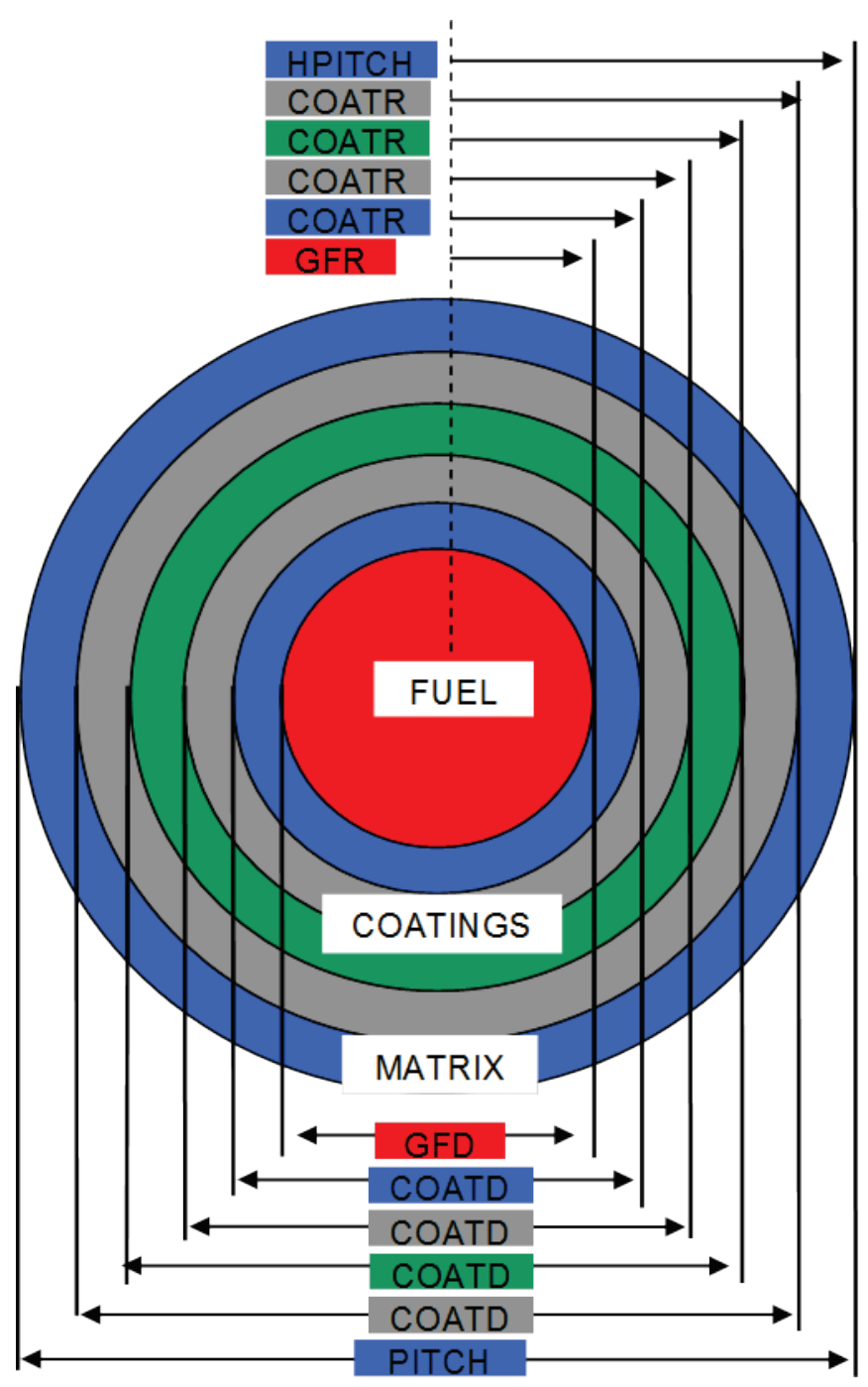

Figure 7.1.7. Arrangement of materials in a grain (first level cell) in a DOUBLEHET unit cell. 
Table 7.1.6. Unit cell specification for DOUBLEHET problems

\begin{tabular}{|c|c|c|c|c|}
\hline $\begin{array}{l}\text { Entry } \\
\text { No. }\end{array}$ & $\begin{array}{c}\text { Input } \\
\text { keyword }\end{array}$ & $\begin{array}{l}\text { Data following } \\
\text { keyword }\end{array}$ & $\begin{array}{l}\text { Entry } \\
\text { requirement }\end{array}$ & Comments \\
\hline 1 & DOUBLEHET & & Always & $\begin{array}{l}\text { The keyword DOUBLEHET is used to } \\
\text { represent cells that exhibit double } \\
\text { heterogeneity. The keyword may be } \\
\text { truncated to any number of characters as long } \\
\text { as the characters presented are identical from } \\
\text { the beginning of the keyword } \\
\text { (i.e., D is acceptable). }\end{array}$ \\
\hline 2 & fuelmix & $\begin{array}{l}\text { Fuel mixture } \\
\text { number }\end{array}$ & Always & $\begin{array}{l}\text { HOMOGENIZED MIXTURE NUMBER. } \\
\text { Enter a unique mixture number to be used for } \\
\text { homogenized grains and matrix material. }\end{array}$ \\
\hline 3 & END & & Always & $\begin{array}{l}\text { The word END is entered to terminate these } \\
\text { data before entering the grain and fuel } \\
\text { element description data. It must not be } \\
\text { entered in columns } 1 \text { through } 3 \text {, and at least } \\
\text { two blanks must separate it from the zone } \\
\text { description. A label can be associated with } \\
\text { this END. The label can be a maximum of } \\
12 \text { characters and is separated from the END } \\
\text { by a single blank. At least two blanks must } \\
\text { follow this entry. }\end{array}$ \\
\hline 4 & $\begin{array}{c}\text { PITCH } \\
\text { or HPITCH }\end{array}$ & Array pitch & Optional & $\begin{array}{l}\text { EQUIVALENT CELL DIMENSION. } \\
\text { This is the equivalent spherical diameter } \\
\text { (or radius), in } \mathrm{cm} \text {, of the "average" unit } \\
\text { cell for this grain, as shown in } \\
\text { Figure } 7.1 .7 . .\end{array}$ \\
\hline 5 & $\begin{array}{l}\text { GFD } \\
\text { or GFR }\end{array}$ & $\begin{array}{l}\text { Outside diameter } \\
\text { or radius of fuel } \\
\text { grain }+ \text { fuel } \\
\text { mixture }\end{array}$ & Always & $\begin{array}{l}\text { OUTSIDE DIMENSION OF FUEL. This is } \\
\text { the outside diameter or radius of the fuel } \\
\text { zone in a grain in cm followed by the } \\
\text { fuel mixture number, as shown in } \\
\text { Figure 7.1.7. }\end{array}$ \\
\hline
\end{tabular}


Table 7.1.6. Unit cell specification for DOUBLEHET problems (continued)

\begin{tabular}{|c|c|c|c|c|}
\hline $\begin{array}{l}\text { Entry } \\
\text { No. }\end{array}$ & $\begin{array}{c}\text { Input } \\
\text { keyword }\end{array}$ & $\begin{array}{l}\text { Data following } \\
\text { keyword }\end{array}$ & $\begin{array}{l}\text { Entry } \\
\text { requirement }\end{array}$ & Comments \\
\hline 6 & $\begin{array}{l}\text { COATD } \\
\text { or COATR }\end{array}$ & $\begin{array}{c}\text { Outside diameter } \\
\text { or radius of fuel } \\
\text { grain coating }+ \\
\text { coating mixture }\end{array}$ & Optional & $\begin{array}{l}\text { OUTSIDE DIMENSION OF COATING. } \\
\text { This is the outside diameter or radius of a } \\
\text { coating zone in cm followed by the } \\
\text { coating mixture number, as shown in } \\
\text { Figure } 7.1 .7 \text {. As many coating-mixture pairs } \\
\text { as desired may be entered. If the coating } \\
\text { dimensions are entered using COATD or } \\
\text { COATR, then the COATT keyword should } \\
\text { not be used. }\end{array}$ \\
\hline
\end{tabular}

THICKNESS OF COATING. This is the thickness of a coating zone in $\mathrm{cm}$ followed by the coating mixture number, as shown in

Thickness of fuel

7 COATT grain coating +

coating mixture
Optional Figure 7.1.7. As many coating-mixture pairs as desired may be entered. If the coating dimensions are entered using COATT, then the COATD or COATR keyword should not be used.

\begin{tabular}{|c|c|c|c|c|}
\hline 8 & MATRIX & $\begin{array}{l}\text { Matrix mixture } \\
\text { number }\end{array}$ & Always & $\begin{array}{l}\text { MIXTURE NUMBER OF THE MATRIX } \\
\text { MATERIAL. This is the mixture number of } \\
\text { the matrix material that encloses the grains. }\end{array}$ \\
\hline 9 & NUMPAR & $\begin{array}{l}\text { Total number } \\
\text { of grains }\end{array}$ & Optional & $\begin{array}{l}\text { NUMBER OF PARTICLES. This is the } \\
\text { number of grains of this type in each fuel } \\
\text { element. }\end{array}$ \\
\hline 10 & VF & $\begin{array}{c}\text { Volume fraction } \\
\text { of grains }\end{array}$ & Optional & $\begin{array}{l}\text { VOLUME FRACTION. This is the volume } \\
\text { fraction of grains of this type in each fuel } \\
\text { element's fuel zone. A fuel element's fuel } \\
\text { zone is entered using the entry number 16- } \\
\text { FUELD (or FUELR). }\end{array}$ \\
\hline 11 & END GRAIN & & Always & $\begin{array}{l}\text { This is used to terminate the grain zone data } \\
\text { for this grain type. At least two blanks must } \\
\text { follow this entry. }\end{array}$ \\
\hline 12 & met & $\begin{array}{l}\text { Type of fuel } \\
\text { element }\end{array}$ & Always & $\begin{array}{l}\text { TYPE OF FUEL ELEMENT (macro cell } \\
\text { type). One of the keywords PEBBLE or } \\
\text { ROD is entered to indicate the type of the fuel } \\
\text { element, i.e., the second layer of } \\
\text { heterogeneity. This data must be entered. The } \\
\text { keyword may NOT be truncated. PEBBLE is } \\
\text { used for spherical fuel elements; ROD is used } \\
\text { for cylindrical fuel elements. }\end{array}$ \\
\hline
\end{tabular}


Table 7.1.6. Unit cell specification for DOUBLEHET problems (continued)

\begin{tabular}{|c|c|c|c|c|}
\hline $\begin{array}{l}\text { Entry } \\
\text { No. }\end{array}$ & $\begin{array}{c}\text { Input } \\
\text { keyword }\end{array}$ & $\begin{array}{c}\text { Data following } \\
\text { keyword }\end{array}$ & $\begin{array}{c}\text { Entry } \\
\text { requirement }\end{array}$ & Comments \\
\hline 13 & ctp & $\begin{array}{l}\text { Macro cell } \\
\text { array type }\end{array}$ & Always & $\begin{array}{l}\text { TYPE OF LATTICE. This defines the type of } \\
\text { lattice or array configuration. Any one of the } \\
\text { following alphanumeric descriptions may be } \\
\text { used. Note that the alphanumeric description } \\
\text { must be separated from subsequent data } \\
\text { entries by one or more blanks. Mixture and } \\
\text { position data are entered using keywords. The } \\
\text { minimum requirement is that a fuel region } \\
\text { and a moderator region are specified. The cell } \\
\text { configurations are specified as shown below. }\end{array}$ \\
\hline
\end{tabular}

SQUAREPITCH is used for an array of cylinders arranged in a square lattice, as shown in Figure 7.1.1. The clad and/or gap can be omitted.

TRIANGPITCH is used for an array of cylinders arranged in a triangular-pitch lattice, as shown in Figure 7.1.2. The clad and/or gap can be omitted.

SPHSQUAREP is used for an array of spheres arranged in a square-pitch lattice. A cross section view through a cell is represented by Figure 7.1.1. The clad and/or gap can be omitted.

SPHTRIANGP is used for an array of spheres arranged in a triangular-pitch (dodecahedral) lattice. A cross section view through a cell is represented by Figure 7.1.2. The clad and/or gap can be omitted.

ASQUAREPITCH is used for an array of annular cylinders arranged in a square lattice, as shown in Figure 7.1.1. The clad and/or gap can be omitted.

ATRIANGPITCH is used for an array of annular cylinders arranged in a triangular-pitch lattice, as shown in Figure 7.1.2. The clad and/or gap can be omitted.

ASPHSQUAREP is used for an array of annular spheres arranged in a square-pitch lattice. A cross section view through a cell is represented by Figure 7.1.1. The clad and/or gap can be omitted.

ASPHTRIANGP is used for an array of annular spheres arranged in a triangular-pitch (dodecahedral) lattice. A cross section view through a cell is represented by Figure 7.1.2.

SYMMSLABCELL is used for an array of slab cells symmetric about the fuel zone. A cross section view through a cell is represented by Figure 7.1.3. The clad and/or gap can be omitted.

ASYMSLABCELL is used for an array of slab cells that are not symmetric about the fuel zone. A cross section view through a cell is represented by Figure 7.1.6. The clad and/or gap can be omitted. 
Table 7.1.6. Unit cell specification for DOUBLEHET problems (continued)

\begin{tabular}{|c|c|c|c|c|}
\hline 14 & $\begin{array}{c}\text { PITCH } \\
\text { or HPITCH }\end{array}$ & $\begin{array}{l}\text { Macro cell array } \\
\text { pitch }+ \\
\text { moderator } \\
\text { mixture }\end{array}$ & Always & $\begin{array}{l}\text { ARRAY PITCH. This is the center-to-center } \\
\text { spacing or half-spacing between the fuel } \\
\text { lumps (pebbles or rods) in cm followed by the } \\
\text { outer moderator material number, as shown in } \\
\text { Figure } 7.1 .1 \text { and Figure } 7.1 .2 \text {. }\end{array}$ \\
\hline 15 & $\begin{array}{c}\text { FUELD } \\
\text { or FUELR }\end{array}$ & $\begin{array}{l}\text { Macro cell fuel } \\
\text { diameter or } \\
\text { radius }\end{array}$ & Always & $\begin{array}{l}\text { OUTSIDE DIMENSION OF FUEL. This is } \\
\text { the outside diameter or radius of the fuel in } \\
\mathrm{cm} \text {, as shown in Figure } 7.1 .1 \text { and } \\
\text { Figure } 7.1 .2 \text {. }\end{array}$ \\
\hline 16 & FUELH & $\begin{array}{l}\text { Macro cell fuel } \\
\text { height }\end{array}$ & Optional & $\begin{array}{l}\text { HEIGHT OF FUEL ROD OR SLAB. This is } \\
\text { the height of the fuel in } \mathrm{cm} \text {. }\end{array}$ \\
\hline 17 & FUELW & $\begin{array}{l}\text { Macro cell fuel } \\
\text { width }\end{array}$ & Optional & $\begin{array}{l}\text { WIDTH OF FUEL SLAB. This is the width } \\
\text { of the fuel in } \mathrm{cm} \text {. }\end{array}$ \\
\hline 18 & $\begin{array}{l}\text { GAPD } \\
\text { or GAPR }\end{array}$ & $\begin{array}{l}\text { Macro cell gap } \\
\text { diameter or } \\
\text { radius + gap } \\
\text { mixture }\end{array}$ & Optional & $\begin{array}{l}\text { OUTSIDE DIMENSION OF GAP. Enter only } \\
\text { if outer gap is present. This is the outside } \\
\text { diameter or radius of the outer gap in } \mathrm{cm} \\
\text { followed by the gap mixture number, as } \\
\text { shown in Figure } 7.1 .1 \text { and Figure } 7.1 .2 \text {. }\end{array}$ \\
\hline 19 & $\begin{array}{c}\text { CLADD } \\
\text { or CLADR }\end{array}$ & $\begin{array}{l}\text { Macro cell clad } \\
\text { diameter or } \\
\text { radius }+ \text { clad } \\
\text { mixture }\end{array}$ & Optional & $\begin{array}{l}\text { OUTSIDE DIMENSION OF CLAD. Enter } \\
\text { ONLY if a clad is present. This is the outside } \\
\text { diameter or radius of the outer clad in } \mathrm{cm} \\
\text { followed by the clad mixture number, as } \\
\text { shown in Figure } 7.1 .1 \text { and Figure } 7.1 .2\end{array}$ \\
\hline 20 & $\begin{array}{l}\text { IMODD } \\
\text { or IMODR }\end{array}$ & $\begin{array}{c}\text { Macro cell inner } \\
\text { moderator } \\
\text { diameter or } \\
\text { radius }+ \\
\text { moderator } \\
\text { mixture }\end{array}$ & Optional & $\begin{array}{l}\text { OUTSIDE DIMENSION OF INNER } \\
\text { MODERATOR. Enter ONLY for annular cells } \\
\text { or asymmetric slab celss }\end{array}$ \\
\hline 21 & $\begin{array}{l}\text { IGAPD } \\
\text { or IGAPR }\end{array}$ & $\begin{array}{l}\text { Macro cell inner } \\
\text { gap diameter or } \\
\text { radius }+ \text { gap } \\
\text { mixture }\end{array}$ & Optional & $\begin{array}{l}\text { OUTSIDE DIMENSION OF INNER GAP } \\
\text { Enter ONLY if Annular cell + Inner Gap } \\
\text { Present }\end{array}$ \\
\hline 22 & $\begin{array}{l}\text { ICLADD } \\
\text { or ICLADR }\end{array}$ & $\begin{array}{l}\text { Macro cell inner } \\
\text { clad diameter or } \\
\text { radius }+ \text { clad } \\
\text { mixture }\end{array}$ & Optional & $\begin{array}{l}\text { OUTSIDE DIMENSION OF INNER CLAD. } \\
\text { Enter ONLY if Annular cell + Inner Clad } \\
\text { Present }\end{array}$ \\
\hline 23 & CELLMIX & $\begin{array}{l}\text { Cell-weighted } \\
\text { mixture number }\end{array}$ & Optional & $\begin{array}{l}\text { CELL-WEIGHTED MIXTURE NUMBER. } \\
\text { Enter ONLY if cell-weighted macro-cell } \\
\text { mixture is to be created (Section 7.1.2.4). }\end{array}$ \\
\hline
\end{tabular}


Table 7.1.6. Unit cell specification for DOUBLEHET problems (continued)

24

END
The word END is entered to terminate the DOUBLEHET data. An optional label can be associated with this END. The label can be as many as 12 characters long and is separated from the END by a single blank. At least two blanks must follow this entry.

\subsubsection{Optional MORE DATA parameter data}

MORE DATA is an optional sub-block of the READ CELL block. MORE DATA parameters allow certain default options in BONAMI and XSDRNPM to be modified for individual cell calculations. Each MORE DATA sub-block applies only to the unit cell immediately preceding it. However a MORE DATA sub-block placed prior to all unit cell definitions applies to all mixtures not assigned to a unit cell, which are treated as infinite homogeneous media. If the default parameters are acceptable, this section of input data should be omitted in its entirety. Non-default values for one or more of the parameters can be specified by entering the words MORE DATA followed by the desired keyword parameters and their associated values. One or more of the parameters can be entered in any order. Default values are used for parameters that are not entered. Each parameter is entered by spelling its name, followed immediately by an equal sign and the value to be entered. There should not be a blank between the parameter name and the equal sign. Each parameter specification must be separated from the rest by at least one blank. For example, if an XSDRNPM calculation is performed for particular unit cell (e.g., cellmix = is specified),

\section{MORE DATA ISN $=16$ EPS $=0.00008$ END MORE}

would result in using an S16 angular quadrature set and tightening the convergence criteria to 0.00008 in the XSDRNPM calculation.

A description of each entry is given. (Also see sections on BONAMI and XSDRNPM input description.)

1. MORE DATA These words, followed by one or more blanks, are entered ONLY if optional parameter data are to be entered. Entries 2 through 42 can be entered in any order.

2. NSENSX This is the XSDRNPM sensitivity output file for TSUNAMI sequences.

3. CROSSEDT BONAMI CROSS SECTION EDIT. Cross section print option for BONAMI 0/1 no/yes (default is 0 ).

4. FFACTEDT BONDARENKO FACTOR EDIT. Bondarenko factor (f-factor) print option 0/1 no/yes (default is 0 ).

5. ISSOPT BONAMI BACKGROUND XSEC OPTIONS. BONAMI background cross section selection option if $>1000$ potential; otherwise, total cross section is used (default is $1)$. 
6. IROPT BONAMI IR/NR CALCULATION OPTION. BONAMI uses intermediate resonance (IR) if iropt $=1$ and narrow resonance (NR) approximation for iropt $=0$ (default is 0 ).

7. BELLOPT BELL FACTOR OPTION. Optional user-defined bell factor calculation option (default is -1).

8. BELLFACT BELL FACTOR. Optional user-defined bell factor for BONAMI (default is 0.0 ).

9. ESCXSOPT ESCAPE CROSS SECTION CALC OPTION. Escape cross section calculation for IR calculations. $0 / 1=$ consistent/inconsis tent (default is 0 ).

10. BONAMIEPS BONAMI CONVERGENCE CRITERIA. BONAMI Bondarenko iteration convergence criteria (default is 0.001 ).

11. LBARIN INPUT MEAN CORD LENGTH. Mean cord length for each zone (default is 0.00).

12. ADJTHERM ADJUST 1D THERMAL CROSS SECTIONS TO MATCH SUM OF 2D CROSS SECTIONS. Flag determining whether 1-D cross sections are scaled to match the 2$\mathrm{D}$ cross sections or the 2-D cross sections are scaled to match the 1-D cross sections.

13. EXSIG

ESCAPE CROSS SECTION. External escape cross section for BONAMI (default is $0.00)$.

14. IEVT

XSDRNPM CALCULATION TYPE. The type of calculation to be performedfixed source, eigenvalue, alpha, zone width search, outer radius search, buckling search, direct buckling search (default is 1).

15. ICLC THEORY OPTION. Number of outer iterations to use an alternative theory (diffusion, infinite medium, or $\mathrm{B}_{\mathrm{N}}$ ) before using discrete ordinates. Negative values indicate alternative theory (default is 0 ).

16. IPVT PARAMETRIC EIGENVALUE SEARCH. 0 - none; 1 - search for eigenvalue equal PV; 2 - alpha search (default is 0 ).

17. IPP

WEIGHTED CROSS SECTION PRINT. 2 -> No print; -1 -> 1-D edit; 0-N - edit through PN cross section arrays (default is 2).

18. IFLU GENERALIZED ADJOINT CALCULATION. 0 is a standard calculation; 1 is a generalized adjoint calculation (default is 0 ).

19. IFSN FISSION SOURCE SUPPRESSION. Non-zero suppresses the fission source in a fixed source calculation (default is 0 ).

20. IQM

VOLUMETRIC FIXED SOURCES. The number of volumetric sources in a fixed source problem (default is 0 ).

21. IPM

BOUNDARY FIXED SOURCES. The number of boundary sources in a fixed source problem (default is 0 ).

22. XNF

SOURCE NORMALIZATION FACTOR. The value used to normalize the problem source (default is 1.0). 
23. VSC

24. EV

25. EQL

26. XNPM

27. ISN

28. SZF

29. IIM

30. ICM

31. EPS

32. PTC

33. BKL
VOID STREAMING CORRECTION. The height of a void streaming path in a cylinder or slab in centimeters (default is 0.0 ).

EIGENVALUE GUESS. Starting eigenvalue guess for a search calculation (default is $0.0)$.

INITIAL SEARCH CONVERGENCE. Initial eigenvalue search convergence (default is 0.0001).

DAMPING FACTOR. Damping factor used in search calculations (default is 0.75).

ORDER OF ANGULAR QUADRATURE FOR XSDRNPM. Quadrature sets are geometry-dependent quantities that are defaulted to order 8 by the XSProc for LATTICECELL and cylindrical MULTIREGION. The default is 32 for MULTIREGION slabs and spheres. See the automatic quadrature generator and Appendix B for a more detailed explanation.

SPATIAL MESH SIZE FACTOR FOR XSDRNPM. The size of the mesh intervals can be adjusted by entering a value for SZF, which is a multiplier of the mesh size. The default value is 1.0. A value between zero and 1.0 yields a finer mesh; a value greater than 1.0 yields a coarser mesh. If $\mathbf{S Z F} \leq 0$, the user specifies the number of mesh intervals in each zone immediately following the MORE DATA block. If $\mathbf{S Z F}=0$, the interval spacing is automatically generated, while if $\mathbf{S Z F}<0$ the intervals are equally spaced intervals in each zone.

MAXIMUM NUMBER OF INNER ITERATIONS FOR XSDRNPM. This is the maximum number of inner iterations to be used in the XSDRNPM calculation. The default value is 20 . See Appendix B for a more detailed explanation.

MAXIMUM NUMBER OF OUTER ITERATIONS FOR XSDRNPM. This is the maximum number of outer iterations to be used in the XSDRNPM calculation. The default value is 25 . If the calculation reaches the outer iteration limit, a larger value should be used. See Appendix B for a more detailed explanation.

OVERALL CONVERGENCE CRITERIA FOR XSDRNPM. This is used by XSDRNPM after each outer iteration to determine if the problem has converged. The default value of EPS is 0.00001 . A value less than 0.00001 tightens the convergence criteria; a larger value loosens the convergence criteria.

POINTWISE CONVERGENCE CRITERIA FOR XSDRNPM. This is the point flux convergence criteria used by XSDRNPM to determine if convergence has been achieved after an inner iteration. The default value for PTC is 0.000001. A smaller value tightens convergence; a larger value loosens it.

BUCKLING FACTOR FOR XSDRNPM. A buckling factor should be used ONLY for a MULTIREGION BUCKLEDSLAB or BUCKLEDCYL problem. Because cylinders are assumed to be infinitely long and slabs are assumed to be infinite in both transverse directions, the analytic sequence may tend to overestimate the total flux for a finite system. A buckling correction can be used to approximate the leakage 
34. IUS

35. $\mathbf{D A N}(\mathrm{mm})$

36. BAL

37. DY

38. DZ

39. $\mathrm{COF}$ from the system in the transverse direction(s). The extrapolation distance factor, BKL, is defaulted to 1.420892 .

UPSCATTER SCALING FLAG for XSDRNPM. This option allows the use of upscatter scaling to accelerate the solution or force convergence. The default value is zero, in which case upscatter scaling is not used. IUS $=1$ facilitates upscatter scaling. Guidelines are not available to indicate when upscatter scaling is needed. Some problems will not converge with it, and some will not converge without it. See Appendix B for a more detailed explanation.

DANCOFF FACTOR for the specified mixtures used in BONAMI and in the CENTRM 2REGION option. This value overrides the internally computed Dancoff factor used in the resonance correction for the specified mixture $\mathrm{mm}$. The Dancoff data are entered in the form $\mathbf{D A N}(\mathrm{mm})=$ Dancoff factor. Note that the parentheses must be entered as part of the data, and the mixture number, mm, must be enclosed in the parentheses. See Appendix B for additional details. (Note: this is not to be confused with the DAN2PITCH parameter in CENTRMDATA)

BALANCE TABLE PRINT FLAG for XSDRNPM. This allows control of the balance table print from XSDRNPM. The default value is FINE. BAL $=$ NONE suppresses the balance table print. $\mathbf{B A L}=\mathbf{A L L}$ prints all of the balance tables. BAL $=$ FINE prints only the fine-group balance tables. See Appendix B for additional details.

FIRST TRANSVERSE DIMENSION for XSDRNPM. This is the first transverse dimension, in $\mathrm{cm}$, used in a buckling correction to calculate the leakage normal to the principal calculation direction (the height of a slab or cylinder). It should only be entered if XSDRNPM is to create cell-weighted cross sections and/or calculate the eigenvalue of a cylinder or slab system of finite height for a LATTICECELL problem. $\mathbf{D Y}=$ is defaulted to an infinite height, or is set to DY for a buckled MULTIREGION cell description. A value entered here overrides any buckling height value entered in the MULTIREGION data.

SECOND TRANSVERSE DIMENSION for XSDRNPM. This is the second transverse dimension, in $\mathrm{cm}$, used for a buckling correction for a slab of finite width. It should only be entered if XSDRNPM is to create cell-weighted cross sections and/or calculate the eigenvalue of a LATTICECELL slab of finite width. DZ= is defaulted to an infinite width, or is set to DZ for a buckled MULTIREGION slab cell of finite width. A value entered here overrides any buckling depth value entered in the MULTIREGION data.

DIFFUSION COEFFICIENT FOR TRANSVERSE LEAKAGE CORRECTIONS IN XSDRNPM. The default value is 3 . The available options are as follows.

$\mathbf{C O F}=0 \quad$ sets a transport-corrected cross section for each zone

$\mathbf{C O F}=1$ use a spatially averaged diffusion coefficient for each zone 
$\mathbf{C O F}=2$ use a diffusion coefficient for all zones that is one-third of the diffusion coefficient determined from the spatially averaged transport cross section for all zones

$\mathbf{C O F}=3$ use a flux and volume weighting across all zones

See Appendix B or XSDRNPM Input/Output Assignments in the XSDRNPM chapter, $3 \$$ array, variable IPN for more details.

40. NT3

41. NT4

42. ADJ

43. NTA

44. NBU

45. NTC

46. NTD

47. FRD

48. FWR

49. WGT

50. $\mathrm{ZMD}(\mathrm{iz})$
UNIT WHERE XSDRNPM WRITES THE WEIGHTED LIBRARY. If XSDRN does a weighting calculation, this is the unit number it uses to write the weighted library on (default is 3 ).

UNIT WHERE XSDRNPM WRITES THE ANGULAR FLUXES. XSDRN writes the angular fluxes on this unit if it is non-zero (default is 16).

Adjoint mode flag for XSDRNPM. Set to 1 to cause XSDRNPM to solve the adjoint problem (default is 0$)$.

UNIT WHERE XSDRNPM WRITES THE ACTIVITIES. XSDRN writes the calculated activities on this unit if it is non-zero (default is 75).

UNIT WHERE XSDRNPM WRITES BALANCE TABLES. If the balance tables file is to be saved, enter the unit number where it is to be written (default is 76).

UNIT WHERE XSDRNPM WRITES THE DERIVED DATA. XSDRN writes the derived input data on this unit if it is non-zero (default is 73).

UNIT WHERE XSDRNPM WRITES THE DATA FOR A SENSITIVITY ANALYSIS. XSDRN writes the data for a sensitivity analysis on this unit if it is nonzero (default is 0$)$.

UNIT WHERE XSDRNPM READS INPUT FLUX GUESS. If greater than 0, a flux guess will be read from this unit.

UNIT WHERE XSDRNPM WRITES OUPUT FLUX. If greater than 0, the spacedependent multigroup scalar flux is written in binary format to this unit.

CROSS SECTION WEIGHTING FLAG for XSDRNPM. The default is 0 , not to perform cross section weighting. To turn on cross section weighting, a positive value should be entered. A value of 1 will weight the cross sections by nuclide; 2 will weight by mixture.

ZONE WIDTH MODIFIERs for an XSDRNPM search problem. This allows entering a zone width modifier for zone iz in the XSDRNPM problem description. The zone width data are entered in the following form:

\section{ZMD(iz) $=$ modifier}

Note that the parentheses must be entered as part of the keyword. The zone number iz, to which the modifier is applied, must be enclosed in the parentheses. The 
modifier is entered after the equal sign. See the "Dimension Search Calculations" description in the XSDRNPM chapter for more information.

51. INT(iz) NUMBER OF MESH INTERVALS FOR ZONE IZ in XSDRNPM. The default is 0 , which causes the number to be calculated. The data are entered in the following form:

\section{$\operatorname{INT}(\mathbf{i z})=$ number}

Note that the parentheses must be entered as part of the keyword. The zone number iz, for which the number of intervals is specified, must be enclosed in the parentheses. The number of intervals is entered after the equal sign.

52. KEF

DESIRED VALUE OF $k_{E F F}$ for an XSDRNPM zone width search. The default value is 1.0. If it is desired to search for some other value, such as 0.9 , then input it here.

53. KFM The first eigenvalue modifier used in an XSDRNPM search. This value is used to make the first change in the XSDRNPM search. The default value is -0.1 . A user may sometimes need to change this to make the search converge.

54. ID1

SCALAR FLUX PRINT CONTROL. The default value is -1 , which suppresses printing the scalar fluxes in XSDRNPM. See the XSDRNPM Input/Output Assignments section in the XSDRNPM chapter, 2\$ array, variable ID1 for allowed values and corresponding actions.

55. ISCT

ORDER OF SCATTERING for XSDRMPM. The default is 5 for all libraries.

56. ICON

TYPE OF WEIGHTING (see Cross-Section Weighting section in the XSDRNPM chapter).

INNERCELL - followed by integer N (zones in the cell). Cell weighting is performed over the $\mathrm{N}$ innermost regions in the problem. Nuclides outside these regions are not weighted.

CELL - $\quad$ cell weighting

ZONE $\quad-\quad$ zone weighting

REGION - $\quad$ region weighting

57. IGMF

NUMBER OF GROUPS IN COLLAPSED LIBRARY. Enter number of groups after equal sign, followed by group lower energy boundaries $(\mathrm{eV})$ in descending order. 
0-19 - $\quad$ cross sections are written only in the AMPX weighted library formats on logical 3. A weighted library is always written when $\mathrm{IFG}=1$.

The various values of ITP (modulo 10) are used to select the different transport cross section weighting options mentioned earlier. The options are as follows:

$\operatorname{ITP}=0,10, \ldots \sqrt{\left(\psi_{1}^{\mathrm{g}}+(D G \psi)\right)^{2}}$,

ITP $=1,11, \ldots$ absolute value of current

ITP $=2,12, \ldots D B^{2} \psi_{g}+$ outside leakage

$\operatorname{ITP}=3,13, \ldots \psi / \Sigma_{\mathrm{t}}^{\mathrm{g}}$

$\mathrm{ITP}=4,14, \ldots D B \psi_{\mathrm{g}}$

ITP $=$ Other values are reserved for future development and should not be used.

59. GAMMA_MT_LIST LIST OF GAMMA 1D REACTIONS ASSOCIATED WITH INPUT. A list of 1-D gamma reactions to be included on a condensed library for later use gamma_mt_list=numberEntries $\mathrm{mt} 1 \mathrm{mt} 2 \ldots \mathrm{mt}$ numberEntries.

60. NEUTRON_MT_LIST LIST OF NEUTRON 1D REACTIONS ASSOCIATED WITH INPUT. A list of 1-D neutron reactions to be included on a condensed library for later use neutron_mt_list=numberEntries $\mathrm{mt} 1 \mathrm{mt} 2 \ldots \mathrm{mt}$ numberEntries.

\section{NEUTRON_2D_LIST}

LIST OF NEUTRON 2D ARRAYS FOR THE MICRO LIBRARY. This list flags the finalizer to place 2-D arrays (currently MT 2, 4, 16) on the micro library for use in SAMS.

\section{ACTIVITY Enter:}

IAZ (number of activities)

IAI (calculate activities by zone or interval)

0 - zone

$1-$ interval

LACFX (unit number to which activities are written)

LAZ (IAZ sets of numbers consisting of the nuclide and process numbers for each activity) 
63. BAND

64. IPRT

65. GRAIN_K

66. SOURCE(iz)
NUMBER OF REBALANCE BANDS for XSDRNPM (default is 1).

CROSS SECTION PRINT CONTROL. The default value is -2 , which suppresses printing the cross sections in XSDRNPM. See XSDRNPM chapter, $2 \$$ array, variable IPRT for allowed value, and corresponding actions.

Flag to control execution of XSDRNPM after each grain calculation for a DOUBLEHET cell.

ZONE SOURCE for an XSDRNPM fixed source problem. This allows entering a source spectrum for zone iz in the XSDRNPM problem description. The source spectrum data are entered in the following form:

\section{SOURCE(iz) $=$ numEntries spectrum_grp_1 ... spectrum_grp_numEntries}

Note that the parentheses must be entered as part of the keyword. The zone number, iz, to which the spectrum is applied, must be enclosed by the parentheses. The numEntries follows the equal sign and must be less than or equal to the number of energy groups for the problem. It is followed by numEntries numbers defining the spectrum for the first numEntries groups for zone iz. Groups not defined are set to zero. The spectrum applies uniformly to zone iz. A different spectrum may be entered for different zones.

67. END MORE Terminate the optional parameter data.

\subsubsection{Optional CENTRM DATA parameter data}

The CENTRM DATA block defines input parameter values for the CENTRM, PMC and CRAWDAD modules. XSProc defines default values for these parameters which are adequate for most applications. If all default values are acceptable, this section of input data can be omitted. The CENTRM DATA block applies only to the unit cell immediately preceding it. CENTRM DATA placed prior to all unit cell data applies to all materials not listed in any unit cell. Parameter values are assigned by entering the words CENTRM DATA followed by the desired keyword parameters and their associated values. One or more parameters can be entered in any order. There should not be a blank between the parameter name and the equal sign. Each parameter specification must be separated from the rest by at least one blank. For example,

\section{CENTRM DATA ISN $=16$ PTC $=0.0008$ N1D $=1$ END CENTRM DATA}

A description of CENTRM DATA parameters is given below.

1. CENTRM DATA These words, followed by one or more blanks, are entered ONLY if optional parameter data are to be entered. They must precede all other optional parameter data. Entries 2 through 42 can be entered in any order. 
2. ISN

3. ISCT

4. IIM

5. IUP

6. NFST

7. NTHR

8. NPXS

9. ISVAR

10. ISCTI

11. NMF6
ORDER OF SN ANGULAR QUADRATURE FOR CENTRM. SN Quadrature sets are geometry-dependent quantities. Default value for ISN is 6 (only used for NFST and NTHR=0; and NPXS=1).

LEGENDRE POLYNOMIAL $\mathrm{P}_{\mathrm{N}}$ ORDER OF SCATTERING. These are used to determine the number of moments calculated for the scattering cross sections. Default value is 0 for 2-D MoC option and 1 for 1-D $S_{n}$, which have been found adequate for nearly all cases.

MAXIMUM NUMBER OF INNER ITERATIONS. This is the maximum number of inner iterations for Sn transport calculations in CENTRM. Default value is 10 .

MAXIMUM NUMBER OF OUTER ITERATIONS IN THERMAL RANGE. This is the maximum number of outer iterations used to converge PW flux changes caused by upscattering in the thermal range. Default value is 3 . More iterations $(\sim 15)$ may be required for higher accuracy in some cases.

FAST RANGE MULTIGROUP CALCULATION OPTION, E > DEMAX. This determines what type of calculation is done above DEMAX. The options are $(0) S_{N}$, (1) diffusion theory, (2) homogenized infinite medium, (3) zonewise infinite medium, or (6) 2D MoC lattice cell [NOTE: NFST=4,5 are deprecated]. Default value is $0\left(\mathrm{~S}_{\mathrm{N}}\right)$.

THERMAL RANGE MULTIGROUP CALCULATION OPTION, E $<$ DEMIN. This determines what type of calculation is done below DEMIN. The options include $(0) \mathrm{S}_{\mathrm{N}}$, (1) diffusion, (2) homogenized infinite medium, (3) zonewise infinite medium, or (6) 2-D MoC lattice cell [NOTE: NTHR=4,5 are deprecated]. Default value is $0\left(\mathrm{~S}_{\mathrm{N}}\right)$.

POINTWISE RANGE MULTIGROUP CALCULATION OPTION, DEMIN $<$ E $<$ DEMAX. This determines what type of calculation is done between DEMIN and DEMAX. The options include (0) MG calculation, (1) 1$\mathrm{D} \mathrm{S}_{\mathrm{N}}$, (2) collision probability, (3) homogenized infinite medium, (4) zonewise infinite medium, (5) two-region, or (6) 2-D MoC lattice cell. Default value is $1\left(\mathrm{~S}_{\mathrm{N}}\right)$, except for square-pitch LATTICECELL where the default is 6 (2D $\mathrm{MoC})$.

LINEARIZATION OPTION. This determines if the MG source and/or the cross sections are linearized in CENTRM calculations. Options for linearizing are (0) neither, (1) source, (2) cross section, or (3) both. Default value is 3.

LEGENDRE POLYNOMIAL $\mathrm{P}_{\mathrm{N}}$ ORDER OF SCATTERING IN THE INELASTIC RANGE. These are used to determine the number of moments calculated for the inelastic scattering cross sections. Default value is 0 , isotropic.

INELASTIC FLAG. This determines if inelastic data are used. The options are to include $(-1)$ no inelastic data, (0) discrete inelastic data, and (1) discrete inelastic and continuum. Default value is -1 . Use of NMF6=1 is not recommended due to long running times. 
12. IPRT

13. ID1

14. KERNEL

15. IPBT

16. IPN

17. IXPRT

18. MLIM

19. NLIM

20. EPS

21. PTC

22. B2

23. DEMIN
MIXTURE CROSS-SECTION OUTPUT OPTION. This determines the output of cross section. The options include $(-3)$ none, (-2) output macro PW cross sections to file "centrm.pw.macroxs", (-1) 1-D MG cross sections, (N) $\mathrm{P}_{0}$ to $\mathrm{P}_{\mathrm{N}}$ MG 2-D matrices. Default value is -3 , none.

FLUX EDIT OPTION. This option determines the output of flux energy spectra. The options are (-1) none, (0) print MG fluxes, (1) also print MG flux moments, (2) save CE fluxes on output file, “_centrm.pw_flux”. Default value is -1 .

BOUND KERNELS. This indicates use of CENTRM PW thermal kernel data $[S(\alpha, \beta)]$ for bound nuclides if $\mathbf{K E R N E L}=1$. If $\mathbf{K E R N E L}=0$, all thermal kernels are treated as free gas; Default is 1, use bound scattering kernels if available.

PRINT GROUP SUMMARY TABLES. Group summary tables for each zone are printed in CENTRM if greater than 0. Default is 0 . Balance ratios are not computed in thermal groups or for MoC option.

GROUP DIFFUSION COEFFICENT. Used for $\mathrm{DB}^{2}$ loss term. See XSDRNPM chapter for more information. Default is 2.

PRINT OPTION FOR CENTRM. This value is $>0$ if more information is printed to output. Default value is 0 , minimum output.

MASS VALUE RESTRICTION ON ORDER OF SCATTERING. Nuclides with mass ratios greater than MLIM are limited to a NLIM order of scattering. Default value is 100 .

ORDER OF SCATTERING RESTRICTION. This is the limiting order of scattering for all nuclides with mass ratios greater than MLIM. Default value is 0 .

INTEGRAL CONVERGENCE CRITERIA. This is used by CENTRM after each outer iteration to determine if the problem has converged. Default value is 0.001 . A value less than 0.0001 tightens the convergence criteria; a larger value loosens the convergence criteria.

POINTWISE CONVERGENCE CRITERIA. This is the point flux convergence criteria used by CENTRM to determine if convergence has been achieved after an inner iteration. Default value is 0.0001. A smaller value tightens convergence; a larger value loosens it.

MATERIAL BUCKLING FACTOR $\left(\mathrm{cm}^{-2}\right)$. This is used with a buckled system. If a buckled system is specified for a unit cell, the code will use this value. Default value is 0.0 .

LOWEST ENERGY OF POINTWISE FLUX CALCULATION. This value is the lowest energy $(\mathrm{eV})$ for which CENTRM calculates PW fluxes. Default is $0.001 \mathrm{eV}$. 
24. DEMAX

25. TOLE

26. FLET

27. DAN2PITCH

28. MRANGE

29. N2D

30. IXTR3

31. NPRT

32. NWT

33. MTT
HIGHEST ENERGY OF POINTWISE FLUX CALCULATION. This value is the highest energy $(\mathrm{eV})$ for which CENTRM calculates PW fluxes. Default is $20,000.0 \mathrm{eV}$, which encompasses the resolved resonance range of all actinides. It is recommended that DEMAX be $<500 \mathrm{keV}$.

CENTRM PW THINNING TOLERANCE. This is the tolerance used to thin the PW material cross sections after they are mixed. Default value is 0.001 .

FRACTIONAL LETHARGY CONSTRAINT. This is the maximum lethargy difference between points in the flux solution energy mesh. Smaller values increase the number of energy points. Default value is 0.1 .

CENTRM DANCOFF FACTOR SEARCH. Fuel Dancoff factor to search for a Dancoff-equivalent pitch used in the CENTRM cell calculation. Only applicable in LATTICECELL cases with fuel in center region, with SN or MoC transport solvers. Default is 0 , which indicates no pitch modification. NOTE! This option should not be used to enter Dancoff factors for the CENTRM 2REGION transport option-use EDAN(m) array in MOREDATA for these values.

PMC GROUP CROSS-SECTION PROCESSING RANGE. This option determines the range over which the group cross sections will be processed. The options are (0) compute new group cross section over the PW range, (1) over the resolved resonance range of each nuclide, or (2) over the PW flux range (DEMAX to DEMIN). Default value is 2 .

PMC ELASTIC MATRIX PROCESSING FLAG. This option determines how MG $\mathrm{P}_{\mathrm{N}}$ elastic scattering matrices are obtained. Options are $(-2)$ perform operations in both (-1) and (2); (-1) compute P0 self-scatter, then renormalize matrix to shielded 1-D elastic values; $(0)$ normalize original scatter matrix to shielded 1-D elastic values; (1) compute new $\mathrm{P}_{\mathrm{N}}$ moments of elastic matrix using scalar flux and S-wave kinematics; or (2) use flux-moments to compute "consistent PN" correction for diagonal elements of elastic $\mathrm{P}_{\mathrm{N}}$ components. Default value is -1 . For unit cell calculations in reactor lattices, option -2 may improve results. NOTE: option 0 is always used in thermal range

PMC $\mathrm{P}_{\mathrm{N}}$ ORDER FLAG. This option determines the maximum order of Legendre moments to be retained on output MG library. The default is 5; i.e., retain scattering moments up to $P_{5}$ if available on the input MG library. If $(-1)$ is entered, all elastic moments on the MG library are included.

PMC PRINT FLAG. This option determines what is printed to output. The options include (-1) minimum output, (0) standard output, (1) print 1-D cross sections, (2) print both $1-\mathrm{D}$ and 2 -D cross sections. Default value is -1 , minimum output.

PMC MULTIGROUP SPATIAL-WEIGHTING FLAG. This option determines if the MG data are (0) zone-weighted or (1) cell-weighted. Default value is 0 .

PMC MT PROCESSING FLAG. This option determines if reaction MTs are processed individually or treat dependencies explicitly. If MTT $=0$ all MTs are 
34. N1D

35. PMC_DILUTE

36. MTOUT

37. IBR

38. IBL

39. ALUMP

40. PMC_OMIT

41. PXSMEM

42. MOCMESH processed independently; if $\mathbf{M T T}=1$, all MTs are processed except 1, 27, and 101. These are then computed as follows: MT101 = sum MT102 - 114, MT27 $=$ MT18 + MT101, MT1 $=$ MT2 + MT4 + MT16 + MT17 + MT27. Default value is 1 .

PMC WEIGHTING FUNCTION FLAG. This is used to determine if (0) flux weighting or (1) current weighting is used to collapse the cross sections. Default value is 0 , flux weighting.

PMC INFINITELY DILUTE BACKGROUND. The background cross section $\left(\sigma_{0}\right)$ value above which materials are considered to be infinitely dilute in PMC. No resonance shielding corrections are performed for materials with background cross sections greater than $p m c$ dilute. Higher values of pmc_dilute result in more nuclides being processed. The default value is $1.0 \mathrm{E} 10$.

PW REACTION TYPES. Reactions included by CRAWDAD on PW library for MG processing in PMC: (0) all; (1) output only MTs 1, 2, 4, 102, 18, 452, 455, 456 (and 107 for ${ }^{10} \mathrm{~B}$ or ${ }^{7} \mathrm{Li}$ ); (2) all from option (1) and all inelastic MTs and 16. Default is MTOUT $=1$ for NMF6 $=-1$ and MTOUT $=2$ for $\mathbf{N M F 6}>-1$.

CENTRM RIGHT BOUNDARY TYPE. Type of boundary condition on right boundary of unit cell for CENTRM LATTICECELL calculations. See allowable IBR values in CENTRM. Default is white $($ IBR $=3)$ for 1D SN; 2D $\mathrm{MoC}$ transport option always uses reflected.

CENTRM LEFT BOUNDARY TYPE. Same as IBR, but for left boundary. Default is reflected $(\mathbf{I B L}=1)$.

MASS LUMPING FRACTION. A value in range [0.0, 1.0] indicates fractional mass lumping criterion for CENTRM. Value of 0 indicates no lumping applied. For example, ALUMP $=0.3$ means that materials are combined into one or more lumps such that their masses are within $+/-30 \%$ of the effective lump mass, while preserving the slowing-down power. This approximation reduces execution time. Default value is 0.2 .

PMC NUCLIDES SKIPPED. PMC normally processes problem-dependent (e.g., self-shielded) $M G$ cross sections for all materials. If PMC_OMIT=1, processing is only performed for materials contained in fuel mixtures. Default value is 0 (all materials processed).

CENTRM PW DATA STORAGE. Option to store PW data in memory or in external file during centrm execution. If PXSMEM=1, PW cross section data are stored by group in external scratch file during CENTRM calculation; if PXSMEM=0 (default value), all PW cross sections are kept in memory.

CENTRM MOC MESH OPTION. Pre-defined space mesh intervals for CENTRM MoC calculation: $0=>$ coarse mesh ( 1 interval per zone); $1=>$ regular mesh ( 4 intervals in fuel, 2 in moderator, 1 in others); $2=>$ fine mesh ( 8 in fuel, 4 in moderator, 1 in others). Default $=0$. 
43. MOCRAY

44. MOCPOL

45. MOCAZI
CENTRM MOC RAY SPACING. Distance between characteristic rays in CENTRM MoC calculation. Default $=0.02$.

CENTRM NUMBER OF MOC POLAR ANGLES. Allowable values are 2, 3, 4. Default=3 (only used for NPXS=6).

CENTRM NUMBER OF MOC AZIMUTHAL ANGLES. Allowable values are $2-16$. Default $=8$ (only used for NPXS $=6$ ).

46. MOCZONE_INT CENTRM MOC MESH BY ZONE. User-defined mesh intervals by zone; e.g., moczone_int $(1)=5$ defines five intervals for zone 1 ; zero value means not used. This overrides the predefined meshs described by MOCMESH

47. ISRC

48. XNF

49. ITERP

50. END CENTRM
CENTRM SOURCE TYPE. CENTRM can use a fission-spectrum source (isrc=1), an input source spectrum (isrc=0), or a combination(isrc=3) for transport. Default=1. spectrum and/or fixed source spectrum) is normalized to this value. Default=1.0.

CRAWDAD TEMPERATURE INTERPOLATION METHOD. Method to use for $\mathrm{CE}$ cross section interpolation $\quad 0=>$ combination of square-root( $\mathrm{T}$ ) and finite-difference; $1=>$ only square-root $(\mathrm{T}) ; 2=>$ only finite-difference. Default is 0 .

The word END is entered to terminate the optional parameter data. A label can be associated with this END. The label can be as long as 12 characters but must be preceded by a single blank. If this END is entered without a label, it must not begin in column 1. At least two blanks must follow this entry.
CENTRM SOURCE NORMALIZATION. The integrated source (fission- 


\subsubsection{References}

1. B. T. Rearden, R. A. Lefebvre, J. P. Lefebvre, K. T. Clarno, M. L. Williams, L. M. Petrie, and U. Mertyurek, "Modernization Enhancements in SCALE 6.2," PHYSOR 2014 - The Role of Reactor Physics Toward a Sustainable Future, Kyoto, Japan, September 28-October 3, 2014, on CD-ROM (2014).

2. B. T. Rearden, R. A. Lefebvre, J. P. Lefebvre, K. T. Clarno, M. A. Williams, L. M. Petrie, U. Mertyurek, B. R. Langley, and A. B. Thompson, "Modernization Strategies for SCALE 6.2," Joint International Conference on Mathematics and Computation (M\&C), Supercomputing in Nuclear Applications (SNA) and the Monte Carlo (MC) Method, Nashville, TN, April 19-23, 2015, on CD-ROM, American Nuclear Society, LaGrange Park, IL (2015).

3. M. L. Williams, "Resonance Self-Shielding Methodologies in SCALE," Nuclear Technology, 174, 149-168 (May 2011).

4. I. I. Bondarenko, Ed., Group Constants for Nuclear Reactor Calculations, Consultants Bureau, New York, 1964.

5. M. L. Williams and M. Asgari, "Computation of Continuous-Energy Neutron Spectra with Discrete Ordinates Transport Theory," Nucl. Sci. \& Engr., 121, 173-201 (1995). 


\section{APPENDIX 7.1.A Standard Composition Examples}

\subsection{A.1. Standard composition fundamentals}

The standard composition specification data are used to define mixtures using standardized engineering data entered in a free-form format. The XSProc uses the standard composition specification data and information from the Standard Composition Library to provide number densities for each nuclide of every defined mixture according to Eq.(7.1.A.1):

$$
\text { 1. } \mathrm{NO}=\frac{\mathrm{RHO} \times \mathrm{AVN} \times \mathrm{C}}{\mathrm{AWT}},
$$

where

NO is the number density of the nuclide in atoms $/ \mathrm{b}-\mathrm{cm}$,

RHO is the actual density of the nuclide in $\mathrm{g} / \mathrm{cm}^{3}$,

AVN is Avogadro's number, $6.02214199 \times 10^{23}$, in atoms $/ \mathrm{mol}$,

C is a constant, $10^{-24} \mathrm{~cm}^{2} / \mathrm{b}$,

AWT is the atomic or molecular weight of the nuclide in $\mathrm{g} / \mathrm{mol}$.

The actual density, RHO, is defined by

$$
\mathrm{RHO}=\mathrm{ROTH} \times \mathrm{VF} \times \mathrm{WGTF},
$$

where

RHO is the actual density of the standard composition in $\mathrm{g} / \mathrm{cm}^{3}$,

ROTH is either the specified density of the standard composition or the theoretical density of the standard composition in $\mathrm{g} / \mathrm{cm}^{3}$,

VF is a density multiplier compatible with ROTH as defined by Eq. (7.1.A.3),

WGTF is the weight fraction of the nuclide in the standard composition. This value is automatically obtained by the code from the Standard Composition Library. WGTF is 1.0 for a single nuclide standard composition.

$$
\mathrm{VF}=\mathrm{DFRAC} \times \mathrm{VFRAC}
$$

where

VF is the density multiplier,

DFRAC is the density fraction,

VFRAC is the volume fraction. 
To illustrate the interaction between ROTH and VF, consider an Inconel having a density of $8.5 \mathrm{~g} / \mathrm{cm}^{3}$. It is $7.0 \%$ by weight iron, $15.5 \%$ chromium, and $77.5 \%$ nickel. The Inconel occupies a volume of $4 \mathrm{~cm}^{3}$.

\section{Method 1:}

To describe the iron, enter 8.5 for ROTH and 0.07 for VF.

To describe the chromium, enter 8.5 for ROTH and 0.155 for VF.

To describe the nickel, enter 8.5 for ROTH and 0.775 for VF.

\section{Method 2:}

Do not enter the density, and by default the theoretical density of each component will be used for ROTH. DFRAC will be the ratio of the specified density to the theoretical density. The specified density of each component is the density of the Inconel $\times$ the weight fraction of that component.

$\begin{array}{cl}\text { Thus, the density of the iron is } & 8.5 \times 0.07=0.595 \mathrm{~g} / \mathrm{cm}^{3} \\ \text { chromium is } & 8.5 \times 0.155=1.318 \mathrm{~g} / \mathrm{cm}^{3} \\ \text { nickel is } & 8.5 \times 0.775=6.588 \mathrm{~g} / \mathrm{cm}^{3} .\end{array}$

To calculate DFRAC, the theoretical density of each material must be obtained from the table Elements and special nuclide symbols in the STDCMP chapter. These values are

$7.86 \mathrm{~g} / \mathrm{cm}^{3}$ for iron

$8.90 \mathrm{~g} / \mathrm{cm}^{3}$ for nickel

$7.20 \mathrm{~g} / \mathrm{cm}^{3}$ for chromium

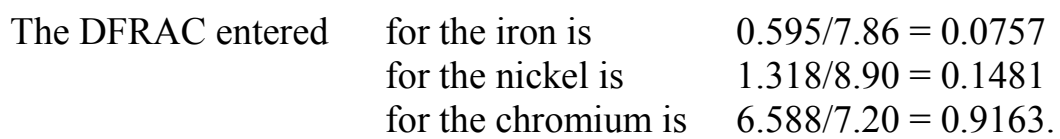

Since there are no volumetric corrections, VFRAC is 1.0 and the values of DFRAC are entered for VF.

\section{Method 3:}

Assume the Inconel, which occupies $4 \mathrm{~cm}^{3}$, is to be spread over a volume of $5 \mathrm{~cm}^{3}$. Then the volume fraction, VFRAC, is $4 \mathrm{~cm}^{3} / 5 \mathrm{~cm}^{3}=0.8$ and can be combined with the density fraction, DFRAC, to obtain the density multiplier, $\mathrm{VF}$, according to Eq.

To describe the iron, $\quad$ enter 8.5 for ROTH and $\quad 0.07 \times 0.8=0.056$ for VF or chromium, enter 8.5 for ROTH and $0.155 \times 0.8=0.124$ for VF for nickel, enter 8.5 for ROTH and $0.775 \times 0.8=0.620$ for VF.

Alternatively, the volume fraction can be applied to the density before it is entered. Then the ROTH can be entered as $8.5 \mathrm{~g} / \mathrm{cm}^{3} \times 0.8=6.8 \mathrm{~g} / \mathrm{cm}^{3}$, and DFRAC is entered for the density multiplier, VF.

To describe the iron, enter 6.8 for ROTH and 0.07 for VF for chromium, enter 6.8 for ROTH and 0.155 for VF for nickel, enter 6.8 for ROTH and 0.775 for VF. 


\section{Method 4:}

Assume the Inconel, which occupies $4 \mathrm{~cm}^{3}$, is to be spread over a volume of $5 \mathrm{~cm}^{3}$. Then the volume fraction, VFRAC, is $4 \mathrm{~cm}^{3} / 5 \mathrm{~cm}^{3}=0.8$. Do not enter the density, and by default the theoretical density of each component will be used for ROTH.

VF is then entered as the product of VFRAC and DFRAC according to Eq. (7.1.A.3). The specified density of each component is the density of the Inconel $\times$ the weight fraction of that component.

$\begin{array}{cl}\text { Thus, the density of the iron is } & 8.5 \times 0.07=0.595 \mathrm{~g} / \mathrm{cm}^{3} \\ \text { chromium is } & 8.5 \times 0.155=1.318 \mathrm{~g} / \mathrm{cm}^{3} \\ \text { nickel is } & 8.5 \times 0.775=6.588 \mathrm{~g} / \mathrm{cm}^{3} .\end{array}$

To calculate DFRAC, the theoretical density of each material must be obtained from Table 7.2.3. These values are

$7.86 \mathrm{~g} / \mathrm{cm}^{3}$ for iron

$8.90 \mathrm{~g} / \mathrm{cm}^{3}$ for nickel

$7.20 \mathrm{~g} / \mathrm{cm}^{3}$ for chromium.

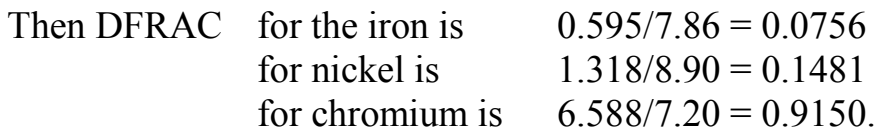

Then VF is DFRAC $\times$ VFRAC

VF for the iron is $\quad 0.0757 \times 0.8=0.0606$

for nickel is $\quad 0.1481 \times 0.8=0.1185$

for chromium is $\quad 0.9150 .8=0.7320$.

\subsection{A.2 Basic standard composition specifications}

EXAMPLE 1. Material name is given. Create a mixture 3 that is Plexiglas.

Since no other information is given, the information on the Standard Composition Library can be assumed to be adequate. Therefore, the only data to be entered are the standard composition name and the mixture number

PLEXIGLAS 3 END

EXAMPLE 2. Material name and density $\left(\mathrm{g} / \mathrm{cm}^{3}\right)$ are given.

Create a mixture 3 that is Plexiglas at a density of $1.15 \mathrm{~g} / \mathrm{cm}^{3}$. Since no other data are specified, the defaults from the Standard Composition Library will be used. Therefore, the only data to be entered are the standard composition name, the mixture number, and the density.

PLEXIGLAS 3 DEN=1.15 END 
EXAMPLE 3. Material name and number density (atoms/b-cm) are given. Create a mixture 2 that is aluminum having a number density of $\mathbf{0 . 0 6 0 2 4 4 .}$

AL $\quad 2 \quad 0 \quad .060244$ END

EXAMPLE 4. Material name, density $\left(\mathrm{g} / \mathrm{cm}^{3}\right)$ and isotopic abundance are given.

Create a mixture 1 that is uranium metal at $18.76 \mathrm{~g} / \mathrm{cm}^{3}$ whose isotopic composition is $93.2 \mathrm{wt} \%{ }^{235} \mathrm{U}$, $5.6 \mathrm{wt} \%{ }^{238} \mathrm{U}$, and $1.0 \mathrm{wt} \%{ }^{234} \mathrm{U}$, and $0.2 \mathrm{wt} \%{ }^{236} \mathrm{U}$. This example uses the DEN= keyword to enter the density and define the standard composition. Example 5 demonstrates another method of defining the standard composition.

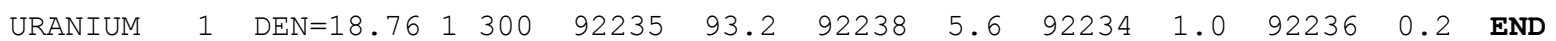

EXAMPLE 5. Material name, density $\left(\mathrm{g} / \mathrm{cm}^{3}\right)$ and isotopic abundance are given.

Create a mixture 7 defining $\mathrm{B}_{4} \mathrm{C}$ with a density of $2.45 \mathrm{~g} / \mathrm{cm}^{3}$. The boron is $40 \mathrm{wt} \%$

${ }^{10} \mathrm{~B}$ and $60 \mathrm{wt} \%{ }^{11} \mathrm{~B}$. This example utilizes the $\mathbf{D E N}=$ keyword. Example 6 illustrates an alternative description.

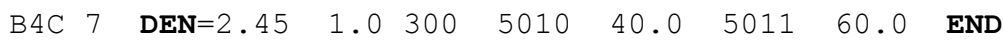

EXAMPLE 6. Material name, density $\left(\mathrm{g} / \mathrm{cm}^{3}\right)$ and isotopic abundance are given.

Create a mixture 7 defining $\mathrm{B}_{4} \mathrm{C}$ with a density of $2.45 \mathrm{~g} / \mathrm{cm}^{3}$. The boron is $40 \mathrm{wt} \%$ ${ }^{10} \mathrm{~B}$ and $60 \mathrm{wt} \%{ }^{11} \mathrm{~B}$. This example incorporates the known density into the density multiplier, $v f$, rather than using the DEN= keyword. The default density for $\mathrm{B}_{4} \mathrm{C}$ given in the COMPOUNDS table in the SCL section 7.2 is equal to $2.52 \mathrm{~g} / \mathrm{cm}^{3}$.

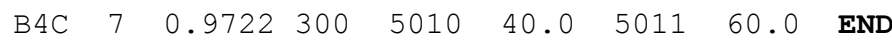

NOTE: In the above examples, the actual density is input for materials containing enriched multiisotope nuclides (uranium in Examples 4 and 5 and boron in Examples 6 and 7). The default density should never be used for enriched materials, especially low atomic mass neutron absorbers such as boron and lithium. The default density is a fixed value for nominal conditions and naturally occurring distributions of isotopes. Use of the default density for enriched materials will likely result in incorrect number densities.

\subsection{A.3 User-defined (arbitrary) chemical compound specifications}

The user-defined compound option allows the user to specify materials that are not found in the Standard Composition Library and can be specified by the number of atoms of each element or isotope that are contained in the molecule. To define a user-defined compound, the first four characters of the standard composition component name must be ATOM. The remaining characters of the standard composition component name are chosen by the user. The maximum length of the standard composition name is 16 characters. All the information that would normally be found in the Standard Composition Library must be entered in the user-defined compound specification. Section 7.1.3.3 contains data input details for arbitrary compounds. 
EXAMPLE 1. Density and chemical equation are given.

Create a mixture 3 that is a hydraulic fluid, $\mathrm{C}_{2} \mathrm{H}_{6} \mathrm{SiO}$, with a density of $0.97 \mathrm{~g} / \mathrm{cm}^{3}$.

The input data for this user-defined compound are given below:

ATOM $\quad 3 \quad 0.97 \quad 460002100161400018000 \quad 1$ END

EXAMPLE 2. Density and chemical equation are given. Create a mixture 7, TBP, also known as phosphoric acid tributyl ester or tributylphosphate, $\left(\mathrm{C}_{4} \mathrm{H}_{9} \mathrm{O}\right)_{3} \mathrm{PO}$, having a density of $0.973 \mathrm{~g} / \mathrm{cm}^{3}$.

$\begin{array}{llllll}\text { ATomtbp } & 7 & 0.973 \quad 41001276000128016415031 & 1 \text { end }\end{array}$

\subsection{A.4. User-defined (arbitrary) mixture/alloy specifications}

The user-defined compound or alloy option allows the user to specify materials that are not found in the Standard Composition Library and are defined by specifying the weight percent of each element or isotope contained in the material. To define a user-defined weight percent mixture, the first four characters of the standard composition component name must be wtpt. The remaining characters of the standard composition component name are chosen by the user. The maximum length of the standard composition name is 16 characters. All the information that would normally be found in the Standard Composition Library must be entered in the arbitrary mixture/alloy specification. Section 7.1.3.3 contains data input details for user-defined compounds.

EXAMPLE 1. Density and weight percents are given.

Create a mixture 5 that defines a borated aluminum that is $2.5 \mathrm{wt} \%$ natural boron. The density of the borated aluminum is $2.65 \mathrm{~g} / \mathrm{cm}^{3}$.

$\begin{array}{lllllllll}\text { WTPTBAL } & 5 & 2.65 & 2 & 5000 & 2.5 & 13027 & 97.5 & \text { END }\end{array}$

EXAMPLE 2. Density, weight percents, and isotopic abundance are given.

Create a mixture 5 that defines a borated aluminum that is $2.5 \mathrm{wt} \%$ boron. The boron is $90 \mathrm{wt} \%{ }^{10} \mathrm{~B}$ and $10 \mathrm{wt} \%{ }^{11} \mathrm{~B}$. The density of the borated aluminum is $2.65 \mathrm{~g} / \mathrm{cm}^{3}$. The minimum generic input specification for this arbitrary material is

$\begin{array}{llllllllllllll}\text { WTPTBAL } & 5 & 2.65 & 2 & 5000 & 2.5 & 13027 & 97.5 & 1 & 293 & 5010 & 90 . & 5011 & 10 . \text { END }\end{array}$

\subsection{A.5 Fissile solution specifications}

Solutions of fissile materials are available in the XSProc. A list of the available solution salts and acids is given in the table Available fissile solution components in section 7.2.3. When the XSProc processes a solution, it breaks the solution into its component parts (basic standard composition specifications) and uses the solution density to calculate the volume fractions.

EXAMPLE 1. Fuel density, excess acid and isotopic abundance are given.

Create a mixture 2 that is a highly enriched uranyl nitrate solution with $415 \mathrm{~g} / \mathrm{L}$ and $0.39 \mathrm{mg}$ of excess nitrate per gram of solution. The uranium isotopic content is $92.6 \mathrm{wt} \%{ }^{235} \mathrm{U}, 5.9 \mathrm{wt} \%{ }^{238} \mathrm{U}, 1.0 \mathrm{wt} \%{ }^{234} \mathrm{U}$, and $0.5 \mathrm{wt} \%{ }^{236} \mathrm{U}$. The temperature is 293 Kelvin. 


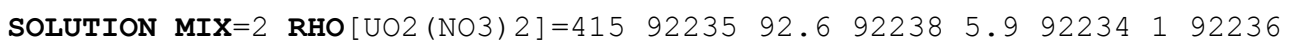

0.5 MASSFRAC $[\mathrm{HNO} 3]=6.339-6$ TEMPERATURE $=293$ END SOLUTION

where

The molecular weight of $\mathrm{NO}_{3}$ is $62.0049 \mathrm{~g} / \mathrm{mole}$, of $\mathrm{H}$ is $1.0078 \mathrm{~g} / \mathrm{mole}$, so the grams of excess $\mathrm{H}$ per gram of solution is $1.0078 / 62.0049 \times(0.39 \mathrm{mg} / \mathrm{g}) \times(1 \mathrm{~g} / 1000 \mathrm{mg})=6.339 \times 10^{-6}$.

\subsection{A.6 Combinations of standard composition materials to define a mixture}

Frequently more than one standard composition is required to define a mixture. This section contains such examples.

\section{EXAMPLE 1. Boral from $\mathrm{B}_{4} \mathrm{C}$ and Aluminum.}

Create a mixture 6 that is Boral, $15 \mathrm{wt} \% \mathrm{~B}_{4} \mathrm{C}$ and $85 \mathrm{wt} \% \mathrm{Al}$, having a density of $2.64 \mathrm{~g} / \mathrm{cm}^{3}$. Natural boron is used in the $\mathrm{B}_{4} \mathrm{C}$. Note that Example 2 demonstrates the use of the keyword DEN= to enter the density of the mixture and avoid having to look up the theoretical density from the table Isotopes in standard composition library, in the section 7.2.2, and calculate the density multiplier (VF)

$\begin{array}{rlll}\text { B4C } & 6 & 0.1571 & \text { END } \\ \text { AL } & 6 & 0.8305 & \text { END }\end{array}$

EXAMPLE 2. Boral from $\mathrm{B}_{4} \mathrm{C}$ and Aluminum.

This is the same problem as Example 1 using a different method of specifying the input data. Create a mixture 6 that is Boral, $15 \mathrm{wt} \% \mathrm{~B}_{4} \mathrm{C}$ and $85 \mathrm{wt} \% \mathrm{Al}$, having a density of $2.64 \mathrm{~g} / \mathrm{cm}^{3}$. Natural boron is used in the $\mathrm{B}_{4} \mathrm{C}$.

B4C $6 \quad$ DEN $=2.64 \quad 0.15 \quad$ END

AL $6 \quad$ DEN $=2.64 \quad 0.85 \quad$ END

\section{EXAMPLE 3. Boral from Boron, Carbon, and Aluminum.}

If neither Boral nor $\mathrm{B}_{4} \mathrm{C}$ were available in the Standard Composition Library, Boral could be described as follows:

Create a mixture 2 that is Boral composed of $35 \mathrm{wt} \% \mathrm{~B}_{4} \mathrm{C}$ and $65 \mathrm{wt} \%$ aluminum with an overall density of $2.64 \mathrm{~g} / \mathrm{cm}^{3}$. The boron is natural boron.

$v f \quad$ is the density multiplier. (The density multiplier is the ratio of actual to theoretical density.) From the Standard Composition Library chapter, table Isotopes in standard composition library, the theoretical density of aluminum is $2.702 \mathrm{~g} / \mathrm{cm}^{3}$; boron is $2.37 \mathrm{~g} / \mathrm{cm}^{3}$; and carbon is $2.1 \mathrm{~g} / \mathrm{cm}^{3}$. The density multiplier, $v f$, for $\mathrm{Al}$ is $(0.65)(2.64) / 2.702=0.63509$. The isotopic abundances in natural boron are known to have some variability. Here it is assumed that natural boron is $18.4309 \mathrm{wt} \%{ }^{10} \mathrm{~B}$ at $10.0129 \mathrm{amu}$ and $81.5691 \mathrm{wt} \%{ }^{11} \mathrm{~B}$ at $11.0096 \mathrm{amu}$. C is $12.000 \mathrm{amu}$.

Convert the weight percents to atom percents for the natural boron where $w$ denotes weight fraction, $a$ denotes atom fraction, and $M$ denotes atomic mass: 


$$
w_{B 10}=0.184309 \equiv \frac{a_{B 10} M_{B 10}}{a_{B 10} M_{B 10}+a_{B 11} M_{B 11}}=\frac{a_{B 10}(10.0129)}{\left.a_{B 10}(10.0129)+\left(1-a_{B 10}\right)\right)(11.0093)}
$$

Solving for $a_{B 10}$ gives:

$$
a_{B 10}=0.184309=\frac{(0.184309)(11.0093)}{(0.184309)(11.0093)-(0.184309)(10.0129)+(10.0129)}=19.900{ }^{10} \mathrm{~B} \mathrm{a} \%
$$

Therefore the atom percent of ${ }^{11} \mathrm{~B}$ is, $a_{B 11}=80.1 \mathrm{a} \%$.

Similarly, the mass of the $\mathrm{B}_{4} \mathrm{C}$ molecule is

$$
[(0.199 \times 4 \times 10.0129)+(0.801 \times 4 \times 11.0093)+(12.000)]=55.24407 \mathrm{amu} .
$$

The mass of the boron is $(55.24407-12.000)=43.24407 \mathrm{amu}$.

The $v f$ of boron would be $\left(\frac{43.24407}{55.24407}\right)\left(\frac{(0.35)(2.64)}{2.37}\right)=0.30519$

The $v f$ of C would be $\left(\frac{12.0000}{55.24407}\right)\left(\frac{(0.35)(2.64)}{2.1}\right)=0.09558$

$$
\left(\frac{12.000}{55.25045}\right)\left[\frac{(0.35)(2.64)}{2.30}\right]=0.08725
$$

The standard composition input data for the Boral follows:

$\begin{array}{llll}\text { AL } & 2 & 0.63509 & \text { END } \\ \text { BORON } & 2 & 0.30519 & \text { END } \\ \text { C } & 2 & 0.09558 & \text { END }\end{array}$

EXAMPLE 4. Boral from ${ }^{10} \mathrm{~B},{ }^{11} \mathrm{~B}$, Carbon, and Aluminum.

Create a mixture 2 that is Boral composed of $35 \mathrm{wt} \% \mathrm{~B}_{4} \mathrm{C}$ and $65 \mathrm{wt} \%$ aluminum. The Boral density is $2.64 \mathrm{~g} / \mathrm{cm}^{3}$. The boron is natural boron.

$v f \quad$ is the density multiplier. Use 0.63581 for $\mathrm{AL}$ and 0.08725 for $\mathrm{C}$ as explained in Example 3 above. From the Standard Composition Library chapter, Isotopes in standard composition library table, the theoretical density of ${ }^{10} \mathrm{~B}$ is $1.00 \mathrm{~g} / \mathrm{cm}^{3}$ and ${ }^{11} \mathrm{~B}$ is $1.00 \mathrm{~g} / \mathrm{cm}^{3}$. As computed in Example 3, the mass of the $\mathrm{B}_{4} \mathrm{C}$ molecule is $55.25045 \mathrm{amu}$, and the boron is 19.764 atom $\%{ }^{10} \mathrm{~B}$ and 80.236 atom $\%{ }^{11} \mathrm{~B}$. The mass of ${ }^{10} \mathrm{~B}$ is $10.0129 \mathrm{amu}$ and the ${ }^{11} \mathrm{~B}$ is 11.0096 . Thus, the $v f$ of ${ }^{10} \mathrm{~B}$ is 


$$
\left(\frac{(4)(0.199)(10.0129)}{55.24407}\right)\left(\frac{(0.35)(2.64)}{1.0}\right)=0.13331
$$

The $v f$ of ${ }^{11} \mathrm{~B}$ is

$$
\left(\frac{(4)(0.801)(11.0093)}{55.24407}\right)\left(\frac{(0.35)(2.64)}{1.0}\right)=0.58998 \text {. }
$$

The standard composition input data for the Boral are given as

$\begin{array}{llll}\text { AL } & 2 & 0.63509 & \text { END } \\ \text { B-10 } & 2 & 0.13331 & \text { END } \\ \text { B-11 } & 2 & 0.58998 & \text { END } \\ \text { C } & 2 & 0.09558 & \text { END }\end{array}$

EXAMPLE 5. Specify all of the number densities in a mixture.

Create a mixture 1 that is vermiculite, defined as

$\begin{array}{ll}\text { hydrogen } & \text { at a number density of } 6.8614-4 \text { atoms } / \mathrm{b}-\mathrm{cm} \\ \text { oxygen } & \text { at a number density of } 2.0566-3 \text { atoms } / \mathrm{b}-\mathrm{cm} \\ \text { magnesium } & \text { at a number density of } 3.5780-4 \text { atoms } / \mathrm{b}-\mathrm{cm} \\ \text { aluminum } & \text { at a number density of } 1.9816-4 \text { atoms } / \mathrm{b}-\mathrm{cm} \\ \text { silicon } & \text { at a number density of } 4.4580-4 \text { atoms } / \mathrm{b}-\mathrm{cm} \\ \text { potassium } & \text { at a number density of } 1.0207-4 \text { atoms } / \mathrm{b}-\mathrm{cm} \\ \text { iron } & \text { at a number density of } 7.7416-5 \text { atoms } / \mathrm{b}-\mathrm{cm}\end{array}$

In this example we use the $2^{\text {nd }}$ syntax option described in section 7.1.3.3, in which the 3rd entry must be 0 . The standard composition input data for the vermiculite are given below:

$\begin{array}{lllll}\mathrm{H} & 1 & 0 & 6.8614-4 & \text { END } \\ \mathrm{O} & 1 & 0 & 2.0566-3 & \text { END } \\ \mathrm{MG} & 1 & 0 & 3.5780-4 & \text { END } \\ \mathrm{AL} & 1 & 0 & 1.9816-4 & \text { END } \\ \mathrm{SI} & 1 & 0 & 4.4580-4 & \text { END } \\ \mathrm{K} & 1 & 0 & 1.0207-4 & \text { END } \\ \mathrm{FE} & 1 & 0 & 7.7416-5 & \text { END }\end{array}$

\subsection{A.7 Combinations of user-defined compound and user-defined mixture/alloy to define a mixture}

Mixtures can usually be created using only basic standard composition specifications. Occasionally, it is convenient to create two or more user-defined materials for a given mixture. This procedure is demonstrated in the following example.

EXAMPLE 1. Specify Boral using a user-defined compound and user-defined mixture/alloy. Create a mixture 6 that is Boral, $15 \mathrm{wt} \% \mathrm{~B}_{4} \mathrm{C}$ and $85 \mathrm{wt} \% \mathrm{Al}$, having a density of $2.64 \mathrm{~g} / \mathrm{cm}^{3}$. Natural boron is used in the $\mathrm{B}_{4} \mathrm{C}$. Boral can be described in several ways. For demonstration purposes, it will be described as a combination of a userdefined compound and user-defined mixture/alloy. This is not necessary, because 
both $\mathrm{B}_{4} \mathrm{C}$ and $\mathrm{Al}$ are available as standard compositions. A method of describing the Boral without using user-defined compounds or user-defined mixtures/alloys is given in Examples 1 and 2 of Section 7.1.A.6. The minimum generic input specifications for this user-defined compound and alloy are

$\begin{array}{llllllllll}\text { ATOM-B4C } & 6 & 2.64 & 2 & 5000 & 4 & 6012 & 1 & 0.15 & \text { END } \\ \text { WTPT-AL } & 6 & 2.64 & 1 & 13027 & & 100.0 & & 0.85 & \text { END }\end{array}$

\subsection{A.8 Combinations of solutions to define a mixture}

This section demonstrates the use of more than one solution definition to describe a single mixture. The assumptions used in processing the cross sections are likely to be inadequate for solutions of mixed oxides of uranium and plutonium. Therefore, this section is given purely for demonstration purposes.

\section{EXAMPLE 1. Solution of uranyl nitrate and plutonium nitrate.}

Note that the assumptions used in processing the cross sections are likely to only be adequate for CENTRM/PMC calculations of mixed-oxide solutions. This example is given purely for demonstration purposes. Create a mixture 1 consisting of a mixture of plutonium nitrate solution and uranyl nitrate solution. The specific gravity of the mixed solution is 1.4828 . The solution contains $325.89 \mathrm{~g}(\mathrm{U}+\mathrm{Pu}) / \mathrm{L}$ soln. The acid molarity of the solution is 0.53 . In this solution $77.22 \mathrm{wt} \%$ of the $\mathrm{U}+\mathrm{Pu}$ is uranium. The isotopic abundance of the uranium is $0.008 \%{ }^{234} \mathrm{U}, 0.7 \%{ }^{235} \mathrm{U}, 0.052 \%{ }^{236} \mathrm{U}$, and $99.24 \%{ }^{238} \mathrm{U}$. The isotopic abundance of the plutonium is $0.028 \%{ }^{238} \mathrm{Pu}, 91.114 \%$ ${ }^{239} \mathrm{Pu}, 8.34 \%{ }^{240} \mathrm{Pu}, 0.426 \%{ }^{241} \mathrm{Pu}$, and $0.092 \%{ }^{242} \mathrm{Pu}$. Note that a single quote in the first column indicates a comment line in SCALE input.

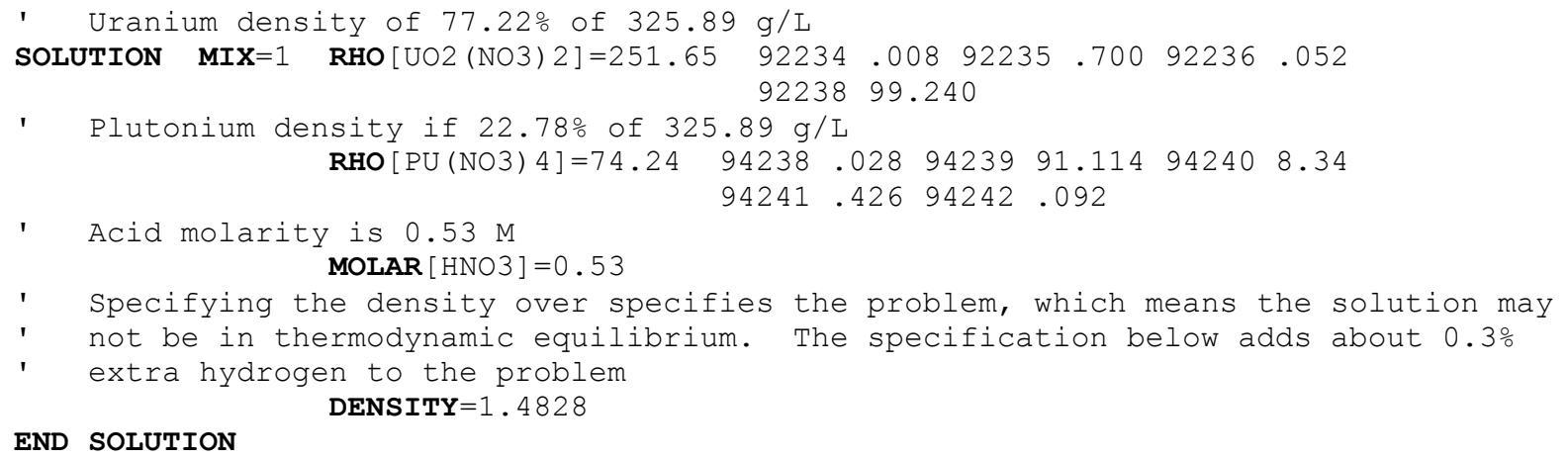

\subsection{A.9 Combinations of basic and user-defined standard compositions to define a mixture}

EXAMPLE 1. Burnable poison from $\mathrm{B}_{4} \mathrm{C}$ and $\mathrm{Al}_{2} \mathrm{O}_{3}$.

Create a mixture 6 that is a burnable poison with a density of $3.7 \mathrm{~g} / \mathrm{cm}^{3}$ and composed of $\mathrm{Al}_{2} \mathrm{O}_{3}$ and $\mathrm{B}_{4} \mathrm{C}$. The material is $1.395 \mathrm{wt} \% \mathrm{~B}_{4} \mathrm{C}$. The boron is natural boron. This material can be easily specified using a combination of user-defined material to describe the $\mathrm{Al}_{2} \mathrm{O}_{3}$ and a simple standard composition to define the $\mathrm{B}_{4} \mathrm{C}$. The minimum generic input specification for this user-defined material and the standard composition are 
The density multiplier of the $\mathrm{B}_{4} \mathrm{C}$ is the density of the material times the weight percent, divided by the theoretical density of $\mathrm{B}_{4} \mathrm{C}[(3.7 \times 0.01395) / 2.52]$ or 0.02048 ; the density multiplier of the $\mathrm{Al}_{2} \mathrm{O}_{3}$ is $1.0-0.01395$ or 0.98605 (the theoretical density of $\mathrm{B}_{4} \mathrm{C}$ was obtained from Isotopes in standard composition library table in the STDCMP chapter).

The input data for the burnable poison are given below:

$\begin{array}{lllllllll}\text { ATOM-AL2O3 } & 6 & 3.70 & 2 & 13027 & 2 & 8016 & 3 & 0.98605\end{array}$ END

B4C $62.048-2$ END

The $\mathrm{B}_{4} \mathrm{C}$ input can be specified using the $\mathbf{D E N}=$ parameter as shown below:

$\begin{array}{lllllllll}\text { ATOM-AL2O3 } & 6 & 3.70 & 2 & 13027 & 2 & 8016 & 3 & 0.98605\end{array}$ END

B4C $6 \quad$ DEN $=3.7 \quad 0.01395 \quad$ END

The fraction of $\mathrm{B}_{4} \mathrm{C}$ in the mixture is $((3.7 \times 0.01395) / 2.52)=0.02048$. The fraction of $\mathrm{Al}_{2} \mathrm{O}_{3}$ in the mixture is $1.0-0.02048=0.979518$. The density of the $\mathrm{Al}_{2} \mathrm{O}_{3}$ can be calculated as shown below.

$$
\begin{aligned}
& \mathrm{F}_{\mathrm{Al}_{2} \mathrm{O}_{3}} * \text { Density } \\
& \text { Densit } \mathrm{O}_{3}+0.020482 * 2.52=3.7=\text { Density of the mixture } \\
& 0.979518 \\
& \mathrm{Al}_{2} \mathrm{O}_{3}
\end{aligned}
$$

Input data using the density of $\mathrm{Al}_{2} \mathrm{O}_{3}$ are given below:

$\begin{array}{lllllllll}\text { ATOM-AL2O3 } & 6 & 3.72467 & 2 & 13027 & 2 & 8016 & 3 & \text { END }\end{array}$

B4C $62.048-2$ END

EXAMPLE 2. Borated water from $\mathrm{H}_{3} \mathrm{BO}_{3}$ and water.

Create a mixture 2 that is borated water at 4350 parts per million (ppm) by weight, resulting from the addition of boric acid, $\mathrm{H}_{3} \mathrm{BO}_{3}$ to water. The density of the borated water is $1.0078 \mathrm{~g} / \mathrm{cm}^{3}$ (see Ref. 1). The solution temperature is $15^{\circ} \mathrm{C}$ and the boron is natural boron.

An easy way to describe this mixture is to use a combination of a user-defined compound to describe the boric acid, and a basic composition to describe the water.

\section{STEP 1. INPUT DATA TO DESCRIBE THE USER-DEFINED COMPOUND}

The generic input data for the boric acid are given below. The actual input data are derived in steps 2 through 5 .

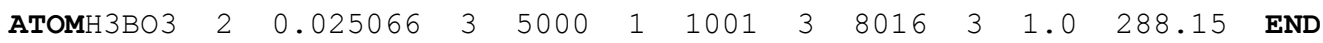


In calculating the molecular weights, use the atomic weights from SCALE, which are available in the table Isotopes in standard composition library in section 7.2.2 of the SCALE manual. The atomic weights used in SCALE may differ from some periodic tables. The SCALE atomic weights used in this problem are listed below:

$\begin{array}{lr}\mathrm{H}(1001) & 1.0078 \\ \mathrm{O}(8016) & 15.9949 \\ { }^{10} \mathrm{~B} & 10.0129 \\ { }^{11} \mathrm{~B} & 11.0093\end{array}$

The natural boron abundance, in weight percent, is defined to be:

$\begin{array}{ll}{ }^{10} \mathrm{~B} & 18.4309 \\ { }^{11} \mathrm{~B} & 81.5691\end{array}$

The molecular weight of natural boron is given by

where:

$$
\begin{aligned}
\text { DEN nat } \mathrm{B} / \mathrm{AWT} \text { nat } \mathrm{B}= & \text { DEN }{ }^{10} \mathrm{~B} / \mathrm{AWT}{ }^{10} \mathrm{~B}+\mathrm{DEN}{ }^{11} \mathrm{~B} / \mathrm{AWT}{ }^{11} \mathrm{~B} \\
& \operatorname{DEN}{ }^{10} \mathrm{~B}=\mathrm{WTF}{ }^{10} \mathrm{~B} \times \mathrm{DEN} \text { nat } \mathrm{B} \\
& \mathrm{DEN}{ }^{11} \mathrm{~B}=\mathrm{WTF}{ }^{11} \mathrm{~B} \times \mathrm{DEN} \text { nat } \mathrm{B}
\end{aligned}
$$

DEN is density in $\mathrm{g} / \mathrm{cm}^{3}$,

AWT is the atomic weight in $\mathrm{g} / \mathrm{mol}$,

WTF is the weight fraction of the isotope.

Substituting,

DEN nat $\mathrm{B} / \mathrm{AWT}$ nat $\mathrm{B}=\mathrm{DEN}$ nat $\mathrm{B} \times\left(\left(\mathrm{WTF}^{10} \mathrm{~B} / \mathrm{AWT}{ }^{10} \mathrm{~B}\right)+\left(\mathrm{WTF}{ }^{11} \mathrm{~B} / \mathrm{AWT}{ }^{11} \mathrm{~B}\right)\right)$

Solving for AWT nat B yields:

AWT nat $\mathrm{B}=1 /\left(\left(\mathrm{WTF}^{10} \mathrm{~B} / \mathrm{AWT}{ }^{10} \mathrm{~B}\right)+\left(\mathrm{WTF}{ }^{11} \mathrm{~B} / \mathrm{AWT}{ }^{11} \mathrm{~B}\right)\right)$

The atomic weight of natural boron is thus

$$
\begin{aligned}
& 1.0 /\left(\left(0.184309 \mathrm{~g}{ }^{10} \mathrm{~B} / \mathrm{g} \text { nat } \mathrm{B} / 10.0129 \mathrm{~g}{ }^{10} \mathrm{~B} / \mathrm{mol}^{10} \mathrm{~B}\right)+\right. \\
& \left.\quad\left(0.815691 \mathrm{~g}{ }^{11} \mathrm{~B} / \mathrm{g} \text { nat } \mathrm{B} / 11.0093 \mathrm{~g} / \mathrm{mol}^{11} \mathrm{~B}\right)\right)=10.81103 \mathrm{~g} \text { nat } \mathrm{B} / \mathrm{mol} \text { nat } \mathrm{B}
\end{aligned}
$$

The molecular weight of the boric acid, $\mathrm{H}_{3} \mathrm{BO}_{3}$ is given by:

$$
(3 \times 1.0078)+10.81103+(3 \times 15.9949)=61.8191
$$

Calculate the grams of boric acid in a gram of solution:

Boric acid, $\mathrm{H}_{3} \mathrm{BO}_{3}$ is $61.8222 \mathrm{~g} / \mathrm{mol}$

Natural boron is $10.81261 \mathrm{~g} / \mathrm{mol}$

$\left(4350 \times 10^{-6} \mathrm{~g} \mathrm{~B} / \mathrm{g} \mathrm{soln}\right) \times(1 \mathrm{~mol} / 10.81261 \mathrm{~g} \mathrm{~B}) \times(61.8191 \mathrm{~g}$ boric acid $/ \mathrm{mol})=$ $0.024874 \mathrm{~g}$ boric $\mathrm{acid} / \mathrm{g}$ soln $(2.4874 \mathrm{wt} \%)$ 
Interpolating from Ref. 1 , the specific gravity of the boric acid solution at 2.4872 weight percent is 1.0087. This value is based on water at $15^{\circ} \mathrm{C}$. The density of pure air free water at $15^{\circ} \mathrm{C}$ is $0.99913 \mathrm{~g} / \mathrm{cm}^{3}$. Therefore, the density of the boric acid solution is $1.0087 \times 0.99913 \mathrm{~g} / \mathrm{cm}^{3}=1.0078 \mathrm{~g}$ $\operatorname{soln} / \mathrm{cm}^{3}$.

Calculate ROTH, the theoretical density of the boric acid.

$1.0078 \mathrm{~g} \mathrm{soln} / \mathrm{cm}^{3} \times 0.024874 \mathrm{~g}$ boric acid $/ \mathrm{g}$ soln $=0.025068 \mathrm{~g}$ boric acid $/ \mathrm{cm}^{3}$

\section{STEP 3. DESCRIBE THE BASIC STANDARD COMPOSITION INPUT DATA}

H2O $20.984507 \quad 288.15 \quad$ END

where the volume fraction $=0.984506$ (see step 4 auxiliary calculations below)

STEP 4. AUXILIARY CALCULATIONS FOR THE BASIC STANDARD COMPOSITION INPUT DATA

Calculate the volume fraction of the water in the solution, assuming 0.9982 is the theoretical density of water from Table 7.2.4. Each gram of solution contains $0.024872 \mathrm{~g}$ of boric acid, so there is $0.975128 \mathrm{~g}$ of water in each gram of solution. The volume fraction of water is then given by:

$$
\left(1.0078 \mathrm{~g} \mathrm{soln} / \mathrm{cm}^{3} \times 0.975128 \mathrm{~g} \text { water } / \mathrm{g} \text { soln }\right) / 0.9982 \mathrm{~g} \text { water } / \mathrm{cm}^{3}=0.984506
$$

STEP 5. CREATE THE MIXTURE FOR BORATED WATER

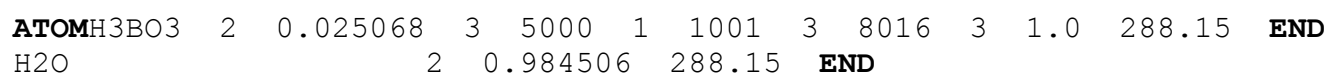

\subsection{A.10 Combinations of basic and solution standard compositions to define a mixture}

The solution specification is the easiest way of specifying the solutions listed in the Available fissile solution components table in section 7.2.3. A combination of solution and basic standard compositions can be used to describe a mixture that contains more than just a solution as demonstrated in the following example.

\section{EXAMPLE 1. Uranyl nitrate solution containing gadolinium.}

Create a $4.306 \%$ enriched uranyl nitrate solution containing $0.184 \mathrm{~g}$ gadolinium per liter. The uranium in the nitrate is $95.65 \%{ }^{238} \mathrm{U}, 0.022 \%{ }^{236} \mathrm{U}, 4.306 \%{ }^{235} \mathrm{U}$, and $0.022 \%{ }^{234} \mathrm{U}$. The uranium concentration is $195.8 \mathrm{~g} \mathrm{U} / \mathrm{L}$ and the specific gravity of the uranyl nitrate is 1.254 . There is no excess acid in the solution. The presence of the gadolinium is assumed to produce no significant change in the solution density. The solution is defined to be mixture 3 . 


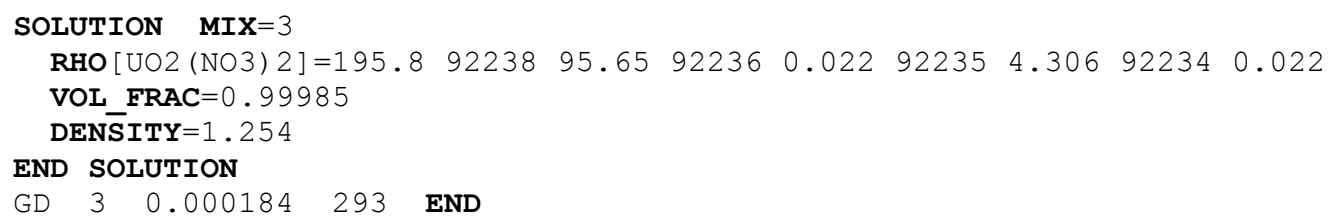

\subsection{A.11 Combinations of user-defined compound and solution to define a mixture}

The solution specification is the easiest way of specifying the solutions listed in the Available fissile solution components table in section 7.2.3 of the SCALE manual. A solution specification and userdefined compound specification can be used to describe a mixture that contains more than just a solution as demonstrated in the following example.

\section{EXAMPLE 1. Uranyl nitrate solution with gadolinium nitrate.}

Create a $4.306 \%$ enriched uranyl nitrate solution containing gadolinium in the form of $\mathrm{Gd}\left(\mathrm{NO}_{3}\right)_{3}$. The uranium in the nitrate is $95.65 \%{ }^{238} \mathrm{U}, 0.022 \%{ }^{236} \mathrm{U}, 4.306 \%{ }^{235} \mathrm{U}$, and $0.022 \%{ }^{234} \mathrm{U}$. The uranium concentration is $195.8 \mathrm{~g} \mathrm{U} / \mathrm{L}$ and the density of the uranyl nitrate is 1.254 . There is no excess acid in the solution. The concentration of the gadolinium is $0.184 \mathrm{~g} / \mathrm{L}$. The volume fraction of the mixture that is uranyl nitrate $(0.99985=1.254 /(1.254+0.000184))$. The solution is defined to be mixture 3 .

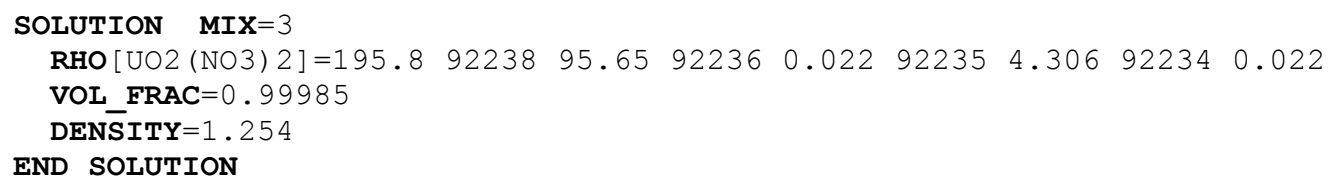

The density of the gadolinium is given as $0.184 \mathrm{~g} / \mathrm{L}$. To describe the user-defined compound, the density of the $\mathrm{Gd}\left(\mathrm{NO}_{3}\right)_{3}$ is needed. The atomic weights from the Standard Composition Library are:

$$
\begin{array}{ll}
\mathrm{Gd} & 157.25 \\
\mathrm{~N} & 14.0067 \\
\mathrm{O} & 15.999
\end{array}
$$

Therefore, the density of the $\mathrm{Gd}\left(\mathrm{NO}_{3}\right)_{3}=0.000184 \mathrm{~g} \mathrm{Gd} / \mathrm{cm}^{3} \times(157.25+3(14.0067+3(15.999)) / 157.25)$ $=0.0004017 \mathrm{~g} / \mathrm{cm}^{3}$.

The input data for this user-defined compound are given below:

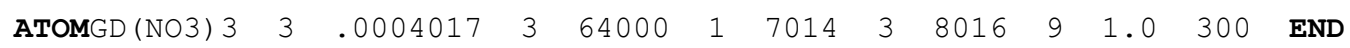

The complete input data for the mixture of uranyl nitrate and gadolinium nitrate are given as:

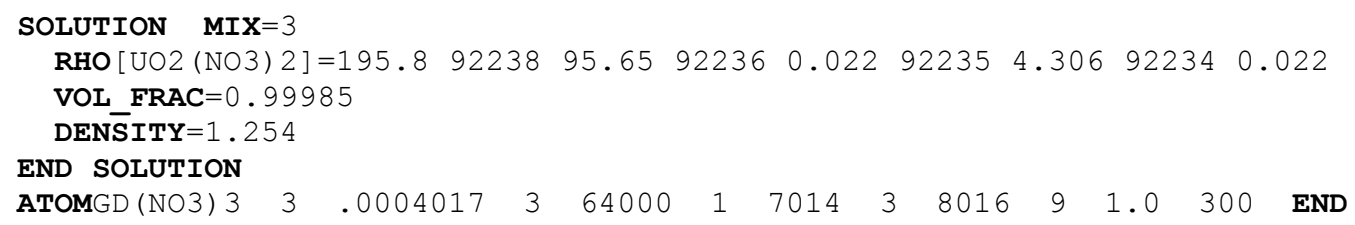


NOTE: Since the default temperature $(300 \mathrm{~K})$ is to be used, it can be omitted from the user-defined compound standard composition. The temperature must be entered if the standard composition contains a multiple-isotope nuclide whose isotopic abundance is to be specified.

1. "Specific Gravity of Boric Acid Solutions," Handbook of Chemistry, 1162, Compiled and Edited by Norbert A. Lange, Ph.D, 1956. 


\section{APPENDIX 7.1.B Standard Composition Examples}

\subsection{B.1. Infinite homogeneous medium unit cell data}

EXAMPLE 1. A single mixture 1.

Consider a single cylindrical configuration of mixture 1 , composed of $10 \%$ enriched $\mathrm{UO}_{2}$ having a radius of $35 \mathrm{~cm}$ and a height of $20 \mathrm{~cm}$. This fuel region is sufficient large to model as an infinite medium. Mixture 100 may be used in subsequent multigroup neutron transport calculations.

INFHOMMEDIUM 1 CELLMIX=10 END

XSDRNPM will calculate the eigenvalue of an infinite mass of $10 \%$ enriched $\mathrm{UO}_{2}$.

\subsection{B.2. LATTICECELL unit cell data}

Examples of "regular" LATTICECELL unit cells are given in Examples 1-5, and examples of "annular" LATTICECELL unit cells are given in Examples 6-10 below.

EXAMPLE 1. SQUAREPITCH (infinitely long cylindrical pins in a square-pitched array).

Consider a large array of $\mathrm{UO}_{2}$ fuel pins having a fuel O.D. of $0.79 \mathrm{~cm}$, a $0.015-\mathrm{cm}$ gap, and a $0.06-\mathrm{cm}$-thick aluminum clad. The array is a square-pitched array with a center-to-center spacing of $1.60 \mathrm{~cm}$ and is completely flooded with water. In the standard composition data, $\mathrm{UO}_{2}$ is defined to be mixture 1 , the aluminum clad is defined to be mixture 2, and the water moderator is defined to be mixture 3 .

LATTICECELL SQUAREPITCH PITCH=1.60 3 FUELD=0.79 1 CLADD=0.94 2 GAPD=0.82 0 END

EXAMPLE 2. TRIANGPITCH (infinitely long cylinders in a triangular-pitched array).

Consider an array of $\mathrm{UO}_{2}$ pins with a diameter of $0.635 \mathrm{~m}$. The outside diameter of the clad is $0.78 \mathrm{~cm}$. There is no gap between the fuel and clad. The array is a triangular-pitched array with a center-to-center spacing of $5.0 \mathrm{~cm}$ and is flooded with water. In the standard composition data, the $\mathrm{UO}_{2}$ is defined to be mixture 1 , the aluminum is defined to be mixture 2 , and the water moderator is defined to be mixture 3 .

LATTICECELL TRIANGPITCH PITCH=5.0 3 FUELD $=.6351$ CLAD $=.78 \quad 2 \quad$ END

EXAMPLE 3. SPHSQUAREP (spheres in a square-pitched array).

Consider a large array of $\mathrm{U}_{3} \mathrm{O}_{8}$ spheres having a fuel O.D. of $18.6 \mathrm{~cm}$, with an aluminum clad that is $0.18 \mathrm{~cm}$ thick. The array is a triangular-pitched array with a center-to-center spacing of $19.0 \mathrm{~cm}$ and is unmoderated. In the standard composition data, the aluminum is defined to be mixture 1 and the $\mathrm{U}_{3} \mathrm{O}_{8}$ is defined to be mixture 2. There is no moderator material, so 0 will be used to represent a void. Also, have XSDRNPM make a cell weighted material 20 from this unit cell. 
LATTICECELL SPHSQUAREP PITCH=19.0 0 FUELD=18.6 2 CLADD=18.96 1 CELLMIX=20 END

EXAMPLE 4. SPHTRIANGP (spheres in a triangular-pitched array).

Consider a large array of $\mathrm{U}_{3} \mathrm{O}_{8}$ spheres having a fuel O.D. of $18.6 \mathrm{~cm}$, with an aluminum clad that is $0.18 \mathrm{~cm}$ thick. The array is a triangular-pitched array with a center-to-center spacing of $19.0 \mathrm{~cm}$ and is flooded with water. In the standard composition data, the aluminum is defined to be mixture 1 , the $\mathrm{U}_{3} \mathrm{O}_{8}$ is defined to be mixture 2 , and the water moderator is defined to be mixture 3 .

LATTICECELL SPHTRIANGP PITCH=19.0 $3 \quad$ FUELD=18.6 2 CLADD=18.96 1 END

EXAMPLE 5. SYMMSLABCELL (slabs repeated in a symmetric fashion).

Consider a system of alternating slabs of $\mathrm{U}_{3} \mathrm{O}_{8}$ and low-density water. Each $\mathrm{U}_{3} \mathrm{O}_{8}$ region is $1.27 \mathrm{~cm}$ thick, and each water region is $15.0 \mathrm{~cm}$ thick. In the standard composition data, the $\mathrm{U}_{3} \mathrm{O}_{8}$ is defined to be mixture 1 , and the low-density water is defined to be mixture 2 .

IATTICECELL SYMMSLABCELL PITCH=16.27 2 FUELD=1.27 1 END

EXAMPLE 5a. SYMMSLABCELL (slabs repeated in a symmetric fashion).

Consider a system of alternating slabs of $\mathrm{U}_{3} \mathrm{O}_{8}$ and low-density water. Each $\mathrm{U}_{3} \mathrm{O}_{8}$ region is $1.27 \mathrm{~cm}$ thick, and each water region is $15.0 \mathrm{~cm}$ thick. The $\mathrm{U}_{3} \mathrm{O}_{8}$ regions have a $0.01-\mathrm{cm}$ gap and $0.24-\mathrm{cm}$-thick aluminum clad on each face. In the standard composition data, the $\mathrm{U}_{3} \mathrm{O}_{8}$ is defined to be mixture 1, the aluminum is defined to be mixture 2, and the low-density water is defined to be mixture 3 . Also, have XSDRNPM make a cell-weighted material 100 from this unit cell.

LATTICECELL SYMMSLABCELL PITCH=16.77 3 FUELD=1.27 1

CLADD $=1.772$ GAPD $=1.290$ CELLMIX $=100$ END

EXAMPLE 6. ASQUAREPITCH (infinitely long annular cylindrical rods in a square-pitched array).

Consider an array of uranium metal pipes having an inside diameter of $5.0 \mathrm{~cm}$ and an outer diameter of $6.75 \mathrm{~cm}$. A gap of $0.025 \mathrm{~cm}$ and a clad of $0.25 \mathrm{~cm}$ exist on both the inner and outer surfaces of the fuel. The fuel rods are arranged in a square-pitched array. The center-to-center spacing is $8.0 \mathrm{~cm}$. The array is completely flooded with water. In the standard composition data, the uranium metal is defined to be mixture 1 , the outer clad is mixture 2 , the inner clad is mixture 7 , the inner moderator is Plexiglas and is mixture 3, the gap is a void, and the external moderator is water, defined to be mixture 4 .

LATTICECELL ASQUAREPITCH PITCH=8.0 4 FUELD=6.75 1 GAPD=6.8 0

CLADD $=7.32$ IMODD $=4.453 \quad$ ICLADD $=4.957 \quad$ IGAPD $=5.0 \quad 0 \quad$ END

EXAMPLE 6a. ASQUAREPITCH (infinitely long annular cylindrical rods in a square-pitched array).

Consider an array of uranium metal pipes having an inside diameter of $5.0 \mathrm{~cm}$ and an outer diameter of $6.75 \mathrm{~cm}$ arranged in a square-pitched array. The center-to-center spacing is $8.0 \mathrm{~cm}$. The array is completely flooded with water. In the standard 
composition data, the uranium metal is defined to be mixture 1 , the water moderator is defined to be mixture 2 , and the inside water moderator is defined as mixture 3 .

LATTICECELL ASQUAREPITCH PITCH=8.0 2 FUELD=6.75 1 IMODD=5.0 3 END

NOTE: This problem defines two water mixtures. If mixture 2 were entered twice, i.e., for both the inner and outer moderator, an error message would be printed and the calculation terminated.

EXAMPLE 7. ATRIANGPITCH (infinitely long annular cylindrical rods in a triangular-pitched array).

Consider an array of uranium metal pipes having an inside diameter of $8.0 \mathrm{~cm}$ and a wall thickness of $0.75 \mathrm{~cm}$ arranged in a square-pitched array. The center-to-center spacing is $9.75 \mathrm{~cm}$. The array is completely flooded with water. A Plexiglas rod fills the center of the uranium pipe. In the standard compositions data, the uranium metal is defined to be mixture 1, the Plexiglas is defined to be mixture 2, and the external water moderator is mixture 3.

IATTICECELL ATRIANGPITCH PITCH=9.75 3 FUELD=9.5 1 IMODD=8.0 2 END

EXAMPLE 8. ASPHSQUAREP (spherical annuli in a square-pitched array).

Consider a large array of hollow $\mathrm{U}_{3} \mathrm{O}_{8}$ spheres having a fuel I.D. of $8.0 \mathrm{~cm}$ and O.D. of $18.6 \mathrm{~cm}$. The centers of the spheres are empty. The external moderator is water. The spheres are stacked in a square-pitched array with a center-to-center spacing of $19.0 \mathrm{~cm}$. In the standard composition data, the $\mathrm{U}_{3} \mathrm{O}_{8}$ is defined to be mixture 1 , and the water is defined to be mixture 2. The centers of the spheres are defined to be void, mixture 0 .

LATTICECELL ASPHSQUAREP HPITCH=9.5 2 FUELR=9.3 1 IMODR=4.0 0 END

EXAMPLE 9. ASPHTRIANGP (spheres in a triangular-pitched array).

Consider a large array of hollow $\mathrm{U}_{3} \mathrm{O}_{8}$ spheres having a fuel I.D. of $8.0 \mathrm{~cm}$ and a fuel O.D. of $18.6 \mathrm{~cm}$. A $0.18-\mathrm{cm}$-thick aluminum clad exists outside the fuel. The interior of each sphere is void. The array is a triangular-pitched array with a centerto-center spacing of $19.0 \mathrm{~cm}$ and is flooded with water. In the standard composition data, the aluminum is defined to be mixture 1 , the $\mathrm{U}_{3} \mathrm{O}_{8}$ is defined to be mixture 2 , and the water moderator is defined to be mixture 3 . The void in the center of each sphere is entered as mixture 0 .

LATTICECELL ASPHTRIANGP HPITCH=9.5 3 FUELR=9.3 2 IMODR=4.0 0 CLADR=9.48 1 END

EXAMPLE 10. ASYMSLABCELL (repeated slabs having different moderator conditions on the left and right boundaries).

Consider an array of $\mathrm{U}_{3} \mathrm{O}_{8}$ slabs with an inner moderator region composed of fulldensity water with a half thickness of $8.0 \mathrm{~cm}$, and a low-density water outer moderator with a $16 \mathrm{~cm}$ half thickness of $16 \mathrm{~cm}$ half thickness. Each $\mathrm{U}_{3} \mathrm{O}_{8}$ slab is $10.54 \mathrm{~cm}$ thick. In the standard composition data, the $\mathrm{U}_{3} \mathrm{O}_{8}$ is defined to be mixture 1 , the full density water is defined to be mixture 2 , and the low-density water is mixture 3. Also, have XSDRNPM create a cell weighted mixture 100 from this unit cell.

LATTICECELL ASYMSLABCELL CELLMIX=100 IMODR=8.0 2 FUELR=18.54 1 HPITCH=34.54 3 END 
EXAMPLE 10a. ASYMSLABCELL (repeated slabs having different moderator conditions on the left and right boundaries).

Consider an array of $\mathrm{U}_{3} \mathrm{O}_{8}$ fuel plates with an inner moderator region of full-density water with a half-thickness of $8.0 \mathrm{~cm}$, and with a $16 \mathrm{~cm}$ thick low-density outer moderator. Each fuel plate includes a $10.54 \mathrm{~cm}$ thick $\mathrm{U}_{3} \mathrm{O}_{8}$ slab with a $0.01 \mathrm{~cm}$ gap and $0.24-\mathrm{cm}$-thick aluminum clad on each face. In the standard composition data, the $\mathrm{U}_{3} \mathrm{O}_{8}$ is defined to be mixture 1 , the full density water is defined to be mixture 2, and the low-density water is mixture 3 , the inner aluminum is mixture 4 , the outer aluminum clad is mixture 5 , and both gaps are voids.

LATTICECELL ASYMSLABCELL IMODR=8.0 2 ICLADR=8.24 5 IGAPR=8.25 0 FUELR=18.79 1

GAPR 18.80 O CLADR 19.044 HPITCH=27.04 3 END

\subsection{B.3. MULTIREGION unit cell data}

Examples of MULTIREGION unit cells follow:

EXAMPLE 1. SLAB.

Consider a 5 -cm-thick slab of fuel (mixture 1) with $0.5 \mathrm{~cm}$ of aluminum (mixture 3 ) and $15 \mathrm{~cm}$ of water (mixture 2) on each face. The unit cell data for this problem could be entered as follows:

MULTIREGION SLAB LEFT_BDY=REFLECTED RIGHT_BDY=VACUUM ORIGIN=0 END

$\begin{array}{lllllllll}1 & 2.5 & 3 & 3.0 & 2 & 18.0 & \text { END ZONE }\end{array}$

\section{EXAMPLE 2. CYLINDRICAL.}

Consider a large array of fuel pins. Each pin is $\mathrm{UO}_{2}$ (mixture 1) with a radius of $0.465 \mathrm{~cm}$, a $0.009-\mathrm{cm}$ gap (mixture 0), and a Zircaloy clad (mixture 9) $0.062 \mathrm{~cm}$ thick, centered in a water (mixture 8) region surrounded by a flooded support structure represented by homogenized water and Zircaloy (mixture 10). The outer radius of the water-Zircaloy region is $0.844 \mathrm{~cm}$ and it is $0.037 \mathrm{~cm}$ thick. This problem cannot be described as a LATTICECELL problem because the LATTICECELL configuration is limited to fuel-gap-clad-cell boundary and this problem is fuel-gap-clad-moderator-outer region. When MULTIREGION is used, lattice effects are accounted for by specifying a WHITE, PERIODIC, or REFLECTED right boundary condition, as long as the CENTRM/PMC selfshielding method is used. MULTIREGION cells should not be used for arrays if BONAMI-only method is specified

MULTIREGION CYLINDRICAL RIGHT_BDY=WHITE END

$\begin{array}{llllllllllll}1 & 0.465 & 0 & 0.474 & 9 & 0.536 & \overline{8} & 0.807 & 10 & 0.844 & \text { END } & \text { ZONE }\end{array}$

EXAMPLE 3. SPHERICAL. 
Describe a bare sphere of uranium metal $8.72 \mathrm{~cm}$ in radius. The uranium metal is defined to be mixture 1. Also, have XSDRNPM create a cell weighted mixture 100 and calculate and eigenvalue. The unit cell data for this problem could be entered as follows:

MULTIREGION SPHERICAL CELLMIX=100 END $1 \quad 8.72 \quad$ END ZONE

EXAMPLE 4. BUCKLEDSLAB.

Consider a plate of fuel $4 \mathrm{~cm}$ thick, reflected by $3 \mathrm{~cm}$ of water on both faces. The plate is $32 \mathrm{~cm}$ tall and $16 \mathrm{~cm}$ deep. The fuel is mixture 1 and the water is mixture 2 .

Also, have XSDRNPM create a cell weighted mixture 100 and calculate and eigenvalue.

MULTIREGION BUCKLEDSLAB CELLMIX=100 LEFT_BDY=REFLECTED RIGHT_BDY=VACUUM

$\mathbf{D Y}=32 \quad \mathbf{D Z}=16.0 \quad$ END $12.0 \quad 2 \quad 5.0 \quad$ END ZONE

EXAMPLE 5. BUCKLEDCYL.

Consider a solution of uranyl nitrate contained in a cylindrical stainless-steel container reflected by $33 \mathrm{~cm}$ of water. The inside dimensions of the steel container are $7.62 \mathrm{~cm}$ in radius and $130.0 \mathrm{~cm}$ tall. The steel is $0.15 \mathrm{~cm}$ thick. The uranyl nitrate is defined to be mixture 1 , the steel is defined to be mixture 2 , and the water is defined to be mixture 3 .

MULTIREGION BUCKLEDCYL DY $=130$ END

$\begin{array}{llllllll}1 & 7.62 & 2 & 7.77 & 3 & 40.77 & \text { END ZONE }\end{array}$

\subsection{B.4. DOUBLEHET unit cell data}

Unit cell data are always required for DOUBLEHET calculations. As many unit cells as needed may be defined in the problem. If CELLMIX $=m x$ is entered after the fuel element (macro cell) description, XSProc calls XSDRNPM to calculate the eigenvalue of the cell and to create a homogenized cellweighted cross section having the characteristics of the doubly-heterogeneous cell configuration.

EXAMPLE 1: A doubly-heterogeneous spherical fuel element with 15,000 $\mathrm{UO}_{2}$ particles in a graphite matrix.

Grain fuel radius is $0.025 \mathrm{~cm}$. Grain contains one coating layer that is $0.009-\mathrm{cm}-$ thick. Pebbles are in a triangular pitch on a 6.4-cm-pitch. Fuel pebble fuel zone is $2.5-\mathrm{cm}$ in radius and contains a $0.5-\mathrm{cm}$-thick graphite clad that contains small amounts of ${ }^{10} \mathrm{~B}$. Pebbles are surrounded by ${ }^{4} \mathrm{He}$. Assume the composition block is below:

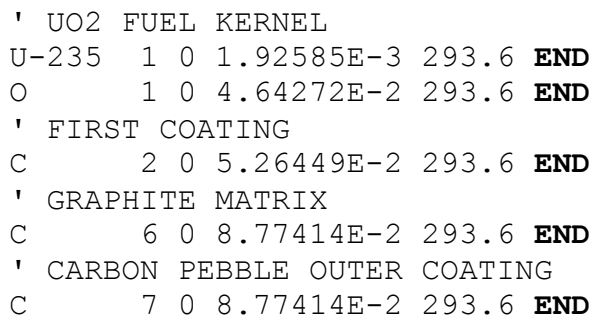


B-10 $7009.64977 E-9 \quad 293.6$ END

$\mathrm{HE}-4 \quad 8 \quad 0 \quad 2.65156 \mathrm{E}-5 \quad 293.6$ END

The cell data for the DOUBLEHET cell follows:

DOUBLEHET FUELMIX $=10$ END

GFR $=0.0251$ COATT $=0.0092$ MATRIX $=6$ NUMPAR $=15000$ END GRAIN

PEBBLE SPHTRIANGP RIGHT_BDY=WHITE HPITCH=3.2 8 FUELR=2.5 CLADR=3.0 7 END

In this case we designated the homogenized mixture as mixture 10. If we have a KENO V.a or KENO-VI input section, we would use mixture 10 in that section. Note that the keyword "FUELR=" is followed by the fuel dimension only, i.e., no mixture number. That is because the fuel mixture number is specified with "FUELMIX=" and therefore need not be repeated.

EXAMPLE 2: Same as Example 1, except volume fraction of the grain type is known and is 0.037732 .

DOUBLEHET RIGHT BDY=WHITE FUELMIX=10 END

GFR $=0.0251 \mathrm{COA} T \mathbf{T}=0.0092$ MATRIX=6 VF=0.037732 END GRAIN

PEBBLE SPHTRIANGP RIGHT_BDY=WHITE HPITCH=3.2 8 FUELR=2.5 CLADR=3.0 7 END

EXAMPLE 3: Same as Example 1, except halfpitch of the grain type is known and is $0.10137 \mathrm{~cm}$.

DOUBLEHET FUELMIX $=10$ END

GFR $=0.0251$ COATT $=0.0092 \mathrm{HPITCH}=0.10137$ MATRIX $=6$ END GRAIN

PEBBLE SPHTRIANGP RIGHT BDY=WHITE HPITCH=3.2 8 FUELR=2.5 CLADR=3.0 7 END

EXAMPLE 4: A doubly-heterogeneous spherical fuel element with 10,000 UO2 particles and $5,000 \mathrm{PuO2}$ particles in a graphite matrix.

Grain fuel radii for $\mathrm{UO}_{2}$ and $\mathrm{PuO}_{2}$ particles are $0.025 \mathrm{~cm}$ and $0.012 \mathrm{~cm}$, respectively. $\mathrm{UO}_{2}$ grains contain one coating layer that is 0.009 -cm-thick. $\mathrm{PuO}_{2}$ grains contain one coating layer that is $0.0095-\mathrm{cm}$-thick. Pebbles are in a triangular pitch on a $6.4-\mathrm{cm}$-pitch. Fuel pebble fuel zone is $2.5-\mathrm{cm}$ in radius and contains a 0.5 -cm-thick graphite clad that contains small amounts of ${ }^{10} \mathrm{~B}$. Pebbles are surrounded by ${ }^{4} \mathrm{He}$. Assume the composition block is given below:

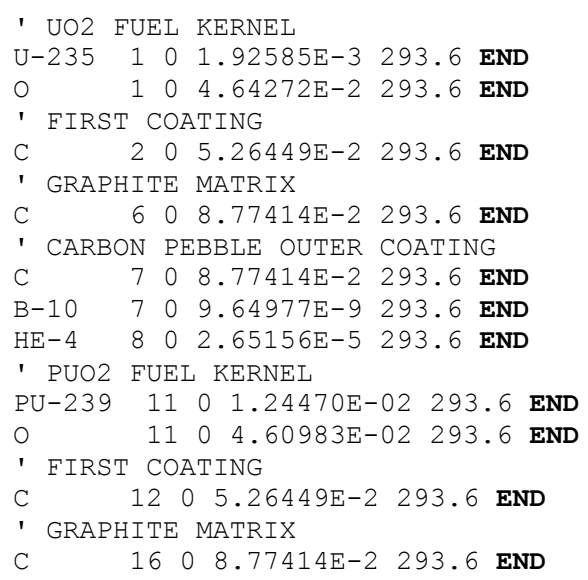

The cell data for the DOUBLEHET cell follows: 
DOUBLEHET FUELMIX $=10$ END

GFR $=0.0251$ COATT $=0.0092$ MATRIX $=6$ NUMPAR $=10000$ END GRAIN

GFR=0.012 11 COATT=0.0095 12 MATRIX=16 NUMPAR=5000 END GRAIN

PEBBLE SPHTRIANGP RIGHT_BDY=WHITE HPITCH=3.2 8 FUELR=2.5 CLADR=3.0 7 END

Since number of particles is entered, the total volume fraction and the pitch can be calculated by the code.

EXAMPLE 5: Same as Example 4 above except the volume fractions of $\mathrm{UO}_{2}$ and $\mathrm{PuO}_{2}$ grains are 0.02511 and 0.00318 , respectively.

DOUBLEHET RIGHT BDY=WHITE FUELMIX $=10$ END

GFR $=0.0251$ COA $\bar{T} T=0.0092$ MATRIX $=6 \quad$ VF $=0.02511$ END GRAIN

GFR $=0.01211$ COATT $=0.009512$ MATRIX $=16$ VF=0.00318 END GRAIN

PEBBLE SPHTRIANGP RIGHT_BDY=WHITE HPITCH=3.2 8 FUELR=2.5 CLADR=3.0 7 END

EXAMPLE 6: Same as Example 4 above except pitch is also known.

$\mathrm{UO}_{2}$ grains have a pitch of $0.25 \mathrm{~cm} . \mathrm{PuO}_{2}$ grains have a pitch of $0.20 \mathrm{~cm}$.

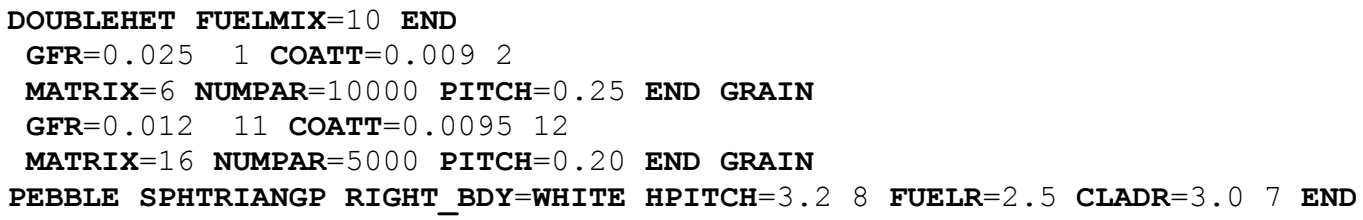

Since number of particles is sufficient to perform the homogenization, it is used. However, instead of calculating the pitch for the 1-D cell calculation for each grain type, the user input pitch is used. Hence, the calculated $k_{\text {eff }}$ of Example 6 will be different from those of Examples 4 and 5.

EXAMPLE 7: Same as Example 6 except the doubly-heterogeneous cell will be cell-weighted.

The final cell-weighted mixture number is 17.

The cell data for the DOUBLEHET cell follows:

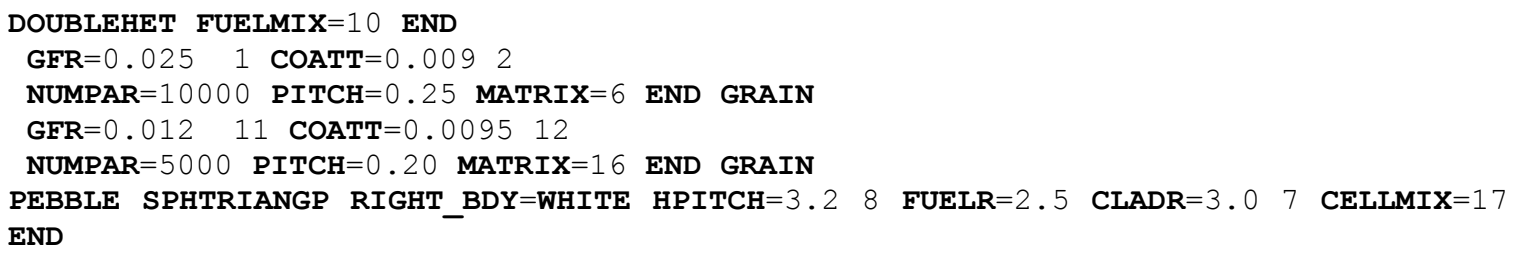

EXAMPLE 8: A doubly-heterogeneous spherical fuel element with $15,000 \mathrm{UO}_{2}$ particles in a graphite matrix.

Grain fuel radius is $0.012 \mathrm{~cm}$. Grain contains four coating layers that are 0.0095 , $0.004,0.0035$, and $0.004-\mathrm{cm}$-thick. Pebbles are in a square pitch on a 6.0-cm-pitch. Fuel pebble fuel zone is $2.5-\mathrm{cm}$ in radius and contains a $0.5-\mathrm{cm}$-thick graphite clad that contains small amounts of ${ }^{10} \mathrm{~B}$. Pebbles are surrounded by ${ }^{4} \mathrm{He}$. Assume the composition block is given below:

' UO2 FUEL KERNEL

U-235 $1001.92585 \mathrm{E}-3 \quad 293.6$ END 


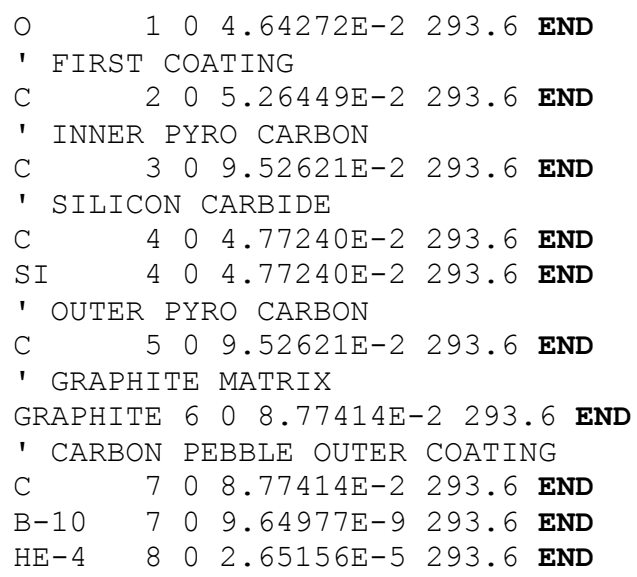

The cell data for the DOUBLEHET cell follows:

DOUBLEHET FUELMIX $=10$ END

GFR=0.012 1 COATT $=0.00952$ COATT $=0.004 \quad 3 \quad$ COATT $=0.00354$ COATT=0.004 5 MATRIX=6 NUMPAR $=15000 \quad \mathrm{VF}=0.0245$ END GRAIN

PEBBLE SPHSQUAREP RIGHT_BDY=WHITE HPITCH=3.0 8 FUELR=2.5 CLADR=3.0 7 END

Note that the grains are overspecified and the numbers are inconsistent. A VF value of 0.0245 results in a total number of particles of 10652.32 which is considerably less than 15,000 . In this case, the code will issue a warning to this effect and will use VF value in the calculations (i.e., ignore NUMPAR $=15000$ entry).

EXAMPLE 9: Similar to Example 8 except radii for grain regions are entered.

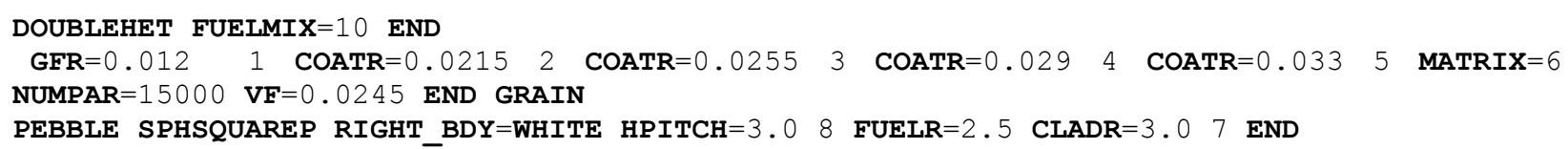

EXAMPLE 10: A doubly-heterogeneous spherical fuel element with two $\mathrm{UO}_{2}$ grain types.

First grain type has a fuel radius of $0.025 \mathrm{~cm}$. Second grain type fuel radius is $0.004 \mathrm{~cm}$. First grain type has one coating that is $0.009-\mathrm{cm}$-thick. Second grain type has two coatings each $0.004-\mathrm{cm}$-thick. Each grain type has a volume fraction of 0.45 . Pebbles are in a triangular pitch on a $7.0-\mathrm{cm}$-pitch. Fuel pebble fuel zone is $2.5-\mathrm{cm}$ in radius and contains a $0.5-\mathrm{cm}$-thick graphite clad that contains small amounts of ${ }^{10} \mathrm{~B}$ and ${ }^{11} \mathrm{~B}$. Pebbles are surrounded by ${ }^{4} \mathrm{He}$. Assume the composition block is given below:

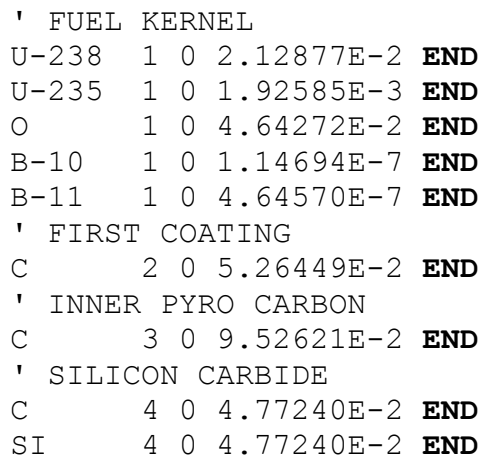




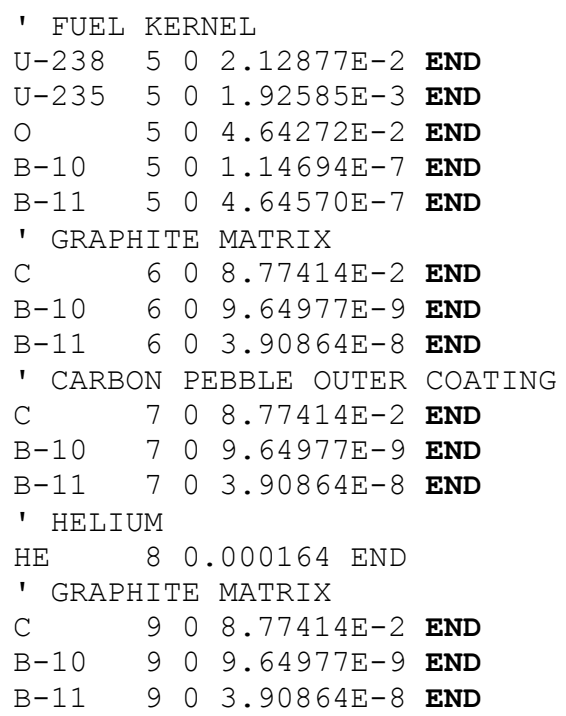

The cell data for the DOUBLEHET cell follows:

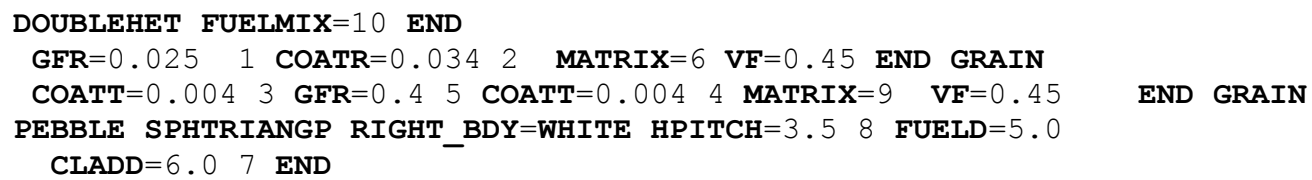

EXAMPLE 11: A doubly-heterogeneous hexagonal block type fuel element with $\mathrm{UO}_{2}$ grains in a cylindrical fuel region.

Grain fuel radius is $0.025 \mathrm{~cm}$. Grain coating is $0.009-\mathrm{cm}$-thick. Grains have a volume fraction of 0.45 . Hexagonal rods are in a $7-\mathrm{cm}$ triangular pitch. Fuel rod fuel zone is $2.5-\mathrm{cm}$ in radius, $10-\mathrm{cm}$-high and contains a $0.5-\mathrm{cm}$-thick graphite clad that contains small amounts of ${ }^{10} \mathrm{~B}$. Assume the composition block is below:

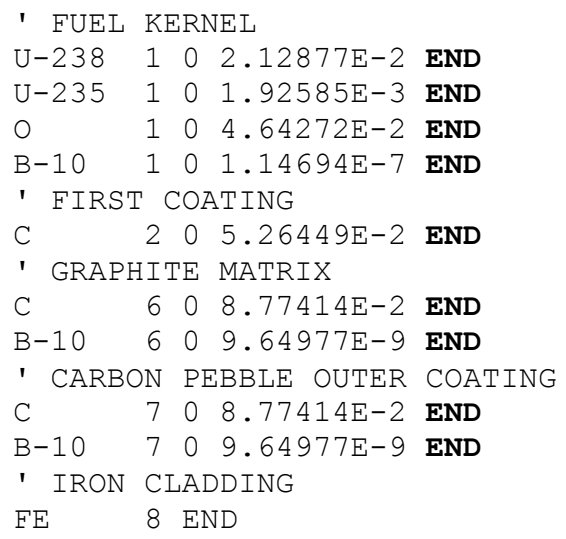

The cell data for the DOUBLEHET cell follows:

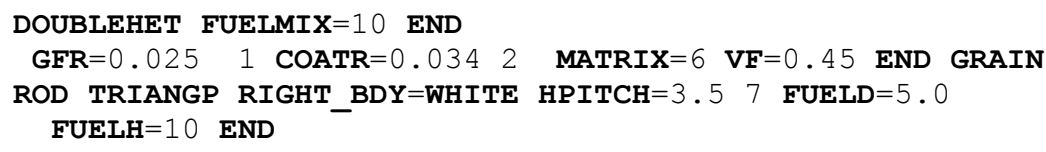


EXAMPLE 12: This is the same as Example 11 except the fuel elements (cylindrical rods) have 0.05-cm-thick iron cladding.

The cell data for the DOUBLEHET cell follows:

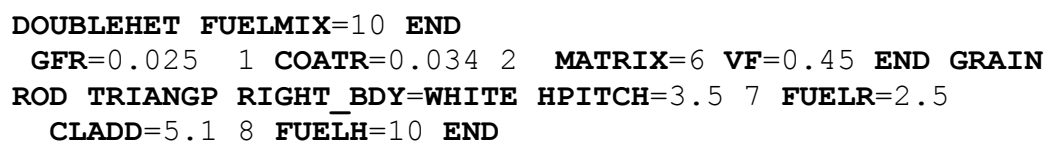

\subsection{B.5. Optional parameter data}

The optional parameter data provide a means of providing additional information or alternative data to the cross-section processing codes. There are two types of optional parameter data. The first type of data is used by XSDRNPM and BONAMI for cross-section processing and cell-weighting cross sections. This type of data is initiated using the keywords MORE DATA and ends with the keywords END MORE. This input is described in Sect. 7.1.3.8. The second type of optional parameter data is used by CENTRM and PMC for cross-section processing. This type of data is initiated using the keywords CENTRM DATA and ends with the keywords END CENTRM. This input is described in Sect. 7.1.3.9. It is possible to input both types of data for a unit cell. The optional parameter data specified apply only to the unit cell that immediately precedes it.

\section{MORE DATA examples}

Consider a problem in which it is desirable to increase the number of inner iterations in XSDRNPM to 30 and to tighten the overall convergence criteria to a value of 0.000075 . This could be accomplished by entering the data as follows:

MORE DATA IIM $=30 \quad$ EPS $=0.000075$ END

The order of the data entry is not important,and it can be continued across several lines. However, a keyword and its value cannot be separated across lines. The terminator for the optional parameter data, END, must not begin in column 1 unless you assign a name to it. An alternative method of entering the above data is given below.

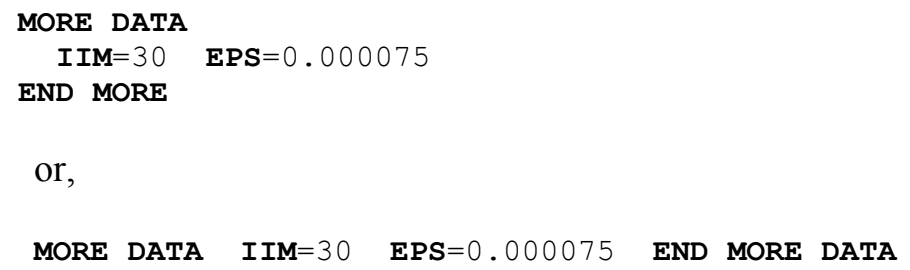

\subsection{B.6. CENTRM DATA examples}

Consider a problem in which it is desirable to increase the upper energy of the CENTRM CE transport calculation from the default of $20000 \mathrm{eV}$ to a value $50000 \mathrm{eV}$, and to extend the default lower energy from $0.001 \mathrm{eV}$ to 0.0001 . This is accomplished by entering the data as follows: 
CENTRM DATA DEMAX $=50000 \quad$ DEMIN $=0.0001$ END CENTRM

As with the MORE DATA block, an alternative method of entering the above data is given below.

CENTRMDATA

DEMAX $=50000 \quad$ DEMIN $=0.0001$

END CENTRMDATA

CENTRM and PMC computation options can also be controlled with CENTRM DATA. A complete description of the CENTRM/PMC computational methods and options can be found the corresponding sections of the SCALE manual. The following example specifies that:

(a) discrete-level inelastic scattering will be used in CENTRM and processed in PMC [nmf6];

(b) the CENTRM 1D discrete $S_{N}$ transport solver will be used in the upper MG energy range [nfst] and the CE energy range [npxs], while the infinitie medium model will be used for the thermal energy range [nthr];

(c) a P3 scattering order [isct] will be used in the transport calculations;

(d) PMC will perform "consistent PN" corrections on Legendre moments of the 2D elastic matrices [n2d];

(e) additional output information will be provided by CENTRM [ixprt] and by PMC [nprt].

CENTRM DATA NMF6 $=0$ NFST $=0$ NTHR $=2 \quad$ ISCT $=3$

N2D $=-2$ IXPRT $=1 \quad$ NPRT $=1$ END CENTRM DATA 


\section{APPENDIX 7.1.C. EXAMPLES of COMPLETE XSPROC INPUT DATA}

\subsection{C.1 Infinite homogeneous medium input data}

Examples of XSProc input data for infinite homogeneous media problems are given below. In these cases the cross section library name "fine_n" indicates that the latest recommended fine-group SCALE library will used in the calculations.

EXAMPLE 1. Default cell definition.

Consider a cylindrical billet of $20 \mathrm{wt} \%$ enriched $\mathrm{UO}_{2}$, having a density of $10.85 \mathrm{~g} / \mathrm{cm}^{3}$ that is $26 \mathrm{~cm}$ in diameter and $26 \mathrm{~cm}$ tall.

The average mean-free path in the uranium dioxide is on the order of $2.5 \mathrm{~cm}$. Because only a small fraction of the billet is within a mean-free path of the surface, the material can be treated as an infinite homogeneous medium; therefore the CELL DATA block can be omitted. The XSProc data follows:

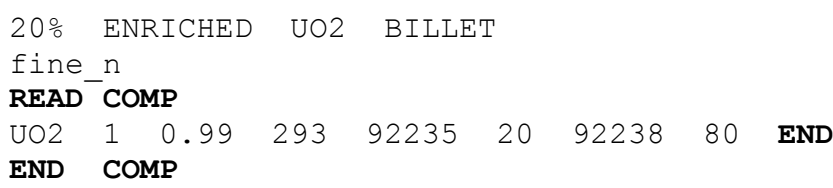

The volume fraction used for the $\mathrm{UO}_{2}, 0.99$, is calculated by dividing the actual density by the theoretical density obtained from the Isotopes in standard composition library table in the STDCMP chapter, $(10.85 / 10.96)$. Since the enrichment was specified as $20 \%$, it is assumed that the remainder is ${ }^{238} \mathrm{U}$.

An alternative input data description follows:

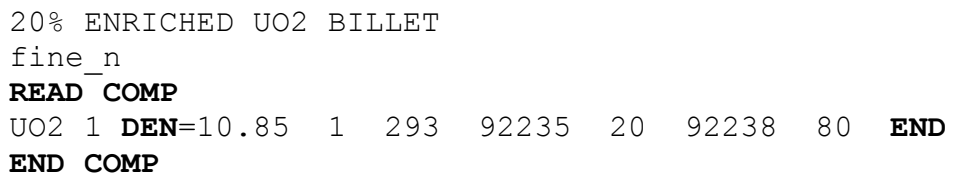

EXAMPLE 2. Specify the cell definition.

Consider a 5-liter Plexiglas bottle with an inner radius of $9.525 \mathrm{~cm}$ and inner height of $17.78 \mathrm{~cm}$ that is filled with highly enriched uranyl nitrate solution at $415 \mathrm{~g} / \mathrm{L}$ and $0.39 \mathrm{mg}$ of excess nitrate per gram of solution. The uranium isotopic content of the nitrate solution is $92.6 \mathrm{wt} \%{ }^{235} \mathrm{U}, 5.9 \mathrm{wt} \%{ }^{238} \mathrm{U}, 1.0 \mathrm{wt} \%{ }^{234} \mathrm{U}$, and $0.5 \mathrm{wt} \%{ }^{236} \mathrm{U}$. Solution density will be calculated from the given data.

The size of the nitrate solution is on the order of 16 to $20 \mathrm{~cm}$ in diameter and height. The average meanfree path in the nitrate solution is on the order of $0.5 \mathrm{~cm}$. Therefore, infinite homogeneous medium is an appropriate choice for this problem. By default BONAMI is used for self-shielding the infinite medium of Plexiglas, while CENTRM is used to shield the infinite medium fissile solution.

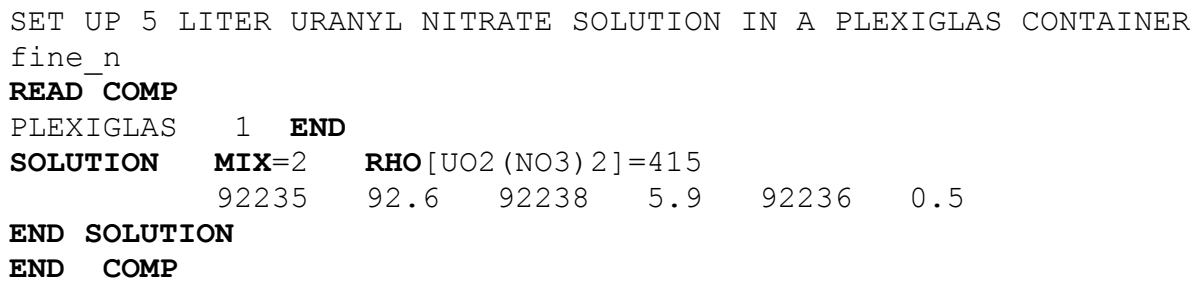


READ CELLDATA

INFHOMMEDIUM 2 END

END CELLDATA

\subsection{C.2 LATTICECELL input data}

Examples of XSProc input data for LATTICECELL problems are given below.

\section{EXAMPLE 1. SQUAREPITCH ARRAY.}

Consider an infinite planar array (infinite in X and $\mathrm{Y}$ and one layer in Z) of $20 \mathrm{wt} \%$ enriched $U$ metal rods with a $1-\mathrm{cm}$ pitch. Each fuel rod is bare uranium metal, $0.75 \mathrm{~cm} \mathrm{OD} \times 30.0 \mathrm{~cm}$ long. The rods are submerged in water.

Because the diameter of the fuel rod, $0.75 \mathrm{~cm}$, is only slightly larger than the average mean-free path in the uranium metal, approximately 0.5 , and because the configuration is a regular array, LATTICECELL is the appropriate choice for proper cross-section processing. The parm field is not provided, so the default CENTRM/PMC self-shielding method is used. XSProc data follows:

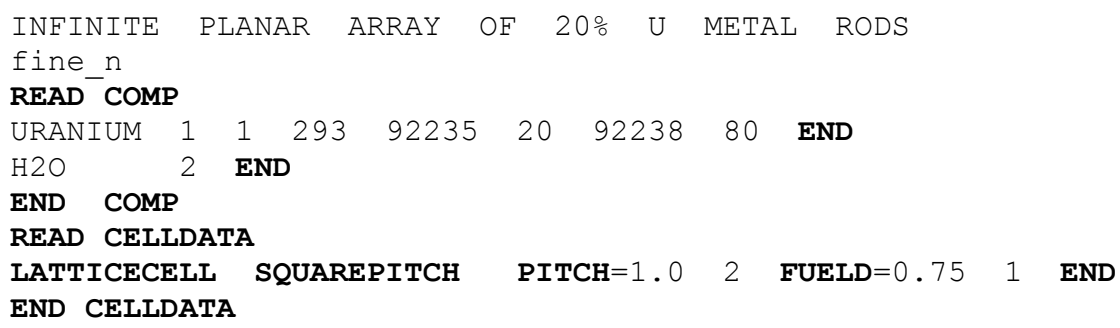

Since the MORE DATA and CENTRM DATA blocks were omitted, default options will be used in the self-shielding calculations. The default CENTRM/PMC computation options for a square pitch lattice cell are the method-of-characteristics (MoC) method with P0 scatter in CENTRM calculations.

EXAMPLE 2. SQUAREPITCH PWR LATTICE.

Consider an infinite, uniform planar array (infinite in $\mathrm{X}$ and $\mathrm{Y}$ and one layer in $\mathrm{Z}$ ) of PWR-like fuel pins of $2.35 \%$ enriched $\mathrm{UO}_{2}$ clad with zirconium. The density of the $\mathrm{UO}_{2}$ is $9.21 \mathrm{~g} / \mathrm{cm}^{3}$. The fuel in each pin is $0.823 \mathrm{~cm}$ in diameter, the clad is $0.9627 \mathrm{~cm}$ in diameter, and the length of each pin is $366 \mathrm{~cm}$. The fuel pins are separated by $0.3124 \mathrm{~cm}$ of water in the horizontal plane.

LATTICECELL is the appropriate choice for cross-section processing. Assume that all defaults are appropriate; thus the CENTRM/PMC methodology is used, and the MORE DATA and CELL DATA blocks are not entered. The input cross section library named "broad_n" indicates that the recommended broad group SCALE library will be used. In this case CENTRM uses the 2D MoC transport solver. The XSProc data follows: 


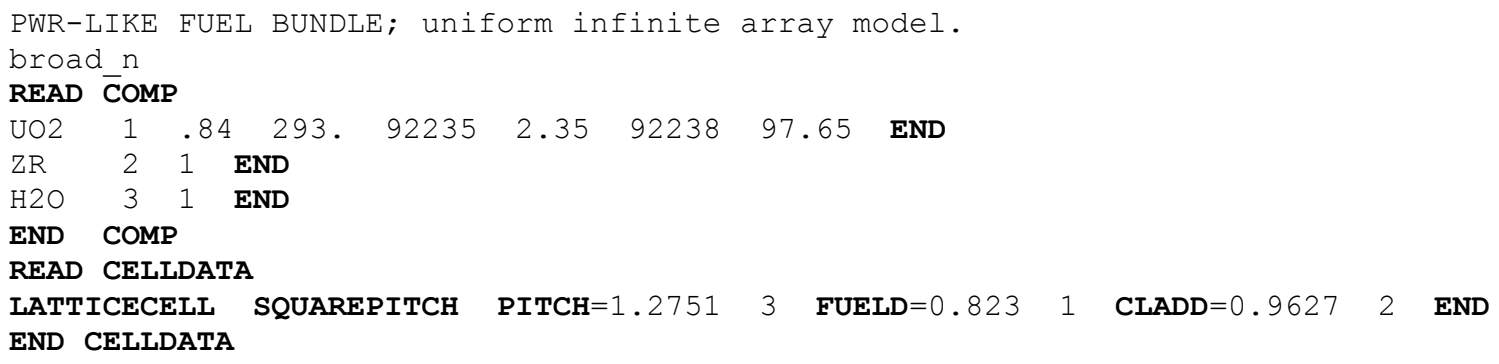

\section{EXAMPLE 3. SQUAREPITCH PWR LATTICE, with non-uniform Dancoff.}

This example is a single PWR assembly of fuel pins of the type described above, contained in a water pool. The interior pins in the assembly can be self-shielded using the same uniform, infinite lattice model in previous example. However self-shielding of the outer boundary-edge pins will be modified to account for being adjacent to a water reflector, rather than surrounded on all sides by similar pins. This requires that the MCDancoff module be executed previously to obtain non-uniform Dancoff factors for the edge pins. The average edge-pin value of 0.61 is used to represent Dancoff factors of all boundary pins. The default CENTRM MoC transport solver is used for both cells, but the original pitch of $1.2751 \mathrm{~cm}$ for the second cell (i.e., boundary pin) is modified to a new pitch corresponding to a Dancoff value of 0.61 .

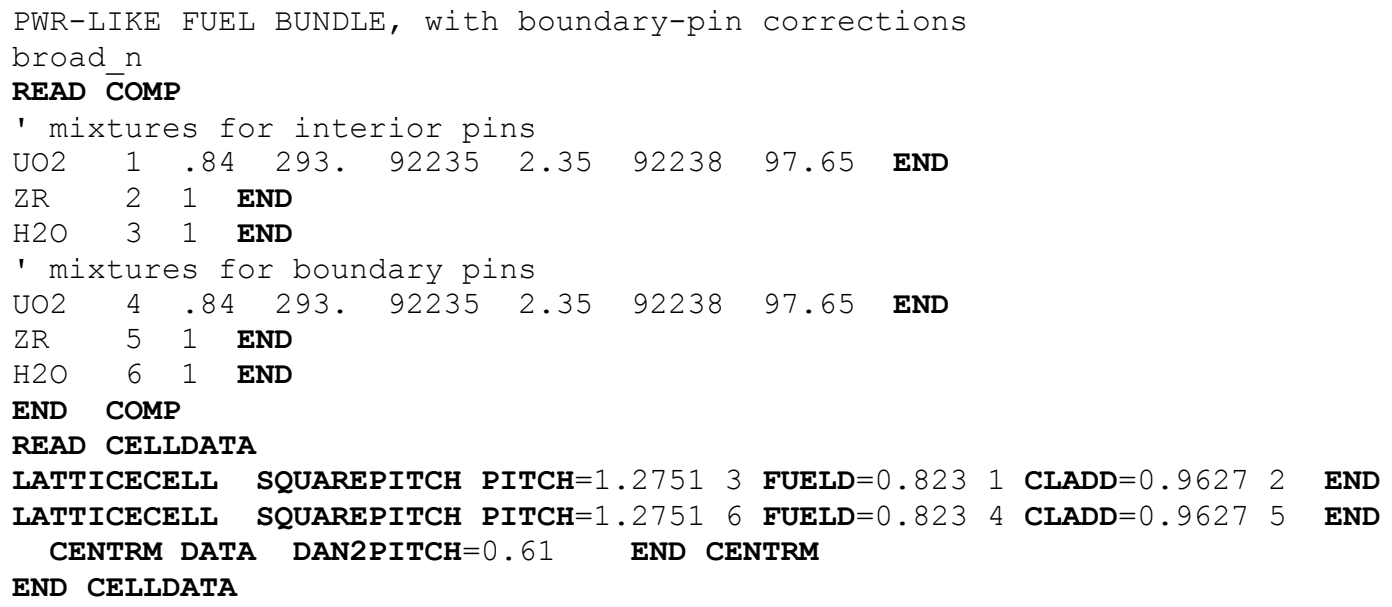

\section{EXAMPLE 6. SPHTRIANGP ARRAY.}

Consider an infinite array of spherical pellets of $2.67 \%$ enriched $\mathrm{UO}_{2}$ with a density of $10.3 \mathrm{~g} / \mathrm{cm}^{3}$ and a diameter of $1.0724 \mathrm{~cm}$ arranged in a "triangular" pitch, flooded with borated water at $4350 \mathrm{ppm}$. The boron is natural boron; the borated water is created by adding boric acid, $\mathrm{H}_{3} \mathrm{BO}_{3}$, and has a density of $1.0078 \mathrm{~g} / \mathrm{cm}^{3}$. The temperature is $15^{\circ} \mathrm{C}$ and the pitch is $1.1440 \mathrm{~cm}$. The standard composition data for the borated water are given in Example 2 of Sect. 7.1.A.9.

Because the diameter of the fuel pellet, $1.0724 \mathrm{~cm}$, is smaller than the average mean-free path in the $\mathrm{UO}_{2}$, approximately $1.5 \mathrm{~cm}$, and because the configuration is a regular array, LATTICECELL is the appropriate choice for proper cross-section processing.

The density fraction for the $\mathrm{UO}_{2}$ is the ratio of actual to theoretical density $(10.3 / 10.96=0.9398)$. Assume that the $\mathrm{U}$ is all ${ }^{235} \mathrm{U}$ and ${ }^{238} \mathrm{U}$. See Sect. 7.1.A.9 for how to define borated water. 
The XSProc data follows:

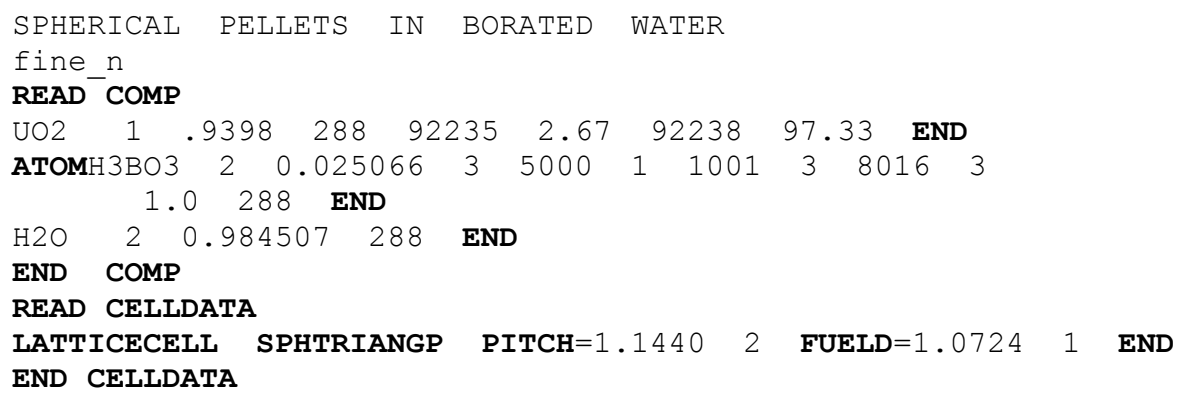

\subsection{C.3 MULTIREGION input data}

Examples of XSProc input data for MULTIREGION problems are given below.

EXAMPLE 1. SPHERICAL.

Consider a small highly enriched uranium sphere supported by a Plexiglas collar in a tank of water. The uranium metal sphere has a diameter of $13.1075 \mathrm{~cm}$, is $97.67 \%$ enriched, and has a density of $18.794 \mathrm{~g} / \mathrm{cm}^{3}$. The cylindrical Plexiglas collar has a $4.1275-\mathrm{cm}$-radius central hole, extends to a radius of $12.7 \mathrm{~cm}$ and is $2.54 \mathrm{~cm}$ thick. The water filled tank is $60 \mathrm{~cm}$ in diameter.

The density fraction of the uranium metal is the ratio of actual to theoretical density, where the theoretical density is obtained from the Isotopes in standard composition library table in section 7.2.1. Thus, the density multiplier is $18.794 / 19.05=0.9866$. The abundance of uranium is not stated beyond $97.67 \%$ enriched, so it is reasonable to assume the remainder is ${ }^{238} \mathrm{U}$. The Plexiglas collar is not significantly different from water and does not surround the fuel, so it can be ignored. If it is ignored, the problem becomes a 1-D geometry that can be defined using the MULTIREGION type of calculation, and the eigenvalue of the system can be obtained without additional data by executing CSAS1. However, the Plexiglas has been included in this data so it can be passed to a code such as KENO V.a which can describe the geometry rigorously. The XSProc data follow:

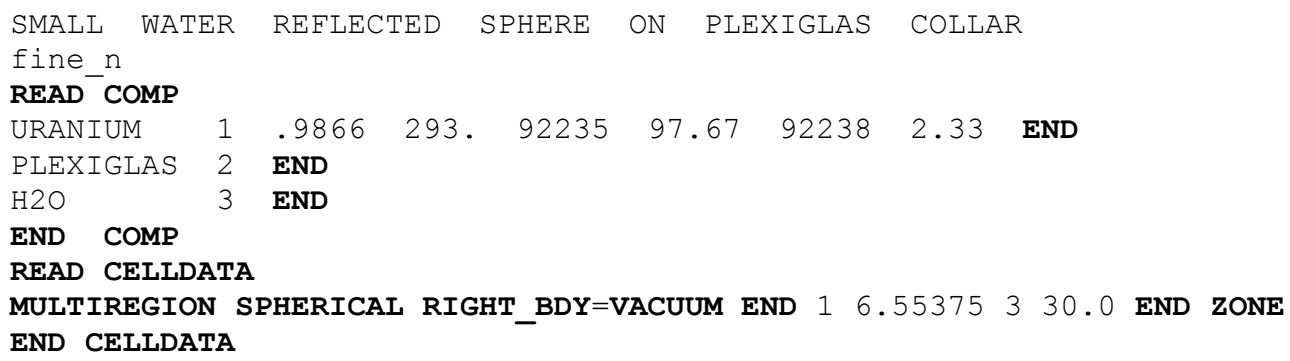

\section{EXAMPLE 2. BUCKLEDSLAB.}

This example features a 93.2\% enriched uranyl-fluoride solution inside a rectangular Plexiglas container immersed in water. The fissile solution contains $578.7 \mathrm{~g}$ of $\mathrm{UO}_{2} \mathrm{~F}_{2}$ per liter and has no excess acid. The critical thickness of the fuel is $5.384 \mathrm{~cm}$. The finite height of the fuel slab is $147.32 \mathrm{~cm}$, and the depth is $71.58 \mathrm{~cm}$. The Plexiglas container is $1.905 \mathrm{~cm}$ thick and is reflected by $20.32 \mathrm{~cm}$ of water. 
The half thickness of the fuel (2.692) will be used with a reflected left boundary and a vacuum right boundary (default). The XSProc data follow:

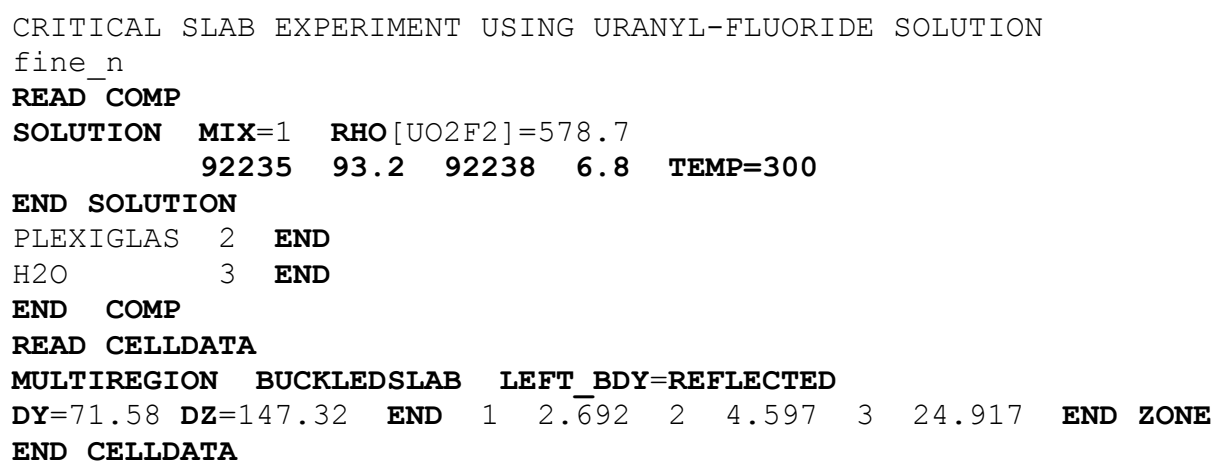

\subsection{C.4 DOUBLEHET input data}

EXAMPLE 1: A doubly-heterogeneous spherical fuel element with $15,000 \mathrm{UO}_{2}$ particles in a graphite matrix.

Grain fuel radius is $0.025 \mathrm{~cm}$. Grain contains one coating layer that is $0.009-\mathrm{cm}-$ thick. Pebbles are in a triangular pitch on a 6.4-cm-pitch. Fuel pebble fuel zone is $2.5-\mathrm{cm}$ in radius and contains a $0.5-\mathrm{cm}$-thick graphite clad that contains small amounts of ${ }^{10} \mathrm{~B}$. Pebbles are surrounded by ${ }^{4} \mathrm{He}$. In this case we designated the homogenized mixture as mixture 10. If we have a KENO V.a or KENO-VI input section, we would use mixture 10 in that section. Note that the keyword "FUELR=" is followed by the fuel dimension only, i.e., no mixture number. That is because the fuel mixture number is specified with "FUELMIX=" and therefore need not be repeated.

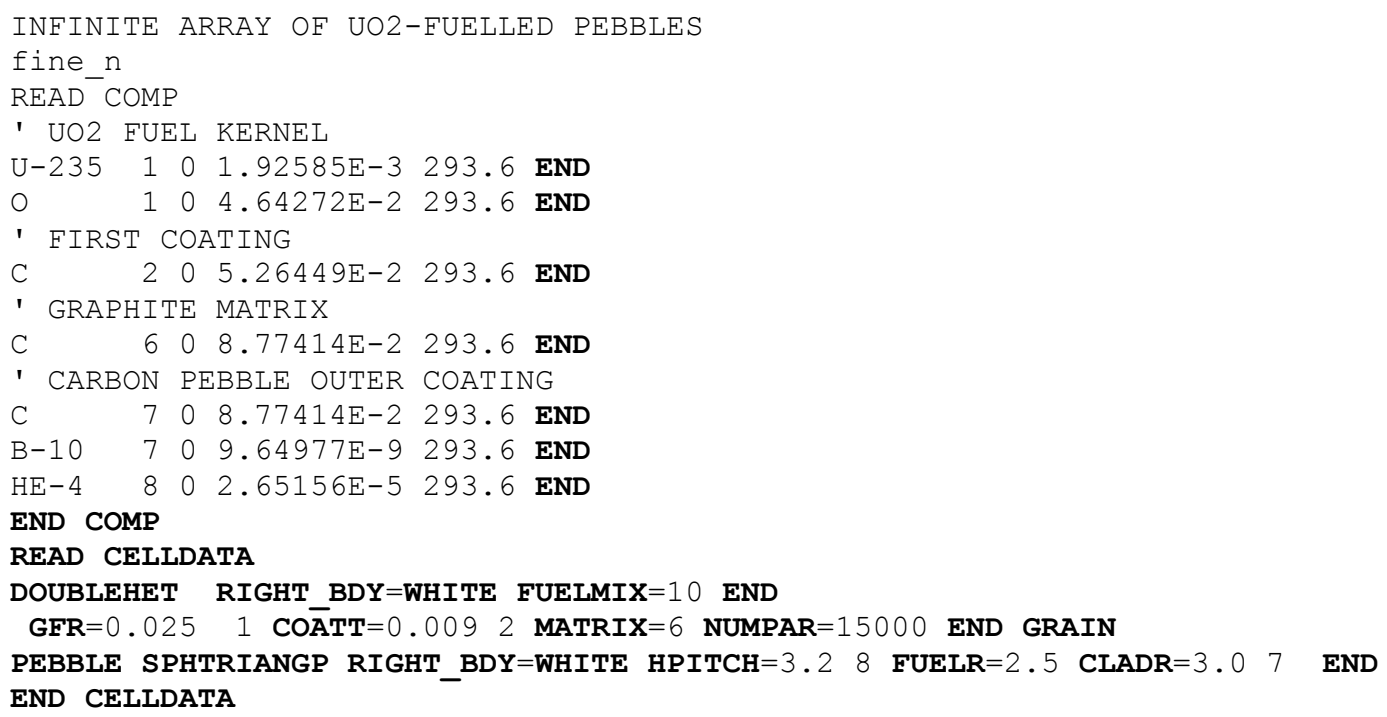


EXAMPLE 2: A doubly-heterogeneous spherical fuel element with $10,000 \mathrm{UO}_{2}$ particles and $5,000 \mathrm{PuO}_{2}$ particles in a graphite matrix.

Grain fuel radii for $\mathrm{UO}_{2}$ and $\mathrm{PuO}_{2}$ particles are $0.025 \mathrm{~cm}$ and $0.012 \mathrm{~cm}$, respectively. $\mathrm{UO}_{2}$ grains contain one coating layer that is 0.009 -cm-thick. $\mathrm{PuO}_{2}$ grains contain one coating layer that is $0.0095-\mathrm{cm}$-thick. Pebbles are in a triangular pitch on a $6.4-\mathrm{cm}-$ pitch. Fuel pebble fuel zone is $2.5-\mathrm{cm}$ in radius and contains a $0.5-\mathrm{cm}$-thick graphite clad that contains small amounts of ${ }^{10} \mathrm{~B}$. Pebbles are surrounded by ${ }^{4} \mathrm{He}$. Since number of particles is entered, the total volume fraction and the pitch can be calculated by the code.

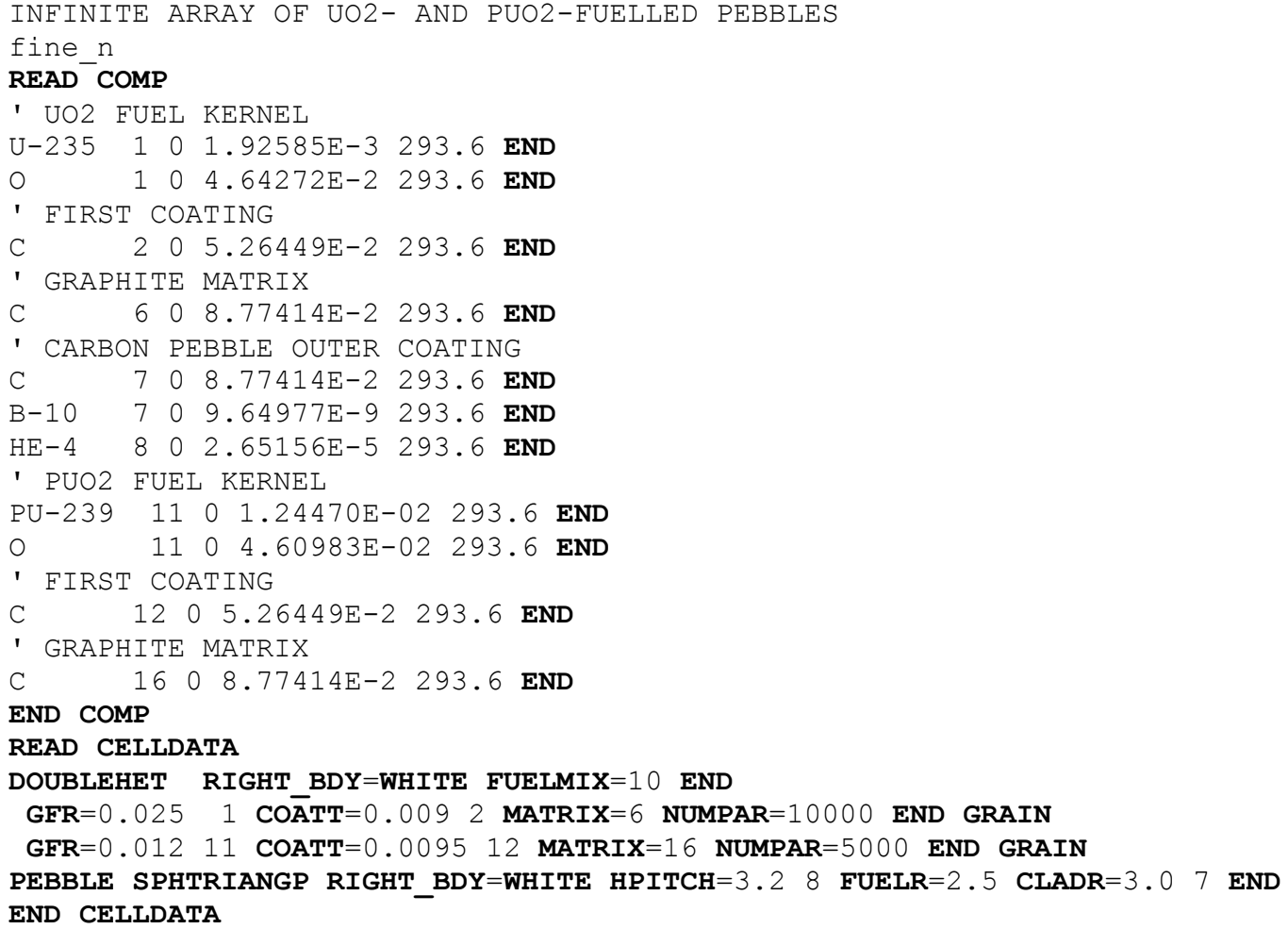

\subsection{C.5 Two methods of specifying a fissile solution}

The standard composition specification data offer flexibility in the choice of input data. This section illustrates two methods of specifying the same fissile solution.

Create a mixture 3 that is aqueous uranyl nitrate solution:

$\mathrm{UO}_{2}\left(\mathrm{NO}_{3}\right)_{2}$, solution density $=1.555 \mathrm{~g} \mathrm{~cm}^{3} /$

$0.2669 \mathrm{~g} \mathrm{U} / \mathrm{g}$-soln., $0.415 \mathrm{~g} \mathrm{U} / \mathrm{cm}^{3}$; excess nitrate $=0.39 \mathrm{mg} / \mathrm{g}$-soln

Uranium isotopic content: $92.6 \mathrm{wt} \% \mathrm{U}-235 \quad 5.9 \mathrm{wt} \% \mathrm{U}-238$

$1.0 \mathrm{wt} \% \mathrm{U}-234$ and $0.5 \mathrm{wt} \% \mathrm{U}-236$

The SCALE atomic weights used in this problem are listed as follows:

$\begin{array}{ll}\mathrm{H} & 1.0078 \\ \mathrm{O} & 15.999\end{array}$




$\begin{array}{ll}\mathrm{N} & 14.0067 \\ \mathrm{U}-234 & 234.041 \\ \mathrm{U}-235 & 235.0439 \\ \mathrm{U}-236 & 236.0456 \\ \mathrm{U}-238 & 238.0508\end{array}$

Two methods of describing the uranyl nitrate solution will be demonstrated. Method 1 is more rigorous, and method 2 is easier and as accurate.

\section{METHOD 1:}

This method involves breaking the solution into its component parts $\left[\left(\mathrm{HNO}_{3}, \mathrm{UO}_{2}\left(\mathrm{NO}_{3}\right)_{2}\right.\right.$, and $\left.\left.\mathrm{H}_{2} \mathrm{O}\right)\right]$ and entering the basic standard composition specifications for each.

1. Calculate the density of the $\mathrm{HNO}_{3} 0.39 \times 10^{-3} \mathrm{~g} \mathrm{NO} / \mathrm{g}$ soln $\times\left[\left(62.997 \mathrm{~g} \mathrm{HNO}_{3} / \mathrm{mole}\right.\right.$ $\left.\mathrm{HNO}_{3}\right) /\left(61.990 \mathrm{~g} \mathrm{NO}_{3} /\right.$ mole $\left.\left.\mathrm{NO}_{3}\right)\right] \times 1.555 \mathrm{~g} \mathrm{soln} / \mathrm{cm}^{3} \mathrm{soln}=6.16 \times 10^{-4} \mathrm{~g} \mathrm{HNO}_{3} / \mathrm{cc}$ soln.

2. Calculate the density fraction of $\mathrm{HNO}_{3}$ (actual density/theoretical density). In the Standard Composition Library the theoretical density of $\mathrm{HNO}_{3}$ is $1.0 .6 .16 \times 10^{-4} / 1.0=6.16 \times 10^{-4}$.

3. Calculate the molecular weight of the uranium

The number of atoms in a mole of uranium is the sum of the number of atoms of each isotope in the mole of uranium.

Let $\mathrm{AU}=$ the average molecular weight of uranium, $\mathrm{g} \mathrm{U} / \mathrm{mole} \mathrm{U}$

$\mathrm{GU}=$ the density of uranium in $\mathrm{g} / \mathrm{cm}^{3}$.

Then the number of atoms in a mol of uranium $=$

$$
\begin{array}{ll} 
& \left(6.023 \times 10^{+23} * 10^{-24} * \mathrm{GU}\right) / \mathrm{AU} \\
\text { or } 0.6023 * \mathrm{GU} / \mathrm{AU} .
\end{array}
$$

The weight fraction of each isotope is the weight $\% * 100$.

Therefore, $\quad F 235=0.926$, the weight fraction of U-235 in the $\mathrm{U}$

F238 $=0.059$, the weight fraction of U-238 in the U

F236 $=0.005$, the weight fraction of U-236 in the U

F234 $=0.010$, the weight fraction of U-234 in the U

A235 $=235.0442$, the molecular weight of U-235

A238 $=238.0510$, the molecular weight of U-238

A236 $=236.0458$, the molecular weight of U-236

A234 $=234.0406$, the molecular weight of U-234.

Then the number of atoms of isotopes in a mol of uranium $=$

$$
\begin{gathered}
6.023 \times 10^{+23} * 10^{-24} *((\mathrm{GU} * \mathrm{~F} 235 / \mathrm{A} 235)+(\mathrm{GU} * \mathrm{~F} 238 / \mathrm{A} 238)+ \\
\mathrm{GU} * \mathrm{~F} 236 / \mathrm{A} 236)+(\mathrm{GU} * \mathrm{~F} 234 / \mathrm{A} 234))
\end{gathered}
$$

or

$$
\begin{aligned}
& 0.6023 * \mathrm{GU} *(0.926 / 235.0442+0.059 / 238.0510+ \\
& 0.005 / 236.0458+0.010 / 234.0406) \text {. }
\end{aligned}
$$


Because the number of atoms of uranium equals the sum of the atoms of isotopes,

$$
\begin{aligned}
& 0.6023 * \mathrm{GU} / \mathrm{AU}=0.6023 * \mathrm{GU} *(0.926 / 235.0442+0.059 / 238.0510+ \\
& 0.005 / 236.0458+0.010 / 234.0406) \\
& 1 / \mathrm{AU}=0.926 / 235.0442+0.059 / 238.0510+0.005 / 236.0458+0.010 / 234.0406 \\
& \mathrm{AU}=235.2144 .
\end{aligned}
$$

4. Calculate the molecular weight of the $\mathrm{UO}_{2}\left(\mathrm{NO}_{3}\right)_{2}$.

$$
235.2144+(8 \times 15.9954)+(2 \times 14.0033)=391.184 \mathrm{~g} \mathrm{UO}_{2}\left(\mathrm{NO}_{3}\right)_{2} / \text { mole }
$$

5. Calculate the density of $\mathrm{UO}_{2}\left(\mathrm{NO}_{3}\right)_{2}$

$0.415 \mathrm{~g} \mathrm{U} / \mathrm{cc} \times\left[\left(391.184 \mathrm{~g} \mathrm{UO}_{2}\left(\mathrm{NO}_{3}\right)_{2} / \mathrm{mol}\right) /(235.2144 \mathrm{~g} \mathrm{U} / \mathrm{mole})\right]=$

$0.69018 \mathrm{~g} \mathrm{UO}_{2}\left(\mathrm{NO}_{3}\right)_{2} / \mathrm{cm}^{3} . \operatorname{soln}$.

Calculate the density fraction (actual density/theoretical density) of $\mathrm{UO}_{2}\left(\mathrm{NO}_{3}\right)_{2}$.

[In the Standard Composition Library the theoretical density of $\mathrm{UO}_{2}\left(\mathrm{NO}_{3}\right)_{2}$ is given as $2.2030 \mathrm{~g} / \mathrm{cm}^{3}$.]

The density fraction is $0.69018 / 2.2030=0.31329$.

6. Calculate the amount of water in the solution

$1.555 \mathrm{~g} \mathrm{soln} / \mathrm{cm}^{3}$. soln $-6.16 \times 10^{-4} \mathrm{~g} \mathrm{HNO}_{3} / \mathrm{cm}^{3}$ soln $-0.69018 \mathrm{~g} \mathrm{UO}_{2}\left(\mathrm{NO}_{3}\right)_{2} \mathrm{LL} / \mathrm{cm}^{3}$. soln $=$ $0.8642 \mathrm{~g} \mathrm{H}_{2} \mathrm{O} / \mathrm{cc}$ soln.

\begin{tabular}{|c|c|c|c|c|c|c|c|}
\hline HNO3 & 3 & $6.16-4$ & END & & & & \\
\hline UO2 (NO3) 2 & 3 & $\begin{array}{c}.31329293 \\
92236\end{array}$ & $\begin{array}{l}92235 \\
0.5\end{array}$ & $\begin{array}{l}92.6 \\
\text { END }\end{array}$ & 92238 & 5.9 & 92234 \\
\hline 20 & 3 & $.86575 \quad 293$ & END & & & & \\
\hline
\end{tabular}

7. Calculate the density fraction (actual density/theoretical density) of water.

(In the Standard Composition Library the theoretical density of water is given as $0.9982 \mathrm{~g} / \mathrm{cm}^{3}$.)

The density fraction is $0.8642 / 0.9982=0.86575$.

The solution specification data follow:

\section{METHOD 2:}

This method utilizes the solution option available in the standard composition specification data. Because the density is specified in the input data, this method should yield correct number densities that should agree with method 1 except for calculational round-off.

1. Calculate the fuel density

$0.415 \mathrm{~g} \mathrm{U} / \mathrm{cc}$ is $415 \mathrm{~g} \mathrm{U} / \mathrm{L}$.

2. The molecular weight of nitrate $\mathrm{NO}_{3}$ is 61.9895 .

3. Calculate the molarity of the solution.

$0.39 \mathrm{mg}$ nitrate $/ \mathrm{g}$ soln $\times 1000 \mathrm{~cm}^{3} \operatorname{soln} / \mathrm{L}$ soln $\times 1 \mathrm{~g} / 1000 \mathrm{mg} \times 1.555 \mathrm{~g}$ soln $/ \mathrm{cm}^{3} \operatorname{soln}=0.60645 \mathrm{~g}$ excess nitrate/L soln. 
A 1-molar solution is 1 mole of acid/L of solution:

(For nitric acid 1 molar is 1 normal because there is only one atom of hydrogen per molecule of acid in $\mathrm{HNO}_{3}$.)

$(0.60645 \mathrm{~g}$ nitrate/L soln $) /\left(61.9895 \mathrm{~g} \mathrm{NO}_{3} /\right.$ mole $\left.\mathrm{NO}_{3}\right)=9.783 \times 10^{-3}$ mole nitrate $/ \mathrm{L}$ is identical to mole of acid/L, which is identical to molarity.

4. The density fraction of the solution is 1.0. Do not try to use the density of the solution divided by the theoretical density of $\mathrm{UO}_{2}\left(\mathrm{NO}_{3}\right)_{2}$ from the Standard Composition Library for your density multiplier. The $\mathrm{UO}_{2}\left(\mathrm{NO}_{3}\right)_{2}$ listed there is the solid, not the solution.

The solution specification data follow:

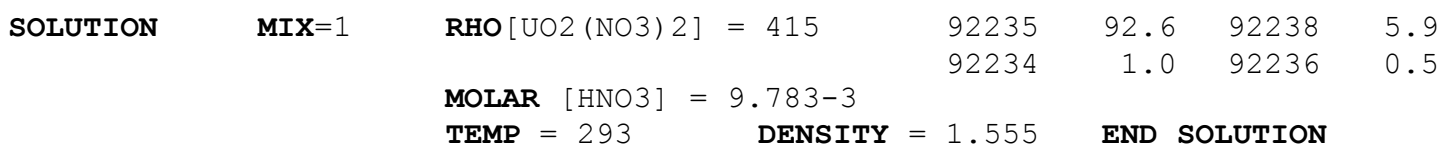

Comparison of number densities from the two methods

The number densities of methods 1 and 2 should agree within the limits of the input data. The density multipliers in method 1 are 5 digits and the density multipliers in method 2 are 4 digits. Therefore, the number densities calculated by the two methods should agree to 4 or 5 digits.

\begin{tabular}{ccc}
\hline & Method 1 & Method 2 \\
\cline { 2 - 3 } Nuclide number & Atom density & Atom density \\
\hline 92235 & $9.84603 \mathrm{E}-04$ & $9.84603 \mathrm{E}-04$ \\
92238 & $6.19415 \mathrm{E}-05$ & $6.19415 \mathrm{E}-05$ \\
92234 & $1.06784 \mathrm{E}-05$ & $1.06784 \mathrm{E}-05$ \\
92236 & $5.29387 \mathrm{E}-06$ & $5.29387 \mathrm{E}-06$ \\
07014 & $2.13092 \mathrm{E}-03$ & $2.13092 \mathrm{E}-03$ \\
08016 & $3.74135 \mathrm{E}-02$ & $3.7410 \mathrm{E}-02$ \\
01001 & $5.77973 \mathrm{E}-02$ & $5.77983 \mathrm{E}-02$ \\
\hline
\end{tabular}

\subsection{C.6 Multiple unit cells in a single problem}

Consider a problem that involves three different $\mathrm{UO}_{2}$ fuel assemblies: a $1.98 \%$-enriched assembly, a $2.64 \%$-enriched assembly, and a $2.96 \%$-enriched assembly. All fuel rods are $\mathrm{UO}_{2}$ at $10.138 \mathrm{~g} / \mathrm{cm}^{3}$ and are $0.94 \mathrm{~cm}$ in diameter. The Zircaloy- 4 clad has an inside radius of $0.4875 \mathrm{~cm}$ and an outside radius of $0.545 \mathrm{~cm}$. The rod pitch is $1.44 \mathrm{~cm}$. Each fuel assembly is a $15 \times 15$ array of fuel pins with water holes, instrumentation holes, and burnable poison rods. For cross-section processing, the presence of the water holes, instrumentation holes, and burnable poison rods in the assemblies are ignored.

The following XSProc input use the CENTRM/PMC method for self-shielding three latticecells with different fuel enrichments. The remaining mixture (SS-304), not specified in a unit cell, is processed as an infinite homogeneous medium using the BONAMI method. Each mixture can appear only in a single 
zone of one unit cell. For square pitch latticecells the default CENTRM transport solver is MoC with P0 scatter; however in this input, the solver for the $3^{\text {rd }}$ cell is modified through CENTRM DATA to use the two-region approximation for the $\mathrm{CE}$ calculation [npxs=5], and discrete $\mathrm{S}_{\mathrm{N}}$ transport calculation with P1 anisotropic scatteringfor the MG solutions in the fast and thermal energy ranges [nfst $=0, \mathrm{nthr}=0]$.

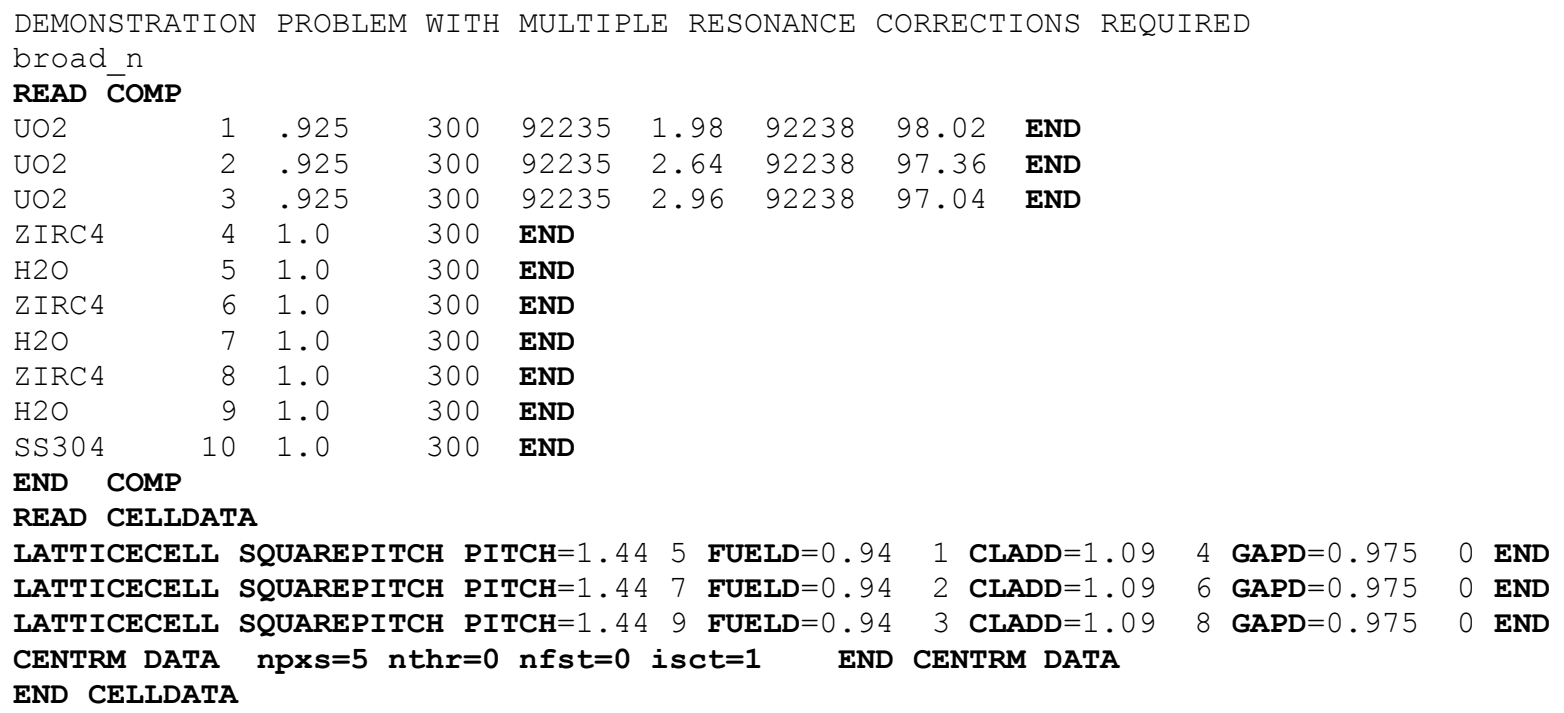

\subsection{C.7 Multiple fissile mixtures in a single unit cell}

The following problem involves large units having the bulk of their fissile material more than one meanfree path away from the surface of the unit. The interaction between the units that occurs in the resonance range is a very small fraction of the total interaction because an overwhelming percentage of the interaction occurs deep within each unit. Therefore, the resonance range interaction between the units can be ignored, and the default infinite homogeneous medium cross-section processing in the resonance range can be considered adequate for this particular application.

Consider a problem that consists of four $20.96-\mathrm{kg} 93.2 \%$-enriched uranium metal cylinders, density $18.76 \mathrm{~g} / \mathrm{cm}^{3}$, and four 5-liters Plexiglas bottles filled with highly enriched uranyl nitrate solution at $415 \mathrm{~g} / \mathrm{L}$, a specific gravity of 1.555 , and $0.39 \mathrm{mg}$ of excess nitrate per gram of solution. The isotopic content of the uranium metal is $93.2 \mathrm{wt} \%{ }^{235} \mathrm{U}, 5.6 \mathrm{wt} \%{ }^{238} \mathrm{U}, 1.0 \mathrm{wt} \%{ }^{234} \mathrm{U}$, and $0.2 \mathrm{wt} \%{ }^{236} \mathrm{U}$. The uranium isotopic content of the nitrate solution is $92.6 \mathrm{wt} \%{ }^{235} \mathrm{U}, 5.9 \mathrm{wt} \%{ }^{238} \mathrm{U}, 1.0 \mathrm{wt} \%{ }^{234} \mathrm{U}$ and $0.5 \mathrm{wt} \%{ }^{236} \mathrm{U}$. The size of the metal cylinders is between 10 and $12 \mathrm{~cm}$ in diameter and height, and the size of the nitrate solution is on the order of 16 and $20 \mathrm{~cm}$ in diameter and height. The average mean-free path in the uranium metal is on the order of $1.5 \mathrm{~cm}$, and the average mean free path in the nitrate solution is on the order of $0.5 \mathrm{~cm}$. Therefore, infinite homogeneous medium is an appropriate choice for this problem and the use of CENTRM/PMC is valid.

See Examples 1-4 of Sect. 7.1.A.2 for data input details for the Plexiglas and uranium metal. See Example 1 of Sect. 7.1.A.5 for data input details for the uranyl nitrate solution. The XSProc data for this problem follow: 


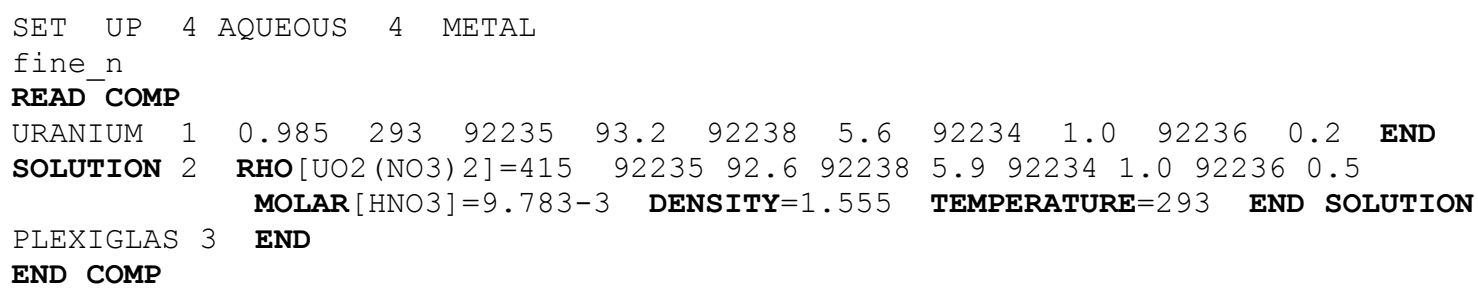

Consider the same materials above except rearrange them so that a $10 \mathrm{~cm}$ diameter uranium metal sphere sits inside a $50 \mathrm{~cm}$ diameter spherical tank of uranyl nitrate solution having a $1-\mathrm{cm}$ thick Plexiglas wall. This problem can be modeled in SCALE but only CENTRM/PMC will treat the resonance processing correctly. This problem is modeled below.

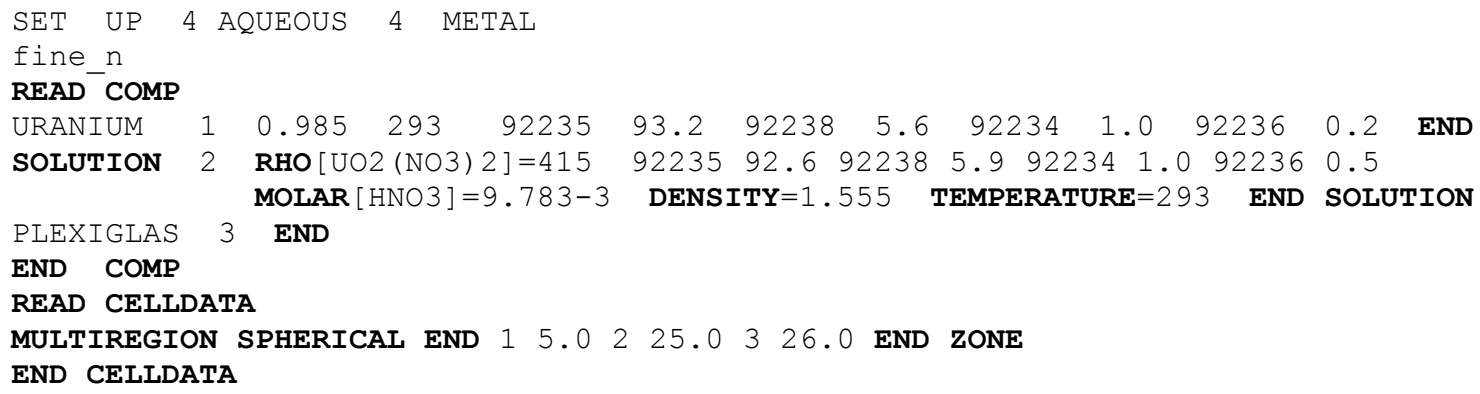

\subsection{C.8 Cell weighting an infinite homogeneous problem}

Cell weighting an infinite homogeneous medium has no effect on the cross sections because there is only one zone and one set of cross sections. However, a cell-weighted mixture number can still be supplied using the keyword CELLMIX= followed by an unique mixture number. This cell-weighted mixture number can be used in subsequent codes and will produce results similar to the cross sections of the original mixture.

\section{EXAMPLE 1}

This problem would probably be run with CSAS1 to provide the k-infinity of $20 \%$-enriched $\mathrm{UO}_{2}$.

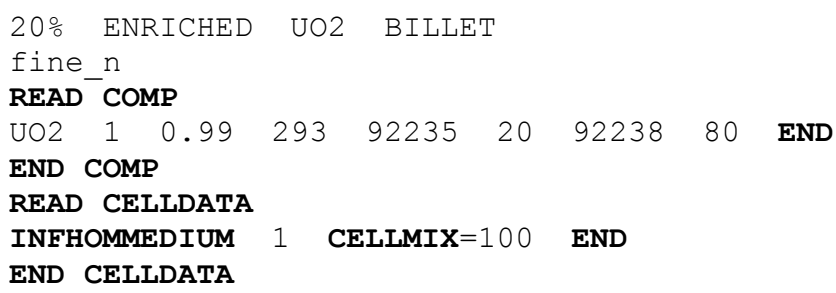

\subsection{C.9 Cell weighting a LATTICECELL problem}

Cell weighting used with a LATTICECELL problem creates cell-weighted homogeneous cross sections that represent the characteristics of the heterogeneous unit cell. This cell-weighted mixture can then be used in a subsequent code for the overall volume where the cells are located without having to mock up the actual 3-D heterogeneous array of cells. This cell-weighted homogeneous mixture is designated by the user with the keyword CELLMIX= immediately followed by an unused mixture number. This needs 
to follow immediately after the cell description. Note that the mixtures used in the unit cell data cannot be used in a subsequent code because they have been flux weighted to create the user specified mixture. Therefore, if a mixture used in the unit cell description is also to be used in a subsequent code, another mixture must be created that is identical except for the mixture number. Every mixture that is to be used in a subsequent code except zero (i.e., void) must be defined in the standard composition data.

A byproduct of the cell-weighting calculation is the eigenvalue (k-effective) of an infinite array of the cell described as the unit cell.

\section{EXAMPLE 1}

Consider a cylindrical stainless steel tank filled with spherical pellets of $2.67 \%$-enriched $\mathrm{UO}_{2}$ arranged in a close-packed "triangular" pitch, flooded with borated water at $4350 \mathrm{ppm}$. The cylindrical stainless tank is sitting in a larger tank filled with borated water at $4350 \mathrm{ppm}$.

The data for the $\mathrm{UO}_{2}$ and borated water were developed in detail in Example 3 of Sect. 7.1.C.2. The stainless steel must be defined, and mixture 3 was chosen because mixture 1 was the $\mathrm{UO}_{2}$ and mixture 2 was the borated water. Because the borated water will be used as a reflector for the stainless steel tank and has been used in the unit cell data, it must be repeated with a different mixture number (in this case, as mixture 4).

In the subsequent calculation, user specified cell mixture 100 will be used to represent the $\mathrm{UO}_{2}$ pellets in the borated water, mixture 3 will represent the stainless steel tank, and mixture 4 will represent the borated water reflector around the stainless-steel tank.

The XSProc data for creating the cell-weighted cross sections on mixture 100 follow:

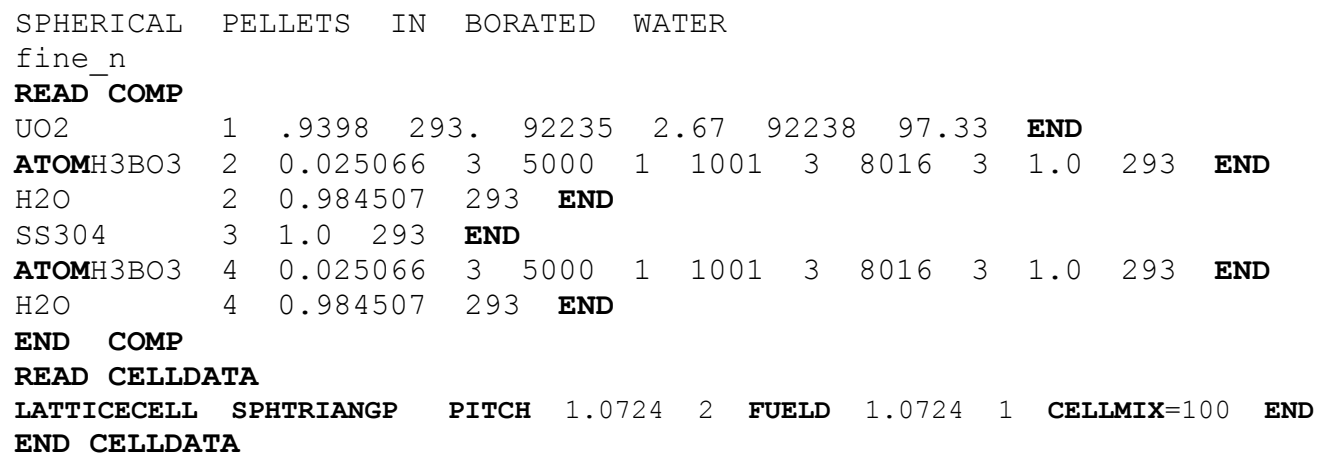

\subsection{C.10 Cell weighting a MULTIREGION problem}

A MULTIREGION problem is cell weighted primarily to obtain a cell-weighted homogeneous cross section that represents the characteristics of the heterogeneous unit cell. The eigenvalue obtained for a MULTIREGION problem with cylindrical or spherical geometry having a white boundary condition specified on the right boundary approximates an infinite array of the cells. A vacuum boundary condition would represent a single cell. A slab with reflected boundary conditions for both boundaries represents an infinite array of slab cells. The cell-weighted cross sections for spherical or cylindrical geometries with a white right boundary condition do not use a Dancoff correction and thus may not be accurate for representing a large array of the specified units. 


\section{EXAMPLE 1}

Consider a small, highly enriched uranium sphere supported by a Plexiglas collar in a tank of water. The uranium metal sphere has a diameter of $13.1075 \mathrm{~cm}$, is $97.67 \%$ enriched, and has a density of $18.794 \mathrm{~g} / \mathrm{cm}^{3}$. The cylindrical Plexiglas collar has a $4.1275-\mathrm{cm}$ radius central hole, extends to a radius of $12.7 \mathrm{~cm}$ and is $2.54 \mathrm{~cm}$ thick. The water-filled tank is $60 \mathrm{~cm}$ in diameter.

The Plexiglas collar is not significantly different from water and does not surround the fuel, so it will be ignored. Because this makes the problem a 1-D geometry, it can be defined using the MULTIREGION type of calculation and the eigenvalue of the system can be obtained without additional data by executing CSAS1 with CENTRM/PMC, if PARM=CENTRM is specified on the command line. The abundance of uranium is not stated beyond $97.67 \%$ enriched, so assume the remainder is ${ }^{238} \mathrm{U}$. The XSProc data follow:

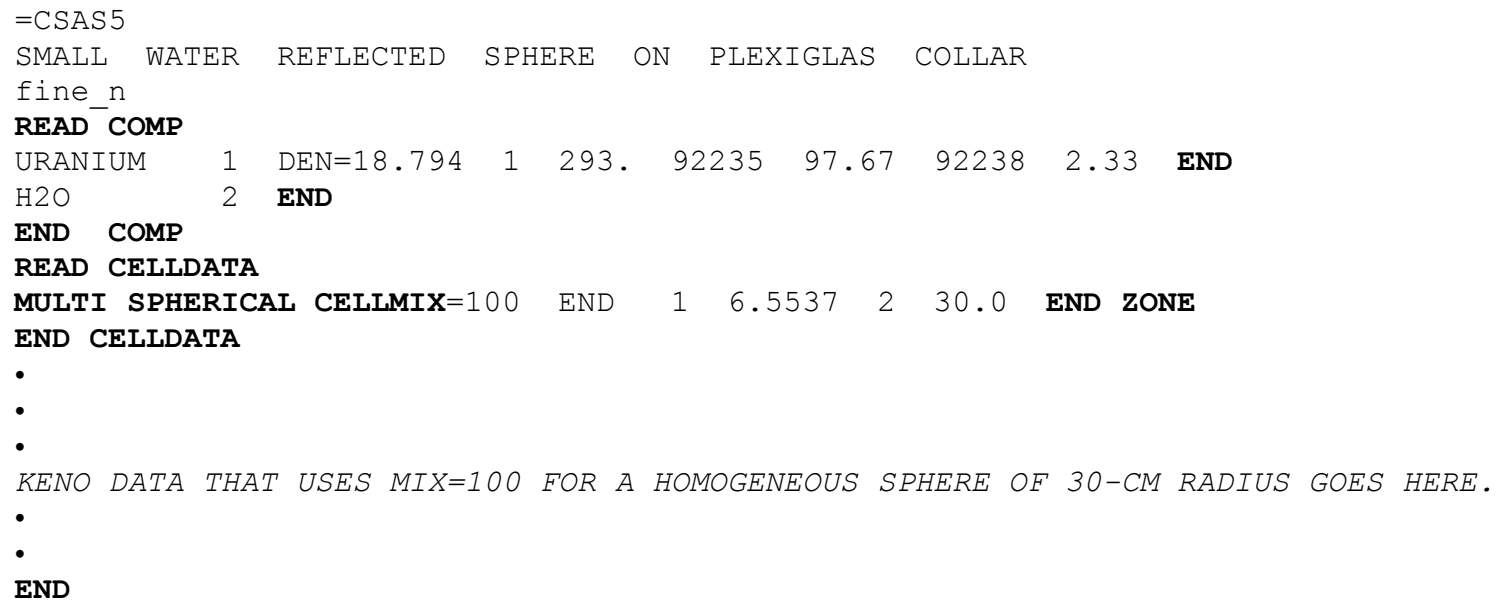




\title{
7.2 STANDARD COMPOSITION LIBRARY
}

L. M. Petrie, R. A. Lefebvre, and D. Wiarda

\begin{abstract}
The SCALE Standard Composition Library provides a flexible and convenient means of generating models that include many types of materials. Users may specify materials as individual nuclides; elements with tabulated natural abundances; compounds, alloys, mixtures, and fissile solutions commonly encountered in engineering practice.
\end{abstract}




\section{TABLE OF CONTENTS}

$\underline{\text { Page }}$

7.2 STANDARD COMPOSITION LIBRARY ............................................................... $7-112$

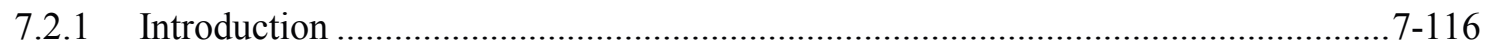

7.2.2 The Standard Composition Library …................................................................ $7-116$

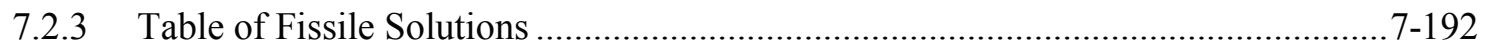

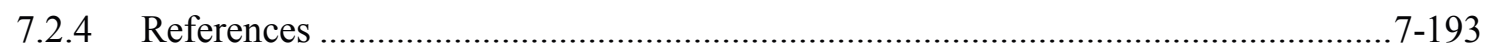




\section{LIST OF TABLES}

$\underline{\text { Page }}$

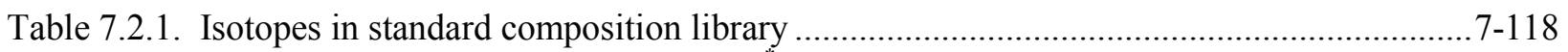

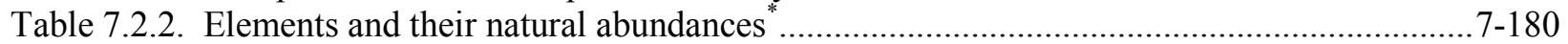

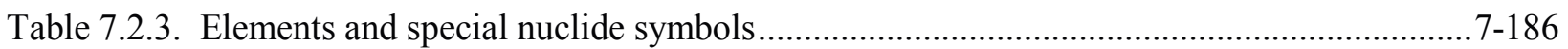

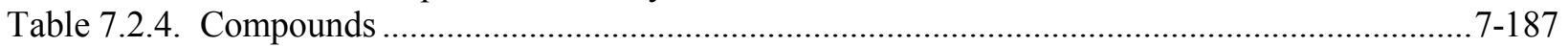

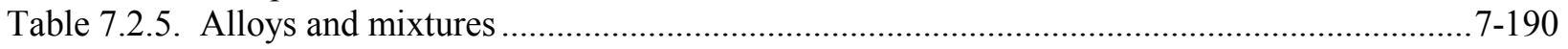

Table 7.2.6. Available fissile solution components ................................................................... $7-193$ 


\section{ACKNOWLEDGMENTS}

This work was originally funded by the U.S. Nuclear Regulatory Commission through the Office of Nuclear Material Safety and Safeguards and the Office of Nuclear Regulatory Research. Continuing support is provided by the U.S. Department of Energy Nuclear Criticality Safety Program. 


\subsubsection{Introduction}

The Standard Composition Library has been included within the SCALE system to provide the user with a simple and straightforward method of specifying the material mixtures for a given problem. The library consists of over 700 mixtures and isotopes commonly used within criticality safety, shielding, and reactor physics models. This library is tabulated in Sect.7.2.2 with the various features of each composition. Additionally, a number of standard fissile solutions available for use are tabulated in Sect. 7.2.3.

The Standard Composition Library is created using the COMPOZ module, and can be updated by users as desired.

\subsubsection{The Standard Composition Library}

The Standard Composition Library describes the various isotopes, elements (both symbols and full name), and compounds/alloys that can be used to define the material mixtures for a given problem. Typically, the alphanumeric description of one or more of these materials will be used to define a material mixture.

When formulating a mixture, it is often necessary to know the density $\left(\mathrm{g} / \mathrm{cm}^{3}\right)$ of the various constituent materials. For convenience, the reference values given in the library have been listed in Table 7.2.2 through Table 7.2.5. Note that the given reference values represent the actual theoretical density, except in the case of isotopes and some individual nuclides where a default value of $1.0 \mathrm{~g} / \mathrm{cm}^{3}$ is used. The actual theoretical densities are fixed values at naturally occurring or nominal conditions. These default densities should not be used for materials containing enriched isotopes, especially light elements with isotopes that are strong absorbers such as boron, $\mathrm{B}_{4} \mathrm{C}$, or lithium. When densities are recalculated by other codes such as KENO, the recalculated densities may differ slightly from those given in the Standard Composition Library depending on the cross-section library specified in the calculation. Note that the recalculated mixture densities are presented for information purposes and are not used in the calculations.

Note that not all nuclides in the Standard Composition Library are available on each cross-section library, and user are encourage to review the code output for warning messages regarding composition data. Refer to the cross section library chapter for listings of available nuclides in each cross-section library.

Multiple sets of iron, nickel, and chromium nuclides are available in the Standard Composition Library. These sets correspond to different weighting functions used in generating the multigroup cross sections. One special weighting function corresponds to $1 /\left[\mathrm{E} \sigma_{t}(\mathrm{E})\right]$, where $\sigma_{t}(\mathrm{E})$ is the total cross section of the referenced nuclide or alloy. Entries have been added to the isotopic distribution table so that standard weighted isotopes will be requested if the desired nuclide is not on the specified library.

The nuclide identifying numbers (IDs) are listed in -. Typically, the ID is $1000 * \mathrm{Z}+\mathrm{A}$, where $\mathrm{Z}$ and $\mathrm{A}$ are the charge and mass numbers for the nuclide (e.g., 1001 for ${ }^{1} \mathrm{H}$ and 8016 for ${ }^{16} \mathrm{O}$ ). Exceptions to this rule include metastable nuclides, nuclides with bound thermal scattering data, and nuclides whose cross sections have a special weighting. Also, elements with isotopic mixtures (typically natural abundance) have IDs of $Z^{*} 1000$.

If a nuclide identifier is listed in Table 7.2.1., it can be accessed and used in a user-specified material (a.k.a., arbitrary material). User-specified materials require the user to provide all the information normally found in the Standard Composition Library. Refer to the XSProc manual for details on how to input user-specified materials.

Several materials contain multiple isotopes of a single element. For these materials, the user is free to specify the isotopic distribution as discussed in the XSProc chapter. Alternatively, the user may elect not 
to enter this data, thereby telling the code to assume the default values shown in the tables. In describing a user-specified material, a multiple isotope ID of $Z^{*} 1000$ can be used to denote the elements of Table 7.2.2. Note that even if the natural abundances from Table 7.2.2 are accessed through elemental specification, not all nuclides are necessarily present on each cross-section library. Refer to the Cross Section Library chapter for listings of available nuclides in each cross-section library.

Atomic masses for the isotopes were taken from "The Ame2003 atomic mass evaluation (II)" by G. Audi et al. The atom percents of the isotopic distribution table were taken from "Isotopic Compositions of the Elements, 2001" by J. K. Böhlke. Densities were taken from several sources, including the CRC Handbook of Chemistry and Physics. Gases and explicit isotopes were changed to all have a theoretical density of $1.0 \mathrm{~g} / \mathrm{cm}^{3}$.

To more fully document the composition of each compound and/or document the assumptions used in producing the associated cross-section data, a brief description is given in the tables where needed. 
Table 7.2.1. Isotopes in standard composition library

\begin{tabular}{|c|c|c|}
\hline Name & Identifier & $\begin{array}{c}\text { Mass } \\
\text { (amu) }\end{array}$ \\
\hline h-1 & 1001 & 1.0078 \\
\hline h-2 & 1002 & 2.0141 \\
\hline h-3 & 1003 & 3.0161 \\
\hline h-4 & 1004 & 4.0278 \\
\hline h-5 & 1005 & 5.0353 \\
\hline h-6 & 1006 & 6.0449 \\
\hline h-7 & 1007 & 7.0528 \\
\hline he-3 & 2003 & 3.016 \\
\hline he-4 & 2004 & 4.0026 \\
\hline he-5 & 2005 & 5.0122 \\
\hline he- 6 & 2006 & 6.0189 \\
\hline he-7 & 2007 & 7.028 \\
\hline he- 8 & 2008 & 8.0339 \\
\hline he-9 & 2009 & 9.044 \\
\hline he-10 & 2010 & 10.0524 \\
\hline li-3 & 3003 & 3.0308 \\
\hline li-4 & 3004 & 4.0272 \\
\hline li-5 & 3005 & 5.0125 \\
\hline li-6 & 3006 & 6.0151 \\
\hline li-7 & 3007 & 7.016 \\
\hline li-8 & 3008 & 8.0225 \\
\hline li-9 & 3009 & 9.0268 \\
\hline li-10 & 3010 & 10.0355 \\
\hline li-11 & 3011 & 11.0438 \\
\hline li-12 & 3012 & 12.0538 \\
\hline be-5 & 4005 & 5.0408 \\
\hline be- 6 & 4006 & 6.0197 \\
\hline be-7 & 4007 & 7.0169 \\
\hline be- 8 & 4008 & 8.0053 \\
\hline be-9 & 4009 & 9.0122 \\
\hline be-10 & 4010 & 10.0135 \\
\hline be-11 & 4011 & 11.0217 \\
\hline be-12 & 4012 & 12.0269 \\
\hline be-13 & 4013 & 13.0357 \\
\hline be-14 & 4014 & 14.0429 \\
\hline be- 15 & 4015 & 15.0535 \\
\hline be-16 & 4016 & 16.0619 \\
\hline$b-6$ & 5006 & 6.0468 \\
\hline$b-7$ & 5007 & 7.0299 \\
\hline$b-8$ & 5008 & 8.0246 \\
\hline b-9 & 5009 & 9.0133 \\
\hline b-10 & 5010 & 10.0129 \\
\hline b-11 & 5011 & 11.0093 \\
\hline b-12 & 5012 & 12.0143 \\
\hline b-13 & 5013 & 13.0178 \\
\hline b-14 & 5014 & 14.0254 \\
\hline$b-15$ & 5015 & 15.0311 \\
\hline b-16 & 5016 & 16.0398 \\
\hline b-17 & 5017 & 17.047 \\
\hline b-18 & 5018 & 18.0562 \\
\hline
\end{tabular}




\begin{tabular}{|c|c|c|}
\hline Name & Identifier & $\begin{array}{c}\text { Mass } \\
\text { (amu) }\end{array}$ \\
\hline b-19 & 5019 & 19.0637 \\
\hline c-8 & 6008 & 8.0377 \\
\hline c-9 & 6009 & 9.031 \\
\hline $\mathrm{c}-10$ & 6010 & 10.0169 \\
\hline c-11 & 6011 & 11.0114 \\
\hline$c-12$ & 6012 & 12.0 \\
\hline$c-13$ & 6013 & 13.0034 \\
\hline c-14 & 6014 & 14.0032 \\
\hline$c-15$ & 6015 & 15.0106 \\
\hline c-16 & 6016 & 16.0147 \\
\hline c-17 & 6017 & 17.0226 \\
\hline c-18 & 6018 & 18.0268 \\
\hline c-19 & 6019 & 19.0348 \\
\hline$c-20$ & 6020 & 20.0403 \\
\hline$c-21$ & 6021 & 21.0493 \\
\hline$c-22$ & 6022 & 22.0572 \\
\hline$n-10$ & 7010 & 10.0416 \\
\hline$n-11$ & 7011 & 11.0261 \\
\hline$n-12$ & 7012 & 12.0186 \\
\hline$n-13$ & 7013 & 13.0057 \\
\hline n-14 & 7014 & 14.0031 \\
\hline$n-15$ & 7015 & 15.0001 \\
\hline$n-16$ & 7016 & 16.0061 \\
\hline$n-17$ & 7017 & 17.0084 \\
\hline$n-18$ & 7018 & 18.0141 \\
\hline n-19 & 7019 & 19.017 \\
\hline$n-20$ & 7020 & 20.0234 \\
\hline$n-21$ & 7021 & 21.0271 \\
\hline$n-22$ & 7022 & 22.0344 \\
\hline$n-23$ & 7023 & 23.0412 \\
\hline$n-24$ & 7024 & 24.051 \\
\hline$n-25$ & 7025 & 25.0607 \\
\hline o-12 & 8012 & 12.0344 \\
\hline $0-13$ & 8013 & 13.0248 \\
\hline $0-14$ & 8014 & 14.0086 \\
\hline $0-15$ & 8015 & 15.0031 \\
\hline $0-16$ & 8016 & 15.9949 \\
\hline $0-17$ & 8017 & 16.9991 \\
\hline o-18 & 8018 & 17.9992 \\
\hline o-19 & 8019 & 19.0036 \\
\hline $0-20$ & 8020 & 20.0041 \\
\hline $0-21$ & 8021 & 21.0087 \\
\hline $0-22$ & 8022 & 22.01 \\
\hline $0-23$ & 8023 & 23.0157 \\
\hline $0-24$ & 8024 & 24.0205 \\
\hline $0-25$ & 8025 & 25.0295 \\
\hline $0-26$ & 8026 & 26.0383 \\
\hline $0-27$ & 8027 & 27.0483 \\
\hline $0-28$ & 8028 & 28.0578 \\
\hline$f-14$ & 9014 & 14.0351 \\
\hline$f-15$ & 9015 & 15.018 \\
\hline$f-16$ & 9016 & 16.0115 \\
\hline
\end{tabular}




\begin{tabular}{|c|c|c|}
\hline Name & Identifier & $\begin{array}{c}\text { Mass } \\
\text { (amu) }\end{array}$ \\
\hline$f-17$ & 9017 & 17.0021 \\
\hline$f-18$ & 9018 & 18.0009 \\
\hline$f-19$ & 9019 & 18.9984 \\
\hline$f-20$ & 9020 & 20.0 \\
\hline$f-21$ & 9021 & 21.0 \\
\hline$f-22$ & 9022 & 22.003 \\
\hline$f-23$ & 9023 & 23.0036 \\
\hline$f-24$ & 9024 & 24.0081 \\
\hline$f-25$ & 9025 & 25.0121 \\
\hline$f-26$ & 9026 & 26.0196 \\
\hline$f-27$ & 9027 & 27.0268 \\
\hline$f-28$ & 9028 & 28.0357 \\
\hline$f-29$ & 9029 & 29.0433 \\
\hline$f-30$ & 9030 & 30.0525 \\
\hline$f-31$ & 9031 & 31.0604 \\
\hline ne-16 & 10016 & 16.0258 \\
\hline ne-17 & 10017 & 17.0177 \\
\hline ne-18 & 10018 & 18.0057 \\
\hline ne-19 & 10019 & 19.0019 \\
\hline ne-20 & 10020 & 19.9924 \\
\hline ne-21 & 10021 & 20.9939 \\
\hline ne-22 & 10022 & 21.9914 \\
\hline ne-23 & 10023 & 22.9945 \\
\hline ne-24 & 10024 & 23.9936 \\
\hline ne-25 & 10025 & 24.9977 \\
\hline ne-26 & 10026 & 26.0005 \\
\hline ne-27 & 10027 & 27.0076 \\
\hline ne-28 & 10028 & 28.0121 \\
\hline ne-29 & 10029 & 29.0194 \\
\hline ne-30 & 10030 & 30.0248 \\
\hline ne-31 & 10031 & 31.0331 \\
\hline ne-32 & 10032 & 32.04 \\
\hline ne-33 & 10033 & 33.0494 \\
\hline ne-34 & 10034 & 34.057 \\
\hline na-18 & 11018 & 18.026 \\
\hline na-19 & 11019 & 19.0139 \\
\hline na-20 & 11020 & 20.0074 \\
\hline na-21 & 11021 & 20.9977 \\
\hline na-22 & 11022 & 21.9944 \\
\hline na-23 & 11023 & 22.9898 \\
\hline na-24 & 11024 & 23.991 \\
\hline na-25 & 11025 & 24.99 \\
\hline na-26 & 11026 & 25.9926 \\
\hline na-27 & 11027 & 26.9941 \\
\hline na-28 & 11028 & 27.9989 \\
\hline na-29 & 11029 & 29.0029 \\
\hline na-30 & 11030 & 30.009 \\
\hline na-31 & 11031 & 31.0136 \\
\hline na-32 & 11032 & 32.0205 \\
\hline na-33 & 11033 & 33.0267 \\
\hline na-34 & 11034 & 34.0352 \\
\hline na-35 & 11035 & 35.0425 \\
\hline
\end{tabular}




\begin{tabular}{|l|l|l|}
\hline \multicolumn{1}{|c|}{ Name } & Identifier & $\begin{array}{c}\text { Mass } \\
\text { (amu) }\end{array}$ \\
\hline na-36 & 11036 & 36.0515 \\
\hline na-37 & 11037 & 37.0593 \\
\hline mg-19 & 12019 & 19.0355 \\
\hline mg-20 & 12020 & 20.0189 \\
\hline mg-21 & 12021 & 21.0117 \\
\hline mg-22 & 12022 & 21.9996 \\
\hline mg-23 & 12023 & 22.9941 \\
\hline mg-24 & 12024 & 23.985 \\
\hline mg-25 & 12025 & 24.9858 \\
\hline mg-26 & 12026 & 25.9826 \\
\hline mg-27 & 12027 & 26.9843 \\
\hline mg-28 & 12028 & 27.9839 \\
\hline mg-29 & 12029 & 28.9886 \\
\hline mg-30 & 12030 & 29.9904 \\
\hline mg-31 & 12031 & 30.9965 \\
\hline mg-32 & 12032 & 31.999 \\
\hline mg-33 & 12033 & 33.0052 \\
\hline mg-34 & 12034 & 34.0095 \\
\hline mg-35 & 12035 & 35.0173 \\
\hline mg-36 & 12036 & 36.023 \\
\hline mg-37 & 12037 & 37.0314 \\
\hline mg-38 & 12038 & 38.0376 \\
\hline mg-39 & 12039 & 39.0468 \\
\hline mg-40 & 12040 & 40.0539 \\
\hline al-21 & 13021 & 21.028 \\
\hline al-22 & 13022 & 22.0195 \\
\hline al-23 & 13023 & 23.0073 \\
\hline al-24 & 13024 & 23.9999 \\
\hline al-25 & 13025 & 24.9904 \\
\hline al-26 & 13026 & 25.9869 \\
\hline al-27 & 13027 & 26.9815 \\
\hline al-28 & 13028 & 27.9819 \\
\hline al-29 & 13029 & 28.9804 \\
\hline al-30 & 13030 & 29.983 \\
\hline al-31 & 13031 & 30.9839 \\
\hline al-32 & 13032 & 31.9881 \\
\hline al-33 & 13033 & 32.9908 \\
\hline al-34 & 13034 & 33.9968 \\
\hline al-35 & 13035 & 34.9999 \\
\hline al-36 & 13036 & 36.0062 \\
\hline al-37 & 13037 & 37.0107 \\
\hline al-38 & 13038 & 38.0172 \\
\hline al-39 & 13039 & 39.023 \\
\hline al-40 & 13040 & 40.0314 \\
\hline al-41 & 13041 & 41.0383 \\
\hline al-42 & 13042 & 42.0469 \\
\hline si-22 & 14022 & 22.0345 \\
\hline si-23 & 14023 & 23.0255 \\
\hline si-24 & 14024 & 24.0116 \\
\hline si-25 & 14025 & 25.0041 \\
\hline si-26 & 14026 & 25.9923 \\
\hline si-27 & 14027 & 26.9867 \\
\hline
\end{tabular}




\begin{tabular}{|l|c|l|}
\hline \multicolumn{1}{|c|}{ Name } & Identifier & $\begin{array}{c}\text { Mass } \\
\text { (amu) }\end{array}$ \\
\hline si-28 & 14028 & 27.9769 \\
\hline si-29 & 14029 & 28.9765 \\
\hline si-30 & 14030 & 29.9738 \\
\hline si-31 & 14031 & 30.9754 \\
\hline si-32 & 14032 & 31.9741 \\
\hline si-33 & 14033 & 32.978 \\
\hline si-34 & 14034 & 33.9786 \\
\hline si-35 & 14035 & 34.9846 \\
\hline si-36 & 14036 & 35.9866 \\
\hline si-37 & 14037 & 36.9929 \\
\hline si-38 & 14038 & 37.9956 \\
\hline si-39 & 14039 & 39.0021 \\
\hline si-40 & 14040 & 40.0059 \\
\hline si-41 & 14041 & 41.0146 \\
\hline si-42 & 14042 & 42.0198 \\
\hline si-43 & 14043 & 43.0287 \\
\hline si-44 & 14044 & 44.0353 \\
\hline p-24 & 15024 & 24.0343 \\
\hline p-25 & 15025 & 25.0203 \\
\hline p-26 & 15026 & 26.0118 \\
\hline p-27 & 15027 & 26.9992 \\
\hline p-28 & 15028 & 27.9923 \\
\hline p-29 & 15029 & 28.9818 \\
\hline p-30 & 15030 & 29.9783 \\
\hline p-31 & 15031 & 30.9738 \\
\hline p-32 & 15032 & 31.9739 \\
\hline p-33 & 15033 & 32.9717 \\
\hline p-34 & 15034 & 33.9736 \\
\hline p-35 & 15035 & 34.9733 \\
\hline p-36 & 15036 & 35.9783 \\
\hline p-37 & 15037 & 36.9796 \\
\hline p-38 & 15038 & 37.9842 \\
\hline p-39 & 15039 & 38.9862 \\
\hline p-40 & 15040 & 39.9913 \\
\hline p-41 & 15041 & 40.9943 \\
\hline p-42 & 15042 & 42.001 \\
\hline p-43 & 15043 & 43.0062 \\
\hline p-44 & 15044 & 44.013 \\
\hline p-45 & 15045 & 45.0192 \\
\hline p-46 & 15046 & 46.0274 \\
\hline s-26 & 16026 & 26.0279 \\
\hline s-27 & 16027 & 27.0188 \\
\hline s-28 & 16028 & 28.0044 \\
\hline s-29 & 16029 & 28.9966 \\
\hline s-30 & 16030 & 29.9849 \\
\hline s-31 & 16031 & 30.9795 \\
\hline s-32 & 16032 & 31.9721 \\
\hline s-33 & 16033 & 32.9715 \\
\hline s-34 & 33.9679 \\
\hline s-35 -36 & 34.969 \\
\hline s-37 & 35.9671 \\
\hline & & 36.9711 \\
\hline
\end{tabular}




\begin{tabular}{|c|c|c|}
\hline Name & Identifier & $\begin{array}{c}\text { Mass } \\
\text { (amu) }\end{array}$ \\
\hline s-38 & 16038 & 37.9712 \\
\hline s-39 & 16039 & 38.9751 \\
\hline$s-40$ & 16040 & 39.9754 \\
\hline $\mathrm{s}-41$ & 16041 & 40.9796 \\
\hline$s-42$ & 16042 & 41.981 \\
\hline$s-43$ & 16043 & 42.9871 \\
\hline s-44 & 16044 & 43.9902 \\
\hline$s-45$ & 16045 & 44.9965 \\
\hline s-46 & 16046 & 46.0008 \\
\hline s-47 & 16047 & 47.0086 \\
\hline s-48 & 16048 & 48.0142 \\
\hline s-49 & 16049 & 49.0236 \\
\hline cl-28 & 17028 & 28.0285 \\
\hline cl-29 & 17029 & 29.0141 \\
\hline cl-30 & 17030 & 30.0048 \\
\hline $\mathrm{cl}-31$ & 17031 & 30.9924 \\
\hline cl-32 & 17032 & 31.9857 \\
\hline cl-33 & 17033 & 32.9775 \\
\hline cl-34 & 17034 & 33.9738 \\
\hline cl-35 & 17035 & 34.9688 \\
\hline cl-36 & 17036 & 35.9683 \\
\hline cl-37 & 17037 & 36.9659 \\
\hline cl-38 & 17038 & 37.968 \\
\hline cl-39 & 17039 & 38.968 \\
\hline $\mathrm{cl}-40$ & 17040 & 39.9704 \\
\hline cl-41 & 17041 & 40.9707 \\
\hline cl-42 & 17042 & 41.9733 \\
\hline cl-43 & 17043 & 42.974 \\
\hline cl-44 & 17044 & 43.9783 \\
\hline $\mathrm{cl}-45$ & 17045 & 44.9803 \\
\hline $\mathrm{cl}-46$ & 17046 & 45.9842 \\
\hline cl-47 & 17047 & 46.9887 \\
\hline cl-48 & 17048 & 47.9949 \\
\hline cl-49 & 17049 & 49.0003 \\
\hline $\mathrm{cl}-50$ & 17050 & 50.0078 \\
\hline cl-51 & 17051 & 51.0145 \\
\hline ar-30 & 18030 & 30.0216 \\
\hline ar-31 & 18031 & 31.0121 \\
\hline ar-32 & 18032 & 31.9976 \\
\hline ar-33 & 18033 & 32.9899 \\
\hline ar-34 & 18034 & 33.9803 \\
\hline ar-35 & 18035 & 34.9753 \\
\hline ar-36 & 18036 & 35.9675 \\
\hline ar-37 & 18037 & 36.9668 \\
\hline ar-38 & 18038 & 37.9627 \\
\hline ar-39 & 18039 & 38.9643 \\
\hline ar-40 & 18040 & 39.9624 \\
\hline ar-41 & 18041 & 40.9645 \\
\hline ar-42 & 18042 & 41.9631 \\
\hline ar-43 & 18043 & 42.9656 \\
\hline ar-44 & 18044 & 43.9649 \\
\hline ar-45 & 18045 & 44.968 \\
\hline
\end{tabular}




\begin{tabular}{|c|c|c|}
\hline Name & Identifier & $\begin{array}{c}\text { Mass } \\
\text { (amu) }\end{array}$ \\
\hline ar-46 & 18046 & 45.9681 \\
\hline ar-47 & 18047 & 46.9722 \\
\hline ar-48 & 18048 & 47.9745 \\
\hline ar-49 & 18049 & 48.9805 \\
\hline ar-50 & 18050 & 49.9844 \\
\hline ar-51 & 18051 & 50.9916 \\
\hline ar-52 & 18052 & 51.9968 \\
\hline ar-53 & 18053 & 53.0049 \\
\hline $\mathrm{k}-32$ & 19032 & 32.0219 \\
\hline $\mathrm{k}-33$ & 19033 & 33.0073 \\
\hline $\mathrm{k}-34$ & 19034 & 33.9984 \\
\hline $\mathrm{k}-35$ & 19035 & 34.988 \\
\hline $\mathrm{k}-36$ & 19036 & 35.9813 \\
\hline $\mathrm{k}-37$ & 19037 & 36.9734 \\
\hline $\mathrm{k}-38$ & 19038 & 37.9691 \\
\hline k-39 & 19039 & 38.9637 \\
\hline $\mathrm{k}-40$ & 19040 & 39.964 \\
\hline $\mathrm{k}-41$ & 19041 & 40.9618 \\
\hline $\mathrm{k}-42$ & 19042 & 41.9624 \\
\hline $\mathrm{k}-43$ & 19043 & 42.9607 \\
\hline $\mathrm{k}-44$ & 19044 & 43.9616 \\
\hline $\mathrm{k}-45$ & 19045 & 44.9607 \\
\hline $\mathrm{k}-46$ & 19046 & 45.962 \\
\hline $\mathrm{k}-47$ & 19047 & 46.9617 \\
\hline $\mathrm{k}-48$ & 19048 & 47.9655 \\
\hline $\mathrm{k}-49$ & 19049 & 48.9674 \\
\hline $\mathrm{k}-50$ & 19050 & 49.9728 \\
\hline $\mathrm{k}-51$ & 19051 & 50.9764 \\
\hline $\mathrm{k}-52$ & 19052 & 51.9826 \\
\hline $\mathrm{k}-53$ & 19053 & 52.9871 \\
\hline $\mathrm{k}-54$ & 19054 & 53.9942 \\
\hline $\mathrm{k}-55$ & 19055 & 54.9997 \\
\hline ca-34 & 20034 & 34.0141 \\
\hline ca-35 & 20035 & 35.0049 \\
\hline ca-36 & 20036 & 35.9931 \\
\hline ca-37 & 20037 & 36.9859 \\
\hline ca-38 & 20038 & 37.9763 \\
\hline ca-39 & 20039 & 38.9707 \\
\hline ca-40 & 20040 & 39.9626 \\
\hline ca-41 & 20041 & 40.9623 \\
\hline ca-42 & 20042 & 41.9586 \\
\hline ca-43 & 20043 & 42.9588 \\
\hline ca-44 & 20044 & 43.9555 \\
\hline ca-45 & 20045 & 44.9562 \\
\hline ca-46 & 20046 & 45.9537 \\
\hline ca-47 & 20047 & 46.9546 \\
\hline ca-48 & 20048 & 47.9525 \\
\hline ca-49 & 20049 & 48.9557 \\
\hline ca-50 & 20050 & 49.9575 \\
\hline ca-51 & 20051 & 50.9615 \\
\hline ca-52 & 20052 & 51.9651 \\
\hline ca-53 & 20053 & 52.9701 \\
\hline
\end{tabular}




\begin{tabular}{|c|c|c|}
\hline Name & Identifier & $\begin{array}{c}\text { Mass } \\
\text { (amu) }\end{array}$ \\
\hline ca-54 & 20054 & 53.9743 \\
\hline ca-55 & 20055 & 54.9805 \\
\hline ca-56 & 20056 & 55.9856 \\
\hline ca-57 & 20057 & 56.9924 \\
\hline sc-36 & 21036 & 36.0149 \\
\hline sc-37 & 21037 & 37.0031 \\
\hline sc-38 & 21038 & 37.9947 \\
\hline sc-39 & 21039 & 38.9848 \\
\hline sc-40 & 21040 & 39.978 \\
\hline sc-41 & 21041 & 40.9692 \\
\hline sc-42 & 21042 & 41.9655 \\
\hline sc-43 & 21043 & 42.9612 \\
\hline sc-44 & 21044 & 43.9594 \\
\hline sc-45 & 21045 & 44.9559 \\
\hline sc-46 & 21046 & 45.9552 \\
\hline sc-47 & 21047 & 46.9524 \\
\hline sc-48 & 21048 & 47.9522 \\
\hline sc-49 & 21049 & 48.95 \\
\hline sc-50 & 21050 & 49.9522 \\
\hline sc-51 & 21051 & 50.9536 \\
\hline sc-52 & 21052 & 51.9567 \\
\hline sc-53 & 21053 & 52.9596 \\
\hline sc-54 & 21054 & 53.9633 \\
\hline sc-55 & 21055 & 54.9682 \\
\hline sc-56 & 21056 & 55.9729 \\
\hline sc-57 & 21057 & 56.9778 \\
\hline sc-58 & 21058 & 57.9837 \\
\hline sc-59 & 21059 & 58.9892 \\
\hline sc-60 & 21060 & 59.9957 \\
\hline ti-38 & 22038 & 38.0098 \\
\hline ti-39 & 22039 & 39.0016 \\
\hline ti-40 & 22040 & 39.9905 \\
\hline ti-41 & 22041 & 40.9832 \\
\hline ti-42 & 22042 & 41.973 \\
\hline ti-43 & 22043 & 42.9685 \\
\hline ti-44 & 22044 & 43.9597 \\
\hline $\mathrm{ti}-45$ & 22045 & 44.9581 \\
\hline ti-46 & 22046 & 45.9526 \\
\hline ti-47 & 22047 & 46.9518 \\
\hline ti-48 & 22048 & 47.9479 \\
\hline ti-49 & 22049 & 48.9479 \\
\hline ti-50 & 22050 & 49.9448 \\
\hline ti-51 & 22051 & 50.9466 \\
\hline ti-52 & 22052 & 51.9469 \\
\hline ti-53 & 22053 & 52.9497 \\
\hline ti-54 & 22054 & 53.951 \\
\hline ti-55 & 22055 & 54.9553 \\
\hline ti-56 & 22056 & 55.9582 \\
\hline ti-57 & 22057 & 56.964 \\
\hline ti-58 & 22058 & 57.967 \\
\hline ti-59 & 22059 & 58.9729 \\
\hline ti-60 & 22060 & 59.9768 \\
\hline
\end{tabular}




\begin{tabular}{|c|c|c|}
\hline Name & Identifier & $\begin{array}{c}\text { Mass } \\
\text { (amu) }\end{array}$ \\
\hline ti-61 & 22061 & 60.9832 \\
\hline ti-62 & 22062 & 61.9875 \\
\hline ti-63 & 22063 & 62.9944 \\
\hline $\mathrm{v}-40$ & 23040 & 40.0111 \\
\hline $\mathrm{v}-41$ & 23041 & 40.9998 \\
\hline $\mathrm{v}-42$ & 23042 & 41.9912 \\
\hline $\mathrm{v}-43$ & 23043 & 42.9807 \\
\hline $\mathrm{v}-44$ & 23044 & 43.9741 \\
\hline $\mathrm{v}-45$ & 23045 & 44.9658 \\
\hline $\mathrm{v}-46$ & 23046 & 45.9602 \\
\hline $\mathrm{v}-47$ & 23047 & 46.9549 \\
\hline $\mathrm{v}-48$ & 23048 & 47.9523 \\
\hline $\mathrm{v}-49$ & 23049 & 48.9485 \\
\hline $\mathrm{v}-50$ & 23050 & 49.9472 \\
\hline $\mathrm{v}-51$ & 23051 & 50.944 \\
\hline $\mathrm{v}-52$ & 23052 & 51.9448 \\
\hline $\mathrm{v}-53$ & 23053 & 52.9443 \\
\hline$v-54$ & 23054 & 53.9464 \\
\hline $\mathrm{v}-55$ & 23055 & 54.9472 \\
\hline $\mathrm{v}-56$ & 23056 & 55.9505 \\
\hline $\mathrm{v}-57$ & 23057 & 56.9526 \\
\hline $\mathrm{v}-58$ & 23058 & 57.9568 \\
\hline v-59 & 23059 & 58.9602 \\
\hline$v-60$ & 23060 & 59.965 \\
\hline $\mathrm{v}-61$ & 23061 & 60.9685 \\
\hline$v-62$ & 23062 & 61.9738 \\
\hline $\mathrm{v}-63$ & 23063 & 62.9776 \\
\hline $\mathrm{v}-64$ & 23064 & 63.9835 \\
\hline$v-65$ & 23065 & 64.9879 \\
\hline cr-42 & 24042 & 42.0064 \\
\hline cr-43 & 24043 & 42.9977 \\
\hline cr-44 & 24044 & 43.9855 \\
\hline cr-45 & 24045 & 44.9796 \\
\hline cr-46 & 24046 & 45.9684 \\
\hline cr-47 & 24047 & 46.9629 \\
\hline cr-48 & 24048 & 47.954 \\
\hline cr-49 & 24049 & 48.9513 \\
\hline cr-50 & 24050 & 49.946 \\
\hline cr-51 & 24051 & 50.9448 \\
\hline cr-52 & 24052 & 51.9405 \\
\hline cr-53 & 24053 & 52.9407 \\
\hline cr-54 & 24054 & 53.9389 \\
\hline cr-55 & 24055 & 54.9408 \\
\hline cr-56 & 24056 & 55.9407 \\
\hline cr-57 & 24057 & 56.9436 \\
\hline cr-58 & 24058 & 57.9444 \\
\hline cr-59 & 24059 & 58.9486 \\
\hline cr-60 & 24060 & 59.9501 \\
\hline cr-61 & 24061 & 60.9547 \\
\hline cr-62 & 24062 & 61.9566 \\
\hline cr-63 & 24063 & 62.9619 \\
\hline cr-64 & 24064 & 63.9644 \\
\hline
\end{tabular}




\begin{tabular}{|c|c|c|}
\hline Name & Identifier & $\begin{array}{c}\text { Mass } \\
\text { (amu) }\end{array}$ \\
\hline cr-65 & 24065 & 64.9702 \\
\hline cr-66 & 24066 & 65.9734 \\
\hline cr-67 & 24067 & 66.9796 \\
\hline mn-44 & 25044 & 44.0069 \\
\hline$m n-45$ & 25045 & 44.9945 \\
\hline $\mathrm{mn}-46$ & 25046 & 45.9867 \\
\hline $\mathrm{mn}-47$ & 25047 & 46.9761 \\
\hline $\mathrm{mn}-48$ & 25048 & 47.9685 \\
\hline$m n-49$ & 25049 & 48.9596 \\
\hline$m n-50$ & 25050 & 49.9542 \\
\hline mn-51 & 25051 & 50.9482 \\
\hline$m n-52$ & 25052 & 51.9456 \\
\hline$m n-53$ & 25053 & 52.9413 \\
\hline mn-54 & 25054 & 53.9404 \\
\hline$m n-55$ & 25055 & 54.938 \\
\hline mn-56 & 25056 & 55.9389 \\
\hline mn-57 & 25057 & 56.9383 \\
\hline$m n-58$ & 25058 & 57.94 \\
\hline mn-59 & 25059 & 58.9404 \\
\hline$m n-60$ & 25060 & 59.9429 \\
\hline mn-61 & 25061 & 60.9446 \\
\hline mn-62 & 25062 & 61.9484 \\
\hline $\mathrm{mn}-63$ & 25063 & 62.9502 \\
\hline$m n-64$ & 25064 & 63.9543 \\
\hline mn-65 & 25065 & 64.9563 \\
\hline mn-66 & 25066 & 65.9611 \\
\hline $\mathrm{mn}-67$ & 25067 & 66.9641 \\
\hline mn-68 & 25068 & 67.9693 \\
\hline mn-69 & 25069 & 68.9728 \\
\hline fe-45 & 26045 & 45.0146 \\
\hline fe-46 & 26046 & 46.0008 \\
\hline fe-47 & 26047 & 46.9929 \\
\hline fe-48 & 26048 & 47.9805 \\
\hline fe-49 & 26049 & 48.9736 \\
\hline fe-50 & 26050 & 49.963 \\
\hline fe-51 & 26051 & 50.9568 \\
\hline fe-52 & 26052 & 51.9481 \\
\hline fe-53 & 26053 & 52.9453 \\
\hline fe-54 & 26054 & 53.9396 \\
\hline fe-55 & 26055 & 54.9383 \\
\hline fe-56 & 26056 & 55.9349 \\
\hline fe-57 & 26057 & 56.9354 \\
\hline fe-58 & 26058 & 57.9333 \\
\hline fe-59 & 26059 & 58.9349 \\
\hline fe-60 & 26060 & 59.9341 \\
\hline fe-61 & 26061 & 60.9367 \\
\hline fe-62 & 26062 & 61.9368 \\
\hline fe-63 & 26063 & 62.9404 \\
\hline fe-64 & 26064 & 63.9412 \\
\hline fe-65 & 26065 & 64.9454 \\
\hline fe-66 & 26066 & 65.9468 \\
\hline fe-67 & 26067 & 66.951 \\
\hline
\end{tabular}




\begin{tabular}{|c|c|c|}
\hline Name & Identifier & $\begin{array}{c}\text { Mass } \\
\text { (amu) }\end{array}$ \\
\hline fe-68 & 26068 & 67.9537 \\
\hline fe-69 & 26069 & 68.9588 \\
\hline fe-70 & 26070 & 69.9615 \\
\hline fe-71 & 26071 & 70.9667 \\
\hline fe-72 & 26072 & 71.9696 \\
\hline co-47 & 27047 & 47.0115 \\
\hline co-48 & 27048 & 48.0018 \\
\hline co-49 & 27049 & 48.9897 \\
\hline co-50 & 27050 & 49.9815 \\
\hline co-51 & 27051 & 50.9707 \\
\hline co-52 & 27052 & 51.9636 \\
\hline co-53 & 27053 & 52.9542 \\
\hline co-54 & 27054 & 53.9485 \\
\hline co-55 & 27055 & 54.942 \\
\hline co-56 & 27056 & 55.9398 \\
\hline co-57 & 27057 & 56.9363 \\
\hline co-58 & 27058 & 57.9357 \\
\hline co-58m & 1027058 & \\
\hline co-59 & 27059 & 58.9332 \\
\hline co-60 & 27060 & 59.9338 \\
\hline co-61 & 27061 & 60.9325 \\
\hline co-62 & 27062 & 61.9341 \\
\hline co-63 & 27063 & 62.9336 \\
\hline co-64 & 27064 & 63.9358 \\
\hline co-65 & 27065 & 64.9365 \\
\hline co-66 & 27066 & 65.9398 \\
\hline co-67 & 27067 & 66.9409 \\
\hline co-68 & 27068 & 67.9449 \\
\hline co-69 & 27069 & 68.9463 \\
\hline co-70 & 27070 & 69.951 \\
\hline co-71 & 27071 & 70.9529 \\
\hline co-72 & 27072 & 71.9578 \\
\hline co-73 & 27073 & 72.9602 \\
\hline co-74 & 27074 & 73.9654 \\
\hline co-75 & 27075 & 74.9683 \\
\hline ni-48 & 28048 & 48.0197 \\
\hline ni-49 & 28049 & 49.0097 \\
\hline ni-50 & 28050 & 49.9959 \\
\hline ni-51 & 28051 & 50.9877 \\
\hline ni-52 & 28052 & 51.9757 \\
\hline ni-53 & 28053 & 52.9685 \\
\hline ni-54 & 28054 & 53.9579 \\
\hline ni-55 & 28055 & 54.9513 \\
\hline ni-56 & 28056 & 55.9421 \\
\hline ni-57 & 28057 & 56.9398 \\
\hline ni-58 & 28058 & 57.9353 \\
\hline ni-59 & 28059 & 58.9343 \\
\hline ni-60 & 28060 & 59.9308 \\
\hline ni-61 & 28061 & 60.9311 \\
\hline ni-62 & 28062 & 61.9283 \\
\hline ni-63 & 28063 & 62.9297 \\
\hline ni-64 & 28064 & 63.928 \\
\hline
\end{tabular}




\begin{tabular}{|c|c|c|}
\hline Name & Identifier & $\begin{array}{c}\text { Mass } \\
\text { (amu) }\end{array}$ \\
\hline ni-65 & 28065 & 64.9301 \\
\hline ni-66 & 28066 & 65.9291 \\
\hline ni-67 & 28067 & 66.9316 \\
\hline ni-68 & 28068 & 67.9319 \\
\hline ni-69 & 28069 & 68.9356 \\
\hline ni-70 & 28070 & 69.9365 \\
\hline ni-71 & 28071 & 70.9407 \\
\hline ni-72 & 28072 & 71.9421 \\
\hline ni-73 & 28073 & 72.9465 \\
\hline ni-74 & 28074 & 73.9481 \\
\hline ni-75 & 28075 & 74.9529 \\
\hline ni-76 & 28076 & 75.9553 \\
\hline ni-77 & 28077 & 76.9605 \\
\hline ni-78 & 28078 & 77.9632 \\
\hline cu-52 & 29052 & 51.9972 \\
\hline cu-53 & 29053 & 52.9855 \\
\hline cu-54 & 29054 & 53.9767 \\
\hline $\mathrm{cu}-55$ & 29055 & 54.966 \\
\hline cu-56 & 29056 & 55.9586 \\
\hline cu-57 & 29057 & 56.9492 \\
\hline cu-58 & 29058 & 57.9445 \\
\hline cu-59 & 29059 & 58.9395 \\
\hline cu-60 & 29060 & 59.9374 \\
\hline cu-61 & 29061 & 60.9335 \\
\hline cu-62 & 29062 & 61.9326 \\
\hline cu-63 & 29063 & 62.9296 \\
\hline cu-64 & 29064 & 63.9298 \\
\hline cu-65 & 29065 & 64.9278 \\
\hline cu-66 & 29066 & 65.9289 \\
\hline cu-67 & 29067 & 66.9277 \\
\hline cu-68 & 29068 & 67.9296 \\
\hline cu-69 & 29069 & 68.9294 \\
\hline $\mathrm{cu}-70$ & 29070 & 69.9324 \\
\hline cu-71 & 29071 & 70.9327 \\
\hline cu-72 & 29072 & 71.9358 \\
\hline cu-73 & 29073 & 72.9367 \\
\hline cu-74 & 29074 & 73.9399 \\
\hline $\mathrm{cu}-75$ & 29075 & 74.9419 \\
\hline $\mathrm{cu}-76$ & 29076 & 75.9453 \\
\hline cu-77 & 29077 & 76.9479 \\
\hline $\mathrm{cu}-78$ & 29078 & 77.952 \\
\hline cu-79 & 29079 & 78.9546 \\
\hline cu-80 & 29080 & 79.9609 \\
\hline zn-54 & 30054 & 53.993 \\
\hline$z n-55$ & 30055 & 54.984 \\
\hline zn-56 & 30056 & 55.9724 \\
\hline$z n-57$ & 30057 & 56.9648 \\
\hline $\mathrm{zn}-58$ & 30058 & 57.9546 \\
\hline zn-59 & 30059 & 58.9493 \\
\hline zn-60 & 30060 & 59.9418 \\
\hline zn-61 & 30061 & 60.9395 \\
\hline$z n-62$ & 30062 & 61.9343 \\
\hline
\end{tabular}




\begin{tabular}{|c|c|c|}
\hline Name & Identifier & $\begin{array}{c}\text { Mass } \\
\text { (amu) }\end{array}$ \\
\hline zn-63 & 30063 & 62.9332 \\
\hline zn-64 & 30064 & 63.9291 \\
\hline $\mathrm{zn}-65$ & 30065 & 64.9292 \\
\hline zn-66 & 30066 & 65.926 \\
\hline zn-67 & 30067 & 66.9271 \\
\hline zn-68 & 30068 & 67.9248 \\
\hline zn-69 & 30069 & 68.9266 \\
\hline $\mathrm{zn}-70$ & 30070 & 69.9253 \\
\hline $\mathrm{zn}-71$ & 30071 & 70.9277 \\
\hline $\mathrm{zn}-72$ & 30072 & 71.9269 \\
\hline zn-73 & 30073 & 72.9298 \\
\hline $\mathrm{zn}-74$ & 30074 & 73.9295 \\
\hline $\mathrm{zn}-75$ & 30075 & 74.9329 \\
\hline $\mathrm{zn}-76$ & 30076 & 75.9333 \\
\hline $\mathrm{zn}-77$ & 30077 & 76.937 \\
\hline zn-78 & 30078 & 77.9384 \\
\hline zn-79 & 30079 & 78.9426 \\
\hline $\mathrm{zn}-80$ & 30080 & 79.9443 \\
\hline $\mathrm{zn}-81$ & 30081 & 80.9505 \\
\hline zn-82 & 30082 & 81.9544 \\
\hline $\mathrm{zn}-83$ & 30083 & 82.961 \\
\hline ga-56 & 31056 & 55.9949 \\
\hline ga-57 & 31057 & 56.9829 \\
\hline ga-58 & 31058 & 57.9743 \\
\hline ga-59 & 31059 & 58.9634 \\
\hline ga-60 & 31060 & 59.9571 \\
\hline ga-61 & 31061 & 60.9495 \\
\hline ga-62 & 31062 & 61.9442 \\
\hline ga-63 & 31063 & 62.9393 \\
\hline ga-64 & 31064 & 63.9368 \\
\hline ga-65 & 31065 & 64.9327 \\
\hline ga-66 & 31066 & 65.9316 \\
\hline ga-67 & 31067 & 66.9282 \\
\hline ga-68 & 31068 & 67.928 \\
\hline ga-69 & 31069 & 68.9256 \\
\hline ga-70 & 31070 & 69.926 \\
\hline ga-71 & 31071 & 70.9247 \\
\hline ga-72 & 31072 & 71.9264 \\
\hline ga-73 & 31073 & 72.9252 \\
\hline ga-74 & 31074 & 73.9269 \\
\hline ga-75 & 31075 & 74.9265 \\
\hline ga-76 & 31076 & 75.9288 \\
\hline ga-77 & 31077 & 76.9292 \\
\hline ga-78 & 31078 & 77.9316 \\
\hline ga-79 & 31079 & 78.9329 \\
\hline ga-80 & 31080 & 79.9365 \\
\hline ga-81 & 31081 & 80.9378 \\
\hline ga-82 & 31082 & 81.943 \\
\hline ga-83 & 31083 & 82.947 \\
\hline ga-84 & 31084 & 83.9527 \\
\hline ga-85 & 31085 & 84.957 \\
\hline ga-86 & 31086 & 85.9631 \\
\hline
\end{tabular}




\begin{tabular}{|c|c|c|}
\hline Name & Identifier & $\begin{array}{c}\text { Mass } \\
\text { (amu) }\end{array}$ \\
\hline ge-58 & 32058 & 57.991 \\
\hline ge-59 & 32059 & 58.9818 \\
\hline ge-60 & 32060 & 59.9702 \\
\hline ge-61 & 32061 & 60.9638 \\
\hline ge-62 & 32062 & 61.9547 \\
\hline ge-63 & 32063 & 62.9496 \\
\hline ge-64 & 32064 & 63.9417 \\
\hline ge-65 & 32065 & 64.9394 \\
\hline ge-66 & 32066 & 65.9338 \\
\hline ge-67 & 32067 & 66.9327 \\
\hline ge-68 & 32068 & 67.9281 \\
\hline ge-69 & 32069 & 68.928 \\
\hline ge-70 & 32070 & 69.9242 \\
\hline ge-71 & 32071 & 70.9249 \\
\hline ge-72 & 32072 & 71.9221 \\
\hline ge-73 & 32073 & 72.9235 \\
\hline ge-74 & 32074 & 73.9212 \\
\hline ge- 75 & 32075 & 74.9229 \\
\hline ge-76 & 32076 & 75.9214 \\
\hline ge-77 & 32077 & 76.9236 \\
\hline ge-78 & 32078 & 77.9229 \\
\hline ge-79 & 32079 & 78.9254 \\
\hline ge- 80 & 32080 & 79.9254 \\
\hline ge- 81 & 32081 & 80.9288 \\
\hline ge- 82 & 32082 & 81.9296 \\
\hline ge- 83 & 32083 & 82.9346 \\
\hline ge-84 & 32084 & 83.9375 \\
\hline ge- 85 & 32085 & 84.943 \\
\hline ge- 86 & 32086 & 85.9465 \\
\hline ge- 87 & 32087 & 86.9525 \\
\hline ge- 88 & 32088 & 87.9569 \\
\hline ge-89 & 32089 & 88.9638 \\
\hline as-60 & 33060 & 59.9931 \\
\hline as-61 & 33061 & 60.9806 \\
\hline as-62 & 33062 & 61.9732 \\
\hline as-63 & 33063 & 62.9637 \\
\hline as-64 & 33064 & 63.9576 \\
\hline as-65 & 33065 & 64.9496 \\
\hline as-66 & 33066 & 65.9447 \\
\hline as-67 & 33067 & 66.9392 \\
\hline as-68 & 33068 & 67.9368 \\
\hline as-69 & 33069 & 68.9323 \\
\hline as-70 & 33070 & 69.9309 \\
\hline as-71 & 33071 & 70.9271 \\
\hline as-72 & 33072 & 71.9268 \\
\hline as-73 & 33073 & 72.9238 \\
\hline as-74 & 33074 & 73.9239 \\
\hline as-75 & 33075 & 74.9216 \\
\hline as-76 & 33076 & 75.9224 \\
\hline as-77 & 33077 & 76.9206 \\
\hline as-78 & 33078 & 77.9218 \\
\hline as-79 & 33079 & 78.921 \\
\hline
\end{tabular}




\begin{tabular}{|c|c|c|}
\hline Name & Identifier & $\begin{array}{l}\text { Mass } \\
\text { (amu) }\end{array}$ \\
\hline as- 80 & 33080 & 79.9225 \\
\hline as-81 & 33081 & 80.9221 \\
\hline as-82 & 33082 & 81.9245 \\
\hline as-83 & 33083 & 82.925 \\
\hline as-84 & 33084 & 83.9291 \\
\hline as-85 & 33085 & 84.932 \\
\hline as-86 & 33086 & 85.9365 \\
\hline as-87 & 33087 & 86.9399 \\
\hline as-88 & 33088 & 87.9449 \\
\hline as-89 & 33089 & 88.9494 \\
\hline as-90 & 33090 & 89.9555 \\
\hline as-91 & 33091 & 90.9604 \\
\hline as-92 & 33092 & 91.9668 \\
\hline se-65 & 34065 & 64.9647 \\
\hline se-66 & 34066 & 65.9552 \\
\hline se-67 & 34067 & 66.9501 \\
\hline se-68 & 34068 & 67.9418 \\
\hline se-69 & 34069 & 68.9396 \\
\hline se-70 & 34070 & 69.9334 \\
\hline se-71 & 34071 & 70.9322 \\
\hline se-72 & 34072 & 71.9271 \\
\hline se-73 & 34073 & 72.9268 \\
\hline se-74 & 34074 & 73.9225 \\
\hline se-75 & 34075 & 74.9225 \\
\hline se-76 & 34076 & 75.9192 \\
\hline se-77 & 34077 & 76.9199 \\
\hline se-78 & 34078 & 77.9173 \\
\hline se-79 & 34079 & 78.9185 \\
\hline se- 80 & 34080 & 79.9165 \\
\hline se-81 & 34081 & 80.918 \\
\hline se-82 & 34082 & 81.9167 \\
\hline se-83 & 34083 & 82.9191 \\
\hline se-84 & 34084 & 83.9185 \\
\hline se- 85 & 34085 & 84.9222 \\
\hline se-86 & 34086 & 85.9243 \\
\hline se- 87 & 34087 & 86.9285 \\
\hline se-88 & 34088 & 87.9314 \\
\hline se-89 & 34089 & 88.9364 \\
\hline se-90 & 34090 & 89.94 \\
\hline se-91 & 34091 & 90.946 \\
\hline se-92 & 34092 & 91.9499 \\
\hline se-93 & 34093 & 92.9563 \\
\hline se-94 & 34094 & 93.9605 \\
\hline br-67 & 35067 & 66.9648 \\
\hline br-68 & 35068 & 67.9585 \\
\hline br-69 & 35069 & 68.9501 \\
\hline br-70 & 35070 & 69.9448 \\
\hline br-71 & 35071 & 70.9387 \\
\hline br-72 & 35072 & 71.9366 \\
\hline br-73 & 35073 & 72.9317 \\
\hline br-74 & 35074 & 73.9299 \\
\hline br-75 & 35075 & 74.9258 \\
\hline
\end{tabular}




\begin{tabular}{|c|c|c|}
\hline Name & Identifier & $\begin{array}{c}\text { Mass } \\
\text { (amu) }\end{array}$ \\
\hline br-76 & 35076 & 75.9245 \\
\hline br-77 & 35077 & 76.9214 \\
\hline br-78 & 35078 & 77.9212 \\
\hline br-79 & 35079 & 78.9183 \\
\hline br- 80 & 35080 & 79.9185 \\
\hline br-81 & 35081 & 80.9163 \\
\hline br- 82 & 35082 & 81.9168 \\
\hline br-83 & 35083 & 82.9152 \\
\hline br-84 & 35084 & 83.9165 \\
\hline br- 85 & 35085 & 84.9156 \\
\hline br-86 & 35086 & 85.9188 \\
\hline br-87 & 35087 & 86.9207 \\
\hline br- 88 & 35088 & 87.9241 \\
\hline br-89 & 35089 & 88.9264 \\
\hline br-90 & 35090 & 89.9306 \\
\hline br-91 & 35091 & 90.934 \\
\hline br-92 & 35092 & 91.9393 \\
\hline br-93 & 35093 & 92.943 \\
\hline br-94 & 35094 & 93.9487 \\
\hline br-95 & 35095 & 94.9529 \\
\hline br-96 & 35096 & 95.9585 \\
\hline br-97 & 35097 & 96.9628 \\
\hline $\mathrm{kr}-69$ & 36069 & 68.9652 \\
\hline $\mathrm{kr}-70$ & 36070 & 69.9553 \\
\hline $\mathrm{kr}-71$ & 36071 & 70.9496 \\
\hline $\mathrm{kr}-72$ & 36072 & 71.9421 \\
\hline $\mathrm{kr}-73$ & 36073 & 72.9393 \\
\hline $\mathrm{kr}-74$ & 36074 & 73.9331 \\
\hline $\mathrm{kr}-75$ & 36075 & 74.9309 \\
\hline $\mathrm{kr}-76$ & 36076 & 75.9259 \\
\hline $\mathrm{kr}-77$ & 36077 & 76.9247 \\
\hline $\mathrm{kr}-78$ & 36078 & 77.9204 \\
\hline $\mathrm{kr}-79$ & 36079 & 78.9201 \\
\hline $\mathrm{kr}-80$ & 36080 & 79.9164 \\
\hline $\mathrm{kr}-81$ & 36081 & 80.9166 \\
\hline $\mathrm{kr}-82$ & 36082 & 81.9135 \\
\hline $\mathrm{kr}-83$ & 36083 & 82.9141 \\
\hline $\mathrm{kr}-84$ & 36084 & 83.9115 \\
\hline $\mathrm{kr}-85$ & 36085 & 84.9125 \\
\hline $\mathrm{kr}-86$ & 36086 & 85.9106 \\
\hline $\mathrm{kr}-87$ & 36087 & 86.9134 \\
\hline $\mathrm{kr}-88$ & 36088 & 87.9145 \\
\hline kr-89 & 36089 & 88.9176 \\
\hline $\mathrm{kr}-90$ & 36090 & 89.9195 \\
\hline kr-91 & 36091 & 90.9234 \\
\hline $\mathrm{kr}-92$ & 36092 & 91.9262 \\
\hline $\mathrm{kr}-93$ & 36093 & 92.9313 \\
\hline kr-94 & 36094 & 93.9344 \\
\hline kr-95 & 36095 & 94.9398 \\
\hline $\mathrm{kr}-96$ & 36096 & 95.9431 \\
\hline kr-97 & 36097 & 96.9486 \\
\hline $\mathrm{kr}-98$ & 36098 & 97.9519 \\
\hline
\end{tabular}




\begin{tabular}{|c|c|c|}
\hline Name & Identifier & $\begin{array}{l}\text { Mass } \\
\text { (amu) }\end{array}$ \\
\hline kr-99 & 36099 & 98.9576 \\
\hline $\mathrm{kr}-100$ & 36100 & 99.9611 \\
\hline $\mathrm{rb}-71$ & 37071 & 70.9653 \\
\hline $\mathrm{rb}-72$ & 37072 & 71.9591 \\
\hline $\mathrm{rb}-73$ & 37073 & 72.9506 \\
\hline $\mathrm{rb}-74$ & 37074 & 73.9443 \\
\hline $\mathrm{rb}-75$ & 37075 & 74.9386 \\
\hline $\mathrm{rb}-76$ & 37076 & 75.9351 \\
\hline rb-77 & 37077 & 76.9304 \\
\hline $\mathrm{rb}-78$ & 37078 & 77.9281 \\
\hline rb-79 & 37079 & 78.924 \\
\hline $\mathrm{rb}-80$ & 37080 & 79.9225 \\
\hline $\mathrm{rb}-81$ & 37081 & 80.919 \\
\hline $\mathrm{rb}-82$ & 37082 & 81.9182 \\
\hline $\mathrm{rb}-83$ & 37083 & 82.9151 \\
\hline $\mathrm{rb}-84$ & 37084 & 83.9144 \\
\hline $\mathrm{rb}-85$ & 37085 & 84.9118 \\
\hline $\mathrm{rb}-86$ & 37086 & 85.9112 \\
\hline $\mathrm{rb}-87$ & 37087 & 86.9092 \\
\hline $\mathrm{rb}-88$ & 37088 & 87.9113 \\
\hline $\mathrm{rb}-89$ & 37089 & 88.9123 \\
\hline rb-90 & 37090 & 89.9148 \\
\hline rb-91 & 37091 & 90.9165 \\
\hline rb-92 & 37092 & 91.9197 \\
\hline rb-93 & 37093 & 92.922 \\
\hline rb-94 & 37094 & 93.9264 \\
\hline rb-95 & 37095 & 94.9293 \\
\hline $\mathrm{rb}-96$ & 37096 & 95.9343 \\
\hline rb-97 & 37097 & 96.9373 \\
\hline rb-98 & 37098 & 97.9418 \\
\hline rb-99 & 37099 & 98.9454 \\
\hline rb-100 & 37100 & 99.9499 \\
\hline rb-101 & 37101 & 100.9532 \\
\hline rb-102 & 37102 & 101.9589 \\
\hline sr-73 & 38073 & 72.966 \\
\hline sr-74 & 38074 & 73.9563 \\
\hline sr-75 & 38075 & 74.95 \\
\hline sr-76 & 38076 & 75.9418 \\
\hline sr-77 & 38077 & 76.938 \\
\hline sr-78 & 38078 & 77.9322 \\
\hline sr-79 & 38079 & 78.9297 \\
\hline sr-80 & 38080 & 79.9245 \\
\hline sr-81 & 38081 & 80.9232 \\
\hline sr-82 & 38082 & 81.9184 \\
\hline sr-83 & 38083 & 82.9176 \\
\hline sr-84 & 38084 & 83.9134 \\
\hline sr-85 & 38085 & 84.9129 \\
\hline sr-86 & 38086 & 85.9093 \\
\hline sr-87 & 38087 & 86.9089 \\
\hline sr-88 & 38088 & 87.9056 \\
\hline sr-89 & 38089 & 88.9074 \\
\hline sr-90 & 38090 & 89.9077 \\
\hline
\end{tabular}




\begin{tabular}{|c|c|c|}
\hline Name & Identifier & $\begin{array}{l}\text { Mass } \\
\text { (amu) }\end{array}$ \\
\hline sr-91 & 38091 & 90.9102 \\
\hline sr-92 & 38092 & 91.911 \\
\hline sr-93 & 38093 & 92.914 \\
\hline sr-94 & 38094 & 93.9154 \\
\hline sr-95 & 38095 & 94.9194 \\
\hline sr-96 & 38096 & 95.9217 \\
\hline sr-97 & 38097 & 96.9261 \\
\hline sr-98 & 38098 & 97.9285 \\
\hline sr-99 & 38099 & 98.9332 \\
\hline sr-100 & 38100 & 99.9353 \\
\hline sr-101 & 38101 & 100.9405 \\
\hline sr-102 & 38102 & 101.943 \\
\hline sr-103 & 38103 & 102.949 \\
\hline sr-104 & 38104 & 103.9523 \\
\hline sr-105 & 38105 & 104.9586 \\
\hline$y-76$ & 39076 & 75.9585 \\
\hline$y-77$ & 39077 & 76.9497 \\
\hline$y-78$ & 39078 & 77.9436 \\
\hline$y-79$ & 39079 & 78.9373 \\
\hline$y-80$ & 39080 & 79.9343 \\
\hline$y-81$ & 39081 & 80.9291 \\
\hline$y-82$ & 39082 & 81.9268 \\
\hline$y-83$ & 39083 & 82.9223 \\
\hline$y-84$ & 39084 & 83.9204 \\
\hline$y-85$ & 39085 & 84.9164 \\
\hline$y-86$ & 39086 & 85.9149 \\
\hline$y-87$ & 39087 & 86.9109 \\
\hline$y-88$ & 39088 & 87.9095 \\
\hline$y-89$ & 39089 & 88.9059 \\
\hline$y-90$ & 39090 & 89.9072 \\
\hline$y-91$ & 39091 & 90.9073 \\
\hline$y-92$ & 39092 & 91.909 \\
\hline$y-93$ & 39093 & 92.9096 \\
\hline$y-94$ & 39094 & 93.9116 \\
\hline$y-95$ & 39095 & 94.9128 \\
\hline$y-96$ & 39096 & 95.9159 \\
\hline$y-97$ & 39097 & 96.9181 \\
\hline$y-98$ & 39098 & 97.9222 \\
\hline$y-99$ & 39099 & 98.9246 \\
\hline$y-100$ & 39100 & 99.9278 \\
\hline$y-101$ & 39101 & 100.9303 \\
\hline$y-102$ & 39102 & 101.9336 \\
\hline$y-103$ & 39103 & 102.9367 \\
\hline$y-104$ & 39104 & 103.941 \\
\hline$y-105$ & 39105 & 104.9449 \\
\hline$y-106$ & 39106 & 105.9498 \\
\hline$y-107$ & 39107 & 106.9541 \\
\hline$y-108$ & 39108 & 107.9595 \\
\hline zr-78 & 40078 & 77.9552 \\
\hline zr-79 & 40079 & 78.9492 \\
\hline $\mathrm{zr}-80$ & 40080 & 79.9404 \\
\hline $\mathrm{zr}-81$ & 40081 & 80.9372 \\
\hline
\end{tabular}




\begin{tabular}{|c|c|c|}
\hline Name & Identifier & $\begin{array}{c}\text { Mass } \\
\text { (amu) }\end{array}$ \\
\hline zr-82 & 40082 & 81.9311 \\
\hline zr-83 & 40083 & 82.9286 \\
\hline$z r-84$ & 40084 & 83.9232 \\
\hline $\mathrm{zr}-85$ & 40085 & 84.9215 \\
\hline zr-86 & 40086 & 85.9165 \\
\hline $\mathrm{zr}-87$ & 40087 & 86.9148 \\
\hline $\mathrm{zr}-88$ & 40088 & 87.9102 \\
\hline zr-89 & 40089 & 88.9089 \\
\hline zr-90 & 40090 & 89.9047 \\
\hline zr-91 & 40091 & 90.9056 \\
\hline zr-92 & 40092 & 91.905 \\
\hline zr-93 & 40093 & 92.9065 \\
\hline zr-94 & 40094 & 93.9063 \\
\hline zr-95 & 40095 & 94.908 \\
\hline zr-96 & 40096 & 95.9083 \\
\hline zr-97 & 40097 & 96.9109 \\
\hline zr-98 & 40098 & 97.9127 \\
\hline zr-99 & 40099 & 98.9165 \\
\hline zr-100 & 40100 & 99.9178 \\
\hline zr-101 & 40101 & 100.9211 \\
\hline zr-102 & 40102 & 101.923 \\
\hline zr-103 & 40103 & 102.9266 \\
\hline zr-104 & 40104 & 103.9288 \\
\hline zr-105 & 40105 & 104.9331 \\
\hline zr-106 & 40106 & 105.9359 \\
\hline zr-107 & 40107 & 106.9408 \\
\hline zr-108 & 40108 & 107.944 \\
\hline zr-109 & 40109 & 108.9492 \\
\hline zr-110 & 40110 & 109.9529 \\
\hline nb-81 & 41081 & 80.949 \\
\hline nb-82 & 41082 & 81.9431 \\
\hline nb-83 & 41083 & 82.9367 \\
\hline nb-84 & 41084 & 83.9336 \\
\hline nb-85 & 41085 & 84.9279 \\
\hline nb-86 & 41086 & 85.925 \\
\hline nb-87 & 41087 & 86.9204 \\
\hline nb-88 & 41088 & 87.9183 \\
\hline nb-89 & 41089 & 88.9134 \\
\hline nb-90 & 41090 & 89.9113 \\
\hline nb-91 & 41091 & 90.907 \\
\hline nb-92 & 41092 & 91.9072 \\
\hline nb-93 & 41093 & 92.9064 \\
\hline nb-94 & 41094 & 93.9073 \\
\hline nb-95 & 41095 & 94.9068 \\
\hline nb-96 & 41096 & 95.9081 \\
\hline nb-97 & 41097 & 96.9081 \\
\hline nb-98 & 41098 & 97.9103 \\
\hline nb-99 & 41099 & 98.9116 \\
\hline $\mathrm{nb}-100$ & 41100 & 99.9142 \\
\hline nb-101 & 41101 & 100.9153 \\
\hline $\mathrm{nb}-102$ & 41102 & 101.918 \\
\hline nb-103 & 41103 & 102.9191 \\
\hline
\end{tabular}




\begin{tabular}{|c|c|c|}
\hline Name & Identifier & $\begin{array}{c}\text { Mass } \\
\text { (amu) }\end{array}$ \\
\hline nb-104 & 41104 & 103.9225 \\
\hline nb-105 & 41105 & 104.9239 \\
\hline nb-106 & 41106 & 105.928 \\
\hline nb-107 & 41107 & 106.9303 \\
\hline nb-108 & 41108 & 107.9348 \\
\hline nb-109 & 41109 & 108.9376 \\
\hline nb-110 & 41110 & 109.9424 \\
\hline nb-111 & 41111 & 110.9456 \\
\hline nb-112 & 41112 & 111.9508 \\
\hline nb-113 & 41113 & 112.9547 \\
\hline mo-83 & 42083 & 82.9487 \\
\hline mo-84 & 42084 & 83.9401 \\
\hline mo-85 & 42085 & 84.9365 \\
\hline mo-86 & 42086 & 85.9307 \\
\hline mo-87 & 42087 & 86.9273 \\
\hline mo-88 & 42088 & 87.922 \\
\hline mo-89 & 42089 & 88.9195 \\
\hline mo-90 & 42090 & 89.9139 \\
\hline mo-91 & 42091 & 90.9118 \\
\hline mo-92 & 42092 & 91.9068 \\
\hline mo-93 & 42093 & 92.9068 \\
\hline mo-94 & 42094 & 93.9051 \\
\hline mo-95 & 42095 & 94.9058 \\
\hline mo-96 & 42096 & 95.9047 \\
\hline mo-97 & 42097 & 96.906 \\
\hline mo-98 & 42098 & 97.9054 \\
\hline mo-99 & 42099 & 98.9077 \\
\hline mo-100 & 42100 & 99.9075 \\
\hline mo-101 & 42101 & 100.9103 \\
\hline mo-102 & 42102 & 101.9103 \\
\hline mo-103 & 42103 & 102.9132 \\
\hline mo-104 & 42104 & 103.9138 \\
\hline mo-105 & 42105 & 104.917 \\
\hline mo-106 & 42106 & 105.9181 \\
\hline mo-107 & 42107 & 106.9217 \\
\hline mo-108 & 42108 & 107.9234 \\
\hline mo-109 & 42109 & 108.9278 \\
\hline mo-110 & 42110 & 109.9297 \\
\hline mo-111 & 42111 & 110.9344 \\
\hline mo-112 & 42112 & 111.9368 \\
\hline mo-113 & 42113 & 112.9419 \\
\hline mo-114 & 42114 & 113.9449 \\
\hline mo-115 & 42115 & 114.9503 \\
\hline tc- -85 & 43085 & 84.9488 \\
\hline tc- 86 & 43086 & 85.9429 \\
\hline tc- 87 & 43087 & 86.9365 \\
\hline tc- 88 & 43088 & 87.9327 \\
\hline tc- 89 & 43089 & 88.9272 \\
\hline tc-90 & 43090 & 89.9236 \\
\hline tc-91 & 43091 & 90.9184 \\
\hline tc-92 & 43092 & 91.9153 \\
\hline tc-93 & 43093 & 92.9102 \\
\hline
\end{tabular}




\begin{tabular}{|c|c|c|}
\hline Name & Identifier & $\begin{array}{l}\text { Mass } \\
\text { (amu) }\end{array}$ \\
\hline tc-94 & 43094 & 93.9097 \\
\hline tc-95 & 43095 & 94.9077 \\
\hline tc-96 & 43096 & 95.9079 \\
\hline tc-97 & 43097 & 96.9064 \\
\hline tc-98 & 43098 & 97.9072 \\
\hline tc-99 & 43099 & 98.9062 \\
\hline tc- 100 & 43100 & 99.9077 \\
\hline tc-101 & 43101 & 100.9073 \\
\hline tc-102 & 43102 & 101.9092 \\
\hline tc-103 & 43103 & 102.9092 \\
\hline tc-104 & 43104 & 103.9115 \\
\hline tc- 105 & 43105 & 104.9117 \\
\hline tc-106 & 43106 & 105.9144 \\
\hline tc-107 & 43107 & 106.9151 \\
\hline tc- 108 & 43108 & 107.9185 \\
\hline tc-109 & 43109 & 108.92 \\
\hline tc-110 & 43110 & 109.9238 \\
\hline tc-111 & 43111 & 110.9257 \\
\hline tc-112 & 43112 & 111.9292 \\
\hline tc-113 & 43113 & 112.9316 \\
\hline tc- 114 & 43114 & 113.9359 \\
\hline tc- 115 & 43115 & 114.9387 \\
\hline tc- 116 & 43116 & 115.9434 \\
\hline tc-117 & 43117 & 116.9465 \\
\hline tc-118 & 43118 & 117.9515 \\
\hline ru-87 & 44087 & 86.9492 \\
\hline ru-88 & 44088 & 87.9403 \\
\hline ru-89 & 44089 & 88.9361 \\
\hline ru-90 & 44090 & 89.9299 \\
\hline ru-91 & 44091 & 90.9263 \\
\hline ru-92 & 44092 & 91.9201 \\
\hline ru-93 & 44093 & 92.9171 \\
\hline ru-94 & 44094 & 93.9114 \\
\hline ru-95 & 44095 & 94.9104 \\
\hline ru-96 & 44096 & 95.9076 \\
\hline ru-97 & 44097 & 96.9076 \\
\hline ru-98 & 44098 & 97.9053 \\
\hline ru-99 & 44099 & 98.9059 \\
\hline ru-100 & 44100 & 99.9042 \\
\hline ru-101 & 44101 & 100.9056 \\
\hline ru-102 & 44102 & 101.9044 \\
\hline ru-103 & 44103 & 102.9063 \\
\hline ru-104 & 44104 & 103.9054 \\
\hline ru-105 & 44105 & 104.9078 \\
\hline ru-106 & 44106 & 105.9073 \\
\hline ru-107 & 44107 & 106.9099 \\
\hline ru-108 & 44108 & 107.9102 \\
\hline ru-109 & 44109 & 108.9132 \\
\hline ru-110 & 44110 & 109.9141 \\
\hline ru-111 & 44111 & 110.9177 \\
\hline ru-112 & 44112 & 111.919 \\
\hline ru-113 & 44113 & 112.9225 \\
\hline
\end{tabular}




\begin{tabular}{|c|c|c|}
\hline Name & Identifier & $\begin{array}{c}\text { Mass } \\
\text { (amu) }\end{array}$ \\
\hline ru-114 & 44114 & 113.9243 \\
\hline ru-115 & 44115 & 114.9287 \\
\hline ru-116 & 44116 & 115.9308 \\
\hline ru-117 & 44117 & 116.9356 \\
\hline ru-118 & 44118 & 117.9378 \\
\hline ru-119 & 44119 & 118.9428 \\
\hline ru-120 & 44120 & 119.9453 \\
\hline rh-89 & 45089 & 88.9488 \\
\hline rh-90 & 45090 & 89.9429 \\
\hline rh-91 & 45091 & 90.9365 \\
\hline rh-92 & 45092 & 91.932 \\
\hline rh-93 & 45093 & 92.9257 \\
\hline rh-94 & 45094 & 93.9217 \\
\hline rh-95 & 45095 & 94.9159 \\
\hline rh-96 & 45096 & 95.9145 \\
\hline rh-97 & 45097 & 96.9113 \\
\hline rh-98 & 45098 & 97.9107 \\
\hline rh-99 & 45099 & 98.9081 \\
\hline rh-100 & 45100 & 99.9081 \\
\hline rh-101 & 45101 & 100.9062 \\
\hline rh-102 & 45102 & 101.9068 \\
\hline rh-103 & 45103 & 102.9055 \\
\hline rh-104 & 45104 & 103.9067 \\
\hline rh-105 & 45105 & 104.9057 \\
\hline rh-106 & 45106 & 105.9073 \\
\hline rh-107 & 45107 & 106.9068 \\
\hline rh-108 & 45108 & 107.9087 \\
\hline rh-109 & 45109 & 108.9087 \\
\hline rh-110 & 45110 & 109.9111 \\
\hline rh-111 & 45111 & 110.9116 \\
\hline rh-112 & 45112 & 111.9144 \\
\hline rh-113 & 45113 & 112.9155 \\
\hline rh-114 & 45114 & 113.9188 \\
\hline rh-115 & 45115 & 114.9203 \\
\hline rh-116 & 45116 & 115.9241 \\
\hline rh-117 & 45117 & 116.926 \\
\hline rh-118 & 45118 & 117.9301 \\
\hline rh-119 & 45119 & 118.9321 \\
\hline rh-120 & 45120 & 119.9364 \\
\hline rh-121 & 45121 & 120.9387 \\
\hline rh-122 & 45122 & 121.9432 \\
\hline pd-91 & 46091 & 90.9491 \\
\hline pd-92 & 46092 & 91.9404 \\
\hline pd-93 & 46093 & 92.9359 \\
\hline pd-94 & 46094 & 93.9288 \\
\hline pd-95 & 46095 & 94.9247 \\
\hline pd-96 & 46096 & 95.9182 \\
\hline pd-97 & 46097 & 96.9165 \\
\hline pd-98 & 46098 & 97.9127 \\
\hline pd-99 & 46099 & 98.9118 \\
\hline pd-100 & 46100 & 99.9085 \\
\hline pd-101 & 46101 & 100.9083 \\
\hline
\end{tabular}




\begin{tabular}{|c|c|c|}
\hline Name & Identifier & $\begin{array}{c}\text { Mass } \\
\text { (amu) }\end{array}$ \\
\hline pd-102 & 46102 & 101.9056 \\
\hline pd-103 & 46103 & 102.9061 \\
\hline pd-104 & 46104 & 103.904 \\
\hline pd-105 & 46105 & 104.9051 \\
\hline pd-106 & 46106 & 105.9035 \\
\hline pd-107 & 46107 & 106.9051 \\
\hline pd-108 & 46108 & 107.9039 \\
\hline pd-109 & 46109 & 108.906 \\
\hline pd-110 & 46110 & 109.9052 \\
\hline pd-111 & 46111 & 110.9077 \\
\hline pd-112 & 46112 & 111.9073 \\
\hline pd-113 & 46113 & 112.9101 \\
\hline pd-114 & 46114 & 113.9104 \\
\hline pd-115 & 46115 & 114.9137 \\
\hline pd-116 & 46116 & 115.9142 \\
\hline pd-117 & 46117 & 116.9178 \\
\hline pd-118 & 46118 & 117.919 \\
\hline pd-119 & 46119 & 118.9231 \\
\hline pd-120 & 46120 & 119.9247 \\
\hline pd-121 & 46121 & 120.9289 \\
\hline pd-122 & 46122 & 121.9305 \\
\hline pd-123 & 46123 & 122.9349 \\
\hline pd-124 & 46124 & 123.9369 \\
\hline ag-93 & 47093 & 92.9498 \\
\hline ag-94 & 47094 & 93.9428 \\
\hline ag-95 & 47095 & 94.9355 \\
\hline ag-96 & 47096 & 95.9307 \\
\hline ag-97 & 47097 & 96.924 \\
\hline ag-98 & 47098 & 97.9216 \\
\hline ag-99 & 47099 & 98.9176 \\
\hline ag-100 & 47100 & 99.9161 \\
\hline ag-101 & 47101 & 100.9128 \\
\hline ag-102 & 47102 & 101.9117 \\
\hline ag-103 & 47103 & 102.909 \\
\hline ag-104 & 47104 & 103.9086 \\
\hline ag-105 & 47105 & 104.9065 \\
\hline ag-106 & 47106 & 105.9067 \\
\hline ag-107 & 47107 & 106.9051 \\
\hline ag-108 & 47108 & 107.906 \\
\hline ag-109 & 47109 & 108.9047 \\
\hline ag-110 & 47110 & 109.9061 \\
\hline ag-110m & 1047110 & \\
\hline ag-111 & 47111 & 110.9053 \\
\hline ag-112 & 47112 & 111.907 \\
\hline ag-113 & 47113 & 112.9066 \\
\hline ag-114 & 47114 & 113.9088 \\
\hline ag-115 & 47115 & 114.9088 \\
\hline ag-116 & 47116 & 115.9114 \\
\hline ag-117 & 47117 & 116.9117 \\
\hline ag-118 & 47118 & 117.9146 \\
\hline ag-119 & 47119 & 118.9157 \\
\hline ag-120 & 47120 & 119.9188 \\
\hline
\end{tabular}




\begin{tabular}{|c|c|c|}
\hline Name & Identifier & $\begin{array}{c}\text { Mass } \\
\text { (amu) }\end{array}$ \\
\hline ag-121 & 47121 & 120.9199 \\
\hline ag-122 & 47122 & 121.9235 \\
\hline ag-123 & 47123 & 122.9249 \\
\hline ag-124 & 47124 & 123.9286 \\
\hline ag-125 & 47125 & 124.9304 \\
\hline ag-126 & 47126 & 125.9345 \\
\hline ag-127 & 47127 & 126.9368 \\
\hline ag-128 & 47128 & 127.9412 \\
\hline ag-129 & 47129 & 128.9437 \\
\hline ag-130 & 47130 & 129.9505 \\
\hline cd-95 & 48095 & 94.9499 \\
\hline cd-96 & 48096 & 95.9398 \\
\hline cd-97 & 48097 & 96.9349 \\
\hline cd-98 & 48098 & 97.9274 \\
\hline cd-99 & 48099 & 98.925 \\
\hline cd-100 & 48100 & 99.9203 \\
\hline cd-101 & 48101 & 100.9187 \\
\hline cd-102 & 48102 & 101.9145 \\
\hline cd-103 & 48103 & 102.9134 \\
\hline cd-104 & 48104 & 103.9099 \\
\hline cd-105 & 48105 & 104.9095 \\
\hline cd-106 & 48106 & 105.9065 \\
\hline cd-107 & 48107 & 106.9066 \\
\hline cd-108 & 48108 & 107.9042 \\
\hline cd-109 & 48109 & 108.905 \\
\hline cd-110 & 48110 & 109.903 \\
\hline cd-111 & 48111 & 110.9042 \\
\hline cd-112 & 48112 & 111.9028 \\
\hline cd-113 & 48113 & 112.9044 \\
\hline cd-114 & 48114 & 113.9034 \\
\hline cd-115 & 48115 & 114.9054 \\
\hline $\mathrm{cd}-115 \mathrm{~m}$ & 1048115 & \\
\hline cd-116 & 48116 & 115.9048 \\
\hline cd-117 & 48117 & 116.9072 \\
\hline cd-118 & 48118 & 117.9069 \\
\hline cd-119 & 48119 & 118.9099 \\
\hline cd-120 & 48120 & 119.9099 \\
\hline cd-121 & 48121 & 120.913 \\
\hline cd-122 & 48122 & 121.9133 \\
\hline cd-123 & 48123 & 122.917 \\
\hline cd-124 & 48124 & 123.9176 \\
\hline cd-125 & 48125 & 124.9212 \\
\hline cd-126 & 48126 & 125.9223 \\
\hline cd-127 & 48127 & 126.9264 \\
\hline cd-128 & 48128 & 127.9278 \\
\hline cd-129 & 48129 & 128.9321 \\
\hline cd-130 & 48130 & 129.9339 \\
\hline cd-131 & 48131 & 130.9407 \\
\hline cd-132 & 48132 & 131.9456 \\
\hline in-97 & 49097 & 96.9495 \\
\hline in-98 & 49098 & 97.9421 \\
\hline in-99 & 49099 & 98.9342 \\
\hline
\end{tabular}




\begin{tabular}{|c|c|c|}
\hline Name & Identifier & $\begin{array}{c}\text { Mass } \\
\text { (amu) }\end{array}$ \\
\hline in-100 & 49100 & 99.9311 \\
\hline in-101 & 49101 & 100.9263 \\
\hline in-102 & 49102 & 101.9241 \\
\hline in-103 & 49103 & 102.9199 \\
\hline in-104 & 49104 & 103.9183 \\
\hline in-105 & 49105 & 104.9147 \\
\hline in-106 & 49106 & 105.9135 \\
\hline in-107 & 49107 & 106.9103 \\
\hline in-108 & 49108 & 107.9097 \\
\hline in-109 & 49109 & 108.9072 \\
\hline in-110 & 49110 & 109.9072 \\
\hline in-111 & 49111 & 110.9051 \\
\hline in-112 & 49112 & 111.9055 \\
\hline in-113 & 49113 & 112.9041 \\
\hline in-114 & 49114 & 113.9049 \\
\hline in-115 & 49115 & 114.9039 \\
\hline in-116 & 49116 & 115.9053 \\
\hline in-117 & 49117 & 116.9045 \\
\hline in-118 & 49118 & 117.9063 \\
\hline in-119 & 49119 & 118.9059 \\
\hline in-120 & 49120 & 119.908 \\
\hline in-121 & 49121 & 120.9079 \\
\hline in-122 & 49122 & 121.9103 \\
\hline in-123 & 49123 & 122.9104 \\
\hline in-124 & 49124 & 123.9132 \\
\hline in- 125 & 49125 & 124.9136 \\
\hline in-126 & 49126 & 125.9165 \\
\hline in-127 & 49127 & 126.9174 \\
\hline in-128 & 49128 & 127.9202 \\
\hline in-129 & 49129 & 128.9217 \\
\hline in-130 & 49130 & 129.925 \\
\hline in-131 & 49131 & 130.9268 \\
\hline in-132 & 49132 & 131.933 \\
\hline in-133 & 49133 & 132.9378 \\
\hline in-134 & 49134 & 133.9442 \\
\hline in-135 & 49135 & 134.9493 \\
\hline sn-99 & 50099 & 98.9493 \\
\hline sn-100 & 50100 & 99.939 \\
\hline sn-101 & 50101 & 100.9361 \\
\hline sn-102 & 50102 & 101.9303 \\
\hline sn-103 & 50103 & 102.9281 \\
\hline sn-104 & 50104 & 103.9231 \\
\hline sn-105 & 50105 & 104.9213 \\
\hline sn-106 & 50106 & 105.9169 \\
\hline sn-107 & 50107 & 106.9156 \\
\hline sn-108 & 50108 & 107.9119 \\
\hline sn-109 & 50109 & 108.9113 \\
\hline sn-110 & 50110 & 109.9078 \\
\hline sn-111 & 50111 & 110.9077 \\
\hline sn-112 & 50112 & 111.9048 \\
\hline sn-113 & 50113 & 112.9052 \\
\hline sn-114 & 50114 & 113.9028 \\
\hline
\end{tabular}




\begin{tabular}{|l|c|c|}
\hline \multicolumn{1}{|c|}{ Name } & Identifier & $\begin{array}{c}\text { Mass } \\
\text { (amu) }\end{array}$ \\
\hline sn-115 & 50115 & 114.9033 \\
\hline sn-116 & 50116 & 115.9017 \\
\hline sn-117 & 50117 & 116.9029 \\
\hline sn-118 & 50118 & 117.9016 \\
\hline sn-119 & 50119 & 118.9033 \\
\hline sn-120 & 50120 & 119.9022 \\
\hline sn-121 & 50121 & 120.9042 \\
\hline sn-122 & 50122 & 121.9034 \\
\hline sn-123 & 50123 & 122.9057 \\
\hline sn-124 & 50124 & 123.9053 \\
\hline sn-125 & 50125 & 124.9078 \\
\hline sn-126 & 50126 & 125.9077 \\
\hline sn-127 & 50127 & 126.9104 \\
\hline sn-128 & 50128 & 127.9105 \\
\hline sn-129 & 50129 & 128.9135 \\
\hline sn-130 & 50130 & 129.914 \\
\hline sn-131 & 50131 & 130.917 \\
\hline sn-132 & 50132 & 131.9178 \\
\hline sn-133 & 50133 & 132.9238 \\
\hline sn-134 & 50134 & 133.9283 \\
\hline sn-135 & 50135 & 134.9347 \\
\hline sn-136 & 50136 & 135.9393 \\
\hline sn-137 & 50137 & 136.946 \\
\hline sb-103 & 51103 & 102.9397 \\
\hline sb-104 & 51104 & 103.9365 \\
\hline sb-105 & 51105 & 104.9315 \\
\hline sb-106 & 51106 & 105.9288 \\
\hline sb-107 & 51107 & 106.9241 \\
\hline sb-108 & 51108 & 107.9222 \\
\hline sb-109 & 51109 & 108.9181 \\
\hline sb-110 & 51110 & 109.9167 \\
\hline sb-111 & 51111 & 110.9132 \\
\hline sb-112 & 51112 & 111.9124 \\
\hline sb-113 & 51113 & 112.9094 \\
\hline sb-114 & 51114 & 113.9093 \\
\hline sb-115 & 51115 & 114.9066 \\
\hline sb-116 & 51116 & 115.9068 \\
\hline sb-117 & 51117 & 116.9048 \\
\hline sb-118 & 51118 & 117.9055 \\
\hline sb-119 & 51119 & 118.9039 \\
\hline sb-120 & 51120 & 119.9051 \\
\hline sb-121 & 51121 & 120.9038 \\
\hline sb-122 & 51122 & 121.9052 \\
\hline sb-123 & 51123 & 122.9042 \\
\hline sb-124 & 51124 & 123.9059 \\
\hline sb-125 & 51125 & 124.9053 \\
\hline sb-126 & 51126 & 125.9072 \\
\hline sb-127 & 51127 & 126.9069 \\
\hline sb-128 & 51128 & 127.9092 \\
\hline sb-129 & 51129 & 128.9091 \\
\hline sb-130 & 51130 & 129.9117 \\
\hline sb-131 & 51131 & 130.912 \\
\hline
\end{tabular}




\begin{tabular}{|l|c|l|}
\hline \multicolumn{1}{|c|}{ Name } & Identifier & $\begin{array}{c}\text { Mass } \\
\text { (amu) }\end{array}$ \\
\hline sb-132 & 51132 & 131.9145 \\
\hline sb-133 & 51133 & 132.9153 \\
\hline sb-134 & 51134 & 133.9204 \\
\hline sb-135 & 51135 & 134.9252 \\
\hline sb-136 & 51136 & 135.9303 \\
\hline sb-137 & 51137 & 136.9353 \\
\hline sb-138 & 51138 & 137.9408 \\
\hline sb-139 & 51139 & 138.946 \\
\hline te-105 & 52105 & 104.9436 \\
\hline te-106 & 52106 & 105.9375 \\
\hline te-107 & 52107 & 106.935 \\
\hline te-108 & 52108 & 107.9294 \\
\hline te-109 & 52109 & 108.9274 \\
\hline te-110 & 52110 & 109.9224 \\
\hline te-111 & 52111 & 110.9211 \\
\hline te-112 & 52112 & 111.917 \\
\hline te-113 & 52113 & 112.9159 \\
\hline te-114 & 52114 & 113.9121 \\
\hline te-115 & 52115 & 114.9119 \\
\hline te-116 & 52116 & 115.9085 \\
\hline te-117 & 52117 & 116.9087 \\
\hline te-118 & 52118 & 117.9058 \\
\hline te-119 & 52119 & 118.9064 \\
\hline te-120 & 52120 & 119.904 \\
\hline te-121 & 52121 & 120.9049 \\
\hline te-122 & 52122 & 121.903 \\
\hline te-123 & 52123 & 122.9043 \\
\hline te-124 & 52124 & 123.9028 \\
\hline te-125 & 52125 & 124.9044 \\
\hline te-126 & 52126 & 125.9033 \\
\hline te-127 & 52127 & 126.9052 \\
\hline te-127m & 1052127 & \\
\hline te-128 & 52128 & 127.9045 \\
\hline te-129 & 52129 & 128.9066 \\
\hline te-129m & 1052129 & \\
\hline te-130 & 52130 & 129.9062 \\
\hline te-131 & 52131 & 130.9085 \\
\hline te-132 & 52132 & 131.9086 \\
\hline te-133 & 52133 & 132.911 \\
\hline te-134 & 52134 & 133.9114 \\
\hline te-135 & 52135 & 134.9164 \\
\hline te-136 & 52136 & 135.9201 \\
\hline te-137 & 52137 & 136.9253 \\
\hline te-138 & 52138 & 137.9292 \\
\hline te-139 & 52139 & 138.9347 \\
\hline te-140 & 52140 & 139.9388 \\
\hline te-141 & 52141 & 140.9447 \\
\hline te-142 & 52142 & 141.9491 \\
\hline i-108 & 53108 & 107.9435 \\
\hline i-109 & 53109 & 108.9381 \\
\hline i-110 & 53110 & 109.9352 \\
\hline i-111 & 53111 & 110.9303 \\
\hline
\end{tabular}




\begin{tabular}{|c|c|c|}
\hline Name & Identifier & $\begin{array}{c}\text { Mass } \\
\text { (amu) }\end{array}$ \\
\hline i-112 & 53112 & 111.928 \\
\hline i-113 & 53113 & 112.9236 \\
\hline i-114 & 53114 & 113.9219 \\
\hline i-115 & 53115 & 114.9181 \\
\hline i-116 & 53116 & 115.9168 \\
\hline i-117 & 53117 & 116.9137 \\
\hline i-118 & 53118 & 117.9131 \\
\hline i-119 & 53119 & 118.9101 \\
\hline i-120 & 53120 & 119.91 \\
\hline i-121 & 53121 & 120.9074 \\
\hline i-122 & 53122 & 121.9076 \\
\hline i-123 & 53123 & 122.9056 \\
\hline i-124 & 53124 & 123.9062 \\
\hline i-125 & 53125 & 124.9046 \\
\hline i-126 & 53126 & 125.9056 \\
\hline i-127 & 53127 & 126.9045 \\
\hline i-128 & 53128 & 127.9058 \\
\hline i-129 & 53129 & 128.905 \\
\hline i-130 & 53130 & 129.9067 \\
\hline i-131 & 53131 & 130.9061 \\
\hline i-132 & 53132 & 131.908 \\
\hline i-133 & 53133 & 132.9078 \\
\hline i-134 & 53134 & 133.9097 \\
\hline i-135 & 53135 & 134.91 \\
\hline i-136 & 53136 & 135.9147 \\
\hline i-137 & 53137 & 136.9179 \\
\hline i-138 & 53138 & 137.9223 \\
\hline i-139 & 53139 & 138.9261 \\
\hline i-140 & 53140 & 139.931 \\
\hline i-141 & 53141 & 140.935 \\
\hline i-142 & 53142 & 141.9402 \\
\hline i-143 & 53143 & 142.9446 \\
\hline i-144 & 53144 & 143.95 \\
\hline xe-110 & 54110 & 109.9443 \\
\hline xe-111 & 54111 & 110.9416 \\
\hline xe-112 & 54112 & 111.9356 \\
\hline xe-113 & 54113 & 112.9333 \\
\hline xe-114 & 54114 & 113.928 \\
\hline xe-115 & 54115 & 114.9263 \\
\hline xe-116 & 54116 & 115.9216 \\
\hline xe-117 & 54117 & 116.9204 \\
\hline xe-118 & 54118 & 117.9162 \\
\hline xe-119 & 54119 & 118.9154 \\
\hline xe-120 & 54120 & 119.9118 \\
\hline xe-121 & 54121 & 120.9115 \\
\hline xe-122 & 54122 & 121.9084 \\
\hline xe-123 & 54123 & 122.9085 \\
\hline xe-124 & 54124 & 123.9059 \\
\hline xe-125 & 54125 & 124.9064 \\
\hline xe-126 & 54126 & 125.9043 \\
\hline xe-127 & 54127 & 126.9052 \\
\hline xe-128 & 54128 & 127.9035 \\
\hline
\end{tabular}




\begin{tabular}{|l|c|l|}
\hline \multicolumn{1}{|c|}{ Name } & Identifier & $\begin{array}{c}\text { Mass } \\
\text { (amu) }\end{array}$ \\
\hline xe-129 & 54129 & 128.9048 \\
\hline xe-130 & 54130 & 129.9035 \\
\hline xe-131 & 54131 & 130.9051 \\
\hline xe-132 & 54132 & 131.9041 \\
\hline xe-133 & 54133 & 132.9059 \\
\hline xe-134 & 54134 & 133.9054 \\
\hline xe-135 & 54135 & 134.9072 \\
\hline xe-136 & 54136 & 135.9072 \\
\hline xe-137 & 54137 & 136.9116 \\
\hline xe-138 & 54138 & 137.914 \\
\hline xe-139 & 54139 & 138.9188 \\
\hline xe-140 & 54140 & 139.9216 \\
\hline xe-141 & 54141 & 140.9267 \\
\hline xe-142 & 54142 & 141.9297 \\
\hline xe-143 & 54143 & 142.9351 \\
\hline xe-144 & 54144 & 143.9385 \\
\hline xe-145 & 54145 & 144.9441 \\
\hline xe-146 & 54146 & 145.9478 \\
\hline xe-147 & 54147 & 146.9536 \\
\hline cs-112 & 55112 & 111.9503 \\
\hline cs-113 & 55113 & 112.9445 \\
\hline cs-114 & 55114 & 113.9415 \\
\hline cs-115 & 55115 & 114.9359 \\
\hline cs-116 & 55116 & 115.9334 \\
\hline cs-117 & 55117 & 116.9287 \\
\hline cs-118 & 55118 & 117.9266 \\
\hline cs-119 & 55119 & 118.9224 \\
\hline cs-120 & 55120 & 119.9207 \\
\hline cs-121 & 55121 & 120.9172 \\
\hline cs-122 & 55122 & 121.9161 \\
\hline cs-123 & 55123 & 122.913 \\
\hline cs-124 & 55124 & 123.9123 \\
\hline cs-125 & 55125 & 124.9097 \\
\hline cs-126 & 55126 & 125.9094 \\
\hline cs-127 & 55127 & 126.9074 \\
\hline cs-128 & 55128 & 127.9078 \\
\hline cs-129 & 55129 & 128.9061 \\
\hline cs-130 & 55130 & 129.9067 \\
\hline cs-131 & 55131 & 130.9055 \\
\hline cs-132 & 55132 & 131.9064 \\
\hline cs-133 & 55133 & 132.9055 \\
\hline cs-134 & 55134 & 133.9067 \\
\hline cs-135 & 55135 & 134.906 \\
\hline cs-136 & 55136 & 135.9073 \\
\hline cs-137 & 55137 & 136.9071 \\
\hline cs-138 & 55138 & 137.911 \\
\hline cs-139 & 55139 & 138.9134 \\
\hline cs-140 & 55140 & 139.9173 \\
\hline cs-142 & 55141 & 140.92 \\
\hline cs-143 & 55142 & 141.9243 \\
\hline cs-144 & 55144 & 142.9274 \\
\hline & & 143.9321 \\
\hline
\end{tabular}




\begin{tabular}{|c|c|c|}
\hline Name & Identifier & $\begin{array}{c}\text { Mass } \\
\text { (amu) }\end{array}$ \\
\hline cs-145 & 55145 & 144.9355 \\
\hline cs-146 & 55146 & 145.9403 \\
\hline cs-147 & 55147 & 146.9442 \\
\hline cs-148 & 55148 & 147.9492 \\
\hline cs-149 & 55149 & 148.9529 \\
\hline cs-150 & 55150 & 149.9582 \\
\hline cs-151 & 55151 & 150.9622 \\
\hline ba-114 & 56114 & 113.9507 \\
\hline ba-115 & 56115 & 114.9474 \\
\hline ba-116 & 56116 & 115.9414 \\
\hline ba-117 & 56117 & 116.9385 \\
\hline ba-118 & 56118 & 117.933 \\
\hline ba-119 & 56119 & 118.9307 \\
\hline ba-120 & 56120 & 119.926 \\
\hline ba-121 & 56121 & 120.924 \\
\hline ba-122 & 56122 & 121.9199 \\
\hline ba-123 & 56123 & 122.9188 \\
\hline ba-124 & 56124 & 123.9151 \\
\hline ba-125 & 56125 & 124.9145 \\
\hline ba-126 & 56126 & 125.9112 \\
\hline ba-127 & 56127 & 126.9111 \\
\hline ba-128 & 56128 & 127.9083 \\
\hline ba-129 & 56129 & 128.9087 \\
\hline ba-130 & 56130 & 129.9063 \\
\hline ba-131 & 56131 & 130.9069 \\
\hline ba-132 & 56132 & 131.9051 \\
\hline ba-133 & 56133 & 132.906 \\
\hline ba-134 & 56134 & 133.9045 \\
\hline ba-135 & 56135 & 134.9057 \\
\hline ba-136 & 56136 & 135.9046 \\
\hline ba-137 & 56137 & 136.9058 \\
\hline ba-138 & 56138 & 137.9052 \\
\hline ba-139 & 56139 & 138.9088 \\
\hline ba-140 & 56140 & 139.9106 \\
\hline ba-141 & 56141 & 140.9144 \\
\hline ba-142 & 56142 & 141.9164 \\
\hline ba-143 & 56143 & 142.9206 \\
\hline ba-144 & 56144 & 143.9229 \\
\hline ba-145 & 56145 & 144.9276 \\
\hline ba-146 & 56146 & 145.9302 \\
\hline ba-147 & 56147 & 146.935 \\
\hline ba-148 & 56148 & 147.9377 \\
\hline ba-149 & 56149 & 148.9426 \\
\hline ba-150 & 56150 & 149.9457 \\
\hline ba-151 & 56151 & 150.9508 \\
\hline ba-152 & 56152 & 151.9543 \\
\hline ba-153 & 56153 & 152.9596 \\
\hline la-117 & 57117 & 116.9501 \\
\hline la-118 & 57118 & 117.9467 \\
\hline la-119 & 57119 & 118.941 \\
\hline la-120 & 57120 & 119.9381 \\
\hline la-121 & 57121 & 120.933 \\
\hline
\end{tabular}




\begin{tabular}{|l|c|c|}
\hline \multicolumn{1}{|c|}{ Name } & Identifier & $\begin{array}{c}\text { Mass } \\
\text { (amu) }\end{array}$ \\
\hline la-122 & 57122 & 121.9307 \\
\hline la-123 & 57123 & 122.9262 \\
\hline la-124 & 57124 & 123.9246 \\
\hline la-125 & 57125 & 124.9208 \\
\hline la-126 & 57126 & 125.9195 \\
\hline la-127 & 57127 & 126.9164 \\
\hline la-128 & 57128 & 127.9156 \\
\hline la-129 & 57129 & 128.9127 \\
\hline la-130 & 57130 & 129.9124 \\
\hline la-131 & 57131 & 130.9101 \\
\hline la-132 & 57132 & 131.9101 \\
\hline la-133 & 57133 & 132.9082 \\
\hline la-134 & 57134 & 133.9085 \\
\hline la-135 & 57135 & 134.907 \\
\hline la-136 & 57136 & 135.9076 \\
\hline la-137 & 57137 & 136.9065 \\
\hline la-138 & 57138 & 137.9071 \\
\hline la-139 & 57139 & 138.9064 \\
\hline la-140 & 57140 & 139.9095 \\
\hline la-141 & 57141 & 140.911 \\
\hline la-142 & 57142 & 141.9141 \\
\hline la-143 & 57143 & 142.9161 \\
\hline la-144 & 57144 & 143.9196 \\
\hline la-145 & 57145 & 144.9216 \\
\hline la-146 & 57146 & 145.9258 \\
\hline la-147 & 57147 & 146.9282 \\
\hline la-148 & 57148 & 147.9322 \\
\hline la-149 & 57149 & 148.9347 \\
\hline la-150 & 57150 & 149.9388 \\
\hline la-151 & 57151 & 150.9417 \\
\hline la-152 & 57152 & 151.9462 \\
\hline la-153 & 57153 & 152.9496 \\
\hline la-154 & 57154 & 153.9545 \\
\hline la-155 & 57155 & 154.9583 \\
\hline ce-119 & 58119 & 118.9528 \\
\hline ce-120 & 58120 & 119.9466 \\
\hline ce-121 & 58121 & 120.9434 \\
\hline ce-122 & 58122 & 121.9379 \\
\hline ce-123 & 58123 & 122.9354 \\
\hline ce-124 & 58124 & 123.9304 \\
\hline ce-125 & 58125 & 124.9284 \\
\hline ce-126 & 58126 & 125.924 \\
\hline ce-127 & 58127 & 126.9227 \\
\hline ce-128 & 58128 & 127.9189 \\
\hline ce-129 & 58129 & 128.9181 \\
\hline ce-130 & 58130 & 129.9147 \\
\hline ce-131 & 58131 & 130.9144 \\
\hline ce-132 & 58132 & 131.9115 \\
\hline ce-134 & 58133 & 132.9115 \\
\hline & 58134 & 133.9089 \\
\hline & 58136 & 134.9091 \\
\hline
\end{tabular}




\begin{tabular}{|c|c|c|}
\hline Name & Identifier & $\begin{array}{l}\text { Mass } \\
\text { (amu) }\end{array}$ \\
\hline ce-137 & 58137 & 136.9078 \\
\hline ce-138 & 58138 & 137.906 \\
\hline ce-139 & 58139 & 138.9066 \\
\hline ce-140 & 58140 & 139.9054 \\
\hline ce-141 & 58141 & 140.9083 \\
\hline ce-142 & 58142 & 141.9092 \\
\hline ce-143 & 58143 & 142.9124 \\
\hline ce-144 & 58144 & 143.9137 \\
\hline ce-145 & 58145 & 144.9172 \\
\hline ce-146 & 58146 & 145.9188 \\
\hline ce-147 & 58147 & 146.9227 \\
\hline ce-148 & 58148 & 147.9244 \\
\hline ce-149 & 58149 & 148.9284 \\
\hline ce-150 & 58150 & 149.9304 \\
\hline ce-151 & 58151 & 150.934 \\
\hline ce-152 & 58152 & 151.9365 \\
\hline ce-153 & 58153 & 152.9406 \\
\hline ce-154 & 58154 & 153.9434 \\
\hline ce-155 & 58155 & 154.948 \\
\hline ce-156 & 58156 & 155.9513 \\
\hline ce-157 & 58157 & 156.9563 \\
\hline pr-121 & 59121 & 120.9554 \\
\hline pr-122 & 59122 & 121.9518 \\
\hline pr-123 & 59123 & 122.946 \\
\hline pr-124 & 59124 & 123.943 \\
\hline pr-125 & 59125 & 124.9378 \\
\hline pr-126 & 59126 & 125.9353 \\
\hline pr-127 & 59127 & 126.9308 \\
\hline pr-128 & 59128 & 127.9288 \\
\hline pr-129 & 59129 & 128.9251 \\
\hline pr-130 & 59130 & 129.9236 \\
\hline pr-131 & 59131 & 130.9203 \\
\hline pr-132 & 59132 & 131.9193 \\
\hline pr-133 & 59133 & 132.9163 \\
\hline pr-134 & 59134 & 133.9157 \\
\hline pr-135 & 59135 & 134.9131 \\
\hline pr-136 & 59136 & 135.9127 \\
\hline pr-137 & 59137 & 136.9107 \\
\hline pr-138 & 59138 & 137.9108 \\
\hline pr-139 & 59139 & 138.9089 \\
\hline pr-140 & 59140 & 139.9091 \\
\hline pr-141 & 59141 & 140.9077 \\
\hline pr-142 & 59142 & 141.91 \\
\hline pr-143 & 59143 & 142.9108 \\
\hline pr-144 & 59144 & 143.9133 \\
\hline pr-145 & 59145 & 144.9145 \\
\hline pr-146 & 59146 & 145.9176 \\
\hline pr-147 & 59147 & 146.919 \\
\hline pr-148 & 59148 & 147.9221 \\
\hline pr-149 & 59149 & 148.9237 \\
\hline pr-150 & 59150 & 149.9267 \\
\hline pr-151 & 59151 & 150.9283 \\
\hline
\end{tabular}




\begin{tabular}{|c|c|c|}
\hline Name & Identifier & $\begin{array}{l}\text { Mass } \\
\text { (amu) }\end{array}$ \\
\hline pr-152 & 59152 & 151.9315 \\
\hline pr-153 & 59153 & 152.9338 \\
\hline pr-154 & 59154 & 153.9375 \\
\hline pr-155 & 59155 & 154.9401 \\
\hline pr-156 & 59156 & 155.9443 \\
\hline pr-157 & 59157 & 156.9474 \\
\hline pr-158 & 59158 & 157.952 \\
\hline pr-159 & 59159 & 158.9555 \\
\hline nd-124 & 60124 & 123.9522 \\
\hline nd-125 & 60125 & 124.9489 \\
\hline nd-126 & 60126 & 125.9432 \\
\hline nd-127 & 60127 & 126.9405 \\
\hline nd-128 & 60128 & 127.9354 \\
\hline nd-129 & 60129 & 128.9332 \\
\hline nd-130 & 60130 & 129.9285 \\
\hline nd-131 & 60131 & 130.9272 \\
\hline nd-132 & 60132 & 131.9233 \\
\hline nd-133 & 60133 & 132.9223 \\
\hline nd-134 & 60134 & 133.9188 \\
\hline nd-135 & 60135 & 134.9182 \\
\hline nd-136 & 60136 & 135.915 \\
\hline nd-137 & 60137 & 136.9146 \\
\hline nd-138 & 60138 & 137.912 \\
\hline nd-139 & 60139 & 138.912 \\
\hline nd-140 & 60140 & 139.9095 \\
\hline nd-141 & 60141 & 140.9096 \\
\hline nd-142 & 60142 & 141.9077 \\
\hline nd-143 & 60143 & 142.9098 \\
\hline nd-144 & 60144 & 143.9101 \\
\hline nd-145 & 60145 & 144.9126 \\
\hline nd-146 & 60146 & 145.9131 \\
\hline nd-147 & 60147 & 146.9161 \\
\hline nd-148 & 60148 & 147.9169 \\
\hline nd-149 & 60149 & 148.9202 \\
\hline nd-150 & 60150 & 149.9209 \\
\hline nd-151 & 60151 & 150.9238 \\
\hline nd-152 & 60152 & 151.9247 \\
\hline nd-153 & 60153 & 152.9277 \\
\hline nd-154 & 60154 & 153.9295 \\
\hline nd-155 & 60155 & 154.9329 \\
\hline nd-156 & 60156 & 155.935 \\
\hline nd-157 & 60157 & 156.939 \\
\hline nd-158 & 60158 & 157.9416 \\
\hline nd-159 & 60159 & 158.9461 \\
\hline nd-160 & 60160 & 159.9491 \\
\hline nd-161 & 60161 & 160.9539 \\
\hline pm-126 & 61126 & 125.9575 \\
\hline pm-127 & 61127 & 126.9516 \\
\hline pm-128 & 61128 & 127.9484 \\
\hline pm-129 & 61129 & 128.9432 \\
\hline pm-130 & 61130 & 129.9404 \\
\hline pm-131 & 61131 & 130.9359 \\
\hline
\end{tabular}




\begin{tabular}{|l|c|l|}
\hline \multicolumn{1}{|c|}{ Name } & Identifier & $\begin{array}{c}\text { Mass } \\
\text { (amu) }\end{array}$ \\
\hline pm-132 & 61132 & 131.9337 \\
\hline pm-133 & 61133 & 132.9298 \\
\hline pm-134 & 61134 & 133.9283 \\
\hline pm-135 & 61135 & 134.9249 \\
\hline pm-136 & 61136 & 135.9236 \\
\hline pm-137 & 61137 & 136.9205 \\
\hline pm-138 & 61138 & 137.9196 \\
\hline pm-139 & 61139 & 138.9168 \\
\hline pm-140 & 61140 & 139.916 \\
\hline pm-141 & 61141 & 140.9136 \\
\hline pm-142 & 61142 & 141.9129 \\
\hline pm-143 & 61143 & 142.9109 \\
\hline pm-144 & 61144 & 143.9126 \\
\hline pm-145 & 61145 & 144.9128 \\
\hline pm-146 & 61146 & 145.9147 \\
\hline pm-147 & 61147 & 146.9151 \\
\hline pm-148 & 61148 & 147.9175 \\
\hline pm-148m & 1061148 & \\
\hline pm-149 & 61149 & 148.9183 \\
\hline pm-150 & 61150 & 149.921 \\
\hline pm-151 & 61151 & 150.9212 \\
\hline pm-152 & 61152 & 151.9235 \\
\hline pm-153 & 61153 & 152.9241 \\
\hline pm-154 & 61154 & 153.9265 \\
\hline pm-155 & 61155 & 154.9281 \\
\hline pm-156 & 61156 & 155.9311 \\
\hline pm-157 & 61157 & 156.933 \\
\hline pm-158 & 61158 & 157.9366 \\
\hline pm-159 & 61159 & 158.939 \\
\hline pm-160 & 61160 & 159.943 \\
\hline pm-161 & 61161 & 160.9459 \\
\hline pm-162 & 61162 & 161.9503 \\
\hline pm-163 & 61163 & 162.9537 \\
\hline sm-128 & 62128 & 127.9581 \\
\hline sm-129 & 62129 & 128.9546 \\
\hline sm-130 & 62130 & 129.9489 \\
\hline sm-131 & 62131 & 130.9461 \\
\hline sm-132 & 62132 & 131.9407 \\
\hline sm-133 & 62133 & 132.9387 \\
\hline sm-134 & 62134 & 133.934 \\
\hline sm-135 & 62135 & 134.9325 \\
\hline sm-136 & 62136 & 135.9283 \\
\hline sm-137 & 62137 & 136.927 \\
\hline sm-138 & 62138 & 137.9232 \\
\hline sm-139 & 62139 & 138.9223 \\
\hline sm-140 & 62140 & 139.919 \\
\hline sm-141 & 62141 & 140.9185 \\
\hline sm-142 & 62142 & 141.9152 \\
\hline sm-143 & 62143 & 142.9146 \\
\hline sm-144 & 62144 & 143.912 \\
\hline sm-145 & 62145 & 144.9134 \\
\hline sm-146 & 62146 & 145.913 \\
\hline
\end{tabular}




\begin{tabular}{|c|c|c|}
\hline Name & Identifier & $\begin{array}{c}\text { Mass } \\
\text { (amu) }\end{array}$ \\
\hline sm-147 & 62147 & 146.9149 \\
\hline sm-148 & 62148 & 147.9148 \\
\hline sm-149 & 62149 & 148.9172 \\
\hline sm-150 & 62150 & 149.9173 \\
\hline sm-151 & 62151 & 150.9199 \\
\hline sm-152 & 62152 & 151.9197 \\
\hline sm-153 & 62153 & 152.9221 \\
\hline sm-154 & 62154 & 153.9222 \\
\hline sm-155 & 62155 & 154.9246 \\
\hline sm-156 & 62156 & 155.9255 \\
\hline sm-157 & 62157 & 156.9284 \\
\hline sm-158 & 62158 & 157.93 \\
\hline sm-159 & 62159 & 158.9332 \\
\hline sm-160 & 62160 & 159.9351 \\
\hline sm-161 & 62161 & 160.9388 \\
\hline sm-162 & 62162 & 161.9412 \\
\hline sm-163 & 62163 & 162.9454 \\
\hline sm-164 & 62164 & 163.9483 \\
\hline sm-165 & 62165 & 164.953 \\
\hline eu-130 & 63130 & 129.9636 \\
\hline eu-131 & 63131 & 130.9577 \\
\hline eu-132 & 63132 & 131.9544 \\
\hline eu-133 & 63133 & 132.9492 \\
\hline eu-134 & 63134 & 133.9465 \\
\hline eu-135 & 63135 & 134.9418 \\
\hline eu-136 & 63136 & 135.9396 \\
\hline eu-137 & 63137 & 136.9356 \\
\hline eu-138 & 63138 & 137.9337 \\
\hline eu-139 & 63139 & 138.9298 \\
\hline eu-140 & 63140 & 139.9281 \\
\hline eu-141 & 63141 & 140.9249 \\
\hline eu-142 & 63142 & 141.9234 \\
\hline eu-143 & 63143 & 142.9203 \\
\hline eu-144 & 63144 & 143.9188 \\
\hline eu-145 & 63145 & 144.9163 \\
\hline eu-146 & 63146 & 145.9172 \\
\hline eu-147 & 63147 & 146.9167 \\
\hline eu-148 & 63148 & 147.9181 \\
\hline eu-149 & 63149 & 148.9179 \\
\hline eu-150 & 63150 & 149.9197 \\
\hline eu-151 & 63151 & 150.9198 \\
\hline eu-152 & 63152 & 151.9217 \\
\hline eu-153 & 63153 & 152.9212 \\
\hline eu-154 & 63154 & 153.923 \\
\hline eu-155 & 63155 & 154.9229 \\
\hline eu-156 & 63156 & 155.9247 \\
\hline eu-157 & 63157 & 156.9254 \\
\hline eu-158 & 63158 & 157.9279 \\
\hline eu-159 & 63159 & 158.9291 \\
\hline eu-160 & 63160 & 159.932 \\
\hline eu-161 & 63161 & 160.9337 \\
\hline eu-162 & 63162 & 161.937 \\
\hline
\end{tabular}




\begin{tabular}{|l|l|l|}
\hline \multicolumn{1}{|c|}{ Name } & Identifier & $\begin{array}{c}\text { Mass } \\
\text { (amu) }\end{array}$ \\
\hline eu-163 & 63163 & 162.9392 \\
\hline eu-164 & 63164 & 163.943 \\
\hline eu-165 & 63165 & 164.9457 \\
\hline eu-166 & 63166 & 165.95 \\
\hline eu-167 & 63167 & 166.9532 \\
\hline gd-134 & 64134 & 133.9554 \\
\hline gd-135 & 64135 & 134.9526 \\
\hline gd-136 & 64136 & 135.9473 \\
\hline gd-137 & 64137 & 136.945 \\
\hline gd-138 & 64138 & 137.9401 \\
\hline gd-139 & 64139 & 138.9382 \\
\hline gd-140 & 64140 & 139.9337 \\
\hline gd-141 & 64141 & 140.9321 \\
\hline gd-142 & 64142 & 141.9281 \\
\hline gd-143 & 64143 & 142.9267 \\
\hline gd-144 & 64144 & 143.923 \\
\hline gd-145 & 64145 & 144.9217 \\
\hline gd-146 & 64146 & 145.9183 \\
\hline gd-147 & 64147 & 146.9191 \\
\hline gd-148 & 64148 & 147.9181 \\
\hline gd-149 & 64149 & 148.9193 \\
\hline gd-150 & 64150 & 149.9187 \\
\hline gd-151 & 64151 & 150.9203 \\
\hline gd-152 & 64152 & 151.9198 \\
\hline gd-153 & 64153 & 152.9218 \\
\hline gd-154 & 64154 & 153.9209 \\
\hline gd-155 & 64155 & 154.9226 \\
\hline gd-156 & 64156 & 155.9221 \\
\hline gd-157 & 64157 & 156.924 \\
\hline gd-158 & 64158 & 157.9241 \\
\hline gd-159 & 64159 & 158.9264 \\
\hline gd-160 & 64160 & 159.927 \\
\hline gd-161 & 64161 & 160.9297 \\
\hline gd-162 & 64162 & 161.931 \\
\hline gd-163 & 64163 & 162.934 \\
\hline gd-164 & 64164 & 163.9359 \\
\hline gd-165 & 64165 & 164.9394 \\
\hline gd-166 & 64166 & 165.9416 \\
\hline gd-167 & 64167 & 166.9456 \\
\hline gd-168 & 64168 & 167.9484 \\
\hline gd-169 & 64169 & 168.9529 \\
\hline tb-136 & 65136 & 135.9614 \\
\hline tb-137 & 65137 & 136.956 \\
\hline tb-138 & 65138 & 137.9532 \\
\hline tb-139 & 65139 & 138.9483 \\
\hline tb-140 & 65140 & 139.9458 \\
\hline tb-141 & 65141 & 140.9415 \\
\hline tb-142 & 65142 & 141.9387 \\
\hline tb-143 & 65143 & 142.9351 \\
\hline tb-144 & 65144 & 143.933 \\
\hline tb-146 & 65145 & 144.9293 \\
\hline & 65146 & 145.9272 \\
\hline
\end{tabular}




\begin{tabular}{|l|c|l|}
\hline \multicolumn{1}{|c|}{ Name } & Identifier & $\begin{array}{c}\text { Mass } \\
\text { (amu) }\end{array}$ \\
\hline tb-147 & 65147 & 146.9241 \\
\hline tb-148 & 65148 & 147.9243 \\
\hline tb-149 & 65149 & 148.9232 \\
\hline tb-150 & 65150 & 149.9237 \\
\hline tb-151 & 65151 & 150.9231 \\
\hline tb-152 & 65152 & 151.9241 \\
\hline tb-153 & 65153 & 152.9234 \\
\hline tb-154 & 65154 & 153.9247 \\
\hline tb-155 & 65155 & 154.9235 \\
\hline tb-156 & 65156 & 155.9247 \\
\hline tb-157 & 65157 & 156.924 \\
\hline tb-158 & 65158 & 157.9254 \\
\hline tb-159 & 65159 & 158.9254 \\
\hline tb-160 & 65160 & 159.9272 \\
\hline tb-161 & 65161 & 160.9276 \\
\hline tb-162 & 65162 & 161.9295 \\
\hline tb-163 & 65163 & 162.9306 \\
\hline tb-164 & 65164 & 163.9333 \\
\hline tb-165 & 65165 & 164.9349 \\
\hline tb-166 & 65166 & 165.938 \\
\hline tb-167 & 65167 & 166.94 \\
\hline tb-168 & 65168 & 167.9436 \\
\hline tb-169 & 65169 & 168.9462 \\
\hline tb-170 & 65170 & 169.9503 \\
\hline tb-171 & 65171 & 170.9533 \\
\hline dy-138 & 66138 & 137.9625 \\
\hline dy-139 & 66139 & 138.9595 \\
\hline dy-140 & 66140 & 139.954 \\
\hline dy-141 & 66141 & 140.9514 \\
\hline dy-142 & 66142 & 141.9464 \\
\hline dy-143 & 66143 & 142.9438 \\
\hline dy-144 & 66144 & 143.9393 \\
\hline dy-145 & 66145 & 144.9374 \\
\hline dy-146 & 66146 & 145.9328 \\
\hline dy-147 & 66147 & 146.9311 \\
\hline dy-148 & 66148 & 147.9272 \\
\hline dy-149 & 66149 & 148.9273 \\
\hline dy-150 & 66150 & 149.9256 \\
\hline dy-151 & 66151 & 150.9262 \\
\hline dy-152 & 66152 & 151.9247 \\
\hline dy-153 & 66153 & 152.9258 \\
\hline dy-154 & 66154 & 153.9244 \\
\hline dy-155 & 66155 & 154.9258 \\
\hline dy-156 & 66156 & 155.9243 \\
\hline dy-157 & 66157 & 156.9255 \\
\hline dy-158 & 66158 & 157.9244 \\
\hline dy-159 & 66159 & 158.9257 \\
\hline dy-160 & 66160 & 159.9252 \\
\hline dy-161 & 66161 & 160.9269 \\
\hline dy-162 & 66162 & 161.9268 \\
\hline dy-163 & 66163 & 162.9287 \\
\hline dy-164 & 66164 & 163.9292 \\
\hline
\end{tabular}




\begin{tabular}{|l|c|c|}
\hline \multicolumn{1}{|c|}{ Name } & Identifier & $\begin{array}{c}\text { Mass } \\
\text { (amu) }\end{array}$ \\
\hline dy-165 & 66165 & 164.9317 \\
\hline dy-166 & 66166 & 165.9328 \\
\hline dy-167 & 66167 & 166.9357 \\
\hline dy-168 & 66168 & 167.9371 \\
\hline dy-169 & 66169 & 168.9403 \\
\hline dy-170 & 66170 & 169.9424 \\
\hline dy-171 & 66171 & 170.9462 \\
\hline dy-172 & 66172 & 171.9488 \\
\hline dy-173 & 66173 & 172.953 \\
\hline ho-140 & 67140 & 139.9685 \\
\hline ho-141 & 67141 & 140.9631 \\
\hline ho-142 & 67142 & 141.9598 \\
\hline ho-143 & 67143 & 142.9546 \\
\hline ho-144 & 67144 & 143.9515 \\
\hline ho-145 & 67145 & 144.9472 \\
\hline ho-146 & 67146 & 145.9446 \\
\hline ho-147 & 67147 & 146.9401 \\
\hline ho-148 & 67148 & 147.9377 \\
\hline ho-149 & 67149 & 148.9338 \\
\hline ho-150 & 67150 & 149.9335 \\
\hline ho-151 & 67151 & 150.9317 \\
\hline ho-152 & 67152 & 151.9317 \\
\hline ho-153 & 67153 & 152.9302 \\
\hline ho-154 & 67154 & 153.9306 \\
\hline ho-155 & 67155 & 154.9291 \\
\hline ho-156 & 67156 & 155.9298 \\
\hline ho-157 & 67157 & 156.9283 \\
\hline ho-158 & 67158 & 157.9289 \\
\hline ho-159 & 67159 & 158.9277 \\
\hline ho-160 & 67160 & 159.9287 \\
\hline ho-161 & 67161 & 160.9279 \\
\hline ho-162 & 67162 & 161.9291 \\
\hline ho-163 & 67163 & 162.9287 \\
\hline ho-164 & 67164 & 163.9302 \\
\hline ho-165 & 67165 & 164.9303 \\
\hline ho-166 & 67166 & 165.9323 \\
\hline ho-166m & 1067166 & \\
\hline ho-167 & 67167 & 166.9331 \\
\hline ho-168 & 67168 & 167.9355 \\
\hline ho-169 & 67169 & 168.9369 \\
\hline ho-170 & 67170 & 169.9396 \\
\hline ho-171 & 67171 & 170.9415 \\
\hline ho-172 & 67172 & 171.9448 \\
\hline ho-173 & 67173 & 172.9473 \\
\hline ho-174 & 67174 & 173.9512 \\
\hline ho-175 & 67175 & 174.9541 \\
\hline er-143 & 68143 & 142.9663 \\
\hline er-144 & 68144 & 143.9604 \\
\hline er-145 & 68145 & 144.9574 \\
\hline er-146 & 68146 & 145.952 \\
\hline er-147 & 68147 & 146.9495 \\
\hline er-148 & 68148 & 147.9445 \\
\hline
\end{tabular}




\begin{tabular}{|c|c|c|}
\hline Name & Identifier & $\begin{array}{c}\text { Mass } \\
\text { (amu) }\end{array}$ \\
\hline er-149 & 68149 & 148.9423 \\
\hline er-150 & 68150 & 149.9379 \\
\hline er-151 & 68151 & 150.9375 \\
\hline er-152 & 68152 & 151.935 \\
\hline er-153 & 68153 & 152.9351 \\
\hline er-154 & 68154 & 153.9328 \\
\hline er-155 & 68155 & 154.9332 \\
\hline er-156 & 68156 & 155.9311 \\
\hline er-157 & 68157 & 156.9319 \\
\hline er-158 & 68158 & 157.9299 \\
\hline er-159 & 68159 & 158.9307 \\
\hline er-160 & 68160 & 159.9291 \\
\hline er-161 & 68161 & 160.93 \\
\hline er-162 & 68162 & 161.9288 \\
\hline er-163 & 68163 & 162.93 \\
\hline er-164 & 68164 & 163.9292 \\
\hline er-165 & 68165 & 164.9307 \\
\hline er-166 & 68166 & 165.9303 \\
\hline er-167 & 68167 & 166.9321 \\
\hline er-168 & 68168 & 167.9324 \\
\hline er-169 & 68169 & 168.9346 \\
\hline er-170 & 68170 & 169.9355 \\
\hline er-171 & 68171 & 170.938 \\
\hline er-172 & 68172 & 171.9394 \\
\hline er-173 & 68173 & 172.9424 \\
\hline er-174 & 68174 & 173.9442 \\
\hline er-175 & 68175 & 174.9478 \\
\hline er-176 & 68176 & 175.9501 \\
\hline er-177 & 68177 & 176.9541 \\
\hline tm-145 & 69145 & 144.9701 \\
\hline $\mathrm{tm}-146$ & 69146 & 145.9664 \\
\hline tm-147 & 69147 & 146.961 \\
\hline tm-148 & 69148 & 147.9578 \\
\hline tm-149 & 69149 & 148.9527 \\
\hline tm-150 & 69150 & 149.95 \\
\hline tm-151 & 69151 & 150.9455 \\
\hline tm-152 & 69152 & 151.9444 \\
\hline tm-153 & 69153 & 152.942 \\
\hline tm-154 & 69154 & 153.9416 \\
\hline tm-155 & 69155 & 154.9392 \\
\hline tm-156 & 69156 & 155.939 \\
\hline tm-157 & 69157 & 156.937 \\
\hline tm-158 & 69158 & 157.937 \\
\hline tm-159 & 69159 & 158.935 \\
\hline tm-160 & 69160 & 159.9353 \\
\hline tm-161 & 69161 & 160.9335 \\
\hline tm-162 & 69162 & 161.934 \\
\hline tm-163 & 69163 & 162.9326 \\
\hline tm-164 & 69164 & 163.9336 \\
\hline tm-165 & 69165 & 164.9324 \\
\hline tm-166 & 69166 & 165.9335 \\
\hline tm-167 & 69167 & 166.9328 \\
\hline
\end{tabular}




\begin{tabular}{|c|c|c|}
\hline Name & Identifier & $\begin{array}{l}\text { Mass } \\
\text { (amu) }\end{array}$ \\
\hline tm-168 & 69168 & 167.9342 \\
\hline tm-169 & 69169 & 168.9342 \\
\hline tm-170 & 69170 & 169.9358 \\
\hline tm-171 & 69171 & 170.9364 \\
\hline tm-172 & 69172 & 171.9384 \\
\hline $\mathrm{tm}-173$ & 69173 & 172.9396 \\
\hline $\mathrm{tm}-174$ & 69174 & 173.9422 \\
\hline tm-175 & 69175 & 174.9438 \\
\hline $\mathrm{tm}-176$ & 69176 & 175.947 \\
\hline tm-177 & 69177 & 176.949 \\
\hline $\mathrm{tm}-178$ & 69178 & 177.9526 \\
\hline tm-179 & 69179 & 178.9553 \\
\hline yb-148 & 70148 & 147.9674 \\
\hline$y b-149$ & 70149 & 148.964 \\
\hline yb-150 & 70150 & 149.9584 \\
\hline$y b-151$ & 70151 & 150.9554 \\
\hline$y b-152$ & 70152 & 151.9503 \\
\hline$y b-153$ & 70153 & 152.9495 \\
\hline yb-154 & 70154 & 153.9464 \\
\hline$y b-155$ & 70155 & 154.9458 \\
\hline$y b-156$ & 70156 & 155.9428 \\
\hline$y b-157$ & 70157 & 156.9426 \\
\hline$y b-158$ & 70158 & 157.9399 \\
\hline yb-159 & 70159 & 158.94 \\
\hline$y b-160$ & 70160 & 159.9375 \\
\hline$y b-161$ & 70161 & 160.9379 \\
\hline$y b-162$ & 70162 & 161.9358 \\
\hline yb-163 & 70163 & 162.9363 \\
\hline$y b-164$ & 70164 & 163.9345 \\
\hline$y b-165$ & 70165 & 164.9353 \\
\hline$y b-166$ & 70166 & 165.9339 \\
\hline$y b-167$ & 70167 & 166.935 \\
\hline$y b-168$ & 70168 & 167.9339 \\
\hline$y b-169$ & 70169 & 168.9352 \\
\hline$y b-170$ & 70170 & 169.9348 \\
\hline$y b-171$ & 70171 & 170.9363 \\
\hline$y b-172$ & 70172 & 171.9364 \\
\hline$y b-173$ & 70173 & 172.9382 \\
\hline yb-174 & 70174 & 173.9389 \\
\hline$y b-175$ & 70175 & 174.9413 \\
\hline$y b-176$ & 70176 & 175.9426 \\
\hline$y b-177$ & 70177 & 176.9453 \\
\hline$y b-178$ & 70178 & 177.9467 \\
\hline$y b-179$ & 70179 & 178.9502 \\
\hline yb-180 & 70180 & 179.9523 \\
\hline$y b-181$ & 70181 & 180.9561 \\
\hline lu-150 & 71150 & 149.9732 \\
\hline lu-151 & 71151 & 150.9676 \\
\hline lu-152 & 71152 & 151.9641 \\
\hline lu-153 & 71153 & 152.9588 \\
\hline lu-154 & 71154 & 153.9575 \\
\hline lu-155 & 71155 & 154.9543 \\
\hline
\end{tabular}




\begin{tabular}{|l|c|l|}
\hline \multicolumn{1}{|c|}{ Name } & Identifier & $\begin{array}{c}\text { Mass } \\
\text { (amu) }\end{array}$ \\
\hline lu-156 & 71156 & 155.953 \\
\hline lu-157 & 71157 & 156.9501 \\
\hline lu-158 & 71158 & 157.9493 \\
\hline lu-159 & 71159 & 158.9466 \\
\hline lu-160 & 71160 & 159.946 \\
\hline lu-161 & 71161 & 160.9436 \\
\hline lu-162 & 71162 & 161.9433 \\
\hline lu-163 & 71163 & 162.9412 \\
\hline lu-164 & 71164 & 163.9413 \\
\hline lu-165 & 71165 & 164.9394 \\
\hline lu-166 & 71166 & 165.9399 \\
\hline lu-167 & 71167 & 166.9383 \\
\hline lu-168 & 71168 & 167.9387 \\
\hline lu-169 & 71169 & 168.9377 \\
\hline lu-170 & 71170 & 169.9385 \\
\hline lu-171 & 71171 & 170.9379 \\
\hline lu-172 & 71172 & 171.9391 \\
\hline lu-173 & 71173 & 172.9389 \\
\hline lu-174 & 71174 & 173.9403 \\
\hline lu-175 & 71175 & 174.9408 \\
\hline lu-176 & 71176 & 175.9427 \\
\hline lu-177 & 71177 & 176.9438 \\
\hline lu-178 & 71178 & 177.946 \\
\hline lu-179 & 71179 & 178.9473 \\
\hline lu-180 & 71180 & 179.9499 \\
\hline lu-181 & 71181 & 180.952 \\
\hline lu-182 & 71182 & 181.955 \\
\hline lu-183 & 71183 & 182.9576 \\
\hline lu-184 & 71184 & 183.9609 \\
\hline hf-153 & 72153 & 152.9707 \\
\hline hf-154 & 72154 & 153.9649 \\
\hline hf-155 & 72155 & 154.9634 \\
\hline hf-156 & 72156 & 155.9594 \\
\hline hf-157 & 72157 & 156.9584 \\
\hline hf-158 & 72158 & 157.9548 \\
\hline hf-159 & 72159 & 158.954 \\
\hline hf-160 & 72160 & 159.9507 \\
\hline hf-161 & 72161 & 160.9503 \\
\hline hf-162 & 72162 & 161.9472 \\
\hline hf-163 & 72163 & 162.9471 \\
\hline hf-164 & 72164 & 163.9444 \\
\hline hf-165 & 72165 & 164.9446 \\
\hline hf-166 & 72166 & 165.9422 \\
\hline hf-167 & 72167 & 166.9426 \\
\hline hf-168 & 72168 & 167.9406 \\
\hline hf-169 & 72169 & 168.9413 \\
\hline hf-170 & 72170 & 169.9396 \\
\hline hf-171 & 72171 & 170.9405 \\
\hline hf-172 & 72172 & 171.9395 \\
\hline hf-173 & 72173 & 172.9405 \\
\hline hf-174 175 & 72174 & 173.94 \\
\hline & 72175 & 174.9415 \\
\hline
\end{tabular}




\begin{tabular}{|c|c|c|}
\hline Name & Identifier & $\begin{array}{l}\text { Mass } \\
\text { (amu) }\end{array}$ \\
\hline hf-176 & 72176 & 175.9414 \\
\hline hf-177 & 72177 & 176.9432 \\
\hline hf-178 & 72178 & 177.9437 \\
\hline hf-179 & 72179 & 178.9458 \\
\hline hf-180 & 72180 & 179.9465 \\
\hline hf-181 & 72181 & 180.9491 \\
\hline hf-182 & 72182 & 181.9505 \\
\hline hf-183 & 72183 & 182.9535 \\
\hline hf-184 & 72184 & 183.9554 \\
\hline hf-185 & 72185 & 184.9588 \\
\hline hf-186 & 72186 & 185.9609 \\
\hline hf-187 & 72187 & 186.9646 \\
\hline hf-188 & 72188 & 187.9668 \\
\hline ta-155 & 73155 & 154.9746 \\
\hline ta-156 & 73156 & 155.9723 \\
\hline ta-157 & 73157 & 156.9682 \\
\hline ta-158 & 73158 & 157.9667 \\
\hline ta-159 & 73159 & 158.963 \\
\hline ta-160 & 73160 & 159.9615 \\
\hline ta-161 & 73161 & 160.9584 \\
\hline ta-162 & 73162 & 161.9573 \\
\hline ta-163 & 73163 & 162.9543 \\
\hline ta-164 & 73164 & 163.9535 \\
\hline ta-165 & 73165 & 164.9508 \\
\hline ta-166 & 73166 & 165.9505 \\
\hline ta-167 & 73167 & 166.9481 \\
\hline ta-168 & 73168 & 167.948 \\
\hline ta-169 & 73169 & 168.946 \\
\hline ta-170 & 73170 & 169.9462 \\
\hline ta-171 & 73171 & 170.9445 \\
\hline ta-172 & 73172 & 171.9449 \\
\hline ta-173 & 73173 & 172.9438 \\
\hline ta-174 & 73174 & 173.9444 \\
\hline ta-175 & 73175 & 174.9437 \\
\hline ta-176 & 73176 & 175.9449 \\
\hline ta-177 & 73177 & 176.9445 \\
\hline ta-178 & 73178 & 177.9458 \\
\hline ta-179 & 73179 & 178.9459 \\
\hline ta-180 & 73180 & 179.9475 \\
\hline ta-181 & 73181 & 180.948 \\
\hline ta-182 & 73182 & 181.9501 \\
\hline ta-183 & 73183 & 182.9514 \\
\hline ta-184 & 73184 & 183.954 \\
\hline ta-185 & 73185 & 184.9556 \\
\hline ta-186 & 73186 & 185.9586 \\
\hline ta-187 & 73187 & 186.9605 \\
\hline ta- 188 & 73188 & 187.9637 \\
\hline ta-189 & 73189 & 188.9658 \\
\hline ta-190 & 73190 & 189.9692 \\
\hline $\mathrm{W}-158$ & 74158 & 157.9746 \\
\hline W-159 & 74159 & 158.9729 \\
\hline $\mathrm{w}-160$ & 74160 & 159.9685 \\
\hline
\end{tabular}




\begin{tabular}{|l|c|l|}
\hline Name & Identifier & $\begin{array}{c}\text { Mass } \\
\text { (amu) }\end{array}$ \\
\hline w-161 & 74161 & 160.9674 \\
\hline w-162 & 74162 & 161.9635 \\
\hline w-163 & 74163 & 162.9625 \\
\hline w-164 & 74164 & 163.959 \\
\hline w-165 & 74165 & 164.9583 \\
\hline w-166 & 74166 & 165.955 \\
\hline w-167 & 74167 & 166.9548 \\
\hline w-168 & 74168 & 167.9518 \\
\hline w-169 & 74169 & 168.9518 \\
\hline w-170 & 74170 & 169.9492 \\
\hline w-171 & 74171 & 170.9494 \\
\hline w-172 & 74172 & 171.9473 \\
\hline w-173 & 74173 & 172.9477 \\
\hline w-174 & 74174 & 173.9461 \\
\hline w-175 & 74175 & 174.9467 \\
\hline w-176 & 74176 & 175.9456 \\
\hline w-177 & 74177 & 176.9466 \\
\hline w-178 & 74178 & 177.9459 \\
\hline w-179 & 74179 & 178.9471 \\
\hline w-180 & 74180 & 179.9467 \\
\hline w-181 & 74181 & 180.9482 \\
\hline w-182 & 74182 & 181.9482 \\
\hline w-183 & 74183 & 182.9502 \\
\hline w-184 & 74184 & 183.9509 \\
\hline w-185 & 74185 & 184.9534 \\
\hline w-186 & 74186 & 185.9544 \\
\hline w-187 & 74187 & 186.9572 \\
\hline w-188 & 74188 & 187.9585 \\
\hline w-189 & 74189 & 188.9619 \\
\hline w-190 & 74190 & 189.9632 \\
\hline w-191 & 74191 & 190.9666 \\
\hline w-192 & 74192 & 191.9682 \\
\hline re-160 & 75160 & 159.9821 \\
\hline re-161 & 75161 & 160.9776 \\
\hline re-162 & 75162 & 161.976 \\
\hline re-163 & 75163 & 162.9721 \\
\hline re-164 & 75164 & 163.9703 \\
\hline re-165 & 75165 & 164.9671 \\
\hline re-166 & 75166 & 165.9658 \\
\hline re-167 & 75167 & 166.9626 \\
\hline re-168 & 75168 & 167.9616 \\
\hline re-169 & 75169 & 168.9588 \\
\hline re-170 & 75170 & 169.9582 \\
\hline re-171 & 75171 & 170.9557 \\
\hline re-172 & 75172 & 171.9554 \\
\hline re-173 & 75173 & 172.9532 \\
\hline re-174 & 75174 & 173.9531 \\
\hline re-175 & 75175 & 174.9514 \\
\hline re-176 & 75176 & 175.9516 \\
\hline re-177 & 75177 & 176.9503 \\
\hline re-178 & 75178 & 177.951 \\
\hline re-179 & 75179 & 178.95 \\
\hline & & \\
\hline
\end{tabular}




\begin{tabular}{|l|c|l|}
\hline \multicolumn{1}{|c|}{ Name } & Identifier & $\begin{array}{c}\text { Mass } \\
\text { (amu) }\end{array}$ \\
\hline re-180 & 75180 & 179.9508 \\
\hline re-181 & 75181 & 180.9501 \\
\hline re-182 & 75182 & 181.9512 \\
\hline re-183 & 75183 & 182.9508 \\
\hline re-184 & 75184 & 183.9525 \\
\hline re-185 & 75185 & 184.953 \\
\hline re-186 & 75186 & 185.955 \\
\hline re-187 & 75187 & 186.9557 \\
\hline re-188 & 75188 & 187.9581 \\
\hline re-189 & 75189 & 188.9592 \\
\hline re-190 & 75190 & 189.9618 \\
\hline re-191 & 75191 & 190.9631 \\
\hline re-192 & 75192 & 191.966 \\
\hline re-193 & 75193 & 192.9675 \\
\hline re-194 & 75194 & 193.9704 \\
\hline os-162 & 76162 & 161.9844 \\
\hline os-163 & 76163 & 162.9827 \\
\hline os-164 & 76164 & 163.978 \\
\hline os-165 & 76165 & 164.9768 \\
\hline os-166 & 76166 & 165.9727 \\
\hline os-167 & 76167 & 166.9716 \\
\hline os-168 & 76168 & 167.9678 \\
\hline os-169 & 76169 & 168.967 \\
\hline os-170 & 76170 & 169.9636 \\
\hline os-171 & 76171 & 170.9632 \\
\hline os-172 & 76172 & 171.96 \\
\hline os-173 & 76173 & 172.9598 \\
\hline os-174 & 76174 & 173.9571 \\
\hline os-175 & 76175 & 174.957 \\
\hline os-176 & 76176 & 175.9548 \\
\hline os-177 & 76177 & 176.955 \\
\hline os-178 & 76178 & 177.9532 \\
\hline os-179 & 76179 & 178.9538 \\
\hline os-180 & 76180 & 179.9524 \\
\hline os-181 & 76181 & 180.9532 \\
\hline os-182 & 76182 & 181.9521 \\
\hline os-183 & 76183 & 182.9531 \\
\hline os-184 & 76184 & 183.9525 \\
\hline os-185 & 76185 & 184.954 \\
\hline os-186 & 76186 & 185.9538 \\
\hline os-187 & 76187 & 186.9557 \\
\hline os-188 & 76188 & 187.9558 \\
\hline os-189 & 76189 & 188.9581 \\
\hline os-190 & 76190 & 189.9585 \\
\hline os-191 & 76191 & 190.9609 \\
\hline os-192 & 76192 & 191.9615 \\
\hline os-193 & 76193 & 192.9642 \\
\hline os-194 & 76194 & 193.9652 \\
\hline os-195 & 76195 & 194.9681 \\
\hline os-196 & 76196 & 195.9696 \\
\hline ir-164 & 77164 & 163.9922 \\
\hline ir-165 & 77165 & 164.9875 \\
\hline
\end{tabular}




\begin{tabular}{|c|c|c|}
\hline Name & Identifier & $\begin{array}{l}\text { Mass } \\
\text { (amu) }\end{array}$ \\
\hline ir-166 & 77166 & 165.9858 \\
\hline ir-167 & 77167 & 166.9817 \\
\hline ir-168 & 77168 & 167.9799 \\
\hline ir-169 & 77169 & 168.9763 \\
\hline ir-170 & 77170 & 169.975 \\
\hline ir-171 & 77171 & 170.9716 \\
\hline ir-172 & 77172 & 171.9705 \\
\hline ir-173 & 77173 & 172.9675 \\
\hline ir-174 & 77174 & 173.9669 \\
\hline ir-175 & 77175 & 174.9641 \\
\hline ir-176 & 77176 & 175.9637 \\
\hline ir-177 & 77177 & 176.9613 \\
\hline ir-178 & 77178 & 177.9611 \\
\hline ir-179 & 77179 & 178.9591 \\
\hline ir-180 & 77180 & 179.9592 \\
\hline ir-181 & 77181 & 180.9576 \\
\hline ir-182 & 77182 & 181.9581 \\
\hline ir-183 & 77183 & 182.9568 \\
\hline ir-184 & 77184 & 183.9575 \\
\hline ir-185 & 77185 & 184.9567 \\
\hline ir-186 & 77186 & 185.9579 \\
\hline ir-187 & 77187 & 186.9574 \\
\hline ir-188 & 77188 & 187.9588 \\
\hline ir-189 & 77189 & 188.9587 \\
\hline ir-190 & 77190 & 189.9606 \\
\hline ir-191 & 77191 & 190.9606 \\
\hline ir-192 & 77192 & 191.9626 \\
\hline ir-193 & 77193 & 192.9629 \\
\hline ir-194 & 77194 & 193.9651 \\
\hline ir-195 & 77195 & 194.966 \\
\hline ir-196 & 77196 & 195.9684 \\
\hline ir-197 & 77197 & 196.9697 \\
\hline ir-198 & 77198 & 197.9723 \\
\hline ir-199 & 77199 & 198.9738 \\
\hline pt-166 & 78166 & 165.9949 \\
\hline pt-167 & 78167 & 166.993 \\
\hline pt-168 & 78168 & 167.9881 \\
\hline pt-169 & 78169 & 168.9867 \\
\hline pt-170 & 78170 & 169.9825 \\
\hline pt-171 & 78171 & 170.9812 \\
\hline pt-172 & 78172 & 171.9774 \\
\hline pt-173 & 78173 & 172.9764 \\
\hline pt-174 & 78174 & 173.9728 \\
\hline pt-175 & 78175 & 174.9724 \\
\hline pt-176 & 78176 & 175.9689 \\
\hline pt-177 & 78177 & 176.9685 \\
\hline pt-178 & 78178 & 177.9657 \\
\hline pt-179 & 78179 & 178.9654 \\
\hline pt-180 & 78180 & 179.963 \\
\hline pt-181 & 78181 & 180.9631 \\
\hline pt-182 & 78182 & 181.9612 \\
\hline pt-183 & 78183 & 182.9616 \\
\hline
\end{tabular}




\begin{tabular}{|c|c|c|}
\hline Name & Identifier & $\begin{array}{c}\text { Mass } \\
\text { (amu) }\end{array}$ \\
\hline pt-184 & 78184 & 183.9599 \\
\hline pt-185 & 78185 & 184.9606 \\
\hline pt-186 & 78186 & 185.9594 \\
\hline pt-187 & 78187 & 186.9606 \\
\hline pt-188 & 78188 & 187.9594 \\
\hline pt-189 & 78189 & 188.9608 \\
\hline pt-190 & 78190 & 189.9599 \\
\hline pt-191 & 78191 & 190.9617 \\
\hline pt-192 & 78192 & 191.961 \\
\hline pt-193 & 78193 & 192.963 \\
\hline pt-194 & 78194 & 193.9627 \\
\hline pt-195 & 78195 & 194.9648 \\
\hline pt-196 & 78196 & 195.965 \\
\hline pt-197 & 78197 & 196.9673 \\
\hline pt-198 & 78198 & 197.9679 \\
\hline pt-199 & 78199 & 198.9706 \\
\hline pt-200 & 78200 & 199.9714 \\
\hline pt-201 & 78201 & 200.9745 \\
\hline pt-202 & 78202 & 201.9757 \\
\hline au-169 & 79169 & 168.9981 \\
\hline au-170 & 79170 & 169.9961 \\
\hline au-171 & 79171 & 170.9919 \\
\hline au-172 & 79172 & 171.99 \\
\hline au-173 & 79173 & 172.9862 \\
\hline au-174 & 79174 & 173.9848 \\
\hline au-175 & 79175 & 174.9813 \\
\hline au-176 & 79176 & 175.9801 \\
\hline au-177 & 79177 & 176.9769 \\
\hline au-178 & 79178 & 177.976 \\
\hline au-179 & 79179 & 178.9732 \\
\hline au-180 & 79180 & 179.9725 \\
\hline au-181 & 79181 & 180.9701 \\
\hline au-182 & 79182 & 181.9696 \\
\hline au-183 & 79183 & 182.9676 \\
\hline au-184 & 79184 & 183.9675 \\
\hline au-185 & 79185 & 184.9658 \\
\hline au-186 & 79186 & 185.9659 \\
\hline au-187 & 79187 & 186.9646 \\
\hline au-188 & 79188 & 187.9653 \\
\hline au-189 & 79189 & 188.9639 \\
\hline au-190 & 79190 & 189.9647 \\
\hline au-191 & 79191 & 190.9637 \\
\hline au-192 & 79192 & 191.9648 \\
\hline au-193 & 79193 & 192.9642 \\
\hline au-194 & 79194 & 193.9654 \\
\hline au-195 & 79195 & 194.965 \\
\hline au-196 & 79196 & 195.9666 \\
\hline au-197 & 79197 & 196.9666 \\
\hline au-198 & 79198 & 197.9682 \\
\hline au-199 & 79199 & 198.9688 \\
\hline au-200 & 79200 & 199.9707 \\
\hline au-201 & 79201 & 200.9717 \\
\hline
\end{tabular}




\begin{tabular}{|c|c|c|}
\hline Name & Identifier & $\begin{array}{l}\text { Mass } \\
\text { (amu) }\end{array}$ \\
\hline au-202 & 79202 & 201.9738 \\
\hline au-203 & 79203 & 202.9752 \\
\hline au-204 & 79204 & 203.9777 \\
\hline au-205 & 79205 & 204.9799 \\
\hline hg-171 & 80171 & 171.0038 \\
\hline hg-172 & 80172 & 171.9988 \\
\hline hg-173 & 80173 & 172.9972 \\
\hline hg-174 & 80174 & 173.9929 \\
\hline hg-175 & 80175 & 174.9914 \\
\hline hg-176 & 80176 & 175.9874 \\
\hline hg-177 & 80177 & 176.9863 \\
\hline hg-178 & 80178 & 177.9825 \\
\hline hg-179 & 80179 & 178.9818 \\
\hline hg-180 & 80180 & 179.9783 \\
\hline hg-181 & 80181 & 180.9778 \\
\hline hg-182 & 80182 & 181.9747 \\
\hline hg-183 & 80183 & 182.9745 \\
\hline hg-184 & 80184 & 183.9717 \\
\hline hg-185 & 80185 & 184.9719 \\
\hline hg-186 & 80186 & 185.9694 \\
\hline hg-187 & 80187 & 186.9698 \\
\hline hg-188 & 80188 & 187.9676 \\
\hline hg-189 & 80189 & 188.9682 \\
\hline hg-190 & 80190 & 189.9663 \\
\hline hg-191 & 80191 & 190.9672 \\
\hline hg-192 & 80192 & 191.9656 \\
\hline hg-193 & 80193 & 192.9667 \\
\hline hg-194 & 80194 & 193.9654 \\
\hline hg-195 & 80195 & 194.9667 \\
\hline hg-196 & 80196 & 195.9658 \\
\hline hg-197 & 80197 & 196.9672 \\
\hline hg-198 & 80198 & 197.9668 \\
\hline hg-199 & 80199 & 198.9683 \\
\hline hg-200 & 80200 & 199.9683 \\
\hline hg-201 & 80201 & 200.9703 \\
\hline hg-202 & 80202 & 201.9706 \\
\hline hg-203 & 80203 & 202.9729 \\
\hline hg-204 & 80204 & 203.9735 \\
\hline hg-205 & 80205 & 204.9761 \\
\hline hg-206 & 80206 & 205.9775 \\
\hline hg-207 & 80207 & 206.9826 \\
\hline hg-208 & 80208 & 207.9859 \\
\hline hg-209 & 80209 & 208.991 \\
\hline hg-210 & 80210 & 209.9945 \\
\hline tl-176 & 81176 & 176.0006 \\
\hline tl-177 & 81177 & 176.9964 \\
\hline tl-178 & 81178 & 177.9949 \\
\hline tl-179 & 81179 & 178.9911 \\
\hline tl-180 & 81180 & 179.9899 \\
\hline tl-181 & 81181 & 180.9863 \\
\hline tl-182 & 81182 & 181.9857 \\
\hline tl-183 & 81183 & 182.9822 \\
\hline
\end{tabular}




\begin{tabular}{|c|c|c|}
\hline Name & Identifier & $\begin{array}{l}\text { Mass } \\
\text { (amu) }\end{array}$ \\
\hline tl-184 & 81184 & 183.9819 \\
\hline tl-185 & 81185 & 184.9788 \\
\hline tl-186 & 81186 & 185.9783 \\
\hline tl-187 & 81187 & 186.9759 \\
\hline tl-188 & 81188 & 187.976 \\
\hline tl-189 & 81189 & 188.9736 \\
\hline tl-190 & 81190 & 189.9739 \\
\hline tl-191 & 81191 & 190.9718 \\
\hline tl-192 & 81192 & 191.9722 \\
\hline tl-193 & 81193 & 192.9707 \\
\hline tl-194 & 81194 & 193.9712 \\
\hline tl-195 & 81195 & 194.9698 \\
\hline tl-196 & 81196 & 195.9705 \\
\hline tl-197 & 81197 & 196.9696 \\
\hline tl-198 & 81198 & 197.9705 \\
\hline tl-199 & 81199 & 198.9699 \\
\hline tl-200 & 81200 & 199.971 \\
\hline tl-201 & 81201 & 200.9708 \\
\hline tl-202 & 81202 & 201.9721 \\
\hline tl-203 & 81203 & 202.9723 \\
\hline tl-204 & 81204 & 203.9739 \\
\hline tl-205 & 81205 & 204.9744 \\
\hline tl-206 & 81206 & 205.9761 \\
\hline tl-207 & 81207 & 206.9774 \\
\hline tl-208 & 81208 & 207.982 \\
\hline tl-209 & 81209 & 208.9854 \\
\hline tl-210 & 81210 & 209.9901 \\
\hline tl-211 & 81211 & 210.9935 \\
\hline tl-212 & 81212 & 211.9982 \\
\hline pb-178 & 82178 & 178.0038 \\
\hline pb-179 & 82179 & 179.0022 \\
\hline $\mathrm{pb}-180$ & 82180 & 179.9979 \\
\hline pb-181 & 82181 & 180.9966 \\
\hline pb-182 & 82182 & 181.9927 \\
\hline pb-183 & 82183 & 182.9919 \\
\hline pb-184 & 82184 & 183.9881 \\
\hline $\mathrm{pb}-185$ & 82185 & 184.9876 \\
\hline pb-186 & 82186 & 185.9842 \\
\hline pb-187 & 82187 & 186.9839 \\
\hline pb-188 & 82188 & 187.9809 \\
\hline pb-189 & 82189 & 188.9808 \\
\hline pb-190 & 82190 & 189.9781 \\
\hline pb-191 & 82191 & 190.9783 \\
\hline pb-192 & 82192 & 191.9758 \\
\hline pb-193 & 82193 & 192.9762 \\
\hline pb-194 & 82194 & 193.974 \\
\hline pb-195 & 82195 & 194.9745 \\
\hline pb-196 & 82196 & 195.9728 \\
\hline pb-197 & 82197 & 196.9734 \\
\hline pb-198 & 82198 & 197.972 \\
\hline pb-199 & 82199 & 198.9729 \\
\hline $\mathrm{pb}-200$ & 82200 & 199.9718 \\
\hline
\end{tabular}




\begin{tabular}{|c|c|c|}
\hline Name & Identifier & $\begin{array}{l}\text { Mass } \\
\text { (amu) }\end{array}$ \\
\hline pb-201 & 82201 & 200.9729 \\
\hline pb-202 & 82202 & 201.9722 \\
\hline pb-203 & 82203 & 202.9734 \\
\hline pb-204 & 82204 & 203.973 \\
\hline pb-205 & 82205 & 204.9745 \\
\hline pb-206 & 82206 & 205.9745 \\
\hline pb-207 & 82207 & 206.9759 \\
\hline pb-208 & 82208 & 207.9767 \\
\hline pb-209 & 82209 & 208.9811 \\
\hline pb-210 & 82210 & 209.9842 \\
\hline pb-211 & 82211 & 210.9887 \\
\hline pb-212 & 82212 & 211.9919 \\
\hline pb-213 & 82213 & 212.9966 \\
\hline pb-214 & 82214 & 213.9998 \\
\hline pb-215 & 82215 & 215.0048 \\
\hline bi-184 & 83184 & 184.0011 \\
\hline bi-185 & 83185 & 184.9976 \\
\hline bi-186 & 83186 & 185.9966 \\
\hline bi-187 & 83187 & 186.9932 \\
\hline bi-188 & 83188 & 187.9923 \\
\hline bi-189 & 83189 & 188.9892 \\
\hline bi-190 & 83190 & 189.9883 \\
\hline bi-191 & 83191 & 190.9858 \\
\hline bi-192 & 83192 & 191.9855 \\
\hline bi-193 & 83193 & 192.983 \\
\hline bi-194 & 83194 & 193.9828 \\
\hline bi-195 & 83195 & 194.9807 \\
\hline bi-196 & 83196 & 195.9807 \\
\hline bi-197 & 83197 & 196.9789 \\
\hline bi-198 & 83198 & 197.9792 \\
\hline bi-199 & 83199 & 198.9777 \\
\hline bi-200 & 83200 & 199.9781 \\
\hline bi-201 & 83201 & 200.977 \\
\hline bi-202 & 83202 & 201.9777 \\
\hline bi-203 & 83203 & 202.9769 \\
\hline bi-204 & 83204 & 203.9778 \\
\hline bi-205 & 83205 & 204.9774 \\
\hline bi-206 & 83206 & 205.9785 \\
\hline bi-207 & 83207 & 206.9785 \\
\hline bi-208 & 83208 & 207.9797 \\
\hline bi-209 & 83209 & 208.9804 \\
\hline bi-210 & 83210 & 209.9841 \\
\hline bi-211 & 83211 & 210.9873 \\
\hline bi-212 & 83212 & 211.9913 \\
\hline bi-213 & 83213 & 212.9944 \\
\hline bi-214 & 83214 & 213.9987 \\
\hline bi-215 & 83215 & 215.0018 \\
\hline bi-216 & 83216 & 216.0063 \\
\hline bi-217 & 83217 & 217.0095 \\
\hline bi-218 & 83218 & 218.0143 \\
\hline po-188 & 84188 & 187.9994 \\
\hline po-189 & 84189 & 188.9985 \\
\hline
\end{tabular}




\begin{tabular}{|c|c|c|}
\hline Name & Identifier & $\begin{array}{l}\text { Mass } \\
\text { (amu) }\end{array}$ \\
\hline po-190 & 84190 & 189.9951 \\
\hline po-191 & 84191 & 190.9946 \\
\hline po-192 & 84192 & 191.9913 \\
\hline po-193 & 84193 & 192.991 \\
\hline po-194 & 84194 & 193.9882 \\
\hline po-195 & 84195 & 194.9881 \\
\hline po-196 & 84196 & 195.9855 \\
\hline po-197 & 84197 & 196.9857 \\
\hline po-198 & 84198 & 197.9834 \\
\hline po-199 & 84199 & 198.9837 \\
\hline po-200 & 84200 & 199.9818 \\
\hline po-201 & 84201 & 200.9823 \\
\hline po-202 & 84202 & 201.9808 \\
\hline po-203 & 84203 & 202.9814 \\
\hline po-204 & 84204 & 203.9803 \\
\hline po-205 & 84205 & 204.9812 \\
\hline po-206 & 84206 & 205.9805 \\
\hline po-207 & 84207 & 206.9816 \\
\hline po-208 & 84208 & 207.9812 \\
\hline po-209 & 84209 & 208.9824 \\
\hline po- 210 & 84210 & 209.9829 \\
\hline po-211 & 84211 & 210.9866 \\
\hline po-212 & 84212 & 211.9889 \\
\hline po-213 & 84213 & 212.9929 \\
\hline po-214 & 84214 & 213.9952 \\
\hline po-215 & 84215 & 214.9994 \\
\hline po-216 & 84216 & 216.0019 \\
\hline po-217 & 84217 & 217.0063 \\
\hline po-218 & 84218 & 218.009 \\
\hline po-219 & 84219 & 219.0137 \\
\hline po-220 & 84220 & 220.0166 \\
\hline at-193 & 85193 & 192.9998 \\
\hline at-194 & 85194 & 193.9987 \\
\hline at-195 & 85195 & 194.9963 \\
\hline at-196 & 85196 & 195.9958 \\
\hline at-197 & 85197 & 196.9932 \\
\hline at-198 & 85198 & 197.9928 \\
\hline at-199 & 85199 & 198.9905 \\
\hline at -200 & 85200 & 199.9904 \\
\hline at-201 & 85201 & 200.9884 \\
\hline at-202 & 85202 & 201.9886 \\
\hline at-203 & 85203 & 202.9869 \\
\hline at-204 & 85204 & 203.9872 \\
\hline at-205 & 85205 & 204.9861 \\
\hline at-206 & 85206 & 205.9867 \\
\hline at-207 & 85207 & 206.9858 \\
\hline at -208 & 85208 & 207.9866 \\
\hline at-209 & 85209 & 208.9862 \\
\hline at -210 & 85210 & 209.9872 \\
\hline at-211 & 85211 & 210.9875 \\
\hline at-212 & 85212 & 211.9908 \\
\hline at-213 & 85213 & 212.9929 \\
\hline
\end{tabular}




\begin{tabular}{|l|c|c|}
\hline \multicolumn{1}{|c|}{ Name } & Identifier & $\begin{array}{c}\text { Mass } \\
\text { (amu) }\end{array}$ \\
\hline at-214 & 85214 & 213.9964 \\
\hline at-215 & 85215 & 214.9987 \\
\hline at-216 & 85216 & 216.0024 \\
\hline at-217 & 85217 & 217.0047 \\
\hline at-218 & 85218 & 218.0087 \\
\hline at-219 & 85219 & 219.0112 \\
\hline at-220 & 85220 & 220.0154 \\
\hline at-221 & 85221 & 221.0181 \\
\hline at-222 & 85222 & 222.0223 \\
\hline at-223 & 85223 & 223.0252 \\
\hline rn-195 & 86195 & 195.0054 \\
\hline rn-196 & 86196 & 196.0021 \\
\hline rn-197 & 86197 & 197.0016 \\
\hline rn-198 & 86198 & 197.9987 \\
\hline rn-199 & 86199 & 198.9984 \\
\hline rn-200 & 86200 & 199.9957 \\
\hline rn-201 & 86201 & 200.9956 \\
\hline rn-202 & 86202 & 201.9933 \\
\hline rn-203 & 86203 & 202.9934 \\
\hline rn-204 & 86204 & 203.9914 \\
\hline rn-205 & 86205 & 204.9917 \\
\hline rn-206 & 86206 & 205.9902 \\
\hline rn-207 & 86207 & 206.9907 \\
\hline rn-208 & 86208 & 207.9896 \\
\hline rn-209 & 86209 & 208.9904 \\
\hline rn-210 & 86210 & 209.9897 \\
\hline rn-211 & 86211 & 210.9906 \\
\hline rn-212 & 86212 & 211.9907 \\
\hline rn-213 & 86213 & 212.9939 \\
\hline rn-214 & 86214 & 213.9954 \\
\hline rn-215 & 86215 & 214.9987 \\
\hline rn-216 & 86216 & 216.0003 \\
\hline rn-217 & 86217 & 217.0039 \\
\hline rn-218 & 86218 & 218.0056 \\
\hline rn-219 & 86219 & 219.0095 \\
\hline rn-220 & 86220 & 220.0114 \\
\hline rn-221 & 86221 & 221.0155 \\
\hline rn-222 & 86222 & 222.0176 \\
\hline rn-223 & 86223 & 223.0218 \\
\hline rn-224 & 86224 & 224.0241 \\
\hline rn-225 & 86225 & 225.0284 \\
\hline rn-226 & 86226 & 226.0309 \\
\hline rn-227 & 86227 & 227.0354 \\
\hline rn-228 & 86228 & 228.038 \\
\hline fr-199 & 87199 & 199.0073 \\
\hline fr-200 & 87200 & 200.0066 \\
\hline fr-205 & 87201 & 201.0039 \\
\hline
\end{tabular}




\begin{tabular}{|c|c|c|}
\hline Name & Identifier & $\begin{array}{c}\text { Mass } \\
\text { (amu) }\end{array}$ \\
\hline fr-207 & 87207 & 206.9969 \\
\hline fr-208 & 87208 & 207.9971 \\
\hline fr-209 & 87209 & 208.996 \\
\hline fr-210 & 87210 & 209.9964 \\
\hline fr-211 & 87211 & 210.9955 \\
\hline fr-212 & 87212 & 211.9962 \\
\hline fr-213 & 87213 & 212.9962 \\
\hline fr-214 & 87214 & 213.999 \\
\hline fr-215 & 87215 & 215.0003 \\
\hline fr-216 & 87216 & 216.0032 \\
\hline fr-217 & 87217 & 217.0046 \\
\hline fr-218 & 87218 & 218.0076 \\
\hline fr-219 & 87219 & 219.0092 \\
\hline fr-220 & 87220 & 220.0123 \\
\hline fr-221 & 87221 & 221.0143 \\
\hline fr-222 & 87222 & 222.0175 \\
\hline fr- 223 & 87223 & 223.0197 \\
\hline fr-224 & 87224 & 224.0233 \\
\hline fr-225 & 87225 & 225.0256 \\
\hline fr-226 & 87226 & 226.0294 \\
\hline fr-227 & 87227 & 227.0318 \\
\hline fr-228 & 87228 & 228.0357 \\
\hline fr-229 & 87229 & 229.0385 \\
\hline fr-230 & 87230 & 230.0425 \\
\hline fr-231 & 87231 & 231.0454 \\
\hline fr-232 & 87232 & 232.0498 \\
\hline ra-202 & 88202 & 202.0099 \\
\hline ra-203 & 88203 & 203.0093 \\
\hline ra-204 & 88204 & 204.0065 \\
\hline ra-205 & 88205 & 205.0063 \\
\hline ra-206 & 88206 & 206.0038 \\
\hline ra-207 & 88207 & 207.0038 \\
\hline ra-208 & 88208 & 208.0018 \\
\hline ra-209 & 88209 & 209.002 \\
\hline ra-210 & 88210 & 210.0005 \\
\hline ra-211 & 88211 & 211.0009 \\
\hline ra-212 & 88212 & 211.9998 \\
\hline ra-213 & 88213 & 213.0004 \\
\hline ra-214 & 88214 & 214.0001 \\
\hline ra-215 & 88215 & 215.0027 \\
\hline ra-216 & 88216 & 216.0035 \\
\hline ra-217 & 88217 & 217.0063 \\
\hline ra-218 & 88218 & 218.0071 \\
\hline ra-219 & 88219 & 219.0101 \\
\hline ra-220 & 88220 & 220.011 \\
\hline ra-221 & 88221 & 221.0139 \\
\hline ra-222 & 88222 & 222.0154 \\
\hline ra-223 & 88223 & 223.0185 \\
\hline ra-224 & 88224 & 224.0202 \\
\hline ra-225 & 88225 & 225.0236 \\
\hline ra-226 & 88226 & 226.0254 \\
\hline ra-227 & 88227 & 227.0292 \\
\hline
\end{tabular}




\begin{tabular}{|c|c|c|}
\hline Name & Identifier & $\begin{array}{l}\text { Mass } \\
\text { (amu) }\end{array}$ \\
\hline ra-228 & 88228 & 228.0311 \\
\hline ra-229 & 88229 & 229.035 \\
\hline ra-230 & 88230 & 230.0371 \\
\hline ra-231 & 88231 & 231.0412 \\
\hline ra-232 & 88232 & 232.0436 \\
\hline ra-233 & 88233 & 233.0481 \\
\hline ra-234 & 88234 & 234.0507 \\
\hline ac-206 & 89206 & 206.0145 \\
\hline ac-207 & 89207 & 207.0119 \\
\hline ac-208 & 89208 & 208.0116 \\
\hline ac-209 & 89209 & 209.0095 \\
\hline ac- 210 & 89210 & 210.0094 \\
\hline ac-211 & 89211 & 211.0077 \\
\hline ac-212 & 89212 & 212.0078 \\
\hline ac-213 & 89213 & 213.0066 \\
\hline ac-214 & 89214 & 214.0069 \\
\hline ac-215 & 89215 & 215.0065 \\
\hline ac-216 & 89216 & 216.0087 \\
\hline ac-217 & 89217 & 217.0094 \\
\hline ac-218 & 89218 & 218.0116 \\
\hline ac-219 & 89219 & 219.0124 \\
\hline ac-220 & 89220 & 220.0148 \\
\hline ac-221 & 89221 & 221.0156 \\
\hline ac-222 & 89222 & 222.0178 \\
\hline ac- 223 & 89223 & 223.0191 \\
\hline ac-224 & 89224 & 224.0217 \\
\hline ac- 225 & 89225 & 225.0232 \\
\hline ac-226 & 89226 & 226.0261 \\
\hline ac-227 & 89227 & 227.0278 \\
\hline ac- 228 & 89228 & 228.031 \\
\hline ac-229 & 89229 & 229.033 \\
\hline ac- 230 & 89230 & 230.0363 \\
\hline ac-231 & 89231 & 231.0386 \\
\hline ac-232 & 89232 & 232.042 \\
\hline ac-233 & 89233 & 233.0446 \\
\hline ac-234 & 89234 & 234.0484 \\
\hline ac- 235 & 89235 & 235.0512 \\
\hline ac-236 & 89236 & 236.0553 \\
\hline th-209 & 90209 & 209.0177 \\
\hline th- 210 & 90210 & 210.0151 \\
\hline th-211 & 90211 & 211.0149 \\
\hline th-212 & 90212 & 212.013 \\
\hline th-213 & 90213 & 213.013 \\
\hline th-214 & 90214 & 214.0115 \\
\hline th- 215 & 90215 & 215.0117 \\
\hline th-216 & 90216 & 216.0111 \\
\hline th-217 & 90217 & 217.0131 \\
\hline th-218 & 90218 & 218.0133 \\
\hline th-219 & 90219 & 219.0155 \\
\hline th- 220 & 90220 & 220.0157 \\
\hline th- 221 & 90221 & 221.0182 \\
\hline th- 222 & 90222 & 222.0185 \\
\hline
\end{tabular}




\begin{tabular}{|l|c|l|}
\hline \multicolumn{1}{|c|}{ Name } & Identifier & $\begin{array}{c}\text { Mass } \\
\text { (amu) }\end{array}$ \\
\hline th-223 & 90223 & 223.0208 \\
\hline th-224 & 90224 & 224.0215 \\
\hline th-225 & 90225 & 225.024 \\
\hline th-226 & 90226 & 226.0249 \\
\hline th-227 & 90227 & 227.0277 \\
\hline th-228 & 90228 & 228.0287 \\
\hline th-229 & 90229 & 229.0318 \\
\hline th-230 & 90230 & 230.0331 \\
\hline th-231 & 90231 & 231.0363 \\
\hline th-232 & 90232 & 232.0381 \\
\hline th-233 & 90233 & 233.0416 \\
\hline th-234 & 90234 & 234.0436 \\
\hline th-235 & 90235 & 235.0475 \\
\hline th-236 & 90236 & 236.0499 \\
\hline th-237 & 90237 & 237.0539 \\
\hline th-238 & 90238 & 238.0565 \\
\hline pa-212 & 91212 & 212.0232 \\
\hline pa-213 & 91213 & 213.0211 \\
\hline pa-214 & 91214 & 214.0209 \\
\hline pa-215 & 91215 & 215.0192 \\
\hline pa-216 & 91216 & 216.0191 \\
\hline pa-217 & 91217 & 217.0183 \\
\hline pa-218 & 91218 & 218.02 \\
\hline pa-219 & 91219 & 219.0199 \\
\hline pa-220 & 91220 & 220.0219 \\
\hline pa-221 & 91221 & 221.0219 \\
\hline pa-222 & 91222 & 222.0237 \\
\hline pa-223 & 91223 & 223.024 \\
\hline pa-224 & 91224 & 224.0256 \\
\hline pa-225 & 91225 & 225.0261 \\
\hline pa-226 & 91226 & 226.028 \\
\hline pa-227 & 91227 & 227.0288 \\
\hline pa-228 & 91228 & 228.0311 \\
\hline pa-229 & 91229 & 229.0321 \\
\hline pa-230 & 91230 & 230.0345 \\
\hline pa-231 & 91231 & 231.0359 \\
\hline pa-232 & 91232 & 232.0386 \\
\hline pa-233 & 91233 & 233.0403 \\
\hline pa-234 & 91234 & 234.0433 \\
\hline pa-235 & 91235 & 235.0454 \\
\hline pa-236 & 91236 & 236.0487 \\
\hline pa-237 & 91237 & 237.0511 \\
\hline pa-238 & 91238 & 238.0545 \\
\hline pa-239 & 91239 & 239.0573 \\
\hline pa-240 & 91240 & 240.061 \\
\hline u-217 & 92217 & 217.0244 \\
\hline u-218 & 92218 & 218.0235 \\
\hline u-219 & 92219 & 219.0249 \\
\hline u-220 & 92220 & 220.0247 \\
\hline u-221 & 92221 & 221.0264 \\
\hline u-222 & 92222 & 222.0261 \\
\hline$u-223$ & 92223 & 223.0277 \\
\hline
\end{tabular}




\begin{tabular}{|c|c|c|}
\hline Name & Identifier & $\begin{array}{l}\text { Mass } \\
\text { (amu) }\end{array}$ \\
\hline $\mathrm{u}-224$ & 92224 & 224.0276 \\
\hline $\mathrm{u}-225$ & 92225 & 225.0294 \\
\hline $\mathrm{u}-226$ & 92226 & 226.0293 \\
\hline $\mathrm{u}-227$ & 92227 & 227.0312 \\
\hline $\mathrm{u}-228$ & 92228 & 228.0314 \\
\hline $\mathrm{u}-229$ & 92229 & 229.0335 \\
\hline $\mathrm{u}-230$ & 92230 & 230.0339 \\
\hline $\mathrm{u}-231$ & 92231 & 231.0363 \\
\hline $\mathrm{u}-232$ & 92232 & 232.0372 \\
\hline $\mathrm{u}-233$ & 92233 & 233.0396 \\
\hline $\mathrm{u}-234$ & 92234 & 234.041 \\
\hline $\mathrm{u}-235$ & 92235 & 235.0439 \\
\hline $\mathrm{u}-236$ & 92236 & 236.0456 \\
\hline $\mathrm{u}-237$ & 92237 & 237.0487 \\
\hline $\mathrm{u}-238$ & 92238 & 238.0508 \\
\hline $\mathrm{u}-239$ & 92239 & 239.0543 \\
\hline $\mathrm{u}-240$ & 92240 & 240.0566 \\
\hline $\mathrm{u}-241$ & 92241 & 241.0603 \\
\hline $\mathrm{u}-242$ & 92242 & 242.0629 \\
\hline np-225 & 93225 & 225.0339 \\
\hline np-226 & 93226 & 226.0352 \\
\hline np-227 & 93227 & 227.035 \\
\hline np-228 & 93228 & 228.0362 \\
\hline np-229 & 93229 & 229.0363 \\
\hline np-230 & 93230 & 230.0378 \\
\hline np-231 & 93231 & 231.0383 \\
\hline np-232 & 93232 & 232.0401 \\
\hline np-233 & 93233 & 233.0407 \\
\hline np-234 & 93234 & 234.0429 \\
\hline np-235 & 93235 & 235.0441 \\
\hline np-236 & 93236 & 236.0466 \\
\hline np-237 & 93237 & 237.0482 \\
\hline np-238 & 93238 & 238.0509 \\
\hline np-239 & 93239 & 239.0529 \\
\hline np-240 & 93240 & 240.0562 \\
\hline np-241 & 93241 & 241.0582 \\
\hline np-242 & 93242 & 242.0616 \\
\hline np-243 & 93243 & 243.0643 \\
\hline np-244 & 93244 & 244.0679 \\
\hline pu-228 & 94228 & 228.0387 \\
\hline pu-229 & 94229 & 229.0401 \\
\hline pu-230 & 94230 & 230.0397 \\
\hline pu-231 & 94231 & 231.0411 \\
\hline pu-232 & 94232 & 232.0412 \\
\hline pu-233 & 94233 & 233.043 \\
\hline pu-234 & 94234 & 234.0433 \\
\hline pu-235 & 94235 & 235.0453 \\
\hline pu-236 & 94236 & 236.0461 \\
\hline pu-237 & 94237 & 237.0484 \\
\hline pu-238 & 94238 & 238.0496 \\
\hline pu-239 & 94239 & 239.0522 \\
\hline pu-240 & 94240 & 240.0538 \\
\hline
\end{tabular}




\begin{tabular}{|c|c|c|}
\hline Name & Identifier & $\begin{array}{c}\text { Mass } \\
\text { (amu) }\end{array}$ \\
\hline pu-241 & 94241 & 241.0569 \\
\hline pu-242 & 94242 & 242.0587 \\
\hline pu-243 & 94243 & 243.062 \\
\hline pu-244 & 94244 & 244.0642 \\
\hline pu-245 & 94245 & 245.0677 \\
\hline pu-246 & 94246 & 246.0702 \\
\hline pu-247 & 94247 & 247.0741 \\
\hline am-231 & 95231 & 231.0456 \\
\hline am-232 & 95232 & 232.0466 \\
\hline am-233 & 95233 & 233.0464 \\
\hline am-234 & 95234 & 234.0478 \\
\hline am-235 & 95235 & 235.0479 \\
\hline am-236 & 95236 & 236.0496 \\
\hline am-237 & 95237 & 237.05 \\
\hline am-238 & 95238 & 238.052 \\
\hline am-239 & 95239 & 239.053 \\
\hline am-240 & 95240 & 240.0553 \\
\hline am-241 & 95241 & 241.0568 \\
\hline am-242 & 95242 & 242.0596 \\
\hline $\mathrm{am}-242 \mathrm{~m}$ & 1095242 & \\
\hline am-243 & 95243 & 243.0614 \\
\hline am-244 & 95244 & 244.0643 \\
\hline $\mathrm{am}-244 \mathrm{~m}$ & 1095244 & \\
\hline am-245 & 95245 & 245.0665 \\
\hline am-246 & 95246 & 246.0698 \\
\hline am-247 & 95247 & 247.0721 \\
\hline am-248 & 95248 & 248.0757 \\
\hline am-249 & 95249 & 249.0785 \\
\hline $\mathrm{cm}-233$ & 96233 & 233.0508 \\
\hline $\mathrm{cm}-234$ & 96234 & 234.0502 \\
\hline $\mathrm{cm}-235$ & 96235 & 235.0514 \\
\hline $\mathrm{cm}-236$ & 96236 & 236.0514 \\
\hline $\mathrm{cm}-237$ & 96237 & 237.0529 \\
\hline $\mathrm{cm}-238$ & 96238 & 238.053 \\
\hline $\mathrm{cm}-239$ & 96239 & 239.055 \\
\hline $\mathrm{cm}-240$ & 96240 & 240.0555 \\
\hline $\mathrm{cm}-241$ & 96241 & 241.0576 \\
\hline $\mathrm{cm}-242$ & 96242 & 242.0588 \\
\hline $\mathrm{cm}-243$ & 96243 & 243.0614 \\
\hline $\mathrm{cm}-244$ & 96244 & 244.0627 \\
\hline $\mathrm{cm}-245$ & 96245 & 245.0655 \\
\hline $\mathrm{cm}-246$ & 96246 & 246.0672 \\
\hline $\mathrm{cm}-247$ & 96247 & 247.0703 \\
\hline $\mathrm{cm}-248$ & 96248 & 248.0724 \\
\hline $\mathrm{cm}-249$ & 96249 & 249.0759 \\
\hline $\mathrm{cm}-250$ & 96250 & 250.0784 \\
\hline $\mathrm{cm}-251$ & 96251 & 251.0823 \\
\hline $\mathrm{cm}-252$ & 96252 & 252.0849 \\
\hline bk-235 & 97235 & 235.0566 \\
\hline bk-236 & 97236 & 236.0573 \\
\hline bk-237 & 97237 & 237.057 \\
\hline bk-238 & 97238 & 238.0583 \\
\hline
\end{tabular}




\begin{tabular}{|l|l|l|}
\hline \multicolumn{1}{|c|}{ Name } & Identifier & $\begin{array}{c}\text { Mass } \\
\text { (amu) }\end{array}$ \\
\hline bk-239 & 97239 & 239.0583 \\
\hline bk-240 & 97240 & 240.0598 \\
\hline bk-241 & 97241 & 241.0602 \\
\hline bk-242 & 97242 & 242.062 \\
\hline bk-243 & 97243 & 243.063 \\
\hline bk-244 & 97244 & 244.0652 \\
\hline bk-245 & 97245 & 245.0664 \\
\hline bk-246 & 97246 & 246.0687 \\
\hline bk-247 & 97247 & 247.0703 \\
\hline bk-248 & 97248 & 248.0731 \\
\hline bk-249 & 97249 & 249.075 \\
\hline bk-250 & 97250 & 250.0783 \\
\hline bk-251 & 97251 & 251.0808 \\
\hline bk-252 & 97252 & 252.0843 \\
\hline bk-253 & 97253 & 253.0869 \\
\hline bk-254 & 97254 & 254.0906 \\
\hline cf-237 & 98237 & 237.0621 \\
\hline cf-238 & 98238 & 238.0614 \\
\hline cf-239 & 98239 & 239.0624 \\
\hline cf-240 & 98240 & 240.0623 \\
\hline cf-241 & 98241 & 241.0637 \\
\hline cf-242 & 98242 & 242.0637 \\
\hline cf-243 & 98243 & 243.0654 \\
\hline cf-244 & 98244 & 244.066 \\
\hline cf-245 & 98245 & 245.0681 \\
\hline cf-246 & 98246 & 246.0688 \\
\hline cf-247 & 98247 & 247.071 \\
\hline cf-248 & 98248 & 248.0722 \\
\hline cf-249 & 98249 & 249.0748 \\
\hline cf-250 & 98250 & 250.0764 \\
\hline cf-251 & 98251 & 251.0796 \\
\hline cf-252 & 98252 & 252.0816 \\
\hline cf-253 & 98253 & 253.0851 \\
\hline cf-254 & 98254 & 254.0873 \\
\hline cf-255 & 98255 & 255.091 \\
\hline cf-256 & 98256 & 256.0934 \\
\hline es-240 & 99240 & 240.0689 \\
\hline es-241 & 99241 & 241.0685 \\
\hline es-242 & 99242 & 242.0697 \\
\hline es-243 & 99243 & 243.0695 \\
\hline es-244 & 99244 & 244.0709 \\
\hline es-245 & 99245 & 245.0713 \\
\hline es-246 & 99246 & 246.0729 \\
\hline es-247 & 99247 & 247.0737 \\
\hline es-248 & 99248 & 248.0755 \\
\hline es-249 & 99249 & 249.0764 \\
\hline es-250 & 99250 & 250.0786 \\
\hline es-251 & 99251 & 251.08 \\
\hline es-253 & 99252 & 252.083 \\
\hline es-254 255 & 99253 & 253.0848 \\
\hline
\end{tabular}




\begin{tabular}{|c|c|c|}
\hline Name & Identifier & $\begin{array}{c}\text { Mass } \\
\text { (amu) }\end{array}$ \\
\hline es-256 & 99256 & 256.0936 \\
\hline es-257 & 99257 & 257.096 \\
\hline es-258 & 99258 & 258.0995 \\
\hline $\mathrm{fm}-242$ & 100242 & 242.0734 \\
\hline $\mathrm{fm}-243$ & 100243 & 243.0744 \\
\hline $\mathrm{fm}-244$ & 100244 & 244.0741 \\
\hline $\mathrm{fm}-245$ & 100245 & 245.0754 \\
\hline $\mathrm{fm}-246$ & 100246 & 246.0753 \\
\hline $\mathrm{fm}-247$ & 100247 & 247.0768 \\
\hline $\mathrm{fm}-248$ & 100248 & 248.0772 \\
\hline $\mathrm{fm}-249$ & 100249 & 249.079 \\
\hline fm-250 & 100250 & 250.0795 \\
\hline $\mathrm{fm}-251$ & 100251 & 251.0816 \\
\hline $\mathrm{fm}-252$ & 100252 & 252.0825 \\
\hline $\mathrm{fm}-253$ & 100253 & 253.0852 \\
\hline $\mathrm{fm}-254$ & 100254 & 254.0869 \\
\hline $\mathrm{fm}-255$ & 100255 & 255.09 \\
\hline $\mathrm{fm}-256$ & 100256 & 256.0918 \\
\hline fm-257 & 100257 & 257.0951 \\
\hline $\mathrm{fm}-258$ & 100258 & 258.0971 \\
\hline $\mathrm{fm}-259$ & 100259 & 259.1006 \\
\hline $\mathrm{fm}-260$ & 100260 & 260.1027 \\
\hline md-245 & 101245 & 245.0808 \\
\hline md-246 & 101246 & 246.0819 \\
\hline md-247 & 101247 & 247.0816 \\
\hline md-248 & 101248 & 248.0828 \\
\hline md-249 & 101249 & 249.083 \\
\hline md-250 & 101250 & 250.0844 \\
\hline md-251 & 101251 & 251.0848 \\
\hline md-252 & 101252 & 252.0866 \\
\hline md-253 & 101253 & 253.0873 \\
\hline md-254 & 101254 & 254.0897 \\
\hline md-255 & 101255 & 255.0911 \\
\hline md-256 & 101256 & 256.0941 \\
\hline md-257 & 101257 & 257.0956 \\
\hline md-258 & 101258 & 258.0984 \\
\hline md-259 & 101259 & 259.1005 \\
\hline md-260 & 101260 & 260.1036 \\
\hline md-261 & 101261 & 261.1057 \\
\hline md-262 & 101262 & 262.1089 \\
\hline no-248 & 102248 & 248.0866 \\
\hline no-249 & 102249 & 249.0878 \\
\hline no- 250 & 102250 & 250.0875 \\
\hline no-251 & 102251 & 251.089 \\
\hline no-252 & 102252 & 252.089 \\
\hline no-253 & 102253 & 253.0907 \\
\hline no-254 & 102254 & 254.091 \\
\hline no-255 & 102255 & 255.0932 \\
\hline no- 256 & 102256 & 256.0943 \\
\hline no-257 & 102257 & 257.0969 \\
\hline no- 258 & 102258 & 258.0982 \\
\hline no-259 & 102259 & 259.101 \\
\hline
\end{tabular}




\begin{tabular}{|c|c|c|}
\hline Name & Identifier & $\begin{array}{l}\text { Mass } \\
\text { (amu) }\end{array}$ \\
\hline no-260 & 102260 & 260.1026 \\
\hline no-261 & 102261 & 261.1057 \\
\hline no-262 & 102262 & 262.1073 \\
\hline no-263 & 102263 & 263.1106 \\
\hline no-264 & 102264 & 264.1123 \\
\hline $\operatorname{lr}-251$ & 103251 & 251.0944 \\
\hline lr-252 & 103252 & 252.0954 \\
\hline lr-253 & 103253 & 253.0952 \\
\hline lr-254 & 103254 & 254.0965 \\
\hline lr-255 & 103255 & 255.0967 \\
\hline lr-256 & 103256 & 256.0986 \\
\hline lr-257 & 103257 & 257.0995 \\
\hline lr-258 & 103258 & 258.1018 \\
\hline lr-259 & 103259 & 259.1029 \\
\hline lr-260 & 103260 & 260.1055 \\
\hline lr-261 & 103261 & 261.1069 \\
\hline lr-262 & 103262 & 262.1096 \\
\hline lr-263 & 103263 & 263.1113 \\
\hline lr-264 & 103264 & 264.114 \\
\hline lr-265 & 103265 & 265.1158 \\
\hline lr-266 & 103266 & 266.1193 \\
\hline $\mathrm{rf}-253$ & 104253 & 253.1007 \\
\hline rf-254 & 104254 & 254.1002 \\
\hline rf-255 & 104255 & 255.1013 \\
\hline rf-256 & 104256 & 256.1012 \\
\hline rf-257 & 104257 & 257.103 \\
\hline $\mathrm{rf}-258$ & 104258 & 258.1035 \\
\hline rf-259 & 104259 & 259.1057 \\
\hline rf-260 & 104260 & 260.1064 \\
\hline rf-261 & 104261 & 261.1088 \\
\hline rf-262 & 104262 & 262.1099 \\
\hline rf-263 & 104263 & 263.1125 \\
\hline rf-264 & 104264 & 264.114 \\
\hline rf-265 & 104265 & 265.1167 \\
\hline rf-266 & 104266 & 266.118 \\
\hline $\mathrm{rf}-267$ & 104267 & 267.1215 \\
\hline rf-268 & 104268 & 268.1236 \\
\hline $\mathrm{db}-255$ & 105255 & 255.1074 \\
\hline $\mathrm{db}-256$ & 105256 & 256.1081 \\
\hline $\mathrm{db}-257$ & 105257 & 257.1077 \\
\hline $\mathrm{db}-258$ & 105258 & 258.1092 \\
\hline $\mathrm{db}-259$ & 105259 & 259.1096 \\
\hline $\mathrm{db}-260$ & 105260 & 260.1113 \\
\hline $\mathrm{db}-261$ & 105261 & 261.1121 \\
\hline $\mathrm{db}-262$ & 105262 & 262.1141 \\
\hline $\mathrm{db}-263$ & 105263 & 263.115 \\
\hline $\mathrm{db}-264$ & 105264 & 264.1174 \\
\hline $\mathrm{db}-265$ & 105265 & 265.1186 \\
\hline $\mathrm{db}-266$ & 105266 & 266.121 \\
\hline $\mathrm{db}-267$ & 105267 & 267.1224 \\
\hline $\mathrm{db}-268$ & 105268 & 268.1255 \\
\hline db-269 & 105269 & 269.1275 \\
\hline
\end{tabular}




\begin{tabular}{|c|c|c|}
\hline Name & Identifier & $\begin{array}{c}\text { Mass } \\
\text { (amu) }\end{array}$ \\
\hline $\mathrm{db}-270$ & 105270 & 270.1307 \\
\hline sg-258 & 106258 & 258.1132 \\
\hline sg-259 & 106259 & 259.1145 \\
\hline sg-260 & 106260 & 260.1144 \\
\hline sg-261 & 106261 & 261.1161 \\
\hline sg-262 & 106262 & 262.1164 \\
\hline sg-263 & 106263 & 263.1183 \\
\hline sg-264 & 106264 & 264.1189 \\
\hline sg-265 & 106265 & 265.1211 \\
\hline sg-266 & 106266 & 266.1221 \\
\hline sg-267 & 106267 & 267.1244 \\
\hline sg-268 & 106268 & 268.1256 \\
\hline sg-269 & 106269 & 269.1288 \\
\hline sg-270 & 106270 & 270.1303 \\
\hline sg-271 & 106271 & 271.1335 \\
\hline sg-272 & 106272 & 272.1352 \\
\hline sg-273 & 106273 & 273.1382 \\
\hline bh-260 & 107260 & 260.122 \\
\hline bh-261 & 107261 & 261.1217 \\
\hline bh-262 & 107262 & 262.1229 \\
\hline bh-263 & 107263 & 263.123 \\
\hline bh-264 & 107264 & 264.1246 \\
\hline bh-265 & 107265 & 265.1252 \\
\hline bh-266 & 107266 & 266.127 \\
\hline bh-267 & 107267 & 267.1277 \\
\hline bh-268 & 107268 & 268.1298 \\
\hline bh-269 & 107269 & 269.1307 \\
\hline bh-270 & 107270 & 270.1336 \\
\hline bh-271 & 107271 & 271.1352 \\
\hline bh-272 & 107272 & 272.138 \\
\hline bh-273 & 107273 & 273.1396 \\
\hline bh-274 & 107274 & 274.1424 \\
\hline bh-275 & 107275 & 275.1443 \\
\hline hs-263 & 108263 & 263.1286 \\
\hline hs-264 & 108264 & 264.1284 \\
\hline hs-265 & 108265 & 265.1301 \\
\hline hs-266 & 108266 & 266.1301 \\
\hline hs-267 & 108267 & 267.1318 \\
\hline hs-268 & 108268 & 268.1322 \\
\hline hs-269 & 108269 & 269.1341 \\
\hline hs -270 & 108270 & 270.1346 \\
\hline hs-271 & 108271 & 271.1377 \\
\hline hs-272 & 108272 & 272.139 \\
\hline hs-273 & 108273 & 273.142 \\
\hline hs-274 & 108274 & 274.1431 \\
\hline hs- 275 & 108275 & 275.1459 \\
\hline hs-276 & 108276 & 276.1472 \\
\hline hs-277 & 108277 & 277.1498 \\
\hline mt-265 & 109265 & 265.1361 \\
\hline mt-266 & 109266 & 266.1373 \\
\hline mt-267 & 109267 & 267.1373 \\
\hline mt-268 & 109268 & 268.1387 \\
\hline
\end{tabular}




\begin{tabular}{|l|l|l|}
\hline \multicolumn{1}{|c|}{ Name } & Identifier & $\begin{array}{c}\text { Mass } \\
\text { (amu) }\end{array}$ \\
\hline mt-269 & 109269 & 269.1391 \\
\hline mt-270 & 109270 & 270.1407 \\
\hline mt-271 & 109271 & 271.1411 \\
\hline mt-272 & 109272 & 272.1437 \\
\hline mt-273 & 109273 & 273.1449 \\
\hline mt-274 & 109274 & 274.1475 \\
\hline mt-275 & 109275 & 275.1487 \\
\hline mt-276 & 109276 & 276.1512 \\
\hline mt-277 & 109277 & 277.1524 \\
\hline mt-278 & 109278 & 278.1548 \\
\hline mt-279 & 109279 & 279.1562 \\
\hline ds-267 & 110267 & 267.1443 \\
\hline ds-268 & 110268 & 268.1438 \\
\hline ds-269 & 110269 & 269.1451 \\
\hline ds-270 & 110270 & 270.1447 \\
\hline ds-271 & 110271 & 271.1461 \\
\hline ds-272 & 110272 & 272.1463 \\
\hline ds-273 & 110273 & 273.1489 \\
\hline ds-274 & 110274 & 274.1495 \\
\hline ds-275 & 110275 & 275.1522 \\
\hline ds-276 & 110276 & 276.153 \\
\hline ds-277 & 110277 & 277.1556 \\
\hline ds-278 & 110278 & 278.1565 \\
\hline ds-279 & 110279 & 279.1589 \\
\hline ds-280 & 110280 & 280.1598 \\
\hline ds-281 & 110281 & 281.162 \\
\hline rg-272 & 111272 & 272.1536 \\
\hline rg-273 & 111273 & 273.1537 \\
\hline rg-274 & 111274 & 274.1557 \\
\hline rg-275 & 111275 & 275.1561 \\
\hline rg-276 & 111276 & 276.1585 \\
\hline rg-277 & 111277 & 277.1595 \\
\hline rg-278 & 111278 & 278.1616 \\
\hline rg-279 & 111279 & 279.1625 \\
\hline rg-280 & 111280 & 280.1645 \\
\hline rg-281 & 111281 & 281.1654 \\
\hline rg-282 & 111282 & 282.1675 \\
\hline rg-283 & 111283 & 283.1684 \\
\hline sg-277 & 112277 & 277.1639 \\
\hline sg-278 & 112278 & 278.1643 \\
\hline sg-279 & 112279 & 279.1666 \\
\hline sg-280 & 112280 & 280.1671 \\
\hline sg-281 & 112281 & 281.1693 \\
\hline sg-282 & 112282 & 282.1698 \\
\hline sg-283 & 112283 & 283.1718 \\
\hline sg-284 & 112284 & 284.1724 \\
\hline sg-285 & 112285 & 285.1741 \\
\hline uut-283 & 113283 & 283.1765 \\
\hline uut-284 & 113284 & 284.1781 \\
\hline uut-285 & 113285 & 285.1787 \\
\hline uut-286 & 113286 & 286.1805 \\
\hline uut-287 & 113287 & 287.1811 \\
\hline
\end{tabular}




\begin{tabular}{|l|l|l|}
\hline Name & Identifier & $\begin{array}{c}\text { Mass } \\
\text { (amu) }\end{array}$ \\
\hline uuq-285 & 114285 & 285.1837 \\
\hline uuq-286 & 114286 & 286.1839 \\
\hline uuq-287 & 114287 & 287.1856 \\
\hline uuq-288 & 114288 & 288.1857 \\
\hline uuq-289 & 114289 & 289.1873 \\
\hline uup-287 & 115287 & 287.1912 \\
\hline uup-288 & 115288 & 288.1925 \\
\hline uup-289 & 115289 & 289.1927 \\
\hline uup-290 & 115290 & 290.1942 \\
\hline uup-291 & 115291 & 291.1944 \\
\hline uuh-289 & 116289 & 289.1989 \\
\hline uuh-290 & 116290 & 290.1986 \\
\hline uuh-291 & 116291 & 291.2 \\
\hline uuh-292 & 116292 & 292.1998 \\
\hline uus-291 & 117291 & 291.2066 \\
\hline uus-292 & 117292 & 292.2076 \\
\hline uuo-293 & 118293 & 293.2147 \\
\hline
\end{tabular}


Table 7.2.2. Elements and their natural abundances"

\begin{tabular}{|c|c|c|c|c|c|c|}
\hline ID & Symbol & Name & $\begin{array}{c}\text { Mass } \\
(\mathrm{AMU})\end{array}$ & Density & $\begin{array}{c}\text { Isotopic } \\
\text { distribution }\end{array}$ & Atom \% \\
\hline \multirow[t]{2}{*}{1000} & $\mathrm{~h}$ & hydrogen & 1.0079 & 1.0 & 1001 & 99.9885 \\
\hline & & & & & 1002 & 0.0115 \\
\hline \multirow[t]{2}{*}{2000} & he & helium & 4.0026 & 1.0 & 2003 & 0.0001 \\
\hline & & & & & 2004 & 99.9999 \\
\hline \multirow[t]{2}{*}{3000} & li & lithium & 6.941 & 0.534 & 3006 & 7.59 \\
\hline & & & & & 3007 & 92.41 \\
\hline 4000 & be & beryllium & 9.0122 & 1.85 & 4009 & 100.0 \\
\hline \multirow[t]{2}{*}{5000} & $\mathrm{~b}$ & boron & 10.811 & 2.37 & 5010 & 19.9 \\
\hline & & & & & 5011 & 80.1 \\
\hline \multirow[t]{2}{*}{6000} & $\mathrm{c}$ & carbon & 12.0107 & 2.1 & 6012 & 98.93 \\
\hline & & & & & 6013 & 1.07 \\
\hline \multirow[t]{2}{*}{7000} & $\mathrm{n}$ & nitrogen & 14.0067 & 1.0 & 7014 & 99.636 \\
\hline & & & & & 7015 & 0.364 \\
\hline \multirow[t]{3}{*}{8000} & $\mathrm{O}$ & oxygen & 15.9994 & 1.0 & 8016 & 99.757 \\
\hline & & & & & 8017 & 0.038 \\
\hline & & & & & 8018 & 0.205 \\
\hline 9000 & $\mathrm{f}$ & fluorine & 18.9984 & 1.0 & 9019 & 100.0 \\
\hline \multirow[t]{3}{*}{10000} & ne & neon & 20.1797 & 1.0 & 10020 & 90.48 \\
\hline & & & & & 10021 & 0.27 \\
\hline & & & & & 10022 & 9.25 \\
\hline 11000 & na & sodium & 22.9898 & 0.97 & 11023 & 100.0 \\
\hline \multirow[t]{3}{*}{12000} & $\mathrm{mg}$ & magnesium & 24.305 & 1.74 & 12024 & 78.99 \\
\hline & & & & & 12025 & 10.0 \\
\hline & & & & & 12026 & 11.01 \\
\hline 13000 & al & aluminum & 26.9815 & 2.702 & 13027 & 100.0 \\
\hline \multirow[t]{3}{*}{14000} & si & silicon & 28.0855 & 2.33 & 14028 & 92.223 \\
\hline & & & & & 14029 & 4.685 \\
\hline & & & & & 14030 & 3.092 \\
\hline 15000 & $\mathrm{p}$ & phosphorus & 30.9738 & 1.82 & 15031 & 100.0 \\
\hline \multirow[t]{4}{*}{16000} & $\mathrm{~s}$ & sulfur & 32.065 & 2.07 & 16032 & 94.99 \\
\hline & & & & & 16033 & 0.75 \\
\hline & & & & & 16034 & 4.25 \\
\hline & & & & & 16036 & 0.01 \\
\hline \multirow[t]{2}{*}{17000} & $\mathrm{cl}$ & chlorine & 35.453 & 1.0 & 17035 & 75.76 \\
\hline & & & & & 17037 & 24.24 \\
\hline \multirow[t]{3}{*}{18000} & ar & argon & 39.948 & 1.0 & 18036 & 0.3365 \\
\hline & & & & & 18038 & 0.0632 \\
\hline & & & & & 18040 & 99.6003 \\
\hline \multirow[t]{3}{*}{19000} & $\mathrm{k}$ & potassium & 39.0983 & 0.86 & 19039 & 93.2581 \\
\hline & & & & & 19040 & 0.0117 \\
\hline & & & & & 19041 & 6.7302 \\
\hline \multirow[t]{6}{*}{20000} & $\mathrm{ca}$ & calcium & 40.078 & 1.55 & 20040 & 96.941 \\
\hline & & & & & 20042 & 0.647 \\
\hline & & & & & 20043 & 0.135 \\
\hline & & & & & 20044 & 2.086 \\
\hline & & & & & 20046 & 0.004 \\
\hline & & & & & 20048 & 0.187 \\
\hline 21000 & $\mathrm{sc}$ & scandium & 44.9559 & 2.989 & 21045 & 100.0 \\
\hline 22000 & ti & titanium & 47.867 & 4.5 & 22046 & 8.25 \\
\hline
\end{tabular}




\begin{tabular}{|c|c|c|c|c|c|c|}
\hline ID & Symbol & Name & $\begin{array}{c}\text { Mass } \\
\text { (AMU) }\end{array}$ & Density & $\begin{array}{c}\text { Isotopic } \\
\text { distribution }\end{array}$ & Atom \% \\
\hline & & & & & 22047 & 7.44 \\
\hline & & & & & 22048 & 73.72 \\
\hline & & & & & 22049 & 5.41 \\
\hline & & & & & 22050 & 5.18 \\
\hline \multirow[t]{2}{*}{23000} & $\mathrm{~V}$ & vanadium & 50.9415 & 5.96 & 23050 & 0.25 \\
\hline & & & & & 23051 & 99.75 \\
\hline \multirow[t]{4}{*}{24000} & $\mathrm{cr}$ & chromium & 51.9961 & 7.2 & 24050 & 4.345 \\
\hline & & & & & 24052 & 83.789 \\
\hline & & & & & 24053 & 9.501 \\
\hline & & & & & 24054 & 2.365 \\
\hline 25000 & $\mathrm{mn}$ & manganese & 54.938 & 7.2 & 25055 & 100.0 \\
\hline \multirow[t]{4}{*}{26000} & fe & iron & 55.845 & 7.86 & 26054 & 5.845 \\
\hline & & & & & 26056 & 91.754 \\
\hline & & & & & 26057 & 2.119 \\
\hline & & & & & 26058 & 0.282 \\
\hline 27000 & co & cobalt & 58.9332 & 8.9 & 27059 & 100.0 \\
\hline \multirow[t]{5}{*}{28000} & ni & nickel & 58.6934 & 8.9 & 28058 & 68.0769 \\
\hline & & & & & 28060 & 26.2231 \\
\hline & & & & & 28061 & 1.1399 \\
\hline & & & & & 28062 & 3.6345 \\
\hline & & & & & 28064 & 0.9256 \\
\hline \multirow[t]{2}{*}{29000} & $\mathrm{cu}$ & copper & 63.546 & 8.92 & 29063 & 69.15 \\
\hline & & & & & 29065 & 30.85 \\
\hline \multirow[t]{5}{*}{30000} & $\mathrm{zn}$ & zinc & 65.38 & 7.14 & 30064 & 48.268 \\
\hline & & & & & 30066 & 27.975 \\
\hline & & & & & 30067 & 4.102 \\
\hline & & & & & 30068 & 19.024 \\
\hline & & & & & 30070 & 0.631 \\
\hline \multirow[t]{2}{*}{31000} & ga & gallium & 69.723 & 5.904 & 31069 & 60.108 \\
\hline & & & & & 31071 & 39.892 \\
\hline \multirow[t]{5}{*}{32000} & ge & germanium & 72.64 & 5.35 & 32070 & 20.38 \\
\hline & & & & & 32072 & 27.31 \\
\hline & & & & & 32073 & 7.76 \\
\hline & & & & & 32074 & 36.72 \\
\hline & & & & & 32076 & 7.83 \\
\hline 33000 & as & arsenic & 74.9216 & 5.73 & 33075 & 100.0 \\
\hline \multirow[t]{6}{*}{34000} & se & selenium & 78.96 & 4.81 & 34074 & 0.89 \\
\hline & & & & & 34076 & 9.37 \\
\hline & & & & & 34077 & 7.63 \\
\hline & & & & & 34078 & 23.77 \\
\hline & & & & & 34080 & 49.61 \\
\hline & & & & & 34082 & 8.73 \\
\hline \multirow[t]{2}{*}{35000} & br & bromine & 79.904 & 3.12 & 35079 & 50.69 \\
\hline & & & & & 35081 & 49.31 \\
\hline \multirow[t]{6}{*}{36000} & $\mathrm{kr}$ & krypton & 83.798 & 1.0 & 36078 & 0.355 \\
\hline & & & & & 36080 & 2.286 \\
\hline & & & & & 36082 & 11.593 \\
\hline & & & & & 36083 & 11.5 \\
\hline & & & & & 36084 & 56.987 \\
\hline & & & & & 36086 & 17.279 \\
\hline \multirow[t]{2}{*}{37000} & $\mathrm{rb}$ & rubidium & 85.4678 & 1.532 & 37085 & 72.17 \\
\hline & & & & & 37087 & 27.83 \\
\hline
\end{tabular}




\begin{tabular}{|c|c|c|c|c|c|c|}
\hline ID & Symbol & Name & $\begin{array}{c}\text { Mass } \\
(\mathrm{AMU})\end{array}$ & Density & $\begin{array}{c}\text { Isotopic } \\
\text { distribution }\end{array}$ & Atom \% \\
\hline \multirow[t]{4}{*}{38000} & $\mathrm{sr}$ & strontium & 87.62 & 2.6 & 38084 & 0.56 \\
\hline & & & & & 38086 & 9.86 \\
\hline & & & & & 38087 & 7.0 \\
\hline & & & & & 38088 & 82.58 \\
\hline 39000 & $\mathrm{y}$ & yttrium & 88.9059 & 4.469 & 39089 & 100.0 \\
\hline \multirow[t]{5}{*}{40000} & $\mathrm{zr}$ & zirconium & 91.224 & 6.49 & 40090 & 51.45 \\
\hline & & & & & 40091 & 11.22 \\
\hline & & & & & 40092 & 17.15 \\
\hline & & & & & 40094 & 17.38 \\
\hline & & & & & 40096 & 2.8 \\
\hline 41000 & $\mathrm{nb}$ & niobium & 92.9064 & 8.57 & 41093 & 100.0 \\
\hline \multirow[t]{7}{*}{42000} & mo & molybdenum & 95.96 & 10.2 & 42092 & 14.77 \\
\hline & & & & & 42094 & 9.23 \\
\hline & & & & & 42095 & 15.9 \\
\hline & & & & & 42096 & 16.68 \\
\hline & & & & & 42097 & 9.56 \\
\hline & & & & & 42098 & 24.19 \\
\hline & & & & & 42100 & 9.67 \\
\hline \multirow[t]{7}{*}{44000} & $\mathrm{ru}$ & ruthenium & 101.07 & 12.3 & 44096 & 5.54 \\
\hline & & & & & 44098 & 1.87 \\
\hline & & & & & 44099 & 12.76 \\
\hline & & & & & 44100 & 12.6 \\
\hline & & & & & 44101 & 17.06 \\
\hline & & & & & 44102 & 31.55 \\
\hline & & & & & 44104 & 18.62 \\
\hline 45000 & rh & rhodium & 102.9055 & 12.4 & 45103 & 100.0 \\
\hline \multirow[t]{6}{*}{46000} & $\mathrm{pd}$ & palladium & 106.42 & 12.02 & 46102 & 1.02 \\
\hline & & & & & 46104 & 11.14 \\
\hline & & & & & 46105 & 22.33 \\
\hline & & & & & 46106 & 27.33 \\
\hline & & & & & 46108 & 26.46 \\
\hline & & & & & 46110 & 11.72 \\
\hline \multirow[t]{2}{*}{47000} & $\mathrm{ag}$ & silver & 107.8682 & 10.5 & 47107 & 51.839 \\
\hline & & & & & 47109 & 48.161 \\
\hline \multirow[t]{8}{*}{48000} & $\mathrm{~cd}$ & cadmium & 112.411 & 8.642 & 48106 & 1.25 \\
\hline & & & & & 48108 & 0.89 \\
\hline & & & & & 48110 & 12.49 \\
\hline & & & & & 48111 & 12.8 \\
\hline & & & & & 48112 & 24.13 \\
\hline & & & & & 48113 & 12.22 \\
\hline & & & & & 48114 & 28.73 \\
\hline & & & & & 48116 & 7.49 \\
\hline \multirow[t]{2}{*}{49000} & in & indium & 114.818 & 7.3 & 49113 & 4.29 \\
\hline & & & & & 49115 & 95.71 \\
\hline \multirow[t]{8}{*}{50000} & sn & tin & 118.71 & 7.31 & 50112 & 0.97 \\
\hline & & & & & 50114 & 0.66 \\
\hline & & & & & 50115 & 0.34 \\
\hline & & & & & 50116 & 14.54 \\
\hline & & & & & 50117 & 7.68 \\
\hline & & & & & 50118 & 24.22 \\
\hline & & & & & 50119 & 8.59 \\
\hline & & & & & 50120 & 32.58 \\
\hline
\end{tabular}




\begin{tabular}{|c|c|c|c|c|c|c|}
\hline ID & Symbol & Name & $\begin{array}{c}\text { Mass } \\
\text { (AMU) }\end{array}$ & Density & $\begin{array}{c}\text { Isotopic } \\
\text { distribution }\end{array}$ & Atom \% \\
\hline & & & & & 50122 & 4.63 \\
\hline & & & & & 50124 & 5.79 \\
\hline \multirow[t]{2}{*}{51000} & $\mathrm{sb}$ & antimony & 121.76 & 6.684 & 51121 & 57.21 \\
\hline & & & & & 51123 & 42.79 \\
\hline \multirow[t]{8}{*}{52000} & te & tellurium & 127.6 & 6.25 & 52120 & 0.09 \\
\hline & & & & & 52122 & 2.55 \\
\hline & & & & & 52123 & 0.89 \\
\hline & & & & & 52124 & 4.74 \\
\hline & & & & & 52125 & 7.07 \\
\hline & & & & & 52126 & 18.84 \\
\hline & & & & & 52128 & 31.74 \\
\hline & & & & & 52130 & 34.08 \\
\hline 53000 & $\mathrm{i}$ & iodine & 126.9045 & 4.93 & 53127 & 100.0 \\
\hline \multirow[t]{9}{*}{54000} & xe & xenon & 131.293 & 1.0 & 54124 & 0.0952 \\
\hline & & & & & 54126 & 0.089 \\
\hline & & & & & 54128 & 1.9102 \\
\hline & & & & & 54129 & 26.4006 \\
\hline & & & & & 54130 & 4.071 \\
\hline & & & & & 54131 & 21.2324 \\
\hline & & & & & 54132 & 26.9086 \\
\hline & & & & & 54134 & 10.4357 \\
\hline & & & & & 54136 & 8.8573 \\
\hline 55000 & cs & cesium & 132.9055 & 1.879 & 55133 & 100.0 \\
\hline \multirow[t]{7}{*}{56000} & ba & barium & 137.327 & 3.51 & 56130 & 0.106 \\
\hline & & & & & 56132 & 0.101 \\
\hline & & & & & 56134 & 2.417 \\
\hline & & & & & 56135 & 6.592 \\
\hline & & & & & 56136 & 7.854 \\
\hline & & & & & 56137 & 11.232 \\
\hline & & & & & 56138 & 71.698 \\
\hline \multirow[t]{2}{*}{57000} & la & lanthanum & 138.9055 & 6.145 & 57138 & 0.09 \\
\hline & & & & & 57139 & 99.91 \\
\hline \multirow[t]{4}{*}{58000} & ce & cerium & 140.116 & 6.657 & 58136 & 0.185 \\
\hline & & & & & 58138 & 0.251 \\
\hline & & & & & 58140 & 88.45 \\
\hline & & & & & 58142 & 11.114 \\
\hline 59000 & $\mathrm{pr}$ & praseodymium & 140.9077 & 6.773 & 59141 & 100.0 \\
\hline \multirow[t]{7}{*}{60000} & nd & neodymium & 144.242 & 6.8 & 60142 & 27.2 \\
\hline & & & & & 60143 & 12.2 \\
\hline & & & & & 60144 & 23.8 \\
\hline & & & & & 60145 & 8.3 \\
\hline & & & & & 60146 & 17.2 \\
\hline & & & & & 60148 & 5.7 \\
\hline & & & & & 60150 & 5.6 \\
\hline \multirow[t]{7}{*}{62000} & $\mathrm{sm}$ & samarium & 150.36 & 7.52 & 62144 & 3.07 \\
\hline & & & & & 62147 & 14.99 \\
\hline & & & & & 62148 & 11.24 \\
\hline & & & & & 62149 & 13.82 \\
\hline & & & & & 62150 & 7.38 \\
\hline & & & & & 62152 & 26.75 \\
\hline & & & & & 62154 & 22.75 \\
\hline 63000 & $\mathrm{eu}$ & europium & 151.964 & 5.243 & 63151 & 47.81 \\
\hline
\end{tabular}




\begin{tabular}{|c|c|c|c|c|c|c|}
\hline ID & Symbol & Name & $\begin{array}{c}\text { Mass } \\
(\mathrm{AMU})\end{array}$ & Density & $\begin{array}{c}\text { Isotopic } \\
\text { distribution }\end{array}$ & Atom \% \\
\hline & & & & & 63153 & 52.19 \\
\hline \multirow[t]{7}{*}{64000} & $\mathrm{gd}$ & gadolinium & 157.25 & 7.9 & 64152 & 0.2 \\
\hline & & & & & 64154 & 2.18 \\
\hline & & & & & 64155 & 14.8 \\
\hline & & & & & 64156 & 20.47 \\
\hline & & & & & 64157 & 15.65 \\
\hline & & & & & 64158 & 24.84 \\
\hline & & & & & 64160 & 21.86 \\
\hline 65000 & $\mathrm{tb}$ & terbium & 158.9254 & 8.229 & 65159 & 100.0 \\
\hline \multirow[t]{7}{*}{66000} & dy & dysprosium & 162.5 & 8.55 & 66156 & 0.056 \\
\hline & & & & & 66158 & 0.095 \\
\hline & & & & & 66160 & 2.329 \\
\hline & & & & & 66161 & 18.889 \\
\hline & & & & & 66162 & 25.475 \\
\hline & & & & & 66163 & 24.896 \\
\hline & & & & & 66164 & 28.26 \\
\hline 67000 & ho & holmium & 164.9303 & 8.795 & 67165 & 100.0 \\
\hline \multirow[t]{6}{*}{68000} & er & erbium & 167.259 & 9.006 & 68162 & 0.139 \\
\hline & & & & & 68164 & 1.601 \\
\hline & & & & & 68166 & 33.503 \\
\hline & & & & & 68167 & 22.869 \\
\hline & & & & & 68168 & 26.978 \\
\hline & & & & & 68170 & 14.91 \\
\hline 69000 & $\mathrm{tm}$ & thulium & 168.9342 & 9.321 & 69169 & 100.0 \\
\hline \multirow[t]{7}{*}{70000} & $\mathrm{yb}$ & ytterbium & 173.054 & 6.965 & 70168 & 0.13 \\
\hline & & & & & 70170 & 3.04 \\
\hline & & & & & 70171 & 14.28 \\
\hline & & & & & 70172 & 21.83 \\
\hline & & & & & 70173 & 16.13 \\
\hline & & & & & 70174 & 31.83 \\
\hline & & & & & 70176 & 12.76 \\
\hline \multirow[t]{2}{*}{71000} & lu & lutetium & 174.9668 & 9.84 & 71175 & 97.41 \\
\hline & & & & & 71176 & 2.59 \\
\hline \multirow[t]{6}{*}{72000} & hf & hafnium & 178.49 & 13.31 & 72174 & 0.16 \\
\hline & & & & & 72176 & 5.26 \\
\hline & & & & & 72177 & 18.6 \\
\hline & & & & & 72178 & 27.28 \\
\hline & & & & & 72179 & 13.62 \\
\hline & & & & & 72180 & 35.08 \\
\hline \multirow[t]{2}{*}{73000} & ta & tantalum & 180.9479 & 16.6 & 73180 & 0.012 \\
\hline & & & & & 73181 & 99.988 \\
\hline \multirow[t]{5}{*}{74000} & W & tungsten & 183.84 & 19.35 & 74180 & 0.12 \\
\hline & & & & & 74182 & 26.5 \\
\hline & & & & & 74183 & 14.31 \\
\hline & & & & & 74184 & 30.64 \\
\hline & & & & & 74186 & 28.43 \\
\hline \multirow[t]{2}{*}{75000} & re & rhenium & 186.207 & 20.53 & 75185 & 37.4 \\
\hline & & & & & 75187 & 62.6 \\
\hline \multirow[t]{4}{*}{76000} & os & osmium & 190.23 & 22.48 & 76184 & 0.02 \\
\hline & & & & & 76186 & 1.59 \\
\hline & & & & & 76187 & 1.96 \\
\hline & & & & & 76188 & 13.24 \\
\hline
\end{tabular}




\begin{tabular}{|c|c|c|c|c|c|c|}
\hline ID & Symbol & Name & $\begin{array}{c}\text { Mass } \\
(\mathrm{AMU})\end{array}$ & Density & $\begin{array}{c}\text { Isotopic } \\
\text { distribution }\end{array}$ & Atom \% \\
\hline & & & & & 76189 & 16.15 \\
\hline & & & & & 76190 & 26.26 \\
\hline & & & & & 76192 & 40.78 \\
\hline \multirow[t]{2}{*}{77000} & ir & iridium & 192.217 & 22.421 & 77191 & 37.3 \\
\hline & & & & & 77193 & 62.7 \\
\hline \multirow[t]{6}{*}{78000} & $\mathrm{pt}$ & platinum & 195.084 & 21.45 & 78190 & 0.014 \\
\hline & & & & & 78192 & 0.782 \\
\hline & & & & & 78194 & 32.967 \\
\hline & & & & & 78195 & 33.832 \\
\hline & & & & & 78196 & 25.242 \\
\hline & & & & & 78198 & 7.163 \\
\hline 79000 & $\mathrm{au}$ & gold & 196.9666 & 18.88 & 79197 & 100.0 \\
\hline \multirow[t]{7}{*}{80000} & hg & mercury & 200.59 & 13.546 & 80196 & 0.15 \\
\hline & & & & & 80198 & 9.97 \\
\hline & & & & & 80199 & 16.87 \\
\hline & & & & & 80200 & 23.1 \\
\hline & & & & & 80201 & 13.18 \\
\hline & & & & & 80202 & 29.86 \\
\hline & & & & & 80204 & 6.87 \\
\hline \multirow[t]{2}{*}{81000} & tl & thallium & 204.3833 & 11.85 & 81203 & 29.52 \\
\hline & & & & & 81205 & 70.48 \\
\hline \multirow[t]{4}{*}{82000} & $\mathrm{pb}$ & lead & 207.2 & 11.344 & 82204 & 1.4 \\
\hline & & & & & 82206 & 24.1 \\
\hline & & & & & 82207 & 22.1 \\
\hline & & & & & 82208 & 52.4 \\
\hline 83000 & bi & bismuth & 208.9804 & 9.8 & 83209 & 100.0 \\
\hline 90000 & th & thorium & 232.0381 & 11.7 & 90232 & 100.0 \\
\hline 91000 & $\mathrm{pa}$ & protactinium & 231.0359 & 15.37 & 91231 & 100.0 \\
\hline \multirow[t]{3}{*}{92000} & $\mathrm{u}$ & uranium & 238.0289 & 19.05 & 92234 & 0.0054 \\
\hline & & & & & 92235 & 0.7204 \\
\hline & & & & & 92238 & 99.2742 \\
\hline \multicolumn{7}{|c|}{$\begin{array}{l}\text { If the column for the chemical symbol has a value, the name and the chemical symbol refer to the same } \\
\text { composition. Otherwise, the chemical symbol refers to a different composition. In case of monoisotopic elements, } \\
\text { such as }{ }^{209} \mathrm{Bi} \text {, the chemical symbol refers directly to SCALE ID } 83209 \text { instead of } 83000 \text {. See Table } 7.2 .3 \text { for } \\
\text { details. }\end{array}$} \\
\hline
\end{tabular}


Table 7.2.3. Elements and special nuclide symbols

\begin{tabular}{|c|c|c|c|}
\hline Name & Description & ID & Density \\
\hline activities & & 900 & 1.0 \\
\hline $1 /$ vabsorber & & 999 & 1.0 \\
\hline $\mathrm{d}$ & Deuterium in heavy water with $S(\alpha, \beta)$ thermal kernel & 1002 & 1.0 \\
\hline h-liquid_ch4 & Liquid methane at $100 \mathrm{~K}$ & 1001001 & 1.0 \\
\hline albound & $\mathrm{Al}$ metal with $\mathrm{S}(\alpha, \beta)$ thermal kernel & 1013027 & 2.702 \\
\hline zr90-zr5h8 & $\begin{array}{l}\text { Zr-90 with the } S(\alpha, \beta) \text { thermal kernel for } \mathrm{Zr} \text { in } \mathrm{zrh} 2 \text { and } \\
\mathrm{zr} 5 \mathrm{~h} 8\end{array}$ & 1040090 & 1.0 \\
\hline zr91-zr5h8 & $\begin{array}{l}\text { Zr-91 with the } S(\alpha, \beta) \text { thermal kernel for } \mathrm{Zr} \text { in } \mathrm{zrh} 2 \text { and } \\
\text { zr5h8 }\end{array}$ & 1040091 & 1.0 \\
\hline zr92-zr5h8 & $\begin{array}{l}\text { Zr-92 with the } S(\alpha, \beta) \text { thermal kernel for } \mathrm{Zr} \text { in } \mathrm{zrh} 2 \text { and } \\
\text { zr5h8 }\end{array}$ & 1040092 & 1.0 \\
\hline zr93-zr5h8 & $\begin{array}{l}\text { Zr-93 with the } S(\alpha, \beta) \text { thermal kernel for } \mathrm{Zr} \text { in } \mathrm{zrh} 2 \text { and } \\
\mathrm{zr} 5 \mathrm{~h} 8\end{array}$ & 1040093 & 1.0 \\
\hline zr94-zr5h8 & $\begin{array}{l}\text { Zr-94 with the } S(\alpha, \beta) \text { thermal kernel for } \mathrm{Zr} \text { in } \mathrm{zrh} 2 \text { and } \\
\text { zr5h8 }\end{array}$ & 1040094 & 1.0 \\
\hline zr95-zr5h8 & $\begin{array}{l}\text { Zr-95 with the } S(\alpha, \beta) \text { thermal kernel for } \mathrm{Zr} \text { in } \mathrm{zrh} 2 \text { and } \\
\text { zr5h8 }\end{array}$ & 1040095 & 1.0 \\
\hline zr96-zr5h8 & $\begin{array}{l}\text { Zr-96 with the } S(\alpha, \beta) \text { thermal kernel for } \mathrm{Zr} \text { in } \mathrm{zrh} 2 \text { and } \\
\text { zr5h8 }\end{array}$ & 1040096 & 1.0 \\
\hline h-solid_ch4 & Solid methane at $22 \mathrm{~K}$ & 2001001 & 1.0 \\
\hline bebound & Beryllium metal with a $S(\alpha, \beta)$ thermal kernel & 3004009 & 1.85 \\
\hline h-cryo_ortho & $\mathrm{H}$ at cryogenic temperatures with ortho form & 4001001 & 1.0 \\
\hline d-cryo_ortho & D at cryogenic temperatures with ortho form & 4001002 & 1.0 \\
\hline h-cryo para & $\mathrm{H}$ at cryogenic temperatures with para form & 5001001 & 1.0 \\
\hline d-cryo_para & D at cryogenic temperatures with para form & 5001002 & 1.0 \\
\hline be-beo & Beryllium in beryllium oxide with a $S(\alpha, \beta)$ thermal kernel & 5004009 & 1.0 \\
\hline o-beo & Oxygen in beryllium oxide with a $S(\alpha, \beta)$ thermal kernel & 5008016 & 1.0 \\
\hline h-benzene & Benzene with a $S(\alpha, \beta)$ thermal kernel & 6001001 & 1.0 \\
\hline h-zrh2 & $\begin{array}{l}\text { Hydrogen in zirconium hydride with a } S(\alpha, \beta) \text { thermal } \\
\text { kernel }\end{array}$ & 7001001 & 1.0 \\
\hline hfreegas & Hydrogen with a free gas thermal kernel & 8001001 & 1.0 \\
\hline dfreegas & Deuterium with a free gas thermal kernel & 8001002 & 1.0 \\
\hline h-poly & Hydrogen in polyethylene with a $S(\alpha, \beta)$ thermal kernel & 9001001 & 1.0 \\
\hline
\end{tabular}


Table 7.2.4. Compounds

\begin{tabular}{|c|c|c|c|c|}
\hline Name & Description & Density & ID & $\begin{array}{c}\text { Atoms per } \\
\text { molecule }\end{array}$ \\
\hline \multirow[t]{2}{*}{ al2o3 } & & 3.97 & 13000 & 2 \\
\hline & & & 8000 & 3 \\
\hline \multirow[t]{2}{*}{$\mathrm{b} 4 \mathrm{c}$} & $\begin{array}{l}\text { Boron carbide: } \mathrm{B}_{4} \mathrm{C} ; \text { natural isotopic } \\
\text { distribution obtained by default }\end{array}$ & 2.52 & 5000 & 4 \\
\hline & & & 6000 & 1 \\
\hline \multirow[t]{3}{*}{ balsa } & Balsa wood: $\mathrm{C}_{6} \mathrm{H}_{10} \mathrm{O}_{5}$ & 0.125 & 6000 & 6 \\
\hline & & & 1000 & 10 \\
\hline & & & 8000 & 5 \\
\hline \multirow[t]{2}{*}{ benzene } & Benzene with a $S(\alpha, \beta)$ thermal kernel & 0.8765 & 5006000 & 6 \\
\hline & & & 6001001 & 6 \\
\hline \multirow[t]{2}{*}{ beo } & Beryllium oxide with a $S(\alpha, \beta)$ thermal kernel & 3.0 & 5009 & 1 \\
\hline & & & 5008016 & 1 \\
\hline \multirow[t]{2}{*}{$\mathrm{d} 2 \mathrm{o}$} & Heavy water: $\mathrm{D}_{2} \mathrm{O}$ & 1.1054 & 1002 & 2 \\
\hline & & & 8000 & 1 \\
\hline \multirow[t]{2}{*}{ gd2o3 } & & 7.07 & 64000 & 2 \\
\hline & & & 8000 & 3 \\
\hline graphite & Graphite carbon & 2.3 & 3006000 & 1 \\
\hline \multirow[t]{2}{*}{ h2o } & $\begin{array}{l}\text { Water with only }{ }^{1} \mathrm{H} \text { and }{ }^{16} \mathrm{O} \text { with } \mathrm{S}(\alpha, \beta) \\
\text { thermal kernels }\end{array}$ & 0.9982 & 1000 & 2 \\
\hline & & & 8000 & 1 \\
\hline \multirow[t]{2}{*}{ h2o-x(e)-hr } & & 0.9982 & 3001001 & 2 \\
\hline & & & 8000 & 1 \\
\hline \multirow[t]{2}{*}{ hfacid } & Hydrafluoric acid: HF & 1.0 & 1000 & 1 \\
\hline & & & 9000 & 1 \\
\hline \multirow[t]{3}{*}{ hno3 } & Nitric acid: $\mathrm{HNO}_{3}$ & 1.0 & 1000 & 1 \\
\hline & & & 7000 & 1 \\
\hline & & & 8000 & 3 \\
\hline \multirow[t]{2}{*}{ norpar(h2o) } & $\begin{array}{l}\text { Normal Paraffin 13: } \mathrm{C}_{13} \mathrm{H}_{28} \text {, uses hydrogen } \\
\text { in water thermal kernel }\end{array}$ & 0.76 & 1001 & 28 \\
\hline & & & 6000 & 13 \\
\hline \multirow[t]{2}{*}{ norpar13 } & Normal paraffin 13: $\mathrm{C}_{13} \mathrm{H}_{28}$ & 0.76 & 9001001 & 28 \\
\hline & & & 6000 & 13 \\
\hline \multirow[t]{3}{*}{ oak } & Oak wood: $\mathrm{C}_{6} \mathrm{H}_{10} \mathrm{O}_{5}$ & 0.7 & 6000 & 6 \\
\hline & & & 1000 & 10 \\
\hline & & & 8000 & 5 \\
\hline \multirow[t]{2}{*}{ para(h2o) } & $\begin{array}{l}\text { Paraffin: } \mathrm{C}_{25} \mathrm{H}_{52} \text {, uses hydrogen in water } \\
\mathrm{S}(\alpha, \beta) \text { thermal kernel }\end{array}$ & 0.9 & 1001 & 52 \\
\hline & & & 6000 & 25 \\
\hline \multirow[t]{2}{*}{ paraffin } & $\begin{array}{l}\text { Paraffin: } \quad \mathrm{C}_{25} \mathrm{H}_{52}, \text { uses hydrogen in } \\
\text { polyethylene } \mathrm{S}(\alpha, \beta) \text { thermal kernel }\end{array}$ & 0.9 & 9001001 & 52 \\
\hline & & & 6000 & 25 \\
\hline \multirow[t]{3}{*}{ plexiglas } & Plexiglas: $\mathrm{C}_{5} \mathrm{H}_{8} \mathrm{O}_{2}$ & 1.18 & 1000 & 8 \\
\hline & & & 6000 & 5 \\
\hline & & & 8000 & 2 \\
\hline \multirow[t]{3}{*}{ plexiglass } & $\begin{array}{l}\text { Plexiglas: } \mathrm{C}_{5} \mathrm{H}_{8} \mathrm{O}_{2} \\
\text { Maintained for backward compatibility }\end{array}$ & 1.18 & 1000 & 8 \\
\hline & & & 6000 & 5 \\
\hline & & & 8000 & 2 \\
\hline
\end{tabular}




\begin{tabular}{|c|c|c|c|c|}
\hline Name & Description & Density & ID & $\begin{array}{c}\text { Atoms per } \\
\text { molecule }\end{array}$ \\
\hline \multirow[t]{2}{*}{ poly(h2o) } & $\begin{array}{l}\text { Polyethylene: } \mathrm{CH}_{2} \text {, uses hydrogen in water } \\
\mathrm{S}(\alpha, \beta) \text { thermal kernel }\end{array}$ & 0.92 & 1001 & 2 \\
\hline & & & 6000 & 1 \\
\hline \multirow[t]{2}{*}{ polyethylene } & $\begin{array}{l}\text { Polyethylene: } \mathrm{CH}_{2}, \text { uses hydrogen in } \\
\text { polyethylene } \mathrm{S}(\alpha, \beta) \text { thermal kernel }\end{array}$ & 0.92 & 9001001 & 2 \\
\hline & & & 6000 & 1 \\
\hline \multirow[t]{3}{*}{ polyvinylcl } & $\begin{array}{l}\text { Polyvinyl chloride: } \mathrm{C}_{2} \mathrm{H}_{3} \mathrm{Cl} \text {, uses hydrogen } \\
\text { in polyethylene } \mathrm{S}(\alpha, \beta) \text { thermal kernel }\end{array}$ & 1.6 & 17000 & 1 \\
\hline & & & 9001001 & 3 \\
\hline & & & 6000 & 2 \\
\hline \multirow[t]{3}{*}{$\mathrm{pu}(\mathrm{no} 3) 4$} & Plutonium nitrate: $\mathrm{Pu}\left(\mathrm{NO}_{3}\right)_{4}$ & 2.447 & 94000 & 1 \\
\hline & & & 7000 & 4 \\
\hline & & & 8000 & 12 \\
\hline \multirow[t]{2}{*}{ puc } & Plutonium carbide: $\mathrm{PuC}$ & 13.6 & 94000 & 1 \\
\hline & & & 6000 & 1 \\
\hline \multirow[t]{2}{*}{ puf4 } & Plutonium tetrafluoride: $\mathrm{PuF}_{4}$ & 7.0 & 94000 & 1 \\
\hline & & & 9000 & 4 \\
\hline \multirow[t]{2}{*}{ pun } & Plutonium nitride: PuN & 14.25 & 94000 & 1 \\
\hline & & & 7000 & 1 \\
\hline \multirow[t]{2}{*}{ puo2 } & Plutonium oxide: $\mathrm{PuO}_{2}$ & 11.46 & 94000 & 1 \\
\hline & & & 8000 & 2 \\
\hline \multirow[t]{3}{*}{ pve } & $\begin{array}{l}\text { Polyvinyl chloride: } \mathrm{C}_{2} \mathrm{H}_{3} \mathrm{Cl} \text {, uses hydrogen } \\
\text { in polyethylene } \mathrm{S}(\alpha, \beta) \text { thermal kernel }\end{array}$ & 1.6 & 17000 & 1 \\
\hline & & & 9001001 & 3 \\
\hline & & & 6000 & 2 \\
\hline \multirow[t]{3}{*}{ pve(h2o) } & $\begin{array}{l}\text { Polyvinyl chloride: } \mathrm{C}_{2} \mathrm{H}_{3} \mathrm{Cl} \text { uses hydrogen in } \\
\text { water } \mathrm{S}(\alpha, \beta) \text { thermal kernel }\end{array}$ & 1.6 & 17000 & 1 \\
\hline & & & 1001 & 3 \\
\hline & & & 6000 & 2 \\
\hline \multirow[t]{3}{*}{ redwood } & Redwood: $\mathrm{C}_{6} \mathrm{H}_{10} \mathrm{O}_{5}$ & 0.387 & 6000 & 6 \\
\hline & & & 1000 & 10 \\
\hline & & & 8000 & 5 \\
\hline \multirow[t]{4}{*}{ tbp } & $\begin{array}{l}\text { Tributyl phosphate: }\left(\mathrm{C}_{4} \mathrm{H}_{9}\right)_{3} \mathrm{PO}_{4} \quad \text { uses } \\
\text { hydrogen in polyethylene } \mathrm{S}(\alpha, \beta) \text { thermal } \\
\text { kernel }\end{array}$ & 0.9724 & 9001001 & 27 \\
\hline & & & 6000 & 12 \\
\hline & & & 8000 & 4 \\
\hline & & & 15000 & 1 \\
\hline \multirow[t]{4}{*}{ tbp(h2o) } & $\begin{array}{l}\text { Tributyl phosphate: }\left(\mathrm{C}_{4} \mathrm{H}_{9}\right)_{3} \mathrm{PO}_{4}, \text { uses } \\
\text { hydrogen in water } \mathrm{S}(\alpha, \beta) \text { thermal kernel }\end{array}$ & 0.9724 & 1001 & 27 \\
\hline & & & 6000 & 12 \\
\hline & & & 8016 & 4 \\
\hline & & & 15031 & 1 \\
\hline \multirow[t]{3}{*}{$\operatorname{th}($ no3) 4} & & 2.3752 & 90000 & 1 \\
\hline & & & 7000 & 4 \\
\hline & & & 8000 & 12 \\
\hline \multirow[t]{2}{*}{ thf4 } & & 6.1 & 90000 & 1 \\
\hline & & & 9000 & 4 \\
\hline \multirow[t]{2}{*}{ u3o8 } & Uranium oxide (yellowcake): $\mathrm{U}_{3} \mathrm{O}_{8}$ & 8.3 & 92000 & 3 \\
\hline & & & 8000 & 8 \\
\hline \multirow[t]{2}{*}{$\mathrm{uc}$} & Uranium carbide: UC & 13.63 & 92000 & 1 \\
\hline & & & 6000 & 1 \\
\hline
\end{tabular}




\begin{tabular}{|c|c|c|c|c|}
\hline Name & Description & Density & ID & $\begin{array}{c}\text { Atoms per } \\
\text { molecule }\end{array}$ \\
\hline \multirow[t]{2}{*}{ uf4 } & Uranium tetrafluoride: $\mathrm{UF}_{4}$ & 6.7 & 92000 & 1 \\
\hline & & & 9000 & 4 \\
\hline \multirow[t]{2}{*}{ uf6 } & Uranium hexafluoride: $\mathrm{UF}_{6}$ & 4.68 & 92000 & 1 \\
\hline & & & 9000 & 6 \\
\hline \multirow[t]{2}{*}{ un } & Uranium nitride: UN & 14.31 & 92000 & 1 \\
\hline & & & 7000 & 1 \\
\hline \multirow[t]{2}{*}{ uo2 } & Uranium dioxide: $\mathrm{UO}_{2}$ & 10.96 & 92000 & 1 \\
\hline & & & 8000 & 2 \\
\hline \multirow[t]{3}{*}{ uo2(no3)2 } & Uranyl nitrate: $\mathrm{UO}_{2}\left(\mathrm{NO}_{3}\right)_{2}$ & 2.203 & 92000 & 1 \\
\hline & & & 7000 & 2 \\
\hline & & & 8000 & 8 \\
\hline \multirow[t]{3}{*}{ uo2f2 } & Uranyl fluoride: $\mathrm{UO}_{2} \mathrm{~F}_{2}$ & 6.37 & 92000 & 1 \\
\hline & & & 8000 & 2 \\
\hline & & & 9000 & 2 \\
\hline \multirow[t]{2}{*}{ uo3 } & Uranium trioxide: $\mathrm{UO}_{3}$ & 7.29 & 92000 & 1 \\
\hline & & & 8000 & 3 \\
\hline \multirow[t]{2}{*}{ water } & $\begin{array}{l}\mathrm{H}_{2} \mathrm{O} \text {, with natural abundance hydrogen and } \\
\text { oxygen and the } \mathrm{S}(\alpha, \beta) \text { thermal kernel for } \\
\text { hydrogen in water }\end{array}$ & 0.9982 & 1000 & 2 \\
\hline & & & 8000 & 1 \\
\hline \multirow[t]{2}{*}{ zr5h8 } & $\begin{array}{l}\text { Zirconium hydride as a mixture of } \mathrm{ZrH} \text { and } \\
\mathrm{ZrH}_{2} \text {, with an effective composition of } \mathrm{Zr}_{5} \mathrm{H}_{8}\end{array}$ & 5.61 & 1040000 & 5 \\
\hline & & & 7001001 & 8 \\
\hline \multirow[t]{2}{*}{ zrh2 } & Zirconium hydride: $\mathrm{ZrH}_{2}$ & 5.61 & 1040000 & 1 \\
\hline & & & 7001001 & 2 \\
\hline
\end{tabular}


Table 7.2.5. Alloys and mixtures

\begin{tabular}{|c|c|c|c|c|}
\hline Name & Description & Density & ID & Weight \% \\
\hline \multirow[t]{2}{*}{ carbonsteel } & Carbon steel & 7.8212 & 26000 & 99.0 \\
\hline & & & 6000 & 1.0 \\
\hline \multirow[t]{3}{*}{ dry-air } & & $1.20000-3$ & 6000 & 0.0126 \\
\hline & & & 7000 & 76.5081 \\
\hline & & & 8000 & 23.4793 \\
\hline \multirow[t]{13}{*}{ granite } & & 2.66 & 1000 & 0.0336 \\
\hline & & & 8000 & 47.8286 \\
\hline & & & 9000 & 0.0901 \\
\hline & & & 11000 & 2.2501 \\
\hline & & & 12000 & 0.1449 \\
\hline & & & 13000 & 7.4752 \\
\hline & & & 14000 & 32.8046 \\
\hline & & & 15000 & 0.0393 \\
\hline & & & 19000 & 5.0108 \\
\hline & & & 20000 & 1.1876 \\
\hline & & & 22000 & 0.252 \\
\hline & & & 25000 & 0.0465 \\
\hline & & & 26000 & 2.8367 \\
\hline \multirow[t]{5}{*}{ inconel } & Inconel & 8.3 & 14000 & 2.5 \\
\hline & & & 22000 & 2.5 \\
\hline & & & 404024000 & 15.0 \\
\hline & & & 404026000 & 7.0 \\
\hline & & & 404028000 & 73.0 \\
\hline \multirow[t]{5}{*}{ inconels } & $\begin{array}{l}\text { Inconel (maintained for } \\
\text { compatibility) }\end{array}$ & 8.3 & 14000 & 2.5 \\
\hline & & & 22000 & 2.5 \\
\hline & & & 24000 & 15.0 \\
\hline & & & 26000 & 7.0 \\
\hline & & & 28000 & 73.0 \\
\hline \multirow[t]{2}{*}{ kero(h2o) } & $\begin{array}{l}\text { Average kerosene (uses hydrogen for water } \\
S(\alpha, \beta) \text { thermal kernel) }\end{array}$ & 0.82 & 6000 & 84.0 \\
\hline & & & 1001 & 16.0 \\
\hline \multirow[t]{2}{*}{ kerosene } & $\begin{array}{l}\text { Average kerosene (uses hydrogen for } \\
\text { polyethylene } S(\alpha, \beta) \text { thermal kernel) }\end{array}$ & 0.82 & 6000 & 84.0 \\
\hline & & & 9001001 & 16.0 \\
\hline \multirow[t]{13}{*}{ limestone } & & 2.15 & 1000 & 0.0863 \\
\hline & & & 6000 & 11.3548 \\
\hline & & & 8000 & 49.6925 \\
\hline & & & 11000 & 0.0371 \\
\hline & & & 12000 & 4.7656 \\
\hline & & & 13000 & 0.4294 \\
\hline & & & 14000 & 2.4299 \\
\hline & & & 15000 & 0.0175 \\
\hline & & & 16000 & 0.0251 \\
\hline & & & 19000 & 0.2744 \\
\hline & & & 20000 & 30.4731 \\
\hline & & & 22000 & 0.036 \\
\hline & & & 26000 & 0.3783 \\
\hline \multirow[t]{2}{*}{ mgconcrete } & Magnuson's Concrete & 2.147 & 26000 & 0.5595 \\
\hline & & & 1000 & 0.3319 \\
\hline
\end{tabular}




\begin{tabular}{|c|c|c|c|c|}
\hline \multirow[t]{13}{*}{ Name } & Description & Density & ID & Weight \% \\
\hline & & & 6000 & 10.5321 \\
\hline & & & 8000 & 49.943 \\
\hline & & & 11000 & 0.1411 \\
\hline & & & 12000 & 9.42 \\
\hline & & & 13000 & 0.7859 \\
\hline & & & 14000 & 4.2101 \\
\hline & & & 16000 & 0.2483 \\
\hline & & & 17000 & 0.0523 \\
\hline & & & 19000 & 0.9445 \\
\hline & & & 20000 & 22.6318 \\
\hline & & & 22000 & 0.1488 \\
\hline & & & 25000 & 0.0512 \\
\hline \multirow[t]{10}{*}{ orconcrete } & Oak Ridge Concrete & 2.2994 & 26000 & 0.7784 \\
\hline & & & 1000 & 0.6187 \\
\hline & & & 6000 & 17.52 \\
\hline & & & 8000 & 41.02 \\
\hline & & & 11000 & 0.0271 \\
\hline & & & 12000 & 3.265 \\
\hline & & & 13000 & 1.083 \\
\hline & & & 14000 & 3.448 \\
\hline & & & 19000 & 0.1138 \\
\hline & & & 20000 & 32.13 \\
\hline \multirow[t]{5}{*}{ pyrex } & Pyrex & 2.23 & 5000 & 3.7 \\
\hline & & & 13000 & 1.0 \\
\hline & & & 8000 & 53.5 \\
\hline & & & 14000 & 37.7 \\
\hline & & & 11000 & 4.1 \\
\hline \multirow[t]{7}{*}{ reg-concrete } & $\begin{array}{l}\text { Regulatory Concrete (developed for U.S. } \\
\text { NRC) }\end{array}$ & 2.3 & 26000 & 1.4 \\
\hline & & & 1000 & 1.0 \\
\hline & & & 13000 & 3.4 \\
\hline & & & 20000 & 4.4 \\
\hline & & & 8000 & 53.2 \\
\hline & & & 14000 & 33.7 \\
\hline & & & 11000 & 2.9 \\
\hline \multirow[t]{13}{*}{ rfconcrete } & Rocky Flats Concrete & 2.321 & 26000 & 1.01 \\
\hline & & & 1000 & 0.75 \\
\hline & & & 6000 & 5.52 \\
\hline & & & 7000 & 0.02 \\
\hline & & & 8000 & 48.49 \\
\hline & & & 11000 & 0.63 \\
\hline & & & 12000 & 1.25 \\
\hline & & & 13000 & 2.17 \\
\hline & & & 14000 & 15.5 \\
\hline & & & 16000 & 0.19 \\
\hline & & & 19000 & 1.37 \\
\hline & & & 20000 & 23.0 \\
\hline & & & 22000 & 0.1 \\
\hline \multirow[t]{4}{*}{ ss304 } & $\begin{array}{l}\text { Stainless steel-304 (using nuclide with special } \\
\text { weighting for ENDF/B-V data only) }\end{array}$ & 7.94 & 6000 & 0.08 \\
\hline & & & 14000 & 1.0 \\
\hline & & & 15000 & 0.045 \\
\hline & & & 304024000 & 19.0 \\
\hline
\end{tabular}




\begin{tabular}{|c|c|c|c|c|}
\hline Name & Description & Density & ID & Weight \% \\
\hline & & & 25000 & 2.0 \\
\hline & & & 304026000 & 68.375 \\
\hline & & & 304028000 & 9.5 \\
\hline \multirow[t]{7}{*}{ ss304s } & $\begin{array}{l}\text { Stainless steel-304 (using standard nuclides } \\
\text { instead of special weighted nuclides) }\end{array}$ & 7.94 & 6000 & 0.08 \\
\hline & & & 14000 & 1.0 \\
\hline & & & 15000 & 0.045 \\
\hline & & & 24000 & 19.0 \\
\hline & & & 25000 & 2.0 \\
\hline & & & 26000 & 68.375 \\
\hline & & & 28000 & 9.5 \\
\hline \multirow[t]{8}{*}{ ss316 } & $\begin{array}{l}\text { Stainless steel-316 (using nuclide with special } \\
\text { weighting for ENDF/B-V data only) }\end{array}$ & 8.03 & 6000 & 0.08 \\
\hline & & & 14000 & 1.0 \\
\hline & & & 15000 & 0.045 \\
\hline & & & 304024000 & 17.0 \\
\hline & & & 25000 & 2.0 \\
\hline & & & 304026000 & 65.375 \\
\hline & & & 304028000 & 12.0 \\
\hline & & & 42000 & 2.5 \\
\hline \multirow[t]{8}{*}{ ss316s } & $\begin{array}{l}\text { Stainless steel-316 (using standard nuclides } \\
\text { instead of special weighted nuclides) }\end{array}$ & 8.03 & 6000 & 0.08 \\
\hline & & & 14000 & 1.0 \\
\hline & & & 15000 & 0.045 \\
\hline & & & 24000 & 17.0 \\
\hline & & & 25000 & 2.0 \\
\hline & & & 26000 & 65.375 \\
\hline & & & 28000 & 12.0 \\
\hline & & & 42000 & 2.5 \\
\hline \multirow[t]{2}{*}{$\mathrm{u}(.27)$ metal } & $\begin{array}{l}\text { Depleted uranium metal having a fixed } \\
\text { isotope distribution [to specify a different } \\
\text { distribution, the user should use URANIUM } \\
\text { instead of U(.27)METAL] }\end{array}$ & 19.05 & 92235 & 0.27 \\
\hline & & & 92238 & 99.73 \\
\hline zircalloy & No longer available & 6.56 & 302040000 & 100 \\
\hline \multirow[t]{6}{*}{ zirc2 } & Zircaloy-2 & 6.56 & 40000 & 98.25 \\
\hline & & & 50000 & 1.45 \\
\hline & & & 26000 & 0.135 \\
\hline & & & 24000 & 0.1 \\
\hline & & & 28000 & 0.055 \\
\hline & & & 72000 & 0.01 \\
\hline \multirow[t]{5}{*}{ zirc4 } & Zircaloy-4 & 6.56 & 40000 & 98.23 \\
\hline & & & 50000 & 1.45 \\
\hline & & & 26000 & 0.21 \\
\hline & & & 24000 & 0.1 \\
\hline & & & 72000 & 0.01 \\
\hline
\end{tabular}

\subsubsection{Table of Fissile Solutions}

The Standard Composition Library ( through ) describes the various compounds, alloys, elements, and isotopes one may use in defining the material mixtures for a given problem. In addition to the various 
materials listed there, one is also free to use any of the fissile solutions listed in Table 7.2.6. Indeed, the user is encouraged to treat the solutions listed in Table 7.2.6 as he would any other standard composition. Using empirical fits to experimental data, the code will then automatically calculate the density of the solution, or the user can explicitly specify the density. The code then calculates the volume fraction corresponding to the heavy metal, acid, and water components of the solution. A fissile solution starts with the keyword, SOLUTION, after which one or two salts and the corresponding acid may be specified. Input specifications for fissile solutions can be found in the XSProc chapter.

Table 7.2.6. Available fissile solution components

\begin{tabular}{lc}
\hline $\begin{array}{c}\text { Name of } \\
\text { component }\end{array}$ & $\begin{array}{c}\text { Nuclides in } \\
\text { component }\end{array}$ \\
\hline & Nitrate solutions \\
\hline $\mathrm{UO}_{2}\left(\mathrm{NO}_{3}\right)_{2}$ & 9200070008000 \\
\hline $\mathrm{Pu}\left(\mathrm{NO}_{3}\right)_{4}$ & 9400070008000 \\
\hline $\mathrm{Th}\left(\mathrm{NO}_{3}\right)_{4}$ & 9000070008000 \\
\hline $\mathrm{HNO}_{3} \mathrm{ACID}$ & 100070008000 \\
\hline & Fluoride solutions \\
\hline $\mathrm{UO}_{2} \mathrm{~F}_{2}$ & 9200080009000 \\
\hline $\mathrm{PuF}_{4}$ & 940009000 \\
\hline $\mathrm{ThF}_{4}$ & 900009000 \\
\hline $\mathrm{HFACID}$ & 10009000 \\
\hline
\end{tabular}

\subsubsection{References}

1 G. Audi, A. H.Wapstra, and C.Thibault, "The Ame2003 Atomic Mass Evaluation (II)," Nucl. Phys. A729, 337-676, December 22, 2003.

2 J. K. Böhlke, J. R. de Laeter, P. De Bievre, H. Hidaka, H. S. Peiser, K. J. R. Rosman, and P. D. P. Taylor, "Isotopic Compositions of the Elements, 2001," J. Phys. Chem. Ref. Data 34(1), 2005.

3 CRC Handbook of Chemistry and Physics, 64th Edition, CRC Press, Boca Raton, Florida, 1983-1984. 


\title{
7.3 BONAMI: RESONANCE SELF-SHIELDING BY THE BONDARENKO METHOD
}

U. Mertyurek and M. L. Williams

\begin{abstract}
BONAMI is a module of the SCALE code system that is used to perform Bondarenko calculations for resonance self-shielding. BONAMI obtains problem-independent cross sections and Bondarenko shielding factors from a multigroup (MG) AMPX master library, and it creates a MG AMPX working library of self-shielded, problem-dependent cross sections. Several options may be used to compute the background cross section values using the narrow resonance or intermediate resonance approximations, with and without Bondarenko iterations. A novel interpolation scheme is used that avoids many of the problems exhibited by other interpolation methods for the Bondarenko factors. BONAMI is most commonly used in automated SCALE sequences and is fully integrated within the SCALE cross section processing module, XSProc.
\end{abstract}




\section{TABLE OF CONTENTS}

Page

7.3 BONAMI: Resonance Self-Shielding by the Bondarenko Method ...................................... 7-194

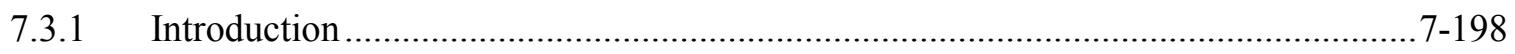

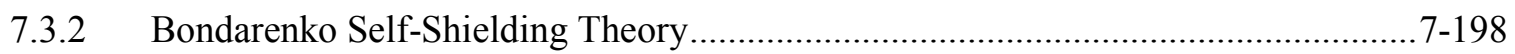

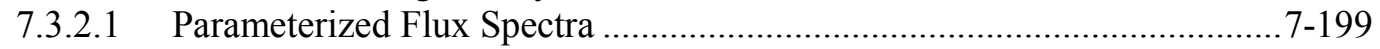

7.3.2.2 Self-Shielded Cross Section Data in SCALE Libraries .............................7-202

7.3.2.3 Background Cross Section Options in BONAMI .................................... 7-203

7.3.2.4 Self-Shielded Cross Sections for Heterogeneous Media ............................ 7-204

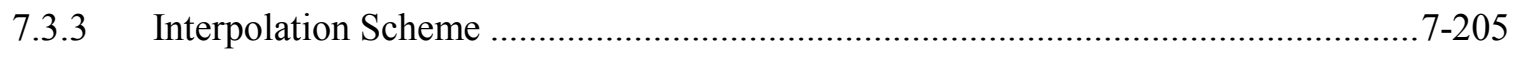

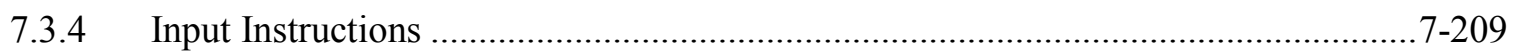

7.3.4.1 Notes on input ...............................................................................

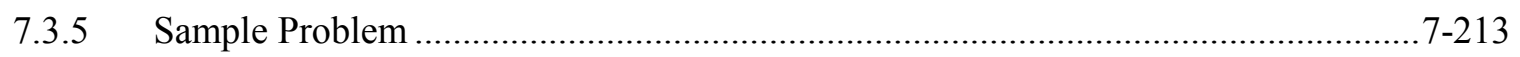

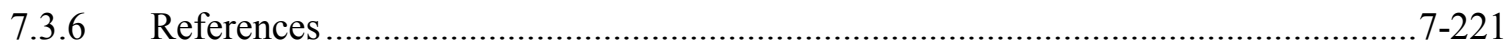




\section{LIST OF FIGURES}

$\underline{\text { Page }}$

Figure 7.3.1. Plot of f-factor variation for ${ }^{238} \mathrm{U}$ capture reaction ...................................................206

Figure 7.3.2. Illustration of the effects of varying "powers" in the Greene interpolation method. ..........207

Figure 7.3.3. Use of Greene's method to fit the $\sigma_{0}$ variation of Bondarenko factors for case 1 ..............208

Figure 7.3.4. Use of Greene's method to fit the $\sigma_{0}$ variation of Bondarenko factors for case 2 ..............209 


\section{ACKNOWLEDGMENTS}

The authors express gratitude to B. T. Rearden and M. A. Jessee for their supervision of the SCALE project and review of the manuscript. The authors acknowledge N. M. Greene, formerly of ORNL, for his original development of and contributions to the BONAMI module and methodology. Finally, the authors wish to thank Sheila Walker for the completion and publication of this document. 


\subsubsection{Introduction}

BONAMI (BONdarenko AMPX Interpolator) is a SCALE module that performs resonance self-shielding calculations based on the Bondarenko method. ${ }^{1}$ It reads Bondarenko shielding factors ("f-factors") and infinitely dilute microscopic cross sections from a problem-independent nuclear data library processed by the AMPX system, ${ }^{2}$ interpolates the tabulated shielding factors to appropriate temperatures and background cross sections for each nuclide in the system, and produces a self-shielded, problemdependent data set.

The code performs self-shielding for an arbitrary number of mixtures using either the narrow resonance (NR) or intermediate resonance (IR) approximation. ${ }^{3}$ The latter capability was introduced in SCALE 6.2. BONAMI has several options for computing background cross sections, which may include Bondarenko iterations to approximately account for the impact of resonance interference for multiple resonance absorbers. Heterogeneous effects are treated using equivalence theory based on an "escape cross section" for arrays of slabs, cylinders, or spheres. During the execution of a typical SCALE computational sequence using XSProc, Dancoff factors for uniform lattices of square- or triangular-pitched units are calculated automatically for BONAMI by numerical integration over the chord length distribution. However, for non-uniform lattices - such as those containing water holes, control rods, and so on - the SCALE module MCDancoff can be run to compute Dancoff factors using Monte Carlo for an arbitrary $3 \mathrm{D}$ configuration, and these values are then provided in the sequence input.

The major advantages of the Bondarenko approach are its simplicity and speed compared with SCALE's more rigorous CENTRM/PMC self-shielding method, which performs a pointwise (PW) deterministic transport calculation "on the fly" to compute multigroup (MG) self-shielded cross sections. With the availability of IR theory in BONAMI, accurate results can be obtained for a variety of system types without the computation expense of CENTRM/PMC.

\subsubsection{Bondarenko Self-Shielding Theory}

In MG resonance self-shielding calculations, one is interested in calculating effective cross sections of the form

$$
\sigma_{\mathrm{X}, \mathrm{g}}^{(\mathrm{r})}=\frac{\int_{\mathrm{g}} \sigma_{\mathrm{X}}^{(\mathrm{r})}(\mathrm{E}) \Phi(\mathrm{E}) \mathrm{dE}}{\int_{\mathrm{g}} \Phi(\mathrm{E}) \mathrm{dE}}
$$

where $\sigma_{\mathrm{X}, \mathrm{g}}^{(\mathrm{r})}$ is the shielded MG cross section for reaction type $X$ of resonance nuclide $r$ in group $g ; \sigma_{\mathrm{X}}^{(\mathrm{r})}(\mathrm{E})$ is a PW cross section; and $\Phi(\mathrm{E})$ is the PW weighting function, which approximates the flux spectrum per unit of energy for the system of interest. PW cross section values are known from processing evaluated data in ENDF/B files; therefore, resonance self-shielding depends mainly on determining the problemdependent flux spectrum $\Phi(\mathbf{E})$, which may exhibit significant fine structure variations as a result of resonance reactions.

The essence of the Bondarenko method is to parameterize the flux spectrum corresponding to varying degrees of self-shielding, represented by the background cross section parameter $\sigma_{0}$ (called "sigmazero") and the Doppler broadening temperature $T$. Hence, 


$$
\Phi(\mathbf{E}) \rightarrow \Phi\left(\mathbf{E} ; \sigma_{0, \mathrm{~g}}^{(\mathrm{r})}, \mathrm{T}\right), \mathbf{E} \in \mathbf{g} ; \text { and } \sigma_{\mathrm{X}, \mathrm{g}}^{(\mathrm{r})} \rightarrow \sigma_{\mathrm{X}, \mathrm{g}}^{(\mathrm{r})}\left(\sigma_{0, \mathrm{~g}}^{(\mathrm{r})}, \mathrm{T}\right)
$$

With this approach, it is possible to preprocess MG data for different background cross sections representing varying degrees of resonance self-shielding. This allows the MG averaging to be performed during the original MG library processing, so that BONAMI can do a simple interpolation on the background cross section and temperature to obtain self-shielded cross sections. This procedure is much faster than the CENTRM/PMC method in SCALE, which computes a PW flux spectrum by solving the neutron transport equation on a PW energy mesh in CENTRM and then evaluates Eq. (7.3.1) in PMC "on the fly" during a sequence execution.

BONAMI performs two main tasks: (a) computation of background cross sections for all nuclides in each mixture in the system and (b) interpolation of shielded cross sections from the library values tabulated vs. background cross sections and temperature. The BONAMI calculation is essentially isolated from the computation of the tabulated shielded cross sections, which is performed by the AMPX processing code system - the only connection is through the definition of the background cross section used in processing the library values. Various approximations can be used to parameterize the flux spectrum in terms of a background XS, as required by the Bondarenko method. We will first consider several approaches to representing the flux in an infinite medium, which lead to different definitions of the background cross section. BONAMI's use of equivalence theory to extend the homogeneous methods to address heterogeneous systems, such as reactor lattices, is discussed in the following section.

\subsubsection{Parameterized Flux Spectra}

Several approximations can be applied to the infinite medium transport equation to parameterize the flux spectrum in terms of a background XS, as required by the Bondarenko method. The resulting homogeneous spectra are used in AMPX to process MG cross sections which can also can be applied to heterogeneous systems (i.e., lattices) by using equivalence theory; thus the key step is determining approximations that provide parameterized solutions for homogeneous media. The neutron transport equation for a homogeneous medium at temperature $T$, containing a resonance nuclide $r$ mixed with other nuclides can be expressed as

$$
\left(\Sigma_{\mathrm{t}}^{(\mathrm{r})}(\mathrm{E}, \mathrm{T})+\sum_{j \neq r} \Sigma_{\mathrm{t}}^{(\mathrm{j})}(\mathrm{E}, \mathrm{T})\right) \Phi(\mathrm{E}, \mathrm{T})=\mathrm{S}^{(\mathrm{r})}(\mathrm{E}, \mathrm{T})+\sum_{j \neq r} \mathrm{~S}^{(\mathrm{j})}(\mathrm{E}, \mathrm{T})
$$

where $\Sigma_{t}^{(\mathrm{r})}(\mathrm{E}, \mathrm{T}), \mathrm{S}^{(\mathrm{r})}(\mathrm{E}, \mathrm{T})$ are the macroscopic total XS and elastic scattering source for $r$, respectively; and $\Sigma_{\mathrm{t}}^{(\mathrm{j})}(\mathrm{E}, \mathrm{T}), \mathrm{S}^{(\mathrm{j})}(\mathrm{E}, \mathrm{T})$ are the macroscopic total cross section and elastic source, respectively, for a nuclide $j$. The cross sections in all these expressions are Doppler-broadened to the temperature of the medium. The nuclides in the summations (i.e., all nuclides except $r$ ) are called background nuclides for the resonance absorber $r$.

The NR approximation can be used to approximate scattering sources of nuclides for which the neutron energy loss is large compared with the practical widths of resonances for the absorber materials of interest. Applying the NR approximation for the scattering source of background material $j$ gives

$$
\mathrm{S}^{(\mathrm{j})}(\mathrm{E}, \mathrm{T}) \rightarrow \Sigma_{\mathrm{p}}^{(\mathrm{j})} C(E) ; \text { for } \mathrm{j}=\mathrm{a} \text { NR-scatterer nuclide }
$$


where $\mathrm{C}(\mathrm{E})$ is a slowly varying function representative of the asymptotic (i.e., no absorption) flux in a homogeneous medium, which approximates the flux between resonances. In the resolved resonance range of most important resonance absorbers, the asymptotic flux per unit energy is represented as,

$$
C(\mathrm{E})=\frac{\Phi_{\infty}}{E}
$$

where $\Phi_{\infty}$ is an arbitrary normalization constant that cancels from the MG cross section expression. In the thermal range a Maxwellian spectrum is used for $\mathrm{C}(\mathrm{E})$, and in the fast range a fission spectrum is used. The SCALE Cross Section Libraries section of the SCALE documentation gives analytical expressions for $\mathrm{C}(\mathrm{E})$ used in AMPX to process MG data with the NR approximation. AMPX also has an option to input numerical values for $\mathrm{C}(\mathrm{E})$, obtained for example from a $\mathrm{PW}$ slowing-down calculation with CENTRM. This method has been used to process MG data for some nuclides on the SCALE libraries.

Conversely, the wide resonance (WR) approximation has been used to represent elastic scattering sources of nuclides for which the neutron energy loss is small compared with the practical width of the resonance. This approximation tends to be more accurate for heavy nuclides and for lower energies. The limit of infinite mass is usually assumed, so the WR approximation is sometimes called the infinite mass (IM) approximation. Because of the assumption of IM, there is no energy loss due to collisions with WR scatterers. Applying the WR approximation for the slowing-down source of background nuclide $j$ gives

$$
\mathrm{S}^{(\mathrm{j})}(\mathrm{E}, \mathrm{T}) \rightarrow \Sigma_{\mathrm{s}}^{(\mathrm{j})}(\mathrm{E}, \mathrm{T}) \Phi(\mathrm{E}, \mathrm{T}) ; \quad \text { for } j=\text { a WR-scatterer nuclide } .
$$

The IR approximation was proposed in the 1960s for scatterers with slowing-down properties intermediate between those of NR and WR scatterers. ${ }^{3}$ The IR method represents the scattering source for arbitrary nuclide $j$ by a linear combination of NR and WR expressions. This is done by introducing an IR parameter usually called lambda, such that

$$
\mathrm{S}^{(\mathrm{j})}(\mathrm{E}, \mathrm{T}) \rightarrow \underbrace{\lambda_{\mathrm{g}}^{(\mathrm{j})} \sum_{\mathrm{p}}^{(\mathrm{j})} C(E)}_{\text {NR scatterer }}+\left(1-\lambda_{\mathrm{g}}^{(\mathrm{j})}\right) \underbrace{\sum_{\mathrm{s}}^{(\mathrm{j})}(\mathrm{E}, \mathrm{T}) \Phi(\mathrm{E}, \mathrm{T})}_{\text {WR scatterer }} ; \mathrm{E} \in \mathrm{g} .
$$

A value of $\lambda=1$ reduces Eq. (7.3.7) to the NR expression, whereas $\lambda=0$ reduces the equation to the WR expression. Fractional $\lambda$ 's are for IR scatterers. Since the type of scatterer can change with the energy, the IR lambdas are functions of the energy group as well as the nuclide. The $\lambda$ values represent the moderation "effectiveness" of a given nuclide, compared to hydrogen. The AMPX module LAMBDA was used to compute the IR parameters on the SCALE libraries. (See AMPX documentation distributed with SCALE) Substituting Eq. (7.3.7) into Eq. (7.3.3) and then dividing by the absorber number density $N^{(r)}$ gives the following IR approximation for the infinite medium transport equation in energy group $\mathrm{g}$

$$
\left(\sigma_{\mathrm{t}}^{(\mathrm{r})}(\mathrm{E}, \mathrm{T})+\sigma_{0}^{(\mathrm{r})}(\mathrm{E}, \mathrm{T})\right) \Phi^{(\mathrm{r})}(\mathrm{E}, \mathrm{T})=\frac{1}{\mathrm{~N}^{(\mathrm{r})}} \mathrm{S}^{(\mathrm{r})}(\mathrm{E}, \mathrm{T})+\frac{1}{\mathrm{~N}^{(\mathrm{r})}} \sum_{j \neq r} \lambda_{\mathrm{g}}^{(\mathrm{j})} \sum_{\mathrm{p}}^{(\mathrm{j})} C(E),
$$

where the background cross section of $r$ in the homogeneous medium is defined as

$$
\sigma_{0}^{(\mathrm{r})}(\mathrm{E}, \mathrm{T})=\frac{1}{\mathrm{~N}^{(\mathrm{r})}} \sum_{j \neq r}\left(\sum_{\mathrm{a}}^{(\mathrm{j})}(\mathrm{E}, \mathrm{T})+\lambda_{\mathrm{g}}^{(\mathrm{j})} \sum_{\mathrm{s}}^{(\mathrm{j})}(\mathrm{E}, \mathrm{T})\right)
$$


Although Eq. (7.3.8) provides the flux spectrum as a function of the background cross section $\sigma_{0}^{(\mathrm{r})}(\mathrm{E}, \mathrm{T})$, it is not in a form that can be preprocessed when the MG library is generated, because the energy variation of $\sigma_{0}^{(\mathrm{r})}(\mathrm{E}, \mathrm{T})$ must be known. If the total cross sections of the background nuclides in Eq. (7.3.9) have different energy variations, the shape of $\sigma_{0}^{(\mathrm{r})}(\mathrm{E}, \mathrm{T})$ depends on their relative concentrations - which are not known when the MG library is processed. However, if the cross sections in Eq. (7.3.9) are independent of energy, so that the background cross section is constant, Eq. (7.3.8) can be solved for any arbitrary value of $\sigma_{0}^{(\mathrm{r})}$ as a parameter. This obviously occurs for the special case in which nuclide $r$ is the only resonance nuclide in the mixture; i.e., the background materials are nonabsorbing moderators for which the total cross section is equal to the potential cross section. In this case, $\sigma_{0}^{(\mathrm{r})}(\mathrm{E}, \mathrm{T}) \rightarrow \sigma_{0, \mathrm{~g}}^{(\mathrm{r})}$, where

$$
\sigma_{0, \mathrm{~g}}^{(\mathrm{r})}=\frac{1}{\mathrm{~N}^{(\mathrm{r})}} \sum_{\mathrm{j} \neq \mathrm{i}} \mathrm{N}^{(\mathrm{j})} \lambda_{\mathrm{g}}^{(\mathrm{j})} \sigma_{\mathrm{p}}^{(\mathrm{j})}
$$

If the mixture contains multiple resonance absorbers, as is usually the case, other approximations must be made to obtain a constant background cross section.

The approximation of "no resonance interference" assumes that resonances of background nuclides do not overlap with those of nuclide $r$, so their total cross sections can be approximated by the potential values within resonances of $r$ where self-shielding occurs. In this approximation, the expression in Eq. (7.3.10) is also used for the background cross section.

Another approximation is to represent the energy-dependent cross sections of the background nuclides by their group-averaged (i.e., self-shielded cross) values; thus

$$
\sigma_{\mathrm{a}}^{(\mathrm{j})}(\mathrm{E}, \mathrm{T}) \rightarrow \sigma_{\mathrm{a}, \mathrm{g}}^{(\mathrm{j})} ; \quad \sigma_{\mathrm{s}}^{(\mathrm{j})}(\mathrm{E}, \mathrm{T}) \rightarrow \sigma_{\mathrm{s}, \mathrm{g}}^{(\mathrm{j})} \text { for } \mathrm{E} \in \mathrm{g} \cdot
$$

In this case, the background cross section in Eq. (7.3.9) for nuclide $r$ is the group-dependent expression,

$$
\sigma_{0, g}^{(\mathrm{r})}=\frac{1}{\mathrm{~N}^{(\mathrm{r})}} \sum_{j \neq r}\left(\Sigma_{\mathrm{a}, \mathrm{g}}^{(\mathrm{j})}+\lambda_{\mathrm{g}}^{(\mathrm{j})} \Sigma_{\mathrm{s}, \mathrm{g}}^{(\mathrm{j})}\right)
$$

An equation similar to Eq. (7.3.12) is used for the background cross sections of all resonance nuclides; thus the self-shielded cross sections of each resonance absorber depend on the shielded cross sections of all other resonance absorbers in the mixture. When self-shielding operations are performed with BONAMI for this approximation, "Bondarenko" iterations are performed to account for the interdependence of the shielded cross sections.

Assuming that $\sigma_{0}^{(\mathrm{r})}$ is represented as a groupwise-constant based on one of the previous approximations, several methods can be used to obtain a parameterized flux spectrum for preprocessing Bondarenko data in the MG libraries. In the simpliest approach, the scattering source of the resonance nuclide $r$ in Eq. (7.3.8) is represented by the NR approximation, $\mathrm{S}^{(\mathrm{r})}(\mathrm{E}, \mathrm{T}) \rightarrow \Sigma_{\mathrm{p}}^{(\mathrm{r})} C(E)$. In this case, Eq. (7.3.8) can be solved analytically to obtain the following expression for the flux spectrum used to process MG data as a function of $\sigma_{0}^{(\mathrm{r})}$ : 


$$
\Phi^{(\mathrm{r})}\left(\mathrm{E} ; \sigma_{0}^{(\mathrm{r})}, \mathrm{T}\right)=\frac{\sigma_{\mathrm{p}}^{(\mathrm{r})}+\frac{1}{\mathrm{~N}^{(\mathrm{r})}} \sum_{j \neq r} \Sigma_{\mathrm{p}}^{(\mathrm{j})}}{\sigma_{\mathrm{t}}^{(\mathrm{r})}(\mathrm{E}, \mathrm{T})+\sigma_{0}^{(\mathrm{r})}} C(E) \rightarrow \frac{C(E)}{\sigma_{\mathrm{t}}^{(\mathrm{r})}(\mathrm{E}, \mathrm{T})+\sigma_{0}^{(\mathrm{r})}},
$$

where $C(E)$ includes is an arbitrary constant multiplier that cancels from eq. (7.3.1).

A more accurate approach that does not require using the NR approximation is to directly solve the IR form of the neutron transport equation using PW cross sections, with the assumption of no interference between mixed absorber resonances. The IRFfactor module of AMPX uses XSProc to calculate the selfshielded flux spectrum for MG data processing using one of two options:

(a) A homogeneous model corresponding to an infinite medium of the resonance nuclide mixed with hydrogen, in which the ratio of the absorber to hydrogen number densities is varied in CENTRM to obtain the desired background cross section values;

(b) A heterogeneous model corresponding to a 2D unit cell from an infinite lattice, in which the cell geometry (e.g., pitch) as well as the absorber number density is varied in CENTRM to obtain the desired background cross section values.

Both of these models provide a numerical solution for the flux spectrum $\Phi\left(E ; \sigma_{0}^{(r)}, T\right)$. Details on these approaches are given in reference 2 .

\subsubsection{Self-Shielded Cross Section Data in SCALE Libraries}

The AMPX code system processes self-shielded cross sections using the flux expressions described in the preceding section. For MG libraries in SCALE-6.2 and later versions, the NR approximation in Eq. (7.3.13) is used to represent the flux spectrum for nuclides with masses below $A=40$, since the NR approximation is generally accurate for low-mass nuclides and/or high energies. The standard AMPX weight functions are used to represent $\mathrm{C}(\mathrm{E})$ over the entire energy range for all nuclides with $\mathrm{A}<40$, except for hydrogen and oxygen which use a calculated C(E) from CENTRM. The NR approximation with a calculated $C(E)$ function is also used to represent the spectrum above the resolved resonance range for nuclides with $\mathrm{A}>40$; but in the resolved resonance range of these nuclides, AMPX processes shielded cross sections with flux spectra obtained from CENTRM calculations using either a homogeneous or heterogeneous model. Regardless of the method used to obtain the flux spectrum, the parameterized shielded cross sections for absorber nuclide " $r$ " are computed from the expression,

$$
\sigma_{\mathrm{X}, \mathrm{g}}^{(\mathrm{r})}\left(\sigma_{0}^{(\mathrm{r})}, \mathrm{T}\right)=\frac{\int_{\mathrm{g}} \sigma_{\mathrm{X}}^{(\mathrm{r})}(\mathrm{E}, \mathrm{T}) \Phi\left(\mathrm{E} ; \sigma_{0}^{(\mathrm{r})}, \mathrm{T}\right) \mathrm{dE}}{\int_{\mathrm{g}} \Phi\left(\mathrm{E} ; \sigma_{0}^{(\mathrm{r})}, \mathrm{T}\right) \mathrm{dE}},
$$

where $\Phi\left(\mathrm{E} ; \sigma_{0}^{(\mathrm{r})}, \mathrm{T}\right)$ is the flux for a given value of $\sigma_{0}^{(\mathrm{r})}$ and $T$.

Rather than storing self-shielded cross sections in the master library, AMPX converts them to Bondarenko shielding factors, also called f-factors, defined as the ratio of the shielded cross section to the infinitely dilute cross section. Thus the MG libraries in SCALE contain Bondarenko data consisting of f-factors defined as 


$$
\mathrm{f}_{\mathrm{X}, \mathrm{g}}^{(\mathrm{r})}\left(\sigma_{0}, \mathrm{~T}\right)=\frac{\sigma_{\mathrm{X}, \mathrm{g}}^{(\mathrm{r})}\left(\sigma_{0}, \mathrm{~T}\right)}{\sigma_{\mathrm{X}, \mathrm{g}}^{(\mathrm{r})}(\infty)},
$$

and infinitely dilute cross sections defined as,

$$
\sigma_{\mathrm{X}, \mathrm{g}}^{(\mathrm{r})}(\infty)=\sigma_{\mathrm{X}, \mathrm{g}}^{(\mathrm{r})}\left(\sigma_{0}=\infty, \mathrm{T}=\mathrm{T}_{\mathrm{ref}}\right) \rightarrow \frac{\int_{\mathrm{g}} \sigma_{\mathrm{X}}^{(\mathrm{r})}\left(\mathrm{E}, \mathrm{T}_{\mathrm{ref}}\right) \mathrm{C}(\mathrm{E}) \mathrm{dE}}{\int_{\mathrm{g}} \mathrm{C}(\mathrm{E}) \mathrm{dE}}
$$

In AMPX, the reference temperature for the infinitely dilute cross section is normally taken to be $293 \mathrm{~K}$. Bondarenko data on SCALE libraries are provided for all energy groups and for five reaction types: total, radiative capture, fission, within-group scattering, and elastic scatter. Recent SCALE libraries include ffactors at $\sim 10-30$ background cross section values (depending on nuclide) ranging from $\sim 10^{-3}$ to $\sim 10^{10}$ barns, which span the range of self-shielding conditions. Typically the f-factor data are tabulated at five temperature values. Background cross sections and temperatures available for each nuclide in the SCALE MG libraries are given in the SCALE Cross Section Libraries chapter.

\subsubsection{Background Cross Section Options in BONAMI}

To compute self-shielded cross sections for nuclide $r$, BONAMI first computes the appropriate background cross section for the system of interest and then interpolates the library Bondarenko data to obtain the f-factor corresponding to this $\sigma_{0}$ and nuclide temperature. Several options are available in BONAMI to compute the background cross section, based on Eqs. (7.3.10) and (7.3.12) in the preceding section. The options are specified by input parameter "iropt" and have the following definitions:

(a) iropt $=0 \Rightarrow$ NR approximation with Bondarenko iterations:

Background cross sections for all nuclides are computed using Eq. (7.3.12) with $\lambda=1$; therefore,

$$
\sigma_{0}^{(\mathrm{r})}=\frac{1}{\mathrm{~N}^{(\mathrm{r})}} \sum_{j \neq r} \sum_{\mathrm{t}, \mathrm{g}}^{(\mathrm{j})} .
$$

Since the background cross section for each nuclide depends on the shielded total cross sections of all other nuclides in the mixture, "Bondarenko iterations" are performed in BONAMI to obtain a consistent set of shielded cross sections. Bondarenko iterations provide a crude method of accounting for resonance interference effects that are ignored by the approximation for $\sigma_{0}^{(\mathrm{r})}$ in Eq. (7.3.10). The BONAMI iterative algorithm generally converges in a few iterations. Prior to SCALE-6.2, this option was the only one available in BONAMI, and it is still the default for XSProc.

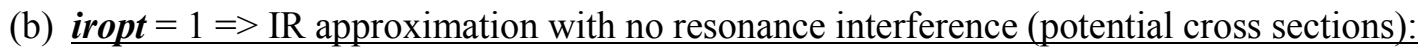
Background cross sections for all nuclides are computed using Eq. (7.3.10). No Bondarenko iterations are needed.

(c) iropt $\mathrm{t}=2 \Rightarrow \mathrm{IR}$ approximation with Bondarenko iterations, but no resonance scattering:

Background cross sections for all nuclides are computed using Eq. (7.3.12) with the scattering cross section approximated by the potential value; therefore, 


$$
\sigma_{0}^{(\mathrm{r})}=\frac{1}{\mathrm{~N}^{(\mathrm{r})}} \sum_{j \neq r}\left(\Sigma_{\mathrm{a}, \mathrm{g}}^{(\mathrm{j})}+\lambda_{\mathrm{g}}^{(\mathrm{j})} \Sigma_{\mathrm{p}}^{(\mathrm{j})}\right) .
$$

Since the background cross section for each resonance nuclide includes the shielded absorption cross sections of all other nuclides, Bondarenko interactions are performed.

(d) iropt $=3 \Rightarrow$ IR approximation with Bondarenko iterations:

Background cross sections for all nuclides are computed using the full IR expression in Eq. (7.3.12). Bondarenko interactions are performed.

Computation of the background cross sections in BONAMI generally requires group-dependent values for the IR parameter $\lambda$. These are calculated by a module in AMPX during the library process and are stored in the MG libraries under the reaction identifier (MT number), MT=2000.

\subsubsection{Self-Shielded Cross Sections for Heterogeneous Media}

Equivalence theory can be used to obtain shielded cross sections for heterogeneous systems containing one or more "lumps" of resonance absorber mixtures separated by moderators, such as reactor lattices. It can be shown that if the fuel escape probability is represented by the Wigner rational approximation, the collision probability formulation of the neutron transport equation for an absorber body in a heterogeneous medium can be reduced to a form identical to Eq. (7.3.3). This can be done for an "equivalent" infinite homogeneous medium consisting of the same absorber body mixture plus an additional NR scatterer with a constant cross section called the "escape cross section." "Equivalence theory states that the self-shielded cross section for resonance absorber $r$ in the heterogeneous medium is equal to the self-shielded cross section of $r$ in the equivalent infinite homogeneous medium; therefore the f-factors that were calculated for homogenous mixtures can also be used to compute self-shielded cross sections for heterogeneous media by simply interpolating the tabulated f-factors in the library to the modified sigma-zero value of

$$
\hat{\sigma}_{0}^{(\mathrm{r})}=\sigma_{0}^{(\mathrm{r})}+\sigma_{\mathrm{esc}}^{(\mathrm{r})}
$$

where

$\hat{\sigma}_{0}^{(r)} \quad=$ background cross section of $r$ in the absorber lump of the heterogeneous system;

$\sigma_{0}^{(\mathrm{r})}=$ background cross section defined in Section 7.3.2.1 for an infinite homogeneous medium of the absorber body mixture;

$\sigma_{\text {esc }}^{(\mathrm{r})}=$ microscopic escape cross section for nuclide $r$, defined as

$$
\sigma_{\text {esc }}^{(\mathrm{r})}=\frac{\Sigma_{\text {esc }}}{\mathrm{N}^{(\mathrm{r})}}
$$

$\Sigma_{\text {esc }} \quad=$ macroscopic escape cross section for the absorber lump defined in BONAMI as

$$
\Sigma_{\text {esc }}=\frac{(1-\mathrm{c}) \mathrm{A}}{\bar{\ell}[1+(\mathrm{A}-1) \mathrm{c}]},
$$

where

$\bar{\ell} \quad=$ average chord length of the absorber body $=4 \times \frac{\text { volume }}{\text { surface area }}$; 
A $\quad=$ Bell factor, used to improve the accuracy of the Wigner rational approximation;

$\mathrm{c} \quad=$ lattice Dancoff factor, which is equal to the probability that a neutron escaping from one absorber body will reach another absorber body before colliding in the intervening moderator.

Values for the mean chord length $\bar{\ell}$ are computed in BONAMI for slab, sphere, and cylinder absorber bodies. In the most common mode of operation where BONAMI is executed through the XSProc module in SCALE, Dancoff factors for uniform lattices are computed automatically and provided as input to BONAMI. For nonuniform lattices — such as those containing water holes, control rods, etc. - it may be desirable for the user to run the SCALE module MCDancoff to compute Dancoff factors using Monte Carlo for an arbitrary 3D configuration. In this case the values are provided in the MORE DATA input block of XSProc. The Bell factor "A" is a correction factor to account for errors caused by use of the Wigner rational approximation to represent the escape probability from a lump. Two optional Bell factor corrections are included in BONAMI. The first uses expressions developed by Otter ${ }^{5}$ that essentially force the Wigner escape probability for an isolated absorber lump to agree with the exact escape probability for the particular geometry by determining a value of $\mathrm{A}$ as a function of $\Sigma_{\mathrm{T}} \bar{\ell}$ for slab, cylindrical, or spherical geometries. Since the Otter expression was developed for isolated bodies, it does not account for errors in the Wigner rational approximation due to lattice effects. BONAMI also includes a Bell factor correction based on a modified formulation developed by Leslie ${ }^{6}$ that is a function of the Dancoff factor.

\subsubsection{Interpolation Scheme}

After the background cross section for a system has been computed, BONAMI interpolates f-factors at the appropriate $\sigma_{0}$ and temperature from the tabulated values in the library. Figure 7.3.1 shows a typical variation of the f-factor vs. background cross sections for the capture cross section of ${ }^{238} \mathrm{U}$ in the SCALE 252 group library. 


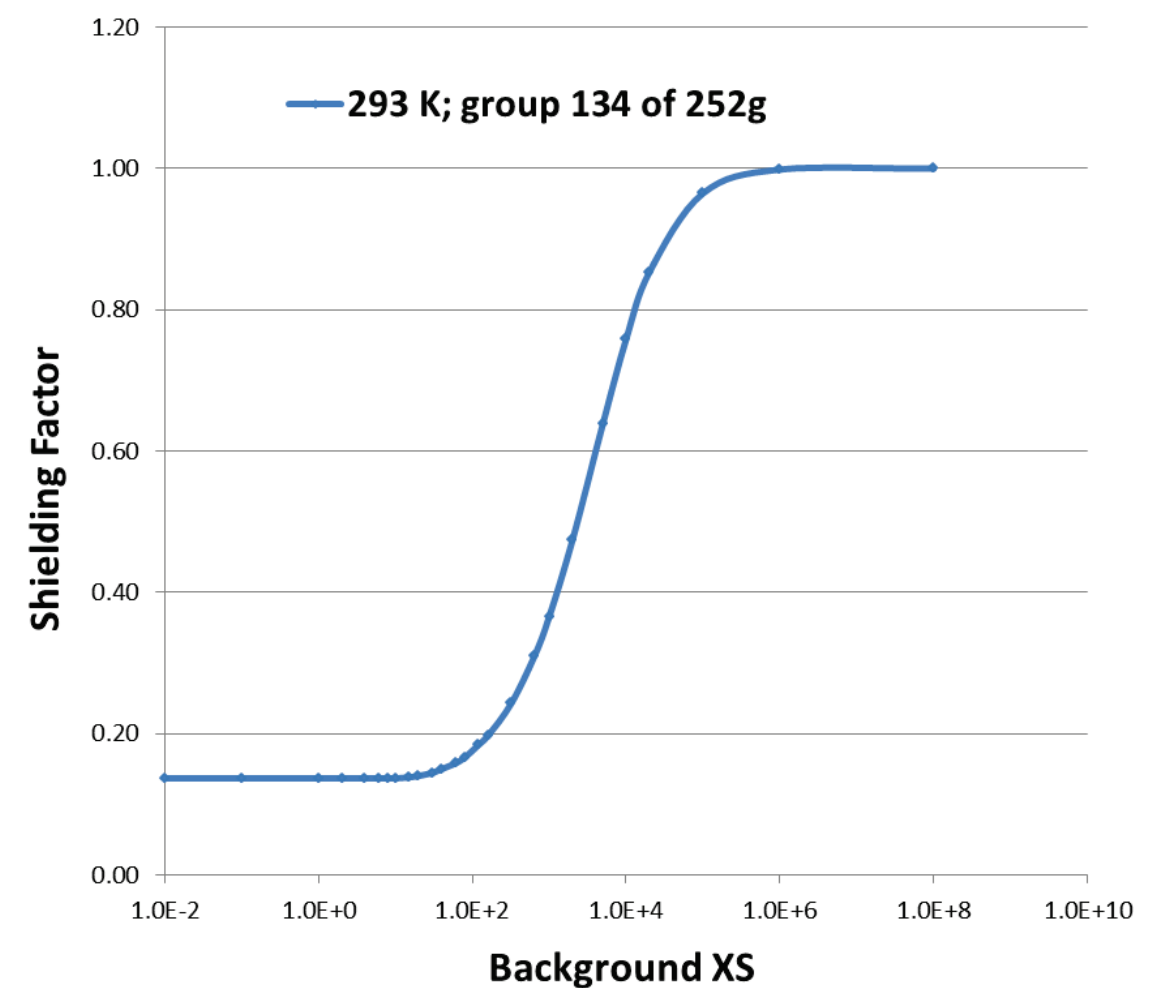

Figure 7.3.1. Plot of f-factor variation for ${ }^{238} \mathrm{U}$ capture reaction

Interpolation of the f-factors can be problematic, and several different schemes have been developed for this purpose. Some of the interpolation methods that have been used in other codes are constrained Lagrangian, ${ }^{7}$ arc-tangent fitting, ${ }^{8}$ and an approach developed by Segev. ${ }^{9}$ All of these were tested and found to be inadequate for use with the SCALE libraries, which may have multiple energy groups within a single resonance. BONAMI uses a unique interpolation method developed by Greene, which is described in Ref. 10. Greene's interpolation method is essentially a polynomial approach in which the powers of the polynomial terms can vary within a panel, as shown in Eq. (7.3.25):

$$
\mathrm{f}(\sigma)=\mathrm{f}\left(\sigma_{1}\right)+\frac{\sigma^{\mathrm{q}(\sigma)}-\sigma_{1}^{\mathrm{q}(\sigma)}}{\sigma_{2}^{\mathrm{q}(\sigma)}-\sigma_{1}^{\mathrm{q}(\sigma)}}\left(\mathrm{f}\left(\sigma_{2}\right)-\mathrm{f}\left(\sigma_{1}\right)\right)
$$

where

$$
\mathrm{q}(\sigma)=\mathrm{q}\left(\sigma_{1}\right)+\frac{\sigma-\sigma_{1}}{\sigma_{2}-\sigma_{1}}\left(\mathrm{q}\left(\sigma_{2}\right)-\mathrm{q}\left(\sigma_{1}\right)\right)
$$

Figure 7.3.2 illustrates the expected behavior of Eq. (7.3.22) caused by varying the powers in a panel. 
By allowing the power $q$ to vary as a function of independent variable $\sigma$, we can move between the various monotonic curves on the graph in a monotonic fashion. Note that when $p$ crosses the $p=1$ curve, the shape changes from concave to convex, or vice versa. This shape change means that we can use the scheme to introduce an inflection point, which is exactly the situation needed for interpolating f-factors.

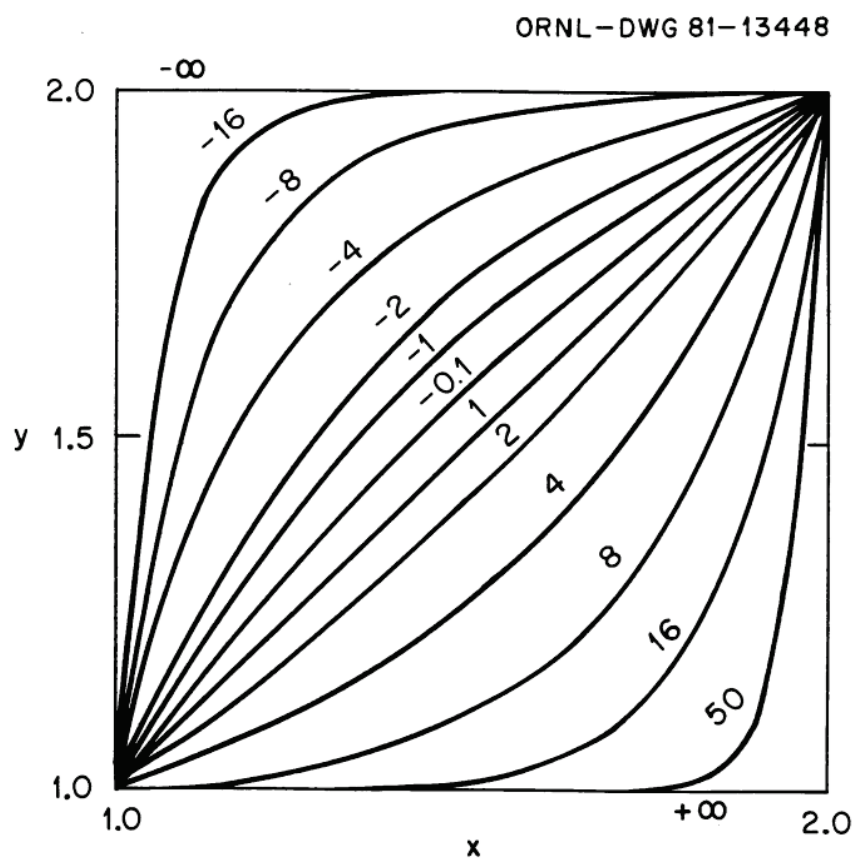

Figure 7.3.2. Illustration of the effects of varying "powers" in the Greene interpolation method.

Figure 7.3.3 and Figure 7.3.4 show typical "fits" of the f-factors using the Greene interpolation scheme for two example cases. Note, in particular, that since this scheme has guaranteed monotonicity, it easily accommodates the end panels that have the smooth asymptotic variation. Even considering the extra task of having to determine the powers for temperature and $\sigma_{0}$ interpolations, the method is not significantly more time-consuming than the alternative schemes for most applications. 


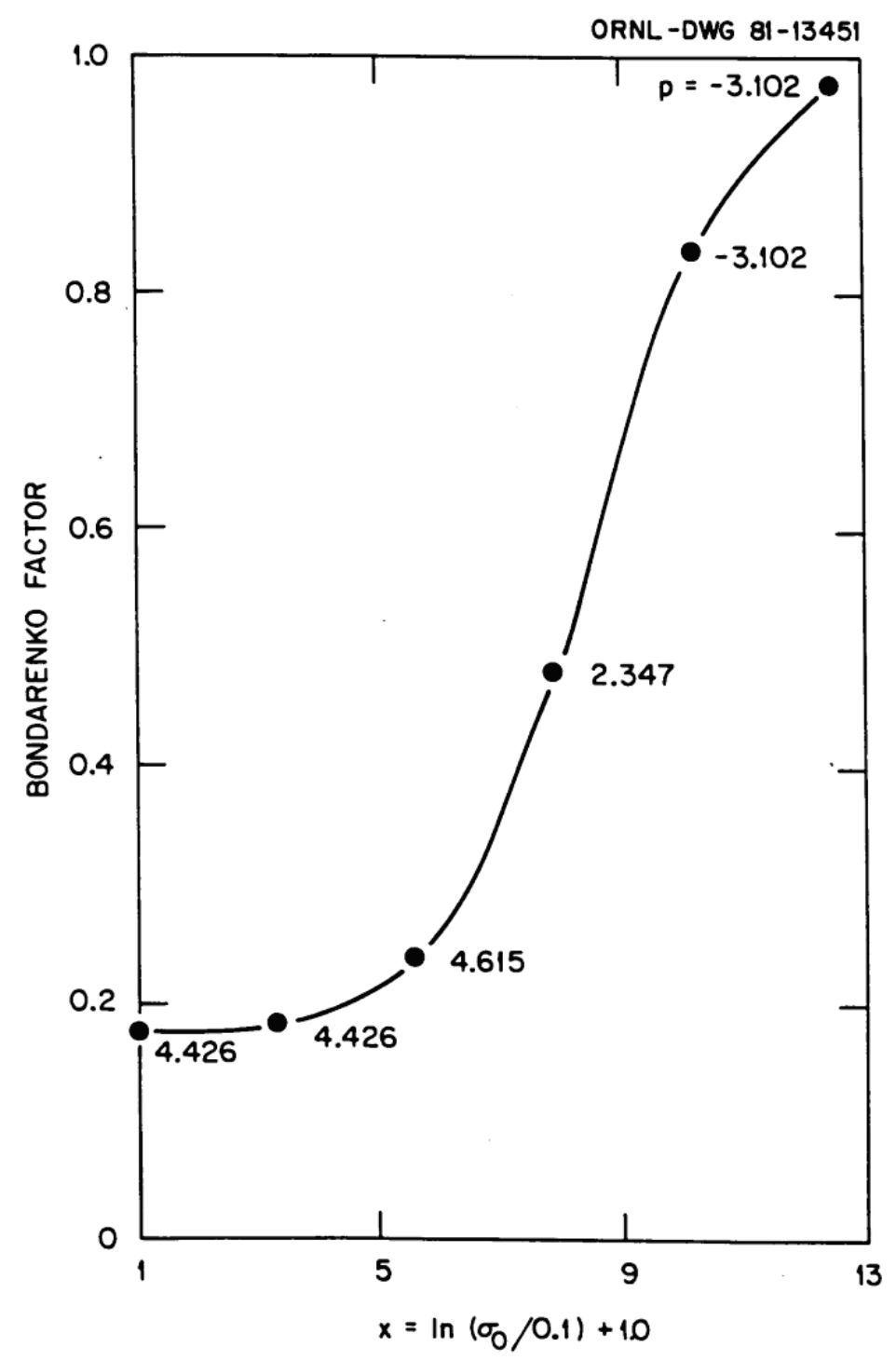

Figure 7.3.3. Use of Greene's method to fit the $\sigma_{0}$ variation of Bondarenko factors for case 1. 


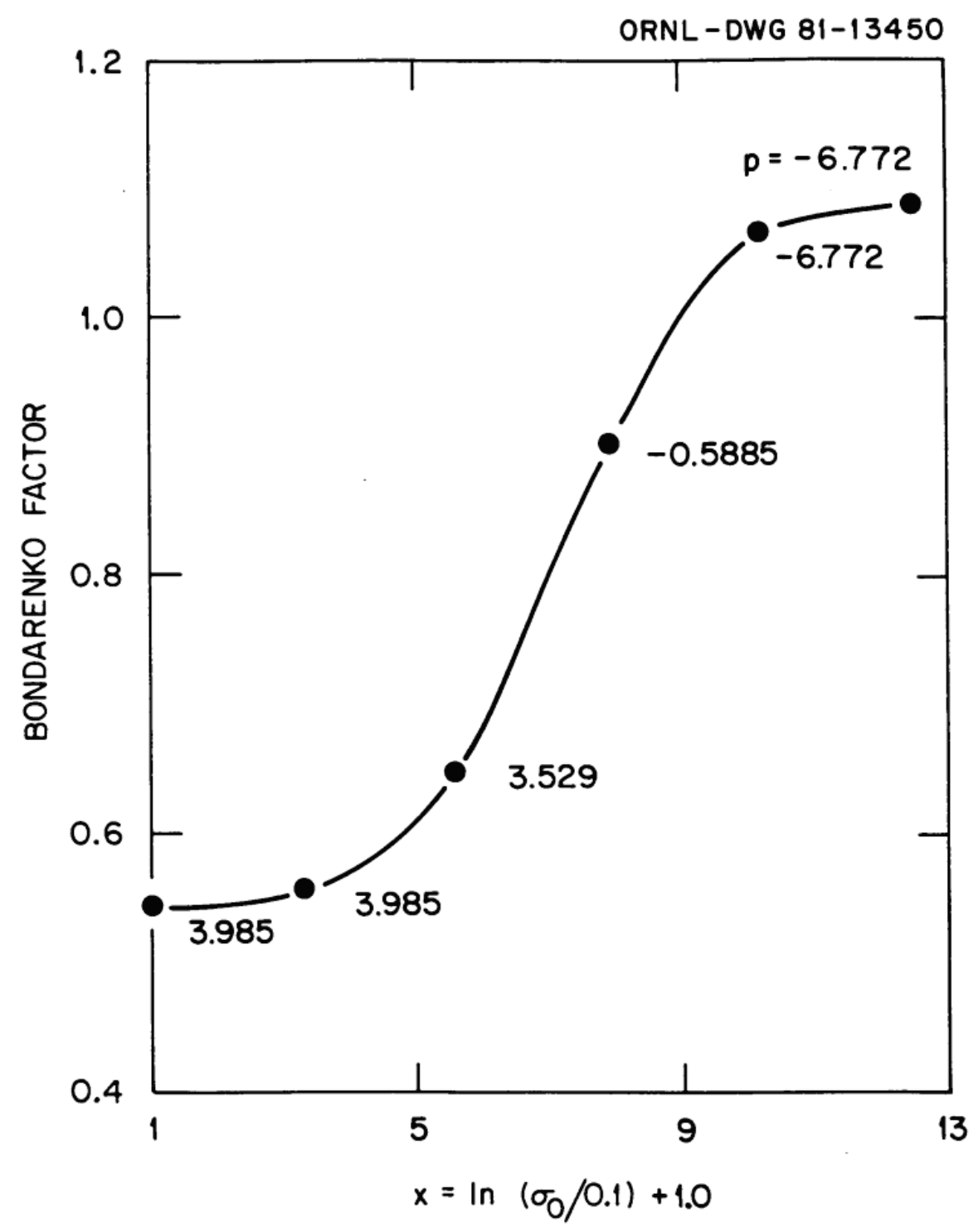

Figure 7.3.4. Use of Greene's method to fit the $\sigma_{0}$ variation of Bondarenko factors for case 2.

\subsubsection{Input Instructions}

BONAMI is most commonly used as an integral component of XSProc through SCALE automated analysis sequences. XSProc automatically prepares all the input data for BONAMI and links it with the other self-shielding modules. During a SCALE sequence execution, the data are provided directly to BONAMI in memory through XSProc. Some of the input parameters can be modified in the MOREDATA block in XSProc.

However, the legacy interface to execute stand-alone BONAMI calculations has been preserved for expert users. The legacy input to BONAMI uses the FIDO schemes described in the FIDO chapter of the SCALE manual. The BONAMI input for standalone execution is given below, where the MOREDATA input keywords are marked in bold. 
Block 1

0\$ Logical Unit Assignments [4]

1. masterlib - input master library (Default $=23$ )

2. mwt-not used

3. msc-not used

4. newlib—output master library (Default $=22)$

$1 \$ \quad$ Case Description [6]

1. cellgeometry-geometry description 0 homogeneous

$\begin{array}{ll}1 & \text { slab } \\ 2 & \text { cylinder } \\ 3 & \text { sphere }\end{array}$

2. numzones - number of zones or material regions

3. mixlength - mixing table length. This is the total number of entries needed to describe the concentrations of all constituents in all mixtures in the problem.

4. ib-not used

5. crossedt-output edit option 0 no output (Default)

1 input echo

2 iteration list, timing

3 background cross section calculation details

6. issopt-not used

4 shielded cross sections, Bondarenko factors

7. iropt-resonance approximation option $0 \mathrm{NR}$ (Default)

(Bondarenko iterations)]

1 IR with potential scattering

2 IR with absorption and potential scattering

(Bondarenko iterations)

3 IR with absorption and elastic scattering

factor calculation option 0 Otter

(Bondarenko iterations)

8. bellopt-Bell

1 Leslie (Default)

9. escxsopt-escape cross section calculation option 0 consistent

1 inconsistent (Default)

2* $\quad$ Floating-Point Constants [2]

1. bonamieps - convergence criteria for the Bondarenko iteration $($ Default $=0.001)$

2. bellfact - geometrical escape probability adjustment factor. See notes below on this parameter (Default $=0.0)$.

T Terminate Data Block 1.

Data Block 2

3\$ Mixture numbers in the mixing table [mixlength]

$4 \$$ Component (nuclide) identifiers in the mixing table [mixlength]

5* Concentrations (atoms $/ \mathrm{b}-\mathrm{cm}$ ) in the mixing table [mixlength]

$6 \$$ Mixtures by zone [numzones]

7* Outer radii $(\mathrm{cm})$ by zone [numzones]

8* Temperature $(\mathrm{k})$ by zone [numzones]

9* Escape cross section $\left(\mathrm{cm}^{-1}\right)$ by zone [numzones] 
$11 \$ \quad$ Not used

12* Temperature $(\mathrm{K})$ of the nuclide in a one-to-one correspondence with the mixing table arrays.

13* Dancoff factors by zone [numzones]

14* $\quad$ Lbar $(\bar{\ell})$ factors by zone [numzones]

T Terminate Data Block 2.

This concludes the input data required by BONAMI.

\subsubsection{Notes on input}

In the $1 \$$ array, cellgeometry specifies the geometry. The geometry information is used in conjunction with the $7^{*}$ array to calculate mean chord length Lbar if it is not provided by the user in the $14^{*}$ array.

numzones, the number of zones, may or may not model a real situation. It may, for example, be used to specify numzones independent media to perform a cell calculation in parallel with one or more infinite medium calculations. The geometry description in $1 \$$ array applies only to mean chord length calculations unless it is provided in $14^{*}$.

In the $2^{*}$ array, bonamieps is used to specify the convergence expected on all macroscopic total values by zone, that is, each $\Sigma_{\mathrm{t}}(\mathrm{g}, \mathrm{j})$ in group $\mathrm{g}$ and zone $\mathrm{j}$ is converged so that

$$
\frac{\left|\Sigma_{t}^{\mathrm{i}}(\mathrm{g}, \mathrm{j})-\Sigma_{\mathrm{t}}^{\mathrm{i}-1}(\mathrm{~g}, \mathrm{j})\right|}{\Sigma_{\mathrm{t}}^{\mathrm{i}}(\mathrm{g}, \mathrm{j})} \leq \text { bonamieps }
$$

The "Bell" factor in the $2^{*}$ array is the parameter used to adjust the Wigner rational approximation for the escape probability to a more correct value. It has been suggested that if one wishes to use one constant value, the Bell factor should be 1.0 for slabs and 1.35 otherwise. In the ordinary case, BONAMI defaults the Bell factor to zero and uses a prescription by Otter ${ }^{5}$ to determine a cross-section geometry-dependent value of the Bell factor for isolated absorber bodies. It uses a prescription by Leslie ${ }^{6}$ to determine the Dancoff factor-dependent values of the Bell factor for lattices, which are much more accurate than the single value. The user who wishes to determine the constant value can, however, use it by inputting a value other than zero.

The $3 \$, 4 \$$, and $5^{*}$ arrays are used to specify the concentrations of the constituents of all mixtures in the problem as follows:

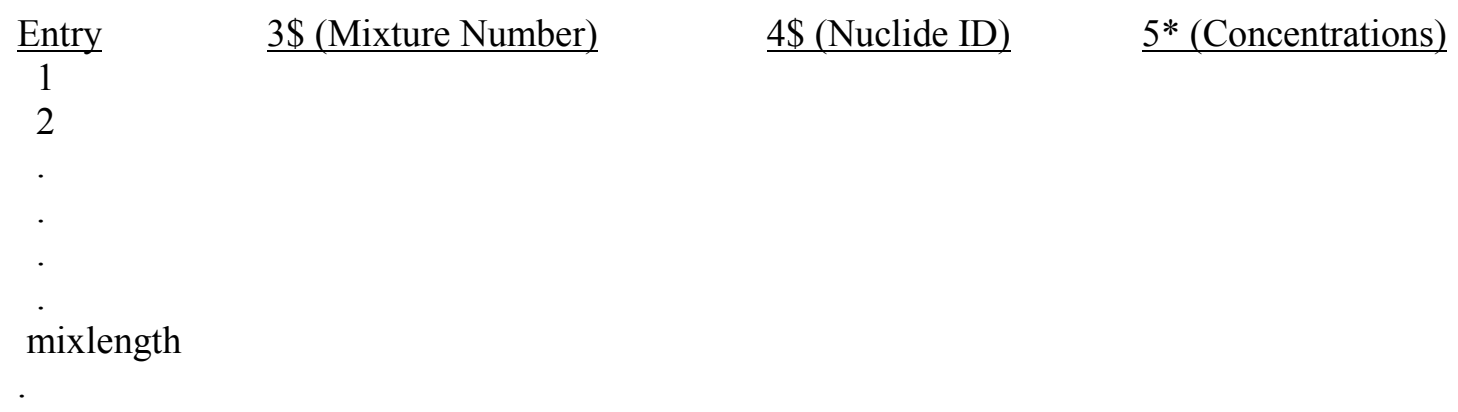


Because of the manner in which BONAMI references the nuclides in a calculation, each nuclide in the problem must have a unique entry in the mixing table. Thus one cannot specify a mixture and subsequently load it into more than one zone, as can be the case with many modules requiring this type of data.

The $12 *$ array is used to allow varying the temperatures by nuclide within a zone. In the event this array is omitted, the $12 *$ array will default by nuclide to the temperature of the zone containing the nuclide.

The mixture numbers in each zone are specified in the $6 \$$ array. Mixture numbers are arbitrary and need only match up with those used in the $3 \$$ array.

The radii in the $7^{*}$ array are referenced to a zero value at the left boundary of the system.

In the event the temperatures in the $8^{*}$ array are not bounded by temperature values in the Bondarenko tables, BONAMI will extrapolate using the three temperature points closest to the value. For example, a request for $273 \mathrm{~K}$ for a nuclide with Bondarenko sets at 300, 900, and 2,100 K would use the polynomial fit from those three temperature points to extrapolate the $273 \mathrm{~K}$ value.

The escape cross sections in the $9^{*}$ array allow a macro escape cross section ( $\Sigma_{\mathrm{e}}^{\text {input }}$ ) to be specified by zone. (This array can be ignored if Dancoff factors are provided.) If the Dancoff factor for a zone is specified as -1 in the input, then the user-specified escape cross section is used in calculating the background cross sections $\sigma_{0}$ as follows:

$$
\sigma_{0}=\frac{\sum_{\mathrm{n} \neq \mathrm{i}} \mathrm{N}_{\mathrm{n}} \sigma_{\mathrm{t}}^{\mathrm{n}}+\sum_{\mathrm{e}}^{\mathrm{input}}}{\mathrm{N}_{\mathrm{i}}} .
$$




\subsubsection{Sample Problem}

In most cases, the input data to BONAMI are simple and obvious because the complicated parameters are determined internally based on the options selected. The user describes his geometry, the materials contained therein, the temperatures, and a few options.

This problem is for a system of iron-clad uranium $\left(\mathrm{U}^{238}-\mathrm{U}^{235}\right)$ fuel pins arranged in a square lattice in a water pool.

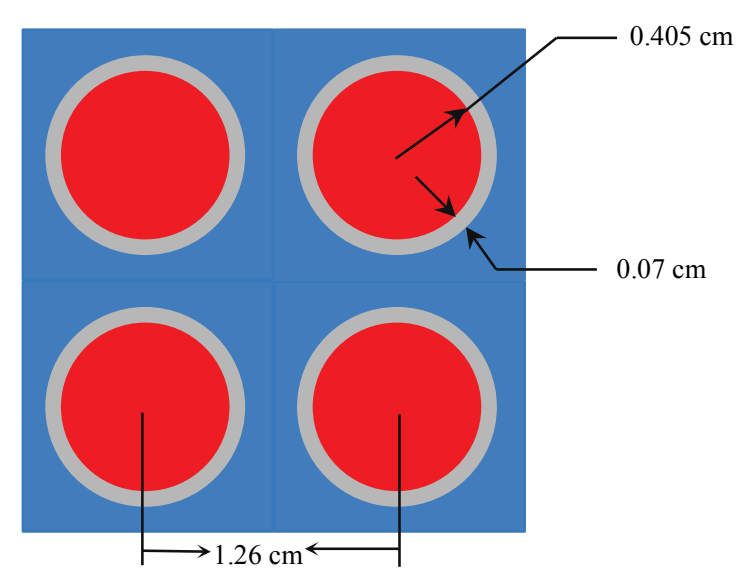

Our number densities are

Fuel:

$$
\begin{aligned}
& \mathrm{N}_{235}=1.4987 \times 10^{-4} \\
& \mathrm{~N}_{238} \mathrm{U}=2.0664 \times 10^{--2}
\end{aligned}
$$

Clad:

$$
\mathbf{N}_{56} \mathrm{Fe}=9.5642 \times 10^{-5}
$$

Water:

$$
\begin{array}{ll}
\mathrm{N}_{\mathrm{H}} & =6.6662 \times 10^{-2} \\
\mathrm{~N}_{\mathrm{O}} & =3.3331 \times 10^{-2}
\end{array}
$$

For the problem, we choose iropt $=1$ (IR approximation with scattering approximated by $\lambda \Sigma_{\mathrm{p}}$ ) and crossedt $=4$ for the most detailed output edits. An 8-group test library is used for fast execution and a short output file.

The XSProc/CSAS1X SCALE sequence input file, the corresponding i_bonami FIDO input file created by the sequence under the temporary working directory, and an abbreviated copy of the output from this case follows. 


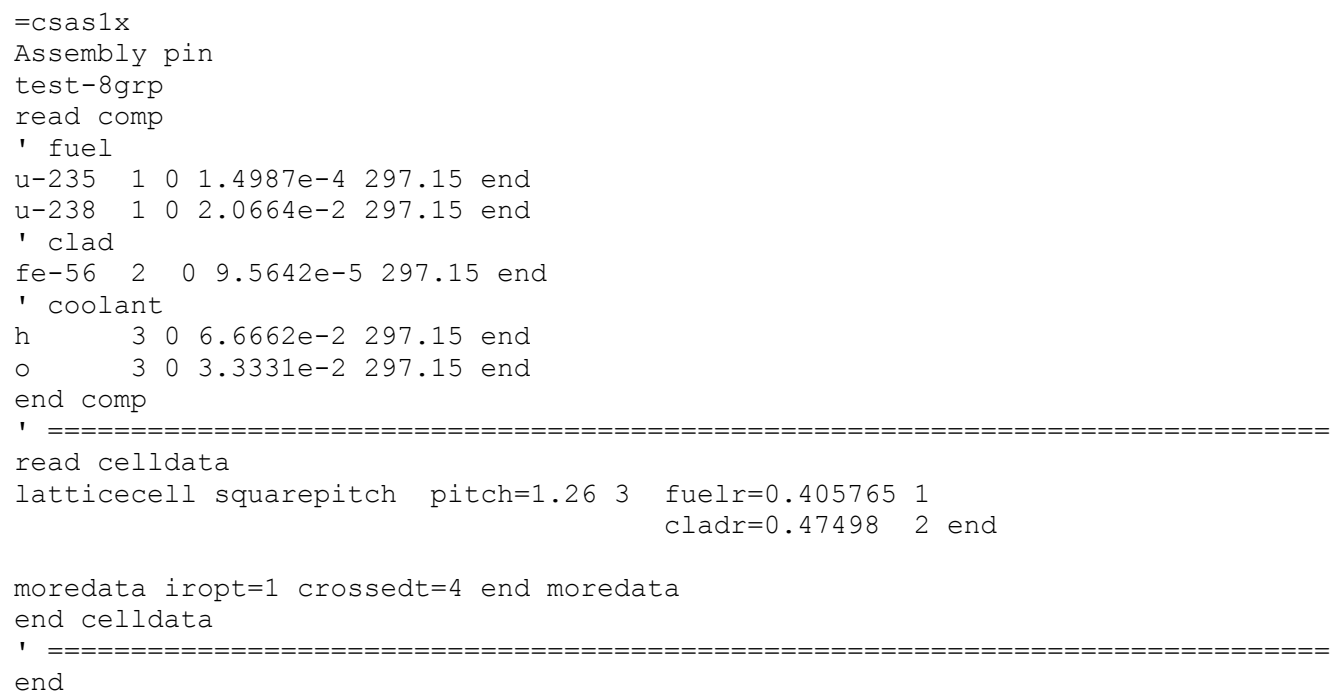

\section{FIDO input i_bonami}

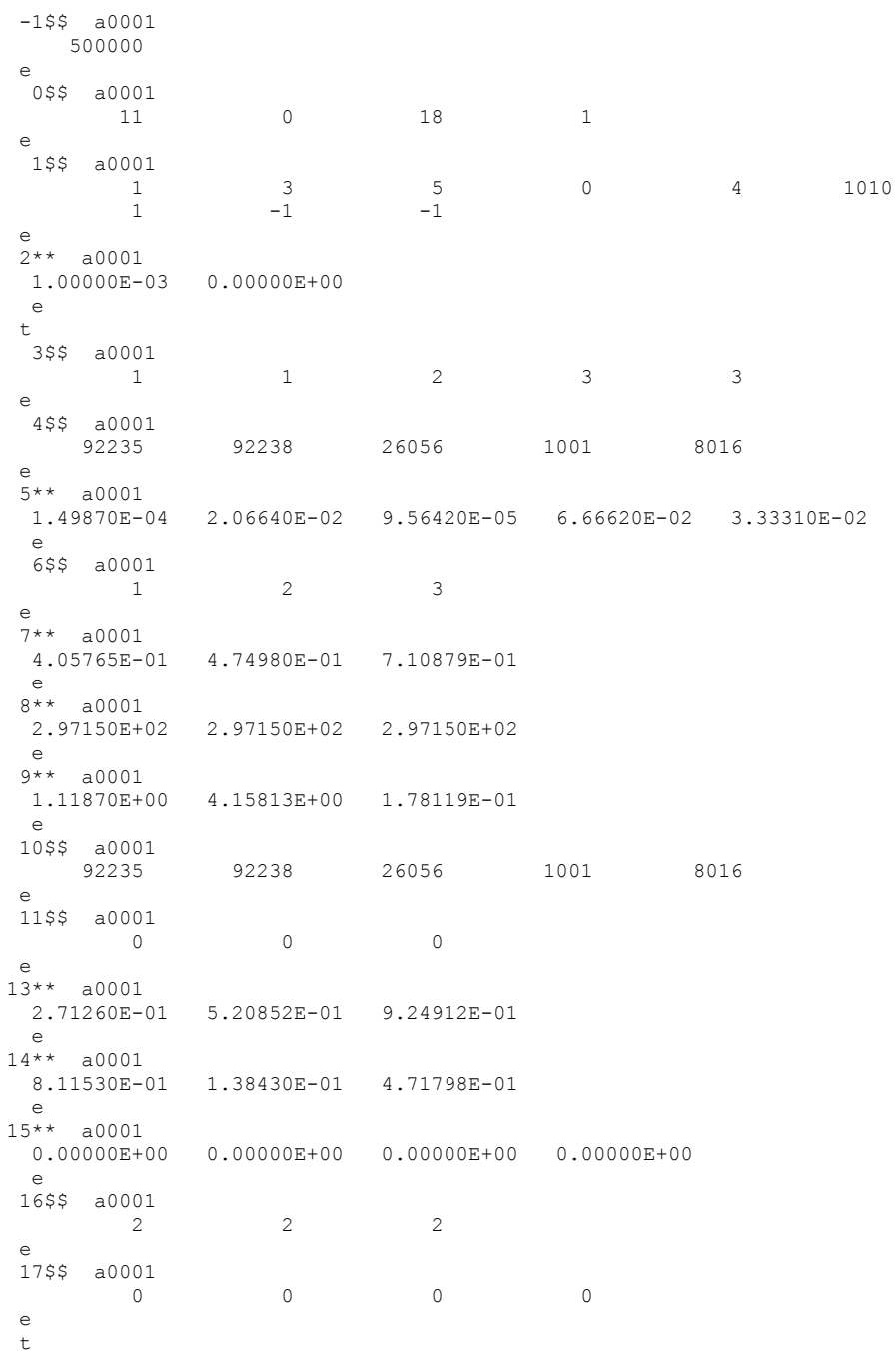




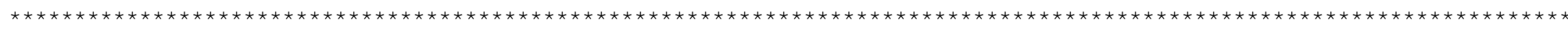

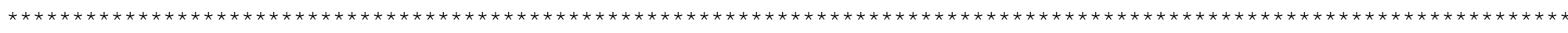

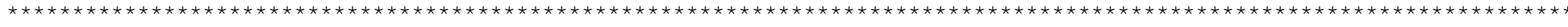
$\star \star \star \star \star \star * \quad$ program verification information

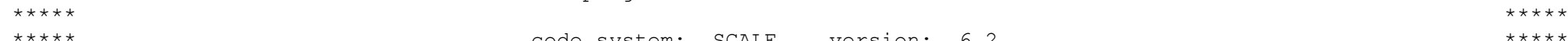

$\star * \star \star *$ Code system: SCALE version: 6.2

*****

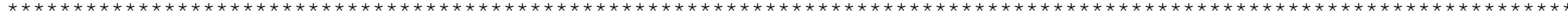

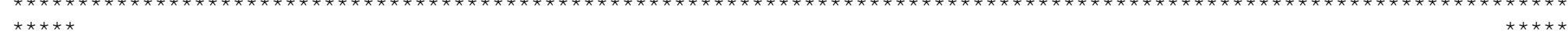

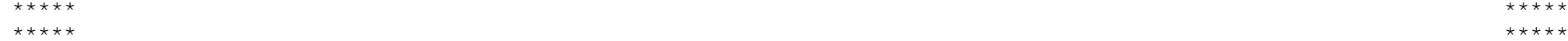

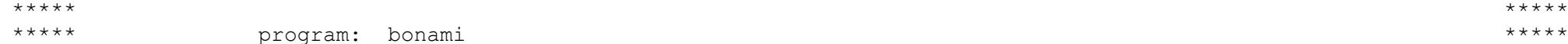

$* \star \star * \star \quad$ creation date: unknown

*****

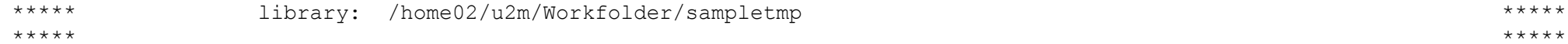

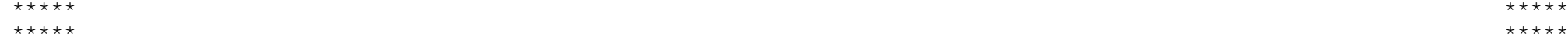

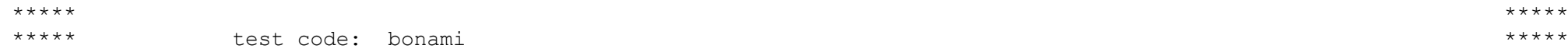

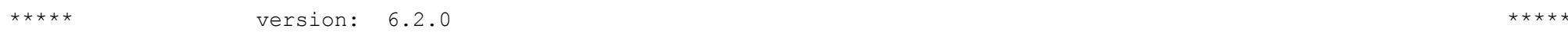

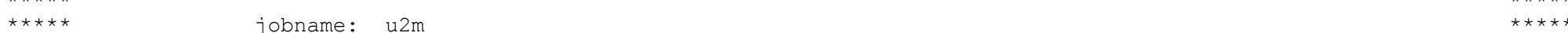

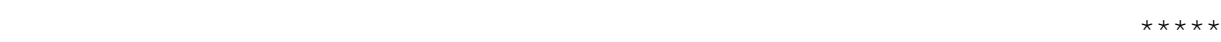

$\star \star \star \star \star *$ machine name: node22.ornl.gov $\star * \star \star \star *$

$+4 * * * *$

$\star * \star \star \star *$ date of execution: 04_dec_2013 *****

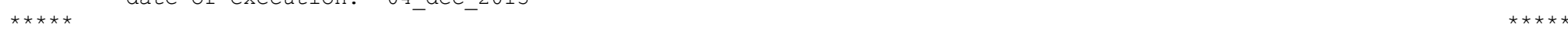

$* * * * *$ time of execution: 21:43:54.38 $* * * * \star$

*t***

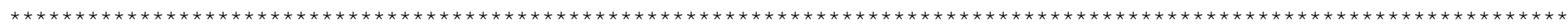

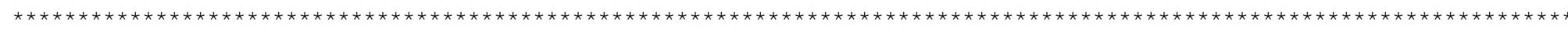

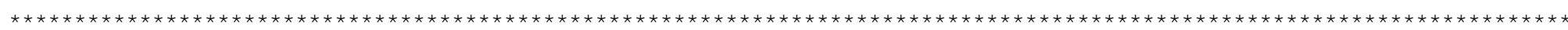


1

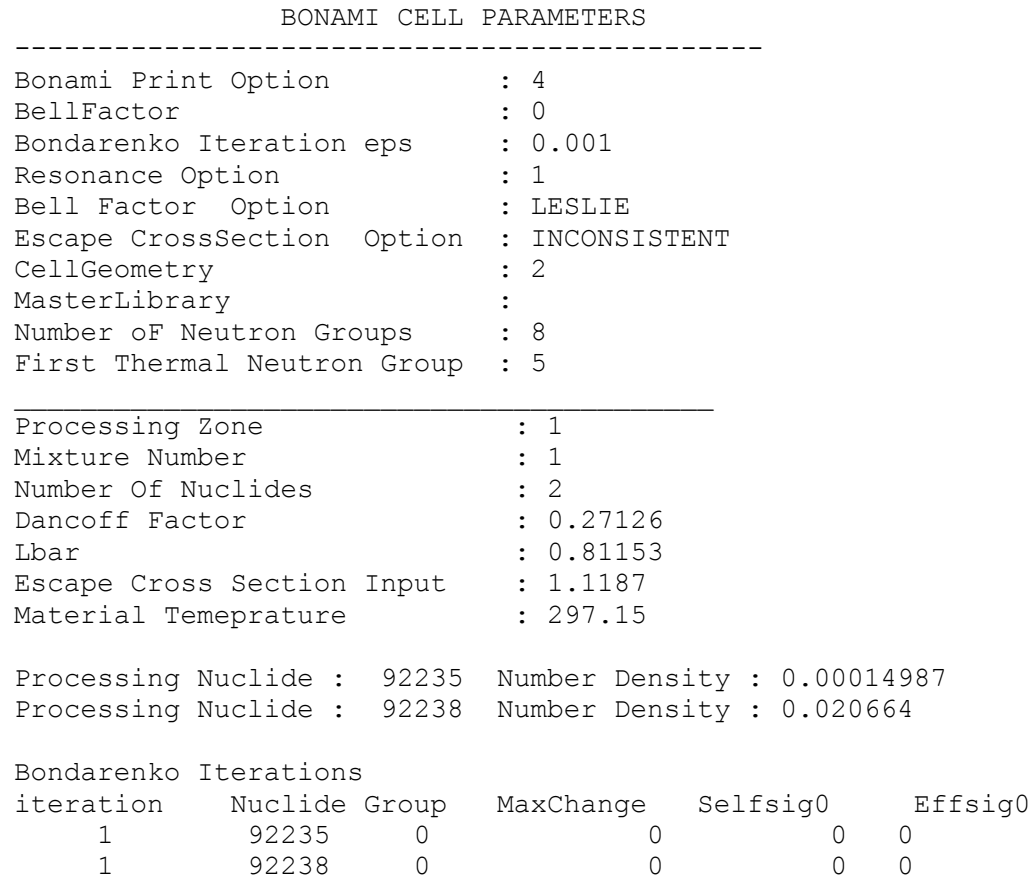

Total number of Bondarenko Iterations : 1

Max Change in Group

: 0

$\begin{array}{ccc}\text { Group } & \text { Eff Macro Sig0 } & \text { Escape Xsec } \\ 1 & 0.2351032 & 0.9075513 \\ 2 & 0.2351032 & 0.9075513 \\ 3 & 0.2351032 & 0.9075513 \\ 4 & 0.2351032 & 0.9075513 \\ 5 & 0.2351032 & 0.9075513 \\ 6 & 0.2351032 & 0.9075513 \\ 7 & 0.2351032 & 0.9075513 \\ 8 & 0.2351032 & 0.9075513\end{array}$

Shielding Nuclide 92235

mt

$\begin{array}{lrc} & \text { Group } & \text { sig0 } \\ 1 & 1 & 7612.7187 \\ 1 & 2 & 7612.7187 \\ 1 & 3 & 7612.7187 \\ 1 & 4 & 7612.7187 \\ 1 & 5 & 7612.7187 \\ 1 & 6 & 7612.7187 \\ 1 & 7 & 7612.7187 \\ 1 & 8 & 7612.7187\end{array}$

mt

$\begin{array}{lrc} & \text { Group } & \text { sig0 } \\ 2 & 1 & 7612.71875 \\ 2 & 2 & 7612.71875 \\ 2 & 3 & 7612.71875 \\ 2 & 4 & 7612.71875 \\ 2 & 5 & 7612.71875 \\ 2 & 6 & 7612.71875 \\ 2 & 7 & 7612.71875 \\ 2 & 8 & 7612.71875\end{array}$

mt

$18 \quad \begin{array}{rr}\text { Group } \\ 18\end{array}$ 7.19131

10.2521

24.9361

75.1109

56.0286

198.645

347.945

761.257

infDiluted Xsec 3.71448

7.63235

11.841

11.5408

12.5449

14.2501

14.8125

15.1274

infDiluted Xsec

1.21846

1. 40834

$$
\begin{array}{rc}
\multicolumn{1}{c}{\text { f-factor }} & \text { shielded Xsec } \\
0.999998 & 7.19129 \\
0.999616 & 10.2481 \\
1.00241 & 24.9963 \\
1.05902 & 79.5436 \\
1.00205 & 56.1434 \\
1.0008 & 198.805 \\
1.00024 & 348.028 \\
1.0066 & 766.282
\end{array}
$$

f-factor shielded Xsec

$0.999999 \quad 3.71448$

$0.99935 \quad 7.62739$

$0.999444 \quad 11.8345$

$1.00561 \quad 11.6055$

$1.00001 \quad 12.545$

$1.00007 \quad 14.2511$

$1.00003 \quad 14.8128$

$1.00015 \quad 15.1297$

$\begin{array}{cc}\text { f-factor } & \text { shielded Xsec } \\ 0.999996 & 1.21846 \\ 1.0002 & 1.40862\end{array}$ 


\begin{tabular}{|c|c|c|c|c|c|}
\hline 18 & 3 & 7612.71875 & 8.92885 & 1.00132 & 8.94062 \\
\hline 18 & 4 & 7612.71875 & 39.2086 & 1.06274 & 41.6686 \\
\hline 18 & 5 & 7612.71875 & 32.7026 & 1.00105 & 32.737 \\
\hline 18 & 6 & 7612.71875 & 153.511 & 1.00089 & 153.647 \\
\hline 18 & 7 & 7612.71875 & 285.775 & 1.00026 & 285.848 \\
\hline 18 & 8 & 7612.71875 & 636.445 & 1.00655 & 640.611 \\
\hline mt & Group & $\operatorname{sig} 0$ & infDiluted Xsec & f-factor & shielded Xsec \\
\hline 102 & 1 & 7612.71875 & 0.060296 & 1 & 0.0602962 \\
\hline 102 & 2 & 7612.71875 & 0.317627 & 1.00352 & 0.318746 \\
\hline 102 & 3 & 7612.71875 & 4.16593 & 1.01325 & 4.22113 \\
\hline 102 & 4 & 7612.71875 & 24.3615 & 1.07832 & 26.2695 \\
\hline 102 & 5 & 7612.71875 & 10.781 & 1.00749 & 10.8618 \\
\hline 102 & 6 & 7612.71875 & 30.8844 & 1.00074 & 30.9073 \\
\hline 102 & 7 & 7612.71875 & 47.3579 & 1.0002 & 47.3671 \\
\hline 102 & 8 & 7612.71875 & 109.685 & 1.00781 & 110.542 \\
\hline mt & Group & sigo & infDiluted Xsec & f-factor & shielded Xsec \\
\hline 1007 & 1 & 7612.71875 & 0 & 0 & 0 \\
\hline 1007 & 2 & 7612.71875 & 0 & 0 & 0 \\
\hline 1007 & 3 & 7612.71875 & 0 & 0 & 0 \\
\hline 1007 & 4 & 7612.71875 & 0 & 0 & 0 \\
\hline 1007 & 5 & 7612.71875 & 12.5448 & 1.00001 & 12.5449 \\
\hline 1007 & 6 & 7612.71875 & 14.2501 & 1.00007 & 14.2511 \\
\hline 1007 & 7 & 7612.71875 & 14.8125 & 1.00003 & 14.8129 \\
\hline 1007 & 8 & 7612.71875 & 15.1278 & 1.00015 & 15.13 \\
\hline \multicolumn{6}{|c|}{-------------------------------------------------- } \\
\hline \multicolumn{6}{|c|}{ Shielding Nuclide 92238} \\
\hline mt & Group & $\operatorname{sig} 0$ & infDiluted Xsec & f-factor & shielded Xsec \\
\hline 1 & 1 & 44.0034676 & 7.33815 & 0.999983 & 7.33803 \\
\hline 1 & 2 & 44.0034676 & 10.3566 & 1.00418 & 10.3999 \\
\hline 1 & 3 & 44.0034676 & 15.0517 & 0.976844 & 14.7032 \\
\hline 1 & 4 & 44.0034676 & 15.951 & 0.983793 & 15.6925 \\
\hline 1 & 5 & 44.0034676 & 9.43867 & 1.00002 & 9.43887 \\
\hline 1 & 6 & 44.0034676 & 10.1008 & 1.00008 & 10.1015 \\
\hline 1 & 7 & 44.0034676 & 10.7744 & 1.00004 & 10.7748 \\
\hline 1 & 8 & 44.0034676 & 12.2124 & 1.00145 & 12.2301 \\
\hline mt & Group & $\operatorname{sig} 0$ & infDiluted Xsec & f-factor & shielded Xsec \\
\hline 2 & 1 & 44.0034676 & 4.0228 & 0.999974 & 4.0227 \\
\hline 2 & 2 & 44.0034676 & 9.05886 & 1.00575 & 9.11093 \\
\hline 2 & 3 & 44.0034676 & 14.0213 & 0.979923 & 13.7398 \\
\hline 2 & 4 & 44.0034676 & 11.9032 & 0.98795 & 11.7598 \\
\hline 2 & 5 & 44.0034676 & 8.86555 & 0.999984 & 8.86541 \\
\hline 2 & 6 & 44.0034676 & 9.24452 & 1.00002 & 9.24471 \\
\hline 2 & 7 & 44.0034676 & 9.2797 & 1.00002 & 9.27987 \\
\hline 2 & 8 & 44.0034676 & 9.3077 & 1.00009 & 9.30853 \\
\hline mt & Group & sig0 & infDiluted Xsec & f-factor & shielded Xsec \\
\hline 18 & 1 & 44.0034676 & 0.376356 & 1.00001 & 0.376361 \\
\hline 18 & 2 & 44.0034676 & 0.000528746 & 1.00019 & 0.000528845 \\
\hline 18 & 3 & 44.0034676 & 0.000308061 & 0.966052 & 0.000297603 \\
\hline 18 & 4 & 44.0034676 & $4.75014 e-06$ & 0.967842 & $4.59738 e-06$ \\
\hline 18 & 5 & 44.0034676 & $2.60878 e-06$ & 1.00006 & $2.60893 e-06$ \\
\hline 18 & 6 & 44.0034676 & $5.27139 e-06$ & 1.00071 & $5.27512 e-06$ \\
\hline 18 & 7 & 44.0034676 & $9.3235 e-06$ & 1.00018 & $9.32514 e-06$ \\
\hline 18 & 8 & 44.0034676 & $1.81868 e-05$ & 1.00588 & $1.82937 e-05$ \\
\hline mt & Group & sig0 & infDiluted Xsec & f-factor & shielded Xsec \\
\hline 102 & 1 & 44.0034676 & 0.0554327 & 1.00006 & 0.0554359 \\
\hline 102 & 2 & 44.0034676 & 0.17972 & 0.978628 & 0.175879 \\
\hline 102 & 3 & 44.0034676 & 1.03011 & 0.934934 & 0.963087 \\
\hline 102 & 4 & 44.0034676 & 4.04777 & 0.971568 & 3.93268 \\
\hline 102 & 5 & 44.0034676 & 0.573119 & 1.0006 & 0.573462 \\
\hline 102 & 6 & 44.0034676 & 0.856257 & 1.00068 & 0.856839 \\
\hline 102 & 7 & 44.0034676 & 1.49471 & 1.00017 & 1.49497 \\
\hline 102 & 8 & 44.0034676 & 2.90465 & 1.00586 & 2.92168 \\
\hline
\end{tabular}




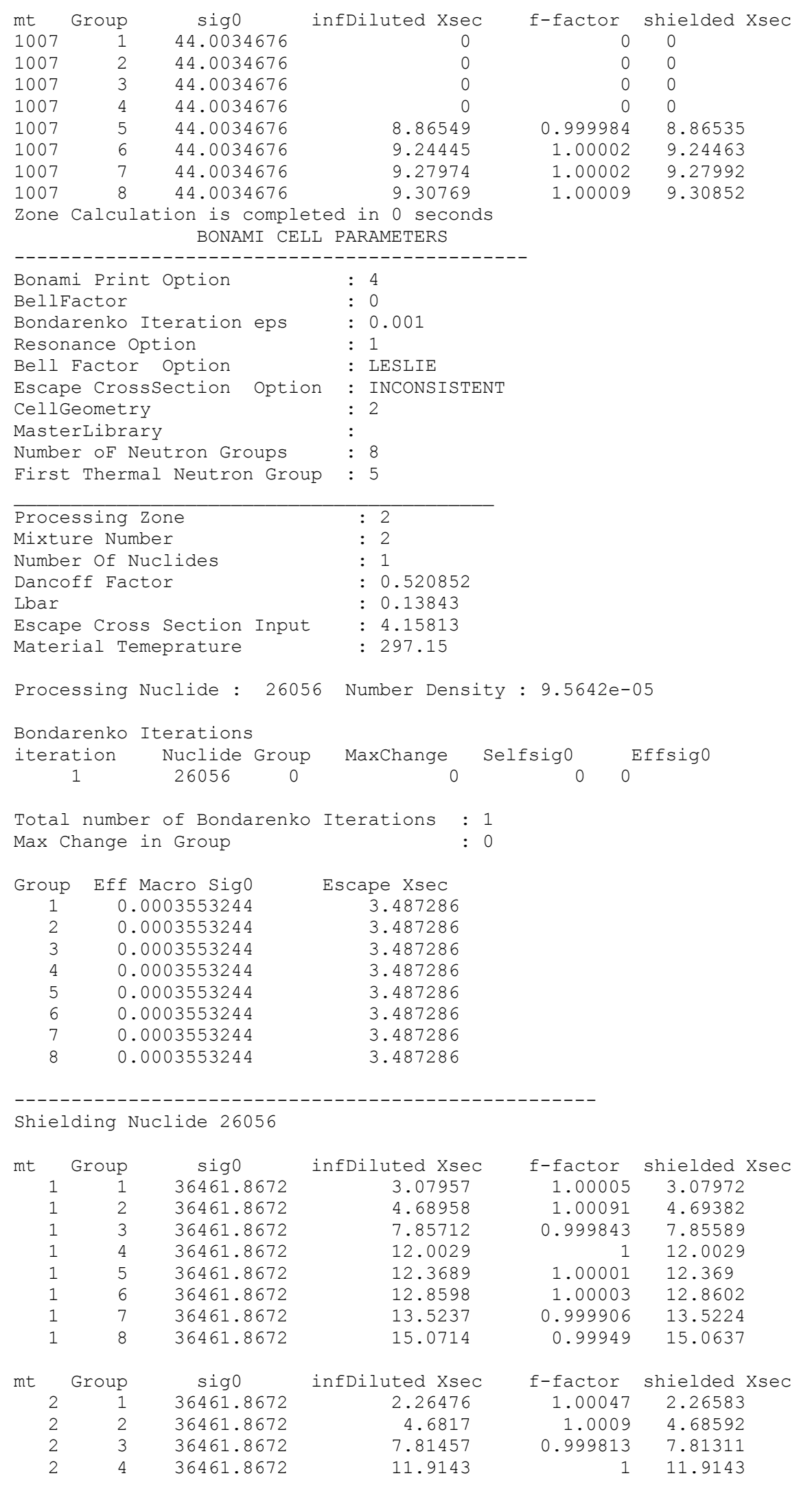




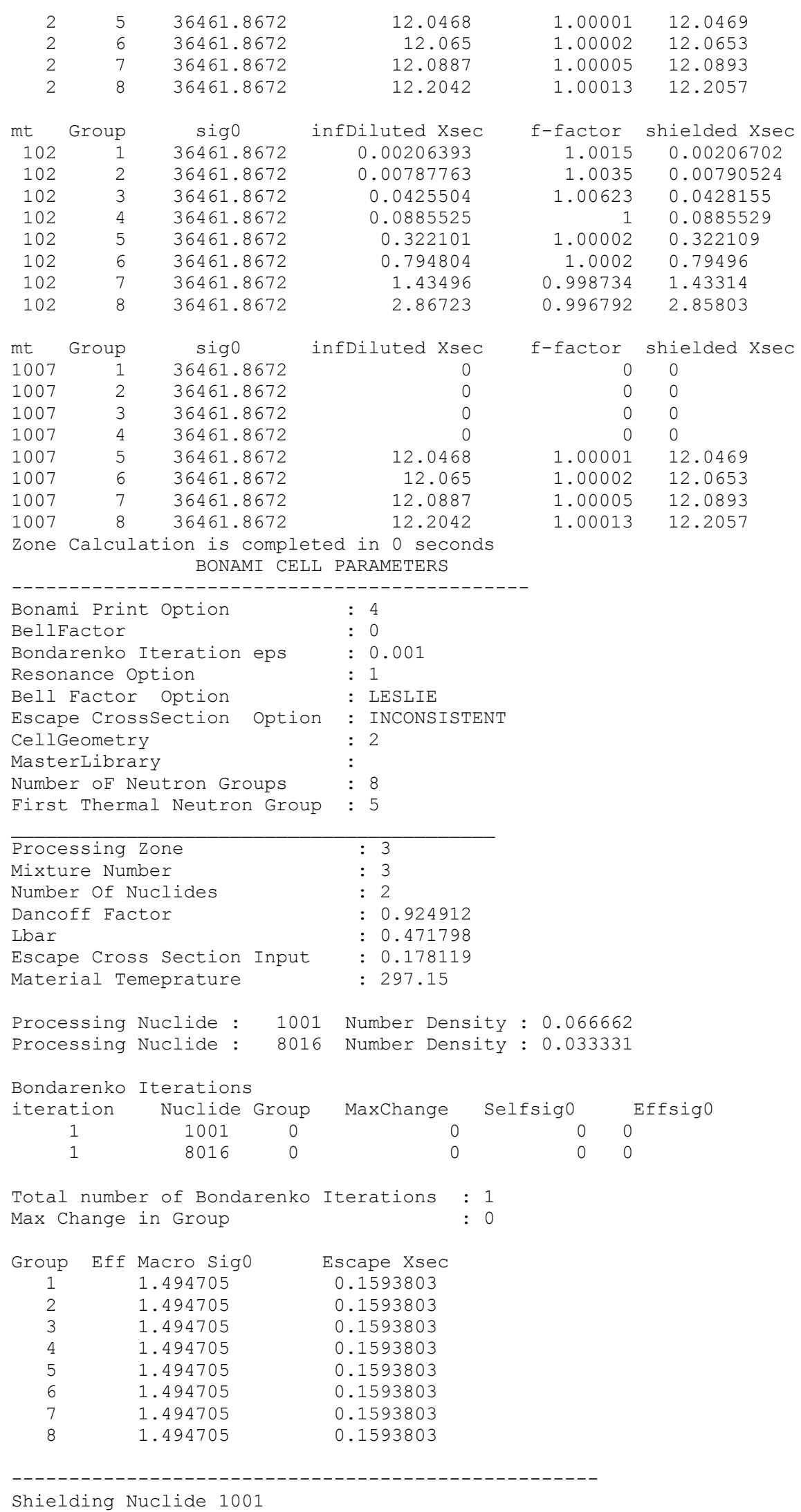




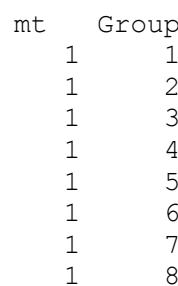

sig0

4. 33502197

4. 33502197

4. 33502197

4.33502197

4. 33502197

4.33502197

4. 33502197

4. 33502197

$$
\text { sig0 }
$$

4. 33502197

4. 33502197

4. 33502197

4. 33502197

4.33502197

4. 33502197

4.33502197

4. 33502197

\section{sig0}

4.33502197

4. 33502197

4. 33502197

4.33502197

4. 33502197

4.33502197

4. 33502197

4. 33502197

$$
\text { sig0 }
$$

4. 33502197

4. 33502197

4. 33502197

4. 33502197

4. 33502197

4. 33502197

4.33502197

4. 33502197

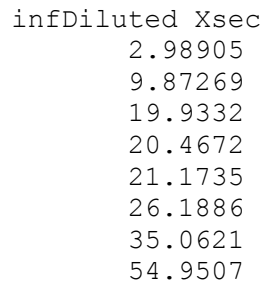

infDiluted Xsec 2. 98901

9.8726

19.9315

20.4556

21.1321

26.0865

34.8778

54.5786

infDiluted Xsec

$3.56422 e-05$

$8.96827 e-05$

0.00171679

0.0116042

0.0413709

0.102043

0.184322

0.372079

infDiluted Xsec

0
0
0

21.1312

26.0871

34.8779

54.5802

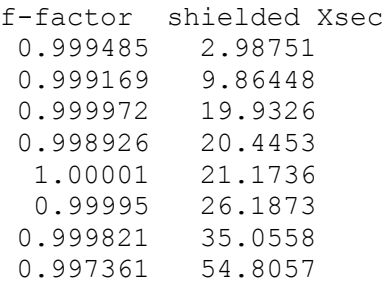

f-factor shielded Xsec

0
0
0
0

$1.00005 \quad 21.1322$

$0.999927 \quad 26.0852$

$0.999826 \quad 34.8718$

$0.997367 \quad 54.4365$

Shielding Nuclide 8016

mt

$\begin{array}{lr} & \text { Group } \\ 1 & 1 \\ 1 & 2 \\ 1 & 3 \\ 1 & 4 \\ 1 & 5 \\ 1 & 6 \\ 1 & 7 \\ 1 & 8\end{array}$

mt

$\begin{array}{rr} & \text { Group } \\ 2 & 1 \\ 2 & 2 \\ 2 & 3 \\ 2 & 4 \\ 2 & 5 \\ 2 & 6 \\ 2 & 7 \\ 2 & 8\end{array}$

mt Group

$102 \quad 1$

$102 \quad 2$

$102 \quad 3$

1024 sig0

45.7377472

45.7377472

45.7377472

45.7377472

45.7377472

45.7377472

45.7377472

45.7377472

sig0 45.7377472

45.7377472

45.7377472

45.7377472

45.7377472

45.7377472

45.7377472

45.7377472

sig0

45.7377472

45.7377472

45.7377472

45.7377472

infDiluted Xsec
2.36081
3.95917
3.84394
3.85289
3.85531
3.8648
3.89139
4.01909

$$
\begin{array}{rc}
\text { f-factor } & \text { shielded Xsec } \\
0.980071 & 2.31376 \\
0.995755 & 3.94236 \\
1 & 3.84394 \\
1 & 3.85291 \\
1.00002 & 3.85537 \\
1.00006 & 3.86502 \\
1.00014 & 3.89194 \\
1.00036 & 4.02056
\end{array}
$$

infDiluted Xsec

f-factor shielded Xsec $\begin{array}{lll}2.34636 & 0.979939 & 2.29929\end{array}$ $\begin{array}{lll}3.95907 & 0.995756 & 3.94226\end{array}$

$\begin{array}{lll}3.84393 & 1 & 3.84393\end{array}$

3.85288

3.85529

3.86474

3. 89128

4.01888

$1.00006 \quad 3.86496$

1.000143 .89183

$1.00037 \quad 4.02035$

$\begin{array}{rrc}\text { infDiluted Xsec } & \text { f-factor } & \text { shielded Xsec } \\ 0.00010111 & 1.0002 & 0.00010113 \\ 0.000101635 & 0.991292 & 0.00010075 \\ 7.10826 e-06 & 1.00036 & 7.11084 \mathrm{e}-06 \\ 7.2788 \mathrm{e}-06 & 1.00001 & 7.27885 \mathrm{e}-06\end{array}$




\begin{tabular}{|c|c|c|c|c|c|}
\hline 102 & 5 & 45.7377472 & $2.38519 e-05$ & 1.00003 & $2.38526 e-05$ \\
\hline 102 & 6 & 45.7377472 & $5.83622 e-05$ & 1.00019 & $5.83734 e-05$ \\
\hline 102 & 7 & 45.7377472 & 0.000105195 & 0.998729 & 0.000105061 \\
\hline 102 & 8 & 45.7377472 & 0.000209981 & 0.996663 & 0.00020928 \\
\hline mt & Group & $\operatorname{sig} 0$ & infDiluted Xsec & f-factor & shielded Xsec \\
\hline 1007 & 1 & 45.7377472 & 0 & 0 & 0 \\
\hline 1007 & 2 & 45.7377472 & 0 & 0 & 0 \\
\hline 1007 & 3 & 45.7377472 & 0 & 0 & 0 \\
\hline 1007 & 4 & 45.7377472 & 0 & 0 & 0 \\
\hline 1007 & 5 & 45.7377472 & 3.85523 & 1.00003 & 3.85535 \\
\hline 1007 & 6 & 45.7377472 & 3.86483 & 1.00003 & 3.86496 \\
\hline 1007 & 7 & 45.7377472 & 3.89119 & 1.00016 & 3.89183 \\
\hline 1007 & 8 & 45.7377472 & 4.01794 & 1.0006 & 4.02035 \\
\hline
\end{tabular}

\subsubsection{References}

1. I. I. Bondarenko, ed., Group Constants for Nuclear Reactor Calculations, Consultants Bureau, New York, 1964.

2. D. Wiarda, M. L. Williams, C. Celik, and M. E. Dunn, "AMPX: A Modern Cross Section Processing System for Generating Nuclear Data Libraries," Proceedings of International Conference on Nuclear Criticality Safety, Charlotte, NC, Sept. 13-17 2015.

3. R. Goldstein and E. R. Cohen, "Theory of Resonance Absorption of Neutrons," Nucl. Sci. Engr. 13, 132 (1962).

4. J. R. Lamarsh, Introduction to Nuclear Reactor Theory, Addison-Wesley Publishers, Boston, 1966.

5. J. M. Otter, Escape Probability Approximations in Lumped Resonance Absorbers, NAA-SR-9744, Office of Technical Services, Washington, DC, August 1964.

6. D. C. Leslie, J. G. Hill, and A. Jonsson, "Improvements to the Theory of Resonance Escape in Heterogeneous Fuel I: Regular Arrays of Fuel Rods,” Nuc Sci Engr 22, 78 (1965).

7. W. J. Davis, M. B. Yarbrough, and A. B. Bortz, SPHINX-A One-Dimensional Diffusion and Transport Nuclear Cross Section Processing Code, WAPD-XS-3045-17 (UC-79d), Westinghouse Advanced Reactor Division, August 1977.

8. R. B. Kidman, "Improved F-Factor Interpolation Scheme for 1DX," Transactions 18, 156-157 (June 23, 1974).

9. M. Segev, “Interpolation of Resonance Integrals,” Nuc Sci Engr 22, 79 (1981).

10. N. M. Greene, “A Method for Interpolating in Bondarenko Factor Tables”, Proceedings: Thermal Reactor Calculations, Techniques, Results, and Applications, Electric Power Research Institute, EPRI NP-2655, Feb. 1983. 


\title{
7.4 CENTRM: A NEUTRON TRANSPORT CODE FOR COMPUTING CONTINUOUS- ENERGY SPECTRA IN GENERAL ONE-DIMENSIONAL GEOMETRIES AND TWO- DIMENSIONAL LATTICE CELLS
}

M. L. Williams

\begin{abstract}
CENTRM computes continuous-energy neutron spectra for infinite media, general one-dimensional (1D) systems, and two-dimensional (2D) unit cells in a lattice, by solving the Boltzmann transport equation using a combination of pointwise and multigroup nuclear data. Several calculational options are available, including a slowing-down computation for homogeneous infinite media, 1D discrete ordinates in slab, spherical, or cylindrical geometries; a simplified two-region solution; and 2D method of characteristics for a unit cell within a square-pitch lattice. In SCALE, CENTRM is used mainly to calculate problem-specific fluxes on a fine energy mesh (10,000-70,000 points), which may be used to generate self-shielded multigroup cross sections for subsequent radiation transport computations.
\end{abstract}




\section{TABLE OF CONTENTS}

$\underline{\text { Page }}$

7.4 CENTRM: A NEUTRON TRANSPORT CODE FOR COMPUTING CONTINUOUSENERGY SPECTRA IN GENERAL ONE-DIMENSIONAL GEOMETRIES AND TWO-

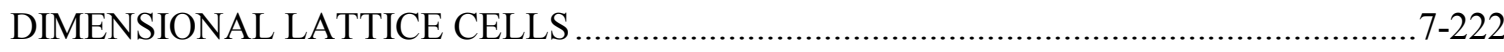

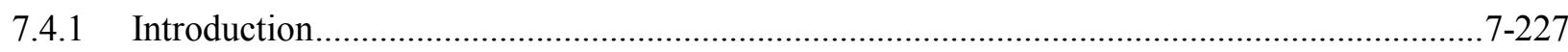

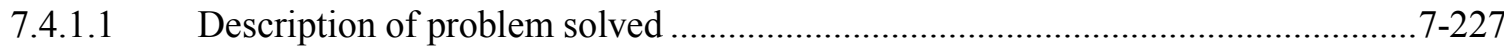

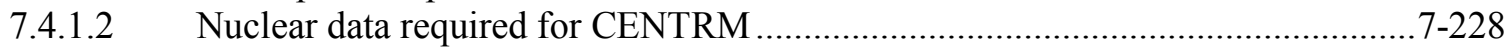

7.4.1.3 Code assumptions and features ............................................................

7.4.2 Theory and Analytical Models......................................................................................... 7-230

7.4.2.1 Energy/lethargy ranges for MG and PW calculations ..................................... 7-230

7.4.2.2 The Boltzmann equation for neutron transport ............................................... $7-232$

7.4.2.3 Legendre moments of the scattering source ..........................................................

7.4.2.4 Sub-moment expansion of the epithermal scattering source ................................ 7-240

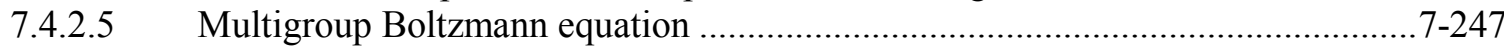

7.4.2.6 The Boltzmann equation within the PW range................................................. 7-248

7.4.2.7 Determination of energy mesh for PW flux calculation....................................... 7-258

7.4.2.8 CENTRM cross sections and fixed sources .................................................... 7-259

7.4.3 Available Methods for Solving Transport Equation .........................................................259

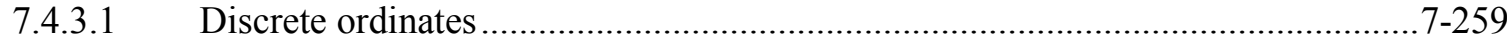

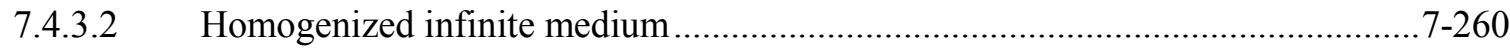

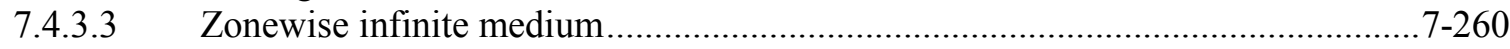

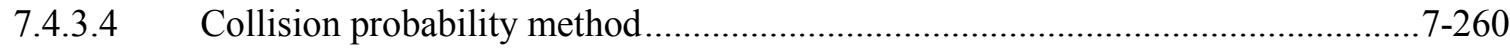

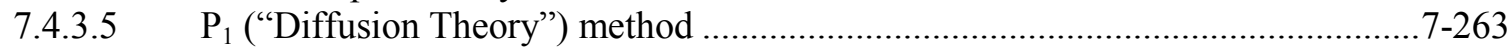

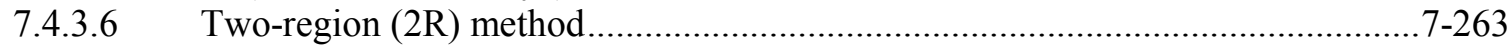

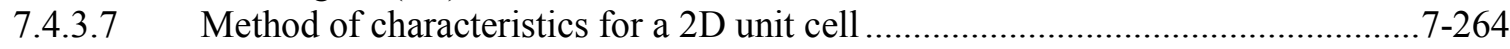

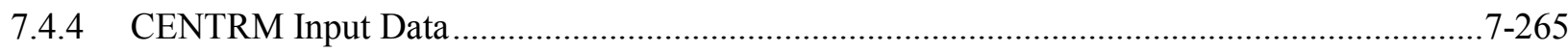

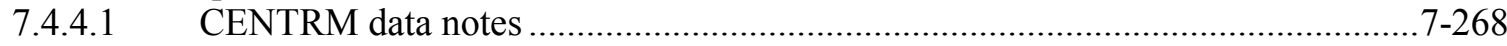

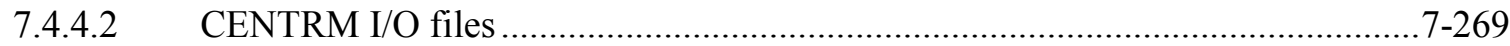

7.4.4.3 Description of the CENTRM CE cross section file............................................... 7-270

7.4.4.4 Description of the CENTRM output PW flux file............................................... 7-272

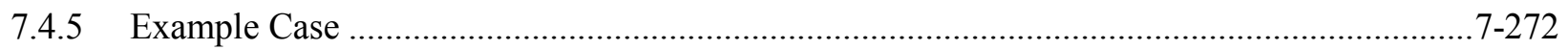

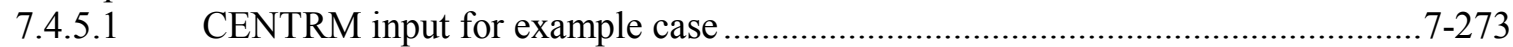

7.4.5.2 CENTRM output for example case ................................................................

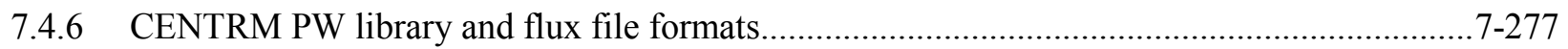

7.4.6.1 Description of the CENTRM CE cross section file............................................ $7-277$

7.4.6.2 Description of the CENTRM output PW flux file................................................ 7-280

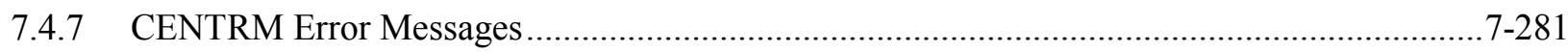

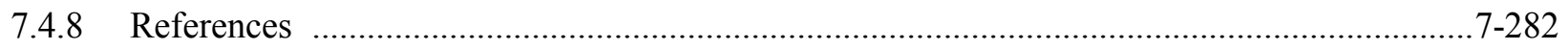




\section{LIST OF FIGURES}

Page

Figure 7.4.1. Definition of UMR, PW, and LMR energy ranges................................................. $7-229$

Figure 7.4.2. Definition of High and Transition regions in upper multigroup region. ....................... 7-231

Figure 7.4.3. Definition of cumulative integral elements.............................................................. 7-251 


\section{LIST OF TABLES}

$\underline{\text { Page }}$

Table 7.4.1. Constants appearing in Legendre polynomials ........................................................... 7-242

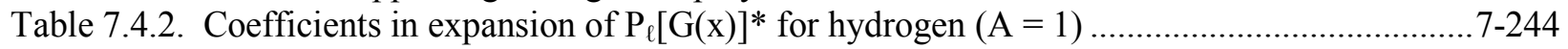

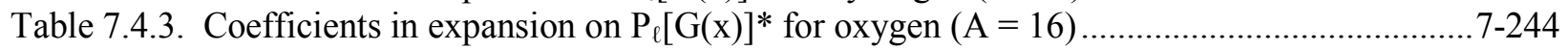

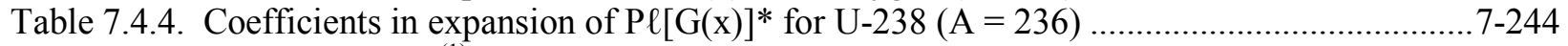

Table 7.4.5. Fractional error ${ }^{(1)}$ in series expansion [Eq. (F18.2.25)] of Legendre polynomials .......... 7-245

Table 7.4.6. Number of moments and submoments as function of scattering order...........................7-246

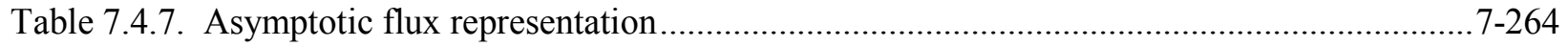

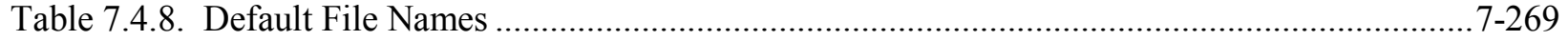




\section{ACKNOWLEDGMENTS}

Several current and former ORNL staff made valuable contributions to the CENTRM development. The author acknowledges the contributions of former ORNL staff members D. F. Hollenbach and N. M. Greene; as well as current staff member L. M. Petrie. Special thanks go to Kang-Seog Kim who developed the 2D method of characteristics option for CENTRM. Portions of the original code development were performed by $\mathrm{M}$. Asgari as partial fulfillment of his $\mathrm{PhD}$ dissertation research at Louisiana State University (LSU); and Riyanto Raharjo from LSU made significant programming contributions for the inelastic scattering and thermal calculations. 


\subsubsection{Introduction}

CENTRM (Continuous ENergy TRansport Module) computes “continuous-energy" neutron spectra using various deterministic approximations to the Boltzmann transport equation. Computational methods are available for infinite media, general one-dimensional (1-D) geometries, and two-dimensional (2D) unit cells in a square-pitch lattice. The purpose of the code is to provide fluxes and flux moments for applications that require a high resolution of the fine-structure variation in the neutron energy spectrum. The major function of CENTRM is to determine problem-specific fluxes for processing multigroup (MG) data with the XSProc self-shielding module (Introduction in XSProc chapter), which is executed by all SCALE MG sequences. XSProc calls an application program interface (API) to perform a CENTRM calculation for a representative model (e.g., a unit cell in a lattice), and then utilizes the spectrum as a problem-dependent weight function for MG averaging. The MG data processing is done in XSProc by calling an API for the PMC code, which uses the CENTRM continuous-energy (CE) flux spectra and cross-section data to calculate group-averaged cross sections over some specified energy range. The resulting application-specific library is used for MG neutron transport calculations within SCALE sequences. In this approach the CENTRM/PMC cross-section processing in XSProc becomes an active component in the overall transport analysis. CENTRM can also be executed as a standalone code, if the user provides all required input data and nuclear data libraries; but execution through XSProc is much simpler and less prone to error.

\subsubsection{Description of problem solved}

CENTRM uses a combination of MG and pointwise (PW) solution techniques to solve the neutron transport equation over the energy range $\sim 0$ to $20 \mathrm{MeV}$. The calculated $\mathrm{CE}$ spectrum consists of PW values for the flux per unit lethargy defined on a discrete energy mesh, for which a linear variation of the flux between energy points is assumed. Depending on the specified transport approximation, the flux spectrum may vary as a function of space and direction, in addition to energy. Spherical harmonic moments of the angular flux, which may be useful in processing MG matrices for higher order moments of the scattering cross section, can also be determined as a function of space and energy mesh.

CENTRM solves the fixed-source (inhomogeneous) form of the transport equation, with a user-specified fixed source term. The input source may correspond to MG histogram spectra for volumetric or surface sources or it may be a "fission source" which has a continuous-energy fission-spectrum distribution (computed internally) appropriate for each fissionable mixture. Note that eigenvalue calculations are not performed in CENTRM - these must be performed by downstream MG transport codes that utilize the self-shielded data processed with the CENTRM spectra. 


\subsubsection{Nuclear data required for CENTRM}

A MG cross section library, a CE cross section library, and a CE thermal kernel $[S(\alpha, \beta)]$ library are required for the CENTRM transport calculation. During XSProc execution for a given unit cell in the CELL DATA block, the MG library specified in the input is processed by BONAMI prior to the CENTRM calculation, in order to provide self-shielded data based on the Bondarenko approximation for the MG component of the CENTRM solution. The shielded MG cross sections are also used in CENTRM to correct infinitely dilute $\mathrm{CE}$ data in the unresolved resoance range. The CRAWDAD module is executed by XSProc to generate the CENTRM CE cross section and thermal kernel libraries, respectively, by concatenating discrete PW data read from individual files for the nuclides in the unit cell mixtures. CE resonance profiles are based strictly on specifications in the nuclear data evaluations; e.g., Reich-Moore formalism is specified for most materials in ENDF/B-VII ${ }^{1}$. PW data in the CENTRM library are processed such that values at any energy can be obtained by linear interpolation within some error tolerance specified during the library generation (usually $\sim 0.1 \%$ or less). CRAWDAD also interpolates the CE cross section data and the Legendre moments of the thermal scattering kernels to the appropriate temperatures for the unit cell mixtures. The format of the CENTRM library is described in Sect. 7.4.6.1

\subsubsection{Code assumptions and features}

As shown in Figure 7.4.1, the energy range of interest is divided into three intervals called the Upper Multigroup Range (UMR), Pointwise Range (PW), and Lower Multigroup Range (LMR), respectively, which are defined by input. MG fluxes are computed using standard multigroup techniques for the UMR and LMR, and these values are then divided by the group lethargy width to obtain the average flux per lethargy within each group. This "pseudo-pointwise" flux is assigned to the midpoint lethargy of the group, so that there is one energy point per group in the UMR and LMR energy intervals. However, for each group in the PW range there are generally several, and possibly many, energy points for which CENTRM computes flux values. In this manner a problem-dependent spectrum is obtained over the entire energy range.

The default PW range goes from $0.001 \mathrm{eV}$ to $20 \mathrm{keV}$, but the user can modify the PW limits. The energy range for the PW transport calculation is usually chosen to include the interval where the important absorber nuclides have resolved resonances, while MG calculations are performed where the cross sections characteristically have a smoother variation or where shielding effects are less important. In the SCALE libraries the thermal range is defined to be energies less than $5.0 \mathrm{eV}$. Above thermal energies, scattering kinematics are based on the stationary nucleus model, while molecular motion and possible chemical binding effects are taken into account for thermal scattering, which can result in an incease in the neutron incident energy. The CENTRM thermal calculation uses Legendre coefficients from the CE kernel library that describes point-to-point energy transfers for incoherent and coherent scattering, as function of temperature, for all moderators that have thermal scattering law data provided in ENDF/B. Thermal kernels for all other materials are generated internally by CENTRM based on the free-gas model. 


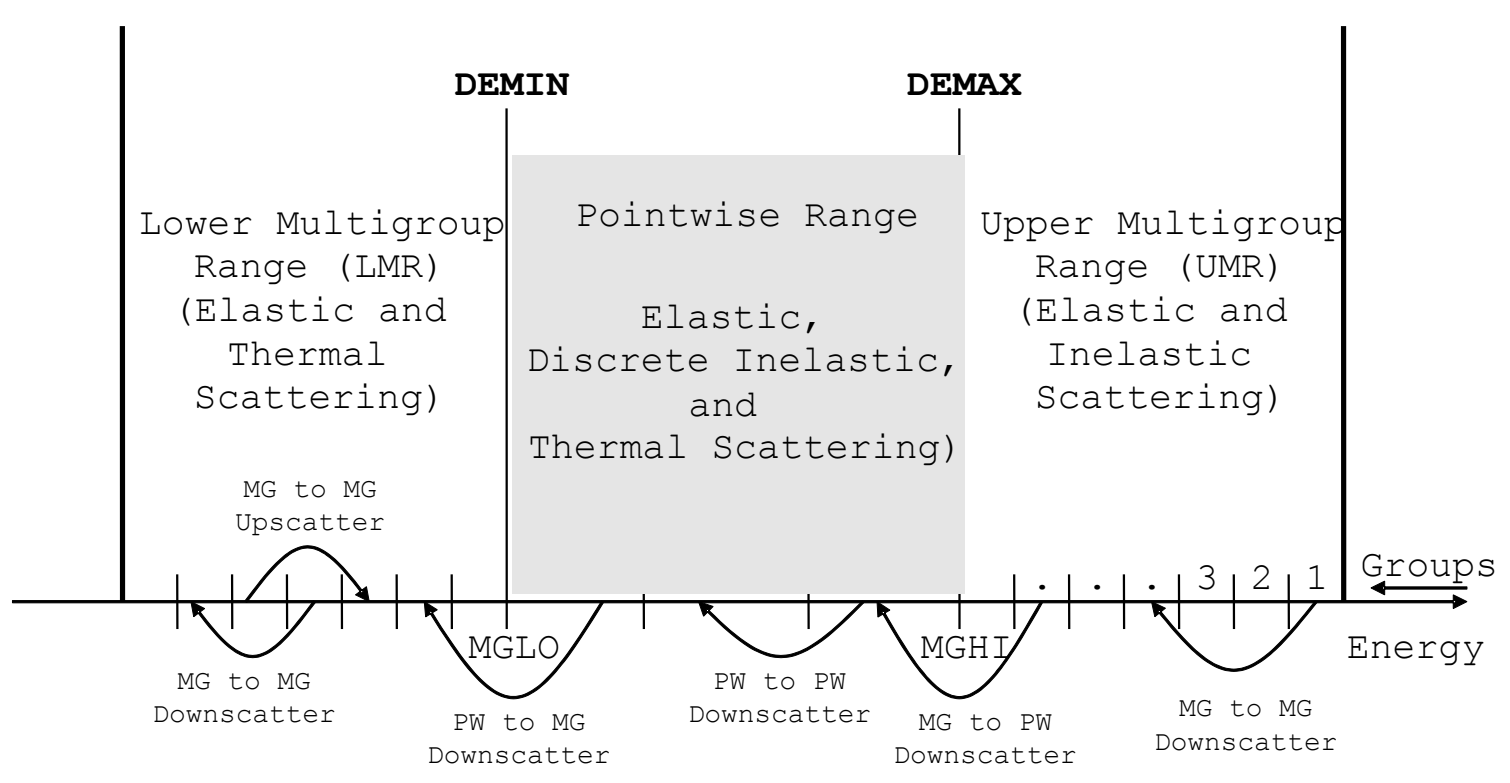

Figure 7.4.1. Definition of UMR, PW, and LMR energy ranges.

Several transport computation methods are available for both MG and PW calculations. These include a space-independent slowing down calculation for infinite homogeneous media, 1D discrete ordinates or P1 methods for slab, spherical, and cylindrical geometries, and a 2D method of chracateristics (MoC) method for lattice unit cells. A simplified two-region collision-probablity method is also available for ther pointwise solution. In general the user may specify different transport methods for the UMR, PW, and LMR, respectively; however, if the $2 \mathrm{D} \mathrm{MoC}$ method is specified for any range, it will be used for all.

The CENTRM 1D discrete ordinates calculation option has many of the same features as the SCALE MG code XSDRNPM. It represents the directional dependence of the angular flux with an arbitrary symmetric-quadrature order, and uses Legendre expansions up to $\mathrm{P}_{5}$ to represent the scattering source. No restrictions are placed on the material arrangement or the number of spatial intervals in the calculation, and general boundary conditions (vacuum, reflected, periodic, albedo) can be applied on either boundary of the 1D geometry. Lattice cells are represented in the CENTRM discrete ordinates option by a 1D Wigner-Sitz cylindrical or spherical model with a white boundary condition on the outer surface.

Starting with SCALE-6.2, CENTRM also includes a 2D MoC solver for lattice cell geometries consisting of a cylindrical fuel rod (fuel/gap/clad) contained within a rectangular moderator region. The MoC calculation is presently limited to square lattices. The $2 \mathrm{D}$ unit cell uses a reflected boundary condition on the outer square surface, which provides a more rigorous treatment than the 1D Wigner-Seitz model; however the MoC option requires a longer execution time than the 1D discrete ordinates method. The $\mathrm{MoC}$ option has been found to improve results compared to the 1D Wigner-Seitz cell model for many cases, but in other cases the improvement is marginal.

A variable PW energy mesh is generated internally to accurately represent the fine-structure flux spectrum for the system of interest. This gives CENTRM the capability to rigorously account for resonance interference effects in systems with multiple resonance absorbers. Because CENTRM calculates the space-dependent PW flux spectrum, the spatial variation of the self-shielded cross sections 
within an absorber body can be obtained. A radial temperature distribution can also be specified, so that space-dependent Doppler broadening can be treated in the transport solution. Within the epithermal PW range, the slowing-down source due to elastic and discrete-level inelastic reactions is computed with the analytical scatter kernel based upon the neutron kinematic relations for $s$-wave scattering. Continuum inelastic scatter is approximated by an analytical evaporation spectrum, assumed isotropic in the laboratory system. For many thermal reactor and criticality safety problems, self-shielding of inelastic cross sections has a minor impact, and by default these options are turned off for faster execution. As previously discussed, the thermal scatter kernel is based on the ENDF/B scattering law data for bound moderators, and uses the free-gas model for other materials.

\subsubsection{Theory and Analytical Models}

This section describes the coupled MG and PW techniques used to solve the neutron ttransport equation.

\subsubsection{Energy/lethargy ranges for MG and PW calculations}

The combined MG/PW CENTRM calculation is performed over the energy range spanned by the group structure in the input MG library. The energy boundaries for the "IGM" neutron groups specified on the MG library divide the entire energy range into energy intervals. The lowest energy group contained in the UMR is defined to be "MGHI"; while the highest energy group in the LMR is designated "MGLO." The boundary between the PW and UMR energy intervals is set by the energy value "DEMAX," while "DEMIN" is the boundary between the PW and LMR. The default values of $0.001 \mathrm{eV}$ and $20 \mathrm{keV}$ for DEMIN and DEMAX, respectively, can be modified by user input, but the input values are altered by the code to correspond to the closest group boundaries. Hence, DEMAX is always equal to the lower energy boundary of group MGHI and DEMIN the upper energy boundary of MGLO. The PW calculation is performed in terms of lethargy $(u)$, rather than energy $(E)$. The origin $(u=0)$ of the lethargy coordinate corresponds to the energy E=DEMAX, which is the top of the PW range. See Figure 7.4.2.

The highest energy group of the thermal range is defined by the parameter "IFTG," obtained from the MG library. If DEMIN is less than the upper energy boundary of IFTG, the PW range extends into thermal. In this case, scattering in the PW region of the thermal range is based on the PW scattering kernel data; and the LMR calculation uses 2D transfer matrices for incoherent and coherent scattering on the MG library. Coupling between the MG and PW thermal calculations is treated, and outer iterations are required to address effects of upscattering. 


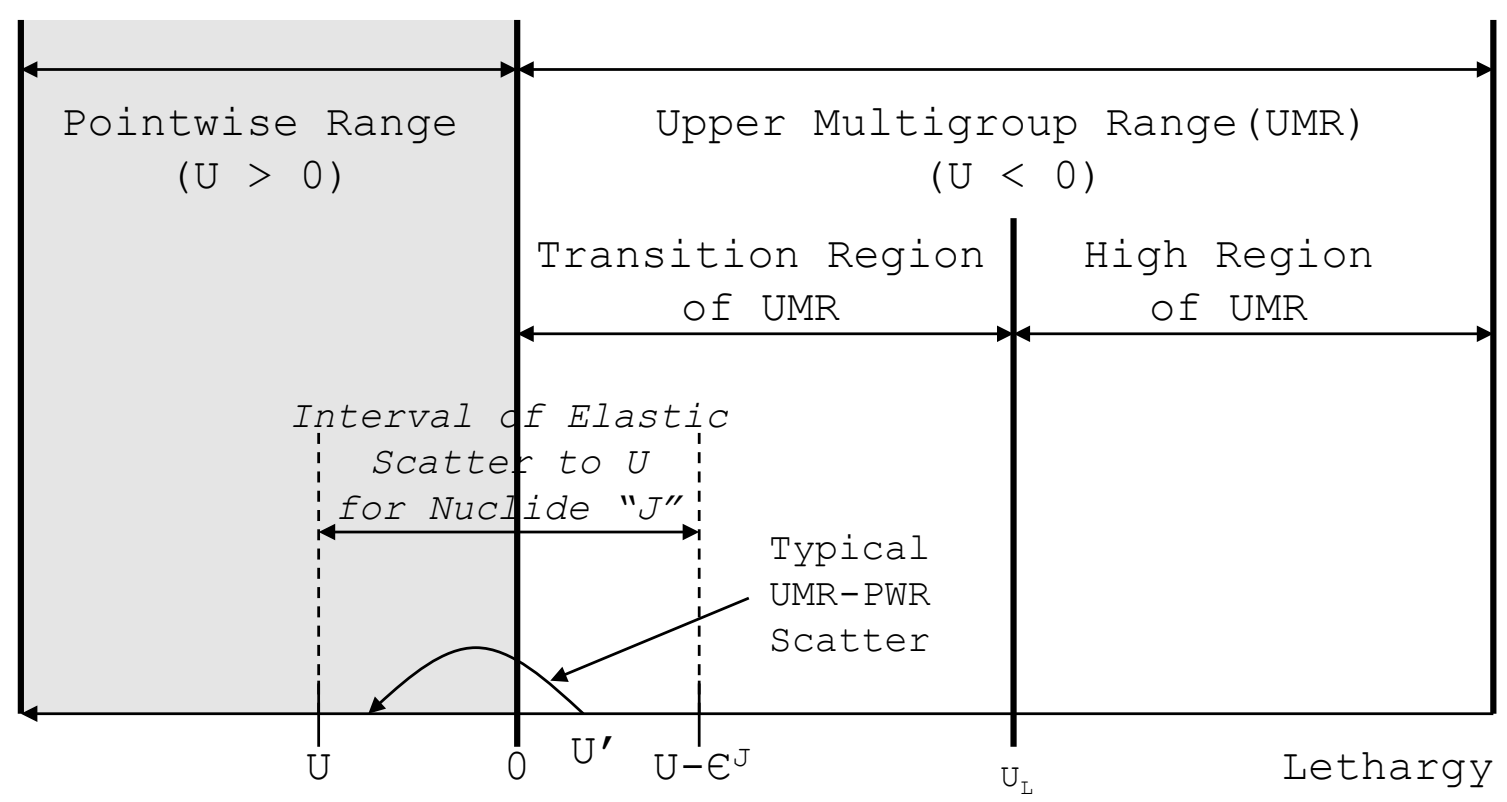

Figure 7.4.2. Definition of High and Transition regions in upper multigroup region.

With the exception of hydrogen moderation, elastic down-scattering coupling the UMR and PW ranges, occurs only within a limited sub-range of the UMR called the "transition region". The highest energy group in the transition region is designated "MGTOP." The precise definition of the transition region is given in Sect. 7.4.2.6.1.

Energy boundaries of the group structure on the input MG library correspond to the IGM +1 values, $\left\{G_{1}\right.$, $\left.\mathrm{G}_{2}, \ldots \mathrm{G}_{\mathrm{g},} \mathrm{G}_{\mathrm{g}+1}, \ldots, \mathrm{G}_{\mathrm{IGM}+1}\right\}$. It is convenient to designate the number of groups in the $\mathrm{UMR}, \mathrm{PW}$, and LMR ranges equal to $\mathrm{NG}_{U}, \mathrm{NG}_{\mathrm{P}}$, and $\mathrm{NG}_{\mathrm{L}}$, respectively, so that $\mathrm{IGM}=\mathrm{NG}_{\mathrm{U}}+\mathrm{NG}_{\mathrm{P}}+\mathrm{NG}_{\mathrm{L}}$; or in terms of the parameters MGHI and MGLO introduced previously:

$$
\mathrm{NG}_{\mathrm{U}}=\mathrm{MGHI} ; \mathrm{NG}_{\mathrm{P}}=\mathrm{MGLO}-\mathrm{MGHI}-1 ; \mathrm{NG}_{\mathrm{L}}=\mathrm{IGM}-\mathrm{MGLO}+1 .
$$

The flux per unit lethargy is calculated for a discrete energy (or lethargy) mesh spanning the MG structure. Groups in the UMR and LMR each contain a single energy mesh point, while groups in the PW range generally contain several points. The number of mesh points in the UMR, PW, and LMR is equal respectively to $\mathrm{NG}_{\mathrm{U}}, \mathrm{N}_{\mathrm{P}}$, and $\mathrm{NG}_{\mathrm{L}}$; and the total number of points in the entire energy mesh is designated as " $\mathrm{N}_{\mathrm{T}}$," which is equal to $\mathrm{NG}_{\mathrm{U}}+\mathrm{N}_{\mathrm{P}}+\mathrm{NG}_{\mathrm{L}}$. Thus the lethargy (u) mesh consists of the set of points:

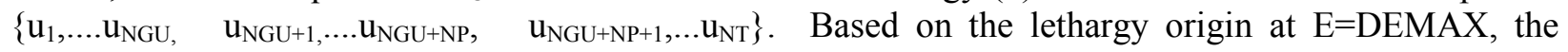
lethargy " $u_{n}$ " associated with any energy point " $E_{n}$ " is equal to,

$$
\mathrm{u}_{\mathrm{n}}=\ln \left(\mathrm{DEMAX} / \mathrm{E}_{\mathrm{n}}\right) \text {. }
$$

Lethargy points are arranged in order of increasing value. The lethargy origin is at point $\mathrm{NG}_{\mathrm{U}}+1$, the lower energy boundary of group MGHI; i.e., $\mathbf{u}_{\mathrm{NGU}+1}=0$. Note that the entire UMR (E $>$ DEMAX) corresponds to negative lethargy values. Lethargy values for the first $\mathrm{NG}_{\mathrm{U}}$ and the last $\mathrm{NG}_{\mathrm{L}}$ points in the mesh are defined to be the midpoint lethargies of groups in the UMR and LMR ranges, respectively. For example, for the $\mathrm{NG}_{\mathrm{U}}$ groups within the UMR, 


$$
\begin{aligned}
\mathrm{u}_{1} & =0.5\left[\ln \left(\text { DEMAX }_{\mathrm{G}_{1}}\right)+\ln \left(\text { DEMAX }_{\mathrm{G}_{2}}\right)\right] \\
\mathrm{u}_{\mathrm{NGU}} & =0.5\left[\ln \left(\text { DEMAX } / \mathrm{G}_{\mathrm{MGHI}}\right)+\ln \left(\text { DEMAX } / \mathrm{G}_{\mathrm{MGHI}+1}\right)\right]
\end{aligned}
$$

and similarly for the $\mathrm{NG}_{\mathrm{L}}$ groups in the LMR,

$$
\begin{aligned}
\mathrm{u}_{\mathrm{NGU}+\mathrm{NP}+1} & =0.5\left[\ln \left(\mathrm{DEMAX} / \mathrm{G}_{\mathrm{MGLO}}\right)+\ln \left(\mathrm{DEMAX} / \mathrm{G}_{\mathrm{MGLO}+1}\right)\right] \\
\mathrm{u}_{\mathrm{NT}} & =0.5\left[\ln \left(\mathrm{DEMAX} / \mathrm{G}_{\mathrm{IGM}}\right)+\ln \left(\mathrm{DEMAX} / \mathrm{G}_{\mathrm{IGM}+1}\right)\right]
\end{aligned}
$$

The remaining $\mathrm{N}_{\mathrm{P}}$ points in the mesh (i.e., values $\mathrm{u}_{\mathrm{NGU}+1}$ to $\mathrm{u}_{\mathrm{NGU}+\mathrm{NP}}$ ) are contained within the $\mathrm{NG}_{\mathrm{P}}$ groups that span the PW range. By definition the first point in the PW range is the lower energy boundary of group MGHI. The other mesh points are computed internally by CENTRM, based on the behavior of the macroscopic PW total cross sections and other criteria.

The neutron flux, as a function of space and direction, is calculated for each energy/lethargy point in the mesh by solving the Boltzmann transport equation. The transport equation at each lethargy point generally includes a source term representing the production rate due to elastic and inelastic scatter from other lethargies, which couples the solutions at different lethargy mesh points. Except in the thermal range, neutrons can only gain lethargy (lose energy) in a scattering reaction; thus the PW flux is computed by solving the transport equation at successive mesh points, sweeping from low to high lethargy values.

\subsubsection{The Boltzmann equation for neutron transport}

The steady state neutron transport equation shown below represents a particle balance-per unit phase space, at an arbitrary point $\rho$ in phase space,

$$
\Omega \cdot \nabla \Psi(\rho)+\sum_{\mathrm{t}}(\mathrm{r}, \mathrm{u}) \Psi(\rho)=\int_{0}^{\infty} \int_{0}^{4 \pi} \sum\left(\mathrm{u}^{\prime} \rightarrow \mathrm{u} ; \mu_{0}\right) \Psi\left(\mathrm{u}^{\prime}, \Omega^{\prime}\right) \mathrm{du}^{\prime}+\mathrm{Q}_{\mathrm{ext}}(\rho)
$$

where:

$$
\begin{aligned}
\psi(\mathrm{p}) & =\text { angular flux (per lethargy) at phase space coordinate } \rho ; \\
\rho=(\mathrm{r}, \mathrm{u}, \Omega) & =\text { phase space point defined by the six independent variables; } \\
\mathrm{r}=\left(\mathrm{x}_{1}, \mathrm{x}_{2}, \mathrm{x}_{3}\right) & =\text { space coordinates; } \\
\mathrm{u}=\ln \left(\mathrm{E}_{\mathrm{ref}} / \mathrm{E}\right) & =\text { lethargy at energy } \mathrm{E}, \text { relative to an origin }(\mathrm{u}=0) \text { at } \mathrm{E}_{\mathrm{ref}} ; \\
\Omega=(\mu, \zeta) & =\text { neutron direction defined by polar cosine } \mu \text { and azimuthal angle } \zeta ; \\
\Sigma_{\mathrm{t}}(\mathrm{r}, \mathrm{u}) & =\text { macroscopic total cross section; } \\
\Sigma\left(\mathrm{u}^{\prime} \rightarrow \mathrm{u} ; \mu_{0}\right) & =\text { double differential scatter cross section; } \\
\mu_{0} & =\text { cosine of scatter angle, measured in laboratory coordinate system; } \\
\mathrm{Q}_{\mathrm{ext}}(\rho) & =\text { external source term, including fission source; }
\end{aligned}
$$


The left and right sides of Eq. (7.4.1) respectively, are equal to the neutron loss and production rates, per unit volume-direction-lethargy. In CENTRM the spatial distribution of the fission source is input as a component of the external source Q; hence, a fixed source rather than an eigenvalue calculation is required for the transport solution.

The angular dependence of the double-differential macroscopic scatter cross section of an arbitrary nuclide " $\mathrm{j}$ " is represented by a finite Legendre expansion of arbitrary order L:

$$
\Sigma^{(\mathrm{j})}\left(\mathrm{u}^{\prime} \rightarrow \mathrm{u} ; \mu_{0}\right)=\sum_{\ell=0}^{\mathrm{L}} \frac{2 \ell+1}{2} \Sigma_{\ell}^{(\mathrm{j})}\left(\mathrm{u}^{\prime} \rightarrow \mathrm{u}\right) \quad \mathrm{P}_{\ell}\left(\mu_{0}\right)
$$

where $\mathrm{P}_{\ell}\left(\mu_{0}\right)=$ Legendre polynomial evaluated at the laboratory scattering cosine $\mu_{0}$; and

$$
\begin{aligned}
\sum_{\ell}^{(\mathrm{j})}\left(\mathrm{u}^{\prime} \rightarrow \mathrm{u}\right)= & \text { cross-section moments of nuclide } \mathrm{j} \text {, defined by the expression } \\
& \sum_{\ell}^{(\mathrm{j})}\left(\mathrm{u}^{\prime} \rightarrow \mathrm{u}\right)=\int_{-1}^{1} \sum^{(\mathrm{j})}\left(\mathrm{u}^{\prime} \rightarrow \mathrm{u} ; \mu_{0}\right) \mathrm{P}_{\ell}\left(\mu_{0}\right) \mathrm{d} \mu_{0}
\end{aligned}
$$

After substitution of the above Legendre expansions for the scattering data of each nuclide, and applying the spherical harmonic addition theorem in the usual manner, the scattering source on the right side of Eq. (7.4.1) becomes ${ }^{1}$

$$
\mathrm{S}(\mathrm{r}, \mathrm{u}, \Omega) \equiv \int_{0}^{\infty} \int_{0}^{4 \pi} \Sigma\left(\mathrm{u}^{\prime} \rightarrow \mathrm{u} ; \mu_{0}\right) \Psi\left(\mathrm{u}^{\prime}, \Omega^{\prime}\right) \mathrm{d} \Omega^{\prime} \mathrm{du}^{\prime}=\sum_{\ell \mathrm{k}=1}^{\mathrm{LK}} \frac{2 \ell+1}{2} \mathrm{Y}_{\ell \mathrm{k}}(\Omega) \mathrm{S}_{\ell \mathrm{k}}(\mathrm{r}, \mathrm{u})
$$

where in,

$$
\begin{aligned}
\mathrm{Y}_{\ell \mathrm{k}}(\Omega)=\mathrm{Y}_{\ell \mathrm{k}}(\mu, \zeta) & =\text { the spherical harmonic function evaluated at direction } \Omega, \\
\mathrm{S}_{\ell k} & =\text { spherical harmonic moments of the scatter source, per unit letharagy. }
\end{aligned}
$$

The summation index " $\ell \mathrm{k}$ " indicates a double sum over $\ell$ and $\mathrm{k}$ indices; in the most general case it is defined as:

$$
\sum_{\ell \mathrm{k}=1}^{\mathrm{LK}}=\sum_{\ell=0}^{\mathrm{L}} \sum_{\mathrm{k}=0}^{\ell}
$$

where " $L "$ is the input value for the maximum order of scatter (input parameter "ISCT").

Due to symmetry conditions, some of the source moments may be zero. The parameter LK is defined to be the total number of non-zero moments (including scalar flux) for the particular geometry of interest, and is equal to,

$$
\begin{array}{cl}
\mathrm{LK}=\mathrm{L}+1 & \text { for 1D slabs and spheres; } \\
\mathrm{LK}=\mathrm{L}^{*}(\mathrm{~L}+4) / 4+1 & \text { for 1D cylinders, and } \\
\mathrm{LK}=\mathrm{L}^{*}(\mathrm{~L}+3) / 2+1 & \text { for 2D MoC cells }
\end{array}
$$

More details concerning the 1-D Boltzmann equation can be found in the XSDRNPM chapter of the SCALE manual. 


\subsubsection{Legendre moments of the scattering source}

The $\mathrm{S}_{\ell \mathrm{k}}$ moments in Eq. (7.4.4) correspond to expansion coefficients in a spherical harmonic expansion of the scatter source. These can be expressed in terms of the cross section and flux moments by

$$
\mathrm{S}_{\ell \mathrm{k}}(\mathrm{u})=\sum_{\mathrm{j}} \int_{\mathrm{u}^{\prime}} \mathrm{S}_{\ell \mathrm{k}}{ }^{(\mathrm{j})}\left(\mathrm{u}^{\prime} \rightarrow \mathrm{u}\right) \mathrm{du}^{\prime}=\sum_{\mathrm{j}} \int_{\mathrm{u}^{\prime}} \Sigma_{\ell}{ }^{(\mathrm{j})}\left(\mathrm{u}^{\prime} \rightarrow \mathrm{u}\right) \Psi_{\ell \mathrm{k}}\left(\mathrm{u}^{\prime}\right) \mathrm{du} \mathrm{u}^{\prime}
$$

where $\quad \psi_{\ell \mathrm{k}}(\mathrm{u})=$ spherical harmonic moments of the angular flux;

$$
=\int_{0}^{4 \pi} \mathrm{Y}_{\ell \mathrm{k}}(\Omega) \quad \Psi(\Omega) \mathrm{d} \Omega
$$

and $\mathrm{S}_{\ell \mathrm{k}}{ }^{\mathrm{j})}\left(\mathrm{u}^{\prime} \rightarrow \mathrm{u}\right)=$ moments of the differential scatter rate from lethargy $\mathrm{u}^{\prime}$ to $\mathrm{u}$, for nuclide " $\mathrm{j}$ ”;

$$
=\Sigma_{\ell}^{(\mathrm{j})}\left(\mathrm{u}^{\prime} \rightarrow \mathrm{u}\right) \quad \Psi_{\ell \mathrm{k}}\left(\mathrm{u}^{\prime}\right)
$$

The $\psi_{\ell \mathrm{k}}$ flux moments are the well known coefficients appearing in a spherical harmonic expansion of the angular flux. These usually are the desired output from the transport calculation. In particular, the $\ell=0, \mathrm{k}=0$ moment corresponds to the scalar flux [indicated here as $\Phi(\mathrm{r}, \mathrm{u})$ ],

$$
\Psi_{0,0}(\mathrm{r}, \mathrm{u})=\Phi(\mathrm{r}, \mathrm{u})=\int_{0}^{4 \pi} \Psi(\mathrm{r}, \mathrm{u}, \Omega) d \Omega
$$

In general the epithermal component of the scatter source in Eq. (7.4.4) contains contributions from both elastic and inelastic scatter reactions; however, inelastic scatter is only possible above the threshold energy corresponding to the lowest inelastic level. The inelastic $\mathrm{Q}$ values for most materials are typically above $40 \mathrm{keV}$; therefore, elastic scatter is most important for slowing down calculations in the resolved resonance range of most absorber materials of interest. For example, the inelastic $Q$ values of ${ }^{238} \mathrm{U}$, iron, and oxygen are approximately $45 \mathrm{keV}, 846 \mathrm{keV}$, and $6 \mathrm{MeV}$, respectively; while the upper energy of the ${ }^{238} \mathrm{U}$ resolved resonance range is $20 \mathrm{keV}$ in ENDF/B-VII. The inelastic thresholds of some fissile materials like ${ }^{235} \mathrm{U}$ and ${ }^{239} \mathrm{Pu}$ are on the order of $10 \mathrm{keV}$; however, with the exception of highly enriched fast systems, these inelastic reactions usually contribute a negligible amount to the overall scattering source. CENTRM assumes that continuum inelastic scatter is isotropic in the laboratory system, while discrete level inelastic scatter is isotropic in the center of mass (CM) coordinate system.

Over a broad energy range, elastic scatter from most moderators can usually be assumed isotropic ( $s$-wave) in the neutron-nucleus CM coordinate system. In the case of hydrogen, this is true up to approximately $13 \mathrm{MeV}$; for carbon up to $2 \mathrm{MeV}$; and for oxygen up to $100 \mathrm{keV}$. However, it is well known that isotropic CM scatter does not result in isotropic scattering in the laboratory system. For $s$-wave elastic scatter the average scatter-cosine in the laboratory system is given by: $\bar{\mu}_{0}=0.667 / \mathrm{A} ;^{3}$ where "A" is the mass number (in neutron mass units) of the scattering material. This relation indicates that $s$-wave, elastic scattering from low A materials tends to be more anisotropic in the laboratory, and that the laboratory scattering distribution approaches isotropic $\left(\bar{\mu}_{0}=0 ; \theta_{0}=90^{\circ}\right)$ as $\mathrm{A}$ becomes large. For example, the $\bar{\mu}_{0}$ of hydrogen is $0.667\left(48.2^{\circ}\right)$; while it is about $0.042\left(87.6^{\circ}\right)$ for oxygen. Because $s$-wave scattering from heavy materials is nearly isotropic in the laboratory system, the differential scattering cross section (and thus the scattering source) can usually be expressed accurately by a low 
order Legendre expansion. On the other hand light moderators like hydrogen may require more termsdepending on the flux anisotropy - to accurately represent the elastic scatter source in the laboratory system. The default settings in CENTRM are to use P0 (isotropic lab scatter) for mass numbers greater than $\mathrm{A}=100$, and $\mathrm{P} 1$ for lighter masses.

An analytical expression can be derived for the cross-section moments in the case of two-body reactions, such as elastic and discrete-level inelastic scattering from "stationary" nuclei. Stationary here implies that the effect of nuclear motion on neutron scattering kinematics is neglected. [NOTE: The stationary nucleus approximation for treating scattering kinematics does not imply that the effect of nuclear motion on Doppler broadening of resonance cross sections is ignored, since this effect is included in the PW cross-section data].

In CENTRM the stationary nucleus approximation is applied above the thermal cutoff, typically around 3-5 eV, but is not valid for low energy neutrons. CENTRM has the capability to perform a PW transport calculation in the thermal energy range using tabulated thermal scattering law data for bound molecules, combined with the analytical free-gas kernel for other materials. In this case the cross-section moments appearing in Eq. (7.4.3) include upscattering effects. The expressions used in CENTRM to compute the PW scatter source moments in the thermal range are given in Sect. 7.4.2.6.

The following two sections discuss the evaluation of the scatter source moments for epithermal elastic and inelastic reactions, respectively.

\subsection{Epithermal Elastic Scatter}

Consider a neutron with energy $E^{\prime}$, traveling in a direction $\Omega^{\prime}$, that scatters elastically from an arbitrary material " $\mathrm{j}$," having a mass $\mathrm{A}^{(\mathrm{j})}$ in neutron-mass units. Conservation of kinetic energy and momentum requires that there be a unique relation between the angle that the neutron scatters (relative to the initial direction) and its final energy $\mathrm{E}$ after the collision. If the nucleus is assumed to be stationary in the laboratory coordinate system, then the cosine $\left(\mu_{0}\right)$ of the scatter angle $\left(\theta_{0}\right)$ measured in the laboratory system, as a function of the initial and final energies, is found to be

$$
\mu_{0} \equiv \Omega^{\prime} \cdot \Omega=\mathrm{G}^{(\mathrm{j})}\left(\mathrm{E}^{\prime}, \mathrm{E}\right)
$$

where the kinematic correlation function $\mathrm{G}$ relating $\mathrm{E}^{\prime}, \mathrm{E}$, and $\mu_{0}$ for elastic scatter is equal to

$$
\begin{aligned}
& \mathrm{G}^{(\mathrm{j})}\left(\mathrm{E}^{\prime}, \mathrm{E}\right)=\mathrm{a}_{1}{ }^{(\mathrm{j})} \times\left[\mathrm{E} / \mathrm{E}^{\prime}\right]^{\frac{1}{2}}-\mathrm{a}_{2}{ }^{(\mathrm{j})} \times\left[\mathrm{E}^{\prime} / \mathrm{E}\right]^{\frac{1}{2}} ; \\
& \text { and } \mathrm{a}_{1}{ }^{(\mathrm{j})}=\left(\mathrm{A}^{(\mathrm{j})}+1\right) / 2 ; \mathrm{a}_{2}{ }^{(\mathrm{j})}=\left(\mathrm{A}^{(\mathrm{j})}-1\right) / 2
\end{aligned}
$$

The final energy E of an elastically scattered neutron is restricted to the range,

$$
\alpha^{(j)} E^{\prime} \leq E \leq E^{\prime}
$$

where $\alpha^{(j)}=$ maximum fractional energy lost by elastic scatter $=\left[a_{2}{ }^{(j)} / a_{1}^{(j)}\right]^{2}$.

The corresponding range of scattered neutrons in terms of lethargy is equal to

$$
\mathrm{u}^{\prime} \leq \mathrm{u} \leq \mathrm{u}^{\prime}+\varepsilon^{(\mathrm{j})}
$$


where

$$
\begin{aligned}
& \mathrm{u}, \mathrm{u}^{\prime}=\mathrm{u}(\mathrm{E}), \mathrm{u}^{\prime}\left(\mathrm{E}^{\prime}\right)=\text { lethargies corresponding to } \mathrm{E} \text { and } \mathrm{E}^{\prime} \text {, respectively; and } \\
& \varepsilon^{(\mathrm{j})}=\text { maximum increase in lethargy, per elastic scatter }=\ln \left[1 / \alpha^{(\mathrm{j})}\right]
\end{aligned}
$$

The double-differential scatter kernel of nuclide $\mathrm{j}$ (per unit lethargy and solid angle) for $s$-wave elastic scatter of neutrons from stationary nuclei, is equal to ${ }^{1}$

$$
\begin{array}{rlrl}
\Sigma^{(\mathrm{j})}\left(\mathrm{u}^{\prime} \rightarrow \mathrm{u} ; \mu_{0}\right) & =\frac{\mathrm{E} \Sigma^{(\mathrm{i})}\left(\mathrm{u}^{\prime}\right)}{\mathrm{E}^{\prime}\left(1-\alpha^{(\mathrm{j})}\right)} \delta\left[\mu_{0}-\mathrm{G}^{(\mathrm{j})}\left(\mathrm{E}^{\prime}, \mathrm{E}\right)\right], & \text { for } \mathrm{u}^{\prime} \leq \mathrm{u} \leq \mathrm{u}^{\prime}+\varepsilon^{(\mathrm{j})} \\
& =0 & \mathrm{u}<\mathrm{u}^{\prime} \text { or } \mathrm{u}>\mathrm{u}^{\prime}+\varepsilon^{(\mathrm{j})}
\end{array}
$$

The presence of the Dirac delta function completely correlates the angle of scatter and the values of the initial and final energies. Substituting the double differential cross-section expression from Eq. (7.4.15) into Eq. (7.4.3) gives the single-differential Legendre moments of the cross section, per final lethargy:

$$
\begin{aligned}
\Sigma_{\ell}^{(\mathrm{j})}\left(\mathrm{u}^{\prime} \rightarrow \mathrm{u}\right) & =\frac{\mathrm{EP}_{\ell}\left[\mathrm{G}^{(\mathrm{j})}\right] \Sigma^{(\mathrm{j})}\left(\mathrm{u}^{\prime}\right)}{\mathrm{E}^{\prime}\left(1-\alpha^{(\mathrm{j})}\right)}, & \text { for } \mathrm{u}^{\prime} \leq \mathrm{u} \leq \mathrm{u}^{\prime}+\varepsilon^{(\mathrm{j})} \\
& =0 & \mathrm{u}^{\prime} \text { or } \mathrm{u}>\mathrm{u}^{\prime}+\varepsilon^{(\mathrm{j})}
\end{aligned}
$$

where $P_{\ell}=$ Legendre polynomial evaluated at argument $G^{(j)}$ equal to the scatter cosine.

When the above expressions are used in Eq. (7.4.5), the following is obtained for the $\ell \mathrm{k}$ moment of the epithermal elastic scattering source at lethargy $\mathrm{u}$ :

$$
\mathrm{S}_{\ell \mathrm{k}}^{(\mathrm{j})}(\mathrm{u})=\sum_{\mathrm{j}} \int_{\mathrm{u}-\varepsilon^{(j)}}^{\mathrm{u}} \mathrm{S}_{\ell \mathrm{k}}\left(\mathrm{u}^{\prime} \rightarrow \mathrm{u}\right) \mathrm{du} \mathrm{u}^{\prime}=\sum_{\mathrm{j}} \int_{\mathrm{u}-\varepsilon^{(j)}}^{\mathrm{u}} \frac{\mathrm{E} \Sigma^{(\mathrm{j})}\left(\mathrm{u}^{\prime}\right) \mathrm{P}_{\ell}\left[\mathrm{G}^{(\mathrm{j})}\right]}{\mathrm{E}^{\prime}\left(1-\alpha^{(\mathrm{j})}\right)} \Psi_{\ell \mathrm{k}}\left(\mathrm{u}^{\prime}\right) \mathrm{du} \mathrm{u}^{\prime} .
$$

\subsection{Epithermal Inelastic Scatter}

If the input value of DEMAX is set above the inelastic threshold of some materials in the problem, then inelastic scattering can occur in the PW range. The pointwise transport calculation may optionally include discrete-level and continuum inelastic reactions in computing the PW scatter source moments. The multigroup calculations always consider inelastic reactions.

Discrete-level inelastic reactions are two-body interactions, so that kinematic relations can be derived relating the initial and final energies and the angle of scatter. It can be shown that the kinematic correlation function for discrete-level inelastic scatter can be written in a form identical to that for elastic scatter by redefining the parameter $a_{1}$ in Eq. (7.4.10) to be the energy dependent function, ${ }^{2}$

$$
a_{1}^{(m, j)}=\frac{\left(A^{(j)}+1\right)}{2}+\frac{\left(-Q^{(m, j)}\right) A^{(j)}}{2 E} ;
$$

The parameter $\mathrm{Q}^{(\mathrm{m}, \mathrm{j})}$ is the $\mathrm{Q}$-value for the $\mathrm{m}_{\mathrm{th}}$ level of nuclide “ $\mathrm{j}$ ”. The $\mathrm{Q}$ value is negative for inelastic scattering, while it is zero for elastic scatter. The threshold energy in the laboratory coordinate system is proportional to the Q-value of the inelastic level, and is given by: 


$$
\mathrm{E}_{\mathrm{T}}^{(\mathrm{m}, \mathrm{j})}=\frac{\mathrm{A}^{(\mathrm{j})}+1}{\mathrm{~A}^{(\mathrm{j})}} \times\left(-\mathrm{Q}^{(\mathrm{m}, \mathrm{j})}\right)
$$

The range of energies that can contribute to the scatter source at $\mathrm{E}$, due to inelastic scatter from the $\mathrm{m}_{\mathrm{th}}$ level of nuclide $\mathrm{j}$ is defined to be $\left[\mathrm{E}_{\mathrm{L}}, \mathrm{E}_{\mathrm{H}}\right]$, where $\mathrm{E}_{\mathrm{H}}>\mathrm{E}_{\mathrm{L}}>\mathrm{E}_{\mathrm{T}}$. This energy range has a corresponding lethargy range of $\left[\mathrm{u}_{\mathrm{LO}}, \mathrm{u}_{\mathrm{HI}}\right]$ which is equal to, ${ }^{2}$

$$
\begin{aligned}
& u_{\mathrm{LO}}=u-\ln \left(\frac{1}{\alpha_{1}^{(j)}\left(E_{\mathrm{H}}\right)}\right)=u-\varepsilon_{1}^{(j)} \\
& \mathrm{u}_{\mathrm{HI}}=\mathrm{u}-\ln \left(\frac{1}{\alpha_{2}^{(\mathrm{j})}\left(E_{\mathrm{L}}\right)}\right)=u-\varepsilon_{2}^{(\mathrm{j})} .
\end{aligned}
$$

The energy-dependent alpha parameters in the above expressions are defined as,

$$
\begin{aligned}
& \alpha_{1}(\mathrm{E})=\left(\frac{\mathrm{A} \Delta^{(\mathrm{m}, \mathrm{j})}(\mathrm{E})-1}{\mathrm{~A}+1}\right)^{2} \\
& \alpha_{2}(\mathrm{E})=\left(\frac{\mathrm{A} \Delta^{(\mathrm{m}, \mathrm{j})}(\mathrm{E})+1}{\mathrm{~A}+1}\right)^{2},
\end{aligned}
$$

where

$$
\Delta^{(\mathrm{m}, \mathrm{j})}(\mathrm{E})=\sqrt{1-\frac{\mathrm{E}_{\mathrm{T}}^{(\mathrm{m}, \mathrm{j})}}{\mathrm{E}}}
$$

Modifying the epithermal elastic scatter source in Eq. (7.4.17) to include discrete-level inelastic scatter gives the following expression

$$
\mathrm{S}_{\ell \mathrm{k}}(\mathrm{u})=\sum_{m, j} \int_{u_{L O}}^{u_{H I}} \frac{\mathrm{E}}{\mathrm{E}^{\prime}} \frac{\Sigma^{(\mathrm{m}, \mathrm{j})}\left(\mathrm{E}^{\prime}\right) \mathrm{P}_{\ell}\left[\mathrm{G}^{(\mathrm{m}, \mathrm{j})}\right]}{\left(1-\alpha^{\mathrm{j}}\right) \Delta^{(\mathrm{m}, \mathrm{j})}\left(\mathrm{E}^{\prime}\right)} \Psi_{\ell \mathrm{k}}\left(\mathrm{u}^{\prime}\right) \mathrm{du}^{\prime}
$$

Detailed expressions for the lethargy limits are given in Ref. 2. Since $\Delta^{(\mathrm{m}, \mathrm{j})}$ is equal to unity for elastic scatter, the above equation reduces to Eq. (7.4.17) if there is no discrete-level inelastic contribution.

At high energies, the inelastic levels of the nucleus become a continuum. In this case CENTRM represents the energy distribution of the scattered neutrons by an evaporation spectrum with an isotropic angular distribution in the lab system; thus, only the $\mathrm{P}_{0}$ moment appears in the continuum inelastic scattering source. Including continuum inelastic reactions in the PW calculation usually has a small impact on the spectrum used for resonance self-shielding, and may adversely impact the computer memory requirements and execution time. Therefore, by default, CENTRM does not include continuum inelastic reactions in the pointwise solution; however, it is always included in the UMR solution. 


\subsection{Thermal Scatter}

Since thermal neutrons have energies comparable to the mean kinetic energy of molecules in thermal equilibrium, the scattering kernels must account for molecular motion. The scatter moments include both downscatter as well as upscatter contributions; hence, the integration limits appearing in Eq. (7.4.17) must be extended from the lowest to the highest energy of the thermal range. Furthermore the cross-section moments correspond to the Legendre expansion coefficients of the thermal scatter kernel, which has a substantially different form than the epithermal kernel discussed in the previous two sections. In general the $\ell_{\text {th }}$ Legendre moment of the thermal scattering kernel at temperature $T$, describing scattering from $E$ to $\mathrm{E}^{\prime}$, is given by

$$
\sigma_{\ell}\left(\mathrm{E}^{\prime} \rightarrow \mathrm{E} ; \mathrm{T}\right)=\frac{\sigma_{b}}{T} \sqrt{\frac{\mathrm{E}}{\mathrm{E}^{\prime}}} e^{-\frac{\beta\left(\mathrm{E}^{\prime} \rightarrow \mathrm{E}\right)}{2}} \int \mathrm{P}_{\ell}\left(\mu_{0}\right) \mathrm{S}[\alpha, \beta ; T] d \mu_{0}
$$

where $\beta\left(E^{\prime} \rightarrow E\right)$ and $\alpha\left(E^{\prime}, E, \mu_{0}\right)$ are dimensionless variables (functions of temperature) defining the energy and momentum exchange, respectively, of the collision; ${ }^{1} \sigma_{\mathrm{b}}$ is the rigidly bound scatter cross section, which is proportional to the free atom cross section; and $S(\alpha, \beta ; T)$ describes the temperature-dependent thermal scattering law.

If atomic bonding effects are neglected, the atoms of a material behave like a gas in thermal equilibrium at the temperature of the medium. In this case $S(\alpha, \beta)$ can be expressed by an analytical function. CENTRM uses the free gas model for all nuclides except those materials that have thermal scattering laws available in the ENDF/B nuclear data files. The ENDF/B scattering law data account for the effects of molecular bonding and possible polyatomic crystalline structure. While free-gas kernels are computed internally in CENTRM, the kernel moments describing bound thermal scatterers are stored in a data file that can be accessed by CENTRM.

\subsection{Bound thermal kernels}

Thermal scattering from bound atoms is classified either as an "inelastic reaction," in which the neutron energy may change, or an "elastic reaction," in which the neutron changes direction, but does not change energy. In ENDF/B the former reactions are treated as incoherent inelastic scattering with a doubly differential kernel describing the secondary neutron energy and angle distribution. The latter reactions are usual treated as coherent elastic scatter characterized by diffractive interference of the scattered deBroglie waves, although a few materials are modeled by the incoherent elastic approximation. Legendre moments for thermal elastic kernels describe the secondary angular distribution with no energy exchange, at a given neutron energy. Bound scatter kernels have been processed by the AMPX code system for most of the $\sim 25$ compounds with thermal scatter laws in ENDF/B, and are stored in individual kinematics files distributed with the SCALE code system. These include materials such as: $\mathrm{H}$ in water, $\mathrm{H}$ and $\mathrm{C}$ in polyethylene, $\mathrm{H}$ and $\mathrm{Zr}$ in $\mathrm{ZrH}, \mathrm{C}$ in graphite, deuterium in heavy water, $\mathrm{Be}$ metal, $\mathrm{Be}$ in $\mathrm{BeO}$, etc. The CRAWDAD module processes scattering kernel data for individual nuclides into a combined library used in CENTRM, and also interpolates the kernels to the appropariate temperatures.

The bound scatter kernels are tabulated at different energy points from the flux solution mesh; therefore it is necessary to map the data onto the desired energy mesh in the CENTRM calculation. Because thermal elastic scattering results in no energy loss, the elastic moments only appear in the within-point term of the scattering source in the CENTRM thermal calculation. Thus the coherent elastic data is easily interpolated since it only involves a single energy index and temperature. However, the incoherent inelastic moments are 2-D arrays in terms of the initial and final energies, so that a 2-D interpolation must be done for each temperature. CENTRM uses a simple type of "unit-base transform" method to 
interpolate incoherent inelastic kernels onto the flux solution mesh. The method attempts to preserve the absolute peak of the secondary energy distribution, at given initial energy. For water-bound hydrogen and several other moderators, this is quite adequate, since the kernel generally has only a single maximum. However, if more than one local extrema is present, such as for graphite, the other local peaks are not explicitly preserved in the interpolation method. For this reason it is necessary to include a fairly dense set of initial energies in the tabulated kernels of graphite and similar materials, to avoid gross changes in the kernel shape at adjacent initial energy panels.

\section{Free gas thermal kernels}

CENTRM computes free-gas kernels using the approach proposed by Robinson ${ }^{3}$ as a modification to the original FLANGE ${ }^{4}$ methodology. Legendre moments of the free-gas scatter kernel per unit lethargy are expressed as,

$$
\Sigma_{\ell}^{(\mathrm{j})}\left(\mathrm{u}^{\prime} \rightarrow \mathrm{u}\right)=\mathrm{A}^{(\mathrm{j})} \Sigma_{\text {free }}^{(\mathrm{j})} \frac{\mathrm{E}}{\mathrm{E}^{\prime}} \mathrm{e}^{-\beta / 2} \sum_{\mathrm{n}=0}^{\ell} \mathrm{W}_{\ell \mathrm{n}} \mathrm{H}_{\mathrm{n}}\left(\mathrm{E}^{\prime}, \mathrm{E}\right)
$$

where $\mathrm{W}_{\ell \mathrm{n}}$ are constant coefficients associated with the Legendre polynomial of order $\ell$; $\Sigma_{\text {free }}$ is the constant free-atom cross section for the material; and $\mathrm{H}_{\mathrm{n}}$ are the $\alpha$-moments of the free-gas scatter law, given as

$$
H_{n}\left(E^{\prime}, E\right)=\frac{1}{\sqrt{\pi}} \int_{\alpha_{L}}^{\alpha_{H}} \alpha^{n} \times\left(\frac{e^{-\frac{\alpha^{2}+\beta^{2}}{4 \alpha}}}{2 \sqrt{\alpha}}\right) d \alpha
$$

The limits on the above integral correspond to:

$$
\alpha_{L}\left(E^{\prime}, E\right)=\alpha\left(E^{\prime}, E, \mu_{0}=-1\right) \text { and } \alpha_{H}\left(E^{\prime}, E\right)=\alpha\left(E^{\prime}, E, \mu_{0}=1\right) \text {. }
$$

The alpha moments for $\mathrm{n}>0$ can be evaluated very efficiently using a recursive relation: ${ }^{2}$

$$
H_{n}\left(E^{\prime}, E\right)=2(2 n-1) H_{n-1}+\beta^{2} H_{n-2}-\left[F_{n}\left(\sqrt{\alpha_{H}}, \beta\right)-F_{n}\left(\sqrt{\alpha_{L}}, \beta\right)\right]
$$

where $F_{n}$ is the function,

$$
F_{n}(t, \beta)=\frac{t^{2 n-1} e^{-\frac{1}{4}\left(\frac{\beta^{2}}{t^{2}}+t^{2}\right)}}{\sqrt{\pi} / 2}
$$

Analytical expressions for the initial two moments, $\mathrm{H}_{0}$ and $\mathrm{H}_{-1}$, are given in Ref. 3 . 
The standard free-gas kernel is based on the assumption of a constant free atom cross section. When averaged over the molecular velocity distribution, this gives a $1 / \mathrm{v}$ variation in the effective free-gas cross section at low energies. To approximately account for nuclear structure effects on the energy dependence of the thermal cross section (e.g., low energy resonances), the free-gas moments are multiplied by the ratio $\sigma_{\mathrm{s}}(\mathrm{E}) / \sigma_{\mathrm{FG}}(\mathrm{E})$, where $\sigma_{\mathrm{s}}$ is the Doppler broadened scatter cross section processed from $\mathrm{ENDF} / \mathrm{B}$ data; and $\sigma_{\mathrm{FG}}$ is the effective free-gas cross section,

$$
\sigma_{\mathrm{FG}}\left(\mathrm{E}^{\prime}\right)=\frac{\sigma_{\text {free }}}{\mathrm{y}^{2}}\left[\left(\mathrm{y}^{2}+1 / 2\right) \operatorname{erf}(\mathrm{y})+\frac{\mathrm{y} \mathrm{e}^{-\mathrm{y}^{2}}}{\sqrt{\pi}}\right]
$$

where $\mathrm{y}^{2}=\mathrm{A} \frac{\mathrm{E}}{\mathrm{kT}}$.

\subsubsection{Sub-moment expansion of the epithermal scattering source}

One difficulty in computing the epithermal scatter source moments is that the Legendre polynomial in the integrand of Eqs. (7.4.17) and (7.4.20) is a function of both the initial and final lethargy (or energy) of the scattered neutrons, due to the correlation function $G^{(j)}\left(E, E^{\prime}\right)$. At each lethargy $u$ this requires that the $u^{\prime}-$ integral be recomputed over all lower lethargies, for every nuclide and moment. A more efficient algorithm would be possible if the differential scattering moments appearing in the integrand could be factored into a product of a function of $u$ multiplied by a function of $\mathrm{u}^{\prime}$ such as

$$
\mathrm{S}_{\ell \mathrm{k}}{ }^{(\mathrm{j})}\left(\mathrm{u}^{\prime} \rightarrow \mathrm{u}\right)=\mathrm{F}^{(\mathrm{j})}\left(\mathrm{u}^{\prime}\right) \quad \mathrm{H}^{(\mathrm{j})}(\mathrm{u})
$$

where $\mathrm{F}$ and $\mathrm{H}$ are the two factors (to be specified later).

If this is done, the u-function can be factored from the scatter source integrals, leaving only integrals over the $\mathrm{u}^{\prime}$-function as shown below:

$$
\mathrm{S}^{(\mathrm{j})}(\mathrm{u})=\int_{\mathrm{u}^{\prime}} \mathrm{S}_{\ell \mathrm{k}}{ }^{(\mathrm{j})}\left(\mathrm{u}^{\prime} \rightarrow \mathrm{u}\right) \mathrm{du}^{\prime}=\mathrm{H}^{(\mathrm{j})}(\mathrm{u}) \int_{\mathrm{u}^{\prime}} \mathrm{F}^{(\mathrm{j})}\left(\mathrm{u}^{\prime}\right) \mathrm{du^{ \prime }}
$$

Because the factored integrand does not depend on the variable $\mathrm{u}$, a running summation over all $\mathrm{u}^{\prime}$ points can be accumulated and saved as the calculation sweeps from low to high lethargy. For example, note that the $\ell=0$ moment in Eq. (7.4.17) is already separable into a product of $u$ times $\mathrm{u}^{\prime}$ because $\mathrm{P}_{0}$ is equal to one at all values of $\mathrm{G}$. Thus the isotropic component of the elastic differential scatter rate (per unit lethargy) from $\mathrm{u}^{\prime}$ to $\mathrm{u}$ is proportional to $\mathrm{E} / \mathrm{E}^{\prime}$, where

$$
\mathrm{E}=\mathrm{E}(\mathrm{u})=\mathrm{E}_{\mathrm{ref}} \mathrm{e}^{-\mathrm{u}}, \quad \text { and } \quad \mathrm{E}^{\prime}=\mathrm{E}^{\prime}\left(\mathrm{u}^{\prime}\right)=\mathrm{E}_{\text {ref }} \mathrm{e}^{-\mathrm{u}^{\prime}}
$$

Therefore, the two separable factors in the lowest moment, $\mathrm{S}_{0.0}^{(\mathrm{j})}\left(\mathrm{u}^{\prime} \rightarrow \mathrm{u}\right)$, are identified as,

$$
\begin{aligned}
& \mathrm{H}(\mathrm{u})=\mathrm{E} /\left(1-\alpha^{(\mathrm{j})}\right), \quad \text { and } \\
& \mathrm{F}\left(\mathrm{u}^{\prime}\right)=\Sigma^{(\mathrm{j})}\left(\mathrm{u}^{\prime}\right) \Psi_{00}\left(\mathrm{u}^{\prime}\right) / \mathrm{E}^{\prime}
\end{aligned}
$$

However, the higher order Legendre moments contain the term $P_{\ell}(G)$ in the integrand; and the expression for $G\left(E^{\prime}, E\right)$ is a difference of two terms that depend on both $E$ and $E^{\prime}$. A new method called a 
"sub-moment expansion" has been developed for CENTRM that allows the Legendre polynomials appearing in the differential scatter moments to be factored into the desired separable form. Each spherical harmonic moment of the scattering source appears expanded in a series of factored "sub-moments."

The Legendre polynomial of order $\ell$ is a polynomial containing terms up to the $\ell^{\text {th }}$ power. Applying the binomial expansion theorem and some algebraic manipulation, the standard expression for $\mathrm{P}_{\ell}$ evaluated at "G" can be expressed as

$$
P_{\ell}(G)=\frac{E^{\prime}}{E} \times a_{1}^{\prime} \sum_{K=-f}^{\ell} \tilde{g}_{\ell, K}(E) h_{K}(E) h_{K}^{-1}\left(E^{\prime}\right)
$$

where $\mathrm{h}_{\mathrm{k}}(\mathrm{E})=\mathrm{E}^{1+\mathrm{K} / 2}$; and the expansion coefficients $\tilde{\mathrm{g}}_{\ell, \mathrm{k}}$ are equal to, $\tilde{\mathrm{g}}_{\ell \mathrm{K}}=\frac{\mathrm{g}_{\ell \mathrm{K}}}{N, \times \alpha_{1}^{\ell}}$ where the $\mathrm{g}_{\ell, \mathrm{K}}($ no tilde) parameters were defined in Ref. 5 to be:

$$
\begin{aligned}
\mathrm{g}_{\ell, \mathrm{K}} & =\frac{\left(1+(-1)^{\ell+\mathrm{K}}\right)}{2} \sum_{K^{\prime}=0}^{\frac{\ell-K}{2}}(-1)^{K^{\prime}} & b_{2 K^{\prime}+K, \ell}\left(\begin{array}{r}
2 K^{\prime}+K \\
K^{\prime}
\end{array}\right) a_{1}^{K+K^{\prime}} a_{2}^{K^{\prime}} ; & \text { for } \quad \mathrm{K} \geq 0 \\
& =\left(-\mathrm{a}_{2} / \mathrm{a}_{1}\right)^{|\mathrm{K}|} \mathrm{g}_{\ell,|\mathrm{K}|} & \quad \text { and } & \text { for } \mathrm{K}<0 .
\end{aligned}
$$

In Eqs. (7.4.23)-(7.4.24), the constants $\mathrm{b}_{\mathrm{m}, \ell}$ and $\mathrm{N}_{\ell}$ are the standard Legendre constants and normalization factors, respectively, which are tabulated in Table 7.4.1 for orders through $\mathrm{P}_{7}$; and $\left(\begin{array}{c}\mathrm{m} \\ \mathrm{i}\end{array}\right)=$ the binomial expansion coefficients $^{(20)}=\frac{m !}{(m-i) ! i !}$ 
Table 7.4.1. Constants appearing in Legendre polynomials

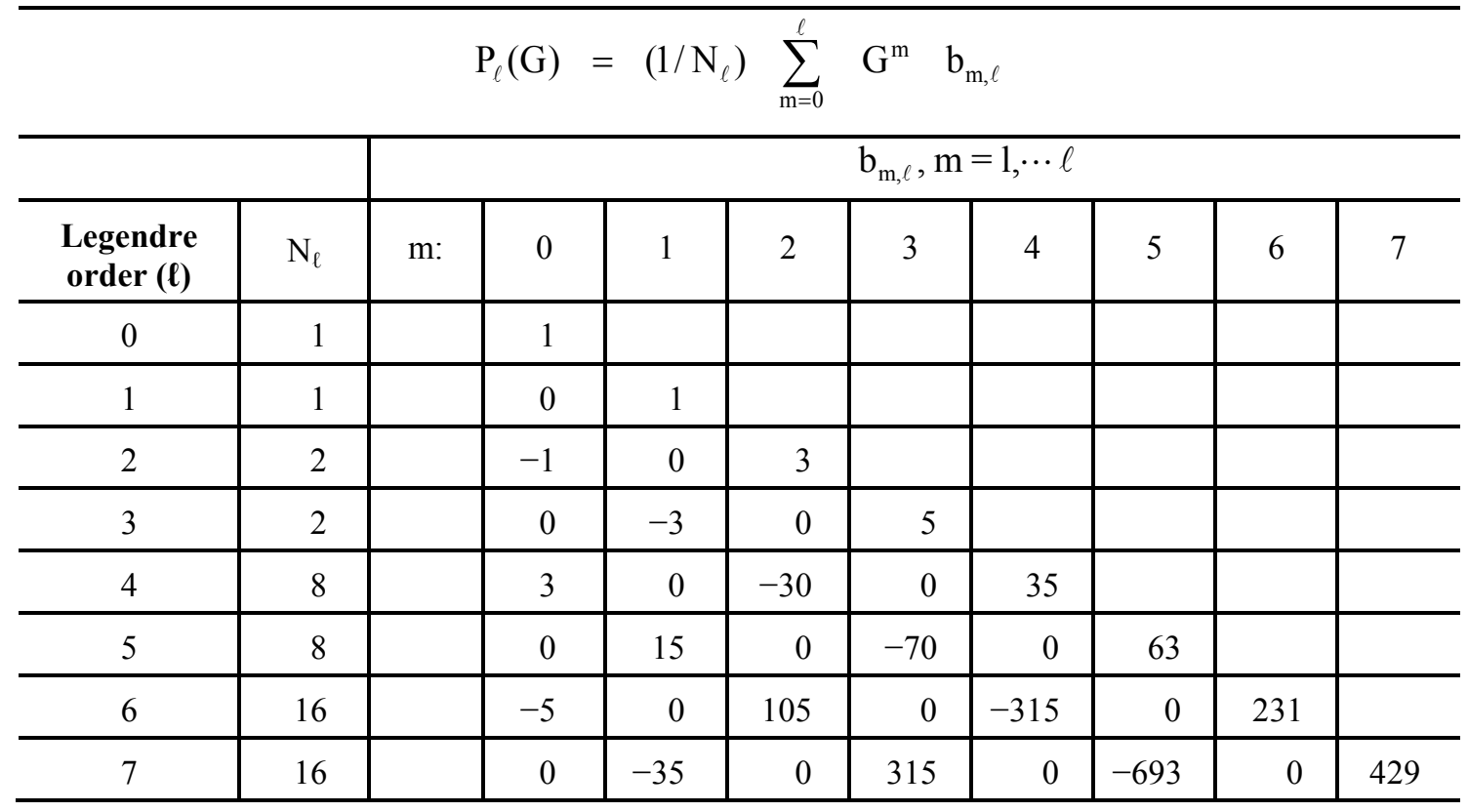

The explicit dependence of the constants $a_{1}$ and $a_{2}$ on the nuclide index $j$ [see Eq. (7.4.10)] has been suppressed to simplify notation. For discrete-level inelastic scatter the parameter $a_{1}$ is an energy dependent function given by Eq. (7.4.18), but for elastic scatter this reduces to the constant in

Eq. (7.4.10). Note that the $g_{\ell, K}$ value is zero unless $\ell$ and $K$ are both even or both odd, respectively, so that about half the terms appearing in the summation of Eq. (7.4.23) vanish. Table 7.4.2 through Table 7.4.4 give values for the submoment expansion coefficients for several nuclides.

The sub-moment expansion of the scattering source, including both elastic and discrete-level inelastic reactions, is obtained by substituting the expansion of the Legendre polynomial from Eq. (7.4.23) into Eq. (7.4.21), giving ${ }^{2}$

$$
\mathrm{S}_{\ell \mathrm{k}}(\mathrm{u})=\sum_{m, j} \sum_{K=-\ell}^{\ell} \mathrm{Z}_{\ell, \mathrm{K}}^{(\mathrm{m}, \mathrm{j})}(\mathrm{E}) \mathrm{h}_{\mathrm{K}}(\mathrm{E}) \int_{u_{L O}^{(m, j)}}^{u_{H,}^{(m)}} \psi_{\ell \mathrm{k}}\left(\mathrm{u}^{\prime}\right) \Sigma^{(\mathrm{m}, \mathrm{j})}\left(\mathrm{E}^{\prime}\right) \frac{\mathrm{h}_{\mathrm{K}}^{-1}\left(\mathrm{E}^{\prime}\right)}{\Delta^{(\mathrm{m}, \mathrm{j})}\left(\mathrm{E}^{\prime}\right)} \mathrm{du} \mathrm{u}^{\prime}
$$

where $Z_{Z_{K}}^{(m, j)}(E)=a_{1}^{\ell}(E) \frac{\tilde{g}_{\ell, K}^{(m, j)}(E)}{\left(1-\alpha^{(j)}\right)}$. For elastic scatter, the $\mathrm{Z}$ coefficients are independent of energy.

With this approach the scatter source moments in Eq. (7.4.26) have been further expanded into a summation of "submoments" identified by index K (although some of these terms are equal to zero, due to the behavior of the $\mathrm{g}_{\ell, \mathrm{K}}$ coefficients). Each term has the desired factored form expressed in Eq. (7.4.22) ; i.e., separable in terms of the variables $u$ and $u^{\prime}$ with

$$
\mathrm{H}_{\ell, \mathrm{K}}^{(\mathrm{j})}(\mathrm{u})=\mathrm{Z}_{\ell, \mathrm{K}}^{(\mathrm{j})}(\mathrm{E}) \mathrm{h}_{\mathrm{K}}(\mathrm{E}), \text { and } \mathrm{F}_{\ell \mathrm{k}, \mathrm{K}}^{(\mathrm{j})}\left(\mathrm{u}^{\prime}\right)=\frac{\mathrm{h}_{\mathrm{K}}^{-1}\left(\mathrm{E}^{\prime}\right) \Psi_{\ell \mathrm{k}}\left(\mathrm{u}^{\prime}\right) \Sigma^{(\mathrm{j})}\left(\mathrm{u}^{\prime}\right)}{\Delta^{(\mathrm{m}, \mathrm{j})}\left(\mathrm{E}^{\prime}\right)}
$$


so that the $1 \mathrm{k}_{\mathrm{th}}$ moment of the scatter source can be written as ${ }^{5}$

$$
\mathrm{S}_{\ell \mathrm{k}}(\mathrm{u})=\sum_{m, j} \sum_{K=-1}^{\ell} \mathrm{H}_{\ell, \mathrm{K}}^{(\mathrm{j})}(\mathrm{u}) \int_{\mathrm{u}_{\mathrm{LO}}}^{(\mathrm{m}, \mathrm{j})} \mathrm{F}_{\ell \mathrm{k}, \mathrm{K}}^{(\mathrm{m}, \mathrm{j})}\left(\mathrm{u}^{\prime}\right) \mathrm{du}
$$

\subsection{Characteristics and Properties of the Sub-Moment Expansion}

The expansion in Eq. (7.4.23) becomes numerically unstable for heavy nuclides (large A), with high Legendre orders. Using double precision arithmetic, it was found that the accuracy of the expansion begins to break down for heavy nuclides $(A \gtrsim 100)$ if the order of scatter exceeds $\mathrm{P}_{5}$; although the expansion for lighter nuclides (viz, moderators) is very accurate even for scattering orders as high as $\mathrm{P}_{7}$ or more. For this reason CENTRM has an option to restrict the Legendre expansion to lower orders for heavy masses, while using the input value of "ISCT" for lighter nuclides. The restricted Legendre order and mass cut-off value can be controlled by user input, but the default is $\mathrm{P}_{0}$ (i.e., isotropic lab scattering) for $\mathrm{A}>100$. Table 7.4.5 shows the maximum error observed in the series representation of Legendre polynomials up to $\mathrm{P}_{5}$, for selected mass numbers. These values were obtained by evaluating the series expansion for $\mathrm{P}_{\ell}(\mathrm{G}(\mathrm{x}))$ in Eq. (7.4.23), and comparing to the exact value computed at 11 equally spaced values for $\mathrm{E} / \mathrm{E}^{\prime}$. The observed error in the $\mathrm{P}_{5}$ polynomial expansion is $<1 \%$ even for heavy materials such as ${ }^{238} \mathrm{U}$, while nuclides whose mass is $<100$ are computed nearly exactly by the expansion.

Although the accuracy of the submoment expansion is good through $\mathrm{P}_{7}$ scattering in moderators, Legendre expansions above P3 are not recommended because the number of terms in the expansion increases rapidly with increasing scattering order, especially for $2 \mathrm{D} \mathrm{MoC}$ and $1 \mathrm{D}$ cylindrical systems. The number of spherical harmonic moments appearing in the scattering source depends on the order ( $\mathrm{L}=\mathrm{ISCAT})$ of the Legendre expansion used to represent the differential scatter cross section, as well as on the type of geometry (slab, spherical, cylindrical, or 2D MoC) used in the transport calculation. The submoment method further expands each source moment. Table 7.4.6 shows the number of moments in the cross-section expansion, and the number of moments and submoments in the scatter source expansion, as a function of scatter order and geometry type. Although the use of cumulative integrals discussed below allows the sub-moments to be evaluated rapidly, the large number of terms becomes prohibative for high scattering orders. Fortunately a $\mathrm{P}_{1}$ Legendre order is sufficient for most self-shielding calculations, and orders beyond $\mathrm{P}_{2}$ should seldom be required for reactor physics and criticality applications. 
Table 7.4.2. Coefficients in expansion of $P_{\ell}[G(x)]^{*}$ for hydrogen $(A=1)$

\begin{tabular}{|c|c|c|c|c|c|c|c|c|}
\hline \multirow[b]{2}{*}{$\begin{array}{c}\text { Legendre } \\
\text { order }(\ell)\end{array}$} & \multicolumn{8}{|c|}{$\tilde{g}_{1, K}, \quad \mathrm{~K}=-1, \ldots, 1$} \\
\hline & $\mathrm{K}:$ & -3 & -2 & -1 & 0 & 1 & 2 & 3 \\
\hline 0 & & & & & 1 & & & \\
\hline 1 & & & & 0 & 0 & 1 & & \\
\hline 2 & & & 0 & 0 & -0.5 & 0 & 1.5 & \\
\hline 3 & & & 0 & 0 & 0 & -1.5 & 0 & 2.5 \\
\hline
\end{tabular}

*For $\mathrm{A}=1, \mathrm{G}(\mathrm{x})=\mathrm{x} ;\left[\mathrm{x}=\left(\mathrm{E} / \mathrm{E}^{\prime}\right)^{1 / 2} ;\right.$ where $\mathrm{E}^{\prime}, \mathrm{E}=$ initial and final energies.

Table 7.4.3. Coefficients in expansion on $P_{\ell}[G(x)]^{*}$ for oxygen $(A=16)$

\begin{tabular}{|c|c|c|c|c|c|c|c|c|}
\hline & \multicolumn{8}{|c|}{$\tilde{g}_{l, K}, \mathrm{~K}=-1, \ldots, 1$} \\
\hline $\begin{array}{c}\text { Legendre } \\
\text { order }(\ell)\end{array}$ & K & -3 & -2 & -1 & 0 & 1 & 2 & 3 \\
\hline 0 & & & & & 1 & & & \\
\hline 1 & & & & -0.88235294 & 0 & 1 & & \\
\hline 2 & & & 1.16782007 & 0 & -2.6539724 & 0 & 1.5 & \\
\hline 3 & & -1.7173824 & 0 & 5.85741909 & 0 & -6.6384083 & 0 & 2.5 \\
\hline
\end{tabular}

*For $A=16, G(x)=\frac{8.5}{x}-7.5 x:\left[x=\left(E / E^{\prime}\right)^{1 / 2}\right.$; where $E^{\prime}, E=$ initial and final energies.

Table 7.4.4. Coefficients in expansion of $P \ell[G(x)]^{*}$ for $U-238(A=236)$

\begin{tabular}{|c|c|c|c|c|c|c|c|c|}
\hline & \multicolumn{8}{|c|}{$\tilde{g}_{1, K}, \quad \mathrm{~K}=-1, \ldots, 1$} \\
\hline $\begin{array}{l}\text { Legendre } \\
\text { order }(\ell)\end{array}$ & K & -3 & -2 & -1 & 0 & 1 & 2 & 3 \\
\hline 0 & & & & & 1 & & & \\
\hline 1 & & & & -0.99156118 & 0 & 1 & & \\
\hline 2 & & & 1.47479036 & 0 & -2.97471915 & 0 & 1.5 & \\
\hline 3 & & -2.43724146 & 0 & 7.37405774 & 0 & -7.43681568 & 0 & 2.5 \\
\hline
\end{tabular}

*For $A=236, G(x)=\frac{118.5}{x}-117.5 x:\left[x=\left(E / E^{\prime}\right)^{1 / 2}\right.$; where $E^{\prime}, E=$ initial and final energies. 
Table 7.4.5. Fractional error ${ }^{(1)}$ in series expansion [Eq. (F18.2.25)] of Legendre polynomials

\begin{tabular}{c|c|c|c|c|c|c}
\hline \multirow{2}{*}{$\begin{array}{c}\text { Legendre } \\
\text { order }(\boldsymbol{\ell})\end{array}$} & \multicolumn{6}{|c}{ Mass number in neutron mass units (A) } \\
\cline { 2 - 7 } & 1 & 12 & 56 & 100 & 150 & 236 \\
\hline 1 & $0^{(*)}$ & 0 & 0 & 0 & 0 & 0 \\
\hline 2 & 0 & 0 & 0 & 0 & 0 & 0 \\
\hline 3 & 0 & 0 & 0 & 0 & 0 & 0 \\
\hline 4 & 0 & 0 & 0 & 0 & 0 & $1.2 \times 10^{-5}$ \\
\hline 5 & 0 & 0 & 0 & $2.1 \times 10^{-5}$ & $3.9 \times 10^{-3}$ & $8.4 \times 10^{-3}$ \\
\hline
\end{tabular}

(1) Fractional error $=\mid 1.0-$ (approximate value/exact value) $\mid$.

${ }^{(*)} 0$ error indicates $<10^{-6}$. 
Table 7.4.6. Number of moments and submoments as function of scattering order

\begin{tabular}{|c|c|c|c|c|}
\hline $\begin{array}{l}\text { Legendre order } \\
(\ell) \text { of scattering }\end{array}$ & $\begin{array}{c}\text { Geometry } \\
\text { type }\end{array}$ & $\begin{array}{c}\text { Number of } \\
\text { scattering } \\
\text { x-section } \\
\text { moments } \\
\end{array}$ & $\begin{array}{c}\text { Number of } \\
\text { scattering } \\
\text { source } \\
\text { moments } \\
\end{array}$ & $\begin{array}{l}\text { Number of } \\
\text { scattering source } \\
\text { submoments }\end{array}$ \\
\hline \multirow{3}{*}{0} & sphere, slab & 1 & 1 & 1 \\
\hline & cylinder & 1 & 1 & 1 \\
\hline & 2D MoC & 1 & 1 & 1 \\
\hline \multirow{3}{*}{1} & sphere, slab & 2 & 2 & 3 \\
\hline & cylinder & 2 & 2 & 3 \\
\hline & 2D MoC & 2 & 3 & 5 \\
\hline \multirow{3}{*}{2} & sphere, slab & 3 & 3 & 6 \\
\hline & 1D cylinder & 3 & 4 & 9 \\
\hline & 2D MoC & 3 & 6 & 14 \\
\hline \multirow{3}{*}{3} & sphere, slab & 4 & 4 & 10 \\
\hline & 1D cylinder & 4 & 6 & 17 \\
\hline & 2D MoC & 4 & 10 & 30 \\
\hline \multirow{3}{*}{4} & sphere, slab & 5 & 5 & 15 \\
\hline & 1D cylinder & 5 & 9 & 36 \\
\hline & 2D MoC & 5 & 15 & 55 \\
\hline \multirow{3}{*}{5} & sphere, slab & 6 & 6 & 21 \\
\hline & 1D cylinder & 6 & 12 & 50 \\
\hline & 2D MoC & 6 & 21 & 91 \\
\hline
\end{tabular}

\subsection{Scattering moments expressed with cumulative integral operator}

It will be convenient to express the scatter source moments in terms of an integral operator $\mathbb{C}$, designated here as the "cumulative integral." The domain of this operator is the vector space of all integrable lethargy functions. The operator is defined for an arbitrary domain element $f\left(u^{\prime}\right)$, at an arbitrary lethargy limit U, to be:

$$
\mathbb{C}(\mathrm{f} ; \mathrm{U})=\int_{\mathrm{u}_{0}}^{\mathrm{U}} \mathrm{f}\left(\mathrm{u}^{\prime}\right) \mathrm{du}
$$

where $\mathrm{u}_{0}$ is an arbitrary reference point. In implementing this method in CENTRM, it is convenient to set $\mathrm{u}_{0}=\mathrm{u}_{\mathrm{L}}$; i.e., the negative lethargy value corresponding to highest energy of the transition range. 
The cumulative integral at some lethargy mesh point $u_{n}$ is related to the value at the previous lethargy mesh point $\mathrm{u}_{\mathrm{n}-1}$ by the expression

$$
\mathbb{C}\left(f ; u_{n}\right)=\mathbb{C}\left(f ; u_{n-1}\right)+\int_{u_{n-1}}^{u_{n}} f\left(u^{\prime}\right) d u^{\prime}
$$

where $u_{n}>u_{n-1}$.

Note that only a single panel of integration over the interval $\left[\mathrm{u}_{\mathrm{n}-1}, \mathrm{u}_{\mathrm{n}}\right]$ must be performed to update the cumulative integrals.

The sub-moment expansion of the scatter source in Eq. (7.4.25) can be expressed in terms of the cumulative integral operator as follows: ${ }^{5}$

$$
\mathrm{S}_{\ell \mathrm{k}}^{(\mathrm{j})}(\mathrm{u})=\sum_{j} \sum_{K=-\ell}^{\ell} \mathrm{H}_{\ell, \mathrm{K}}^{(\mathrm{j})}(\mathrm{u}) \times\left[\mathbb{C}\left(\mathrm{F}_{\ell \mathrm{k}, \mathrm{K}}^{(\mathrm{j})} ; u_{H I}^{(m, j)}\right)-\mathbb{C}\left(\mathrm{F}_{\ell \mathrm{k}, \mathrm{K}}^{(\mathrm{j})} ; u_{L O}^{(m, j)}\right)\right]
$$

For elastic scatter the value of $\mathrm{u}_{\mathrm{LO}}^{(\mathrm{m}, \mathrm{j})}$ is equal to $\left(\mathrm{u}-\varepsilon^{(\mathrm{j})}\right)$, and $\mathrm{u}_{\mathrm{HI}}^{(\mathrm{m}, \mathrm{j})}$ is equal to $\mathrm{u}$.

\subsubsection{Multigroup Boltzmann equation}

The MG form of the transport equation used in the UMR and LMR is derived by integrating Eq. (7.4.1) over the energy intervals defined by the group structure in the MG library. Details concerning the MG transport equation, including its solution using the discrete ordinates method, can be found in the SCALE documentation of XSDRNPM. The CENTRM MG solution is similar to the XSDRNPM method: however; the outer iteration loop in CENTRM is limited to the thermal groups, since no eigenvalue calculation is performed in CENTRM. The MG scatter source in the thermal range has upscatter contributions that depend on group fluxes from lower energy groups in the LMR, so that outer iterations are performed over thermal groups in the LMR until the upscatter portion of the MG scatter source converges.

\subsection{Multigroup data for CENTRM calculation}

Group cross-section data for the MG calculations are taken from the input MG library which should include a combined 2D transfer matrix representing all pertinent scatter reactions (viz, elastic, inelastic, coherent and incoherent thermal reactions, n-2n, etc). MG cross sections also should be problemdependent values. This is done by processing the data with BONAMI prior to the CENTRM calculation. BONAMI converts the problem-independent cross-sections into problem-dependent values by using the Bondarenko factors on the MG library.

\subsection{Conversion of multigroup fluxes to pseudo-pointwise values}

The MG flux solution provides the integrated flux over lethargy, for each group interval. The average flux within a group is assumed to approximate the value of the flux per unit lethargy at the midpoint lethargy of the group; thus a set of "pseudo-pointwise" angular fluxes and moments can be obtained for the $\mathrm{NG}_{\mathrm{U}}$ and $\mathrm{NG}_{\mathrm{L}}$ mesh points in the UMR and LMR, respectively. For lethargy point $\mathrm{u}_{\mathrm{n}}$, corresponding to the midpoint lethargy of group g contained within the LMR and UMR, a PW flux value is computed from the expression,

$$
\Psi\left(\mathrm{u}_{\mathrm{n}}\right)=\Psi_{\mathrm{g}} / \Delta \mathrm{u}_{\mathrm{g}}
$$


where $\Delta \mathrm{u}_{\mathrm{g}}$ is the lethargy width of group g. Equation (7.4.31) provides PW flux values for lethargy mesh points,

$$
\mathrm{u}_{1} \ldots . . \mathrm{u}_{\mathrm{NGU}} \text {, and } \mathrm{u}_{\mathrm{NGU}+\mathrm{NP}+1} \ldots . . \mathrm{u}_{\mathrm{NT}} \text {. }
$$

A linear variation of the flux per unit lethargy is assumed between lethargy points to obtain a continuous representation in the UMR and LMR.

\subsubsection{The Boltzmann equation within the PW range}

In contrast to the "pseudo-pointwise" fluxes obtained from the MG transport calculation, a true PW solution is performed for the $\mathrm{N}_{\mathrm{P}}$ lethargy points between DEMAX and DEMIN. The PW solution is performed within a loop over energy groups: i.e., for each of the $\mathrm{NG}_{\mathrm{P}}$ groups in the $\mathrm{PW}$ range there is an additional loop over all lethargy mesh points contained inside the group. This approach facilitates coupling of the scatter source from the UMR to the PW range and from the PW and LMR.

Evaluating Eq. (7.4.1) at each of the $\mathrm{N}_{\mathrm{P}}$ energy mesh-points in the PW range gives a system of integrodifferential equations that can be solved to obtain the PW flux moments, per lethargy, for the $\mathrm{N}_{\mathrm{P}}$ energy mesh points in the range DEMAX to DEMIN-which correspond to the lethargy points, $\mathrm{U}_{\mathrm{NGU}+1}, \ldots \mathrm{U}_{\mathrm{NGU}+\mathrm{NP}}$. Again linear variation of the flux between lethargy points is assumed, to obtain a continuous spectrum. Substituting Eq. (7.4.4) into Eq. (7.4.1), the PW transport equation at mesh point $n$ is found to be,

$$
\Omega \bullet \nabla \Psi_{\mathrm{n}}(\mathrm{r}, \Omega)+\Sigma_{\mathrm{t}, \mathrm{n}}(\mathrm{r}) \Psi_{\mathrm{n}}(\mathrm{r}, \Omega)=\sum_{\ell \mathrm{k}} \frac{2 \ell+1}{2} \mathrm{Y}_{\ell \mathrm{k}}(\Omega) \mathrm{S}_{\ell \mathrm{k}, \mathrm{n}}(\mathrm{r})+\mathrm{Q}_{\mathrm{n}}(\mathrm{r}, \Omega)
$$

for $\mathrm{n}=\left(\mathrm{NG}_{\mathrm{U}}+1\right), \ldots .,\left(\mathrm{NG}_{\mathrm{U}}+\mathrm{N}_{\mathrm{P}}\right)$

where

$$
\begin{gathered}
\sum_{\mathrm{t}, \mathrm{n}}(\mathbf{r})=\sum_{\mathrm{t}}\left(\mathbf{r}, \mathrm{u}_{\mathrm{n}}\right) \\
\Psi_{\mathrm{n}}(\mathbf{r}, \Omega)=\Psi\left(\mathbf{r}, \Omega, \mathrm{u}_{\mathrm{n}}\right) \\
\mathrm{S}_{\ell \mathrm{k}, \mathrm{n}}(\mathbf{r})=\mathrm{S}_{\ell \mathrm{k}}\left(\mathbf{r}, \mathrm{u}_{\mathrm{n}}\right)
\end{gathered}
$$

Aside from the definition of the cross-section data, the above equation appears identical in form to the MG transport equation, and can be solved with virtually the same algorithm as the MG solution, once the scatter source moments are determined. The same computer routines in CENTRM calculate both the MG and PW fluxes. However, a major conceptual difference between the PW and MG transport equations is that the PW equation describes a differential neutron balance per unit lethargy at an energy point, while the MG equation represents an integral balance over an interval of lethargy points. Although this type of point solution is not inherently conservative over the intervals defined by the energy mesh, the particle balance for each interval has been found to be very good. It should also be noted that exact particle conservation is not a strict requirement for this type of application where flux spectra rather than particle balances are primarily of interest. 
In the PW range the scatter source is composed of (a) MG-to-PW scatter from the UMR and possibly upscatter from the LMR if the PW range extends into thermal, and (b) PW-to-PW scatter from points in the PW range. The submoment expansion method described previously is used in CENTRM to provide an efficient method of evaluating the PW-to-PW downscatter source for the epithermal range, which includes most of the resolved resonances.

\subsection{Scattering sources for the PW range}

In the case of elastic scatter from nuclide " $\mathrm{j}$," only the lethargy interval below $\mathrm{u}_{\mathrm{n}}-\varepsilon^{(\mathrm{j})}$ can scatter to a lethargy point $u_{n}$ in the PW range. If $u_{n}-\varepsilon^{(j)}$ is negative, then some portion of the source at $u_{n}$ is due to UMR-to-PW from energies above DEMAX, since zero-lethargy is equal to the top energy of the PW range. Otherwise, the elastic source is entirely PW-to-PW.

For any given nuclide $\mathrm{j}$, the lowest lethargy in the UMR range that contributes to the elastic scatter source in the PW range is equal to $-\varepsilon^{(j)}$. Let " $\mathrm{jL}$ " represent the lightest non-hydrogen nuclide (i.e., having the smallest A value greater than unity) in the system. The associated fractional energy loss for this material is indicated as $\alpha_{\mathrm{L}}$, so that the highest energy neutron in the UMR range that can scatter into the PW range from an elastic collision with any non-hydrogenous moderator will have an energy equal to DEMAX $/ \alpha_{\mathrm{L}}$. The corresponding lethargy is equal to be the negative value $-\varepsilon^{(\mathrm{L})}$, or $-\ln \left(1 / \alpha_{\mathrm{L}}\right)$. The value of $-\varepsilon^{(\mathrm{L})}$ is actually adjusted in CENTRM to coincide with the immediately preceding multigroup boundary, which has a lethargy value designated as $u_{L}$. The interval of negative lethargy in the UMR between $u_{L}$ and 0 has been defined previously to be transition range, because the elastic slowing-down source from this interval provides a transition between the UMR and PW solutions, respectively. The transition range always contains an integer number of groups, corresponding to MGTOP to MGHI. The total downscatter source from the UMR to lethargy $\mathrm{u}_{\mathrm{n}}$ is composed of elastic and inelastic contributions from the transition range between $\left[\mathrm{u}_{\mathrm{L}}, 0\right]$; and contributions from the "high" energy range from lethargies below $\mathrm{u}_{\mathrm{L}}$. The high contribution comes from inelastic and hydrogen elastic reactions in the energy interval above the transition range.

The downscatter source at $\mathrm{u}_{\mathrm{n}}$ in the PW range can thus be expressed as the sum of three distinct contributions $-\mathrm{S}_{\mathrm{HI}}, \mathrm{S}_{\mathrm{Tr}}$, and $\mathrm{S}_{\mathrm{PW}}-$, that correspond to scatter from the high region of the UMR, the transition region of the UMR, and the PW ranges, respectively. The source moments appearing in Eq. (7.4.32) can thus be expressed as:

$$
\begin{aligned}
\mathrm{S}_{\ell \mathrm{k}, \mathrm{n}}(\mathrm{r}) & =\mathrm{S}_{\ell \mathrm{k}, \mathrm{HI}}\left(\mathrm{r}, \mathrm{u}_{\mathrm{n}}\right)+\mathrm{S}_{\ell \mathrm{k}, \mathrm{Tr}}\left(\mathrm{r}, \mathrm{u}_{\mathrm{n}}\right)+\mathrm{S}_{\ell \mathrm{k}, \mathrm{PR}}\left(\mathrm{r}, \mathrm{u}_{\mathrm{n}}\right) \\
& =\int_{-\infty}^{\mathrm{u}_{\mathrm{L}}} \mathrm{S}_{\ell \mathrm{k}}\left(\mathrm{u}^{\prime} \rightarrow \mathrm{u}\right) d \mathrm{u}^{\prime}+\int_{\mathrm{u}_{\mathrm{L}}}^{0} \mathrm{~S}_{\ell \mathrm{k}}\left(\mathrm{u}^{\prime} \rightarrow \mathrm{u}\right) \mathrm{du}^{\prime}+\int_{0}^{\mathrm{u}_{\mathrm{n}}} \mathrm{S}_{\ell \mathrm{k}}\left(\mathrm{u}^{\prime} \rightarrow \mathrm{u}\right) d \mathrm{u}^{\prime} .
\end{aligned}
$$

\subsection{Downscatter source from high region of the UMR to the PW range $\left(S_{H 1}\right)$}

The high region of the UMR corresponds to groups 1 through MGTOP-1. The MG-to-PW scattering source $\left(\mathrm{S}_{\mathrm{HI}}\right)$ from high energy region originates in the energy range above DEMAX/ $\alpha_{\mathrm{L}}$; i.e., lethargies below $u_{L}$ (see Figure 7.4.2). In this region, inelastic reactions may scatter neutrons to the PW range; but due to the definition of $u_{\mathrm{L}}$, the only elastic reactions that scatter to the PW range are due to hydrogen. Therefore in general, the MG matrices describing scatter from groups in high region to groups in the PW range correspond to discrete and continuum inelastic reactions, and elastic scatter from hydrogen. If $\mathrm{g}^{\prime}$ is an arbitrary group in the UMR range above the transition interval and $\mathrm{g}$ is a fixed group interval in the $\mathrm{PW}$ range, then the rate that neutrons scatter from all groups $\mathrm{g}^{\prime}$ in the high region to all energy points 
in $\mathrm{g}$, for a given direction $\Omega$, is obtained from the usual expression for MG-to-MG transfers, and is equal to

$$
\mathrm{S}_{\mathrm{g}}(\mathrm{r}, \Omega)=\sum_{\ell \mathrm{k}} \frac{2 \ell+1}{2} \mathrm{Y}_{\ell \mathrm{k}}(\Omega) \quad \mathrm{S}_{\ell \mathrm{k}, \mathrm{g}}
$$

where $\mathrm{MGLO}>\mathrm{g}>\mathrm{MGHI}$, and the MG source moments are,

$$
\mathrm{S}_{\ell \mathrm{k}, \mathrm{g}}=\sum_{\mathrm{g}^{\prime}=1}^{\mathrm{MGTOP}-1} \Sigma_{\ell, \mathrm{g}^{\prime} \rightarrow \mathrm{g}} \Psi_{\ell \mathrm{k}, \mathrm{g}^{\prime}} .
$$

while Eq. (7.4.34) gives the moments of the overall scatter rate from all groups in the high range into the entire PW group g, it is necessary to determine how the group source should be distributed over the PW energy mesh contained within the group; i.e., it is desired to extract the PW source moments, from the group moments by applying some "intra-group" distribution $\mathrm{H}_{\ell \mathrm{k}, \mathrm{g}}(\mathrm{E})$ such that,

$$
\mathrm{S}_{\ell \mathrm{k}, \mathrm{HI}}(\mathrm{u})=\mathrm{S}_{\ell \mathrm{k}, \mathrm{g}} \mathrm{H}_{\ell \mathrm{k}, \mathrm{g}}(\mathrm{E}), \quad \text { for } \mathrm{u}(\mathrm{E}) \varepsilon \text { group } \mathrm{g} \text {. }
$$

The intra-group distribution has units of "per unit lethargy," and its integral over the group is normalized to unity. This form of the scatter source preserves the MG moments $S_{\ell k, g}$, whenever $S_{\ell k, H I}(u)$ is integrated over group g, insuring that the correct number of neutrons (as determined from the UMR calculation) will always be transferred from the high range into the PW group. Only the distribution within the group is approximate.

Recall that the scatter source of concern here is due only to elastic scatter from hydrogen and inelastic scatter from all other materials. In the case of $s$-wave elastic scatter from hydrogen, the $\mathrm{P}_{0}$ and $\mathrm{P}_{1}$ moments per unit lethargy, respectively, can be rigorously expressed in the form of Eq. (7.4.35) with

$$
\mathrm{H}_{0} \propto \mathrm{E}, \text { and } \mathrm{H}_{1} \propto \mathrm{E}^{3 / 2}
$$

These expressions can be inferred directly from the moments of the scatter kernel in Eq. (7.4.16). The higher order scatter moments for hydrogen have a somewhat more complicated form containing sums of energy functions; but since these moments are usually less important than the first two moments, a less rigorous treatment of their intra-group distribution is used. The intra-group distribution due to inelastic scatter depends on the Q values for the individual levels, and these are not available on the multigroup libraries. Fortunately, the scatter source in the PW range is not very sensitive to the assumed intra-group distribution for inelastic scatter, as long as the total inelastic source for the group is computed correctly. As a reasonable trade-off between rigor and complexity, the high energy component of the UMR-to-PW scatter source is approximated using $\mathrm{H}_{0}$ for the intra-group distribution of all $\mathrm{P}_{0}$ moments, and $\mathrm{H}_{1}$ for all higher order moments. This approximation produces the correct intra-group variation for the lowest two moments of the hydrogen scatter source, but the higher order moments of hydrogen and the inelastic scatter source are not distributed exactly throughout the group. However, the integrated source moments are correct in all cases. Again, it should be emphasized that the approximations discussed here only apply to the UMR-to-PW component designated as $\mathrm{S}_{\mathrm{HI}}$, which comes from reactions above the transition range (energies above $\mathrm{E}_{\mathrm{HI}} / \alpha_{\mathrm{L}}$ ). This is often a small contribution to the overall PW source term. 


\subsection{Scattering sour ces from UMR transition region and epithermal PW range}

Most coupling between the UMR and the PW range is due usually to elastic scatter from energies immediately above DEMAX. The contribution to the PW source due to downscatter source from this transition range has been designated $\mathrm{S}_{\mathrm{Tr}}\left(\mathrm{u}_{\mathrm{n}}\right)$. The other component of the PW source, $\mathrm{S}_{\mathrm{PW}}\left(\mathrm{u}_{\mathrm{n}}\right)$, accounts for the scattering source coming from all lethargies lower than $\mathrm{u}_{\mathrm{n}}$ in the PW range. It is convenient to combine the two sources together as the PW epithermal source called " $\mathrm{S}_{\mathrm{Ep}}$," which has an $1 \mathrm{k}_{\mathrm{th}}$ moment given by Eq. (7.4.22),

$$
\begin{aligned}
S_{\ell k, E p} & =\int_{\mathrm{u}_{\mathrm{L}}}^{\mathrm{u}_{\mathrm{n}}} \mathrm{S}_{\ell \mathrm{k}}\left(\mathrm{r}, \mathrm{u}^{\prime} \rightarrow \mathrm{u}\right) \mathrm{du}^{\prime} \\
& =\sum_{\mathrm{j}} \sum_{\mathrm{K}=-\ell}^{\ell} \mathrm{Z}_{\ell, \mathrm{k}}^{(\mathrm{j})} \mathrm{h}_{\mathrm{K}}(\mathrm{E}) \int_{\mathrm{u}_{\mathrm{n}}-\varepsilon^{(\mathrm{j})}}^{\mathrm{u}_{\mathrm{n}}} \Psi_{\ell \mathrm{k}}\left(\mathrm{u}^{\prime}\right) \Sigma^{(\mathrm{j})}\left(\mathrm{u}^{\prime}\right) \mathrm{h}_{\mathrm{K}}^{-1}\left(\mathrm{E}^{\prime}\right) \mathrm{du} \mathrm{u}^{\prime}
\end{aligned}
$$

This is done because CENTRM uses the submoment expansion technique to compute both the PW-to-PW epithermal source from the PW range as well as the MG-to-PW source from the transition range of the UMR. Note that elastic scattering from the transition range only impacts the PW scatter source at the initial mesh points in the PW range; i.e., those contained in the interval $0<\mathrm{u}_{\mathrm{n}}<\varepsilon^{(\mathrm{j})}$, for nuclide j. Beyond these mesh points the elastic scatter source is due only to PW-to-PW scatter, as illustrated in Figure 7.4.2.

The epithermal elastic source at $u_{n}$, coming from the range $u_{L}$ to $u_{n}$, is expressed as an integral over the immediately preceding lethargy mesh interval from $u_{n-1}$ to $u_{n}$ plus the integral over the remaining lethargy interval, as illustrated in Figure 7.4.3. The former integral is designated as $I\left(u_{n-1}, u_{n}\right)$ and the latter as $\mathrm{I}\left(\mathrm{u}_{\mathrm{L}}, \mathrm{u}_{\mathrm{n}-1}\right)$, so that

$$
\mathrm{S}_{\ell \mathrm{k}, \mathrm{E} \ell}\left(\mathrm{u}_{\mathrm{n}}\right)=\mathrm{I}\left(\mathrm{u}_{\mathrm{n}-1}, \mathrm{u}_{\mathrm{n}}\right)+\mathrm{I}\left(\mathrm{u}_{\mathrm{L}}, \mathrm{u}_{\mathrm{n}-1}\right) .
$$

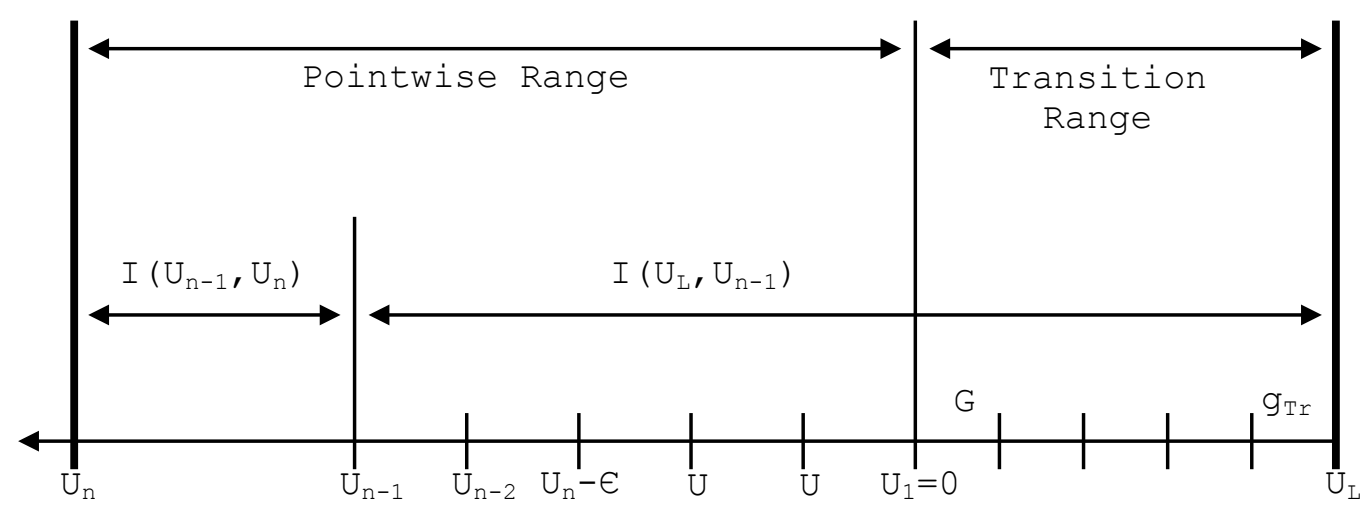

Figure 7.4.3. Definition of cumulative integral elements.

The lethargy mesh in CENTRM is constrained such that the maximum lethargy gain in an elastic reaction $\left(\varepsilon^{(j)}\right)$ is always greater than the maximum mesh interval size, which insures that $\mathrm{I}\left(\mathrm{u}_{\mathrm{n}-1}, \mathrm{u}_{\mathrm{n}}\right)$ always includes the full panel from $u_{n-1}$ to $u_{n}$. In the above and subsequent equations the explicit dependence of $S_{E p}$ on independent variables other than lethargy is not shown for notational convenience. The integral $I\left(u_{n-1}, u_{n}\right)$ is evaluated approximately by applying the trapezoidal rule, which leads to, 


$$
\mathrm{I}\left(\mathrm{u}_{\mathrm{n}}, \mathrm{u}_{\mathrm{n}-1}\right)=\int_{\mathrm{u}_{\mathrm{n}-1}}^{\mathrm{u}_{\mathrm{n}}} \mathrm{S}_{\ell \mathrm{k}}\left(\mathrm{u}^{\prime} \rightarrow \mathrm{u}_{\mathrm{n}}\right) d \mathrm{u}^{\prime} \sim \frac{\left[\mathrm{S}_{\ell \mathrm{k}}\left(\mathrm{u}_{\mathrm{n}} \rightarrow \mathrm{u}_{\mathrm{n}}\right)+\mathrm{S}_{\ell \mathrm{k}}\left(\mathrm{u}_{\mathrm{n}-1} \rightarrow \mathrm{u}_{\mathrm{n}}\right)\right]}{2} \times\left(\mathrm{u}_{\mathrm{n}}-\mathrm{u}_{\mathrm{n}-1}\right)
$$

Using the submoment expansion from Eq. (7.4.25), Eq. (7.4.38) can be written for elastic scatter as, ${ }^{5}$

$$
\mathrm{I}\left(\mathrm{u}_{\mathrm{n}-1}, \mathrm{u}_{\mathrm{n}}\right)=\Sigma_{\mathrm{n} \rightarrow \mathrm{n}} \Psi_{\ell \mathrm{k}, \mathrm{n}}+\sum_{\mathrm{K}} \mathrm{Z}_{\ell \mathrm{K}}{ }^{(\mathrm{j})} \mathrm{h}_{\mathrm{K}}\left(\mathrm{E}_{\mathrm{n}}\right) \mathrm{h}_{\mathrm{K}}^{-1}\left(\mathrm{E}_{\mathrm{n}-1}\right) \Sigma^{(\mathrm{j})}\left(\mathrm{u}_{\mathrm{n}-1}\right) \Psi_{\ell \mathrm{k}, \mathrm{n}-1} \frac{\Delta \mathrm{u}_{\mathrm{n}-1}}{2} .
$$

The first term on the right side of Eq. (7.4.39) corresponds to the "within-point" component of elastic scatter from $u_{n}$ to $u_{n}$, which only occurs for straight ahead scatter $\left(\mu_{0}=1\right)$. The within-point cross section is defined as,

$$
\Sigma_{n \rightarrow n}=\frac{\Delta u_{n-1}}{2} \sum_{j} \frac{\Sigma_{n}^{(j)}}{\left(1-\alpha^{(j)}\right)}
$$

In deriving this term the following relation has been used for each nuclide:

$$
\sum_{\mathrm{K}} \mathrm{Z}_{\ell \mathrm{K}}=\frac{1}{1-\alpha}
$$

The $\mathrm{I}\left(\mathrm{u}_{\mathrm{L}}, \mathrm{u}_{\mathrm{n}-1}\right)$ portion of the integral in Eq. (7.4.37) is equal to

$$
\mathrm{I}\left(\mathrm{u}_{\mathrm{L}}, \mathrm{u}_{\mathrm{n}-1}\right)=\sum_{j} \sum_{K=-\ell}^{\ell} \mathrm{Z}_{\ell, \mathrm{K}}^{(\mathrm{j})} \mathrm{h}_{\mathrm{K}}\left(\mathrm{E}_{\mathrm{n}}\right) \int_{\mathrm{u}_{\mathrm{n}}-\varepsilon^{(j)}}^{\mathrm{u}_{\mathrm{n}-1}} \Psi_{\ell \mathrm{k}}\left(\mathrm{u}^{\prime}\right) \Sigma^{(\mathrm{j})}\left(\mathrm{u}^{\prime}\right) \mathrm{h}_{\mathrm{K}}^{-1}\left(\mathrm{E}^{\prime}\right) d \mathrm{u}^{\prime}
$$

Note that the lower lethargy limit of the integral is restricted to $u_{n}-\varepsilon^{(j)}$, since this is the maximum limit of lethargy that can scatter to $u_{n}$ in an elastic reaction. In terms of the cumulative integral operator, the integral in Eq. (7.4.42) over the interval $\left[u_{n}-\varepsilon^{(j)}, u_{n-1}\right]$ is equal to

$$
\int_{\mathrm{u}_{\mathrm{n}}-\varepsilon(\mathrm{j})}^{\mathrm{u}_{\mathrm{n}}-1} \Psi_{\ell \mathrm{k}}\left(\mathrm{u}^{\prime}\right) \Sigma^{(\mathrm{j})}\left(\mathrm{u}^{\prime}\right) \mathrm{h}_{\mathrm{K}}\left(\mathrm{E}^{\prime}\right)^{-1} \mathrm{du} \mathrm{u}^{\prime}=\left[\mathbb{C}\left(\mathrm{F}_{\ell \mathrm{k}, \mathrm{K}}^{(\mathrm{j})} ; \mathrm{u}_{\mathrm{n}-1}\right)-\mathbb{C}\left(\mathrm{F}_{\ell \mathrm{k}, \mathrm{K}}^{(\mathrm{j})} ; \mathrm{u}_{\mathrm{n}}-\varepsilon^{(\mathrm{j})}\right)\right]
$$

where $\mathrm{F}_{\ell \mathrm{k}, \mathrm{K}}$ has been defined in Eq. (7.4.26). In order to evaluate Eq. (7.4.43) it is necessary to determine the cumulative integral values at $u_{n-1}$ and $u_{n}-\varepsilon^{(j)}$. The lethargy $u_{n-1}$ will always correspond to a mesh point value, but in general $u_{n}-\varepsilon^{(j)}$ can fall between mesh points. Evaluation of the cumulative integrals at an arbitrary limit such as $u_{n}-\varepsilon^{(j)}$ is performed in CENTRM by interpolation of previously calculated values stored for all the mesh points below $u_{n}$ during the transport calculation at lower lethargies. The interpolated value of the cumulative integral at $u_{n}-\varepsilon^{(j)}$ that is subtracted in Eq. (7.4.43) is called the "excess integral" in CENTRM. At each lethargy point, excess integrals must be found as a function of space, nuclide, moment, and submoment. Also note that for some initial mesh points (i.e., $u_{n}<\varepsilon^{(j)}$ ) the value $u_{n}-\varepsilon^{(j)}$ can be negative, indicating that a portion of the PW scatter source at $u_{n}$ is due to elastic scattering from the negative lethargy range above DEMAX. This means that cumulative integrals must be known for mesh intervals in the transition as well as in the PW range. Values of the cumulative integrals at all points within the transition range are first computed from the results from the UMR calculation, prior to the PW transport calculation (but after the UMR calculation). Additional cumulative 
integrals are then calculated successively during the PW transport solution at all mesh points and are stored as the calculation proceeds from low to high lethargy. Thus in evaluating $S_{k \mathrm{k}, \mathrm{Ep}}\left(\mathrm{u}_{\mathrm{n}}\right)$, the cumulative integrals at every space interval already will have been stored at all energy points up to (n-1), in an array called $\mathrm{CUM}^{(\mathrm{j})}{ }_{\ell \mathrm{k}, \mathrm{K}}$, for each nuclide $\mathrm{j}$, moment $\ell \mathrm{k}$, and submoment $\mathrm{K}$ :

$$
\mathrm{CUM}_{\ell \mathrm{k}, \mathrm{K}}^{(\mathrm{j})}=\left\{\mathbb{C}\left(\mathrm{F}_{\ell \mathrm{k}, \mathrm{K}}^{(\mathrm{j})} ; \mathrm{u}_{\mathrm{n}^{\prime}}\right), \mathrm{n}^{\prime}=1, \mathrm{n}-1\right\}
$$

so that the excess integral values can be interpolated from the above array. The first $\mathrm{N}_{\mathrm{Tr}}$ elements of the array $\mathrm{CUM}^{(\mathrm{j})}{ }_{\mathrm{k}, \mathrm{K}}$ correspond to lethargy points in the transition range, and the remainder are in the PW range, where

$$
\begin{aligned}
& \mathrm{N}_{\mathrm{Tr}}=\mathrm{G}_{\mathrm{U}}-\mathrm{g}_{\mathrm{Tr}}+1 ; \\
& \mathrm{g}_{\mathrm{Tr}}=\begin{array}{l}
\text { MGTOP, the highest energy group in the transition range; (i.e., the group whose high } \\
\text { energy boundary corresponds to } \mathrm{u}_{\mathrm{L}} \text { ); }
\end{array} \\
& \mathrm{G}_{\mathrm{U}}=\text { Lowest energy group in the transition range. }
\end{aligned}
$$

Elastic cumulative integrals contained in array $\mathrm{CUM}^{(\mathrm{j})}{ }_{\ell \mathrm{k}, \mathrm{K}}$ are calculated at each lethargy point $\mathrm{u}_{\mathrm{n}}$ with the expression:

$$
\begin{aligned}
& \mathbb{C}\left(\mathrm{F}_{\ell \mathrm{k}, \mathrm{K}}^{(\mathrm{j})} ; \mathrm{u}_{\mathrm{n}}\right)=\mathbb{C}\left(\mathrm{F}_{\ell \mathrm{k}, \mathrm{K}}^{(\mathrm{j})} ; \mathrm{u}_{\mathrm{n}-1}\right)+\int_{\mathrm{u}_{\mathrm{n}-1}}^{\mathrm{u}_{\mathrm{n}}} \mathrm{F}_{\ell \mathrm{k}, \mathrm{K}}(\mathrm{j})\left(\mathrm{u}^{\prime}\right) \mathrm{du}^{\prime} \\
& =\mathbb{C}\left(\mathrm{F}_{\ell \mathrm{k}, \mathrm{K}}^{(\mathrm{j})} ; \mathrm{u}_{\mathrm{n}-1}\right)+\int_{\mathrm{u}_{\mathrm{n}}-1}^{\mathrm{u}_{\mathrm{n}}} \Psi_{\ell \mathrm{k}}\left(\mathrm{u}^{\prime}\right) \Sigma^{(\mathrm{j})}\left(\mathrm{u}^{\prime}\right) \mathrm{h}_{\mathrm{K}}\left(\mathrm{E}^{\prime}\right)^{-1} \mathrm{du}^{\prime}
\end{aligned}
$$

After completing the calculation of PW angular fluxes and moments at $u_{n}$ the integral over the most current lethargy panel $\left[\mathrm{u}_{\mathrm{n}-1}, \mathrm{u}_{\mathrm{n}}\right]$ is evaluated with the trapezoidal approximation, resulting in an updated cumulative integral array containing the value at lethargy $u_{n}$ :

$$
\mathbb{C}\left(\mathrm{F}_{\ell \mathrm{k}, \mathrm{K}}^{(\mathrm{j})} ; \mathrm{u}_{\mathrm{n}}\right) \simeq \mathbb{C}\left(\mathrm{F}_{\ell \mathrm{k}, \mathrm{K}}^{(\mathrm{j})} ; \mathrm{u}_{\mathrm{n}-1}\right)+\Delta \mathrm{u}_{\mathrm{n}-1} \frac{\left[\mathrm{h}_{\mathrm{K}}{ }^{-1}\left(\mathrm{E}_{\mathrm{n}-1}\right) \sum_{\mathrm{n}-1}^{(\mathrm{j})} \Psi_{\ell \mathrm{k}, \mathrm{n}-1}+\mathrm{h}_{\mathrm{K}}{ }^{-1}\left(\mathrm{E}_{\mathrm{n}}\right) \sum_{\mathrm{n}}^{(\mathrm{j})} \Psi_{\ell \mathrm{k}, \mathrm{n}}\right]}{2}
$$

where the cumulative integrals at the preceding mesh point are known from the previous calculation, and the flux moments $\Psi_{\ell \mathrm{k}}\left(\mathrm{u}_{\mathrm{n}}\right)$ are determined from the transport calculation at the current lethargy point. Only a single panel of integration is required to update the cumulative integrals, significantly reducing the amount of computation compared to recomputing the entire summation again at each new energy point. The integration is performed rapidly with the trapezoidal approximation, which should be accurate since the energy mesh is defined to reproduce the macroscopic cross sections linearly between mesh points. In order to avoid loss of numerical significance, the set of stored cumulative integrals is periodically "renormalized," by translating to a new reference lethargy point (recall that only the differences of cumulative integrals is needed). 
Elastic cumulative integrals for the transition range are calculated with a slightly different expression, using MG flux moments obtained in the UMR calculation. Because the transition interval is part of the $\mathrm{UMR}$, it is convenient to evaluate cumulative integrals at lethargy values corresponding to group boundaries. This requires approximating the energy distribution of the flux spectrum within each group in the transition range. To evaluate the cumulative interval in the transition range of some nuclide $j$, the scalar flux per energy (at a given space location) within a transition group is approximated as: $\Phi(E)=M^{(j)} / E$, where $M^{(j)}$ is a normalization constant defined so that the MG outscatter rate (i.e., slowingdown density) from the group is preserved. It can be shown that this normalization condition requires that, ${ }^{5}$

$$
\mathbf{M}^{(\mathrm{j})}=\frac{\left[\Sigma_{t, g^{\prime}}^{(j)}-\Sigma_{a, g^{\prime}}^{(j)}-\Sigma_{g^{\prime}, g^{\prime}}^{(j)}\right] \Delta u_{g^{\prime}}}{\xi^{(j)} \Sigma_{s, g^{\prime}}^{(j)}} \times\left[\phi_{g^{\prime}} / \Delta u_{g^{\prime}}\right]
$$

where $\xi$ is the average lethargy gain in an elastic reaction and $\Sigma_{g^{\prime} g^{\prime}}$, is the within-group MG scatter cross section. Thus the scalar flux per unit lethargy used to evaluate cumulative integrals of nuclide $\mathrm{j}$ is:

$$
\phi\left(\mathrm{u}^{\prime}\right)=\mathrm{M}^{(\mathrm{j})} ; \quad \text { for } \mathrm{u}^{\prime} \varepsilon \mathrm{g}^{\prime}, \text { and } \mathrm{g}^{\prime} \varepsilon \text { Transition region of } \mathrm{UMR} \text {, }
$$

Within-group energy spectra for the higher order flux-moments could be approximated in similar manner by preserving the higher order Legendre moments of the slowing-down density, but CENTRM simply uses the same form in Eq. (7.4.47) for all flux moments, so that in general the within-group energy distribution for any $\ell \mathrm{k}_{\mathrm{th}}$ moment in the transition range is approximated as,

$$
\Psi_{\ell k}\left(u^{\prime}\right)=\frac{\left[\Sigma_{t, g^{\prime}}^{(j)}-\Sigma_{a, g^{\prime}}^{(j)}-\Sigma_{g^{\prime} g^{\prime}}^{(j)}\right] \Delta u_{g^{\prime}}}{\xi^{(j)} \Sigma_{s, g^{\prime}}^{(j)}} \times\left[\Psi_{\ell k, g^{\prime}} / \Delta u_{g^{\prime}}\right]
$$

for $\mathrm{u}^{\prime} \mathrm{\varepsilon g}^{\prime}$, and $\mathrm{g}^{\prime} \varepsilon$ transition region of UMR. Therefore the following integrals can be evaluated:

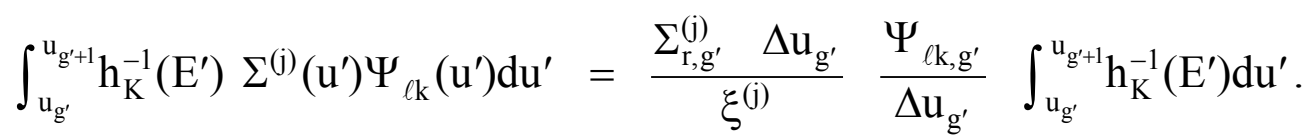

Integration of the $\mathrm{h}_{\mathrm{k}}{ }^{-1}$ function is performed analytically to give the cumulative integral at any group boundary $\mathrm{u}_{\mathrm{g}}$ in the transition range:

$$
\begin{aligned}
& \mathbb{C}\left(\mathrm{F}_{\ell \mathrm{k}, \mathrm{K}}^{(\mathrm{j})} ; \mathrm{u}_{\mathrm{g}}\right) \simeq \sum_{\mathrm{g}^{\prime}=\mathrm{g}}^{\mathrm{G}_{\mathrm{U}}} \frac{\sum_{\mathrm{r}, \mathrm{g}^{\prime}}^{(\mathrm{j})} \Delta \mathrm{u}_{\mathrm{g}^{\prime}}}{\xi^{(\mathrm{j})}} \frac{\Psi_{\ell \mathrm{k}, \mathrm{g}^{\prime}}}{\Delta \mathrm{u}_{\mathrm{g}^{\prime}}} \times\left[\frac{2}{\mathrm{~K}+2}\right]\left[\mathrm{h}_{\mathrm{K}}{ }^{-1}\left(\mathrm{E}_{\mathrm{g}^{\prime}+1}\right)-\mathrm{h}_{\mathrm{K}}{ }^{-1}\left(\mathrm{E}_{\mathrm{g}^{\prime}}\right)\right] \\
& \mathrm{g}=\mathrm{g}_{\mathrm{Tr}}, \mathrm{g}_{\mathrm{Tr}+1}, \ldots . . \mathrm{G}_{\mathrm{U}}
\end{aligned}
$$

Equation (7.4.50) is used to obtain the initial $\mathrm{N}_{\mathrm{Tr}}$ values of the cumulative integrals, corresponding to the transition range. If the lower limit of the integral in Eq. (7.4.43) is negative, then the cumulative integral at $u_{n}-\varepsilon^{(j)}$ is interpolated from among the set of $\mathrm{N}_{T r}$ tabulated values generated by Eq. (7.4.50); otherwise it is interpolated from the values that were computed with Eq. (7.4.46). The following algorithm is used to interpolate cumulative integrals for negative lethargy arguments (i.e., in the transition range ): 


$$
\begin{aligned}
& \mathbb{C}\left(\mathrm{F}_{\ell \mathrm{k}, \mathrm{K}}^{(\mathrm{j})} ; \mathrm{u}\right)=\mathbb{C}\left(\mathrm{F}_{\ell \mathrm{k}, \mathrm{K}}^{(\mathrm{j})} ; \mathrm{u}_{\mathrm{g}}\right)+\frac{\mathrm{h}_{\mathrm{K}}{ }^{-1}(\mathrm{E})-\mathrm{h}_{\mathrm{K}}{ }^{-1}\left(\mathrm{E}_{\mathrm{g}}\right)}{\mathrm{h}_{\mathrm{K}}^{-1}\left(\mathrm{E}_{\mathrm{g}+1}\right)-\mathrm{h}_{\mathrm{K}}{ }^{-1}\left(\mathrm{E}_{\mathrm{g}}\right)} \\
& \times\left[\mathbb{C}\left(F_{\ell k, K}^{(j)} ; u_{g+1}\right)-\mathbb{C}\left(F_{\ell k, K}^{(j)} ; u_{g}\right)\right]
\end{aligned}
$$

For $\mathrm{u}(\mathrm{E}) \varepsilon \mathrm{g}$; and $\mathrm{g} \varepsilon$ transition range of UMR.

Because the energy mesh in the PW range is very fine, simple linear interpolation of the cumulative integrals is used for positive lethargy arguments.

The complete epithermal elastic scatter source $S\left(r, \Omega, u_{n}\right)$ appearing in Eq. (7.4.32) at any mesh point $u_{n}$ corresponds to a spherical harmonic expansion using the previously derived moments of $\mathrm{S}_{\mathrm{HI}}$ and $\mathrm{S}_{\mathrm{Ep}}$. This angular scatter source is equal to, ${ }^{5}$

$$
\begin{aligned}
& \mathrm{S}\left(\mathbf{r}, \boldsymbol{\Omega}, \mathrm{u}_{\mathrm{n}}\right)=\Sigma_{\mathrm{n} \rightarrow \mathrm{n}} \Psi_{\mathrm{n}}(\mathbf{r}, \boldsymbol{\Omega}) \\
& +\sum_{\ell \mathrm{k}} \frac{2 \ell+1}{2} \mathrm{Y}_{\ell \mathrm{k}}(\Omega)\left\{\mathrm{H}_{\ell}\left(\mathrm{E}_{\mathrm{n}}\right) \sum_{\mathrm{g}^{\prime}=1}^{\mathrm{g}_{\mathrm{Tr}}-1} \sum_{\ell, \mathrm{g}^{\prime} \rightarrow \mathrm{g}} \Psi_{\ell \mathrm{k}, \mathrm{g}^{\prime}}\right. \\
& \left.+\sum_{\mathrm{j}} \sum_{\mathrm{K}} \mathrm{Z}_{\ell \mathrm{K}}{ }^{(\mathrm{j})} \mathrm{h}_{\mathrm{K}}\left(\mathrm{E}_{\mathrm{n}}\right)\left[0.5 \Delta \mathrm{u}_{\mathrm{n}-1} \mathrm{~F}_{\ell \mathrm{k}, \mathrm{K}}^{(\mathrm{j})}\left(\mathrm{u}_{\mathrm{n}-1}\right)+\mathbb{C}\left(\mathrm{F}_{\ell \mathrm{k}, \mathrm{K}}^{(\mathrm{j})} ; \mathrm{u}_{\mathrm{n}-1}\right)-\mathbb{C}\left(\mathrm{F}_{\ell \mathrm{k}, \mathrm{K}}^{(\mathrm{j})} ; \mathrm{u}_{\mathrm{n}}-\varepsilon^{(\mathrm{j})}\right)\right]\right\} .
\end{aligned}
$$

The above expression was written explicitly for the case of elastic scatter; however, the discrete level inelastic PW source can be incorporated with little modification. The only changes are that additional cumulative integral terms corresponding to each inelastic level will appear in Eq. (7.4.52); the cumulative integrals for the inelastic levels must be computed by integrating the more general expression in Eq. (7.4.26); and the lethargy arguments for the inelastic cumulative integrals are the generalized lethargy limits $\mathrm{u}_{\mathrm{LO}}$ and $\mathrm{u}_{\mathrm{HI}}$ defined in Sect. 7.4.2.4 and Ref. 2.

Note that Eq. (7.4.52) contains the term $\Sigma_{\mathrm{n} \rightarrow \mathrm{n}} \Psi_{\mathrm{n}}(\mathrm{r}, \Omega)$ which can be subtracted from both sides of the transport equation in Eq. (7.4.32) to give a slightly altered form of the PW transport equation that contains a modified scatter source and a modified total cross section. The modified source component is identical to the expression in Eq. (7.4.52) with the within-point term $\Sigma_{n \rightarrow n} \Psi_{n}(r, \Omega)$ removed. The modified total cross section, represented by $\tilde{\Sigma}_{\mathrm{t}, \mathrm{n}}$, has the appearance of a "transport-corrected" cross section given below:

$$
\tilde{\Sigma}_{\mathrm{t}, \mathrm{n}}=\Sigma_{\mathrm{t}, \mathrm{n}}-\Sigma_{\mathrm{n} \rightarrow \mathrm{n}}
$$

An interesting and significant consequence of this operation is that the right side of Eq. (7.4.52) no longer contains the unknown flux $\Psi_{\mathrm{n}}(\mathrm{r}, \Omega)$ since the within-point term is eliminated. The resulting modified transport equation has the same form as a purely absorbing medium with a known source term; and thus can be solved without requiring scatter-source iterations in the epithermal range. However, iterations may still be required for cell cases with two reflected or albedo boundary conditions.

\subsection{PW thermal scatter source}

There are significant differences in the CENTRM epithermal and thermal PW transport solutions. In the epithermal range neutrons can only lose energy in scattering reactions, so that a single sweep from high to 
low energy (i.e., low to high lethargy) is required in the solution. On the other hand, since low energy neutrons may gain as well as lose energy in scattering reactions, outer iterations are required to converge the thermal scattering source. Furthermore, the PW scatter kernels $\Sigma_{\ell}\left(u^{\prime} \rightarrow u\right)$ in the epithermal range represent two-body interactions (such as elastic and discrete-level inelastic reactions) between a neutron and a stationary nucleus. The simple kinematic relations for these cases allow the efficient sub-moment expansion method to be utilized in computing scattering source moments. Thermal scattering reactions are not two body reactions, but rather represent an effective average over the molecular velocity distribution; thus, there is no simple kinematic relationship between neutron energy loss and the angle of scatter relative to its initial direction. In solving the transport equation for thermal neutrons, the scatter source at lethargy $\mathrm{u}_{\mathrm{n}}$ is approximated as a summation over the "N" mesh points in the thermal range,

$$
\int_{\text {thermal }} \sum_{\ell}{ }^{(\mathrm{j})}\left(\mathrm{u}^{\prime} \rightarrow \mathrm{u}_{\mathrm{n}}\right) \Psi_{\ell \mathrm{k}}\left(\mathrm{u}^{\prime}\right) \mathrm{du}^{\prime}=\sum_{\mathrm{m}=1}^{\mathrm{N}} \mathrm{W}_{\mathrm{m}} \Sigma_{\ell}^{(j)}\left(\mathrm{u}_{\mathrm{m}} \rightarrow \mathrm{u}_{\mathrm{n}}\right) \Psi_{\ell \mathrm{k}}\left(\mathrm{u}_{\mathrm{m}}\right)
$$

where

$$
\begin{aligned}
& \mathrm{m}=1 \text { is the thermal/epithermal boundary point; } \\
& \mathrm{m}=\mathrm{N} \text { is the lowest thermal energy point; and }
\end{aligned}
$$

$\mathrm{W}_{\mathrm{m}}$ are standard quadrature weights for trapezoidal integration with $\mathrm{N}-1$ lethargy panels:

$$
\begin{aligned}
\mathrm{W}_{\mathrm{m}}=0.5 \times\left(\Delta \mathrm{u}_{\mathrm{m}}+\Delta \mathrm{u}_{\mathrm{m}+1}\right) & ; \text { for } \mathrm{m}=2,3, \ldots \mathrm{N}-1 \\
0.5 \times \Delta \mathrm{u}_{\mathrm{m}} & ; \text { for } \mathrm{m}=1 \text { or } \mathrm{N}
\end{aligned}
$$

Point-to-point cross-section moments in the thermal range are computed from the free-gas or bound kernels evaluated at the desired initial $\left(\mathrm{u}_{\mathrm{m}}\right)$ and final $\left(\mathrm{u}_{\mathrm{n}}\right)$ lethargy mesh points. For a given outer iteration, the summation in Eq. (7.4.54) is evaluated usi ng the most recently computed flux moments. In many instances the main purpose of the CENTRM calculation will be to obtain a PW spectrum for resonance self-shielding calculations. In these cases the thermal flux does not have to be converged very tightly to obtain a reasonable thermal spectrum for self-shielding low energy resonances, so that only a few outer iterations are typically employed.

An additional complication in the thermal calculation is that inner iterations are necessary to converge the "within-point" (no energy loss) contribution of the thermal scattering source, due to the presence of PW flux moments at lethargy point $\mathrm{m}=\mathrm{n}$. No inner iterations are required to converge the within-point elastic scatter term in the epithermal PW calculation because there can be no change in the neutron direction if there is no energy loss, unlike the thermal range.

A space-dependent rebalance calculation for the entire thermal energy band is performed between outer iterations in order to speed up convergence of the solution. Reaction rates and leakage values appearing in the thermal-band rebalance equation are obtained by integrating PW values over the thermal range. Other acceleration techniques, such as over-relaxation, extrapolation, and renormalization, are also employed.

\subsection{Downscatter source from the epithermal PW range to the LMR}

MG transport calculations performed in the energy range below DEMIN, which includes the thermal energy range, are coupled to the epithermal PW range transport calculations by the slowing down source. The epithermal PW-to-LMR scatter source represents the contribution to the multigroup source in some 
fixed group g contained in the LMR, from scatter reactions in the epithermal range above DEMIN. The lethargy value corresponding to the energy DEMIN (i.e., the bottom energy of the PW range) will be indicated as $\mathrm{u}_{\mathrm{PW}}$, thus $\mathrm{u}_{\mathrm{PW}}=\ln (\mathrm{DEMAX} / \mathrm{DEMIN})$; while the lethargy corresponding to the thermal energy boundary will be designated as $\mathrm{u}_{\mathrm{TH}}$. The cut-off lethargy for the epithermal PW range will correspond to: $u_{c u t}=\min \left(u_{P W}, u_{T H}\right)$. If there is no PW thermal calculation in CENTRM, then $u_{c u t}=u_{P W}$; otherwise, $\mathrm{u}_{\mathrm{cut}}=\mathrm{u}_{\mathrm{TH}}$. For a given nuclide $\mathrm{j}$, the lowest lethargy in the epithermal PW range from which a neutron can scatter elastically into the LMR is equal to $\left(u_{c u t}-\varepsilon^{(j)}\right)$. If the value of $\left(u-\varepsilon^{(j)}\right)$ is greater than $\mathrm{u}_{\text {cut }}$, then an elastic collision with nuclide $\mathrm{j}$ cannot moderate an epithermal neutron from the PW range to $\mathrm{u}$. Therefore in general for a given material zone, only a limited number of nuclides (possibly none) and a limited portion of the epithermal PW energy range may be able to scatter neutrons elastically to any particular group in the LMR. Utilizing the elastic scatter kernel and applying a sub-moment expansion to the resulting expression, the source moment describing scatter from the PW epithermal range to a lethargy $\mathrm{u}$ in the LMR is found to be

$$
\mathrm{S}_{\ell \mathrm{k}}{ }^{(\mathrm{j})}(\mathrm{u})=\sum_{\mathrm{K}} Z_{\ell \mathrm{K}}{ }^{(\mathrm{j})} \mathrm{h}_{\mathrm{K}}(\mathrm{E}) \quad \int_{\mathrm{u}-\varepsilon^{\mathrm{j})}}^{\mathrm{u}_{\mathrm{cut}}} \mathrm{F}_{\ell \mathrm{k}, \mathrm{K}}^{(\mathrm{j})}\left(\mathrm{u}^{\prime}\right) \mathrm{du} \mathrm{u}^{\prime}
$$

where $\mathrm{u}$ in group $\mathrm{g}$; and $\mathrm{g} \varepsilon \mathrm{LMR}$.

The integral in the above expression can be evaluated from cumulative integrals stored during the epithermal PW transport calculation. Thus the source moment per unit lethargy at $u$ in the LMR range, due to epithermal scattering from nuclide $\mathrm{j}$, can be written as,

$$
\mathrm{S}_{\ell \mathrm{k}}^{(\mathrm{j})}(\mathrm{u})=\sum_{\mathrm{K}} \mathrm{Z}_{\ell \mathrm{K}}{ }^{(\mathrm{j})} \mathrm{h}_{\mathrm{K}}(\mathrm{E}) \quad\left[\mathbb{C}\left(\mathrm{F}_{\ell \mathrm{k}, \mathrm{K}}^{(\mathrm{j})} ; \mathrm{u}_{\text {cut }}\right)-\mathbb{C}\left(\mathrm{F}_{\ell \mathrm{k}, \mathrm{K}}^{(\mathrm{j})} ; \mathrm{u}-\varepsilon^{(\mathrm{j})}\right)\right]
$$

for $\mathrm{u} \varepsilon$ group $g$ and $\mathrm{u}-\varepsilon(\mathrm{j})<\mathrm{u}_{\mathrm{PW}}$.

The source per unit lethargy in Eq. (7.4.55) is integrated over the "sink group" $g$ in the LMR to determine the desired MG scatter source moment due to reactions in the epithermal PW range. The actual integral over group g is performed numerically by introducing a three-point (two panel) integration mesh within the group, as follows:

$$
\begin{aligned}
\mathrm{u}_{\mathrm{I}} & =\text { initial integration point in group } \mathrm{g}=\text { lethargy at top energy of group } \mathrm{g}=\mathrm{u}_{\mathrm{g}} ; \\
\mathrm{u}_{\mathrm{F}}^{(\mathrm{j})} & =\text { final integration point in group } \mathrm{g}, \\
& =\operatorname{MIN}\left\{\mathrm{u}_{\mathrm{g}+1} ; \mathrm{u}_{\text {cut }}+\varepsilon^{(\mathrm{j})}\right\} \text {, where } \mathrm{u}_{\mathrm{g}+1}=\text { lethargy at bottom of energy of group } \mathrm{g} ; \\
\mathrm{u}_{\mathrm{A}}^{(\mathrm{j})} & =\text { middle integration point in group } \mathrm{g}=0.5\left(\mathrm{u}_{\mathrm{F}}^{(\mathrm{j})}+\mathrm{u}_{\mathrm{I}}\right) .
\end{aligned}
$$

Note that the final and middle points of integration (i.e., $\mathrm{u}_{\mathrm{F}}{ }^{(\mathrm{j})}$ and $\mathrm{u}_{\mathrm{A}}{ }^{(\mathrm{j})}$ ) may be nuclide dependent; and if $\left(u_{I}-\varepsilon^{(j)}\right)>u_{\text {cut }}$, then nuclide $\mathrm{j}$ does not contribute to the pointwise-to-LMR scatter source in $g$. Applying the two-panel Simpson's approximation for integration over group g results in

$$
\mathrm{S}_{\ell \mathrm{k}, \mathrm{g}}^{(\mathrm{j})}=\Delta^{(\mathrm{j})} / 3\left[\mathrm{~S}_{\ell \mathrm{k}}{ }^{(\mathrm{j})}\left(\mathrm{u}_{\mathrm{I}}\right)+4 \mathrm{~S}_{\ell \mathrm{k}}{ }^{(\mathrm{j})}\left(\mathrm{u}_{\mathrm{A}}{ }^{(\mathrm{j})}\right)+\mathrm{S}_{\ell \mathrm{k}}{ }^{(\mathrm{j})}\left(\mathrm{u}_{\mathrm{F}}{ }^{(\mathrm{j})}\right)\right]
$$

where $\Delta^{(j)}=0.5\left(u_{F}^{(j)}-u_{I}\right)$. 
The values for $\mathrm{S}_{\ell \mathrm{k}}{ }^{(\mathrm{j})}\left(\mathrm{u}_{\mathrm{I}}\right), \mathrm{S}_{\ell \mathrm{k}}{ }^{(\mathrm{j})}\left(\mathrm{u}_{\mathrm{A}}{ }_{\mathrm{A}}^{(\mathrm{j})}\right)$, and $\mathrm{S}_{\ell \mathrm{k}}{ }^{(\mathrm{j})}\left(\mathrm{u}_{\mathrm{F}}{ }^{(\mathrm{j})}\right)$ in Eq. (7.4.56) are obtained by evaluating Eq. (7.4.55) at the lethargy values $\mathrm{u}_{\mathrm{I}}, \mathrm{u}_{\mathrm{A}}(\mathrm{j})$, and $\mathrm{u}_{\mathrm{F}}{ }^{(\mathrm{j})}$, respectively. Use of more than two panels for the group integration was found to have an insignificant impact.

The complete epithermal PW-to-LMR source in group g is finally obtained by summing Eq. (7.4.55) over all nuclides and then substituting the spherical harmonic moments into the Legendre expansion of the MG scatter source, resulting in

$$
\mathrm{S}_{\mathrm{PW} \rightarrow \mathrm{g}}=\sum_{\ell \mathrm{k}} \frac{2 \ell+1}{2} \mathrm{Y}_{\ell \mathrm{k}}(\Omega) \sum_{\mathrm{j}} \Delta^{(\mathrm{j}) / 3} \quad\left[\mathrm{~S}_{\ell \mathrm{k}}{ }^{(\mathrm{j})}\left(\mathrm{u}_{\mathrm{I}}\right)+4 \mathrm{~S}_{\ell \mathrm{k}}{ }^{(\mathrm{j})}\left(\mathrm{u}_{\mathrm{A}}^{(\mathrm{j})}\right)+\mathrm{S}_{\ell \mathrm{k}}{ }^{(\mathrm{j})}\left(\mathrm{u}_{\mathrm{F}}^{(\mathrm{j})}\right)\right] \text {. }
$$

\subsection{Thermal scatter sources from LMR and PW range}

If the value of DEMIN is specified to be below the thermal energy boundary, the portion of the PW range between DEMIN and the thermal cutoff, as well as the entire LMR, will be contained in the thermal range. In this case thermal neutrons will downscatter from the thermal PW range to the LMR, and upscatter from the LMR to the thermal PW range.

The latter thermal source (LMR-to-PW) is computed in exactly the same manner as used to compute the UMR-to-PW source $\mathrm{S}_{\mathrm{HI}}$, described in section 7.4.2.6.2. On the other hand, the scatter source from the thermal PW to the LMR is computed with a similar approach as given in the previous section for epithermal PW-to-LMR scatter. In this case Eq. (7.4.57) is used as before, except the source moments are not obtained from the submoment expansion in Eq. (7.4.55), but rather by evaluating the PW thermal scatter expression in Eq. (7.4.54).

In performing the transport calculation for any group $\mathrm{g}$ in the LMR range, the PW-to-MG source component in Eq. (7.4.57) is added to the MG-to-MG scattering into $g$ from all groups in the UMR and LMR ranges, respectively, to obtain the total scatter source.

\subsubsection{Determination of energy mesh for PW flux calculation}

The energy mesh for the PW flux computation is determined for a specific problem as follows: (a) for each zone-composition, microscopic cross-section data are interpolated (if necessary) to the desired zonetemperature, and a union energy mesh is formed from the energy meshes of PW total cross sections of all materials in that zone, plus the MG boundaries; (b) macroscopic total cross sections are computed for the union meshes in each zone; (c) union meshes for each zone are thinned (i.e., some energy points eliminated) in a manner that allows the zone macroscopic cross section to be interpolated linearly, within some input error tolerance; (d) a union mesh is created from the thinned energy meshes for each zone thus producing a "global" energy mesh; (e) the global mesh is checked to insure that it still contains group boundaries and midpoint-energies of the input MG library, and finally, (f) still more points may be added to constrain the maximum interval width between successive lethargy points to be less than some fraction of the maximum lethargy gained by elastic scatter from a fictitious nuclide having a mass of approximately 400 . The fraction used in limiting the maximum size of any lethargy interval can be set by the input value of "FLET," but is defaulted to a value of $1 / 3$.

The mesh thinning procedure is effective in reducing the number of energy points in the PW transport calculation, while preserving essential features of the macroscopic cross-section data that affect the flux spectrum; viz, the mesh is typically fine in energy regions corresponding to important resonances, but coarser where there is little variation in the macroscopic cross-section data. The default thinning tolerance is $0.1 \%$. A less stringent thinning tolerance may give a large reduction in computation time, but also can affect the accuracy. 


\subsubsection{CENTRM cross sections and fixed sources}

\subsection{CENTRM PW cross-section libraries}

SCALE includes CE nuclear data for all materials and all reaction types available in ENDF/B, processed for several different temperatures. The $\mathrm{CE}$ data, spanning the energy range from $10^{-5} \mathrm{eV}$ to $20 \mathrm{MeV}$, are stored in separate files for individual nuclides, which can be used for CENTRM as well as CE Monte Carlo calculations. The CRAWDAD module reads these files and merges the data to form a single CENTRM formatted library containing only the particular materials, cross section types, temperatures, and energy range needed for a given calculation (see section 8.1.5). In general the CENTRM library includes CE data for the unresolved, as well as the resolved, resonance range. Unresolved resonance data typically have rather smooth variations, but in reality the cross sections represent average values for very closely spaced resoances that can not be measureed individually

\subsection{Linearization of MG cross sections and fixed sources}

Shielded group cross sections from the input MG library are always required for the UMR and LMR portions of the CENTRM calculation. Two approaches are available to translate multigroup cross sections into pseudo-PW data at energy points within a group. The first is a "step" approximation in which $\sigma\left(E_{n}\right)=\sigma_{g}$, where $E_{n}$ is any energy point contained in group g. This leads to a histogram representation of $\sigma(\mathrm{E})$ that is discontinuous at the group boundaries. If the multigroup data show significant variation between adjacent groups, then the histogram approach can introduce discontinuities and oscillations into the pointwise flux. An alternative approach is to "linearize" the multigroup cross sections, using a linear representation that preserves the group-average values and is continuous at the group boundaries. Although the resulting cross section is continuous and does not cause distortions in the flux spectrum, the data does not necessarily represent the actual energy variation of the cross section.

Input fixed source terms are treated in a similar manner. The multigroup spectra that are input by the user may be converted either to a discontinuous histogram function in lethargy; or may be linearized by group. In the latter case the resulting groupwise-linear function is evaluated at the energy mesh points to obtain the pointwise source term.

\subsubsection{Available Methods for Solving Transport Equation}

CENTRM offers several calculation options for solving the Boltzmann equation. Some of these are only available for either the MG or PW calculations, respectively. In the case of the MG methods, the calculation procedures are similar to those described in the XSDRNPM documentation. The following sections briefly describe the PW transport approximations available in CENTRM.

\subsubsection{Discrete ordinates}

The discrete ordinates method can be used for both MG and PW solutions. The main difference in the solution is the computation of the scattering sources: the multigroup method uses group-to-group scatter matrices, while the PW method uses the submoment expansion technique described earlier. Also, as previously discussed, the pointwise discrete ordinates equation has the same form as the transport equation for a purely absorbing medium; so that inner iterations are not required to converge the pointwise scattering source. The XSDRNPM documentation shows the finite-difference form of the discrete ordinate equations, and includes a discussion of $\mathrm{S}_{\mathrm{N}}$ quadratures, the weighted-difference model, angular streaming coefficients, treatment of boundary conditions, and other standard procedures used in the CENTRM 1-D discrete ordinates solution. 


\subsubsection{Homogenized infinite medium}

A homogenized infinite medium calculation can be performed for either the MG or the PW energy ranges. This method is essentially a "zero-dimensional" model that has no spatial or angular variation in the flux (only energy dependence). The materials contained in all zones are "smeared" into a single homogenized mixture using volume weighting of the number densities, and the effective external source is defined to be the volume-weighted source density. The resulting homogenized composition is then solved as an infinite medium, so that the PW scalar flux is equal to,

$$
\Phi\left(\mathrm{u}_{\mathrm{n}}\right)=\frac{\int \tilde{\Sigma}\left(u^{\prime} \rightarrow \mathrm{u}_{\mathrm{n}}\right) \Phi\left(u^{\prime}\right) d u^{\prime}+\tilde{Q}_{e x t}(E)}{\tilde{\Sigma}_{t}\left(\mathrm{u}_{\mathrm{n}}\right)}=\frac{\tilde{S}\left(\mathrm{u}_{\mathrm{n}}\right)+\tilde{Q}_{e x t}\left(\mathrm{u}_{\mathrm{n}}\right)}{\tilde{\Sigma}_{t}\left(\mathrm{u}_{\mathrm{n}}\right)}
$$

where $\tilde{\Sigma}_{\mathrm{t}}, \tilde{\mathrm{S}}, \tilde{\mathrm{Q}}_{\mathrm{ext}},=$ homogenized cross section, scatter source, and external source, respectively.

The total cross section in the PW calculation is reduced by the value of the "within-point" cross section, as discussed in Sect. 7.4.2.6. The PW scattering source is computed from a $\mathrm{P}_{0}$ submoment expansion using cumulative integrals, as described in Sect. 7.4.2.4. The scalar flux at all space-intervals and the angular flux in all directions are set to the above value, while higher order flux moments are equal to zero.

\subsubsection{Zonewise infinite medium}

The zonewise infinite medium solution is an option for the both PW and MG calculations. This method is similar to the homogenized infinite medium option, except each zone is treated independently as an infinite medium, so that no spatial homogenization is required; i.e., Eq. (7.4.58) is solved for each individual zone. The zonewise source corresponds to the input external source within the respective zone. NOTE: THERE IS NO COUPLING BETWEEN THE CALCULATIONS FOR EACH ZONE. Hence, any zones that do not contain an input external source will have zero fluxes, since there is no leakage between zones in the zonewise infinite medium model. To avoid this problem the user must either input a volumetric source, or specify a fission source and add a minute amount of fissionable material to generate a fission spectrum source.

\subsubsection{Collision probability method}

A collision probability (CP) solution of the 1-D integral transport equation is available in CENTRM as an alternative to the pointwise discrete ordinates approach. This option is only available for the pointwise solution, and has the following additional restrictions compared to the pointwise $\mathrm{S}_{\mathrm{N}}$ method:

(a) only 1-D cylindrical or slab geometry is treated (no spherical geometry);

(b) periodic boundary conditions have not been implemented;

(c) interior surface sources cannot be treated;

(d) $\mathrm{P}_{0}$ scattering (isotropic laboratory scatter) is assumed; no transport correction is applied to the pointwise cross sections in CENTRM.

(e) due to assumption (d), computation of the angular flux is not required in order to obtain the scalar flux; therefore, only the $\mathrm{P}_{0}$ flux moment (scalar flux) of the angular flux is calculated.

(f) PW thermal calculations are not supported for the CP method.

This method has only had a limited amount of testing, and has not been validated as extensively as the discrete ordinates and MoC options. 
The PW scattering source for the integral transport equation is similar to that for the discrete ordinates method, except that only the $\mathrm{P}_{0}$ source moment is considered, and the "within point" correction is not applied to the total cross section for the collision probability method. Therefore only the spatial and directional treatment is substantially different in the two transport approaches. In the collision probability method the total interaction rate within a space interval is expressed in terms of collision probabilities $\mathrm{P}_{\mathrm{i}^{\prime} \rightarrow \mathrm{i}}$ corresponding to the probability that neutrons born uniformly in volume $\mathrm{V}_{\mathrm{i}^{\prime}}$, at lethargy $\mathrm{u}_{\mathrm{n}}$ will collide in interval $i$ with volume $V_{i}$. The scalar flux in space interval " $i$," at lethargy point $u_{n}$ obeys the integral transport equation,

$$
\Sigma_{\mathrm{i}}\left(\mathrm{u}_{\mathrm{n}}\right) \phi_{\mathrm{i}}\left(\mathrm{u}_{\mathrm{n}}\right) \mathrm{V}_{\mathrm{i}}=\sum_{\mathrm{i}^{\prime}} \mathrm{P}_{\mathrm{i}^{\prime} \rightarrow \mathrm{i}}\left(\mathrm{u}_{\mathrm{n}}\right)\left[\sum_{\mathrm{i}^{\prime} ; \mathrm{n} \rightarrow \mathrm{n}} \Phi_{\mathrm{i}}\left(\mathrm{u}_{\mathrm{n}}\right)+\mathrm{Q}_{\mathrm{eff}, \mathrm{i}^{\prime}}\left(\mathrm{u}_{\mathrm{n}}\right)\right] \mathrm{V}_{\mathrm{i}^{\prime}}
$$

where

$\sum_{\mathrm{i}^{\prime}, \mathrm{n} \rightarrow \mathrm{n}} \quad$ is the within-point cross section at $\mathrm{i}^{\prime}$, defined in Eq. (7.4.40); and

$\mathrm{Q}_{\text {eff }, \mathrm{i}^{\prime}} \quad$ is the external source $\left(\mathrm{Q}_{\mathrm{n}}\right)$ plus the $\mathrm{P}_{0}$ downscatter source in $\mathrm{i}^{\prime}$ and at $\mathrm{u}_{\mathrm{n}}$, which is computed in a similar manner as for the discrete ordinates solution.

The boundary condition at the center of a cylinder is assumed to be reflected; while the outer boundary condition can either be vacuum or albedo. An albedo boundary condition returns a fraction of the outward leakage, using a cosine distribution for the return-current. An albedo of unity corresponds to a "white" boundary condition.

Unlike the integro-differential transport equation used by the discrete ordinates method, the integral equation at space interval $i$, contains the unknown fluxes at all other space intervals $i^{\prime}$. This requires that inner iterations be performed for the PW solution using the $\mathrm{CP}$ option.

Collision Probabilities (CP) in 1-D cylindrical geometry are computed using the method developed by Carlvik. ${ }^{6}$ The CPs for a vacuum outer boundary condition are first computed. In this case the probability that a neutron born in annular interval $i$ with outer radius $R_{i}$, will have its next collision in annular interval $j$ with outer radius $R_{j}$, is computed from the expression:

$$
P_{i \rightarrow j}=\delta_{i j} \sum_{j} V_{j}+2\left(S_{i-1, j-1}-S_{i-1, j}-S_{i, j-1}-S_{i, j}\right)
$$

where $\delta_{\mathrm{ij}}$ represents the Kronecker delta function and

$$
S_{i j}=\int_{0}^{R_{t}}\left[K i_{3}\left(\tau_{i j}{ }^{+}\right)-K i_{3}\left(\tau_{i j}^{-}\right)\right] d y
$$

In the above expression $\mathrm{Ki}_{3}$ corresponds to a 3 rd order Bickley function, and $\tau_{\mathrm{ij}}{ }^{+}$and $\tau_{\mathrm{ij}}{ }^{-}$are,

$$
\tau_{i f}^{ \pm}=\Sigma \times\left(\sqrt{R_{j}^{2}-y^{2}} \pm \sqrt{R_{i}^{2}-y^{2}}\right) .
$$

Integration of the Bickley functions is performed numerically using Gauss-Jacobi quadrature. 
The probability that a neutron born in interval "i" will escape uncollided from a cell with a vacuum outer boundary is equal to one minus the probability that it has a collision in any interval of the cell, so that

$$
P_{e s c, i}=1-\sum_{j} P_{i \rightarrow j} .
$$

The "blackness" $\left(\Gamma_{\mathrm{i}}\right)$ of interval $\mathrm{i}$ is defined to be the probability that neutrons entering the cell with a cosine-current angular distribution on the surface will collide within interval i. The total blackness $(\Gamma)$ is the probability that a neutron entering the outer boundary will collide anywhere in the cell. The blackness is computed from the escape probability:

$$
\Gamma_{i}=\frac{4 V_{i} \Sigma_{i}}{S} \times P_{e s c, i} ; \text { and } \Gamma=\sum_{i} \Gamma_{i}
$$

where $\mathrm{S}$ is the outer surface area of the cell.

The vacuum CPs can be modified to account for the effect of an albedo condition on the outer surface. In these cases a fraction of the neutrons reaching the outer boundary are returned isotropically back into the cell, and "bounce" back and forth between the two boundaries, with each traverse reducing the number of uncollided neutrons. Mathematically this corresponds to a converging geometric series that accounts for the cumulative effect of the "infinite" number of traverses through the cell. Evaluating the geometric sum, the collision probability $\left(\mathrm{P}^{\mathrm{A}}\right)$ for an albedo of $\alpha$ can be expressed as

$$
P_{i \rightarrow j}^{A}=P_{i \rightarrow j}+\frac{S}{4 V_{i} \Sigma_{i}} \times \frac{\alpha \Gamma_{i} \Gamma_{j}}{1-\alpha(1-\Gamma)} .
$$

In the case of a slab with two vacuum boundaries, the CP's are expressed in terms of transmission probabilities $T_{i \rightarrow j}$, corresponding to the probability that a neutron born in volume $i$ will reach surface $j$ without a collision. The transmission probability is equal to,

$$
T_{i \rightarrow j}=\frac{E_{3}\left(\tau_{i j}\right)-E_{3}\left(\tau_{i j}+\tau_{i}\right)}{2 \tau_{i}} .
$$

where:

$\tau_{i j}$ is the optical distance between surface $S_{j}$ and the surface volume $V_{i}$ that is closest to $S_{j}$;

$\tau_{\mathrm{i}} \quad$ is the optical thickness of volume $\mathrm{V}_{\mathrm{i}}$; and

$\mathrm{E}_{3}$ is the third order Exponential Function.

In a slab with vacuum boundaries, the $\mathrm{CP}$ for a neutron born in interval $\mathrm{i}$ to have its next collision in some interval $\mathrm{j}$ bounded by surfaces $\mathrm{S}_{\mathrm{j}}$ and $\mathrm{S}_{\mathrm{j}+1}$, is given by the expression,

$$
P_{i \rightarrow j}=T_{i \rightarrow j}-T_{i \rightarrow j+1} .
$$

A slab with a specular-reflected boundary condition on one side and a vacuum on the other can be represented as an "expanded" geometry with two vacuum boundaries, simply by adding the cell's mirror image at the reflected boundary. Hence, collision probabilities for these cases can also be obtained from the expressions for two vacuum boundaries. For slabs with an albedo (or white) boundary on one side 
and a reflected boundary on the other, the same expression in Eq. (7.4.64) presented earlier for cylinders can also be used to obtain albedo-corrected collision probabilities from the vacuum values. However, unlike cylindrical geometry, a slab may have specular-reflected boundary conditions on both sides, corresponding to an infinite array of repeating cells. In CENTRM the collision probabilities for a doublyreflected slab geometry are obtained by explicitly including two reflected cells in addition to the "primary" cell, and then applying a white boundary condition on the outer surface of the expanded geometry. The resulting modified geometry, consisting of three cells (i.e., the primary plus two reflected cells) with a reflected center and outer albedo boundary, can be treated as described previously.

\subsubsection{5 $\quad P_{1}$ ("Diffusion Theory") method}

The CENTRM MG calculation has an option for a $\mathrm{P}_{1}$ calculation; however, this option is not available for the PW calculation. The method is essentially identical to that used in XSDRNPM. The $\mathrm{P}_{1}$ option is called diffusion theory in both XSDRNPM and CENTRM input descriptions; but since the $\mathrm{P}_{1}$ component of the scatter source is explicitly treated and since Fick's Law is not assumed, the method is actually more closely related to the $\mathrm{P}_{1}$ spherical harmonic solution approach. The $\mathrm{P}_{1}$ method is also used in CENTRM to generate a flux guess for the thermal flux distribution in an $\mathrm{S}_{\mathrm{N}}$ calculation. Several outer iterations over the thermal groups are always performed with $\mathrm{P}_{1}$ theory, prior to beginning the discrete ordinates calculation for the thermal range. In the case of PW thermal calculations, the multigroup thermal flux guess is converted in PW flux values by multiplying by the ratio of MG to PW cross-section values at each lethargy point.

\subsubsection{Two-region (2R) method}

A two-region (2R) calculation similar to the Nordheim method is also available as a PW option in CENTRM. In general the PW $\mathrm{S}_{\mathrm{N}}$ or MoC solutions provide a more rigorous approach to compute selfshielded cross sections than the $2 \mathrm{R}$ method; however the $2 \mathrm{R}$ approximation gives accurate results for a wide range of "conventional cases." Verification studies have shown that the $2 \mathrm{R}$ option produces eigenvalues comparable to those obtained with the $\mathrm{S}_{\mathrm{N}}$ and $\mathrm{MoC}$ options for numerous cases of interest, and the execution time is often significantly faster since only the zone-averaged PW scalar flux is computed. Thus the CENTRM 2R option is an adequate and attractive alternative for many applications.

The CENTRM 2R solution provides several advantages over the original Nordheim method. CENTRM uses pre-processed PW nuclear data, rather than the Nordheim approach of using input resonance parameters for a built-in resonance formula (Breit-Wigner). This allows the CENTRM-2R calculation to utilize PW cross sections processed from Reich-Moore resonance data in ENDF/B, while the conventional Nordheim method is limited to Breit-Wigner resonance formulae that do not treat level-level interference. Other advantages of the CENTRM-2R calculation include a rigorous treatment of resonance overlap from mixed absorbers, ability to address mixtures of arbitrary moderators with energy dependent cross sections, and capability to include inelastic and thermal scattering effects in the flux calculation.

The $2 \mathrm{R}$ approximation, which is a simplified version of the general collision probability method, represents the system by an interior region containing the mixture of materials to be self-shielded, and an exterior moderator region where the asympototic flux per lethargy is approximated by a simple analytical expression (e.g., constant for epithermal energies).

CENTRM performs a separate 2R calculation for each zone. For example, if a problem consists of fuel and moderator zones, then two 2R calculations are done: one has fuel as the interior region and moderator as the exterior, and the other has moderator as interior and fuel as exterior. In the case of lattices, multiple bodies composed of the same mixture are addressed by introducing a Dancoff factor. 
The 2R equation solved by CENTRM for interior region "I" is equal to,

$$
\Sigma_{\mathrm{I}}\left(\mathrm{u}_{\mathrm{n}}\right) \phi_{\mathrm{I}}\left(\mathrm{u}_{\mathrm{n}}\right)=\left(1-\mathrm{P}_{\mathrm{I}}^{(e s c)}\left(\mathrm{u}_{\mathrm{n}}\right)\right)\left(\mathrm{S}_{\mathrm{I}}\left(\mathrm{u}_{\mathrm{n}}\right)+\mathrm{Q}_{\mathrm{I}}^{\text {ext }}\left(\mathrm{u}_{\mathrm{n}}\right)\right)+\Sigma_{\mathrm{I}}\left(\mathrm{u}_{\mathrm{n}}\right) \mathrm{P}_{\mathrm{I}}^{(e s c)}\left(\mathrm{u}_{\mathrm{n}}\right) \varphi_{\text {asy }}\left(\mathrm{u}_{\mathrm{n}}\right)
$$

In the above equation, $\mathrm{S}_{\mathrm{I}}$ and $\mathrm{Q}_{\mathrm{I}}$ are the scatter and fixed sources, respectively, for interior region "I"; and $\mathrm{P}_{\mathrm{I}}^{(e s c)}$ is the probability that a neutron born in the interior region will escape and have its next collision in the external region. For an interior region consisting of multiple bodies of the same composition separated by an exterior region, the escape probability is equal to

$$
\mathrm{P}_{\mathrm{I}}^{(e s c)}\left(\mathrm{u}_{\mathrm{n}}\right)=\frac{\mathrm{P}_{0}^{(e s c)}\left(\mathrm{u}_{\mathrm{n}}\right)\left[1-C_{\mathrm{I}}\right]}{\left[1-C_{\mathrm{I}}\right]+C_{\mathrm{I}} \bar{\ell}_{\mathrm{I}} \Sigma_{\mathrm{I}}\left(\mathrm{u}_{\mathrm{n}}\right) \mathrm{P}_{0}^{(e s c)}\left(\mathrm{u}_{\mathrm{n}}\right)}
$$

where $\mathrm{P}_{0}^{(e s c)}$ is the escape probability from a single, isolated body in the interior region; $\bar{\ell}_{\mathrm{I}}$ is the average chord length of bodies in the interior region; and $C_{I}$ is the Dancoff factor, corresponding to the probability that a neutron escaping one interior body will pass through the exterior region and have its next collision in another body of the interior region. Values for $\mathrm{P}_{0}^{(e s c)}$ are computed internally by the code at each energy mesh point, but Dancoff factors must be provided by input for each zone. In the standard XSProc computational sequence, Dancoff values are automatically computed and provided to CENTRM.

The asymptotic flux $\varphi_{\text {asy }}$ for the exterior region is represented by analytical expressions in the fast, epithermal, and thermal energy ranges, respectively, as summarized in Table 7.4.7. The constants $\mathrm{C}_{1}, \mathrm{C}_{2}$ and $\mathrm{C}_{3}$ are defined to impose continuity at the energy boundaries, and also include the overall normalization condition that the PW asymptotic flux at DEMAX is equal to the MG flux for group MGHI obtained from the UMR calculation.

Table 7.4.7. Asymptotic flux representation

\begin{tabular}{llll}
\hline Upper energy & Description & $\begin{array}{c}\text { Distribution } \\
\text { (per unit energy) }\end{array}$ & $\begin{array}{c}\text { Distribution } \\
\text { (per unit lethargy) }\end{array}$ \\
\hline $20 \mathrm{MeV}$ & Fission spectrum & $\mathrm{C}_{3} \mathrm{E}^{1 / 2} \mathrm{e}^{-\mathrm{E} / \theta}\left(^{*}\right)$ & $\mathrm{C}_{3} \mathrm{E}^{3 / 2} \mathrm{e}^{-\mathrm{E} / \theta}(*)$ \\
$200 \mathrm{keV}$ & Slowing-down & $\mathrm{C}_{2} / \mathrm{E}$ & $\mathrm{C}_{2}$ \\
$5 \mathrm{kT}$ & Maxwellian & $\mathrm{C}_{1} \mathrm{E} \mathrm{e}^{-\mathrm{E} / \mathrm{kT}}$ & $\mathrm{C}_{1} \mathrm{E}^{2} \mathrm{e}^{-\mathrm{E} / \mathrm{kT}}$ \\
\hline
\end{tabular}

(*) $\theta=$ fission spectrum "temperature" $=1 \mathrm{MeV}$.

Equation (7.4.67) is solved similarly to the zonewise infinite medium equation; the only difference being that the interior sources are multiplied by $\left(1-\mathrm{P}_{0}^{(e s c)}\right)$, and the presence of an inhomogeneous source term coming from the exterior region. Like all CENTRM transport options, the PW scattering source is computed using cumulative integrals as given in Eq. (7.4.30). In the $2 \mathrm{R}$ option, only the $\mathrm{P}_{0}$ scatter-source moment is needed.

\subsubsection{Method of characteristics for a 2D unit cell}

The 2D MoC option is available for square-lattice unit cell cases, with both multigroup and pointwisecross sections. The geometry for the $\mathrm{MoC}$ cell calculation consists of a number of concentric 
cylindrical zones contained within a square outer surface with reflected boundary conditions. The MoC calculation is not currently functional for triangular lattices. A multiregion cell may be used in the MoC option, but it must correspond to a similar geometry as a square lattice cell. Because the 2D MoC solution is limited to 2D rectangular lattice cell geometries, the code performs a number of internal checks to verify the input geometry is permitted. The energy mesh generation, scattering source calculation, etc. are performed in the same manner as for the discrete ordinates option, except that only $\mathrm{P}_{0}$ scatter is treated. Unlike the CENTRM discrete ordinates method which solves the 1D transport equation, the MoC option solves the 2D transport equation for planar XY slice through a square-pitch lattice of cylindrical pins. Because the the MoC calculation treats the outer rectangular boundary of the unit cell correctly, it provides a more rigorous calculation than using an equivalent 1D cylindrical Wigner-Seitz cell. The geometry input for the MoC calculation is identical to the input for the 1D cylindrical model--the code internally converts the input value for the equivalent cylindrical radius into the outer rectangular cell dimension (pitch) by preserving the total cell area. Thus the length (L) of a side of the rectangular cell in the MoC calculation is computed form the relation $L=\sqrt{\pi R_{e q}^{2}}$, where $R_{e q}$ is the equivalent outer radius of the Wigner-Seitz cell, given in the CENTRM input array of radial dimensions. Due to symmetry conditions, the MoC calculation is performed for only $1 / 8$ of the rectangular cell (i.e., a 45 degree sector) and for the upward directions.

MoC uses a integrating factor to convert the divergence term in the transport equation into a directional derivative along the direction of neutron transport $(\Omega)$. For a given direction in the angular quadrature set, the spatial domain is spanned by a set of parallel characteristic rays originating at one boundary and terminating at the opposite boundary. The default separation distance between the parallel rays is 0.02 $\mathrm{cm}$. in CENTRM. The angular flux in given direction is computed by integrating the directional derivative along the characteristic direction. A reflected boundary condition is normally applied along the rectangular outer surface of the unit cell. The default directional quadrature, which defines the characterstic directions and is used for integration, consists of 8 azimuthal angles for the 45 degree sector in the $\mathrm{X}$-Y plane and 3 postive polar angles relative to the $\mathrm{Z}$-axis.

The spatial domain of the unit cell is defined by zones of uniform mixtures (e.g., fuel, clad, moderator) in which the cross section at a fixed energy point or group is constant. These uniform composition regions may be further divided into sufficiently small "flat-source" sub-divisons in which the sources (scattering and external) can be approximated as being constant. The $\mathrm{MoC}$ computes the average flux in the flatsource region by summing over all characteristics in the volume.

Reference [7] gives more information about the CENTRM MoC solution method.

\subsubsection{CENTRM Input Data}

The standard mode for executing CENTRM is through the XSProc self-shielding module which automatically defines the CENTRM options, mixing table, and geometry, and also prepares the necessary MG and PW nuclear data files and passes the CENTRM PW flux results to the downstream code PMC [see section describing XSProc]. However CENTRM can also be executed in standalone using the FIDO input provided in this section. When executed through XSProc, the user can set values for most CENTRM input parameters given in this section by using keywords in the CENTRM DATA block [see XSProc section]. Note that if CENTRM is run as a standalone module, the input data files defined in Table 7.4.8 must be provided, and the default values for some input parameters are different. 
Title Card - (72 Characters)

\section{DATA BLOCK 1 : GENERAL PROBLEM DATA}

1\$\$ GENERAL PROBLEM DESCRIPTION (18 entries. Defaults shown in parenthesis)

1. IGE $=$ Problem Geometry:

$0 / 1 / 2 / 3=$ inf. hom. medium $/$ plane/ cylinder/ sphere

2. $\mathrm{IZM}=$ Number of Zones

3. IM = Number of Spatial Intervals

4. $\mathrm{IBL}=$ Left Boundary Condition:

$0 / 1 / 2 / 3=$ vacuum $/$ reflected $/$ periodic/albedo

5. $\mathrm{IBR}=$ Right Boundary Condition:

$0 / 1 / 2 / 3=$ vacuum $/$ reflected $/$ periodic/albedo

6. $\mathrm{MXX}=$ Number of Mixtures

7. $\mathrm{MS}=$ Mixing Table Length

8. ISN $=\mathrm{S}_{\mathrm{N}}$ Quadrature Order

9. ISCT $=$ Order of Elastic Scattering

10. ISRC $=$ Problem Type: $0 / 1 / 2=$ fixed source/input fission source/both $(0)$ [see Note 1]

11. IIM = Inner Iteration Maximum (10)

12. IUP $=$ Upscatter Outer Iterations in Thermal Range (3) [see Note 2]

13. NFST $=$ Multigroup Calculation Option in Upper MG Range [E $>$ DEMAX] (3)

$0 / 1 / 2 / 3 / 4 / 5 / 6=\mathrm{S}_{\mathrm{N}} /$ diffusion $/$ homogenized infinite medium $/$

zonewise infinite medium/(option 5 deprecated)/2D MoC cell

[see Note 3]

14. NTHR $=$ Multigroup Calculation Option in Lower MG Range $[\mathrm{E}<\mathrm{DEMIN}]$ (3)

$0 / 1 / 2 / 3 / 4 / 5 / 6=S_{N} /$ diffusion $/$ homogenized infinite medium $/$

zonewise infinite medium/(option 5 deprecated)/2D MoC cell

[see Note 3]

15. NPXS $=$ Calculation Option in PW Range [DEMIN $<$ E $<$ DEMAX] (4)

0/1/2/3/4/5/6 = none (do NFST multigroup calculation) $/ \mathrm{S}_{\mathrm{N}} /$ collision-probability /homogenized infinite medium /zonewise infinite medium/2-region/ 2D MoC cell method of characteristics (only for square lattice cells) [see Note 3]

16. ISVAR $=$ Multigroup Source \& Cross-Section Linearization Option (3) $0 / 1 / 2 / 3=$ none /linearize MG source /linearize MG XS's /both [see Note 4]

17. mocMesh $=$ Mesh Options for MoC Calculation; npxs $=6$ only:

$0 / 1 / 2=$ coarse/regular/fine mesh/input by zone ( 0 )

18. $\operatorname{mocPol}=$ Number of Polar Angles for MoC Quadrature; npxs $=6$ only:

$2 / 3 / 4=$ allowable values 
19. $\operatorname{mocAzi}=$ Number of Azimuthal Angles for MoC Quadrature; npxs $=6$ only:

$$
1->16=\text { allowable values }
$$

20. kern $=$ Thermal Neutron Scattering Treatment:

$0 / 1=$ all free-gas kernels/use bound S(alpha, beta) data if available (1)

21. ISCTI $=\mathrm{P}_{\mathrm{N}}$ Order of Scattering for PW inelastic $[<=\mathrm{ISCT}]$ (1)

22. NMF6 = PW Inelastic Scatter Option (-1)

$-1 / 0 / 1=$ no inelastic/ discrete level inelastic/ discrete and continuum

2\$\$ EDITING AND OTHER OPTIONS (12 entries. Defaults shown in parenthesis)

1. IPRT $=$ Mixture Cross-Section Print Option: $(-3)$

$-3 /-2 /-1 / \mathrm{N}=$ none / write PW macro cross sections to output file, in tab1 format/ print 1-D MG cross sections /print $\mathrm{P}_{0} \rightarrow \mathrm{P}_{\mathrm{N}} \mathrm{MG}$ scatter matrices

2. ID1 $=$ Flux Print/Punch Options $(-1)$

$-1 / 0 / 1 / 2 /=$ none / print MG flux / also print M.G moments /save PW zone-average flux in ascii file

3. IPBT = Balance Tables Print Option (PW thermal B.T. edit not functional)

$0 / 1=$ none $/$ print balance tables

4. $\mathrm{IQM}=$ Use Volumetric Sources: $0 / \mathrm{N}=$ No $/$ Yes $(0)$

5. IPM = Use Boundary Source: $0 / \mathrm{N}=$ No $/$ Yes $(0)$

6. IPN = Group Diffusion Coefficient Option (2)

$0 / 1 / 2=[$ see XSDRNPM input $]$

7. $\mathrm{IDFM}=$ Use Density Factors $0 / 1=$ No $/$ Yes $(0)$

8. IXPRT $=$ Extra Print Option:

$0 / 1=$ minimum print /regular print $(0)$

9. MLIM = Mass Value Restriction on Order of Scattering (100)

$0 / \mathrm{M}=$ no effect $/$ restrict nuclides with mass $\geq \mathrm{M}$ to have scatter order $\leq$ NLIM

10. $\mathrm{NLIM}=$ Restrictive Scatter Order (0)

3** CONVERGENCE CRITERIA AND OTHER CONSTANTS ( 9 entries. Defaults in parenthesis)

1. $\mathrm{EPS}=$ Upscatter Integral Convergence Criterion $(0.001)$

2. $\mathrm{PTC}=$ Point Convergence Criterion (0.001) [see Note 5]

3. $\mathrm{XNF}=$ Source Normalization Factor (1.0) [see Note 1]

4. $\mathrm{B} 2=$ Material Buckling Value [units of $\mathrm{cm}^{-2}$ ] $(0.0)$

5. DEMIN $=$ Lowest Energy of Pointwise Flux Calculation, in eV (0.001)

6. DEMAX $=$ Highest Energy of Pointwise Flux Calculation, in eV (2.0E4)

7. TOLE $=$ Tolerance Used in Thinning Pointwise Cross Sections (0.001) [see Note 7]

8. MOCRAY $=$ Distance $(\mathrm{cm})$ Between MoC Rays; only for npxs $=6(0.02)$

9. $\mathrm{FLET}=$ Lethargy-Gain Fraction For Determining Energy Mesh (0.1) [see Note 7]

10. ALUMP $=0.0 \rightarrow 1.0$, Criterion for Lumping of Materials by Mass $(0.0)$ [see Notes]

T [TERMINATE DATA BLOCK 1] 


\section{DATA BLOCK 2: MIXING TABLE}

$12 \$$ COMPOSITION NUMBERS $[$ AS IN MG LIBRARY] (MS entries)

13\$\$ MIXTURE NUMBERS (MS entries)

$14 \$$ NUCLIDE IDENTIFIERS [AS IN MG LIBRARY] (MS entries)

[Note on 14\$\$: Negative entry excludes material from PW treatment; i.e., MG data used]

$15 *$ NUCLIDE CONCENTRATIONS (MS entries)

T [TERMINATE DATA BLOCK 2]

\section{DATA BLOCK 3: SOURCE DATA}

30\$\$ SOURCE NO. BY INTERVAL (IM entries, if IQM or IPM >0)

31** VOLUMETRIC MULTIGROUP SOURCE SPECTRA (IQM*IGM entries, if IQM >0)

32** MULTIGROUP BOUNDARY ANGULAR SOURCE SPECTRA (IPM*IGM*MM, if IPM $>0$ )

34** SPACE-DEPENDENT FISSION SOURCE (IM entries, if ISRC >0)

T [ TERMINATE DATA BLOCK 3 ]

DATA BLOCK 4: OTHER INPUT ARRAYS

35** INTERVAL BOUNDARIES (IM+1 entries)

36\$\$ ZONE NUMBER BY INTERVAL (IM entries)

38** DENSITY FACTORS (IM entries, if IDFM $>0$ )

39\$\$ MIXTURE NUMBER BY ZONE (IZM entries)

41** TEMPERATURE [kelvin] BY ZONE (IZM entries: Default $=$ F300.0)

47** RIGHT BOUNDARY ALBEDOS $($ IGM entries if $\mathrm{IBR}=3$ : Default $=\mathrm{F} 1.0)$

48** LEFT BOUNDARY ALBEDOS (IGM entries if $\mathrm{IBL}=3$ : Default $=\mathrm{F} 1.0$ )

49** DANCOFF FACTOR BY ZONE $\quad($ IZM entries: Default $=0.0)$

T [ TERMINATE DATA BLOCK 4 ]

$* * * * * * * \quad$ END OF CENTRM INPUT DATA $* * * * * * *$

\subsubsection{CENTRM data notes}

1. If ISRC $=0$, an external volumetric or boundary source is used, as specified in the $30 \$ \$, 31^{* *}$, and $32 * *$ arrays (these are similar to same arrays in XSDRNPM). This option is only available for 
standalone CENTRM calculations; it is not an option for XSProc execution. If ISRC $=1$, the magnitude of the fission source density (neutrons $/ \mathrm{cm}^{3}$-s) is input in the $34^{* *}$ array, and the source energy spectrum is assumed to be $\chi(\mathrm{E})$ for the interval (if no fissionable material is present in an interval, then $\chi=0$ regardless of value in $34^{* *}$ array). Both external and fission sources may be used if $\mathrm{ISRC}=2$. The source normalization parameter XNF in the $3^{*}$ array applies to the combined sources specified in data block 3 .

2. If the value of DEMIN in the $3^{*}$ array is set lower than the thermal cutoff energy of the AMPX MG library, a PW thermal calculation is performed over the energy range between DEMIN and the thermal cutoff. A MG thermal calculation is performed over the remainder of the thermal range. PW thermal calculations always start with ten outer iterations of MG theory to obtain an initial flux guess. The value "IUP" indicates how many additional outer iterations are to be performed.

3. The solutions in the upper and lower MG ranges, as well as the PW solution, provide several calculational options in addition to the default method. These are described in Sect. 7.4.3.

4. The parameter ISVAR indicates how MG values for sources and cross sections are mapped onto the PW energy mesh. See Sect. 7.4.2.8.

5. "Point Convergence" refers to the worst convergence over all space intervals, between successive iterations. It is applied to (a) inner iterations for $\mathrm{MG} \mathrm{S}_{\mathrm{N}}$ solution; (b) inner iterations of doublyreflected boundary conditions in PW $\mathrm{S}_{\mathrm{N}}$ solution; and (c) outer iterations of MG and PW solution in thermal range.

6. The values for DEMAX and DEMIN determine the energy range of the PW calculation. If the purpose of the CENTRM calculation is to obtain PW fluxes for resonance self-shielding calculations with PMC, then the PW energy range should at least span the resolved resonance ranges of all materials for which self shielding is significant.

7. The energy mesh for the PW flux calculation is based on several factors, as described in Sect. 7.4.2.7.

8. If parameter alump $>0$, epithermal and thermal scattering sources for individual nuclides with similar masses are combined into macroscopic "lumps," based on a fractional mass deviation of "alump." For example, alump $=0.2$ means that materials are combined into one or more lumps such that their masses are within $+/-20 \%$ of the effective mass of the lump. The effective mass of the lump is defined to preserve the macroscopic slowing down power. Mass lumping up to 0.2 will often reduce execution time with little impact on the results.

\subsubsection{CENTRM I/O files}

Table 7.4.8 shows filenames used by CENTRM. Some files are not required for some calculations.

Table 7.4.8. Default File Names

\begin{aligned} \hline File Name & Description \\ \hline ft04f001 & Input MG library (only for standalone execution) \\ ft81f001 & Input PW cross-section library from Crawdad PW \\ lib_cen_kern & Input PW thermal scatter kernels from Crawdad \\ centrm.pw.flux & Output PW flux by zone (only for standalone) \\ _centrm.pw_macro_xs & Output PW macro cross-section (optional) \end{aligned}




\subsubsection{Description of the CENTRM CE cross section file}

The CENTRM CE cross section library is typically created using the CRAWDAD module. CRAWDAD is executed automatically during XSProc cases; but when CENTRM run standalone, the CE library must be pre-generated prior to the CENTRM calculation (e.g., by running CRAWDAD). CRAWDAD reads the SCALE CE data files for individual nuclides, and creates a combined CENTRM library in the binary format shown below.

[17 Header Records Described Below]

\begin{tabular}{|c|c|c|}
\hline $\begin{array}{l}\text { Record } \\
\text { No. }\end{array}$ & Parameters & Description \\
\hline 1 & IDTAPE & Tape Identifier. \\
\hline 1 & NNUC & Number of Nuclides on pointwise file. \\
\hline 1 & MTMP & Maximum Number of Temperatures for any Nuclide on file. \\
\hline 1 & MAXMT & Maximum Number of Reactions for any Nuclide on file. \\
\hline 1 & MAXPT & $\begin{array}{l}\text { Maximum Number of Energy Points for any Nuclide (any MT) } \\
\text { on File. }\end{array}$ \\
\hline 1 & $\operatorname{IX}(10)$ & Unused for Now. \\
\hline 1 & TITLE & Title (Character*72) \\
\hline 2 & NZAPT(NNUC) & ZA's for Each Nuclide. \\
\hline 3 & IDPT(NNUC) & MAT ID's for Each Nuclide. \\
\hline 4 & NTEM(NNUC) & Number of Temperatures for Each Nuclide. \\
\hline 5 & EMAX(NNUC) $)^{(\mathrm{DP})}$ & Highest Energy of Pointwise Data for Each Nuclide. \\
\hline 6 & EMIN(NNUC) ${ }^{(\mathrm{DP})}$ & Lowest Energy of Pointwise Data for Each Nuclide. \\
\hline 7 & EUPUR(NNUC) ${ }^{(\mathrm{DP})}$ & Upper Energy of Unresolved range. \\
\hline 8 & EUPRR(NNUC) ${ }^{(\mathrm{DP})}$ & Upper Energy of Resolved range \\
\hline 9 & ELORR(NNUC) ${ }^{(\mathrm{DP})}$ & Lower Energy of Resolved range. \\
\hline 10 & MPT(NNUC) & $\begin{array}{l}\text { Number of Energy Points for Total Cross Sections of Each } \\
\text { Nuclide. }\end{array}$ \\
\hline 11 & MPS(NNUC) & $\begin{array}{l}\text { Number of Energy Points for Elastic Scatter Cross Sections of } \\
\text { Each Nuclide. }\end{array}$ \\
\hline 12 & NFIRST(NNUC) & Starting Record Number of Data for Each Nuclide. \\
\hline 13 & MPIN(NNUC) & Number of inelastic levels for Each Nuclide. \\
\hline 14 & POTXS(NNUC) & Potential Cross Sections for Each Nuclide. \\
\hline 15 & MAX_INEL(NNUC) & $\begin{array}{l}\text { Maximum Number of Energy Points for any Inelastic Discrete } \\
\text { or Continuum Level. }\end{array}$ \\
\hline 16 & NTHERM(NNUC) & Thermal Scattering Kernel IDs for Each Nuclide ( $0=$ free gas). \\
\hline 17 & Free & Not Used \\
\hline
\end{tabular}


Nuclide Dependent Records (repeat for each nuclide)

[NUCLIDE DIRECTORY : 1 RECORD/NUCLIDE]

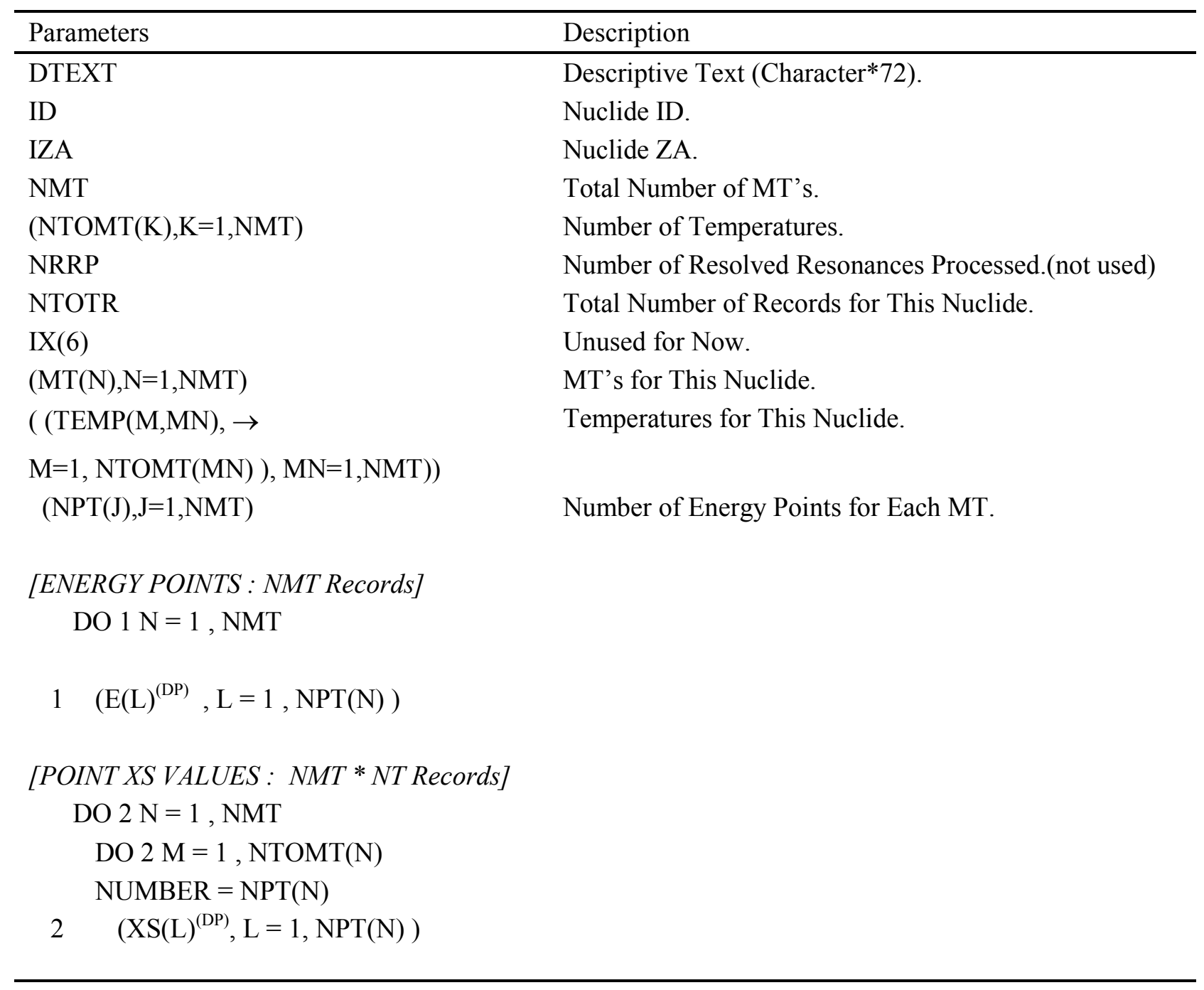

(DP) DOUBLE PRECISION ARRAYS 


\subsubsection{Description of the CENTRM output PW flux file}

[3 Header Records Described Below]

\begin{tabular}{|c|c|c|}
\hline Record No. & Parameters & Description \\
\hline 1 & IGE & Type of Geometry \\
\hline 1 & MMT & Number of Neutron Groups \\
\hline 1 & JT & Number of Flux Moments \\
\hline 1 & IZM & Number of Zones \\
\hline 1 & IZMT & Total Number of Nuclides in all Zones \\
\hline 1 & IM & Number of Space Intervals \\
\hline 1 & IBR & Right Boundary Condition \\
\hline 1 & IBL & Left Boundary Condition \\
\hline 1 & MGHI & Lowest Energy Group in UMR \\
\hline 1 & MGLO & Highest Energy Group in LMR \\
\hline 1 & MT & Number of Materials in Problem \\
\hline 1 & IFTG & First Thermal Group \\
\hline 1 & NTOT & Number of Points in PW Energy Mesh \\
\hline 1 & NTOTP & Number of Points in Full Energy Mesh (UMR+PW+LMR) \\
\hline 1 & MULT & Flag for Double or Single Precision Word Length \\
\hline 1 & IX (10) & Dummy array of 10 integers \\
\hline 1 & DEMAX $^{(\mathrm{DP})}$ & Upper Energy of PW Calculation \\
\hline 1 & DEMIN $^{(\mathrm{DP})}$ & Lowest Energy of PW Calculation \\
\hline 1 & ETHRM $^{(\mathrm{DP})}$ & Energy Corresponding to Thermal Cutoff \\
\hline 1 & $\mathrm{RX}(10)$ & Dummy Array of 10 Single-Precision Real Numbers \\
\hline 2 & NIDS(MT) & Nuclide IDs \\
\hline 2 & $\mathrm{NZA}(\mathrm{MT})$ & Nuclide ZA Numbers \\
\hline 2 & IRCUM(IZM) & Cumulative Number of Nuclides in Each Zone \\
\hline 2 & MBYZ(IZMT) & List of All Nuclides in All Zones \\
\hline 2 & ZTEMP(IZM) & Zone Temperatures \\
\hline 2 & $\mathrm{RD}(\mathrm{IZMT})$ & Number Densities of all Nuclides in All Zones \\
\hline 2 & $\mathrm{R}(\mathrm{IM})$ & Interval Volumes \\
\hline 2 & MA(IM) & Zone Number by Interval \\
\hline 2 & DF(IM) & Density Factor \\
\hline 3 & $\mathrm{DEN}(\mathrm{IGP})^{(\mathrm{DP})}$ & Group Energy Boundaries \\
\hline 3 & $\mathrm{E}(\mathrm{NTOTP})^{(\mathrm{DP})}$ & Energies Corresponding to PW Energy Mesh \\
\hline 3 & $\mathrm{U}(\mathrm{NTOTP})^{(\mathrm{DP})}$ & Lethargies Corresponding to PW Energy Mesh \\
\hline
\end{tabular}

[ IZM Records Containing Zone-Averaged PW Fluxes and Moments]

DO $1 \mathrm{~N}=1, \mathrm{IZM}$

1 PXJ( NTOTP , M), M=1, JT+1)

(DP) DOUBLE PRECISION ARRAYS

\subsubsection{Example Case}

In this section an example standalone CENTRM calculation is demonstrated for a 1-D slab geometry model of a highly enriched uranium solution with an iron-56 reflector. To execute CENTRM in standalone mode, nuclear data libraries must be provided for MG cross sections (file ft04f001), PW cross 
sections (file ft81f001), and PW thermal scatter kernels (lib_cen_kernel). The shell script (=shell) in front of the CENTRM input is used to link the necessary nuclear data libraries to the CENTRM calculation. Here it is assumed that the Crawdad module has been previously run to produce the PW cross section and thermal kernel libraries, which are located in the same directory from which that the job is submitted, while the MG library is the 8 group test library from the SCALE data directory.

\subsubsection{CENTRM input for example case}

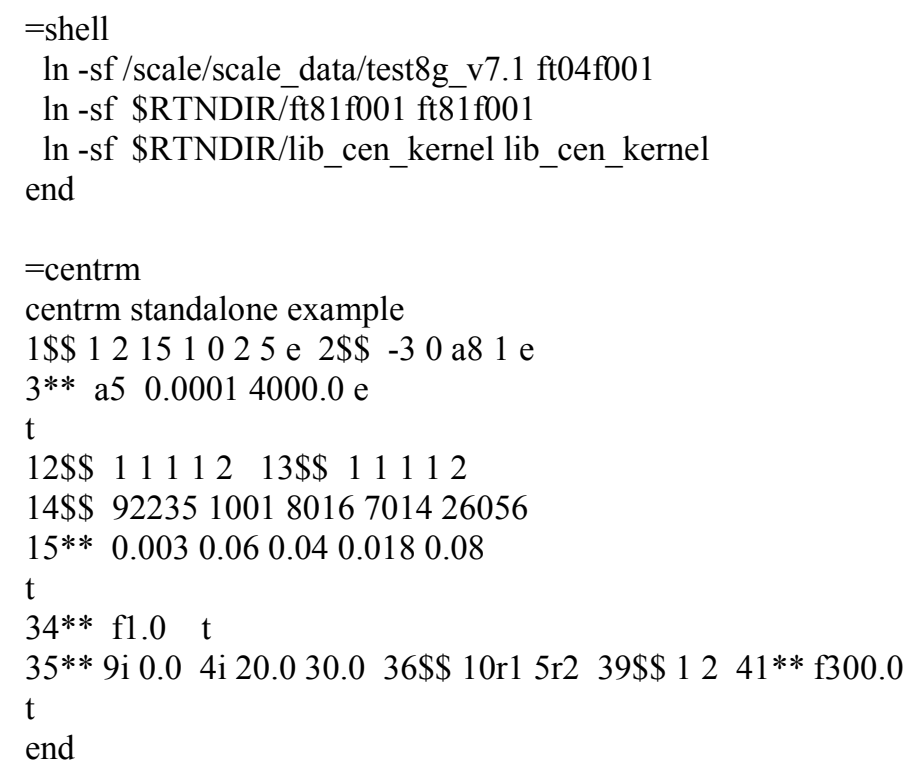

\subsubsection{CENTRM output for example case}

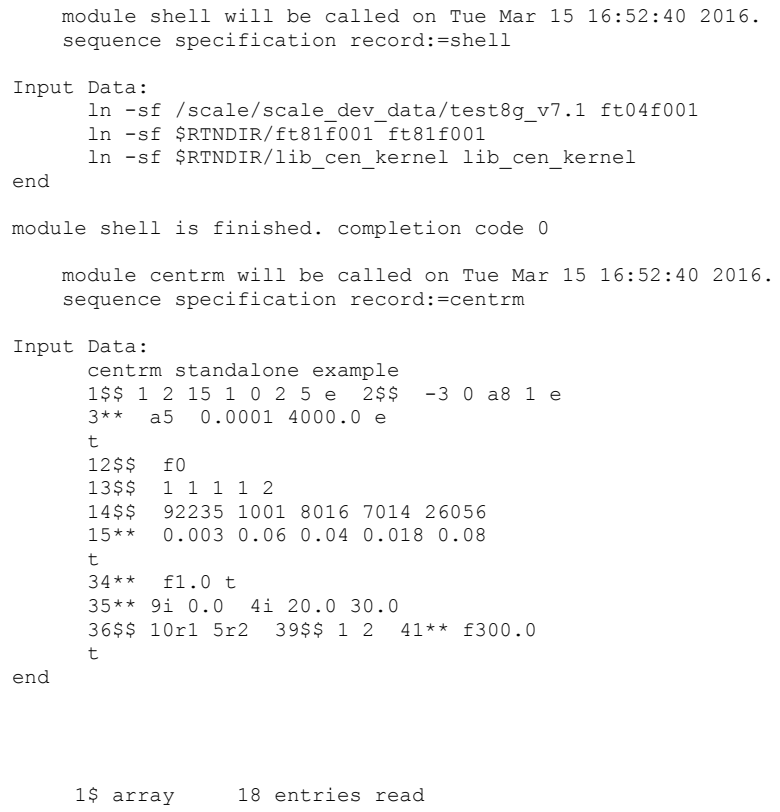




\begin{tabular}{|c|c|c|c|c|}
\hline $2 \$$ & array & 12 & entries & read \\
\hline $3 *$ & array & 9 & entries & read \\
\hline $0 t$ & & & & \\
\hline $12 \$$ & array & 5 & entries & read \\
\hline $13 \$$ & array & 5 & entries & read \\
\hline $14 \$$ & array & 5 & entries & read \\
\hline $15 *$ & array & 5 & entries & read \\
\hline $0 t$ & & & & \\
\hline 34 * & array & 15 & entries & read \\
\hline $0 t$ & & & & \\
\hline $35 *$ & array & 16 & entries & read \\
\hline $36 \$$ & array & 15 & entries & read \\
\hline $39 \$$ & array & 2 & entries & read \\
\hline 41 * & array & 2 & entries & read \\
\hline
\end{tabular}

CENTRM MATERIALS

\begin{tabular}{ccc}
\multicolumn{3}{c}{ nuclides on } \\
& multi-grp lib. & composition \\
1 & 92235 & 0 \\
2 & 1001 & 0 \\
3 & 8016 & 0 \\
4 & 7014 & 0 \\
5 & 26056 & 0
\end{tabular}

elapsed time .00 min.

=time after return from setup_centrm.

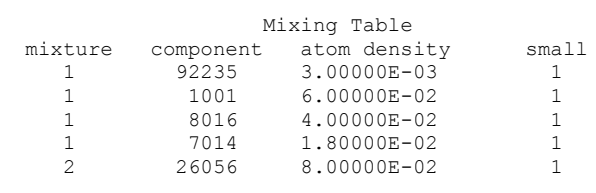

ft81f001 = pointwise XS library from Crawdad

MULTIGROUP STRUCTURE

$\begin{array}{llll}\text { igm } & = & 8 & \text { number of energy groups } \\ \text { mmt } & = & 8 & \text { number of neutron groups } \\ \text { mcr } & = & 0 & \text { number of gamma groups } \\ \text { iftg } & = & 5 & \text { first thermal group number }\end{array}$

GENERAL PROBLEM DATA

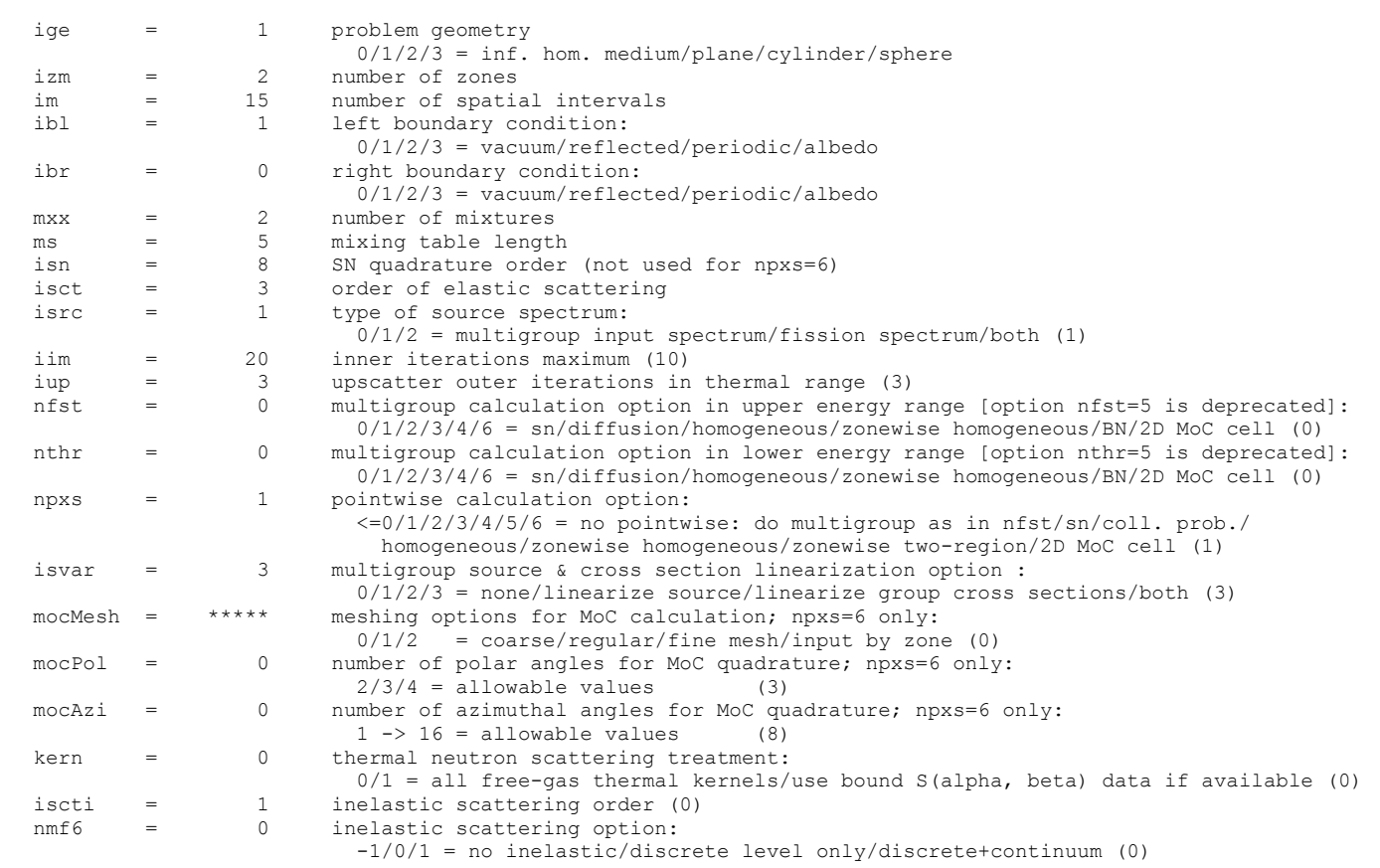

EDITTING AND OTHER OPTIONS 


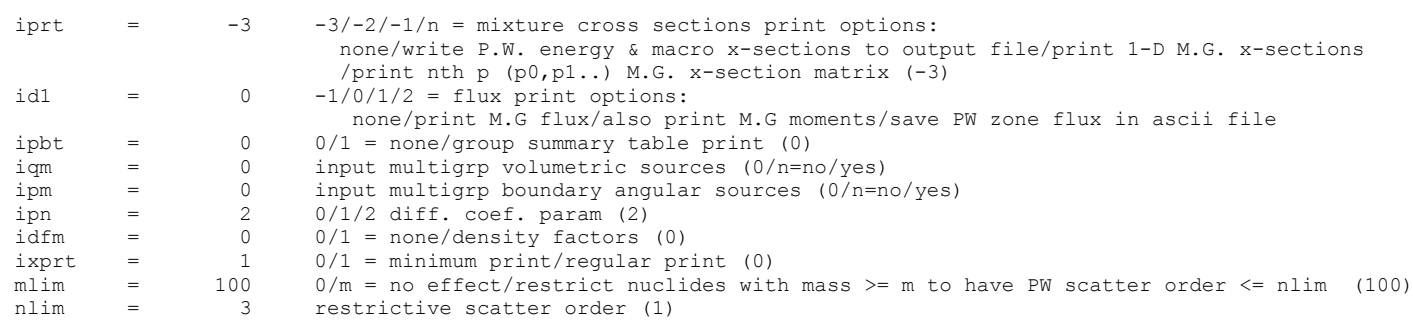

FLOATING POINT VALUES

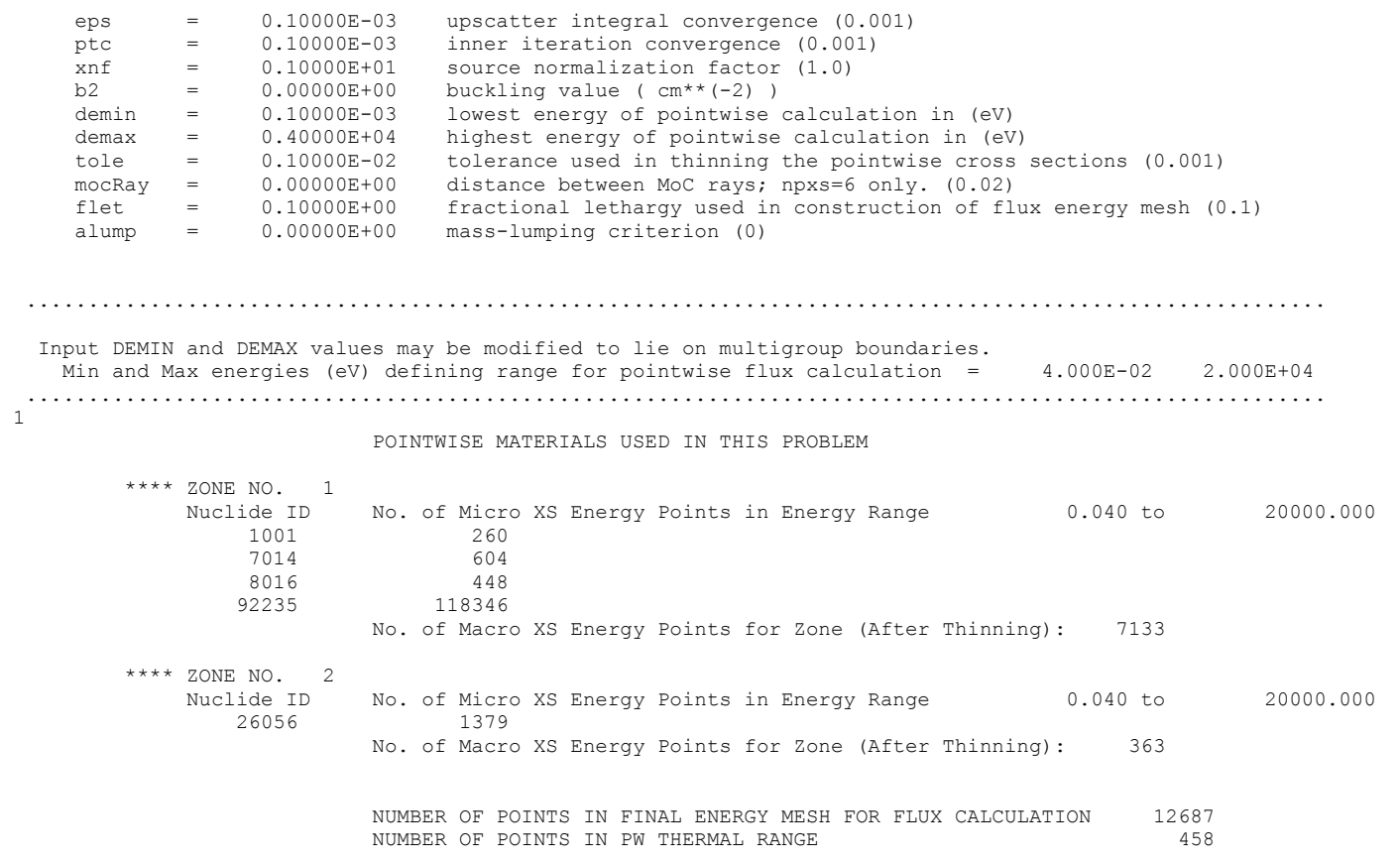

No unit supplied for PW kinematics data.

All PW thermal scatter kernels are treated as Free Gas

NEUTRON MULTIGROUP PARAMETERS

calc
type
0
0
1
1
1
1
1
0

right left

$1.00000 \mathrm{E}-05 \quad 2.76310 \mathrm{E}+01$

DESCRIPTION OF ZONES

$\begin{array}{cccc} & \begin{array}{c}\text { mixture } \\ \text { by zone }\end{array} & \begin{array}{c}\text { temperature } \\ \text { by zone }\end{array} & \begin{array}{c}\text { Dancoff factr } \\ \text { by zone }\end{array} \\ 1 & 1 & 3.00000 \mathrm{E}+02 & 0 \\ 2 & 2 & 3.00000 \mathrm{E}+02 & 0\end{array}$

SN QUADRATURE CONSTANTS

$\begin{array}{lccccc} & \text { weights } & \text { directions } & \text { refl direc } & \text { wt } x \cos \\ 1 & 0 & -1.00000 \mathrm{E}+00 & 9 & 0 \\ 2 & 8.69637 \mathrm{E}-02 & -9.30568 \mathrm{E}-01 & 9 & -8.09257 \mathrm{E}-02 \\ 3 & 1.63036 \mathrm{E}-01 & -6.69991 \mathrm{E}-01 & 8 & -1.09233 \mathrm{E}-01 \\ 4 & 1.63036 \mathrm{E}-01 & -3.30009 \mathrm{E}-01 & 7 & -5.38035 \mathrm{E}-02 \\ 5 & 8.69637 \mathrm{E}-02 & -6.94318 \mathrm{E}-02 & 6 & -6.03805 \mathrm{E}-03 \\ 6 & 8.69637 \mathrm{E}-02 & 6.94318 \mathrm{E}-02 & 5 & 6.03805 \mathrm{E}-03 \\ 7 & 1.63036 \mathrm{E}-01 & 3.30009 \mathrm{E}-01 & 4 & 5.38035 \mathrm{E}-02\end{array}$




$\begin{array}{rrrr}8 & 1.63036 \mathrm{E}-01 & 6.69991 \mathrm{E}-01 & 3 \\ 9 & 8.69637 \mathrm{E}-02 & 9.30568 \mathrm{E}-01 & 2 \\ \text { CONSTANTS FOR P( } 3) & \text { SCATTERING IN } & \text { SN CALCULATION } \\ \text { angl } & \text { set } 1 & \text { set 2 } & \text { set } 3 \\ 1 & -1.000 \mathrm{E}+00 & 1.000 \mathrm{E}+00 & -1.000 \mathrm{E}+00 \\ 2 & -9.306 \mathrm{E}-01 & 7.989 \mathrm{E}-01 & -6.187 \mathrm{E}-01 \\ 3 & -6.700 \mathrm{E}-01 & 1.733 \mathrm{E}-01 & 2.531 \mathrm{E}-01 \\ 4 & -3.300 \mathrm{E}-01 & -3.366 \mathrm{E}-01 & 4.052 \mathrm{E}-01 \\ 5 & -6.943 \mathrm{E}-02 & -4.928 \mathrm{E}-01 & 1.033 \mathrm{E}-01 \\ 6 & 6.943 \mathrm{E}-02 & -4.928 \mathrm{E}-01 & -1.033 \mathrm{E}-01 \\ 7 & 3.300 \mathrm{E}-01 & -3.366 \mathrm{E}-01 & -4.052 \mathrm{E}-01 \\ 8 & 6.700 \mathrm{E}-01 & 1.733 \mathrm{E}-01 & -2.531 \mathrm{E}-01 \\ 9 & 9.306 \mathrm{E}-01 & 7.989 \mathrm{E}-01 & 6.187 \mathrm{E}-01\end{array}$

elapsed time .02 min.

time at after return from drtran... calling calc.

\begin{tabular}{rrccccc}
\multicolumn{7}{c}{ GEOMETRY DESCRIPTION } \\
1 int & radii & mid pts & zone no. & areas & volumes \\
1 & 0 & $1.00000 \mathrm{E}+00$ & 1 & $1.00000 \mathrm{E}+00$ & $2.00000 \mathrm{E}+00$ \\
2 & $2.00000 \mathrm{E}+00$ & $3.00000 \mathrm{E}+00$ & 1 & $1.00000 \mathrm{E}+00$ & $2.00000 \mathrm{E}+00$ \\
3 & $4.00000 \mathrm{E}+00$ & $5.00000 \mathrm{E}+00$ & 1 & $1.00000 \mathrm{E}+00$ & $2.00000 \mathrm{E}+00$ \\
4 & $6.00000 \mathrm{E}+00$ & $7.00000 \mathrm{E}+00$ & 1 & $1.00000 \mathrm{E}+00$ & $2.00000 \mathrm{E}+00$ \\
5 & $8.00000 \mathrm{E}+00$ & $9.00000 \mathrm{E}+00$ & 1 & $1.00000 \mathrm{E}+00$ & $2.00000 \mathrm{E}+00$ \\
6 & $1.00000 \mathrm{E}+01$ & $1.10000 \mathrm{E}+01$ & 1 & $1.00000 \mathrm{E}+00$ & $2.00000 \mathrm{E}+00$ \\
7 & $1.20000 \mathrm{E}+01$ & $1.30000 \mathrm{E}+01$ & 1 & $1.00000 \mathrm{E}+00$ & $2.00000 \mathrm{E}+00$ \\
8 & $1.40000 \mathrm{E}+01$ & $1.50000 \mathrm{E}+01$ & 1 & $1.00000 \mathrm{E}+00$ & $2.00000 \mathrm{E}+00$ \\
9 & $1.60000 \mathrm{E}+01$ & $1.70000 \mathrm{E}+01$ & 1 & $1.00000 \mathrm{E}+00$ & $2.00000 \mathrm{E}+00$ \\
10 & $1.80000 \mathrm{E}+01$ & $1.90000 \mathrm{E}+01$ & 1 & $1.00000 \mathrm{E}+00$ & $2.00000 \mathrm{E}+00$ \\
11 & $2.00000 \mathrm{E}+01$ & $2.10000 \mathrm{E}+01$ & 2 & $1.00000 \mathrm{E}+00$ & $2.00000 \mathrm{E}+00$ \\
12 & $2.20000 \mathrm{E}+01$ & $2.30000 \mathrm{E}+01$ & 2 & $1.00000 \mathrm{E}+00$ & $2.00000 \mathrm{E}+00$ \\
13 & $2.40000 \mathrm{E}+01$ & $2.50000 \mathrm{E}+01$ & 2 & $1.00000 \mathrm{E}+00$ & $2.00000 \mathrm{E}+00$ \\
14 & $2.60000 \mathrm{E}+01$ & $2.70000 \mathrm{E}+01$ & 2 & $1.00000 \mathrm{E}+00$ & $2.00000 \mathrm{E}+00$ \\
15 & $2.80000 \mathrm{E}+01$ & $2.90000 \mathrm{E}+01$ & 2 & $1.00000 \mathrm{E}+00$ & $2.00000 \mathrm{E}+00$ \\
16 & $3.00000 \mathrm{E}+01$ & & & $1.00000 \mathrm{E}+00$ &
\end{tabular}

dens fact

EXTERNAL AND FISSION SOURCE DATA

\begin{tabular}{|c|c|c|c|}
\hline int & radii & fixd src spec & normalized fiss \\
\hline 1 & 0 & $1.00000 \mathrm{E}+00$ & $5.00000 \mathrm{E}-02$ \\
\hline 2 & $2.00000 \mathrm{E}+00$ & $3.00000 \mathrm{E}+00$ & $5.00000 \mathrm{E}-02$ \\
\hline 3 & $4.00000 \mathrm{E}+00$ & $5.00000 \mathrm{E}+00$ & $5.00000 \mathrm{E}-02$ \\
\hline 4 & $6.00000 \mathrm{E}+00$ & $7.00000 \mathrm{E}+00$ & $5.00000 \mathrm{E}-02$ \\
\hline 5 & $8.00000 \mathrm{E}+00$ & $9.00000 \mathrm{E}+00$ & $5.00000 \mathrm{E}-02$ \\
\hline 6 & $1.00000 \mathrm{E}+01$ & $1.10000 \mathrm{E}+01$ & $5.00000 \mathrm{E}-02$ \\
\hline 7 & $1.20000 \mathrm{E}+01$ & $1.30000 \mathrm{E}+01$ & $5.00000 \mathrm{E}-02$ \\
\hline 8 & $1.40000 \mathrm{E}+01$ & $1.50000 \mathrm{E}+01$ & $5.00000 \mathrm{E}-02$ \\
\hline 9 & $1.60000 \mathrm{E}+01$ & $1.70000 \mathrm{E}+01$ & $5.00000 \mathrm{E}-02$ \\
\hline 10 & $1.80000 \mathrm{E}+01$ & $1.90000 \mathrm{E}+01$ & $5.00000 \mathrm{E}-02$ \\
\hline 11 & $2.00000 \mathrm{E}+01$ & $2.10000 \mathrm{E}+01$ & 0 \\
\hline 12 & $2.20000 \mathrm{E}+01$ & $2.30000 \mathrm{E}+01$ & 0 \\
\hline 13 & $2.40000 \mathrm{E}+01$ & $2.50000 \mathrm{E}+01$ & 0 \\
\hline 14 & $2.60000 \mathrm{E}+01$ & $2.70000 \mathrm{E}+01$ & 0 \\
\hline 15 & $2.80000 \mathrm{E}+01$ & $2.90000 \mathrm{E}+01$ & 0 \\
\hline 16 & $3.00000 \mathrm{E}+01$ & & \\
\hline
\end{tabular}

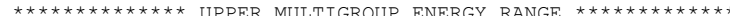
multi \# inner mfd spatial ms max. scale group iterations int. convergence int. factor

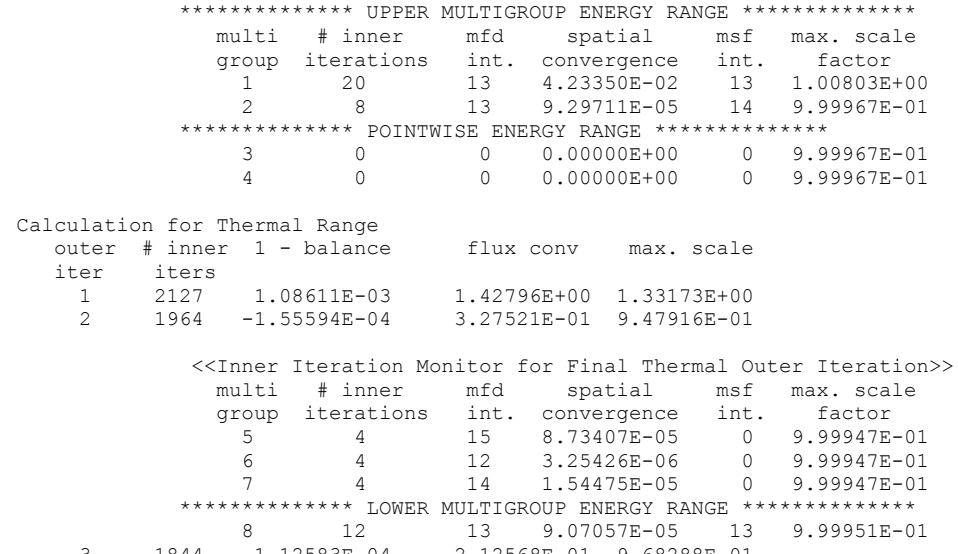

Calculation for Thermal Range $\begin{array}{ccccc}\begin{array}{l}\text { outer } \\ \text { iter }\end{array} & \begin{array}{c}\text { inners } \\ \text { iters }\end{array} & 1 \text { - balance } & \text { flux conv } & \text { max. Scale } \\ 1 & 2127 & 1.08611 \mathrm{E}-03 & 1.42796 \mathrm{E}+00 & 1.33173 \mathrm{E}+00 \\ 2 & 1964 & -1.55594 \mathrm{E}-04 & 3.27521 \mathrm{E}-01 & 9.47916 \mathrm{E}-01\end{array}$

8.09257E-02

$3 \quad 1844 \quad-1.12583 \mathrm{E}-04 \quad 2.12568 \mathrm{E}-01 \quad 9.68288 \mathrm{E}-01$

***Thermal Outer Iteration Limit Reached!

***Worst Inner Convergence For Epithermal Groups Was 4.23350E-02

$* * *$ Worst Inner Convergence For Thermal Flux Was $9.96724 \mathrm{E}-05$

$\star \star \star$ Outer Convergence of Thermal Flux (MG/PW) Was $2.12568 \mathrm{E}-012.38437 \mathrm{E}-01$ 


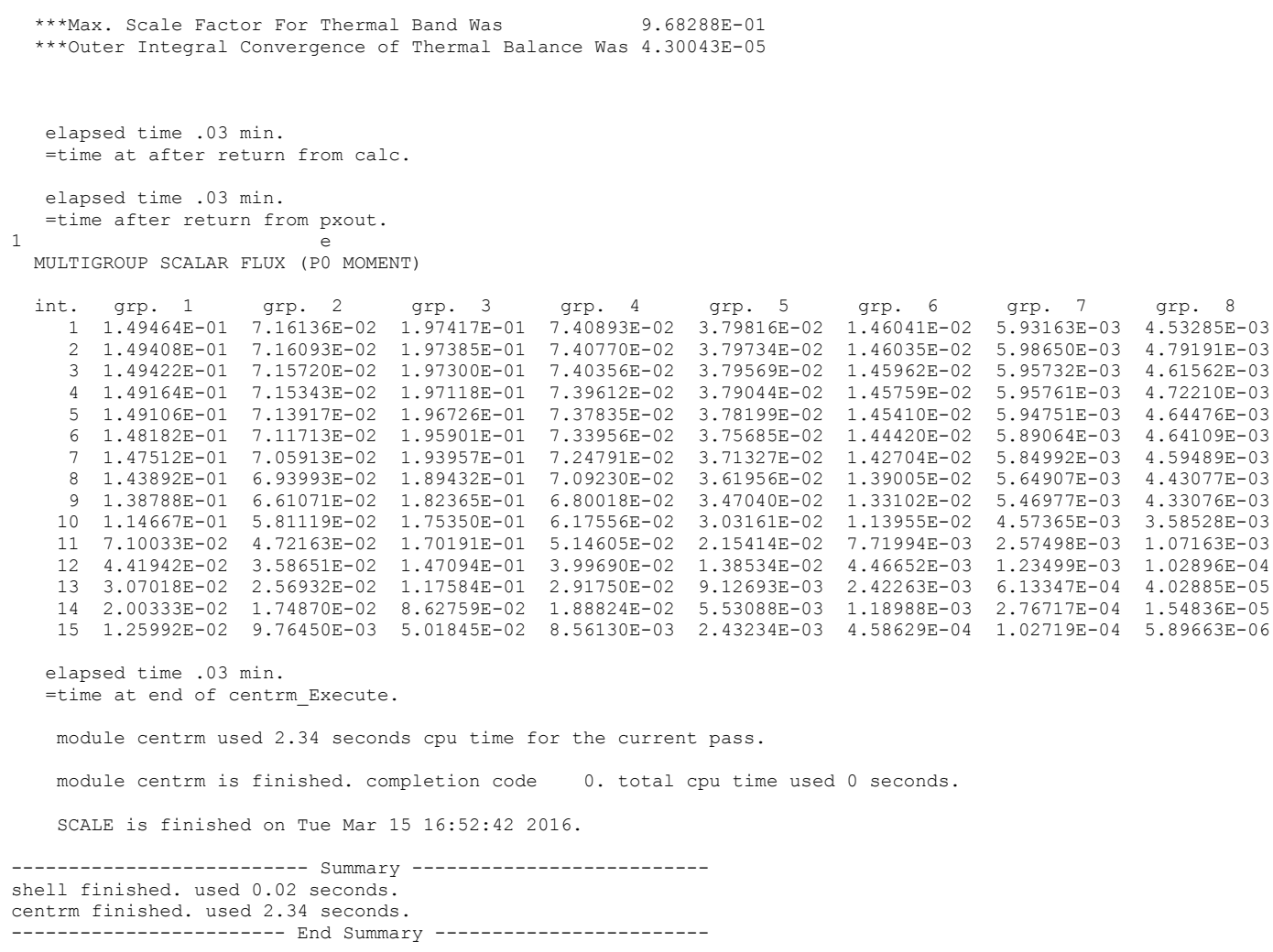

\subsubsection{CENTRM PW library and flux file formats}

Section 7.4.6.1 below describes the format for the input CENTRM PW data library (binary). The subsequent Sect. 7.4.6.2, describes the format of the output PW flux file (binary) produced by CENTRM, which is input to the PMC code.

\subsubsection{Description of the CENTRM CE cross section file}

The CENTRM CE cross section library is typically created using the CRAWDAD module in SCALE. CRAWDAD reads the SCALE CE data files for individual nuclides, and creates the combined CENTRM library. 


\begin{tabular}{|c|c|c|}
\hline $\begin{array}{l}\text { Record } \\
\text { No. }\end{array}$ & Parameters & Description \\
\hline 1 & IDTAPE & Tape Identifier. \\
\hline 1 & NNUC & Number of Nuclides on PW file. \\
\hline 1 & MTMP & Maximum Number of Temperatures for any Nuclide on file. \\
\hline 1 & MAXMT & Maximum Number of Reactions for any Nuclide on file. \\
\hline 1 & MAXPT & $\begin{array}{l}\text { Maximum Number of Energy Points for any Nuclide (any MT) } \\
\text { on File. }\end{array}$ \\
\hline 1 & $\operatorname{IX}(10)$ & Unused for Now. \\
\hline 1 & TITLE & Title (Character*72) \\
\hline 2 & NZAPT(NNUC) & ZA’s for Each Nuclide. \\
\hline 3 & IDPT(NNUC) & MAT ID's for Each Nuclide. \\
\hline 4 & NTEM(NNUC) & Number of Temperatures for Each Nuclide. \\
\hline 5 & EMAX(NNUC) $)^{(\mathrm{DP})}$ & Highest Energy of Pointwise Data for Each Nuclide. \\
\hline 6 & EMIN(NNUC) ${ }^{(\mathrm{DP})}$ & Lowest Energy of Pointwise Data for Each Nuclide. \\
\hline 7 & EUPUR(NNUC) ${ }^{(\mathrm{DP})}$ & Upper Energy of Unresolved range. \\
\hline 8 & EUPRR(NNUC) ${ }^{(\mathrm{DP})}$ & Upper Energy of Resolved range \\
\hline 9 & ELORR(NNUC) ${ }^{(\mathrm{DP})}$ & Lower Energy of Resolved range. \\
\hline 10 & MPT(NNUC) & $\begin{array}{l}\text { Number of Energy Points for Total Cross Sections of Each } \\
\text { Nuclide. }\end{array}$ \\
\hline 11 & MPS(NNUC) & $\begin{array}{l}\text { Number of Energy Points for Elastic Scatter Cross Sections of } \\
\text { Each Nuclide. }\end{array}$ \\
\hline 12 & NFIRST(NNUC) & Starting Record Number of Data for Each Nuclide. \\
\hline 13 & MPIN(NNUC) & Number of inelastic levels for Each Nuclide. \\
\hline 14 & POTXS(NNUC) & Potential Cross Sections for Each Nuclide. \\
\hline 15 & MAX_INEL(NNUC) & $\begin{array}{l}\text { Maximum Number of Energy Points for any Inelastic Discrete } \\
\text { or Continuum Level. }\end{array}$ \\
\hline 16 & NTHERM(NNUC) & Thermal Scattering Kernel IDs for Each Nuclide ( $0=$ free gas). \\
\hline 17 & Free & Not Used \\
\hline
\end{tabular}


Nuclide Dependent Records (repeat for each nuclide)

[NUCLIDE DIRECTORY : 1 RECORD/NUCLIDE]

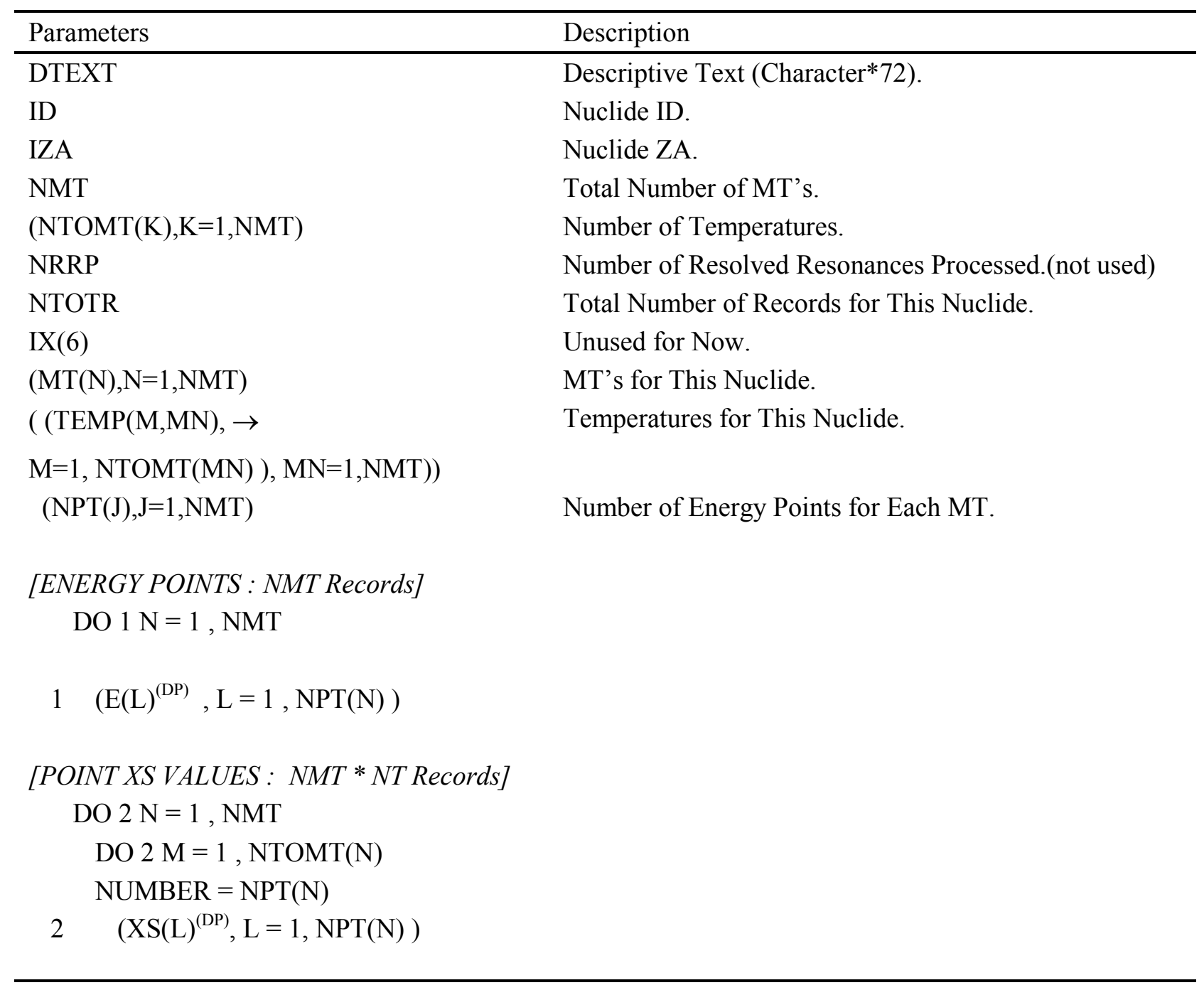

(DP) DOUBLE PRECISION ARRAYS 


\subsubsection{Description of the CENTRM output PW flux file}

[3 Header Records Described Below]

\begin{tabular}{|c|c|c|}
\hline Record No. & Parameters & Description \\
\hline 1 & IGE & Type of Geometry \\
\hline 1 & MMT & Number of Neutron Groups \\
\hline 1 & JT & Number of Flux Moments \\
\hline 1 & IZM & Number of Zones \\
\hline 1 & IZMT & Total Number of Nuclides in all Zones \\
\hline 1 & IM & Number of Space Intervals \\
\hline 1 & IBR & Right Boundary Condition \\
\hline 1 & IBL & Left Boundary Condition \\
\hline 1 & MGHI & Lowest Energy Group in UMR \\
\hline 1 & MGLO & Highest Energy Group in LMR \\
\hline 1 & MT & Number of Materials in Problem \\
\hline 1 & IFTG & First Thermal Group \\
\hline 1 & NTOT & Number of Points in PW Energy Mesh \\
\hline 1 & NTOTP & Number of Points in Full Energy Mesh (UMR+PW+LMR) \\
\hline 1 & MULT & Flag for Double or Single Precision Word Length \\
\hline 1 & IX (10) & Dummy array of 10 integers \\
\hline 1 & DEMAX $^{(\mathrm{DP})}$ & Upper Energy of PW Calculation \\
\hline 1 & $\operatorname{DEMIN}^{(\mathrm{DP})}$ & Lowest Energy of PW Calculation \\
\hline 1 & ETHRM $^{(\mathrm{DP})}$ & Energy Corresponding to Thermal Cutoff \\
\hline 1 & $\mathrm{RX}(10)$ & Dummy Array of 10 Single-Precision Real Numbers \\
\hline 2 & NIDS(MT) & Nuclide IDs \\
\hline 2 & $\mathrm{NZA}(\mathrm{MT})$ & Nuclide ZA Numbers \\
\hline 2 & IRCUM(IZM) & Cumulative Number of Nuclides in Each Zone \\
\hline 2 & MBYZ(IZMT) & List of All Nuclides in All Zones \\
\hline 2 & ZTEMP(IZM) & Zone Temperatures \\
\hline 2 & $\mathrm{RD}(\mathrm{IZMT})$ & Number Densities of all Nuclides in All Zones \\
\hline 2 & $\mathrm{R}(\mathrm{IM})$ & Interval Volumes \\
\hline 2 & MA(IM) & Zone Number by Interval \\
\hline 2 & $\mathrm{DF}(\mathrm{IM})$ & Density Factor \\
\hline 3 & $\mathrm{DEN}(\mathrm{IGP})^{(\mathrm{DP})}$ & Group Energy Boundaries \\
\hline 3 & $\mathrm{E}(\mathrm{NTOTP})^{(\mathrm{DP})}$ & Energies Corresponding to PW Energy Mesh \\
\hline 3 & U(NTOTP) $)^{(\mathrm{DP})}$ & Lethargies Corresponding to PW Energy Mesh \\
\hline
\end{tabular}

[ IZM Records Containing Zone-Averaged P.W. Fluxes and Moments, Described Below]

DO $1 \mathrm{~N}=1, \mathrm{IZM}$

1 PXJ( NTOTP , M), M=1, JT+1)

(DP) DOUBLE PRECISION ARRAYS 


\subsubsection{CENTRM Error Messages}

CENTRM prints several of the same error messages as the XSDRNPM code. Users should refer to the XSDRNPM chapter for these. CENTRM also prints the additional error messages shown below.

\begin{tabular}{|c|c|}
\hline STATEMENT IN PRINTOUT & USER ACTION \\
\hline $\begin{array}{l}\text { ERROR. DEMIN exceeds maximum energy in working library } \\
\text { STOP } 400\end{array}$ & Reduce input value of DEMIN \\
\hline $\begin{array}{l}\text { ERROR. DEMAX exceeds DEMIN } \\
\text { STOP } 401\end{array}$ & $\begin{array}{l}\text { Check input values for PW energy } \\
\text { limits }\end{array}$ \\
\hline $\begin{array}{l}* * * \text { CALCULATION TERMINATED. } \\
\text { No neutron groups in working library }\end{array}$ & Check AMPX working library \\
\hline $\begin{array}{l}\text { WARNING: Thermal Source is } 0 \text { in ACCEL -OR- } \\
\text { WARNING: Thermal Absorption + Leakage is } 0 \text { in ACCEL }\end{array}$ & $\begin{array}{l}\text { Check input source and thermal } \\
\text { cross-section data (often caused by } \\
\text { using Zonewise Infinite Medium } \\
\text { option with no source in some } \\
\text { zone) }\end{array}$ \\
\hline \multirow[t]{2}{*}{ Requests nuclide $X X X$ which is not on your working library. } & $\begin{array}{l}\text { Check nuclide ID's and } \\
\text { composition numbers in mixing } \\
\text { table to make sure they are on MG } \\
\text { library; Verify that PW and MG }\end{array}$ \\
\hline & libraries are consistent \\
\hline
\end{tabular}




\subsubsection{References}

1. G. I. Bell and S. Glasstone, Nuclear Reactor Theory, Van Nostrand, Princeton, NJ, 1970.

2. M. L. Williams, "Submoment Expansion of Neutron-Scattering Sources," Nucl. Sci. Eng. 134, 218-226 (2000).

3. G. S. Robinson, "Notes on the AAEC Version of XLACS-II," Australian Atomic Energy Commission, 1981.

4. H. C. Honeck and D. R. Finch, "FLANGE-II, A Code to Process Thermal Neutron Data from an ENDF/B Tape,” DP-1278 (ENDF-152), Du Pont De Nemours Co., Savannah River Laboratory, 1971.

5. M. L. Williams and M. Asgari, "Computation of Continuous-Energy Neutron Spectra with Discrete Ordinates Transport Theory,” Nucl. Sci. Eng. 121, 173-201 (1995).

6. I. Carlvik, "A Method for Calculating Collision Probabilities in General Cylindrical Geometry and Applications to Flux Distributions and Dancoff Factors," p. 681, in Proc. Int. Conf. on Peaceful Use of Atomic Energy, 1964.

7. K. S. Kim, M. L. Williams, "The Method Of Characteristics For 2-D Multigroup And Pointwise Transport Calculations In Centrm," PHYSOR 2012 - Advances in Reactor Physics - Linking Research, Industry, and Education Knoxville, Tennessee, USA, April 15-20, 2012. 


\title{
7.5 PMC: A PROGRAM TO PRODUCE MULTIGROUP CROSS SECTIONS USING POINTWISE ENERGY SPECTRA FROM CENTRM
}

M. L. Williams, D. F. Hollenbach, U. Merteryuk

\begin{abstract}
PMC generates problem-dependent multigroup cross sections from an existing multigroup cross-section library, a pointwise nuclear data library, and a pointwise neutron flux file produced by the CENTRM continuous-energy transport code. In the SCALE sequences, PMC is a computational module called from XSProc to produce self-shielded multigroup (MG) cross-sections over a specified energy range (e.g., resolved resonance range) of individual nuclides in the system of interest. The self-shielded cross sections are obtained by integrating the pointwise (PW) nuclear data using the CENTRM problemspecific, continuous-energy flux as a weight function for each spatial mixture in the system. Several options are available in PMC to specify various types of weighting methods for the one-dimensional and two-dimensional MG data. PMC outputs problem-dependent self-shielded cross sections that can be used in XSDRNPM, KENO, NEWT or other MG transport codes.
\end{abstract}




\section{TABLE OF CONTENTS}

$\underline{\text { Page }}$

\subsection{PMC: A PROGRAM TO PRODUCE MULTIGROUP CROSS SECTIONS USING}

POINTWISE ENERGY SPECTRA FROM CENTRM ..................................................... 7-283

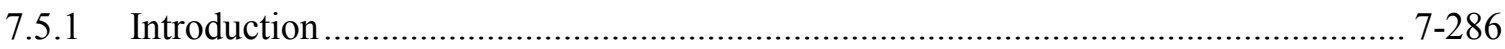

7.5.1.1 Description of PMC input nuclear data ............................................... 7-286

7.5.1.2 Description of PMC input pointwise flux data....................................... 7-286

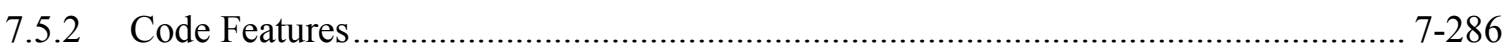

7.5.2.1 Options for treatment of 1-D cross sections ......................................... 7-287

7.5.2.2 Spatial averaging of 1D cross sections............................................. 7-288

7.5.2.3 Energy ranges for multigroup weighting............................................ 7-28

7.5.2.4 Options for treatment of 2-D cross sections ........................................ 7-289

7.5.3 Calculation of Problem-Dependent Multigroup Cross Sections ................................ 7-290

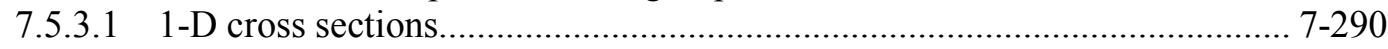

7.5.3.2 2-D scattering cross sections ........................................................... 7-290

7.5.3.3 Problem-dependent fission spectra...................................................... 7-293

7.5.3.4 Definition of background cross sections ..................................................... 7-294

7.5.4 PMC Input Data .............................................................................................. 7-294

7.5.4.1 Notes for PMC users (See PMC chapter for additional details)................. 7-297

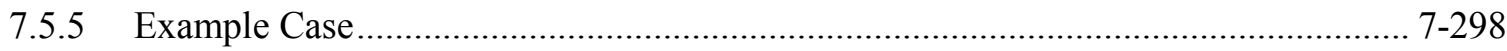

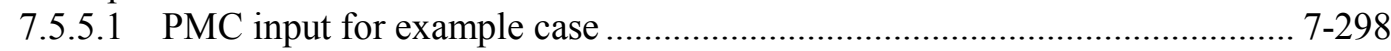

7.5.5.2 PMC output for example case ............................................................ 7-298

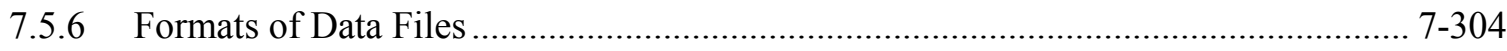

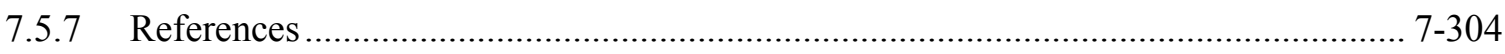

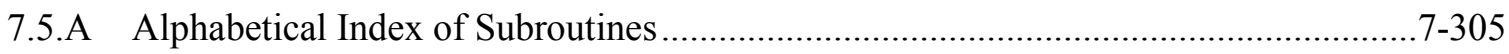

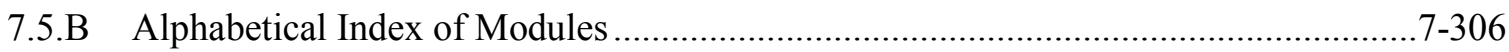




\section{ACKNOWLEDGMENTS}

The authors acknowledge the suggestions and direct contributions of L. M. Petrie of Oak Ridge National Laboratory, and former ORNL staff N. M. Greene, and R. M. Westfall. 


\subsubsection{Introduction}

PMC is a computational module used for the CENTRM/PMC self-shielding method performed by the XSProc driver module [see XSProc chapter of SCALE manual]. It can also be run in standalone mode. PMC (Produce Multigroup Cross sections) computes multigroup (MG) cross sections by utilizing the pointwise (PW) neutron spectra calculated in CENTRM $^{1}$ to weight cross sections in a continuous-energy (CE) library file. This provides problem-dependent, self-shielded MG data representative of the finestructure variation in the neutron energy spectrum for the system of interest. PMC only computes shielded cross sections within the energy interval of the CENTRM PW flux calculation, defined by the energy limits DEMIN and DEMAX. By default the lower limit is DEMIN $=0.001 \mathrm{eV}$, and the upper energy is DEMAX $=20,000 \mathrm{eV}$; however these parameters can be modified by the user in the CENTRM DATA input block. Outside of this energy interval, the shielded cross sections previously computed with the Bondarenko method in BONAMI are retained. PMC is automatically called from the XSProc driver module during execution of a SCALE sequence, and the resulting zone-averaged, problemdependent cross sections can be passed to MG transport solvers (e.g., KENO, NEWT, XSDRNPM, etc.) called by the sequence.

\subsubsection{Description of PMC input nuclear data}

The nuclear data input to PMC consists of both MG and CE cross sections. Input MG data are obtained from the MG library specified in the sequence input. During SCALE execution, CE data used by both CENTRM and PMC are prepared by the code CRAWDAD [section 8.6], which reads CE files for individual nuclides, interpolates the data to the appropriate temperatures for the specified mixtures, and concatenates the data into a one problem-specific, multiple-nuclide CENTRM PW library. In general each nuclide has its own unique energy mesh defined such that the cross section at any energy value can be interpolated linearly from the library point data to accuracy better than $0.1 \%$. Although cross sections in the original $\mathrm{CE}$ data files include values over the full energy range of $0-20 \mathrm{MeV}$, CRAWDAD reduces the energy range to interval of the CENTRM PW calculation (i.e., DEMIN $\rightarrow$ DEMAX). It is this combined PW library that is accessed by PMC. The format of the CENTRM PW library is described in the CENTRM chapter.

\subsubsection{Description of PMC input pointwise flux data}

In addition to the input nuclear data, PMC also requires PW flux values calculated in CENTRM to be provided. Depending on the CENTRM transport approximation, the flux data includes the PW scalar flux spectrum as a function of energy and spatial-zone, and also may include PW spherical harmonic moments of the angular flux (e.g., the current), which can be used in processing MG scattering matrices for higher-order Legendre moments. The non-uniform energy-mesh of the PW flux is determined during the CENTRM calculation in order to represent the spectrum variation with a minimum number of energy points. Like the CE cross section data, the flux spectrum at any energy value can be obtained within a specified tolerance by linear interpolation of the PW flux values.

\subsubsection{Code Features}

Two types of MG data are processed by PMC: 1-D cross sections and 2-D scatter matrices. The 1-D cross sections are weighted-average values over each energy group, by nuclide and reaction type. If there are "G" energy groups on the input library, then the 1-D cross section for each reaction type can be viewed as a 1-D vector with G values (of course some may be zero). Depending on the options and PW energy range specified, PMC will generally only re-compute and replace some of the G-group data. The 
2-D cross sections correspond to group-to-group transfers (and corresponding Legendre moments) associated with various types of scatter reactions. These data can be arranged into a 2-D G by G matrix. For most materials this matrix is quite sparse. The 2-D data depend not only on the cross-section data, but also on the energy/angular distributions of the secondary neutrons, which are represented by Legendre moments. PMC always re-normalizes the 2-D elastic and inelastic scattering matrices (including moments) to be consistent with the respective self-shielded 1-D data. In the case of elastic scattering, PMC also has rigorous options that can be used to modify the secondary energy distribution to account for self-shielding effects, such as by correcting the group removal cross section.

\subsubsection{Options for treatment of 1-D cross sections}

PMC computes new MG data for each reaction type (MT) and each nuclide on the input MG library, which also has CE data on the CENTRM PW library. Cross sections for reactions on the input MG library which do not have corresponding PW reaction data are not replaced; i.e., the original MG values are retained. SCALE CE library files for individual nuclides contain all reaction types included in the ENDF/B data; however the CRAWDAD module, executed prior to PMC, only includes certain ones when it produces the problem-specific CENTRM library. By default the CENTRM PW nuclear data library always includes cross sections for the total (MT-1), radiative capture (MT-102), and elastic scattering reactions (MT-2) of all nuclides; as well as fission (MT-18), and prompt, delayed, and totalnubar values (MTs-456, 455, 452, respectively) for fissionable nuclides. The (n,alpha) cross sections (MT-107) for B-10 and Li-6 are also always included if these nuclides are present in a mixture. If the CENTRM PW transport calculation includes the inelastic scattering option, indicated by CENTRM input parameter nmf6 $>=0$, the discrete-level PW inelastic (MTs 50-90) and continuum inelastic (MT-91) data are also included in the CENTRM PW library.

PW data for the unresolved resonance range are infinitely dilute on the CENTRM library; therefore PMC does not use PW cross sections to compute self-shielded data for the unresolved range. Instead, selfshielded cross sections in the unresolved range are calculated using the Bondarenko method in BONAMI prior to the CENTRM and PMC calculations. This step is automatically performed by XSProc in the SCALE calculation sequences.

PMC offers two methods to compute the total cross section. In the first method the MG value for the total cross section $(\mathrm{MT}=1)$ is processed directly from the PW MT-1 data on the CENTRM library. Total cross sections are generally considered the most accurate type of evaluated reaction data (due to measurement techniques); however if PW data for MT-1 are processed as an independent cross section, there is no guarantee that the sum of the partial cross sections will sum to the total. These small imbalances in cross sections affect the neutron balance, and may impact eigenvalue calculations. For this reason the PMC default option does not compute the total cross section by weighting the MT-1 PW data, but rather by summing the MG partial cross sections (including the original MG data not reprocessed in PMC).

The 1-D cross sections can be weighted using either the $\mathrm{P}_{0}$ (scalar flux) or $\mathrm{P}_{1}$ (current) $\mathrm{PW}$ Legendre moment. In almost all cases flux weighting is more desirable, since resonance reaction rates are usually the dominant factor in the PW range. However, current-weighting may be more accurate for certain problems where spatial transport and leakage strongly influence the spectrum in the resonance range, such as when the leakage spectrum is greatly impacted by cross section interference minima such as occur in iron media. The current-weighting option has been successfully applied for criticality calculations involving mixtures of highly-enriched uranium and iron. An alternative approach to using the current-weighted total cross section is to include a Legendre expansion of the angular-flux-weighted total cross section, which modifies the diagonal elements of the $2 \mathrm{D}$ elastic scattering moments. ${ }^{7}$ This option is specified by setting PMC input parameter $\mathrm{n} 2 \mathrm{~d}= \pm 2$, as discussed in section 7.5.2.4. 


\subsubsection{Spatial averaging of $1 \mathrm{D}$ cross sections}

PMC computes MG microscopic cross sections for each material mixture in a given CENTRM calculation, using the spatially averaged PW spectrum within the mixture. In SCALE this method is called "zone-weighting", and it is the default for PMC. Zone-weighted cross sections are generated for every mixture zone in the unit cell. In configurations containing fuel/absorber mixtures (e.g., lattices) in multiple unit cells, CENTRM/PMC calculations may be performed for each mixture, resulting in multiple mixture-weighted cross sections for the same nuclide ID. For this reason, both the nuclide ID and a mixture number are generally required to uniquely identify any specific cross section data generated by PMC.

PMC also has an option to calculate "cell-weighted" (i.e., homogenized) MG data, which applies disadvantage factors to preserve the cell-averaged reaction rates for the entire unit cell. This is not typically done, except for treating doubly-heterogeneous cells with SCALE. In this case the PMC cellweighting option is performed to produce homogenized $\mathrm{MG}$ cross sections for the low level heterogeneity (e.g., fuel grain in a fuel pebble). The XSProc control module automatically sets the correct PMC weighing flag based on the type of unit cell.

\subsubsection{Energy ranges for multigroup weighting}

The energy range of the MG and CE libraries in SCALE typically spans $10^{-5}$ to $2 * 10^{7} \mathrm{eV}$. In general this encompasses the (a) thermal region where upscatter is treated, (b) resolved and unresolved resonance ranges, and (c) high energy region above the resonance ranges. The thermal range for the current SCALE libraries is defined to be below $5 \mathrm{eV}$. Energy limits for the resolved and unresolved resonance ranges are defined by the individual ENDF/B evaluations for each nuclide, and these limits are included in the CENTRM PW library.

As discussed in section 8.3, the CENTRM PW flux file contains values of the zone-flux (and moments) per unit lethargy, calculated over the entire energy range $10^{-5} \mathrm{eV}$ to $20 \mathrm{MeV}$; however, only the fluxes in the energy range from DEMAX to DEMIN are computed from the PW transport solution and exhibit the spectral fine-structure due to resonance reactions. The flux outside interval [DEMAX, DEMIN] is represented by the smoother "pseudo-pointwise" values obtained from CENTRM's MG solution. PMC provides two options to define the nuclide-specific energy range for computing problem-dependent $\mathrm{MG}$ data:

Option (1). Compute MG cross sections of a given nuclide only over the resolved resonance range of the nuclide. If the CENTRM PW calculation does not encompass the entire resolved resonance range for the nuclide, pseudo-point fluxes are be used in the self-shielding calculations for some groups in the resolved regions. The pseudo-point fluxes are generally a good representation for the gross spectrum shape, but do not reflect fine-structure effects caused by resonance absorption; therefore with this option, the user should take care that the CENTRM PW limits are appropriate for the resonance nuclides of interest.

Option (2). Compute MG cross sections for a given nuclide over the entire energy range for which PW flux values are calculated in the CENTRM. In this case PMC computes MG cross sections only over the portion of the PW data that is contained within the PW flux range; i.e., the pseudo PW spectrum is not used to process any data. Shielded cross sections for groups not included in the PW calculation are based on the BONAMI self-shielding method.

Option (2) above is default in PMC. SCALE-6.2 has DEMIN and DEMAX default values of $0.001 \mathrm{eV}$ and $20 \mathrm{keV}$. This is sufficient for resonance self-shielding of essentially all actinide and important 
fission product nuclides; but some structural materials such as iron have resonances above $20 \mathrm{keV}$ which would be shielded by BONAMI

\subsubsection{Options for treatment of 2-D cross sections}

The input parameter N2D defines five PMC options for processing problem-dependent, 2-D elastic scattering matrices. The first approach, $\mathrm{N} 2 \mathrm{D}=0$, simply multiplies the elastic scattering matrices by the ratio of the new to old 1-D elastic cross sections for the specified reaction process, where the "old" data are the 1-D values in the original MG library, and the "new" data are the problem-dependent MG cross sections processed using the PW flux as described above. The $\mathrm{P}_{\ell}$ Legendre moments as well as the $\mathrm{P}_{0}$ matrix are scaled by the same ratio for a given group. This method is also always used for discretelevel and continuum inelastic cross sections, as well as any other 2-D data other than elastic. The basic assumption is that the relative group-to-group scattering distribution does not change from the distribution in the original MG library, which is processed with an infinitely dilute spectrum - i.e., selfshielding only affects the total scatter rate. This approach gives good results for many applications, and is very efficient computationally. However, for intermediate and high mass materials, the elastic removal rate from a group may be sensitive to the problem-dependent $\mathrm{CE}$ spectrum. In these cases the scaling approximation may not give the correct elastic removal rate from the group, because the withingroup elastic cross section will be in error. In these cases the alternate approaches described below can be used.

The option $\mathrm{N} 2 \mathrm{D}=-1$ corrects for the impact of resonance self-shielding on the elastic removal from an energy group. This option recomputes a new value for the within-group cross section by applying a correction factor based on the ratio of shielded versus unshielded removal probabilities for $s$-wave scatter (isotropic center-of-mass scatter). The $\mathrm{P}_{0}$ out-scattering cross sections are then renormalized to give the correct 1D shielded cross section for the group. This approach provides a reasonable and computationally efficient approximation to process $2 \mathrm{D}$ elastic matrices in the resolved resonance range of actinide nuclides. However the assumption of s-wave scatter may not be valid in the resolved resonance range of a structural material such as iron; therefore users should beware when applying the approximation if the PW range is extended above $50 \mathrm{keV}$, for systems with large sensitivity to structural materials.

Option N2D=1 uses the CENTRM PW flux to recompute the entire set of group-to-group scatter data (including Legendre moments) assuming $s$-wave kinematics. Since the CENTRM PW flux is used as the weighting function, this approach is sometimes more accurate for groups with large spectral gradients as discussed above. As with the $\mathrm{N} 2 \mathrm{D}=-1$ option, the main limitation is the $s$-wave scattering approximation for the secondary energy distribution. This option requires more computation time than the N2D methods discussed previously, and usually gives similar results as $\mathrm{N} 2 \mathrm{D}=-1$.

A rigorous derivation of the $\mathrm{MG}$ transport equation from the $\mathrm{CE}$ equation results in a directionally dependent total cross section. PMC option $\mathrm{N} 2 \mathrm{D}=2$ uses the method in reference 2 to address this effect by modifying the Legendre moments of the $2 \mathrm{D}$ elastic matrix. For cross section moment " $n$ ", the diagonal term (i.e., within-group scatter) is modified by adding a term equal to the difference in the MG total cross section weighted with the PW scalar flux and the MG total cross section weighted with the $\mathrm{n}_{\text {th }}$ Legendre moment of the PW flux.

Option $\mathrm{N} 2 \mathrm{D}=-2$ is essentially a combination of options $\mathrm{N} 2 \mathrm{D}=2$ and $\mathrm{N} 2 \mathrm{D}=-1$. This option applies the elastic removal correction to the diagonal term of the $\mathrm{P}_{0}$ moment of the elastic $2 \mathrm{D}$ matrix, and applies the PL correction described above to the diagonal term of the PL Legendre moment of the elastic matrix. 
The thermal energy range presents a particularly difficult challenge for processing problem-dependent 2-D scattering data, due to the complicated kinematics associated with molecular motion, chemical binding, and coherent scattering effects. PMC currently the scaling approximation ( $2 \mathrm{D}=0$ option) for the thermal energy range, regardless of the input value of N2D.

\subsubsection{Calculation of Problem-Dependent Multigroup Cross Sections}

\subsubsection{1-D cross sections}

The 1D MG cross section is defined as the weighted group-average of the CE cross-section data. PMC uses either the scalar flux or the absolute value of the neutron current from a CENTRM PW calculation as the weighting function, the latter being preferable only in exceptional situations where leakage spectra are very important. Defining $\Phi_{z}(E)$ as the zone-average, PW flux from the CENTRM calculation and $\sigma_{z, \mathrm{r}}(\mathrm{E})$ to be the CE cross-section data from the CENTRM nuclear data library, the zone-averaged MG cross section in group " $\mathrm{g}$ " for reaction type " $r$ " of nuclide " $\mathrm{j}$ ", for zone " $\mathrm{z}$ " (=>specified temperature) is expressed as,

$$
\sigma_{z, r, g}^{j}=\frac{\int_{\Delta E_{g}} \sigma_{z, r}^{j}(E) \Phi_{z}(E) d E}{\int_{\Delta E_{g}} \Phi_{z}(E) d E}=\frac{\int_{\Delta E_{g}} \sigma_{z, r}^{j}(E) \Phi_{z}(E) d E}{\Phi_{z, g}}
$$

where

$\Phi_{z, \mathrm{~g}}$ is the multigroup zone flux,

$\sigma_{z, r, g}^{j}$ is the zone-average, group cross section, and

$\Delta \mathrm{E}_{\mathrm{g}}$ is the energy interval of group $\mathrm{g}$.

The integration in Eq. (7.5.1) is performed by summing over a discrete energy mesh within the group boundaries. Since the CE cross section and the PW flux generally have different energy grids, the integration mesh for the numerator is formed by taking the union of the two. The CE cross sections and the PW flux are mapped onto the union mesh, and the integral is evaluated using the trapezoidal method. Equation (7.5.1) is used to compute weighted group data for all MT's for which CE data are available on the CENTRM library, except in the case of the fission neutron yield $v$. Instead of using the PW scalar flux as the weighting function, the MG value for $v$ is weighted by the product of the PW flux and the PW fission cross section for the material.

\subsubsection{2-D scattering cross sections}

The 2-D MG cross section moments are defined as the weighted group-average of terms appearing in a Legendre (PL) expansion of the CE double-differential scatter cross section, which describes the transfer of neutrons from one energy to another, for a given angle of scatter. The PL Legendre moments on the original MG library are fully consistent with the ENDF/B kinematic specifications. Thus the specified anisotropy in elastic or inelastic data in the center-of-mass (CM) system is reflected in the PL scattering matrices; however the library MG data are processed with an infinitely dilute flux spectrum. PMC provides several options for modifying these data to correct for problem-specific spectral effects, such as self-shielding. First, consider the scaling method $(\mathrm{N} 2 \mathrm{D}=0)$ in which all the elements of the original scatter matrix (i.e., on the input Master library) for a given initial group are multiplied by the ratio of 1-D scatter cross sections. This has the effect of normalizing the original scatter matrix to the problem- 
dependent value calculated for the 1-D scatter data. In this case the $1_{\text {th }}$ Legendre moment of the 2-D multigroup cross section for reaction type " $s$ " of nuclide " $\mathrm{j}$ " in zone " $\mathrm{z}$ " (at a specified temperature), for scatter from initial group $\mathrm{g}^{\prime}$ to final group $\mathrm{g}$, is computed by:

$$
\sigma_{l, z, s, g^{\prime} \rightarrow g}^{j}=\frac{\left(\sigma_{z, s, g^{\prime}}^{j}\right)_{\text {new }}}{\left(\sigma_{s, g^{\prime}}^{j}\right)_{\text {orig }}} \times\left(\sigma_{l, s, g^{\prime} \rightarrow g}^{j}\right)_{\text {orig }},
$$

where the subscripts "orig" and "new," respectively, refer to the original MG data on the Master library, and the new problem-dependent data computed by PMC. The types of reactions for which problemdependent 2-D cross sections may be processed using the scaling method are elastic (MT=2), discretelevel inelastic (MT's 50-89), continuum inelastic $(\mathrm{MT}=90)$, and $(\mathrm{n}, 2 \mathrm{n})(\mathrm{MT}=16)$. This approach is also applied to obtain problem-dependent thermal scatter matrices, which contain upscatter as well as downscatter reactions. The CENTRM nuclear data libraries include PW cross sections for incoherent $(\mathrm{MT}=1007)$ and coherent $(\mathrm{MT}=1008$, if available) thermal scattering reactions, which can be processed into 1-D MG data by PMC in the same manner as other reaction types. The 1-D weighted thermal scattering data are then used to normalize the 2-D thermal matrices on the input Master library. For materials with both coherent and incoherent thermal scatter data, each matrix is scaled by the corresponding type of 1-D data. The coherent scattering matrix only contains within-group terms.

The option $\mathrm{N} 2 \mathrm{D}=-1$ recomputes the $\mathrm{P}_{0}$ within-group elastic cross section based on the assumption of swave scatter kinematics, and scales the other terms of the original P0 elastic matrix by the modified removal rate. This procedure approximately corrects for effects of resonance self-shielding on the group removal probability, without having to recompute the entire matrix assuming $s$-wave scatter, as done for $\mathrm{N} 2 \mathrm{D}=1$. Suppressing the zone index for simplicity, the $\mathrm{P}_{0}$ within-group XS is defined as:

$$
\sigma_{\mathrm{g}, \mathrm{g}} \equiv \frac{\int_{\mathrm{g}} \sigma_{\mathrm{s}}(\mathrm{E})\left[1-\mathrm{p}_{\mathrm{r}}(\mathrm{E})\right] \Phi(\mathrm{E}) \mathrm{dE}}{\int_{\mathrm{g}} \Phi(\mathrm{E}) \mathrm{dE}}
$$

where $\mathrm{p}_{\mathrm{r}}(\mathrm{E})$ is the probability that a neutron at energy $\mathrm{E}$, within group $\mathrm{g}$, will scatter to an energy below the lower boundary of the group. For $s$-wave scattering this equation becomes,

$$
\sigma_{g, g}=\frac{\int_{\mathrm{E}_{\mathrm{Lo}}}^{\min \left(\mathrm{E}_{\mathrm{Hi}}, \frac{\mathrm{E}_{\mathrm{Lo}}}{\alpha}\right)} \sigma_{\mathrm{s}}(\mathrm{E})\left[\frac{\mathrm{E}-\mathrm{E}_{\mathrm{L}}}{\mathrm{E}(1-\alpha)}\right] \Phi(\mathrm{E}) \mathrm{dE}}{\int_{\mathrm{g}} \Phi(\mathrm{E}) \mathrm{dE}}
$$

The $\mathrm{N} 2 \mathrm{D}=-1$ option recomputes a modified $\mathrm{P}_{0}$ within-group cross section from the expression,

$$
\left(\sigma_{\mathrm{g}, \mathrm{g}}\right)_{\text {new }}=\frac{{\widetilde{\sigma_{g}, \mathrm{~g}}}_{(\boldsymbol{\varphi})}}{\widetilde{\sigma}_{\mathrm{g}, \mathrm{g}}^{\infty}}\left(\sigma_{\mathrm{g}, \mathrm{g}}\right)_{\text {orig }}
$$


here $\left(\sigma_{\mathrm{g}, \mathrm{g}}\right)_{\text {orig }}$ is the original within-group cross section on the MG library, based on actual kinematics and weighted with an infinitely dilute spectrum;

$\widetilde{\sigma}_{\mathrm{g}, \mathrm{g}}^{(\infty)}$ is the infinitely dilute within-group cross section based on s-wave kinematics, which is computed from Eq. (7.5.4) using an infinitely dilute spectrum

$\widetilde{\sigma}_{\mathrm{g}, \mathrm{g}}^{(\varphi)} \quad$ is the self-shielded within-group based on s-wave kinematics, computed from Eq. (7.5.4) using $\Phi(E) \rightarrow$ CENTRM PW flux.

If the effects of resonance self-shielding are small, then there will be little change in the original withingroup value, since in this case $\widetilde{\sigma}_{\mathrm{g}, \mathrm{g}}^{(\varphi)} \sim \widetilde{\sigma}_{\mathrm{g}, \mathrm{g}}^{(\infty)}$.

The $\mathrm{P}_{0}$ group-to-group out-scatter terms for $\mathrm{N} 2 \mathrm{D}=-1$ are scaled as follows:

$$
\sigma_{g \rightarrow g^{\prime}}=\frac{\left(\sigma_{\mathrm{s}, \mathrm{g}}\right)_{\text {new }}-\widetilde{\sigma}_{\mathrm{g}, \mathrm{g}}^{(\varphi)}}{\left(\sigma_{\mathrm{s}, \mathrm{g}}^{\infty}\right)_{\text {new }}-\widetilde{\sigma}_{\mathrm{g}, \mathrm{g}}^{\infty}} \times\left(\sigma_{\mathrm{g} \rightarrow \mathrm{g}^{\prime}}\right)_{\text {orig }}
$$

Again if there is little self-shielding, the change in off-diagonal matrix elements is small, so that the original secondary energy distribution is preserved. Finally the entire modified $\mathrm{P}_{0}$ scatter matrix is renormalized to correspond to the self-shielded 1-D scatter cross section.

For the option $\mathrm{N} 2 \mathrm{D}=1$, an entirely new PL elastic scattering matrix is computed. The $1_{\text {th }}$ Legendre moment of the 2-D MG elastic cross section of nuclide " $\mathrm{j}$ " in zone " $\mathrm{z}$ " (at a specified temperature), for scattering from initial group $\mathrm{g}$ ' to final group $\mathrm{g}$ is rigorously defined $\mathrm{as},{ }^{2}$

$$
\sigma_{l, g^{\prime} \rightarrow g}^{j}=\frac{\int_{\Delta E_{g}} \int_{\Delta E_{g^{\prime}}} \sigma_{l}^{j}\left(E^{\prime} \rightarrow E\right) \Phi_{l, z}\left(E^{\prime}\right) d E^{\prime} d E}{\int_{\Delta E_{g^{\prime}}} \Phi_{l, z}\left(E^{\prime}\right) d E^{\prime}}=\frac{\int_{\Delta E_{g}} \int_{\Delta E_{g^{\prime}}} \sigma^{j}\left(E^{\prime}\right) f_{l}^{j}\left(E^{\prime} \rightarrow E\right) \Phi_{l, z}\left(E^{\prime}\right) d E^{\prime} d E}{\int_{\Delta E_{g^{\prime}}} \Phi_{l, z}\left(E^{\prime}\right) d E^{\prime}}
$$

where $\sigma_{z}(E)$ is the CE elastic cross-section data from the CENTRM nuclear data file, evaluated at the appropriate temperature for zone $\mathrm{z} ; f_{l}^{j}\left(\mathrm{E}^{\prime} \rightarrow \mathrm{E}\right)$ is the secondary neutron energy distribution from elastic scattering; and $\Phi_{1, \mathrm{Z}}(\mathrm{E})$ is the 1th PW flux moment averaged over zone Z. PMC assumes $s$-wave scattering from stationary nuclei to evaluate the scattering distribution, and uses the $\mathrm{P}_{0}$ flux moment (i.e., scalar flux) as for the weighting function for all PL matrices; therefore the expression evaluated by PMC for $\mathrm{N} 2 \mathrm{D}=1$ is:

$$
\sigma_{l, z, g^{\prime} \rightarrow g}^{j}=\frac{\int_{g^{\prime}} \int_{g} \frac{\sigma_{z}^{j}(\mathrm{E}) \Phi_{z}\left(E^{\prime}\right) P_{l}\left(G^{j}\right)}{\left(1-\alpha^{j}\right) E^{\prime}} d E^{\prime} d E}{\int_{g} \Phi_{z}\left(E^{\prime}\right) d E^{\prime}}
$$


here $\mathrm{P}_{l}$ is the $l_{\text {th }}$ order Legendre polynomial; and $\mathrm{G}^{\mathrm{j}}$ is the kinematics relation expressing the cosine of the scattering angle as a function of $E$ and $E$ ', for elastic scattering from nuclear mass $A^{j}$. The kinematics function for nuclide $\mathrm{j}$ is defined as,

$$
G^{j}\left(E^{\prime}, E\right)=\frac{A^{j}+1}{2} \sqrt{\frac{E}{E^{\prime}}}-\frac{A^{j}-1}{2} \sqrt{\frac{E^{\prime}}{E}},
$$

where $\mathrm{G}^{\mathrm{j}}\left(\mathrm{E}^{\prime}, \mathrm{E}\right)$ is equal to the cosine of the angle of scatter between the initial and final directions. The integral over the final group ( $\mathrm{g}$ ) is evaluated analytically using routines developed by J. A. Bucholz. ${ }^{3}$ Integration over the initial group $\left(\mathrm{g}^{\prime}\right)$ is then performed numerically using the same method as for evaluating the problem-dependent $1-\mathrm{D}$ cross sections.

Option $\mathrm{N} 2 \mathrm{D}=2$ adds the following term to the diagonal of the $l_{t h}$ moment of the PL elastic scatter matrix,

$$
\left(\sigma_{l ; g, g}^{j}\right)_{\text {new }}=\left(\sigma_{l, g, g}^{j}\right)_{\text {origg }}+\sigma_{t, g}^{j}-\sigma_{t, l ; g}^{j} ; 0<l<i s c t
$$

where isct is the order of scatter specified in CENTRM calculation [see section 8.3]; $\sigma_{t ; g}^{j}$ is the standard $1 \mathrm{D}$ total cross section weighted with the scalar flux, and $\sigma_{t, l ; g}^{j}$ is the total cross section weighted with the $l_{t h}$ Legendre moment of the angular flux; i.e.,

$$
\sigma_{t, l ; g}^{j}=\frac{\int_{g} \sigma_{t}^{j}(E) \Phi_{l}(E) d E}{\int_{g} \Phi_{l}(E) d E}
$$

\subsubsection{Problem-dependent fission spectra}

Fission spectra (chi) describing the energy distribution of secondary neutrons produced by fission depend upon the energy of the neutron causing the fission, thus the MG chi data should be a 2-D matrix, $\chi_{\mathrm{g} \rightarrow \mathrm{g}^{\prime}}$. However, neutron transport codes in SCALE expect a 1-D distribution, $\chi_{\mathrm{g}^{\prime}}$; therefore the production of fission neutrons in group $\mathrm{g}^{\prime}$ by neutrons in group $\mathrm{g}$ is approximated as,

$$
\mathrm{P}_{\mathrm{g} \rightarrow \mathrm{g}^{\prime}}=\chi_{\mathrm{g}^{\prime}} \nu_{\mathrm{g}} \sigma_{\mathrm{f}, \mathrm{g}} \Phi_{\mathrm{g}}
$$

and the total number of secondary neutrons generated in group g' is,

$$
\mathrm{P}_{\mathrm{g}^{\prime}}=\chi_{\mathrm{g}^{\prime}} \sum_{\mathrm{g}} v_{\mathrm{g}} \sigma_{\mathrm{f}, \mathrm{g}} \Phi_{\mathrm{g}}
$$

SCALE MG libraries contain "generic" 1-D chi distributions for each fissionable nuclide. These are processed from the evaluated ENDF/B fission data, weighted by the standard weighting function used to process the SCALE MG libraries (i.e., Maxwellian in thermal energy range, 1/E in epithermal range, 
fission spectrum in fast range). The SCALE MG libraries also contain 2-D chi distributions processed from ENDF/B fission data, can be processed with a problem-dependent weighting function to create a more representative 1-D chi. This procedure is done in PMC for each fissionable nuclide, using the following equation that preserves the secondary neutron energy distribution:

$$
\chi_{\mathrm{g}^{\prime}}=\frac{\sum_{\mathrm{g}} \chi_{\mathrm{g} \rightarrow \mathrm{g}^{\prime}} v_{\mathrm{g}} \sigma_{\mathrm{f}, \mathrm{g}} \Phi_{\mathrm{g}}}{\sum_{\mathrm{g}} v_{\mathrm{g}} \sigma_{\mathrm{f}, \mathrm{g}} \Phi_{\mathrm{g}}}
$$

In the above equation, $v_{\mathrm{g}}, \sigma_{\mathrm{f}, \mathrm{g}}$, and $\Phi_{\mathrm{g}}$ are problem-dependent 1-D data computed by PMC using the PW fluxes calculated by CENTRM, and $\chi_{\mathrm{g} \rightarrow \mathrm{g}^{\prime}}$ are the 2-D MG fission spectra data on the AMPX multigroup Master library. The 1D prompt chi computed by PMC includes all fission components (firstchance-fission, second-chance-fission, etc) given in the ENDF/B files, weighted by the relative fissionsource fraction associated with each channel. PMC also computes an effective delayed neutron fission spectra, and this is combined with the prompt chi, using the appropriate delayed neutron fraction, to obtain the final 1-D fission spectra. The 1-D chi computed by PMC replaces the generic 1-D values for MT-1018 that were originally in the Master library.

\subsubsection{Definition of background cross sections}

The value of the "background cross section $\left(\sigma_{0}\right)$ " may be used in PMC to determine which materials are considered to be infinitely dilute, in which case no cross section processing is done for the material. No processing is performed for material " $\mathrm{j}$ " if its background cross section exceeds the value of input parameter XS_dilute; i.e., if $\sigma_{0}^{(\mathrm{j})}>$ XS_dilute. The expression used in PMC to compute the background cross section $\sigma_{0}^{(\mathrm{j})}$ is given in the BONAMI chapter.

\subsubsection{PMC Input Data}

The Fido input blocks shown in this section are only required when executing PMC as a standalone module. In the more typical case where PMC is executed through the XSProc module during a SCALE sequence calculation, the default parameter values are automatically defined within XSProc. Default values for XSProc execution can be overridden using keyword input in the CENTRM DATA block (see section 8.I). The keyword input names correspond to the variable names given in this section.

\section{DATA BLOCK 1}

0\$\$ LOGICAL UNIT ASSIGNMENTS (8 entries. Default values given in parenthesis)*

1. $\mathrm{LIBM}=$ Input AMPX Master nuclear data library

2. $\mathrm{LIBX}=$ Input CENTRM pointwise nuclear data library

3. $\mathrm{LIBF}=$ Pointwise flux file produced by CENTRM

4. LIBNM $=$ Output problem-dependent Master library created by PMC (92)

5. LIBSC $=$ Scratch unit (18) 
6. LIBSX $=$ Scratch unit (24)

(*) Parameters in the $0 \$ \$$ array cannot be modified for XSProc execution.

1\$\$ INTEGER PARAMETERS (10 entries)

1. MRANGE $=0$, obsolete option

$=1$, Compute new group cross sections over resolved resonance range of pointwise nuclides [from EUPR to ELOR given in CENTRM data library]

$=2$, Compute new group cross sections over pointwise flux range [from DEMAX to DEMIN in CENTRM flux calculation] (2).

2. N2D = -2, Apply removal correction to P0 elastic scatter matrix AND apply consistent PN correction to higher order Legendre components; normalize to 1D.

-1, Apply elastic removal correction to P0 elastic scatter matrix; normalize to 1D.

$=0$, Normalize $\mathrm{P}_{\mathrm{N}}$ components of original elastic scattering matrix to new 1-D elastic value.

$=1$, Compute new $\mathrm{P}_{\mathrm{N}}$ components of elastic matrix, using scalar flux as weighting function.

$=2$, Modify diagonal elements of the PN moments of the elastic matrix using the consistent PN method (-1).

3. NTHRM $=0$ Treatment of thermal scatter kernels [not functional] (0)

4. NPRT = $=-1$, Minimum printed output;

$=0$, Standard print out;

$=1$, Also print new weighted cross sections for MT's 1, 2, 18, and 102 .

$=2$, Maximum amount of printed output includes $2 \mathrm{D}$ matrices $(-1)$.

5. NWT $=0$, Generate zone-weighted multigroup data;

$=1$, Generate cell-weighted multigroup data $(0)$.

6. MTT $=0$, Process all MT's included in LIBX. [NOTE: With this option, total
[NT cross section may not equal to sum of partials];

$=1$, Process all MT's except 1,27, 101; then compute: 


$$
\begin{aligned}
& \text { MT } 101=\text { sum of MT's 102-114, } \\
& \text { MT } 27=\text { sum of MT's } 18 \text { and 101, } \\
& \text { MT } 1=\text { sum of MT's 2, 4, 16, 17, and } 27
\end{aligned}
$$

7. PMC_OMIT $=0$, Process all pointwise nuclides used in CENTRM calculation;

$=1$, Process only nuclides in fuel zones.

$>1$, Process all materials except those in $2 \$ \$$ array

8. IXTR2 $=0$, PMC run in CSAS standard sequence;

$=1$, PMC run in stand-alone mode (1);

$=2$ PMC run in CSAS double-heterogeneous cell sequence

9. IXTR3 $=-1$, Process new data for all Legendre components on the input AMPX master library up to $\mathrm{P}_{7}$.

$=\mathrm{N}$, Process new data through $\mathrm{P}_{\mathrm{N}}$ moments. [N=Scattering Order +1$](-1)$.

10. N1D = 0 Use CENTRM scalar flux for weighting function;

$=1$, Use the absolute value of CENTRM current for weighting function (0).

1** REAL PARAMETERS $(10$ entries)

1. XS_DILUTE $=$ background cross section (barns) considered to be infinitely dilute $\left(10^{10}\right)$

2-10. Fill with 0.0

T [ TERMINATE DATA BLOCK 1$]$ 


\title{
DATA BLOCK 2 : INDIVIDUAL NUCLIDES OMITTED FROM PROCESSING
}

\author{
NOTE: this data cannot be entered for XSProc execution.
}

2\$\$ ISOTOPE IDENTIFIERS (PMC_OMIT entries). Only enter PMC_OMIT $>1$

[IDs of nuclides to be omitted from pointwise processing]

\section{T ITERMINATE DATA BLOCK}

\section{END OF PMC INPUT DATA}

\subsubsection{Notes for PMC users (See PMC chapter for additional details)}

1. N2D specifies the method used to process the $\mathrm{P}_{\mathrm{N}}$ components of the 2-D elastic scattering matrices. In the option $\mathrm{N} 2 \mathrm{D}=0$, the $\mathrm{P}_{\mathrm{N}}$ components of the original elastic scattering matrix are simply re-normalized using the new, problem-dependent 1-D elastic values. This simple scaling approach often works well, but it does not account for the impact of resonance self-shielding on the group removal probability. The default option $\mathrm{N} 2 \mathrm{D}=-1$ approximately corrects the $\mathrm{P} 0$ elastic matrix for removal self-shielding effects on and is usually preferred to $\mathrm{N} 2 \mathrm{D}=0$, except for fast systems. Option $\mathrm{N} 2 \mathrm{D}=1$ re-computes all the $\mathrm{P}_{\mathrm{N}}$ components of 2-D elastic cross sections using the scalar flux as a weighting function, along with the assumption of $s$-wave scattering within the PW energy range. This approach takes significantly more execution time than $\mathrm{N} 2 \mathrm{D}=-1$, and usually is not necessary. Option $\mathrm{N} 2 \mathrm{D}=2$ corrects the diagonal terms of the Legendre moments, using the consistent $\mathrm{PN}$ expression. Option $\mathrm{N} 2 \mathrm{D}=-2$ is similar to $\mathrm{N} 2 \mathrm{D}=2$, except the elastic removal correction is applied to the $\mathrm{P} 0$ moment (Like for $\mathrm{N} 2 \mathrm{D}=-1$ ). Option $\mathrm{N} 2 \mathrm{D}=-2$ has been found to improve results for many infinite lattice cases.

2. NWT specifies whether the new multigroup cross sections are zone-weighted or cell-weighted. When PMC is executed through XSProc, nuclides are always zone-weighted unless the doubleheterogeneous option is specified in the CELLDATA block of the sequence input. Except for double-heterogeneous cells, cell-weighting of the MG cross sections should be done by the multigroup XSDRNPM calculation.

3. PMC_OMIT is used to indicate which pointwise nuclides are processed when computing new group cross sections. If PMC_OMIT=1, only nuclides in fuel mixtures are processed. Fuel mixtures are defined as having at least one material with $Z \geq 90$. Option PMC_OMIT $>1$ only works for PMC standalone runs, since there is no mechanism for inputting the $2 \$ \$$ array in sequences.

4. IXTR3 is used to indicate through what Legendre order the scattering matrices are to be processed. By default, in stand-alone mode all $\mathrm{P}_{\mathrm{N}}$ moments on the Master library are processed, where as in a SCALE sequence only through order $\mathrm{N}=5$ are processed. With few exceptions, the SCALE multigroup libraries contain scattering data through $\mathrm{P}_{5}$. 
5. If input parameter XS_DILUTE $>0.0$, PMC computes background cross sections $\left(\sigma_{0}\right)$ for each material, and bypasses processing materials with $\sigma_{0}>$ XS_DILUTE. The default of XS_DILUTE $=10^{10}$ barns causes essentially all materials to be processed regardless of dilution. Smaller XS_DILUTE values may reduce the number of materials being processed, and hence reduce the execution time; however, XS_DILUTE should not be so low that important absorbers are not shielded.

\subsubsection{Example Case}

Usually PMC is executed through one of the automated SCALE sequences such as CSAS or TRITON where it is called by XSProc in conjunction with other SCALE modules, such as CRAWDAD which provides the pointwise nuclear data library and CENTRM which provides pointwise fluxes. In such cases the user does not have to prepare input directly for PMC.

\subsubsection{PMC input for example case}

An example of PMC stand-alone execution is given below, but it should be noted that this PMC case cannot be executed unless it is linked to the output data files produced by other modules. The example problem given in the CENTRM chapter shows the coupled execution of several stand-alone modules , including PMC, which mimics the function of XSProc.

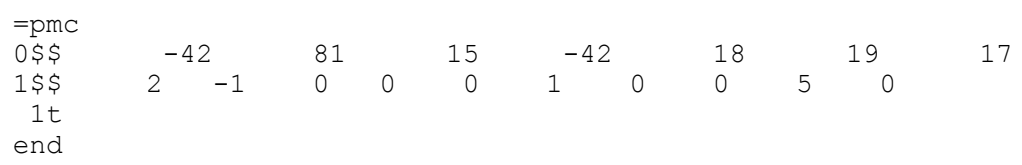

\subsubsection{PMC output for example case}

Only the printed output produced by PMC for the example problem is shown here. In this case the "standard" PMC editing option (NPRT=0) was specified. The XSProc default of "minimum" print in the SCALE sequences produces considerably less output.

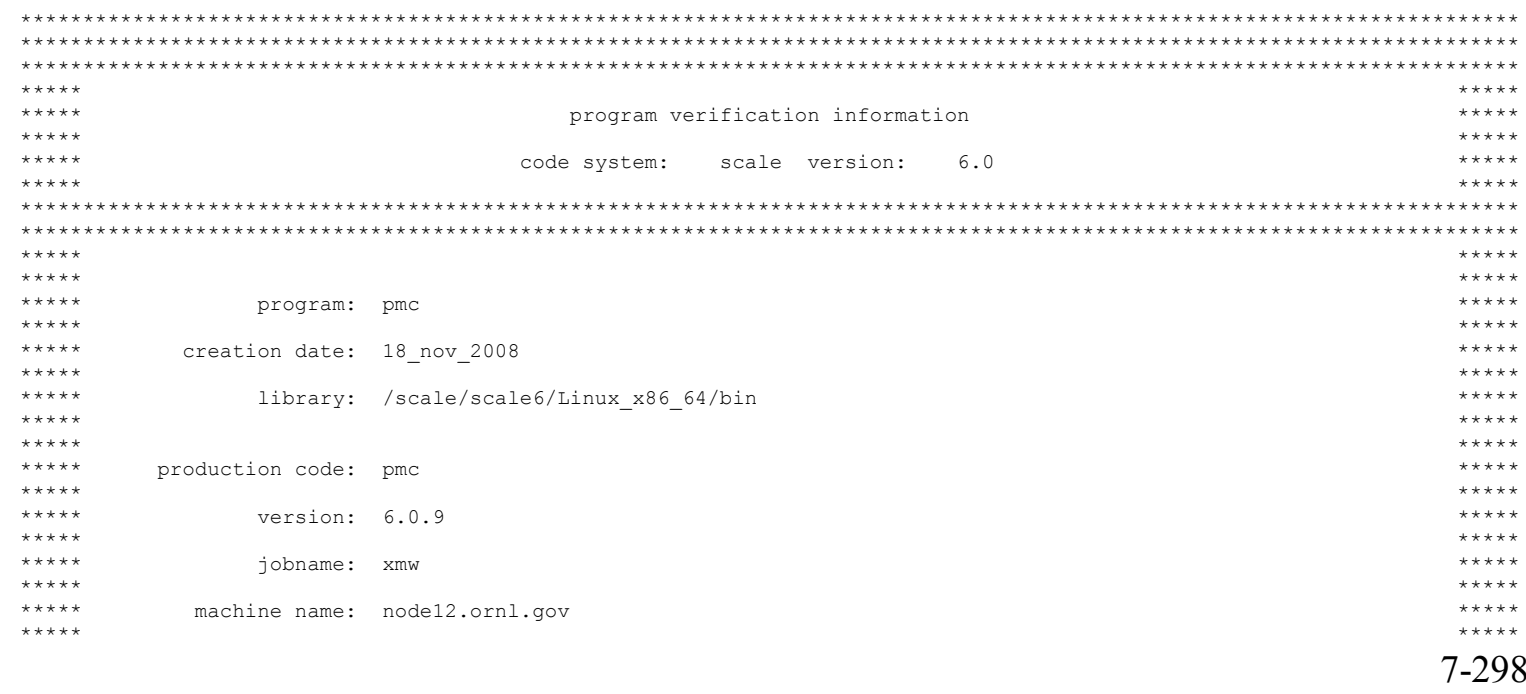


$1 \mathrm{t}$

$$
\star \star \star \star \text { LOGICAL UNITS } \star \star \star \star *
$$

nin $=5$ Card Image Input Unit

nout $=6$ Print Output Unit

libm $=-42 \quad$ Input Master Library

libx = 81 Input Pointwise XS Library

libf $=15$ Input Pointwise Flux File

libnm $=-42 \quad$ Output Master Library

libsc $=18 \quad$ Scratch Unit 1

libsx $=19 \quad$ Scratch Unit

libsm $=17$ scratch unit (master library)

$\star \star \star *$ INPUT PARAMETERS $* * \star \star$

mrange $=2$ Option for choosing energy range

$\mathrm{n} 2 \mathrm{~d}=-1$ Option for 2-D scat. calculation

nthrm $=0$ Option for thermal scatter kernal (NOT FUNCTIONAL)

nprt $=0$ Option for PMC print output

nwt $=0$ Option for XS averaging

mtt $=1$ Option for total $\mathrm{XS}$ calculation

$\operatorname{ixtr}(1)=0$ Option for Processing PW Materials

$\operatorname{ixtr}(2)=0 \quad$ Option for calculation sequence

ixtr(3)= 5 Legendre expansion order

n1d $=0$ Option for $1-\mathrm{D}$ cross-sections
Averaging over pointwise xs limits

Averaging over resolved resonance range

Averaging over pointwise flux limits

Recompute self-scatter, then normalize 2-D elastic data to shielded 1-D value

Normalize 2-D elastic data to shielded 1-D value

Recompute 2-D elastic using flux and s-wave kernel

2 Recompute 2-D moments with flux-moments weighting

Minimum data printed

Standard printed output

Print 1-D XSs

Print both 1-D and 2-D XSs

Zone average

Cell average

Average independently

As sum of partial XS

Process all Pointwise Materials Used in CENTRM

Omit $N$ Materials

CSAS Standard Sequence

Independant (stand-alone) Execution

CSAS Doubly-Heterogeneous Cell Sequence

Process all Legendre expansion moments found on AMPX LIB. Process only up through PN moments

Weight using using scalar flux

Weight using using abs value of current (1st moment)

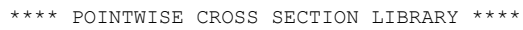

tape identifier

No. of nuclides

Max no. of temperatures

Max no. of processes

Max no. of energy points

66666

666
9

174194

$\star * * *$ POINTWISE FLUX FILE $* * * \star$

No. of nuclides

No. flux moments

No. of zones

No. of energy points

Upper energy limit, demax

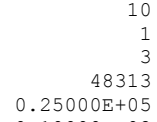

Lower energy limit, demin $0.10000 \mathrm{E}-02$

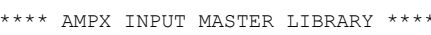




\begin{tabular}{|c|c|c|c|c|c|}
\hline & \multicolumn{3}{|l|}{238000} \\
\hline \multirow{2}{*}{$\begin{array}{l}\text { No. } \\
\text { No. }\end{array}$} & \multicolumn{2}{|l|}{ of nuclides } & \multicolumn{3}{|l|}{10} \\
\hline & \multicolumn{2}{|c|}{ of neutron groups } & 238 & & \\
\hline \multirow[t]{2}{*}{ No. } & \multicolumn{2}{|c|}{ of gamma groups } & \multicolumn{2}{|l|}{0} & \\
\hline & \multicolumn{4}{|c|}{$\star * \star *$ POINTWISE CROSS SECTION DIRECTORY $* * * *$} & \\
\hline $\mathrm{ZA}$ & Pointwise & Pointwise & Unresolved & Resolved & Resolved \\
\hline 8016 & $0.2500 \mathrm{E}+05$ & $0.1000 \mathrm{E}-02$ & $0.0000 \mathrm{E}+00$ & $0.0000 \mathrm{E}+00$ & $0.0000 \mathrm{E}+00$ \\
\hline & $0.2500 \mathrm{E}+05$ & $0.1000 \mathrm{E}-02$ & $0.4000 \mathrm{E}+06$ & $0.6000 E+05$ & $0.0000 \mathrm{E}+00$ \\
\hline 1 & $0.2500 \mathrm{E}+05$ & $0.1000 \mathrm{E}-02$ & $0.1000 \mathrm{E}+06$ & $0.2000 \mathrm{E}+05$ & $0.0000 \mathrm{E}+00$ \\
\hline 0092 & $0.2500 \mathrm{E}+05$ & $0.1000 \mathrm{E}-02$ & $0.1000 \mathrm{E}+06$ & $0.7100 \mathrm{E}+05$ & $0.0000 \mathrm{E}+00$ \\
\hline & $0.2500 \mathrm{E}+05$ & $0.1000 \mathrm{E}-02$ & $0.1000 \mathrm{E}+06$ & $0.9000 E+05$ & $0.0000 \mathrm{E}+00$ \\
\hline & $0.2500 \mathrm{E}+05$ & $0.1000 \mathrm{E}-02$ & $0.1000 \mathrm{E}+06$ & $0.1000 \mathrm{E}+06$ & $0.0000 \mathrm{E}+00$ \\
\hline & $0.2500 \mathrm{E}+05$ & $0.1000 \mathrm{E}-02$ & $0.2500 \mathrm{E}+05$ & $0.2250 \mathrm{E}+04$ & $0.0000 \mathrm{E}+00$ \\
\hline & $0.2500 \mathrm{E}+05$ & $0.1000 \mathrm{E}-02$ & $0.1490 \mathrm{E}+06$ & $0.2000 \mathrm{E}+05$ & $0.0000 \mathrm{E}+00$ \\
\hline 1001 & $0.2500 \mathrm{E}+05$ & $0.1000 \mathrm{E}-02$ & $0.0000 \mathrm{E}+00$ & $0.0000 \mathrm{E}+00$ & $0.0000 \mathrm{E}+00$ \\
\hline
\end{tabular}

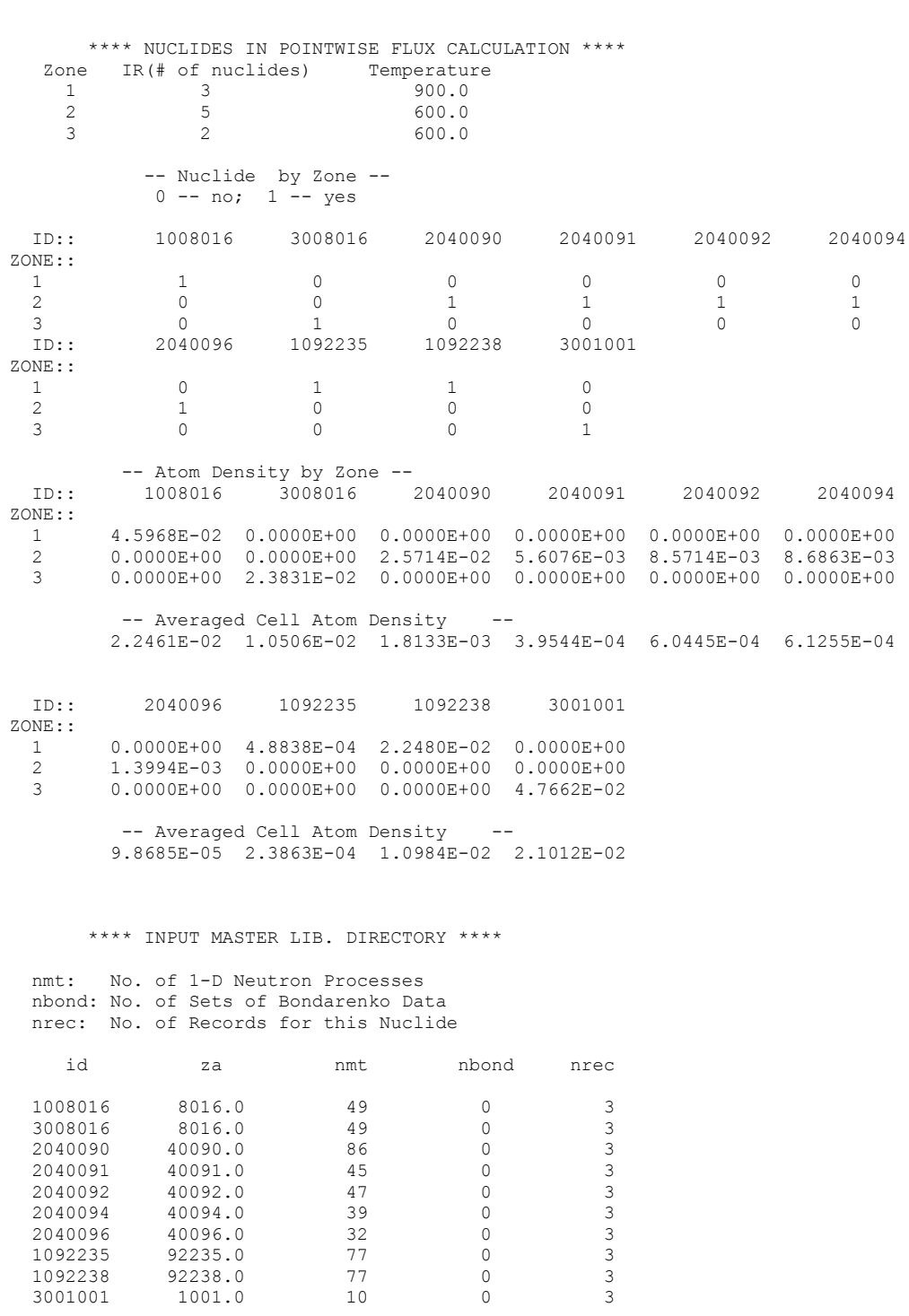

Processing N u c lide 1008016

Energy Range for Multigroup Averaging of this Data $\mathrm{EH}=2.50000 \mathrm{E}+04 \quad \mathrm{EL}=1.00000 \mathrm{E}-03$

INFORMATION ON CENTRM POINTWISE XS LIB:

$\begin{array}{rcl}\text { MT } & \text { ENERGY POINTS } & \text { TEMPERATURE (K) } \\ 1 & 459 & 600.0\end{array}$




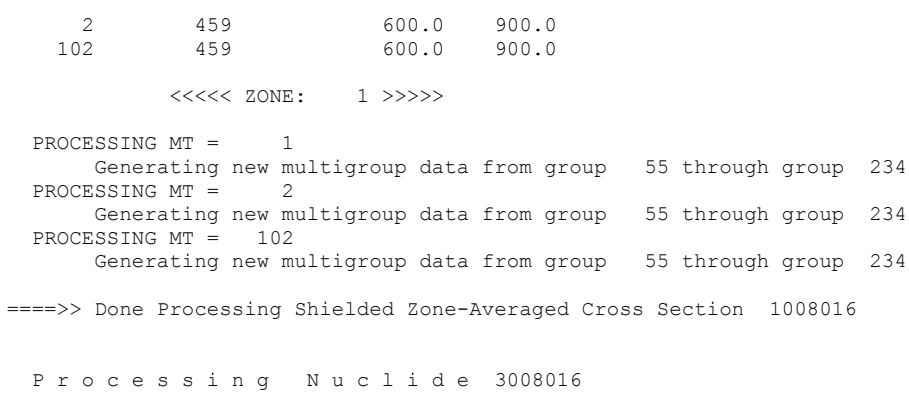




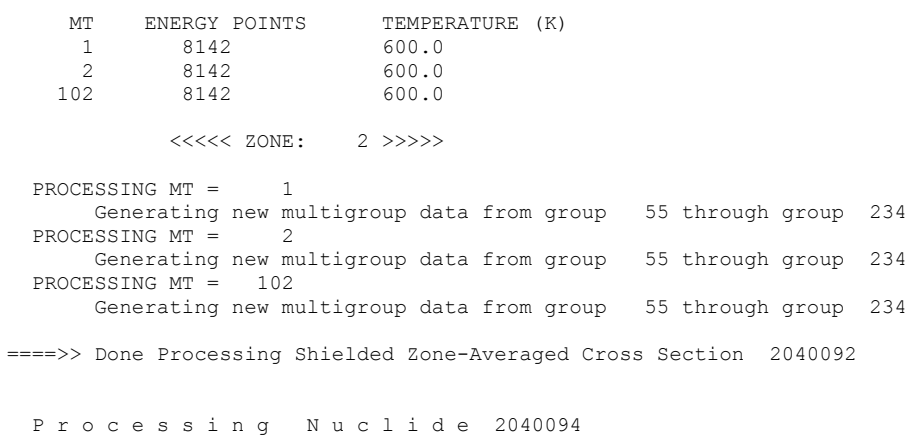

$\begin{array}{rccl}\text { MT } & \text { ENERGY POINTS } & \text { TEMPERATURE }(\mathrm{K}) \\ 1 & 59851 & 900.0 & \\ 2 & 59851 & 900.0 & \\ 18 & 59851 & 900.0 & \\ 102 & 59851 & 900.0 & 0.0 \\ 51 & 94 & 0.0 \\ 52 & 74 & 0.0 \\ 452 & 48 & 0.0 \\ 455 & 6 & 0.0 \\ 456 & 48 & \\ & & \end{array}$




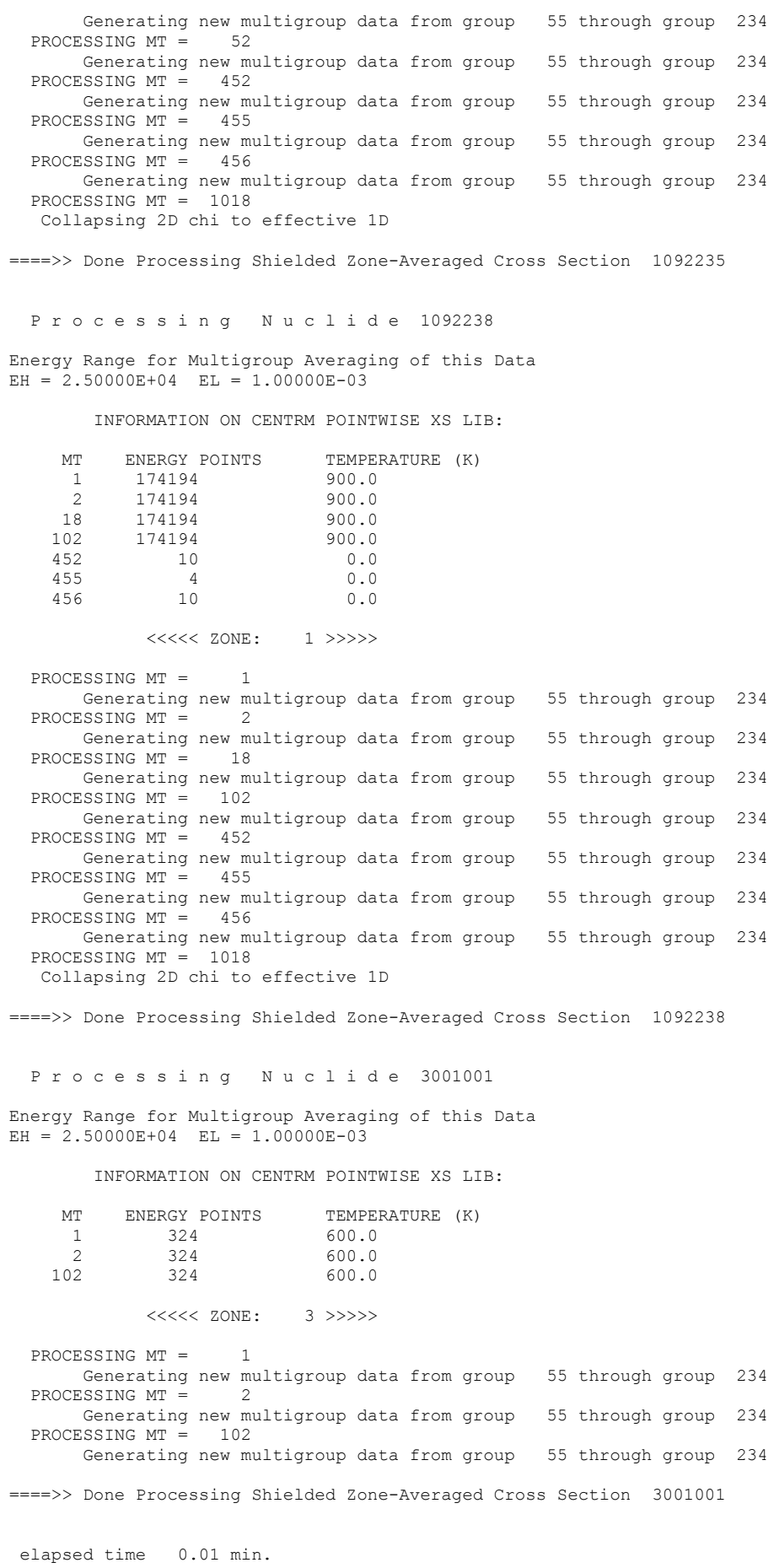



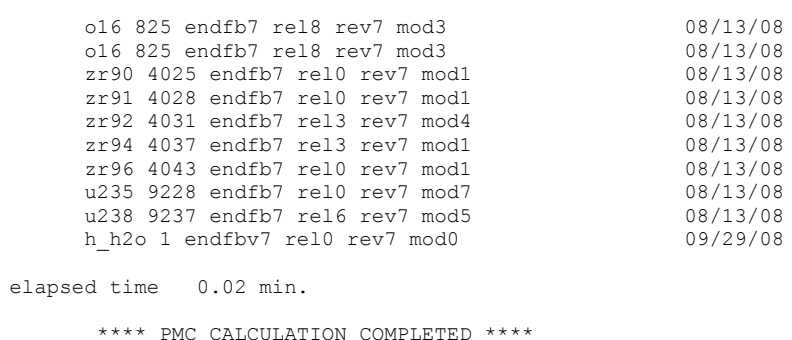

1008016

3008016

2040091

2040092

2040094

2040096

1092235

1092238

3001001

**** PMC CALCULATION COMPLETED ****

\subsubsection{Formats of Data Files}

The CENTRM chapter of the SCALE manual describes the format for the CENTRM PW nuclear data library and the format of the output PW flux file produced by CENTRM, which is input to the PMC code.

\subsubsection{References}

1. M. L. Williams and M. Asgari, "Computation of Continuous-Energy Neutron Spectra with Discrete Ordinates Transport Theory,” Nucl. Sci. Eng., 121, 173-201 (1995).

2. G. I. Bell and S. Glasstone, Nuclear Reactor Theory, Van Nostrand, Princeton, NJ, 1970.

3. J. A. Bucholz, A Method of Generating Multigroup Transfer Matrices Using an Analytical Angular Integration Free of Truncation Error, ORNL/NUREG/CSD-8, Union Carbide Corporation (Nuclear Division), Oak Ridge National Laboratory, September 1978. 


\subsection{A Alphabetical Index of Subroutines}

This section provides a convenient alphabetical index of the subroutines used in PMC, the subroutines that call them and the subroutines they call.

\begin{tabular}{|c|c|c|}
\hline $\begin{array}{l}\text { Subroutine } \\
\text { Name }\end{array}$ & $\begin{array}{l}\text { Calling } \\
\text { Subroutine }\end{array}$ & $\begin{array}{l}\text { Called } \\
\text { Subroutine }\end{array}$ \\
\hline $\operatorname{add} 2 \mathrm{~d}$ & xscal & pint \\
\hline addpxs & xscal & \\
\hline boss & pmc & $\begin{array}{l}\text { flxrec } \\
\text { process } \\
\text { replace } \\
\text { set } \\
\text { zonefl }\end{array}$ \\
\hline capy & replace & $\begin{array}{l}\text { cellav2 } \\
\text { factor } \\
\text { normal } \\
\text { repl2d }\end{array}$ \\
\hline cellav2 & capy & \\
\hline cellxs & process & \\
\hline dilutxs & xscal & \\
\hline factor & capy & iset \\
\hline flxrec & boss & \\
\hline normal & capy & iset \\
\hline pgen & pint & \\
\hline pint & $\operatorname{add} 2 d$ & $\begin{array}{l}\text { pgen } \\
\text { pinte } \\
\text { pinto }\end{array}$ \\
\hline pinte & pint & \\
\hline pinto & pint & \\
\hline pmc & & $\begin{array}{l}\text { boss } \\
\text { setup }\end{array}$ \\
\hline print & process & prtid \\
\hline process & boss & $\begin{array}{l}\text { cellxs } \\
\text { print } \\
\text { sumtot } \\
\text { xscal }\end{array}$ \\
\hline reorder & xscal & \\
\hline repl2d & capy & \\
\hline
\end{tabular}




\begin{tabular}{lll}
\hline $\begin{array}{l}\text { Subroutine } \\
\text { Name }\end{array}$ & $\begin{array}{l}\text { Calling } \\
\text { Subroutine }\end{array}$ & $\begin{array}{l}\text { Called } \\
\text { Subroutine }\end{array}$ \\
\hline replace & boss & Capy \\
\hline setup & pmc & mesage \\
\hline xscal & process & $\begin{array}{l}\text { add2d } \\
\text { addpxs } \\
\text { dilutxs } \\
\text { reorder }\end{array}$ \\
& & \\
& & \\
zonefl & boss & - \\
\hline
\end{tabular}

\subsection{B Alphabetical Index of Modules}

This section provides a list of the modules used in PMC and the subroutines that reference them.

\begin{tabular}{ll}
\hline Module & Referencing \\
Name & Subroutine \\
\hline antplu_c & pgen \\
& pinte \\
pinto
\end{tabular}




\begin{tabular}{|c|c|}
\hline $\begin{array}{l}\text { Module } \\
\text { Name }\end{array}$ & $\begin{array}{l}\text { Referencing } \\
\text { Subroutine }\end{array}$ \\
\hline vmgcwrd & $\begin{array}{l}\text { cellav2 } \\
\text { factor } \\
\text { normal } \\
\text { repl2d }\end{array}$ \\
\hline vtime & $\begin{array}{l}\text { boss } \\
\text { setup }\end{array}$ \\
\hline
\end{tabular}


7.6 CHOPS: MODULE TO COMPUTE POINTWISE DISADVANAGE FACTORS AND PRODUCE A CELL-HOMOGENIZED CENTRM LIBRARY

M. L. Williams and L. M. Petrie 


\section{TABLE OF CONTENTS}

$\underline{\text { Page }}$

7.6 CHOPS: Module to Compute Pointwise Disadvanage Factors and Produce a

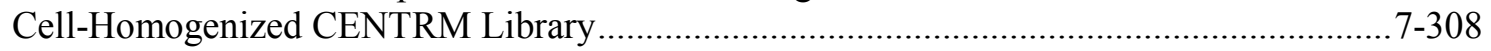

7.6.1 Introduction............................................................................................. 7-312

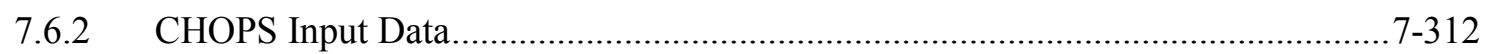

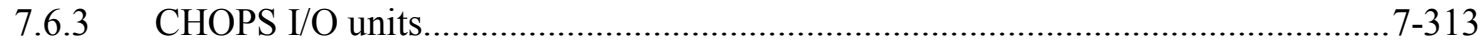

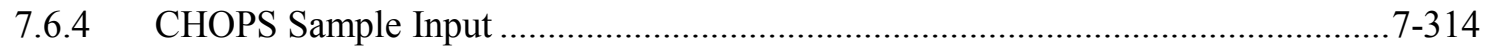




\section{LIST OF FIGURES}

$\underline{\text { Page }}$

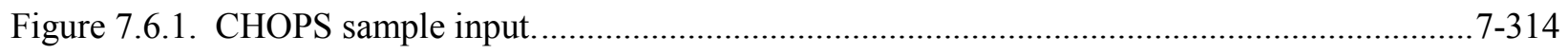




\section{LIST OF TABLES}

$\underline{\text { Page }}$

Table 7.6.1. Default I/O unit assignments for CHOPS ................................................................. 7-314 


\subsubsection{Introduction}

CHOPS (Compute HOmogenized Pointwise Stuff) computes pointwise (PW) disadvantage factors from the PW zone fluxes on a CENTRM output file, and then multiples the disadvantage factors by continuous-energy (CE) cross section data in a CENTRM library to generate a new cell-homogenized CENTRM CE library. The PW disadvantage factor for zone "Z", as a function of energy E, is calculated from the expression,

$$
D_{Z}(E)=\frac{\Phi_{Z}(E)}{\Phi_{C}(E)}
$$

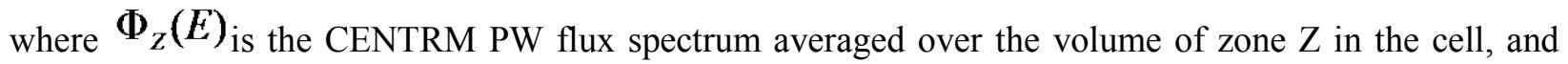
$\Phi_{C}(E)_{\text {is }}$ the PW flux averaged over the entire cell volume. The cell-homogenized CE cross section for a nuclide " $\mathrm{j}$ " is equal to

$$
\sigma_{C}^{(j)}(E)=\sum_{Z} F_{Z}^{(j)} D_{Z}(E) \sigma_{Z}^{(j)}(E)
$$

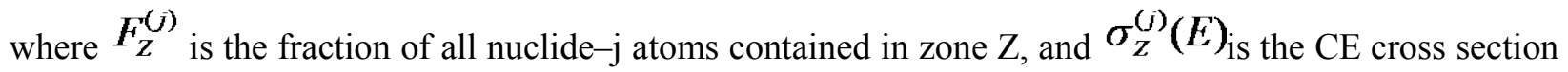
for nuclide-j at the temperature of zone $Z$. When multiplied by the cell-homogenized number density of nuclide-j and by the cell-average flux, the cross section expression in eq. 8.5.1 gives the correct average reaction rate at energy $\mathrm{E}$.

CHOPS is used in the automated double heterogeneity sequence in SCALE, in which a low-level heterogeneity, such as microspheres in a granular fuel element, are smeared into a homogenized absorber region appearing in the second level heterogeneity, such as fuel pellet or pebble appearing in a lattice. The disadvantage factors provide for flux weighting of the PW XS data so that the spatial self-shielding is treated correctly in the homogenized geometry. A second CENTRM PW transport calculation is performed with the cell-averaged PW library output by CHOPS in order to account for the additional selfshielding of the absorber pellets/pebbles in the lattice. CHOPS is called automatically by the XSProc module for double-heterogeneous unit cells, or it can run as a standalone code.

\subsubsection{CHOPS Input Data}

CHOPS input is read using the FIDO input processor. Only one input data block is required, as described below.

\section{DATA BLOCK 1}

0\$\$ LOGICAL UNIT ASSIGNMENTS (10 entries. Default values given in parentheses)

1. lold -- logical unit number of input CENTRM XS library

2. Inew -- logical unit number of output CENTRM homogenized XS library

3. Iflx -- logical unit number of input CENTRM PW flux library

4. 1dis -- logical unit number for edit of PW disadvantage factors

5. n15 -- logical unit number for scratch

6. n16 -- logical unit number for scratch

7. n17 -- logical unit number for scratch

8. n18 -- logical unit number for scratch 
9. n19 -- logical unit number for scratch

10. nsq -- sequence number used in filename on unit "lnew"

[Example: if $\operatorname{lnew}=11$ and $n s q=3$ : output filename of homogenized library $=f t 11 f 003]$

1\$\$ INTEGER PARAMETERS (5 entries)

1. idtap -- identifier for the new library

[for macro library, the value of idtap is made negative]

2. nprt $\quad-$ output print option: $0=>\min$ print; $1=>$ normal; $2=>\max$ print

3. iden $\quad--$ if $=0=>$ define homogenized XS id $=$ id on CENTRM flux file if $>0=>$ define homogenized XS id to be, $\left(\right.$ iden $\left.* 10^{6}+\mathrm{ZA}\right)$

4. macr -- type of XS output: $0=>$ microscopic ; $1=>$ macroscopic

5. icorr -- not used

$2^{* *}$ REAL PARAMETERS (3 entries)

1. tole -- tolerance used to thin pointwise cross-sections

( 0.0 means no thinning is done )

2. cleth -- maximum lethargy between thinned pointwise cross-sections points that allow a point to be discarded

3. vfrac -- multiplier applied to all output XS's [eg, grain fraction]

$\mathrm{T}$ [ TERMINATE DATA BLOCK 1 ]

\subsubsection{CHOPS I/O units}

Table 7.6.1 shows default logical unit numbers used by CHOPS. These values may be changed in the $0 \$ \$$ array of input. 
Table 7.6.1. Default I/O unit assignments for CHOPS

\begin{tabular}{cl}
\hline Unit number & \multicolumn{1}{c}{ Description } \\
\hline 1 & Input CENTRM CE data library \\
2 & Output homogenized CENTRM CE data library \\
3 & Input pointwise CENTRM flux file \\
15 & Scratch file \\
16 & Scratch file \\
17 & Scratch file \\
18 & Scratch file \\
19 & Scratch file \\
\hline
\end{tabular}

\subsubsection{CHOPS Sample Input}

The sample case in Figure 7.6.1 first executes a CENTRM unit cell geometry calculation using the CSAS-MG sequence, which by default generates the PW flux file on unit 15, as well as the CE nuclear data library on unit 81 for input to CHOPS. The standalone CHOPS code then computes a cellhomogenized CE library for the unit cell. The new homogenized CENTRM CE library is output on unit 91with filename: $f t 91 f 001$

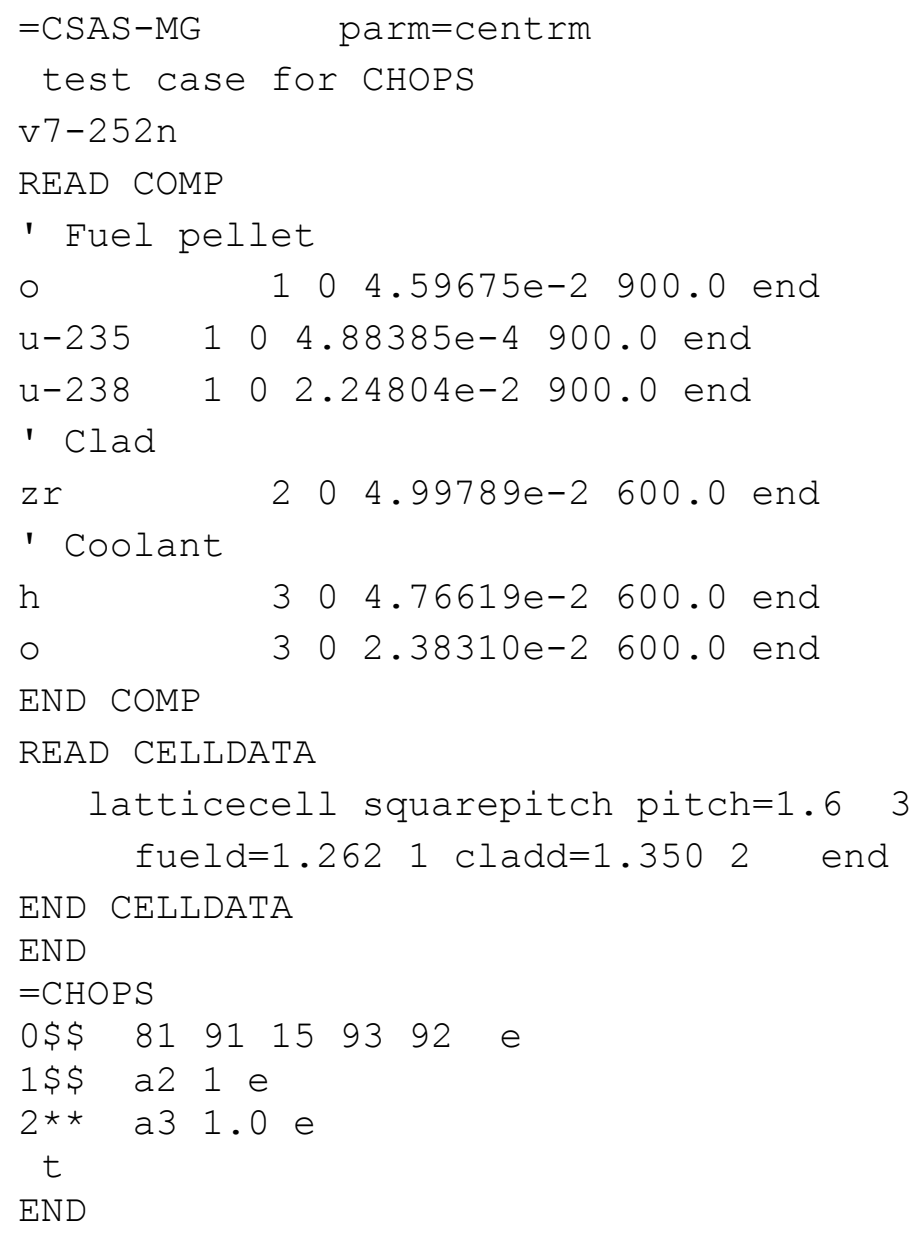

Figure 7.6.1. CHOPS sample input. 
7.7 CRAWDAD: MODULE TO PRODUCE CENTRM-FORMATTED CONTINUOUS-ENERGY NUCLEAR DATA LIBRARIES

M. L. Williams, D. Wiarda, and S. W. D. Hart 


\section{TABLE OF CONTENTS}

$\underline{\text { Page }}$

7.7 CRAWDAD: Module to Produce CENTRM-Formatted Continuous-Energy Nuclear

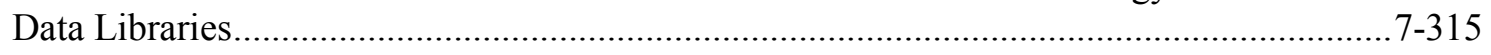

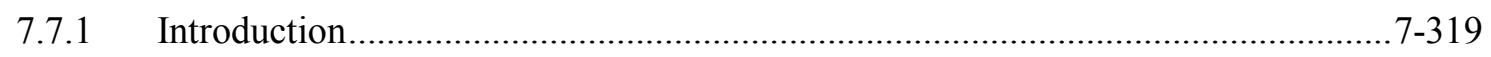

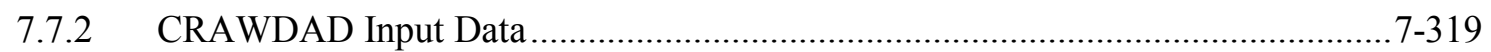

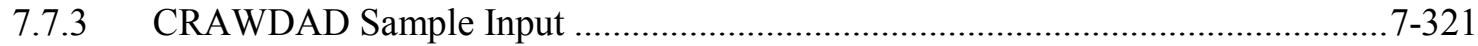

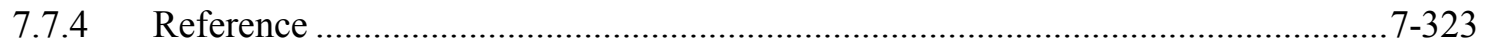




\section{LIST OF FIGURES}

$\underline{\text { Page }}$

Figure 7.7.1. CRAWDAD input generated by CSAS1 sample.................................................... 7-321

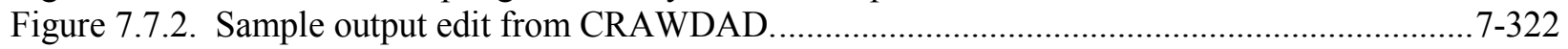




\section{ACKNOWLEDGMENTS}

The authors would like to acknowledge the important contributions to CRAWDAD made by former ORNL staff N. M. Greene and D. F. Hollenbach. 


\subsubsection{Introduction}

SCALE uses the code CRAWDAD (Code to Read And Write DAta for Discretized solution) to read nuclear data from the SCALE-6 continuous (CE) library files, and write it to an output file in the particular format needed for the discretized energy solution in CENTRM. Prior to SCALE-6, the CE data used by the CENTRM and PMC modules were distributed directly in the CENTRM library format. However beginning with SCALE-6, the same CE data are used by both CENTRM/PMC and by the CE versions of the KENO and Monaco Monte Carlo codes. CE nuclear data for each nuclide are stored in individual files contained in the SCALE permanent data directory. CRAWDAD reads the files for each material appearing in a problem and combines all data into a single problem-dependent CENTRM library file stored in the temporary directory for execution.

All SCALE-6 calculations that use modules CENTRM and PMC for self-shielding multigroup (MG) cross sections must first execute the CRAWDAD computational module. During execution of SCALE sequences, the XSProc self-shielding module automatically executes CRAWDAD whenever the CENTRM/PMC method is specified. CRAWDAD also can be run in stand-alone mode to process and save a CENTRM-formatted library for subsequent CENTRM/PMC calculations.

PMC allows the energy range of the CE data to be selected, as well as which reactions are placed on the output CENTRM library. The output CENTRM library always contains the following "standard" nuclear data types for all materials: total (1); elastic (2); complete inelastic (4); radiative capture (102); fission (18); total/prompt/delayed nubars $(452,455,456)$; and $(\mathrm{n}, \alpha)$ cross section for $10 \mathrm{~B}$ and $7 \mathrm{Li}$. In this list, the number shown in parenthesis corresponds to the ENDF/B "mt numbers."

$\mathrm{CE}$ data are obtained for arbitrary energies by linear interpolation of discrete cross sections defined on a pointwise (PW) energy mesh. The PW energy mesh for a given nuclide is sufficiently fine that error introduced by linear interpolation between any two points is less than $0.1 \%$. CRAWDAD also interpolates the $\mathrm{CE}$ data to the specific temperatures needed for the problem. The default temperature interpolation method uses square-root of temperature below 1200 Kelvin and a finite difference procedure above this temperature ${ }^{(1)}$.

\subsubsection{CRAWDAD Input Data}

For standalone CRAWDAD execution, the user prepares the FIDO input deck as described below. However during a SCALE sequence computation, the XSProc module always executes CRAWDAD for CENTRM/PMC self-shielding calculations, and defines appropriate CRAWDAD parameter values based on specified CENTRM and PMC options. This is the recommended mode of operation. Some XSProc default values for CRAWDAD can be changed using keywords in the CENTRM DATA block; e.g., see parameters $m$ tout $=$ and $k e r n e l=$ in section 8.1.3.9. Several options available for stand-alone execution cannot be controlled by keywords in the sequence runs, as these are set automatically

\section{CRAWDAD STANDALONE INPUT}

\section{$* * * * * * * * * * * * * * \quad$ DATA BLOCK 1}

0\$\$ LOGICAL UNIT ASSIGNMENTS [4 entries. Default values given in parentheses]

$\begin{array}{lll}\text { Entry Number } & \text { Variable Name Description Default Value } \\ 1 & \text { lcen } & \text { logical unit number of output CENTRM library } \\ 2 & \text { n17 } & \text { logical unit for scratch }(17) \\ 3 & \text { n18 } & \text { logical unit for scratch }(18)\end{array}$

3 n18 logical unit for scratch (18) 
$4 \quad$ n19 logical unit for reading CE-KENO libraries

$1 \$ \$$ INTEGER PARAMETERS [10 entries ]

$1 \quad$ num_nucs number of PW nuclides to process (1)

2 idtap ${ }^{-}$identifier placed on header of output CENTRM library (66666)

3 iprt print out option (1)

-1 no print out AT ALL

0 hardly any print

1 normal print

2 debug print

4 obsolete feature

5 iterp temperature interpolation method for PW cross sections (0)

0 square-root-T interpolation for $\mathrm{T}<1200 \mathrm{~K}$ and finite difference for $\mathrm{T}>1200 \mathrm{~K}$

1 square-root-T interpolation for all temperatures

2 finite difference interpolation for all temperatures

6 libth create CENTRM thermal kernel library for bound moderators (1)

0 no

1 yes (output kernel file is named lib_cen_kern)

7-10 N/A extra integer parameters (not used) (0)

$1 * * \quad$ REAL PARAMETERS [10 entries]

1 teps tolerance on temperature differences (5.0)

( temperatures within $+/-$ "teps" are assumed equal)

2 tole not implemented

3-10 N/A extra real parameters (not used) $(0.0)$

$\mathrm{T} \quad$ terminate data block 1

\section{$* * * * * * * * * * * * * * \quad$ DATA BLOCK 2}

***** Repeat data block(s) 2 and 3, stacked "num_nucs" times to create a new CENTRM library containing specified temperatures and reaction types

2\$\$ NUCLIDE INFORMATION [5 entries]

Entry Number Variable Name Description Default Value

1 za zaid for this nuclide in PW XS library

2 lver version number of evaluated nuclear data (e.g, 7 for ENDF/B-VII)

3 mod desired mod number of evaluated nuclear $(-1)$

$-1=>$ use latest mod

4 inum desired number of temperatures for this nuclide (0)

0 - put all available temperatures on output CENTRM library

$\mathrm{n}$ - include data at the " $\mathrm{n}$ " temperatures in $4 * *$ array

5 mtout MTS to be included on output CENTRM PW library (2)

0 - output PW data for all available MTs

1 - output PW data only for default standard MTs:

$1,2,4,102,18,452,455,456$ for all materials; and 107 for $10 \mathrm{~B}$ and $7 \mathrm{Li}$ 
2 - output standard MTs, plus inelastic levels and (n,2n)

3 - standard MTs plus those listed in $5 \$ \$$ array

-3 - out all MTs EXCEPT those listed in $5 \$ \$$

6 kmod mod number for ENDF thermal scattering law data (-1)

$\geq 0$ - use cross section data with this thermal mod number

-1 - use cross section data with latest thermal mod and kernel (if available)

-2 - do not include bound kernel data (i.e., free-gas scattering will be used in CENTRM)

$7 \quad$ lsrc Source of nuclear data (0 only allowed at present)

0/1/2/3/4 $\Rightarrow$ ENDF/JEF/JENDL/BROND/CENDL

$3 * *$ ENERGY LIMITS [2 entries]

1 pemin minimum energy for PW data $(0.0001 \mathrm{eV})$

2 pemax maximum energy for PW data $(20 \mathrm{MeV})$

$\mathrm{T}$ terminate data block 2

$* * * * * * * * * * * * * * \quad$ DATA BLOCK 3

$* * * * *$ Only enter if inum $>0$, or mtout $=+/-3$ )

4** DESIRED TEMPERATURES for this nuclide [inum entries]

$5 \$ \$$ MT VALUES (if mtout $=+/-3$ ) [always end with an "E"]

$\mathrm{T}$ terminate data block 3

Optional 72 character title for the CENTRM library

\subsubsection{CRAWDAD Sample Input}

Figure 7.7.1 shows an example input file for standalone execution of CRAWDAD. The CRAWDAD output for this case is shown in Figure 7.7.2. In more typical cases where CRAWDAD is executed automatically by the XSProc module as part of a SCALE sequence calculation, no CRAWDAD input is needed, but similar CRAWDAD output will be printed.

Figure 7.7.1. CRAWDAD input generated by CSAS1 sample.

\begin{tabular}{|c|c|c|c|c|c|c|c|c|}
\hline \multicolumn{9}{|c|}{$=$ crawdad } \\
\hline $0 \$ \$$ & 81 & 17 & \multicolumn{2}{|c|}{18} & \multicolumn{2}{|l|}{77} & & e \\
\hline $1 \$ \$$ & 5 & 66666 & & 0 & 0 & & 1 & e \\
\hline 1 ** & \multirow{2}{*}{\multicolumn{3}{|c|}{$5.00 \mathrm{E}+00$}} & & & & & e \\
\hline \multicolumn{5}{|c|}{ 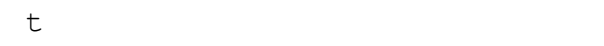 } & & & & \\
\hline $2 \$ \$$ & \multirow{2}{*}{$\begin{array}{r}8016 \\
* \quad 1 .\end{array}$} & 7 & 3 & 2 & 2 & -1 & 0 & \\
\hline \multirow{2}{*}{\multicolumn{2}{|c|}{$\begin{array}{l}3 * * \\
4 * *\end{array}$}} & \multicolumn{2}{|c|}{$1.00-03$} & \multicolumn{3}{|c|}{$1.30+04$} & & $2 t$ \\
\hline & & $00+02$ & & \multicolumn{3}{|c|}{$9.00+02$} & & $3 t$ \\
\hline $2 \$ \$$ & \multicolumn{3}{|c|}{$13027 \quad 7$} & 1 & 2 & -1 & 0 & \\
\hline \multicolumn{2}{|c|}{$3 * \star$} & \multicolumn{2}{|c|}{$1.00 \mathrm{E}-03$} & \multirow{2}{*}{\multicolumn{4}{|c|}{$1.30 \mathrm{E}+04$}} & $2 t$ \\
\hline \multirow{2}{*}{\multicolumn{3}{|c|}{$\begin{array}{l}4^{* *} \quad 6.50 \mathrm{E}+02 \\
\$ 922357\end{array}$}} & & & & & & $3 t$ \\
\hline & & & 7 & 1 & 2 & -1 & & 0 \\
\hline
\end{tabular}




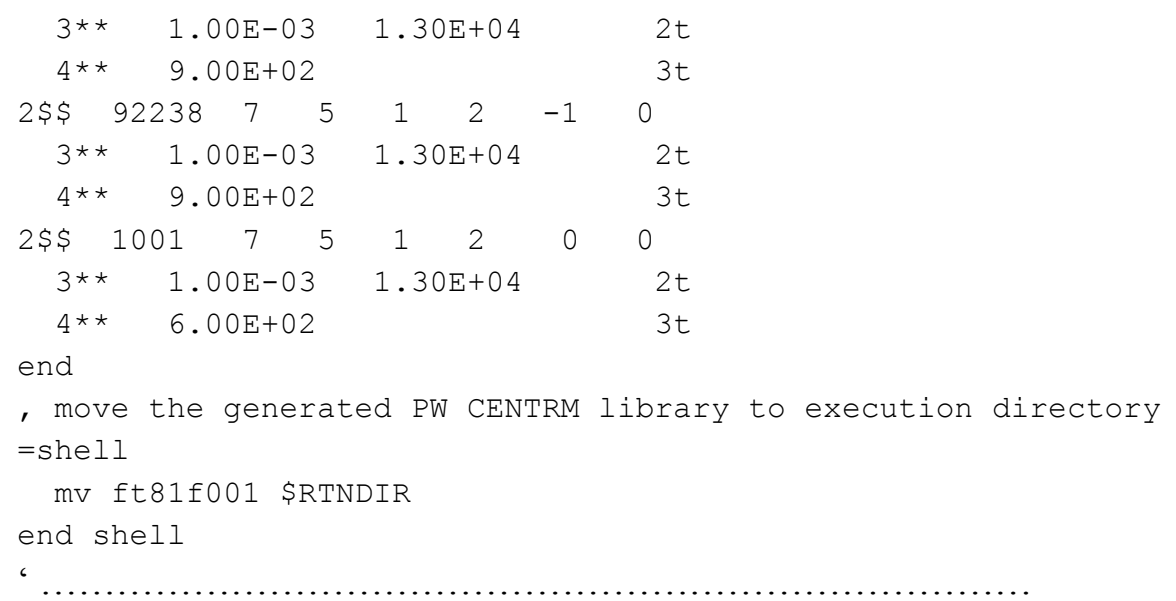

Figure 7.7.2. Sample output edit from CRAWDAD.

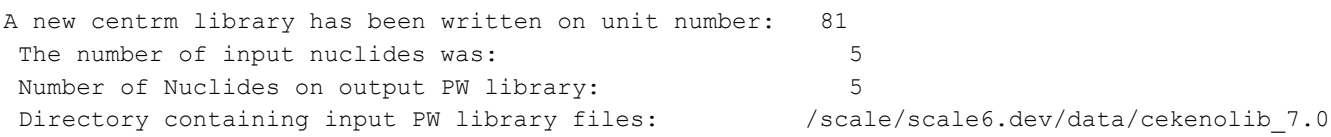

\begin{tabular}{|c|c|c|c|c|c|c|c|}
\hline Entry & $\mathrm{ZA}$ & Data SrC & Vers No. & Mod No. & MT-Optn & Thermal ID & XS temperatures \\
\hline---- & ---- & -------- & -------- & ------- & ------- & ---------- & 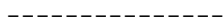 \\
\hline \multirow[t]{2}{*}{1} & 8016 & endf & 7 & 3 & 2 & 0 & 600.00 \\
\hline & & & & & & & 900.00 \\
\hline 2 & 13027 & endf & 7 & 1 & 2 & 0 & 650.00 \\
\hline 3 & 92235 & endf & 7 & 7 & 2 & 0 & 900.00 \\
\hline 4 & 92238 & endf & 7 & 5 & 2 & 0 & 900.00 \\
\hline 5 & 1001 & endf & 7 & 5 & 2 & 7000001 & 600.00 \\
\hline
\end{tabular}

Nuclides in Problem-Dependent Thermal Kernel Library

$\begin{array}{rr}\text { Library Identifier: } & 901 \\ \text { Number of kernels: } & 1 \\ \text { Order of Scattering: } & 6 \\ \text { ber of Temperatures: } & 9\end{array}$

Maximum Order of Scattering:
9 aximum Number of Temperatures:

Library Directory

Nuclide Identifier Sigfree File

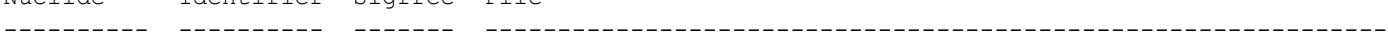

h(h20) $\quad 7000001 \quad 20.48$ endf_b/vers7/1-0

logical 18 (problem dependent centrm thermal kernel library)

dataset name: /usr/tmp/xmw.9890/lib_cen_kernel volume:

CRAWDAD has terminated normall 


\subsubsection{Reference}

1. S. W. D. Hart, C. Celik, G. I Maldonado, L. Leal, "Creation of problem-dependent Doppler-broadened cross sections in the KENO Monte Carlo code," Annals of Nuclear Energy Vol 88, p49, Feb. 2016. 


\title{
7.8 MCDANCOFF DATA GUIDE
}

L. M. Petrie, B. T. Rearden

\begin{abstract}
The MCDancoff program is used to calculate Dancoff factors in complicated, three-dimensional (3-D) geometries using Monte Carlo integrations. The geometries are standard SCALE geometry descriptions, with the current restriction that Dancoff factors can only be calculated for regions bounded by cuboids, spheres, or cylinders. Multiple Dancoff factors can be calculated with one input file.
\end{abstract}




\section{TABLE OF CONTENTS}

$\underline{\text { Page }}$

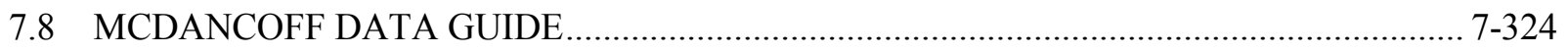

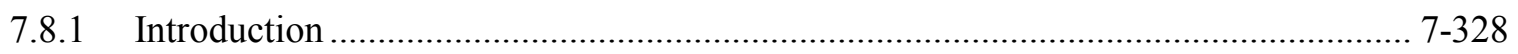

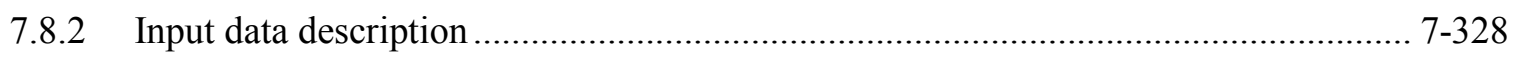

7.8.3 Calculation and use of 3-d Dancoff factors ...................................................... 7-329

7.8.4 Example Case ......................................................................................... 7-330 


\section{LIST OF FIGURES}

$\underline{\text { Page }}$

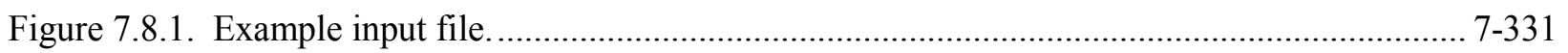

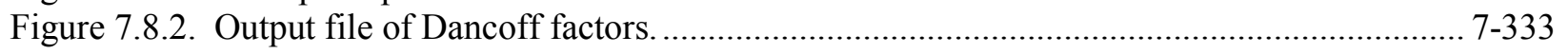




\section{ACKNOWLEDGMENT}

This work was sponsored in part by Atomic Energy of Canada, Ltd. The contribution of S. J. Poarch in preparing this document is gratefully acknowledged. 


\subsubsection{Introduction}

MCDancoff (Monte Carlo Dancoff) is a program that calculates Dancoff factors for complicated, threedimensional geometries. Its input is a slight modification of a CSAS6 input file which uses the standard SCALE geometry as detailed for KENO-VI. The modifications to the input involve different input in the START data block describing which Dancoff factors are to be calculated. The calculation involves starting histories isotropically on the surface of the region for which the Dancoff factor is to be calculated and following the path of each history until it has encountered all the elements of the material in the region, or until it has exited the system. A one group cross-section library is used to determine the total cross sections of the mixtures in the problem.

A current restriction of MCDancoff is that it can only calculate Dancoff factors for regions bounded by cylinders, spheres, or cuboids. Other simple bodies could be added in the future, but a general bounding surface would be impractical.

The Dancoff factors are used in SCALE to correctly self-shield multigroup cross sections for a given problem; either as input to BONAMI or to determine an equivalent cell for CENTRM. This is most typically accomplished through the MORE DATA and CENTRM DATA blocks documented in Sect. M7.

The Dancoff factors are actually calculated by a modified version of the KENO-VI code called KENO_Dancoff. All printed output from these calculations is suppressed by default. If there is a need to see this output (for example, to find an error message), it can be turned on by setting an environment variable print_dancoff $=$ yes.

\subsubsection{Input data description}

MCDancoff input data is the same as CSAS6 input data with the following exceptions. A special one group cross-section library will be used. It can be specified as $\boldsymbol{x n 0 1}$ in the input but will be set to this if anything else is entered for the library. Because MCDancoff is running a fixed source problem, and the Dancoff factor doesn't need to be calculated with the same accuracy as an eigenvalue, there are useful changes that can be made to the parameters in the PARAMETER data block. Section 7.8.3 discusses this in more detail. Finally, the START data block is used to define which Dancoff factors will be calculated. This data block is defined below.

READ START Begins the data block

1. dancoff begins defining a new Dancoff factor. Always start relative to the global unit in the geometry.

2. array step into an array contained in the current unit - followed by karray, nbx, nby, $\mathbf{n b z}$ where karray is the region containing the array in the current unit, $\mathbf{n b x}$ is the $\mathrm{x}$ position in the array of the next unit, nby is the y position in the array of the next unit, and $\mathbf{n b z}$ is the $\mathrm{z}$ position in the array of the next unit.

3. hole step into a hole contained in the current unit - followed by nhole, the hole number relative to the current unit.

4. unit final unit in the nesting chain - followed by $\mathbf{n n}$, the unit number 
5. region region to calculate the Dancoff factor for - followed by $\mathbf{k}$, the relative geometry word in unit nn defining the outer bound of the region.

6. nst if input, must be 0 (defaults to 0 ).

Repeat 2 and 3 to get from the global unit to the final unit $\mathbf{n n}$.

Repeat $1-5$ for each Dancoff factor to be calculated.

END START $\quad$ Ends the data block

\subsubsection{Calculation and use of 3-d Dancoff factors}

1. The 3-D Dancoff factors are computed with KENO-VI geometry. If beginning with CSAS5 model, use C5TOC6 to convert to CSAS6.

2. Change sequence name from CSAS6 to MCDancoff and change cross-section library to $\boldsymbol{x n 0 1}$.

3. Input appropriate parameter data.

Since the Dancoff calculation is fixed source integration, there is no need to skip generations, and nsk should be set to 0 . Since small changes to the Dancoff have very minor effects on the cross sections, fewer histories are probably needed for calculating the Dancoff than for calculating $k_{\text {eff. }}$. Thirty thousand histories divided as 100 generations of 300 histories per generation has produced Dancoff factors with deviations of less than 1 percent. It may be advantageous to turn off plots at this point. Since the same parameters can be entered more than once, with the final entry being the one used, adding a separate record with these values immediately before the end parameter keywords would override the original KENO-VI parameters.

Example:

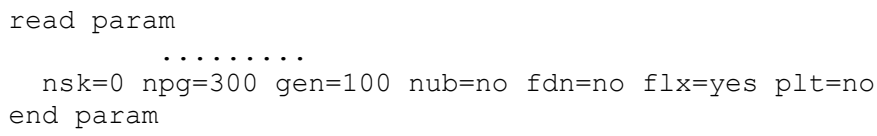

4. Identify the region for which Dancoff factors are desired in START data.

The start type needs to be set to $\mathbf{0}$ for the Dancoff calculation (this is the default). All KENO-VI START data should be removed or commented out by placing an apostrophe in column 1. Each region for which a Dancoff calculation is desired then starts with the keyword dancoff. This is followed by data that specify the relationship of the global unit to the specific geometry description of the region. If the region is nested inside an array, then the keyword, array, is specified, followed by four integers. The first integer is the indices of the media record specifying the array relative to the current unit. The next 3 integers are the $\mathrm{X}, \mathrm{Y}$, and $\mathrm{Z}$ indexes of the position of the next unit in the array. If the region is nested in a hole, then the keyword, hole, is specified, followed by the relative count of the correct hole in the unit. The preceding data are repeated (in the correct nesting order starting with the global unit) until reaching the unit where the region is located. Then the keyword, unit, followed by the unit number is given, followed by the keyword, region, followed by the relative index of the geometry keyword describing the desired region with respect to that unit. Currently, only cylinders, spheres, and cuboids are programmed for calculating Dancoff factors. 
Examples:

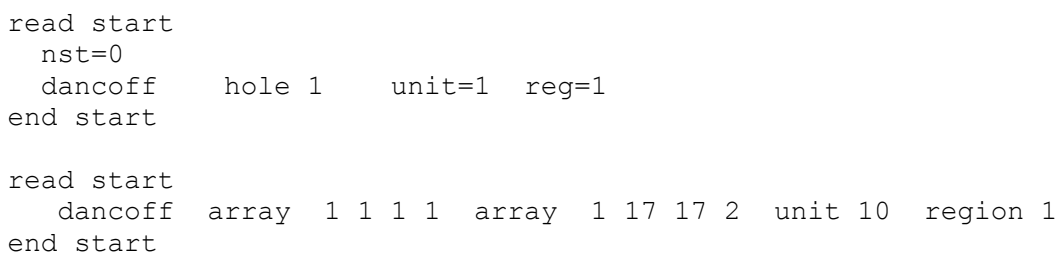

5. Execute MCDANCOFF filename.input file like any other SCALE input file.

6. Examine filename.dancoff file, which will contain Dancoff factors for each nuclide in the specified region

$\begin{array}{rrrr}\text { index } & \text { nuclide } & \text { dancoff } & \text { deviation } \\ 1 & 92234 & 3.36340 \mathrm{E}-01 & 1.81134 \mathrm{E}-03 \\ 2 & 92235 & 3.36340 \mathrm{E}-01 & 1.81134 \mathrm{E}-03 \\ 3 & 92236 & 3.36340 \mathrm{E}-01 & 1.81134 \mathrm{E}-03 \\ 4 & 92238 & 3.36340 \mathrm{E}-01 & 1.81134 \mathrm{E}-03 \\ 5 & 8016 & 1.00000 \mathrm{E}+00 & 0.00000 \mathrm{E}+00\end{array}$

7. Once all desired Dancoff factors are obtained, return to original model and enter CENTRM DATA for each cell with dan2pitch(mix) specified.

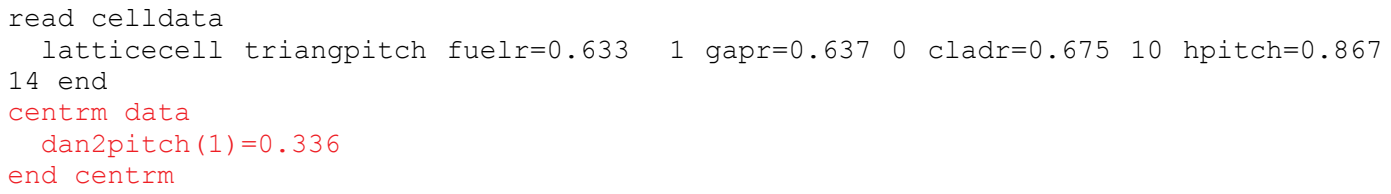

8. If executing TSUNAMI-3D, additional steps are necessary because TSUNAMI-3D does not treat the dan2pitch input parameter.

Return to the original TSUNAMI-3D input file and replace the sequence name to "CSAS-MG PARM $=$ CHECK" and delete all data after the unit cell data to quickly obtain revised pitch values. (Note: CSAS will not modify cell dimensions to more than $20 \mathrm{~cm}$, so a revised moderator density may need to be entered to obtain the desired Dancoff factor.) Search for the word "desired" in output file to find new pitch values for each cell.

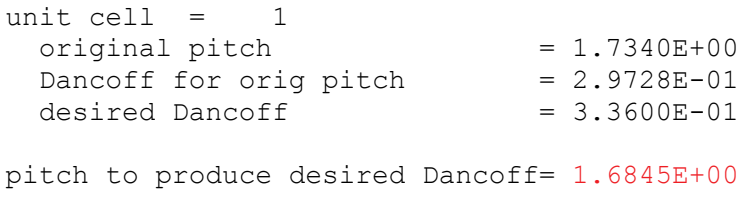

9. Enter revised pitch and revised moderator density (for cell calculation only, not for geometry model) in TSUNAMI model.

latticecell triangpitch fuelr=0.633 1 gapr=0.637 0 cladr=0.675 10 pitch=1.6845 14 end

\subsubsection{Example Case}

The following is a contrived case to illustrate an input file using both holes, arrays, and multiple sets of Dancoff factors (although both factors apply to the same pin, so only one set can be used). The case 
represents two fuel assemblies in a cylindrical tank, each assembly having a poisoned central pin, and four water holes. The Dancoff factors are calculated for each central pin. The input file is listed in Figure 7.8.1.

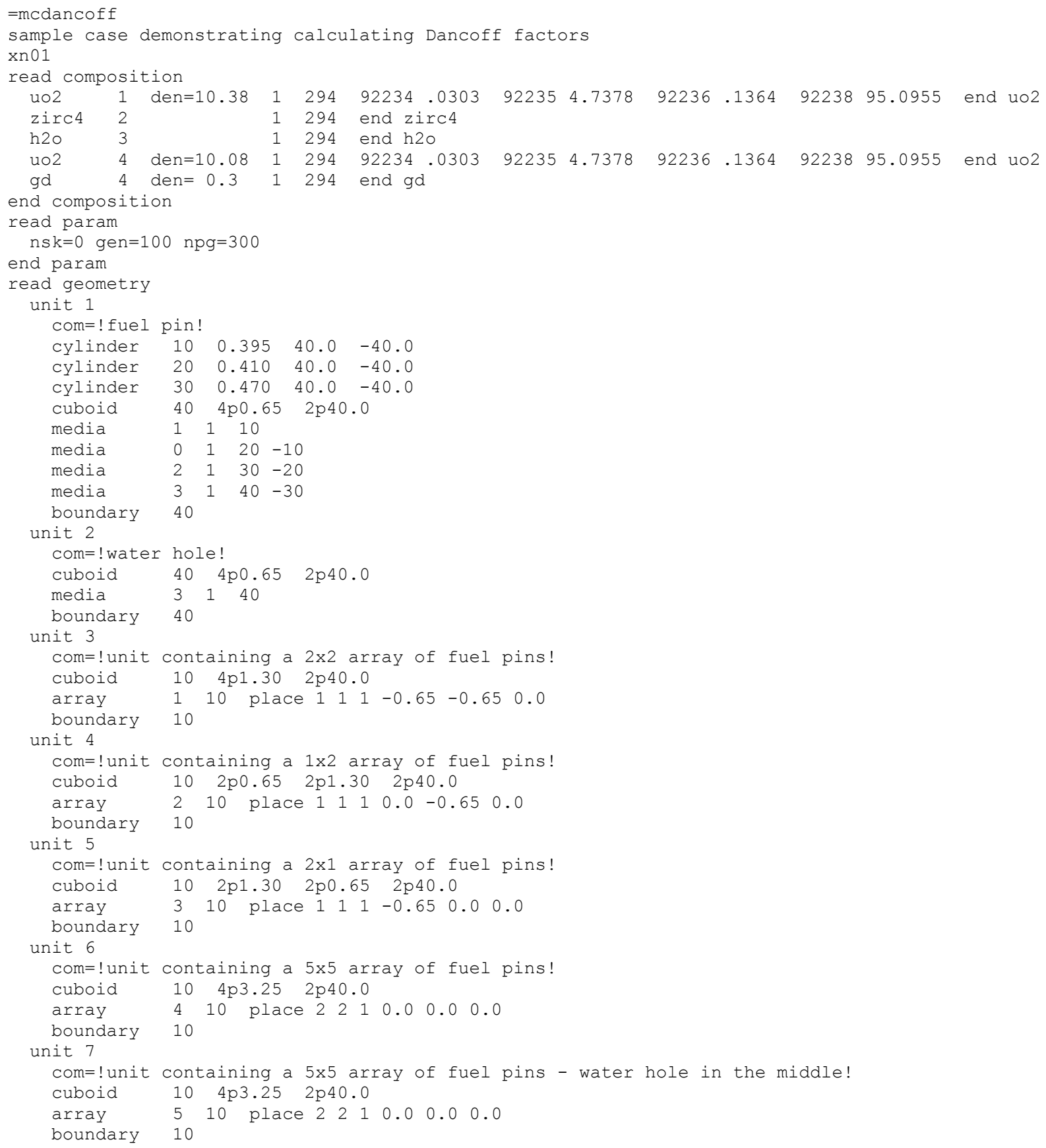

Figure 7.8.1. Example input file. 


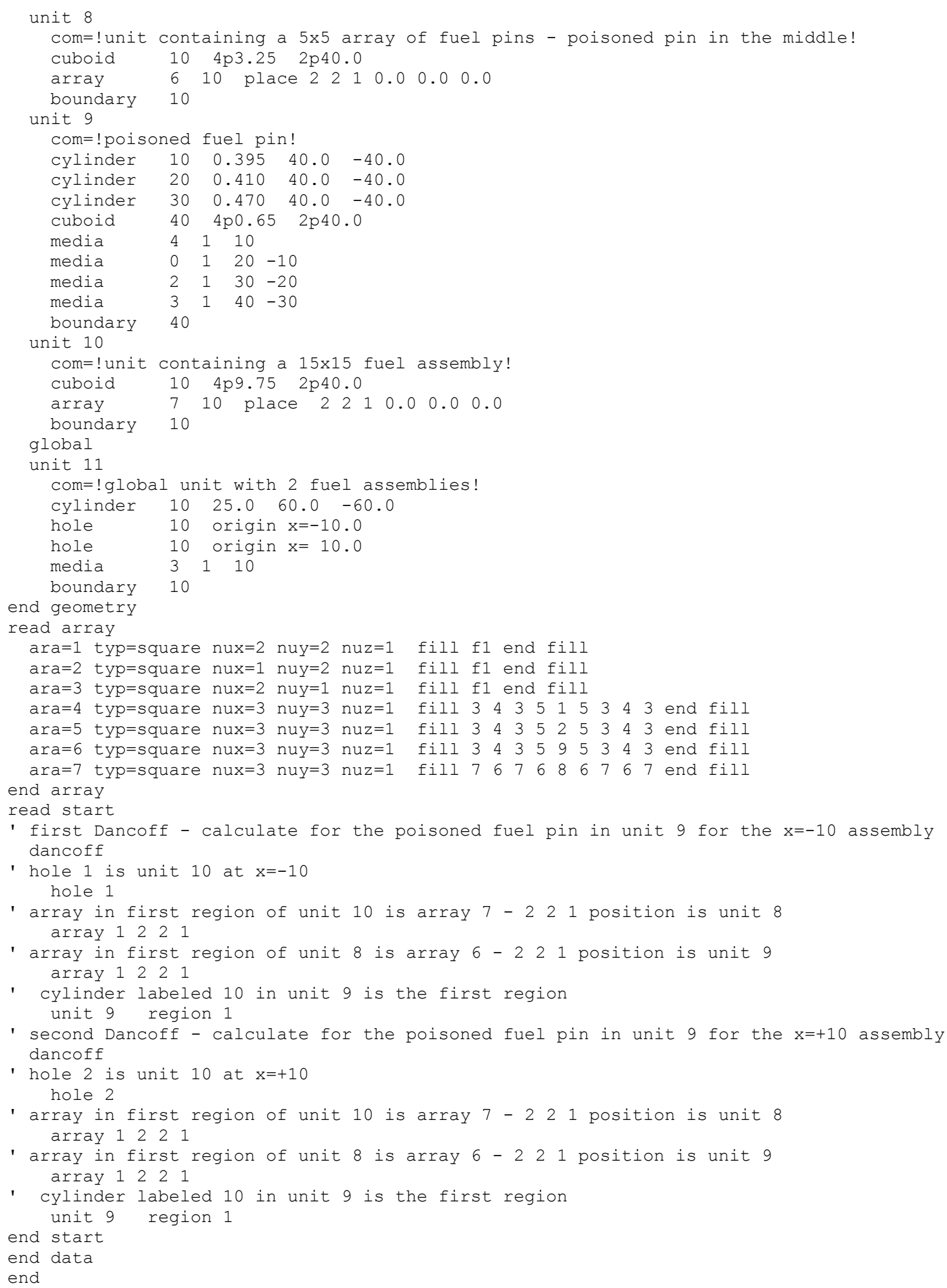

Figure 7.8.1. Example input file (continued). 
This input file creates two files of Dancoff factors. The first such file is listed in Figure 7.8.2.

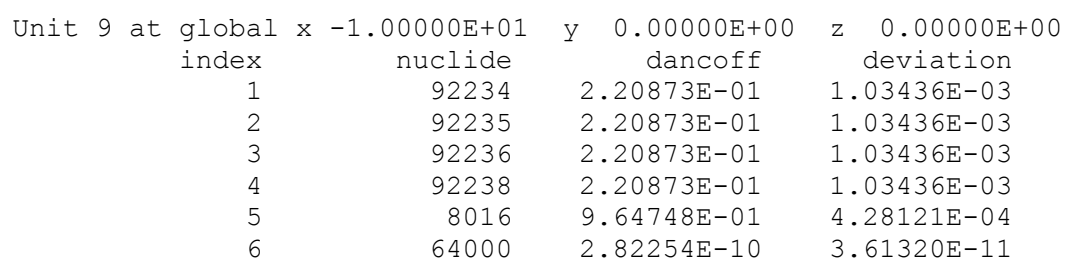

Figure 7.8.2. Output file of Dancoff factors.

The second file is statistically the same, as it solved for the mirror image Dancoff factor. 
7.9 CAJUN: MODULE FOR COMBINING AND MANIPULATING CENTRM CONTINUOUSENERGY LIBRARIES

L. M. Petrie and N. M. Greene 


\section{TABLE OF CONTENTS}

$\underline{\text { Page }}$

7.9 CAJUN: Module for Combining and Manipulating CENTRM Continuous-Energy

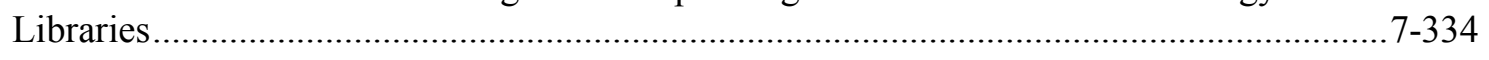

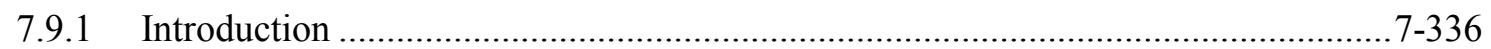

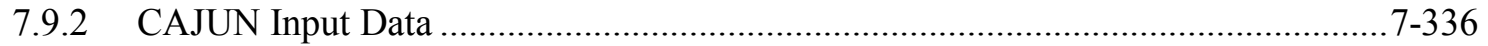

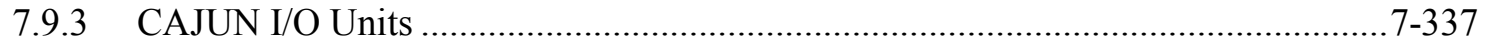




\subsubsection{Introduction}

CAJUN is a program used to combine continuous-energy (CE) cross section libraries for use in the crosssection processing codes CENTRM and PMC. It is used primarily by SCALE sequences when processing pointwise cross section data for DOUBLEHET unit cells with the XSProc module (see section 8.1.1), although it can also be run standalone in conjunction with CRAWDAD. CAJUN combines multiple CE libraries into a single library, adds selected nuclides from one library into another library, deletes nuclides from a specified library, or renames nuclides in a library. CAJUN performs an analogous function for CE libraries as AJAX does for multigroup libraries.

In order for input CE libraries to be read by CAJUN, they must be assigned a unit number from 1 to 99. The SHELL module can be used to link individual CE libraries to appropriate file names that are accessible by unit number.

\subsubsection{CAJUN Input Data}

Input data for CAJUN is read into the program using FIDO type input. The data is divided into three data blocks. The first data block provides the output file number and number of libraries processed. The second data block provides input library numbers, number of nuclides in each library, and whether nuclides are selected by MAT or ZA number. The third data block provides current nuclide MAT or ZA

numbers and new nuclide MAT or ZA numbers. Detailed description of the CAJUN input data is provided below.

\section{Data Block 1}

$0 \$$ unit assignments (1)

1. lcen - logical unit number of output CE CENTRM library (1)

$1 \$ \$$ number of files to process (2)

1. nfile - the number of CENTRM CE libraries to process (1)

2. idtap - identifier for the new library (0)

$\mathrm{T}$ terminate data block 1

$* * *$ repeat data block(s) 2 and 3 "nfile" times to create new library

***************************************************************************

Data Block 2

$2 \$$ file selection and treatment option (4)

1. $\log$ - logical unit number of input CENTRM CE library (77)

2. inum - number of nuclides selected from this library (0)

0 - select all nuclides on the library as is.

$-n-$ select all nuclides on the library as is except for those indicated in the $3 \$ \$$ array.

$\mathrm{n}-$ select the "n" nuclides on the library listed in the $3 \$ \$$ array.

3. iopt - select nuclides by 'mat' or 'nza' number (1)

0 - mat 
$1-$ nza (default)

4. nsq-sequence number of file opened on unit LOG (1)

$\mathrm{T}$ terminate data block 2

Data Block 3 (enter if inum is non-zero)

$3 \$$ nuclide selection list (inum)

enter a positive identifier to select a nuclide.

enter a negative identifier to exclude a nuclide.

$4 \$$ new nuclide identifiers (inum)

enter the new nuclide identifiers in the locations corresponding to the positive identifier entry in the $3 \$$ array.

$5 \$$ version of the data $(-1,0,1,2,3,4 / \mathrm{unk}, \mathrm{ENDF}, J E F, J E N D L, B R O N D, C E N D L)$ (inum)

6\$ 8-Character Identifier for Thermal Kinematics Data (inum)

$7 \$$ ZA-override Values. Non-zero values will replace the ZA values in the Header Record. The ZA values in the Data Directory records are not changed. (inum)

$\mathrm{T}$ terminate data block 3

\subsubsection{CAJUN I/O Units}

CAJUN requires the following $\mathrm{I} / \mathrm{O}$ devices.

$\begin{array}{rll}\text { Unit No. } & & \text { Purpose } \\ 5 & & \text { Standard definition input } \\ 6 & & \text { Output } \\ 18 & \text { Scratch file }\end{array}$




\subsection{MONTE CARLO TRANSPORT}

\section{Introduction by B. T. Rearden}

SCALE Monte Carlo transport capabilities enable criticality safety, shielding, depletion, and sensitivity and uncertainty analysis. ${ }^{1}$ SCALE provides separate Monte Carlo capabilities for eigenvalue neutronics and fixed-source coupled neutron-gamma calculations, in the KENO code ${ }^{2}$ and fixed-source coupled neutron-gamma calculations in the Monaco code. ${ }^{3}$ Although the eigenvalue and fixed-source capabilities are provided in separate codes, many capabilities are shared between them, including physics and geometry packages. The foundational features shared between the codes are described below, with specific implementations provided in subsequent sections. Generally, the use of the Monte Carlo transport solvers in SCALE are best accessed through the capability-specific sequences: CSAS and Sourcerer for criticality safety, MAVRIC for shielding, TRITON for depletion, TSUNAMI-3D for sensitivity and uncertainty analysis, and MCDancoff for three-dimensional Dancoff factor calculations.

\section{Multigroup Physics}

The multigroup treatment implemented in SCALE has been in use since the 1960s and provides efficient, effective solutions with superior runtime performance. Problem-dependent multigroup cross section data are temperature interpolated and resonance self-shielded by other SCALE modules before they are used in each Monte Carlo calculation. Without proper resonance self-shielding, accurate multigroup calculations would not be possible for thermal or intermediate energy spectrum systems. After self-shielding has been accomplished and the two-dimensional expansions have been summed into a Legendre expansion of the total group-to-group transfer arrays, individual nuclide cross sections are multiplied by their densities and summed into mixtures. These mixture cross sections can then be used by the deterministic transport codes for their calculations. The Monte Carlo codes convert the Legendre expansion of the transfer arrays into probability distributions for the group-to-group transfers and for the discrete scattering angles and probabilities that preserve the moments of the Legendre expansion of each group-to-group transfer. These transfer probabilities, angles, and angle probabilities are then transformed so that the new group and angle of scatter are efficiently selected through two random numbers with only one multiplication and one addition operation. If the selected new group is negative, it is reset to positive, and the new direction is chosen isotropically. If the problem is run with $\mathrm{P}_{1}$ scattering, the scattering angle is chosen from a continuous distribution. For higher order scattering, the polar scatter angle is discrete, and the azimuthal angle is randomly selected from a uniform distribution. Multigroup physics is implemented for neutron, photon, and neutron-photon coupled particle transport modes.

\section{Continuous-energy Physics}

The continuous energy treatment in SCALE provides high resolution solution strategies with explicit physics representation. The continuous energy data represent thermal scattering using free gas and $s(\alpha, \beta)$, with explicit point-to-point data provided through the thermal region. The resolved resonance region is represented by pointwise data where the energy point density is optimized for each reaction of each nuclide. Data in the unresolved resonance region are represented by probability tables, and data above the unresolved region implement pointwise data with explicit point-to-point representation for secondary particles. Photon yield data represent each discrete photon. Continuous energy physics contains non-transport data handling to support various flux, reaction rate, point detector tallies, and

sensitivity analysis. In addition, continuous energy data are converted from a double differential data format to a lab format in a process where fast look-up tables are provided during library generation. In SCALE 6.0-6.1, calculations are performed only at temperatures available on the data libraries by selecting the library temperature nearest to the desired temperature for the calculation. Resonance 
upscattering techniques are implemented via the Doppler Broadened Rejection Correction method. ${ }^{4}$ With SCALE 6.2, problem-dependent continuous energy cross sections at the user specified temperature are generated at the beginning of the calculation. Continuous energy physics is implemented for neutron, photon, and neutron-photon coupled particle transport modes.

\section{Geometry Packages}

Two variants of KENO provide identical solution capabilities with different geometry packages. KENO V.a implements a simple and efficient geometry package sufficient for modeling many systems of interest to criticality safety and reactor physics analysts. KENO-VI implements the SCALE Generalized Geometry Package (SGGP), which provides a quadratic-based geometry system with much greater flexibility in solution modeling. Monaco implements only the SGGP geometry package. Both packages are based on solid bodies organized into reusable objects called units that are constructed of material regions. Units can be conveniently arranged in rectangular or hexagonal arrays of repeating units. Additionally, nesting is available so that one unit can contain another unit as a hole, or an array can be nested inside of a unit, which itself can be repeated in another array. There is no limit to the number of nesting levels available, so very complex systems can be quickly generated.

KENO V.a models are constructed from regions of specific shapes following strict rules which provide great efficiency in geometry tracking. Allowed shapes are cubes, cuboids (rectangular parallelepipeds), spheres, cylinders, hemispheres, and hemicylinders. These shapes must be oriented along orthogonal axes, and they can be translated, but they cannot be rotated. A major restriction applied to KENO V.a geometry is that intersections are not allowed, and each region of a unit must fully enclose the preceding region. An exception to this rule is in the use of holes through which many units can be placed within an enclosing unit. However, there is a runtime penalty in geometry tracking for this flexibility, so this feature should be used judiciously. KENO V.a provides rectangular arrays where the outer body of each unit contained in the array must have a cuboidal shape, and adjacent faces must have the same dimensions. The entire array must be fully enclosed by the region in which it is placed.

SGGP is a quadratic-based geometry system that provides predefined bodies including cone, cuboid, cylinder, dodecahedron, ecylinder (elliptical cylinders), ellipsoid, hexprism, hopper (truncated pyramid), parallelepiped, planes, rhombohedron, rhexprism (rotated hexprisms), sphere, and wedge. Bodies not directly provided with SGGP can be constructed from quadratic surfaces defined with coefficients entered by the user. All bodies and surfaces can be rotated and translated to any orientation and position within their respective unit. SGGP also provides intersecting regions.

SGGP arrays may be composed of cuboids, hexprisms, rhexprisms, or dodecahedrons. Like KENO V.a, the faces of adjacent units in an array must have the same dimensions. An array boundary must be specified for each array, and only the portion of the array within the boundary is considered a part of the system. Also, the specified array must fill the entire volume in the specified array boundary. The array boundary may be any shape that can be specified using quadratic equations.

The use of holes is more flexible in SGGP than in KENO V.a. Within a unit, holes cannot intersect other holes or the unit boundary, but they can intersect region boundaries. The use of holes is not necessary to build complex geometries; they are used primarily to more efficiently build complex geometries and improve the tracking efficiency of the simulation. In SGGP the distance to each surface in the unit must be calculated after each collision. By moving some of the surfaces in a unit into another unit that is included as a hole, all the surfaces in the hole unit except the outer boundary are removed from the containing unit. The judicious use of holes in SGGP can significantly speed up the calculation. 


\section{Eigenvalue Analysis}

KENO performs eigenvalue calculations for neutron transport primarily to calculate multiplication factors and flux distributions of fissile systems in continuous energy and multigroup modes. Both codes allow explicit geometric representation with their respective geometry packages. KENO provides a multigroup adjoint capability which is especially useful for sensitivity analysis. KENO implements standard variance reduction techniques such as implicit capture, splitting, and Russian roulette.

The initial fission source distribution in KENO can be specified with nine options. These options include the default option of a uniform distribution throughout the fissile material; an axially varying distribution input by the user or defined as $\cos (Z)$ or $(1-\cos (Z))^{2}$, where $Z$ is the axial position; several options to initialize the source at a given position (within a given volume, a given unit, or a unit at a specified array index); or to specifically provide the coordinates of each starting point.

KENO approximates the real $k_{\text {eff }}$ variance using an iterative approach and lagging covariance data between generations. ${ }^{5}$ KENO provides a $\chi^{2}$ test for the normality of $k_{\text {eff }}$ and provides plots of $k_{\text {eff }}$ by active and inactive generations. KENO reports a best estimate of $k_{\text {eff }}$ that is computed as the minimum variance of $k_{\text {eff }}$ based on generations skipped and generations run.

KENO provides track-length tallies for scalar flux and angular flux moments needed for sensitivity analysis. Additionally, tallies are provided for reaction rates, with isotopic tallies available only in CE calculations. KENO also provides mesh tallies based on a user-input orthogonal grid.

Matrix $k_{\text {eff }}$ calculations provide an additional method of calculating the $k_{\text {eff }}$ of the system. Cofactor $k_{\text {eff }}$ and source vectors, which describe the contribution to the system $k_{\text {eff }}$ from each unit, hole, or array, are available.

KENO provides plots of $k_{\text {eff }}$ by generation and average $k_{\text {eff }}$ for visual inspection of source convergence, followed by a $\chi^{2}$ statistical assessment of convergence. Fission source convergence diagnostic techniques are implemented in KENO to provide improved confidence in the computed results and to reduce the simulation time for some cases. Confirming the convergence of the fission source distribution is especially useful to avoid the false convergence of $k_{\text {eff }}$ that can be caused by insufficient sampling of important portions of the system. ${ }^{6}$ KENO source convergence diagnostics rely on Shannon entropy statistics of the mesh-based fission source data.

Parallel computation capabilities are available in both versions of KENO to provide reductions in wall clock time, especially for sensitivity analysis or Monte Carlo depletion on computer clusters. By introducing a simple master-slave approach via message passing interface (MPI), KENO runs different random walks concurrently on the replicated geometry within the same generation. The fission source and other tallied quantities are gathered at the end of each generation by the master process, and then they are processed either for final edits or next generations.

\section{Shielding Analysis}

Monaco is a fixed-source Monte Carlo shielding code that calculates neutron and photon fluxes and response functions for specific geometry regions, point detectors, and mesh tallies. Monaco has variance reduction capabilities, such as source biasing and weight windows, which can be automated via the MAVRIC sequence. MAVRIC performs radiation transport on problems that are too challenging for standard, unbiased Monte Carlo methods. Monaco provides multiple methods to enter the radioactive source descriptions. Spatial distribution options include volumetric sources and mesh sources which can be generated by other codes such as KENO. Energy distributions can be entered by the user or imported directly from emission data provided by ORIGEN. Spent fuel analysis is simplified through direct coupling with the ORIGEN binary concentration files. 


\section{References}

1. B. T. Rearden, L. M. Petrie, D. E. Peplow, K. B. Bekar, D. Wiarda, C. Celik, C. M. Perfetti, A. M. Ibrahim, S. W. D. Hart, M. E. Dunn, and W. J. Marshall, "Monte Carlo Capabilities of the SCALE Code System," Annals of Nuclear Energy, 82, 130-141 (2015).

2. S. Goluoglu, L. M. Petrie, Jr., M. E. Dunn, D. F. Hollenbach, and B. T. Rearden, "Monte Carlo Criticality Methods and Analysis Capabilities in SCALE," Nucl. Technol. 174(2), 214-235 (2011).

3. D. E. Peplow, "Monte Carlo Shielding Analysis Capabilities with MAVRIC," Nucl. Technol., 174(2), 289-313 (2011).

4. S. Hart, "Implementation of the Doppler Broadening Rejection Correction in KENO," Trans. Am. Nucl. Soc., 108, 423-426 (June 2013).

5. T. Ueki, et al., "Error Estimations and Their Biases in Monte Carlo Eigenvalue Calculations," Nucl. Sci. Eng., 125, 1 (1997).

6. T. Ueki and F. B. Brown, "Stationarity Modeling and Informatics-Based Diagnostics in Monte Carlo Criticality Calculations," Nucl. Sci. Eng., 149, 38-50 (2005). 


\title{
8.1 KENO: A MONTE CARLO CRITICALITY PROGRAM
}

\author{
L. M. Petrie, K. B. Bekar, C. Celik, D. F. Hollenbach, ${ }^{1}$ C. M. Perfetti, S. Goluoglu, ${ }^{1}$ N. F. Landers, ${ }^{1}$ \\ M. E. Dunn, B. T. Rearden
}

\begin{abstract}
KENO is a three-dimensional (3D) Monte Carlo criticality transport program developed and maintained for use as part of the SCALE Code System. It can be used as part of a sequence or as a standalone program. There are two versions of the code currently supported in SCALE. KENO V.a is the older of the two. KENO-VI contains all current KENO V.a features plus a more flexible geometry package known as the SCALE Generalized Geometry Package. The geometry package in KENO-VI is capable of modeling any volume that can be constructed using quadratic equations. In addition, such features as geometry intersections, body rotations, hexagonal and dodecahedral arrays, and array boundaries have been included to make the code more flexible.

The simpler geometry features supported by KENO V.a allow for significantly shorter execution times than KENO-VI, while the additional geometry features supported in KENO-VI make the code appropriate for cases where geometry modeling is not possible with KENO V.a. In particular, KENO-VI allows intersections, body truncations with planes, and a much wider variety of geometrical bodies. KENO-VI also has the ability to rotate bodies so that volumes no longer must be positioned parallel to a major axis. Hexagonal arrays are available in KENO-VI and dohecahedral arrays enable the code to model pebble bed reactors and other systems composed of close packed spheres. The use of array boundaries makes it possible to fill a non-cuboidal volume with an array, specifying the boundary where a particle leaves and enters the array.
\end{abstract}

Except for geometry capabilities, the two versions of KENO share most of the computational capabilities and the input flexibility specific to most SCALE modules. They can both operate in multigroup or continuous energy mode, run as standalone codes, or integrated in computational sequences such as CSAS, TSUNAMI-3D, or TRITON. Both versions of the code are continually updated and are written in FORTRAN 90.

Computational capabilities shared by the two versions of KENO include the determination of k-effective, neutron lifetime, generation time, energy-dependent leakages, energy- and region-dependent absorptions, fissions, the system mean-free-path, the region-dependent mean-free-path, average neutron energy, flux densities, fission densities, reaction rate tallies, mesh tallies, source convergence diagnostics, problemdependent continuous energy temperature treatments, parallel calculations, restart capabilities, and many more.

\footnotetext{
${ }^{1}$ Formerly with Oak Ridge National Laboratory
} 


\section{ACKNOWLEDGMENTS}

Many individuals have contributed significantly to the development of KENO. Special recognition is given to G. E. Whitesides, former Director of the Computing Applications Division, who was responsible for the concept and development of the original KENO code. He has also contributed significantly to some of the techniques used in both KENO versions. The late J. T. Thomas offered many ideas that have been implemented in the code. R. M. Westfall, retired from ORNL, provided early consultation, encouragement, and benchmarks for validating the code. The special abilities of J. R. Knight, retired from ORNL, contributed substantially to debugging early versions of the code. S. W. D. Hart was instrumental in implementing continuous energy temperature treatments. W. J. Marshall has provided substantial validation and quality assurance reviews. Appreciation is expressed to C. V. Parks and S. M. Bowman for their support of KENO and the KENO3D visualization tool. The late P. B. Fox provided many of the figures in this document. D. Ilas, B. J. Marshall, and D. E. Mueller consolidated the previous KENO V.a and KENO-VI manuals into this present form. The efforts of L. F. Norris (retired), W. C. Carter (retired), S. J. Poarch, D. J. Weaver (retired), S. Y. Walker and R. B. Raney in preparing this document are gratefully acknowledged.

The authors thank the U. S. Nuclear Regulatory Commission and the DOE Nuclear Criticality Safety Program for sponsorship of the continuous energy, source convergence diagnostics, and grid geometry features in the current version. 


\section{TABLE OF CONTENTS}

Page

8.1 KENO: A MONTE CARLO CRITICALITY PROGRAM ..................................................... $8-5$

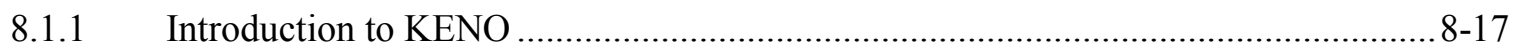

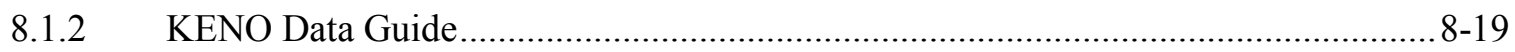

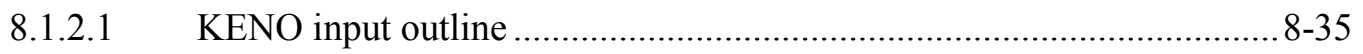

8.1.2.2 Procedure for data input .............................................................. $8-38$

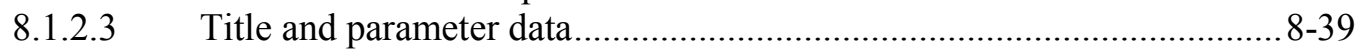

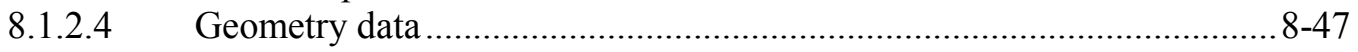

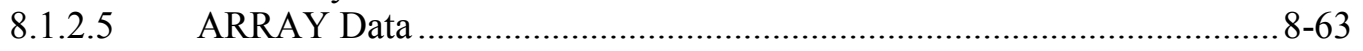

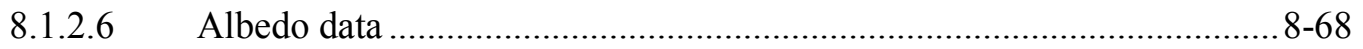

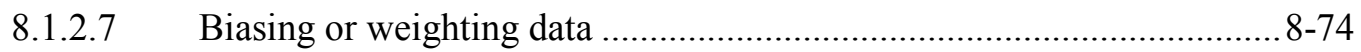

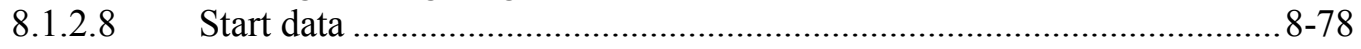

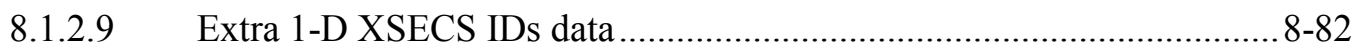

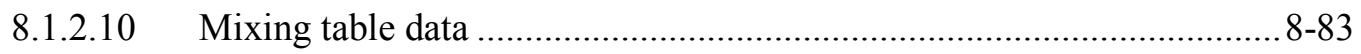

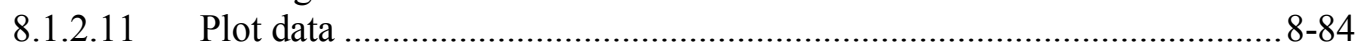

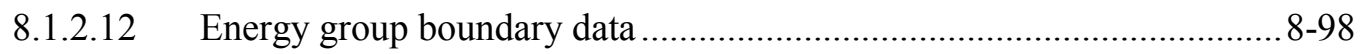

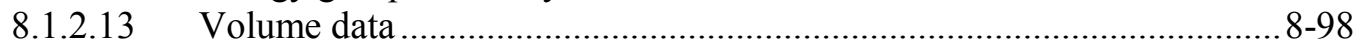

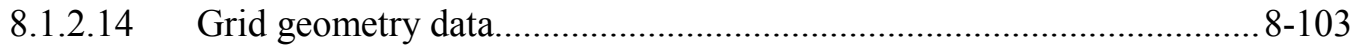

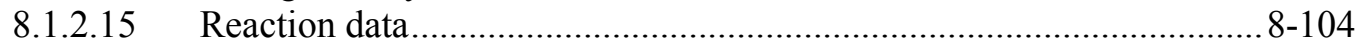

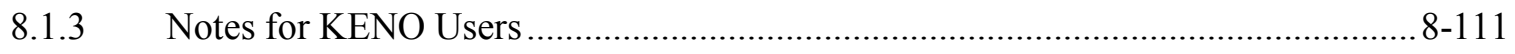

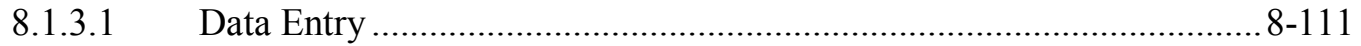

8.1.3.2 Default logical unit numbers for KENO ............................................ 8-114

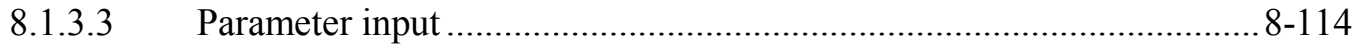

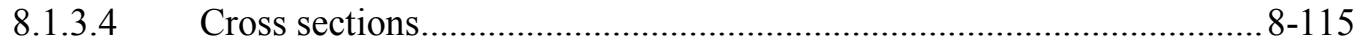

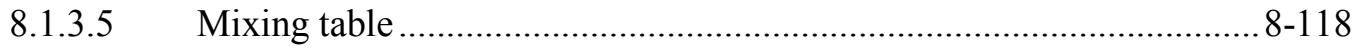

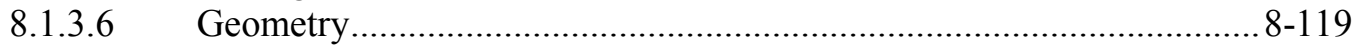

8.1.3.7 Alternative sample problem mockups.............................................. 8-224

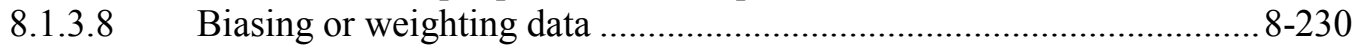

8.1.3.9 Color plots................................................................................ 8-234

8.1.3.10 KENO Multiple Mesh and Mesh-based Quantity Specifications ......... 8-267

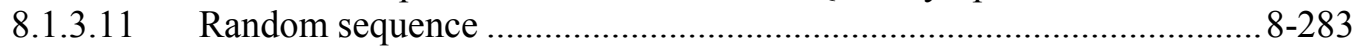

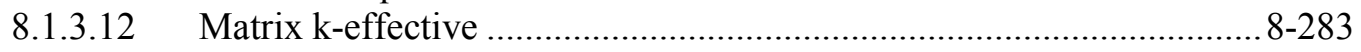

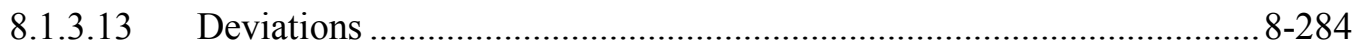

8.1.3.14 Generation time and lifetime.......................................................... 8-285

8.1.3.15 Energy of the Average Lethargy of Fission ...................................... 8-285

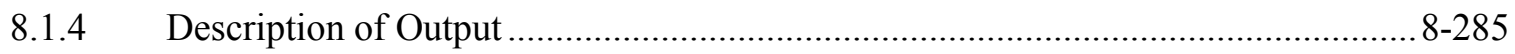

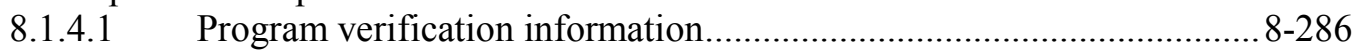

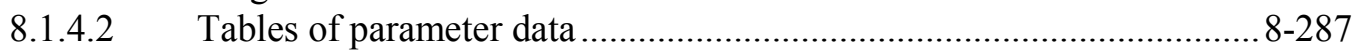

8.1.4.3 Unprocessed geometry input data .................................................... 8-290

8.1.4.4 Table of data sets used in the problem ............................................. 8-290

8.1.4.5 Table of additional information ................................................... 8-291

8.1.4.6 Mixing table data ........................................................................ 8-293

8.1.4.7 Albedo cross section correspondence …......................................... 8-294 


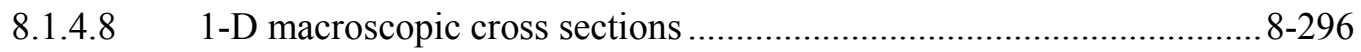

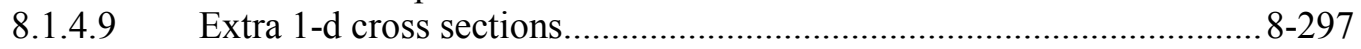

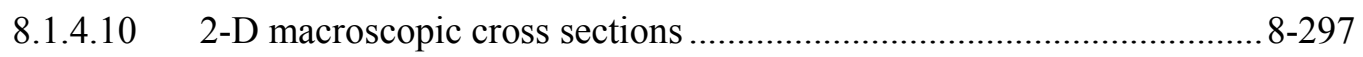

8.1.4.11 Probabilities and angles ............................................................... 8-298

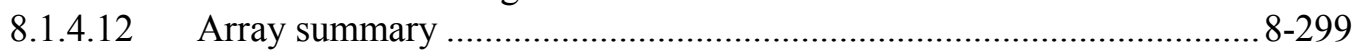

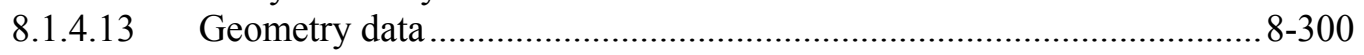

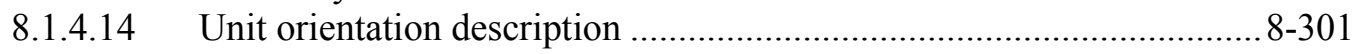

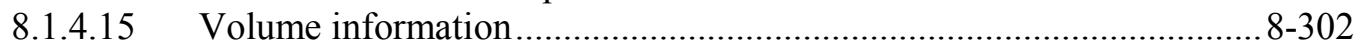

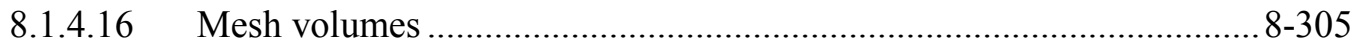

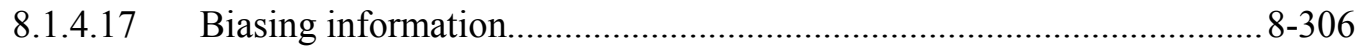

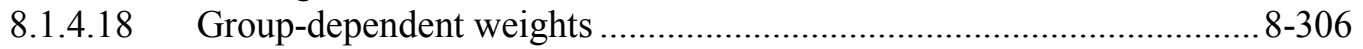

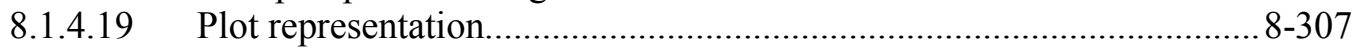

8.1.4.20 Initial source and final pretracking edits .......................................... 8-311

8.1.4.21 Reference center for flux moment/angular flux transform................... 8-311

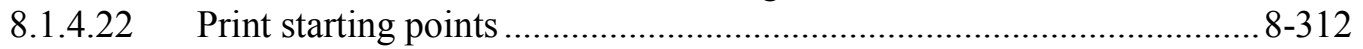

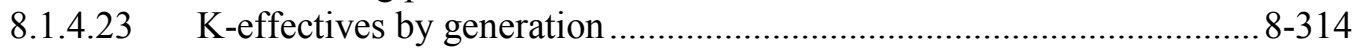

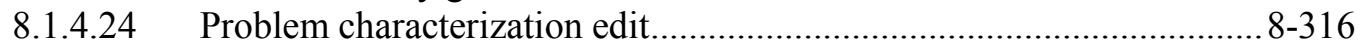

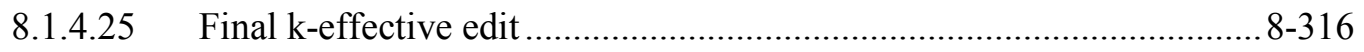

8.1.4.26 Plot of average k-effective by generation run ..................................... 8-317

8.1.4.27 Plot of average k-effective by generations skipped ............................ 8-319

8.1.4.28 Final edit of fissions, absorptions, and leakage.................................. 8-320

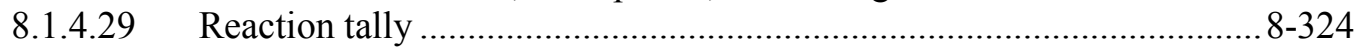

8.1.4.30 Source convergence diagnostics ..................................................... 8-325

8.1.4.31 Matrix k-effective by position index................................................ 8-325

8.1.4.32 Fission production by position index matrix....................................... 8-326

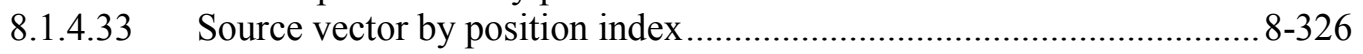

8.1.4.34 Cofactor k-effective by position index.............................................. 8-327

8.1.4.35 Matrix k-effective by unit number ............................................... 8-327

8.1.4.36 Fission production by unit number matrix ........................................ 8-328

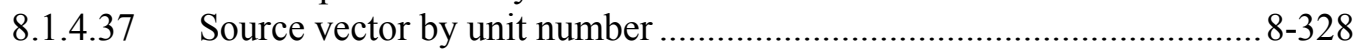

8.1.4.38 Cofactor k-effective by unit number ................................................ 8-329

8.1.4.39 Matrix k-effective by hole number ................................................... 8-329

8.1.4.40 Fission production by hole number matrix ....................................... 8-330

8.1.4.41 Source vector by hole number .................................................... 8-330

8.1.4.42 Cofactor k-effective by hole number ............................................. 8-330

8.1.4.43 Matrix k-effective by array number .............................................. 8-331

8.1.4.44 Fission production by array number matrix ........................................ 8-331

8.1.4.45 Source vector by array number ................................................... 8-331

8.1.4.46 Cofactor k-effective by array number ............................................ 8-332

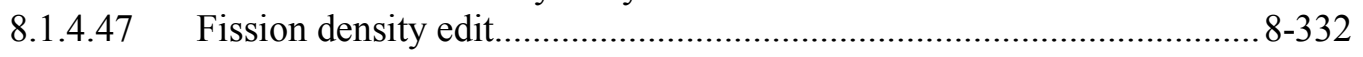

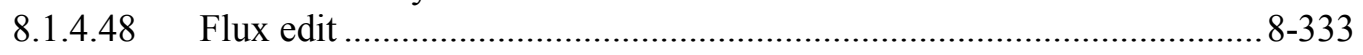

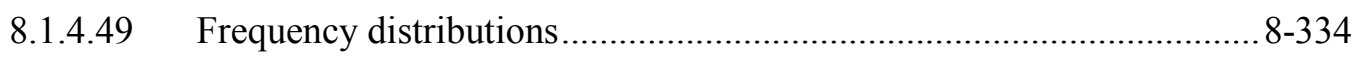

8.1.4.50 Summary of parallel performance ….............................................. 8-335

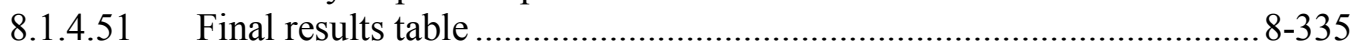

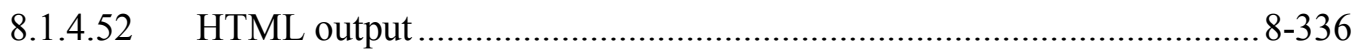

8.1.5 Warning messages and error messages......................................................... 8-371

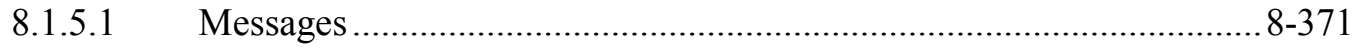

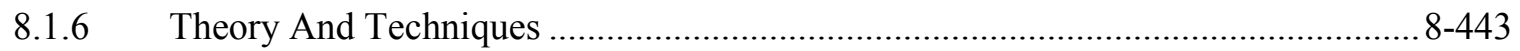

8.1.6.1 The transport equation ............................................................. 8-443 
8.1.6.2 Continuous energy mode solution procedure..................................... 8-444

8.1.6.3 Multigroup mode solution procedure .............................................. 8-474

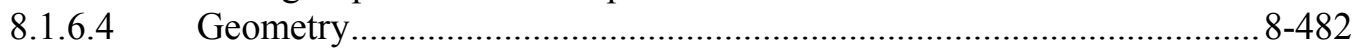

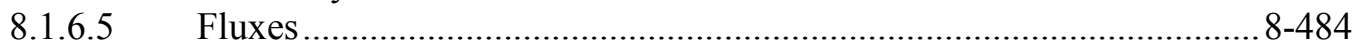

8.1.6.6 Reaction Rate and Few Group Micro Cross Section Calculations........ 8-486

8.1.6.7 Source Convergence Diagnostics..................................................... 8-486

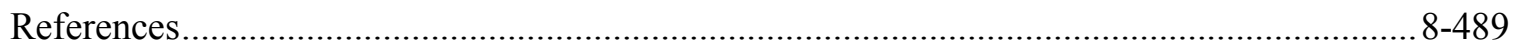

8.1.A Appendix A KENO V.a Shape Descriptions .......................................................... 8-491

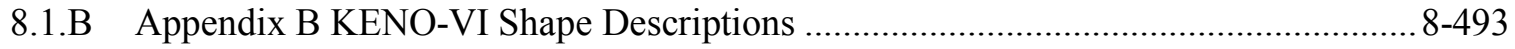

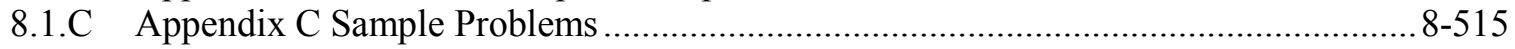

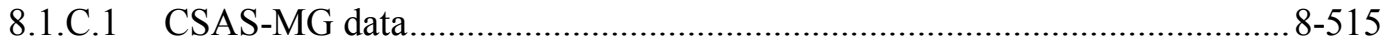

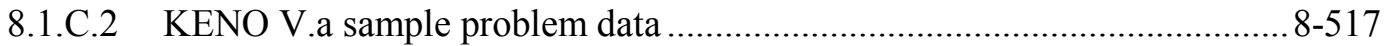

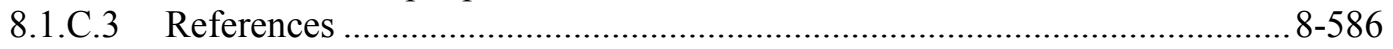




\section{LIST OF FIGURES}

Fig. 8.1.1. Examples of geometry allowed in KENO-VI but not allowed in KENO V a

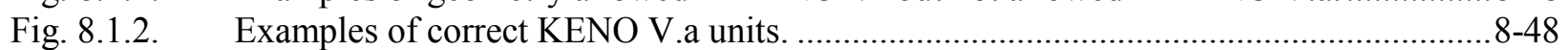

Fig. 8.1.3. Example demonstrating HOLE capability in KENO................................................ $8-49$

Fig. 8.1.4. Example of triangular pitched ARRAY construction................................................. $8-50$

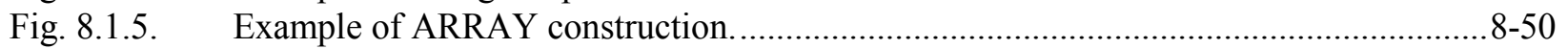

Fig. 8.1.6. Example of an ARRAY composed of UNITs containing ARRAYs and HOLEs.......... 8-51

Fig. 8.1.7. Partial hemisphere or hemicylinder; less than half exists (less than half is defined

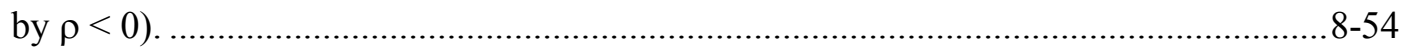

Fig. 8.1.8. Partial hemisphere of hemicylinder; more than half exists (more than half is

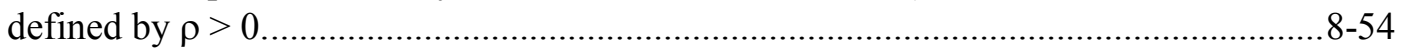

Fig. 8.1.9. Examples of the CHORD option in KENO-VI. ..............................................................

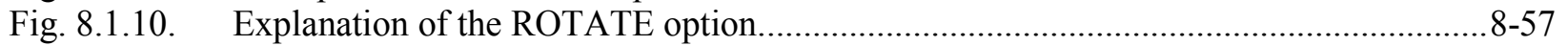

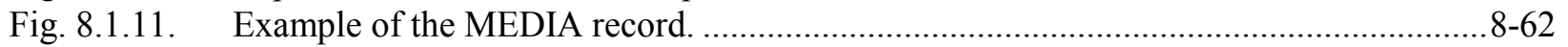

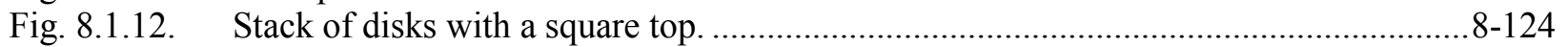

Fig. 8.1.13. Two large cylinders joined axially by a small cylinder..............................................8-137

Fig. 8.1.14. Two large cylinders radially connected by a small cylinder. ......................................8-138

Fig. 8.1.15. KENO V.a approximations of cylindrical intersections. ...........................................8-138

Fig. 8.1.16. Two small cylinders joined axially by a large cylinder..........................................8 8 -142

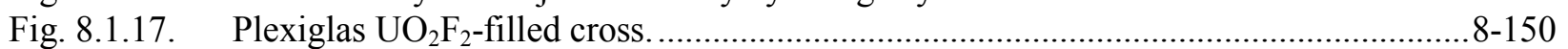

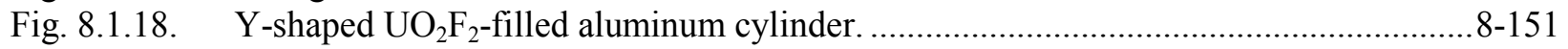

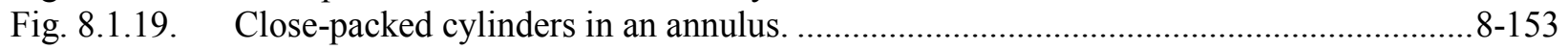

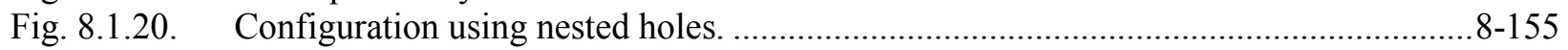

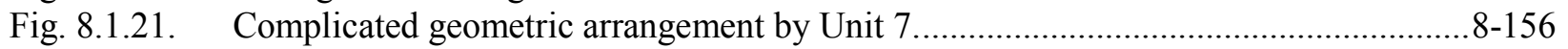

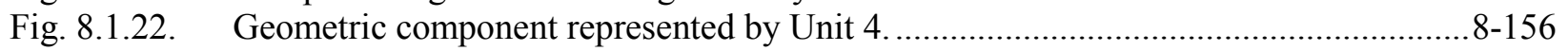

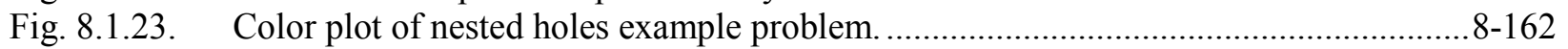

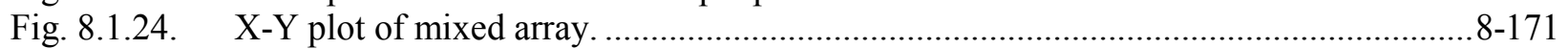

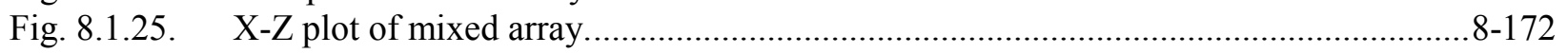

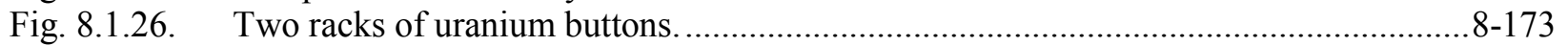

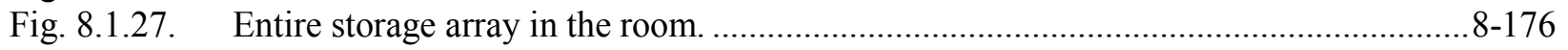

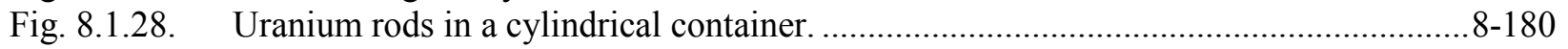

Fig. 8.1.29. The $10 \times 10$ array of 32 uranium rods and 68 void cuboids with the array

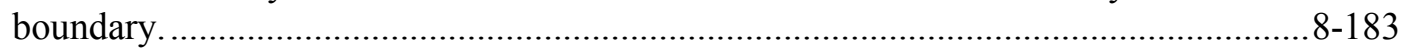

Fig. 8.1.30. X-Y slice of uranium rods in a cylindrical container. .............................................. $8-185$

Fig. 8.1.31. $\quad \mathrm{X}-\mathrm{Z}$ slice of uranium rods in a cylindrical container.............................................. $8-186$

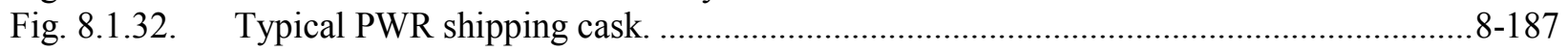

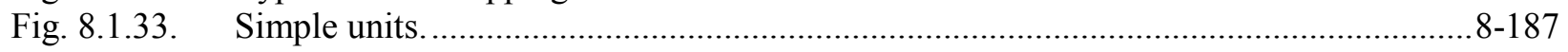

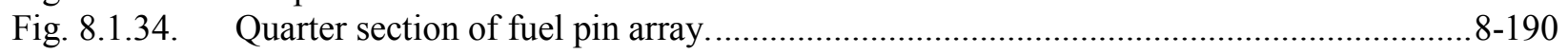

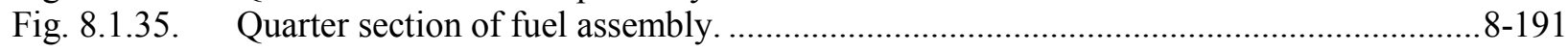

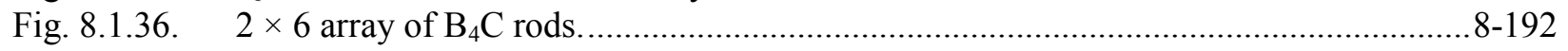

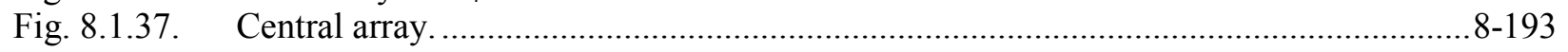

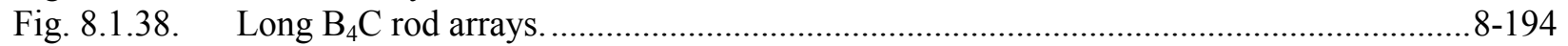

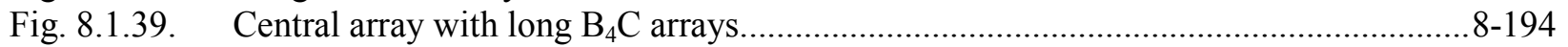

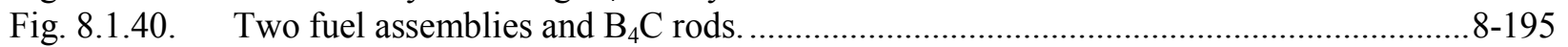

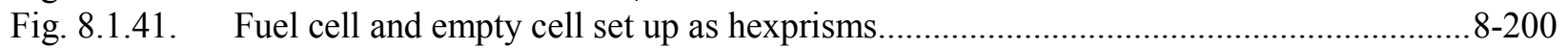

Fig. 8.1.42. Seven cylinders stacked in a HEXAGONAL array with cylindrical array

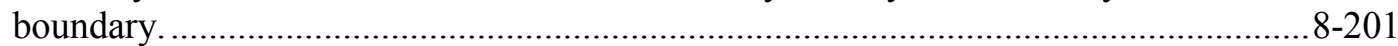


Fig. 8.1.43. Seven cylinders stacked in a SHEXAGONAL array with a cylindrical array boundary

Fig. 8.1.44.

$\mathrm{X}-\mathrm{Y}$ slice of 7 cylinders in a triangular pitch array.

$8-202$

Fig. 8.1.45.

Fuel cell and empty cell set up as rotated hexprism (RHEXPRISM).

$8-204$

Fig. 8.1.46.

Seven cylinders stacked in a RHEXAGONAL array with a cylindrical array

boundary.

Fig. 8.1.47.

$\mathrm{X}-\mathrm{Y}$ slice of 7 cylinders in a rotated hexagonal pitched array.

$8-206$

Fig. 8.1.48.

Bare triangular pitched ARRAY.

$8-208$

Fig. 8.1.49.

Units used to describe a bare triangular pitched ARRAY.

$8-208$

Fig. 8.1.50.

UNITs to complete the triangular pitched ARRAY.

8-209

Fig. 8.1.51.

Completed ARRAY.

$8-210$

Fig. 8.1.52.

Triangular pitched ARRAY within a cylinder.

$8-210$

Fig. 8.1.53.

$4 \times 2 \times 1$ array to be placed within a cylinder.

$8-211$

Fig. 8.1.54.

$4 \times 2 \times 1$ ARRAY within a cylinder.

$8-212$

Fig. 8.1.55.

Partially completed triangular pitched ARRAY in a cylinder.

$8-213$

Fig. 8.1.56.

Completed triangular pitched array in a cylinder.

$8-214$

Fig. 8.1.57.

Array to be placed in a cylinder.

8-216

Fig. 8.1.58.

Partially completed array in a cylinder.

$8-217$

Fig. 8.1.59.

Description of UNITs 10 and 11 for Example 2b.

8-217

Fig. 8.1.60.

Partially completed triangular pitched ARRAY in a cylinder.

$8-218$

Fig. 8.1.61.

Final configuration of triangular pitched ARRAY in a cylinder.

$8-219$

Fig. 8.1.62.

Fuel cell and empty cell set up as dodecahedra.

$8-221$

Fig. 8.1.63.

$\mathrm{X}-\mathrm{Y}$ slice of dodecahedral array at even level $\mathrm{Z}=2$.

$8-222$

Fig. 8.1.64.

Fig. 8.1.65.

$\mathrm{X}-\mathrm{Y}$ slice of dodecahedral array at odd level $\mathrm{Z}=3$.

$8-223$

Mixture color plot for single unit with centered origin. ............................................... 8-237

Fig. 8.1.66.

Fig. 8.1.67.

Fig. 8.1.68.

Fig. 8.1.69.

Fig. 8.1.70.

Fig. 8.1.71.

Fig. 8.1.72.

Fig. 8.1.73.

Fig. 8.1.74.

Fig. 8.1.75.

Fig. 8.1.76.

Fig. 8.1.77.

Fig. 8.1.78.

Fig. 8.1.79.

Fig. 8.1.80.

Fig. 8.1.81.

Fig. 8.1.82.

Fig. 8.1.83.

Fig. 8.1.84.

Fig. 8.1.85.

Fig. 8.1.86.

Fig. 8.1.87.

Fig. 8.1.88.

Unit map color plot for single unit with centered origin.

$8-238$

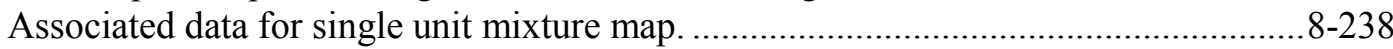

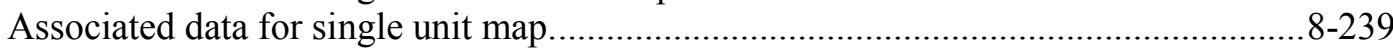

Associated data for mixture map of single unit with offset origin. .............................8-241

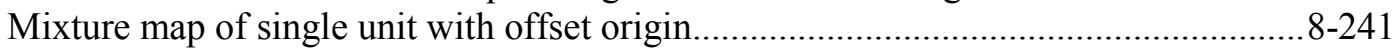

Associated data for unit map of single unit with offset origin. .................................. 8-242

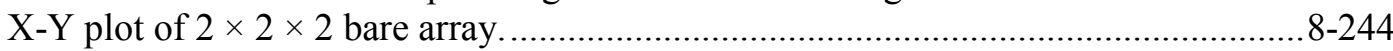

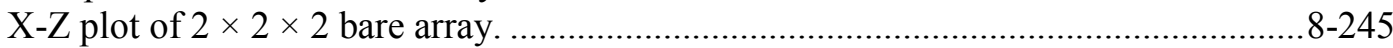

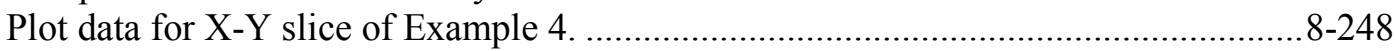

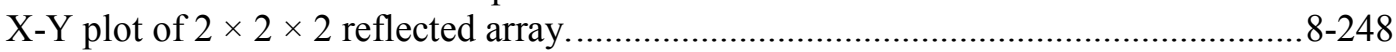

Plot data for enlarged X-Y slice of Example 4 ..................................................... 8-249

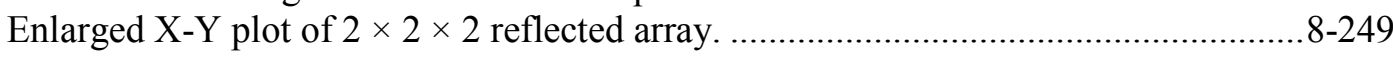

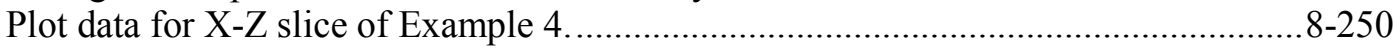

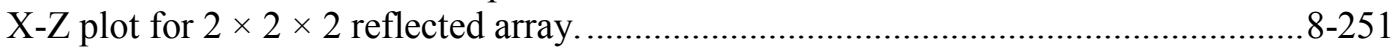

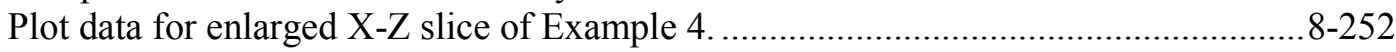

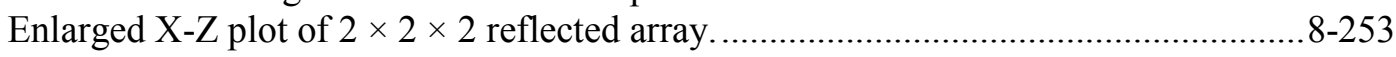

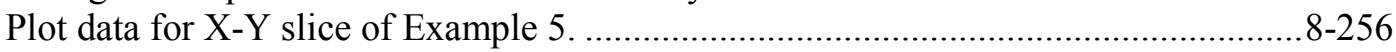

$\mathrm{X}-\mathrm{Y}$ plot of $2 \times 2 \times 2$ reflected array with centered origin...................................... 8-256

Plot data for an enlarged X-Y slice of Example 5 .................................................... 8-257

Enlarged X-Y plot of $2 \times 2 \times 2$ reflected array with centered origin. ..........................8-257

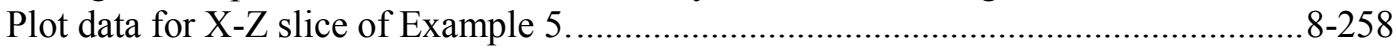

$\mathrm{X}-\mathrm{Z}$ plot of reflected $2 \times 2 \times 2$ array with centered origin. ...................................... 8 -258

Pot data for enlarged $X-Z$ slice of Example 5. …............................................. $8-25$

Fig. 8.1.90. Mixture plot of nested holes problem.................................................................. $8-260$ 
Fig. 8.1.91.

Fig. 8.1.92.

Fig. 8.1.93.

Fig. 8.1.94.

Fig. 8.1.95.

Fig. 8.1.96.

Fig. 8.1.97.

Fig. 8.1.98.

Fig. 8.1.99.

Fig. 8.1.100.

Fig. 8.1.101.

Fig. 8.1.102.

Fig. 8.1.103.

Fig. 8.1.104.

Fig. 8.1.105.

Fig. 8.1.106.

Fig. 8.1.107.

Fig. 8.1.108.

Fig. 8.1.109.

Fig. 8.1.110.

Fig. 8.1.111.

Fig. 8.1.112.

Fig. 8.1.113.

Fig. 8.1.114.

Fig. 8.1.115.

Fig. 8.1.116.

Fig. 8.1.117.

Fig. 8.1.118.

Fig. 8.1.119.

Fig. 8.1.120.

Fig. 8.1.121.

Fig. 8.1.122.

Fig. 8.1.123.

Fig. 8.1.124.

Fig. 8.1.125.

Fig. 8.1.126.

Fig. 8.1.127.

Fig. 8.1.128.

Fig. 8.1.129.

Fig. 8.1.130.

Fig. 8.1.131.

Plot data for unit plot of nested holes.

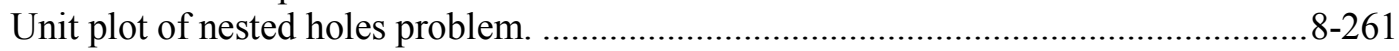

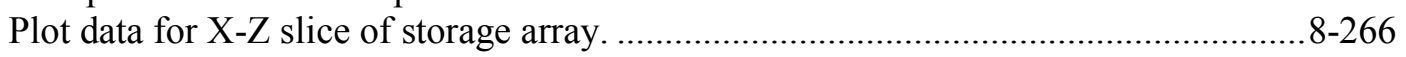

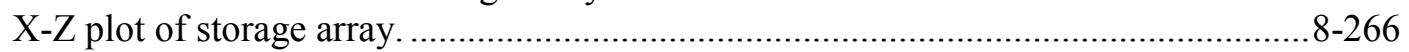

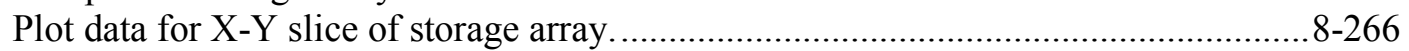

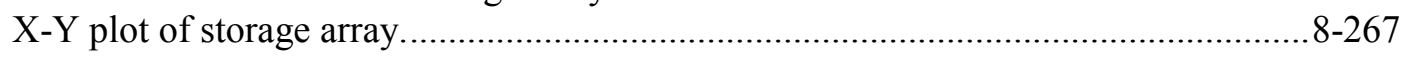

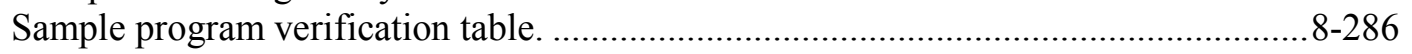

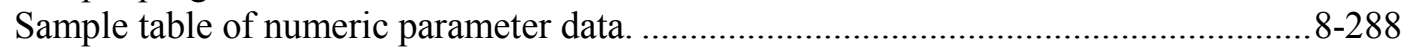

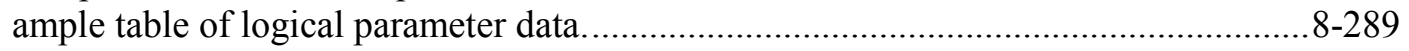

Sample table of data sets used in the problem........................................................ $8-290$

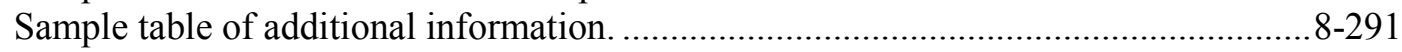

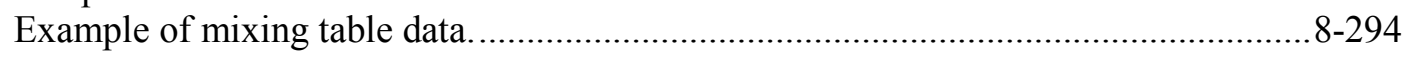

Cumulative probabilities for correlating the albedo energy group to the cross

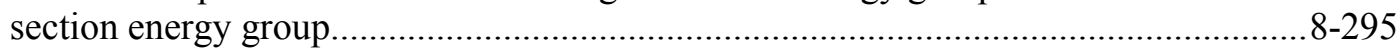

Cumulative probabilities for correlating the cross section energy group to the

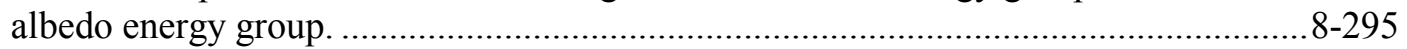

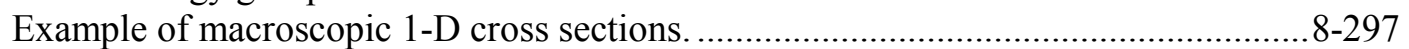

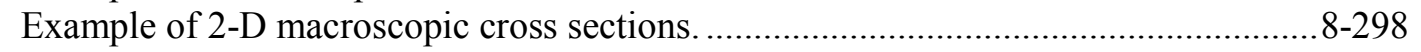

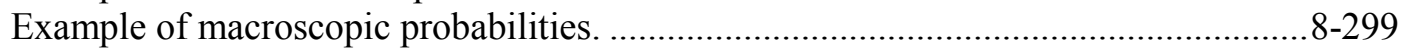

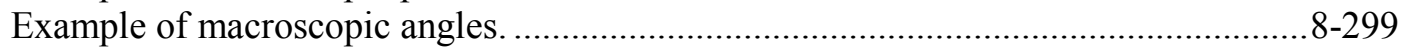

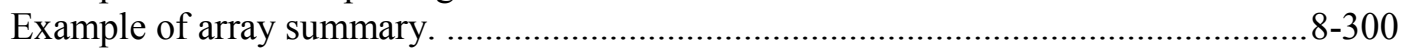

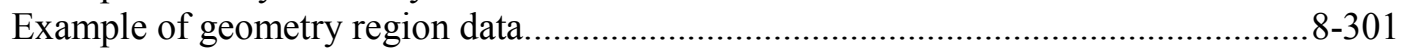

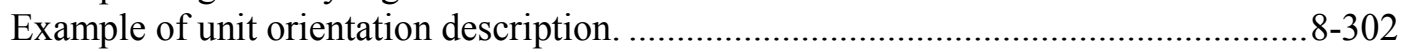

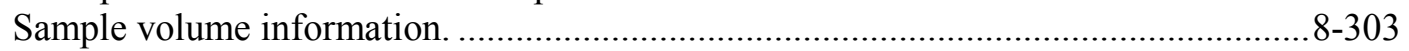

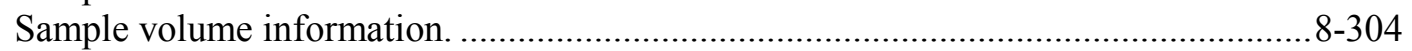

Sample sum of mesh volumes edit. .......................................................... 8-305

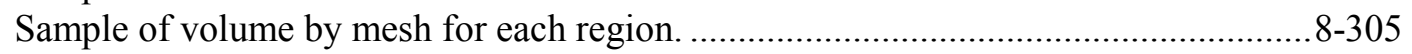

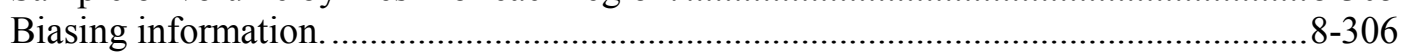

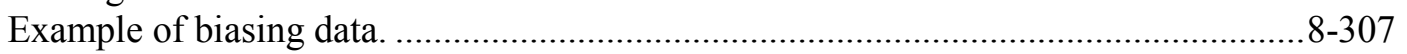

Summary of character plot symbols, coordinates, and data. .................................. 8-308

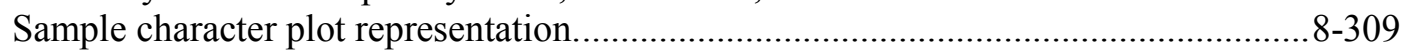

Summary of color plot symbols, coordinates, and data............................................... $8-310$

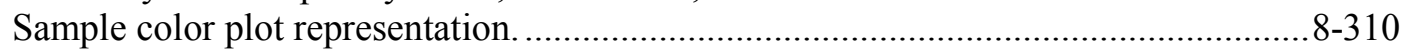

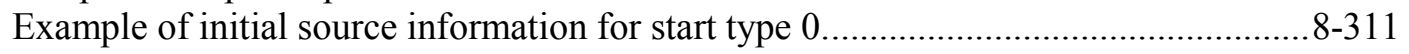

Example fuel center and reference center edits.................................................. 8-312

Example of initial source points (KENO V.a edit) .................................................. $8-313$

Example of initial source points (KENO-VI edit) ...................................................... $8-314$

Example of k-effectives and source entropy by generation.................................... 8-315

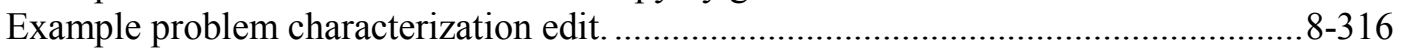

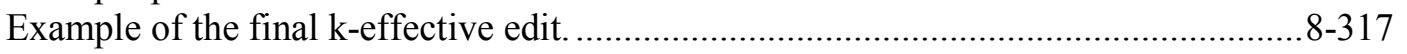

Sample plot of average k-effective by generation run.............................................. 8-319

Sample plot of average k-effective by generations skipped. ...................................... 8-320

Sample of the final edit of fissions, absorptions, and leakage with all

region-dependent information suppressed................................................................. 8-321

Fig. 8.1.132. Sample of the final edit of region-dependent fissions, absorptions, and leakage

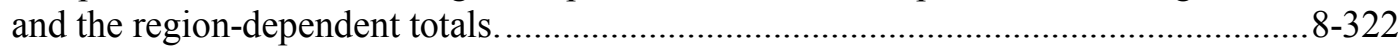

Fig. 8.1.133. Sample of the final edit of fissions, absorptions, and leakage and the region-dependent totals with print by region suppressed. ......................................... 8-323

Fig. 8.1.134. xample of reaction tally summary table. ............................................................ $8-324$

Fig. 8.1.135. Summary of fission source convergence diagnostics............................................ 8-325 
Fig. 8.1.136. Example of matrix k-effective by position index. …............................................ 8-326

Fig. 8.1.137. Sample fission production matrix by position index. ............................................ 8-326

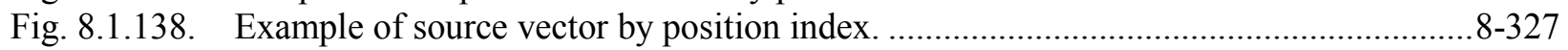

Fig. 8.1.139. Example of cofactor k-effective by position index. ................................................... 8-327

Fig. 8.1.140. Example of matrix k-effective by unit number. .................................................. 8-328

Fig. 8.1.141. An example of the fission probability matrix by unit................................................ 8-328

Fig. 8.1.142. Example of the source vector by unit. ..................................................................... 8-329

Fig. 8.1.143. Example of cofactor k-effective by unit number...................................................... 8-329

Fig. 8.1.144. Example of matrix k-effective by hole number....................................................... 8-329

Fig. 8.1.145. Example of fission production matrix by hole. ...................................................... 8-330

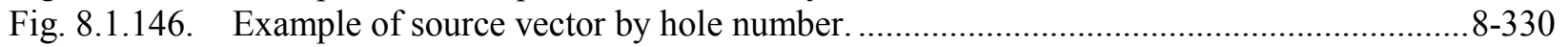

Fig. 8.1.147. Example of cofactor k-effective by hole number. ................................................... 8-330

Fig. 8.1.148. Example of matrix k-effective by array number. ..................................................... 8-331

Fig. 8.1.149. An example of the fission production matrix by array number................................. 8-331

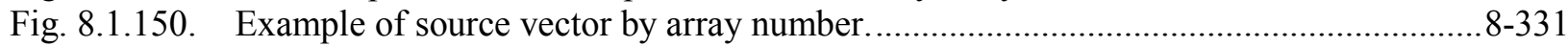

Fig. 8.1.151. Example of cofactor k-effective by array number................................................ 8-332

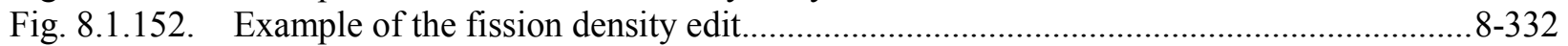

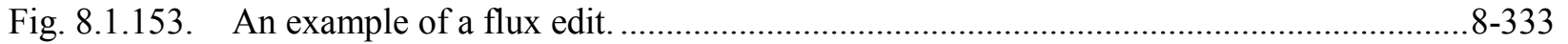

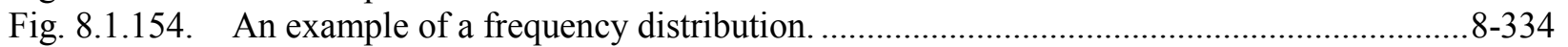

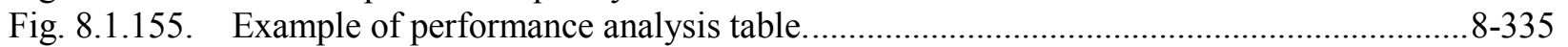

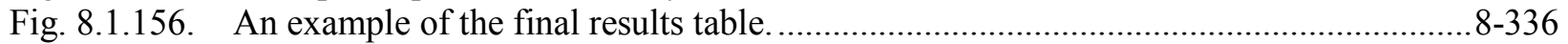

Fig. 8.1.157. Sample program verification information in HTML output.....................................8-336

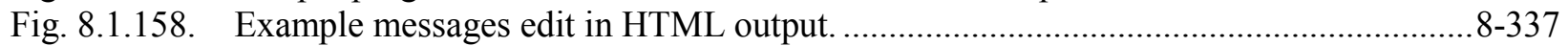

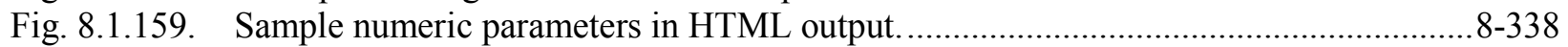

Fig. 8.1.160. Sample logical parameters in HTML output. ............................................................... 8-339

Fig. 8.1.161. Sample table of additional information in HTML output.........................................8-340

Fig. 8.1.162. Example of mixing table data in HTML output. .................................................... $8-341$

Fig. 8.1.163. Example 1-D macroscopic cross section in HTML output. .......................................... 8-342

Fig. 8.1.164. Example of 2-D macroscopic cross sections in HTML output....................................8-343

Fig. 8.1.165. Example of macroscopic probabilities in HTML output...........................................8-344

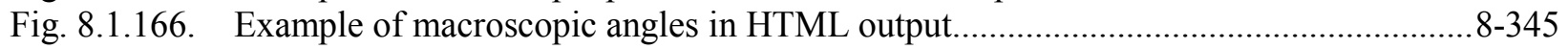

Fig. 8.1.167. Example of geometry region data in HTML output............................................... 8-346

Fig. 8.1.168. Sample volume information in HTML output............................................................ 8-347

Fig. 8.1.169. Sample sum of mesh volumes in HTML output.......................................................8-349

Fig. 8.1.170. Sample of volume by mesh for each region in HTML output..................................8-350

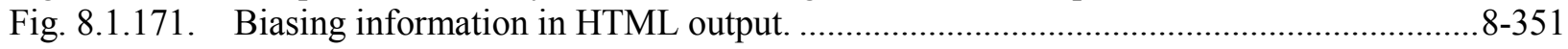

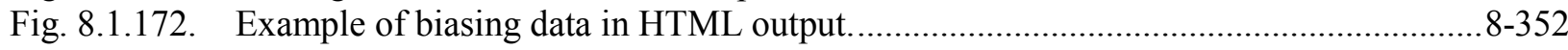

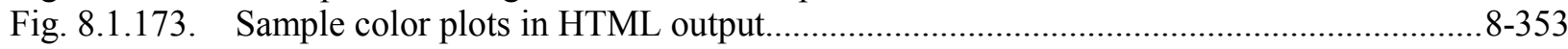

Fig. 8.1.174. Example of initial source information for start type 6 in HTML output.....................8-354

Fig. 8.1.175. Example of fuel center and reference center edits in HTML output. ..........................8-355

Fig. 8.1.176. Example of k-effective by generation in HTML output.......................................... 8-356

Fig. 8.1.177. Example problem characterization edit in HTML output.......................................... 8-357

Fig. 8.1.178. Example of the final k-effective edit in HTML output. .............................................. 8-358

Fig. 8.1.179. Sample plot of average k-effective by generations run in HTML output.................... 8-358

Fig. 8.1.180. Sample plot of average k-effective by generations skipped in HTML output..............8-359

Fig. 8.1.181. Sample fissions, absorptions and leakage data in HTML output. ................................8-360

Fig. 8.1.182. Sample fissions and absorptions plot in HTML output............................................... 8-360

Fig. 8.1.183. Example of matrix k-effective by position index edit in HMTL output.......................8-361

Fig. 8.1.184. Sample fission production matrix by position index edit in HMTL output..................8-361

Fig. 8.1.185. Example of source vector by position index edit in HTML output............................. 8-362 
Fig. 8.1.186. Example cofactor k-effective by position index edit in HTML output. ......................8-362

Fig. 8.1.187. Example of source vector by position index edit in HTML output.............................8-363

Fig. 8.1.188. Example matrix by unit edit in HTML output....................................................... 8-364

Fig. 8.1.189. Example matrix k-effective by hole edit in HTML output......................................... 8 -365

Fig. 8.1.190. Example matrix by array edit in HTML output................................................... $8-366$

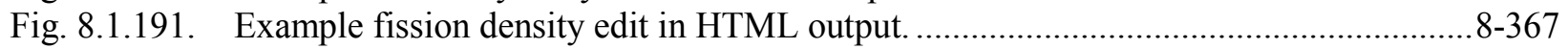

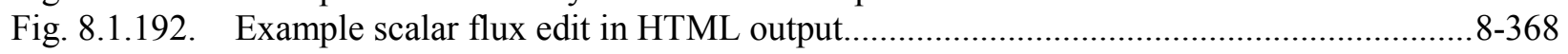

Fig. 8.1.193. Example flux plot in HTML output...................................................................... 8-369

Fig. 8.1.194. Example frequency of generations plot in HTML output. ......................................... 8-370

Fig. 8.1.195. An example of the final results table in HTML output. ...........................................8-370

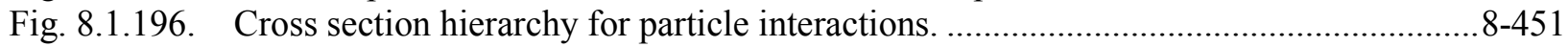

Fig. 8.1.197. Interpolation diagram for secondary angle-energy data..........................................8-459

Fig. 8.1.198. Range of incident energies $\left(\Delta E_{\text {double }}\right)$ for double valued region as a function of

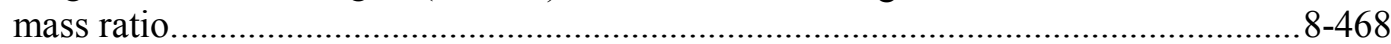

Fig. 8.1.199. $\quad{ }^{7} \mathrm{Li}$ exit energy distribution for the first discrete level inelastic scattering reaction $(\mathrm{MT}=51) . E_{\text {threshold }}=5.4672 \times 10^{5} \mathrm{eV} ; \Delta E_{\text {double }}=1.1539 \times 10^{4} \mathrm{eV} \ldots \ldots \ldots \ldots \ldots \ldots . .8 .470$

Fig. 8.1.200. Analytic estimate of the relationship between WTLOW and the variance, $\sigma_{\mathrm{k}}^{2}$,

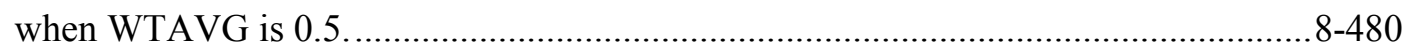

Fig. 8.1.201. Analytic estimate of the relationship between WTAVG and the variance, $\sigma_{\mathrm{k}}^{2}$,

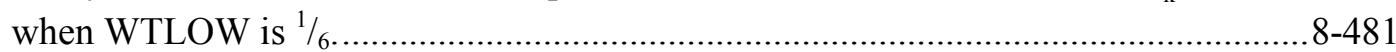

Fig. 8.1.202. Coordinate transform used for quadrature direction and spherical harmonics terms.

Fig. 8.1.B.1. Fig. 8.1.B.2.

Example of cone construction. $8-493$

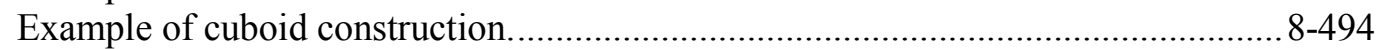

Fig. 8.1.B.3.

Example of cylinder construction.

$8-495$

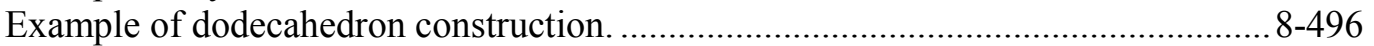

Fig. 8.1.B.4.

Fig. 8.1.B.5.

Fig. 8.1.B.6.

Fig. 8.1.B.7.

Fig. 8.1.B.8.

Fig. 8.1.B.9.

Fig. 8.1.B.10.

Fig. 8.1.B.11.

Fig. 8.1.B.12.

Fig. 8.1.B.13.

Example of elliptical cylinder construction.

8-497

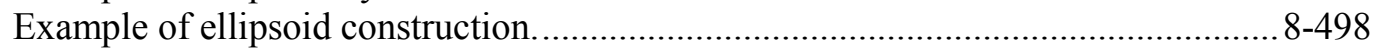

Example of hexprism construction.......................................................... 8-499

Example of hopper construction. ............................................................. 8-500

Example of parallelepiped construction................................................... 8-501

Example of pentagon construction................................................................ $8-502$

Example of plane construction..................................................................... 8-503

Example of rotated hexprism construction....................................................... 8-504

Example of ring construction. ........................................................................ 8-505

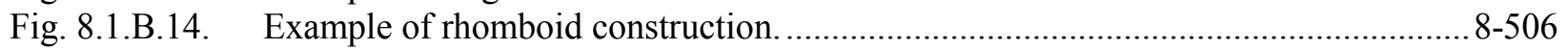

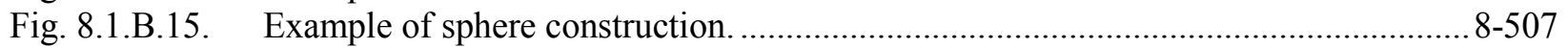

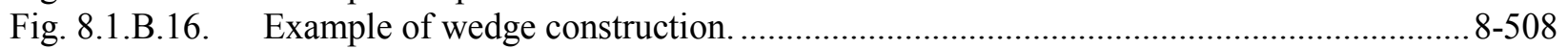

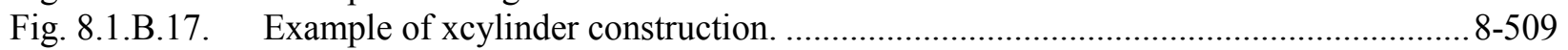

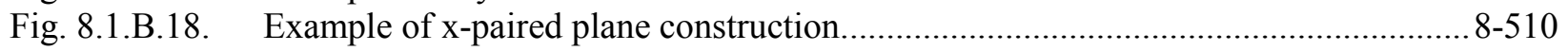

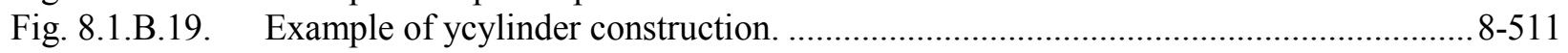

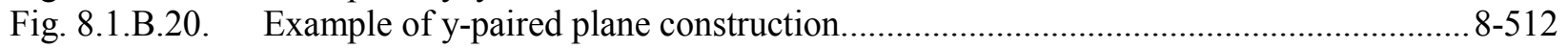

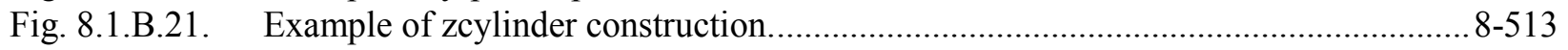

Fig. 8.1.B.22. Example of z-paired plane construction....................................................... 8-514

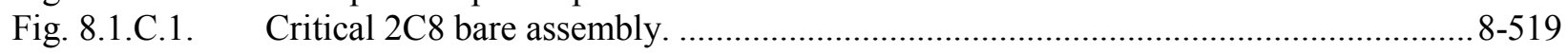

Fig. 8.1.C.2. Half of the paraffin reflected $2 \mathrm{C} 8$ assembly before the top reflector was added....... $8-522$

Fig. 8.1.C.3. Critical assembly of 4 solution units and 4 metal units.......................................... $8-533$ 
Fig. 8.1.C.4. Drawing of two cuboids in an annulus critical assembly...................................... 8-536

Fig. 8.1.C.5. Drawing of the cylinder in an annulus critical assembly. ....................................... 8-538

Fig. 8.1.C.6. Drawing of a critical assembly consisting of a uranium sphere on a Plexiglas

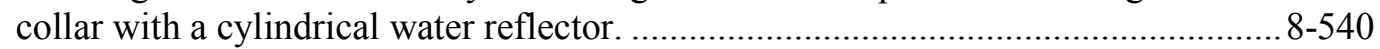

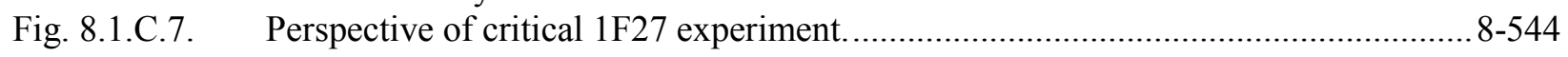

Fig. 8.1.C.8. View of a 27-unit array with $2.54 \mathrm{~cm}$ thick Plexiglas reflector on five sides and a $15.24 \mathrm{~cm}$. thick paraffin base.

Fig. 8.1.C.9. Typical arrangement for critical experiments with interacting arrays of aluminum cylinders containing enriched ${ }^{235} \mathrm{U}$ solutions.

Fig. 8.1.C.10. Schematic of bare partially filled sphere experiment inside a $9.5 \mathrm{ft}$. diameter, $9 \mathrm{ft}$. high steel tank.

Fig. 8.1.C.11. Critical Oralloy sphere.

Fig. 8.1.C.12. Horizontal slice through a critical triangular pitched array of partially flooded $1.95 \%$ enriched uranium metal annular billets. $8-583$

Fig. 8.1.C.13. Vertical slice through the center of a critical triangular pitched array of partially flooded $1.9 \%$ enriched uranium metal annular billets.

Fig. 8.1.C.14. 1.95\% Enriched uranium metal annular billet used in critical experiments 8-585 


\section{LIST OF TABLES}

$\underline{\text { Page }}$

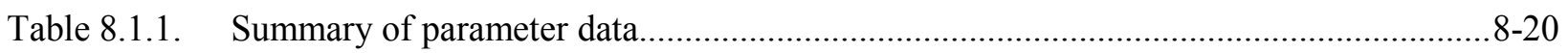

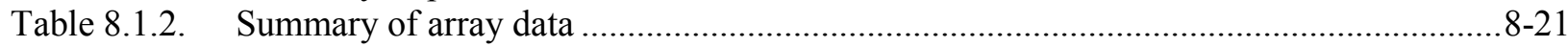

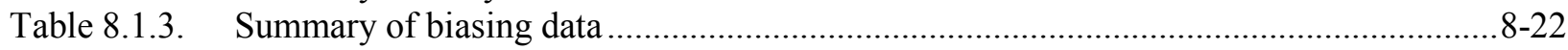

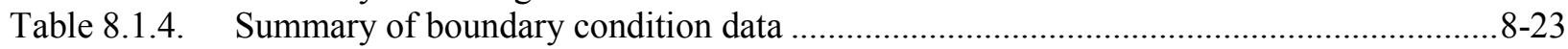

Table 8.1.5. Summary of boundary condition data specific to KENO-VI ........................................8-24

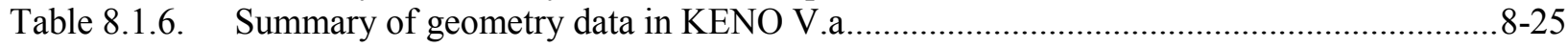

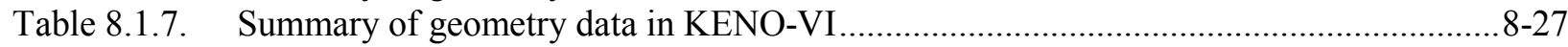

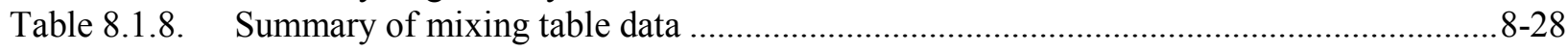

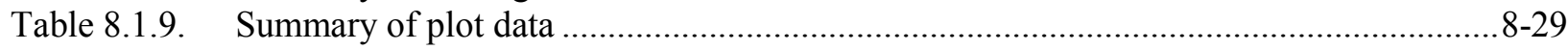

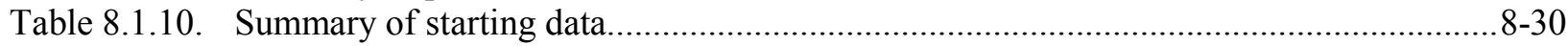

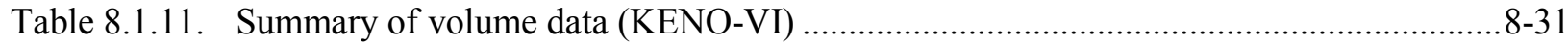

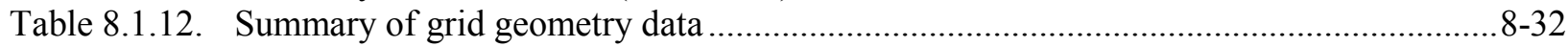

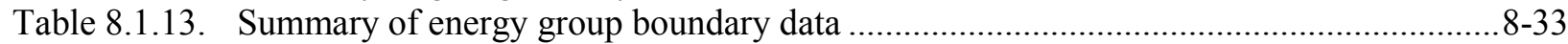

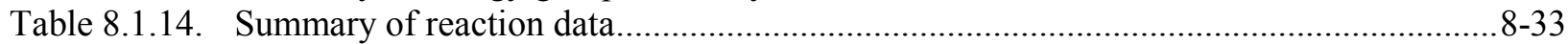

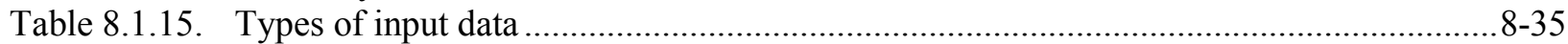

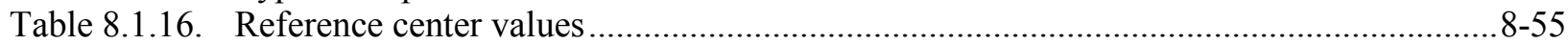

Table 8.1.17. FIDO-like input for mixed box orientation fill option .....................................................6-67

Table 8.1.18. Face codes and surface numbers for entering boundary (albedo) conditions..................8-70

Table 8.1.19. Albedo names available on the KENO albedo library for use with the face codes*......8-73

Table 8.1.20. IDs, group structure and incremental thickness for weighting data available on

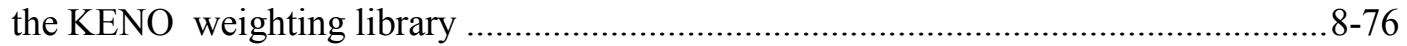

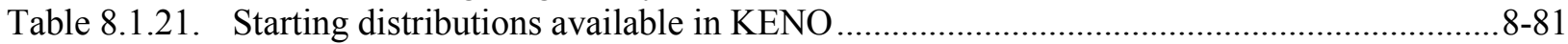

Table 8.1.22. Default color specifications for the color plot display method......................................8-90

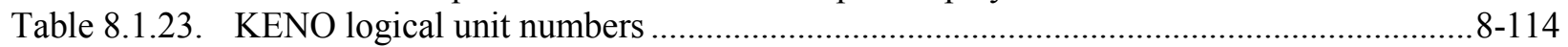

Table 8.1.24. Array index guide for a sample $2 \times 2 \times 2$ array................................................... 8-283

Table 8.1.25. Kinematics data structure for isotropic angular distributions...................................... 8-463

Table 8.1.26. Kinematics data structure for coherent and incoherent elastic scattering.....................8-466

Table 8.1.27. Secondary energy distribution format for elastic and discrete-level inelastic

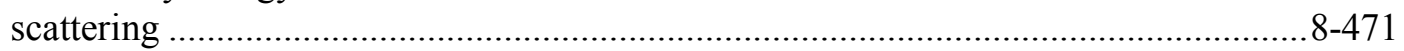

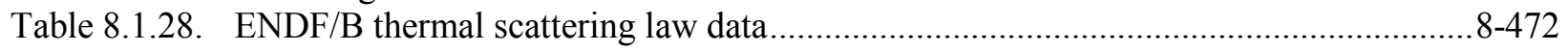




\subsubsection{Introduction to KENO}

KENO, a functional module in the SCALE system, is a Monte Carlo criticality program used to calculate $k_{\text {eff, }}$ fluxes, reaction rates, and other data for three-dimensional (3-D) systems. Special features include multigroup or continuous energy mode, simplified data input, the ability to specify origins for spherical and cylindrical geometry regions, a $\mathrm{P}_{\mathrm{n}}$ scattering treatment, and restart capability.

The KENO data input features flexibility in the order of input. The only restrictions are that the sequence identifier, title, and cross section library must be entered first. A large portion of the data has been assigned default values that have been found to be adequate for many problems. This feature enables the user to run a problem with a minimum of input data.

In addition to the features listed above, KENO-VI uses the SCALE Generalized Geometry Package (SGGP) which contains a much larger set of geometrical bodies, including cuboids, cylinders, spheres, cones, dodecahedrons, elliptical cylinders, ellipsoids, hoppers, parallelepipeds, planes, rhomboids, and wedges. The code's flexibility is increased by allowing: intersecting geometry regions; hexagonal, dodecahedral, and cuboidal arrays; bodies and holes rotated to any angle and translated to any position; and a specified array boundary that contains only that portion of the array located inside the boundary. Users should be aware that the added geometry features in KENO-VI can result in significantly longer run times than KENO V.a. A KENO-VI problem that can be modeled in KENO V.a will typically run about four times as long with KENO-VI as it does with KENO V.a. Therefore KENO-VI is not a replacement for KENO V.a, but rather an additional version for more complex geometries that could not be modeled previously.

Blocks of input data are entered in the form

\section{READ XXXX input_data END XXXX,}

where $\mathbf{X X X X}$ is the keyword for the type of data being entered. The types of data entered include parameters, geometry region data, array definition data, biasing or weighting data, albedo boundary conditions, starting distribution information, the cross section mixing table, extra one-dimensional (1-D) (reaction rate) cross section IDs for special applications, energy group boundaries for tallying in the continuous energy mode, a mesh grid for collecting flux moments, and printer plot information.

A block of data can be omitted unless it is needed or desired for the problem. Within the blocks of data, most of the input is activated by using keywords to override default values.

The treatment of the energy variable can be either multigroup or continuous. Changing the calculation mode from multigroup to continuous energy or vice versa is established by simply changing the cross section library used. All available calculated entities in the multigroup mode can also be calculated in the continuous energy mode. If the calculated entity is energy or group dependent, it is automatically tallied into the appropriate group structure in the continuous energy mode.

The KENO V.a geometry input consists of spheres, hemispheres, cylinders, hemicylinders, and cuboids. Although the origin of the cylinders, hemicylinders, spheres, and hemispheres is zero by default, they may be specified to any value that will allow the geometry to fit in the problem. This feature allows the use of nonconcentric cylindrical and spherical shapes and provides a great deal of freedom in positioning them. Another feature that expands the generality of the code is the ability to place the cut surface of the hemicylinders and hemispheres at any distance between the radius and the origin. 
An additional convenience is the availability of an alternative method for specifying the array definition unit-location data. This method uses FIDO-like options for filling the array.

As mentioned above, KENO-VI uses the SGGP, which contains a much more flexible geometry package than the one in KENO V.a. In KENO-VI, geometry regions are constructed and processed as sets of quadratic equations. A set of geometric shapes (including all of those used in KENO V.a plus others) is available in KENO-VI, as well as the ability to build more complex geometric shapes using sets of quadratic equations. Unlike KENO V.a, KENO-VI allows intersections between geometry regions within a unit, and it provides the ability to specify an array boundary that intersects the array.

The most flexible KENO V.a geometry features are the "ARRAY-of-ARRAYs" and "HOLEs" capabilities. The ARRAY-of-ARRAYs option allows the construction of ARRAYs from other ARRAYs. The depth of nesting is limited only by computer space restrictions. This option greatly simplifies the setup for ARRAYs involving different UNITs at different spacings. The HOLE option allows a UNIT or an ARRAY to be placed at any desired location within a geometry region. The emplaced UNIT or ARRAY cannot intersect any geometry region and must be wholly contained within a region. As many HOLEs as will snugly fit without intersecting can be placed in a region. This option is especially useful for describing shipping casks and reflectors that have gaps or other geometrical features. Any number of HOLEs can be described in a problem, and HOLEs can be nested to any depth.

The primary difference between the KENO V.a and KENO-VI geometry input is the methodology used to represent the geometry/material regions in a unit. KENO-VI uses two geometry records (cards) to describe a region. The first record, called the GEOMETRY record, contains the geometry (shape) keyword, region boundary definitions, and any geometry modification data. Using geometry modification data, regions can be rotated and translated to any angle and position within a unit. The second record, the CONTENT record, contains the MEDIA keyword; the material, HOLE, or ARRAY ID number; the bias ID number; and the region definition vector. KENO-VI requires that a GLOBAL UNIT be specified in all problems, including single unit problems.

In addition to the cuboidal ARRAYs available in KENO V.a, hexagonal ARRAYs and dodecahedral ARRAYs can be directly constructed in KENO-VI. Also, the ability to specify an ARRAY boundary that intersects the ARRAY makes it possible to construct a lattice in a cylinder using one ARRAY in KENOVI instead of multiple ARRAYs and HOLEs as would be required in KENO V.a.

Anisotropic scattering is treated by using discrete scattering angles. The angles and associated probabilities are generated in a manner that preserves the moments of the angular scattering distribution for the selected group-to-group transfer. These moments can be derived from the coefficients of a $P_{n}$ Legendre polynomial expansion. All moments through the $2 \mathrm{n}-1$ moment are preserved for $\mathrm{n}$ discrete scattering angles. A one-to-one correspondence exists such that $\mathrm{n}$ Legendre coefficients yield $\mathrm{n}$ moments. The cases of zero and one scattering angle are treated in a special manner. Even when the user specifies multiple scattering angles, KENO can recognize that the distribution is isotropic, and therefore KENO selects from a continuous isotropic distribution. If the user specifies one scattering angle, the code selects the scattering angle from a linear function if it is positive between -1 and +1 , and otherwise it performs semicontinuous scattering by picking scattering angle cosines uniformly over some range between -1 and +1 . The probability is zero over the rest of the range.

The KENO restart option is easy to activate. Certain changes can be made when a problem is restarted, including using a different random sequence or turning off certain print options such as fluxes or the fissions and absorptions by region. 
KENO can also compute angular fluxes and flux moments in multigroup calculations, which are required to compute scattering terms for generation of sensitivity coefficients with the SAMS module or the TSUNAMI-3D control module. Fluxes can also be accumulated in a Cartesian mesh that is superimposed over the user-defined geometry in an automated manner.

KENO can perform Monte Carlo transport calculations concurrently on a number of computational nodes. By introducing a simple master-slave approach via MPI, KENO runs different random walks concurrently on the replicated geometry within the same generation. Fission source and other tallied quantities are gathered at the end of each generation by the master process and are then processed either for final edits or subsequent generations. Code parallel performance is strongly dependent on the size of the problem simulated and the size of the tallied quantities.

\subsubsection{KENO Data Guide}

KENO may be run stand alone or as part of a SCALE criticality safety or sensitivity and uncertainty analysis sequence. If KENO is run stand alone in the multigroup mode, cross section data can be used from an AMPX $^{1}$ working format library or from a Monte Carlo format cross section library. If KENO uses an AMPX working format library, a mixing table data block must be entered. If a Monte Carlo format library is used, a mixing table data block is not entered, and the mixtures specified in the KENO geometry description must be consistent with the mixtures created on the Monte Carlo format library file.

If KENO is run stand alone in the continuous energy mode, a mixing table data block must be provided unless the restart option is used.

If KENO is run as part of a SCALE criticality safety or sensitivity and uncertainty analysis sequence, the mixtures are defined in the CSAS or TSUNAMI-3D input, and a mixing table data block cannot be entered in KENO. Furthermore, the mixture numbers used in the KENO geometry description must correspond to those defined in the composition data block of the CSAS or TSUNAMI-3D input. To use a cell-weighted mixture in KENO, the keyword "CELLMIX=," followed by a unique mixture number, must be specified in the unit cell data of the CSAS or TSUNAMI-3D sequence. Unit cell data are applicable only in the multigroup mode. The mixture number used in the KENO input is the unique mixture number immediately following the keyword "CELLMIX=." A cell-weighted mixture is available only in SCALE sequences that use XSDRN to perform a cell-weighting calculation using a multigroup cross section library. Table 8.1.1 through Table 8.1.14 summarize the KENO input data blocks. These input data blocks are discussed in detail in the following sections.

In order to run KENO parallel (standalone execution), the user must provide a name with the "\%" prefix in the input file (=\%kenovi). Control modules like CSAS, TRITON, and TSUNAMI-3D automatically initiate parallel KENO execution if the user provides the required arguments while running this code. 
Table 8.1.1. Summary of parameter data

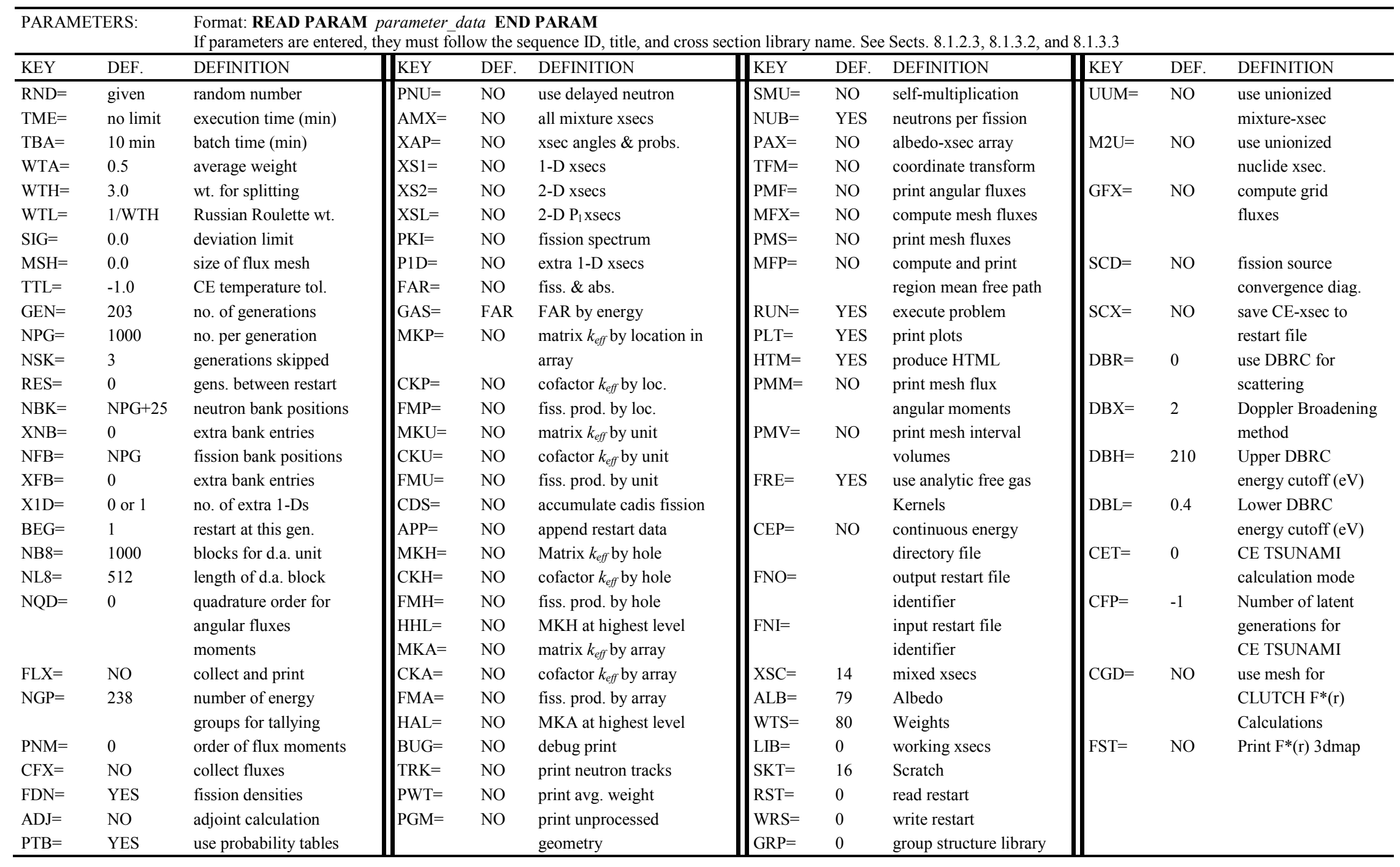


Table 8.1.2. Summary of array data

\begin{tabular}{|c|c|c|c|c|c|}
\hline \multirow[t]{11}{*}{$\overline{\text { ARRAY }}$} & \multicolumn{5}{|c|}{$\begin{array}{l}\text { Repeat the sequence ARRAY PARAMETERS DATA TYPE ORIENTATION DATA for each array used in the problem. } \\
\text { ARRAY PARAMETERS }\end{array}$} \\
\hline & \multicolumn{2}{|c|}{$\overline{\text { KEYWORD DEFAULT }}$} & $\begin{array}{c}\text { DEFINITION } \\
\end{array}$ & & \\
\hline & $\overline{\mathrm{ARA}}=$ & none $^{\mathrm{a}}$ & no. defining the array & & \\
\hline & TYP= & Cuboidal $^{\mathrm{c}}$ & array type (cuboidal or square, hexagonal or & & \\
\hline & NUX $=$ & none $^{\mathrm{a}}$ & no. of units in $\mathrm{X}$ direction & & \\
\hline & $\mathrm{NUY}=$ & none $^{a}$ & no. of units in $\mathrm{Y}$ direction & & \\
\hline & $\mathrm{NUZ}=$ & none $^{\mathrm{a}}$ & no. of units in $\mathrm{Z}$ direction & & \\
\hline & $\mathrm{GBL}=$ & none $^{\mathrm{b}}$ & global or overall array number & & \\
\hline & $\mathrm{PRT}=$ & YES & print any label & & \\
\hline & $\mathrm{COM}=$ & none & delim comment delim & & \\
\hline & & & optional, maximum of 132 characters & & \\
\hline \multicolumn{4}{|c|}{ ORIENTATION DATA FOR FILL } & \multicolumn{2}{|c|}{ ORIENTATION DATA FOR LOOP } \\
\hline \multicolumn{4}{|c|}{$\begin{array}{l}\text { Enter unit numbers to define every position in the array. When entering data using the options in this } \\
\text { table, the count and option fields must be adjacent with no imbedded blanks. The operand field may be } \\
\text { separated from the option field by one or more blanks. Orientation data for FILL is terminated by } \\
\text { entering END FILL. }\end{array}$} & \multicolumn{2}{|r|}{$\begin{array}{l}\text { Enter the unit number and nine numbers that define the position(s) of that unit. Data for each of } \\
\text { these ten entries are repeated until every position in the array has been defined. Orientation data } \\
\text { for LOOP are terminated by entering END LOOP. } \\
\text { ENTER DATA IN THE FORM: }\end{array}$} \\
\hline COUNT & OPTION & OPERAND & & DATA & 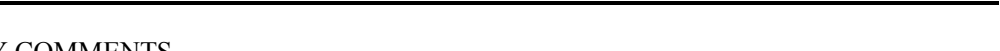 \\
\hline \multirow[t]{2}{*}{ FIELD } & FIELD & FIELD & COMMENTS & ENTRY & C COMMENTS \\
\hline & & $\bar{j}$ & stores $\mathrm{j}$ at the current position in the array & LTYPE & The unit type. LTYPE must be greater than 0 . \\
\hline \multirow[t]{3}{*}{$\mathrm{i}$} & $\mathrm{R}, *, \$$ & $\mathrm{j}$ & stores $\mathrm{j}$ in the next $\mathrm{i}$ positions in the array & & Starting position in the $\mathrm{X}$ direction. IX1 must be at least 1 and no larger than \\
\hline & $\mathrm{F}$ & $\mathrm{j}$ & fills remainder of the array with unit no. $\mathrm{j}$ starting with the & & the value entered for NUX. \\
\hline & A & $\mathrm{j}$ & $\begin{array}{l}\text { current array position } \\
\text { sets the current position in the array to } j\end{array}$ & & $\begin{array}{l}\text { Ending position in the X direction. IX } 2 \text { must be at least } 1 \text { and no larger than } \\
\text { the value of NUX. }\end{array}$ \\
\hline \multirow[t]{2}{*}{$\mathrm{i}$} & $\mathrm{S}$ & & increments current position in the array by $\mathrm{i}$ & INCX & The number of units by which increments are made in the $\mathrm{X}$ direction. \\
\hline & & & $\begin{array}{l}\text { (This allows skipping i positions. The value of i may be } \\
\text { positive or negative.) }\end{array}$ & IY1 & $\begin{array}{l}\text { The starting position in the Y direction. IY1 must be at least } 1 \text { and less than } \\
\text { the value entered for NUY. }\end{array}$ \\
\hline \multirow[t]{3}{*}{1} & Q & $\mathrm{j}$ & repeats the previous $j$ entries $i$ times. The default value of $i$ is 1 & & Ending position in the Y direction. IY2 must be at least 1 and no larger than \\
\hline & $\mathrm{N}$ & $\mathrm{j}$ & repeats the previous $\mathrm{j}$ entries $\mathrm{i}$ times, inverting the sequence & & the value of NUY. \\
\hline & & & each time. The default value of $i$ is 1 . & INCY & The number of units by which increments are made in the positive $\mathrm{Y}$ direction. \\
\hline \multirow[t]{2}{*}{$\mathrm{i}$} & B & $\mathrm{j}$ & starting with the entry at $-\mathrm{i}$ from the current position, & & Starting position in the $Z$ direction. IZ1 must be at least 1 and no larger than NUZ. \\
\hline & & & $\begin{array}{l}\text { store entries in inverse order until position }-(\mathrm{i}+\mathrm{j}) \text { is reached. } \\
\text { Default value of } \mathrm{i}=1 \text {. }\end{array}$ & $\begin{array}{l}\text { IZ2 } \\
\text { INCZ }\end{array}$ & $\begin{array}{l}\text { Ending position in the } Z \text { direction. IZ2 must be at least } 1 \text { and no larger than NUZ. } \\
\text { The number of units by which increments are made in the positive } Z \text { direction. }\end{array}$ \\
\hline $\mathrm{i}$ & I & $\mathrm{j} \mathrm{k}$ & $\begin{array}{l}\text { provides the end points }(\mathrm{j}, \mathrm{k}) \text { with } \mathrm{i} \text { entries linearly interpolated } \\
\text { between them (i.e., a total of } \mathrm{i}+2 \text { points). At least one blank } \\
\text { must separate } \mathrm{j} \text { and } \mathrm{k} \text {. When used for an integer array, the } \\
\text { i option should only be used to generate integer steps } \\
\text { (i.e., }(\mathrm{k}-\mathrm{j}) /(\mathrm{i}+1) \text { should be a whole number). }\end{array}$ & & \\
\hline
\end{tabular}

${ }^{b}$ In KENO V.a the default is the largest array number, maxara

${ }^{c}$ This is a KENO-VI parameter. In KENO V.a the array type is always cuboidal (square). 
Table 8.1.3. Summary of biasing data

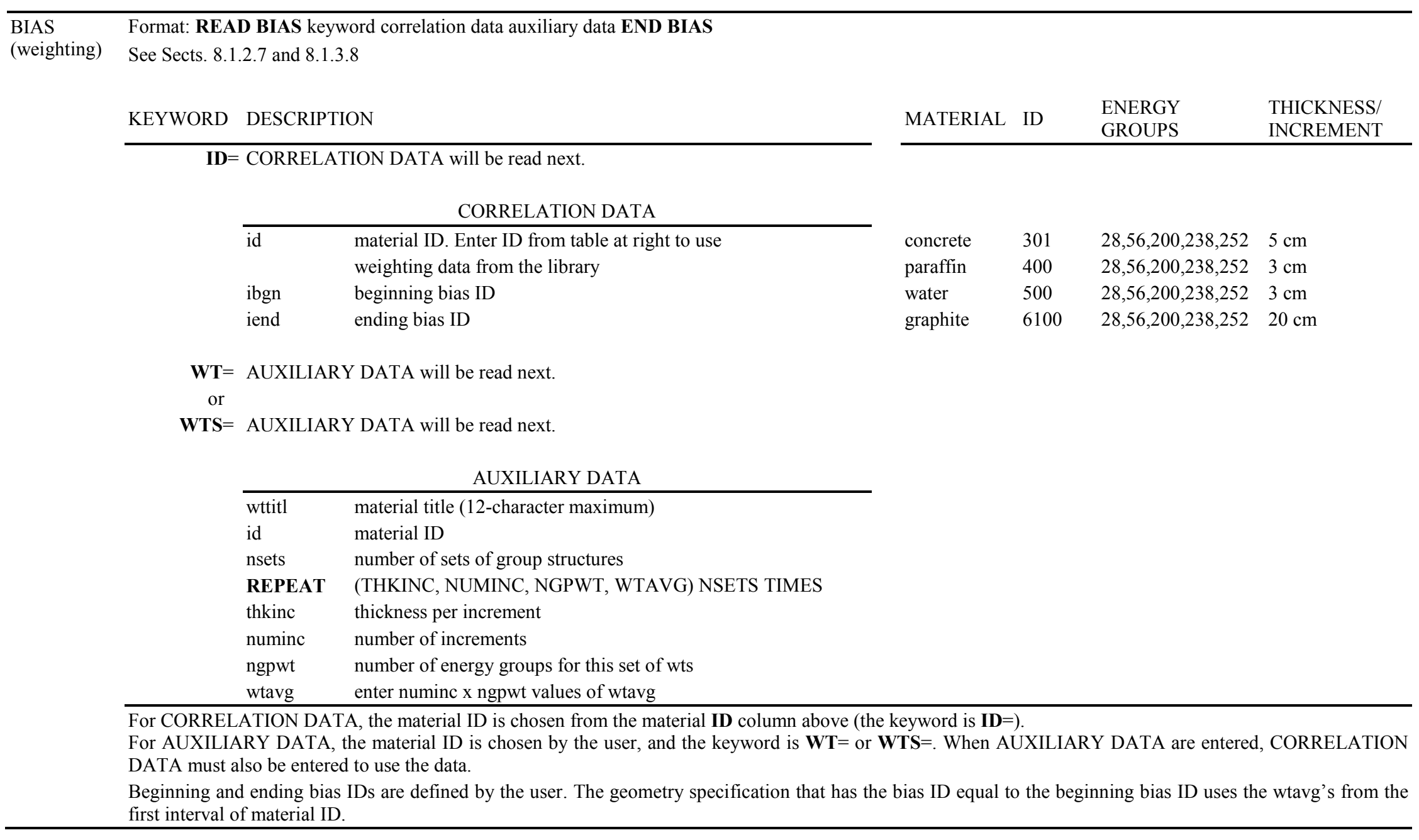




\section{Table 8.1.4. Summary of boundary condition data}

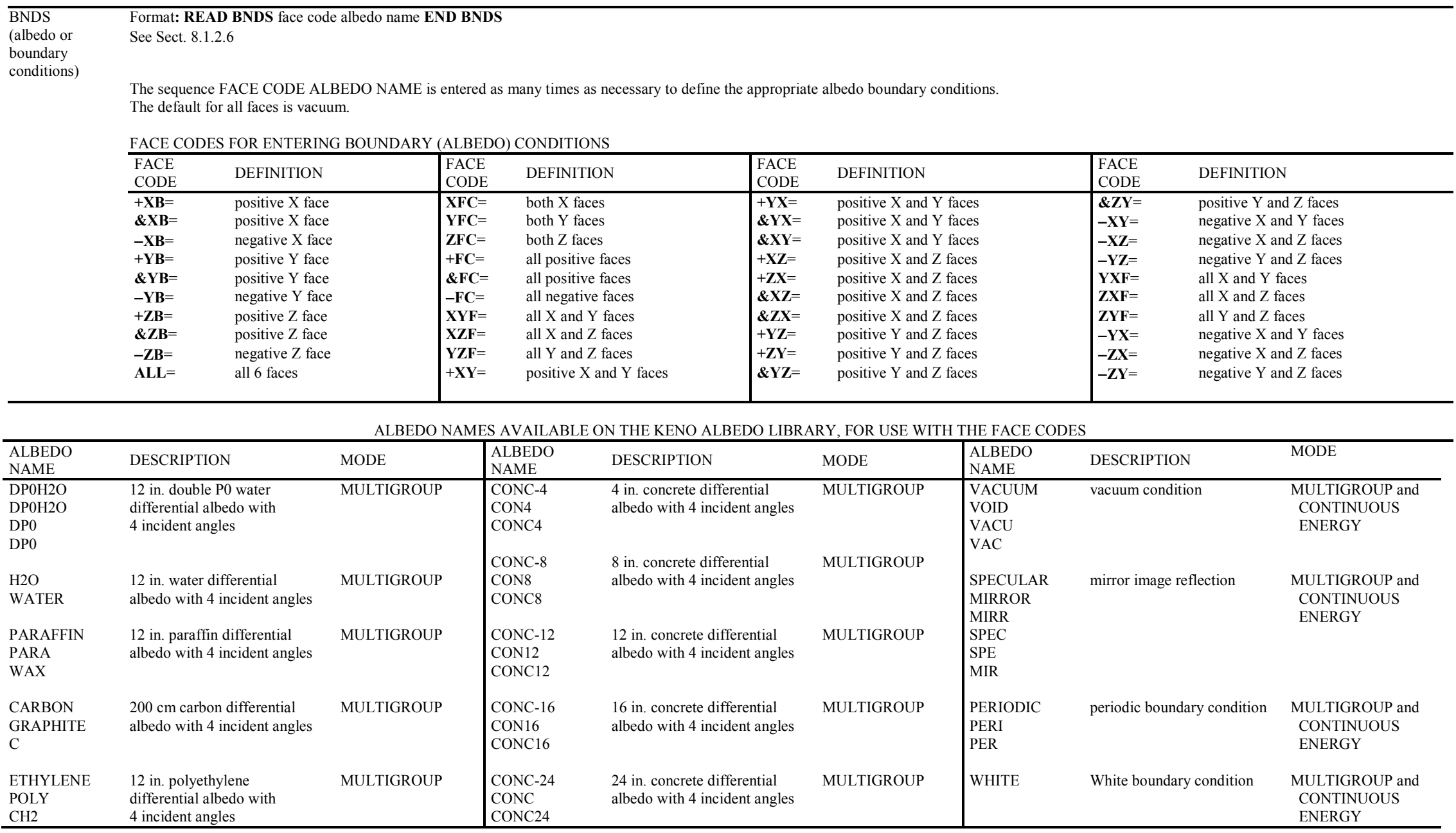


Table 8.1.5. Summary of boundary condition data specific to KENO-VI

\begin{tabular}{|c|c|c|c|c|c|c|c|c|c|c|c|c|}
\hline \multicolumn{13}{|c|}{ ALBEDO SURFACE NUMBERS RELATED TO KENO-VI GEOMETRY BODIES } \\
\hline $\begin{array}{l}\text { GEOMETRY } \\
\text { BODY }\end{array}$ & 1 & 2 & 3 & 4 & 5 & 6 & 7 & 8 & 9 & 10 & 11 & 12 \\
\hline CONE & Radial & $+\mathrm{Z}$ & $-\mathrm{Z}$ & & & & & & & & & \\
\hline CUBOID & $+\mathrm{X}$ & $-\mathrm{X}$ & $+\mathrm{Y}$ & $-\mathrm{Y}$ & $+\mathrm{Z}$ & $-Z$ & & & & & & \\
\hline CYLINDER & Radial & $+\mathrm{Z}$ & $-Z$ & & & & & & & & & \\
\hline DODECAHEDRON & $+\mathrm{X}$ & $-\mathrm{X}$ & $+\mathrm{Y}$ & $-\mathrm{Y}$ & $+\mathrm{X}+\mathrm{Y}+\mathrm{Z}$ & $-\mathrm{X}-\mathrm{Y}-\mathrm{Z}$ & $-\mathrm{X}+\mathrm{Y}+\mathrm{Z}$ & $+\mathrm{X}-\mathrm{Y}-\mathrm{Z}$ & $-\mathrm{X}-\mathrm{Y}+\mathrm{Z}$ & $+\mathrm{X}+\mathrm{Y}-\mathrm{Z}$ & $+\mathrm{X}-\mathrm{Y}+\mathrm{Z}$ & $-\mathrm{X}+\mathrm{Y}-\mathrm{Z}$ \\
\hline ECYLINDER & Radial & $+\mathrm{Z}$ & $-Z$ & & & & & & & & & \\
\hline ELLIPSIOD & Radial & & & & & & & & & & & \\
\hline HEXPRISM & $+\mathrm{X}$ & $-X$ & $+\mathrm{X}+\mathrm{Y}$ & $-\mathrm{X}-\mathrm{Y}$ & $-\mathrm{X}+\mathrm{Y}$ & $+\mathrm{X}-\mathrm{Y}$ & $+\mathrm{Z}$ & $-Z$ & & & & \\
\hline HOPPER & $+\mathrm{X}$ & $-X$ & $+\mathrm{Y}$ & $-\mathrm{Y}$ & $+Z$ & $-Z$ & & & & & & \\
\hline PENTAGON & $-\mathrm{Y}$ & $+\mathrm{X}-\mathrm{Y}$ & $+\mathrm{X}+\mathrm{Y}$ & $-\mathrm{X}+\mathrm{Y}$ & $-\mathrm{X}-\mathrm{Y}$ & $+\mathrm{Z}$ & $-\mathrm{Z}$ & & & & & \\
\hline PLANE & Surface & & & & & & & & & & & \\
\hline QUADRATIC & Surface & & & & & & & & & & & \\
\hline RHEXPRISM & $+\mathrm{Y}$ & $-\mathrm{Y}$ & $-X+Y$ & $+\mathrm{X}-\mathrm{Y}$ & $+\mathrm{X}+\mathrm{Y}$ & $-\mathrm{X}-\mathrm{Y}$ & $+Z$ & $-Z$ & & & & \\
\hline RING & $\begin{array}{l}\text { Inner } \\
\text { Radius }\end{array}$ & $\begin{array}{l}\text { Outer } \\
\text { Radius }\end{array}$ & $+\mathrm{Z}$ & $-\mathrm{Z}$ & & & & & & & & \\
\hline SPHERE & Radial & & & & & & & & & & & \\
\hline WEDGE & $-\mathrm{Y}$ & $-\mathrm{X}+\mathrm{Y}$ & $+\mathrm{X}+\mathrm{Y}$ & $+Z$ & $-Z$ & & & & & & & \\
\hline XCYLINDER & Radial & $+\mathrm{X}$ & $-\mathrm{X}$ & & & & & & & & & \\
\hline XPPLANE & $+\mathrm{X}$ & $-\mathrm{X}$ & & & & & & & & & & \\
\hline YCYLINDER & Radial & $+\mathrm{Y}$ & $-\mathrm{Y}$ & & & & & & & & & \\
\hline YPPLANE & $+\mathrm{Y}$ & $-\mathrm{Y}$ & & & & & & & & & & \\
\hline ZCYLINDER & Radial & $+\mathrm{Z}$ & $-\mathrm{Z}$ & & & & & & & & & \\
\hline ZPPLANE & $+\mathrm{Z}$ & $-Z$ & & & & & & & & & & \\
\hline
\end{tabular}

Surfaces refer to the pre-rotation surface of the body that occurs in the indicated quadrant.

Refer to Figs.9.B.1-9.B.22 for illustrations of each geometry body. 


\section{Table 8.1.6. Summary of geometry data in KENO V.a}

\begin{tabular}{|c|c|c|c|c|c|c|c|}
\hline \multirow{29}{*}{$\begin{array}{l}\text { GEOMETRY } \\
\text { (region) }\end{array}$} & \multicolumn{7}{|c|}{ Format: READ GEOM enter geometry region data here END GEOM } \\
\hline & \multicolumn{7}{|c|}{ See Sect.8.1.2.4, 8.1.3.1.2, 8.1.3.6,and 8.1.3.7 } \\
\hline & \multicolumn{7}{|c|}{ GEOMETRY REGION DATA consist of SIMPLE GEOMETRY REGION DATA and EXTENDED GEOMETRY REGION DATA. } \\
\hline & \multicolumn{7}{|c|}{ ENTER GEOMETRY DATA IN THE FOLLOWING FORM: } \\
\hline & \multicolumn{7}{|c|}{ OPTIONAL GLOBAL SPECIFICATION } \\
\hline & \multicolumn{7}{|c|}{ UNIT n } \\
\hline & \multicolumn{7}{|c|}{ OPTIONAL GEOMETRY COMMENT } \\
\hline & \multicolumn{7}{|c|}{ GEOMETRY REGION DATA and/or EXTENDED GEOMETRY REGION DATA } \\
\hline & \multicolumn{7}{|c|}{ 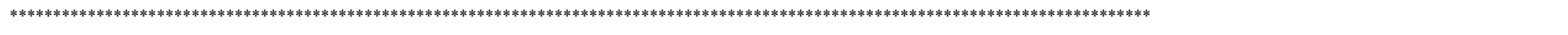 } \\
\hline & \multicolumn{7}{|c|}{ ENTER SIMPLE REGION DATA IN THE FOLLOWING FORM: } \\
\hline & \multicolumn{7}{|c|}{ GLOBAL Enter only to specify this unit as the global unit. } \\
\hline & \multicolumn{7}{|l|}{ UNIT n } \\
\hline & \multicolumn{7}{|c|}{ COM=delim comment delim This optional comment can be up to 132 characters long. It must begin and end with a delimiter (i.e. “, ‘, *, , or !) } \\
\hline & \multicolumn{7}{|c|}{ fgeom mix no. bias ID dimensions optional origin data (ORIGIN coordinates) optional chord data (CHORD distance) optional center data (CENTER type X-offset Y-offset Z-offset) } \\
\hline & \multicolumn{7}{|c|}{ Enter as many geometry description specifications as necessary to describe the unit and as many units as necessary to describe the system. } \\
\hline & \multicolumn{7}{|c|}{$\begin{array}{ll}\text { SIMPLE GEOMETRY REGION INPUT DATA REQUIREMENTS } \\
\end{array}$} \\
\hline & TYPE OF DATA & TYPE 1 DATA & TYPE 2 DATA & TYPE 3 DATA & TYPE 4 DATA & TYPE 5 DATA & TYPE 6 DATA \\
\hline & \multirow[t]{8}{*}{$\overline{\text { fgeom }}$} & SPHERE & XCYLINDER & YCYLINDER & CYLINDER & CUBE & CUBOID \\
\hline & & HEMISPHERE & XHEMICYL+Y & YHEMICYL $+X$ & ZCYLINDER & & \\
\hline & & HEMISPHE $+\mathrm{X}$ & XHEMICYL-Y & YHEMICYL-X & ZHEMICYL+X & & \\
\hline & & HEMISPHE-X & XHEMICYL+Z & YHEMICYL+Z & ZHEMICYL-X & & \\
\hline & & HEMISPHE+Y & XHEMICYL-Z & YHEMICYL-Z & ZHEMICYL+Y & & \\
\hline & & HEMISPHE-Y & & & ZHEMICYL-Y & & \\
\hline & & HEMISPHE+Z & & & & & \\
\hline & & HEMISPHE-Z & & & & & \\
\hline & dimensions & $\mathrm{R}$ (radius) & $\mathrm{R}+\mathrm{X}-\mathrm{X}$ & $R+Y-Y$ & $\mathrm{R}+\mathrm{Z}-\mathrm{Z}$ & $+\mathrm{X}-\mathrm{X}$ & $+\mathrm{X}-\mathrm{X}+\mathrm{Y}-\mathrm{Y}+\mathrm{Z}-\mathrm{Z}$ \\
\hline & optional origin coordinates* & $\begin{array}{l}\text { Enter the X Y Z coordinates } \\
\text { of origin }\end{array}$ & $\begin{array}{l}\mathrm{s} \text { Enter the } \mathrm{Y} \mathrm{Z} \text { coordinates } \\
\text { of centerline }\end{array}$ & $\begin{array}{l}\text { Enter the } \mathrm{X} \mathrm{Z} \text { coordinates } \\
\text { of centerline }\end{array}$ & $\begin{array}{l}\text { Enter the X Y coordinates } \\
\text { of centerline }\end{array}$ & omit & omit \\
\hline & optional chord data** & Enter the dist. to plane & Enter the dist. to plane & Enter the dist. to plane & Enter the dist. to plane & omit & omit \\
\hline & optional center data*** & Center type & X-offset & X-offset & Z-offset & omit & omit \\
\hline
\end{tabular}

*Enter ORIG or ORIGIN for fgeom.

**Enter CHORD for fgeom.

***Center data are only applicable when flux moments ( $\mathrm{PNM}>0$ ) or angular fluxes (NQD $>0)$ are requested with the coordinate transform (TFM) set to YES. NOTE: Chord data are not applicable for SPHERE, XCYLINDER, YCYLINDER, CYLINDER, ZCYLINDER, CUBE, or CUBOID.
Origin data are not applicable for a CUBE or CUBOID. 


\section{Fig. 8.1.6. Summary of geometry data in KENO V.a (continued)}

\begin{tabular}{|c|c|c|c|c|}
\hline \multirow[t]{9}{*}{$\begin{array}{l}\text { GEOMETRY } \\
\text { (region) } \\
\text { (continued) }\end{array}$} & \multicolumn{4}{|c|}{$\begin{array}{l}\text { ENTER GEOMETRY DATA IN THE FOLLOWING FORM: } \\
\text { fgeom ref. ID bias ID thickness per region origin coordinates nreg }\end{array}$} \\
\hline & \multicolumn{4}{|c|}{ EXTENDED GEOMETRY REGION INPUT DATA REQUIREMENTS } \\
\hline & $\begin{array}{l}\text { TYPE OF } \\
\text { DATA }\end{array}$ & $\begin{array}{l}\text { TYPE } 1 \\
\text { DATA }\end{array}$ & $\begin{array}{l}\text { TYPE } 2 \\
\text { DATA }\end{array}$ & $\begin{array}{l}\text { TYPE } 3 \\
\text { DATA }\end{array}$ \\
\hline & fgeom & ARRAY & HOLE & $\begin{array}{l}\text { REPLICATE } \\
\text { REFLECTOR }\end{array}$ \\
\hline & ref. ID & array no. & emplaced unit number & mixture number in generated regions \\
\hline & bias ID & omit for ARRAY & omit & first bias ID \\
\hline & thickness / region & omit & omit & variable $^{a}$ \\
\hline & origin coordinates & $\begin{array}{l}\text { Enter the X Y Z coordinates of the most negative point } \\
\text { of the array }\end{array}$ & Enter the X Y Z coordinates of the origin & omit \\
\hline & nreg & omit & omit & number of regions to be generated \\
\hline
\end{tabular}


Table 8.1.7. Summary of geometry data in KENO-VI

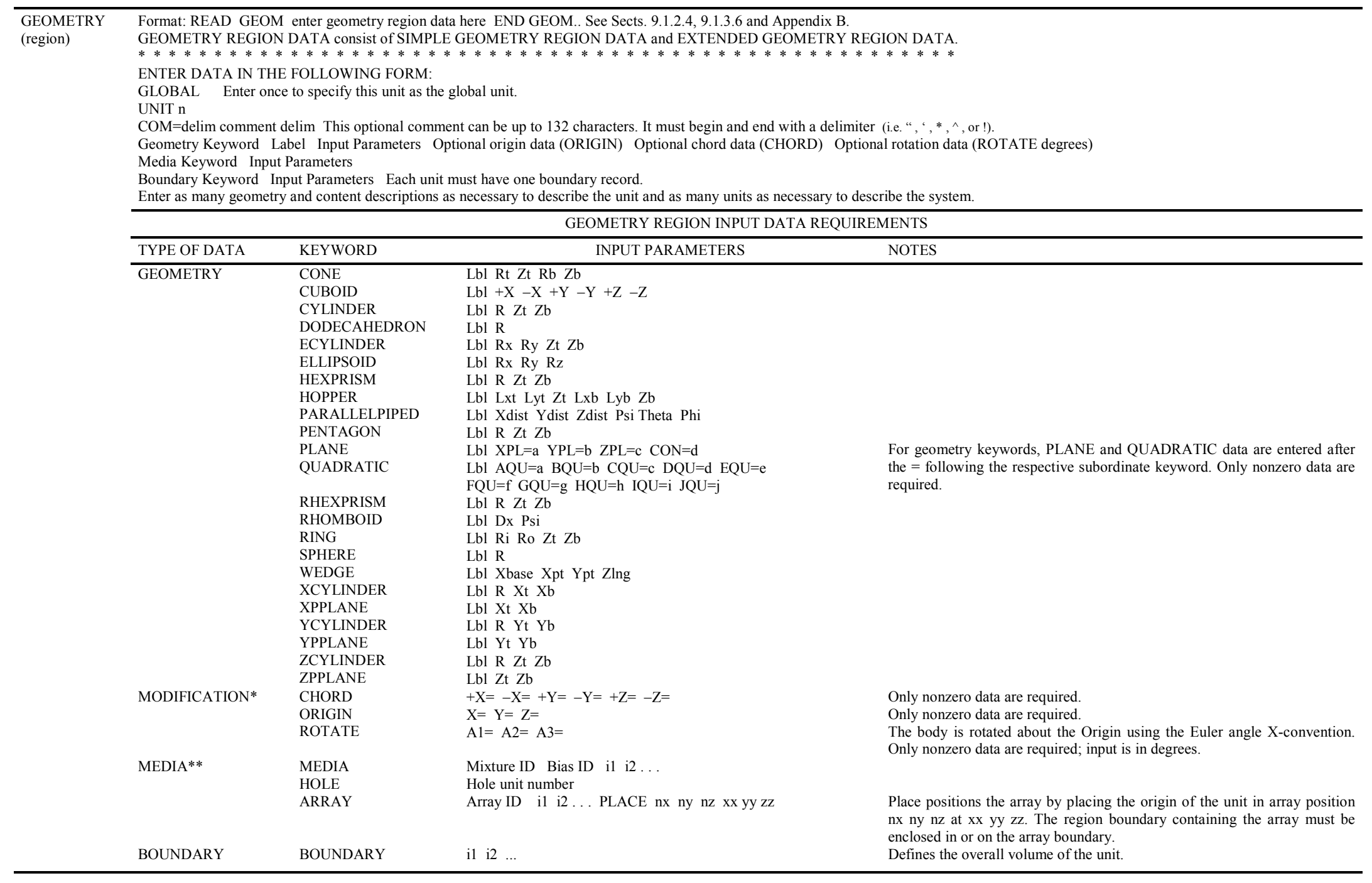

*Modification data can be entered in any order. Any combination of modification data can follow any geometry record.

**The Region definition vector, il i2 ..., should contain references to all geometry records which share a boundary with the region being defined. 


\section{Table 8.1.8. Summary of mixing table data}

\begin{tabular}{|c|c|c|c|c|}
\hline \multirow{28}{*}{ MIXTURES } & \multicolumn{4}{|c|}{ Format: READ MIXT xsec parameters mixing table data. END MIXT } \\
\hline & \multirow{2}{*}{\multicolumn{4}{|c|}{$\begin{array}{l}\text { Multigroup mode: These data are entered only if an AMPX working format library is being used. (LIB=) } \\
\text { (XSC=) in the parameter data. } \\
\text { Continuous energy mode: Only the mixing table data are entered. See Sects. 8.1.2.10 and 8.1.3.5. }\end{array}$}} \\
\hline & & & & \\
\hline & \multirow[t]{9}{*}{ XSEC PARAMETERS } & \multicolumn{3}{|c|}{ consists of keywords and associated values. } \\
\hline & & \multicolumn{3}{|c|}{ These parameters, if entered, only need to be entered once. } \\
\hline & & KEYWORD & DEFAULT & DEFINITION \\
\hline & & \multirow[t]{5}{*}{$\overline{\mathrm{SCT}}=$} & \multirow[t]{5}{*}{1} & number of discrete scattering angles \\
\hline & & & & 0 is isotropic \\
\hline & & & & 1 is $\mathrm{P} 1$ \\
\hline & & & & 2 is $\mathrm{P} 3$ \\
\hline & & & & 3 is $\mathrm{P} 5$ \\
\hline & & $\mathrm{EPS}=$ & 0.1 & $\begin{array}{l}\text { cross section message cutoff value used to suppress message } \\
\text { numbers K5-060 and K6-060. }\end{array}$ \\
\hline & \multirow[t]{16}{*}{$\overline{\text { MIXING TABLE DATA consists of }}$} & \multicolumn{3}{|c|}{ (1) a keyword and mixture ID for the mixture } \\
\hline & & \multicolumn{3}{|c|}{ The keyword is MIX= } \\
\hline & & \multicolumn{3}{|c|}{ The desired mixture number follows the keyword } \\
\hline & & \multicolumn{3}{|c|}{ (1a) optionally, a keyword and nuclide mixture ID for the rest of the current mixture } \\
\hline & & \multicolumn{3}{|c|}{ The keyword is $\mathrm{NCM}=$} \\
\hline & & \multicolumn{3}{|c|}{ The desired nuclide mixture ID follows the keyword } \\
\hline & & \multicolumn{3}{|c|}{ (1b) optionally, a keyword and temperature for the mixture (used for continuous energy problems) } \\
\hline & & \multicolumn{3}{|c|}{ The keyword is TMP $=$ or TEM $=$} \\
\hline & & \multicolumn{3}{|c|}{ The desired temperature in Kelvin follows the keyword } \\
\hline & & \multicolumn{3}{|c|}{ (2) nuclide ID** } \\
\hline & & \multicolumn{3}{|c|}{ (2a) optionally a keyword and nuclide mixture ID for this nuclide } \\
\hline & & \multicolumn{3}{|c|}{ The keyword is NCM= and the desired nuclide mixture ID follows the keyword } \\
\hline & & \multicolumn{3}{|c|}{ (3) number density** } \\
\hline & & \multicolumn{3}{|c|}{ (4) continuous energy cross section filename** } \\
\hline & & \multicolumn{3}{|c|}{ ** The sequence (2) (3) (4) is repeated for each nuclide in the mixture. Entry (4) is optional and entered with XS= name where name is the filename. } \\
\hline & & \multicolumn{3}{|c|}{ REPEAT the sequence (1) (2)s (3)s (4)s until all the mixtures have been described. } \\
\hline
\end{tabular}


Table 8.1.9. Summary of plot data

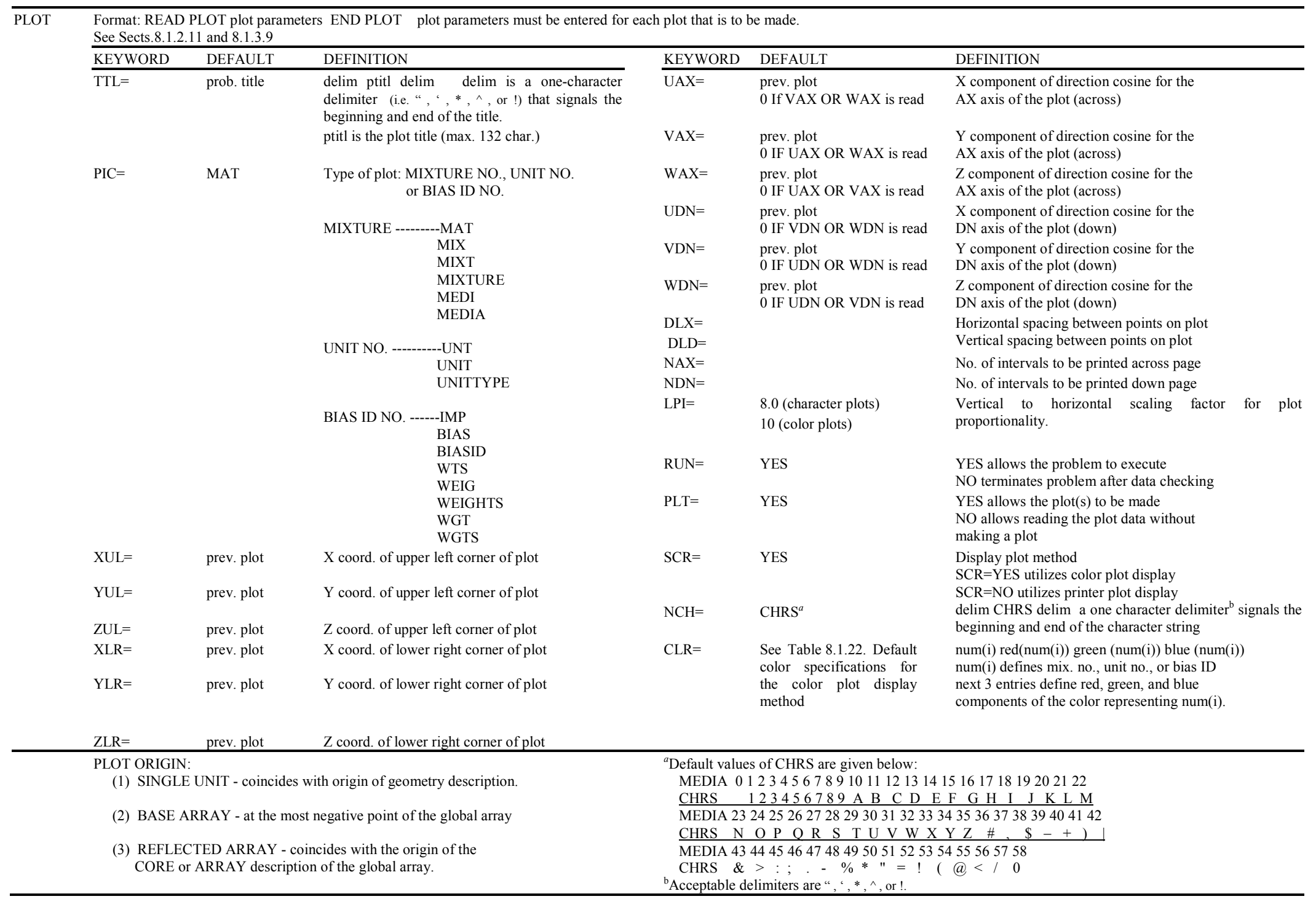


Table 8.1.10. Summary of starting data

\begin{tabular}{|c|c|c|c|c|c|c|c|c|c|c|c|}
\hline \multirow[t]{2}{*}{ START } & \multicolumn{8}{|c|}{$\begin{array}{l}\text { Format: READ START enter start data here END START } \\
\text { The default value of start type is zero. See Sect. 8.1.2.8. }\end{array}$} & \multirow[b]{2}{*}{ KEYWORD } & \multirow[b]{2}{*}{ DEFAULT } & \multirow[b]{2}{*}{ DEFINITION } \\
\hline & $\begin{array}{l}\text { START } \\
\text { TYPE }\end{array}$ & $\begin{array}{l}\text { REQUIRED } \\
\text { DATA }\end{array}$ & $\begin{array}{l}\text { OPTIONAL } \\
\text { DATA }\end{array}$ & STARTING & $\begin{array}{l}\text { START } \\
\text { TYPE }\end{array}$ & $\begin{array}{l}\text { REQUIRED } \\
\text { DATA }\end{array}$ & $\begin{array}{l}\text { OPTIONAL } \\
\text { DATA }\end{array}$ & $\begin{array}{l}\text { STARTING } \\
\text { DISTRIBUTION }\end{array}$ & & & \\
\hline & 0 & none & NST & uniform & 3 & NST & KFS & spike & $\mathrm{NST}=$ & 0 & start type \\
\hline & & & XSM & & & TFX & PSP & & $\mathrm{TFX}=$ & 0.0 & $\mathrm{X}$ coordinate \\
\hline & & & XSP & & & TFY & & & $\mathrm{TFY}=$ & 0.0 & Y coordinate \\
\hline & & & YSM & & & TFZ & & & $\mathrm{TFZ}=$ & 0.0 & $\mathrm{Z}$ coordinate \\
\hline & & & YSP & & & NXS & & & $\mathrm{NXS}=$ & 0 & $\mathrm{X}$ index of unit pos. \\
\hline & & & ZSM & & & NYS & & & NYS $=$ & 0 & Y index of unit pos. \\
\hline & & & ZSP & & & NZS & & & $\mathrm{NZS}=$ & 0 & $\mathrm{Z}$ index of unit pos. \\
\hline & & & RFL & & & & & & $\mathrm{KFS}=$ & & fissile mixture no. \\
\hline & & & PSP & & 4 & NST & KFS & multiple & $\mathrm{LNU}=$ & 0 & number of last neutron \\
\hline & & & & & & TFX & PSP & spikes & $\mathrm{NBX}=$ & 0 & source unit number \\
\hline & 1 & NST & XSM & cosine & & TFY & & & $\mathrm{FCT}=$ & 0 & fraction \\
\hline & & & XSP & & & TFZ & & & $\mathrm{XSM}=$ & $-\mathrm{X}$ & $-\mathrm{X}$ of source cuboid \\
\hline & & & YSM & & & NBX & & & $\mathrm{XSP}=$ & $+\mathrm{X}$ & $+\mathrm{X}$ of source cuboid \\
\hline & & & YSP & & & & & & $\mathrm{YSM}=$ & $-\mathrm{Y}$ & -Y of source cuboid \\
\hline & & & ZSM & & 5 & NST & PSP & in specified & $\mathrm{YSP}=$ & $+\mathrm{Y}$ & $+\mathrm{Y}$ of source cuboid \\
\hline & & & ZSP & & & NBX & & units & $\mathrm{ZSM}=$ & $-Z$ & $-\mathrm{Z}$ of source cuboid \\
\hline & & & RFL & & & & & & $\mathrm{ZSP}=$ & $+\mathrm{Z}$ & $+Z$ of source cuboid \\
\hline & & & PSP & & 6 & NST & NXS & arbitrary & $\mathrm{RFL}=$ & NO & start in reflector \\
\hline & & & & & & TFX & NYS & points & PS6 $=$ & NO & print start 6 input \\
\hline & 2 & NST & XSM & cosine with & & TFY & NZS & & $\mathrm{PSP}=$ & NO & print starting points \\
\hline & & NXS & XSP & fraction & & TFZ & KFS & & $\mathrm{RDU}=$ & 0 & unit containing start data \\
\hline & & NYS & YSM & specified unit & & LNU* & PS6 & & WS6 $=$ & 0 & unit to write start data \\
\hline & & NZS & YSP & & & & PSP & & & & \\
\hline & & FCT & ZSM & & & & RDU & & & & \\
\hline & & & ZSP & & & & WS6 & & & & \\
\hline & & & RFL & & & & & & & & \\
\hline & & & PSP & & 7 & NST & XSM & uniform in $\mathrm{X}$ - and & & & \\
\hline & & & & & & & XSP & Y-dimension and & & & \\
\hline & & & & & & & YSM & $(1-\cos )^{2}$ in & & & \\
\hline & & & & & & & YSP & Z-dimension & & & \\
\hline & & & & & & & ZSM & & & & \\
\hline & & & & & & & ZSP & & & & \\
\hline & & & & & 8 & NST & XSM & flat in $\mathrm{X}$ - and $\mathrm{Y}$ - & & & \\
\hline & & & & & & ZSM & XSP & dimension and & & & \\
\hline & & & & & & ZSP & YSM & segmented in $\mathrm{Z}$ & & & \\
\hline & & & & & & FCT & YSP & & & & \\
\hline & & & & & & LNU & & & & & \\
\hline
\end{tabular}


Table 8.1.11. Summary of volume data (KENO-VI)

\begin{tabular}{|c|c|c|c|c|}
\hline \multirow{2}{*}{$\begin{array}{l}\text { KEYWORD } \\
\text { TYPE= }\end{array}$} & \multicolumn{4}{|c|}{$\begin{array}{l}\text { Format: READ VOLUME enter volume data here END VOLUME } \\
\text { The default type for volume calculation type is NONE. See Sect. Volume data }\end{array}$} \\
\hline & $\begin{array}{l}\text { TYPE } \\
\text { NONE }\end{array}$ & $\begin{array}{c}\text { REQUIRED } \\
\text { DATA } \\
\text { none }\end{array}$ & $\begin{array}{c}\text { OPTIONAL } \\
\text { DATA } \\
\text { none }\end{array}$ & DEFAULT \\
\hline & RANDOM & none & $\begin{array}{l}\text { BATCHES }= \\
\text { POINTS }= \\
\mathrm{XP}= \\
\mathrm{XM}= \\
\mathrm{YP}= \\
\mathrm{YM}= \\
\mathrm{ZP}= \\
\mathrm{ZM}= \\
\text { SAMPLE_DEN }=\end{array}$ & $\begin{array}{l}500 \\
1000 \\
\text { global unit boundary } \\
\text { global unit boundary } \\
\text { global unit boundary } \\
\text { global unit boundary } \\
\text { global unit boundary } \\
\text { global unit boundary } \\
\text { points sampled per cubic cm }\end{array}$ \\
\hline & TRACE & none & $\begin{array}{l}\mathrm{NRAYS}= \\
\mathrm{XP}= \\
\mathrm{XM}= \\
\mathrm{YP}= \\
\mathrm{YM}= \\
\mathrm{ZP}= \\
\mathrm{ZM}= \\
\mathrm{IFACE}=\end{array}$ & $\begin{array}{l}100,000 \\
\text { global unit boundary } \\
\text { global unit boundary } \\
\text { global unit boundary } \\
\text { global unit boundary } \\
\text { global unit boundary } \\
\text { global unit boundary } \\
\text { smallest face }\end{array}$ \\
\hline
\end{tabular}


Table 8.1.12. Summary of grid geometry data

\begin{tabular}{|c|c|c|c|}
\hline GRID & $\begin{array}{l}\text { Format: READ GRID } \\
\text { See Sect. 8.1.2.14 }\end{array}$ & here END C & \\
\hline & KEYWORD & DEFAULT & DEFINITION \\
\hline & None & -1 & grid ID number, required if multiple mesh is defined \\
\hline & NXCELLS $=$ & & \\
\hline & NUMXCELLS $=$ & 1 & number of cells in the the $\mathrm{X}$ direction \\
\hline & $\mathrm{XMIN}=$ & 0 & minimum cell boundary in the $\mathrm{X}$ direction \\
\hline & $\mathrm{XMAX}=$ & 1 & maximum cell boundary in the $\mathrm{X}$ direction \\
\hline & $\begin{array}{c}\text { or } \\
\text { XPLANES }\end{array}$ & {$[0.11]$} & the cell houndaries in the $\mathrm{X}$ direction \\
\hline & XLINEAR & & ncells; $x \min ;$ xmax \\
\hline & & & Number of cells in the $\mathrm{X}$ direction; $\mathrm{X}$ minimum; $\mathrm{X}$ maximum \\
\hline & NYCELLS $=$ & & \\
\hline & NUMYCELLS $=$ & 1 & number of cells in the $\mathrm{Y}$ direction \\
\hline & $\mathrm{YMIN}=$ & 0 & minimum cell boundary in the $\mathrm{Y}$ direction \\
\hline & $\mathrm{YMAX}=$ & 1 & maximum cell boundary in the $\mathrm{Y}$ direction \\
\hline & $\begin{array}{c}\text { or } \\
\text { YPLANES }=\end{array}$ & {$[0,1]$} & the cell boundaries in the $\mathrm{Y}$ direction \\
\hline & YLINEAR & & ncells; ymin; ymax \\
\hline & & & Number of cells in the $Y$ direction; $Y$ minimum; $Y$ maximum \\
\hline & NZCELLS $=$ & & \\
\hline & NUMZCELLS $=$ & 1 & number of cells in the $\mathrm{Z}$ direction \\
\hline & $\mathrm{ZMIN}=$ & 0 & minimum cell boundary in the $\mathrm{Z}$ direction \\
\hline & $\mathrm{ZMAX}=$ & 1 & maximum cell boundary in the $\mathrm{Z}$ direction \\
\hline & & & \\
\hline & ZPLANES $=$ & {$[0,1]$} & the cell boundaries in the $\mathrm{Z}$ direction \\
\hline & ZLINEAR & & ncells; zmin; zmax \\
\hline & & & Number of cells in the $\mathrm{Z}$ direction; $\mathrm{Z}$ minimum; $\mathrm{Z}$ maximum \\
\hline & TITLE $=$ & 11 & a title for this grid mesh \\
\hline & TOLERANCE $=$ & $1.0 \mathrm{E}-6$ & For removing duplicate planes from *PLANES or *LINEAR \\
\hline
\end{tabular}




\section{Table 8.1.13. Summary of energy group boundary data}

\begin{tabular}{ll}
\hline & Format: READ ENERGY energy group boundaries END ENERGY \\
ENERGY & $\begin{array}{l}\text { Enter upper energy boundary for each group in eV. The last entry is the lower energy } \\
\text { boundary of the last group. For } \mathrm{N} \text { groups, there are } \mathrm{N}+1 \text { entries. Entries must be in } \\
\text { descending order and in units of eV. }\end{array}$
\end{tabular}

\section{Table 8.1.14. Summary of reaction data}

\begin{tabular}{|c|c|c|c|c|}
\hline REACTION & \multicolumn{4}{|c|}{$\begin{array}{l}\text { Format: READ REACTION } \\
\text { Tally type (required) } \\
\text { Reaction filters (required) } \\
\text { Energy group boundaries (optional) } \\
\text { Output edits (optional) } \\
\text { END REACTION } \\
\text { Parameters for defining the reaction filters and tally type must be entered to enable the reaction tally calculations. } \\
\text { Parameters for defining energy group boundaries and output edits are optional. } \\
\text { Tally type, reaction filters, energy group boundaries, and output edits data blocks may be entered in any order. } \\
\text { The reaction tally calculations is only available for the continuous energy mode, see Sect. 8.1.2.15. }\end{array}$} \\
\hline & TYPE OF DATA & KEYWORD & DEFAULT & DEFINITION \\
\hline & REACTION FILTERS $^{1}$ & $\begin{array}{l}\text { MIX }= \\
\text { NUC }= \\
\text { MT }= \\
\text { MIXLIST } \\
\text { NUCLIST } \\
\text { MTLIST }\end{array}$ & - & $\begin{array}{l}\text { Mixture number } \\
\text { Nuclide identifier } \\
\text { Reaction MT } \\
\text { List of mixture numbers, terminated with "end" } \\
\text { List of nuclide identifiers, terminated with "end" } \\
\text { List of reaction MTs, terminated with "end" }\end{array}$ \\
\hline & TALLY TYPE & $\begin{array}{l}\text { XSTALLY= } \\
\text { RRTALLY= } \\
\text { MIXFLX= }\end{array}$ & $\begin{array}{l}\text { NO } \\
\text { NO } \\
\text { NO }\end{array}$ & $\begin{array}{l}\text { Tally reaction cross sections } \\
\text { Tally reaction rates } \\
\text { Tally mixture fluxes }\end{array}$ \\
\hline & OUTPUT EDITS & $\begin{array}{l}\text { PRNTXS }= \\
\text { PRNTRR }= \\
\text { PRNTFLX }=\end{array}$ & $\begin{array}{l}\text { NO } \\
\text { NO } \\
\text { NO }\end{array}$ & $\begin{array}{l}\text { Write reaction cross sections into separate file for each mixture } \\
\text { Write reaction rates into separate file for each mixture } \\
\text { Write mixture fluxes into separate file for each mixture }\end{array}$ \\
\hline & $\begin{array}{l}\text { ENERGY GROUP } \\
\text { BOUNDARIES }\end{array}$ & $\begin{array}{l}\text { ENER_XS } \\
\text { ENER_FLX }\end{array}$ & $\begin{array}{l}\text { default energy group } \\
\text { boundaries }^{3} \\
\text { default energy group } \\
\text { boundaries }^{3}\end{array}$ & $\begin{array}{l}\text { Energy group boundaries for cross section or reaction rate tally }{ }^{4} \text {, terminated with } \\
\text { "end" } \\
\text { Energy group boundaries for mixture flux tally }{ }^{4} \text {, terminated with "end" }\end{array}$ \\
\hline 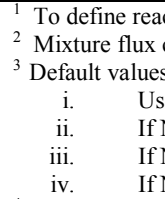 & $\begin{array}{l}\text { filters, sets of mix (or mixlis } \\
\text { llations also require a reactio } \\
\text { the energy group boundaries } \\
\text { ergy group boundaries from } F \\
\text { is specified (in READ PAR } \\
\text { is specified (in READ PAR } \\
\text { is not specified and there is }\end{array}$ & $\begin{array}{l}\text { st), mt (or mtlist) } \\
\text { n with REACTIC } \\
\text { calculations are } \\
\text { Y block if specifi } \\
\text { it matches the nu } \\
\text { NGP does not me } \\
\text { RGY block, use } t\end{array}$ & $\begin{array}{l}\text { ed in this order. See Sect. } 8 \\
\text { the following order: } \\
\text { The number of the entry } \\
\text { y groups in one of the SCA } \\
\text { er of energy groups in one } \\
8 \text { group library energy stru }\end{array}$ & $\begin{array}{l}\text { READ ENERGY block is NGP+1. } \\
\text { SCAtron cross section libraries, use the energy structure from that library. } \\
\text { SCALE neutron cross section libraries, use NGP equal lethargy bins. }\end{array}$ \\
\hline
\end{tabular}




\subsubsection{KENO input outline}

The data input for KENO is outlined below. Default data for KENO have been found to be adequate for many problems. These values should be carefully considered when entering data.

Blocks of input data are entered in the form:

\section{READ XXXX input_data END XXXX}

where $\mathbf{X X X X}$ is the keyword for the type of data being entered. The keywords that can be used are listed in Table 8.1.15. A minimum of four characters is required for a keyword, and some keyword names may be as long as twelve characters (READ PARAMETER, READ GEOMETRY, etc.). Keyword inputs are not case sensitive. Data input is activated by entering the words READ XXXX followed by one or more blanks. All input data pertinent to $\mathbf{X X X X}$ are then entered. Data for $\mathbf{X X X X}$ are terminated by entering END XXXX followed by two or more blanks. Note that multiple READ GRID blocks are used if multiple grid definitions are needed.

Table 8.1.15. Types of input data

\begin{tabular}{ll}
\hline \multicolumn{1}{c}{ Type of data } & \multicolumn{1}{c}{ First four characters } \\
\hline Parameters & PARA or PARM \\
Geometry & GEOM \\
Biasing & BIAS \\
Boundary conditions & BOUN or BNDS \\
Start & STAR or STRT \\
Energy & ENER \\
Array (unit orientation) & ARRA \\
Extra 1-D cross sections & X1DS \\
Cross section mixing table ${ }^{a}$ & MIXT or MIX \\
Plot ${ }^{a}$ & PLOT or PLT or PICT \\
Volumes & VOLU \\
Grid geometry & GRID \\
Reactions & REAC \\
\hline${ }^{a}$ MIX and PLT must include a trailing blank, which is \\
considered part of the keyword.
\end{tabular}

Three data records must be entered for every problem: first the SCALE sequence identifier, then the problem title, and then the END DATA to terminate the problem.

(1) KENO is typically run using one of the SCALE CSAS or TSUNAMI sequences, but it may also be run stand alone using KENO V.a or KENO-VI. The sequence identifier is specified using one line similar to: 
=kenovi

This line may also include additional runtime directives that are described throughout the SCALE manual. For example:

$=$ kenova $\quad$ parm $=$ check

The following guidance generally assumes the user is running KENO stand alone. If KENO is to be run using of the other sequences (e.g., CSAS5), see the appropriate manual section for additional guidance.

\section{(2) problem title}

Enter a problem title (limit 80 characters, including blanks; extra characters will be discarded). A title must be entered. See Sect. 8.1.2.3.

\section{(3) READ PARA parameter data END PARA}

Enter parameter input as needed to describe a problem. If parameter data are desired in standalone KENO calculations (i.e., non-CSAS), they must immediately follow the problem title. Default values are assigned to all parameters. A problem can be run without entering any parameter data if the default values are acceptable.

Parameter data must begin with the words READ PARA, READ PARM, or READ PARAMETER. Parameter data may be entered in any order. If a parameter is entered more than once, the last value is used. The words END PARA or END PARM, or END PARAMETER terminate the parameter data. See Sect. 8.1.2.3.

$\left(n_{1}\right) \ldots\left(n_{13}\right)$ The following data may be entered in any order. Data not needed to describe the problem may be omitted.

\section{$\left(\mathrm{n}_{1}\right)$ READ GEOM all geometry_region_data END GEOM}

Geometry region data must be entered for every problem that is not a restart problem. Geometry data must begin with the words READ GEOM or READ GEOMETRY. The words END GEOM or END GEOMETRY terminate the geometry region data. See Sect. 8.1.2.4.

$\left(\mathrm{n}_{2}\right)$ READ ARRA array_definition_data END ARRA

Enter array definition data as needed to describe the problem. Array definition data define the array size and position units (defined in the geometry data) in a 3-D lattice that represents the physical problem being analyzed. Array data must begin with the words READ ARRA or READ ARRAY and must terminate with the words END ARRA or END ARRAY. See Sect. 8.1.2.5.

$\left(\mathrm{n}_{4}\right)$ READ BOUN albedo_boundary_conditions END BOUN

Enter albedo boundary conditions as needed to describe the problem. Albedo data must begin with the words READ BOUN, READ BNDS, READ BOUND, or READ BOUNDS, and it 
must terminate with the words END BOUN, ENDS BNDS, END BOUND, or END BOUNDS. See Sect. 8.1.2.6.

$\left(\mathrm{n}_{3}\right)$ READ BIAS biasing_information END BIAS

The biasing_information is used to define the weight given to a neutron surviving Russian roulette. Biasing data must begin with the words READ BIAS. The words END BIAS terminate the biasing data. See Sect. 8.1.2.7.

$\left(\mathrm{n}_{5}\right)$ READ STAR starting_distribution_information END STAR

Enter starting information data for starting the initial source neutrons only if a uniform starting distribution is undesirable. Start data must begin with the words READ STAR, READ STRT or READ START, and it must terminate with the words END STAR, END STRT or END START. See Sect. 8.1.2.8.

$\left(\mathrm{n}_{6}\right)$ READ ENER energy_group_boundaries END ENER

Enter upper energy boundaries for each neutron energy group to be used for tallying in the continuous energy mode. Energy bin data begin with the words READ ENER or READ ENERGY and terminate with the words END ENER or END ENERGY. The last entry is the lower energy boundary of the last group. The values must be in descending order. This block is only applicable to continuous energy KENO calculations. See Sect. 8.1.2.12.

$\left(\mathrm{n}_{7}\right)$ READ MIXT cross_section_mixing_table END MIXT

Enter a mixing table to define all the mixtures to be used in the problem. The mixing table must begin with the words READ MIXT or READ MIX and must end with the words END MIXT or END MIX. Do not enter mixing table data if KENO is being executed as a part of a SCALE sequence. See Sect. 8.1.2.10.

$\left(\mathrm{n}_{8}\right)$ READ X1DS extra_1-D_cross_section_IDs END X1DS

Enter the IDs of any extra 1-D cross sections to be used in the problem. These must be available on the mixture cross section library. Extra 1-D cross section data must begin with the words READ X1DS and terminate with the words END X1DS. See Sect. 8.1.2.9.

$\left(\mathrm{n}_{9}\right)$ READ PLOT plot_data END PLOT

Enter the data needed to provide a 2-D character or color plot of a slice through a specified portion of the 3-D geometrical representation of the problem. Plot data must begin with the words READ PLOT, READ PLT, or READ PICT and terminate with the words END PLOT, END PLT, or END PICT. See Sect. 8.1.2.11.

\section{$\left(\mathrm{n}_{10}\right)$ READ VOLU volume_data END VOLU}

Enter the data needed to specify the volumes of the geometry data. Volume data must begin with the words READ VOLU or READ VOLUME and end with the words END VOLU or END VOLUME. See Sect.Volume data. 
$\left(\mathrm{n}_{11}\right)$ READ GRID mesh_grid_data END GRID

Enter the data needed to specify a simple Cartesian grid over either the entire problem or part of the problem geometry for tallying fluxes, moments, fission sources, etc. Grid data may be entered using the keywords READ GRID, READ GRIDGEOM, or READ GRIDGEOMETRY, and they are terminated with either END GRID, END GRIDGEOM, or END GRIDGEOMETRY. Multiple grids may be defined by repeating the READ GRID block several times, specifying a different mesh grid identification number for each so defined grid. See Sect. Grid geometry data for further information.

\section{$\left(\mathrm{n}_{12}\right)$ READ REAC reaction_data END REAC}

Enter the data needed to specify filters for the reaction tally calculations. Reaction data must begin with the words READ REAC and terminate with END REAC. This block is only applicable to calculations in the continuous energy mode. See Sect.8.1.2.15.

\section{$\left(\mathrm{n}_{13}\right)$ END DATA must be entered}

Terminate the data for the problem.

\subsubsection{Procedure for data input}

For a standalone KENO problem, the first data records must be the sequence identifier (e.g., =kenovi or =kenova) and the title. The next block of data must be the parameters if they are to be entered. A problem can be run without entering the parameters, which causes KENO to use default values for input parameters. The remaining blocks of data can be entered in any order.

BOLD TYPE specifies keywords. A keyword is used to identify the data that follow it. When a keyword is used, it must be entered exactly as shown in the data guide. All keywords except those ending with an equal sign must be followed by at least one blank.

small_italics correlate data with a program variable name. The actual values are entered in place of the program variable name and are terminated by a blank or a comma.

CAPITAL ITALICS identify general data items. General data items are general classes of data including

(1) geometry data such as UNIT INITIALIZATION and UNIT NUMBER DEFINITION, GEOMETRY REGION DESCRIPTION, GEOMETRY WORD, MIXTURE NUMBER, BIAS ID, and REGION DIMENSIONS,

(2) albedo data such as FACE CODES and ALBEDO NAMES,

(3) weighting data such as BIAS ID NUMBERS, etc.

Square brackets The square brackets, [ and ], are used to show that an entry is optional.

Broken line The broken line, |, is used as a logical "or" symbol to show that the entries to its left and right are alternatives that cannot be used simultaneously. 


\subsubsection{Title and parameter data}

A title, a character string, must be entered at the top of the input file. The syntax is:

title a string of characters with a length of up to 80 characters, including blanks.

The PARAMETER block may contain parameter initializations for those parameters that need to be changed from their default value. The syntax for the PARAMETER block is:

READ PARA[METER] $p_{1} \ldots p_{N}$ END PARA[METER]

or

READ PARM $p_{1} \ldots p_{N}$ END PARM

$p_{1} \ldots p_{N} \quad$ are $N(N$ greater than or equal to zero) keyworded parameters that together make up the PARAMETER DATA

The commonly changed parameters are TME, GEN, NSK, and NPG. Seldom changed parameters are NBK, NFB, XNB, XFB, WTH, WTL, TBA, BUG, TRK, and LNG.

The PARAMETER DATA, $p_{1} \ldots p_{N}$, consists of one or more of the parameters described below.

Floating point parameters

$\mathbf{R N D}=$ rndnum $\quad$ input hexadecimal random number, a default value is provided

$\mathbf{T M E}=\operatorname{tmax} \quad$ execution time (in minutes) for the problem, default $=0.0$ (no limit)

TBA $=$ tbtch time allotted for each generation (in minutes), default $=10$ minutes. If $t b t c h$ is exceeded in any generation, the problem is assumed to be looping. Execution is terminated, and final edits are performed. The problem can loop indefinitely on a computer if the system-dependent routine to interrupt the problem (PULL) is not functional. TBA= is also used to set the amount of time available for generating the initial starting points.

$\mathbf{S I G}=$ tsigma $\quad$ if entered and $>0.0$, this is the standard deviation at which the problem will terminate, default $=0.0$, which means do not check sigma.

WTA $=d w t a v \quad$ the default average weight given a neutron that survives Russian roulette, $d w \operatorname{tav}$ default $=0.5$.

WTH $=$ wthigh $\quad$ the default value of wthigh is 3.0 and should be changed only if the user has a valid reason to do so. The weight at which splitting occurs is defined to be wthigh $x$ wtavg, where wtavg is the weight given to a neutron that survives Russian roulette.

$\mathbf{W T L}=$ wtlow $\quad$ Russian roulette is played when the weight of a neutron is less than wtlow $x$ wtavg. The wtlow default $=1.0 /$ wthigh .

NOTE: The default values of wthigh and wtlow have been determined to minimize the deviation per unit running time for many problems. 
MSH $=$ mesh_size Length $(\mathrm{cm})$ of one side of a cubic mesh for tallying fluxes. Default $=0.0$. A positive non-zero value must be entered if MFX=YES and READ GRID input is not entered.

TTL = temperature_tolerance The continuous energy cross sections must be within the temperature tolerance (in degrees Kelvin) of the requested temperature for the problem to run. A negative value specifies the use of the closest temperature to that requested. TTL is ignored when DBX is nonzero. The default $=-1.0$.

DBH $=d b r c$ high the energy cutoff (in $\mathrm{eV}$ ) up to which the Doppler Broadening Rejection Correction (DBRC) method will be used on nuclides for which DBRC is enabled, and cross section libraries are available. DBH is only used in CE simulations. Default $=210.0 \mathrm{eV}$.

$\mathbf{D B L}=$ dbrc_low the energy cutoff (in $\mathrm{eV}$ ) down to which DBRC will be used on nuclides for which DBRC is enabled and cross section libraries are available. Only used in $\mathrm{CE}$ simulations. Default $=0.4 \mathrm{eV}$.

Integer parameters

$$
\begin{aligned}
& \text { GEN }=n b a \quad \text { number of generations to be run, default }=203 . \\
& \mathbf{N P G}=n p b \quad \text { number of neutrons per generation, default }=1000 . \\
& \text { NSK = nskip number of generations (1 through nskip) to be omitted when collecting } \\
& \text { results, default }=3 \text {. } \\
& \mathbf{R E S}=n r s t r t \quad \text { number of generations between writing restart data, default }=0 \text {. If } \mathbf{R E S} \text { is } \\
& \text { NBK }=\text { nbank number of positions in the neutron bank, default }=n p b+25 . \\
& \mathbf{X N B}=n x n b k \quad \text { number of extra entries in the neutron bank, default }=0 . \\
& \mathbf{N F B}=n f b n k \quad \text { number of positions in the fission bank, default }=n p b . \\
& \mathbf{X F B}=n x f b k \quad \text { number of extra entries in the fission bank, default }=0 . \\
& \mathbf{X 1 D}=\text { numx } 1 d \quad \text { number of extra 1-D cross sections, default }=0 . \\
& \mathbf{B E G}=\text { nbas } \quad \text { beginning generation number, default }=1 \text {. If } \mathbf{B E G} \text { is greater than 1, restart } \\
& \text { data must be available. BEG must be } 1 \text { greater than the number of } \\
& \text { generations retrieved from the restart file. }
\end{aligned}
$$$$
\text { NB8 }=n b 8 \quad \text { number of blocks allocated for the first direct-access unit, default }=1000 .
$$ 


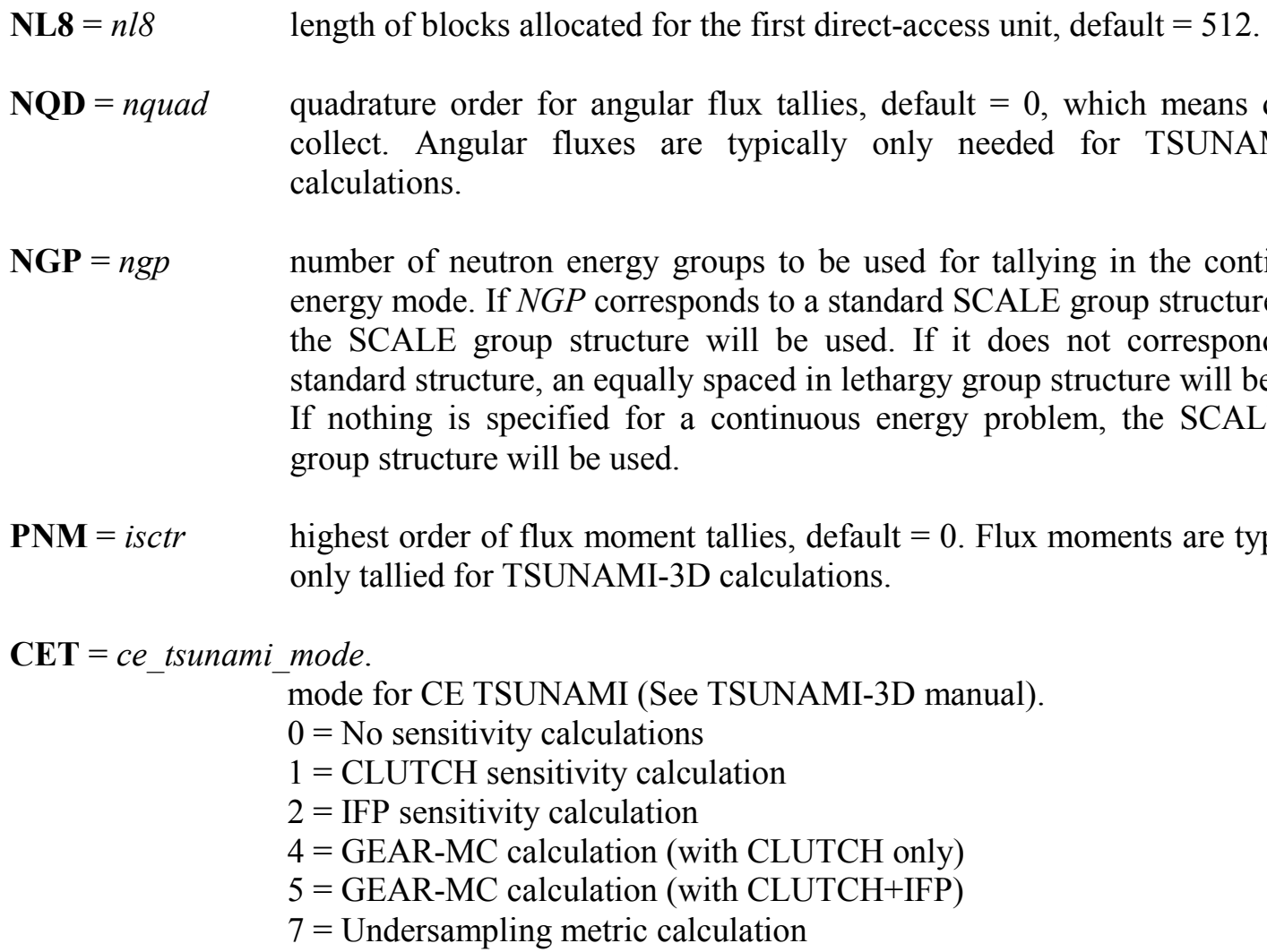
group structure will be used. only tallied for TSUNAMI-3D calculations.

$\mathbf{C E T}=$ ce_tsunami_mode.

mode for CE TSUNAMI (See TSUNAMI-3D manual).

$0=$ No sensitivity calculations

$1=$ CLUTCH sensitivity calculation

$2=$ IFP sensitivity calculation

$4=$ GEAR-MC calculation (with CLUTCH only)

$5=$ GEAR-MC calculation (with CLUTCH+IFP)

$7=$ Undersampling metric calculation

$\mathbf{N G P}=n g p \quad$ number of neutron energy groups to be used for tallying in the continuous energy mode. If $N G P$ corresponds to a standard SCALE group structure, then the SCALE group structure will be used. If it does not correspond to a standard structure, an equally spaced in lethargy group structure will be used. If nothing is specified for a continuous energy problem, the SCALE 238

PNM = isctr $\quad$ highest order of flux moment tallies, default $=0$. Flux moments are typically

$\mathbf{C F P}=$ number_of latent generations

number of latent generations used for IFP sensitivity or $F^{*}(r)$ calculations. Note:

- If $\mathrm{CET}=1$ and $\mathrm{CFP}=-1$ then $F^{*}(r)$ is assumed to equal one everywhere.

- If CET $=4$ and $\mathrm{CFP}=-1$ then $F^{*}(r)$ is assumed to equal zero everywhere.

DBR = lusedbrc $\quad$ use Doppler Broadening Rejection Correction method. See Sect. 8.1.6.2.9 for more details. Only used in CE simulations. Default $=2$.

$$
\begin{aligned}
& 0=\text { No DBRC } \\
& 1=\text { DBRC for }{ }^{238} \mathrm{U} \text { only } \\
& 2=\text { DBRC for all available nuclides }\left({ }^{232} \mathrm{Th},{ }^{234} \mathrm{U},{ }^{235} \mathrm{U},{ }^{236} \mathrm{U},{ }^{238} \mathrm{U},{ }^{237} \mathrm{~Np},\right. \\
& \left.{ }^{239} \mathrm{Pu},{ }^{240} \mathrm{Pu}\right)
\end{aligned}
$$

$\mathbf{D B X}=d b \_x s \_$mode

option for performing problem-dependent or on-the-fly Doppler Broadening. See Sect. 8.1.6.2.10 for more details. Default $=2$.

$0=$ No problem-dependent or on-the-fly Doppler Broadening

$1=$ Perform problem-dependent Doppler Broadening for 1D cross sections only.

$2=$ Perform problem-dependent Doppler Broadening for both 1D and 2D (thermal scattering data) cross sections.

Alphanumeric parameter data 
$\mathbf{C E P}=$ lcep $\quad$ key for choosing the calculation mode in stand alone KENO calculations. The parameter is set to the appropriate value by the calling sequence if not stand alone KENO. For stand alone KENO, enter NO for multigroup mode, or enter the continuous energy directory filename for the continuous energy mode. The directory file is the file containing pointers to files significant for the continuous energy run.

FNI $=$ mode _in extra field in the input restart file name [restart_mode_in.keno_input] and [restart_mode_in.keno_calculated]. The default is an empty field.

FNO $=$ mode_out extra field in the output restart filename [restart_mode_out.keno_input] and [restart_mode_out.keno_calculated]. The default is an empty field.

Logical parameter data ... enter YES or $N O$
$\mathbf{A P P}=$ lappend $\quad$ key for appending the restart data, default $=$ NO.
$\mathbf{F L X}=n f l x \quad$ key for collecting and printing fluxes, default $=$ NO.
$\mathbf{F D N}=n f d e n \quad$ key for collecting and printing fission densities, default $=$ YES.
ADJ $=$ nadj $\quad$ key for running adjoint calculation, default $=$ NO. Adjoint cross sections must be available to run an adjoint problem. If $\mathrm{LIB}=$ is specified, the cross sections will be adjointed by the code. If $\mathrm{XSC}=$ is specified, the cross sections must already be in adjoint order.

PTB $=p t b \quad$ key for using probability tables in the continuous energy mode, default $=$ YES

$\mathbf{P N U}=$ lpromptn $u$ key for using delayed or prompt $v$ in the continuous energy mode, default $=$ $\mathrm{NO}$ - use total.

$\mathbf{F R E}=$ lfree_analytic $\quad$ key for using free analytic gas treatment, default $=$ YES
$\mathbf{A M X}=a m x$
key for printing all mixture cross section data. This is the same as activating $X A P, X S 1, X S 2, P K I$, and $P 1 D$. If any of these are entered in addition to $A M X$, that portion of $A M X$ will be overridden, default $=\mathrm{NO}$.
$\mathbf{X A P}=$ prtap $\quad$ key for printing discrete scattering angles and probabilities for the mixture cross sections, default $=$ NO.
$\mathbf{X S 1}=\operatorname{prtp0} \quad$ key for printing mixture $1-\mathrm{D}$ cross sections, default $=$ NO.
$\mathbf{X S 2}=$ prt $\quad$ key for printing mixture $2-\mathrm{D}$ cross sections, default $=$ NO.
$\mathbf{X S L}=p r t \quad \quad$ key for printing mixture $2-\mathrm{D} \mathrm{P}_{\mathrm{L}}$ cross sections, default $=\mathrm{NO}$. The Legendre expansion order $\mathrm{L}$ is automatically read from the cross section library.
$\mathbf{P K I}=$ prtchi $\quad$ print input fission spectrum, default $=$ NO. 
$\mathbf{P 1 D}=$ prtex $\quad$ print extra $1-\mathrm{D}$ cross sections, default $=$ NO.

$\mathbf{F A R}=l f a \quad$ key for generating region-dependent fissions and absorptions for each energy group, default $=$ NO.

$\mathbf{G A S}=\lg a \mathrm{~s} \quad$ key for printing region-dependent fissions and absorptions by energy group, applicable only if FAR $=$ YES. Default $=$ FAR . GAS $=$ YES prints regiondependent data by energy group. GAS $=$ NO suppresses region-dependent data by energy group.

$\mathbf{M K P}=$ larpos $\quad$ calculate and print matrix k-effective by unit location, default $=$ NO. Unit location may also be referred to as array position or position index.

$\mathbf{C K P}=l c k p \quad$ calculate and print cofactor k-effective by unit location, default $=$ NO. Unit location may also be referred to as array position or position index.

$\mathbf{F M P}=$ pmapos $\quad$ print fission production matrix by array position, default $=$ NO.

$\mathbf{M K U}=$ lunit $\quad$ calculate and print matrix k-effective by unit type, default $=$ NO.

$\mathbf{C K U}=l c k u \quad$ calculate and print cofactor k-effective by unit type, default $=$ NO.

$\mathbf{F M U}=$ pmunit $\quad$ print fission production matrix by unit type, default $=$ NO.

MKH $=$ lmhole $\quad$ calculate and print matrix k-effective by hole number, default $=$ NO.

$\mathbf{C K H}=l c k h \quad$ calculate and print cofactor $\mathrm{k}$-effective by hole number, default $=$ NO.

$\mathbf{F M H}=$ pmhole $\quad$ print fission production matrix by hole number, default $=\mathrm{NO}$.

HHL $=$ lhhgh collect matrix information by hole number at the highest hole nesting level, default $=$ NO.

MKA $=$ lmarry $\quad$ calculate and print matrix k-effective by array number, default $=$ NO.

$\mathbf{C K A}=l c k a \quad$ calculate and print cofactor k-effective by array number, default $=$ NO.

FMA = pmarry $\quad$ print fission production matrix by array number, default $=$ NO.

HAL = langh collect matrix information by array number at the highest array nesting level, default $=$ NO.

$\mathbf{B U G}=$ ldbug print debug information, default $=$ NO. Enter $Y E S$ for code debug purposes only.

TRK $=$ ltrk print tracking information, default $=$ NO. Enter YES for code debug purposes only.

$\mathbf{P W T}=l p w t \quad$ print weight average array, default $=$ NO. 

$\mathbf{P G M}=$ lgeom $\quad$ print unprocessed geometry as it is read, default $=$ NO.
$\mathbf{S M U}=$ lmult $\quad$ calculate the average self-multiplication of a unit, default $=$ NO.
NUB = nubar calculate the average number of neutrons per fission and the average energy group at which fission occurred, default $=$ YES.
PAX $=$ lcorsp $\quad$ print the arrays defining the correspondence between the cross section energy group structure and the albedo energy group structure, default $=$ NO.
TFM $=l t f m \quad$ perform coordinate transform for flux moments and angular flux calculations, default $=$ NO.
$\mathbf{P M F}=$ prtmore $\quad$ print angular fluxes or flux moments if calculated, default $=$ NO.
$\mathbf{C F X}=n f l x \quad$ collect fluxes, default $=$ NO.

$\mathbf{U U M}=$ IUnionizedMix $\quad$ use unionized mixture cross section, default $=$ NO. Only used in CE simulations. See Sect. 8.1.6.2.3 for further details.

M2U = luseMap2Union

store cross sections for each nuclide on a unionized energy grid, default=NO. Only used in CE simulations. See Sect. 8.1.6.2.3 for further details.

$\mathbf{S C X}=$ lxsecSave

save $\mathrm{CE}$ cross sections to restart file, default $=\mathrm{NO}$.

MFX $=$ make_mesh_flux

compute mesh fluxes on intervals defined by MSH above or by READ GRID data block, default $=$ NO.

$\mathbf{P M S}=$ print_mesh $\_l$ lux

print mesh fluxes if computed, default $=$ NO.

MFP = mean-free-path

$\mathbf{H T M}=$ html_output

$\mathbf{P M M}=$ print_mesh_moments

$\mathbf{P M V}=$ print_mesh_volumes

$\mathbf{F S T}=$ lprint_FStar

$\mathbf{R U N}=$ lrun

$\mathbf{P L T}=l p l o t$ compute and print the mean-free-path of a neutron by region, default $=$ NO.

produce HTML formatted output for interactive browsing, sorting, and plotting of results, default = YES.

print the angular moments of the mesh flux, if computed, default $=$ NO.

print the volume of each mesh interval, if computed. Default $=$ NO.

Create a .3 dmap file that contains the $F^{*}(\mathrm{r})$ mesh used by a CETSUNAMI CLUTCH sensitivity calculation.

key for determining if the problem is to be executed when data checking is complete, default $=$ YES.

key for drawing specified plots of the problem geometry, default $=$ YES. 
NOTE: The parameters RUN and PLOT can also be entered in the PLOT data. See Sect. 8.1.2.11. It is recommended that these parameters be entered only in the parameter data in order to ensure that the data printed in the Logical Parameters table is actually performed. If RUN and/or PLT are entered in both the parameter data and plot data, the results vary depending on whether the problem is run (1) stand alone, (2) as a restarted problem, (3) as CSAS with parm $=$ check, or (4) as CSAS without parm $=$ check. These conditions are detailed below.

KENO standalone and CSAS with PARM=CHECK

\section{Restarted KENO}

CSAS Without PARM $=$ CHECK
The values of RUN and/or PLT entered in KENO parameter data are printed in the Logical Parameters table of the problem output. However, values for RUN and/or PLT entered in the KENO plot data will override the values entered in the parameter data.

The values of RUN and/or PLT printed in the Logical Parameters table of the problem output are the final values from the parent problem unless those values are overridden by values entered in the KENO parameter data of the restarted problem. If the problem is restarted at generation 1, KENO plot data can be entered, and the values for RUN and/or PLT will override the values printed in the Logical Parameters table.

The values of RUN and/or PLT entered in the KENO parameter data override values entered in the KENO plot data. The values printed in the Logical Parameters table control whether the problem is to be executed and whether a plot is performed.

\section{Parameters that are either Integer or Logical}

$\mathbf{S C D}=l$ ScnvgDiag

$\mathbf{G F X}=$ lGridFlux

MFX $=$ lMeshFlux enable fission source convergence diagnostics (ScnvgDiag), default=YES. See Sect. Source Convergence Diagnostics for further details.
$\mathbf{C D S}=$ lcadis/lGridFissions
accumulate neutron fissions to use as fission source in subsequent MAVRIC/Monaco shielding calculation or for visualization, default $=\mathrm{NO}$

compute grid fluxes averaged over the volume of the mesh on intervals defined by a READ GRID data block, default $=$ NO.

compute mesh fluxes averaged over the volume of mixtures/materials in a mesh on intervals defined by MSH above or by READ GRID data block, default $=$ NO. 
NOTE: The KENO codes in previous SCALE versions allowed for only one mesh definition in the user input with either MSH parameter or READ GRID data block, and (2) calculation of a single mesh-based quantity, such as MFX (mesh fluxes) or CDS (fission source accumulation on a mesh), per KENO simulation.

The option to define multiple spatial meshes during a single simulation has been implemented in the KENO codes to add flexibility to mesh-based quantity calculations. The new implementation requires that each mesh definition in the READ GRID block should have a unique NUMBER (grid ID), which is used for mesh assignment. Users can assign any number of mesh-based quantities by setting the mesh parameters SCD, CDS, GFX, MFX and CGD to this grid NUMBER.

To support these former and new definition formats, the parameters SCD, CDS, GFX, MFX and CGD have been redesigned to allow either integer or logical entries. Integer entries are required if multiple mesh-based quantities are requested by the user. In this case, each integer entry must point to a grid ID specified in any READ GRID data block. See Sect. 8.1.2.14. for several examples for the use of these parameter definitions. These entries are detailed below.

SCD=yes Enable source convergence diagnostics using the fission source accumulation on the default mesh, which is $5 \times 5 \times 5$ Cartesian mesh overlaying the whole problem geometry, generated automatically. See Sect. 8.1.6.7.

$\mathbf{S C D}=i d \quad$ Enable source convergence diagnostics using the fission source accumulation on the mesh defined with READ GRID data block with grid ID, id.

MFX=yes Compute mesh fluxes (fluxes averaged over each region volume in a voxel) on intervals defined by MSH above or by the first specified READ GRID data block.

$\mathbf{M F X}=i d \quad$ Compute mesh fluxes on intervals defined by the READ GRID data block with grid ID, id.

CDS=yes Accumulate fission sources on intervals defined by the first specified READ GRID data block.

$\mathbf{C D S}=i d$ Accumulate fission source on intervals defined by the specified READ GRID data block with grid ID, $i d$. 
GFX $=i d \quad$ Compute grid fluxes (fluxes averaged over a voxel volume) on intervals defined by the READ GRID data block with grid ID, id.

CGD $=i d \quad$ Enable a mesh grid defined by the READ GRID data block with grid ID, id for CLUTCH $F^{*}(r)$ calculations.

All of the above quantities may be requested in a single input using either the same or different grids. See Sect. 8.1.2.14 for further details.

I/O Unit Numbers

$\mathbf{X S C}=$ xsecs $\quad \mathrm{I} / \mathrm{O}$ unit number for a Monte Carlo format mixed cross section library. When $\mathrm{LIB} \neq 0$, default $=14$. To read a mixed cross section library from a Monte Carlo format library file or CSASI, XSC must be specified.

$\mathbf{A L B}=$ albdo $\quad$ I/O unit number for albedo data, default $=79$.

$\mathbf{W T S}=w t s \quad \mathrm{I} / \mathrm{O}$ unit number for weights, default $=80$.

$\mathbf{L I B}=l i b \quad \mathrm{I} / \mathrm{O}$ unit number for $A M P X$ working format cross section library, default $=0$.

SKT $=s k r t \quad \mathrm{I} / \mathrm{O}$ unit number for scratch space, default $=16$.

$\mathbf{R S T}=r s t r t \quad \mathrm{I} / \mathrm{O}$ unit number for reading restart data, default $=0$.

Enter a logical unit number to restart if $B E G>1$.

$\mathbf{W R S}=w s t r t \quad \mathrm{I} / \mathrm{O}$ unit number for writing restart data, default $=0$.

A non-zero value must be entered if $R E S>0$.

$\mathbf{G R P}=$ grpbs $\quad$ I/O unit number for an energy group boundary library, default $=77$.

Example: READ PARAM NPG=203 FLX=YES END PARAM

\subsubsection{Geometry data}

The GEOMETRY_DATA consists of a series of UNIT descriptions, one of which may be the GLOBAL UNIT. The UNIT is the basic geometry piece in KENO and often corresponds to a well-defined physical entity (e.g., a fuel pin). A UNIT, therefore, may consist of multiple material regions. Each UNIT has its own, local coordinate system. The UNITs are assembled to construct the problem's global geometry for KENO. The GEOMETRY_DATA must be entered unless the problem is being restarted. See Sect. 8.1.3.6 for detailed examples.

\subsection{UNITS}

Geometric arrangements in KENO are achieved in a manner similar to using a child's building blocks. Each building block is called a UNIT. An ARRAY or lattice is constructed by stacking these UNITs. Once an ARRAY or lattice has been constructed, it can be placed in a UNIT by using an ARRAY specification. 
Each UNIT in an ARRAY or lattice has its own coordinate system. In KENO V.a, all coordinate systems in all UNITs must have the same orientation. This restriction is removed in KENO-VI. All geometry data used in a problem are correlated to the absolute coordinate system by specifying a GLOBAL UNIT. UNITs are constructed of combinations from several allowed shapes or geometric regions. These regions can be placed anywhere within a UNIT. In KENO V.a the regions are oriented along the coordinate system of the UNIT and do not intersect other regions. This means, for example, that a CYLINDER must have its axis parallel to one of the coordinate axes, while a rectangular parallelepiped must have its faces perpendicular to a coordinate axis. The most stringent KENO V.a geometry restriction is that none of the options allow geometry regions to intersect. In KENO V.a, each region in a unit must entirely contain each preceding region. The orientation, intersection, and containment restrictions are eliminated in KENO-VI. Fig. 8.1.1 shows some situations that are not allowed in KENO V.a, but are allowed in KENO-VI.

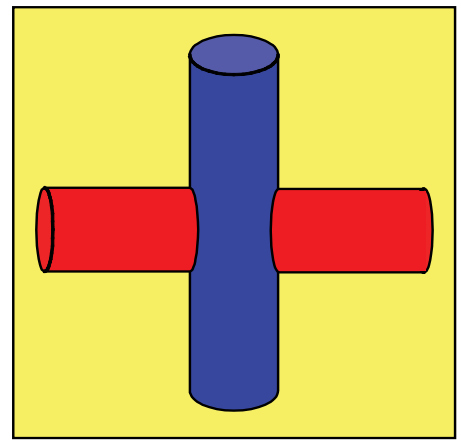

INTERSECTION REGIONS

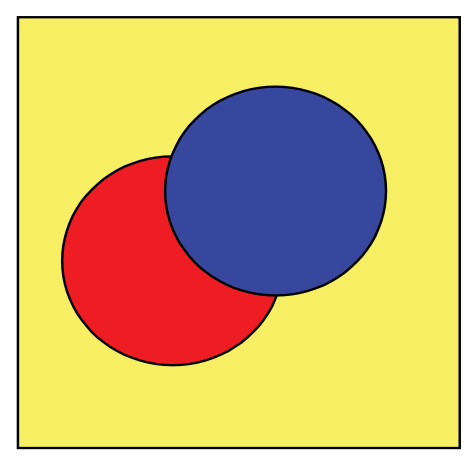

INTERSECTION REGIONS

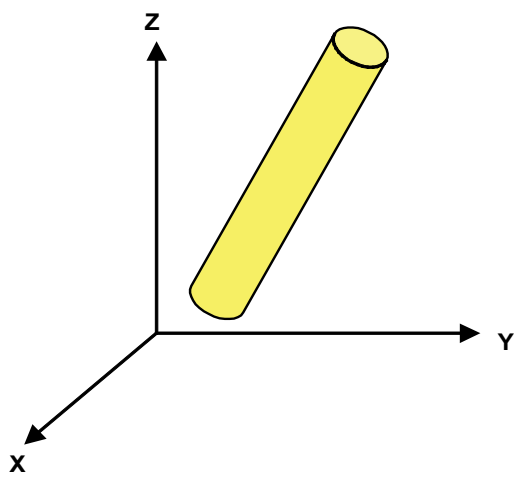

ROTATED REGION

Fig. 8.1.1. Examples of geometry allowed in KENO-VI but not allowed in KENO V.a.

For KENO V.a, unless special options are invoked, each geometric region in a UNIT must completely enclose each interior region. Regions may touch at points of tangency and may share faces. See Fig. 8.1.2 for examples of allowable situations.

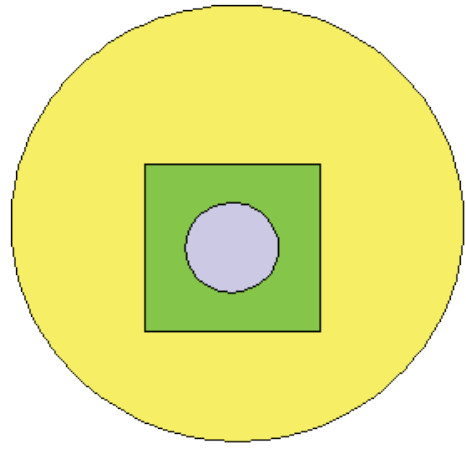

REGIONS ENCOMPASSING INTERIOR REGIONS

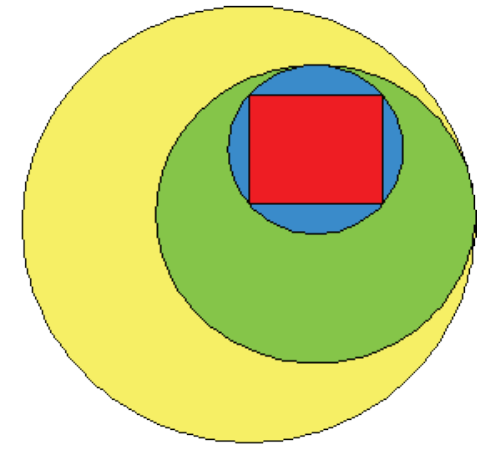

REGIONS ENCOMPASSING REGIONS AND TOUCHING

Fig. 8.1.2. Examples of correct KENO V.a units.

Special options are provided to circumvent the complete enclosure restriction in KENO V.a or to enhance the basic geometry package in KENO-VI. These options include ARRAY and HOLE descriptions. The 
HOLE option is the simplest of these and allows placing a UNIT anywhere within a region of another UNIT. In KENO V.a, HOLEs are not allowed to intersect the region into which they are placed; this restriction does not apply in KENO-VI (see Fig. 8.1.3). In both geometry packages, a HOLE cannot intersect the UNIT boundary. It is recommended that the outer boundary of a UNIT used as a HOLE should not be tangent to or share a boundary with another HOLE or a region of the UNIT containing the HOLE because the code may find that the regions are intersecting due to precision and round-off. Since a particle must check every region to determine its location within a UNIT, using HOLEs to contain complex sections of a problem may decrease the CPU time needed for the problem in KENO-VI. Inclusion of HOLEs increases run-time in KENO V.a, but in many cases cannot be avoided. An arbitrary number of HOLEs can be placed in a region in combination with a series of surrounding regions. The only restrictions on HOLEs are (1) when they are placed in a UNIT, they must be entirely contained within the UNIT, and (2) they cannot intersect other HOLEs or nested ARRAYs. HOLEs in KENO V.a cannot intersect an ARRAY; in KENO-VI, the HOLE cannot intersect the ARRAY boundary.

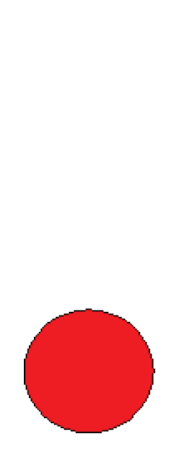

UNIT 1

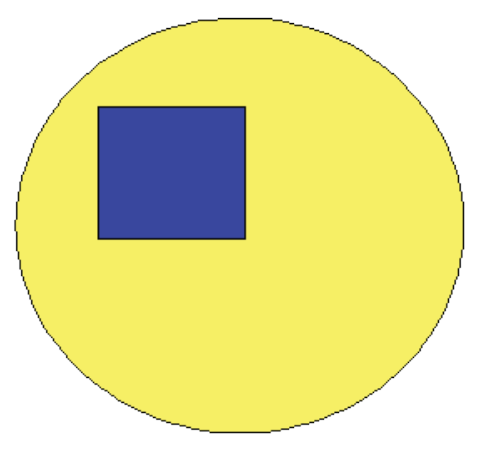

UNIT 2

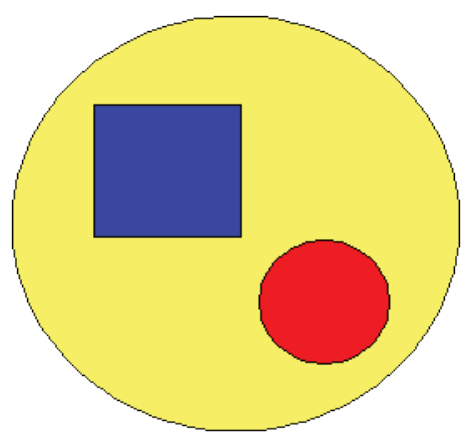

UNIT 2 WITH UNIT 1 PLACED IN IT AS A HOLE

Fig. 8.1.3. Example demonstrating HOLE capability in KENO.

Lattices or arrays are created by stacking UNITs. In KENO V.a, only rectangular parallelepipeds can be organized in an ARRAY. HEXPRISMs and DODECAHEDRONs are allowed in KENO-VI to construct triangular pitched or closed-packed dodecahedral ARRAYs, respectively. The adjacent faces of adjacent UNITs stacked in this manner must match exactly. See Sect. 8.1.3.6.4 for additional clarification and Fig. 8.1.4 and Fig. 8.1.5 for typical examples. 


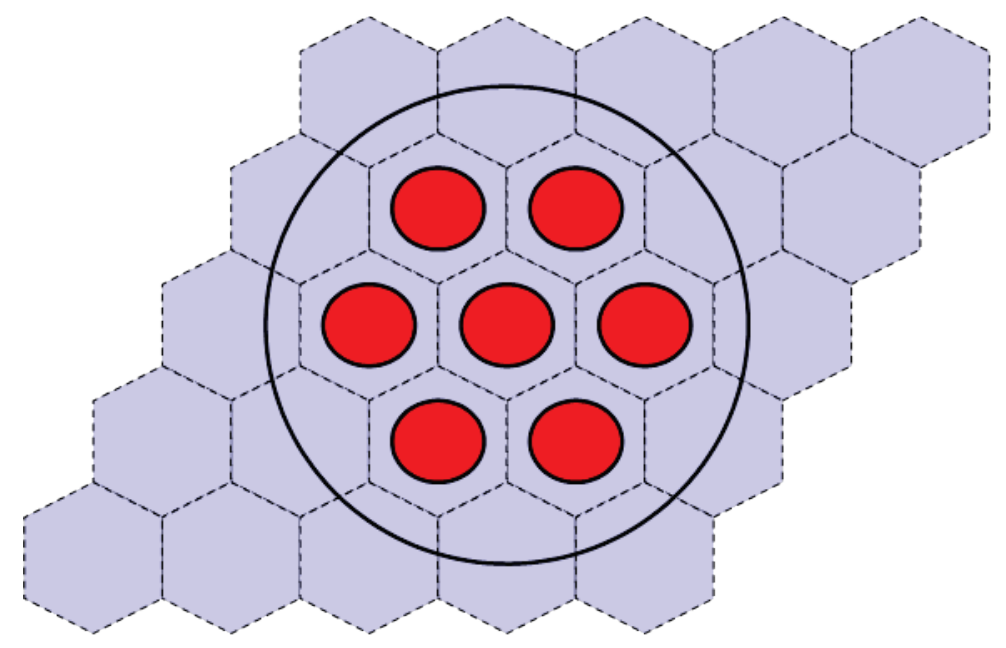

Fig. 8.1.4. Example of triangular pitched ARRAY construction.

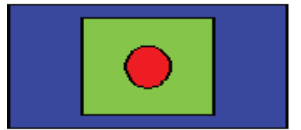

UNIT 1

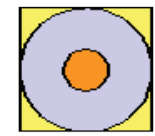

UNIT 2

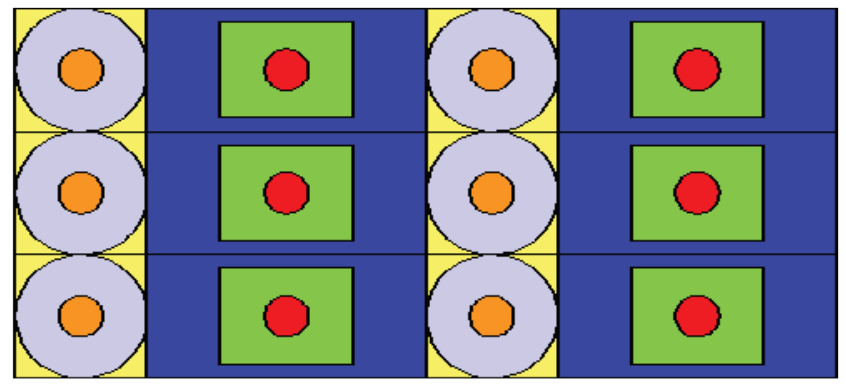

STACK THE UNIT TO FORM AN ARRAY

Fig. 8.1.5. Example of ARRAY construction.

The ARRAY option is provided to allow for placing an ARRAY or lattice within a UNIT. In KENO-VI, an ARRAY is placed in a UNIT by inserting it directly into a geometry/material region as a content record. In KENO V.a, the ARRAY is placed directly in the unit like a CUBOID: it must be the first region in the UNIT, or the ARRAY elements must intersect with the smaller region. Subsequent regions in the UNIT containing the ARRAY must contain it entirely. In KENO-VI, the reverse is true: the region boundary containing the ARRAY must coincide with or be contained within the ARRAY boundary. Therefore, in KENO-VI the region boundary becomes the ARRAY boundary, with the problem ignoring any part of the ARRAY outside the boundary. A particle enters or leaves the ARRAY when the region boundary is crossed. In KENO V.a, only one ARRAY can be placed directly in a UNIT. However, multiple ARRAYs can be placed within a UNIT by using HOLEs. When an ARRAY is placed in a UNIT via a HOLE, the UNIT that contains the ARRAY (rather than the ARRAY itself) is placed in the UNIT. ARRAYs of dissimilar ARRAYs can be created by stacking UNITs that contain ARRAYs. In KENO-VI, it is possible to place multiple ARRAYs in a UNIT by placing them in separate regions. Also in KENO-VI, using HOLEs to insert ARRAYs allows the ARRAYs to be rotated when placed. See Fig. 8.1.6 for an example of an ARRAY composed of UNITs containing HOLEs and ARRAYs. 


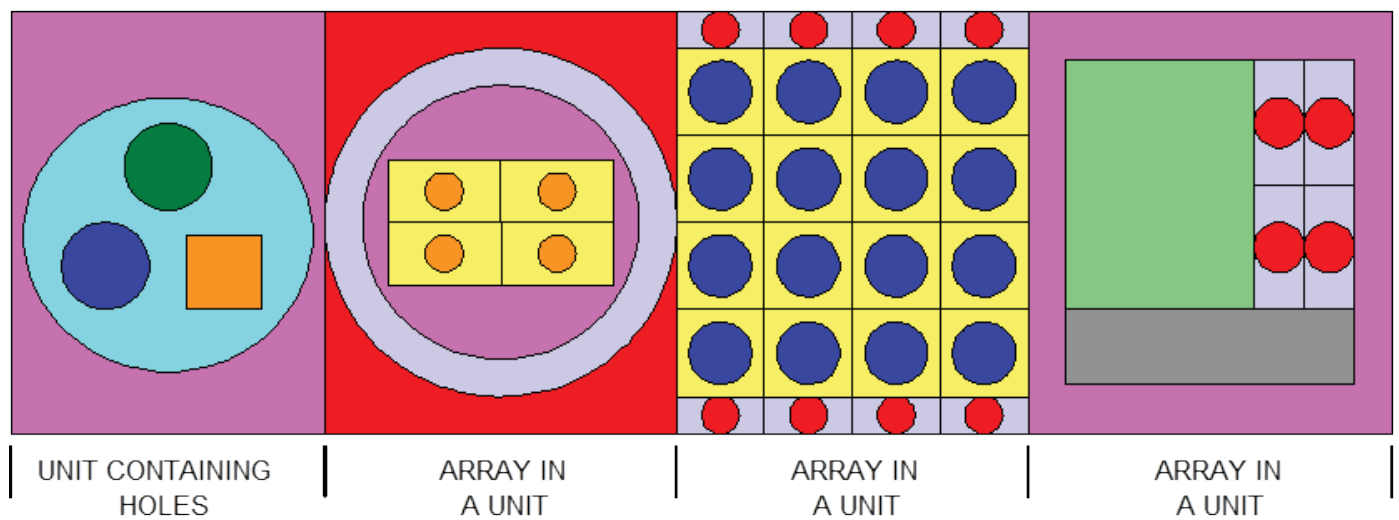

Fig. 8.1.6. Example of an ARRAY composed of UNITs containing ARRAYs and HOLEs.

The method of entering GEOMETRY_DATA in the geometry data block follows:

READ GEOM GEOMETRY_DATA END GEOM

\subsection{UNIT initialization}

The description of a UNIT starts out with the UNIT INITIALIZATION and is terminated by encountering another UNIT INITIALIZATION or END GEOM.

The UNIT INITIALIZATION has the following format:

[GLOBAL] UNIT $u$

$u$

is the identification number (positive integer) assigned to the particular UNIT. It may be used later to reference a UNIT previously constructed that the user wishes to place in a HOLE, or it may be used in an ARRAY (see below for more details).

GLOBAL is an attribute that specifies that the respective UNIT is the most comprehensive UNIT in the KENO problem to be solved, the UNIT that includes all the other UNITs and defines the overall geometric boundaries of the problem. In general, a GLOBAL UNIT must be entered for each problem.

In KENO V.a, the GLOBAL specification is optional. If it is used, it can precede either a UNIT command or an ARRAY PLACEMENT_DESCRIPTION. If it is not entered and the problem does not contain ARRAY data, UNIT 1 is the default GLOBAL UNIT. If there is no GLOBAL UNIT specified and UNIT 1 is absent from the geometry description, an error message is printed. If the geometry description contains an ARRAY, KENO V.a defaults the global array to the array referenced by the last ARRAY PLACEMENT_DESCRIPTION that is not immediately preceded by a unit description. Otherwise, it is the largest array number specified in the array data (Sect. 8.1.2.5). 
Examples of initiating a UNIT:

1. Initiate input data for UNIT No. 6.

UNIT 6

2. Initiate input data for the GLOBAL UNIT which is UNIT No. 4.

\section{GLOBAL UNIT 4}

For each UNIT, the UNIT's DESCRIPTION follows the UNIT's INITIALIZATION. The DESCRIPTION is realized by combining the commands listed below. The basic principles for constructing a UNIT are different between KENO V.a and KENO-VI. A brief discussion of these principles, together with a few examples, is presented at the end of this section following the description of the basic input used to build the geometry of a UNIT. The keywords that may be used to define a UNIT in KENO are as follows:

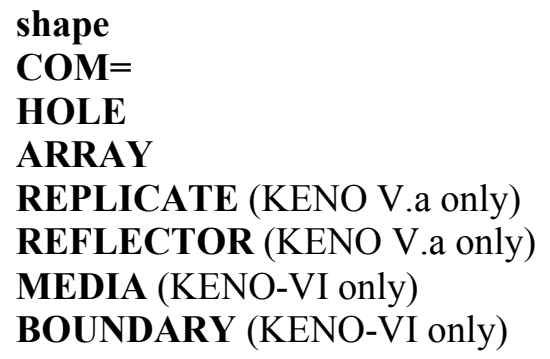

\subsection{Shape}

Shape is a generic keyword used to describe a basic geometric shape that may be used in building the geometry of a particular UNIT. The general format varies between KENO V.a and KENO-VI. In KENO V.a, the shape defines a region containing a material, so the user is required to provide both a material and a bias ID. In KENO-VI the shape is used strictly as a surface, which is later used to define the monomaterial regions (using the MEDIA card). The user is therefore required to enter a label for this surface so that the shape can be referenced later.

KENO V.a:

shape $m b d_{1} \ldots d_{N}\left[a_{1} \ldots\left[a_{M}\right] \ldots\right]$

KENO-VI:

shape $l d_{1} \ldots d_{N}\left[a_{1} \ldots\left[a_{M}\right] \ldots\right]$

shape is a generic keyword that describes a basic predefined KENO shape (e.g., CUBOID, CYLINDER) that is used to build the geometry of the UNIT. The predefined shapes differ between KENO V.a and KENO-VI. See Appendix A for a description of the KENO V.a basic shapes and Appendix B for the KENO-VI shapes.

$m$ is the mixture number of the material (positive integer) that fills the particular shape in KENO V.a UNIT description. A material number of zero indicates a void region (i.e., no material is present in the volume defined by the shape).

$b$ is the bias identification number (bias $I D$, a positive integer) assigned to the particular region defined by the shape in the KENO V.a UNIT description. 

description. This label is used later to define a certain mono-material region within the UNIT.

$d_{1} \ldots d_{N} \quad$ represent the $N$ dimensions (floating point numbers) that define the particular shape (e.g., radius of a sphere or cylinder). See Appendixes A and B for the particular value of $N$ and how each shape is described.

$a_{1} \ldots a_{M} \quad$ are $M$ optional ATTRIBUTES for the shape. The attributes provide additional flexibility in the shape description. The attributes that may be used with either KENO V.a or KENO-VI are described below (see shape ATTRIBUTES).

shape $A T T R I B U T E S$

The ATTRIBUTES that can be used to enhance the shape description are CHORD, ORIG[IN], CENTER, and ROTATE (KENO-VI only).

The CHORD attribute

This attribute has different formats in KENO V.a and KENO-VI. The user will notice that it is more restrictive in KENO V.a. Only the HEMISPHERE and HEMICYLINDER shapes can be CHORDed in KENO V.a, but all 3-D shapes may be CHORDed in KENO-VI.

KENO V.a:

CHORD $\rho$

KENO-VI:

CHORD $\left[+\mathbf{X}=x_{+}\right]\left[-\mathbf{X}=x_{-}\right]\left[+\mathbf{Y}=y_{+}\right]\left[-\mathbf{Y}=y_{-}\right]\left[+\mathbf{Z}=z_{+}\right]\left[-\mathbf{Z}=z_{-}\right]$

$\rho$

$+\mathrm{X}=,-\mathrm{X}=,+\mathrm{Y}=,-\mathrm{Y}=,+\mathrm{Z}=,-\mathrm{Z}=$

$x_{+}, x_{-}, y_{+}, y_{-}, z_{+}, z_{-}$ is the distance $\rho$ from the cut surface to the center of the sphere or the axis of a hemicylinder. See Fig. 8.1.7 and Fig. 8.1.8. Negative values of $\rho$ indicate that less than half of the shape is retained, while positive values indicate that more than half of the shape will be retained.

are subordinate keywords that define the axis parallel to the chord. The "+" and "-" signs are used to define the side of the chord which is included in the volume. A " + " in the keyword indicates that the more positive side of the chord is included in the volume. A "-" in the keyword indicates that the more negative side of the chord is included in the volume.

are the coordinates of the plane perpendicular to the chord. For each chord added to a body, the keyword CHORD must be used, followed by one of the subordinate keywords and its dimension.

In KENO V.a, the CHORD attribute is applicable for only hemispherical and hemicylindrical shapes, not for SPHERE, XCYLINDER, YCYLINDER, CYLINDER, ZCYLINDER, CUBE, or CUBOID. 


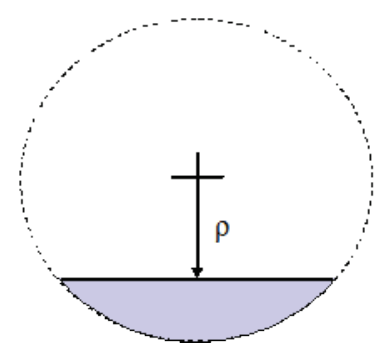

Fig. 8.1.7. Partial hemisphere or hemicylinder; less than half exists (less than half is defined by $\rho<0$ ).

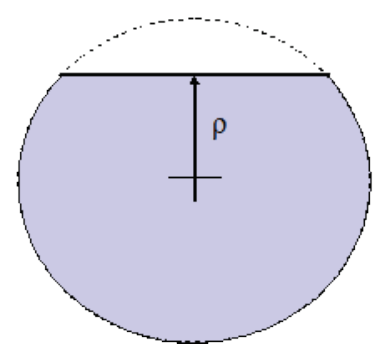

Fig. 8.1.8. Partial hemisphere of hemicylinder; more than half exists (more than half is defined by $\rho>0$ ).

Fig. 8.1.9 provides two examples of the use of the CHORD option in KENO-VI.
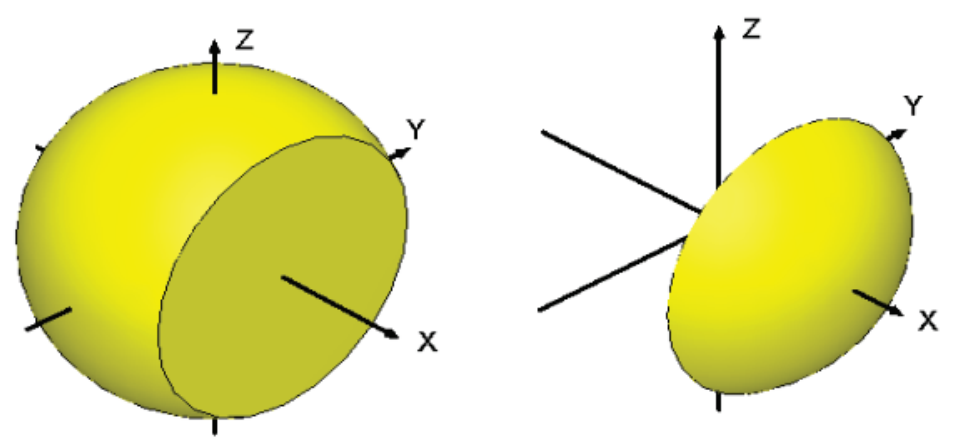

SPHERE LABEL 10.0 CHORD $-\mathrm{X}=5.0$

SPHERE LABEL 10.0 CHORD $+X=5.0$

Fig. 8.1.9. Examples of the CHORD option in KENO-VI. 


\section{The ORIG[IN] attribute}

The format is slightly different between KENO V.a and KENO-VI. Since the entries in KENO-VI are key worded, the user has more flexibility in choosing the order of these entries or in using default values. Only non-zero values must be entered in KENO-VI, but all applicable values, whether zero or non-zero, must be entered in KENO V.a.

KENO V.a:

ORIG[IN] $a b[c]$

KENO-VI:

ORIGIN $\left[\mathbf{X}=x_{0}\right]\left[\mathbf{Y}=y_{0}\right]\left[\mathbf{Z}=z_{0}\right]$

$a$

$b$

$c$

$\mathbf{X}=, \mathbf{Y}=, \mathbf{Z}=$

$x_{0}, y_{0}, z_{0}$ is the $\mathrm{X}$ coordinate of the origin of a sphere or hemisphere; the $\mathrm{X}$ coordinate of the centerline of a $\mathrm{Z}$ or $\mathrm{Y}$ cylinder or hemicylinder; the $\mathrm{Y}$ coordinate of the centerline of an $X$ cylinder or hemicylinder.

is the $\mathrm{Y}$ coordinate of the origin of a sphere or hemisphere; the $\mathrm{Y}$ coordinate of the centerline of a $\mathrm{Z}$ cylinder or hemicylinder; the $\mathrm{Z}$ coordinate of the centerline of an $\mathrm{X}$ or $\mathrm{Y}$ cylinder or hemicylinder.

is the $\mathrm{Z}$ coordinate of the origin of a sphere or hemisphere; it must be omitted for all cylinders or hemicylinders.

are the subordinate keywords used to define the new position of the origin of the shape. If the a subordinate keyword appears more than once after the ORIGIN keyword, the values are summed. If the new value is zero, the particular coordinate does not need to be specified.

are the values for the new coordinates where the origin of the shape is to be translated.

\section{The CENTER attribute}

This attribute establishes the reference center for the flux moment calculations, which can be useful in TSUNAMI calculations. The syntax for this attribute is:

CENTER center_type $[u][x y z]$

center_type

is the reference center value, as described in

Table 8.1.16. The default value is global.

$u$

$x, y, z$ is the UNIT number to be used as a reference center for this region when the center type is unit.

are the offset from the point specified by the center_type. The default is 0.0 for all three entries.

Table 8.1.16. Reference center values 
global Reference is defined as system origin-i.e., $(0,0,0)$ point of the GLOBAL UNITplus the offset defined by $x, y$, and $z$.

local Reference is defined as the origin of the current UNIT plus the offset defined by $x, y$, and $z$.

fuelcenter Reference is defined as the center of all fissile material in the system plus the offset defined by $x, y$, and $z$.

wholeunit When entered for the first region in a unit, the reference for all regions in the unit are defined as the origin of the current unit plus the offset defined by $x, y$, and $z$.

The ROTATE attribute.

This attribute can only be used in the KENO-VI input. It allows for the rotation of the shape or HOLE to which it is applied. If ORIGIN and ROTATE data follow the same shape or HOLE record, the shape is always rotated prior to translation, regardless of the order in which the data appear. Fig. 8.1.10 provides an example of the use of the ROTATE option. Its syntax is:

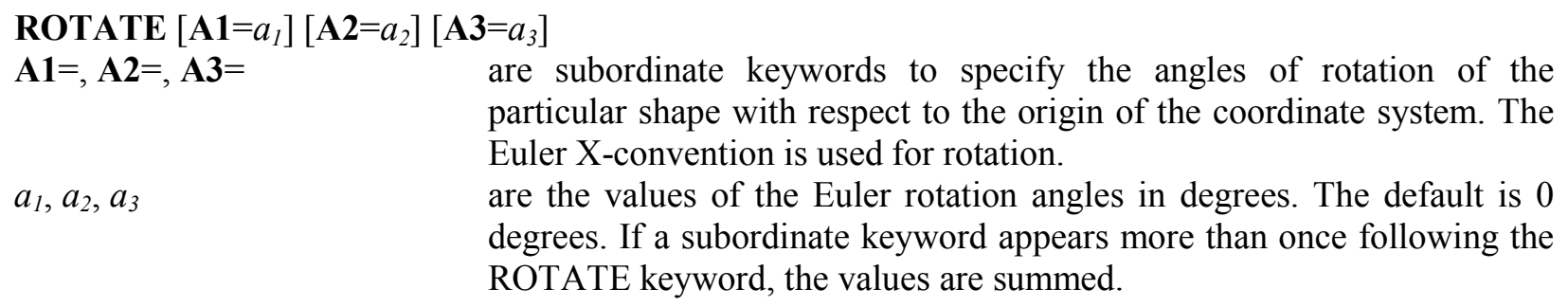
particular shape with respect to the origin of the coordinate system. The Euler X-convention is used for rotation.

$a_{1}, a_{2}, a_{3}$ are the values of the Euler rotation angles in degrees. The default is 0 degrees. If a subordinate keyword appears more than once following the ROTATE keyword, the values are summed. 


\section{CYLINDER Label R Zt Zb ROTATE $A 1=\alpha \quad A 2=\beta \quad A 3=\gamma$}

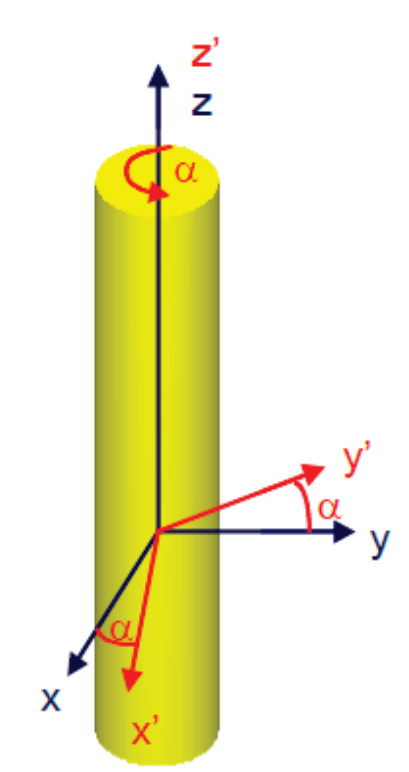

First rotation, $\mathrm{A} 1=\alpha$, is counter-clockwise about the $z$ axis

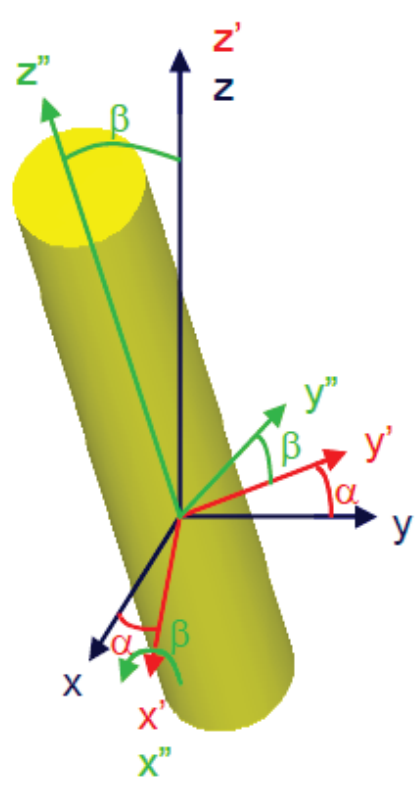

Second rotation, $\mathrm{A} 2=\beta$, is counter-clockwise about the $x^{\prime}$ axis

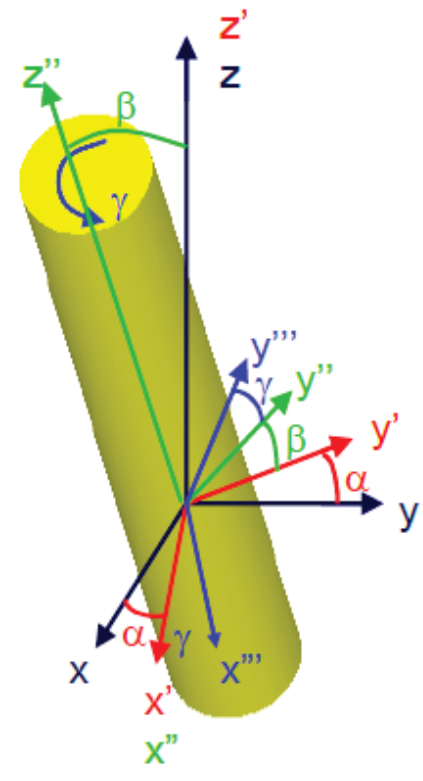

Third rotation, $\mathbf{A} 3=\gamma$, is counter-clockwise about the $z^{\prime \prime}$ axis

Fig. 8.1.10. Explanation of the ROTATE option.

Examples of shapes:

1. Specify a hemisphere labeled 10 , containing material 2 with a radius of $5.0 \mathrm{~cm}$ which contains only material where $Z>2.0$ within the sphere centered at the origin, and its origin translated to $\mathrm{X}=1.0, \mathrm{Y}=1.5$, and $\mathrm{Z}=3.0$.

KENO V.a (no label, but material and bias ID are the first two numerical entries):

HEMISPHERE 213.0 CHORD -2.0 ORIGIN $1.0 \quad 1.5 \quad 3.0$

or

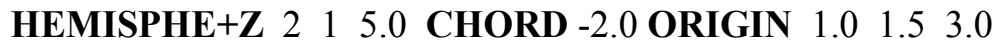

KENO-VI (no material; this is to be specified with MEDIA):

SPHERE $10 \quad 5.0 \quad$ CHORD $+Z=2.0 \quad$ ORIGIN $\quad \mathbf{X}=1.0 \quad \mathbf{Y}=1.5 \quad \mathbf{Z}=3.0$

2. Specify a hemicylinder labeled 10 , containing material 1 , having a radius of $5.0 \mathrm{~cm}$ and a length extending from $Z=2.0 \mathrm{~cm}$ to $Z=7.0 \mathrm{~cm}$. The hemicylinder has been truncated perpendicular to the $X$ axis at $X=-3$ such that material 1 does not exist between $X=-3$ and $X=-5$. Position the origin of the truncated hemicylinder at $X=10 \mathrm{~cm}$ and $Y=15 \mathrm{~cm}$ with respect to the origin of the unit, and rotate it (in KENO-VI input) so it is in the $\mathrm{YZ}$ plane at $\mathrm{X}=10$ and at $45^{\circ}$ angle with the $\mathrm{Y}$ plane. 
KENO V.a (no rotation possible, no label):

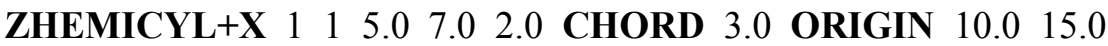

KENO-VI (no material; this is to be specified with MEDIA card):

CYLINDER 10 5.0 7.0 2.0 CHORD $+\mathbf{X}=-3.0$ ORIGIN $\mathbf{X}=10.0 \quad \mathbf{Y}=15.0$ ROTATE $\mathbf{A 2}=-45$

\subsection{3 $C O M=$}

The keyword $\mathbf{C O M = ~ s i g n a l s ~ t h a t ~ a ~ c o m m e n t ~ i s ~ t o ~ b e ~ r e a d . ~ T h e ~ o p t i o n a l ~ c o m m e n t ~ c a n ~ b e ~ p l a c e d ~ a n y w h e r e ~}$ within a unit definition. Its syntax is:

$\mathbf{C O M}=$ delim comment delim

delim is the delimiter, which may be any one of “, ', *, ^, or !.

comment is the comment string, up to 132 characters long.

Example of comment within a UNIT:

$\mathbf{C O M = " T h i s ~ i s ~ a ~ f u e l ~ p i n " ~}$

\subsection{HOLE}

This entry is used to position a UNIT within a surrounding UNIT relative to the origin of the surrounding UNIT. HOLEs may share surfaces with but may not intersect other HOLEs, the BOUNDARY of the UNIT which contains the HOLE, or an ARRAY boundary. In KENO-VI, the BOUNDARY record of a UNIT placed in a HOLE may contain more than one geometry label, but all labels must be positive, indicating inside the respective geometry bodies. The syntax for HOLE is:

KENO V.a:

HOLE $u x y z$

KENO-VI: 
HOLE $u\left[a_{1} \ldots\left[a_{M}\right] \ldots\right]$

$u \quad$ is the unit previously defined that is to be placed within the HOLE.

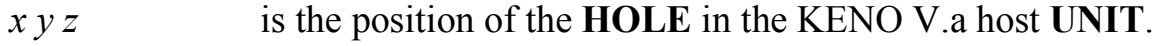

$a_{1} \ldots a_{M} \quad$ are optional KENO-VI ATTRIBUTES for the HOLE. The ATTRIBUTES can be ORIGIN or ROTATE and follow the same syntax previously defined for KENO-VI shape ATTRIBUTES. These ATTRIBUTES allow for the translation and/or rotation of the HOLE within the host region.

Examples of HOLE use:

Place UNIT 2 in the surrounding UNIT such that the ORIGIN of UNIT 2 is at $\mathbf{X}=3, \mathbf{Y}=3.5, \mathbf{Z}=4$ relative to the origin of the surrounding UNIT.

KENO V.a:

HOLE 233.54

KENO-VI:

HOLE 2 ORIGIN $\mathbf{X}=3.0 \quad \mathrm{Y}=3.5 \quad \mathrm{Z}=4.0$

\subsection{ARRAY}

When used within a UNIT description, this entry provides an ARRAY placement description. In KENO V.a, it always starts a new UNIT and generates a rectangular parallelepiped that fits the outer boundaries of the specified ARRAY. The specified ARRAY is positioned in the UNIT according to the most negative point in the ARRAY with respect to the coordinate system of the surrounding UNIT. Thus, the location of the minimum $\mathrm{x}$, minimum $\mathrm{y}$, and minimum $\mathrm{z}$ point in the array is specified in the coordinate system of the UNIT into which the ARRAY is being placed.

In KENO-VI, the ARRAY keyword is used to position an ARRAY within a region in a surrounding UNIT relative to the origin of the surrounding UNIT. When the subordinate keyword PLACE is entered, it is followed by six numbers that precisely locate the ARRAY within the surrounding UNIT as shown in the example below. The first three numbers consist of the element in the ARRAY of the UNIT selected to position the ARRAY. The next three numbers consist of the position of the origin of the selected UNIT in the surrounding UNIT. Higher level ARRAY boundaries may intersect lower level ARRAY boundaries as long as they do not intersect HOLEs in the UNITs contained in the ARRAY or in UNITs contained in lower level ARRAYs.

The syntax for the ARRAY card is as follows:

KENO V.a:

ARRAY array_id $x y z$ 


\begin{abstract}
KENO-VI:
ARRAY array_id $l_{1} \ldots l_{N}\left[\right.$ PLACE $\left.N_{x} N_{y} N_{z} x y z z\right]$

array_id is the label that identifies the array to be placed.

$l_{1} \ldots l_{N} \quad$ is the REGION DEFINITION VECTOR. These are previously defined shape labels, and together they define the region in which the array array_id is to be placed. This is used only in KENO-VI.

$N_{x} N_{y} N_{z} \quad$ are three integers that define the element in the ARRAY of the UNIT selected to position the ARRAY. This is used only in KENO-VI.

$x y z \quad$ specify the position of the ARRAY in the UNIT.

In KENO V.a, the $\mathrm{x}, \mathrm{y}$, and $\mathrm{z}$ values are the point where the most negative $\mathrm{x}, \mathrm{y}$, and $z$ point of the ARRAY is to be located in the UNIT's coordinates.

In KENO-VI, the $\mathrm{x}, \mathrm{y}$, and $\mathrm{z}$ values are the point where the origin of the UNIT specified by $N_{x}, N_{y}$, and $N_{z}$ is to be located in the shape specified by the $R E G I O N$ DEFINITION VECTOR.
\end{abstract}

Example of ARRAY use:

In KENO V.a, position the most negative point of ARRAY 6 at $\mathrm{X}=2.0, \mathrm{Y}=3.0, \mathrm{Z}=4.0$ relative to the origin of the containing UNIT.

ARRAY $6 \quad 2.0 \quad 3.0 \quad 4.0$

In KENO-VI, position instead the origin of UNIT $(1,2,3)$ of ARRAY 6 at $\mathrm{X}=2.0, \mathrm{Y}=3.0, \mathrm{Z}=4.0$ and specify the ARRAY boundary to be the region that is inside the geometry shapes labeled 10 and 20 and outside the geometry shape labeled 30 used to describe the surrounding UNIT.

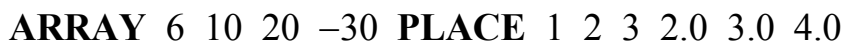

\title{
8.1.2.4.1.6 REPLICATE and REFLECTOR
}

These keywords specific to KENO V.a are used to generate additional geometry regions having the shape of the previous region. The geometry keyword REFLECTOR is a synonym for REPLICATE. The desired weighting functions can be applied to those regions by specifying biasing data as described in Sect. 8.1.2.7. The total thickness generated for each surface is the thickness per region for that surface times the number of regions to be generated, nreg.

The replicate specification is frequently used to generate weighting regions external to an ARRAY placement description. Thus an ARRAY placement description followed by a REPLICATE description would generate regions of a cuboidal shape. A cylindrical reflector could be generated by following the ARRAY placement description with a CYLINDER and then a REPLICATE. A HOLE cannot immediately follow a REPLICATE.

Extra regions using default weights can be generated by specifying the first importance region, imp, to be one that was not defined in the BIASING INFORMATION provided in a READ BIAS block. This 
capability can be used to generate extra regions for collecting information such as fluxes, leakage, etc.

Multiple replicate descriptions can be used in any problem. This capability can be used to model different reflector materials of different thicknesses on different faces.

The number of appropriate region dimensions needed for specifying REPLICATE is determined by the preceding region. For example, if the previous region were a SPHERE, one entry (i.e., $t_{l}$ ) would be required. If the previous region were a CYLINDER, the first entry, $t_{l}$, would be the thickness/region in the radial direction, the second entry, $t_{2}$, would the thickness/region in the positive length direction, the third entry, $t_{3}$, would be the thickness/region in the negative length direction, etc. The REPLICATE specification requirements for a CUBE are the same as for a CUBOID.

Syntax:

REPLICATE|REFLECTOR $m b t_{1} \ldots t_{N} n r e g$

$m \quad$ is the number of the material (non-negative integer) that fills the particular REPLICATE/REFLECTOR region in the UNIT description. A material of zero indicates a void region (i.e., no material is present in the volume defined by the shape).

$b \quad$ is the bias identification number (positive integer) assigned to the particular region defined by the shape in the KENO V.a UNIT description. If the specified bias ID is defined in a READ BIAS block, the bias ID number will be incremented automatically, increasing one for each additional region up to $\mathrm{nreg}$.

$t_{1} \ldots t_{N} \quad$ represent the thickness (floating point number) per region for each of the $N$ surfaces that define the particular shape. If the specified bias ID is one that is defined in the READ BIAS block, the region thicknesses should be consistent with the thicknesses used to generate the bias data being used. See Sect. 9.1.2.7.

nreg is the number of regions (integer) to be generated.

Example:

Create five regions of material 4 , each being $3 \mathrm{~cm}$ thick, outside a cuboid region (a cuboid has six dimensions). The inner-most of the five generated regions has a bias id of 2 . The following four regions have bias id of 3, 4, 5, and 6 .

REPLICATE $426 * 3.05$

\subsection{MEDIA}

This card is used in the KENO-VI input file to define the location of a mixture relative to the geometric shapes in the UNIT. Fig. 8.1.11 shows the input for a set of three intersecting SPHEREs in a CUBOID. The total volume data for a region in the problem may be entered as the last entry on the MEDIA card by 
using the keyword "VOL=" keyword immediately followed by the volume in $\mathrm{cm}^{3}$. The volume entered is the volume of the region in the unit multiplied by the number of times the unit occurs in the problem minus any volume excluded from the problem by ARRAY boundaries and HOLEs. The volumes for any or all regions may be entered. If the volume is entered here, this value will be used even if volumes are also entered as a file or calculated (See Sect. 8.1.2.13). Volumes not entered will be determined by the input specified in the VOLUME DATA block. If no volume is supplied, the KENO-VI default volume of -1 will be used. This only affects volume-averaged quantities, i.e., not $k_{\text {eff }}$.

Syntax:

MEDIA $m b l_{l} \ldots l_{N}[$ VOL $=v]$

$m \quad$ is the material (positive integer or zero for vacuum) that fills the region defined by MEDIA.

$b \quad$ is the bias id for the material sector being defined.

$l_{1} \ldots l_{N} \quad$ is the region definition vector ( $N$ integers). These are $N$ previously defined shape labels that together define the material sector.

VOL $=\quad$ is an optional sub-keyword used to input the material sector volume.

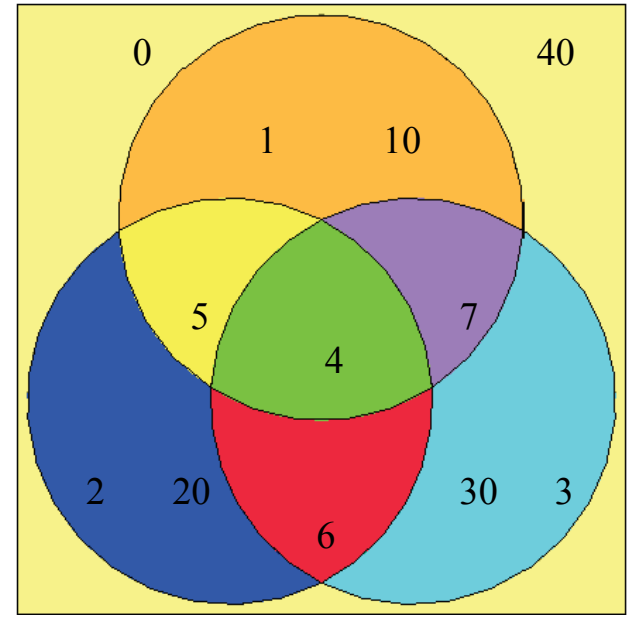

GLOBAL UNIT 1

SPHERE 1010.0 ORIGIN $Z=4.5$

SPHERE 2010.0 ORIGIN $\mathbf{Y}=-4.5 \mathbf{Z}=-4.5$

SPHERE 3010.0 ORIGIN $\mathbf{Y}=4.5 \mathrm{Z}=-4.5$

CUBOID 40 6P15.0

MEDIA $11110-20-30 \quad$ VOL $=2210.8$

MEDIA $2120-10-30 \quad$ VOL 12210.8

MEDIA $3130-10-20 \quad$ VOL $=2210.8$

MEDIA $41102030 \quad$ VOL=672.39

MEDIA $511020 \quad-30 \quad$ VOL $=652.8$

MEDIA $612030-10 \quad$ VOL $=652.8$

MEDIA $711030-20 \quad$ VOL $=652.8$

MEDIA $00140-10-20$-30 VOL=17736.81

BOUNDARY 40

Fig. 8.1.11. Example of the MEDIA record. 


\subsection{BOUNDARY}

This card is used in KENO-VI to define the outer boundary of the UNIT. In KENO V.a, the outer boundary of the UNIT is implicitly defined by the last shape in the UNIT. Each UNIT must have one and only one BOUNDARY card.

Syntax:

BOUNDARY $l_{l} \ldots l_{N}$

$l_{l} \ldots l_{N} \quad$ is the UNIT BOUNDARY DEFINITION VECTOR ( $N$ integers). These are $N$ previously defined shape labels that together define the outer boundary of the UNIT. All entries must be positive for the UNIT being defined to be used subsequently as a HOLE.

\subsubsection{ARRAY Data}

The array definition data block is used to define the size of an ARRAY and to position UNITs (defined in the geometry data) in a 3-D lattice that represents the ARRAY being described. As many arrays as are necessary can be described in a problem, subject to computer storage limitations. In KENO V.a, only one ARRAY may be placed directly in a UNIT, but as many ARRAYs as are needed may be placed in the UNIT by using HOLEs. In KENO-VI, any number of arrays can be placed in any UNIT either directly or indirectly using HOLEs. There is no default global array. If a global array is desired it must be explicitly defined.

The ARRAY definition data is entered as:

READ ARRAY ARRAY_DATA END ARRAY

The ARRAY_DATA consists of ARRAY_PARAMETERS and UNIT_ORIENTATION_DESCRIPTION.

\subsection{ARRAY parameters}

The ARRAY parameters that can be used in the definition of an ARRAY are:
ARA $=$
GBL $=$
$\mathbf{N U X}=, \mathbf{N U Y}=, \mathbf{N U Z}=$
PRT $=$
$\mathrm{COM}=$
$\mathbf{T Y P}=($ KENO-VI only $)$ 


\subsection{1 $A R A=$}

The ARA= parameter defines a reference number for an ARRAY. It has no default in KENO-VI. In KENO V.a, if is missing, the default is 1.

Syntax:

$\mathbf{A R A}=a$

$a$

is the reference number for the ARRAY. It has no default in KENO-VI. In KENO V.a, if is missing, the default is 1.

\subsection{2 $G B L=$}

This is used to input the number of the global array.

Syntax:

$\mathbf{G B L}=g$

$g$

is the reference number for the global ARRAY. In KENO V.a it must not be entered more than once. The default is the largest value for $a$, the reference number for the ARRAY. In KENO-VI it is no default value and if entered more than once, the last value is used.

\subsection{PRT $=$}

This entry is used to enable printing the ARRAY of UNIT numbers.

Syntax:

PRT $=$ print

print is a logical constant which defaults to YES, indicating that the ARRAY of UNIT numbers is printed. If the value is NO, then a summary table is printed instead containing the number of times each unit is used in each array.

\subsection{NUX=, NUY=, NUZ=}

These entries are used to input the number of units in the $\mathrm{X}, \mathrm{Y}$, and $\mathrm{Z}$ directions, respectively.

Syntax:

$\mathbf{N U X}=n_{x} \mathbf{N U Y}=n_{y} \mathbf{N U Z}=n_{z}$ 
$n_{x} n_{y} n_{z} \quad$ are the number of units in the $\mathrm{X}, \mathrm{Y}$, and $\mathrm{Z}$ directions, respectively. There is no default in KENO-VI. In KENO V.a, each of them defaults to 1.

\subsection{TYP=}

This entry is used to specify the type of ARRAY and is specific to KENO-VI, where more than one type of arrays can be used. It cannot be used in KENO V.a.

Syntax:

$\mathbf{T Y P}=$ atyp

atyp type of array (cuboidal or square, hexagonal or triangular, rhexagonal, shexagonal, dodecahedral), default $=$ cuboidal

\subsection{6 $\mathrm{COM}=$}

This keyword is used to enter a comment.

Syntax:

$\mathbf{C O M}=$ delim comment delim

delim is a delimiter. Acceptable delimiters are “, ‘, *, ^, or !.

comment is the comment string. Maximum comment length is 132 characters.

\subsection{ARRAY orientation data}

There are two methods to enter the UNIT numbers constituting an ARRAY: LOOP and FILL.

\subsection{LOOP construct}

The LOOP construct resembles a FORTRAN DO-loop construct. The arrangement of UNITs may be considered as consisting of a 3-D matrix of UNIT numbers, with the UNIT position increasing in the positive $\mathrm{X}, \mathrm{Y}$, and $\mathrm{Z}$ directions, respectively.

Syntax:

LOOP $u i x_{1} i x_{2}$ incx $i y_{1} i y_{2}$ incy $i z_{1} i z_{2} i n c z$ END LOOP

$u \quad$ is the UNIT identification number (a positive integer).

$i x_{1} \quad$ is the starting position in the X direction; $i x_{1}$ must be at least 1 and less than or equal to $n_{x}$ of section 8.1.2.5.1.4. 
$i x_{2}$ is the ending position in the X direction; $i x_{2}$ must be at least 1 and less than or equal to $n_{x}$.

incx is the number of UNITs by which increments are made in the positive $\mathrm{X}$ direction; incx must be greater than zero and less than or equal to $n_{x}$.

$i y_{1} \quad$ is the starting position in the Y direction; $i y_{l}$ must be at least 1 and less than or equal to $n_{y}$.

$i y_{2}$ is the ending position in the Y direction; $i y_{2}$ must be at least 1 and less than or equal to $n_{y}$.

incy is the number of UNITs by which increments are made in the positive Y direction; incy must be greater than zero and less than or equal to $n_{y}$.

$i z_{l} \quad$ is the starting position in the $\mathrm{Z}$ direction; $i z_{l}$ must be at least 1 and less than or equal to $n_{z}$.

$i z_{2} \quad$ is the ending position in the $\mathrm{Z}$ direction; $i z_{2}$ must be at least 1 and less than or equal to $n_{z}$.

incz is the number of UNITs by which increments are made in the positive $\mathrm{Z}$ direction; incz must be greater than zero and less than or equal to $n_{z}$.

The syntax for ending the LOOP construct is:

\section{END LOOP}

The sequence $u$ through incz is repeated until the entire ARRAY is described. If any portion of an ARRAY is defined in a conflicting manner, the last entry to define that portion will determine the ARRAY's configuration. To use this feature, fill the entire ARRAY with the most relevant UNIT number and superimpose the other UNIT numbers in their proper places. An example showing the use of the LOOP option is given below. This $5 \times 4 \times 3$ ARRAY of UNITs is a matrix of UNITs that has 5 UNITs stacked in the $X$ direction, 4 UNITs in the Y direction, and 3 UNITs in the $Z$ direction. $X$ increases from left to right, and $\mathrm{Y}$ increases from bottom to top. Each $\mathrm{Z}$ layer is shown separately.

Given:

\begin{tabular}{|c|c|c|}
\hline 12121 & 21212 & 11111 \\
\hline 11111 & 22222 & 13331 \\
\hline 11111 & 22222 & 13331 \\
\hline 12121 & 21212 & 11111 \\
\hline Z Layer 1 & Z Layer 2 & Z Layer 3 \\
\hline
\end{tabular}

The data for this array could be entered using the following entries.

(1) $1151141131 \quad$ This fills the entire array with 1s.

(2) $2252143111 \quad$ This loads the four $2 \mathrm{~s}$ in the first $\mathrm{Z}$ layer.

(3) $21512231221 \quad$ This loads the second and third rows of $2 \mathrm{~s}$ in the second $\mathrm{Z}$ layer.

(4) $2152143221 \quad$ This loads the desired $2 \mathrm{~s}$ in the first and fourth rows of the second Z layer.

(5) $3241231331 \quad$ This loads the $3 \mathrm{~s}$ in the third $\mathrm{Z}$ layer and completes the array data input. 
The second layer could have been defined by substituting the following data for entries (3) and (4):

(3) $2151141221 \quad$ This completely fills the second layer with $2 \mathrm{~s}$.

(4) $1242143221 \quad$ This loads the four $1 \mathrm{~s}$ in the second layer.

When using the LOOP option, there is no single correct method of entering the data. If a UNIT is improperly positioned in the ARRAY or if some positions in the ARRAY are left undefined, it is often easier to add data to correctly define the ARRAY than to try to correct the existing data.

\subsection{FILL construct}

The FILL construct enters data by stringing in UNIT numbers starting at $X=1, Y=1, Z=1$, and varying $X$, then Y, and then $\mathrm{Z}$ to fill the ARRAY. $n_{x} \mathrm{x} n_{y} \mathrm{x} n_{z}$ entries are required. FIDO-like input options specified in Table 8.1.17 are also available for filling the ARRAY.

Syntax:

FILL $u_{1} \ldots u_{N}\{$ END FILL $\} \mid$ T

$u_{1} \ldots u_{N} \quad$ are the $N=n_{x} \times n_{y} \times n_{z}$ UNIT numbers that make up the ARRAY

The syntax for ending the FILL construct is

\section{END FILL}

An alternative to end the UNIT data in FILL is by entering the letter T.

Table 8.1.17. FIDO-like input for mixed box orientation fill option

\begin{tabular}{|c|c|c|c|}
\hline $\begin{array}{l}\text { Count } \\
\text { field }\end{array}$ & $\begin{array}{l}\text { Option } \\
\text { field }\end{array}$ & $\begin{array}{l}\text { Operand } \\
\text { field }\end{array}$ & Function \\
\hline & & $j$ & stores $j$ at the current position in the array \\
\hline$i$ & $\mathrm{R}$ & $j$ & stores $j$ in the next $i$ positions in the array \\
\hline$i$ & $*$ & $j$ & stores $j$ in the next $i$ positions in the array \\
\hline \multirow[t]{3}{*}{$i$} & $\$$ & $j$ & stores $j$ in the next $i$ positions in the array \\
\hline & $\mathrm{F}$ & $j$ & $\begin{array}{l}\text { fills the remainder of the array with unit number } j \text {, starting with the } \\
\text { current position in the array }\end{array}$ \\
\hline & A & $j$ & sets the current position in the array to $j$ \\
\hline$i$ & $\mathrm{~S}$ & & $\begin{array}{l}\text { increments the current position in the array by } i \text { (This allows for } \\
\text { skipping } i \text { positions; } i \text { may be positive or negative.) }\end{array}$ \\
\hline
\end{tabular}




\begin{tabular}{|c|c|c|}
\hline Q & $j$ & repeats the previous $j$ entries $i$ times (default value of $i$ is 1 ) \\
\hline $\mathrm{N}$ & $j$ & $\begin{array}{l}\text { repeats previous } j \text { entries } i \text { times, inverting the sequence each time. } \\
\text { (default value of } i \text { is } 1 \text { ) }\end{array}$ \\
\hline B & $j$ & $\begin{array}{l}\text { backs } i \text { entries. From that position, repeats the previous } j \text { entries in } \\
\text { reverse order (default value of } i \text { is } 1 \text { ) }\end{array}$ \\
\hline I & $j k$ & $\begin{array}{l}\text { provides the end points } \mathrm{j} \text { and } \mathrm{k} \text {, with } \mathrm{i} \text { entries linearly interpolated } \\
\text { between them (i.e., a total of } \mathrm{i}+2 \text { points). At least one blank } \\
\text { must separate } \mathrm{j} \text { and } \mathrm{k} \text {. When used for an integer array, the } \\
\text { I option should only be used to generate integer steps- } \\
\text { i.e., }(\mathrm{k}-\mathrm{j}) /(\mathrm{i}+1) \text { should be a whole number }\end{array}$ \\
\hline $\mathrm{T}$ & & terminates the data reading for the array \\
\hline
\end{tabular}

NOTE: When entering data using the options in this table, the count field and option field must be adjacent with no imbedded blanks. The operand field may be separated from the option field by one or more blanks.

Example: Consider a $3 \times 3 \times 1$ ARRAY filled with 8 UNIT 1 s and a UNIT 2, as shown below.

$\begin{array}{lll}1 & 1 & 1 \\ 1 & 2 & 1 \\ 1 & 1 & 1\end{array}$

The input data to describe this ARRAY could be entered as follows:

(1) $111121111 \mathbf{T} \quad$ This fills the array one position at a time, starting at the lower left corner. The $\mathbf{T}$ terminates the data.

or

(2) F1 A5 2 END FILL The F1 fills the entire array with 1s, the A5 locates the fifth position in the array, and the 2 loads a 2 in that position. The END FILL terminates the data.

\subsubsection{Albedo data}

Albedo boundary conditions are entered by assigning an albedo condition to each face of the outermost boundary. The default value for each face is vacuum. The default values are overridden only on faces for which other albedo names are specified. Albedo boundary conditions are applied only to the outermost region of a problem. In KENO V.a this geometry region must be a rectangular parallelepiped. The outer boundary can be any shape (or combination of shapes) in KENO-VI. Material-specific albedos (e.g., $\mathrm{H} 2 \mathrm{O}$ and $\mathrm{CONC}$ ) may not be used in continuous energy calculations.

KENO-VI users need to be aware that when a neutron reaches a surface with a vacuum albedo, that neutron is lost. If a model contains features that are reentrant, that is a neutron could exit the model and reenter the model on the other side of an unmodeled region, all neutrons passing through the problem boundary are lost when they reach the unmodeled region. Neutrons are not "transported" across unmodeled areas between reentrant surfaces. It is not possible to create a KENO V.a model with reentrant problem outer boundary surfaces. 
The syntax for entering the albedo boundary conditions is:

READ BOUNDS $\mathbf{f c}_{1}=a_{1}\left[\mathbf{f c}_{2}=a_{2} \ldots\left[\mathbf{f c}_{\mathbf{N}}=a_{N}\right] \ldots\right]$ END BOUNDS

$\mathbf{f c}_{\mathbf{1}} \ldots \mathbf{f c}_{\mathbf{N}} \quad$ are $\mathbf{N}$ face codes as defined in Table 8.1.18.

$a_{1} \ldots a_{N} \quad$ are the albedo types as defined in Table 8.1.19.

Table 8.1.19 lists some material-specific albedo sets. Care must be exercised when using material-specific albedo types. These data sets were generated using a real problem, and they implicitly reflect the neutron energy spectrum, materials, and geometry from that model. Where neutron energy spectra, materials, and geometry vary from that model, the material-specific albedos may give significantly incorrect results.

This may be checked by comparing results from a sample of calculations performed with both explicitly modeled reflectors and material-specific albedos. In general, use of material-specific albedos is not recommended. 
Table 8.1.18. Face codes and surface numbers for entering boundary (albedo) conditions

\begin{tabular}{|c|c|}
\hline Face code & Faces defined by face codes \\
\hline$+\mathrm{XB}=$ & Positive $\mathrm{X}$ face \\
\hline$\& \mathrm{XB}=$ & Positive $\mathrm{X}$ face \\
\hline$-\mathrm{XB}=$ & Negative $\mathrm{X}$ face \\
\hline$+\mathrm{YB}=$ & Positive Y face \\
\hline$\& \mathrm{YB}=$ & Positive Y face \\
\hline$-\mathrm{YB}=$ & Negative $Y$ face \\
\hline$+\mathrm{ZB}=$ & Positive $\mathrm{Z}$ face \\
\hline$\& \mathrm{ZB}=$ & Positive $\mathrm{Z}$ face \\
\hline$-\mathrm{ZB}=$ & Negative $\mathrm{Z}$ face \\
\hline $\mathrm{ALL}=$ & All six faces \\
\hline $\mathrm{XFC}=$ & Both positive and negative $\mathrm{X}$ faces \\
\hline $\mathrm{YFC}=$ & Both positive and negative $\mathrm{Y}$ faces \\
\hline $\mathrm{ZFC}=$ & Both positive and negative $\mathrm{Z}$ faces \\
\hline$+\mathrm{FC}=$ & Positive $\mathrm{X}, \mathrm{Y}$, and $\mathrm{Z}$ faces \\
\hline$\& \mathrm{FC}=$ & Positive $\mathrm{X}, \mathrm{Y}$, and $\mathrm{Z}$ faces \\
\hline$-\mathrm{FC}=$ & Negative $X, Y$, and $Z$ faces \\
\hline $\mathrm{XYF}=$ & Positive and negative $\mathrm{X}$ and $\mathrm{Y}$ faces \\
\hline $\mathrm{XZF}=$ & Positive and negative $\mathrm{X}$ and $\mathrm{Z}$ faces \\
\hline $\mathrm{YZF}=$ & Positive and negative $\mathrm{Y}$ and $\mathrm{Z}$ faces \\
\hline$+\mathrm{XY}=$ & Positive $\mathrm{X}$ and $\mathrm{Y}$ faces \\
\hline$+\mathrm{YX}=$ & Positive $\mathrm{X}$ and $\mathrm{Y}$ faces \\
\hline$\& X Y=$ & Positive $\mathrm{X}$ and $\mathrm{Y}$ faces \\
\hline$\& Y Z=$ & Positive $\mathrm{X}$ and $\mathrm{Y}$ faces \\
\hline$+X Z=$ & Positive $\mathrm{X}$ and $\mathrm{Z}$ faces \\
\hline$+\mathrm{ZX}=$ & Positive $\mathrm{X}$ and $\mathrm{Z}$ faces \\
\hline$\& X Z=$ & Positive $\mathrm{X}$ and $\mathrm{Z}$ faces \\
\hline$\& Z X=$ & Positive $\mathrm{X}$ and $\mathrm{Z}$ faces \\
\hline$+\mathrm{YZ}=$ & Positive $\mathrm{Y}$ and $\mathrm{Z}$ faces \\
\hline$+\mathrm{ZY}=$ & Positive $\mathrm{Y}$ and $\mathrm{Z}$ faces \\
\hline$\& Y Z=$ & Positive $\mathrm{Y}$ and $\mathrm{Z}$ faces \\
\hline$\& Z Y=$ & Positive $\mathrm{Y}$ and $\mathrm{Z}$ faces \\
\hline$-X Y=$ & Negative $\mathrm{X}$ and $\mathrm{Y}$ faces \\
\hline$-\mathrm{XZ}=$ & Negative $\mathrm{X}$ and $\mathrm{Z}$ faces \\
\hline$-\mathrm{YZ}=$ & Negative $\mathrm{Y}$ and $\mathrm{Z}$ faces \\
\hline $\mathrm{YXF}=$ & Positive and negative $\mathrm{X}$ and $\mathrm{Y}$ faces \\
\hline $\mathrm{ZXF}=$ & Positive and negative $\mathrm{X}$ and $\mathrm{Z}$ faces \\
\hline $\mathrm{ZYF}=$ & Positive and negative $\mathrm{Y}$ and $\mathrm{Z}$ faces \\
\hline$-\mathrm{YX}=$ & Negative $\mathrm{X}$ and $\mathrm{Y}$ faces \\
\hline$-Z X=$ & Negative $\mathrm{X}$ and $\mathrm{Z}$ faces \\
\hline$-\mathrm{ZY}=$ & Negative $\mathrm{Y}$ and $\mathrm{Z}$ faces \\
\hline $\mathrm{BODY}=\mathrm{x}$ & $\mathrm{x}$ is the body's geometry label in the global unit (KENO-VI only) \\
\hline $\operatorname{SURFACE}($ ii $)=$ & Boundary condition for surface number ii of body $\mathrm{x}$ (KERNO-VI) only \\
\hline
\end{tabular}


Table 8.1.18. Face codes and surface numbers for entering boundary (albedo) conditions (continued)

\begin{tabular}{|c|c|c|c|c|c|c|c|c|c|c|c|c|}
\hline \multirow{2}{*}{$\begin{array}{l}\text { Geometry } \\
\text { body }\end{array}$} & \multicolumn{12}{|c|}{ Albedo surface numbers related to KENO-VI geometry bodies } \\
\hline & 1 & 2 & 3 & 4 & 5 & 6 & 7 & 8 & 9 & 10 & 11 & 12 \\
\hline Cone & Radial & $+\mathrm{Z}$ & $-\mathrm{Z}$ & & & & & & & & & \\
\hline Cuboid & $+\mathrm{X}$ & $-\mathrm{X}$ & $+\mathrm{Y}$ & $-\mathrm{Y}$ & $+\mathrm{Z}$ & $-\mathrm{Z}$ & & & & & & \\
\hline Cylinder & Radial & $+\mathrm{Z}$ & $-\mathrm{Z}$ & & & & & & & & & \\
\hline Dodecahedron & $+\mathrm{X}$ & $-\mathrm{X}$ & $+\mathrm{Y}$ & $-\mathrm{Y}$ & $\begin{array}{l}+\mathrm{X} \\
+\mathrm{Y} \\
+\mathrm{Z}\end{array}$ & $\begin{array}{l}-X \\
-Y \\
-Z\end{array}$ & $\begin{array}{l}-\mathrm{X} \\
+\mathrm{Y} \\
+\mathrm{Z}\end{array}$ & $\begin{array}{l}+\mathrm{X} \\
-\mathrm{Y} \\
-\mathrm{Z}\end{array}$ & $\begin{array}{l}-X \\
-Y \\
+Z\end{array}$ & $\begin{array}{l}+\mathrm{X} \\
+\mathrm{Y} \\
-\mathrm{Z}\end{array}$ & $\begin{array}{l}+\mathrm{X} \\
-\mathrm{Y} \\
+\mathrm{Z}\end{array}$ & $\begin{array}{l}-\mathrm{X} \\
+\mathrm{Y} \\
-\mathrm{Z}\end{array}$ \\
\hline Ecylinder & Radial & $+Z$ & $-Z$ & & & & & & & & & \\
\hline Ellipsoid & Radial & & & & & & & & & & & \\
\hline Hexprism & $+\mathrm{X}$ & $-\mathrm{X}$ & $\begin{array}{l}+\mathrm{X} \\
+\mathrm{Y} \\
\end{array}$ & $\begin{array}{l}-\mathrm{X} \\
-\mathrm{Y} \\
\end{array}$ & $\begin{array}{l}-\mathrm{X} \\
+\mathrm{Y} \\
\end{array}$ & $\begin{array}{l}+\mathrm{X} \\
-\mathrm{Y}\end{array}$ & $+\mathrm{Z}$ & $-Z$ & & & & \\
\hline Hopper & $+\mathrm{X}$ & $-X$ & $+Y$ & $-Y$ & $+Z$ & $-\mathrm{Z}$ & & & & & & \\
\hline Pentagon & $-Y$ & $\begin{array}{l}+\mathrm{X} \\
-\mathrm{Y}\end{array}$ & $\begin{array}{l}+\mathrm{X} \\
+\mathrm{Y}\end{array}$ & $\begin{array}{l}-\mathrm{X} \\
+\mathrm{Y}\end{array}$ & $\begin{array}{l}-\mathrm{X} \\
-\mathrm{Y}\end{array}$ & $+Z$ & $-Z$ & & & & & \\
\hline $\begin{array}{l}\text { Paralellepiped } \\
\text { Ppiped }\end{array}$ & $+\mathrm{X}$ & $-\mathrm{X}$ & $+Y$ & $-Y$ & $+Z$ & $-\mathrm{Z}$ & & & & & & \\
\hline Plane & Surface & & & & & & & & & & & \\
\hline Quadratic & Surface & & & & & & & & & & & \\
\hline Rhexprism & $+\mathrm{Y}$ & $-Y$ & $\begin{array}{l}-\mathrm{X} \\
+\mathrm{Y} \\
\end{array}$ & $\begin{array}{l}+\mathrm{X} \\
-\mathrm{Y} \\
\end{array}$ & $\begin{array}{l}+\mathrm{X} \\
+\mathrm{Y} \\
\end{array}$ & $\begin{array}{l}-\mathrm{X} \\
-\mathrm{Y} \\
\end{array}$ & $+\mathrm{Z}$ & $-Z$ & & & & \\
\hline Rhomboid & $+\mathrm{X}$ & $-\mathrm{X}$ & $+\mathrm{Y}$ & $-\mathrm{Y}$ & $+Z$ & $-\mathrm{Z}$ & & & & & & \\
\hline Sphere & Radial & & & & & & & & & & & \\
\hline Wedge & $-Y$ & $\begin{array}{l}-\mathrm{X} \\
+\mathrm{Y}\end{array}$ & $\begin{array}{l}+\mathrm{X} \\
+\mathrm{Y}\end{array}$ & $+Z$ & $-Z$ & & & & & & & \\
\hline Xcylinder & Radial & $+\mathrm{X}$ & $-X$ & & & & & & & & & \\
\hline Xpplane & $+\mathrm{X}$ & $-\mathrm{X}$ & & & & & & & & & & \\
\hline
\end{tabular}


Table 8.1.18. Face codes and surface numbers for entering boundary (albedo) conditions (continued)

\begin{tabular}{|l|c|c|c|c|c|c|c|c|c|c|c|c|}
\hline $\begin{array}{c}\text { Geometry } \\
\text { body }\end{array}$ & \multicolumn{10}{|c|}{ Albedo surface numbers related to KENO-VI geometry bodies } \\
\hline & 1 & 2 & 3 & 4 & 5 & 6 & 7 & 8 & 9 & 10 & 11 & 12 \\
\hline Ycylinder & Radial & $+\mathrm{Y}$ & $-\mathrm{Y}$ & & & & & & & & & \\
\hline Ypplane & $+\mathrm{Y}$ & $-\mathrm{Y}$ & & & & & & & & & & \\
\hline Zcylinder & Radial & $+\mathrm{Z}$ & $-\mathrm{Z}$ & & & & & & & & & \\
\hline Zpplane & $+\mathrm{Z}$ & $-\mathrm{Z}$ & & & & & & & & & & \\
\hline
\end{tabular}

Surfaces refer to the surface of the body that occurs in the indicated quadrant.

Refer to Figs. 9.B.1-9.B.22 for illustrations of each geometry body. 
Table 8.1.19. Albedo names available on the KENO albedo library for use with the face codes*

\begin{tabular}{|c|c|}
\hline $\begin{array}{l}\mathrm{DP} 0 \mathrm{H} 2 \mathrm{O} \\
\mathrm{DPOH} 2 \mathrm{O} \\
\text { DP0 } \\
\text { DPO }\end{array}$ & $\begin{array}{l}12 \text { in. }(30.48 \mathrm{~cm}) \text { double } \mathrm{P}_{0} \text { water differential albedo with } \\
4 \text { incident angles }\end{array}$ \\
\hline $\begin{array}{l}\mathrm{H} 2 \mathrm{O} \\
\text { WATER }\end{array}$ & $\begin{array}{l}12 \text { in. }(30.48 \mathrm{~cm}) \text { water differential albedo with } 4 \text { incident } \\
\text { angles }\end{array}$ \\
\hline $\begin{array}{l}\text { PARAFFIN } \\
\text { PARA } \\
\text { WAX }\end{array}$ & $\begin{array}{l}12 \text { in. }(30.48 \mathrm{~cm}) \text { paraffin differential albedo with } \\
4 \text { incident angles }\end{array}$ \\
\hline $\begin{array}{l}\text { CARBON } \\
\text { GRAPHITE } \\
\text { C }\end{array}$ & $\begin{array}{l}78.74 \text { in. }(200.00 \mathrm{~cm}) \text { carbon differential albedo with } \\
4 \text { incident angles }\end{array}$ \\
\hline $\begin{array}{l}\text { ETHYLENE } \\
\text { POLY } \\
\text { CH2 }\end{array}$ & $\begin{array}{l}12 \text { in. }(30.48 \mathrm{~cm}) \text { polyethylene differential albedo with } \\
4 \text { incident angles }\end{array}$ \\
\hline $\begin{array}{l}\text { CONC-4 } \\
\text { CON4 } \\
\text { CONC4 }\end{array}$ & $\begin{array}{l}4 \text { in. }(10.16 \mathrm{~cm}) \text { concrete differential albedo with } \\
4 \text { incident angles }\end{array}$ \\
\hline $\begin{array}{l}\text { CONC-8 } \\
\text { CON8 } \\
\text { CONC8 }\end{array}$ & $\begin{array}{l}8 \text { in. }(20.32 \mathrm{~cm}) \text { concrete differential albedo with } \\
4 \text { incident angles }\end{array}$ \\
\hline $\begin{array}{l}\text { CONC-12 } \\
\text { CON12 } \\
\text { CONC12 } \\
\end{array}$ & $\begin{array}{l}12 \text { in. }(30.48 \mathrm{~cm}) \text { concrete differential albedo with } \\
4 \text { incident angles }\end{array}$ \\
\hline $\begin{array}{l}\text { CONC-16 } \\
\text { CON16 } \\
\text { CONC16 }\end{array}$ & $\begin{array}{l}16 \text { in. }(40.64 \mathrm{~cm}) \text { concrete differential albedo with } \\
4 \text { incident angles }\end{array}$ \\
\hline $\begin{array}{l}\text { CONC-24 } \\
\text { CON24 } \\
\text { CONC24 } \\
\end{array}$ & $\begin{array}{l}24 \text { in. }(60.96 \mathrm{~cm}) \text { concrete differential albedo with } \\
4 \text { incident angles }\end{array}$ \\
\hline $\begin{array}{l}\text { VACUUM } \\
\text { VOID }\end{array}$ & Vacuum condition \\
\hline $\begin{array}{l}\text { SPECULAR } \\
\text { MIRROR } \\
\text { REFLECT } \\
\end{array}$ & Mirror image reflection \\
\hline PERIODIC & Periodic boundary condition \\
\hline WHITE & White boundary condition \\
\hline
\end{tabular}

The BODY and SURFACE keywords are unique to KENO-VI. The face code BODY= refers to the body label in global unit input. For example, assume the GLOBAL UNIT boundary record in a KENO-VI input consisted of the following: BOUNDARY $10-30$ 20. In this case BODY=10 would refer to the geometry record labeled $10, \mathbf{B O D Y}=20$ would refer to the geometry record labeled 20, and $\mathbf{B O D Y}=30$ would refer to the geometry record labeled 30. All surface numbers following the BODY keyword apply 
to that body. The default value of BODY is the first geometry label listed in the GLOBAL UNIT boundary record.

All the face codes, listed in the first part of Table 8.1.18, except BODY= and SURFACE(ii)= were intended to apply only to cuboids (KENO V.a). However, when used with non-cuboidal surfaces (KENOVI) they will fill in the first six surface positions of a body in the following order, $+\mathrm{X},-\mathrm{X},+\mathrm{Y},-\mathrm{Y},+\mathrm{Z}$, $-Z$. The ALL face code will apply the listed boundary conditions to all surfaces of the body currently being considered.

Albedo boundary conditions may be entered on each GLOBAL UNIT boundary surface multiple times. The boundary condition that applies to the surface is the last one entered. If no boundary data are entered or if no albedo boundary condition is applied to a GLOBAL UNIT boundary surface, then the boundary surface is assumed to have a void or vacuum boundary condition. Any CHORDed surfaces that are GLOBAL UNIT boundaries will use the default (void) boundary condition, and it cannot be changed. This restriction may need to be considered when building the geometry of the GLOBAL UNIT.

Example: $\quad$ Use a 24 in. concrete albedo boundary condition on the $-Z$ face of a problem with a cuboidal boundary and use mirror image reflection on the $+X$ and $-X$ faces of the cuboid to represent an infinite linear array on a $2 \mathrm{ft}$. thick concrete pad.

\section{READ BOUNDS - ZB=CON24 XFC=MIRROR END BOUNDS}

Example: Use a 24 in. concrete albedo boundary condition on the $-\mathrm{Z}$ face of a problem with a hexagonal boundary, and use mirror image reflection on all side faces of the hexprism to represent an infinite planar array on a $2 \mathrm{ft}$. thick concrete pad.

READ BOUNDS

SURFACE $(1)=$ MIRROR SURFACE $(2)=$ MIRROR SURFACE $(3)=M I R R O R$

SURFACE(4)=MIRROR SURFACE $(5)=$ MIRROR SURFACE $(6)=$ MIRROR

SURFACE $(7)=$ VACUUM SURFACE $(8)=$ CONC 24

END BOUNDS

Example: The outer boundary of the global unit consists of a cuboid (body label 10) and a sphere (body label 20). The sphere is large enough to cut the corners of the cuboid leaving most of the cuboid intact. Use a $24 \mathrm{in}$. concrete albedo boundary condition on the $-\mathrm{Z}$ face of the cuboid to represent a $2 \mathrm{ft}$. thick concrete pad. Use the DP0H2O on the other surfaces to represent an infinite water reflector.

READ BOUNDS

BODY $=10$ ALL $=$ DP0H $2 \mathrm{O}-\mathbf{Z B}=\mathrm{CON} 24$

BODY $=20$ SURFACE $(1)=\mathrm{DP} 0 \mathrm{H} 2 \mathrm{O}$

END BOUNDS

WARNING: The user should thoroughly understand material-specific albedos (e.g., DP0H2O, CON24, etc.) before attempting to use these reflectors. Missapplication of these problem-specific albedo data can cause the code to produce incorrect results without obvious symptoms.

\subsubsection{Biasing or weighting data}

The biasing data block is used (in only multigroup mode) to define the weight that is given to a neutron surviving Russian roulette. The average weight of a neutron that survives Russian roulette, wtavg, is 
defaulted to $d w \operatorname{tav}$ (WTA= in the parameter data, Sect. 8.1.2.3) for all BIAS IDs and can be overridden by entering biasing information.

The biasing information is used to relate a BIAS ID to the desired energy-dependent values of wtavg. This concept is similar to the way the MIXTURE ID, mat, is related to the macroscopic cross section data.

The weighting functions used in KENO are energy-dependent values of wtavg that are applicable over a given thickness interval of a material. For example, the weighting function for water is composed of sets of energy-dependent values of wtavg for 11 intervals, each interval being $3 \mathrm{~cm}$ thick. The first set of wtavg's is for the $0-3 \mathrm{~cm}$ interval of water, the second set of wtavg's is for the 3-6 cm interval of water, etc. The eleventh set of wtavg's is for the $30-33 \mathrm{~cm}$ interval of water.

To input biasing information, a BIAS ID must be assigned to correspond to a set of wtavg. Biasing data can specify a MATERIAL ID from the existing KENO V.a weighting library or from the AUXILIARY DATA input. The materials available from the KENO weighting library are listed in Table 8.1.20.

The biasing information is entered in one of the following two forms. The first set is said to input the CORRELATION DATA, while the second form is said to input the AUXILIARY DATA.

READ BIAS ID $=m$ ib ie

or

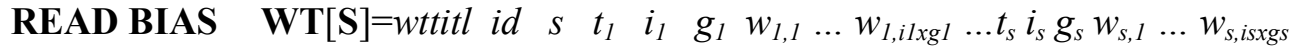

END BIAS

END BIAS

ID = specifies that CORRELATION DATA will be entered next.

$\mathbf{W T}=$ or $\mathbf{W T S}=$ specifies that $A U X I L I A R Y$ DATA will be entered next.

$m$

$i b$

ie

wttitl

id

$S$

$t_{1} \ldots t_{s}$

$i_{1} \ldots i_{s}$

$g_{1} \ldots g_{s}$

$w_{1, i 1 \times g l} \ldots w_{s, i s x g s}$

is the identification (material ID) for the material whose weighting function is to be used. A material ID can be chosen from the existing KENO weighting library (Table 8.1.20) or from the auxiliary data input using the second form of the BIAS block as described later. If a material ID appears in both the KENO weighting library and the auxiliary data, the weights from the auxiliary data will be used.

is the bias ID of the weighting function for the first interval of material $m$. The geometry record having the bias ID equal to $i b$ will use the group-dependent weights from the first interval of material $m$. is the bias ID of the group-dependent weights from the $(i e-i b+1) t h$ interval of material $m$.

is an arbitrary title name (12 characters maximum), such as CONCRETE, WATER, SPECIALH2O, etc., to identify the material for which the user is entering data. Embedded blanks are not allowed.

is an identification number (material ID). The value is arbitrary. However, if the data are to be utilized in the problem, this ID must also be used at least once in the first form of the BIAS block.

is the number of sets of group structures for which weights will be read for this ID.

are $s$ thicknesses of each increment for which weights will be read for this ID.

are $s$ numbers of increments for which weights will be read for this ID.

are $s$ numbers of energy groups for which weights will be read.

are $s$ sets of weights, each set containing a number of weights equal to the product of number of increments times the number of groups for that set. The group index varies the fastest. 
Table 8.1.20. IDs, group structure and incremental thickness for weighting data available on the KENO weighting library

\begin{tabular}{|c|c|c|c|c|}
\hline Material & $\begin{array}{c}\text { Material } \\
\text { ID }\end{array}$ & $\begin{array}{l}\text { Group structure } \\
\text { for which } \\
\text { weights } \\
\text { are available }\end{array}$ & $\begin{array}{l}\text { Increment }^{a} \\
\text { thickness } \\
(\mathrm{cm})\end{array}$ & $\begin{array}{c}\text { Total } \\
\text { number of } \\
\text { increments } \\
\text { available }\end{array}$ \\
\hline \multirow[t]{6}{*}{ Concrete } & \multirow[t]{6}{*}{301} & 27 & 5 & 20 \\
\hline & & 28 & 5 & 20 \\
\hline & & 56 & 5 & 20 \\
\hline & & 200 & 5 & 20 \\
\hline & & 238 & 5 & 20 \\
\hline & & 252 & 5 & 20 \\
\hline \multirow[t]{6}{*}{ Paraffin } & \multirow[t]{6}{*}{400} & 27 & 3 & 10 \\
\hline & & 28 & 3 & 10 \\
\hline & & 56 & 3 & 10 \\
\hline & & 200 & 3 & 10 \\
\hline & & 238 & 3 & 10 \\
\hline & & 252 & 3 & 10 \\
\hline \multirow[t]{6}{*}{ Water } & \multirow[t]{6}{*}{500} & 27 & 3 & 10 \\
\hline & & 28 & 3 & 10 \\
\hline & & 56 & 3 & 10 \\
\hline & & 200 & 3 & 10 \\
\hline & & 238 & 3 & 10 \\
\hline & & 252 & 3 & 10 \\
\hline \multirow[t]{6}{*}{ Graphite } & \multirow[t]{6}{*}{6100} & 27 & 20 & 10 \\
\hline & & 28 & 20 & 10 \\
\hline & & 56 & 20 & 10 \\
\hline & & 200 & 20 & 10 \\
\hline & & 238 & 20 & 10 \\
\hline & & 252 & 20 & 10 \\
\hline
\end{tabular}

${ }^{a}$ Group-dependent weight averages are supplied for each increment of the specified incremental thickness (i.e., for any given material) the first $n g p$ (number of energy groups) weights apply to the first increment of the thickness specified in Table 8.1.20, the next $n g p$ weights apply to the next increment of that thickness, etc. CAUTION--If bias IDs defined in the weighting information data are used in the geometry, the region thickness should be consistent with the incremental thickness of the weighting data in order to avoid overbiasing or underbiasing.

WARNING: The user should thoroughly understand weighted tracking before attempting to generate and use auxiliary data for biasing. Incorrect weighting can cause the code to produce incorrect results without obvious symptoms.

\section{CAUTIONS:}


1. Each set of AUXILIARY or CORRELATION data must be completely described in conjunction with its keyword. Complete sets of these data can be interspersed in an arbitrary order but data within each set must be entered in the specified order.

2. AUXILIARY DATA: If the same $m$ is specified in more than one set of data, the last set having the group structure used in the problem is the set that will be utilized. When AUXILIARY DATA are entered, CORRELATION DATA must also be entered in order to use the AUXILIARY DATA.

3. CORRELATION DATA: If biasing data define the same bias ID (from the geometry data) more than once, the value that is entered last supersedes previous entries. Be well aware that multiple definitions for the same bias ID can cause erroneous answers due to overbiasing.

4. Bias data may not be used in continuous energy mode.

Examples:

1. Use the first form of the BIAS block to utilize the water biasing factors in bias IDs 2 through 11 . From Table 8.1.20, water has material ID $\mathrm{m}=500$ and has bias parameters for 10 intervals that are each $3 \mathrm{~cm}$ thick.

\section{READ BIAS ID $=500211 \quad$ END BIAS}

2. Use the second form of the BIAS block to specify biasing factors for SPECIALWATER to be used in bias IDs 6 and 7. The SPECIALWATER biasing factors have a value of 0.69 for BIAS ID 6 and 0.86 for bias ID 7 in each energy group. Sixteen-group cross sections are being used. Each weighting region is $3.048 \mathrm{~cm}$ thick. The material ID is arbitrarily chosen to be 510 . Note that the first form of the BIAS block must be entered to allow the second form of the BIAS block to be used for BIAS IDs 6 and 7.

READ BIAS WT=SPECIALWATER $510103.048 \quad 2 \quad 16 \quad 16^{*} 0.69 \quad 16^{*} 0.86 \quad$ ID=510 67 END BIAS

3. An example of multiple definitions for the same bias ID follows:

\section{READ BIAS ID=400 27 ID=500 57 END BIAS .}

The data for paraffin (ID=400) will be used for bias IDs 2, 3, and 4, and the data for water $(\mathbf{I D}=500)$ will be used for bias IDs 5, 6, and 7. The paraffin data for bias IDs 5, 6, and 7 have been overwritten by water data.

Multiple definitions for the same bias ID are not necessarily incorrect, but the user should be cautious about using multiple definitions and should ensure that the desired biasing or weighting functions are used in the desired geometry regions.

4. An example of how the bias ID relates to the energy-dependent values of weights is given below.

Assume that a paraffin reflector is to be used, and it is desirable to use the weighting function from the KENO weighting library to minimize the running time for the problem. Also assume that these weighting functions are to be used in the volumes defined in the geometry records having the bias ID (defined on a shape or MEDIA card for KENO V.a and KENO-VI, respectively) equal to 6, 7, 8, and 9. Correlation data are then entered and auxiliary data will not be entered. 
The biasing data would be:

\section{READ BIAS ID=400 69 END BIAS.}

The results of these data are

(1) the group-dependent weights for the $0-3 \mathrm{~cm}$ interval of paraffin will be used in the volume defined by the geometry region having bias $I D=6$.

(2) the group-dependent weights for the 3-6 cm interval of paraffin will be used in the volume defined by the geometry region having bias $I D=7$.

(3) the group-dependent weights for the 6-9 $\mathrm{cm}$ interval of paraffin will be used in the volume defined by the geometry region having bias $I D=8$.

(4) the group-dependent weights for the 9-12 cm interval of paraffin will be used in the volume defined by the geometry region having bias $I D=9$.

\subsubsection{Start data}

Special start options are available for controlling the initial neutron distribution. The default starting distribution for an array is flat over the overall array dimensions, in fissile material only. The default starting distribution for a single unit is flat over the system, in fissile material only. See Table 8.1.21 for the starting distributions available in KENO. The syntax for the START block is:

\section{READ START $p_{1} \ldots p_{N}$ END START}

$p_{1} \ldots p_{N} \quad$ are $N$ initializations for the parameters listed below.

The starting information that can be entered is given below. Enter only the data necessary to describe the desired starting distribution.

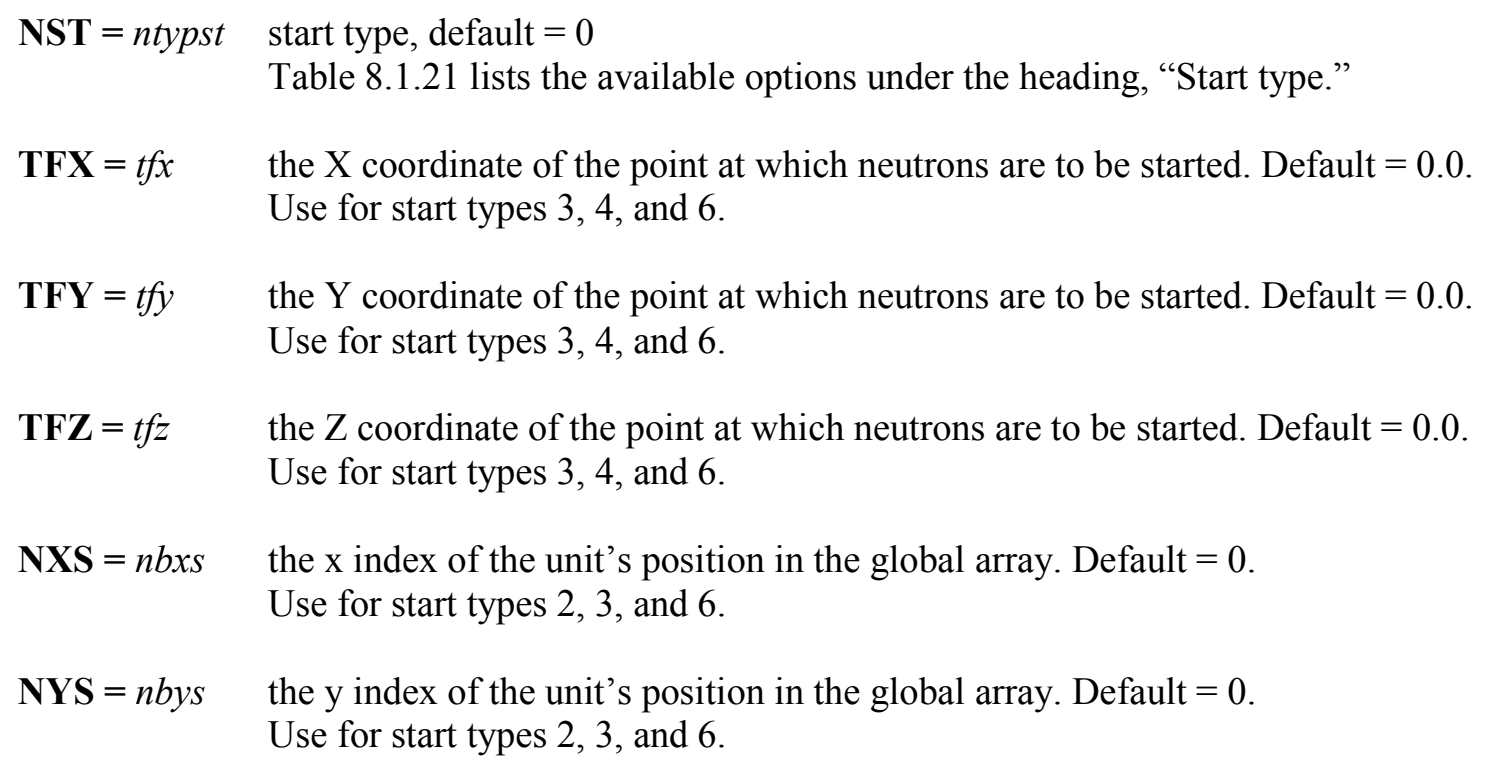

$\mathbf{T F Z}=t f z \quad$ the $\mathrm{Z}$ coordinate of the point at which neutrons are to be started. Default $=0.0$. Use for start types 3,4 , and 6 .

$\mathbf{N X S}=$ nbxs $\quad$ the $\mathrm{x}$ index of the unit's position in the global array. Default $=0$.

Use for start types 2,3 , and 6 .

NYS $=$ nbys $\quad$ the $y$ index of the unit's position in the global array. Default $=0$.

Use for start types 2,3 , and 6 . 
$\mathbf{N Z S}=n b z s \quad$ the $\mathrm{z}$ index of the unit's position in the global array. Default $=0$.

Use for start types 2,3 , and 6 .

$\mathbf{K F S}=k f i s \quad$ the mixture whose fission spectrum is to be used for starting neutrons that are not in a fissionable medium. Defaulted to the fissionable mixture having the smallest mixture number. Available for start types 3, 4, and 6.

$\mathbf{L N U}=$ lfin $\quad$ the final neutron to be started at a point. Default $=0$. Each $l$ fin should be greater than zero and less than or equal to NPG. Each successive lfin should be greater than the previous one. Use for start types 6 and 8.

$\mathbf{N B X}=$ nboxst the unit in which neutrons will be started. Default $=0$.

Use for start types 4 and 5.

$\mathbf{F C T}=$ fract $\quad$ the fraction of neutrons that will be started as a spike. Default $=0$.

Use for start type 2 .

$\mathbf{X S M}=x s m \quad$ the $-\mathrm{X}$ dimension of the cuboid in which the neutron will be started. For an array problem, XSM is defaulted to the minimum $X$ coordinate of the global array. If the reflector key RFL is YES, then and the outer reflector region is a cube or cuboid, XSM is defaulted to the minimum X coordinate of the outer reflector region. If RFL is YES and the outer region of the reflector is not a cube or cuboid, then XSM must be entered in the start data and must fit inside the outer reflector region.

Available for start types $0,1,2$, and 8 .

$\mathbf{X S P}=x s p \quad$ the $+\mathrm{X}$ dimension of the cuboid in which the neutrons will be started. For an array problem, XSP is defaulted to the maximum X coordinate of the global array. If the reflector key RFL is YES, then and the outer reflector region is a cube or cuboid, XSP is defaulted to the maximum X coordinate of the outer reflector region. If RFL is YES and the outer region of the reflector is not a cube or cuboid, then XSP must be entered in the data and must fit inside the outer reflector region.

Available for start types $0,1,2$, and 8 .

$\mathbf{Y S M}=y s m$ the $-\mathrm{Y}$ dimension of the cuboid in which the neutron will be started. For an array problem, YSM is defaulted to the minimum Y coordinate of the global array. If the reflector key RFL is YES, then YSM is defaulted to the minimum Y coordinate of the outer reflector region, provided that region is a cube or cuboid. If RFL is YES and the outer region of the reflector is not a cube or cuboid, then YSM must be entered in the start data and must fit inside the outer reflector region.

Available for start types $0,1,2$, and 8 .

$\mathbf{Y S P}=y s p \quad$ the $+\mathrm{Y}$ dimension of the cuboid in which the neutrons will be started. For an array problem, YSP is defaulted to the maximum Y coordinate of the global array. If the reflector key RFL is YES, then YSP is defaulted to the maximum Y coordinate of the outer reflector region, provided that region is a cube or cuboid. If RFL is YES and the outer region of the reflector is not a cube or cuboid, then YSP must be entered in the start data and must fit inside the outer reflector region.

Available for start types $0,1,2$, and 8 . 
$\mathbf{Z S M}=z s m$ the $-\mathrm{Z}$ dimension of the cuboid in which the neutrons will be started. For an array problem, ZSM is defaulted to the minimum $Z$ coordinate of the global array. If the reflector key RFL is YES, then ZSM is defaulted to the minimum $Z$ coordinate of the outer reflector region, provided that region is a cube or cuboid. If RFL is YES and the outer region of the reflector is not a cube or cuboid, then ZSM must be entered in the start data and must fit inside the outer reflector region.

Available for start types $0,1,2$, and 8 .

$\mathbf{Z S P}=z s p \quad$ the $+Z$ dimension of the cuboid in which the neutrons will be started. For an array problem, ZSP is defaulted to the maximum $\mathrm{Z}$ coordinate of the global array. If the reflector key RFL is YES, then ZSP is defaulted to the maximum $Z$ coordinate of the outer reflector region, provided that region is a cube or cuboid. If RFL is YES and the outer region of the reflector is not a cube or cuboid, then ZSP must be entered in the start data and must fit inside the outer reflector region.

Available for start types $0,1,2$, and 8 .

$\mathbf{R F L}=$ rflkey the reflector key. If the reflector key is YES, then neutrons can be started in the reflector. If it is NO, then all the neutrons will be started in the array. Enter YES or NO. Default = NO.

Available for start types 0,1 , and 2 .

PS6 $=$ lprt6 the key for printing start type 6 input data. If the key is YES, then start type 6 data are printed. If it is NO, then start type 6 data are not printed. Enter YES or NO. Default $=$ NO.

Available for start type 6 .

$\mathbf{P S P}=$ lpstp $\quad$ the key for printing the neutron starting points using the tracking format. If the key is YES, then print the neutron starting points. If it is NO, then do not print the starting points. Enter YES or NO. Default $=$ NO.

Available for all start types.

$\mathbf{R D U}=r d u \quad$ the file from which ASCII start data are to be read for start type 6 .

WS6 = ws6 the file to which ASCII start data are written.

MSS = filename.msl the file from which ASCII start data are to be read. filename may include a valid pathname. Available for start type 9. 
Table 8.1.21. Starting distributions available in KENO

\begin{tabular}{|c|c|c|c|}
\hline $\begin{array}{l}\text { Start } \\
\text { type }\end{array}$ & $\begin{array}{l}\text { Required } \\
\text { data }\end{array}$ & $\begin{array}{l}\text { Optional } \\
\text { data }\end{array}$ & Starting distribution \\
\hline 0 & None & $\begin{array}{c}\text { NST } \\
\text { XSM } \\
\text { XSP } \\
\text { YSM } \\
\text { YSP } \\
\text { ZSM } \\
\text { ZSP } \\
\text { RFL } \\
\text { PSP }\end{array}$ & $\begin{array}{l}\text { Uniform throughout fissile material within the volume defined by } \\
\text { (1) the outer region of a single unit, (2) the outer region of a } \\
\text { reflected array having the reflector key set true, ( } 3 \text { ) the boundary } \\
\text { of the global array, or (4) a cuboid specified by XSM, XSP, } \\
\text { YSM, YSP, ZSM, and ZSP. }\end{array}$ \\
\hline 1 & NST & $\begin{array}{c}\text { XSM } \\
\text { XSP } \\
\text { YSM } \\
\text { YSP } \\
\text { ZSM } \\
\text { ZSP } \\
\text { RFL } \\
\text { PSP } \\
\end{array}$ & $\begin{array}{l}\text { The starting points are chosen according to a cosine distribution } \\
\text { throughout the volume of a cuboid defined by XSM, XSP, YSM, } \\
\text { YSP, ZSM, and ZSP. Points that are not in fissile material are } \\
\text { discarded. }\end{array}$ \\
\hline 2 & $\begin{array}{l}\text { NST } \\
\text { NXS } \\
\text { NYS } \\
\text { NZS } \\
\text { FCT }\end{array}$ & $\begin{array}{c}\text { XSM } \\
\text { XSP } \\
\text { YSM } \\
\text { YSP } \\
\text { ZSM } \\
\text { ZSP } \\
\text { RFL } \\
\text { PSP } \\
\end{array}$ & $\begin{array}{l}\text { An arbitrary fraction (FCT) of neutrons are started uniformly in } \\
\text { the unit located at position NXS, NYS, NZS in the global array. } \\
\text { The remainder of the neutrons is started in fissile material, from } \\
\text { points chosen from a cosine distribution throughout the volume } \\
\text { of a cuboid defined by XSM, XSP, YSM, YSP, ZSM, ZSP. }\end{array}$ \\
\hline 3 & $\begin{array}{c}\text { NST } \\
\text { TFX } \\
\text { TFY } \\
\text { TFZ } \\
\text { NXS } \\
\text { NYS } \\
\text { NZS } \\
\end{array}$ & $\begin{array}{l}\text { KFS } \\
\text { PSP }\end{array}$ & $\begin{array}{l}\text { All neutrons are started at position TFX, TFY, TFZ within the } \\
\text { unit located at position NXS, NYS, NZS in the global array. }\end{array}$ \\
\hline 4 & $\begin{array}{l}\text { NST } \\
\text { TFX } \\
\text { TFY } \\
\text { TFZ } \\
\text { NBX }\end{array}$ & $\begin{array}{l}\text { KFS } \\
\text { PSP }\end{array}$ & $\begin{array}{l}\text { All neutrons are started at position TFX, TFY, TFZ within units } \\
\text { NBX in the global array. }\end{array}$ \\
\hline
\end{tabular}


Table 8.1.21. Starting distributions available in KENO (continued)

\begin{tabular}{|c|c|c|c|}
\hline $\begin{array}{l}\text { Start } \\
\text { type }\end{array}$ & $\begin{array}{l}\text { Required } \\
\text { data }\end{array}$ & $\begin{array}{l}\text { Optional } \\
\text { data }\end{array}$ & Starting distribution \\
\hline 5 & $\begin{array}{l}\text { NST } \\
\text { NBX }\end{array}$ & PSP & $\begin{array}{l}\text { Neutrons are started uniformly in fissile material in units NBX in } \\
\text { the global array. }\end{array}$ \\
\hline 6 & $\begin{array}{l}\text { NST } \\
\text { TFX } \\
\text { TFY } \\
\text { TFZ } \\
\text { LNU }^{a}\end{array}$ & $\begin{array}{l}\text { NXS } \\
\text { NYS } \\
\text { NZS } \\
\text { KFS } \\
\text { PS6 } \\
\text { PSP } \\
\text { RDU }\end{array}$ & $\begin{array}{l}\text { The starting distribution is arbitrarily input. LNU is the final } \\
\text { neutron to be started at a point TFX, TFY, TFZ relative to the } \\
\text { global coordinate system or at a point TFX, TFY, TFZ, relative } \\
\text { to the unit located at the global array position NXS, NYS, NZS. }\end{array}$ \\
\hline 7 & & $\begin{array}{c}\text { XSM } \\
\text { XSP } \\
\text { YSM } \\
\text { YSP } \\
\text { ZSM } \\
\text { ZSP }\end{array}$ & $\begin{array}{l}\text { The starting points are chosen according to a flat distribution in } \\
\text { the X-and Y-dimensions and a }(1.0-\cos (\mathrm{z}))^{2} \text { distribution in the } \\
\text { Z-dimension throughout the volume of a cuboid defined by } \\
\text { XSM, XSP, YSM, YSP, ZSM, and ZSP. Points that are not in } \\
\text { fissile material are discarded. }\end{array}$ \\
\hline 8 & $\begin{array}{l}\text { NST } \\
\text { ZSM } \\
\text { ZSP } \\
\text { FCT }\end{array}$ & $\begin{array}{l}\text { XSM } \\
\text { XSP } \\
\text { YSM } \\
\text { YSP }\end{array}$ & $\begin{array}{l}\text { Neutrons are started with flat distribution in } \mathrm{X} \text { and } \mathrm{Y} \text {, and a } \\
\text { segmented distribution in Z, with the X-Y limits defined by } \\
\text { XSM, XSP, YSM, YSP and the relative fraction in ZSP-ZSM } \\
\text { defined by FCT. FCT must be the last thing entered for each } \\
\text { segment. }\end{array}$ \\
\hline 9 & $\begin{array}{l}\text { NST } \\
\text { MSS }\end{array}$ & & $\begin{array}{l}\text { Mesh source from Sourcerer. The starting distribution is read } \\
\text { from a previously created mesh source file declared with } \\
\text { MSS=filename.msl, where filename may include a valid } \\
\text { pathname. See Sourcerer section of SCALE manual for more } \\
\text { details. }\end{array}$ \\
\hline \multicolumn{4}{|c|}{$\begin{array}{l}\text { When entering data for start } 6 \text {, LNU must be the last entry for each set of data and the LNU in each } \\
\text { successive set of data must be larger than the previous value of LNU. A set of data consists of required and } \\
\text { optional data. The last LNU entered should be equal to the number per generation (parameter NPG= in the } \\
\text { parameter input, Sect. 8.1.2.3). }\end{array}$} \\
\hline
\end{tabular}

\subsubsection{Extra 1-D XSECS IDs data}

Extra 1-D cross section IDs are not required. They are allowed as input in order to simplify future modifications to calculate reaction rates, etc., as well as for compatibility with other SCALE codes. The syntax for the extra 1-D cross section data block is:

\section{READ X1DS NEUTRON $i_{1} \ldots i_{x l d} \quad$ END X1DS}

NEUTRON is a keyword to indicate that the following ID identifies a neutron interaction.

$i_{1} \ldots i_{x I d} \quad$ X1D 1-D identification numbers or keyword identifiers for the 1-D cross section to be used. These cross sections must be available on the mixture cross section library. X1D entries are expected to be read (see integer PARAMETER data). 


\subsubsection{Mixing table data}

A cross section mixing table must be entered if KENO is being run stand alone and a Monte Carlo cross section format library is not being used in the multigroup mode, or KENO is being run stand alone in the continuous energy mode. If the parameter $\mathbf{L I B}=($ Sect. 8.1.2.3) is entered, then mixing table data must be entered. A cross section mixing table is entered using the following syntax:

\section{READ MIXT $p_{1} \ldots p_{N}$ END MIXT}

$p_{1} \ldots p_{N} \quad$ are $N$ parameters that might or might not be keyworded.

The possible parameters that can be used in a MIXT block are described below.

SCT $=n s c t \quad$ is used to input the number of scattering angles and only applies in multigroup mode. $n s c t$ is the number of discrete scattering angles, default $=$ 1. The number of scattering angles specifies the number of discrete scattering angles to be used for the cross sections. If SCT is not set (i.e., SCT $=-1$ ), then the number of scattering angles is determined from the cross section library specified. The number of scattering angles defaults to (ncoef +1$) / 2$, where ncoef is the largest Legendre polynomial order used in the problem. It needs to be entered only once for a problem. If more than one value is entered, the last one is used for the problem. For assistance in determining the number of discrete scattering angles for the cross sections, see Sect. 8.1.3.4.3.

$\mathbf{E P S}=p b x s \quad$ is used to enter the cross section message cutoff value, and it only applies in multigroup mode. pbxs is the value of the $\mathrm{P}_{0}$ cross section for each transfer, above which generated warning messages will be printed, default $=3 \times 10^{-5}$. The primary purpose of entering this cutoff value is to suppress printing these messages when they are generated during cross section processing. For assistance in determining a value for EPS, see Sect. 8.1.3.4.4.

MIX $=\operatorname{mix}$

$\mathbf{N C M}=n c m x$

is used to input the identification number of the mixture being described. mix defines the mixture being described.

is used to input the nuclide mixture IDs to be used for this mixture. ncmx defines the nuclide mixture ID. When MIX=mix is read, ncmx is defaulted to mix also. Then, as long as all the nuclides that need to be mixed into mix already have mix specified as their nuclide mixture (frequently the case when using SCALE), the user does not need to specify NCM. The most usual case where NCM must be specified is when the mixtures were specified as a different mixture number when they were created in SCALE as compared to the mixture number used for them in KENO. Cell homogenized mixtures also need NCM specified.

TMP $\mid$ TEM = temperature is used to input the desired temperature of the CE cross section data.

nucl

is the nuclide ID number from the AMPX working format cross section library. 
The sequence "nucl $\mathbf{N C M}=n c m x$ [XS=fname] dens" may be repeated until the mixture defined by MIX=mix has been completely described.

The sequence "MIX = mix $\mathbf{N C M}=$ ncm $x$ TMP $\mid \mathbf{T E M}=$ temperature nucl $\mathbf{N C M}=$ ncmx $[\mathbf{X S}=$ fname $]$ dens" may be repeated until all the mixtures have been described.

NOTE: If a given nuclide ID is entered more than once in the same mixture, then the number densities for that nuclide are summed.

If a mixture number is used as a nuclide ID, then it is treated as a nuclide and the number density associated with it is used as a density modifier. (If the density is entered as 1 , then the mixture is mixed in at full density. If it is entered as 0.5 , the mixture is mixed in at one half of its full density.) A Monte Carlo formatted cross section library is generated on the unit defined by the parameter $\mathbf{X S C}=$. If this data set is saved, subsequent cases can utilize these mixtures without remixing.

The entry $\mathbf{X S}=$ fname is optional. If a nuclide is entered more than once in a mixture and this entry is specified, then they must be the same (i.e., cannot use more than one continuous energy cross section sets for a nuclide in a given mixture). Different mixtures may have the same nuclide with different continuous energy cross section sets.

\subsubsection{Plot data}

Plots of slices specified through the geometry can be generated and displayed (1) as character plots using alphanumeric characters to represent mixture numbers, unit numbers or bias ID numbers or (2) as color plots which generate a PNG file using colors to represent mixture numbers, unit numbers or bias ID numbers. Color plots require an independent program to display the PNG file to a PC or workstation monitor or to convert the file to be displayed using a plotting device. The keyword $\mathbf{S C R}=$ is used to control the plot display method. SCR=YES, the default value, uses the color plot display method. $\mathbf{S C R}=$ NO uses the character plot display method. The value of SCR determines the plot display method for all the plots specified in a problem. If $\mathbf{S C R}=$ is entered more than once, the last entry determines the plot display method. In other words, all plots generated by a problem will be either character plots or color plots.

The plot data can include the data for any or all types of plots. A plot by mixture number is the default. The kind of plot is defined by the parameter $\mathbf{P I C}=$. Character plots are printed after the volumes are printed and before the final preparations for tracking are completed. Plot data are not required for a problem, but theyb can be used to verify the problem description. The actual plotting of the picture can be suppressed by entering PLT $=$ NO in the parameter data or plot data. This allows plot data to be kept in the problem input for reference purposes without actually plotting the picture(s). Entering a value for PLT in the plot data will override any value entered in the parameter data. However, if a problem is restarted, the value of PLT from the parameter data is used. The upper left and lower right coordinates of the plot must be specified relative to the origin of the problem. See Sect. 8.1.3.9 for a discussion of plot origins and plot data. 
Enter the plot data using the following syntax:

\section{READ PLOT $p_{1} \ldots p_{N}$ END PLOT}

$p_{1} \ldots p_{N}$

$\mathbf{P I C}=w r d$

MAT

MIX[T[URE]]

MEDI[A]

UNT

UNIT[TYPE]

IMP

BIAS[ID]

WTS

WEIG[HTS]

WGT[S]

TYP=

Plot coordinates

Upper left coordinates are $N$ parameters entered using keywords followed by the appropriate data. The plot title and the plot character string must be contained within delimiters. Enter as many picture parameters as necessary to describe the plot. Multiple sets of plot data can be entered. The parameter input for each plot is terminated by a labeled or unlabeled END. The labeled END cannot use the word PLOT as the first four characters of the label. For example, END PLT1 is a valid label, but END PLOT1 is not. If an unlabeled END is used, it cannot start in column 1.

The possible parameters that can be used in a PLOT block are described below.

Enter a one-character delimiter delim to signal the beginning of the title (132 characters maximum). The title is terminated when delim is encountered the second time. Acceptable delimiters include “, ', *, , , or!.

Default $=$ title of the KENO case.

The plot type, wrd, is followed by one or more blanks and must be one of the keywords listed below. The plot type is initialized to MAT; the default is the value from the previous plot.

These keywords will cause the plot to represent the mixture numbers used in the specified geometry slice.

These keywords will cause the plot to represent the units used in the specified geometry slice. In the legend of the color plot, the material number actually refers to the units.

These keywords will cause the plot to represent the bias ID numbers used in the specified geometry slice. In the legend of the color plot, the material number actually refers to the bias ID numbers.

Enter the type desired.

$X Y$ for an X-Y plot

$\mathrm{XZ}$ for an $\mathrm{X}-\mathrm{Z}$ plot

YZ for a Y-Z plot

Direction cosines do not need to be entered if TYP is entered.

Enter values for the upper left and lower right coordinates of the plot as described below. Data must be entered for all nonzero coordinates unless all six values from the previous plot are to be used.

Enter the $\mathrm{X}, \mathrm{Y}$, and $\mathrm{Z}$ coordinates of the upper left-hand corner of the plot. 
$\mathbf{X U L}=x u l$

$\mathbf{Y U L}=y u l$

$\mathbf{Z U L}=z u l$

Lower right coordinates

$\mathbf{X L R}=x l r$

$\mathbf{Y L R}=y l r$

$\mathbf{Z L R}=z l r$

Direction cosines across the plot

Direction cosines down the plot is used to enter the X coordinate of the upper left-hand corner of the plot. Default $=$ value from previous plot; initialized to zero if any other coordinates are entered.

is used to enter the Y coordinate of the upper left-hand corner of the plot. Default $=$ value from previous plot; initialized to zero if any other coordinates are entered.

is used to enter the $\mathrm{Z}$ coordinate of the upper left-hand corner of the plot. Default $=$ value from previous plot; initialized to zero if any other coordinates are entered.

Enter the $\mathrm{X}, \mathrm{Y}$, and $\mathrm{Z}$ coordinates of the lower right-hand corner of the plot.

is used to enter the $\mathrm{X}$ coordinate of the lower right-hand corner of the plot. Default $=$ value from previous plot; initialized to zero if any other coordinates are entered.

is used to enter the Y coordinate of the lower right-hand corner of the plot. Default $=$ value from previous plot; initialized to zero if any other coordinates are entered.

is used to enter the $\mathrm{Z}$ coordinate of the lower right-hand corner of the plot. Default $=$ value from previous plot; initialized to zero if any other coordinates are entered.

Enter direction numbers proportional to the direction cosines for the AX axis of the plot. The AX axis is from left to right across the plot. If any one of the AX direction cosines is entered, the other two are set to zero. The direction cosines are normalized by the code.

$\mathbf{U A X}=$ uax is used to enter the $\mathrm{X}$ component of the direction cosines for the $\mathrm{AX}$ axis of the plot. Default $=$ value from previous plot; initialized to zero if any other direction cosines are entered.

$\mathbf{V A X}=\operatorname{vax}$ is used to enter the $\mathrm{Y}$ component of the direction cosines for the $\mathrm{AX}$ axis of the plot. Default $=$ value from previous plot; initialized to zero if any other direction cosines are entered.

$\mathbf{W A X}=$ wax is used to enter the $\mathrm{Z}$ component of the direction cosines for the AX axis of the plot. Default $=$ value from previous plot; initialized to zero if any other direction cosines are entered.

Enter direction numbers proportional to the direction cosines for the DN axis of the plot. The DN axis is from top to bottom down the plot. If any one of the DN direction cosines is entered, the other two are set to zero. The direction cosines are normalized by the code. 
Scaling parameters

$\mathbf{D L X}=d l x$

$\mathbf{D L D}=d l d$

$\mathbf{N A X}=\operatorname{nax}$

$\mathbf{N D N}=n d n$

Global scaling parameter

$\mathbf{L P I}=l p i$
$\mathbf{U D N}=u d n$ is used to enter the $\mathrm{X}$ component of the direction cosines for the $\mathrm{DN}$ axis of the plot. Default = value from previous plot; initialized to zero if any other direction cosines are entered.

$\mathrm{VDN}=v d n$ is used to enter the $\mathrm{Y}$ component of the direction cosines for the $\mathrm{DN}$ axis of the plot. Default $=$ value from previous plot; initialized to zero if any other direction cosines are entered.

$\mathbf{W D N}=w d n$ is used to enter the $\mathrm{Z}$ component of the direction cosines for the $\mathrm{DN}$ axis of the plot. Default $=$ value from previous plot; initialized to zero if any other direction cosines are entered.

Enter one or more scaling parameters to define the size of the plot.

NOTE: If any of the scaling parameters are entered for a plot, the value of those that were not entered is recalculated. If none of the scaling parameters are specified for a plot, the values from the previous plot are used.

is used to input the horizontal spacing between points on the plot. Default $=$ value from previous plot; initialized to zero if NAX or NDN is entered.

is used to input the vertical spacing between points on the plot. Default $=$ value from previous plot; initialized to zero if NAX or NDN is entered.

NOTE: If either DLX or DLD is entered, the code will calculate the value of the other. If both are entered, the plot may be distorted.

is used to input the number of intervals to be printed across the plot. Default $=$ value from previous plot; initialized to zero if DLX or DLD is entered.

is used to input the number of intervals to be printed down the plot. Default $=$ value from previous plot; initialized to zero if DLX or DLD is entered.

is used to input a scaling factor used to control the horizontal to vertical proportionality of a plot or plots. SCALE 4.3 and later versions allow lpi to be input as a floating point number. For an undistorted character plot, lpi should be specified as the number of characters down the page that occupy the same distance as ten characters across the page. For an undistorted color plot, lpi should be entered as ten times the ratio of the vertical pixel dimension to the horizontal pixel dimension. The default value of lpi is 8.0 for a character plot and 10.0 for a color plot. $l p i=10$ will usually display an undistorted color plot. 
Miscellaneous

parameters

$\mathbf{R U N}=$ run

$\mathbf{P L T}=p l t$

$\mathbf{S C R}=s r c$

$\mathbf{N C H}=$ delim char delim
The value entered for lpi applies to all plot data following it until a new value of lpi is specified.

NOTE: Plot data must include the specification of the upper left corner of the plot and the direction cosines across and down the plot.

Additional data required to generate a plot are one of the following combinations:

1. the lower right corner of the plot, the global scaling parameter, LPI, and one of the scaling parameters (DLX, DLD, NAX, NDN).

2. the lower right corner of the plot, one of the scaling parameters related to the horizontal specifications of the plot (DLX or NAX), and one of the scaling parameters related to the vertical specification of the plot (DLD or NDN). LPI, even if specified will not be used.

3. NAX and NDN and any two of LPI, DLX, and DLD. If LPI, DLX, and DLD are all specified, LPI is not used.

The data required to generate a plot may be supplied from (1) defaulted values, (2) data from the previous plot, or (3) data that are specifically entered for the current plot.

Enter miscellaneous parameters

is used to determine if the problem is executed or is terminated after data checking. A value of YES for run means the problem will be executed if all the data were acceptable. A value of NO specifies the problem will be terminated after data checking is completed. The default value of RUN is YES.

is used to specify if a plot is to be made. A value of YES for plt specifies that a plot is to be made. If plot data are entered, PLT is defaulted to YES.

NOTE: The parameters RUN and PLT can also be entered in the PARAMETER data. See Sect. 8.1.2.3. It is recommended that these parameters be entered only in the parameter data block in order to ensure that the data printed in the "Logical Parameters" table are what is actually performed.

This is used to determine the plot display method. The plot display method is specified by entering either YES or NO for $s r c$. The default value is YES. $\mathbf{S C R}=$ YES uses the color plot display method. $\mathbf{S C R}=\mathrm{NO}$ uses the character plot display method. If $\mathbf{S C R}$ is entered more than once in a problem, the last value entered is the one that is used.

Enter only if plots are to be made utilizing the character plot display method $(\mathbf{S C R}=\mathrm{NO})$. Enter a delimiter (i.e., ", ' , $*, \wedge$, or !) to signal the beginning of character string char. The character string is terminated when the delim character is encountered the second time. Do not use the initial delimiter in 
the char string, as it will be read as terminating the string. char is a character string with each entry representing a plottable quantity (i.e., media \{mixture\} number, unit number, or bias ID). These are the characters that will be used in the plot. The first entry represents media, unit, or bias ID zero; the second entry represents the smallest media, unit, or bias ID used in the problem; the third entry represents the next larger media, unit, or bias ID used in the problem; etc. For example, assume PIC=MAT is specified, and 15 mixtures are defined in the mixing table, and the geometry data use only mixtures 3 and 7. By default, a blank will be printed for mixture zero, a 1 will be printed for mixture 3 , and a 2 will be printed for mixture 7 . If you wish to print a zero for a void (mixture 0), a 3 for mixture 3, and a 7 for mixture 7, enter $\mathrm{NCH}={ }^{\circ} 037$ '.

The default values of $C H A R$ are the following:

$\begin{array}{lllllllllllllllll}\text { Quantity } & 0 & 1 & 2 & 3 & 4 & 5 & 6 & 7 & 8 & 9 & 10 & 11 & 12 & 13 & 14 & 15 \\ \text { SYMBOL } & & 1 & 2 & 3 & 4 & 5 & 6 & 7 & 8 & 9 & \mathrm{~A} & \mathrm{~B} & \mathrm{C} & \mathrm{D} & \mathrm{E} & \mathrm{F} \\ & & & & & & & & & & & & & & & & \\ \text { Quantity } & 16 & 17 & 18 & 19 & 20 & 21 & 22 & 23 & 24 & 25 & 26 & 27 & 28 & 29 & 30 & 31 \\ \text { SYMBOL } & \mathrm{G} & \mathrm{H} & \mathrm{I} & \mathrm{J} & \mathrm{K} & \mathrm{L} & \mathrm{M} & \mathrm{N} & \mathrm{O} & \mathrm{P} & \mathrm{Q} & \mathrm{R} & \mathrm{S} & \mathrm{T} & \mathrm{U} & \mathrm{V} \\ & & & & & & & & & & & & & & & & \\ \text { Quantity } & 32 & 33 & 34 & 35 & 36 & 37 & 38 & 39 & 40 & 41 & 42 & 43 & 44 & 45 & 46 & \\ \text { SYMBOL } & \mathrm{W} & \mathrm{X} & \mathrm{Y} & \mathrm{Z} & \# & , & \$ & - & + & ) & \mid & \& & > & : & ; & \\ & & & & & & & & & & & & & & & & \\ \text { Quantity } & 47 & 48 & 49 & 50 & 51 & 52 & 53 & 54 & 55 & 56 & 57 & 58 & & & & \\ \text { SYMBOL } & \cdot & - & \% & * & \cdots & = & ! & ( & 0 & < & / & 0 & & & & \end{array}$

$\mathbf{C L R}=n_{1} r\left(n_{l}\right) g\left(n_{l}\right) b\left(n_{1}\right) \ldots n_{N} r\left(n_{N}\right) g\left(n_{N}\right) b\left(n_{N}\right)$ END COLOR

this entry is used to define the colors to be used by the color plot. It may be entered only if plots are to be made utilizing the color plot display method (SCR=YES). After entering the keyword $\mathbf{C L R}=, 4$ numbers are entered $N$ times. The first number, $n_{l}$, represents a media (mixture) number, unit number, or bias ID. The next three numbers, whose values can range from 0 through 255 , define the red, green, and blue components of the color that will represent this $n_{l}$ in the plot. The sequence of 4 numbers is repeated until the colors associated with all of the media (mixture) numbers, unit numbers, or bias IDs used in the problem have been defined. The smallest number that can be entered for $n_{i}$ is -1 , representing undefined regions in the plot. An $n_{i}$ of 0 represents void regions; $n_{i}$ of 1 represents the smallest media, unit, or bias ID used in the problem; $n_{i}$ of 2 represents the next larger media, unit, or bias ID used in the problem, etc. The color plot definition data are terminated by entering the keywords END COLOR. A total of 256 default colors are provided in Table 8.1.22. Two of those colors represent undefined regions, $n_{i}=-1$, as black and void regions, and $n_{i}=0$ as gray. The remaining 254 colors represent the default values for mixtures, bias IDs, or unit numbers used in the problem. If num is entered as $\mathbf{- 1}$, the next three numbers define the color that will be used to represent undefined regions of the plot. The default color for undefined regions is black, represented as 000 . If $n_{i}$ is entered as 0 , the next three numbers define the color that will represent void 
regions in the plot. The default color for void is gray, represented as 200200200 . For example, assume a color plot is to be made for a problem that uses void regions and mixture numbers 1, 3, and 5. By default, the undefined regions (Index -1) will be black; void regions (Index 0) will be gray; the first mixture, mixture 1 (Index 1), will be medium blue; the next larger mixture, mixture 3 (Index 2), will be turquoise2; and the last mixture, mixture 5 (Index 3 ), will be green2. If these values are acceptable, data do not need to be entered for $\mathbf{C L R}=$. If the user decides to define void to be white (255 255 255), mixture 1 to be red (25500), mixture 3 to be bright blue ( 00255$)$, and mixture 5 to be green $(02550)$, then the following data could be entered:

\section{CLR=0 255255255125500200255302550 END COLOR}

In this example, the first number (0) defines the void, and the next three numbers are the red, green, and blue components that combine as the color white. The fifth number (1) represents the smallest mixture number (mixture 1), and the next three numbers are the red, green, and blue components of red. The ninth number (2) represents the next larger mixture number (mixture 3), and the next three numbers are the red, green, and blue components of bright blue. The thirteenth number (3) represents the next larger mixture number (mixture 5), and the next three numbers are the red, green, and blue components of green. The END COLOR terminates the color definition data. Because color data were not entered for $n_{i}$ of -1 , undefined regions will be represented by the color black, the default specification from Table 8.1.22. The red, green, and blue components of some bright colors are listed below.

\begin{tabular}{|c|c|c|c|}
\hline Display Color & red & green & $\underline{\text { blue }}$ \\
\hline black & 0 & 0 & $\overline{0}$ \\
\hline white & 255 & 255 & 255 \\
\hline "default void gray" & 200 & 200 & 200 \\
\hline red & 255 & 0 & 0 \\
\hline green & 0 & 255 & 0 \\
\hline brightest blue & 0 & 0 & 255 \\
\hline yellow & 255 & 255 & 0 \\
\hline brightest cyan & 0 & 255 & 255 \\
\hline magenta & 255 & 0 & 255 \\
\hline
\end{tabular}

The 256 default colors are listed in Table 8.1.22.

Table 8.1.22. Default color specifications for the color plot display method

\begin{tabular}{|r|r|r|r|l|}
\hline Mixture number & \multicolumn{1}{|c|}{ Red } & \multicolumn{1}{c|}{ Green } & \multicolumn{1}{c|}{ Blue } & Color \\
\hline-1 & 0 & 0 & 0 & \\
\hline 0 & 200 & 200 & 200 & $\square$ \\
\hline 1 & 0 & 0 & 205 & $\square$ \\
\hline 2 & 0 & 229 & 238 & $\square$ \\
\hline 3 & 0 & 238 & 0 & $\square$ \\
\hline 4 & 205 & 205 & 0 & $\square$ \\
\hline 5 & 238 & 0 & 0 & $\square$ \\
\hline
\end{tabular}




\begin{tabular}{|c|c|c|c|c|}
\hline Mixture number & Red & Green & Blue & Color \\
\hline 6 & 145 & 44 & 238 & \\
\hline 7 & 150 & 150 & 150 & \\
\hline 8 & 240 & 200 & 220 & \\
\hline 9 & 0 & 191 & 255 & \\
\hline 10 & 224 & 255 & 255 & \\
\hline 11 & 0 & 255 & 127 & \\
\hline 12 & 255 & 255 & 224 & \\
\hline 13 & 255 & 0 & 0 & \\
\hline 14 & 255 & 0 & 255 & \\
\hline 15 & 67 & 110 & 238 & \\
\hline 16 & 174 & 238 & 238 & \\
\hline 17 & 180 & 238 & 180 & \\
\hline 18 & 238 & 220 & 130 & \\
\hline 19 & 238 & 99 & 99 & \\
\hline 20 & 238 & 122 & 233 & \\
\hline 21 & 25 & 25 & 112 & \\
\hline 22 & 0 & 0 & 128 & \\
\hline 23 & 100 & 149 & 237 & \\
\hline 24 & 72 & 61 & 139 & \\
\hline 25 & 106 & 90 & 205 & \\
\hline 26 & 123 & 104 & 238 & \\
\hline 27 & 30 & 144 & 255 & \\
\hline 28 & 135 & 206 & 235 & \\
\hline 29 & 135 & 206 & 250 & \\
\hline 30 & 70 & 130 & 180 & \\
\hline 31 & 176 & 196 & 222 & \\
\hline 32 & 176 & 224 & 230 & \\
\hline 33 & 0 & 206 & 209 & \\
\hline 34 & 72 & 209 & 204 & \\
\hline 35 & 95 & 158 & 160 & \\
\hline 36 & 102 & 205 & 170 & \\
\hline 37 & 127 & 255 & 212 & \\
\hline 38 & 0 & 100 & 0 & \\
\hline 39 & 85 & 107 & 47 & \\
\hline 40 & 143 & 188 & 143 & \\
\hline
\end{tabular}




\begin{tabular}{|c|c|c|c|c|}
\hline Mixture number & Red & Green & Blue & Color \\
\hline 41 & 60 & 179 & 113 & \\
\hline 42 & 32 & 178 & 170 & \\
\hline 43 & 152 & 251 & 152 & \\
\hline 44 & 0 & 255 & 0 & \\
\hline 45 & 127 & 255 & 0 & \\
\hline 46 & 0 & 250 & 154 & \\
\hline 47 & 173 & 255 & 47 & \\
\hline 48 & 50 & 205 & 50 & \\
\hline 49 & 154 & 205 & 50 & \\
\hline 50 & 34 & 139 & 34 & \\
\hline 51 & 107 & 142 & 35 & \\
\hline 52 & 189 & 183 & 107 & \\
\hline 53 & 240 & 230 & 140 & \\
\hline 54 & 238 & 232 & 170 & \\
\hline 55 & 250 & 250 & 210 & \\
\hline 56 & 255 & 255 & 224 & \\
\hline 57 & 255 & 255 & 0 & \\
\hline 58 & 255 & 215 & 0 & \\
\hline 59 & 238 & 221 & 130 & \\
\hline 60 & 184 & 134 & 11 & \\
\hline 61 & 188 & 143 & 143 & \\
\hline 62 & 205 & 92 & 92 & \\
\hline 63 & 139 & 69 & 19 & \\
\hline 64 & 160 & 82 & 45 & \\
\hline 65 & 205 & 133 & 63 & \\
\hline 66 & 222 & 184 & 135 & \\
\hline 67 & 245 & 245 & 220 & \\
\hline 68 & 245 & 222 & 179 & \\
\hline 69 & 244 & 164 & 96 & \\
\hline 70 & 210 & 105 & 30 & \\
\hline 71 & 178 & 34 & 34 & \\
\hline 72 & 165 & 42 & 42 & \\
\hline 73 & 233 & 150 & 122 & \\
\hline 74 & 250 & 128 & 114 & \\
\hline 75 & 255 & 160 & 122 & \\
\hline
\end{tabular}




\begin{tabular}{|c|c|c|c|c|}
\hline Mixture number & Red & Green & Blue & Color \\
\hline 76 & 255 & 165 & 0 & \\
\hline 77 & 255 & 140 & 0 & \\
\hline 78 & 255 & 127 & 80 & \\
\hline 79 & 240 & 128 & 128 & \\
\hline 80 & 255 & 99 & 71 & \\
\hline 81 & 255 & 69 & 0 & \\
\hline 82 & 255 & 0 & 0 & \\
\hline 83 & 255 & 105 & 180 & \\
\hline 84 & 255 & 20 & 147 & \\
\hline 85 & 255 & 192 & 203 & \\
\hline 86 & 255 & 182 & 193 & \\
\hline 87 & 219 & 112 & 147 & \\
\hline 88 & 176 & 48 & 96 & \\
\hline 89 & 199 & 21 & 133 & \\
\hline 90 & 208 & 32 & 144 & \\
\hline 91 & 238 & 130 & 238 & \\
\hline 92 & 221 & 160 & 221 & \\
\hline 93 & 218 & 112 & 214 & \\
\hline 94 & 153 & 50 & 204 & \\
\hline 95 & 148 & 0 & 211 & \\
\hline 96 & 186 & 85 & 211 & \\
\hline 97 & 138 & 43 & 226 & \\
\hline 98 & 160 & 32 & 240 & \\
\hline 99 & 147 & 112 & 219 & \\
\hline 100 & 216 & 191 & 216 & \\
\hline 101 & 238 & 233 & 233 & \\
\hline 102 & 238 & 229 & 222 & \\
\hline 103 & 238 & 223 & 204 & \\
\hline 104 & 238 & 213 & 183 & \\
\hline 105 & 238 & 203 & 173 & \\
\hline 106 & 238 & 207 & 161 & \\
\hline 107 & 238 & 233 & 191 & \\
\hline 108 & 238 & 232 & 205 & \\
\hline 109 & 238 & 238 & 224 & \\
\hline 110 & 224 & 238 & 224 & \\
\hline
\end{tabular}




\begin{tabular}{|c|c|c|c|c|}
\hline Mixture number & Red & Green & Blue & Color \\
\hline 111 & 238 & 224 & 229 & \\
\hline 112 & 238 & 213 & 210 & \\
\hline 113 & 224 & 238 & 238 & \\
\hline 114 & 122 & 103 & 238 & \\
\hline 115 & 67 & 110 & 238 & \\
\hline 116 & 0 & 0 & 238 & \\
\hline 117 & 28 & 134 & 238 & \\
\hline 118 & 92 & 172 & 238 & \\
\hline 119 & 0 & 178 & 238 & \\
\hline 120 & 126 & 192 & 238 & \\
\hline 121 & 164 & 211 & 238 & \\
\hline 122 & 185 & 211 & 238 & \\
\hline 123 & 188 & 210 & 238 & \\
\hline 124 & 178 & 223 & 238 & \\
\hline 125 & 209 & 238 & 238 & \\
\hline 126 & 174 & 238 & 238 & \\
\hline 127 & 142 & 229 & 238 & \\
\hline 128 & 0 & 238 & 238 & \\
\hline 129 & 141 & 238 & 238 & \\
\hline 130 & 118 & 238 & 198 & \\
\hline 131 & 180 & 238 & 180 & \\
\hline 132 & 78 & 238 & 148 & \\
\hline 133 & 144 & 238 & 144 & \\
\hline 134 & 0 & 238 & 118 & \\
\hline 135 & 0 & 238 & 0 & \\
\hline 136 & 118 & 238 & 0 & \\
\hline 137 & 179 & 238 & 58 & \\
\hline 138 & 188 & 238 & 104 & \\
\hline 139 & 238 & 230 & 133 & \\
\hline 140 & 238 & 220 & 130 & \\
\hline 141 & 238 & 238 & 209 & \\
\hline 142 & 238 & 238 & 0 & \\
\hline 143 & 238 & 201 & 0 & \\
\hline 144 & 238 & 180 & 34 & \\
\hline 145 & 238 & 173 & 14 & \\
\hline
\end{tabular}




\begin{tabular}{|c|c|c|c|c|}
\hline Mixture number & Red & Green & Blue & Color \\
\hline 146 & 238 & 180 & 180 & \\
\hline 147 & 238 & 99 & 99 & \\
\hline 148 & 238 & 121 & 66 & \\
\hline 149 & 238 & 197 & 145 & \\
\hline 150 & 238 & 216 & 174 & \\
\hline 151 & 238 & 154 & 73 & \\
\hline 152 & 238 & 118 & 33 & \\
\hline 153 & 238 & 44 & 44 & \\
\hline 154 & 238 & 59 & 59 & \\
\hline 155 & 238 & 130 & 98 & \\
\hline 156 & 238 & 149 & 114 & \\
\hline 157 & 238 & 154 & 0 & \\
\hline 158 & 238 & 118 & 0 & \\
\hline 159 & 238 & 106 & 80 & \\
\hline 160 & 238 & 92 & 66 & \\
\hline 161 & 238 & 64 & 0 & \\
\hline 162 & 238 & 0 & 0 & \\
\hline 163 & 238 & 18 & 137 & \\
\hline 164 & 238 & 106 & 167 & \\
\hline 165 & 238 & 169 & 184 & \\
\hline 166 & 238 & 162 & 173 & \\
\hline 167 & 238 & 121 & 159 & \\
\hline 168 & 238 & 48 & 167 & \\
\hline 169 & 238 & 58 & 140 & \\
\hline 170 & 238 & 0 & 238 & \\
\hline 171 & 238 & 122 & 233 & \\
\hline 172 & 238 & 174 & 238 & \\
\hline 173 & 209 & 95 & 238 & \\
\hline 174 & 178 & 58 & 238 & \\
\hline 175 & 145 & 44 & 238 & \\
\hline 176 & 159 & 121 & 238 & \\
\hline 177 & 238 & 210 & 238 & \\
\hline 178 & 255 & 250 & 250 & \\
\hline 179 & 139 & 137 & 137 & \\
\hline 180 & 255 & 245 & 238 & \\
\hline
\end{tabular}




\begin{tabular}{|c|c|c|c|c|}
\hline Mixture number & Red & Green & Blue & Color \\
\hline 181 & 255 & 228 & 196 & \\
\hline 182 & 255 & 218 & 185 & \\
\hline 183 & 255 & 250 & 205 & \\
\hline 184 & 255 & 248 & 220 & \\
\hline 185 & 255 & 255 & 240 & \\
\hline 186 & 240 & 255 & 240 & \\
\hline 187 & 255 & 240 & 245 & \\
\hline 188 & 255 & 228 & 225 & \\
\hline 189 & 240 & 255 & 255 & \\
\hline 190 & 131 & 111 & 255 & \\
\hline 191 & 72 & 118 & 255 & \\
\hline 192 & 30 & 144 & 255 & \\
\hline 193 & 99 & 184 & 255 & \\
\hline 194 & 0 & 191 & 255 & \\
\hline 195 & 135 & 206 & 255 & \\
\hline 196 & 176 & 226 & 255 & \\
\hline 197 & 198 & 226 & 255 & \\
\hline 198 & 202 & 225 & 255 & \\
\hline 199 & 191 & 239 & 255 & \\
\hline 200 & 224 & 255 & 255 & \\
\hline 201 & 187 & 255 & 255 & \\
\hline 202 & 152 & 245 & 255 & \\
\hline 203 & 0 & 245 & 255 & \\
\hline 204 & 151 & 255 & 255 & \\
\hline 205 & 127 & 255 & 212 & \\
\hline 206 & 193 & 255 & 193 & \\
\hline 207 & 84 & 255 & 159 & \\
\hline 208 & 154 & 255 & 154 & \\
\hline 209 & 0 & 255 & 127 & \\
\hline 210 & 127 & 255 & 0 & \\
\hline 211 & 192 & 255 & 62 & \\
\hline 212 & 202 & 255 & 112 & \\
\hline 213 & 255 & 246 & 143 & \\
\hline 214 & 255 & 236 & 139 & \\
\hline 215 & 255 & 255 & 224 & \\
\hline
\end{tabular}




\begin{tabular}{|c|c|c|c|c|}
\hline Mixture number & Red & Green & Blue & Color \\
\hline 216 & 255 & 215 & 0 & \\
\hline 217 & 255 & 193 & 37 & \\
\hline 218 & 255 & 185 & 15 & \\
\hline 219 & 255 & 193 & 193 & \\
\hline 220 & 255 & 106 & 106 & \\
\hline 221 & 255 & 130 & 71 & \\
\hline 222 & 255 & 211 & 155 & \\
\hline 223 & 255 & 231 & 186 & \\
\hline 224 & 255 & 165 & 79 & \\
\hline 225 & 255 & 127 & 36 & \\
\hline 226 & 255 & 48 & 48 & \\
\hline 227 & 255 & 64 & 64 & \\
\hline 228 & 255 & 140 & 105 & \\
\hline 229 & 255 & 160 & 122 & \\
\hline 230 & 255 & 165 & 0 & \\
\hline 231 & 255 & 127 & 0 & \\
\hline 232 & 255 & 114 & 86 & \\
\hline 233 & 255 & 99 & 71 & \\
\hline 234 & 255 & 69 & 0 & \\
\hline 235 & 255 & 20 & 147 & \\
\hline 236 & 255 & 110 & 180 & \\
\hline 237 & 255 & 181 & 197 & \\
\hline 238 & 255 & 174 & 185 & \\
\hline 239 & 255 & 130 & 171 & \\
\hline 240 & 255 & 52 & 179 & \\
\hline 241 & 255 & 62 & 150 & \\
\hline 242 & 255 & 131 & 250 & \\
\hline 243 & 255 & 187 & 255 & \\
\hline 244 & 224 & 102 & 255 & \\
\hline 245 & 191 & 62 & 255 & \\
\hline 246 & 155 & 48 & 255 & \\
\hline 247 & 171 & 130 & 255 & \\
\hline 248 & 255 & 225 & 255 & \\
\hline 249 & 139 & 0 & 139 & \\
\hline 250 & 139 & 0 & 0 & \\
\hline
\end{tabular}




\begin{tabular}{|r|r|r|r|l|}
\hline Mixture number & \multicolumn{1}{|c|}{ Red } & \multicolumn{1}{c|}{ Green } & \multicolumn{1}{l|}{ Blue } & Color \\
\hline 251 & 0 & 139 & 0 & $\square$ \\
\hline 252 & 0 & 0 & 139 & \\
\hline 253 & 0 & 139 & 139 & $\square$ \\
\hline 254 & 139 & 139 & 0 & $\square$ \\
\hline
\end{tabular}

\subsubsection{Energy group boundary data}

Upper energy group boundary data in $\mathrm{eV}$ are entered to determine the groups into which the tallies will be collected in the continuous energy mode. For $G$ groups $G+1$ entries are entered. The last entry is the lower energy boundary of the last group. The values must be in descending order. The parameter NGP is set equal to the number of entries-1. The syntax is:

\section{READ ENERGY $u_{1} \ldots u_{G} u_{G+l}$ END ENERGY}

$u_{1} \ldots u_{G} \quad$ are the upper energy limits of energy groups $1 \ldots G$, respectively.

$u_{G+1} \quad$ is the lower energy limit of energy group $G$.

\section{Example: READ ENERGY}

\section{2e7 $1 \mathrm{e} 5$ 1 $1 \mathrm{e}-5$}

\section{END ENERGY}

Defines a 3-group structure with group $1(2 \mathrm{e}+7 \mathrm{eV}$ to $1 \mathrm{e}+5 \mathrm{eV})$, group $2(1 \mathrm{e}+5 \mathrm{eV}$ to $1 \mathrm{eV})$, and group 3 $(1 \mathrm{eV}$ to $1 \mathrm{e}-5 \mathrm{eV})$ and sets $\mathbf{N G P}=3$.

Energy group boundary data are optional. Default values for the energy group boundaries in the calculations are determined as in the following order:

i. Use energy group boundaries from READ ENERGY block if specified in the input. The number of entries in the READ ENERGY block is NGP+1.

ii. If only NGP is specified (in READ PARAMETER) in the input and NGP is equal to the number of energy groups in one of the SCALE neutron cross section libraries, the energy group structure from that library will be used.

iii. If only NGP is specified (in READ PARAMETER) in the input and NGP is not equal to the number of energy groups in one of the SCALE neutron cross section libraries, NGP equal lethargy bins will be used.

iv. Use SCALE 238 group structure as default, $\mathbf{N G P}=238$.

\subsubsection{Volume data}

If volumes are needed (for calculating fission densities, fluxes, etc.), then the data necessary to determine them are entered. The syntax for this block is:

READ VOLUME $p_{1} \ldots p_{N}$ END VOLUME

$p_{1} \ldots p_{N}$ are $N$ parameters entered using keywords followed by the appropriate data.

The possible parameters that can be used in a VOLUME block are described below. 
READVOL $=v o l$

TYPE $=$ vcalc

NRAYS $=$ ntotal

BATCHES $=n l o o p$

POINTS $=$ nplp

$\mathbf{X P}=x p$
$\mathbf{X M}=x m$
$\mathbf{Y}=y p$
$\mathbf{Y} \mathbf{M}=y m$
$\mathbf{Z} \mathbf{P}=z p$
$\mathbf{Z M}=z m$

SAMPLE_DEN=sampleden

IFACE $=$ fname used to input the file name (up to 256 characters) of the file from which user-specified volumes are read. This is an optional parameter and only works for KENO-VI. The data are read in sections for each UNIT contained in the problem. First the keyword "UNIT" is read, followed by the UNIT number. For that UNIT the data for each region containing material in the order shown in the input is read as follows: the keyword "MEDIA" is read, followed by the mixture number, followed by the keyword "VOL=", followed by the total volume for that region. Regions containing ARRAYs and HOLEs are skipped. An example of the data contained in a volume file is given later in this section.

used to determine the type of volume calculation. vcalc can have the values:

NONE: (only works in KENO-VI, where it is also the default) No volume calculation, volumes are set to -1.0 (only in KENO-VI).

TRACE: A trapezoidal integration will be performed (only in KENO-VI).

RANDOM: A Monte Carlo integration will be performed.

the number of intervals used in the trapezoidal integration (default $100,000)$. Used only with TYPE=TRACE (KENO-VI).

the number of batches to be used in the Monte Carlo integration (default 500). Used only with TYPE=RANDOM

the number of points per batch used in the Monte Carlo integration (default 1000). Used only with TYPE=RANDOM

the plus $\mathrm{X}$ face of the encompassing cuboid.

the minus $X$ face of the encompassing cuboid.

the plus $Y$ face of the encompassing cuboid.

the minus $Y$ face of the encompassing cuboid.

the plus $Z$ face of the encompassing cuboid.

the minus $Z$ face of the encompassing cuboid.

the density of sampling points per $\mathrm{cm}^{3}$ per batch. Used only with TYPE=RANDOM.

the face of the enclosing cuboid where the trapezoidal integration will be performed. Enter either XFACE, YFACE, or ZFACE. KENO-VI will integrate over the face with the smallest area by default. This allows specifying a different face. Used only for TYPE=TRACE (KENO-VI).

The volume parameters include specifying the type of calculation to determine the volumes and additional parameters needed for the selected type. In KENO-VI the default type is NONE (i.e., no volume calculation will be performed), and the volumes for regions not containing HOLEs or ARRAYs will be set to -1.0. No other data are needed for this type.

In KENO-VI the volume data may be entered for any or all regions within the geometry data by placing the keyword VOL $=$ followed by the total volume of that region in the problem at the end of the MEDIA card. See Sect. 8.1.2.4 (Geometry Data) for more details.

For KENO-VI, in the same problem, volumes may be entered using a combination of three methods: (1) in the geometry data using $\mathbf{V O L}=$, (2) read from a volume file, and (3) calculated. The calculated volumes (method 3) are obtained for both the regions, and the meshes are defined by a grid (such as in TSUNAMI runs). As for KENO V.a, the mesh volumes must always be calculated (i.e., there is no method to input the mesh volumes). If volumes are entered or calculated using more than one method, the following hierarchy is used to determine which volume is used for the regions. 
1. Volumes entered as part of a MEDIA card using $\mathbf{V O L}=$ are always used.

2. Volumes read from the volume file are used if that volume for the region was not specified using $\mathrm{VOL}=$ following a MEDIA card.

3. Calculated volumes are used if they are not specified using $\mathbf{V O L}=$ and if there is no volume file or data for that region on the volume file.

4. Volumes that have not been set or calculated will be set to -1.0 . This may result in negative fluxes and fission densities for these regions.

5. Volumes are only calculated for regions containing material. Regions containing ARRAYs or HOLEs have no volume. Those volumes are associated with the UNIT contained in the ARRAY or HOLE.

In KENO V.a, the region volumes are always calculated by the code without the user's intervention. This is possible because KENO V.a has no region intersections, so calculation of the volumes is always possible using analytical methods. The use of the (RANDOM) calculated volumes using the VOLUME block is then only justified when the user needs to calculate the volumes defined by a grid, such as for TSUNAMI calculations.

When volumes are calculated using either RANDOM or TRACE, then a file containing volumes and named_volxxxx (where xxxx is an 18-digit number with the leftmost unused digits padded with zeros) is created in the temporary directory. The program searches the temporary directory for a file name beginning with _vol. If it is not found, the volume file that is created is named_vol000000000000000000. If a file exists, then a new file will be created where the file number is the largest number associated with a previous volume file incremented by 1 . The file is automatically copied to the user directory with the input file base name prepended to it, such as inputfile.volxxxx.volumes.

Below is an example of the VOLUME data block associated with a case in which volumes are being calculated using ray tracing. The number of rays used is set to one million, and if the outer unit volume is not a cuboid, then a cuboid will be placed around the global region prior to calculating volumes.

READ VOLUME

TYPE $=$ TRACE NRAYS $=1000000$

$\mathbf{X P}=10 \quad \mathbf{X M}=-15 \quad \mathbf{Y P}=15 \quad \mathbf{Y M}=-15 \quad \mathbf{Z P}=15 \quad \mathbf{Z M}=-15$

END VOLUME

Below is an example of the VOLUME data block associated with a case where volumes are being calculated using random sampling. The number of particles per batch is set to 100,000, and the number of batches used is set to 500. After being calculated, the volume data will be written to a file in the temporary directory as discussed above.

READ VOLUME

TYPE $=$ RANDOM POINTS $=100000$ BATCHES $=500$

$\mathbf{X P}=10 \quad \mathbf{X M}=-10 \quad \mathbf{Y P}=15 \quad \mathbf{Y M}=-15 \quad \mathbf{Z P}=25 \quad \mathbf{Z M}=15$

END VOLUME

Below is an example of the VOLUME data block associated with a case where volumes are both read in from the file VOLUME_DATA and calculated using random sampling. The number of particles per generation is set to $1,000,000$, and the number of generations used is set to 500. The file VOLUME_DATA must be formatted as shown below. The calculated volume data are written in the temporary working directory to a file as discussed above. Calculating volume data for some volume 
regions and providing input volume data for others may be useful if only part of the volume data is known and the remaining data need to be calculated.

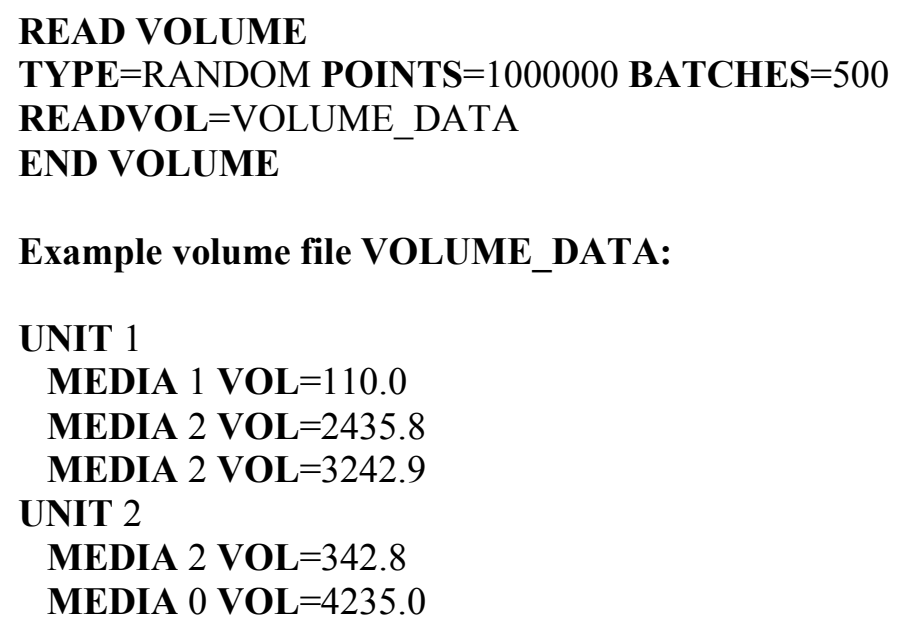

Below is an example of a sample problem in which volumes are being calculated using random sampling. The number of particles per generation is set to 100,000 , and the number of generations used is set to 500 . After being calculated, the VOLUME data will be written as described above.

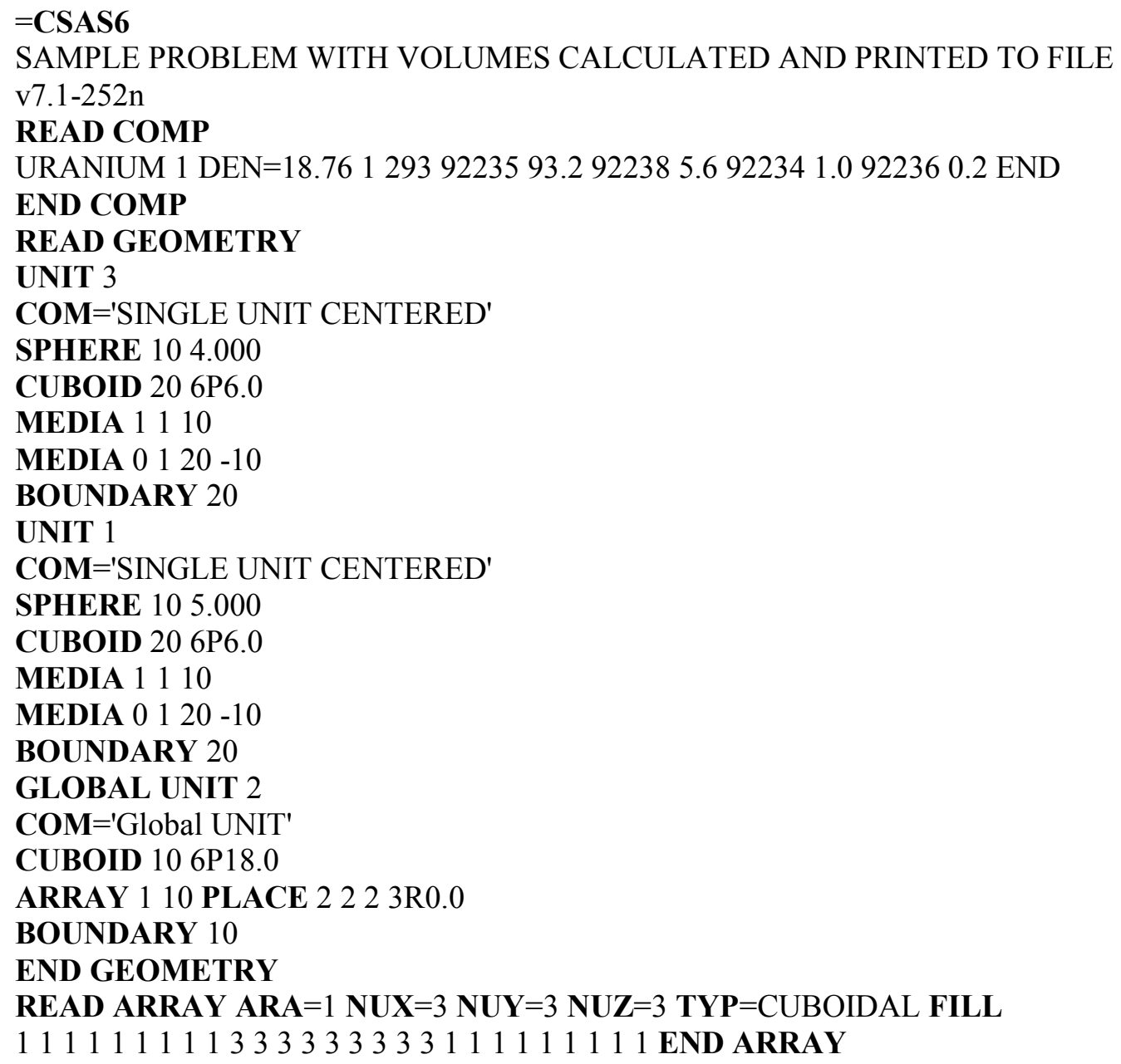




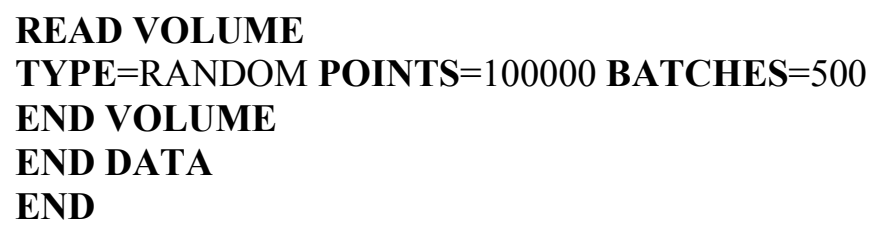

Below is an example of a problem with the volumes entered in the geometry data block using $\mathbf{V O L}=$ followed by the volume for all MEDIA type content records. Note that the keyword "VOL=" should never follow a HOLE or ARRAY content record.

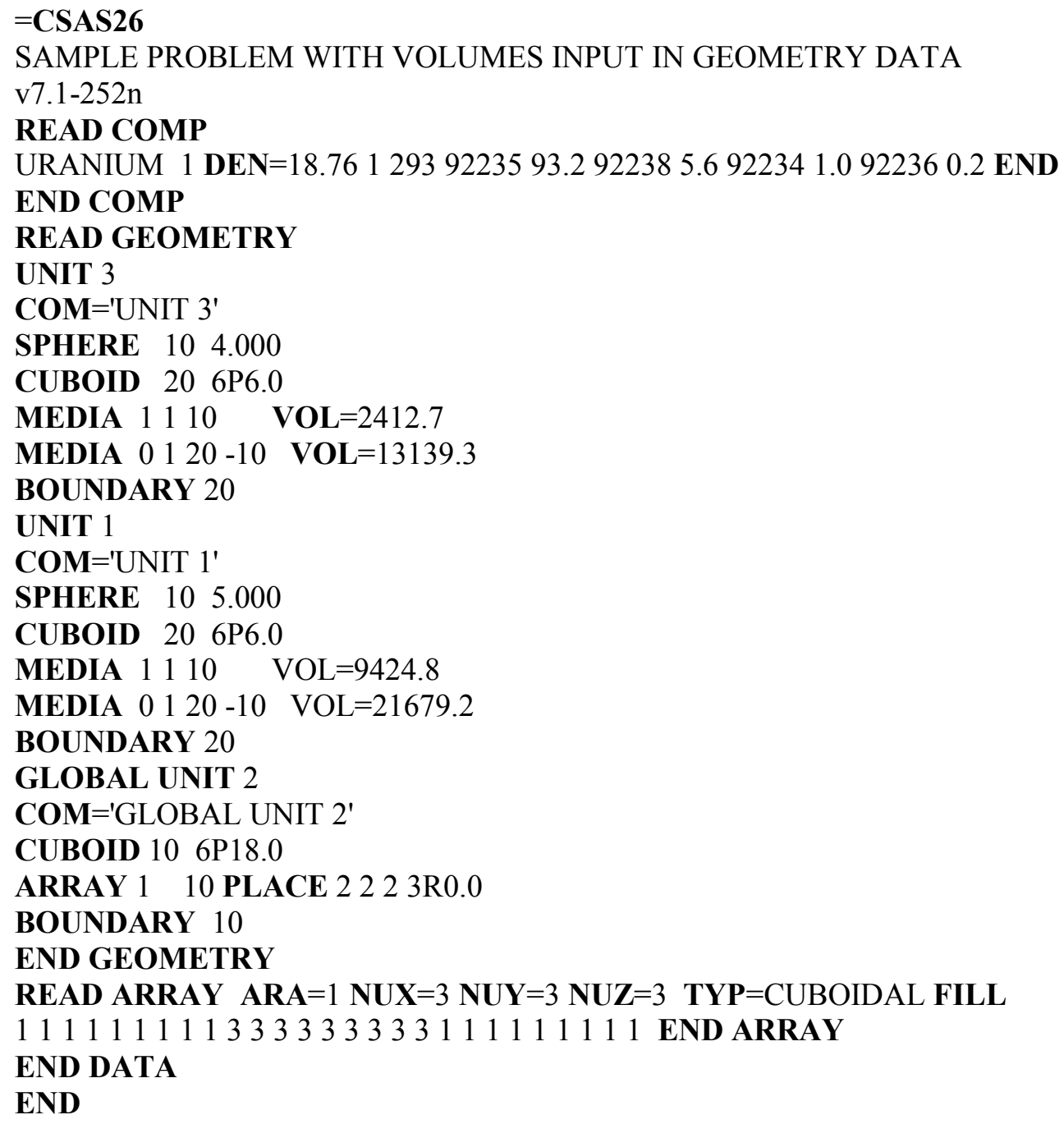




\subsubsection{Grid geometry data}

This data block is used to input the data needed to define a Cartesian grid for tallying purposes.

READ GRID $\boldsymbol{N} p_{1} \ldots p_{L}$ END GRID

$N$

$p_{1} \ldots p_{L}$

$\mathbf{N}[\mathbf{U M}] \mathbf{X C E L L S}=n u m x$

$\mathbf{N}[\mathbf{U M}] \mathbf{Y C E L L S}=n u m y$

$\mathbf{N}[\mathbf{U M}] \mathbf{Z C E L L S}=$ numz

$\mathbf{X M I N}=x \min$

XMAX $=x \max$

YMIN $=y \min$

$\mathbf{Y M A X}=y \max$

$\mathbf{Z M I N}=z \min$

ZMAX $=z \max$

XPLANES=xplanes

YPLANES $=$ yplanes

ZPLANES $=z$ planes

XLINEAR $=$ numcells $x x_{\min } x_{\max }$

YLINEAR $=$ numcellsy $y_{\min } y_{\max }$

ZLINEAR=numcellsz $z_{\min } z_{\max }$

TITLE$=$ title mesh grid identifier, always entered.

are $L$ parameters chosen from the list below. The parameters are entered using keywords followed by the appropriate data, except for the grid identifier, which is always entered first as an integer.

number of cells in the $\mathrm{x}$ direction, default $=1$.

number of cells in the y direction, default $=1$.

number of cells in the $\mathrm{z}$ direction, default $=1$.

minimum cell boundary in the $\mathrm{x}$ direction, default $=0$.

maximum cell boundary in the $\mathrm{x}$ direction, default $=1$.

minimum cell boundary in the y direction, default $=0$.

maximum cell boundary on the y direction, default $=1$.

minimum cell boundary in the $\mathrm{z}$ direction, default $=0$.

maximum cell boundary in the $\mathrm{z}$ direction, default $=1$.

the cell boundaries in the $\mathrm{x}$ direction followed by end, default $=0,1$ end.

the cell boundaries in the $y$ direction followed by end, default $=0,1$ end.

the cell boundaries in the $\mathrm{z}$ direction followed by end, default $=0,1$ end.

generate $\mathrm{y}-\mathrm{z}$ planes from $\mathrm{x}_{\min }$ to $\mathrm{x}_{\max }$ creating numcellsx intervals.

generate $\mathrm{x}-\mathrm{z}$ planes from $\mathrm{y}_{\min }$ to $\mathrm{y}_{\max }$, creating numcellsy intervals.

generate $x-y$ planes from $z_{\min }$ to $z_{\max }$, creating numcellsz intervals.

optional title for this mesh grid. Only used in KENO if an error in the grid causes a debug print.

If numx, xmin, xmax are entered, then the code will calculate numx equally spaced cells in the $\mathrm{x}$ direction between $x \min$ and $x \max$.

If xplanes is entered, then the code will count the number of unique xplanes, and order them from minimum to maximum, deleting any duplicates.

If the user inputs both sets of data, then the code will use the xplanes data.

If xplanes and xlinear are both entered, then the code will retain all unique planes from xplanes and all xlinear entries provided. The above also applies to $\mathrm{Y}$ and $\mathrm{Z}$.

NOTE: The user MUST set the minimum and maximum values in each direction so that the actual geometry is totally covered by the mesh for mesh flux tally that is used in TSUNAMI sensitivity calculations.

KENO checks for and eliminates duplicate or nearly duplicate planes.

The user may specify multiple mesh grids; each must be defined in separate READ GRID blocks. In this case, each grid should have different $N$ (grid ID number). See Sect. 8.1.3.10 for details and samples. 


\subsubsection{Reaction data}

The reaction data block is used to specify the type of tally (e.g., reaction rates, flux, and few group reaction cross sections) and the reaction/nuclide pairs in any mixture used in the problem for reaction tally calculations. This block is operational only with the continuous energy mode, and it provides the specifications for reaction rate, neutron flux, and reaction cross section tallies. See Sect. 8.1.6.6 for more details. For multigroup KENO calculations, use KMART5 or KMART6, which are described in the KMART section of the SCALE manual.

A reaction data block consists of REACTION FILTERS, TALLY TYPE, ENERGY GROUP BOUNDARIES, and OUTPUT EDITS. These data types can be entered in any order. A combination of parameters for describing the REACTION FILTERS and TALLY TYPE must be entered for any reaction or cross section tally calculation. ENERGY GROUP BOUNDARIES and OUTPUT EDITS data are optional. Tally calculations can be performed for multiple reactions specified by the REACTION FILTERS. Only one energy grid, either specified with the data in ENERGY GROUP BOUNDARIES or from the READ ENERGY block or from the code defaults, is used for all reaction tally calculations. To provide data for the continuous energy depletion calculations, another energy grid can be specified and used for tallying only the mixture flux.

Enter REACTION DATA in the form:

READ REACTION REACTION FILTERS [TALLY TYPE] [ENERGY GROUP BOUNDARIES] [OUTPUT EDITS] END REACTION

REACTION FILTERS define a reaction map that is used in reaction tally calculations. The REACTION FILTERS must be entered in the following order; mixture data (MIX or MIXLIST) followed by nuclide data (NUC or NUCLIST) followed by reaction IDs (MT or MTLIST). Each filter is defined using a combination of the following keywords:

MIX=mixnum Mixture number, no default value. Specified mixture number must exist in the mixing table and be used in the problem for a valid filter generation. A wildcard "*" can be used to define a filter applicable for all mixtures in the problem.

MIXLIST mixnum mixnum $_{2} \ldots$ mixnum $_{N}$ END A list of mixture numbers followed by end, no default values. Specified mixture numbers must exist in the mixing table and be used in the problem for a valid reaction tally calculation. Within each filter, use either MIX or MIXLIST, but not both.

NUC=nucid Nuclide identifier, no default value. Specified nuclide must be a constituent of the mixtures used in this filter definition (specified with MIX or MIXLIST). A wildcard "*" can be used to define a filter applicable for all nuclides in each mixture in this filter definition. Nuclide identifiers are listed for all isotopes in the Standard Composition Library section of the SCALE manual (see Sect. 7.2).

NUCLIST nucid $_{1}$ nucid $_{2} \ldots$ nucid $_{N}$ END A list of nuclide identifiers followed by end, no default values. Specified nuclides must be the constituents of the mixtures used in this filter definition (specified by MIX or MIXLIST). Within each filter, use either NUC or NUCLIST, but not both. 
MT $=m t \quad$ Reaction MT number, no default value. Specified reaction MT number should be available for the nuclides defined in this filter definition (specified by NUC or NUCLIST). Otherwise, the code skips the filter definition with this given reaction MT. A wildcard "*" can be used to define a filter with all reaction MTs. Valid SCALE library MT values are listed in the SCALE Cross Section Libraries section of the SCALE manual (see Appendix A of Sect. 11.1).

MTLIST $m t_{1} m t_{2} \ldots m t_{N}$ END A list of reaction MT numbers followed by end, no default values. Specified MT numbers should be available for the nuclides defined in this filter definition (specified by MIX or MIXLIST). Otherwise, KENO skips that reaction specified in the filter for the reaction tally calculations. Within each filter, use either MT or MTLIST, but not both.

A reaction filter consists of either single or multiple mixture, nuclide and reaction definitions. A valid reaction filter starts with mixture specification, followed by nuclide specification, and ends with reaction specification. Mixture(s) must be specified with either MIX or MIXLIST keywords. Nuclide(s) in these mixtures must be entered with either NUC or NUCLIST, and reactions for each nuclide must be specified with either MT or MTLIST.

Mixture, nuclide, and reaction number are required for mixture average fluxes, even though the nuclide and reaction numbers are not used for the neutron flux tallies.

Multiple reaction filter definitions are allowed. KENO processes all the definitions and creates a reaction map based on them. The following examples demonstrate the reaction filter specifications for different problems. In these examples, reaction filters are specified based on the following composition data used in the problem:

\begin{tabular}{lc} 
mixture & $\begin{array}{c}\text { compositions in the example problem } \\
\text { nuclides }\end{array}$ \\
\hline 10 & $92235,92238,8016$ \\
20 & $92238,94239,8016$ \\
30 & $92235,92238,8016$ \\
40 & 1001,8016 \\
100 & $1001,8016,5010,5011$
\end{tabular}

Example-1:

READ REACTION

MIX $=10$ NUC $=92235$ MT $=18$

END REACTION

Defines a reaction filter used to tally only fission reaction $(\mathbf{M T}=18)$ of ${ }^{235} \mathrm{U}$ in mixture 10 .

Example-2:

READ REACTION

MIX $=10$ NUC $=92235$ MT $=*$

END REACTION

Defines a reaction filter used to tally all available reactions of ${ }^{235} \mathrm{U}$ in mixture 10 . 


\section{Example-3: \\ READ REACTION \\ MIX=10 NUC=92235 MTLIST 218102 END \\ END REACTION}

Defines a reaction filter used to tally the elastic scattering $(\mathrm{mt}=2)$, fission $(\mathrm{mt}=18)$, and capture $(\mathrm{mt}=102)$ reactions of ${ }^{235} \mathrm{U}$ in mixture 10 .

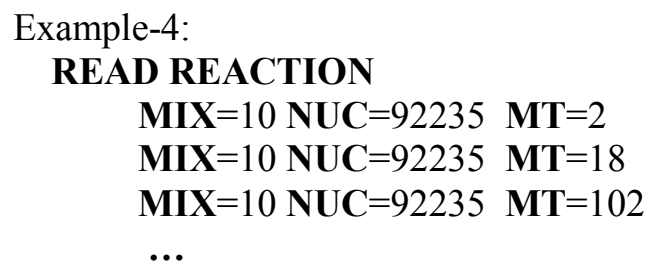

\section{END REACTION}

Defines a reaction filter used to tally the elastic scattering $(\mathbf{M T}=2)$, fission $(\mathbf{M T}=18)$, and capture (MT=102) reactions of ${ }^{235} \mathrm{U}$ in mixture 10. Reaction filter definition in this example is identical to the filter definition given in Example-3.

\section{Example-5:}

READ REACTION

MIX $=10$ NUC $=* \quad$ MT $=18$

$\cdots$

\section{END REACTION}

Defines a reaction filter used to tally the fission reaction $(\mathbf{M T}=18)$ of ${ }^{235} \mathrm{U}$ and ${ }^{238} \mathrm{U}$ in mixture 10. Code skips the reaction tally request for ${ }^{16} \mathrm{O}$ since the requested reaction is not available for this nuclide in the data library.

Example-6:

READ REACTION

MIX $=10$ NUC $=92235 \quad$ MT $=18$

MIX $=10$ NUC $=92238$ MT $=18$

...

\section{END REACTION}

Defines a reaction filter used to tally the fission reaction $(\mathbf{M T}=18)$ of ${ }^{235} U$ and ${ }^{238} \mathrm{U}$ in mixture 10 . Reaction filter definition in this example is identical to the filter definition given in Example-5.

\section{Example-7:}

READ REACTION

MIX $=*$ NUC $=8016$ MT $=102$

\section{END REACTION}

Defines a reaction filter used to tally the capture reaction $(\mathbf{M T}=102)$ of ${ }^{16} \mathrm{O}$ in all mixtures. 
Example-8:

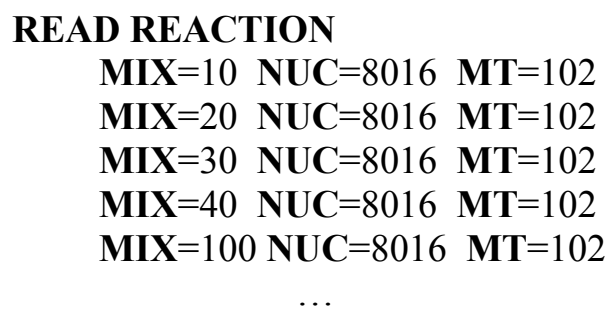

\section{END REACTION}

Defines a reaction filter used to tally the capture reaction $(\mathbf{M T}=102)$ of ${ }^{16} \mathrm{O}$ in mixtures $10,20,30,40$, and 100 respectively. Reaction filter definition in this example is identical to the filter definition given in Example-7.

Example-9:

\section{READ REACTION}

MIXLIST 10203040100 END NUC=8016 MT=102

\section{END REACTION}

Defines a reaction filter used to tally the capture reaction $(\mathbf{M T}=102)$ of ${ }^{16} \mathrm{O}$ in mixtures $10,20,30,40$, and 100 respectively. Reaction filter definition in this example is identical to the filter definition given in Examples 7 and 8.

Example-10:

\section{READ REACTION}

MIXLIST 102030 END NUC $=92238$ MT $=102$

MIX $=20 \quad$ NUC $=94239$ MT $=18$

MIX $=40$ NUC $=1001$ MT $=*$

MIX $=* \quad$ NUC $=8016 \quad$ MT $=2$

MIX $=* \quad$ NUC $=* \quad$ MT $=27$

\section{END REACTION}

Defines a complex reaction filter used to tally:

a) Capture reaction (MT=102) of ${ }^{238} \mathrm{U}$ in mixtures 10, 20 and 30 respectively,

b) Fission reaction (MT $=18)$ of ${ }^{239} \mathrm{Pu}$ in mixture 20,

c) All reactions of ${ }^{1} \mathrm{H}$ in mixture 40,

d) Elastic scattering reaction of ${ }^{16} \mathrm{O}$ in all mixtures,

e) Total absorption reaction of all nuclides in all mixtures.

Parameters of TALLY TYPE are logical parameters used to select quantities (reaction cross section, reaction rate, and mixture flux) that are tallied for the given problem. The user specifies any combination of these TALLY TYPEs once for all filters: 
XSTALLY $=l C E X S T$ Tally

Enter YES or NO. A value of YES specifies that reaction cross sections be tallied for the reactions listed in REACTION FILTERS. The default value of XSTALLY is NO. Computed reaction cross sections are saved in a file named BASENAME_keno_micro_xs.0 in RTNDIR, which is a SCALE environment variable for the directory from where the calculation was started. BASENAME is a SCALE environment variable that is the base name of the input file. (BASENAME is equal to "mytest" if the SCALE input name is "mytest.inp.")

RRTALLY $=l C E R R T a l l y$

Enter YES or NO. A value of YES specifies that reaction rates be tallied for the reactions listed in REACTION FILTERS. The default value of RRTALLY is NO. Computed reaction rates are saved in a file named BASENAME_keno_micro_rr.0 in RTNDIR.

NOTE: KENO combines and saves reaction rate and reaction cross section tallies to the same file, named BASENAME_keno_micro_xs_rr.0 in RTNDIR, if both XSTALLY and RRTALLY are set to YES.

MIXFLX=lCEMixFlux

Enter YES or NO. A value of YES specifies that mixture fluxes are to be tallied for the mixtures listed in REACTION FILTERS. The default value of MIXFLX is NO. Computed mixture fluxes are saved in a file named BASENAME_keno_mixture flux.0 in RTNDIR.

Mixture, nuclide, and reaction number are required for mixture average fluxes, even though the nuclide and reaction numbers are not used for the neutron flux tallies.

Example-11:

READ REACTION

MIX $=10$ NUC $=92235$ MT $=18$

XSTALLY=YES

$\ldots$

END REACTION

Defines a reaction filter, which uses fission reaction $(\mathbf{M T}=18)$ of ${ }^{235} \mathrm{U}$ in mixture 10 , for tallying reaction cross sections.

NOTE: Computed data are saved in a file named BASENAME_keno_micro_xs.0

Example-12:

READ REACTION

MIX $=10$ NUC $=92235$ MT $=18$

XSTALLY=YES RRTALLY $=$ YES

END REACTION 
Defines a reaction filter, which uses fission reaction $(\mathbf{M T}=18)$ of ${ }^{235} \mathrm{U}$ in mixture 10 , for tallying reaction rates as well as the reaction cross sections.

NOTE: Computed data are saved in files named BASENAME_keno_micro_xs_rr.0

Example-13:

READ REACTION

MIX $=10$ NUC $=92235 \quad$ MT $=18$

XSTALLY=YES MIXFLX=YES

\section{END REACTION}

Defines a reaction filter, which uses fission reaction $(\mathbf{M T}=18)$ of ${ }^{235} \mathrm{U}$ in mixture 10 , for tallying reaction cross sections. In addition, mixture flux is tallied for mixture 10 given in this reaction filter.

NOTE: Computed data are saved in files named BASENAME_keno_micro_xs.0, and BASENAME_keno_mixture_flux.0, respectively.

ENERGY GROUP BOUNDARIES data define energy group structure other than the defaults for tallying both reaction cross sections/reaction rates and mixture fluxes.

ENER_XS $e_{1} e_{2} e_{3} \ldots$ END Upper energy boundary for each group. The last entry is the lower energy boundary of the last group. For $\mathrm{N}$ groups, there are $\mathrm{N}+1$ entries. Entries must be in descending order. This may be specified once in the REACTION block and, if used, is applied to all cross section and reaction rate tallies.

ENER_FLX $e_{1} e_{2} e_{3} \ldots$ END Upper energy boundary for each group, default is NGP-group data. The last entry is the lower energy boundary of the last group. For $\mathrm{N}$ groups, there are $\mathrm{N}$ +1 entries. Entries must be in descending order. This may be specified once in the REACTION block and, if used, is applied to all mixture flux tallies.

NOTE: Default values for the energy group boundaries in reaction tally calculations are determined as in the order described in Sect. Energy group boundary data.

Example-14: (no READ ENERGY block, no NGP in READ PARAMETER block, no energy group boundaries in READ REACTION block)

\section{READ REACTION \\ MIX=10 NUCLIST 9223592238 END \\ MTLIST 161718 END \\ MIXFLX=YES XSTALLY=YES}

\section{END REACTION}

Default SCALE 238-group energy structure is used for tallying both mixture flux and reaction cross sections.

Example-15: (energy group bounds specified in READ ENERGY block) 


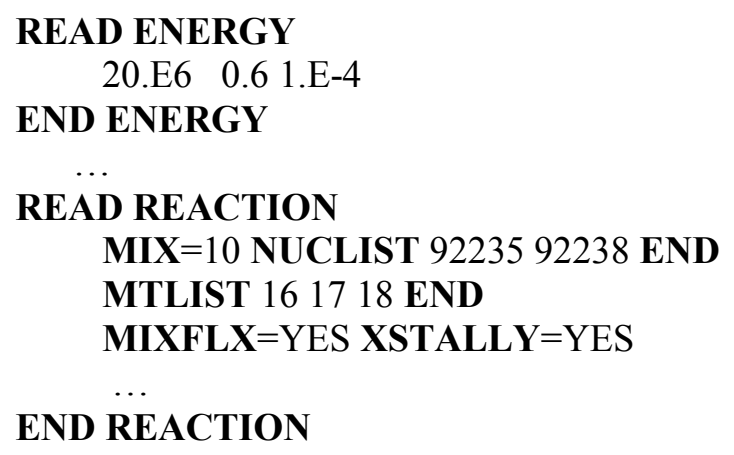

2-group energy structure given in READ ENERGY block is used for tallying both mixture flux and reaction cross sections.

Example-16: (NGP is set in READ PARAMETER block)

READ PARAMETER

NGP $=4$

END PARAMETER

READ REACTION

MIX=10 NUCLIST 9223592238 END

MTLIST 161718 END

MIXFLX=YES XSTALLY=YES

END REACTION

4-group energy group structure (4-equal lethargy bins) is used for tallying both mixture flux and reaction cross sections.

Example-17:

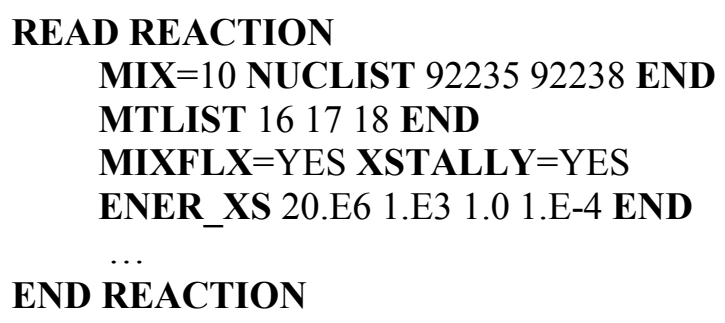

3-group energy group structure given in READ REACTION block is used for tallying reaction cross sections, and default SCALE 238 group energy structure is used for tallying mixture flux.

Example-18:

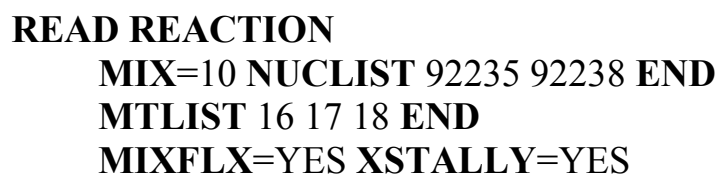


ENER_FLX 20.E6 1.E3 1.0 1.E-4 END

ENER_XS 20.E6 1.0 1.E-4 END

\section{END REACTION}

2-group energy group structure given in READ REACTION block is used for tallying reaction cross sections, and 3-group energy group structure given in READ REACTION block is used for tallying mixture flux.

Parameters of OUTPUT EDITS are logical parameters used to print reaction tallies and mixture fluxes in separate files. These parameters are optional parameters.

PRNTXS $=l C E$ print $X S \quad$ Enter YES or NO. A value of YES specifies that reaction cross sections tallies for each mixture be written in separate files in RTNDIR (BASENAME_keno_micro_xs_mix\{mixnum\}.0, mixnum is the mixture numbers specified in the reaction filters). The default value of PRNTXS is NO.

PRNTRR $=l C E$ printRR Enter YES or NO. A value of YES specifies that reaction rate tallies for each mixture be written in separate files in RTNDIR (BASENAME_keno_micro_rr_mix $\{$ mixnum $\} .0$, mixnum is the mixture numbers specified in the reaction filters). The default value of PRNTRR is NO.

PRNTFLX=lCEprintMixFlux

Enter YES or NO. A value of YES specifies that mixture flux tallies for each mixture be written in separate files in RTNDIR (BASENAME keno mixture flux mix\{mixnum\}.0, mixnum is the mixture numbers specified in the reaction filters). The default value of PRNTFLX is NO.

\subsubsection{Notes for KENO Users}

This section provides assorted tips designed to assist the KENO user with problem mockups. Some information concerning methods used by KENO is also included.

\subsubsection{Data Entry}

The KENO data is entered in blocks that begin and end with keywords as described in Sect. 8.1.2.1. Only one set of parameter data can be entered for a problem. However, for other data blocks, it is possible to enter more than one block of the same kind of data. When this is done, only the last block of that kind of data is retained for use by the problem, except for the GRID block for which all blocks are retained.

Within data blocks, a number, $\mathrm{x}$, can be repeated $\mathrm{n}$ times by specifying $\mathrm{nRx}, \mathrm{n}^{*} \mathrm{x}$, or $\mathrm{n} \$ \mathrm{x}$.

Numbers in engineering notation may be specified with or without an "E" between the base and the exponent. For example; 0.0011 may be specified as 1.1e-3 or as 1.1-3.

\subsection{Multiple and scattered entries in the mixing table}

In the following examples, assume 1001 is the nuclide ID for hydrogen, 8016 is the nuclide ID for oxygen, 92235 is the nuclide ID for ${ }^{235} \mathrm{U}$, and 92238 is the nuclide ID for ${ }^{238} \mathrm{U}$. If a given nuclide ID is used more than once in the same mixture, the result is the summing of all the number densities associated with that nuclide. For example: 
MIX=1 92235 4.3e-2 92238 2.6e-3 $10013.7 \mathrm{e}-2 \quad 92235$ 1.1e-3 8016 1.8e-2

would be the same as entering:

MIX=1 92235 4.41e-2 92238 2.6e-3 $10013.7 \mathrm{e}-2 \quad 8016$ 1.8e-2

A belated entry for a mixture can be made as follows:

MIX=1 1001 6.6e-2 MIX=2 92235 4.3e-2 92238 2.6e-3 MIX=1 8016 3.3e-2

This is the same as entering:

MIX=1 1001 6.6e-2 8016 3.3e-2 MIX=2 92235 4.3e-2 92238 2.6e-3

\subsection{Multiple entries in geometry data}

Individual geometry regions cannot be replaced by adding an additional description. However, entire unit descriptions can be replaced by adding a new description having the same unit number. The last description entered for a unit is used in the calculation. For example, the following geometry descriptions are equivalent in KENO V.a and KENO-VI, respectively:

In KENO V.a:

READ GEOM UNIT 1 SPHERE 115.0 CUBE $0110.0-10.0$

UNIT 2 CYLINDER 1112.05 .0 -5.0 CUBE 011 10.0 - 10.0

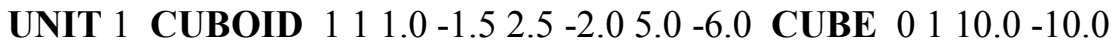

END GEOM

is the same as entering:

READ GEOM UNIT 1 CUBOID 1111.0 - 1.5 2.5 -2.05 .0 -6.0

CUBE $0 \begin{array}{lllll} & 10.0 & -10.0\end{array}$

UNIT 2 CYLINDER 112.05 .0 -5.0 CUBE 0110.0 -10.0 END GEOM

or

READ GEOM UNIT 2 CYLINDER 112.05 .0 -5.0 CUBE $0110.0-10.0$

UNIT 1 CUBOID $1111.0-1.52 .5-2.05 .0-6.0$ CUBE $00110.0-10.0$

END GEOM

In KENO-VI:

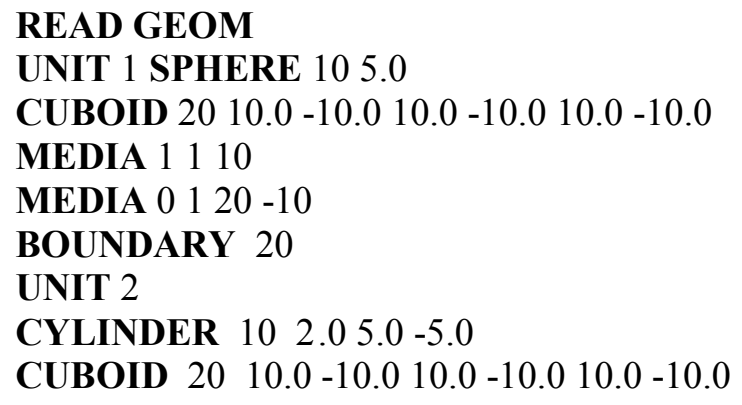


MEDIA 1110

MEDIA 01120 - 10

BOUNDARY 20

UNIT 1

$\begin{array}{llllllll}\text { CUBOID } & 10 & 1.0 & -1.5 & 2.5 & -2.0 & 5.0 & -6.0\end{array}$

CUBOID $20 \quad 10.0-10.010 .0-10.010 .0-10.0$

MEDIA 1110

MEDIA $001-1020$

BOUNDARY 20

END GEOM

is the same as entering

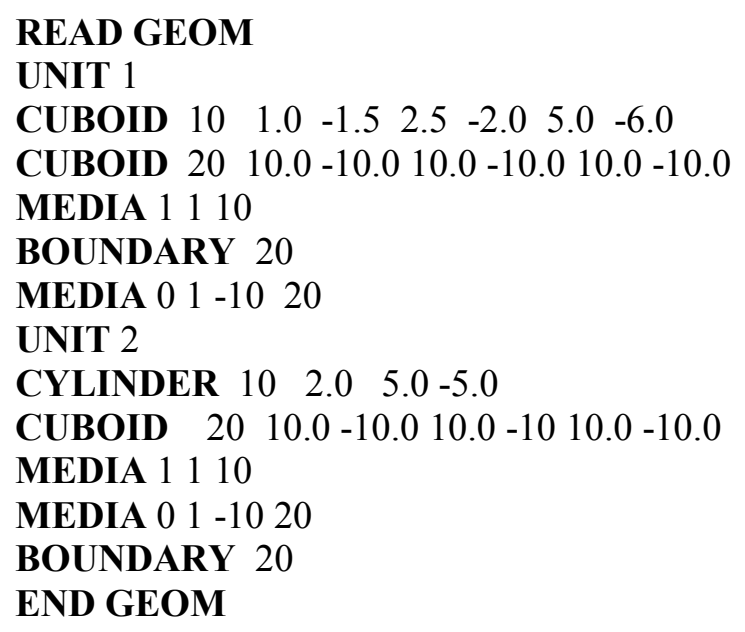

READ GEOM

UNIT 1

$\begin{array}{lllllllll}\text { CUBOID } & 10 & 1.0 & -1.5 & 2.5 & -2.0 & 5.0 & -6.0\end{array}$

CUBOID $20 \quad 10.0-10.010 .0-10.010 .0-10.0$

MEDIA 1110

BOUNDARY 20

MEDIA $01-1020$

UNIT 2

CYLINDER $10 \quad 2.0 \quad 5.0-5.0$

CUBOID $20 \quad 10.0-10.010 .0-10 \quad 10.0-10.0$

MEDIA 1110

MEDIA 0 1 $1-1020$

BOUNDARY 20

END GEOM

or

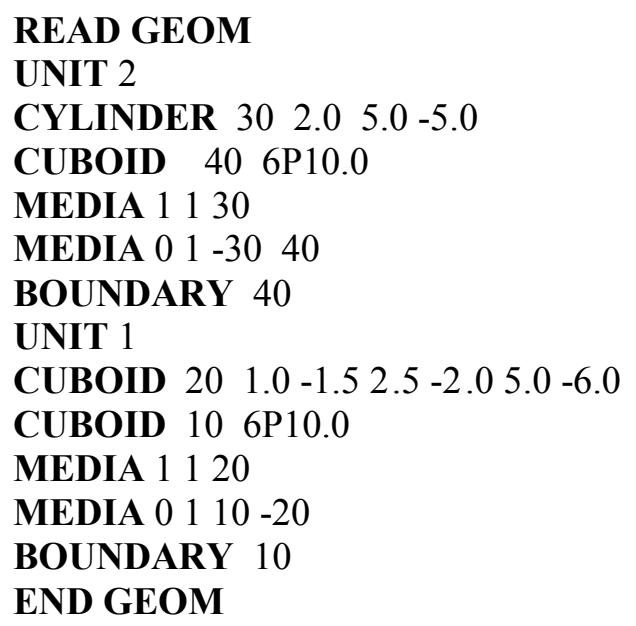

The order of entry for UNIT descriptions is not important because the UNIT number is assigned as the value following the word UNIT. They do not need to be entered sequentially, and they do not need to be numbered sequentially. It is perfectly acceptable to enter UNITs 2, 3, and 5, omitting Units 1 and 4 as long as UNITs 1 and 4 are not referenced in the problem. It is also acceptable to scramble the order of entry as in entering UNITs 3, 2, and 5. 


\subsubsection{Default logical unit numbers for KENO}

The logical unit numbers for data used by KENO are listed in Table 8.1.23.

Table 8.1.23. KENO logical unit numbers

\begin{tabular}{|c|c|c|c|}
\hline Function & $\begin{array}{c}\text { Parameter } \\
\text { name }\end{array}$ & $\begin{array}{c}\text { Unit } \\
\text { number }\end{array}$ & $\begin{array}{c}\text { Variable } \\
\text { name }\end{array}$ \\
\hline Problem input data (ASCII) & & 5 & INPT \\
\hline Problem input data (binary) & & 95 & BIN \\
\hline Program output (ASCII) & & 6 & OUTPT \\
\hline Albedo data & $\mathrm{ALB}=$ & 79 & ALBDO \\
\hline Scratch unit & $\mathrm{SKT}=$ & 16 & SKRT \\
\hline \multirow[t]{2}{*}{ Read restart data } & $\mathrm{RST}=$ & $0^{a}$ & RSTRT \\
\hline & & $34^{b}$ & RSTRT \\
\hline \multirow[t]{2}{*}{ Write restart data } & $\mathrm{WRS}=$ & $0^{a}$ & WSTRT \\
\hline & & $35^{c}$ & WSTRT \\
\hline Direct access storage for input data & & 8 & DIRECT(1) \\
\hline Direct access storage for supergrouped data & & 9 & DIRECT(2) \\
\hline Direct access storage for cross section mixing & & 10 & DIRECT(3) \\
\hline Mixed cross section data set & $\mathrm{XSC}=$ & $14^{d}$ & ICEXS \\
\hline Group-dependent weights & $\mathrm{WTS}=$ & 80 & WTS \\
\hline AMPX working format cross sections & $\mathrm{LIB}=$ & $0^{a}$ & AMPXS \\
\hline Group boundary Library (KENO-VI) & $\mathrm{GRP}=$ & 77 & GRPBS \\
\hline
\end{tabular}

\subsubsection{Parameter input}

When the parameter data block is entered for a problem, the same keyword may be entered several times. The last value that is entered is used in the problem. Data may be entered as follows:

READ PARAM FLX=YES NPG $=1000$ TME $=0.5$ TME $=1.0$

$\mathbf{N P G}=50 \mathrm{TME}=10.0 \mathrm{FLX}=\mathrm{NO}$

NPG $=500$

END PARA

This will result in the problem having $\mathbf{F L X}=\mathbf{N O}, \mathbf{T M E}=\mathbf{1 0 . 0}$, and $\mathbf{N P G}=\mathbf{5 0 0}$. It may be more convenient for the user to insert a new value than to change the existing data.

Certain parameter default values should not be overridden unless the user has a very good reason to do so. These parameters are as follows:

1. $\quad \mathbf{X 1 D}=$ which defines the number of extra 1-D cross sections. The use of extra 1-D cross sections - other than the use of the fission cross section for calculating the average number of neutrons per fission-requires programming changes to the code;

2. $\mathbf{N F B}=$ which defines the number of neutrons that can be entered in the fission bank (the fission bank is where the information related to a fission is stored); 
3. $\mathbf{X F B}=$ which defines the number of extra positions in the fission bank;

4. $\mathbf{N B K}=$ which defines the number of neutrons that can be entered in the neutron bank (the neutron bank contains information about each history);

5. $\mathbf{X N B}=$ which defines the number of extra positions in the neutron bank;

6. $\quad \mathbf{W T H}=$ which defines the factor that determines when splitting occurs;

7. WTA= which defines the default average weight given to a neutron that survives Russian roulette;

8. $\mathbf{W T L}=$ which defines the factor that determines when Russian roulette is played; and

9. $\quad \mathbf{L N G}=$ which sets the maximum words of storage available to the program.

It is recommended that $\mathbf{B U G}=$, the flag for printing debug information, never be set to YES. The user would have to look at the FORTRAN coding to determine what information is printed. BUG $=$ YES prints massive amounts of sparsely labeled information. The user should only rarely consider using TRK=YES. This generates thousands of lines of well-labeled output that provides information about each history at key locations during the tracking procedure. All other parameters can be changed at will to provide features the user wishes to activate.

\subsubsection{Cross sections}

In multi-energy group mode, KENO always uses cross sections from a mixed cross section data file. The format of this file is the Monte Carlo processed cross section file. A mixed cross section file can be created by previous KENO run, or by using an AMPX working format library and entering mixing table data in KENO.

\subsection{Use a mixed cross section Monte Carlo format library}

A mixed cross section Monte Carlo format library (premixed cross section data file) from a previous KENO case may be used. This file is specified using the parameter $\mathbf{X S C}=$. If a mixing table data block is entered, the premixed cross section data file will be rewritten. Therefore, a mixing table should not be entered if a premixed cross section data file is used. The user should verify that the mixtures created by a previous KENO case are consistent with those used in the geometry data of the problem.

\subsection{Use an AMPX working format library}

When an AMPX working format library is used, it must be specified using the parameter $\mathbf{L I B}=$, and mixing table data must always be entered. IDs used in the mixing table must match the IDs on the AMPX working format library. The user must provide a file with the correct cross sections with a name that matches the pattern ftNNf001, with NN being the number of the logical unit.

\subsection{Number of scattering angles}

The number of scattering angles is defaulted to 1 (defaulted to 2 when KENO-VI is run as part of the CSAS6 sequence). This stand alone default is not adequate for many applications. The user should 
specify the scattering angle to be consistent with the cross sections being used. The number of scattering angles is entered in the cross section mixing table by using the keyword $\mathbf{S C T}=$. See Sect. 8.1.2.10.

The order of the last Legendre coefficient to be preserved in the scattering distribution is equal to $(2 \times \mathrm{SCT}-1)$. $\mathbf{S C T}=1$ could be used with a $\mathrm{P}_{1}$ cross section set such as the 16-group Hansen-Roach cross section library, and $\mathbf{S C T}=2$, for a $\mathrm{P}_{3}$ cross section set such as the SCALE 27-group cross section library. ENDF/B-V cross section libraries such as the 44-group or 238-group libraries contain many nuclides having $\mathrm{P}_{5}$ cross section sets. Isotropic scattering is achieved by entering $\mathbf{S C T}=0$.

\subsection{Cross section message cutoff}

The cross section message cutoff value, pbxs, is defaulted to $3 \times 10^{-5}$. Warning messages generated when errors are encountered in the $\mathrm{P}_{\mathrm{L}}$ expansion of the group-to-group transfers will be suppressed if the $\mathrm{P}_{0}$ cross section for that particular energy transfer is less than pbxs. The value of pbxs is specified in the cross section mixing table by using the keyword $\mathbf{E P S}=$. See Sect. 8.1.2.10.

The default value of pbxs is sufficient to assure that warning messages will not be printed for most of the SCALE $\mathrm{P}_{1}$ and $\mathrm{P}_{3}$ cross section libraries. However, the v7.0-238n library may print a few errors if $\mathrm{P}_{5}$ cross sections are specified.

The error messages below were printed for a problem using the 238-group cross section library and $p b x s=3.0 \times 10^{-5}$. If the default value of pbxs allows too many warning messages to be printed, a value can be determined which does not print the error messages from the printed messages by choosing a number larger than the $\mathrm{P}_{0}$ component on the first line, as shown below.

THE LEGENDRE EXPANSION OF THE CROSS SECTION $\left(\mathrm{P}_{0}-\mathrm{P}_{\mathrm{N}}\right)$ IS

$\underline{\left(\mathrm{P}_{0}\right)} \quad \underline{\left(\mathrm{P}_{1}\right)} \quad \underline{\left(\mathrm{P}_{2}\right)} \quad \cdots \quad \underline{\left(\mathrm{P}_{\mathrm{n}}\right)}$

THE MOMENTS CORRESPONDING TO THIS DISTRIBUTION ARE

$\underline{\left(\mathrm{M}_{1}\right)} \underline{\underline{\left(\mathrm{M}_{2}\right)}} \quad \cdots \quad \underline{\left(\mathrm{M}_{\mathrm{n}}\right)}$

THE MOMENTS CORRESPONDING TO THE GENERATED DISTRIBUTION ARE

$\underline{\left(\mathrm{M}_{1}\right)} \quad \underline{\left(\mathrm{M}_{2}\right)} \quad \cdots \quad \underline{\left(\mathrm{M}_{\mathrm{n}}\right)}$

THE LEGENDRE EXPANSION CORRESPONDING TO THESE MOMENTS IS

$\underline{\left(\mathrm{P}_{0}\right)} \quad \underline{\left(\mathrm{P}_{1}\right)} \quad \underline{\left(\mathrm{P}_{2}\right)} \quad \cdots \quad \underline{\left(\mathrm{P}_{\mathrm{n}}\right)}$

MOMENTS WERE ACCEPTED

For the following messages, EPS $=6.9 \mathrm{e}-5$ would cause all three messages to be suppressed. A value less than $5.615159 \mathrm{e}-5$ and greater than $4.767635 \mathrm{e}-5$ would suppress the second message, and a value less than $6.855362 \mathrm{e}-5$ and greater than $5.615159 \mathrm{e}-5$ would suppress the first two messages.

KMSG060 THE ANGULAR SCATTERING DISTRIBUTION FOR MIXTURE 2 HAS BAD MOMENTS FOR THE TRANSFER FROM GROUP 28 TO GROUP 72

1 MOMENTS WERE ACCEPTED

THE LEGENDRE EXPANSION OF THE CROSS SECTION (PO-PN) IS

$5.615159 \mathrm{E}-05 \quad 1.155527 \mathrm{E}-06 \quad-2.804013 \mathrm{E}-05 \quad-1.732067 \mathrm{E}-06$ 
THE MOMENTS CORRESPONDING TO THIS DISTRIBUTION ARE

$$
\text { 2.057870E-02 4.234578E-04 8.710817E-06 }
$$

THE MOMENTS CORRESPONDING TO THE GENERATED DISTRIBUTION ARE

$$
2.057870 \mathrm{E}-02 \quad 4.235078 \mathrm{E}-04 \quad 8.710817 \mathrm{E}-06
$$

THE LEGENDRE EXPANSION CORRESPONDING TO THESE MOMENTS IS

$$
\begin{aligned}
& 5.615159 \mathrm{E}-05 \quad 1.155527 \mathrm{E}-06 \quad-2.804011 \mathrm{E}-05 \quad-1.732066 \mathrm{E}-06 \\
& \text { THE WEIGHTS/ANGLES FOR THIS DISTRIBUTION ARE } \\
& 9.999995 \mathrm{E}-01 \quad 5.268617 \mathrm{E}-07 \\
& 2.057881 \mathrm{E}-02-1.973451 \mathrm{E}-01
\end{aligned}
$$

THE MOMENTS CORRESPONDING TO THIS DISTRIBUTION ARE
$2.057870 \mathrm{E}-02$
$4.235078 \mathrm{E}-04$
$8.710817 \mathrm{E}-06$

KMSG060 THE ANGULAR SCATTERING DISTRIBUTION FOR MIXTURE 2 HAS BAD MOMENTS FOR THE TRANSFER FROM GROUP 31 TO GROUP 75

1 MOMENTS WERE ACCEPTED

THE LEGENDRE EXPANSION OF THE CROSS SECTION (PO-PN) IS

$4.767635 \mathrm{E}-05 \quad 7.834378 \mathrm{E}-07 \quad-2.381887 \mathrm{E}-05 \quad-1.174626 \mathrm{E}-06$

THE MOMENTS CORRESPONDING TO THIS DISTRIBUTION ARE

1.643242E-02 2.700205E-04 4.451724E-06

THE MOMENTS CORRESPONDING TO THE GENERATED DISTRIBUTION ARE

1.643242E-02 2.700282E-04 4.437279E-06

THE LEGENDRE EXPANSION CORRESPONDING TO THESE MOMENTS IS

$4.767635 \mathrm{E}-05 \quad 7.834378 \mathrm{E}-07 \quad-2.381885 \mathrm{E}-05 \quad-1.174627 \mathrm{E}-06$

THE WEIGHTS/ANGLES FOR THIS DISTRIBUTION ARE

$9.999858 \mathrm{E}-01 \quad 1.420136 \mathrm{E}-05$

$1.643265 \mathrm{E}-02-2.334324 \mathrm{E}-07$

THE MOMENTS CORRESPONDING TO THIS DISTRIBUTION ARE

$1.643242 \mathrm{E}-02 \quad 2.700282 \mathrm{E}-04 \quad 4.437279 \mathrm{E}-06$

KMSG060THE ANGULAR SCATTERING DISTRIBUtion FOR MIXTURE 2 HAS BAD MOMENTS FOR THE TRANSFER FROM GROUP 32 TO GROUP 74

1 MOMENTS WERE ACCEPTED

THE LEGENDRE EXPANSION OF THE CROSS SECTION (PO-PN) IS

$6.855362 \mathrm{E}-05 \quad 1.341944 \mathrm{E}-06 \quad-3.423741 \mathrm{E}-05 \quad-2.011613 \mathrm{E}-06$

THE MOMENTS CORRESPONDING TO THIS DISTRIBUTION ARE

$1.957510 \mathrm{E}-02 \quad 3.831484 \mathrm{E}-04 \quad 7.601939 \mathrm{E}-06$

THE MOMENTS CORRESPONDING TO THE GENERATED DISTRIBUTION ARE (7)

1.957510E-02 3.832207E-04 7.502292E-06

THE LEGENDRE EXPANSION CORRESPONDING TO THESE MOMENTS IS

6.855362E-05 1.341944E-06 -3.423740E-05 -2.011629E-06

THE WEIGHTS/ANGLES FOR THIS DISTRIBUTION ARE

$9.999056 \mathrm{E}-01 \quad 9.437981 \mathrm{E}-05$

$1.957695 \mathrm{E}-02-1.848551 \mathrm{E}-06$

THE MOMENTS CORRESPONDING TO THIS DISTRIBUTION ARE

$1.957510 \mathrm{E}-02 \quad 3.832207 \mathrm{E}-04 \quad 7.502292 \mathrm{E}-06$

The user does not need to attempt to suppress all these messages. They are printed to inform the user of the fact that the moments of the angular distribution are not moments of a valid probability distribution. The original $P_{n}$ coefficients and their moments are listed in lines 3-6 of the message. Lines 7-10 list the new corrected moments and their corresponding $\mathrm{P}_{\mathrm{n}}$ coefficients. 
The weights and angles printed in lines 11-13 were generated from the corrected moments. The last two lines of the message list the moments generated from those weights and angles. They should match line 8 , which lists the moments corresponding to the generated distribution.

For most criticality problems, the first moment contributions are much more significant than the contributions of the higher order moments. Thus, the higher order moments may not affect the results significantly. The user may compare the original moments and corrected moments to make a judgment as to the significance of the change in the moments.

\subsubsection{Mixing table}

Mixtures can be used in defining other mixtures. When defining mixture numbers, care should be taken to avoid using a mixture number that is identical to a nuclide ID number if the mixture is to be used in defining another mixture. If a mixture number is defined more than once, it results in a summing effect.

The nuclide mixing loop is done before the mixture mixing loop, which performs mixing in the order of data entry. Thus, the order of mixing mixtures into other mixtures is important because a mixture must be defined before it can be used in another mixture. Some examples of correct and incorrect mixing are shown below, using 1001 as the nuclide ID for hydrogen, 8016 as the nuclide ID for oxygen, 92235 as the nuclide ID for ${ }^{235} \mathrm{U}$, and 92238 as the nuclide ID for ${ }^{238} \mathrm{U}$.

\section{EXAMPLES OF CORRECT USAGE}

\section{READ MIXT \\ MIX=1 $10016.6 \mathrm{e}-2 \quad 80163.3 \mathrm{e}-2$ \\ MIX $=210.5$ \\ END MIXT}

This results in mixture 1 being full-density water and mixture 2 being half-density water.

\section{READ MIXT}

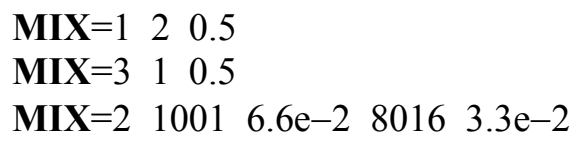

This results in mixture 1 being half-density water, mixture 2 being full-density water, and mixture 3 being quarter-density water. Because the nuclide mixing loop is done first, mixture 2 is created first and is available to create mixture 1 , which is then available to create mixture 3 .

\section{READ MIXT}

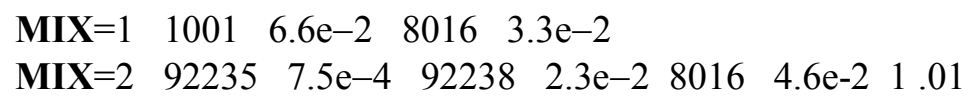

This results in mixture 1 being full-density water and mixture 2 being uranium oxide containing 0.01 density water. 


\section{READ MIXT \\ MIX $=1 \quad 1001 \quad 6.6 \mathrm{e}-2 \quad 8016 \quad 3.3 \mathrm{e}-2$ \\ $\mathbf{M I X}=292235 \quad 4.4 \mathrm{e}-2 \quad 92238 \quad 2.6 \mathrm{e}-3$ \\ MIX=1 110.5 \\ END MIXT}

This results in mixture 1 being water at 1.5 density (1001 9.9e-2 and $80164.95 \mathrm{e}-2)$ and mixture 2 is highly enriched uranium metal.

\section{EXAMPLES OF INCORRECT USAGE}

1. READ MIXT

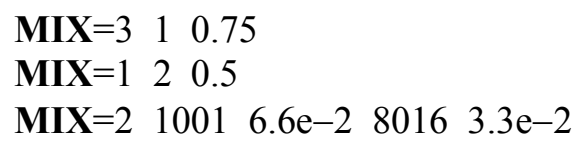

Here the intent is for mixture 2 to be full-density water, mixture 1 to be half-density water, and mixture 3 to be $3 / 8(0.75 \times 0.5)$ density water. Instead, the result for mixture 3 is a void, mixture 1 is half-density water, and mixture 2 is full-density water. This is because the nuclide mixing loop is done first, thus defining mixture 2. The mixture mixing loop is done next. Mixture 3 is defined to be mixture 1 multiplied by 0.75 , but since mixture 1 has not been defined yet, 0.75 of zero is zero. Mixture 1 is then defined to be mixture 2 multiplied by 0.5 . If the definition of mixture 1 preceded the definition of mixture 3 , as in item (2) under examples of correct usage, it would work correctly.

\section{READ MIXT \\ $\mathbf{M I X}=1 \quad 1001 \quad 6.6 \mathrm{e}-2 \quad 8016 \quad 3.3 \mathrm{e}-2$ \\ MIX=1001 $92235 \quad 4.4 \mathrm{e}-2 \quad 92238 \quad 2.6 \mathrm{e}-3$ \\ MIX $=2 \quad 1001 \quad 0.5$ \\ END MIXT}

This results in mixture 1 being full-density water, mixture 1001 being uranium metal, and mixture 2 being hydrogen with a number density of 0.5 because 1001 is the nuclide ID number for hydrogen. When a mixture number is identical to a nuclide ID and is used in mixing, that number is assumed to be a nuclide ID rather than a mixture number. The intent was for mixture 1 to be full-density water, mixture 1001 to be uranium metal, and mixture 2 to be half-density uranium metal.

\subsubsection{Geometry}

In general, KENO geometry descriptions consist of (1) geometry data (Sect. 8.1.2.4) defining the geometrical shapes present in the problem, and (2) array data (Sect. 8.1.2.5) defining the placement of the units that were defined in the geometry data. The geometry data block is prefaced by READ GEOM, and the array data block is prefaced by READ ARRAY.

When a 3-D geometrical configuration is described as KENO geometry data, it may be necessary to describe portions of the configuration individually. These individual partial descriptions of the configuration are called UNITs. KENO geometry modeling is subject to the following restrictions: 
1. Units are composed of regions. These regions are created using geometric bodies and surfaces (shapes) that are previously defined.

1.1. In KENO-VI the geometric bodies and surfaces may intersect. Regions are defined relative to the geometric bodies and surfaces in a UNIT. HOLEs provide a means of creating complex geometries in a UNIT and then inserting the UNIT into existing UNITs. For complex geometries the use of HOLEs may decrease the CPU time required for the problem.

1.2. In KENO V.a, each geometry region in a UNIT must completely enclose all geometry regions which precede it. Boundaries of the surfaces of the regions may be shared or tangent, but they must not intersect. The use of HOLEs in KENO V.a provides an exception to this complete enclosure rule. The use of HOLEs in KENO V.a will increase the CPU time required for the problem.

2. All geometrical surfaces must be describable in KENO V.a as spheres, hemispheres, cylinders, hemicylinders, cubes, cuboids, or as a set of quadratic equations in KENO-VI.

3. When specifying an ARRAY, each UNIT used in a cuboidal ARRAY must have a CUBE or CUBOID as its outer region (this is the only option in KENO V.a); a hexprism as the outer boundary if it is a hexagonal, triangular, or shexagonal ARRAY; a rhexprism as the outer boundary if it is a rhexagonal ARRAY; and a dodecahedron as the outer boundary if it is a dodecahedral ARRAY. In addition, the outer boundary of a UNIT cannot be rotated or translated in KENO V.a.

4. When several UNITs are used to describe an ARRAY, the adjacent faces of the UNITs in contact with each other must be the same size and shape.

5. UNITs are placed directly into regions using HOLEs. As many HOLEs as will fit without intersecting other HOLEs, nested ARRAYs or HOLEs, or the UNIT BOUNDARY can be placed in a UNIT without intersecting each other. In KENO V.a, HOLEs cannot intersect any of the regions within the UNIT in which they are placed. HOLEs are described in more detail in Sect. 8.1.3.6.1, and nested HOLEs are described in Sect. 8.1.3.6.2.

6. Multiple ARRAYs may be required to describe a complicated system. In KENO V.a, only one ARRAY may be placed directly into a UNIT. However, multiple ARRAYs may be placed in a UNIT by placing the ARRAYs in other UNITs and placing those UNITs in the original UNIT using HOLEs. Multiple ARRAYs may be placed directly into a UNIT in KENO-VI. These UNITs may then be used to create other ARRAYs, or they may be placed in other UNITs using HOLEs. UNITs placed in ARRAYs or HOLEs that are contained in other HOLEs or ARRAYs are said to be nested. The nesting level of a UNIT is the number of ARRAYs and HOLEs between the ARRAY or HOLE in the GLOBAL UNIT or ARRAY and the UNIT. Multiple ARRAYs are described in more detail in Sect. 8.1.3.6.3.

The KENO V.a geometry package allows any applicable shape to be enclosed by any other applicable shape, subject only to the complete enclosure restriction. The KENO-VI geometry package allows any shape describable using quadratic equations to be enclosed or intersected by any other allowable shape. The implication of this type of description is that the entire volume between two adjacent geometrical surfaces contains only one mixture, HOLE, or ARRAY. A void is specified by a mixture ID of zero. If HOLEs are present in the volume between two surfaces, the volume of that region is reduced by the HOLE's volume(s). 
In KENO V.a geometry, if the problem requires several UNITs to describe its geometrical characteristics, each UNIT that is used in an ARRAY must have a rectangular parallelepiped as its outer surface. This restriction is relaxed in KENO-VI, where the outer surface may be a rectangular parallelepiped, a hexagonal prism, a $90^{\circ}$ rotated hexagonal prism, or a dodecahedron, but all units placed in an array must use the same shape as their outer BOUNDARY. In order to describe the composite overall geometrical characteristics of the problem, these UNITs may be arranged in a rectangular ARRAY for KENO V.a geometry, or in either a rectangular, hexagonal, shexagaonal, rhexagonal, or dodecahedral ARRAY for KENO-VI geometry. This is done by specifying the number of units in the $\mathrm{X}, \mathrm{Y}$, and $\mathrm{Z}$ directions. If more than one UNIT is involved, data must be entered to define the number assigned to the ARRAY and the placement of the individual UNITs in the ARRAY. The ARRAY number, the number of UNITs in the $\mathrm{X}, \mathrm{Y}$, and $\mathrm{Z}$ directions, and the placement data are called array data (Sect. 8.1.2.5).

In the KENO V.a geometry description, the surrounding regions of any shape may be placed around an ARRAY, and they may consist of any number of regions in any order subject to the complete enclosure restriction. ARRAYs are positioned relative to the UNIT ORIGIN by specifying the location of the most negative point in the array, i.e. the most negative $\mathrm{X}$, most negative $\mathrm{Y}$, and most negative $\mathrm{Z}$ corner of the ARRAY. In KENO-VI geometry, an ARRAY may be placed in a region of any shape if the region boundary is contained within the ARRAY or shares the ARRAY boundary and does not cut across nested ARRAYs or HOLEs. In this case, only the section of the ARRAY contained within the region is recognized by the problem. ARRAYs are positioned relative to the UNIT ORIGIN by placing the ORIGIN of a specified UNIT in the ARRAY at a specified location in the UNIT.

To create a geometry mockup from a physical configuration, the user should keep the restrictions mentioned earlier in mind. There may be several ways of correctly describing the same physical configuration. Careful analysis of the system can result in a simpler mockup and shorter computer running time. A mockup with fewer geometry regions may run faster than the same mockup with extraneous regions. The number of UNITs used can affect the running time, because a transformation of coordinates must be made every time a history moves from one UNIT into another. Thus, if the size of a UNIT is small relative to the mean free path, a larger percentage of time is spent processing the transformation of coordinates. Because all boundaries in a UNIT must be checked for crossings, it may be more efficient to break up complex UNITs into several smaller, simpler UNITs. The trade-off involves the time required to process more boundary crossings vs the time required to transform coordinate systems when UNIT boundaries are crossed.

Geometry dimensions: The use of FIDO syntax may help simplify the description of the geometry. For example, a $20 \times 20 \times 2.5 \mathrm{~cm}$ rectangular parallelepiped would have been described as CUBOID 1110.0

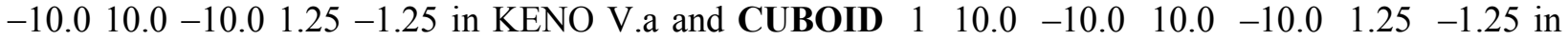
KENO-VI. By using the P option (see Table 8.1.17), the same rectangular parallelepiped could be described as CUBOID 11 4P10.0 2P1.25 in KENO V.a and or CUBOID 1 4P10.0 2P1.25 in KENO-VI, where the last 6 entries describe the geometry. The P option simply repeats the dimension following the $\mathrm{P}$ for the number of times stated before the $\mathrm{P}$, and it reverses the sign every time. Therefore, 6P8.0 is equivalent to $8.0-8.08 .0-8.08 .0-8.0$.

Geometry comments: One comment can be entered for each UNIT in the geometry region data. Similarly, one comment can be entered for each ARRAY in the array definition data. A comment can be entered using the keyword $\mathbf{C O M}=$. This is followed by a comment with a maximum length of 132 characters. The comment must be preceded and terminated by a delimiter character. Acceptable delimiters include “ , ' , *,, , or !. One comment is allowed for each UNIT in the geometry region data. If multiple comments are entered for a UNIT, the last comment is used. The comment can be entered anywhere after the UNIT number where a keyword is expected (Sect. 8.1.2.4). See the following example. 
KENO V.a:

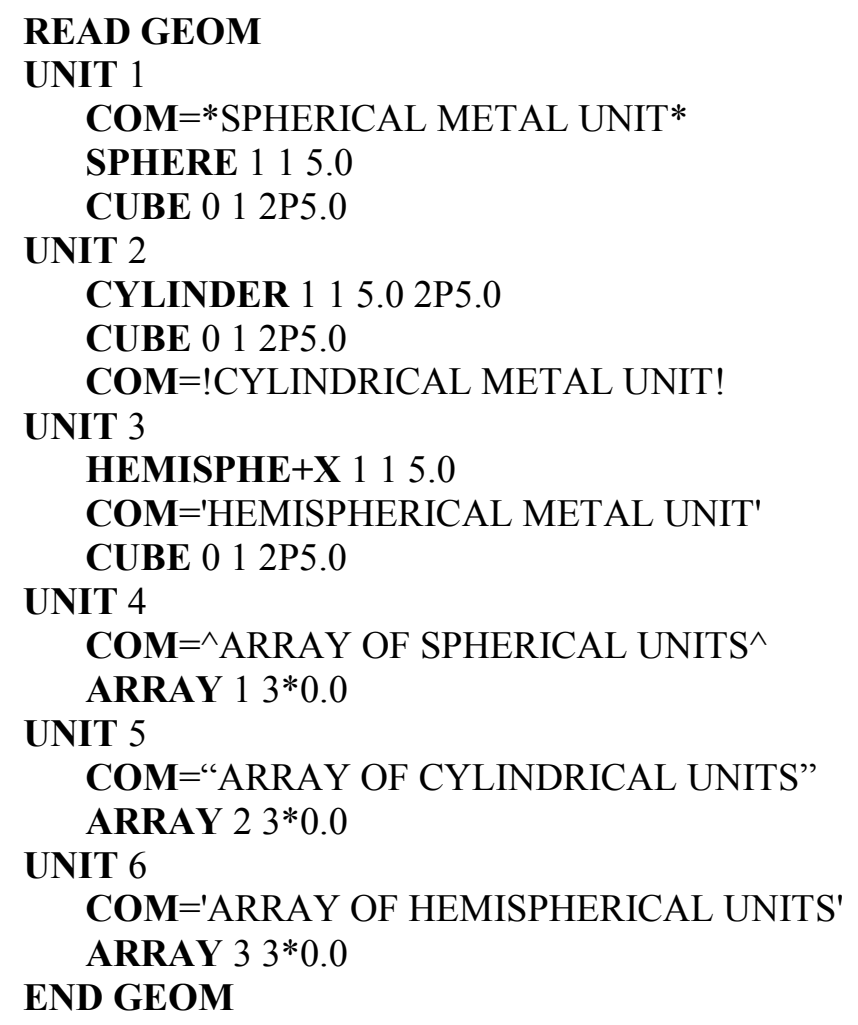




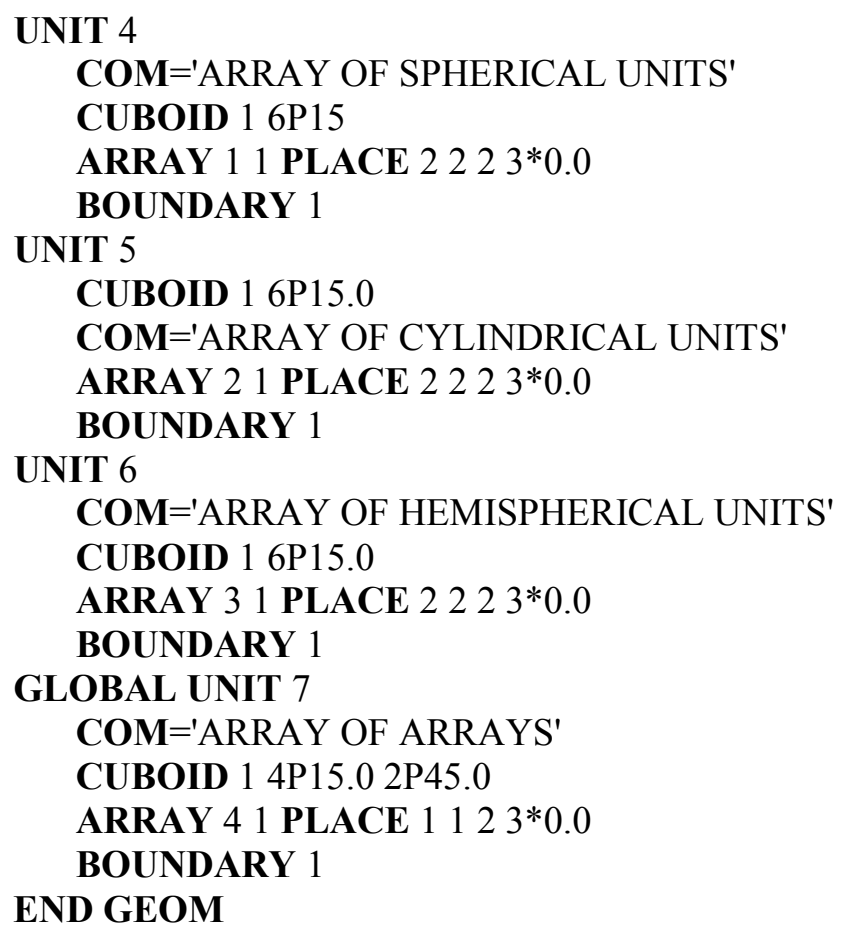

One comment is allowed for each array in the array definition data. The rules governing these comments are the same as those listed above. However, the comment for an ARRAY must precede the UNIT arrangement description, and it can precede the ARRAY number (Sect. 8.1.2.5). Examples of correct ARRAY comments are given below.

KENO V.a:

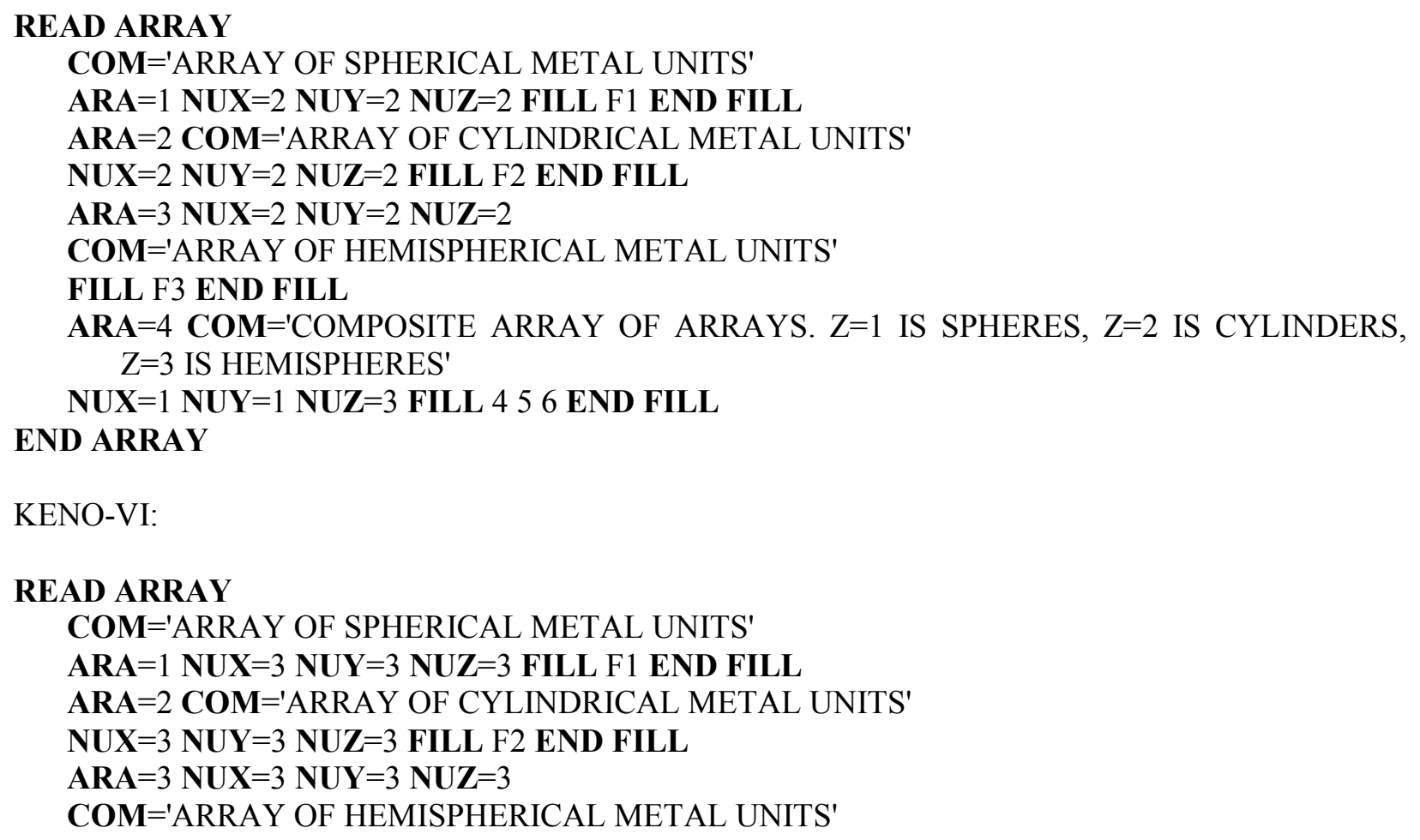


FILL F3 END FILL

ARA=4 COM='COMPOSITE ARRAY OF ARRAYS. $Z=1$ IS SPHERES, $Z=2$ IS CYLINDERS, $\mathrm{Z}=3$ IS HEMISPHERES'

\section{NUX=1 NUY=1 NUZ=3 FILL 456 END FILL} END ARRAY

Some of the basics of KENO geometry are illustrated in the following examples:

EXAMPLE 1. Assume a stack of six cylindrical disks each measuring $5 \mathrm{~cm}$ in radius and $2 \mathrm{~cm}$ thick. The bottom disk is composed of material 1, and the next disk is composed of material 2, etc., alternating throughout the stack. A square plate of material 3 that is $20 \mathrm{~cm}$ on a side and $2.5 \mathrm{~cm}$ thick is centered on top of the stack. This configuration is shown in Fig. 8.1.12.

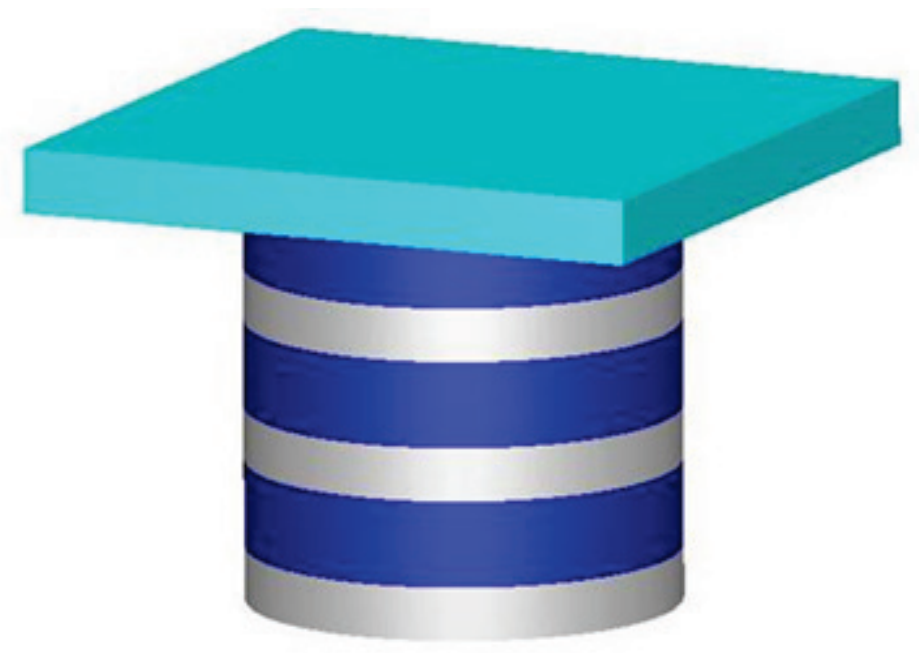

Fig. 8.1.12. Stack of disks with a square top.

This problem can be described as a single UNIT problem, describing the cylindrical portion first. In this instance, the origin has been chosen at the center bottom of the bottom disk. The bottom disk is defined by the first cylinder description, and the next disk is defined by the difference between the first and second cylinder descriptions. Since both disks have a radius of 5.0 and a $-Z$ length of 0.0 , the first cylinder containing material 1 exists from $Z=0.0$ to $Z=2.0$, and the second cylinder containing material 2 exists from $Z=2.0$ to $Z=4.0$. When all the disks have been described, a void cuboid having the same $\mathrm{X}$ and $\mathrm{Y}$ dimensions as the square plate and the same $\mathrm{Z}$ dimensions as the stack of disks is defined. The square plate of material 3 is then defined on top of the stack. Omission of the first cuboid description would result in the stack of disks being encased in a solid cuboid of material 3 instead of having a flat plate on top of the stack. The geometry input is shown below. 
Data description 1, Example 1.

KENO V.a:

\begin{tabular}{|c|c|c|}
\hline \\
\hline \multicolumn{3}{|l|}{ CYLINDER 11} \\
\hline CYLINDER 21 & 5.04 .0 & 0.0 \\
\hline CYLINDER 11 & 5.06 .0 & 0.0 \\
\hline CYLINDER 21 & 5.08 .0 & 0.0 \\
\hline CYLINDER 11 & 5.010 .0 & 0.0 \\
\hline CYLINDER 21 & 5.012 .0 & 0.0 \\
\hline CUBOID 0110 . & 0.010 .0 & -10.0 \\
\hline CUBOID 3110 & 10 & 0.0 \\
\hline
\end{tabular}

KENO-VI:

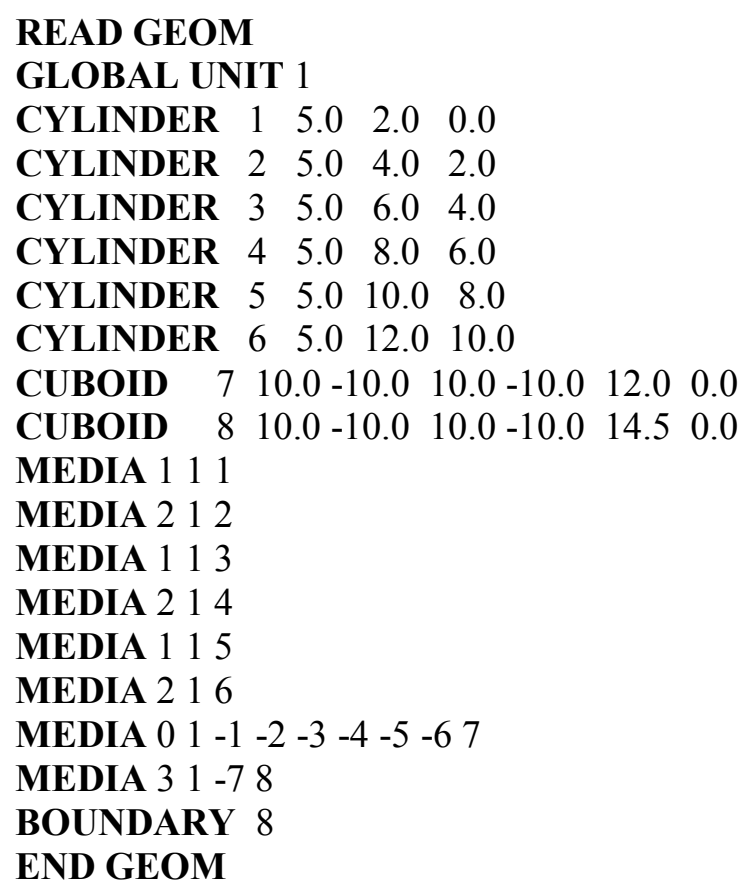

An alternative description of the same example is given below. The origin has been chosen at the center of the disk of material 1 nearest the center of the stack. This disk of material 1 is defined by the first cylinder description, and the disks of material 2 on either side of it are defined by the second cylinder description. The top and bottom disks of material 1 are defined by the third cylinder, and the top disk of material 2 is defined by the last cylinder. The square plate is defined by the two cuboids. This description is more efficient than the previous one because there are fewer surfaces to check for crossings. 
Data description 2, Example 1.

KENO V.a:

READ GEOM

CYLINDER $1115.0 \quad 1.0-1.0$

CYLINDER $21 \quad 5.0 \quad 3.0-3.0$

CYLINDER $1115.0 \quad 5.0-5.0$

CYLINDER $21 \quad 5.0 \quad 7.0-5.0$

CUBOID $00110.0-10.010 .0-10.07 .0$-5.0

CUBOID $3110.0-10.010 .0-10.09 .5-5.0$

KENO-VI:
READ GEOM
GLOBAL UNIT 1
MEDIA 1110
MEDIA 2 1 -10 20
MEDIA $111-2030$
MEDIA $21-3040$
MEDIA $0 \begin{array}{llll}0 & -40 & 50\end{array}$
MEDIA $31-5060$
BOUNDARY 60
END GEOM

CYLINDER $10 \quad 5.0 \quad 1.0-1.0$

CYLINDER $20 \quad 5.0 \quad 3.0-3.0$

CYLINDER $30 \quad 5.0 \quad 5.0-5.0$

CYLINDER $40 \quad 5.0 \quad 7.0-5.0$

CUBOID $5010.0-10.010 .0-10.07 .0-5.0$

CUBOID $6010.0-10.010 .0-10.0 \quad 9.5-5.0$

Example 1 can also be described as an ARRAY. Define three different UNIT types. UNIT 1 will define a disk of material 1, UNIT 2 will define a disk of material 2, and UNIT 3 will define the square plate of material 3. The origin of each UNIT is defined at the center bottom of the disk or plate being described. As mentioned earlier, only UNITs with a CUBE or CUBOID as their outer boundary can be placed in a cuboidal ARRAY. The geometry input for this arrangement is shown below.

Data description 3, Example 1.

KENO V.a:

READ GEOM

UNIT 1

$\begin{array}{llllll}\text { CYLINDER } & 1 & 1 & 5.0 & 2.0 & 0.0\end{array}$

CUBOID $00110.0-10.010 .0-10.02 .0 \quad 0.0$

UNIT 2

CYLINDER $2115.0 \quad 2.0 \quad 0.0$

CUBOID $\quad 0 \quad 1 \quad 10.0-10.0 \quad 10.0-10.02 .00 .0$

UNIT 3

$\begin{array}{llllllllll}\text { CUBOID } 3 & 1 & 10.0 & -10.0 & 10.0 & -10.0 & 2.5 & 0.0\end{array}$

END GEOM 
READ ARRAY NUX=1 NUY=1 NUZ=7 FILL 12122123 END ARRAY

NOTE: The ARRAY is assumed to be the GLOBAL ARRAY because only a single ARRAY is defined.

KENO-VI:

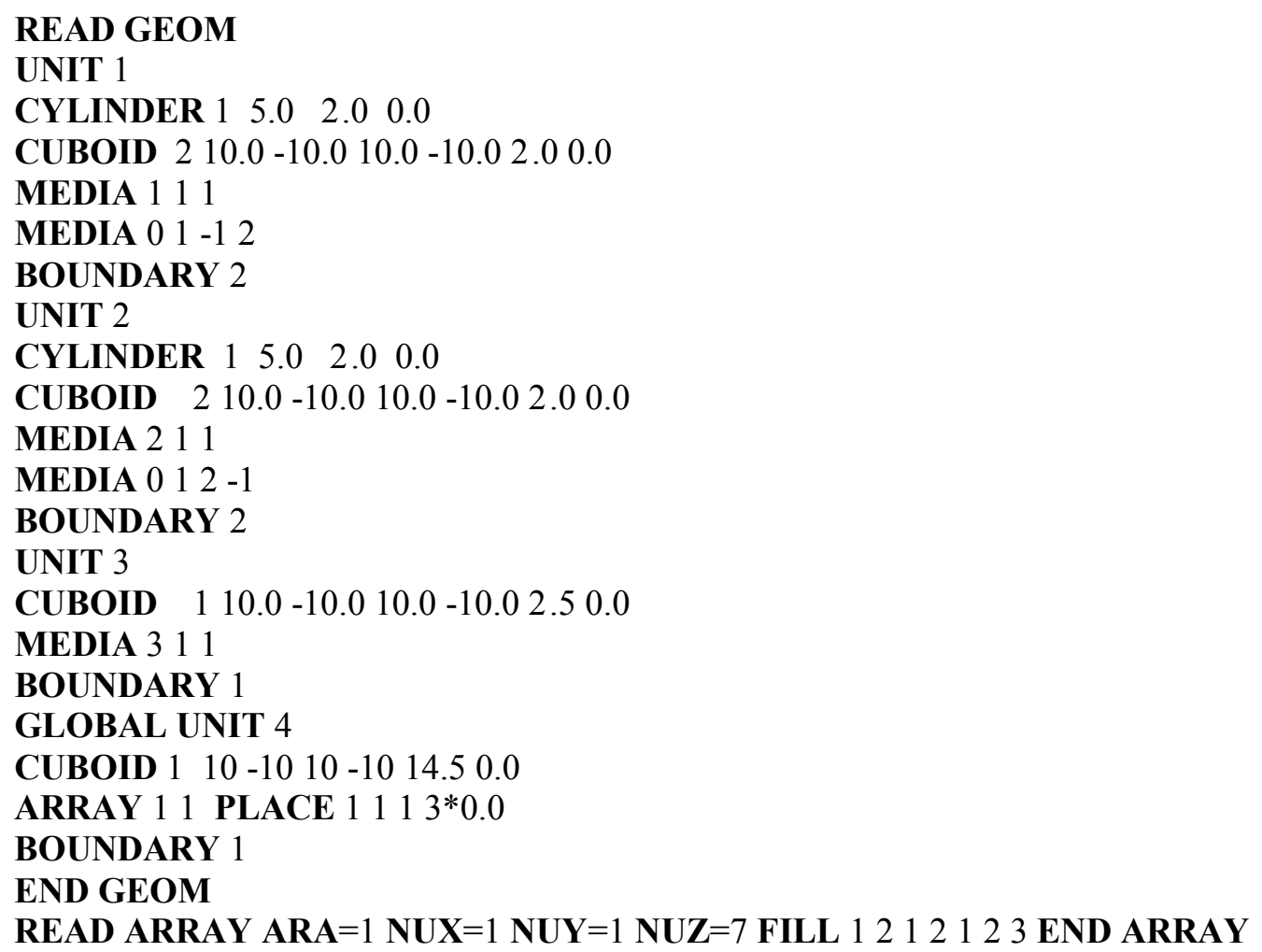

If the user prefers for the origin of each unit to be at its center, the geometry region data can be entered as shown below. The array data would be identical to that of data description 3, Example 1.

Data description 4, Example 1.

KENO V.a:

READ GEOM

UNIT 1

CYLINDER $1115.0 \quad 1.0-1.0$

CUBOID $\quad 0 \quad 1 \quad 10.0-10.010 .0-10.01 .0-1.0$

UNIT 2

CYLINDER $2115.0 \quad 1.0-1.0$

CUBOID $\quad 0 \quad 1 \quad 10.0-10.010 .0-10.01 .0-1.0$

UNIT 3

CUBOID $3110.0-10.010 .0-10.01 .25-1.25$

END GEOM

KENO-VI: 


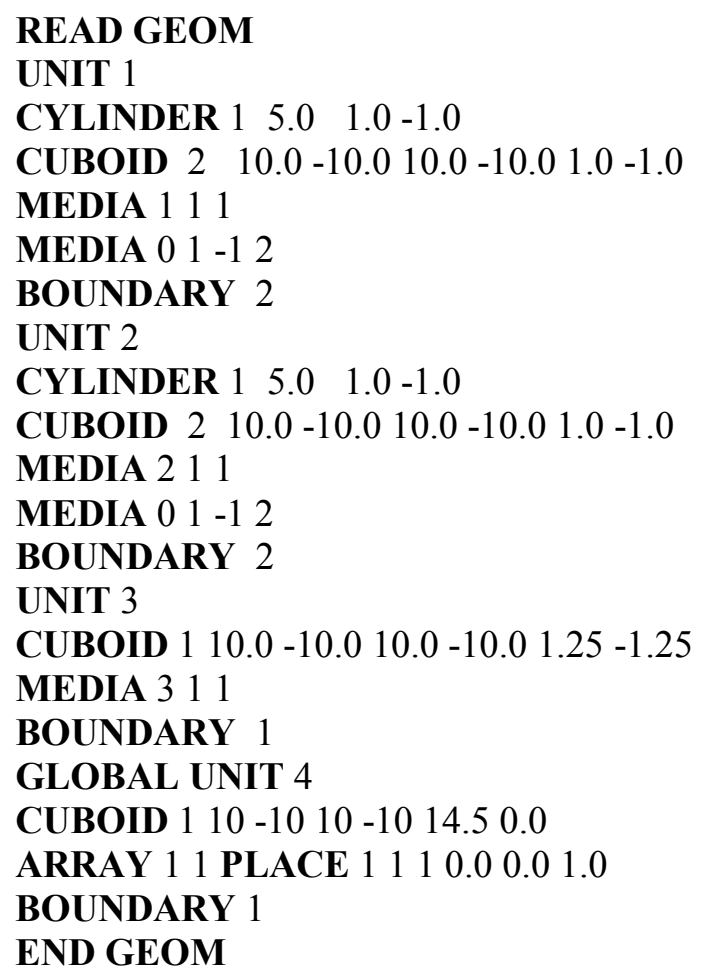

Be aware that each UNIT in a geometry description can have its origin defined independent of the other UNITs. It would be correct to use UNITs 1 and 3 from data descriptions 3, and UNIT 2 from data description 4. The array data would remain the same as data description 3, Example 1. The user should define the origin of each unit to be as convenient as possible for the chosen description. Care should be taken when assigning coordinates to the UNIT used to PLACE the ARRAY in its surrounding region.

Another method of describing Example 1 as a bare array is to define UNIT 1 to be a disk of material 1 topped by a disk of material 2. The origin has been chosen at the center bottom of the disk of material 1. UNIT 2 is the square plate of material 3 with the origin at the center of the UNIT. The ARRAY consists of three UNIT 1s, topped by a UNIT 2, as shown below.

Data description 5, Example 1.

KENO V.a:

READ GEOM

UNIT 1

$\begin{array}{llllll}\text { CYLINDER } & 1 & 1 & 5.0 & 2.0 & 0.0\end{array}$

CYLINDER $2115.0 \quad 4.0 \quad 0.0$

CUBOID $\quad 0 \quad 1 \quad 10.0-10.010 .0-10.04 .00 .0$

UNIT 2

CUBOID $3110.0-10.010 .0-10.01 .25-1.25$

END GEOM

READ ARRAY NUX=1 NUY=1 NUZ=4 FILL 3R 12 END ARRAY

KENO-VI: 


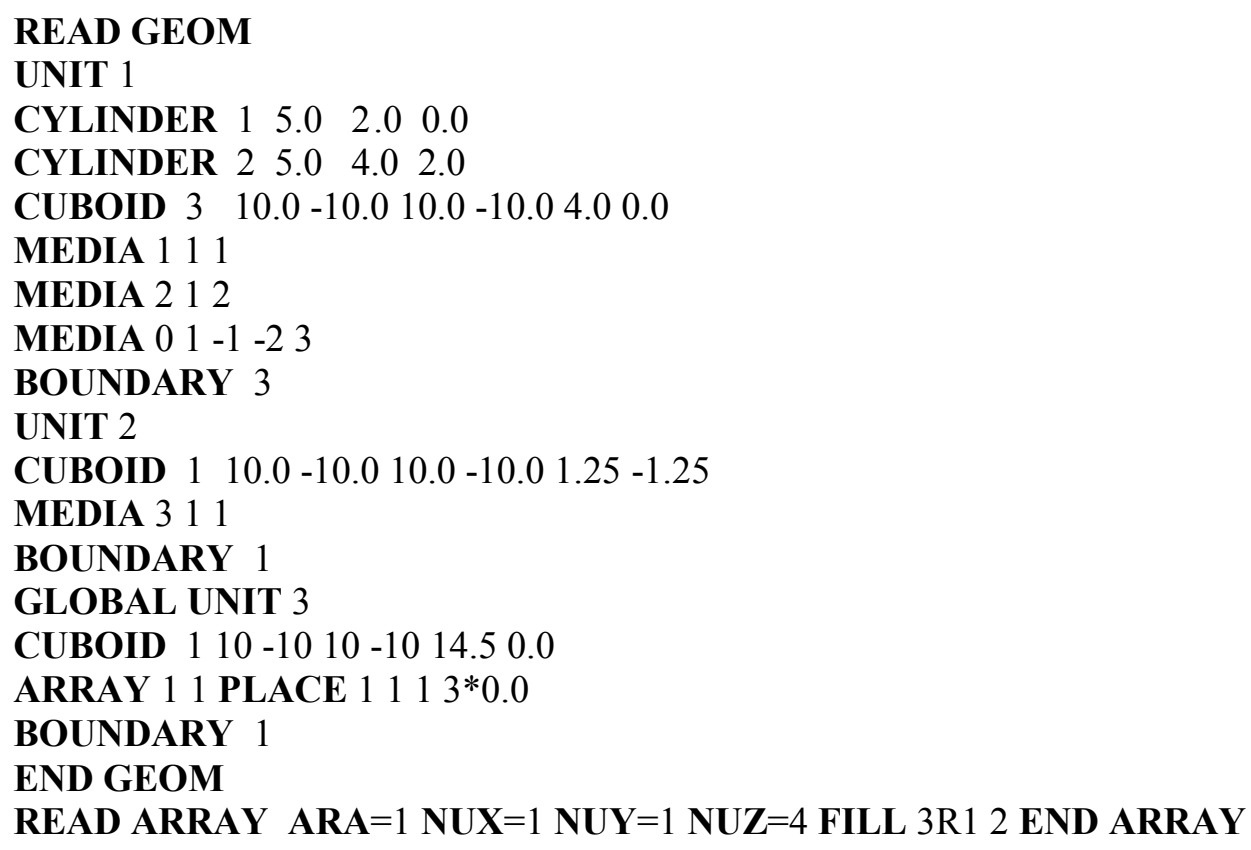

Example 1 can be described as a reflected ARRAY by treating the square plate as a reflector in the positive $\mathrm{Z}$ direction. One means of describing this situation is to define UNITs 1 and 2 as in data description 3, Example 1. The origin of the GLOBAL UNIT is defined to be at the center of the ARRAY. The corresponding input geometry is shown below.

Data description 6, Example 1.

KENO V.a:

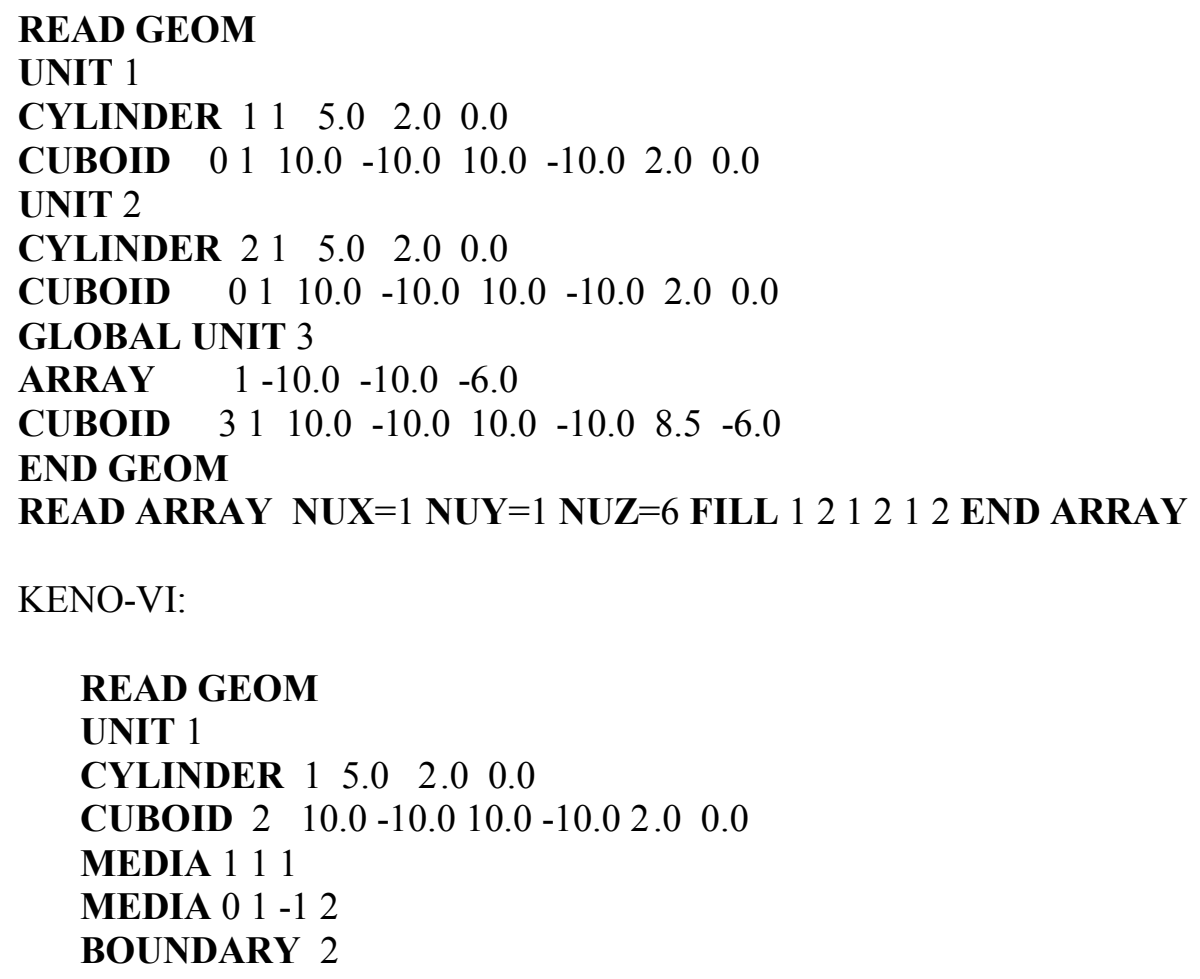


UNIT 2

$\begin{array}{lllll}\text { CYLINDER } & 1 & 5.0 & 2.0 & 0.0\end{array}$

$\begin{array}{llllllll}\text { CUBOID } 2 & 10.0 & -10.0 & 10.0 & -10.0 & 2.0 & 0.0\end{array}$

MEDIA 211

MEDIA $001-12$

BOUNDARY 2

GLOBAL UNIT 3

CUBOID $110.0-10.010 .0-10.06 .0-6.0$

CUBOID $210.0-10.010 .0-10.08 .5-6.0$

ARRAY 11 PLACE $11110.00 .0-6.0$

MEDIA $31-12$

BOUNDARY 2

END GEOM

READ ARRAY ARA=1 NUX=1 NUY=1 NUZ=6 FILL 12121212 END ARRAY

The user could have chosen the origin of the ARRAY boundary to be at the center bottom of the ARRAY, in which case the geometry description for the GLOBAL UNIT would be:

KENO V.a:

ARRAY $\quad 1-10.0-10.0 \quad 0.0$

$\begin{array}{lllllllll}\text { CUBOID } & 3 & 1 & 10.0 & -10.0 & 10.0 & -10.0 & 14.5 & 0.0\end{array}$

or

ARRAY $\quad 1 \quad-10.0 \quad-10.00 .0$

REPLICATE $3 \quad 4 * 0.02 .5 \quad 01$

The reflector region at the top of the array can be added by using a CUBOID or by using a REPLICATE description in KENO V.a. Recall that there is no REPLICATE function in KENO-VI.

KENO-VI:

CUBOID $110.0-10.010 .0-10.012 .0 \quad 0.0$

CUBOID $2 \quad 10.0-10.010 .0-10.014 .5 \quad 0.0$

ARRAY 11 PLACE $11113 * 0.0$

MEDIA 3 1 $1-12$

BOUNDARY 2

A simpler method of describing Example 1 as a reflected array is to define only one unit as in data description 5, Example 1. The square plate is treated as a reflector, as in data description 6, Example 1. The input for this arrangement is given below.

Data description 7, Example 1.

KENO V.a:

READ GEOM

UNIT 1

CYLINDER $111 \quad 5.0 \quad 2.0 \quad 0.0$

$\begin{array}{llllll}\text { CYLINDER } & 2 & 1 & 5.0 & 4.0 & 0.0\end{array}$ 
$\begin{array}{lllllllll}\text { CUBOID } & 0 & 1 & 10.0 & -10.0 & 10.0 & -10.0 & 4.0 & 0.0\end{array}$

GLOBAL UNIT 2

ARRAY $\quad 1 \quad-10.0 \quad-10.0 \quad 0.0$

$\begin{array}{lllllllll}\text { CUBOID } & 3 & 1 & 10.0 & -10.0 & 10.0 & -10.0 & 14.5 & 0.0\end{array}$

END GEOM

READ ARRAY NUX $=1$ NUY $=1$ NUZ $=3$ END ARRAY

KENO-VI:

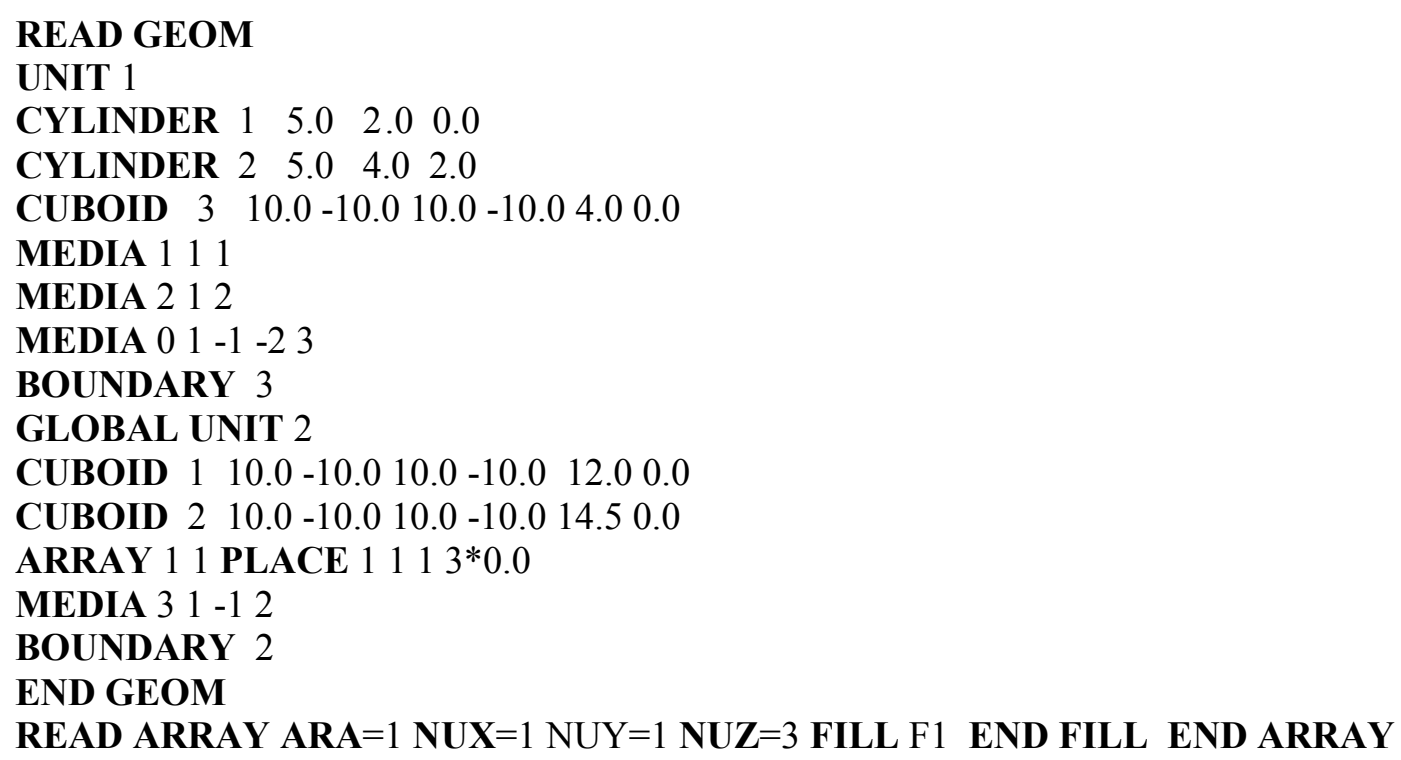

EXAMPLE 2. Assume that the stack of six disks in Example 1 is placed at the center bottom of a cylindrical container composed of material 6 whose inside diameter is $16.0 \mathrm{~cm}$. The bottom and sides of the container are $0.25 \mathrm{~cm}$ thick, the top is open, and the total height of the container is $18.25 \mathrm{~cm}$. Assume the square plate of Example 1 is centered on top of the container.

The geometry input can be described utilizing most of the data description methods associated with Example 1. One method of describing Example 2 as a single UNIT is given below.

Data description 1, Example 2.

KENO V.a:

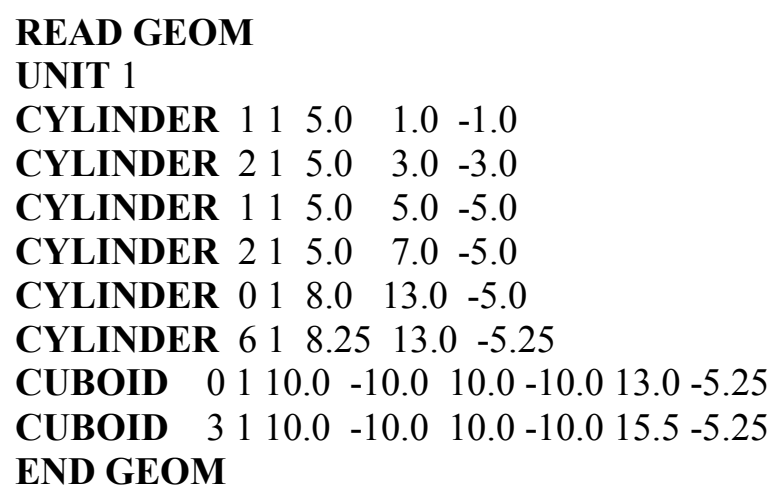


KENO-VI:

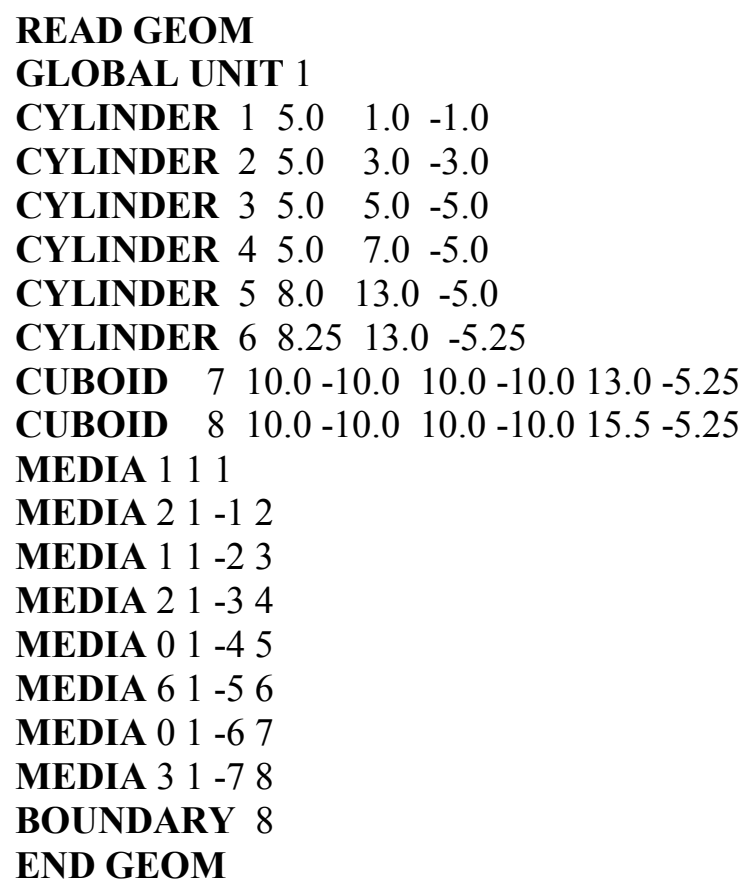

In the above description, the origin is defined to be at the center of the disk of material 1 nearest the center of the stack. This disk is defined by the first cylinder description. The disks of material 2 above and below it are defined by the second cylinder description. The disks of material 1 above and below them are defined by the third cylinder description. The top disk of material 2 is defined by the fourth cylinder description. The void interior of the container is defined by the fifth cylinder description. The container is defined by the last cylinder description. The first cuboid description is used to define a void whose $\mathrm{X}$ and $\mathrm{Y}$ dimensions are the same as the square plate and whose $\mathrm{Z}$ dimensions are the same as the container. The last cuboid description defines the square plate. Omission of the first cuboid description would result in the container being encased in a solid cuboid of material 3 . Thus, both cuboids are necessary to properly define the square plate.

Example 2 can be described as a reflected ARRAY. One of the descriptions uses only one UNIT and is similar to data description 7, Example 1. This description is shown below.

Data description 2, Example 2.

KENO V.a:

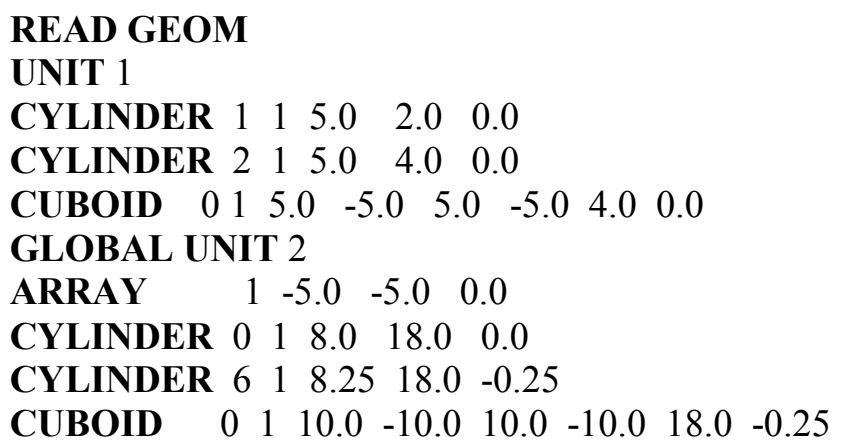


$\begin{array}{lllllllll}\text { CUBOID } & 3 & 1 & 10.0 & -10.0 & 10.0 & -10.0 & 20.5 & -0.25\end{array}$

END GEOM

READ ARRAY NUX=1 NUY $=1$ NUZ $=3$ END ARRAY

KENO-VI:

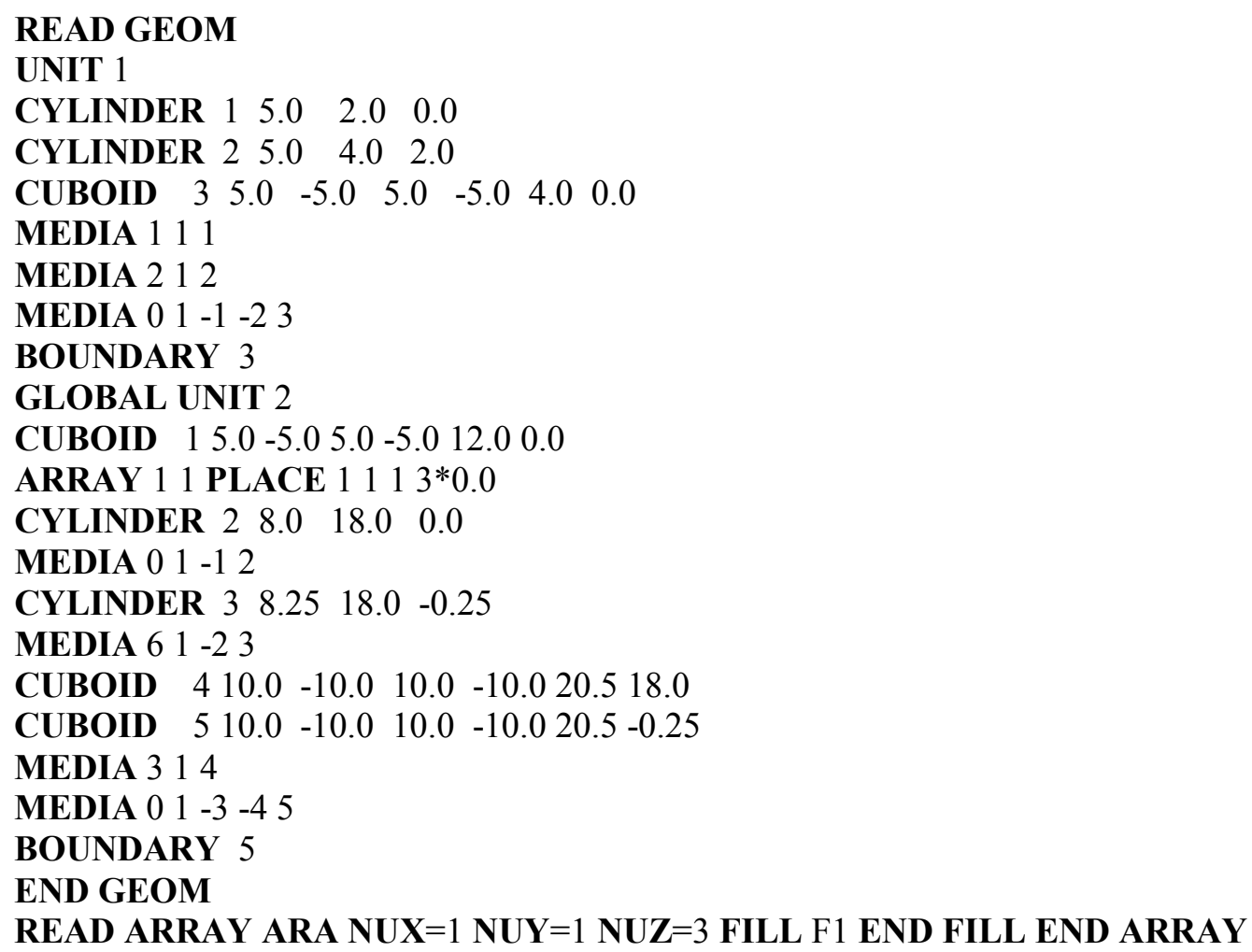

In the above data description, the first two cylinder descriptions define a disk of material 1 with a disk of material 2 directly on top of it. A tight fitting void cuboid is placed around them so they can be stacked three high to achieve the stack of disks shown in Example 1, Fig. 8.1.12. This array comprises the array portion of the geometry region description. The origin of the array boundary, a tight fitting cube or cuboid that encompasses the array, is defined by the ARRAY description. Everything after the ARRAY record is considered part of the reflector. The first cylinder after the ARRAY record defines the void interior of the cylindrical container. The next cylinder defines the walls of the container. The second-to-last cuboid defines a void volume outside the container from its bottom to its top and having the same $\mathrm{X}$ and $\mathrm{Y}$ dimensions as the square plate. The last cuboid defines the square plate of material 3 that is sitting on top of the container.

Another method to describe Example 2 is as an array composed of units that contain both the stack and container. This description requires a minimum of four units to describe the problem. This configuration is given below in data description 3, Example 2. 
Data description 3, Example 2.

KENO V.a:

READ GEOM

UNIT 1

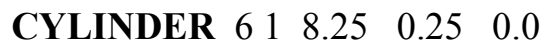

$\begin{array}{lllllllll}\text { CUBOID } & 0 & 1 & 10.0 & -10.0 & 10.0 & -10.0 & 0.25 & 0.0\end{array}$

UNIT 2

$\begin{array}{llllll}\text { CYLINDER } & 1 & 1 & 5.0 & 2.0 & 0.0\end{array}$

$\begin{array}{llllll}\text { CYLINDER } & 2 & 1 & 5.0 & 4.0 & 0.0\end{array}$

$\begin{array}{llllll}\text { CYLINDER } & 0 & 1 & 8.0 & 4.0 & 0.0\end{array}$

$\begin{array}{llllll}\text { CYLINDER } & 6 & 1 & 8.25 & 4.0 & 0.0\end{array}$

$\begin{array}{llllllllll}\text { CUBOID } & 0 & 1 & 10.0 & -10.0 & 10.0 & -10.0 & 4.0 & 0.0\end{array}$

UNIT 3

$\begin{array}{llllll}\text { CYLINDER } & 0 & 1 & 8.0 & 3.0 & -3.0\end{array}$

$\begin{array}{llllll}\text { CYLINDER } & 6 & 1 & 8.25 & 3.0 & -3.0\end{array}$

CUBOID $\quad \begin{array}{lllllllll}0 & 1 & 10.0 & -10.0 & 10.0 & -10.0 & 3.0 & -3.0\end{array}$

$\begin{array}{llllllllll}\text { CUBOID } & 3 & 1 & 10.0 & -10.0 & 10.0 & -10.0 & 5.5 & -3.0\end{array}$

END GEOM

READ ARRAY NUX=1 NUY=1 NUZ=5 FILL 1 3R2 3 END ARRAY

KENO-VI:

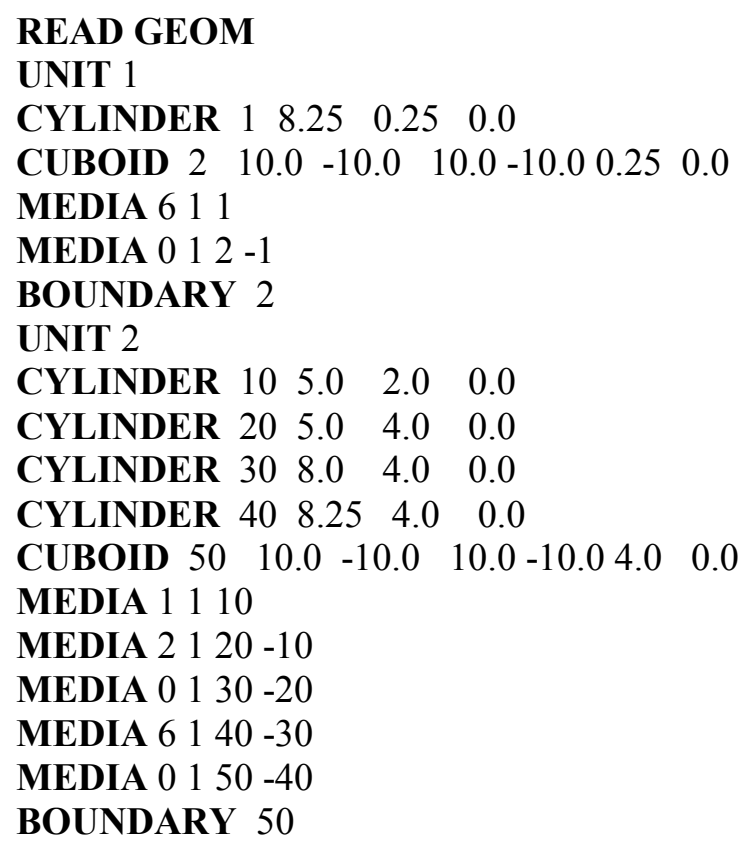


UNIT 3

$\begin{array}{lllll}\text { CYLINDER } & 1 & 8.0 & 3.0 & -3.0\end{array}$

$\begin{array}{lllll}\text { CYLINDER } & 2 & 8.25 & 3.0 & -3.0\end{array}$

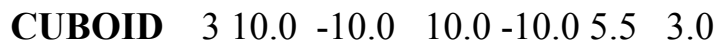

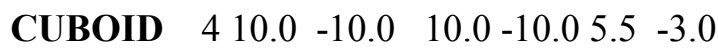

MEDIA $0 \begin{array}{llll}0 & 1 & 1\end{array}$

MEDIA $6 \begin{array}{llll} & 1 & 2 & -1\end{array}$

MEDIA $3 \quad 13$

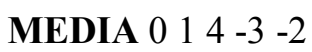

BOUNDARY 4

GLOBAL UNIT 4

CUBOID $110.0-10.010 .0-10.020 .750 .0$

ARRAY 11 PLACE $11113 * 0.0$

BOUNDARY 1

END GEOM

READ ARRAY NUX=1 NUY=1 NUZ=5 FILL 1 3R2 3 END ARRAY

In the above description, UNIT 1 is the bottom of the cylindrical container. The void CUBOID is only as tall as the bottom of the container, and its X and Y dimensions are the same as the square plate on top of the container. If all the UNITs in the ARRAY use these same dimensions in the X and Y directions, the requirement that adjacent faces of units in contact with each other must be the same size and shape is satisfied. This ARRAY is stacked in the Z direction, so all UNITs must have the same overall dimensions in the $\mathrm{X}$ direction and in the Y direction. UNIT 2 will be used in the ARRAY three times to create the stack of disks. It contains a disk of material 1 topped by a disk of material 2 . The portion of the container that contains the disks and the cuboid that defines the outer boundaries of the unit are included in UNIT 2. UNIT 3 describes the empty top portion of the container and the square plate on top of it. The $\mathrm{Z}$ dimensions of UNIT 3 were determined by subtracting three times the total Z dimension of UNIT 2 from the inside height of the container $[18.0-(3 \times 4.0)=6.0]$. This can also be determined from the overall height of the container by subtracting off the bottom thickness of the container and three times the height of UNIT 2 [18.25 $-0.25-(3 \times 4.0)=6.0]$. The origin of UNIT 3 is located at the center of this distance. For the KENO-VI input, a GLOBAL UNIT is also provided, UNIT 4, containing the ARRAY built with UNITs 1, 2, 3.

EXAMPLE 3. Refer to Example 1, Fig. 8.1.12, and imagine a HOLE $1.5 \mathrm{~cm}$ in diameter is drilled along the centerline of the stack through the disks and the square plate. In KENO V.a this HOLE would eliminate the possibility of describing the system as a single unit because the HOLE in the center of the alternating materials of the stack cannot be described in a manner that allows each successive geometry region to encompass the regions interior to it. Therefore, it must be described as an ARRAY. The square plate on the top of the disks is defined as a UNIT in the ARRAY. In the geometry description given below, the square plate is defined in UNIT 3. KENO-VI can easily describe this configuration as a single UNIT. 
Data description 1, Example 3.

KENO V.a:

READ GEOM

UNIT 1

$\begin{array}{llllll}\text { CYLINDER } & 0 & 1 & 0.75 & 2.0 & 0.0\end{array}$

$\begin{array}{llllll}\text { CYLINDER } & 1 & 1 & 5.0 & 2.0 & 0.0\end{array}$

CUBOID $\quad 0 \quad 1110.0 \quad-10.010 .0-10.02 .00 .0$

UNIT 2

$\begin{array}{llllll}\text { CYLINDER } & 0 & 1 & 0.75 & 2.0 & 0.0\end{array}$

$\begin{array}{llllll}\text { CYLINDER } & 2 & 1 & 5.0 & 2.0 & 0.0\end{array}$

CUBOID $\quad \begin{array}{lllllllll}0 & 1 & 10.0 & -10.0 & 10.0 & -10.0 & 2.0 & 0.0\end{array}$

UNIT 3

$\begin{array}{llllll}\text { CYLINDER } & 0 & 1 & 0.75 & 2.5 & 0.0\end{array}$

$\begin{array}{llllllllll}\text { CUBOID } & 3 & 1 & 10.0 & -10.0 & 10.0 & -10.0 & 2.5 & 0.0\end{array}$

END GEOM

READ ARRAY NUX=1 NUY=1 NUZ=7 FILL 12 2Q2 3 END FILL END ARRAY

KENO-VI:

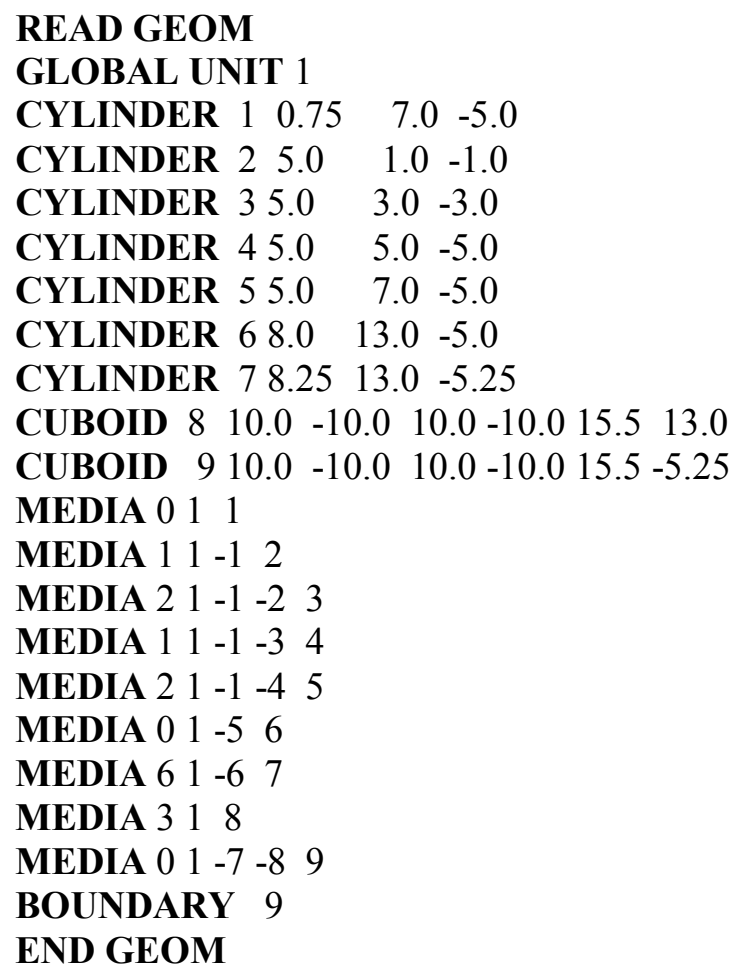

In data description 1, Example 3 above, KENO V.a input, UNIT 1 describes a disk of material 1 with a HOLE through its centerline. The first CYLINDER defines the HOLE, the second defines the rest of the disk, and the CUBOID defines the size of the UNIT to be consistent with the square plate so they can be stacked together in an ARRAY. UNIT 2 describes a disk of material 2 in similar fashion. UNIT 3 describes the square plate of material 3 with a HOLE through its center. The CYLINDER defines the HOLE and the CUBOID defines the square plate. These three UNITs are stacked in the Z direction to achieve the composite system. This is represented by FILL 12 2Q2 3. The 2Q2 repeats the two entries 
preceding the 2Q2 twice. Alternatively, this can be achieved by entering FILL $1 \begin{array}{llllllll}1 & 2 & 1 & 2 & 1 & 2 & 3 & \text { END }\end{array}$ FILL. The same ARRAY can also be achieved using the LOOP option. An example of the data for this option is:

\section{LOOP 1 6R1 1522 6R1 2623 6R1 771 END LOOP.}

UNIT 1 is placed at the $X=1, Y=1$, and $Z=1,3,5$ positions of the ARRAY by entering $16 R 1152$. UNIT 2 is positioned at the $X=1, Y=1$ and $Z=2,4,6$ positions in the ARRAY by entering $26 R 1262$. UNIT 3 is placed at the $X=1, Y=1, Z=7$ position of the ARRAY by entering $36 R 1771$. See Sect. 8.1.2.5 for additional information regarding ARRAY specifications.

For the KENO-VI input, UNIT 1 contains the entire problem description. The first CYLINDER describes the $1.5 \mathrm{~cm}$ diameter hole through the stack. The next four CYLINDERs define the stack. The sixth and seventh CYLINDERs describe the void and container. The two CUBOIDs describe the top plate and surrounding global region of void. The MEDIA cards are used to place the materials in the appropriate regions.

EXAMPLE 4. Assume two large cylinders that are $2.5 \mathrm{~cm}$ in radius and $5 \mathrm{~cm}$ long are connected by a smaller cylinder that is $0.5 \mathrm{~cm}$ in radius and $10 \mathrm{~cm}$ long, as shown in Fig. 8.1.13. All three cylinders are composed of material 1. By starting the geometry description in the small cylinder, this system can be described as a single unit.

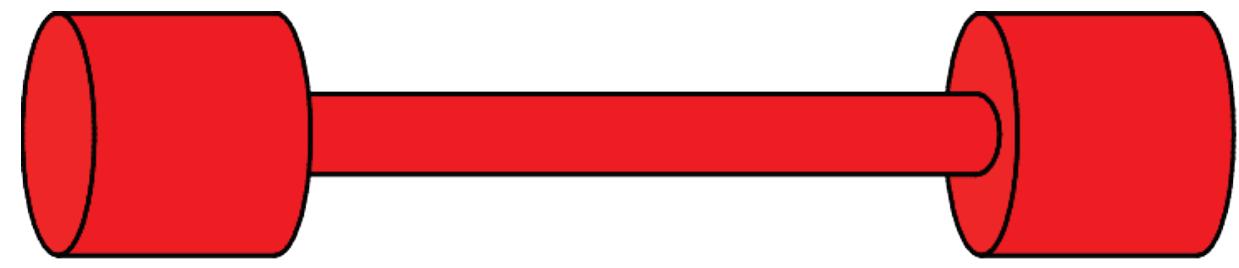

Fig. 8.1.13. Two large cylinders joined axially by a small cylinder.

Data description 1, Example 4.

KENO V.a:

READ GEOM

$\begin{array}{llllll}\text { CYLINDER } & 1 & 1 & 0.5 & 5.0 & -5.0\end{array}$

$\begin{array}{llllll}\text { CYLINDER } & 0 & 1 & 2.5 & 5.0 & -5.0\end{array}$

CYLINDER $112.510 .0-10.0$

END GEOM

KENO-VI:

READ GEOM

GLOBAL UNIT 1

$\begin{array}{lllll}\text { XCYLINDER } & 1 & 0.5 & 5.0 & -5.0\end{array}$

$\begin{array}{lllll}\text { XCYLINDER } & 2 & 2.5 & 5.0 & -5.0\end{array}$

XCYLINDER $32.510 .0-10.0$

MEDIA 111 
MEDIA $0 \begin{array}{llll}0 & 1 & 2 & -1\end{array}$

MEDIA 1113 - 1

BOUNDARY 3

END GEOM

The origin is at the center of the small cylinder, which is described by the first cylinder description. The second cylinder description defines a void cylinder surrounding the small cylinder. Its radius is the same as the large cylinders, and its height (length) coincides with that of the small cylinder. The last cylinder description defines the large cylinders on either end of the small cylinder. In KENO V.a, because this problem does not specify otherwise, the length of the CYLINDERs is assumed to coincide with the $\mathrm{Z}$ axis. In KENO-VI, because the problem was created using XCYLINDERs, the long axes of the CYLINDERs coincide with the $\mathrm{X}$ axis.

EXAMPLE 5. Assume two large cylinders with a center-to-center spacing of $15 \mathrm{~cm}$, each having a radius of $2.5 \mathrm{~cm}$ and length of $5 \mathrm{~cm}$, are connected radially by a small cylinder having a radius of $1.5 \mathrm{~cm}$, as shown in Fig. 8.1.14

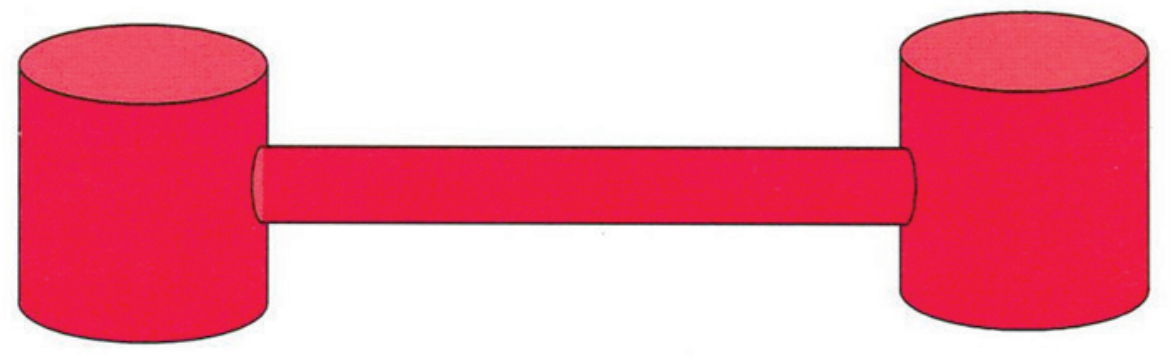

Fig. 8.1.14. Two large cylinders radially connected by a small cylinder.

This system cannot be described rigorously in KENO V.a geometry because the intersection of the cylinders cannot be described. However, it can be approximated two ways, as shown in Fig. 8.1.15. The top approximation is described in data description 1, Example 5. The bottom approximation is described in data description 2, Example 5, and data description 3, Example 5. These may be poor approximations for criticality safety calculations.

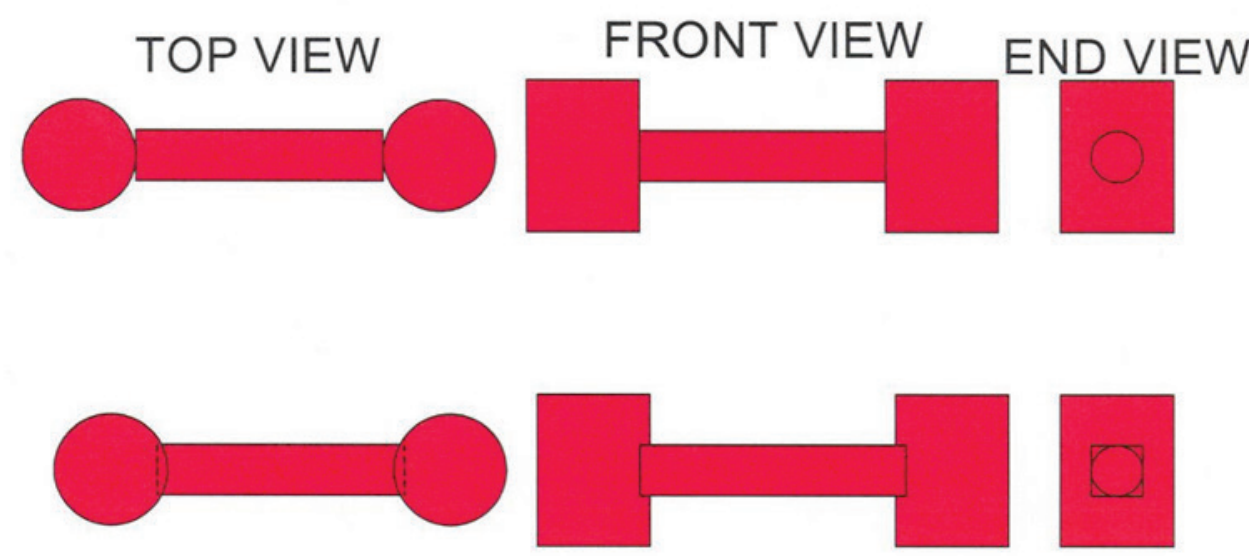

Fig. 8.1.15. KENO V.a approximations of cylindrical intersections. 
Data description 1, Example 5.

KENO V.a:

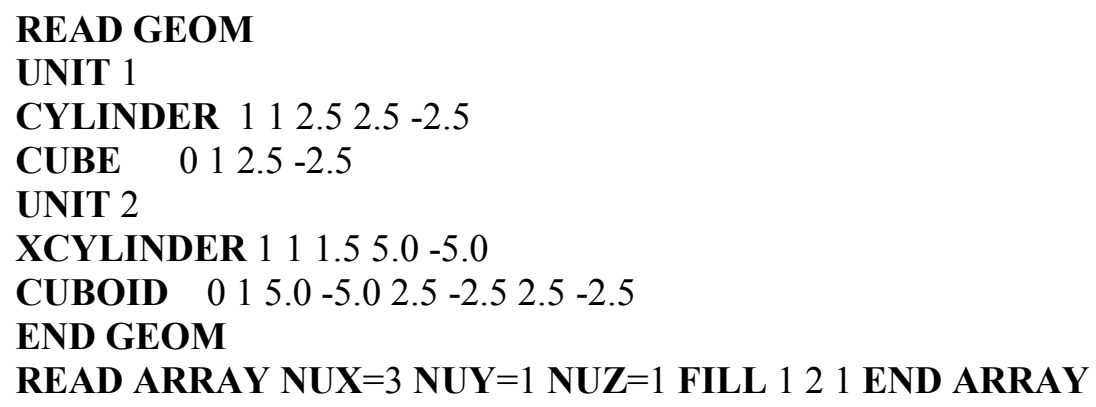

UNIT 1 defines a large CYLINDER, and UNIT 2 describes the small CYLINDER. In both UNITs the origin is at the center of the CYLINDER. The large CYLINDERs have their centerlines along the $Z$ axis and the small CYLINDER has its length along the $\mathrm{X}$ axis.

Data description 2, Example 5.

KENO V.a:

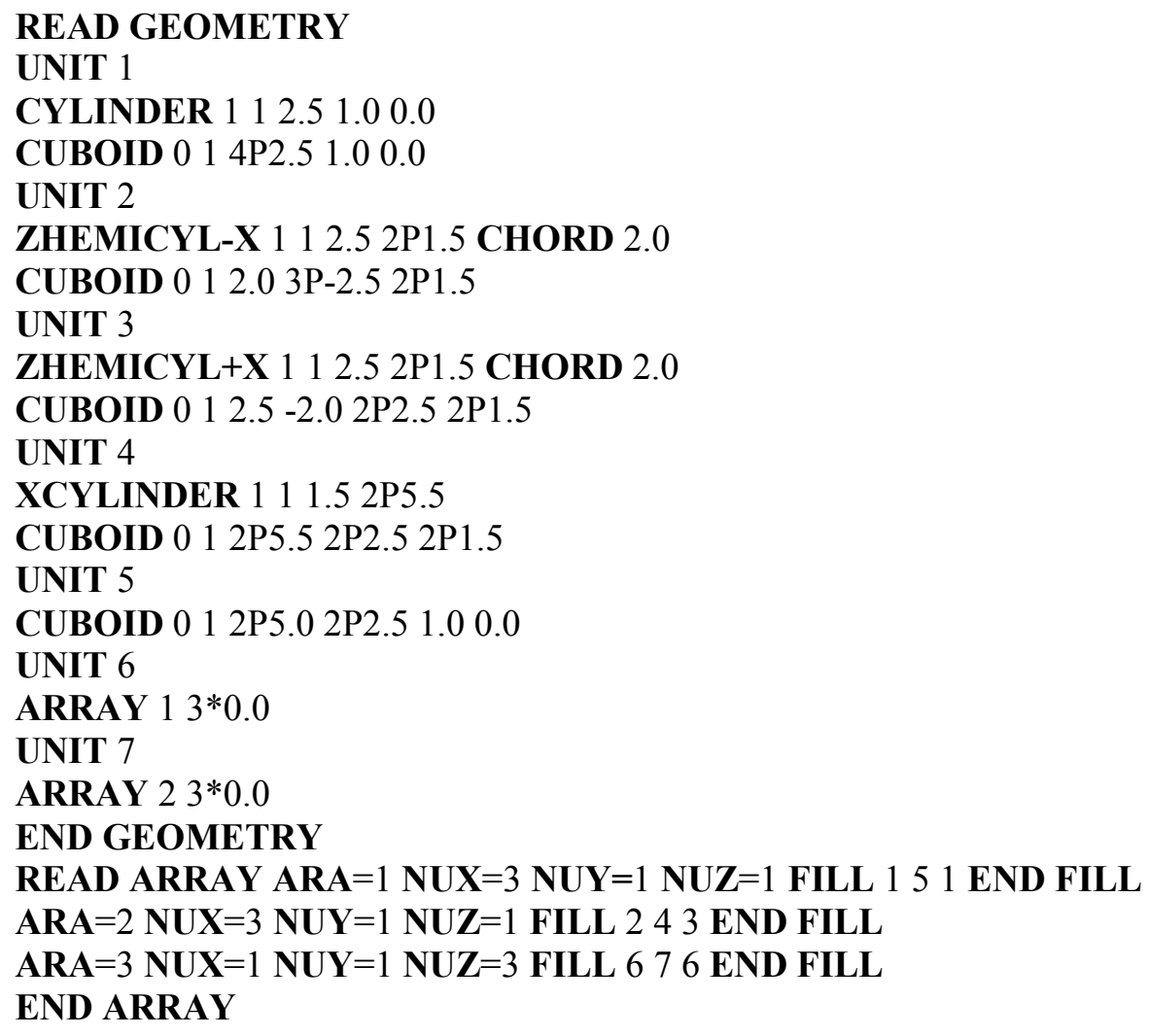

The above geometry description uses ARRAYs of ARRAYs (see Sect. 8.1.3.6.3) to describe the bottom approximation of Fig. 8.1.15. UNIT 1 defines a large CYLINDER that has a radius of $2.5 \mathrm{~cm}$ and a height of $10 \mathrm{~cm}$ inside a close-fitting CUBOID. This is used in both large CYLINDERs as the portion of the large CYLINDER that exists above and below the region where the small CYLINDER joins it. 
UNIT 5 is the spacing between the tops of the two large CYLINDERs and the spacing between the bottoms of the two large CYLINDERs. ARRAY 1 thus defines the bottom of the system: two short CYLINDERs (UNIT 1s) separated by $10 \mathrm{~cm}$ (UNIT 5 is the separation). UNIT 6 contains ARRAY 1.

UNIT 2 is the left hemicylinder that adjoins the horizontal CYLINDER, and UNIT 3 is the right hemicylinder that adjoins the horizontal CYLINDER. UNIT 4 defines the horizontal CYLINDER. ARRAY 2 contains UNITs 2, 4, and 3 from left to right. This defines the central portion of the system where the horizontal CYLINDER adjoins the two hemicylinders. These hemicylinders are larger than half CYLINDERs. UNIT 7 contains ARRAY 2. The entire system is achieved by stacking a UNIT 6 above and below the UNIT 7 as defined in ARRAY 3, the GLOBAL ARRAY.

Data description 3, Example 5.

KENO V.a:

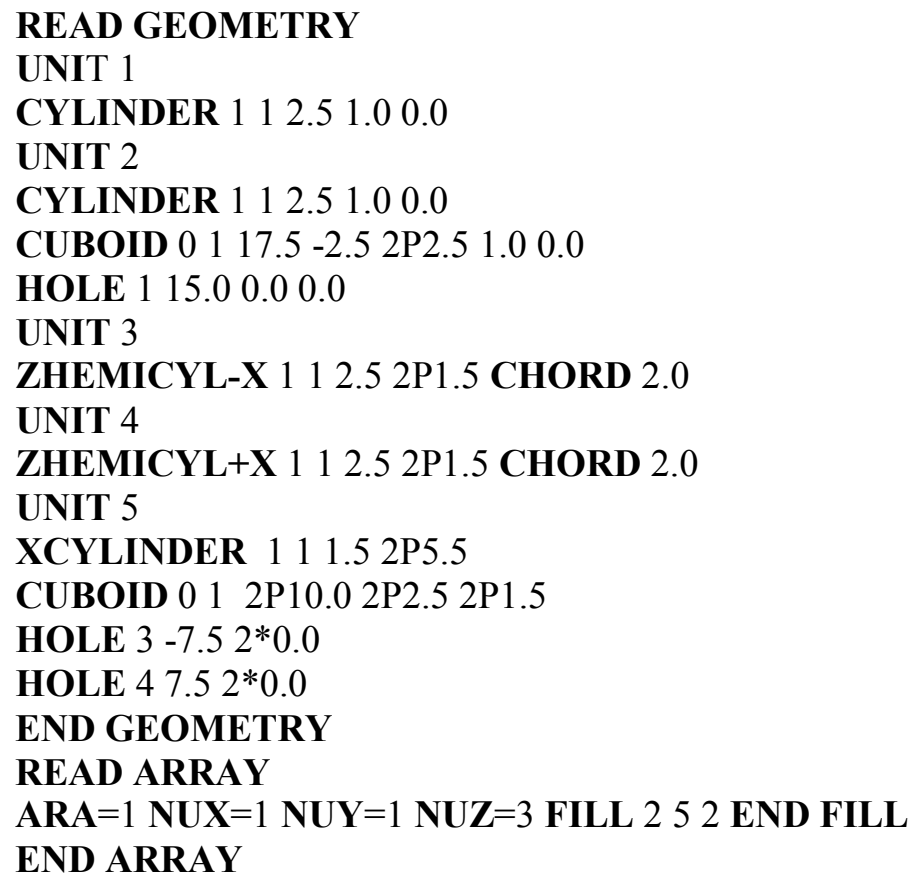

The above geometry description uses HOLEs (see Sect. 8.1.3.6.1) to describe the bottom approximation of Fig. 8.1.15. UNIT 1 defines a large CYLINDER with a radius of $2.5 \mathrm{~cm}$ and a height of $1.0 \mathrm{~cm}$. UNIT 2 defines the same CYLINDER within a CUBOID that extends from $X=-2.5$ to $X=17.5$, from $\mathrm{Y}=-2.5$ to $\mathrm{Y}=2.5$, and $\mathrm{Z}=0.0$ to $\mathrm{Z}=1.0$. The origin of the CYLINDER is at $(0.0,0.0,0.0)$. Thus UNIT 2 describes the top and bottom of the CYLINDER on the left. UNIT 1 is positioned within this CUBOID as a HOLE with its origin at $(15.0,0.0,0.0)$ to describe the top and bottom of the CYLINDER on the right. UNIT 3 is the left hemicylinder that adjoins the horizontal CYLINDER, and UNIT 4 is the right hemicylinder that adjoins the horizontal CYLINDER. UNIT 5 defines the horizontal CYLINDER with its origin at the center within a CUBOID that extends from $\mathrm{X}=-10.0$ to $\mathrm{X}=+10.0, \mathrm{Y}=-2.5$ to $\mathrm{Y}=$ 2.5 , and $Z=-1.5$ to $Z=1.5$. UNIT 3 is positioned to the left of the horizontal CYLINDER, and UNIT 4 is positioned to the right of the horizontal CYLINDER by using HOLEs. The entire system is achieved by stacking a UNIT 2 above and below UNIT 5 as shown in the ARRAY data. 
This same geometry description can be used with UNIT 2 redefined, having its origin defined so that it extends from $\mathrm{X}=-10$ to $\mathrm{X}=10, \mathrm{Y}=-2.5$ to $\mathrm{Y}=2.5$, and $\mathrm{Z}=0.0$ to $\mathrm{Z}=1$. In this instance, the geometry data would be identical except for UNIT 2. This alternative description of UNIT 2 is

KENO V.a:

UNIT 2

CYLINDER 112.51 .0 0.0 ORIGIN -7.5 0.0

CUBOID $012 \mathrm{P} 10.02 \mathrm{P} 2.51 .00 .0$

HOLE 17.50 .00 .0

This system can be easily described in KENO-VI geometry because intersections are allowed. The small CYLINDER is rotated in data description 1, Example 5.

Data description 1, Example 5.

KENO-VI:

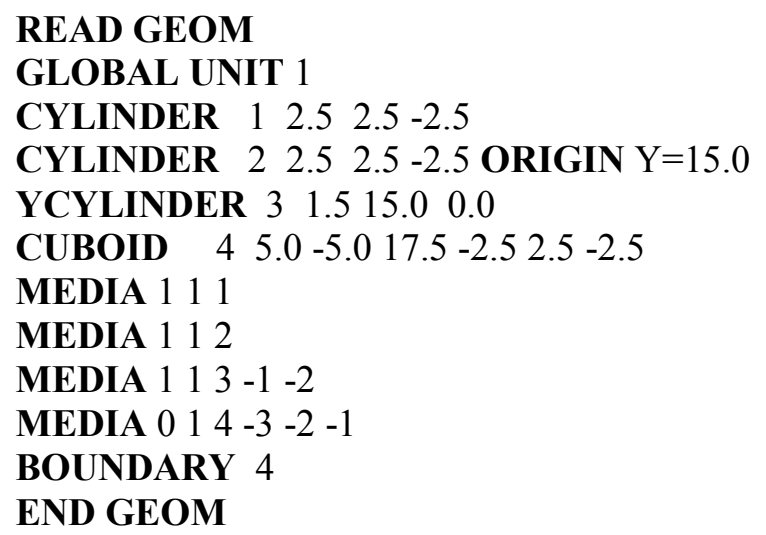

The first and second CYLINDERs define the two large CYLINDERs, and the third CYLINDER describes the small connecting CYLINDER. The two large CYLINDERs are oriented along the $\mathrm{Z}$ axis. The second large cylinder is translated so its origin is at position $(0.0,15.0,0.0)$. The small CYLINDER is oriented along the $\mathrm{Y}$ axis. Region 1 consists of the material in the first large CYLINDER. Region 2 consists of the material in the second large CYLINDER. Region 3 consists of the material in the small CYLINDER but not in either of the large CYLINDERs. Region 4 is the BOUNDARY region.

EXAMPLE 6. Assume 2 small cylinders $1.0 \mathrm{~cm}$ in radius and $10 \mathrm{~cm}$ long are connected by a large cylinder $2.5 \mathrm{~cm}$ in radius and $5 \mathrm{~cm}$ long as shown in Fig. 8.1.16. 


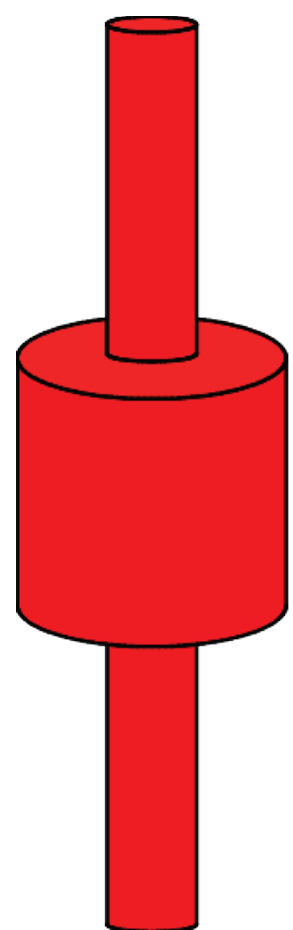

Fig. 8.1.16. Two small cylinders joined axially by a large cylinder.

This problem is very similar to Example 4. It can be described as a single UNIT in KENO-VI, but not in KENO V.a where it must be described as an array. In KENO V.a, UNIT 1 defines the large cylinder, and UNIT 2 defines the small cylinder. The origin of each UNIT is at its center. The composite system consists of two UNIT 2s and one UNIT 1 as shown below. In KENO-VI, CYLINDER 1 defines the long thin cylinder, and CYLINDER 2 defines the short thick cylinder. The origin of each cylinder is at its center. In both KENO V.a and KENO-VI, the centerline of the cylinders lies along the $\mathrm{Z}$ axis.

Data description 1, Example 6.

KENO V.a:

\section{READ GEOM}

UNIT 1

CYLINDER $112.52 .5-2.5$

CUBE $\quad 0 \quad 1 \quad 2.5 \quad-2.5$

UNIT 2

CYLINDER $1111.05 .0-5.0$

CUBOID $0012.5-2.52 .5-2.5 \quad 5.0-5.0$

END GEOM

READ ARRAY NUX=1 NUY=1 NUZ=3 FILL 212 END ARRAY

KENO-VI: 
READ GEOM

GLOBAL UNIT 1

$\begin{array}{lllll}\text { CYLINDER } & 1 & 1.0 & 12.5 & -12.5\end{array}$

$\begin{array}{lllll}\text { CYLINDER } & 2 & 2.5 & 2.5 & -2.5\end{array}$

$\begin{array}{lllll}\text { CUBOID } 3 & 4 \mathrm{P} 2.5 & 12.5 & -12.5\end{array}$

MEDIA 1111

MEDIA $1 \begin{array}{llll}1 & 1 & 2 & -1\end{array}$

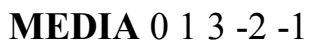

BOUNDARY 3

END GEOM

EXAMPLE 7. Assume an $11 \times 5 \times 3$ square-pitched array of spheres of material 1, radius $3.75 \mathrm{~cm}$, with a center-to-center spacing of $10 \mathrm{~cm}$ in the $\mathrm{X}, \mathrm{Y}$, and $\mathrm{Z}$ directions. The data for this system are given below.

Data description 1, Example 7.

KENO V.a:

READ GEOM

SPHERE 113.75

CUBE $\quad 0 \quad 1 \quad 5.0-5.0$

END GEOM

READ ARRAY NUX $=11$ NUY $=5$ NUZ $=3$ END ARRAY

KENO-VI:

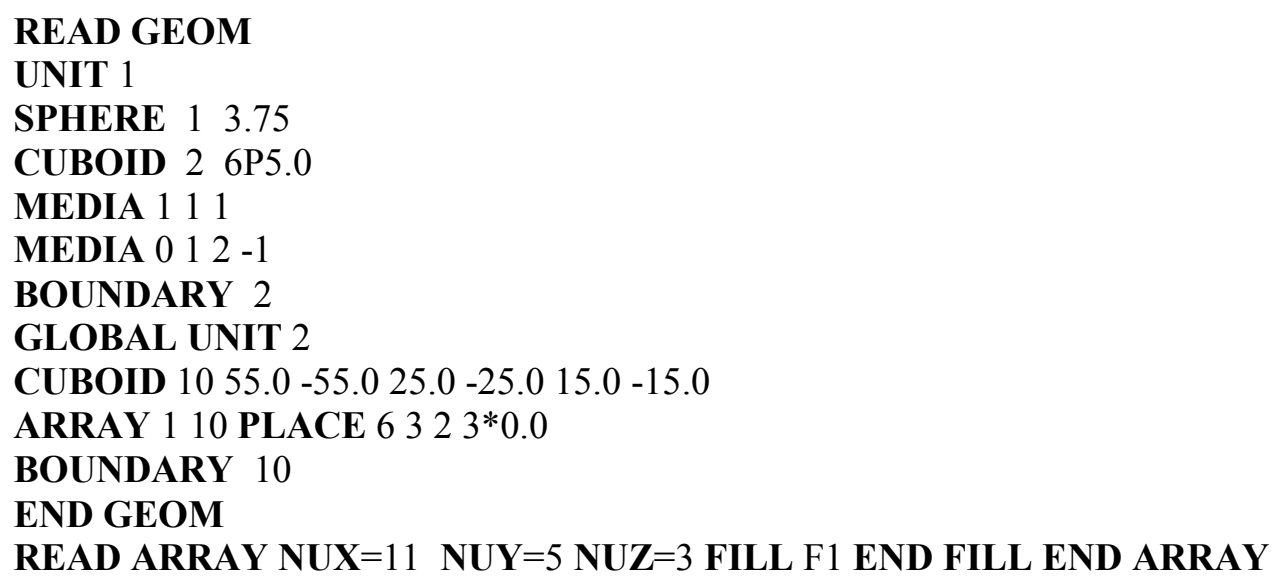

EXAMPLE 8. Assume an $11 \times 5 \times 3$ rectangular-pitched array of spheres of material 1 , whose radius is $3.75 \mathrm{~cm}$ and whose center-to-center spacing is $10 \mathrm{~cm}$ in the $\mathrm{X}$ direction, $15 \mathrm{~cm}$ in the $\mathrm{Y}$ direction, and $20 \mathrm{~cm}$ in the $\mathrm{Z}$ direction. The input for this geometry is given below.

Data description 1, Example 8.

KENO V.a:

READ GEOM 
SPHERE 113.75

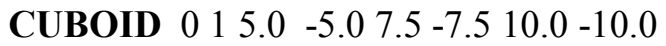

END GEOM

READ ARRAY NUX $=11$ NUY $=5$ NUZ $=3$ END ARRAY

KENO-VI:

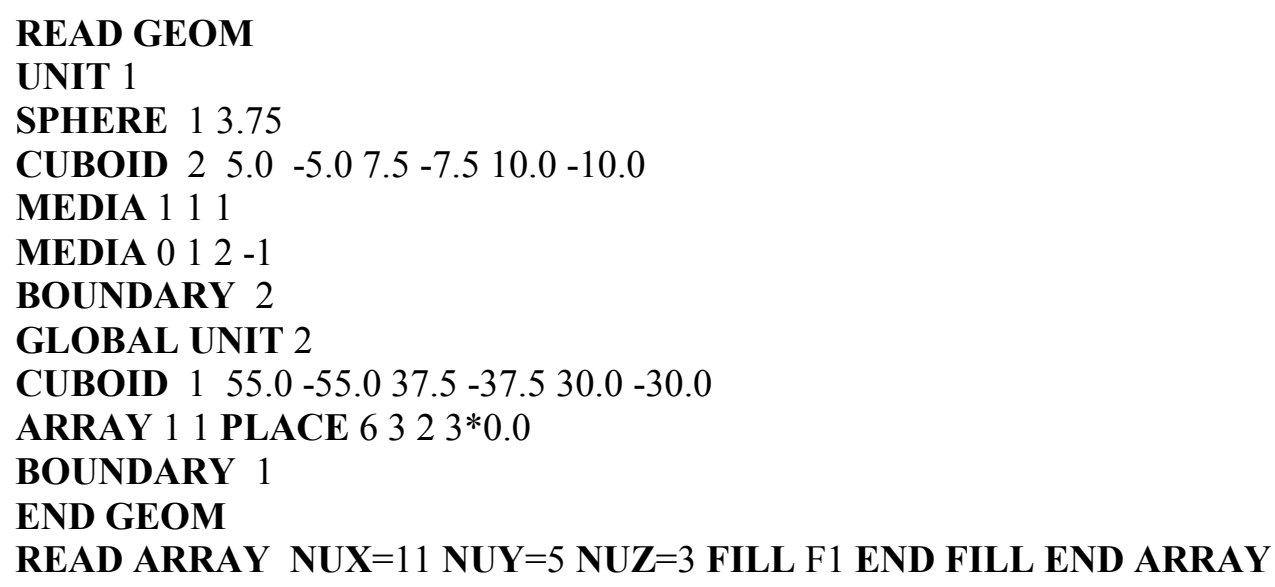

EXAMPLE 9. Assume an $11 \times 5 \times 3$ square-pitched array of spheres of material 1 with a $3.75 \mathrm{~cm}$ radius and $10 \mathrm{~cm}$ center-to-center spacing in the $\mathrm{X}, \mathrm{Y}$, and $\mathrm{Z}$ directions. This array is reflected by $30 \mathrm{~cm}$ of material 2 (water) on all faces, and weighted tracking (biasing) is to be used in the water reflector. The array spacing defines the perpendicular distance from the outer layer of spheres to the reflector to be $5 \mathrm{~cm}$ in the $\mathrm{X}, \mathrm{Y}$, and $\mathrm{Z}$ directions. The geometry input for this system is given below.

Data description 1, Example 9.

KENO V.a:

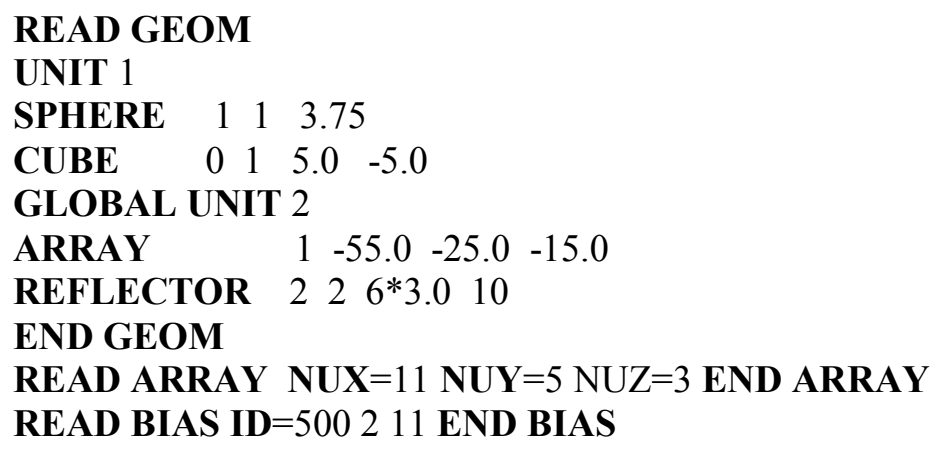

KENO-VI:

READ GEOM

UNIT 1

SPHERE 13.75

CUBOID 2 6P5.0

MEDIA 1111

MEDIA $0 \begin{array}{llll}0 & 1 & 2 & -1\end{array}$

BOUNDARY 2 


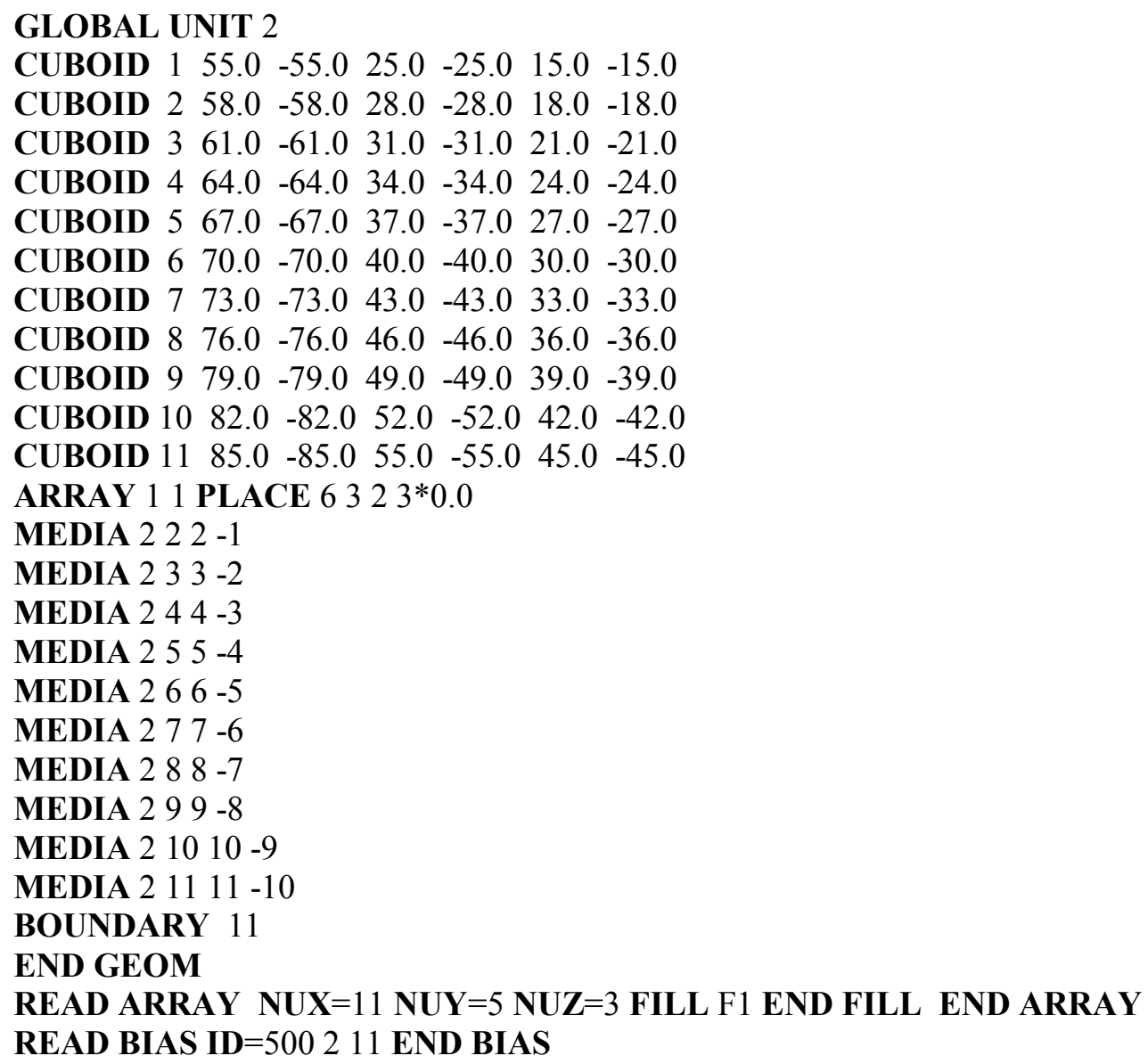

In the KENO V.a input, the ARRAY boundary defines the origin of the REFLECTOR to be at the center of the ARRAY. The 6*3.0 in the REFLECTOR description repeats the 3.0 six times. The REFLECTOR record is used to generate ten REFLECTOR regions, each of which is $3.0 \mathrm{~cm}$ thick, on all six faces of the ARRAY.

In the KENO-VI input, the basic UNIT used to construct the ARRAY is defined in UNIT 1. The ARRAY is positioned in UNIT 2 (the GLOBAL UNIT) using the ARRAY card and the PLACE option. The ARRAY is then surrounded by ten REFLECTOR regions, each $3.0 \mathrm{~cm}$ thick, on all sides.

The first bias ID is 2, so the last bias ID will be 11 if 10 regions are created. The biasing data block is necessary to apply the desired weighting or biasing function to the reflector. The biasing material ID is obtained from Table 8.1.20. IDs, group structure and incremental thickness for weighting data available on the KENO weighting library. If the biasing data block is omitted from the problem description, the 10 reflector regions will not have a biasing function applied to them, and the default value of the average weight will be used. This may cause the problem to execute more slowly, and therefore require the use of more computer time.

EXAMPLE 10. Assume the reflector in Example 9 is present only on both $X$ faces, both $Y$ faces, and the negative $\mathrm{Z}$ face. The reflector is only $15.24 \mathrm{~cm}$ thick on these faces. The top of the array (positive $\mathrm{Z}$ face) is unreflected.

Data description 1, Example 10. 
KENO V.a:

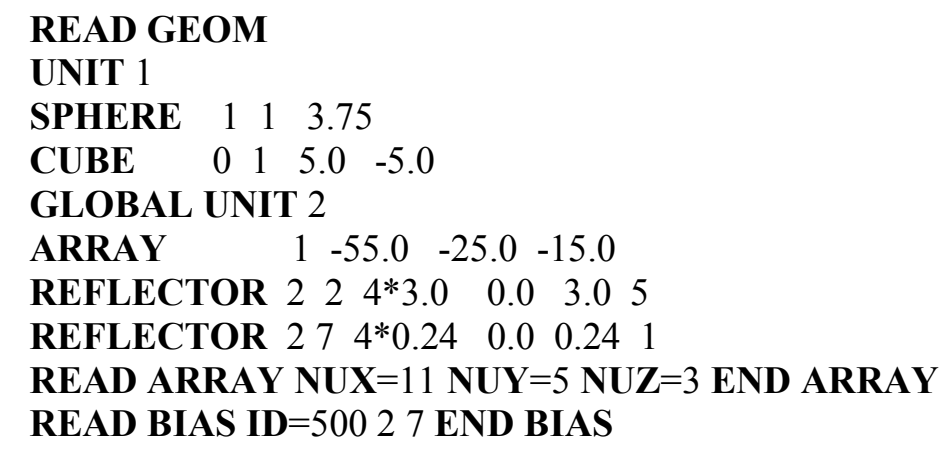

KENO-VI:

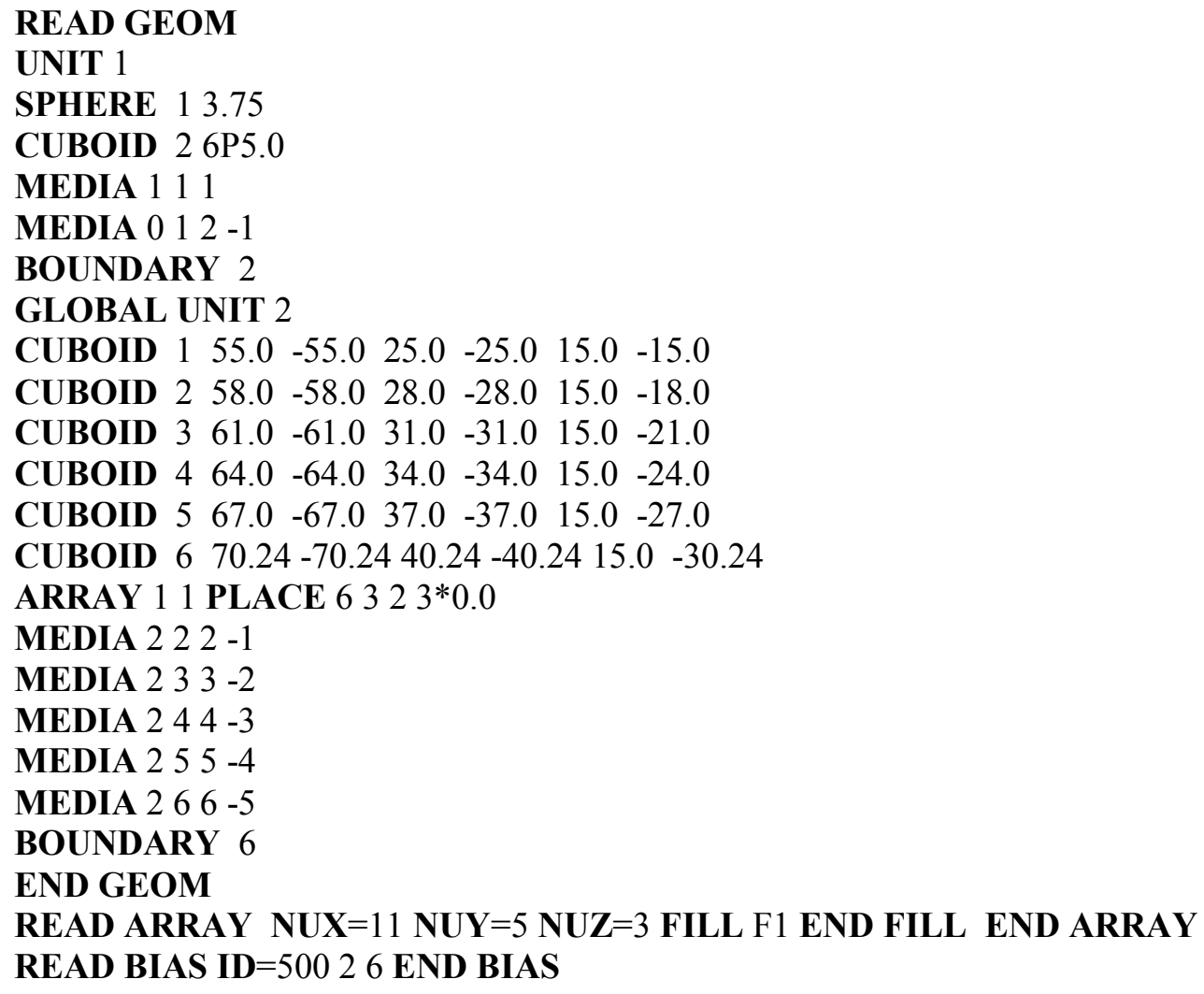

In the KENO V.a input, the first REFLECTOR description generates five regions around the ARRAY, each region being $3.0 \mathrm{~cm}$ thick in the $+\mathrm{X},-\mathrm{X},+\mathrm{Y},-\mathrm{Y}$, and $-\mathrm{Z}$ directions, and of zero thickness in the $+Z$ direction. This defines a total thickness of $15 \mathrm{~cm}$ of reflector material on the appropriate faces. The second REFLECTOR description generates the last $0.24 \mathrm{~cm}$ of material 2 on those faces. Thus, the total reflector thickness is $15.24 \mathrm{~cm}$ on each face of the array, except the top which has no reflector. Five reflector regions were generated by the first REFLECTOR description, and one was generated by the second REFLECTOR description; so, six biasing regions must be defined in the biasing data. Thus, the beginning bias ID is 2, and the ending bias ID is 7.

In the KENO-VI input, the first CUBOID in Unit 2 represents the boundary for the ARRAY. The next four CUBOIDs represent the first four regions around the ARRAY, each region being $3.0 \mathrm{~cm}$ thick in the 
$+\mathrm{X}, \mathrm{X},+\mathrm{Y},-\mathrm{Y}$, and $-\mathrm{Z}$ directions, and of zero thickness in the $+\mathrm{Z}$ direction. A total thickness of $12 \mathrm{~cm}$ of reflector material is on the appropriate faces. The last CUBOID represents the last $3.24 \mathrm{~cm}$ of material 2 on those faces. Thus, the total reflector thickness is $15.24 \mathrm{~cm}$ on each face of the array, except the top which has no reflector. The beginning bias ID is 2, and the ending bias ID is 6 . The last region could either be larger or smaller than the recommended thickness to complete the reflector.

The biasing material ID and thickness per region are obtained from Table 8.1.20. The thickness per region should be very nearly the thickness per region from the table to avoid over biasing in the reflector. Partial increments at the outer region of a reflector are exempt from this recommendation. If a biasing function is not to be applied to a region generated by the REFLECTOR record, the thickness per region can be any desired thickness and the biasing data block is omitted.

EXAMPLE 11. Assume the array of Example 7 has the central unit of the array replaced by a cylinder of material 4, $5 \mathrm{~cm}$ in radius and $10 \mathrm{~cm}$ tall. Assume a $20 \mathrm{~cm}$ thick spherical reflector of material 3 (concrete) is positioned so its inner radius is $65 \mathrm{~cm}$ from the center of the array. The minimum inner radius of a spherical reflector for this array is $62.25 \mathrm{~cm}\left(\sqrt{55^{2}+25^{2}+15^{2}}\right)$. If the inner radius is smaller than this, the problem cannot be described using KENO V.a geometry.

Data description 1, Example 11.

KENO V.a:

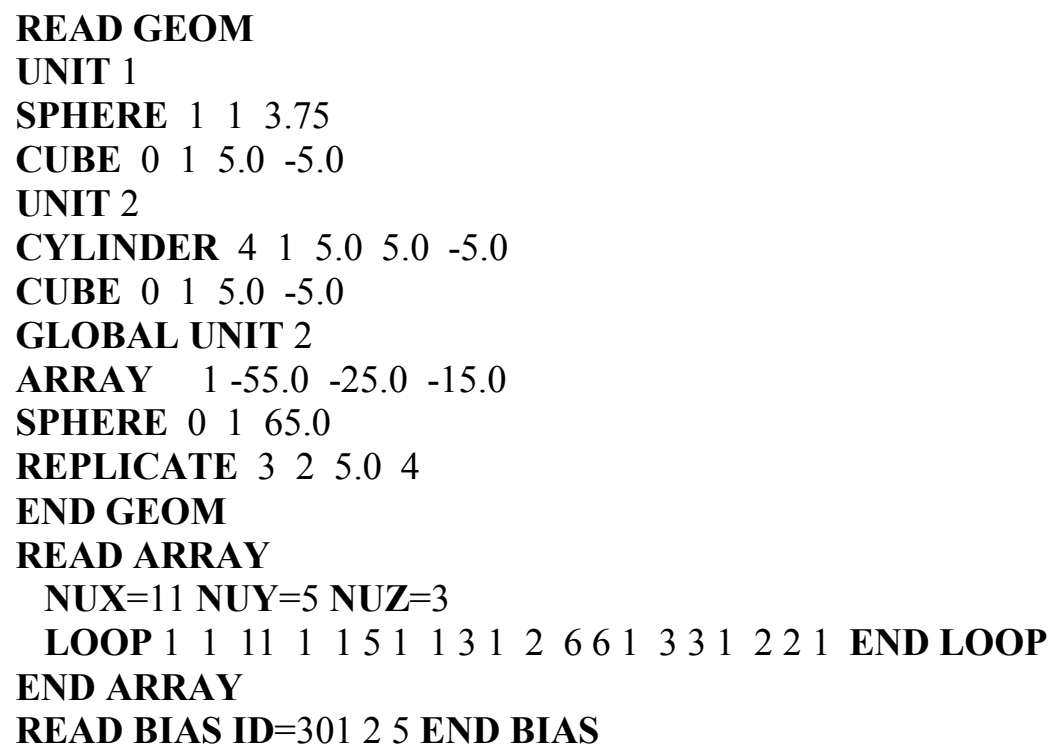

KENO-VI:

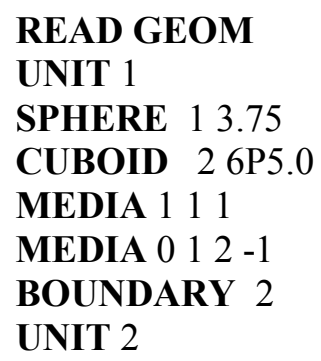


CYLINDER $15.05 .0-5.0$

CUBOID 2 6P5.0

MEDIA 411

MEDIA $0 \begin{array}{llll} & 1 & 2 & -1\end{array}$

BOUNDARY 2

GLOBAL UNIT 3

CUBOID 155.0 -55.0 25.0 -25.0 15.0 - 15.0

SPHERE 265.0

SPHERE 370.0

SPHERE 475.0

SPHERE 580.0

SPHERE 685.0

ARRAY 11 PLACE $6323 * 0.0$

MEDIA $0 \begin{array}{llll}0 & 1 & 2 & -1\end{array}$

MEDIA $3 \quad 2 \quad 3 \quad-2$

MEDIA $3 \begin{array}{llll} & 3 & 4 & -3\end{array}$

MEDIA 345 - 4

MEDIA 35 r 6 - 5

BOUNDARY 6

END GEOM

READ ARRAY

$\mathbf{N U X}=11$ NUY $=5$ NUZ $=3$

LOOP 111111115113312661331221 END LOOP

END ARRAY

READ BIAS ID=3012 5 END BIAS

UNIT 1 describes the SPHERE and spacing used in the ARRAY. UNIT 2 defines the CYLINDER located at the center of the ARRAY. In KENO V.a, the ARRAY record defines the origin of the reflector to be at the center of the ARRAY, while in KENO-VI it defines the origin of the ARRAY to be at the center of the GLOBAL UNIT. The first SPHERE in the GLOBAL UNIT defines the inner radius of the reflector. The next four SPHERE and four MEDIA records of the KENO-VI input and the REPLICATE record of the KENO V.a input will generate four spherical regions of material 3, each $5.0 \mathrm{~cm}$ thick. The data for the BIAS block is generated in a similar manner to previous examples, except that concrete $(\mathrm{ID}=301)$ is used. The recommended reflector thickness is $5 \mathrm{~cm}$; this thickness is incorporated explicitly in the KENO-VI model and with 4 repetitions of the $5 \mathrm{~cm}$ thick reflector via REPLICATE in the KENO V.a model. The first 10 entries following the word LOOP fills the $11 \times 5 \times 3$ ARRAY with UNITs 1 . The next 10 entries position UNIT 2 at the center of the ARRAY $(X=6, Y=3$, and $Z=2$ ), replacing the UNIT 1 that had been placed there by the first 10 entries.

EXAMPLE 12. Assume a data profile such as fission densities is desired in a cylinder at $0.5 \mathrm{~cm}$ intervals in the radial direction and $1.5 \mathrm{~cm}$ intervals axially. The cylinder is composed of material 1 and has a radius of $5 \mathrm{~cm}$ and a height of $15 \mathrm{~cm}$. The REPLICATE or REFLECTOR description can be used to generate these regions in KENO V.a. A biasing data block is not entered because default biasing is desired throughout the cylinder.

Data description 1, Example 12.

KENO V.a:

READ GEOM

CYLINDER 110.51 .50 
REFLECTOR 110.51 .509

END GEOM

KENO-VI:

READ GEOM

GLOBAL UNIT 1

CYLINDER 10.51 .50

CYLINDER 21.03 .00

CYLINDER 31.54 .50

CYLINDER 42.06 .00

CYLINDER 52.57 .50

CYLINDER 63.09 .00

CYLINDER 73.510 .50

CYLINDER 84.012 .00

CYLINDER 94.513 .50

CYLINDER 105.015 .00

MEDIA 1111

MEDIA $1 \begin{array}{lllll}1 & 2 & -1\end{array}$

MEDIA 1113 - 1

MEDIA 1114 - 14

MEDIA $1115-4$

MEDIA 1116 - 16

MEDIA 1117 - 6

MEDIA 1118 -

MEDIA 1119 - 18

MEDIA 11110 -9

BOUNDARY 10

END GEOM

EXAMPLE 13. (KENO-VI due to pipe junctions) Assume a cross composed of two Plexiglas cylinders (material 3) having an inner diameter of $13.335 \mathrm{~cm}$ and an outer diameter of $16.19 \mathrm{~cm}$. The bottom and side legs of the cross are closed by a $3.17 \mathrm{~cm}$ thick piece of Plexiglas. From the center of the intersection, the bottom and side legs are $91.44 \mathrm{~cm}$ long and the top leg is $121.92 \mathrm{~cm}$ long. The cross is filled with a $\mathrm{UO}_{2} \mathrm{~F}_{2}$ solution (material 1) to a height of $28.93 \mathrm{~cm}$ above the center of the cylinder intersection. The cross is then surrounded by a water reflector (material 2) that extends from the center of the intersection: $111.74 \mathrm{~cm}$ in the $\pm X$ directions, $20.64 \mathrm{~cm}$ in the $\pm Y$ directions, $29.03 \mathrm{~cm}$ in the $+Z$ direction, and $118.428 \mathrm{~cm}$ in the $-Z$ direction. A schematic of the assembly is shown in Fig. 8.1.17. 


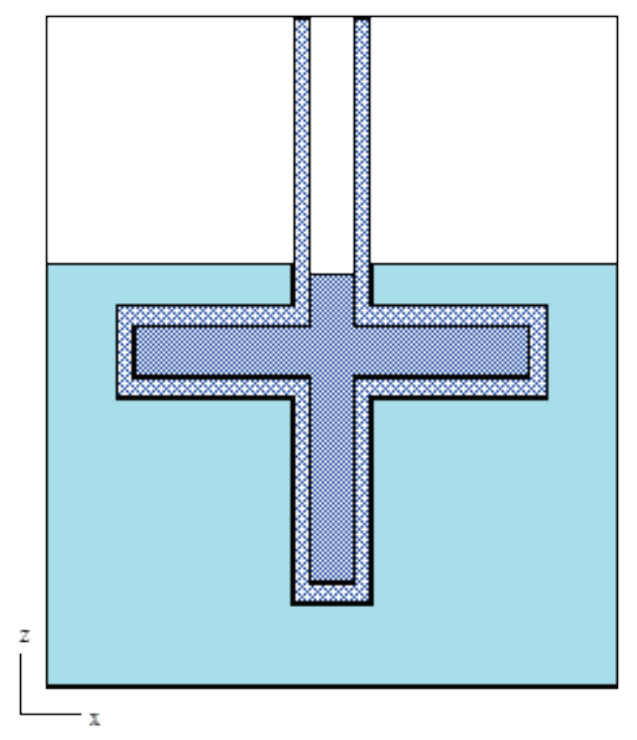

Fig. 8.1.17. Plexiglas $\mathrm{UO}_{2} \mathrm{~F}_{2}$-filled cross.

Data description of Example 13 (KENO-VI only).

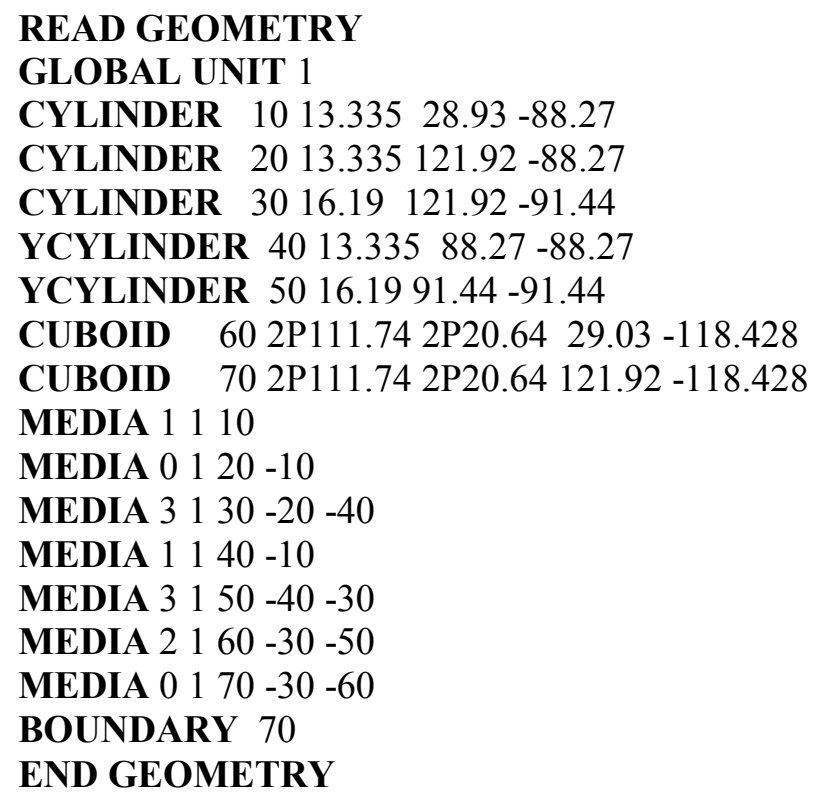

EXAMPLE 14. (KENO-VI only because of rotation) Assume a Y-shaped aluminum cylinder (material 2) with a $13.95 \mathrm{~cm}$ inner radius and a $0.16 \mathrm{~cm}$ wall thickness is filled with a $\mathrm{UO}_{2} \mathrm{~F}_{2}$ solution (material 1). From the center where the Y intersects the cylinder, the bottom leg is $76.7 \mathrm{~cm}$ long, the top leg is $135.4 \mathrm{~cm}$ long, and the Y leg is $126.04 \mathrm{~cm}$ long, canted at a 29.26-degree angle. The bottom of the bottom leg and the top of the Y leg are sealed with $1.3 \mathrm{~cm}$ caps. The $\mathrm{Y}$ cylinder is filled to a height of $52.8 \mathrm{~cm}$ above the center where the $\mathrm{Y}$ leg intersects the vertical cylinder. The cylinder is surrounded by a water reflector (material 3) that extends out $37.0 \mathrm{~cm}$ in the $\pm \mathrm{X}$ direction, 100.0 and $-37.0 \mathrm{~cm}$ in the 
$\pm Y$ direction, and 135.4 and -99.6 in the $\pm Z$ direction. A schematic of the assembly is shown in Fig. 8.1.18.

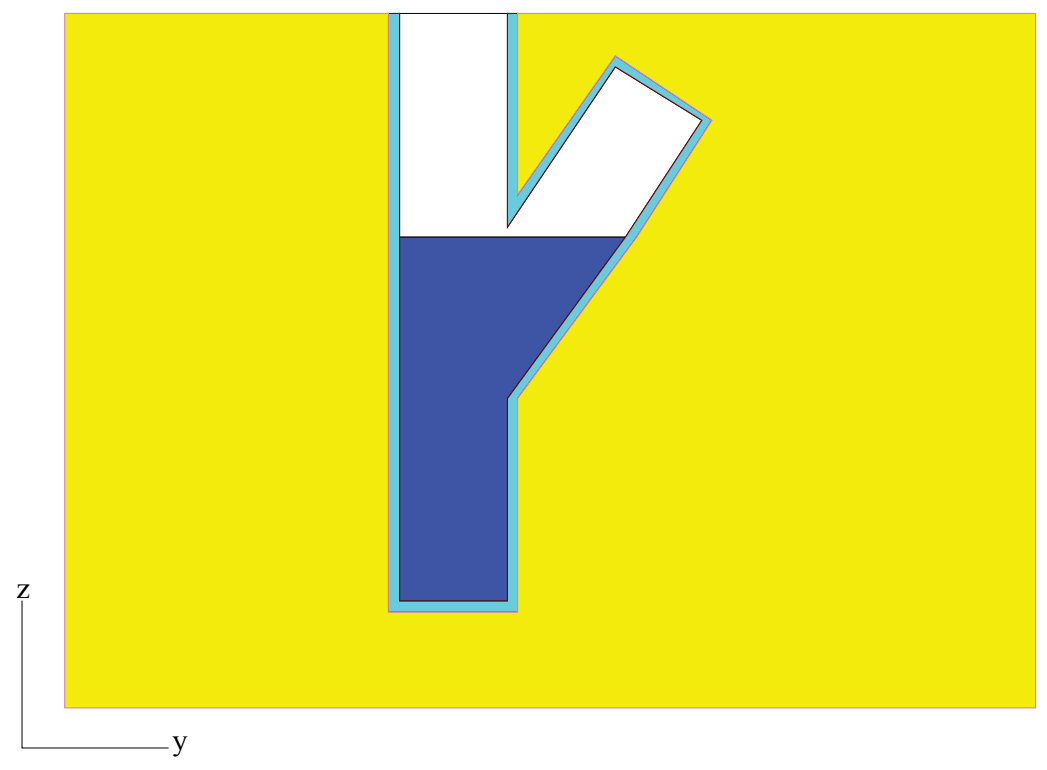

Fig. 8.1.18. Y-shaped $\mathrm{UO}_{2} \mathrm{~F}_{2}$-filled aluminum cylinder.

Data description of Example 14 (KENO-VI only).
READ GEOMETRY
GLOBAL UNIT 1
MEDIA 111050
MEDIA $2120-10-30$
MEDIA $1130 \begin{array}{llll}1 & 30 & -10\end{array}$
MEDIA $2140-30-20$
MEDIA $0 \begin{array}{lllll}0 & 1 & 10 & -50\end{array}$
MEDIA $0130-50$ - 130
MEDIA 3160 -20 -40
BOUNDARY 60
END GEOMETRY

COM='30 DEG Y CYLINDER'

CYLINDER $1013.95135 .4-75.4$

CYLINDER $2014.11135 .4-76.7$

CYLINDER 3013.95124 .740 .0 ROTATE A2=-29.26

CYLINDER 4014.11126 .04 0.0 ROTATE A2=-29.26

CUBOID 50 2P37.0 $100.0-37.052 .8$-75.4

CUBOID 60 2P37.0 $100.0-37.0135 .4-99.6$ 


\subsection{Use of holes in the geometry}

Section 8.1.3.6 tells how each KENO V.a geometry region in a UNIT must completely enclose all previously described regions in that UNIT and how KENO-VI geometry allows regions in a UNIT to intersect, thus eliminating the need for HOLEs. HOLEs can be used to circumvent the complete enclosure restriction in KENO V.a to some degree. In KENO-VI, they can be useful in simplifying the input of a problem and decreasing the total CPU time needed for a problem. A HOLE is a means of placing an entire UNIT within a geometry region. A separate HOLE description is required for every location in a geometry region where a UNIT is to be placed. The information contained in a HOLE description is (1) the keyword HOLE, (2) the UNIT number of the UNIT to be placed, and (3) any modification data needed to correctly position and rotate (in KENO-VI) the specified UNIT within the containing UNIT. In KENO V.a, a HOLE is placed inside the geometry region that precedes it. This excludes HOLEs ... (i.e., if a CUBE geometry region is followed by four HOLE descriptions, all four HOLEs are located within the CUBE). In KENO V.a, HOLEs are subject to the restriction that they cannot intersect any other geometry region. HOLEs can be nested to any depth (see Sect. 8.1.3.6.2). It is not advisable to use HOLEs tangent to other HOLEs or geometry, because round-off error may cause them to overlap. It is not uncommon for a problem that runs on one type of computer to fail on another type using the same data. Therefore, it is recommended that tangency and boundaries shared with HOLEs be avoided. This may be accomplished by separating the otherwise collocated or tangent surfaces by a very small (i.e., $10^{-6} \mathrm{~cm}$ ) distance.

In KENO V.a, tracking in regions that contain holes is less efficient than tracking in regions that do not contain holes. Therefore, holes should be used only when the system cannot be easily described by conventional methods. One example of the use of holes is shown in Fig. 8.1.19, representing nine closepacked rods in an annulus.

In KENO-VI, tracking in regions that contain HOLEs can be more efficient than tracking in regions that do not contain HOLEs because every region boundary in a UNIT must be checked for a crossing whenever a crossing is possible. Putting small but complex geometries in a hole will lessen the number of boundaries that need to be checked for possible crossings. However, the indiscriminate use of holes is not advised since the particle must change coordinate systems every time a hole is entered or exited. Therefore, holes should be used carefully and only when the system can be simplified significantly by their use.

EXAMPLE 15. One example of a unit that requires holes in KENO V.a is better described not using holes in KENO-VI as shown in Fig. 8.1.19, representing nine close-packed rods in an annulus. The large rods are $1.4 \mathrm{~cm}$ in radius and composed of mixture 3 . The small rods are $0.6 \mathrm{~cm}$ in radius and composed of mixture 1. The inside radius of the annulus is $3.6 \mathrm{~cm}$, and the outside radius is $3.8 \mathrm{~cm}$. The annulus is made of mixture 2. The rods and annulus are both $30 \mathrm{~cm}$ long. The annulus is centered in a cuboid having an $8 \mathrm{~cm}^{2}$ cross section and a length of $32 \mathrm{~cm}$. The black and gray areas in Fig. 8.1.19 are void. 


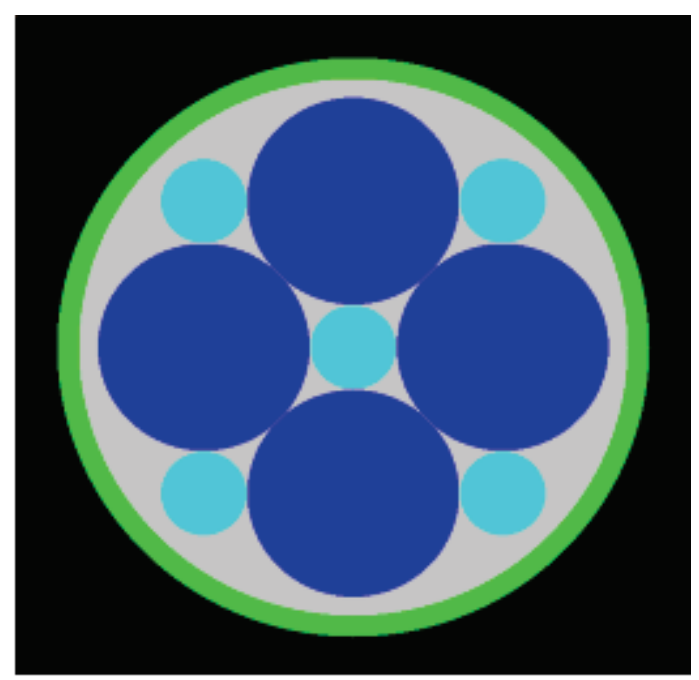

Fig. 8.1.19. Close-packed cylinders in an annulus.

Data description of Example 15.

KENO V.a:

READ GEOM

UNIT 1

CYLINDER 110.6 2P15.0

UNIT 2

CYLINDER 311.4 2P15.0

GLOBAL UNIT 3

CYLINDER 110.6 2P15.0

CYLINDER 013.6 2P 15.0

HOLE $20.0-2.00 .0$

HOLE $12.0-2.00 .0$

HOLE 22.00 .00 .0

HOLE 12.02 .00 .0

HOLE 20.02 .00 .0

HOLE 1 -2.0 2.00 .0

HOLE $2-2.00 .00 .0$

HOLE $1-2.0-2.00 .0$

CYLINDER 213.8 2P15.0

CUBOID $0014 \mathrm{P} 4.0$ 2P16.0

END GEOM

KENO-VI:

READ GEOM

GLOBAL UNIT 1

CYLINDER 10.6 2P15.0 
CYLINDER 20.6 2P15.0 ORIGIN X=2.0 Y=-2.0

CYLINDER 30.6 2P15.0 ORIGIN $\mathbf{X}=2.0 \quad \mathrm{Y}=2.0$

CYLINDER 40.6 2P15.0 ORIGIN $\mathbf{X}=-2.0 \mathrm{Y}=2.0$

CYLINDER 5 0.6 2P15.0 ORIGIN X=-2.0 Y=-2.0

CYLINDER 61.4 2P15.0 ORIGIN X=2.0

CYLINDER 71.4 2P15.0 ORIGIN Y $=2.0$

CYLINDER 81.4 2P15.0 ORIGIN X=-2.0

CYLINDER 91.4 2P15.0 ORIGIN Y=-2.0

CYLINDER 103.6 2P15.0

CYLINDER 113.8 2P15.0

CUBOID 12 4P4.0 2P16.0

MEDIA 1111

MEDIA 1112

MEDIA 1113

MEDIA 1114

MEDIA 1115

MEDIA 316

MEDIA 317

MEDIA 3118

MEDIA 319

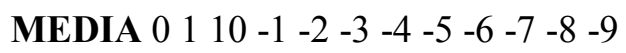

MEDIA 2

MEDIA 0112

BOUNDARY 12

END GEOM

The first HOLE description in the KENO V.a input represents the bottom large rod. It takes UNIT 2 and places its ORIGIN at $(0.0,-2.0,0.0)$ relative to the ORIGIN of UNIT 3. The second HOLE description represents the small rod to the right of the large rod just discussed. It places the origin of UNIT 1 at $(2.0,-2.0,0.0)$ in UNIT 3. The third HOLE description represents the large rod to the right. It places the origin of UNIT 2 at $(2.0,0.0,0.0)$ in UNIT 3. This procedure is repeated in a counterclockwise direction until all eight rods have been placed within the region that defines the inner surface of the annulus. The CYLINDER that defines the outer surface of the annulus is described after all the HOLEs for the previous region have been placed. Then the outer CUBOID is described. This example illustrates that a UNIT that is to be placed using a HOLE description need not have a CUBE or CUBOID as its last region. Note that including the central rod directly in UNIT 3 reduces the CPU time required for transport compared to the case of all 9 rods being inserted as HOLEs. It is also important that the 9 HOLEs are inserted after the void cylinder into which they are inserted. Entering the HOLEs in any other position in the input would generated region intersection errors. The order of the HOLE records in any given region is not important, as they can be interchanged with each other randomly. However, they must always appear immediately after the region in which they are placed.

The KENO-VI input does not need to use HOLEs. The first CYLINDER description in this case represents the middle small rod. The next four CYLINDER records describe the four remaining small rods surrounding the middle rod. The ORIGIN attribute is used to shift the origin of each CYLINDER to the appropriate location. The following four CYLINDER records represent the four large rods. Again, the ORIGIN attribute is used to shift the ORIGIN of each CYLINDER to the appropriate location. Only the nonzero dimensions need to be entered in the ORIGIN data. The tenth CYLINDER record is the void in the annulus that contains the rods. The last CYLINDER record defines the outer surface of the annulus. Finally, the CUBOID record describes the surrounding UNIT boundary. 


\section{In KENO V.a, the}

In KENO-VI, holes may not extend across any array outer boundary, may not intersect with other holes, and may not cross the host UNIT outer boundary. Thus a hole may be placed so that it crosses several regions within an array. The hole description replaces the unit description within the hole domain. Since the holes are placed using the host UNIT coordinate system, the location of the hole record in the unit definition is not relevant.

An array of the arrangement shown in Fig. 8.1.19 can be easily described by altering the array description data. For example, a $5 \times 3 \times 2$ array of these shapes with a center-to-center spacing of $8 \mathrm{~cm}$ in $\mathrm{X}$ and $\mathrm{Y}$ and $32 \mathrm{~cm}$ in $\mathrm{Z}$ can be achieved by using the following array data:

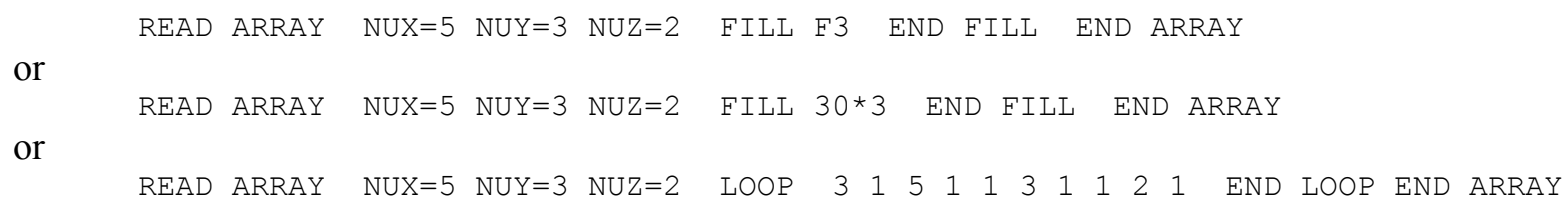

\subsection{Nesting holes}

This section illustrates how holes are nested. Holes can be nested to any level. Consider the configuration illustrated in Fig. 8.1.19 and replace the large rods with a complicated geometric arrangement. The resulting Fig. is shown in Fig. 8.1.20. Fig. 8.1.21 shows the complicated geometric arrangement that replaced the large rods of Fig. 8.1.19. Fig. 8.1.22 shows a component of the arrangement shown in Fig. 8.1.20.

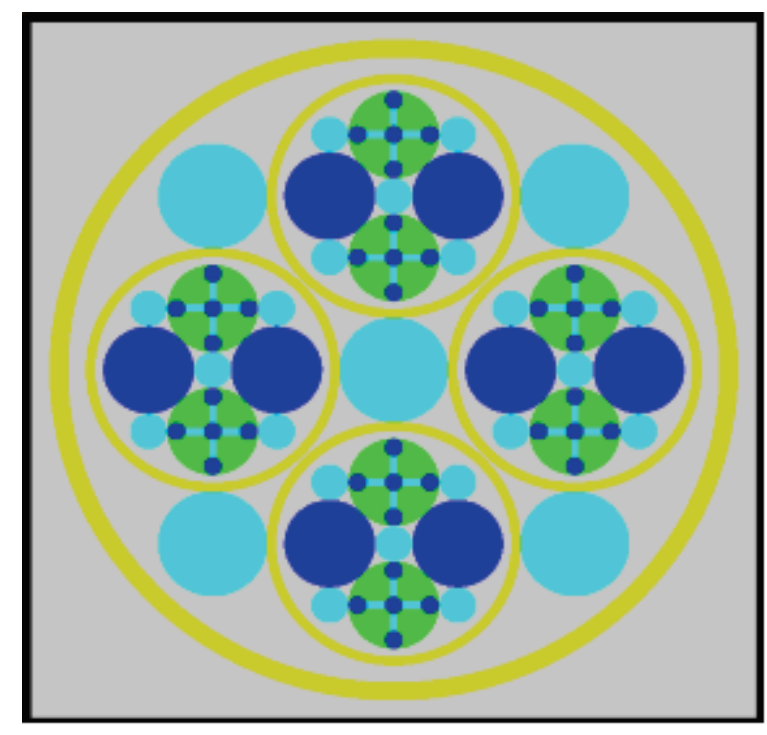

Fig. 8.1.20. Configuration using nested holes. 


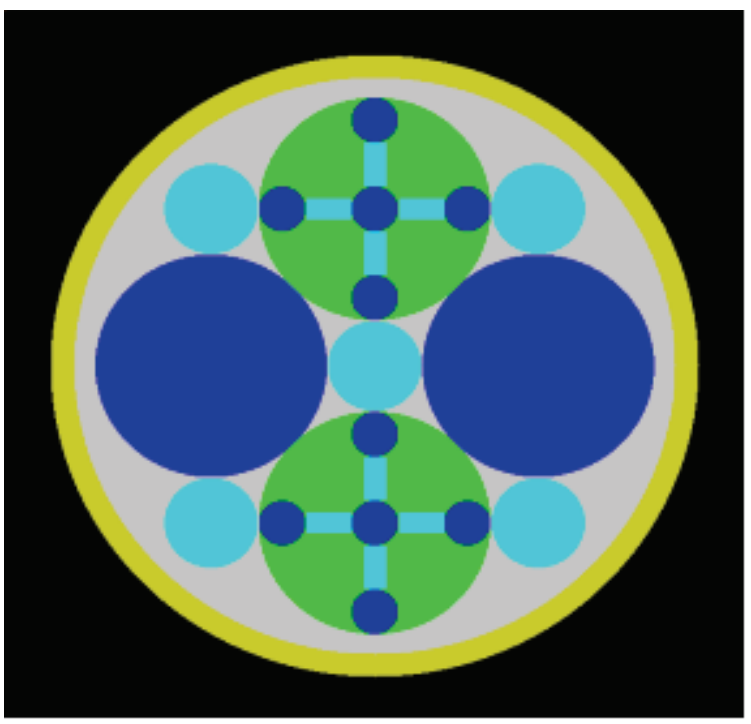

Fig. 8.1.21. Complicated geometric arrangement by Unit 7.

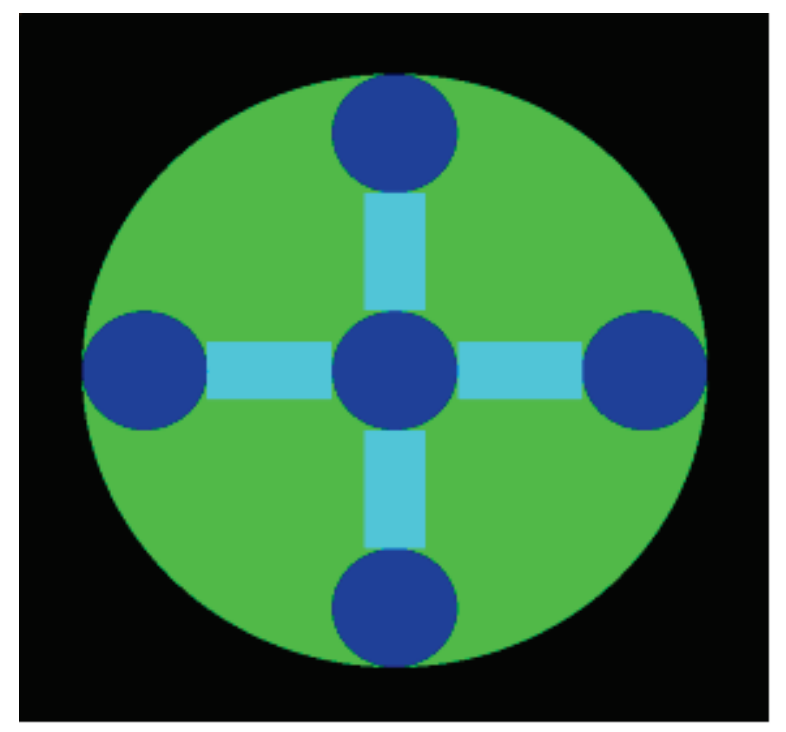

Fig. 8.1.22. Geometric component represented by Unit 4 .

EXAMPLE 16. There is no predetermined preferred method to create a geometry mockup for a given physical system. The user should determine the most convenient order. To describe the configuration shown in Fig. 8.1.20 using nested HOLEs, it is likely most convenient to start the geometry mockup at the deepest nesting level, as shown in Fig. 8.1.22. The small CYLINDERs are composed of mixture 1, and they are each $0.1 \mathrm{~cm}$ in radius and $30 \mathrm{~cm}$ long. There are five small CYLINDERs used in Fig. 8.1.22. Their centers are located at $(0,0,0)$ for the central one, at $(0,-0.4,0)$ for the bottom one, at $(0.4,0,0)$ for the right one, at $(0,0.4,0)$ for the top one, and at $(-0.4,0,0)$ for the left one. The rectangular parallelepipeds (CUBOIDs) are composed of mixture 2. Each one is $30 \mathrm{~cm}$ long and $0.1 \mathrm{~cm}$ by $0.2 \mathrm{~cm}$ in cross section. The large CYLINDER containing the configuration is composed of mixture 3 , is $30 \mathrm{~cm}$ long and has a radius of $0.5 \mathrm{~cm}$. 
A possible geometry mockup for this system is described as follows in KENO V.a:

(1) define a small cylinder to be UNIT 1,

(2) define a small CUBOID with its length in the X direction to be UNIT 2,

(3) define a small CUBOID with its length in the Y direction to be UNIT 3,

(4) define UNIT 4 to be the large cylinder and place the CYLINDERs and CUBOIDs in it using HOLEs.

UNIT 1

CYLINDER $110.12 \mathrm{P} 15.0$

UNIT 2

CUBOID $212 \mathrm{P} 0.1$ 2P0.05 2P15.0

UNIT 3

CUBOID $212 \mathrm{P} 0.05$ 2P0.12P15.0

UNIT 4

CYLINDER $1110.12 \mathrm{P} 15.0$

CYLINDER 310.5 2P15.0

HOLE $10.0-0.40 .0$

HOLE 10.40 .00 .0

HOLE 10.00 .40 .0

HOLE $1-0.40 .00 .0$

HOLE $2-0.20 .00 .0$

HOLE 20.20 .00 .0

HOLE 3 0.0 -0.20 .0$

HOLE 30.00 .20 .0

The first cylinder description in UNIT 4 places the central rod, the second cylinder description in UNIT 4 places the outer cylinder, the first HOLE places the bottom CYLINDER, the second HOLE places the CYLINDER at the right, the third HOLE places the top CYLINDER, the fourth HOLE places the CYLINDER at the left, the fifth HOLE places the left CUBOID whose length is in X, the sixth HOLE places the right CUBOID whose length is in X, the seventh HOLE places the bottom CUBOID whose length is in Y, and the eighth HOLE places the top CUBOID whose length is in Y.

A possible geometry mockup for this system is described as follows in KENO-VI:

1. define UNIT 1 to contain the five small cylinders and four blocks,

2. define UNIT 2 to contain the next two larger-sized cylinders and UNIT 1 as HOLEs, and

3. define GLOBAL UNIT 3 to contain the large cylinders and UNIT 2 as HOLEs.

\section{UNIT 1}

CYLINDER $10.12 \mathrm{P} 15.0$ 
CYLINDER 20.1 2P15.0 ORIGIN Y=-0.4

CYLINDER 30.1 2P15.0 ORIGIN X=0.4

CYLINDER 40.1 2P15.0 ORIGIN Y $=0.4$

CYLINDER 50.1 2P15.0 ORIGIN $\mathrm{X}=-0.4$

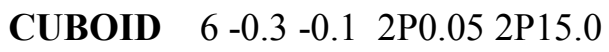

CUBOID $70.30 .12 \mathrm{P} 0.052 \mathrm{P} 15.0$

CUBOID 8 2P0.05 $-0.3-0.12 \mathrm{P} 15.0$

CUBOID 9 2P0.05 $0.30 .12 \mathrm{P} 15.0$

CYLINDER 100.5 2P15.0

MEDIA 1111

MEDIA 1112

MEDIA 1113

MEDIA 1114

MEDIA 1115

MEDIA 216

MEDIA 217

MEDIA 218

MEDIA 219

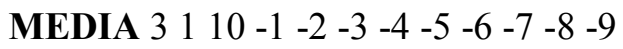

BOUNDARY 10

geometry record 1 places the central rod, geometry record 2 places the bottom CYLINDER, geometry record 3 places the CYLINDER at the right, geometry record 4 places the top CYLINDER, geometry record 5 places the CYLINDER at the left, geometry record 6 places the left CUBOID whose length is in X, geometry record 7 places the right CUBOID whose length is in $\mathrm{X}$, geometry record 8 places the bottom CUBOID whose length is in $\mathrm{Y}$, geometry record 9 places the top CUBOID whose length is in Y, and geometry record 10 is the surrounding CYLINDER that defines the unit boundary.

In Fig. 8.1.21, the large plain cylinders are composed of mixture 1 and are $0.5 \mathrm{~cm}$ in radius and $30 \mathrm{~cm}$ long. The cylindrical component of UNIT 4 for KENO V.a or UNIT 1 for KENO-VI is the same size: an outer radius of $0.5 \mathrm{~cm}$ and a length of $30 \mathrm{~cm}$. The small cylinders located in the interstices between the large cylinders are composed of mixture 2 , are $0.2 \mathrm{~cm}$ in radius, and are $30 \mathrm{~cm}$ long. The annulus is composed of mixture 4 , has a $1.3 \mathrm{~cm}$ inside radius and a $1.4 \mathrm{~cm}$ outer radius. The volume between the inner cylinders is void. The large cylinders each have a radius of $0.5 \mathrm{~cm}$ and are tangent. Therefore, their origins are offset from the origin of the UNIT by 0.707107 . This is from $\mathrm{X}^{2}+\mathrm{Y}^{2}=1.0$, where $\mathrm{X}$ and $\mathrm{Y}$ are equal.

For KENO V.a, define UNIT 5 to be the large plain cylinder, UNIT 6 to be the small cylinder, and UNIT 7 as the annulus that contains the cylinders. Its origin is at its center. The geometry mockup for this portion of the problem follows: 
KENO V.a:

UNIT 5

CYLINDER 110.5 2P15.0

UNIT 6

CYLINDER 210.2 2P15.0

UNIT 7

CYLINDER 210.2 2P15.0

CYLINDER 0111.3 2P15.0

HOLE 50.7071070 .00 .0

HOLE $\quad 60.7071070 .7071070 .0$

HOLE 40.00 .7071070 .0

HOLE $\quad 6-0.7071070 .7071070 .0$

HOLE $5-0.7071070 .00 .0$

HOLE $6-0.707107-0.7071070 .0$

HOLE $40.0-0.7071070 .0$

HOLE $60.707107-0.7071070 .0$

CYLINDER 411.4 2P15.0

The first HOLE places the larger CYLINDER of mixture 1 at the right with its origin at $(0.707107,0.0,0.0)$,

the second HOLE places the small CYLINDER in the upper right quadrant,

the third HOLE places the top CYLINDER that contains the geometric component defined in UNIT 4,

the fourth HOLE places the small CYLINDER in the upper left quadrant,

the fifth HOLE places the larger CYLINDER of mixture 1 at the left,

the sixth HOLE places the small CYLINDER in the lower lower left quadrant,

the seventh HOLE places the bottom CYLINDER that contains the geometric component defined in UNIT 4, and

the eighth HOLE places the small CYLINDER in the lower right quadrant.

The last CYLINDER defines the outer surface of the annulus.

For KENO-VI, UNIT 2 is the annulus that contains the cylinders.

KENO-VI:

UNIT 2

CYLINDER 10.2 2P15.0

CYLINDER 20.2 2P15.0 ORIGIN X $=0.707107 \quad \mathbf{Y}=0.707107$

CYLINDER 30.2 2P15.0 ORIGIN $\mathbf{X}=-0.707107 \mathbf{Y}=0.707107$

CYLINDER 40.2 2P15.0 ORIGIN $X=-0.707107 \mathbf{Y}=-0.707107$

CYLINDER 50.2 2P15.0 ORIGIN $\mathbf{X}=0.707107 \quad \mathbf{Y}=-0.707107$

CYLINDER 60.5 2P15.0 ORIGIN X=0.707107

CYLINDER 70.5 2P15.0 ORIGIN $\mathrm{X}=-0.707107$

CYLINDER 101.3 2P15.0

CYLINDER 111.4 2P15.0

MEDIA 211

MEDIA 212

MEDIA 213

MEDIA 214

MEDIA 215

MEDIA 116 
MEDIA 117

HOLE 1 ORIGIN Y=0.707107

HOLE 1 ORIGIN $Y=-0.707107$

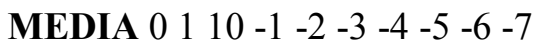

MEDIA 4 1 $111-10$

BOUNDARY 11

In Unit 2 of the above KENO-VI geometry,

CYLINDER 1 places a small CYLINDER of mixture 2 at the origin,

CYLINDER 2 places the small CYLINDER of mixture 2 in the upper right quadrant,

CYLINDER 3 places the small CYLINDER of mixture 2 in the upper left quadrant,

CYLINDER 4 places the small CYLINDER of mixture 2 in the lower left quadrant,

CYLINDER 5 places the small CYLINDER of mixture 2 in the lower right quadrant,

CYLINDER 6 places the larger CYLINDER of mixture 1 at the right with its origin at $(0.707107,0.0,0.0)$,

CYLINDER 7 places the larger CYLINDER of mixture 1 at the left with its origin at (0.0,0.707107.0.0),

CYLINDER 10 defines the inner surface of the annulus,

CYLINDER 11 defines the outer surface of the annulus and the UNIT boundary,

the first HOLE places the top CYLINDER that contains the geometric component defined in UNIT 1, and

the second HOLE places the bottom CYLINDER that contains the geometric component defined in UNIT 1.

To complete the geometry mockup, consider Fig. 8.1.20.

For KENO V.a geometry, define UNIT 8 to be a cylinder of mixture 2 having a radius of $0.6 \mathrm{~cm}$ and a length of $30 \mathrm{~cm}$. Define UNIT 9 to be the central rod and the large annulus of $3.6 \mathrm{~cm}$ inner radius, $3.8 \mathrm{~cm}$ outer radius, and $30 \mathrm{~cm}$ length centered in a CUBOID having an $8 \mathrm{~cm}^{2}$ cross section and being $32 \mathrm{~cm}$ long.

KENO V.a:

UNIT 8

CYLINDER 210.6 2P15.0

UNIT 9

CYLINDER 210.6 2P15.0

CYLINDER 013.6 2P15

HOLE 72.00 .00 .0

HOLE $82 * 2.00 .0$

HOLE 70.02 .00 .0

HOLE $8-2.02 .00 .0$

HOLE $7-2.02 * 0.0$

HOLE $82 *-2.00 .0$

HOLE $70.0-2.00 .0$

HOLE 8 2P2.0 0.0

CYLINDER 413.8 2P15.0

CUBOID 0 1 4P4.0 2P16.0 
In UNIT 9 of the KENO V.a description, the first CYLINDER defines the rod of mixture 2, centered in the annulus. The second CYLINDER defines the void volume between the central rod and the annulus.

The first HOLE places the composite annulus of UNIT 7 to the right of the central rod, the second HOLE places a rod defined by UNIT 8 in the upper right quadrant of the annulus, the third HOLE places the composite annulus of UNIT 7 above the central rod, the fourth HOLE places a rod defined by UNIT 8 in the upper left quadrant of the annulus, the fifth HOLE places the composite annulus of UNIT 7 to the left of the central rod, the sixth HOLE places a rod defined by UNIT 8 in the lower left quadrant, the seventh HOLE places the composite annulus of UNIT 7 below the central rod, and the eighth HOLE places a rod defined by UNIT 8 in the lower right quadrant.

The last CYLINDER defines the outer surface of the annulus. The outer CUBOID is the last region.

For KENO-VI geometry, define UNIT 3 to be the central rod and four outer rods of $0.6 \mathrm{~cm}$ radius and $30.0 \mathrm{~cm}$ length, and the large annulus of $3.6 \mathrm{~cm}$ inner radius, $3.8 \mathrm{~cm}$ outer radius, and $30 \mathrm{~cm}$ length centered in a cuboid having an $8 \mathrm{~cm}^{2}$ cross section and a length of $32 \mathrm{~cm}$.

KENO-VI:

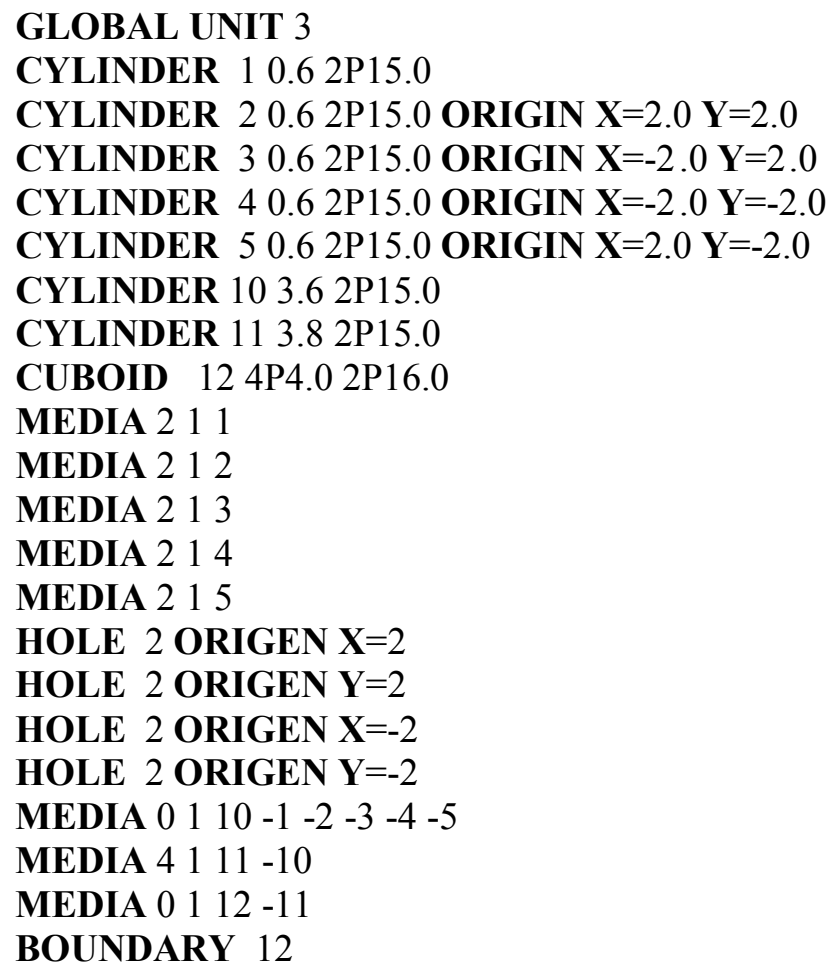


In UNIT 3 of the above KENO-VI description,

CYLINDER $l$ defines the rod of mixture 2, centered in the annulus,

CYLINDER 2 places a rod of mixture 2 in the upper right quadrant of the annulus,

CYLINDER 3 places a rod of mixture 2 in the upper left quadrant of the annulus,

CYLINDER 4 places a rod of mixture 2 in the lower left quadrant,

CYLINDER 5 places a rod of mixture 2 in the lower right quadrant,

CYLINDER 10 defines the void volume between the central rod and the annulus,

CYLINDER 11 defines the outer surface of the annulus,

CUBOID 12 defines the unit boundary,

the first HOLE places UNIT 2 to the right of the central rod,

the second HOLE places UNIT 2 above the central rod,

the third HOLE places UNIT 2 to the left of the central rod, and

the fourth HOLE places UNIT 2 below the central rod.

This problem illustrates three levels of HOLE nesting. The total input data for the problem is given below. The geometry description accurately recreates the geometry arrangement of Fig. 8.1.20. The 2-D color plot output is shown in Fig. 8.1.23

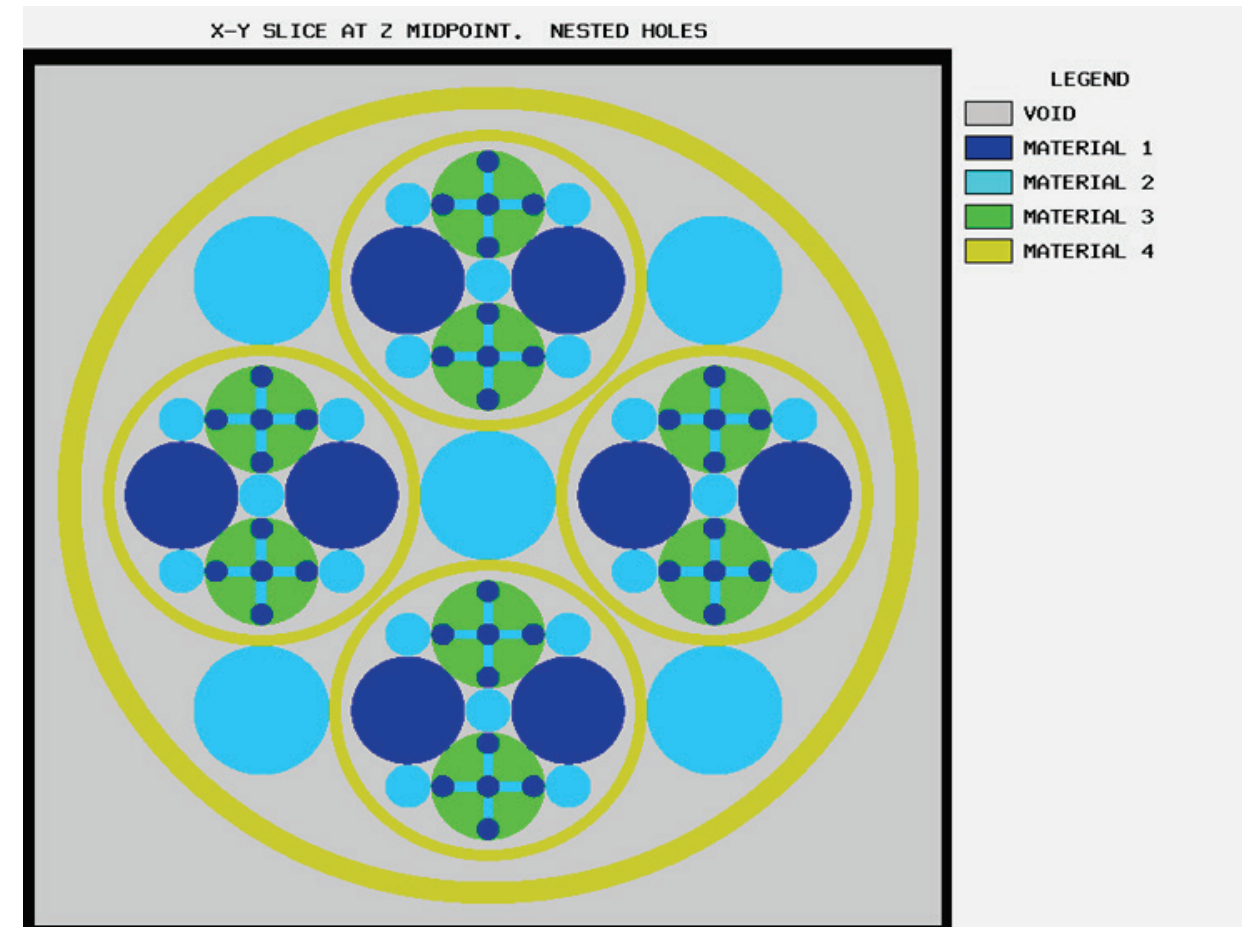

Fig. 8.1.23. Color plot of nested holes example problem.

KENO V.a:

NESTED HOLES SAMPLE

READ GEOM

UNIT 1

CYLINDER $110.12 \mathrm{P} 15.0$ 
UNIT 2

CUBOID 21 2P0.1 2P0.05 2P15.0

UNIT 3

CUBOID 21 2P0.05 2P0.1 2P15.0

UNIT 4

CYLINDER $110.12 \mathrm{P} 15.0$

CYLINDER 310.5 2P15.0

HOLE $10.0-0.40 .0$

HOLE $1 \quad 0.4 \quad 0.00 .0$

HOLE $10.0 \quad 0.40 .0$

HOLE $1-0.4 \quad 0.0 \quad 0.0$

HOLE $2-0.2 \quad 0.0 \quad 0.0$

HOLE 20.20 .00 .0

HOLE $3 \quad 0.0-0.20 .0$

HOLE $3 \quad 0.0 \quad 0.20 .0$

UNIT 5

CYLINDER 110.5 2P15.0

UNIT 6

CYLINDER 210.2 2P15.0

UNIT 7

CYLINDER 210.2 2P15.0

CYLINDER 011.3 2P15.0

HOLE $50.7071072 * 0.0$

HOLE 60.7071070 .7071070 .0

HOLE 40.00 .7071070 .0

HOLE $6-0.7071070 .7071070 .0$

HOLE $5-0.7071070 .00 .0$

HOLE $6-0.707107-0.7071070 .0$

HOLE $40.0-0.7071070 .0$

HOLE $60.707107-0.7071070 .0$

CYLINDER 411.4 2P15.0

UNIT 8

CYLINDER 210.6 2P15.0

GLOBAL UNIT 9

CYLINDER 210.6 2P15.0

CYLINDER 013.6 2P15.0

HOLE 72.00 .00 .0

HOLE $82 * 2.00 .0$

HOLE 70.02 .00 .0

HOLE 8 -2.0 2.0 0.0

HOLE $7-2.02 * 0.0$

HOLE $82 *-2.00 .0$

HOLE $70.0-2.00 .0$

HOLE 8 2P2.0 0.0

CYLINDER 413.8 2P15.0

CUBOID 014 P 4.0 2P16.0

END GEOM

READ PLOT

TTL $=$ 'X-Y SLICE AT Z MIDPOINT. NESTED HOLES'

$\mathbf{X U L}=-0.1 \quad$ YUL $=8.1 \quad \mathbf{Z U L}=16.0$

$\mathbf{X L R}=8.1 \mathbf{Y L R}=-0.1 \mathbf{Z L R}=16$ 


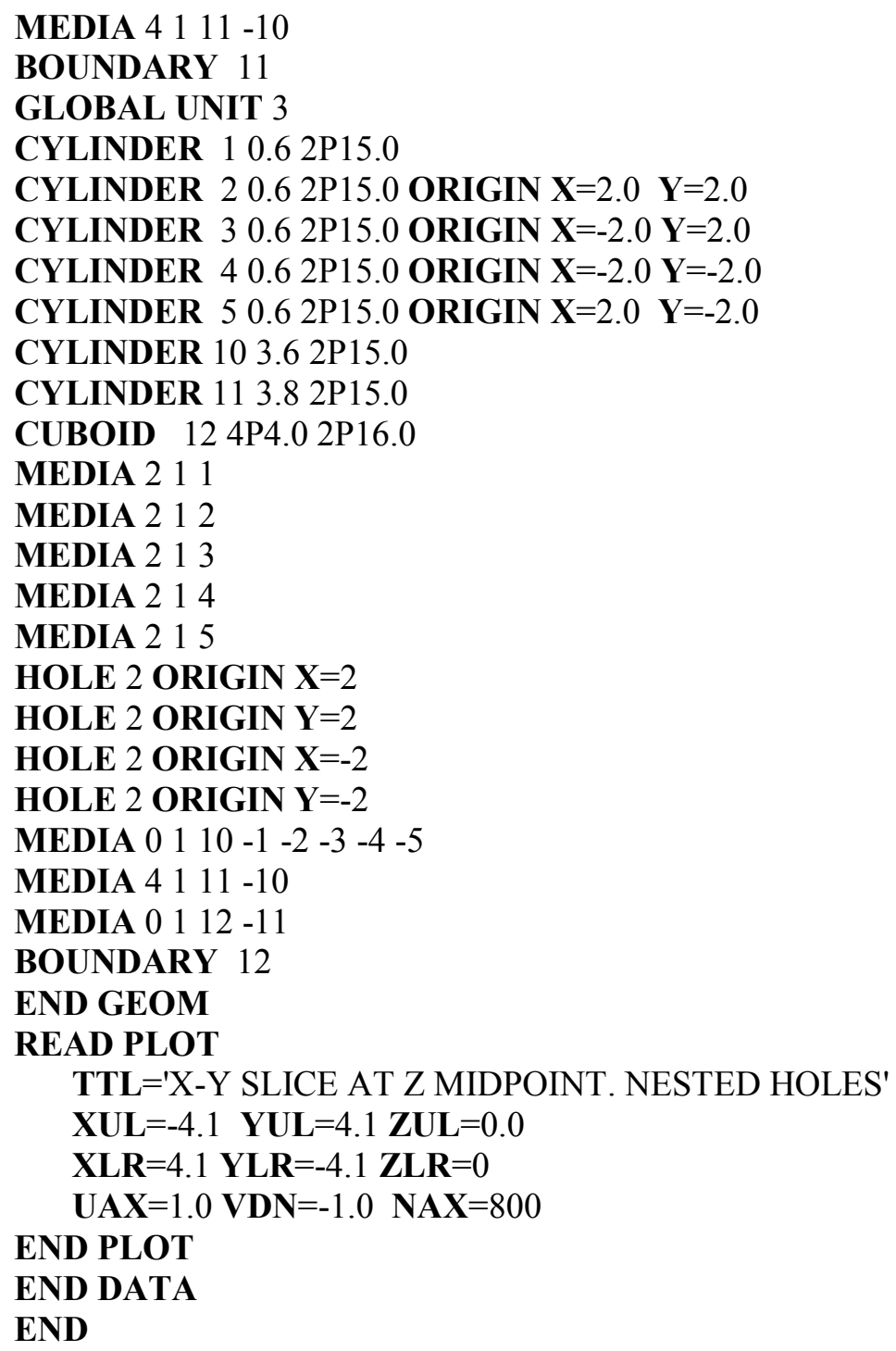

\subsection{Multiple arrays}

EXAMPLE 17. Section 8.1.3.6 demonstrates how UNITs are composed of geometry regions and how these UNITs can be stacked in an ARRAY. This same procedure can be extended to create multiple ARRAYs. Furthermore, ARRAYs can be used as building blocks within other ARRAYs.

Consider Sample Problem 19 from Appendix C. This problem is a critical experiment consisting of a composite array ${ }^{2,3}$ of four highly enriched uranium metal cylinders and four cylindrical Plexiglas containers filled with uranyl nitrate solution. A photograph of the experiment is given in Fig. 9.1.C.3. The coordinate system is defined to be $\mathrm{Z}$ up the page, $\mathrm{Y}$ across the page, and $\mathrm{X}$ out of the page.

The Plexiglas containers have an inside radius of $9.525 \mathrm{~cm}$ and an outside radius of $10.16 \mathrm{~cm}$. The inside height is $17.78 \mathrm{~cm}$, and the outside height is $19.05 \mathrm{~cm}$. Four of these containers are stacked with a centerto-center spacing of $21.75 \mathrm{~cm}$ in the $\mathrm{Y}$ direction and $20.48 \mathrm{~cm}$ in the $\mathrm{Z}$ direction (vertical). This arrangement of four Plexiglas containers can be described as follows: mixture 2 is the uranyl nitrate 
and mixture 3 is Plexiglas, so the Plexiglas container with its appropriate spacing CUBOID can be described as UNIT 1. This considers the ARRAY to be bare and suspended with no supports.

KENO V.a:

UNIT 1

CYLINDER 219.525 2P8.89

CYLINDER 3110.16 2P9.525

CUBOID $\quad 014 \mathrm{P} 10.875$ 2P10.24

KENO-VI:

UNIT 1

CYLINDER 19.525 2P8.89

CYLINDER 210.16 2P9.525

CUBOID 3 4P10.875 2P10.24

MEDIA 211

MEDIA $3 \quad 1 \quad 2 \quad-1$

MEDIA $0 \begin{array}{llll}0 & 3 & -2\end{array}$

BOUNDARY 3

The ARRAY of four Plexiglas containers can be described as ARRAY 1 in the array data as follows:

\section{ARA=1 NUX=1 NUY=2 NUZ=2 FILL F1 END FILL}

The four metal cylinders, comprised of mixture 1, each have a radius of $5.748 \mathrm{~cm}$ and are $10.765 \mathrm{~cm}$ tall. They have a center-to-center spacing of $13.18 \mathrm{~cm}$ in the $\mathrm{Y}$ direction and $12.45 \mathrm{~cm}$ in the $\mathrm{Z}$ direction (vertical). Thus, one of the metal cylinders with its appropriate spacing CUBOID can be described as UNIT 2. This ARRAY is also considered to be bare and unsupported.

KENO V.a:

UNIT 2

CYLINDER 115.748 2P5.3825

CUBOID 0014 P 6.59 2P6.225

KENO-VI:

UNIT 2

CYLINDER 15.748 2P5.3825

CUBOID 2 4P6.59 2P6.225

MEDIA 1111

MEDIA $0 \begin{array}{llll}0 & 2 & -1\end{array}$

BOUNDARY 2

The array of four metal cylinders can be described as ARRAY 2 in the array data.

ARA $=2$ NUX $=1 \quad$ NUY=2 NUZ=2 FILL F2 END FILL 
Now two ARRAYs have been described. The overall dimensions of the ARRAY of Plexiglas containers are $21.75 \mathrm{~cm}$ in $\mathrm{X}, 43.5 \mathrm{~cm}$ in $\mathrm{Y}$, and $40.96 \mathrm{~cm}$ in $\mathrm{Z}$. The overall dimensions of the ARRAY of metal cylinders are $13.18 \mathrm{~cm}$ in $X, 26.36 \mathrm{~cm}$ in $Y$, and $24.9 \mathrm{~cm}$ in $Z$.

In order to describe the composite ARRAY, these two ARRAYs must be positioned within UNITs and stacked together into one ARRAY. In order for them to be stacked into one ARRAY, the adjacent faces must match. This is accomplished by defining a UNIT 3 which contains ARRAY 1, the ARRAY of Plexiglas solution containers. The overall dimensions of this UNIT are $21.75 \mathrm{~cm}$ in X, $43.5 \mathrm{~cm}$ in Y, and $40.96 \mathrm{~cm}$ in Z. These dimensions are calculated by the code and need not be specified. UNIT 3 is defined as follows:

KENO V.a:

UNIT 3

ARRAY $13 * 0.0$

KENO-VI:

UNIT 3

CUBOID 1 2P10.875 2P21.75 2P20.48

ARRAY 11 PLACE $1110.0-10.875-10.24$

BOUNDARY 1

The ARRAY of metal cylinders will be defined to be UNIT 4. However, this ARRAY is $17.14 \mathrm{~cm}$ smaller in the $\mathrm{Y}$ and $16.06 \mathrm{~cm}$ smaller in the $\mathrm{Z}$ dimensions than the ARRAY of Plexiglas UNITs. Therefore, a void region must be placed around the ARRAY in those directions so UNIT 4 and UNIT 3 will be the same size in $\mathrm{Y}$ and $\mathrm{Z}$.

KENO V.a:

UNIT 4

ARRAY $23 * 0.0$

REPLICATE $\quad 0112 * 0.0 \quad 2 * 8.57 \quad 2 * 8.03 \quad 1$

KENO-VI:

UNIT 4

CUBOID $12 \mathrm{P} 6.59$ 2P13.18 2P12.45

CUBOID 2 2P6.59 2P21.75 2P20.48

ARRAY 21 PLACE $1110.0-6.59-6.225$

MEDIA 0112

BOUNDARY 2

Now that UNIT 3 and UNIT 4 have been defined, they must be placed in the global or universe ARRAY to define the physical arrangement of the eight pieces. This procedure is implemented via a GLOBAL ARRAY in KENO V.a, while KENO-VI uses a GLOBAL UNIT 3 as follows:

KENO V.a:

GBL $=3$ ARA $=3 \quad$ NUX=2 NUY=1 NUZ=1 FILL 43 END FILL 
KENO-VI:

GLOBAL UNIT 5

CUBOID 134.930 .043 .50 .040 .960 .0

ARRAY 31 PLACE 1116.5921 .7520 .48

BOUNDARY 1

The description of ARRAY 3 in KENO-VI is identical to that shown for KENO V.a.

This completes the geometry description for the problem. The complete geometry input description for the problem is given below.

KENO V.a:

$=$ KENOVA

SAMPLE PROBLEM 194 AQUEOUS 4 METAL ARRAY OF ARRAYS

READ PARAM LIB $=4$ RUN=NO END PARAM

READ MIXT SCT $=1$

MIX $=1$

$1092238 \quad 3.2275 \mathrm{e}-3$

$1092235 \quad 4.4802 \mathrm{e}-2$

MIX $=2$

$20011023 \quad 5.81 \mathrm{e}-2$

$2007014 \quad 1.9753 \mathrm{e}-3$

$2008016 \quad 3.6927 \mathrm{e}-2$

$20092235 \quad 9.8471 \mathrm{e}-4$

$20092238 \quad 7.7697 \mathrm{e}-5$

MIX $=3$

$11006012 \quad 3.5552 \mathrm{e}-2$

$11011023 \quad 5.6884 \mathrm{e}-2$

$11008016 \quad 1.4221 \mathrm{e}-2$

END MIXT

READ GEOM

UNIT 1

CYLINDER $219.5258 .89-8.89$

CYLINDER 3110.162 P9.525

CUBOID 014 P 10.875 2P10.24

UNIT 2

CYLINDER 115.748 2P5.3825

CUBOID 01 4P6.59 2P6.225

UNIT 3

ARRAY $13 * 0.0$

UNIT 4

ARRAY $23 * 0.0$

REPLICATE $012 * 0.02 * 8.572 * 8.031$

END GEOM

READ ARRAY

ARA $=1 \mathbf{N U X}=1 \quad \mathbf{N U Y}=2 \mathbf{N U Z}=2$

FILL F1 END FILL

$A R A=2$ NUX $=1$ NUY $=2 \mathbf{N U Z}=2$

FILL F2 END FILL

GBL $=3$ ARA $=3$ NUX=2 NUY=1 NUZ=1 FILL 43 END FILL

END ARRAY 


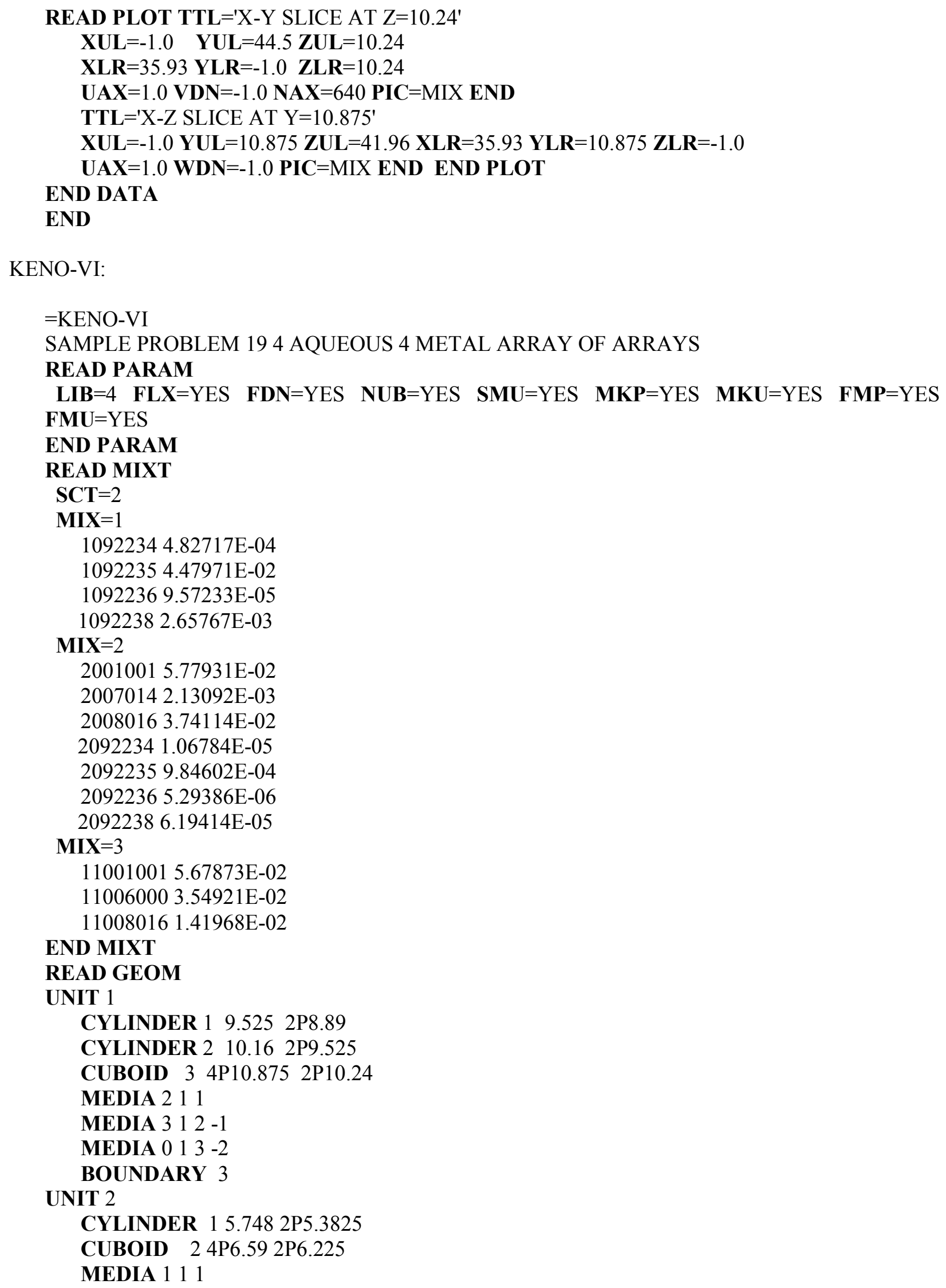




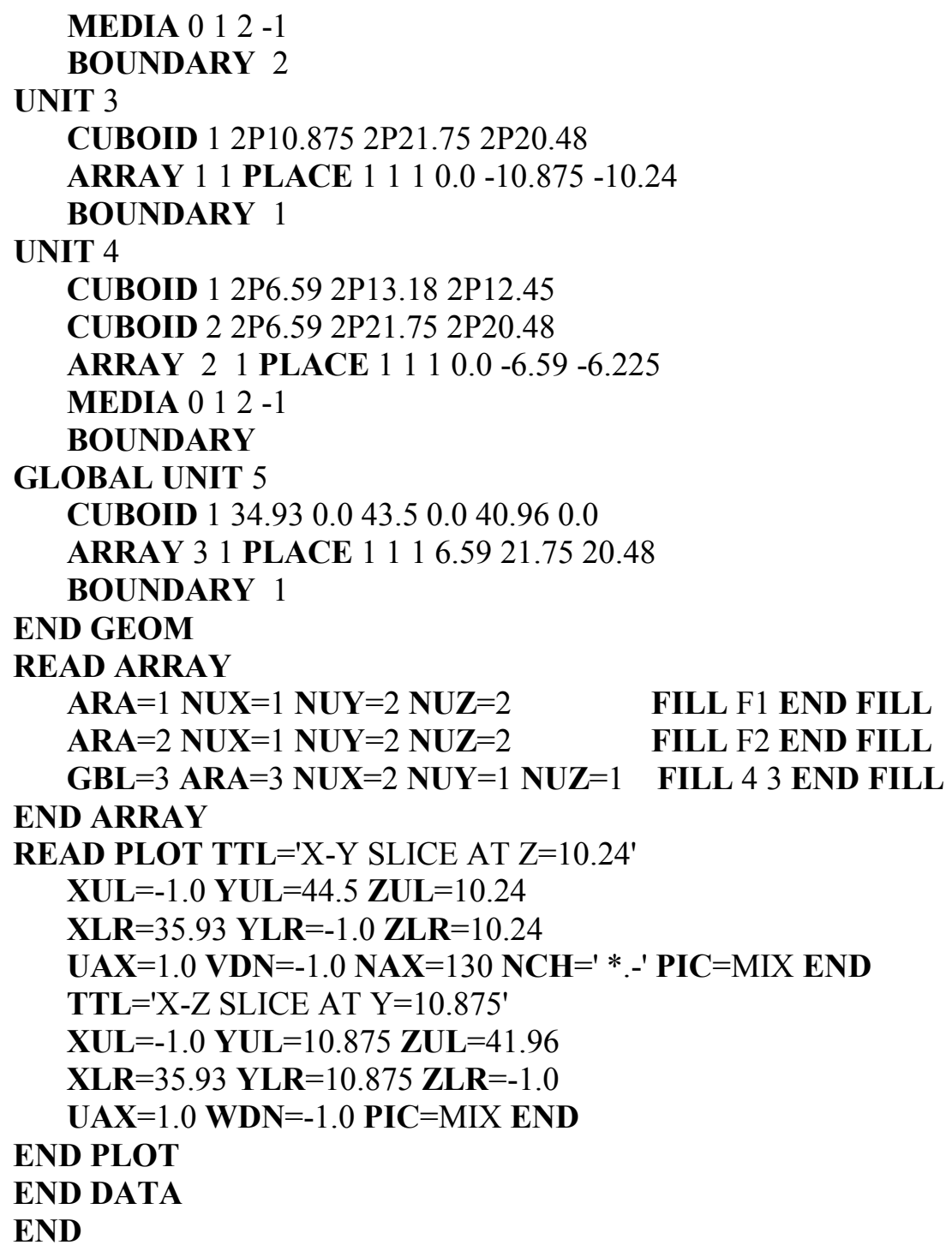

A plot of an X-Y slice taken through the bottom layer of the array is shown in Fig. 8.1.24. A plot of an X$\mathrm{Z}$ slice taken through the $+\mathrm{Y}$ half of the array is shown in Fig. 8.1.25. These plots were used to verify the geometry mockup. 


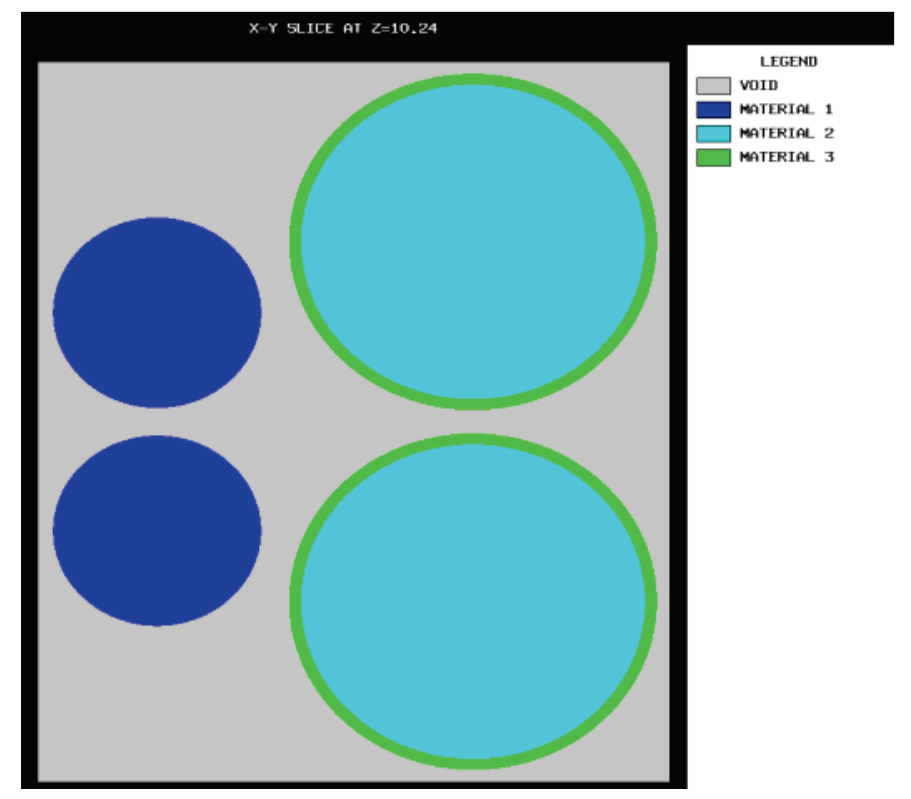

Fig. 8.1.24. X-Y plot of mixed array. 


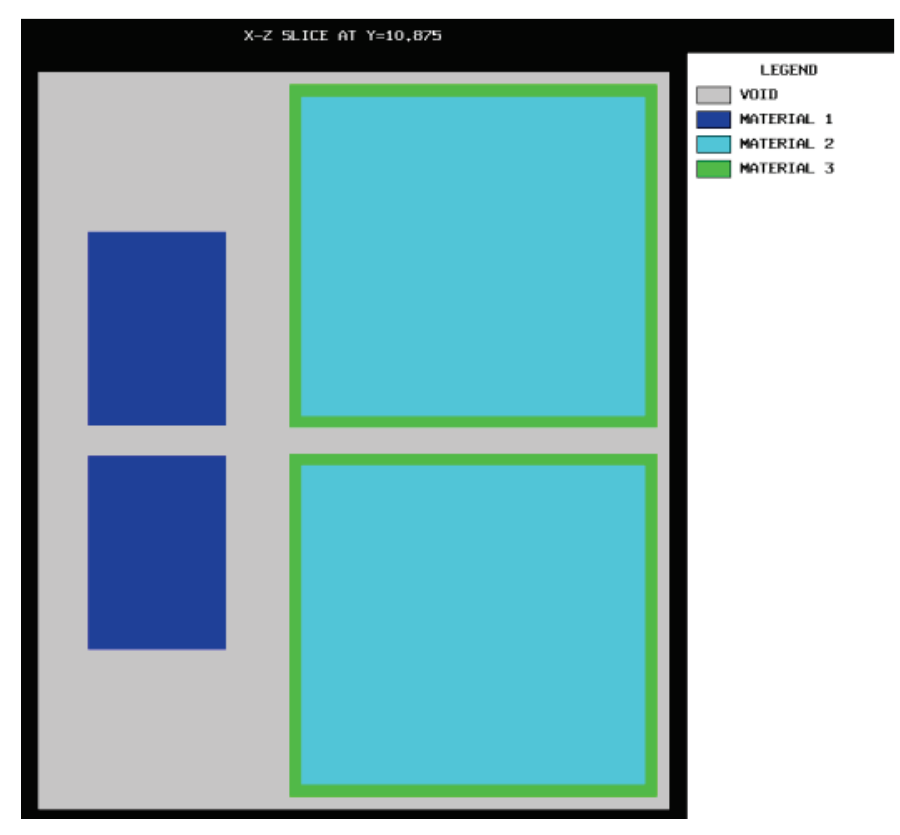

Fig. 8.1.25. X-Z plot of mixed array.

\section{STORAGE ARRAY}

EXAMPLE 18. Consider a storage array of highly enriched uranium buttons, each 1 in. tall and 4 in. in diameter. These buttons are stored on stainless steel shelves with a center-to-center spacing of $60.96 \mathrm{~cm}$ ( $2 \mathrm{ft})$ between them in the $\mathrm{Y}$ direction, and only one button on each shelf in the $\mathrm{X}$ direction. The shelves are $0.635 \mathrm{~cm}\left({ }^{1} / 4\right.$ in.) thick ( $\mathrm{Z}$ dimension), $45.72 \mathrm{~cm}$ (18 in.) wide ( $\mathrm{X}$ dimension), $609.6 \mathrm{~cm}(20 \mathrm{ft}) \operatorname{long}(\mathrm{Y}$ dimension), and are $45.72 \mathrm{~cm}$ (18 in.) from the top of a shelf to the bottom of the shelf above it. Each rack of storage shelves is four shelves high, with the first shelf being $15.24 \mathrm{~cm}(6 \mathrm{in}$.) above the floor. The storage room is $586.56 \mathrm{~cm}(19.5 \mathrm{ft})$ in the $\mathrm{X}$ direction by $1293.44 \mathrm{~cm}(43 \mathrm{ft})$ in the $\mathrm{Y}$ direction with $365.76 \mathrm{~cm}$ (12 ft. ) ceilings in the $\mathrm{Z}$ direction. The walls, ceiling, and floor are composed of concrete, $30.48 \mathrm{~cm}(1 \mathrm{ft})$ thick. All the aisles between the storage racks are $91.44 \mathrm{~cm}(3 \mathrm{ft})$ wide. The racks are arranged with their length in the $\mathrm{Y}$ direction and an aisle between them. The arrays of racks are arranged with two in the $\mathrm{Y}$ direction and five in the $\mathrm{X}$ direction. Mixture 1 is the uranium metal, mixture 2 is the stainless steel, and mixture 3 is the concrete.

The metal button and its center-to-center spacing are described first. The void vertical spacing has arbitrarily been chosen to extend from the bottom of the button to the next shelf above the button. The shelf of stainless steel is described under the button.

KENO V.a:

UNIT 1

COM='METAL BUTTONS'

CYLINDER 115.082 .540 .0

CUBOID 012 2P22.86 2P30.48 45.720 .0

CUBOID 21 2P22.86 2P30.48 $45.72-0.635$ 
KENO-VI:

UNIT 1

CYLINDER $15.08 \quad 2.54 \quad 0.0$

$\begin{array}{llllll}\text { CUBOID } & 2 & 2 \mathrm{P} 22.86 & 2 \mathrm{P} 30.48 & 45.72 & 0.0\end{array}$

$\begin{array}{llllll}\text { CUBOID } & 3 & 2 \mathrm{P} 22.86 & 2 \mathrm{P} 30.48 & 45.72 & -0.635\end{array}$

MEDIA 1111

MEDIA 0012

MEDIA 2

BOUNDARY 3

ARRAY 1 creates an ARRAY of these buttons that fills one shelf. UNIT 2 then contains one of the shelves shown in Fig. 8.1.26.
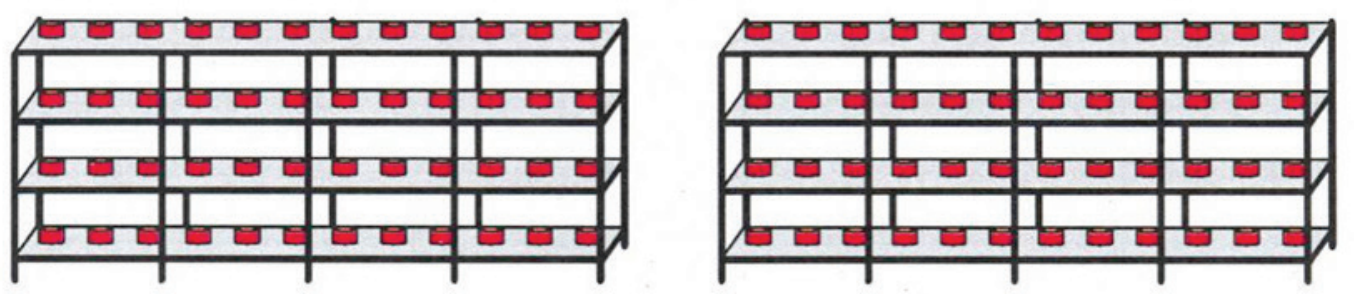

Fig. 8.1.26. Two racks of uranium buttons.

KENO V.a:

ARA $=1$ COM='SINGLE SHELF CONTAINING 10 METAL BUTTONS'

$\mathbf{N U X}=1$ NUY=10 NUZ=1 FILL F1 END FILL

UNIT 2

COM='SINGLE SHELF (1 X 10 X 1 ARRAY OF METAL BUTTONS ON A SHELF)'

ARRAY $13 * 0.0$

KENO-VI: 


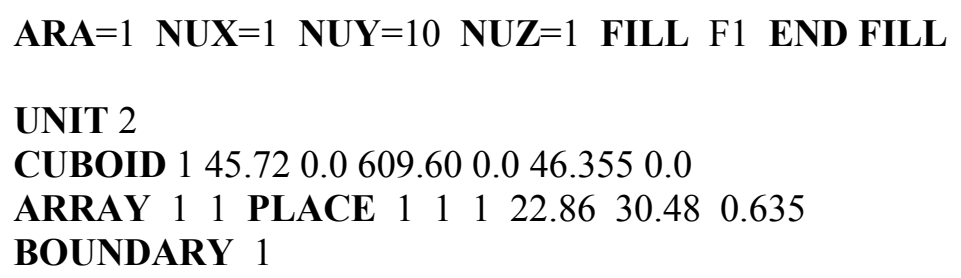

NOTE: The origin of UNIT 2 is on the bottom of the bottom shelf; it has been moved from the bottom of the button. The $\mathrm{X}$ and Y position of the origin is at the front, left-hand corner of the bottom of this lowest shelf.

Stack four UNIT 2 s vertically to obtain one of the racks shown in Fig. 8.1.26. One rack is defined by array 2 .

\section{ARA $=2 \mathbf{C O M}=$ 'SINGLE RACK OF 4 SHELVES' \\ NUX=1 NUY=1 NUZ=4 FILL F2 END FILL}

Generate a UNIT 3 that contains a rack of shelves and a UNIT 4 that is the aisle between the ends of the two racks in the Y direction.

KENO V.a:

UNIT 3

COM='SINGLE RACK (4 SHELVES TALL)'

ARRAY $23 * 0.0$

UNIT 4

$\mathbf{C O M}=$ 'CENTRAL AISLE UNIT SAME HEIGHT AS 4 SHELVES'

CUBOID 01 2P22.86 2P45.72 185.42 0.0

KENO-VI:

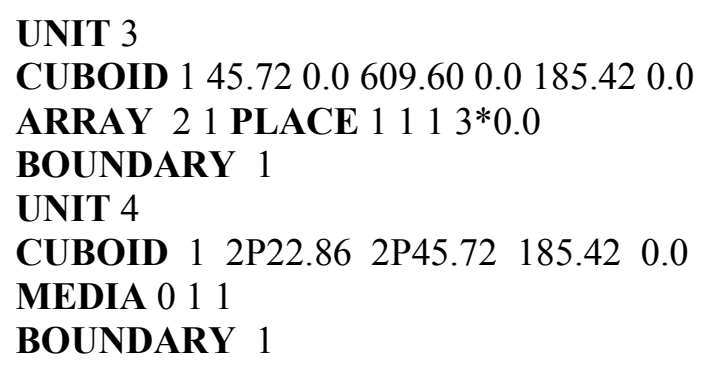

Stack UNITs 3 and 4 together in the Y direction to create UNIT 5 which contains both racks in the $\mathrm{Y}$ direction and the aisle between them. This configuration is shown in Fig. 8.1.26.

KENO V.a:

ARA=3 COM='TWO RACKS END TO END WITH CENTRAL AISLE'

NUX=1 NUY=3 NUZ=1 FILL 343 END FILL

UNIT 5

$\mathbf{C O M = ' S E T ~ O F ~ T W O ~ R A C K S ~ E N D ~ T O ~ E N D ~ S E P A R A T E D ~ B Y ~ T H E ~ C E N T R A L ~ A I S L E ' ~}$ 
ARRAY $33 * 0.0$

KENO-VI:

ARA=3 NUX=1 NUY=3 NUZ=1 FILL 343 END FILL

UNIT 5

CUBOID 145.720 .01310 .640 .0185 .420 .0

ARRAY 31 PLACE $11113 * 0.0$

BOUNDARY 1

Create a UNIT 6, which is an aisle $91.44 \mathrm{~cm}(3 \mathrm{ft})$ wide in the $\mathrm{X}$ direction and $1310.64 \mathrm{~cm}(43 \mathrm{ft})$ in the $\mathrm{Y}$ direction (full length of the room).

KENO V.a:

UNIT 6

COM='AISLE BETWEEN ADJACENT SETS OF TWO RACKS \& CENTRAL AISLE (UNITS 5)'

CUBOID 0191.440 .01310 .640 .0185 .420 .0

KENO-VI:

UNIT 6

CUBOID $191.440 .0 \quad 1310.640 .0 \quad 185.420 .0$

MEDIA 0111

BOUNDARY 1

Stack UNITs 5 and 6 in the $\mathrm{X}$ direction to achieve the array of racks in the room. Then put the 6 in. spacing below the bottom of the racks, the spacing between the top of the top rack and the ceiling, and add the concrete floor, walls, and ceiling around the array. ARRAY 4 describes the array of racks in the room. ARRAY record (first CUBOID description in KENO-VI) encompasses this ARRAY, and the first REFLECTOR (second CUBOID in KENO-VI) descriptions are used to add the spacing between the top rack and the ceiling. The last two REFLECTOR (CUBOIDs 3 through 9 in KENO-VI) descriptions add the ceiling, walls, and floor in $5.0 \mathrm{~cm}$ increments to bias the concrete. A perspective of the room is shown in Fig. 8.1.27.

KENO V.a:

ARA $=4$ COM $=$ 'ENTIRE STORAGE ARRAY'

NUX $=9$ NUY=1 NUZ=1 FILL 56 3Q2 5 END FILL

GLOBAL

UNIT 7

COM='STORAGE ARRAY IN THE ROOM WITH WALLS, FLOOR AND CEILING'

ARRAY $43 * 0.0$

$\begin{array}{lllllllll}\text { REFLECTOR } & 0 & 1 & 4 * 0.0 & 165.1 & 15.24 & 1\end{array}$

REFLECTOR $326 * 5.06$

REFLECTOR $3 \quad 8 \quad 6 * 0.48 \quad 1$

KENO-VI:

GBL=4 ARA=4 NUX=9 NUY=1 NUZ=1 FILL 56 3Q2 5 END FILL 
GLOBAL UNIT 7

CUBOID $1594.36 \quad 0.0 \quad 1310.64 \quad 0.0 \quad 185.42 \quad 0.0$

$\begin{array}{lllllll}\text { CUBOID } 2594.36 & 0.0 & 1310.64 & 0.0 & 350.52 & -15.24\end{array}$

CUBOID $3599.36-5.001315 .64-5.00355 .52-20.24$

CUBOID $4604.36-10.001320 .64-10.00360 .52-25.24$

CUBOID $5609.36-15.001325 .64-15.00365 .52-30.24$

CUBOID $6614.36-20.001330 .64-20.00370 .52-35.24$

CUBOID $7619.36-25.001335 .64-25.00375 .52-40.24$

CUBOID 8624.36 -30.00 1340.64 -30.00 380.52 -45.24

CUBOID $9624.84-30.481341 .12-30.48381 .00-45.72$

ARRAY 4 1 PLACE $11113 * 0.0$

MEDIA $0 \begin{array}{llll} & 1 & 2 & -1\end{array}$

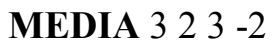

MEDIA $3 \quad 3 \quad 4 \quad-3$

MEDIA 3 4 5 - 5 - 4

MEDIA 35 r 6 - 5

MEDIA $367-6$

MEDIA 378 - 7

MEDIA 38 \& 9 -8

BOUNDARY 9

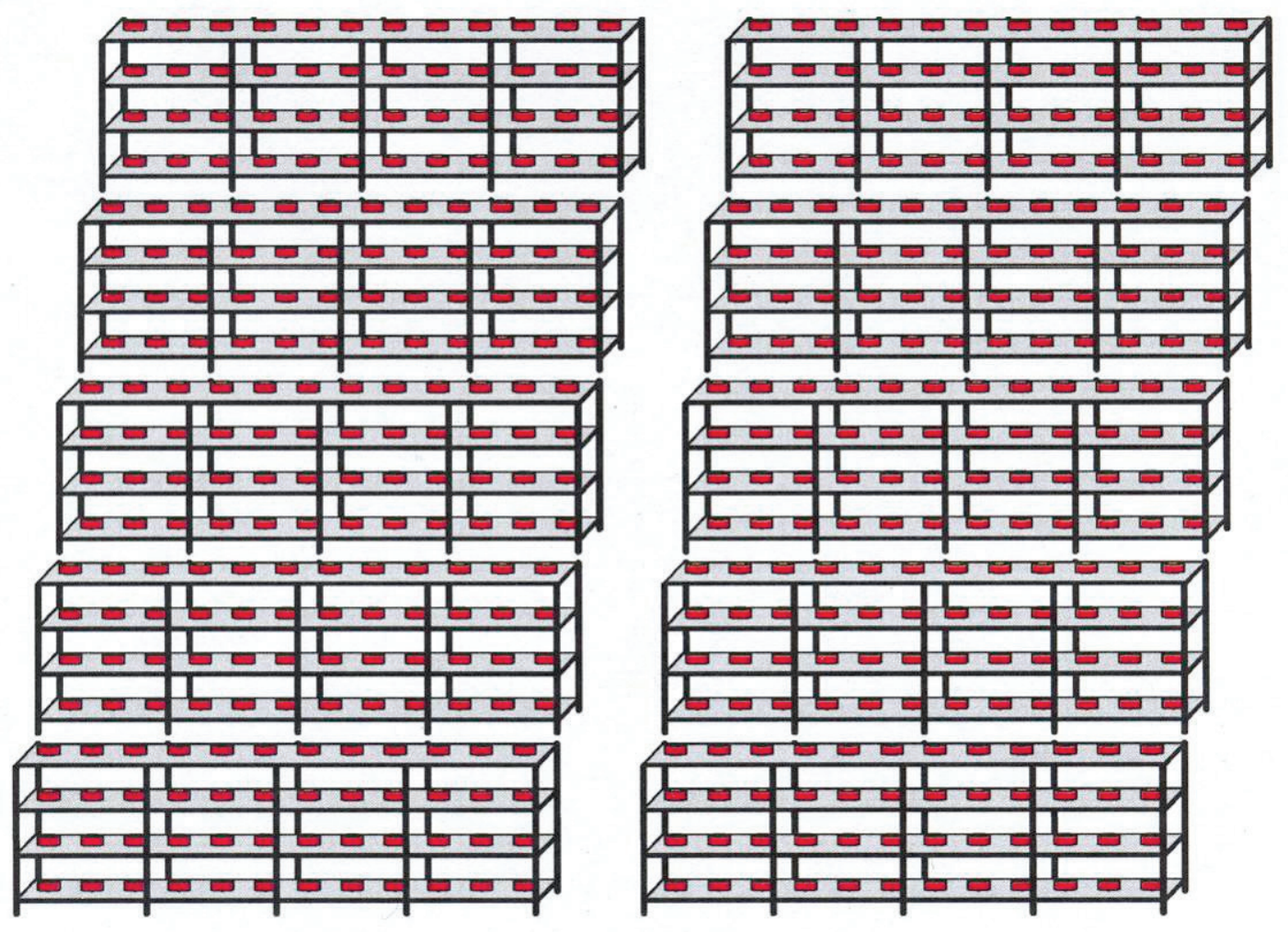

Fig. 8.1.27. Entire storage array in the room. 
The complete input for this room is given below: The plots for this problem must be quite large in order to see all the detail because the array is sparse and the shelves are thin. Therefore, the plots for this system are not included as Fig.s. The user can generate the plots if it is desirable to see them. The nuclide IDs used in this problem are for the 16-group Hansen-Roach working format library, which is no longer distributed with SCALE.

KENO V.a:

$=$ KENO5A

STORAGE ARRAY

READ PARAMETERS FDN=YES LIB $=41$

END PARAMETERS

READ MIXT SCT=1 MIX=1 92500 4.48006e-2 92800 2.6578e-3 92400 4.827e-4

92600 9.57e-5 MIX=2 2001.0 MIX=3 3011 END MIXT

READ GEOMETRY

UNIT 1

COM='METAL BUTTONS'

CYLINDER 115.082 .540 .0

CUBOID $012 \mathrm{P} 22.86$ 2P30.48 45.720 .0

CUBOID $212 \mathrm{P} 22.86$ 2P30.48 $45.72-0.635$

UNIT 2

COM='SINGLE SHELF ( 1 X 10 X 1 ARRAY OF METAL BUTTONS ON A SHELF)'

ARRAY $13 * 0.0$

UNIT 3

$\mathbf{C O M}=$ 'SINGLE RACK (4 SHELVES TALL)'

ARRAY $23 * 0.0$

UNIT 4

COM='CENTRAL AISLE UNIT SAME HEIGHT AS 4 SHELVES'

CUBOID $012 \mathrm{P} 22.86$ 2P45.72 185.420 .0

UNIT 5

COM='SET OF TWO RACKS END TO END SEPARATED BY THE CENTRAL AISLE'

ARRAY $33 * 0.0$

UNIT 6

$\mathbf{C O M = ' A I S L E ~ B E T W E E N ~ A D J A C E N T ~ S E T S ~ O F ~ T W O ~ R A C K S ~ \& ~ C E N T R A L ~ A I S L E ~ ( U N I T S ~ 5 ) ' ~}$

CUBOID 0191.440 .01310 .640 .0185 .420 .0

GLOBAL

UNIT 7

COM='STORAGE ARRAY IN THE ROOM WITH WALLS, FLOOR AND CEILING'

ARRAY $43 * 0.0$

REFLECTOR $014 * 0.0165 .115 .241$

REFLECTOR $326 * 5.0 \quad 6$

REFLECTOR $386 * 0.481$

END GEOMETRY

READ ARRAY

ARA=1 COM='SINGLE SHELF CONTAINING 10 METAL BUTTONS'

NUX $=1$ NUY $=10$ NUZ $=1$ FILL F1 END FILL

ARA $=2$ COM $=$ 'SINGLE RACK OF 4 SHELVES'

$\mathbf{N U X}=1 \mathbf{N U Y}=1 \mathbf{N U Z}=4$ FILL F2 END FILL

ARA $=3 \mathbf{C O M}=$ 'TWO RACKS END TO END WITH CENTRAL AISLE'

NUX=1 NUY=3 NUZ=1 FILL 343 END FILL

ARA $=4$ COM='ENTIRE STORAGE ARRAY' 
NUX $=9$ NUY=1 NUZ=1 FILL 56 3Q2 5 END FILL

END ARRAY

READ BIAS ID $=30128$ END BIAS

READ START NST $=5$ NBX $=5$ END START

READ PLOT TTL $=$ 'X-Z SLICE AT Y $=30.48$ WITH Z ACROSS AND X DOWN'

$\mathbf{X U L}=594.8$ YUL=30.48 ZUL=-1.0 XLR=-0.5 YLR=30.48 ZLR=186.0

WAX $=1.0$ UDN $=-1.0$ NAX $=640$ END

TTL $=$ 'Y-Z SLICE OF LEFT RACKS, $X=22.86$ WITH Z ACROSS AND Y DOWN'

$\mathbf{X U L}=22.86 \mathbf{Y U L}=1311.0 \mathbf{Z U L}=-0.5 \mathbf{X L R}=22.86 \mathbf{Y L R}=-3.0 \mathbf{Z L R}=186.0$

WAX $=1.0$ VDN $=-1.0$ NAX $=640$ END

TTL $=$ 'X-Y SLICE OF ROOM THROUGH SHELF Z $=0.3175$ WITH X ACROSS AND Y DOWN'

$\mathbf{X U L}=-1.0 \quad \mathbf{Y U L}=1312.0 \mathbf{Z U L}=0.3175 \mathbf{X L R}=596.0 \mathbf{Y L R}=-2.5 \mathbf{Z L R}=0.3175$

$\mathbf{U A X}=1.0$ VDN $=-1.0 \mathbf{N A X}=320$ END

END PLOT

END DATA

END

KENO-VI:

$=\mathrm{KENOVI}$

STORAGE ARRAY

READ PARAMETERS FDN=YES LIB=41 END PARAMETERS

READ MIXT SCT $=1$

MIX=1 92500 4.48006e-2 92800 2.6578e-3 92400 4.827e-4 92600 9.57e-5

MIX=2 2001.0 MIX=3 3011

END MIXT

READ GEOMETRY

UNIT 1

$\begin{array}{llll}\text { CYLINDER } 1 & 5.08 & 2.54 & 0.0\end{array}$

$\begin{array}{lllllll}\text { CUBOID } & 2 & 2 \mathrm{P} 22.86 & 2 \mathrm{P} 30.48 & 45.72 & 0.0\end{array}$

$\begin{array}{lllllll}\text { CUBOID } & 3 & 2 \mathrm{P} 22.86 & 2 \mathrm{P} 30.48 & 45.72 & -.635\end{array}$

MEDIA 1111

MEDIA $0 \begin{array}{llll}0 & 1 & 2 & -1\end{array}$

MEDIA $2 \begin{array}{llll} & 1 & 3 & -2\end{array}$

BOUNDARY 3

UNIT 2

CUBOID 145.720 .0609 .600 .046 .3550 .0

$\begin{array}{llllllllll}\text { ARRAY } & 1 & 1 & \text { PLACE } & 1 & 1 & 1 & 22.86 & 30.48 & 0.635\end{array}$

BOUNDARY 1

UNIT 3

CUBOID 145.720 .0609 .600 .0185 .420 .0

ARRAY 21 PLACE $11113 * 0.0$

BOUNDARY 1

UNIT 4

$\begin{array}{llllll}\text { CUBOID } & 1 & 2 \mathrm{P} 22.86 & 2 \mathrm{P} 45.72 & 185.42 & 0.0\end{array}$

MEDIA 011 


\section{BOUNDARY 1}

UNIT 5

CUBOID 145.720 .01310 .640 .0185 .420 .0

ARRAY 31 PLACE $11113 * 0.0$

BOUNDARY 1

UNIT 6

CUBOID $191.440 .0 \quad 1310.640 .0 \quad 185.420 .0$

MEDIA 011

BOUNDARY 1

GLOBAL UNIT 7

CUBOID $1594.36 \quad 0.01310 .64 \quad 0.0185 .42 \quad 0.0$

CUBOID $2594.36 \quad 0.01310 .64 \quad 0.0350 .52-15.24$

CUBOID $3599.36-5.001315 .64-5.00355 .52-20.24$

CUBOID $4604.36-10.001320 .64-10.00360 .52-25.24$

CUBOID $5609.36-15.001325 .64-15.00365 .52-30.24$

CUBOID $6614.36-20.001330 .64-20.00370 .52-35.24$

CUBOID $7619.36-25.001335 .64-25.00375 .52-40.24$

CUBOID $8624.36-30.001340 .64-30.00380 .52-45.24$

CUBOID $9624.84-30.481341 .12-30.48381 .00-45.72$

ARRAY 41 PLACE $11113 * 0.0$

MEDIA $0 \begin{array}{llll}0 & 1 & 2 & -1\end{array}$

MEDIA 32

MEDIA 3 3 34 -

MEDIA 3 4 $45-4$

MEDIA $356-5$

MEDIA $367-6$

MEDIA 378 - 7

MEDIA $3 \quad 8 \quad 9 \quad-8$

BOUNDARY 9

END GEOMETRY

READ ARRAY

ARA $=1$ NUX $=1$ NUY=10 NUZ=1 FILL F1 END FILL

ARA $=2$ NUX $=1$ NUY $=1$ NUZ $=4$ FILL F2 END FILL

ARA $=3$ NUX $=1$ NUY=3 NUZ=1 FILL 343 END FILL

$\mathbf{G B L}=4$ ARA $=4$ NUX=9 NUY=1 NUZ=1 FILL 56 3Q 25 END FILL

END ARRAY

READ BIAS ID $=30128$ END BIAS

READ START NST $=5$ NBX $=5$ END START

READ PLOT PLT $=$ YES TTL $=$ 'X-Z SLICE AT Y=30.48 WITH Z ACROSS AND X DOWN'

$\mathbf{X U L}=594.8$ YUL=30.48 ZUL=-1.0 XLR=-0.5 YLR=30.48 ZLR=186.0

$\mathbf{W A X}=1.0$ UDN $=-1.0 \mathrm{NAX}=640$ END

$\mathbf{T T L}=' \mathrm{Y}-\mathrm{Z}$ SLICE OF LEFT RACKS, $\mathrm{X}=22.86$ WITH Z ACROSS AND Y DOWN'

$\mathbf{X U L}=22.86 \mathbf{Y U L}=1311.0 \mathbf{Z U L}=-0.5 \mathbf{X L R}=22.86 \mathbf{Y L R}=-3.0 \mathbf{Z L R}=186.0$

$\mathbf{W A X}=1.0 \mathrm{VDN}=-1.0 \mathrm{NAX}=640 \mathbf{E N D}$ 
END

\subsection{Arrays and holes}

Sections 8.1.3.6.1 and 8.1.3.6.2 describe the use of HOLEs, and Sect. 8.1.3.6.3 describes multiple ARRAYs and ARRAYs of ARRAYs. HOLEs can be used to place ARRAYs at locations in other UNITs. This section contains examples to illustrate the combined use of ARRAYs and HOLEs.

\section{EXAMPLE 19. A SIMPLE CASK}

This example consists of cylindrical mild steel container with an inside radius of $4.15 \mathrm{~cm}$ and a radial wall thickness of $0.45 \mathrm{~cm}$. The thickness of the ends of the container is $1.27 \mathrm{~cm}$, and the inside height is $10.1 \mathrm{~cm}$. Highly enriched uranium rods $1 \mathrm{~cm}$ in diameter and $10 \mathrm{~cm}$ long are banded together into square bundles of four. These bundles are then positioned in the mild steel container as shown in Fig. 8.1.28. The rods sit on the floor of the container and have a $0.1 \mathrm{~cm}$ gap between their tops and the top of the container.

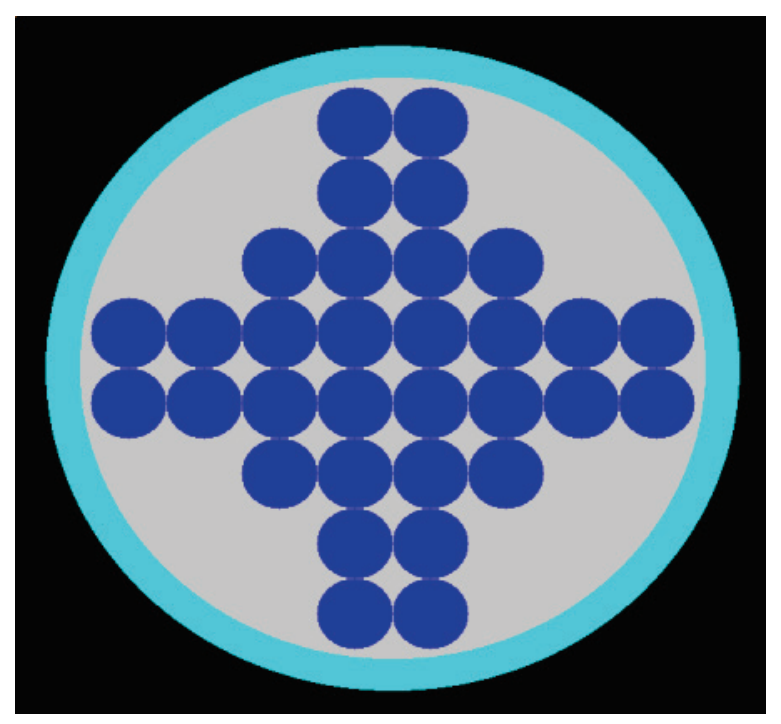

Fig. 8.1.28. Uranium rods in a cylindrical container.

To generate the geometry description for this system, UNIT 1 is defined as one uranium rod and its associated square-pitch close-packed spacing region. 
KENO V.a:

UNIT 1

CYLINDER $1110.5 \quad 2 \mathrm{P} 5.0$

CUBOID $\quad 0 \quad 1 \quad 4$ P 0.5 2P5.0

KENO-VI:

UNIT 1

CYLINDER 10.5 2P5.0

CUBOID 2 4P0.5 2P5.0

MEDIA 1111

MEDIA 0012 - 1

BOUNDARY 2

From here, the geometry description diverges between KENO V.a and KENO-VI.

KENO V.a:

ARRAY 1 is defined to be the central square ARRAY consisting of four bundles of rods.

\section{ARA $=1$ NUX=4 NUY=4 NUZ=1 FILL F1 END FILL}

ARRAY 2 is defined to be a bundle of four rods.

\section{ARA $=2$ NUX=2 NUY=2 NUZ=1 FILL F1 END FILL}

ARRAY 2 is placed in UNIT 2. This defines the outer boundaries of an imaginary CUBOID that contains the ARRAY. It is convenient to have the origin of the ARRAY at its center, so the most negative point of the array will be $(-1,-1,-5)$.

\section{UNIT 2}

ARRAY 2 - $1.0-1.0-5.0$

An ARRAY record is used to place ARRAY 1 in the GLOBAL UNIT. Then the cylindrical container is described around it and HOLEs are used to place the four outer bundles around the central ARRAY.

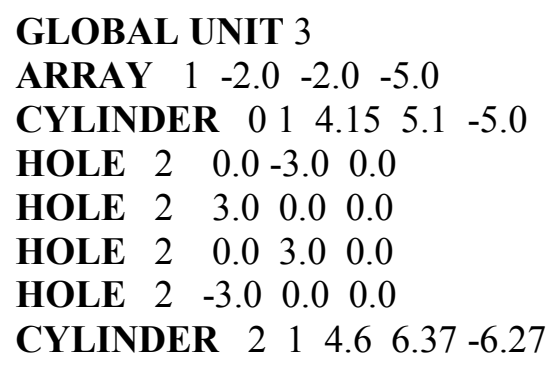

The first HOLE places the bottom bundle of four rods, the second HOLE places the bundle of four rods at the right, the third HOLE places the top bundle of rods and the fourth HOLE places the left bundle of rods.

KENO-VI: 
Define UNIT 2 to be a void CUBOID with the same square pitch as the rod square pitch.

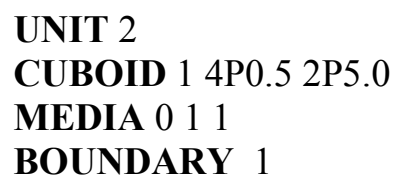

ARRAY 1 is defined to be the central square $10 \times 10$ ARRAY consisting of 32 rods and 68 void positions that can be used to represent the array shown in

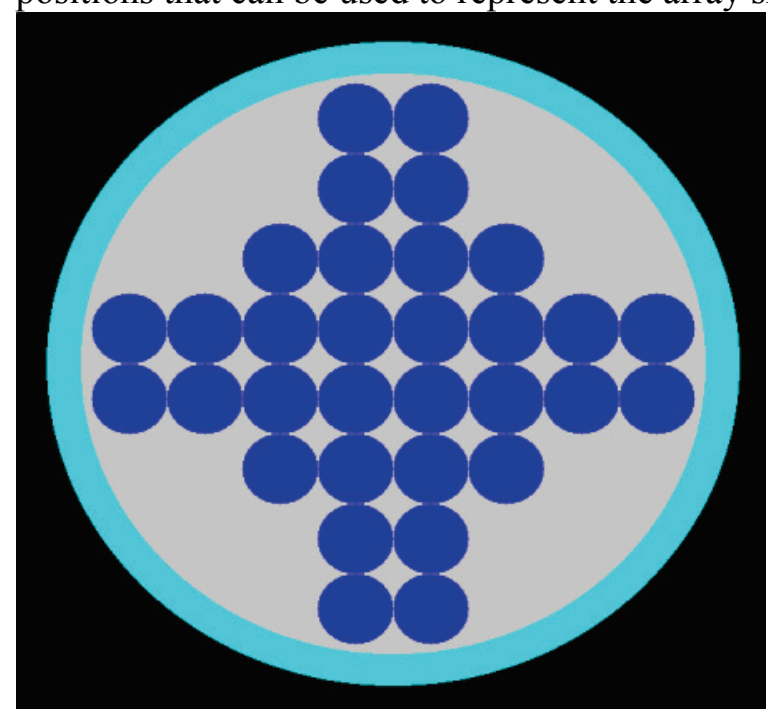

Fig. 8.1.28.

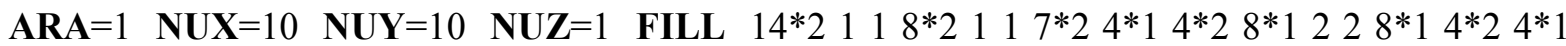
7*2 $1118 * 21114 * 2$ END FILL

ARRAY 1 is placed in UNIT 3. The first CYLINDER card defines the ARRAY BOUNDARY. Everything external to this boundary is not considered part of the problem. The positions in the ARRAY that do not contain rods are filled with cuboids consisting of void. The ARRAY boundary must either coincide with the outer boundary of the ARRAY or be contained within the ARRAY. An exterior void region is placed around the array boundary to coincide with the size of the interior radius of the container. The $10 \times 10$ ARRAY with the ARRAY boundary is shown in Fig. 8.1.29.

\section{UNIT 3}

CYLINDER 14.15 5.0 -5.0

CYLINDER $24.155 .1-5.0$

ARRAY 11 PLACE $551 \quad-0.5-0.5-0.0$

MEDIA $0 \begin{array}{llll} & 1 & 2 & -1\end{array}$

BOUNDARY 2 


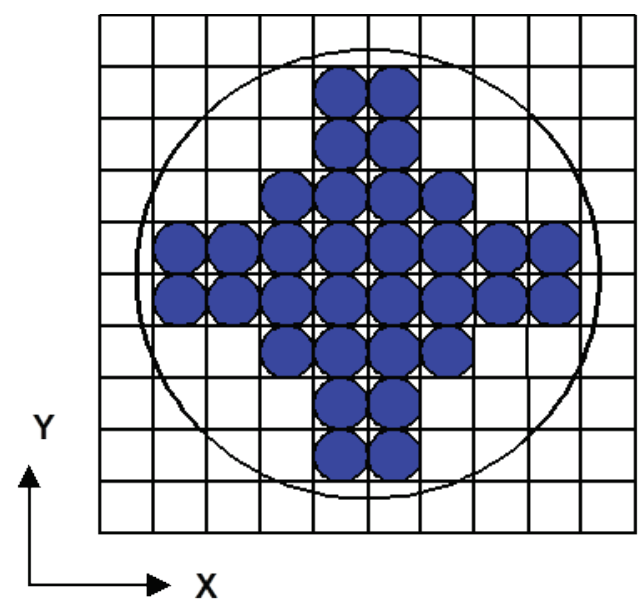

Fig. 8.1.29. The $10 \times 10$ array of 32 uranium rods and 68 void cuboids with the array boundary.

The UNIT containing the ARRAY is now placed within the global unit using a HOLE content record. The location of the HOLE is determined using ORIGIN data to match the origin of the UNIT in the HOLE with an X, Y, Z position in the surrounding UNIT. In this problem, the origin of the UNIT must be at position $(0,0,0)$. Since only nonzero data must be entered, ORIGIN data are not needed for this problem. The boundary region consists of the steel container.

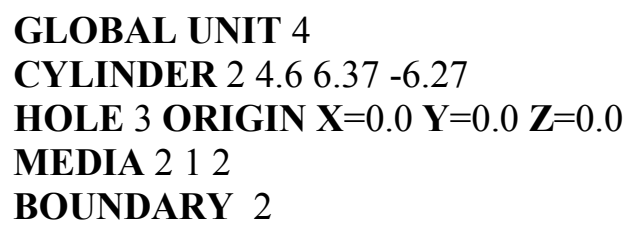

The overall problem description is shown below. Two of the color plots used for verification of this mockup are shown in Fig. 8.1.30 and Fig. 8.1.31. The black outside border of the two color plots indicates volume outside the global unit. The plot can be extended just outside the global unit boundary to ensure that the entire problem is included in the plot. This results in a black area surrounding the actual problem.

\section{KENO V.a:}

$=$ KENOVA

CASK ARRAY

READ PARAMETERS FDN $=$ YES LIB $=41$ GEN $=10$

END PARAMETERS

READ MIXT SCT=1 MIX=1 92500 4.48006e-2 92800 2.6578e-3 92400 4.827e-4

92600 9.57e-5 MIX=2 1001.0 END MIXT

READ GEOMETRY

UNIT 1

CYLINDER 110.5 2P5.0

CUBOID 014 P0.5 2P5.0

UNIT 2

ARRAY 2 - 1.0 - 1.0 -5.0

GLOBAL UNIT 3 
ARRAY $1-2.0-2.0-5.0$

CYLINDER $014.155 .1-5.0$

HOLE $20.0-3.00 .0$

HOLE 23.00 .00 .0

HOLE 20.03 .00 .0

HOLE 2 -3.0 0.00 .0

CYLINDER $214.66 .37-6.27$

END GEOM

READ ARRAY

ARA $=1$ NUX $=4$ NUY $=4$ NUZ $=1$ FILL F1 END FILL

ARA $=2$ NUX $=2$ NUY $=2$ NUZ $=1$ FILL F1 END FILL

END ARRAY

READ PLOT TTL $=$ 'X-Z SLICE AT Y $=0.25$ WITH X ACROSS AND Z DOWN'

$\mathbf{X U L}=-5.0 \quad \mathbf{Y U L}=0.25 \mathrm{ZUL}=6.5 \mathrm{XLR}=5.0 \mathrm{YLR}=0.25 \mathbf{Z L R}=-6.5$

UAX $=1.0$ WDN $=-1.0$ NAX $=640$ END

TTL $=$ 'X-Y SLICE AT Z $=0.0$ WITH X ACROSS AND Y DOWN'

$\mathbf{X U L}=-5.0 \mathrm{YUL}=5.0 \mathrm{ZUL}=0.0 \mathrm{XLR}=5.0 \mathrm{YLR}=-5.0 \mathrm{ZLR}=0.0$

$\mathbf{U A X}=1.0 \mathrm{VDN}=-1.0 \mathrm{NAX}=640 \mathbf{E N D}$

END PLOT

END DATA

END

KENO-VI:

$=$ KENO VI

CASK ARRAY

READ PARAMETERS TME $=1.0$ FDN=YES LIB=41 GEN=10 END PARAMETERS

READ MIXT SCT $=1$

MIX=1 92500 4.48006e-2 92800 2.6578e-3 92400 4.827e-4 92600 9.57e-5

MIX=2 1001.0 END MIXT

READ GEOMETRY

UNIT 1

CYLINDER 10.5 2P5.0

CUBOID 2 4P0.5 2P5.0

MEDIA 1111

MEDIA 0 H 12 -

BOUNDARY 2

UNIT 2

CUBOID $14 \mathrm{P} 0.5$ 2P5.0

MEDIA 011

BOUNDARY 1

UNIT 3

CYLINDER 14.15 5.0 -5.0

CYLINDER 24.15 5.1 -5.0

ARRAY 11 PLACE $551 \quad-0.5-0.5-0.0$

MEDIA $0 \begin{array}{lll}0 & 1 & -1\end{array}$

BOUNDARY 2

GLOBAL UNIT 4

CYLINDER $24.66 .37-6.27$

HOLE 3 ORIGIN $X=0.0 \quad Y=0.0 \mathrm{Z}=0.0$ 
MEDIA 212

BOUNDARY 2

END GEOM

READ ARRAY

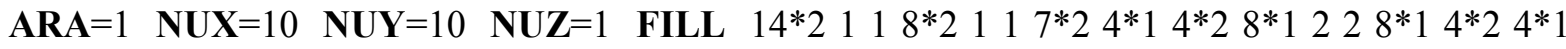

$7 * 211$

$8 * 21114 * 2$ END FILL

END ARRAY

READ PLOT TTL $=$ 'X-Z SLICE AT Y $=0.25$ WITH X ACROSS AND Z DOWN'

$\mathbf{X U L}=-5.0 \mathrm{YUL}=0.25 \mathrm{ZUL}=6.5 \mathrm{XLR}=5.0 \mathrm{YLR}=0.25 \mathrm{ZLR}=-6.5$

$\mathbf{U A X}=1.0 \mathrm{WDN}=-1.0 \mathrm{NAX}=640$ END

TTL $=$ 'X-Y SLICE AT Z $=0.0$ WITH X ACROSS AND Y DOWN'

$\mathbf{X U L}=-5.0 \mathrm{YUL}=5.0 \mathrm{ZUL}=0.0 \mathrm{XLR}=5.0 \mathrm{YLR}=-5.0 \mathrm{ZLR}=0.0$

$\mathbf{U A X}=1.0 \mathbf{V D N}=-1.0 \mathrm{NAX}=640 \mathbf{E N D}$

END PLOT

END DATA

END

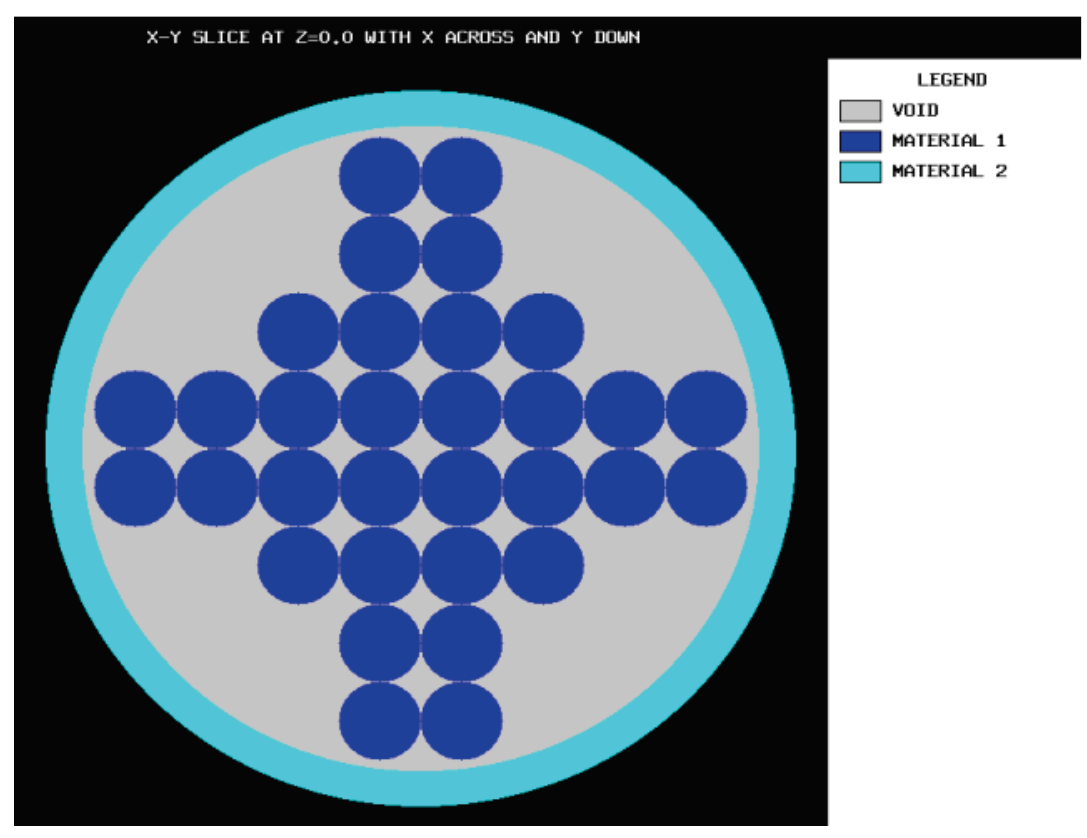

Fig. 8.1.30. X-Y slice of uranium rods in a cylindrical container. 


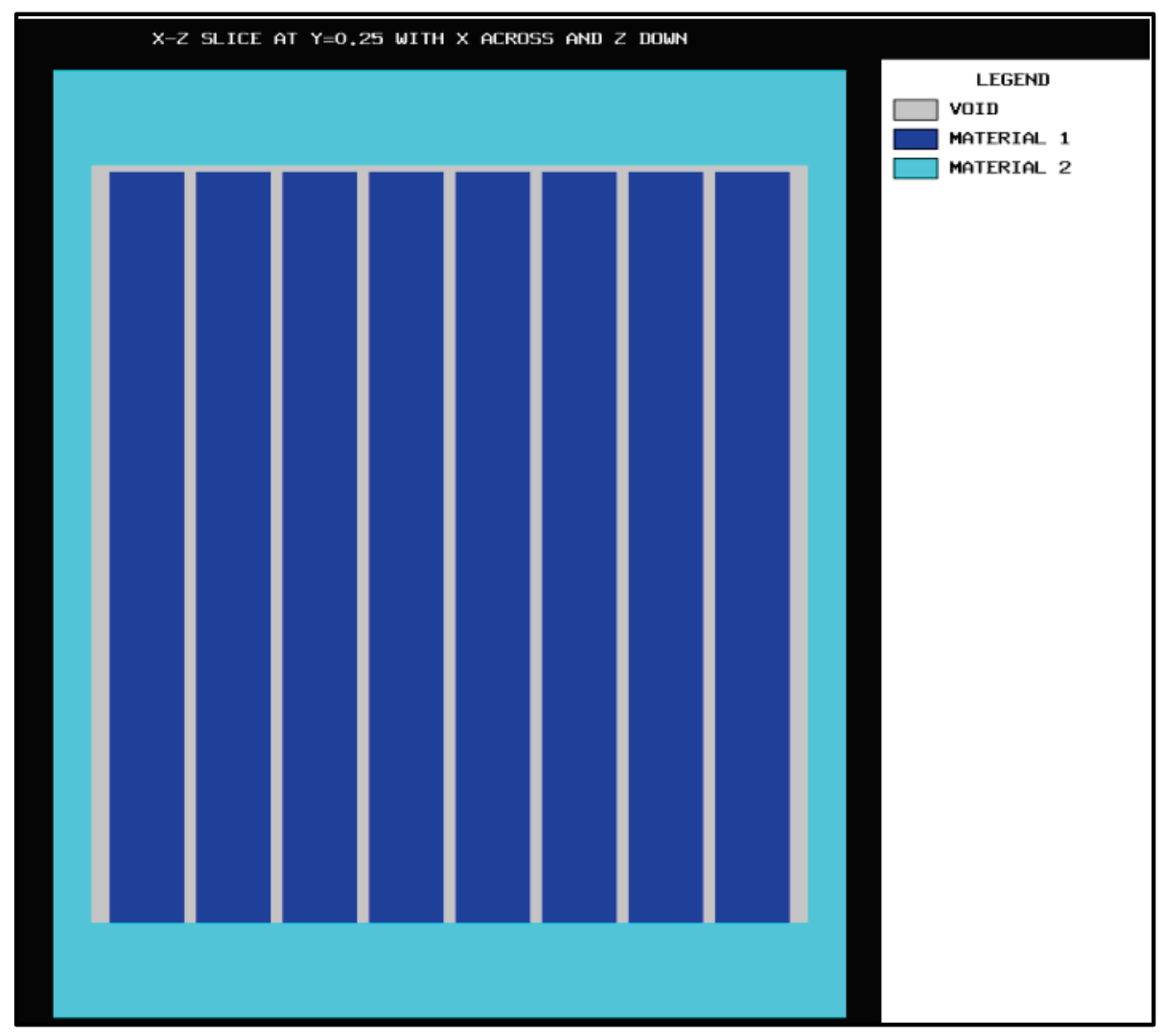

Fig. 8.1.31. X-Z slice of uranium rods in a cylindrical container.

\section{EXAMPLE 20. A TYPICAL PRESSURIZED WATER REACTOR (PWR) SHIPPING CASK}

A typical PWR shipping cask is illustrated in Fig. 8.1.32. The interior and exterior shell of the cask is carbon steel (mixture 7), and a depleted uranium gamma shield (mixture 6) is present in the annulus between the steel layers. The shipping cask contains seven PWR fuel assemblies. Each assembly is a $17 \times 17$ ARRAY of fuel rods with water holes. Each assembly is contained in a stainless steel (mixture 5) box. Each fuel rod is composed of $4 \%$ enriched $\mathrm{UO}_{2}$ (mixture 1) clad with Zircaloy (mixture 2). Rods of $\mathrm{B}_{4} \mathrm{C}$ clad (mixture 4) with stainless steel are positioned between the fuel assemblies. The entire cask is filled with water (mixture 3 ). 


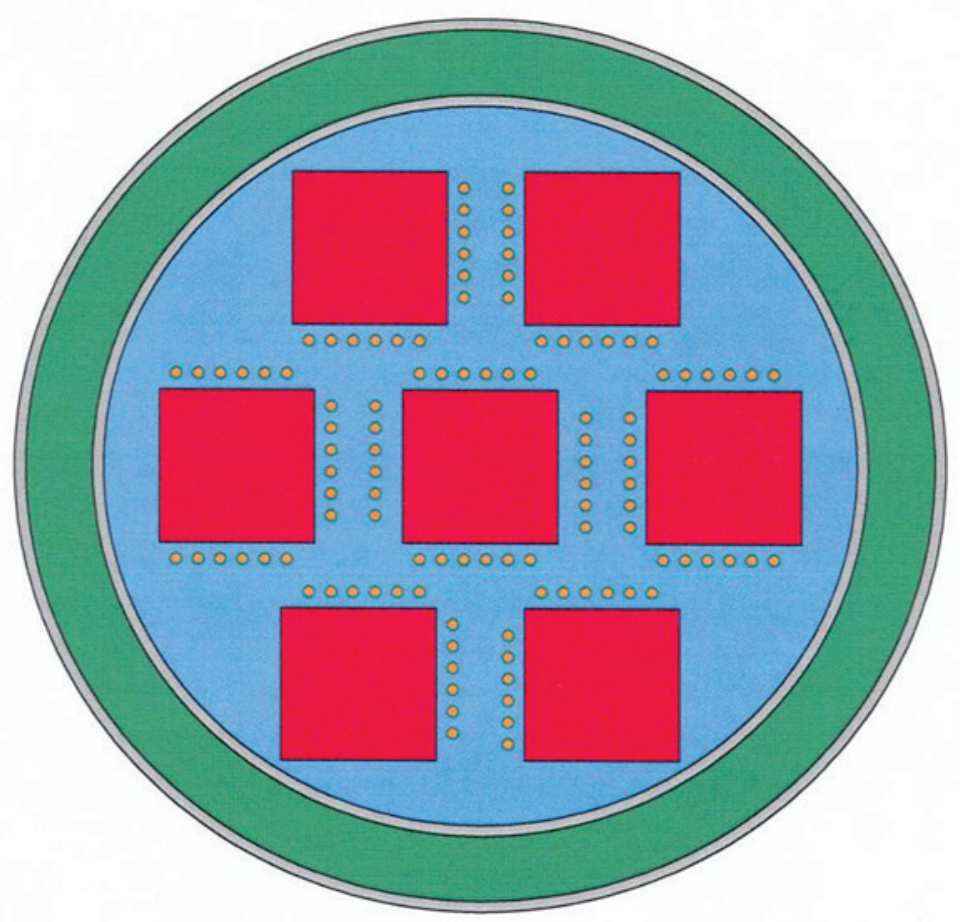

Fig. 8.1.32. Typical PWR shipping cask.

To describe the geometry of the cask, some simple units are defined as shown in Fig. 8.1.33. UNIT 1 represents a fuel rod and its associated square pitch spacing region. UNIT 2 represents a water hole in a fuel assembly. UNITs 3, 4, and 6 represent the $\mathrm{B}_{4} \mathrm{C}$ rods with their various spacings, and UNIT 5 is a water hole that is used in association with some of the $\mathrm{B}_{4} \mathrm{C}$ rods.

UNIT 1

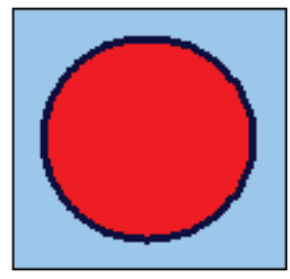

UNIT 2

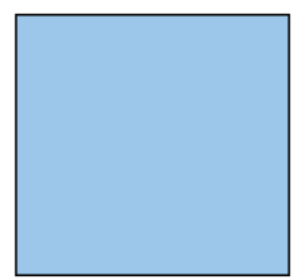

UNIT 3

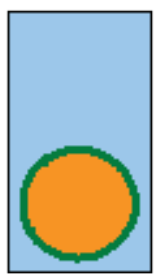

UNIT 4

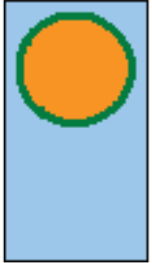

UNIT 5

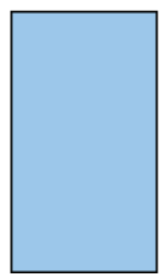

UNIT 6

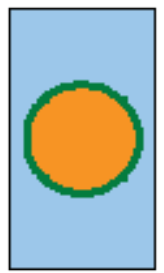

Fig. 8.1.33. Simple units.

\section{KENO V.a:}

UNIT 1

CYLINDER 11.41148365 .760 .0

CYLINDER 21.48133365 .760 .0

CUBOID $31.63754-.63754 .63754-.63754365 .760 .0$

UNIT 2

CUBOID $31.63754-63754.63754-63754365.760 .0$ 
UNIT 3

CYLINDER 41.584365 .760 .0

CYLINDER 51.635365 .760 .0

CUBOID $\quad 3 \quad 1.9912-.99122 .2352-1.27365 .760 .0$

UNIT 4

CYLINDER 41.584365 .760 .0

CYLINDER 51.635365 .760 .0

CUBOID $\quad 3 \quad 1.9912-.99121 .2702-2.235365 .760 .0$

UNIT 5

CUBOID $31.9912-.99121 .7526-1.7526365 .760 .0$

UNIT 6

CYLINDER 41.584365 .760 .0

CYLINDER 51.635365 .760 .0

CUBOID $311.1875215-1.18752151 .883706-1.883706365 .760 .0$ 
KENO-VI:

UNIT 1

CYLINDER 1.41148365 .760 .0

CYLINDER 2.48133365 .760 .0

CUBOID $3.63754-63754.63754-63754365.760 .0$

MEDIA 1111

MEDIA 2 r 12 r

MEDIA 3 1 13 -

BOUNDARY 3

UNIT 2

$\begin{array}{llllllll}\text { CUBOID } & 1 & 1.63754 & -63754.63754 & -63754 & 365.76 & 0.0\end{array}$

MEDIA 311

BOUNDARY 1

UNIT 3

CYLINDER $1 \quad .584365 .760 .0$

CYLINDER $2 \quad .635365 .760 .0$

$\begin{array}{lllllll}\text { CUBOID } & 3 & .9912 & -.9912 & 2.2352 & -1.27365 .76 & 0.0\end{array}$

MEDIA 411

MEDIA 5 1 12 -

MEDIA $3 \quad 1 \quad 3-2$

BOUNDARY 3

UNIT 4

CYLINDER $1 \quad .584365 .760 .0$

CYLINDER 2.635365 .760 .0

$\begin{array}{llllllll}\text { CUBOID } & 3 & .9912 & -.9912 & 1.2702 & -2.235 & 365.76 & 0.0\end{array}$

MEDIA 4 1 11

MEDIA 5 1 12 -

MEDIA $3 \quad 1 \quad 3 \quad-2$

BOUNDARY 3

UNIT 5

CUBOID 1 .9912 -.9912 $1.7526-1.7526365 .760 .0$

MEDIA 311

BOUNDARY 1

UNIT 6

CYLINDER 1.584365 .760 .0

CYLINDER 2.635365 .760 .0

CUBOID $3 \quad 1.1875215-1.18752151 .883706-1.883706365 .760 .0$

MEDIA 4 1 11

MEDIA 5 1 12 2

MEDIA 3 1 13 -

BOUNDARY 3 
UNITs 1 and 2 are stacked together into ARRAY 1 to form the ARRAY of fuel pins and water holes in a fuel assembly as shown in Fig. 8.1.34. This ARRAY is then encompassed with a layer of water and a layer of stainless steel to complete a fuel assembly in a storage cell (UNIT 7) as shown in Fig. 8.1.35.

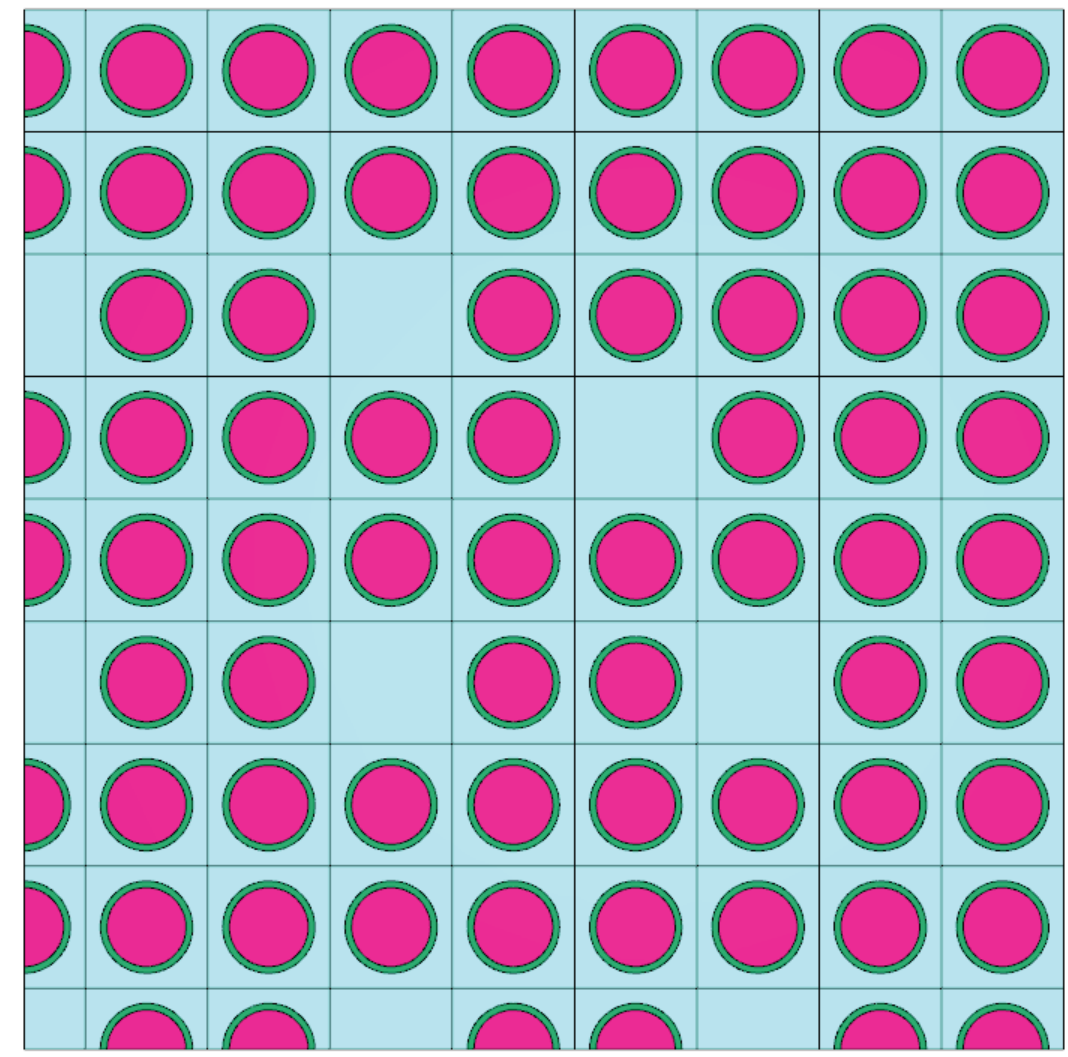

Fig. 8.1.34. Quarter section of fuel pin array. 


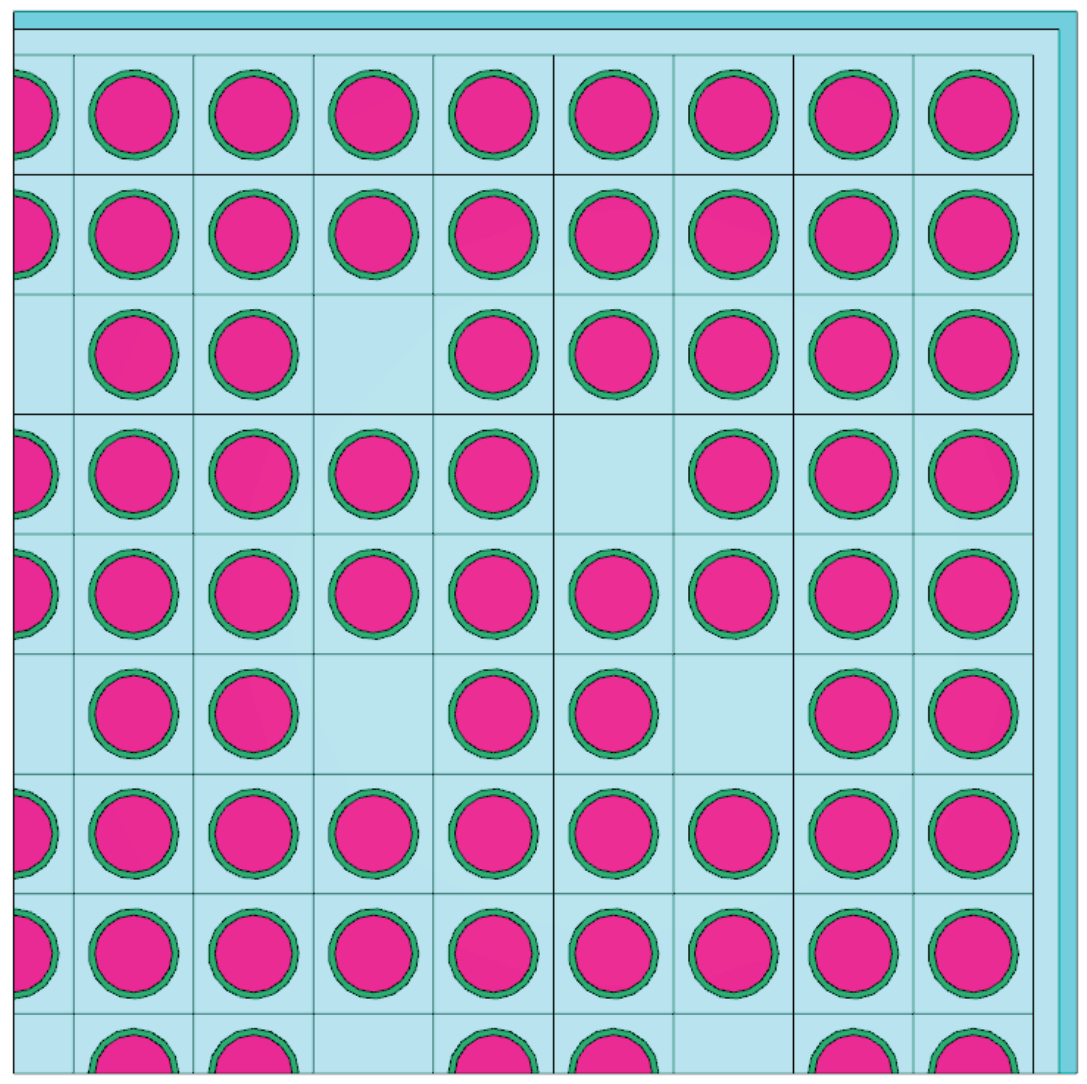

Fig. 8.1.35. Quarter section of fuel assembly.

KENO V.a:

ARA $=1$ NUX $=17$ NUY $=17$ NUZ $=1$ FILL

39R1 2 2Q3 8R1 2 9R1 2 22R1 2 4Q3 38R1 2 4Q3

Q51 22R1 2 Q10 Q9 2Q3 39R1 END FILL

UNIT 7

ARRAY $1-10.83818-10.838180 .0$

CUBOID $3 \quad 1 \quad 11.112495-11.11249511 .112495-11.112495365 .760 .0$

CUBOID $8 \quad 111.302238-11.30223811 .302238-11.302238365 .760 .0$

KENO-VI:

ARA $=1$ NUX $=17$ NUY $=17$ NUZ $=1$ FILL

39R1 2 2Q3 8R1 2 9R1 2 22R1 2 4Q3 38R1 2 4Q3

Q51 22R1 2 Q10 Q9 2Q3 39R1

END FILL

UNIT 7

CUBOID $110.83818-10.8381810 .83818-10.83818365 .760 .0$

CUBOID $211.112495-11.11249511 .112495-11.112495365 .760 .0$

CUBOID $311.302238-11.30223811 .302238-11.302238365 .760 .0$

ARRAY 11 PLACE $9913 * 0.0$

MEDIA 3 1 2 2 -1 
MEDIA 513 -2

BOUNDARY 3

An array of UNIT $6 \mathrm{~s}$ is created to represent the array of $\mathrm{B}_{4} \mathrm{C}$ rods that is positioned between the fuel assemblies. In KENO V.a, the array of $\mathrm{B}_{4} \mathrm{C}$ rods shown in Fig. 8.1.36 is contained in UNIT 8 for further use. KENO-VI geometry description does not need the placement of ARRAY 2 in a separate UNIT.

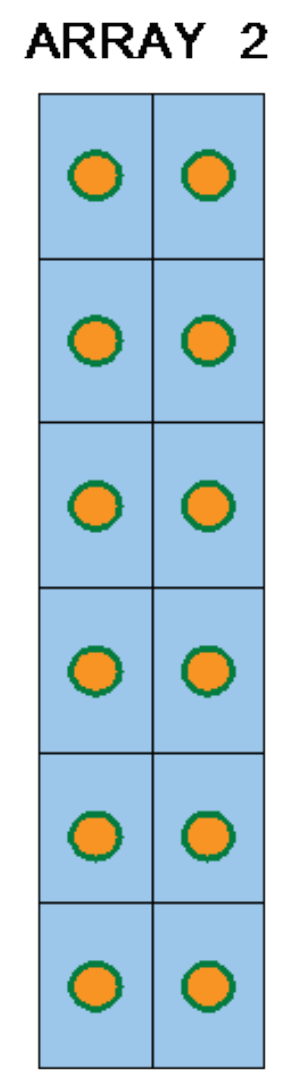

Fig. 8.1.36. $2 \times 6$ array of $\mathrm{B}_{4} \mathrm{C}$ rods.

KENO V.a:

ARA $=2$ NUX $=2$ NUY $=6$ NUZ $=1 \quad$ FILL F6 END FILL

UNIT 8

ARRAY 20000

KENO-VI:

\section{ARA $=2$ NUX $=2$ NUY $=6$ NUZ $=1 \quad$ FILL F6 END FILL}

The next step is to create the central array of three fuel assemblies with $\mathrm{B}_{4} \mathrm{C}$ rods between them. In KENO V.a, this is done by stacking fuel assemblies in storage cells (UNIT 7) and $\mathrm{B}_{4} \mathrm{C}$ rod arrays (UNIT 8) into an array (ARRAY 3) and placing it in a UNIT (UNIT 9). In KENO-VI description, UNIT 7 (fuel assembly in storage cell) and the array of $\mathrm{B}_{4} \mathrm{C}$ rods (ARRAY 2) are directly placed into a UNIT (UNIT 8). The resultant geometry is shown in Fig. 8.1.37. 


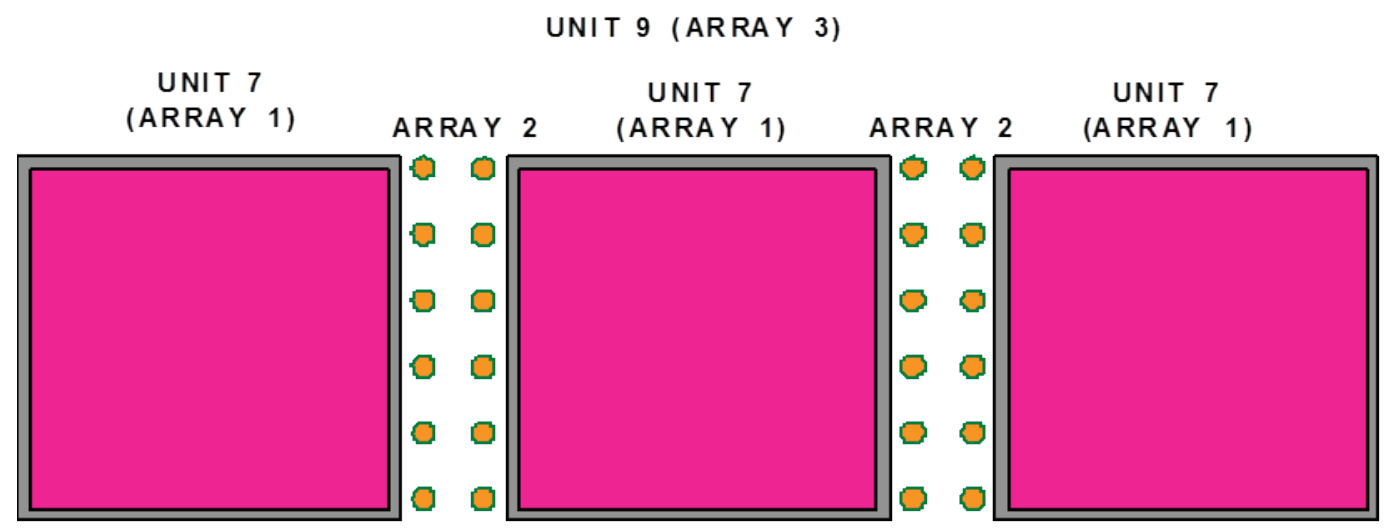

Fig. 8.1.37. Central array.

KENO V.a:

ARA $=3$ NUX=5 NUY=1 NUZ=1 FILL 78787 END FILL

UNIT 9 ARRAY 3000

KENO-VI:

UNIT 8

CUBOID $4-11.302238-16.05232411 .302238-11.302238365 .760 .0$

CUBOID $5 \quad 16.05232411 .30223811 .302236-11.302236365 .760 .0$

CUBOID $6 \quad 38.052324-38.05232411 .302236-11.302236365 .760 .0$

HOLE 7

HOLE 7 ORIGIN $X=-27.354562$

HOLE 7 ORIGIN $X=27.354562$

ARRAY 24 PLACE $1111-14.8648025 \quad-9.4185300 .0$

ARRAY 25 PLACE $111112.4897595 \quad-9.4185300 .0$

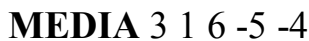

BOUNDARY 6

UNITs 3, 4, and 5 are used to define the arrays of $\mathrm{B}_{4} \mathrm{C}$ rods that fit above and below the central array, as shown in Fig. 8.1.38.

ARRAY 4

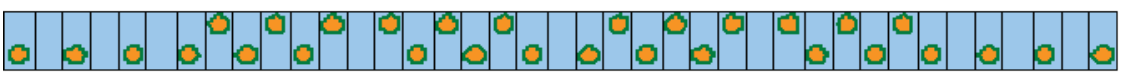

ARRAY 5

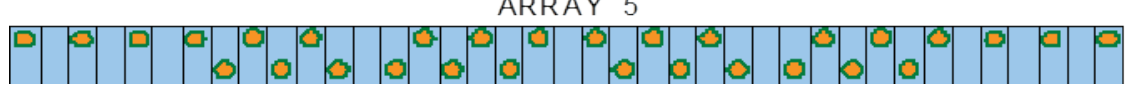


Fig. 8.1.38. Long $\mathrm{B}_{4} \mathrm{C}$ rod arrays.

ARA=4 NUX=39 NUY=1 NUZ=1 FILL 35 2Q2 34 2Q2 543 2Q2 534 2Q2 543 2Q2 5 2Q2 3 END FILL

ARA=5 NUX=39 NUY=1 NUZ=1 FILL 45 2Q2 43 2Q2 534 2Q2 543 2Q2 534 2Q2 5 2Q2 4 END FILL

In KENO V.a these ARRAYs are placed in UNITs (UNITs 10 and 11) for further use.

KENO V.a:

UNIT 10 ARRAY 4000

UNIT 11 ARRAY 50000

UNITs 9, 10, and 11 in the KENO V.a description, or UNIT 8 and ARRAYs 3 and 4 in the KENO-VI description, are stacked to form the central array with $\mathrm{B}_{4} \mathrm{C}$ rods as shown in Fig. 8.1.39.

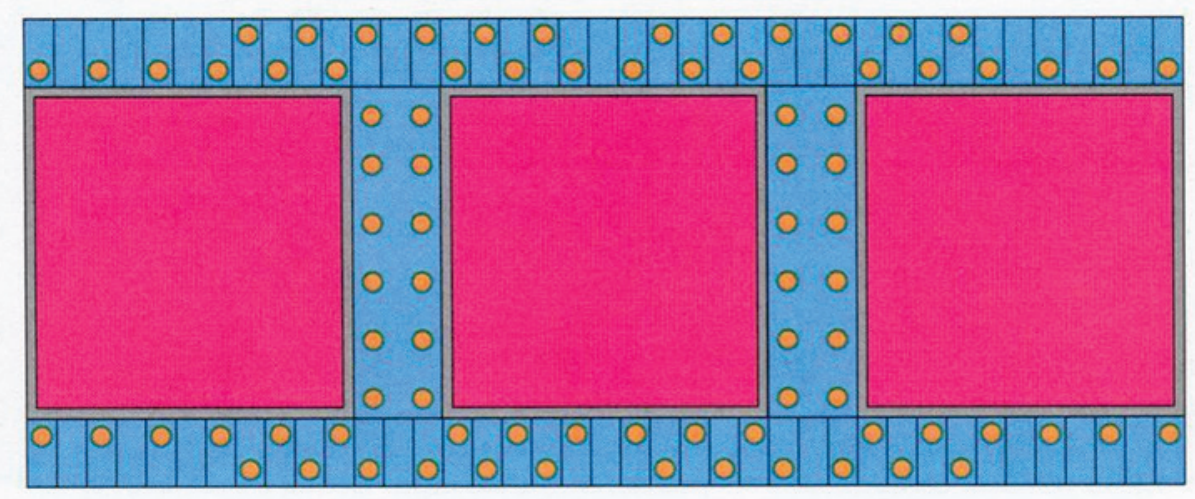

Fig. 8.1.39. Central array with long $\mathrm{B}_{4} \mathrm{C}$ arrays.

KENO V.a:

ARA $=6$ NUX=1 NUY=3 NUZ=1 FILL 11910 END FILL

KENO-VI:

CUBOID $238.052324-38.05232414 .80743611 .302236365 .760 .0$

CUBOID $338.052324-38.05232411 .302236-14.807436365 .760 .0$

HOLE 8

ARRAY 32 PLACE 20110.013 .0548360 .0

ARRAY 43 PLACE 20110.013 .0548360 .0

This completes the three central fuel assemblies and all the $\mathrm{B}_{4} \mathrm{C}$ rods associated with them. Next, UNITs 7 and 8 in KENO V.a geometry, UNIT 7 and ARRAY 2 in the KENO-VI geometry, are stacked together to form the array of two fuel assemblies separated by $\mathrm{B}_{4} \mathrm{C}$ rods as shown in Fig. 8.1.40. This is designated as ARRAY 7 and UNIT 12 in KENO V.a, and UNIT 9 in KENO-VI. The origin of UNIT 12 
for KENO V.a is specified at the center of the array in the X and Y directions and the bottom of the fuel assemblies $(Z=27.94 \mathrm{~cm})$. The origin of UNIT 9 in the KENO-VI description is specified at the center of the $\mathrm{B}_{4} \mathrm{C}$ array in the $\mathrm{X}$ and $\mathrm{Y}$ directions and the bottom of the array in the $\mathrm{Z}$ direction.

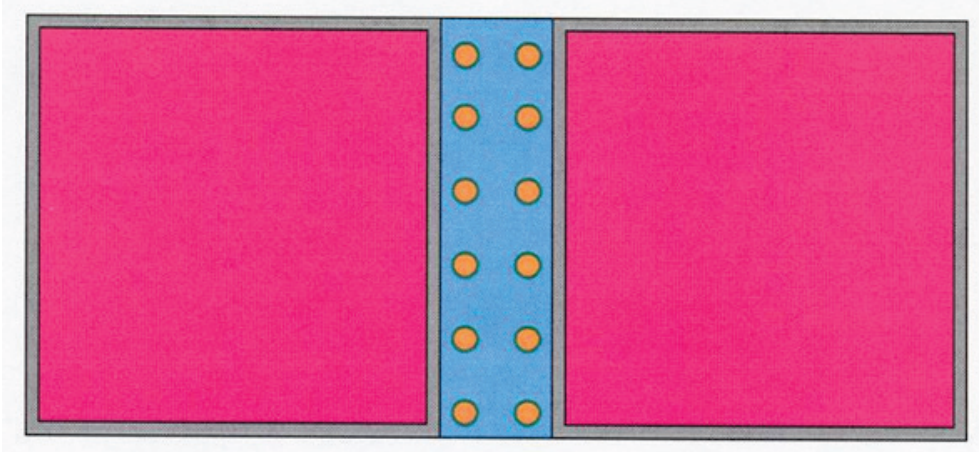

Fig. 8.1.40. Two fuel assemblies and $\mathrm{B}_{4} \mathrm{C}$ rods.

KENO V.a:
ARA $=7$ NUX $=3$ NUY $=1$ NUZ $=1$
FILL 787
END FILL

UNIT 12 ARRAY 7 -24.979519-11.302238 27.94

KENO-VI:

UNIT 9

CUBOID $12.375043-2.37504311 .302236-11.302236365 .760 .0$

CUBOID $424.979519-23.791997511 .302238-11.302238365 .760 .0$

ARRAY 21 PLACE $1111-1.1875215$-9.418530 0.0

HOLE 7 ORIGIN $X=-13.67728$

HOLE 7 ORIGIN $X=13.67728$

MEDIA 0 1 14 - 1

BOUNDARY 4

In KENO V.a, UNIT 13 is simply a cylindrical lid that fits on top of the shipping cask. It is described relative to the origin of the shipping cask and is made of depleted uranium.

KENO V.a:

\section{UNIT 13 \\ CYLINDER 6147.625457 .2449 .58}

All necessary subassemblies that make up the shipping cask have been built. The shipping cask is completed by specifying the origin of the central section (ARRAY 6 in KENO V.a) (see Fig. 8.1.39) to be at the center of the array in $\mathrm{X}$ and $\mathrm{Y}$ and the bottom of the array in the $\mathrm{Z}$ direction. A cylinder of water defining the interior of the shipping cask is described around the array. In the KENO V.a geometry, a HOLE is used to place UNIT 12 (Fig. 8.1.40) below the array, and a second HOLE is used to place another UNIT 12 above the array. In the KENO-VI geometry, UNIT 9 is placed as a HOLE above and below the central array. Then a cylinder of steel is placed around the water, which is in turn encased by 
depleted uranium. The depleted uranium is then contained in the outer steel cylinder of the shipping cask. In KENO V.a description, a third HOLE is used to place the depleted uranium lid (UNIT 13) on the shipping cask. This completes the shipping cask description of Fig. 8.1.32.

The geometry data for this shipping cask are shown below. The plot data have been included for verification of the geometry description. However, the plot generated by this data is quite large and is therefore not included in this document.

KENO V.a:

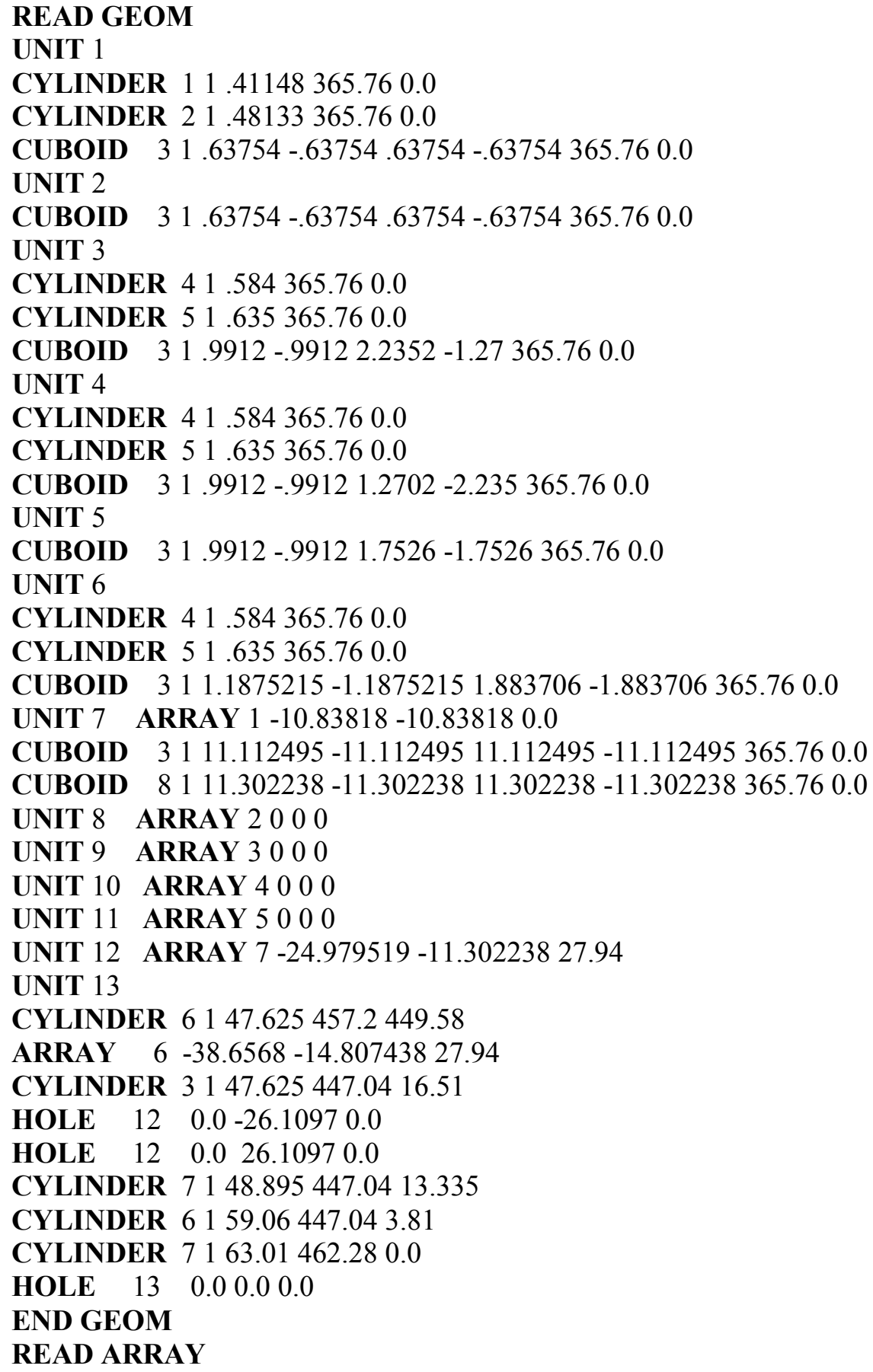




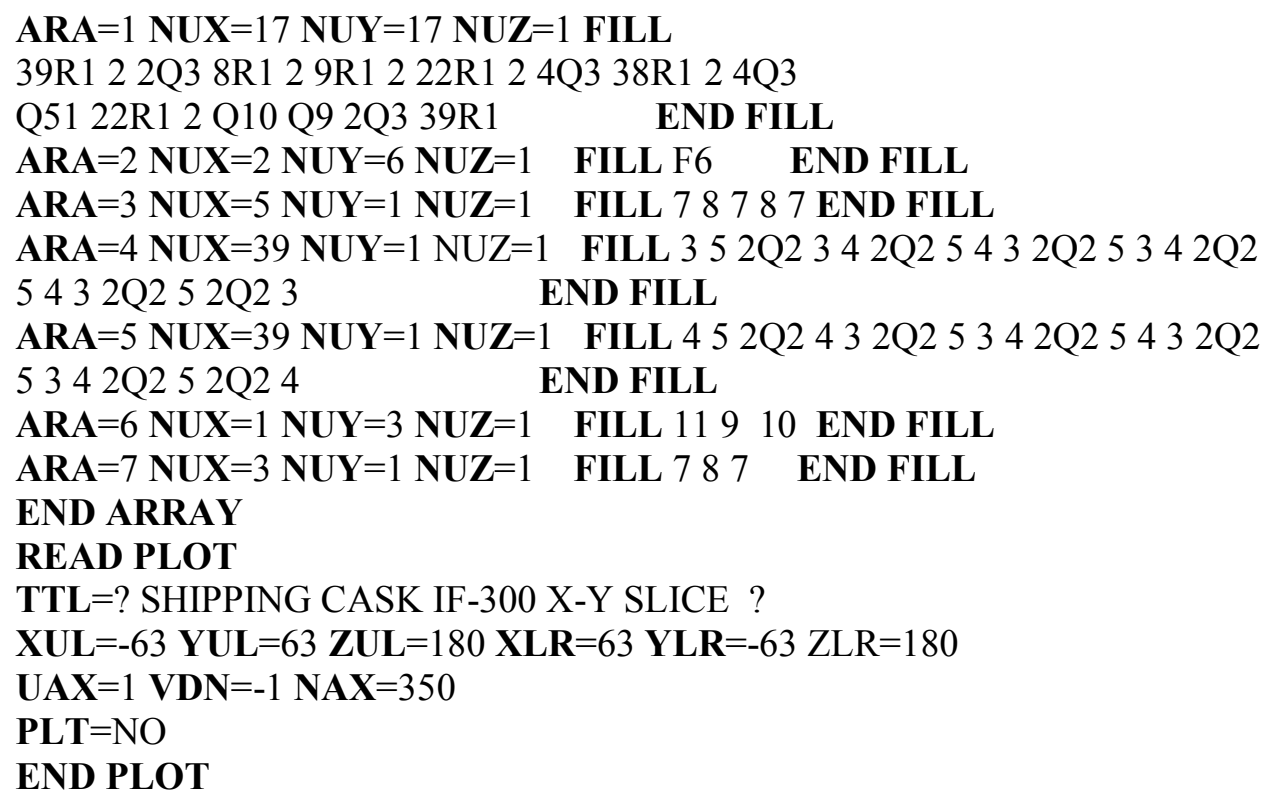


MEDIA 411

MEDIA 5 1 12 -

MEDIA 3 1 13 -

BOUNDARY 3

UNIT 5

CUBOID $1.9912-.99121 .7526-1.7526365 .760 .0$

MEDIA 311

BOUNDARY 1

UNIT 6

CYLINDER $1 \quad .584365 .760 .0$

CYLINDER $2 \quad .635365 .760 .0$

CUBOID $3 \quad 1.1875215-1.18752151 .883706-1.883706365 .760 .0$

MEDIA 411

MEDIA 5 1 12 -

MEDIA 3 1 13 -

BOUNDARY 3

UNIT 7

CUBOID $110.83818-10.8381810 .83818-10.83818365 .760 .0$

CUBOID $211.112495-11.11249511 .112495-11.112495365 .760 .0$

CUBOID $311.302238-11.30223811 .302238-11.302238365 .760 .0$

ARRAY 11 PLACE $9913 * 0.0$

MEDIA $3 \quad 1 \quad 2 \quad-1$

MEDIA 8 r 13 -

BOUNDARY 3

UNIT 8

CUBOID $4-11.302238-16.05232411 .302238-11.302238365 .760 .0$

CUBOID $5 \quad 16.05232411 .30223811 .302236-11.302236365 .760 .0$

CUBOID $6 \quad 38.052324-38.05232411 .302236-11.302236365 .760 .0$

HOLE 7

HOLE 7 ORIGIN $X=-27.354562$

HOLE 7 ORIGIN $X=27.354562$

ARRAY 24 PLACE $1111-14.8648025-9.4185300 .0$

ARRAY 25 PLACE 111112.4897595 -9.418530 0.0

MEDIA $0 \begin{array}{lllll} & 1 & 6 & -5 & -4\end{array}$

BOUNDARY 6

UNIT 10

CUBOID $11.1875215-1.187521511 .302236-11.302236365 .760 .0$

CUBOID $423.7919975-23.791997511 .302238-11.302238365 .760 .0$

ARRAY 21 PLACE $11113 * 0.0$

HOLE 7 ORIGIN $X=-12.4897595$

HOLE 7 ORIGIN $X=12.4897595$

MEDIA 0 l 14 - 1

BOUNDARY 4

GLOBAL UNIT 11 
CUBOID $238.052324-38.05232413 .284638 \quad 11.302236 \quad 365.760 .0$

CUBOID $338.052324-38.05232411 .302236-13.284638365 .760 .0$

CYLINDER $647.625419 .10-11.43$

CYLINDER $7 \quad 48.895419 .10-14.605$

CYLINDER $859.06419 .10-24.13$

CYLINDER 947.625429 .26421 .64

CYLINDER $10 \quad 63.01434 .34-27.94$

HOLE 8

ARRAY 32 PLACE 20110.012 .2934380 .0

ARRAY 43 PLACE 20110.012 .2934380 .0

HOLE 9 ORIGIN Y=24.586876

HOLE 9 ORIGIN $Y=-24.586876$

MEDIA $3 \quad 1 \quad 6 \quad-3-2$

MEDIA 7 $17-6-6-2$

MEDIA 618 - 7

MEDIA 719

MEDIA 6110 - 10 -8

BOUNDARY 10

END GEOM

READ ARRAY

ARA $=1$ NUX $=17$ NUY $=17$ NUZ $=1$ FILL

39R1 2 2Q3 8R1 2 9R1 2 22R1 2 4Q3 38R1 2 4Q3

Q51 22R1 2 Q10 Q9 2Q3 39R1

END FILL

ARA $=2$ NUX $=2$ NUY $=6$ NUZ $=1 \quad$ FILL F6 END FILL

ARA $=3$ NUX=5 NUY=1 NUZ=1 FILL 78787 END FILL

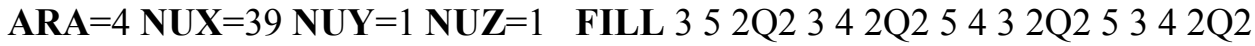

$5432 \mathrm{Q} 252 \mathrm{Q} 23$

END FILL

ARA=5 NUX=39 NUY=1 NUZ=1 FILL 45 2Q2 43 2Q2 5534 2Q2 54 \begin{tabular}{lll}
\hline & $2 Q 2$
\end{tabular}

$5342 \mathrm{Q} 252 \mathrm{Q} 24$ END FILL

ARA $=6 \mathbf{N U X}=1$ NUY=3 NUZ=1 FILL 11910 END FILL

ARA $=7$ NUX $=3$ NUY=1 NUZ=1 FILL $787 \quad$ END FILL

END ARRAY

READ PLOT

TTL=? SHIPPING CASK IF-300 X-Y SLICE ?

$\mathbf{X U L}=-63 \mathbf{Y U L}=63 \mathbf{Z U L}=180 \mathbf{X L R}=63 \mathbf{Y L R}=-63 \mathbf{Z L R}=180$

$\mathbf{U A X}=1$ VDN $=-1 \mathbf{N A X}=350$

PLT $=$ NO

END PLOT 


\subsection{Triangular pitched arrays in KENO-VI}

EXAMPLE 21. Triangular pitched ARRAYs can be described in KENO-VI by defining the UNITs that make up the ARRAY as HEXPRISM and in the array data block setting TYP=TRIANGULAR, HEXAGONAL, SHEXAGONAL, or RHEXAGONAL. This includes close-packed triangular pitched arrays. Since the ARRAYs are constructed by stacking hexprisms, care must be taken to ensure that the ARRAY boundary is completely enclosed within the stacked UNIT. Below is an example of a triangular pitched ARRAY.

The first and second UNITs are the HEXPRISMs that make up the ARRAY. UNIT 1 is the fuel cell stacked in a triangular pitched or hexagonal lattice. UNIT 2 is a dummy UNIT used to fill in the ARRAY so the array boundary is contained within the stacked UNITs. Since the ARRAY is not moderated, UNIT 2 contains void. Fig. 8.1.41 shows an X-Y cross section schematic of UNITs 1 and 2.

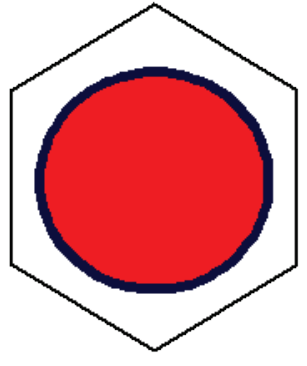

UNIT 1

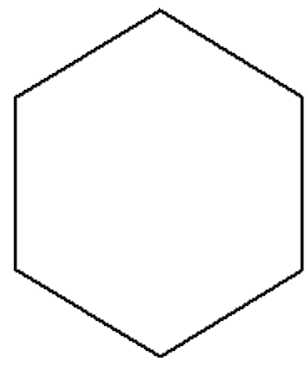

UNIT 2

Fig. 8.1.41. Fuel cell and empty cell set up as hexprisms.

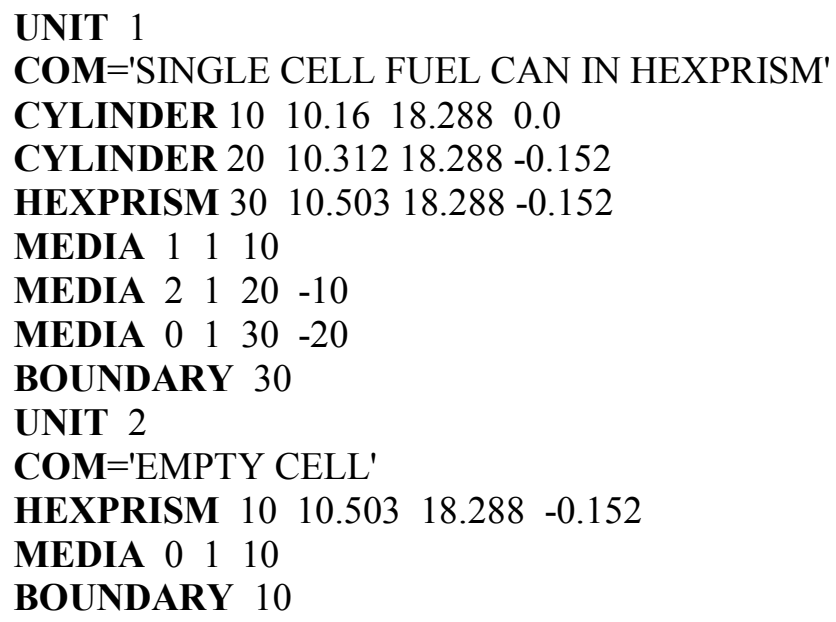


UNIT 3 is the GLOBAL UNIT that contains the ARRAY and ARRAY BOUNDARY. The ARRAY is an unmoderated triangular pitched assembly of 7 cells. Unrotated triangular or hexagonal pitched arrays can be set up in two array configurations. The first configuration, selected using the $\mathbf{T Y} \mathbf{P}=$ followed by keyword HEXAGONAL or TRIANGULAR, stacks the UNITs so that the faces perpendicular to the X axis meet. Each consecutive row in the Y direction begins $1 / 2$ of the face-to-face dimension farther over in the positive $\mathrm{X}$ direction than in the previous row. The second configuration, selected using the $\mathbf{T Y P}=$ followed by keyword SHEXAGONAL, also stacks the UNITs so that the faces perpendicular to the X axis meet. However, for this type of ARRAY, the odd numbered rows in the Y direction $(1,3$, 5, etc.) begin at the left edge of the ARRAY, and the even numbered rows in the Y direction $(2,4,6$, etc.) begin $1 / 2$ of the face-to-face dimension to the right of the left edge of the ARRAY. Fig. 8.1.42 and Fig. 8.1.43 show X-Y cross section schematics of the assembly for the two different unrotated hexagonal ARRAY types.

\section{GLOBAL UNIT 3}

COM='7 CYLINDERS IN A CIRCLE WITH CYLINDRICAL BOUNDARY'

CYLINDER $1032.000 \quad 18.288-0.152$

ARRAY 110 PLACE $3313 * 0.0$

BOUNDARY 10

READ ARRAY GBL $=1$ ARA $=1$ TYP $=$ HEXAGONAL NUX $=5$ NUY $=5$ NUZ $=1$

FILL $7 * 22 * 12 * 23 * 12 * 22 * 17 * 2$ END FILL END ARRAY

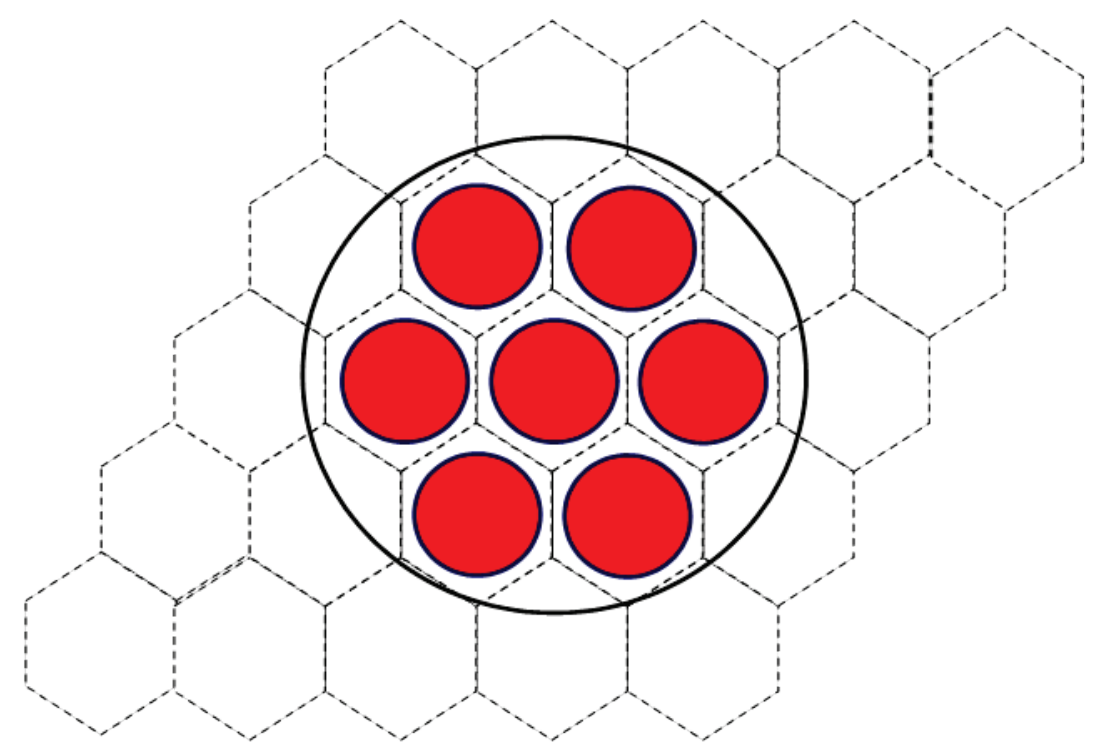

Fig. 8.1.42. Seven cylinders stacked in a HEXAGONAL array with cylindrical array boundary. 


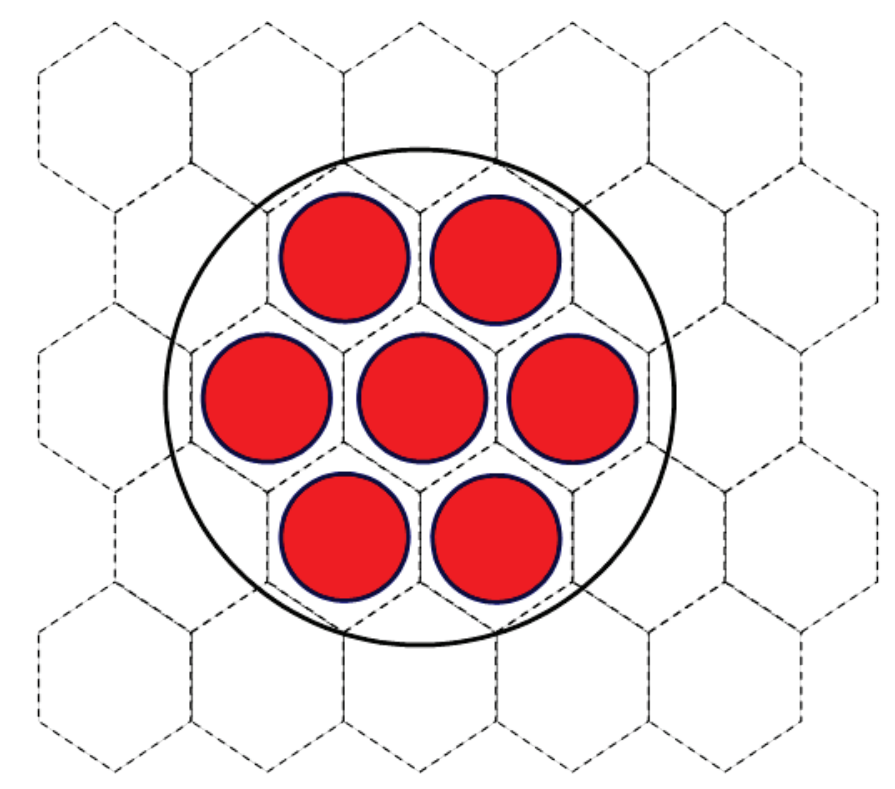

Fig. 8.1.43. Seven cylinders stacked in a SHEXAGONAL array with a cylindrical array boundary.

The overall problem description is shown below. The cross section library would be generated in a separate CSAS-MG step. An X-Y cross section color plot used for verification of this mockup is shown in Fig. 8.1.44.

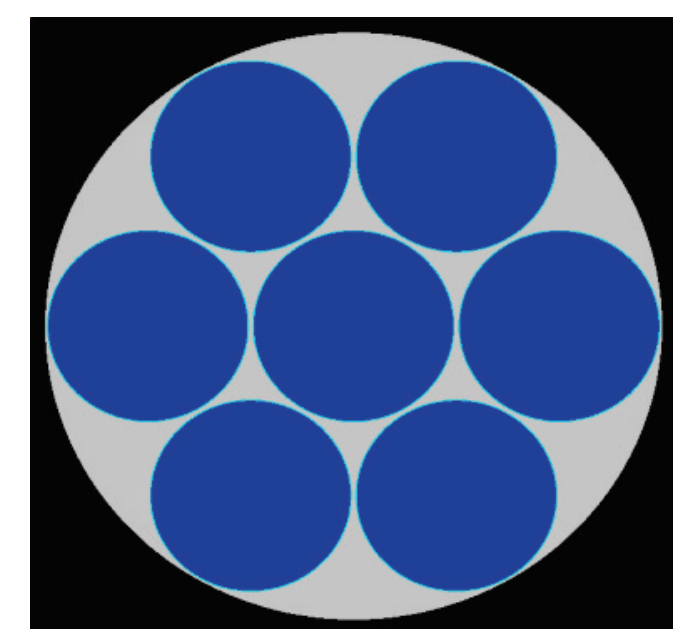

Fig. 8.1.44. X-Y slice of 7 cylinders in a triangular pitch array.

Data description of Example 21.

$=$ KENOVI

TRIANGULAR PITCHED ARRAY 7 PINS IN A CIRCLE

READ PARAMETERS LNG $=20000$ LIB=4 END PARAMETERS

READ MIXT SCT $=2$

MIX=1 NCM=8 92235 1.37751E-03 92238 9.92354E-05 8016 3.32049E-02

9019 2.95349E-03 $10016.05028 \mathrm{E}-02$ 


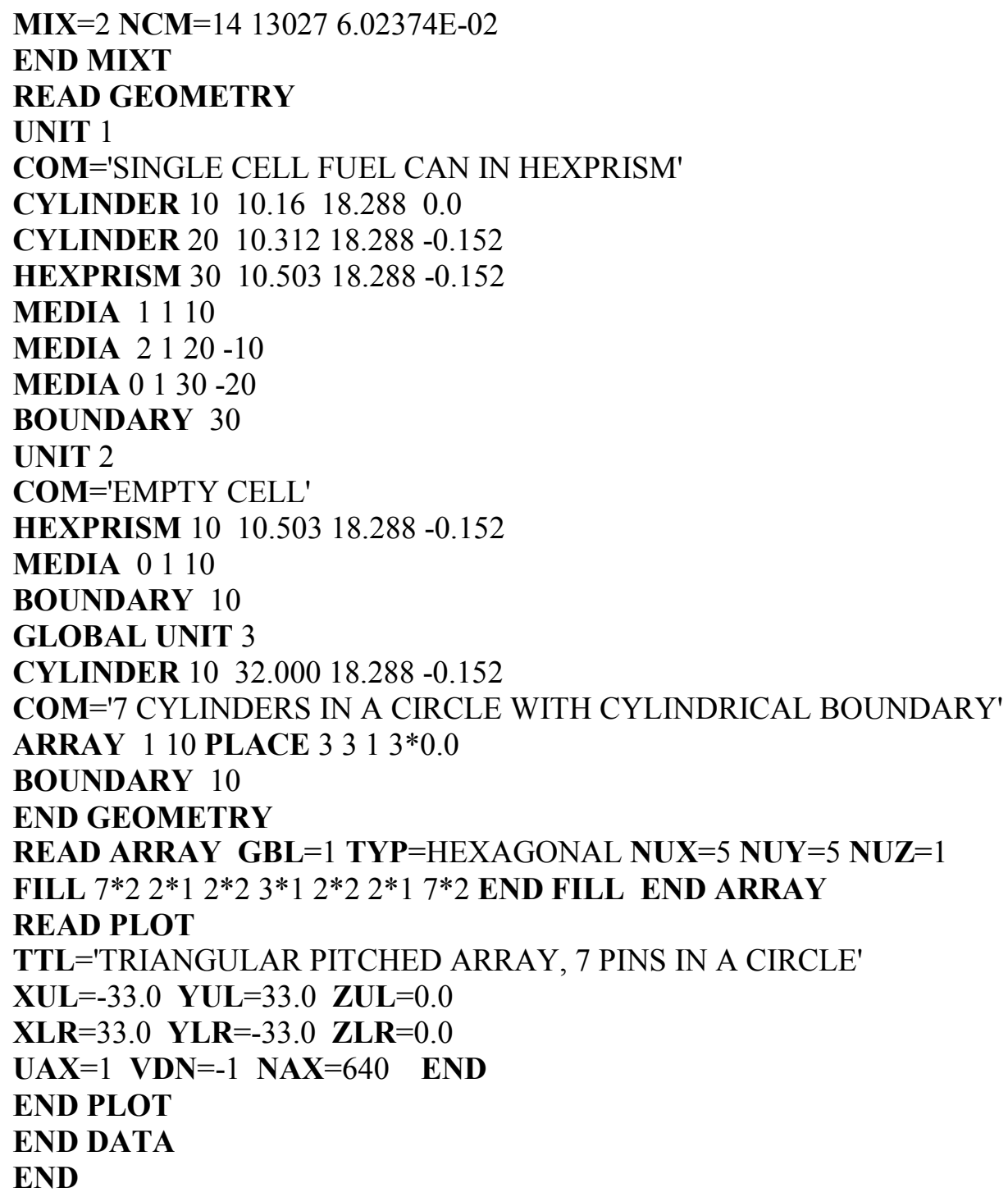

\section{EXAMPLE 21a}

Another hexagonal ARRAY type involves stacking rotated hexprisms, which are hexprisms rotated $30^{\circ}$ \% $90^{\circ}$ so that the flat faces perpendicular to the $\mathrm{Y}$ axis now meet. Rotated hexagonal arrays are specified by setting TYP=RHEXAGONAL in the array data block. Because the ARRAYs are constructed by stacking hexprisms, care must be taken to ensure the array boundary is completely enclosed within the stacked UNIT. Below is an example of a rotated hexagonal pitched ARRAY.

The first and second UNITs are the rotated hexprisms that make up the ARRAY. They are specified using the geometry keyword RHEXPRISM. UNIT 1 is the fuel cell that is stacked in a rotated hexagonal lattice. UNIT 2 is a dummy UNIT used to fill in the ARRAY so that the ARRAY BOUNDARY is contained within the stacked UNITs. Since the ARRAY is not moderated, UNIT 2 contains void. Fig. 8.1.45 shows an X-Y cross section schematic of UNITs 1 and 2. 


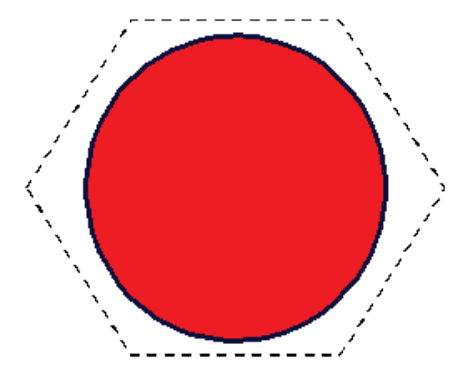

UNIT 1

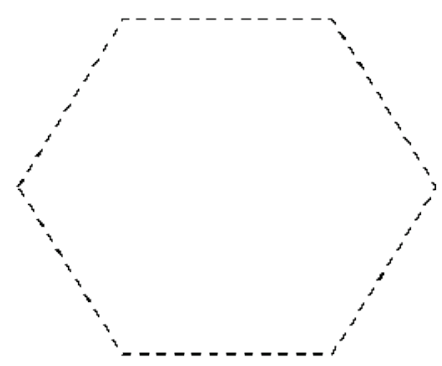

UNIT 2

Fig. 8.1.45. Fuel cell and empty cell set up as rotated hexprism (RHEXPRISM).

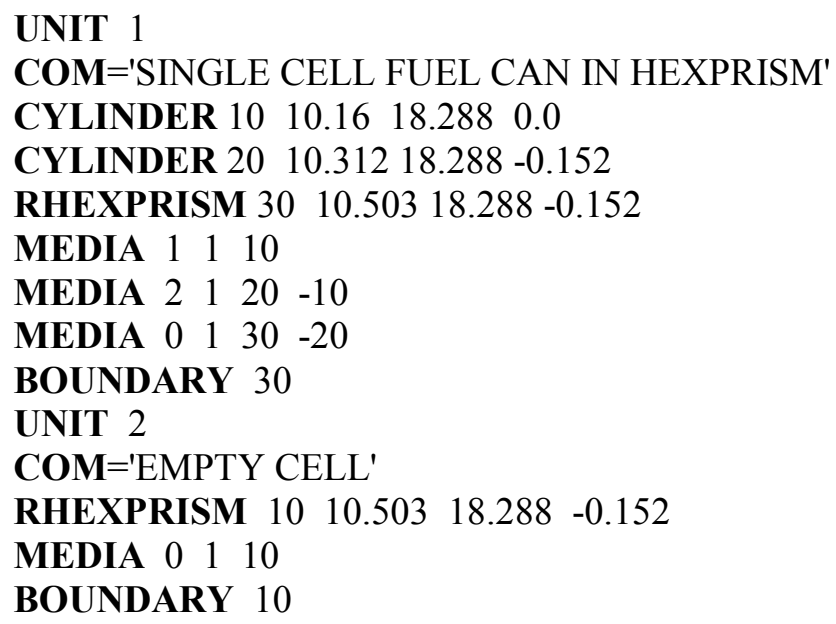

UNIT 3 is the GLOBAL UNIT that contains the ARRAY and ARRAY BOUNDARY. The ARRAY is an unmoderated rotated hexagonal pitched assembly of 7 cells. The rotated hexagonal array type is specified in the array data block using TYP= with the keyword RHEXAGONAL. This ARRAY type stacks the UNITs so the faces perpendicular to the Y axis meet. In the odd numbered columns (i.e., 1, 3, 5 , etc.), the UNITs are stacked so the columns begin at the lower edge of the array and in the even numbered columns (i.e., 2, 4, 6, etc.), the UNITs are stacked so the columns begin $1 / 2$ of the face-to-face dimension above the lower edge of the ARRAY. Fig. 8.1.46 shows the X-Y cross section schematic of the assembly for the rotated hexagonal ARRAY type. 


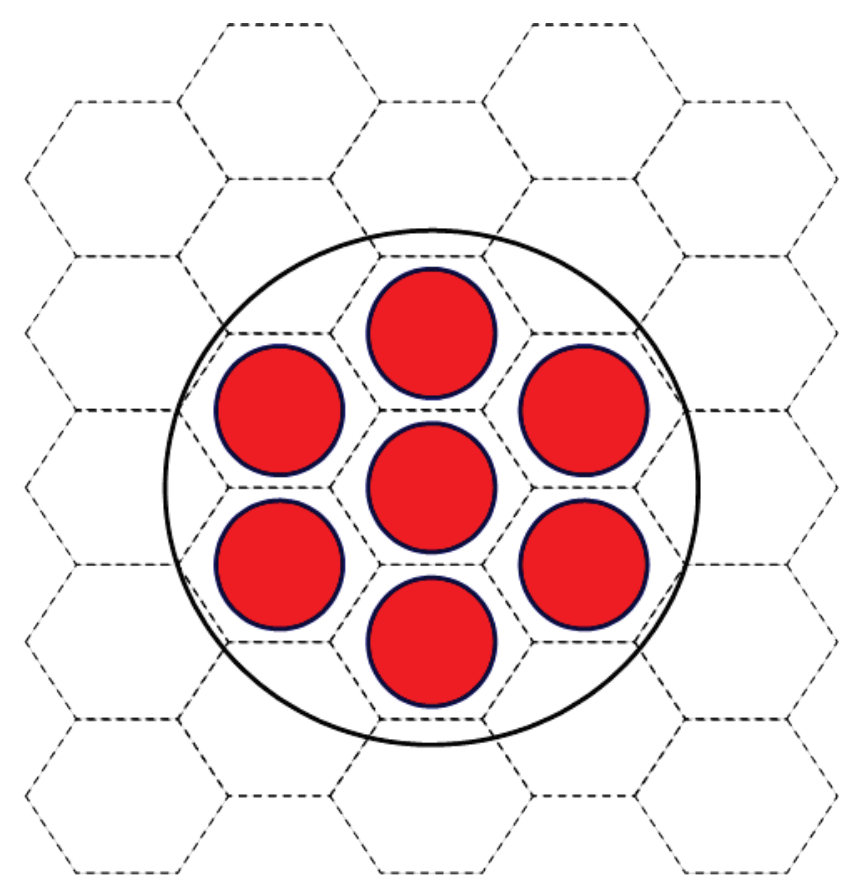

Fig. 8.1.46. Seven cylinders stacked in a RHEXAGONAL array with a cylindrical array boundary.

\section{GLOBAL UNIT 3}

COM='7 CYLINDERS IN A CIRCLE WITH CYLINDRICAL BOUNDARY'

CYLINDER $1032.000 \quad 18.288-0.152$

ARRAY 110 PLACE $3313^{*} 0.0$

BOUNDARY 10

READ ARRAY GBL $=1$ ARA $=1$ TYP=RHEXAGONAL NUX $=5$ NUY $=5$ NUZ $=1$

FILL $6 * 23 * 12 * 23 * 13 * 21 * 17 * 2$ END FILL END ARRAY

The overall problem description is shown below. The cross section library would be generated in a separate CSAS-MG step. An X-Y cross section color plot used for verification of this mockup is shown in Fig. 8.1.47. 


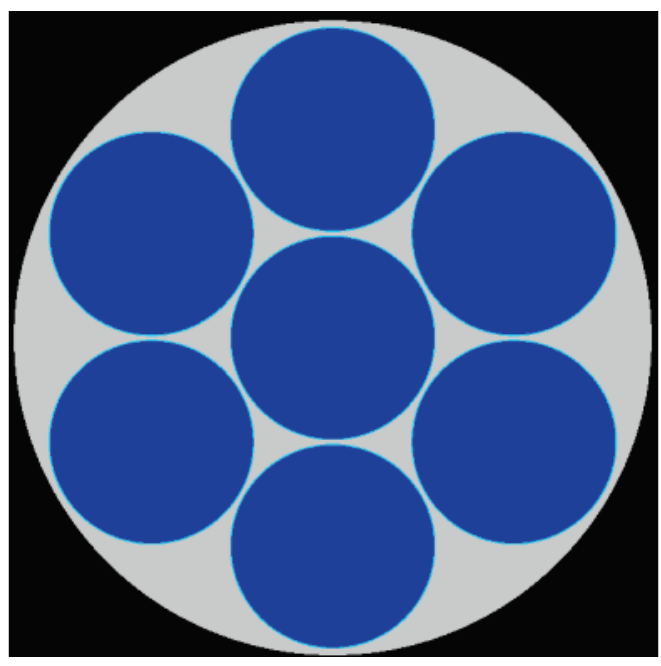

Fig. 8.1.47. X-Y slice of 7 cylinders in a rotated hexagonal pitched array.

Data description of Example 21a.

$=$ KENOVI

TRIANGULAR PITCHED ARRAY 7 PINS IN A CIRCLE

READ PARAMETERS LNG $=20000$ LIB $=4$ END PARAMETERS

READ MIXT SCT $=2$

MIX=1 NCM=8 92235 1.37751E-03 92238 9.92354E-05 8016 3.32049E-02

$90192.95349 \mathrm{E}-03 \quad 10016.05028 \mathrm{E}-02$

MIX=2 NCM=14 13027 6.02374E-02

END MIXT

READ GEOMETRY

UNIT 1

$\mathbf{C O M = ' S I N G L E ~ C E L L ~ F U E L ~ C A N ~ I N ~ H E X P R I S M ' ~}$

CYLINDER $10 \quad 10.16 \quad 18.288 \quad 0.0$

CYLINDER $2010.31218 .288-0.152$

RHEXPRISM $30 \quad 10.50318 .288-0.152$

MEDIA 1110

MEDIA $2120-10$

MEDIA 0130 -20

BOUNDARY 30

UNIT 2

COM='EMPTY CELL'

RHEXPRISM $10 \quad 10.50318 .288-0.152$

MEDIA 0110

BOUNDARY 10

GLOBAL UNIT 3

CYLINDER $1032.00018 .288-0.152$

$\mathbf{C O M}=' 7$ CYLINDERS IN A CIRCLE WITH CYLINDRICAL BOUNDARY'

ARRAY 110 PLACE $3313 * 0.0$

BOUNDARY 10

END GEOMETRY

READ ARRAY GBL $=1$ TYP $=$ RHEXAGONAL NUX $=5$ NUY $=5$ NUZ $=1$

FILL $6 * 23 * 12 * 23 * 13 * 21 * 17 * 2$ END FILL END ARRAY 


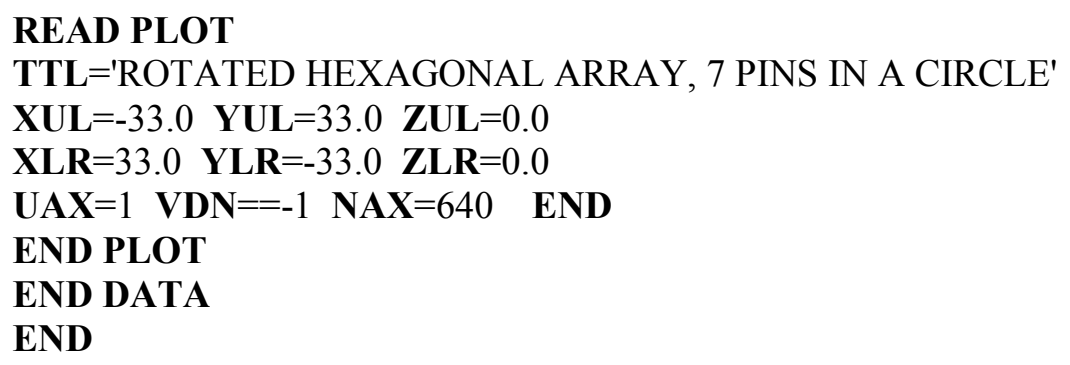

\subsection{Triangular pitched Arrays in KENO V.a}

Triangular pitched arrays can be described in KENO V.a geometry by properly defining the basic unit from which the array can be built. This includes close-packed triangular pitched arrays. Two geometry configurations are described below.

EXAMPLE 1. Bare Triangular pitched Array

Fig. 8.1.48 illustrates a small close-packed triangular pitched ARRAY. Each rod in the ARRAY has a radius of $2.0 \mathrm{~cm}$, and the pitch of the ARRAY is $4 \mathrm{~cm}$. To create this ARRAY, describe five units as defined in Fig. 8.1.49.

Assume the rods described in the ARRAY are each $2.0 \mathrm{~cm}$ in radius and $100 \mathrm{~cm}$ tall. The rods are composed of mixture 1. The geometry descriptions for the first four UNITs are given below.

UNIT 1

ZHEMICYL-Y 112.050 .0 -50.0

UNIT 2

ZHEMICYL+Y 112.050 .0 -50.0

UNIT 3

ZHEMICYL-X 112.050 .0 -50.0

UNIT 4

ZHEMICYL+X $112.050 .0-50.0$ 


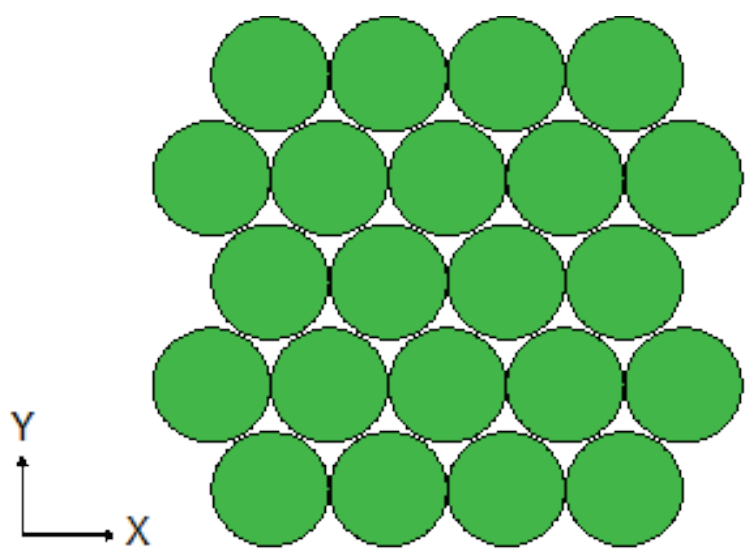

Fig. 8.1.48. Bare triangular pitched ARRAY.
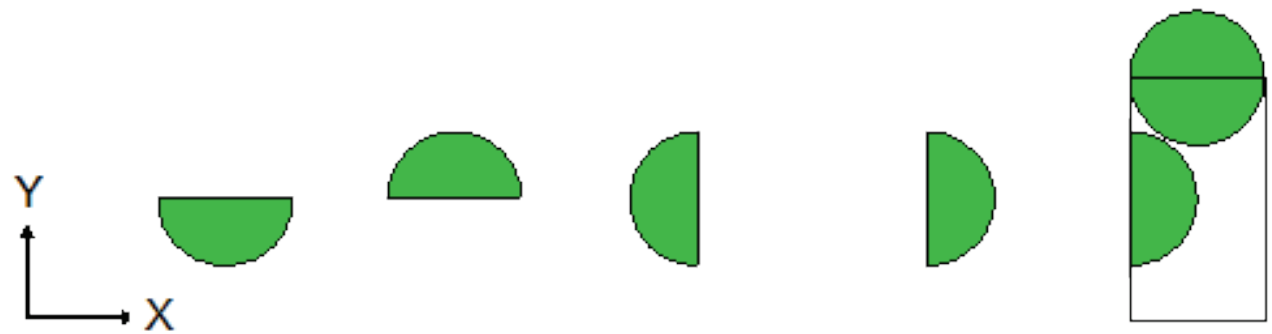

Fig. 8.1.49. Units used to describe a bare triangular pitched ARRAY.

To describe UNIT 5, the origin of the UNIT is defined to be at its center. One of the hemicylinders is built into the box, and the other three are added as holes. In this example, the $+\mathrm{X}$ hemicylinder is built into the box, and the other hemicylinders are inserted as holes. (Because the $+\mathrm{X}$ hemicylinder is built into UNIT 5, UNIT 4 is not used in the problem.) The half dimension of the box in the X dimension is equal to the radius, $2.0 \mathrm{~cm}$. The half dimension of the box in the $\mathrm{Y}$ direction is $\frac{\sqrt{3}}{2}$ times the pitch $(0.866025 *$ 4.0) or $3.46411 \mathrm{~cm}$. UNIT 5 is described below.

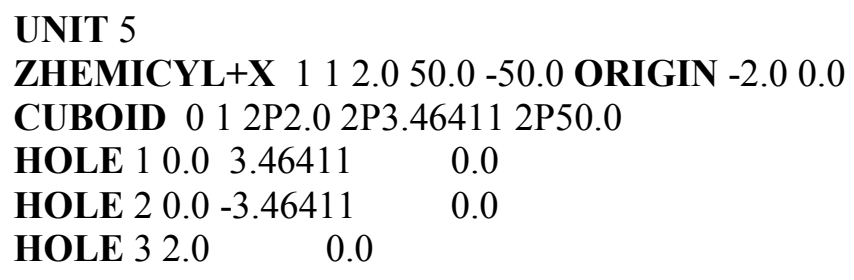

In the description of UNIT 5, the ZHEMICYL+X places the hemicylinder at the left of the box. HOLE 1 places the top hemicylinder, HOLE 2 places the bottom hemicylinder, and HOLE 3 places the hemicylinder at the right of the box.

Next, a UNIT 6 is defined that can be used to complete the lower rod of UNIT 5. A UNIT 7 is defined that can be used to complete the upper rod of UNIT 5. UNIT 8 is defined to complete the left rod of UNIT 5, and UNIT 9 is defined to complete the right rod of UNIT 5. UNIT 10 is defined to complete the corners of the overall ARRAY. The input data for these UNITs are given below and are illustrated in Fig. 8.1.50. 


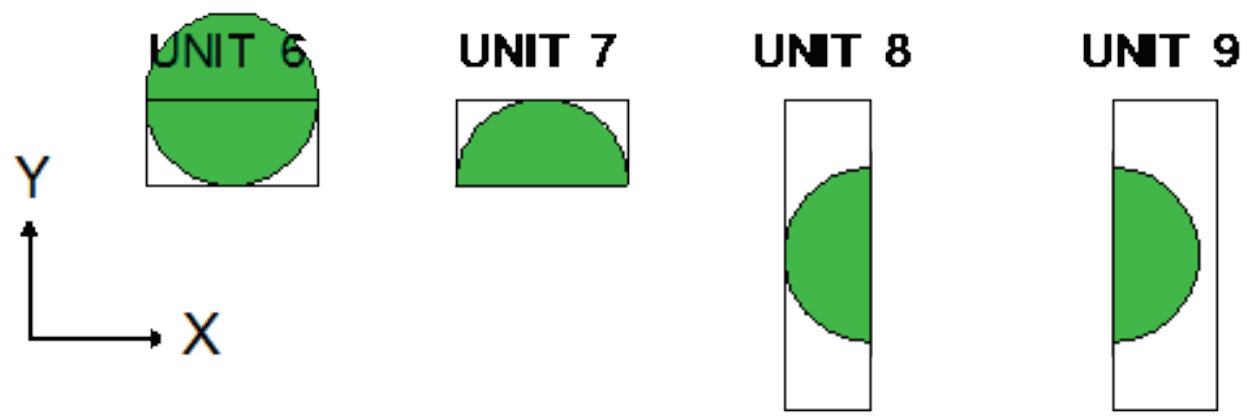

Fig. 8.1.50. UNITs to complete the triangular pitched ARRAY.

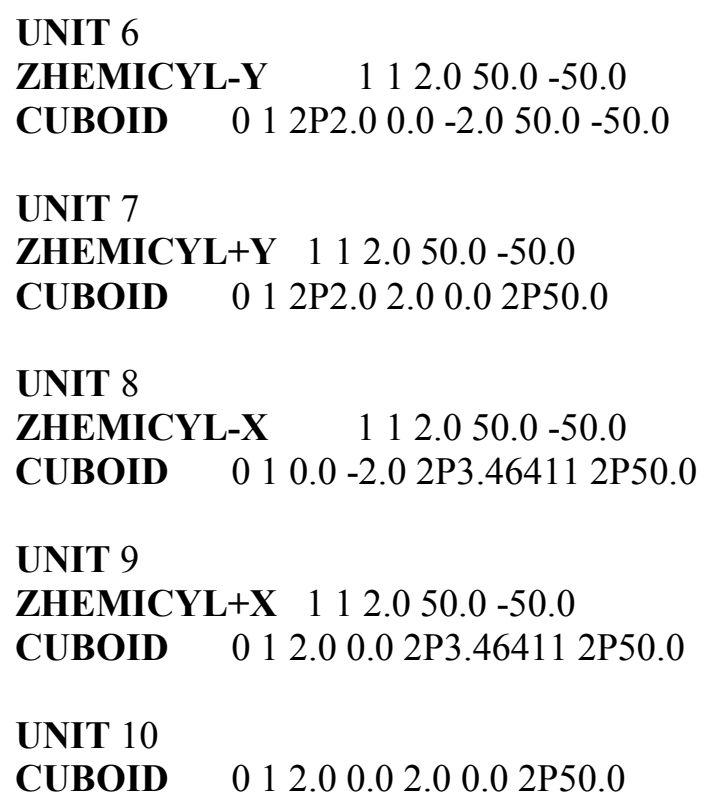

Fig. 8.1.51 shows the arrangement of the UNITs to complete the ARRAY. The data to describe the ARRAY are shown below.

$$
\text { ARA=1 NUX=6 NUY=4 NUZ=1 FILL } 104 R 61084 R 59 \text { 1Q6 } 104 R 710 \text { END FILL }
$$

The bottom row of the ARRAY is described by the data entries 104 R6 10. The second row of the ARRAY is described by the data entries $84 R 59$. The third row is filled by repeating the previous six entries (1Q6). It could also have been described by entering 8 4R5 9. The top row of the ARRAY is described by the data entries $104 \mathrm{R} 710$. 


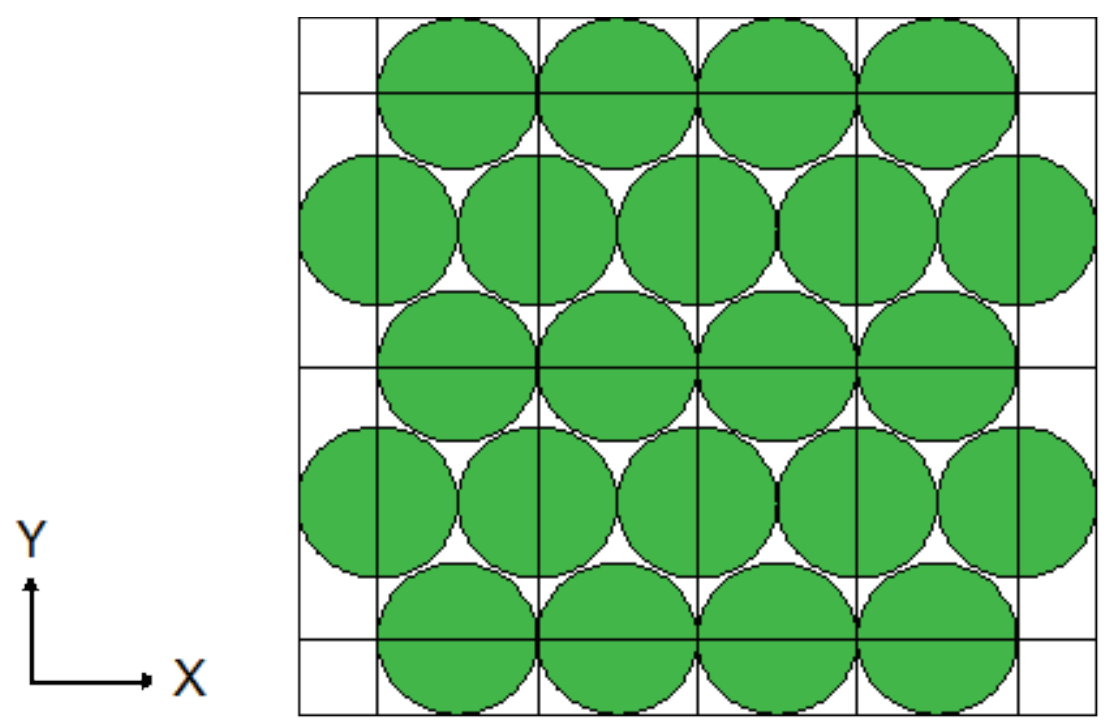

Fig. 8.1.51. Completed ARRAY.

EXAMPLE 2a. Triangular Pitched ARRAY in a Cylinder

Fig. 8.1.52 illustrates a close-packed triangular pitched ARRAY in a cylinder. This array may be described by defining five basic units that are the same as those of Example 1 shown in Fig. 8.1.49.

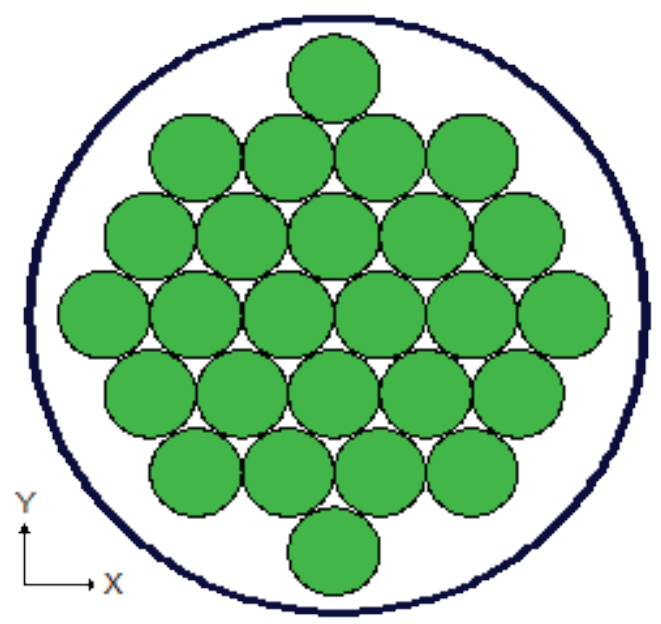

Fig. 8.1.52. Triangular pitched ARRAY within a cylinder.

UNIT 1

ZHEMICYL-Y $112.050 .0-50.0$

UNIT 2

ZHEMICYL+Y $112.050 .0-50.0$ 


\section{UNIT 3}

ZHEMICYL-X $112.050 .0-50.0$

UNIT 4

ZHEMICYL+X $112.050 .0-50.0$

To describe UNIT 5, the origin of the UNIT to be at its center is defined. One of the hemicylinders is built into the box, and the other three are added as HOLEs. In this example, the $+\mathrm{X}$ hemicylinder is built into the box, and the other hemicylinders are inserted as HOLEs. The half dimension of the box in the $\mathrm{X}$ dimension is equal to the radius, $2.0 \mathrm{~cm}$. The half dimension of the box in the $\mathrm{Y}$ direction is $\frac{\sqrt{3}}{2}$ times the pitch $(0.866025 * 4.0)$ or $3.46411 \mathrm{~cm}$. UNIT 5 is described below.

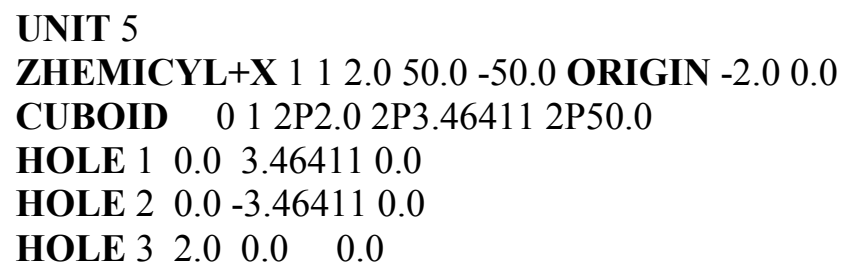

UNIT 5

ZHEMICYL+X 112.050 .0 -50.0 ORIGIN -2.0 0.0

CUBOID $\quad 012 \mathrm{P} 2.02 \mathrm{P} 3.464112 \mathrm{P} 50.0$

HOLE $1 \quad 0.0 \quad 3.464110 .0$

HOLE $20.0-3.464110 .0$

HOLE $3 \quad 2.0 \quad 0.0 \quad 0.0$

In the description of UNIT 5, the ZHEMICYL+X places the hemicylinder at the left of the box. HOLE 1 places the top hemicylinder, HOLE 2 places the bottom hemicylinder, and HOLE 3 places the hemicylinder at the right of the box.

To describe the base ARRAY of the problem, UNITs 5 is stacked in a $4 \times 2 \times 1$ array as shown in Fig. 8.1.53. The input data for the ARRAY are the following:

\section{ARA $=1$ NUX=4 NUY=2 NUZ=1 FILL F5 END FILL}

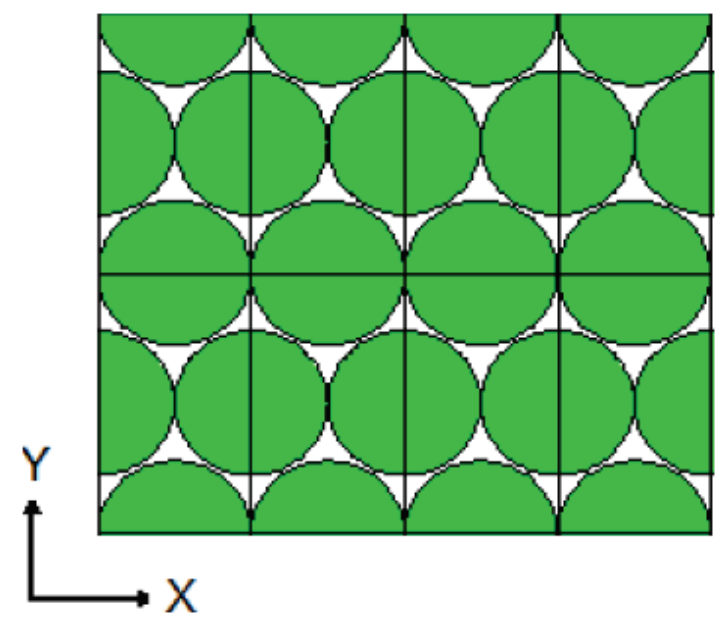

Fig. 8.1.53. $4 \times 2 \times 1$ array to be placed within a cylinder.

Next, the ARRAY is placed within the cylinder. This is done by placing the ARRAY in a UNIT, defined here to be UNIT 6. The origin of the cylinder has been defined to be at the center of the ARRAY. The resulting geometry is shown in Fig. 8.1.54. 


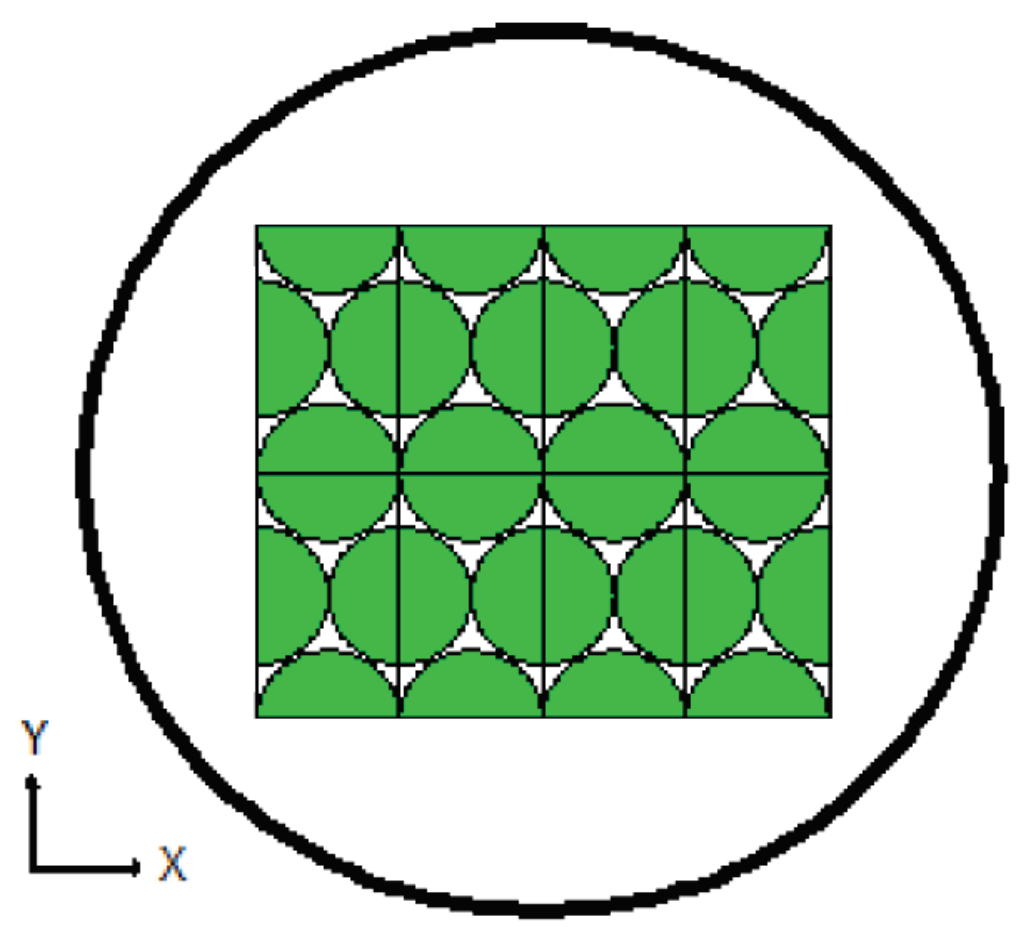

Fig. 8.1.54. $4 \times 2 \times 1$ ARRAY within a cylinder.

\section{UNIT 6}

ARRAY $1-8.0-6.92822-50.0$

CYLINDER 0112.4 2P50.0

CYLINDER 2112.65 2P50.0

Next, the hemicylinders necessary to complete all of the half cylinders remaining in Fig. 8.1.54 are added. This is done by placing four UNITs 1 at the appropriate positions along the bottom of the ARRAY, four UNITs 2 at the top of the ARRAY, two UNITs 3 at the left of the ARRAY, and two UNITs 4 at the right of the ARRAY. The input data are shown below, and the resulting configuration is shown in Fig. 8.1.55. In UNIT 6, the first HOLE 1 places a UNIT 1 under the lower left UNIT of the ARRAY. The second HOLE 1 places a UNIT 1 under the next ARRAY UNIT to the right of the first one. This procedure is repeated for the next two lower ARRAY UNITs, thus completing the lower row of cylinders. Similarly, the first HOLE 2 places a UNIT 2 above the upper left UNIT of the ARRAY. The second HOLE 2 places a UNIT 1 to the right of the first one, etc., until the four cylinders at the top of the ARRAY are completed. The first HOLE 3 places a UNIT 3 at the lower left side of the ARRAY to complete that rod. The second HOLE 3 completes the rod above it. The first HOLE 4 completes the lower rod on the right side of the ARRAY. The second HOLE 4 completes the rod above it. The geometry data listed below result in the configuration shown in Fig. 8.1.55. 


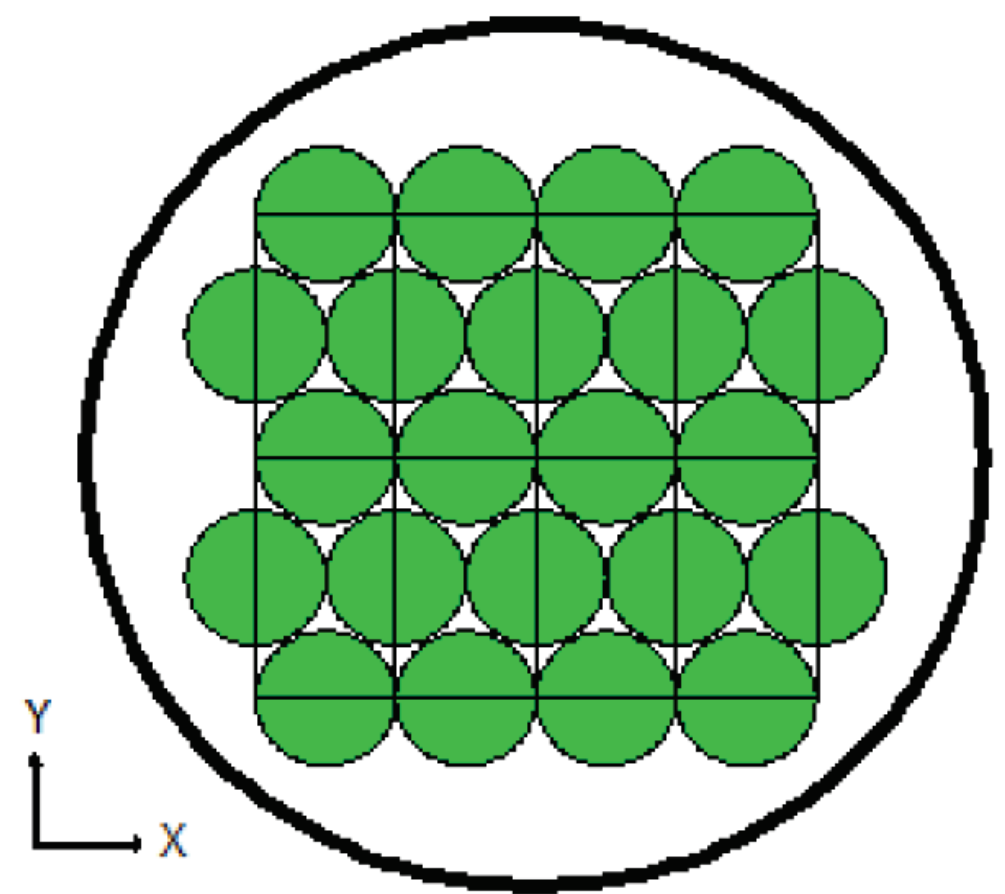

Fig. 8.1.55. Partially completed triangular pitched ARRAY in a cylinder.
UNIT 6
ARRAY 1 -8.0 -6.92822 -50.0
CYLINDER 0112.4 2P50.0
HOLE $1-6.0-6.928220 .0$
HOLE $1-2.0-6.928220 .0$
HOLE $1 \quad 2.0-6.928220 .0$
HOLE $1 \quad 6.0-6.928220 .0$
HOLE $2-6.06 .928220 .0$
HOLE $2-2.0 \quad 6.928220 .0$
HOLE $2 \quad 2.06 .928220 .0$
HOLE $2 \quad 6.06 .928220 .0$
HOLE $3-8.0-3.464110 .0$
HOLE 3 - 8.0 3.46411 0.0
HOLE 4 8.0 -3.464110 .0$
HOLE $4 \quad 8.03 .464110 .0$
CYLINDER 2112.65 2P50.0

To complete the desired configuration, a cylinder is defined, UNIT 7, and it is placed at the four appropriate positions as shown below. The first HOLE 7 places the cylinder of UNIT 7 at the left of the ARRAY, the second HOLE 7 places the cylinder at the top of the ARRAY, the third HOLE 7 places the cylinder at the right of the ARRAY, and the fourth HOLE 7 places the cylinder at the bottom of the ARRAY. The completed configuration is shown in Fig. 8.1.56. It is not necessary for UNIT 7 to precede UNIT 6. It is allowable to place UNIT 7 after UNIT 6 in the input data. Because the final configuration is defined in UNIT 6, it must be designated as the GLOBAL UNIT. The total geometry input for this example is listed below. 


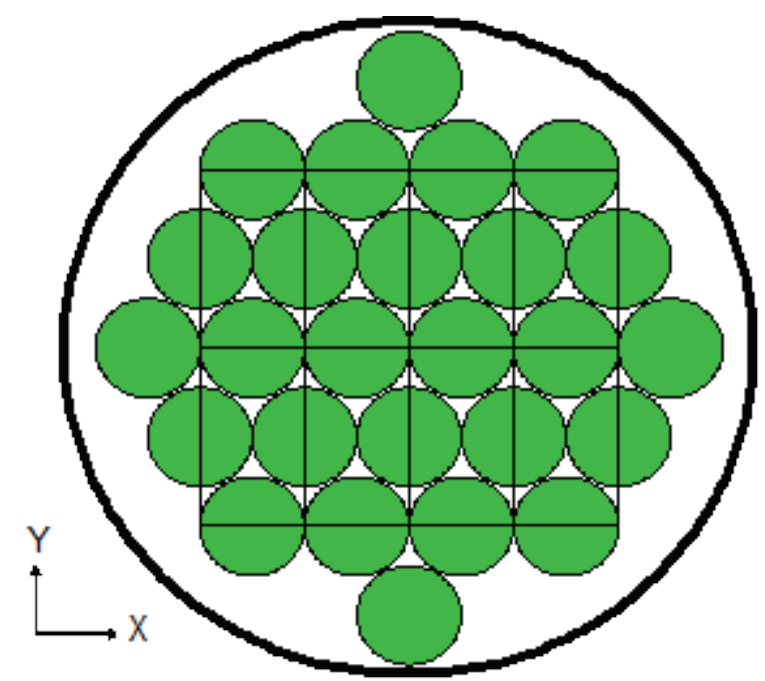

Fig. 8.1.56. Completed triangular pitched array in a cylinder.

\author{
READ GEOM \\ UNIT 1 \\ ZHEMICYL-Y $112.050 .0-50.0$ \\ UNIT 2 \\ ZHEMICYL+Y $112.050 .0-50.0$ \\ UNIT 3 \\ ZHEMICYL-X 112.050 .0 -50.0 \\ UNIT 4 \\ ZHEMICYL+X $112.050 .0-50.0$
}

UNIT 5

ZHEMICYL+X 112.050 .0 -50.0 ORIGIN -2.0 0.0

CUBOID $\quad 012$ 2P2.0 2P3.46411 2P50.0

HOLE 10.03 .464110 .0

HOLE $20.0-3.464110 .0$

HOLE $3 \quad 2.0 \quad 0.0 \quad 0.0$

UNIT 7

CYLINDER 112.0 2P50.0

\title{
GLOBAL UNIT 6
}

ARRAY 1 -8.0 -6.92822 -50.0

CYLINDER 0112.4 2P50.0

HOLE $1-6.0-6.928220 .0$

HOLE $1-2.0-6.928220 .0$

HOLE $1 \quad 2.0-6.928220 .0$

HOLE $1 \quad 6.0-6.928220 .0$ 
HOLE $2-6.0 \quad 6.928220 .0$

HOLE $2-2.0 \quad 6.928220 .0$

HOLE $22.0 \quad 6.928220 .0$

HOLE $2 \quad 6.0 \quad 6.928220 .0$

HOLE $3 \quad-8.0 \quad-3.464110 .0$

HOLE $3 \quad-8.0 \quad 3.464110 .0$

HOLE $4 \quad 8.0 \quad-3.464110 .0$

HOLE $4 \quad 8.0 \quad 3.464110 .0$

HOLE 7 -10.0 $\quad 0.0 \quad 0.0$

HOLE $7 \quad 0.0 \quad 10.392330 .0$

$\begin{array}{llll}\text { HOLE } 7 & 10.0 & 0.0 & 0.0\end{array}$

HOLE $7 \quad 0.0-10.392330 .0$

CYLINDER 2112.65 2P50.0

END GEOM

READ ARRAY

ARA $=1$ NUX=4 NUY=2 NUZ=1 FILL F5 END FILL

END ARRAY

EXAMPLE 2b. Alternative Mockup of Triangular Pitched ARRAY in a Cylinder

Consider the triangular pitched ARRAY shown in Fig. 8.1.52. Another method of describing this configuration is given below. Four basic UNITs are defined, as listed below. These are the same UNITs shown in Fig. 8.1.49.

UNIT 1

ZHEMICYL-Y $112.050 .0-50.0$

UNIT 2

ZHEMICYL+Y $112.050 .0-50.0$

UNIT 3

ZHEMICYL-X $112.050 .0-50.0$

UNIT 4

ZHEMICYL+X $112.050 .0-50.0$

UNIT 5 is the same as previously defined in Example 2a, and pictured in Fig. 8.1.49.

UNIT 5

ZHEMICYL+X 112.050 .0 -50.0 ORIGIN -2.0 0.0

CUBOID $\quad 012 \mathrm{P} 2.02 \mathrm{P} 3.464112 \mathrm{P} 50.0$

HOLE 10.03 .464110 .0

HOLE $20.0-3.464110 .0$

HOLE $3 \quad 2.0 \quad 0.0 \quad 0.0$

To describe the basic array for the problem, UNITs 5 is stacked in a $4 \times 2 \times 1$ as shown in Fig. 8.1.53. The input data for the ARRAY are the following:

ARA $=1$ NUX=4 NUY=2 NUZ=1 FILL F5 END FILL 
Next, the ARRAY (ARRAY 1) is placed in UNIT 6, and UNITs 7 and 8 are defined to be placed to the left and right of it (see Fig. 8.1.57). UNIT 7 will complete the two rods at the left boundary of the ARRAY and will contain half of the far left rod in the completed configuration. In the description of UNIT 7, the ZHEMICYL+X is half of the far right rod and is located with its cut face at the left boundary of a box that is as tall as the entire ARRAY of Fig. 8.1.53. The first HOLE 3 in UNIT 7 completes the lower left rod of that ARRAY, and the second HOLE 3 completes the upper left rod. UNIT 8 is constructed in similar fashion to complete the two rods at the right of the ARRAY shown in Fig. 8.1.53. UNIT 8 is the mirror image of UNIT 7. UNITs 6, 7, and 8 are stacked in an ARRAY (ARRAY 2) to achieve the configuration shown in Fig. 8.1.57. The data to accomplish this are listed below.

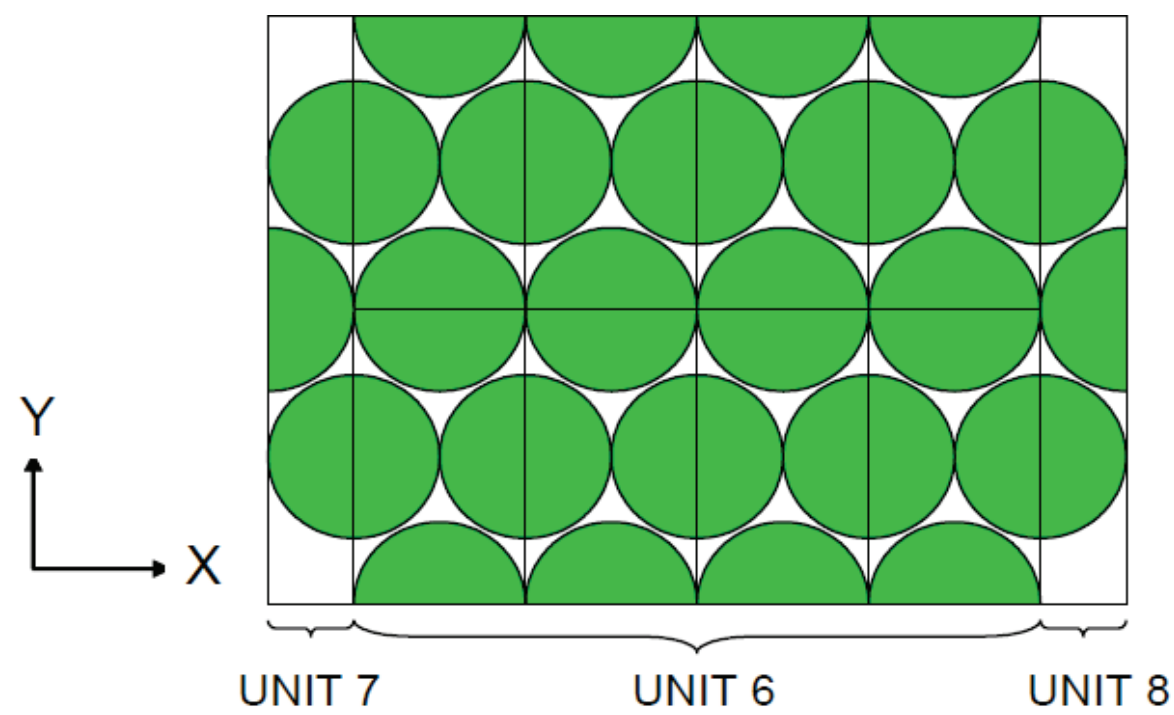

Fig. 8.1.57. Array to be placed in a cylinder.

UNIT 6

ARRAY $1-8.0-6.92822-50.0$

GLOBAL

UNIT 7

ZHEMICYL+X $112.050 .0-50.0$

CUBOID $\quad 0 \quad 12.00 .02 \mathrm{P} 6.928222 \mathrm{P} 50.0$

HOLE $32.0-3.464110 .0$

HOLE 32.03 .464110 .0

UNIT 8

ZHEMICYL-X $112.050 .0-50.0$

CUBOID $\quad 0 \quad 1 \quad 0.0-2.0 \quad 2 \mathrm{P} 6.928222 \mathrm{P} 50.0$

HOLE 4 -2.0 -3.464110 .0$

HOLE 4 -2.0 3.464110 .0

ARA $=2$ NUX=3 NUY=1 NUZ=1 FILL 768 END FILL 
Next, ARRAY 2 is placed in the cylinder as shown in Fig. 8.1.58. The data are listed below.

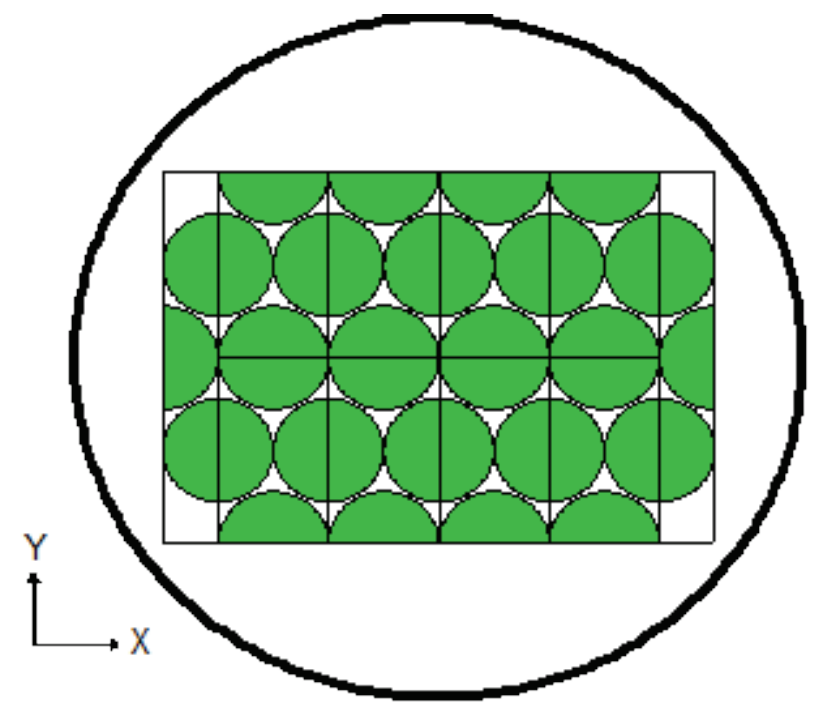

Fig. 8.1.58. Partially completed array in a cylinder.

UNIT 9

ARRAY $2-10.0-6.92822-50.0$

CYLINDER 0112.4 2P50.0

CYLINDER 2112.65 2P50.0

Now UNITs 10 and 11 are described and placed above and below the ARRAY. These UNITs are shown in Fig. 8.1.59. UNIT 10 is described to complete the two central rods at the top of the ARRAY of Fig. 8.1.58. Fig. 8.1.59 and Fig. 8.1.60 illustrate these UNITs. The ZHEMICYL-Y is placed at the top of the UNIT to describe half of the rod at the very top of the ARRAY. The first HOLE 2 completes the left center rod at the top of the ARRAY pictured in Fig. 8.1.58. The second HOLE 2 completes the right center rod at the top of the ARRAY. UNIT 11 is described in similar fashion. It is the mirror image of UNIT 10 and is placed below the ARRAY of Fig. 8.1.58. The resulting configuration is shown in Fig. 8.1.59.

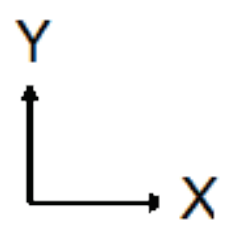

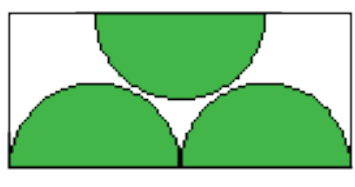

UNIT 10

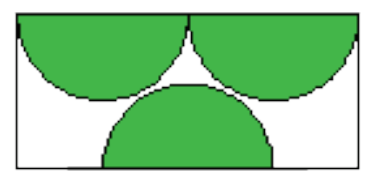

UNIT 11

Fig. 8.1.59. Description of UNITs 10 and 11 for Example 2b. 
UNIT 10

ZHEMICYL-Y 112.0 2P50.0

CUBOID 012 2P4.0 0.0 -3.46411 2P50.0

HOLE 2 -2.0 -3.46411 0.0 2P50.0

HOLE 2 2.0 -3.464110 .0$

UNIT 11

ZHEMICYL+Y $112.02 \mathrm{P} 50.0$

CUBOID 01 2P4.0 3.46411 0.0 2P50.0

HOLE 1 -2.0 3.464110 .0

HOLE 12.03 .464110 .0

Now UNITs 10 and 11 are placed above and below the ARRAY of Fig. 8.1.58 to obtain the configuration shown in Fig. 8.1.60.

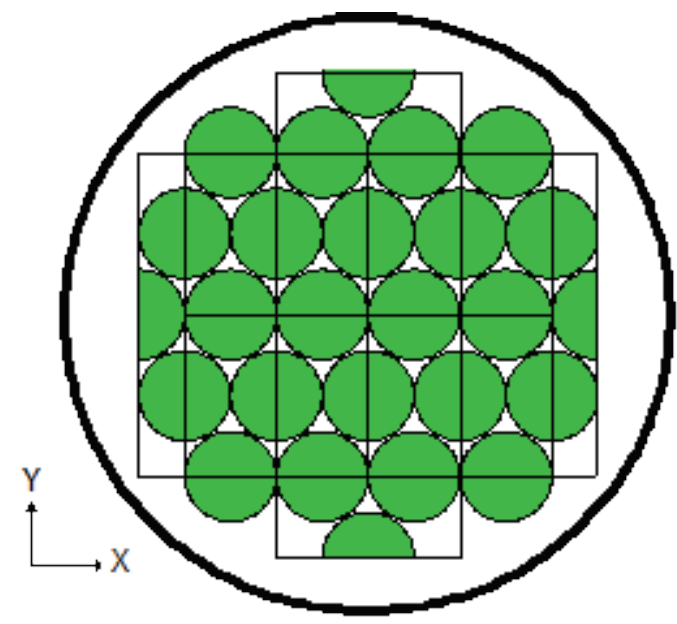

Fig. 8.1.60. Partially completed triangular pitched ARRAY in a cylinder.

\section{UNIT 9}

ARRAY 2 -10.0 -6.92822 -50.0

CYLINDER 0112.4 2P50.0

HOLE 100.010 .392330 .0

HOLE $110.0-10.392330 .0$

CYLINDER 2112.65 2P50.0

To complete the array, the remaining half cylinders must be entered as HOLEs, as shown below. The first HOLE 1 completes the half rod at the lower left of Fig. 8.1.60. The second HOLE 1 completes the half rod at the lower center, and the third HOLE 1 completes the half rod at the lower right of Fig. 8.1.60. Similarly, the first HOLE 2 completes the half rod at the upper left of Fig. 8.1.60. The second HOLE 2 completes the half rod at the upper center, and the third HOLE 2 completes the half rod at the upper right. 
HOLE 3 completes the half rod at the left of Fig. 8.1.60, and HOLE 4 completes the half rod at the right. The final geometry configuration is shown in Fig. 8.1.61. UNIT 9 must be specified as the GLOBAL UNIT because it defines the overall configuration.

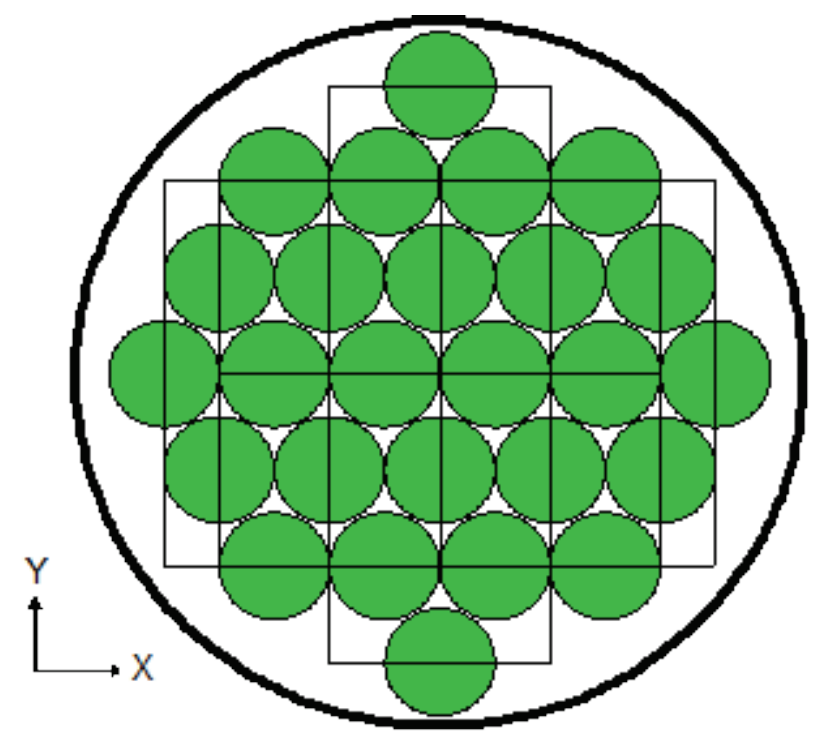

Fig. 8.1.61. Final configuration of triangular pitched ARRAY in a cylinder.

GLOBAL UNIT 9

ARRAY 2 -10.0 -6.92822 -50.0

CYLINDER $0112.42 \mathrm{P} 50.0$

HOLE $10 \quad 0.010 .392330 .0$

HOLE $110.0-10.392330 .0$

HOLE $1-6.0-6.928220 .0$

HOLE $1 \quad 0.0-10.392330 .0$

HOLE $1 \quad 6.0-6.928220 .0$

HOLE $2-6.0 \quad 6.928220 .0$

HOLE $2 \quad 0.0 \quad 10.392330 .0$

HOLE $2 \quad 6.0 \quad 6.928220 .0$

HOLE $3-10.0 \quad 0.0 \quad 0.0$

$\begin{array}{llll}\text { HOLE } 4 & 10.0 & 0.0 & 0.0\end{array}$

CYLINDER 2112.65 2P50.0

The geometry data for Example $2 \mathrm{~b}$ are given below.

\section{READ GEOM}

UNIT 1

ZHEMICYL-Y $112.050 .0-50.0$

UNIT 2

ZHEMICYL+Y $112.050 .0-50.0$ 
UNIT 3

ZHEMICYL-X $112.050 .0-50.0$

UNIT 4

ZHEMICYL+X $112.050 .0-50.0$

UNIT 5

ZHEMICYL+X 112.050 .0 -50.0 ORIGIN -2.0 0.0

CUBOID $\quad 012 \mathrm{P} 2.02 \mathrm{P} 3.464112 \mathrm{P} 50.0$

HOLE 10.03 .464110 .0

HOLE $20.0-3.464110 .0$

HOLE $3 \quad 2.0 \quad 0.0 \quad 0.0$

UNIT 6

ARRAY 1 -8.0 -6.92822 -50.0

UNIT 7

ZHEMICYL+X $112.050 .0-50.0$

CUBOID $\quad 012.00 .02 \mathrm{P} 6.92822$ 2P50.0

HOLE $32.0-3.464110 .0$

HOLE 32.03 .464110 .0

UNIT 8

ZHEMICYL-X $112.050 .0-50.0$

CUBOID $\quad 0 \quad 1 \quad 0.0-2.02$ 2P6.92822 2P50.0

HOLE $30.0-3.464110 .0$

HOLE 30.03 .464110 .0

UNIT 10

YZHEMICYL-Y 112.0 2P50.0

CUBOID 01 2P4.0 0.0 -3.46411 2P50.0

HOLE 2 - 2.0 - 3.464110 .0

HOLE 2 2.0 -3.464110 .0$

UNIT 11

YZHEMICYL+Y 112.02 P50.0

CUBOID 012 2P4.0 3.46411 0.0 2P50.0

HOLE 1 -2.0 3.464110 .0

HOLE 12.03 .464110 .0

GLOBAL

UNIT 9

ARRAY 2 -10.0 -6.92822 -50.0

CYLINDER 0112.4 2P50.0

$\begin{array}{llll}\text { HOLE } 10 & 0.0 & 10.39233 & 0.0\end{array}$

$\begin{array}{llll}\text { HOLE } 11 & 0.0 & -10.39233 & 0.0\end{array}$

$\begin{array}{llll}\text { HOLE } 1 & -6.0 & -6.92822 & 0.0\end{array}$

$\begin{array}{llll}\text { HOLE } 1 & 0.0 & -10.39233 & 0.0\end{array}$

$\begin{array}{llll}\text { HOLE } 1 & 6.0 & -6.92822 & 0.0\end{array}$

$\begin{array}{llll}\text { HOLE } 2 & -6.0 & 6.92822 & 0.0\end{array}$ 


\begin{tabular}{lcccc} 
HOLE 2 & 0.0 & \multicolumn{2}{c}{10.39233} & 0.0 \\
HOLE 2 & 6.0 & 6.92822 & 0.0 \\
HOLE 3 & -10.0 & 0.0 & 0.0 & \\
HOLE 4 & 10.0 & 0.0 & 0.0 &
\end{tabular}

CYLINDER 2112.65 2P50.0

END GEOM

READ ARRAY

ARA $=1$ NUX $=4$ NUY=2 NUZ $=1$ FILL F5 END FILL

ARA $=2$ NUX=3 NUY=1 NUZ=1 FILL 768 END FILL

END ARRAY

\subsection{Dodecahedral pitched arrays}

EXAMPLE 22. Dodecahedral pitched ARRAYs can be described in KENO-VI by defining the UNITs that make up the ARRAY as dodecahedra and in the ARRAY data block setting TYP=DODECAHEDRAL. Since the ARRAYs are constructed by stacking dodecahedra, care must be taken to ensure the ARRAY boundary is completely enclosed within the stacked unit. Below is an example of a dodecahedral ARRAY that represents a close packed ARRAY of spheres.

The first and second UNITs are the dodecahedra that make up the ARRAY. UNIT 1 is the fuel sphere stacked in a dodecahedral lattice. UNIT 2 is a dummy UNIT used to fill in the ARRAY so the ARRAY boundary is contained within the stacked UNITs. Since the ARRAY is not moderated, UNIT 2 contains void. Fig. 8.1.62 shows an isometric, cross section view of UNITs 1 and 2.

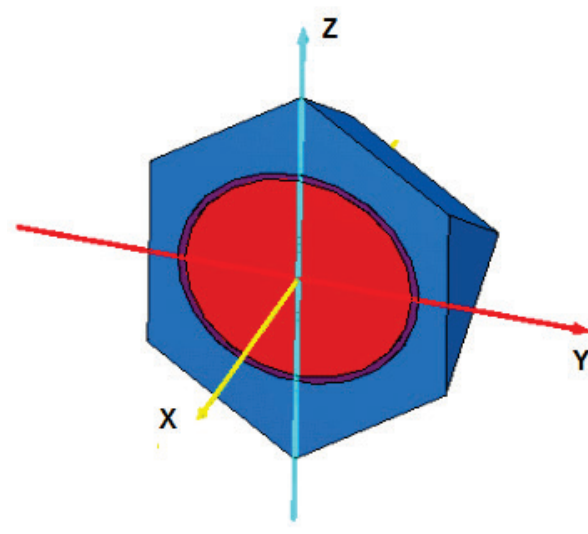

UNIT 1

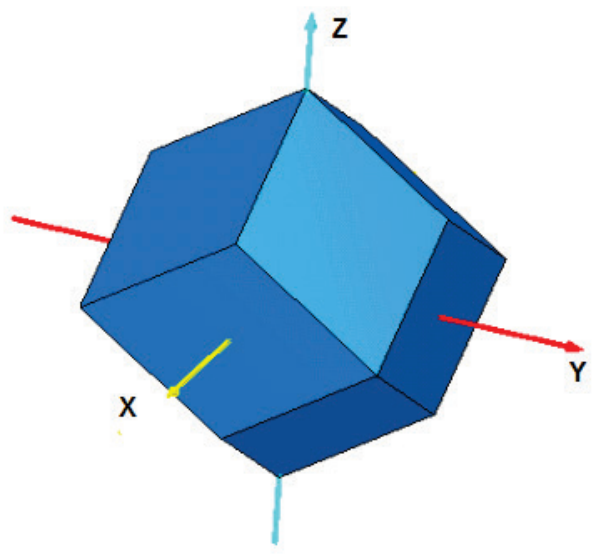

UNIT 2

Fig. 8.1.62. Fuel cell and empty cell set up as dodecahedra.

UNIT 1

$\mathbf{C O M}=$ 'SINGLE CELL FUEL CAN IN DODECAHDRON'

SPHERE $10 \quad 8.0$

SPHERE $20 \quad 8.5$

DODECAHEDRON 3010.5

MEDIA 11110

MEDIA $2 \begin{array}{llll}2 & 1 & 20 & -10\end{array}$

MEDIA $\quad \begin{array}{lllll}0 & 1 & 30 & -20\end{array}$ 


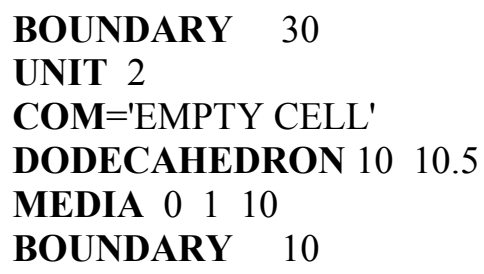

UNIT 3 is the GLOBAL UNIT that contains the ARRAY and ARRAY BOUNDARY. The ARRAY is an unmoderated triangular pitched assembly of 17 fuel spheres. Dodecahedral ARRAYs are specified by using $\mathbf{T Y P}=$ followed by keyword dodecahedral in the array data block. The $\mathrm{X}$ and $\mathrm{Y}$ coordinates are stacked together as a square pitched ARRAY with the pitch equal to twice the dodecahedron radius. In the $Z$ dimension, the odd $Z$ planes $(Z=1,3$, 5, etc.) have the $X$ and $Y$ UNITs begin at the most negative edge of the ARRAY, while the even $Z$ planes $(Z=2,4,6$, etc.) have the $X$ and Y UNITs begin one dodecahedron inscribed sphere radius in the positive direction from the most negative edge of the ARRAY. Also, the Z distance between the centers of the UNITs in successive Z planes is the square root of 2.0 times the dodecahedron radius (or the dodecahedron diameter divided by the square root of 2.0). Fig. 8.1.63 and Fig. 8.1.64 show X-Y cross sectional color plots of the assembly at an odd and even Z plane.

\section{GLOBAL UNIT 3}

$\mathbf{C O M = ' 1 7 ~ C L O S E ~ P A C K E D ~ F U E L ~ S P H E R E S ~ I N ~ A ~ C Y L I N D E R ' ~}$

CYLINDER $10 \quad 41.0 \quad 44.5 \quad 0.0$

CYLINDER $20 \quad 42.0 \quad 44.5 \quad-1.0$

ARRAY 110 PLACE $3313 * 0.0$

MEDIA $3 \quad 20 \quad-10$

BOUNDARY 20
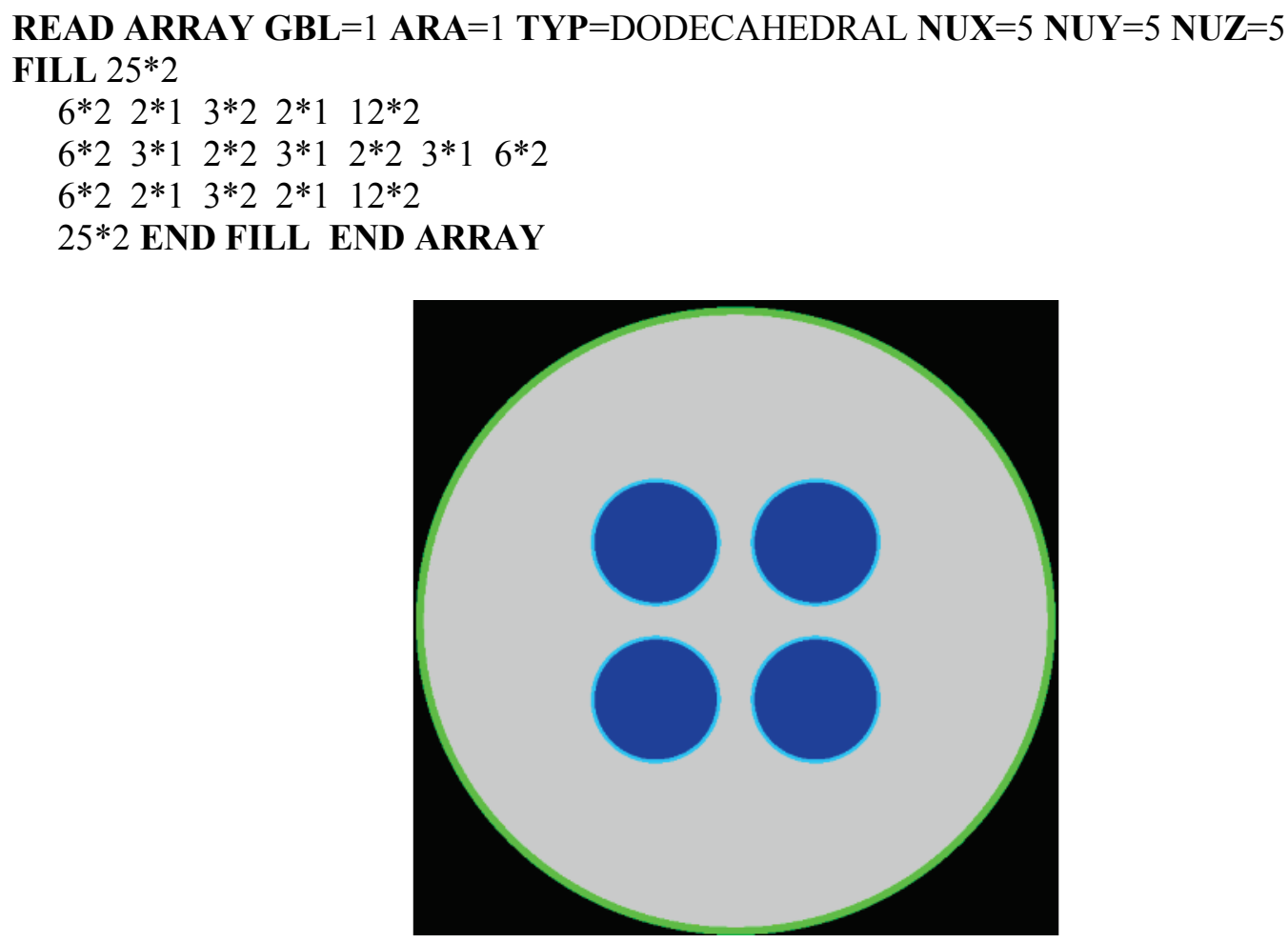

Fig. 8.1.63. $\mathrm{X}-\mathrm{Y}$ slice of dodecahedral array at even level $\mathrm{Z}=2$. 


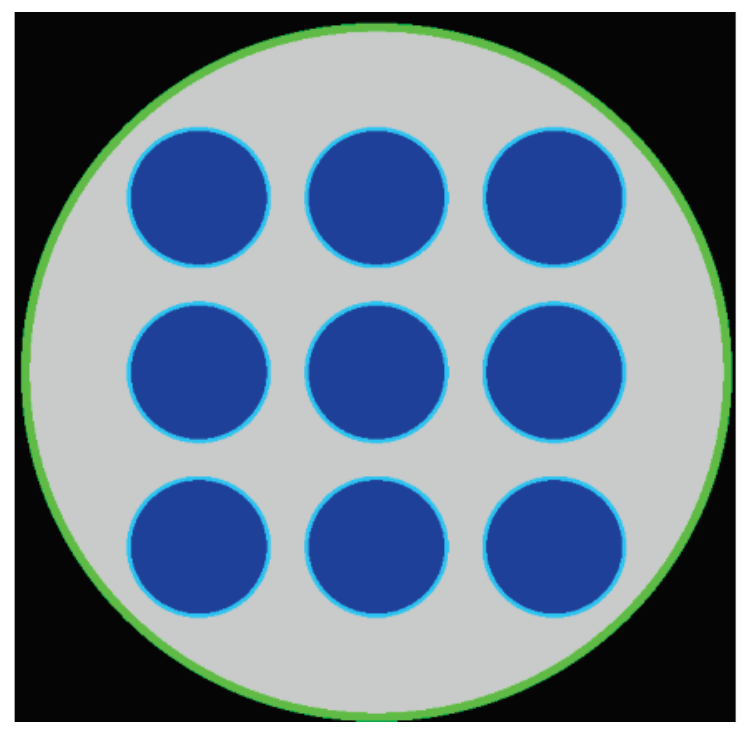

Fig. 8.1.64. $\mathrm{X}-\mathrm{Y}$ slice of dodecahedral array at odd level $\mathrm{Z}=3$.

The overall problem description is shown below. The cross section library would be generated in a separate CSAS-MG step.

Data description of Example 22.

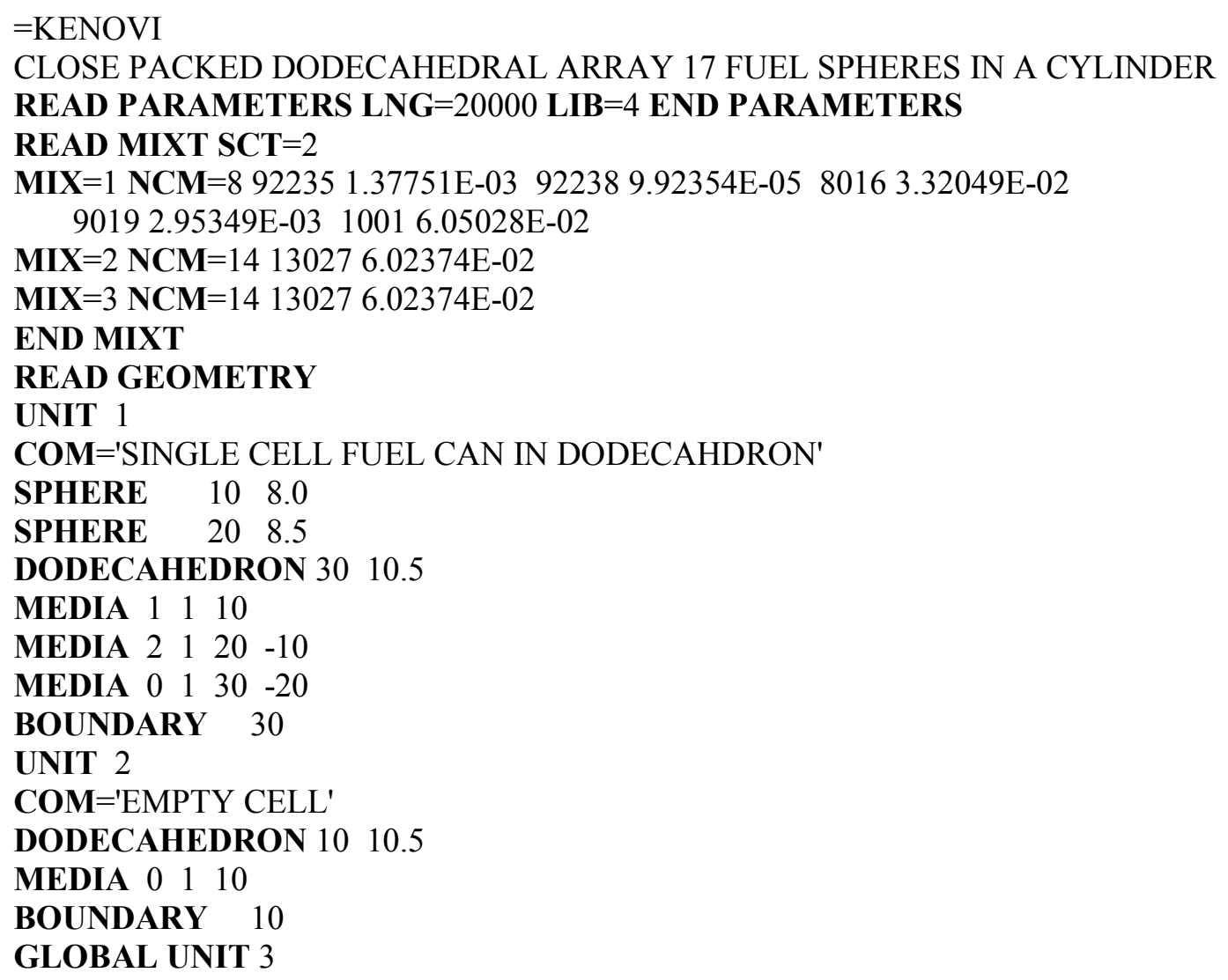


$\mathbf{C O M}=$ '9 CLOSE PACKED FUEL SPHERES IN A CYLINDER'

$\begin{array}{lllll}\text { CYLINDER } & 10 & 41.0 & 44.5 & 0.0\end{array}$

CYLINDER $2042.0 \quad 44.5-1.0$

ARRAY 110 PLACE $3313 * 0.0$

MEDIA $3 \quad 1 \quad 20 \quad-10$

BOUNDARY 20

END GEOMETRY

READ ARRAY GBL $=1$ ARA $=1$ TYP $=$ DODECAHEDRAL NUX $=5$ NUY $=5$ NUZ $=5$

FILL $25 * 2$

$\begin{array}{llllllll}6 * 2 & 2 * 1 & 3 * 2 & 2 * 1 & 12 * 2\end{array}$

$6 * 2 \quad 3 * 1 \quad 2 * 2 \quad 3 * 1 \quad 2 * 2 \quad 3 * 1 \quad 6 * 2$

$\begin{array}{llllll}6 * 2 & 2 * 1 & 3 * 2 & 2 * 1 & 12 * 2\end{array}$

$25 * 2$ END FILL END ARRAY

READ PLOT

TTL='DODECAHEDRAL ARRAY, 4 SPHERES - Z LEVEL $=2 '$

$\mathbf{X U L}=-43.0 \quad$ YUL $=43.0 \quad \mathbf{Z U L}=14.85 \quad \mathbf{X L R}=43.0 \quad \mathbf{Y L R}=-43.0 \quad \mathbf{Z L R}=14.85$

$\mathbf{U A X}=1$ VDN $=-1 \quad$ NAX $=640$ END PLT0

TTL='DODECAHEDRAL ARRAY, 9 SPHERES - Z LEVEL $=3$ '

$\mathbf{X U L}=-43.0 \quad$ YUL=43.0 ZUL=29.70 $\quad$ XLR=43.0 $\quad$ YLR=-43.0 $\quad \mathbf{Z L R}=29.70$

UAX $=1$ VDN $=-1$ NAX $=640$ END PLT1

END PLOT

END DATA

END

\subsubsection{Alternative sample problem mockups}

The geometry data for KENO can often be described correctly in several ways. Some alternative geometry descriptions are given here for sample problems C.12 and C.13. (See Appendix C of the KENO manual.)

\subsection{Sample Problem C.12, First Alternative}

This mockup maintains the same overall unit dimensions that were used in sample problem C.12. In sample problem C.12, the origin of UNIT 1, the solution cylinder, is at the center of the unit; the origin of UNITs 2, 3, 4, and 5, the metal cylinders, are at the center of the cylinders. In this mockup, the unit numbers remain the same and the origin of each unit is at the center of the unit. In each unit the cylinder is offset by specifying the position of its centerline relative to the origin of the UNIT.

KENO V.a:

\section{READ GEOM}

UNIT 1

CYLINDER $219.5258 .89-8.89$

CYLINDER $3110.169 .525-9.525$

CUBOID $\quad 0 \quad 110.875-10.87510 .875-10.87510 .24-10.24$

UNIT 2

CYLINDER 115.748 9.3975 -1.3975 ORIG 4.285 4.285 
CUBOID $\quad 0 \quad 1 \quad 10.875-10.87510 .875-10.87510 .24-10.24$

UNIT 3

CYLINDER 115.748 9.3975 -1.3675 ORIG 4.285 -4.285

CUBOID $\quad 0110.875-10.87510 .875-10.87510 .24-10.24$

UNIT 4

CYLINDER 115.748 1.3675 -9.3975 ORIG 4.285 4.285

CUBOID $\quad 0 \quad 1 \quad 10.875-10.87510 .875-10.87510 .24-10.24$

UNIT 5

CYLINDER 115.748 1.3675 -9.3975 ORIG 4.285 -4.285

CUBOID $\quad 0 \quad 1 \quad 10.875-10.87510 .875-10.875$ 10.24 -10.24

END GEOM

READ ARRAY NUX=2 NUY=2 NUZ=2 FILL 21314151 END ARRAY

KENO-VI:

\section{READ GEOM}

UNIT 1

CYLINDER $19.5258 .89-8.89$

CYLINDER $210.169 .525-9.525$

CUBOID $3 \quad 10.875-10.87510 .875-10.87510 .24-10.24$

MEDIA 211

MEDIA 3 1 12 -

MEDIA $0 \begin{array}{llll}0 & 1 & 3 & -2\end{array}$

BOUNDARY 3

UNIT 2

CYLINDER 15.748 9.3975 -1.3675 ORIG X=4.285 Y=4.285

CUBOID $210.875-10.87510 .875-10.87510 .24-10.24$

MEDIA 111

MEDIA $0 \begin{array}{llll} & 1 & 2 & -1\end{array}$

BOUNDARY 2

UNIT 3

CYLINDER 15.7489 .3975 -1.3675 ORIG X=4.285 Y=-4.285

CUBOID $210.875-10.87510 .875-10.87510 .24-10.24$

MEDIA 1111

MEDIA $0 \begin{array}{llll}0 & 1 & -1\end{array}$

BOUNDARY 2

UNIT 4

CYLINDER 15.748 1.3675 -9.3975 ORIG X=4.285 Y=4.285

CUBOID $210.875-10.87510 .875-10.87510 .24-10.24$

MEDIA 1111

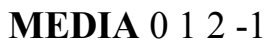

BOUNDARY 2

UNIT 5

CYLINDER 15.7481 .3675 -9.3975 ORIG X=4.285 Y=-4.285 
CUBOID $210.875-10.87510 .875-10.87510 .24-10.24$

MEDIA 1111

MEDIA 0012 - 1

BOUNDARY 2

GLOBAL UNIT 6

CUBOID $121.75-21.7521 .75-21.7520 .48-20.48$

ARRAY 1 PLACE $1111-10.875-10.875-10.24$

BOUNDARY 1

END GEOM

READ ARRAY ARA=1 NUX=2 NUY=2 NUZ=2 FILL 213144151 END ARRAY

\subsection{Sample Problem C.12, Second Alternative}

In this mockup, the outer boundaries of the system are made as close fitting as possible on all six faces.

The origin of each UNIT is located at the center of the cylinder. UNITs 1, 3, 5, and 7 contain the metal cylinders. UNITs 2, 4, 6, and 8 contain the solution cylinders.

KENO V.a:

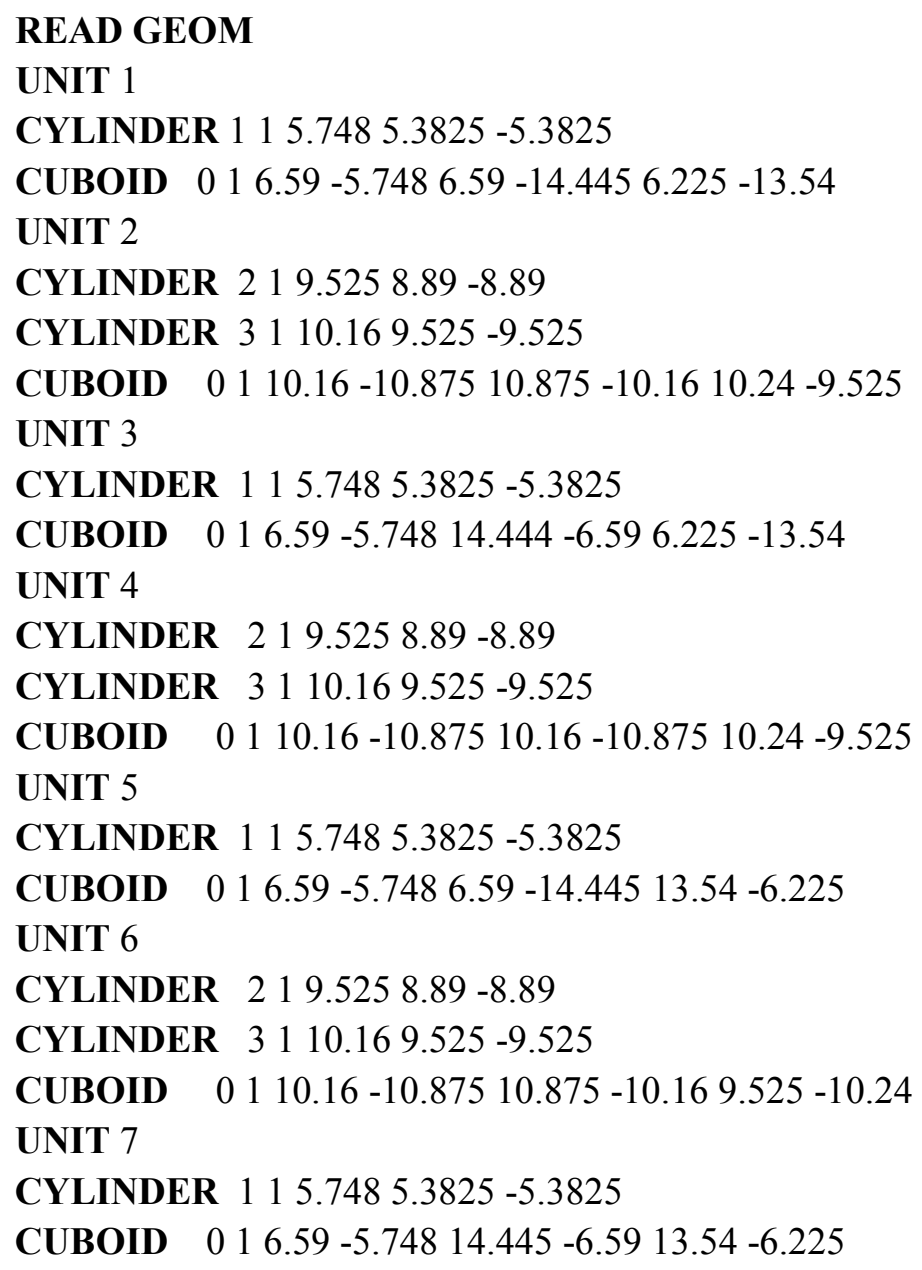


UNIT 8

CYLINDER $219.5258 .89-8.89$

CYLINDER $3110.169 .525-9.525$

CUBOID $\quad 0 \quad 1 \quad 10.16-10.87510 .16-10.8759 .525-10.24$

READ ARRAY NUX=2 NUY=2 NUZ=2 FILL 6I1 8 END FILL END ARRAY

KENO-VI:

READ GEOM

UNIT 1

CYLINDER $15.7485 .3825-5.3825$

CUBOID $26.59-5.7486 .59-14.4456 .225-13.54$

MEDIA 1111

MEDIA $0 \begin{array}{llll} & 1 & 2 & -1\end{array}$

BOUNDARY 2

UNIT 2

CYLINDER $19.5258 .89-8.89$

CYLINDER $210.169 .525-9.525$

CUBOID $3 \quad 10.16-10.87510 .875-10.1610 .24-9.525$

MEDIA 211

MEDIA 3 1 12 -

MEDIA $0 \begin{array}{lll}1 & 3 & -2\end{array}$

BOUNDARY 3

UNIT 3

CYLINDER $15.7485 .3825-5.3825$

CUBOID $2 \quad 6.59-5.748 \quad 14.444-6.596 .225-13.54$

MEDIA 1111

MEDIA $0 \begin{array}{lll}0 & 2 & -1\end{array}$

BOUNDARY 2

UNIT 4

CYLINDER $19.5258 .89-8.89$

CYLINDER $210.169 .525-9.525$

CUBOID $3 \quad 10.16-10.87510 .16-10.87510 .24-9.525$

MEDIA 211

MEDIA $3 \quad 1 \quad 2-1$

MEDIA $0 \begin{array}{llll}0 & 1 & -2 & -2\end{array}$

BOUNDARY 3

UNIT 5

CYLINDER $15.7485 .3825-5.3825$

$\begin{array}{lllllll}\text { CUBOID } 2 & 6.59 & -5.748 & 6.59 & -14.445 & 13.54 & -6.225\end{array}$

MEDIA 1111

MEDIA $0 \begin{array}{llll} & 1 & 2 & -1\end{array}$

BOUNDARY 2

UNIT 6 
CYLINDER $19.5258 .89-8.89$

CYLINDER $210.169 .525-9.525$

CUBOID $3 \quad 10.16-10.87510 .875-10.169 .525-10.24$

MEDIA 211

MEDIA 3 1 12 -

MEDIA $0 \begin{array}{llll}0 & 1 & 3 & -2\end{array}$

BOUNDARY 3

UNIT 7

CYLINDER $15.7485 .3825-5.3825$

CUBOID $2 \quad 6.59-5.748 \quad 14.445-6.5913 .54-6.225$

MEDIA 111 MEDIA 0112

BOUNDARY 2

UNIT 8

CYLINDER $19.5258 .89-8.89$

CYLINDER $210.169 .525-9.525$

CUBOID $3 \quad 10.16-10.87510 .16-10.8759 .525-10.24$

MEDIA 211

MEDIA $3 \quad 1 \quad 2 \quad-1$

MEDIA $0 \begin{array}{llllll} & 1 & 3 & -2\end{array}$

BOUNDARY 3

GLOBAL UNIT 9

CUBOID $120.035-12.74820 .67-20.6719 .765-19.765$

ARRAY 11 PLACE $1111-7.00-6.225-6.225$

BOUNDARY 1

END GEOM

READ ARRAY ARA=1 NUX=2 NUY=2 NUZ=2 FILL 6I 18 END FILL END ARRAY

\subsection{Sample Problem C.13, Alternative}

This mockup maintains the same overall UNIT dimensions that were used in sample problem C.13, (KENO Manual, Appendix C). In sample problem C.13, the origin of UNITs 1, 2, and 3 is located at the center of the base of the uranium metal cuboid. In this mockup, the origin of UNITs 1 and 2 is located at the center of the cylinder. In UNIT 3, the origin is at the center of the UNIT.

KENO V.a:

READ GEOM

UNIT 1

CUBOID $1110.2566-12.44346 .35-6.353 .81-3.81$

CYLINDER $0113.973 .81-3.81$

CYLINDER $1119.053 .81-3.81$

CUBOID $0119.05-19.0519 .05-19.053 .81-3.81$

UNIT 2

CUBOID $1112.4434-0.2566 \quad 6.35-6.354 .28-4.28$ 
CYLINDER $0113.974 .28-4.28$

CYLINDER $1119.054 .28-4.28$

CUBOID $0119.05-19.0519 .05-19.054 .28-4.28$

UNIT 3

CUBOID $1112.4434-0.25666 .35-6.351 .308-1.308$

CUBOID $0119.05-19.0519 .05-19.051 .308-1.308$

END GEOM

READ ARRAY NUX=1 NUY=1 NUZ=3 FILL 123 END ARRAY

KENO-VI:

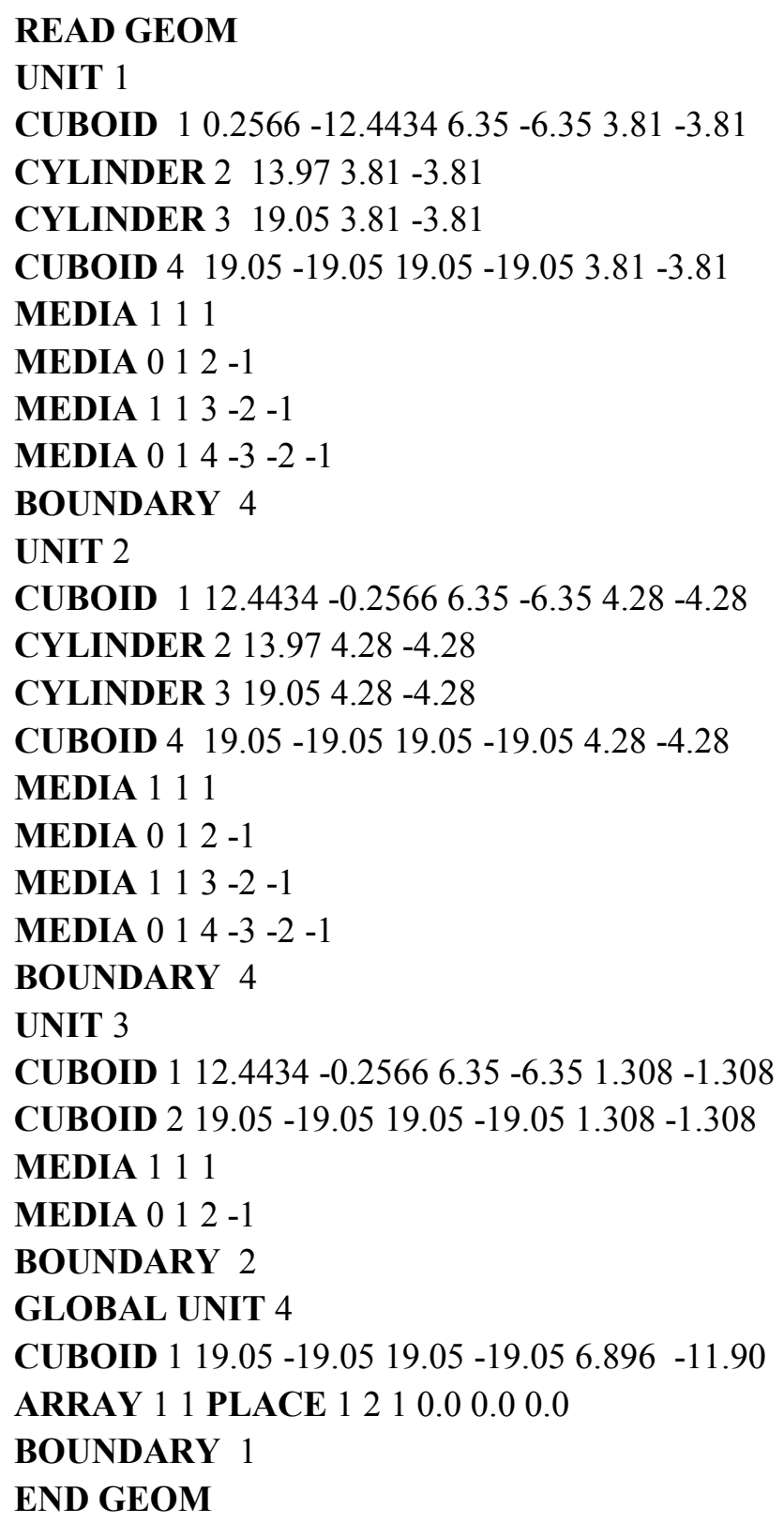




\subsubsection{Biasing or weighting data}

Section 8.1.6.3 discusses the basis of weighting or biasing. The use of biasing data in reflected problems has been illustrated in Examples 9, 10, and 11 of Sect. 8.1.3.6. Section 8.1.2.7 discusses the input directions for entering biasing data.

Every shape card in KENO V.a, or MEDIA card in KENO-VI requires a bias ID to associate that geometry region with a biasing or weighting function. A biasing or weighting function is a set of energydependent values of the average weight that are applicable in a given region. The default function for all bias IDs is constant through all energy groups and is defined to be the default value of weight average which can be specified in the parameter data. A bias ID can be associated with a biasing function (other than default) by specifying it in the biasing input data. This function can be chosen from the weighting library, or it can be entered from records. Table 8.1.20 lists the materials and energy group structures for biasing functions available from the weighting library.

In general, the use of biasing should be restricted to external reflectors unless the user has generated correct biasing functions for other applications. Improper use of biasing functions can result in erroneous answers without giving any indication that they are invalid. Caution should be exercised in the generation and use of biasing functions.

Biasing functions are most applicable to thick external reflectors. Their use can significantly reduce the amount of computer time required to obtain answers in KENO. If the user wishes to use a biasing function for a concrete reflector, for example, the following steps must be included in preparing the input data:

1. The geometry region data must define the shape and dimensions of the reflector using the mixture ID for concrete and a sequence of bias IDs that associate the geometry region with the appropriate interval of the concrete weighting function. CAUTION: THE THICKNESS AND SEQUENTIAL LOCATION OF EACH REGION USING BIASING FUNCTIONS MUST MATCH OR VERY NEARLY MATCH THE INCREMENT THICKNESS AND ORDER OF THE WEIGHTING DATA. NO CHECK IS MADE ON THE REQUIREMENT. IT IS THE USER'S RESPONSIBILITY TO ENSURE CONSISTENCY.

2. Biasing data must be entered. This must include the material ID for the reflector material (from Table 8.1.20 or as specified on records) and a beginning and ending bias ID. The beginning bias ID is used to select the first set of energy-dependent average weights, and the subsequent sets of energy-dependent average weights are assigned consecutive IDs until the ending bias ID is reached.

Small deviations in reflector region thickness are allowed, such as using three generated regions with a thickness per region of $5.08 \mathrm{~cm}$ to generate a $15.24 \mathrm{~cm}$ thick reflector of concrete, or using five generated regions with a thickness per region of $3.048 \mathrm{~cm}$ to generate a $15.24 \mathrm{~cm}$ thick reflector of water. See Table 8.1.20 for a list of the increment thicknesses for the materials in the weighting library. It is acceptable for the thickness of the last reflector region to be significantly different than the increment thickness. For example, a reflector record specifying five generated regions with a thickness per region of $3.0 \mathrm{~cm}$ could be followed by a reflector record specifying one region with a thickness per region of 
$0.24 \mathrm{~cm}$. Assuming that material 2 is water and a $15.24 \mathrm{~cm}$ thick cuboidal reflector of water is desired, the required reflector description and biasing data could be entered as follows:

KENO V.a:

REFLECTOR $226 * 3.05$

REFLECTOR $276 * 0.241$

READ BIAS ID=500 27 END BIAS

KENO-VI:

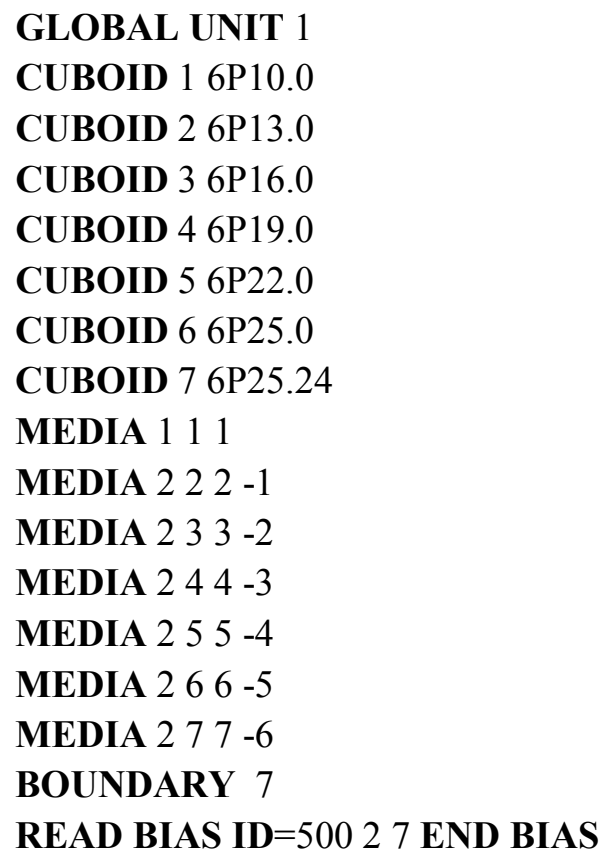

The same $15.24 \mathrm{~cm}$ thick reflector can be described by including the extra $0.24 \mathrm{~cm}$ in the last region as shown below:

KENO V.a:

REFLECTOR $226 * 3.04$

REFLECTOR 26 6*3.24 1

READ BIAS ID $=50026$ END BIAS

KENO-VI:

GLOBAL UNIT 1

CUBOID $16 \mathrm{P} 10.0$

CUBOID 2 6P13.0 
CUBOID 3 6P16.0

CUBOID 4 6P19.0

CUBOID 5 6P22.0

CUBOID 6 6P25.24

MEDIA 111

MEDIA 222

MEDIA 2

MEDIA 2 4 4 -

MEDIA 255

MEDIA 266 -5

BOUNDARY 6

READ BIAS ID=500 26 END BIAS

Here the weighting functions associated with bias IDs 2, 3, 4, and 5 each have a thickness of $3.0 \mathrm{~cm}$, corresponding exactly to the increment thickness for water in Table 8.1.20 Bias ID 6 is used for the last generated region which is $3.24 \mathrm{~cm}$ thick.

The following examples illustrate the use of biasing data. Suppose the user wishes to use the weighting function for water from Table 8.1.20 for bias IDs 2 through 6 . The biasing input data would then be:

\section{READ BIAS ID=500 26 END BIAS}

The energy-dependent values of weight average for the first $3 \mathrm{~cm}$ interval of water will be used for weighting the geometry regions that specify a bias ID of 2 . The energy-dependent values of weight average for the second $3 \mathrm{~cm}$ interval of water will be used for geometry regions that specify a bias ID of 3 , etc. Thus, the energy-dependent values of weight average for the fifth $3 \mathrm{~cm}$ interval of water will be used for geometry regions that specify a bias ID of 6 . Geometry regions that use bias IDs other than 2,3 , 4,5 , and 6 will use the default value of weight average that is constant for all energies as a biasing function.

Several sets of biasing data can be entered at once. Assume the user wishes to use the weighting function for concrete from Table 8.1.20 for bias IDs 2 through 4 and the weighting function for water for bias IDs 5 through 7 . The appropriate input data block is the following:

\section{READ BIAS ID=301 24 ID=500 57 END BIAS}

The energy-dependent values of weight average for the first $5 \mathrm{~cm}$ interval of concrete will be used for the geometry regions that specify a bias ID of 2, the energy-dependent values of weight average for the second $5 \mathrm{~cm}$ interval of concrete will be used for the geometry regions that specify a bias ID of 3 , and the energy-dependent values of weight average for the third $5 \mathrm{~cm}$ interval of concrete will be used for the geometry regions that specify a bias ID of 4 . The energy-dependent values of weight average for the first $3 \mathrm{~cm}$ interval of water will be used for geometry regions that specify a bias ID of 5, the values for the second $3 \mathrm{~cm}$ interval of water will be used for geometry regions that specify a bias ID of 6 , and the values for the third $3 \mathrm{~cm}$ interval of water will be used for geometry regions that specify a bias ID of 7 . The default value of weight average will be used for all bias IDs outside the range of 2-7. 
If the biasing data block defines the same bias ID more than once, the value that is entered last supersedes previous entries. Assuming that the following data block is entered,

\title{
READ BIAS ID=400 27 ID=500 57 END BIAS
}

the data for paraffin (ID=400) will be used for bias IDs 2, 3, and 4, and the data for water (ID=500) will be used for bias IDs 5, 6, and 7 .

\section{EXAMPLE 1. USE OF BIASING DATA}

It is assumed that a $5 \mathrm{~cm}$ radius sphere of material 2 is reflected by a $20 \mathrm{~cm}$ thickness of material 1 (concrete). The concrete reflector is spherical and close fitting upon the sphere of material 2. The mixing table must specify material 1 and material 2 . Material 1 must be defined as concrete. The geometry and biasing data should be entered as follows:

KENO V.a:

\section{READ GEOM}

SPHERE 215.0

REPLICATE 125.04

END GEOM

READ BIAS ID=3012 5 END BIAS

\section{KENO-VI:}

\author{
READ GEOM \\ GLOBAL UNIT 1 \\ SPHERE 15.0 \\ SPHERE 210.0 \\ SPHERE 315.0 \\ SPHERE 420.0 \\ SPHERE 525.0 \\ MEDIA 211

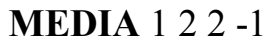 \\ MEDIA 13 H 3 - \\ MEDIA 14 4 4 - 3 \\ MEDIA 155 - 5 \\ BOUNDARY 5 \\ END GEOM \\ READ BIAS ID=301 25 END BIAS
}

The bias ID for the first generated region is 2 , the second is 3 , the third is 4 , and the fourth is 5 . The biasing data block specifies that the biasing function for material ID 301 (concrete) will be used from the weighting library. The bias ID to which the energy-dependent weighting function for the first $5.0 \mathrm{~cm}$ interval of concrete is applied is 2; the energy-dependent weighting function for the fourth $5 \mathrm{~cm}$ interval of concrete is applied to the fourth generated geometry region. This generated region has a bias ID of 5 . 
In KENO V.a, Example 1 can also be described without using a reflector record as shown below. The records that are generated by the reflector record in the previous set of data are identical to the last four spheres in this mockup.

EXAMPLE 1. Use of biasing without a reflector record.

KENO V.a:

\author{
READ GEOM \\ SPHERE 215.0 \\ SPHERE 1210.0 \\ SPHERE 1315.0 \\ SPHERE 1420.0 \\ SPHERE 1525.0 \\ END GEOM \\ READ BIAS ID=3012 5 END BIAS
}

\title{
8.1.3.9 Color plots
}

Plots are generated only if a plot data block has been entered for the problem and PLT $=$ NO has not been entered in the parameter data or the plot data. See Sect.8.1.2.11 for a description of plot data. When a plot is to be made, the user MUST correctly specify the upper left-hand corner of the plot with respect to the origin of the plot. The origin of a plot is defined as the origin of the GLOBAL UNIT.

Plots can represent mixture numbers, unit numbers, or bias ID numbers. A title can be entered for each plot. If plot titles are omitted, the title of the KENO case will be printed for each plot title until a plot title is entered. If a plot title is entered and a subsequent plot title is omitted, the last plot title prior to the omitted one will be used for the omitted one.

The upper left and lower right coordinates define the area (i.e., the slice and its location) for which the plot is to be made. The direction cosines across the plot and the direction cosines down the plot define the direction of the vector across the plot and the vector down the plot with respect to the geometry coordinate system. One of the simplest ways of generating a plot is to specify the desired coordinates of the upper left and lower right corners of the plot. Then one must determine which plot axis is to be across the plot and which is to be down. The sign of the direction cosine should be consistent with the direction of that component when moving from the upper left to lower right corner. For example, to draw a plot of an $\mathrm{X}-\mathrm{Z}$ slice at $\mathrm{Y}=5.0$ with $\mathrm{X}$ across the plot and $\mathrm{Z}$ down the plot for a system whose $\mathrm{X}$ coordinates ranges from 0.0 to 10.0 and whose $Z$ coordinates range from 0.0 to 20.0, the upper left coordinate could be $\mathbf{X U L}=0.0 \quad \mathbf{Y U L}=5.0 \quad \mathbf{Z U L}=20.0$ and the lower right coordinates could be $\mathbf{X L R}=10.0 \quad \mathbf{Y L R}=5.0$ $\mathbf{Z L R}=0.0$. Since $X$ is to be plotted across the plot with $X=0.0$ at the left and $X=10.0$ at the right, only the X component of the direction cosines across the plot need be entered. It should be positive because going from 0.0 to 10.0 is moving in the positive direction. Thus, $\mathbf{U A X}=1.0$ would be entered for the direction cosines across the plot. VAX and WAX could be omitted. $Z$ is to be plotted down the plot with $\mathrm{Z}=20.0$ at the top and $\mathrm{Z}=0.0$ at the bottom. Therefore, only the $\mathrm{Z}$ component of the direction cosines 
down the plot needs to be defined. It should be negative because moving from 20.0 to 0.0 is moving in the negative direction. Thus, WDN $=-1.0$ would be entered for the direction cosines down the plot. UDN and VDN could be omitted. The sign of the direction cosines should be consistent with the coordinates of the upper left and lower right corners in order to get a plot.

It is not necessary that the plot be made for a slice orthogonal to one of the axes. Plots can be made of slices cut at any desired angle, but the user should exercise caution and be well aware of the distortion of shapes that can be introduced. (Nonorthogonal slices through cylinders plot as ellipses.)

The user can specify the horizontal and vertical spacing between points on the plot. It is usually advisable to enter one or the other. Entering both can cause distortion of the plot. DLX= is used to specify the horizontal spacing between points and $\mathbf{D L D}=$ is used to specify the vertical spacing between points. When only one of them is specified, the code calculates the correct value of the other so the plot will not be distorted. DLX or DLD can be specified by the user to be small enough to show the desirable detail in the plot. The plot is generated by starting at the upper left corner of the plot and generating a point every DLX across the plot; then moving down DLD and repeating the generation of the points across the plot.

NAX specifies the number of intervals (pixels) that will be printed across the plot. NDN specifies the number of intervals (pixels) that will be printed down the plot. If both NAX and NDN are entered, the plot may be distorted. If one of them is entered, the value of the other will be calculated so the plot will not be distorted.

When a plot is being made, the first pixel represents the coordinates of the upper left corner. The value of DELV is added to the coordinate that is to be printed across the plot, and the next pixel is printed. DELV is added to that value to determine the location of the next pixel, that is, a point is determined every DELV across the plot and a pixel is printed for each point. When a line has been completed, a new line is begun DELU from the first line. This procedure is repeated until the plot is complete.

\section{EXAMPLE 1. SINGLE UNIT WITH CENTERED ORIGIN}

Consider two concentric cylinders in a cuboid. The inner cylinder is $5.2 \mathrm{~cm}$ in diameter. The outer cylinder has an inside diameter of $7.2 \mathrm{~cm}$ and an outside diameter of $7.6 \mathrm{~cm}$. Both cylinders are $30 \mathrm{~cm}$ high. They are contained in a tight-fitting box with a wall thickness of $0.5 \mathrm{~cm}$ and top and bottom thickness of $1.0 \mathrm{~cm}$. The inner cylinder is composed of mixture 1, the outer cylinder is made of mixture 4 , and the box is made of mixture 2 . The problem can be described with its origin at the center of the inner cylinder. The problem description for this arrangement is shown below:

KENO V.a:

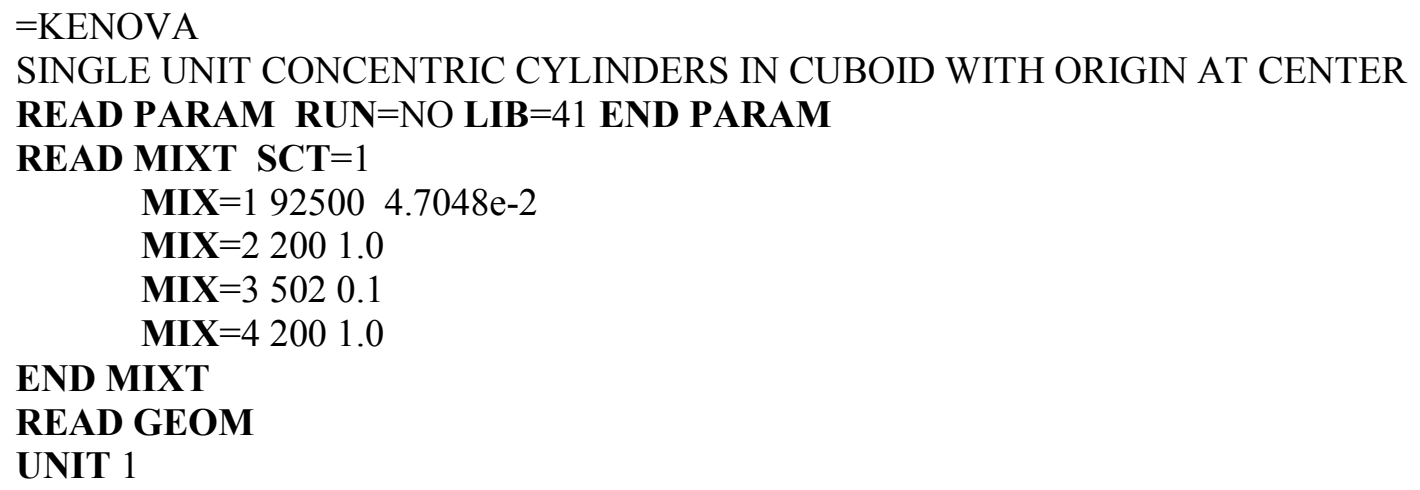


CYLINDER 112.6 2P15.0

CYLINDER 013.6 2P15.0

CYLINDER 413.8 2P15.0

CUBOID 014 P 3.8 2P15.0

CUBOID $214 \mathrm{P} 4.32 \mathrm{P} 16.0$

END GEOM

READ PLOT

TTL $=$ 'X-Y SLICE AT Z MIDPOINT. SINGLE UNIT CONCENTRIC CYLS'

$\mathbf{X U L}=-4.6 \quad \mathrm{YUL}=4.6 \quad \mathrm{ZUL}=0.0 \quad \mathrm{XLR}=4.6 \quad \mathrm{YLR}=-4.6 \quad \mathbf{Z L R}=0.0$

$\mathbf{U A X}=1.0 \quad$ VDN $=-1.0 \quad$ NAX $=640 \quad$ END

PIC $=$ UNIT END

END PLOT

END DATA

END

KENO-VI:

$=$ KENOVI

SINGLE UNIT CONCENTRIC CYLINDERS IN CUBOID WITH ORIGIN AT CENTER

READ PARAM RUN=NO LIB $=41$ TME $=0.5$ END PARAM

READ MIXT SCT=1 MIX=1 92500 4.7048-2 MIX=2 2001.0 MIX=3 5020.1

MIX=4 2001.0

END MIXT

READ GEOM

GLOBAL UNIT 1

CYLINDER 12.6 2P15.0

CYLINDER 23.6 2P15.0

CYLINDER 33.8 2P15.0

CUBOID 4 4P3.8 2P15.0

CUBOID 5 4P4.3 2P16.0

MEDIA 1111

MEDIA $0 \begin{array}{llll}0 & 1 & 2 & -1\end{array}$

MEDIA 4 1 13 -

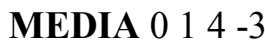

MEDIA $2 \quad 1 \quad 5 \quad-4$

BOUNDARY 5

END GEOM

\section{READ PLOT}

TTL $=$ 'X-Y SLICE AT Z MIDPOINT. SINGLE UNIT CONCENTRIC CYLS.'

$\mathbf{X U L}=-4.6 \quad \mathbf{Y U L}=4.6 \quad \mathbf{Z U L}=0.0 \quad \mathbf{X L R}=4.6 \quad \mathbf{Y L R}=-4.6 \quad \mathbf{Z L R}=0.0$

$\mathbf{U A X}=1.0$ VDN $=-1.0 \quad$ NAX $=640$ END

PIC $=$ UNIT END

END PLOT

END DATA

END 
The plot data blocks included above are set up to draw a mixture map of an X-Y slice taken at the half height $(Z=0.0)$ and a unit map for the same slice. In the above examples, the geometry dimensions extend from $X=-4.3$ to $X=4.3$, from $Y=-4.3$, to $Y=4.3$, and from $Z=-16.0$ to $Z=16.0$. An $X-Y$ slice is be printed at the half height $(Z=0.0)$. The desired plot data sets the upper left-hand corner of the plot to be $\mathrm{X}=-4.6$ and $\mathrm{Y}=4.6$. The lower right-hand corner of the plot is specified as $\mathrm{X}=4.6$ and $\mathrm{Y}=-4.6$. These data are entered by specifying the upper left-hand corner as $\mathbf{X U L}=-4.6 \mathbf{Y U L}=4.6 \quad \mathbf{Z U L}=0.0$ and the lower right-hand corner as $\mathbf{X L R}=4.6 \mathbf{Y L R}=-4.6 \mathbf{Z L R}=0.0$. It is desired to print $\mathrm{X}$ across the plot and $\mathrm{Y}$ down the plot. Therefore, the $\mathrm{X}$ direction cosine is specified across the plot, in the direction from $\mathrm{X}=-4.6$ to $\mathrm{X}=4.6$ as $\mathbf{U A X}=1.0$. The $\mathrm{Y}$ direction cosine is specified down the plot, from $\mathrm{Y}=4.6$ to $\mathrm{Y}=-4.6$ as $\mathrm{VDN}=-1.0$.

A black border will be printed for points outside the range of the problem geometry description. By setting the plot dimension slightly larger than the geometry dimension, a black border will be printed around the specified plot. This verifies that the outer boundaries of the geometry are contained within the plot dimensions. NAX is the number of pixels across for a color plot. An initial recommended range for NAX is between 600 and 800 pixels. The resulting plots are shown in Fig. 8.1.65 and Fig. 8.1.66. The associated data for both plots are shown in Fig. 8.1.67 and Fig. 8.1.68.

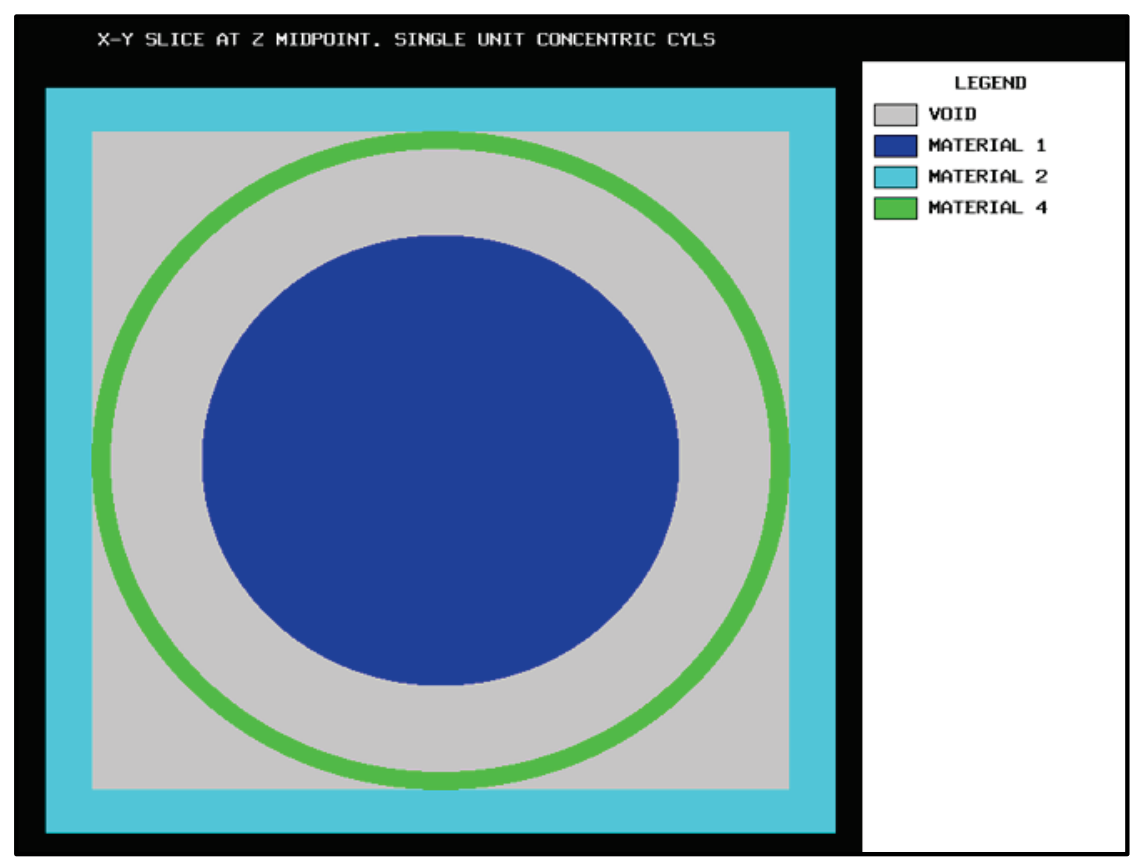

Fig. 8.1.65. Mixture color plot for single unit with centered origin. 


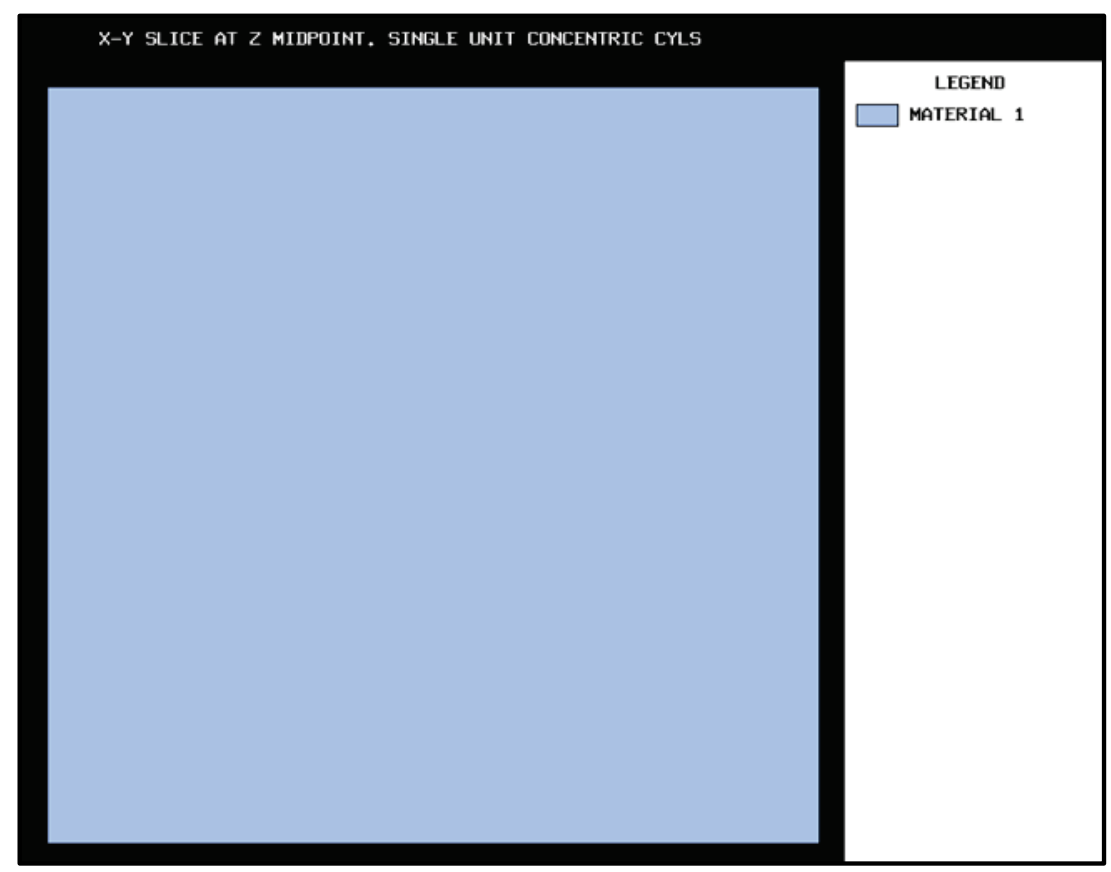

Fig. 8.1.66. Unit map color plot for single unit with centered origin.

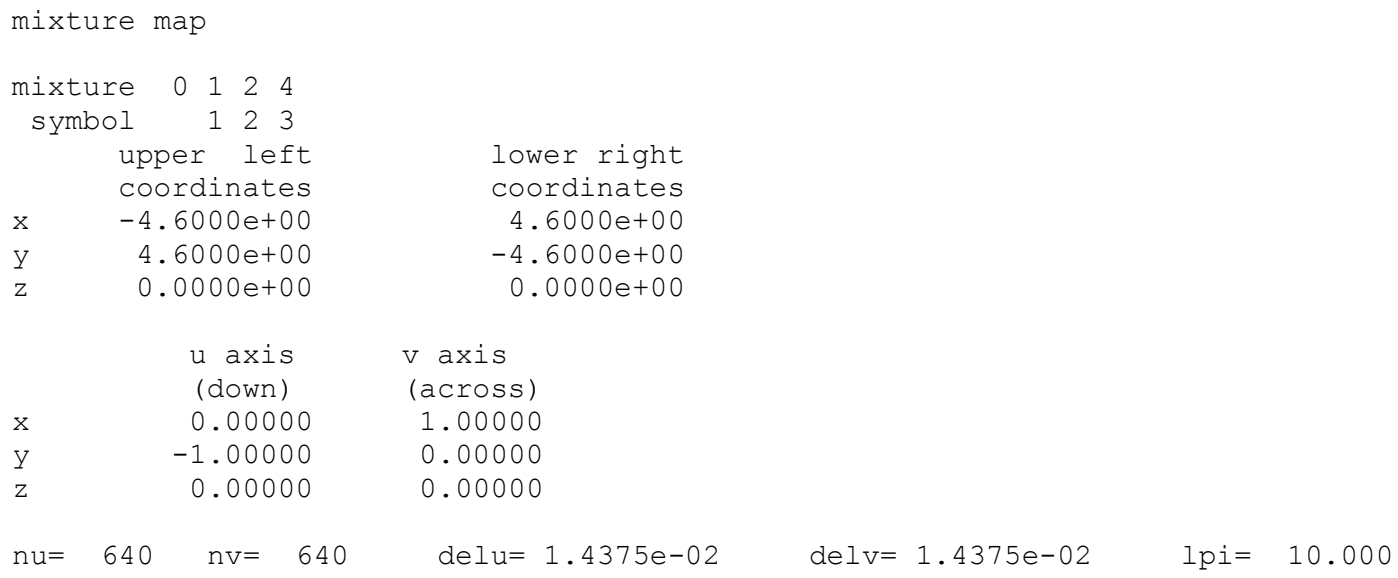

Fig. 8.1.67. Associated data for single unit mixture map. 


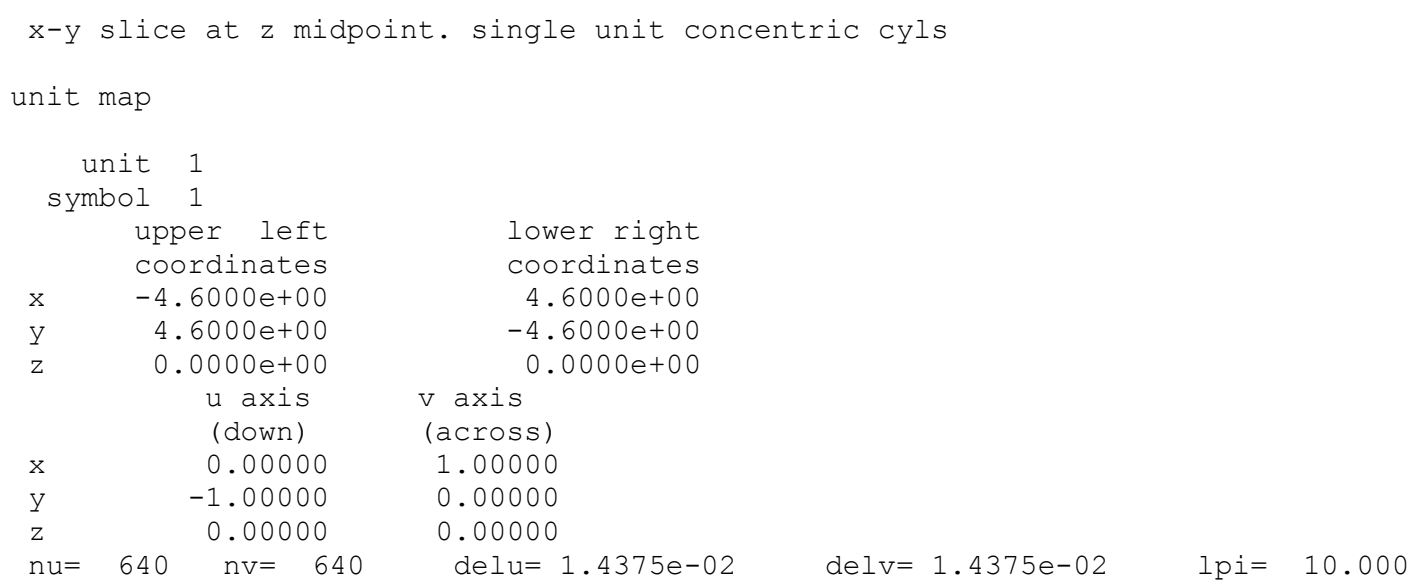

Fig. 8.1.68. Associated data for single unit map.

\section{EXAMPLE 2. SINGLE UNIT WITH OFFSET ORIGIN}

The physical problem is the same as that described in Example 1: two concentric cylinders in a cuboid. The dimensions are exactly the same, and the difference is in the choice of the origin. In this geometry description, the origin was specified as the most negative point of the unit. Thus, the cylinders must have an origin specified to center them in the cuboid, and the cuboid extends from 0.0 to 8.6 in $\mathrm{X}$ and $\mathrm{Y}$ and from 0.0 to 32 in $\mathrm{Z}$ as shown in the problem description below.

KENO V.a:

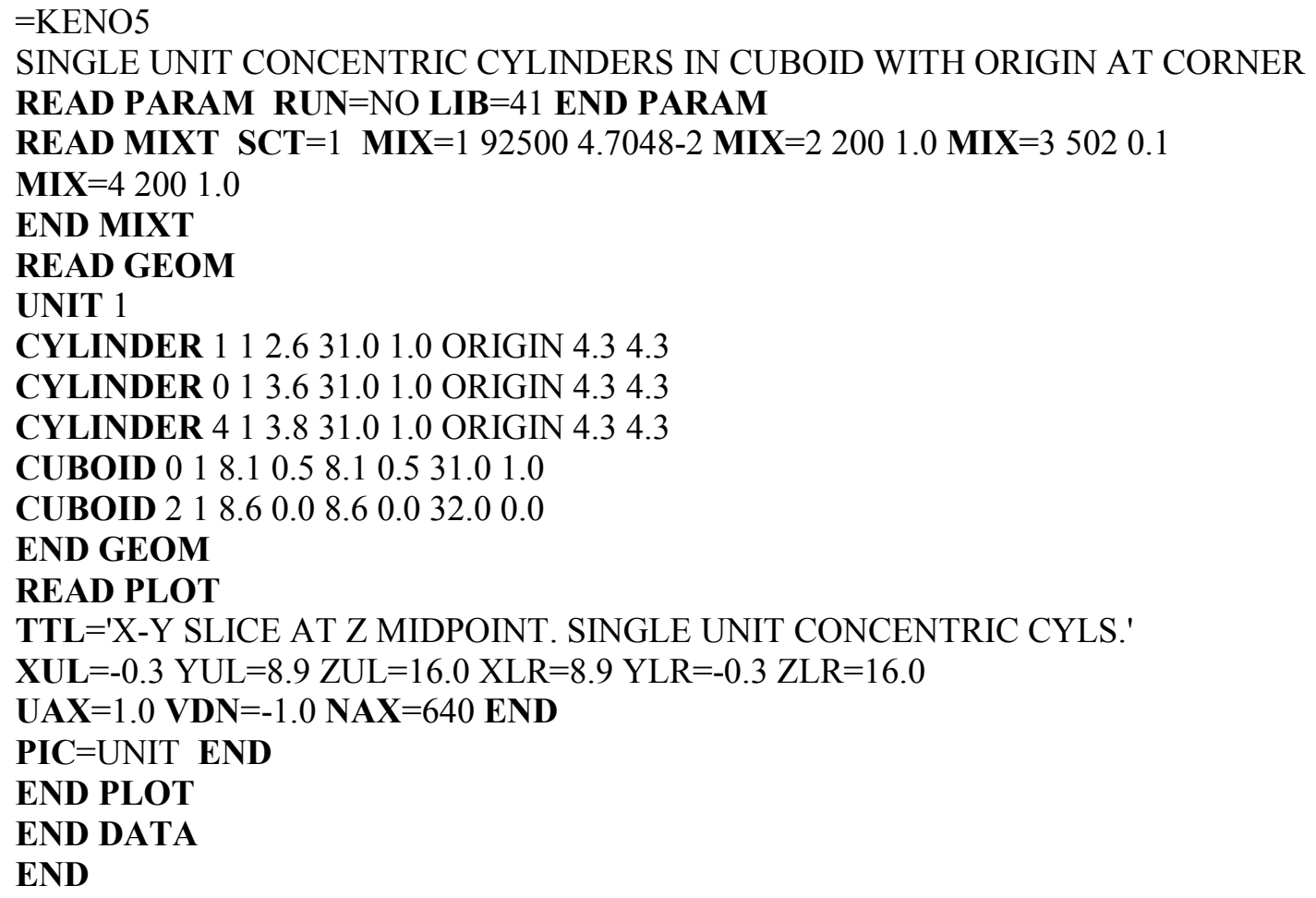


KENO-VI:

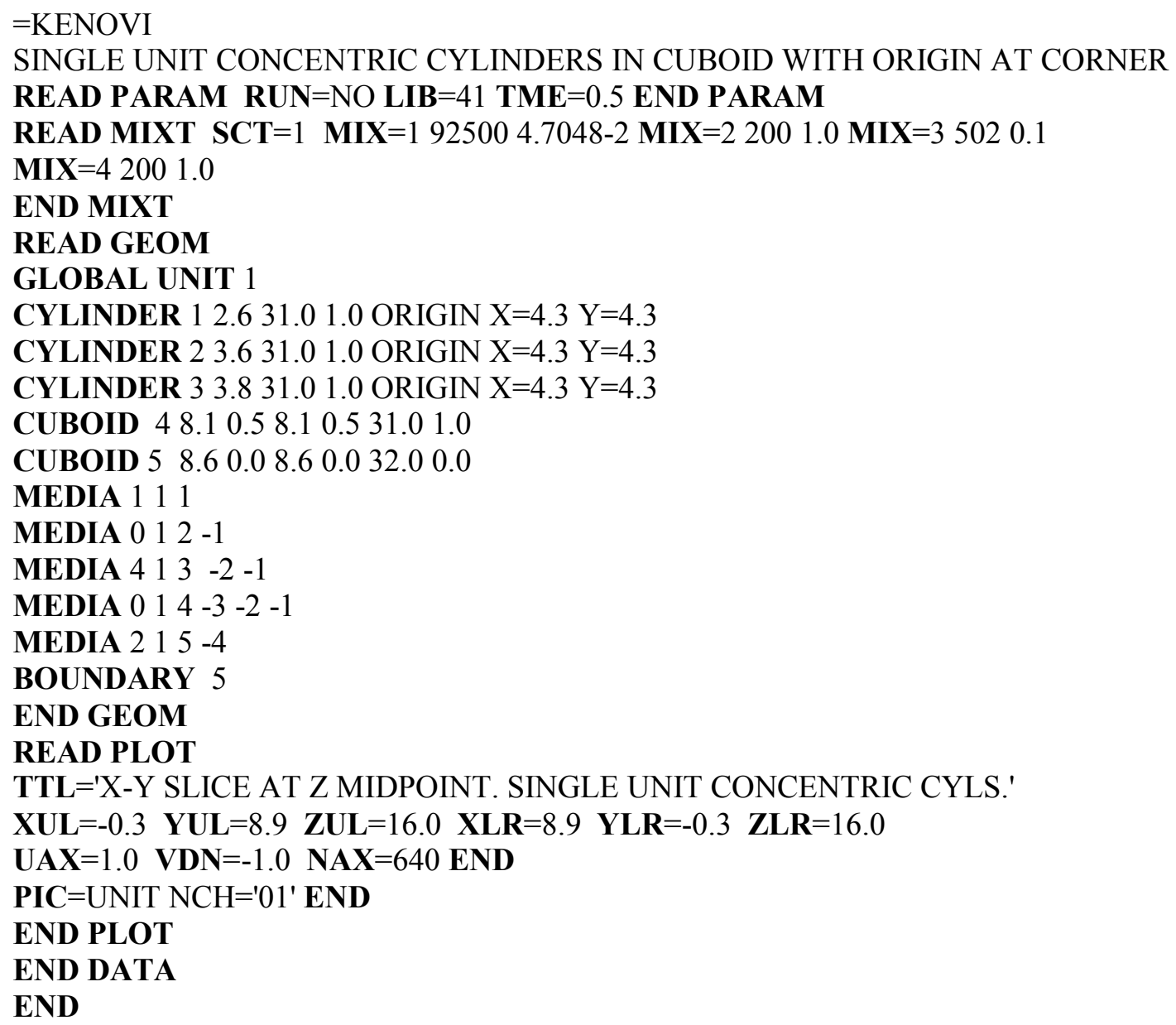

The plot data included above will draw a mixture map of an $X-Y$ slice taken at the half height $(Z=16.0)$. It will also draw a unit map of the same slice. The plot dimensions extend $0.3 \mathrm{~cm}$ beyond the problem dimensions to provide a black border around the plot. The associated plot data specification for the mixture map is shown in Fig. 8.1.69, the mixture map is shown in Fig. 8.1.70, and the associated plot data for the unit map is shown in Fig. 8.1.71. The unit map is identical to Fig. 8.1.66 and is not included. 
x-y slice at $z$ midpoint. single unit concentric cyls.

mixture map

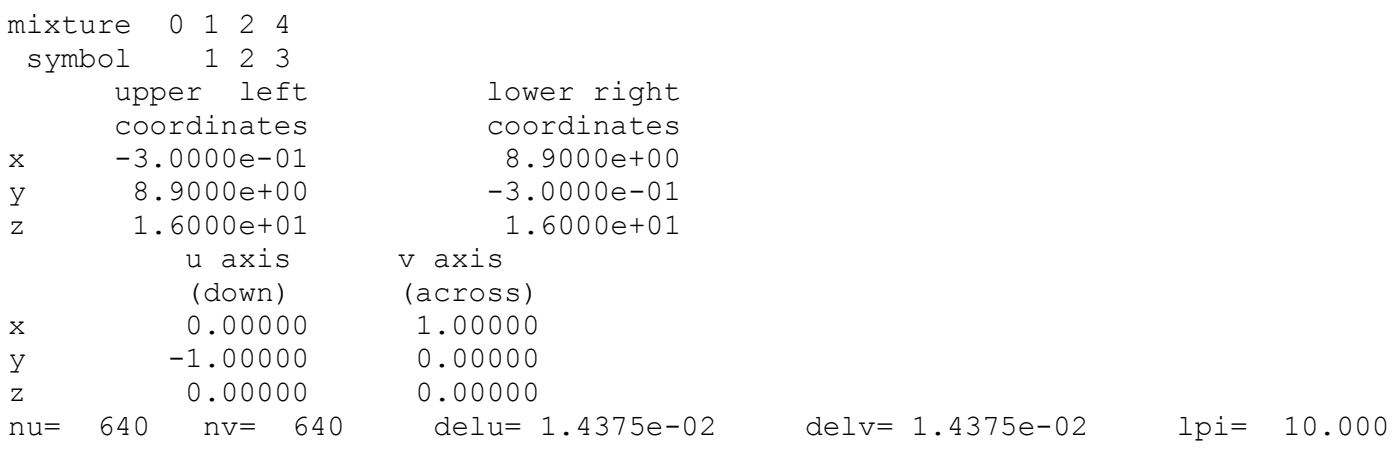

Fig. 8.1.69. Associated data for mixture map of single unit with offset origin.

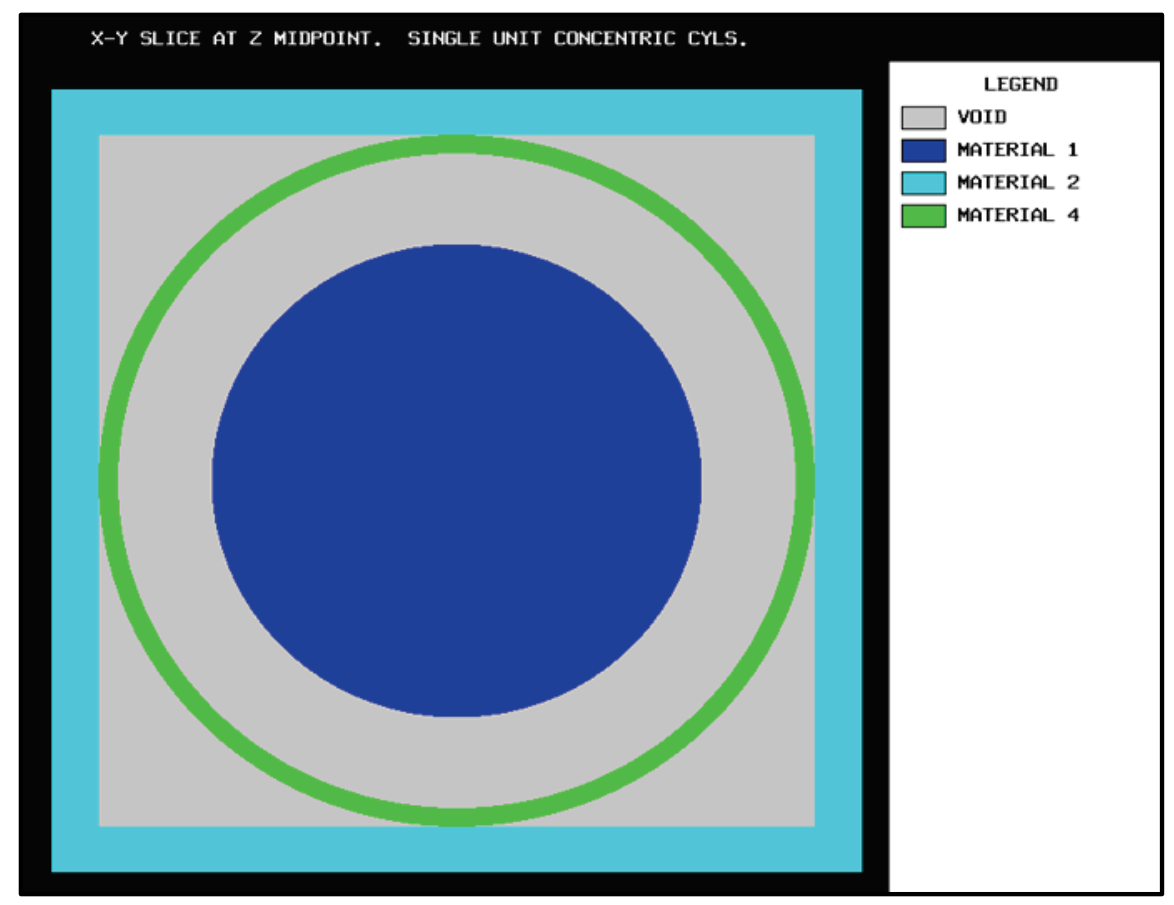

Fig. 8.1.70. Mixture map of single unit with offset origin. 


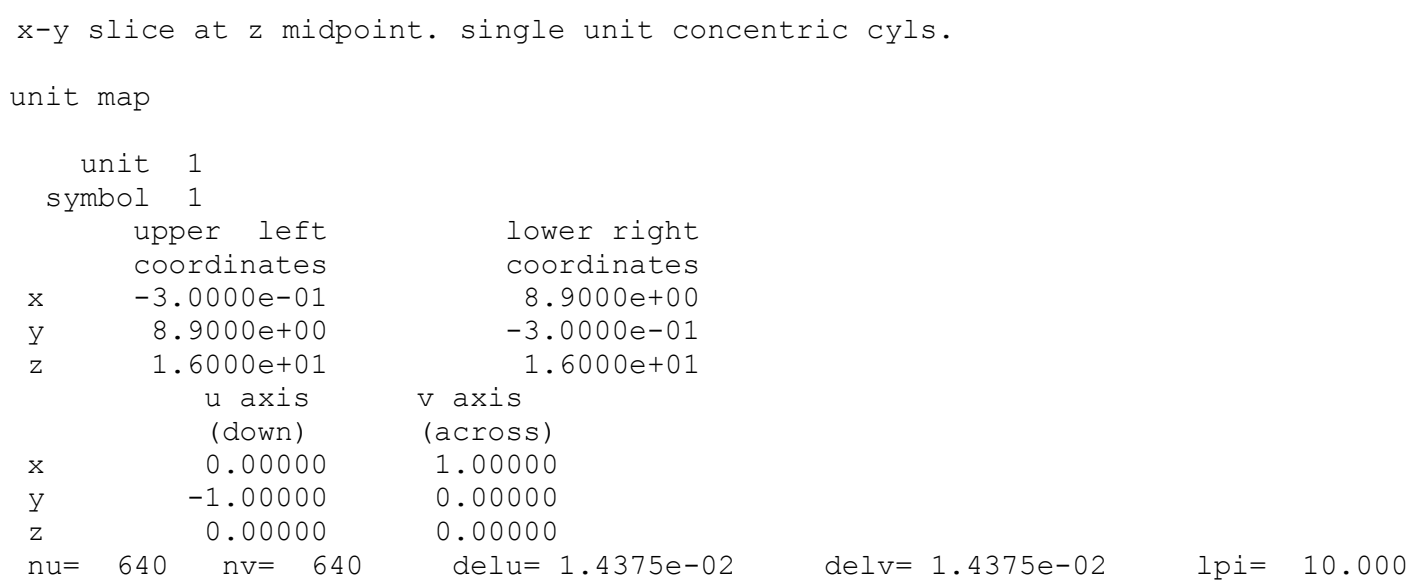

Fig. 8.1.71. Associated data for unit map of single unit with offset origin.

EXAMPLE 3. A $2 \times 2 \times 2$ UNREFLECTED ARRAY OF CONCENTRIC CYLINDERS IN CUBOIDS

The physical representation of this example is a $2 \times 2 \times 2$ array of the configuration described in Example 1 of this section. The input data description for this array is given below:

KENO V.a:

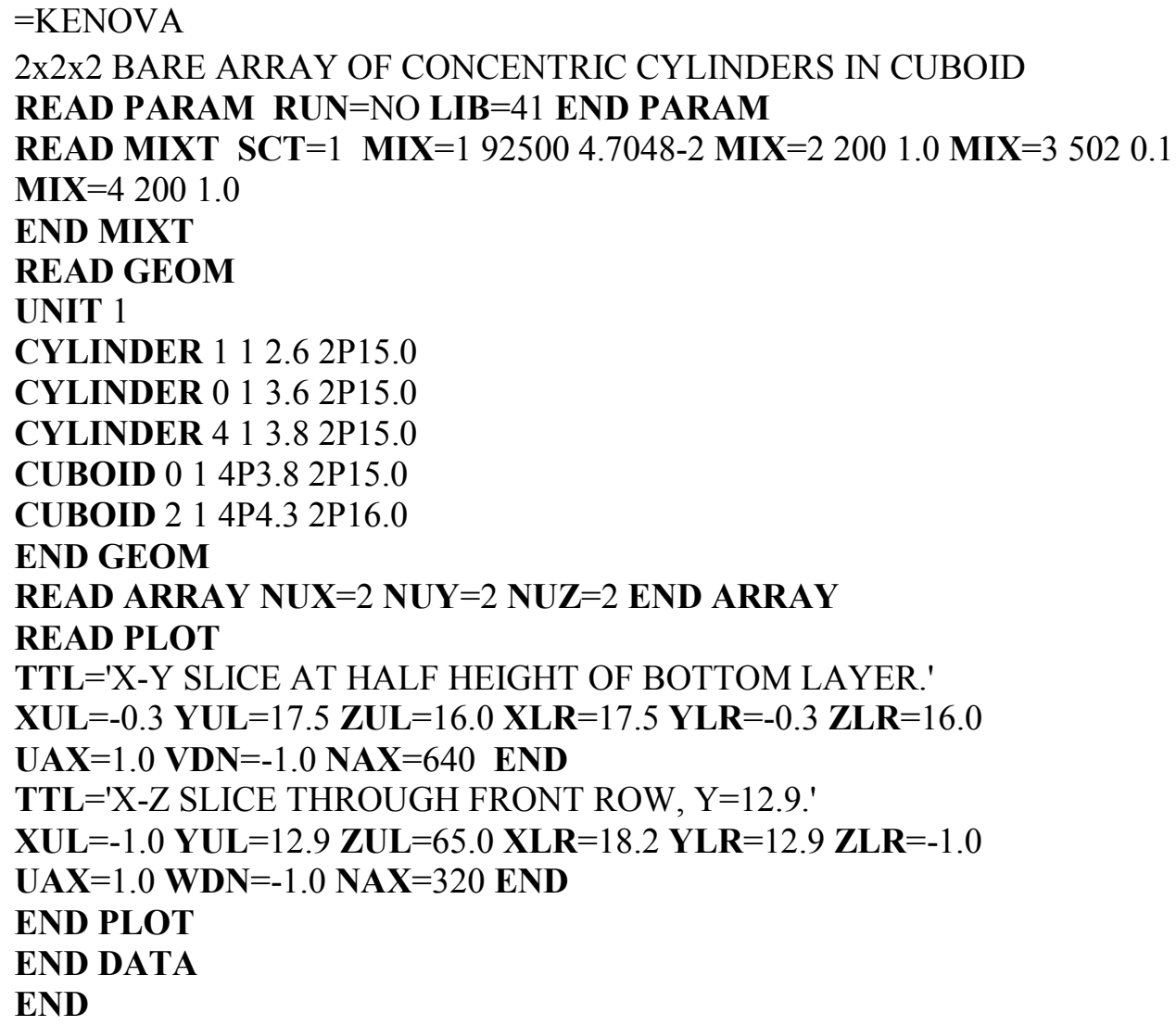


KENO-VI:

$=$ KENOVI

$2 \times 2 \times 2$ BARE ARRAY OF CONCENTRIC CYLINDERS IN CUBOID

READ PARAM RUN=NO LIB $=41$ TME $=8.5$ END PARAM

READ MIXT SCT=1 MIX=1 92500 4.7048-2 MIX=2 2001.0 MIX=3 5020.1

MIX=4 2001.0

END MIXT

READ GEOM

UNIT 1

CYLINDER 12.6 2P15.0

CYLINDER 23.6 2P15.0

CYLINDER 33.8 2P15.0

CUBOID 4 4P3.8 2P15.0

CUBOID 5 4P4.3 2P16.0

MEDIA 111

MEDIA 0012

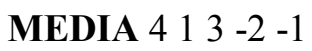

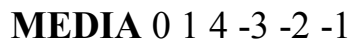

MEDIA 2 2 $1-5$ - 5 - 4

BOUNDARY 5

GLOBAL UNIT 2

CUBOID 117.20 .017 .20 .064 .00 .0

ARRAY 11 PLACE 1114.34 .316 .0

BOUNDARY 1

END GEOM

READ ARRAY ARA $=1$ NUX=2 NUY=2 NUZ $=2$ FILL F1 END FILL END ARRAY

READ PLOT

TTL $=$ 'X-Y SLICE AT HALF HEIGHT OF BOTTOM LAYER.'

$\mathbf{X U L}=-0.3 \mathbf{Y U L}=17.5 \mathbf{Z U L}=16.0 \mathbf{X L R}=17.5 \quad \mathrm{YLR}=-0.3 \mathbf{Z L R}=16.0$

$\mathbf{U A X}=1.0 \mathrm{VDN}=-1.0 \mathrm{NAX}=640 \mathbf{E N D}$

TTL $=$ 'X-Z SLICE THROUGH FRONT ROW, Y=12.9.'

$\mathbf{X U L}=-1.0 \quad \mathbf{Y U L}=12.9 \mathbf{Z U L}=65.0 \mathbf{X L R}=18.2 \mathbf{Y L R}=12.9 \mathbf{Z L R}=-1.0$

$\mathbf{U A X}=1.0 \mathbf{W D N}=-1.0 \mathrm{NAX}=320 \mathbf{E N D}$

END PLOT

END DATA

END 
As stated at the beginning of Sect. 8.1.3.9, the origin of a plot is defined as the origin of the GLOBAL UNIT. Each individual unit in the array is $8.6 \mathrm{~cm}$ wide in $X$ and $Y$ and $32 \mathrm{~cm}$ high in Z. Since the array has two units stacked in each direction, the array is $17.2 \mathrm{~cm}$ wide in $\mathrm{X}$ and $\mathrm{Y}$ and is $64 \mathrm{~cm}$ high. Therefore, the array exists from $\mathrm{X}=0.0$ to $\mathrm{X}=17.2$, from $\mathrm{Y}=0.0$ to $\mathrm{Y}=17.2$ and from $\mathrm{Z}=0.0$ to $\mathrm{Z}=64.0$.

The first color plot is to generate an X-Y slice through the array at the half height $(Z=16.0 \mathrm{~cm})$ of the first layer as shown in Fig. 8.1.72. It is desirable to create an image with a larger extent than the global unit to ensure that the boundaries are as expected. This is achieved by setting the boundaries of the plot larger than the array. In this case, the boundaries were arbitrarily set $0.3 \mathrm{~cm}$ larger than the array, resulting in a black border around the array. If the plot were to exclude everything external to the array, the following coordinates could have been entered: $\mathbf{X U L}=0.0 \quad \mathbf{Y U L}=17.2 \mathrm{ZUL}=16.0 \mathrm{XLR}=17.2 \mathrm{YLR}=0.0$ $\mathbf{Z L R}=16.0$. This would have eliminated the black border. The existing plot was made using $\mathbf{X U L}=-0.3$ $\mathbf{Y U L}=17.5 \mathbf{Z U L}=16.0 \mathrm{XLR}=17.5 \mathbf{Y L R}=-0.3 \mathbf{Z L R}=16.0$.

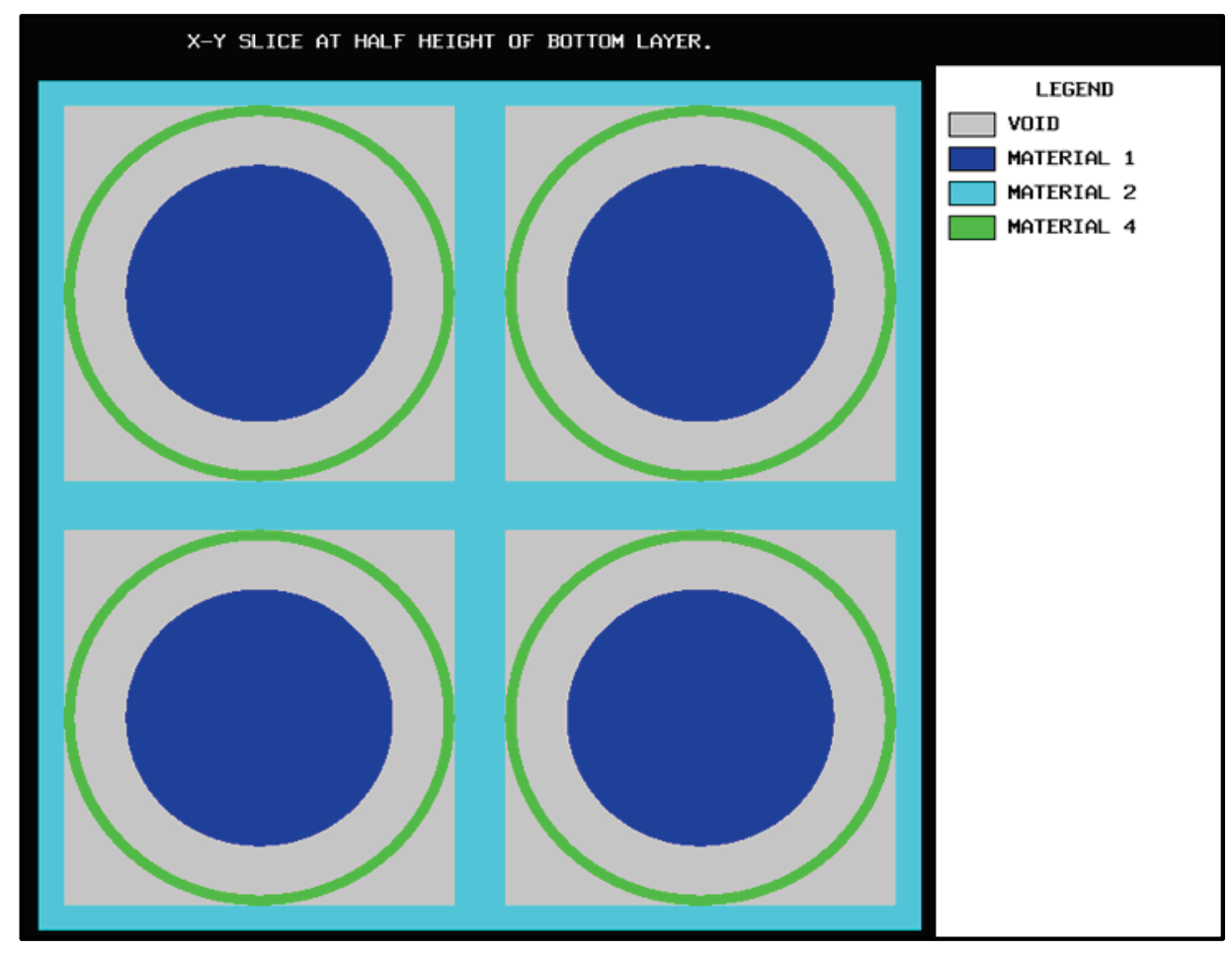

Fig. 8.1.72. X-Y plot of $2 \times 2 \times 2$ bare array. 
The second color plot is to generate an X-Z slice through the center of the front row of the array. In order to obtain a black border, the coordinates of $\mathrm{X}$ and $\mathrm{Z}$ were arbitrarily set $1.0 \mathrm{~cm}$ larger than the boundaries of the array. The center of the front row occurs at $\mathrm{Y}=12.9$. The coordinates of the plot were: $\mathbf{X U L}=-1.0$ $\mathbf{Z U L}=65.0 \quad \mathbf{Y U L}=12.9 \quad \mathbf{X L R}=18.2 \quad \mathbf{Z L R}=-1.0 \quad \mathbf{Y L R}=12.9$. The resulting mixture map is shown in Fig. 8.1.73.

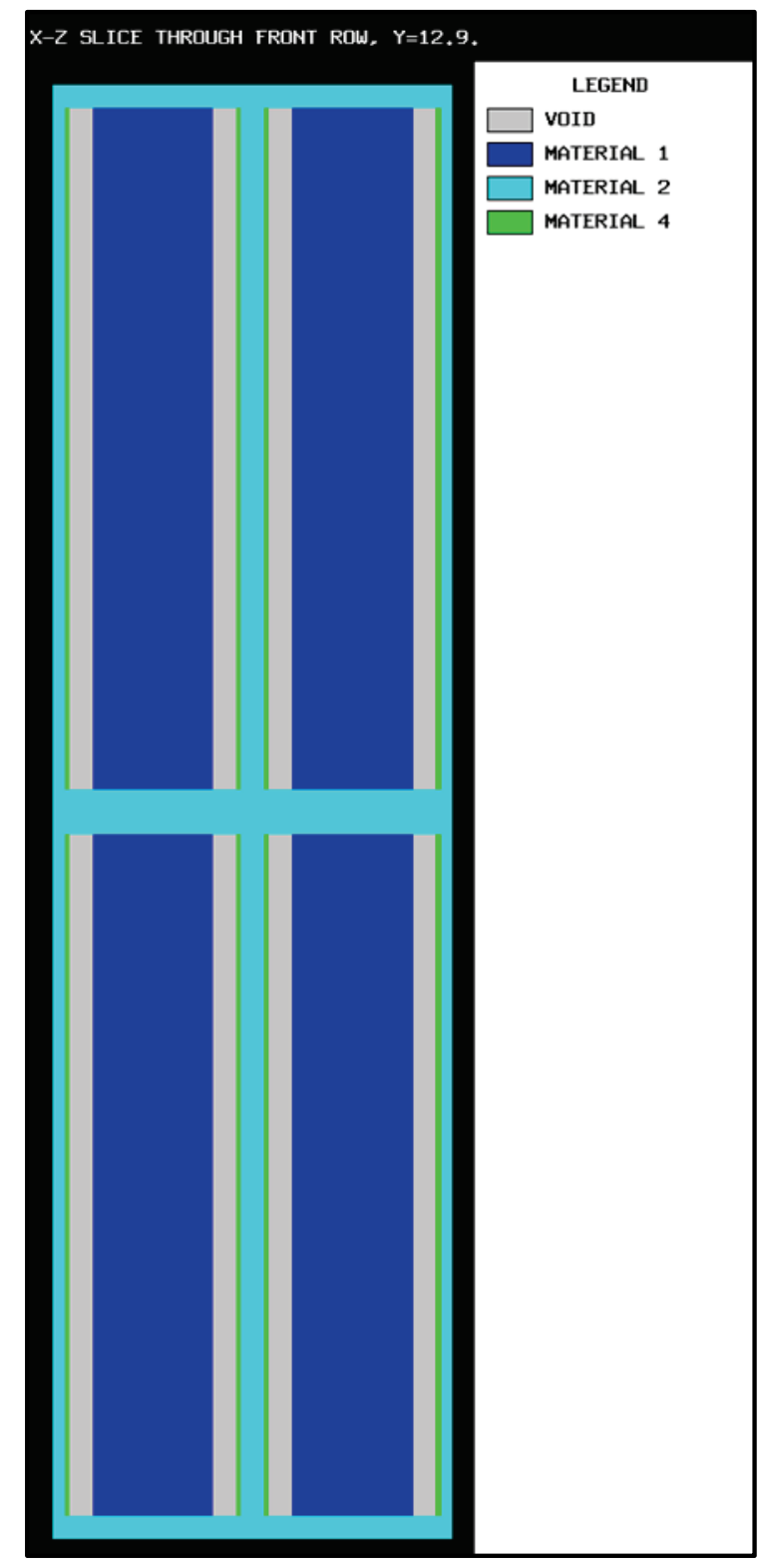

Fig. 8.1.73. X-Z plot of $2 \times 2 \times 2$ bare array.

EXAMPLE 4. A $2 \times 2 \times 2$ REFLECTED ARRAY WITH THE ORIGIN AT THE MOST NEGATIVE POINT OF THE ARRAY

The ARRAY is described in Example 3 of this section with a 6 in. concrete reflector on all faces. The input data description for this array is given below. 
KENO V.a:

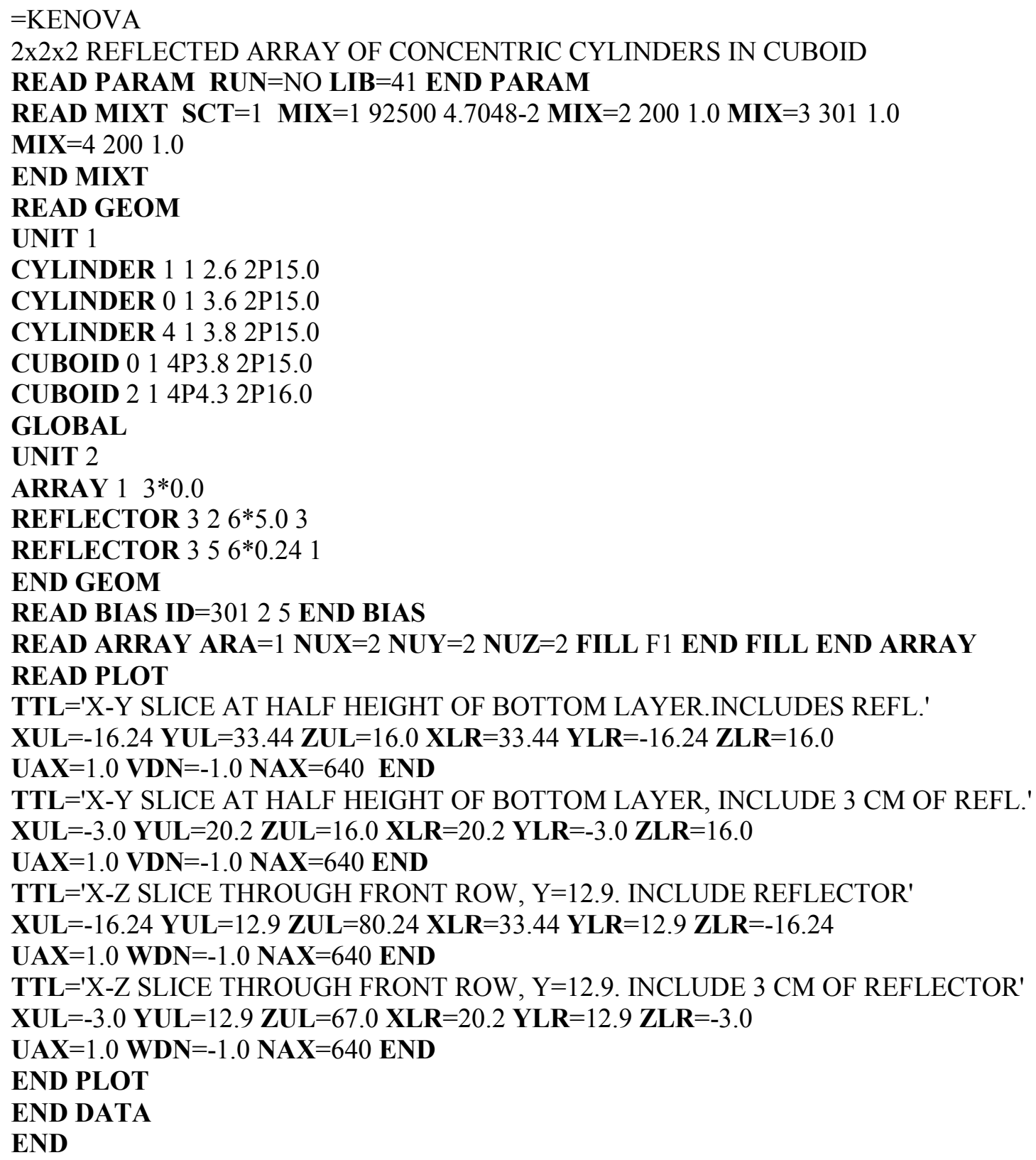


CYLINDER 12.6 2P15.0

CYLINDER 23.6 2P15.0

CYLINDER 33.8 2P15.0

CUBOID 4 4P3.8 2P15.0

CUBOID 5 4P4.3 2P16.0

MEDIA 1111

MEDIA $0 \begin{array}{llll}0 & 1 & 2 & -1\end{array}$

MEDIA $4 \begin{array}{lll}1 & 3 & -2\end{array}$

MEDIA $0 \begin{array}{llll} & 1 & 4 & -3\end{array}$

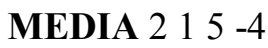

BOUNDARY 5

GLOBAL UNIT 2

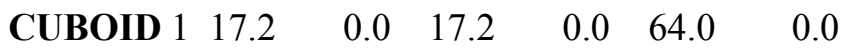

$\begin{array}{lllllll}\text { CUBOID } 2 & 22.20 & -5.00 & 22.20 & -5.00 & 69.00 & -5.00\end{array}$

$\begin{array}{lllllll}\text { CUBOID } 3 & 27.20 & -10.00 & 27.20 & -10.00 & 74.00 & -10.00\end{array}$

$\begin{array}{lllllll}\text { CUBOID } 4 & 32.20 & -15.00 & 32.20 & -15.00 & 79.00 & -15.00\end{array}$

$\begin{array}{lllllll}\text { CUBOID } 5 & 32.44 & -15.24 & 32.44 & -15.24 & 79.24 & -15.24\end{array}$

ARRAY 11 PLACE 11114.34 .316 .0

MEDIA $3 \quad 2 \quad 2 \quad-1$

MEDIA $3 \quad 3 \quad 3 \quad-2$

MEDIA $3 \quad 4 \quad 4 \quad-3$

MEDIA $3 \quad 5 \quad 5 \quad-4$

BOUNDARY 5

END GEOM

READ BIAS ID $=30125$ END BIAS

READ ARRAY ARA=1 NUX=2 NUY=2 NUZ=2 FILL F1 END FILL END ARRAY READ PLOT

TTL $=$ 'X-Y SLICE AT HALF HEIGHT OF BOTTOM LAYER.INCLUDES REFL.'

$\mathbf{X U L}=-16.24 \quad$ YUL=33.44 ZUL=16.0 XLR=33.44 YLR=-16.24 ZLR=16.0

$\mathbf{U A X}=1.0 \quad$ VDN $=-1.0 \quad$ NAX $=640$ END

TTL $=$ 'X-Y SLICE AT HALF HEIGHT OF BOTTOM LAYER, INCLUDE 3 CM OF REFL.'

$\mathbf{X U L}=-3.0 \quad$ YUL $=20.2 \quad \mathbf{Z U L}=16.0 \quad \mathbf{X L R}=20.2 \quad \mathbf{Y L R}=-3.0 \quad \mathbf{Z L R}=16.0$

$\mathbf{U A X}=1.0 \quad \mathrm{VDN}=-1.0 \quad \mathrm{NAX}=640 \mathrm{END}$

TTL $=$ 'X-Z SLICE THROUGH FRONT ROW, Y=12.9. INCLUDE REFLECTOR'

$\mathbf{X U L}=-16.24 \quad$ YUL=12.9 ZUL=80.24 XLR=33.44 YLR=12.9 ZLR=-16.24

$\mathbf{U A X}=1.0$ WDN $=-1.0 \quad$ NAX $=640$ END

TTL $=$ 'X-Z SLICE THROUGH FRONT ROW, Y=12.9. INCLUDE 3 CM OF REFLECTOR'

$\mathbf{X U L}=-3.0 \quad$ YUL=12.9 $\quad$ ZUL=67.0 $\quad \mathbf{X L R}=20.2 \quad \mathbf{Y L R}=12.9 \quad \mathbf{Z L R}=-3.0$

$\mathbf{U A X}=1.0 \quad$ WDN $=-1.0 \quad \mathbf{N A X}=640$ END

END PLOT

END DATA

END

The ARRAY record specifies the array number and the coordinates of the most negative point of the array to be $(0.0,0.0,0.0)$ and the coordinates of the most positive point to be $(17.2,17.2,64.0)$. Thus the reflected array extends from $-15.24 \mathrm{~cm}$ to $+32.44 \mathrm{~cm}$ in $\mathrm{X}$ and $\mathrm{Y}$ and from -15.24 to $+79.24 \mathrm{in} \mathrm{Z}$. 
The first color plot for this example is to show an X-Y slice through the array and reflector at the half height of the bottom layer. A black border is used to verify that the entire reflector has been shown. This is accomplished by arbitrarily setting the plot boundaries $1 \mathrm{~cm}$ beyond the reflector boundaries. The coordinates used for this plot are $\mathbf{X U L}=-16.24 \quad$ YUL $=33.44 \quad$ ZUL $=16.0 \quad$ XLR $=33.44$ YLR = 16.24 ZLR = 16.0. The plot data description is shown in Fig. 8.1.74, and the plot is shown in Fig. 8.1.75.

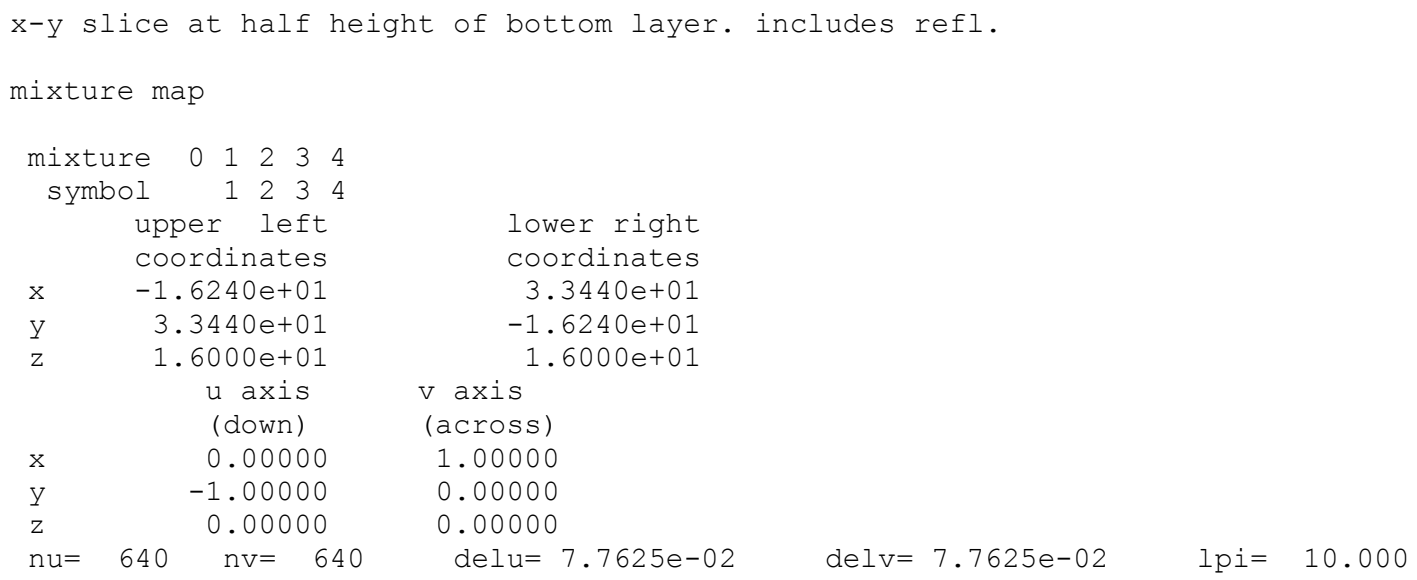

Fig. 8.1.74. Plot data for X-Y slice of Example 4.

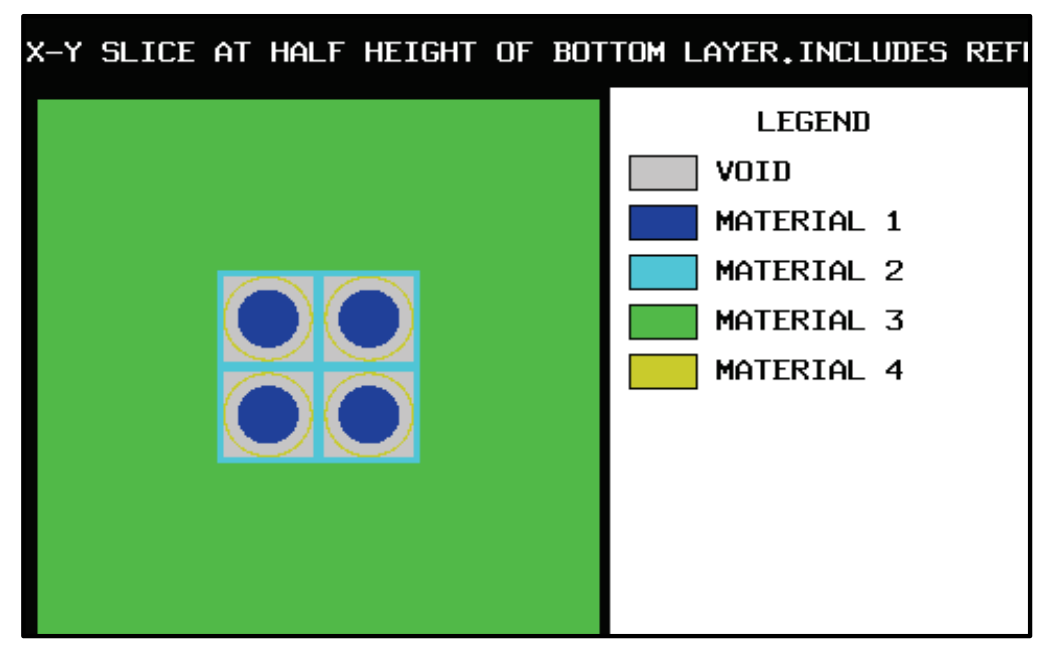

Fig. 8.1.75. X-Y plot of $2 \times 2 \times 2$ reflected array.

The next color plot is the same as the previous plot, except this plot includes only the first $3 \mathrm{~cm}$ of the reflector in order to show more detail. The coordinates used for this plot are $\mathbf{X U L}=-3.0 \mathbf{Y U L}=20.2$ $\mathbf{Z U L}=16.0 \mathbf{X L R}=20.2 \mathbf{Y L R}=-3.0 \mathbf{Z L R}=16.0$. This plot data description is given in Fig. 8.1.76, and the plot is shown in Fig. 8.1.77. 


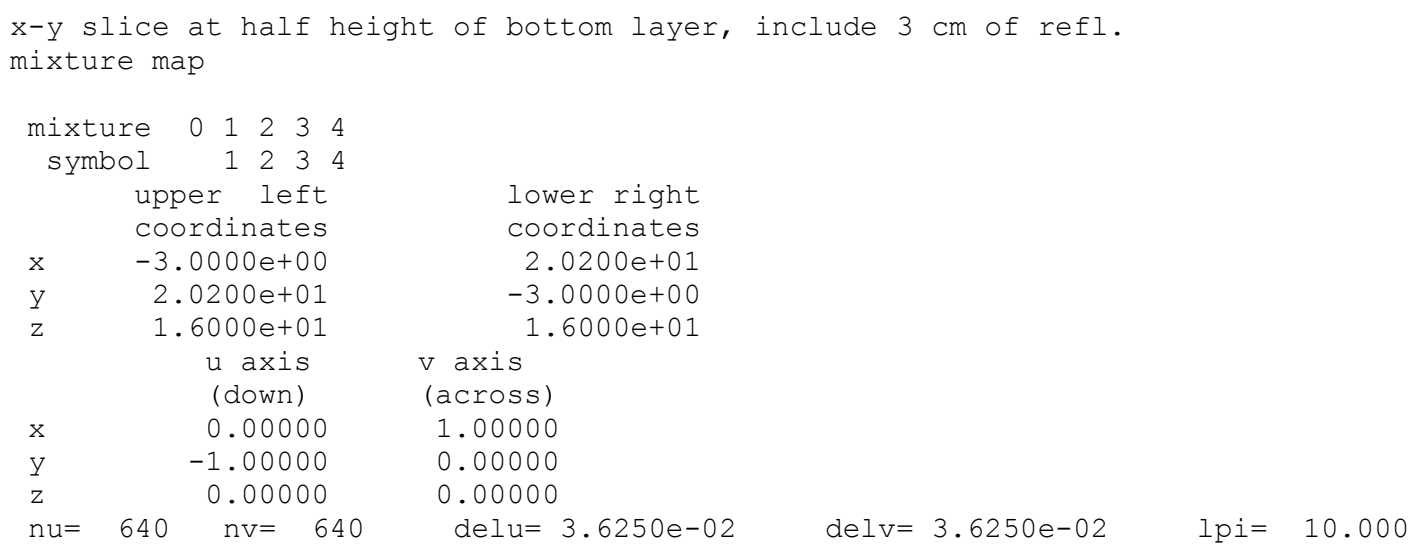

Fig. 8.1.76. Plot data for enlarged X-Y slice of Example 4.

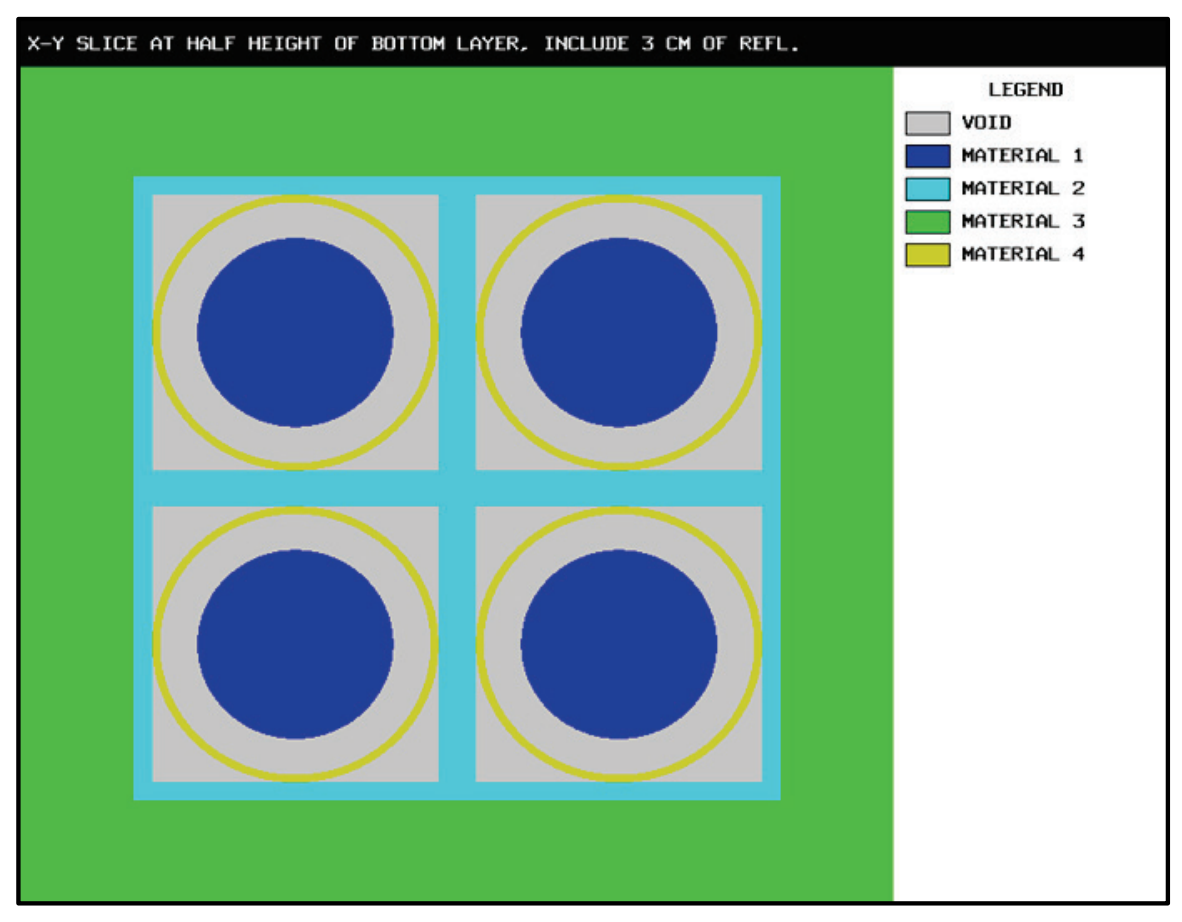

Fig. 8.1.77. Enlarged X-Y plot of $2 \times 2 \times 2$ reflected array.

The third color plot for this example is an X-Z slice through the center of the front row. An extra $1 \mathrm{~cm}$ is included in the coordinates to provide a black border around the plot. The coordinates are: $\mathbf{X U L}=-16.24$ $\mathbf{Y U L}=12.9 \mathbf{Z U L}=80.24 \mathbf{X L R}=33.44 \mathbf{Y L R}=12.9 \mathbf{Z L R}=-16.24$. The resultant plot data and plot are shown in Fig. 8.1.78 and Fig. 8.1.79. X-Z plot for $2 \times 2 \times 2$ reflected array.. 


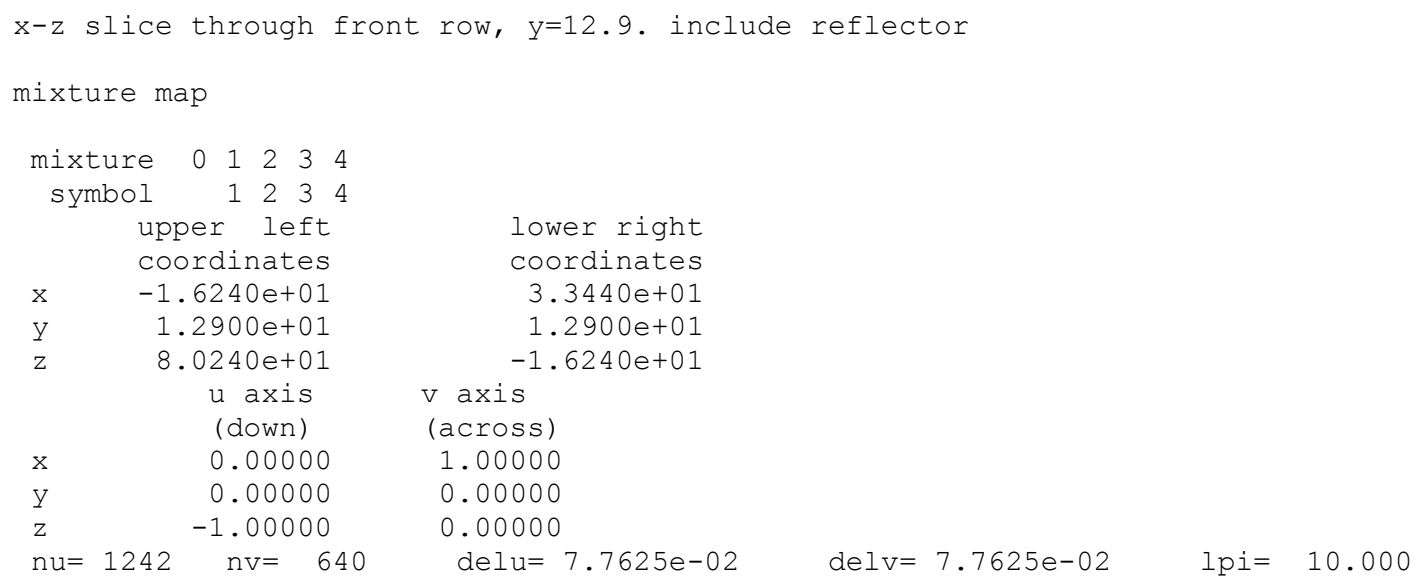

Fig. 8.1.78. Plot data for X-Z slice of Example 4. 


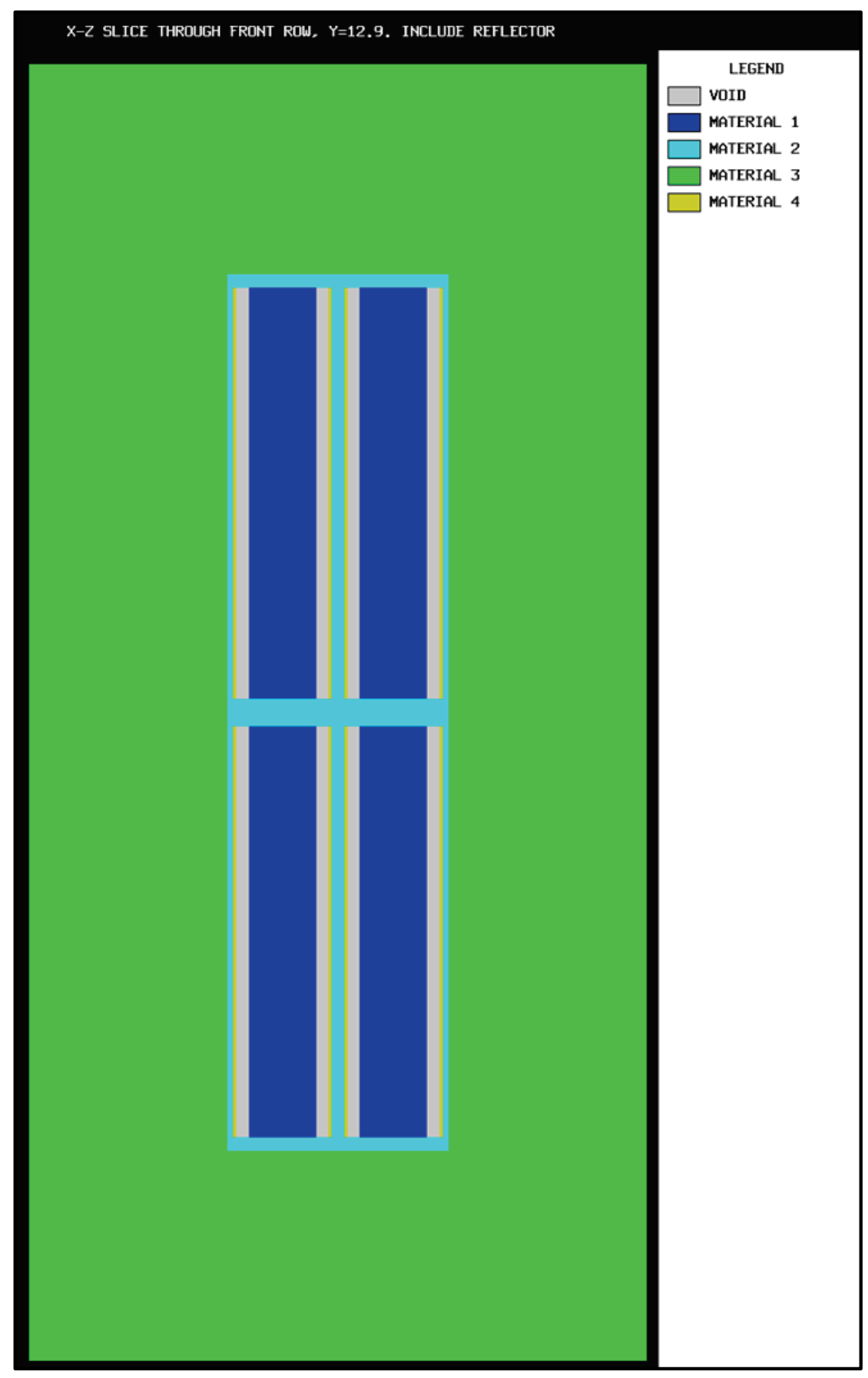

Fig. 8.1.79. X-Z plot for $2 \times 2 \times 2$ reflected array.

The last color plot for this example is the same as the previous one, except only $3 \mathrm{~cm}$ of the reflector is included in the plot. The plot data and associated plot are shown in Fig. 8.1.80 and Fig. 8.1.81. 


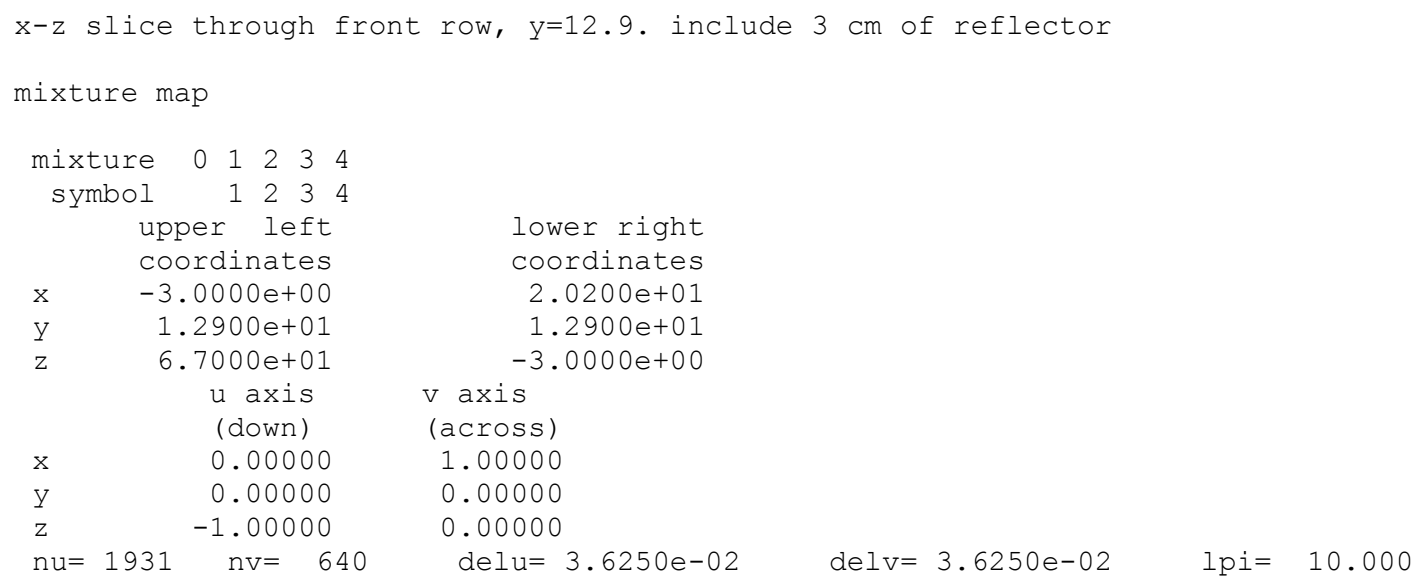

Fig. 8.1.80. Plot data for enlarged X-Z slice of Example 4. 


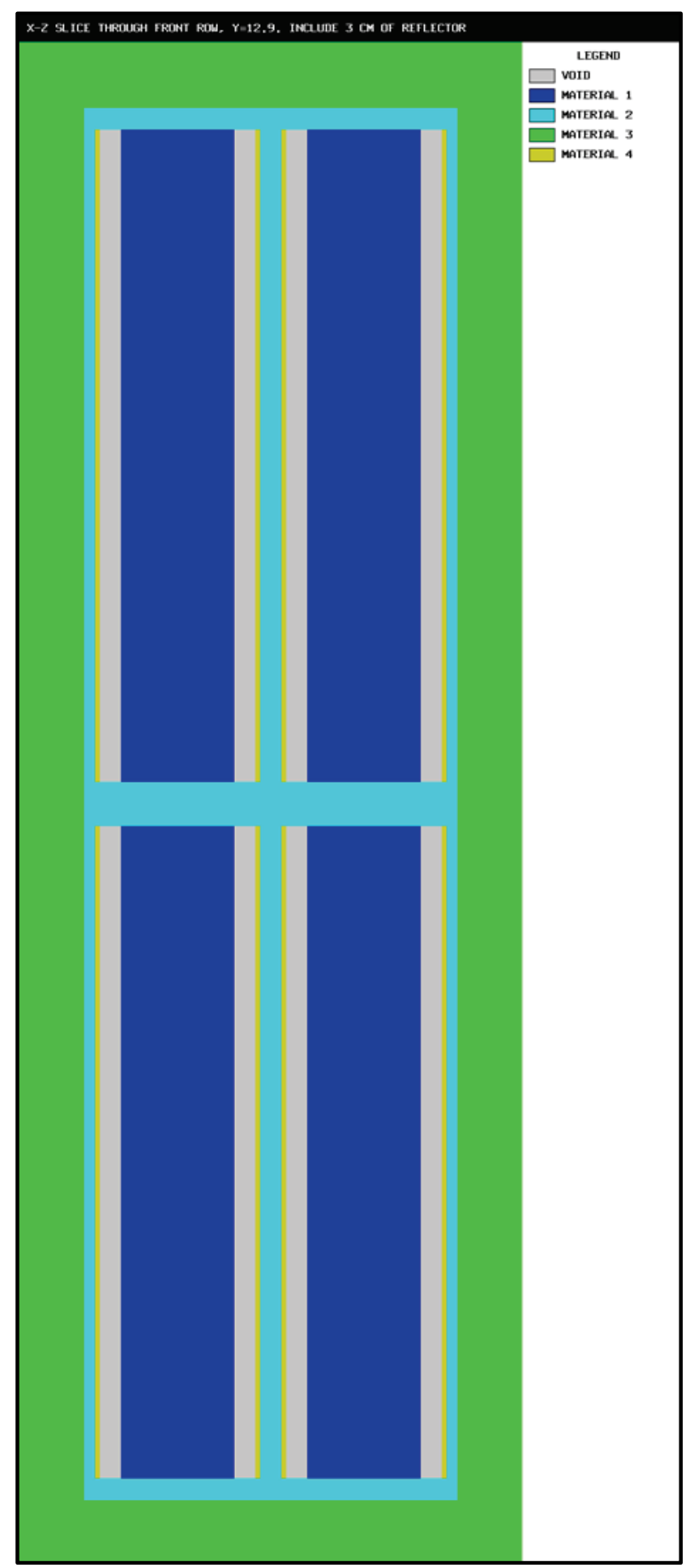

Fig. 8.1.81. Enlarged X-Z plot of $2 \times 2 \times 2$ reflected array. 
This example is physically identical to Example 4. The difference is in the specification of the origin. The bare array is $17.2 \mathrm{~cm}$ wide in $\mathrm{X}$ and $\mathrm{Y}$ and $64 \mathrm{~cm}$ high. The origin $(0,0,0)$ can be placed at the exact center of the array by specifying the most negative point of the array as $\mathrm{X}=-8.6, \mathrm{Y}=-8.6$ and $\mathrm{Z}=-32.0$. This is done using the ARRAY description in the geometry block. Because the origin is located at a different position, the coordinates of the plots will also be different. The input data description for this example is given below.

KENO V.a:

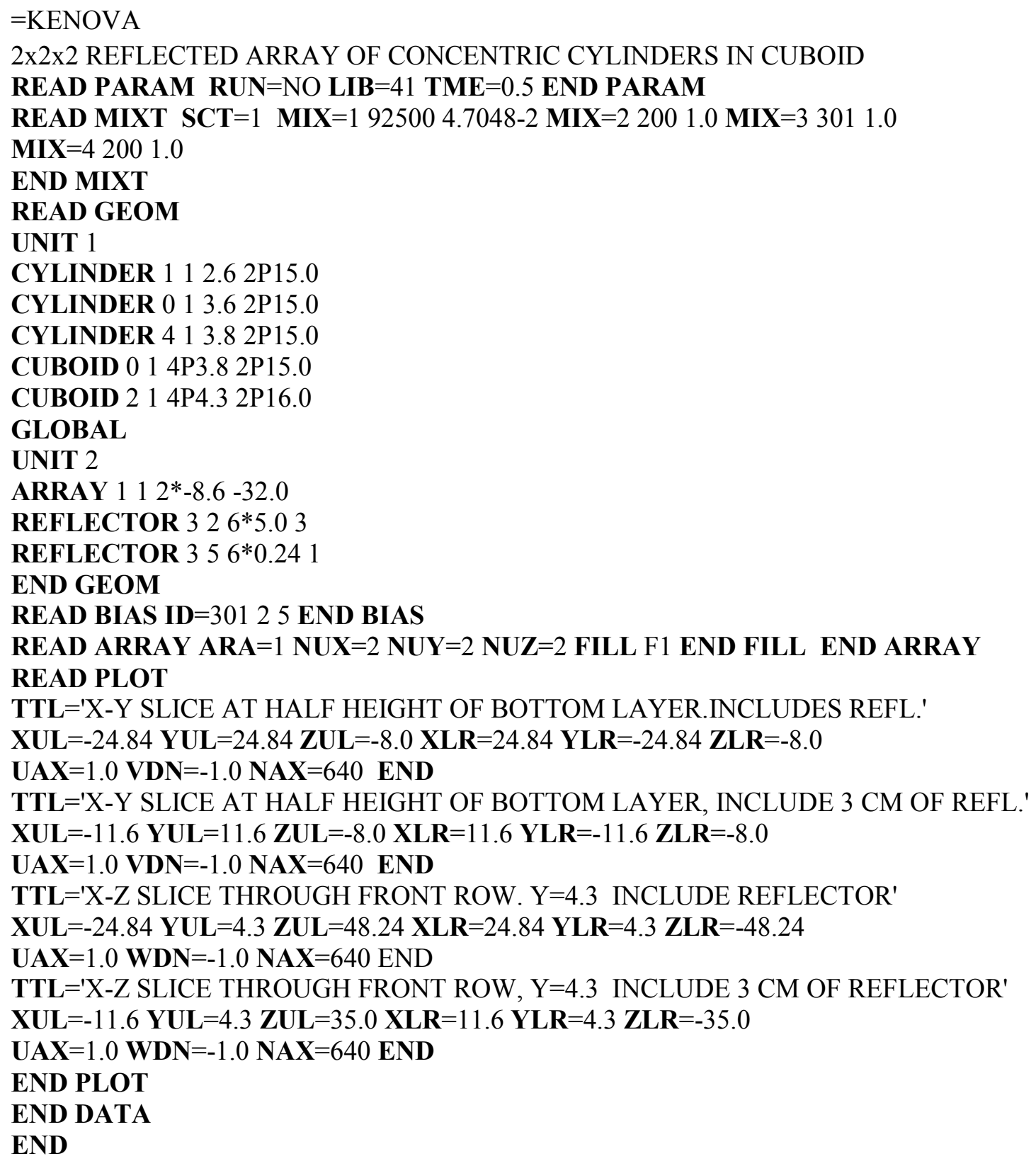


KENO-VI:

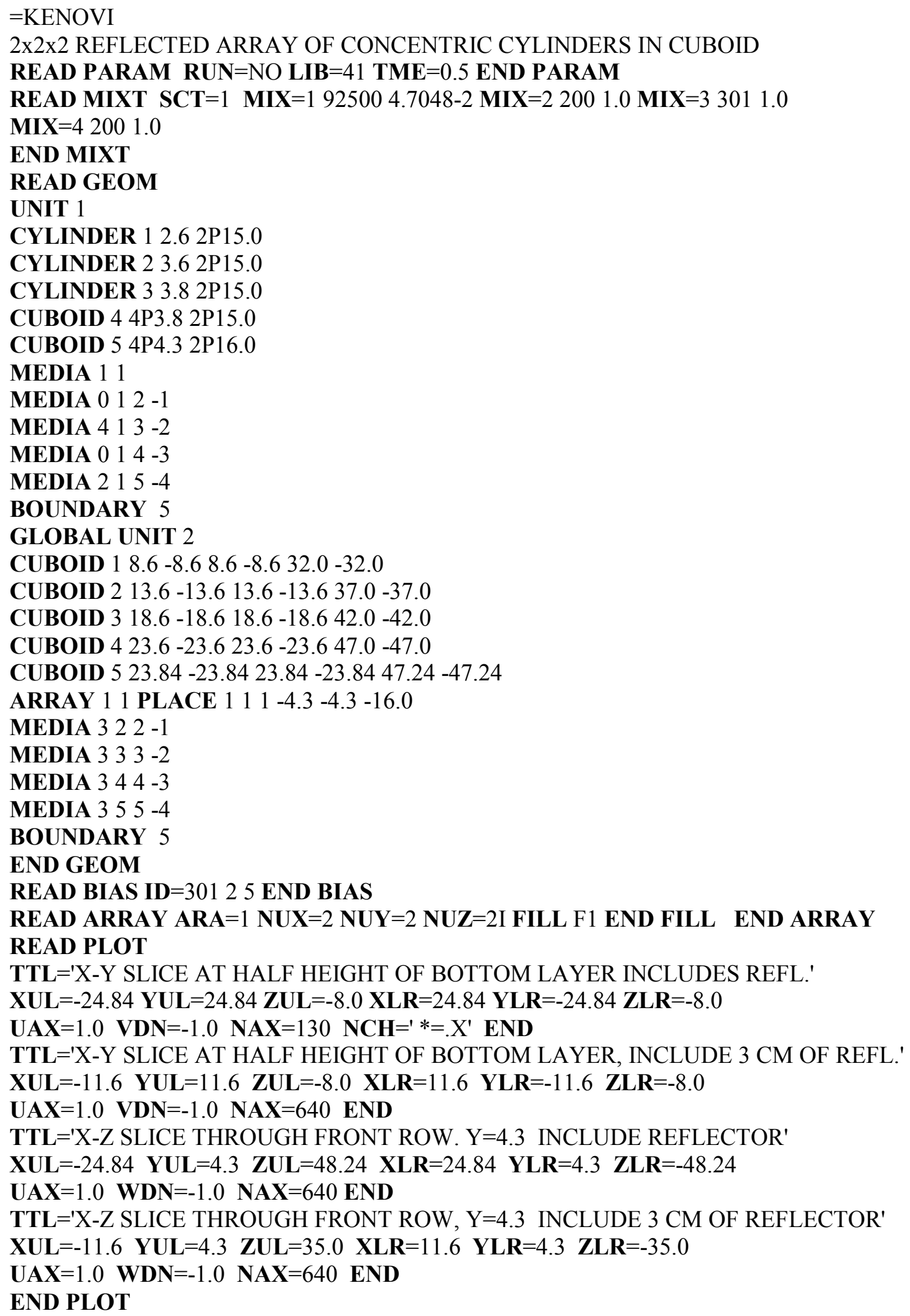




\section{END DATA \\ END}

The first color plot for this example covers exactly the same area as the first plot for Example 4. The plot data and the plot are given in Fig. 8.1.82 and Fig. 8.1.83, respectively.

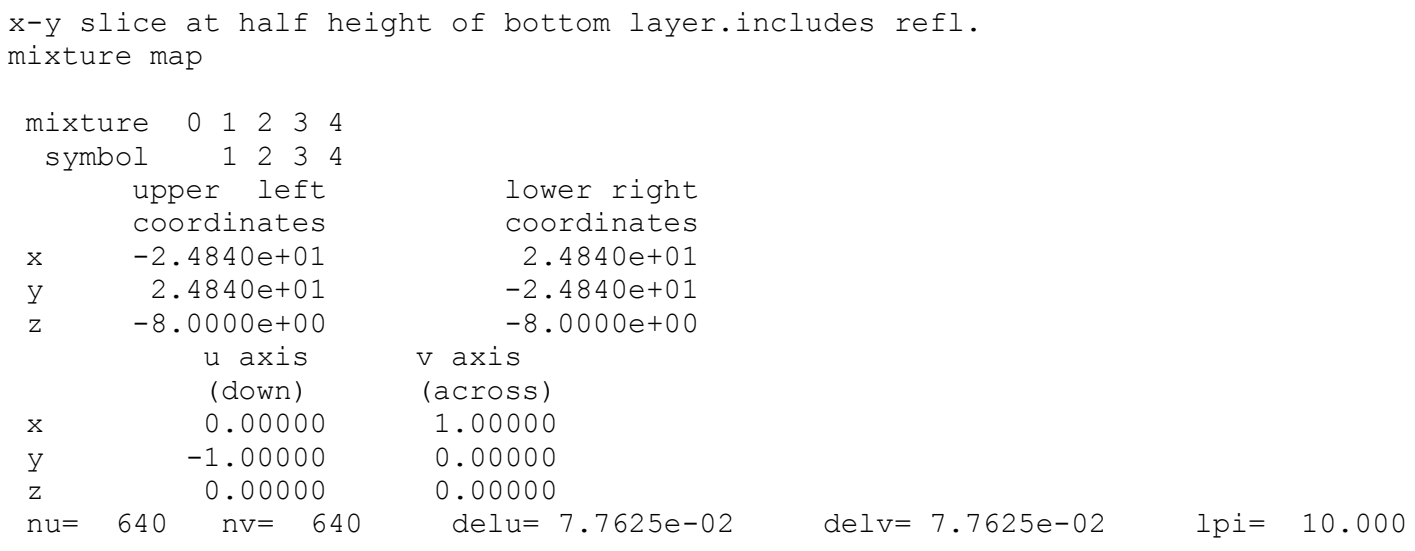

Fig. 8.1.82. Plot data for X-Y slice of Example 5.

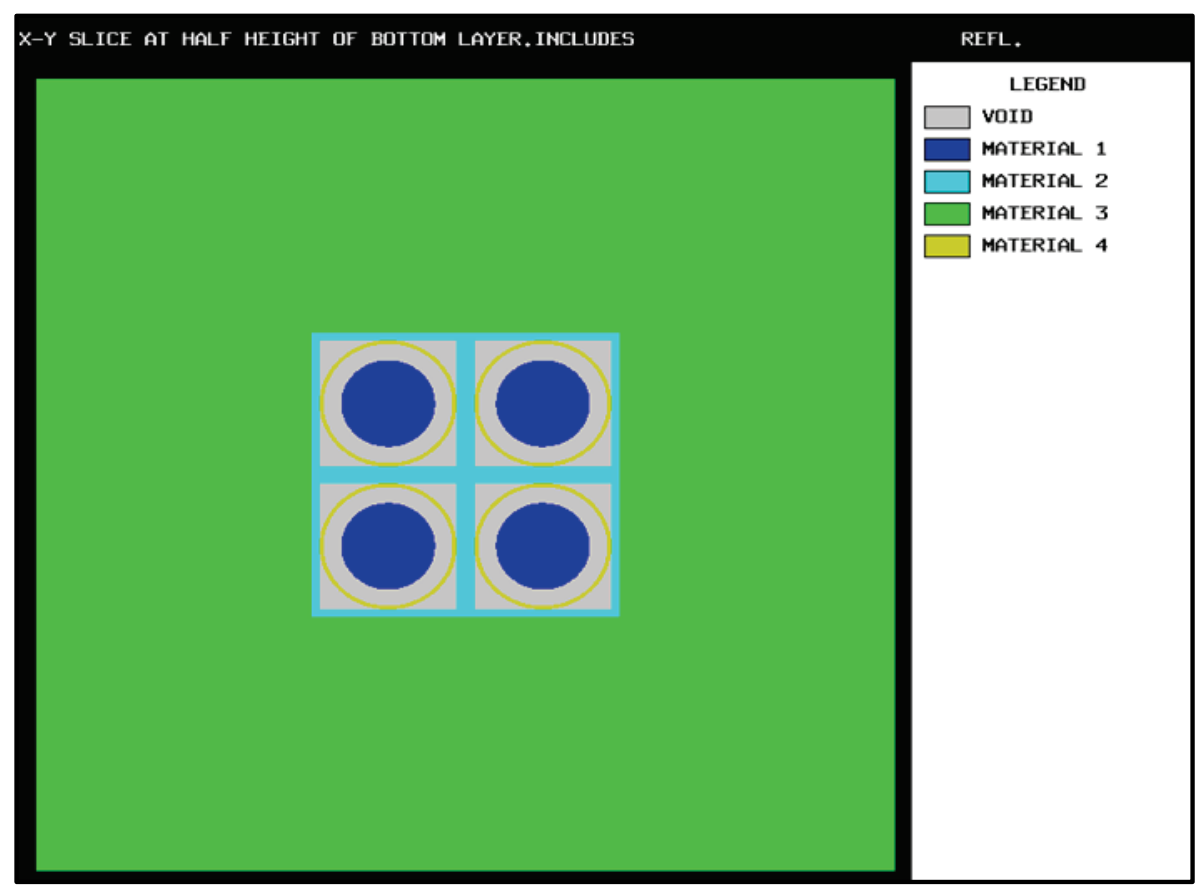

Fig. 8.1.83. X-Y plot of $2 \times 2 \times 2$ reflected array with centered origin. 
The Example 5 plot data and associated plots for an enlarged X-Y plot, an X-Z plot and an enlarged X-Z Plot are given in Fig. 8.1.84-Fig. 8.1.89.

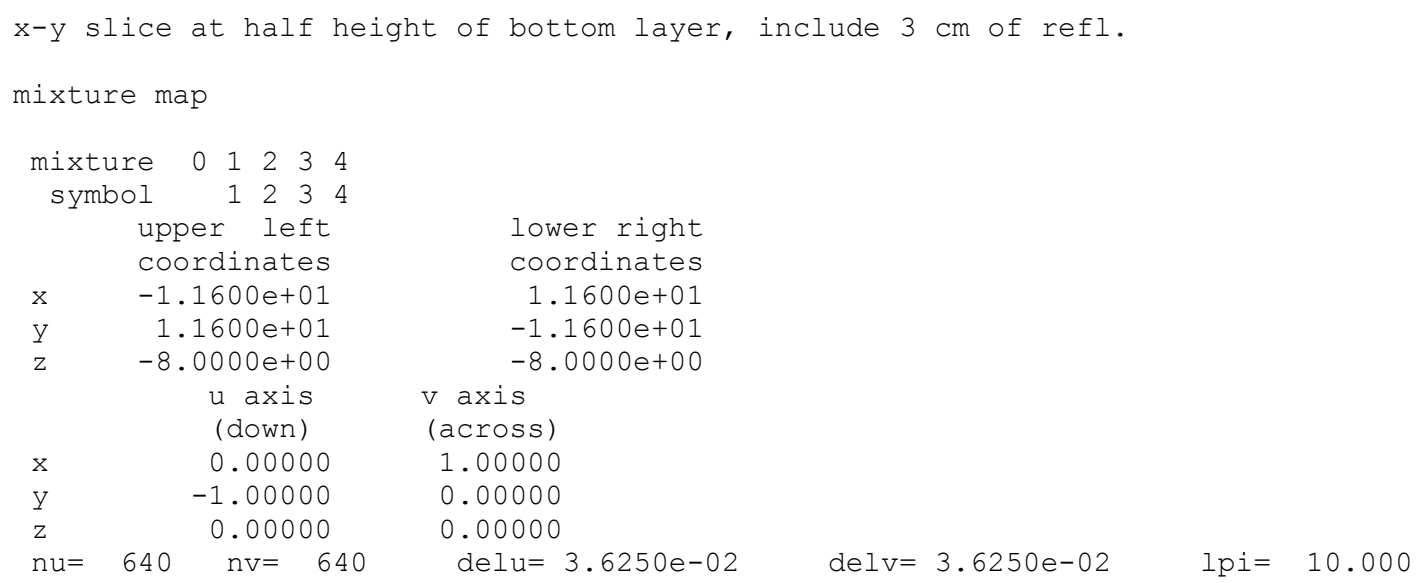

Fig. 8.1.84. Plot data for an enlarged X-Y slice of Example 5.

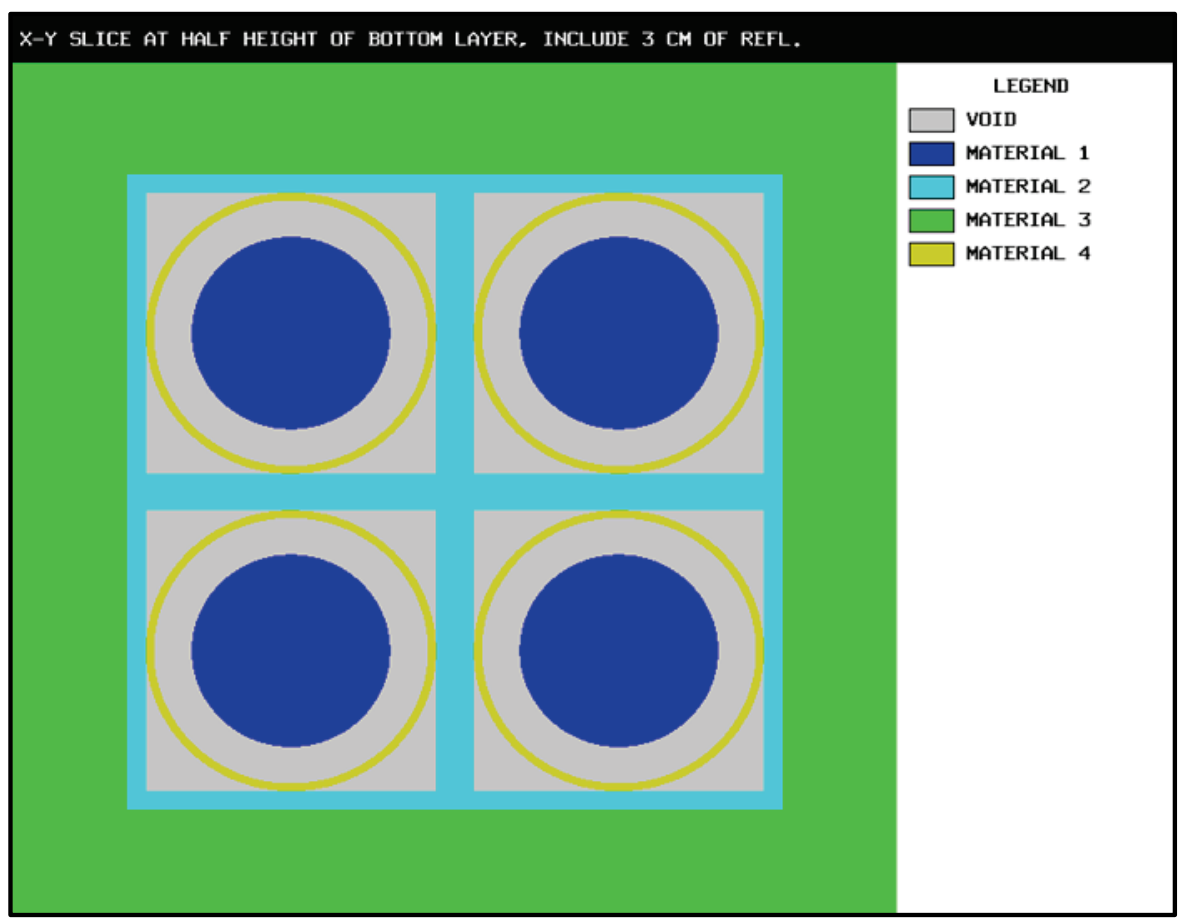

Fig. 8.1.85. Enlarged X-Y plot of $2 \times 2 \times 2$ reflected array with centered origin. 
$x-z$ slice through front row. $y=4.3$ include reflector

mixture map

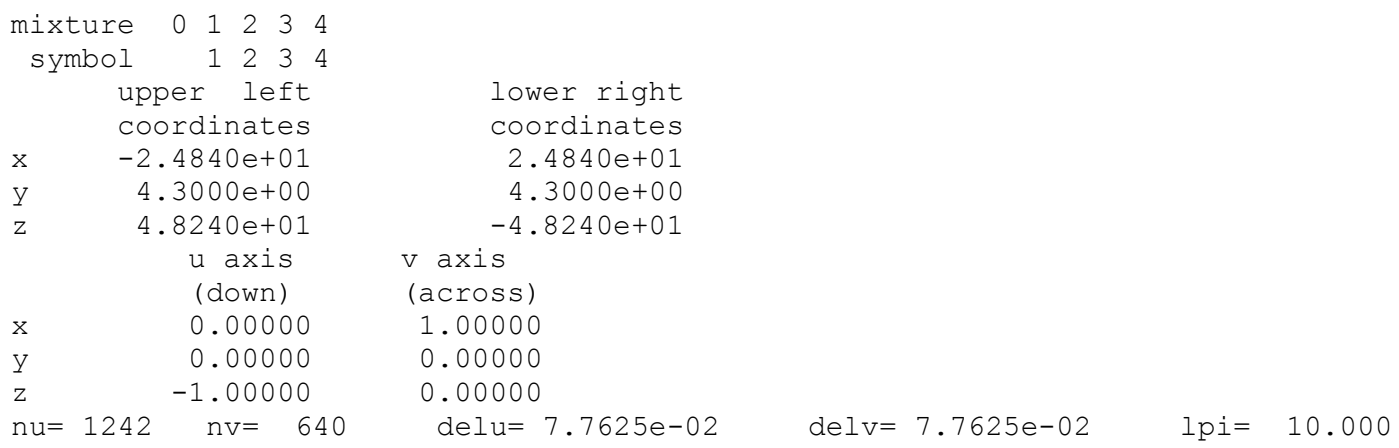

Fig. 8.1.86. Plot data for X-Z slice of Example 5.

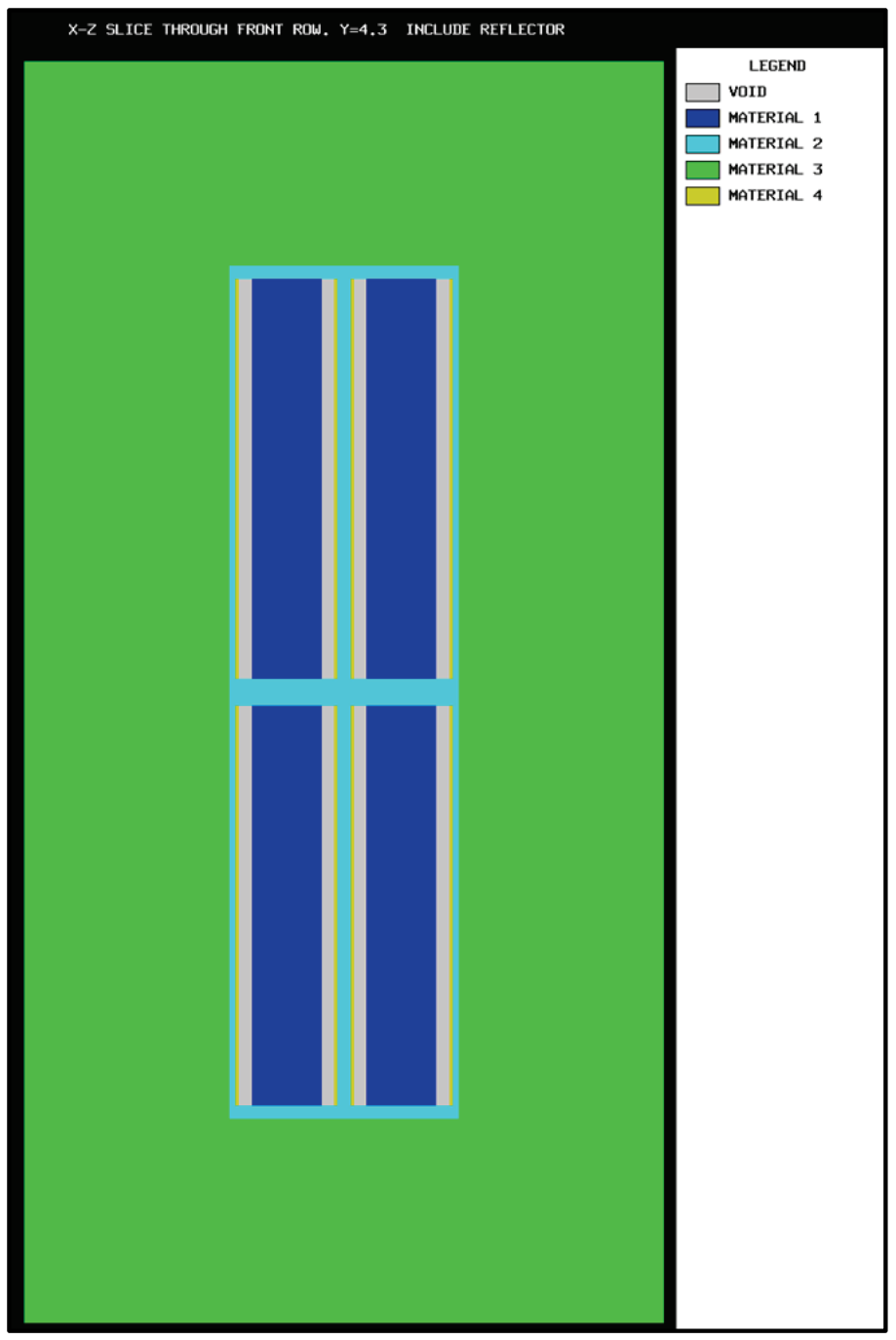

Fig. 8.1.87. X-Z plot of reflected $2 \times 2 \times 2$ array with centered origin. 
$x-z$ slice through front row, $y=4.3$ include $3 \mathrm{~cm}$ of reflector

mixture map

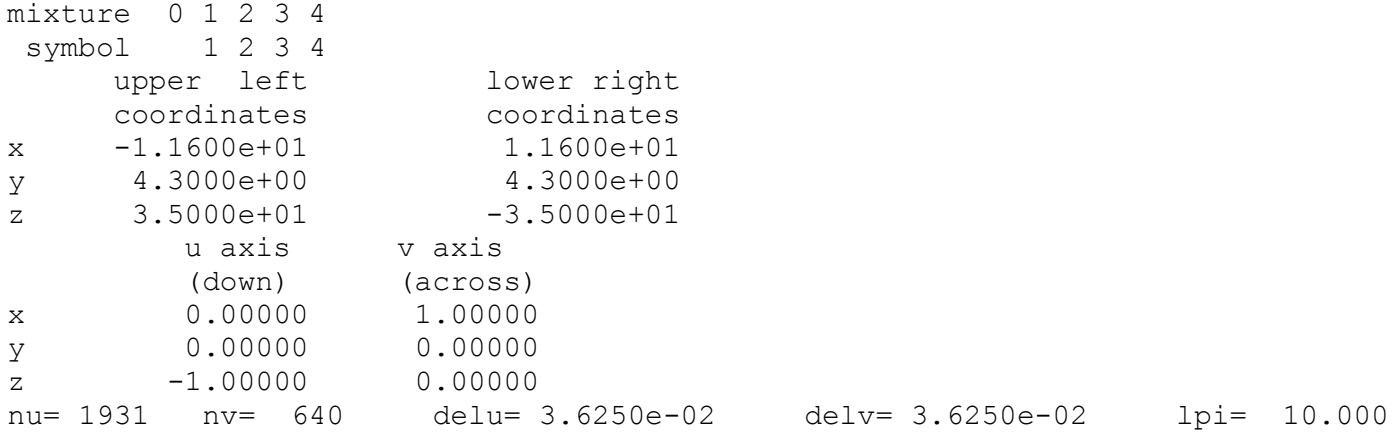

Fig. 8.1.88. Plot data for enlarged X-Z slice of Example 5.

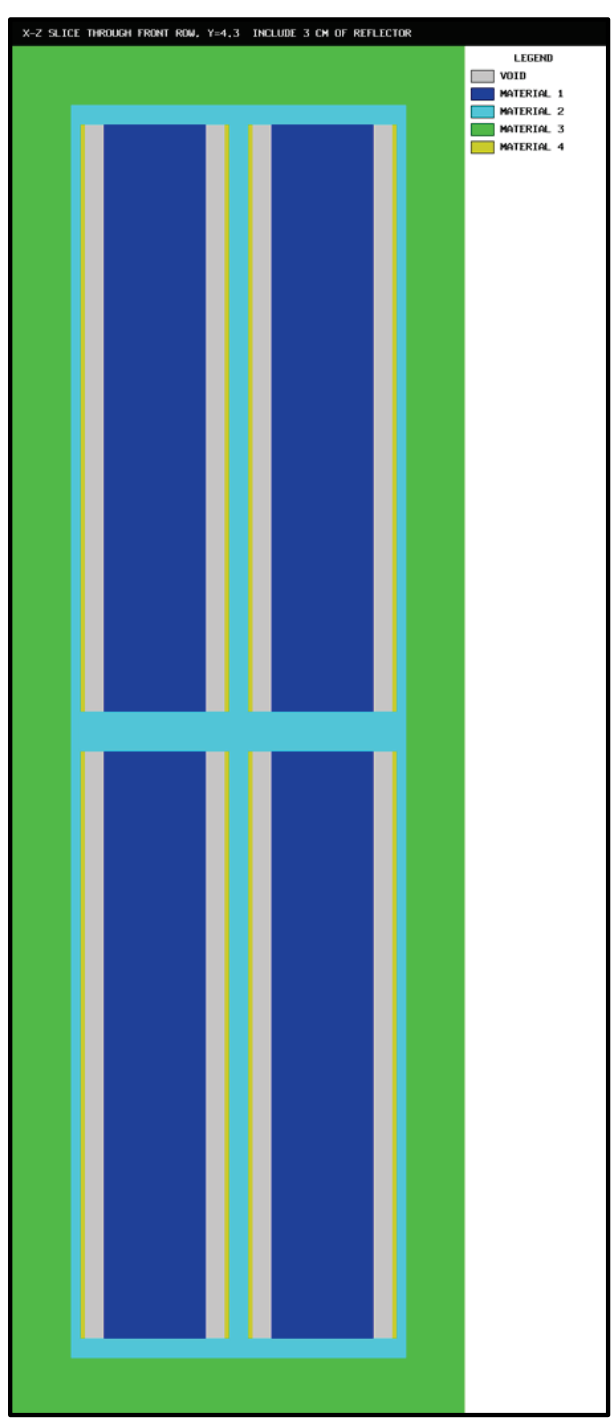

Fig. 8.1.89. Enlarged X-Z plot of reflected $2 \times 2 \times 2$ array with centered origin. 


\section{EXAMPLE 6. NESTED HOLES}

The nested hole description is provided in Sect. 8.1.3.6.2. Because this example involves a complicated placement of units, it is helpful useful to the user to generate a mixture plot and/or a unit plot for the problem. The resultant mixture plot for this problem is shown in Fig. 8.1.90, and the data description for Example 6 follows.

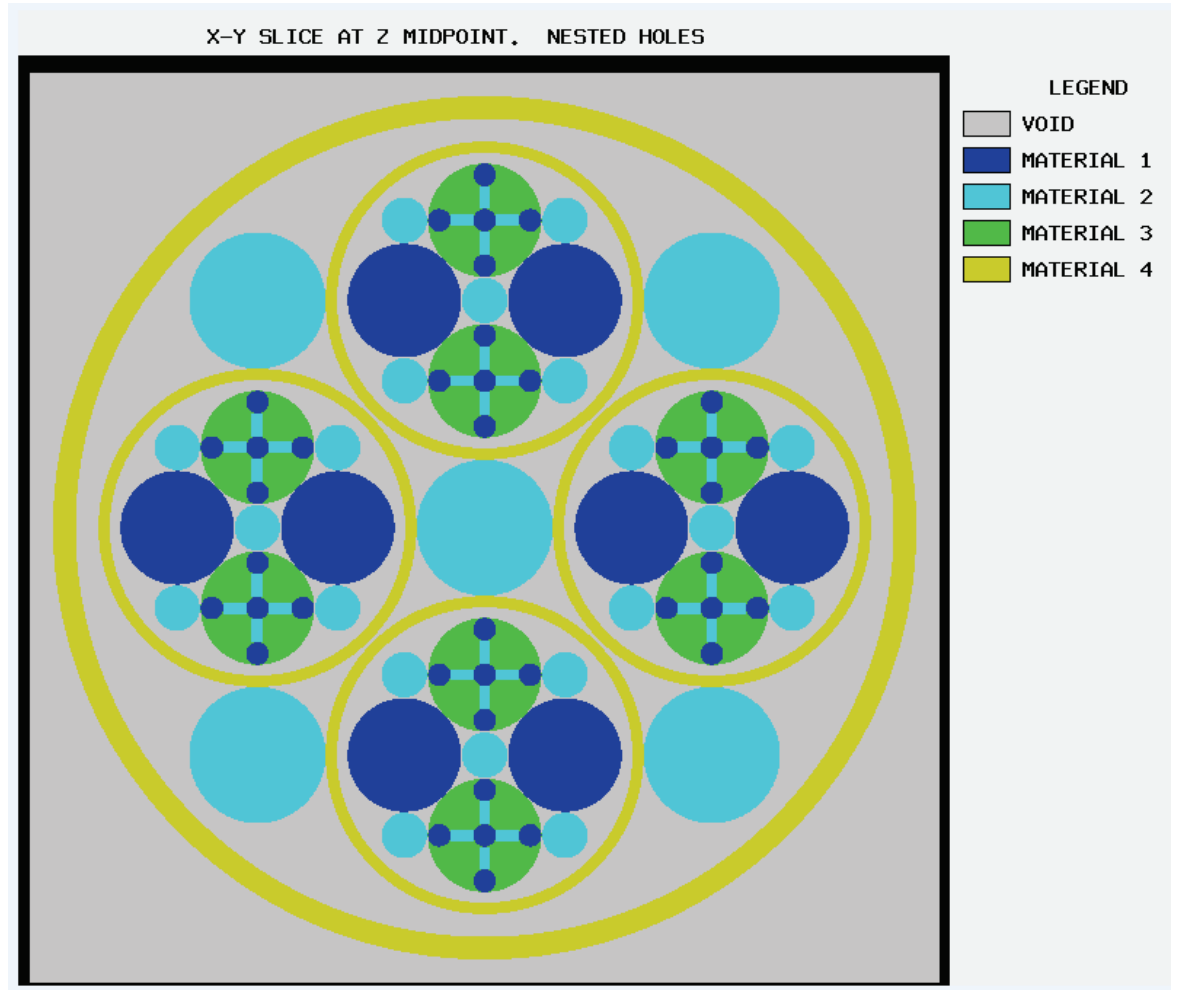

Fig. 8.1.90. Mixture plot of nested holes problem.

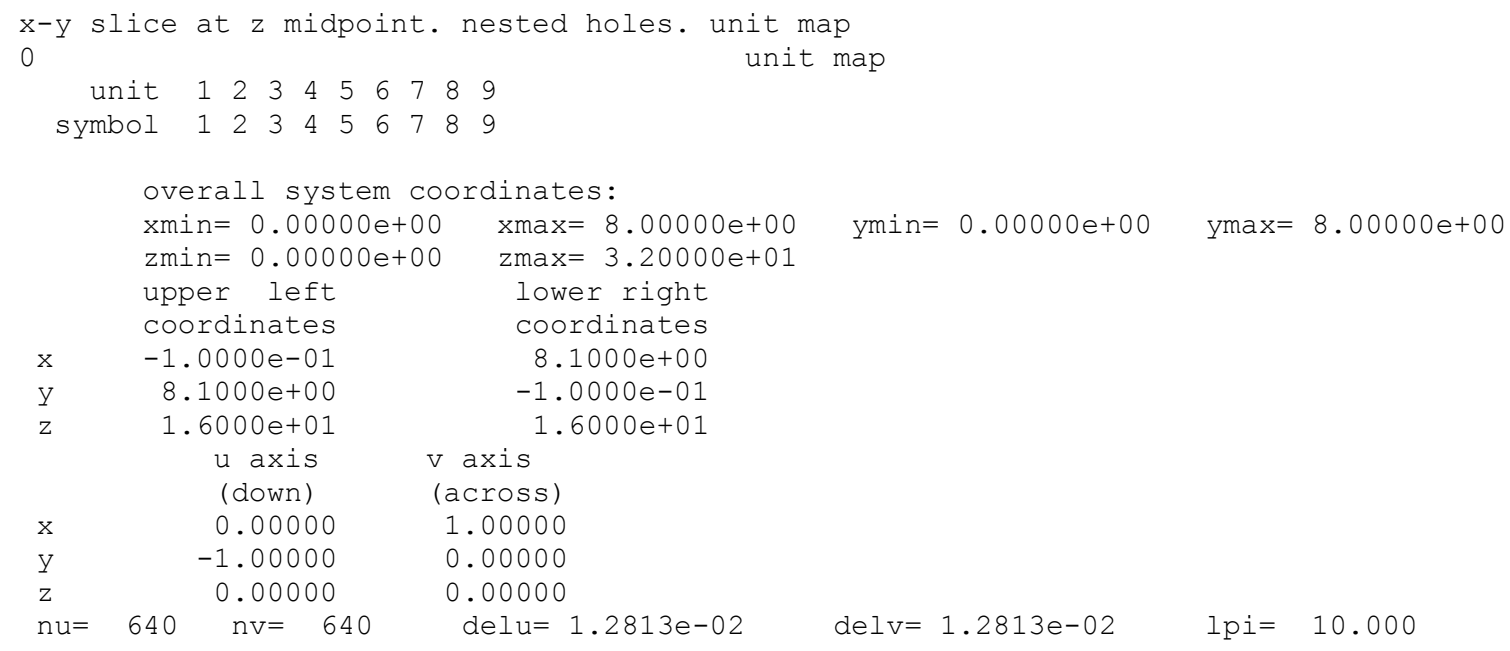

Fig. 8.1.91. Plot data for unit plot of nested holes. 
$X-Y$ SLICE AT Z MIDPOINT. NESTED HOLES. UNIT MAP

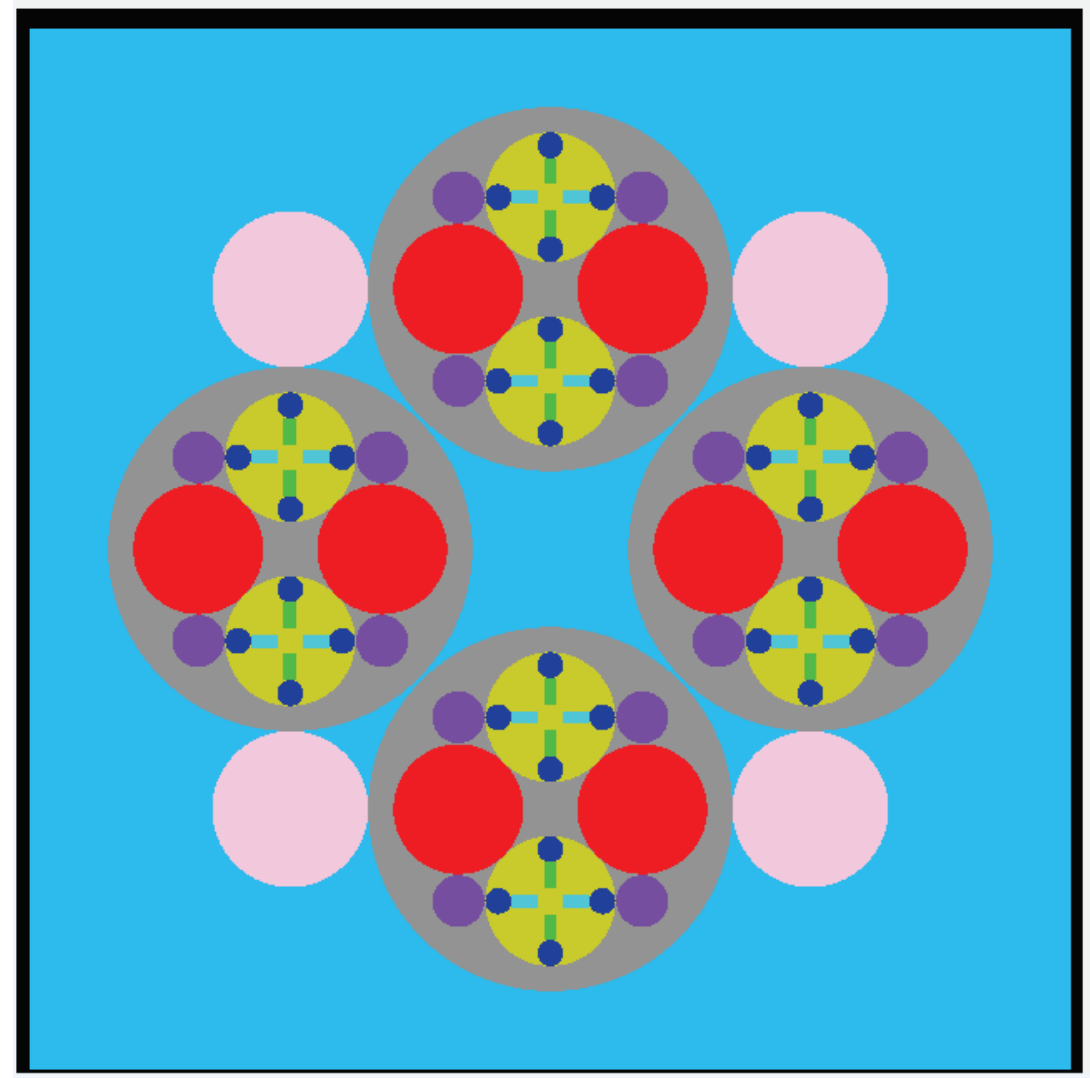

LEGEND

MATERIAL 1

MATERIAL 2

MATERIAL 3

MATERIAL 4

MATERIAL 5

MATERIAL 6

MATERIAL 7

MATERIAL 8

MATERIAL 9

Fig. 8.1.92. Unit plot of nested holes problem. 
KENO V.a:

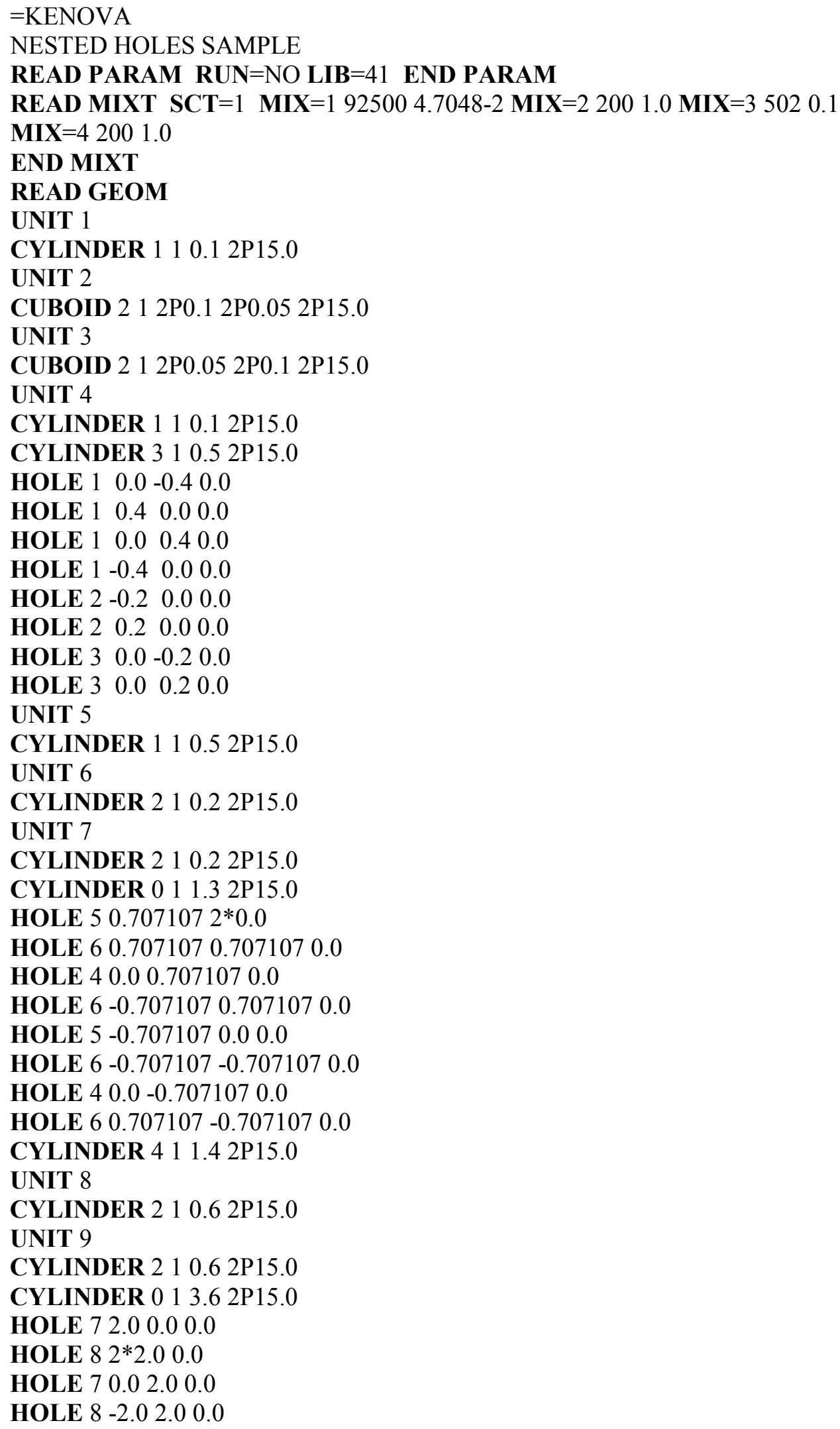


HOLE $7-2.02 * 0.0$

HOLE $82 *-2.00 .0$

HOLE $70.0-2.00 .0$

HOLE 8 2P2.0 0.0

CYLINDER 413.8 2P15.0

CUBOID 014 P4.0 2P16.0

END GEOM

READ ARRAY ARA $=1$ NUX $=1$ NUY $=1$ NUZ=1 FILL 9 END ARRAY

READ PLOT

TTL $=$ 'X-Y SLICE AT Z MIDPOINT. NESTED HOLES'

$\mathbf{X U L}=-0.1 \mathbf{Y U L}=8.1 \mathbf{Z U L}=16.0 \mathbf{X L R}=8.1 \quad \mathbf{Y L R}=-0.1 \mathbf{Z L R}=16$

$\mathbf{U A X}=1.0 \mathrm{VDN}=-1.0 \mathrm{NAX}=640$ END

TTL $=$ 'X-Y SLICE AT Z MIDPOINT. NESTED HOLES. UNIT MAP'

PIC $=$ UNIT END

END PLOT

END DATA

END

KENO-VI:

$=\mathrm{KENOVI}$

NESTED HOLES SAMPLE

READ PARAM RUN $=$ NO LIB $=41$ TME $=0.5$ END PARAM

READ MIXT SCT=1 MIX=1 92500 4.7048-2 MIX=2 2001.0 MIX=3 5020.1

MIX=4 2001.0

END MIXT

READ GEOM

UNIT 1

CYLINDER $10.12 \mathrm{P} 15.0$

CYLINDER 20.1 2P15.0 ORIGIN Y=-0.4

CYLINDER 30.1 2P15.0 ORIGIN $\mathbf{X}=0.4$

CYLINDER 40.1 2P15.0 ORIGIN Y $=0.4$

CYLINDER 50.1 2P15.0 ORIGIN $\mathrm{X}=-0.4$

CUBOID 6 2P0.1 2P0.05 2P15.0 ORIGIN $\mathrm{X}=-0.2$

CUBOID 7 2P0.1 2P0.05 2P15.0 ORIGIN X=0.2

CUBOID 8 2P0.05 2P0.1 2P15.0 ORIGIN $Y=-0.2$

CUBOID 9 2P0.05 2P0.1 2P15.0 ORIGIN Y $=0.2$

CYLINDER 10 0.5 2P15.0

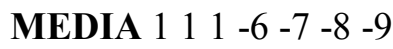

MEDIA 1112 - 12

MEDIA $1 \begin{array}{lllll}1 & 3 & -7\end{array}$

MEDIA 11014 - 14

MEDIA 1115 - 6

MEDIA 2 2 16 r

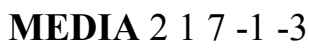

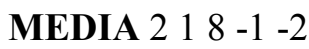

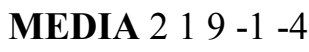

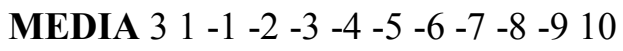

BOUNDARY 10

UNIT 2 
CYLINDER 10.2 2P15.0

CYLINDER 2 0.2 2P15.0 ORIGIN X $=0.707107 \mathbf{Y}=0.707107$

CYLINDER 3 0.2 2P15.0 ORIGIN $X=-0.707107 \quad \mathbf{Y}=0.707107$

CYLINDER 4 0.2 2P15.0 ORIGIN $\mathbf{X}=-0.707107 \mathbf{Y}=-0.707107$

CYLINDER 5 0.2 2P15.0 ORIGIN X=0.707107 Y=-0.707107

CYLINDER 60.5 2P15.0 ORIGIN X $=0.707107$

CYLINDER 70.5 2P15.0 ORIGIN $X=-0.707107$

CYLINDER 80.5 2P15.0 ORIGIN Y $=0.707107$

CYLINDER 90.5 2P15.0 ORIGIN Y=-0.707107

CYLINDER 101.3 2P15.0

CYLINDER 11 1.4 2P15.0

MEDIA 211

MEDIA 212

MEDIA 2

MEDIA 214

MEDIA 215

MEDIA $1110 \begin{array}{llll}1 & 6 & -8 & -9\end{array}$

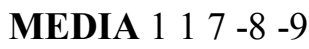

HOLE 18 -3 -6 ORIGIN Y=0.707107

HOLE $19-6-7$ ORIGIN Y=-0.707107

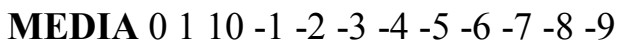

MEDIA $41111-10$

BOUNDARY 11

GLOBAL UNIT 3

CYLINDER 10.6 2P15.0

CYLINDER 20.6 2P15.0 ORIGIN X=2.0 Y=2.0

CYLINDER 30.6 2P15.0 ORIGIN $\mathbf{X}=-2.0 \quad \mathrm{Y}=2.0$

CYLINDER 40.6 2P15.0 ORIGIN $\mathbf{X}=-2.0 \quad \mathrm{Y}=-2.0$

CYLINDER 50.6 2P15.0 ORIGIN X=2.0 Y $=-2.0$

CYLINDER 61.4 2P15.0 ORIGIN $X=2.0$

CYLINDER 71.4 2P15.0 ORIGIN Y $=2.0$

CYLINDER 8 1.4 2P15.0 ORIGIN $\mathbf{X}=-2.0$

CYLINDER 91.4 2P15.0 ORIGIN Y=-2.0

CYLINDER 103.6 2P15.0

CYLINDER 113.8 2P15.0

CUBOID 12 4P4.0 2P16.0

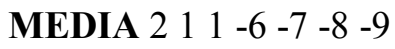

MEDIA $2 \begin{array}{lllll}1 & 2 & -6 & -7\end{array}$

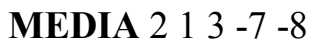

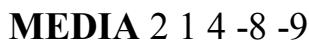

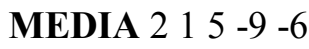

HOLE 2 - 6 - 1 - -5 - 2 ORIGIN $\mathbf{X}=2$

HOLE $27-1-2$ - 3 ORIGIN Y=2

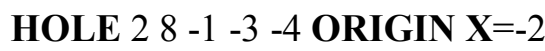

HOLE 2 - $9-4-5$ ORIGIN Y=-2

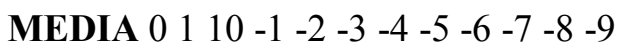

MEDIA $41111-10$

MEDIA 0112 -1 11

BOUNDARY 12

END GEOM

READ PLOT 
TTL $=$ 'X-Y SLICE AT Z MIDPOINT. NESTED HOLES'

$\mathbf{X U L}=-4.1 \quad$ YUL=4.1 ZUL=0.0 XLR=4.1 YLR=-4.1 ZLR=0

$\mathbf{U A X}=1.0 \mathrm{VDN}=-1.0 \quad \mathrm{NAX}=640 \mathbf{E N D}$

TTL $=$ 'X-Y SLICE AT Z MIDPOINT. NESTED HOLES, UNIT MAP.'

PIC $=$ UNIT END

END PLOT

END DATA

END

The plot data description for the UNIT plot of nested holes is shown in Fig. 8.1.91. The UNIT plot is shown in Fig. 8.1.92. The user can reference this map to verify the correct placement of the UNITs. Note that the UNIT map plots the UNITs present at the deepest nesting level for the 2-D slice. It does not show any detail within a UNIT. The apparent detail within UNIT 7 includes UNITs 1-6, which were placed there via the hole option. In the legend of the plot, the material number actually refers to the UNIT number.

\section{EXAMPLE 7. LARGE STORAGE ARRAY}

The storage array described Example 18 in Sect. 8.1.3.6.3 and Fig. 8.1.27 is such a sparse array that the mixture map had to be very large in order to show the detail of the shelves and uranium buttons. The mixture maps for this configuration were not presented in Sect. 8.1.3.6.3, but the data description was listed so the user can generate them. It may be useful to generate a UNIT map for this kind of problem. The input data for generating unit maps for this storage array is given below.

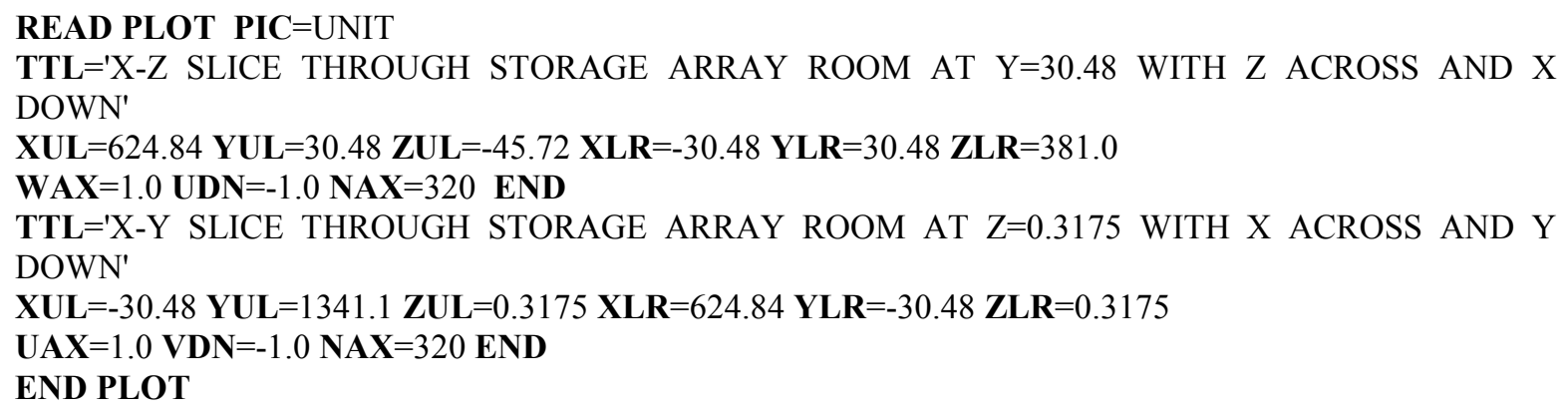

The plot data and UNIT map for an X-Z slice through the array at $Y=30.48 \mathrm{~cm}$ is given in Fig. 8.1.93 andFig. 8.1.94. The $Z$ direction, which extends from $-45.72 \mathrm{~cm}$ to $381.0 \mathrm{~cm}$, is plotted in 320 pixels across the plot. This UNIT map was created with $\mathrm{Z}$ across the plot and $\mathrm{X}$ down the plot.

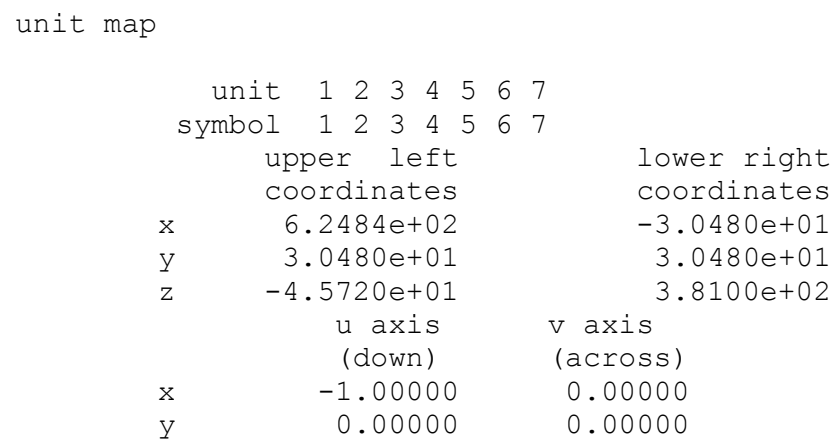




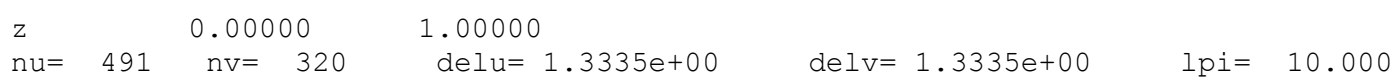

Fig. 8.1.93. Plot data for X-Z slice of storage array.

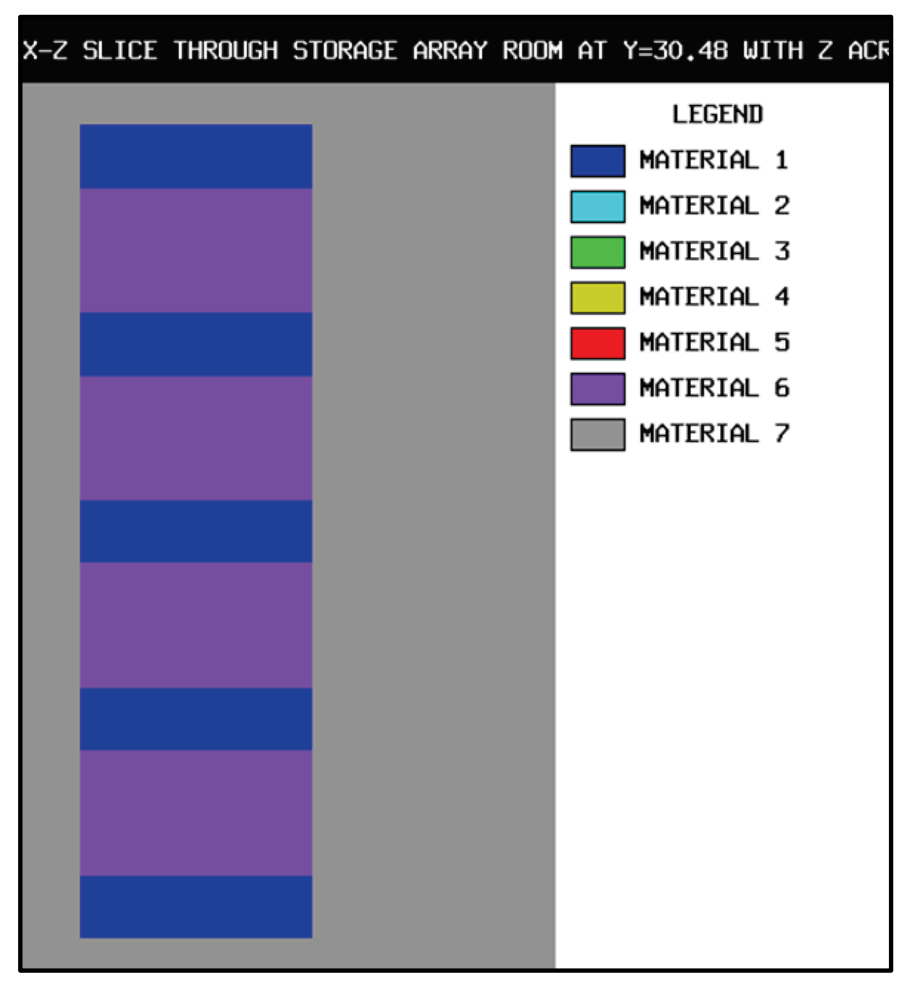

Fig. 8.1.94. X-Z plot of storage array.

The plot data and UNIT map for an X-Y slice through the shelf are given in Fig. 8.1.95 and Fig. 8.1.96. This UNIT map was created with $\mathrm{X}$ across the plot and $\mathrm{Y}$ down the plot. This shows five rows of shelves in the $\mathrm{X}$ direction.

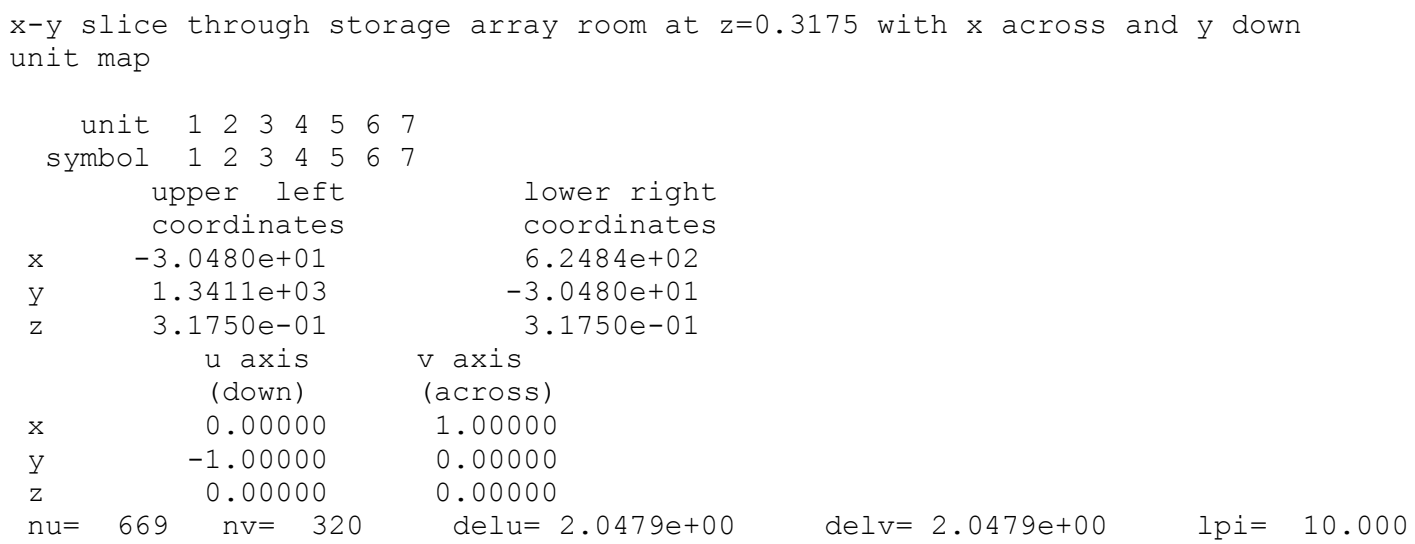

Fig. 8.1.95. Plot data for X-Y slice of storage array. 


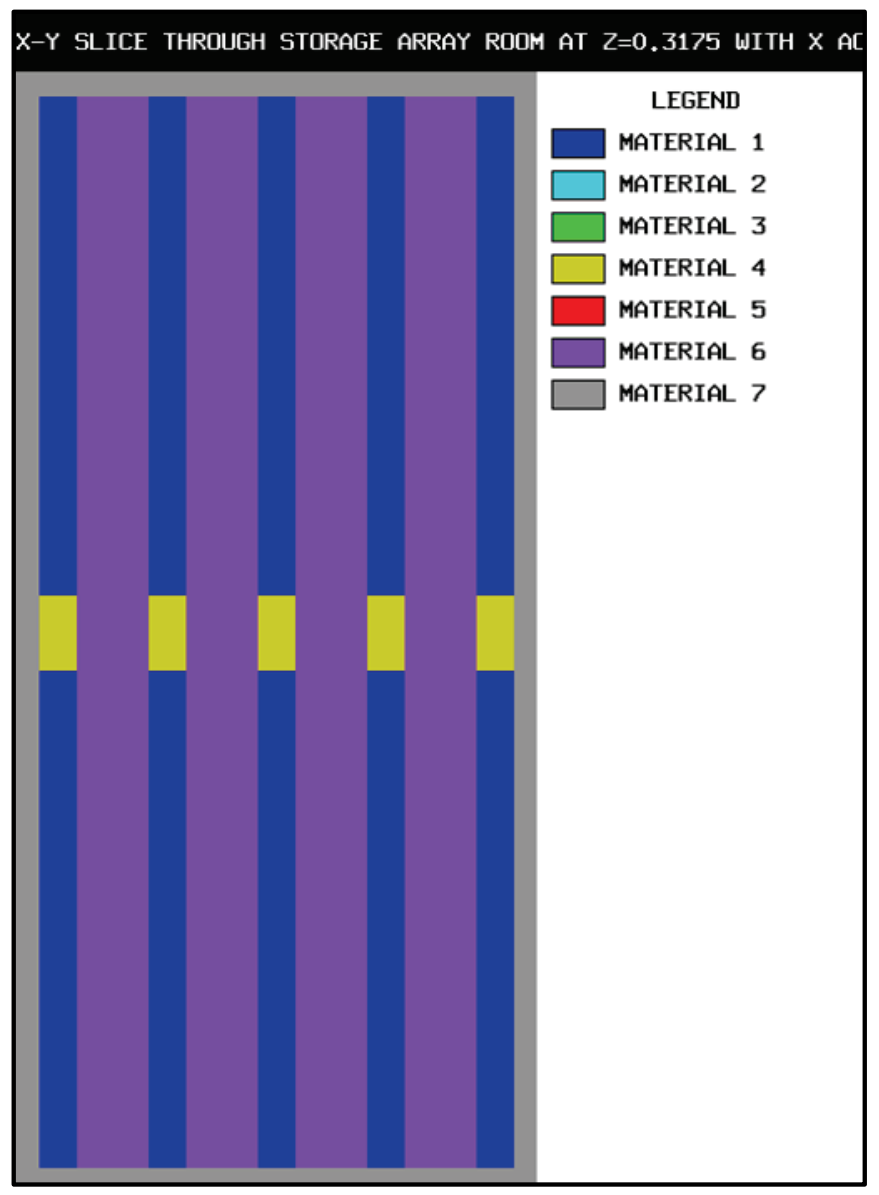

Fig. 8.1.96. X-Y plot of storage array.

\subsubsection{KENO Multiple Mesh and Mesh-based Quantity Specifications}

KENO creates a grid and stores several mesh definitions in this object. The following mesh definitions are supported by KENO:

i. $\quad$ Default mesh $(\operatorname{grid}$ ID $=10001)$ :

KENO generates a $5 \times 5 \times 5$ Cartesian mesh which overlays the entire geometry. This mesh is always used for fission source convergence diagnostics if the user does not specify any mesh for this quantity. Currently users cannot enter grid IDs greater than 9999.

ii. $\quad$ Simple mesh $($ grid ID $=20001)$ :

KENO generates a uniform simple mesh with the mesh size specified by the user with the MSH parameter, which is introduced in Table 9.1.1. This mesh definition is only used for mesh flux calculations.

iii. $\quad$ Mesh with READ GRID block (grid ID = NUMBER):

The user can specify either a mesh with a single READ GRID block or multiple meshes by repeating the READ GRID block with different mesh definitions. Note that the entire block, including READ GRID 
and END GRID, must be repeated each time; this behavior is different from all other blocks of KENO input.

After setting up all mesh, KENO tries to match the IDs specified in the parameter block with the keywords SCD, MFX, CDS, CGD, and GFX (See Sect. 8.1.2.3) to the grid ID in each grid definition. Each mesh-based quantity requested by the user is associated with a grid if the requested grid ID exists.

The following examples demonstrate the mesh features in KENO:

\section{EXAMPLE 1: SOURCE CONVERGENCE DIAGNOSTICS WITH DEFAULT MESH}

KENO always generates a default mesh for source convergence diagnostics if the user does not specify any mesh for this quantity. The following example (only KENO-VI version is shown) will be used in all examples in this section to summarize several mesh definition scenarios with several mesh-based quantity requests. In this sample problem, a bounding box is defined to enclose the entire geometry with $x \min =-13.01, x \max =13.01, y \min =-13.74, y \max =13.74, \mathrm{zmin}=-13.74$, and $\mathrm{zmax}=13.74$. KENO uses this bounding box and creates a $5 \times 5 \times 5$ grid for source convergence diagnostics.

KENO-VI:

$=$ CSAS6

MESH TEST - CHECK DIFFERENT MESH DEFINITIONS WITH SEVERAL MESH QUANTITIES. V7.1-252n

READ COMP

URANIUM 1 DEN=18.7613009223593.2922385.692234 1.0922360.2 END

END COMP

READ PARAMETERS

FDN $=$ YES HTM $=$ NO

END PARAMETERS

READ MIXT SCT $=2$ EPS $=1.0$ END MIXT

READ GEOMETRY

UNIT 1

COM='-Y HALF OF UNIT 3'

CYLINDER $105.7485 .3825-5.3825$ CHORD -X=0.0 ROTATE A1 $=90$ A2 $=90$

CUBOID $206.505-6.5050 .0-6.876 .87-6.87$

MEDIA $1110 \quad$ VOL $=2234.742156$

MEDIA 0120 -10 VOL=2677.511196

BOUNDARY 20

UNIT 2

$\mathbf{C O M}='+Y$ HALF OF UNIT 3'

CYLINDER $105.7485 .3825-5.3825$ CHORD +X=0.0 ROTATE A1 $=90$ A2 $=90$

CUBOID $206.505-6.5056 .870 .06 .87-6.87$

MEDIA $1110 \quad$ VOL $=2234.742156$

MEDIA 0120 -10 VOL=2677.511196 
BOUNDARY 20

UNIT 3

$\mathbf{C O M = ' C Y L I N D E R ~ C O M P O S E D ~ O F ~ E Q U A L ~ H A L V E S ~ ( X H E M I C Y L I N D E R S ~ W I T H ~ Y ~ R A D I I ) ' ~}$

CUBOID $106.505-6.5056 .87-6.876 .87-6.87$

ARRAY 110 PLACE 1110.00 .00 .0

BOUNDARY 10

UNIT 4

$\mathbf{C O M}='-Y$ PORTION (MORE THAN HALF) OF UNIT 6'

CYLINDER 105.748 5.3825 -5.3825 CHORD -X=3.0 ROTATE A1=90 A2=90

CUBOID $206.505-6.5053 .0-6.876 .87-6.87$

MEDIA $1110 \quad$ VOL $=2234.742156$

MEDIA $0120-10$ VOL=2677.511196

BOUNDARY 20

UNIT 5

$\mathbf{C O M}='+Y$ PORTION (LESS THAN HALF) OF UNIT 6'

CYLINDER 105.748 5.3825 -5.3825 CHORD +X=3.0 ROTATE A1=90 A2=90

CUBOID $206.505-6.5056 .873 .06 .87-6.87$

MEDIA $1110 \quad$ VOL $=2234.742156$

MEDIA $0120-10$ VOL=2677.511196

BOUNDARY 20

UNIT 6

COM='CYLINDER COMPOSED OF UNEQUAL HALVES (XHEMICYLINDERS WITH Y RADII)'

CUBOID $106.505-6.5056 .87-6.876 .87-6.87$

ARRAY 210 PLACE $11113 * 0.0$

BOUNDARY 10

UNIT 7

$\mathbf{C O M}=$ 'CYLINDER OF A SINGLE XHEMICYLINDER IN THE -Y DIRECTION'

CYLINDER $105.7485 .3825-5.3825$ CHORD -X=5.748 ROTATE A1=90 A2=90

CUBOID $206.505-6.5056 .87-6.876 .87-6.87$

MEDIA $1110 \quad$ VOL $=2234.742156$

MEDIA 0120 -10 VOL=2677.511196

BOUNDARY 20

UNIT 8

$\mathbf{C O M}=$ 'CYLINDER OF A SINGLE XHEMICYLINDER IN THE +Y DIRECTION'

CYLINDER 105.748 5.3825 -5.3825 CHORD +X=-5.748 ROTATE A1=90 A2=90

CUBOID $206.505-6.5056 .87-6.876 .87-6.87$

MEDIA $1110 \quad$ VOL $=2234.742156$

MEDIA $0120-10$ VOL=2677.511196

BOUNDARY 20

UNIT 9

$\mathbf{C O M}=$ '-Z HALF OF UNIT 11'

CYLINDER 10 5.748 5.3825 -5.3825 CHORD -Y=0.0 ROTATE A1=90 A2=90

CUBOID $206.505-6.5056 .87-6.870 .0-6.87$

MEDIA $1110 \quad$ VOL=2234.742156 
MEDIA 0120 -10 VOL=2677.511196

BOUNDARY 20

UNIT 10

COM='+Z HALF OF UNIT 11'

CYLINDER 105.748 5.3825 -5.3825 CHORD +Y=0.0 ROTATE A1=90 A2=90

CUBOID $206.505-6.5056 .87-6.876 .870 .0$

MEDIA $1110 \quad$ VOL $=2234.742156$

MEDIA 0120 -10 VOL=2677.511196

BOUNDARY 20

UNIT 11

COM='CYLINDER COMPOSED OF EQUAL HALVES (XHEMICYLINDERS WITH Z RADII)'

CUBOID $106.505-6.5056 .87-6.876 .87-6.87$

ARRAY 310 PLACE 1110.00 .00 .0

BOUNDARY 10

UNIT 12

COM='-Z PORTION (MORE THAN HALF) OF UNIT 14'

CYLINDER 105.7485 .3825 -5.3825 CHORD -Y=3.0 ROTATE A1=90 A2=90

CUBOID $206.505-6.5056 .87-6.873 .0-6.87$

MEDIA $1110 \quad$ VOL=2234.742156

MEDIA 0120 -10 VOL=2677.511196

BOUNDARY 20

UNIT 13

$\mathbf{C O M}='+Z$ PORTION (LESS THAN HALF) OF UNIT 14'

CYLINDER 105.748 5.3825 -5.3825 CHORD +Y=3.0 ROTATE A1=90 A2=90

CUBOID $206.505-6.5056 .87-6.876 .873 .0$

MEDIA $1110 \quad$ VOL=2234.742156

MEDIA $0120-10$ VOL=2677.511196

BOUNDARY 20

UNIT 14

$\mathbf{C O M = ' C Y L I N D E R ~ C O M P O S E D ~ O F ~ U N E Q U A L ~ H A L V E S ~ ( X H E M I C Y L I N D E R S ~ W I T H ~ Z ~ R A D I I ) ' ~}$

CUBOID $106.505-6.5056 .87-6.876 .87-6.87$

ARRAY 410 PLACE $11113 * 0.0$

BOUNDARY 10

UNIT 15

$\mathbf{C O M}=$ 'CYLINDER OF A SINGLE XHEMICYLINDER IN THE -Z DIRECTION'

CYLINDER 105.748 5.3825 -5.3825 CHORD -Y=5.748 ROTATE A1=90 A2=90

CUBOID $206.505-6.5056 .87-6.876 .87-6.87$

MEDIA $1110 \quad$ VOL=2234.742156

MEDIA 0120 -10 VOL=2677.511196

BOUNDARY 20

UNIT 16

$\mathbf{C O M}={ }^{\prime}$ 'CYLINDER OF A SINGLE XHEMICYLINDER IN THE + Z DIRECTION'

CYLINDER 105.748 5.3825 -5.3825 CHORD +Y=-5.748 ROTATE A1=90 A2=90

CUBOID $206.505-6.5056 .87-6.876 .87-6.87$ 


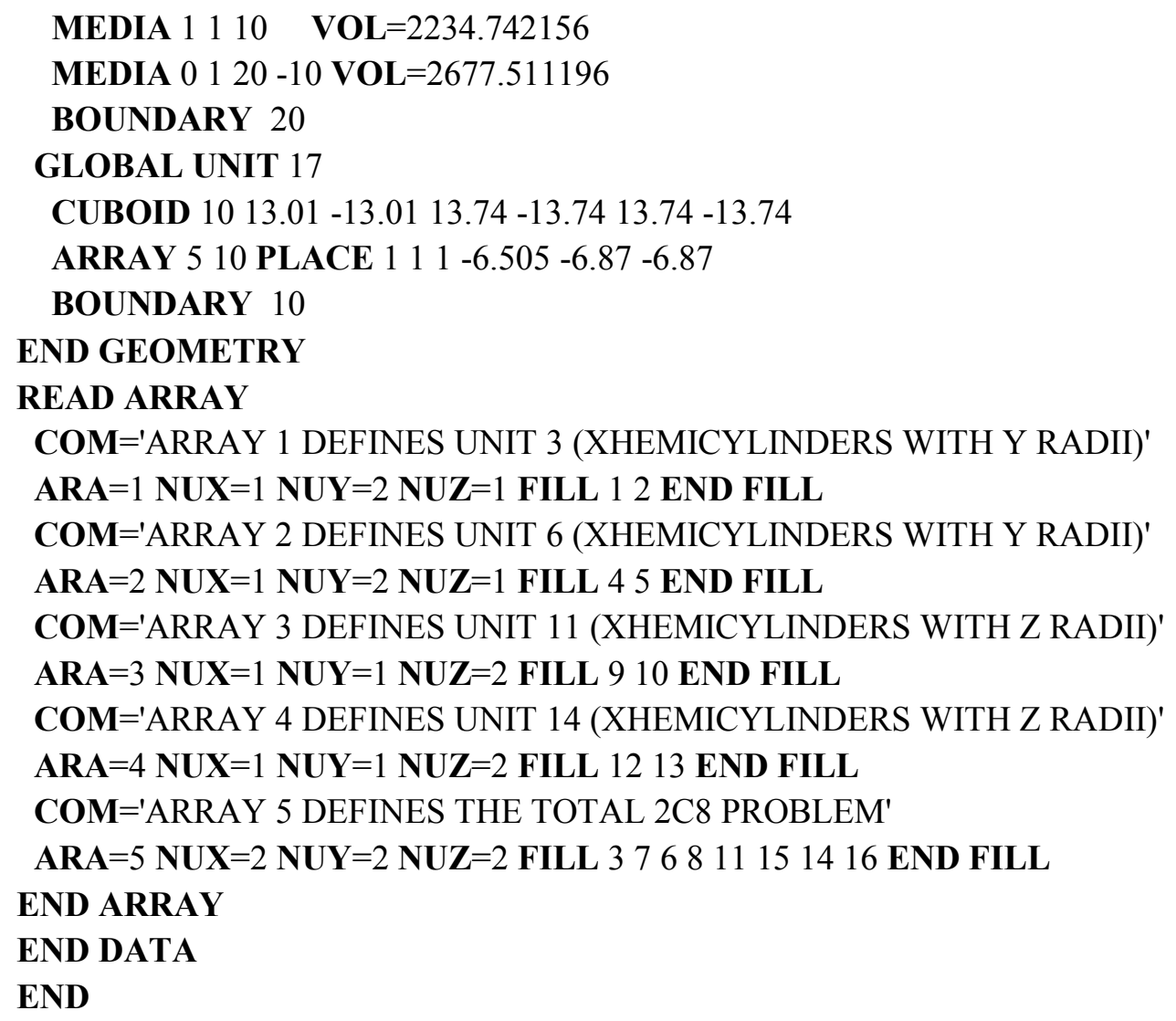

In the output, KENO notifies users about the default mesh usage for source convergence diagnostics with the message $\mathrm{kN}-316$. The default grid definition and the source entropy are shown in the output edits as follows:

$* * * * *$ warning $* * * * *$ keno message number k6-316 follows:

No mesh provided for Source Convergence Diagnostics (SCD). Continue with default mesh. ...

....... finished in Keno-VI before tracking

0.00183 minutes were used processing data.

$===>$ Grid Geometry: 10001

$===>$ title: Default mesh grid with $\mathrm{nx}=5$, ny $=5$, and $\mathrm{nz}=5$

$===>\quad$ Plane Summary

$===\Rightarrow \quad \mathrm{x}: 5$ cells from $-1.30100 \mathrm{E}+01$ to $1.30100 \mathrm{E}+01$

$===>\quad \mathrm{y}: 5$ cells from $-1.37400 \mathrm{E}+01$ to $1.37400 \mathrm{E}+01$

$===>\quad$ z: 5 cells from $-1.37400 \mathrm{E}+01$ to $1.37400 \mathrm{E}+01$

$===>$ Total number of cells: 125

volume fraction of fissile material in the system $=4.54932 \mathrm{E}-01$

start type 0 was used.

the neutrons were started with a flat distribution in a cuboid defined by: 


$$
+\mathrm{x}=1.30100 \mathrm{E}+01-\mathrm{x}=-1.30100 \mathrm{E}+01+\mathrm{y}=1.37400 \mathrm{E}+01 \quad-\mathrm{y}=-1.37400 \mathrm{E}+01+\mathrm{z}=1.37400 \mathrm{E}+01-\mathrm{z}=-1.37400 \mathrm{E}+01
$$

0.00017 minutes were required for starting. total elapsed time is 0.00200 minutes.

1 mesh test - scd with default mesh $(5 \times 5 \times 5)$, scd=yes (default)

\begin{tabular}{ccccccccc}
\multicolumn{2}{c}{ generation } & \multicolumn{2}{c}{ average } & \multicolumn{2}{c}{ avg k-eff } & \multicolumn{2}{c}{ generation } & \multicolumn{2}{c}{ matrix } & \multicolumn{2}{c}{ matrix k-eff } \\
generation & k-effective & k-effective & deviation & entropy & k-effective & deviation \\
1 & $9.17432 \mathrm{E}-01$ & $1.00000 \mathrm{E}+00$ & $0.00000 \mathrm{E}+00$ & $6.58169 \mathrm{E}+00$ & $0.00000 \mathrm{E}+00$ & $0.00000 \mathrm{E}+00$ \\
2 & $9.44195 \mathrm{E}-01$ & $1.00000 \mathrm{E}+00$ & $0.00000 \mathrm{E}+00$ & $6.54091 \mathrm{E}+00$ & $0.00000 \mathrm{E}+00$ & $0.00000 \mathrm{E}+00$
\end{tabular}

\section{EXAMPLE 2: SOURCE CONVERGENCE DIAGNOSTICS WITH USER-DEFINED MESH}

The user can request the accumulation of the fission source on a different grid rather than the default grid for source convergence diagnostics. In this example, the sample problem specified in EXAMPLE 1 is modified for this purpose. A new 6x6x6 grid is defined over the entire geometry in a READ GRID block with a grid ID 12, and source convergence diagnostics are requested by setting SCD parameter to 12 in the parameter block that matches the NUMBER specification in the READ GRID block.

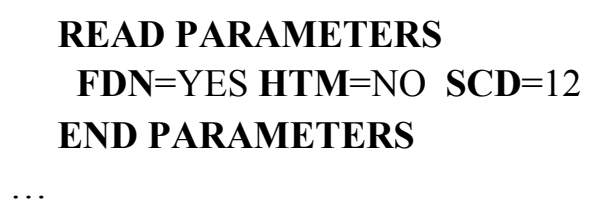

\section{READ GRID}

12

TITLE "TEST SCD WITH THIS MESH"

NUMXCELLS $=6$ NUMYCELLS $=6$ NUMZCELLS $=6$

$\mathbf{X M I N}=-13.01$ XMAX $=13.01$

YMIN $=-13.74$ YMAX $=13.74$

ZMIN $=-13.74$ ZMAX $=13.74$

END GRID

1

The output prints for the new mesh for source convergence diagnostics:

\section{....... finished in Keno-VI before tracking …....}

0.00200 minutes were used processing data.

\footnotetext{
$===>$ Grid Geometry: 12

$===>$ title: test SCD with this mesh

$===>\quad$ Plane Summary

$===>\quad \mathrm{x}: 6$ cells from $-1.30100 \mathrm{E}+01$ to $1.30100 \mathrm{E}+01$

$===>\quad \mathrm{y}: 6$ cells from $-1.37400 \mathrm{E}+01$ to $1.37400 \mathrm{E}+01$

$===>\quad \mathrm{z}: 6$ cells from $-1.37400 \mathrm{E}+01$ to $1.37400 \mathrm{E}+01$

$====>$ Total number of cells: 216
} 
volume fraction of fissile material in the system $=4.54932 \mathrm{E}-01$

start type 0 was used.

the neutrons were started with a flat distribution in a cuboid defined by:

$+\mathrm{x}=1.30100 \mathrm{E}+01-\mathrm{x}=-1.30100 \mathrm{E}+01+\mathrm{y}=1.37400 \mathrm{E}+01 \quad-\mathrm{y}=-1.37400 \mathrm{E}+01+\mathrm{z}=1.37400 \mathrm{E}+01-\mathrm{z}=-1.37400 \mathrm{E}+01$

\section{EXAMPLE 3: SOURCE CONVERGENCE DIAGNOSTICS WITH NON-EXISTING MESH}

This example demonstrates the code behavior if there is a mismatch between the NUMBER entry in READ GRID data block and the SCD parameter in the READ PARAMETER data block. The sample input in EXAMPLE 2 was modified as (a) a single mesh is defined with a grid ID $=12$, and (b) source convergence diagnostics is intended to use a grid with grid $\mathbf{I D}=99$, which does not exist.

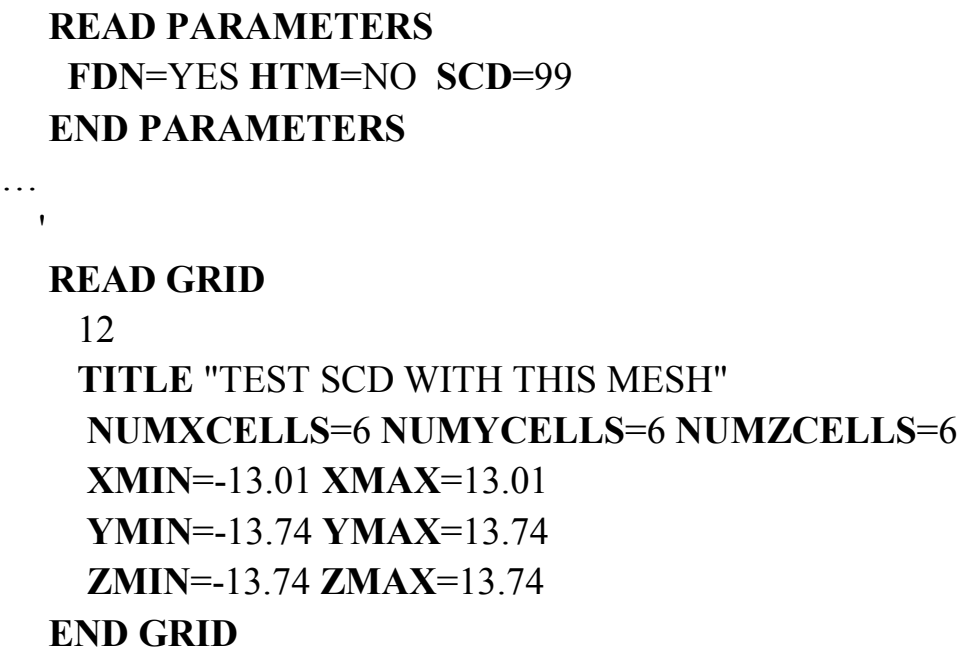

In this case, calculation is stopped with an error message since the requested grid definition for source convergence diagnostics does not exist in the input.

$* * * * * * * * * * * * * * *$ data reading completed $* * * * * * * * * * * * * * *$

....... finished preparing the keno-vi input data

finished loading the data

$* * * * *$ error $* * * * *$ csas message number cs-315 follows:

Mesh $<99>$ specified for Source Convergence Diagnostics(SCD) is not found in input. .

finished processing csas input data 


\section{EXAMPLE 4: MESH FLUX TALLY WITH A MESH DEFINITION WITH MSH PARAMETER}

In this example, we slightly modify the sample problem described in EXAMPLE 1 to request a mesh flux tally calculations over a uniform mesh defined with the MSH parameter. In this case, the default mesh (grid ID=10001) is used for the source convergence diagnostics, and the simple uniform mesh (grid ID=20001) generated with the MSH parameter is used for a mesh flux tally.

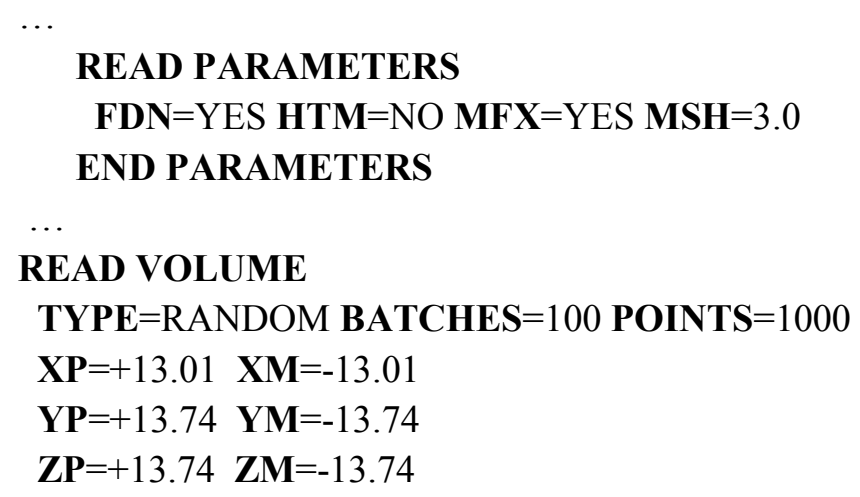

\section{END VOLUME}

The output for this sample problem has the following sections for mesh definitions/mesh quantities:

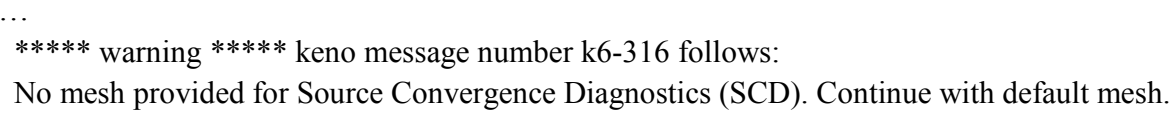

volumes for those units utilized in this problem

volumes not specified in the input were set to -1.0

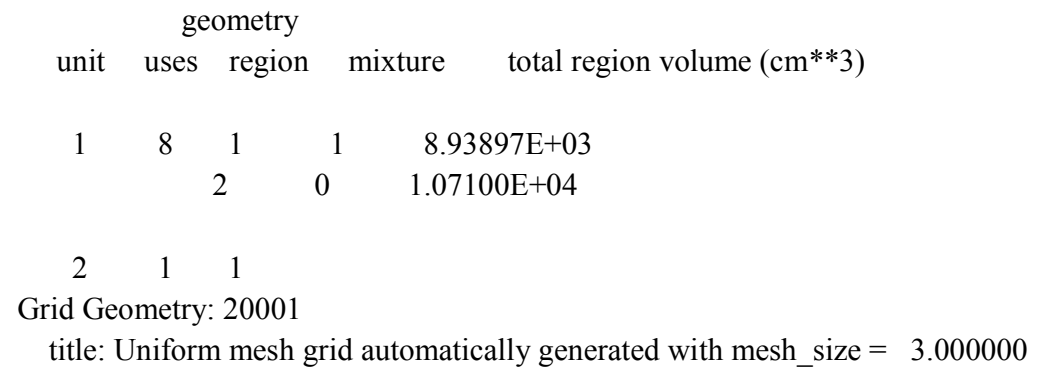




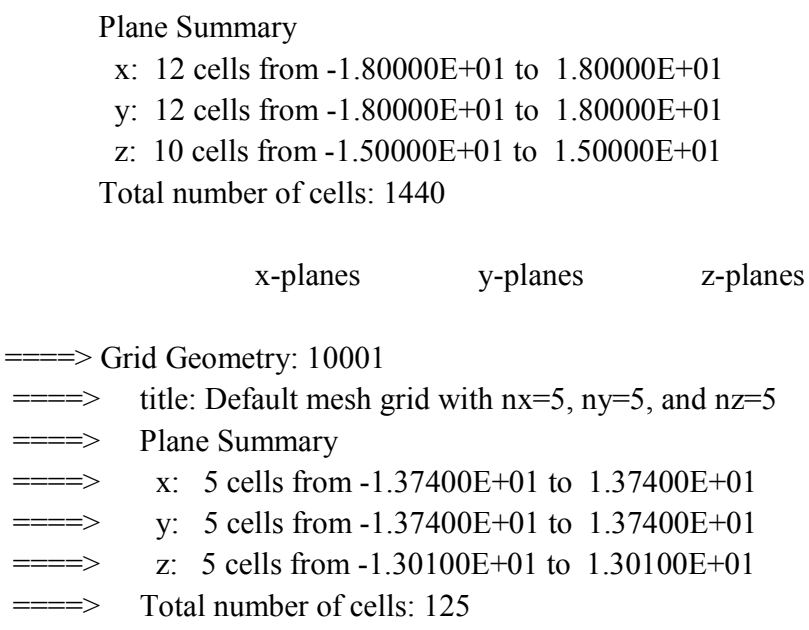

EXAMPLE 5: MESH FLUX TALLY WITH A MESH DEFINITION IN READ GRID DATA BLOCK

In this example, we slightly modify the sample problem described in EXAMPLE 1 to request a mesh flux tally calculation over a mesh defined in the READ GRID data block. In this case, the default mesh (grid $\mathbf{I D}=10001$ ) is used for the source convergence diagnostics, and the user defined mesh (grid $\mathbf{I D}=12$ ) is used for the mesh flux tally.

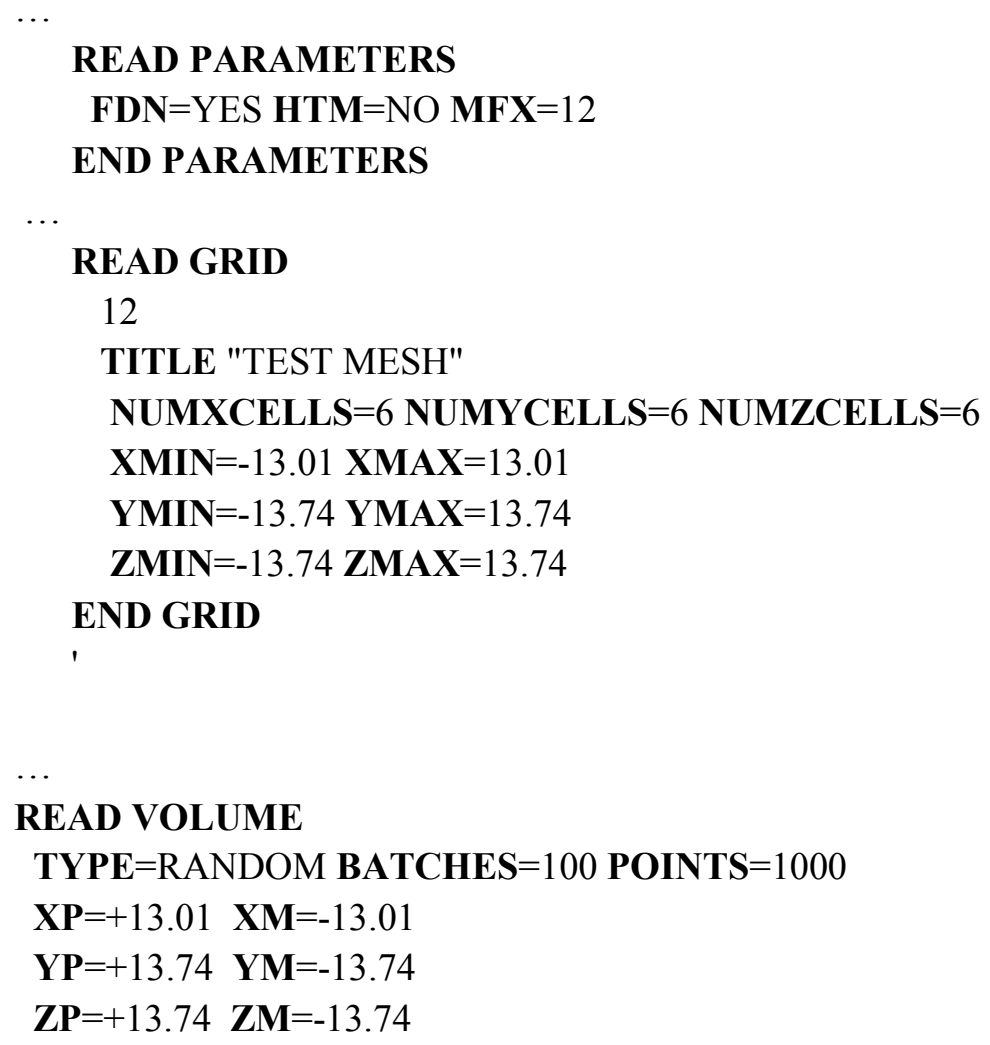


The output for this sample problem has the following sections for mesh definitions/mesh quantities:

***** warning $* * * * *$ keno message number k6-316 follows:

No mesh provided for Source Convergence Diagnostics (SCD). Continue with default mesh.

1

kenovi sample problem 1 case $2 \mathrm{c} 8$ bare

volumes for those units utilized in this problem

volumes not specified in the input were set to -1.0

\begin{tabular}{cccccc}
\multicolumn{7}{c}{ geometry } \\
unit & uses & region & mixture & total region volume $(\mathrm{cm} * * 3)$ \\
1 & 8 & 1 & 1 & $8.93897 \mathrm{E}+03$ \\
& & 2 & 0 & $1.07100 \mathrm{E}+04$
\end{tabular}

$\begin{array}{lll}2 & 1 & 1\end{array}$

Grid Geometry: 12

title: test - mesh

Plane Summary

$\mathrm{x}$ : 6 cells from $-1.37400 \mathrm{E}+01$ to $1.37400 \mathrm{E}+01$

$\mathrm{y}$ : 6 cells from $-1.37400 \mathrm{E}+01$ to $1.37400 \mathrm{E}+01$

z: 6 cells from $-1.30100 \mathrm{E}+01$ to $1.30100 \mathrm{E}+01$

Total number of cells: 216

$====>$ Grid Geometry: 10001

$===>$ title: Default mesh grid with $\mathrm{nx}=5$, ny $=5$, and $\mathrm{nz}=5$

$===>$ Plane Summary

$===>\quad \mathrm{x}: 5$ cells from $-1.37400 \mathrm{E}+01$ to $1.37400 \mathrm{E}+01$

$===>\quad \mathrm{y}: 5$ cells from $-1.37400 \mathrm{E}+01$ to $1.37400 \mathrm{E}+01$

$===>\quad$ z: 5 cells from $-1.30100 \mathrm{E}+01$ to $1.30100 \mathrm{E}+01$

$====$ Total number of cells: 125

\section{EXAMPLE 6: MESH FLUX TALLY WITH A MESH DEFINITION IN READ GRID DATA BLOCK}

In this example, the sample input in EXAMPLE 5 is used with the old-style definition, mesh flux tally is requested with setting MFX parameter to yes. In this case, the default mesh (grid $\mathbf{I D}=10001$ ) is used for the source convergence diagnostics, and the first defined user defined mesh (grid $\mathbf{I D}=12$ ) is used for the mesh flux tally.

$\cdots$

\section{READ PARAMETERS \\ FDN=YES HTM=NO MFX $=$ YES \\ END PARAMETERS}

...

\section{READ GRID}

12

TITLE "TEST MESH" 


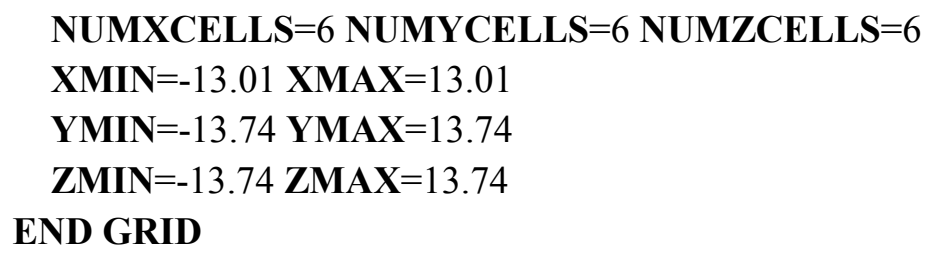

END VOLUME

The output for this sample problem has the following sections for mesh definitions/mesh quantities:

$* * * * *$ warning $* * * * *$ keno message number k6-316 follows:

No mesh provided for Source Convergence Diagnostics (SCD). Continue with default mesh.

volumes for those units utilized in this problem

volumes not specified in the input were set to -1.0

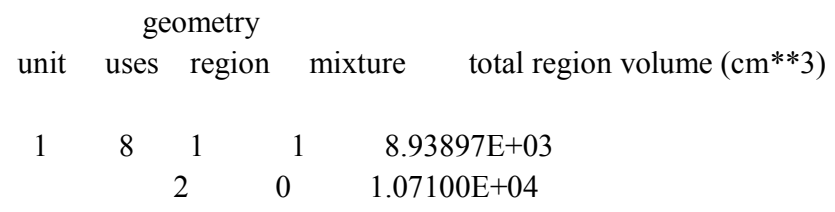


In this example, the sample problem described in EXAMPLE 1 is modified to request a mesh flux tally calculation over a mesh over a part of geometry defined in the READ GRID data block. In this case, the default mesh (grid ID=10001) is used for the source convergence diagnostics, and the user-defined mesh (grid ID=14) is used for the mesh flux tally. Code execution is terminated since KENO requires the mesh definition to cover the whole geometry for the mesh flux tally.

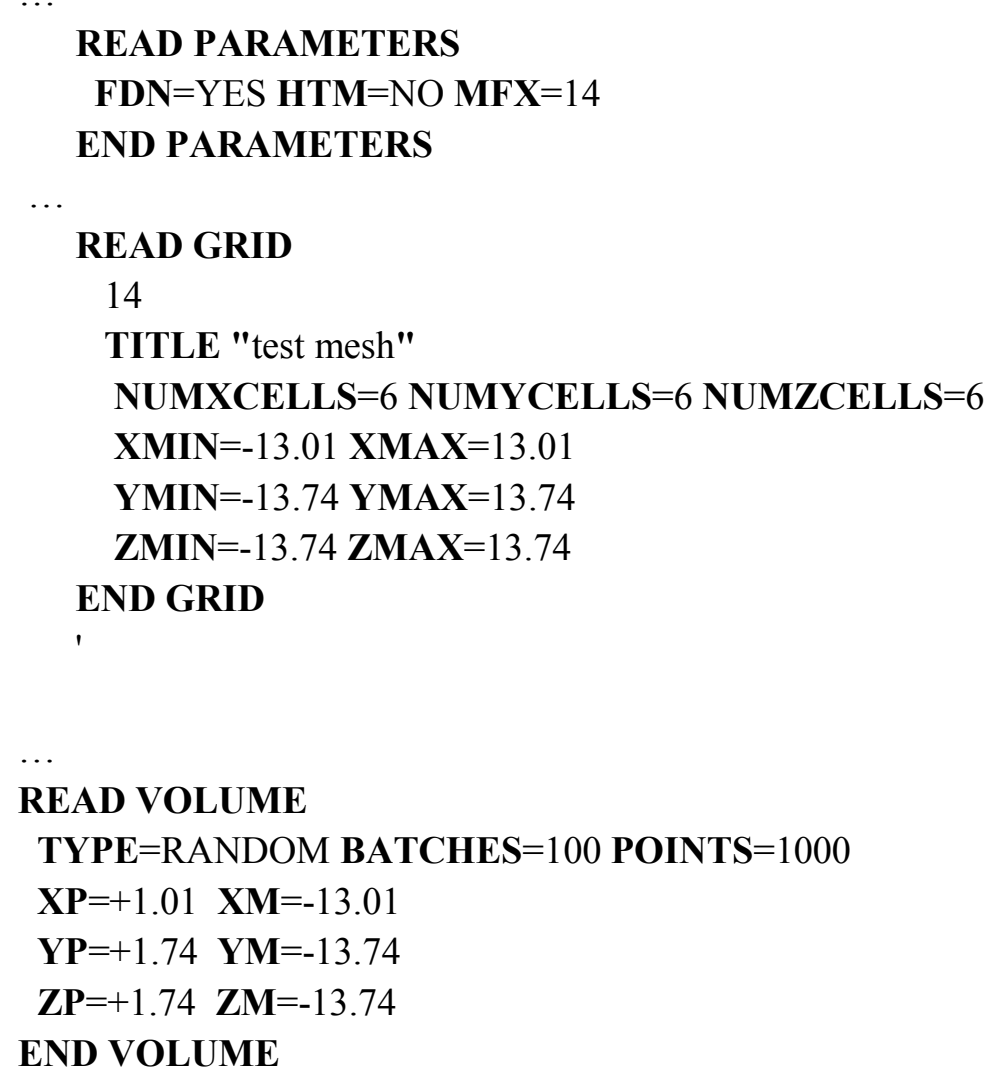

The output prints for this sample problem have the following sections for mesh definitions/mesh quantities:

No mesh provided for Source Convergence Diagnostics (SCD). Continue with default mesh.

Mesh Volume Sampling Parameters

The number of points per batch was specified as 1000

This gives a sampling density of 5.08931E-02 points per cc per batch.

The number of batches is 100

$* * * * *$ error $* * * * *$ csas message number cs-305 follows:

Mesh fluxes have been specified, but the mesh does not completely cover the geometry.

The point $\mathrm{x}=3.85512 \mathrm{E}+00 \mathrm{y}=1.90999 \mathrm{E}+00 \mathrm{z}=-9.70580 \mathrm{E}+00$ lies outside the mesh.

The problem will not be run. Fix the mesh and resubmit the case. 


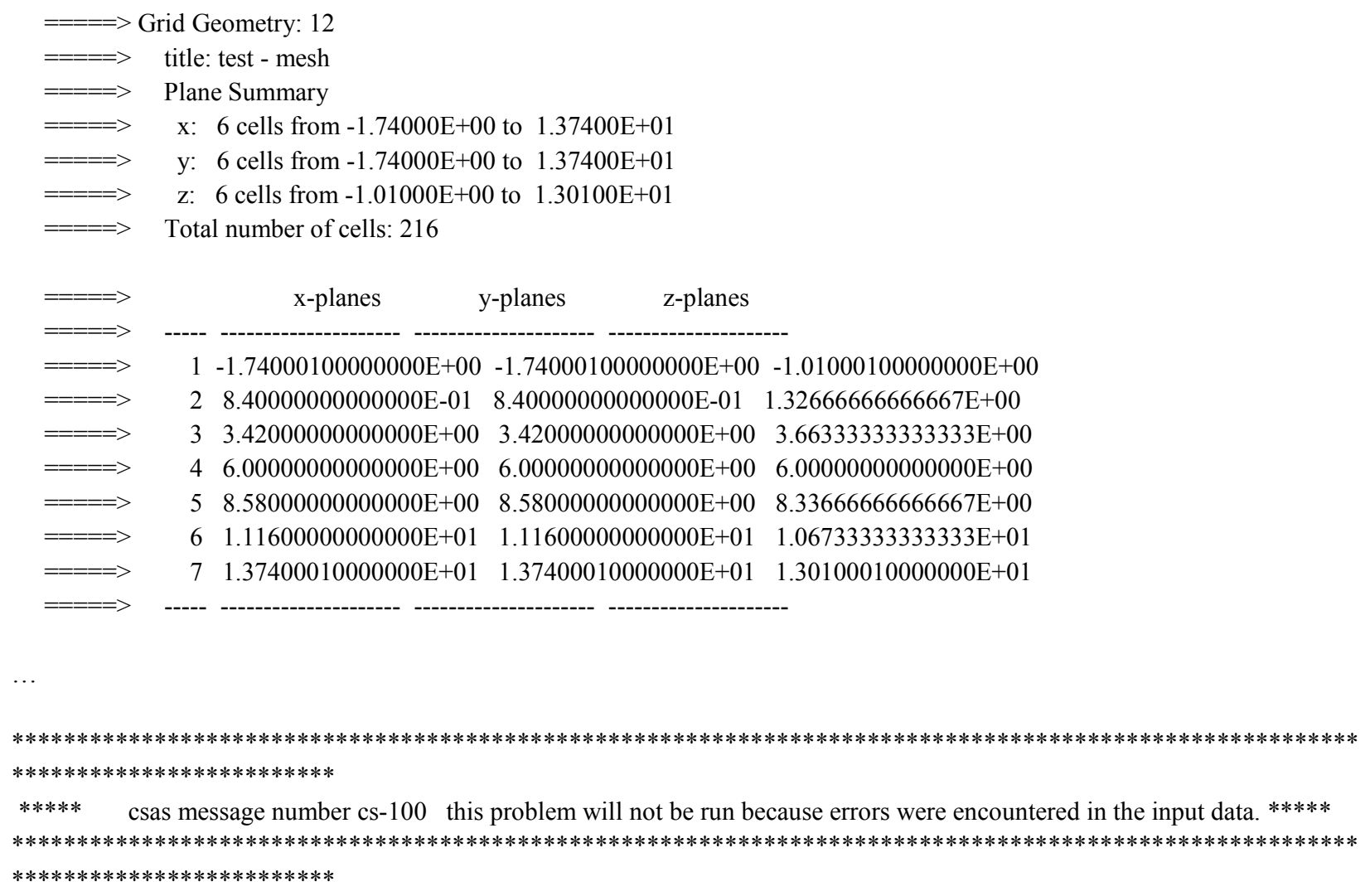


This example demonstrates the multiple mesh definition for multiple mesh-based quantities. Our sample problem is modified so that (1) two grids have been defined with the READ GRID data block, and (2) both the source convergence diagnostics and mesh flux tally are requested. In this sample, the grid with grid ID = 12 is assigned to the source convergence diagnostics, and the grid with grid ID = 1 is assigned to the mesh flux tally.

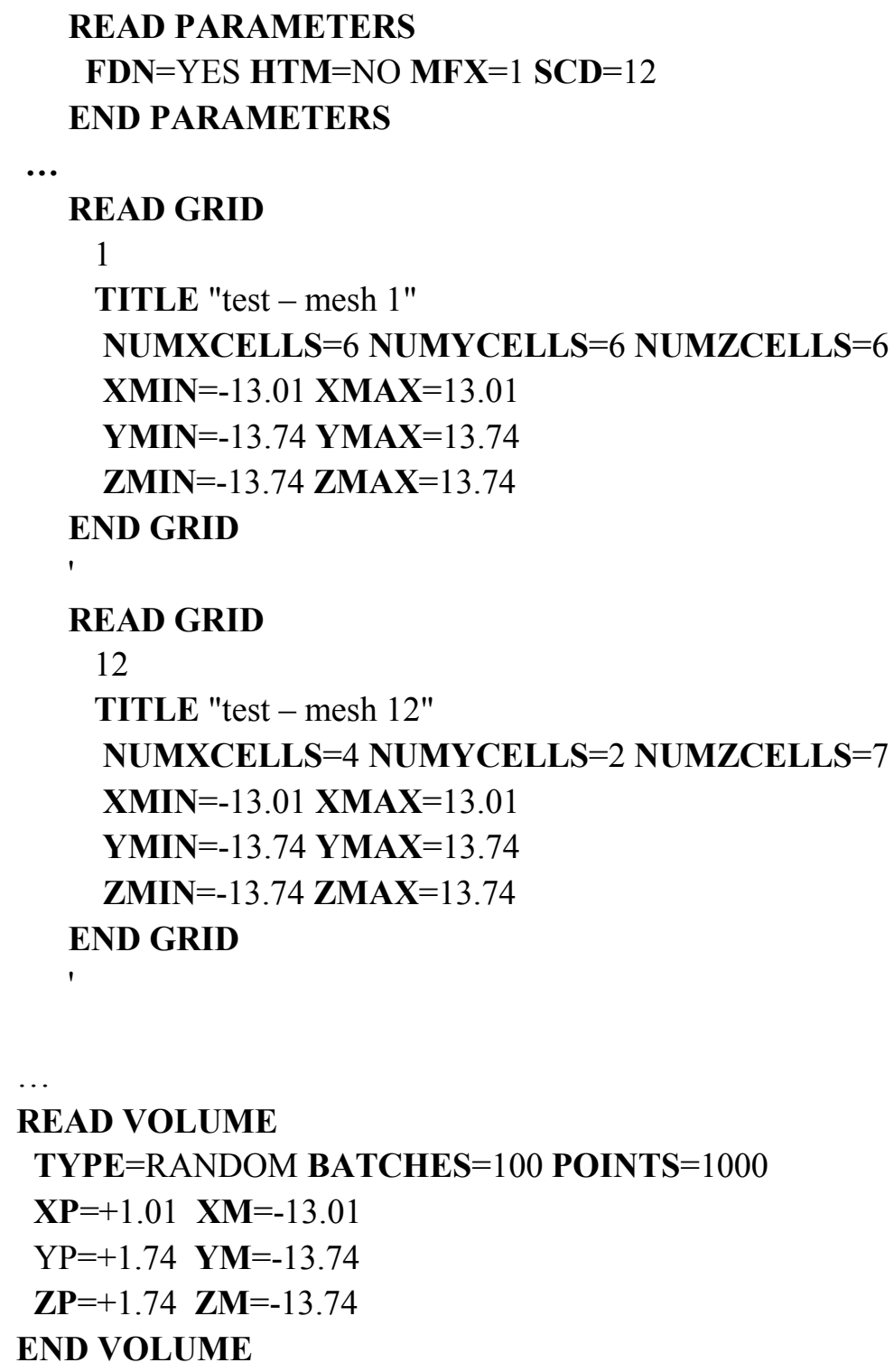

KENO prints the following sections for mesh definitions/mesh quantities in the output:

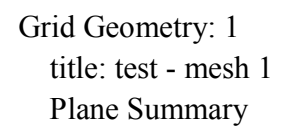




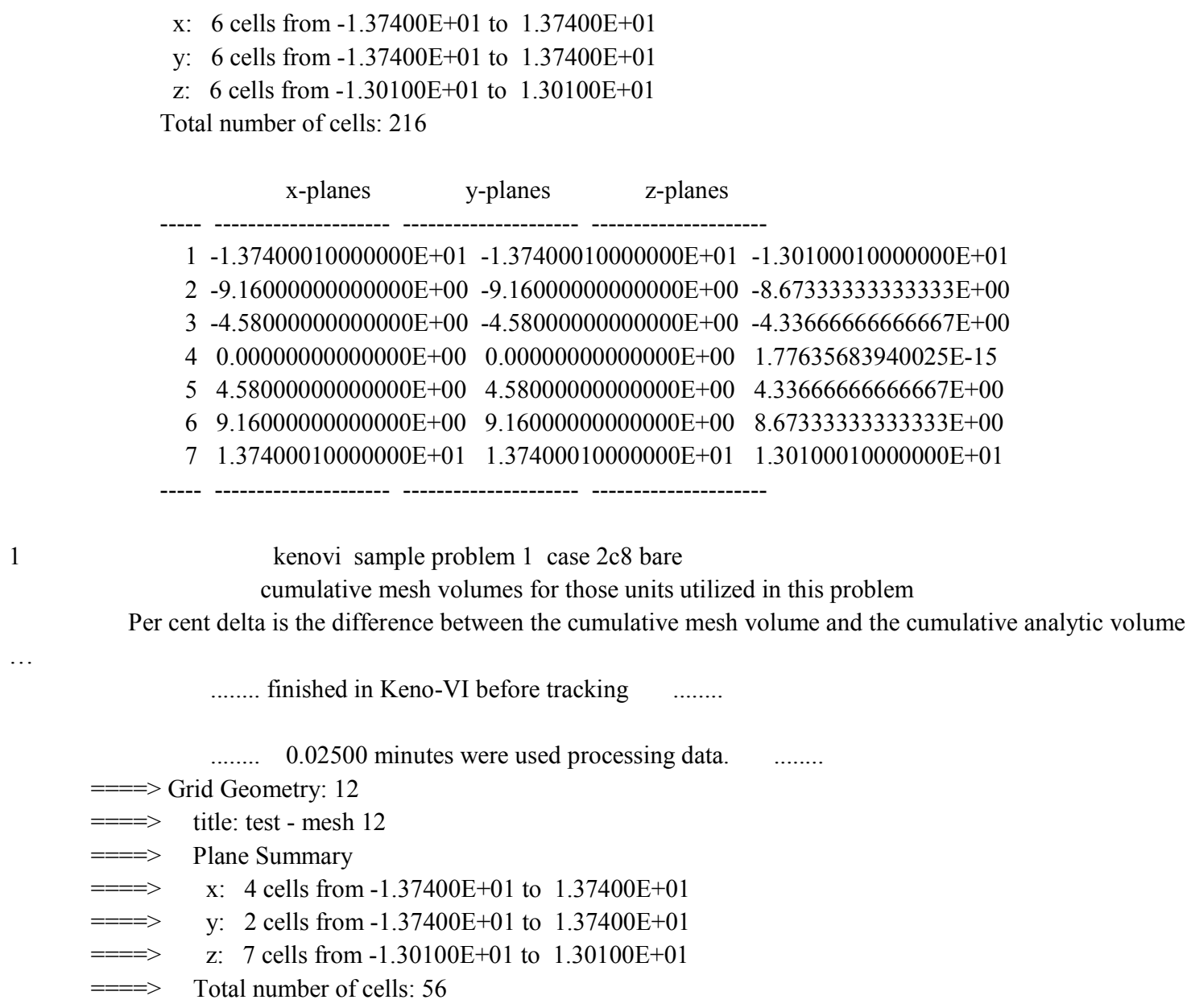

\section{EXAMPLE 9: MULTIPLE MESH QUANTITY WITH A SINGLE MESH DEFINITION}

This example demonstrates that the same grid may be used for multiple mesh-based quantities. Our sample problem is modified as (1) a single grid has been defined with READ GRID data block, and (2) both source convergence diagnostics and mesh flux tally are requested. In this sample, the grid with grid ID $=1$ is assigned to both the source convergence diagnostics and the mesh flux tally. KENO calculates the specified quantities using the same grid data.

\section{READ PARAMETERS \\ FDN $=$ YES HTM $=$ NO MFX $=1$ SCD $=1$ \\ END PARAMETERS}

\section{READ GRID}

1

TITLE "test - mesh 1"

NUMXCELLS $=6$ NUMYCELLS $=6$ NUMZCELLS $=6$

$\mathbf{X M I N}=-13.01$ XMAX=13.01

YMIN=-13.74 YMAX $=13.74$ 


\section{ZMIN=-13.74 ZMAX=13.74 \\ END GRID \\ 1}

\section{・. \\ READ VOLUME \\ $\mathbf{X P}=+1.01 \quad \mathbf{X M}=-13.01$ \\ $\mathbf{Y P}=+1.74 \quad \mathbf{Y M}=-13.74$ \\ $\mathbf{Z P}=+1.74 \mathbf{Z M}=-13.74$}

TYPE $=$ RANDOM BATCHES $=100$ POINTS $=1000$

END VOLUME

KENO prints the following sections for mesh definitions/mesh quantities in the output:

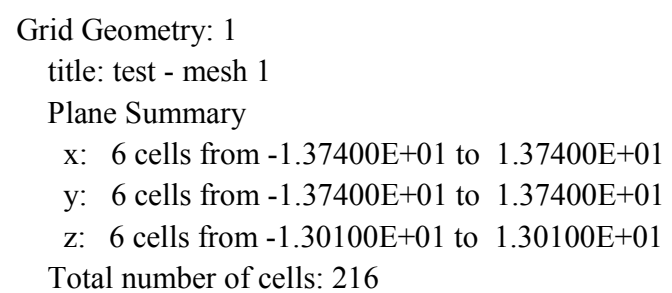

Total number of cells: 216

\begin{tabular}{rrrrr} 
& \multicolumn{1}{c}{ x-planes } & y-planes & \multicolumn{2}{c}{ z-planes } \\
\hline 1 & $-1.37400010000000 \mathrm{E}+01$ & $-1.37400010000000 \mathrm{E}+01$ & $-1.30100010000000 \mathrm{E}+01$ \\
2 & $-9.16000000000000 \mathrm{E}+00$ & $-9.16000000000000 \mathrm{E}+00$ & $-8.67333333333333 \mathrm{E}+00$ \\
3 & $-4.58000000000000 \mathrm{E}+00$ & $-4.58000000000000 \mathrm{E}+00$ & $-4.33666666666667 \mathrm{E}+00$ \\
4 & $0.00000000000000 \mathrm{E}+00$ & $0.00000000000000 \mathrm{E}+00$ & $1.77635683940025 \mathrm{E}-15$ \\
5 & $4.58000000000000 \mathrm{E}+00$ & $4.58000000000000 \mathrm{E}+00$ & $4.33666666666667 \mathrm{E}+00$ \\
6 & $9.16000000000000 \mathrm{E}+00$ & $9.16000000000000 \mathrm{E}+00$ & $8.6733333333333 \mathrm{E}+00$ \\
7 & $1.37400010000000 \mathrm{E}+01$ & $1.37400010000000 \mathrm{E}+01$ & $1.30100010000000 \mathrm{E}+01$
\end{tabular}

1

kenovi sample problem 1 case $2 \mathrm{c} 8$ bare cumulative mesh volumes for those units utilized in this problem

Per cent delta is the difference between the cumulative mesh volume and the cumulative analytic volume ....... finished in Keno-VI before tracking 0.02500 minutes were used processing data.

\footnotetext{
$===>$ Grid Geometry: 1

$===>$ title: test - mesh 1

$===>\quad$ Plane Summary

$===>\quad \mathrm{x}: 6$ cells from $-1.37400 \mathrm{E}+01$ to $1.37400 \mathrm{E}+01$

$===>\quad \mathrm{y}: 6$ cells from $-1.37400 \mathrm{E}+01$ to $1.37400 \mathrm{E}+01$

$===>\quad \mathrm{z}: 6$ cells from $-1.30100 \mathrm{E}+01$ to $1.30100 \mathrm{E}+01$

$====$ Total number of cells: 216
} 


\subsubsection{Random sequence}

The random-number package used by KENO always starts with the same seed and thus always reproduces the same sequence of random numbers. Any random number except the one printed as the starting random number in the parameter table can be used to activate a different random sequence. The user can rerun a problem with a different random sequence by simply entering a hexadecimal random number other than the starting random number in the parameter data. For example, by entering $\mathrm{RND}=\mathrm{A} 10 \mathrm{C} 1893 \mathrm{E} 6 \mathrm{D} 5$ in the parameter data, the problem will be run with a different random sequence.

\subsubsection{Matrix k-effective}

Matrix k-effective calculations provide an alternative method of calculating the k-effective of the system. Cofactor k-effectives and source vectors are additional information that can be provided when the matrix k-effective is calculated. The necessary source and fission weight data are collected during the neutron tracking procedure. This information is converted to a FISSION PRODUCTION MATRIX, which is the number of next generation neutrons produced at $\mathrm{J}$ by a neutron born at $\mathrm{I}$. The principal eigenvalue of the fission probability matrix is the matrix k-effective. KENO offers four alternatives when calculating matrix k-effective as discussed below:

(1) If MKP=YES is specified in the parameter data, the fission production matrix is collected by array position or position index in the GLOBAL ARRAY. The position index is used to reference a given location in a 3-D lattice. For a $2 \times 2 \times 2$ array, there are nine unique position indices as shown below. Position zero contains everything outside the GLOBAL ARRAY.

Table 8.1.24. Array index guide for a sample $2 \times 2 \times 2$ array

\begin{tabular}{llll}
\hline & \multicolumn{3}{l}{ POSITION } \\
\cline { 2 - 4 } & $\mathrm{X}$ & $\mathrm{Y}$ & $\mathrm{Z}$ \\
\hline 0 & 0 & 0 & 0 \\
1 & 1 & 1 & 1 \\
2 & 2 & 1 & 1 \\
3 & 1 & 2 & 1 \\
4 & 2 & 2 & 1 \\
5 & 1 & 1 & 2 \\
6 & 2 & 1 & 2 \\
7 & 1 & 2 & 2 \\
8 & 2 & 2 & 2 \\
\hline
\end{tabular}

The fission production matrix is the number of next generation neutrons produced at index $\mathrm{J}$ by a neutron born at index I. This matrix is used to calculate the matrix k-effective, cofactor k-effectives and the source vector by position index. Because the size of the fission probability matrix is the square of the array size (for a $4 \times 4 \times 4$ array there are 4,096 entries), it can use vast amounts of computer memory. 
(2) If $\mathbf{M K U}=\mathrm{YES}$ is specified in the parameter data, the fission production matrix is collected by UNIT. It is the number of next generation neutrons produced in UNIT J by a neutron born in UNIT I. This matrix is used to calculate the matrix k-effective, cofactor k-effectives and source vector by UNIT.

(3) If MKH=YES is specified in the parameter data, the fission production matrix is collected by the HOLE number. Matrix information can be collected at either the highest HOLE nesting level (first level of nesting) or the deepest HOLE nesting level. HHL=YES specifies that the matrix information will be collected at the first nesting level. By default, the matrix information is collected at the deepest nesting level. The fission production matrix is the number of next generation neutrons produced in HOLE J by a neutron born in HOLE I. This matrix is used to calculate the matrix k-effective, cofactor k-effectives and the source vector by HOLE.

(4) If MKA=YES is specified in the parameter data, the fission production matrix is collected by ARRAY number. It can be collected at the highest ARRAY level (first level of nesting) or at the deepest ARRAY level. HAL=YES specifies that the matrix information will be collected at the first nesting level. By default, the matrix information is collected at the deepest nesting level. The fission production matrix is the number of next generation neutrons produced in ARRAY J by a neutron born in ARRAY I. This matrix is used to calculate the matrix k-effective, cofactor k-effectives and the source vector by ARRAY.

The user can simultaneously implement all methods of calculating the matrix k-effective. The results are labeled in the printout. Matrix k-effectives cannot be calculated for a single unit problem. If the user wishes to do so, the geometry description must have a cube or cuboid as its outer region, and the problem description should include READ ARRAY END ARRAY. These two actions convert the single unit problem into a $1 \times 1 \times 1$ array.

A cofactor k-effective is the eigenvalue of the fission production matrix reduced by the row and column that references the specified UNIT or position index. The difference between the k-effective for the system and the cofactor k-effective for a UNIT or position index is an indication of the in situ k-effective of that UNIT or the contribution that UNIT makes to the k-effective of the system. The cofactor k-effective of a UNIT devoid of fissile material should approximate the k-effective of the system.

\subsubsection{Deviations}

When a deviation is calculated by KENO, it is the standard deviation of the mean. This assumes a large sample having a normal distribution. KENO calculates the real variance using an iterative approach and lag covariance data between generations as follows: ${ }^{4,5}$

1. The sample variance and covariance estimates are calculated.

2. The apparent variance is set equal to the sample variance and the apparent covariance is set equal to the sample covariance.

3. The real covariance is set equal to the apparent covariance and the real variance is calculated.

4. Using the real variance and apparent covariance calculate the real covariance.

5. The real variance is recalculated.

6. Steps 4 and 5 are repeated until the real variance converges within a preset tolerance. 
The covariance estimates are only calculated for the previous 20 generations. A maximum of 50 iterations are allowed for the real variance to converge.

\subsubsection{Generation time and lifetime}

The generation time and lifetime calculations use the average velocity. The validity of these calculations is determined by how accurately the average velocity represents the spectrum over the range of the energy group. The lifetime and generation time calculated by KENO are not kinetics parameters. The lifetime is the average life span of a neutron (in seconds) from the time it is born until it is absorbed or leaks from the system. The generation time is the average time (in seconds) between successive neutron generations.

\subsubsection{Energy of the Average Lethargy of Fission}

The energy of the average lethargy of neutrons causing fission $(E A L F)$ is a parameter calculated in KENO to characterize the neutron energy spectrum or fastness of a system. The $E A L F$ is given in units of eV in the KENO output. An $E A L F$ value that is high $(>100 \mathrm{keV})$ indicates that most fissions in the system are being caused by fast neutrons, and an $E A L F$ value that is low $(<1 \mathrm{eV})$ indicates that most fissions are induced by thermal neutrons.

The $E A L F$ is calculated by determining the lethargy, $u$, a measure of how much neutron energy changes from its initial or birth energy:

$$
u=\ln \left(\frac{E_{0}}{E}\right)
$$

where $E$ is the energy of the neutron colliding with a nucleus and $E_{0}$ is the maximum possible energy of fission neutrons (assumed in KENO to be $10 \mathrm{MeV}$ ). The average lethargy of all fission events is calculated by weighting the lethargy for each collision by the probability that the collision will create a fission event, and averaging this quantity. Using a log-scale parameter like lethargy to represent the fastness of systems is more convenient than directly averaging the energy of neutrons causing fission both because of the wide range of neutrons in a problem (potentially more than seven orders of magnitude), and because fast neutrons that are slowing down lose about the same fraction of their energy during each collision.

$$
E A L F=E_{o} e^{-u_{a v g}}
$$

Equation (8.1.2) is then applied again to transform the average lethargy of neutrons causing fission into a neutron energy that corresponds to the average lethargy of neutrons causing fission (this is the EALF); this transformation back to units of energy is done because energy has a much more intuitive meaning than units of lethargy.

\subsubsection{Description of Output}

This section contains a brief description and explanation of the KENO output. Portions of the printout will not be printed for every problem. Some printout is optional, as noted in this section. This section 
provides representative samples of the output format. The actual data contained in this section are not necessarily consistent with results computed by the current version of KENO.

KENO offers an HTML output format including a series of files that can be viewed in a standard web browser. The HTML formatted output offers interactive output that is easy to read and navigate. Many of the tables of data can be sorted in ascending or descending order by clicking on the heading of the column for which sorting is desired. Interactive plotting of fluxes, fission production, absorption, and k-effective values are also available within the HTML output through an applet version of the Javapeño plotting package. In this section, the standard text output description is followed by a description of the optional HTML formatted output. The HTML formatted output can be deactivated by entering HTM=NO in the parameter data section.

\subsubsection{Program verification information}

Program verification information (Fig. 8.1.97) is printed after the header page. It lists the name of the program, the date the load module was created, the library that contains the load module, the computer code name from the configuration control table, and the revision number. The job name, date, and time of execution are also printed. This information may be used for quality assurance purposes.

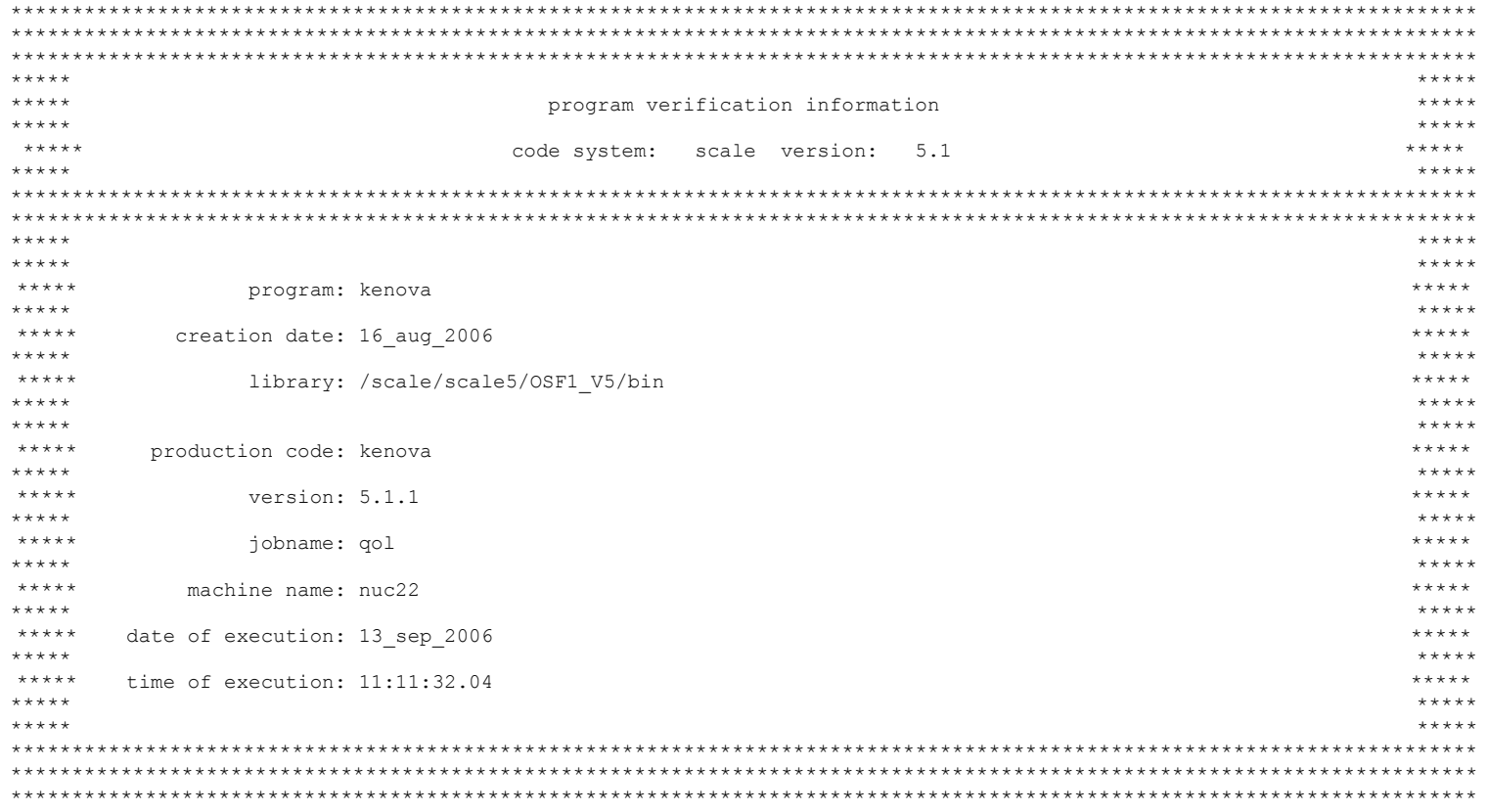

Fig. 8.1.97. Sample program verification table.

The program verification information is the first page shown in the KENO HTML output, after selecting KENO from the SCALE HTML index page. This page can also be displayed by selecting the Program Verification Information link under the General Information submenu and is shown in Fig. 8.1.97. 


\subsubsection{Tables of parameter data}

The first two tables printed by KENO list the numeric parameters and logical parameters used in the problem. The user should always verify that the parameter data block was entered as desired. An example of numeric parameters table is shown in Fig. 8.1.98. An example of the logical parameters table is shown in Fig. 8.1.99.

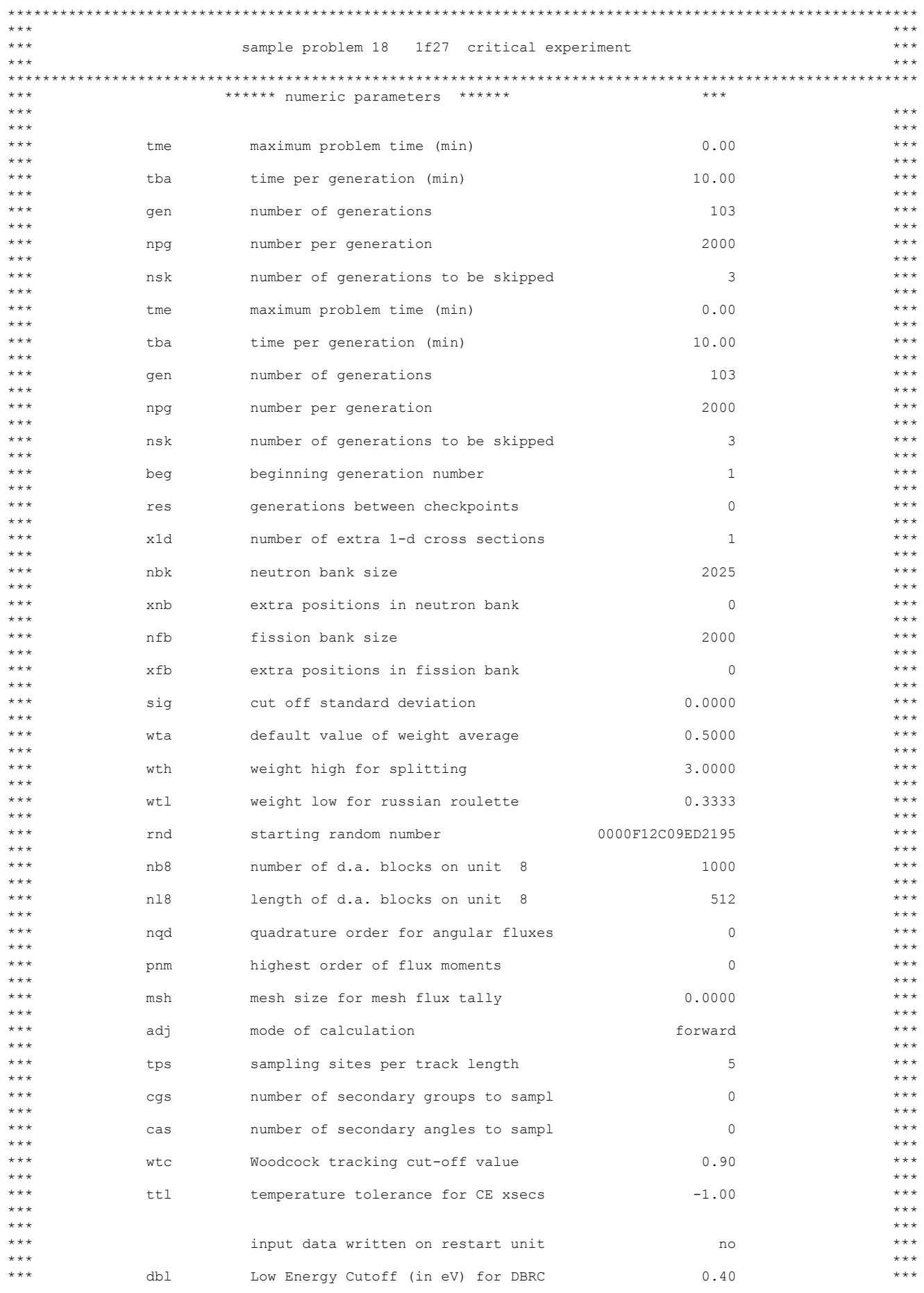




\begin{tabular}{|c|c|c|c|c|}
\hline 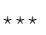 & & & & *** \\
\hline $\begin{array}{ll}* \star \star \\
\star \star \star \star *\end{array}$ & $\mathrm{dbh}$ & High Energy Cutoff (in eV) for DBRC & 210.00 & $\begin{array}{l}\star \star \star \\
\star \star \star *\end{array}$ \\
\hline$\star \star \star$ & cet & CE-TSUNAMI sensitivity method & NONE & 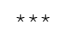 \\
\hline * & $\operatorname{cfp}$ & Number of latent gens in CE-TSUNAMI & -1 & 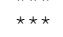 \\
\hline$\star \star \star * \star *$ & $d b r$ & DBRC isotope selection & 0 & ** \\
\hline ** & $d b x$ & Doppler Broadening Method & 0 & 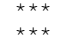 \\
\hline & & & & \\
\hline
\end{tabular}

Fig. 8.1.98. Sample table of numeric parameter data. 


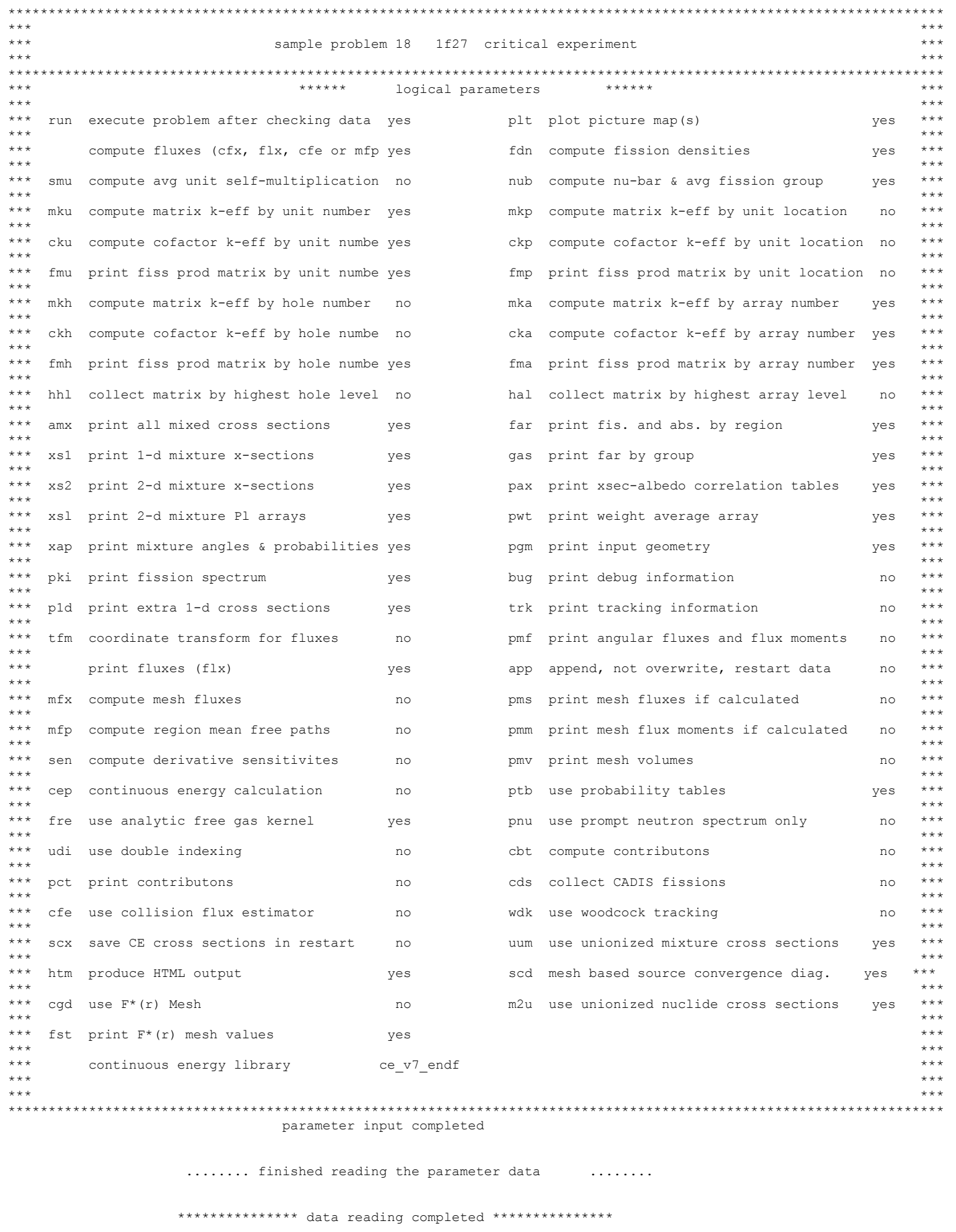

Fig. 8.1.99. Sample table of logical parameter data. 
For the logical parameter data table, messages concerning the parameter data may be printed at the bottom of the table. If the problem is being restarted, the title of the parent case is printed at the bottom of the table. If the restart title or messages are not printed, the bottom section of the table is omitted.

\subsubsection{Unprocessed geometry input data}

This printout is optional and is usually used to locate code difficulties, to show all the geometry input data when only part of it is used in the problem, or to show the order in which units were entered. It is considered debug information and is printed only if PGM=YES is specified in the parameter input data. Standard KENO use does not require printing these data because the processed geometry that is used in the problem is always printed. See Sects. 8.1.4.52.9 and 8.1.4.13 for examples of the standard printed KENO geometry data.

When the unprocessed geometry input is printed, the problem title is located at the top of the page, followed by the heading "GEOMETRY DESCRIPTION INPUT." The region-dependent geometry information is then printed. If the problem contains a unit orientation array, the problem title is printed again, followed by the unit orientation. This is followed by a statement affirming the completion of the data input.

\subsubsection{Table of data sets used in the problem}

This table is the third table of data printed by KENO. It should be carefully scrutinized to verify the desired data set name is associated with the proper unit number and volume. An example of this table is shown in Fig. 9.1.100.

Fig. 8.1.100

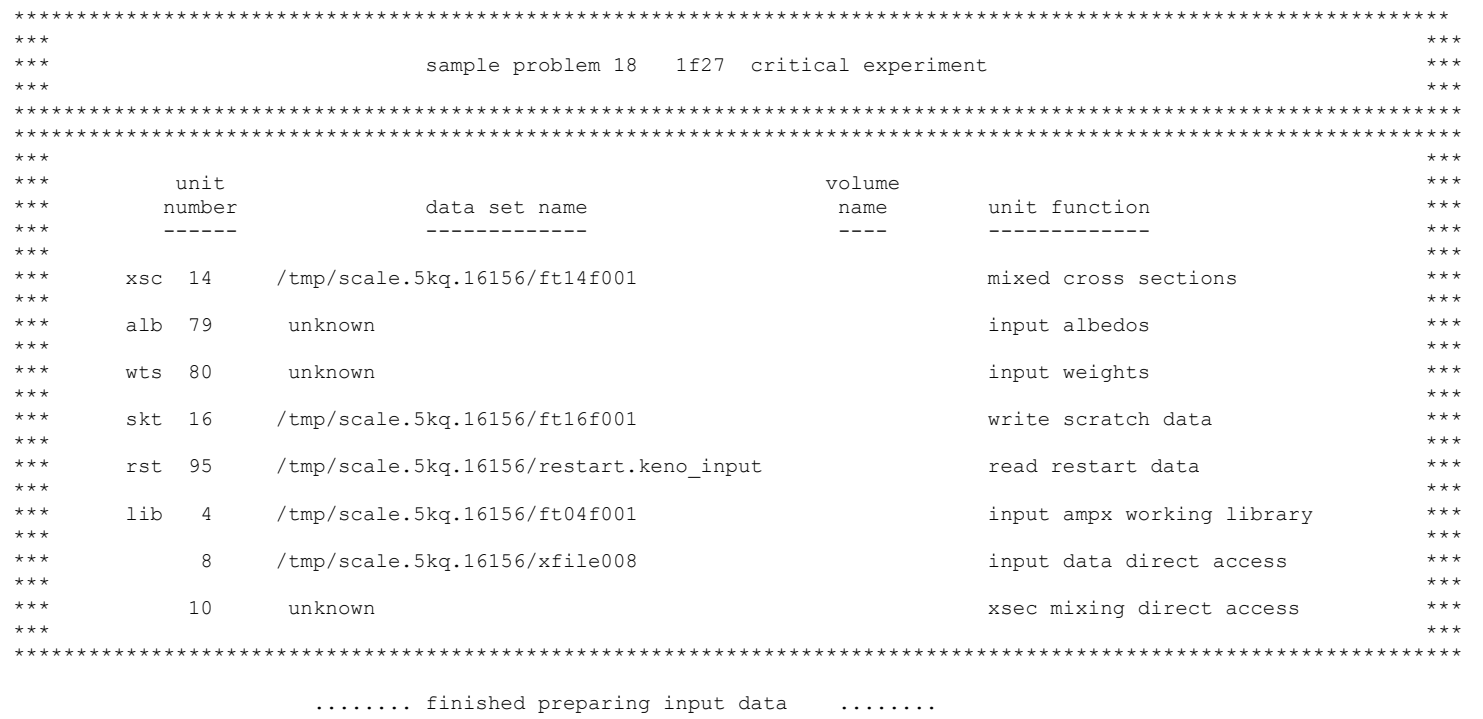

Fig. 8.1.100. Sample table of data sets used in the problem.

This table lists unit numbers specified in the parameter data or that are defaulted in the code, along with the information pertinent to them. This information is given in the following order, left to right: (1) the keyword used in the parameter data to define the unit number, (2) the unit number, (3) the data set name, (4) the name of the volume on which the data set resides, and (5) the type of data contained on the data set. This table can be useful for quality assurance purposes. Information for units for which default values have not been overridden is printed even though they may not be used in the problem. Information for 
every unit specified in the parameter data is also printed. Units 8 and 10 are the direct-access devices, and their unit numbers are fixed within the code. When this table is printed, Unit 10 has not yet been defined. This causes its data set names to be listed as FT10F001 or as "UNKNOWN" on some systems. If KENO is run as part of a CSAS sequence, this table will include two entries for Unit 95: one for binary input data, and one for read restart data.

\subsubsection{Table of additional information}

The fourth table of data printed by KENO contains additional information determined from the input data. An example of this table is shown in Fig. 8.1.101.

This table should be used to verify the problem input. The NUMBER OF ENERGY GROUPS for a multigroup problem is read either from the Monte Carlo formatted library, identified by the keyword XSC and the unit function name MIXED CROSS SECTIONS from the Table of Data Sets in Sect. 8.1.4.4, or from the restart unit, identified by the keyword RST and the unit function name, READ RESTART DATA. The NO. OF FISSION SPECTRUM SOURCE GROUP is the number of different energy groups for which a fission spectrum is defined. In the present version, this number should always be 1 . The NO. OF SCATTERING ANGLES IN XSECS is the number of scattering angles to be used in processing the cross sections. The default value is one, and it may be overridden by specifying the parameter $\mathbf{S C T}=$ in the mixing table input. One scattering angle yields $\mathrm{P}_{1}$ cross sections, two scattering angles yield $\mathrm{P}_{3}$ cross sections, three scattering angles yield $\mathrm{P}_{5}$ cross sections, etc. ENTRIES/NEUTRON IN THE NEUTRON BANK specifies the number of pieces of data that are banked for each history during tracking. ENTRIES/NEUTRON IN THE FISSION BANK is the amount of data stored for each source neutron for each generation.

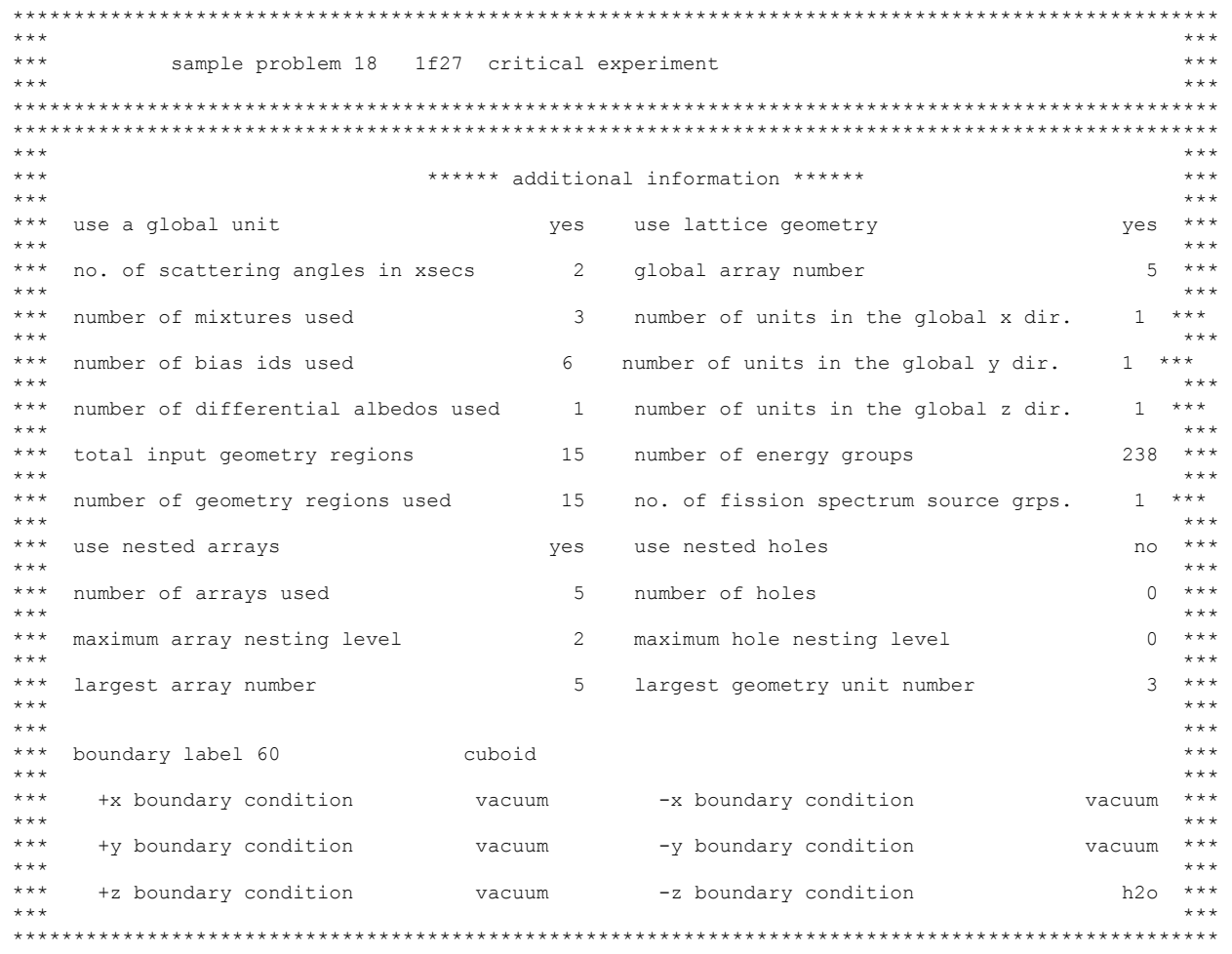

Fig. 8.1.101. Sample table of additional information. 
The NUMBER OF MIXTURES USED is the number of different mixtures (media) used in the geometry data used by the problem. This may be less than the total number of different mixtures specified in the geometry data if portions of the geometry data are not used in the problem.

The NUMBER OF BIAS IDS USED is the number of different biasing regions used in the problem. This will always be one unless a biasing data block is entered.

The NUMBER OF DIFFERENTIAL ALBEDOS USED is the number of different differential albedo reflectors used in the problem. This will always be zero unless the boundary condition data specify the use of differential albedo reflection on one or more faces of the system as described in Sect. 8.1.4.7. The BOUNDARY CONDITION data printed in this table should also be checked. The number of different differential albedos specified on the faces should be consistent with the NUMBER OF DIFFERENTIAL ALBEDOS USED. Specular, mirror, vacuum, and periodic are not differential albedos. Several different keywords may be used to specify the same differential albedo.

The TOTAL INPUT GEOMETRY REGIONS is the number of geometry regions specified in the problem input. This excludes UNIT label and comments provided using $\mathrm{COM}=$, but it includes the array boundary description. It excludes the automatic reflector description, but it includes the geometry regions generated by it. The NUMBER OF GEOMETRY REGIONS USED is the number of geometry regions used in the problem. It may be less than or equal to the TOTAL INPUT GEOMETRY REGIONS. The LARGEST GEOMETRY UNIT NUMBER is the largest unit number defined in the geometry data, including unused units and implicitly defined units. Implicitly defined unit numbers are created when a core boundary specification is not immediately preceded by a specification. The unit number is assigned by the code by adding one to the largest unit number encountered in the geometry region data. For example, if two such core boundary specifications are contained in a problem whose largest explicitly defined unit number is 7 , then a unit number of 8 is assigned to the first one, and a unit number of 9 is assigned to the second one. A value of 9 would be printed for the LARGEST GEOMETRY UNIT NUMBER. The LARGEST ARRAY NUMBER is the largest array number specified in the array data.

USE LATTICE GEOMETRY is determined by the logical flag that indicates whether or not the problem is a single unit problem. This should be YES for any problem that is not a single unit problem and NO for a single unit problem. By definition, a single unit problem does not use array data in any form. Section 8.1.4.12 describes array data. The GLOBAL ARRAY NUMBER is the number of the array designated as the global, overall, or universal array. The global array can be thought of as the array that defines the overall system.

The NUMBER OF UNITS IN THE GLOBAL X/Y/Z DIR. defines the size of the global array in terms of the number of units that are located along the edge of the array boundaries in the $\mathrm{X} / \mathrm{Y} / \mathrm{Z}$ directions. For a single unit, all three of these should be zero. For a simple $1 \times 1 \times 1$ array consisting of one unit type, all three of these numbers should be 1 .

USE GLOBAL REFLECTOR indicates if the global array is reflected.

USE NESTED HOLES is set YES if holes are nested deeper than one level.

NUMBER OF HOLES is the number of HOLES that are entered in the geometry region data.

The MAXIMUM HOLE NESTING LEVEL is the deepest level of hole nesting.

USE NESTED ARRAYS is set YES if arrays are nested deeper than one level. 
The NUMBER OF ARRAYS USED is the number of array descriptions actually used in the problem description.

MAXIMUM ARRAY NESTING LEVEL is the deepest level of array nesting.

Six BOUNDARY CONDITIONs are printed near the bottom of the table. They show the type of boundary condition that is applied to each face of the system. These should all be VACUUM unless albedo boundary conditions are applied to one or more faces of the system. One should refer to the NUMBER OF DIFFERENTIAL ALBEDOS USED, as discussed previously in the description of this table of information.

\subsubsection{Mixing table data}

If $\mathbf{L I B}=$ is entered in the KENO parameter data and a mixing table data block is provided to KENO, mixing table data will be printed. This output edit is not considered optional because it cannot be suppressed if the necessary data are present. Sample mixing table data are shown in Fig. 8.1.102 In the HTML output, the mixing table data can be accessed with the Mixing Table link in the Input Data section.

The data printed in this table include the problem title, the number of scattering angles, and the cross section message threshold. Data are then printed for each mixture. First the mixture number, density, and temperature are printed, followed by a table of the nuclides which make up the mixture. This table contains the following data: nuclide ID number, nuclide mixture ID number, atom density, weight fraction of nuclide in mixture, ZA number, atomic weight, temperature, and nuclide title. Mixture temperature is the same as the nuclides' temperatures for the multigroup calculations, but it may be different for the continuous energy calculations if Doppler broadening is not enabled since the nuclides are loaded from libraries with the closest temperature. After all mixture data have been printed, a table of nuclides and descriptive titles is printed for all nuclides included in the mixtures. If extra 1-D cross sections were specified in the problem (see $\mathbf{X 1 D}=$, Sect. 8.1.2.3), the extra 1-D cross section IDs will be printed under the heading "1-D CROSS SECTION ARRAY ID NUMBERS." If $\bar{v}$ is to be calculated (parameter NUB=YES), six MT numbers will be printed. The MT number for the total cross section $\left(\Sigma_{\mathrm{T}}\right)$ is 1 ; the MT number for the sum of the transfer array normalized by $\Sigma_{\mathrm{T}}$ is 2002 ; the MT number for the normalized fission-product cross section $\left(v \Sigma_{\mathrm{f}} / \Sigma_{\mathrm{t}}\right)$ is 1452 ; the MT number for the normalized absorption cross section $\left(\Sigma_{\text {abs }} / \Sigma_{\mathrm{T}}\right)$ is 27 ; the MT number for the normalized fission cross section $\left(\Sigma_{\mathrm{f}} / \Sigma_{\mathrm{T}}\right)$ is 18 ; and the MT number for the fission spectrum $(\chi)$ is 1018. $\chi$ is summed and normalized to 1.0. Other MT numbers in this list have been specified by the user. If the number of blocks on the direct access data set are insufficient to hold the cross section data, a message is printed stating THE NUMBER OF DIRECT ACCESS BLOCKS ON UNIT HAS BEEN INCREASED TO

If the problem is to write a restart data set (parameter $\mathbf{R E S =}$ ), a message is printed stating that restart information was written, and the restart I/O unit number is specified. This is followed by a statement of the number of I/Os used in preparing the cross sections. The user should examine the mixing table carefully to verify that the proper nuclides are specified for the proper mixtures and that all the data are correct. The mixing table is printed in subroutine PRTMIX. 


\begin{tabular}{|c|c|c|c|c|c|c|c|}
\hline \multirow{3}{*}{$\begin{array}{l}\text { mixture }= \\
\text { nuclide }\end{array}$} & \multicolumn{6}{|c|}{$\begin{aligned} \text { number of scattering angles } & =2 \\
\text { cross section message threshold } & =1.0 \mathrm{E}+00\end{aligned}$} & \\
\hline & 1 & density (g & $(\mathrm{cC})=1.5549$ & & tem & perature $(\mathrm{K})=$ & \\
\hline & nucmix & atom-dens. & wgt. frac. & $\mathrm{za}$ & awt & nuclide title & \\
\hline 1001 & 1 & $5.77931 \mathrm{E}-02$ & $6.22019 \mathrm{E}-02$ & 1001 & 1.0078 & h h2o 1 fast: h1 endf/b7 relo rev7 modo & $12 / 17 / 09$ \\
\hline 7014 & 1 & $2.13092 \mathrm{E}-03$ & $3.18663 \mathrm{E}-02$ & 7014 & 14.0031 & n'14 725 endf/b7 rel8 rev7 modo & $12 / 17 / 09$ \\
\hline 8016 & 1 & $3.74114 \mathrm{E}-02$ & $6.39037 \mathrm{E}-01$ & 8016 & 15.9949 & 016825 endf/b7 rel8 rev7 mod3 & $12 / 17 / 09$ \\
\hline 92234 & 1 & $1.06784 \mathrm{E}-05$ & $2.66894 \mathrm{E}-03$ & 92234 & 234.0410 & u234 9225 endf/b7 rel5 rev7 mod2 & $12 / 17 / 09$ \\
\hline 92235 & 1 & $9.84603 \mathrm{E}-04$ & $2.47144 \mathrm{E}-01$ & 92235 & 235.0439 & u235 9228 endf $/ \mathrm{b} 7$ rel0 $\mathrm{rev} 7 \bmod 7$ & $12 / 17 / 09$ \\
\hline 92236 & 1 & $5.29387 \mathrm{E}-06$ & $1.33447 \mathrm{E}-03$ & 92236 & 236.0456 & u236 9231 endf $/ \mathrm{b} 7$ rel0 rev7 mod1 & $12 / 17 / 09$ \\
\hline 92238 & 1 & $6.19415 \mathrm{E}-05$ & $1.57468 \mathrm{E}-02$ & 92238 & 238.0508 & u238 9237 endf/b7 rel6 rev7 mod5 & $12 / 17 / 09$ \\
\hline mixture $=$ & 2 & density ( $g$ & $(\mathrm{CC})=1.1800$ & & tem & perature $(\mathrm{K})=$ & \\
\hline nuclide & nucmix & atom-dens. & wgt. frac. & $\mathrm{za}$ & awt & nuclide title & \\
\hline 1001 & 2 & $5.67888 \mathrm{E}-02$ & $8.05411 \mathrm{E}-02$ & 1001 & 1.0078 & h h20 1 fast: $h 1$ endf $/ \mathrm{b} 7$ relo rev 7 modo & $12 / 17 / 09$ \\
\hline 6000 & 2 & $3.54930 \mathrm{E}-02$ & $5.99899 \mathrm{E}-01$ & 6000 & 12.0107 & $\mathrm{c}^{-} 600$ endf/b7 rel6 rev 7 modo & $12 / 17 / 09$ \\
\hline 8016 & 2 & $1.41972 \mathrm{E}-02$ & $3.19560 \mathrm{E}-01$ & 8016 & 15.9949 & 016 825 endf/b7 rel8 rev7 mod3 & $12 / 17 / 09$ \\
\hline mixture $=$ & 3 & density (g & $(\mathrm{cc})=0.90000$ & & tem & perature $(\mathrm{K})=$ & \\
\hline nuclide & nucmix & atom-dens. & wgt. frac. & $\mathrm{za}$ & awt & nuclide title & \\
\hline 9001001 & 3 & $7.99139 \mathrm{E}-02$ & $1.48599 \mathrm{E}-01$ & 1001 & 1.0078 & h_ch2 37 fast: $h 1$ endf $/ \mathrm{b} 7$ rel0 rev 7 modo & $12 / 17 / 09$ \\
\hline 6000 & 3 & $3.84202 \mathrm{E}-02$ & $8.51401 \mathrm{E}-01$ & 6000 & 12.0107 & $\mathrm{c}^{-} 600$ endf/b7 rel 6 rev 7 modo & $12 / 17 / 09$ \\
\hline mixture $=$ & 4 & density ( $g$ & $(\mathrm{cC})=0.99820$ & E-09 & tem & perature $(\mathrm{K})=$ & \\
\hline nuclide & nucmix & atom-dens. & wgt. frac. & $\mathrm{za}$ & awt & nuclide title & \\
\hline 1001 & 4 & $6.67530 \mathrm{E}-11$ & $1.11915 \mathrm{E}-01$ & 1001 & 1.0078 & h_h2o 1 fast: h1 endf/b7 relo rev7 modo & $12 / 17 / 09$ \\
\hline 8016 & 4 & $3.33765 \mathrm{E}-11$ & $8.88085 \mathrm{E}-01$ & 8016 & 15.9949 & ০16 825 endf/b7 rel8 rev7 mod3 & $12 / 17 / 09$ \\
\hline
\end{tabular}

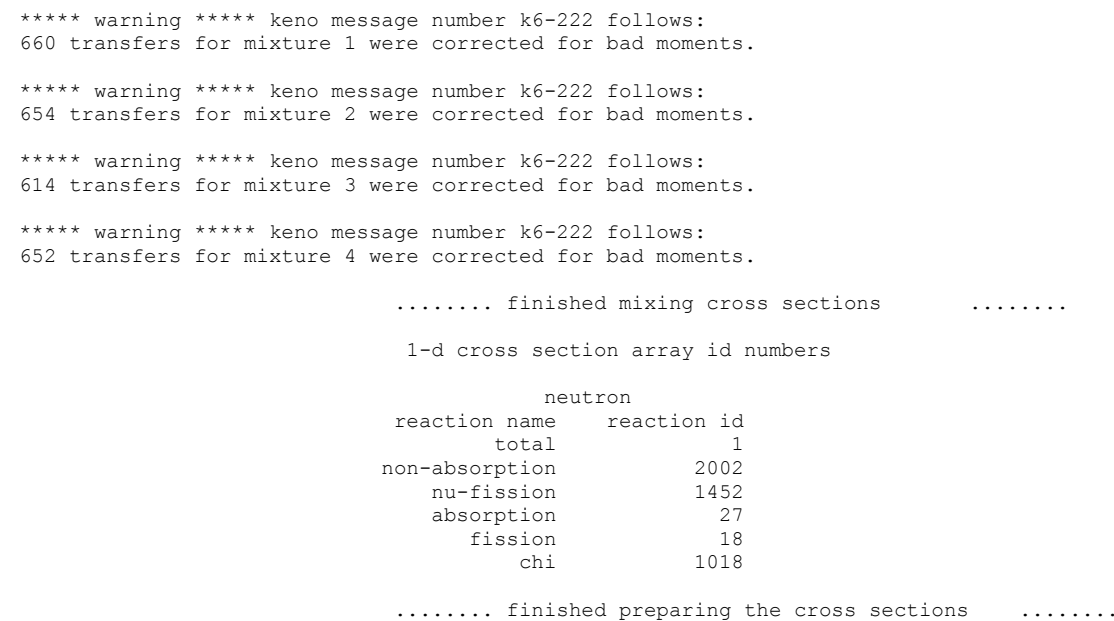

Fig. 8.1.102. Example of mixing table data.

\subsubsection{Albedo cross section correspondence}

Printing the albedo cross section correspondence tables is optional. The headings for the tables are printed in subroutine CORRE, and then subroutine RATIO prints the data. These tables are printed only if PAX $=$ YES is specified in the parameter data as described in Sect. 8.1.2.3. Examples of these tables are shown in Fig. 8.1.103 and Fig. 8.1.104.

The table shown in Fig. 8.1.103 contains, left to right, the cross section energy group, the lower and upper lethargy bounds, the corresponding albedo energy groups, and the cumulative probability associated with each albedo energy group for choosing the albedo energy group corresponding to the cross section energy group. The table shown in Fig. 8.1.104 is the inverse of the table shown in Fig. 8.1.103. It provides the cumulative probabilities for choosing the cross section energy group corresponding to the albedo energy group. The information in these tables is automatically generated by KENO. 

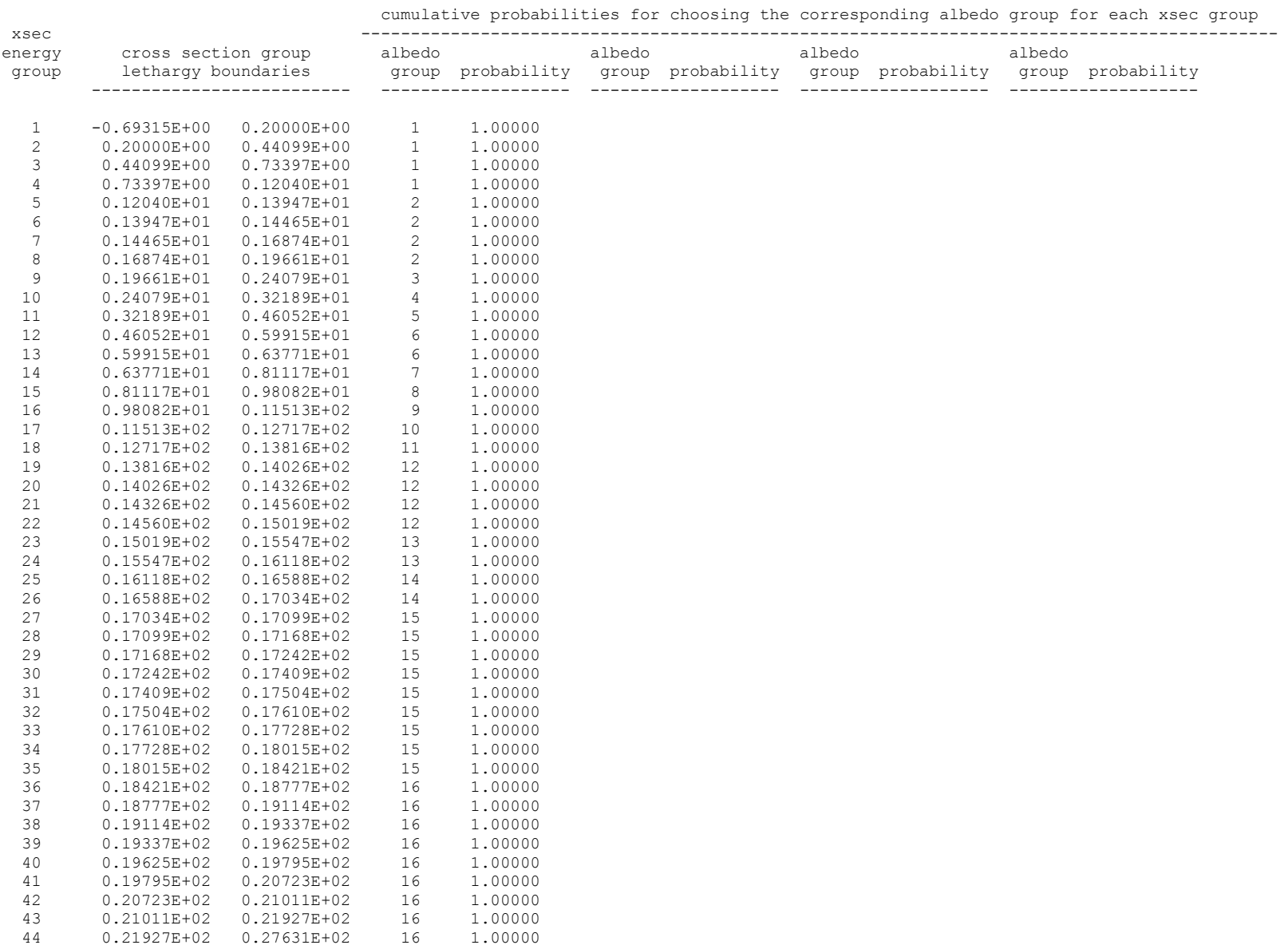

Fig. 8.1.103. Cumulative probabilities for correlating the albedo energy group to the cross section energy group.

\begin{tabular}{|c|c|c|c|c|c|c|c|c|c|c|}
\hline \multirow{2}{*}{$\begin{array}{c}\text { albedo } \\
\text { energy } \\
\text { group }\end{array}$} & \multirow{2}{*}{\multicolumn{2}{|c|}{$\begin{array}{l}\text { albedo group } \\
\text { lethargy boundaries }\end{array}$}} & & & & & & & & \\
\hline & & & $\begin{array}{r}\text { xsec } \\
\text { group }\end{array}$ & probability & $\begin{array}{r}\text { xsec } \\
\text { group }\end{array}$ & probability & $\begin{array}{r}\text { xsec } \\
\text { group }\end{array}$ & probability & $\begin{array}{r}\text { xsec } \\
\text { group }\end{array}$ & probabilit \\
\hline 1 & $-0.40547 \mathrm{E}+00$ & $0.12040 \mathrm{E}+01$ & 1 & 0.37620 & 2 & 0.52593 & 3 & 0.70797 & 4 & 1.00000 \\
\hline 2 & $0.12040 \mathrm{E}+01$ & $0.19661 \mathrm{E}+01$ & 5 & 0.25029 & 6 & 0.31818 & 7 & 0.63430 & 8 & 1.00000 \\
\hline 3 & $0.19661 \mathrm{E}+01$ & $0.24079 \mathrm{E}+01$ & 9 & 1.00000 & & & & & & \\
\hline 4 & $0.24079 \mathrm{E}+01$ & $0.32189 \mathrm{E}+01$ & 10 & 1.00000 & & & & & & \\
\hline 5 & $0.32189 \mathrm{E}+01$ & $0.46052 E+01$ & 11 & 1.00000 & & & & & & \\
\hline 6 & $0.46052 \mathrm{E}+01$ & $0.63771 E+01$ & 12 & 0.78235 & 13 & 1.00000 & & & & \\
\hline 7 & $0.63771 \mathrm{E}+01$ & $0.81117 E+01$ & 14 & 1.00000 & & & & & & \\
\hline 8 & $0.81117 \mathrm{E}+01$ & $0.98082 E+01$ & 15 & 1.00000 & & & & & & \\
\hline 9 & $0.98082 \mathrm{E}+01$ & $0.11513 E+02$ & 16 & 1.00000 & & & & & & \\
\hline 10 & $0.11513 E+02$ & $0.12717 E+02$ & 17 & 1.00000 & & & & & & \\
\hline 11 & $0.12717 E+02$ & $0.13816 \mathrm{E}+02$ & 18 & 1.00000 & & & & & & \\
\hline 12 & $0.13816 \mathrm{E}+02$ & $0.15019 E+02$ & 19 & 0.17502 & 20 & 0.42428 & 21 & 0.61832 & 22 & 1.00000 \\
\hline 13 & $0.15019 \mathrm{E}+02$ & $0.16118 \mathrm{E}+02$ & 23 & 0.48027 & 24 & 1.00000 & & & & \\
\hline & $0.16118 \mathrm{E}+02$ & $0.17034 \mathrm{E}+02$ & 25 & 0.51294 & 26 & 1.00000 & & & & \\
\hline \multirow[t]{2}{*}{15} & $0.17034 \mathrm{E}+02$ & $0.18421 \mathrm{E}+02$ & 27 & 0.04655 & 28 & 0.09632 & 29 & 0.14978 & 30 & 0.27029 \\
\hline & & & $\begin{array}{l}31 \\
35\end{array}$ & $\begin{array}{l}0.33904 \\
1.00000\end{array}$ & 32 & 0.41504 & 33 & 0.50000 & 34 & 0.70752 \\
\hline \multirow[t]{3}{*}{16} & $0.18421 E+02$ & $0.23026 \mathrm{E}+02$ & 36 & 0.07745 & 37 & 0.15052 & 38 & 0.19897 & 39 & 0.26144 \\
\hline & & & 40 & 0.29844 & 41 & 0.50000 & 42 & 0.56247 & 43 & 0.76144 \\
\hline & & & 44 & 1.00000 & & & & & & \\
\hline
\end{tabular}

Fig. 8.1.104. Cumulative probabilities for correlating the cross section energy group to the albedo energy group. 


\subsubsection{1-D macroscopic cross sections}

The decision to print the 1-D mixture cross sections is optional. They are printed only if XS1=YES is specified in the parameter data. When the 1-D cross sections are to be printed, they are printed a group at a time for each mixture. The 1-D mixture cross sections for a mixture are shown in Fig. 8.1.105.

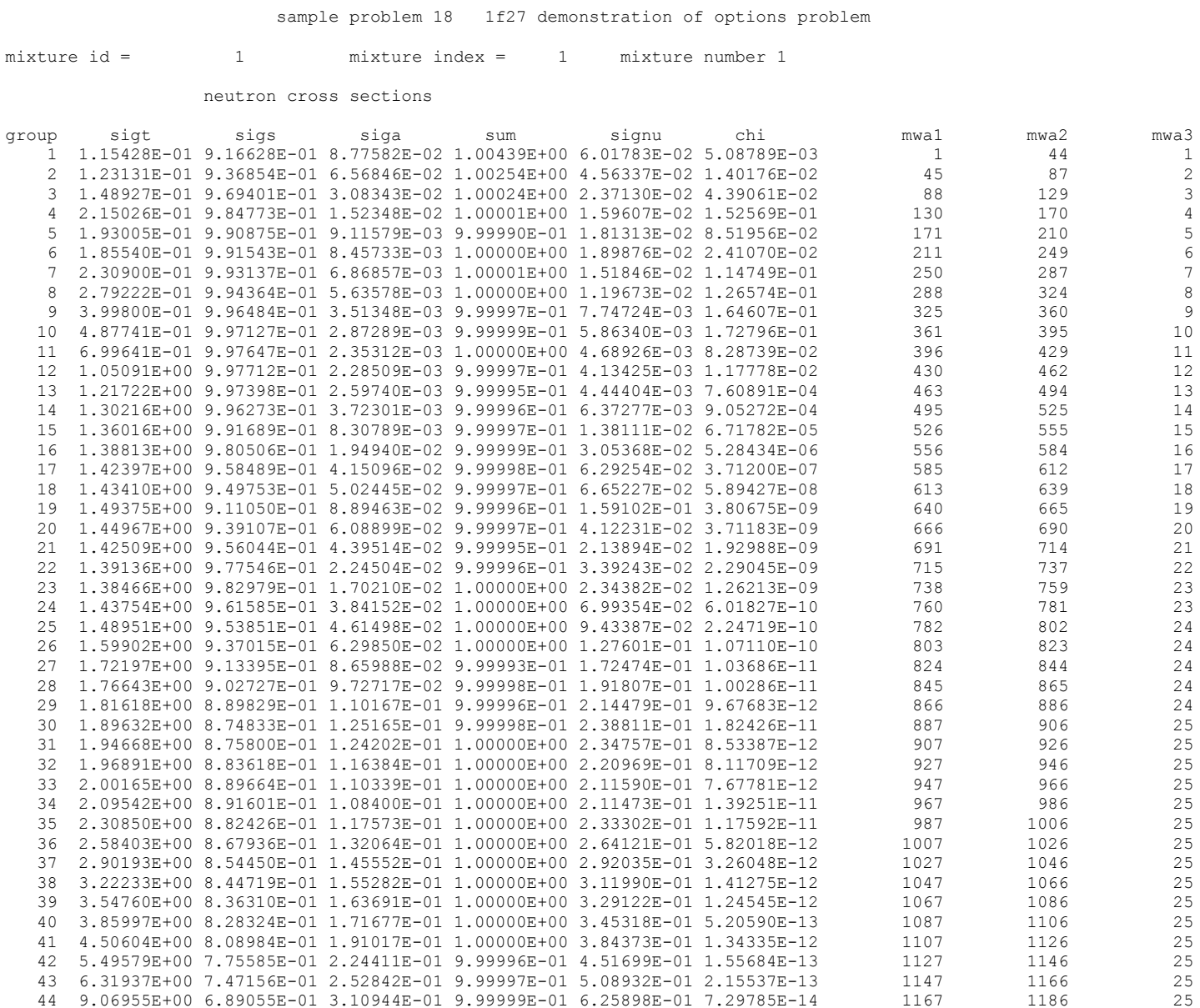

$\begin{array}{rr}\text { group } & \begin{array}{r}\text { fission } \\ \text { reaction }\end{array} \\ 1 & 1.59471 \mathrm{E}-02 \\ 2 & 1.33170 \mathrm{E}-02 \\ 3 & 7.54948 \mathrm{E}-03 \\ 4 & 5.55928 \mathrm{E}-03 \\ 5 & 6.63986 \mathrm{E}-03 \\ 6 & 7.06642 \mathrm{E}-03 \\ 7 & 5.74782 \mathrm{E}-03 \\ 8 & 4.61959 \mathrm{E}-03 \\ 9 & 3.04435 \mathrm{E}-03 \\ 10 & 2.34878 \mathrm{E}-03 \\ 11 & 1.90913 \mathrm{E}-03 \\ 12 & 1.69467 \mathrm{E}-03 \\ 13 & 1.82380 \mathrm{E}-03 \\ 14 & 2.61534 \mathrm{E}-03 \\ 15 & 5.66797 \mathrm{E}-03 \\ 16 & 1.25321 \mathrm{E}-02 \\ 17 & 2.58241 \mathrm{E}-02 \\ 18 & 2.73003 \mathrm{E}-02 \\ 19 & 6.52940 \mathrm{E}-02 \\ 20 & 1.69176 \mathrm{E}-02 \\ 21 & 8.78070 \mathrm{E}-03 \\ 22 & 1.39222 \mathrm{E}-02 \\ 23 & 9.61884 \mathrm{E}-03 \\ 24 & 2.87009 \mathrm{E}-02 \\ 25 & 3.87158 \mathrm{E}-02 \\ 26 & 5.23664 \mathrm{E}-02 \\ 27 & 7.07818 \mathrm{E}-02 \\ 28 & 7.87160 \mathrm{E}-02 \\ 29 & 8.80204 \mathrm{E}-02 \\ 30 & 9.80058 \mathrm{E}-02\end{array}$




$$
\begin{aligned}
& 9.63423 \mathrm{E}-02 \\
& 9.06838 \mathrm{E}-02 \\
& 8.68348 \mathrm{E}-02 \\
& 8.67866 \mathrm{E}-02 \\
& 9.57453 \mathrm{E}-02 \\
& 1.08393 \mathrm{E}-01 \\
& 1.19849 \mathrm{E}-01 \\
& 1.28038 \mathrm{E}-01 \\
& 1.35069 \mathrm{E}-01 \\
& 1.41716 \mathrm{E}-01 \\
& 1.57743 \mathrm{E}-01 \\
& 1.85373 \mathrm{E}-01 \\
& 2.08861 \mathrm{E}-01 \\
& 2.56863 \mathrm{E}-01
\end{aligned}
$$

Fig. 8.1.105. Example of macroscopic 1-D cross sections.

When the 1-D mixture cross sections are printed, the problem title is printed at the top of the page. The mixture ID, mixture index, and mixture number are then printed. ID is the mixture number from the mixing table, and mixture index is the index used to reference it and mixture number is its identifier. This step is followed by a heading to identify the different 1-D cross sections. GROUP is the energy group, sigt is the total cross section for the mixture, sigs is the nonabsorption probability, siga is the absorption probability, signu is the production probability, chi is the fission spectrum, mwal is the pointer for the first position of the cross sections for the energy group, mwa 2 is the pointer for the last position of the cross sections for the energy group, and $m w a 3$ contains the group for the transfer corresponding to the first position. $S U M$ is the sum of the absorption probability and the nonabsorption probability. The absorption probability is defined as the absorption cross section divided by the total cross section. The nonabsorption probability is the sum of the group-to-group transfers for this group, divided by the total cross section. The production probability is defined as the fission production cross section divided by the total cross section $\left(v \Sigma_{\mathrm{f}} / \Sigma_{\mathrm{T}}\right)$. The nonabsorption probability and the production probability are not true probabilities in that they may be greater than 1 . This is because the nonabsorption probability has the $(n, 2 n)$ transfer array summed into the total transfer array twice, and the $(n, 3 n)$ is summed three times, etc.

\subsubsection{Extra 1-d cross sections}

Printing the extra 1-D cross sections is optional. They are printed if P1D=YES is specified in the parameter data. Extra 1-D cross sections are not used in KENO unless NUB=YES is specified in the parameter data or the user has altered the code to access and utilize other 1-D cross sections. If NUB=YES is specified, the extra 1-D cross section is the fission cross section, which is used to calculate the average number of neutrons per fission. This is printed only for fissile mixtures as shown in Fig. 8.1.105. The fission cross section heading follows the table of 1-D cross sections. The fission cross section heading is XSEC ID 18, and it follows the table of 1-D cross sections.

\subsubsection{2-D macroscopic cross sections}

The decision to print the 2-D mixture cross sections is optional. They are printed only if XS2=YES is specified in the parameter data. They are printed after the 1-D cross sections for the mixture. A heading is printed, followed by the transfer data. An example of the 2-D mixture cross sections is given in Fig. 8.1.106.

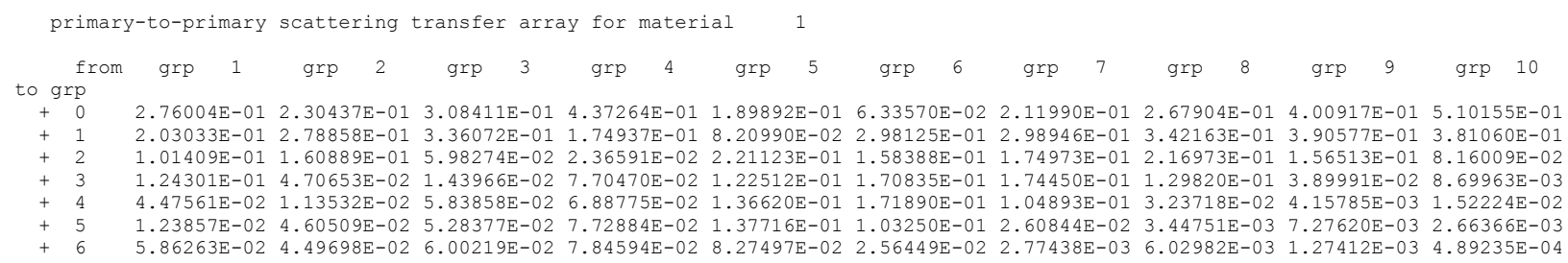




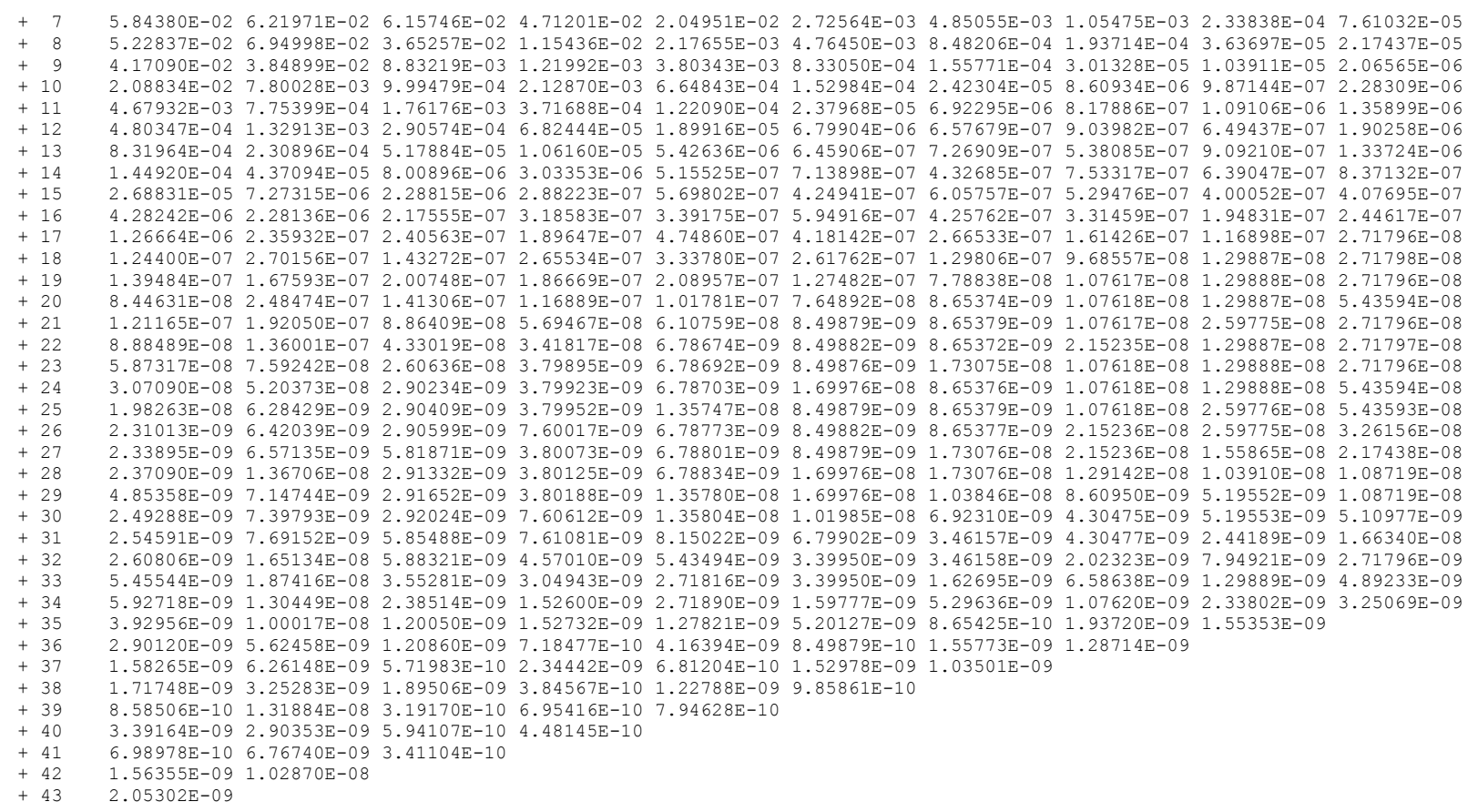

Fig. 8.1.106. Example of 2-D macroscopic cross sections.

\subsubsection{Probabilities and angles}

Printing the probabilities and angles is optional. They are printed if the number of scattering angles is greater than zero and $\mathbf{X A P}=$ YES is specified in the parameter data. Examples of the probabilities are shown in Fig. 8.1.107. Examples of the angles are shown in Fig. 8.1.108. If the group-to-group transfer for a mixture is isotropic, the first angle for that transfer will be set to -2.0 as a flag to the code.

\begin{tabular}{|c|c|c|c|c|c|c|c|c|c|c|}
\hline from & $\operatorname{grp}$ & grp & $\operatorname{grp}$ & $\operatorname{grp}$ & $g r p$ & grp & grp & grp & grp & grp \\
\hline $\mathrm{p}$ & & & & & & & & & & \\
\hline 0 & $8.52241 \mathrm{E}-01$ & $30759 \mathrm{E}-01$ & $08166 \mathrm{E}-01$ & $14207 \mathrm{E}-01$ & $01140 \mathrm{E}-01$ & $25173 \mathrm{E}-01$ & $8.36425 \mathrm{E}$ & $76545 \mathrm{E}-$ & $7.82953 \mathrm{E}$ & $7.94158 \mathrm{E}-$ \\
\hline 1 & $6.61685 \mathrm{E}-01$ & $6.42141 \mathrm{E}-01$ & $33639 \mathrm{E}-01$ & $5.61329 \mathrm{E}-01$ & $6.65762 \mathrm{E}-01$ & $7.51642 \mathrm{E}-01$ & & & & \\
\hline 2 & $4934 \mathrm{E}-01$ & $9.85130 \mathrm{E}-01$ & $93821 \mathrm{E}-01$ & 8.072331 & $98629 \mathrm{E}-01$ & $76556 \mathrm{E}-01$ & $9.87838 \mathrm{E}$ & & & \\
\hline 3 & $9.30280 \mathrm{E}-01$ & $9.91115 \mathrm{E}-01$ & $9.93502 \mathrm{E}-01$ & $9.89998 \mathrm{E}-01$ & $9.94645 \mathrm{E}-01$ & $9.91922 \mathrm{E}-01$ & $9.76019 \mathrm{E}-01$ & $9.32118 \mathrm{E}-01$ & $8.43266 \mathrm{E}-01$ & $9.13831 \mathrm{E}-01$ \\
\hline 4 & $8.71709 \mathrm{E}-01$ & $9.89244 \mathrm{E}-01$ & $9.89845 \mathrm{E}-01$ & $9.88632 \mathrm{E}-01$ & $9.90806 \mathrm{E}-01$ & $9.81214 \mathrm{E}-01$ & $9.62138 \mathrm{E}-01$ & $9.63139 \mathrm{E}-01$ & $9.93653 \mathrm{E}-01$ & $6.19199 \mathrm{E}-01$ \\
\hline 5 & $8.30505 E-01$ & $9.83908 \mathrm{E}-01$ & $.83380 \mathrm{E}-01$ & $9.83574 \mathrm{E}-01$ & $9.80597 \mathrm{E}-01$ & $9.67755 \mathrm{E}-01$ & $97 \mathrm{E}-01$ & $35 E-01$ & & \\
\hline+6 & $7.78710 \mathrm{E}-01$ & $9.47570 \mathrm{E}-01$ & $9.70721 \mathrm{E}-01$ & $9.71325 \mathrm{E}-01$ & $9.67987 \mathrm{E}-01$ & $9.74418 \mathrm{E}-01$ & $9.91481 \mathrm{E}-01$ & $9.54305 \mathrm{E}-01$ & $9.62199 \mathrm{E}-01$ & $7.14936 \mathrm{E}-01$ \\
\hline 7 & $7.10370 \mathrm{E}-01$ & $8.48230 \mathrm{E}-01$ & $48712 \mathrm{E}-01$ & $969 \mathrm{E}-01$ & $9.72320 \mathrm{E}-01$ & $9.89297 \mathrm{E}-01$ & $9.67976 \mathrm{E}-01$ & $9.72428 \mathrm{E}-01$ & $3 E-01$ & \\
\hline 8 & $7.07306 \mathrm{E}-01$ & $7.76263 \mathrm{E}-01$ & $9.26753 \mathrm{E}-01$ & $9.55943 \mathrm{E}-01$ & $9.85543 \mathrm{E}-01$ & $9.68681 \mathrm{E}-01$ & $9.76689 \mathrm{E}-01$ & $2 \mathrm{E}-01$ & 9.92 & \\
\hline 9 & $7.97797 \mathrm{E}-01$ & $7.68471 \mathrm{E}-01$ & $9.07553 \mathrm{E}-01$ & 9.68550 & $9.71040 \mathrm{E}-01$ & $9.72539 \mathrm{E}-01$ & $91 \mathrm{E}-01$ & $7 E-01$ & 9.9 & -01 \\
\hline 10 & $E-01$ & 8.70 & $24619 \mathrm{E}-01$ & 9.63 & $4 \mathrm{E}-01$ & & & & & \\
\hline+11 & $8.83152 \mathrm{E}-01$ & 9.2247 & $6.56381 \mathrm{E}-01$ & 9.9394 & $9.99738 \mathrm{E}-01$ & 9.983 & 9.99 & $E-01$ & 9.9 & $4 E-01$ \\
\hline+12 & $9.39710 \mathrm{E}-01$ & $9.45242 \mathrm{E}-01$ & $6.35618 \mathrm{E}-01$ & 9.9964 & $9.99927 \mathrm{E}-01$ & $4 \mathrm{E}-01$ & $5 E-01$ & $6 \mathrm{E}-01$ & $E-01$ & $9 \mathrm{E}-01$ \\
\hline 13 & 9.67 & 9.55 & $8.68720 \mathrm{E}-01$ & 9.99 & 9.999 & $E-01$ & 9.9 & $4 \mathrm{E}-01$ & 9.9 & \\
\hline 14 & 9.85 & 9.35 & $E-01$ & 9.99 & 9.998 & 9.9 & & 9. & & $D E-01$ \\
\hline 15 & -01 & 8.91 & $E-01$ & 9.99 & 9.99 & 9.9 & 9.9 & $E-01$ & & \\
\hline+16 & $9.62903 \mathrm{E}-01$ & $8.33435 \mathrm{E}-01$ & $9.96696 \mathrm{E}-01$ & $9.99582 \mathrm{E}-01$ & $9.99852 \mathrm{E}-01$ & $5 \mathrm{E}-01$ & 9.99 & $6 \mathrm{E}-01$ & $E-01$ & $0 \mathrm{E}-01$ \\
\hline 17 & 9.38 & 7.85 & $9.96433 \mathrm{E}-01$ & 9.99 & -01 & 5.4 & & $3 E-01$ & 9.9 & -01 \\
\hline 18 & $E-01$ & 7.6808 & $9.96072 \mathrm{E}-01$ & 9.99 & $9.99780 \mathrm{E}-01$ & $E-01$ & $7 E-01$ & $0 \mathrm{E}-01$ & 9.9 & -01 \\
\hline+19 & $9.06506 \mathrm{E}-01$ & $7.46502 \mathrm{E}-01$ & $9.95475 \mathrm{E}-01$ & 9.9929 & $9.99717 \mathrm{E}-01$ & $E-01$ & $1 \mathrm{E}-01$ & $E-01$ & 9.99 & $1 \mathrm{E}-01$ \\
\hline+20 & 8.95 & 7.19 & 9.9445 & 9.990 & $E-01$ & 5.0 & 9.9 & DE-01 & E-01 & $E-01$ \\
\hline+21 & $8.79642 \mathrm{E}-01$ & $6.81376 \mathrm{E}-01$ & $9.92628 \mathrm{E}-01$ & 9.98 & $9.99544 \mathrm{E}-01$ & $E-01$ & 9.9 & $0 \mathrm{E}-01$ & 9. & -01 \\
\hline+22 & & & & & & & & & & \\
\hline+23 & -01 & 5.86 & -01 & 9.98 & 9.99 & & 9. & 9. & & \\
\hline+24 & $7.80584 \mathrm{E}-01$ & $5.47732 \mathrm{E}-01$ & $9.86505 \mathrm{E}-01$ & $9.98290 \mathrm{E}-01$ & $9.99373 \mathrm{E}-01$ & $5.00000 \mathrm{E}-01$ & $9.99988 \mathrm{E}-01$ & $9.99973 \mathrm{E}-01$ & $9.99989 \mathrm{E}-01$ & $9.99808 \mathrm{E}-01$ \\
\hline+25 & & & $9.85597 \mathrm{E}-01$ & & & & & & & \\
\hline+26 & 7.14 & 5.22 & $9.85561 \mathrm{E}-01$ & 9.98 & 9.99 & 5.0 & 9.9 & 9.9 & 9. & \\
\hline+27 & 7.08 & 5.16 & $9.84702 \mathrm{E}-01$ & 9.98 & 9.99 & & & 9. & & \\
\hline & $7.01707 \mathrm{E}-01$ & & $9.83687 \mathrm{E}-01$ & & 9.99 & & & & & \\
\hline+29 & $6.93003 E-01$ & $5.01514 \mathrm{E}-01$ & $9.83158 \mathrm{E}-01$ & $9.97769 \mathrm{E}$ & $9.99250 \mathrm{E}-01$ & $0 E-01$ & $56 \mathrm{E}-01$ & $72 \mathrm{E}-01$ & $2 E-01$ & $81 \mathrm{E}-01$ \\
\hline & & & & & & & & & & \\
\hline+31 & $6.76098 \mathrm{E}-01$ & $5.16147 \mathrm{E}-01$ & $9.80437 \mathrm{E}-01$ & $9.97058 \mathrm{E}-01$ & $9.98751 \mathrm{E}-01$ & & $72 \mathrm{E}-01$ & 9.99 & 9.9 & \\
\hline+32 & $6.69503 \mathrm{E}-01$ & $5.29078 \mathrm{E}-01$ & $9.76923 \mathrm{E}-01$ & $9.96482 \mathrm{E}-01$ & $9.98494 \mathrm{E}-01$ & $5.00000 \mathrm{E}-01$ & $9.99968 \mathrm{E}-01$ & $9.99891 \mathrm{E}-01$ & $9.99976 \mathrm{E}-01$ & $5.00000 \mathrm{E}-01$ \\
\hline 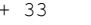 & $E-01$ & 5.4 & $9.71776 \mathrm{E}-01$ & & 9.98 & $0 E-01$ & & & & \\
\hline 34 & $6.20059 \mathrm{E}-01$ & $5.69269 \mathrm{E}-01$ & $9.66677 \mathrm{E}-01$ & $9.95214 \mathrm{E}-01$ & $9.98337 \mathrm{E}-01$ & $5.00000 \mathrm{E}-01$ & $9.99948 \mathrm{E}-01$ & $09 \mathrm{E}-01$ & $9.99969 \mathrm{E}-01$ & $00 \mathrm{E}-01$ \\
\hline
\end{tabular}




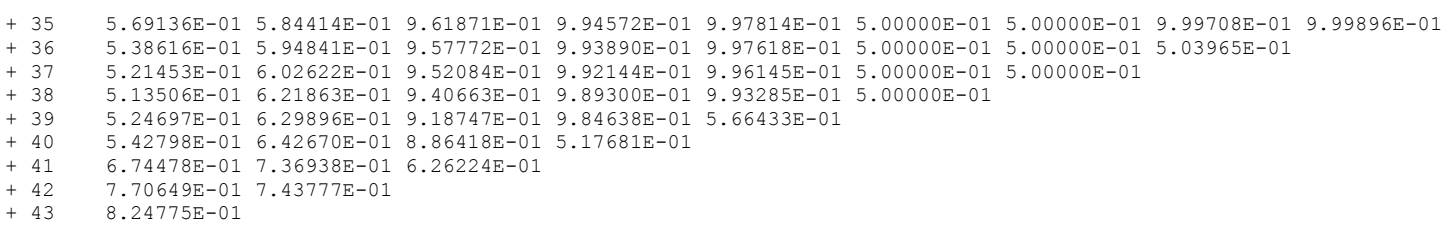

Fig. 8.1.107. Example of macroscopic probabilities.

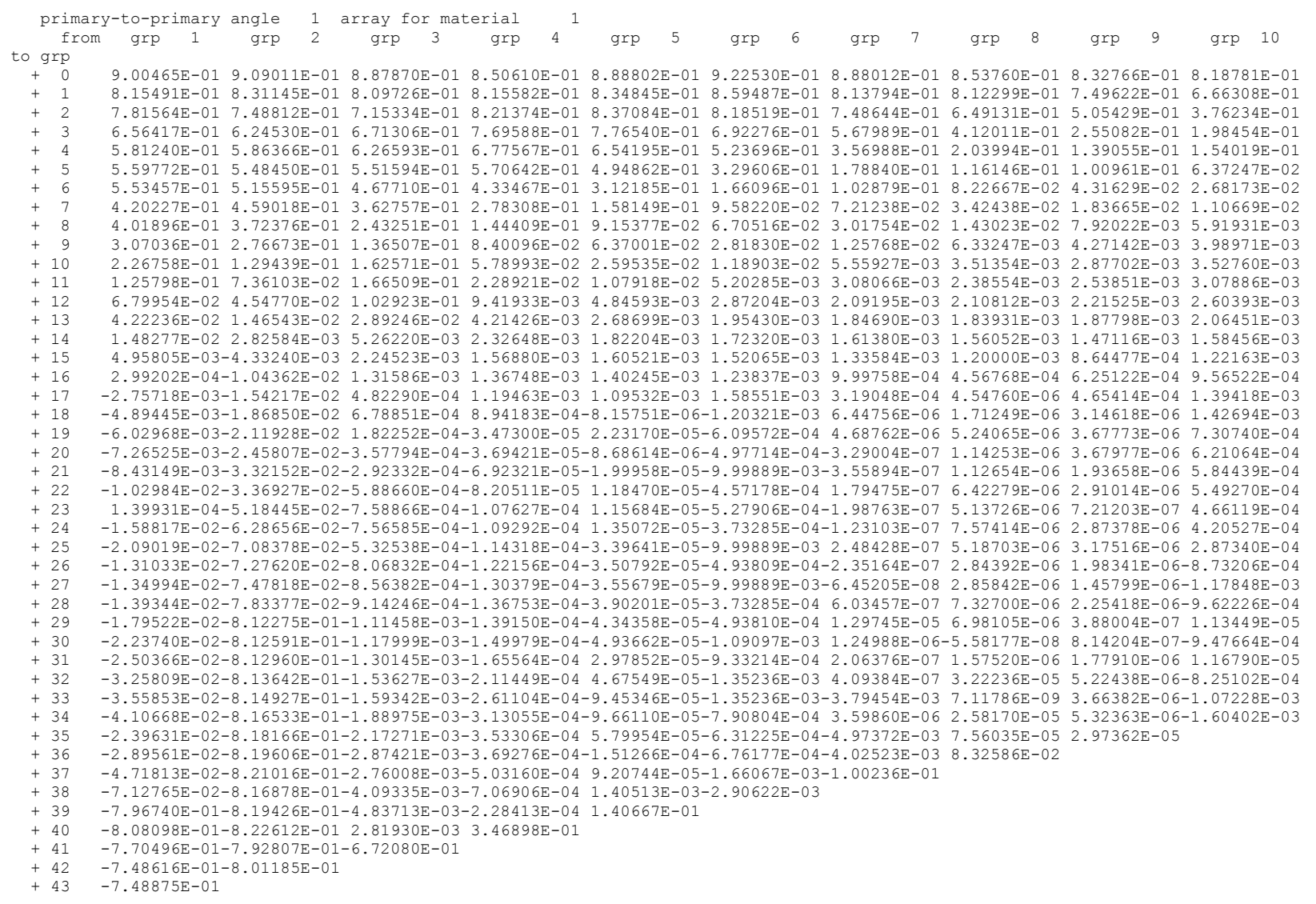

Fig. 8.1.108. Example of macroscopic angles.

\subsubsection{Array summary}

The arrays that are used in the problem are summarized in the table shown in Fig. 8.1.109. This table is printed whenever more than one array is used in the problem.

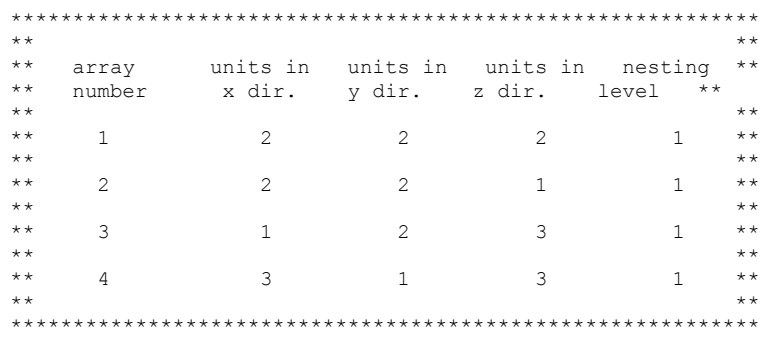


Fig. 8.1.109. Example of array summary.

The ARRAY NUMBER is the number by which the array is designated in the input data. The number of units in the X, Y, and Z directions is listed for each array. The NESTING LEVEL indicates the level of nesting for each array. The global, overall, or universe array is flagged by the word GLOBAL. The global array should always appear at the first nesting level. Arrays that have been placed in the global reflector by using holes should also appear at the first nesting level. A nesting level of one is the highest or first nesting levels. The larger the number in the nesting level column, the deeper the nesting level will be.

\subsubsection{Geometry data}

The geometry region data used by the problem are always printed and cannot be suppressed. They should be carefully examined by the user to verify the mixture number, bias ID, and geometry specifications used in the problem. If geometry region data are entered but are not referenced in the unit orientation array data, they will not be printed here. An example would be to enter geometry region data describing Units 1, 2, 3, and 4 and to use only Units 1, 3, and 4 in the unit orientation array. Then the geometry region data for Unit 2 will not be printed. An example of the KENO V.a geometry region printout for a problem is given in Fig. 9.1.110.

Fig. 8.1.110

The problem title and a heading are printed at the top of each page. REGION is the region number within a unit. Each unit has its regions numbered sequentially, beginning with one. MEDIA NUM is the mixture number or mixture ID that occupies the volume defined by the region. BIAS ID is the bias ID that corresponds to the desired set of weight average for biasing the region. The unit number is printed at the top of each unit's geometry region description near the center of the page. The data printed for each geometry region include (1) the region number relative to the unit (numbered sequentially within the unit), (2) the shape of the geometry region, (3) the mixture ID of the material within the volume defined by the region, (4) the bias ID to define the average weight of a neutron in the region, and (5) the dimensions defining the outer boundaries of the geometry region. If additional geometry surrounds an array, a heading is printed stating: UNIT EXTERNAL TO LATTICE . The lattice number is the number of the array that is surrounded by the specified geometry. The unit number is the unit that contains the specified geometry. In the case of an external reflector for the global array, the unit number is assigned by the code.

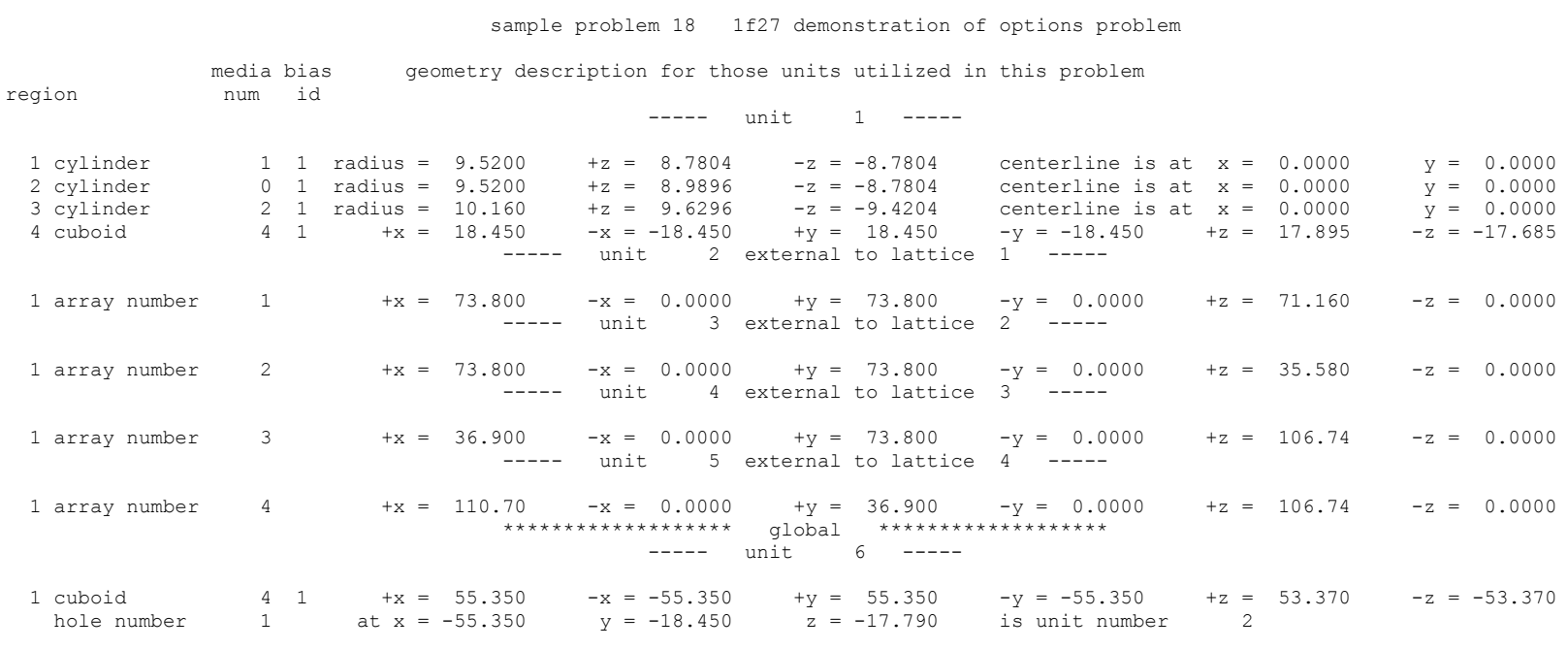




\begin{tabular}{|c|c|c|c|c|c|c|c|c|c|c|c|}
\hline hole number & 2 & & at $x=$ & -55.350 & $y=-18.450$ & $\mathrm{z}=$ & -53.370 & is unit number & & 3 & \\
\hline hole number & 3 & & at $\mathrm{x}=$ & 18.450 & $y=-18.450$ & $z=$ & -53.370 & is unit number & & 4 & \\
\hline hole number & 4 & & at $\mathrm{x}=$ & -55.350 & $y=-55.350$ & $z=$ & -53.370 & is unit number & & 5 & \\
\hline 2 cuboid & 3 & 2 & $+x=$ & 58.350 & $-x=-58.350$ & $+y=$ & 58.350 & $-y=-58.350$ & $+z=$ & $=56.370$ & $-z=-56.370$ \\
\hline 3 cuboid & 3 & 3 & $+x=$ & 61.350 & $-x=-61.350$ & $+y=$ & 61.350 & $-y=-61.350$ & $+z=$ & $=59.370$ & $-z=-59.370$ \\
\hline 4 cuboid & 3 & 4 & $+x=$ & 64.350 & $-x=-64.350$ & $+y=$ & 64.350 & $-y=-64.350$ & $+z=$ & $=62.370$ & $-z=-62.370$ \\
\hline 5 cuboid & 3 & 5 & $+x=$ & 67.350 & $-x=-67.350$ & $+y=$ & 67.350 & $-y=-67.350$ & $+z=$ & $=65.370$ & $-z=-65.370$ \\
\hline 6 cuboid & 3 & 6 & $+x=$ & 70.350 & $-x=-70 \cdot 350$ & $+y=$ & 70.350 & $-y=-70.350$ & $+z=$ & $=68.370$ & $-z=-68.370$ \\
\hline 7 cuboid & 3 & 7 & $+x=$ & 70.590 & $-x=-70.590$ & $+y=$ & 70.590 & $-y=-70.590$ & $+z=$ & $=68.610$ & $-z=-68.610$ \\
\hline
\end{tabular}

Fig. 8.1.110. Example of geometry region data.

\subsubsection{Unit orientation description}

Each unit orientation description defines the location of units in the 3-D lattice that represent the specified array. The array described is identified in the heading UNIT ORIENTATION DESCRIPTION FOR ARRAY _. The arrays used in the problem are stacked together to represent the physical problem being analyzed. The unit orientation description is not printed if only Unit 1 is described in the problem. The user should carefully examine the unit orientation descriptions to ensure proper placement of the units in each lattice. A sample unit orientation description is shown in Fig. 8.1.111.

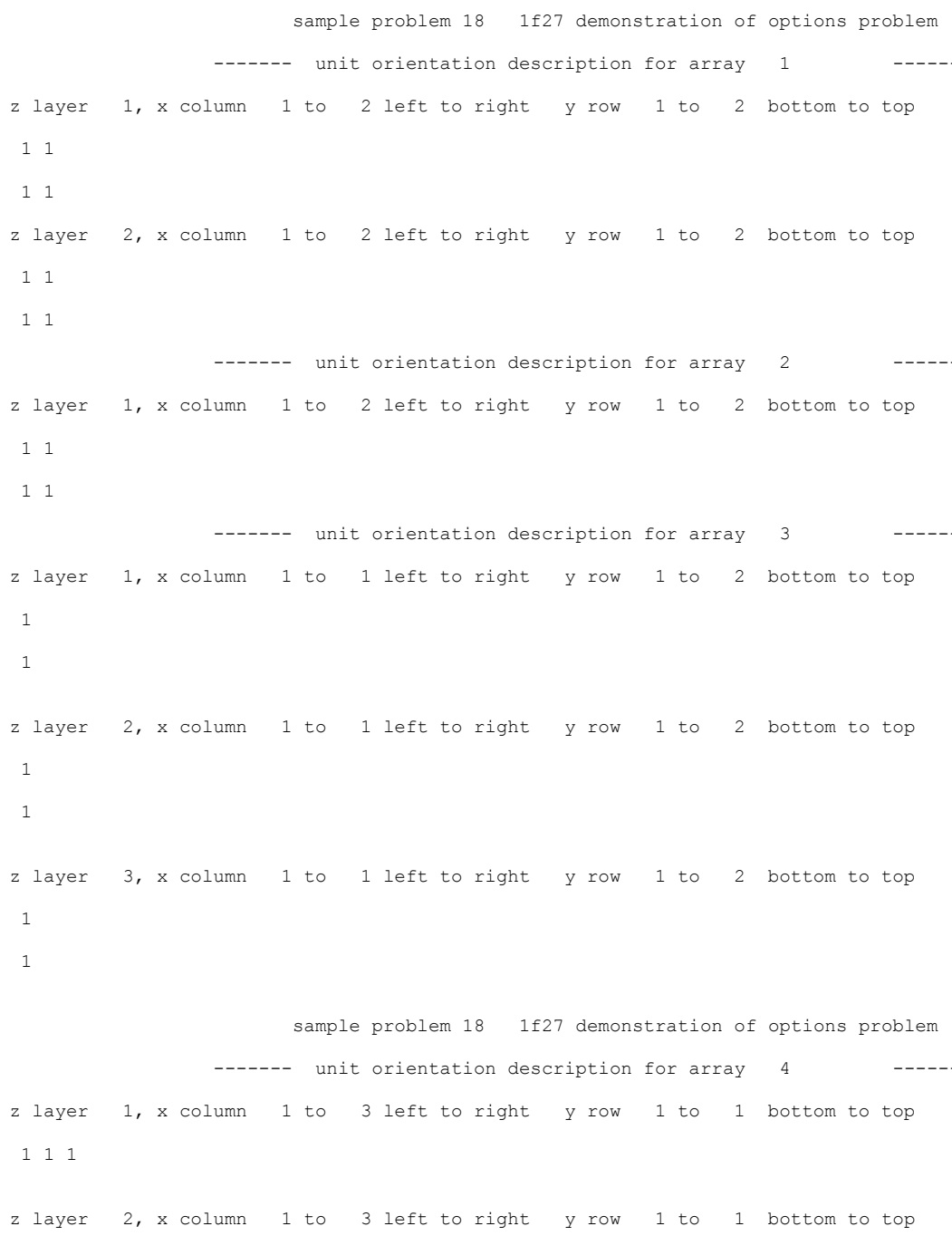




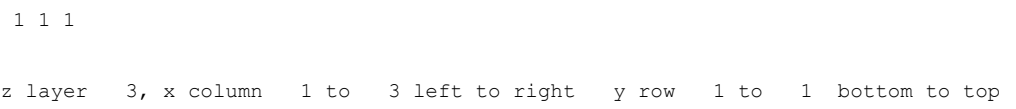

Fig. 8.1.111. Example of unit orientation description.

If a very large array is used by the problem, its unit orientation description may be spread over several pages. When checking the printout, the user should pay careful attention to the headings that indicate the portion of each lattice being printed. The detailed printout of an array may be suppressed using the ARRAY parameter PRT=no.

\subsubsection{Volume information}

The volume information printout differs between KENO V.a and KENO-VI. Because it does not allow intersections of bodies, KENO V.a is always able to calculate analytically the volume of any region in the geometry. KENO-VI, on the other hand, never calculates volumes analytically. In fact, KENO-VI never calculates volumes unless it is directed to do so, but it can read volumes off the MEDIA cards or off a VOLUME file (Sect. 8.1.2.13).

\subsection{KENO V.a}

Three tables of volumes are always printed and cannot be suppressed. The problem title is printed at the top of the page, followed by the heading "VOLUMES FOR THOSE UNITS UTILIZED IN THIS PROBLEM." An example of the volume printout is given in Fig. 8.1.112.

The first table is arranged by ascending unit number. It includes (1) the unit number, (2) the region number within the unit, (3) the overall geometry region number, (4) the net volume of each individual region, and (5) the cumulative volume through each region in the unit. The cumulative volume of the last region in a unit is the total volume of the unit. The unit number is printed under the heading UNIT. Data listed under the heading REGION refer to the number of the geometry region within the unit. The geometry regions within a unit are numbered sequentially starting with 1 . Data entered under the heading GEOMETRY REGION refer to the entry number of the individual geometry region. These are numbered sequentially, starting with 1, through the TOTAL INPUT GEOMETRY REGIONS defined in Fig. 8.1.101. The net volume of each individual region is calculated by subtracting the volume of the interior region from the volume of the region and is listed under the heading VOLUME. The data listed under the heading CUMULATIVE VOLUME are calculated from the dimensions of the region. A simple example demonstrating how volumes are calculated can be given by assuming a unit that is composed of three concentric cubes. Region 1 is a cube $3 \mathrm{~cm}$ on a side, region 2 is a cube $4 \mathrm{~cm}$ on a side, and region 3 is a cube $5 \mathrm{~cm}$ on a side. The cumulative volume of region 1 is $27 \mathrm{~cm}^{3}\left(3^{3}\right)$; the cumulative volume of region 2 is $64 \mathrm{~cm}^{3}\left(4^{3}\right)$; the cumulative volume of region 3 is $125 \mathrm{~cm}^{3}\left(5^{3}\right)$. The volume of region 1 is $27 \mathrm{~cm}^{3}\left(3^{3}\right)$, the volume of region 2 is $37 \mathrm{~cm}^{3}(64-27)$, and the volume of region 3 is $61 \mathrm{~cm}^{3}(125-64)$.

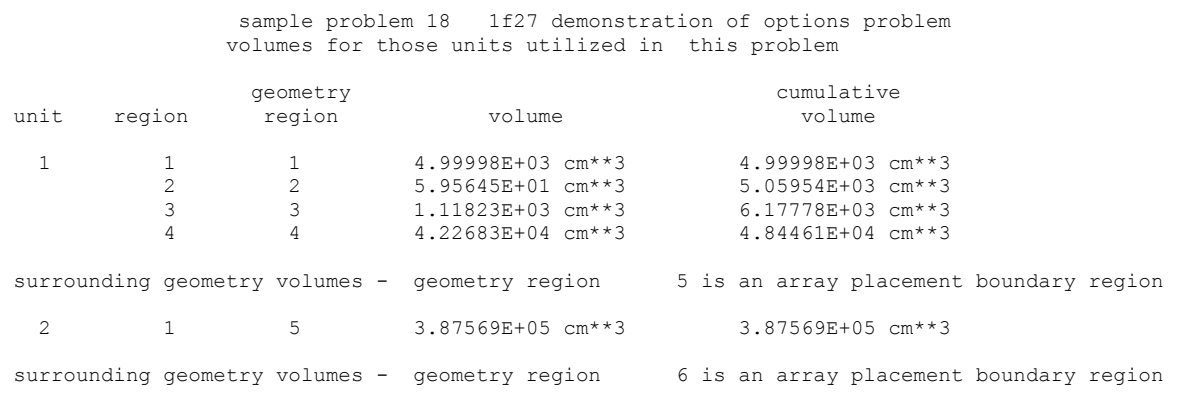




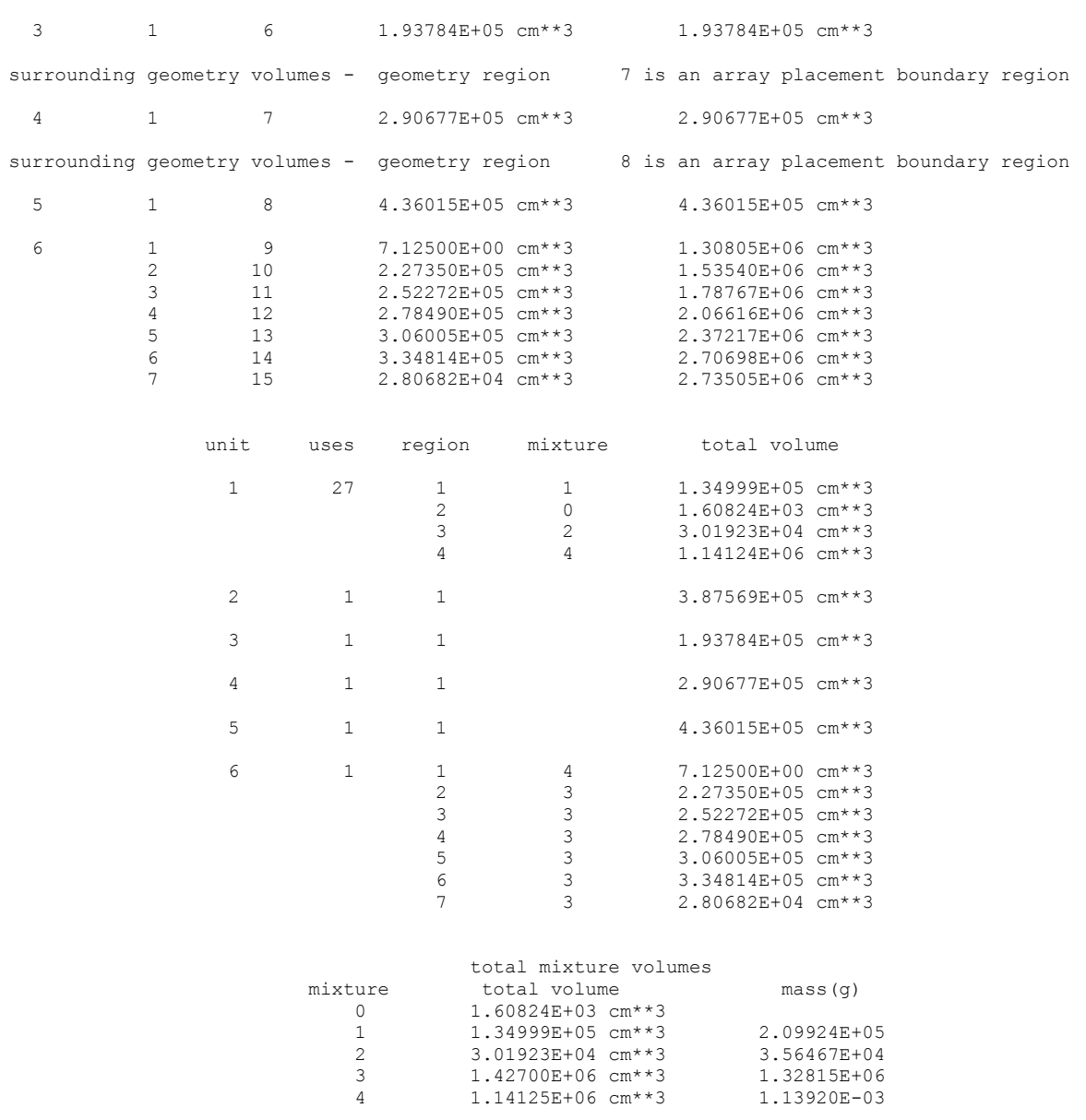

Fig. 8.1.112. Sample volume information.

The second table contains (1) the unit number, (2) the number of times the unit is used in the problem, (3) the region number within the unit, (4) the mixture number present in the region, and (5) the total volume associated with the region in the whole problem. The unit number is printed under the heading UNIT, data printed under the heading USES indicates the number of times the unit is used in the problem. Data printed under the heading MIXTURE indicates the mixture number used in the region. The total volume of each region is printed under the heading TOTAL VOLUME and is determined by multiplying the VOLUME of the region listed in the first table by the number of times the unit containing that region is used in the problem.

The third table is printed following the heading "TOTAL MIXTURE VOLUMES." In this table, the mixtures used in the problem are listed with their associated total volume and total mass. The mixture numbers are printed under the heading MIXTURE, the total volume of each mixture is printed under the heading TOTAL VOLUME, and the mass of each mixture is printed under the heading MASS(G). All masses will be printed as zero if the working format cross section library does not contain the data required to calculate the densities of the mixtures used in the problem.

\subsection{KENO-VI}

Two tables of volumes are printed by subroutine VOLUME and cannot be suppressed. If a volume calculation type of NONE is specified and no volumes are read in for the regions, then the volumes will 
be set to -1.0 , which will result in negative fluxes and fission densities. For the calculated volumes, an error estimate is printed after the volumes. The first table will list the number of times each unit is used in the problem and the total volume of each region throughout the entire problem description. The second table will list the total volume and mass of each mixture used in the problem. An example of the volume printout is given in Fig. 8.1.113.

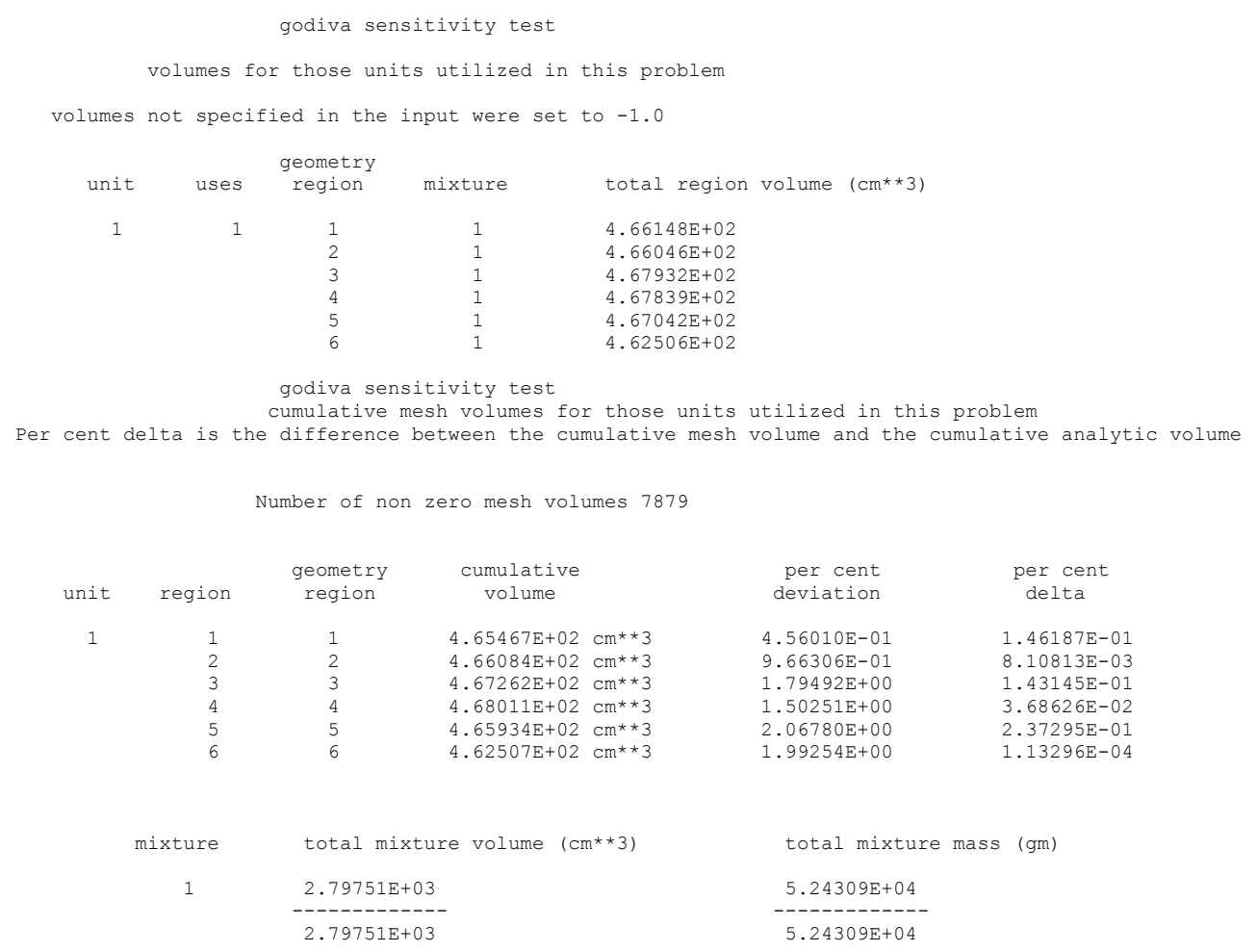

Fig. 8.1.113. Sample volume information.

The unit number is printed under the heading UNIT. The REGION refers to the number of the geometry region within the unit determined by the order of the CONTENT records in the unit. USES indicates the number of times the unit is used in the problem. MIXTURE is the mixture number used in the region. A blank indicates an array or unit, placed in a hole, in that region. TOTAL VOLUME is the total volume of that region followed by $+/-$ and an error estimate if the volume for the region was calculated.

If mesh volumes are used, there will be a table listing the cumulative volumes generated by summing the mesh volumes, the deviations, and the deltas between the summed volumes and the overall volumes for the regions. If the overall volume has been calculated, then the delta should be zero, but if it was entered, then the delta will show the difference.

In the last table, the mixtures used in the problem are listed along with their total volumes and masses. Along with the total volumes and masses, an error estimate is printed for any mixtures having regions that were calculated using either the RANDOM or TRACE volume estimates. 


\subsubsection{Mesh volumes}

With the mesh flux accumulator, fluxes are tabulated for each region of each unit in a cuboidal mesh. KENO computes the volume of each region in each mesh interval so that fluxes per unit volume can be determined. For models where the mesh flux accumulator is activated by setting MFX=YES and the mesh size set to a positive value with $\mathbf{M S H}=$, the cumulative volume of all mesh intervals for a given region and the number of meshes used in each region are printed as shown in Fig. 8.1.114

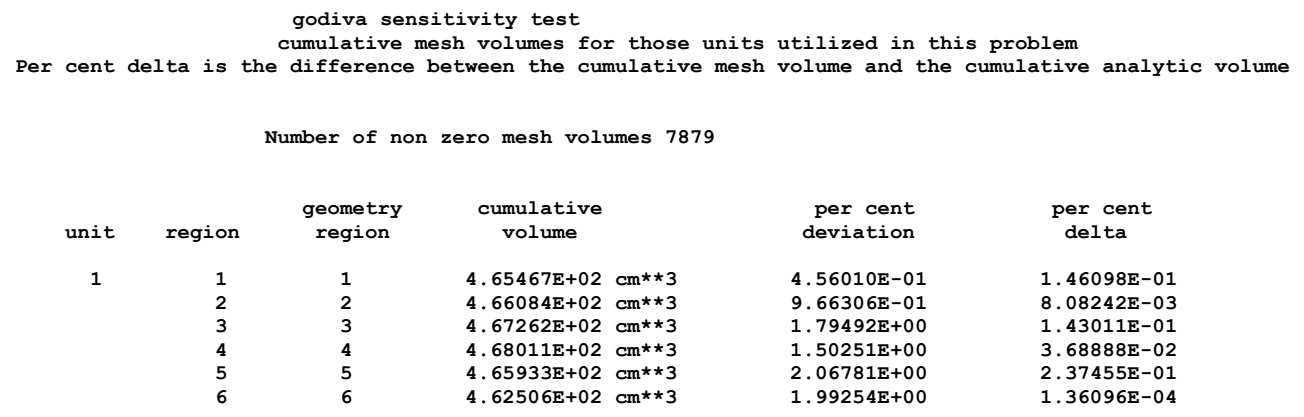

Fig. 8.1.114. Sample sum of mesh volumes edit.

An optional edit of volumes for each mesh interval for each region can be activated by entering $\mathbf{P M V}=$ YES in the parameter input. This edit can be very large, especially if a small mesh size is used with a large model. A sample of the volume by mesh for each region is shown in Fig. 8.1.115

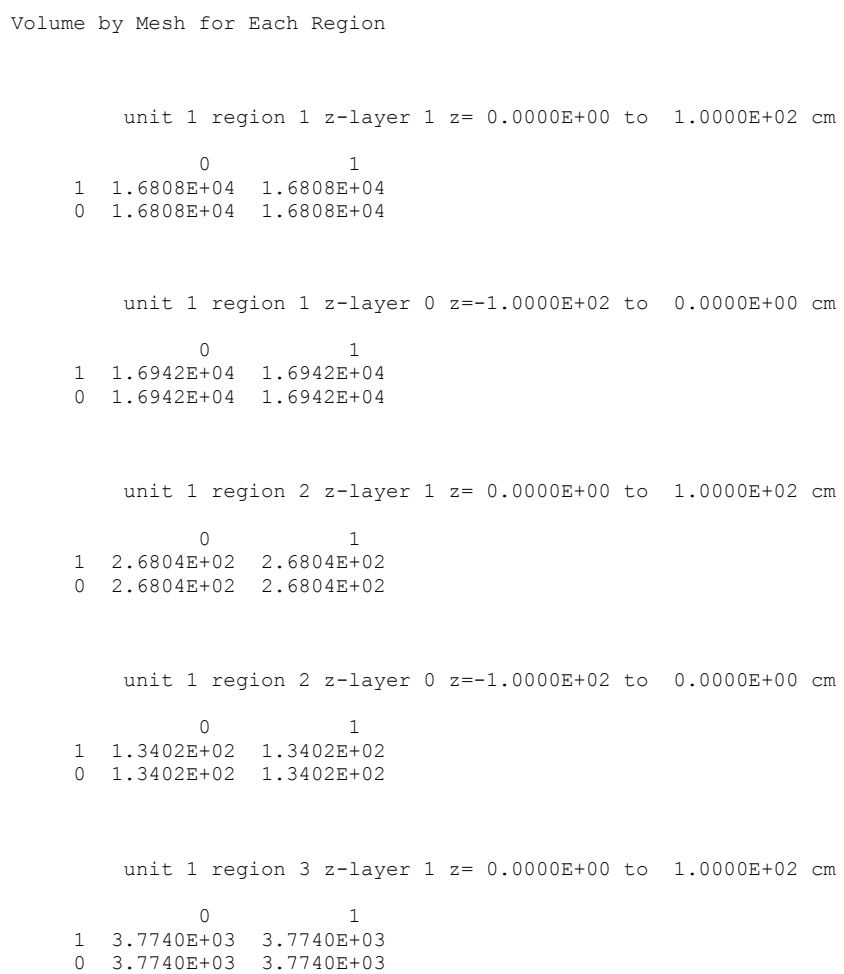

Fig. 8.1.115. Sample of volume by mesh for each region. 


\subsubsection{Biasing information}

This table specifies the weighting or biasing data to be used in the problem. An example of biasing information is given in Fig. 8.1.116.

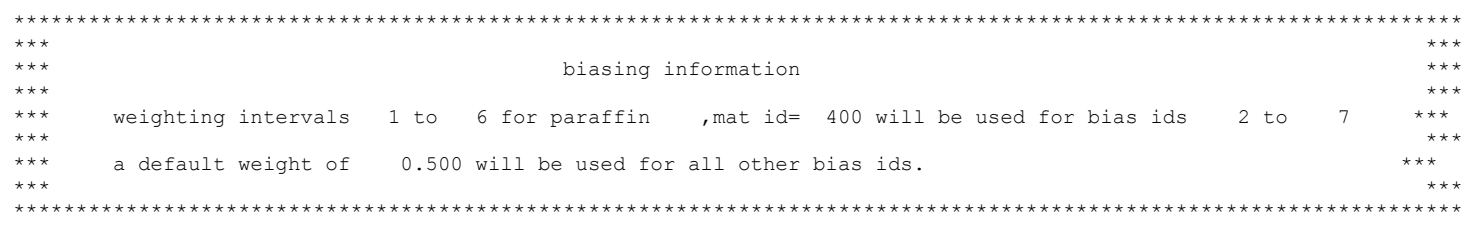

Fig. 8.1.116. Biasing information.

The user is responsible for determining from the input data whether the group-dependent weights (wtavg) for the specified material(s) were obtained from the weighting library or were entered by the user. The group-dependent weights can be printed for verification purposes as shown in Sect. 8.1.4.18.

\subsubsection{Group-dependent weights}

Printing the group-dependent weights is optional. They are printed if PWT=YES is entered in the parameter data. An example of the printed group-dependent weights is shown in Fig. 8.1.117. 
group dependent weights

\begin{tabular}{|c|c|c|c|}
\hline $\begin{array}{l}\text { bias } \\
\text { id } 4\end{array}$ & $\begin{array}{l}\text { bias } \\
\text { id } 5\end{array}$ & $\begin{array}{l}\text { bias } \\
\text { id }\end{array}$ & $\begin{array}{l}\text { bias } \\
\text { id }\end{array}$ \\
\hline----- & ---- & -_- - - & \\
\hline $1.27953 \mathrm{E}+00$ & $1.79963 \mathrm{E}+00$ & $2.54822 \mathrm{E}+00$ & $3.62191 \mathrm{E}+00$ \\
\hline $1.27093 \mathrm{E}+00$ & $1.86077 \mathrm{E}+00$ & $2.76980 \mathrm{E}+00$ & $4.16192 \mathrm{E}+00$ \\
\hline $1.21142 \mathrm{E}+00$ & $1.85960 \mathrm{E}+00$ & $2.93788 \mathrm{E}+00$ & $4.71945 \mathrm{E}+00$ \\
\hline $1.38278 \mathrm{E}+00$ & $2.35268 \mathrm{E}+00$ & $4.18040 \mathrm{E}+00$ & $7.61880 \mathrm{E}+00$ \\
\hline $1.51145 \mathrm{E}+00$ & $2.77730 \mathrm{E}+00$ & $5.39596 \mathrm{E}+00$ & $1.08420 \mathrm{E}+01$ \\
\hline $1.54519 \mathrm{E}+00$ & $2.90888 \mathrm{E}+00$ & $5.81680 \mathrm{E}+00$ & $1.20707 \mathrm{E}+01$ \\
\hline $1.62838 \mathrm{E}+00$ & $3.19784 \mathrm{E}+00$ & $6.71280 \mathrm{E}+00$ & $1.46796 \mathrm{E}+01$ \\
\hline $1.78398 \mathrm{E}+00$ & $3.79208 \mathrm{E}+00$ & $8.72478 \mathrm{E}+00$ & $2.10917 \mathrm{E}+01$ \\
\hline $2.03660 \mathrm{E}+00$ & $4.82428 \mathrm{E}+00$ & $1.25518 \mathrm{E}+01$ & $3.46445 \mathrm{E}+01$ \\
\hline $2.52715 \mathrm{E}+00$ & $7.05251 \mathrm{E}+00$ & $2.19482 \mathrm{E}+01$ & $7.30304 \mathrm{E}+01$ \\
\hline $3.60867 \mathrm{E}+00$ & $1.24307 \mathrm{E}+01$ & $4.75318 \mathrm{E}+01$ & $1.91658 \mathrm{E}+02$ \\
\hline $4.88448 \mathrm{E}+00$ & $1.89880 \mathrm{E}+01$ & $7.88949 \mathrm{E}+01$ & $3.34568 \mathrm{E}+02$ \\
\hline $5.55442 \mathrm{E}+00$ & $2.22849 \mathrm{E}+01$ & $9.38038 \mathrm{E}+01$ & $3.99407 \mathrm{E}+02$ \\
\hline $6.10317 \mathrm{E}+00$ & $2.47702 \mathrm{E}+01$ & $1.04624 \mathrm{E}+02$ & $4.45818 \mathrm{E}+02$ \\
\hline $7.15390 \mathrm{E}+00$ & $2.96382 \mathrm{E}+01$ & $1.25893 \mathrm{E}+02$ & $5.37071 \mathrm{E}+02$ \\
\hline $8.03687 \mathrm{E}+00$ & $3.37577 \mathrm{E}+01$ & $1.43807 \mathrm{E}+02$ & $6.13781 \mathrm{E}+02$ \\
\hline $8.97808 \mathrm{E}+00$ & $3.80495 \mathrm{E}+01$ & $1.62325 \mathrm{E}+02$ & $6.92943 \mathrm{E}+02$ \\
\hline $9.20905 \mathrm{E}+00$ & $3.91797 \mathrm{E}+01$ & $1.67220 \mathrm{E}+02$ & $7.13870 \mathrm{E}+02$ \\
\hline $1.12404 \mathrm{E}+01$ & $4.79024 \mathrm{E}+01$ & $2.04480 \mathrm{E}+02$ & $8.72943 \mathrm{E}+02$ \\
\hline $8.13714 \mathrm{E}+00$ & $3.46882 \mathrm{E}+01$ & $1.48076 \mathrm{E}+02$ & $6.32151 \mathrm{E}+02$ \\
\hline $8.67361 \mathrm{E}+00$ & $3.69873 \mathrm{E}+01$ & $1.57894 \mathrm{E}+02$ & $6.74064 \mathrm{E}+02$ \\
\hline $1.12536 \mathrm{E}+01$ & $4.80027 \mathrm{E}+01$ & $2.04921 \mathrm{E}+02$ & $8.74827 \mathrm{E}+02$ \\
\hline $1.14781 \mathrm{E}+01$ & $4.89758 \mathrm{E}+01$ & $2.09079 \mathrm{E}+02$ & $8.92578 \mathrm{E}+02$ \\
\hline $1.36168 \mathrm{E}+01$ & $5.81160 \mathrm{E}+01$ & $2.48101 \mathrm{E}+02$ & $1.05917 \mathrm{E}+03$ \\
\hline $1.53591 \mathrm{E}+01$ & $6.55621 \mathrm{E}+01$ & $2.79891 \mathrm{E}+02$ & $1.19488 \mathrm{E}+03$ \\
\hline $1.57871 \mathrm{E}+01$ & $6.73937 \mathrm{E}+01$ & $2.87711 \mathrm{E}+02$ & $1.22827 \mathrm{E}+03$ \\
\hline $1.58550 \mathrm{E}+01$ & $6.76852 \mathrm{E}+01$ & $2.88955 \mathrm{E}+02$ & $1.23358 \mathrm{E}+03$ \\
\hline $1.58135 \mathrm{E}+01$ & $6.75082 \mathrm{E}+01$ & $2.88199 \mathrm{E}+02$ & $1.23035 \mathrm{E}+03$ \\
\hline $1.57530 \mathrm{E}+01$ & $6.72506 \mathrm{E}+01$ & $2.87100 \mathrm{E}+02$ & $1.22566 \mathrm{E}+03$ \\
\hline $1.56921 \mathrm{E}+01$ & $6.69908 \mathrm{E}+01$ & $2.85991 \mathrm{E}+02$ & $1.22093 \mathrm{E}+03$ \\
\hline $1.57880 \mathrm{E}+01$ & $6.74006 \mathrm{E}+01$ & $2.87740 \mathrm{E}+02$ & $1.22839 \mathrm{E}+03$ \\
\hline $1.59923 \mathrm{E}+01$ & $6.82725 \mathrm{E}+01$ & $2.91463 \mathrm{E}+02$ & $1.24429 \mathrm{E}+03$ \\
\hline $1.62479 \mathrm{E}+01$ & $6.93639 \mathrm{E}+01$ & $2.96122 \mathrm{E}+02$ & $1.26418 \mathrm{E}+03$ \\
\hline $1.66638 \mathrm{E}+01$ & $7.11398 \mathrm{E}+01$ & $3.03703 \mathrm{E}+02$ & $1.29654 \mathrm{E}+03$ \\
\hline $1.73469 \mathrm{E}+01$ & $7.40559 \mathrm{E}+01$ & $3.16153 \mathrm{E}+02$ & $1.34969 \mathrm{E}+03$ \\
\hline $1.79855 \mathrm{E}+01$ & $7.67823 \mathrm{E}+01$ & $3.27792 \mathrm{E}+02$ & $1.39938 \mathrm{E}+03$ \\
\hline $1.84479 \mathrm{E}+01$ & $7.87566 \mathrm{E}+01$ & $3.36221 \mathrm{E}+02$ & $1.43536 \mathrm{E}+03$ \\
\hline $1.87423 \mathrm{E}+01$ & $8.00137 \mathrm{E}+01$ & $3.41588 \mathrm{E}+02$ & $1.45828 \mathrm{E}+03$ \\
\hline $1.89521 \mathrm{E}+01$ & $8.09093 \mathrm{E}+01$ & $3.45412 \mathrm{E}+02$ & $1.47460 \mathrm{E}+03$ \\
\hline $1.91140 \mathrm{E}+01$ & $8.16004 \mathrm{E}+01$ & $3.48363 \mathrm{E}+02$ & $1.48720 \mathrm{E}+03$ \\
\hline $1.93645 \mathrm{E}+01$ & $8.26699 \mathrm{E}+01$ & $3.52929 \mathrm{E}+02$ & $1.50670 \mathrm{E}+03$ \\
\hline $1.96886 \mathrm{E}+01$ & $8.40529 \mathrm{E}+01$ & $3.58831 \mathrm{E}+02$ & $1.53189 \mathrm{E}+03$ \\
\hline $1.98916 \mathrm{E}+01$ & $8.49188 \mathrm{E}+01$ & $3.62524 \mathrm{E}+02$ & $1.54764 \mathrm{E}+03$ \\
\hline & & $3.68566 \mathrm{E}+02$ & $1.57327 \mathrm{E}+03$ \\
\hline
\end{tabular}

Fig. 8.1.117. Example of biasing data.

The title is printed at the top of the table. The average weight (wtavg) is printed for each energy group and each BIAS ID. The BIAS ID number printed at the top of the column corresponds to the BIAS ID used in the geometry region description or MEDIA record and printed in the biasing information.

\subsubsection{Plot representation}

Plots representing 2-D slices through the geometrical description of the problem are optional. They are created if plot data are entered unless PLT $=\mathrm{NO}$ is specified either in the plot data or the parameter data. Plots can be generated and displayed as (1) character plots with alphanumeric characters representing mixture numbers, unit numbers, or bias ID numbers or (2) color plots with colors representing mixture numbers, unit numbers, or bias ID numbers. Color plots generate a PNG file and require an independent program to be displayed.

An example of the output generated using the character plot method is given in Fig. 8.1.118 and Fig. 8.1.119. An example of the output generated using the color plot method is given in Fig. 8.1.120 and Fig. 8.1.121.

Fig. 8.1.118 summarizes the data used to generate the character plot. Fig. 8.1.119 is an example of a character plot of the 2-D slice specified through the geometrical description of the problem. In 
Fig. 8.1.118, the plot title is printed at the top of the page, followed by a statement that "THE FOLLOWING WILL BE A CHARACTER PLOT." If a plot title was not entered in the plot data, the plot title is defaulted to the problem title. The title is followed by a heading specifying the type of plot (MIXTURE MAP, BIAS ID MAP, or UNIT MAP). This is followed by a table that correlates the symbols to be used in the character plot with the mixture numbers, bias ID numbers, or unit numbers that were used in the problem. If the problem is a bare array, the overall system coordinates are printed. Then the coordinates of the upper left corner and lower right corner of the plot are printed. This is followed by the direction cosines down and across the plot. The remaining plot parameters (including both input data and calculated values) are then printed. NU is the number of characters printed in the $U$ (down) direction, $\mathrm{NV}$ is the number of characters printed in the $\mathrm{V}$ (across) direction, DELU is the incremental distance, in $\mathrm{cm}$, represented by each character in the $U$ (down) direction, DELV is the incremental distance, in $\mathrm{cm}$, represented by each character in the V (across) direction, and LPI is the vertical to horizontal scaling factor for plot proportionality.

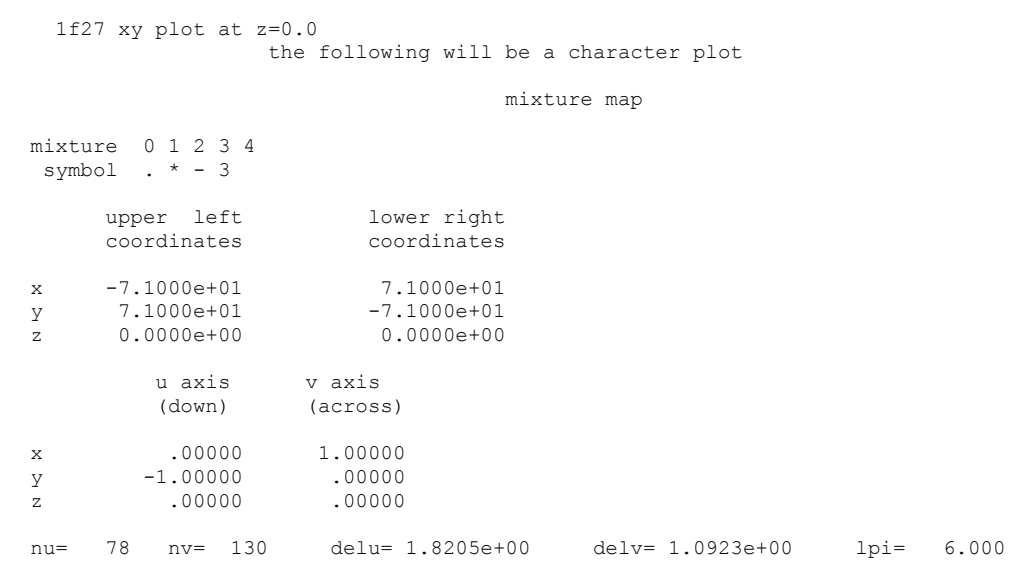

Fig. 8.1.118. Summary of character plot symbols, coordinates, and data.

Fig. 8.1.119 shows a character plot of a 2-D slice specified through the geometrical description of the problem. These plots aid the user in verifying that the problem is described correctly. Any number of plots can be made in one problem.

Fig. 8.1.120 summarizes the data used to generate the color plot. Fig. 8.1.121 is an example of a color plot of the 2-D slice specified through the geometrical description of the problem. This plot does not appear in the KENO printout. It is generated from a PNG file that is created when a color plot has been specified in the KENO input data and requires special processing by the user.

In Fig. 8.1.121, the plot title is printed at the top of the page, followed by a statement that "THE FOLLOWING WILL BE A COLOR PLOT." If a plot title was not entered in the plot data, the plot title is defaulted to the problem title. The title is followed by a heading specifying the type of plot (MIXTURE MAP, BIAD ID MAP, or UNIT MAP). If the problem is a bare array, the overall system coordinates are printed. Then the coordinates of the upper left corner and lower right corner of the plot are printed. This is followed by the direction cosines down and across the plot. The remaining plot parameters (including both input data and calculated values) are then printed. NU is the number of characters printed in the $U$ (down) direction, $\mathrm{NV}$ is the number of characters printed in the $\mathrm{V}$ (across) direction, DELU is the incremental distance, in $\mathrm{cm}$, represented by each character in the $U$ (down) direction, DELV is the incremental distance, in $\mathrm{cm}$, represented by each character in the $\mathrm{V}$ (across) direction, and LPI is the vertical to horizontal scaling factor for plot proportionality. 


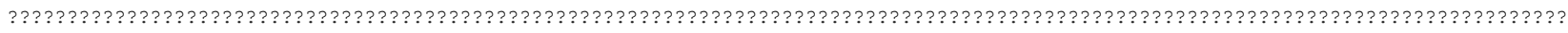

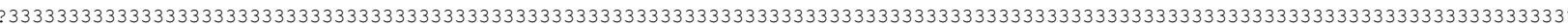

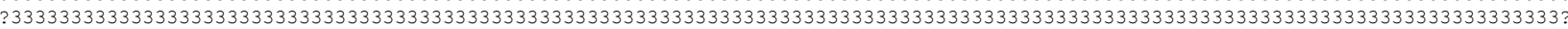

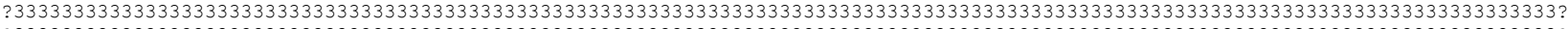

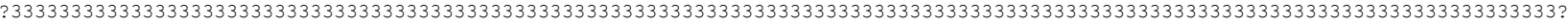

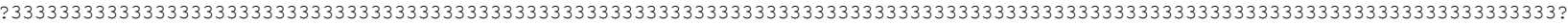

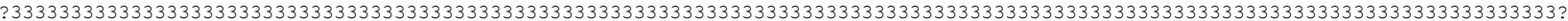
23333333333333333333333333333333333333333333333333333333333333333333333333333333333333333333333333333333333333333333333333333333333332 23333333333333333333333333333333333333333333333333333333333333333333333333333333333333333333333333333333333333333333333333333333332 333333333333333 333333333333333

33333333333333

?33333333333333

?33333333333333

?33333333333333

?3333333333333

?33333333333333

?3333333333333

?33333333333333

?3333333333333

233333333333333

33333333333333

?33333333333333

?3333333333333

?33333333333333

?33333333333333

?33333333333333

?3333333333333

?33333333333333

?33333333333333

233333333333333

.33333333333333

?33333333333333

?3333333333333

?33333333333333

?33333333333333

?33333333333333

?33333333333333

?3333333333333

333333333333333

233333333333333

?333333333

?3333333333333

?33333333333333

?3333333333333

?33333333333333

?3333333333333

?33333333333333

?3333333333333

23333333333333

.33333333333333

233333333333333

233333333333333

?33333333333333

?33333333333333

?33333333333333

?3333333333333

?33333333333333

?33333333333333

?33333333333333

233333333333333

?33333333333333

.33333333333333

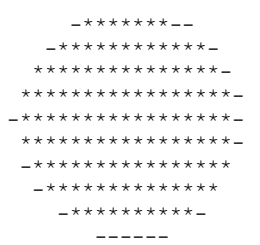

$-\star \star \star \star \star * \star * \star *-$

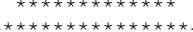

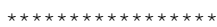

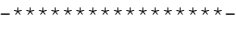

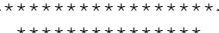

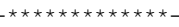

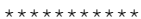

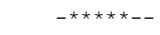

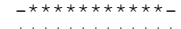

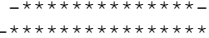

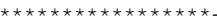

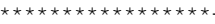

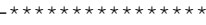

-

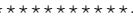

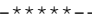

$--* * * * *-1$

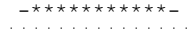

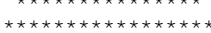

-

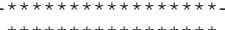

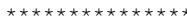

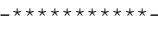

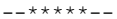
33333333333333 ? 3333333333333 ? 33333333333333 ? 33333333333333 ? 33333333333333 ? 33333333333333 ? 33333333333333 ? 33333333333333? 33333333333333 ? 33333333333333 ? 33333333333333? 33333333333333 ? 33333333333333 ? 33333333333333? 33333333333333? 33333333333333 ? 33333333333333 ? 33333333333333? 33333333333333 ? 33333333333333 ? 33333333333333? 33333333333333 33333333333333? 33333333333333 ? 33333333333333 33333333333333? 33333333333333? 33333333333333 33333333333333 ? 33333333333333? 33333333333333 ? 33333333333333? 33333333333333 ? 33333333333333 ? 33333333333333 ? 33333333333333 ? 33333333333333 33333333333333 ? 33333333333333? 33333333333333 ? 33333333333333 ? 33333333333333 ? 333333333333333? 33333333333333 ? 33333333333333? 33333333333333 ? 33333333333333 33333333333333 ? 33333333333333? 33 3333333333333 ? 33333333333333? 33333333333333? 33333333333333 ? 33333333333333 ? 33333333333333? 33333333333333 ? 33333333333333? 33333333333333? 33333333333333 ? 33333333333333? 33333333333333 ? 33333333333333 ? 33333333333333

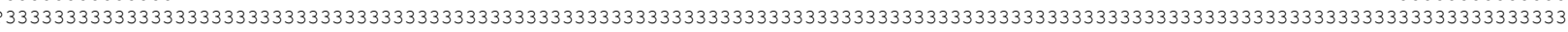

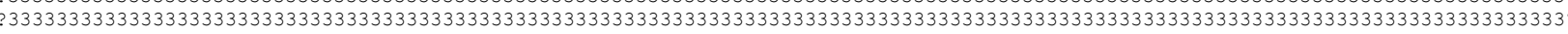

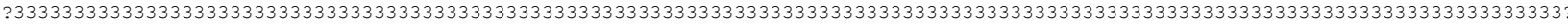

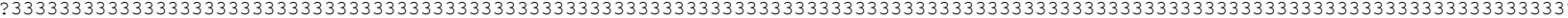

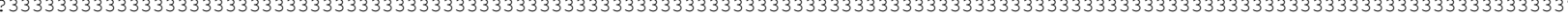

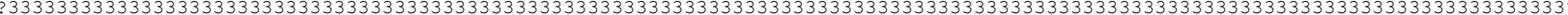

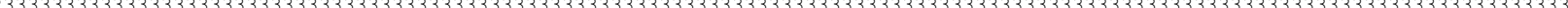

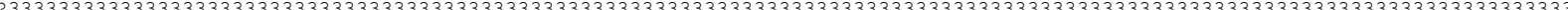

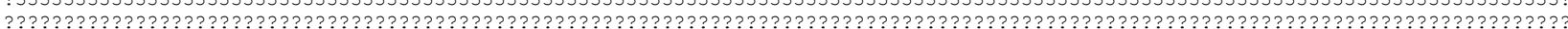

Fig. 8.1.119. Sample character plot representation. 


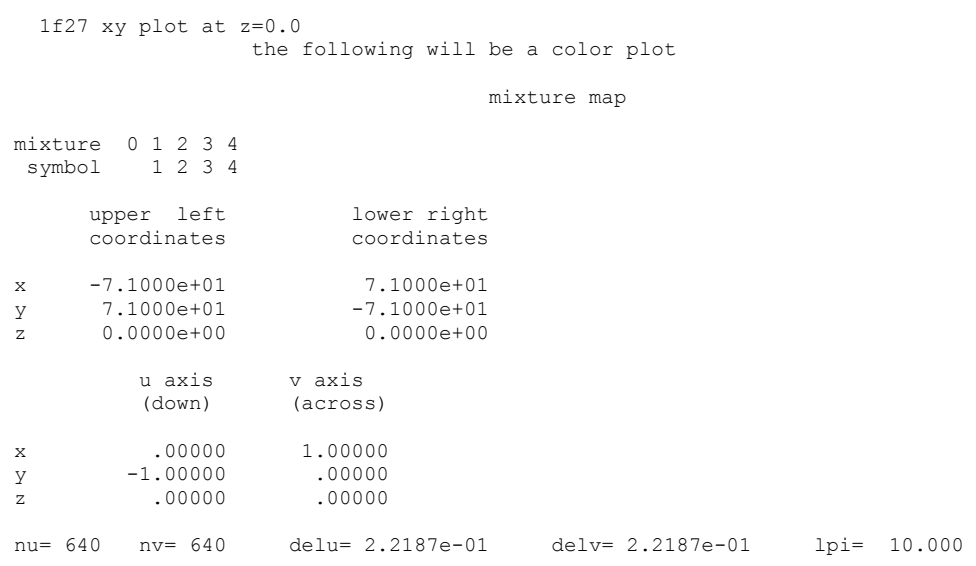

Fig. 8.1.120. Summary of color plot symbols, coordinates, and data.

Fig. 8.1.121 shows an example of a color plot of a 2-D slice specified through the geometrical description of the problem. Any number of plots can be made in one problem. The color plots can be valuable tools to assist the user in verifying that a problem is described correctly.

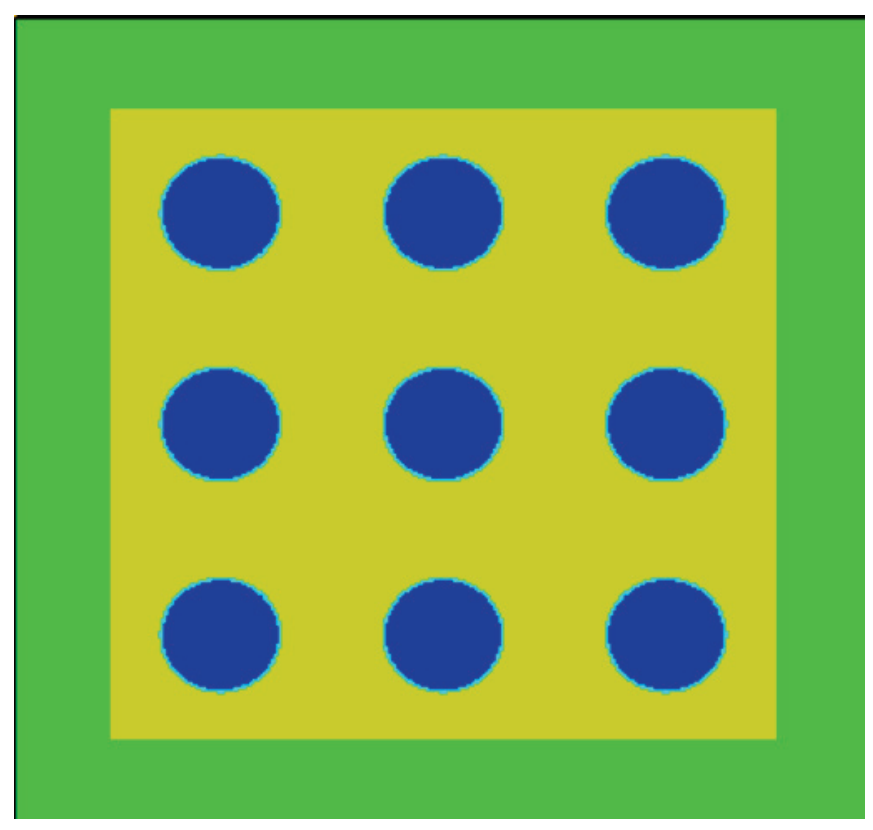

Fig. 8.1.121. Sample color plot representation. 


\subsubsection{Initial source and final pretracking edits}

Prior to calculating the $k_{\text {eff }}$ values for each generation, KENO prints the number of I/Os used before tracking and the number of minutes used processing the data. If the problem is not a restart problem, initial source information follows those two lines as shown in Fig. 8.1.122 (KENO V.a example).

The VOLUME FRACTION OF FISSILE MATERIAL IN THE CORE is the first line of data printed. If $\mathbf{R F L}=$ YES was specified in the start data, the volume fraction message is changed to VOLUME FRACTION OF FISSILE MATERIAL IN THE SYSTEM. This is followed by the start type, other data used to generate the initial source distribution, and finally, the amount of time used to generate the initial source distribution and the total elapsed time. Fig. 8.1.122 illustrates starting information printed for start type 0 . The cuboid for choosing starting points is (1) the array boundary for either a bare array or a reflected array with the key to start in the reflector turned off $(\mathbf{R F L}=\mathrm{NO})$, (2) the overall system for a reflected array if the key to start in the reflector is turned on (RFL=YES) and the outer region is a cuboid, or (3) a cuboid specified by the user via the keywords $\mathbf{X S M}=, \mathbf{X S P}=, \mathbf{Y S M}=, \mathbf{Y S P}=, \mathbf{Z S M}=$, and $\mathbf{Z S P}=$. For each start type for which the reflector key, $\mathbf{R F L}=$ is applicable, a statement is printed stating that "the flag to start neutrons in the reflector is turned 'on' or 'off."'

Fig. 8.1.122 illustrates typical starting data for start type 0 (a flat start in fissile material). In this example, the starting cuboid is the core boundary of a bare array. The default value of RFL was not overridden. The parameter used in this example was $\mathbf{N S T}=0$.

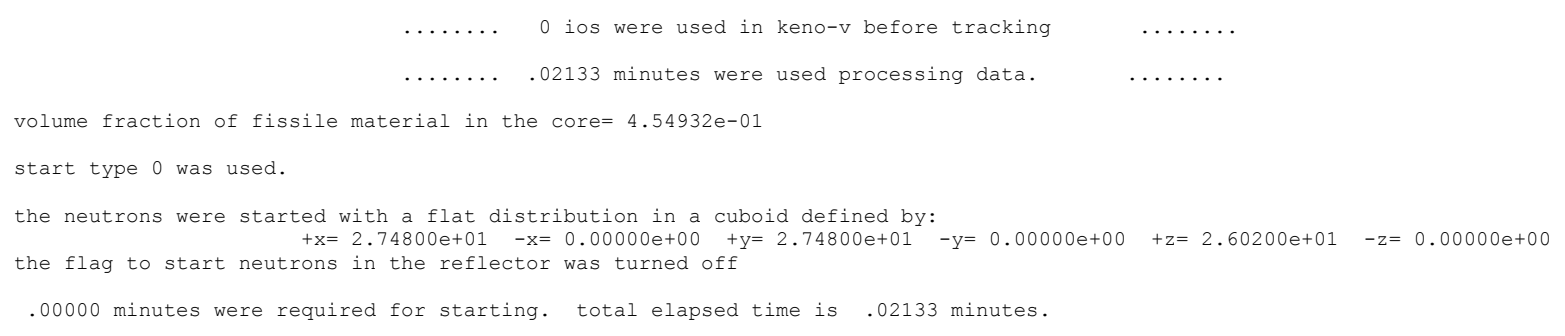

Fig. 8.1.122. Example of initial source information for start type 0 .

\subsubsection{Reference center for flux moment/angular flux transform}

When TFM=YES is entered, the flux moments and angular fluxes are computed in a transformed coordinate system, relative to a "reference center" point. The default position for the reference center is the center of all fueled regions in the model. However, the center can be specified by the user for each region defined in the system model using the "center" modifier on a region input card. Edits of the fuel center and the position of the reference center for each region are shown when TFM=YES as shown in Fig. 8.1.123.

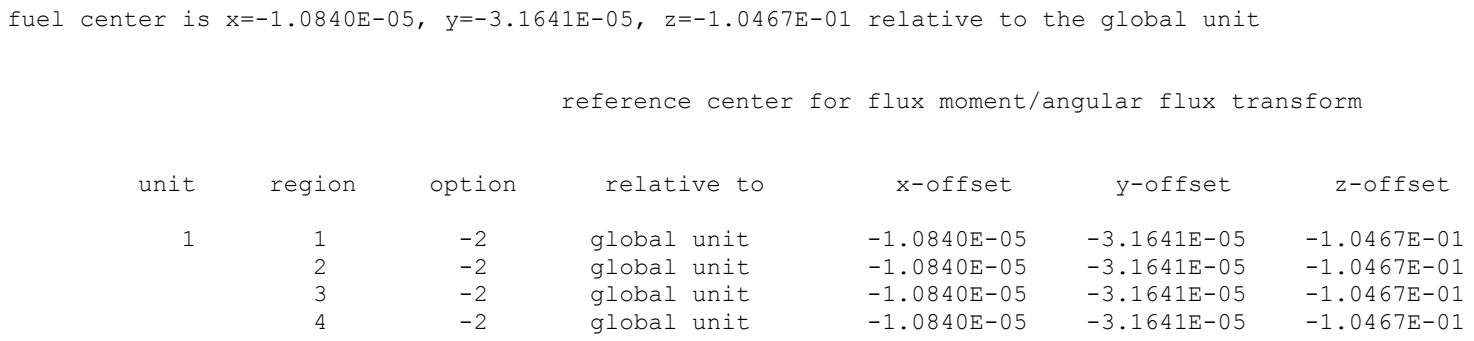

relative to

global unit

global unit

global unit

global unit

$x$-offset

$-1.0840 \mathrm{E}-05$

$-1.0840 \mathrm{E}-05$

$-1.0840 \mathrm{E}-05$

$-1.0840 \mathrm{E}-05$

$y$-offset

$-3.1641 \mathrm{E}-05$

$-3.1641 \mathrm{E}-05$

$-3.1641 \mathrm{E}-05$

$-3.1641 \mathrm{E}-05$ 


\begin{tabular}{|c|c|c|c|c|c|c|}
\hline 2 & 1 & 0 & global unit & $0.0000 \mathrm{E}+00$ & $0.0000 \mathrm{E}+00$ & $0.0000 \mathrm{E}+00$ \\
\hline 3 & 1 & 0 & global unit & $0.0000 \mathrm{E}+00$ & $0.0000 \mathrm{E}+00$ & $0.0000 \mathrm{E}+00$ \\
\hline 4 & 1 & 0 & global unit & $0.0000 \mathrm{E}+00$ & $0.0000 \mathrm{E}+00$ & $0.0000 \mathrm{E}+00$ \\
\hline 5 & 1 & 0 & global unit & $0.0000 \mathrm{E}+00$ & $0.0000 \mathrm{E}+00$ & $0.0000 \mathrm{E}+00$ \\
\hline 6 & $\begin{array}{l}1 \\
2 \\
3 \\
4 \\
5 \\
6 \\
7\end{array}$ & $\begin{array}{l}-2 \\
-2 \\
-2 \\
-2 \\
-2 \\
-2 \\
-2\end{array}$ & $\begin{array}{l}\text { global unit } \\
\text { global unit } \\
\text { global unit } \\
\text { global unit } \\
\text { global unit } \\
\text { global unit } \\
\text { global unit }\end{array}$ & $\begin{array}{l}-1.0840 \mathrm{E}-05 \\
-1.0840 \mathrm{E}-05 \\
-1.0840 \mathrm{E}-05 \\
-1.0840 \mathrm{E}-05 \\
-1.0840 \mathrm{E}-05 \\
-1.0840 \mathrm{E}-05 \\
-1.0840 \mathrm{E}-05\end{array}$ & $\begin{array}{l}-3.1641 \mathrm{E}-05 \\
-3.1641 \mathrm{E}-05 \\
-3.1641 \mathrm{E}-05 \\
-3.1641 \mathrm{E}-05 \\
-3.1641 \mathrm{E}-05 \\
-3.1641 \mathrm{E}-05 \\
-3.1641 \mathrm{E}-05\end{array}$ & $\begin{array}{l}-1.0467 \mathrm{E}-01 \\
-1.0467 \mathrm{E}-01 \\
-1.0467 \mathrm{E}-01 \\
-1.0467 \mathrm{E}-01 \\
-1.0467 \mathrm{E}-01 \\
-1.0467 \mathrm{E}-01 \\
-1.0467 \mathrm{E}-01\end{array}$ \\
\hline
\end{tabular}

Fig. 8.1.123. Example fuel center and reference center edits.

\subsubsection{Print starting points}

This printout is optional and is used to verify the initial source starting points. This option is activated by specifying the parameter PSP=YES. Examples of this information are given in Fig. 8.1.124 and Fig. 8.1.125.

The information pertinent to the initial source distribution is printed two lines at a time and appears under the designated headings. For example, the coordinates $\mathrm{X}, \mathrm{Y}$, and $\mathrm{Z}$ are printed on one line and the direction cosines $\mathrm{U}, \mathrm{V}$, and $\mathrm{W}$ are printed directly under them. The data printed for each source neutron include the following:

NEUTRON is the ID number of the neutron.

$\mathrm{X}, \mathrm{Y}$, and $\mathrm{Z}$ are the coordinates of the starting point relative to the coordinate system of the unit.

$\mathrm{K}$ is the region number that contains the point $\mathrm{X}, \mathrm{Y}, \mathrm{Z}$.

ETAUSD is the amount of the distance to collision already traversed (KENO-VI edit).

NOW is the array number.

NBX, NBY, NBZ are the coordinates of the unit within the array.

LSTC is the last unit where the neutron resided (KENO-VI edit).

NHOL is the hole where the neutron currently resides (KENO-VI edit).

LL is the unit number located at NBX, NBY, NBZ.

$\mathrm{KR}$ is the mixture number present at the starting point.

$\mathrm{KI}$ is the bias ID number or importance region at the starting point.

WT is the current weight (WT is always 1.0 for a neutron when it is started).

$\mathrm{U}, \mathrm{V}$, and $\mathrm{W}$ are the direction cosines defining the direction the history is traveling.

IG is the energy group.

$\mathrm{KCOR}$ is the region in the unit that contains the array where the neutron is located.

$\mathrm{K} 1$ is the region number of the first region in unit LL.

$\mathrm{K} 2$ is the region number of the last region in unit LL.

IGEO is an integer that defines the geometry shape of the region. 
KCOL is the region number in which the last collision occurred.

NSIG is the position of the group IG in the supergroup.

$\mathrm{SG} \quad$ is the supergroup number.

RANDOM NUMBER is the current random number.

When starting points are printed, many of the above named variables have not been initialized. For starting, the variables of interest are X, Y, Z, U, V, W, NBX, NBY, NBZ, and LL.

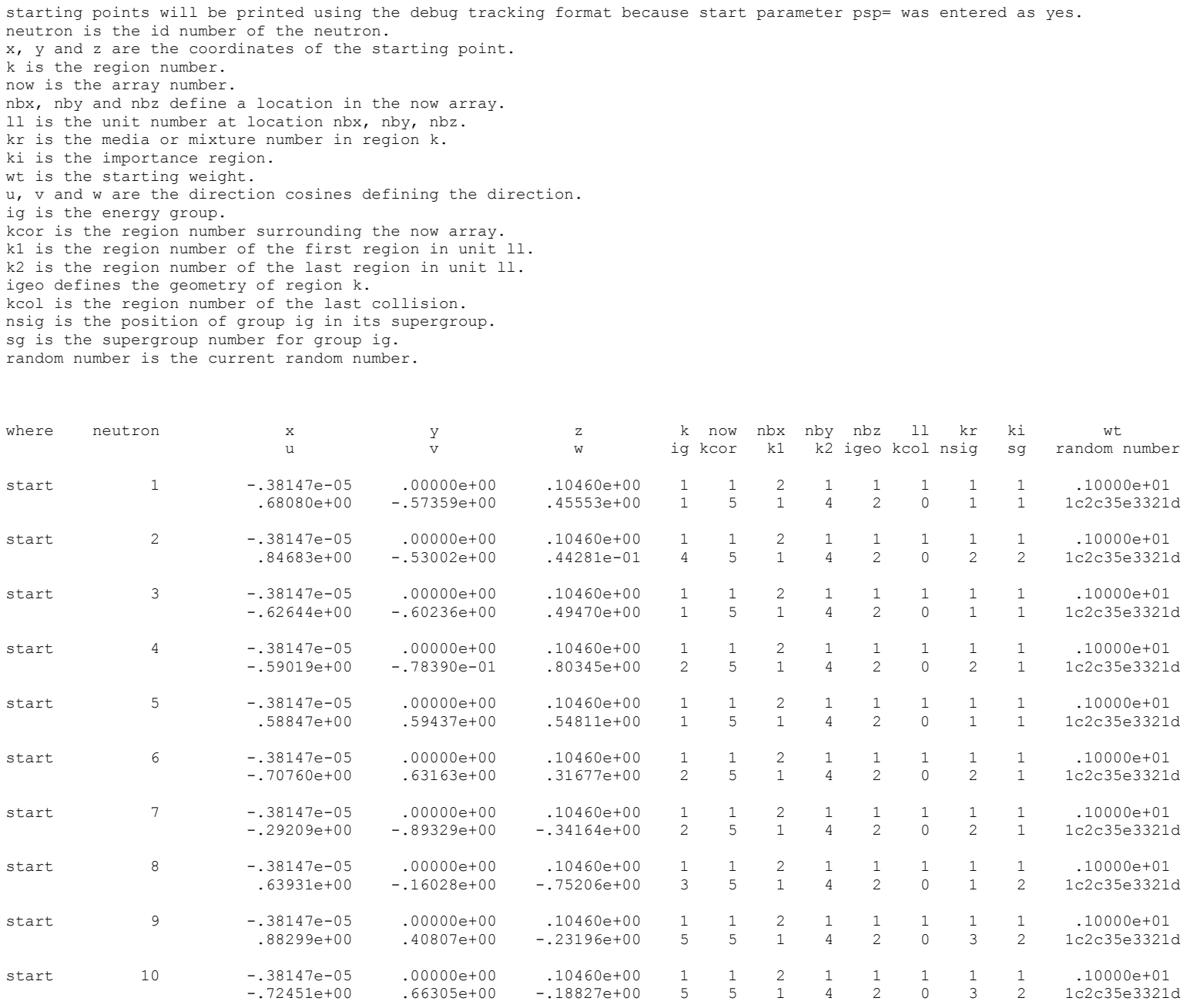

Fig. 8.1.124. Example of initial source points (KENO V.a edit). 


\begin{tabular}{|c|c|c|c|c|c|c|c|c|c|c|c|c|c|c|}
\hline \multirow{2}{*}{ where } & $\begin{array}{l}\text { neutron } \\
\text { random number }\end{array}$ & $\mathrm{x}$ & $\mathrm{y}$ & z & wt & k & $\mathrm{nbx}$ & nby & $\mathrm{nbz}$ & lstc & nhol & 11 & $\mathrm{kr}$ & $\mathrm{ki}$ \\
\hline & random number & $\mathrm{u}$ & $\mathrm{v}$ & $\mathrm{w}$ & etausd & now & kcor & $11 \mathrm{c}$ & $\mathrm{kcol}$ & lsth & ig & nsig & $\mathrm{sg}$ & \\
\hline \multirow[t]{2}{*}{ start } & 350 & $-2.93389 \mathrm{E}+00$ & $4.55407 \mathrm{E}+00$ & $4.68300 \mathrm{E}+00$ & $1.00000 \mathrm{E}+00$ & 1 & 2 & 1 & 3 & 0 & 1 & 1 & 1 & 0 \\
\hline & $0000334768 \mathrm{~F} 44690$ & $3.78162 \mathrm{E}-01$ & $4.60779 \mathrm{E}-01$ & $8.02917 \mathrm{E}-01$ & $0.00000 \mathrm{E}+00$ & 4 & 8 & 0 & 0 & 3 & 3 & 1 & & \\
\hline \multirow[t]{2}{*}{ start } & 349 & $-4.00210 \mathrm{E}+00$ & $6.15422 \mathrm{E}+00$ & $5.93966 \mathrm{E}+00$ & $1.00000 \mathrm{E}+00$ & 1 & 3 & 1 & 2 & 0 & 1 & 1 & 1 & 0 \\
\hline & $0000334768 \mathrm{~F} 44690$ & $2.08141 \mathrm{E}-01$ & $2.01154 \mathrm{E}-01$ & $-9.57191 \mathrm{E}-01$ & $0.00000 \mathrm{E}+00$ & 4 & 8 & 0 & 0 & 1 & 1 & 1 & & \\
\hline \multirow[t]{2}{*}{ start } & 348 & $-2.80915 E+00$ & $2.29649 \mathrm{E}+00$ & $3.72475 \mathrm{E}+00$ & $1.00000 \mathrm{E}+00$ & 1 & 2 & 1 & 1 & 0 & 1 & 1 & 1 & 0 \\
\hline & $0000334768 \mathrm{~F} 44690$ & $-7.35305 \mathrm{E}-01$ & $1.49356 \mathrm{E}-01$ & $6.61074 \mathrm{E}-01$ & $0.00000 \mathrm{E}+00$ & 4 & 6 & 0 & 0 & 1 & 1 & 1 & & \\
\hline \multirow[t]{2}{*}{ start } & 347 & $3.94031 \mathrm{E}+00$ & $-6.48469 \mathrm{E}+00$ & $1.03994 \mathrm{E}+00$ & $1.00000 \mathrm{E}+00$ & 1 & 1 & 1 & 1 & 0 & 1 & 1 & 1 & 0 \\
\hline & $0000334768 \mathrm{~F} 44690$ & $3.18453 \mathrm{E}-01$ & $-2.49655 \mathrm{E}-01$ & $-9.14473 \mathrm{E}-01$ & $0.00000 \mathrm{E}+00$ & 4 & 6 & 0 & 0 & 4 & 4 & 1 & & \\
\hline \multirow[t]{2}{*}{ start } & 346 & $5.68195 \mathrm{E}+00$ & $-4.41095 \mathrm{E}-01$ & $-3.78734 \mathrm{E}+00$ & $1.00000 \mathrm{E}+00$ & 1 & 2 & 2 & 2 & 0 & 1 & 1 & 1 & 0 \\
\hline & $0000334768 \mathrm{~F} 44690$ & $3.83747 \mathrm{E}-01$ & $-8.02679 \mathrm{E}-01$ & $4.56557 \mathrm{E}-01$ & $0.00000 \mathrm{E}+00$ & 4 & 5 & 0 & 0 & 4 & 4 & 1 & & \\
\hline \multirow[t]{2}{*}{ start } & 345 & $-1.29827 \mathrm{E}+00$ & $-1.33806 \mathrm{E}+00$ & $-5.75073 \mathrm{E}+00$ & $1.00000 \mathrm{E}+00$ & 1 & 1 & 1 & 2 & 0 & 1 & 1 & 1 & 0 \\
\hline & $0000334768 \mathrm{~F} 44690$ & $-3.05448 \mathrm{E}-01$ & $-9.31668 \mathrm{E}-01$ & $-1.96713 \mathrm{E}-01$ & $0.00000 \mathrm{E}+00$ & 4 & 7 & 0 & 0 & 2 & 2 & 1 & & \\
\hline \multirow{2}{*}{ start } & 344 & $3.16313 \mathrm{E}-02$ & $8.62766 \mathrm{E}+00$ & $3.12925 \mathrm{E}+00$ & $1.00000 \mathrm{E}+00$ & 1 & 2 & 1 & 1 & 0 & 1 & 1 & 1 & 0 \\
\hline & $0000334768 \mathrm{~F} 44690$ & $-8.19429 \mathrm{E}-01$ & $2.34186 \mathrm{E}-01$ & $5.23157 \mathrm{E}-01$ & $0.00000 \mathrm{E}+00$ & 4 & 8 & 0 & 0 & 4 & 4 & 1 & & \\
\hline \multirow[t]{2}{*}{ start } & 343 & $2.31603 \mathrm{E}+00$ & $-3.41755 \mathrm{E}+00$ & $8.47753 \mathrm{E}+00$ & $1.00000 \mathrm{E}+00$ & 1 & 3 & 1 & 1 & 0 & 1 & 1 & 1 & 0 \\
\hline & $0000334768 \mathrm{~F} 44690$ & $2.50993 \mathrm{E}-01$ & $-9.44899 \mathrm{E}-01$ & $-2.10163 \mathrm{E}-01$ & $0.00000 \mathrm{E}+00$ & 4 & 8 & 0 & 0 & 2 & 2 & 1 & & \\
\hline \multirow[t]{2}{*}{ start } & 342 & $7.92013 \mathrm{E}+00$ & $-3.31669 \mathrm{E}+00$ & $-6.54100 \mathrm{E}+00$ & $1.00000 \mathrm{E}+00$ & 1 & 1 & 1 & 1 & 0 & 1 & 1 & 1 & 0 \\
\hline & $0000334768 \mathrm{~F} 44690$ & $1.75156 \mathrm{E}-02$ & $9.23284 \mathrm{E}-01$ & $3.83718 \mathrm{E}-01$ & $0.00000 \mathrm{E}+00$ & 4 & 8 & 0 & 0 & 3 & 3 & 1 & & \\
\hline \multirow[t]{2}{*}{ start } & 341 & $8.17152 \mathrm{E}+00$ & $7.80831 \mathrm{E}-01$ & $4.88753 \mathrm{E}+00$ & $1.00000 \mathrm{E}+00$ & 1 & 2 & 1 & 2 & 0 & 1 & 1 & 1 & 0 \\
\hline & $0000334768 \mathrm{~F} 44690$ & $-4.58036 \mathrm{E}-01$ & $8.74804 \mathrm{E}-01$ & $-1.57864 \mathrm{E}-01$ & $0.00000 \mathrm{E}+00$ & 4 & 8 & 0 & 0 & 1 & 1 & 1 & & \\
\hline \multirow[t]{2}{*}{ start } & 340 & $2.25991 \mathrm{E}+00$ & $-7.97285 \mathrm{E}+00$ & $1.27218 \mathrm{E}+00$ & $1.00000 \mathrm{E}+00$ & 1 & 1 & 2 & 1 & 0 & 1 & 1 & 1 & 0 \\
\hline & $0000334768 \mathrm{~F} 44690$ & $4.04771 \mathrm{E}-01$ & $4.06303 \mathrm{E}-01$ & $8.19193 \mathrm{E}-01$ & $0.00000 \mathrm{E}+00$ & 4 & 5 & 0 & 0 & 1 & 1 & 1 & & \\
\hline \multirow[t]{2}{*}{ start } & 339 & $-7.11336 \mathrm{E}+00$ & $-5.01182 \mathrm{E}+00$ & $-1.35331 \mathrm{E}-01$ & $1.00000 \mathrm{E}+00$ & 1 & 1 & 2 & 2 & 0 & 1 & 1 & 1 & 0 \\
\hline & $0000334768 \mathrm{~F} 44690$ & $-8.35758 \mathrm{E}-01$ & $4.92574 \mathrm{E}-01$ & $-2.42650 \mathrm{E}-01$ & $0.00000 \mathrm{E}+00$ & 4 & 5 & 0 & 0 & 2 & 2 & 1 & & \\
\hline \multirow[t]{2}{*}{ start } & 338 & $-4.42523 \mathrm{E}+00$ & $1.73051 \mathrm{E}+00$ & $4.17569 \mathrm{E}+00$ & $1.00000 \mathrm{E}+00$ & 1 & 1 & 2 & $\perp$ & 0 & 1 & 1 & 1 & 0 \\
\hline & $0000334768 \mathrm{~F} 44690$ & $2.08661 \mathrm{E}-01$ & $-9.69024 \mathrm{E}-01$ & $1.32109 \mathrm{E}-01$ & $0.00000 \mathrm{E}+00$ & 4 & 7 & 0 & 0 & 2 & 2 & 1 & & \\
\hline
\end{tabular}

Fig. 8.1.125. Example of initial source points (KENO-VI edit).

\subsubsection{K-effectives by generation}

At the completion of each generation, KENO prints the $k_{\text {eff }}$ for that generation and associated information. An example of this printout is given in

the matrix k-effective is the largest eigenvalue of the fission production by unit number matrix.

Fig. 8.1.126.

\begin{tabular}{|c|c|c|c|c|c|c|}
\hline & generation & average & avg $k$-eff & generation & matrix & matrix k-eff \\
\hline generation & k-effective & k-effective & deviation & entropy & k-effective & deviation \\
\hline 1 & $9.94682 \mathrm{E}-01$ & $1.00000 \mathrm{E}+00$ & $0.00000 \mathrm{E}+00$ & $6.00366 \mathrm{E}+00$ & $9.94682 \mathrm{E}-01$ & $0.00000 \mathrm{E}+00$ \\
\hline 2 & $1.02075 \mathrm{E}+00$ & $1.00000 \mathrm{E}+00$ & $0.00000 \mathrm{E}+00$ & $5.94690 \mathrm{E}+00$ & $1.02075 \mathrm{E}+00$ & $0.00000 \mathrm{E}+00$ \\
\hline 3 & $1.00770 \mathrm{E}+00$ & $1.00770 \mathrm{E}+00$ & $0.00000 \mathrm{E}+00$ & $5.90382 \mathrm{E}+00$ & $1.00770 \mathrm{E}+00$ & $0.00000 \mathrm{E}+00$ \\
\hline 4 & $9.72514 \mathrm{E}-01$ & $9.90106 \mathrm{E}-01$ & $1.75912 \mathrm{E}-02$ & $5.94194 \mathrm{E}+00$ & $9.72514 \mathrm{E}-01$ & $0.00000 \mathrm{E}+00$ \\
\hline 5 & $9.78521 \mathrm{E}-01$ & $9.86244 \mathrm{E}-01$ & $1.08656 \mathrm{E}-02$ & $5.93816 \mathrm{E}+00$ & $9.75518 \mathrm{E}-01$ & $3.00344 \mathrm{E}-03$ \\
\hline 6 & $1.01509 \mathrm{E}+00$ & $9.93455 \mathrm{E}-01$ & $1.05371 \mathrm{E}-02$ & $5.90826 \mathrm{E}+00$ & $9.88708 \mathrm{E}-01$ & $1.33038 \mathrm{E}-02$ \\
\hline 7 & $9.71119 \mathrm{E}-01$ & $9.84311 \mathrm{E}-01$ & $1.46850 \mathrm{E}-02$ & $5.91640 \mathrm{E}+00$ & $9.84311 \mathrm{E}-01$ & $1.03842 \mathrm{E}-02$ \\
\hline 8 & $1.01561 \mathrm{E}+00$ & $9.90571 \mathrm{E}-01$ & $4.16922 \mathrm{E}-02$ & $5.94791 \mathrm{E}+00$ & $9.90571 \mathrm{E}-01$ & $1.01926 \mathrm{E}-02$ \\
\hline 9 & $9.60771 \mathrm{E}-01$ & $9.85604 \mathrm{E}-01$ & $2.11744 \mathrm{E}-02$ & $5.90329 \mathrm{E}+00$ & $9.85604 \mathrm{E}-01$ & $9.69159 \mathrm{E}-03$ \\
\hline 10 & $9.77417 \mathrm{E}-01$ & $9.84435 \mathrm{E}-01$ & $1.54731 \mathrm{E}-02$ & $5.79472 \mathrm{E}+00$ & $9.84435 \mathrm{E}-01$ & $8.27397 \mathrm{E}-03$ \\
\hline 11 & $1.00763 \mathrm{E}+00$ & $9.87334 \mathrm{E}-01$ & $1.10333 \mathrm{E}-02$ & $5.83135 \mathrm{E}+00$ & $9.87334 \mathrm{E}-01$ & $7.72996 \mathrm{E}-03$ \\
\hline 12 & $1.01826 \mathrm{E}+00$ & $9.90770 \mathrm{E}-01$ & $8.65604 \mathrm{E}-03$ & $5.92968 \mathrm{E}+00$ & $9.90770 \mathrm{E}-01$ & $7.63401 \mathrm{E}-03$ \\
\hline 13 & $1.00976 \mathrm{E}+00$ & $9.92669 \mathrm{E}-01$ & $9.19308 \mathrm{E}-03$ & $5.88436 \mathrm{E}+00$ & $9.92669 \mathrm{E}-01$ & $7.08710 \mathrm{E}-03$ \\
\hline 14 & $1.02742 \mathrm{E}+00$ & $9.95828 \mathrm{E}-01$ & $1.03148 \mathrm{E}-02$ & $5.89267 \mathrm{E}+00$ & $9.95828 \mathrm{E}-01$ & $7.14661 \mathrm{E}-03$ \\
\hline 15 & $9.88217 \mathrm{E}-01$ & $9.95193 \mathrm{E}-01$ & $9.10658 \mathrm{E}-03$ & $5.92788 \mathrm{E}+00$ & $9.95193 \mathrm{E}-01$ & $6.55469 \mathrm{E}-03$ \\
\hline 16 & $9.92170 \mathrm{E}-01$ & $9.94961 \mathrm{E}-01$ & $8.12596 \mathrm{E}-03$ & $5.92451 \mathrm{E}+00$ & $9.94961 \mathrm{E}-01$ & $6.03392 \mathrm{E}-03$ \\
\hline 17 & $1.00415 \mathrm{E}+00$ & $9.95617 \mathrm{E}-01$ & $7.35047 \mathrm{E}-03$ & $6.00362 \mathrm{E}+00$ & $9.95617 \mathrm{E}-01$ & $5.62474 \mathrm{E}-03$ \\
\hline 18 & $9.79078 \mathrm{E}-01$ & $9.94515 \mathrm{E}-01$ & $6.96679 \mathrm{E}-03$ & $5.92450 \mathrm{E}+00$ & $9.94515 \mathrm{E}-01$ & $5.35118 \mathrm{E}-03$ \\
\hline 19 & $1.00053 \mathrm{E}+00$ & $9.94890 \mathrm{E}-01$ & $6.49482 \mathrm{E}-03$ & $5.91204 \mathrm{E}+00$ & $9.94890 \mathrm{E}-01$ & $5.01965 \mathrm{E}-03$ \\
\hline 20 & $9.82400 \mathrm{E}-01$ & $9.94155 \mathrm{E}-01$ & $6.28256 \mathrm{E}-03$ & $5.94278 \mathrm{E}+00$ & $9.94155 \mathrm{E}-01$ & $4.77204 \mathrm{E}-03$ \\
\hline 21 & $9.99761 \mathrm{E}-01$ & $9.94467 \mathrm{E}-01$ & $5.89924 \mathrm{E}-03$ & $5.94086 \mathrm{E}+00$ & $9.94467 \mathrm{E}-01$ & $4.50989 \mathrm{E}-03$ \\
\hline 22 & $1.01911 \mathrm{E}+00$ & $9.95764 \mathrm{E}-01$ & $5.48740 \mathrm{E}-03$ & $5.87810 \mathrm{E}+00$ & $9.95764 \mathrm{E}-01$ & $4.45870 \mathrm{E}-03$ \\
\hline 23 & $1.06847 \mathrm{E}+00$ & $9.99399 \mathrm{E}-01$ & $7.59384 \mathrm{E}-03$ & $5.83725 \mathrm{E}+00$ & $9.99399 \mathrm{E}-01$ & $5.57749 \mathrm{E}-03$ \\
\hline 24 & $9.56023 \mathrm{E}-01$ & $9.97334 \mathrm{E}-01$ & $5.98529 \mathrm{E}-03$ & $5.83523 \mathrm{E}+00$ & $9.97334 \mathrm{E}-01$ & $5.69317 \mathrm{E}-03$ \\
\hline 25 & $9.86202 \mathrm{E}-01$ & $9.96828 \mathrm{E}-01$ & $5.71783 \mathrm{E}-03$ & $5.99533 \mathrm{E}+00$ & $9.96828 \mathrm{E}-01$ & $5.45176 \mathrm{E}-03$ \\
\hline 26 & $9.74280 \mathrm{E}-01$ & $9.95847 \mathrm{E}-01$ & $5.89085 \mathrm{E}-03$ & $5.96158 \mathrm{E}+00$ & $9.95847 \mathrm{E}-01$ & $5.30077 \mathrm{E}-03$ \\
\hline 27 & $9.84275 \mathrm{E}-01$ & $9.95365 \mathrm{E}-01$ & $5.69765 \mathrm{E}-03$ & $5.82695 \mathrm{E}+00$ & $9.95365 \mathrm{E}-01$ & $5.09796 \mathrm{E}-03$ \\
\hline 28 & $1.01790 \mathrm{E}+00$ & $9.96267 \mathrm{E}-01$ & $5.18387 \mathrm{E}-03$ & $5.90530 \mathrm{E}+00$ & $9.96267 \mathrm{E}-01$ & $4.97220 \mathrm{E}-03$ \\
\hline 29 & $1.02889 \mathrm{E}+00$ & $9.97521 \mathrm{E}-01$ & $5.14081 \mathrm{E}-03$ & $5.97433 \mathrm{E}+00$ & $9.97521 \mathrm{E}-01$ & $4.93914 \mathrm{E}-03$ \\
\hline 30 & $1.03543 \mathrm{E}+00$ & $9.98925 \mathrm{E}-01$ & $5.81888 \mathrm{E}-03$ & $5.92559 \mathrm{E}+00$ & $9.98925 \mathrm{E}-01$ & $4.95576 \mathrm{E}-03$ \\
\hline 31 & $9.59335 \mathrm{E}-01$ & $9.97511 \mathrm{E}-01$ & $5.16841 \mathrm{E}-03$ & $5.87863 \mathrm{E}+00$ & $9.97511 \mathrm{E}-01$ & $4.98041 \mathrm{E}-03$ \\
\hline 32 & $9.89604 \mathrm{E}-01$ & $9.97239 \mathrm{E}-01$ & $4.98842 \mathrm{E}-03$ & $5.87271 \mathrm{E}+00$ & $9.97239 \mathrm{E}-01$ & $4.81334 \mathrm{E}-03$ \\
\hline 33 & $9.79932 \mathrm{E}-01$ & $9.96662 \mathrm{E}-01$ & $4.85023 \mathrm{E}-03$ & $5.94115 \mathrm{E}+00$ & $9.96662 \mathrm{E}-01$ & $4.68577 \mathrm{E}-03$ \\
\hline 34 & $1.00370 \mathrm{E}+00$ & $9.96889 \mathrm{E}-01$ & $4.69165 \mathrm{E}-03$ & $5.83387 \mathrm{E}+00$ & $9.96889 \mathrm{E}-01$ & $4.53779 \mathrm{E}-03$ \\
\hline 35 & $9.77272 \mathrm{E}-01$ & $9.96276 \mathrm{E}-01$ & $4.58174 \mathrm{E}-03$ & $5.86485 \mathrm{E}+00$ & $9.96276 \mathrm{E}-01$ & $4.43626 \mathrm{E}-03$ \\
\hline
\end{tabular}




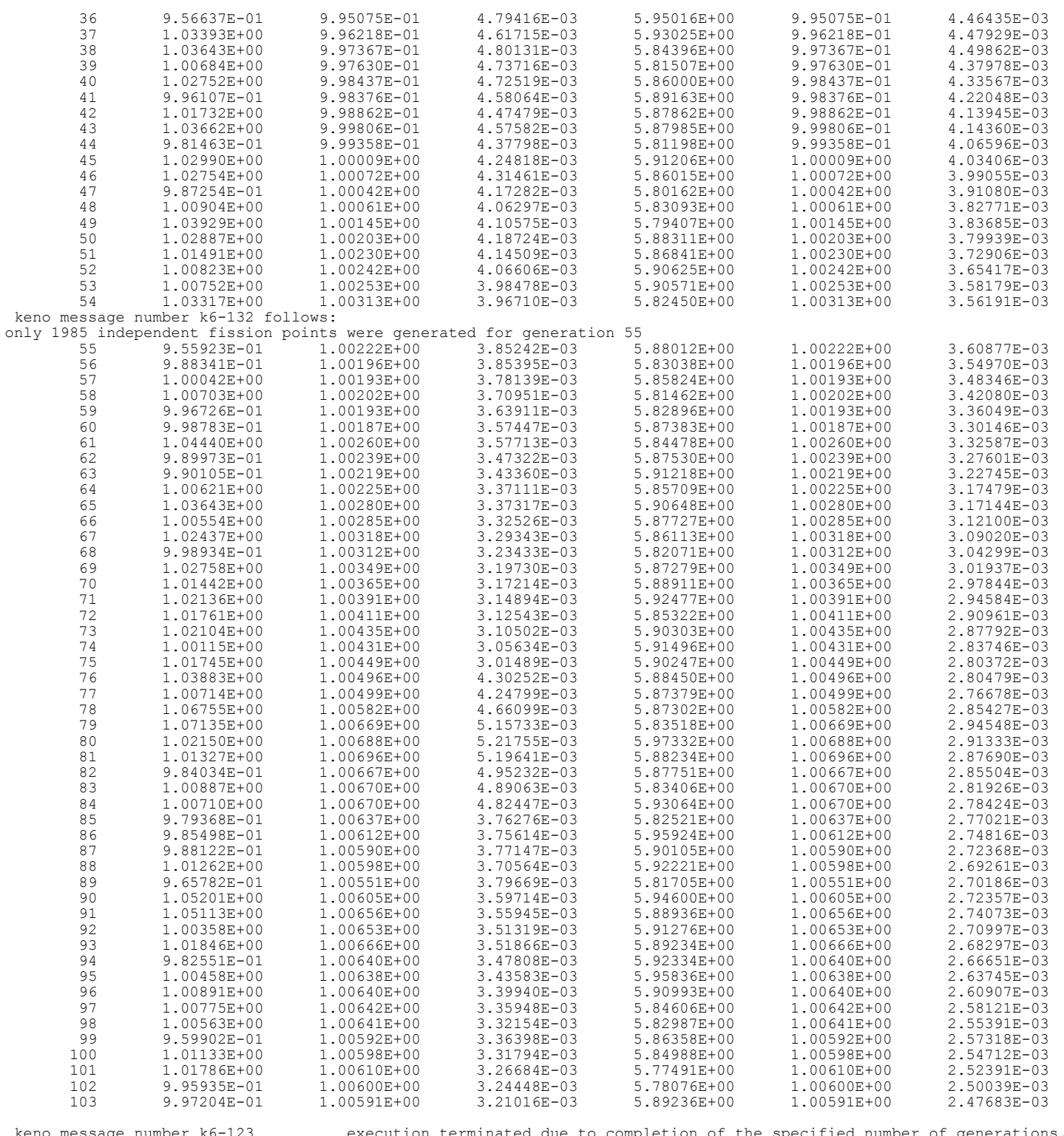

the matrix k-effective is the largest eigenvalue of the fission production by unit number matrix.

Fig. 8.1.126. Example of k-effectives and source entropy by generation.

The data printed include (1) the generation number, (2) the k-effective calculated for the generation, (3) the average value of k-effective through the current generation (excluding the nskip- 1 generations), (4) the deviation associated with the average k-effective, (5) Shannon entropy for the generation, (6) the matrix k-effective for the generation, and (7) the deviation associated with the matrix k-effective. Column 5 is omitted if the user disabled source convergence diagnostics. The last two columns are filled with zeros if the user did not specify matrix k-effective calculations. The matrix k-effective is the largest eigenvalue of the fission production matrix. Matrix information can be calculated based on (1) position index, (2) unit number, (3) hole number, and (4) array number. The matrix k-effective printed in the sixth column is based on this order. If the matrix k-effective is calculated by position index, it is the one printed in the sixth column. The matrix k-effective by unit number is given second preference, followed by hole number and then array number. 
After the last generation, a message is printed to indicate why execution was terminated. If matrix k-effectives were calculated, this is followed by a message stating the method used to determine the matrix k-effective.

The user should examine this portion of the printed results to ensure that the two methods of calculating k-effective are in acceptable agreement and to verify that the average value of k-effective has become relatively stable. If the k-effectives appear to be oscillating or drifting significantly, then the user should consider rerunning the problem with a larger number of histories per generation.

If a problem is restarted, then the generation numbers and k-effectives are printed and the words FROM RESTART UNIT are printed in the elapsed time column. All other columns are blank. When the generation at which the problem is to be restarted is reached, the print reverts to the normal format as shown in Fig. 8.1.126.

\subsubsection{Problem characterization edit}

The problem characterization edit follows the k-effective by generation edit. The title is printed at the top of the page, followed by the lifetime and the generation time and their associated deviations. The lifetime is the average lifespan of a neutron (in seconds) from the time it is born until it is absorbed or leaks from the system. The generation time is the average time (in seconds) between successive neutron generations. If $\mathbf{N U B}=$ YES is specified in the parameter data, (Sect. 8.1.2.3) the average number of neutrons per fission, NU BAR, and its associated deviation are printed and the AVERAGE FISSION GROUP (the average energy group at which fission occurs) and its associated deviation are printed. Then the ENERGY(EV) OF THE AVERAGE LETHARGY OF NEUTRONS CAUSING FISSION and its associated deviation are printed, followed by the system mean free path. If SMU=YES is specified in the parameter data, the average self-multiplication of a unit and its associated deviation is printed. This selfmultiplication results from fissions caused by neutrons born in the unit. Fissions caused by neutrons that exit the unit and return are not included. The problem characterization edit is shown in Fig. 8.1.127.

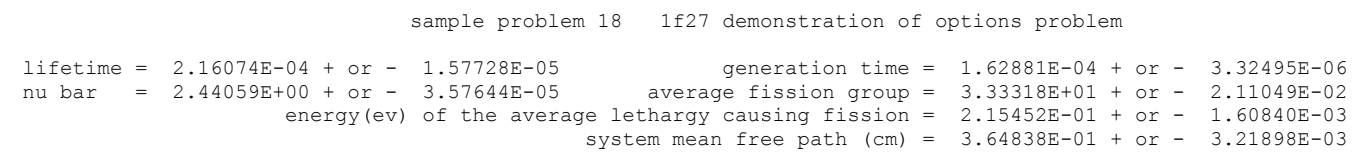

Fig. 8.1.127. Example problem characterization edit.

\subsubsection{Final k-effective edit}

The final k-effective edit prints the average k-effective and its associated deviation and the limits of k-effective for the 67,95 , and $99 \%$ confidence intervals. The number of histories used in calculating the average k-effective is also printed. This is done skipping various numbers of generations. The user should carefully examine the final k-effective edit to determine if the average k-effective is relatively stable. If a noticeable drift is apparent as the number of initial generations skipped increases, it may indicate a problem in converging the source. If this appears to be the case, the problem should be rerun with a better initial source distribution and should be run for a sufficient number of generations that the average k-effective becomes stable. Then the final k-effective edit is printed as shown in Fig. 8.1.128.

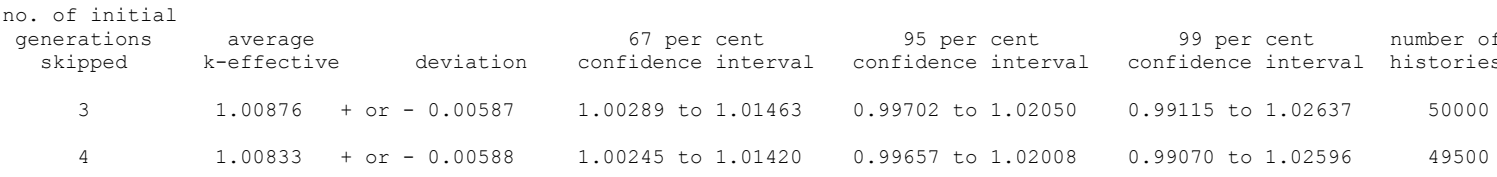




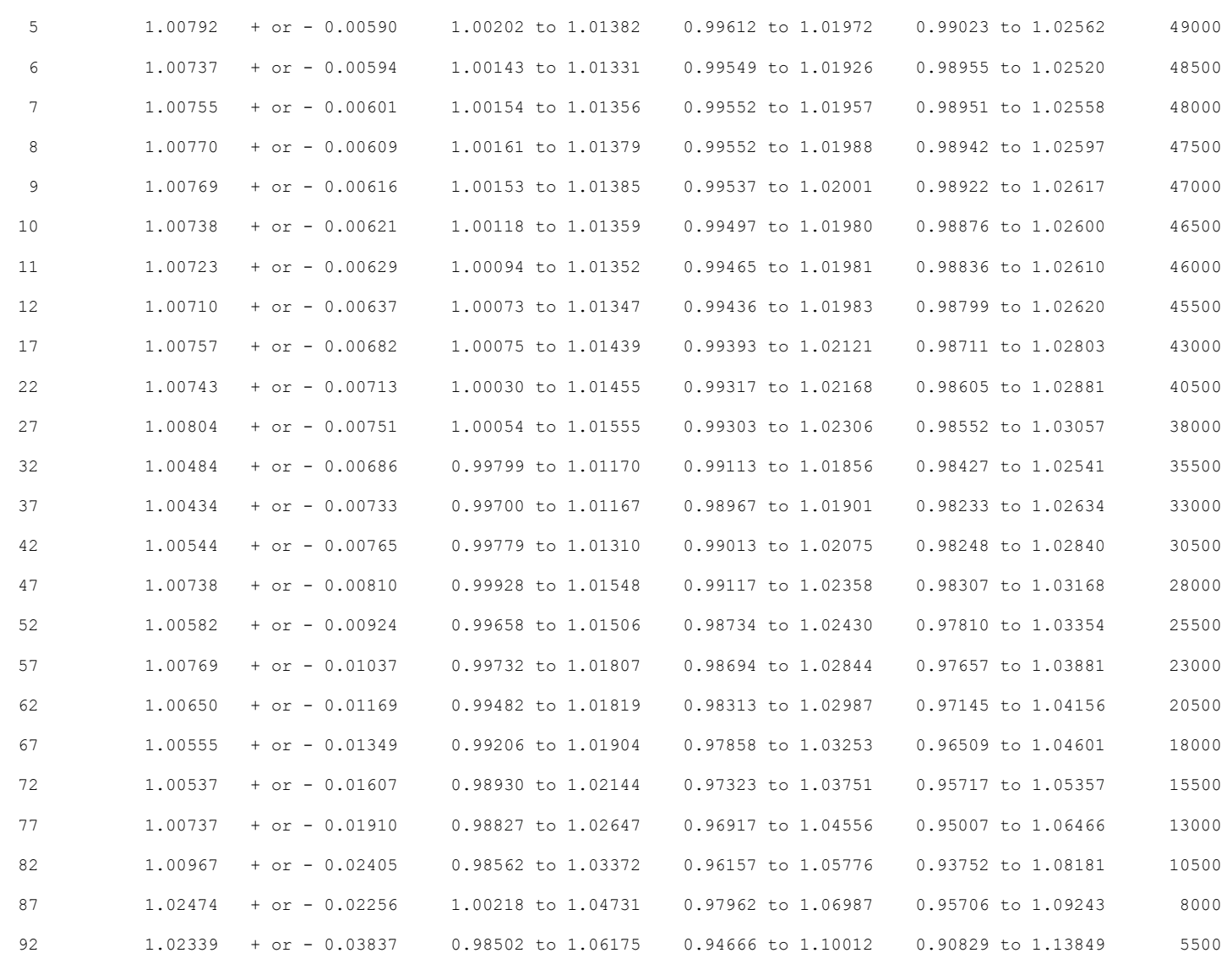

Fig. 8.1.128. Example of the final k-effective edit.

\subsubsection{Plot of average k-effective by generation run}

This plot consists of average k-effectives plotted versus the number of generations run. The limits of one standard deviation are plotted on either side of each average k-effective. These average k-effectives are not necessarily the same as the average k-effectives described in Sect. 8.1.4.23. The code omits the k-effectives of the first nskip generations when the average k-effectives for this plot are calculated. Although the k-effective of the nskip generation is summed into the average k-effective, it is not plotted because standard deviations cannot be calculated for a single point. Thus, if nskip is 3 (i.e., the first three generations are skipped), the first value plotted is the average k-effective corresponding to the fifth generation. The dotted line represents the value of the average k-effective corresponding to the smallest deviation when the average k-effective and its deviation are computed for each generation over the range of nskip through the total number of generations.

Fig. 8.1.129 is an example of this type of plot in the text output. The primary use for this plot is to determine if the problem has source convergence difficulties.

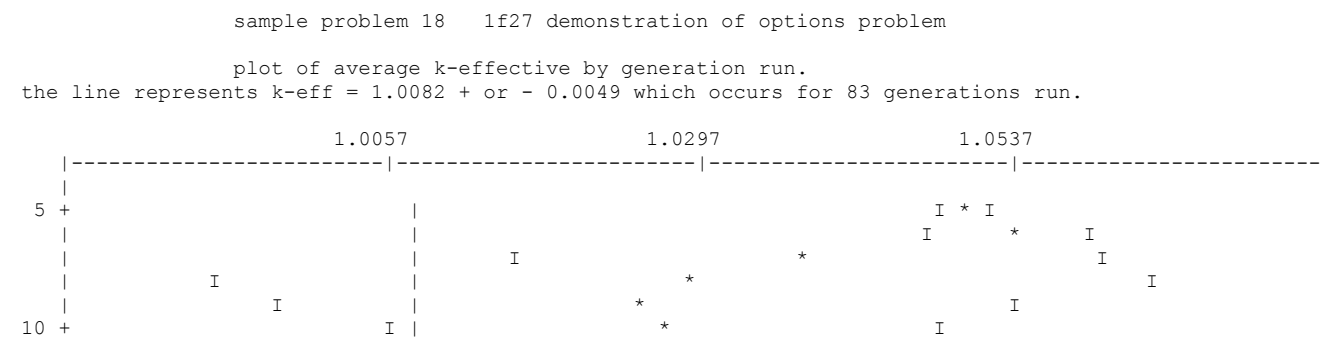




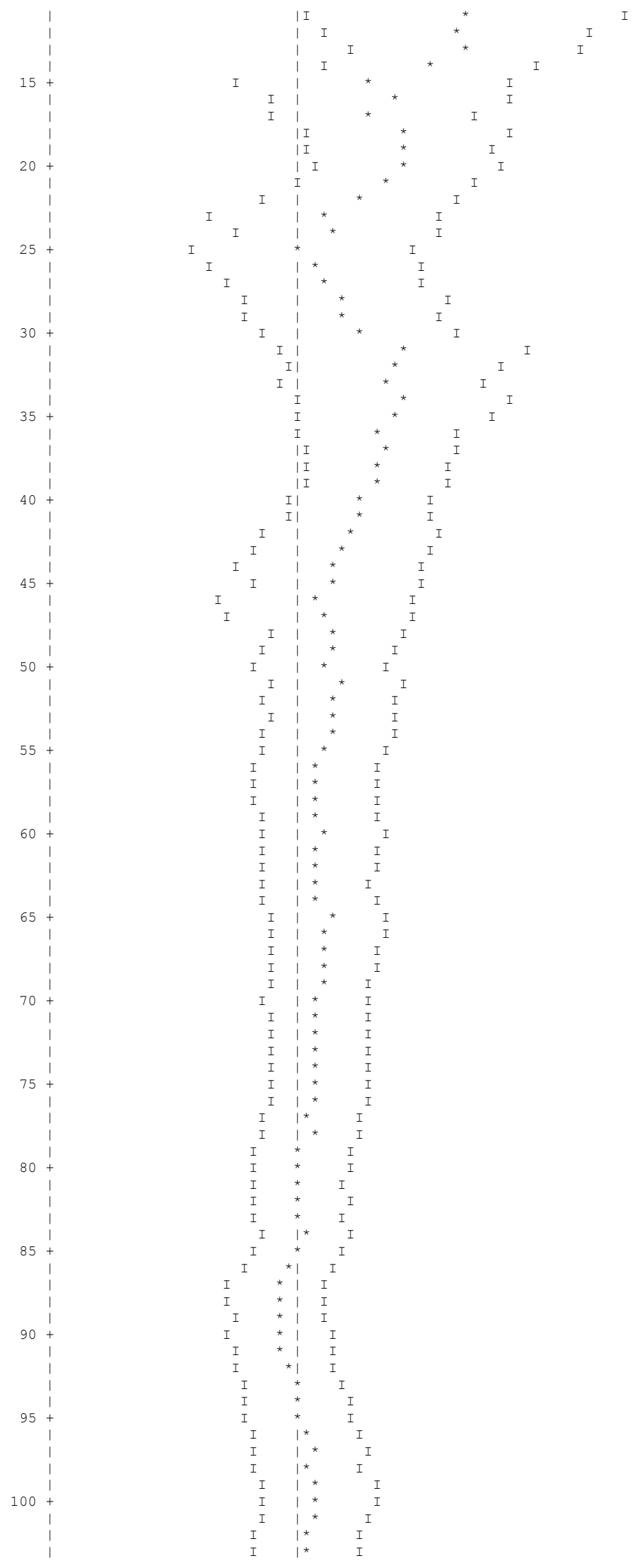

8-318 
Fig. 8.1.129. Sample plot of average k-effective by generation run.

\subsubsection{Plot of average k-effective by generations skipped}

This plot illustrates the average k-effective versus the number of generations skipped as shown in Fig. 8.1.130. The limits of one standard deviation are plotted on either side of the average k-effective. The dotted line represents the value of the average k-effective corresponding to the smallest deviation when the average k-effective and its deviation are computed for the number of generations skipped over the range of nskip+1 through $2 / 3$ the total number of generations calculated. The plot is essentially a plot of the data described in sect. 8.1.4.25. It is useful for determining if source convergence has been achieved.

Fig. 8.1.130

sample problem $18 \quad 1$ f27 demonstration of options problem

plot of average k-effective by generation skipped.

the line represents $k$ eff $=1.0087+$ or -0.0058 which occurs for 3 generations skipped.

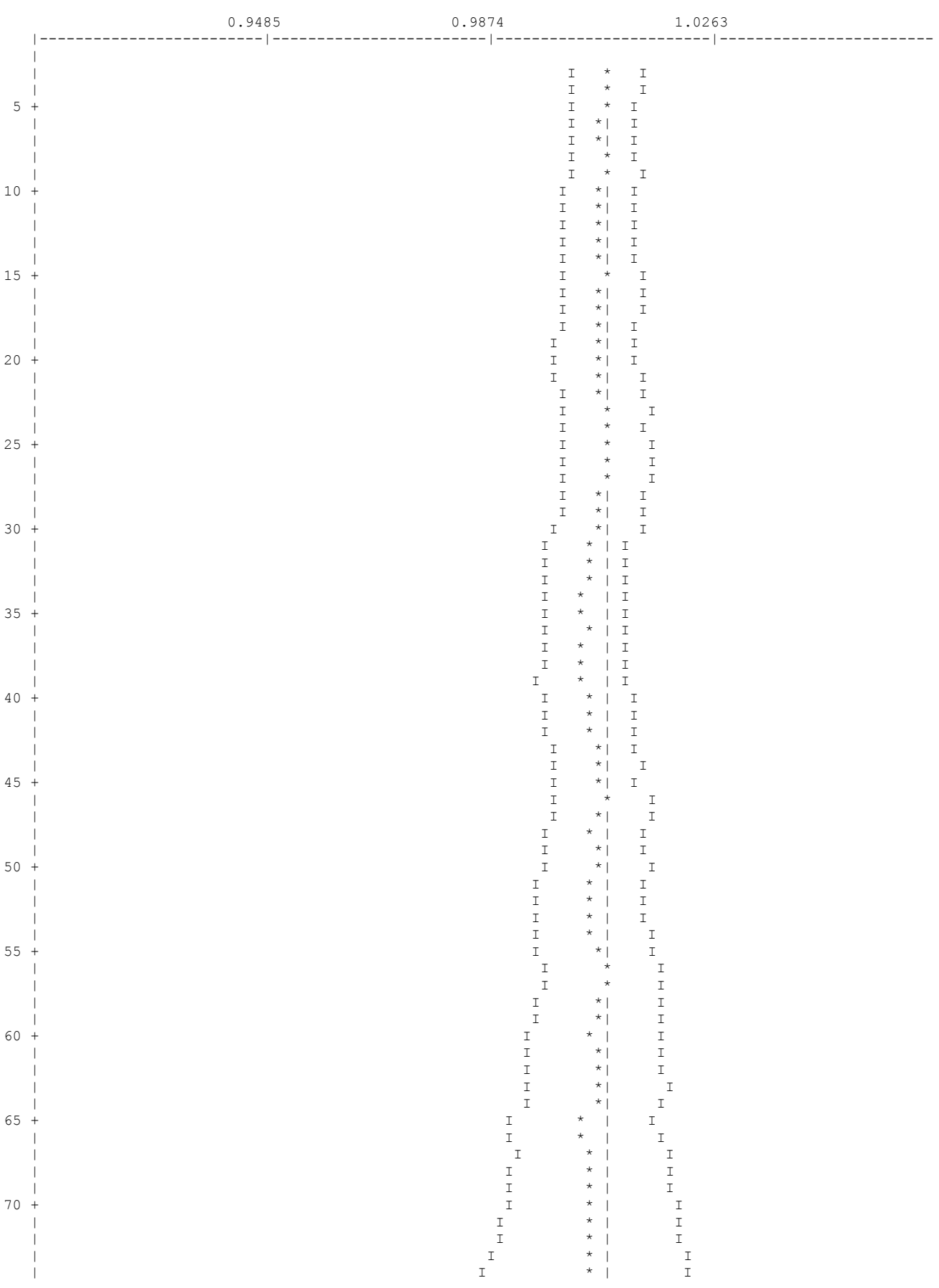




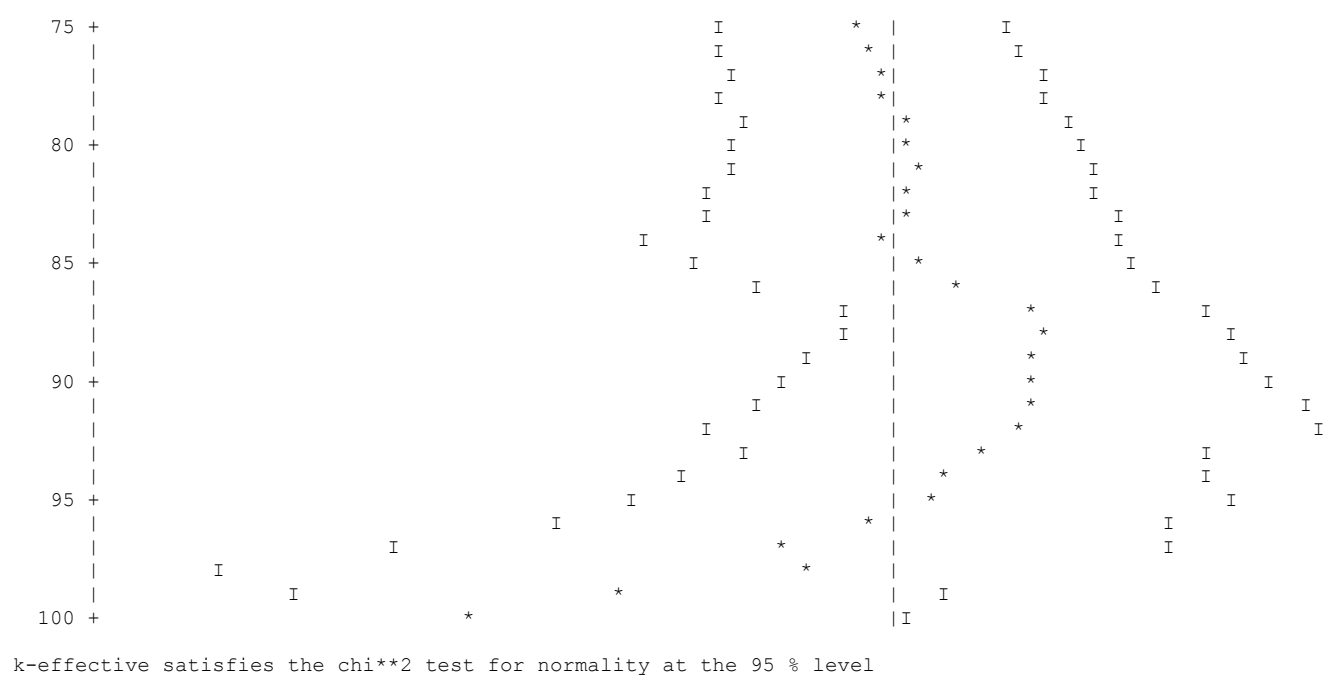

Fig. 8.1.130. Sample plot of average k-effective by generations skipped.

\subsubsection{Final edit of fissions, absorptions, and leakage}

The final edit of fissions, absorptions, and leakage follows the final k-effective edit and includes the fission fraction for each group and the fission production, absorptions, and leakage, each with its associated percent deviation. Examples of the final edits of fissions, absorptions, and leakage are shown in Fig. 8.1.131-Fig. 8.1.133.

If $\mathbf{F A R}=\mathrm{NO}$ is specified, region-dependent fissions and absorptions are not printed. Fig. 8.1.131 demonstrates the printout if $\mathbf{F A R}=\mathrm{NO}$. 


\begin{tabular}{|c|c|c|c|c|c|c|c|c|c|}
\hline \multirow[b]{2}{*}{ group } & \multirow[b]{2}{*}{$\begin{array}{l}\text { fission } \\
\text { fraction }\end{array}$} & \multirow[b]{2}{*}{ unit } & \multicolumn{2}{|c|}{ sample problem } & \multirow{2}{*}{$\begin{array}{c}2 \mathrm{c} 8 \text { bare } \\
\text { percent } \\
\text { deviation }\end{array}$} & & & \multirow[t]{2}{*}{ skipping 3 generations } \\
\hline & & & region & fissions & & absorptions & $\begin{array}{c}\text { percent } \\
\text { deviation }\end{array}$ & leakage & \\
\hline 1 & .1439 & & & $1.43327 e-01$ & 1.6542 & $5.06130 e-02$ & 1.6542 & $7.82282 e-02$ & 2.0448 \\
\hline 2 & .2391 & & & $2.38112 e-01$ & .8605 & $9.69963 e-02$ & .8605 & $1.44757 e-01$ & 1.3068 \\
\hline 3 & .1429 & & & $1.42343 e-01$ & 1.6270 & $6.15892 e-02$ & 1.6270 & $8.82221 e-02$ & 1.8458 \\
\hline 4 & .2303 & & & $2.29375 e-01$ & 1.2187 & $1.04317 e-01$ & 1.2187 & $1.50874 e-01$ & 1.0063 \\
\hline 5 & .1940 & & & $1.93177 e-01$ & 1.3393 & $9.17772 e-02$ & 1.3393 & $9.41416 e-02$ & 1.5240 \\
\hline 6 & .0490 & & & $4.87624 e-02$ & 2.5599 & $2.53366 e-02$ & 2.5599 & $1.25316 e-02$ & 3.3854 \\
\hline 7 & .0007 & & & $7.31275 e-04$ & 21.1490 & $3.94460 e-04$ & 21.1490 & $1.12024 \mathrm{e}-04$ & 32.0667 \\
\hline 8 & .0000 & & & $0.00000 e+00$ & .0000 & $0.00000 e+00$ & .0000 & $0.00000 \mathrm{e}+00$ & .0000 \\
\hline 9 & .0000 & & & $0.00000 e+00$ & .0000 & $0.00000 e+00$ & .0000 & $0.00000 e+00$ & .0000 \\
\hline 10 & .0000 & & & $0.00000 e+00$ & .0000 & $0.00000 e+00$ & .0000 & $0.00000 e+00$ & .0000 \\
\hline 11 & .0000 & & & $0.00000 e+00$ & .0000 & $0.00000 e+00$ & .0000 & $0.00000 \mathrm{e}+00$ & .0000 \\
\hline 12 & .0000 & & & $0.00000 e+00$ & .0000 & $0.00000 e+00$ & .0000 & $0.00000 e+00$ & .0000 \\
\hline 13 & .0000 & & & $0.00000 e+00$ & .0000 & $0.00000 e+00$ & .0000 & $0.00000 \mathrm{e}+00$ & .0000 \\
\hline 14 & .0000 & & & $0.00000 e+00$ & .0000 & $0.00000 e+00$ & .0000 & $0.00000 e+00$ & .0000 \\
\hline 15 & .0000 & & & $0.00000 e+00$ & .0000 & $0.00000 e+00$ & .0000 & $0.00000 e+00$ & .0000 \\
\hline 16 & .0000 & & & $0.00000 e+00$ & .0000 & $0.00000 e+00$ & .0000 & $0.00000 e+00$ & .0000 \\
\hline system & n total $=$ & & & $9.95826 e-01$ & .4520 & $4.31024 \mathrm{e}-01$ & .4528 & $5.68867 e-01$ & .3422 \\
\hline elapse & ed time & .21333 & minutes & & & & & & \\
\hline rand & n number= & 6 & & & & & & & \\
\hline
\end{tabular}

Fig. 8.1.131. Sample of the final edit of fissions, absorptions, and leakage with all region-dependent information suppressed. 
sample problem 18 ff27 demonstration of options problem

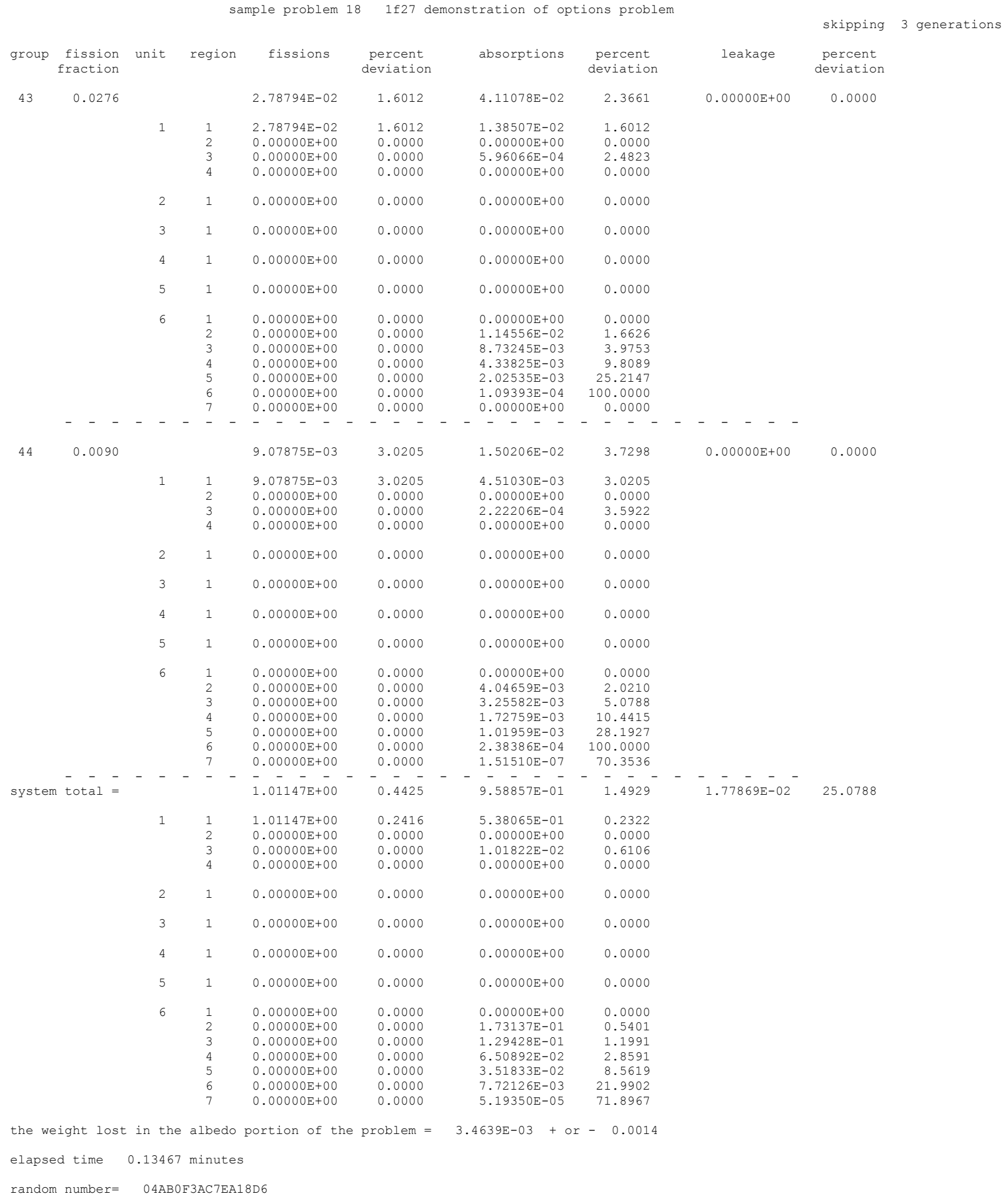

Fig. 8.1.132. Sample of the final edit of region-dependent fissions, absorptions, and leakage and the regiondependent totals. 
sample problem $18 \quad 1$ f27 demonstration of options problem

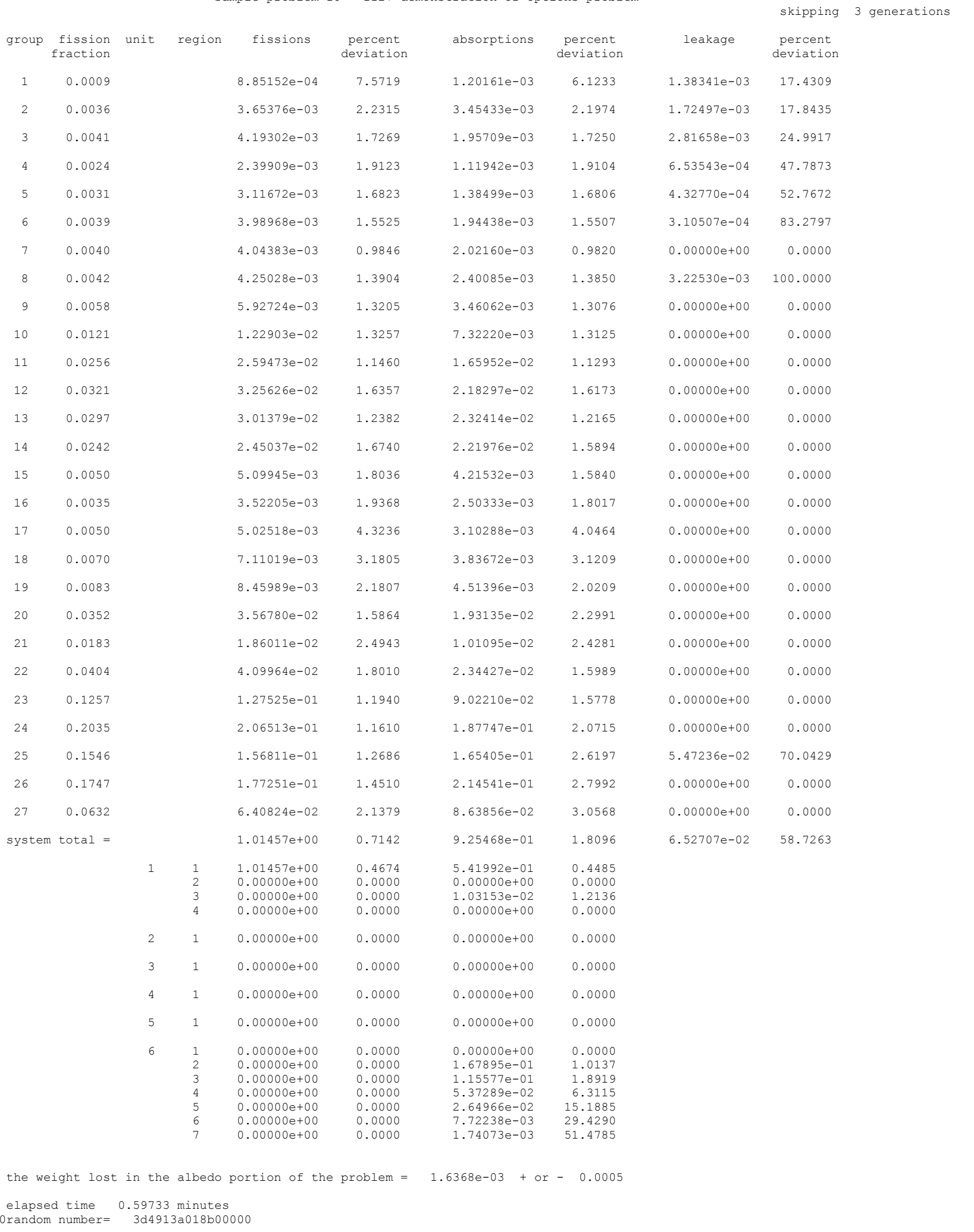

Fig. 8.1.133. Sample of the final edit of fissions, absorptions, and leakage and the region-dependent totals with print by region suppressed. 
If $\mathbf{F A R}=$ YES is specified in the parameter data, the fissions and absorptions for each geometry region used in the problem are calculated for each energy group. Leakage is not collected by geometry region, but rather it represents the leakage from the system. GROUP is the energy group number; FISSION FRACTION is the fraction of the fissions that occur in that energy group. The percent deviation for the fission fraction is the same as that of the fissions in the same group. The heading UNIT refers to the unit, and REGION is the region number within the specified unit. The geometry regions are numbered sequentially within each unit, starting with 1 . The sum of the fissions for every region for a given energy group is the total printed for that energy group. The same is true of absorptions. The fissions, absorptions, and leakages are given in units of per source neutron. The SYSTEM TOTAL is the sum over all the energy groups of the fissions, absorptions, and leakage. The associated percent deviation is printed for each.

The parameter GAS is used to control printing of fission productions and absorptions for each geometry region by energy group as shown in Fig. 8.1.131. GAS $=$ YES causes this data to be printed, provided $\mathbf{F A R}=$ YES is also specified. GAS=NO turns off this print by group as shown in Fig. 8.1.133.

The sum of the leakage and absorptions printed for the system total should be close to 1 . The fissions printed for the system total should be the same as the first k-effective printed in the final k-effective edit described in Sect. 8.1.4.25. If differential albedos are used, the leakage does not include the weight lost in the albedo reflection. A message stating the weight lost in the albedo is printed. This is the weight lost due to absorptions in the albedo reflector and leakage from the albedo reflector. No leakage is associated with faces having specular, mirror image, or periodic reflection. Thus there is no leakage associated with an infinite problem. The total elapsed time and final random number are printed at the end of this edit.

\subsubsection{Reaction tally}

Reaction tally calculations are performed if the required data is specified in the reaction data block (Sect. 8.1.2.15). KENO prints a table in the output that summarizes the parameter specifications in reaction tally calculations. An example of the parameter table for the reaction tally calculations are is in Fig. 8.1.134.

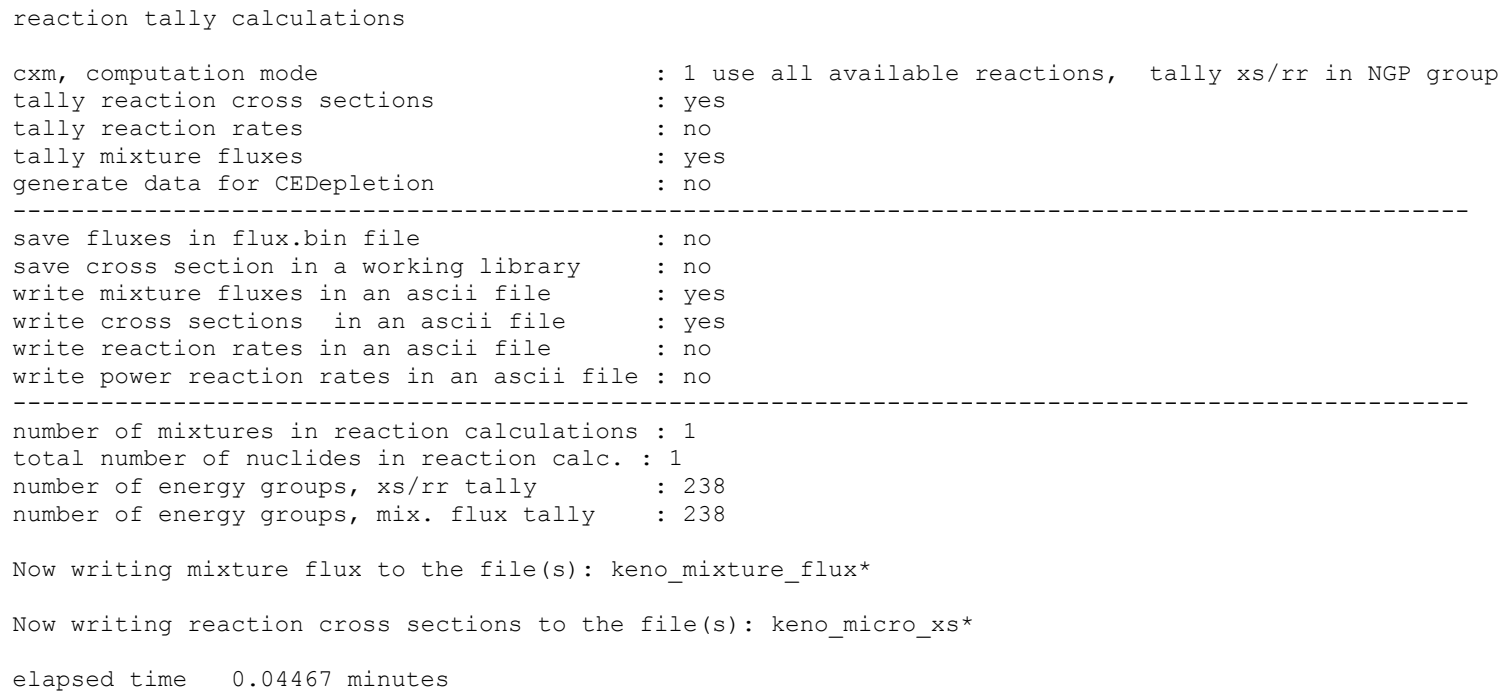

Fig. 8.1.134. Example of reaction tally summary table. 


\subsubsection{Source convergence diagnostics}

KENO calculates the Shannon entropy of the fission source distribution at each generation and reports this in the problem output (Sect. 8.1.4.23). At the end of calculation, KENO performs three tests to check the fission source convergence and reports these test results in the output. (See Sect. 8.1.6.7 for the definition of these tests.) An example of the fission source convergence diagnostics is given in Fig. 8.1.135.

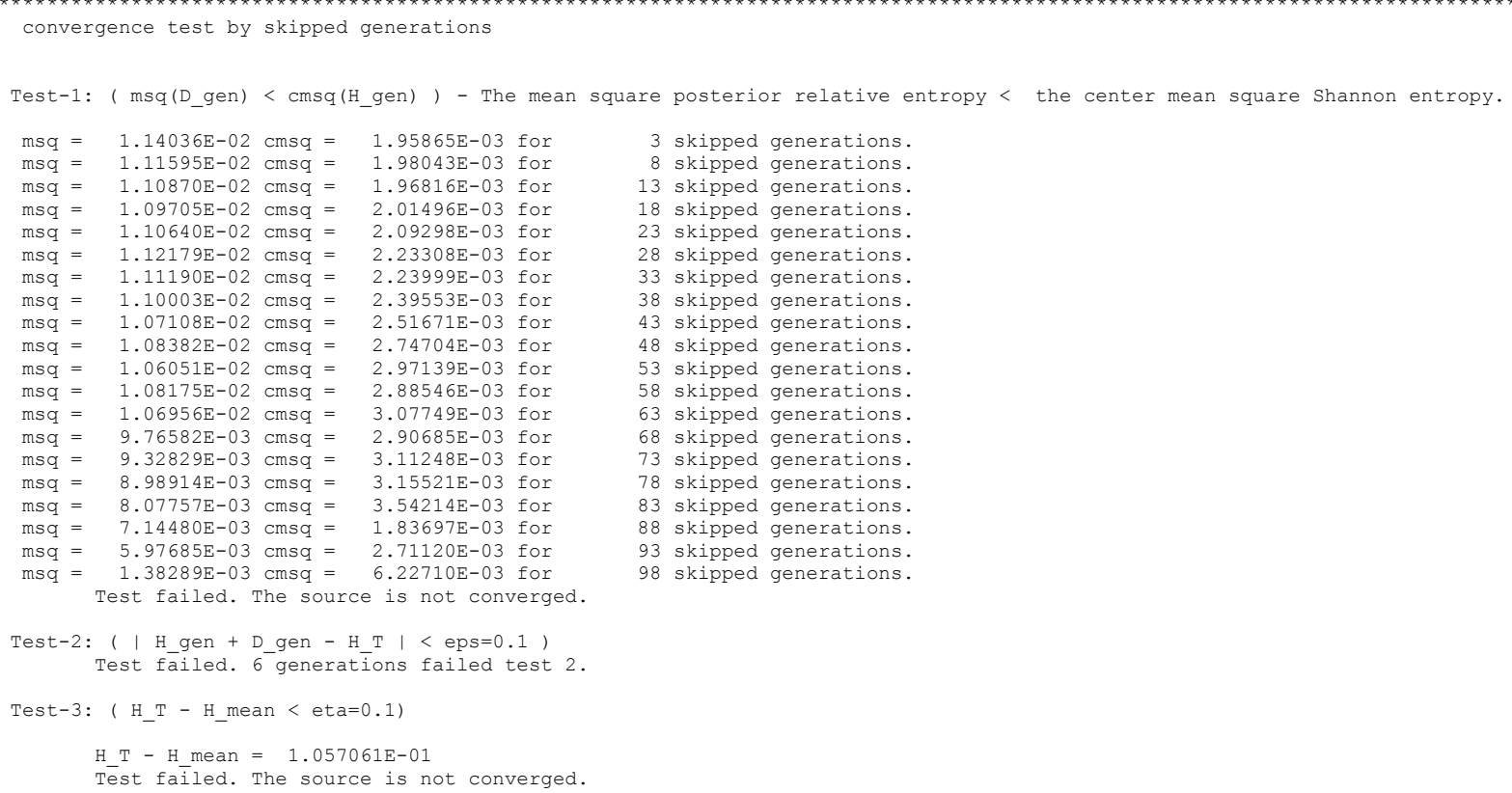

Fig. 8.1.135. Summary of fission source convergence diagnostics.

\subsubsection{Matrix k-effective by position index}

The matrix k-effective by unit location (also referred to as array position or position index) is calculated if MKP $=$ YES is specified in the parameter data and a global array is present in the model. It is the largest eigenvalue of the fission production matrix collected by position index. Section 8.1.3.12 provides a complete discussion of matrix k-effective. The POSITION INDEX is a number referencing a position in a 3-D lattice. POSITION is the $\mathrm{X}, \mathrm{Y}$, and $\mathrm{Z}$ location within the lattice. UNIT is the unit located at the specified location in the lattice. Thus, in Fig. 8.1.139, UNIT 1 is located at the lower left-hand front corner of the array or 3-D lattice representing the problem $(X=1, Y=1, Z=1)$ and the corresponding POSITION INDEX is 1 . POSITION INDEX 8 is the top right-hand back corner of the lattice, POSITION $\mathrm{X}=2, \mathrm{Y}=2, \mathrm{Z}=2$ and the unit located at that position is UNIT 8. An example of the matrix k-effective by position is given in Fig. 8.1.136. The text output is contained within two rows of asterisks to draw attention to it. 
Fig. 8.1.136. Example of matrix k-effective by position index.

\subsubsection{Fission production by position index matrix}

To obtain fission production by position information, the user must specify MKP=YES and FMP=YES in the parameter data. The number of entries in the fission production matrix by position index is the square of the array size. Thus for a $2 \times 2 \times 2$ array there are 64 entries, and for a $4 \times 4 \times 4$ array there are 4,096 entries in the fission production matrix by position index. An example of the fission production matrix by position index for a $2 \times 2 \times 2$ array is shown in Fig. 8.1.137.

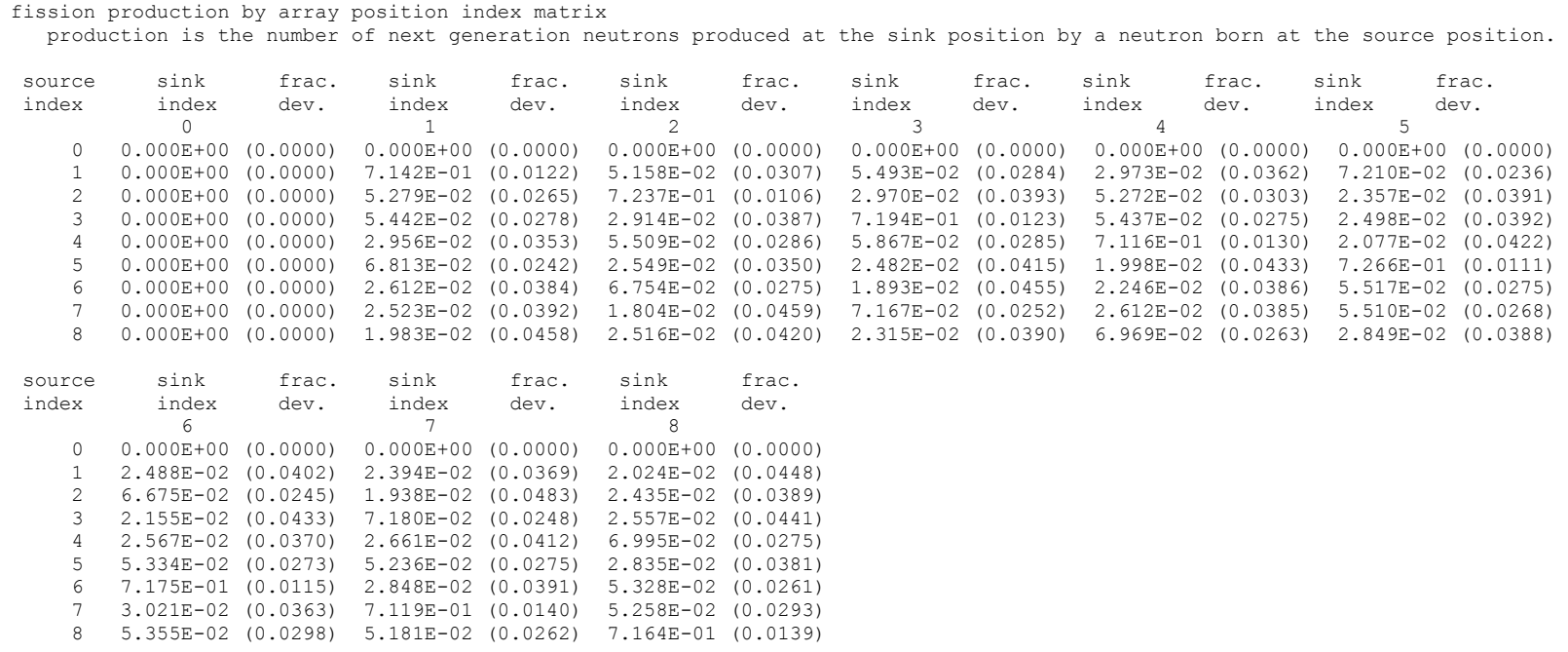

Fig. 8.1.137. Sample fission production matrix by position index.

For each position index in the array, the number of next-generation neutrons produced at position index $J$ per neutron born at position index I is determined. The fission production matrix by position index is used to determine the matrix k-effective, cofactor k-effective and source vector by position index.

\subsubsection{Source vector by position index}

Source vector by position index information is printed if MKP=YES is specified in the parameter data. The source vector by position index is the eigenvector of the fission production matrix by position index and should sum to 1.0. It represents the fission source for the specified locations in the 3-D lattice representing the physical problem being analyzed. Position zero contains all material outside the global array. An example of the source vector by position index is shown in Fig. 8.1.138. The average selfmultiplication by array position is the overall average of the self-multiplication of all units used in the problem. 


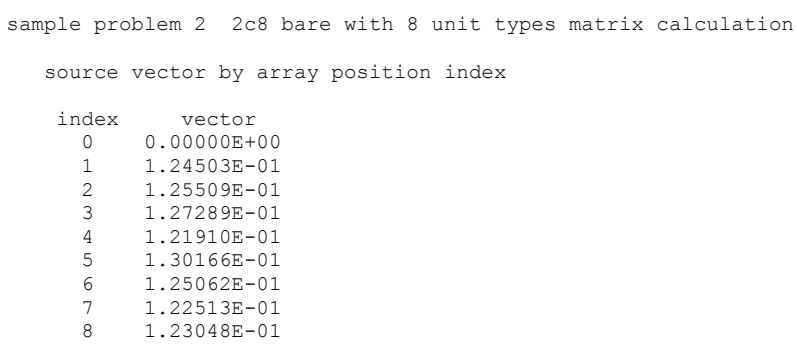

Fig. 8.1.138. Example of source vector by position index.

\subsubsection{Cofactor k-effective by position index}

The cofactor k-effective by position index edit is printed if MKP=YES is specified in the parameter data. This means that the fission production matrix is collected by position index. Calculating and printing cofactor k-effectives by position index can be avoided by specifying $\mathbf{C K P}=\mathrm{NO}$ in the parameter data. An example of the cofactor k-effective by position index is shown in Fig. 8.1.139. See KENO Appendix C for a description of the problem used for the example.

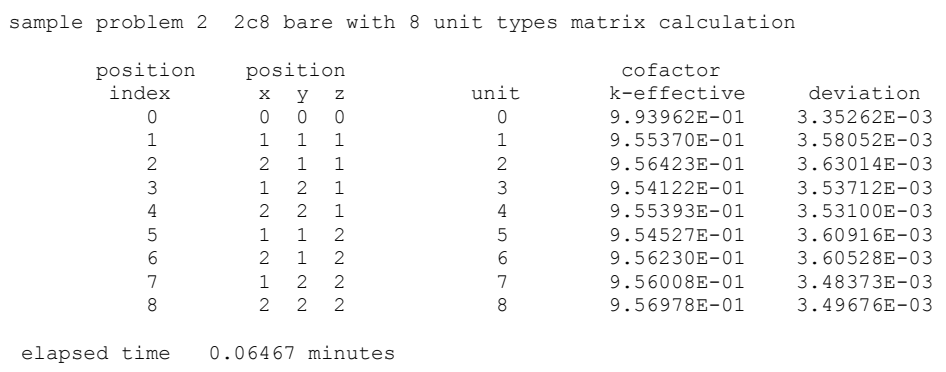

Fig. 8.1.139. Example of cofactor k-effective by position index.

The cofactor k-effective for a given position index is the largest eigenvalue of the fission production matrix collected by position index, reduced by the row and column associated with that position index. Thus the cofactor k-effective is the value of k-effective for the system calculated without the fission source of the unit located at the specified position index.

\subsubsection{Matrix k-effective by unit number}

The matrix k-effective by unit number (unit k-effective) is the largest eigenvalue of the fission production by unit matrix. It is calculated only if MKU $=$ YES is specified in the parameter data. An example of the matrix k-effective by unit is given in Fig. 8.1.140. 
sample problem 2 2c8 bare with 8 unit types matrix calculation

unit $\mathrm{k}$-effective $=1.00115 \mathrm{E}+00+$ or $-3.16228 \mathrm{E}-03$

the unit k-effective is the largest eigenvalue of the fission production by unit number matrix.

elapsed time 0.04967 minutes

Fig. 8.1.140. Example of matrix k-effective by unit number.

\subsubsection{Fission production by unit number matrix}

The fission production by unit number matrix is computed and printed if $\mathbf{M K U}=\mathrm{YES}$ and $\mathbf{F M U}=\mathrm{YES}$ are specified in the parameter data. Thus, for each unit in the array, the number of next-generation neutrons produced in Unit $J$ per neutron born in Unit I is determined. This is the fission production matrix by unit, and it is used to determine the matrix k-effective by unit, the cofactor k-effective by unit, and the source vector by unit. An example of the fission production matrix by unit is shown in Fig. 8.1.141 for text output.

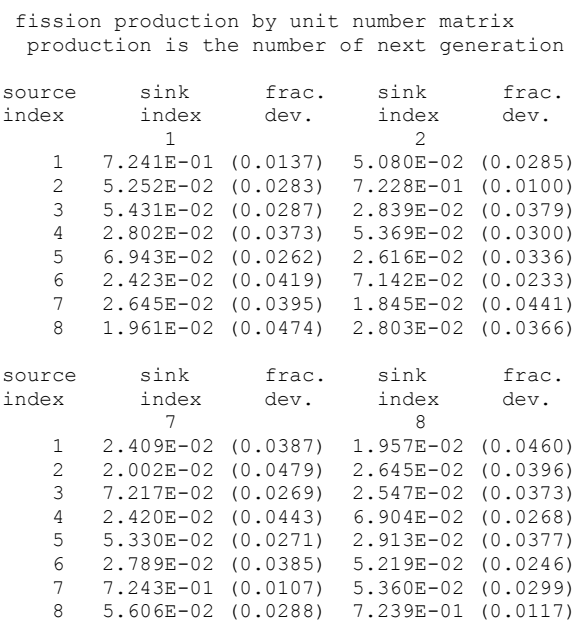

Fig. 8.1.141. An example of the fission probability matrix by unit.

\subsubsection{Source vector by unit number}

The source vector by unit is the eigenvector of the fission production matrix by unit and is printed if $\mathbf{M K U}=$ YES is specified in the parameter data. It represents the fission source for the units used in the problem. The components of the source vector should sum to 1.0. An example of the source vector by unit is given in Fig. 8.1.142. The average self-multiplication by unit is printed following the source vector. This value of self-multiplication includes those histories born in the unit which cause fissions in the same unit regardless of whether or not it exited and then returned. Therefore, this value will not agree with the value printed for the self-multiplication of the unit as described in Sect. 8.1.4.33 if the problem uses multiple units, if the system is reflected, or if a differential albedo is used in the problem. 


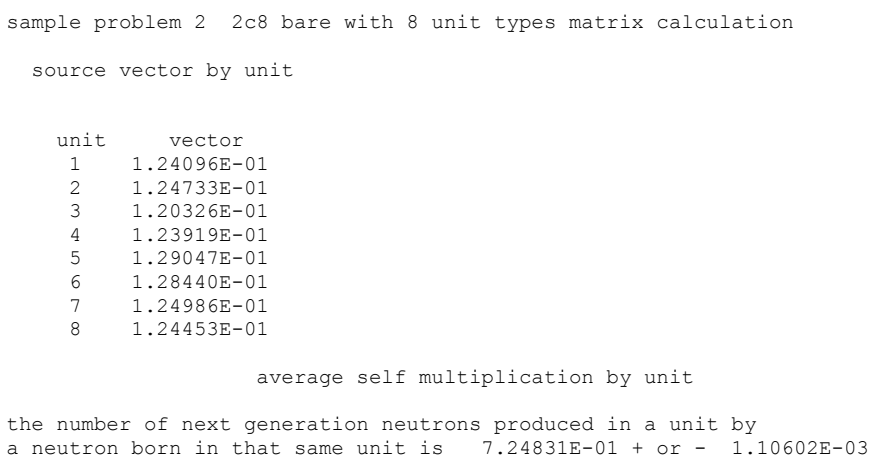

Fig. 8.1.142. Example of the source vector by unit.

\subsubsection{Cofactor k-effective by unit number}

The cofactor k-effective for a given unit is the k-effective of the system calculated without the fission source of that unit. Cofactor k-effectives are printed if MKU $=$ YES is specified in the parameter data. Calculating and printing cofactor k-effectives by unit can be avoided by specifying $\mathbf{C K U}=\mathrm{NO}$ in the parameter data. This step is accomplished by determining the eigenvalue of the fission production matrix by unit after it has been reduced by the row and column associated with that unit. An example of the cofactor k-effective by unit is given in Fig. 8.1.143.

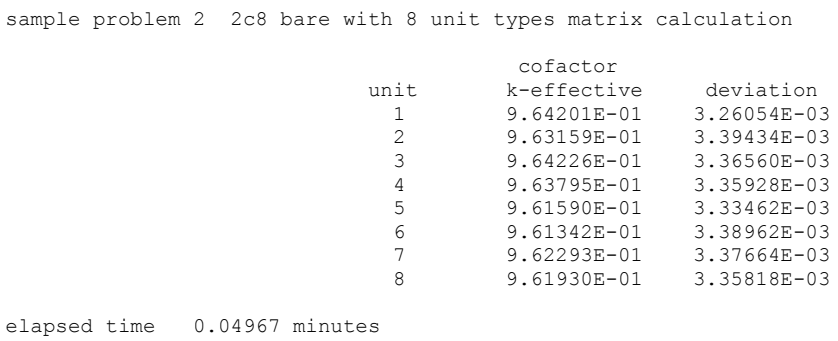

Fig. 8.1.143. Example of cofactor k-effective by unit number.

\subsubsection{Matrix k-effective by hole number}

The matrix k-effective by hole number is the largest eigenvalue of the fission production matrix collected by hole number and is calculated if $\mathbf{M K H}=$ YES was specified in the parameter data. An example of the matrix k-effective by hole number is given in Fig. 8.1.144.

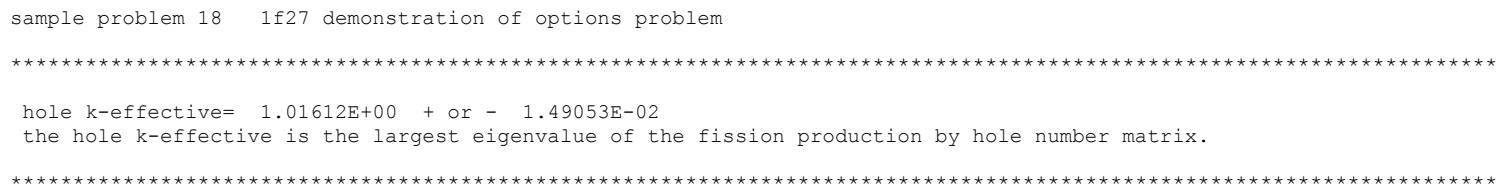

elapsed time 0.13550 minutes

Fig. 8.1.144. Example of matrix k-effective by hole number. 


\subsubsection{Fission production by hole number matrix}

This is the fission production matrix collected by hole number. It is printed only if MKH=YES and FMH=YES were specified in the parameter data. An example of this fission production matrix is given in Fig. 8.1.145. This matrix indicates the number of next generation neutrons produced in HOLE number J by a neutron born in HOLE number I.

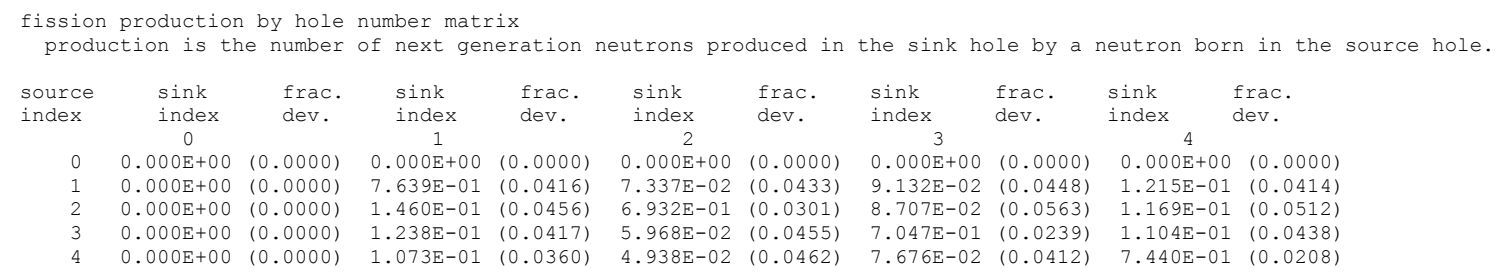

Fig. 8.1.145. Example of fission production matrix by hole.

\subsubsection{Source vector by hole number}

The source vector by hole is the eigenvalue of the fission production matrix by hole number and is printed if $\mathbf{M K H}=$ YES is specified in the parameter data. The source vector should sum to 1.0. An example of the source vector by hole is shown in Fig. 8.1.146. The average self-multiplication by hole is the overall average of the self-multiplication of all the holes in the problem.

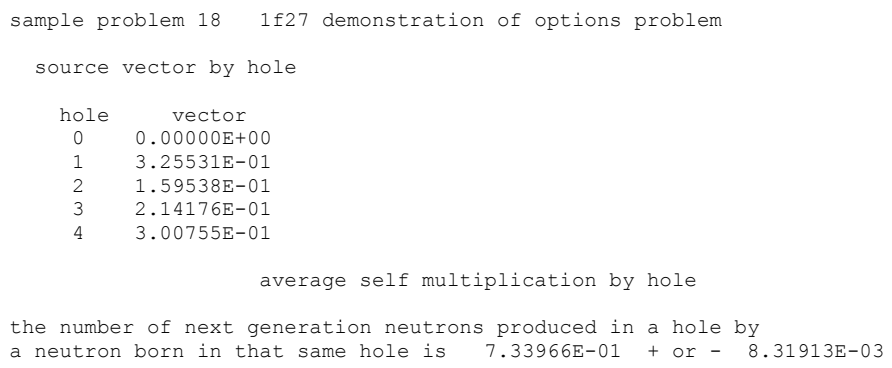

Fig. 8.1.146. Example of source vector by hole number.

\subsubsection{Cofactor k-effective by hole number}

The cofactor k-effective for a given hole is the k-effective of the system calculated without the fission source of that hole and is calculated if $\mathbf{C K H}=$ YES is entered in the parameter data. These values are computed by determining the eigenvalue of the fission production matrix by hole after it has been reduced by the row and column associated with that hole. An example of the cofactor k-effective by hole number is given in Fig. 8.1.147.

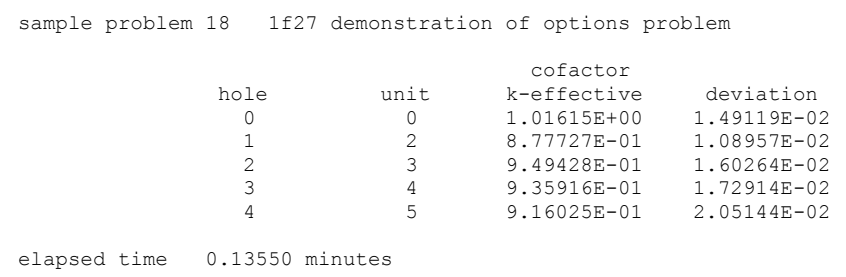

Fig. 8.1.147. Example of cofactor k-effective by hole number. 


\subsubsection{Matrix k-effective by array number}

The matrix k-effective by array number is the largest eigenvalue of the fission production matrix collected by array number and is calculated if MKA $=$ YES is entered in the parameter data. An example is given in Fig. 8.1.148. The number of next generation neutrons produced in array number $\mathrm{J}$ by a neutron born in array number I is given in this fission production matrix.

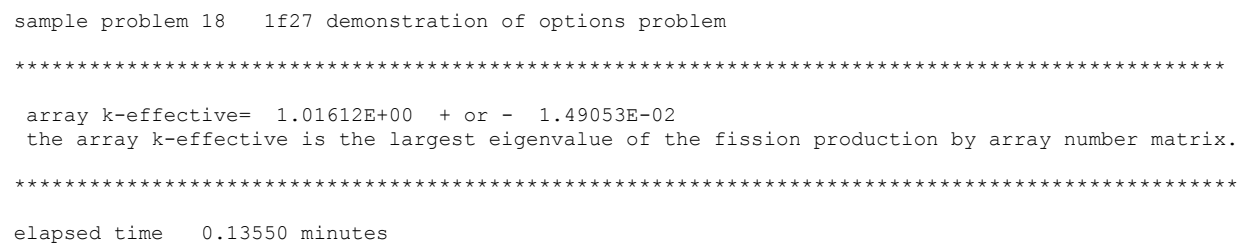

Fig. 8.1.148. Example of matrix k-effective by array number.

\subsubsection{Fission production by array number matrix}

The fission production matrix collected by array number is shown in Fig. 8.1.149. It is printed only if MKA $=$ YES and FMA=YES are specified in the parameter data.

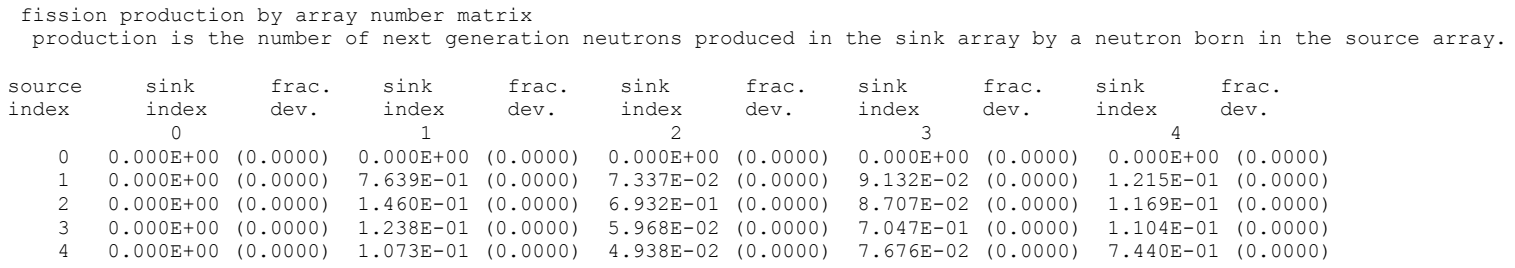

Fig. 8.1.149. An example of the fission production matrix by array number.

\subsubsection{Source vector by array number}

The source vector by array number is the eigenvector of the fission production matrix by array number and is printed if MKA=YES is specified in the parameter data. The source vector should sum to 1.0. An example of the source vector by array number is shown in Fig. 8.1.150. The average selfmultiplication by array number is the overall self-multiplication of all the arrays in the problem.

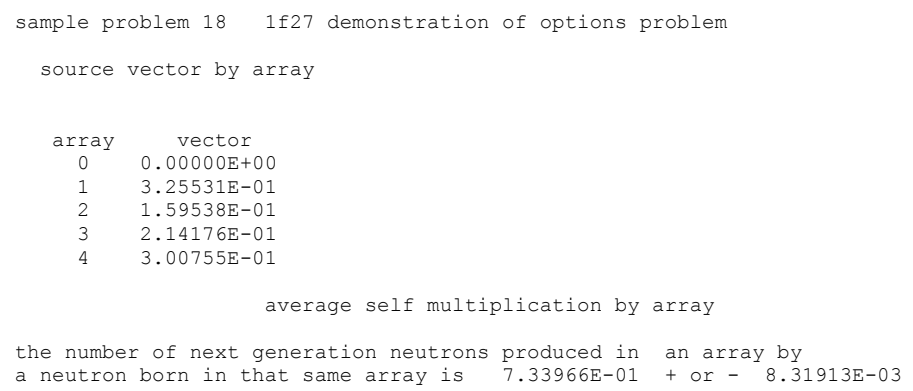

Fig. 8.1.150. Example of source vector by array number. 


\subsubsection{Cofactor k-effective by array number}

The cofactor k-effective by array number is the k-effective of the system calculated without the fission source of that array and is calculated if $\mathbf{C K A}=$ YES is entered in the parameter data. This is achieved by determining the eigenvector of the fission production matrix by array after reducing it by the row and column associated with the specified array. Fig. 8.1.151 is an example of the cofactor k-effective by array number.

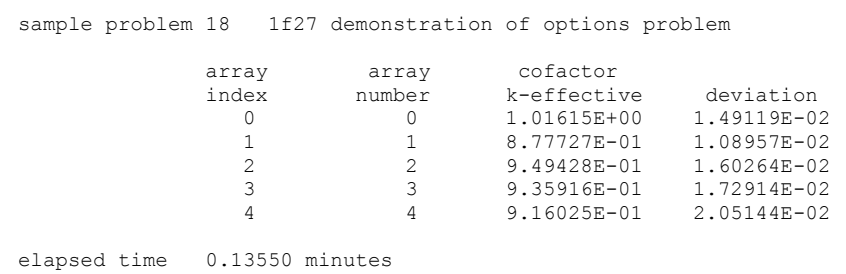

Fig. 8.1.151. Example of cofactor k-effective by array number.

\subsubsection{Fission density edit}

The fission density edit is optional. Subroutine KEDIT prints the neutron production density and the fission density for each geometry region if $\mathbf{F D N}=$ YES and $\mathbf{N U B}=$ YES is specified in the parameter data (these are the default values). If $\mathbf{N U B}=\mathrm{NO}$ is specified, but $\mathbf{F D N}=\mathrm{YES}$, then only the production density will be given. An example of the fission density edit is shown in Fig. 8.1.152.

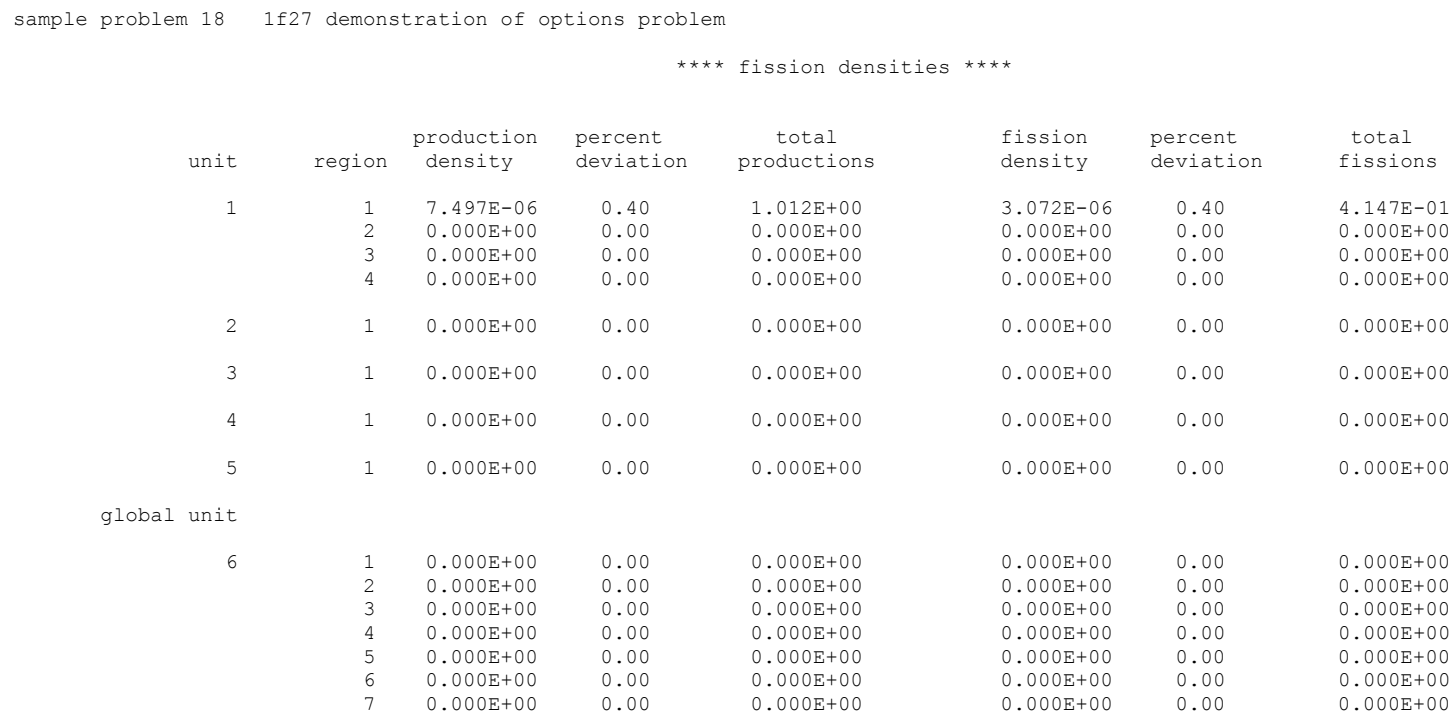

Fig. 8.1.152. Example of the fission density edit.

The UNIT is the unit number from the geometry data, the REGION is the region number relative to the unit, the PRODUCTION DENSITY is the neutrons produced per $\mathrm{cm}^{3}$ per source-neutron, and the FISSION DENSITY is the fissions per $\mathrm{cm}^{3}$ per source-neutron for that geometry region, the PERCENT DEVIATION is 100 times the fractional standard deviation associated with the production density and/or the fission density, the TOTAL PRODUCTIONS is the total number of neutrons produced per source 
neutron, and the TOTAL FISSIONS is the total number of fissions per source neutron in the geometry region.

\subsubsection{Flux edit}

Printing the fluxes is optional; they are only printed if FLX=YES is specified in the parameter data. The fluxes are printed for each unit and each geometry region in the unit for every energy group. A sample of a flux edit is given in Fig. 8.1.153.

The title of the problem is printed at the top of the page. The heading FLUXES FOR UNIT indicates the geometry unit for which fluxes are being printed. The region numbers relative to the unit are identified by the heading REGION . The geometry regions within each unit are numbered sequentially, beginning with 1 . GROUP is the heading for the energy groups. The headings FLUX and PERCENT DEVIATION are printed for each geometry region in the unit. The flux and its associated percent deviation are printed for every energy group and every geometry region. The flux is in units of neutrons $/ \mathrm{cm}^{2} /$ source neutron.

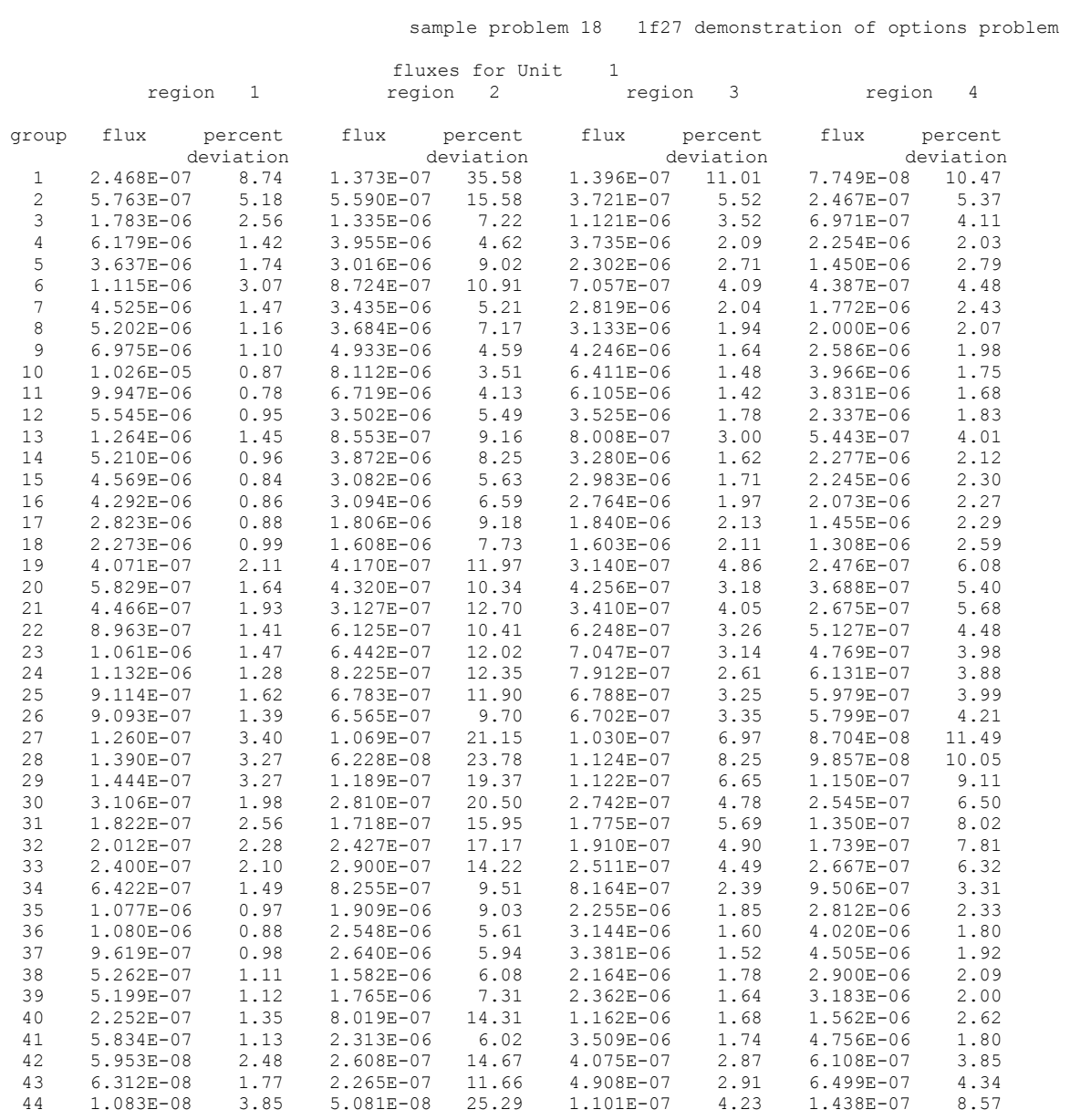

Fig. 8.1.153. An example of a flux edit. 


\subsubsection{Frequency distributions}

A frequency distribution consists of a bar graph indicating the number of generations having k-effective in a specified interval. The intervals are determined by the code, based on the upper and lower limits of the k-effectives calculated for the generations. In this example, one asterisk is printed for each generation k-effective. Four frequency distributions are printed as shown in Fig. 8.1.154.

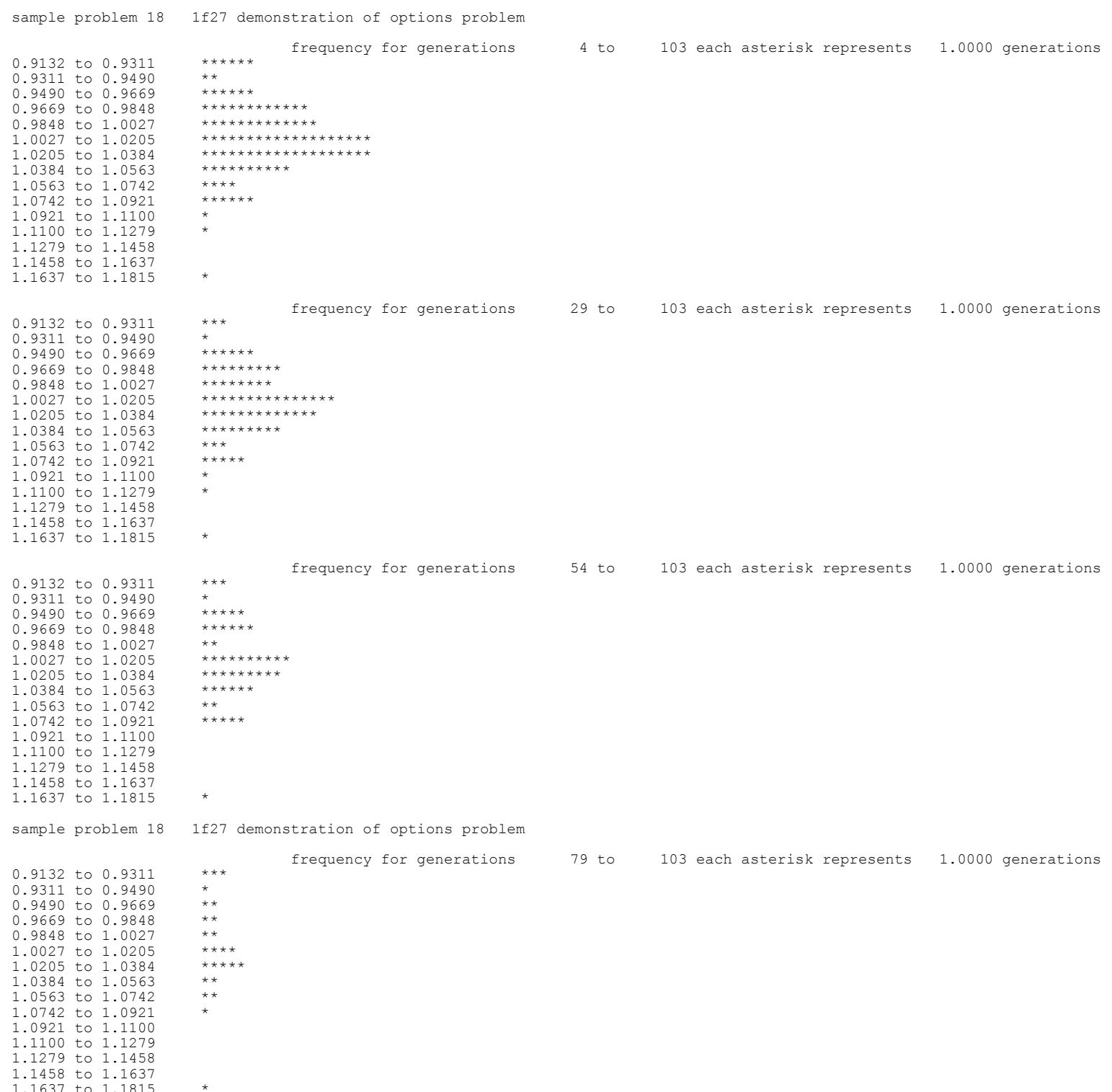

Fig. 8.1.154. An example of a frequency distribution. 


\subsubsection{Summary of parallel performance}

KENO summarizes parallel performance in the output if parallel execution is requested by the user. The performance table, shown in Fig. 8.1.155, can be used to evaluate the code parallel performance with the given problem parameters. The quantities like speedup, parallel efficiency, and wall-clock time for each section (serial section, parallel section, and communication time among the processors) can be used to estimate resource requirement for similar problems to accomplish a faster execution.

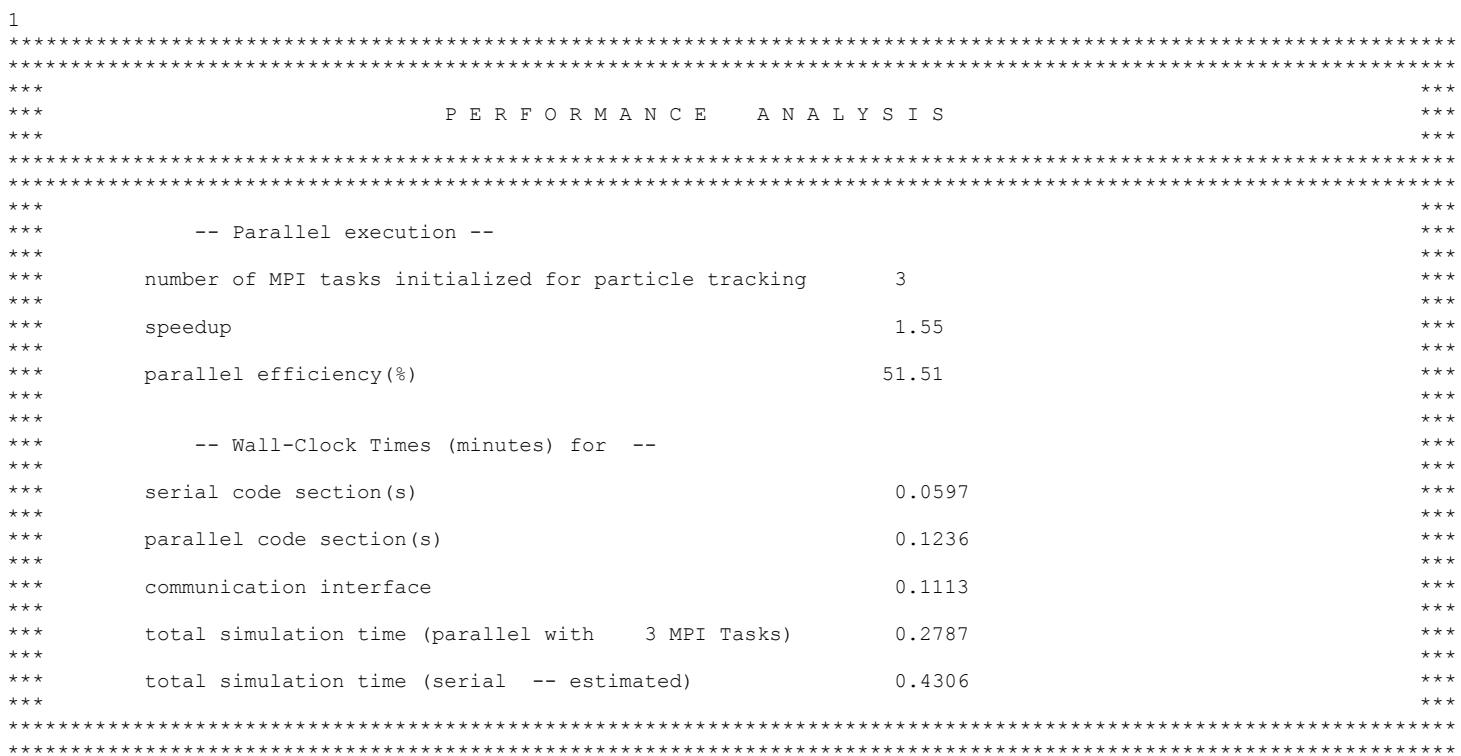

Fig. 8.1.155. Example of performance analysis table.

\subsubsection{Final results table}

The final results table contains a summary of the most important physics parameters of the system and the number of error and warning messages generated during execution. The table contains the best-estimate system k-effective with one standard deviation, the energy of the average lethargy of fission, the average system nu-bar, the average mean free path of a neutron throughout the system, the number of warning and error messages generated during code execution, and a final statement on the convergence of the $\chi^{2}$ test results. An example of this table is shown in Fig. 8.1.156. Also shown is the terminal edit of KENO, detailing the time required to traverse the "perilous path" through KENO. 


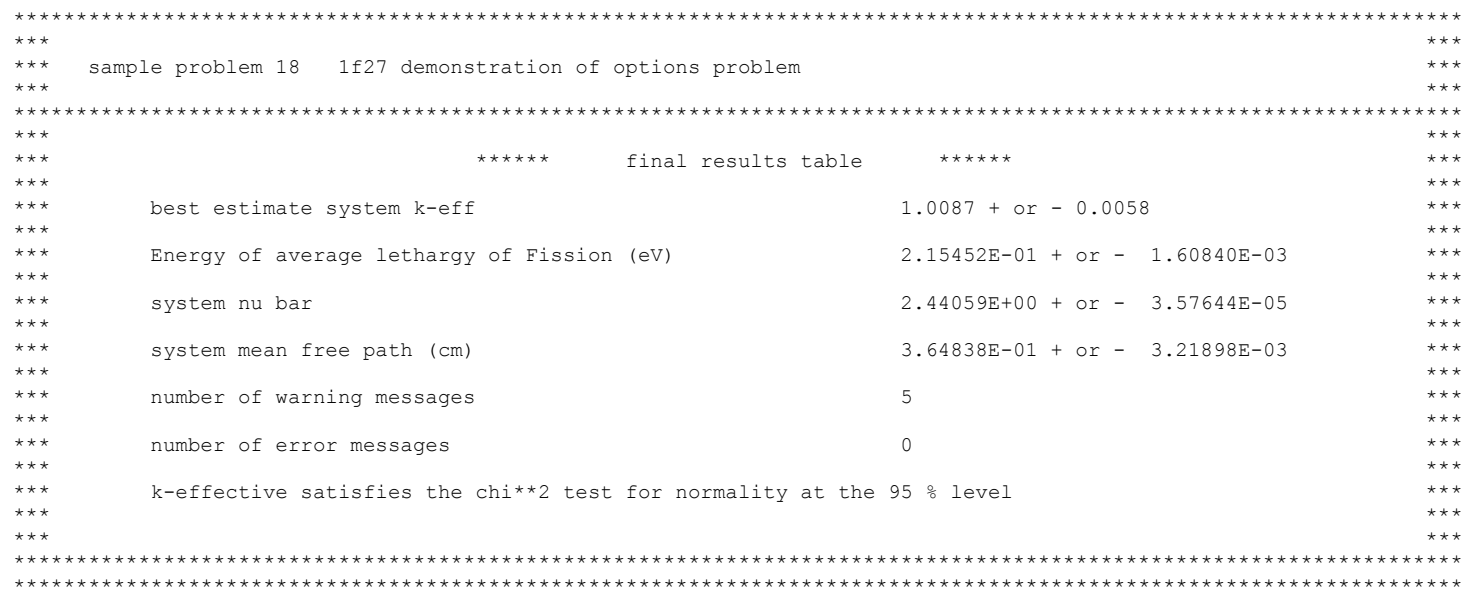

congratulations! you have successfully traversed the perilous path through keno $\mathrm{v}$ in $0.80217 \mathrm{minutes}$

Fig. 8.1.156. An example of the final results table.

\subsubsection{HTML output}

\subsection{Program verification information}

The program verification information is the first page shown in the KENO HTML output, after selecting KENO from the SCALE HTML index page. This page can also be displayed by selecting the Program Verification Information link under the General Information submenu and is shown in Fig. 8.1.157.

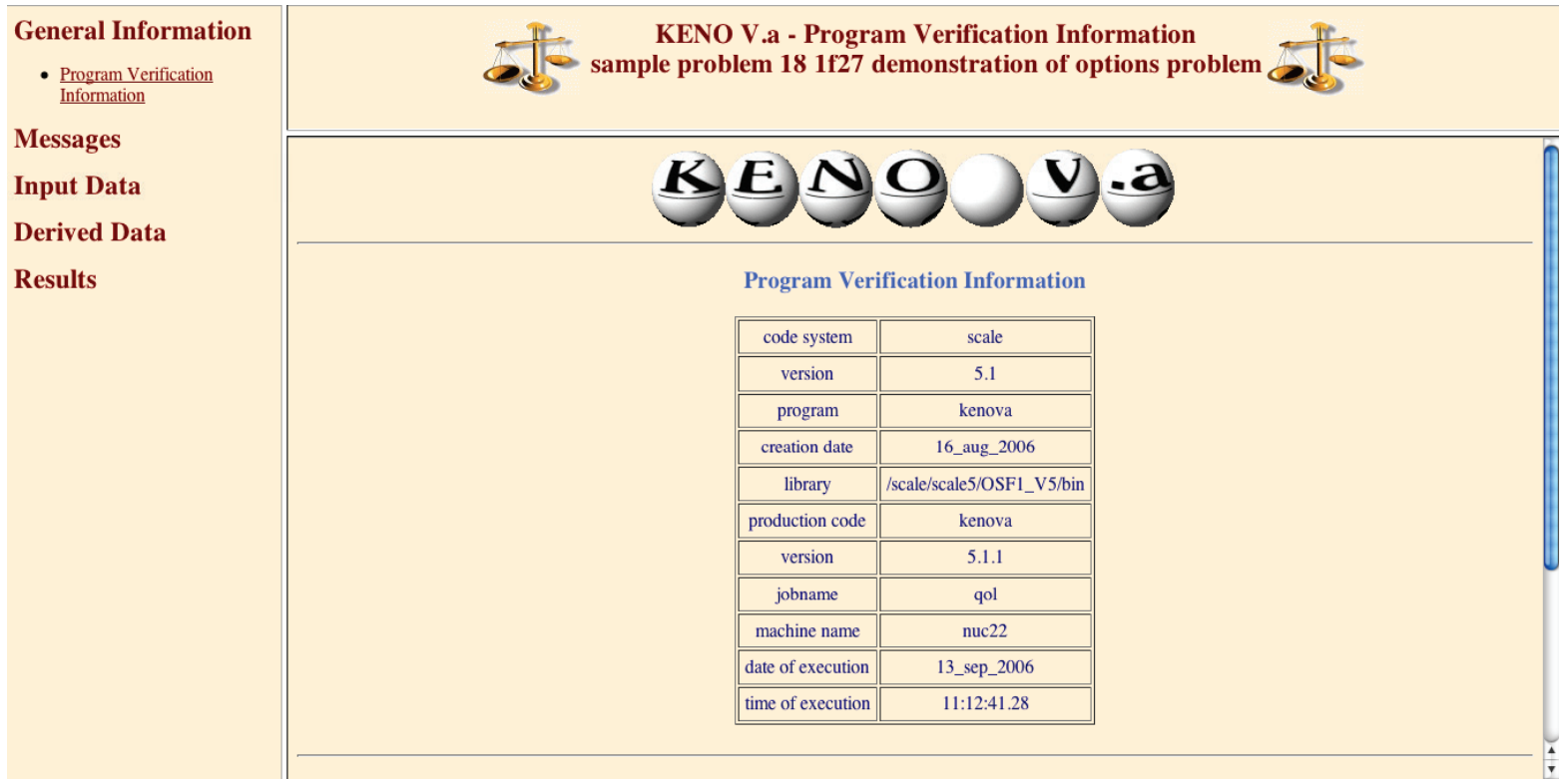

Fig. 8.1.157. Sample program verification information in HTML output. 


\subsection{Messages}

In the HTML output, all error and warning messages are consolidated into the Messages section of the output. Error Messages and Warning Messages links are available to the user as needed. An example edit with warning messages is shown in Fig. 8.1.158.

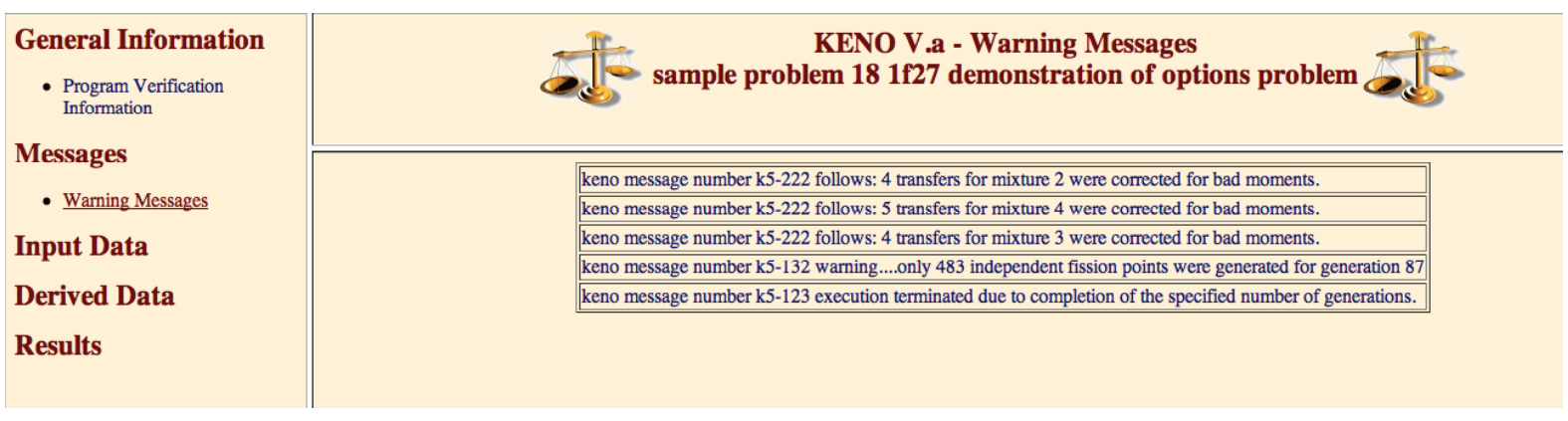

Fig. 8.1.158. Example messages edit in HTML output.

\subsection{Tables of parameter data}

The first two tables generated by KENO HTML output list the numeric parameters and logical parameters that are used in the problem. The user should always verify that the parameter data block was input as desired. An example of numeric parameters table is shown in Fig. 8.1.159. An example of the logical parameters table is shown in Fig. 8.1.160. The HTML output is accessed with the Numeric Parameters and Logical Parameters links in the Input Data section. 


\section{Keno-V.a - Numeric Parameters sample problem $181 \mathrm{f} 27$ demonstration of options problem}

\begin{tabular}{|c|c|c|}
\hline Parameter & Description & Value \\
\hline tme & maximum problem time (min) & 0.00 \\
\hline tba & time per generation (min) & 10.00 \\
\hline gen & number of generations & 103 \\
\hline npg & number per generation & 500 \\
\hline nsk & number of generations to be skipped & 3 \\
\hline beg & beginning generation number & 1 \\
\hline res & generations between checkpoints & 0 \\
\hline $\mathrm{x} 1 \mathrm{~d}$ & number of extra 1-d cross sections & 1 \\
\hline nbk & neutron bank size & 525 \\
\hline $\mathrm{xnb}$ & extra positions in neutron bank & 0 \\
\hline $\mathrm{nfb}$ & fission bank size & 500 \\
\hline $\mathrm{xfb}$ & extra positions in fission bank & 0 \\
\hline sig & cut off standard deviation & 0.0000 \\
\hline wta & default value of weight average & 0.5000 \\
\hline wth & weight high for splitting & 3.0000 \\
\hline wtl & weight low for russian roulette & 0.3333 \\
\hline rnd & starting random number & 0000F12C09ED2195 \\
\hline nb8 & number of d.a. blocks on unit 8 & 1000 \\
\hline nl8 & length of d.a. blocks on unit 8 & 512 \\
\hline nqd & quadrature order for angular fluxes & 0 \\
\hline pnm & highest order of flux moments & 0 \\
\hline $\mathrm{msh}$ & mesh size for mesh flux tally & 0.0000 \\
\hline adj & mode of calculation & forward \\
\hline tps & sampling sites per track length & 5 \\
\hline cgs & number of secondary groups to sampl & 0 \\
\hline cas & number of secondary angles to sampl & 0 \\
\hline
\end{tabular}

Fig. 8.1.159. Sample numeric parameters in HTML output. 


\begin{tabular}{|c|c|c|}
\hline Parameter & Description & Value \\
\hline run & execute problem after checking data & yes \\
\hline \multirow[t]{2}{*}{ plt } & plot picture map(s) & yes \\
\hline & compute fluxes (cfx, flx, cfe or mfp) & yes \\
\hline fdn & compute fission densities & yes \\
\hline smu & compute avg unit self-multiplication & no \\
\hline nub & compute nu-bar \& avg fission group & yes \\
\hline mku & compute matrix k-eff by unit number & yes \\
\hline cku & compute cofactor k-eff by unit number & no \\
\hline fmu & print fiss prod matrix by unit number & yes \\
\hline mkp & compute matrix k-eff by unit location & no \\
\hline ckp & compute cofactor k-eff by unit location & no \\
\hline fmp & print fiss prod matrix by unit location & no \\
\hline mkh & compute matrix k-eff by hole number & yes \\
\hline ckh & compute cofactor $\mathrm{k}$-eff by hole number & no \\
\hline $\mathrm{fmh}$ & print fiss prod matrix by hole number & yes \\
\hline hhl & collect matrix by highest hole level & no \\
\hline mka & compute matrix k-eff by array number & yes \\
\hline cka & compute cofactor k-eff by array number & no \\
\hline fma & print fiss prod matrix by array number & yes \\
\hline hal & collect matrix by highest array level & no \\
\hline $\operatorname{amx}$ & print all mixed cross sections & yes \\
\hline $\mathrm{xs} 1$ & print 1 - $\mathrm{d}$ mixture $\mathrm{x}$-sections & yes \\
\hline $\mathrm{xs} 2$ & print 2-d mixture $\mathrm{x}$-sections & yes \\
\hline $\mathrm{xsl}$ & print 2-d mixture $\mathrm{Pl}$ arrays & yes \\
\hline xap & print mixture angles \& probabilities & yes \\
\hline pki & print fission spectrum & yes \\
\hline
\end{tabular}

Fig. 8.1.160. Sample logical parameters in HTML output.

\subsection{Table of additional information}

The fourth table of data printed by KENO contains additional information determined from the input data. An example of this table is shown in Fig. 8.1.161. The HTML output is accessed with the Additional Information link in the Input Data section. 


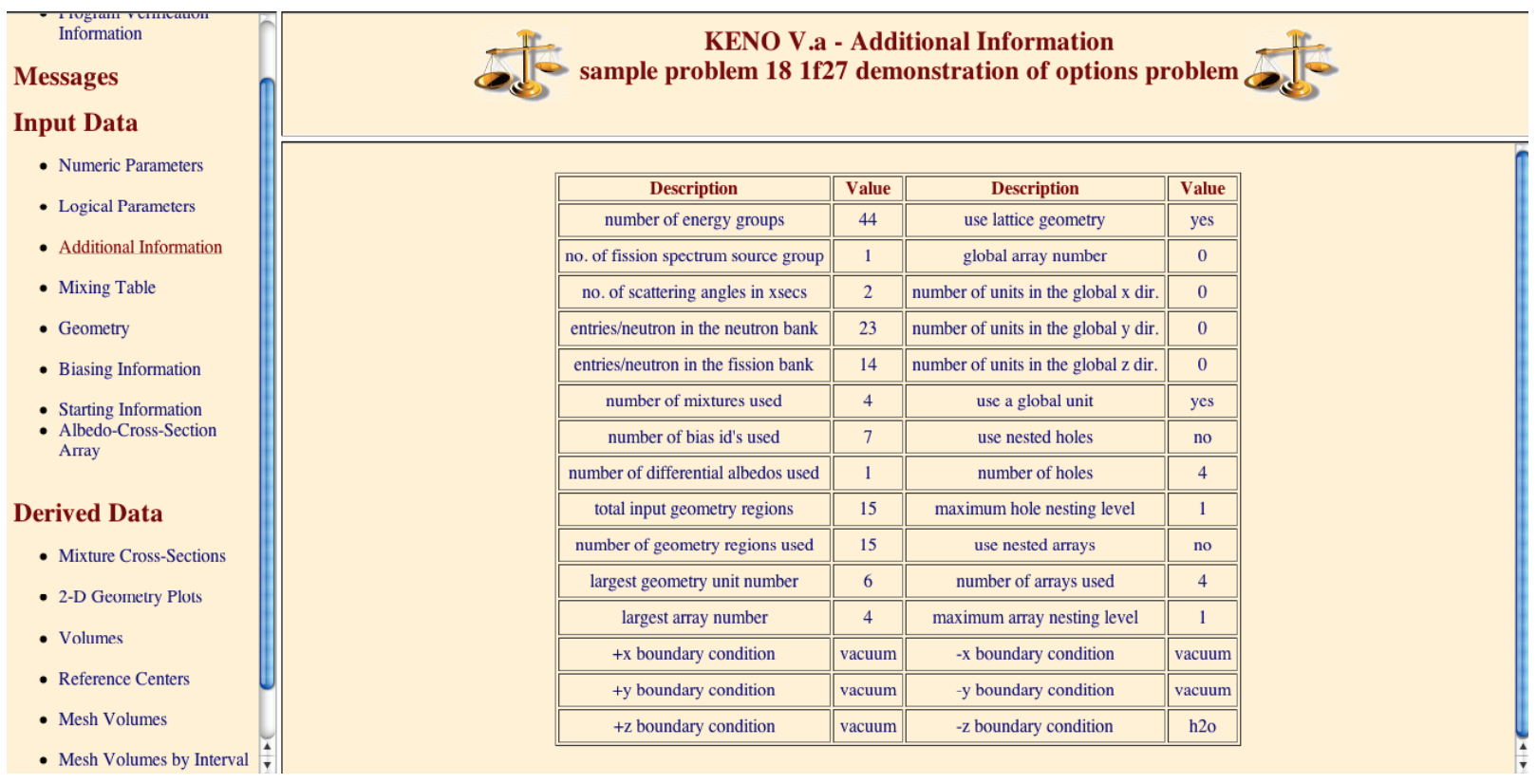

Fig. 8.1.161. Sample table of additional information in HTML output.

\subsection{Mixing table data}

If $\mathbf{L I B}=$ is entered in the KENO parameter data and a mixing table data block is provided to KENO, then mixing table data will be printed. Sample mixing table data are shown in Fig. 8.1.162. In the HTML output, the mixing table data can be accessed with the Mixing Table link in the Input Data section. 


\begin{tabular}{|c|c|c|c|c|c|c|c|c|}
\hline $\begin{array}{l}\text { General } \\
\text { Information } \\
\text { Messages }\end{array}$ & & $\frac{\pi r}{6}$ & imple I & roblem & $\begin{array}{l}\text { KEN } \\
181 \mathrm{f}\end{array}$ & V.a - & $\begin{array}{l}\text { lixing Table } \\
\text { stration of options }\end{array}$ & $\operatorname{lem} \frac{\pi}{0}$ \\
\hline $\begin{array}{l}\text { Input Data } \\
\text { - Numeric Parameters } \\
\text { - Logical Parameters } \\
\text { - Additional } \\
\text { Information }\end{array}$ & & & cross se & $\begin{array}{r}\text { mber of s } \\
\text { ction mes } \\
\text { Density }\end{array}$ & $\begin{array}{l}\text { catter } \\
\text { sage t } \\
\text { lixtur } \\
1.55\end{array}$ & $\begin{array}{l}\text { ng angle } \\
\text { reshold } \\
\text { e } 1 \\
50 \mathrm{~g} / \mathrm{cm}^{3}\end{array}$ & $\begin{array}{l}s=2 \\
=1.0 \mathrm{E}+00\end{array}$ & $\begin{array}{l}\text { Mixture } \\
\text { - Mixture 1 } \\
\text { - Mixture 2 } \\
\text { - Mixture 3 }\end{array}$ \\
\hline - Geometry & $\begin{array}{c}\text { Nuclide } \\
\text { ID }\end{array}$ & Nuclide & $\begin{array}{c}\text { Atom } \\
\text { Density }\end{array}$ & \begin{tabular}{|c|} 
Weight \\
Fraction
\end{tabular} & ZA & \begin{tabular}{|l|} 
Atomic \\
Weight
\end{tabular} & Nuclide Title & \\
\hline - Biasing Information & 1001001 & ${ }^{1} \mathrm{H}$ & \begin{tabular}{|c|}
$5.77931 \mathrm{E}-$ \\
02
\end{tabular} & \begin{tabular}{|c|}
$6.21990 \mathrm{E}-$ \\
02
\end{tabular} & 1001 & 1.0078 & $\begin{array}{c}\text { hydrogen in water } 1301 / 1002 \\
\text { mod1 } 11 / 23 / 92\end{array}$ & \\
\hline - Albedo-Cross- & 1007014 & ${ }^{14} \mathrm{~N}$ & \begin{tabular}{|c|}
$2.13092 \mathrm{E}-$ \\
03
\end{tabular} & \begin{tabular}{|l|}
$3.18658 \mathrm{E}-$ \\
02
\end{tabular} & 7014 & 14.0031 & $\begin{array}{c}\text { 7n } 14 \text { lasl evaljul73 p.young d.fost } \\
\bmod 211 / 28 / 88\end{array}$ & \\
\hline sectón Anay & 1008016 & ${ }^{16} \mathrm{O}$ & \begin{tabular}{|c|}
$3.74114 \mathrm{E}-$ \\
02
\end{tabular} & \begin{tabular}{|c|}
$6.39046 \mathrm{E}-$ \\
01
\end{tabular} & 8016 & 15.9954 & 8016 from version 6 evaluation & \\
\hline $\begin{array}{l}\text { Derived Data } \\
\text { Results }\end{array}$ & 1092234 & ${ }^{234} \mathrm{U}$ & $\begin{array}{c}1.06784 \mathrm{E}- \\
05\end{array}$ & $\begin{array}{c}2.66890 \mathrm{E}- \\
03\end{array}$ & 92234 & 234.0405 & $\begin{array}{l}\text { 92U 234 BNL HEDL + } \\
\text { EVALJUL78 DIVADEENAM } \\
\text { MANN MOD3 01/10/91 }\end{array}$ & \\
\hline & 1092235 & ${ }^{235} \mathrm{U}$ & $\begin{array}{c}9.84602 \mathrm{E}- \\
04\end{array}$ & $\begin{array}{c}2.47140 \mathrm{E}- \\
01\end{array}$ & 92235 & 235.0441 & $\begin{array}{l}\text { 92u } 235 \text { bnl evalapr77 m.r.bhat } \\
\bmod 302 / 28 / 89\end{array}$ & \\
\hline & 1092236 & ${ }^{236} \mathrm{U}$ & $\begin{array}{c}5.29386 \mathrm{E}- \\
06\end{array}$ & $\begin{array}{c}1.33445 \mathrm{E}- \\
03\end{array}$ & 92236 & 236.0458 & $\begin{array}{l}\text { 92U } 236 \text { BNL HEDL }+ \\
\text { EVALJUL78 DIVADEENAM } \\
\text { MANN MOD } 301 / 23 / 91\end{array}$ & \\
\hline & 1092238 & ${ }^{238} \mathrm{U}$ & $\begin{array}{c}6.19414 \mathrm{E}- \\
05\end{array}$ & $\begin{array}{c}1.57465 \mathrm{E}- \\
02\end{array}$ & 92238 & 238.0510 & $\begin{array}{c}\text { 92U } 238 \text { ANL+ EVALJUN77 } \\
\text { E.PENNINGTON A. MOD3 } \\
02 / 13 / 92\end{array}$ & \\
\hline
\end{tabular}

Fig. 8.1.162. Example of mixing table data in HTML output. 


\subsection{1-D macroscopic cross sections}

The decision to print the 1-D mixture cross sections is optional. They are printed only if XS1=YES is specified in the parameter data. The 1-D mixture cross sections for a mixture are shown in Fig. 8.1.163. The HTML output is accessed with the Mixture Cross Sections link in the Derived Data section. The top menu on the right side of the window is used to set the mixture displayed in the bottom menu on the right side of the window. The bottom menu contains hyperlinks to all available edits for mixture cross sections. The 1-D cross sections are accessed with the Neutron Cross Sections link in the bottom menu for the desired mixture.

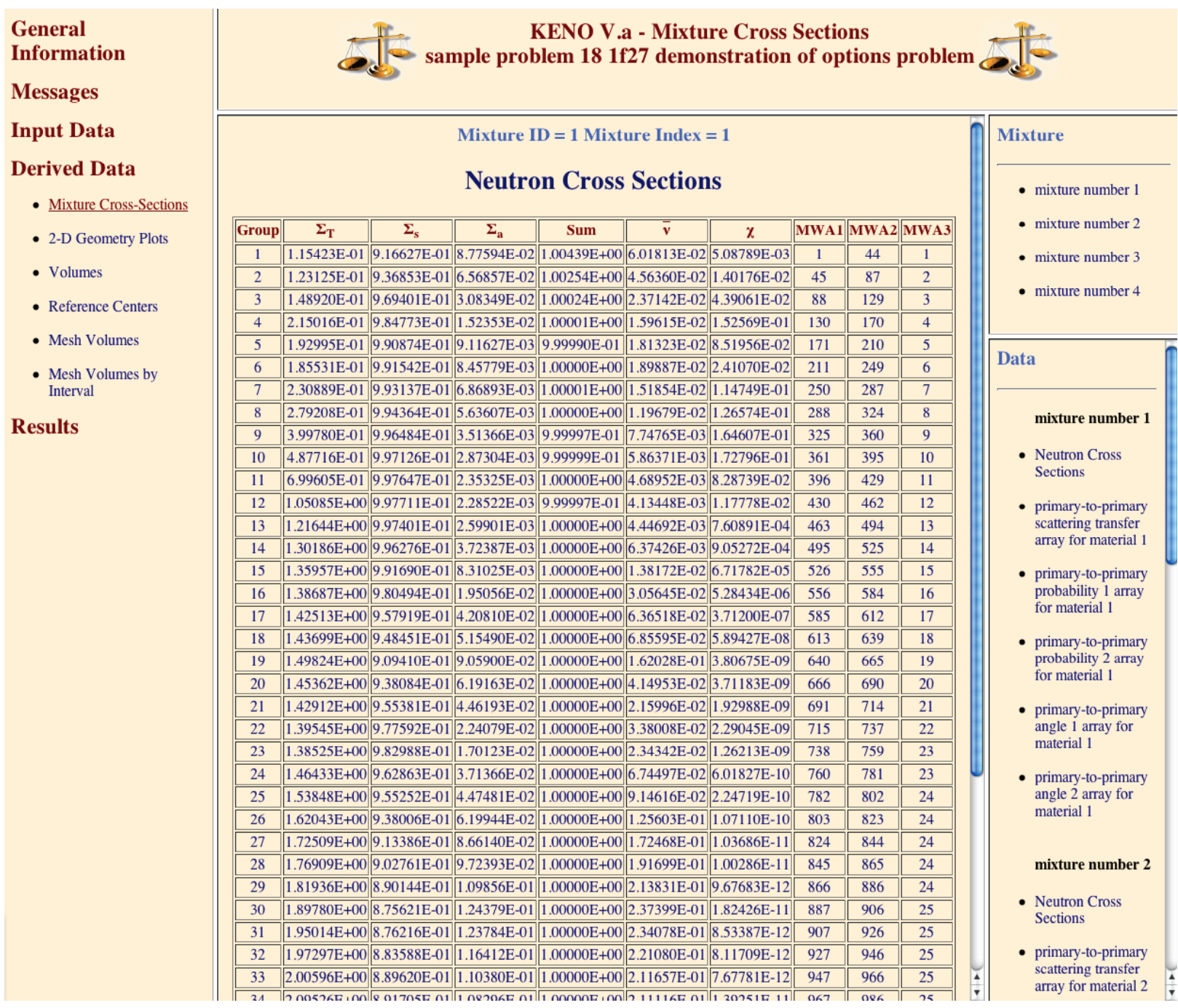

Fig. 8.1.163. Example 1-D macroscopic cross section in HTML output.

When the 1-D mixture cross sections are printed, the problem title is printed at the top of the page. The mixture ID, mixture index and mixture number are then printed. ID is the mixture number from the mixing table and mixture index is the index used to reference it and mixture number is its identifier. This step is followed by a heading to identify the different 1-D cross sections. GROUP is the energy group, sigt is the total cross section for the mixture, sigs is the nonabsorption probability, siga is the absorption probability, signu is the production probability, chi is the fission spectrum, mwal is the pointer for the 
first position of the cross sections for the energy group, mwa 2 is the pointer for the last position of the cross sections for the energy group, and mwa3 contains the group for the transfer corresponding to the first position. $S U M$ is the sum of the absorption probability and the nonabsorption probability. The nonabsorption probability and the production probability are not true probabilities in that they may be greater than 1. This is because the nonabsorption probability has the $(n, 2 n)$ transfer array summed into the total transfer array twice, and the $(\mathrm{n}, 3 \mathrm{n})$ is summed three times, etc. The absorption probability is defined as the absorption cross section divided by the total cross section. The nonabsorption probability is the sum of the group-to-group transfers for this group divided by the total cross section. The production probability is defined as the fission production cross section divided by the total cross section $\left(v \Sigma_{\mathrm{f}} / \Sigma_{\mathrm{T}}\right)$.

\subsection{2-D macroscopic cross sections}

The decision to print the 2-D mixture cross sections is optional. An example of the 2-D mixture cross sections is given in Fig. 8.1.164. The HTML output is accessed with the Mixture Cross Sections link in the Derived Data section. The top menu on the right side of the window is used to set the mixture displayed in the bottom menu on the right side of the window. The bottom menu contains hyperlinks to all available edits for mixture cross sections. The 1-D macroscopic cross sections are accessed with the primary-to-primary scattering transfer array link in the bottom menu for the desired mixture.

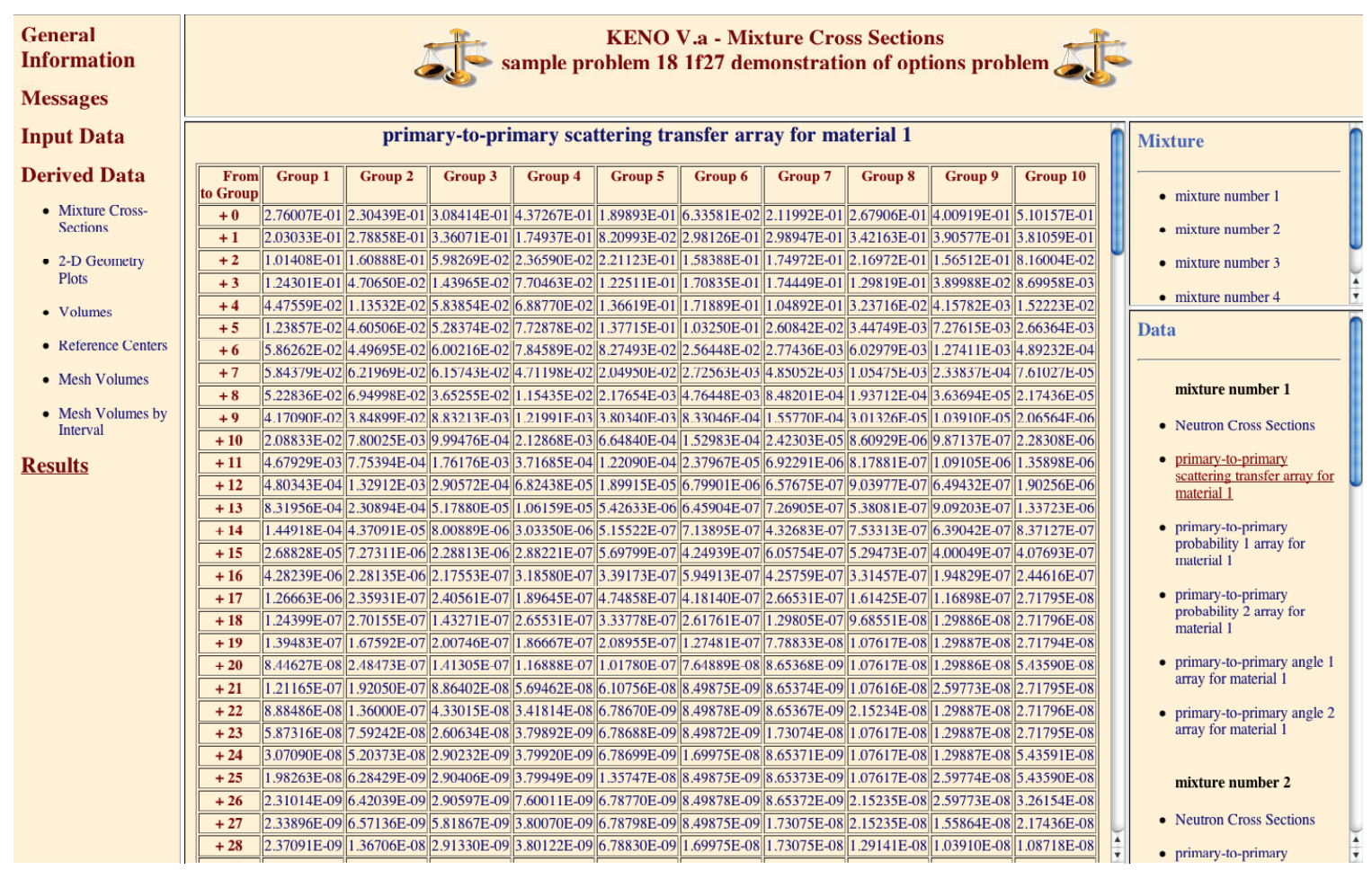

Fig. 8.1.164. Example of 2-D macroscopic cross sections in HTML output.

\subsection{Probabilities and angles}

Printing the probabilities and angles is optional. The HTML output, Fig. 8.1.165, is accessed with the Mixture Cross Sections link in the Derived Data section. The top menu on the right side of the window is used to set the mixture displayed in the bottom menu on the right side of the window. The bottom menu contains hyperlinks to all available edits for mixture cross sections. The probabilities and angles edits, 
shown in Fig. 8.1.166, are accessed with the primary-to-primary probability link in the bottom menu for the desired mixture.

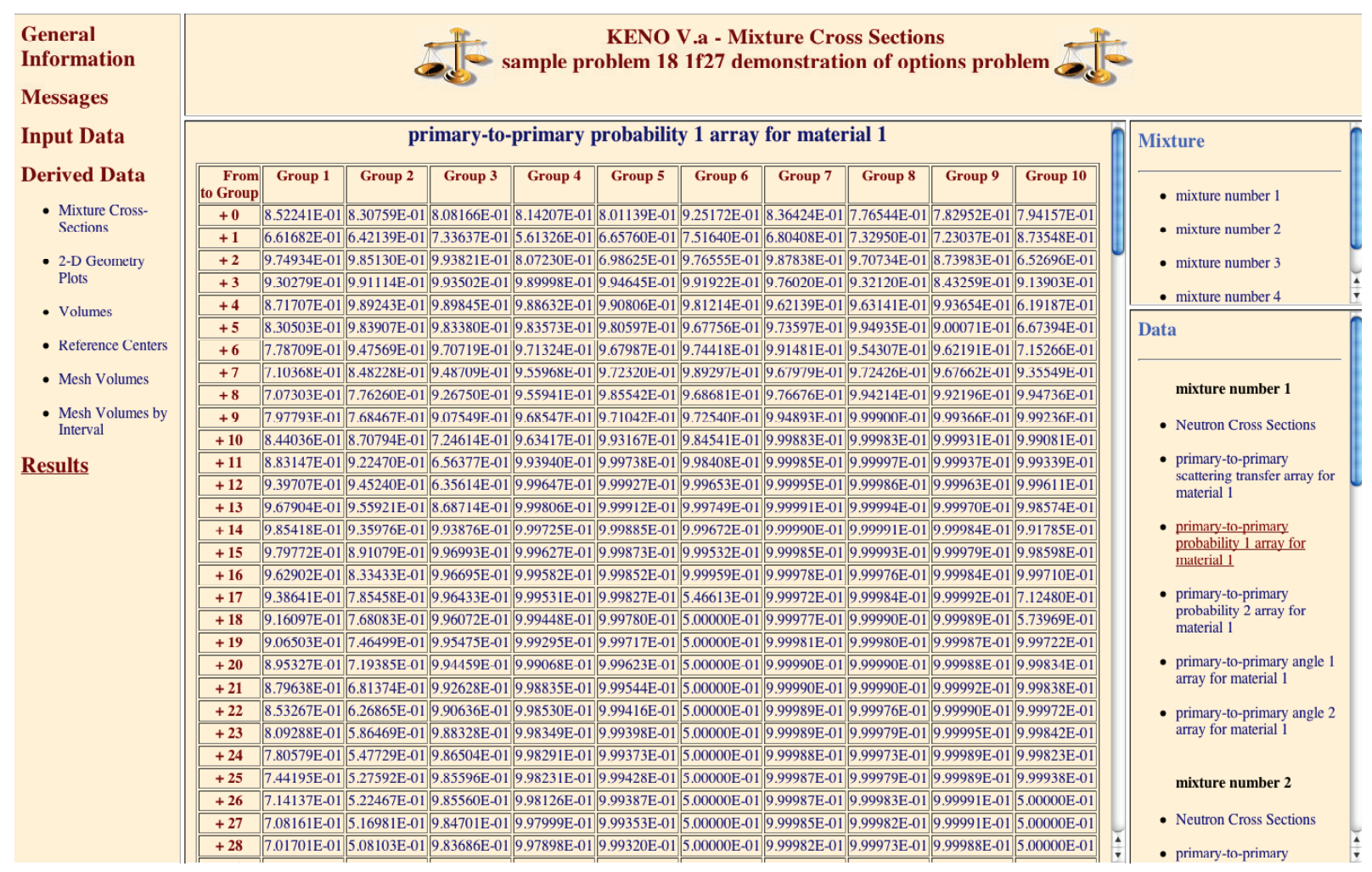

Fig. 8.1.165. Example of macroscopic probabilities in HTML output. 


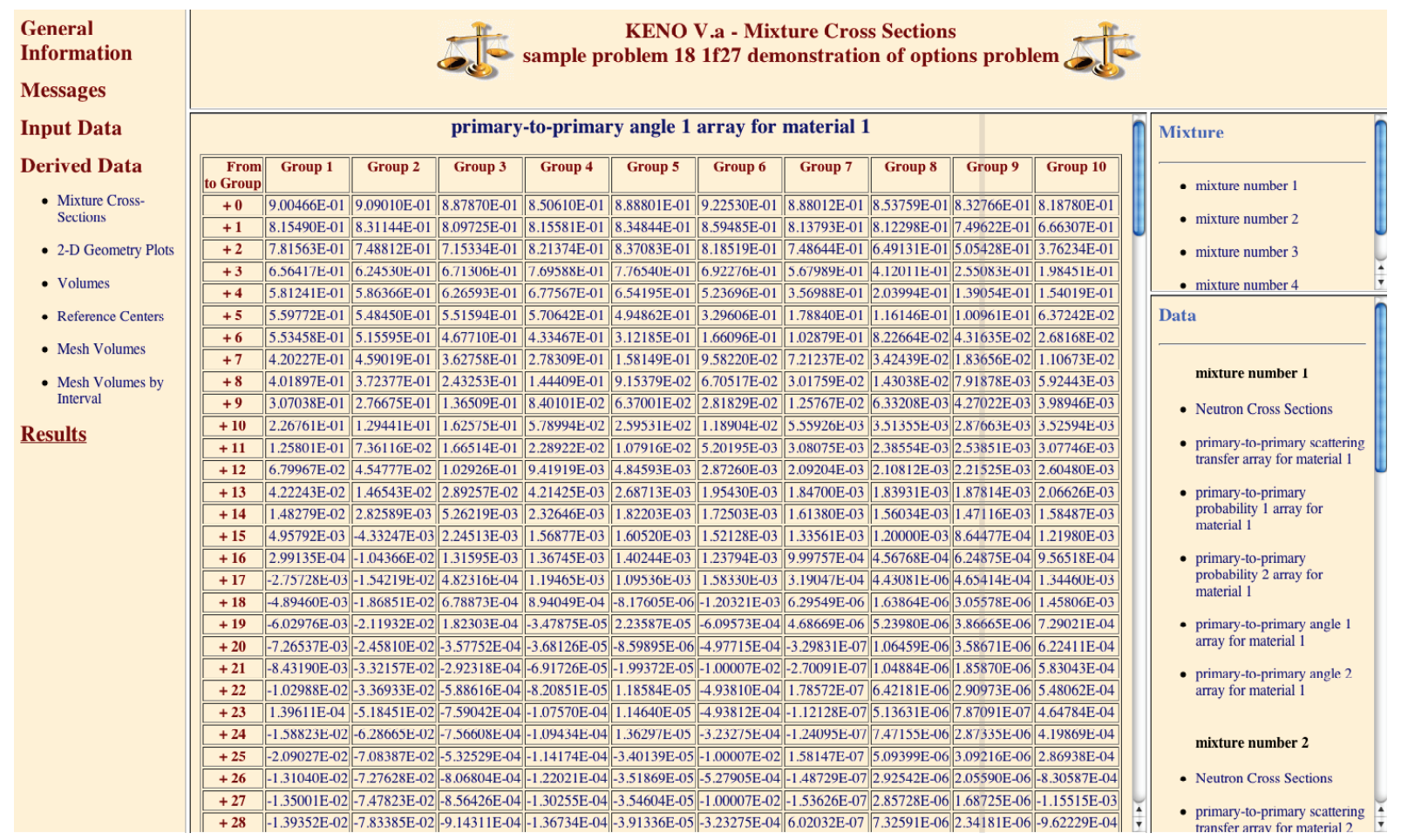

Fig. 8.1.166. Example of macroscopic angles in HTML output.

\subsection{Geometry data}

The KENO V.a HTML version of the geometry region printout is shown in Fig. 8.1.167. The HTML edits of geometry are accessed with the Geometry link in the Input Data section. 


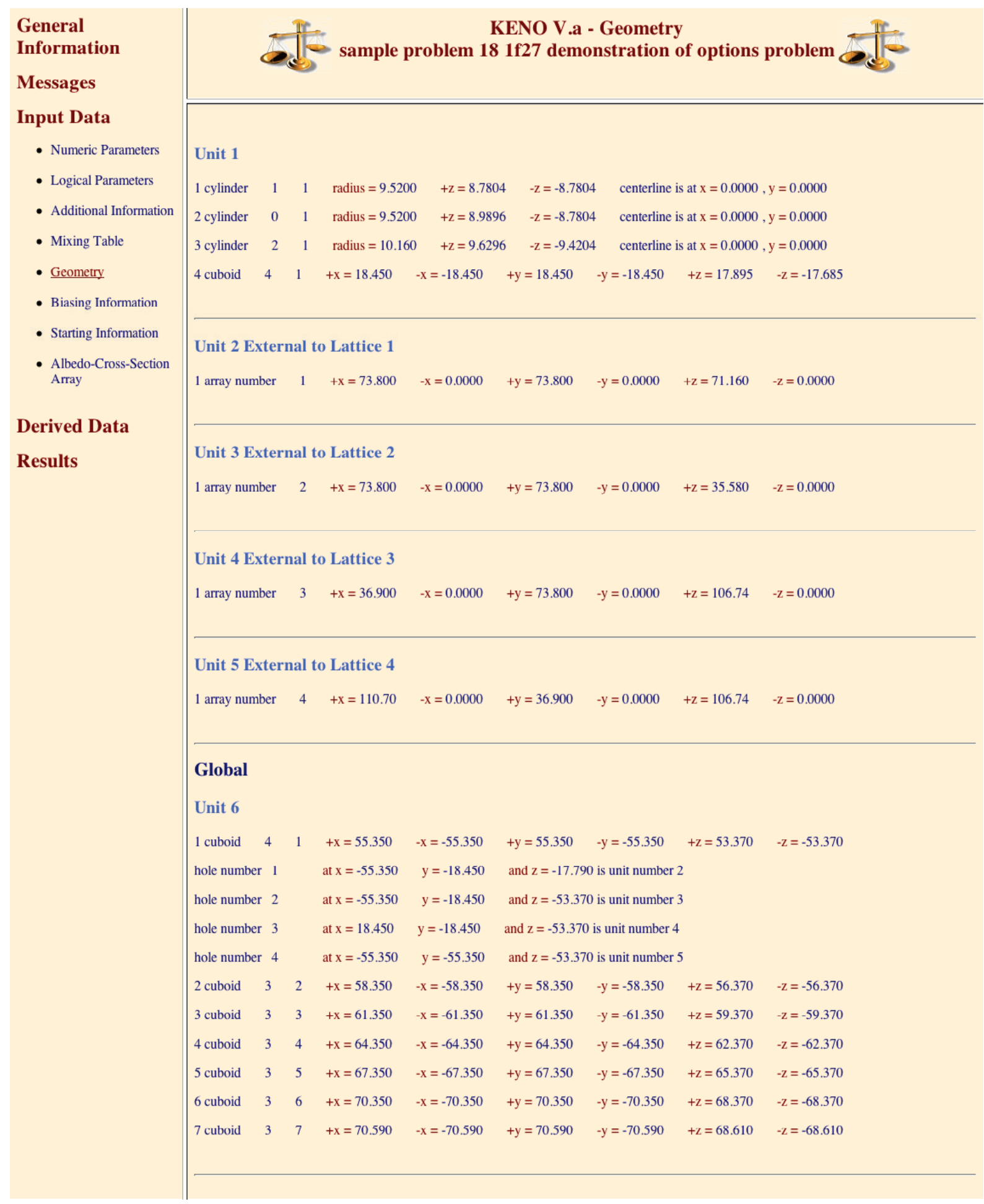

Fig. 8.1.167. Example of geometry region data in HTML output. 


\subsection{Volume information}

\subsection{KENO V.a}

Three tables of volumes are always printed. An example of the volume printout is given in Fig. 8.1.168. The HTML edit is accessed with the Volumes link in the Derived Data section.

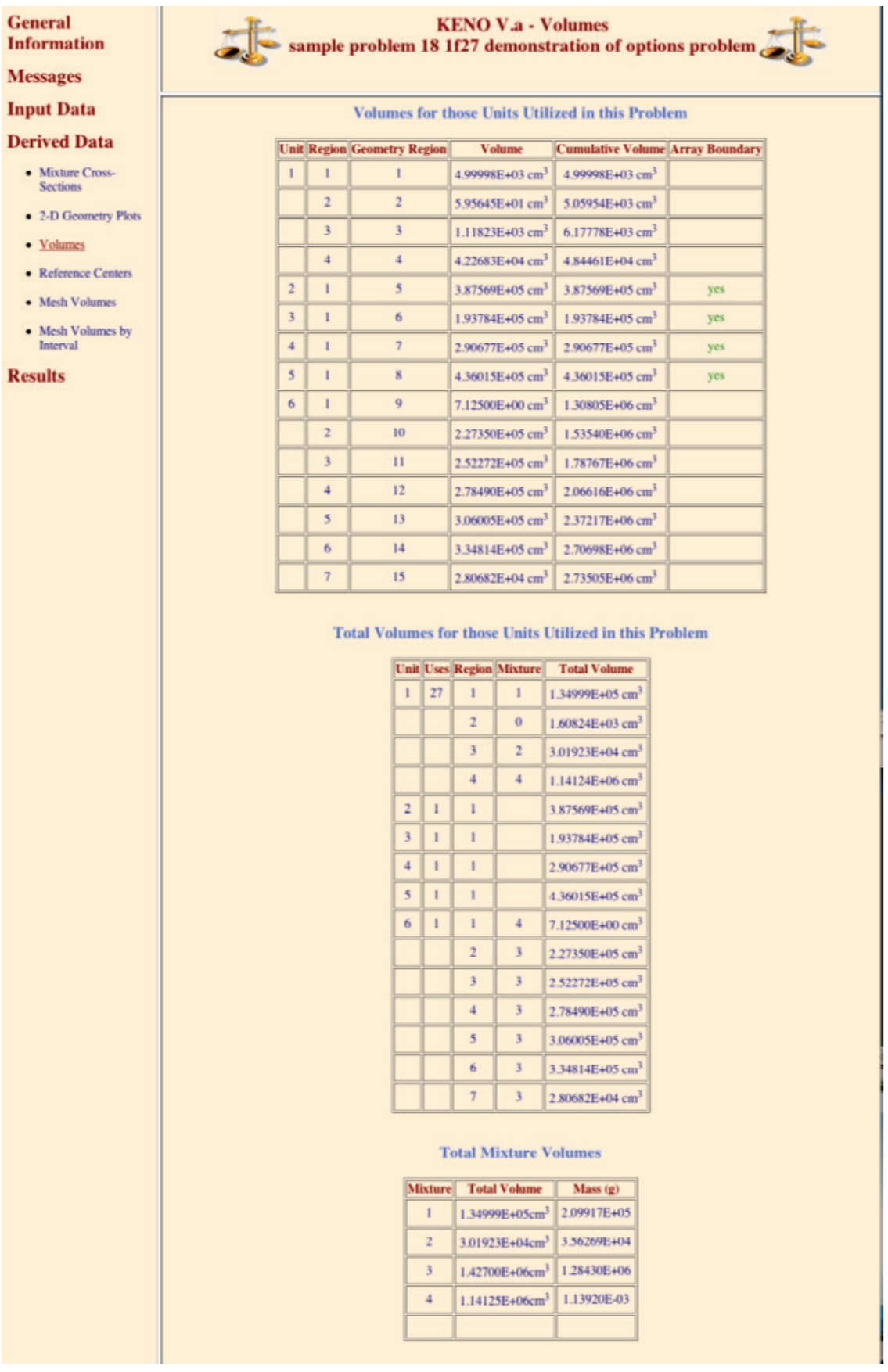

Fig. 8.1.168. Sample volume information in HTML output. 


\subsection{KENO-VI}

Two tables of volumes are always printed. The first table will list the number of times each unit is used in the problem and the total volume of each region throughout the entire problem description. The second table will list the total volume and mass of each mixture used in the problem.

\subsection{Mesh volumes}

With the mesh flux accumulator, fluxes are tabulated for each region of each unit in a cubic mesh. For models in which the mesh flux accumulator is activated by setting MFX=YES and the mesh size set to a positive value with $\mathbf{M S H}=$, the cumulative volume of all mesh intervals for a given region and the number of meshes used in each region are printed as shown in Fig. 8.1.169. The HTML edit is accessed with the Mesh Volumes link in the Derived Data section. 


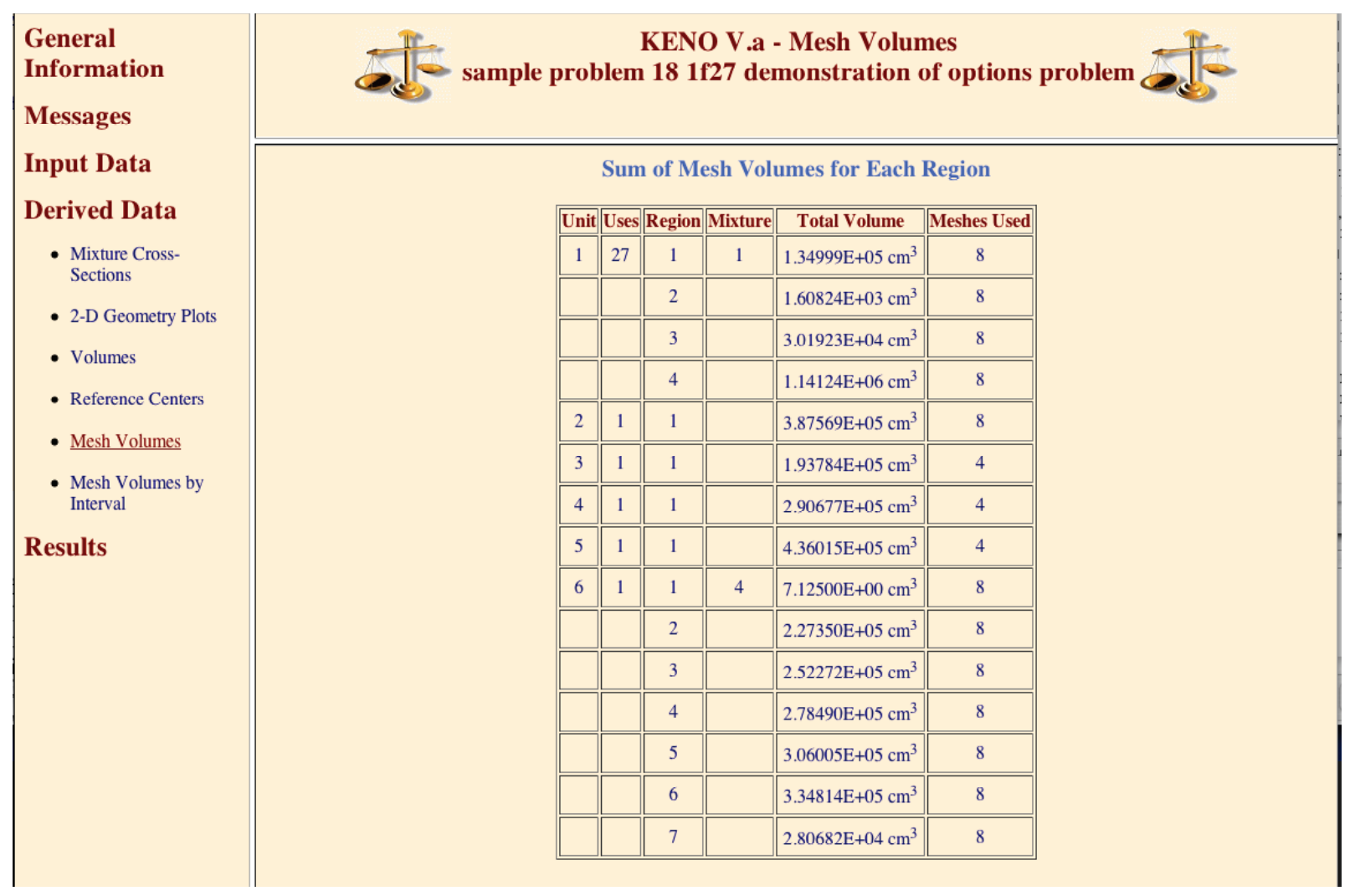

Fig. 8.1.169. Sample sum of mesh volumes in HTML output.

An optional edit of volumes for each mesh interval for each region can be activated by entering PMV=YES in the parameter input. This edit can be very large, especially if a small mesh size is used with a large model. A sample of the volume by mesh for each region is shown in Fig. 8.1.170. This edit is found in under Derived Data with the Mesh Volumes by Interval link. For the HTML output, the links on the right side of the screen control what data are displayed in the center pane. Clicking on the unit number in the pane labeled UNIT places the pane labeled Region at that unit. Clicking on a region number in the region pane places the volume by mesh for the selected unit in the center pane. 


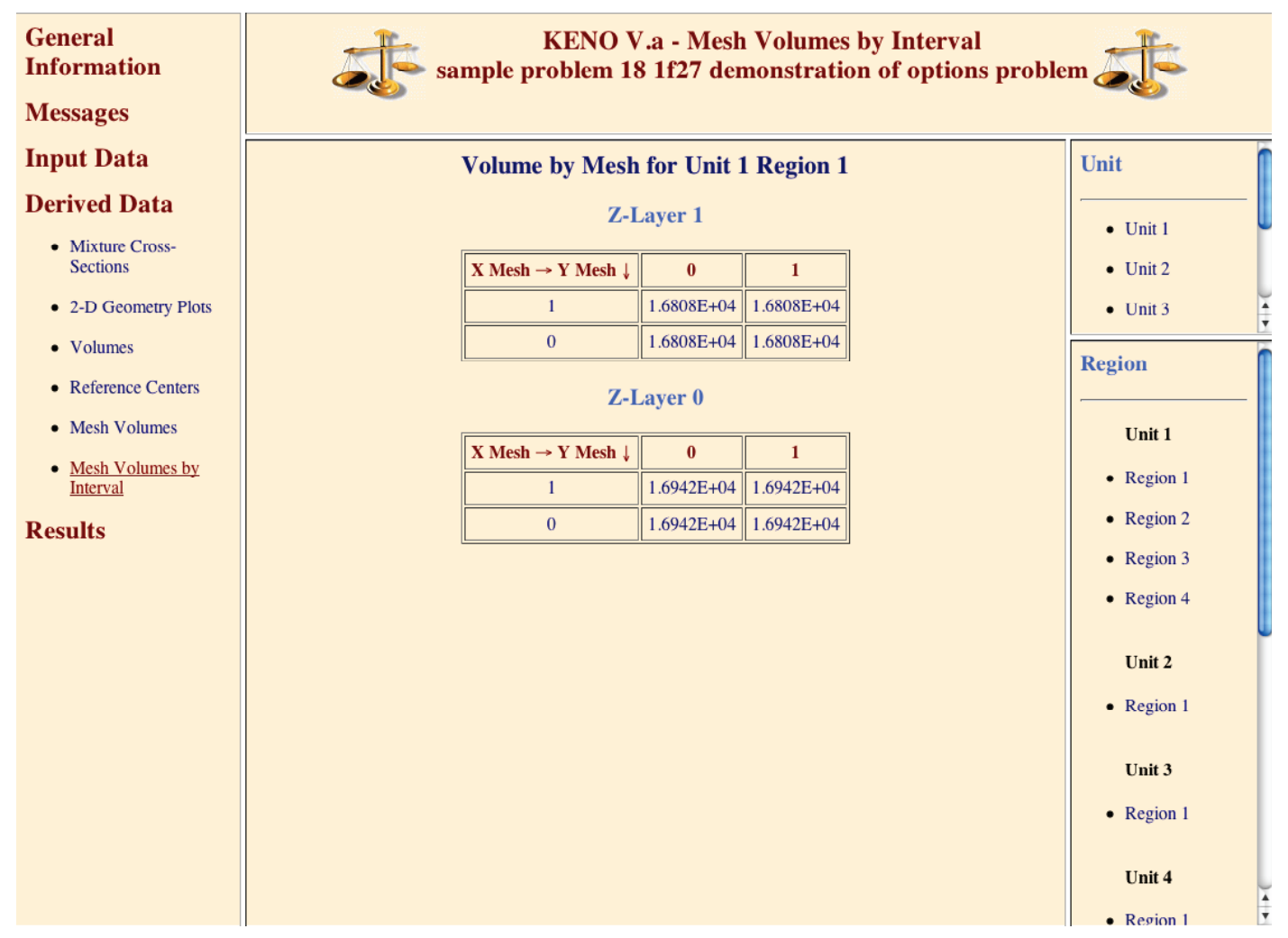

Fig. 8.1.170. Sample of volume by mesh for each region in HTML output.

\subsection{Biasing information}

This table specifies the weighting or biasing data to be used in the problem. An example of biasing information is given in Fig. 8.1.171. 


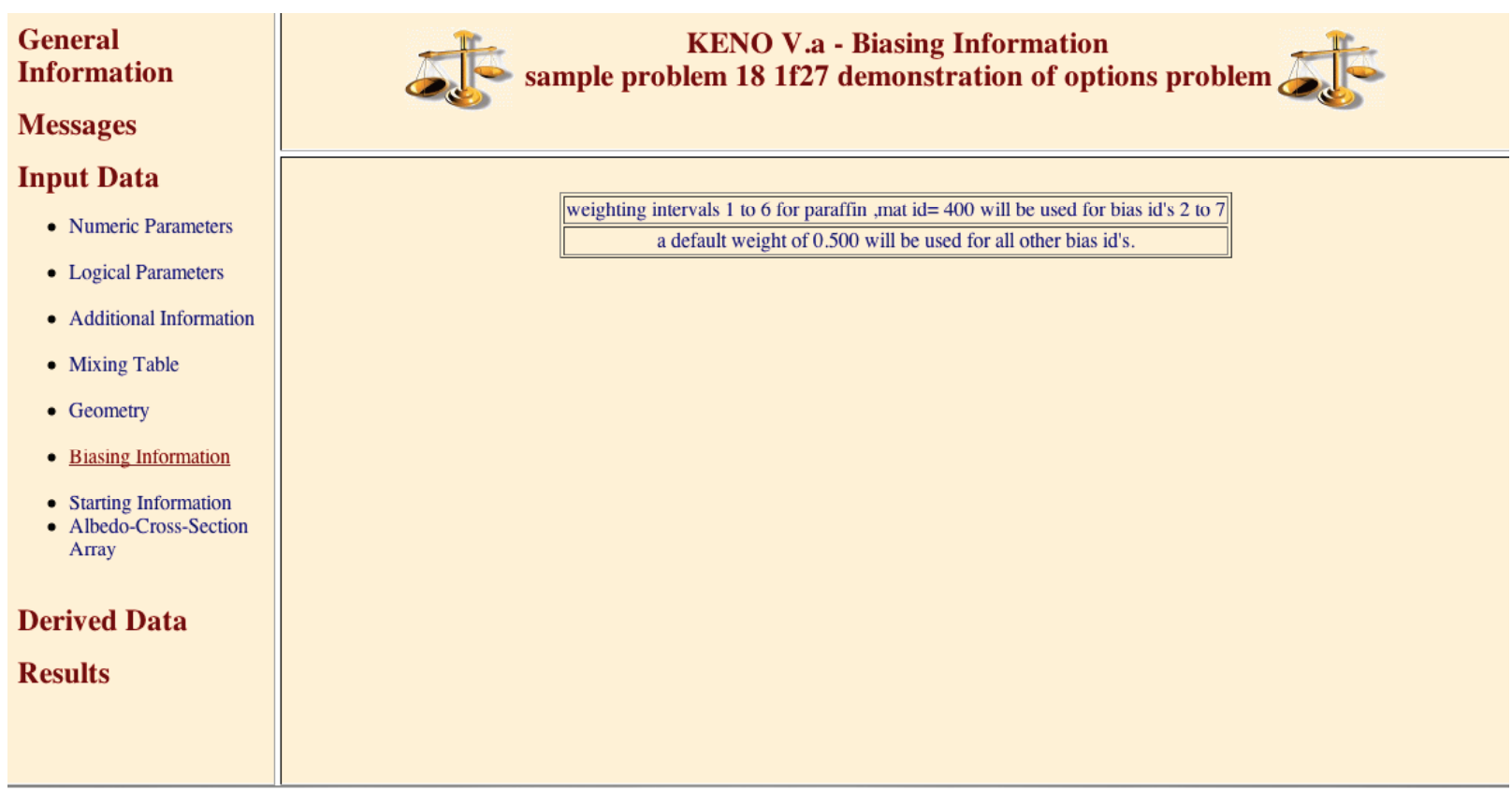

Fig. 8.1.171. Biasing information in HTML output. 


\subsection{Group-dependent weights}

Printing the group-dependent weights is optional. They are printed if PWT=YES is entered in the parameter data. This edit is available in the HTML output under Results in the Average Weight link, as shown in Fig. 8.1.172.

\begin{tabular}{|c|c|c|}
\hline \multirow{3}{*}{$\begin{array}{l}\text { General } \\
\text { Information } \\
\text { Messages } \\
\text { Input Data } \\
\text { Derived Data }\end{array}$} & \multicolumn{2}{|c|}{$\begin{array}{c}\text { KENO V.a - Average Weights } \\
\text { If sample problem } 181 \text { f27 demonstration of options problem }\end{array}$} \\
\hline & \multicolumn{2}{|r|}{ Group Dependent Weights } \\
\hline & energy group & bias id \\
\hline \multirow{3}{*}{$\begin{array}{l}\text { - Mixture Cross- } \\
\text { Sections }\end{array}$} & 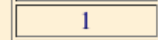 & $5.00000 \mathrm{E}-016.45620 \mathrm{E}-01$ 9.18370E-01 1.27953E+00 1.79963E+00 2.54822E+00 3.62191E+00 \\
\hline & 2 & $5.00000 \mathrm{E}-01 \quad 6.30150 \mathrm{E}-018.90506 \mathrm{E}-011.27093 \mathrm{E}+001.86077 \mathrm{E}+002.76980 \mathrm{E}+004.16192 \mathrm{E}+00$ \\
\hline & 3 & $5.00000 \mathrm{E}-015.97447 \mathrm{E}-01 \quad 8.27164 \mathrm{E}-011.21142 \mathrm{E}+001.85960 \mathrm{E}+002.93788 \mathrm{E}+004.71945 \mathrm{E}+00$ \\
\hline \multirow{3}{*}{$\begin{array}{l}\text { - 2-D Geometry Plots } \\
\text { - Volumes }\end{array}$} & 4 & $5.00000 \mathrm{E}-016.02319 \mathrm{E}-018.72352 \mathrm{E}-011.38278 \mathrm{E}+002.35268 \mathrm{E}+004.18040 \mathrm{E}+007.61880 \mathrm{E}+00$ \\
\hline & 5 & $5.00000 \mathrm{E}-016.03416 \mathrm{E}-018.99907 \mathrm{E}-011.51145 \mathrm{E}+002.77730 \mathrm{E}+005.39596 \mathrm{E}+001.08420 \mathrm{E}+01$ \\
\hline & 6 & $5.00000 \mathrm{E}-016.02020 \mathrm{E}-019.03990 \mathrm{E}-011.54519 \mathrm{E}+002.90888 \mathrm{E}+005.81680 \mathrm{E}+001.20707 \mathrm{E}+01$ \\
\hline \multirow{5}{*}{$\begin{array}{l}\text { - Reference Centers } \\
\text { - Mesh Volumes } \\
\text { - Mesh Volumes by } \\
\text { Interval }\end{array}$} & 7 & $5.00000 \mathrm{E}-01 \quad 6.03913 \mathrm{E}-019.22489 \mathrm{E}-011.62838 \mathrm{E}+003.19784 \mathrm{E}+006.71280 \mathrm{E}+001.46796 \mathrm{E}+01$ \\
\hline & 8 & $5.00000 \mathrm{E}-01 \quad 6.04658 \mathrm{E}-019.51760 \mathrm{E}-011.78398 \mathrm{E}+003.79208 \mathrm{E}+008.72478 \mathrm{E}+002.10917 \mathrm{E}+01$ \\
\hline & 9 & $5.00000 \mathrm{E}-016.06036 \mathrm{E}-019.98160 \mathrm{E}-012.03660 \mathrm{E}+004.82428 \mathrm{E}+001.25518 \mathrm{E}+013.46445 \mathrm{E}+01$ \\
\hline & 10 & $5.00000 \mathrm{E}-016.07775 \mathrm{E}-011.08248 \mathrm{E}+002.52715 \mathrm{E}+007.05251 \mathrm{E}+002.19482 \mathrm{E}+017.30304 \mathrm{E}+01$ \\
\hline & 11 & $5.00000 \mathrm{E}-016.25788 \mathrm{E}-011.27469 \mathrm{E}+003.60867 \mathrm{E}+001.24307 \mathrm{E}+014.75318 \mathrm{E}+011.91658 \mathrm{E}+02$ \\
\hline \multirow{6}{*}{$\begin{array}{l}\text { Results } \\
\text { - Final Results } \\
\text { - Problem } \\
\text { Characterization }\end{array}$} & 12 & $5.00000 \mathrm{E}-016.48768 \mathrm{E}-011.49488 \mathrm{E}+004.88448 \mathrm{E}+001.89880 \mathrm{E}+017.88949 \mathrm{E}+013.34568 \mathrm{E}+02$ \\
\hline & 13 & $5.00000 \mathrm{E}-016.60420 \mathrm{E}-01 \quad 1.61202 \mathrm{E}+005.55442 \mathrm{E}+002.22849 \mathrm{E}+019.38038 \mathrm{E}+013.99407 \mathrm{E}+02$ \\
\hline & 14 & $5.00000 \mathrm{E}-016.82387 \mathrm{E}-01 \quad 1.72650 \mathrm{E}+006.10317 \mathrm{E}+002.47702 \mathrm{E}+011.04624 \mathrm{E}+024.45818 \mathrm{E}+02$ \\
\hline & 15 & $5.00000 \mathrm{E}-017.13029 \mathrm{E}-011.92603 \mathrm{E}+007.15390 \mathrm{E}+002.96382 \mathrm{E}+011.25893 \mathrm{E}+025.37071 \mathrm{E}+02$ \\
\hline & 16 & $5.00000 \mathrm{E}-017.23974 \mathrm{E}-012.07297 \mathrm{E}+008.03687 \mathrm{E}+003.37577 \mathrm{E}+011.43807 \mathrm{E}+026.13781 \mathrm{E}+02$ \\
\hline & 17 & $5.00000 \mathrm{E}-017.33870 \mathrm{E}-012.23252 \mathrm{E}+008.97808 \mathrm{E}+003.80495 \mathrm{E}+011.62325 \mathrm{E}+026.92943 \mathrm{E}+02$ \\
\hline \multirow{5}{*}{$\begin{array}{l}\text { - } \mathrm{k}_{\mathrm{eff}} \text { Results } \\
\text { - Fluxes } \\
\text { - Fissions and } \\
\text { Absorptions }\end{array}$} & 18 & $5.00000 \mathrm{E}-017.00287 \mathrm{E}-012.23813 \mathrm{E}+009.20905 \mathrm{E}+003.91797 \mathrm{E}+011.67220 \mathrm{E}+027.13870 \mathrm{E}+02$ \\
\hline & 19 & $5.00000 \mathrm{E}-017.97973 \mathrm{E}-012.69722 \mathrm{E}+001.12404 \mathrm{E}+014.79024 \mathrm{E}+012.04480 \mathrm{E}+028.72943 \mathrm{E}+02$ \\
\hline & 20 & $5.00000 \mathrm{E}-015.87908 \mathrm{E}-01 \quad 1.94657 \mathrm{E}+008.13714 \mathrm{E}+003.46882 \mathrm{E}+01 \quad 1.48076 \mathrm{E}+026.32151 \mathrm{E}+02$ \\
\hline & 21 & $5.00000 \mathrm{E}-016.07528 \mathrm{E}-012.06750 \mathrm{E}+008.67361 \mathrm{E}+003.69873 \mathrm{E}+011.57894 \mathrm{E}+026.74064 \mathrm{E}+02$ \\
\hline & 22 & $5.00000 \mathrm{E}-017.48539 \mathrm{E}-012.67353 \mathrm{E}+001.12536 \mathrm{E}+014.80027 \mathrm{E}+012.04921 \mathrm{E}+028.74827 \mathrm{E}+02$ \\
\hline \multirow{3}{*}{$\begin{array}{l}\text { - Average Weight } \\
\text { - Matrix by Hole }\end{array}$} & 23 & $5.00000 \mathrm{E}-017.41377 \mathrm{E}-012.71578 \mathrm{E}+001.14781 \mathrm{E}+014.89758 \mathrm{E}+012.09079 \mathrm{E}+028.92578 \mathrm{E}+02$ \\
\hline & 24 & $5.00000 \mathrm{E}-018.36856 \mathrm{E}-01 \quad 3.21015 \mathrm{E}+001.36168 \mathrm{E}+015.81160 \mathrm{E}+012.48101 \mathrm{E}+021.05917 \mathrm{E}+03$ \\
\hline & 25 & $5.00000 \mathrm{E}-01$ 9.09954E-01 3.61098E+00 1.53591E+01 6.55621E+01 2.79891E+02 1.19488E+03 \\
\hline \multirow{10}{*}{$\begin{array}{l}\text { - Matrix by Unit } \\
\text { - Matrix by Array }\end{array}$} & 26 & $5.00000 \mathrm{E}-019.21096 \mathrm{E}-013.70575 \mathrm{E}+001.57871 \mathrm{E}+016.73937 \mathrm{E}+012.87711 \mathrm{E}+021.22827 \mathrm{E}+03$ \\
\hline & 27 & $5.00000 \mathrm{E}-019.19665 \mathrm{E}-013.71912 \mathrm{E}+001.58550 \mathrm{E}+016.76852 \mathrm{E}+012.88955 \mathrm{E}+021.23358 \mathrm{E}+03$ \\
\hline & 28 & $5.00000 \mathrm{E}-019.16251 \mathrm{E}-01 \quad 3.70863 \mathrm{E}+001.58135 \mathrm{E}+016.75082 \mathrm{E}+012.88199 \mathrm{E}+021.23035 \mathrm{E}+03$ \\
\hline & 29 & $5.00000 \mathrm{E}-019.11580 \mathrm{E}-013.69380 \mathrm{E}+001.57530 \mathrm{E}+016.72506 \mathrm{E}+012.87100 \mathrm{E}+021.22566 \mathrm{E}+03$ \\
\hline & 30 & $5.00000 \mathrm{E}-019.04871 \mathrm{E}-01 \quad 3.67861 \mathrm{E}+001.56921 \mathrm{E}+016.69908 \mathrm{E}+012.85991 \mathrm{E}+021.22093 \mathrm{E}+03$ \\
\hline & 31 & $5.00000 \mathrm{E}-019.05042 \mathrm{E}-013.70027 \mathrm{E}+001.57880 \mathrm{E}+016.74006 \mathrm{E}+012.87740 \mathrm{E}+021.22839 \mathrm{E}+03$ \\
\hline & 32 & $5.00000 \mathrm{E}-019.12037 \mathrm{E}-013.74764 \mathrm{E}+001.59923 \mathrm{E}+016.82725 \mathrm{E}+012.91463 \mathrm{E}+021.24429 \mathrm{E}+03$ \\
\hline & 33 & $5.00000 \mathrm{E}-019.22067 \mathrm{E}-013.80714 \mathrm{E}+001.62479 \mathrm{E}+016.93639 \mathrm{E}+012.96122 \mathrm{E}+021.26418 \mathrm{E}+03$ \\
\hline & 34 & $5.00000 \mathrm{E}-019.38872 \mathrm{E}-013.90409 \mathrm{E}+001.66638 \mathrm{E}+017.11398 \mathrm{E}+013.03703 \mathrm{E}+021.29654 \mathrm{E}+03$ \\
\hline & 35 & $5.00000 \mathrm{E}-01$ 9.64627E-01 4.06345E+00 1.73469E+01 7.40559E+01 3.16153E+02 1.34969E+03 \\
\hline
\end{tabular}

Fig. 8.1.172. Example of biasing data in HTML output.

\subsection{Plot representation}

Plots representing 2-D slices through the geometrical description of the problem are optional. They are created if plot data are entered unless PLT $=\mathrm{NO}$ is specified either in the plot data or the parameter data. Character plots are not available in the HTML output, but the color plots are easily viewed as shown in Fig. 8.1.173. The user-requested plots can be viewed under Derived Data in the 2-D Geometry Plots link. 
Fig. 8.1.173 shows two color plots in a single HTML page. The color plots do not appear in the KENO text printout, but they are an integral part of the HTML output. They are generated from PNG files created when a color plot has been specified in the KENO input data. Any number of plots can be made in one problem. The color plots can be valuable tools to assist the user in verifying that a problem is described correctly.

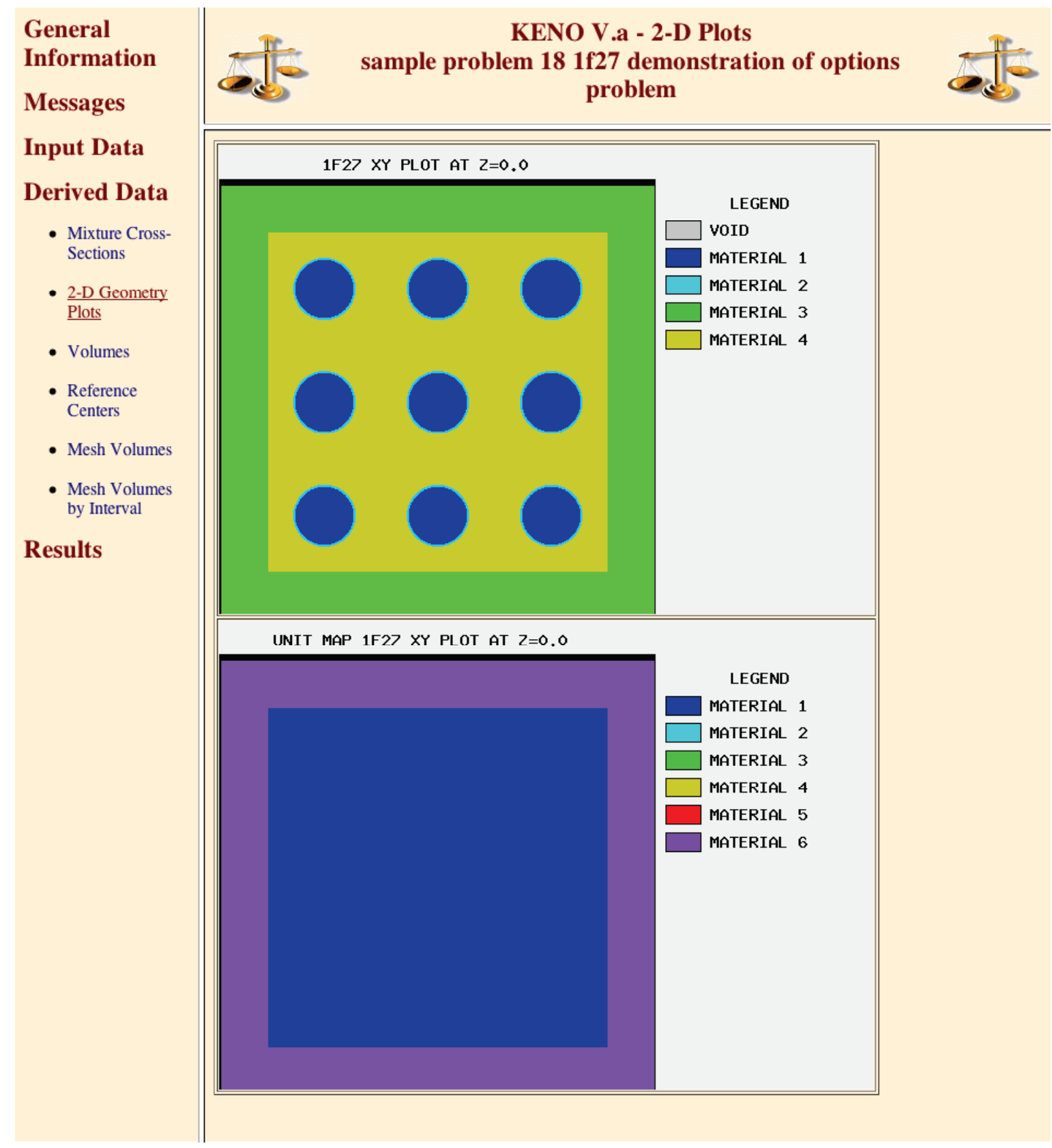

Fig. 8.1.173. Sample color plots in HTML output. 


\subsection{Initial source and final pretracking edits}

Prior to calculating the k-effs for each generation, KENO prints the final input data edit. The starting information is available in the HTML output under Input Data with the Starting Information link. An example of the HTML edit is shown in Fig. 8.1.174.

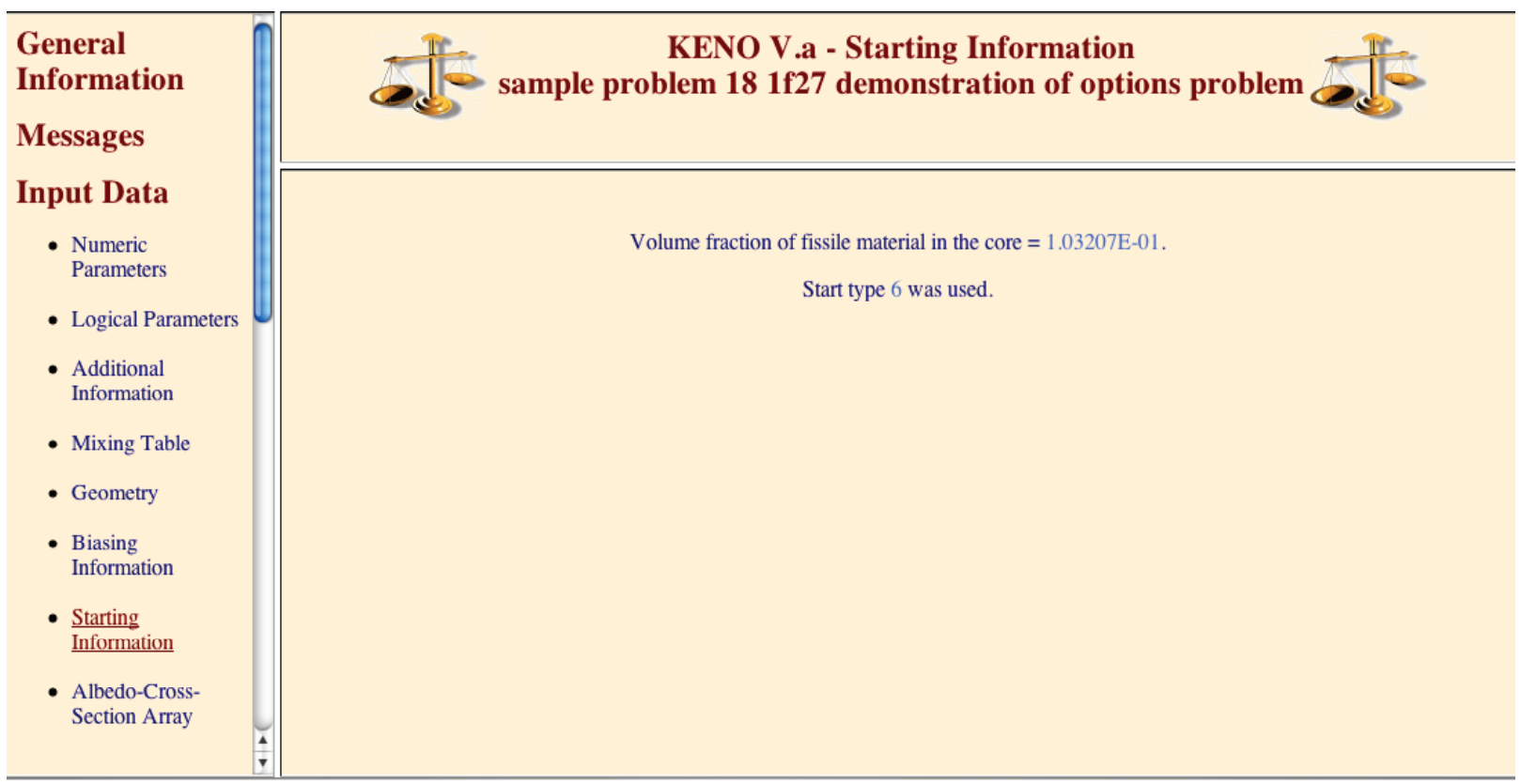

Fig. 8.1.174. Example of initial source information for start type 6 in HTML output.

\subsection{Reference center for flux moment/angular flux tranform}

When TFM=YES is entered, the flux moments and angular fluxes are computed in a transformed coordinate system relative to a reference center point. Edits of the fuel center and the position of the reference center for each region are shown when TFM=YES in Fig. 8.1.175. The HTML output edit is available under Derived Data in the Reference Centers link. 


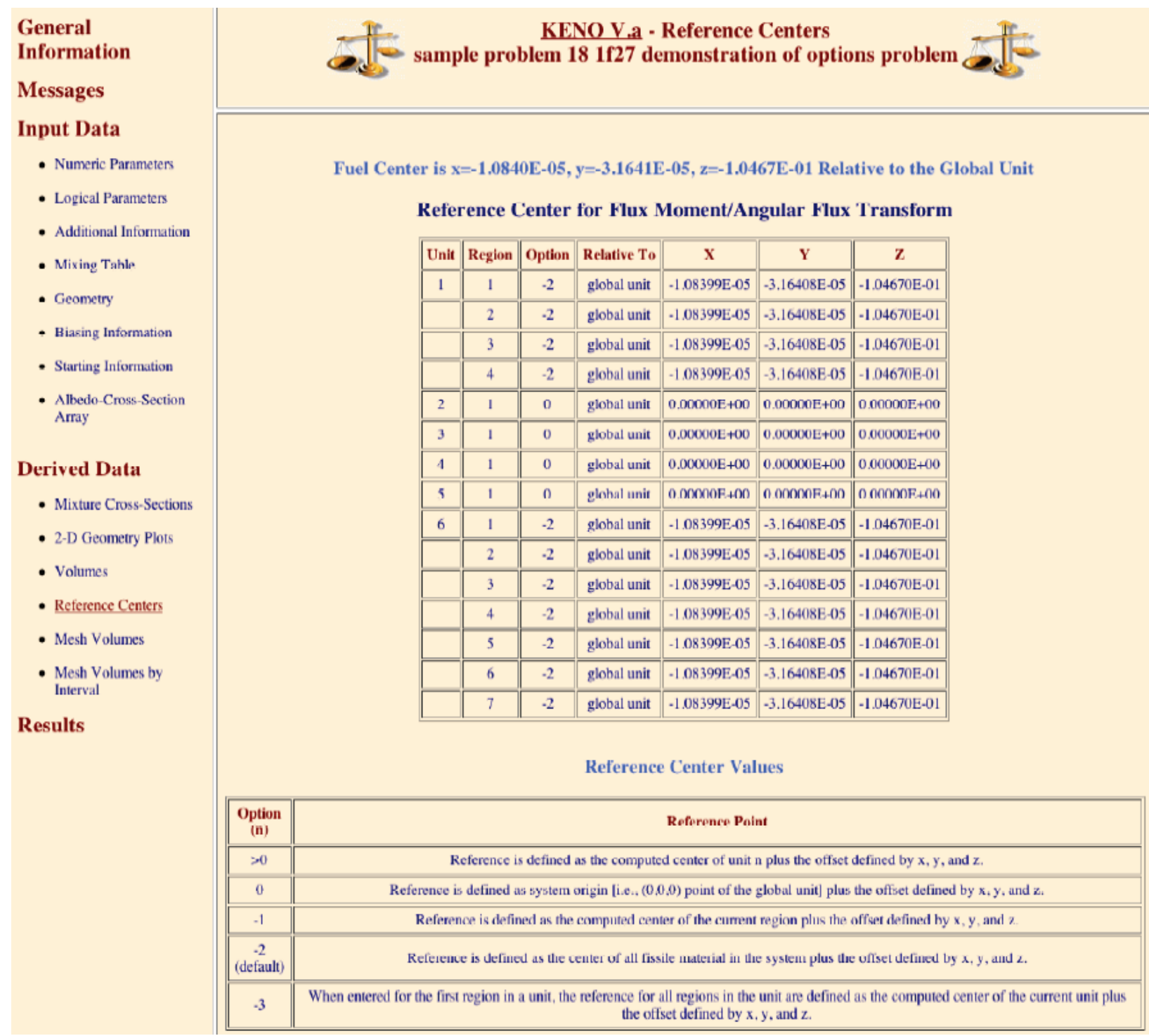

Fig. 8.1.175. Example of fuel center and reference center edits in HTML output. 


\subsection{K-effectives by generation}

At the completion of each generation, KENO prints the k-effective for that generation and associated information. An example of this printout is given in Fig. 8.1.176 for HTML output. This HTML output edit is available under Results in the section of $k_{\text {eff }}$ Results in the $k_{\text {eff }}$ by Generations Run link.

Example fuel center and reference center edits in HTML output.

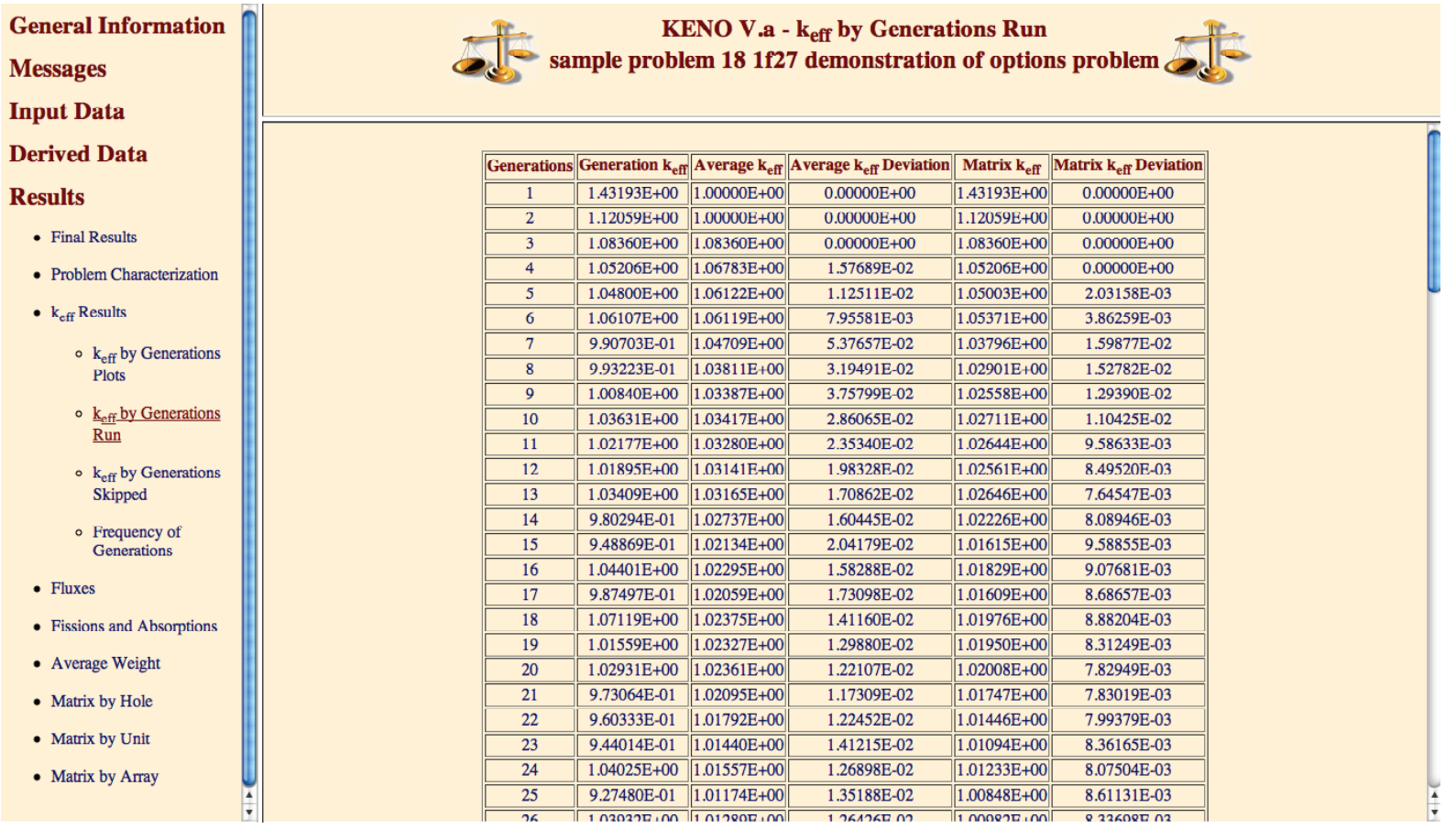

Fig. 8.1.176. Example of k-effective by generation in HTML output.

The problem title is printed at the top of the page. A descriptive heading is printed at the top of each column of data. The data printed include (1) the generation number, (2) the k-effective calculated for the generation, (3) the average value of k-effective through the current generation (excluding the first two generations), (4) the deviation associated with the average k-effective, and (5) the matrix k-effective for the generation and the deviation associated with the matrix k-effective. The last two columns are filled with zeros if the user did not specify matrix k-effective calculations. The matrix k-effective is the largest eigenvalue of the fission production matrix. Matrix information can be calculated based on (1) position index, (2) unit number, (3) hole number, and (4) array number. The matrix k-effective printed in the sixth column is based on this order. If the matrix k-effective is calculated by position index, it is the one printed in the sixth column. The matrix k-effective by unit number is given second preference, followed by hole number and then array number. After the last generation, a message is printed to indicate why execution was terminated. If matrix k-effectives were calculated, this is followed by a message stating the method used to determine the matrix k-effective.

The user should examine this portion of the results to ensure that the two methods of calculating k-effective are in acceptable agreement and to verify that the average value of k-effective has become relatively stable. If the k-effectives appear to be oscillating or drifting significantly, the user should consider rerunning the problem with a larger number of histories per generation. 
If a problem is restarted, the generation numbers and k-effectives are printed and the words FROM RESTART UNIT are printed in the elapsed time column. All other columns are blank. When the generation at which the problem is to be restarted is reached, the print reverts to the normal format as shown in Fig. 8.1.126.

\subsection{Problem characterization edit}

The problem characterization edit is shown in Fig. 8.1.177 for HTML output.

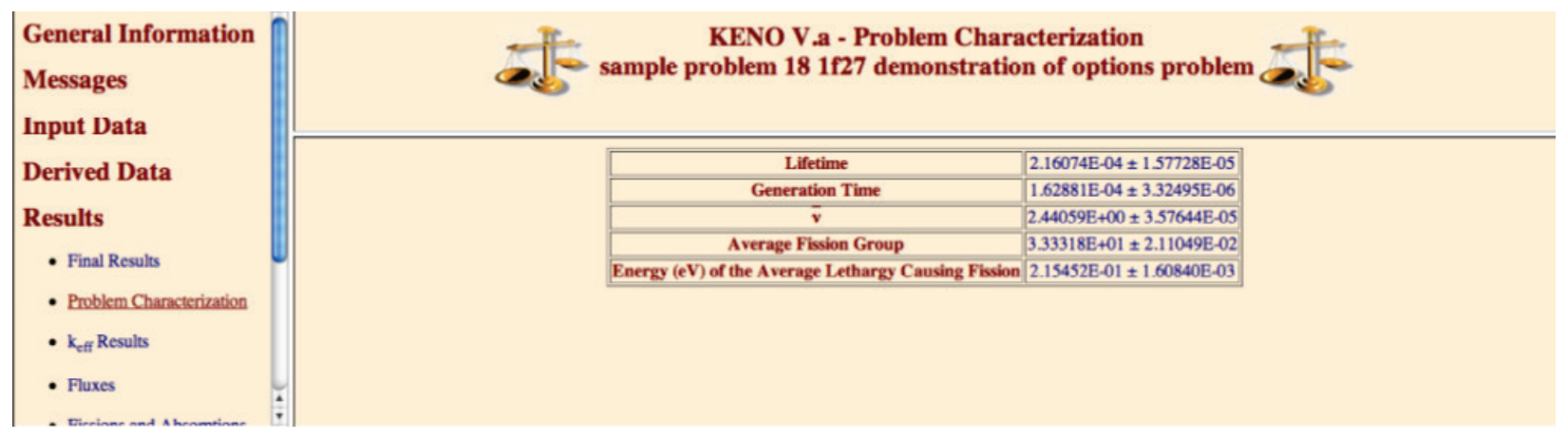

Fig. 8.1.177. Example problem characterization edit in HTML output.

\subsection{Final k-effective edit}

The final k-effective edit prints the average k-effective and its associated deviation and the limits of k-effective for the 67,95 , and $99 \%$ confidence intervals. The final k-effective edit is printed as shown in Fig. 8.1.178. This HTML output edit is available under Results in the section of $k_{\text {eff }}$ Results in the $k_{\text {eff }} b y$ Generations Skipped link.

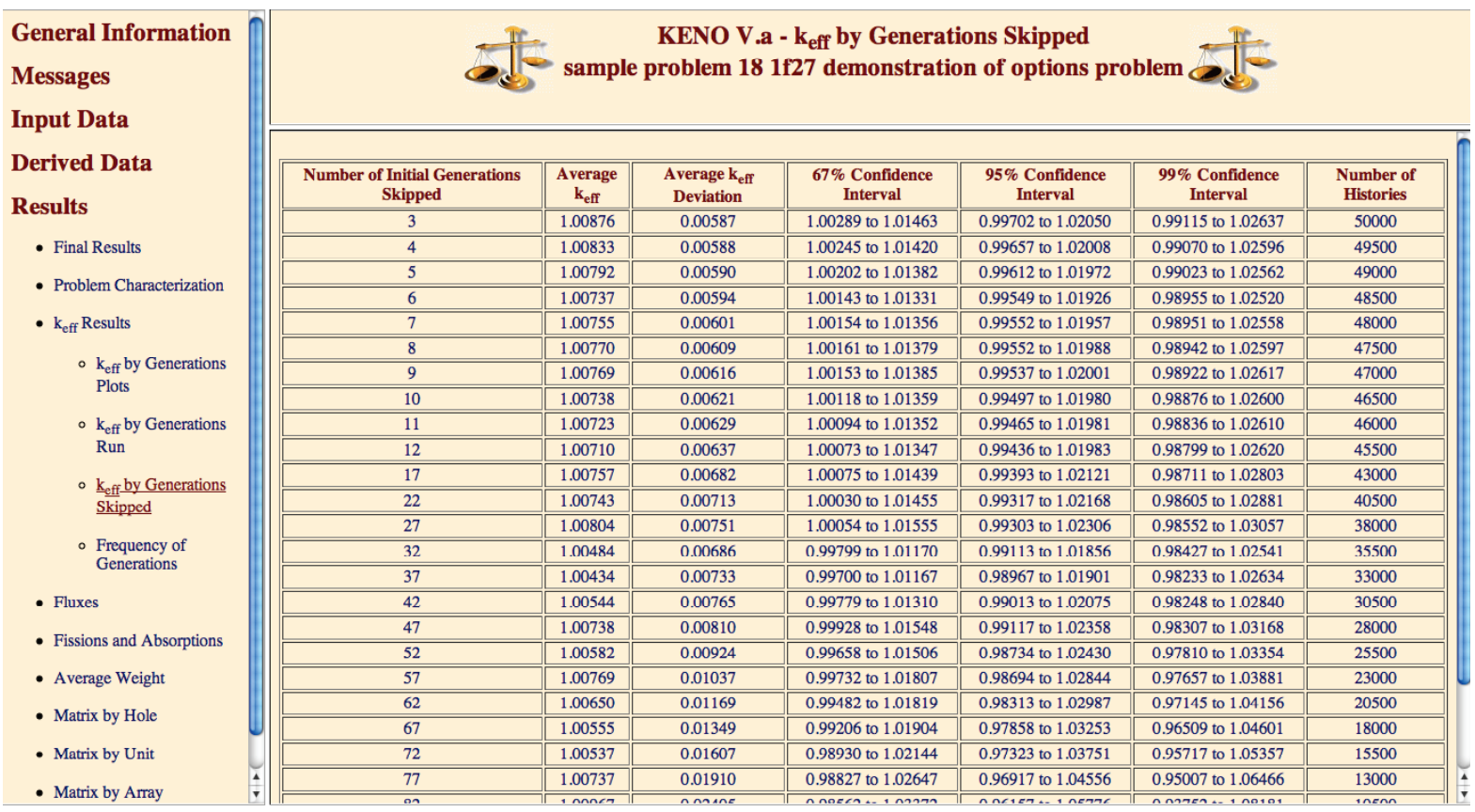


Fig. 8.1.178. Example of the final k-effective edit in HTML output.

\subsection{Plot of average k-effective by generation run}

This plot consists of average k-effectives plotted versus the number of generations run. For the HTML output, this plot is presented as an embedded Javapeño applet plot, as shown in Fig. 8.1.179. The data shown in the plot are the same as those shown in the text output, with the addition of $95 \%$ and $99 \%$ confidence curves. With this interactive plot, the user can zoom in and out of the data, remove curves and utilize all of the features of Javapeño for data visualization and manipulation. This plot is available as the first plot on the page accessed with the $k_{\text {eff }}$ by Generations Plots link under $k_{\text {eff }}$ Results in the Results section of the HTML output.

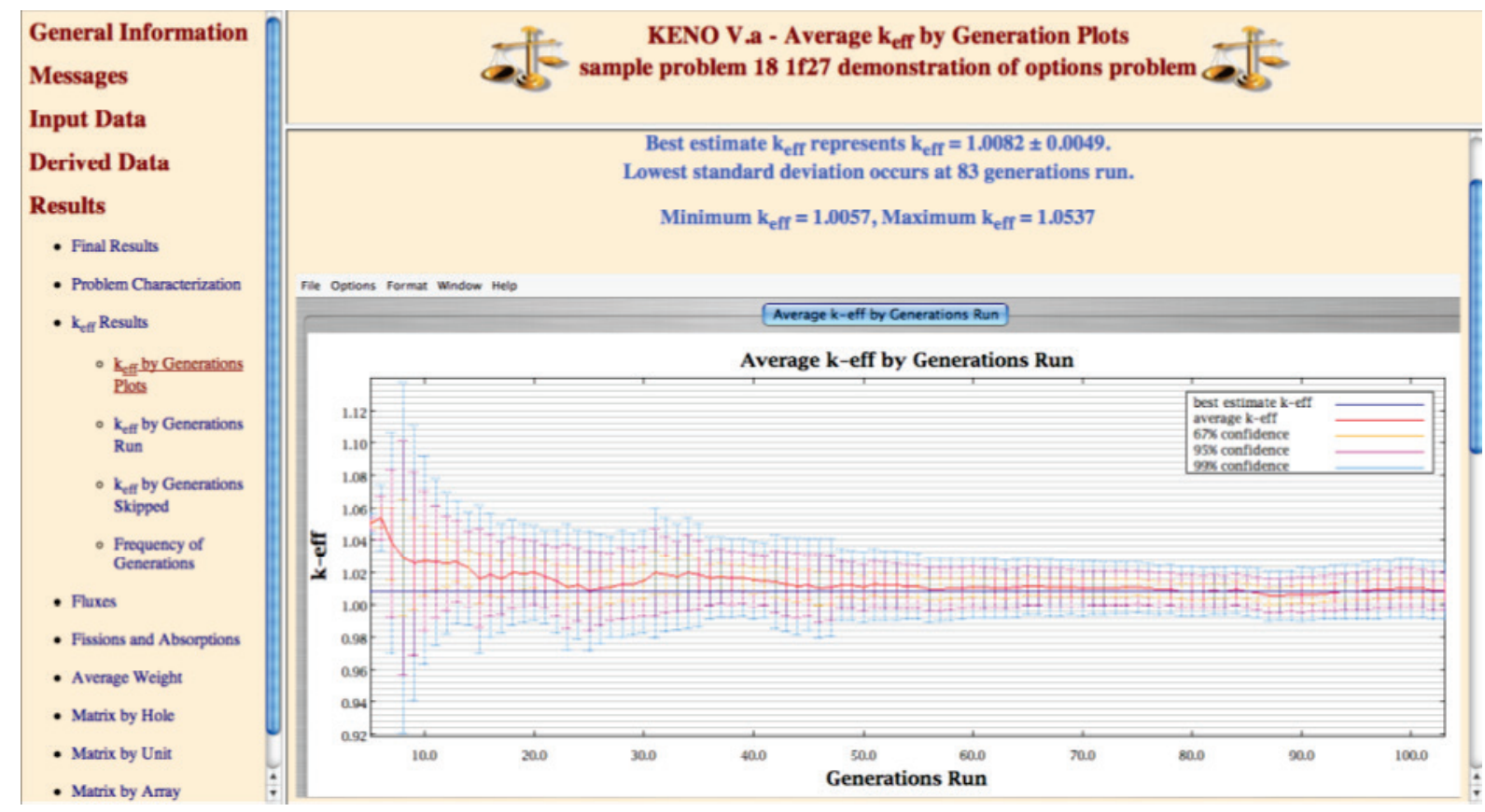

Fig. 8.1.179. Sample plot of average k-effective by generations run in HTML output.

\subsection{Plot of average k-effective by generations skipped}

This plot illustrates the average k-effective versus the number of generations skipped. For the HTML output, this plot is presented as an embedded Javapeño applet plot, as shown in Fig. 8.1.180. The data shown in the plot are the same as those shown in the text output, with the addition of $95 \%$ and $99 \%$ confidence curves. With this interactive plot, the user can zoom in and out of the data, remove curves and utilize all of the features of Javapeño for data visualization and manipulation. This plot is available as the second plot on the page accessed with the $k_{\text {eff }}$ by Generations Plots link under $k_{\text {eff }}$ Results in the Results section of the HTML output. 


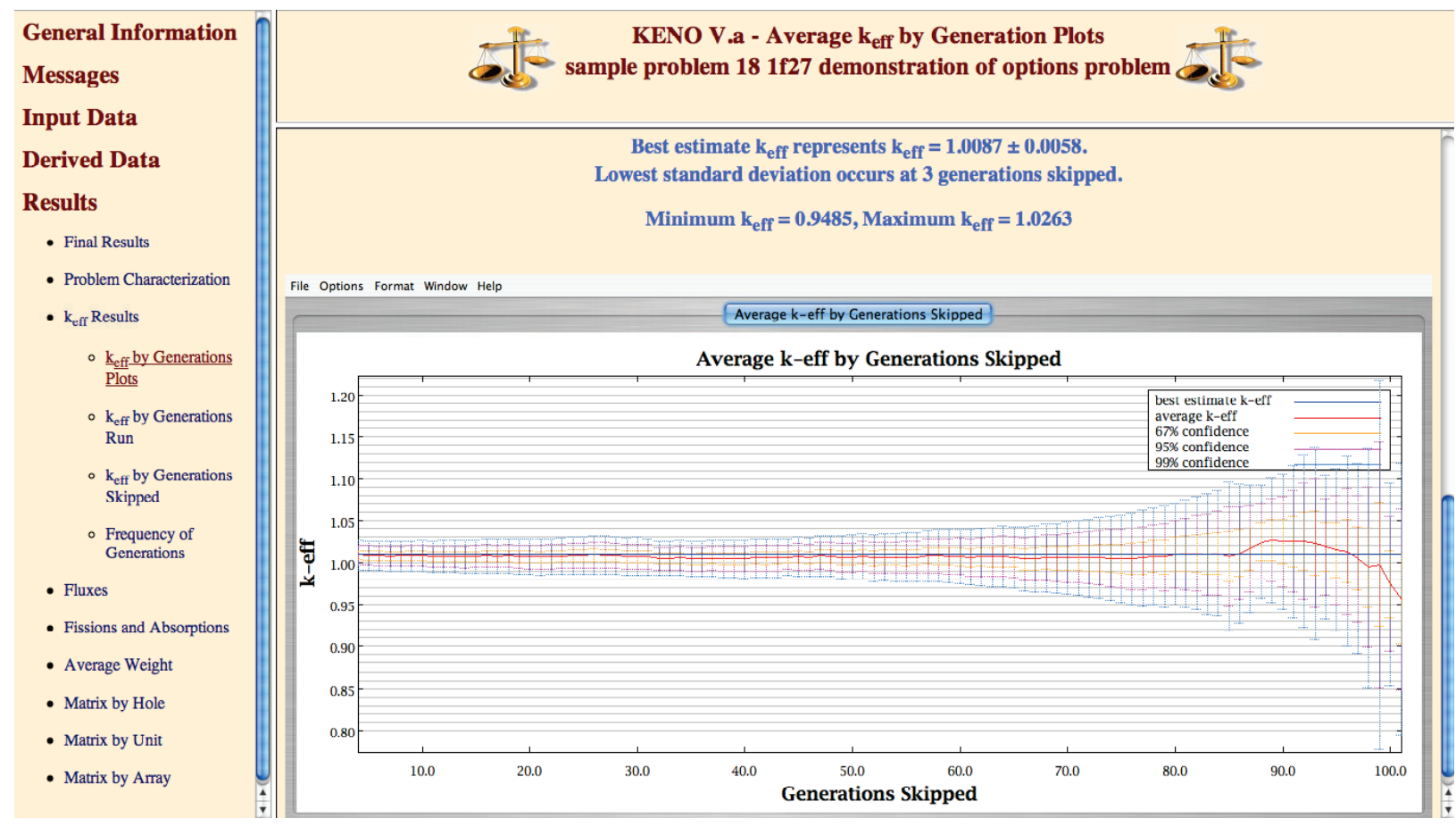

Fig. 8.1.180. Sample plot of average k-effective by generations skipped in HTML output.

\subsection{Final edit of fissions, absorptions, and leakage}

The final edit of fissions, absorptions, and leakage follows the final k-effective edit and includes the fission fraction for each group and the fission production, absorptions, and leakage, each with its associated percent deviation.

Tables corresponding to those available in the text output are also available in the HTML output. These data are accessed with the Fissions and Absorptions Data link under Fissions and Absorptions in the Results section, as illustrated in Fig. 8.1.181, where the region and group data (FAR=YES and $\mathbf{G A S}=$ YES) are present. To allow quicker loading of the large amounts of data in the HTML output, the data have been subdivided into several separate tables, which are accessed with the menu on the right side of the window.

Where the group data are accumulated, plots of fissions and absorptions are available through the use of the Javapeño applet. The plot of fissions and absorptions is available through the Fission and Absorption Plots link under Fissions and Absorptions in the Results section of the output. An example plot is shown in Fig. 8.1.182. This page loads with no data plotting in Javapeño. Double-clicking on the title of the data on the right side of the plot will cause the corresponding data to appear. All features of Javapeño are available to the user in the interactive plot. 


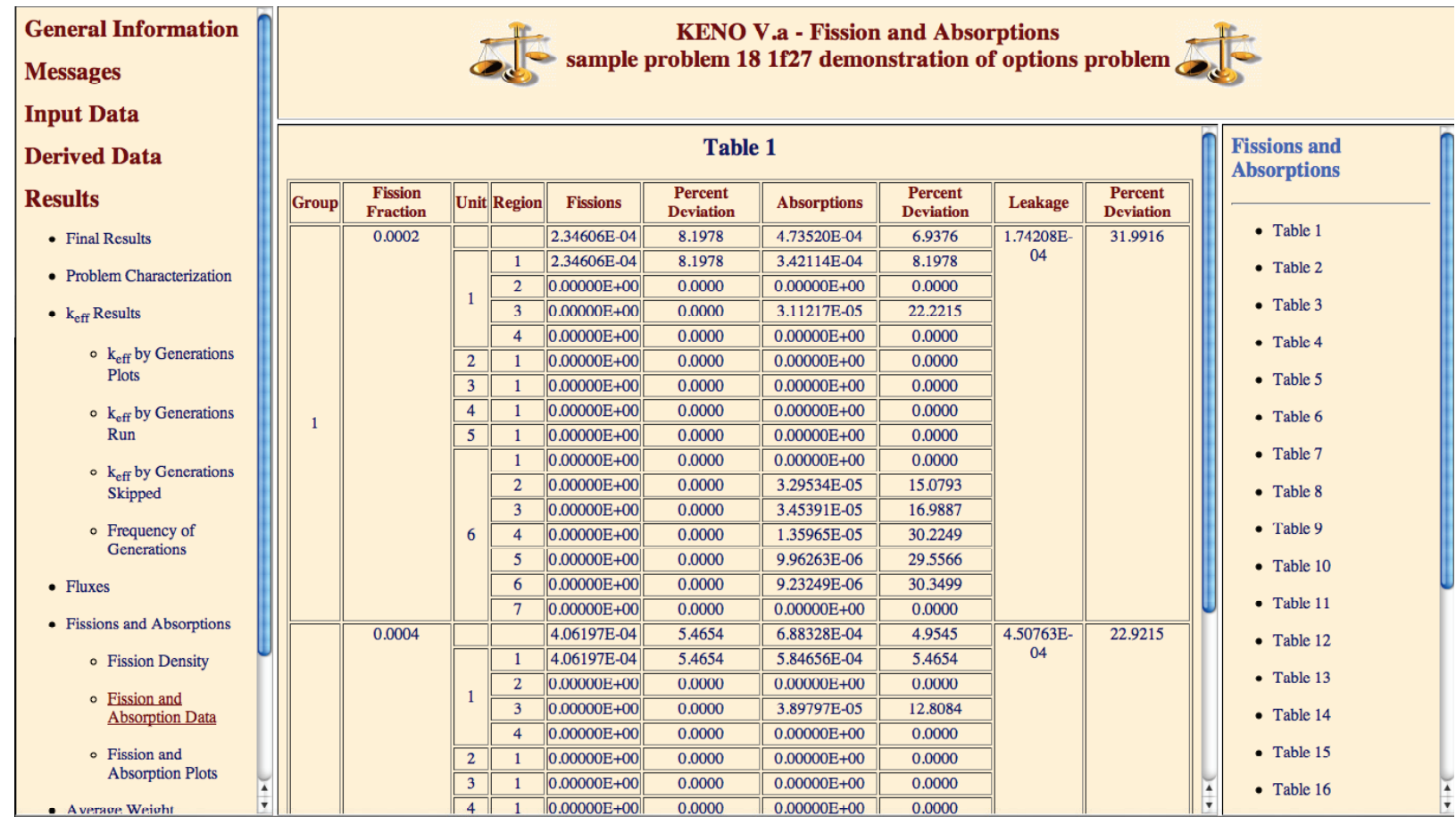

Fig. 8.1.181. Sample fissions, absorptions and leakage data in HTML output.

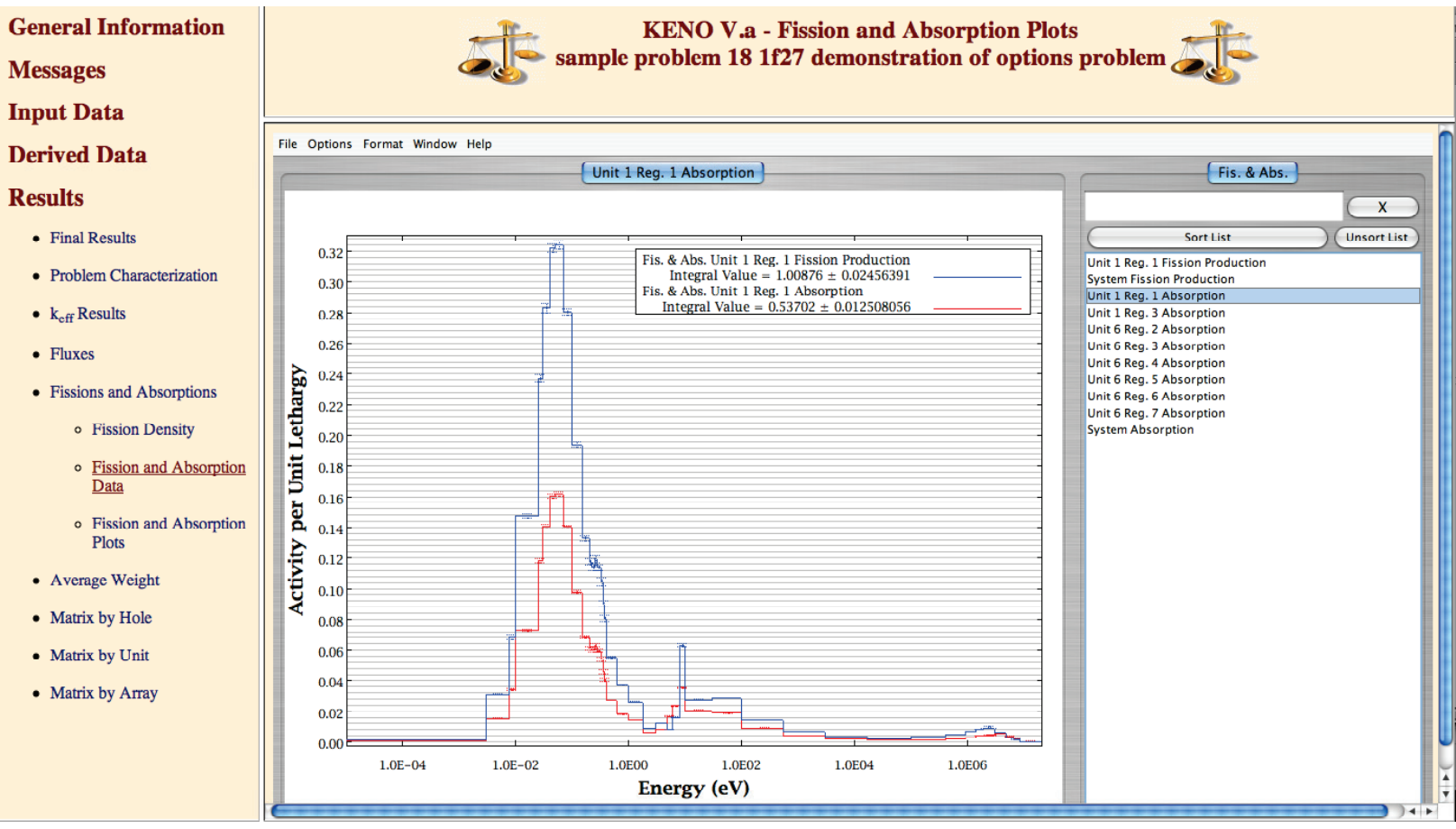

Fig. 8.1.182. Sample fissions and absorptions plot in HTML output.

\subsection{Matrix k-effective by position index}

The matrix k-effective by unit location (also referred to as array position or position index) is calculated if $\mathbf{M K P}=$ YES is specified in the parameter data and a global array is present in the model. An example of 
the matrix k-effective by position is given in Fig. 8.1.183. In the HTML output, the k-effective by position data is available in the Matrix by Position link in the Results section.

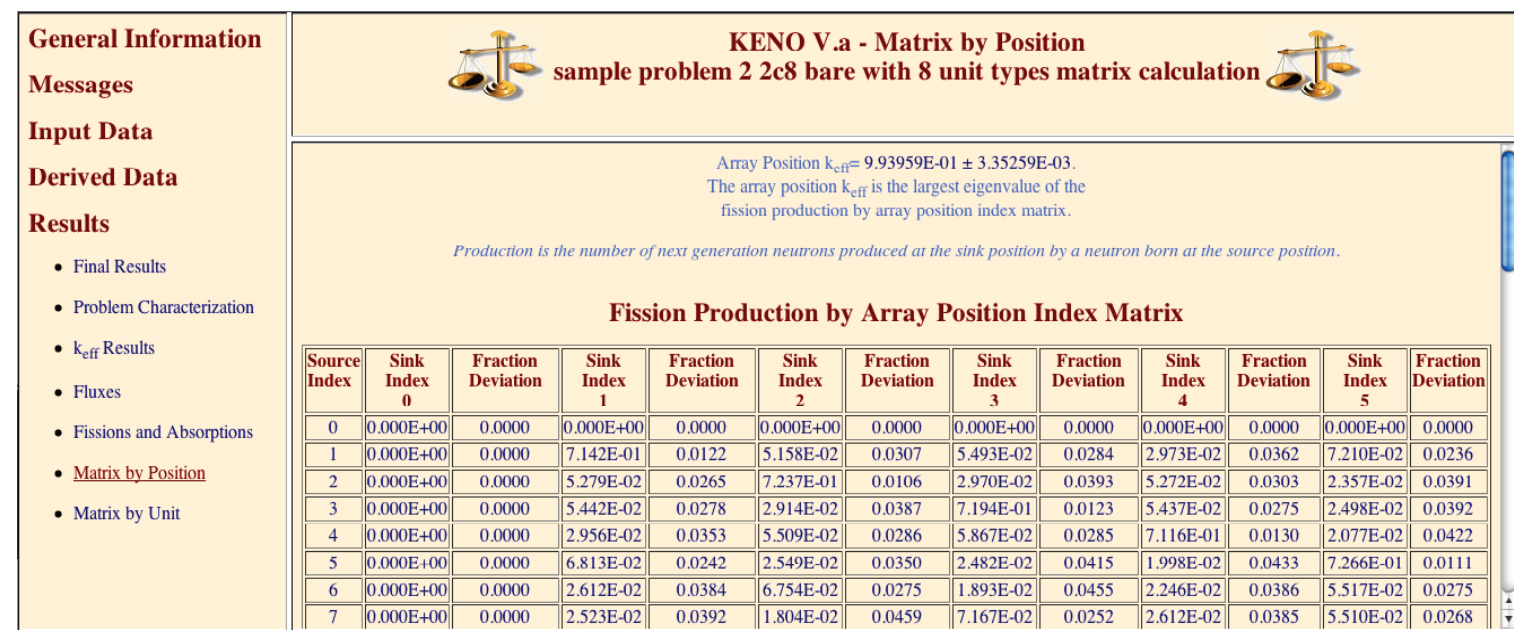

Fig. 8.1.183. Example of matrix k-effective by position index edit in HMTL output.

\subsection{Fission production by position index matrix}

To obtain fission production by position information, the user must specify MKP $=$ YES and $\mathbf{F M P}=$ YES in the parameter data. An example of the fission production matrix by position index for a $2 \times 2 \times 2$ array is shown in Fig. 8.1.184.

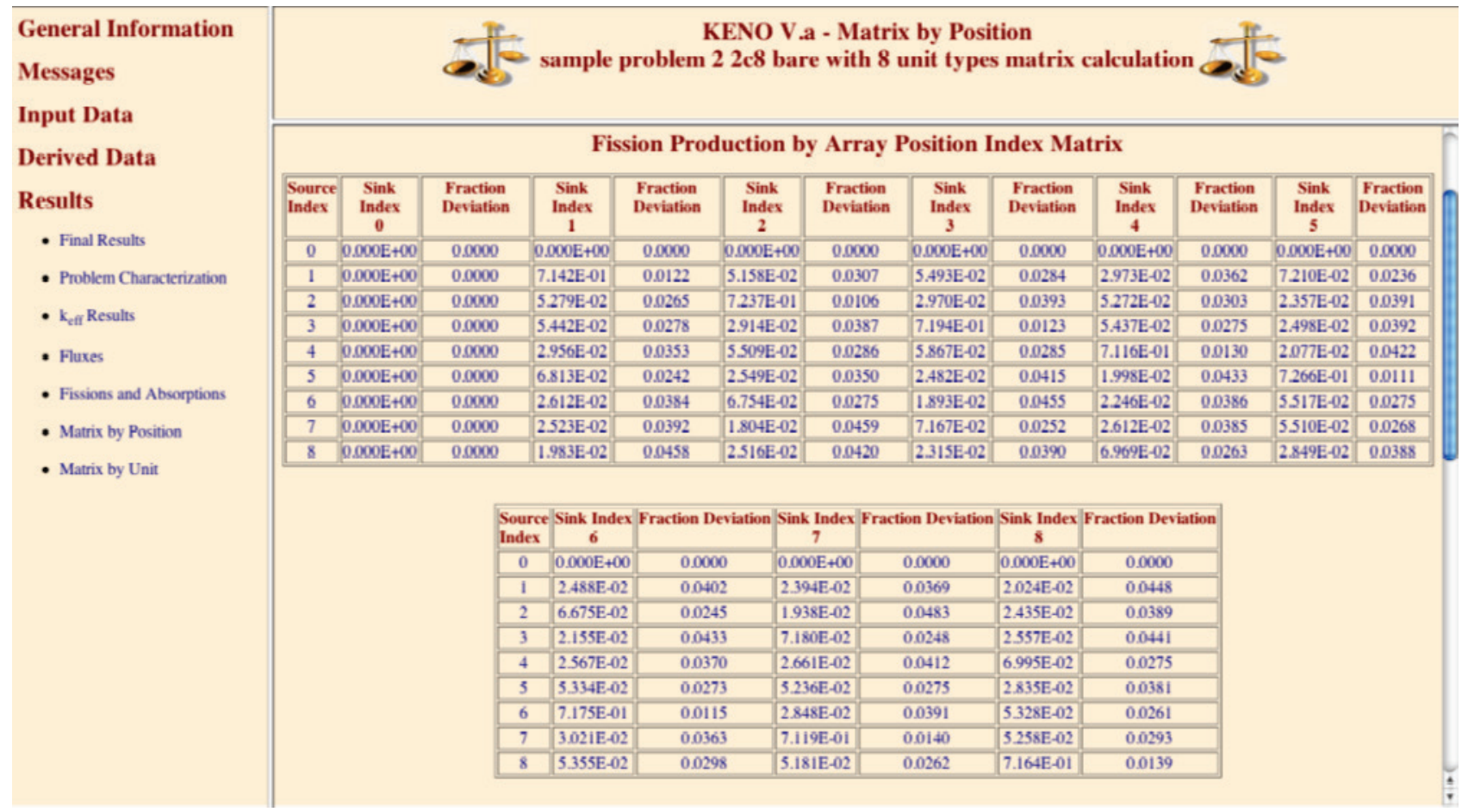

Fig. 8.1.184. Sample fission production matrix by position index edit in HMTL output. 
For each position index in the array, the number of next-generation neutrons produced at position index $\mathrm{J}$ per neutron born at position index I is determined. The fission production matrix by position index is used to determine the matrix k-effective, cofactor k-effective and source vector by position index.

\subsection{Source vector by position index}

Source vector by position index information is printed if MKP=YES is specified in the parameter data. An example of the source vector by position index is shown in Fig. 8.1.185. The average selfmultiplication by array position is the overall average of the self-multiplication of all units used in the problem.

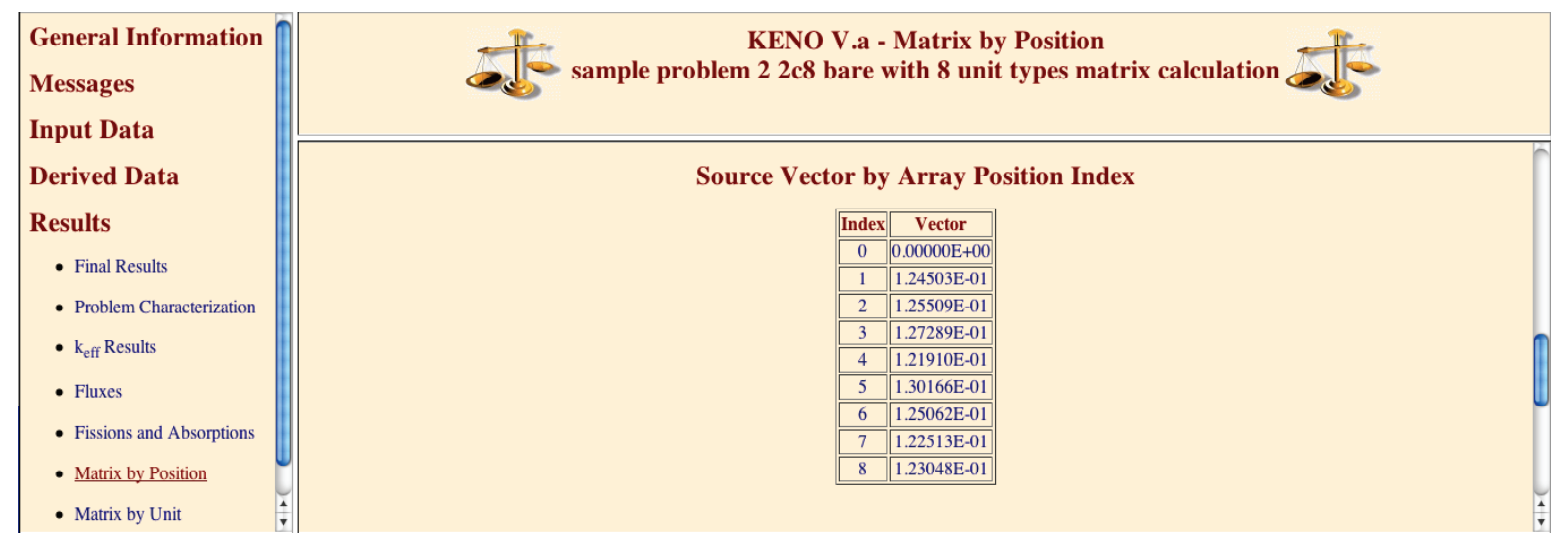

Fig. 8.1.185. Example of source vector by position index edit in HTML output.

\subsection{Cofactor k-effective by position index}

The cofactor k-effective by position index edit is printed if MKP=YES is specified in the parameter data. An example of the cofactor k-effective by position index is shown in Fig. 8.1.186 and an example of the source vector by position index is shown in Fig. 8.1.187.

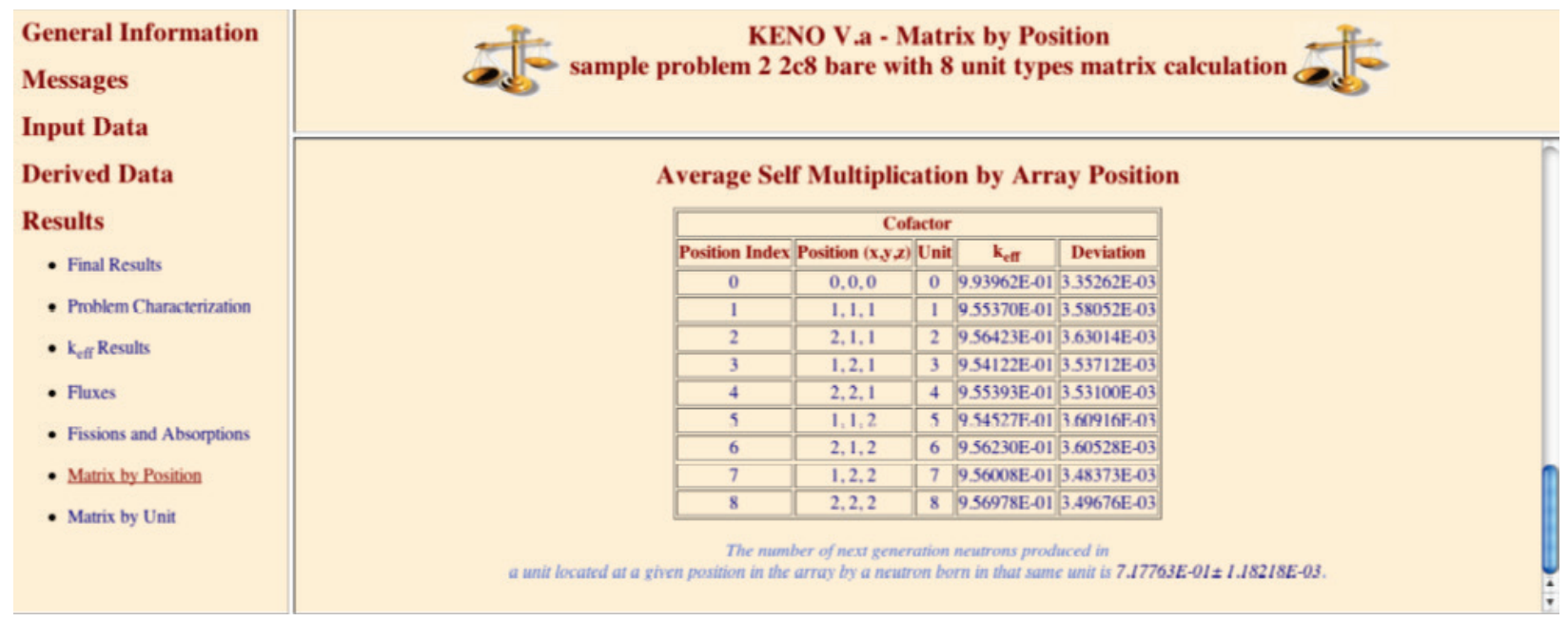

Fig. 8.1.186. Example cofactor k-effective by position index edit in HTML output. 


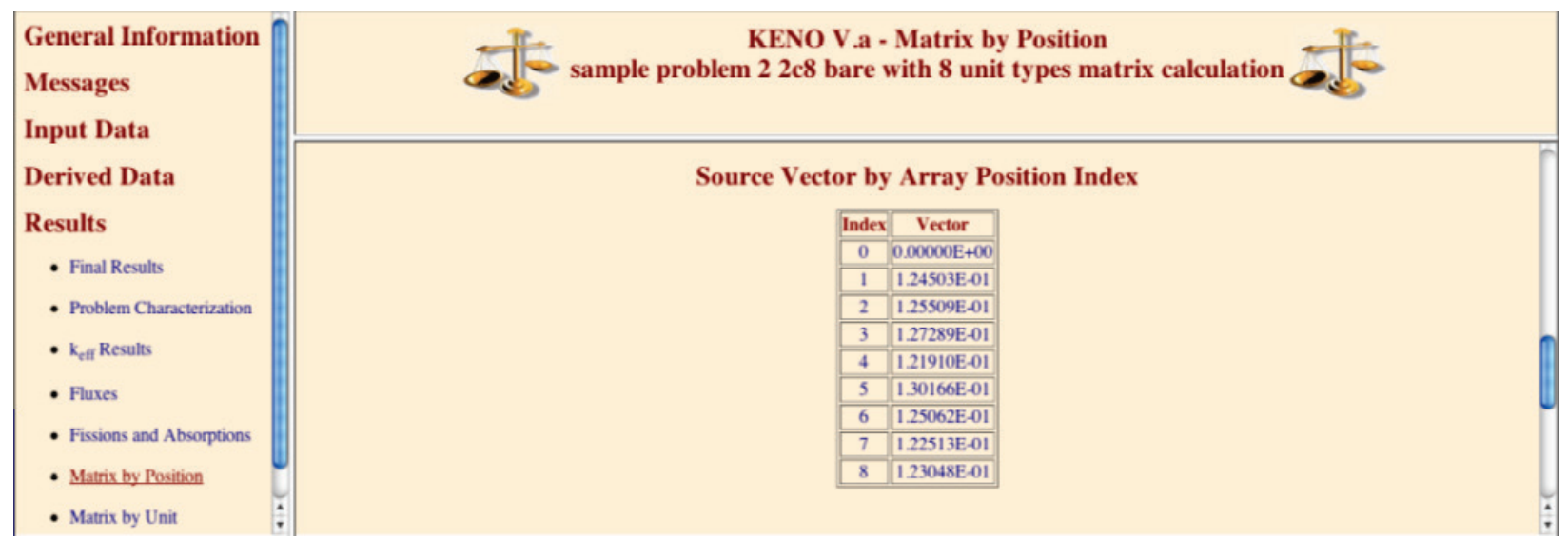

Fig. 8.1.187. Example of source vector by position index edit in HTML output.

\subsection{Matrix k-effective, fission production, source vector and cofactor $k$-effective by unit number}

The matrix k-effective by UNIT number (unit k-effective) is the largest eigenvalue of the fission production by unit matrix. It is calculated only if MKU=YES is specified in the parameter data. An example of the matrix k-effective by UNIT is given in Fig. 8.1.188. Fission production, source vector and average self-multiplication by UNIT are all printed on the same page as the matrix k-effective by UNIT. The matrix k-effective HTML data by unit is available in the Matrix by Unit link in the Results section. 


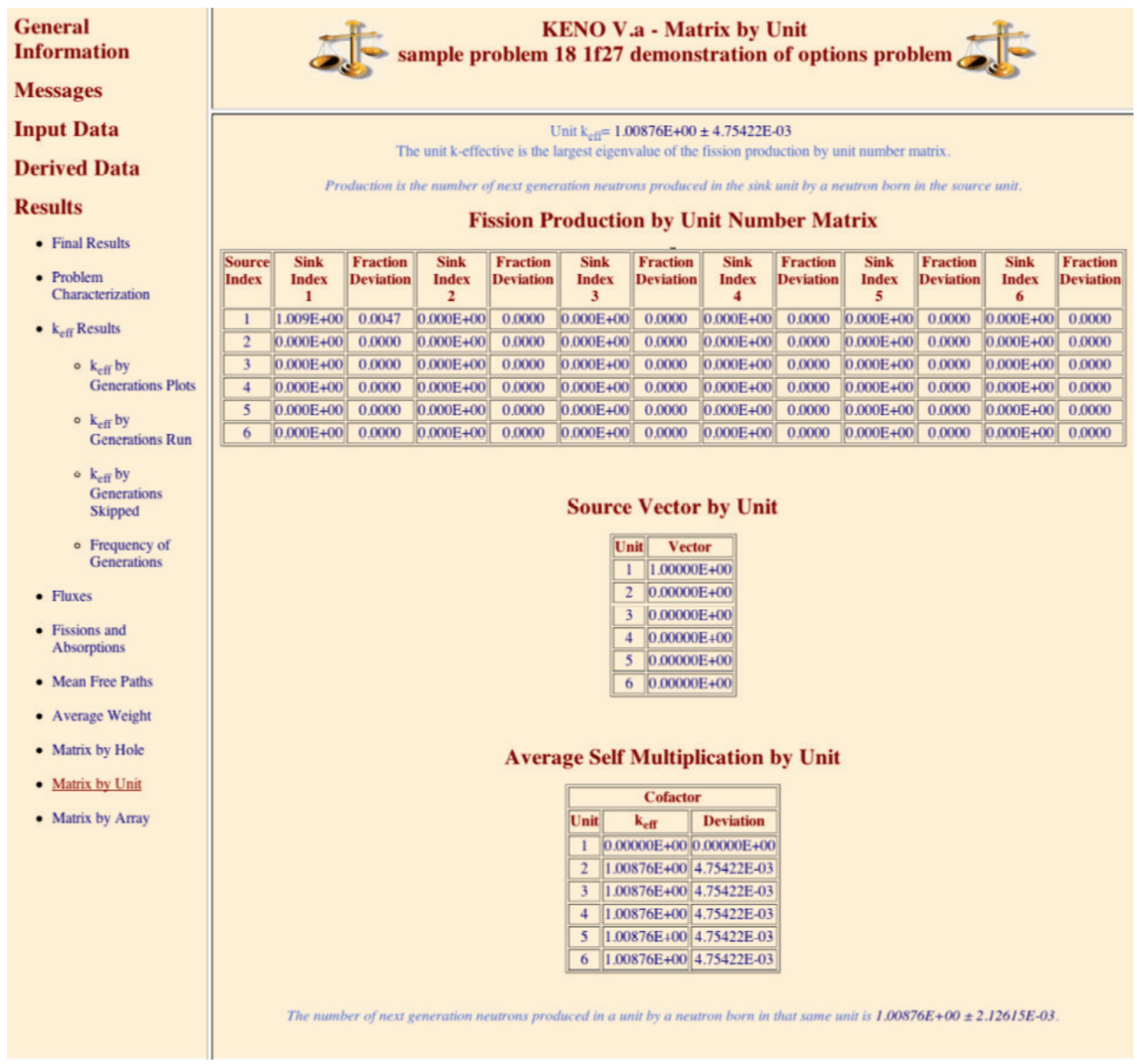

Fig. 8.1.188. Example matrix by unit edit in HTML output.

\subsection{Matrix k-effective, fission production, source vector and cofactor k-effective by hole number}

The matrix k-effective by HOLE number is the largest eigenvalue of the fission production matrix collected by HOLE number and is calculated if MKH=YES was specified in the parameter data. An example of the matrix k-effective by HOLE number is given in Fig. 8.1.189. Fission production, source vector and average self-multiplication by HOLE are all printed on the same page as the matrix k-effective by HOLE. The matrix k-effective HTML data by HOLE is available in the Matrix by Hole link in the Results section. 


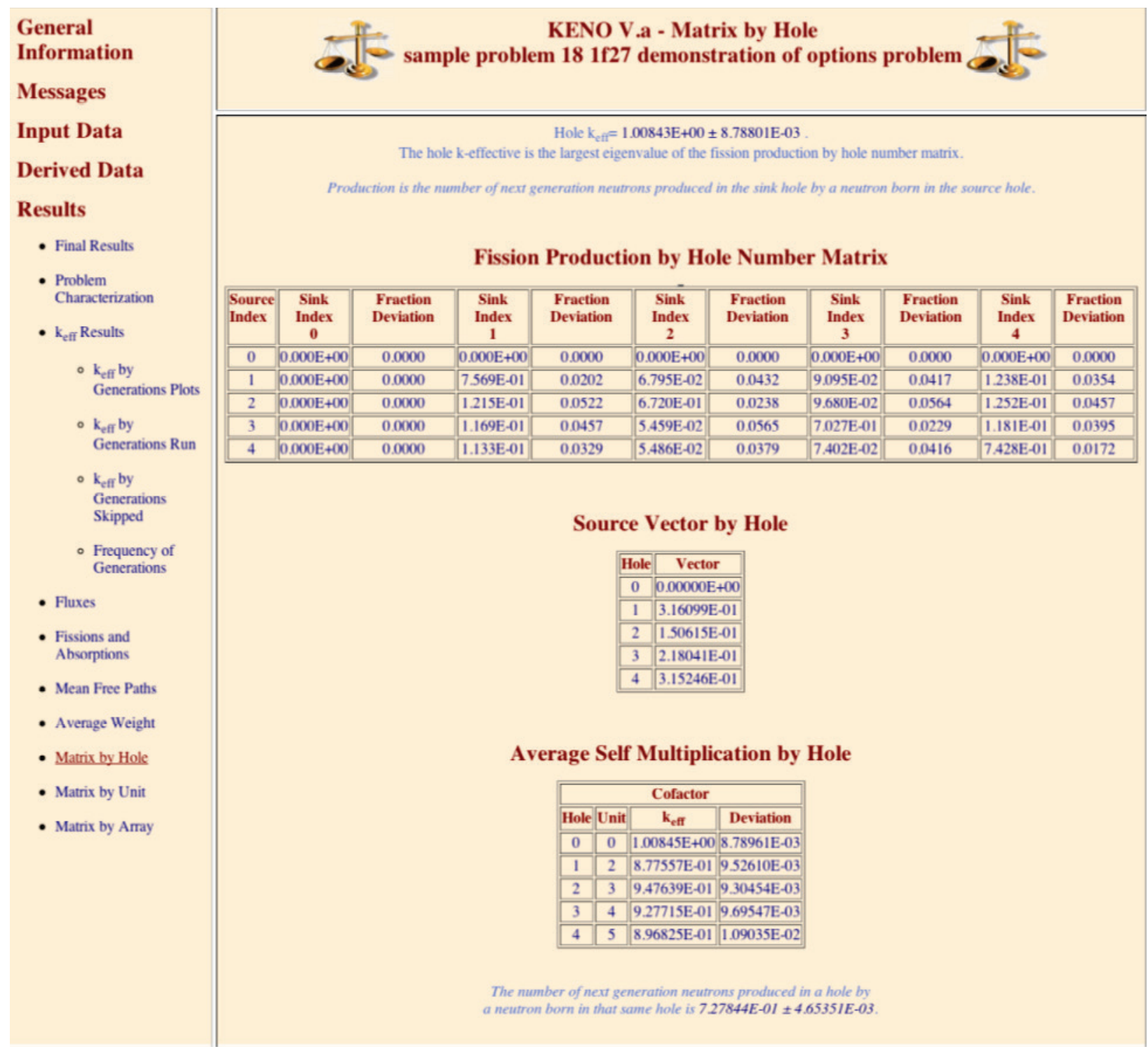

Fig. 8.1.189. Example matrix k-effective by hole edit in HTML output.

\subsection{Matrix k-effective, fission production, source vector and cofactor k-effective by array number}

The matrix k-effective by array number is the largest eigenvalue of the fission production matrix collected by array number and is calculated if MKA=YES is entered in the parameter data. The number of next generation neutrons produced in array number $J$ by a neutron born in array number $I$ is given in this fission production matrix.

The matrix k-effective HTML data by array is available in the Matrix by Array link in the Results section, with an example shown in Fig. 8.1.190. Fission production, source vector and average self-multiplication by ARRAY number are all printed on the same page as the matrix k-effective by ARRAY number. 


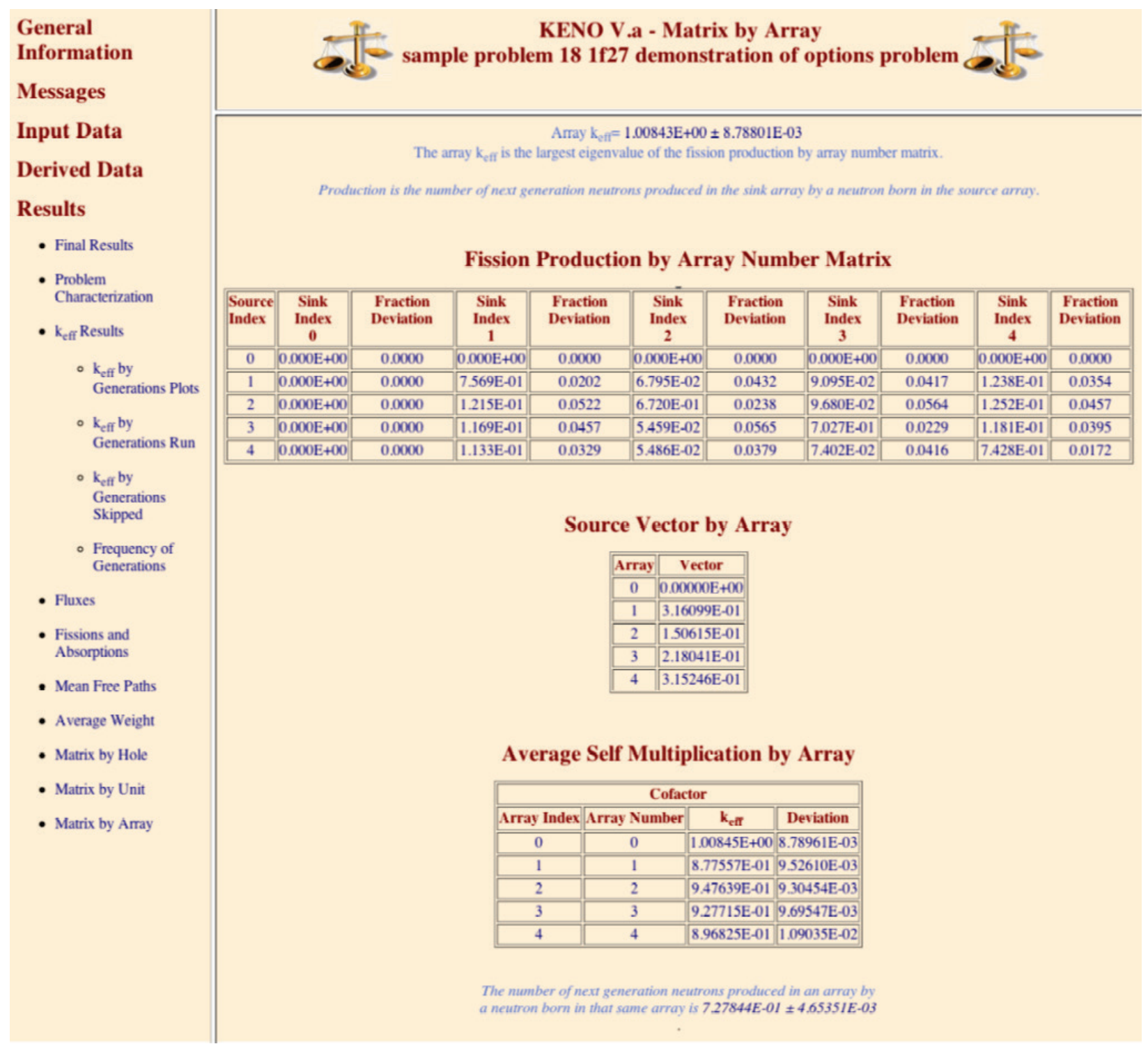

Fig. 8.1.190. Example matrix by array edit in HTML output.

\subsection{Fission density edit}

The fission density edit is optional. In the HTML output, the fission density edit is available in the Fission Density link of Fissions and Absorptions under the Results section, as shown in Fig. 8.1.191. 


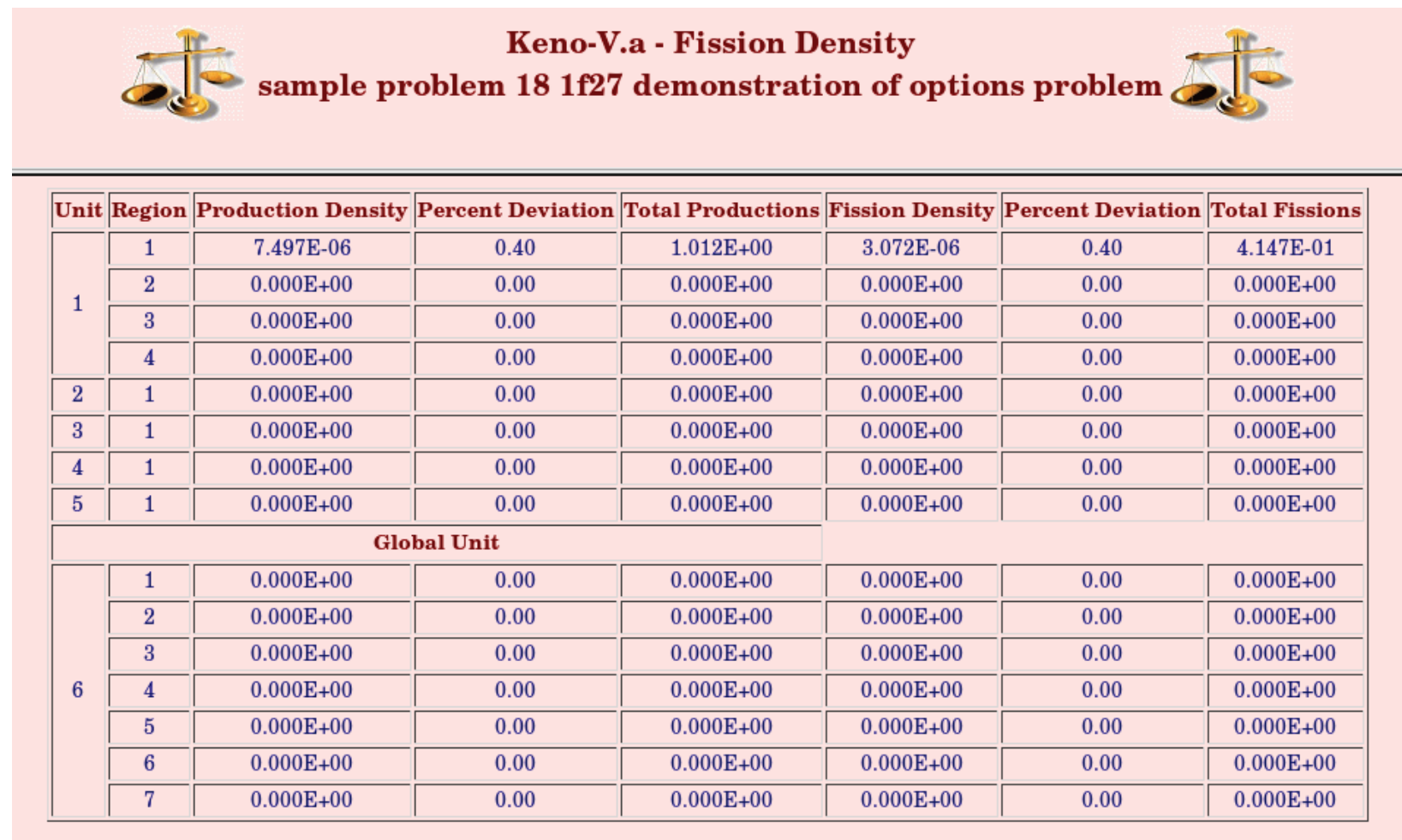

Fig. 8.1.191. Example fission density edit in HTML output.

\subsection{Flux edit}

Printing the fluxes is optional; they are only printed if FLX=YES is specified in the parameter data. The fluxes are printed for each unit and each geometry region in the unit for every energy group.

In the HTML output, a table of flux data can be accessed with the Scalar Fluxes under Fluxes in the Results section, as shown in Fig. 8.1.192. The fluxes for each unit in the problem are accessed from links on the right side of the page. Separate links are presented for Angular Fluxes and Flux Moments if they are requested in the problem. 


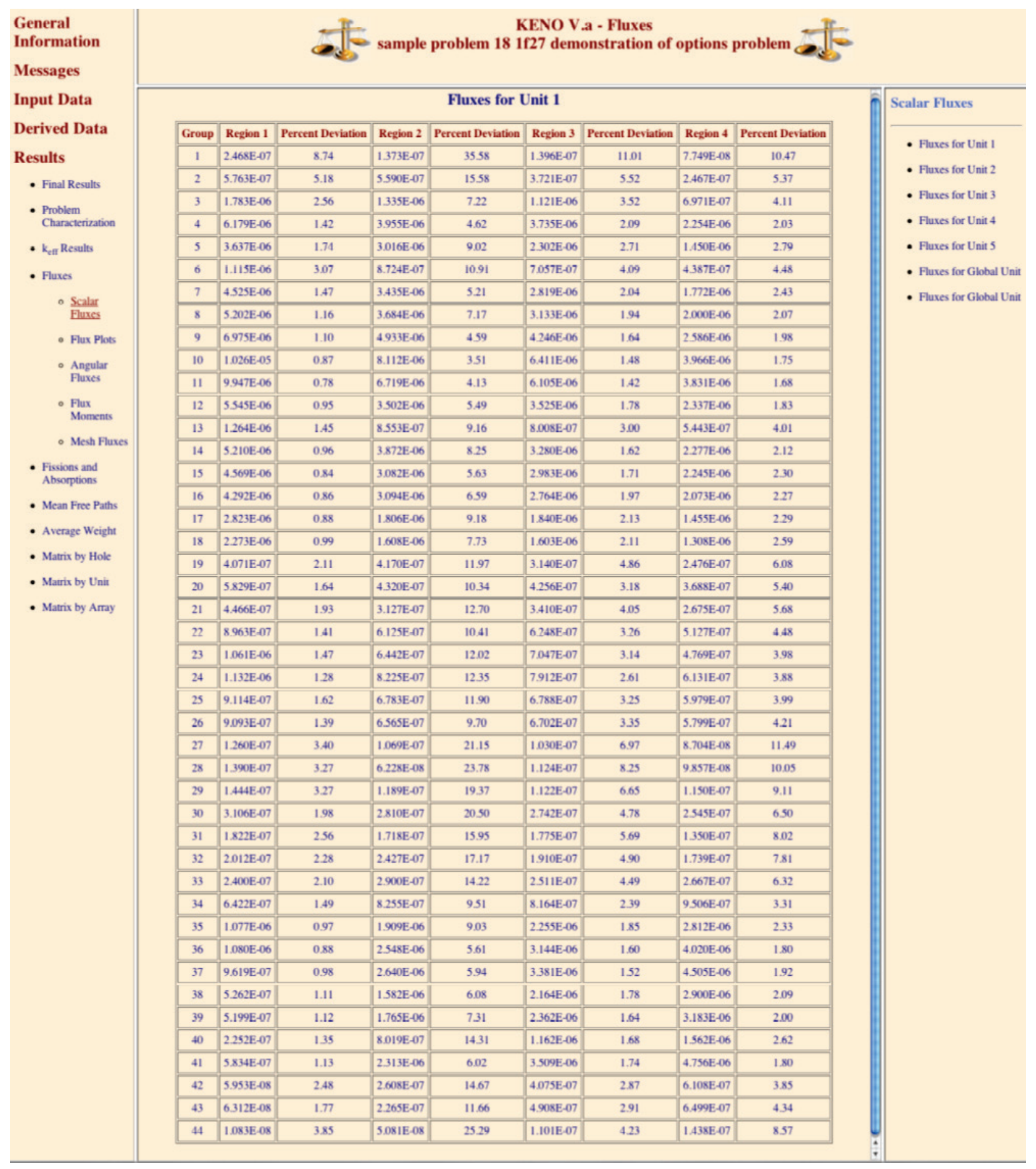

Fig. 8.1.192. Example scalar flux edit in HTML output.

\subsection{Flux plotting}

In the HTML output, the applet version of Javapeño is used for plotting flux data. Clicking on the Flux Plots link under Fluxes in the Results reveals a page with the Javapeño applet with the flux data file pre-loaded. Double-clicking on a data title on the right side of the plot window will cause the corresponding data to be plotted. An example of this page with some flux data plotted is shown in 
Fig. 8.1.193. Here, the scalar flux for each region of each unit is listed, followed by the flux moments. The flux moments are identified according to their spherical harmonics components, not the Legendre order. Moments 1-3 correspond to the three first order components of the expansion, moments 4-8 correspond to the second order components of the expansion and moments 9-15 correspond to the third order components of the expansion. For the example data shown in Fig. 8.1.193, only scalar fluxes $\left(0^{\text {th }}\right.$ order expansion $)$ and first order expansion terms were computed.

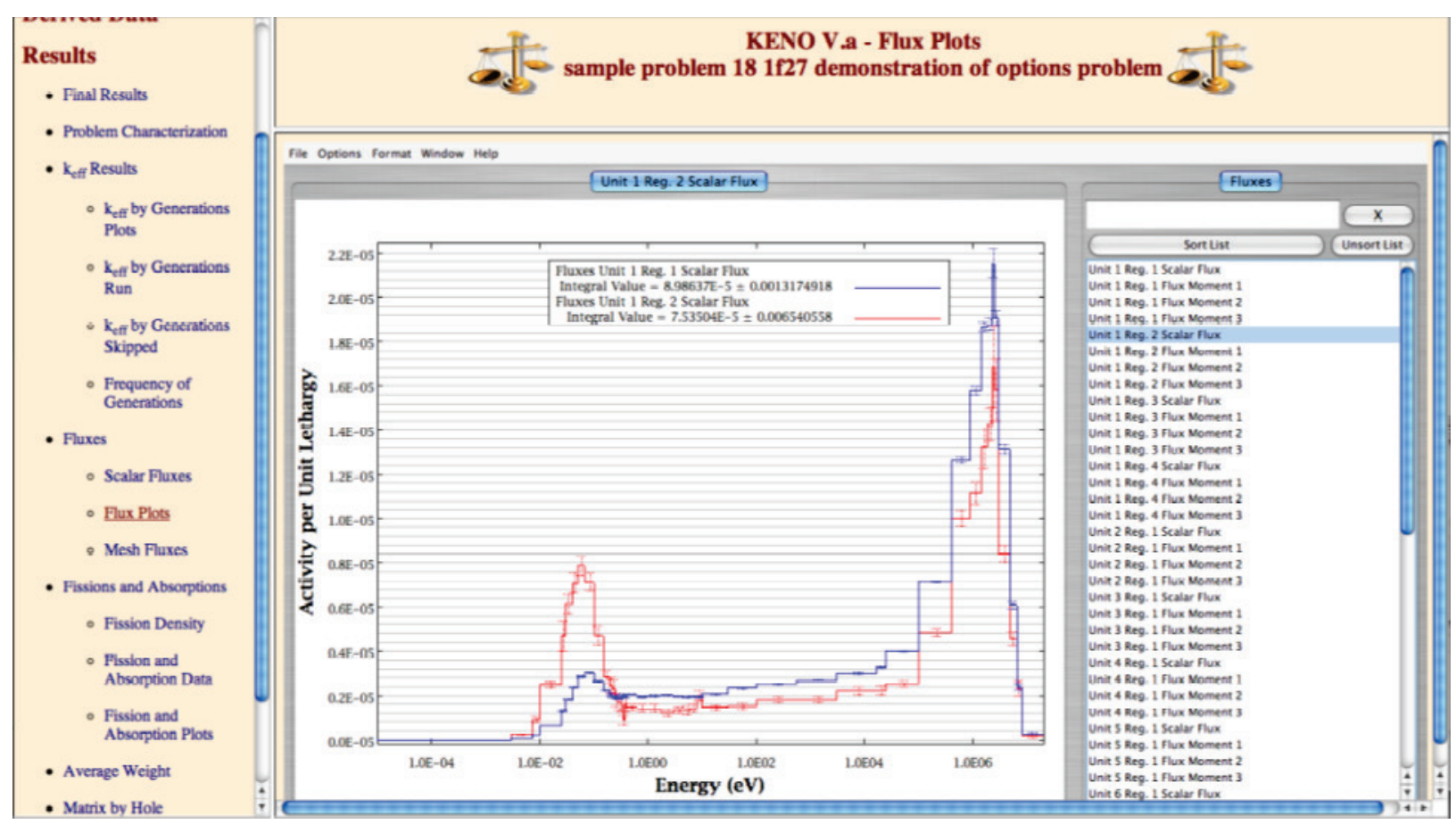

Fig. 8.1.193. Example flux plot in HTML output.

\subsection{Frequency distributions}

A frequency distribution consists of a bar graph indicating the number of generations having k-effective in a specified interval. The intervals are determined by the code based on the upper and lower limits of the k-effectives calculated for the generations. In the HTML output, the Javapeño applet is used to display the frequency distributions, as shown in Fig. 8.1.194. 


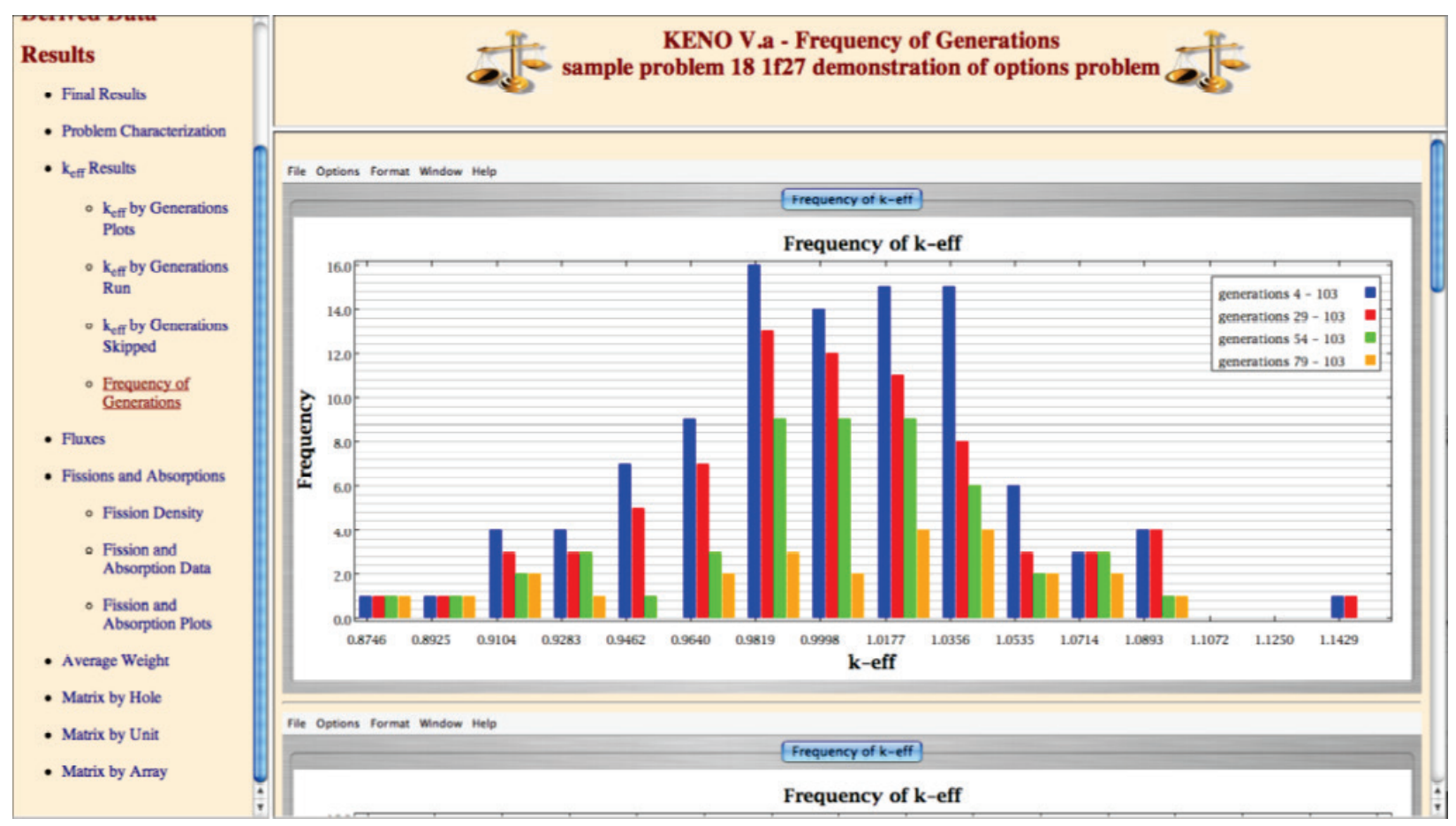

Fig. 8.1.194. Example frequency of generations plot in HTML output.

\subsection{Final results table}

The final results table contains a summary of the most important physics parameters of the system and the number of error and warning messages generated during execution.

In the HTML output, the final results table is accessed with the Final Results link in the Results section, as shown in Fig. 8.1.195.

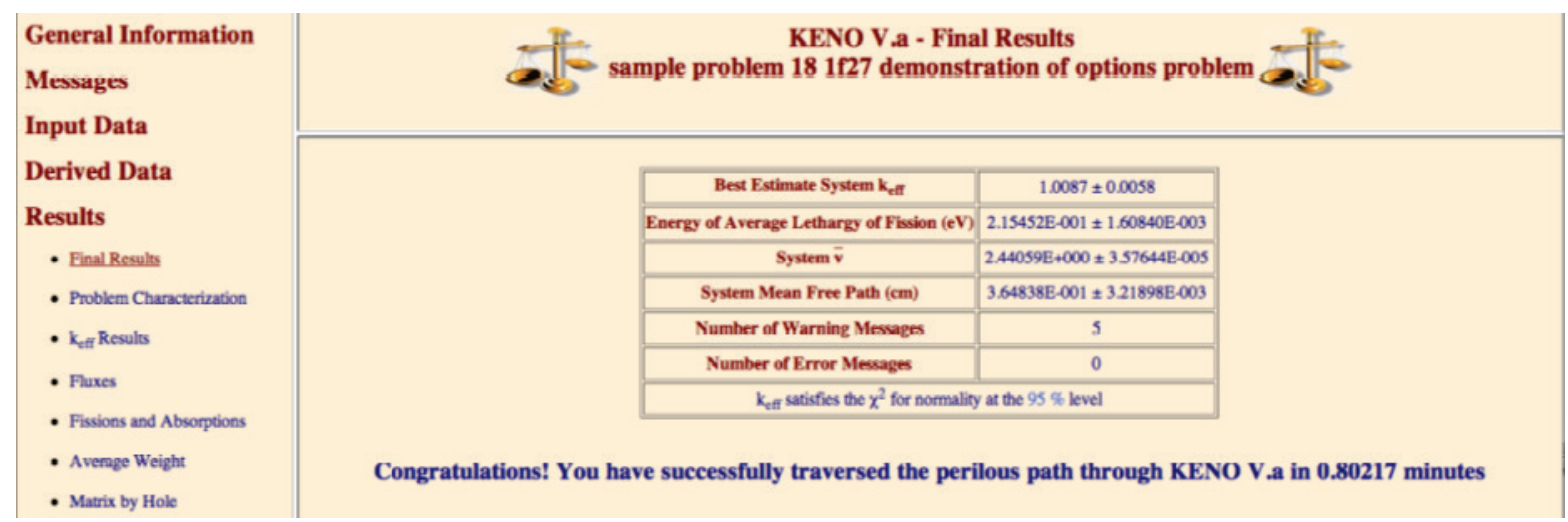

Fig. 8.1.195. An example of the final results table in HTML output. 


\subsubsection{Warning messages and error messages}

KENO prints warning and error messages that are identified by K5- or K6- for KENO V.a and KENO-VI, respectively, followed by a unique number (i.e., K5-001 is the identifier of the first message for KENO V.a). The generic symbol for messages originating in KENO V.a and KENO-VI is K?, where the question mark stands for 5 or 6 . For additional information concerning the message, refer to the identifier number in this section.

Warning messages appear when a possible error is encountered. If the code alters data, that fact is stated in the message. It is the responsibility of the user to verify correct usage when a warning message is printed.

When an error is encountered, the error flag MFLAG is set true and an error message is printed. The code stops if the error is too severe to continue. The warning and error messages in this section may show an underscore___ or a numbered underscore (1) where data will be printed by the code. The explanation of the message will show an underscore or a numbered underscore to indicate the corresponding data.

\subsubsection{Messages}

KMSG001

***** WARNING ***** K?-001 FOLLOWS:

READ FLAG NOT FOUND. ASSUME PARAMETER DATA FOLLOWS.

This message occurs in subroutine INITAL. It indicates that the word READ was not the first word of data encountered after the title card. If a parameter data block is to be entered, the code expects the words READ PARAMETERS to precede the parameter input data. If the word READ is not the first word, the code expects parameter input data immediately.

KMSG002

***** ERROR ***** K?-002 FOLLOWS:

THE NUMBER OF ENERGY GROUPS IS

OUT OF RANGE FOR THE CROSS SECTION LIBRARY ON UNIT THE INPUT MAY NOT SPECIFY A VALID DATA SET ON THIS UNIT OR THE MODULE THAT WAS TO CREATE THE CROSS SECTION LIBRARY ON THIS UNIT MAY HAVE FAILED

This message occurs in subroutine INITAL after subroutine PARAM has been executed. Unit number should be checked to ensure that it was properly specified in the job control language. The data set name associated with this unit number should also be verified. This information is given in the printout in the third table. The module that generated the cross sections should be verified as having executed properly and that the data were saved or passed correctly. When this message is printed for an AMPX working format library, a STOP 108 is executed. When this message is printed for a mixed cross section format library, a STOP 109 is executed.

KMSG003 
***** WARNING ***** K?-003 FOLLOWS:

MAT ID $=\quad$ WAS NOT FOUND IN THE WEIGHTS LIBRARY FOR THE SPECIFIED

ENERGY GROUP STRUCTURE.

This message is printed in subroutine LODWTS when loading biasing factors from the direct access scratch file. It indicates that weights library did not contain biasing factors for this ID for the group structure that corresponds to the cross sections (multigroup), or to the binning structure (continuous energy). Default biasing factors will be substituted. The location of this set of biasing parameters should be checked to verify that the substitution does not cause a problem.

KMSG004

***** ERROR ***** K?-004 FOLLOWS:

INVALID INPUT PARAMETER NAME

This message comes from subroutine PARAM and indicates that the keyword for entering parameter data was misspelled or in error in some other way. A list of allowed keywords is given in Table 8.1.1 in the KENO input outline.

KMSG005

***** ERROR ***** K?-005 FOLLOWS:

AN ERROR WAS ENCOUNTERED IN THE ALPHANUMERIC PARAMETER DATA. THE DATA

WAS

This message comes from subroutine PARAM and indicates that the keyword for the alphanumeric parameter data was entered correctly, but the data associated with it were not YES or NO as is required. The in the error message could be something like $\mathbf{F L X}=$ YEX instead of FLX=YES.

KMSG006

***** WARNING ***** K?-006 FOLLOWS:

READ FLAG FOUND WHEN LOOKING FOR END FLAG. PARAMETER INPUT ASSUMED COMPLETE

This message occurs in subroutine PARAM. It indicates that the keywords END PARAMETERS were not found. The keywords READ were found instead. The code assumes the parameter data are complete and proceeds normally.

KMSG007

***** ERROR ***** K?-007 FOLLOWS:

ATTEMPT TO FIND END PARAMETER FLAG WAS UNSUCCESSFUL.

This message from subroutine PARAM occurs during the reading of the parameter data if the word END is found and it is not followed by the word PARAMETERS. A STOP 118 may be executed when this message is printed.

KMSG008 
***** ERROR ***** K?-008 FOLLOWS:

AN END OF FILE WAS ENCOUNTERED WHILE ATTEMPTING TO READ PARAMETER DATA

This message is from subroutine PARAM. A STOP 118 may be executed when this message is printed.

KMSG009

***** WARNING ***** K?-009 FOLLOWS:

DUE TO INCONSISTENCIES BETWEEN INPUT AND RESTART DATA, FISSIONS AND ABSORPTIONS BY REGION WILL BE CALCULATED BUT NOT PRINTED. INPUT DATA SET FAR=NO, BUT DATA FROM THE RESTART UNIT SPECIFIED YES.

This message occurs in subroutine PARAM and is mostly self-explanatory. The original problem (parent case) that wrote the restart data specified data inconsistent with the parameter data input to the restarted problem. The title of the parent case is given at the end of the parameter tables. The specification of the restart unit RST is given in the third table of the KENO output.

KMSG010

***** WARNING ***** K?-010 FOLLOWS:

DUE TO INCONSISTENCIES BETWEEN INPUT AND RESTART DATA, FLUXES WILL BE

CALCULATED BUT NOT PRINTED.

INPUT DATA SET FLX=NO, BUT DATA FROM THE RESTART UNIT SPECIFIED YES.

This message occurs in subroutine PARAM. The original problem (parent case) that wrote the restart data specified data did not agree with the parameter data input to the restarted problem. The title of the parent case is given at the end of the parameter tables. The specification of the restart unit RST is given in the third table of the KENO output.

KMSG011

***** ERROR ***** K6-011 FOLLOWS:

A BOUNDARY GEOMETRY WORD WAS NOT SPECIFIED FOR UNIT

This KENO-VI message is from subroutine KENOG. A BOUNDARY card must be associated with each unit identifying the outermost region of the unit.

\section{KMSG012}

***** WARNING ***** K?-012 FOLLOWS:

INPUT PARAMETER NBK WAS ENTERED AS _ _ IT WAS CHANGED TO

AT LEAST _ POSITIONS ARE NECESSARY TO ACCOMMODATE THE NEUTRON BANK DATA.

This self-explanatory message is from subroutine PARAM. NBK should not be entered as input data unless it is known that the default value is inadequate. 
KMSG013

$* * * * *$ ERROR ***** K6-013 FOLLOWS:

MEDIA DATA MUST BE ENTERED FOR UNIT

This KENO-VI message is from subroutine KENOG. Either another UNIT card or an END GEOM card was encountered prior to inputting MEDIA data for the current unit.

KMSG014

***** ERROR ***** K?-014 FOLLOWS:

ERROR - KEYWORD IS NOT A VALID MIXING TABLE KEYWORD.

This message is from subroutine MIXIT. It can only be encountered if a mixing table is expected (i.e., READ MIX or READ MIXT has been entered as data). At this point the only valid keywords are $\mathbf{M I X}=, \mathbf{E P S}=, \mathbf{S C T}=$ or $\mathbf{N C M}=$. The keyword that was entered is printed in the message. See Sect. 8.1.2.10 for assistance in setting up the mixing table data.

KMSG015

***** ERROR ***** K5-015 FOLLOWS:

AN ERROR WAS FOUND IN THE HEMISPHERE DESIGNATION

This KENO V.a message was printed by subroutine KENOG. It signifies that the geometry keyword has been destroyed after it was read. This indicates a code bug.

\section{KMSG016}

$* * * * *$ ERROR $* * * * * \mathrm{~K}$ ?-016 FOLLOWS:

$* * *$ ERROR $* * *$ A VALUE MUST BE ENTERED FOR LIB IN THE PARAMETER INPUT SO CROSS SECTIONS CAN BE MIXED.

This message is from subroutine DATAIN. It occurs when a mixing table has been read but the unit number for the AMPXS working library is undefined. This is corrected by entering $\mathbf{L I B}=$ in the parameter input data and making sure the desired AMPXS working library is properly defined as being on that unit in the job control language.

KMSG017

***** ERROR ***** K?-017 FOLLOWS:

UNRECOGNIZABLE GEOMETRY WORD

This message is from subroutine KENOG. In the process of reading the geometry data, the word was encountered when a geometry word was expected. Several of these messages may be generated. A message is generated for each word of data that is read, until a valid geometry word is found. The data are out of phase or the geometry word is misspelled. The previous geometry word should be checked to ensure that it has the correct entries. See Appendices A and B for lists of accepted geometry words.

KMSG018 
$* * * * *$ ERROR ***** K?-018 FOLLOWS:

INVALID INDEX INTO KEYWRD ARRAY. INP=

CHECK SUBROUTINE KENOG FOR A CODE ERROR.

This message is from subroutine KENOG. This message usually means that a code error was introduced when changes were made to the program. Subroutine KENOG, function LCOMPR, and KEYWRD data block should be checked.

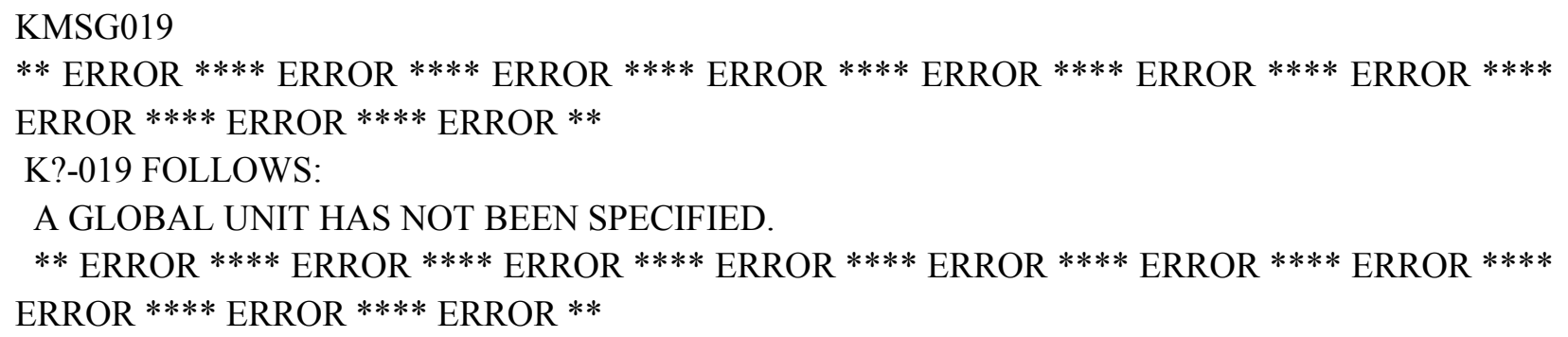

This message from subroutine KENOG indicates that a GLOBAL UNIT has not been specified. The outermost unit in the problem must be specified as the GLOBAL UNIT. See Sect. 8.1.2.4 to determine the correct specification.

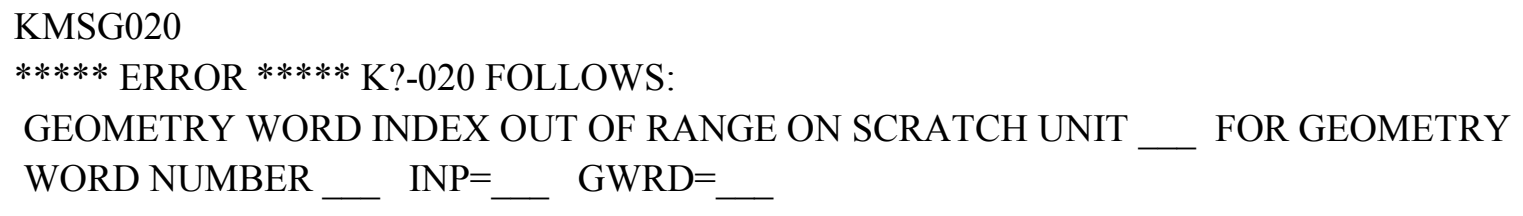

This message from subroutine READGM means that INP is negative or larger than NP when the geometry data are read from the scratch unit, SKRT. This usually means that a code error was introduced when changes were made to the program. True geometry errors should be detected when the scratch unit, SKRT, is written. A STOP 125 is executed when this message is printed.

KMSG021

$* * * * *$ ERROR ***** K?-021 FOLLOWS: IS AN INVALID PARAMETER NAME FOR BIASING DATA.

$\mathrm{ID}=\mathrm{OR} \mathrm{WT}=\mathrm{OR}$ WTS $=$ SHOULD HAVE BEEN ENTERED.

This message is from subroutine RDBIAS. See Sect. 8.1.2.7 for assistance in determining the proper procedure for entering biasing data.

\section{KMSG022}

***** ERROR ***** K?-022 FOLLOWS:

PROBLEM IN READGM, IGEO = IS AN INVALID GEOMETRY TYPE

If this message has not been preceded by other geometry error messages, it indicates a code bug. 
KMSG023

***** ERROR ***** K?-023 FOLLOWS:

REFLECTION OR PERIODIC BOUNDARY CONDITIONS ARE ILLEGAL ON A CURVED BOUNDARY.

Only a vacuum or a white boundary condition is allowed on a curvilinear surface.

KMSG024

K?-024 FOLLOWS:

INCORRECT FLAG RETURNED FROM AREAD. IRET=

This message from subroutine RDBIAS indicates that an error was encountered while reading the biasing data. The biasing data were not entered properly. See Sect. 8.1.2.7 for assistance.

\section{KMSG025}

***** ERROR ***** K?-025 FOLLOWS:

WHITE BOUNDARY CONDITION IS ALLOWED ONLY ON CUBOIDS, CYLINDERS, OR SPHERES

This self-explanatory message should never occur and is an indication of a code bug.

KMSG026

K?-026 - ARRAY DESCRIPTION ERROR MESSAGES

K?-026 FOLLOWS:

SET NUMBER __ OF THE UNIT ORIENTATION DATA CONTAINS _ _ ERROR(S).

This message from subroutine $\mathrm{RDBOX}$ is triggered when input errors are recognized in the unit orientation data. A set of unit orientation data consists of 10 numbers as shown in the companion message KMSG027. The number of errors printed in this message is a lower bound. More errors may actually exist. This message often means that a number was omitted or a blank was omitted when entering the unit orientation data.

KMSG027

K?-027 FOLLOWS:

$\mathrm{LTYPE}=\ldots \mathrm{IX} 1=\ldots \mathrm{IX} 2=\ldots \mathrm{INCX}=\ldots \mathrm{IY} 1=\ldots \mathrm{IY} 2=\ldots \mathrm{INCY}=\ldots \mathrm{IZ} 1=\ldots \mathrm{IZ2}=\ldots \mathrm{INCZ}=$ This message is a companion to KMSG026. It indicates how the unit orientation data description for the set named in KMSG026 was entered. See Sect. 9.1.2.5.2 for information pertaining to unit orientation data.

\section{KMSG028}

K?-028 FOLLOWS:

THE ABOVE UNIT ORIENTATION CARD(S) CONTAIN(S) AT LEAST ONE 
OF THE FOLLOWING ERRORS.

1. IX1,IY1,IZ1,INCX,INCY,OR INCZ IS LESS THAN OR EQUAL TO ZERO

2. IX2 IS LESS THAN IX1,IY2 IS LESS THAN IY1, OR IZ2 IS LESS THAN IZ1

3. IX2 IS GREATER THAN NBXMAX, IY2 IS LARGER THAN NBYMAX, OR IZ2 IS LARGER THAN NBZMAX

\section{LTYPE IS LESS THAN 1 OR GREATER THAN NBOX}

This self-explanatory message is from subroutine RDBOX. It pertains to the input orientation data for LOOP. See Sect. 8.1.2.5 for input instructions.

KMSG029

***** ERROR ***** K?-029 FOLLOWS:

THE ARRAY SIZE HAS BEEN SPECIFIED INCORRECTLY. NBXMAX= NBYMAX $=$ NBZMAX $=$

UNIT ORIENTATION DATA CANNOT BE READ UNLESS NBXMAX, NBYMAX AND NBZMAX ARE GREATER THAN ZERO.

This message from subroutine ARAYIN indicates that the array definition data were incorrectly specified. It occurs only if one or more of NBXMAX, NBYMAX, or NBZMAX is less than 1. In the array information data these are entered in the form $\mathbf{N U X}=$ NUY= $\mathbf{N U Z}=$ See Sect. 8.1.2.5. If

a unit orientation data description is to be entered, NBXMAX, NBYMAX, and NBZMAX must all be greater than zero.

KMSG030

$* * * * *$ ERROR ***** K?-030 FOLLOWS:

END _ _ FLAG WAS NOT FOUND. WAS READ INSTEAD.

This message from subroutine ARAYIN occurs if the unit orientation data description is terminated with the incorrect END flag.

KMSG031

***** ERROR ***** K?-031 FOLLOWS: IS AN INVALID PARAMETER NAME IN THE ARRAY DATA.

This message is written from subroutine ARAYIN if the array data block contains an incorrect keyword. The allowed keywords include $\mathbf{N U X}=$, NUY $=, \mathbf{N U Z}=$, FILL, LOOP, ARA=, TYP=. See Sect. 8.1.2.5 for additional assistance. A STOP 101 is executed when this message is printed.

KMSG032

***** ERROR ***** K?-032 FOLLOWS:

AN ERROR EXISTS IN UNIT ORIENTATION ARRAY NUMBER 
This message from subroutine SORTA is printed when an error is recognized in the array description. The type of error that will trigger the message is for a position in the unit orientation array to be undefined, zero, negative or greater than NBOX, the largest unit number. KMSG033 is a companion message.

KMSG033

$* * * * *$ ERROR ***** K?-033 FOLLOWS:

UNIT _ IS INVALID AT X INDEX $=\_$Y INDEX $=\_Z$ Z INDEX $=$

This message comes from subroutine SORTA. It is printed for each position in the unit orientation array that is in error. The message is printed a maximum of 10 times. Refer to Sect. 8.1.2.5 for assistance in correcting the error(s).

KMSG034

***** ERROR ***** K?-034 FOLLOWS:

THE NUMBER OF MIXTURES REQUESTED IN THE GEOMETRY IS MIXTURE CROSS SECTIONS IS THE NUMBER OF

This message from subroutine FLDATA occurs if the number of mixture cross sections from the restart unit, RSTRT, does not equal the number of mixtures requested in the geometry for a restarted problem.

\section{KMSG035}

$* * * * *$ ERROR ***** K?-035 FOLLOWS:

IN THE ALBEDO INPUT DATA IS AN INVALID FACE CODE NAME.

This message is from subroutine RDREF. It occurs if an invalid face code name was entered in the albedo data. See Table 8.1.18 in Sect. 8.1.2.6 for a list of acceptable face code names.

KMSG036

$* * * * *$ ERROR $* * * * * \mathrm{~K}$ ?-036 FOLLOWS:

A BOUNDARY BODY_ W WAS SPECIFIED THAT IS NOT

ONE OF THE BODIES IN THE GLOBAL UNIT BOUNDARY.

THE PROBLEM WILL NOT BE RUN.

** ERROR $* * * *$ ERROR $* * * *$ ERROR $* * * *$ ERROR $* * * *$ ERROR $* * * *$ ERROR $* * * *$ ERROR $* * * *$ $\operatorname{ERROR} * * * * \operatorname{ERROR} * * * * \operatorname{ERROR} * *$

This message is from subroutine RDBNDS. It indicates that a boundary body was specified to assign boundary conditions that do not exist in the global unit. The bodies specified for boundary conditions should be carefully matched with those specified in the bounds of the global unit.

KMSG037

***** ERROR ***** K?-037 FOLLOWS:

AVERAGE NU-BAR AND AVG. FISSION GROUP WAS SPECIFIED, BUT THE FISSION XSEC ID (18)

WAS NOT FOUND IN THE EXTRA 1-D ARRAY (MT). 
This message is from subroutine IDX1D. It indicates that the parameter data contained NUB=YES, but the corresponding necessary type of data was absent from the extra 1-D array. This can be due to a code error or an error concerning the extra 1-D data $(\mathbf{X 1 D}=$ in the parameter data).

KMSG038

$* * * * *$ ERROR $* * * * * \mathrm{~K}$ ?-038 FOLLOWS:

INPUT DATA INDICATED NO EXTRA 1-D XSEC IDS TO BE READ, BUT A READ FLAG WAS ENCOUNTERED.

This message from subroutine DATAIN is printed when the parameter data did not specify $\mathbf{X 1 D}=$ and the words READ X1DS were encountered later in the data. If extra 1-D data are to be used, X1D= must be entered in the parameter data and appropriate code modifications must be made to properly use the extra 1-D data.

KMSG039

***** ERROR ***** K?-039 FOLLOWS:

INVALID START PARAMETER NAME

This message is from subroutine RDSTRT. It indicates that an invalid start parameter name was encountered when the start data block was being read. A list of allowed start parameter names is contained in Sect. 8.1.2.8.

KMSG040

***** WARNING ***** K?-040 FOLLOWS:

LNU FOR START TYPE 6 WAS ENTERED AS

THE LARGEST VALUE NEEDED FOR LNU IS NPG; THE LARGEST VALUE ALLOWED FOR

LNU IS NFB ( $\left({ }_{\text {) }}\right.$.

BOTH NPG AND NFB ARE PARAMETER DATA.

ENTRIES BEYOND NFB WILL BE IGNORED.

This self-explanatory message is from subroutine RDSTRT. See Sect. 8.1.2.8 for assistance in determining a valid value for LNU. NFB, the fission bank size, is the largest value allowed for LNU. NPG, the number of histories per generation, is the smallest value allowed for LNU.

\section{KMSG041}

***** ERROR ***** K?-041 FOLLOWS:

ALPHANUMERIC START DATA MUST BE ENTERED AS YES OR NO. THE DATA READ WAS

This self-explanatory message is from subroutine RDSTRT. See Sect. 8.1.2.8 for assistance concerning start data.

KMSG042 
***** WARNING ***** K?-042 FOLLOWS:

END

FLAG WAS NOT FOUND.

WAS READ INSTEAD

This message occurs when the READ and END each block must start with READ and end with END do not match. When entering data blocks,

KMSG043

***** ERROR ***** K?-043 FOLLOWS:

AN END OF FILE WAS ENCOUNTERED BEFORE AN END DATA WAS FOUND. THE PROBLEM WILL NOT RUN.

This message is from subroutine DATAIN. It occurs when an end of file is encountered while reading data.

\section{KMSG044}

***** ERROR ***** K?-044 FOLLOWS:

A PLOT OF ZERO HEIGHT AND/OR WIDTH HAS BEEN SPECIFIED.

THE HEIGHT IS AND THE WIDTH IS

THE DELTA X IS _ _ THE DELTA Y IS AND THE DELTA Z IS

THE DIRECTION COSINES DOWN THE PAGE ARE

THE DIRECTION COSINES ACROSS THE PAGE ARE

This message is from subroutine RDPLOT. At least one of the $\mathrm{X}$, Y, or Z components that make up the upper left corner and lower right corner is inconsistent or one of the direction cosines UAX, VAX, WAX, UDN, VDN, or WDN have the wrong sign. Check the plot data for consistency.

\section{KMSG045}

***** ERROR ***** K?-045 FOLLOWS:

ILLEGAL DATA BLOCK IDENTIFIER,

This message from subroutine DATAIN is printed whenever an invalid data block identifier is encountered. This can be caused by having the data out of order, by omitting data or by misspelling data. A block identifier consists of the words READ XXXX where XXXX is a keyword identifying the type of data to be read. Acceptable keywords are listed in Sect. 8.1.2.1.

KMSG046

$* * * * *$ ERROR ***** K?-046 FOLLOWS:

$\mathrm{IPT}=$ IS OUTSIDE THE ALLOWABLE LIMIT OF

This message is from subroutine DATAIN. It is indicative of a code error. IPT is the index into the LPOINT array, which contains the direct access pointers for the various types of data.

LPOINT(1) is the pointer for the geometry region data.

LPOINT(2) is the pointer for the array description (unit orientation) data.

LPOINT(3) is the pointer for the mixing table data. 
LPOINT(4) is the pointer for volume data.

LPOINT(5) is the pointer for the biasing or weighting data.

LPOINT(6) is the pointer for the start data.

LPOINT(7) is the pointer for the albedo data.

LPOINT(8) is the pointer for the mixed cross section data.

LPOINT(9) is the pointer for the energy and inverse velocity data.

LPOINT(10) is the pointer for the plot data.

LPOINT(11) is the pointer for the biasing input data.

LPOINT(12) is the pointer for the grid data for fluxes.

LPOINT(13) is the pointer for importance mapping data.

LPOINT(14) is the pointer for reactions data.

LPOINT(15) is the pointer for the albedo-xsec energy correspondence data.

A STOP 152 is executed when this message is printed.

\section{KMSG047}

***** ERROR ***** K6-047 FOLLOWS:

SECTOR DATA WAS NOT FOUND FOR ARRAY

This KENO-VI message is from subroutine ARRAY. This message usually means that sector data was left off the ARRAY contents record. See Sect. 8.1.2.4 for assistance in specifying an ARRAY.

KMSG048

$* * * * *$ ERROR ***** K?-048 FOLLOWS:

UNABLE TO ALLOCATE DUE TO ERROR STATUS CODE IN SUBROUTINE

This message is associated with allocating memory used to store problem dependent data. The message states the type of data memory was allocated for the error type returned when memory could not be allocated and the current subroutine. This message could be caused by insufficient system memory or a code problem.

KMSG049

***** ERROR ***** K?-049 FOLLOWS:

WEIGHTS ARE THE WRONG LENGTH

EXPECTED LENGTH BUT NEED LENGTH

\section{KMSG050}

***** ERROR ***** K?-050 FOLLOWS:

THE _ POSITION OF ARRAY__ IN UNIT _ W WAS SPECIFIED AS _ WHICH IS LESS THAN 1 OR GREATER THAN _ _, THE NUMBER OF UNITS IN THAT DIRECTION.

This self-explanatory message from subroutine RDICE indicates that more space is needed to store the macroscopic cross sections in Monte Carlo format. A STOP 122 is executed when this message is printed. 
KMSG051

***** ERROR ***** K?-051 FOLLOWS:

SURFACE OF BOUNDARY LABEL REQUESTED A DIFFERENTIAL ALBEDO THAT IS

NOT ON THE ALBEDO DATA SET.

THE REQUESTED ALBEDO NAME IS

This message is from subroutine ALBRD. A list of the albedos that are on the standard albedo data set is given in Table 8.1.19 (Sect. 8.1.2.6).

KMSG052

***** ERROR ***** K?-052 FOLLOWS:

INTERPOLATION CODE SHOULD BE IN THE RANGE 1 TO 5

INT:

This message is from subroutine XSEC_INTERP. This indicates an error in the continuous energy point data.INT is the interpolation code from the data. A STOP 104 is executed in conjunction with this message, and a traceback may be printed from subroutine STOP.

KMSG053

***** ERROR ***** K?-053 FOLLOWS:

LOG INTERPOLATION SHOULD NOT BE USED WITH NEG OR ZERO ARGUMENT

XP:

This message from subroutine XSEC_INTERP points to a problem with the continuous energy point data. $\mathrm{XP}$ is the offending argument. A STOP 104 is executed in conjunction with this message, and a traceback may be printed from subroutine STOP.

KMSG054

$* * * * *$ ERROR $* * * * *$ K6-054 FOLLOWS:

A SURFACE NUMBER _ W WAS SPECIFIED THAT IS LARGER THAN NUMBER OF SURFACES IN THE INDICATED GLOBAL BODY.

THE PROBLEM WILL NOT BE RUN.

This KENO-VI message is from subroutine RDBNDS. It indicates that an incorrect surface was specified to which a boundary condition was to be assigned. The surfaces of the bodies specified for boundary conditions should be carefully matched with those specified in the bounds of the global unit.

KMSG055

***** ERROR ***** K?-055 FOLLOWS:

ERRORS FOUND IN MIXING CROSS SECTIONS

THE FOLLOWING NUCLIDE(S) SPECIFIED IN THE MIXING TABLE WERE

NOT FOUND ON THE CROSS SECTION LIBRARY

ENTRY NUCLIDE ID 
This message is from subroutine MIXMIX. Either a nuclide ID was misspelled or was not in the cross section library.

KMSG056

$* * * * *$ ERROR ***** K?-056 FOLLOWS:

MIXTURE LACKS EITHER NU*FISSION OR CHI DATA

This message from subroutine NORM1D or XSEC1D indicates that a mixture that contains fissile material is missing the nu-fission cross section or the fission spectrum. A STOP 115 is executed when this message is printed.

\section{KMSG057}

***** ERROR ***** K?-057 FOLLOWS:

INVALID BIAS ID _ IN REGION _ _ ABOVE.

This message is from subroutines PRTJOM or READGM. It is printed if a negative or zero bias ID is entered for the specified geometry region. Review Sect. 8.1.2.4 for correct geometry data specification information.

KMSG058

$* * * * *$ ERROR ***** K?-058 FOLLOWS:

INVALID BIAS ID IN REGION ABOVE.

This message is from subroutine PRTJOM. It is printed if a negative or zero bias ID is entered for the specified geometry region. Review Sect. 8.1.2.4 for correct geometry data specification information.

\section{KMSG059}

***** ERROR ***** K?-059 FOLLOWS:

THE CALCULATION WAS TERMINATED BECAUSE OF EXCESSIVE SPLITTING.

This message from subroutine GUIDE is printed only if message K?-128 is printed 50 or more times for a given generation. This indicates that the problem and/or the code is incapable of achieving a reasonable solution. If changes have been made in the code, they should be carefully scrutinized. If a biasing data block has been entered (Sect. 8.1.2.7), it should be checked carefully.

\section{KMSG060 \\ ***** WARNING ***** K?-060 FOLLOWS: \\ THE ANGULAR SCATTERING DISTRIBUTION FOR MIXTURE THE TRANSFER FROM GROUP TO GROUP HAS BAD MOMENTS FOR MOMENTS WERE ACCEPTED}

This message from subroutine BADMOM indicates that the moments from the cross section data are incorrect for the group transfer shown. The code replaces the incorrect moments with acceptable moments and proceeds normally. The moments printed in the last line of the message should match those printed in 
the eighth line. The user can suppress these messages by entering an appropriate value for the cross section message cutoff parameter, EPS= in the mixing table data, Sect. 8.1.2.10. See Sect. 8.1.3.4.4 for assistance in determining an appropriate value.

KMSG061

$* * * * *$ ERROR ***** K?-061 FOLLOWS: IS NOT A VALID UNIT NUMBER, A UNIT NUMBER MUST BE A POSITIVE INTEGER.

This message is from subroutine KENOG. A unit number must be an integer greater than 0 .

KMSG062

***** ERROR ***** K?-062 FOLLOWS:

INSUFFICIENT DATA FOLLOWED THE KEYWORD ARRAY.

This message is from subroutine ARRAY. The data following the keyword ARRAY are misspelled or incomplete. See Sect. 8.1.2.5 for assistance in determining the correct method for inputting array data.

\section{KMSG063}

***** WARNING ***** K?-063 FOLLOWS:

NUCLIDE

HAS NO GAMMA PRODUCTION DATA.

$* * * * *$ WARNING *****

This message from subroutine MIXMIX indicates that somehow KENO has been told to run a coupled neutron-gamma problem, and the indicated nuclide has no neutron to gamma transfer data. This means this nuclide cannot produce any gammas. This message should never occur in KENO, as it should have ignored all gamma data from a coupled library.

KMSG064

***** WARNING ***** K?-064 FOLLOWS:

THE ANGULAR SCATTERING DISTRIBUTION FOR MIXTURE HAS BAD MOMENTS FOR THE TRANSFER FROM GROUP TO GROUP MOMENTS WERE ACCEPTED. THE P0 COEFFICIENT IS

This message from subroutine BADMOM is printed to inform the user that the cross sections were altered by the code because the moments from the cross section data were incorrect for the group transfer shown. The $\mathrm{P}_{0}$ coefficient was larger than the cross section message cutoff parameter EPS, but the relative change in the moments was smaller than EPS.

KMSG065

***** WARNING ***** K?-065 FOLLOWS:

AN AMPX WORKING LIBRARY WAS SPECIFIED ON UNIT BUT NO MIXING DATA WAS READ. WILL BE USED. 
This message from subroutine ICEMIX occurs if the parameter data specified $\mathbf{L I B}=$ but no cross section mixing data block was entered. The cross section mixing data block begins with READ MIXT. See Sect. 8.1.2.3 for parameter data and Sect. 8.1.2.10 for mixing table information.

KMSG066

***** WARNING ***** K?-066 FOLLOWS: MIXTURES WERE REQUESTED IN THE GEOMETRY, BUT ONLY THE MIXED CROSS SECTION LIBRARY.

This message from subroutine ICEMIX indicates that more mixtures were requested in the geometry region data than were available on the mixed cross section library. See Sect. 8.1.2.3 for the specification of the unit number of the mixed cross section library $(\mathbf{X S C}=)$, Sect. 8.1.2.10 for the specification of the mixing table, and Sect. 8.1.2.4 to determine the mixtures used in the geometry region data.

KMSG067

***** ERROR ***** K?-067 FOLLOWS:

THE ADJOINT INPUT PARAMETER WAS _BUT THE ADJOINT INDICATOR FROM THE MIXTURE CROSS SECTION LIBRARY WAS _. KENO WILL NOT EXECUTE.

This message from subroutine ICEMIX occurs if the adjoint input parameter ADJ $=$ specified a forward calculation and the cross sections were adjointed, or the adjoint input parameter specified an adjoint calculation and the cross sections were not adjointed. $\mathrm{T}$ indicates true, $\mathrm{F}$ indicates false.

\section{KMSG069}

IS AN INVALID GEOMETRY TYPE INDEX

This message is companion message to KMSG091, and will follow it.

KMSG070

***** ERROR ***** K?-070 FOLLOWS:

ERROR IN SUBROUTINE WRTRST. NDX=

This message from subroutine WRTRST occurs only if the type of data to be written on the restart (WSTRT) unit is undefined (i.e., NDX is greater than 15). NDX is the index in the LPOINT array as described in messages KMSG046 and KMSG058. This error is usually caused by code errors that were introduced when changes were made to the code. A STOP 133 is executed when this message is printed.

KMSG071

THIS INTERSECTION MAY BE DUE TO ROUNDOFF IN CALCULATING THE ARRAY BOUNDARY DIMENSIONS.

FOR ROUNDOFF ERRORS, INCREASE THE DIMENSIONS OF ALL SUBSEQUENT REGIONS SHARING THAT BOUNDARY. 


$$
+\mathrm{X}-\mathrm{X}+\mathrm{Y}-\mathrm{Y}+\mathrm{Z}-\mathrm{Z}
$$

ARRAY

This message is companion message to KMSG092 and will follow it.

KMSG072

CUBOID

This message is companion message to KMSG092 and will follow it.

KMSG073

***** ERROR ***** K?-073 FOLLOWS: MIXTURES WERE REQUESTED IN THE GEOMETRY DATA, BUT ONLY OF THESE WERE FOUND IN THE MIXED CROSS SECTIONS.

This message indicates a mixture number used in the KENO model was not included in the cross sections defined in the READ MIXT block.

KMSG074 ***** ERROR ***** K?-074 FOLLOWS:

INVALID GEOMETRY TYPE IN VOLCUB. IGEO =

This message from subroutine VOLCUB is probably the result of a code error.

This message is from subroutine MASTER. Either the wrong mixed cross section data set $(\mathbf{X S C}=$ from the parameter data, Sect. 8.1.2.3) is being used, or one or more mixture numbers are in error in the geometry region data (see Sect. 8.1.2.4).

KMSG075

***** ERROR ***** THE PROBLEM WILL NOT RUN

This message is companion message to KMSG188

KMSG076

***** ERROR ***** K?-076 FOLLOWS:

POSIT ERROR --- ILLEGAL GEOMETRY TYPE

$\mathrm{X}=\_\mathrm{Y}=\_\mathrm{Z}=\ldots \mathrm{K} 1=\_\mathrm{K} 2=\ldots \mathrm{K}=\_\mathrm{IGEO}=$

This message indicates that the history at $\mathrm{X}, \mathrm{Y}, \mathrm{Z}$ in region $\mathrm{K}$ in the unit with regions $\mathrm{K} 1$ to $\mathrm{K} 2$ has a geometry type IGEO which is not a valid geometry type. This is caused by a code error.

KMSG077

***** INFORMATION WARNING ***** K?-077 FOLLOWS:

POSIT WARNING --- POINT NOT IN GEOMETRY

$\mathrm{X}=\_\mathrm{Y}=\_\mathrm{Z}=\ldots \mathrm{K} 1=\ldots \mathrm{K} 2=$

This message indicates that point $\mathrm{X}, \mathrm{Y}, \mathrm{Z}$ is not within any region between $\mathrm{K} 1$ and $\mathrm{K} 2$. This is usually 
caused from specifying start data that tries to start histories outside the geometry.

KMSG078

***** ERROR ***** K?-078 FOLLOWS:

INVALID GEOMETRY ENCOUNTERED FOR THE LAST GEOMETRY REGION. IGEO=

This message says the geometry type of the last geometry region of the global unit is invalid. This indicates a code bug.

KMSG079

***** ERROR ***** K6-079 FOLLOWS:

UNABLE TO DETERMINE IF QUADRATIC IS CONVEX OR CONCAVE. CHECK FUNCTION VEXCAV CALLED FROM QUAD.

This self-explanatory KENO-VI message is from function VEXCAV. It indicates a problem with an arbitrary quadratic. KENO-VI is missing the data indicating whether a surface is convex or concave and so was unable to determine this for the current arbitrary quadratic.

KMSG080

***** ERROR ***** K?-080 FOLLOWS:

UNRECOGNIZED GEOMETRY TYPE. IGEOM = IN SUBROUTINE PRTJOM

This message indicates a code bug.

KMSG081

***** ERROR ***** K?-081 FOLLOWS:

ERROR IN HEMISPHERE DESIGNATION. ISET $=$ IN SUBROUTINE PRTJOM

This message indicates a code bug.

KMSG082

***** ERROR ***** K?-082 FOLLOWS:

ERROR IN PRTJOM WITH HEMICYLINDER DESIGNATION. NHCYL =

This message indicates a code bug.

KMSG083

***** ERROR ***** K?-083 FOLLOWS:

FOR ARRAY _ _ THE _ DIMENSIONS OF UNIT _ AT (__ _ _ _ _ DO NOT MATCH THOSE OF UNIT _ AT FOR UNIT $\ldots+{ }_{-}=\ldots$ AND $-_{-}=\ldots$ WHILE FOR UNIT ${ }_{-}+{ }_{-}=\ldots$ AND $-_{-}=$

This message is from subroutine ARASIZ. The common faces of adjacent units must be the same size and shape. This message occurs whenever this requirement is not met. One or more of the dimensions of the 
units specified in the message may be incorrect, or the array definition data may be incorrect. Carefully check the input data relating to the geometry region data and the array definition data as described in Sects. 8.1.2.4 and 8.1.2.5.

KMSG084

***** ERROR ***** K?-084 FOLLOWS:

UNIT__ _ IN UNIT ORIENTATION ARRAY NUMBER __ IS UNDEFINED IN THE INPUT DATA.

This message from subroutine ARASIZ occurs when the array description data block specifies a unit that was not defined in the geometry region data. Verify the array definition data and the geometry region data as described in Sects. 8.1.2.4 and 8.1.2.5.

KMSG085

$* * * * *$ ERROR ***** K?-085 FOLLOWS:

UNIT _ AT POSITION $\mathrm{X}=\ldots \mathrm{Y}=\ldots \mathrm{Z}=\ldots$ IN UNIT ORIENTATION ARRAY NUMBER IS INVALID BECAUSE IT IS LESS THAN ZERO OR LARGER THAN THE LARGEST UNIT NUMBER IN THE INPUT DATA.

This message from subroutine ARASIZ occurs if the unit number named in the message is less than or equal to zero or greater than NBOX (the number of different units). The position of the offending unit is also given. This error usually results from leaving some positions undefined in the unit orientation array or from erroneous data in the unit orientation data. (This includes extra data, mistyped data and omitted data.) See Sect. 8.1.2.5 for additional information.

KMSG086

***** ERROR ***** K?-086 FOLLOWS:

GEOMETRY WORD INDEX OUT OF RANGE. IGEOM = _ IN SUBROUTINE PRTJOM

This message from subroutine PRTJOM is self-explanatory. The printed value of IGEOM must be greater than zero and less than 24 in KENO V.a or less than 27 in KENO-VI to be valid. If the geometry words (see Sect. 8.1.2.4) are correct, this message is due to a code error that has been introduced when changes were made to the code.

KMSG087

***** ERROR ***** K6-087 FOLLOWS:

INSUFFICIENT DATA FOLLOWED THE KEYWORD MEDIA.

This KENO-VI message is from subroutine MEDIA. Either the material, bias ID, or sector data were not included on the media card, or non-integer data were inadvertently entered. See Sect. 8.1.2.4 for additional information on the type of data required on a MEDIA card.

KMSG088

***** ERROR ***** K6-088 FOLLOWS: 


\section{SECTOR DATA WAS NOT FOUND FOR MEDIA}

This KENO-VI message is from subroutine MEDIA. Either the material, bias ID, or sector data were not included on media card or non-integer data were inadvertently entered. See Sect. 8.1.2.4 for additional information on the type of data required on a MEDIA card.

KMSG089

***** ERROR ***** K6-089 FOLLOWS:

VOLUME HAS BEEN MULTIPLY DEFINED.

GENERATION $=\ldots$ NEUTRON $=\ldots$ UNIT $=$

PARTICLE AT POSITION $\mathrm{X}=\ldots \quad \overline{\mathrm{Y}}=\ldots \mathrm{Z}=$

IS LOCATED INSIDE THE FOLLOWING REGIONS IN THE UNIT:

This KENO-VI message is from subroutine PRTPOS out of either subroutine TRACE or TRACK. It is caused by incorrectly specifying the region definition vector in the media cards of a unit. It may be caused by a volume defined as part of more than one media record or incorrectly specifying regions that share a common boundary so the boundary between regions is contained in more than one region. See Sect. 8.1.2.4 for more information concerning vector definition arrays on MEDIA records.

KMSG090

$* * * * *$ ERROR ***** K?-090 FOLLOWS:

THE PROBLEM HAS TERMINATED BECAUSE THE PARTICLE HAS ENTERED AN INFINITE LOOP.

THIS IS POSSIBLY DUE TO A PROBLEM WITH THE CODE.

UNIT _ ARRAY _ HOLE

This self-explanatory message indicates that a history has become totally lost, and this message prevents the code from wasting time in an infinite loop. This message should never occur.

\section{KMSG091}

***** ERROR ***** K?-091 FOLLOWS:

UNIT__ CONTAINS THE FOLLOWING GEOMETRY INCONSISTENCIES:

This message from subroutines JOMCHK, HOLEXT or HOLHOL indicates that one or more intersecting geometry regions were encountered in unit . KMSG092 is the companion message from subroutine JOMCHK and specifies the regions that intersect. KMSG069 is the companion message from subroutine HOLEXT. KMSG163 and KMSG179 are the companion messages from subroutine HOLHOL. The geometry region data should be corrected and the problem resubmitted. Section 8.1.2.4 may provide assistance in correctly specifying the data.

KMSG092

***** ERROR ***** K5-092 FOLLOWS:

REGION NUMBER _ _ INTERSECTS REGION NUMBER 
This KENO V.a message from subroutine JOMCHK is a companion message to K5-91 and K5-94. It specifies the intersecting regions. The user must determine which region is incorrectly specified or if the data are out of order. KENO V.a REQUIRES THAT EACH SUCCESSIVE GEOMETRY REGION MUST COMPLETELY ENCLOSE THE PREVIOUS REGION. THIS DOES ALLOW COMMON FACES AND TANGENCY.

KMSG093

***** ERROR ***** K?-093 FOLLOWS:

INVALID GEOMETRY TYPE. IGEO=

This message is from subroutine JOMCHK. IGEO must be greater than zero and less than 20. If it does not fall in this range, a code error is the probable cause. KENOG or READGM are the subroutines likely to have the code error. Verify that all the geometry words (fgeom, Sect. 8.1.2.4) are correct.

\section{KMSG094}

***** ERROR ***** K5-094 FOLLOWS:

THE REFLECTOR DIMENSIONS ARE INCONSISTENT.

This KENO-VA message is from subroutine JOMCHK. It is a companion to message K?-095 and is printed whenever one or more intersecting geometry regions are encountered in the external reflector.

KMSG095

***** ERROR ***** K?-095 FOLLOWS:

REGION NUMBER __ IN UNIT NUMBER _ _ CONTAINS AN ERROR IN THE DIMENSIONS.

This message from subroutine VOLUME indicates an error in the geometry input data such that the negative dimension specification for a cube or cuboid is larger than the positive dimension specification (i.e., the $-\mathrm{X}$ dimension is greater than the $+\mathrm{X}$ dimension, or the $-\mathrm{Y}$ dimension is greater than the $+\mathrm{Y}$ dimension or the $-\mathrm{Z}$ dimension is greater than the $+\mathrm{Z}$ dimension). This message is also printed if the magnitude of the chord for a hemicylinder or hemisphere is larger than the radius. See Sect. 8.1.2.4 for assistance in specifying the geometry correctly.

KMSG096

***** ERROR ***** K?-096 FOLLOWS:

THE VOLUME DEFINED BY REGION__ IN UNIT __ IS NEGATIVE.

This message from subroutine VOLUME is printed whenever a negative volume is calculated. This can be caused by the positive dimension being smaller than the negative dimension on a face of a geometry region. It can also be caused by having intersecting regions, or it may be the result of round-off when the volumes are calculated. Either the geometry regions are incorrectly specified, the data are out of order, or the dimensions are so tight fitting that round-off causes the net volume of the region to be negative. If the error is caused by round-off, adjust the appropriate dimensions slightly. See Sect. 8.1.2.4.

KMSG097 
***** ERROR ***** K?-097 FOLLOWS:

THE VOLUME FOR UNIT__ IS NEGATIVE.

This message is from subroutine VOLUME. A negative volume for a unit can be caused by having intersecting regions within the unit, or by having a unit consisting of one region and having a positive dimension smaller than the negative dimension on one or more faces. Message K5-95 or K5-96 may accompany this message. See Sect. 8.1.2.4 for assistance in specifying the geometry data correctly.

\section{KMSG098}

***** ERROR ***** K?-098 FOLLOWS:

PROBLEM IN UNIT

THE ARRAY TYPE SPECIFIED IN THIS UNIT IS UNDEFINED. SPECIFY IF THE ARRAY TYPE IS CUBOID, HEXPRISM, OR DODECAHEDRON.

This message is from subroutine ARASIZ. The array type specified in this unit in an unknown type. See 8.1.2.5 for how to enter array definitions.

\section{KMSG099}

***** ERROR ***** K?-099 FOLLOWS:

PROBLEM WITH UNIT

THE OUTERMOST GEOMETRY REGION OF A UNIT UTILIZED IN THE UNIT ORIENTATION DESCRIPTION OF THE ARRAY DATA MUST BE A

This message is from subroutine ARASIZ. It can occur when the boundary region of a unit in an array is not consistent with the array type. The unit specified should be corrected so that the outer boundary is of the type required, or it should be replaced in the unit orientation data with a unit that has the correct outer boundary.

KMSG100

$* * * * *$ ERROR ***** K?-100 FOLLOWS:

THIS PROBLEM WILL NOT BE RUN BECAUSE ERRORS WERE ENCOUNTERED IN THE INPUT DATA.

This message is from subroutine MASTER and indicates that other error messages were printed in the problem output. These messages should be located and the data corrected accordingly. A STOP 129 is executed when this message is printed.

KMSG101

***** ERROR ***** K?-101 FOLLOWS:

NO FISSILE MATERIAL WAS FOUND IN SUBROUTINE START

This message is from subroutine START. It indicates that none of the mixtures used in this problem has an associated fission spectrum. Either the geometry data did not specify a fissionable mixture number, the mixing table is incorrect, the wrong mixed cross section data set was mounted, or the mixed cross section 
data set was incorrectly or incompletely made. A STOP 128 is executed in conjunction with this message, and a traceback may be printed from subroutine STOP.

KMSG102

$* * * * *$ ERROR ***** K?-102 FOLLOWS:

THE START DATA SPECIFIES THAT NEUTRONS CAN BE STARTED IN THE REFLECTOR. HOWEVER NEUTRONS WILL NOT BE STARTED BECAUSE THE OUTER REGION OF THE REFLECTOR IS NOT A CUBE OR CUBOID. NEUTRONS CAN BE STARTED FOR THE EXISTING GEOMETRY IF XSM,XSP,YSM,YSP,ZSM AND ZSP ARE ENTERED AS START DATA. XSM,XSP,YSM,YSP,ZSM AND ZSP MUST FIT WITHIN THE OUTER REGION OF THE REFLECTOR.

This message is from subroutine START. Start type 0 allows starting points throughout noncuboidal regions. If a start type other than 0 or 6 is desired and the outermost region of the reflector is not a cube or cuboid, then data must be entered to specify an imaginary cube or cuboid within this outer region. See Sect. 8.1.2.8 for assistance in specifying this data.

KMSG103

***** ERROR ***** K?-103 FOLLOWS:

START TYPE IS OUT OF RANGE.

This message from subroutine START indicates that the start type was less than zero or greater than 9. The start type is defined by entering the keyword NST= followed by the desired start type in the start data. The available starting options are given inTable 8.1.21, Sect. 8.1.2.8.

KMSG104

$* * * * *$ ERROR ***** K?-104 FOLLOWS:

A POSIT ERROR INDICATES THAT THE POINT $\mathrm{X}=\ldots \mathrm{Y}=\ldots \mathrm{Z}=\ldots$ DOES NOT OCCUR WITHIN UNIT

IF XSM, XSP, YSM, YSP, ZSM, ZSP WERE ENTERED IN THE START DATA, VERIFY THAT THEY FIT WITHIN THE OVERALL COORDINATES OF THE SYSTEM. THE OVERALL COORDINATES MAY NOT BE PRINTED FOR A BARE ARRAY.

If XSM, XSP, YSM, YSP, ZSM, ZSP were entered in the start data, they should fit within the overall coordinates of the system. The overall coordinates may not be printed for a bare array. This message from subroutine START may result from precision difficulties. It is allowed to occur a maximum of five times before being considered fatal. A code error may be the cause of this message if it becomes fatal.

KMSG105

K?-105 ***** WARNING, ONLY INDEPENDENT STARTING POSITIONS WERE GENERATED. 
This message is from subroutine START. KENO must have $n p b$ (NPG=, see parameter data, Sect. 8.1.2.3) starting positions. This message is to inform the user that fewer than $n p b$ starting positions were generated. The remaining starting positions are randomly selected from those that were generated, thus giving duplicate starting positions. If the number of independent starting positions is nearly $n p b$, the starting distribution is probably acceptable. If it is much smaller than $n p b$, a different start type should be used to give a better starting distribution (see Sect. 8.1.2.8). The amount of time allowed to generate the starting positions is controlled by parameter $\mathbf{T B A}=$ (see Sect. 8.1.2.3). If the start data are appropriate, it may be necessary to increase the value of TBA to ensure generating $n p b$ starting positions.

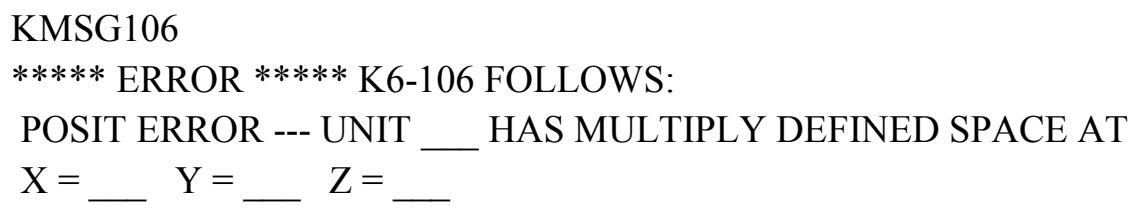

SECTOR INSIDE

This KENO-VI message from subroutine POSIT is usually the result of an incorrectly specified starting point for the initial source distribution when NST=3, 4 or 6 is specified in the start data. The starting point may not be consistent with the unit's position in the global array. The message can also be caused by improperly specifying media records. MEDIA records in the specified units should be checked for multiply defined volumes. $\mathrm{X}, \mathrm{Y}, \mathrm{Z}$ is the location of the neutron, and SECTOR INSIDE is the sectors which contain that point. See Sect. 8.1.2.4 for correct geometry words.

KMSG107

$* * * * *$ ERROR $* * * * * \mathrm{~K}$ ?-107 FOLLOWS:

POSIT ERROR --- UNIT __ HAS AN UNDEFINED SPACE AT

$\mathrm{X}=\ldots \mathrm{Y}=\ldots \mathrm{Z}=$

SECTOR INSIDE

This message from subroutine POSIT may result from not specifying all volumes in the unit using media records. It could also occur from start data if a particle is attempting to start outside the global unit or in an undefined space. Geometry should be checked for the specified unit.

KMSG108

***** ERROR ***** K?-108 FOLLOWS:

POSITION (_________ IS IS NOT VALID FOR THE POSITION OF THE SPIKE FOR START TYPE

2.

This message from subroutine START2 indicates that NXS, NYS or NZS was entered as zero. See Sect. 8.1.2.8 for the correct start data specification.

KMSG109

***** ERROR ***** K?-109 FOLLOWS:

INVALID GEOMETRY TYPE IN START. IGEO =

This message from subroutine STRTSU is likely the result of a code error. 
KMSG110

$* * * * *$ ERROR ***** K?-110 FOLLOWS:

THE PROBLEM WILL NOT BE EXECUTED BECAUSE NO FISSILE MATERIAL WAS FOUND.

This message from subroutine VOLFIS occurs when the volume of fissile material is found to be zero. It should be verified that the fissile material was correctly specified in the geometry data, and the volume of the fissile material in the printout should be nonzero. The mixing table should be verified as being correct or that the correct Monte Carlo mixed cross section data set has been used if a mixing table is not used. If the fissile region is small compared to the rest of the problem and the region volumes are calculated using TRACE or RANDOM types with default number of traces or particles, the fissile region volumes may be incorrect (even zero). If the volumes calculate to be zero, this message may be issued. In this case, the volume calculation (see Sect. 8.1.2.13) should be corrected. A STOP 131 is executed when this message is printed.

KMSG111

***** WARNING ***** K?-111 FOLLOWS:

RESTART DATA IS NOT AVAILABLE FOR RESTARTING WITH GENERATION AS SPECIFIED IN THE INPUT DATA. **

** HOWEVER, AVAILABLE RESTART DATA HAS ALLOWED RESTARTING WITH GENERATION

This message from subroutine RDCALC indicates that $n$ bas $(\mathbf{B E G}=$ in Sect. 8.1.2.3) was not consistent with the set of restart data that was to be used. A set of restart data is written every nrstrt (RES= in Sect. 8.1.2.3). The value of nbas should be 1 greater than one of these generations.

\section{KMSG112}

$* * * * *$ ERROR $* * * * * \mathrm{~K}$ ?-112 FOLLOWS:

ERROR IN RESTART. PARAMETER DATA AND RESTART DATA DO NOT AGREE NUMBER PER GENERATION FROM RESTART, NPBT $=$ _NUMBER PER GENERATION FROM INPUT DATA, NPB= NUMBER OF ENERGY GROUPS FROM RESTART, NGPT $=\ldots$ NUMBER OF ENERGY GROUPS FROM INPUT DATA, NGP=

This message is from subroutine RDCALC. A restarted problem MUST use the same number per generation and the same number of energy groups as the parent problem that wrote the restart data. It should be verified that the correct data set is mounted on unit $r$ strt. $(\mathbf{R S T}=$ in Sect. 8.1.2.3). This message can also be caused by a code error.

KMSG113

$* * * * *$ ERROR $* * * * * \mathrm{~K}$ ?-113 FOLLOWS:

ERROR IN RESTART. PARAMETER DATA AND RESTART DATA DO NOT AGREE. FISSION DENSITIES, FLUXES, OR REGION DEPENDENT FISSIONS AND ABSORPTIONS WERE REQUESTED, BUT THE GEOMETRY DATA IS INCONSISTENT. 
NUMBER OF GEOMETRY REGIONS FROM RESTART, KMAXT= NUMBER OF GEOMETRY REGIONS FROM INPUT DATA, KMAX=

This message is from subroutine RDCALC. It should be verified that the correct data set is mounted on unit $r$ strt $(\mathbf{R S T}=$ in Sect. 8.1.2.3). A code error can also cause this message.

KMSG114

$* * * * *$ ERROR ***** K?-114 FOLLOWS:

PARAMETER DATA SPECIFIED FLUXES BUT THE RESTART DATA DID NOT INCLUDE FLUXES.

This message is from subroutine RDCALC. The restarted problem can turn off fluxes if the parent case that wrote the restart data set calculated fluxes. However, if the parent case did not calculate fluxes, the restarted problem cannot calculate fluxes either. If the correct restart data set was mounted on $r s t r t$ (RST= in Sect. 8.1.2.3), the parameter data FLX=YES must be removed from the input data or $\mathbf{F L X}=\mathrm{NO}$ must be entered later in the parameter data of the restarted problem.

\section{KMSG115}

***** ERROR ***** K?-115 FOLLOWS:

PARAMETER DATA SPECIFIED REGION DEPENDENT FISSIONS AND ABSORPTIONS, BUT THEY WERE NOT INCLUDED ON RESTART

This message is from subroutine RDCALC. The restarted problem specified $\mathbf{F A R}=$ YES in the parameter data block but the parent case that wrote the restart data set did not calculate region-dependent fissions and absorptions. The restarted problem can turn off region-dependent data if the parent case calculated them, but cannot turn them on if they were not calculated by the parent case. It should be verified that the correct restart data set is mounted on rstrt $(\mathbf{R S T}=$ in Sect. 8.1.2.3). FAR=YES should be removed from the parameter data of the restarted problem, or $\mathbf{F A R}=\mathrm{NO}$ should be added later in the parameter data. Section 8.1.2.3 illustrates methods of changing the parameter input data.

KMSG116

$* * * * *$ ERROR ***** K?-116 FOLLOWS:

EXECUTION IS TERMINATED.

This message from subroutine RDCALC is a companion to messages K?-112 through K?-115. A STOP 121 is executed when this message is printed.

KMSG117

***** ERROR ***** K?-117 FOLLOWS:

THE CALCULATION WAS TERMINATED BECAUSE ERRORS WERE ENCOUNTERED IN THE START DATA.

This message is from subroutine GUIDE. It will be accompanied by one or more of messages K?-101 through K?-104 or K?-106 through K?-110. A STOP 130 is executed when this message is printed. 
KMSG118

$* * * * *$ ERROR $* * * * * \mathrm{~K}$ ?-118 FOLLOWS:

EXECUTION TERMINATED. RAKBAR HAS BECOME ZERO OR NEGATIVE.

This message is from subroutine GUIDE. If this message appears without other error messages, a code error is the probable cause.

\section{KMSG1 19}

\section{$\mathrm{k} ?-119 \quad$ JOB PULLED $\quad$ GENERATION $=\ldots \quad$ NEUTRON $=$}

This message from subroutine REPORTIT indicates that the problem is looping, or the time allotted for each generation, $t b t c h(\mathbf{T B A}=$ in Sect. 8.1.2.3) is too small. If $t b t c h(\mathbf{T B A}=)$ is increased significantly and the message occurs again for the same generation and the same neutron, it is due to a code error. Without a functional system-dependent routine (PULL) to interrupt execution, the problem will loop indefinitely, and this message will not be printed.

\section{KMSG120}

***** ERROR ***** K?-120 FOLLOWS:

THE MESH FLUX PLANES DO NOT COMPLETELY COVER THE GEOMETRY IN THE DIRECTION.

THE GEOMETRY IS THAT SPECIFIED IN THE GEOMETRY AND ARRAY DATA BLOCKS, AND/OR POSSIBLY COMPUTED OR SPECIFIED IN THE VOLUME DATA BLOCK.

This message is from subroutine CHECK_MESH_BOUNDS. Mesh fluxes were requested in the input, but the outer bound of the mesh in the specified direction does not completely enclose the geometry. The geometry bounds are determined from the input GEOMETRY block, but they may have been overridden if a volume sampling cuboid was specified in the VOLUME data block. If mesh fluxes are not needed, they should be turned off. Otherwise, the outer mesh bound should be moved so that it completely encloses the geometry in the specified direction, or the sampling bounds specified in the VOLUME data block should be fixed and the problem resubmitted.

\section{KMSG121}

***** ERROR ***** K?-121 FOLLOWS:

EXECUTION TERMINATED DUE TO INSUFFICIENT TIME IN THE JOB STEP. _ SECONDS ARE NEEDED PER GENERATION, BUT ONLY _ _ REMAIN IN THE JOB STEP.

This message is from subroutine GUIDE. If more histories are desired, the job control language should be changed to allow adequate time and resubmit the problem. This message is inaccessible on computers lacking job control language to specify the maximum execution time.

\section{KMSG122}

$* * * * *$ ERROR $* * * * * \mathrm{~K}$ ?-122 FOLLOWS:

EXECUTION TERMINATED DUE TO EXCEEDING THE TIME SPECIFIED FOR THE PROBLEM. 
This message is from subroutine GUIDE. If more histories are desired, tmax (TME= in Sect. 8.1.2.3) should be increased to allow computation of the desired number of histories.

KMSG123

K?-123 EXECUTION TERMINATED DUE TO COMPLETION OF THE SPECIFIED NUMBER OF GENERATIONS.

This message from subroutine GUIDE states that the requested number of histories has been completed. If more histories are desired, the number of generations $(\mathbf{G E N}=$ in Sect. 8.1.2.3) should be increased.

\section{KMSG124}

$* * * * *$ ERROR $* * * * * \mathrm{~K}$ ?-124 FOLLOWS:

THE OPTION TO USE EXTRA 1-DS WAS SPECIFIED, BUT ID NO.__ WAS NOT FOUND IN THE EXTRA 1-D ARRAY.

This self-explanatory message is from function INDX. If extra 1-Ds are specified in the parameter data $(\mathbf{X 1 D}=$, Sect. 8.1.2.3), extra 1-D IDs must be entered as data. See Sect. 8.1.2.9. A STOP 107 is executed when this message is printed.

\section{KMSG126}

$* * * * *$ ERROR ***** K?-126 FOLLOWS:

CROSS ERROR

This message from subroutine CROS indicates a code error. The printed data, left to right, are as follows: IGEO,K,X,Y,Z,X1,Y1,Z1. IGEO is the geometry type, $\mathrm{K}$ is the region number, $\mathrm{X}, \mathrm{Y}, \mathrm{Z}$ is the current position, and $\mathrm{X} 1, \mathrm{Y} 1, \mathrm{Z} 1$ is the end point of the path. A STOP 103 is executed when this message is printed.

\section{KMSG127}

***** ERROR ***** K?-127 FOLLOWS:

NHCYL $=$

This message from subroutine CROS indicates invalid hemicylinder information as the result of a code error. A STOP 104 is executed when this message is printed.

KMSG128

***** ERROR ***** K?-128 FOLLOWS:

NEUTRON BANK IS FULL. SPLITTING NOT ALLOWED

This message from subroutine TRACK indicates that the neutron bank is too small to allow additional splitting. This can occur if the bank size, nbank $(\mathbf{N B K}=$ in Sect. 8.1.2.3) is too small, if the biasing or weighting data are incorrect (Sect. 8.1.2.7), or if the biasing data are incorrectly utilized in the geometry description (Sect. 8.1.2.4). 
KMSG129

***** ERROR ***** K?-129 FOLLOWS:

*** ERROR IN SUBROUTINE ALBEDO *** FACE NUMBER USES

ALBEDO

NUMBER $=$

INCIDENT XSEC ENERGY GROUP= INCIDENT ALBEDO ENERGY GROUP= INCIDENT

ANGLE INDEX=

RANDOM NUMBER $=$

This message from subroutine ALBEDO indicates that a code error was encountered when determining the output energy group during the albedo treatment.

KMSG130

$* * * * *$ ERROR $* * * * * \mathrm{~K}$ ?-130 FOLLOWS:

PROBLEM IN SUBROUTINE ALBEDO: FACE NUMBER __ USES _ ALBEDO NUMBER= RETURNING XSEC ENERGY GROUP= INCIDENT ALBEDO ENERGY GROUP= INCIDENT ANGLE INDEX=

RETURNING ALBEDO ENERGY GROUP= RANDOM NUMBER=

This message from subroutine ALBEDO indicates that a code error was encountered while computing the returning angle in the albedo treatment.

KMSG131

$* * * * *$ ERROR ***** K?-131 FOLLOWS:

NO FISSIONS WERE GENERATED

This message from subroutine NSTART indicates that none of the histories in the generation encountered a fissile material, so no fission points were generated.

\section{KMSG132}

K?-132 FOLLOWS:

ONLY INDEPENDENT FISSION POINTS WERE GENERATED FOR GENERATION

This message from subroutine NSTART indicates that fewer than $n p b$ (NPG $=$ in Sect. 8.1.2.3) fission points were generated during the previous generation. Because $n p b$ fission points are required by the code, the remaining fission points are randomly selected from those that were generated, thus using duplicate fission points. If the k-effective of the system is significantly less than 1.0, several of these messages should be expected in the first few generations. The code attempts to set RAKBAR so the message can be expected to occur about once every 100 generations. The message may occur more frequently in a correctly modeled problem. However, if the number of fission points is considerably less than $n p b$ for most of the generations, the answer can be affected.

KMSG133

***** WARNING ***** K?-133 FOLLOWS: 
THE CALCULATION CONCLUDED WITHOUT PRODUCING RESULTS BECAUSE NO ACTIVE GENERATIONS WERE RUN.

THE NUMBER OF GENERATIONS RUN WAS NOT GREATER THAN THE NUMBER OF

GENERATIONS SKIPPED.

This message from subroutine KEDIT occurs if the number of generations completed is less than nskip +1 $(\mathbf{N S K}=$, Sect. 8.1.2.3). In this instance, the summaries for the problem cannot be printed.

\section{KMSG136}

***** ERROR ***** K?-136 FOLLOWS:

GEOMETRY TYPE - IGEO = - OUT OF RANGE IN

This message from subroutine SRMAX occurs if the geometry indicator is invalid as the result of a code error. A STOP 127 is executed in conjunction with this message and a traceback may be printed from subroutine STOP.

KMSG137

***** ERROR ***** K?-137 FOLLOWS:

THERE ARE MISSING CROSS SECTIONS. CHECK THE OUTPUT FOR OTHER ERROR MESSAGES.

This message from subroutine FILLSG says that not all the cross sections requested have been found. There should be other previous messages giving more detail on what cross sections are missing.

KMSG138

$* * * * *$ WARNING ***** $\mathrm{K}$ ?-138 FOLLOWS:

A WEIGHT OF 0.0 INDICATES THAT WEIGHTS WERE NOT READ OR GENERATED FOR THE BIAS ID.

WEIGHTS OF 0.0 WILL BE DEFAULTED TO 0.5 PRIOR TO EXECUTION.

This message is from subroutine PRTWTS. It is printed to alert the user that weights were not entered, defaulted, or generated. This message may appear as the result of a code error.

KMSG139

***** ERROR ***** K?-139 FOLLOWS:

NO VALID MIXTURES WERE FOUND IN THE GEOMETRY DESCRIPTION.

This message from subroutine ICEMIX indicates that the geometry data did not specify any valid mixtures. The geometry data (Sect. 8.1.2.4) should be checked and any errors that are found should be corrected. This message can also be triggered if the unit orientation data description is not properly entered for geometry having more than one unit.

KMSG140 


\section{***** WARNING ***** THE UNIT THE HOLE IS IN IS NOT USED IN THE MODEL, SO THE PROBLEM WILL NOT BE TERMINATED BECAUSE OF THIS ERROR.}

This message is companion message to KMSG188

KMSG141

***** ERROR ***** K?-141 FOLLOWS:

DATA CANNOT BE CHANGED WHEN A PROBLEM IS RESTARTED AT A GENERATION GREATER THAN ONE.

This message from subroutine DATAIN is printed if data other than parameter data are entered for a problem being restarted at a generation greater than 1 . If data other than certain parameter data are to be changed, the problem must be restarted with the first generation. The error flag is set so the problem will not execute.

KMSG142

$* * * * *$ ERROR ***** K?-142 FOLLOWS:

NO GEOMETRY DATA HAS BEEN SPECIFIED IN THE INPUT DATA.

This message from subroutine DATAIN indicates that a geometry data block was not entered for the problem either as input data or from the restart unit. The data needs to be corrected and the problem resubmitted. A STOP 135 is executed in conjunction with this message, and a traceback may be printed from subroutine STOP.

KMSG143

$* * * * *$ ERROR ***** K?-143 FOLLOWS:

UNIT ORIENTATION DATA IS REQUIRED IF MORE THAN ONE UNIT TYPE IS SPECIFIED IN THE GEOMETRY DATA.

This self-explanatory message is from subroutine DATAIN. Enter an array i or unit orientation data block as described in Sects. 8.1.2.5 and 8.1.3.6. A STOP 136 is executed in conjunction with this message, and a traceback may be printed from subroutine STOP.

KMSG144

***** WARNING ***** K?-144 FOLLOWS:

DUE TO INCONSISTENCIES BETWEEN INPUT AND RESTART DATA, MATRIX INFORMATION BY UNIT TYPE WILL BE CALCULATED BUT NOT PRINTED. INPUT DATA SET MKU=NO, BUT DATA FROM THE RESTART UNIT SPECIFIED YES.

This self-explanatory warning message is from subroutine PARTBL. The matrix information by unit type cannot be eliminated if it was calculated by the original problem (parent case) that wrote the restart data. However, printing it can be avoided. It should be verified that the correct problem is being used for restarting the problem (the title is printed at the bottom of the parameter tables). The specification of the restart unit, RST, in the third table of the output should also be verified. 
KMSG145

***** WARNING ***** K?-145 FOLLOWS:

DUE TO INCONSISTENCIES BETWEEN INPUT AND RESTART DATA, MATRIX INFORMATION BY UNIT LOCATION WILL BE CALCULATED BUT NOT PRINTED. INPUT DATA SET MKP=NO, BUT DATA FROM THE RESTART UNIT SPECIFIED YES.

This self-explanatory warning message is from subroutine PARTBL. The matrix information by unit location (also called array position or position index) cannot be eliminated if it was calculated by the original problem (parent case) that wrote the restart data. However, printing it can be avoided. It should be verified that the correct problem is being used for restarting the problem (the title is printed at the bottom of the parameter tables). The specification of the restart unit, RST, in the third table of the output should also be verified.

\section{KMSG146}

***** ERROR ***** K?-146 FOLLOWS:

PARAMETER DATA SPECIFIED MATRIX INFORMATION BY UNIT TYPE BUT IT WAS NOT FOUND ON THE RESTART UNIT.

This message from subroutine RDCALC is printed if a restarted problem requests matrix information by unit type when it was not requested and calculated by the original problem (parent case) that wrote the restart data. It should be verified that the correct restart data are being used and that the restart unit (RST) is correctly specified. The request for matrix information by unit type ( $\mathbf{M K U}=$, in the parameter data) should be eliminated if it is not necessary. The problem must be restarted with the first generation (BEG $=1$, in the parameter data) if matrix information by unit type is required and was not calculated by the parent case. A STOP 121 is executed in conjunction with this message.

KMSG147

***** ERROR ***** K?-147 FOLLOWS:

PARAMETER DATA SPECIFIED MATRIX INFORMATION BY UNIT LOCATION BUT IT WAS NOT FOUND ON THE RESTART UNIT.

This message from subroutine RDCALC is printed if a restarted problem requests matrix information by unit location (also called array position or position index) when it was not requested and calculated by the original problem (parent case) that wrote the restart data. It should be verified that the correct restart data are being used and that the restart unit (RST) is correctly specified in the first table following the parameter table. The request for matrix information by unit location ( $\mathbf{M K P}=$, in the parameter data) should be eliminated if it is not necessary. If matrix information by unit location is required and it was not calculated by the parent case, the problem must be restarted with the first generation $(\mathbf{B E G}=1$, in the parameter data). A STOP 121 is executed in conjunction with this message. 
KMSG148

***** ERROR ***** K?-148 FOLLOWS:

AN ERROR WAS ENCOUNTERED WHILE ATTEMPTING TO READ RESTART DATA FROM UNIT

$\mathrm{NDX}=\mathrm{NREC}=$

This message from subroutine RDRST is printed if a problem is encountered while reading restart data from the restart file. This may be caused by the specified section of the restart file not being present or the file section being the wrong length. The restart file is corrupted. It should be verified that the problem ran to completion and a restart file was generated. Problems in the input should be corrected, and the problem should be run again. If the problem persists, contact the SCALE helpline.

KMSG149

***** ERROR ***** K?-149 FOLLOWS:

A CROSS SECTION LIBRARY WAS SPECIFIED FOR A RESTARTED PROBLEM, BUT MIXING TABLE DATA WAS NOT AVAILABLE. THE PROBLEM WILL NOT EXECUTE.

This message from subroutine RDRST means that $\mathbf{L I B}=$ was entered in the parameter data block and a mixing table data block was not available. A flag is set to terminate execution when the data reading has been completed. If cross sections are to be used from the restart unit $(\mathbf{R S T}=)$, the $\mathbf{L I B}=$ or $\mathbf{X S C}=$ should be eliminated from the parameter data. If new cross sections are to be mixed, $\mathbf{L I B}=$ must be specified in the parameter data. The IDs in the mixing table must be available on the cross section library specified by $\mathbf{L I B}=$. A problem can be restarted using a new mixed cross section library by specifying $\mathbf{X S C}=$ in the parameter data.

\section{KMSG150}

$* * * * *$ ERROR ***** K5-150 FOLLOWS:

TOO FEW ENTRIES WERE SUPPLIED IN THE REFLECTOR GEOMETRY DESCRIPTION.

This KENO-V.a message from subroutine KENOG indicates that too few data entries were supplied for the geometry word REFLECTOR. The mixture ID, bias ID, one of the thickness/region specifications or the number of regions to be generated was omitted or incorrectly specified. Each REFLECTOR entry requires (1) a mixture ID, (2) a bias ID, (3) N entries for the thickness/region specifications, and (4) the number of regions to be generated. The thickness/region can be obtained from the Increment Thickness column of Table 8.1.20 for the material to be used in the regions generated by the REFLECTOR specification. $\mathrm{N}$ is the number of thickness/region specifications required by the geometry shape: $\mathrm{N}=1$ for spheres or hemispheres, $\mathrm{N}=3$ for cylinders and hemicylinders, and $\mathrm{N}=6$ for cubes, cuboids, and cores. A flag is set to terminate the problem when the input data reading is completed.

\section{KMSG151}

***** ERROR ***** K?-151 FOLLOWS: IS AN INVALID ARRAY TYPE IN THE ARRAY DEFINITION DATA.

This message from subroutine ARAYIN indicates that a parameter name was misspelled or the data were out of order. See Sect. 8.1.2.5 for a list of the array parameter names. A stop 137 is executed in conjunction with this message. 
KMSG152

***** ERROR ***** K?-152 FOLLOWS:

IRET $=\ldots$ A PREMATURE TERMINATION WAS ENCOUNTERED WHILE READING ARRAY

DATA.

IRET=1 INDICATES AN END WAS FOUND. IRET=2 INDICATES AN END OF FILE.

This message from subroutine ARAYIN indicates that an array number was specified without entering the corresponding UNIT ORIENTATION DESCRIPTION. See Sects. 8.1.2.5 and 8.1.3.6 for assistance.

KMSG153

$* * * * *$ ERROR $* * * * *$ IF THE UNIT THIS HOLE IS IN IS USED IN THE MODEL, THE PROBLEM

WILL NOT BE RUN.

This message is companion message to KMSG188

KMSG154

***** ERROR ***** K?-154 FOLLOWS:

ARRAY LEVEL _ _ IS LARGER THAN _ _ THE NUMBER OF ARRAYS.

CHECK THE ARRAY AND GEOMETRY INPUT FOR AN ARRAY OR UNIT THAT IS

RECURSIVELY NESTED.

This message from subroutine SORTA indicates that the array data specified in the problem are recursively nested. An example of this follows:

array 1 contains array 3

array 2 contains array 1

array 3 contains array 2

Thus the definition of array 1 and array 3 are intertwined in a never-ending loop. The array data (Sect. 8.1.2.5) should be corrected and the problem resubmitted. If the input data did not specify recursive nesting, a code error has occurred. A STOP 139 is executed in conjunction with this message.

KMSG155

$* * * * *$ ERROR ***** K?-155 FOLLOWS:

THE NESTING FLAG OR NUMBER OF ARRAY LEVELS HAS BEEN DESTROYED BY A CODE ERROR.

THE ORIGINAL NESTING FLAG WAS SET _ IT IS NOW SET_. THE ORIGINAL NESTING

LEVEL WAS , IT IS NOW

This self-explanatory message is from subroutine LODARA. A STOP 140 is executed in conjunction with this message. 
KMSG156

$* * * * *$ ERROR ***** K?-156 FOLLOWS:

MIXTURE CONTAINS AT LEAST ONE ZERO VALUE FOR THE TOTAL CROSS SECTION.

This message is from subroutine XSEC1D. All the total cross sections must be positive. Zero total cross sections can occur if all the components of a mixture are mixed with a zero-number density. Correct the mixing table for the specified mixture and resubmit the problem.

\section{KMSG157}

***** ERROR ***** K5-157 FOLLOWS:

THE FIRST HOLE IN A UNIT MUST FOLLOW A VALID GEOMETRY REGION.

A STOP 141 accompanies this KENO V.a message from subroutine READGM. If holes are to be used in the geometry region data (Sect. 8.1.2.4), they must follow the region in which they are to be placed. This message indicates that HOLE was the first geometry description in a unit or was placed inside an ARRAY description. Correct the geometry region data and resubmit the problem.

\section{KMSG158}

***** ERROR ***** K?-158 FOLLOWS:

THE NUMBER OF HOLES IS INCORRECT. IHOL= $\mathrm{NUMHOL}=$

This message is from subroutine READGM. A code error is the probable cause of this error.

\section{KMSG159}

***** ERROR ***** K?-159 FOLLOWS:

ARRAY NUMBER SPECIFIED IN THE GEOMETRY REGION DATA WAS NOT ENTERED IN THE ARRAY DATA.

This message from subroutine SORTA or subroutine HOLE occurs if the array number specified for an ARRAY region description of the EXTENDED GEOMETRY data (Sect. 8.1.2.4) did not have the corresponding UNIT ORIENTATION DATA entered in the ARRAY DATA (Sect. 8.1.2.5). A STOP 142 is executed in conjunction with this message when it is printed from subroutine SORTA. The data should be corrected and the problem resubmitted.

KMSG160

$* * * * *$ ERROR ***** K?-160 FOLLOWS:

THE HOLES ARE RECURSIVELY NESTED.

This message from subroutine HOLE indicates that the geometry region data description (Sect. 8.1.2.4) specifies holes that are recursively nested. This can occur if a unit contains a hole whose definition traces back to the same unit or are defined in terms of each other. A simple example of recursive nesting is

KENO V.a: 


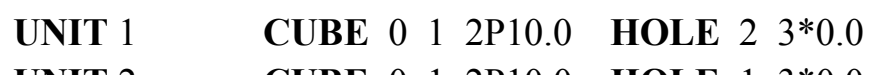

$\begin{array}{lllllllll}\text { UNIT } 2 & \text { CUBE } & 0 & 1 & 2 P 10.0 & \text { HOLE } & 1 & 3 * 0.0\end{array}$

KENO-VI:

UNIT 1 CUBOID 10 6P10.0 BOUNDARY 10 HOLE $213 * 0.0$

UNIT 2 CUBOID 10 6P10.0 BOUNDARY 10 HOLE $113 * 0.0$

Thus UNIT 1 contains UNIT 2 and UNIT 2 contains UNIT 1. The geometry region data should be checked for recursive nesting. In the absence of recursive nesting, a code error is the probable cause of this message. A STOP 143 is executed when this message occurs and a traceback is printed.

KMSG161

***** ERROR ***** K?-161 FOLLOWS:

THE GLOBAL ARRAY SPECIFIED IN THE GEOMETRY REGION DATA IS

BUT THE GLOBAL ARRAY SPECIFIED IN THE ARRAY DATA IS . EXECUTION IS TERMINATED.

This message is from subroutine FLDATA. A STOP 144 is executed in conjunction with it. The global array specified in the array data (Sect. 8.1.2.5) was entered using the keyword $\mathbf{G B L}=$. It was not consistent with the implied global array from the geometry region data (Sect. 8.1.2.4). The global array number in the geometry region data is defined to be the array number in the global unit or of the last ARRAY description that does not immediately follow a UNIT definition (i.e., other geometry definitions occur between UNIT and ARRAY). The data should be corrected and the problem resubmitted.

KMSG162

***** ERROR ***** K?-162 FOLLOWS:

THE GLOBAL ARRAY WAS NOT CONSISTENTLY SPECIFIED. THE ARRAY DATA SPECIFIED ARRAY NUMBER __ AND THE GEOMETRY DATA SPECIFIED ARRAY NUMBER

This message from subroutine SORTA occurs if $\mathbf{G B L}=$ in the array data (Sect. 8.1.2.5) does not agree with the global array number implicitly set in the geometry region data (Sect. 8.1.2.4). The geometry region data define the global array number in the global unit or to be the array number of the last ARRAY description that does not immediately follow a UNIT definition (i.e., other geometry definitions occur between UNIT and ARRAY). A flag is set to terminate execution when the data reading is completed.

KMSG163

$* * * * *$ ERROR ***** K?-163 FOLLOWS:

HOLE NUMBER __ (UNIT NUMBER __ INTERSECTS REGION NUMBER _

This self-explanatory message is from subroutine HOLHOL.

KMSG164

***** ERROR ***** K?-164 FOLLOWS: 
HOLE NUMBER (UNIT NUMBER ) INTERSECTS REGION NUMBER

This self-explanatory message is from subroutine HOLEXT.

KMSG165

$* * * * *$ ERROR ***** K?-165 FOLLOWS:

*** ERROR *** GEOMETRY TYPE OUT OF RANGE IN SUBROUTINE ADJUST. IGEOH =

This message from subroutine ADJUST indicates that the geometry type, IGEO, falls outside the allowable range of 1 through 19. This can occur if a HOLE references an undefined unit number. If all the unit numbers referenced by the holes are valid, a code error is the probable cause.

KMSG166

$* * * * *$ ERROR ***** K?-166 FOLLOWS:

THE PLUS CUBE FACE IS NOT GREATER THAN THE MINUS CUBE FACE

PLUS FACE $=$ MINUS FACE $=$

This self-explanatory message is from subroutine KENOG.

KMSG167

$* * * * *$ ERROR ***** K?-167 FOLLOWS:

ONE OR MORE PLUS FACES OF A CUBOID ARE NOT GREATER THAN THE CORRESPONDING MINUS FACES

$+\mathrm{X}=\quad-\mathrm{X}=\quad+\mathrm{Y}=-\mathrm{Y}=$ $+\mathrm{Z}=$

This self-explanatory message is from subroutine KENOG.

KMSG168

***** ERROR ***** K?-168 FOLLOWS:

THE CYLINDER RADIUS IS NOT GREATER THAN ZERO, OR THE + HEIGHT IS NOT

GREATER THAN THE - HEIGHT

RADIUS = $+\mathrm{H}=$ $-\mathrm{H}=$

This self-explanatory message is from subroutine KENOG.

\section{KMSG169}

***** ERROR ***** K?-169 FOLLOWS:

THE SPHERE RADIUS IS NOT GREATER THAN ZERO - RADIUS =

This self-explanatory message is from subroutine KENOG.

KMSG170

***** ERROR ***** K?-170 FOLLOWS:

A REPLICATE THICKNESS IS LESS THAN ZERO

SURFACE NUMBER $=$ THICKNESS $=$ 
This message from subroutine KENOG specifies that a thickness on a replicate was specified as a negative number. This is not allowed, as it would lead to an intersection.

KMSG171

***** ERROR ***** K?-171 FOLLOWS:

DIRECTION COSINES DOWN THE PAGE WERE ALL INPUT AS ZERO.

This message from subroutine RDPLOT indicates that the values for $\mathbf{U D N}=, \mathbf{V D N}=$ and $\mathbf{W D N}=$ were all zero. A zero value vector does not define a direction, so an error has occurred. See Sect. 8.1.2.11 for information concerning direction cosines down the page.

KMSG172

***** ERROR ***** K?-172 FOLLOWS:

DIRECTION COSINES ACROSS THE PAGE WERE ALL INPUT AS ZERO.

This message from subroutine RDPLOT indicates that the values for $\mathbf{U A X}=, \mathbf{V A X}=$ and $\mathbf{W A X}=$ were all zero. This is an error because a zero-value vector does not define a direction. See Sect. 8.1.2.11 for assistance in defining direction cosines across the page.

KMSG173

***** ERROR ***** K?-173 FOLLOWS:

ERRORS WERE DETECTED IN THE INPUT DATA FOR PLOT . IT WILL NOT BE DRAWN

This message from subroutine RDPLOT is a companion to messages K?-171, K?-172, K?-174, and K?180. The error that triggered the companion message should be corrected and the problem resubmitted.

KMSG174

$* * * * *$ ERROR $* * * * * \mathrm{~K}$ ?-174 FOLLOWS:

ERROR IN KENO PLOT DATASHOULD BE ENTERED AS _ Y YES, OR__ NO

This self-explanatory message is from subroutine RDPLOT. The error should be corrected and the problem resubmitted. See Sect. 8.1.2.11 for assistance.

KMSG175

***** WARNING ***** K?-175 FOLLOWS:

DUE TO INCONSISTENCIES BETWEEN INPUT AND RESTART DATA, MATRIX INFORMATION BY HOLE WILL BE CALCULATED BUT NOT PRINTED. INPUT DATA SET MKH=NO, BUT DATA FROM THE RESTART UNIT SPECIFIED YES.

This self-explanatory warning message is from subroutine PARTBL. The matrix information by hole cannot be eliminated if it was calculated by the original problem (parent case) that wrote the restart data. However, printing it can be avoided. It should be verified that the correct problem is being used for restarting the problem (the title is printed at the bottom of the parameter tables). The specification of the restart unit, RST, in the third table of the computer output should be verified. 
KMSG176

***** WARNING ***** K?-176 FOLLOWS:

DUE TO INCONSISTENCIES BETWEEN INPUT AND RESTART DATA, MATRIX INFORMATION BY ARRAY WILL BE CALCULATED BUT NOT PRINTED. INPUT DATA SET MKA=NO BUT DATA FROM THE RESTART UNIT SPECIFIED YES.

This self-explanatory warning message is from subroutine PARTBL. The matrix information by array cannot be eliminated if it was calculated by the original problem (parent case) that wrote the restart data. However, printing it can be avoided. It should be verified that the correct problem is being used for restarting the problem (the title is printed at the bottom of the parameter tables). Specification of the restart unit, RST, in the third table in the computer output should also be verified.

KMSG177

***** ERROR ***** K?-177 FOLLOWS:

PARAMETER DATA SPECIFIED MATRIX INFORMATION BY HOLE BUT IT WAS NOT FOUND ON THE RESTART UNIT.

This message from subroutine RDCALC is printed if a restarted problem requests matrix information by hole when it was not requested and calculated by the original problem (parent case) that wrote the restart data. It should be verified that the correct restart data file is being used and that the restart unit (RST) is correctly specified. The request for matrix information by hole $(\mathbf{M K H}=$, in the parameter data) should be eliminated if it is not necessary. The problem must be restarted with the first generation ( $\mathbf{B E G}=1$, in the parameter data) if matrix information by hole is required and was not calculated by the parent case. A STOP 121 is executed in conjunction with this message.

KMSG178

***** ERROR ***** K?-178 FOLLOWS:

PARAMETER DATA SPECIFIED MATRIX INFORMATION BY ARRAY BUT IT WAS NOT FOUND ON THE RESTART UNIT.

This message from subroutine RDCALC is printed if a restarted problem requests matrix information by array (also called array position or position index) when it was not requested and calculated by the original problem (parent case) that wrote the restart data. It should be verified that the correct restart data are being used and that the restart unit (RST) is correctly specified in the first table following the parameter tables. The request for matrix information by array $(\mathrm{MKA}=$, in the parameter data) should be eliminated if it is not necessary. If matrix information by array is required and it was not calculated by the parent case, the problem must be restarted with the first generation (BEG=1, in the parameter data). A STOP 121 is executed in conjunction with this message.

KMSG179

***** ERROR ***** K?-179 FOLLOWS:

HOLE NUMBER (UNIT NUMBER ) IN REGION NUMBER ) INTERSECTS HOLE NUMBER (UNIT NUMBER 
This message from subroutine HOLHOL indicates that the specified holes intersect. Check the dimensions and origins of the units being placed in the region.

KMSG180

$* * * * *$ ERROR ***** K?-180 FOLLOWS:

PROBLEM IN KENO PLOT DATA - KEYWORD IS NOT VALID

This message from subroutine RDPLOT indicates that the plot data are out of order or a keyword is incorrectly spelled. See Sect. 8.1.2.11 for a list of correct keywords.

KMSG181

$* * * * *$ ERROR $* * * * * \mathrm{~K}$ ?-181 FOLLOWS:

LPIC IS OUT OF RANGE. LPIC=

This message from subroutine PRTPLT indicates that a code error has occurred or the type of plot $(\mathbf{P I C}=)$ was not properly specified. lpic $=1$ for a mixture map, lpic $=2$ for a unit map, and lpic=3 for a bias ID map. Any other values of lpic are invalid.

KMSG182

***** ERROR ***** K?-182 FOLLOWS:

AN ARRAY WAS SPECIFIED IN THE GLOBAL UNIT, BUT ARRAY DATA WAS NOT ENTERED.

This message from subroutine SORTA indicates that the global unit contains an array specification, but the array was not entered in the array data block. A typo may need to be corrected in the GLOBAL UNIT or in the ARRAY data block.

KMSG183

***** WARNING ***** K?-183 FOLLOWS:

UNIT__ WAS NOT CHECKED FOR GEOMETRIC CONSISTENCY. IT CONTAINS ARRAY _ _ BUT WAS NOT USED IN THE PROBLEM.

This warning message is from subroutine JOMCHK. It indicates that a unit whose first region is an array was described in the extended geometry data, but that unit was not referenced in the unit orientation data (see Sects. 8.1.2.4 and 8.1.2.5). KENO usually checks all the geometry region data to be sure it is correct, even when the unit is not used in the problem. The code is unable to make these checks when a unit containing an array is not used in the problem. It is not necessarily an error, but the user should double check to be sure that unit was intentionally omitted from all the arrays.

KMSG184

$* * * * *$ ERROR ***** K?-184 FOLLOWS:

ALBEDOS WERE SPECIFIED FOR A PROBLEM WHOSE OUTER BOUNDARY IS NOT A CUBE

OR CUBOID.

THE PROBLEM WILL NOT EXECUTE. 
This message from subroutine JOMCHK indicates that a non-vacuum albedo boundary condition was entered for a curvilinear outer surface. This is not allowed, and the problem will not be run.

KMSG185

$* * * * *$ ERROR $* * * * * \mathrm{~K}$ ?-185 FOLLOWS:

*** ERROR *** THE NUMBER OF SETS OF BIASING CORRELATION DATA EXCEEDS THE

NUMBER THAT WAS WRITTEN WHEN THE BIASING DATA WAS

READ. WERE WRITTEN, BUT AN ATTEMPT WAS MADE TO READ

This message from subroutine WAITIN is accompanied by a STOP 148. It indicates a code error unless it is accompanied by error messages related to the biasing input data.

KMSG186

***** ERROR ***** K?-186 FOLLOWS:

THE NUMBER OF SETS OF BIASING AUXILIARY DATA EXCEEDS THE NUMBER THAT WAS WRITTEN WHEN THE BIASING DATA WAS

READ. WERE WRITTEN, BUT AN ATTEMPT WAS MADE TO READ

This message from subroutine WAITIN is accompanied by a STOP 149. It indicates a code error unless it is accompanied by error messages related to the biasing input data.

KMSG187

***** WARNING ***** K?-187 FOLLOWS:

INTERVALS IN THE ABOVE RANGE WERE NOT USED. $\quad * * *$

$* * *$ THIS COULD LEAD TO IMPROPER BIASING. ***** ***

$* * *$

$* * *$

This message from subroutine LODWTS is printed to remind the user that at least one of the specified intervals was not used in the problem. This can result in improper biasing. Biasing should not be used between fissile units. When biasing is used, it should be flat or increasing as distance from the fissile material increases and flat or decreasing as a history moves toward fissile material. See Sect. 8.1.2.7 for additional assistance.

KMSG188

$* * * * *$ ERROR ***** K?-188 FOLLOWS:

HOLE NUMBER __ REFERENCES UNDEFINED UNIT NUMBER

This message is printed by subroutine READGM if the unit number referenced by the hole is less than 1 or greater than the largest unit number in the geometry data. The message is printed by subroutine HOLE if the unit number referenced by the hole is larger than zero and not greater than the largest unit number in the geometry data but is a unit number for which all data are missing. The message is printed by subroutine HOLCHK if the unit number referenced by the hole is undefined. Message K?-165 may 
accompany this message. Specify a valid unit number (lhole - see EXTENDED GEOMETRY DESCRIPTION, Sect. 8.1.2.4) and resubmit the problem.

KMSG189

$* * * * *$ ERROR $* * * * * \mathrm{~K}$ ?-189 FOLLOWS:

HOLE NUMBER __ REFERENCES UNDEFINED UNIT NUMBER

This self-explanatory message is from subroutine READGM.

KMSG190

***** ERROR ***** K?-190 ERROR IN PLOT DATA - OPTION__ IS NOT VALID FOR

KEYWORD PIC $=$.

This message from subroutine RDPLOT indicates an incorrect option associated with the keyword PIC $=$. See Sect. 8.1.2.11. Acceptable options include MAT, MIX, MIXT, MEDI, UNT, UNIT, IMP, BIAS, WTS, WGT, WGTS, or WEIGH.

KMSG191

$* * * * *$ ERROR ***** K?-191 FOLLOWS:

START TYPE _ IS INVALID FOR A PROBLEM THAT

DOES NOT HAVE A GLOBAL ARRAY.

This message from subroutine DATAIN occurs if the start type (NST= in the start data, Sect. 8.1.2.8) is 2, 3,4 , or 5 . A global array is required in order to use the specified start type.

KMSG192

***** WARNING ***** K?-192 FOLLOWS:

MATRIX INFORMATION BY ARRAY WAS SPECIFIED AS YES IN THE PARAMETER DATA

$(\mathrm{MKA}=)$,

BUT IS NOT OF USE UNLESS ARRAYS ARE SPECIFIED.

This warning message from subroutine DATAIN is self-explanatory. The code redefines the problem so matrix information will not be collected by array number.

KMSG193

***** WARNING ***** K?-193 FOLLOWS:

MATRIX INFORMATION BY HOLE WAS SPECIFIED AS YES IN THE PARAMETER, DATA

$(\mathrm{MKH}=)$,

BUT IS NOT OF USE UNLESS HOLES ARE SPECIFIED.

This warning message from subroutine DATAIN is self-explanatory. The code redefines the problem so matrix information will not be collected by hole number. 
KMSG194

***** WARNING ***** K?-194 FOLLOWS:

MATRIX INFORMATION BY UNIT LOCATION WAS SPECIFIED AS YES IN THE PARAMETER DATA $(\mathrm{MKP}=)$, BUT IS NOT ALLOWED BECAUSE A GLOBAL ARRAY WAS NOT SPECIFIED.

This warning message from subroutine DATAIN is self-explanatory. The code redefines the problem so matrix information will not be collected by unit location.

KMSG195

***** ERROR ***** K?-195 FOLLOWS:

CHARACTER STRING EXCEEDS THE SPECIFIED LENGTH. CHECK FOR ENDING

DELIMITER.

This error message is from subroutine RCHRS. It indicates that either the character string exceeds 132 characters or the ending delimiter was omitted for $\mathbf{T T L}=$ (plot title, Sect. 8.1.2.11) or for $\mathbf{C O M}=$ (unit comment, Sect. 8.1.2.4, or array comment, Sect. 8.1.2.5). A STOP 153 is executed when this message is printed.

KMSG196

$* * * * *$ ERROR ***** K?-196 FOLLOWS:

A PROBLEM CANNOT BE RESTARTED WHEN RESTART DATA DO NOT EXIST FOR THE SPECIFIED GENERATION AND THE

NEXT GENERATION FOR WHICH RESTART DATA ARE AVAILABLE IS LARGER THAN THE REQUESTED NUMBER OF GENERATIONS. **

** EXECUTION IS TERMINATED.

This message from subroutine RDCALC indicates that a problem was to be restarted but the requested number of generations (GEN= in Sect. 8.1.2.3) was smaller than the beginning generation number (BEG $=$ in Sect. 8.1.2.3). The beginning generation number for a restarted problem is the generation at which the calculation of k-effectives and associated information is resumed. Therefore, the number of generations to be run must be larger than the beginning generation number. The data should be corrected and the problem resubmitted. A STOP 154 is executed when this message is printed.

KMSG197

$* * * * *$ ERROR ***** K5-197 FOLLOWS:

A VALID GEOMETRY REGION MUST PRECEDE A REPLICATE REGION.

This KENO V.a message from subroutine KENOG indicates that a replicate specification follows an invalid geometry specification (for example, REPLICATE immediately follows a UNIT specification).

KMSG198

$* * * * *$ ERROR $* * * * * \mathrm{~K}$ ?-198 FOLLOWS:

ARRAY__ CONTAINS AN ERROR IN THE INPUT DATA. 
This message from subroutine ARAYIN is printed as the result of an error in the FILL input data for the specified array. One or more messages from the library routine YREAD should immediately precede this message and indicate the nature of the error. The data should be corrected and the problem resubmitted. Messages K?-32, K?-33, and/or K?-85 may also print as a result of this error.

KMSG199

***** ERROR ***** K?-199 FOLLOWS:

THERE IS NO MIXING TABLE

This self-explanatory message comes from subroutine PREMIX-SETUP. A continuous energy problem requires a mixing table, but none was entered.

KMSG200

$* * * * *$ ERROR ***** K?-200 FOLLOWS:

START TYPE 6 WAS SPECIFIED IN THE START DATA, BUT THE STARTING POINTS WERE NOT SPECIFIED.

This message from subroutine RDSTRT indicates that start type 6 was specified but the corresponding starting points were not included in the START data block. The corresponding starting points must be entered or the start type must be changed. See Sect. 8.1.2.8.

KMSG201

$* * * * *$ WARNING $* * * * * \mathrm{~K}$ ?-201 FOLLOWS:

NEUTRON__ SPECIFIED A POSITION IN THE GLOBAL ARRAY.

THE GLOBAL UNIT DID NOT CONTAIN AN ARRAY SO THE POSITION WAS IGNORED $* * *$ WARNING $* * * * * *$ WARNING $* * * * * *$ WARNING $* * * * * *$ WARNING $* * * * * *$ WARNING

$* * * * * *$ WARNING $* * * * * *$ WARNING $* * * * * *$ WARNING $* * * * * *$ WARNING $* * * * * *$ WARNING

$* * *$

This warning message from subroutine START6 indicates that extraneous data were specified in the start type 6 data. It should be verified that the correct global unit is specified.

KMSG202

$* * * * *$ ERROR ***** K?-202 FOLLOWS:

ONLY START TYPES 0,1,6 OR 7 ARE VALID FOR A PROBLEM WITHOUT AN ARRAY IN THE GLOBAL UNIT.

This message from subroutine START indicates that the specified start type is not valid for the problem. An appropriate start type must be chosen. A STOP 157 is executed when this message is printed.

KMSG203

***** ERROR ***** K?-203 FOLLOWS:

UNIDENTIFIED KEYWORD _ _ IN MIXING TABLE 
This message from subroutine MIXING_TABLE indicates that when reading the mixing table, an unrecognized keyword was found. See 8.1. 2.10 for the acceptable keywords.

\section{KMSG204}

K?-204 EXECUTION TERMINATED DUE TO ACHIEVING THE STANDARD DEVIATION SPECIFIED FOR THE PROBLEM.

NOTE THAT AT LEAST 50 OR NSK*2 ACTIVE GENERATIONS ARE ALWAYS RUN.

This message from subroutine GUIDE indicates that the specified standard deviation has been achieved and the problem will therefore terminate at the current generation. If a lower standard deviation is desired the requested standard deviation $(\mathbf{S I G}=$, Sect. 8.1.2.3) must be decreased.

KMSG205

***** ERROR ***** K?-205 FOLLOWS:

A GLOBAL UNIT MUST BE SPECIFIED FOR A SINGLE UNIT PROBLEM.

This message from subroutine FLDATA is printed if a global unit is not specified for a single unit problem. A STOP 164 is executed when the message is printed. If the input data do not specify the global unit, it is defaulted to Unit 1.

KMSG206

***** ERROR ***** K?-206 FOLLOWS:

THE UNIT SPECIFIED FOR STARTING IS NOT USED IN THE PROBLEM OR IS UNDEFINED.

This message from subroutine START indicates that the unit in which neutrons are to be started is undefined. It should be verified that the global unit or array is correctly specified. The start data should be checked for start types 4 and 5 (Sect. 8.1.2.8) to be sure that $\mathbf{N B X}=$ is correctly specified. A STOP 158 is executed when this message is printed.

\section{KMSG207}

***** ERROR ***** K?-207 FOLLOWS:

THE STARTING ARRAY POSITION IS INVALID.

This message from subroutine START indicates that the array position NXS, NYS, or NZS is not valid for start types 3 or 6. NXS, NYS, and NZS must be larger than zero and no larger than NBXMAX, NBYMAX, and NBZMAX of the global array, respectively. It should be verified that the GLOBAL UNIT or ARRAY is correctly specified. The start data (Sect. 8.1.2.8) should be corrected and the problem resubmitted. A STOP 159 is executed when this message is printed.

KMSG208

***** WARNING ***** K?-208 FOLLOWS:

THE FRACTION OF NEUTRONS STARTED AS A SPIKE WAS LESS THAN ZERO. IT HAS BEEN RESET TO ZERO. 
This message from subroutine START indicates that $\mathbf{F C T}=$ was incorrectly specified in the start data for start type 2. The resultant starting distribution is a cosine distribution throughout the volume of a cuboid defined by XSM, XSP, YSM, YSP, ZSM, and ZSP (see Sect. 8.1.2.8). If a spike was desired, FCT= should be set to a positive number between 0.0 and 1.0. If FCT $=0.0$ is specified, a cosine distribution without a spike is used as the starting distribution. If $\mathbf{F C T}=1.0$ is specified, all the neutrons are started as a spike (i.e., they are started uniformly in the unit located at position NXS, NYS, NZS in the global array) as noted in Sect. 8.1.2.8.

KMSG209

***** WARNING ***** K?-209 FOLLOWS:

THE FRACTION OF NEUTRONS STARTED AS A SPIKE WAS GREATER THAN ONE. IT HAS BEEN RESET TO ONE.

This message from subroutine START indicates that $\mathbf{F C T}=$ was incorrectly specified in the start type 2 data. The code reset $\mathbf{F C T}=1$, so all of the neutrons are started as a spike (i.e., they are started uniformly in the unit located at NXS, NYS, NZS in the global array) as noted in Sect. 8.1.2.8.

KMSG210

$* * * * *$ ERROR $* * * * * \mathrm{~K}$ ?-210 FOLLOWS:

THE UNIT SPECIFIED FOR STARTING IS NOT IN THE GLOBAL ARRAY.

This message from subroutine START indicates that the unit specified by $\mathbf{N B X}=$ does not occur in the global array. It should be verified that the global array is correctly specified and that the unit specified by $\mathbf{N B X}=$ is correct (see Sect. 8.1.2.8).

KMSG211

***** ERROR ***** K?-211 FOLLOWS:

THE NUMBER OF SETS OF BIAS FACTORS FROM CARDS EXCEEDS THE NUMBER THAT

WAS WRITTEN WHEN THE BIASING DATA WAS

READ. _ _ WERE WRITTEN, BUT AN ATTEMPT WAS MADE TO READ __.

This message from subroutine WAITIN indicates a code error. A STOP 161 is executed when this message is printed.

\section{KMSG212}

***** ERROR ***** K?-212 FOLLOWS:

THE BIASING DATA SPECIFIED IBGN $=\ldots$ AND IEND $=$ IBGN MUST BE LARGER THAN ZERO AND IEND MUST BE AT LEAST AS LARGE AS IBGN. THE PROBLEM WILL NOT BE RUN.

This message from subroutine RDBIAS indicates an error in the biasing data. The biasing correlation data is order dependent. The order of data entry is $\mathrm{ID}=n n$ ibgn iend. where $n n$ is an ID number from Table 8.1.20 and ibgn is the beginning BIAS ID and iend is the ending BIAS ID (see Sects. 8.1.2.7 and 
8.1.3.8). In order to continue checking the input data, if $i b g n$ is less than or equal to zero, it is set to 1. Similarly, if iend is less than ibgn, it is set to ibgn.

KMSG214

$* * * * *$ ERROR ***** K6-214 FOLLOWS:

THE BASE OF A TRIANGULAR FACE OF A WEDGE CANNOT BE ZERO.

This KENO-VI message is from subroutine WEDGE. The input data for the wedge following the keyword WEDGE are incorrect. See Appendix B.

KMSG215

***** ERROR ***** K6-215 FOLLOWS:

THE Y COORDINATE OF A TRIANGULAR FACE OF A WEDGE CANNOT BE ZERO.

This KENO-VI message is from subroutine WEDGE. The input data for the wedge following the keyword WEDGE are incorrect. See Appendix B.

KMSG216

***** ERROR ***** K?-216 FOLLOWS:

UNIT__ CONTAINS ARRAY__ WHICH WAS NOT DEFINED IN THE INPUT DATA.

This message from subroutine JOMCHK indicates that the unit specified an array, but the array number was not entered in the array data block. The array number specified in the unit should be checked to verify tha tit is correct, or the unit orientation data should be entered in the array data block.

KMSG217

$* * * * *$ ERROR $* * * * * \mathrm{~K}$ ?-217 FOLLOWS:

*** ERROR *** DIFFERENTIAL ALBEDOS CANNOT BE USED IN AN ADJOINT PROBLEM.

This self-explanatory message is from subroutine FLDATA. Reflector material in the mixing table and the geometry should be described instead of using differential albedos, or the problem should be run in the forward mode.

KMSG218

***** ERROR ***** K?-218 FOLLOWS:

AN INPUT DATA ERROR HAS BEEN ENCOUNTERED IN THE DATA ENTERED FOR THIS PROBLEM.

This message is from subroutine DATAIN. Additional error messages should be found and corrected, and the data in the named data block should be checked.

KMSG219

$* * * * *$ ERROR ***** K?-219 FOLLOWS:

THE START DATA SPECIFIED __ STARTING POINTS CHOSEN FROM A COSINE DISTRIBUTION BUT NONE WERE FOUND. 
This message from subroutine START is printed if start type 2 was specified and the code was unable to start any neutrons in the cuboid defined by XSM, XSP, YSM, YSP, ZSM, ZSP. It should be verified that fissile material exists within that cuboid. If it does not, the starting cuboid should be respecified to contain fissile material, or a different start type should be chosen. If message KMSG105 states that only 0 independent starting points were generated, it indicates that the code was unable to start any neutrons in the spike specified by start type 2 . It should be verified that the unit specified for the spike contains fissile material. If only a very small fraction of the volume of this unit is fissile, it may be necessary to enter a larger value for the KENO parameter $\mathbf{T B A}=$ or a different start type may need to be selected. The problem will not be run if message KMSG219 is printed.

KMSG220

$* * * * *$ ERROR ***** K?-220 FOLLOWS:

ERROR IN PLOT DATA.

IF THE COORDINATE OF THE LOWER RIGHT-HAND CORNER IS ENTERED, ONE OF THE PLOT PARAMETERS DLX, DLD, NAX OR NDN MUST BE ENTERED.

CURRENT VALUES ARE LISTED BELOW. TITLE:

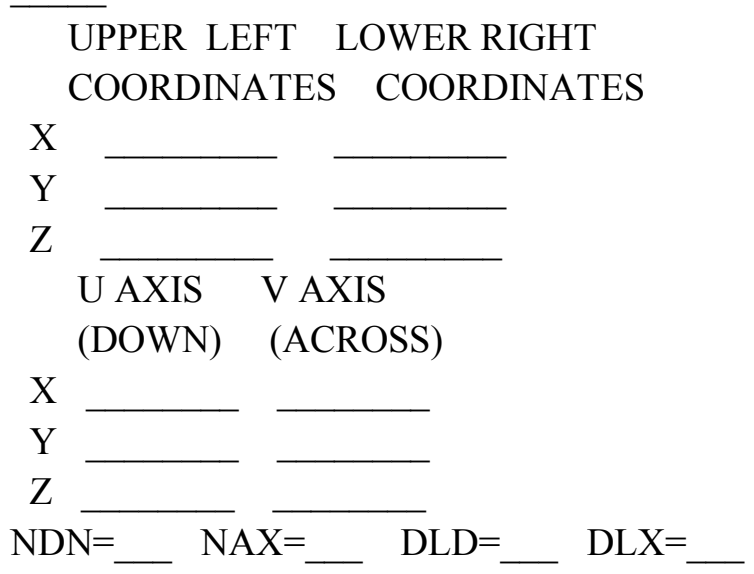

This message from subroutine RDPLOT indicates that the coordinates of the lower right-hand corner of the plot were specified in the input data without specifying one of the following plot parameters: (1) NDN, the number of characters down the page, (2) NAX, the number of characters across the page, (3) DLD, the vertical spacing between points, or (4) DLX, the horizontal spacing between points. The problem will not be run. To correct the error, NDN, NAX, DLD, or DLX should be specified in the plot data and the problem resubmitted. See Sects. 8.1.2.11 and 8.1.3.9 for assistance.

KMSG221

$* * * * *$ ERROR $* * * * *$ K6-221 FOLLOWS:

THE LENGTH OF THE EDGE ALONG THE BASE OF THE X AXIS FOR A RHOMBOID MUST BE GREATER THAN 0.0. CHECK GEOMETRY WORD

This KENO-VI message is from subroutine RHOMB. The data following the keyword RHOMB should be checked. See Appendix B. 
KMSG222

***** WARNING ***** K?-222 FOLLOWS:

TRANSFERS FOR MIXTURE___ WERE CORRECTED FOR BAD MOMENTS.

This message from subroutine MAKANG indicates moments were corrected to eliminate negative probabilities for calculated angles. If the moment was changed by more than EPS times the moment, a $\mathrm{K}$ ?-60 or K?-64 message is printed. If EPS is very small, any change without an accompanying message is trivial. The K?-60 or K?-64 messages can be used to determine if the affected transfer and correction are significant. Most messages are caused by the cross sections being in single precision and the moments calculations being done in double precision. Generally, as the number of energy groups increase, so does the number of corrected transfers for a given mixture.

KMSG224

***** ERROR ***** K6-224 FOLLOWS:

THE HEIGHT OF A WEDGE CANNOT BE ZERO.

This KENO-VI message is from subroutine WEDGE. The input data for the wedge following the keyword WEDGE are incorrect. See Appendix B

KMSG225

$* * * * *$ ERROR $* * * * * \mathrm{~K}$ ?-225 FOLLOWS:

TO START IN A GLOBAL PLANE GEOMETRY, IGEO =

VALUES FOR XSM, XSP, YSM, YSP, ZSM, AND ZSP MUST BE ENTERED AS START DATA.

This error message is from subroutine STRTSU. A GLOBAL PLANE GEOMETRY implies an infinite media. The boundary of the starting points must be entered. See Sect. 8.1.2.8.

KMSG226

***** ERROR ***** K6-226 FOLLOWS:

XDIST, YDIST, AND ZDIST SPECIFIED FOR A PPIPED MUST BE GREATER THAN 0.0. CHECK GEOMETRY WORD

This KENO-VI message is from subroutine PPIPED. The data must be checked following the keyword PPIPED. See Appendix B.

KMSG227

***** ERROR ***** K6-227 FOLLOWS:

PSI, THETA, AND PHI SPECIFIED FOR A PARALLELEPIPED MUST BE GREATER THAN OR EQUAL TO 0.0 AND LESS THAN 90.0. CHECK GEOMETRY WORD

This KENO-VI message is from subroutine PPIPED. The data following the keyword PPIPED should be checked. See Appendix B. 
KMSG228

***** ERROR ***** K?-228 FOLLOWS:

IS INVALID. UNITS MUST BE GREATER THAN 0.

THE PREVIOUS UNIT NUMBER IS . PRINTING THE GEOMETRY AS READ WILL BE

TURNED ON

This message from subroutine KENOG is printed if a unit number, $n n$, is read which is not greater than 0 . This unit followed unit $\mathrm{mm}$ in the input (if $\mathrm{mm}$ is 1 , the unit $n n$ may be the first unit in the geometry). The geometry following this will be printed as read as an aid in locating the error. The error must be corrected, and all unit numbers must be greater than 0 .

KMSG229

***** WARNING ***** K?-229 FOLLOWS:

THE NUMBER OF START TYPE 6 HISTORIES SPECIFIED DOES NOT MATCH THE NUMBER OF HISTORIES PER GENERATION HISTORIES WILL BE RANDOMLY SELECTED FROM THE HISTORIES SPECIFIED.

This message from subroutine START6 is printed if the number of starting points specified (LNU) is less than the number of histories per generation (NPG). The remaining unspecified starting points will be randomly selected from the already specified starting points.

KMSG230

$* * * * *$ ERROR $* * * * * \mathrm{~K}$ ?-230 FOLLOWS:

MATRIX K-EFFECTIVE WAS NOT CONVERGED. K-EFFECTIVE IS CONVERGENCE ERROR IS

This message is printed if the matrix eigenvalue equation did not converge to $0.1 \%$ after a maximum number of iterations. This is likely a code error, but it might be caused by too much variance in the matrix terms.

KMSG231

***** ERROR ***** K6-231 FOLLOWS:

THE CALCULATION WAS TERMINATED BECAUSE ARRAY IS NOT PROPERLY POSITIONED IN REGION OF UNIT

THE ARRAY BOUNDARY IS OUTSIDE POSITION ON THE ARRAY FACE.

This KENO-VI message from subroutine PNTCHK is printed only if an array is placed in a region so that there is space in the region not occupied by the array. The array needs to be repositioned.

KMSG233

***** ERROR ***** K?-233 FOLLOWS:

MIXTURE HAS A NEGATIVE CROSS SECTION FOR REACTION - GROUP THE VALUE IS 
This error message from subroutine NORM1D is printed if an MT number for a specified mixture has a negative cross section value in any group. This is a library or cross section processing problem. This error should be reported to scalehelp@ornl.gov.

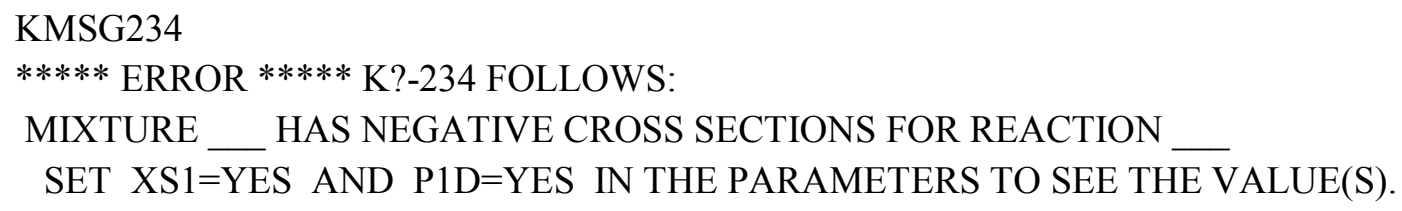

This error message from subroutine FILLSG is printed if an MT number for a specified mixture has a negative cross section value in any group. This is a library or cross section processing problem. This error should be reported to scalehelp@ornl.gov.

\section{KMSG235}

$* * * * *$ ERROR $* * * * * \mathrm{~K}$ ?-235 FOLLOWS:

ERROR IN TYPE OF CENTER FOR REGION IS NOT ALLOWED.

This error message from subroutine VOLUME is related to the flux moments used in SENS. After a geometry record, keyword CENTER may be entered followed by an integer $<=-3$ followed by an X, Y, and $\mathrm{Z}$ offset. The number after CENTER should be checked to ensure that it is between 0 and -3 or a unit number.

KMSG236

$* * * * *$ ERROR ***** K?-236 FOLLOWS:

EITHER NEUTRON OR GAMMA SHOULD HAVE BEEN ENTERED TO SELECT THE TYPE OF 1-D CROSS SECTIONS, BUT WAS ENTERED INSTEAD.

This error message from subroutine IDX1D indicates the user specified extra 1-D cross sections, but specified an invalid type. Valid types are either neutron or gamma. The data must be corrected in the READ X1DS data block.

KMSG237

***** ERROR ***** K?-237 FOLLOWS:

NUMBER OF SCATTERING ANGLES ASKED FOR,_,DO NOT MATCH THE NUMBER ON THE MIXED CROSS SECTION LIBRARY, UNABLE TO PROCESS FURTHER.

This error message from subroutine ICEMIX indicates the user requested a number of angles to be used in the problem, NSCT, that is inconsistent with the premixed cross section library specified for this problem. Either $\mathbf{S C T}=$ should not be specified, or it should be specified by creating a new mixed library.

KMSG238

***** ERROR ***** K?-238 FOLLOWS: 
DELTA -COORDINATE

DIRECTION COSINE ACROSS

DIRECTION COSINE DOWN

ARE INCONSISTENT. IF DELTA IS ZERO, THEN BOTH DIRECTION COSINES SHOULD BE ZERO.

IF DELTA IS NOT ZERO, THEN AT LEAST ONE DIRECTION COSINE SHOULD BE NONZERO, AND THE NONZERO VALUES SHOULD ALL HAVE THE SAME SIGN.

The error message from subroutine rdplot_I.f90 indicates an error in the READ PLOT input data. The direction cosines entered using UAX, VAX, WAX, UDN, VDN, and WDN are inconsistent with one of the following: (1) the lower right-hand corner and upper left-hand corner specified; (2) the delta across (DLX) and delta down (DLD); or (3) the number of points across (NAX) and the number of points down (NDN).

KMSG239
$* * * * *$ WARNING $* * * * *$ K?-239 FOLLOWS:

THE VOLUMES FOR ALL REGIONS ARE NOT SET.

SOME REGION VOLUMES ARE NOT SET BY INPUT, NOT IN THE SPACE FOR WHICH VOLUMES ARE CALCULATED,

MISSED BY THE VOLUME CALCULATION, OR ACTUALLY 0.0. FOR THESE REGIONS, VOLUMES ARE SET TO -1.0

AND THE FLUXES AND FISSION DENSITIES ARE DIVIDED BY -1.0 INSTEAD OF THE REAL VOLUME.

This error message is from subroutine VOLUME. If fluxes or other densities are needed, the real volumes are required. These can be calculated, with some error, or entered with as much precision as needed and the user knows.

KMSG240

$* * * * * *$ ERROR $* * * * * \mathrm{~K}$ ?-240 FOLLOWS:

GEOMETRY WORD UNIT IS REQUIRED BUT WAS READ INSTEAD.

This error message is from subroutine KENOG. Prior to specifying geometry or media data in the GEOMETRY DATA BLOCK, a unit must be specified.

KMSG241

$* * * * * *$ ERROR $* * * * * \mathrm{~K}$ ?-241 FOLLOWS:

NEUTRON__ OF GENERATION _ HAS LEAKED WITHOUT BEING ON THE

SURFACE OF THE BOUNDARY REGION OF UNIT

$\mathrm{X}=\ldots \mathrm{Y}=\ldots \mathrm{Z}=$

$\mathrm{U}=\longleftarrow \mathrm{V}=\longleftarrow \mathrm{W}=-$

This message from subroutine TRACK indicates a history exited the system from somewhere internal to the geometry. The geometry of the unit at the specified point should be checked to ensure that it is correctly defined.

KMSG242

$* * * * * *$ ERROR $* * * * * \mathrm{~K}$ ?-242 FOLLOWS:

NEUTRON IN GENERATION HAS LEAKED FROM UNIT 
$\mathrm{AT} \mathrm{X}=\mathrm{Y}=$

BUT IS STILL WITHIN THE VOLUME DEFINED BY THE UNIT BOUNDARY RECORD.

This error message from subroutine TRACK indicates that the specified particle is inside the volume defined by the unit's boundary record but is not in any region. The media record descriptions should be checked for the specified unit. Also, specifying a screen plot of the problem may help identify the problem.

KMSG243

$* * * * *$ ERROR $* * * * *$ K6-243 FOLLOWS:

IN UNIT _ THE BOUNDARY RECORD CONTAINS LABEL _ _ WHICH DOES NOT CORRESPOND TO A GEOMETRY RECORD.

This KENO-VI error message from subroutine KLANGA indicates that in the specified unit the boundary record contains a geometry record label that is not linked to a geometry record in the unit.

KMSG244

$* * * * *$ ERROR ***** K?-244 FOLLOWS:

UNRECOGNIZABLE VOLUME CALCULATION TYPE SPECIFIED

This error message from subroutine RDVOL indicates an invalid input. TYPE $=$ parameter was encountered in the READ volume data block.

KMSG245

$* * * * *$ ERROR $* * * * * \mathrm{~K}$ ?-245 FOLLOWS:

UNIT REGION REFERENCES UNIT

AN INSTANCE OF THIS REGION HAS OCCURRED WITHOUT FINDING THE REFERENCED UNIT AT ANY NESTING LEVEL.

This error message from subroutine TRACK indicates that the fuel center for the unit and region printed is specified with respect to the referenced unit, but the referenced unit could not be found in the geometry.

KMSG246

$* * * * *$ ERROR ***** K?-246 FOLLOWS:

THE RESTART FILE WAS A __ PROBLEM, BUT THE CURRENT PROBLEM IS THE CURRENT PROBLEM WILL NOT BE RUN

This error message from subroutine PARAM indicates that a restart file to be read for this problem was either forward or adjoint, which conflicted with what was specified in the input. Only a forward restart file can be used for a forward problem, and only an adjoint restart file can be used for an adjoint problem.

KMSG247

***** ERROR ***** K?-247 FOLLOWS:

INVALID KEYWORD __ IN THE VOLUME INPUT DATA ***** ERROR *****

This error message from subroutine RDVOL indicates the keyword printed is not valid in the READ VOLUME data block. See Sect. 8.1.2.13 for guidance on VOLUME inputs.

KMSG249

$* * * * *$ ERROR ***** K?-249 FOLLOWS:

UNDEFINED SPACE IN THE REGION CONTAINING HOLE 
This error message from subroutine TRACE is caused by a unit improperly being placed in a hole, leaving a gap between crossing into a hole and entering the unit in the hole. The hole geometry must be checked.

\section{KMSG250}

\section{K?-250 EXECUTION TERMINATED ON USER SIGNAL.}

This message from subroutine GUIDE indicates that the user has set the flag to KENO so that it will not run further generations and to wrap up execution. The flag is set by creating a file named stop_keno in the working directory.

KMSG251

$* * * * *$ ERROR $* * * * * \mathrm{~K}$ ?-251 FOLLOWS:

INVALID VOLUME CALCULATION TYPE

This error message from subroutine VOLUME indicates that the variable VCALC has been overwritten. This indicates a code error.

KMSG253

$* * * * *$ ERROR $* * * * * \mathrm{~K}$ ?-253 FOLLOWS:

CANNOT SELECT A FACE FOR VOLUME INTEGRATION.

This error message from subroutine PATHVOLS indicates an invalid face was selected for the volume integration. This indicates a code error.

KMSG254

$* * * * *$ ERROR ***** K6-254 FOLLOWS:

THE ANGLE BETWEEN THE Y AXIS AND THE Y EDGE OF THE BASE MUST BE BETWEEN 0 AND 90 DEGREES. CHECK GEOMETRY WORD

This KENO-VI error message from subroutine RHOMB indicates an invalid angle has been entered. The angle must be between 0 and 90 degrees.

KMSG256

$* * * * *$ ERROR $* * * * *$ K6-256 FOLLOWS:

RAY TRACE NUMBER

$\mathrm{AT} X=\ldots \mathrm{Y}=\ldots \mathrm{Z}=$ HAS LEAKED FROM UNIT

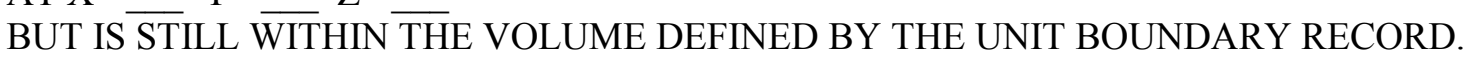

This KENO-VI message from subroutine TRACE occurs when volumes are calculated using ray tracing and an undefined region was encountered by a ray. 
KMSG257

$* * * * *$ ERROR ***** K6-257 FOLLOWS:

$* * * * *$ ERROR $* * * * * \quad$ KEYWORD PLACE NOT FOUND FOR ARRAY

This KENO-VI error message from subroutine ARRAY means an array was improperly placed within a unit. The keyword PLACE, which should immediately follow the array's vector definition array, is missing from the array data. See Sect 8.1.2.5 for more information about array placement.

\section{KMSG258}

$* * * * *$ ERROR ***** K6-258 FOLLOWS:

THE CALCULATION WAS TERMINATED BECAUSE A GEOMETRY WORD IN REGION __ OF UNIT _ PRODUCES AN IMAGINARY BOUNDARY.

This KENO-VI message from subroutine PNTCHK should only be produced by the geometry word QUADRATIC when used as part of an array boundary. The boundary produces imaginary results. The coefficients of the geometry word should be checked.

KMSG259

$* * * * *$ ERROR $* * * * * \mathrm{~K}$ ?-259 FOLLOWS:

NEUTRON__ FROM GENERATION

AT POSITION $\mathrm{X}=\ldots \mathrm{Y}=\ldots \mathrm{Z}=$

IN ARRAY _ BOUNDARY UNIT _ GLOBAL REGION

AT BOUNDARY POSITION XARY $\overline{=}$ YARY $=\ldots$ ZARY $=$

HAS A NEGATIVE DISTANCE TO CROSS THE ARRAY BOUNDARY

THIS COULD BE DUE TO AN ARRAY BOUNDARY OVERLAPPING A HOLE.

This message from subroutine TRACK occurs when a history computes a negative distance to cross an array boundary when it is inside the array. This can be caused by the array boundary intersecting a hole, of by round off in computing the coordinates of the point in the unit containing the array boundary for large dodecahedral arrays. For the latter case, KENO-VI will silently allow up to 5 such events per generation before giving this message and terminating.

KMSG260

$* * * * *$ ERROR ***** K?-260 FOLLOWS:

NEUTRON__ FROM GENERATION

A PARTICLE AT POSITION $X=$

TRAVELING IN DIRECTION U = $\mathrm{Y}=$ LOCATED IN UNIT TRAVELED__CM INSIDE HOLE__ BEFORE CROSSING THE BOUNDARY.

THIS COULD BE DUE TO THE HOLE OVERLAPPING ANOTHER HOLE, UNIT, OR ARRAY BOUNDARY.

This message from subroutine TRACK occurs when a history checks whether it is crossing into a hole and finds it is already inside the hole.

KMSG261

***** ERROR ***** K?-261 FOLLOWS:

NEUTRON__ FROM GENERATION

A PARTICLE AT POSITION $X=$

TRAVELING IN DIRECTION U = $\mathrm{Y}=\mathrm{Z}=$

TRAVELED

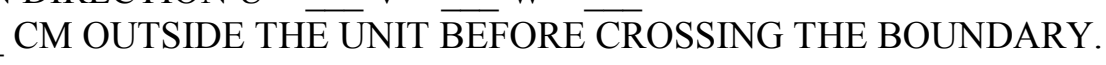




\section{THIS COULD BE DUE TO A HOLE OVERLAPPING THE UNIT BOUNDARY.}

This message from subroutine TRACK occurs when a history goes to check crossing out of a unit and finds that it does not occur at the unit boundary.

KMSG262

$* * * * *$ ERROR $* * * * * \mathrm{~K}$ ?-262 FOLLOWS:

NEUTRON__ FROM GENERATION__ LOCATED IN UNIT

A PARTICLE AT POSITION X =

TRAVELING IN DIRECTION $\mathrm{U}=\mathrm{V}=\mathrm{W}=$

IS OUTSIDE THE ARRAY BOUNDARY WITHOUT CROSSING OUT OF THE ARRAY

THIS COULD BE DUE TO A HOLE OVERLAPPING THE ARRAY BOUNDARY.

This message from subroutine CRSARA occurs when a history computes a negative distance to cross an array boundary when it is inside the array. This can be caused by the array boundary intersecting a hole.

KMSG263

$* * * * *$ ERROR $* * * * *$ K6-263 FOLLOWS:

MULTIPLY DEFINED POINT - LL $=\ldots \mathrm{X}=\ldots \mathrm{Y}=\ldots \mathrm{Z}=$

This KENO-VI message from subroutine TRACE indicates incorrect sector definitions in unit LL at point $\mathrm{X}, \mathrm{Y}, \mathrm{Z}$.

\section{KMSG264}

$* * * * *$ ERROR $* * * * * \mathrm{~K}$ ?-264 FOLLOWS:

HOLE NOT DEFINED IN REGION

This message from subroutine TRACE indicates incorrect geometry specification of holes. See Sect. 8.1.2.4.1.4 for more information on the correction specification of HOLEs.

KMSG265

$* * * * *$ ERROR $* * * * *$ K6-265 FOLLOWS:

BOUNDARY SECTOR DATA WAS NOT FOUND FOR UNIT

This KENO-VI message from subroutine BNDRY indicates that the region definition vector for the unit is missing in the input.

KMSG266

$* * * * *$ ERROR ***** K6-266 FOLLOWS:

A VECTOR DEFINITION ARRAY IN UNIT WHICH HAS NOT BEEN SPECIFIED. REFERENCES GEOMETRY RECORD

This KENO-VI message from subroutine KLANGA indicates that a region definition vector in the unit references an undefined geometry input record.

KMSG267

$* * * * *$ ERROR ***** K6-267 FOLLOWS:

IN UNIT__ THE USE OF GEOMETRY LABEL _ _ IN THE BOUNDARY DEFINITION VECTOR CONFLICTS WITH VECTOR DEFINITON GEOMETRY LABEL 
This KENO-VI message from subroutine KLANGA indicates that a geometry word referenced in a region definition vector in the unit defines the region to be on the wrong side of the unit boundary (the same geometry record is referenced with opposite signs).

KMSG268

$* * * * *$ ERROR ***** K?-268 FOLLOWS:

INVALID KEYWORD IN THE VOLUME INPUT FILE

This self-explanatory message resykts from subroutine GTVOLS, and it occurs when reading a separate volume file. See Sect. 8.1.2.13 for more information regarding the volume input file.

KMSG269

$* * * * *$ ERROR ***** K6-269 FOLLOWS:

FOR UNIT NUMBER __ TOO MANY MEDIA ENTRIES WERE SPECIFIED.

This KENO-VI error message results from subroutine GTVOLS, and it indicates that the separate volume file is inconsistent with the geometry input.

KMSG270

$* * * * * \mathrm{ERROR} * * * * * \mathrm{~K} 6-270$

CYLINDERS/ECYLINDERS/CONES IN THE GLOBAL UNIT BOUNDARY DEFINITION VECTOR NEED TO BE ORIENTED ALONG A MAJOR AXIS.

This KENO-VI message from subroutine VOLCUB indicates that a bounding cuboid for the global unit cannot be calculated because one of the referenced bodies has been rotated off axis. The user can bypass this message by specifying a bounding cuboid in the input in the VOLUME data block.

KMSG271

$* * * * *$ ERROR $* * * * * \mathrm{~K} 6-271$

THE FIRST NON-NEGATIVE BODY SPECIFIED IN THE BOUNDARY DEFINITION VECTOR OF THE

GLOBAL UNIT MUST SPECIFY FINITE VOLUME BODY.

This KENO-VI message from subroutine VOLCUB indicates that a bounding cuboid for the global unit cannot be calculated because the first body in the boundary definition vector does not define a finite volume body. The user can bypass this message by specifying a bounding cuboid in the input in the VOLUME data block.

KMSG273

$* * * * *$ ERROR ***** $\mathrm{K} ?-273$

THE NUMBER OF GROUPS ON THE RESTART LIBRARY DOES NOT MATCH WHAT WAS SET PREVIOUSLY.

THE NUMBER OF GROUPS SET PREVIOUSLY

THE NUMBER OF GROUPS FROM THE RESTART UNIT

This self-explanatory message from subroutine READ_MIXED XSECS indicates an inconsistency between the number of groups for cross sections on the restart unit and the number of groups specified elsewhere.

KMSG274

***** ERROR ***** K?-274 FOLLOWS: 
AN ERROR OCCURED WHILE READING GRIDGEOMETRY DATA. MESSAGES CAPTURED FROM GRIDGEOMETRY CONSTRUCTOR AS FOLLOWS:

This error message comes from subroutine READ_MESH_INPUT, and indicates an error in specifying the mesh grid for the problem. See Sect. 8.1.2.14 for more information about the specification of the mesh grid geometry.

KMSG275

***** ERROR ***** K?-275 FOLLOWS:

ADJOINT SOLUTIONS ARE CURRENTLY NOT AVAILABLE IN CONTINUOUS ENERGY

PROBLEMS.

IF ADJOINT SOLUTION IS DESIRED, THE USER SHOULD CHOOSE THE MULTIGROUP ENERGY TREATMENT

This message from subroutine DATAIN indicates the user turned on adjoint calculation (see parameter ADJ in Sect. 8.1.2.3) for a continuous energy problem. The current version of KENO does not have adjoint continuous energy cross sections. If adjoint solution is desired, the user should choose the multigroup energy treatment.

KMSG276

$* * * * *$ ERROR ***** K?-276 FOLLOWS:

DIFFERENTIAL ALBEDOS ARE CURRENTLY NOT AVAILABLE IN CONTINUOUS ENERGY PROBLEMS.

This message from subroutine DATAIN indicates the user entered differential albedos for a continuous energy problem. The current version of KENO does not have differential albedos for continuous energy treatment. The user should replace the albedo data with appropriate materials.

KMSG277

***** ERROR ***** K6-277 FOLLOWS:

THE UNIT _ BOUNDARY RECORD CONTAINS MORE THAN ONE POSITIVE GEOMETRY RECORD LABEL

This KENO-VI message from subroutine JOMCHK indicates that the unit reported has multiple geometry records defining the boundary. This unit is used in an array, so it is only allowed one boundary geometry record.

KMSG278

$* * * * *$ ERROR ***** K?-278 FOLLOWS:

TOO MANY ERRORS TRACING THROUGH THE GEOMETRY WHILE INTEGRATING VOLUMES.

This self-explanatory message is from subroutine PATHVOLS, and it indicates that the problem is terminated because of errors. There should be error messages previous to this indicating what the problems are. Errors should be corrected and the problem resubmitted. 


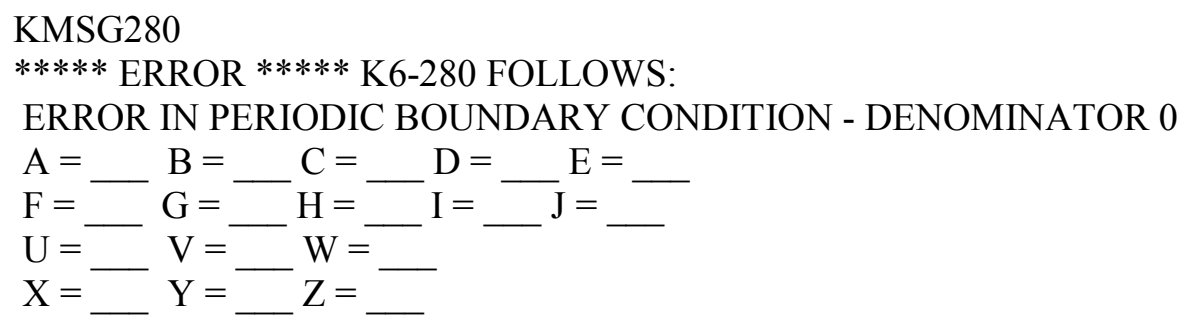

This KENO-VI error message comes from subroutine PERIOD, and it indicates the problem with periodic boundary conditions. A-J are the coefficients of the relevant quadratic equation, U-W are the direction cosines, and $\mathrm{X}-\mathrm{Z}$ is the point on the boundary. The denominator is $\mathrm{AU}^{2}+\mathrm{BV}^{2}+\mathrm{CW}^{2}+\mathrm{DUV}+\mathrm{EUW}+\mathrm{FVW}$.

KMSG281

$* * * * *$ ERROR $* * * * *$ K6-281 FOLLOWS:

IN UNIT_ _ MORE THAN ONE GEOMETRY RECORD IS SPECIFIED AS NUMBER

This self-explanatory KENO-VI message from subroutine READGM indicates that multiple geometry records in the specified unit have been given the specified number. This is not allowed. The data must be corrected and the problem resubmitted.

KMSG282

***** ERROR ***** K?-282 FOLLOWS:

FISSION FLAG IS NONZERO BUT COULD NOT FIND NUBAR DATA FOR NUCLIDE:

This message from subroutine READ_POINTWISE indicates a problem with the cross section data for a certain nuclide. If the nuclide cross section file being used is a standard file that is part of an official and current SCALE system, scalehelp@ornl.gov should be contacted.

KMSG283

***** ERROR ***** K?-283 FOLLOWS:

USER DID NOT PROVIDE A CROSS SECTION DIRECTORY FILE (CE_XXXX). A DIRECTORY FILE THAT CONTAINS THE DATA PATH AND THE CROSS SECTION ID RECORDS MUST BE PROVIDED.

This message from subroutines NUCLIDE_SETUP and PREMIX_SETUP indicates that the filename specified in the library name record is not valid (i.e., the file does not exist in the working directory) for calculations in the continuous energy mode. Links to SCALE-provided libraries are automatically created in the working directory. If one of the default files is being used, the file may not be accessible due to network problems, or access permissions. If it is a user-supplied directory file, it should be verified to exist in the SCALE temporary working directory.

KMSG284

$* * * * *$ ERROR $* * * * * \mathrm{~K}$ ?-284 FOLLOWS:

PROCESSING NUCLIDE _ , ID=

COULD NOT FIND CROSS SECTION FILE. TRIED:

AND 
This message from subroutine NUCLIDE_SETUP indicates the continuous energy cross sections for the specified nuclide are not accessible. This message is issued if the continuous energy cross section filename extension for the nuclide is provided in the input. The code checks the working directory first. If not found, it will check the directory specified in the cross section directory file. Access permissions, network problems, and typographical errors in the input file should be verified. If the nuclide cross section file being used is a standard file that is part of an official and current SCALE system, please contact scalehelp@ornl.gov.

KMSG285

$* * * * *$ ERROR ***** K?-285 FOLLOWS:

PROCESSING NUCLIDE__, ID =

THE SPECIFIED LIBRARY DOES NOT CONTAIN NUCLIDE:

This message from subroutine NUCLIDE_SETUP indicates the nuclide requested does not exist in the continuous energy cross section directory file (ce_xxxx). This may be due to user requesting a nuclide that is not available with the ENDF version and release of the continuous energy cross sections specified in the input. The user should either specify what file to use in the input explicitly or provide a userdefined continuous energy directory file containing the nuclide requested.

\section{KMSG286}

***** ERROR ***** K?-286 FOLLOWS:

PROCESSING NUCLIDE , ID $=$

COULD NOT FIND NUCLIDE:

This message from subroutine NUCLIDE_SETUP indicates the continuous energy cross sections for the specified nuclide are not accessible. This message is issued if continuous energy cross section filename extension for the nuclide is NOT provided in the input (default usage). The code checks the working directory first. If not found, it will check the directory specified in the cross section directory file. Access permissions, network problems, and typographical errors in the input file should be ruled out. If the nuclide cross section file being used is a standard file that is part of an official and current SCALE system, please contact scalehelp@ornl.gov.

KMSG287

***** ERROR ***** K?-287 FOLLOWS:

OUTGOING ANGLE COSINE __ IS GREATER THAN 1

This message from subroutine ROTASZ indicates a problem with the angle cosine when calculating the particle velocity in the continuous energy mode. This error usually indicates a coding error. Please contact scalehelp@ornl.gov.

\section{KMSG288}

***** ERROR ***** K?-288 FOLLOWS:

SAMPLING PROBABILITY TABLE FOR NUCLIDE: 


\section{$\operatorname{ENERGY~(EV):~}$}

This message from subroutine PROB_TABLE is issued if the energy of the particle that is being tracked is higher than the maximum energy of the probabilities available in the probability table that is being sampled. This error may be due to data problems. Please contact scalehelp@ornl.gov.

KMSG290

***** WARNING ***** K?-290 FOLLOWS:

COULD NOT FIND TEMP:_ ON CROSS SECTION FILE. USING CLOSEST

TEMPERATURE:

This warning message from subroutine READ_XSEC is issued to indicate that the temperature selected was not found for the current nuclide and the continuous energy cross sections that are at the closest temperature will be used. This should only be issued with $\mathbf{D B X}=0$, so the user has disabled problemdependent Doppler broadening. See Sect. 8.1.2.3 or Sect. 8.1.6.2.10 for more information.

KMSG291

***** ERROR ***** K?-291 FOLLOWS:

COULD NOT FIND CROSS SECTION FILE.

NOTE THAT THE TEMPERATURE-DEPENDENT AND TEMPERATURE-INDEPENDENT FILES MUST BE IN THE SAME DIRECTORY.

This message from subroutine READ_XSEC is issued to indicate that the file that contains the continuous energy cross sections for the requested temperature is not found. Since the continuous energy cross sections are kept in different files for each temperature, it indicates that the location in which the zero degree file is kept does not contain the file that has the requested temperature. Access permissions should be checked, and it should be verfied that the file is located in the appropriate data directory or the SCALE temporary working directory.

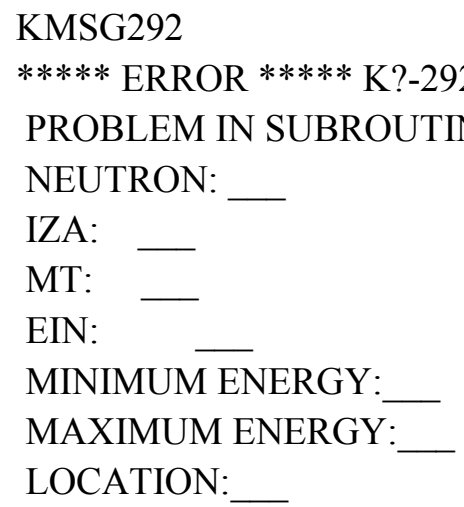

This message from subroutine SAMPLE_COLLISION indicates the energy of the particle being tracked is outside the energy range of the selected reaction for the selected nuclide. The code will reset the particle's energy to correspond to one of the boundaries and will continue tracking. If this message is 
issued, it usually indicates a problem with the continuous energy cross sections. The problem is usually not severe and may be the result of round off. Please contact scalehelp@ornl.gov.

KMSG293

***** ERROR ***** K?-293 FOLLOWS:

PROBLEM IN SUBROUTINE SAMPLE_ENERGY AT:

MT:

EIN:

EOUT:

LOCATION:

This message from subroutine SAMPLE_ENERGY indicates the energy of the particle being tracked is outside the energy range of the selected reaction for the selected nuclide. If this message is issued, it usually indicates a problem with the continuous energy cross sections. Please contact scalehelp@ornl.gov.

KMSG294

***** WARNING ***** K?-294 FOLLOWS:

UNIT _ REGION _ CONTAINS FISSIONABLE MATERIAL, BUT NO FISSIONS OCCURRED

IN IT.

THIS MAY MEAN THE PROBLEM WAS INADEQUATELY SAMPLED.

This message from subroutine KEDIT indicates some of the fissile regions are not adequately sampled. Depending on the problem, the calculated $\mathrm{k}_{\text {eff }}$ value may be too small. The problem should be run again with enough histories or start data to ensure that all regions are sampled adequately.

KMSG295

***** WARNING ***** K?-295 FOLLOWS:

THERE WERE __ COLLISIONS THAT PRODUCED A NEUTRON WITH AN ENERGY OUTSIDE THE GROUP STRUCTURE YOU SELECTED.

THESE WERE TALLIED INTO THE APPROPRIATE END GROUP.

IF YOU WISH TO SEPARATE THESE NEUTRONS, ADD A GROUP ON EACH END WITH LIMITS CORRESPONDING TO THE CROSS SECTIONS.

This warning message from subroutine SAMPLE_ENERGY indicates the energy boundaries of the groups specified for tallying in the continuous energy mode does not cover the possible energy ranges of all reactions for all nuclides in the problem. Group energy boundaries must be adjusted.

\section{KMSG296}

***** ERROR ***** K?-296 FOLLOWS:

IT IS NOT POSSIBLE TO TURN OFF WRITING ARRAYS TO DIRECT ACCESS IN WRTICE.

This error message from subroutine WRITE_ICE indicates that the arrays must be saved in the direct access file. 
KMSG297

***** ERROR ***** K?-297 FOLLOWS:

ENERGY BOUNDARIES MUST BE IN DESCENDING ORDER (EV). EB(__ $)=$ IS GREATER THAN EB $\left(\_\right)=$

This error message from subroutine RDENER indicates the upper energy boundaries specified in the ENERGY data block are not in descending order. The upper energy boundary of the highest energy group (i.e., group 1) must be specified first and then successive energy groups with descending upper energy group boundaries must be specified until all groups are specified.

KMSG298

***** ERROR ***** K?-298 FOLLOWS:

CROSS SECTION FILE FOR NUCLIDE HAS NO 2-D KINEMATICS DATA FOR THERMAL RANGE. THIS IS OK IF FREEGAS TREATMENT IS NOT TURNED OFF.

FOR THIS PROBLEM, FREEGAS TREATMENT HAS BEEN TURNED OFF (FRE=NO IN PARAMETER DATA BLOCK).

EITHER PROVIDE DATA THAT CONTAINS 2-D KINEMATICS FOR THERMAL RANGE OR TURN ON FREEGAS TREATMENT.

This message from subroutine READ_KINEMATICS indicates that no thermal scattering has been specified for the given nuclide. Data must be provided or freegas scattering must be allowed.

KMSG299

***** ERROR ***** K?-299 FOLLOWS:

INVALID KEYWORD IN THE ENERGY BOUNDARIES INPUT DATA.

This message from subroutine RDENER says that an invalid keyword was read while reading the energy boundaries for a continuous energy problem. The energy bounds data block must be checked to correct the error. See Sect. 8.1.2.12 for more information.

KMSG300

***** ERROR ***** K?-300 FOLLOWS:

INVALID THERMAL CUTOFF VALUE CUTOFF VALUE MUST BE GREATER THAN ZERO.

The thermal cutoff value for a continuous energy problem must be greater than zero. The data must be corrected and the problem rerun.

KMSG304 
K?-304 ALTHOUGH THE STANDARD DEVIATION SPECIFIED FOR THE PROBLEM IS ACHIEVED,

AT LEAST 50 OR NSK $* 2=$ EXECUTION WILL CONTINUE.

ACTIVE GENERATIONS HAVE NOT BEEN RUN.

This message from GUIDE indicates that although the standard deviation asked for in the input has been achieved, too few generations have been run to get a fair value for the standard deviation, and more generations will be run.

KMSG305

***** ERROR ***** K?-305 FOLLOWS:

MESH FLUXES HAVE BEEN SPECIFIED, BUT THE MESH DOES NOT COMPLETELY COVER THE GEOMETRY.

THE POINT $\mathrm{X}=\ldots \mathrm{Y}=\ldots \mathrm{Z}=\ldots$ LIES OUTSIDE THE MESH.

THE PROBLEM WILL NOT BE RUN. FIX THE MESH AND RESUBMIT THE CASE.

This self-explanatory message comes from subroutine accumulateTempVolumes or from pntvols.

KMSG306

***** ERROR ***** K?-306 FOLLOWS:

UNIT _ HAS BEEN DEFINED _ _ TIMES.

THIS BEHAVIOR IS NO LONGER ALLOWED AS IT COULD POTENTIALLY MISLEAD THE USERS.

NOTE THAT DEFINING UNITS THAT ARE NOT USED IN THE PROBLEM IS STILL ALLOWED.

IF DUPLICATE UNITS ARE KEPT FOR CONVENIENCE (E.G., SCOPING CALCULATIONS), THEN

SIMPLY USE A UNIT NUMBER THAT IS UNIQUE AND IS NOT UTILIZED IN THE PROBLEM.

THE PROBLEM WILL NOT BE RUN. REMOVE DUPLICATE DEFINITIONS AND RESUBMIT.

This self-explanatory message comes from READGM.

KMSG307

***** ERROR ***** K?-307 FOLLOWS:

INVALID KEYWORD IN THE REACTIONS INPUT DATA. 
This message comes from subroutine READ_REACTIONS_INPUT, and indicates that an invalid keyword was entered in the input. See Sect. 8.1.2.15 for valid reaction block input. The data must be corrected and the problem resubmitted.

KMSG308

***** ERROR ***** K?-308 FOLLOWS:

THE USER ENTERED __ FOR THE _ _ NUMBER.

ONLY LEGAL NUMBERS ( $>0$ ) AND * (WILDCARD) ARE ALLOWED. THE PROBLEM WILL NOT BE RUN.

This message results from subroutine READENTRY, and it indicates invalid reaction input. See Sect. 8.1.2.15 for valid reaction block input; data must be corrected and resubmitted.

Messages KMSG309 through KMSG354 are assumed to be self-explanatory.

KMSG309

$* * * * *$ ERROR $* * * * * \mathrm{~K}$ ?-309 FOLLOWS:

REACTION TALLY CALCULATIONS ARE CURRENTLY ALLOWED FOR ONLY CE TRANSPORT.

KMSG310

$* * * * *$ ERROR $* * * * * \mathrm{~K} ?-310$ FOLLOWS:

THE USER ENTERED FOR THE CUTOFF VALUE FOR WOODCOCK TRACKING.

CUTOFF VALUE FOR WOODCOCK TRACKING MUST BE BETWEEN 0 (ZERO) AND 1 (ONE). THE PROBLEM WILL NOT BE RUN.

KMSG311

$* * * * *$ WARNING $* * * * * \mathrm{~K} ?-311$ FOLLOWS:

THE USER ENTERED FOR THE CUTOFF VALUE FOR WOODCOCK TRACKING.

CUTOFF VALUE FOR WOODCOCK TRACKING MUST BE GREATER THAN 0 (ZERO) AND LESS THAN OR EQUAL TO 1 (ONE).

THE PROBLEM WILL CONTINUE WITHOUT WOODCOCK TRACKING. 
KMSG312

***** ERROR ***** K?-312 FOLLOWS:

OBSOLETE PARAMETER (CXM). USE REACTION DATA BLOCK TO SPECIFY PARAMETERS FOR REACTION TALLY CALCULATIONS.

KMSG313

$* * * * *$ ERROR ***** $\mathrm{K} ?-313$ FOLLOWS:

INVALID COMPUTATIONAL MODE (CXM=_) FOR TALLYING REACTION CROSS SECTIONS FOR CE-DEPLETION.

AVAILABLE MODES ARE:

(1) ALL REACITONS, NGP GROUP,

(2) TRANSMUATION REACTIONS, NGP GROUP,

(3) ALL REACTIONS, 1 GROUP,

(4) TRANSMUTATION REACTIONS, 1 GROUP.

KMSG314

***** ERROR ***** K?-314 FOLLOWS:

NO MESH DEFINITION IN INPUT. THE USER REQUESTED WITHOUT PROVIDING A MESH IN INPUT.

KMSG315

$* * * * *$ ERROR $* * * * * \mathrm{~K}$ ?-315 FOLLOWS:

MESH $<\_>$SPECIFIED FOR _ _ IS NOT FOUND IN INPUT.

KMSG316

***** WARNING ***** $\mathrm{K} ?-316$ FOLLOWS: 
NO MESH PROVIDED FOR SOURCE CONVERGENCE DIAGNOSTICS (SCD). CONTINUE WITH DEFAULT MESH.

KMSG317

***** ERROR ***** K?-317 FOLLOWS:

INCONSISTENT MIXTURE NUMBERS. REACTION DATA BLOCK HAS MORE MIXTURE DEFINITIONS THAN DEPLETION DATA BLOCK.

KMSG318

***** WARNING ***** K?-318 FOLLOWS:

INCONSISTENET MIXTURE NUMBERS. REACTION DATA BLOCK HAS FEWER MIXTURE DEFINITIONS THAN THE DEPLETION DATA BLOCK DOES. CONTINUE DEPLETION CALCULATIONS BY UPDATING REACTION DATA BLOCK WITH RESPECT TO ENTRIES IN THE DEPLETION DATA BLOCK.

KMSG319

***** WARNING ***** K?-319 FOLLOWS:

CFP WAS EITHER NOT SPECIFIED OR A NON-POSITIVE VALUE WAS ENTERED!

THE CE TSUNAMI IFP METHOD REQUIRES A POSITIVE NUMBER OF LATENT GENERATIONS.

$\mathrm{CFP}=5$ WILL BE USED FOR THIS CALCULATION.

KMSG320

$* * * * *$ WARNING $* * * * * \mathrm{~K} ?-320$ FOLLOWS:

CFP>10 DETECTED! THIS CE TSUNAMI IFP RUN MAY PRODUCE A LARGE MEMORY FOOTPRINT!

KMSG321

***** WARNING $* * * * * \mathrm{~K} ?-321$ FOLLOWS: 
CFP $<0$ DETECTED! AN F*(R) MESH WILL NOT BE USED FOR THIS CALCULATION.

THIS CE TSUNAMI CLUTCH CALCULATION MAY NOT PRODUCE ACCURATE SENSITIVITIES.

KMSG322

***** ERROR ***** K?-322 FOLLOWS:

CFP IS GREATER THAN THE NUMBER OF ACTIVE GENERATIONS! CE TSUNAMI IFP SENSITIVITY TALLIES WILL NOT BE PERFORMED. CFP SHOULD BE $<<$ THE NUMBER OF ACTIVE GENERATIONS.

KMSG323

***** ERROR ***** K?-323 FOLLOWS:

CFP IS GREATER THAN THE NUMBER OF INACTIVE GENERATIONS! CE TSUNAMI F*(R) TALLIES CANNOT BE PERFORMED. CFP SHOULD BE $<<$ THE NUMBER OF INACTIVE GENERATIONS.

KMSG324

$* * * * *$ ERROR ***** K?-324 FOLLOWS:

NO F*(R) MESH GRID WAS SPECIFIED!

KMSG325

***** WARNING ***** K?-325 FOLLOWS:

ONLY __ PARTICLE HISTORIES ARE BEING USED TO POPULATE F*(R) TALLIES PER F*(R) MESH INTERVAL. THIS MAY LEAD TO POORLY-CONVERGED F*(R) ESTIMATES AND INACCURATE SENSITIVITY COEFFICIENTS! AT LEAST 10-100 INACTIVE PARTICLE HISTORIES SHOULD BE SIMULATED PER MESH INTERVAL TO OBTAIN ACCURATE F*(R) SCORES.

KMSG326 
$* * * * *$ ERROR $* * * * * \mathrm{~K} ?-326$ FOLLOWS:

THE CE TSUNAMI IFP METHOD CANNOT BE EXECUTED IN PARALLEL.

KMSG327

$* * * * *$ WARNING $* * * * * \mathrm{~K} ?-327$ FOLLOWS:

USER SET MAP-TO-UNION (M2U) TO ON (DEFAULT),

BUT ALSO TURNED ON-THE-FLY DOPPLER PREBROADENING (OTF) TO ON. MAP-TOUNION IS FORCED TO OFF.

KMSG328

$* * * * *$ ERROR $* * * * * \mathrm{~K} ?-328$ FOLLOWS:

NO SOURCE ENTROPY CALCULATIONS! ALL FISSION SOURCE POINTS ARE OUTSIDE OF THE USER-DEFINED MESH.

KMSG329

$* * * * *$ ERROR ***** K?-329 FOLLOWS:

NO SOURCE ENTROPY CALCULATIONS! ALL FISSION SOURCE POINTS ARE SCORED IN THE SAME MESH VOXEL.

USER-DEFINED MESH FOR SOURCE CONVERGENCE DIAGNOSTICS DOES NOT ENCLOSE THE FISSILE REGIONS PROPERLY.

KMSG330

***** WARNING ***** K?-330 FOLLOWS:

DISABLING SOURCE ENTROPY CALCULATIONS. ALL FISSION SOURCE POINTS ARE SCORED IN THE SAME MESH VOXEL.

THE DEFAULT MESH FOR SOURCE CONVERGENCE DIAGNOSTICS DOES NOT ENCLOSE THE FISSILE REGIONS PROPERLY.

KMSG331 
***** WARNING ***** K?-331 FOLLOWS:

ALL FISSION SOURCE POINTS ARE OUTSIDE OF THE USER-DEFINED MESH.

KMSG332

$* * * * *$ WARNING $* * * * * \mathrm{~K} ?-332$ FOLLOWS:

MESH FLUXES HAVE BEEN SPECIFIED, BUT CE TSUNAMI DOES NOT ALLOW MESH FLUX USAGE.

CONTINUE CALCULATIONS WITH DISABLING MESH FLUXES.

KMSG333

$* * * * *$ ERROR ***** K?-333 FOLLOWS:

THE NUMBER OF GENERATIONS SKIPPED IS NOT LESS THAN THE NUMBER OF GENERATIONS TO BE RUN.

$\mathrm{NSK}=$ $-\mathrm{GEN}=$

THE CASE WILL NOT BE RUN.

KMSG334

***** ERROR ***** K?-334 FOLLOWS:

UNABLE TO CREATE A WEIGHTED LIBRARY. CODE COULD NOT INITIALIZE AMPX WORKING LIBRARY TO WRITE REACTION CROSS SECTIONS IN CE DEPLETION CALCULATIONS. CE DEPLETION CALCULATIONS WILL BE TERMINATED.

KMSG335

$* * * * *$ ERROR ***** K?-335 FOLLOWS:

UNABLE TO ADD NUCLIDE _ _ TO THE WEIGHTED LIBRARY.

CE DEPLETION CALCULATIONS WILL BE TERMINATED.

KMSG336 
$* * * * *$ ERROR ***** K?-336 FOLLOWS:

UNABLE TO GET NEUTRON 1D CROSS SECTION FOR THE REACTION, MT= CE DEPLETION CALCULATIONS WILL BE TERMINATED.

KMSG337

***** ERROR ***** K?-337 FOLLOWS:

UNABLE TO CREATE A NEUTRON 1D CROSS SECTION FOR THE WEIGHTING LIBRARY. CE DEPLETION CALCULATIONS WILL BE TERMINATED.

KMSG338

$* * * * *$ ERROR ***** K?-338 FOLLOWS:

ERROR OCCURED WHILE WRITING WEIGHTED LIBRARY, RETURN CODE= CE DEPLETION CALCULATIONS WILL BE TERMINATED.

KMSG339

$* * * * *$ ERROR ***** K?-339 FOLLOWS:

UNABLE TO INITIALIZE OBJECT FOR ENERGY BOUNDS FOR THE WEIGHTING LIBRARY. CE DEPLETION CALCULATIONS WILL BE TERMINATED.

KMSG340

$* * * * *$ ERROR ***** K?-340 FOLLOWS:

UNABLE TO INITIALIZE HEADER OBJECT FOR THE WEIGHTING LIBRARY. CE DEPLETION CALCULATIONS WILL BE TERMINATED.

KMSG341

***** ERROR ***** K?-341 FOLLOWS: 
DATA ENTERED TO THE REACTION BLOCK WITHOUT CALCULATION MODE.

CALCULATION MODE SHOULD BE ENTERED IN REACTION BLOCK WITH ONE OF THE FOLLOWING KEYWORD: (1) XS/XSTALLY=YES, (2) RX/RRTALLY=YES, (3) CEDEPL=YES, AND/OR, (4) MIXFLX=YES.

KMSG342

***** WARNING ***** K?-342 FOLLOWS:

REACTION BLOCK IS CURRENTLY ALLOWED FOR ONLY CE TRANSPORT. SKIP READING DATA FROM REACTION BLOCK.

The READ REACTION block will not function when KENO is run in multigroup cross section mode. Use KMART5 or KMART 6 to generation reaction rates when running KENO in multigroup mode.

KMSG343

***** WARNING ***** K?-343 FOLLOWS:

MISSING REACTION BLOCK FOR REACTIONVCROSS SECTION TALLY CALCULATIONS. CXM HAS BEEN SET _ WITHOUT PROVIDING DATA WITHIN REACTION BLOCK. CONTINUE CALCULATIONS AS IF IT IS A DEPLETION CALCULATION.

KMSG344

$* * * * *$ ERROR ***** K?-344 FOLLOWS:

MISSING CXM PARAMETER IN PARAMETER BLOCK. REACTION BLOCK FOR REACTION/CROSS SECTION TALLY CALCULATIONS IS NOT OPERATIONAL WITHOUT CXM.

KMSG345

***** WARNING ***** $\mathrm{K}$ ?-345 FOLLOWS:

DIRECT S(A,B) METHOD TURNED ON, BUT S(A,B) LIBRARY NOT FOUND FOR THIS NUCLIDE. NOT USING DIRECT S(A,B) METHOD FOR THIS NUCLIDE.

KMSG346

$* * * * *$ ERROR ***** K?-346 FOLLOWS: 
ERROR IN MIXTURE ENTRY IN REACTION BLOCK. MIXTURE NUMBER (MIX= SPECIFIED IN REACTION BLOCK IS NOT USED IN THE PROBLEM.

KMSG347

***** ERROR ***** K?-347 FOLLOWS:

ERROR IN NUCLIDE ENTRY IN REACTION BLOCK. NUCLIDE __[MIXTURE = SPECIFIED IN REACTION BLOCK IS NOT FOUND IN THE MIXING TABLE.

KMSG348

***** WARNING ***** K?-348 FOLLOWS:

SPECIFIED REACTION IS NOT AVAILABLE IN CE DATA LIBRARY FOR THE GIVEN NUCLIDE.

PERFORM REACTION TALLY CALCULATIONS BY SKIPPING THIS REACTION MT= FOR THE GIVEN NUCLIDE,

KMSG349

***** WARNING ***** K?-349 FOLLOWS:

A CE TSUNAMI F*(R) MESH PRINT (FST=YES) WAS REQUESTED BUT AN F*(R) MESH IS NOT BEING USED.

KMSG350

$* * * * *$ ERROR ***** K?-350 FOLLOWS:

A FISSION NEUTRON WAS BORN OUTSIDE OF THE F*(R) MESH AT POSITION:

$$
\mathrm{X}=\_\mathrm{Y}=
$$

THE F*(R) MESH MUST ENCOMPASS ALL FISSILE MATERIAL IN THE SYSTEM.

KMSG351

***** ERROR ***** K?-351 FOLLOWS: 
MISSING REACTION MT SPECIFICATION IN REACTION BLOCK (MT).

KMSG352

$* * * * *$ ERROR ***** K?-352 FOLLOWS:

MISSING NUCLIDE SPECIFICATION IN REACTION BLOCK (NUC).

KMSG353

***** ERROR ***** K?-353 FOLLOWS:

MISSING MIXTURE SPECIFICATION IN REACTION BLOCK (MIX).

KMSG354

***** ERROR ***** K?-354 FOLLOWS:

ERROR IN REACTION BLOCK. NUMBER OF ENERGY GROUPS ( _ ${ }^{\prime}$ AND ENERGY BOUNDS (_ ) DO NOT AGREE

NUMBER OF ENERGY GROUPS ___ _ _ NUMBER OF ENERGY INTERVALS $=$

\subsubsection{Theory And Techniques}

\subsubsection{The transport equation}

The equation KENO solves may be derived in the following manner, starting with the Boltzmann neutron transport equation which may be written as

$$
\begin{aligned}
\frac{1}{\mathrm{v}} \frac{\partial \Phi}{\partial t}(X, E, \Omega, t) & +\Omega \cdot \nabla \Phi(X, E, \Omega, t)+\Sigma_{t}(X, E, \Omega, t) \Phi(X, E, \Omega, t) \\
& =S(X, E, \Omega, t) \\
& +\int_{E^{\prime}} \int_{\Omega^{\prime}} \Sigma_{S}\left(X, E^{\prime} \rightarrow E, \Omega^{\prime} \rightarrow \Omega, t\right) \Phi\left(X, E^{\prime}, \Omega^{\prime}, t\right) d \Omega^{\prime} d \mathrm{E}^{\prime},
\end{aligned}
$$

where

$$
\begin{aligned}
\Phi(\mathrm{X}, \mathrm{E}, \Omega, \mathrm{t})= & \begin{array}{l}
\text { neutron flux }\left(\text { neutrons } / \mathrm{cm}^{2} / \mathrm{s}\right) \text { per unit energy at energy } \mathrm{E} \text { per steradian about } \\
\text { direction } \Omega \text { at position } \mathrm{X} \text { at time } \mathrm{t} \text { moving at speed } \mathrm{v} \text { corresponding to } \mathrm{E} ;
\end{array} \\
\Sigma_{\mathrm{t}}(\mathrm{X}, \mathrm{E}, \Omega, \mathrm{t})= & \begin{array}{l}
\text { macroscopic total cross section of the media }\left(\mathrm{cm}^{-1}\right) \text { at position } \mathrm{X} \text {, energy } \mathrm{E}, \\
\text { direction } \Omega \text { and time } \mathrm{t} ;
\end{array}
\end{aligned}
$$


$\Sigma_{\mathrm{s}}\left(\mathrm{X}, \mathrm{E}^{\prime} \rightarrow \mathrm{E}, \Omega^{\prime} \rightarrow \Omega, \mathrm{t}\right) \quad=$ macroscopic differential cross section of the media $\left(\mathrm{cm}^{-1}\right)$ per unit energy at energy $E^{\prime}$ per steradian about direction $\Omega^{\prime}$ at position $X$, and time $t$, for scattering to energy $\mathrm{E}$ and direction $\Omega$;

$\mathrm{S}(\mathrm{X}, \mathrm{E}, \Omega, \mathrm{t})=$ neutrons $/ \mathrm{cm}^{3} / \mathrm{s}$ born at position $\mathrm{X}$ and time $\mathrm{t}$ per unit energy at energy $\mathrm{E}$ per steradian about direction $\Omega$ (excludes scatter source).

Defining $\mathrm{q}(\mathrm{X}, \mathrm{E}, \Omega, \mathrm{t})$ as the total source resulting from the external source, scattering, fission, and all other contributions, the following relationship can be written.

$$
\mathrm{q}(\mathrm{X}, \mathrm{E}, \Omega, \mathrm{t})=\mathrm{S}(\mathrm{X}, \mathrm{E}, \Omega, \mathrm{t})+\int_{\mathrm{E}^{\prime}} \int_{\Omega^{\prime}} \sum_{\mathrm{s}}\left(\mathrm{X}, \mathrm{E}^{\prime} \rightarrow \mathrm{E}, \Omega^{\prime} \rightarrow \Omega, \mathrm{t}\right) \Phi\left(\mathrm{X}, \mathrm{E}^{\prime}, \Omega^{\prime}, \mathrm{t}\right) \mathrm{d} \Omega^{\prime} \mathrm{dE} \mathrm{E}^{\prime}
$$

Combining Eqs. (9.1.3) and (8.1.4), assuming media to be stationary and ignoring time-dependence, yields

$$
\Omega \cdot \nabla \Phi(X, E, \Omega)+\Sigma_{t}(X, E, \Omega) \Phi(X, E, \Omega)=q(X, E, \Omega)
$$

\subsubsection{Continuous energy mode solution procedure}

Using the relationship $\mathrm{X}^{\prime}=\mathrm{X}-\mathrm{R} \Omega$, using an integrating factor on both sides of Eq. 9.1.5, and defining

$$
\mathrm{T}(\mathrm{R})=\int_{0}^{\mathrm{R}} \Sigma_{\mathrm{t}}\left(\mathrm{X}-\mathrm{R}^{\prime} \Omega, \mathrm{E}\right) \mathrm{dR} \mathrm{R}^{\prime}
$$

the following equation can be written.

$$
\Phi(X, E, \Omega)=\int_{O}^{\infty} q(X-R \Omega, E, \Omega) e^{-T(R)} d R
$$

At this point, the problem becomes an eigenvalue problem. If there is no external source, the source may be defined as

$$
q(X, E, \Omega)=\iint d E^{\prime} d \Omega^{\prime} \Phi\left(X, E^{\prime}, \Omega^{\prime}\right) \Sigma_{s}\left(X, E^{\prime} \rightarrow E, \Omega^{\prime} \cdot \Omega\right)+\frac{1}{k} Q^{\prime}(X, E, \Omega),
$$

where

$\mathrm{k} \quad$ is the largest eigenvalue of the integral equation,

$\mathrm{Q}^{\prime}(\mathrm{X}, \mathrm{E}, \Omega) \quad$ is the fission source at position $\mathrm{X}$ for energy $\mathrm{E}$ and direction $\Omega$ (all fission contributions to point $\mathrm{E}$ from all energy points in the previous generation),

$\Sigma_{\mathrm{s}}\left(\mathrm{X}, \mathrm{E}^{\prime} \rightarrow \mathrm{E}, \Omega^{\prime} \bullet \Omega\right)$ is the scattering cross section for scattering at position $\mathrm{X}$ from energy point $\mathrm{E}^{\prime}$ and direction $\Omega^{\prime}$ to energy point $\mathrm{E}$ and direction $\Omega$. 
Assuming the fission neutrons to be isotropic, the fission source $\mathrm{Q}^{\prime}(\mathrm{X}, \mathrm{E}, \Omega)$ can be written as

$$
\mathrm{Q}^{\prime}(\mathrm{X}, \mathrm{E}, \Omega)=\frac{1}{4 \pi} \int_{\mathrm{E}^{\prime}} \int_{\Omega^{\prime}} \mathrm{d} \mathrm{E}^{\prime} \mathrm{d} \Omega^{\prime} \Phi\left(\mathrm{X}, \mathrm{E}^{\prime}, \Omega^{\prime}\right) \chi\left(\mathrm{X}, \mathrm{E}^{\prime} \rightarrow \mathrm{E}\right) v\left(\mathrm{X}, \mathrm{E}^{\prime}\right) \Sigma_{\mathrm{f}}\left(\mathrm{X}, \mathrm{E}^{\prime}\right)
$$

where

$\chi\left(X, E^{\prime} \rightarrow E\right)$ is the fraction of neutrons born at energy point $E$ from fission at energy point $E^{\prime}$ in the media at position $\mathrm{X}$,

$v\left(X, E^{\prime}\right) \quad$ is the number of neutrons resulting from a fission at energy point $E^{\prime}$ at position $X$,

$\Sigma_{\mathrm{f}}\left(\mathrm{X}, \mathrm{E}^{\prime}\right) \quad$ is the macroscopic fission cross section of the material at position $\mathrm{X}$ for a neutron at energy point $\mathrm{E}^{\prime}$.

Substituting Eq. (9.1.7) into Eq. (8.1.6) yields the following equation:

$$
\begin{gathered}
\Phi(X, E, \Omega)=\int_{0}^{\infty} d R e^{-T(R)}\left\{\frac{1}{k} Q^{\prime}(X-R \Omega, E, \Omega)\right. \\
\left.+\int_{E^{\prime}} \int_{\Omega^{\prime}} d E^{\prime} d \Omega^{\prime} \Phi\left(X-R \Omega, E^{\prime}, \Omega^{\prime}\right) \Sigma_{S}\left(X-R \Omega, E^{\prime} \rightarrow E, \Omega^{\prime} \cdot \Omega\right)\right\}
\end{gathered}
$$

The definition of $\mathrm{k}$ may be given as the ratio of the number of neutrons produced in the $(\mathrm{n}+1) t \mathrm{th}$ generation to the number of neutrons produced in the $\mathrm{n} t h$ generation or the largest eigenvalue of the integral equation. Using Eq. (8.1.8), Eq. (8.1.6) can be written as

$$
\begin{gathered}
\Phi(X, E, \Omega)=\int_{0}^{\infty} d R e^{-T(R)}\left\{\frac{1}{k} \int_{E^{\prime}} \int_{\Omega^{\prime}} v\left(X-R \Omega, E^{\prime}\right) \Sigma_{f}\left(X-R \Omega, E^{\prime}\right) \chi\left(X-R \Omega, E^{\prime} \rightarrow E\right) \Phi\left(X-R \Omega, E^{\prime}, \Omega^{\prime}\right) d \mathrm{E}^{\prime} \frac{d \Omega^{\prime}}{4 \pi}\right. \\
\left.+\int_{E^{\prime}} \int_{\Omega^{\prime}} d \mathrm{E}^{\prime} d \Omega^{\prime} \Phi\left(X-R \Omega, E^{\prime}, \Omega^{\prime}\right) \Sigma_{s}\left(X-R \Omega, E^{\prime} \rightarrow E, \Omega^{\prime} \cdot \Omega\right)\right\}
\end{gathered}
$$

Writing Eq. (9.1.10) in generation notation, multiplying and dividing certain terms by $\Sigma_{\mathrm{t}}(\mathrm{X}, \mathrm{E})$ and multiplying both sides of the equation by $v(\mathrm{X}, \mathrm{E}) \Sigma_{\mathrm{f}}(\mathrm{X}, \mathrm{E})$, yields the following equation, which is solved by KENO $\mathrm{V}$ in the continuous energy mode:

$$
\begin{gathered}
\frac{v(X, E) \Sigma_{f}(X, E)}{\Sigma_{t}(X, E)} \Sigma_{t}(X, E) \Phi_{n}(X, E, \Omega)=\frac{v(X, E) \Sigma_{f}(X, E)}{\Sigma_{t}(X, E)} \Sigma_{t}(X, E) \int_{0}^{\infty} d R e^{-T(R)} \\
\left\{\begin{array}{c}
\frac{1}{k} \int_{E^{\prime}} \int_{\Omega^{\prime}} \frac{v\left(X-R \Omega, E^{\prime}\right) \Sigma_{f}\left(X-R \Omega, E^{\prime}\right)}{\Sigma_{t}\left(X-R \Omega, E^{\prime}\right)} \chi\left(X-R \Omega, E^{\prime} \rightarrow E\right) \Sigma_{t}\left(X-R \Omega, E^{\prime}\right) \Phi_{n-1}\left(X-R \Omega, E^{\prime}, \Omega^{\prime}\right) d \mathrm{E}^{\prime} \frac{d \Omega^{\prime}}{4 \pi} \\
\left.\quad+\int_{E^{\prime}} \int_{\Omega^{\prime}} \frac{\Sigma_{S}\left(X-R \Omega, E^{\prime} \rightarrow E, \Omega^{\prime} \cdot \Omega\right)}{\Sigma_{t}\left(X-R \Omega, E^{\prime}\right)} \Sigma_{t}\left(X-R \Omega, E^{\prime}\right) \Phi_{n}\left(X-R \Omega, E^{\prime}, \Omega^{\prime}\right) d \mathrm{E}^{\prime} d \Omega^{\prime}\right\}
\end{array}\right.
\end{gathered}
$$

where $\mathrm{n}$ indicates the $\mathrm{n}$ th generation and $\mathrm{n}-1$ is the $(\mathrm{n}-1)$ th generation. Note that the left-hand side of the equation, $v(\mathrm{X}, \mathrm{E}) \Sigma_{\mathrm{f}}(\mathrm{X}, \mathrm{E}) \Phi_{\mathrm{n}}(\mathrm{X}, \mathrm{E}, \Omega)$ is the fission production for the $\mathrm{n}$ th generation.

The solution strategy used by KENO solves Eq. (9.1.11) by using an iterative procedure. The fission production at point $\mathrm{X}$ at energy point $\mathrm{E}$ due to neutrons in the $(\mathrm{n}-1)$ th generation, normalized to the system multiplication, is 


$$
\frac{1}{\mathrm{k}} \int_{\mathrm{E}^{\prime}} \int_{\Omega^{\prime}} \frac{v\left(\mathrm{X}, \mathrm{E}^{\prime}\right) \Sigma_{\mathrm{f}}\left(\mathrm{X}, \mathrm{E}^{\prime}\right)}{\Sigma_{\mathrm{t}}\left(\mathrm{X}, \mathrm{E}^{\prime}\right)} \chi\left(\mathrm{X}, \mathrm{E}^{\prime} \rightarrow \mathrm{E}\right) \Sigma_{\mathrm{t}}(\mathrm{X}, \mathrm{E}) \Phi_{\mathrm{n}-1}\left(\mathrm{X}, \mathrm{E}^{\prime}, \Omega^{\prime}\right) \mathrm{d} \mathrm{E}^{\prime} \frac{\mathrm{d} \Omega^{\prime}}{4 \pi}
$$

The collision points used in KENO are chosen by selecting path lengths from the distribution

$$
\mathrm{e}^{-\mathrm{T}(\mathrm{R})}
$$

which is the probability of transport from any position $X-R \Omega$ to position $X$.

The first collision density of neutrons at energy E per unit solid angle about $\Omega$ resulting from the fission source produced by the $(n-1)$ generation, normalized to the system multiplication, is

$$
\begin{aligned}
& \Sigma_{\mathrm{t}}(\mathrm{X}, \mathrm{E}) \int_{0}^{\infty} \mathrm{dR} \mathrm{e}^{-\mathrm{T}(\mathrm{R})} \frac{1}{\mathrm{k}} \int_{\Omega^{\prime}} \int_{\mathrm{E}^{\prime}} \frac{\mathrm{v}\left(\mathrm{X}-\mathrm{R} \Omega, \mathrm{E}^{\prime}\right) \Sigma_{\mathrm{f}}\left(\mathrm{X}-\mathrm{R} \Omega, \mathrm{E}^{\prime}\right)}{\Sigma_{\mathrm{t}}\left(\mathrm{X}-\mathrm{R} \Omega, \mathrm{E}^{\prime}\right)} \\
& \chi\left(\mathrm{X}-\mathrm{R} \Omega, \mathrm{E}^{\prime} \rightarrow \mathrm{E}\right) \Sigma_{\mathrm{t}}\left(\mathrm{X}-\mathrm{R} \Omega, \mathrm{E}^{\prime}\right) \Phi_{\mathrm{n}-1}\left(\mathrm{X}-\mathrm{R} \Omega, \mathrm{E}^{\prime}, \Omega^{\prime}\right) \mathrm{d} \mathrm{E}^{\prime} \frac{\mathrm{d} \Omega^{\prime}}{4 \pi} .
\end{aligned}
$$

The scattering source at position $\mathrm{X}$ emerging at energy $\mathrm{E}$ and direction $\Omega$ resulting from previous collisions in the same generation, is

$$
\int_{E^{\prime}} \int_{\Omega^{\prime}} \frac{\Sigma_{s}\left(X, E^{\prime} \rightarrow E, \Omega^{\prime} \cdot \Omega\right)}{\Sigma_{t}\left(X, E^{\prime}\right)} \Sigma_{t}\left(X, E^{\prime}\right) \Phi_{n}\left(X, E^{\prime}, \Omega^{\prime}\right) d \Omega^{\prime} d E^{\prime}
$$

The collision density at energy E, per solid angle about $\Omega$ is

$$
\Sigma_{t}(X, E) \int_{0}^{\infty} d R e^{-T(R)} \int_{E^{\prime}} \int_{\Omega^{\prime}} \frac{\Sigma_{s}\left(X-R \Omega, E^{\prime} \rightarrow E, \Omega^{\prime} \cdot \Omega\right)}{\Sigma_{t}\left(X-R \Omega, E^{\prime}\right)} \Sigma_{t}\left(X-R \Omega, E^{\prime}\right) \Phi_{n}\left(X-R \Omega, E^{\prime}, \Omega^{\prime}\right) d \Omega^{\prime} d E^{\prime}
$$

The total collision density times $\frac{v_{\mathrm{g}}(\mathrm{X}) \Sigma_{\mathrm{fg}}(\mathrm{X})}{\Sigma_{\mathrm{tg}}(\mathrm{X})}$ is the relationship from which KENO picks the source points for the next generation. 


\subsection{Problem initialization}

In order to use continuous energy cross sections in the random walk, various initialization tasks must be addressed for each problem. Based on the mixture specifications for a problem, KENO reads the microscopic cross section data for each nuclide and dynamically allocates storage for the particular problem. For continuous energy problems, if $\mathbf{U} \mathbf{U M}=$ no then KENO will not allocate macroscopic cross sections for each material, and will instead use the master set of microscopic cross section data (on a nonunionized energy grid). This is the default behavior. Storing cross sections on a material-based unionized energy grid (UUM=yes) results in smaller cross section lookup times and faster KENO runtimes; however, storing unionized cross section data for every material can require a prohibitively large amount of memory for problems with a large number of materials. Users should therefore only set $\mathbf{U U M}=$ yes for relatively small models; experience is the best guide as to what "small" means with respect to available system resources. After finishing the cross section processing, KENO reads the user-specified KENO data (See Sect. 8.1.4) and stores the problem-dependent data for retrieval during the random walk. After the data initialization tasks are complete, the Monte Carlo random walk can be performed according to the procedures that are documented in the subsequent sections.

\subsection{Initial source distribution}

Before the Monte Carlo simulation can be performed, the initial source distribution of neutrons must be sampled. Typically, each mixture in a problem is composed of multiple isotopes, and each mixture must be checked for the presence of fissionable material. For each fissionable mixture, the volume fraction of fissionable material must be calculated on a per isotope basis.

The first source distribution is comprised of the initial spatial coordinates, direction cosines and energy for each neutron within the problem. Regarding the coordinate values, both continuous energy and multigroup KENO use the same start types, and the initial angular distribution is sampled from an isotropic distribution. For the initial energy distribution, the energy of each neutron must be sampled from the continuous energy fission spectrum, $\chi(E)$, of a fissionable isotope within the mixture. If more than one fissionable isotope is present, the $i^{\text {th }}$ isotope can be selected using the following relation:

$$
\sum_{j=1}^{i-1} \Sigma_{f}^{j}<R \sum_{j=1}^{N} \Sigma_{f}^{j} \leq \sum_{j=1}^{i} \Sigma_{f}^{j}
$$

where

$$
\begin{aligned}
& \Sigma_{f}^{j}=\text { macroscopic fission cross section for the } j^{\text {th }} \text { isotope }, \\
& R=\text { random number }[0,1) \\
& N=\text { total number of fissionable isotopes in the mixture. }
\end{aligned}
$$

Note that the relation in Eq. (8.1.12) requires the knowledge of the fission cross section at a particular energy. Therefore, an initial energy of $0.025 \mathrm{eV}$ is assumed for selecting the appropriate isotope to sample. Once the $i^{\text {th }}$ isotope is selected, the initial energy is sampled from the corresponding $\chi_{\mathrm{i}}(E)$. 


\subsection{Collision site selection}

Each neutron must be tracked until the history is terminated via leakage from the system or the particle is "killed" via roulette. The selection of the next collision site is governed by the following probability density function (PDF):

$$
f(x) d x=\Sigma_{t}^{m}(E) e^{-\Sigma_{t}^{m}(E) \mathrm{x}} d x
$$

where

$$
\begin{array}{ll}
\Sigma_{t}^{m}(E) & =\text { macroscopic total cross section for mixture } m \text { at energy } E, \\
x & =\text { spatial variable. }
\end{array}
$$

The PDF in Eq. (8.1.13) describes the probability that a neutron will have an interaction between $x$ and $x+d x$ along its flight path. Integrating Eq. (8.1.13) over the spatial variable yields the following cumulative distribution function (CDF):

$$
F(x)=\int_{0}^{x} \Sigma_{t}^{m}(E) e^{-\Sigma_{t}^{m}(E) x^{\prime}} d x^{\prime}=1-e^{-\Sigma_{t}^{m}(E) x}
$$

and the next collision site is determined by setting the CDF in Eq. (8.1.14) equal to a random number on the interval $[0,1)$ and solving for $x$.

Note that Eq. (8.1.14) requires the total cross section for mixture $m$ at energy $E$ in order to calculate the next collision site. As mentioned in the Sect. 8.1.6.2.1, KENO has two approaches to calculate the macroscopic total cross section for each mixture; (1) use a unionized energy grid (UUM=yes) - once KENO reads the microscopic data for each isotope/nuclide in a mixture, KENO calculates and stores the macroscopic total cross sections for the nuclides in each mixture (2) on-the-fly mixture macroscopic cross section calculation ( $\mathbf{U U M}=\mathrm{no}$ ) - KENO does not store macroscopic cross sections and instead calculates mixture cross sections upon request during particle tracking. Selecting $\mathbf{U U M}=$ yes results in substantially increased memory usage for problems with a large number of materials, making it impossible to perform $\mathrm{CE}$ calculations for some detailed problems. Thus, it is highly recommended to use $\mathbf{U} \mathbf{U M}=$ no, the default setting, for the large problems with multiple mixture definitions. For most cases selecting $\mathbf{U U M}=$ no increases the runtime for KENO CE calculations by approximately $17 \%$, but this feature also expands the code's capability to simulate very large problems with multiple mixture configurations.

Just as the UUM option allows the user to prevent KENO from storing mixture cross sections on a unionized energy grid, the M2U option toggles whether or not KENO stores cross sections on a unionized energy grid for each individual nuclide. The default for this option is $\mathbf{M 2} \mathbf{U}=$ no, which prevents the storage of all the transport cross sections (i.e. inelastic scattering levels) for each nuclide in addition to the major reactions that are already on the unionized energy grid (i.e. total, fission, capture, and scatter) on an energy grid that is unionized for that nuclide, and setting $\mathbf{M 2} \mathbf{U}=y e s$ activates this unionization and storage. $\mathbf{M 2} \mathbf{U}=$ no reduces the nuclear data memory footprint of large models by several gigabytes, but it also increases the runtime of these problems by several percent. It is encouraged to investigate both UUM and M2U options in continuous energy problems to optimize the runtime and memory depending on the user's models and applications. In the continuous energy depletion calculations, UUM parameter is intentionally forced to "no" to minimize the memory requirement of internal data storage. The cross 
section storage and treatment options should be used consistently in both validation and analysis calculations.

If any of the isotopes in the mixture have unresolved-resonance data and corresponding probability-table data, KENO must determine if the neutron energy is in the unresolved resonance range (URR) for each isotope during the random walk. If the neutron energy is in the URR for an isotope, the appropriate probability table must be sampled to obtain the total cross section for each isotope (refer to Sect. 8.1.6.2.6.1 for sampling probability tables). Subsequently, the macroscopic total cross section for mixture $m$ is adjusted to account for sampling the probability tables. Once the revised $\Sigma_{\mathrm{t}}^{\mathrm{m}}(E)$ is determined, Eq. (8.1.14) can be used to select the next collision site within mixture $m$ at energy $E$.

\subsection{Collision treatment}

Once the collision site is determined, the collision can be modeled, and the post collision parameters can be calculated. In the continuous energy approach, each collision is modeled with an individual isotope/nuclide. If a mixture is defined by multiple isotopes and/or nuclides, the target must be selected at each collision site. If there are $N$ different isotopes/nuclides present in a mixture, the following equation can be used to select the $i^{\text {th }}$ target for interaction:

$$
\sum_{j=1}^{i-1} \Sigma_{t}^{j}<R \sum_{j=1}^{N} \Sigma_{t}^{j} \leq \sum_{j=1}^{i} \Sigma_{t}^{j}
$$

where

$$
\Sigma_{t}^{j}=\text { macroscopic total cross section for the } j^{\text {th }} \text { isotope/nuclide. }
$$

As noted in Sects. 8.1.6.2.2 and 8.1.6.2.3, the collision energy may be in the URR of one or more isotopes within the mixture. Consequently, the total cross section for each isotope that has unresolved-resonance data is sampled from the probability table information prior to selecting the next collision site (see discussion in Sect. 8.1.6.2.6.1). Subsequently, the sampled values for the total and partial reactions are stored for retrieval. Therefore, the macroscopic total cross section values that are used in Eq. (8.1.15) are adjusted to account for sampling the probability table data by retrieving the appropriate microscopic total cross section value at energy $E$. Once the corrected values for $\Sigma_{t}^{j}(E)$ are determined, Eq. (8.1.15) can be used to select the target for interaction.

After selecting the collision target, the neutron's weight is reduced by the nonabsorption probability:

$$
w=\frac{\sigma_{s}^{i}(E)}{\sigma_{t}^{i}(E)} w_{b}=P_{n a b s}(E) w_{b},
$$

where

$$
\begin{aligned}
& \sigma_{s}^{i}(E)=\text { microscopic scattering cross section for the } i^{\text {th }} \text { isotope/nuclide at energy } E, \\
& \sigma_{t}^{i}(E)=\text { microscopic total cross section for the } i^{\text {th }} \text { isotope/nuclide at energy E, } \\
& w_{b}=\text { weight before collision. }
\end{aligned}
$$


The absorption and fission weights are calculated using the following relations, respectively:

$$
w_{a}=\frac{\sigma_{a}^{i}(E)}{\sigma_{t}^{i}(E)} w_{b},
$$

where

$$
\sigma_{a}^{i}(E)=\text { microscopic absorption cross section for the } i^{\text {th }} \text { isotope/nuclide at energy } E \text {, }
$$

and

$$
\begin{gathered}
w_{f}=\frac{\bar{v}^{i}(E) \sigma_{f}^{i}(E)}{\sigma_{t}^{i}(E)} w_{b}, \\
\bar{v}^{i}(E)=\text { average number of neutrons released per fission at energy } E, \\
\sigma_{f}^{i}(E)=\text { microscopic fission cross section for the } i^{\text {th }} \text { isotope/nuclide at energy } E .
\end{gathered}
$$

If the collision isotope is in the URR at energy $E$ and probability table data are available, the collision probabilities must be adjusted for sampling the partial reactions from the appropriate probability table. In particular, the revised or sampled values for scattering, absorption and fission must be used to calculate the collision weights as defined by Eqs. (8.1.16)-(8.1.18). Depending on the neutron's weight, splitting and/or Roulette are performed as necessary. Once the appropriate weights are calculated, the collision can be processed to determine the type of interaction and the corresponding exiting energy and angle if secondary neutrons are generated.

Because an explicit collision treatment is dictated by the point cross section data, the type of reaction must be modeled explicitly in the continuous energy version of KENO. Fig. 8.1.196 summarizes the cross section hierarchy and can be used as an aide to understanding the collision treatment in KENO in continuous energy mode. After selecting the isotope/nuclide for interaction according to Eq. (8.1.15) and calculating the weights using Eqs. (8.1.16)-(8.1.18), the collision is modeled using the following procedures:

1. At each collision site, ensure that the type of collision is selected based on the cross section data. Moreover, the $k^{\text {th }}$ reaction can be randomly selected using the following relation:

$$
\sum_{j=1}^{k-1} \sigma_{j}<\mathrm{R} \sum_{j=1}^{N I} \sigma_{j}<\sum_{j=1}^{k} \sigma_{j}
$$

where

$$
\begin{aligned}
& \sigma_{j}(E)= \text { cross section for the } j^{\text {th }} \text { reaction, } \\
& N I=\quad \begin{array}{l}
\text { number of elastic and nonelastic reactions (i.e., excludes neutron } \\
\text { disappearance reactions). }
\end{array}
\end{aligned}
$$


Note that the fission reactions (e.g., first chance fission, second chance fission, etc.) are excluded from Eq. (8.1.19) because an implicit approach is used to treat fission. The details of the fission treatment are provided in Sect. 8.1.6.2.5. If a neutron production reaction with multiple exit neutrons such as $(n, 2 n),(n, 3 n)$ is selected and the reaction is not a fission reaction, then the weight of the neutron is adjusted by the multiplicity to account for the additional neutrons, such as $w=2 w$ for (n, 2n), $w=3 w$ for (n, 3n).

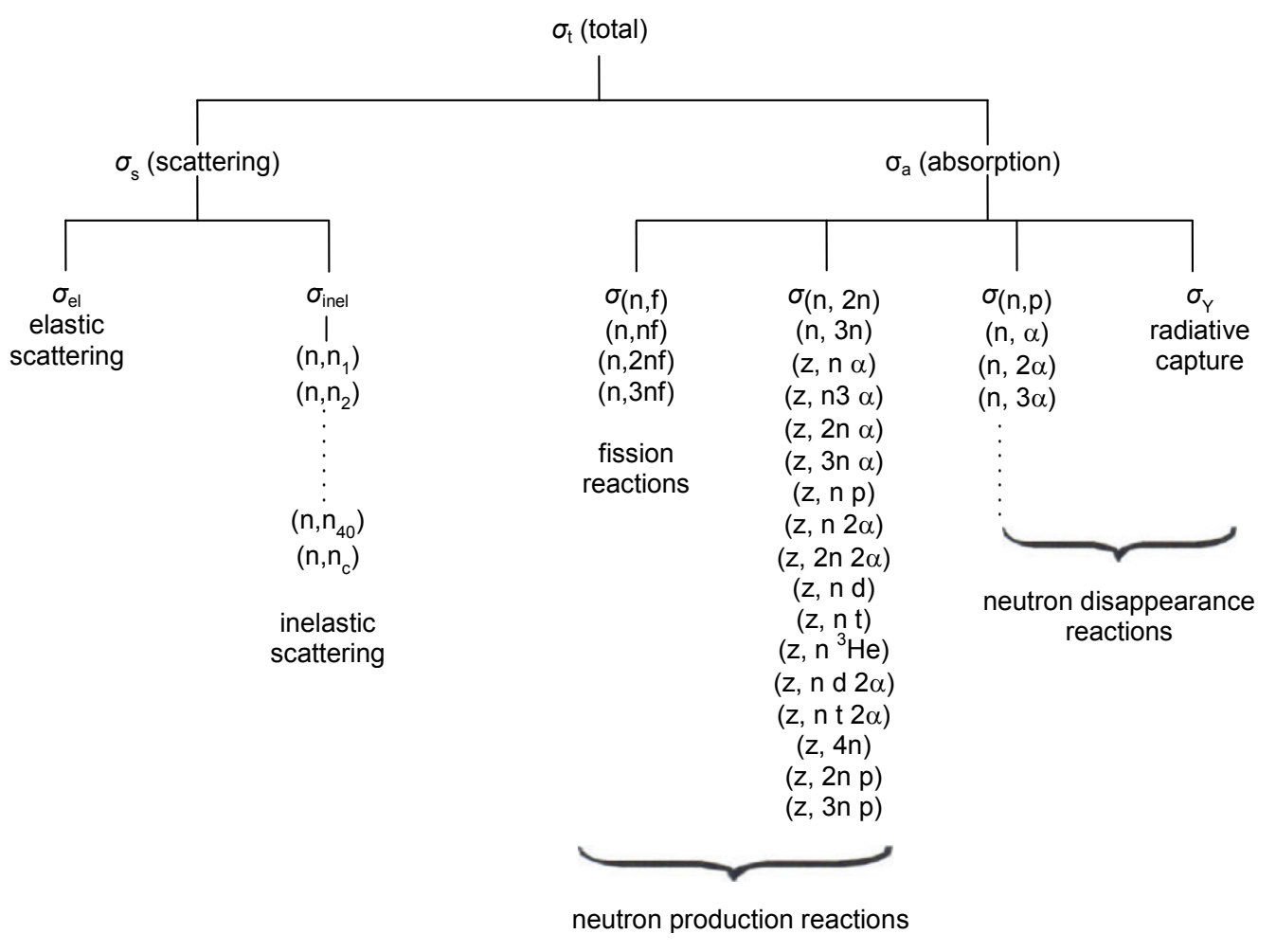

(nonelastic cross section $)^{*}: \sigma_{\mathrm{ne}}=\sigma_{\text {inel }}+$ neutron production cross sections

Fig. 8.1.196. Cross section hierarchy for particle interactions.

2. Select the exiting angle for the collision. The cross section library provides a kinematics data block that provides the angle and energy distributions of secondary particles for a specified reaction. Each record for each section of a reaction provides a flag (NPU) indicating the number of secondary angles for that energy. If the NPU flag is 1 and the corresponding cosine value is -2 (set as a programming flag), then the reaction is isotropic. The exiting angle for the collision can be obtained as follows:

i. Isotropic Emission

If the LAND flag is 0 for the secondary angular distribution, the exiting angle cosine is sampled uniformly between -1 and +1 :

$$
\mu=2 R-1
$$

ii. Anisotropic Emission 
If the LAND flag is 1 for the secondary angular distribution, the exiting angle cosine must be sampled from an anisotropic distribution provided in the cross section library for the specified reaction. Details for sampling the exiting angle from the angular distribution data are provided in Sect. 8.1.6.2.6.

The secondary angular distribution data are provided as a function of incident energy. The initial record of each section for a reaction provides the number of incident energies $(N E)$ for the secondary angular and energy distributions. Corresponding to each incident energy is a secondary angular distribution. Therefore, there will be $N E$ records used to describe the secondary angular data for each section.

For each angular distribution record, there is an $L M U$ flag and a $N P U$ flag that describes the format of the data. The $L M U$ flag designates whether the secondary distribution is provided in equiprobable cosine bins or in nonequiprobable cosine bins. The procedures for sampling the two types of data are provided in Sect. 8.1.6.2.7. The $L M U$ flag must be the same for all $N E$ records within a section. The $N P U$ flag specifies the number of cosine values in the distribution.

Based on the incident energy of the neutron, the angular distribution data are sampled according to the procedures of Sect. 8.1.6.2.7 to obtain the exiting angle cosine, $\mu$, in the lab system.

3. Select the exiting energy for the collision. In addition to the angular distributions of the kinematics data, the cross section library provides the energy distributions of secondary particles for a specified reaction.

The secondary energy distributions are provided as a function of incident energy and secondary angle cosine in each section. Once the exiting angle cosine is selected, the exiting energy is selected from the energy distribution that corresponds to the $(E, \mu)$ pair. The exit energy is determined using the procedures of Sect. 8.1.6.2.7.

For each energy distribution record, there is an $L E$ flag and an NPE flag describing the format of the data. The $L E$ flag designates whether the secondary distribution is provided in equiprobable energy bins or in nonequiprobable energy bins. The procedures for sampling the two types of data are provided in Sect. 8.1.6.2.7. Note that the $L E$ flag must be the same for all $N E$ records within a section for the secondary energy distribution data. The $N P E$ flag specifies the number of secondary energy values in the distribution. For elastic scattering and discrete-level inelastic scattering, the $N P E$ flag will equal the $N P U$ flag due to the one-to-one correspondence between angle and energy.

4. Select the azimuthal angle $\eta$ uniformly between 0 . and $2 \pi$ (i.e., $\eta=2 R \pi$ ).

5. Calculate the new direction cosines in the lab system using the following equations:

$$
\begin{gathered}
u^{\prime}=u \mu-\sqrt{v^{2}+w^{2}} \sqrt{1-\mu^{2}} \cos (\eta), \\
v^{\prime}=v \mu+\frac{u v}{\sqrt{v^{2}+w^{2}}} \cos (\eta) \sqrt{1-\mu^{2}}-\frac{w}{\sqrt{v^{2}+w^{2}}} \sqrt{1-\mu^{2}} \sin (\eta),
\end{gathered}
$$




$$
w^{\prime}=w \mu+\frac{u w}{\sqrt{v^{2}+w^{2}}} \cos (\eta) \sqrt{1-\mu^{2}}+\frac{v}{\sqrt{v^{2}+w^{2}}} \sqrt{1-\mu^{2}} \sin (\eta)
$$

where

$$
\begin{aligned}
u, v, \text { and } w & =\text { initial direction cosines, } \\
u^{\prime}, v^{\prime}, \text { and } w^{\prime} & =\text { exiting direction cosines, } \\
\mu & =\text { cosine of the scattering angle, and } \\
\eta & =\text { cosine of the azimuthal angle. }
\end{aligned}
$$

\subsection{Fission treatment}

As noted in the previous section, an implicit approach is used to treat a fission event. During the collision treatment, the fission weight is calculated using Eq. (8.1.18). After processing the collision, the fission weight is evaluated to determine if the fission treatment should be applied. If the fission weight is greater than zero, the collision occurred in fissile material. During the random walk, several fission source points must be generated to provide an adequate representation of the true source distribution. A minimum production factor is defined at the beginning of each generation to ensure that enough fission points are generated:

$$
m p f=\frac{3.0 \bar{k}}{\sqrt{F G}}
$$

where

$$
\begin{aligned}
& \bar{k}=\text { running average of } k_{\text {eff }} \text { through the current generation, and } \\
& F G=\text { number of histories per generation. }
\end{aligned}
$$

Eq. (8.1.24) represents an estimate of the $99 \%$ lower confidence interval for the distribution of the generation k-effective.

If the fission weight is greater than zero, a pseudo-fission weight is calculated as follows:

$$
p f w=\frac{w_{f}}{R}=\frac{\bar{v}^{i}(E) \sigma_{f}^{i}(E)}{R \sigma_{t}^{i}(E)} w_{b} .
$$

If the pseudo-fission weight is less than the minimum production factor given by Eq. (8.1.24), fission points are not generated and tracking of the particle continues. However, if the pseudo-fission weight is greater than the minimum production factor, $p f w$ is redefined to be the minimum production factor divided by a random number:

$$
p f w=\frac{m p f}{R} .
$$


Once the pseudo-fission weight is redefined, a fission point can be generated. Each time a fission point is generated, the pseudo-fission weight is stored with the point in the fission bank. When a new point is stored in the fission bank, the energy and angular cosine must be selected from the kinematics data. Once the kinematics data are sampled for the new fission point, the fission weight of the history is decremented by the minimum production factor. If the remaining fission weight is greater than zero, the fission treatment procedures are repeated until the fission weight of the history has been exhausted (i.e., $\left.w_{f} \leq 0\right)$.

\subsection{Sampling details}

The preceding sections describe the procedures for the continuous energy random walk for KENO. During the random walk, KENO must sample various tables of data that may include probability tables and angle-energy distributions for secondary particles.

\subsection{Probability tables}

For each isotope with unresolved resonance data, multiple probability tables may be used to describe the URR. The header block for each isotope has an LPTAB flag that provides the number of probability tables for an isotope. Each table is defined for a range of incident energies between $E_{i}$ and $E_{i+1}$, and the energy range for a table should not overlap with another table for the isotope. For a particle with energy $E$, a search must be performed to find the probability table with energy bounds that bracket the particle energy (i.e., $E_{i} \leq E<E_{i+1}$ ). Once the appropriate table is identified, the table can be sampled to obtain the total, elastic scattering, fission and capture cross section values in the URR.

The probability table block provides four separate records that correspond to each reaction within a table; however, the probability table construction is based on the total cross section. Therefore, the probabilities in each table refer to the total cross section band values, and the bands should be sampled based on the total cross section. Once the band is sampled, the corresponding partial reaction cross section values are selected from the same band as the total cross section. The cross section format permits the probability table to be expressed in equiprobable or nonequiprobable cross section bands (i.e., LBND $=0$ or 1, respectively). The procedures for sampling both types of tables are provided in the subsequent subsections.

\subsection{Equiprobable cross section bands}

Each probability record has an NB parameter that designates the number of cross section bands for a table. If the table is constructed with equiprobable cross section bands, the $k^{\text {th }}$ band can be selected as follows:

$$
k=N B^{*} R+1,
$$

where

$$
R=\text { random number }[0 ., 1)
$$

Based on the sampled cross section band, the total cross section corresponding to $k^{\text {th }}$ band is extracted from the table. Likewise, the elastic scattering, fission and capture cross section values that correspond to the $k^{\text {th }}$ band are also extracted from their respective records in the probability-table block. 


\subsection{Nonequiprobable cross section bands}

If the LBND flag is 1 , the cross section bands in the table are not equiprobable, and a different procedure must be used to sample the appropriate cross section band. For this case, the probability values in the table must be constructed as a cumulative distribution function. As noted previously, each probability record has an NB parameter that designates the number of cross section bands for a table, and the $k^{\text {th }}$ band can be selected as follows:

$$
\sum_{j=1}^{k-1} P_{j}<R \sum_{j=1}^{N B} P_{j} \leq \sum_{j=1}^{k} P_{j}
$$

where

$$
P_{j}=\text { probability corresponding to the } \mathrm{j}^{\text {th }} \text { cross section band. }
$$

Based on the sampled cross section band, the total cross section corresponding to the $k^{\text {th }}$ band is extracted from the table. Likewise, the elastic scattering, fission and capture cross section values that correspond to the $k^{\text {th }}$ band are also extracted from their respective records in the probability-table block.

\subsection{Kinematics data}

The kinematics data in the KENO library are provided in the lab or target-at-rest system as opposed to the center-of-mass system. By adhering to the lab coordinate system, KENO does not have to transform between different coordinate systems during the random walk; however, the energy and angle representations for elastic and discrete-level inelastic scattering become more complex in the lab system. For example, an angular distribution that is isotropic in the center-of-mass system is anisotropic in the lab system. Moreover, the secondary energy distribution as a function of exit angle in the lab system can be double valued (i.e., two possible exit energies with respect to a single angle) for energies above the threshold for the reaction. Likewise, a similar double valued distribution is observed for elastic scattering with hydrogen in the lab system if the actual mass ratio is used (i.e., $A=0.99928$ as opposed to $A=1.0$ ). Consequently, special care must be exercised in the construction of the secondary angle and energy distributions in the lab system.

The subsequent sections address the general procedures for sampling the kinematics data with emphasis on the special cases that must be addressed during the random walk. In Sect. 8.1.6.2.7.1, the general procedures for sampling the kinematics data are provided, Sect. 8.1.6.2.7.4 discusses isotropic angular data with energy coupling, while Sect 8.1.6.2.7.5 discusses coherent and incoherent elastic scattering. A discussion is also provided in Sect. 8.1.6.2.7.6 to describe the elastic and discrete-level inelastic treatment.

\subsection{General procedures}

The kinematics data format is designed to accommodate coupled angle energy distributions of secondary particles. The following discussion provides the procedures for sampling the coupled distributions. Special cases such as elastic and discrete level inelastic scattering are discussed after the "General Procedures" section. 
The sampling procedures in the following sections assume that the angle and energy distributions are continuous in nature. As a result, interpolation procedures can be used to obtain intermediate angle or energy values between the tabulated angle or energy values. During the course of KENO development, there may be a need to provide an average angle or energy value for a bin. The kinematics format can be modified as needed to accommodate additional angle and energy representations. Therefore, the following procedures represent the anticipated approach for sampling the current form of the kinematics data.

\subsection{Exit angle cosine}

The first record for each reaction provides the number of sections (NSECT) used to describe the kinematics for the reaction. Within each section, the first record defines the incident energy range for the section. After the first record for a section, the first block of data provides the secondary angular distributions for $N E$ different incident energies within the energy range of the section. Consequently, there will be $N E$ different angular distribution records in the secondary angle cosine block. The kinematics format permits the anisotropic angular distributions to be expressed in either equiprobable or nonequiprobable cosine bins (i.e., $\mathrm{LMU}=0$ or 1 , respectively). Moreover, the formats permit the number of cosine bins to vary as a function of incident energy. In particular, each cosine distribution record can have $N P U$ secondary angles that correspond to $N P U-1$ cosine bins.

Equiprobable Cosine Bins. If the incident energy, $E$, is between $E_{i}$ and $E_{i+1}$, the angle cosine is sampled in both tables, and the exit angle cosine is obtained by interpolating between the two tables. If the angular distribution is provided in equiprobable cosine bins, the $a^{\text {th }}$ bin is selected from the $i^{\text {th }}$ table as follows:

$$
a=\left(N P U_{i}-1\right) * R_{1}+1=N A_{i} * R_{1}+1
$$

In Eq. (8.1.29) $a$ denotes an integer quantity, and $N A_{i}$ is the number of cosine bins for the $i^{\text {th }}$ table. Using a similar procedure, the $b^{\text {th }}$ bin is selected from the $i+1^{\text {st }}$ table:

$$
b=N A_{i+1} * R_{1}+1
$$

As noted for the $i^{\text {th }}$ table, $b$ is an integer quantity in Eq. (8.1.30) and $N A_{i+1}$ is the number of cosine bins for the $i+1^{\text {st }}$ table. Once the cosine bins are selected, the cosine of the exiting angle from the $i^{\text {th }}$ and $i+1^{\text {st }}$ tables is calculated with Eqs. (8.1.31) and (8.1.32), respectively:

$$
\begin{gathered}
\dot{\mu}_{i}=\mu_{i a}+\left(a-N A_{i} * R_{1}\right)\left(\mu_{i a+1}-\mu_{i a}\right), \\
\dot{\mu}_{i+1}=\mu_{i+1 b}+\left(b-N A_{i+1} * R_{1}\right)\left(\mu_{i+1 b+1}-\mu_{i+1 b}\right) .
\end{gathered}
$$

The exiting angle cosine is obtained by interpolating between $i$ and $i+1$ based on the incident energy grid:

$$
\mu=\dot{\mu}_{i}+\left(\frac{E-E_{i}}{E_{i+1}-E_{i}}\right)\left(\dot{\mu}_{i+1}-\dot{\mu}_{i}\right)
$$

Nonequiprobable Cosine Bins. If the angular distribution is in the form of nonequiprobable cosine bins, the $a^{\text {th }}$ bin is selected from the cumulative distribution function for the $i^{\text {th }}$ table:

$$
C_{i a-1}<R_{1} \leq C_{i a},
$$


where

$$
\begin{aligned}
& C_{i a-1}=\text { cumulative probability corresponding to the } a-1^{\text {st }} \text { cosine bin, } \\
& C_{i a}=\text { cumulative probability corresponding to the } a^{\text {th }} \text { cosine bin. }
\end{aligned}
$$

Using a similar procedure, the $b^{\text {th }}$ bin is selected from the $i+1^{\text {st }}$ table:

$$
C_{i+1 b-1}<R_{1} \leq C_{i+1 b}
$$

where

$$
\begin{aligned}
& C_{i b-1}=\text { cumulative probability corresponding to the } b-1^{\text {st }} \text { cosine bin, } \\
& C_{i b}=\text { cumulative probability corresponding to the } b^{\text {th }} \text { cosine bin. }
\end{aligned}
$$

If the cosine bins are not equiprobable and the PDF is represented by a continuous distribution, the bins are selected so that linear interpolation can be performed in the PDF. Because the CDF is obtained by integrating the PDF, the interpolation procedure for the CDF has a quadratic form. The value for $\dot{\mu}_{i}$ is obtained with the following equation:

$$
\dot{\mu}_{i}=\mu_{i a}+\frac{\sqrt{P_{i a}^{2}+2 s_{i}\left(R_{1}-C_{i a-1}\right)}-P_{i a}}{s_{i}},
$$

and

$$
S_{i}=\frac{P_{i a+1}-P_{i a}}{\mu_{i a+1}-\mu_{i a}},
$$

where

$$
\begin{aligned}
P_{i a}= & \text { value of the PDF corresponding to the lower boundary of the } a^{\text {th }} \text { cosine bin in the } \\
& \text { distribution for } E_{i} \text {, and } \\
P_{i a+1}= & \begin{array}{l}
\text { value of the PDF corresponding to the upper boundary of the } a^{\text {th }} \text { cosine bin in the } \\
\text { distribution for } E_{i} .
\end{array}
\end{aligned}
$$

Likewise, the equation for $\dot{\mu}_{i+1}$ is obtained using an equation that is similar to Eq. (9.1.36):

$$
\dot{\mu}_{i+1}=\mu_{i+1 b}+\frac{\sqrt{P_{i+1 b}^{2}+2 s_{i+1}\left(R_{1}-C_{i+1 b-1}\right)}-P_{i+1 b}}{s_{i+1}},
$$

and

$$
S_{i+1}=\frac{P_{i+1 b+1}-P_{i+1 b}}{\mu_{i+1 b+1}-\mu_{i+1 b}},
$$

where 


$$
\begin{aligned}
P_{i+1 b}= & \text { value of the PDF corresponding to the lower value of the } b^{\text {th }} \text { cosine bin in the } \\
& \text { distribution for } E_{i+1} \text {, and } \\
P_{i+1 b+1}= & \text { value of the PDF corresponding to the upper value of the } b^{\text {th }} \text { cosine bin in the } \\
& \text { distribution for } E_{i+1} .
\end{aligned}
$$

The exiting angle cosine is obtained by interpolating between $\dot{\mu}_{i}$ and $\dot{\mu}_{i+1}$ based on the incident energy using Eq. (9.1.33).

\subsection{Exit energy}

In the kinematics data block, each incident energy has a secondary distribution of $N P U$ angle cosines. Therefore, there are $N P U(E, \mu)$ pairs associated with each incident energy. For each $(E, \mu)$ pair, there is a corresponding exit energy distribution that can have $N P E$ exit energies. Because the kinematics data are tabulated in a coupled angle-energy format, the exit energy is obtained by a 2-D interpolation as shown in Fig. 8.1.197.

The objective of the sampling procedure is to sample the exit energy $E^{\prime}$ in conjunction with the sampled angle cosine. Therefore, the interpolation for the exit energy is performed with an equation that is analogous to Eq. (9.1.33):

$$
E^{\prime}=E_{i}^{\prime}+\left(\frac{E-E_{i}}{E_{i+1}-E_{i}}\right)\left(E_{i+1}^{\prime}-E_{i}^{\prime}\right) .
$$

Based on Eq. (9.1.40), the values of $E_{i}^{\prime}$ and $E_{i+1}^{\prime}$ must be obtained in order to calculate the exit energy for the collision. The $a^{\text {th }}$ and $b^{\text {th }}$ cosine bins are selected according to the procedures in Sect. 8.1.6.2.7.1 General Procedures from tables $i$ and $i+1$, respectively. As a result, the secondary energy distributions corresponding to the $a^{\text {th }}$ and $b^{\text {th }}$ cosine bins must be used to sample the exit energies from tables $i$ and $i+1$. The details for sampling the energy bins are deferred for the moment. Once the appropriate energy bins are sampled from the two tables, the interpolation for $E_{i}^{\prime}$ and $E_{i+1}^{\prime}$ is obtained with the following equations:

$$
E_{i}^{\prime}=E_{i a}^{\prime}+\left(\frac{\dot{\mu}_{i}-\mu_{i a}}{\mu_{i a+1}-\mu_{i a}}\right)\left(E_{i a+1}^{\prime}-E_{i a}^{\prime}\right)
$$

and

$$
E_{i+1}^{\prime}=E_{i+1 b}^{\prime}+\left(\frac{\dot{\mu}_{i+1}-\mu_{i+1 b}}{\mu_{i+1 b+1}-\mu_{i+1 b}}\right)\left(E_{i+1 b+1}^{\prime}-E_{i+1 b}^{\prime}\right) .
$$




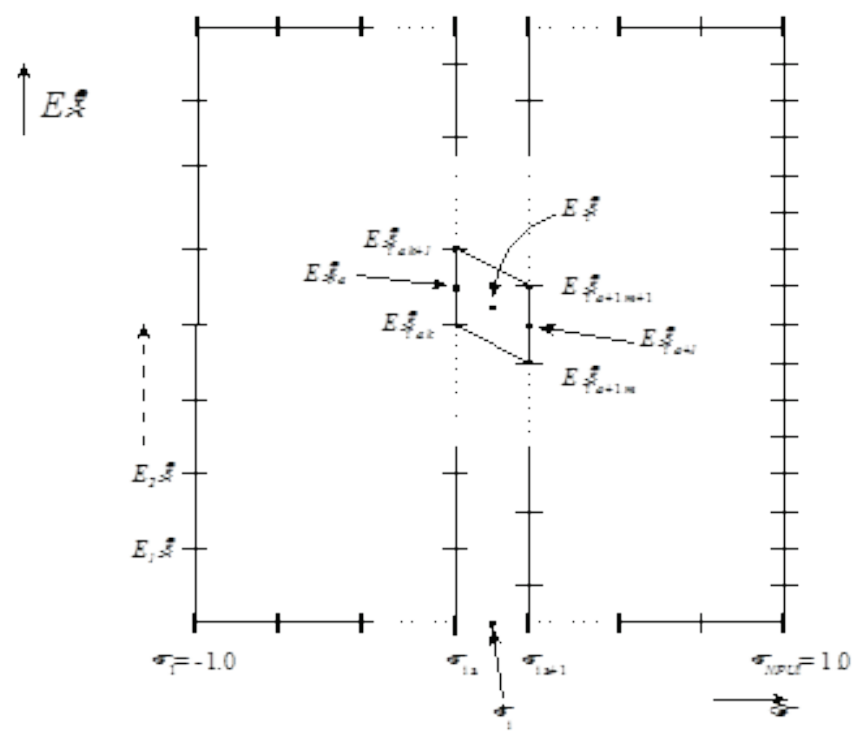

$\underline{\text { Secondary Angle-Energy Table } i \text { for } E_{i}}$

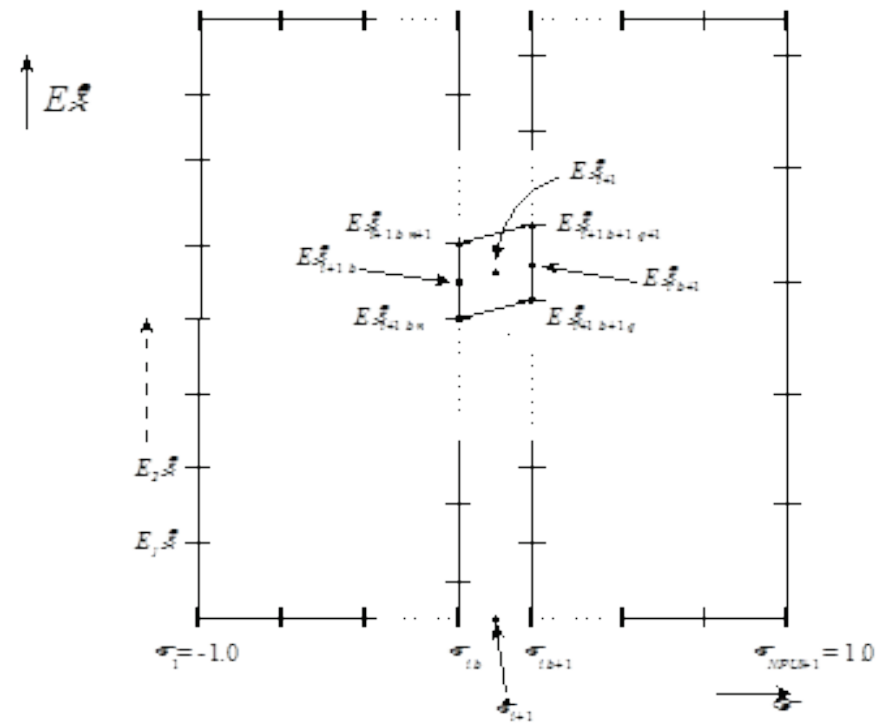

Secondary Angle-Energy Table $i+1$ for $E_{i+1}$

Fig. 8.1.197. Interpolation diagram for secondary angle-energy data. 
Based on Eqs. (8.1.41) and (8.1.42), the values for $E_{i a}^{\prime}, E_{i a+1}^{\prime}, E_{i+1 b}^{\prime}$ and $E_{i+1}^{\prime}{ }_{b+1}$ are needed to calculate $E_{i}^{\prime}$ and $E_{\mathrm{i}+1}^{\prime}$. The values for $E_{i a}^{\prime}$ and $E_{i a+1}^{\prime}$ correspond to secondary energies that are coupled with the $a^{\text {th }}$ and $a+1^{\text {st }}$ angle cosines in Table $i$. Likewise, the values for $E_{i+1}^{\prime} b$ and $E_{i+1}^{\prime} b+1$ correspond to the secondary energies that are coupled with the $b^{\text {th }}$ and $b+1^{\text {st }}$ angle cosines in Table $i+1$. Therefore, the exit energies from Table $i$ are sampled from the secondary energy distributions that correspond to $\left(E, \mu_{i a}\right)$ and $\left(E, \mu_{i a+1}\right)$, and the energies from Table $i+1$ are sampled from the energy distributions for $\left(E, \mu_{i+1} b\right)$ and $\left(E, \mu_{i+1 b+1}\right)$.

As with the angular data, the secondary energy distributions may be provided in equiprobable or nonequiprobable energy bins. The following discussion provides the procedures for sampling the different distributions.

Equiprobable Energy Bins. In Table $i$, the $k^{\text {th }}$ and $m^{\text {th }}$ energy bins are sampled from the energy distributions for $\left(E, \mu_{i a}\right)$ and $\left(E, \mu_{i a+1}\right)$, respectively. The number of energy bins in a distribution is one less than the number of energies in the table (i.e., $N P E-1$ ). If the energy distributions are provided in equiprobable bins, the $k^{\text {th }}$ and $m^{\text {th }}$ bins are selected as follows:

$$
\begin{gathered}
k=\left(N P E_{i a}-1\right) * R_{2}+1, \\
m=\left(N P E_{i a+1}-1\right) * R_{2}+1 .
\end{gathered}
$$

The exiting energy values for $E_{i a}^{\prime}$ and $E_{i a+1}^{\prime}$ are interpolated as follows:

$$
\begin{gathered}
{E^{\prime}}_{i a}=E^{\prime}{ }_{i a k}+\left[k-\left(N P E_{i a}-1\right) * R_{2}\right]\left({E^{\prime}}_{i a k+1}-E^{\prime}{ }_{i a k}\right), \\
E^{\prime}{ }_{i a+1}=E^{\prime}{ }_{i a+1 m}+\left[m-\left(N P E_{i a+1}-1\right) * R_{2}\right]\left({E^{\prime}}_{i a+1 m+1}-E^{\prime}{ }_{i a+1 m}\right),
\end{gathered}
$$

In Table $i+1$, the $n^{\text {th }}$ and $q^{\text {th }}$ energy bins are sampled from the energy distributions for $\left(E, \mu_{i b}\right)$ and $\left(E, \mu_{i b+1}\right)$, respectively. As presented for the $i^{\text {th }}$ table, the $n^{\text {th }}$ and $q^{\text {th }}$ bins are selected from an equiprobable distribution in a manner analogous with Eqs. (8.1.43) and (8.1.44):

$$
\begin{gathered}
n=\left(N P E_{i+1 b}-1\right) * R_{2}+1, \\
q=\left(N P E_{i+1 b+1}-1\right) * R_{2}+1 .
\end{gathered}
$$

The exiting energy values for $E_{i+1 b}^{\prime}$ and $E_{i+1}^{\prime} b+1$ are interpolated as follows:

$$
\begin{gathered}
{E^{\prime}}{ }_{i+1 b}=E^{\prime}{ }_{i+1 b n}+\left[n-\left(N P E_{i+1 b}-1\right) * R_{2}\right]\left({E^{\prime}}{ }_{i+1 b n+1}-E^{\prime}{ }_{i+1 b n}\right) \\
{E^{\prime}}{ }_{i+1 b+1}=E^{\prime}{ }_{i+1 b+1 q}+\left[q-\left(N P E_{i+1 b+1}-1\right) * R_{2}\right]\left({E^{\prime}}{ }_{i+1 b+1 q+1}-E^{\prime}{ }_{i+1 b+1 q}\right) .
\end{gathered}
$$

The exit energy from the $i^{\text {th }}$ table (i.e., $E_{i}^{\prime}$ ) is obtained by substituting the values for $E_{i a}^{\prime}$ and $E_{i a+1}^{\prime}$ into Eq. (8.1.41), and the exit energy from the $i+1^{\text {st }}$ table (i.e., $\left.E^{\prime}{ }_{i+1}\right)$ is calculated by substituting the values for $E_{i b}^{\prime}$ and $E_{i b+1}^{\prime}$ into Eq. (8.1.42). Finally, the exit energy for the collision is obtained by inserting the calculated values for $E_{i}^{\prime}$ and $E_{i+1}^{\prime}$ into Eq. (9.1.40). 
Nonequiprobable Energy Bins. If the energy distributions are provided in nonequiprobable bins, the $k^{\text {th }}$ and $m^{\text {th }}$ bins are sampled according to the cumulative distribution values:

$$
\begin{gathered}
C_{i a k-1}<R_{2} \leq C_{i a k}, \\
C_{i a+1 m-1}<R_{2} \leq C_{i a+1 m},
\end{gathered}
$$

where

$$
\begin{aligned}
C_{i a k-1}= & \text { cumulative probability corresponding to the } k-1^{\text {st }} \text { energy bin in the }\left(E, \mu_{i a}\right) \text { distribution, } \\
\mathrm{C}_{i a k}= & \text { cumulative probability corresponding to the } k^{\text {th }} \text { energy bin in the }\left(E, \mu_{i a}\right) \text { distribution, } \\
\mathrm{C}_{i a+1 m-1}= & \begin{array}{l}
\text { cumulative probability corresponding to the } m-1^{\text {st }} \text { energy bin in the }\left(E, \mu_{i} a+1\right) \\
\text { distribution, and }
\end{array} \\
\mathrm{C}_{i a+1 m}= & \text { cumulative probability corresponding to the } m^{\text {th }} \text { energy bin in the }\left(E, \mu_{i a+1}\right) \text { distribution. }
\end{aligned}
$$

For the nonequiprobable bins, the bins for the secondary energy are selected so that linear interpolation can be performed in the PDF. Since the CDF is obtained by integrating the PDF, the interpolation procedure for the CDF is quadratic in form. As a result, the value for $E_{i a}^{\prime}$ is obtained with the following equation:

$$
E^{\prime}{ }_{i a}=E^{\prime}{ }_{i a k}+\frac{\sqrt{P_{i a k}^{2}+2 s_{i a}\left(R_{2}-C_{i a k-1}\right)}-P_{i a k}}{s_{i a}},
$$

and

$$
s_{i a}=\frac{\sqrt{P_{i a k+1}-P_{i a k}}}{E^{\prime}{ }_{i a k+1}-E^{\prime}{ }_{i a k}},
$$

where

$$
\begin{aligned}
P_{i a k}= & \text { value of the PDF corresponding to the lower boundary of the } k^{\text {th }} \text { energy bin in the } \\
& \left(E, \mu_{i a}\right) \text { distribution, and } \\
P_{i a k+1}= & \text { value of the PDF corresponding to the upper boundary of the } k^{\text {th }} \text { energy bin in the } \\
& \left(E, \mu_{i a}\right) \text { distribution. }
\end{aligned}
$$

Likewise, the value for $E_{i a^{+1}}^{\prime}$ is obtained with the following equation:

$$
E_{i a+1}^{\prime}=E_{i a+1 m}^{\prime}+\frac{\sqrt{P_{i a+1 m}^{2}+2 s_{i a+1}\left(R_{2}-C_{i a+1 m-1}\right)}-P_{i a+1 m}}{s_{i a+1}},
$$


and

$$
s_{i a+1}=\frac{P_{i a+1 m+1}-P_{i a+1 m}}{E^{\prime}{ }_{i a+1 m+1}-E^{\prime}{ }_{i a+1 m}},
$$

where

$$
\begin{aligned}
P_{i a+1 m}= & \text { value of the PDF corresponding to the lower boundary of the } m^{\text {th }} \text { energy bin in the } \\
& \left(E, \mu_{i a+1}\right) \text { distribution, and } \\
P_{i a+1 m+1}= & \text { value of the PDF corresponding to the upper boundary of the } m^{\text {th }} \text { energy bin in the } \\
& \left(E, \mu_{i a+1}\right) \text { distribution. }
\end{aligned}
$$

For nonequiprobable distributions in the $i+1^{\text {st }}$ table, the $n^{\text {th }}$ and $q^{\text {th }}$ bins are sampled according to the cumulative distribution values:

$$
\begin{gathered}
C_{i+1 b n-1}<R_{2} \leq C_{i+1 b n}, \\
C_{i+1 b+1 q-1}<R_{2} \leq C_{i+1 b+1 q},
\end{gathered}
$$

where

$$
\begin{aligned}
& C_{i+1 b n-1}= \begin{array}{l}
\text { cumulative probability corresponding to the } n-1^{\text {st }} \text { energy bin in the }\left(E, \mu_{i+1} b\right) \\
\text { distribution, }
\end{array} \\
& C_{i+1 b n}=\begin{array}{l}
\text { cumulative probability corresponding to the } n^{\text {th }} \text { energy bin in the }\left(E, \mu_{i+1} b\right) \\
\text { distribution, }
\end{array} \\
& C_{i+1 b+1 q-1}=\begin{array}{l}
\text { cumulative probability corresponding to the } q-1^{\text {st }} \text { energy bin in the }\left(E, \mu_{i+1} b+1\right) \\
\text { distribution, and }
\end{array} \\
& C_{i+1 b+1 q}=\begin{array}{l}
\text { cumulative probability corresponding to the } q^{\text {th }} \text { energy bin in the }\left(E, \mu_{i+1} b+1\right) \\
\text { distribution. }
\end{array}
\end{aligned}
$$

As observed for the $i^{\text {th }}$ table, the interpolation procedure for the CDF is quadratic in form, and the value for $E_{i+1 b}^{\prime}$ is obtained as follows:

$$
E^{\prime}{ }_{\mathrm{i}+1 b}=E^{\prime}{ }_{i+1 b n}+\frac{\sqrt{P_{i+1 b n}^{2}+2 s_{i+1 b}\left(R_{2}-C_{i+1 b n-1}\right)}-P_{i+1 b n}}{s_{\mathrm{i}+1 b}},
$$

and

$$
s_{i+1 b}=\frac{P_{i+1 b n+1}-P_{i+1 b n}}{E^{\prime}{ }_{i+1 b n+1}-E^{\prime}{ }_{i+1 b n}},
$$

where 


$$
\begin{aligned}
P_{i+1 b n}= & \text { value of the PDF corresponding to the lower boundary of the } n^{\text {th }} \text { energy bin in the } \\
& \left(E, \mu_{i+1} b\right) \text { distribution, and } \\
P_{i+1 b n+1}= & \text { value of the PDF corresponding to the upper boundary of the } n^{\text {th }} \text { energy bin in the } \\
& \left(E, \mu_{i+1} b\right) \text { distribution. }
\end{aligned}
$$

Likewise, the value for $E_{i+1}^{\prime} b+1$ is obtained with the following equation:

$$
E^{\prime}{ }_{i+1 b+1}=E^{\prime}{ }_{i+1 b+1 q}+\frac{\sqrt{P_{i+1 b+1 q}^{2}+2 s_{i+1 b+1}\left(R_{2}-C_{i+1 b+1 q-1}\right)}-P_{i+1 b+1 q}}{s_{i+1 b+1}}
$$

and

$$
s_{i+1 b+1}=\frac{P_{i+1 b+1 q+1}-P_{i+1 b+1 q}}{E^{\prime}{ }_{i+1 b+1 q+1}-E^{\prime}{ }_{i+1 b+1 q}}
$$

where

$$
\begin{aligned}
P_{i+1 b+1 q}= & \text { value of the PDF corresponding to the lower boundary of the } q^{\text {th }} \text { energy bin in } \\
& \text { the }\left(E, \mu_{i+1} \mathrm{~b}+1\right) \text { distribution, and } \\
P_{i+1 b+1 q+1}= & \begin{array}{l}
\text { value of the PDF corresponding to the upper boundary of the } q^{\text {th }} \text { energy bin in } \\
\text { the }\left(E, \mu_{i+1} b+1\right) \text { distribution. }
\end{array}
\end{aligned}
$$

To obtain the exit energy for the collision, the energy from the $i^{\text {th }}$ table (i.e., $E_{i}^{\prime}$ ) is calculated by substituting the values for $E_{i a}^{\prime}$ and $E_{i a+1}^{\prime}$ into Eq. (8.1.41), and the exit energy from the $i+1^{\text {st }}$ table (i.e., $\left.E_{i+1}^{\prime}\right)$ is calculated by substituting the values for $E_{i b}^{\prime}$ and $E_{i b+1}^{\prime}$ into Eq. (8.1.42). Finally, the exit energy for the collision is obtained by inserting the calculated values for $E_{i}^{\prime}$ and $E_{i+1}^{\prime}$ into Eq. (9.1.40).

\subsection{Isotropic Angular Distributions With Energy Coupling}

The kinematics format can accommodate isotropic angular distributions in the coupled angle energy format. The following discussion describes the special case with the appropriate sampling procedures. If the secondary angular distribution is isotropic at an incident energy $E$, a single exit cosine with a value of -2.0 is specified in the $(E, \mu)$ block. Therefore, the exit angle cosine is sampled uniformly between -1.0 and 1.0:

$$
\mu=2 R_{1}-1
$$

Because there is only one exit cosine specified in the $(E, \mu)$ block, there is a single energy distribution record specified for the $(E, \mu)$ pair. The general structure (i.e., material identifiers and data flags are omitted for clarity) of the kinematics data within a section for a reaction is presented in Table 8.1.25. As a result, the sampling procedure for the exit energy is based on the incident energy.

Table 8.1.25. Kinematics data structure for isotropic angular distributions 


\begin{tabular}{|c|c|c|c|c|c|}
\hline \multicolumn{6}{|c|}{$(E, \mu)$ Data Block } \\
\hline$E$ & $\mathrm{C}_{2}$ & $\mu$ & $\overline{C_{\mu}}$ & $\bar{P} P_{\mu}$ & \\
\hline$E_{1}$ & 0 & -2.0 & 1.0 & 1.0 & \\
\hline$E_{2}$ & 0 & -2.0 & 1.0 & 1.0 & \\
\hline - & $\bullet$ & $\bullet$ & $\bullet$ & $\bullet$ & \\
\hline$\bullet$ & $\bullet$ & $\bullet$ & $\bullet$ & $\bullet$ & \\
\hline$\bullet$ & $\bullet$ & $\bullet$ & $\bullet$ & $\bullet$ & \\
\hline$E_{\mathrm{NE}}$ & 0 & -2.0 & 1.0 & 1.0 & \\
\hline \multicolumn{6}{|c|}{$\left(E, \mu, E^{\prime}\right)$ Data Block } \\
\hline$E$ & $\mu$ & $E^{\prime}(\mathrm{j})$ & $\overline{C_{E^{\prime}}(\mathrm{j})}$ & $P_{E^{\prime}}(\mathrm{j})$ & $\mathrm{j}=1, N P E$ \\
\hline$E_{1}$ & -2.0 & $E^{\prime}(\mathrm{j})$ & $C_{E^{\prime}}(\mathrm{j})$ & $P_{E^{\prime}}(\mathrm{j})$ & $\mathrm{j}=1, N P E$ \\
\hline$E_{2}$ & -2.0 & $E^{\prime}(\mathrm{j})$ & $C_{E^{\prime}}(\mathrm{j})$ & $P_{E^{\prime}}(\mathrm{j})$ & $\mathrm{j}=1, N P E$ \\
\hline$\bullet$ & $\bullet$ & $\bullet$ & $\bullet$ & $\bullet$ & $\bullet$ \\
\hline$\bullet$ & $\bullet$ & $\bullet$ & $\bullet$ & $\bullet$ & $\bullet$ \\
\hline$\bullet$ & $\bullet$ & $\bullet$ & $\bullet$ & $\bullet$ & $\bullet$ \\
\hline$E_{\mathrm{NE}}$ & -2.0 & $E^{\prime}(\mathrm{j})$ & $C_{E^{\prime}}(\mathrm{j})$ & $P_{E^{\prime}}(\mathrm{j})$ & $\mathrm{j}=1, N P E$ \\
\hline
\end{tabular}

Once the angle is sampled according to Eq. (8.1.63), the exit energy is sampled in a manner that is analogous to the procedures of Sect. Exit energy. If the incident energy, $E$, is between $E_{\mathrm{i}}$ and $E_{i+1}$, the $k^{\text {th }}$ energy bin is sampled from the distribution corresponding to $E_{\mathrm{i}}$ using Eq.(8.1.64) or Eq. (8.1.65) for equiprobable or nonequiprobable distributions, respectively.

$$
k=\left(N P E_{i}-1\right) * R_{2}+1,
$$

or

$$
C_{i k-1}<R_{2} \leq C_{i k},
$$

where

$$
\begin{aligned}
N P E_{i}= & \text { number of exit energies corresponding to } E_{i}, \\
C_{i k-1}= & \text { cumulative probability corresponding to the } k-1^{\text {st }} \text { energy bin in the distribution for } \\
& E_{i} \text {, and } \\
\mathrm{C}_{i k}= & \text { cumulative probability corresponding to the } k^{\text {th }} \text { energy bin in the distribution for } E_{i} .
\end{aligned}
$$

For equiprobable energy bins, the exit energy corresponding to $E_{i}$ is calculated as follows:

$$
E^{\prime}{ }_{i}=E^{\prime}{ }_{i k}+\left[k-\left(N P E_{i}-1\right) * R_{2}\right]\left(E^{\prime}{ }_{i k+1}-E^{\prime}{ }_{i k}\right) .
$$


If the secondary energy distributions are provided in nonequiprobable bins the exit energy is calculated using the following equation:

$$
E_{i}^{\prime}=E^{\prime}{ }_{i k}+\frac{P_{i k}^{2}+2 s_{i}\left(R_{2}-C_{i k-1}\right)-P_{i k}}{s_{i}},
$$

and

$$
s_{i}=\frac{P_{i k+1}-P_{i k}}{E^{\prime}{ }_{i k+1}-E^{\prime}{ }_{i k}},
$$

where

$$
\begin{aligned}
& P_{i k}=\quad \begin{array}{l}
\text { value of the PDF corresponding to the lower boundary of the } k^{\text {th }} \text { energy bin in the } \\
\text { distribution for } E_{i} \text {, and }
\end{array} \\
& P_{i k+1}=\quad \begin{array}{l}
\text { value of the PDF corresponding to the upper boundary of the } k^{\text {th }} \text { energy bin in the } \\
\text { distribution for } E_{i} .
\end{array}
\end{aligned}
$$

By replacing $i$ with $i+1$ in Eqs. (8.1.64) through (8.1.68), the exit energy $E_{i+1}^{\prime}$ can be calculated from the secondary energy distribution corresponding to the incident energy $E_{i+1}$. Subsequently, Eq. (9.1.40) can be used to calculate the exit energy.

\subsection{Coherent and incoherent elastic scattering}

If thermal scattering law data are available for a material, the elastic scattering mechanism may be specified as either coherent or incoherent elastic scattering. Consequently, there is no change in energy resulting from either collision. The secondary energy distribution block for each $(E, \mu)$ pair only has one exit energy with a value equal to the incident energy $E$. Therefore, the procedure for coherent or incoherent elastic scattering reduces to sampling the exit angle cosine. The procedures detailed in Sect. 8.1.6.2.7.2 are used to sample the exit angle cosine. The general structure of the kinematics data within a section for coherent or incoherent elastic scattering is presented in Table 8.1.26 with the material identifiers and data flags omitted for clarity. Once the angle cosine is selected, the exit energy is set equal to the incident energy. 
Table 8.1.26. Kinematics data structure for coherent and incoherent elastic scattering

\begin{tabular}{|c|c|c|c|c|c|}
\hline \multicolumn{6}{|c|}{$(E, \mu)$ Data Block } \\
\hline$E$ & $\mathrm{C}_{2}$ & $\mu(\mathrm{j})$ & $C_{\mu}(\mathrm{j})$ & $P_{\mu}(\mathrm{j})$ & $\mathrm{j}=1, N P U$ \\
\hline$E_{1}$ & 0 & $\mu(\mathrm{j})$ & $C_{\mu}(\mathrm{j})$ & $P_{\mu}(\mathrm{j})$ & $\mathrm{j}=1, N P U$ \\
\hline$E_{2}$ & 0 & $\mu(\mathrm{j})$ & $C_{\mu}(\mathrm{j})$ & $P_{\mu}(\mathrm{j})$ & $\mathrm{j}=1, N P U$ \\
\hline • & $\bullet$ & $\bullet$ & $\bullet$ & • & \\
\hline$\bullet$ & $\bullet$ & $\bullet$ & $\bullet$ & $\bullet$ & \\
\hline$\bullet$ & $\bullet$ & $\bullet$ & $\bullet$ & $\bullet$ & \\
\hline$E_{\mathrm{NE}}$ & 0 & $\mu(\mathrm{j})$ & $C_{\mu}(\mathrm{j})$ & $P_{\mu}(\mathrm{j})$ & $\mathrm{j}=1, N P U$ \\
\hline \multicolumn{6}{|c|}{$\left(E, \mu, E^{\prime}\right)$ Data Block } \\
\hline$E$ & $\mu$ & $E^{\prime}$ & $C_{E^{\prime}}$ & $P_{E^{\prime}}$ & \\
\hline$E_{1}$ & $\mu(1)$ & $\overline{E_{1}}$ & 1.0 & 1.0 & \\
\hline$E_{1}$ & $\mu(2)$ & $E_{1}$ & 1.0 & 1.0 & \\
\hline$\bullet$ & $\bullet$ & $\bullet$ & $\bullet$ & $\bullet$ & \\
\hline$\bullet$ & & $\bullet$ & $\bullet$ & $\bullet$ & \\
\hline$\bullet$ & $\bullet$ & $\bullet$ & $\bullet$ & $\bullet$ & \\
\hline$E_{1}$ & $\mu(N P U)$ & $E_{1}$ & 1.0 & 1.0 & \\
\hline \multicolumn{6}{|l|}{ • } \\
\hline$E_{\mathrm{NE}}$ & $\mu(1)$ & $E_{\mathrm{NE}}$ & 1.0 & 1.0 & \\
\hline$E_{\mathrm{NE}}$ & $\mu(2)$ & $E_{\mathrm{NE}}$ & 1.0 & 1.0 & \\
\hline$\bullet$ & $\bullet$ & $\bullet$ & $\bullet$ & $\bullet$ & \\
\hline$\bullet$ & $\bullet$ & $\bullet$ & $\bullet$ & $\bullet$ & \\
\hline$\bullet$ & $\bullet$ & $\bullet$ & $\bullet$ & $\bullet$ & \\
\hline$E_{\mathrm{NE}}$ & $\mu(N P U)$ & $E_{\mathrm{NE}}$ & 1.0 & 1.0 & \\
\hline
\end{tabular}




\subsection{Elastic and discrete-level inelastic scattering}

There is a one-to-one correspondence between the exiting angle and energy for elastic and discrete level inelastic scattering. Once the exiting angle is selected, the exiting energy is already determined based on the kinematics equations documented in most conventional reactor theory text books. However, an obscure fact is that the exiting energy distributions for discrete-level inelastic reactions and hydrogen elastic scattering can be double valued in the lab system. For discrete level inelastic scattering in the lab system, the range of incident energies that can have a double valued exit energy is given by the following expression:

$$
\Delta E_{\text {double }}=\frac{\mathrm{Q}}{A(1-A)},
$$

where

$=$ the excess of the kinetic energy of the product particles over that of the original particles, and

$A=$ atomic mass ratio of the target mass to the mass of a neutron.

Eq. (8.1.69) provides the size in energy of the double valued region above the threshold energy for the reaction. As indicated by Eq. (8.1.69), the range of energies for a double valued region is inversely proportional to the target mass. Using Eq. (8.1.69) and ENDF/B data, the values of $\Delta E_{\text {double }}$ can be calculated for all discrete level inelastic scattering reactions for all isotopes. A plot of $\Delta E_{\text {double }}$ as a function of atomic mass ratio is provided in Fig. 8.1.198 for all possible discrete level inelastic scattering collisions for all isotopes. As shown in Fig. 8.1.198, the size of the double valued region approaches $1 \mathrm{MeV}$ as the mass ratio decreases. Because $\Delta E_{\text {double }}$ can be relatively large, the double valued anomaly cannot be ignored in the collision treatment. The following discussion outlines the properties of the kinematics data and the procedures for treating elastic and discrete level inelastic scattering. 


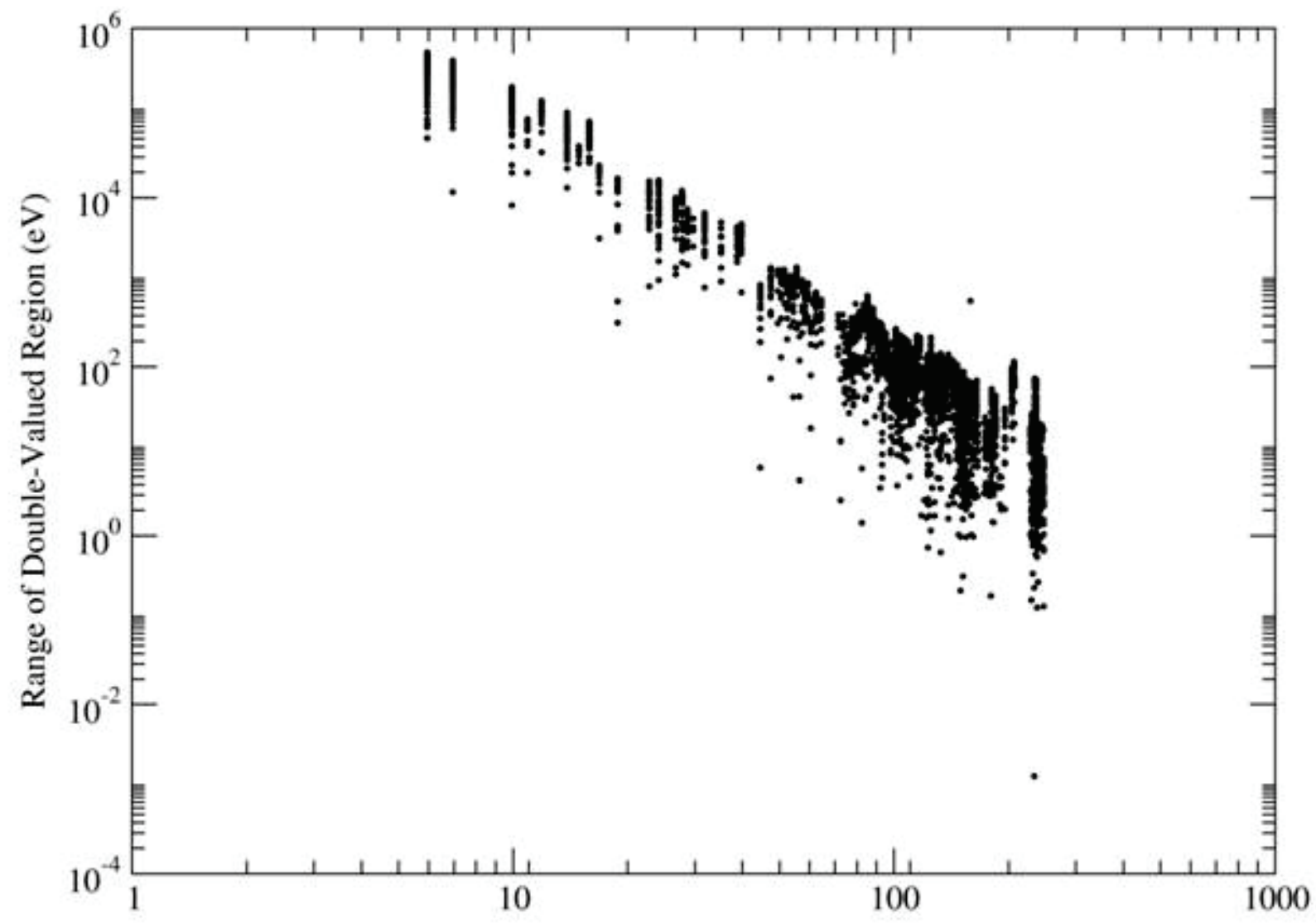

Atomic Mass Ratio (A)

Fig. 8.1.198. Range of incident energies $\left(\Delta E_{\text {double }}\right)$ for double valued region as a function of mass ratio.

As noted previously, NPU secondary angle cosines are provided for each incident energy for a reaction, and the cosine distribution can be represented with either equiprobable or nonequiprobable distributions. Depending on the type of distribution, procedures similar to those of Sect. Exit energy are used to sample an equiprobable or nonequiprobable angular distribution for the exit cosine in the lab system.

The angular data blocks for discrete level inelastic scattering and hydrogen elastic scattering are similar in form to other reactions; however, the exit lab cosines for discrete level inelastic scattering are greater than zero in the double-valued region, and an exit lab cosine may appear twice in the distribution with different probabilities. As an example, discrete level inelastic scattering for ${ }^{7} \mathrm{Li}$ is characterized by a double valued exit energy distribution as a function of exit cosine in the lab system. Fig. 8.1.190 provides the exit energy distribution for an inelastic collision that leaves the ${ }^{7} \mathrm{Li}$ nucleus in the first excited state (i.e., MT $=51$ ) in the lab system. For ${ }^{7} \mathrm{Li}$, the threshold for $\mathrm{MT}=51$ is $5.4672 \times 10^{5} \mathrm{eV}$, and the double valued region extends from the threshold energy up to $5.58259 \times 10^{5} \mathrm{eV}$; therefore, the incident energy range of the double valued region is $\sim 1.15 \times 10^{4} \mathrm{eV}$. As shown in Fig. 8.1.190, the exit energy distributions are provided for different incident energies within the double valued region and an incident energy above the double valued region. As the incident energy exceeds the maximum energy for which two exiting energies can be produced, the secondary energy distribution becomes single valued (i.e., one exit energy for an exit cosine). 
Note that the double valued anomaly does not occur in the center-of-mass system. Therefore, the two exit energies possible for the lab system actually correspond to two different center-of-mass exit cosines. During the preparation of the KENO library, the angular distribution tables for discrete level inelastic scattering and elastic scattering for hydrogen can be constructed in the center-of-mass system and subsequently converted to the lab system. In order to properly construct the angular distribution tables in the lab system, the minimum lab cosine, $\mu^{\min }$ lab, must be determined because exit angles below the minimum lab cosine are not possible.

For discrete level inelastic scattering and elastic scattering, the exit cosine in the lab system can be expressed as a function of the center-of-mass scattering angle, $\mu_{c m}$ :

$$
\mu=\frac{\gamma+\mu_{c m}}{\left(1+2 \gamma \mu_{c m}+\gamma^{2}\right)^{1 / 2}},
$$

where

$$
\frac{1}{\gamma}=\left[A^{2}+\frac{A(A+1) Q}{E}\right]^{1 / 2} .
$$

Physically, the quantity $1 / \gamma$ represents the ratio of the exit velocity of the neutron in the lab system to the center-of-mass velocity. Moreover, the quantity $1 / \gamma$ reduces to $A$ for elastic scattering (i.e., $Q=0$ ). The minimum value for $\mu$ can be obtained by taking the derivative of Eq. (8.1.72) with respect to $\mu_{c m}$ which yields the following expression:

$$
\frac{d \mu}{d \mu_{c m}}=\frac{1+\gamma \mu_{c m}}{\left(1+2 \gamma \mu_{c m}+\gamma^{2}\right)^{3 / 2}} .
$$

Setting Eq. (8.1.72) equal to 0 reveals that the minimum lab cosine $\left(\mu_{\text {lab }}^{\min }\right)$ occurs when $\mu_{c m}=-1 / \gamma$. For elastic scattering, the minimum lab cosine occurs when $\mu_{c m}=-A$. Because hydrogen is the only nuclide with a mass ratio below 1, hydrogen is the only nuclide that has a double valued exit energy distribution in the lab system for elastic scattering.

During the preparation of the KENO library, the lab distributions for discrete level inelastic scattering and hydrogen elastic scattering will be constructed so that $\mu_{\text {lab }}^{\min }$ is a boundary for an angular bin, and no angular cosines in the lab system will be permitted below $\mu{ }_{\text {lab. }}^{\text {min }}$ As shown in Fig. 8.1.199 for ${ }^{7} \mathrm{Li}$, the exit cosines greater than $\mu_{\text {lab }}^{\min }$ will have two possible exit energies in the double valued region. As a result, the angular distribution table in the library will have angles that appear twice with different probabilities. For example, an angle cosine $\mu$ may be present in the table with probabilities $P_{m}$ and $P_{n}$. Although the value of the angle cosine is the same for both probabilities, the corresponding exit energy will be different for each $(\mu, P)$ pair. In other words, the probability for an exit cosine determines the location in the exit energy distribution table for selecting the outgoing energy. 


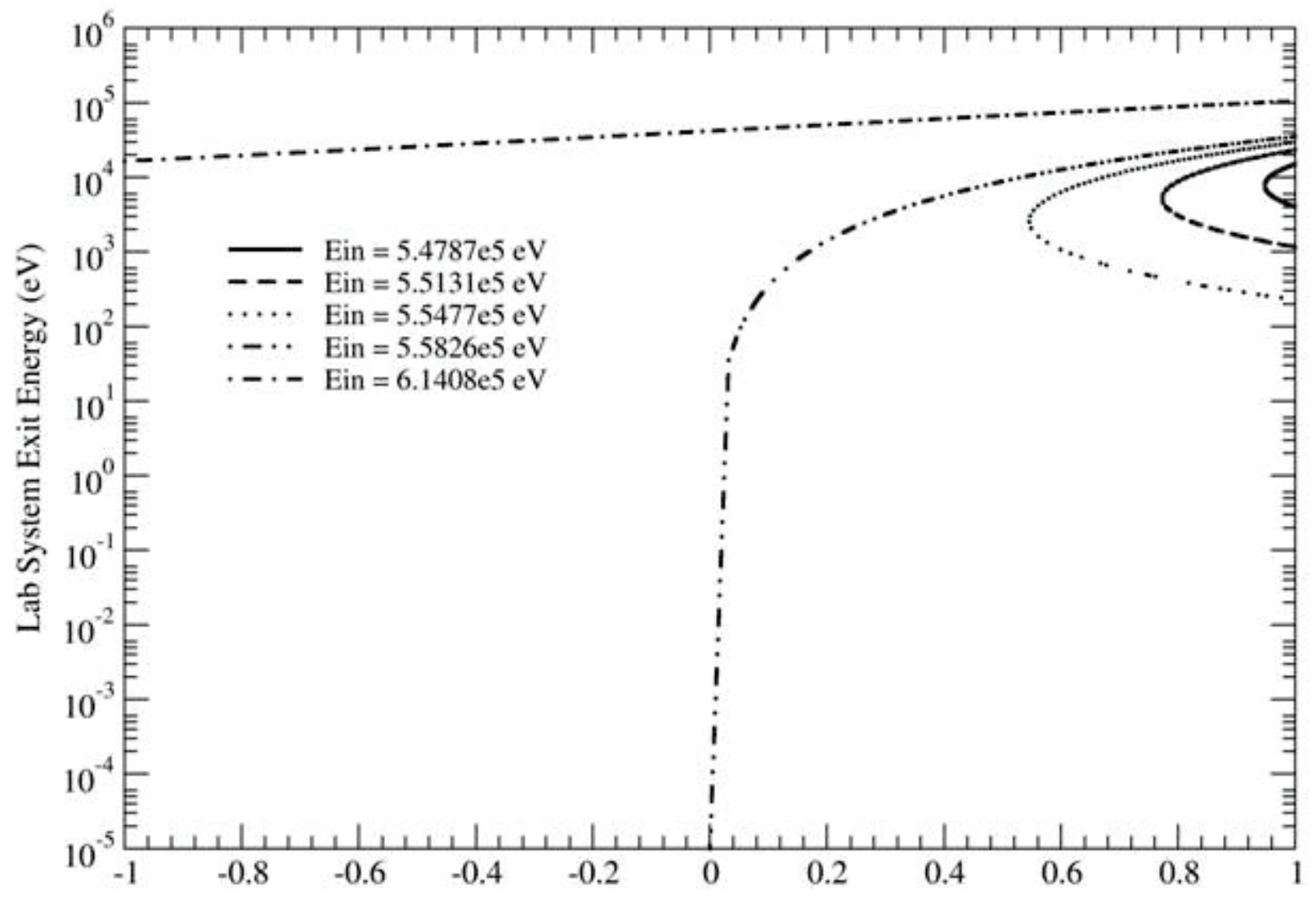

Lab System Exit Cosine

Fig. 8.1.199. ${ }^{7} \mathrm{Li}$ exit energy distribution for the first discrete level inelastic scattering reaction $(\mathrm{MT}=51) . E_{\text {threshold }}=$ $5.4672 \times 10^{5} \mathrm{eV} ; \Delta E_{\text {double }}=1.1539 \times 10^{4} \mathrm{eV}$.

Based on the format of the kinematics data, angle cosine distributions are provided as a function of incident energy. If the incident energy, $E$, is between $E_{i}$ and $E_{i+1}$, then the $i^{\text {th }}$ table is selected with probability $P_{i}$ and the $i+1$ table is selected with probability $P_{i+1}$ :

$$
\begin{gathered}
P_{i}=\frac{E_{i+1}-E}{E_{i+1}-E_{i}}, \\
P_{i+1}=\frac{E-E_{i}}{E_{i+1}-E_{i}} .
\end{gathered}
$$

Once the angular distribution corresponding to the incident energy is selected, the exit angle cosine can be selected using procedures like those in Sect. Exit angle cosine. For example, if the $i^{\text {th }}$ incident energy is selected, either Eq. (8.1.29) is used to select the $a^{\text {th }}$ cosine bin for equiprobable bins, or Eq. (8.1.34) is used for nonequiprobable bins. Subsequently, either Eq. (8.1.31) or Eq. (9.1.36) is used to calculate the exit angle cosine, depending on the type of angular distribution provided. 
The coupled secondary angle-energy data blocks $\left(E, \mu, E^{\prime}\right)$ follow the angular data block in the kinematics data. For elastic scattering and discrete level inelastic scattering, there is one possible exit energy. Therefore, in the exit energy distribution block, a single exit energy $(N P E=1)$ is provided for each $(E, \mu)$ pair, and the general structure (i.e., material identifiers and data flags are omitted for clarity) of the secondary energy distribution data block is presented in Table 8.1.27. Because only one exit energy is provided for each $(E, \mu)$ pair, the probability for the exit energy is 1.0. As indicated in Table 8.1.27, a cumulative probability of 1.0 is stored for each exit energy; however, the parameters stored in the PDF location are the power-interpolation parameters needed to interpolate the exit energy.

Table 8.1.27. Secondary energy distribution format for elastic and discrete-level inelastic scattering

\begin{tabular}{ccccc}
\hline$E$ & $\mu$ & $E^{\prime}$ & $C_{E^{\prime}}$ & $p_{E^{\prime}}$ \\
\hline$E_{1}$ & $\mu_{1}$ & $E_{1}^{\prime}$ & 1.0 & $p_{1}$ \\
$E_{1}$ & $\mu_{2}$ & $E_{2}^{\prime}$ & 1.0 & $p_{2}$ \\
$\bullet$ & $\bullet$ & $\bullet$ & $\bullet$ & $\bullet$ \\
$\bullet$ & $\bullet$ & $\bullet$ & $\bullet$ & $\bullet$ \\
$\bullet$ & $\bullet$ & $\bullet$ & $\bullet$ & $p_{\mathrm{NPU}}$ \\
$E_{1}$ & $\mu_{\mathrm{NPU}}$ & $\bullet$ & 1.0 & \\
& & $\bullet$ & & $p_{1}$ \\
& & $\bullet$ & 1.0 & $p_{2}$ \\
$E_{\mathrm{NE}}$ & $\mu_{1}$ & $E_{1}^{\prime}$ & 1.0 & $\bullet$ \\
$E_{\mathrm{NE}}$ & $\mu_{2}$ & $E_{2}^{\prime}$ & $\bullet$ & $\bullet$ \\
$\bullet$ & $\bullet$ & $\bullet$ & $\bullet$ & $p_{\mathrm{NPU}}$ \\
\hline & $\bullet$ & $\bullet$ & $\bullet$ & \\
$E_{\mathrm{NE}}$ & $\bullet$ & $E_{\mathrm{NPU}}^{\prime}$ & 1.0 & \\
\hline
\end{tabular}

When an exiting angle $\dot{\mu}_{i}$ is sampled between $\mu_{a}$ and $\mu_{a+1}$, the exiting energy values that correspond to $\mu_{a}$ and $\mu_{a+1}$ for the incident energy $E_{i}$ can be used to interpolate the exiting energy $E^{\prime}$ that corresponds to $\dot{\mu}_{i}$. Physically, the interpolation procedures correspond to an interpolation along one of the incident energy curves as depicted in Fig. 8.1.197. In order to perform the interpolation, the $a^{\text {th }}$ and $a+1^{\text {st }}(E, \mu)$ pairs are located in the secondary energy distribution block corresponding to $E_{i}$. Subsequently, the corresponding $a^{\text {th }}$ and $a+1$ exit energy values (i.e., $E_{a}^{\prime}$ and $E_{a+1}^{\prime}$ ) are read from the secondary energy distribution record.

The exiting energy is interpolated with the following relation:

$$
E^{\prime}\left(\dot{\mu}_{i}\right)=E_{a}^{\prime}+\left(\frac{\dot{\mu}_{i}^{P a}-\mu_{a}^{P a}}{\mu_{a+1}^{P a}-\mu_{a}^{P a}}\right)\left(E_{a+1}^{\prime}-E_{a}^{\prime}\right)
$$


where

$$
\begin{aligned}
& \mathrm{p}=\text { is a "power-interpolation" factor for the } a^{\text {th }} \text { cosine bin that is obtained from a fit to the } E^{\prime} \\
& \text { distribution as a function of } \mu \text {. }
\end{aligned}
$$

The interpolation procedure in Eq. (8.1.75) is analogous to the interpolation scheme that has been used successfully to interpolate Bondarenko factors as a function of temperature in the BONAMI module. If the curve is monotonic in nature, the power-interpolation scheme can be used to represent the curvature with a minimum number of points. For elastic and discrete level inelastic scattering, the $E^{\prime}(\mu)$ distribution is monotonic and is suited for the power interpolation scheme. Note that if $p$ is 1 , Eq. (8.1.75) reduces to a linear interpolation in $\mu-E^{\prime}$ space.

\subsection{Thermal scattering effects}

A collision between a neutron and nucleus can be affected by the thermal motion of the target nucleus. Moreover, ENDF provides thermal scattering law data to account for the thermal effects of scattering with a material below $5 \mathrm{eV}$; however, the scattering law data are only available for a select number materials as shown in Table 8.1.28. If thermal scattering law data are not available, elastic scattering is treated with the free gas approximation in the KENO library. For heavy nuclides with $0 \mathrm{~K}$ scattering libraries available, the Doppler Broadening Rejection Correction (DBRC) method is used instead. For DBRCenabled nuclides, thermal scattering effects are taken into account up to $210 \mathrm{eV}$ (although this parameter can be controlled by the user). More information on DBRC is given in Sect. 8.1.6.2.9.

Table 8.1.28. ENDF/B thermal scattering law data

\begin{tabular}{lccccc}
\hline \multicolumn{1}{c}{ Symbol } & MAT & $\begin{array}{c}\text { Principal Scatterer } \\
\mathrm{S}(\alpha, \beta)\end{array}$ & $\begin{array}{c}\text { Coherent } \\
\text { Elastic }\end{array}$ & $\begin{array}{c}\text { Incoherent } \\
\text { Elastic }\end{array}$ & $\begin{array}{c}\text { Incoherent } \\
\text { Inelastic S }(\alpha, \beta)\end{array}$ \\
\hline Be & 26 & $\mathrm{Be}$ & $\checkmark$ & $\checkmark$ \\
Bemetal & 26 & $\mathrm{Be}$ & $\checkmark$ & $\checkmark$ \\
benzine $\left(\mathrm{C}_{6} \mathrm{H}_{6}\right)$ & 40 & $\mathrm{H}$ & & $\checkmark$ \\
beo & 27 & $\mathrm{BeO}$ & $\checkmark$ & $\checkmark$ \\
d_d2o & 11 & ${ }_{1}^{2} \mathrm{H}$ & & $\checkmark$ \\
graphite & 31 & $\mathrm{C}$ & $\checkmark$ & $\checkmark$ \\
h_ch2 & 37 & $\mathrm{H}$ & $\checkmark$ & $\checkmark$ \\
h_h2o & 1 & $\mathrm{H}$ & $\checkmark$ & $\checkmark$ \\
h_zrh & 7 & $\mathrm{ZrH}$ & & $\checkmark$ \\
lch4 & 33 & $\mathrm{H}$ & $\checkmark$ \\
orthod & 13 & ${ }_{1}^{2} \mathrm{H}$ & $\checkmark$ \\
orthoh & 3 & $\mathrm{H}$ & $\checkmark$ \\
parad & 12 & ${ }_{1}^{2} \mathrm{H}$ & & $\checkmark$ \\
parah & 2 & $\mathrm{H}$ & & $\checkmark$ \\
\end{tabular}




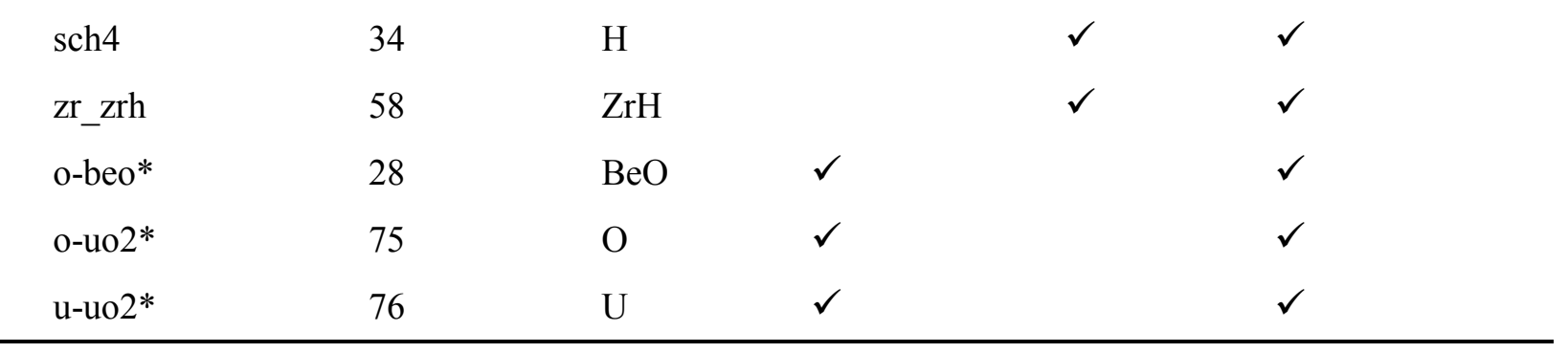

*ENDF/B-VII only.

The continuous energy cross section library is processed to account for the appropriate scattering effects. As a result, the KENO cross section library inherently accounts for thermal scattering using either the thermal scattering law data or the free gas approximation. In other words, the kinematics data based on thermal effects are combined with the fast data to represent the collision kinematics for the material. As noted previously, the kinematics data block for a reaction is divided into sections that describe the collision kinematics for a range of incident energies. As a result, the kinematics data structure has a modular format that is beneficial for data library production. Because of the kinematics data structure, the thermal scattering law data, which are typically applied up to $5 \mathrm{eV}$, can be processed independently with AMPX to obtain the angle energy probability distributions. Subsequently, one or more sections in the kinematics data block can be used to represent the thermal angle -energy distributions for energies up to $5 \mathrm{eV}$. Moreover, the fast kinematics data can be represented with one or more sections for energies above $5 \mathrm{eV}$. As part of the library preparation, the thermal kinematics data are combined with the fast kinematics data block to complete the collision representation for the entire range of incident energies. Because the thermal scattering effects are treated during the processing of the cross section data for a material, the thermal effects are inherently treated in KENO. During the Monte Carlo random walk, the procedures provided in Sect. 8.1.6.2.7.2 and 8.1.6.2.7.3 are used to sample the exit angle and energy for the collision.

\subsection{Doppler broadening rejection correction method}

When colliding with a heavy nuclide, the thermal motion of the target nuclide can significantly affect the exit angle and energy of the neutron. The thermal motion of the target nuclide was previously ignored, but this assumption has been proven inadequate by Dagan and Becker. ${ }^{6}$

A new sampling equation has been implemented to allow for Doppler broadening of the scattering kernel. Removing this approximation can have a significant impact on the critical eigenvalue of systems by allowing neutrons to be upscattered into absorption resonances. Currently, DBRC is enabled in KENO only for ${ }^{238} \mathrm{U}$, and this feature can be activated by setting the parameter $\mathbf{D B R}=1$.

\subsection{Doppler broadening methods}

CE KENO calculations will perform temperature adjustment of neutron cross sections by default. The cross sections provided with SCALE are typically supplied in $300 \mathrm{~K}$ intervals, and additional cross section temperature resolution may be desired to model non-room temperature systems or systems with significant temperature effects.

The capability to perform problem-dependent Doppler broadening of nuclear cross sections has been implemented in KENO. This feature is controlled by the DBX parameter, which causes KENO to perform Doppler broadening on the cross sections in all compositions in a problem before simulating particle 
histories. When this occurs, the cross sections for each material are broadened to the temperature assigned to that material in the READ COMP block, as long as the requested temperature is more than $4 \mathrm{~K}$ from the library temperature. Within this $\pm 4 \mathrm{~K}$ band, the library temperature is used to avoid difficulties with numerical instabilities in small cross section adjustments. The default for this feature is $\mathbf{D B X}=2$, which performs Doppler broadening for both the 1-D and 2-D cross sections. Current options available are as follows:

- $\mathbf{D B X}=0$ - Doppler broadening is not performed. KENO selects the library at the closest temperature. If desired, the user can use the TTL parameter to force a job abort if the library temperature is too far from the requested temperature. By default, TTL $=-1$, so the closest library will be used regardless of the temperature difference.

- DBX = 1 -Doppler broadening is performed using a finite difference method on the onedimensional cross sections that are temperature dependent. Logarithmic interpolation is also used to broaden the probability table data. When this option is used, the collision probabilities are recalculated after the Doppler broadening is complete to ensure consistency.

- $\mathbf{D B X}=2$-The same actions as $\mathbf{D B X}=1$ are performed, and Doppler broadening is also performed on the thermal moderator data by conducting interpolation on the double differential probability data present on the cross section library.

Nuclide level energy unionization is automatically disabled $(\mathbf{M} \mathbf{2} \mathbf{U}=\mathrm{NO})$ when Doppler broadening is enabled in order to reduce the memory usage. At this writing, research is in progress to allow KENO to perform on-the-fly cross section Doppler broadening as particle histories are being simulated.

\subsubsection{Multigroup mode solution procedure}

Combining Eqs. (9.1.3) and (8.1.4), assuming the media to be isotropic, ignoring the time dependence of the cross sections and converting the equation to multigroup form yields the following:

$$
\frac{1}{\mathrm{v}_{g}} \frac{\partial \Phi_{g}}{\partial t}(X, \Omega, t)+\Omega \cdot \nabla \Phi_{g}(X, \Omega, t)+\Sigma_{t g}(X) \Phi_{g}(X, \Omega, t)=\mathrm{q}_{\mathrm{g}}(X, \Omega, t)
$$

where

$$
\begin{aligned}
& \mathrm{g}= \text { is the energy group of interest, } \\
& \mathrm{v}_{\mathrm{g}}= \text { is the average velocity of the neutrons in group } \mathrm{g}, \\
& \Phi_{\mathrm{g}}(\mathrm{X}, \Omega, \mathrm{t})= \begin{array}{l}
\text { is the angular flux of neutrons having their energies in group } \mathrm{g}, \text { at position } \mathrm{X} \text { and } \\
\text { time } \mathrm{t} \text {, and }
\end{array} \\
& \Sigma_{\mathrm{tg}}(\mathrm{X}) \quad \begin{array}{l}
\text { is the macroscopic total cross section of the media at position } \mathrm{X} \text { for group } \mathrm{g}, \\
\text { corresponding to }
\end{array}
\end{aligned}
$$




$$
\Sigma_{\mathrm{tg}}(\mathrm{X})=\frac{\int_{\Delta \mathrm{E}_{\mathrm{g}}} \Sigma_{\mathrm{t}}(\mathrm{X}, \mathrm{E}) \Phi(\mathrm{X}, \mathrm{E}, \Omega, \mathrm{t}) \mathrm{dE}}{\int_{\Delta \mathrm{E}_{\mathrm{g}}} \Phi(\mathrm{X}, \mathrm{E}, \Omega, \mathrm{t}) \mathrm{dE}}
$$

where
$\Delta \mathrm{E}_{\mathrm{g}} \quad$ defines group $\mathrm{g}$, and
$\mathrm{q}_{\mathrm{g}}(\mathrm{X}, \Omega, \mathrm{t})$ is the total source contributing to energy group $\mathrm{g}$ at position $\mathrm{X}$, and time $\mathrm{t}$ in direction $\Omega$.

Using the relationship $\mathrm{X}^{\prime}=\mathrm{X}-\mathrm{R} \Omega$, defining the problem to be time independent using an integrating factor on both sides of Eq. (9.1.76), and defining

$$
\mathrm{T}(\mathrm{R})=\int_{0}^{\mathrm{R}} \Sigma_{\mathrm{tg}}\left(\mathrm{X}-\mathrm{R}^{\prime} \Omega\right) \mathrm{dR}^{\prime}
$$

the following equation can be written:

$$
\Phi_{g}(X, \Omega)=\int_{O}^{\infty} q_{g}(X-R \Omega, \Omega) e^{-T(R)} d R .
$$

At this point, the problem becomes an eigenvalue problem. If there is no external source, the source may be defined as

$$
\mathrm{q}_{\mathrm{g}}(X, \Omega)=\sum_{g^{\prime}} \int \mathrm{d} \Omega^{\prime} \Phi_{g^{\prime}}\left(X, \Omega^{\prime}\right) \Sigma_{s}\left(X, g^{\prime} \rightarrow g, \Omega^{\prime} \cdot \Omega\right)+\frac{1}{\mathrm{k}} \mathrm{Q}_{g}^{\prime}(X, \Omega),
$$

where

$\mathrm{k} \quad$ is the largest eigenvalue of the integral equation,

$\mathrm{Q}_{\mathrm{g}}^{\prime}(\mathrm{X}, \Omega) \quad$ is the fission source at position $\mathrm{X}$ for energy group $\mathrm{g}$ and direction $\Omega$ (all fission contributions to group $\mathrm{g}$ from all energy groups in the previous generation), and

$\Sigma_{\mathrm{s}}\left(\mathrm{X}, \mathrm{g}^{\prime} \rightarrow \mathrm{g}, \Omega^{\prime} \cdot \Omega\right)$ is the scattering cross section for scattering at position $\mathrm{X}$ from group $\mathrm{g}^{\prime}$ and direction $\Omega^{\prime}$ to group $g$ and direction $\Omega$. 
In terms of energy, the scatter can be defined as

$$
\Sigma_{s}\left(X, g^{\prime} \rightarrow g, \Omega^{\prime} \cdot \Omega\right)=\frac{\int_{\Delta E_{g}} \int_{\Delta E_{g^{\prime}}} \Sigma_{s}\left(X, E^{\prime} \rightarrow E, \Omega^{\prime} \cdot \Omega\right) \Phi\left(X, E^{\prime}, \Omega^{\prime}\right) \mathrm{dE} \mathrm{dE}^{\prime}}{\int_{\Delta E_{g^{\prime}}} \Phi\left(X, E^{\prime}, \Omega^{\prime}\right) \mathrm{dE}^{\prime}},
$$

where

$$
\begin{aligned}
& \Delta \mathrm{E}_{\mathrm{g}} \quad \text { is the energy-range-defining energy group g, and } \\
& \Delta \mathrm{E}_{\mathrm{g}}{ }^{\prime} \quad \text { is the energy-range-defining energy group } \mathrm{g}^{\prime} \text {. }
\end{aligned}
$$

Assuming the fission neutrons to be isotropic, the fission source $\mathrm{Q}_{\mathrm{g}^{\prime}}(\mathrm{X}, \Omega)$ can be written as

$$
\mathrm{Q}_{\mathrm{g}}^{\prime}(\mathrm{X}, \Omega)=\frac{1}{4 \pi} \sum_{\mathrm{g}^{\prime}} \int_{\Omega^{\prime}} \mathrm{d} \Omega^{\prime} \Phi_{\mathrm{g}^{\prime}}\left(\mathrm{X}, \Omega^{\prime}\right) \chi\left(\mathrm{X}, \mathrm{g}^{\prime} \rightarrow \mathrm{g}\right) v_{\mathrm{g}^{\prime}}(\mathrm{X}) \Sigma_{\mathrm{fg}}(\mathrm{X})
$$

where

$\chi\left(\mathrm{X}, \mathrm{g}^{\prime} \rightarrow \mathrm{g}\right)$ is the fraction of neutrons born in energy group $\mathrm{g}$ from fission in energy group $\mathrm{g}$ in the media at position $\mathrm{X}$,

$v_{\mathrm{g}^{\prime}}(\mathrm{X}) \quad$ is the number of neutrons resulting from a fission in group $\mathrm{g}^{\prime}$ at position $\mathrm{X}$, and

$\Sigma_{\mathrm{fg}^{\prime}}(\mathrm{X}) \quad$ is the macroscopic fission cross section of the material at position $\mathrm{X}$ for a neutron in energy group $\mathrm{g}^{\prime}$.

Substituting Eq. (9.1.78) into Eq. (8.1.77) yields the following equation:

$$
\begin{gathered}
\Phi_{g}(X, \Omega)=\int_{0}^{\infty} d R e^{-T(R)}\left\{\frac{1}{k} Q_{g}^{\prime}(X-R \Omega, \Omega)\right. \\
\left.+\sum_{g^{\prime}}\left[\int_{\Omega^{\prime}} d \Omega^{\prime} \Phi_{g^{\prime}}\left(X-R \Omega, \Omega^{\prime}\right) \Sigma_{s}\left(X-R \Omega, g^{\prime} \rightarrow g, \Omega^{\prime} \cdot \Omega\right)\right]\right\}
\end{gathered}
$$

The definition of k may be given as the ratio of the number of neutrons in the $(n+1)$ th generation to the number of neutrons in the $\mathrm{n}$ th generation or the largest eigenvalue of the integral equation. Using Eq. (8.1.80), Eq. (9.1.81) can be written as

$$
\begin{gathered}
\Phi_{g}(X, \Omega)=\int_{0}^{\infty} d R e^{-T(R)}\left\{\sum_{g^{\prime}} \frac{1}{k} \int_{\Omega^{\prime}} v_{g^{\prime}}(X-R \Omega) \Sigma_{f g^{\prime}}(X-R \Omega) \chi\left(X-R \Omega, g^{\prime} \rightarrow g\right) \Phi_{g}\left(X-R \Omega, \Omega^{\prime}\right) \frac{d \Omega^{\prime}}{4 \pi}\right. \\
\left.+\sum_{g^{\prime}}\left[\int_{\Omega^{\prime}} d \Omega^{\prime} \Sigma_{t g^{\prime}}\left(X-R \Omega, \Omega^{\prime}\right) \Sigma_{s}\left(X-R \Omega, g^{\prime} \rightarrow g, \Omega^{\prime} \cdot \Omega\right)\right]\right\}
\end{gathered}
$$

Writing Eq. 9.1.82 in generation notation, multiplying and dividing certain terms by $\Sigma_{\mathrm{t}}(\mathrm{X})$, and multiplying both sides of the equation by $v_{\mathrm{g}}(\mathrm{X}) \Sigma_{\mathrm{fg}}(\mathrm{X})$ yields the following equation, which is solved by KENO: 


$$
\begin{gathered}
\frac{v_{g}(X) \Sigma_{f g}(X)}{\Sigma_{t g}(X)} \Sigma_{t g}(X) \Phi_{g, n}(X, \Omega)=\frac{v_{g}(X) \Sigma_{f g}(X)}{\Sigma_{t g}(X)} \Sigma_{t g}(X) \int_{0}^{\infty} d R e^{-T(R)} \\
\left\{\frac{1}{k} \sum_{g^{\prime}}\left[\int_{\Omega^{\prime}} \frac{v_{g^{\prime}}(X-R \Omega) \Sigma_{f g^{\prime}}(X-R \Omega)}{\Sigma_{t g^{\prime}}(X-R \Omega)} \chi\left(X-R \Omega, g^{\prime} \rightarrow g\right) \Sigma_{t g^{\prime}}(X-R \Omega) \Phi_{g^{\prime}, n-1}\left(X-R \Omega, \Omega^{\prime}\right) \frac{d \Omega^{\prime}}{4 \pi}\right]\right. \\
\left.+\sum_{g^{\prime}}\left[\int_{\Omega^{\prime}} \frac{\Sigma_{S}\left(X-R \Omega, g^{\prime} \rightarrow g, \Omega^{\prime} \cdot \Omega\right)}{\Sigma_{t g^{\prime}}(X-R \Omega)} \Sigma_{t g^{\prime}}(X-R \Omega) \Phi_{g^{\prime}, n}\left(X-R \Omega, \Omega^{\prime}\right) d \Omega^{\prime}\right]\right\},
\end{gathered}
$$

where $\mathrm{n}$ indicates the $\mathrm{n}$ th generation and $\mathrm{n}-1$ is the $(\mathrm{n}-1)$ th generation. Note that the left-hand side of the equation $-v_{\mathrm{g}}(\mathrm{X}) \Sigma_{\mathrm{fg}}(\mathrm{X}) \Phi_{\mathrm{g}, \mathrm{n}}(\mathrm{X}, \Omega)$ - is the fission production for the $\mathrm{n} t h$ generation.

The solution strategy used by KENO solves Eq. (8.1.83) by using an iterative procedure. The fission production at point $\mathrm{X}$ in energy group $\mathrm{g}$ due to neutrons in the $(\mathrm{n}-1)$ th generation, normalized to the system multiplication, is

$$
\frac{1}{\mathrm{k}} \sum_{\mathrm{g}^{\prime}} \int_{\Omega^{\prime}} \frac{v_{\mathrm{g}^{\prime}}(\mathrm{X}) \Sigma_{\mathrm{fg}^{\prime}}(\mathrm{X})}{\Sigma_{\mathrm{tg}^{\prime}}(\mathrm{X})} \chi\left(\mathrm{X}, \mathrm{g}^{\prime} \rightarrow \mathrm{g}\right) \Sigma_{\mathrm{tg}^{\prime}}(\mathrm{X}) \Phi_{\mathrm{g}^{\prime}, \mathrm{n}-1}\left(\mathrm{X}, \Omega^{\prime}\right) \frac{\mathrm{d} \Omega^{\prime}}{4 \pi}
$$

The collision points used in KENO are chosen by selecting path lengths from the distribution

$$
\mathrm{e}^{-\mathrm{T}(\mathrm{R})} \text {, }
$$

which is the probability of transport from any position $\mathrm{X}-\mathrm{R} \Omega$ to position $\mathrm{X}$.

The first collision density of neutrons in group g per unit solid angle about $\Omega$ resulting from the fission source produced by the $(n-1)$ generation, normalized to the system multiplication, is

$$
\begin{aligned}
& \Sigma_{\mathrm{tg}}(\mathrm{X}) \int_{0}^{\infty} \mathrm{dR \textrm {R } ^ { - \mathrm { T } ( \mathrm { R } ) }} \frac{1}{\mathrm{k}} \int_{\Omega^{\prime}} \sum_{\mathrm{g}^{\prime}} \frac{v_{\mathrm{g}^{\prime}}(\mathrm{X}-\mathrm{R} \Omega) \Sigma_{\mathrm{fg}}(\mathrm{X}-\mathrm{R} \Omega)}{\Sigma_{\mathrm{tg}^{\prime}}(\mathrm{X}-\mathrm{R} \Omega)} \\
& \chi\left(\mathrm{X}-\mathrm{R} \Omega, \mathrm{g}^{\prime} \rightarrow \mathrm{g}\right) \Sigma_{\mathrm{tg}}(\mathrm{X}-\mathrm{R} \Omega) \Phi_{\mathrm{g}^{\prime}, \mathrm{n}-1}\left(\mathrm{X}-\mathrm{R} \Omega, \Omega^{\prime}\right) \frac{\mathrm{d} \Omega^{\prime}}{4 \pi} .
\end{aligned}
$$

The scattering source at position $\mathrm{X}$ emerging in group $\mathrm{g}$ and direction $\Omega$ resulting from previous collisions in the same generation, is

$$
\sum_{g^{\prime}} \int_{\Omega^{\prime}} \frac{\Sigma_{s}\left(X, g^{\prime} \rightarrow g, \Omega^{\prime} \cdot \Omega\right)}{\Sigma_{t g^{\prime}}(X)} \Sigma_{t g^{\prime}}(X) \Phi_{g^{\prime}, n}(X, \Omega) d \Omega^{\prime}
$$

The collision density in group g, per solid angle about $\Omega$ is

$$
\Sigma_{t g} \int_{0}^{\infty} d R e^{-T(R)} \sum_{g^{\prime}} \int_{\Omega^{\prime}} \frac{\Sigma_{S}\left(X-R \Omega, g^{\prime} \rightarrow g, \Omega^{\prime} \cdot \Omega\right)}{\Sigma_{t g^{\prime}}(X-R \Omega)} \Sigma_{t g^{\prime}}(X-R \Omega) \Phi_{g^{\prime}, n}\left(X-R \Omega, \Omega^{\prime}\right) d \Omega^{\prime}
$$


The total collision density times $\frac{v_{\mathrm{g}}(\mathrm{X}) \Sigma_{\mathrm{fg}}(\mathrm{X})}{\Sigma_{\mathrm{tg}}(\mathrm{X})}$ is the relationship from which KENO picks the source points for the next generation.

\subsection{Collision treatment}

A collision occurs in a geometrical region when a history exhausts its mean-free-path length within the boundaries of the region. For each collision, the absorbed weight and the fission weight are tabulated, then the weight is modified by the nonabsorption probability. This new weight is checked for splitting and Russian roulette, and if it survives, the history is scattered. A new energy group is selected from the cumulative transfer probability distribution. This group-to-group transfer determines an angular scattering distribution, usually expressed as a Legendre expansion of the cross section transfer array. A set of discrete angles and probabilities are generated by a generalized Gaussian quadrature procedure, preserving the moments of the Legendre expansion of the angular scattering distribution. KENO treats $\mathrm{P}_{0}$ and $\mathrm{P}_{1}$ Legendre expansions as special cases. If the scattering distribution is isotropic, a flag is set to randomly select new direction cosines from an isotropic distribution, instead of using discrete scattering angles. If the distribution is a $\mathrm{P}_{1}$ expansion, KENO randomly selects the cosine of the scattering angle according to

(1) $|\bar{\mu}|<\frac{10^{-10}}{3}:$ scattering distribution is isotropic,

(2) $|\bar{\mu}| \leq 1 / 3: \mu=\left(\sqrt{1+6 \zeta \bar{\mu}+(3 \bar{\mu})^{2}}-1\right) / 3 \bar{\mu}$,

or

(3) $|\bar{\mu}|>1 / 3: \mu=\zeta(1-|\bar{\mu}|)+\bar{\mu}$,

$\begin{array}{cl}\text { where } & \zeta \quad \text { is a uniform random variable between }-1 \text { and }+1 \text { and } \\ \bar{\mu} & \text { is the mean cosine of the scattering angle. }\end{array}$

Otherwise, KENO randomly selects one of the discrete scattering angles $(\mu)$. New direction cosines are then calculated according to the following relationships where $\mathrm{u}, \mathrm{v}$, and $\mathrm{w}$ are the initial direction cosines and $\mathrm{u}^{\prime}, \mathrm{v}^{\prime}$, and $\mathrm{w}^{\prime}$ are the direction cosines after the collision:

$$
\begin{aligned}
& \mathrm{u}^{\prime}=\mathrm{u} \cos \psi-\sqrt{\mathrm{v}^{2}+\mathrm{w}^{2}} \sin \psi \cos \eta \\
& \mathrm{v}^{\prime}=\mathrm{v} \cos \psi+\frac{\mathrm{uv}}{\sqrt{\mathrm{v}^{2}+\mathrm{w}^{2}}} \cos \eta \sin \psi-\frac{\mathrm{w}}{\sqrt{\mathrm{v}^{2}+\mathrm{w}^{2}}} \sin \psi \sin \eta, \\
& \mathrm{w}^{\prime}=\mathrm{w} \cos \psi+\frac{\mathrm{uw}}{\sqrt{\mathrm{v}^{2}+\mathrm{w}^{2}}} \cos \eta \sin \psi+\frac{\mathrm{v}}{\sqrt{\mathrm{v}^{2}+\mathrm{w}^{2}}} \sin \psi \sin \eta,
\end{aligned}
$$

where 


$$
\begin{aligned}
& \sin \psi=\sqrt{1-\mu^{2}}, \\
& \cos \psi=\mu=\text { cosine of the scattering angle, } \\
& \eta \quad=\text { a random azimuthal angle between } 0 \text { and } 2 \pi .
\end{aligned}
$$

\subsection{Fission point selection}

In order for a fission to occur, a neutron must first have a collision. The fission weight, fisw, is defined as the neutron weight, $w t$, times the $v$-fission probability, $f n f p$ :

$$
f i s w=w t \times f n f p
$$

Two important variables used in the processing of fission points are (1) FWR, which is defined as the fission weight, fisw, divided by a random number, and (2) RAKBAR, which is defined as a factor times the running average value of k-effective, AKBAR. This factor is a function of the square root of the number of neutrons per generation and was chosen because it usually produces an adequate number of independent fission points and does not produce so many that an excessive amount of time is spent choosing from the fission points produced.

The following procedure for generating fission points is repeated until FWR is less than RAKBAR. A fission point is generated only if FWR is greater than RAKBAR. Multiple fissions at the same point are allowed only if $f i s w$ is greater than RAKBAR. If $f i s w$ is greater than RAKBAR, a fission point is stored with FWR set equal to RAKBAR divided by a random number and fisw is decremented by RAKBAR. Then the energy group of fission is chosen randomly from the fission spectrum of the mixture in which the fission occurred. The energy group of fission, the $\mathrm{X}, \mathrm{Y}$, and $\mathrm{Z}$ position, the location of the unit within the array, the region number, the value of FWR, the region number of the array boundary, and the nesting data for holes and/or arrays are stored in the fission bank. The quantity of fission points kept to be used as fission positions for the next generation is limited to the number of positions in the fission bank (input parameter $\mathbf{N F B}=$ ). Typically $\mathbf{N F B}$ is equal to the input parameter NPG, the number of neutrons per generation. If a fission occurs and the fission counter is less than NFB, the fission point information is stored in the fission bank. If a fission occurs and the fission counter is greater than or equal to the number per generation, a search is made to find the smallest stored value of FWR. If FWR of the newly fissioned neutron is less than the smallest FWR in the table, it is discarded. Otherwise, the information from the newly fissioned neutron replaces that associated with the smallest value of FWR found in the table.

When the next generation is ready to be processed, data are transferred from the fission bank into the neutron bank to be used as starting positions for the fission neutrons. If more than NPG neutrons are saved in the fission bank, NPG of those having the highest values of FWR will be used. If too few fission positions were stored (less than the number per generation), a warning message to that effect (K?-132) is printed, and additional fission points are randomly chosen from those that were stored until the number of fission points available to start the next generation is equal to the number of neutrons per generation.

\subsection{Biasing or weighting}

In order to minimize the statistical deviation of k-effective per unit computer time, KENO uses weighted tracking rather than analog tracking. Weighted tracking accounts for absorption by reducing the neutron weight rather than allowing the neutron history to be terminated by absorption. To prevent expending 
excessive computer time tracking low-weight neutrons, Russian roulette is played when the weight of the neutron drops below a preset weight, WTLOW. Neutrons that survive Russian roulette are assigned a weight, WTAVG. The value of WTLOW and WTAVG can be assigned as a function of position and energy. The values used by KENO are

DWTAV $=0.5$, the default value of WTAVG,

WTAVG $=$ DWTAV, the weight given a neutron that survives Russian roulette, and

WTLOW $=$ WTAVG/3.0, the value of weight at which Russian roulette is played.

A study $^{7}$ by Hoffman shows these default values to be reasonable for bare critical assemblies. Fig. 8.1.200 from this study shows the analytic relationship between the variance and WTLOW when WTAVG is 0.5 . Note that the default value of 0.167 for WTLOW is very close to the minimum point on the curve. Experimental results of actual Monte Carlo calculations ${ }^{7}$ provide further assurance that 0.167 is an optimum choice for WTLOW when WTAVG is 0.5 .

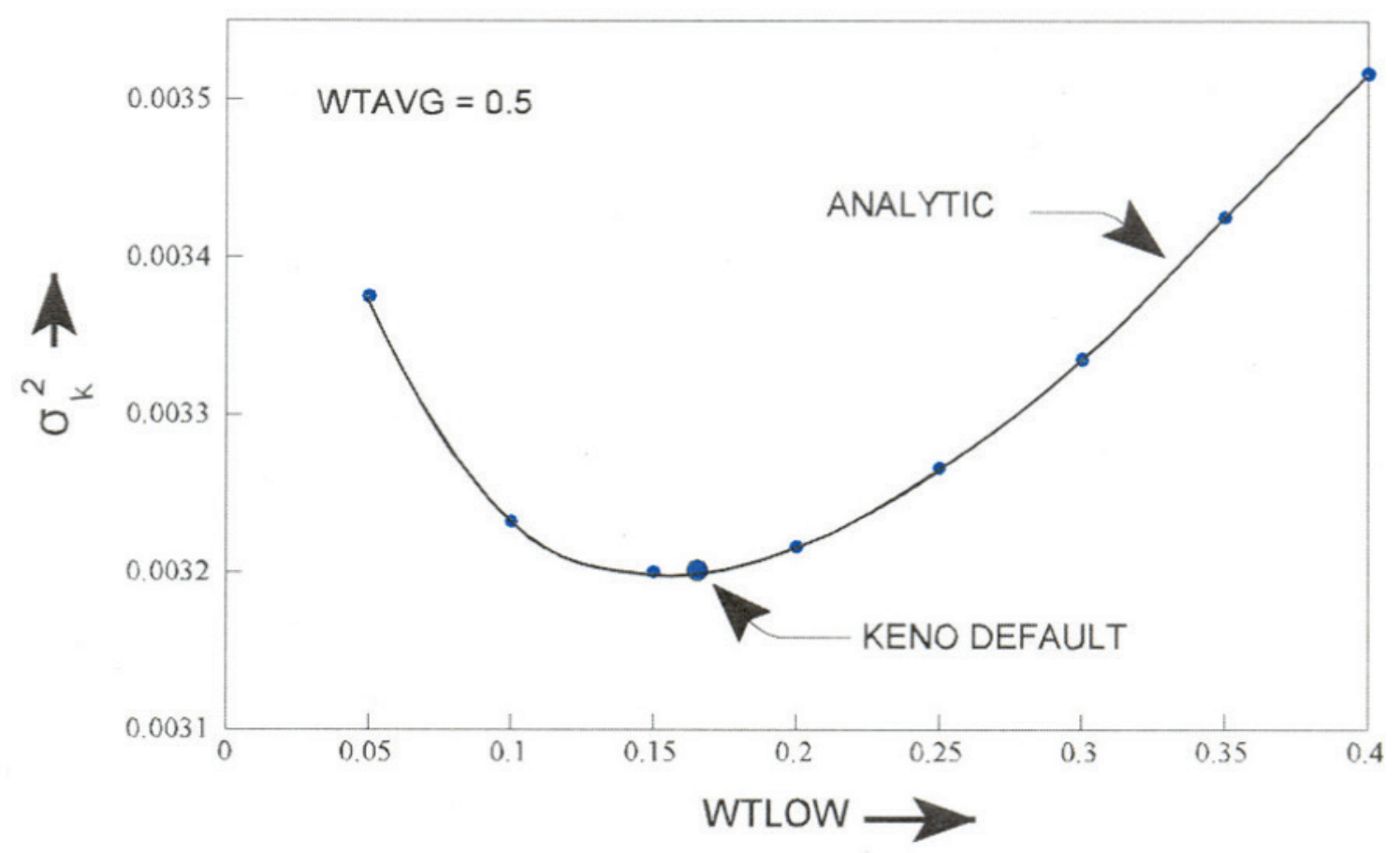

Fig. 8.1.200. Analytic estimate of the relationship between WTLOW and the variance, $\sigma_{\mathrm{k}}^{2}$, when WTAVG is 0.5 .

Fig. 8.1.201, also from the Hoffman study, shows the analytic relationship between the variance and the value chosen for WTAVG for a value of WTLOW $=0.167$. Although the KENO default value for WTAVG is not the optimum, a close examination of the data shows that the variance was changing relatively slowly as a function of WTAVG. Even though this study shows a value near 0.26 to be optimum for this system, further studies of other systems are needed before changing the default value of WTAVG from 0.5 as it has been used in previous versions of KENO. 


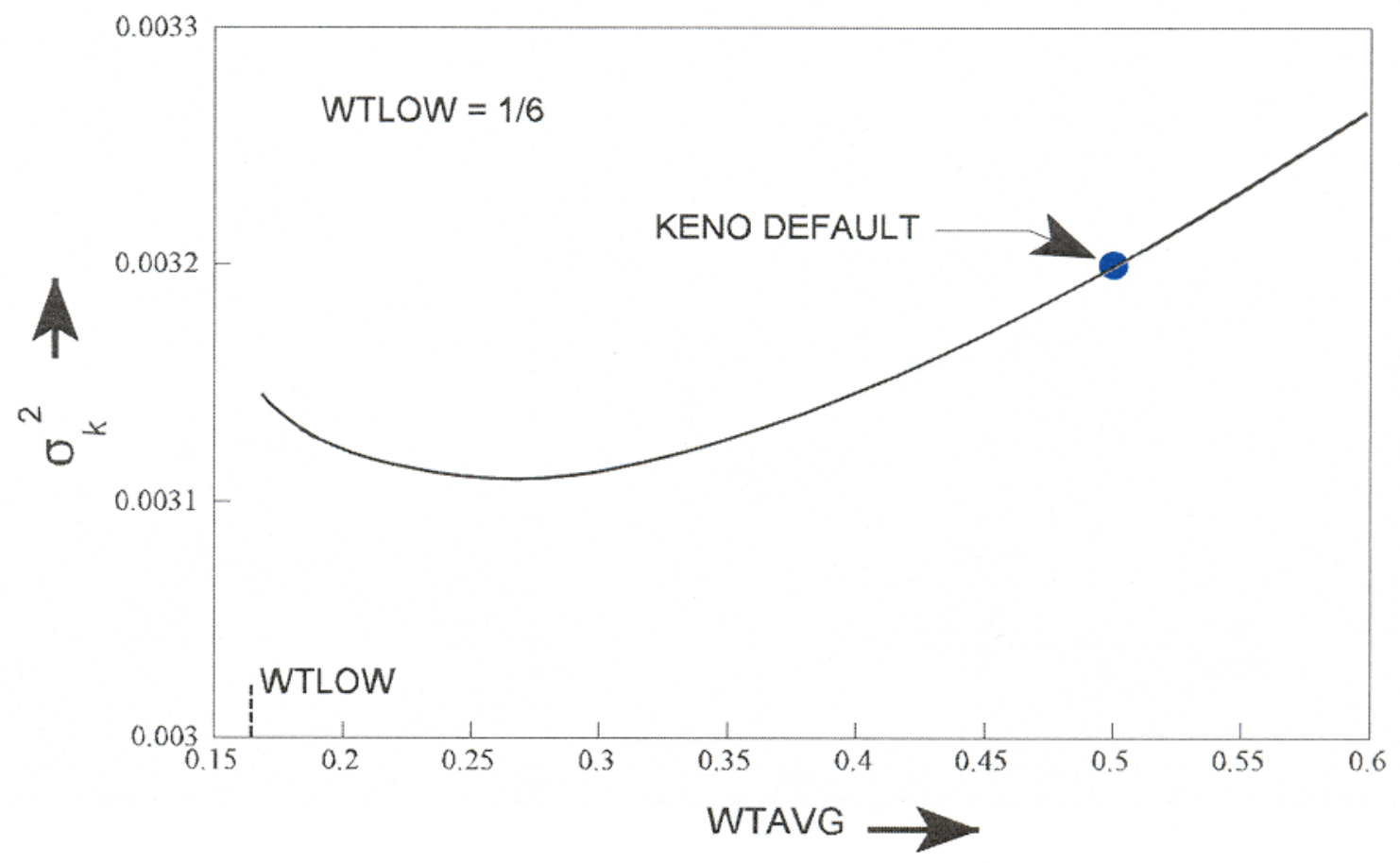

Fig. 8.1.201. Analytic estimate of the relationship between WTAVG and the variance, $\sigma_{\mathrm{k}}^{2}$, when WTLOW is $1 / 6$.

Inside a fissile core, the importance of a neutron is a slowly varying function in terms of energy and position. Hence, for many systems, the standard defaults for WTLOW and WTAVG are good values to use. For reflectors, however, the worth of a neutron varies as a function of distance from the fissile material and as a function of energy. As a neutron in the reflector becomes less important relative to a neutron in the fissile region, it becomes desirable to spend less time tracking it. Therefore a space- and energy-dependent weighting or biasing function is used in KENO to allow the user to minimize the variance in k-effective per unit of tracking time. When a biasing function is used in a reflector, it becomes possible for a neutron to move from one importance region into another in which the WTLOW is greater than the weight of the neutron. When this occurs, Russian roulette is played to reduce the number of neutrons tracked. When the reverse occurs, that is, when the neutron moves to a region of higher importance, its weight may be much higher than WTAVG for that region. When the weight of the neutron is greater than a preset value, WTHI, the neutron is split into two neutrons, each having a weight equal to one-half the weight of the original neutron. This procedure is repeated until the weight of the split neutron is less than WTHI. The default value for WTHI is WTAVG*3.0. WTHI is the weight at which splitting occurs.

The weighting or biasing function for a given core material and reflector material can be obtained by using the adjoint solution from $S_{n}$ type programs for a similar (usually simplified) problem. This adjoint flux gives the relative contribution of a neutron at a given energy and position to the total fissions in the system. The weighting function for KENO is thus proportional to the reciprocal of the adjoint flux. Although such a function can be difficult to obtain, the savings gained makes the effort worthwhile for many of the materials that are frequently used as reflectors. Biasing functions ${ }^{8}$ have been prepared for several reflector materials commonly used in KENO calculations. The use of biasing to minimize the 
variance in k-effective per unit of computer time will usually increase the variance in other parameters such as leakage or absorption in the reflector.

\subsection{Differential albedos}

Arrays reflected by thick layers of material having a small absorption to the scattering ratio may require large amounts of computer time to determine k-effective ${ }^{4}$ because of the relatively long time a history may spend in the reflector. A differential albedo technique was developed for use with the KENO codes to eliminate tracking in the reflector. This involves returning a history at the point it impinges on the reflector and selecting an emergent energy and polar angle from a joint density function dependent upon the incident energy and polar angle. The weight of the history is adjusted by the functional return from the reflector, which is also based on the incident energy and angle.

The characteristics of a differential albedo emulate the attributes of the reflector material and are independent of the material or materials adjacent to the reflector. Thus, a differential albedo that is generated for a given reflector material can be used with any array, regardless of the type of fuel or fissile material contained within the array.

For many calculations involving reflected arrays of fissile material, the differential albedo treatment is a powerful tool that can significantly reduce the computing time required to determine k-effective. The savings will vary depending on the importance of the reflector to the system. A substantial effort is required to generate a differential albedo, but the savings gained were well worth the effort for commonly used reflector materials. The savings are not worth the applicability questions on modern computing platforms; the differential albedo capability is maintained only for backwards compatibility.

To generate the differential albedo information for a material, a fixed-source calculation must be made for each incident energy and angle. The data presently available for use with KENO were generated by 1-D discrete ordinates calculations for slab geometry representing infinite slabs. Consequently, for a finite reflector, these data will not correctly treat histories that enter the reflector near an edge. Past experience with differential albedo reflectors indicates that k-effective appears to be conservative for small faces and will tend toward the correct result as the face becomes large relative to the area near the corners. Care must be taken to ensure that any surface to which a differential albedo is applied is large enough that the errors at the edges can be ignored.

Because differential albedos are expensive and time consuming to generate, those corresponding to the Hansen-Roach 16-energy-group structure are the only differential albedos available for use with KENO at this writing. In the past, their use was limited to problems using cross sections having the Hansen-Roach 16-energy-group structure. KENO extends the use of differential albedos to other energygroup structures by allowing appropriate energy transfers. This is accomplished by creating lethargy boundary tables for the albedo group structure and the cross section group structure and determining the lethargy interval corresponding to the desired transfer (cross section group structure to albedo group structure or vice versa) based on a uniform lethargy distribution over the interval. When the energy-group boundaries of the cross sections and albedos are different, the results should be scrutinized by the user to evaluate the effects of the approximations.

\subsubsection{Geometry}

KENO V.a geometry is restricted to the use of specific shapes. These shapes are called geometry regions or regions. Allowed shapes in KENO V.a are cubes, cuboids (rectangular parallelepipeds), spheres, cylinders, hemispheres, and hemicylinders. These shapes must be oriented along orthogonal axes and cannot be rotated in KENO V.a. They can be translated. Hemispheres and hemicylinders are not limited to half spheres and half cylinders; the definitive plane can be positioned by entering a chord. The value of 
this chord can range from the positive magnitude of the radius (giving a complete sphere or cylinder) to the negative magnitude of the radius (giving a zero volume, nonexistent sphere or cylinder).

KENO-VI geometry can model any geometric shape that can be described using quadratic equations. These geometric shapes are stacked together forming regions. The set of regions is then used to build units. A set of predefined shapes that include cones, cuboids (rectangular parallelepipeds), cylinders, dodecahedrons, ecylinders (extruded elliptical cylinders), ellipsoids, hexprisms, hoppers, parallelepipeds, planes, rhombohedrons, spheres, wedges (triangular prisms), as well as others is used to construct regions. In addition, the keyword QUADRATIC is provided which allows additional shapes to be constructed by specifying the quadratic equations that describe the shape. These shapes can be rotated and translated to any orientation and position within their respective units. Hemispheres and hemicylinders can be constructed using spheres and cylinders with a chord. Regions are rotated by providing the nonzero angles associated with the Euler X-convention.

A major restriction applied to KENO V.a geometry is that intersections are not allowed. Furthermore, each successive geometry region must completely enclose the preceding region. Tangency and shared faces are allowed. The volume of a region is the volume of the specified shape minus the volume of the preceding region shape and any holes contained in the region. To alleviate the complete enclosure restriction, KENO V.a allows multiple sets of geometry regions, with each set independently governed by this restriction. Each set of these multiple geometry regions is called a unit. Units can be stacked together in a 3-D rectangular parallelepiped called an array or lattice, just as children's blocks can be stacked. Units that are to be stacked together in this manner must have a rectangular parallelepiped outer region, and the adjacent faces of adjacent units must be the same size and shape. An array can be treated as a building block and used as a unit within another array.

A major improvement in KENO-VI is the ability to intersect regions. Region volumes are no longer calculated due to the complexity involved with intersecting regions. Each set of multiple geometry regions is called a unit. KENO-VI allows multiple sets of geometry regions (i.e., units), and each set has an independent coordinate system. A global unit must be specified for every problem, including singleunit problems. Units with cuboidal outer boundaries where the adjoining faces have the same dimensions can be stacked together in a 3-D rectangular parallelepiped called an array or lattice, just as children's blocks can be stacked. Unlike KENO V.a, units having hexagonal or dodecahedral outer boundaries where the adjoining faces have the same dimensions can also be stacked together in an array. An array boundary must be specified that either coexists with the outermost edge of the array or that is entirely within the array. The array boundary can be any shape that is definable using quadratic equations. An array can be treated as a building block and used as a unit within another array.

The use of holes in KENO allows a unit to be emplaced within another unit. This feature allows the addition of a complex structure, previously defined as a unit, to be directly placed within another unit. In KENO V.a a hole is not allowed to intersect other holes or regions, but holes may intersect multiple regions in KENO-VI.

Multiple arrays can be described in KENO. The global array in an unreflected problem is the outermost array in the problem geometry description. The global array in a reflected problem is the array referenced by surrounding geometry regions following the last array placement description that does not immediately follow a unit number description. In KENO-VI, the outermost boundary is always specified as the global unit boundary. If the outermost boundary is to be the array boundary, a global unit must still be specified with the global unit boundary coinciding with the array boundary. Unlike past versions of KENO, KENOVI cannot run a single unit problem without specifying a global unit. 


\subsubsection{Fluxes}

Fluxes are computed in KENO with a track length estimator. The scalar flux in region $z$ for energy group $g$ for a single generation is computed as

$$
\Phi_{g, z}=\frac{\sum_{k=1}^{K} W_{k, z} l_{k, z}}{V_{z} \sum_{k=1}^{K} W_{k, 0}},
$$

where

$$
\begin{aligned}
& l_{k, z}=\text { distance traversed by particle } k \text { while within region } \mathrm{z} \text { and energy group } g, \\
& W_{k, z}=\text { weight of particle } k \text { while traversing region } z, \\
& V_{z}=\text { volume of region } z, \\
& W_{k, 0}=\text { initial weight of particle } k, \text { and } \\
& K \quad=\text { total number of histories in the generation. }
\end{aligned}
$$

The average fluxes for all active generations and the standard deviation in the averages are also computed. Scaler fluxes computed by KENO are reported in units of neutrons per $\mathrm{cm}^{2}$ per initial source neutron.

KENO is also capable of computing the angular flux for a level symmetric quadrature set. The angular flux for energy group $\mathrm{g}$ in region $\mathrm{z}$ for quadrature direction $n$ is computed as

$$
\Phi_{g, z}^{n}=\frac{\sum_{k=1}^{K} W_{k, z} l_{k, z, n}}{V_{z} \sum_{k=1}^{K} W_{k, 0}},
$$

where

$$
\begin{aligned}
& l_{k, z, n}= \text { the distance traversed by particle } k \text { while within region } z \text { and energy group } g \text { within } \\
& \text { the quadrature direction } n \text {. }
\end{aligned}
$$

The angular flux can be expanded to flux moments using an appropriate spherical harmonics expansion. KENO has even number symmetric level quadrature sets $S_{2}-S_{16}$.

Flux moments can also be computed directly in KENO. The $j^{\text {th }}$ moment, which corresponds to real valued spherical harmonics functions ${ }^{9}$ for a single generation for energy group $g$ in region $z$, is computed as 


$$
\tilde{\phi}_{g, z}^{j}=\frac{\sum_{k=1}^{K} R_{k}^{j} W_{k, z} l_{k, z}}{V_{z} \sum_{k=1}^{K} W_{k, 0}},
$$

where

$$
\begin{aligned}
\mathrm{R}_{\mathrm{k}}^{\mathrm{j}}= & \text { real valued spherical harmonics function for moment index } j \text { corresponding to the } \\
& \text { direction of particle } k \text {. }
\end{aligned}
$$

KENO offers the option of computing the angular fluxes and flux moments using a transformed coordinate system so that the moments are based on a polar rather than a Cartesian position vector. This is a 3-D extension of the 1-D method for calculating the flux moments in terms of Legendre polynomials based only on the direction cosine with respect to the spatial coordinate.

This coordinate transform is illustrated in Fig. 8.1.193. Here, $\hat{\mathbf{i}}, \hat{\mathbf{j}}$, and $\hat{\mathrm{k}}$ represent the directional coordinate system axes, $\mu, \eta, \xi$ represent the direction cosines, and $\theta$ and $\rho$ represent the polar and azimuthal angles of the "normal" coordinate system. The same symbols primed represent the transformed coordinate system. The transformed polar or $\hat{k^{\prime}}$ axis is co-linear, with the position vector $\vec{r}$ directed from the center of the region for which moments are desired to the point at which the flux tally occurs. By using the center of the region as a reference point, consistency of the moment calculation is assured with differing models of the same system. The $\hat{i}^{\prime}$ and $\hat{j}^{\prime}$ axes are chosen to form an orthogonal coordinate system, with $\hat{i}^{\prime}$ held in the plane formed by $\hat{i}$ and $\hat{j}$. The use of constraints other than the restriction of $\hat{i}^{\prime}$ to this plane may be explored in future studies. If an additional constraint is not placed on either $\hat{i}^{\prime}$ or $\hat{j}^{\prime}$, the transform would be able to rotate about $\hat{\mathrm{k}}^{\prime}$, and the consistency of consecutive transformations of the same direction could not be assured. With the transform computed, the position and direction of travel of the particle remain unchanged, but the quadrature directions and/or spherical harmonics terms are calculated using the transformed coordinate system. With the direction cosines consistently transformed for each history, the new polar and azimuthal angles can be computed and the spherical harmonics functions can be calculated for each history.

KENO offers a mesh flux tally in which the fluxes are computed in a user-defined cubic mesh superimposed on the geometry model. The mesh is defined from the origin of the global unit and is oriented with the Cartesian axes. At the end of each particle track, appropriate track lengths are assigned to the mesh intervals that were crossed for the region in which the track occurred. The length of the actual particle track is equal to the sum of the lengths assigned to the meshes encountered by the particle track. Because the coordinates of the mesh are defined in terms of the global unit, fluxes for regions in repeated units (i.e., units in arrays or holes) may be stored separately for each occurrence of the unit. The volumes of each region in each mesh interval are computed so that the fluxes are appropriately normalized on a per unit volume basis, consistent with Eq. (8.1.85). When the mesh flux option is activated, all requested fluxes, scalar, angular and/or moment, are computed for each mesh interval. 


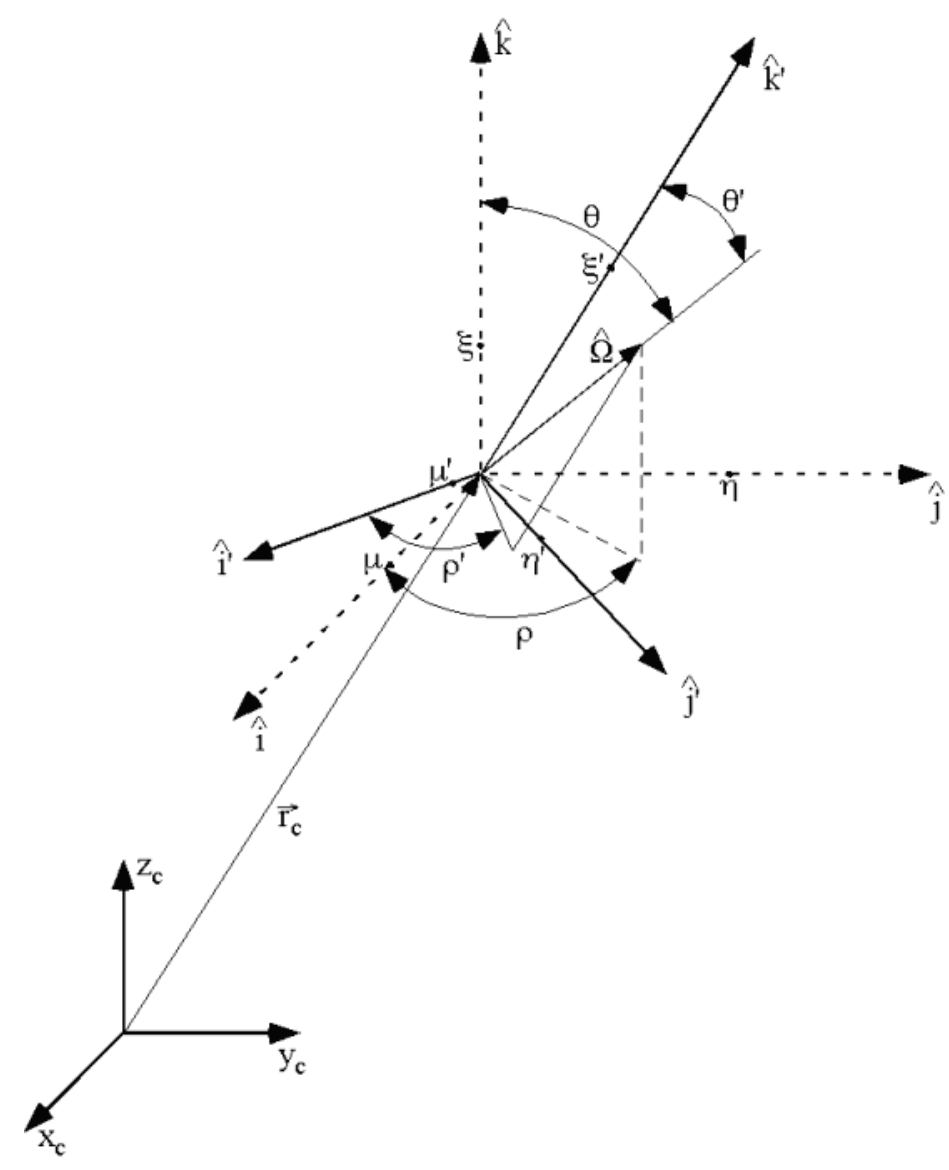

Fig. 8.1.202. Coordinate transform used for quadrature direction and spherical harmonics terms.

\subsubsection{Reaction Rate and Few Group Micro Cross Section Calculations}

A few-group microscopic reaction cross section calculation capability is included in the KENO codes to provide this data especially for the CE depletion calculations in SCALE. This new method produces multigroup cross sections and reaction rates directly in $\mathrm{CE}$ mode calculations rather than using a postprocessing approach. In each generation, KENO uses track length estimators for the reaction rate tallies for all isotopes in each specified regions. At the end of each generation, a subsequent calculation is performed to compute few group microscopic reaction cross sections for all isotopes in a region as the ratio of the computed reaction rates to the flux averaged over this cell. Finally, KENO computes mean values and statistical uncertainties for all these quantities, and saves them in a file, which could be used by the depletion modules in SCALE.

Reaction tally calculations can be enabled by entering the required data in the READ REACTION block described in Sect. 8.1.2.15.

\subsubsection{Source Convergence Diagnostics}

Fission source convergence detection techniques are implemented in KENO to provide improved 
confidence in the computed $k_{\text {eff }}$ and tally results. The dominance ratio of a system is equal to the ratio of the eigenvalue of the first nonfundamental mode to that of the fundamental mode, $k_{l} / k_{0}$. The dominance ratio is strongly related to the convergence rate of the fission source for systems, and problems with larger dominance ratios will require more inactive generations to produce reliable $k_{\text {eff }}$ and reaction rate estimates. A converged $k_{\text {eff }}$ estimate does not necessarily guarantee a converged fission source distribution, and the fission source and flux distribution may continue to evolve well after $k_{\text {eff }}$ convergence is reached. Convergence of the fission source distribution is necessary to ensure that all regions containing fissile material in a model are adequately represented in the final $k_{\text {eff }}$ estimate. Typically, the $k_{\text {eff }}$ estimate converges much faster than the fission source and neutron flux distributions, and simulations may undersample important regions and produce inaccurate flux and reaction rate estimates if the user includes only enough inactive generations for the eigenvalue to converge. This is particularly important in models where one region is physically small but substantially more reactive than others (i.e. the k-effective of the World problem $^{10}$ ). The highly reactive region may not be adequately sampled, which may result in an underprediction of $k_{\text {eff }}$. Thus, it is essential to monitor the convergence of both the fission source distribution and $k_{e f f}$; versions of KENO included in SCALE versions prior to SCALE 6.2 only monitored the convergence of $k_{\text {eff }}$, but diagnostic tools to identify unconverged fission source distributions are included in SCALE 6.2 and beyond.

NOTE: Due to limited experience with these tests in KENO, the Shannon entropy test results should be used as a supplement to guarantee that a problem's fission source is converged. Failure of Shannon entropy tests typically indicates insufficient convergence of fission site source distributions. Whenever Shannon entropy tests report failure, users should review the plots of $k_{\text {eff }}$ as a function of generation skipped and $k_{\text {eff }}$ as a function of generation completed (Sects. 8.1.4.26 and 8.1.4.27) to ensure that the $k_{\text {eff }}$ value is adequately converged. If it is not clear that the $k_{\text {eff }}$ value is converged, users should rerun the calculation using a different number of neutrons per generation (using the NPG parameter) or using a different random number seed value (using the RND parameter) or a different starting source term distribution (using the READ START block).

It may also be possible to pass the Shannon entropy tests by changing the source convergence mesh (using the SCD parameter and the READ GRID block) or changing the number skipped generations (using the NSK parameter).

For KENO calculations that are used for other purposes (such as producing neutron flux and reaction rate distributions, fuel depletion calculations, or fission distributions for radiation transport calculations) in which the spatial dependence of the neutron flux and fission source may be more important, KENO calculations with failed Shannon entropy tests should be rerun because these spatially dependent distributions may not be adequately converged.

\subsection{Shannon Entropy Statistics}

The fission source distribution, $S$, is measured at the beginning of each generation using given spatial meshes. The state of source distribution at the $j^{\text {th }}$ generation can then be characterized by Shannon entropy;

$$
H\left(S^{j}\right)=-\sum_{i}^{M}\left[S_{i}^{j} \log _{2}\left(S_{i}^{j}\right)\right]
$$

where $S_{i}^{j}$ is the fraction of the fission source distribution from the $j^{t h}$ 'generation tallied in the spatial bin $i$, and $M$ is the total number of spatial bins (typically represented using a spatial mesh). The value of $H$ 
ranges from a maximum value of $\log _{2} M$ when the source distribution is uniform, and to a minimum value of zero when the whole source is located in a single bin. By computing $H$ for each generation, the distribution of the fission source is used to generate a score for $H$ for each generation, and the convergence of the fission source can be assessed by tracking the convergence and randomness of the values of $H$ over each successive generation. Random fluctuations in the generation-to-generation Shannon entropy tallies make it difficult to determine if and when the Shannon entropy of a system has converged, so several metrics and tests are typically applied to examine the Shannon entropy and identify convergence in a system ${ }^{11}$.

The first test for fission source convergence is a comparison between the mean square of posterior relative entropy, and the centered mean square Shannon entropy. The posterior relative entropy, $D\left(S^{j} \| T\right)$, is defined as the statistical distance between the binned fission source $S_{i}^{j}$ and the average fission source over the second half of the active generations, $T$, and is given by

$$
D\left(S^{j} \| T\right)=\sum_{i}^{M}\left[S_{i}^{j} \log _{2}\left(S_{i}^{j} / T_{i}\right)\right]
$$

where $T$ is defined by,

$$
T_{i}=\frac{2}{N} \sum_{j=N / 2+1}^{N} S_{i}^{j}
$$

where $N$ is the number of active generations. The posterior relative entropy is non-negative and becomes zero only when $S^{j}=T$, and achieves its maximum value when $S^{j}$ is uniform.

The mean square posterior relative entropy is defined as,

$$
m s q(D)=\frac{2}{N} \sum_{j=N / 2+1}^{N} D\left(S_{i}^{j} \| T_{i}\right)^{2}
$$

and the centered mean square Shannon entropy is defined as,

$$
\operatorname{cms} q(H)=\frac{2}{N-2} \sum_{j=N / 2+1}^{N}\left[H\left(S_{i}^{j}\right)-\bar{H}\right]^{2}
$$

where $\bar{H}$ is defined as,

$$
\bar{H}=\frac{2}{N} \sum_{j=N / 2+1}^{N} H\left(S_{i}^{j}\right)
$$

This test compares the measure of fluctuations of Shannon entropy $(c m s q(H))$ with the measure of the magnitude of the penalty incurred by assuming $T(m s q(D))$ and states that the fission source is converged if $m s q(D)<c m s q(H)$. This test is useful to determine whether the source is converged by the end of the calculation. Failure of this test indicates that the fission source was still moving during the active generations, which indicates that the number of skipped generations was not sufficient to converge the fission source. 
The second test is given by

$$
\mid D\left(S^{j} \| T\right)-\left[H\left(T-H\left(S^{j}\right)\right] \leq \varepsilon\right.
$$

where $\varepsilon$ has been set to 0.1 . This test verifies that the Shannon entropy of each active generation does not vary significantly from the average Shannon entropy of the system and should be valid over all active generations. For example, this test could detect an unconverged fission source for a problem with an undersampled, highly fissile fuel region, as any neutrons that reached this region would produce an outlier in the Shannon entropy tally for that generation. This test is especially useful for reporting the generation at which the source converged. Once the converged generation is determined, the user can repeat the calculation with the number of inactive generations (skipped generations) set to be the identified generation or greater to ensure that only contributions from generations with a converged source contribute to flux and reaction rate tallies. This test complements the first test since it will not be affected by whether or not the binned source is uniform.

The last test calculates whether

$$
H_{\eta}=H\left(S^{j}\right)-H_{b} \leq \eta
$$

where $H_{b}$ is the average Shannon Entropy over the second half of active generations, and $\eta$ has been set to 0.1 . This test verifies that the average Shannon entropy of all active generations does not differ significantly from the Shannon entropy of the last half of the active generations. This test is useful for detecting fission source convergence in problems where an inadequate number of inactive generations was sampled, as the Shannon entropy would continue to change during the active generations until it eventually converges.

\subsection{Source Convergence Diagnostic Input}

Because the processing of the source tallies for fission source convergence calculations is quite fast and produces small memory requirements, these source convergence diagnostics have been permanently enabled in KENO and require no additional input parameters. However, the KENO input specifications were updated to allow users to specify the spatial mesh data for the source convergence tests via the SCD parameter and READ GRID data block.

\section{References}

1. M. E. Dunn and N. M. Greene, "AMPX-2000: A Cross-Section Processing System for Generating Nuclear Data for Criticality Safety Applications,” Trans. Am. Nucl. Soc. 86, 118-119 (2002).

2. J. T. Thomas, "Critical Three-Dimensional Arrays of U(93.2)-Metal Cylinders," Nucl. Sci. Eng. 52, 350 (November 1973). 
3. J. T. Thomas, Critical Three-Dimensional Arrays of Neutron-Interacting Units, Part II, ORNL/TM-868, Union Carbide Corporation (Nuclear Division), Oak Ridge National Laboratory, July 1964.

4. T. Ueki, T. Mori, and M. Nakagawa, "Error Estimations and Their Biases in Monte Carlo Eigenvalue Calculations," Nucl. Sci. Eng. 125, 1 (January 1997).

5. L. Demaret, A. Nouri, L. Carraro, and O. Jacquet, "Accurate Determination of Confidence Intervals in Monte Carlo Eigenvalue Calculations," ICNC 99, Sixth International Conference on Nuclear Criticality Safety, Vol. 1, 66 (September 1999).

6. B. Becker, R. Dagan, G. Lohnert, "Proof and implementation of the stochastic formula for ideal gas, energy dependent scattering kernel," Annals of Nuclear Energy, 36, pp. 470-474 (2009).

7. T. J. Hoffman, The Optimization of Russian Roulette Parameters for KENO V.a, ORNL/TM-7539, Union Carbide Corporation (Nuclear Division), Oak Ridge National Laboratory, 1982.

8 R. D. O`Dell and R. E. Alcouffe, Transport Calculations for Nuclear Analyses: Theory and Guidelines for Effective Use of Transport Codes, LA-10983-MS, Los Alamos National Laboratory, 1987.

9. L. Demaret, A. Nouri, L. Carraro, and O. Jacquet, "Accurate Determination of Confidence Intervals in Monte Carlo Eigenvalue Calculations," ICNC 99, Sixth International Conference on Nuclear Criticality Safety, Vol. 1, 66 (September 1999).

10. G. E. Whitesides, "A Difficulty in Computing the k-effective of the World," Trans. Am. Nucl. Soc., 14[2], 680 (1971).

11. T. Ueki, "Information Theory and Undersampling Diagnostics for Monte Carlo Simulation of Nuclear Criticality," Nuclear Science and Engineering, 151, pp. 283-292 (2005). 


\section{APPENDIX 8.1.A: KENO V.a Shape Descriptions}

The geometry shapes allowed in KENO V.a geometry description are:

$\begin{array}{llll}\text { CUBE, CUBOID, SPHERE, CYLINDER, ZCYLINDER, XCYLINDER, YCYLINDER, } \\ \text { HEMISPHERE, } & \text { HEMISPHE+X, } & \text { HEMISPHE-X, } & \text { HEMISPHE+Y, } \\ \text { HEMISPHE-Y, } & \text { HEMISPHE+Z, } & \text { HEMISPHE-Z, } & \text { XHEMICYL+Y, } \\ \text { XHEMICYL-Y, } & \text { XHEMICYL+Z, } & \text { XHEMICYL-Z, } & \text { YHEMICYL+X, } \\ \text { YHEMICYL-X, } & \text { YHEMICYL+Z, } & \text { YHEMICYL-Z, } & \text { ZHEMICYL+X, } \\ \text { ZHEMICYL-X, } & \text { ZHEMICYL+Y, } & \text { ZHEMICYL-Y } & \end{array}$

CUBE

specifies a cube. It sets $+X=+Y=+Z$ and $-X=-Y=-Z$. Note that the $+X$ dimension need not equal the $-X$ dimension of the cube (i.e., the origin need not be at the center of the cube).

CUBOID is a rectangular parallelepiped and may be described anywhere relative to the origin.

SPHERE specifies a sphere that is centered about the origin, unless otherwise specified by the optional region origin data.

CYLINDER specifies a cylinder that has its length described along the $\mathrm{Z}$ axis. Its centerline must lie on the $\mathrm{Z}$ axis, unless otherwise specified by the optional region origin data.

ZCYLINDER specifies a cylinder that has its length described along the $\mathrm{Z}$ axis. Its centerline must lie on the $\mathrm{Z}$ axis, unless otherwise specified by the optional region origin data.

XCYLINDER specifies a cylinder that has its length described along the $\mathrm{X}$ axis. Its centerline must lie on the $\mathrm{X}$ axis, unless otherwise specified by the optional region origin data.

YCYLINDER specifies a cylinder that has its length described along the Y axis. Its centerline must lie on the $\mathrm{Y}$ axis, unless otherwise specified by the optional region origin data.

HEMISPHERE is used to specify a spherical segment of one base whose spherical surface exists in the positive $\mathrm{Z}$ direction. The base or flat portion of the spherical segment is centered about a point that may be specified in the optional region origin data. By default, the center of the spherical surface is the origin and the distance to the base from the center of the spherical surface is zero.

HEMISPHE $b c$ is used to specify a spherical segment of one base whose spherical surface exists in the $b c$ direction $(\mathrm{b}=+$ or $-, \mathrm{c}=\mathrm{x}, \mathrm{y}$, or $\mathrm{z})$. The base or flat portion of the spherical segment is located a distance $\rho$ from the center of the spherical surface, and the center may be specified in the optional region origin data. HEMISPHE $+\mathbf{Z}$ is the same as the previously described HEMISPHERE and HEMISPHE- $Z$ is the mirror image of HEMISPHE $+Z$, therefore existing only in the negative $Z$ direction. By default the center of the spherical surface is the origin and the distance of the base from the center of the spherical surface is zero. 
$b$ HEMICYL $c d$ is used to specify a cylindrical segment whose axis is in the $b$ direction $(\mathrm{b}=\mathrm{x}, \mathrm{y}$, or $\mathrm{z})$ and whose cylindrical surface exists only in the $c$ direction from a plane perpendicular to the $d$ axis ( $\mathrm{c}=+$ or $-, \mathrm{d}=\mathrm{x}, \mathrm{y}$, or $\mathrm{z}$ ). The position of the plane (cut surface) can be specified in the optional region chord data. This plane cuts the cylinder parallel to the axis at some distance, $\rho$, from the axis. By default, the axis passes through the origin and $\rho$ is zero. (Examples: ZHEMICYL+X, YHEMICYL-Z, XHEMICYL+Y)

Up to 6 dimensions follow the shape keyword [ $x x(1)$ through $x x(6)]$. These entries are separated by one or more blanks and define the size of the region. Dimensions must be given in $\mathrm{cm}$.

$x x$ (1) Radius for a sphere, cylinder, hemisphere or hemicylinder, $+\mathrm{X}$ dimension for a cube or cuboid.

$x x(2) \quad-X$ dimension for cube or cuboid, $+\mathrm{Z}$ for cylinder or $\mathrm{Z}$ cylinder, $+X$ for $X$ cylinder, $+\mathrm{Y}$ for $\mathrm{Y}$ cylinder, +length for hemicylinder, omit $x x(2)$ for a sphere or hemisphere.

$x x(3)+Y$ dimension for cuboid, $-\mathrm{Z}$ for cylinder or $\mathrm{Z}$ cylinder, $-X$ for $\mathrm{X}$ cylinder, -Y for Y cylinder, -length for hemicylinder, omit $x x$ (3) for a sphere, hemisphere, or cube.

$x x(4)-Y$ dimension for cuboid, omit for all other geometry types.

$x x(5)+Z$ dimension for cuboid, omit for all other geometry types.

$x x$ (6) $-Z$ dimension for cuboid, omit for all other geometry types. 


\section{APPENDIX 8.1.B: KENO VI Shape Descriptions}

The geometry shapes allowed in KENO-VI geometry description are:

CONE, CUBOID, CYLINDER, DODECAHEDRON, ECYLINDER, ELLIPSOID, HEXPRISM, HOPPER, PARALLELEPIPED, PPIPED, PENTAGON, PLANE, QUADRATIC, RHEXPRISM, RHOMBOID, SPHERE, WEDGE，XCYLINDER, XPPLANE, YCYLINDER, YPPLANE, ZCYLINDER, ZPPLANE

CONE specifies a body consisting of one nappe of a right circular cone. It is defined by specifying the top radius of the cone, $R_{t}$, the $Z$ coordinate of the top face, $Z_{t}$, the bottom radius of the cone, $R_{b}$, and the $\mathrm{Z}$ coordinate of the bottom face, $\mathrm{Z}_{\mathrm{b}}$. Figure 8.1.B.1 shows the correct input sequence for a cone.

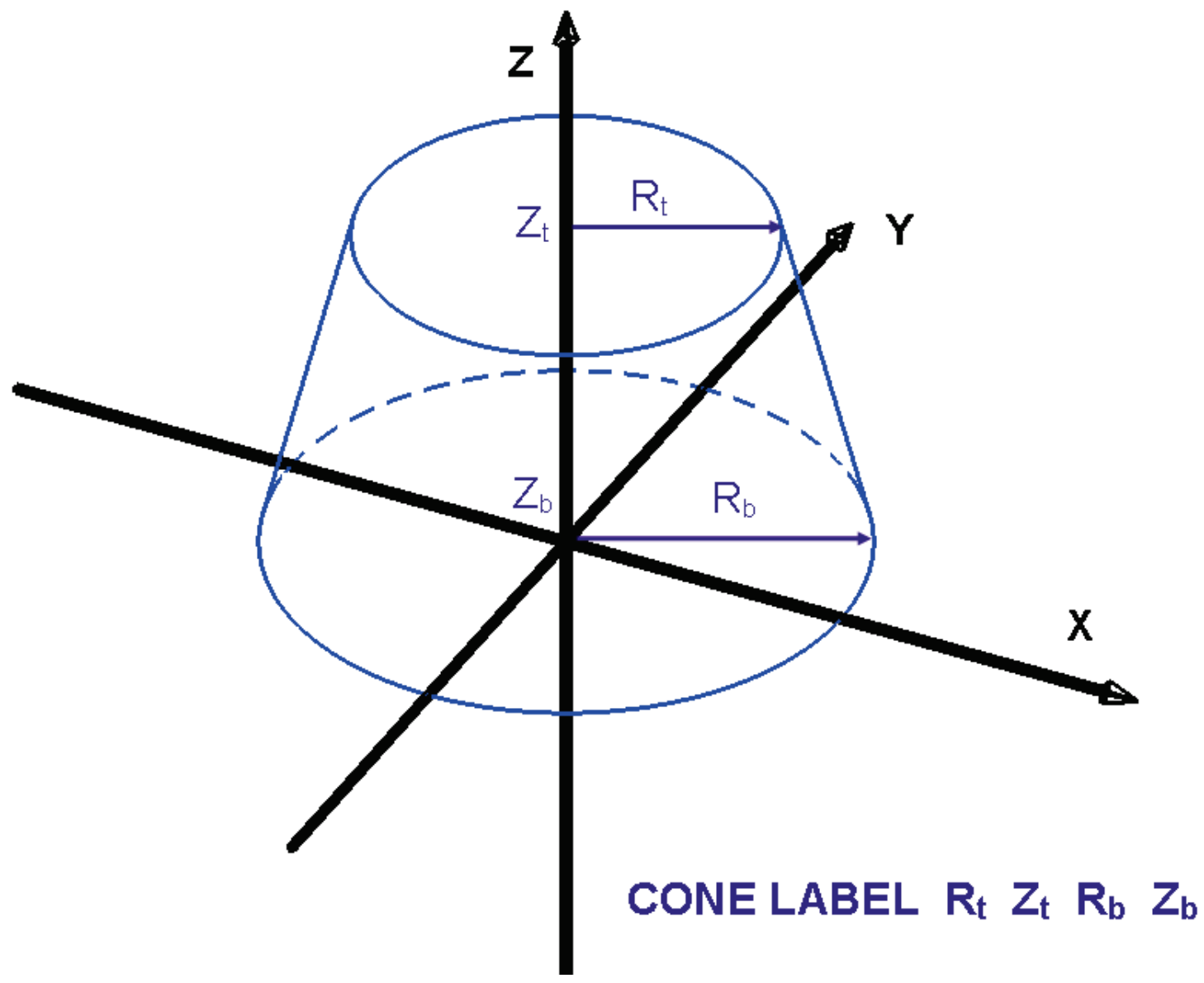

Figure 8.1.B.1. Example of cone construction. 
CUBOID specifies a rectangular parallelepiped. It is defined by specifying the $+X$ dimension, $-\mathrm{X}$ dimension, $+\mathrm{Y}$ dimension, $-\mathrm{Y}$ dimension, $+\mathrm{Z}$ dimension, $-\mathrm{Z}$ dimension. It is perpendicular to the $\mathrm{X}, \mathrm{Y}$, and $\mathrm{Z}$ axes unless otherwise specified by the option geometry modification data. Figure 8.1.B.2 shows the correct input sequence for a cuboid.

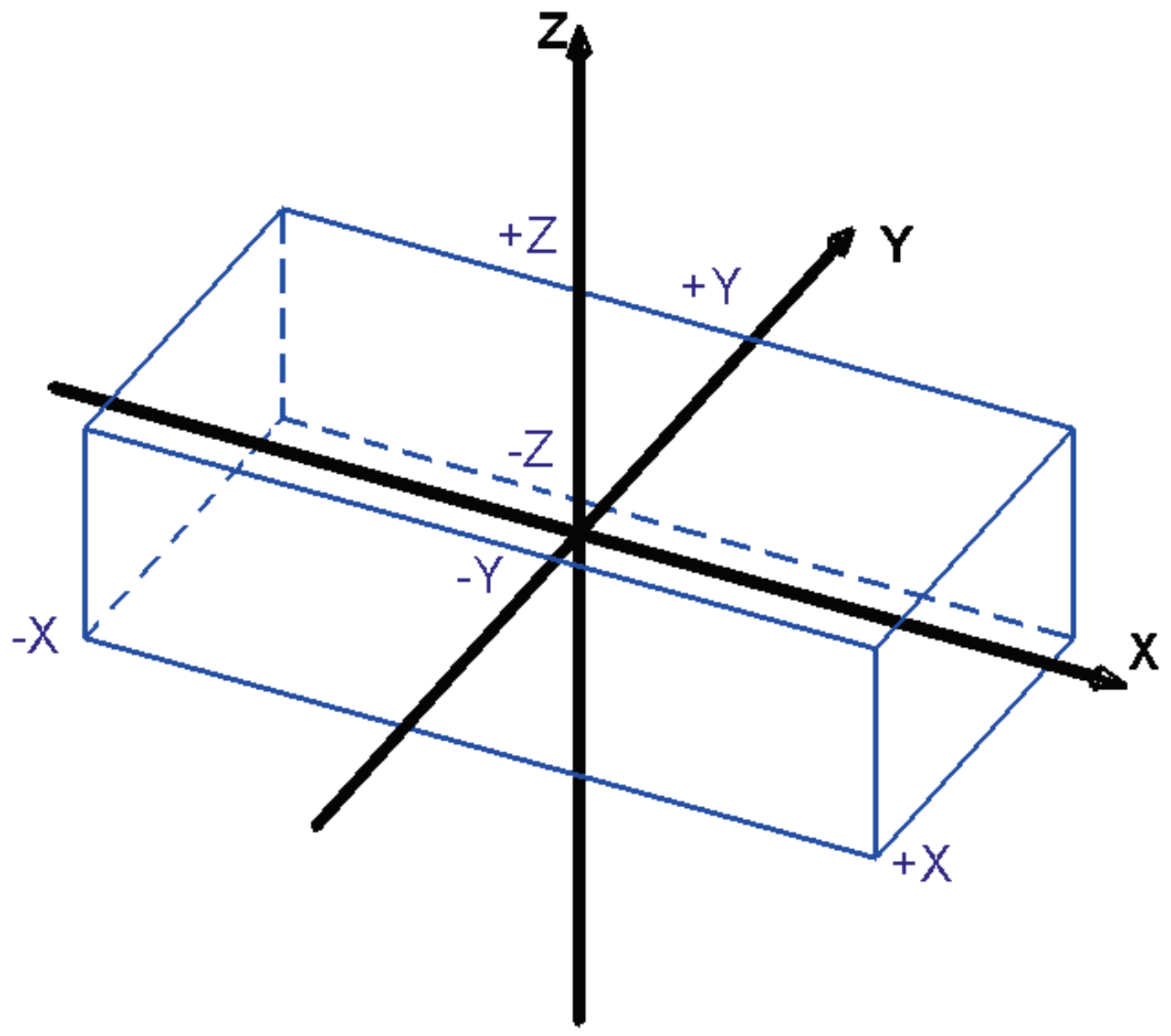

CUBOID LABEL $+X-X+Y-Y+Z-Z$

Figure 8.1.B.2. Example of cuboid construction. 
CYLINDER specifies a right circular cylinder. It is defined by specifying the radius of the cylinder, R, the $\mathrm{Z}$ coordinate of the top face, $\mathrm{Z}_{\mathrm{t}}$, and the $\mathrm{Z}$ coordinate of the bottom face, $\mathrm{Z}_{\mathrm{b}}$. Its centerline must lie on the $Z$ axis, unless otherwise specified by the optional geometry modification data. Figure 8.1.B.3 shows the correct input sequence for a cylinder.

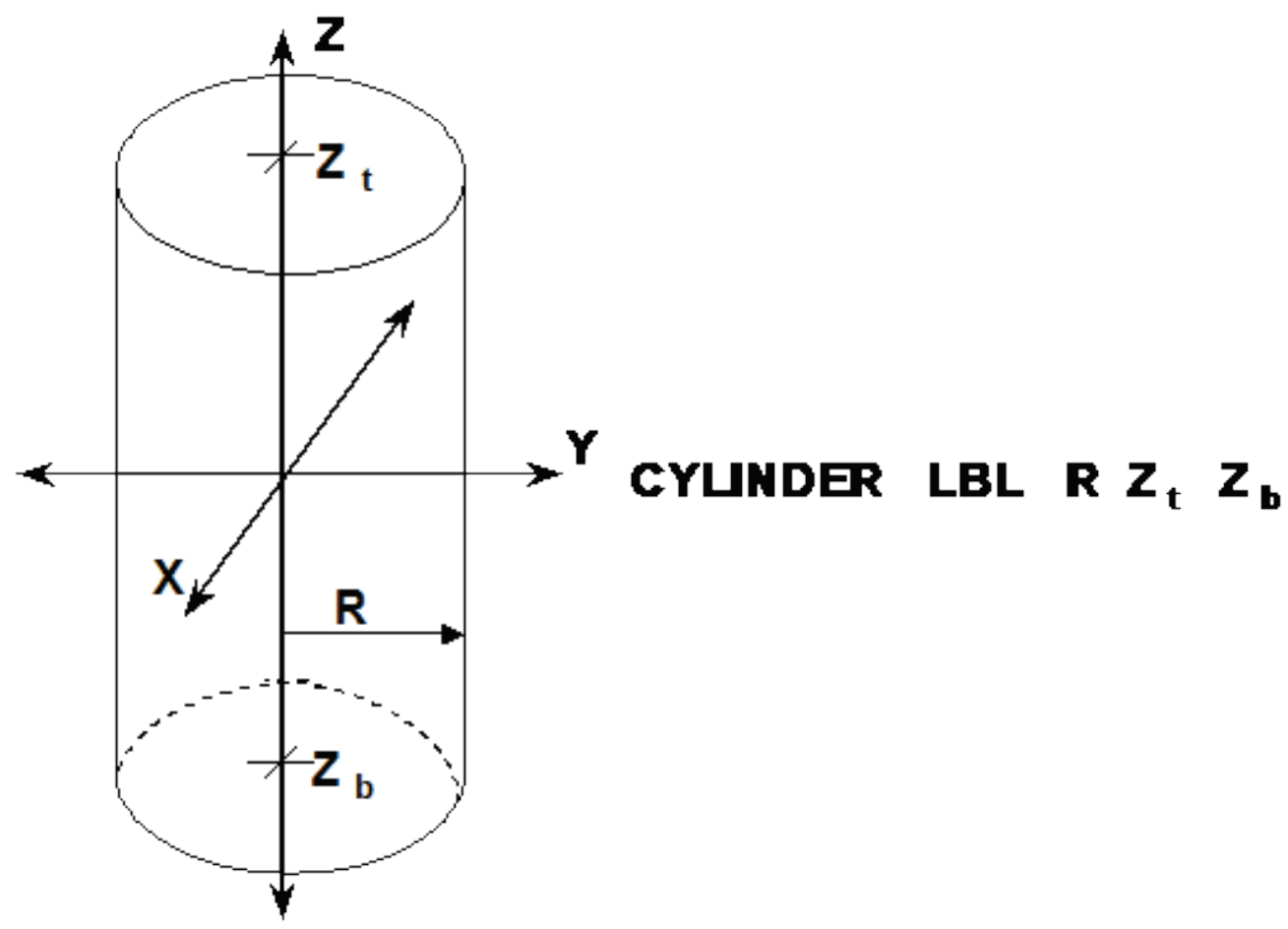

Figure 8.1.B.3. Example of cylinder construction. 
DODECAHEDRON specifies a body whose surface consists of 12 rhombuses of the same size and shape. It is defined by specifying the radius of the inscribed sphere, $R$. It is centered on the origin in a fixed orientation unless otherwise specified by the optional geometry modification data. Figure 8.1.B.4 shows the correct input sequence for a dodecahedron.
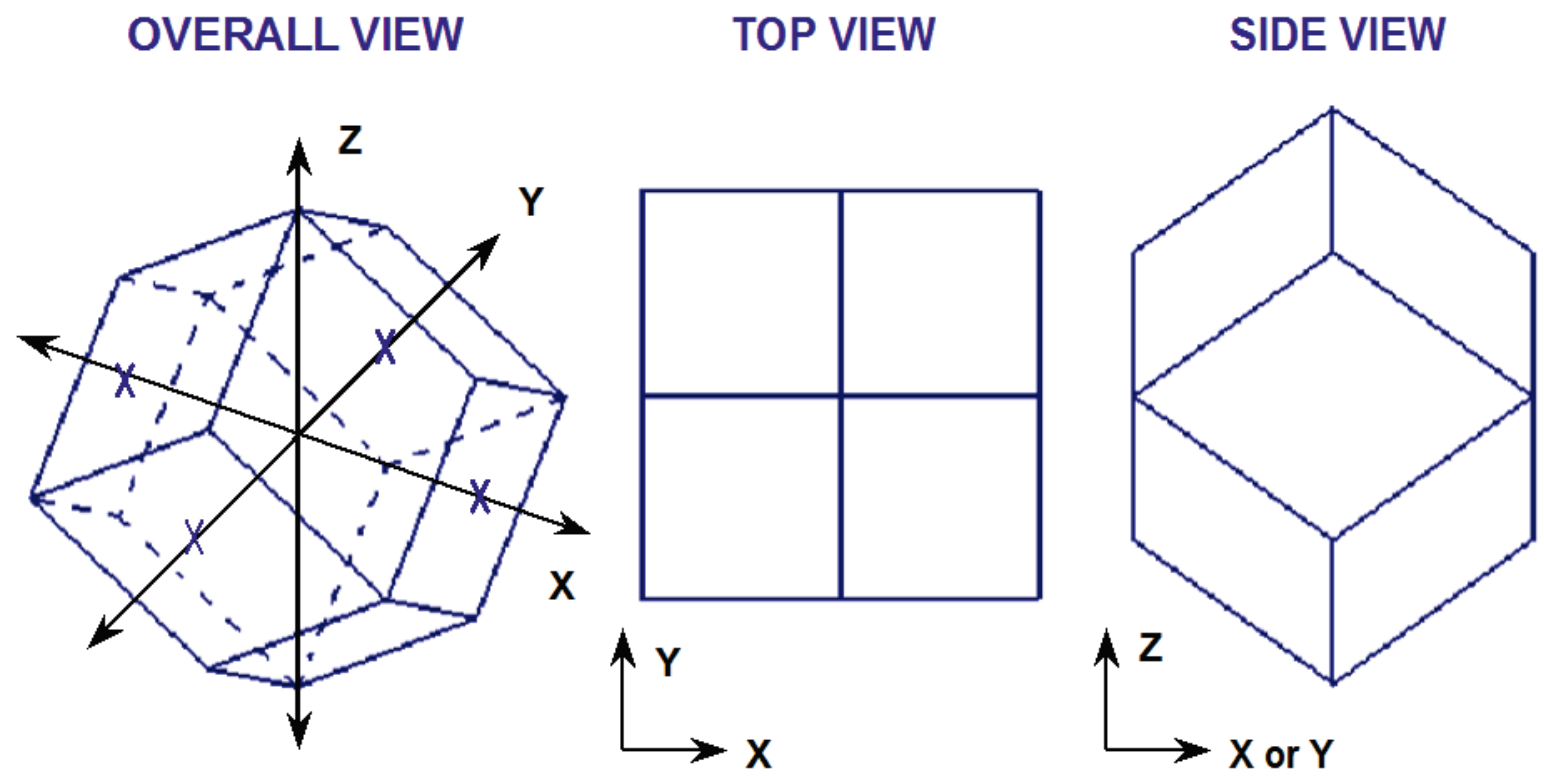

\section{DODECAHEDRON LABEL $R$ ( $R$ is the Inscribed Radius )}

Figure 8.1.B.4. Example of dodecahedron construction. 
ECYLINDER specifies a right cylinder with an elliptical cross section. It is defined by specifying the semiradius along the $X$-axis, $R_{x}$, the semiradius along the $Y$-axis, $R_{y}$, the $Z$ coordinate of the top face, $Z_{t}$, and the $Z$ coordinate of the bottom face, $Z_{b}$. Its centerline must lie on the $\mathrm{Z}$ axis, unless otherwise specified by the optional geometry modification data. Figure 8.1.B.5 shows the correct input sequence for an elliptical cylinder.

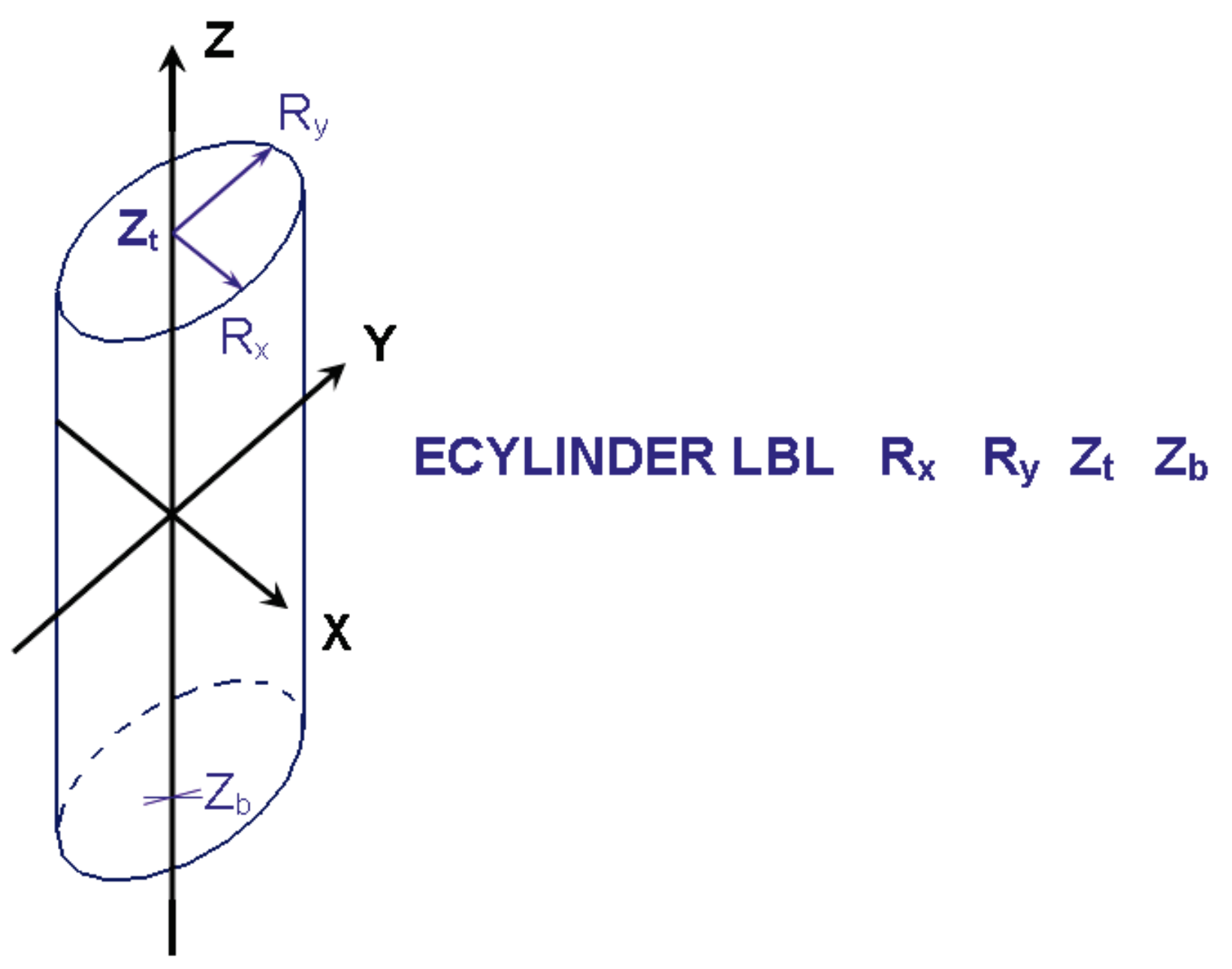

Figure 8.1.B.5. Example of elliptical cylinder construction. 
ELLIPSOID specifies a body whose cross-section slices parallel to each of the coordinate axes are ellipses. It is defined by specifying the semiradius along the $\mathrm{X}$-axis, $\mathrm{R}_{\mathrm{x}}$, the semiradius along the $\mathrm{Y}$-axis, $\mathrm{R}_{\mathrm{y}}$, and the semiradius along the $\mathrm{Z}$-axis, $\mathrm{R}_{\mathrm{z}}$. It is centered about the origin, unless otherwise specified by the optional geometry modification data. Figure 8.1.B.6 shows the correct input sequence for an ellipsoid.
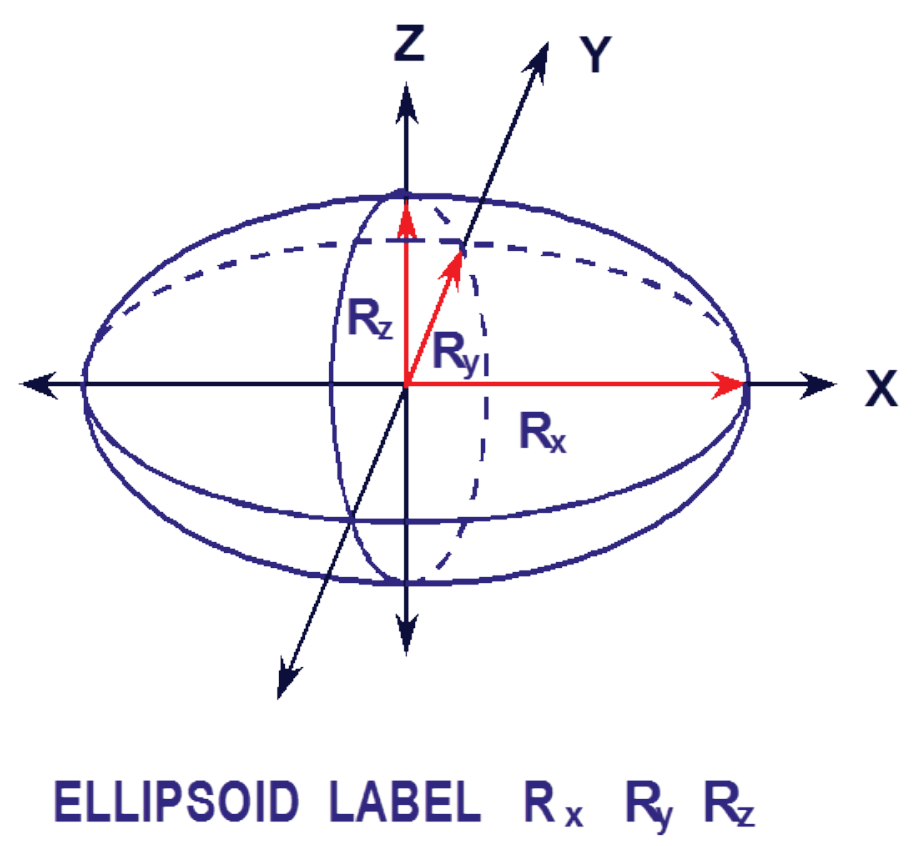

Figure 8.1.B.6. Example of ellipsoid construction. 
HEXPRISM specifies a body whose top and bottom faces are hexagons that have the same orientation and are perpendicular to the $Z$ axis. It is defined by specifying the inscribed radius, $R$, the $Z$ coordinate of the top face, $Z_{t}$, and the $Z$ coordinate of the bottom face, $Z_{b}$. Figure 8.1.B.7 is an example input for a hexprism.

\section{OVERALL VIEW}

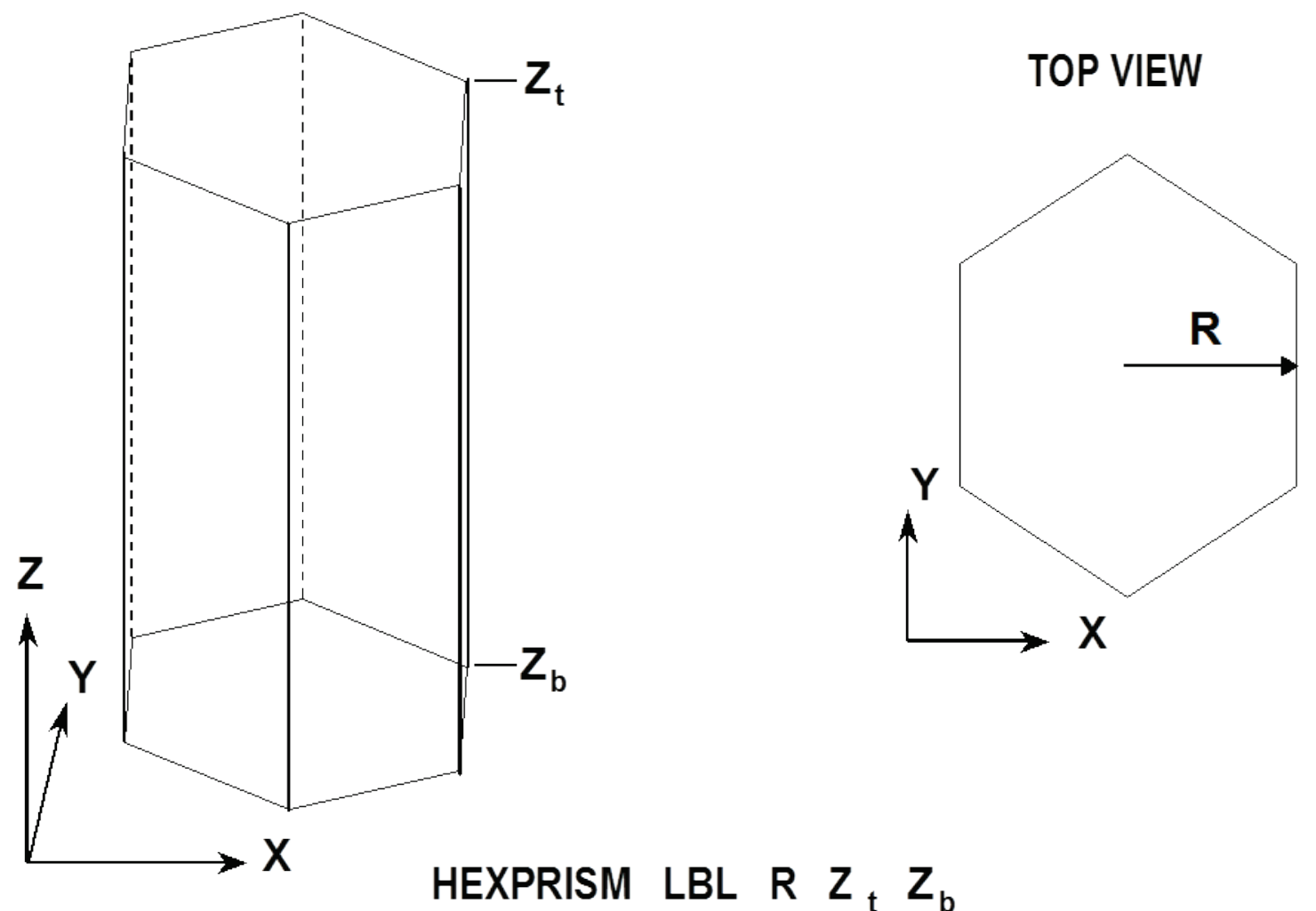

Figure 8.1.B.7. Example of hexprism construction. 
HOPPER specifies a body whose top and bottom faces are rectangular parallelepipeds centered about the $\mathrm{Z}$-axis and parallel to the $\mathrm{X}$ and $\mathrm{Y}$ axes. It is defined by specifying the half-length of the top face along the $X$-axis, $X_{t}$, the half-length of the top face along the $Y$-axis, $Y_{t}$, the $Z$ coordinate of the top face, $Z_{t}$, the half-length of the bottom face along the $X$-axis, $X_{b}$, the half-length of the bottom face along the $\mathrm{Y}$-axis, $\mathrm{Y}_{\mathrm{b}}$, and the $\mathrm{Z}$ coordinate of the bottom face, $Z_{b}$. Its centerline must lie on the $\mathrm{Z}$ axis unless otherwise specified by the optional geometry modification data. Figure 8.1.B.8 shows the correct input sequence for a hopper.

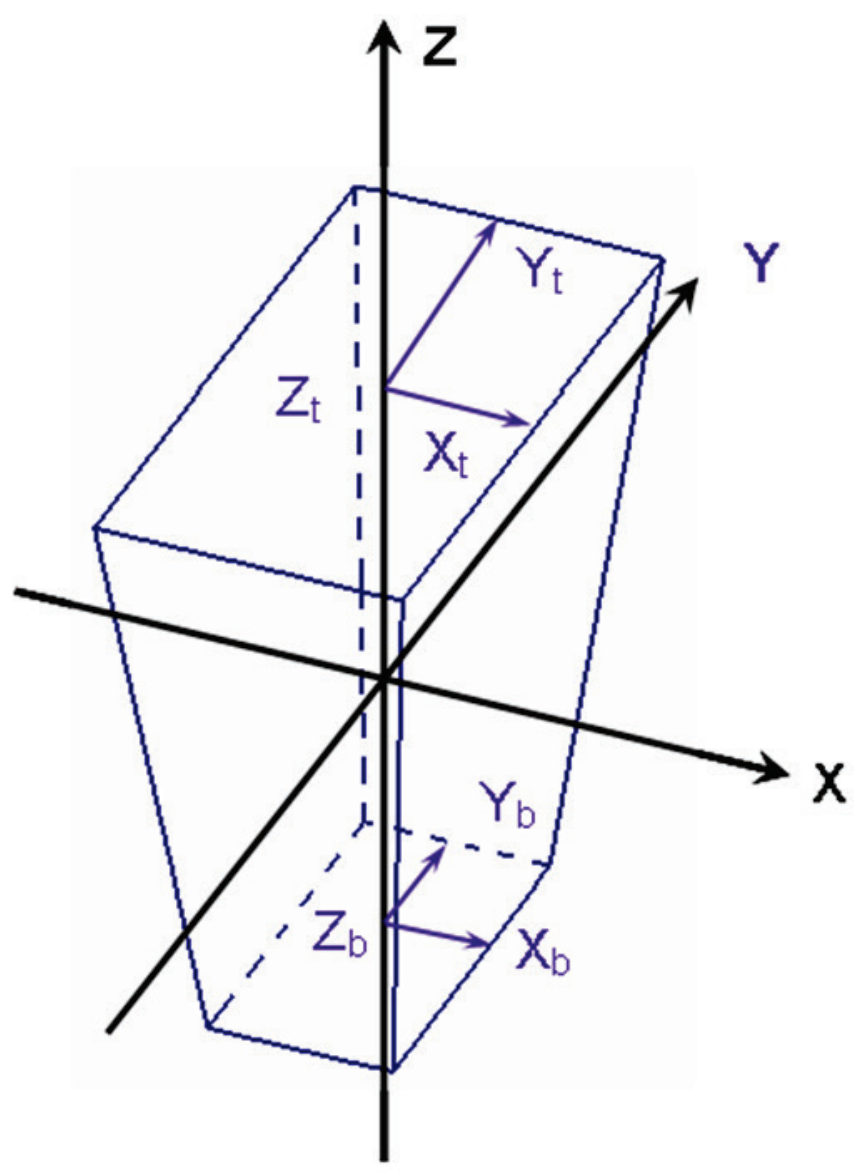

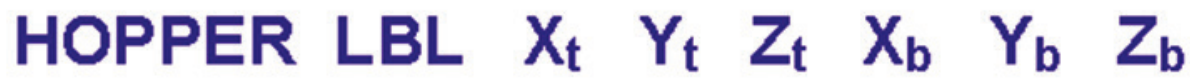

Figure 8.1.B.8. Example of hopper construction. 
PARALLELEPIPED is a body with six faces composed of parallelograms, whose opposing faces are or PPIPED parallel. It is defined by specifying the length of the faces in the $\mathrm{X}$ direction, XDIST, the length of the faces in the Y direction, YDIST, the length of the faces in the $\mathrm{Z}$ direction, ZDIST, the angle between the $\mathrm{X}$-face and the $\mathrm{Y}$-axis, PSI, the angle between the Y-face and the Z-axis, THETA, and the angle between the projection of the top corner nearest the Z-axis onto the X-Y plane and the $\mathrm{X}$-axis, PHI. The bottom face must lie on the $\mathrm{X}-\mathrm{Y}$ plane at $\mathrm{Z}=0$ with a corner at the origin unless otherwise specified by the optional geometry modification data. Figure 8.1.B.9 shows the correct input sequence for a parallelepiped. The angles psi, theta, and phi must be in the range 0 to $90^{\circ}$.

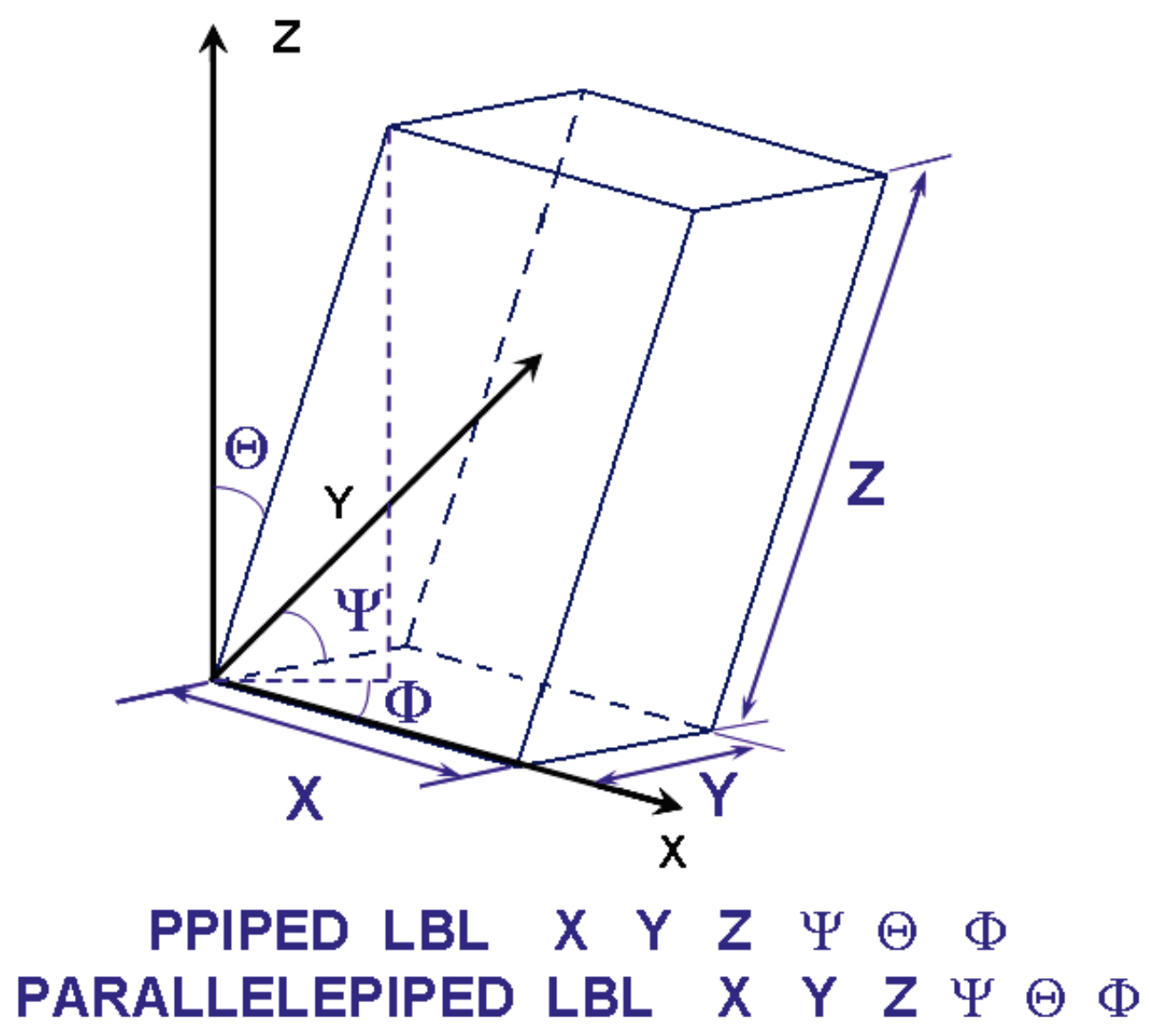

Figure 8.1.B.9. Example of parallelepiped construction. 
PENTAGON specifies a body whose top and bottom faces are pentagons that have the same orientation and are perpendicular to the $Z$ axis. It is defined by specifying the inscribed radius, $R$, the $\mathrm{Z}$ coordinate of the top face, $\mathrm{Z}_{\mathrm{t}}$, and the $\mathrm{Z}$ coordinate of the bottom face, $\mathrm{Z}_{\mathrm{b}}$.

Figure 8.1.B.10 is an example input for a pentagon.

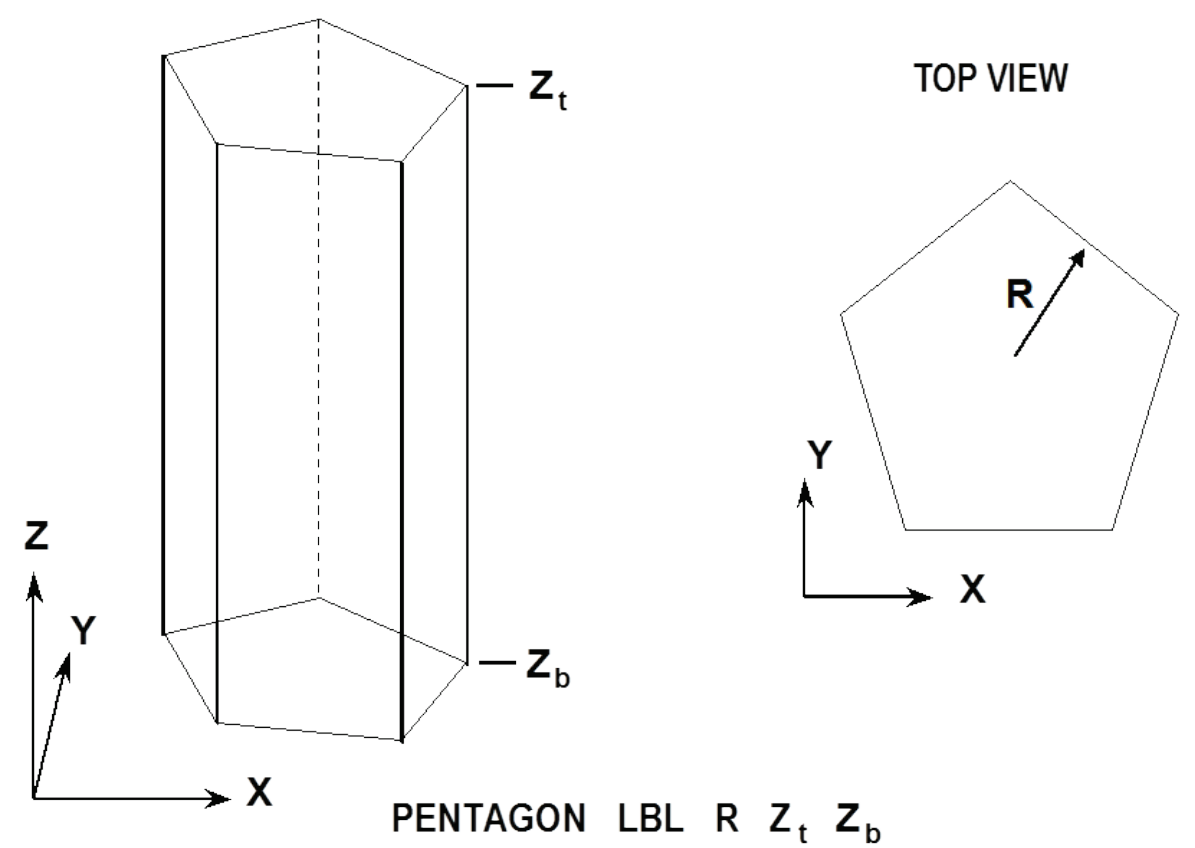

Figure 8.1.B.10. Example of pentagon construction. 
PLANE is a surface where any two points can be connected by a straight line entirely contained within a plane that divides all space into two regions. The positive side of the plane is the side the normal points to or where the equation $\mathrm{aX}+\mathrm{bY}+\mathrm{cZ}+\mathrm{d}>0$. It is defined by specifying the coefficients of the equation $a X+b Y+c Z+d=0$ using the keywords $\mathrm{XPL}=\mathrm{a}, \mathrm{YPL}=\mathrm{b}, \mathrm{ZPL}=\mathrm{c}$, and $\mathrm{CON}=\mathrm{d}$. Only the nonzero coefficients of the equation need to be specified. Figure 8.1.B.11 shows the correct input sequence for a plane.

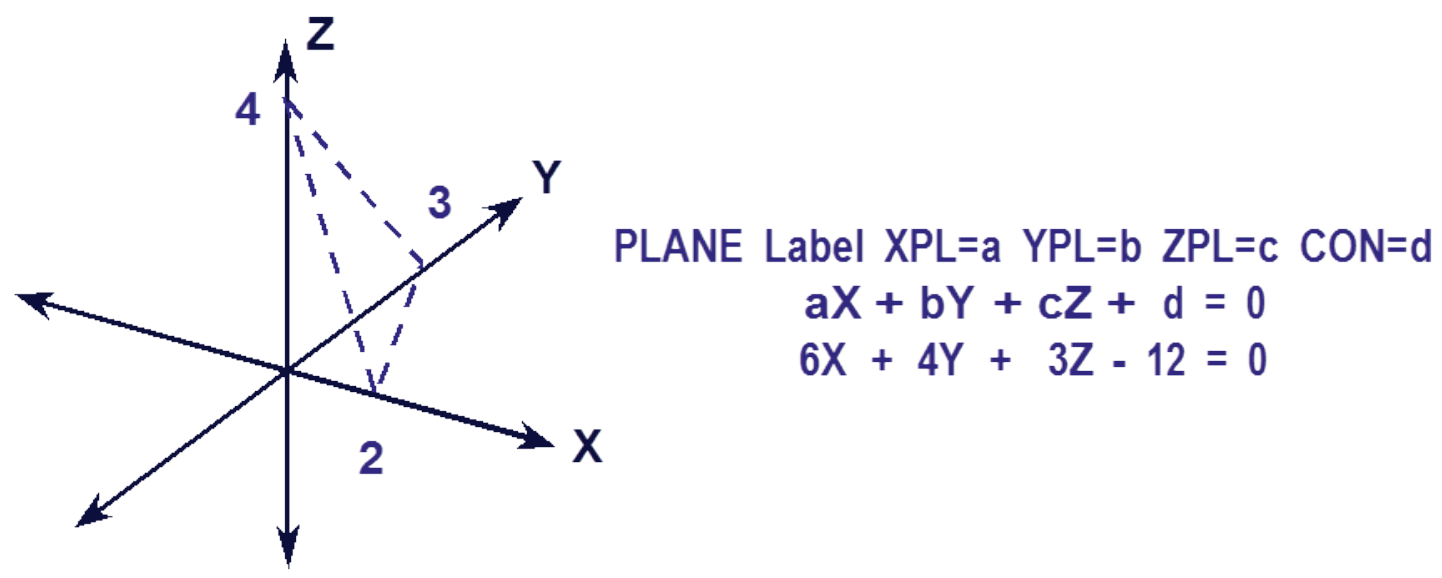

Figure 8.1.B.11. Example of plane construction.

QUADRATIC specifies a surface using a quadratic equation of the form:

$a X^{2}+b Y^{2}+c Z^{2}+d X Y+e X Z+f Y Z+g X+h Y+i Z+j=0$.

It is defined by specifying the coefficients of the above equation using the keywords $\mathrm{AQU}=\mathrm{a}, \mathrm{BQU}=\mathrm{b}, \mathrm{CQU}=\mathrm{c}, \mathrm{DQU}=\mathrm{d}, \mathrm{EQU}=\mathrm{e}, \mathrm{FQU}=\mathrm{f}, \mathrm{GQU}=\mathrm{g}, \mathrm{HQU}=\mathrm{h}, \mathrm{IQU}=\mathrm{i}$, and $\mathrm{JQU}=\mathrm{j}$. Only the nonzero coefficients of the equation need to be specified.

RHEXPRISM specifies a body whose top and bottom faces are rotated hexagons that have the same orientation and are perpendicular to the $Z$ axis. It is defined by specifying the inscribed radius, $R$, the $Z$ coordinate of the top face, $Z_{t}$, and the $Z$ coordinate of the bottom face, $Z_{b}$. Figure 8.1.B.12 is an example input for a rotated hexprism. 


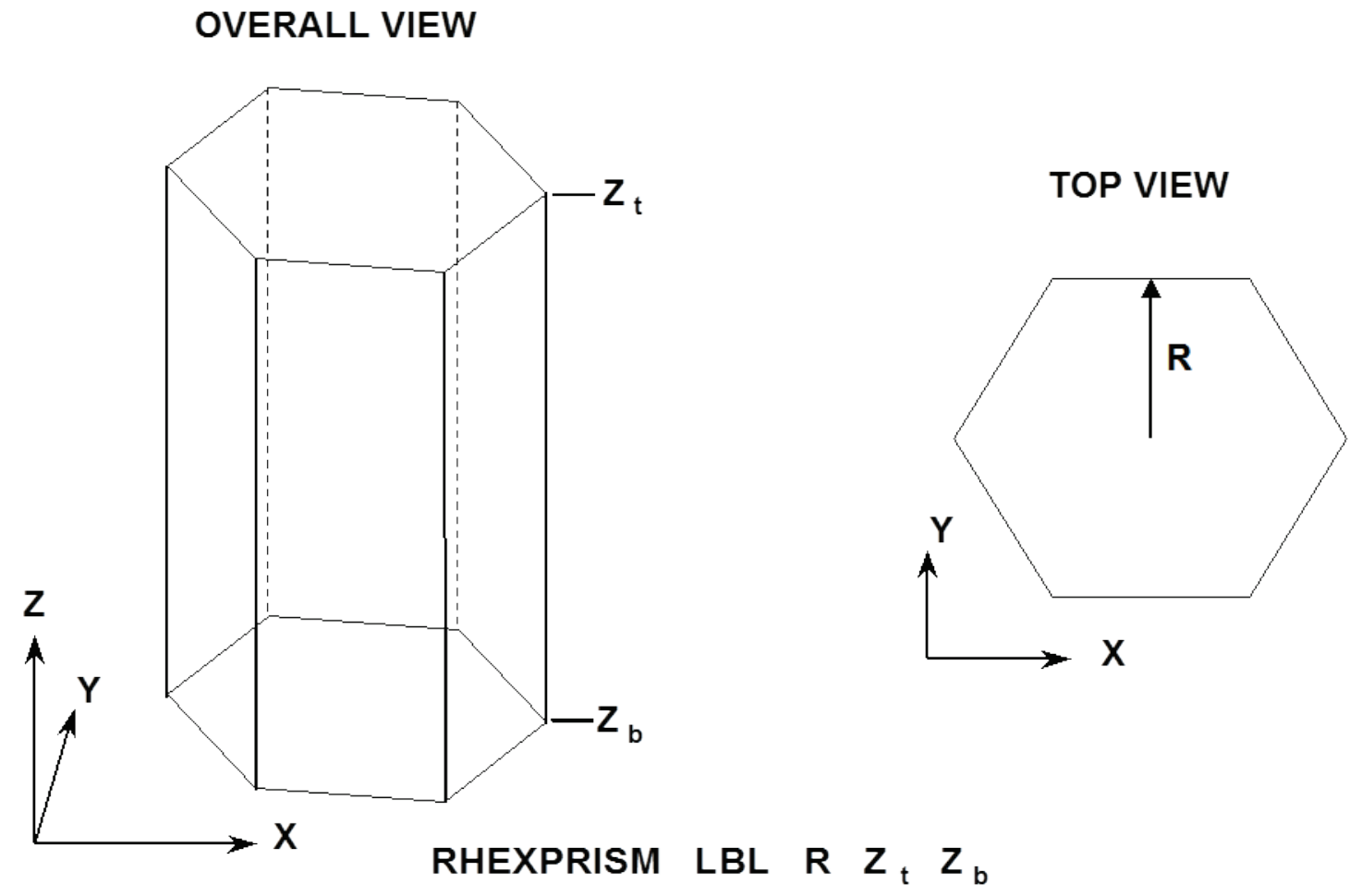

Figure 8.1.B.12. Example of rotated hexprism construction. 
RING is a body composed of the space between 2 concentric cylinders. It is defined by specifying the radius Rin of the inner cylinder and Rout of the outer cylinder, and the coordinate $\mathrm{Zt}$ of the top and $\mathrm{Zb}$ of the bottom of the annulus. Its center line lies on the $\mathrm{Z}$ axis unless specified by the optional geometry modification data. Figure 8.1.B.13 shows the correct input sequence for a ring.

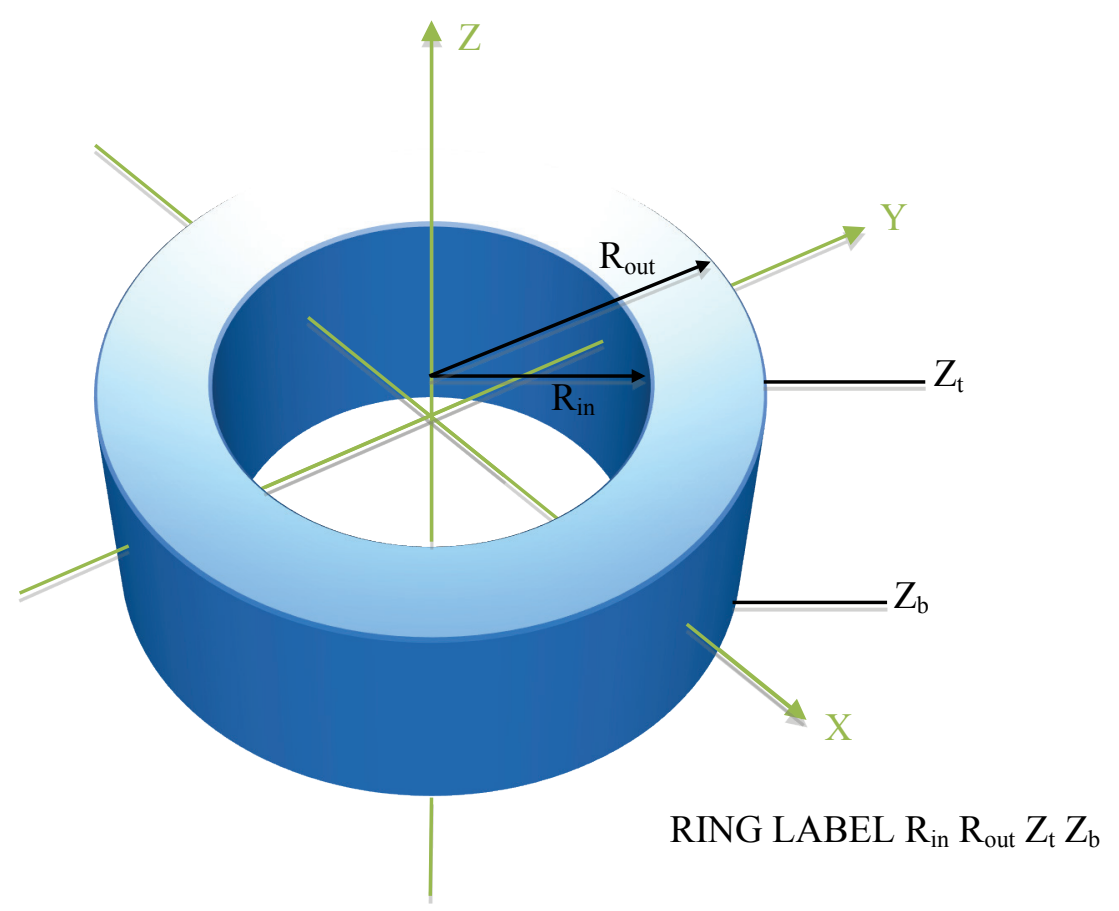

Figure 8.1.B.13. Example of ring construction. 
RHOMBOID is a body composed of six identical faces, each one a rhombus. It is defined by specifying the length of the edge of the base along the X-axis, DX and the angle between Y edge of the base and the $Y$-axis, $\Psi$. Its base is in the $X Y$ plane at $Z=0$, with a corner at the origin unless otherwise specified by the optional geometry modification data. Figure 8.1.B.14 shows the correct input sequence for a rhomboid.

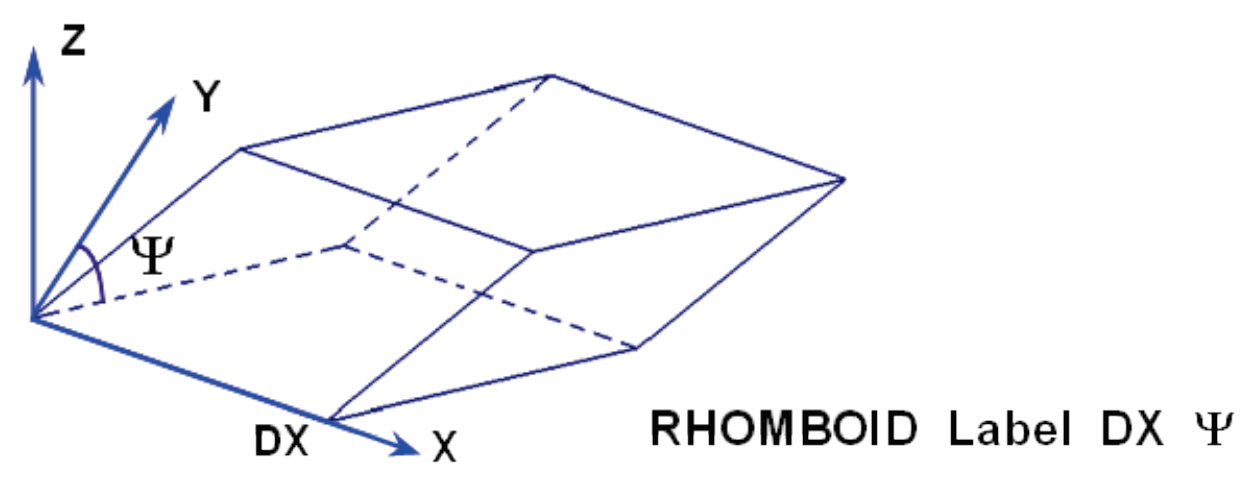

Figure 8.1.B.14. Example of rhomboid construction. 
SPHERE specifies a sphere. It is defined by specifying the radius, R. It is centered about the origin, unless otherwise specified by the optional geometry modification data. Figure 8.1.B.15 shows the correct input sequence for a sphere.

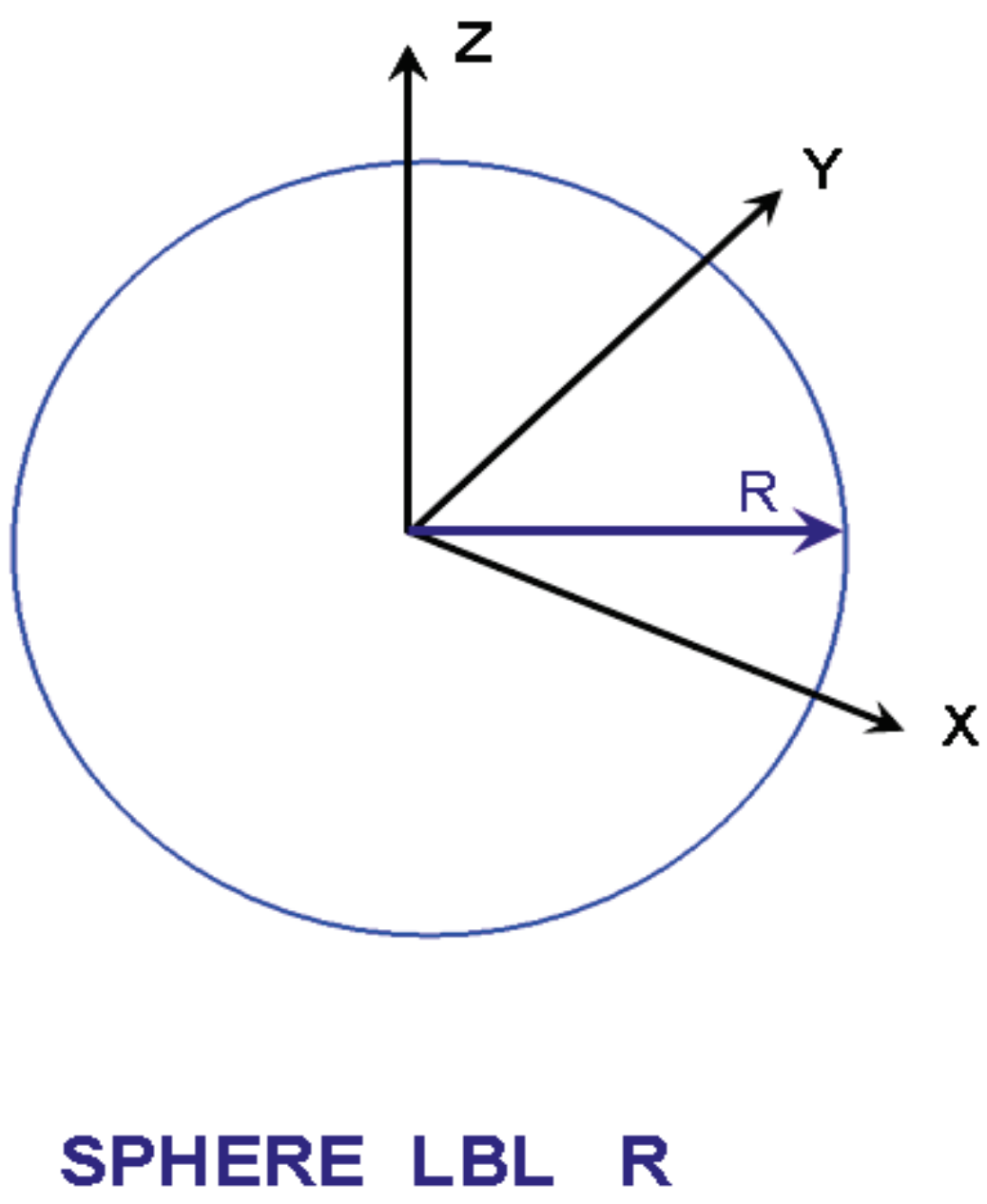

Figure 8.1.B.15. Example of sphere construction. 
WEDGE is a right-triangular prism having five faces. The two ends are triangles, and the three sides are rectangles. It is defined by specifying the length of the base along the X-axis, XBASE, the $\mathrm{X}$ and $\mathrm{Y}$ coordinate where the other two sides meet, $\mathrm{Xpt}$ and $\mathrm{Ypt}$, and the length along the Z-axis, ZLNG. One side is in the XZ plane at $\mathrm{Y}=0$, and the bottom face is in the $\mathrm{XY}$ plane at $\mathrm{Z}=0$, with a corner at the origin unless otherwise specified by the optional geometry modification data. Figure 8.1.B.16 shows the correct input sequence for a wedge.

\section{WEDGE LABEL XBASE Xpt Ypt ZLNG}

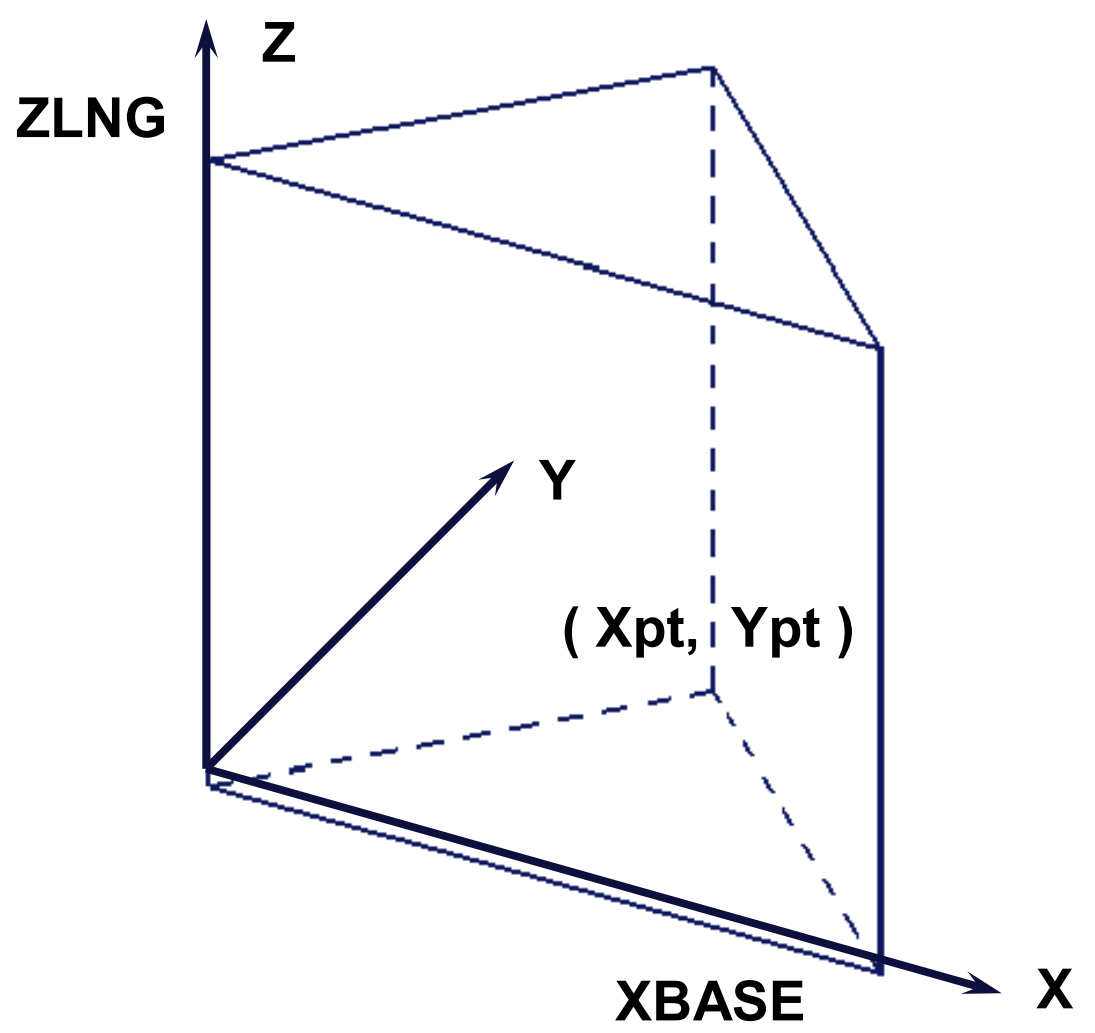

Figure 8.1.B.16. Example of wedge construction. 
XCYLINDER specifies a right circular cylinder oriented about the X-axis. It is defined by specifying the radius of the cylinder, $\mathrm{R}$, the $\mathrm{X}$ coordinate of the top face, $\mathrm{X}_{\mathrm{t}}$, and the $\mathrm{X}$ coordinate of the bottom face, $X_{b}$. Its centerline must lie on the $X$ axis, unless otherwise specified by the optional geometry modification data. Figure 8.1.B.17 shows the correct input sequence for a cylinder.

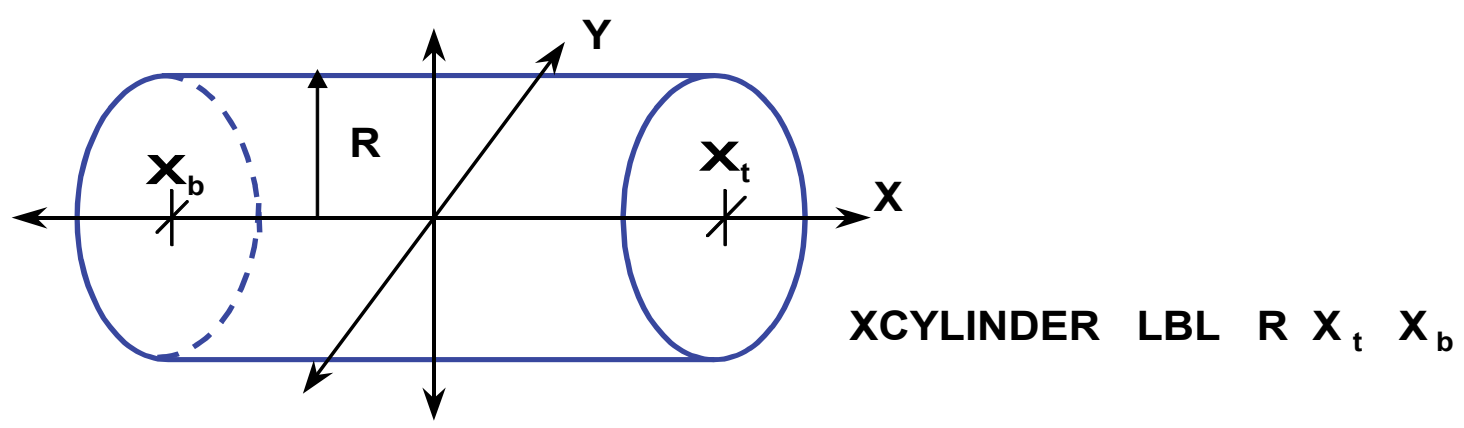

Figure 8.1.B.17. Example of xcylinder construction. 
XPPLANE is a set of flat parallel surfaces where any two points in one of the surfaces can be connected by a straight line entirely contained within that surface. These surface planes divide space into three sections; one section between the two planes which is considered inside the surfaces, one section on the negative side of the negative plane which is considered outside the surfaces, and one section on the positive side of the positive plane which is considered outside the surfaces. The set of parallel planes are defined by the keyword XPPLANE, which places the planes perpendicular to the X-axis, the Xintercept between the more positive plane and the $\mathrm{X}$-axis $\left(\mathrm{X}_{+}\right)$and the $\mathrm{X}$-intercept between the more negative plane and the $\mathrm{X}$-axis $\left(\mathrm{X}_{-}\right)$. Figure 8.1.B.18 shows the correct input sequence for the set of paired planes.

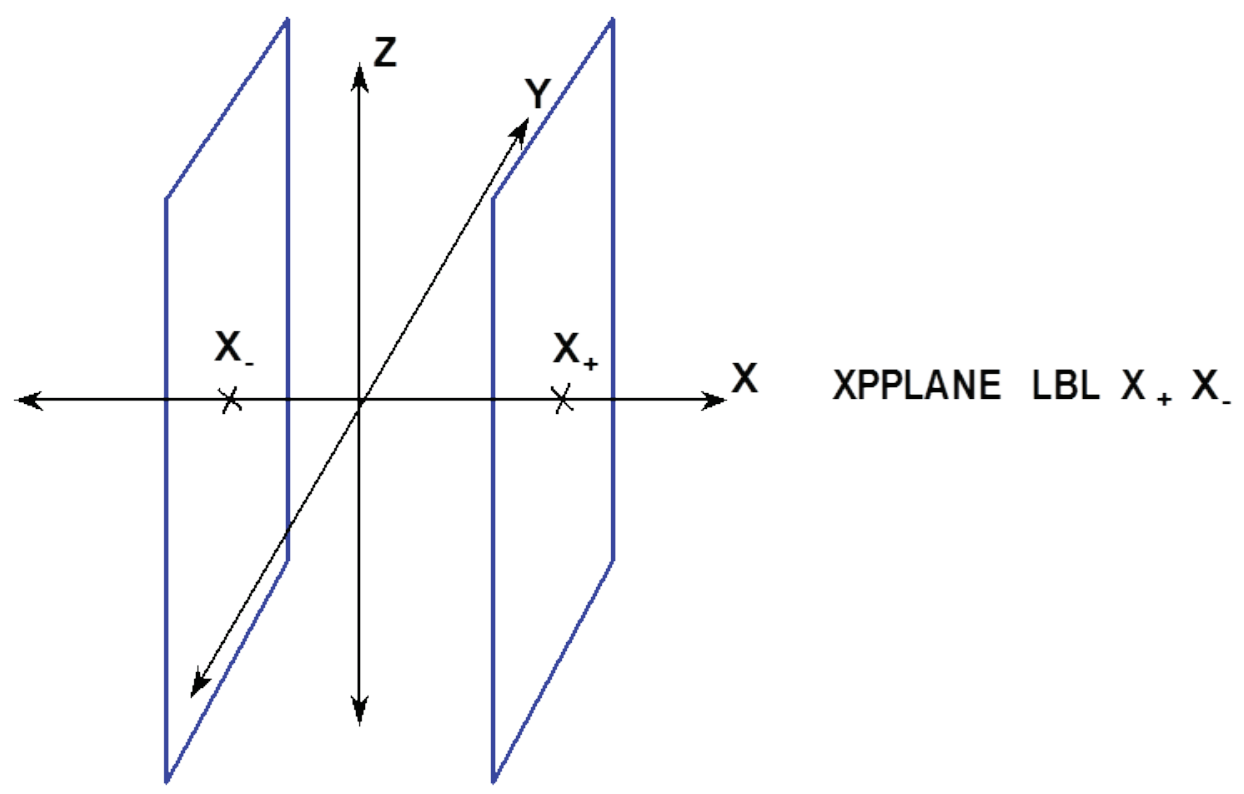

Figure 8.1.B.18. Example of x-paired plane construction. 
YCYLINDER specifies a right circular cylinder oriented about the Y-axis. It is defined by specifying the radius of the cylinder, $\mathrm{R}$, the $\mathrm{Y}$ coordinate of the top face, $\mathrm{Y}_{\mathrm{t}}$, and the $\mathrm{Y}$ coordinate of the bottom face, $Y_{b}$. Its centerline must lie on the $Y$ axis, unless otherwise specified by the optional geometry modification data. Figure 8.1.B.19 shows the correct input sequence for a cylinder.

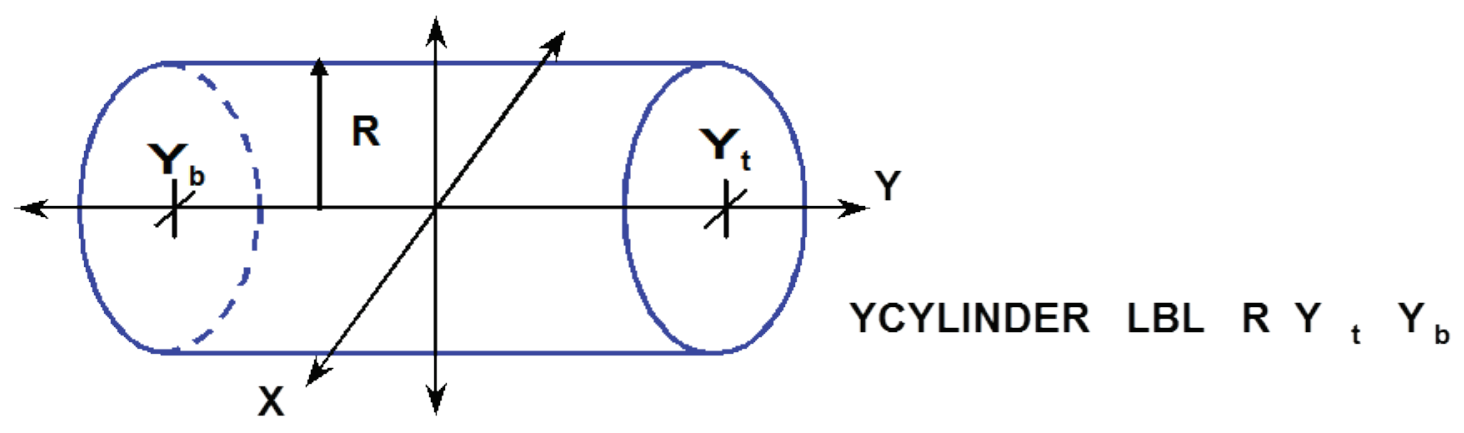

Figure 8.1.B.19. Example of ycylinder construction. 
YPPLANE is a set of flat parallel surfaces where any two points in one of the surfaces can be connected by a straight line entirely contained within that surface. These surface planes divide space into three sections; one section between the two planes which is considered inside the surfaces, one section on the negative side of the negative plane which is considered outside the surfaces, and one section on the positive side of the positive plane which is considered outside the surfaces. The set of parallel planes are defined by the keyword YPPLANE, which places the planes perpendicular to the Y-axis, the Y-intercept between the more positive plane and the $\mathrm{Y}$-axis $\left(\mathrm{Y}_{+}\right)$and the $\mathrm{Y}$-intercept between the more negative plane and the $\mathrm{Y}$-axis $\left(\mathrm{Y}_{-}\right)$. Figure 8.1.B.20 shows the correct input sequence for the set of paired planes.

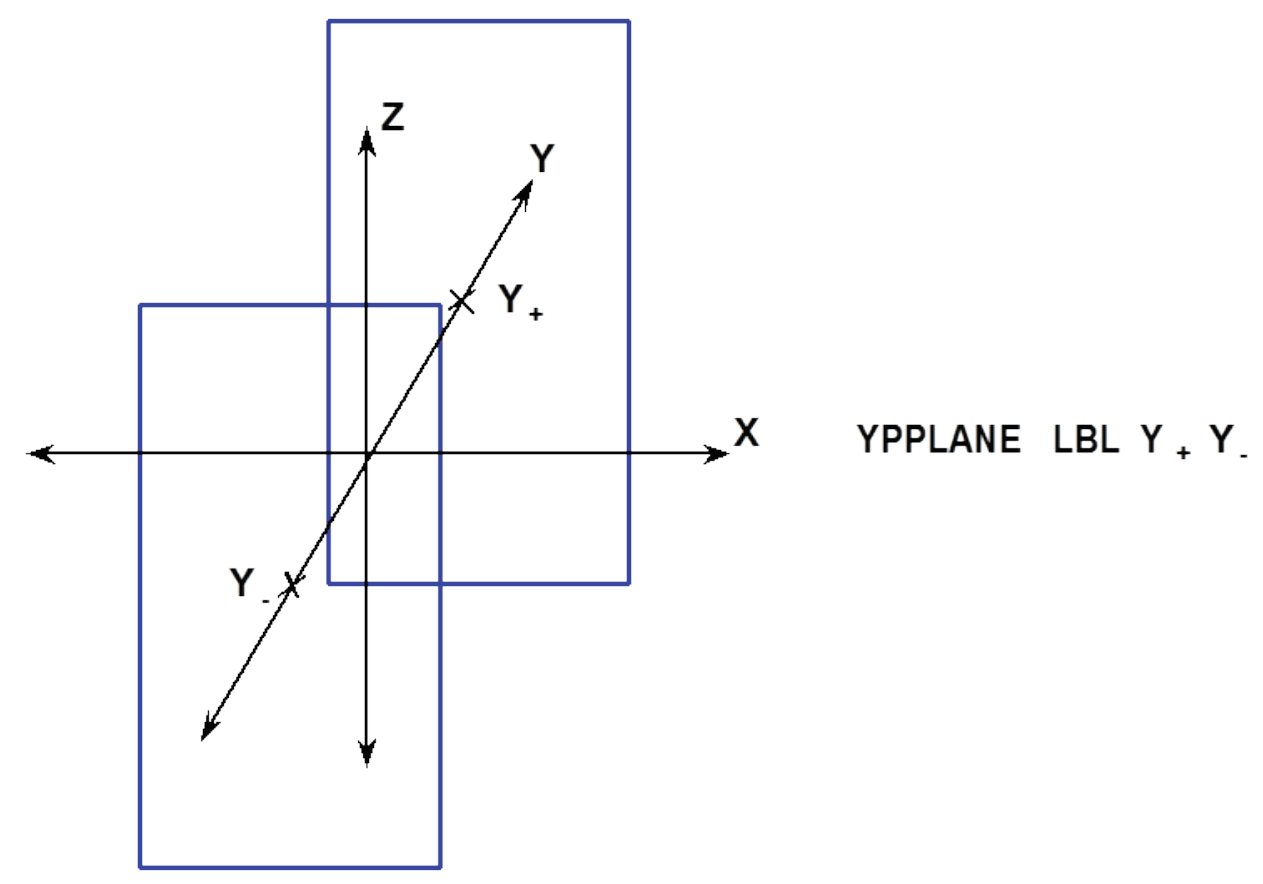

Figure 8.1.B.20. Example of y-paired plane construction. 
ZCYLINDER specifies a right circular cylinder oriented about the Z-axis. It is defined by specifying the radius of the cylinder, $R$, the $Z$ coordinate of the top face, $Z_{t}$, and the $Z$ coordinate of the bottom face, $Z_{b}$. Its centerline must lie on the $Z$-axis, unless otherwise specified by the optional geometry modification data. The keyword ZCYLINDER is the same as CYLINDER. It is included to be consistent with the XCYLINDER and YCYLINDER keywords. Figure 8.1.B.21 shows the correct input sequence for a zcylinder.

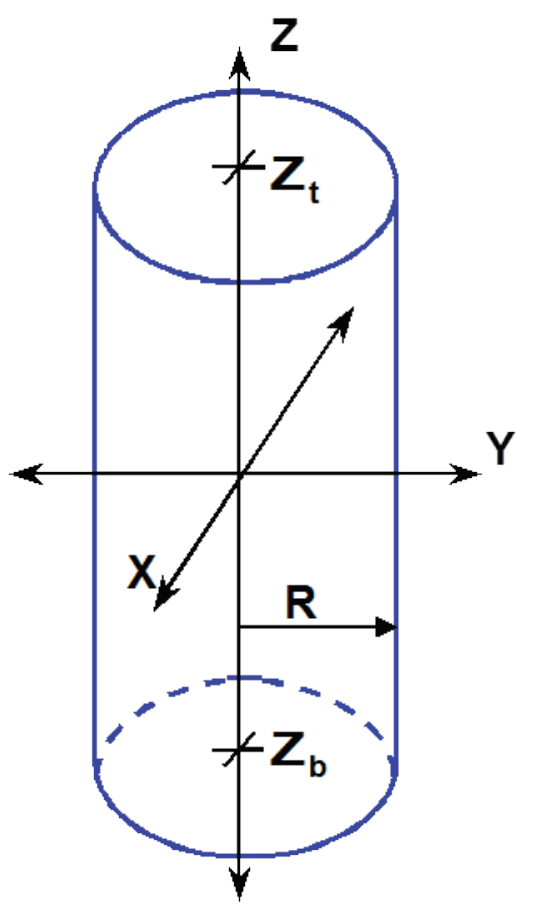

ZCYLINDER LBL $R Z_{t} \mathbf{z}_{\mathrm{b}}$

Figure 8.1.B.21. Example of zcylinder construction. 
ZPPLANE is a set of flat parallel surfaces where any two points in one of the surfaces can be connected by a straight line entirely contained within that surface. These surface planes divide space into three sections; one section between the two planes which is considered inside the surfaces, one section on the negative side of the negative plane which is considered outside the surfaces, and one section on the positive side of the positive plane which is considered outside the surfaces. The set of parallel planes are defined by the keyword ZPPLANE, which places the planes perpendicular to the Z-axis, the Z-intercept between the more positive plane and the $\mathrm{Z}$-axis $\left(\mathrm{Z}_{+}\right)$and the $\mathrm{Z}$-intercept between the more negative plane and the $Z$-axis $\left(Z_{-}\right)$. Figure 8.1.B.22 shows the correct input sequence for the set of paired planes.

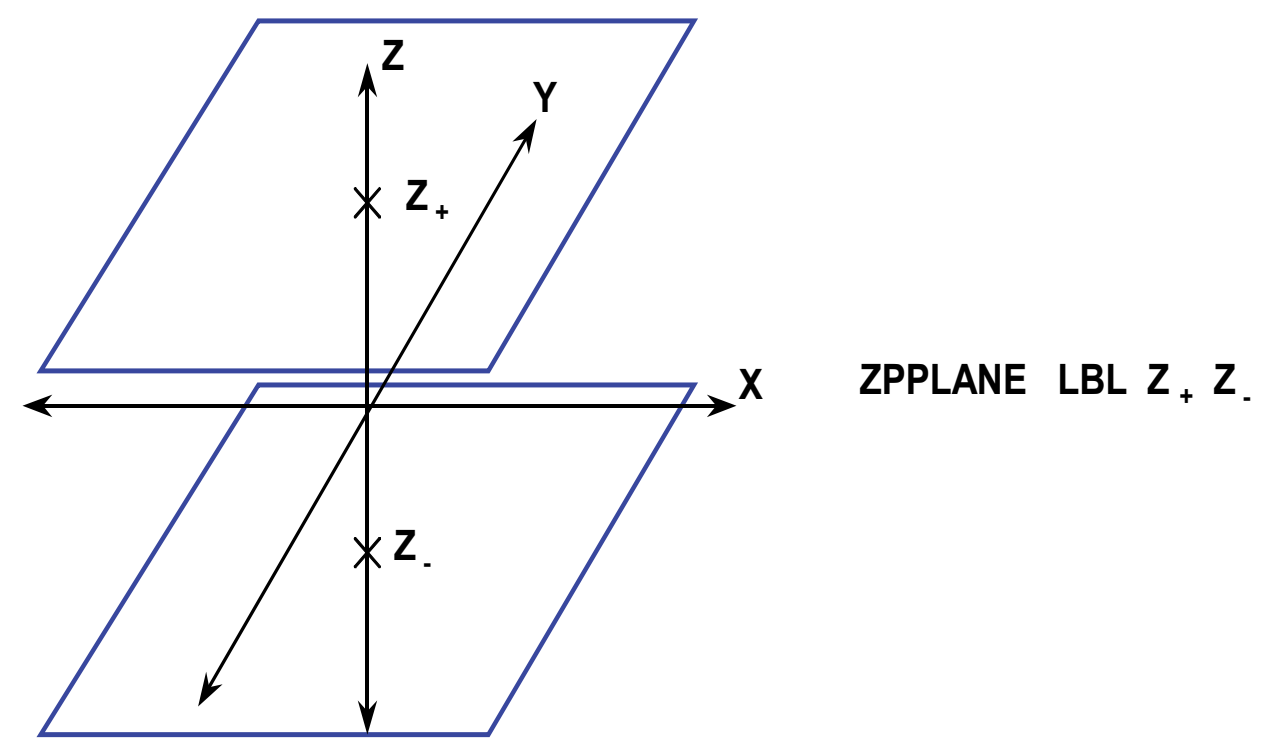

Figure 8.1.B.22. Example of z-paired plane construction. 


\subsection{Sample Problems}

This section contains sample problems to demonstrate some of the options available in KENO in standalone mode. Because stand-alone KENO has no means to read standard composition information and process for use, the problem-dependent cross section library must be prepared before executing KENO in the multigroup mode. The MIXTURE data block (See Sect. 9.1.2.1) is used to provide the mixing table. In the continuous energy mode, the cross sections are directly used and therefore no problem-dependent library is needed. The mixing table is required in the continuous energy mode as well. If KENO is executed as part of CSAS5 or CSAS6 sequence, generation of the problem-dependent library (for the multigroup mode) and the mixing table is automatically performed by the sequence.

A total of 33 KENO V.a different case inputs and 27 KENO-VI inputs are provided as multigroup mode KENO sample problems in a single input file "kenova.input" and "kenovi.input" for KENO V.a and KENO-VI, respectively. This input file contains an initial CSAS-MG input to create the problemdependent cross section library to be used in the sample problems in the input file. Although KENO does not run stacked cases, when KENO is run as part of SCALE, the driver allows KENO to be executed each time it encounters an "=KENO5" or "=KENOVA", respectively "=KENO6" or "=KENOVI." The ".input" file contains all 33/27 problems one after the other. A similar input file "cekenova.input", respectively "cekenovi.input" is also provided for continuous energy mode of calculations. The changes required to create the continuous energy mode input file from the multigroup mode input file are simple. The continuous energy mode file does not have (or need) the CSAS-MG input at the beginning. In addition, all "lib=4" entries in the PARAMETER data block are changed to "cep=ce_v7_endf" to indicate the mode of calculation is continuous energy and the continuous energy cross section directory file is "ce_v7_endf" indicating ENDF/B-VII-based cross sections. The mixing table entry SCT is not applicable in the continuous energy mode, so it has been deleted from the continuous energy input file. Finally, the nuclide IDs in the mixing table are modified to remove the mixture number prefix because the continuous energy mode cross section file for a nuclide or isotope is the same regardless of where that nuclide is used.

The same 33/27 problems are also executed as individual cases with filenames "k5smp??.input", respectively "k6smp??.input", where ?? stands for sample problem number (01 through 33 or 27). Since each one of these sample problems needs a problem-dependent cross section library (multigroup mode only) and a mixing table, these problems have been converted to run as CSAS5/6 problems. Similar input files are also provided to be run in the continuous energy mode and the files are named "cek5smp??.input", respectively "cek6smp??.input", where ?? again stands for sample problem number (01 through 33 or 27). The change required to create the continuous energy mode inputs from the multigroup mode inputs is very simple: the cross-section library name is changed from "v7-238" to "ce_v7".

In the following section the input for each case is listed assuming the multigroup mode of calculation in KENO. The KENO input is also listed in the file corresponding ".input" file. The CSAS-MG input file for these cases is in the next section.

\subsection{C.1 CSAS-MG data}

The multigroup mode KENO sample problems use nuclide IDs that are consistent with the SCALE CSAS5/6 nuclide ID naming convention. Nuclides are identified by the ZA number plus 1000000 times the mixture number. CSAS-MG can be used to create a problem-dependent working format cross-section library suitable for use with the sample problems. CSAS-MG can (1) be run alone with problem- 
dependent working library on logical Unit 4 saved for later use with the KENO sample problems, or (2) be placed in front of the KENO sample problems.

The CSAS-MG SCALE control module calculates the necessary resonance data required to create the problem-dependent AMPX working format library using SCALE standard composition input.

The multigroup mode KENO sample problem input data are independent of energy group structure. To use a different energy group structure, simply supply the desired master cross-section library name in the CSAS-MG or CSAS5/6 data. See XSProc, Standard Composition and CSAS5/6 chapters for additional information and examples. See the XSLib chapter for information about the master format cross-section libraries that are available in SCALE.

Data for CSAS-MG are provided to create a problem-dependent AMPX working format cross-section library suitable for use with the multigroup mode KENO sample problems. These data include all of the mixtures used in the KENO sample problems and will create an AMPX working format cross-section library with nuclide IDs matching those in the KENO sample problem mixing tables. This cross-section library is problem-specific and is not appropriate for use with other problems.

The CSAS-MG input data to produce an AMPX working format cross-section library for the multigroup mode KENO V.a sample problems are given below.

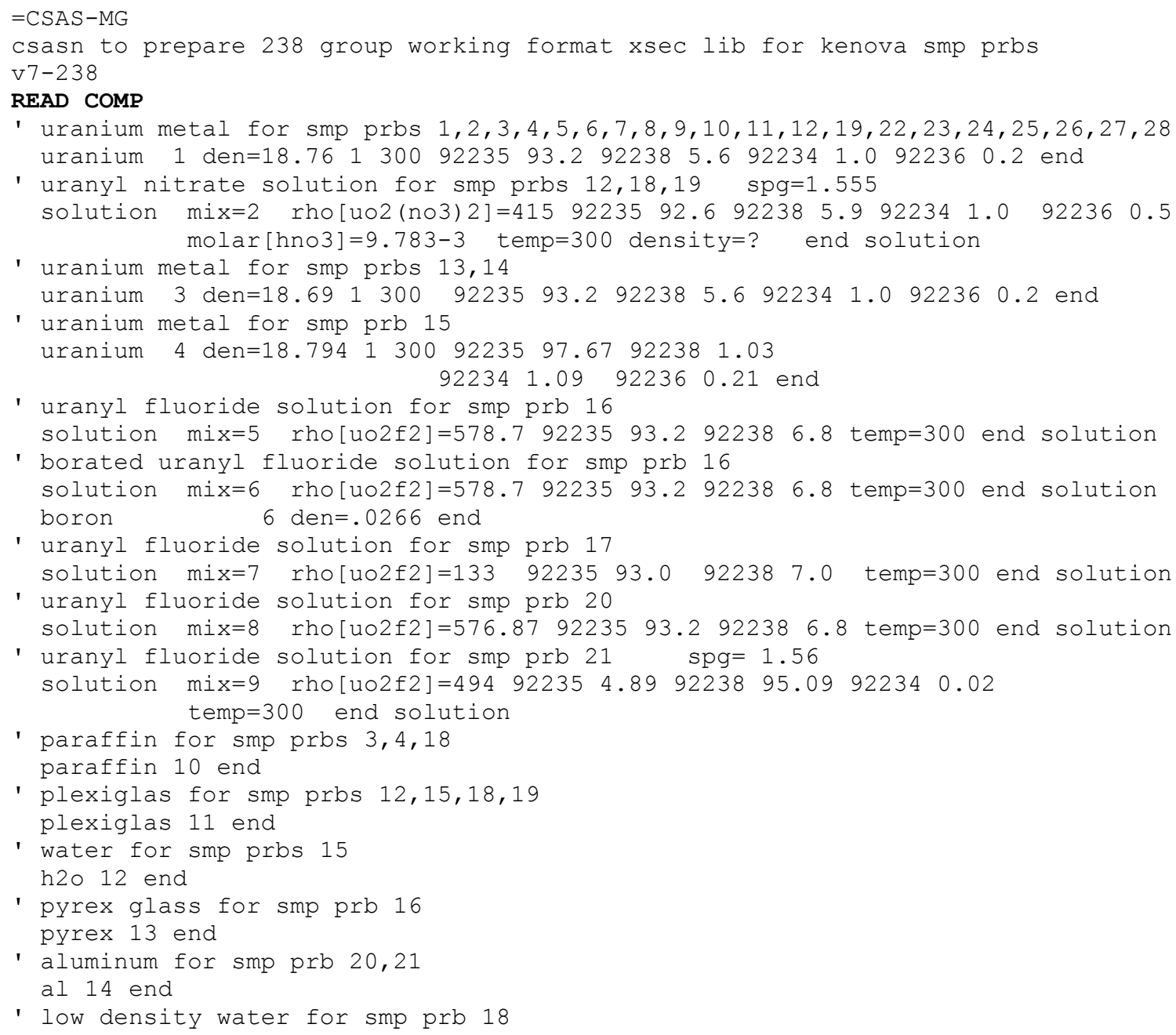




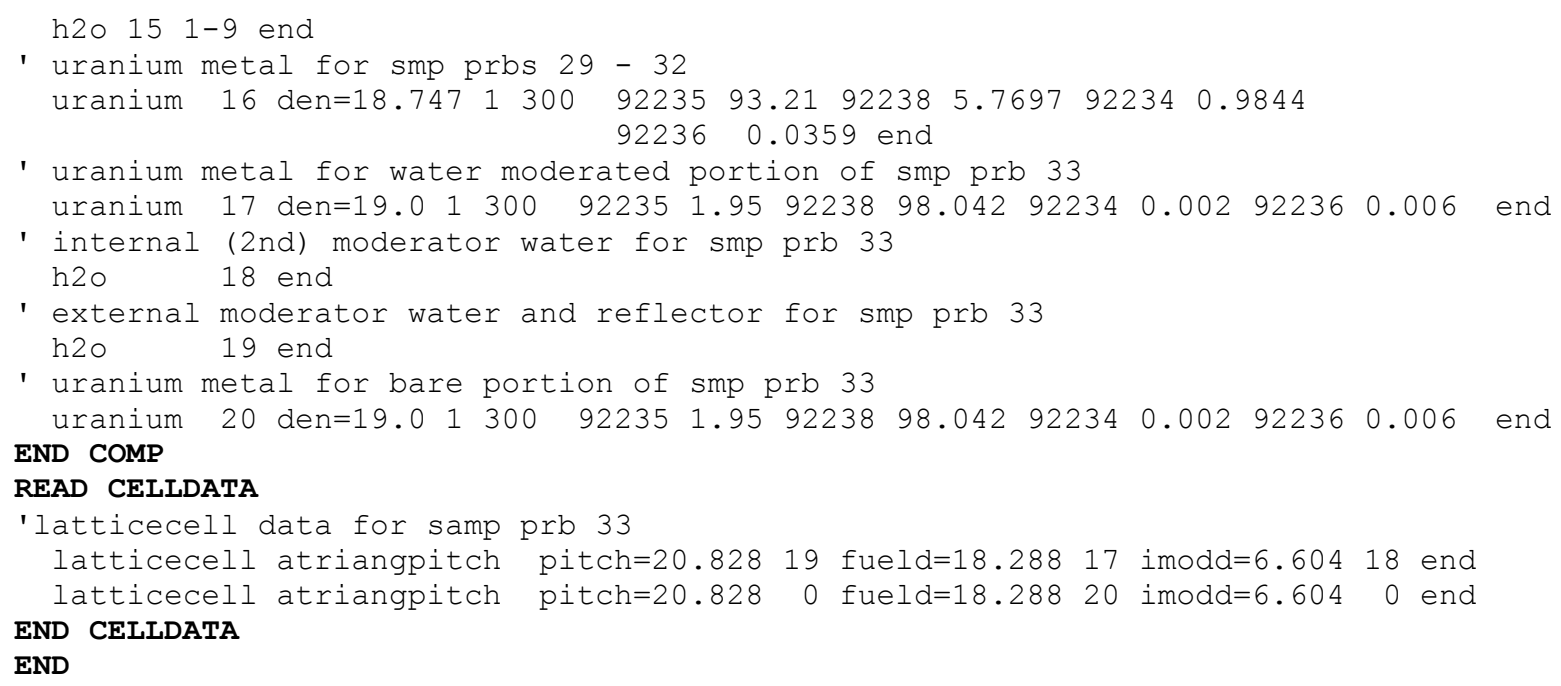

\subsection{C.2 KENO V.a sample problem data}

A brief problem description and the associated input data are included for each multigroup mode KENO sample problem. Different options may be easily activated by making changes in the data. These problems are set up using an AMPX working format library which was created by a CSAS-MG case just prior to the KENO V.a cases. The nuclide identifiers for this library are consistent with the SCALE identifiers created by CSAS-MG. Input data to create this library are given in Sect. 8.1.C.1. The unit number is defined by the parameter $\mathrm{LIB}=$ in the parameter data.

\subsection{C.2.1 Sample Problem 1 2C8 BARE}

This is a simple $2 \times 2 \times 2$ array of uranium metal cylinders as described in the article, "Critical ThreeDimensional Arrays of U(93.2)-Metal Cylinders," by J.T. Thomas. This critical experiment is designated in Table II of that article as cylinder index 11 and reflector index 1. Figure 8.1.C.1 shows the critical experiment.

\section{Input Data}

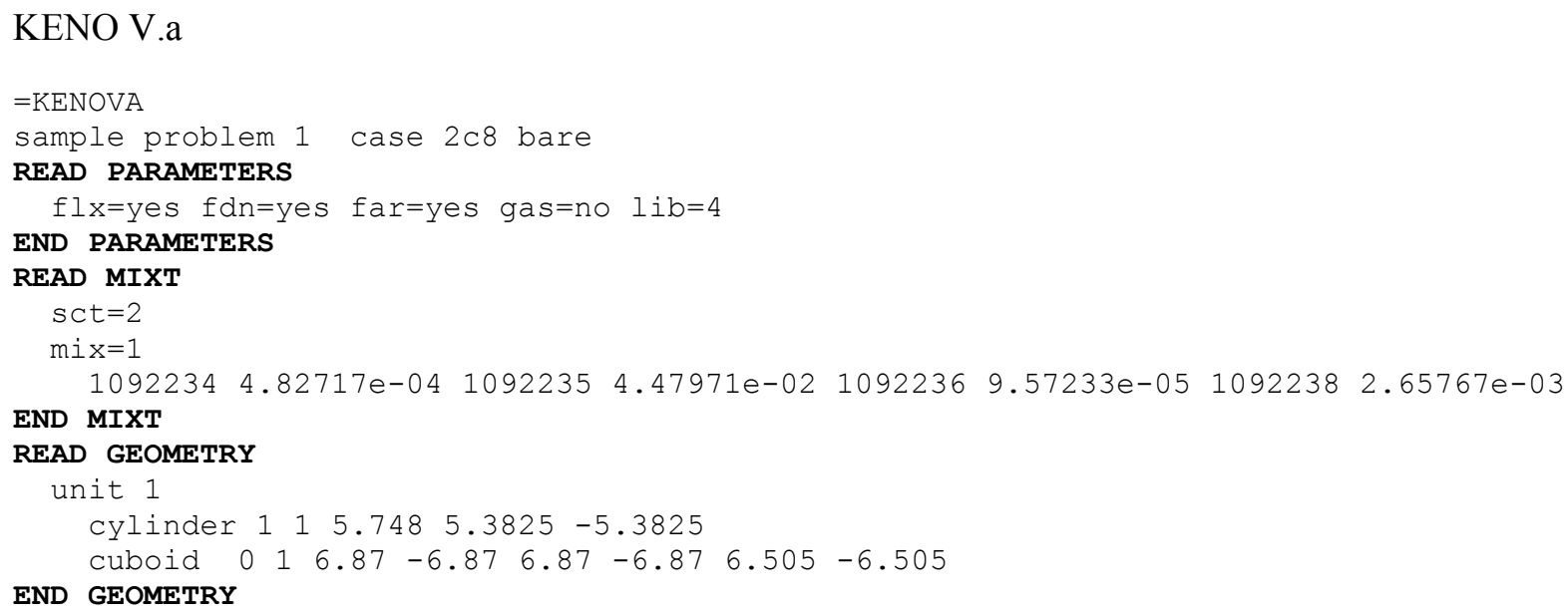


READ ARRAY

nux=2 nuy=2 nuz=2

END ARRAY

END DATA

END

\section{KENO-VI}

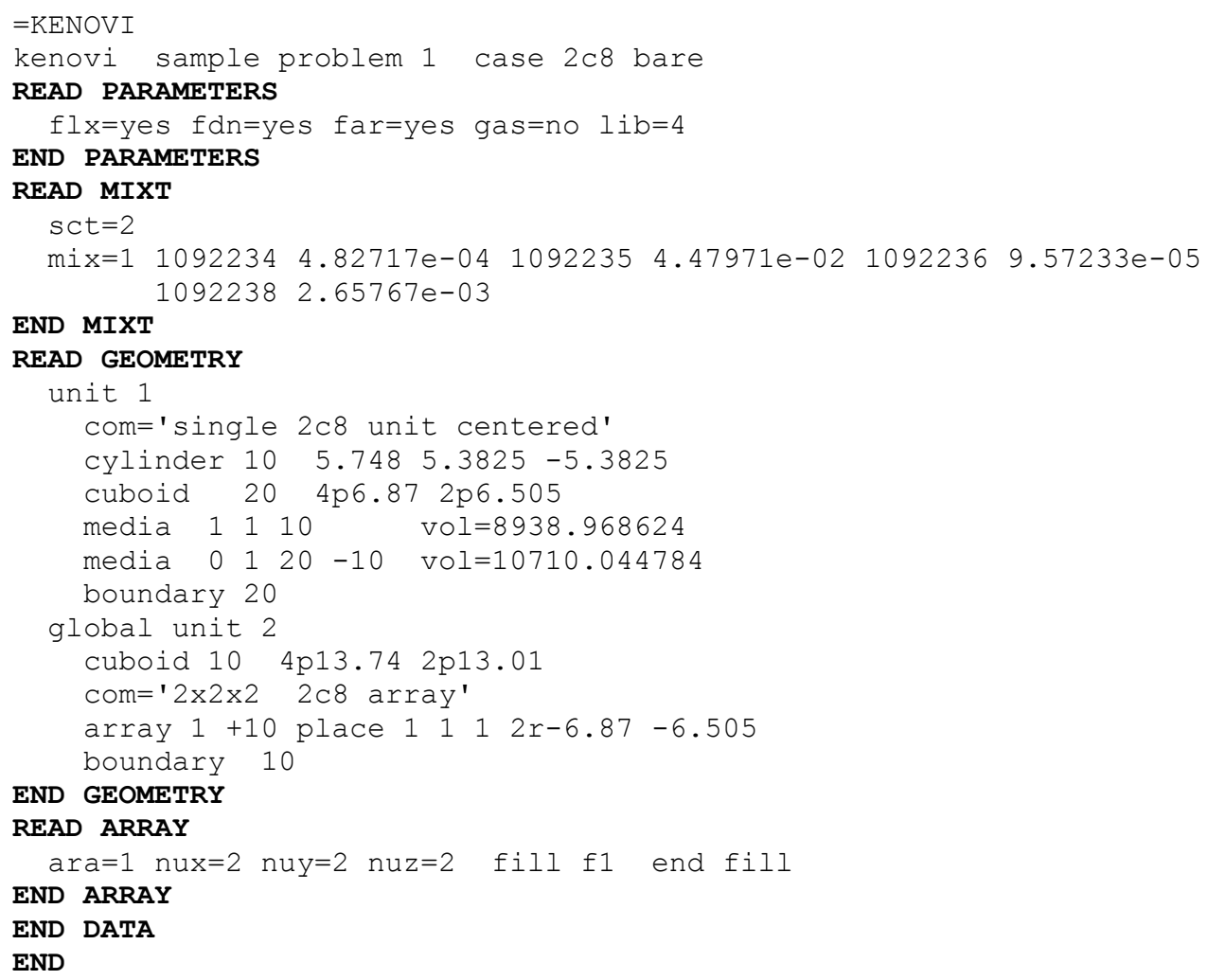




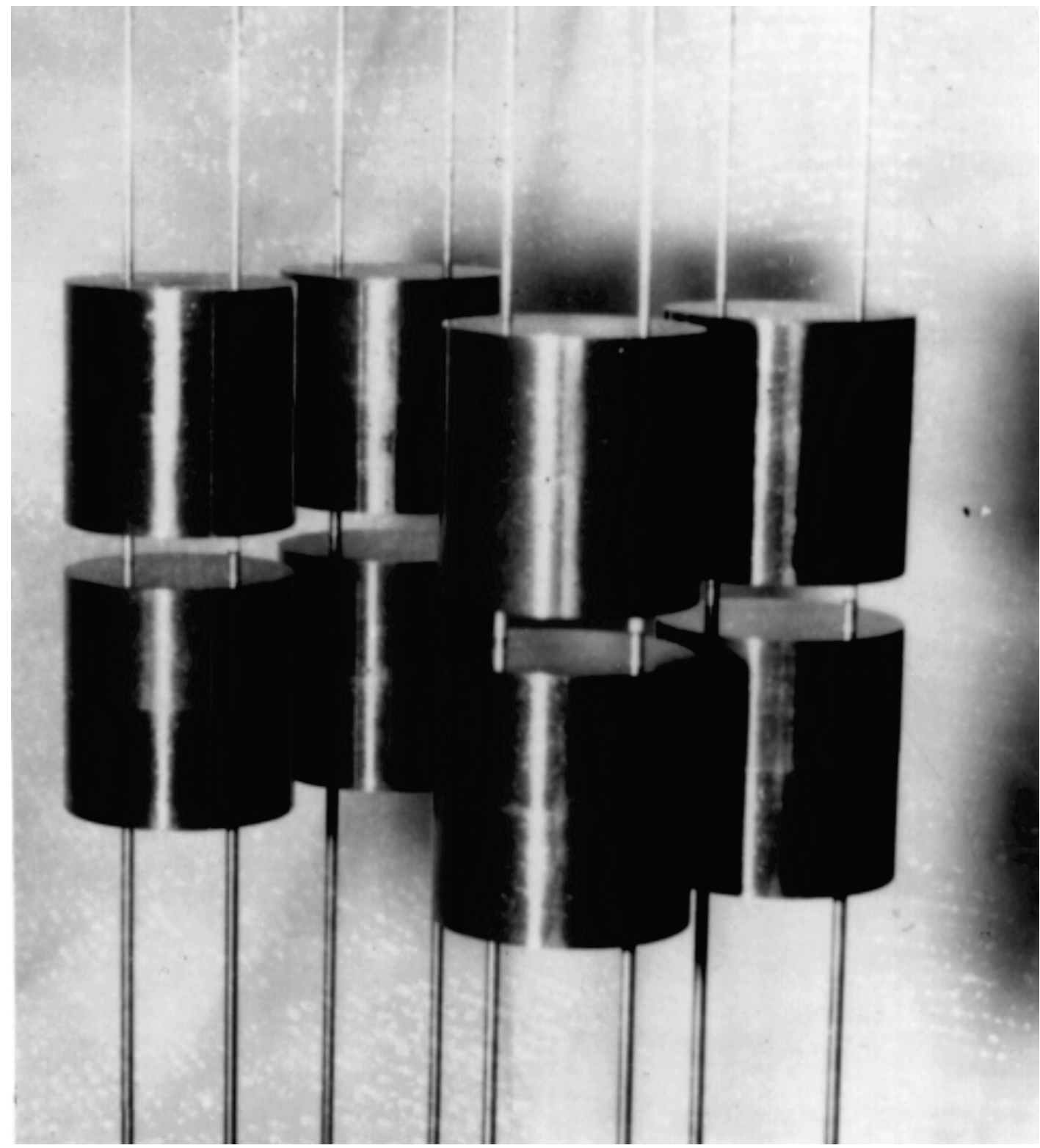

Figure 8.1.C.1. Critical $2 \mathrm{C8}$ bare assembly.

\subsection{C.2.2 Sample Problem 2 CASE 2C8 BARE WITH 8 UNIT TYPES MATRIX CALCULATION}

This problem is the same as sample problem 1 except it is set up as a mixed unit problem with each unit of the array defined as a different unit type. Matrix k-effectives will be calculated for this problem by both unit type and array position. The print flags are set to print all matrix data. 


\section{Input Data}

\section{KENO V.a}

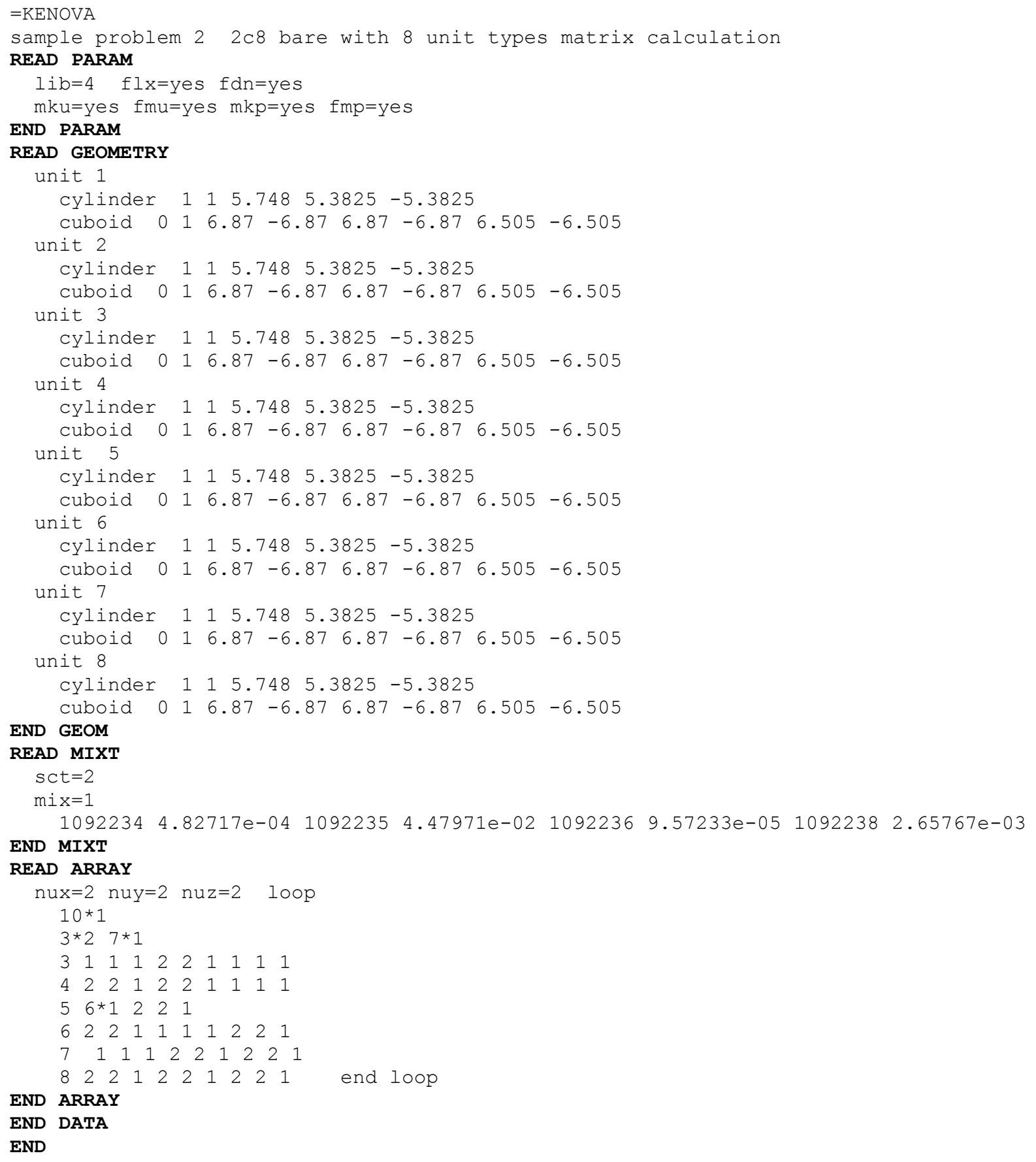

KENO-VI

$=\mathrm{KENOVI}$

kenovi sample problem 2 case 2 c 8 bare with 8 unit types matrix cal READ PARAM

$\mathrm{lib}=4 \mathrm{flx}=y e s$ fdn=yes mku=yes cku=yes fmulyes mkp=yes ckp=yes fmp=yes 


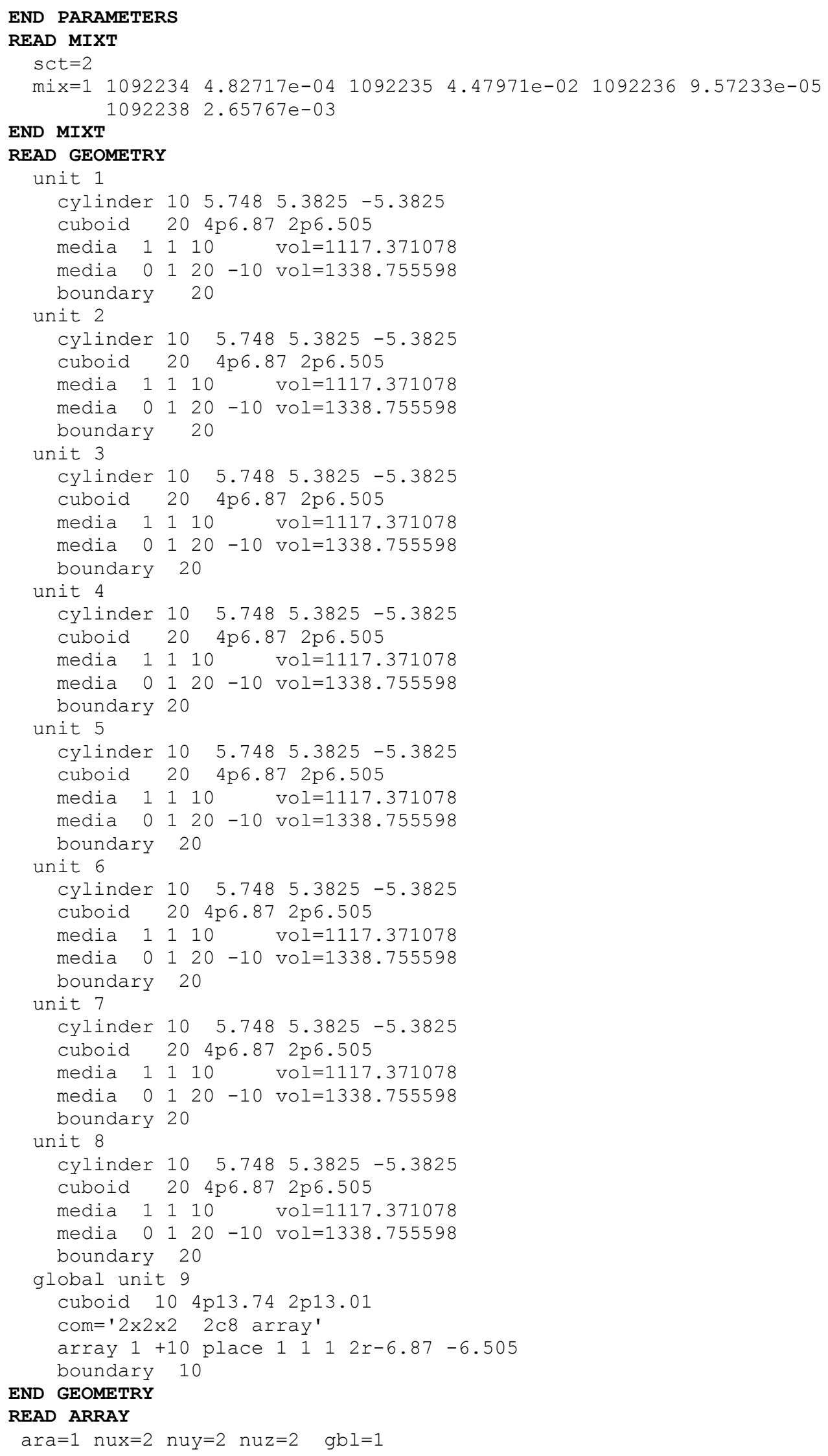




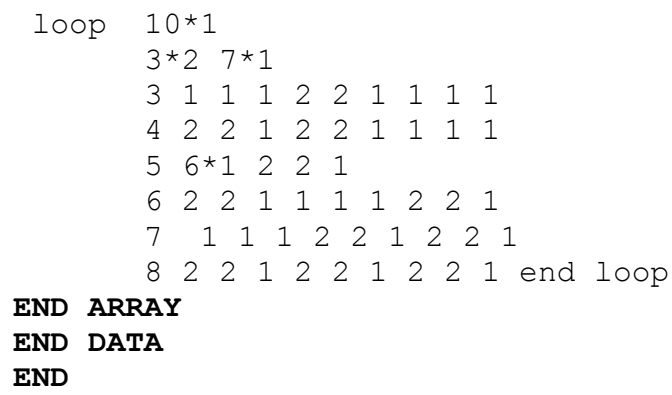

\subsection{C.2.3 Sample Problem 3 2C8 15.24-CM PARAFFIN REFL}

A $2 \times 2 \times 2$ array of uranium metal cylinders is reflected by 6 in. of paraffin on all faces (Figure 8.1.C.1). This critical experiment ${ }^{1}$ is designated as cylinder index 11 and reflector index 5 in Table II of Ref. 1. Figure 8.1.C.2 shows half of the critical experiment, which consisted of the half shown and the mirror image of it. These two assemblies were moved together to achieve criticality. The top reflector is missing in Figure 8.1.C.2, but was present when criticality was achieved.

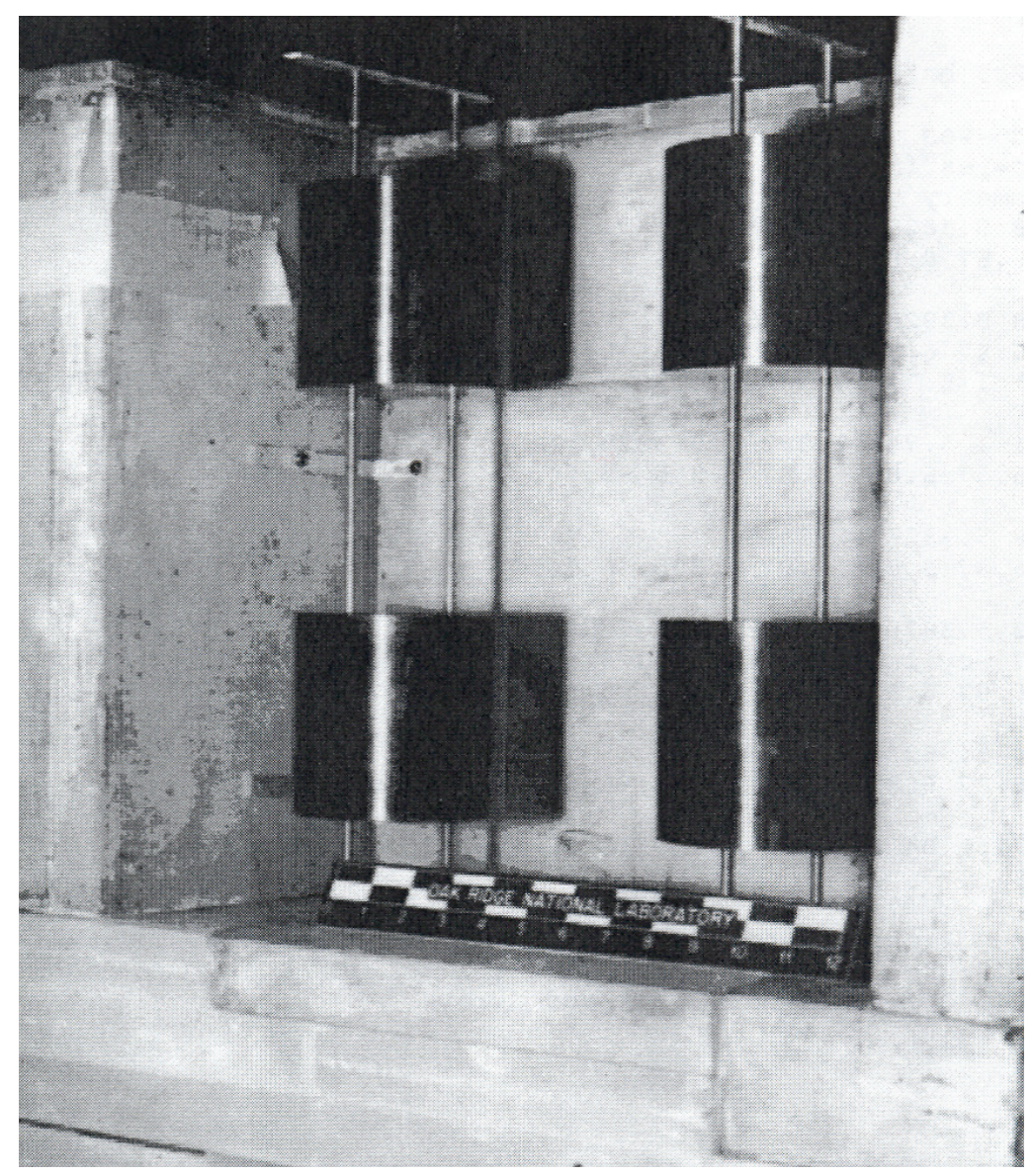

Figure 8.1.C.2. Half of the paraffin reflected $2 \mathrm{C} 8$ assembly before the top reflector was added. 


\section{Input Data}

\section{KENO V.a}

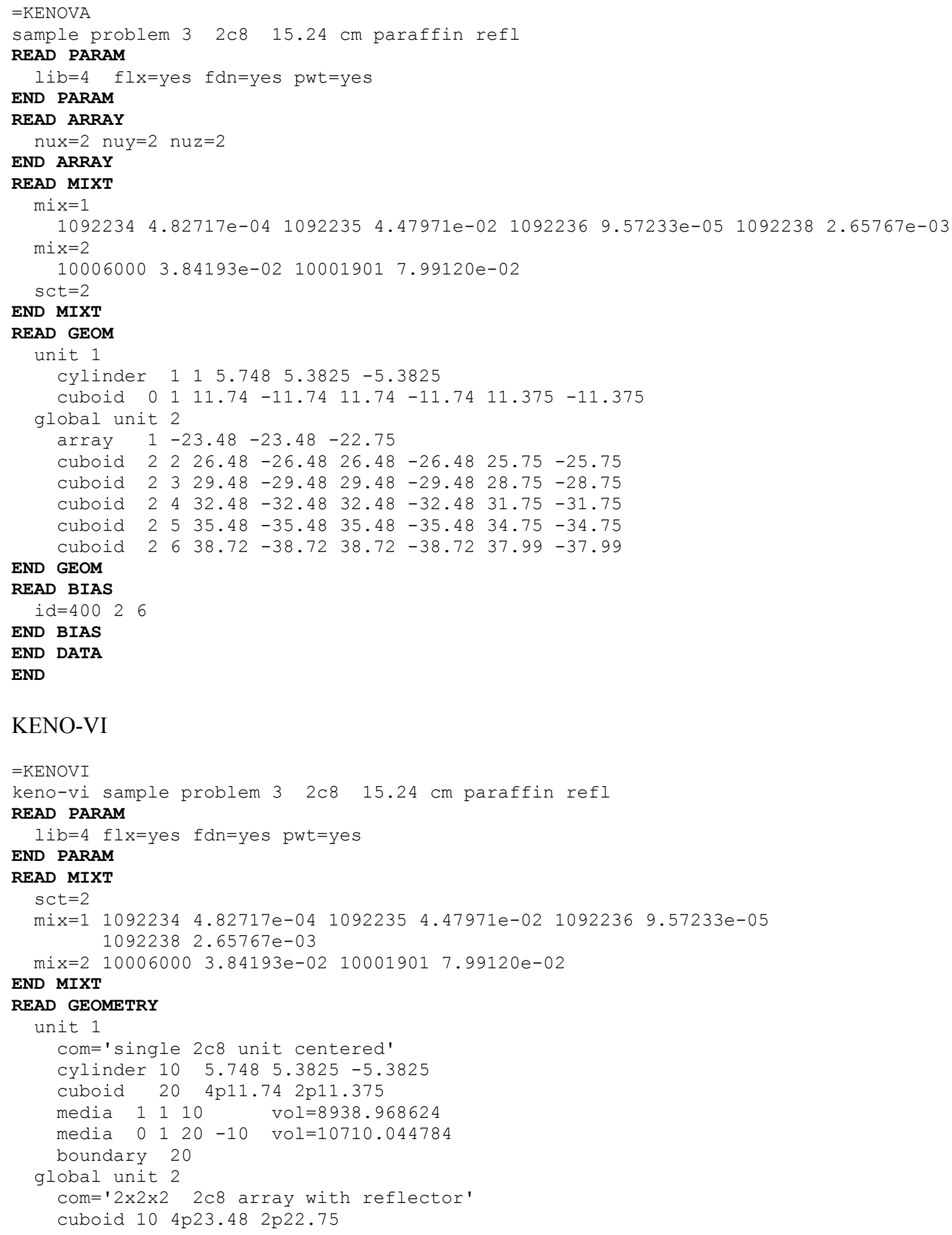

\section{KENO-VI}




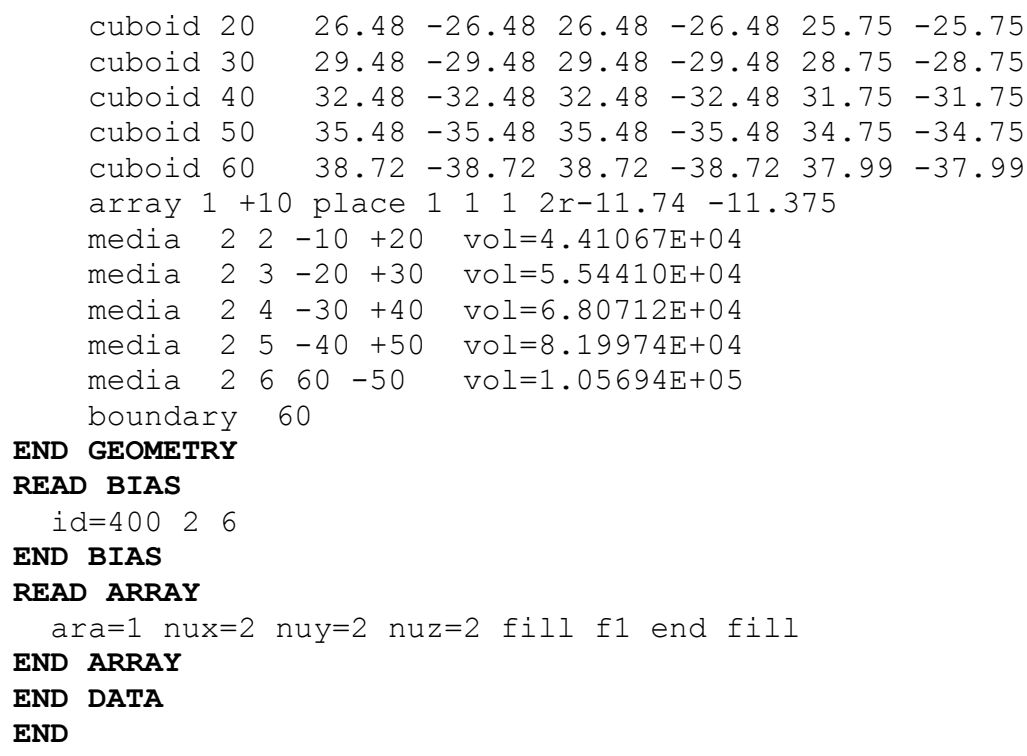

\subsection{C.2.4 Sample Problem 4 2C8 15.24-CM PARAFFIN REFL AUTOMATIC REFL}

This problem is the same as sample problem 3 except it is set up using more reflector regions.

\section{Input Data}

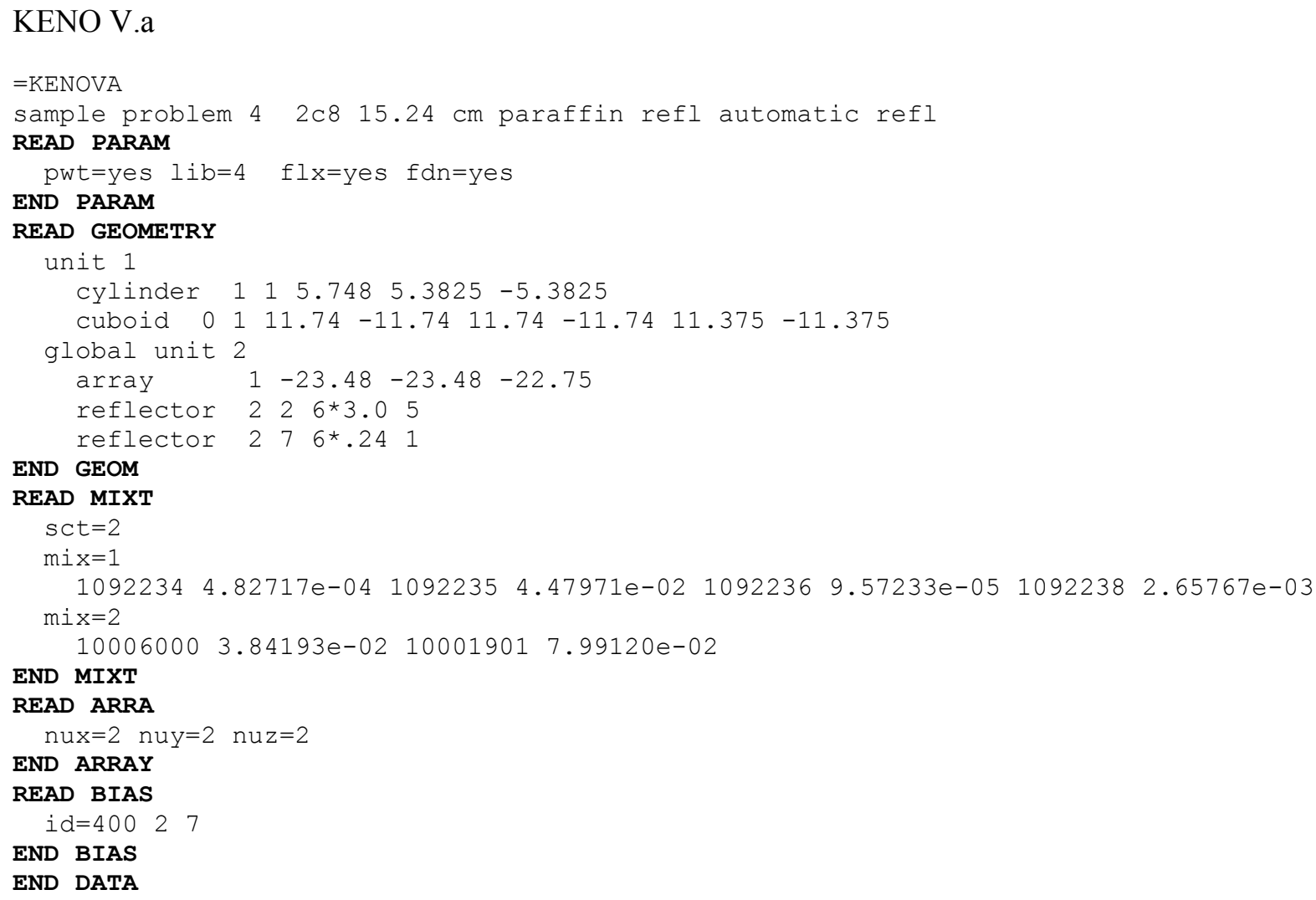




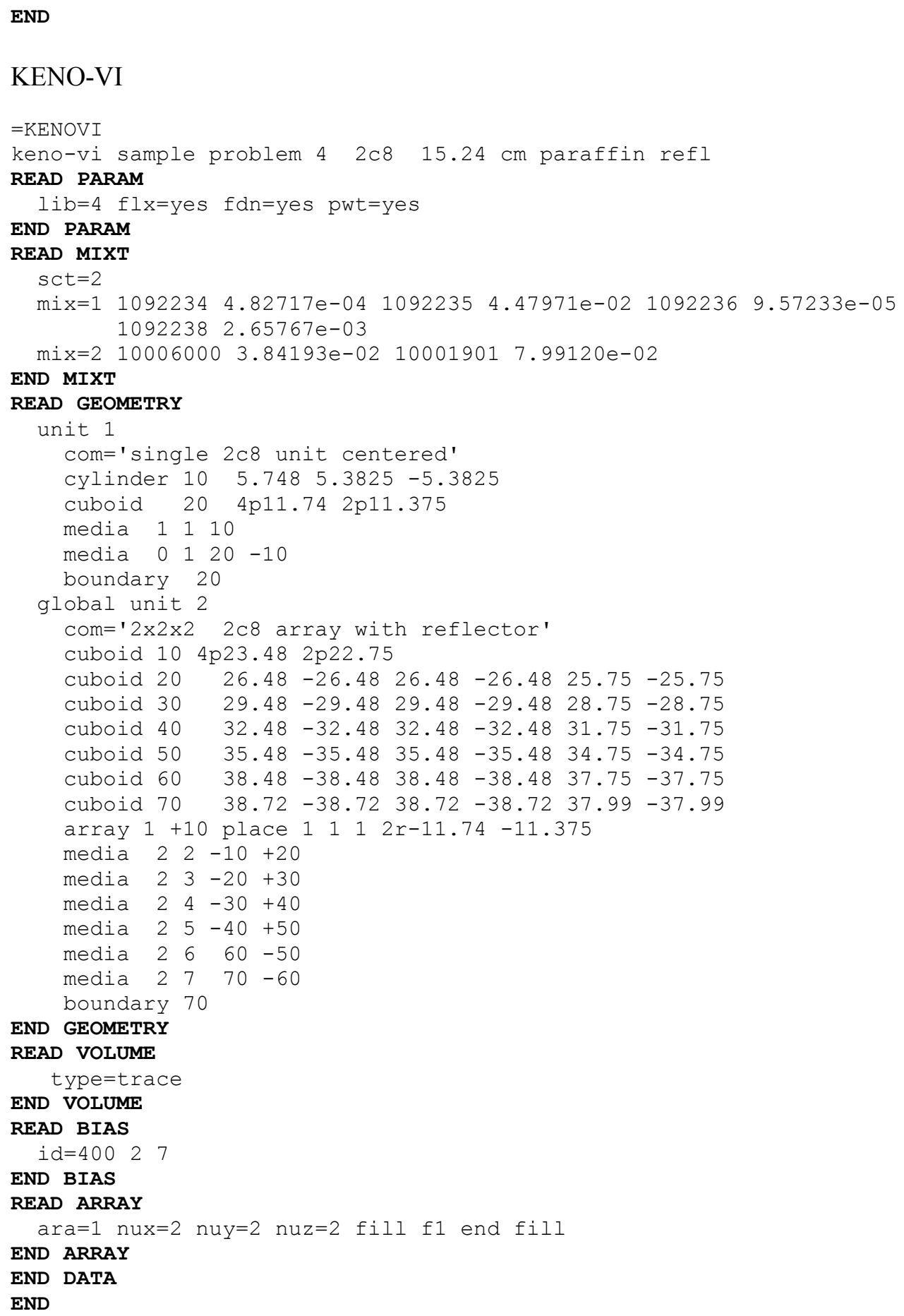

\subsection{C.2.5 Sample Problem 5 2C8 12-INCH PARAFFIN ALBEDO REFLECTOR}

This problem is the same as samples problems 3 and 4 except the reflector is represented by a 12-in. paraffin albedo. Note the decrease in execution time when using an albedo reflector instead of doing actual tracking. Note also that k-effective is somewhat higher for this system, probably due to the small edge size of the system. ${ }^{2}$ 


\section{Input Data}

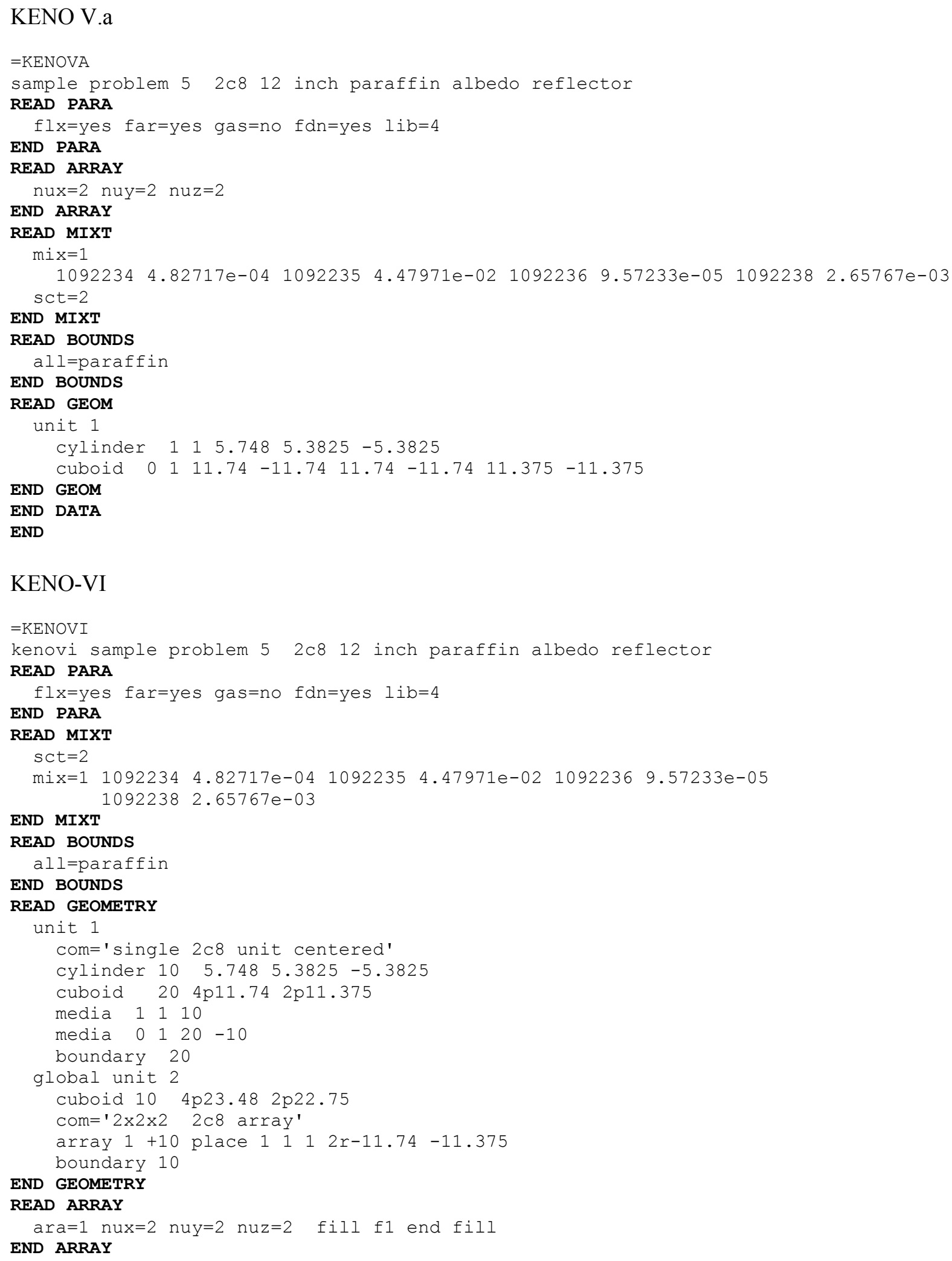


READ VOLUME

type $=$ random

END VOLUME

END DATA

END

\subsection{C.2.6 Sample Problem 6 ONE 2C8 UNIT (SINGLE UNIT)}

One of the $2 \mathrm{C}$ units ${ }^{1}$ is described and run as a single-unit problem, and its k-effective is calculated.

\section{Input Data}

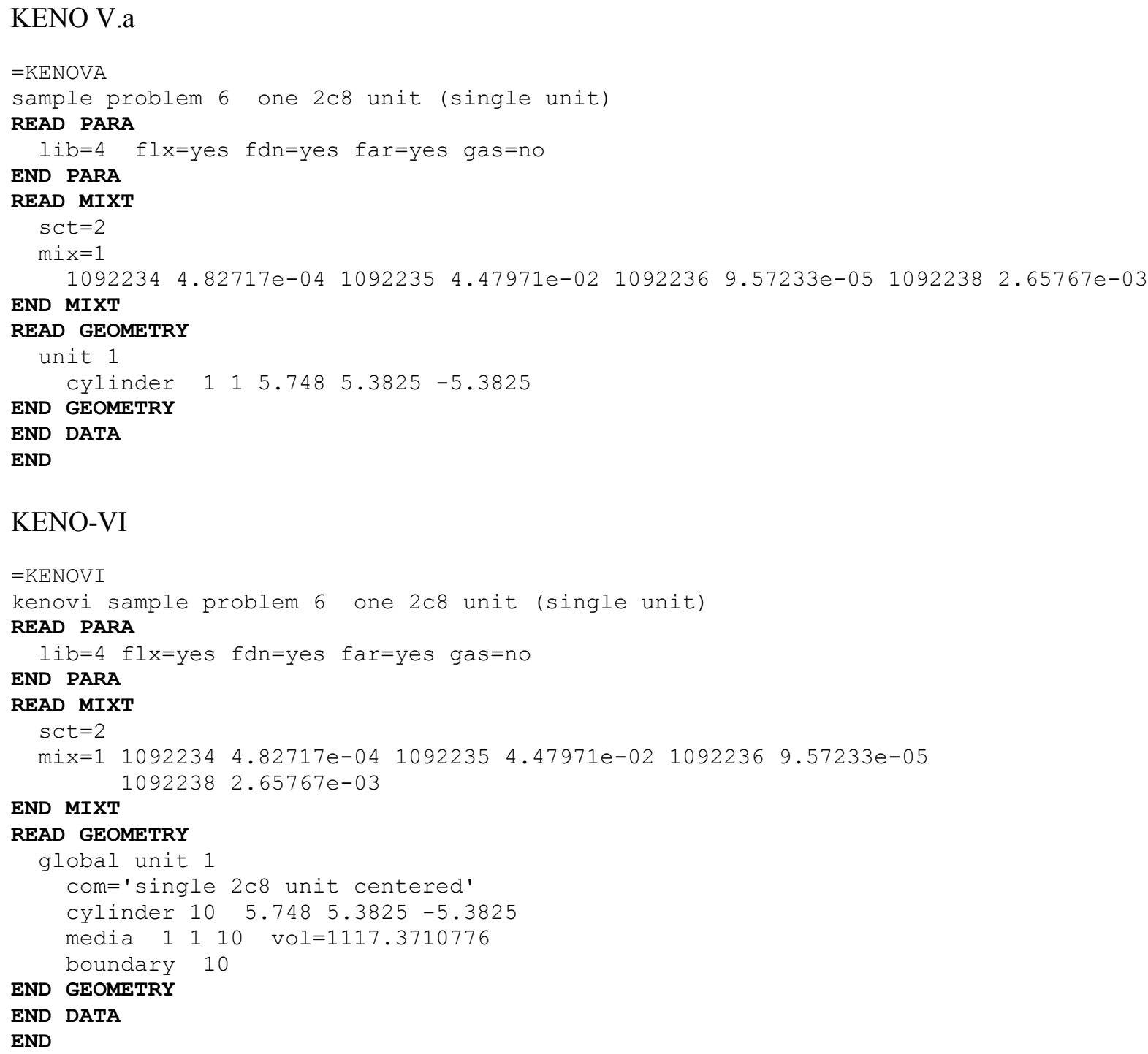




\subsection{C.2.7 Sample Problem 7 BARE 2C8 USING SPECULAR REFLECTION}

One of the $2 \mathrm{C}$ units $^{1}$ is described and the $2 \times 2 \times 2$ array is simulated by using specular reflection on the positive $\mathrm{X}, \mathrm{Y}$, and $\mathrm{Z}$ faces of the unit. This is a simulation of sample problem 1 .

\section{Input Data}

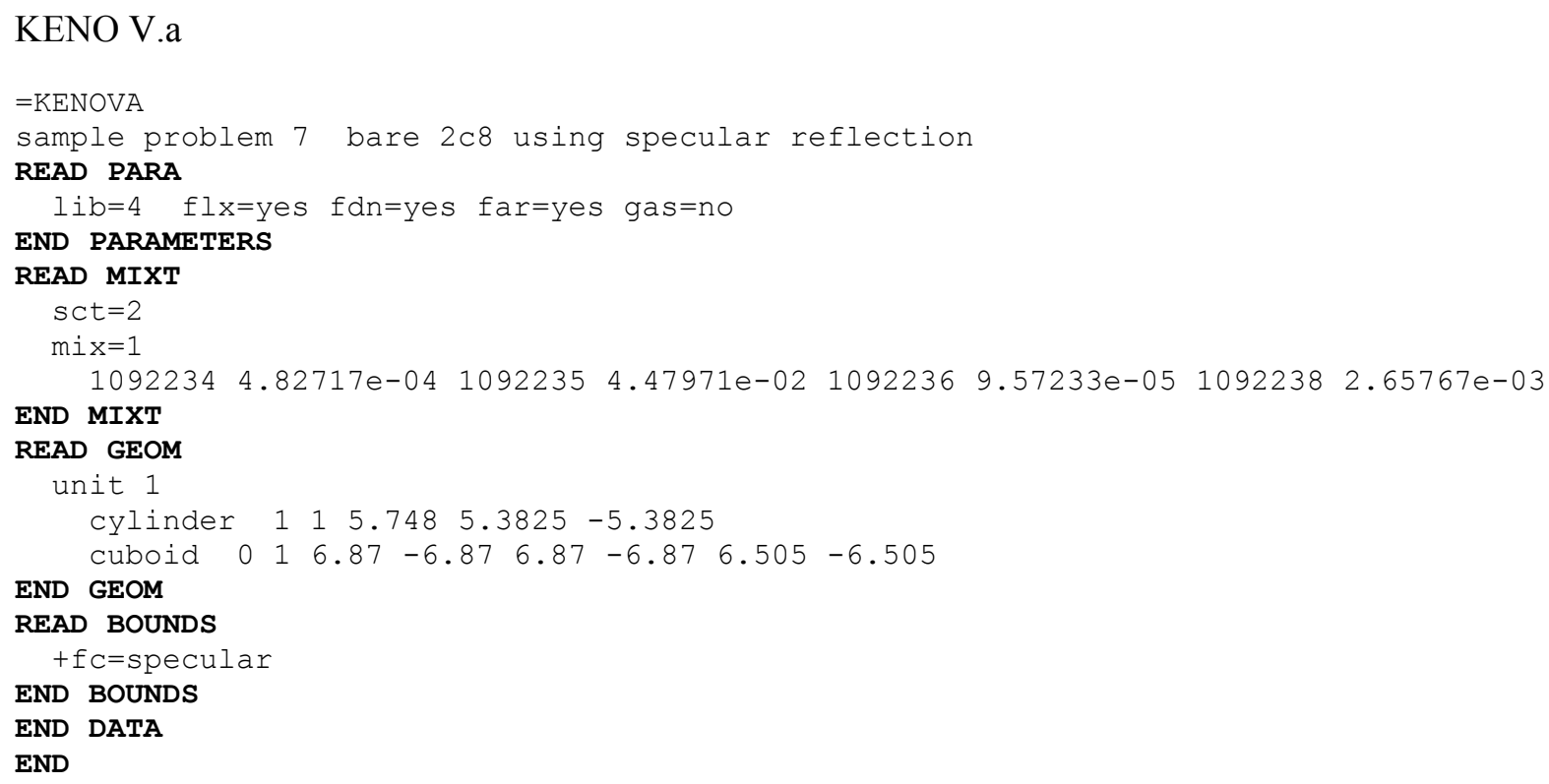




\subsection{C.2.8 Sample Problem 8 INFINITELY LONG CYLINDER FROM 2C8 UNIT}

The fuel and cylinder radius from sample problem 1 is used. The length of the cylinder is arbitrarily chosen to be $20 \mathrm{~cm}$, and the unit is specularly reflected on the top and bottom to create an infinitely long cylinder.

\section{Input Data}

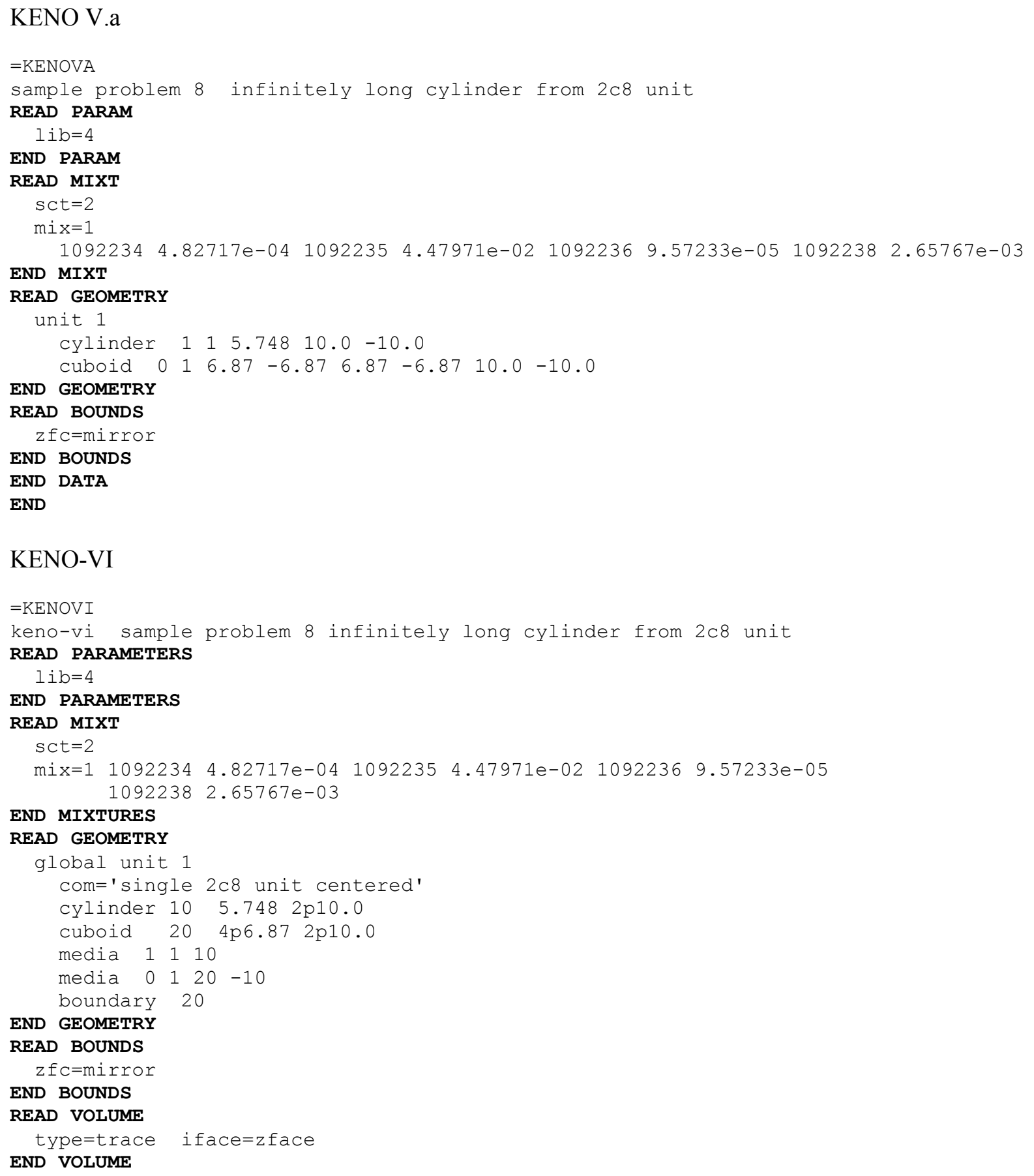


END BOUNDS

END DATA

END

\subsection{C.2.10 Sample Problem 10 2C8 BARE WRITE RESTART}

This problem is the same as sample problem 1 , a $2 \times 2 \times 2$ array of metal cylinders. Restart information is written on unit 94 after the completion of every fifth generation.

\section{Input Data}

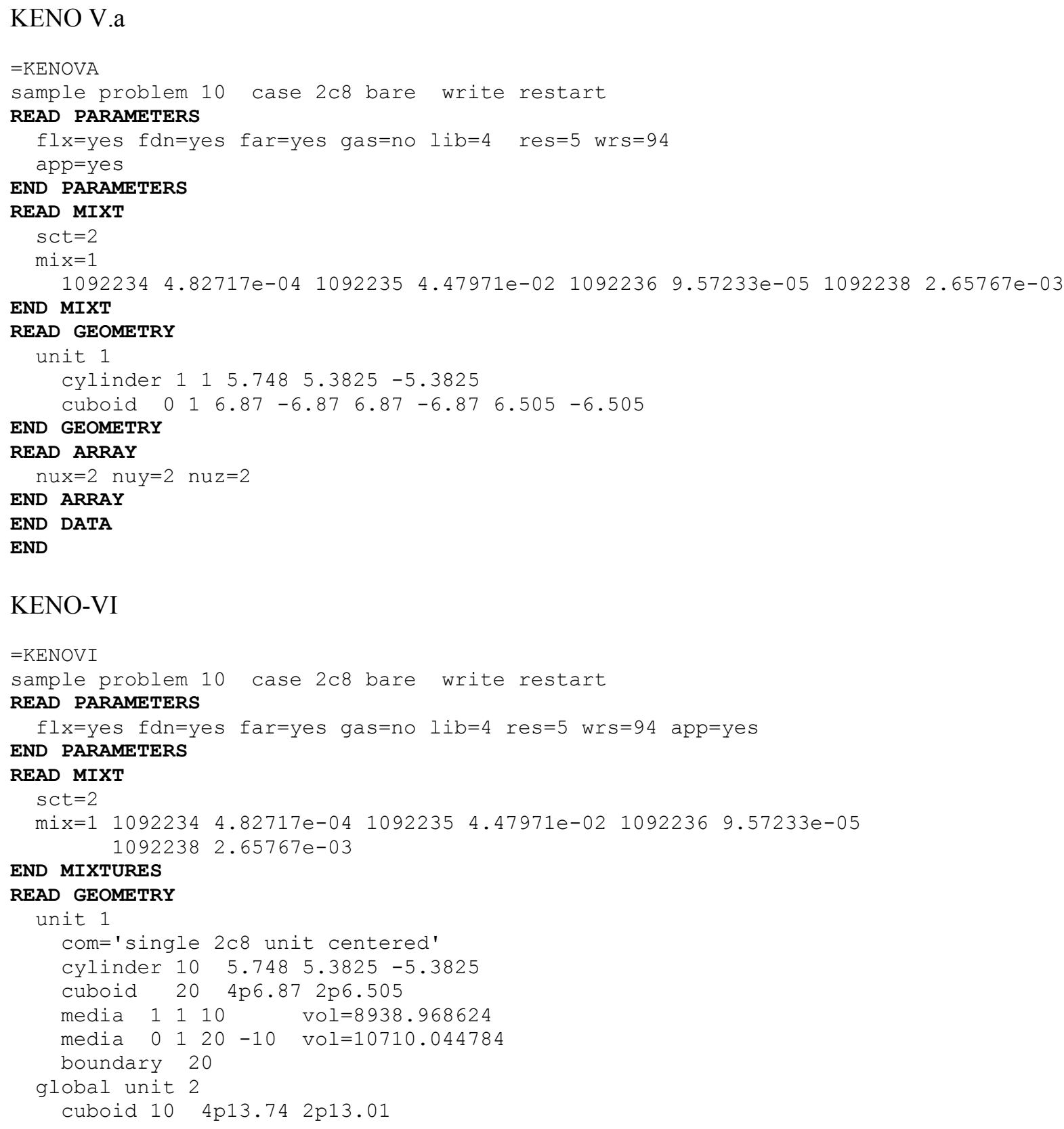




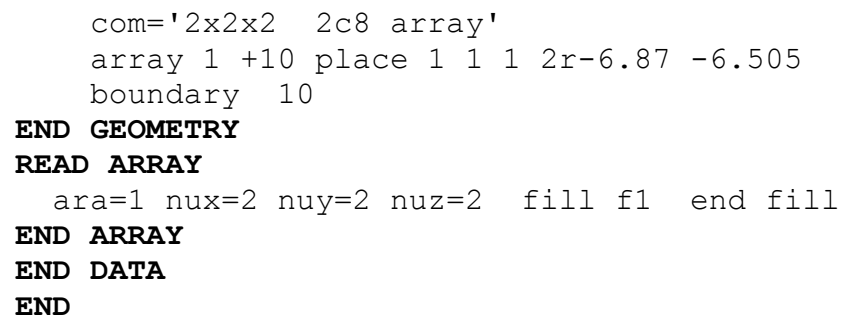

\subsection{C.2.11 Sample Problem 11 2C8 BARE READ RESTART DATA}

This problem is a restart of sample problem 10. The problem is restarted from the tenth set of restart data that was written by sample problem 10 (i.e., it restarts with the fifty-first generation).

\section{Input Data}

KENO V.a

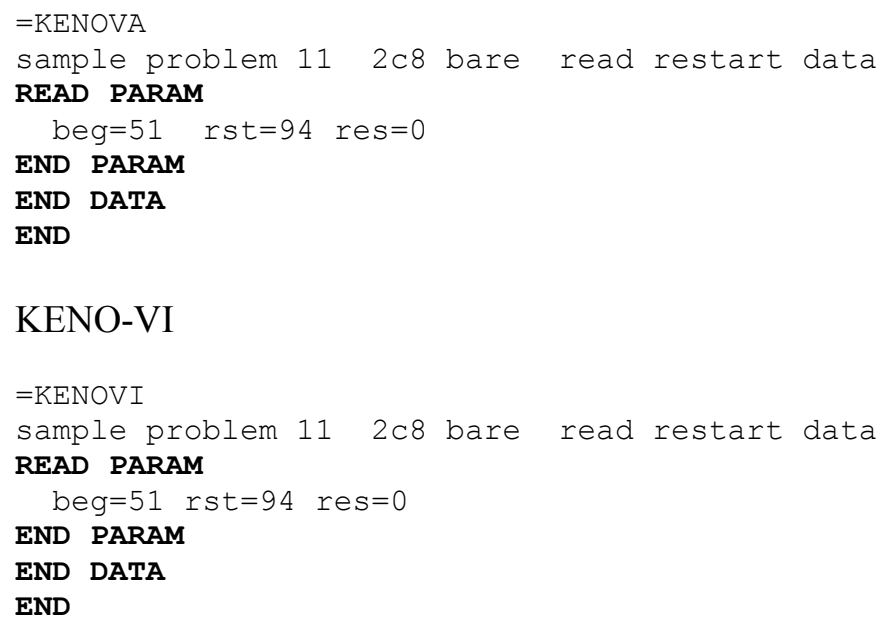

8.1.C.2.12 Sample Problem 124 AQUEOUS 4 METAL 
This problem is a critical experiment consisting of a composite array ${ }^{1}$ of four highly enriched uranium metal cylinders and four cylindrical Plexiglas containers filled with uranyl nitrate solution. The metal units in this experiment are designated in Table II of Ref. 1 as cylinder index 11 and reflector index 1. A photograph of the experiment is given in Figure 8.1.C.3.

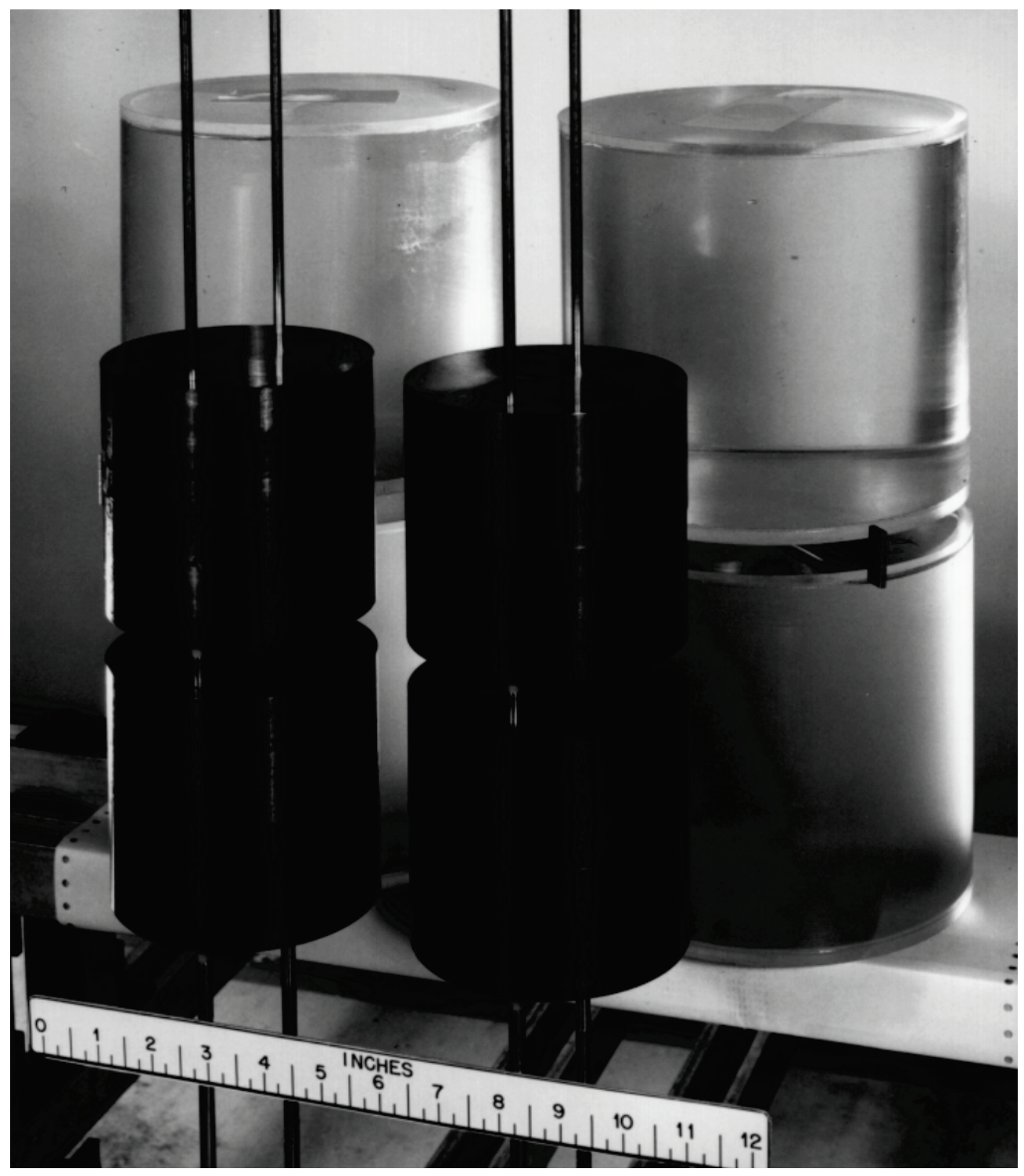

Figure 8.1.C.3. Critical assembly of 4 solution units and 4 metal units. 


\section{Input Data}

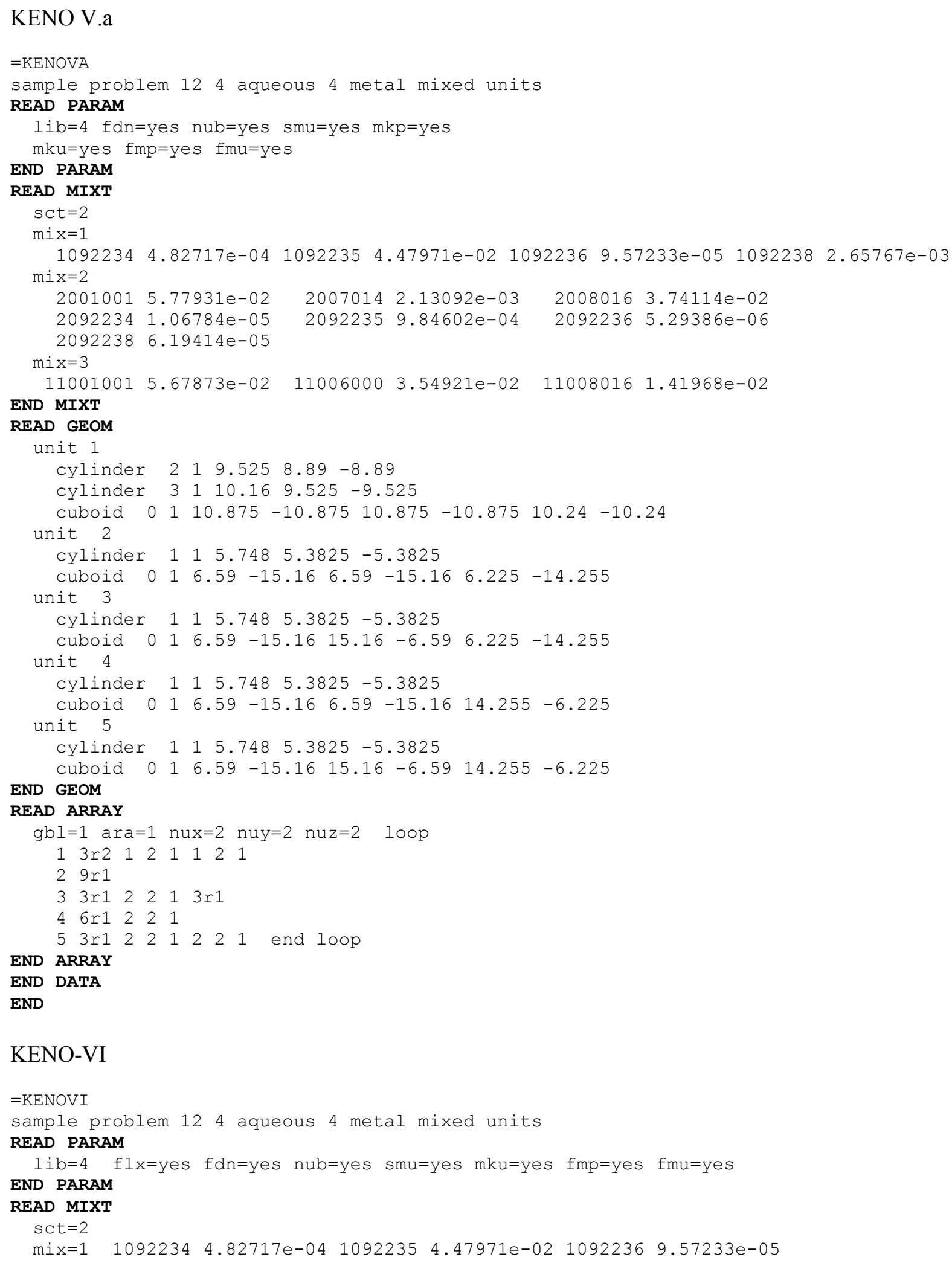




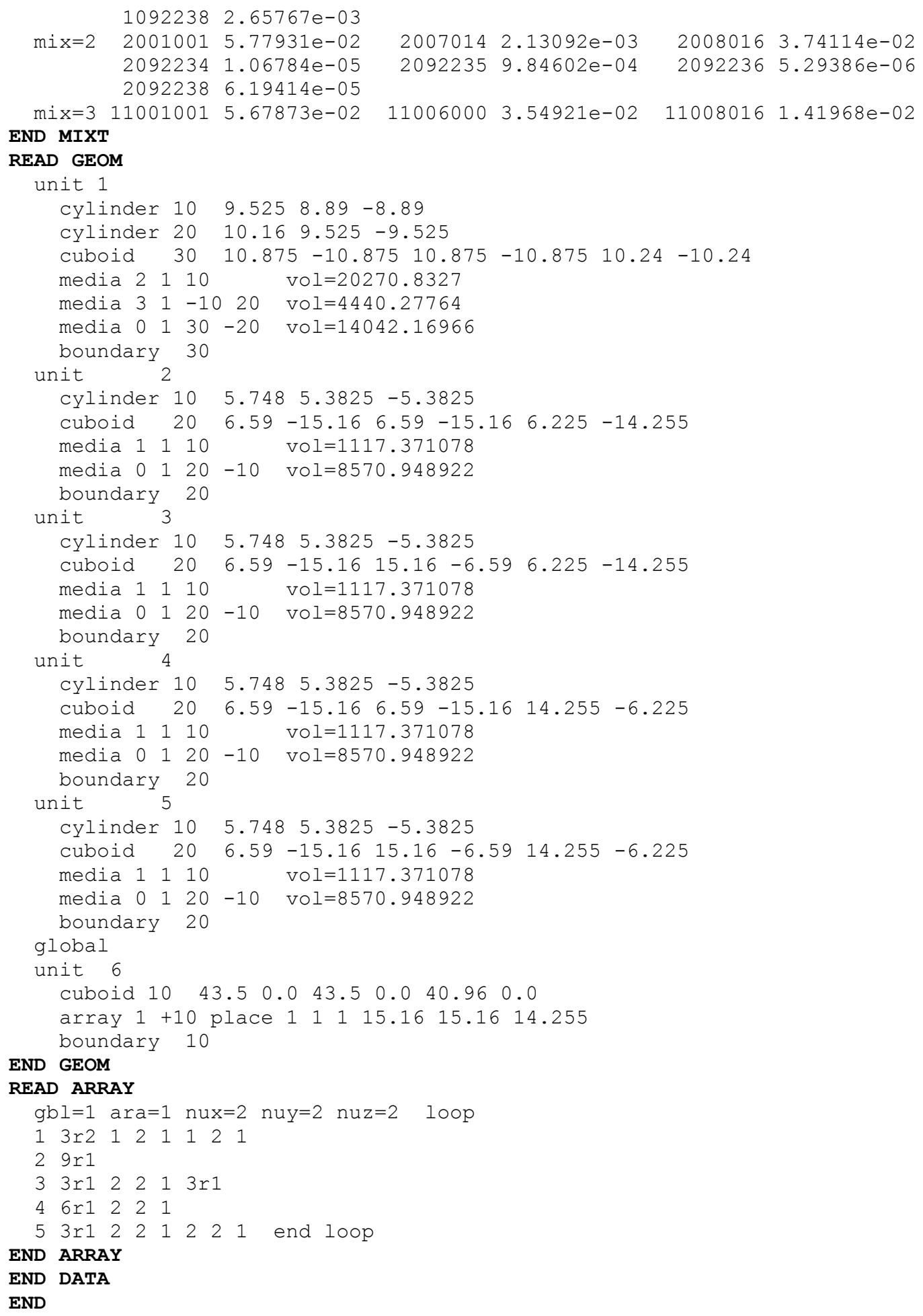

\subsection{C.2.13 Sample Problem 13 TWO CUBOIDS IN A CYLINDRICAL ANNULUS}

This critical experiment ${ }^{3}$ consists of two assemblies of $93.2 \%{ }^{235} \mathrm{U}$-enriched uranium metal $(\rho=18.69 \mathrm{~g} / \mathrm{cc})$ stacked vertically. The bottom assembly contains a uranium metal cuboid offset to the 
left within a uranium metal cylindrical annulus. The top assembly contains a uranium metal cuboid offset to the right within a uranium metal cylindrical annulus. The cuboid extends above the annulus. A drawing of the two sections and the total assembly is given in Figure 8.1.C.4.

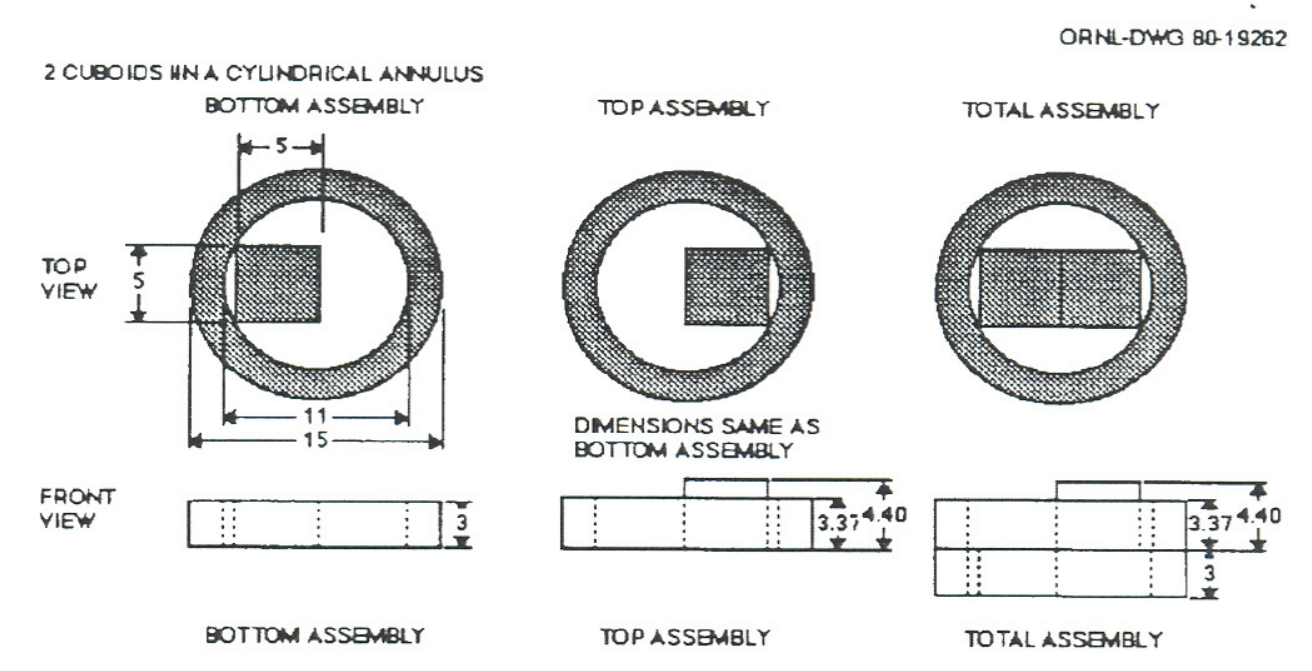

\section{ALL DIMENSIONS ARE IN INCHES}

Figure 8.1.C.4. Drawing of two cuboids in an annulus critical assembly. 


\section{Input Data}

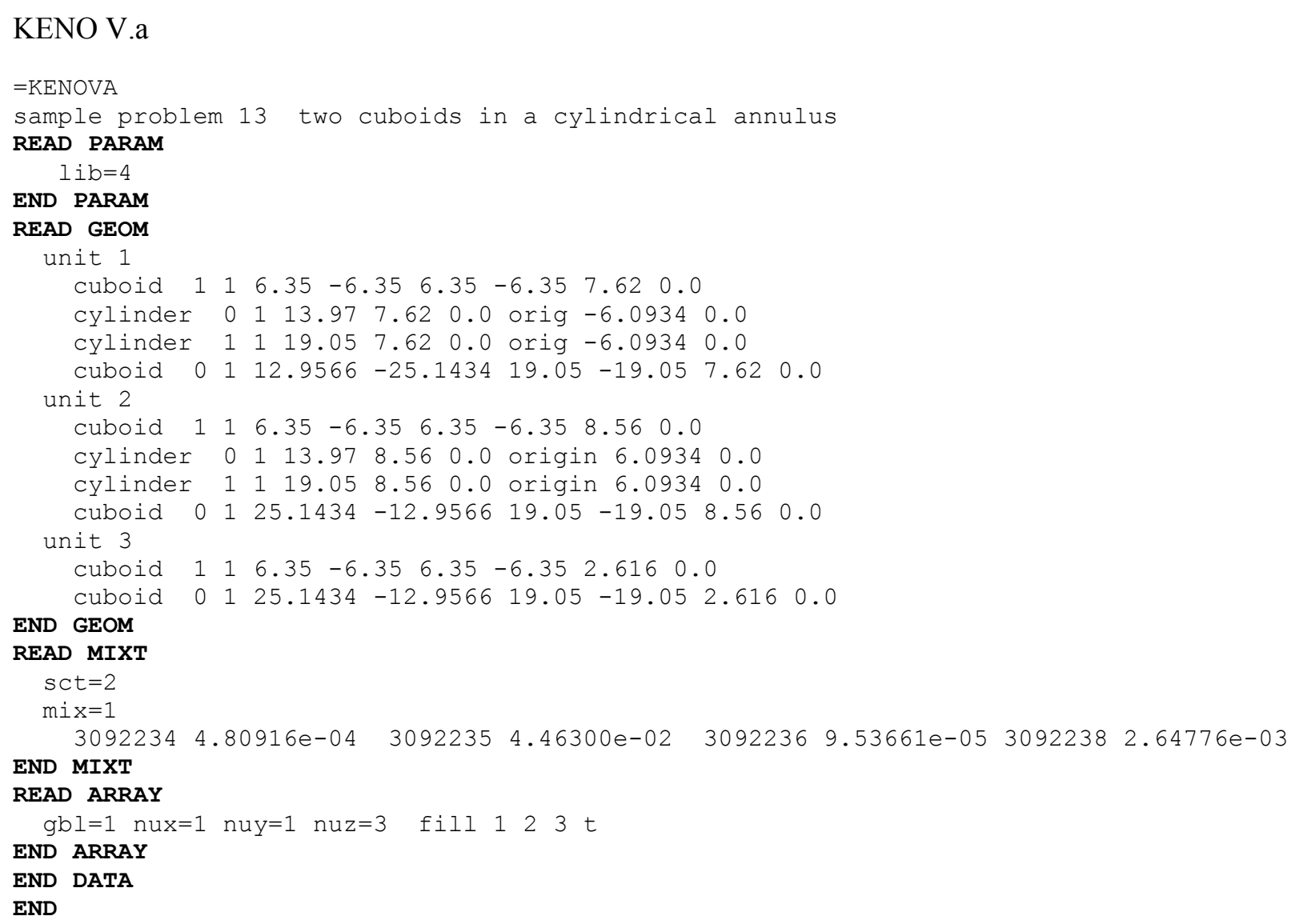

\section{KENO-VI}

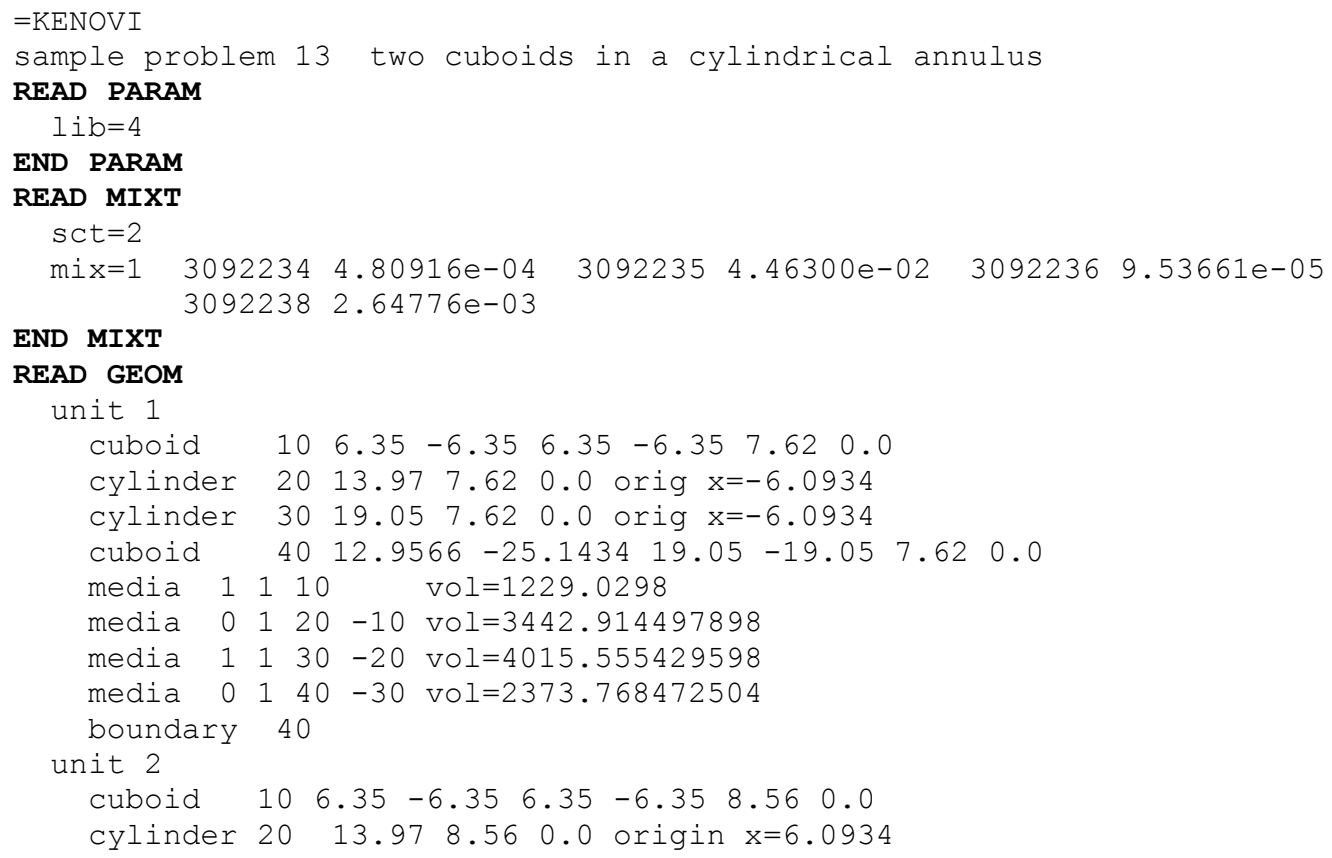




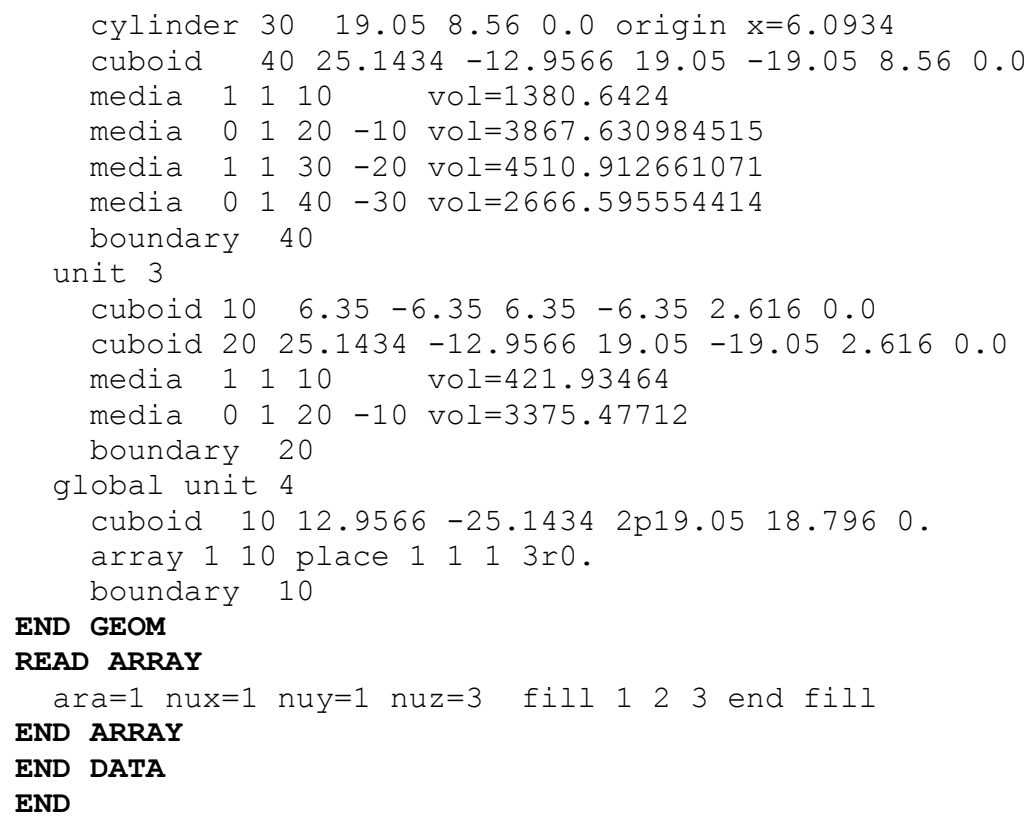

\subsection{C.2.14 Sample Problem 14 U METAL CYLINDER IN AN ANNULUS}

This critical experiment ${ }^{3}$ consists of a $93.2{ }^{235} \mathrm{U}$-enriched uranium metal cylinder within a cylindrical annulus of the same material as shown in Figure 8.1.C.5. The uranium metal specification is identical to that used in sample problem 13.

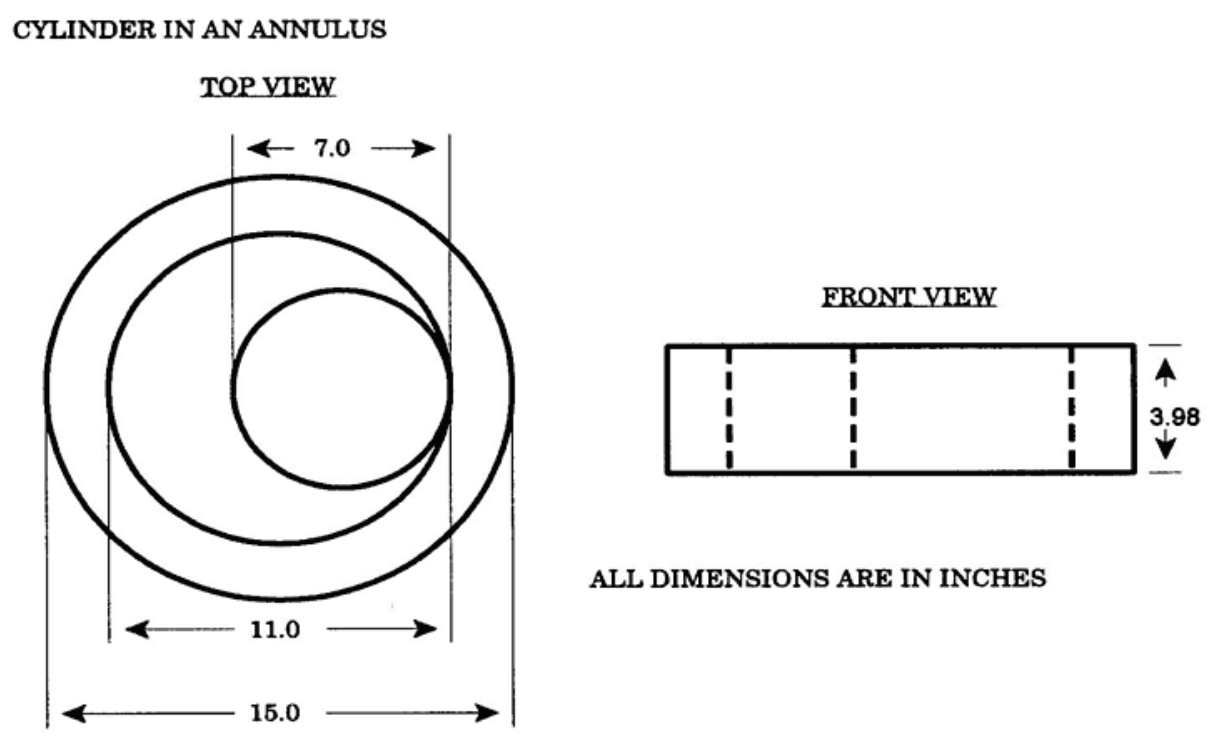

Figure 8.1.C.5. Drawing of the cylinder in an annulus critical assembly. 


\section{Input Data}

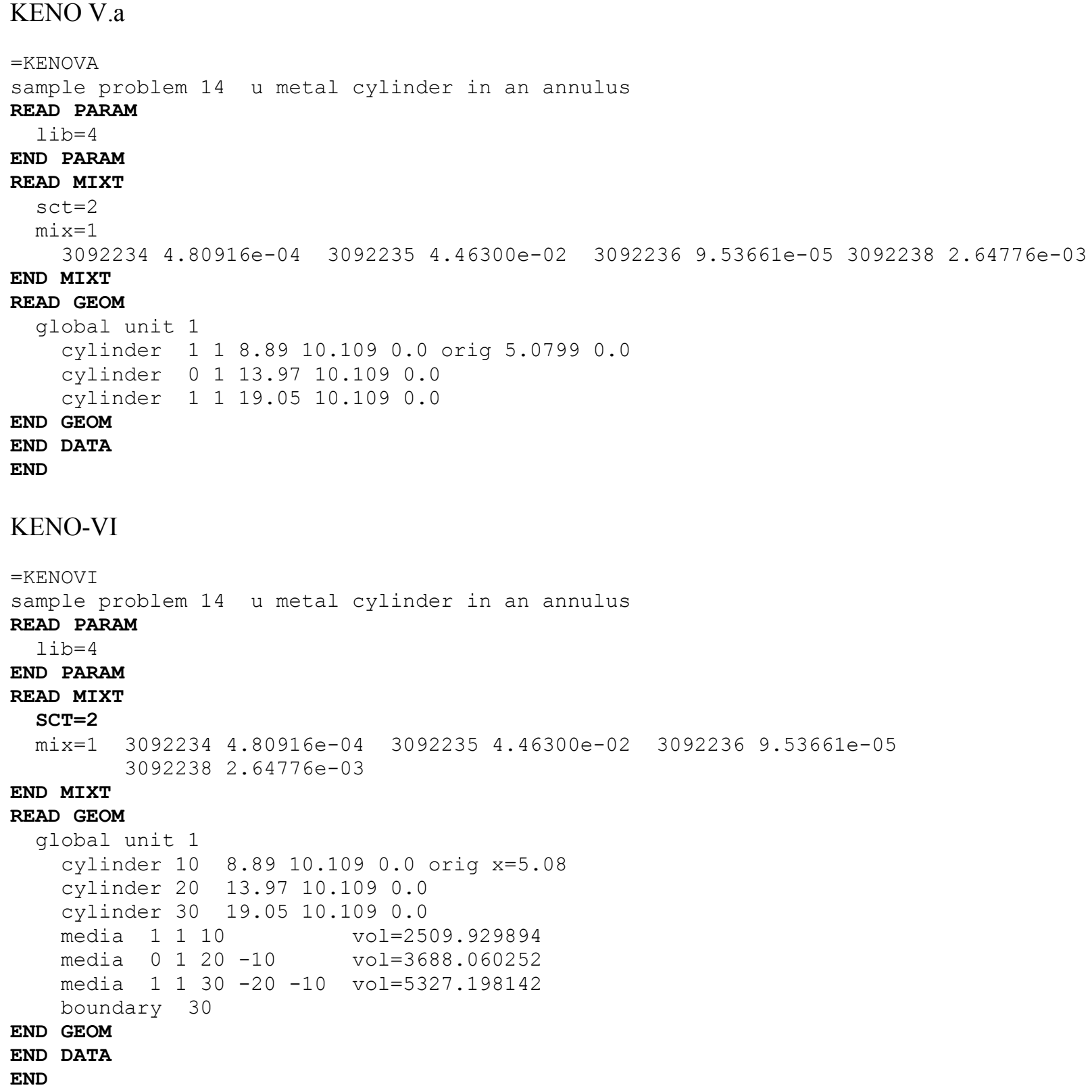

\subsection{C.2.15 Sample Problem 15 SMALL WATER REFLECTED SPHERE ON PLEXIGLAS COLLAR}

This critical experiment ${ }^{4}$ is a small highly enriched uranium sphere supported by a Plexiglas doughnut in a tank of water. The sphere extends down through the hole of the doughnut. However, the KENO geometry package cannot rigorously describe a doughnut (torus) with either KENO V.a or KENO-VI. Therefore, the KENO mockup of this problem describes the doughnut as an annular cylindrical plate and the sphere is supported by it. Both are contained in a cylindrical tank of water. A drawing of the 
experiment is given in Figure 8.1.C.6. This drawing shows the sphere above the cylindrical collar for the sake of clarity. The sphere is actually supported by the collar and extends into the opening in its center. The actual experiment utilized a torus or doughnut instead of a cylindrical collar.

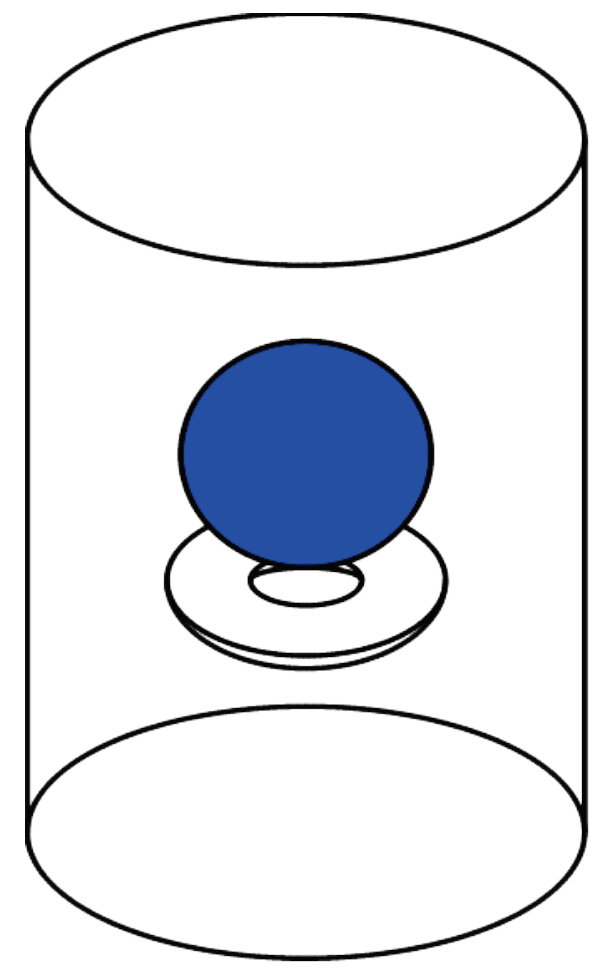

Figure 8.1.C.6. Drawing of a critical assembly consisting of a uranium sphere on a Plexiglas collar with a cylindrical water reflector.

\section{Input Data}

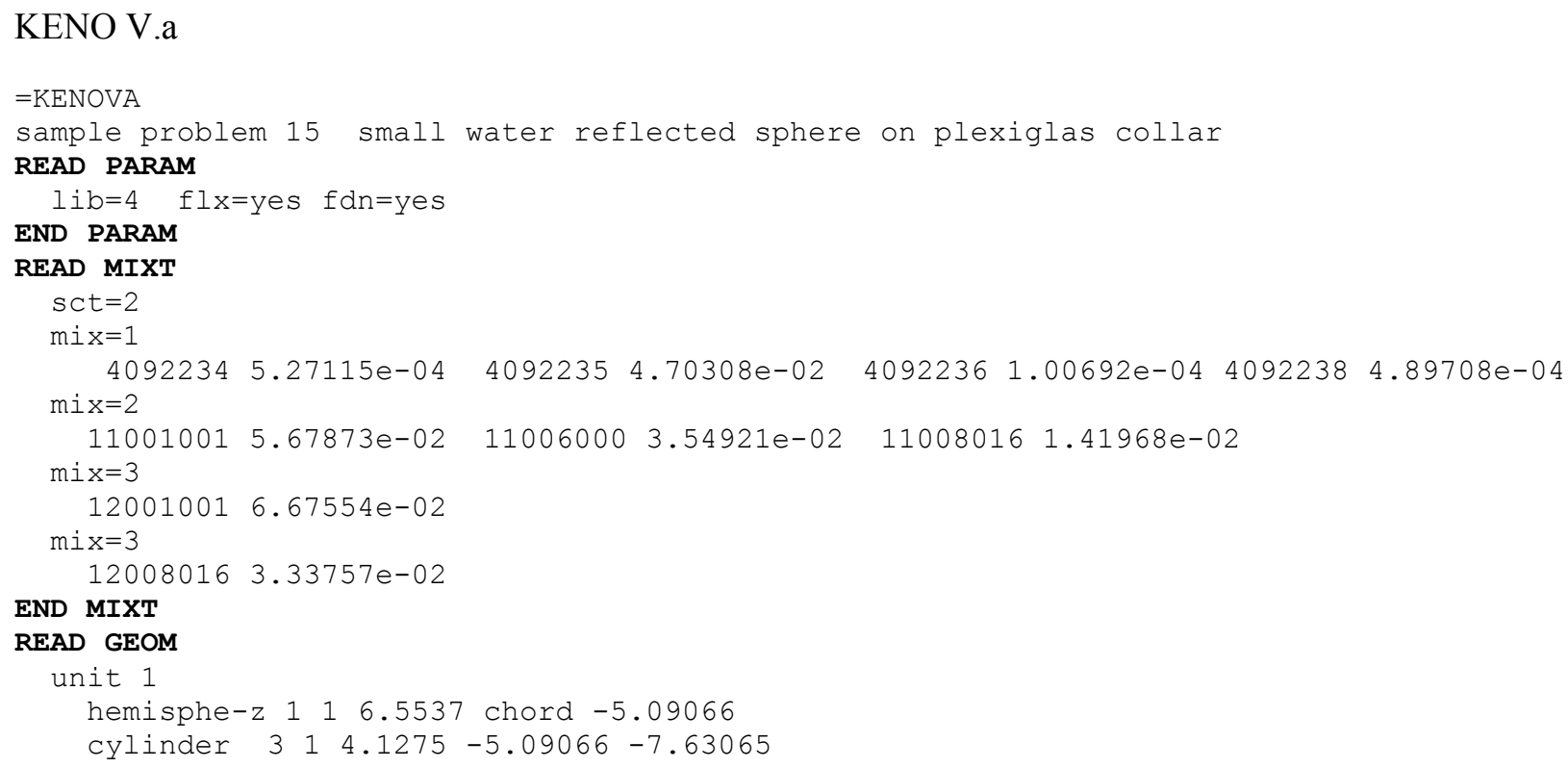




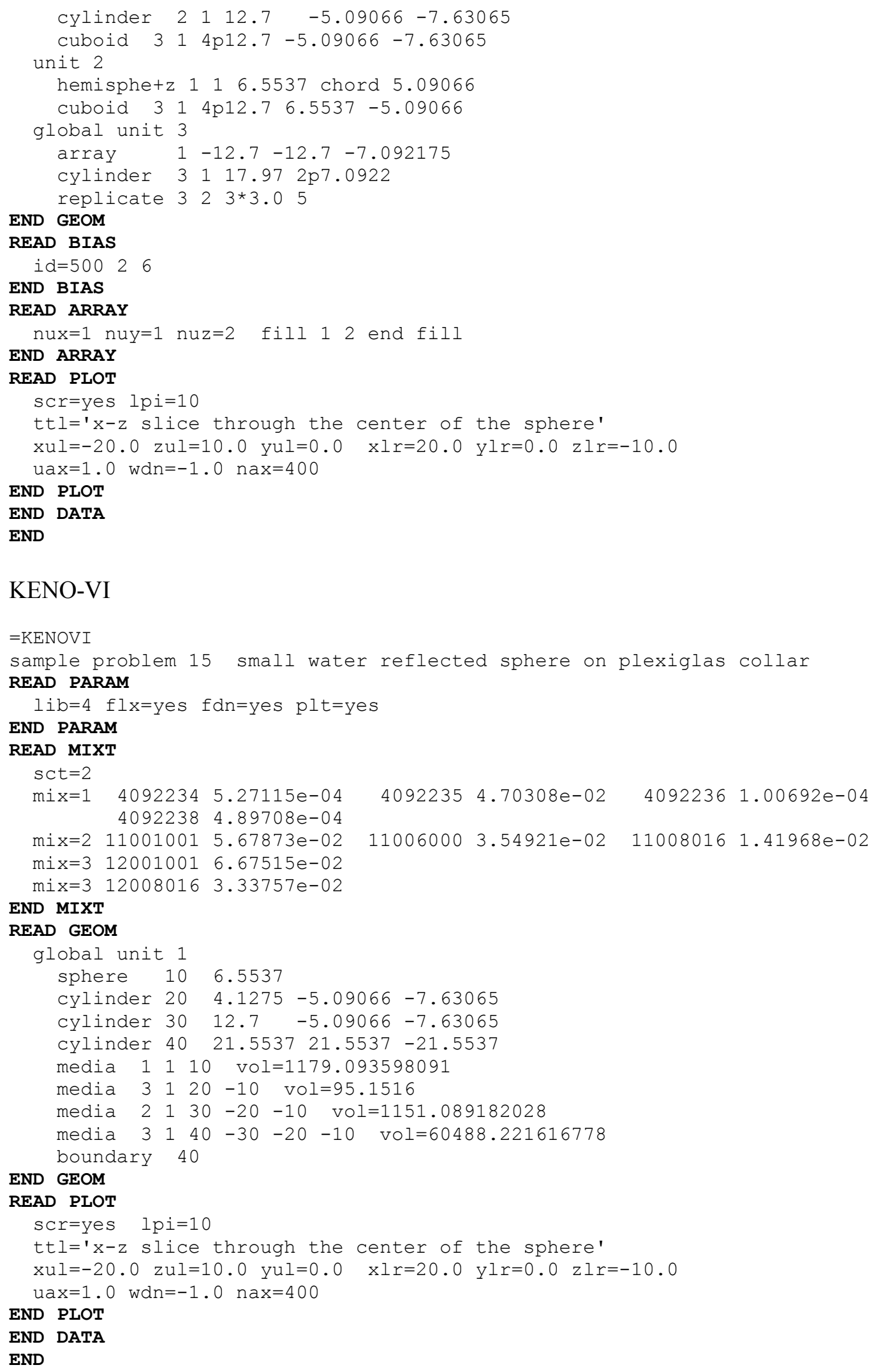




\subsection{C.2.16 Sample Problem 16 UO2F2 INFINITE SLAB K-INFINITY}

This problem solves for the k-infinity of an infinite number of slabs of uranyl fluoride solution contained in Pyrex glass and separated by borated uranyl fluoride solution. The uranyl fluoride slab is $4.958 \mathrm{~cm}$ thick, 93.2\% enriched, and has a density of $578.7 \mathrm{~g} \mathrm{U} / 1$. The Pyrex glass is $1.27 \mathrm{~cm}$ thick and is present on both faces of the uranyl fluoride solution. A total of $27.46 \mathrm{~cm}$ of borated solution separates the Pyrex glass of adjacent slabs of solution. $1.482 \times 10^{-27}$ atoms of boron per milliliter are present in the borated solution.

\section{Input Data}

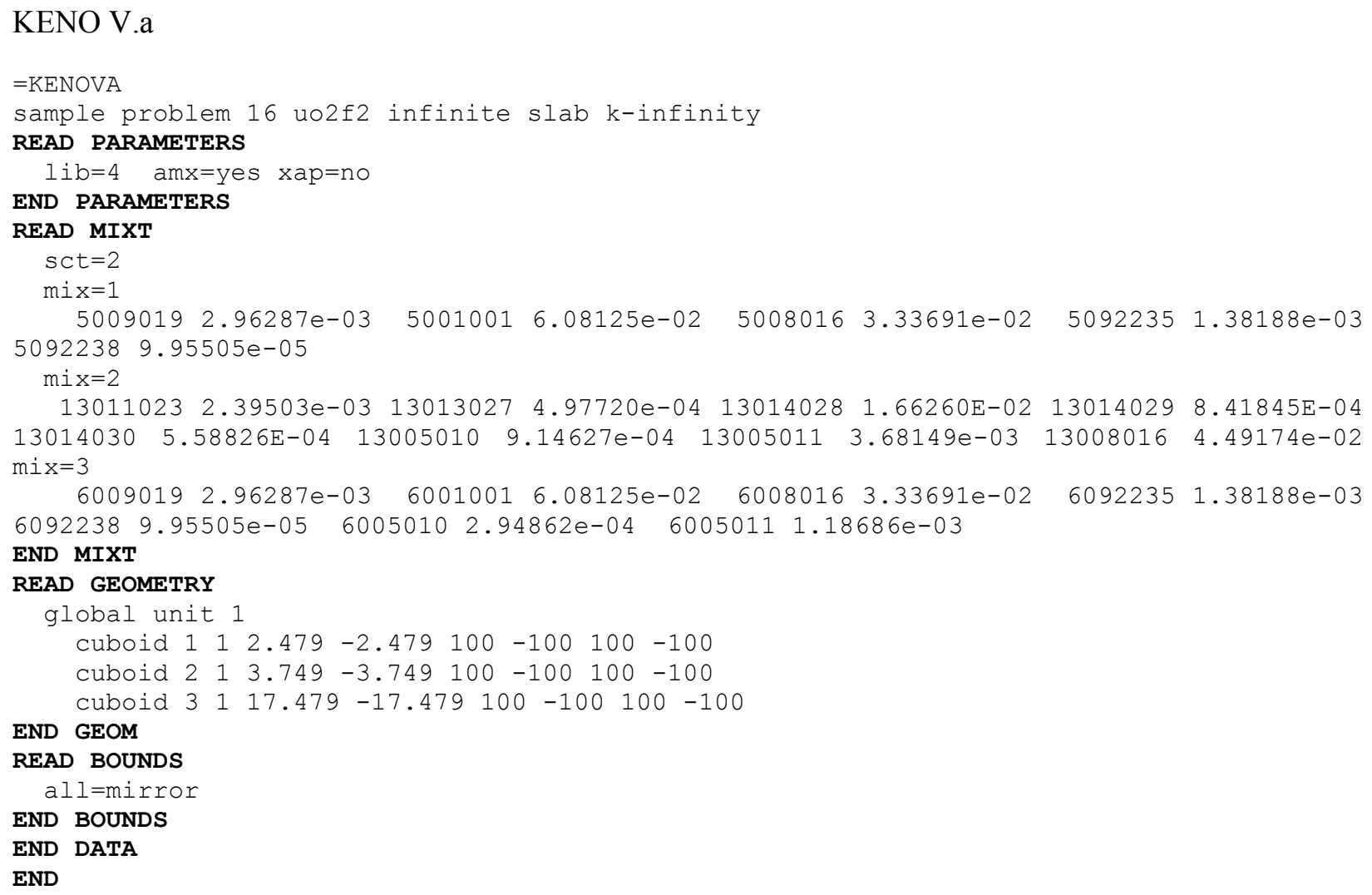

\section{KENO-VI}

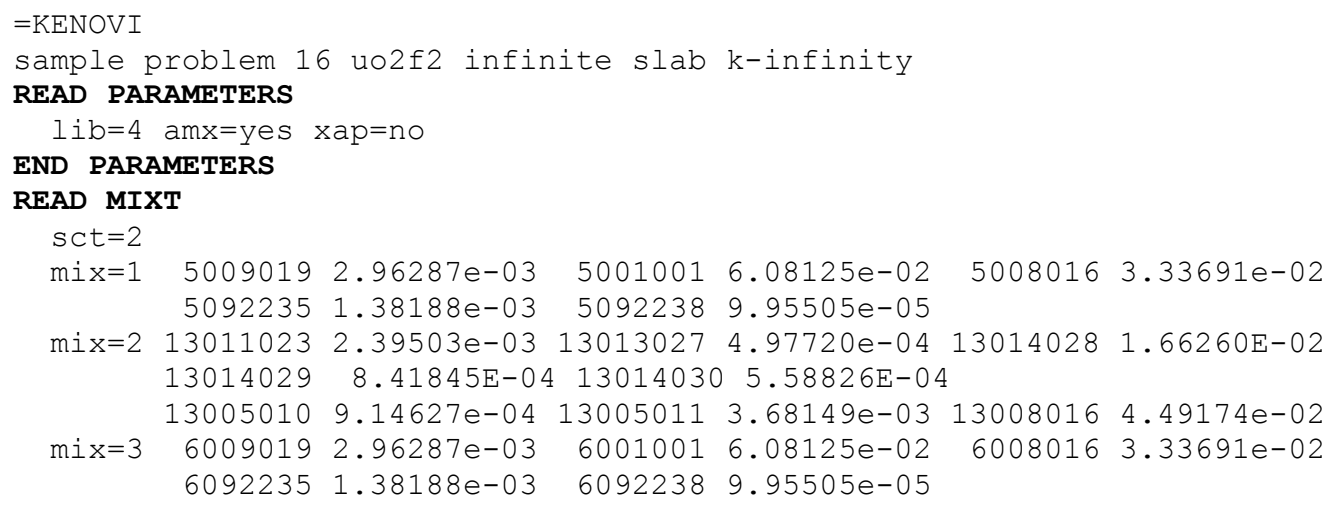




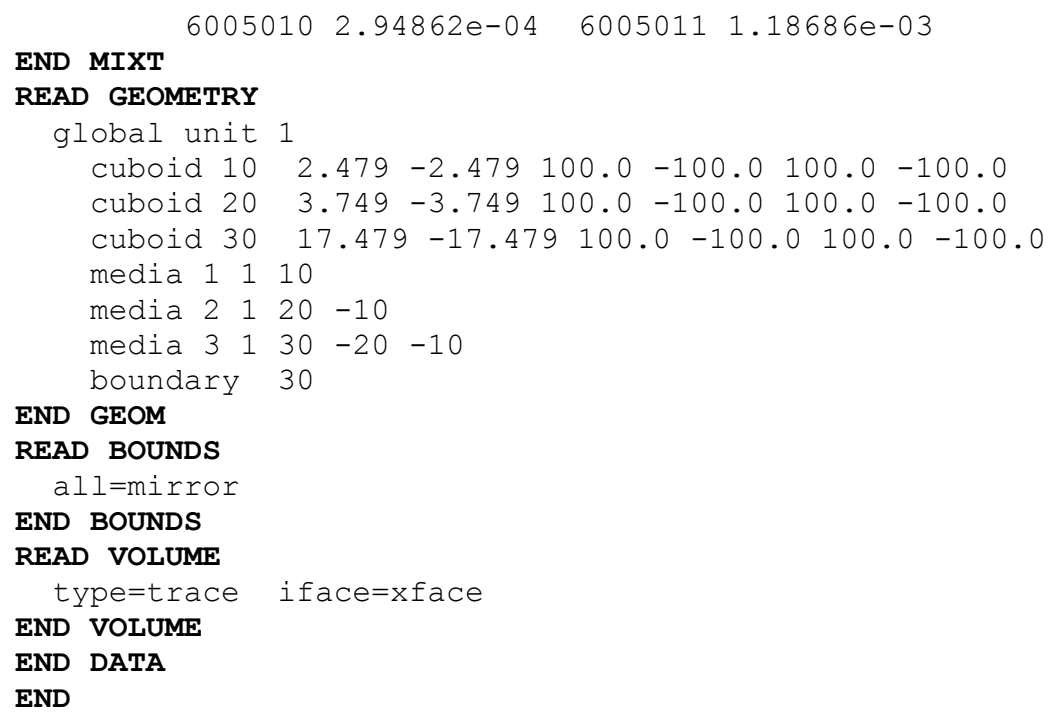

\subsection{C.2.17 Sample Problem 17 93\% UO2F2 SOLUTION SPHERE ADJOINT CALCULATION}

A single 93\% enriched uranyl fluoride sphere is run as an adjoint calculation. The result for the forward and adjoint k-effectives should be the same within statistical error when the problem is run both ways.

\section{Input Data}

\section{KENO V.a}

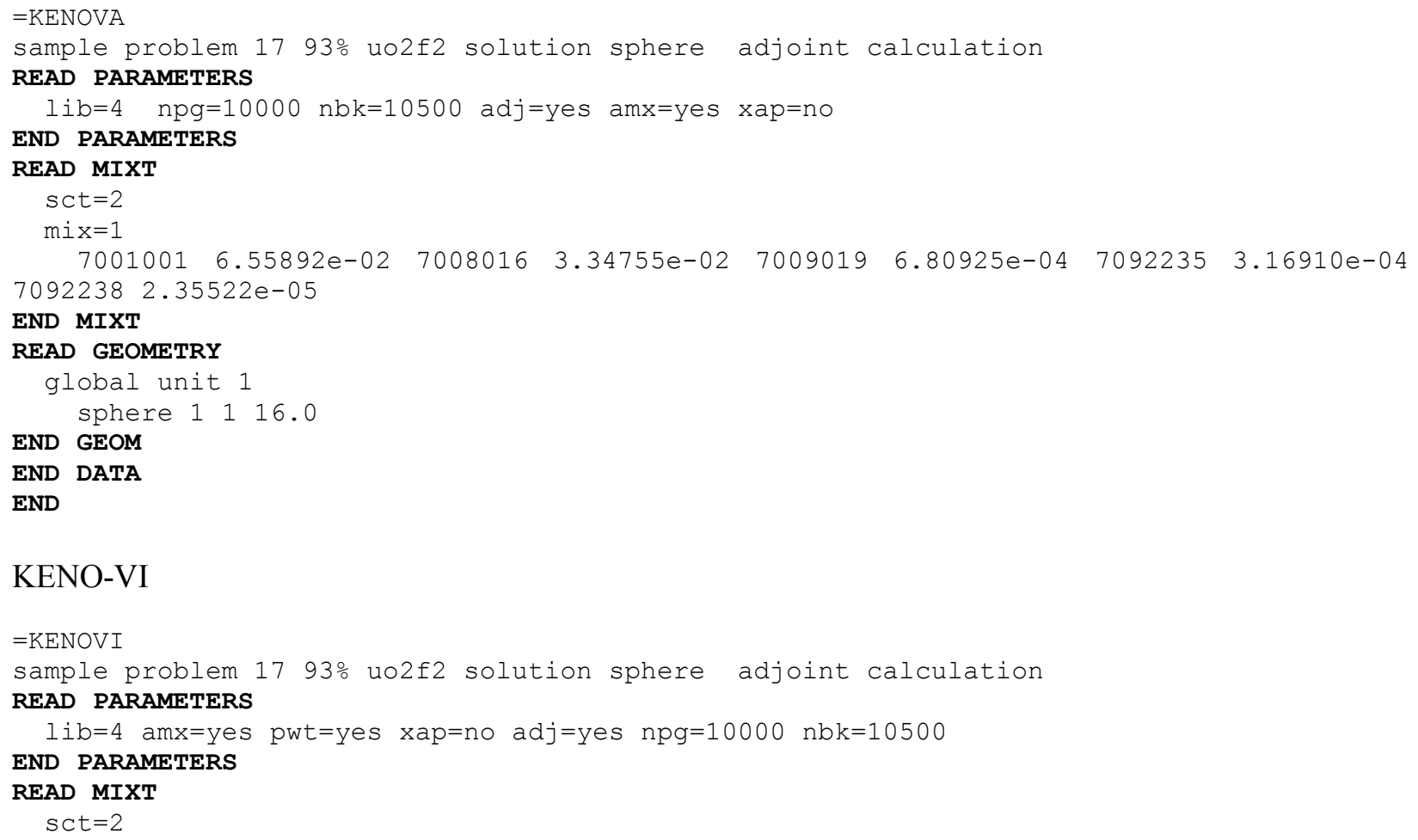




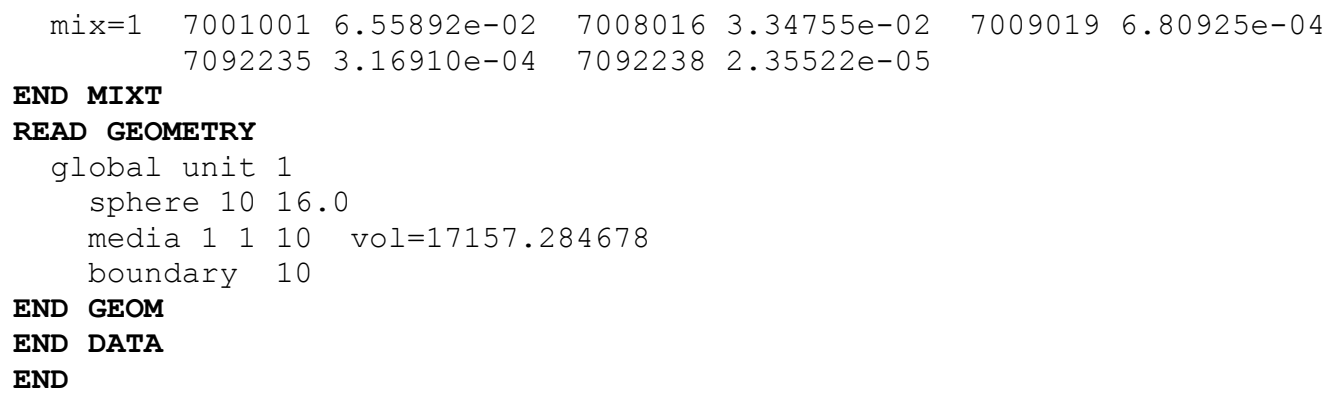

\subsection{C.2.18 Sample Problem 18 1F27 DEMONSTRATION OF OPTIONS}

A reflected cubic array of 27 cylinders of aqueous uranyl nitrate in Plexiglas bottles. ${ }^{5}$ The walls of the bottles were $0.64-\mathrm{cm}$ thick, and each bottle was filled with 5 liters of $92.6 \%$ enriched solution at a concentration of $415 \mathrm{~g} / \mathrm{L}$, a specific gravity of 1.555 and $0.39 \mathrm{mg}$ excess nitrate $/ \mathrm{g}$ soln. ${ }^{*}$ The $3 \times 3 \times 3$ array was surrounded by a 6 -in. paraffin reflector. Most of the print options available in KENO are exercised in this problem. A perspective of this critical experiment is shown in Figure 9.1.A.7. A photograph of one of the experiments utilized 27 of the Plexiglas bottles is shown in Figure 9.1.A.8. Sample problem 18 has $15.24 \mathrm{~cm}$ of paraffin on all six faces rather than the 2.54-cm Plexiglas shown on five faces.

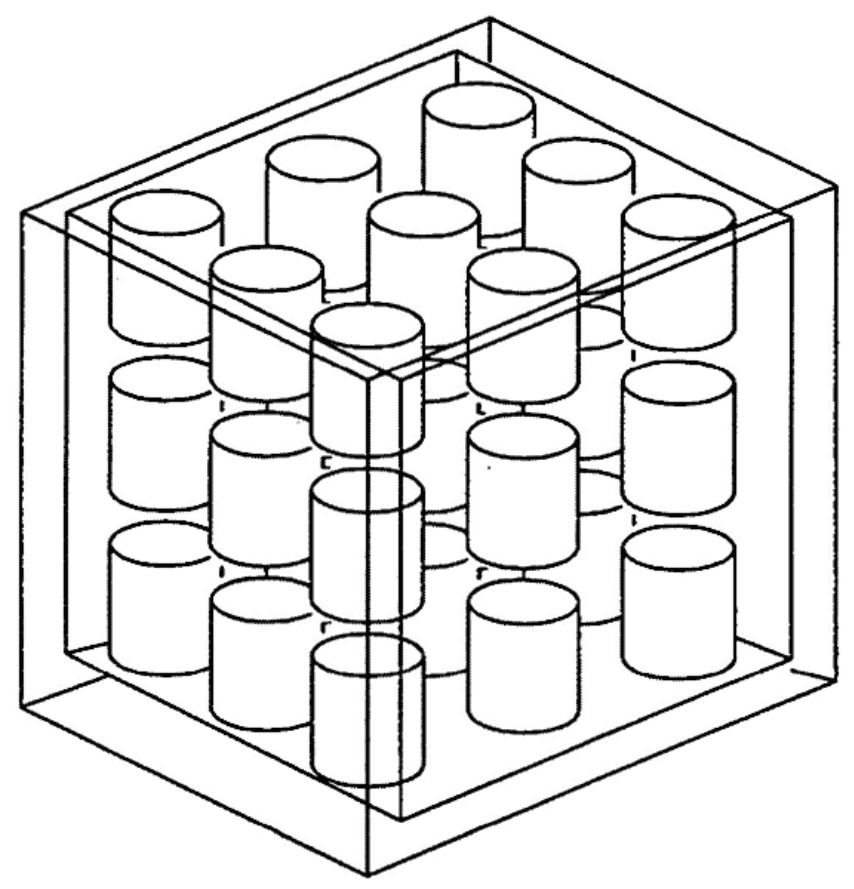

Figure 8.1.C.7. Perspective of critical 1F27 experiment.

${ }^{*}$ From experimental facility documents. Not reported in ORNL/TM-719. 


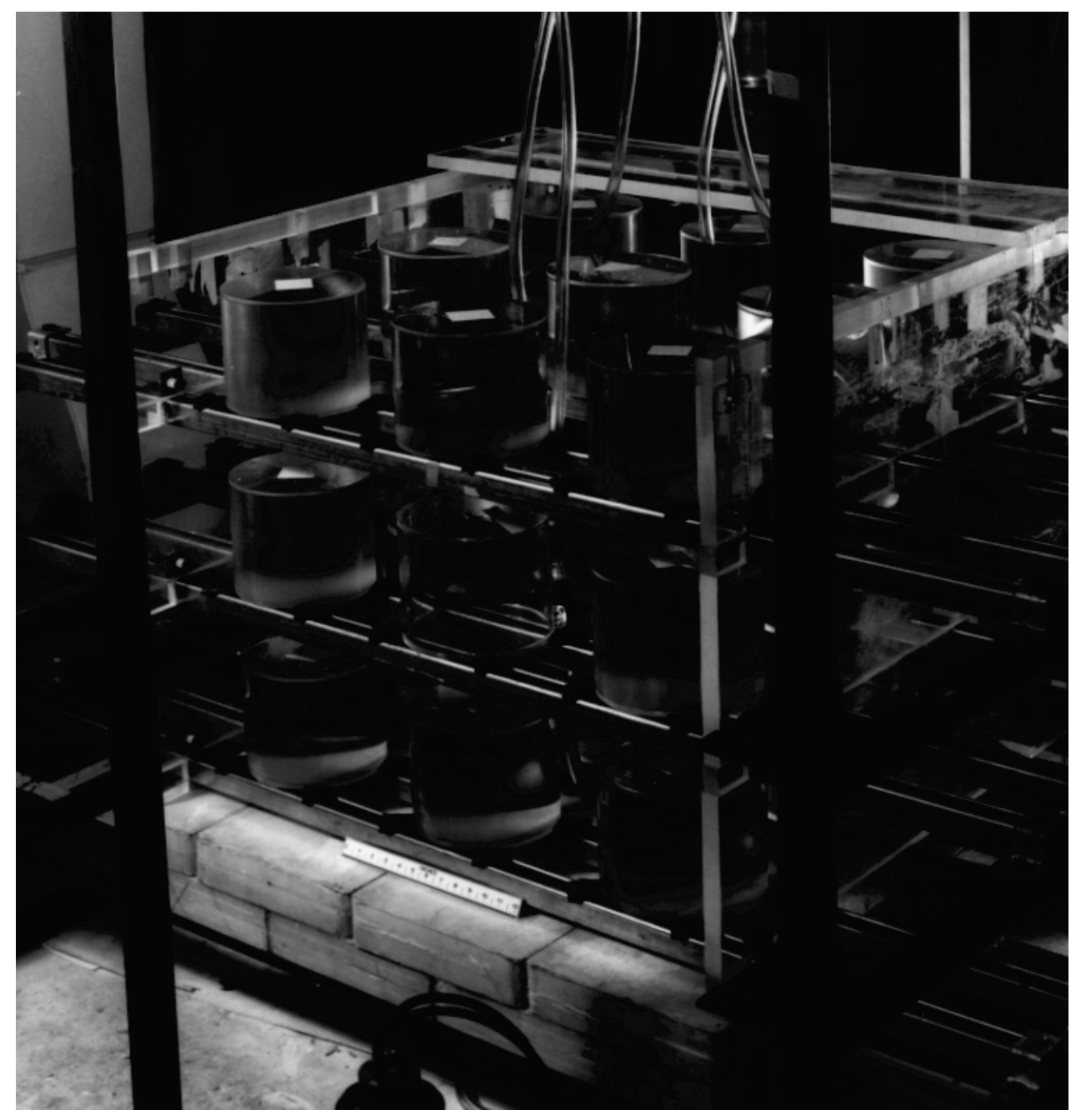

Figure 8.1.C.8. View of a 27-unit array with 2.54-cm. thick Plexiglas reflector on five sides and a 15.24-cm. thick paraffin base. 


\section{Input Data}

\section{KENO V.a}

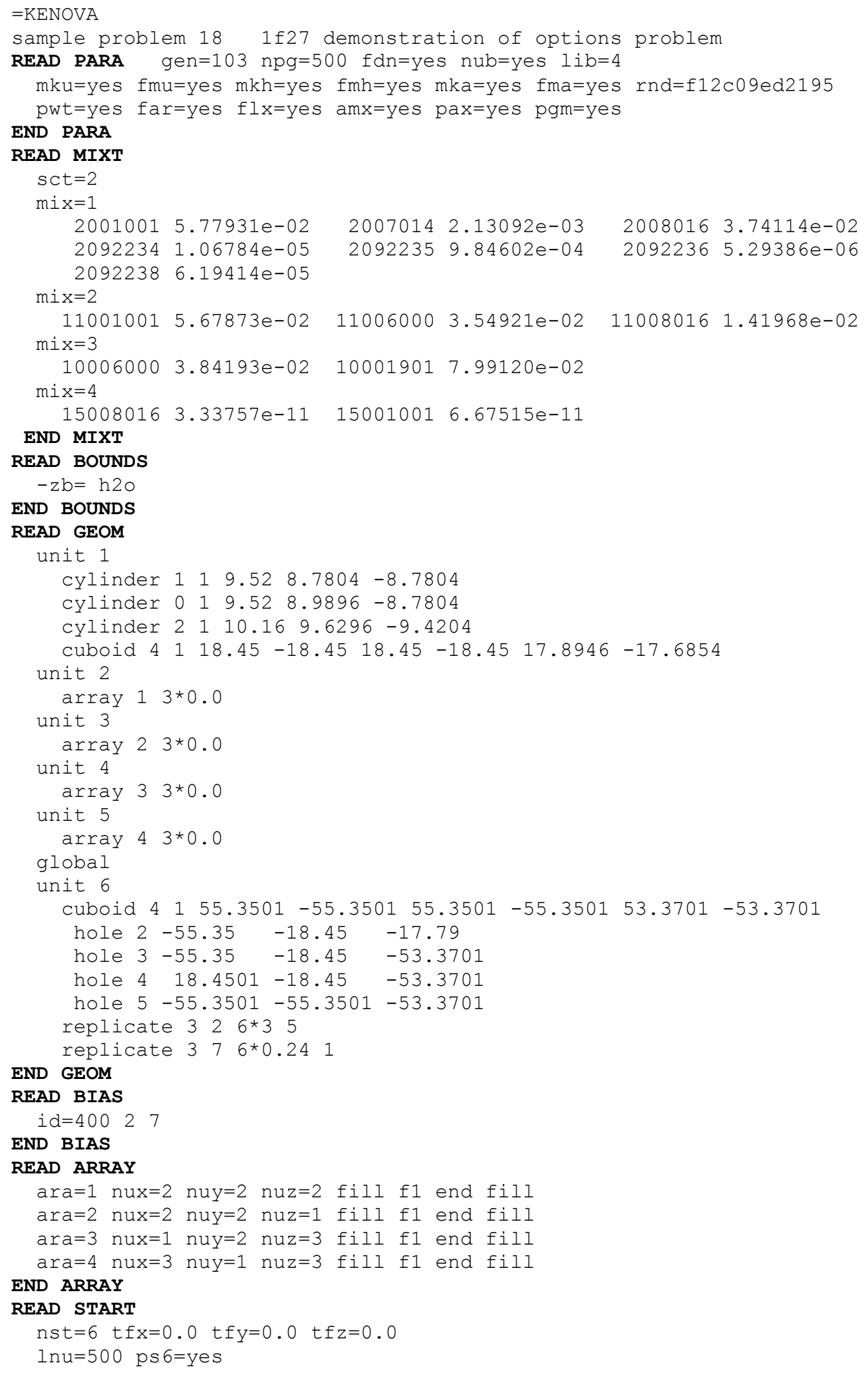




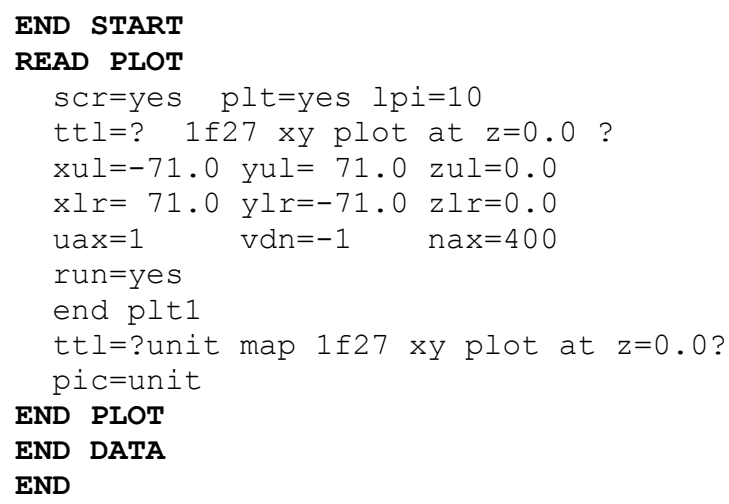

\section{KENO-VI}

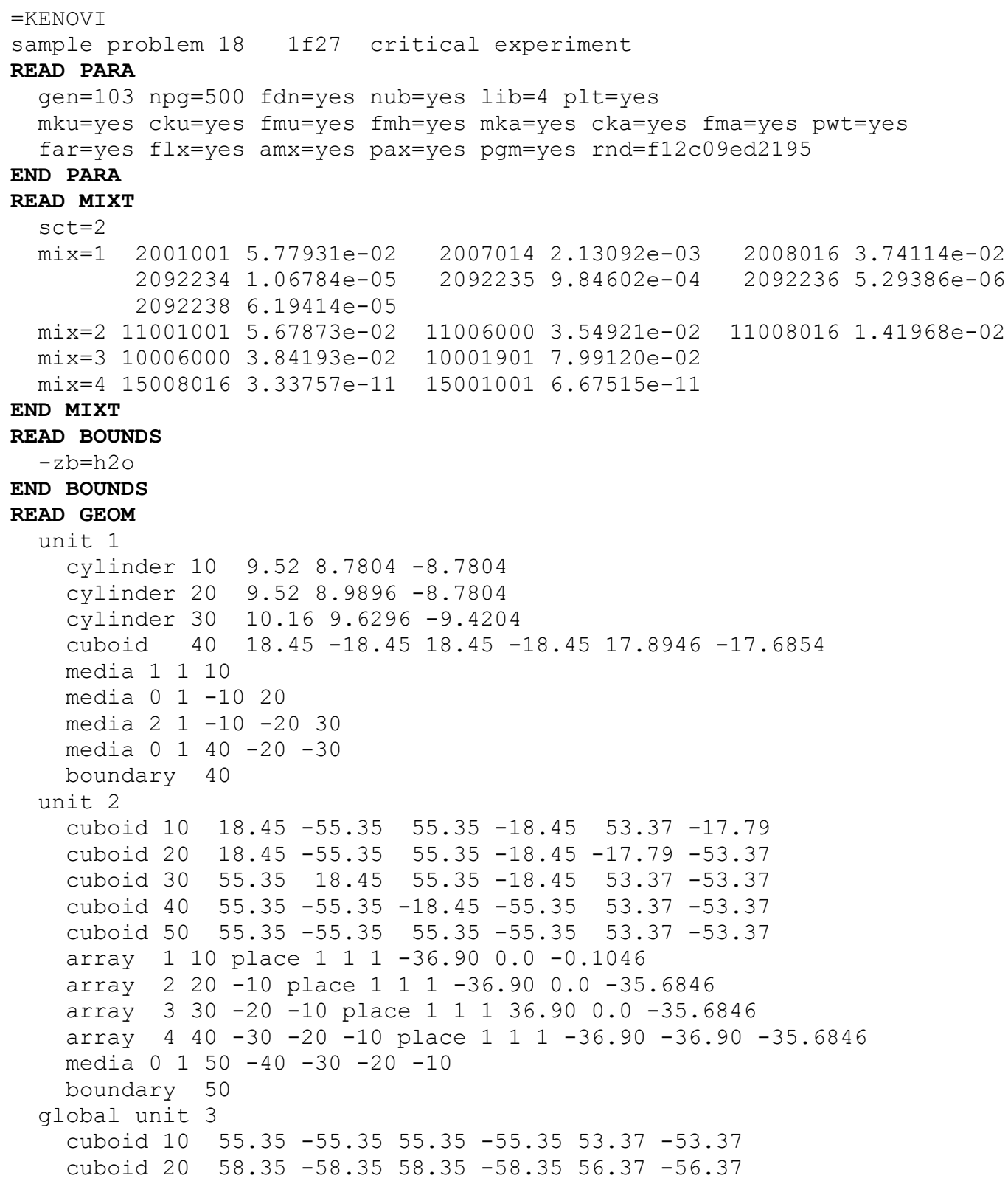




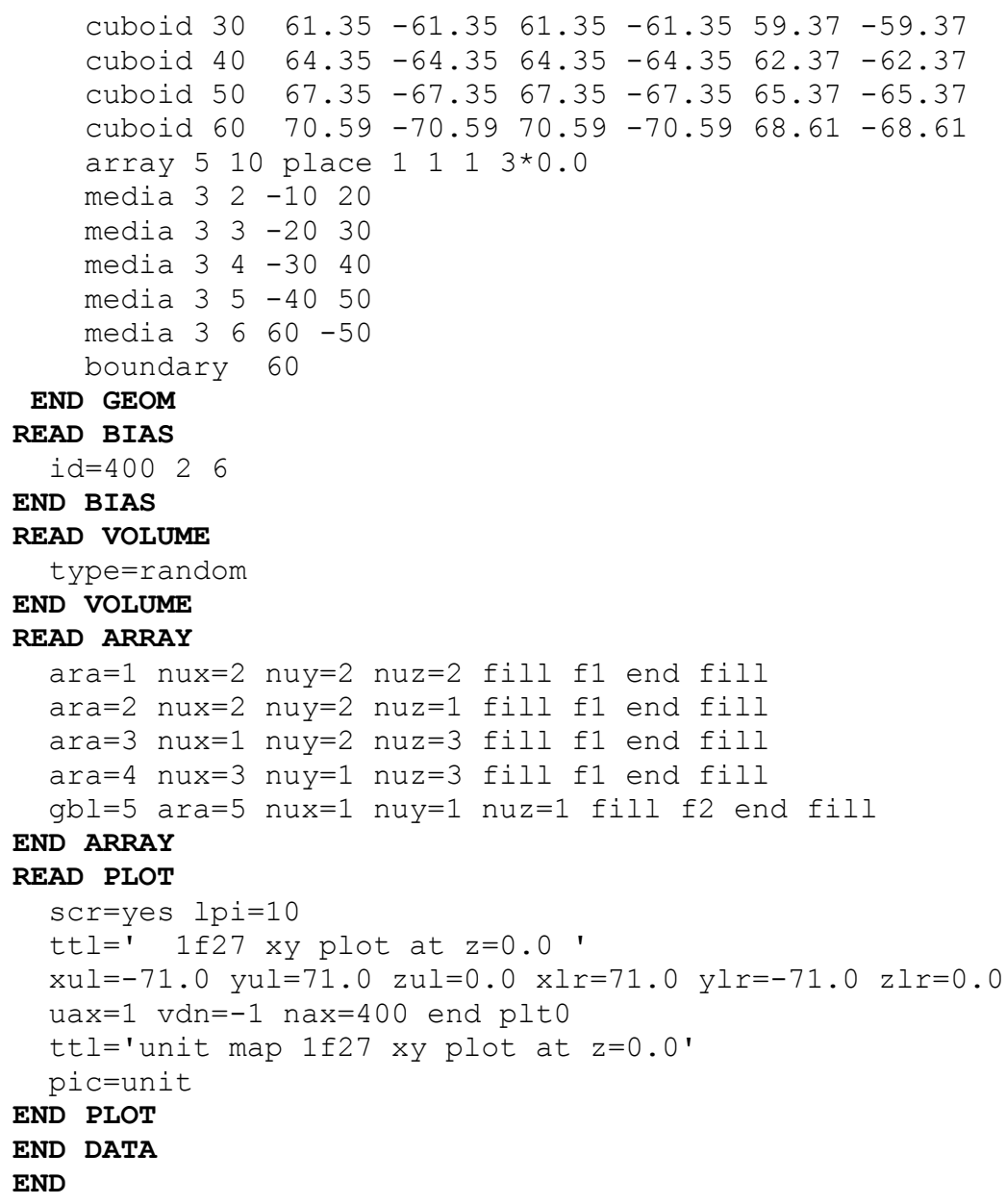

\subsection{C.2.19 Sample Problem 194 AQUEOUS 4 METAL ARRAY OF ARRAYS (SAMP PROB 12)}

This critical experiment was described previously as SAMPLE PROBLEM 12. The input data given below utilize the array of arrays option. See Figure 8.1.C.3.

\section{Input Data}

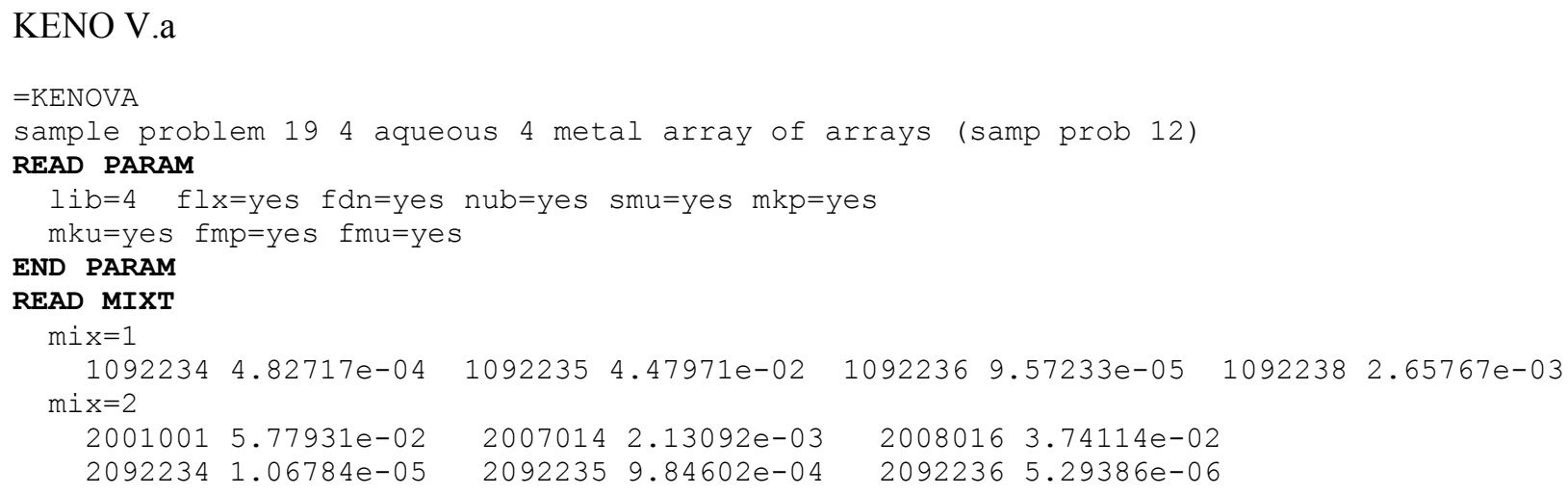




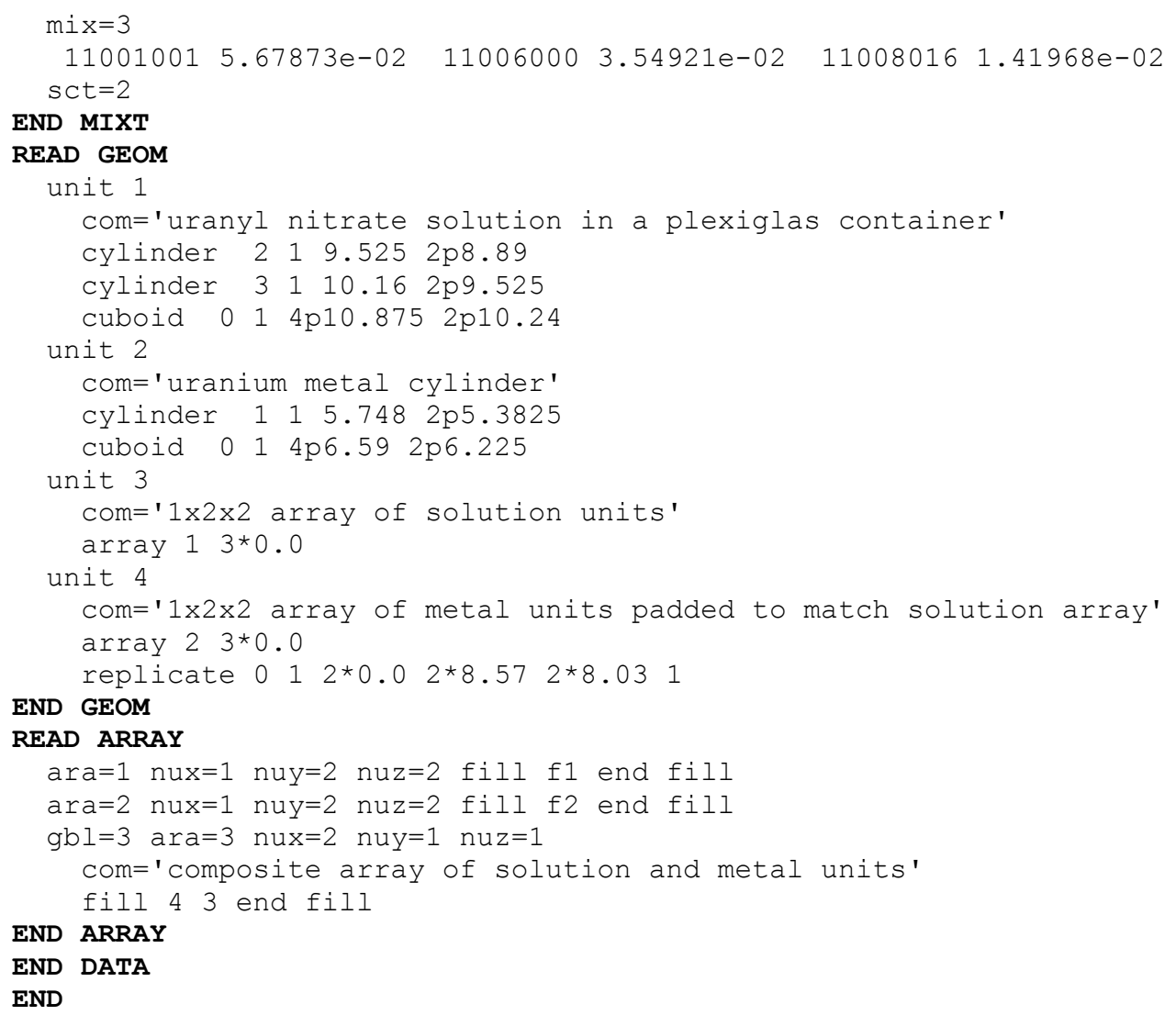

\section{KENO-VI}

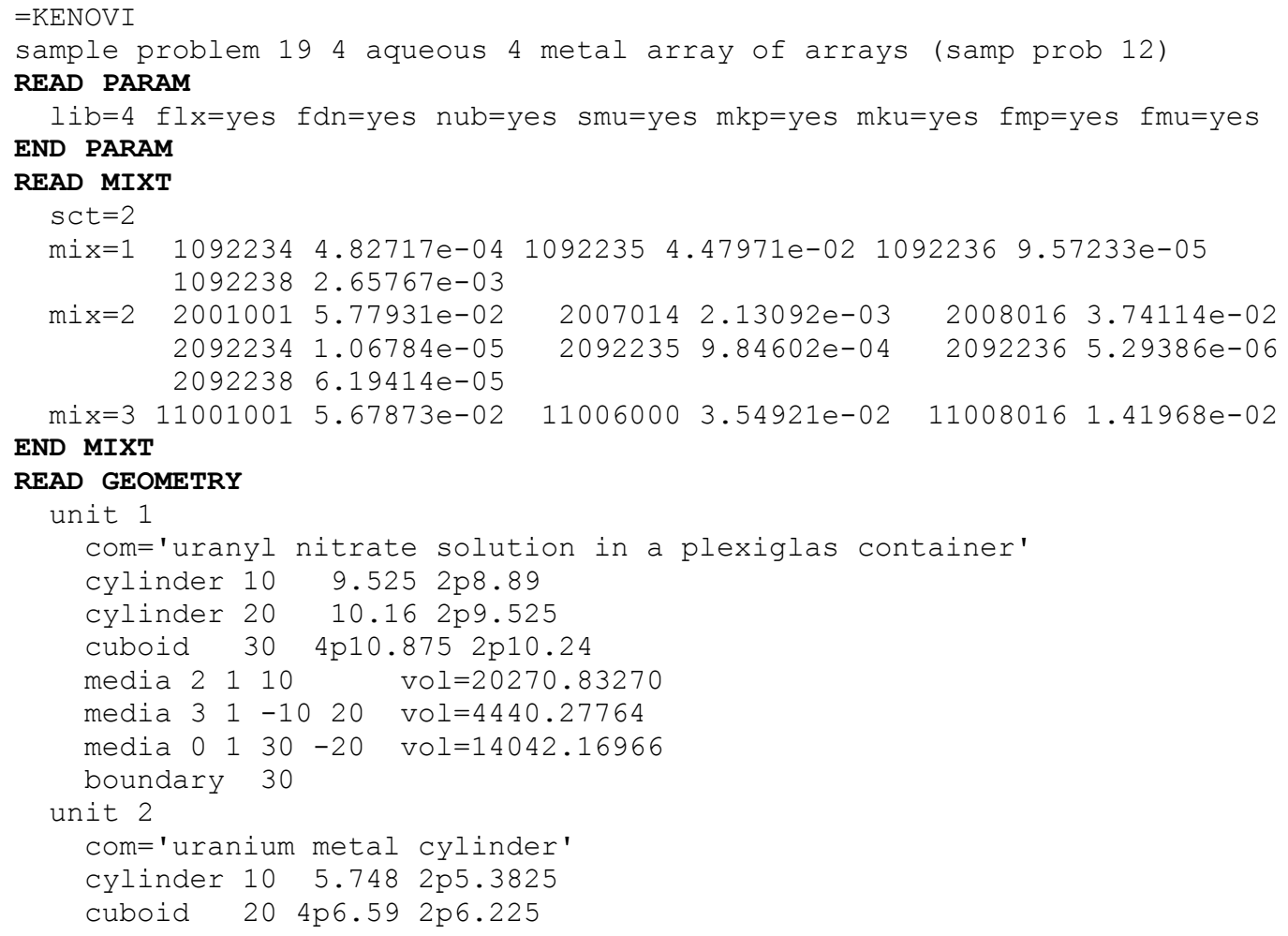




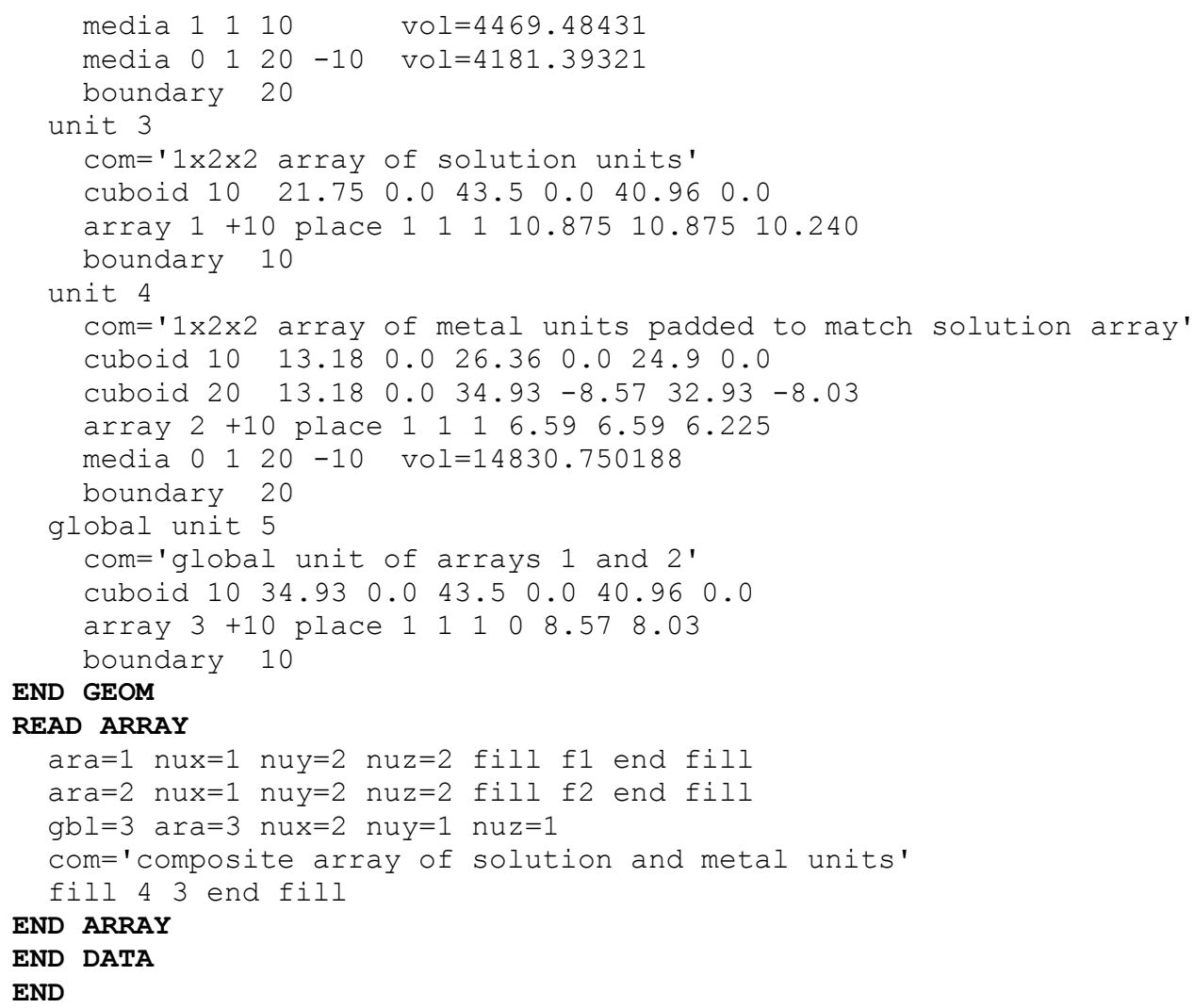

\subsection{C.2.20 Sample Problem 20 TRIANGULAR PITCHED ARRAY}

This problem is a critical experiment 14 consisting of seven cylinders in a triangular-pitched unreflected array. The central cylinder has six cylinders arranged around it. The surface-to-surface separation between the units is 0.15 in. Each unit consists of a 60-mil-thick aluminum can with an 8-in. inside diameter, filled with a solution of $93.2 \%$ enriched uranyl fluoride with a $\mathrm{H} / 235 \mathrm{U}$ atomic ratio of 44.3 and a density of $576.87 \mathrm{~g} \mathrm{U} / \mathrm{L}$. The apparatus for conducting this experiment is shown in Figure 8.1.C.9. 


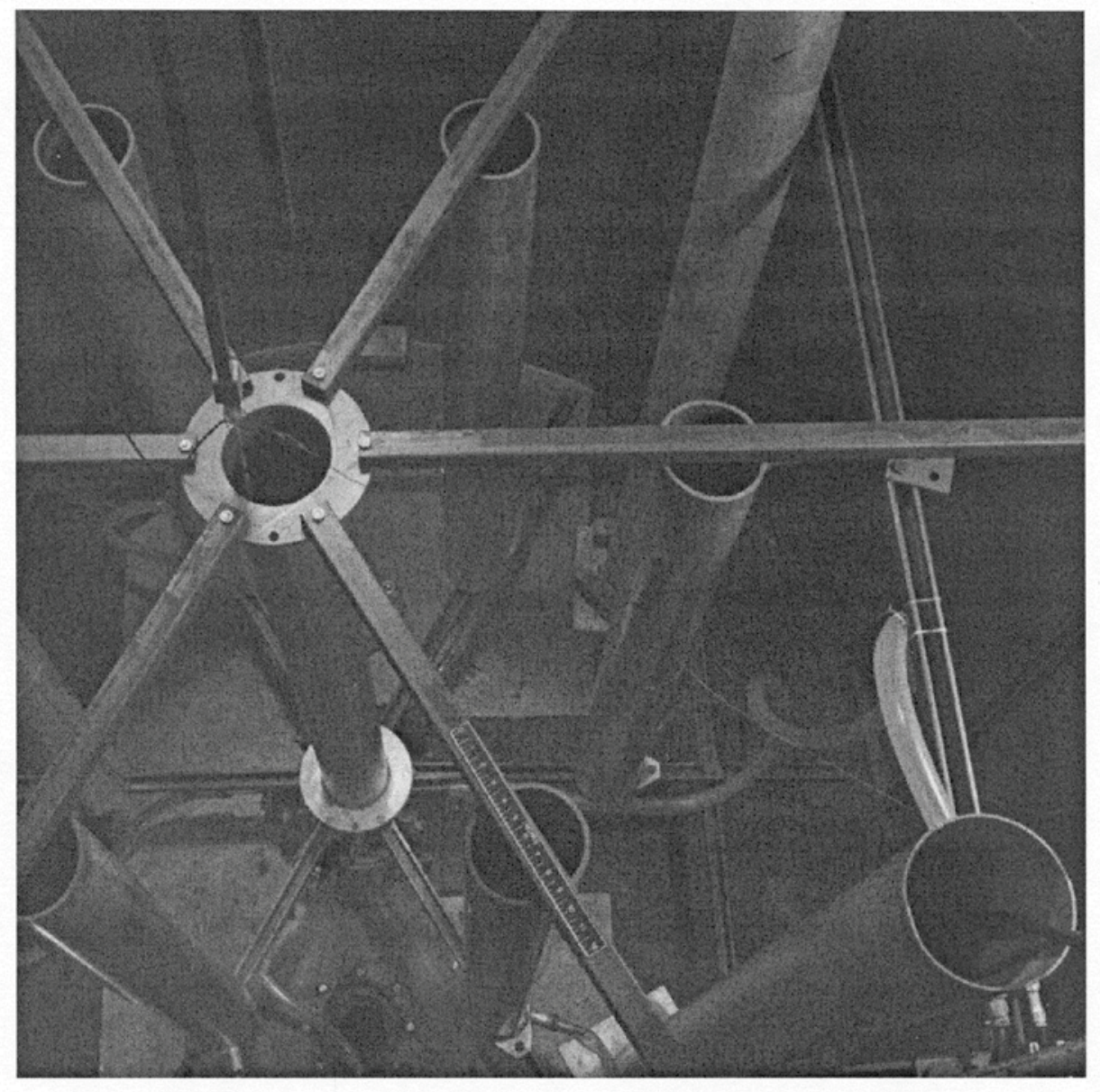

Figure 8.1.C.9. Typical arrangement for critical experiments with interacting arrays of aluminum cylinders containing enriched ${ }^{235} \mathrm{U}$ solutions. 


\section{Input Data}

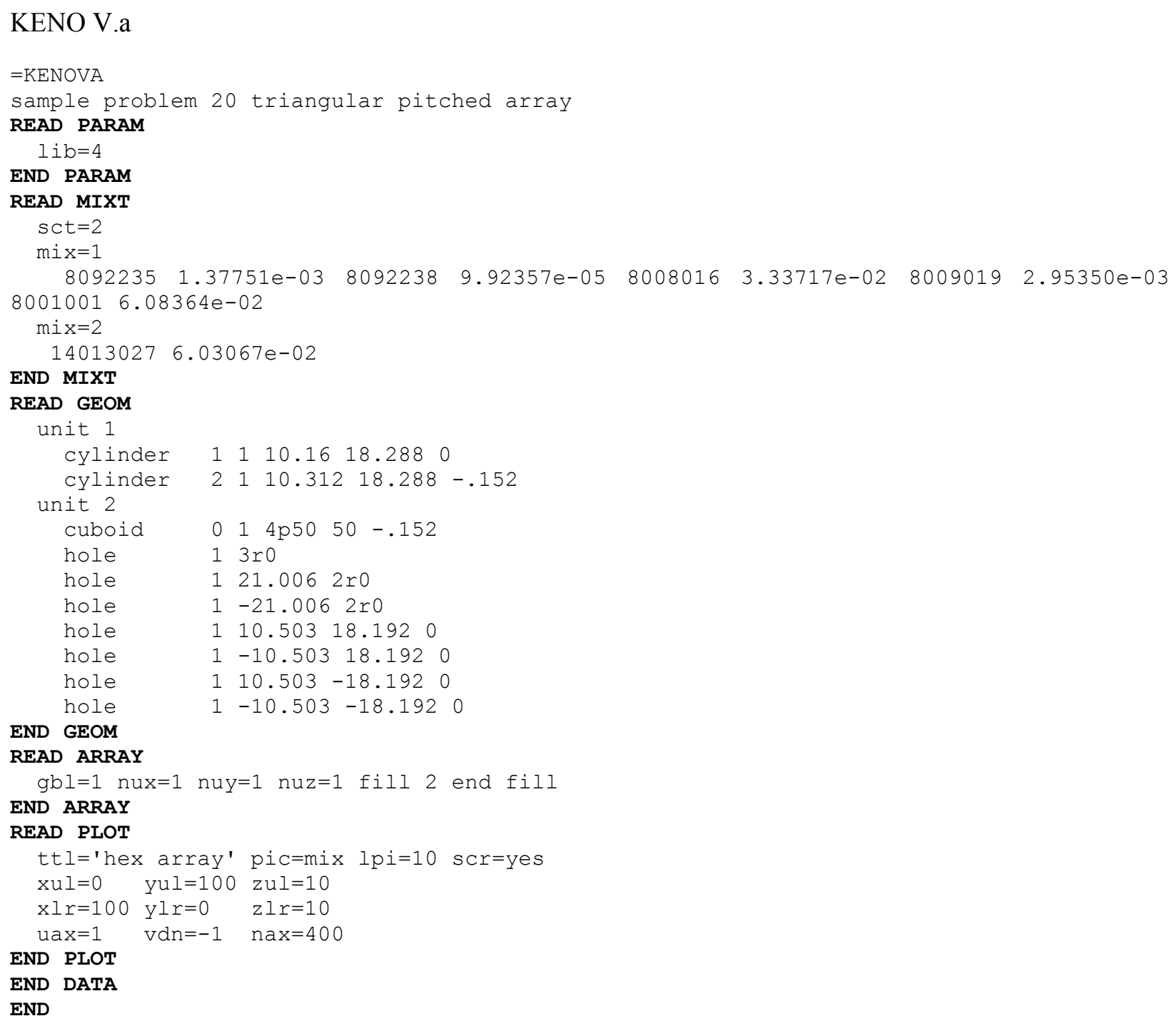

\section{KENO-VI}

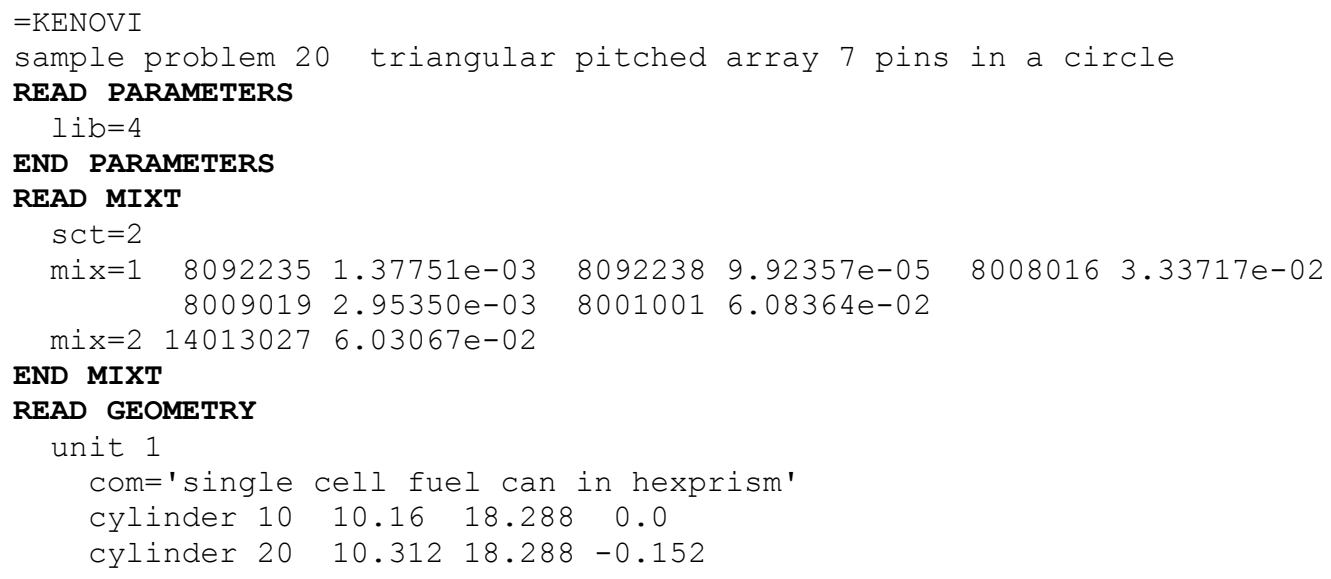




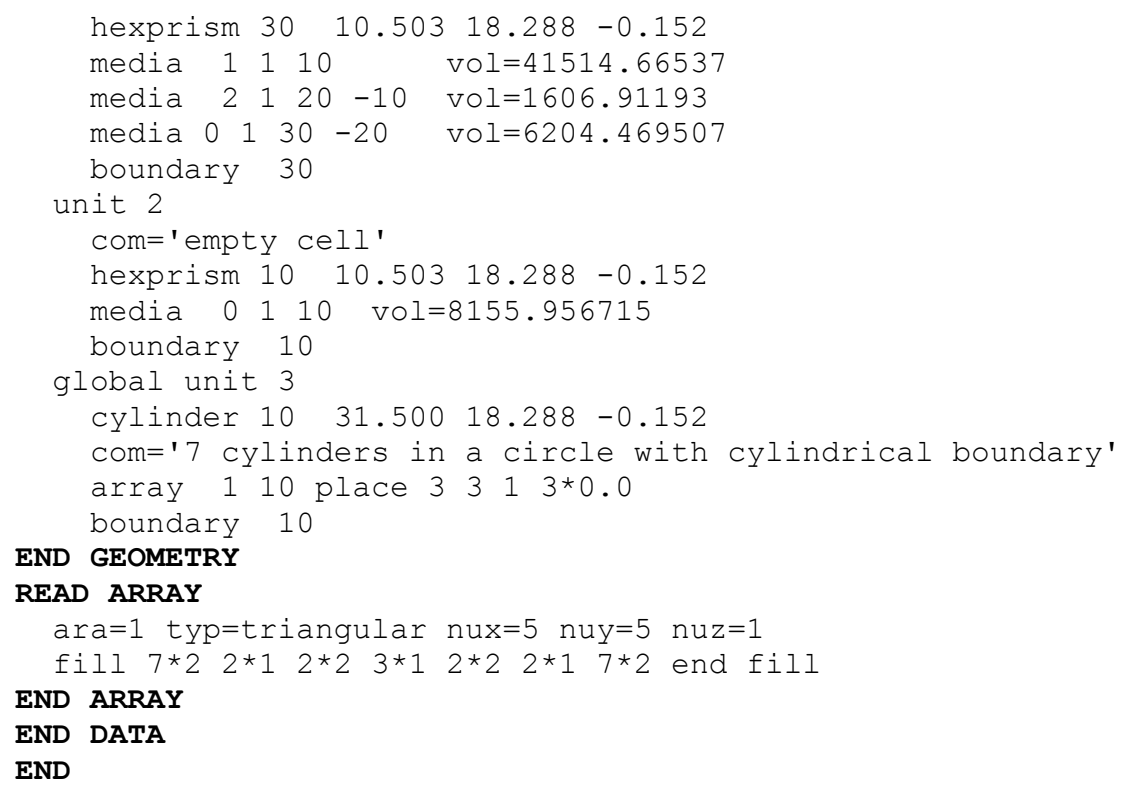

\subsection{C.2.21 Sample Problem 21 PARTIALLY FILLED SPHERE}

This critical experiment ${ }^{15}$ consisted of a partially filled, unreflected spherical container. This aluminum container had an inside diameter of $27.244 \mathrm{in}$. and a wall thickness of 1/16 in. It is referred to in the report as the 27.3-in.-diameter vessel. The sphere was $98 \%$ filled with uranyl fluoride at an enrichment of $4.89 \%$ with an $\mathrm{H} /{ }^{235} \mathrm{U}$ atomic ratio of 1099 . The height of the solution in the sphere was $64.6 \mathrm{~cm}$ above the bottom of the sphere. A schematic diagram of the apparatus used in the experiment is given in Figure 9.1.A.10. The steel tank was ignored.

\section{Input Data}

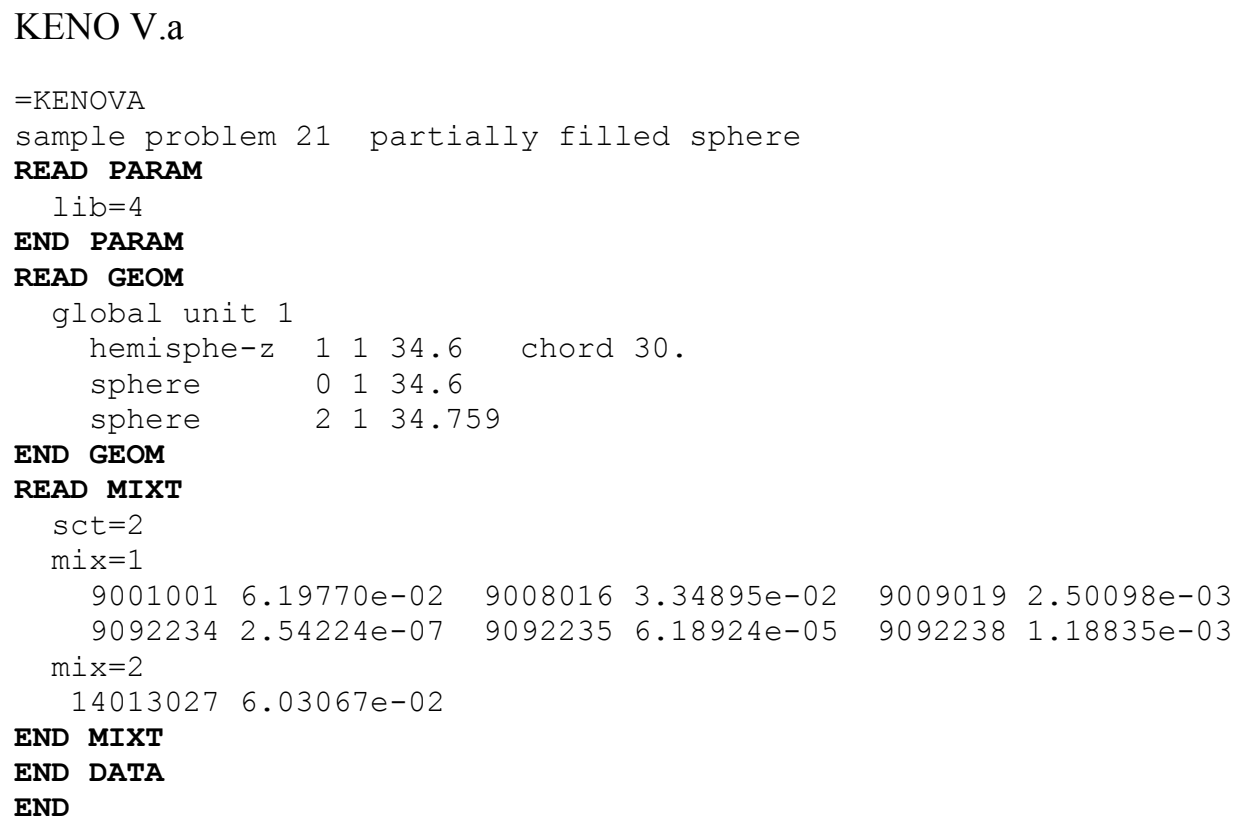



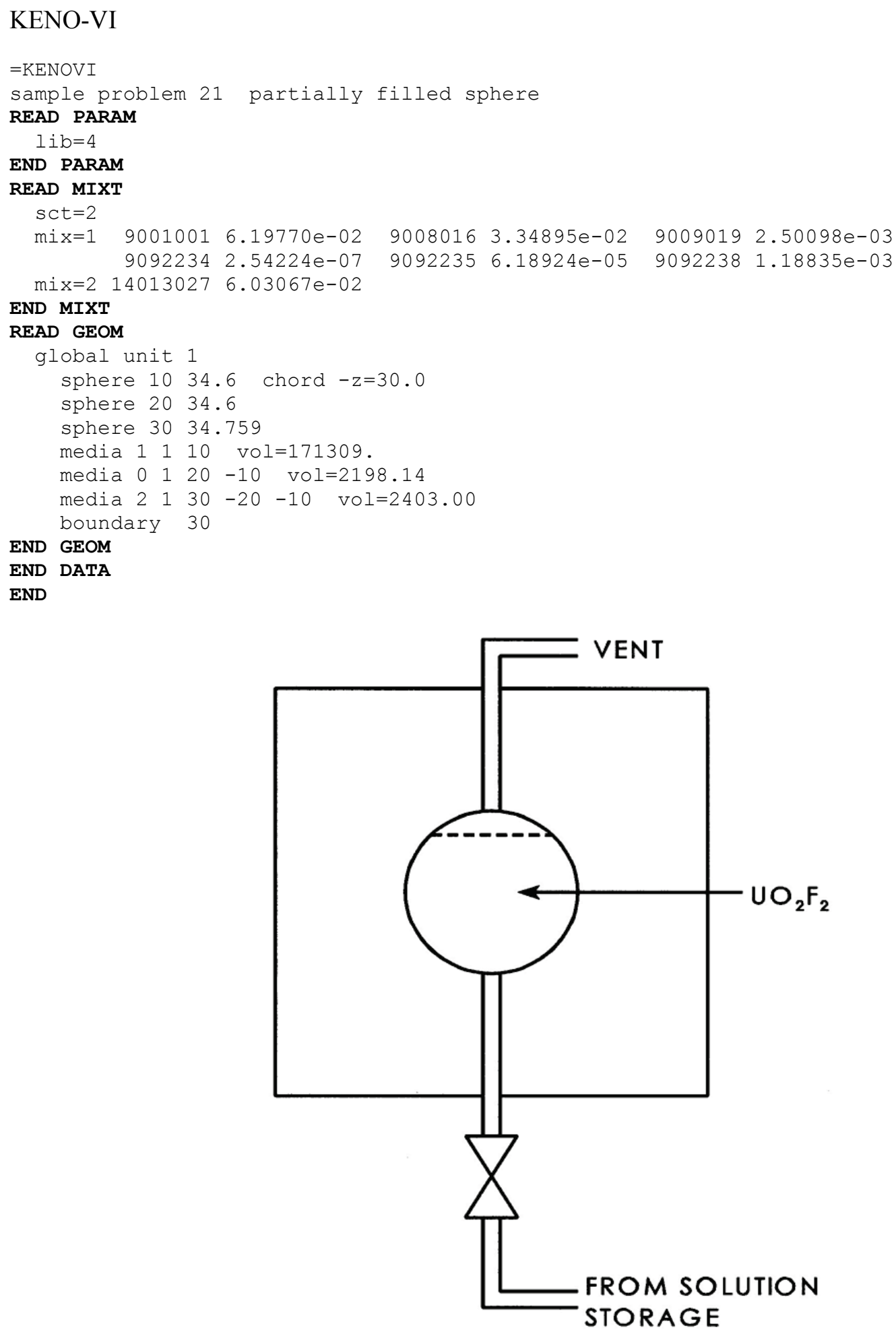

Figure 8.1.C.10. Schematic of bare partially filled sphere experiment inside a 9.5-ft-diameter, 9-ft-high steel tank. 


\subsection{C.2.22 Sample Problem 22 CASE 2C8 BARE WITH 3 NESTED HOLES, EACH IS EQUAL VOLUME}

The physical representation of this sample problem is the critical experiment described in sample problem 1. It is a simple $2 \times 2 \times 2$ array of $93.2 \% \mathrm{wt}$ enriched uranium metal cylinders. This sample problem defines a uranium cylinder in a void spacing cuboid using nested holes. Eight of these units are stacked together in a $2 \times 2 \times 2$ array.

\section{Input Data}

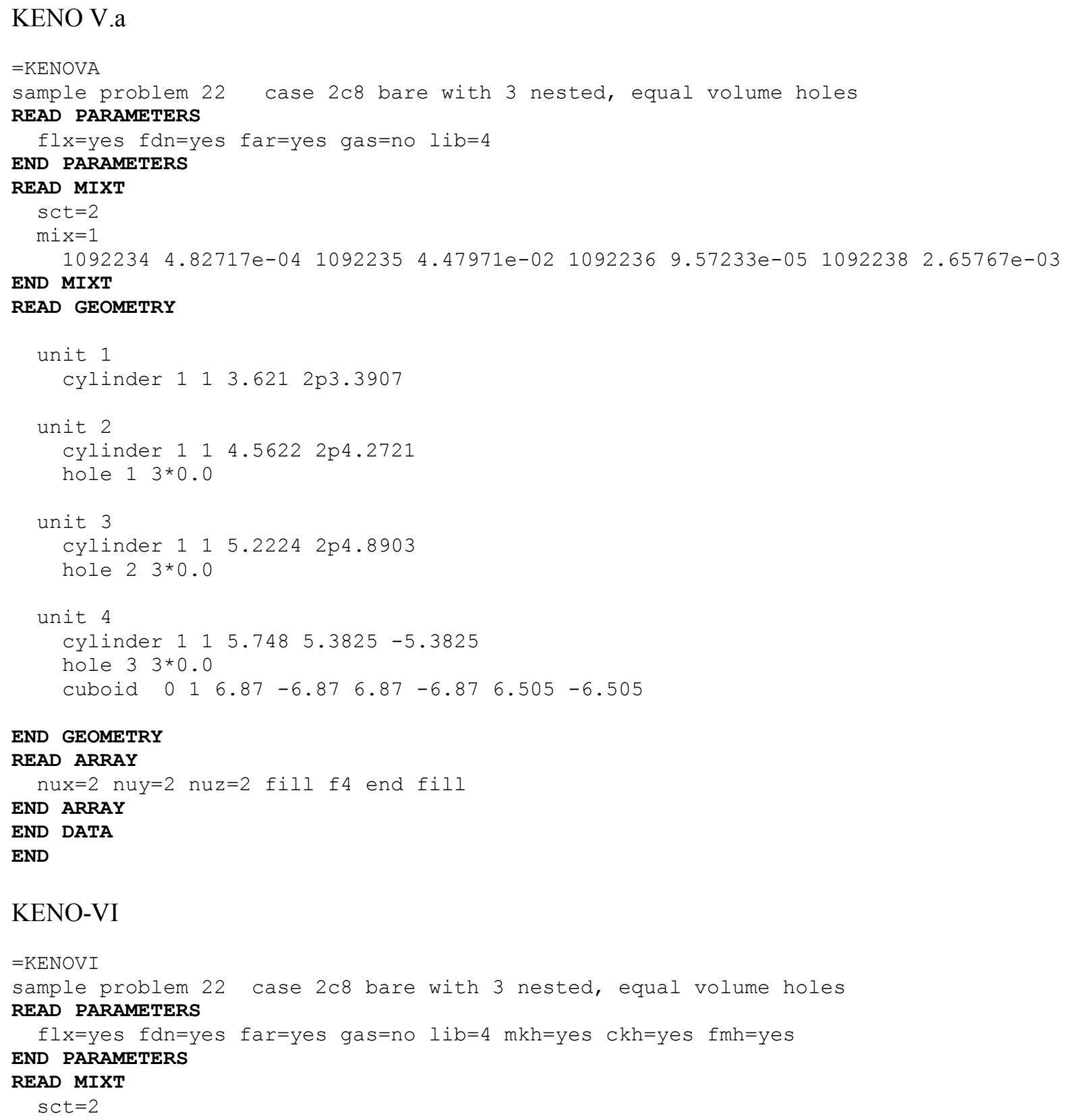




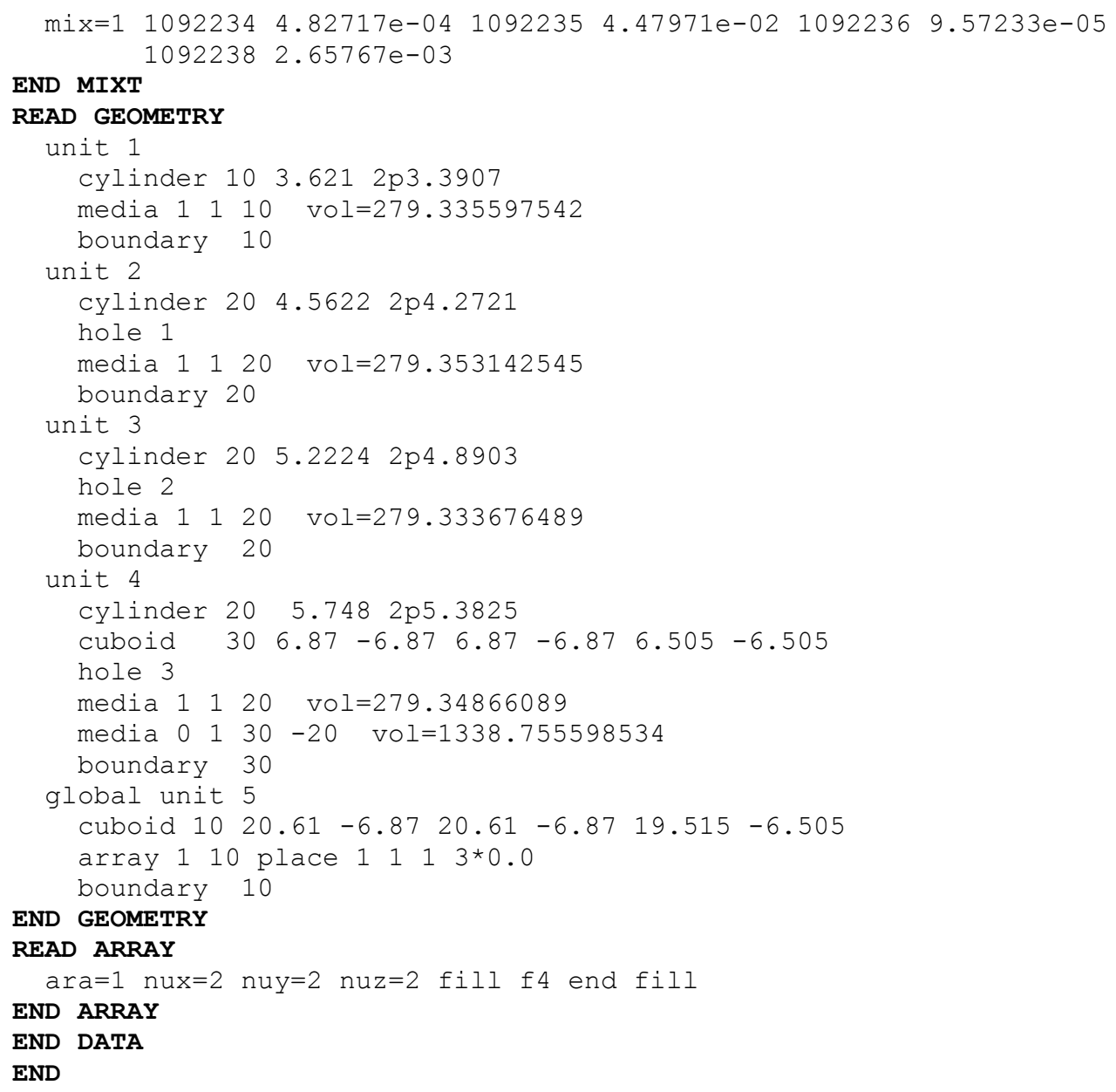

\subsection{C.2.23 Sample Problem 23 CASE 2C8 BARE AS STACKED CYLINDERS}

The physical representation of this sample problem is the critical experiment described in sample problem 1. This sample problem describes each of the eight units in the critical $2 \times 2 \times 2$ array using $Z$ hemicylinders (in KENO V.a) or hemicylinders with different chord sizes and directions (in KENO-VI).

\section{Input Data}

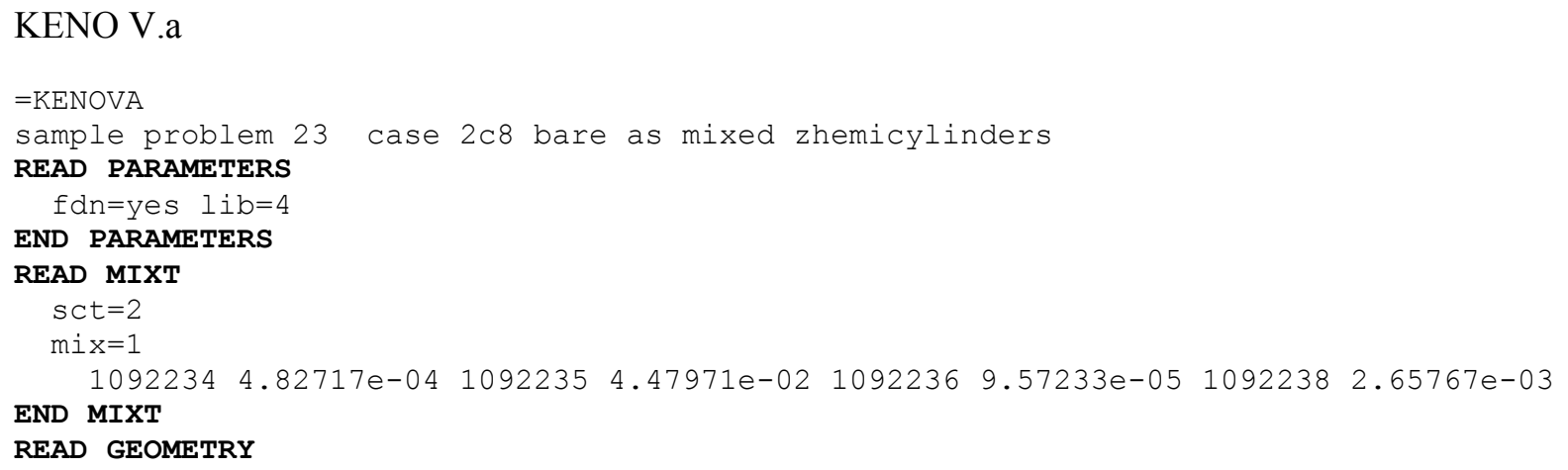




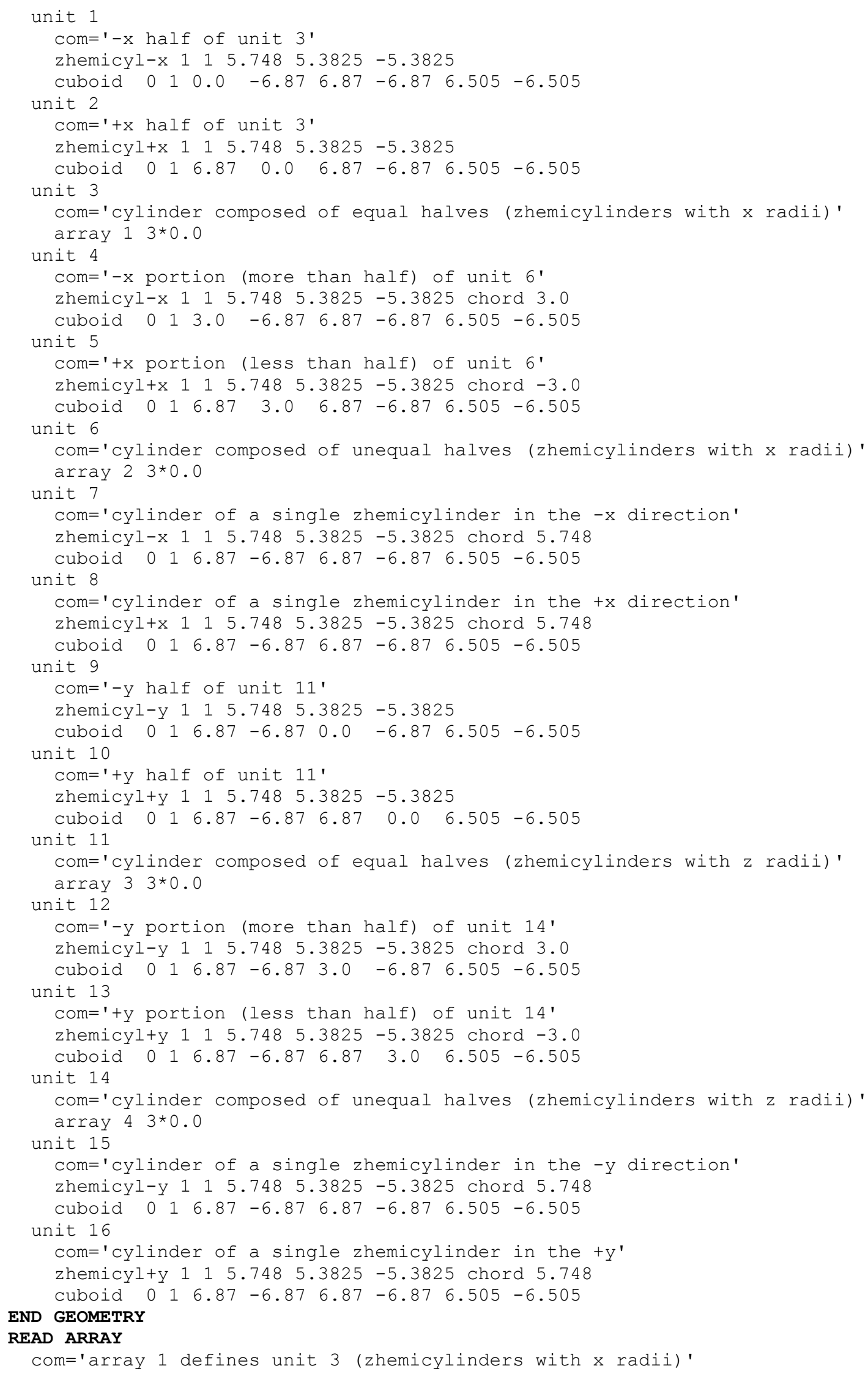




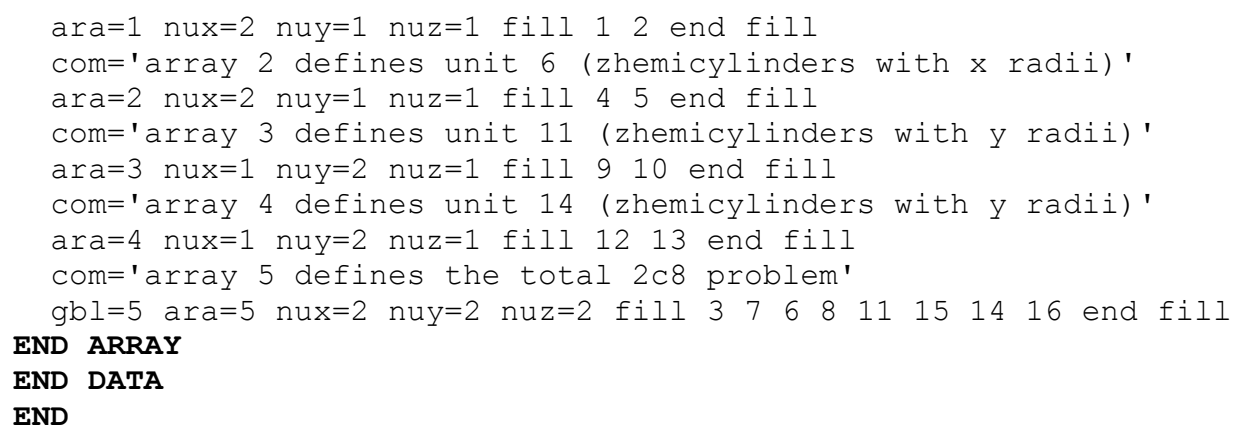

\section{KENO-VI}

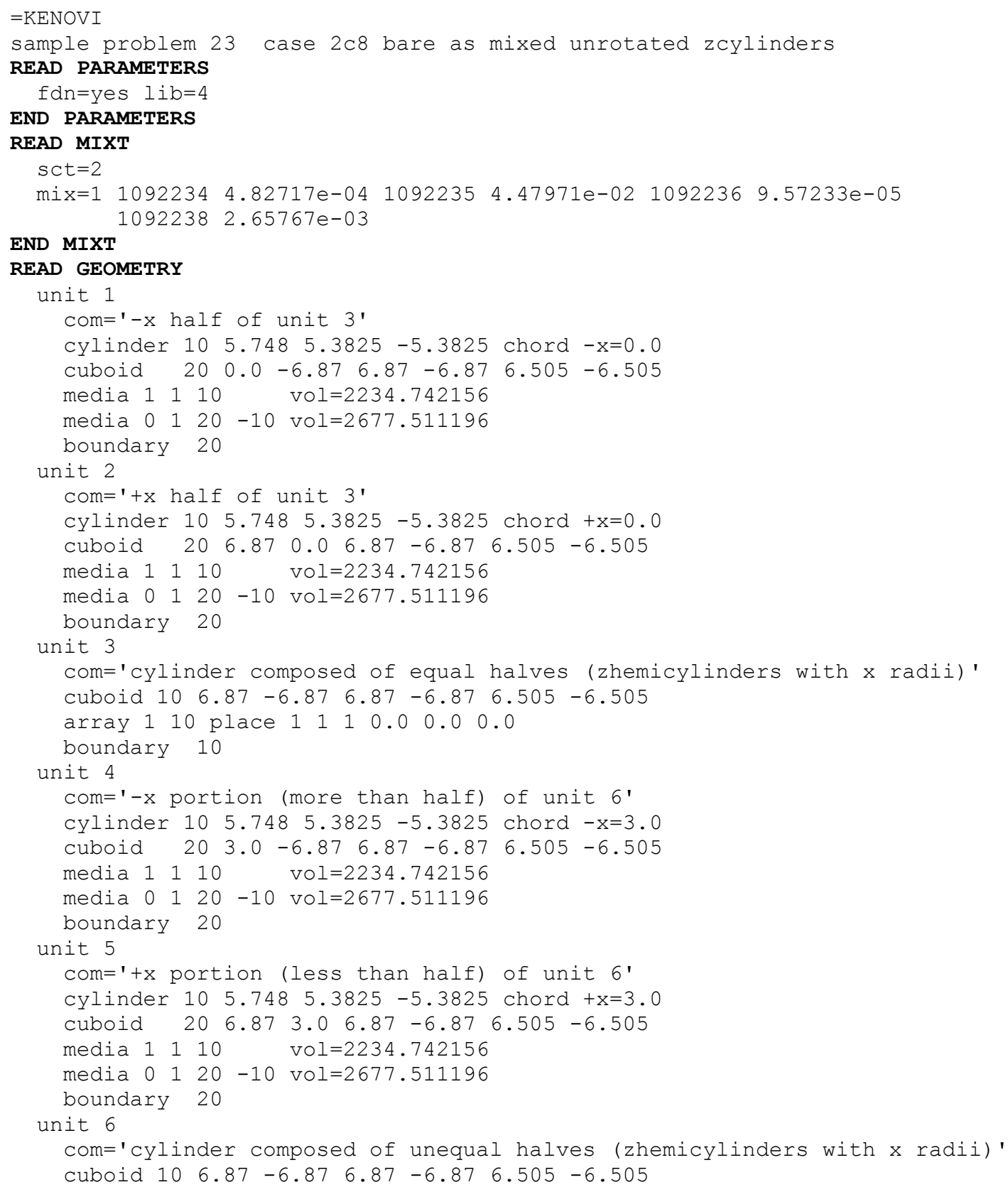




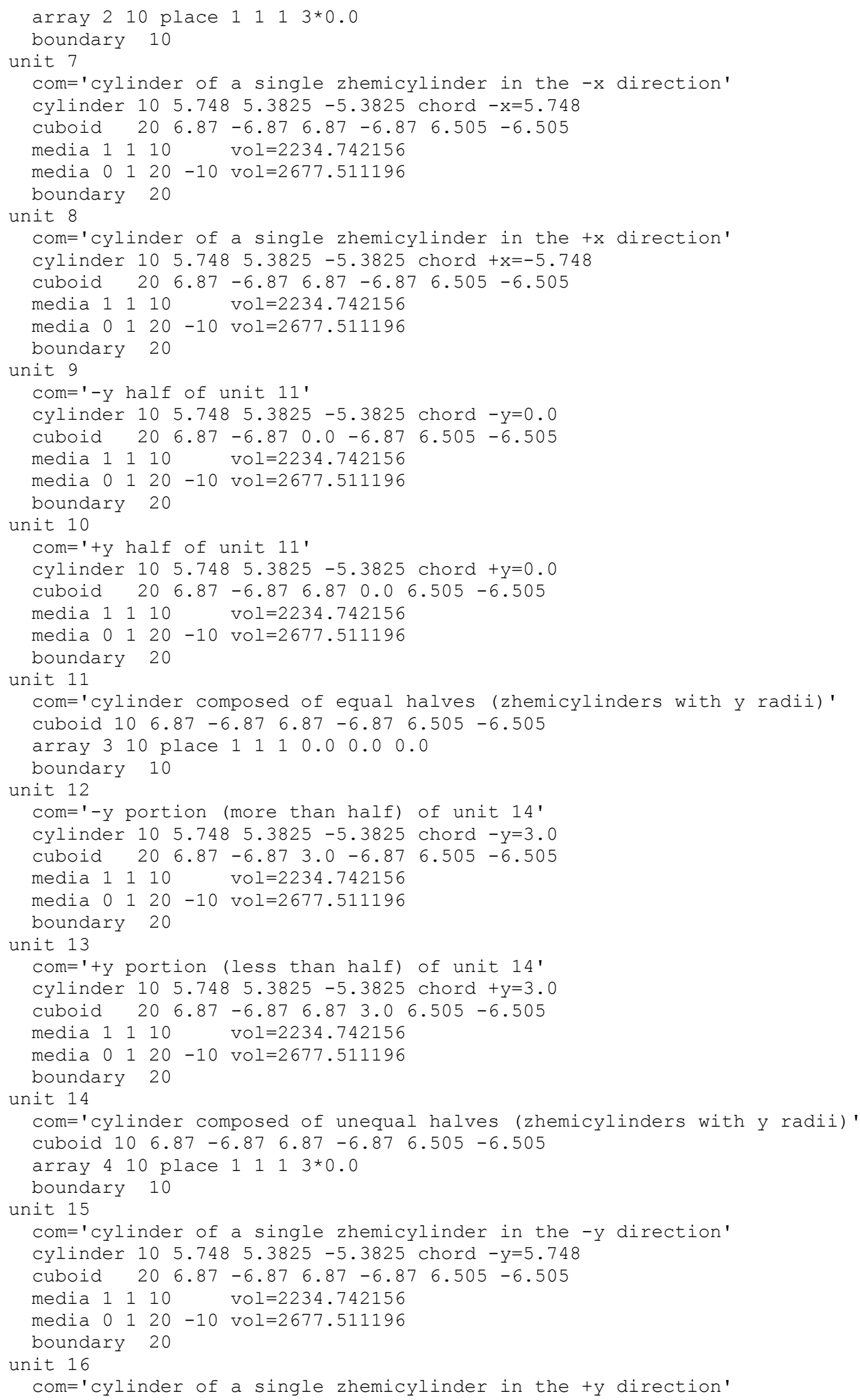




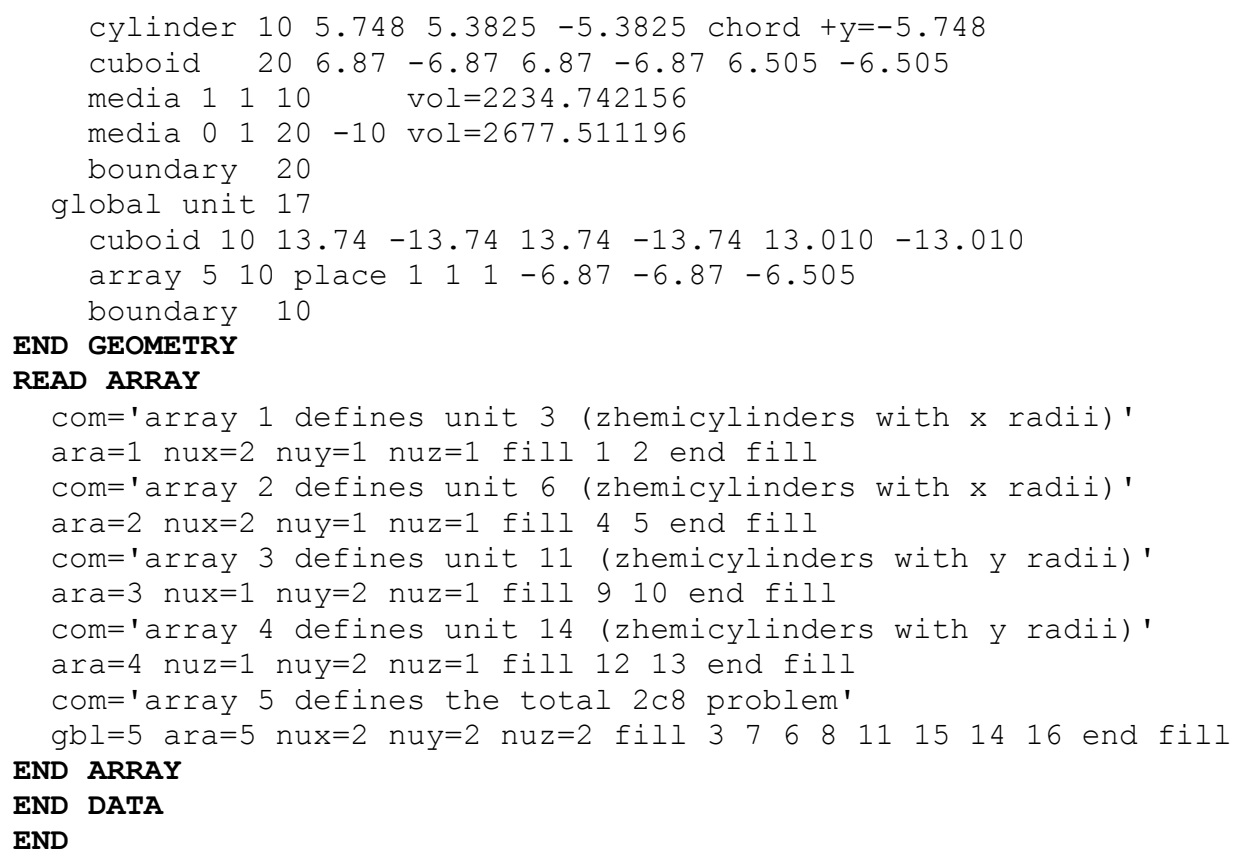

\subsection{C.2.24 Sample Problem 24 CASE 2C8 BARE AS STACKED ROTATED CYLINDERS}

The physical representation of this sample problem is the critical experiment described in sample problem 1. This sample problem describes each of the eight units in the critical $2 \times 2 \times 2$ array using hemicylinders whose axes are in the $\mathrm{x}$ direction. In KENO V.a this is realized using xhemicylinders, while in KENO-VI the hemycylinders with different chord sizes are rotated in the X-direction.

\section{Input Data}

\section{KENO V.a}

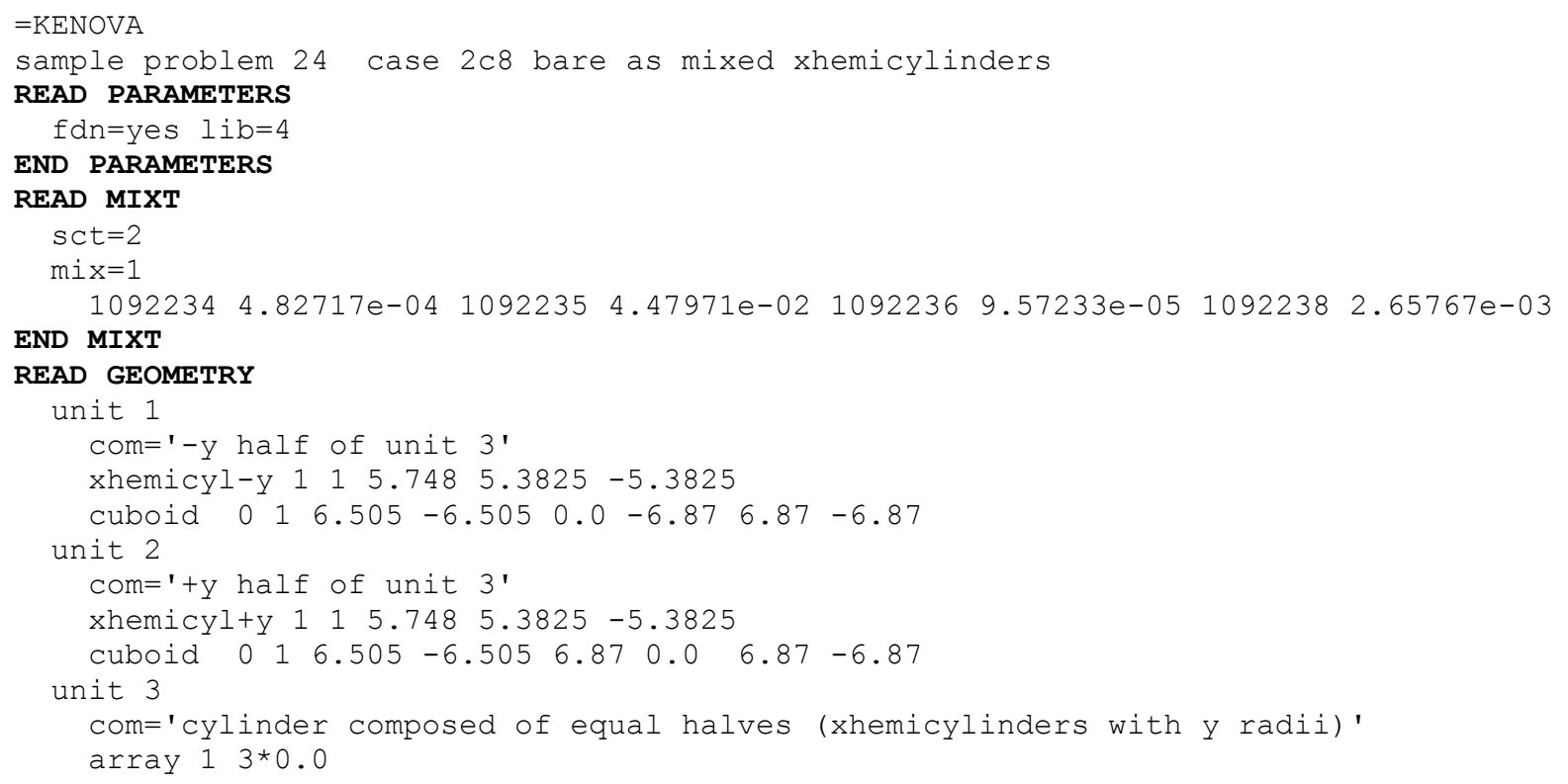




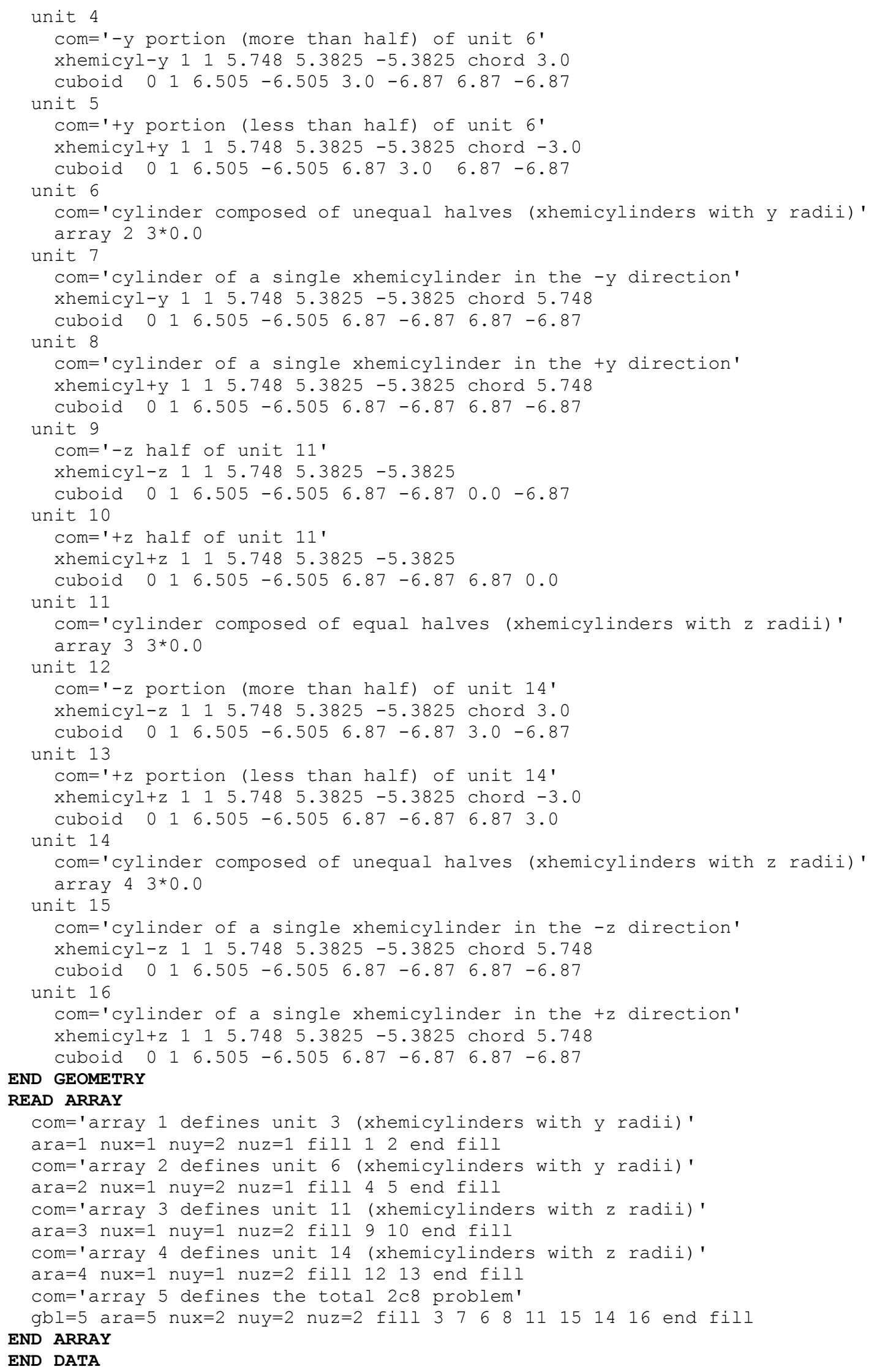




\section{KENO-VI}

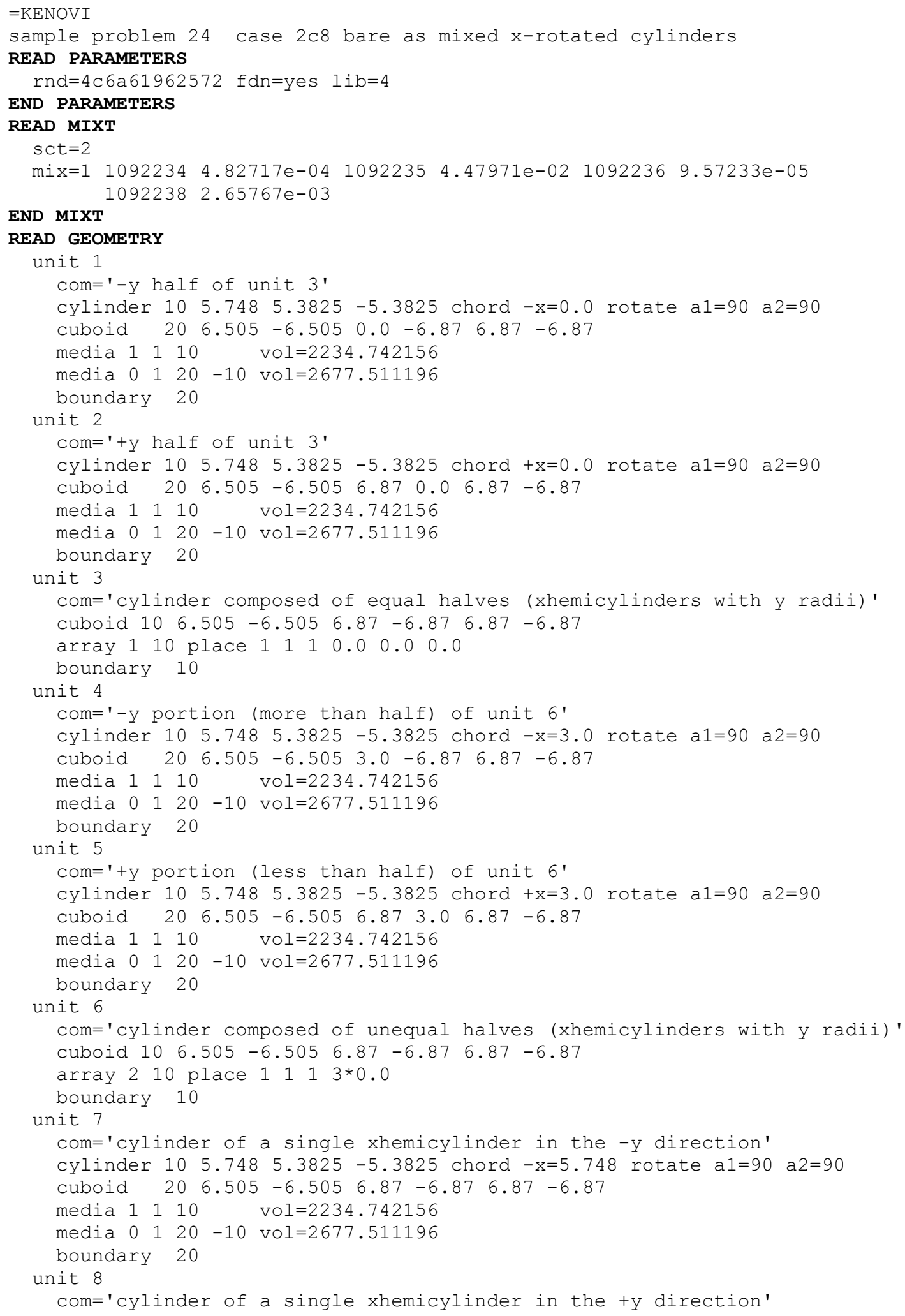




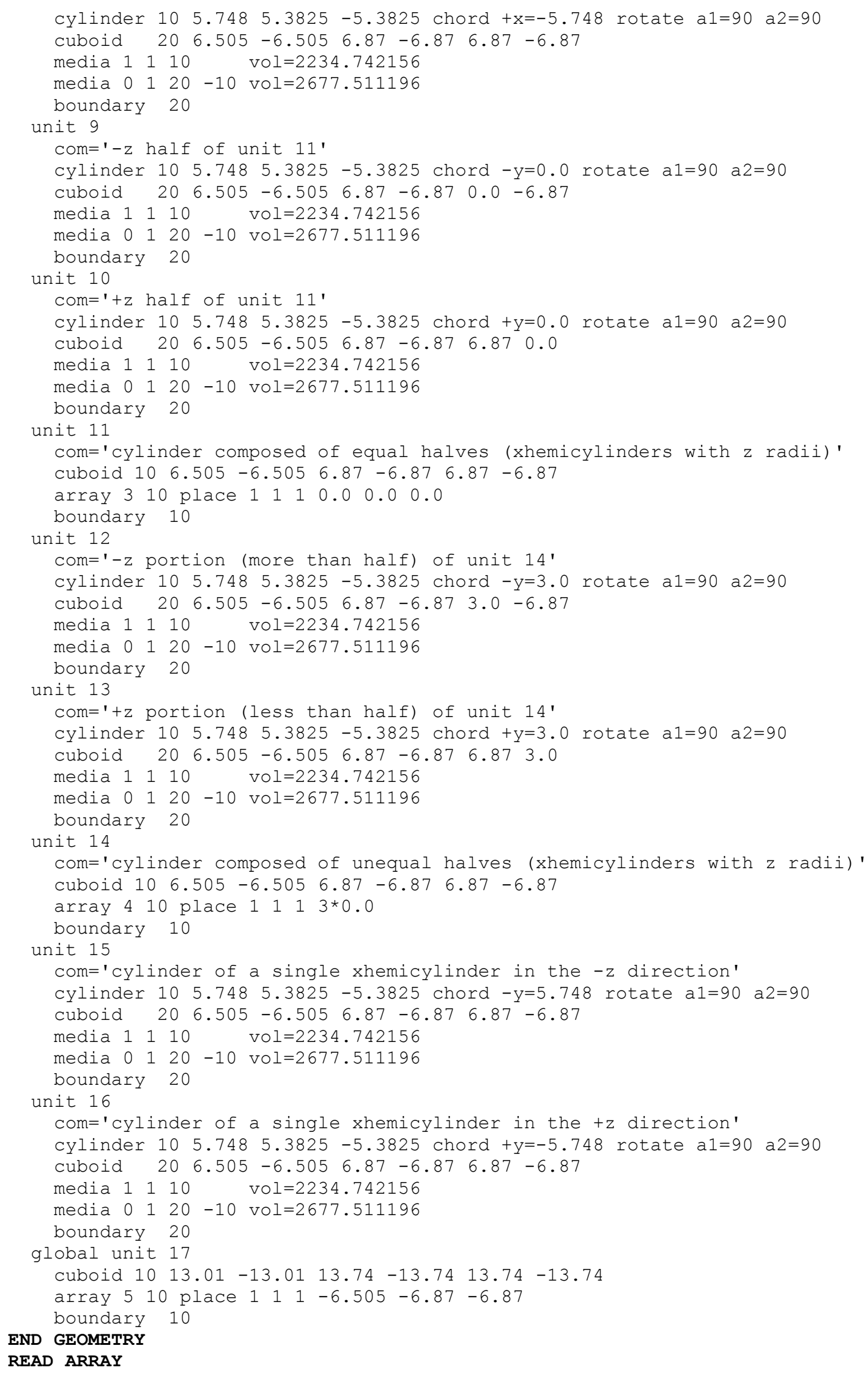




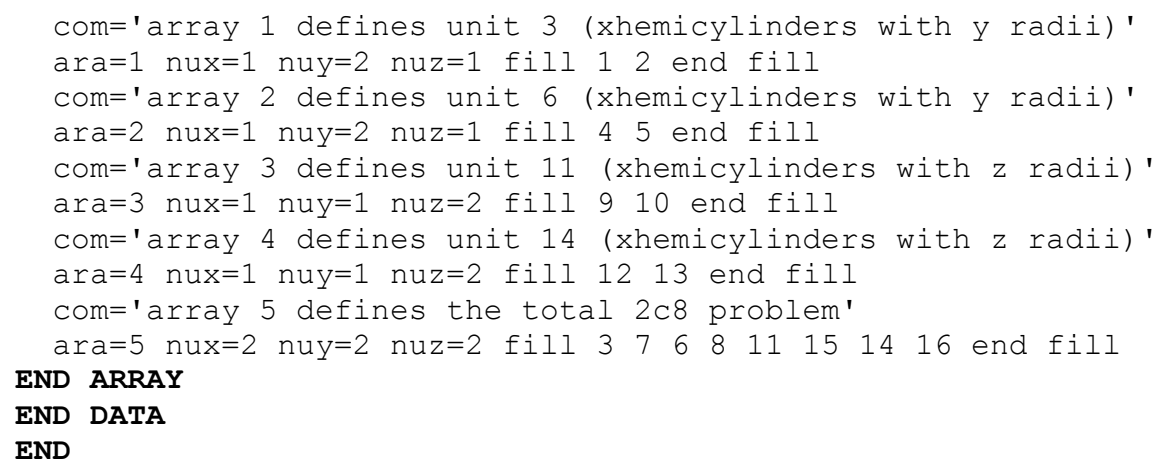

\subsection{C.2.25 Sample Problem 25 CASE 2C8 BARE AS MIXED YHEMICYLINDERS}

The physical representation of this sample problem is the critical experiment described in sample problem 1. This sample problem describes each of the eight units in the critical $2 \times 2 \times 2$ array using hemicylinders whose axes are in the y direction. This is realized in KENO V.a by using yhemicylinders, while in KENO-VI it is realized using hemicylinders with different chord sizes and directions whose long axes are rotated in the Y-direction.

\section{Input Data}

\section{KENO V.a}

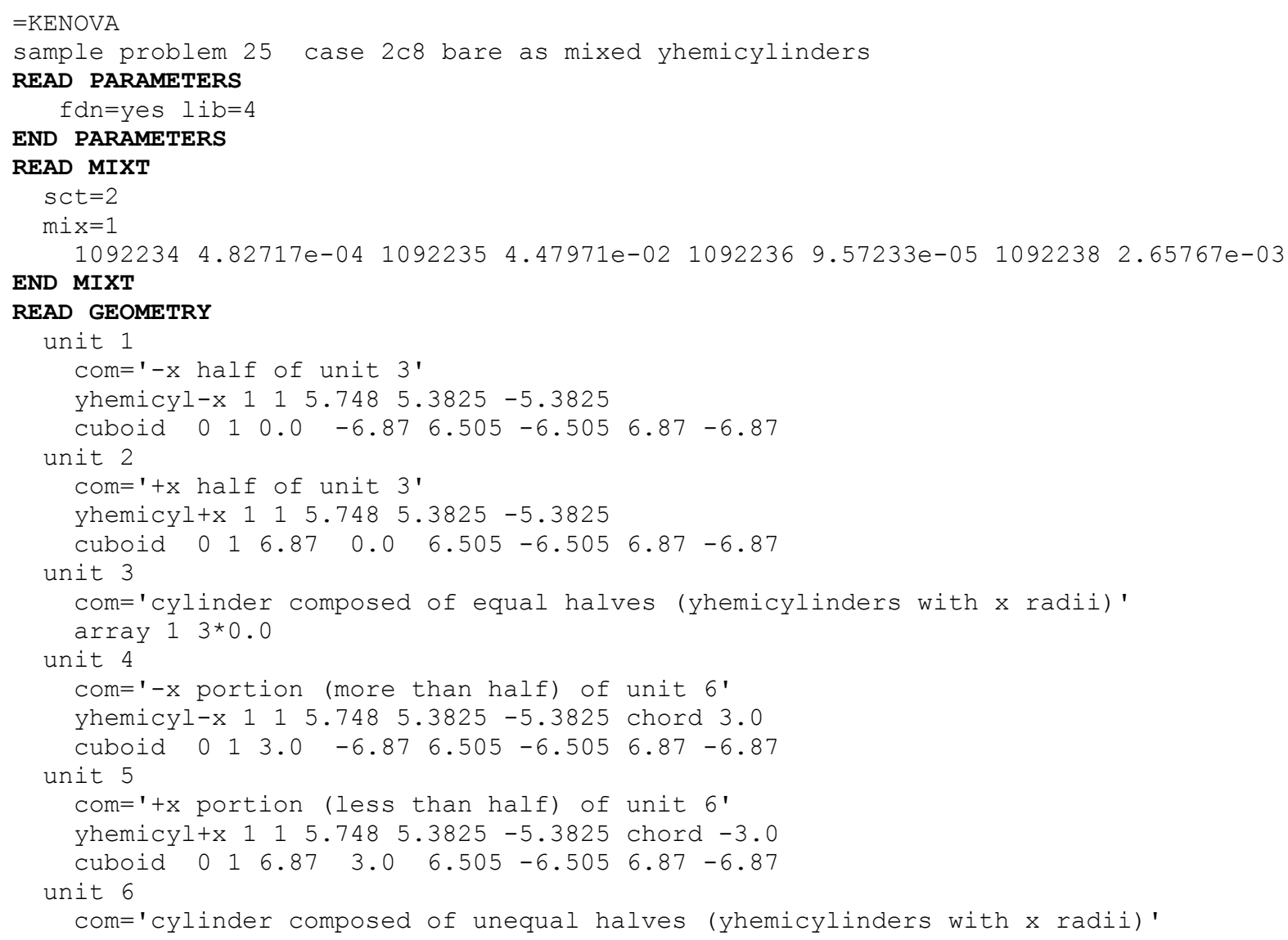




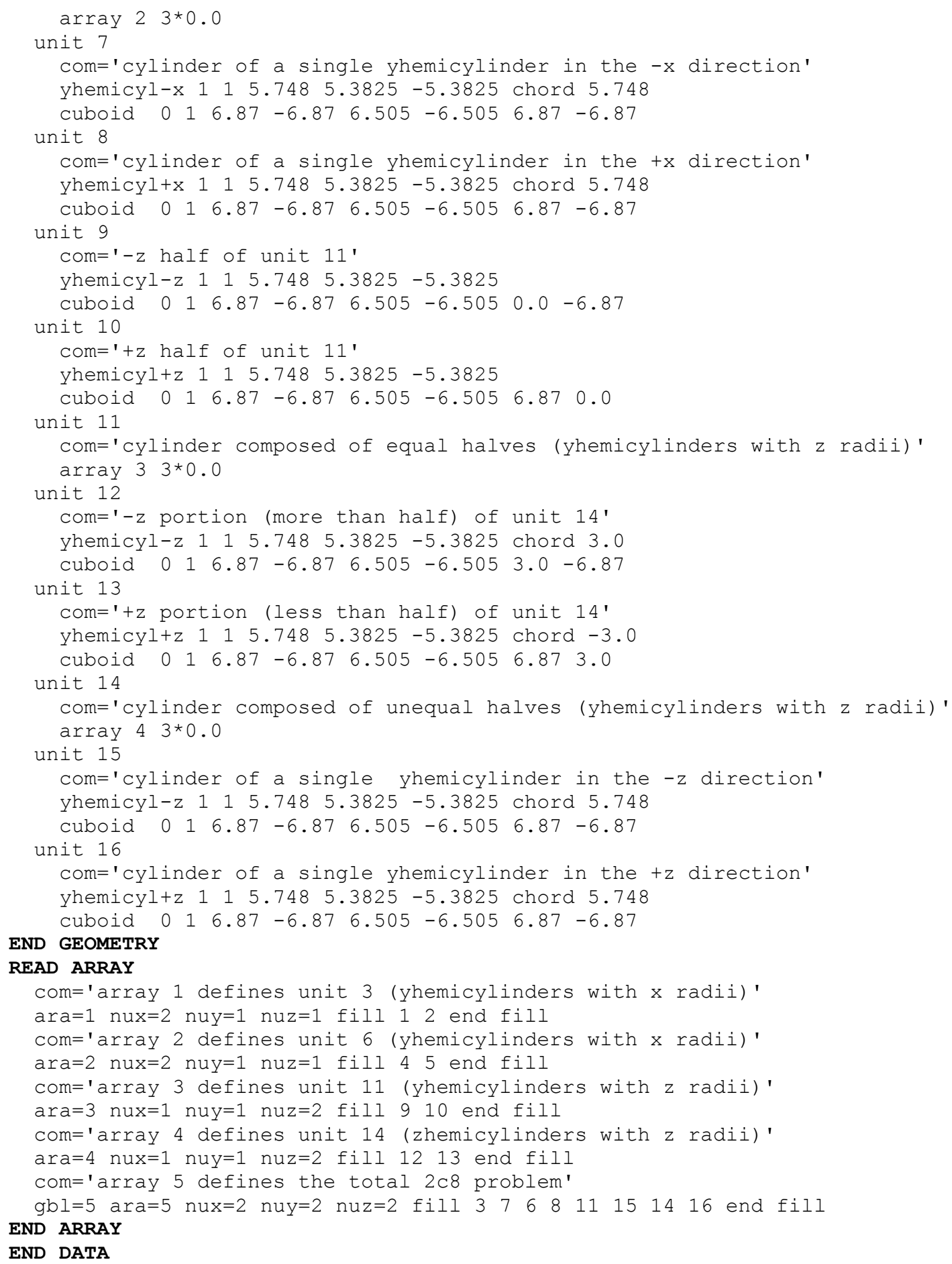

\section{KENO-VI}

$=\mathrm{KENOVI}$

sample problem 25 case 2c8 bare as mixed y-rotated cylinders

READ PARAMETERS

fdn=yes $l i b=4$

END PARAMETERS 


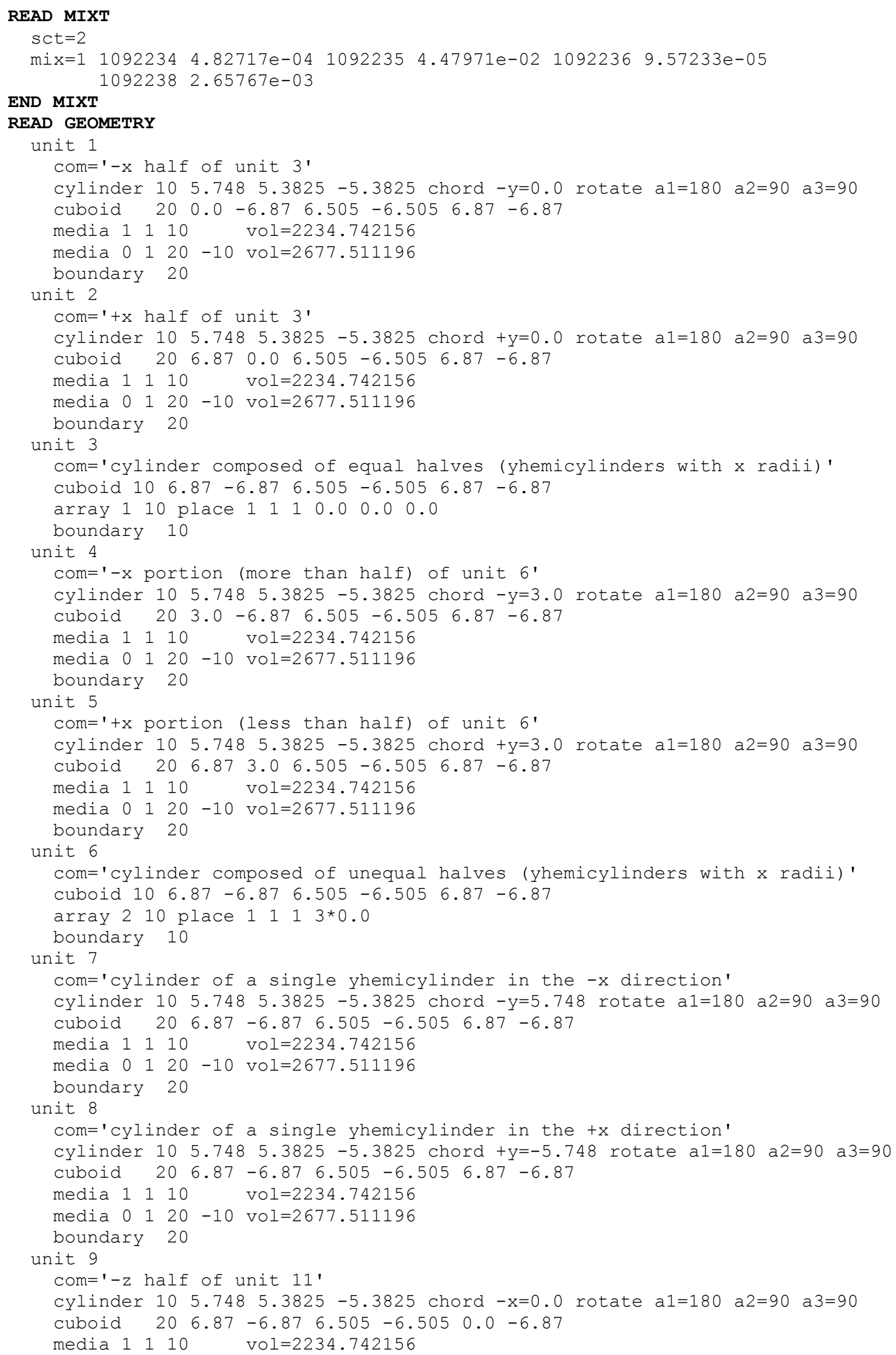




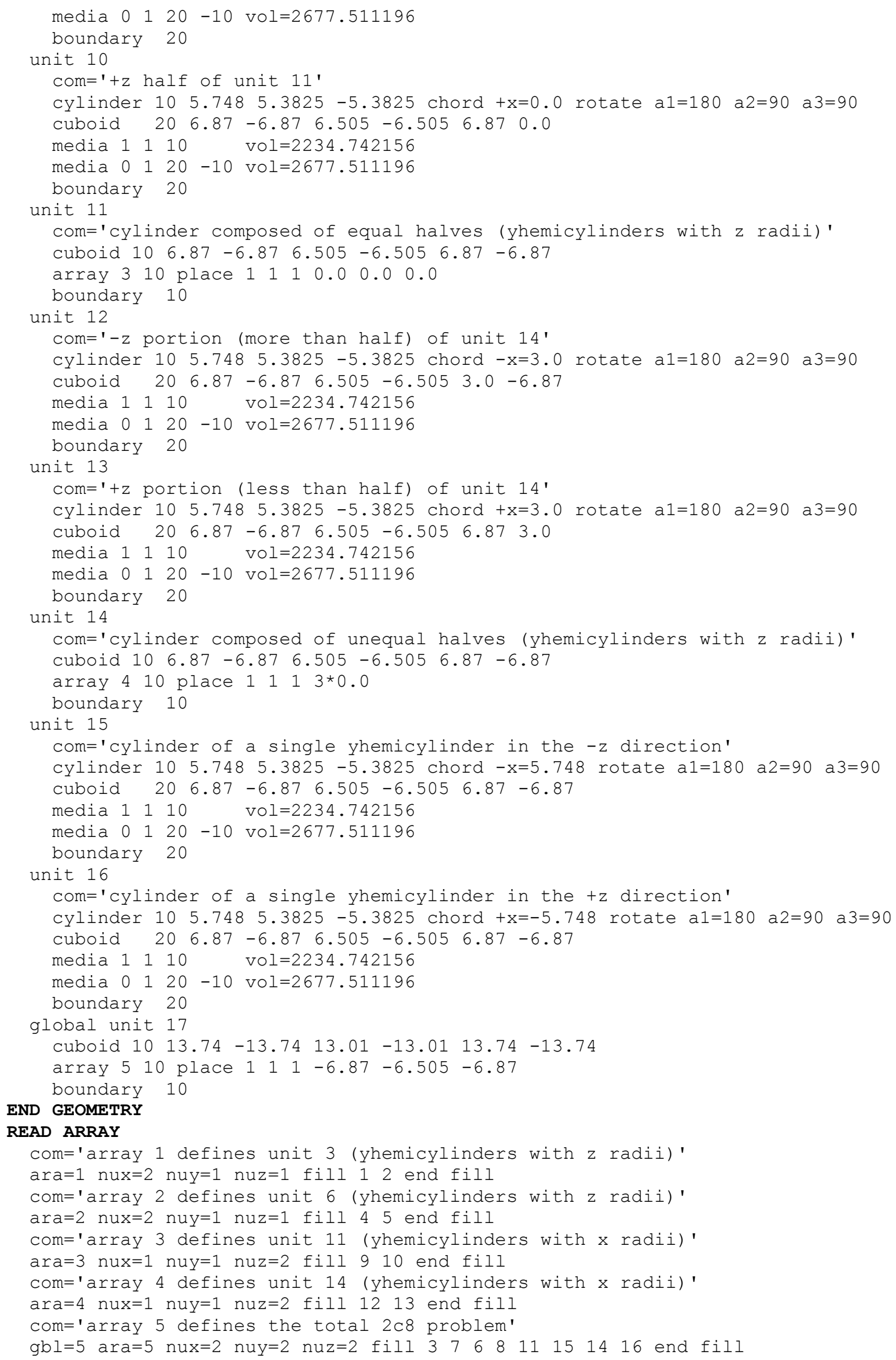




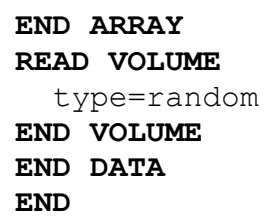

\subsection{C.2.26 Sample Problem 26 (KENO V.a ONLY) CASE 2C8 BARE AS MIXED ZHEMICYLINDERS WITH ORIGINS}

The physical representation of this sample problem is the critical experiment described in sample problem 1. This sample problem describes each of the eight units in the critical $2 \times 2 \times 2$ array using zhemicylinders with origins.

\section{Input Data}

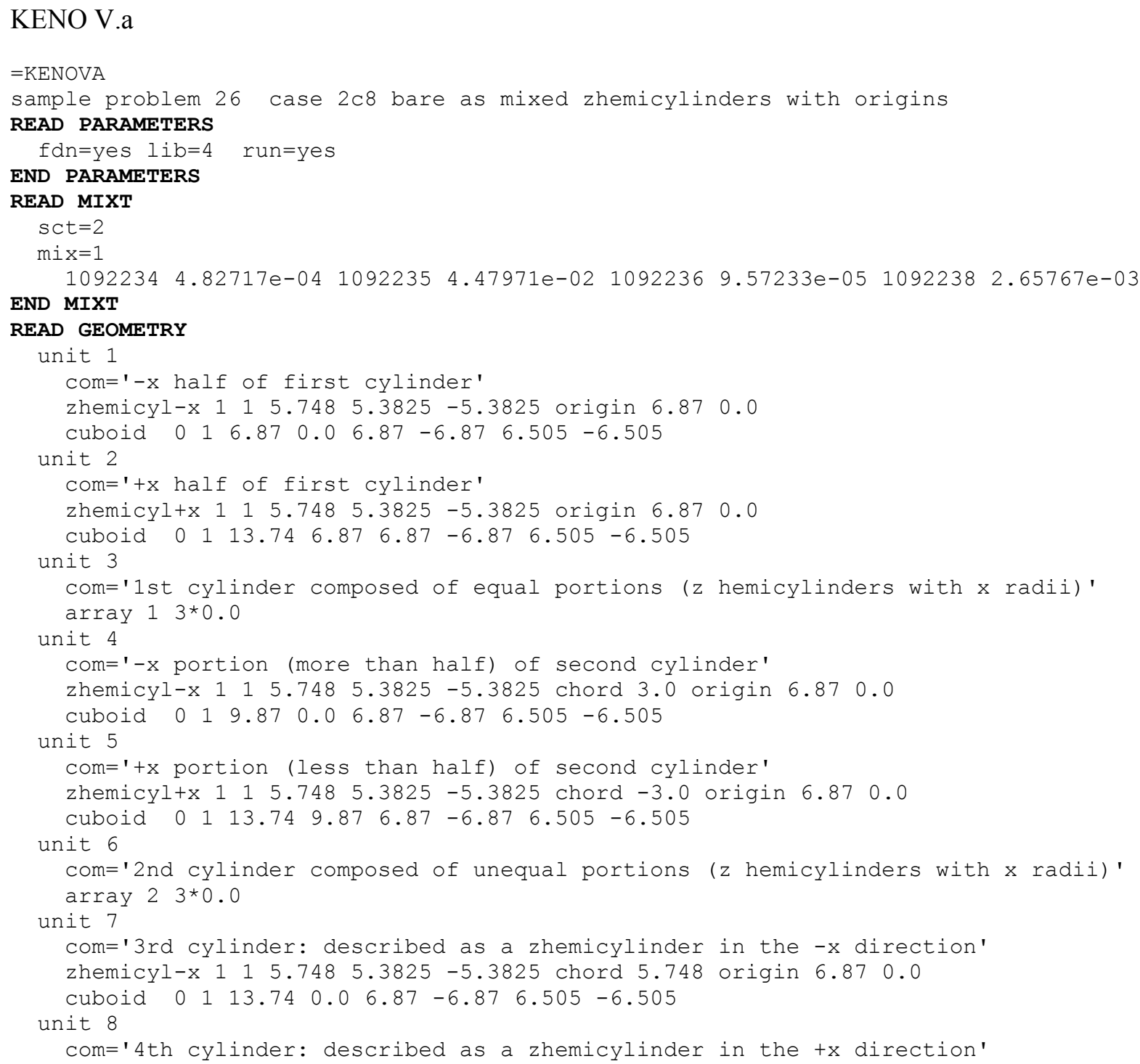




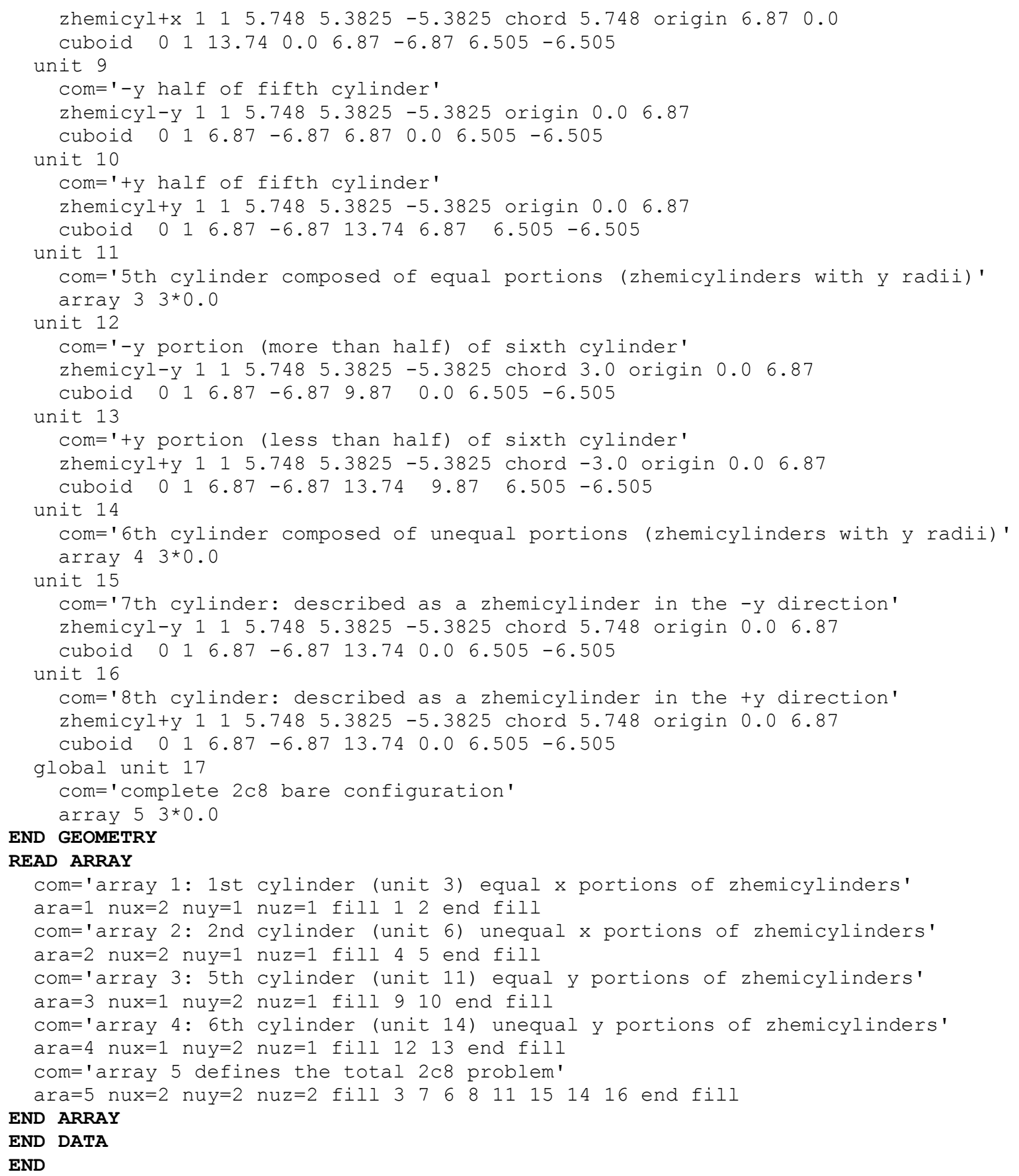

\subsection{C.2.27 Sample Problem 27 (KENO V.a oONLY) CASE 2C8 BARE AS MIXED XHEMICYLINDERS WITH ORIGINS}

The physical representation of this sample problem is the critical experiment described in sample problem 1. This sample problem describes each of the eight units in the critical $2 \times 2 \times 2$ array using hemicylinders whose axes are in the $\mathrm{x}$ direction. Origins are specified for each hemicylinder. 


\section{Input Data}

\section{KENO V.a}

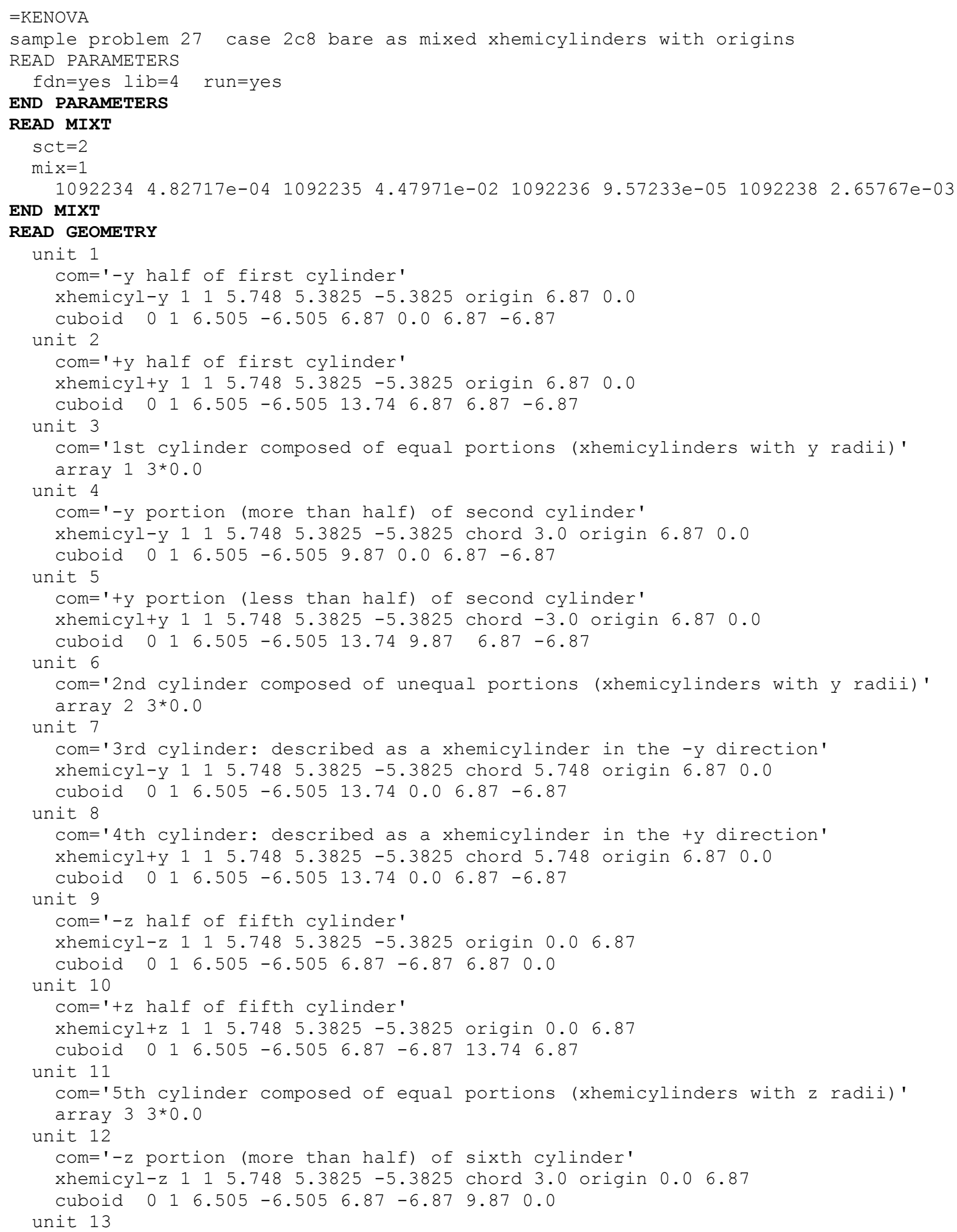




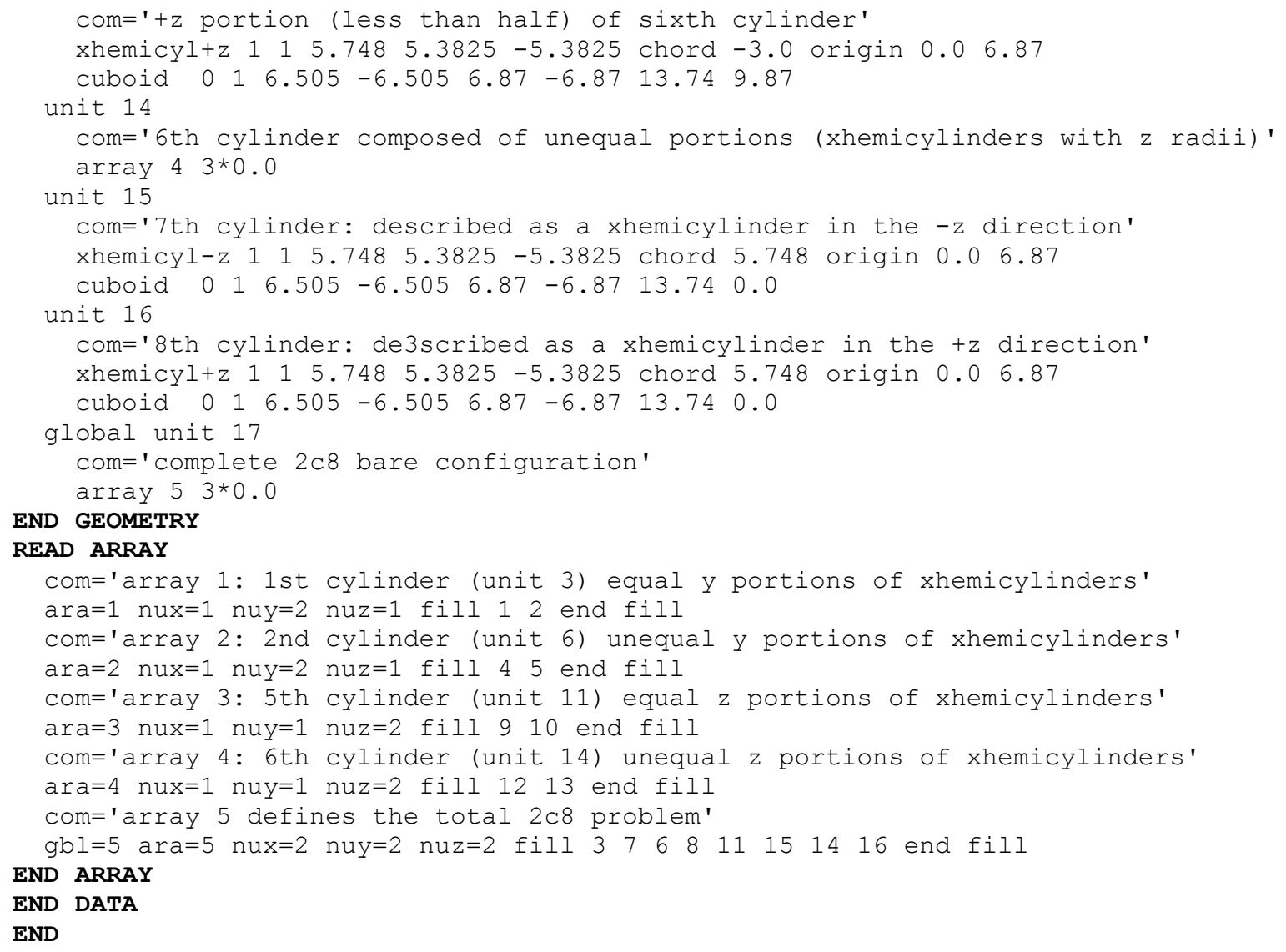




\subsection{C.2.28 Sample Problem 28 (KENO V.a oONLY) CASE 2C8 BARE AS MIXED YHEMICYLINDERS WITH ORIGINS}

The physical representation of this sample problem is the critical experiment described in sample problem 1. This sample problem describes each of the eight units in the critical $2 \times 2 \times 2$ array using hemicylinders whose axes are in the y direction. Origins are specified for each hemicylinder.

\section{Input Data}

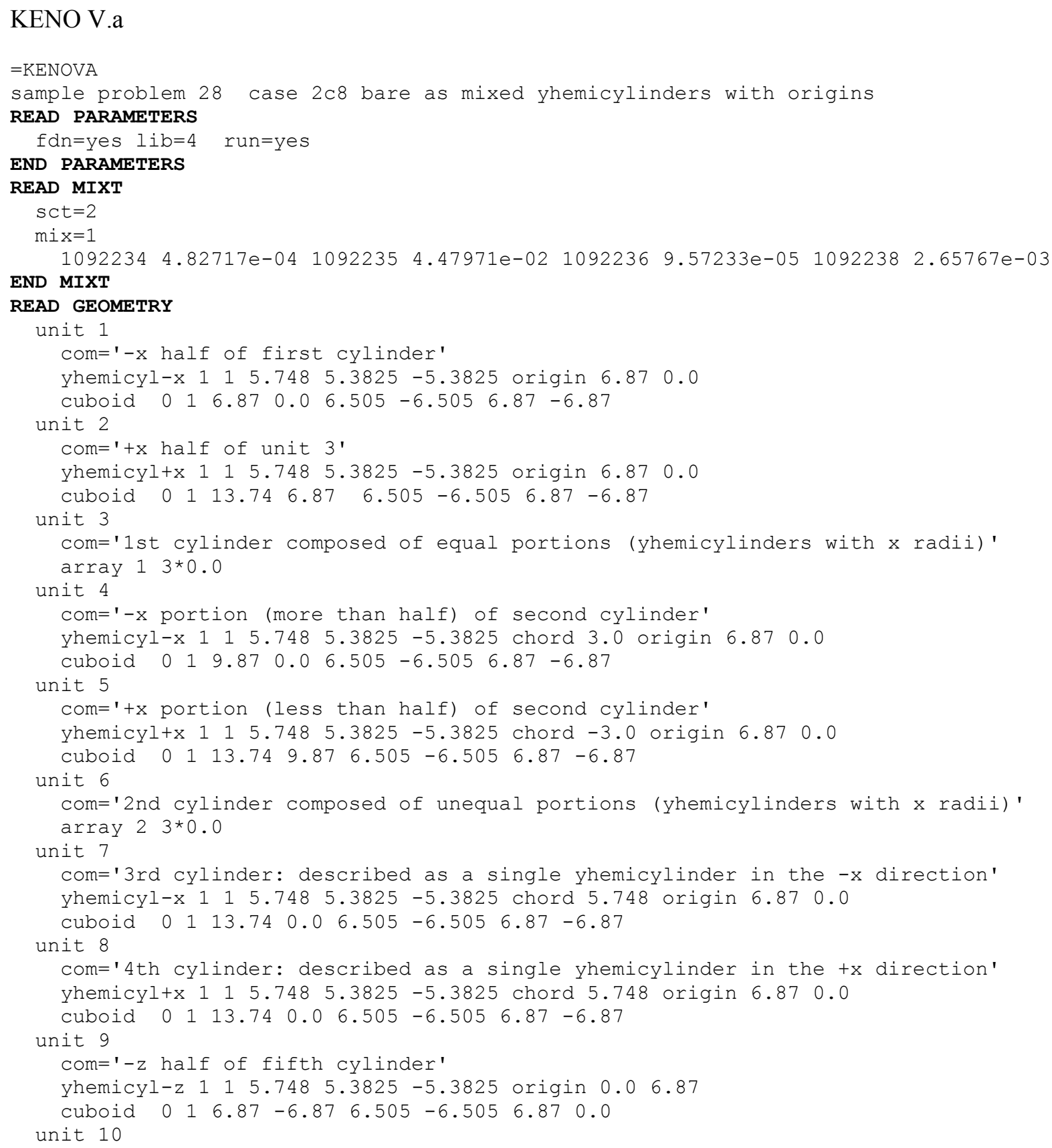




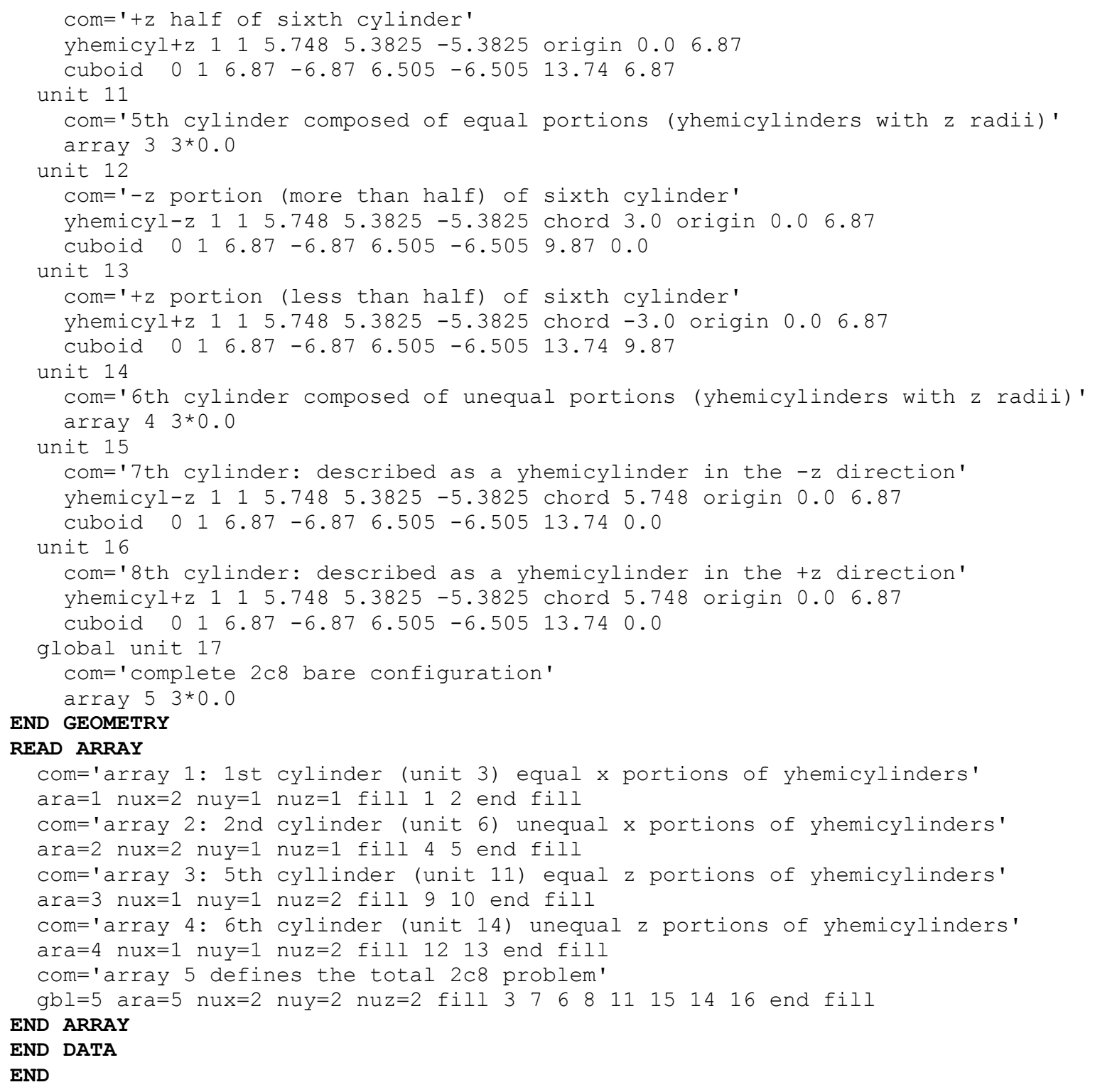

\subsection{C.2.29 Sample Problem 29 BARE CRITICAL SPHERE 3.4420-IN. RADIUS}

This problem is a critical experiment ${ }^{6}$ consisting of a critical Oralloy sphere. The density of the Oralloy is $18.747 \mathrm{~g} / \mathrm{cc}$, and the isotopic enrichment (wt \%) is $93.21 \%{ }^{235} \mathrm{U}, 5.7697 \%{ }^{238} \mathrm{U}, 0.9844 \%{ }^{234} \mathrm{U}$, and $0.0359 \%{ }^{236} \mathrm{U}$. The critical radius was $8.74268 \mathrm{~cm}$. A photograph of the experiment is given in Figure 8.1.C.11. The support structure was ignored in the input data. 


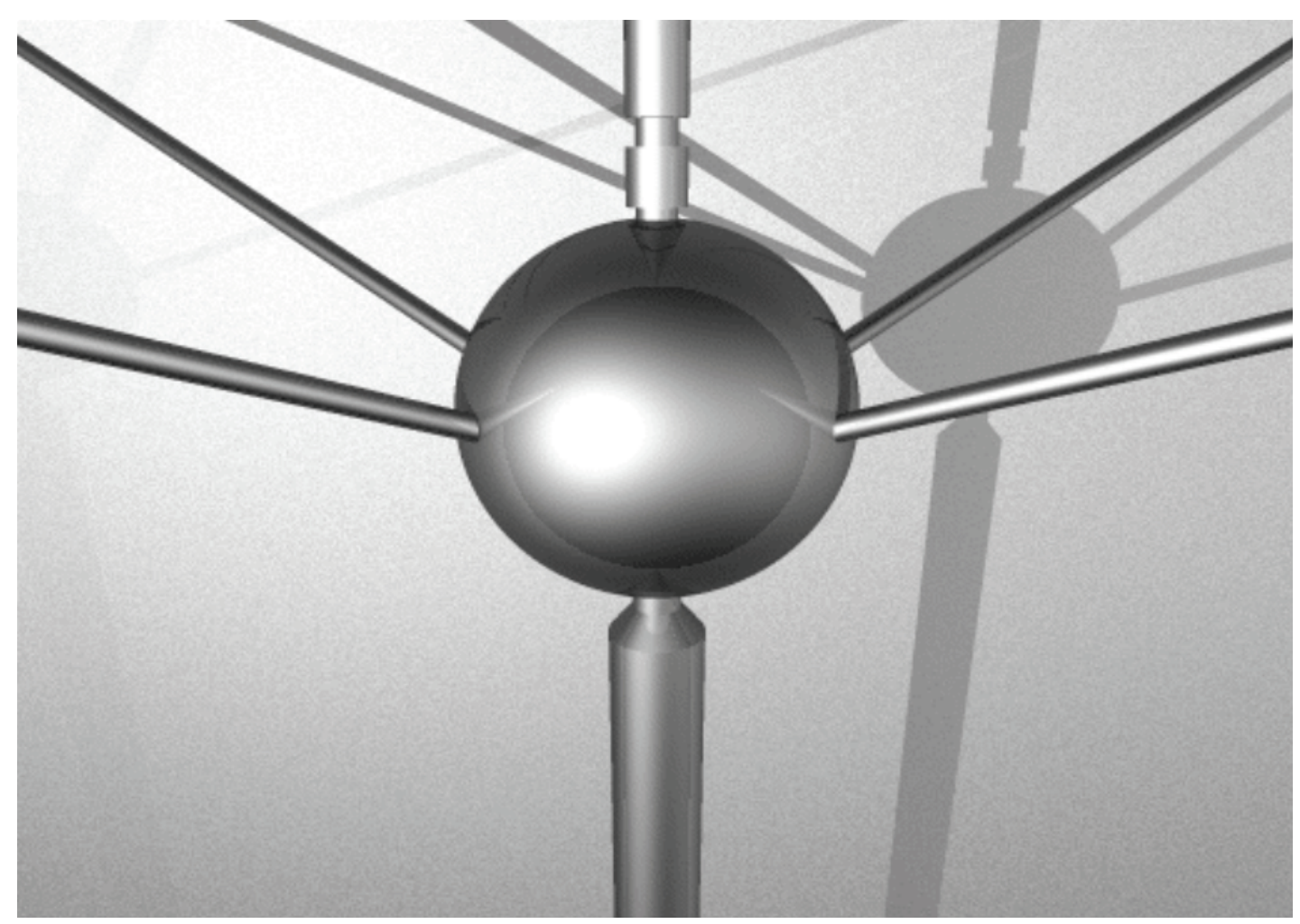

Figure 8.1.C.11. Critical Oralloy sphere.

\section{Input Data}

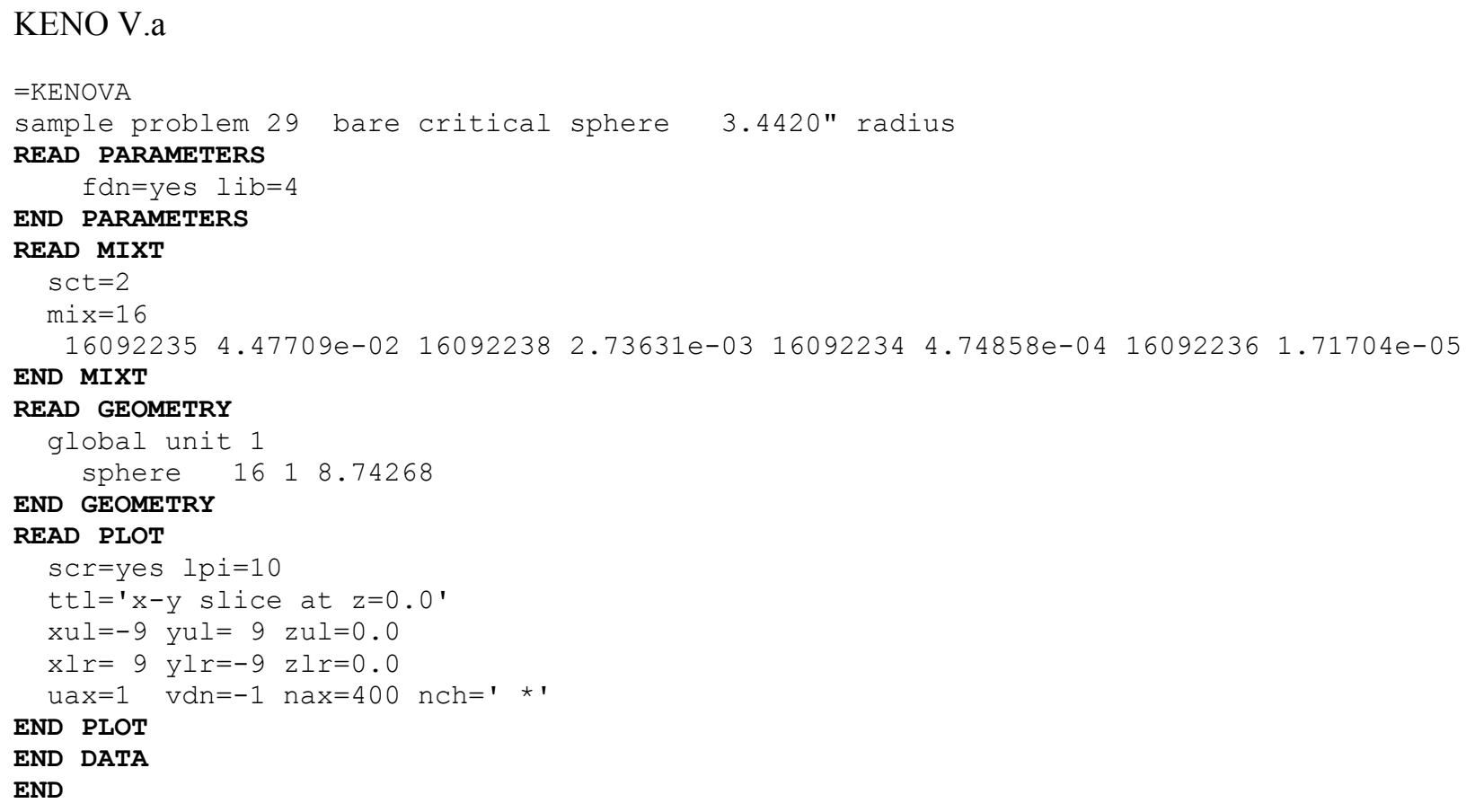




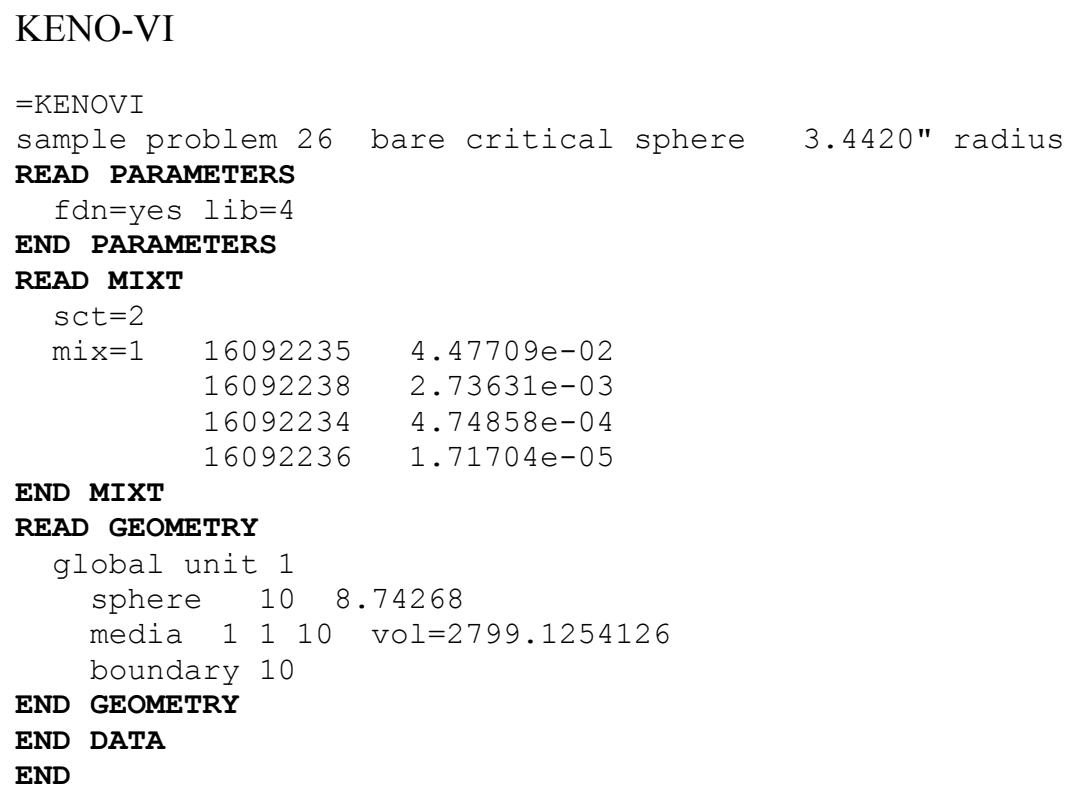

\subsection{C.2.30 Sample Problem 30 (KENO V.a ONLY) BARE CRITICAL SPHERE Z HEMISPHERE MODEL 3.4420-IN. RADIUS}

The physical representation of this sample problem is the critical experiment described in sample problem 29. This sample problem describes the sphere as two $\mathrm{Z}$ hemispheres, each with a chord and origin specified. One of the hemispheres is placed using the hole geometry option.

\section{Input Data}

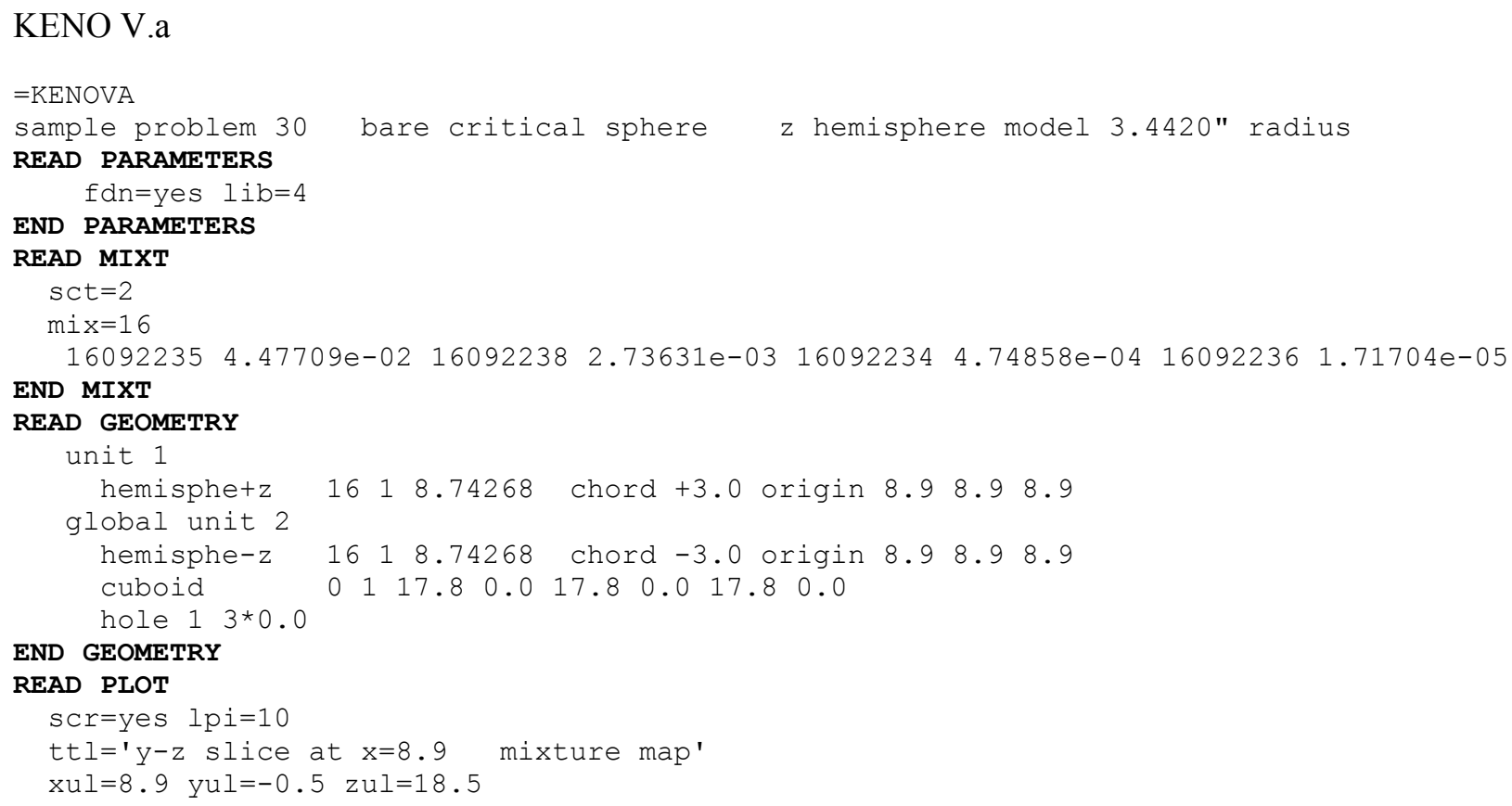


$\mathrm{xlr}=8.9$ ylr=18.5 $\mathrm{zlr}=-0.5$

$\operatorname{vax}=1 \quad \mathrm{wdn}=-1 \quad \operatorname{nax}=400$ end plt 1

tt $l=' y-z$ slice at $x=8.9$ unit map'

pic=box end plt2

END PLOT

END DATA

END 


\subsection{C.2.31 Sample Problem 31 (KENO V.a ONLY) BARE CRITICAL SPHERE X HEMISPHERE MODEL 3.4420-IN. RADIUS}

The physical representation of this sample problem is the critical experiment described in sample problem 29. This sample problem describes the sphere as two $\mathrm{X}$ hemispheres, each with a chord and origin specified. One of the hemispheres is placed using the hole geometry option.

\section{Input Data}

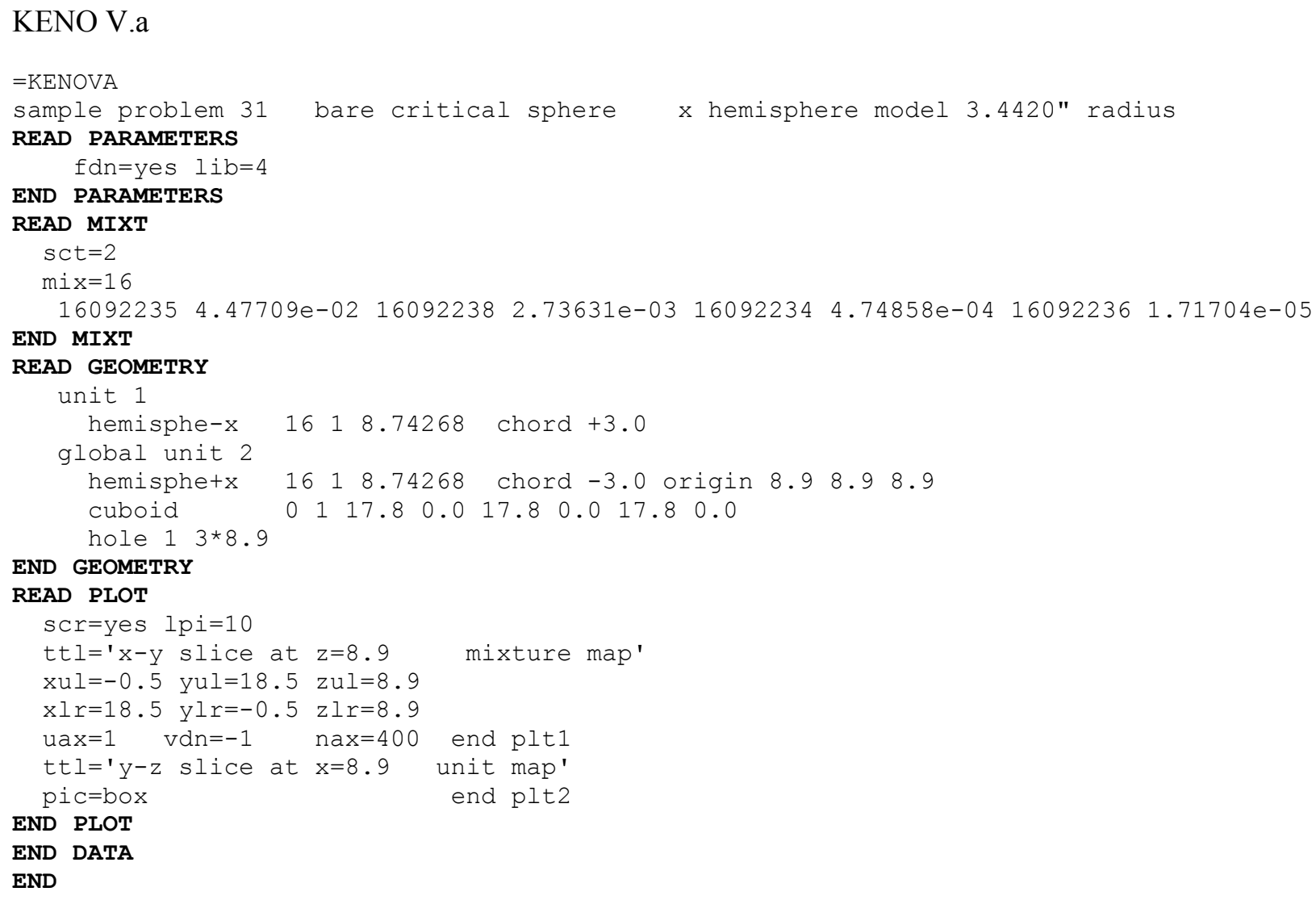




\subsection{C.2.32 Sample Problem 32 (KENO V.a ONLY) BARE CRITICAL SPHERE Y HEMISPHERE MODEL 3.4420-IN. RADIUS}

The physical representation of this sample problem is the critical experiment described in sample problem 29. This sample problem describes the sphere as two Y hemispheres, each with a chord and origin specified. One of the hemispheres is placed using the hole geometry option.

\section{Input Data}

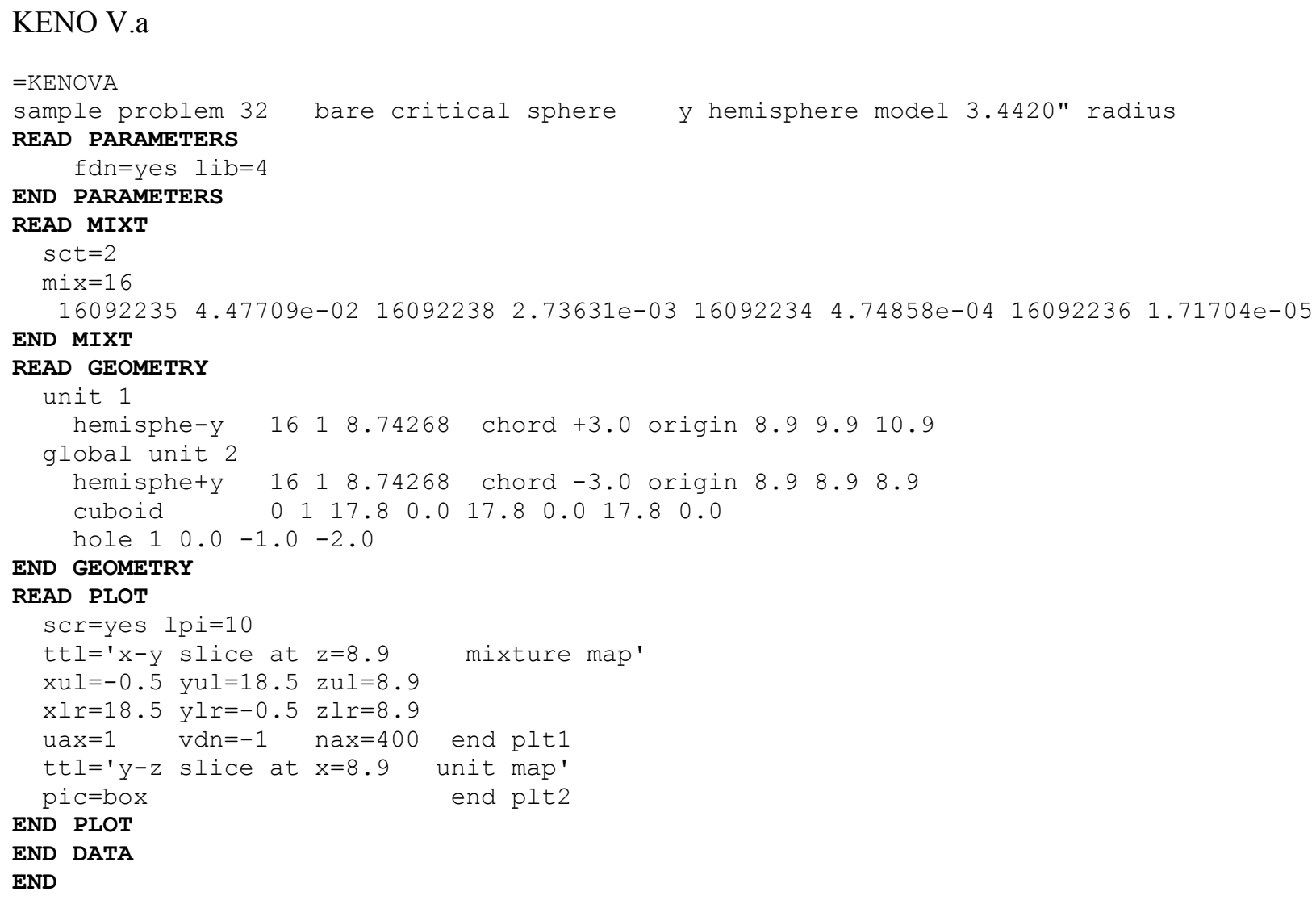




\subsection{C.2.33 Sample Problem 33 CRITICAL TRIANGULAR PITCHED ARRAY OF ANNULAR RODS}

This sample problem represents a critical experiment ${ }^{7,8}$ that consists of a partially flooded array of 19 low enriched uranium metal cylindrical annuli billets arranged in a triangular pitched array. The density of the uranium metal was $19.0 \mathrm{~g} / \mathrm{cc}$, and the isotopic enrichment in weight percent was $1.95 \%{ }^{235} \mathrm{U}, 98.02 \%$ ${ }^{238} \mathrm{U}, 0.006 \%{ }^{236} \mathrm{U}$, and $0.002 \%{ }^{234} \mathrm{U}$. The cylindrical annuli had an inside diameter of $6.604 \mathrm{~cm}$, an outside diameter of $18.288 \mathrm{~cm}$, and were placed with a pitch of $20.828 \mathrm{~cm}$. Each billet was $101.6 \mathrm{~cm}$ long. The array was positioned in a very large tank. This configuration was critical when the tank was filled to a height of $47.7 \mathrm{~cm}$ on a scale whose zero point was $0.6 \mathrm{~cm}$ below the bottom of the billets. The bottom of the billets was $21.6 \mathrm{~cm}$ above the bottom of the tank. The tank and all support structures have been ignored in this model. The model utilizes only $15.24 \mathrm{~cm}$ of water reflector on all sides of the array. Figure 8.1.C.12 and Figure 8.1.C.13 provide a representation of the model. A photograph of a single annular billet is shown in Figure 8.1.C.14.

\section{Input Data}

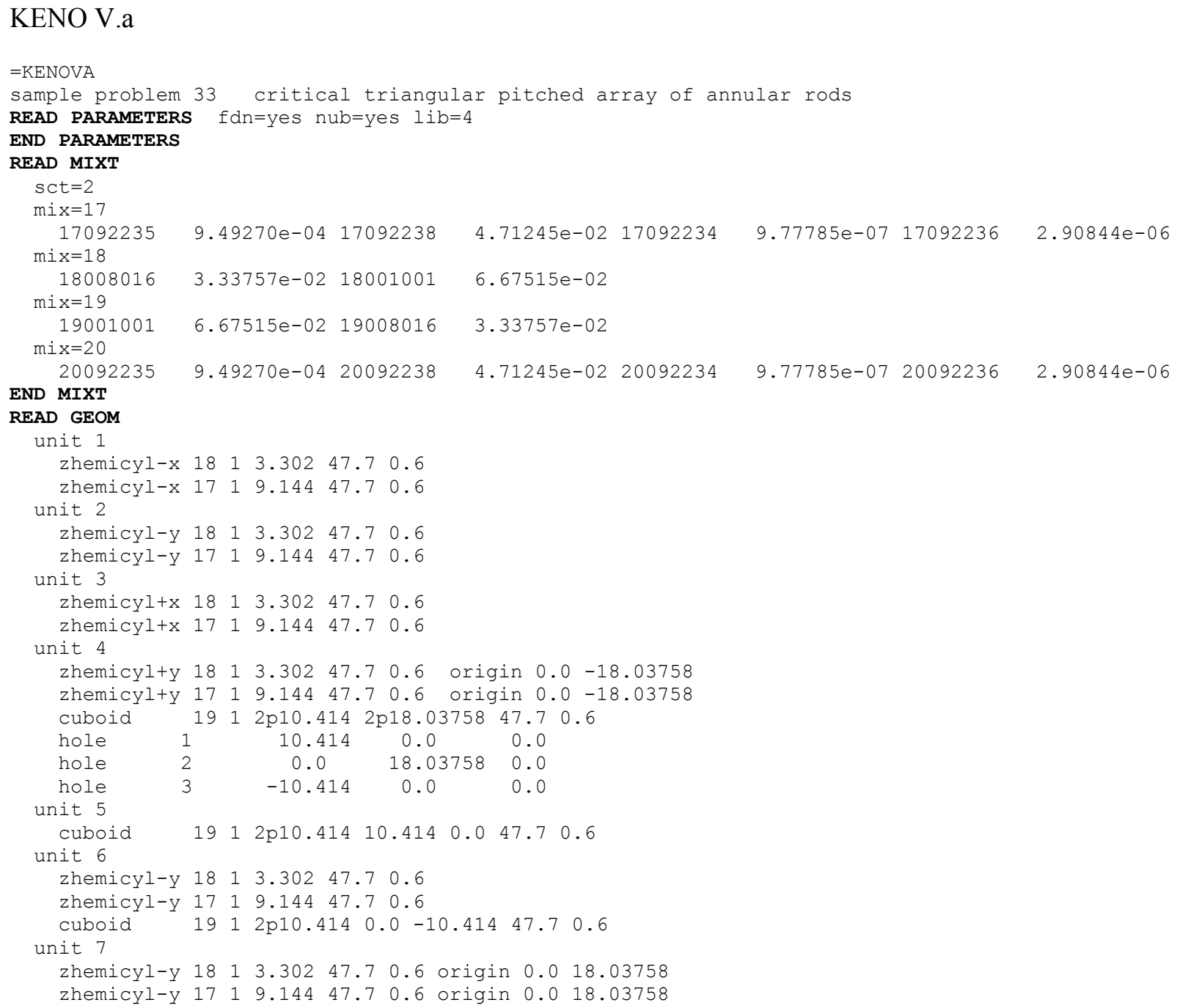




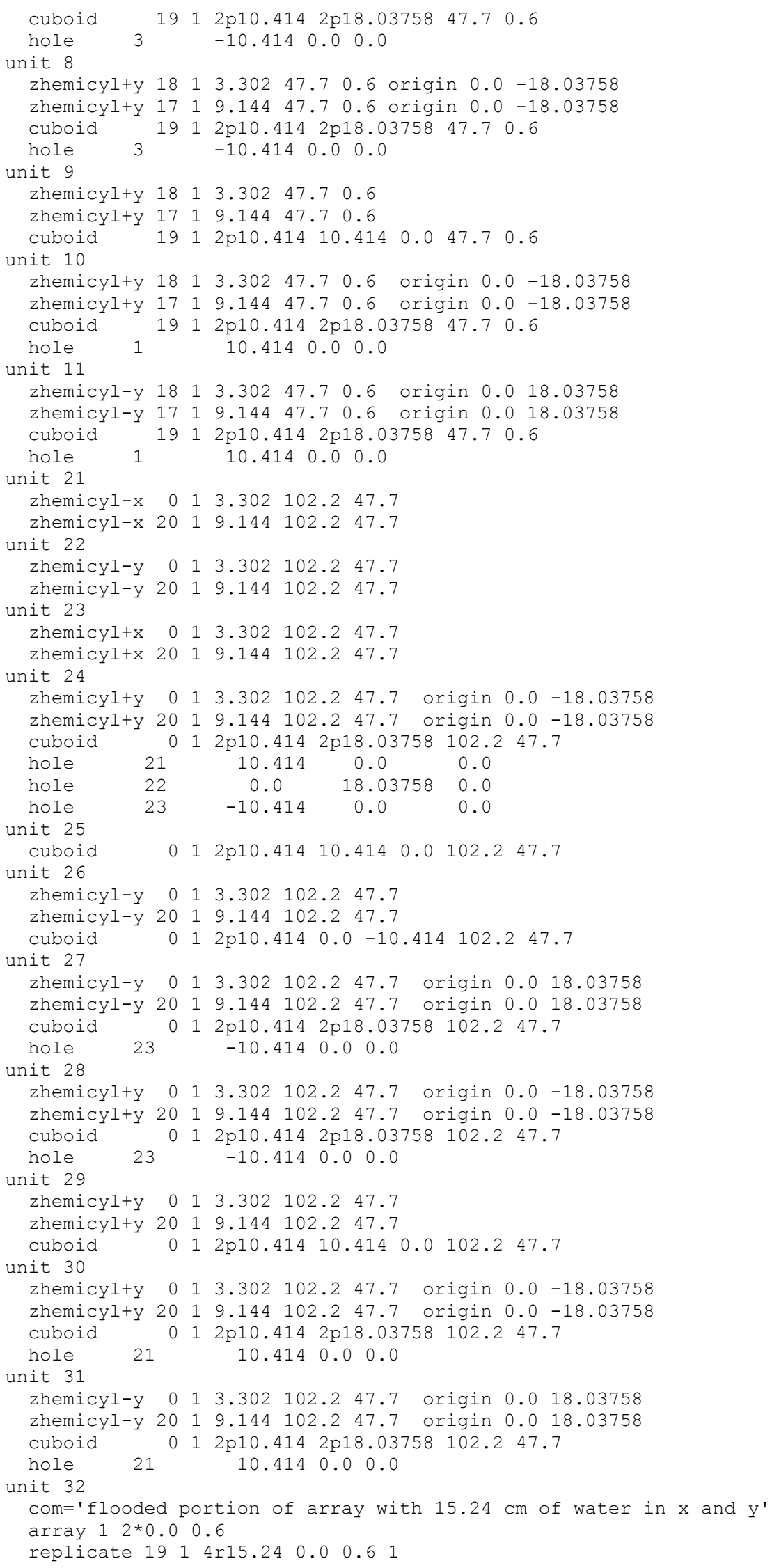




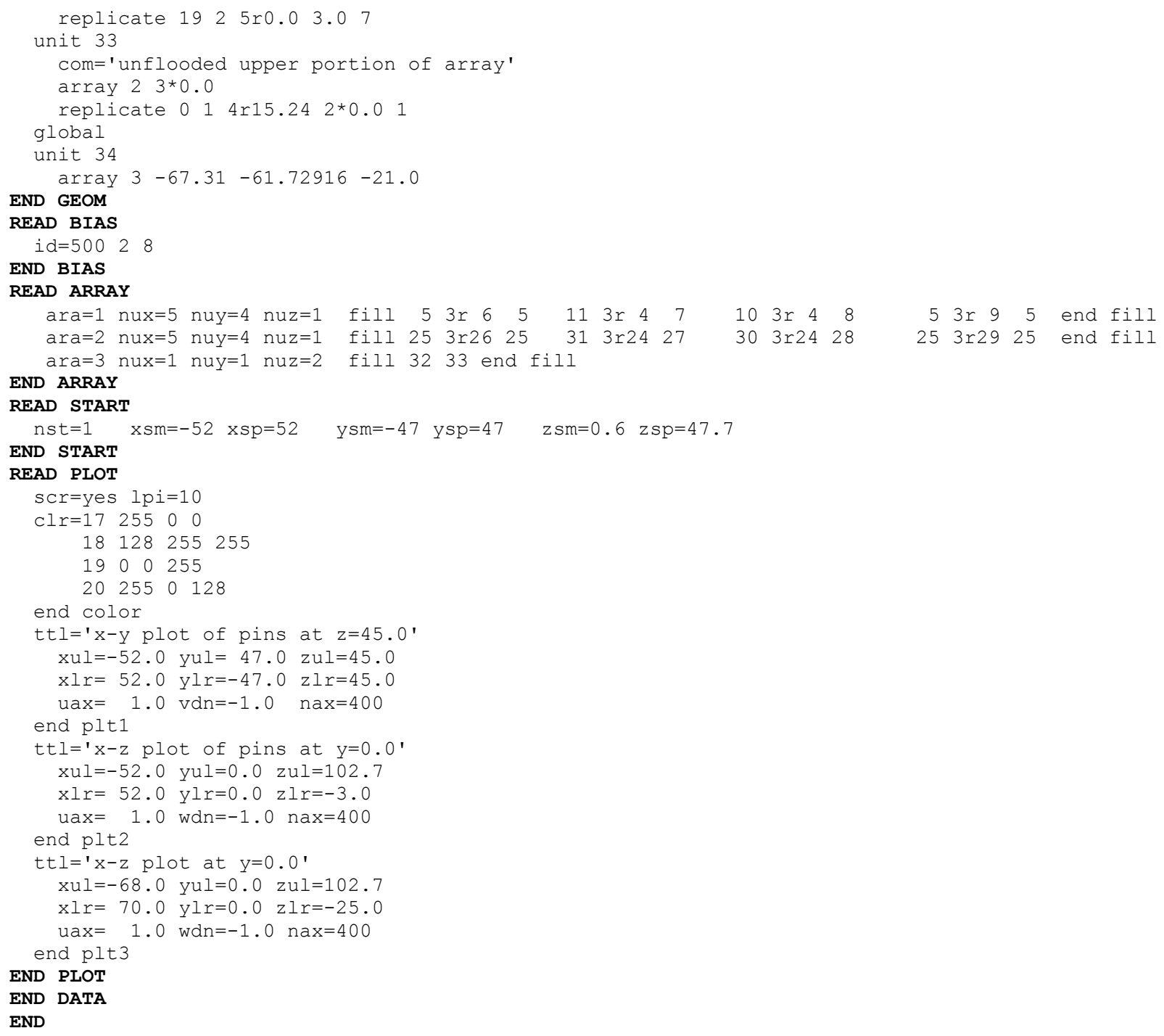

\section{KENO-VI}

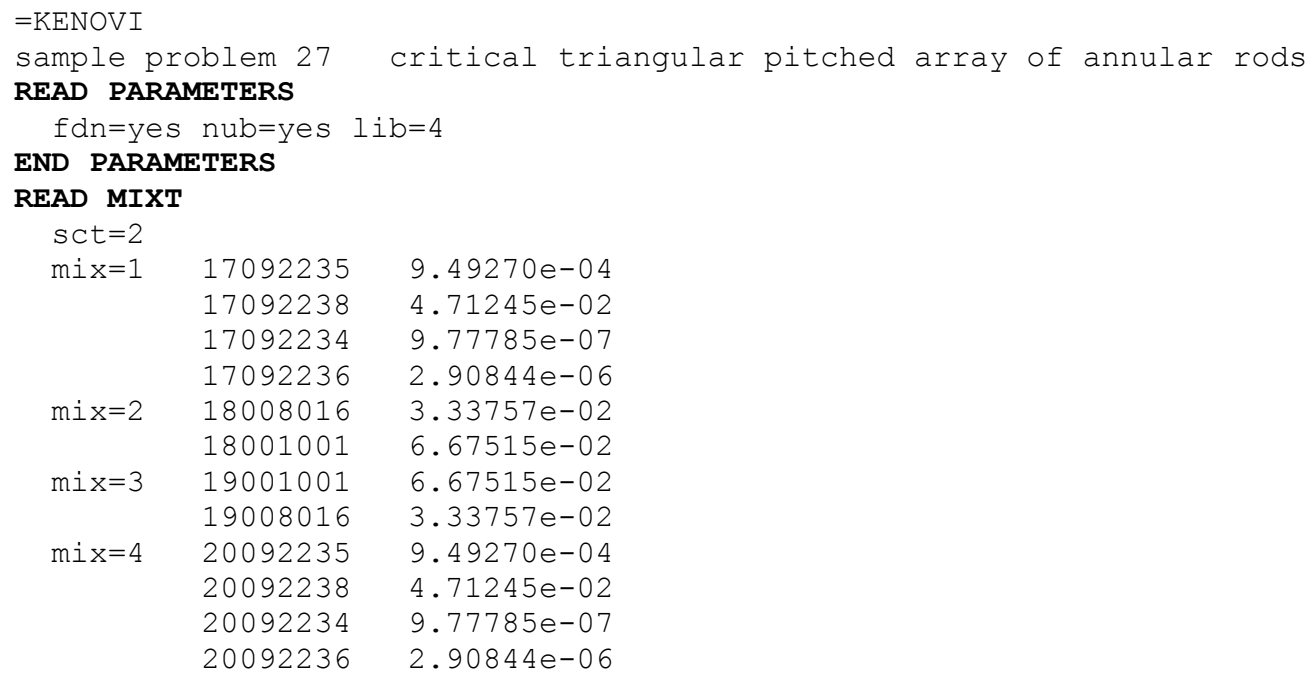




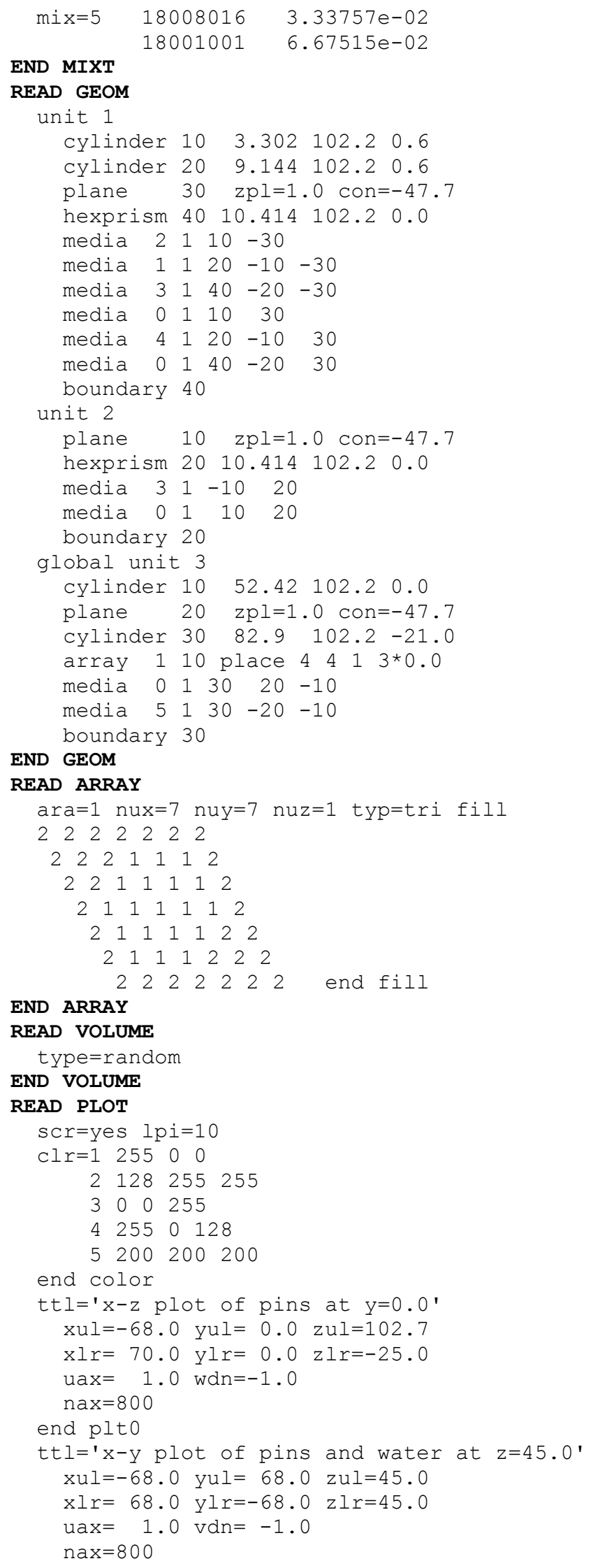




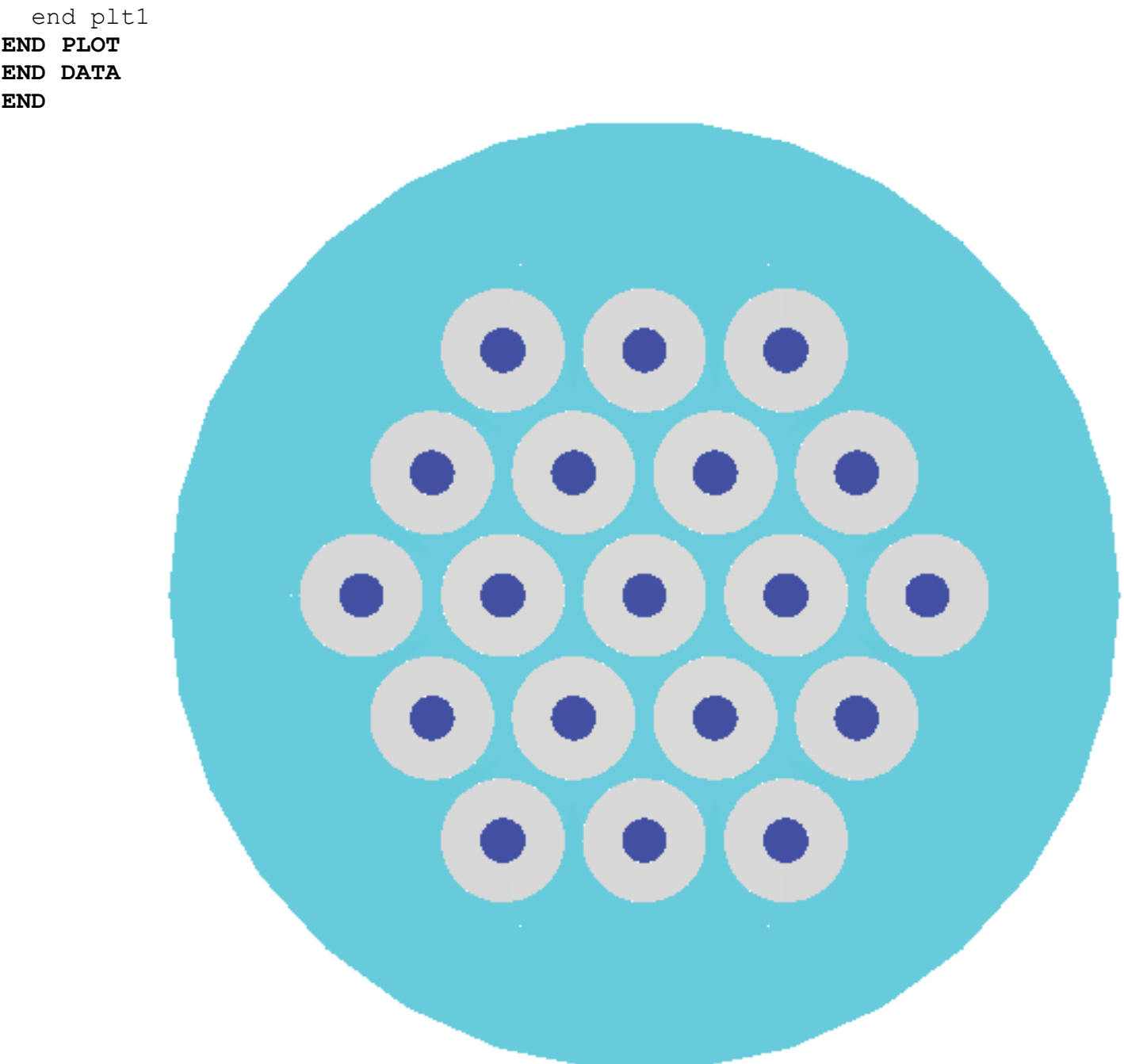

Figure 8.1.C.12. Horizontal slice through a critical triangular pitched array of partially flooded $1.95 \%$ enriched uranium metal annular billets. 


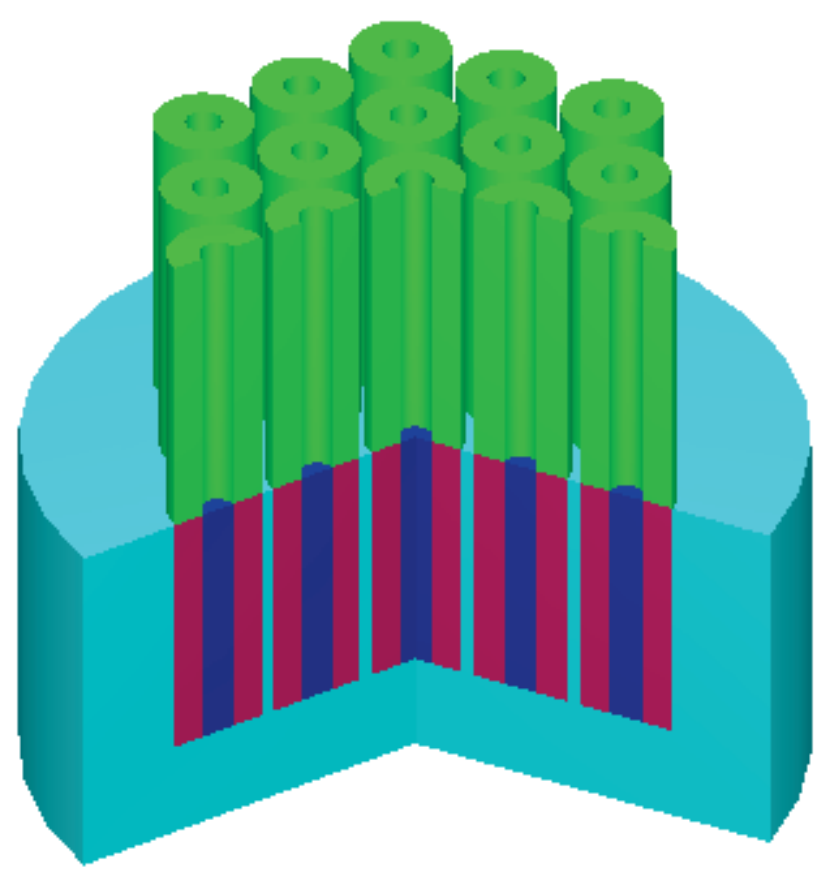

Figure 8.1.C.13. Vertical slice through the center of a critical triangular-pitched array of partially flooded $1.9 \%$ enriched uranium metal annular billets. 


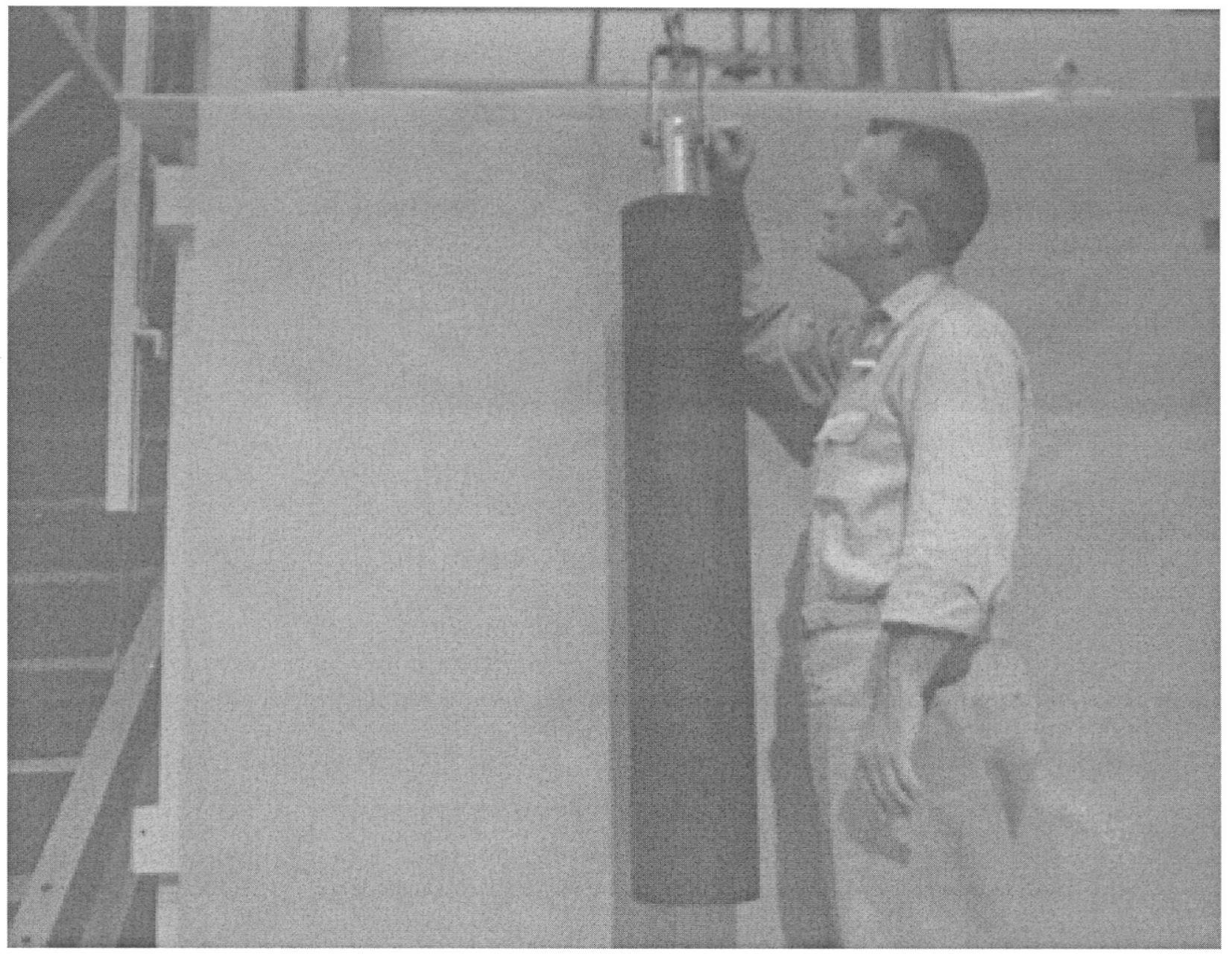

Figure 8.1.C.14. 1.95\% Enriched uranium metal annular billet used in critical experiments. 


\subsection{C.3 References}

1. J. T. Thomas, "Critical Three-Dimensional Arrays of U(93.2)-Metal Cylinders," Nucl. Sci. Eng. 52, 350 (November 1973).

2. G. E. Whitesides and J. T. Thomas, "The Use of Differential Current Albedos in Monte Carlo Criticality Calculations,” Trans. Am. Nucl. Soc. 12, 889 (November 1969).

3. D. C. Irving and J. T. Mihalczo, "Monte Carlo Calculations for Enriched Uranium Metal Assemblies," Trans. Am. Nucl. Soc. 7, 284 (November 1964).

4. Cleo C. Byers et al., "Critical Measurements of a Water-Reflected Enriched Uranium Sphere," Trans. Am. Nucl. Soc. 27, 412 (November 1977).

5. J. T. Thomas, Critical Three-Dimensional Arrays of Neutron-Interacting Units, Part II, ORNL/TM868, Union Carbide Corporation (Nuclear Division), Oak Ridge National Laboratory (July 1964).

6. J. T. Mihalczo, J. J. Lynn, J. R. Taylor, and G. E. Hansen, "Measurements with an Unreflected Uranium (93.2\%) Metal Sphere," pp. 26-33, in Proc. 1993 Topical Meeting on Physics and Methods in Criticality Safety, September 19-23, 1993.

7. E. B. Johnson, "Critical Parameters of U(1.95) Metal Cylindrical Annuli," Neutron Physics Division Annual Progress Report, September 1996, ORNL-3973, Union Carbide Corporation (Nuclear Division), Oak Ridge National Laboratory, 1966.

8. E. B. Johnson, "Critical Parameters of U(1.95) Metal Cylindrical Annuli," Trans. Am. Nucl. Soc. 9, 185-186 (1966). 


\title{
8.2 MONACO: A FIXED-SOURCE MONTE CARLO TRANSPORT CODE FOR SHIELDING APPLICATIONS
}

D. E. Peplow and C. Celik

\begin{abstract}
Monaco is a general-purpose, fixed-source, Monte Carlo shielding code for the SCALE package. It is a functional module that uses either AMPX cross sections or continuous energy libraries to calculate neutron and photon fluxes and responses to specific geometry regions, to point detectors and to mesh tallies. Basic, multigroup transport methods are inherited from Monaco's predecessor, MORSE. Continuous energy physics has been incorporated into the code with a new physics package that uses the same CE data as CE-KENO-VI, with extensions for simulating photons. Variance reduction capabilities include source biasing and weight windows, either by geometry region or by using a mesh-based importance map. User input includes the cross section file unit number; the geometry description using the SCALE General Geometry Package; source description as a function of position, energy, and direction; tally descriptions (fluxes in which regions, at what point detectors, or over what mesh grids); and response functions (functions of energy). Output consists of tables detailing the region and point detector fluxes (and their responses), as well as files for mesh tallies.
\end{abstract}




\section{CONTENTS}

$\underline{\text { Page }}$

8.2 Monaco: A Fixed-Source Monte Carlo Transport Code for Shielding Applications ..............8-587

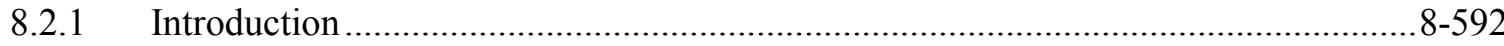

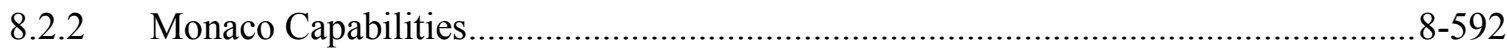

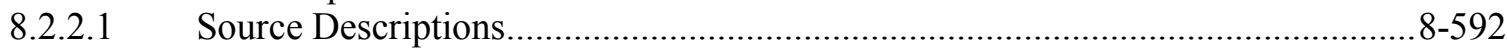

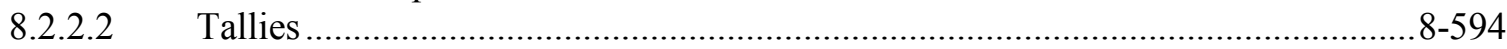

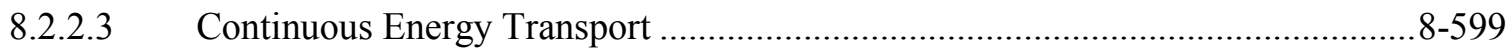

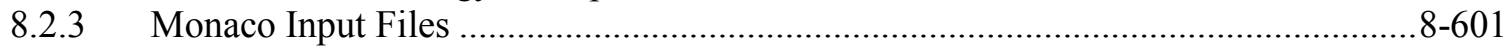

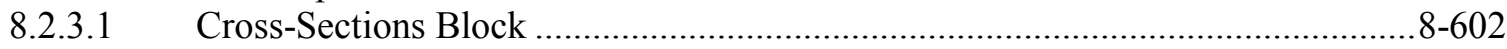

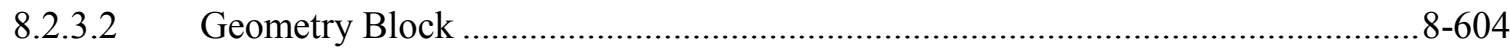

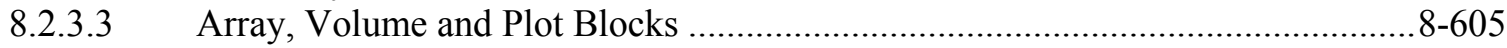

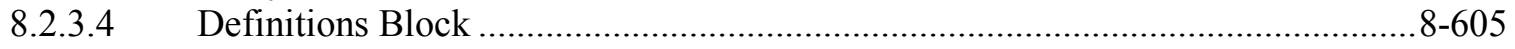

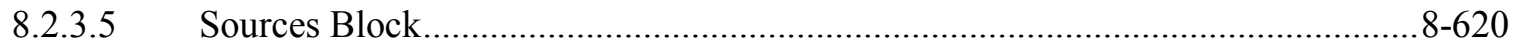

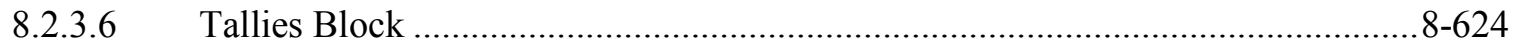

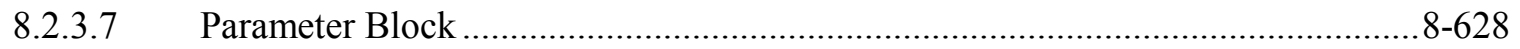

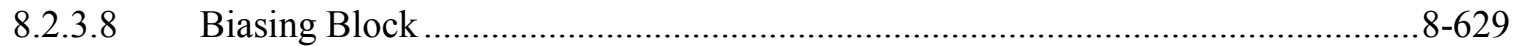

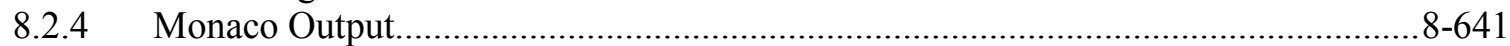

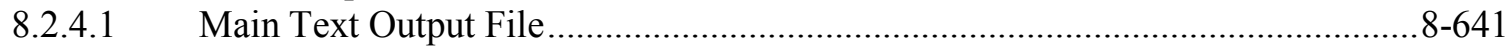

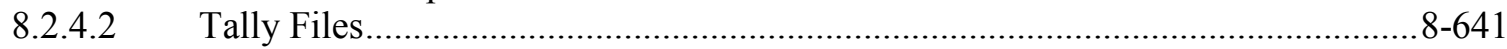

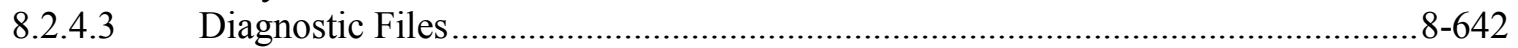

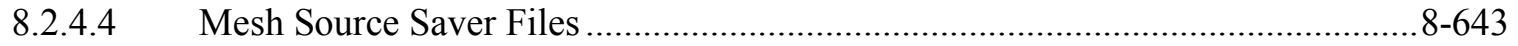

8.2.4.5 Statistical Checks on Point Detector and Region Tallies .....................................8-643

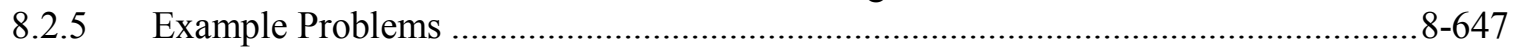

8.2.5.1 Neutron Transmission Through an Iron Sphere ............................................ $8-647$

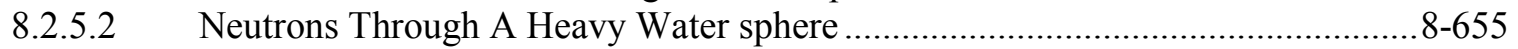

8.2.5.3 Activation Rate from a Neutron Howitzer ........................................................... 8-661

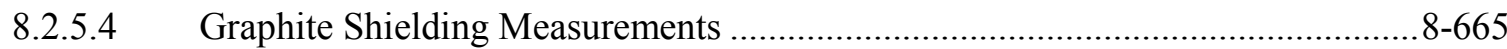

8.2.5.5 Simple shielding Demonstration with Line Spectra ........................................... 8-669

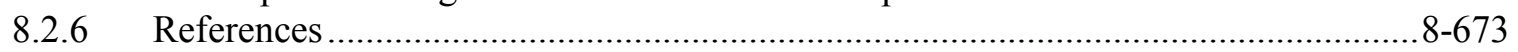




\section{LIST OF FIGURES}

$\underline{\text { Page }}$

Figure 8.2.1. The multigroup representation of a cobalt-60 source.

$8-599$

Figure 8.2.2.

Photon total cross section in tungsten. The energies of the cobalt-60 are

displayed as lines at 1173230 and $1332490 \mathrm{eV}$.

Figure 8.2.3. Photon total cross section in tungsten, near the cobalt lines. The energies of the cobalt-60 are displayed as lines at 1173230 and $1332490 \mathrm{eV}$.............................. 8-601

Figure 8.2.4.

Histogram-type response

$8-606$

Figure 8.2.5.

Value/function pair response.

$8-606$

Figure 8.2.6.

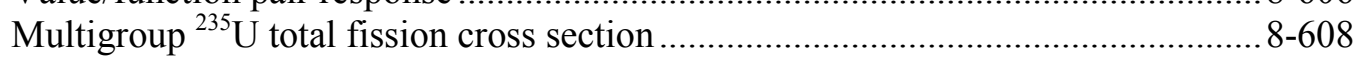

Figure 8.2.7.

$\mathrm{CE}{ }^{235} \mathrm{U}$ total fission cross section.

$8-608$

Figure 8.2.8.

ANSI 1991 neutron CE (left is log-log, right is linear-linear) ................................. 8-610

Figure 8.2.9.

ANSI 1991 neutron MULTIGROUP (left is log-log, right is linear-linear)............. 8-610

Figure 8.2.10.

Figure 8.2.11.

Figure 8.2.12.

ANSI 1991 photon CE (left is log-log, right is linear-linear) ................................ 8-610

Figure 8.2.13.

ANSI 1991 photon MULTIGROUP (left is log-log, right is linear-linear) .............. 8-610

Figure 8.2.14.

Sampling tests for the distribution examples.

$8-616$

Sampling tests for the special (built-in) distribution examples. .............................. 8-619

Figure 8.2.15.

Tally values for a well-converged tally.

$8-644$

Final check for a well-converged tally. .......................................................... 8-644

Figure 8.2.16.

Tally values for a not-yet-converged tally.

$8-645$

Figure 8.2.17.

Final check for a not-yet-converged tally.

$8-645$

Figure 8.2.18.

Behavior of two tallies as a function of number of particle batches..

$8-646$

Figure 8.2.19.

Total neutron flux in $\mathrm{n} / \mathrm{cm}^{2} / \mathrm{s}$.

$8-652$

Figure 8.2.20.

Neutron dose rate in rem $/ \mathrm{hr}$.

$8-652$

Figure 8.2.21.

Comparison of Monaco results using the ENDF/B-VII.0 27n/19g library and the

measured values...

$8-653$

Figure 8.2.22.

Comparison of Monaco results using the new ENDF/B-VII.0 200n/47g library

and the measured values.

$8-653$

Figure 8.2.23. Comparison of Monaco results using the ENDF/B-VII 238n library and the measured values.....

Figure 8.2.24. Comparison of Monaco results using the ENDF/B-VII 252n library and the measured values.

Figure 8.2.25. Comparison of Monaco results using the ENDF/B-VII CE n/p library (binned

using the 200n group structure) and the measured values.................................... 8-655

Figure 8.2.26. Experimental setup.

Figure 8.2.27.

Comparison of Monaco calculated results using the ENDF/B-VII.0 27n/19g

library to the measured values.

Figure 8.2.28.

Comparison of Monaco calculated results using the ENDF/B-VII.0 200n/47g

library to the measured values.

Figure 8.2.29. Comparison of Monaco calculated results using the ENDF/B-V 238n library to the measured values.

Figure 8.2.30.

Comparison of Monaco calculated results using the ENDF/B-V 252n library to

the measured values.

Figure 8.2.31.

Figure 8.2.32.

Figure 8.2.33.

Comparison of Monaco calculated results using the ENDF/B-VII CE n/p library

(binned using the 200n group structure) to the measured values.

$8-661$

Idealized geometry for a neutron howitzer.

$8-662$

Response 1.

$8-665$

Figure 8.2.34.

Distribution 1.

$8-665$ 
Figure 8.2.35. Images (*.png files) generated with the "read plot" block. .................................. 8-668

Figure 8.2.36. Mesh tally showing neutron dose rate (rem/hr) ................................................... 8-669

Figure 8.2.37. Simple slab geometry with a source $(\mathrm{S})$ on the left and a tally region $(\mathrm{T})$ on the right......................................................................................................... 8-670

Figure 8.2.38. Point detector fluxes for the cobalt-60/tungsten problem using different energy

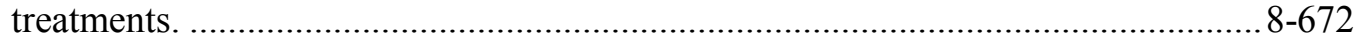

Figure 8.2.39. Region tally fluxes for the cobalt-60/tungsten problem using different energy treatments.. 


\section{LIST OF TABLES}

Page

Table 8.2.1. Available source shapes and their allowed degenerate cases .................................... 8-593

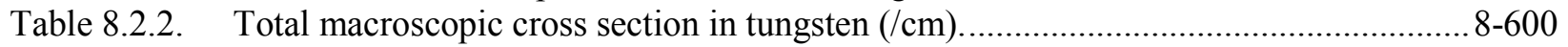

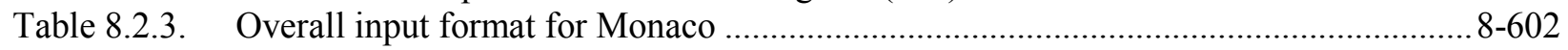

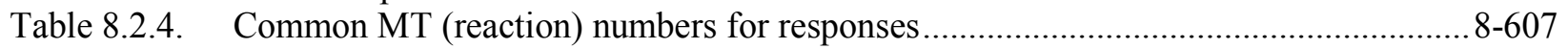

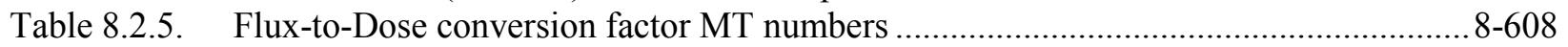

Table 8.2.6. Energy ranges of the original Flux-to-Dose responses.............................................. 8-609

Table 8.2.7. Use of the "specialDose=" and "doseData=" keywords........................................ 8-611

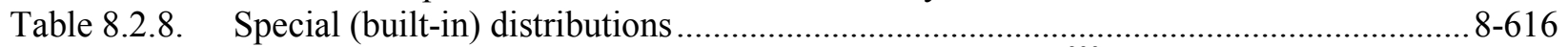

Table 8.2.9. Watt spectrum parameters for neutron induced fission of ${ }^{233} \mathrm{U}$ (from

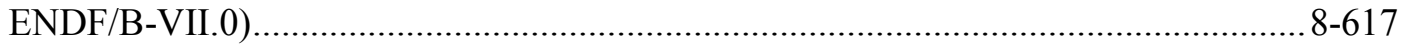

Table 8.2.10. Directions available for path-length stretching …................................................ 8-631

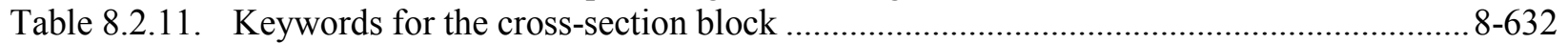

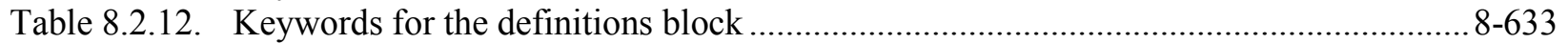

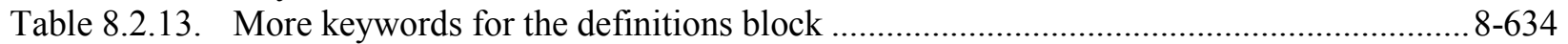

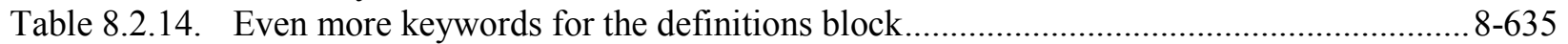

Table 8.2.15. Special distribution keywords for the definitions block .............................................. 8-636

Table 8.2.16. Continuous energy keywords for the definitions block .......................................... 8-636

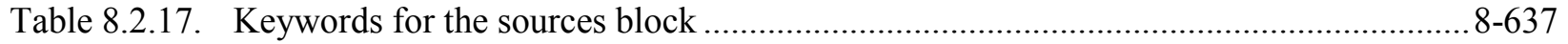

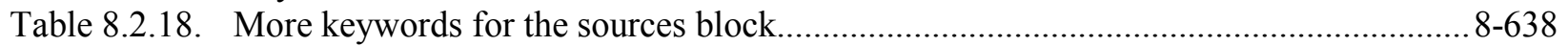

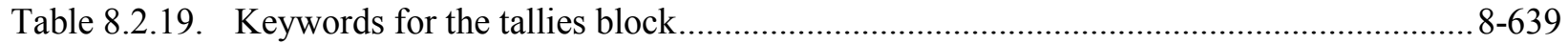

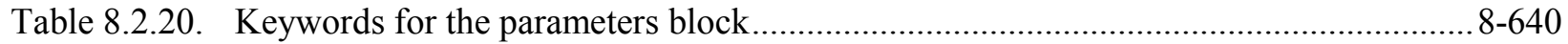

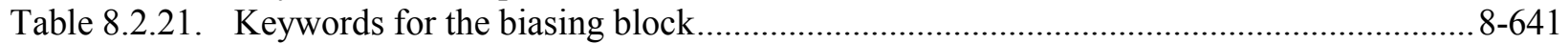

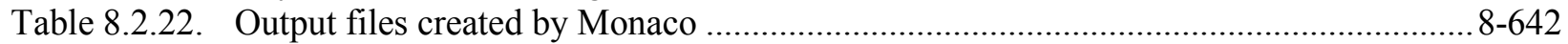

Table 8.2.23. Group-by-group details of the three flux tallies (ENDF/B-VII.0 27n19g) ................. 8-651

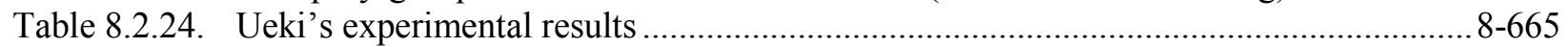

Table 8.2.25. Measured and calculated dose attenuation rate for graphite......................................... 8-668

Table 8.2.26. Dose rates for the cobalt-60/tungsten problem using different energy treatments........8-672

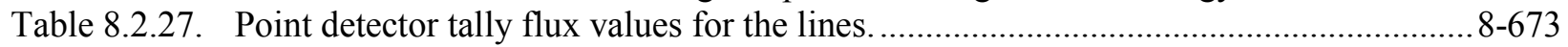

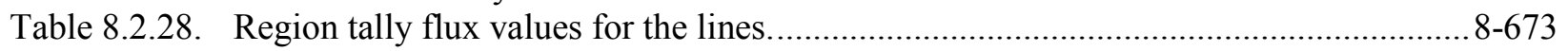




\subsubsection{Introduction}

Monaco is a neutron/photon, fixed-source Monte Carlo shielding code for the SCALE code package. Monaco uses the SCALE General Geometry Package (SGGP) - the same geometry description as KENOVI. Monaco has many options available to the user for specifying source distributions, many tally options, and many variance reduction capabilities. Monaco was originally based on the MORSE Monte Carlo code but has been extensively modified to modernize the coding, increase the number of capabilities in terms of sources and tallies, and allow for either multigroup or continuous energy (CE) transport through the use of the new SCALE CE Modular Physics Package (SCEMPP).

Monaco was developed to address a number of long-term goals for the Monte Carlo shielding capabilities in SCALE. The principal goals for this project included (1) unification of geometric descriptions between the SCALE shielding and criticality Monte Carlo codes, (2) implementation of a mesh-based importance map and mesh-based biased source distribution so that automated variance reduction could be used, and (3) establishment of a code using modern programming practices from which to continue future development. The addition of a continuous-energy transport capability is a significant change as well.

Monaco is the key component of the MAVRIC sequence, which also uses Denovo to create the meshbased importance map and mesh-based biased source distribution for general 3-D automated variance reduction. See the MAVRIC chapter for more information.

\subsubsection{Monaco Capabilities}

Monaco has a wide range of source descriptions and tallies for performing general radiation transport calculations. Note that Monaco can work with either the AMPX-based multigroup libraries or the newer AMPX-based CE libraries. Note that for CE calculations, tallies still employ a multigroup energy structure to store and report results.

\subsubsection{Source descriptions}

Multiple sources can be defined for a Monaco calculation. Sampling of the different sources can be biased by the user. Each source is specified by its spatial distribution, its energy distribution, its directional distribution, and its strength. Distributions defined by the user can also be biased and can be used multiple times by different sources. The Monaco tallies assume that the sources all have units of particles/second. If the source strengths are given in other units, the user will have to incorporate the proper conversion to the tally results and remember to interpret the results accordingly.

\subsection{Distributions}

Two types of basic distributions are used by Monaco - binned histograms and a set of value/function pairs. The binned histogram type is defined by $n+1$ bin boundaries and $n$ values, representing the integrated amount in each bin. For the true distribution $f(x)$, the bin boundaries $\left[x_{0}, x_{1}, \ldots, x_{n}\right]$ and the integrated amounts $F_{i}=\int_{x_{i-1}}^{x_{i}} f(x) d x$ are given. The distribution will be normalized by Monaco after reading. The user can optionally bias a binned histogram distribution by supplying one of the following: the biased sampling distribution amounts, $G_{i}=\int_{x_{i-1}}^{x_{i}} g(x) d x$; the importance of each bin, $I_{i}$; or the suggested weight for each bin, $w_{i}$.

Based on what type of input is given, Monaco will compute a properly normalized probability distribution function for sampling. If the importances are given, the sampling distribution is computed as 


$$
G_{i}=\frac{I_{i} F_{i}}{\sum_{i} I_{i} F_{i}}
$$

If suggested weights are given, then the sampling distribution is computed as

$$
G_{i}=\frac{F_{i} / w_{i}}{\sum_{i} F_{i} / w_{i}}
$$

for bins with non-zero weight. The sampling distribution for bins with a suggested weight of zero are set to $G_{i}=0$. When sampled, particles are assigned a weight of $F_{i} / G_{i}$.

The second type of distribution that a user can define is for a series of point values of a function. For a set of $n+1$ point pairs, $\left(x_{i}, f_{i}\right)$ for $i \in[0 \ldots n]$, defining $n$ intervals, a distribution can be made by linearly interpolating between adjacent point pairs. This type of distribution can also be biased by supplying one of the following: the biased sampling distribution function value $g_{i}$ at each point, the importance of each point, $I_{i}$; or the suggested weight for each point, $w_{i}$. Similar to above, if importances or weights are given, Monaco computes the biased distribution for sampling. For the value/function point pairs type of distribution, the weight assigned to the sampled particle is a continuous function.

Some commonly used distributions are built into Monaco and can be used by simple keywords. Monaco can produce a graph of any distribution so that the user can verify that the input was entered correctly.

\subsection{Spatial, energy and directional distributions}

Each Monaco source is described by three separable components: spatial, energy and directional.

The spatial component of a source in Monaco is simple but very flexible. First, the general shape of the source region is defined in global coordinates. The basic solid shapes and their allowed degenerate cases are listed in Table 8.2.1. The user can reference any of the defined distributions to describe the source distribution in any coordinate ( $x, y$, and $z$ for cuboids, $r$ and $z$ for cylinders and $r$ for spheres) to use for sampling or leave the source distribution as uniform over each dimension for the solid shape. The source region can be limited by the underlying SGGP geometry variables of unit, media, and mixture. This way, source volumes (or planes, lines, or points) can be defined that are independent or dependent on the model geometry. A cylinder or cylindrical shell region can be oriented with its axis in any direction.

Table 8.2.1. Available source shapes and their allowed degenerate cases

\begin{tabular}{ll}
\hline \multicolumn{1}{c}{ Shape } & \multicolumn{1}{c}{ Allowable degenerate cases } \\
\hline cuboid & rectangular plane, line, point \\
cylinder & circular plane, line, point \\
cylindrical shell & cylinder, planar annulus, circular plane, cylindrical surface, line, ring, point \\
sphere & point \\
spherical shell & sphere, spherical surface, point \\
\hline
\end{tabular}

Monaco samples the source position using either the given distributions or uniformly over the basic solid shape and then uses rejection if any of the optional SGGP geometry limiters have been specified. For 
sources that are confined to a particular unit, media, or mixture, users should make sure the basic solid shape tightly bounds the desired region for efficient sampling.

For the energy component of each source, either type of distribution described above can be used. Biasing can be used in the energy component of the source as well. The Watt spectrum is a built-in distribution which uses the Froehner and Spencer ${ }^{1}$ method for sampling. If the defined energy distribution has point(s) that are out of the problem's energy range for a CE problem, these points will be rejected in the source energy sampling and an error message will be generated. The warnings will be suppressed if the number of rejected source points exceeds a pre-defined threshold (1000).

Distributions can be used to define the directional component of the source. A function of the cosine of the polar angle, with respect to some reference direction in global coordinates, can be used by Monaco. If no directional distribution is specified, the default is an isotropic distribution (one directional bin from $\mu=-1$ to $\mu=1)$. The default reference direction is the positive $z$-axis $(<0,0,1>)$.

\subsection{Monaco mesh source map files}

An alternative to specifying the separate spatial and energy distributions, a Monaco mesh source file can be used. A mesh source consists of a 3D Cartesian mesh that overlay the geometry. Each mesh cell has some probability of emitting a source particle, and within each mesh cell, a different energy distribution can be sampled. Position within each mesh cell is sampled uniformly, and the emission direction is sampled from the standard directional distribution. Monaco mesh source files are typically produced by the MAVRIC sequence or by other Monaco calculations (see the mesh source saver option in the source input). For a source constructed from the separable spatial and energy distributions, Monaco can create a mesh source file which can then be visualized using the Mesh File Viewer. This is a convenient way to ensure that the source being used is what was intended.

\subsubsection{Tallies}

Monaco offers three tally types: point detectors, region tallies, and mesh tallies. Each is useful in determining quantities of interest in the simulation. Any number of each can be used, up to the limit of machine memory. The tallies will compute flux for each group, the total neutron and total photon fluxes, and any number of dose-like responses. A typical dose-like response, $R$, is the integral over energy of the product of a response function, $f(E)$, and the flux, $\phi(E)$.

$$
R=\int f(E) \phi(E) d E
$$

In multigroup calculations, the total response would be expressed as the sum over all groups $R=\sum f_{g} \phi_{g}$. For CE calculations, tallies can be segmented into energy and time bins which can be thought of as "groups". All three of the tally types can be scaled with a constant - for example, to account for units conversions.

\subsection{Tally statistics}

The three Monaco tallies are really just collections of simple and extended tallies for each group, each total, and each group contribution to a response or total response. The simple tally works in the following way: a history score $h_{i}$ is zeroed out at the start of history $i$. During the course of the history, when an event occurs during substep $j$, a score consisting of some contribution $c_{i j}$ weighted by the current particle weight $w_{i j}$ is calculated and added to $h_{i}$. At the end of the history, the history score is the total weighted score for each substep $j$ in the history. 


$$
h_{i}=\sum_{j} w_{i j} c_{i j}
$$

Note that the values for the contribution $c_{i j}$ and when it is added to the accumulator are determined by the tally type. At the end of the each history, the history score is added to two accumulators (power sums) the first accumulator is for finding the tally average, $S_{1}$, and the second accumulator is for finding the uncertainty in the tally average, $S_{2}$.

$$
\begin{aligned}
& S_{1}=\sum_{i} h_{i} \\
& S_{2}=\sum_{i} h_{i}^{2}
\end{aligned}
$$

At the end of all $N$ histories, the second sample central moment is found from the power sums

$$
m_{2}=\frac{S_{2}}{N}-\frac{S_{1}^{2}}{N^{2}}
$$

and then the tally average is computed as $\bar{x}=S_{1} / N$ and the uncertainty in the tally average is $u=$ $\sqrt{m_{2} / N}$.

The extended tally uses four accumulators - the first and second are the same as the simple tally - with the third and fourth accumulators used for finding the variance of the variance (VOV). These extra accumulators, $S_{3}$ and $S_{4}$, are calculated as

$$
\begin{aligned}
& S_{3}=\sum_{i} h_{i}^{3} \\
& S_{4}=\sum_{i} h_{i}^{4}
\end{aligned}
$$

At the end of all $N$ histories, the tally average $\bar{x}$ and uncertainty in the tally average $u$ are found in the same way as a simple tally. For the VOV calculation, the third and fourth sample central moments are found as

$$
\begin{gathered}
m_{3}=\frac{S_{3}}{N}-\frac{3 S_{1} S_{2}}{N^{2}}+\frac{2 S_{1}^{3}}{N^{3}} \\
m_{4}=\frac{S_{4}}{N}-\frac{4 S_{1} S_{3}}{N^{2}}+\frac{6 S_{1}^{2} S_{2}}{N^{3}}-\frac{3 S_{1}^{4}}{N^{4}}
\end{gathered}
$$

and then the $\mathrm{VOV}^{2}$ and figure-of-merit (FOM) are found using

$$
\mathrm{VOV}=\frac{m_{4}-m_{2}^{2}}{N m_{2}^{2}}
$$




$$
\mathrm{FOM}=\frac{1}{(u / \bar{x})^{2} T}
$$

where $T$ is the calculation time (in minutes).

Extended tallies are used for the total neutron flux, total photon flux and any responses for the Monaco tallies. Simple tallies are used for each group's flux and each group's contribution to a response.

Detailed, group-wise results for each tally are saved to separate files at the end of each batch of particles. Users can view these files (in the SCALE temporary directory) as the Monaco simulation progresses. Summaries of the extended tallies appear in the final Monaco output file.

\subsection{Statistical tests}

Statistical tests are performed on the extended tallies at the end of each batch. Results for each batch are stored in files and the results for the final batch are shown in the main output tally summary. The six tests are:

\begin{tabular}{llllc}
\hline & Quantity & Test & Goal & Within \\
\hline 1. & mean & relative slope of linear fit & $=0.00$ & \pm 0.10 \\
2. & standard deviation & exponent of power fit & $=-0.50$ & $R^{2}>0.99$ \\
3. & relative uncertainty & final value & $<0.05$ & \\
4. & relative VOV & exponent of power fit & $=-1.00$ & $R^{2}>0.95$ \\
5. & relative VOV & final value & $<0.10$ & \\
6. & figure-of-merit & relative slope of linear fit & $=0.00$ & \pm 0.10 \\
\hline
\end{tabular}

For the tests that are fit to a function with respect to batch $(1,2,4$, and 6$)$, only the last half of the simulation is used. The basis for these tests is that in a well-behaved Monte Carlo, the mean should not increase or decrease as a function of the number of histories $(N)$, the standard deviation should decrease with $1 / \sqrt{N}$, the variance of the variance should decrease with $1 / N$ and the figure-of-merit should neither increase or decrease as a function of the number of histories (proportional to time). For tests 2 and 4, the coefficient of determination, $R^{2}$, from a forced fit to a function with the right exponent is used as the tally test.

\subsection{Point detector tallies}

Point detectors are a form of variance reduction in computing the flux or response at a specific point. At the source emission site and at every interaction in the particle's history, an estimate is made of the probability of the particle striking the position of the point detector. For each point detector, Monaco tallies the uncollided and total flux for each energy group, the total for all neutron groups, and the total for all photon groups. Any number of optional dose-like responses can be calculated as well.

\subsection{Mutli-Group}

After a source particle of group $g$ is started, the distance $R$ between the source position and the detector position is calculated. Along the line connecting the source and detector positions, the sum of the distance $s_{j}$ through each region $j$ multiplied by the total cross section $\Sigma_{j}^{g}$ for that region is also calculated. The contribution $c_{g}$ to the uncollided flux estimator is then made to the tally for group $g$. 


$$
c_{g}=\frac{1}{4 \pi R^{2}} \exp \left(-\sum_{j} s_{j} \Sigma_{j}^{g}\right)
$$

\subsection{Continuous Energy}

After a source particle with energy $E$ is started, the distance $R$ between the source position and the detector position is calculated. For each bin $g$ of the tally energy structure, a specific energy $E_{g}$ is sampled uniformly within the bin. Along the line connecting the source and detector positions, the sum of the distance $s_{j}$ through each region $j$ multiplied by the total cross section $\Sigma_{j}\left(E_{g}\right)$ for that region. The contribution $c_{g}$ to the uncollided flux estimator is then made to the tally for group $g$. total cross section $\Sigma_{j}(E)$ :

$$
c_{g}=\frac{1}{4 \pi R^{2}} \exp \left(-\sum_{j} s_{j} \Sigma_{j}(E)\right)
$$

Only source particles contribute to the uncollided flux tally. At each interaction point during the life of the particle, similar contributions are made to each of the tallies. For each group $g^{\prime}$ that the particle could scatter into and reach the detector location, a contribution is made that also includes the probability to scatter from the current direction towards the detector and having the energy change from group $g$ to group $g^{\prime}$.

This type of tally is costly, since ray-tracing through the geometry from the current particle position to the detector location is required many times over the particle history. Point detectors should be located in regions made of void material, so that contributions from interactions arbitrarily close to the point detector cannot overwhelm the total estimated flux $\left(\right.$ as $\left.1 / 4 \pi R^{2} \rightarrow \infty\right)$.

Care must be taken in using point detectors in deep penetration problems to ensure that the entire phase space that could contribute has been well sampled - so that the point detector is not underestimating the flux by leaving out areas far from the source but close to the point detector position. One way to check this is by examining how the tally average and uncertainty change with each batch of particles used in the simulation. Large fluctuations in either quantity could indicate that the phase space is not being sampled well.

\subsection{Region tallies}

Region tallies are used for calculating the flux and/or responses over one of the regions listed in the SGGP geometry. Both the track-length estimate of the flux and the collision density estimate of the flux are calculated-and for each, the region tally contains simple tallies for finding flux in each group, the total neutron flux, and the total photon flux. For each of the optional response functions, the region tally also contains simple tallies for each group and the total response.

For the track-length estimate of flux, each time a particle of energy $E$ moves through the region of interest, a contribution of $l$ (the length of the step in the region) is made to the history score for the simple tally for flux for tally group $g$. The same contribution is made for the history score for the simple tally for total particle flux, neutron or photon, depending on the particle type.

If any optional response functions were requested with the tally, then the contribution of $l f(E)$ is made for the response group, where $f(E)$ is the response function value for energy $E$. The history score for the total response function is also incremented using $l f(E)$. 
At the end of all of the histories, the averages and uncertainties of all of the simple tallies for fluxes are found for every group and each total. These results then represent the average track-length over the region. To determine flux, these results are divided by the volume of the region. If the volume $V$ of the region was not given in the geometry input nor calculated by Monaco, then the tally results will be just the average track lengths and their uncertainties. A reminder message is written to the tally detail file if the volume of the region was not set.

For the collision density estimate of the flux, each time a particle of energy $E$ has a collision in the region of interest, a contribution of $1 / \Sigma$ (the reciprocal of the total macroscopic cross section) is made to the history scores for the simple tally for flux for tally energy group $g$ and for the total particle flux. At the end of the simulation, the averages and uncertainties of all of the simple tallies for every group flux and total flux are found and then divided by the region volume, if available.

Similar to the point detector tallies, region tallies produce a file listing the tally average and uncertainty at the end of each batch of source particles ( $a *$.chart file). This file can be plotted using the simple 2-D plotter (ChartPlot) to observe the tally convergence behavior.

\subsection{Mesh tallies}

For a 3-D Cartesian mesh or a cylindrical mesh (independent of the SGGP geometry), Monaco can calculate the track-length estimate of the flux. Since the number of cells (voxels) in a mesh can become quite large, the mesh tallies are not updated at the end of each history but are instead updated at the end of each batch of particles. This prevents the mesh tally accumulation from taking too much time but means that the estimate of the statistical uncertainty is slightly low.

Like the other tallies, mesh tallies can calculate optional response functions.

Since a mesh tally consists of many actual tallies, the statistical tests are a bit more complex than for the region and point detector tallies. Several statistical quantities and tests are used in Monaco similar to those in several recent studies ${ }^{3,4}$ which look at a distribution of relative variances over the mesh tally. In Monaco, the basis of the statistical tests center on the distribution of relative uncertainties and its mean, $\bar{r}$, of the voxels $(V)$ with score.

$$
\bar{r}=\frac{1}{V} \sum R_{i}
$$

where $R_{i}$ is the relative uncertainty of the flux or dose in voxel $i$. If every voxel has been sampled well and its relative uncertainty $R_{i} \propto 1 / \sqrt{N}$, then the mean relative uncertainty of the voxels should also behave as $1 / \sqrt{N}$. The variance of the mean relative uncertainty can be calculated and a figure of merit (FOM) for the mesh tally can be constructed using

$$
F O M=\frac{1}{\bar{r}^{2} T}
$$

with the time $T$ in minutes. The four tests measure over the simulation: 1) if $\zeta$, the fraction of voxels with non-zero score, is constant; 2 ) if the mean relative uncertainty is decreasing as $1 / \sqrt{N}$ (as measured by the coefficient of determination, $R^{2}$, of a fit to a curve with power of -0.5 ); 3 ) if the variance of the mean relative uncertainty is decreasing with $1 / N$; and 4 ) if the FOM is constant. 


\begin{tabular}{lllcc}
\hline & Quantity & Test & Goal & Within \\
\hline 1. & $\zeta$, fraction with score & relative slope of linear fit & $=0.00$ & \pm 0.10 \\
2. & $\bar{r}$, mean relative uncertainty & exponent of power fit & $=-0.50$ & $R^{2}>0.99$ \\
3. & variance of $\bar{r}$ & exponent of power fit & $=-1.00$ & $R^{2}>0.95$ \\
4. & figure-of-merit & relative slope of linear fit & $=0.00$ & \pm 0.10 \\
\hline
\end{tabular}

For non-uniform meshes (especially cylindrical), these tests may not be the best measure of performance since different size voxels will have a wider variety of relative uncertainties. The user is also cautioned that if there are individual voxels within the mesh tally that have relative uncertainties that are not decreasing as $1 / \sqrt{N}$, then the mesh tally statistical tests will not be meaningful. It is ultimately up to the user to decide if the mesh tally is performing well (is the goal of the mesh tally just to calculate dose, not flux?; are all spatial areas of the mesh tally equally important?; are all magnitudes of the flux or response values equally important?; etc.)

Mesh tallies can be viewed with the Mesh File Viewer, a Java utility that can be run from GeeWiz (on PC systems) or can be run separately (on any system). The Mesh File Viewer will show the flux for each group, the total flux for each type of particle and the optional responses. Uncertainties and relative uncertainties can also be shown for mesh tallies using the Mesh File Viewer. For more information on the Mesh File Viewer, see its on-line documentation.

\subsubsection{Continuous energy transport}

Using multigroup data in Monte Carlo transport calculations is generally sufficient for most problems (both shielding and criticality). Many of the reaction cross sections vary slowly with energy, so energy "groups" can be made with one set of properties for the group. Multigroup treatments can further simplify radiation transport by combining the different types of reactions that can occur into a simple scattering matrix - particles then have certain probabilities to scatter from their current energy group to another energy group. If the user is not interested in knowing which specific type of interaction happened at each collision, this simplification can increase calculation efficiency.

One major drawback of the multigroup approach is in representing discrete gammas, such as the decay radiation from common isotopic sources. Consider a simple shielding simulation using cobalt- 60 . This isotope gives off two high-energy gamma rays when it decays (1173230 eV with intensity $99.85 \%$ and $1332490 \mathrm{eV}$ with intensity $99.9826 \%$ ). In the SCALE multigroup calculations, a cobalt-60 source spectrum is represented by a broad pdf, controlled by the group structure. This is shown in Figure 8.2.1. for the fine 47-group structure and the broad 19-group structure.

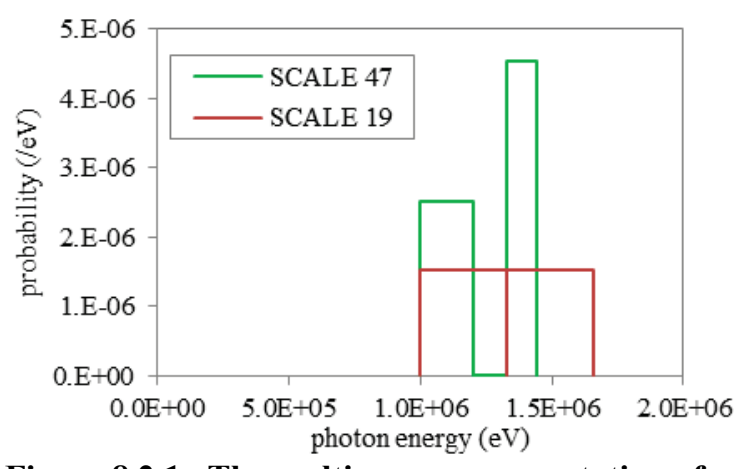

Figure 8.2.1. The multigroup representation of a cobalt-60 source.

Note that in both group structures, $1.33 \mathrm{MeV}$ is a group boundary, so the $1332490 \mathrm{eV}$ line is represented by group that covers higher energies. The cross section for that group is lower than the cross section for the specific line, so multigroup transport calculations will tend to overestimate the number of photons penetrating a shield, which will overestimate dose rates. 
Using CE and the two multigroup libraries, the total cross sections for the cobalt lines are listed in Table 8.2.2. Figure 8.2.2. shows the total cross section of photons in tungsten, in both CE and the two SCALE multigroup structures. On the whole, the multigroup data represents the $\mathrm{CE}$ data well. Figure 8.2.3. shows the same cross section information near the two cobalt lines, which shows how the multigroup cross sections average over quite large energy ranges.
Table 8.2.2. Total macroscopic cross section in tungsten (/cm).

\begin{tabular}{l|cc}
\hline & $1173230 \mathrm{eV}$ & $1332490 \mathrm{eV}$ \\
\hline SCALE CE & 1.03353 & 0.94864 \\
SCALE 47 & 1.09066 & 0.92743 \\
SCALE 19 & 1.05167 & 0.89289 \\
\hline
\end{tabular}

The small differences in cross section can make large differences in the transport. Consider just $5 \mathrm{~cm}$ of tungsten. Using the cross sections in Table 8.2.2, the attenuation $\left(e^{-\mu x}\right)$ of either line can vary by $30 \%$.

In addition to source representation problems, multigroup transport is not adequate for applications where line spectra are measured. Because of the group structure, tally results will be averaged out within a group. With the fixed boundaries, specific lines in the tallies will not be able to be seen. For examples, in the 19-group library, there is no group around the $511 \mathrm{keV}$ annihilation gammas - they are averaged in with other photons from 400 to $600 \mathrm{keV}$. No multigroup structure could contain thin groups around every line of interest.

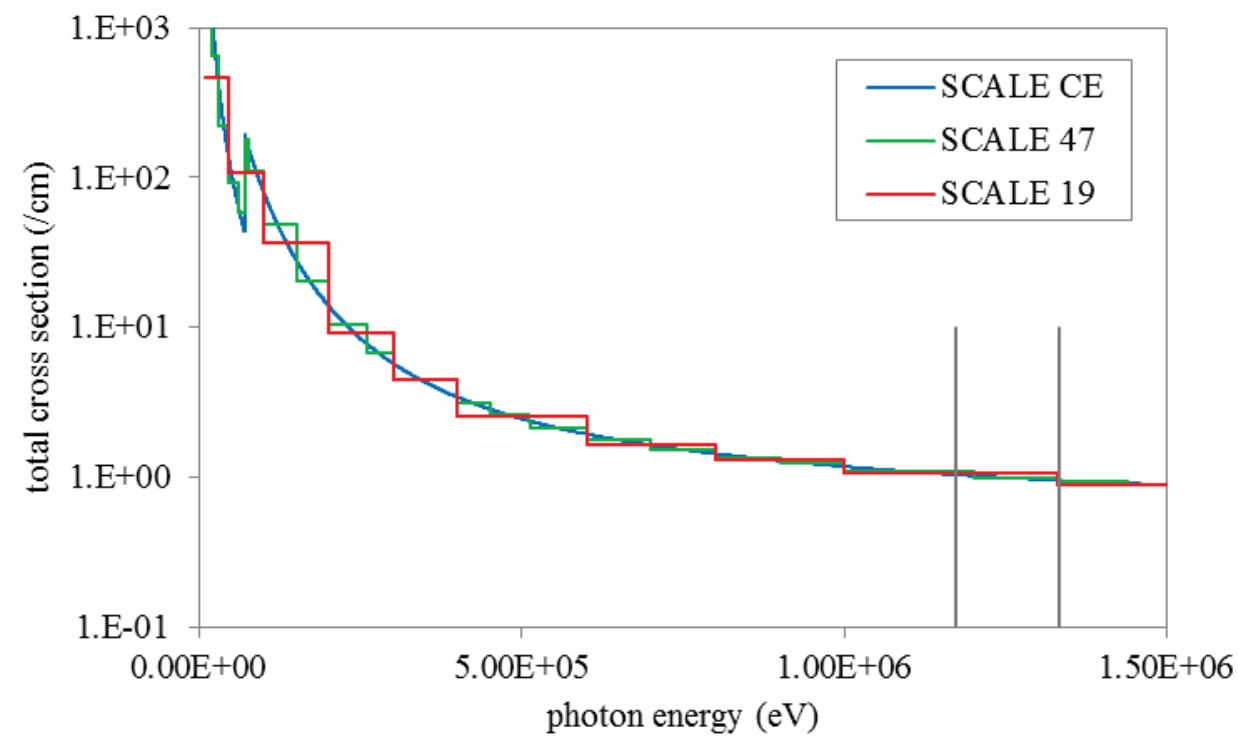

Figure 8.2.2. Photon total cross section in tungsten. The energies of the cobalt-60 are displayed as lines at 1173230 and $1332490 \mathrm{eV}$. 


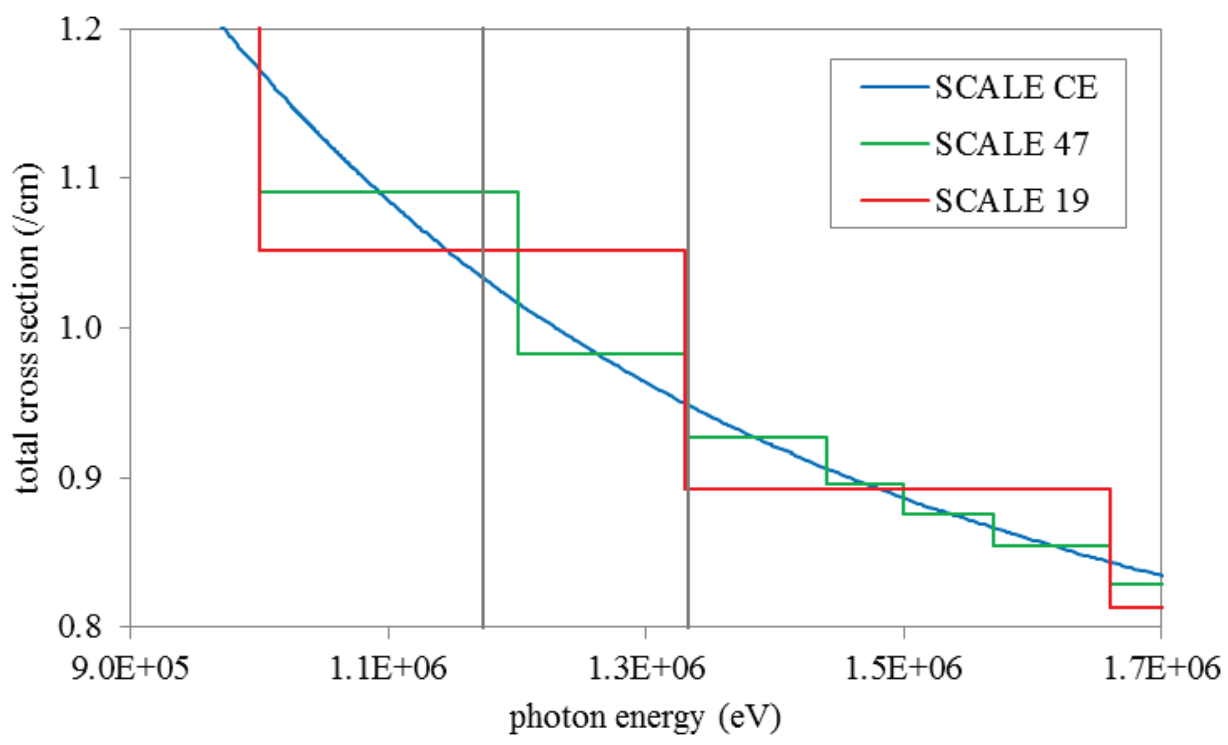

Figure 8.2.3. Photon total cross section in tungsten, near the cobalt lines. The energies of the cobalt-60 are displayed as lines at 1173230 and $1332490 \mathrm{eV}$.

A sample problem involving a cobalt source and a slab of tungsten will compare the use of continuousenergy transport to multigroup transport, to demonstrate the large difference in results for single-line sources. For distributions, differences between multigroup and continuous-energy may not be very significant.

\subsubsection{Monaco Input Files}

The input file for Monaco consists of two lines of text ("=monaco" command line and one for the problem title) and then several blocks, with each block starting with "read xxxx" and ending with "end xxxx". There are three blocks that are required and seven blocks that are optional. The cross section and geometry blocks must be listed first and in the specified order. Other blocks may be listed in any order.

Blocks (must be in this order):

- Cross Sections - (required) lists the cross-section file and the mixing table information

- Geometry - (required) SCALE general geometry description

- Array - optional addition to the above geometry description

- Volume - optional calculation or listing of region volumes

- Plot - create 2D slices of the SGGP geometry

Other Blocks (any order, following the blocks listed above):

- Definitions - defines locations, response functions, grid geometries, cylindrical mesh geometries, energy bin boundaries, time bin boundaries and various distributions used by other blocks

- Source - (required) description of multiple sources; with the spatial, energy, and directional distributions and particle type for each

- Tallies - description of what to calculate: point detector tallies, region tallies, or mesh tallies

- Parameters - how to perform the simulation (random number seed, how many histories, etc.)

- Biasing - data for reducing the variance of the simulation 
The physical model blocks (Geometry, Array, Volume and Plot) follow the standard SCALE format. See the other SCALE references as noted in the following sections for details.

For the other six blocks, scalar variables are set by "keyword=value", fixed length arrays are set with "keyword value ${ }_{1} \ldots$ value $_{N}$ ", variable length arrays are set with "keyword value ${ }_{1} \ldots$ value $_{N}$ end", and some text and filenames are read in as quoted strings. Single keywords to set options are also used in some instances. The indention, comment lines, and upper/lower case shown in this document are not required - they are used in the examples only for clarity. Except for strings in quotes (like filenames), SCALE is not case sensitive.

After all of the blocks are listed, a single line with "end data" should be listed. A final "end" should also be listed, to signify the end of all Monaco input. See Table 8.2.3 for an overview of the Monaco input file structure.

\subsubsection{Cross-sections block}

Monaco does its own mixing, so it needs a mixing table. For each element of each mixture, an identifier and a number density must be supplied. These can be found in the output of whatever sequence was used to make the cross-section file, such as CSAS-MG. Two coupled neutron/photon multigroup libraries were created specifically for shielding problems from ENDF/B-VII.0 data - the v7-200n47g fine-group and the $\mathrm{v} 7-27 \mathrm{n} 19 \mathrm{~g}$ coarse-group libraries. CE libraries made from ENDF/BVII.0 are also available in SCALE.

Table 8.2.3. Overall input format for Monaco

\begin{tabular}{|c|c|}
\hline input file & Comment \\
\hline $\begin{array}{l}\text { =monaco } \\
\text { Some title for this problem } \\
\text { read crosssections } \\
\quad \text {. . } \\
\text { end crosssections } \\
\text { read geometry } \\
\quad \text {. . } \\
\text { end geometry } \\
\text { read array } \\
\quad \text {. . } \\
\text { end array } \\
\text { read volume } \\
\quad \text {. . } \\
\text { end volume } \\
\text { read plot } \\
\quad \text {. . } \\
\text { end plot } \\
\text { read definitions } \\
\quad \text {. . } \\
\text { end definitions } \\
\text { read sources } \\
\text { … } \\
\text { end sources } \\
\text { read tallies } \\
\text { end tallies } \\
\text { read parameters } \\
\text { end parameters } \\
\text { read biasing } \\
\text { end biasing } \\
\text { end data } \\
\text { end }\end{array}$ & 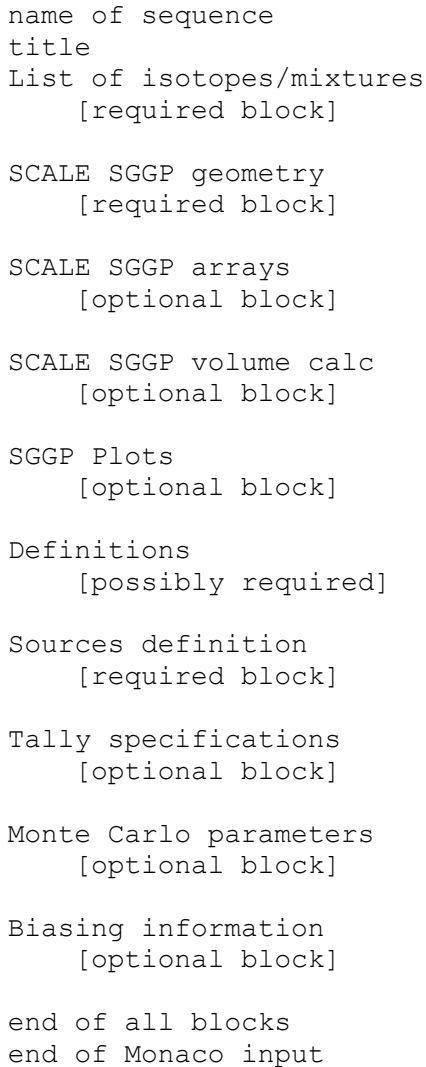 \\
\hline
\end{tabular}


For example, if CSAS-MG was used to produce an AMPX file using the following input,

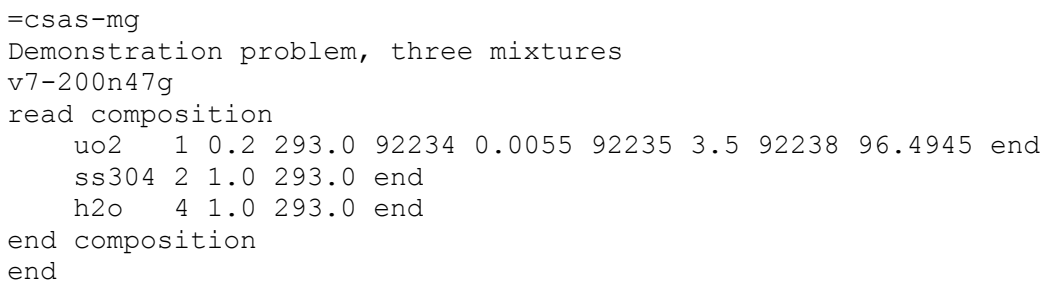

in addition to creating an AMPX file, the output would include a tables similar to

\begin{tabular}{|c|c|c|c|c|c|c|c|c|c|}
\hline entry & & mixture & & isotope & number density & new & identifier & explicit $t$ & temperature \\
\hline 1 & & 1 & & 92234 & $2.73451 E-07$ & & 92234 & & 293.0 \\
\hline 2 & & 1 & & 92235 & $1.73272 \mathrm{E}-04$ & & 92235 & & 293.0 \\
\hline 3 & & 1 & & 92238 & $4.71674 \mathrm{E}-03$ & & 92238 & & 293.0 \\
\hline 4 & & 1 & & 8016 & $9.78057 \mathrm{E}-03$ & & 8016 & & 293.0 \\
\hline$m$ i $x$ & i & $n g$ & E a & $\mathrm{b} 1 \mathrm{e}$ & $($ THREAD $=00)$ & & & & \\
\hline $\begin{array}{r}\text { entry } \\
1\end{array}$ & & $\begin{array}{c}\text { mixture } \\
2\end{array}$ & & $\begin{array}{l}\text { isotope } \\
6000\end{array}$ & $\begin{array}{r}\text { number density } \\
3.18488 \mathrm{E}-04\end{array}$ & new & $\begin{array}{r}\text { identifier } \\
6000\end{array}$ & explicit t & $\begin{array}{c}\text { temperature } \\
293.0\end{array}$ \\
\hline 2 & & 2 & & 14028 & $1.57010 \mathrm{E}-03$ & & 14028 & & 293.0 \\
\hline 3 & & 2 & & 14029 & $7.97625 E-05$ & & 14029 & & 293.0 \\
\hline 4 & & 2 & & 14030 & $5.26416 \mathrm{E}-05$ & & 14030 & & 293.0 \\
\hline 5 & & 2 & & 15031 & $6.94688 E-05$ & & 15031 & & 293.0 \\
\hline 6 & & 2 & & 24050 & $7.59178 \mathrm{E}-04$ & & 24050 & & 293.0 \\
\hline 7 & & 2 & & 24052 & $1.46400 \mathrm{E}-02$ & & 24052 & & 293.0 \\
\hline 8 & & 2 & & 24053 & $1.66006 \mathrm{E}-03$ & & 24053 & & 293.0 \\
\hline 9 & & 2 & & 24054 & $4.13224 \mathrm{E}-04$ & & 24054 & & 293.0 \\
\hline 10 & & 2 & & 25055 & $1.74072 \mathrm{E}-03$ & & 25055 & & 293.0 \\
\hline 11 & & 2 & & 26054 & $3.42190 \mathrm{E}-03$ & & 26054 & & 293.0 \\
\hline 12 & & 2 & & 26056 & $5.37166 \mathrm{E}-02$ & & 26056 & & 293.0 \\
\hline 13 & & 2 & & 26057 & $1.24055 \mathrm{E}-03$ & & 26057 & & 293.0 \\
\hline 14 & & 2 & & 26058 & $1.65094 \mathrm{E}-04$ & & 26058 & & 293.0 \\
\hline 15 & & 2 & & 28058 & $5.26873 E-03$ & & 28058 & & 293.0 \\
\hline 16 & & 2 & & 28060 & $2.02951 E-03$ & & 28060 & & 293.0 \\
\hline 17 & & 2 & & 28061 & $8.82212 \mathrm{E}-05$ & & 28061 & & 293.0 \\
\hline 18 & & 2 & & 28062 & $2.81288 E-04$ & & 28062 & & 293.0 \\
\hline 19 & & 2 & & 28064 & $7.16357 \mathrm{E}-05$ & & 28064 & & 293.0 \\
\hline$m$ i $x$ & $i$ & $\mathrm{n} g$ & $t$ & $\mathrm{a} b \mathrm{~b} e$ & $($ THREAD $=00)$ & & & & \\
\hline entry & & mixtur & & isotope & number density & new & identifier & explicit & temperature \\
\hline 1 & & 4 & & 1001 & $6.67531 \mathrm{E}-02$ & & 1001 & & 293.0 \\
\hline 2 & & 4 & & 8016 & $3.33765 E-02$ & & 8016 & & 293.0 \\
\hline
\end{tabular}

which can be used to construct the Monaco cross-section block mixing table.

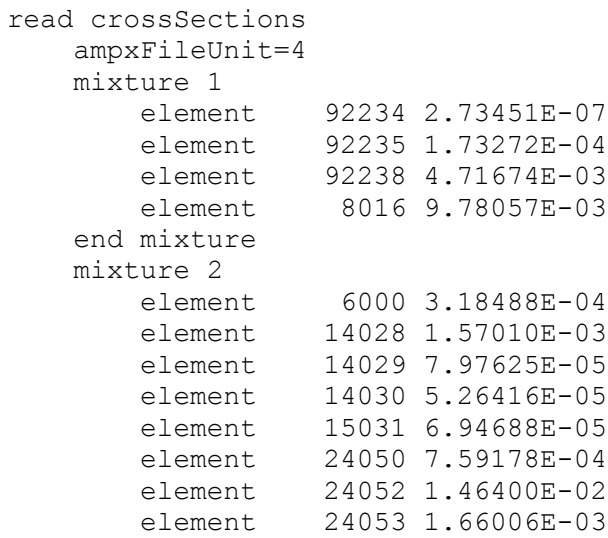




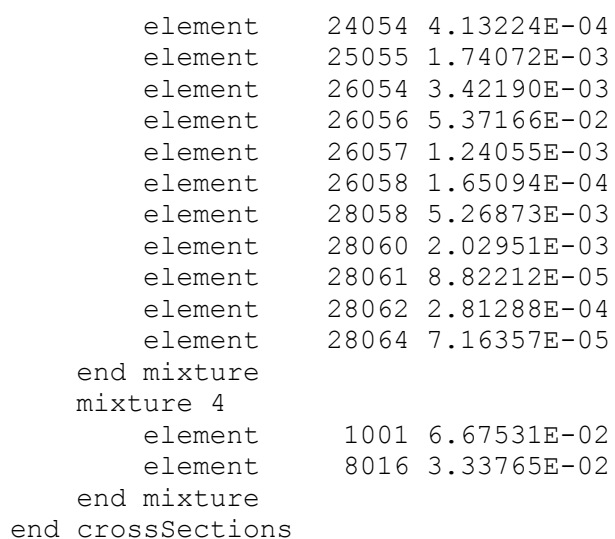

For a CE calculation, instead of the keyword "ampxFileUnit=" (which refers to a given AMPX library), the keyword "ceLibrary=" should be used with a CE library name, enclosed in quotes. Also for CE, a default temperature can be set before any mixtures are defined using the "ceTempDefault=" temperature (in Kelvins). With each mixture, a specific temperature can be set using "temperature."

Other keywords that can be used in the cross-section block for multigroup problems include flags to turn on printing of different aspects of the cross-section mixing process ("printTotals", "printScatters", "printAngleProb", "printFissionChi", "printExtra", and "printLegendre"). The keyword "fullyCoupled" can be used to specify all groups to be treated as primary groups. These keywords do not work in CE problems since the point wise data contain an enormous number of points.

Users are encouraged to use Monaco by running the MAVRIC sequence, which creates the cross-section mixing table automatically, for both multigroup and CE calculations.

\subsubsection{Geometry block}

The geometry input uses the standard SGGP, similar to KENO-VI. Input instructions can be found in Geometry Data in the KENO-VI chapter of the SCALE manual.

Shielding calculations (Monaco, MAVRIC, SAS4) differ from their criticality cousins (KENO V.a, KENO-VI) in a very special way-sources and detectors can be located outside of the materials where the transport takes place. To accommodate this fact in Monaco and MAVRIC, make sure that a void region (a media record using mixture 0 ) surrounds the source area and any point detectors, if they are not located in a region of the actual geometry.

For example, if the objective is to calculate the effectiveness of a simple slab shield, the model geometry would consist of just one slab of material. The source would be on one side of the slab, and a detector would be on the other side of the slab. In Monaco (and the MAVRIC sequence), the input should list at least two regions: (1) the slab itself and (2) a void region outside of the slab containing both the source and detector positions.

Monaco tracks particles through the SGGP geometry as well as other geometries used for mesh tallies or mesh importance maps. Because Monaco must track through all of these geometries at the same time, users should not use the reflective boundary capability in the SGGP geometry.

The graphical user interfaces GeeWiz and Keno3D can be used on Windows platforms to develop and view the geometry. 


\subsubsection{Array, volume and plot blocks}

Geometry array input uses the standard SGGP, similar to KENO-VI. Input instructions can be found in KENO-VI chapter on Array Data of the SCALE manual.

Volumes of various geometry regions are used to calculate fluxes for those regions. Volumes can be input as part of the geometry input block above, or calculated by the SGGP using one of two different methods. See KENO-VI chapter on Volume Data for instructions.

The "read plot" block allows users to create a 2-D character or color plots of slices through a specified portion of the 3-D geometrical representation of the problem. These images can be saved as *.png files. For more information, see the KENO-VI chapter on Plot Data.

\subsubsection{Definitions block}

The definitions block defines different types of data (locations, detector response functions, grid geometries, cylindrical geometries, distributions, energy bin boundaries and time bin boundaries) that are used by some of the other blocks in Monaco. Individual data can be listed in any order. Identification numbers must be positive integers and unique within that type of data. Each type of data begins with a keyword and ends with an "end" and that same keyword. All of the different data types can have an optional title using the keyword "title=".

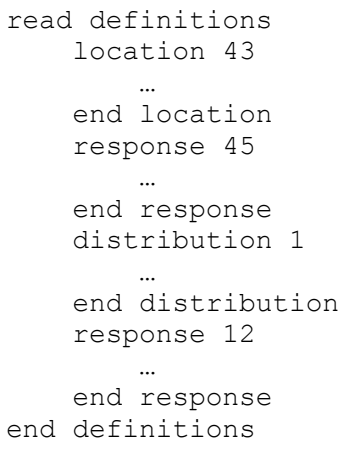

\subsection{Locations}

Locations ("location") require an identification number and the physical position in global coordinates using the "position" keyword (a fixed length array). A position is specified by listing its $x, y$, and $z$ coordinates.

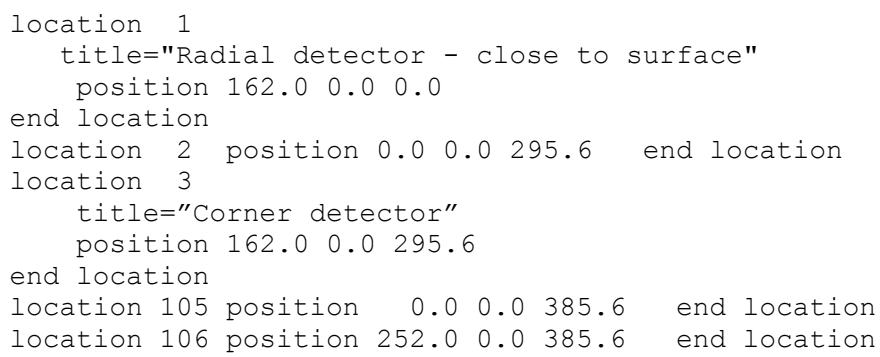

\subsection{Response functions}

Response functions ("response") require an identification number and information on how to build an energy dependent response function. There are three basic types of responses: 1) the general userdefined response, 2) a response based on cross-section data, and 3) a response based on a specific flux-to- 
dose conversion factor. For multigroup calculations, a fourth type of a response simply listing multigroup values is also available. Responses must be defined as either a neutron response or a photon response.

Type 1. A general user-defined response function can be either a binned histogram function $(n+1$ energies and $n$ values) or a set of value/function pairs that will be linearly interpolated ( $n+1$ energies and $n+1$ values). The energies (in $\mathrm{eV}$ ) are set using the "bounds ... end" keyword. The response values are entered with the "values ... end" keyword. The energies can be entered from low energy to high energy order or the traditional high energy to low energy order but must be monotonic. The values array of the response is interpreted to correspond to the order of the bounds array. These two examples

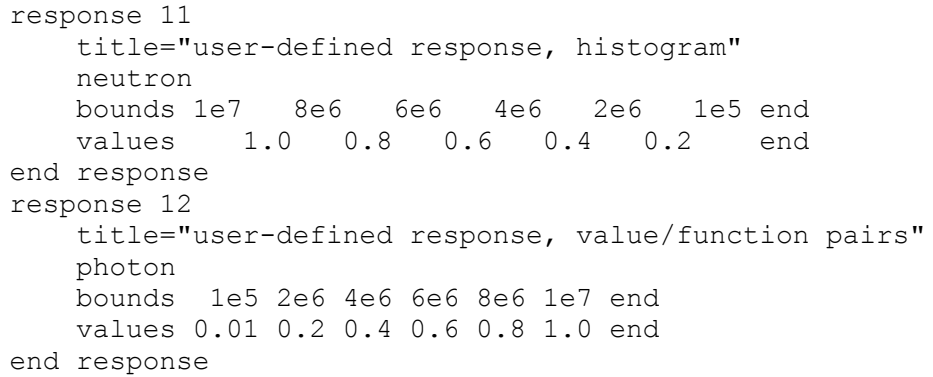

are shown in Figure 8.2.4 and Figure 8.2.5.

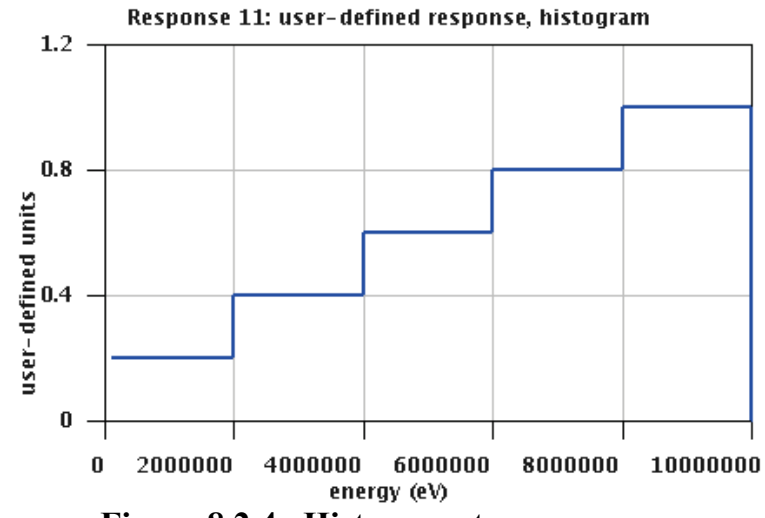

Figure 8.2.4. Histogram-type response

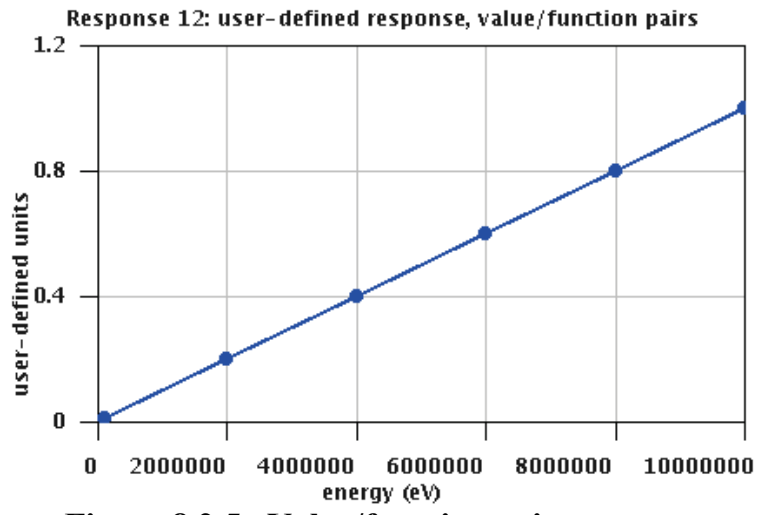

Figure 8.2.5. Value/function pair response

Type 2. Data from the cross-section library can also be used to define a response, for example in finding reaction rates. For the cross section (with units of barns) for a single isotope, the user specifies a material/ZAID/MT combination. The keyword "macro" can be used to multiply the cross section by the atom density of the ZAID in the material (which converts the units of the response from barns to $/ \mathrm{cm}$ ). Users can also specify just the material and MT numbers, to produce the macroscopic cross section of reaction MT for the entire material (with units of $/ \mathrm{cm}$ ). A partial list of common MT numbers is shown in Table F23.3.2 (the full list is in XSECLIB M04, Appendix B). To match some other sequences in SCALE, users can also use text strings to specify the ZAID and MT by using keywords "nuclide=" (for example, nuclide=U-235) and "reaction=" (for example reaction=fission). If the user requested a microscopic cross section response for a reaction in a CE problem, the response will be generated for the nuclide from the AMPX CE libraries even if the nuclide itself is not included in any of the material definitions in the problem. Available reaction lists depend on the nuclide and the list will be printed as a warning message in the output if a non-existing reaction is requested. 


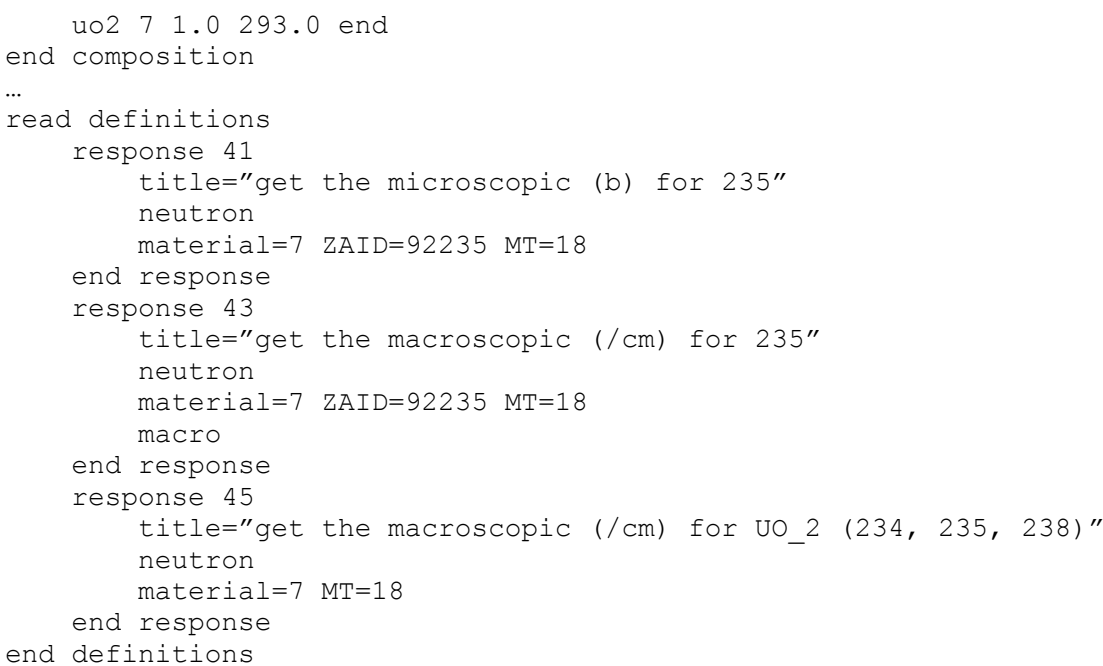

For the examples above, response 41 is shown in Figure 8.2.6. and Figure 8.2.7. for both MULTIGROUP and CE.

Table 8.2.4. Common MT (reaction) numbers for responses

\begin{tabular}{rllrl}
\hline MT & Description & & MT & Description \\
\hline 1 & Total cross section & 501 & Total photon interaction cross section \\
18 & Total fission cross section & 502 & Photon coherent scattering \\
27 & Absorption cross section $(\mathrm{MT}=18$ and 101) & 504 & Photon incoherent scattering \\
101 & Neutron disappearance & 516 & Pair production, nuclear and electron field \\
102 & $(\mathrm{n}, \gamma)$ radiative capture cross section & & 518 & Photofission $(\gamma, \mathrm{f})$ \\
103 & $(\mathrm{n}, \mathrm{p})$ cross section & & 522 & Photoelectric \\
104 & $\left(\mathrm{n},{ }^{2} \mathrm{H}\right)$ cross section & & \\
105 & $\left(\mathrm{n},{ }^{3} \mathrm{H}\right)$ cross section & & \\
106 & $\left(\mathrm{n},{ }^{3} \mathrm{He}\right)$ cross section & & & \\
107 & $\left(\mathrm{n},{ }^{4} \mathrm{He}\right)$ cross section & & & \\
1452 & Product of $v$ times the fission cross section & &
\end{tabular}




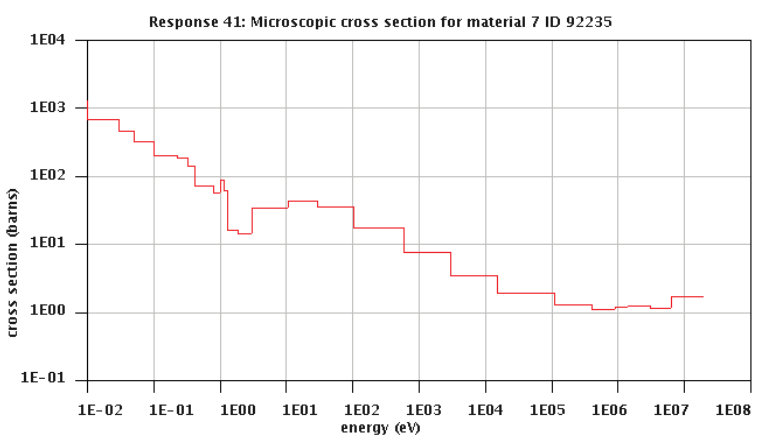

Figure 8.2.6. Multigroup ${ }^{235} \mathrm{U}$ total fission cross section

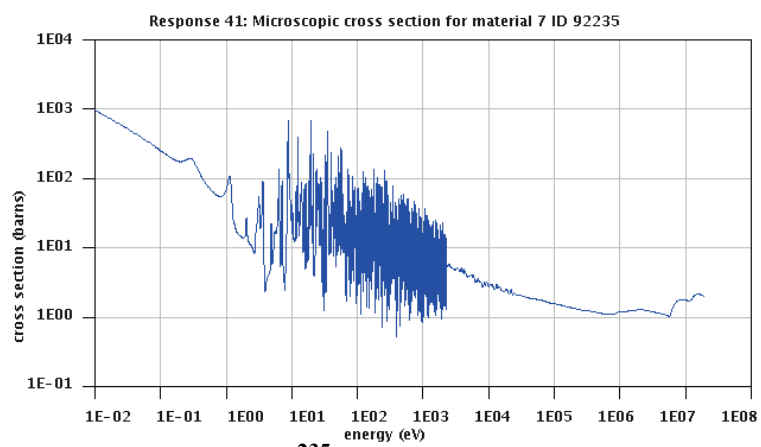

Figure 8.2.7. $C E{ }^{235} \mathrm{U}$ total fission cross section

Type 3. Flux-to-dose conversion factors are a little different in multigroup and continuous-energy implementations. The AMPX multigroup shielding libraries contain neutron and photon dose responses from several sources. These have been processed by the AMPX system (the jergens module). To form the multigroup values for the libraries, the original data was extrapolated to cover the entire energy range of the shielding libraries and was then collapsed into the group structures using a weighting spectrum. These dose responses can be accessed through Monaco/MAVRIC by defining a response object that uses the keyword "specialDose=" and then providing the MT number of the particular response. The dose responses available in the shielding libraries in are shown in Table 8.2.5. Note that the coupled responses in SCALE 6.1 are no longer used by Monaco, since responses are now defined to be either a neutron response or a photon response. When using the "specialDose=" keyword, the "neutron" or "photon" designation is ignored, since the particle type is inherent with the MT number.

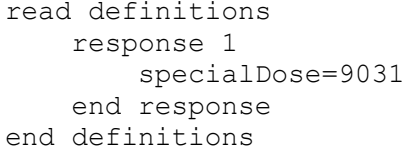

Table 8.2.5. Flux-to-Dose conversion factor MT numbers

\begin{tabular}{|c|c|c|}
\hline \multirow[b]{2}{*}{ Response } & Neutron & Photon \\
\hline & Units & Units \\
\hline Henderson conversion factors & $9027(\mathrm{rad} / \mathrm{h}) /\left(\mathrm{n} / \mathrm{cm}^{2} / \mathrm{s}\right)$ & $9502(\mathrm{rad} / \mathrm{h}) /\left(\mathrm{p} / \mathrm{cm}^{2} / \mathrm{s}\right)$ \\
\hline Claiborne-Trubey conversion factors & & $9503(\mathrm{rad} / \mathrm{h}) /\left(\mathrm{p} / \mathrm{cm}^{2} / \mathrm{s}\right)$ \\
\hline ANSI standard (1977) flux-to-dose-rate factors & $9029(\mathrm{rem} / \mathrm{h}) /\left(\mathrm{n} / \mathrm{cm}^{2} / \mathrm{s}\right)$ & $9504(\mathrm{rem} / \mathrm{h}) /\left(\mathrm{p} / \mathrm{cm}^{2} / \mathrm{s}\right)$ \\
\hline ANSI standard (1991) flux-to-dose-rate factors & $9031(\mathrm{rem} / \mathrm{h}) /\left(\mathrm{n} / \mathrm{cm}^{2} / \mathrm{s}\right)$ & $9505(\mathrm{rem} / \mathrm{h}) /\left(\mathrm{p} / \mathrm{cm}^{2} / \mathrm{s}\right)$ \\
\hline ICRU-44 Table B.3 (air) Kerma & $9032(\mathrm{~Gy} / \mathrm{h}) /\left(\mathrm{n} / \mathrm{cm}^{2} / \mathrm{s}\right)$ & \\
\hline & $9033(\mathrm{rad} / \mathrm{h}) /\left(\mathrm{n} / \mathrm{cm}^{2} / \mathrm{s}\right)$ & \\
\hline ICRU-57 Table A.21 (air) Kerma & & $9506(\mathrm{~Gy} / \mathrm{h}) /\left(\mathrm{p} / \mathrm{cm}^{2} / \mathrm{s}\right)$ \\
\hline & & $9507(\mathrm{rad} / \mathrm{h}) /\left(\mathrm{p} / \mathrm{cm}^{2} / \mathrm{s}\right)$ \\
\hline Ambient dose equivalent (ICRU-57) & $9034 \quad(\mathrm{~Sv} / \mathrm{h}) /\left(\mathrm{n} / \mathrm{cm}^{2} / \mathrm{s}\right)$ & $9508 \quad(\mathrm{~Sv} / \mathrm{h}) /\left(\mathrm{p} / \mathrm{cm}^{2} / \mathrm{s}\right)$ \\
\hline & $9035(\mathrm{rem} / \mathrm{h}) /\left(\mathrm{n} / \mathrm{cm}^{2} / \mathrm{s}\right)$ & $9509(\mathrm{rem} / \mathrm{h}) /\left(\mathrm{p} / \mathrm{cm}^{2} / \mathrm{s}\right)$ \\
\hline Effective dose (ICRU-57) & $9036 \quad(\mathrm{~Sv} / \mathrm{h}) /\left(\mathrm{n} / \mathrm{cm}^{2} / \mathrm{s}\right)$ & $9510 \quad(\mathrm{~Sv} / \mathrm{h}) /\left(\mathrm{p} / \mathrm{cm}^{2} / \mathrm{s}\right)$ \\
\hline & $9037(\mathrm{rem} / \mathrm{h}) /\left(\mathrm{n} / \mathrm{cm}^{2} / \mathrm{s}\right)$ & $9511(\mathrm{rem} / \mathrm{h}) /\left(\mathrm{p} / \mathrm{cm}^{2} / \mathrm{s}\right)$ \\
\hline
\end{tabular}


The standard flux-to-dose conversion factors have not been made part of the continuous-energy libraries. Routines have been added to the Monaco code base to generate data points to allow users to define responses based on the original references. Note that the responses in these references were defined over different energy ranges, as shown in Table 8.2.6.

Table 8.2.6. Energy ranges of the original Flux-to-Dose responses

\begin{tabular}{l|cc|cc}
\hline & \multicolumn{2}{|c|}{$\begin{array}{c}\text { Neutron Energy } \\
\text { Response }\end{array}$} & \multicolumn{2}{|c}{$\begin{array}{c}\text { Photon Energy } \\
\text { Range (MeV) }\end{array}$} \\
\hline Henderson conversion factors & 0.01 & 18 & 0.01 & 10 \\
Claiborne-Trubey conversion factors & & & 0.02 & 16 \\
ANSI standard (1977) flux-to-dose-rate factors & $2.5 \mathrm{E}-08$ & 20 & 0.01 & 15 \\
ANSI standard (1991) flux-to-dose-rate factors & $2.5 \mathrm{E}-08$ & 14 & 0.01 & 12 \\
ICRU-44 Table B.3 (air) Kerma & $2.5 \mathrm{E}-08$ & 29 & & \\
ICRU-57 Table A.21 (air) Kerma & & & 0.01 & 10 \\
Ambient dose equivalent (ICRU-57) & $1.0 \mathrm{E}-09$ & 20.1 & 0.01 & 10 \\
Effective dose (ICRU-57) & $1.0 \mathrm{E}-09$ & 18 & 0.01 & 10 \\
\hline
\end{tabular}

The keyword "doseData=" can be used to create a response using the original, point-wise data (except for Claiborne-Trubey where the original data is a histogram). Data points are also extrapolated to cover the energy range of $10^{-5}$ to $2 \times 10^{7} \mathrm{eV}$ for neutrons and up to $20 \mathrm{MeV}$ for photons. (The optional keyword "noExtrapolation" can be used to get just the original data without the extrapolations.) The final response is formed by interpolating (lin-lin) between these points. For multigroup problems, these keywords will collapse the original data (with or without extrapolation) into a multigroup structure but without the weighting function used to create the dose factors in the multigroup libraries. This will not match the multigroup responses in the those libraries.

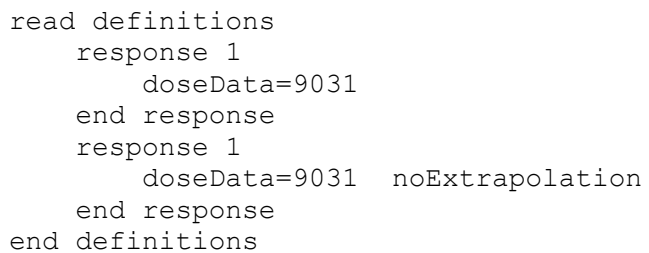

As an example of the various forms of a flux-to-dose conversion factor, the ANSI 1991 values (MT=9031 and 9505) are shown in Figure 8.2.8-Figure 8.2.11. 

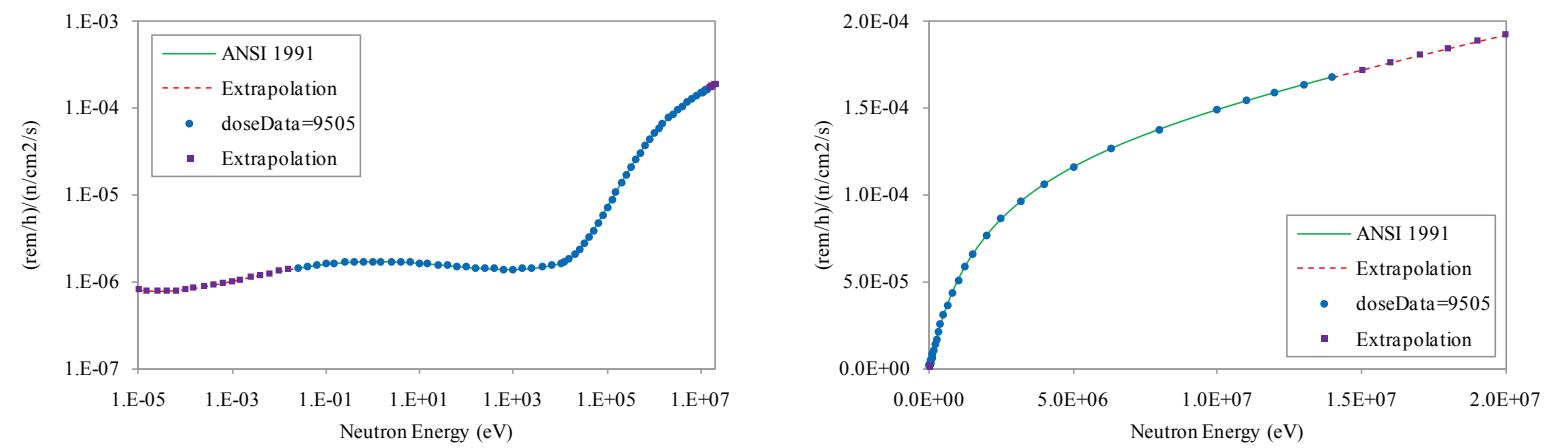

Figure 8.2.8. ANSI 1991 neutron CE (left is log-log, right is linear-linear)
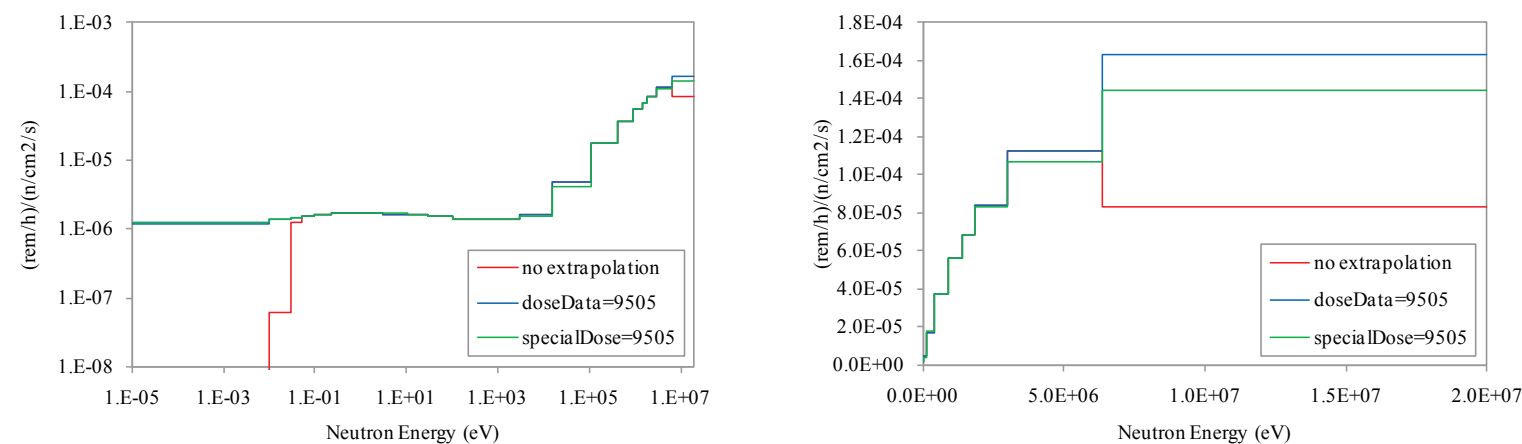

Figure 8.2.9. ANSI 1991 neutron MULTIGROUP (left is log-log, right is linear-linear)
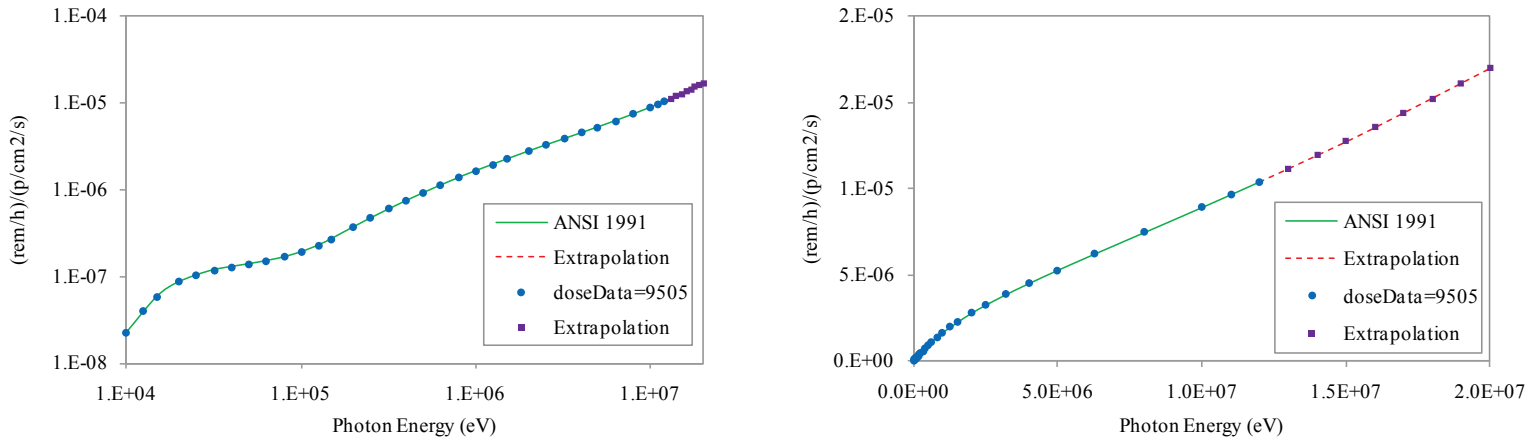

Figure 8.2.10. ANSI 1991 photon CE (left is log-log, right is linear-linear)
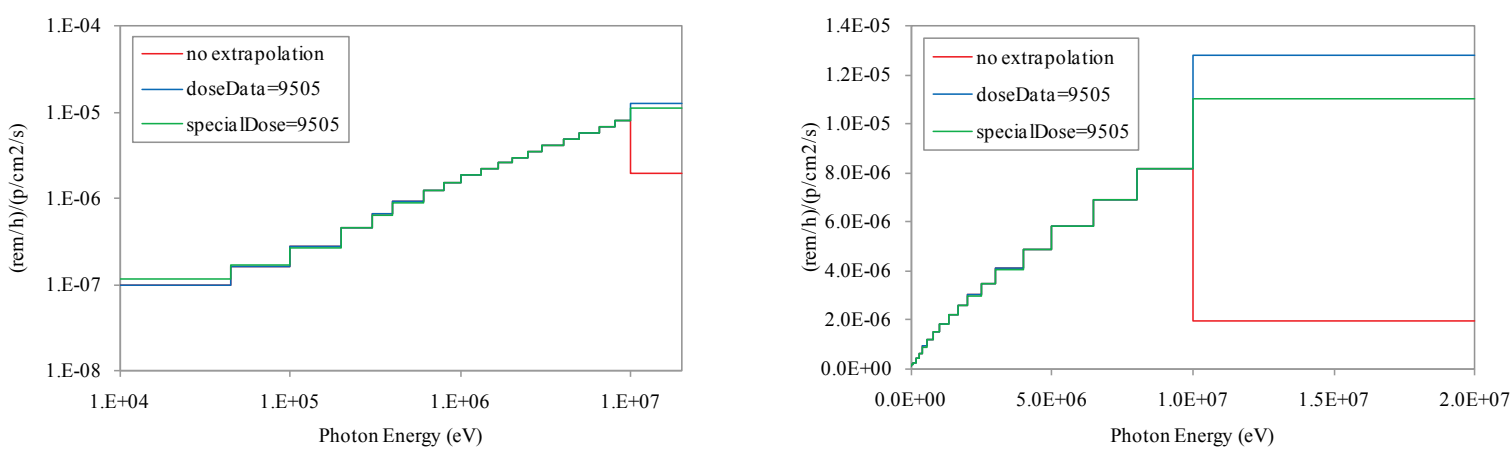

Figure 8.2.11. ANSI 1991 photon MULTIGROUP (left is log-log, right is linear-linear) 
The use of the "specialDose=" and "doseData=" keywords is summarized in Table 8.2.7. Users should understand that the only way to get the 'true' response described in the original references is to use the "doseData=" and "noExtrapolation" keywords. The traditional approach in SCALE has been to extrapolate the original data over the entire energy range of the problem, yielding higher dose rates than the 'true' response would.

Table 8.2.7. Use of the "specialDose $=$ " and "doseData=" keywords.

\begin{tabular}{l|l|l}
\hline keyword(s) & \multicolumn{1}{|c}{ Multi-Group (MG) } & \multicolumn{1}{c}{ Continuous Energy (CE) } \\
\hline specialDose $=M T$ & $\begin{array}{l}\text { uses pre-computed responses contained } \\
\text { in the MG library: data points from } \\
\text { reference, extrapolated to energy range } \\
\text { of the library and collapsed to the library } \\
\text { group structure using a weighting } \\
\text { spectrum }\end{array}$ & $\begin{array}{l}\text { this keyword is not allowed since } \\
\text { there are no pre-computed dose } \\
\text { responses contained within the } \\
\text { CE libraries }\end{array}$ \\
\hline doseData $=M T$ & $\begin{array}{l}\text { data points from reference, extrapolated } \\
\text { over the range of 1.0d-11 to 20.0 MeV } \\
\text { and collapsed to the library group } \\
\text { structure without a weighting spectrum }\end{array}$ & $\begin{array}{l}\text { data points from reference, } \\
\text { extrapolated over the range of } \\
1.0 \mathrm{~d}-11 \text { to 20.0 MeV }\end{array}$ \\
\hline doseData $=M T$ & $\begin{array}{l}\text { data points from reference and collapsed } \\
\text { to the library group structure without a } \\
\text { neighting spectrum }\end{array}$ & $\begin{array}{l}\text { data points from reference using } \\
\text { only the original energy range }\end{array}$ \\
\hline
\end{tabular}

Type 4. For multigroup calculations, since the energy structure is already known, a response can be defined by listing just the values for each group using the keyword "values ... end". The array length of this type of response should match the number of energy groups for that particle type in the cross-section library. Values should be entered in the standard multigroup order - from high energy to low energy. The shortcut keyword "unity" places a value of 1.0 as the response for each group.

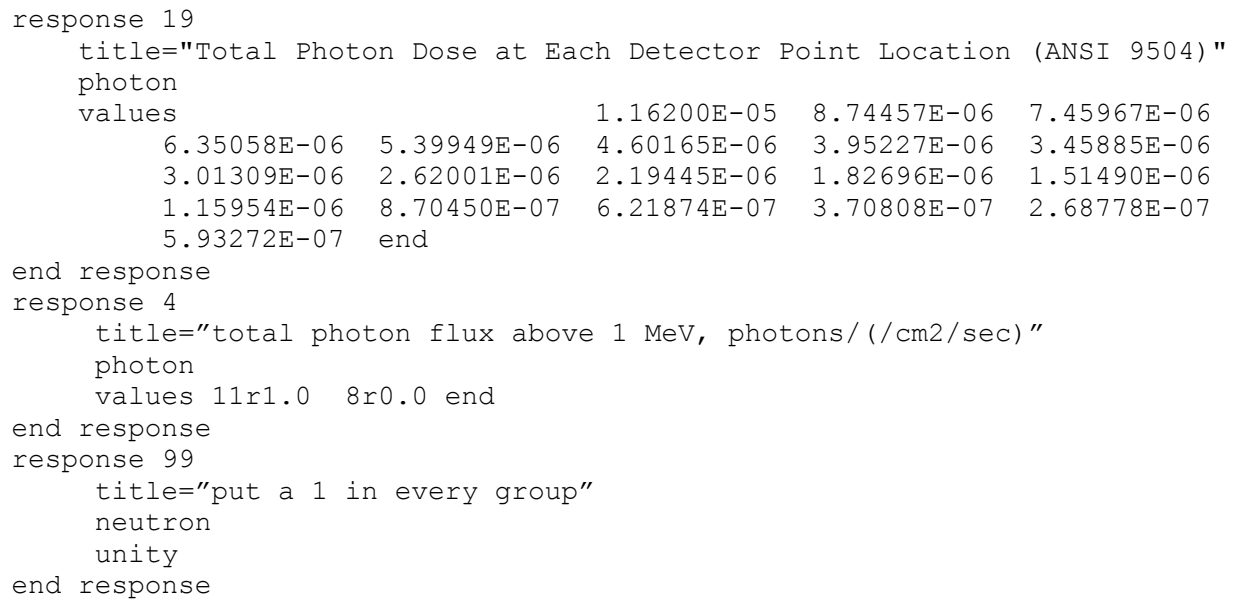

The different response types all share some optional keywords. The keyword "makeChart" can be used to produce a *.chart file (called 'outputName.respid.chart') so that the response can be plotted with the ChartPlot 2D plotter. To create files for every response, use the keyword "makeCharts" inside the definitions block but outside any particular response definition. The keyword "multiplier=" can be used 
with any type of response, which is useful for things such as units conversions. Multiple uses of the "multiplier=" keyword within one response definition will apply the product of all multipliers to that response. Using the keyword "multiplier=" in the definitions block but outside any particular response will apply that multiplier to all responses. Keywords "eHigh=" and "eLow=" can be used to only keep the response values in a range between eHigh and eLow (both in eV). The keyword "lessOutput" can be used to suppress response data echoing in the output file and minimize output file size particularly for CE responses that can have fine point-wise data. It will cause to print only the first five and the last five points of the data if the number of bins is greater than twenty for binned histogram and value/function pairs type of responses.

The original flux-to-dose conversion factor references that were incorporated into Monaco are:

ANSI/ANS-6.1.1-1977 (N666) "American National Standard Neutron and Gamma-Ray Flux-to-DoseRate Factors," Prepared by the American Nuclear Society Standards Committee Working Group ANS6.1.1, Published by the American Nuclear Society, 555 North Kensington Avenue LaGrange Park, Illinois 60525, Approved March 17, 1977 by the American National Standards Institute, Inc.

ANSI/ANS·6.1.1-1991, "American National Standard for Neutron and Gamma-Ray Fluence-to-Dose Factors," Prepared by the American Nuclear Society Standards Committee Working Group ANS-6.1.1, Published by the American Nuclear Society, 555 North Kensington Avenue LaGrange Park, Illinois 60525 USA, Approved August 26, 1991 by the American National Standards Institute, Inc.

H. C. Claiborne and D. K. Trubey, "Dose Rates in a Slab Phantom from Monoenergetic Gamma Rays," Nuclear Applications \& Technology, Vol. 8, May 1970.

B. J. Henderson, "Conversion of Neutron or Gamma Ray Flux to Absorbed Dose Rate," ORNL Report No. XDC-59-8-179, August 14, 1959.

International Commission of Radiation Units and Measurements, ICRU Report 44: Tissue Substitutes in Radiation Dosimetry and Measurement, Bethesda, MD, 1989.

International Commission of Radiation Units and Measurements, ICRU Report 57: Conversion Coefficients for use in Radiological Protection Against External Radiation, Bethesda, MD, August 1, 1998.

\subsection{Grid geometries}

Grid geometries ("gridGeometry") require an identification number and then a description of a 3-D rectangular mesh by specifying the bounding planes of the cells in each of the $x, y$, and $z$ dimensions. The keyword "xplanes ... end" can be used to list plane values (in any order). The keyword "xLinear $n a$ $b$ " can be used to specify $n$ cells between $a$ and $b$. The keywords "xplanes" and "xLinear" can be used together and multiple times - they will simply add planes to any already defined for that dimension. Any duplicate planes will be removed. Similar keywords are used for the $y$-and $z$-dimensions.

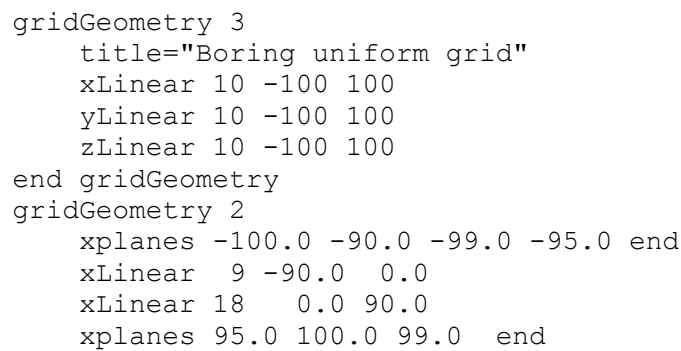




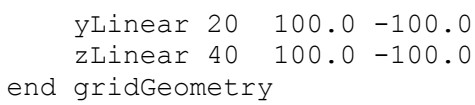

When using multiple instances of the keyword *Linear and *planes for a given dimension, duplicates should be removed from the final list. In some cases, double precision math will leave two planes that are nearly identical but not removed (for example: 6.0 and 5.9999999). To prevent this, a default tolerance is set to remove planes that are within $10^{-6} \mathrm{~cm}$ of each other. The user is free to change this by using the keyword "tolerance=" and specifying something else. Note that the tolerance can be reset to a different value in between each use of *Linear or *planes.

The keyword "make3dmap" for a particular grid geometry definition will create a file called 'outputName.gridid.3dmap' which can be visualized using the Java Mesh File Viewer. Using the keyword "make3dmaps" in the definitions block but outside any particular gridGeometry definition will create a geometry file for each gridGeometry defined.

\subsection{Cylindrical mesh geometries}

Cylindrical geometries ("cylGeometry") require an identification number and then a description of a 3-D cylindrical mesh by specifying the bounding planes of the cells in each of the $r, \theta$, and $z$ dimensions. The keywords "radii ... end", "thetas ... end", and "zplanes ... end" can be used to list the plane values in any order. The keywords "radiusLinear $n a b$ ", "thetaLinear $n a b$ ", and "zLinear $n a b$ " can be used to specify $n$ cells between $a$ and $b$. Note that the keywords "thetas" and "thetaLinear" expect values between 0 and $2 \pi$. For entering values between 0 and $360^{\circ}$, use the keywords "degrees" and "degreeLinear" instead. The keywords for each dimension can be used together and multiple times - they will simply add planes to any already defined for that dimension. Any duplicate planes will be removed. Cylindrical meshes are oriented along the positive z-axis by default. To change this, the user can specify the axis of the cylinder using the keyword "zaxis $u v w$ " and specify the perpendicular direction where $\theta$ $=0$ using "xaxis $u v w$ ". To change the base position of the cylinder, use the keyword "position $x y z$ ". Some examples of cylindrical mesh geometries include:

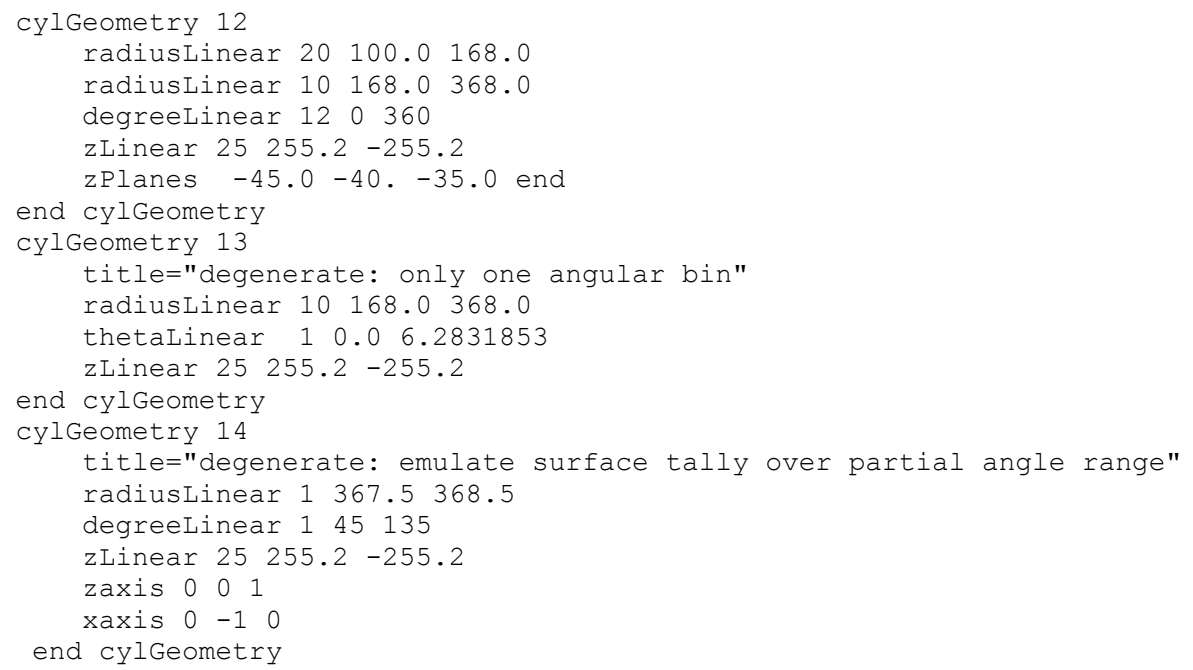

Similar to the grid geometries, the user can use the keyword "tolerance=" to specify how close duplicate planes can be when being considered for removal. The keyword "makeCylMap" for a particular cylindrical geometry definition will create a file called 'outputName.cylid.3dmap' which can be visualized using the Java Mesh File Viewer. Using the keyword "makeCylMaps" in the definitions block but outside any particular gridGeometry definition will create a geometry file for each gridGeometry 
defined. The Mesh File Viewer is written for rectilinear geometries and will not display circles. The only view that works in the Mesh File Viewer for cylindrical meshes is the $x-z$ view, which will correctly show an $r-z$ slice. The slider (marked " $y$ ") will control which $\theta$ value to display (from 0 to $2 \pi$ ).

Cylindrical meshes can only be used for tallies. They cannot be used for making mesh sources or for any importance calculations in MAVRIC.

\subsection{Distributions}

Distributions ("distribution") require an identification number and several other keywords depending on the type of distribution. For a binned histogram distribution over $n$ intervals, the keyword "abscissa ... end" is used to list the $n+1$ bin boundaries and the keyword "truePDF ... end" is used to list the $n$ values of the pdf integrated over those bins. For a pdf defined using a series of evaluated points over $n$ intervals, use the keywords "abscissa ... end" and "truePDF ... end" listing the $n+1$ values for each. The "truePDF" values should be the value of the pdf evaluated at the corresponding point in the abscissa array. The abscissa array should either be in increasing order or decreasing order - monotonic either way - with the truePDF array ordered accordingly.

For either the binned histogram or the value/function point pairs distributions, biasing can also be specified for a given distribution using the "biasedPDF ... end" keyword, the "weight ... end" keyword, or the "importance ... end" keyword, with a length that matches the truePDF array. Weights specify the suggested sampling weights for particles and importances specify the suggested importance. For biasing, the user only needs to specify just one of "biasedPDF", "weight" or "importance". The other arrays will be computed by Monaco.

For discrete distributions (such as gamma line sources), use the keyword "discrete ... end" to list the discrete abscissa values and use the keyword "truePDF ... end" to list the probabilities. The "biasedPDF ... end", "trueCDF ... end", and "biasedCDF ... end" keywords can also be used. Each array should have the same length - the number of discrete lines.

To visualize a distribution, add the keyword "runSampleTest" and a *.chart file will be produced showing the true pdf, the pdf used for sampling (the biased pdf) and the results of a sampling test using $10^{6}$ samples. The file will be named using the output name of the SCALE job and the distribution identification number 'outputName.distid.chart' and can be viewed with the ChartPlot 2D Interactive Plotter. To perform a sampling test and create a *.chart file for all of the distributions in the definitions block, use the keyword "runSampleTests" inside the definitions block but outside any particular distribution.

Some example distribution inputs are listed below and shown in Figure 8.2.12.

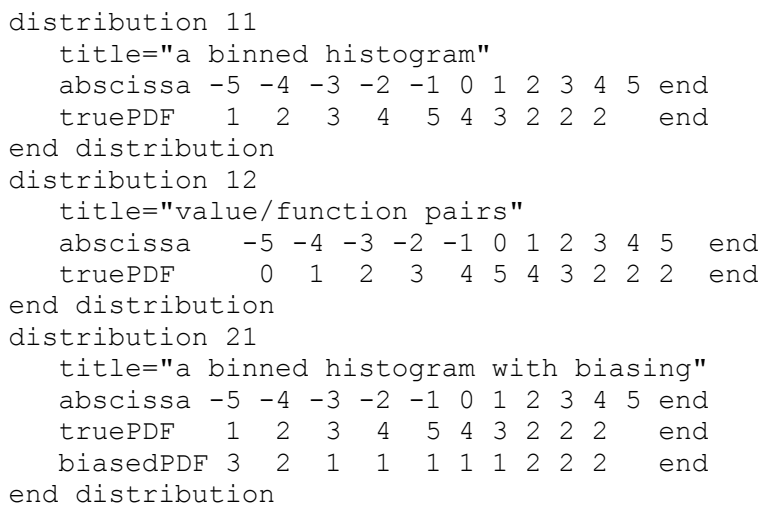




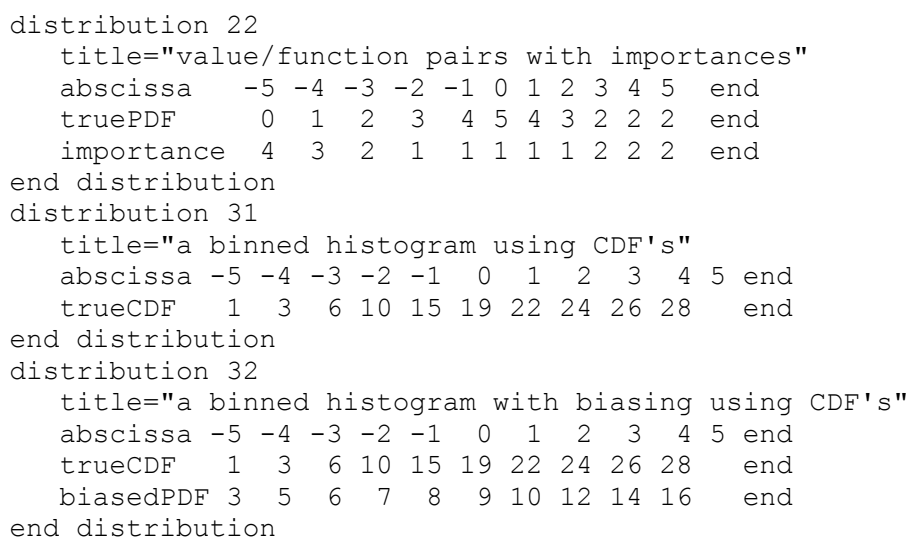

Other notes on distributions:

1) Binned histogram distributions can also be specified using cdf's (keywords "trueCDF" and "biasedCDF").

2) For distributions that will be used for source energy sampling, use abscissa values of eV.

3) For multigroup calculations using histograms, the keywords "neutronGroups" or "photonGroups" can be used instead of specifying the abscissa values. In this case, be sure to list the binned pdf values in order from the highest energy group to the lowest energy group.

4) For CE calculations, instead of specifying abscissa values, the bin boundaries of an energyBounds object (see next section) can be specified using "energyBoundsID=".
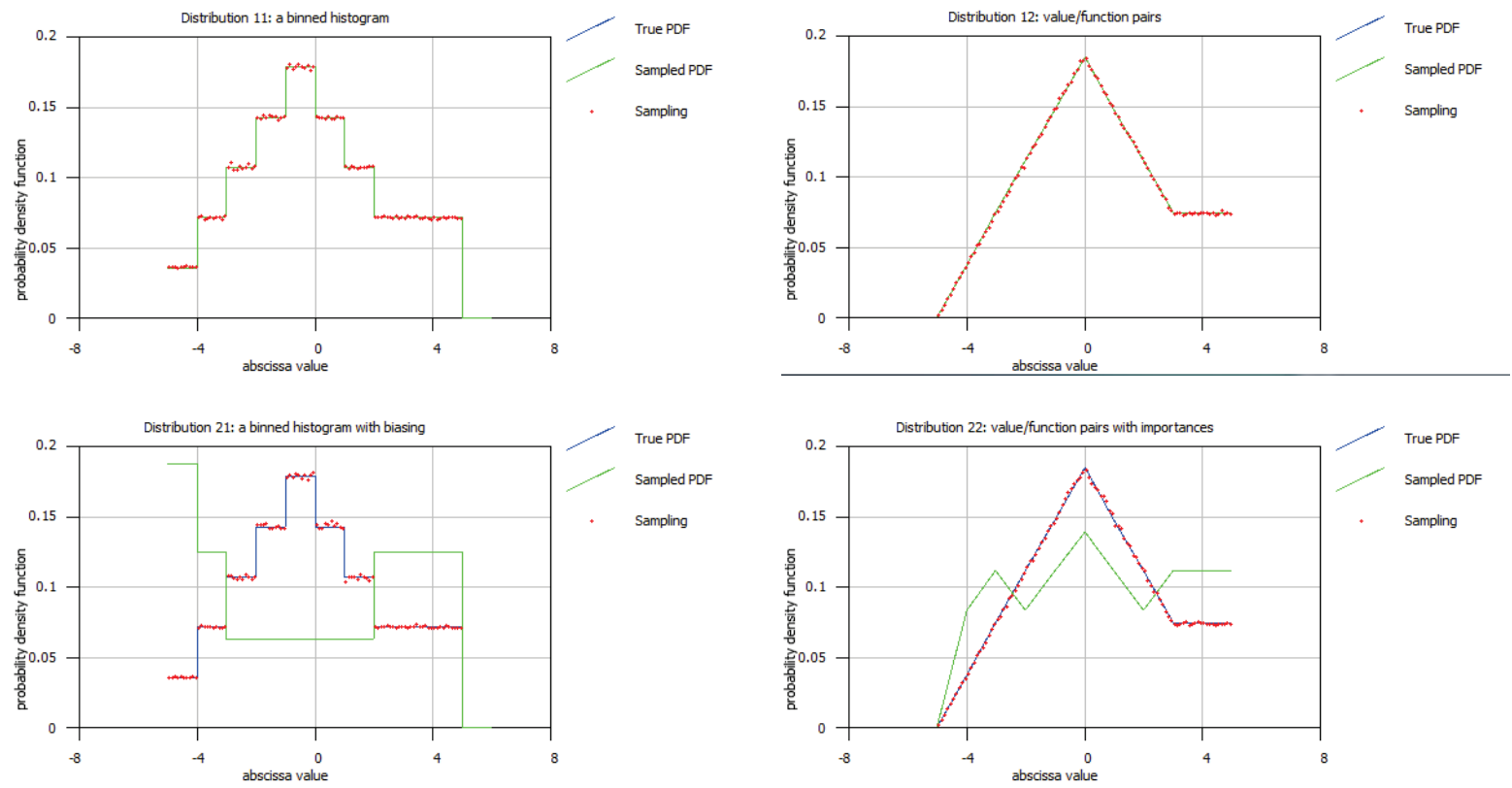

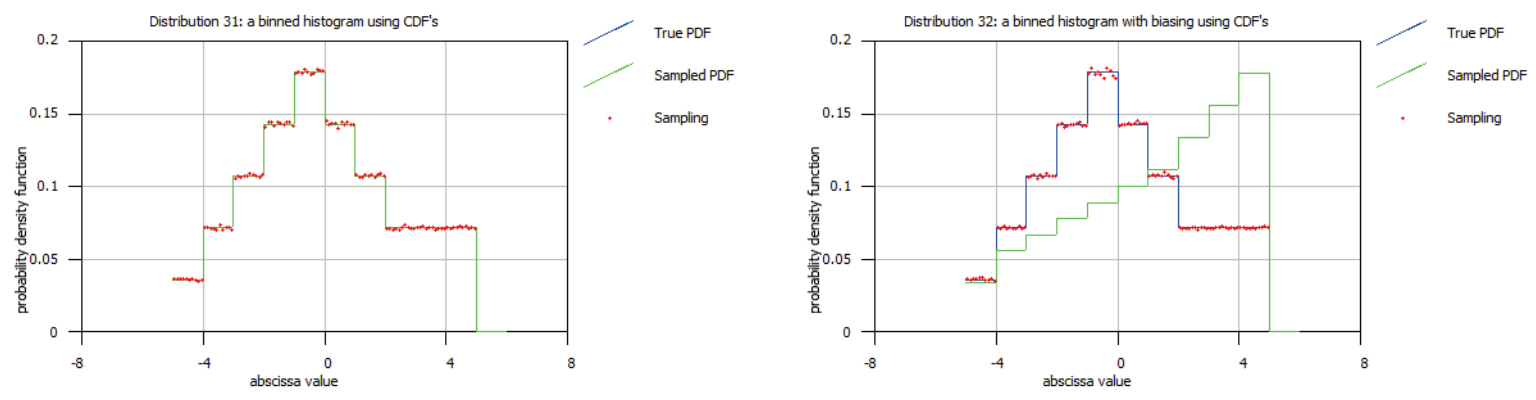

Figure 8.2.12. Sampling tests for the distribution examples.

Several special (built-in) distributions are available in Monaco. To use one of these, specify the keyword "special=" with a distribution name in quotes and the keyword "parameters ... end" (if required) for that type of distribution. These special distributions are summarized in Table 8.2.8.

The Watt spectrum has the form

$$
p(E)=c e^{-E / a} \sinh (\sqrt{b E})
$$

with the parameters $a$ and $b$ (with $c$ as a normalization constant). For spontaneous fission of ${ }^{252} \mathrm{Cf}$, values typically used are $a=1.025 \mathrm{MeV}$ and $b=2.926 / \mathrm{MeV}$. For thermal fission of ${ }^{235} \mathrm{U}$, the parameters are $a=1.028 \mathrm{MeV}$ and $b=2.249 / \mathrm{MeV}$. For induced fission, the parameters $a$ and $b$ are, in general, functions of incident neutron energy. See Table 8.2.9 for an example. The Watt spectrum distribution will be displayed in the *.chart plot as a histogram distribution using the cross-section energy structure neutron groups but when sampled in Monaco, the continuous Froehner and Spencer ${ }^{1}$ method is used to select an energy of source particles using a Watt spectrum distribution.

Table 8.2.8. Special (built-in) distributions

\begin{tabular}{|c|c|c|}
\hline Distribution & Parameters & Description \\
\hline "wattSpectrum" & $a b n$ & $\begin{array}{l}\text { Watt spectrum distribution. Units are: } a \text { in } \mathrm{MeV}, b \text { in } \\
\text { /MeV. Optional parameter } n \text { specifies how many } \\
\text { subintervals in each neutron group to use in } \\
\text { integrating the pdf (default 100) for the histogram } \\
\text { representation in the sampling test and mesh source } \\
\text { representation. }\end{array}$ \\
\hline "fissionNeutrons" & $m Z A I D$ & $\begin{array}{l}\text { Spectrum of fission neutrons from the } \\
\text { MULTIGROUP cross-section library for material } m \\
\text { and nuclide ZAID. }\end{array}$ \\
\hline "fissionPhotons" & $Z A I D$ & Spectrum of fission photons from nuclide $Z A I D$. \\
\hline "origensBinaryConcentrationFile" & $c s$ & $\begin{array}{l}\text { Spectrum from an ORIGEN-S binary concentration } \\
\text { file case number } c \text {, spectra type } s \text {. For the spectra } \\
\text { type } s \text {, values are: } 1 \text { - total neutron, } 2-\text { spontaneous } \\
\text { fission, } 3-(\alpha, n) \text {, and } 4-\text { delayed neutrons, } 5- \\
\text { photons. The ORIGEN-S filename should be } \\
\text { supplied with the keyword filename }=\text { "..." and the } \\
\text { path/filename in quotes. }\end{array}$ \\
\hline "cosine" & $n$ & $\begin{array}{l}\text { Cosine function from }-\pi / 2 \text { to } \pi / 2 \text {. Optional } \\
\text { parameter } n \text { (default } 100 \text { ) is the number of }\end{array}$ \\
\hline
\end{tabular}




\begin{tabular}{|c|c|c|}
\hline "pwrNeutronAxialProfile" & none & $\begin{array}{l}\text { value/function pairs to show in the sampling test. } \\
\text { Typical neutron PWR axial profile. }\end{array}$ \\
\hline "pwrGammaAxialProfile" & none & Typical gamma PWR axial profile. \\
\hline "pwrNeutronAxialProfileReverse" & none & $\begin{array}{l}\text { Typical neutron PWR axial profile, reversed top to } \\
\text { bottom. }\end{array}$ \\
\hline "pwrGammaAxialProfileReverse" & none & $\begin{array}{l}\text { Typical gamma PWR axial profile, reversed top to } \\
\text { bottom. }\end{array}$ \\
\hline "exponential" & $a n$ & $\begin{array}{l}\text { Exponential function } e^{a x} \text { from }-1 \text { to } 1 \text {. Optional } \\
\text { parameter } n \text { (default 100) is the number of } \\
\text { value/function pairs to show in the sampling test. }\end{array}$ \\
\hline "origensDiscreteGammas" & $z a m$ & $\begin{array}{l}\text { Discrete gammas from the ORIGEN mpdkxgam } \\
\text { database for isotope of atomic number } z \text {, mass } a \text { and } \\
\text { metastable state } m \text {. (default is } \mathrm{m}=0 \text { ) }\end{array}$ \\
\hline
\end{tabular}

For the ORIGEN-S binary concentration sources, the ORIGEN input file should be specified using the filename $=$ "..." with the path/filename in quotes. Note that the ORIGEN calculation has to be set to save the neutron or photon data will be used as a Monaco distribution. This can be done by specifying the number of photon or neutron groups on the $3 \$$ (library integer constants) array and specifying the energy bin boundaries on the 83* and 84* (group structure) arrays. In Monaco, to show all of the cases in the binary concentration file, ask for case 0 . To show what data is available for a particular case, ask for that case number and spectra type 0 .

Other notes on special distributions: 1) Fission neutron distributions use MT=1018 for the specified ZAID of the specified isotope from the cross-section library. 2) Fission photon distributions are not read from the cross-section file but are instead read from a separate file containing only ENDF/B-VII.0 fission photon data. 3) The neutron and photon axial profile distributions come from the SCALE 5.1 SAS4 manual, Table S4.4.5. 4) Fission neutron distributions are not allowed in the CE problems, users are advised to use "wattSpectum" in order to get a similar distribution.

Table 8.2.9. Watt spectrum parameters for neutron induced fission of ${ }^{233} \mathrm{U}$

(From ENDF/B-VII.0)

\begin{tabular}{ccc}
\hline $\begin{array}{c}\text { Energy } \\
(\mathrm{MeV})\end{array}$ & $\begin{array}{c}\mathrm{a} \\
(\mathrm{MeV})\end{array}$ & $\begin{array}{c}\mathrm{b} \\
(/ \mathrm{MeV})\end{array}$ \\
\hline $1 . \mathrm{E}-11$ & 0.977 & 2.546 \\
1.5 & 0.977 & 2.546 \\
2 & 0.980 & 2.532 \\
5 & 1.010 & 2.412 \\
7 & 0.970 & 2.571 \\
10 & 0.980 & 2.474 \\
12.2 & 1.010 & 2.612 \\
15 & 1.000 & 2.652 \\
18 & 1.040 & 2.689 \\
20 & 1.060 & 2.620 \\
30 & 1.060 & 2.620 \\
\hline
\end{tabular}


Some example special distribution inputs are listed below and shown in Figure 8.2.13.

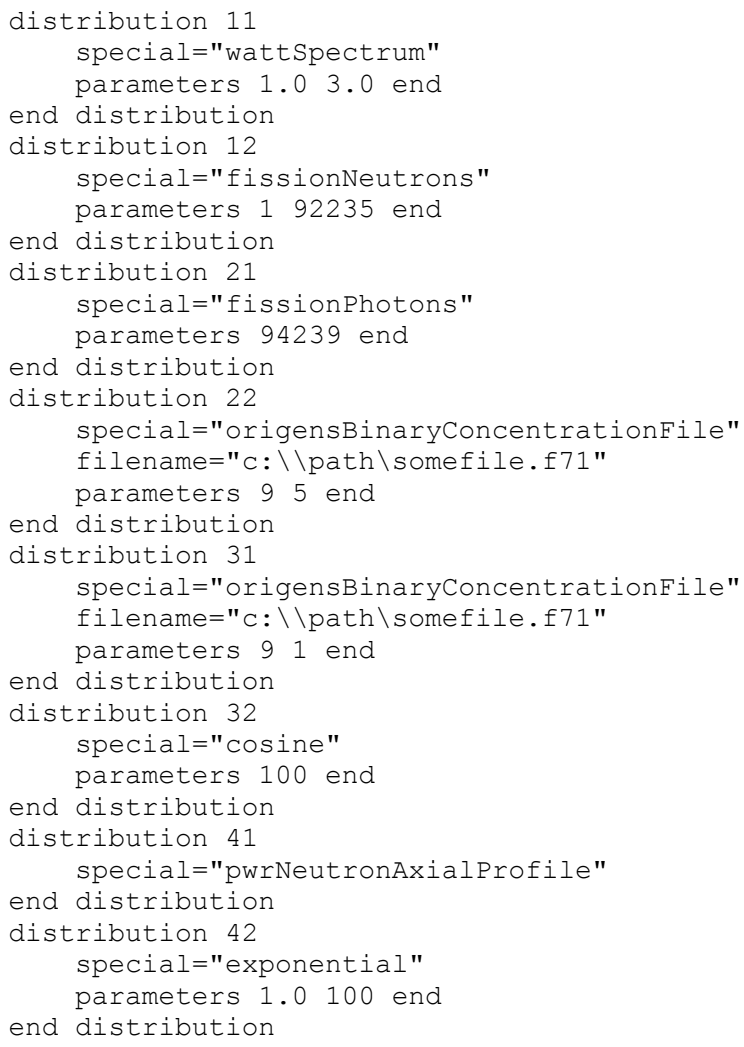



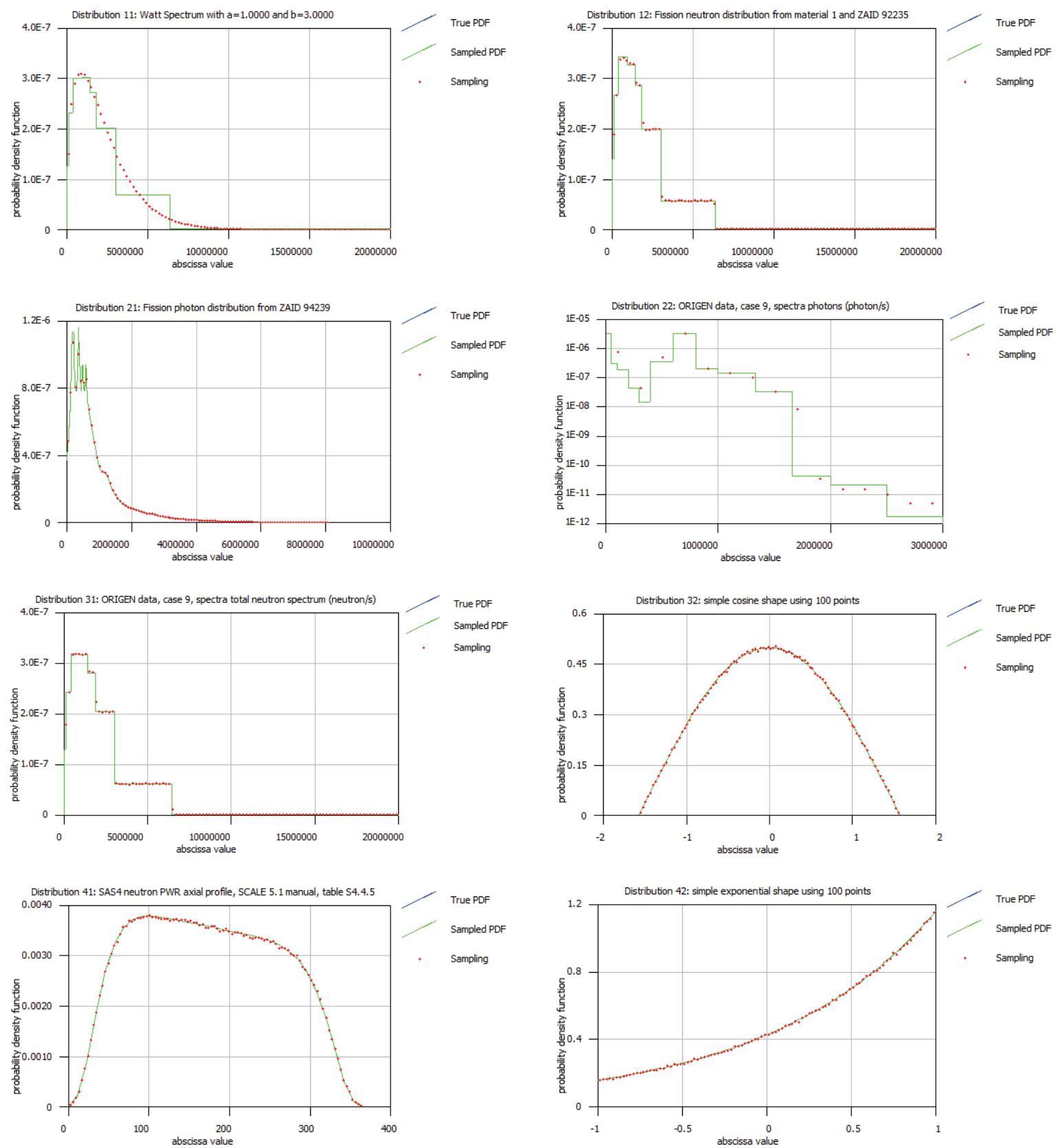

Figure 8.2.13. Sampling tests for the special (built-in) distribution examples.

\subsection{Energy boundaries}

Energy boundaries ("energyBounds") require an identification number and a specification of a set of bin boundaries in energy $(\mathrm{eV})$. Energy bounds objects are typically used in CE calculations for specifying and energy grid for tallies. The keyword "bounds ... end" can be used to list energy values (in eV, in any order). The keyword "linear $n a b$ " can be used to specify $n$ bins between $a$ and $b$. Likewise, the keyword "logarithmic $n a b$ " can be used for $n$ bins logarithmically spaced between $a$ and $b$. The keywords "bounds", "linear" and "logarithmic" can be used together and multiple times - they will 
simply add energy boundaries to any already defined. Any duplicate planes will be removed using the absolute tolerance, specified with the keyword "tolerance=". To specify one of the more common SCALE energy structures (handy for doing tallies one a standard structure in CE calculations), one of the following shortcut keywords can be used: "252n", "238n", "200n”, "56n", “47p", “44n”, "27n", or "19p". These keywords will cause to load the energy structures from the MG cross-section libraries aliased in the "FileNameAliases.txt" with names of "xn252", "xn238", "xn200", "xn56", "xg47”, "xn44", "xn27”, and "xg19" relatively. If required energy structure is for neutrons and there is no alias for MG cross-section library or the library is missing, MG JEFF reaction data library will be searched as " $n\{N G\}$.reaction.data" to load the energy structure. These can be used in combination with the other keywords to use existing structures supplemented with extra boundaries.

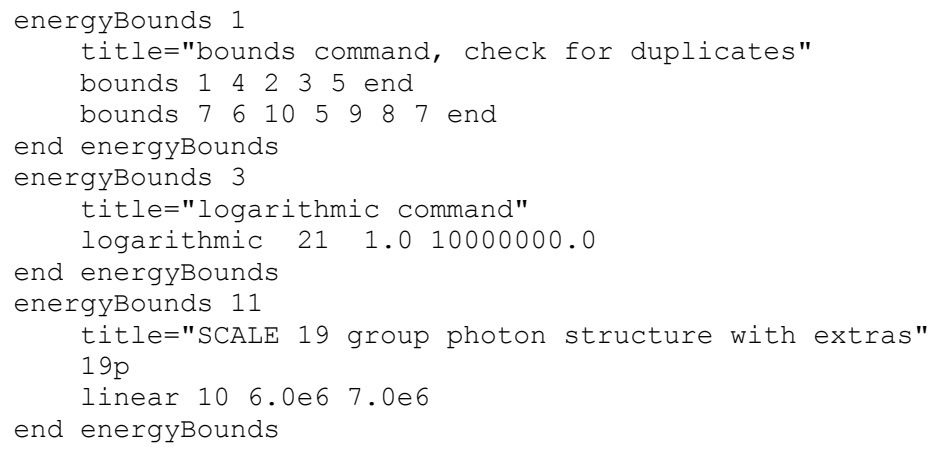

An energyBounds object can also be used to set the energy bin boundaries for a response (type1) instead of using the "bounds ... end" keyword. This is done by using with the keyword "energyBoundsID=" and referencing a defined energyBounds object. Likewise for distributions, instead of specifying the "abscissa ... end" keyword and listing abscissa values, an energyBounds object can be used. This allows the user to define a set of energy bin boundaries once and re-use them across multiple responses and definitions. When using the "energyBoundsID=" keyword, the data values should be entered in the standard multigroup order - from high energy to low energy. For a stand-alone multigroup Monaco calculation, do not use ID numbers of 1 or 2 for energyBounds objects - these ID numbers are reserved.

\subsection{Time boundaries}

Time boundaries ("timeBounds") are similar to energy bin boundaries but take values in seconds. These objects are only used in tallies in CE calculations.

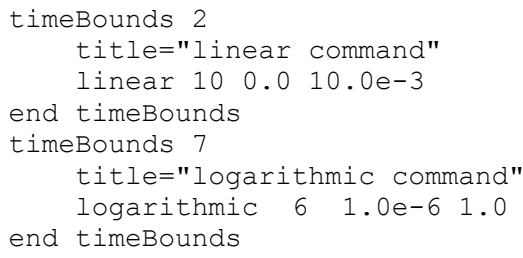

\subsubsection{Sources block}

The sources block specifies what sources to use. Multiple sources are allowed and each is sampled according to its strength, relative to the total strength of all sources. Each source description must be contained with a "src id" and an "end src" (where the id is the source identification number). The sources block must contain at least one source.

For each user-defined source, the user can specify the spatial distribution, the energy distribution and the directional distribution separately. Many options for each distribution are available and defaults are used 
for most if the user does not specify anything. The source strength is set using the keyword "strength=" and the type of source is set using the keyword "neutron" or "photon". The "strength=" keyword is required for each source.

When using more than one source, the user can set the true strength of each using the keyword "strength=" and can also specify how often to sample each source using the keyword "biasedStrength=". The true strengths of the sources will be combined to form the true source distribution PDF. The biased strengths of sources will be combined to form a PDF from which to sample. The weights of the source particles will be properly weighted to account for the biased sampling strengths. For example, consider two sources of strengths $10^{9}$ and $9 \times 10^{9} / \mathrm{sec}$ that should be sampled in a ratio of $4: 1$. The biased sampling strengths are then set to 4 and 1 . Monaco will sample the first source $80 \%$ of the time and the particles will be born with a weight of 0.125 . The second source will be sampled $20 \%$ of the time and its particles will be born with weights of 4.5 .

\subsection{Spatial distribution}

For the spatial distribution, Monaco first defines a bounding simple geometric solid body, limited by the SGGP unit, region or mixture number. The available solid bodies, their required parameters and their degenerate cases are listed below:

\begin{tabular}{llll}
\hline \multicolumn{1}{c}{ Keyword } & \multicolumn{2}{c}{ Parameters } & \multicolumn{1}{c}{ Possible degenerate cases } \\
\hline cuboid & $x_{\max } x_{\min } y_{\max } y_{\min } z_{\max } z_{\min }$ & $\begin{array}{l}\text { rectangular plane, line, point } \\
\text { xCylinder }\end{array}$ \\
$r x_{\max } x_{\min }$ & circular plane, line, point \\
yCylinder & $r y_{\max } y_{\min }$ & circular plane, line, point \\
ZCylinder & $r z_{\max } z_{\min }$ & circular plane, line, point \\
xShellCylinder & $r_{1} r_{2} x_{\max } x_{\min }$ & cyl., planar annulus, cyl. surface, line, ring, point \\
yShellCylinder & $r_{1} r_{2} y_{\max } y_{\min }$ & cyl., planar annulus, cyl. surface, line, ring, point \\
zShellCylinder & $r_{1} r_{2} z_{\max } z_{\min }$ & cyl., planar annulus, cyl. surface, line, ring, point \\
sphere & $r$ & & point \\
shellSphere & $r_{1} r_{2}$ & sphere, spherical surface, point \\
\hline
\end{tabular}

Note that other than the shell-type solids, the parameters are the same as the SGGP geometry specification of those solids. The SGGP keyword "origin" (followed by at least one of " $x=$ ", " $y=$ ", and/or " $z=$ ") is available for all of the different source solid bodies. For the cylinder based solid bodies, the direction of the axis of the cylinder can be set by using the keyword "cylinderAxis $u v w$ ", where $u, v$, and $w$ are the direction cosines with respect to the global $x$-, $y$-, and $z$-directions.

The source can be limited to only be from the parts of the solid body that are inside a specific unit ("unit="), inside a specific region ("region=") within the specified unit, or made of a certain material ("mixture="). A mixture and a unit/region cannot both be specified since that would either be redundant or mutually exclusive.

If no source spatial information is provided by the user, the default is a point source located at the origin (in global coordinates). Like SGGP input, the geometry keywords used for the bounding shape are fixed lengths arrays and do not have an "end" terminator. They must be followed by the correct number of parameters. 
The spatial distribution in each dimension of the cuboid shape is specified by using the keywords "xDistributionID=", "yDistributionID=", or "zDistributionID=" and pointing to a distribution defined in the definitions block. For the cylindrical shapes, "rDistributionID=" and "zDistributionID=" can be used. For spherical shapes, only the "rDistributionID=" can be specified. Distributions defined using abscissa values that are different than the length of the simple geometry bounding shape can still be used if the keyword "xScaleDist" (or " $y$ ", "z", or "r") is used. This linearly scales the distribution abscissa values to the length of the simple geometry bounding shape. Note that for cylindrical sources, since the axis can point in any direction, the $\mathrm{z}$ distribution is interpreted as the length along the axis, with the base position as $\mathrm{z}=0$.

\subsection{Energy distribution}

The energy distribution of the source is specified by using the keyword "eDistributionID=" and pointing to one of the distributions defined in the definitions block. Energies will be sampled from the distribution in a continuous manner. For MULTIGROUP calculations, that energy will then be mapped onto the group structure of the cross-section library being used by Monaco. Each source should have an energy distribution that has abscissa values in units of $\mathrm{eV}$. If no energy distribution is given, $1 \mathrm{MeV}$ (translated to the current group structure if a multigroup problem) will be used.

To use the total of an energy distribution as the source strength, use the keyword "useNormConst" without either "strength=" or "fissions=". This will set the strength to be equal to the normalization constant of the distribution - the total of the distribution before it was normalized into a pdf. An optional "multiplier=" keyword can be used to increase or decrease that strength. For example, consider a case using the neutron spectrum information from a case of an ORIGEN-S binary concentration file that used a basis of an entire core. If the Monaco source was just one of the 200 assemblies, then the "multiplier=" keyword can be set to 0.005 so that the source strength is scaled appropriately.

\subsection{Directional distribution}

The directional distribution of the source is specified by using the keyword "dDistributionID=" and pointing to one of the distributions defined in the definitions block. The distribution will be used to sample the cosine of the polar angle, $\mu$, from the reference direction. The reference direction, where $\mu=1$, is set with the keyword "direction $u v w$ ", where $u, v$, and $w$ are the direction cosines with respect to the global $x$-, $y$-, and $z$-directions. The default value for the reference direction is the positive $z$-axis $(<0,0,1>)$. The keyword "dScaleDist" can be used to linearly scale the distribution abscissa values to the range of $\mu \in[-1,1]$. If no directional distribution is specified with the keyword "dDistributionID=", then an isotropic directional distribution will be used.

\subsection{Using a Monaco mesh source map file}

The user can alternatively specify an existing Monaco mesh source map file - a binary file created by a previous MAVRIC or Monaco calculation. The mesh source map must be a binary file using the Monaco mesh source map format (a *.msm file). This option is specified with the "meshSourceFile=" keyword and the file name (and full path if necessary) in quotes.

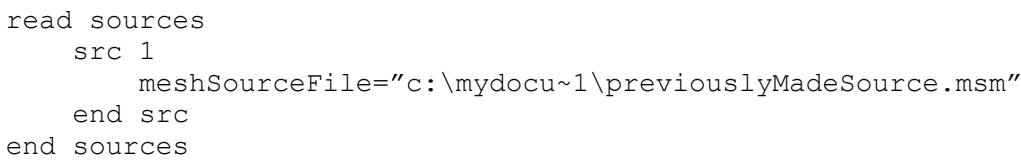

If the "meshSourceFile=" keyword is used, all energy distribution keywords and most spatial distribution keywords will be ignored. Source keywords that can be used with a mesh source include "strength=" to override the source strength in the mesh source; "biasedStrength=" to set the sampling strength; "origin", 
" $\mathrm{x}=$ ", " $\mathrm{y}=$ ", and " $\mathrm{z}=$ " to place the origin of the mesh source file at a particular place in the current global coordinate system; and the keywords for describing the directional distribution - "dDistributionID=", "direction $u v w$ " and "dScaleDist".

Mesh sources are sampled using the following algorithm: First, a direction is sampled. Second, a voxel is sampled and a position is picked uniformly within the voxel. If that position does not match the optional limiters (unit, region, material specified in the mesh source), a new position is chosen within the voxel until a match is made. If a position cannot be found within the voxel after 10000 tries, Monaco will stop. (This can occur if the mesh voxel contained just a sliver of source volume when generated. For this case, the keyword "allowResampling" can be used to select a new voxel instead of stopping. In general, this keyword should not be used.)

\subsection{Creating a mesh source}

To create a mesh source out of the source definition, use the "meshSourceSaver" subblock inside the sources block. It is quite handy to visualize the sources and ensure they are what were intended. You must specify which one of the defined grid geometries to use (keyword "gridGeometryID=") and a filename for the resulting mesh source file (keyword "filename=" with the filename in quotes "path \name.msm"). For more than one source, each will be stored separately and the filename will include the source id number.

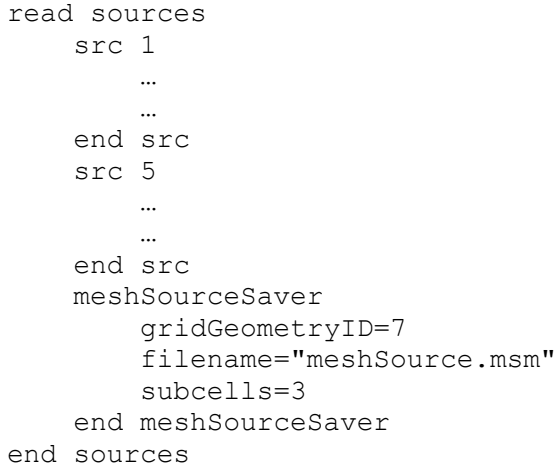

To create the mesh source, Monaco determines if the defined source exists within each cell. This is done by dividing each mesh cell into $n \times n \times n$ subcells (from the keyword subCells $=n$ with a default of $n=2$ ) and testing each subcell center. For every subcell center that is a valid source position (within the spatial solid and meets the optional unit, region, or mixture requirements), an amount of source proportional to the subcell volume is assigned to the mesh cell. The keyword subCells $=$ can be used to better refine how much source is computed for the mesh cells at the boundary of a curved source region. Of course, more subcell testing takes more time. If a given source is degenerate in any dimension (point, line, or plane), that information will be stored in the resulting mesh source so that particles will not be sampled over the entire corresponding voxel but will have closer to the original spatial distribution. Likewise, if the original source had restrictions based on unit, region or mixture, those restrictions will be stored as part of the resulting mesh source.

The above process may miss small sources or degenerate sources (surfaces, lines, points) that do not lay on the tested subcell centers. If none of the mesh cells contain any source after the subcell method, then random sampling of the source is used. A number of source positions are sampled from the source (set by the "sourceTrials=" keyword, default of 1000000) and then placed into the proper mesh cell. If this method is used, the resulting mesh source file should be visualized to ensure that the statistical nature of the source trials method does not unduly influence the overall mesh source. To skip the subcell method and go directly to the source trials method, use "subCells=0". 
The keyword "makeTotal" will make a single mesh source file which is the composite of all of the individual sources. Geometric degeneracies or restrictions to only sample particles from a specified unit, region or material will only be kept if they are the same for all of the sources. For this reason, users may not wish to use a mesh source using the "makeTotal" keyword for transport but rather use it to verify that all of the sources have been input properly.

The keyword "reduce" can be used to only save the smallest rectangular portion of the mesh surrounding the voxels with non-zero source amounts. This can result in much smaller file sizes for sources that are small compared to the extents of the grid geometry.

Monaco mesh source files (*.msm) can be viewed with the Mesh File Viewer. Plots can be made showing the source values for each group (or total). The viewer can also show the geometry regions or material mixtures as well. Using the viewer is an easy way to confirm that the source definition was entered correctly. Note that the *.msm files actually only store the biased sampling distribution and the initial weights (to speed up the sampling process). So, in the viewer the "true" source is computed as the product of the sampled distribution and the weights. If groups with real source are set to zero importance, the viewer cannot recreate the original true source. The true source shown by the viewer is the amount of true source only in groups that have non-zero importance.

\subsection{Mesh source advanced features}

Two advanced features exist in the meshSourceSaver subblock - mainly used by the MAVRIC sequence when the importance map calculations use a different cross-section library than the final Monaco calculation.

The keyword "sampleFromMesh" can be used to tell Monaco to sample from the created mesh file(s) instead of the standard source definition. This can be useful in determining if the mesh source is fine enough to accurately represent the original source definition. If the "makeTotal" keyword was used, then Monaco will sample from the total mesh source file.

The keyword "meshBiasFile=" can be optionally be used when "sampleFromMesh" is on. This tells Monaco to sample from the mesh source file(s) version of the source definition that has been modified using just the importance information from the named mesh source file. For example, using a 27-group biased mesh source for a Watt spectrum source may not represent the high energy tail very well. In this case, it would be better to do a 200-group Monaco calculation but still use the importance information from 27-group mesh source file using "sampleFromMesh" and "meshBiasFile=".

\subsubsection{Tallies block}

The tallies block tells Monaco what to compute: fluxes at certain points in space (point detectors), fluxes in certain geometry regions, or fluxes in each voxel of a mesh grid. The computed fluxes can also be integrated with response functions to compute dose, reaction rate or some other dose-like quantity. Any number of optional response functions can be evaluated with each tally.

Each tally type begins with a keyword ("pointDetector", "regionTally", or "meshTally") and ends with an "end" and that same keyword. Individual tallies can be listed in any order. Identification numbers for each tally are required and must be positive integers and unique among the tally type. All three of the tally types can have an optional title using the keyword "title=" followed by the title enclosed in double quotes. Tallies should be defined as either a neutron tally or a photon tally. 


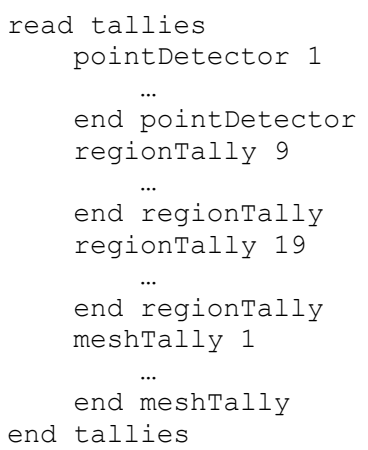

Each tally computes the fluxes in each tally bin and the total flux. For multigroup calculations, the multigroup cross section group structure is used for all tallies. For CE calculations, each tally can use a different "energyBoundsID=", which points to one of the energy bounds defined in the definitions block. $\mathrm{CE}$ calculations can also use the keyword "timeBoundsID=" to specify a set of time bin boundaries. For one response function to integrate the fluxes with, the keyword "responseID=" can be used, where the value corresponds to the identification number of one of the response functions defined in the definitions block. For multiple response functions, the keyword array "responseIDs ... end" can be used.

Each tally type can be multiplied by using the "multiplier=" keyword. This is useful for units conversions or other types of scaling. Multiple uses of the multiplier keyword within one tally definition will apply the product of all multipliers to that tally. Using the keyword "multiplier=" inside the tallies block but outside any particular tally will apply that multiplier to all tallies.

\subsection{Point detector tallies}

A point detector tally computes the uncollided and total flux at a given location in space. This tally requires exactly one location and can use any number of optional response functions. The "locationID=" keyword is used to specify one of the locations listed in the definitions block. Point detectors should only use locations that are in void regions of the geometry.

Because point detectors estimate the flux at the location using a ray-trace from every collision site during the life of the particle, they can be quite expensive. For particles very far away from the detector location, the contributions to the tally can be quite small. Point detectors can be made to use the importance of the current particle location/energy to decide whether or not to make a contribution to the tally using the keyword "minSampProb=" and a value such as 0.1 or 0.01 . This keyword specifies the minimum sampling probability for a given point detector. As the particle is transported, the probability $p$ of making a contribution to the point detector tally is set by using the current weight of the particle, $w$, to be

$$
p=\left(w_{\min } / w\right)^{a}
$$

where the power $a$ for each point detector was determined at the start of the simulation using the minimum sampling probability $p_{\min }$ to be

$$
a=\frac{\ln \left(p_{\min }\right)}{\ln \left(w_{\min } / w_{\text {max }}\right)}
$$

where the minimum and maximum target weights, $w_{\min }$ and $w_{\min }$, were determined from either the region-based weight targets or the mesh-based importance map weight targets. So, when the current 
particle weight approaches the minimum target weight of the problem (in very important areas), the point detector contribution is made nearly $100 \%$ of the time. When the particle weight approaches the maximum target weight (in very unimportant areas), the contribution to the point detector tally is made with probability $p_{\min }$, saving quite a bit of computer time.

This option should only be used if the point detector location is in the area of high importance. For point detectors in areas of low importance, using this option may severely undersample the point detector where contributions would actually be the most significant, causing an underestimation of the flux. The default value for the minimum sampling probability is $p_{\min }=1$, giving a default value of $a=0$ so that the point detector contribution is made at every collision, independent of the current particle weight.

The keyword "minSampProb" used in the tallies block but outside any particular point detector tally specification will be applied to all point detectors.

Each point detector is summarized in the main output file. The uncollided and total flux for each particle type is listed as well as the values for the optional integrated response functions. Along with each of these quantities is a list of the standard deviation of the quantity, the relative uncertainty, the figure-ofmerit and a summary of list of the statistical checks (passed or not). Group-by-group values of the fluxes and responses are listed in a separate file named "outputName.pdid.txt" where outputName is the name the user chose for his output file and " $i d$ " is the identification number corresponding to the point detector tally specification. This file also contains more information about the six statistical checks for flux and each response - their pass/fail values with each batch of simulated particles and their final numerical values at the end of the simulation. A second file called "outputName.pdid.chart" is also created which can be displayed using the ChartPlot 2D Interactive Plotter, to visually check the convergence behavior of the tally. The total neutron flux, the total photon flux, and the total response function value for each response as a function of batch can be viewed.

\subsection{Region tallies}

A region tally computes both the track-length estimate and the collision density estimate of the flux over a given geometry region (an SGGP "media"). This tally uses the keywords "unit=", "region=", and "mixture=" to limit the tally to one or more of those aspects. For example, "unit=2" and "region=3" are used to specify a region tally for the 3rd media listed for unit 2 of the SGGP geometry input. A mixture and a unit/region cannot both be specified since that would either be redundant or mutually exclusive. If the volume of the region is not given (or calculated) in the SGGP input, then instead of flux, the tally will compute average track length and average collision density.

Each region tally is summarized in the main output file. The total flux for each particle type is listed as well as the values for the optional integrated response functions. Along with each of these quantities is a list of the standard deviation of the quantity, the relative uncertainty, the figure-of-merit and a summary of list of the statistical checks (passed or not). Group-by-group values of the fluxes and responses are listed in a separate file named "outputName.rtid.txt" where outputName is the name the user chose for his output file and " $i d$ " is the identification number corresponding to the region tally specification. This file also contains more information about the six statistical checks for flux and each response - their pass/fail values with each batch of simulated particles and their final numerical values at the end of the simulation. A second file called "outputName.rtid.chart" is also created which can be displayed using the ChartPlot 2D Interactive Plotter, to visually check the convergence behavior of the tally. The total neutron flux, the total photon flux, and the total response function value for each response as a function of batch can be viewed. 


\subsection{Mesh tallies}

A mesh tally computes the track-length estimate of the flux for every cell in a grid (mesh) geometry. This tally requires exactly one grid geometry or cylindrical geometry and can use any number of optional response functions. The "gridGeometryID=" or "cylGeometryID=" keyword is used to specify one of the mesh geometries listed in the definitions block. The group-by-group flux values, the total flux values and the total for each response are kept in memory during the simulation. Group-by-group contributions to the responses are not tallied during the simulation.

Mesh tallies can be limited to only save contributions to the voxel flux from track lengths through a certain unit, region or material using the keywords "unit=", "region=", and "mixture=". For example, to compute a mesh tally of a reaction rate of a specific isotope, the response function should only be multiplied by the amount of flux in the voxel that resulted in contributions from the material that contained that isotope. In this case, the keyword "mixture=" should be used so that the fluxes in each voxel represent only the flux from that material that holds the desired isotope.

A mesh tally saves the flux for each group, as well as the integrated response functions for each listed response for every cell of the grid geometry to a file called "outputName.mtid.3dmap" where outputName is the name the user chose for his output file and " $i d$ " is the identification number corresponding to the mesh tally specification. This file contains the group flux values and their absolute uncertainties. If any response functions were specified, then the responses and their uncertainties will be computed and stored in the same file. Monaco mesh tally files can be viewed with the Mesh File Viewer.

With each mesh tally, files are also created with the statistical test information - "outputName.mtid.flux" and "outputName.mtid.respxx.txt" where $x x$ is the responseID. The statistical tests can be turned off with the keyword "noStatChecks".

The Mesh File Viewer can be used to show the value, uncertainty, or relative uncertainty of any of the group fluxes, total fluxes, or responses tallied. For characterizing the mesh tally, the viewer can be used to display histograms of the relative errors - showing what fraction of the mesh cells had less than some amount of relative uncertainty. Images from the Mesh File Viewer can be saved as *.jpg, *.bmp, *.gif or *.png files or exported to other applications, such as MS Word.

Mesh tally files can become quite large - if the group-by-group fluxes are not important for a given problem, the keyword "noGroupFluxes" can be added to the mesh tally input. Instead of the group fluxes, only the total neutron and total photon fluxes will be written to the mesh tally file. If the group-by-group values for the response functions are required, the keyword "saveRespDetails" can be used to create separate mesh tally files of each response called "outputName.mtid.respid.3dmap" where the second "id" is the response identification number. Note that for CE calculations, these group-by-group results are formed using the response function mapped onto the energy grid of the tally, since separate tallies of response by group are not made during the simulation.

The mesh tally keyword "weightless" will instruct Monaco to not include the particle weight in the contribution to the mesh tally for track lengths that cross the voxels of the mesh. Instead of a flux tally, this will compute the Monte Carlo particle density - a measure of the number of particles simulated by Monaco in each mesh cell and in each energy group.

Mesh tallies can use a cylindrical mesh instead of a rectilinear mesh. Use the keyword "cylGeometryID=" instead of "gridGeometryID=" and reference one of the defined cylindrical meshes defined in the definitions block. Both cannot be specified at the same time. The Java Mesh File Viewer can only show the $r-z$ view of a cylindrical mesh tally. 


\subsubsection{Parameter block}

The parameter block sets the Monte Carlo parameters used by Monaco. Items can be listed in any order. The initial random number ("randomSeed=") is given as a 16-digit hexadecimal number. The number of histories per batch ("perBatch=") and the number of batches ("batches=") can be specified. After every batch of source particles, the tally files are saved to disk. To prevent long run times, a maximum run time in minutes ("maxMinutes=") can also be specified. Defaults are 10 batches of 1000 histories each, with no time limit. The value of batches is used to allocate arrays for the tally statistical tests - so do not make this overly large, even when using maxMinutes to control termination.

For MULTIGROUP, the particles contained in the library will be transported, unless turned off using the keywords "noNeutron" or "noPhoton." In CE, to prevent loading large amounts of unneeded crosssection information into memory, the user should specify which particles to transport, using the keywords "neutron" and/or "photon." Monaco also supports Doppler pre-broadening of the CE neutron cross sections. This is controlled by the "dopplerBroaden=" parameter. Integer options are 0 (disabled, default), 1 (broaden 1D cross sections only), 2 (broaden 1D and 2D cross sections), and 3 (broaden 2D cross sections normally and broaden 1D cross sections using a less robust, but faster, interpolation method).

The default behavior for Monaco is to create neutrons from fission events and create secondary gammas from neutron collisions. To turn off the creation of fission neutrons in all multiplying media (for example, when the source already includes them), use the keyword "fissionMult=0". For problems where the library has photon data but none of the tallies require photons, use the keyword "secondaryMult $=0$ " to stop the creation of secondary photons from neutrons. In CE problems, the number of fission neutrons and secondary gammas can be simulated as one particle of each type having a high weight ("fissionMult=1", "secondaryMult=1") or as many particles as the physical yield for the reaction would dictate ("fissionMult=2", "secondaryMult=2"), each with a weight corresponding to their probability of emission. If the user requests option 2 and the particle bank becomes too large, Monaco will drop the settings back to option 1 . In MULTIGROUP, values of 1 and 2 are both treated as 1 .

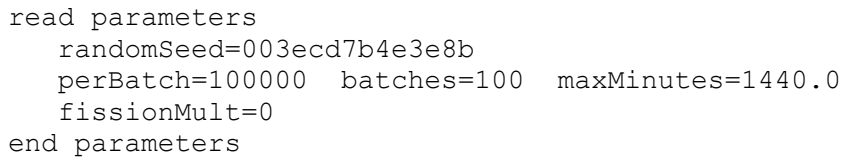

In complex geometries, particles can sometimes "get lost" due to round-off errors in the ray-tracing. This would normally result in the code stopping, since lost particles usually indicate an undefined or doubly defined region of space. In order to tolerate a few lost particles without stopping the code, use the keyword "maximumLost=". Care should be taken not to increase this just to get around poorly defined geometry. To aid in geometry testing, the keyword "voidAllRegions" can be used. This keyword sets every region material to void so that tracks can stream through without interacting (faster). A large source and this keyword can be used to test a geometry input for gaps and overlaps. When the "voidAllRegions" keyword is used, mesh tally files will not contain material information, only the unit and region information.

Note that for both CE and MULTIGROUP, using fissionMult $=0$ only turns off the creation of neutrons from fission. The fission photons are controlled by the secondaryMult setting because some of the ENDF data evaluations do not separate fission gammas from other neutron collision gammas. When using a mesh-based importance map, if a particle is outside the importance map, the code will stop with the message "Could not find particle importance. The particle is outside of the importance map." If the importance map does not cover the entire problem, then the tallies may be missing part of their final 
values. If the user intends to use an importance map that does not cover the entire geometry and wants particles outside the importance map to have zero importance (they will then be killed), then the keyword "allowShortImpMap" can be used to allow the use of a "short" importance map. Users must be sure that areas outside the importance map are unimportant to the problem.

\subsubsection{Biasing block}

The optional biasing block lists the parameters for the standard Monaco variance reduction tools: forced collisions, region-based weight windows, and path-length stretching. This block also allows for the use of a previously made Monaco mesh importance map, such as those produced by the MAVRIC sequence.

\subsection{Forced collisions}

Forced collisions are one of the simplest variance reduction techniques. This makes a particle have a collision along its current flight direction before leaving the geometry. The collision is forced and the particle weight is reduced by the true probability of having a collision within the geometry. This is helpful in small or low-density geometries where many particles leave without interacting but can add computation time to ordinary problems. To use forced collisions, specify the "forcedCollisions" keyword. This requires the use of Russian roulette ("targetWeights" and "lowerWeights", see below).

\subsection{Weight windows}

Monaco can use Russian roulette for preventing low particle weights from being followed and splitting to prevent the production of very high weight particles. Either of these requires the target weight values ("targetWeights ... end") for each energy group and for each region to be listed. For CE calculations, the energy bin boundaries are specified with the keywords "nEnergyBoundsID=" and "pEnergyBoundsID=". For Russian roulette, the lower weight bounds must be specified ("lowerWeights ... end") and for splitting the upper weight bounds are listed ("upperWeights ... end"). The different weight arrays can have a length matching (1) the product of the number of energy groups and the number of regions, (2) the number of regions, or (3) the number of energy groups. In case 2, the values are repeated for each energy group. In case 3, the values are repeated for each region. For example, to specify only Russian roulette in a coupled neutron-photon problem with target and lower weights the same in each region the following is used. An example using the $27 / 19$ multigroup library would be

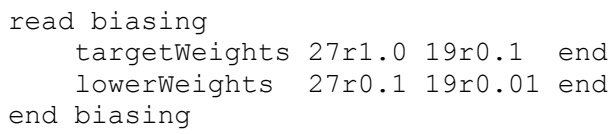

and for a CE calculation

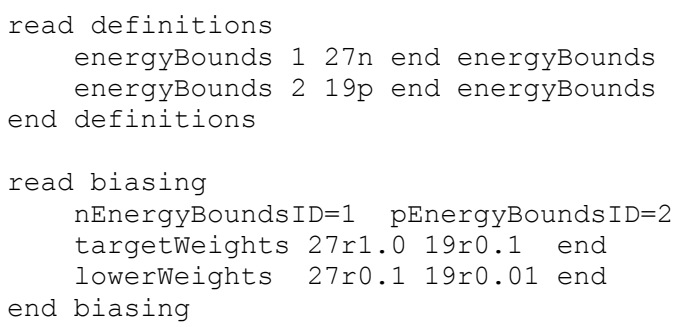

Alternatively, to use Russian roulette and splitting, the target weights and a window ratio ("windowRatio=") can be specified. The window ratio is simply the ratio of the weight window upper bound to the weight window lower bound, with the target weight being the average of the upper and lower. If target weights $\bar{w}$ and a window ratio $r$ are supplied, then the lower and upper weight bounds are found by using 


$$
\begin{aligned}
& w_{\text {min }}=\frac{2}{r+1} \bar{w} \\
& w_{\text {max }}=\frac{2 r}{r+1} \bar{w}
\end{aligned}
$$

If only the window ratio is supplied, both Russian roulette and splitting will be turned on with the target weights for every energy group and every region set to 1 .

A target weight of 0 will prevent particles of that energy group in that region from being transported. For example, to perform a neutron-only calculation using a coupled neutron-photon library, simply set the target weight values for all of the photon groups in every region to 0 . The user should be careful not to "turn off" energy groups or regions that may impact (bias incorrectly) the final tally results.

Monaco always uses the implicit capture technique - at collision sites absorption is not simulated but instead the particle weight is reduced by the ratio of the scatter probability to the total interaction probability. Particles only stop if they escape the defined geometry. This generally produces tally results with lower uncertainties in less time, but for highly scattering or very large geometries, particles with very low weights will be tracked until their weight reaches the lower limit of real numbers in double precision. This is not typically what the user wants. So, for problems that are not using any weight windows or importance map, Russian roulette and splitting are automatically turned on using the a target weight of 1 for every energy group and every region and a window ratio of 5.

\subsection{Path-length stretching}

Path-length stretching allows particles going a certain direction to travel farther (with reduced weight) before interacting. Seventeen different directions are available, as listed in the Table 8.2.10. One of the directions is specified by using the "direction=" keyword and one of the direction strings listed in Table 8.2.10, in quotes. The amount of stretching is specified using the "pathStretch ... end" array, with values between 0 (no stretching) and 1 (lots of stretching), for each energy group and region. Items can be listed in any order. Similar to the weight window arrays, the "pathStretch" array can have a length matching (1) the product of the number of energy groups and the number of regions, (2) the number of regions, or (3) the number of energy groups. Values are repeated to fill in all of the regions and groups.

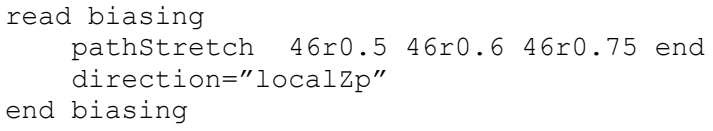

When stretching toward a given location (direction="location"), then the location ID number must be specified using the "locationID=" keyword. For CE calculations, energy boundary objects need to be defined using the keywords "nEnergyBoundsID=" and "pEnergyBoundsID=". 
Table 8.2.10. Directions available for path-length stretching

\begin{tabular}{l|l}
\hline Direction & Comment \\
\hline localXp & +x direction in local coordinates \\
localYp & $+y$ direction in local coordinates \\
localZp & + Z direction in local coordinates \\
localXm & - x direction in local coordinates \\
localYm & $-y$ direction in local coordinates \\
localZm & $-\mathrm{Z}$ direction in local coordinates \\
localYZ & cylindrically away from $x$-axis (local) \\
localXZ & cylindrically away from $y$-axis (local) \\
localXY & cylindrically away from $z$-axis (local) \\
\hline
\end{tabular}

\begin{tabular}{l|c}
\hline Direction & Comment \\
\hline globalXp & +x direction in global coordinates \\
globalYp & $+y$ direction in global coordinates \\
globalZp & $+z$ direction in global coordinates \\
globalXm & - x direction in global coordinates \\
globalYm & $-y$ direction in global coordinates \\
globalZm & - Z direction in global coordinates \\
outward & spherically outward (local) \\
location & in the direction of a given location \\
& (requires a locationID) \\
\hline
\end{tabular}

\subsection{Mesh-based importance map}

The user can alternatively specify an existing Monaco mesh-based importance map - a binary file created by a previous MAVRIC calculation. The mesh importance map must be a binary file using the Monaco mesh importance map format (a *.mim file). This option is specified with the "meshImpMapFile=" keyword and the file name (and full path if necessary) in quotes.

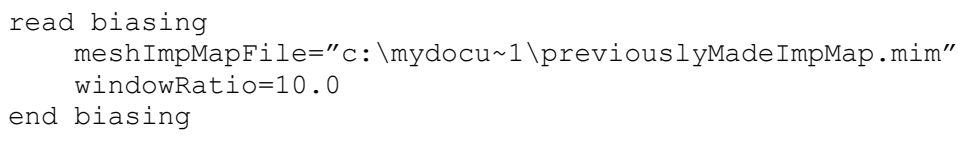

If the "meshImpMapFile=" keyword is used, most other biasing block keywords cannot be used. The keyword "windowRatio=" can be used and its default value is five.

If particles leave the importance map but are still in the defined geometry, the simulation will be stopped. If the user wants to allow importance maps that do not cover the entire problem, the keyword "allowShortImpMap" should be used in the parameters block. In that case, areas outside the mesh importance map will be treated as completely unimportant — particles will be killed outside the mesh.

Note that for the most effective use of an importance map, the source should be biased to match. This is what the MAVRIC sequence does - it produces a biased source such that sampled particles are born with a weight matching the target weight of the importance map.

\subsection{Monaco input summary}

below summarize the Monaco blocks and their available keywords. 
Table 8.2.11. Keywords for the cross-section block

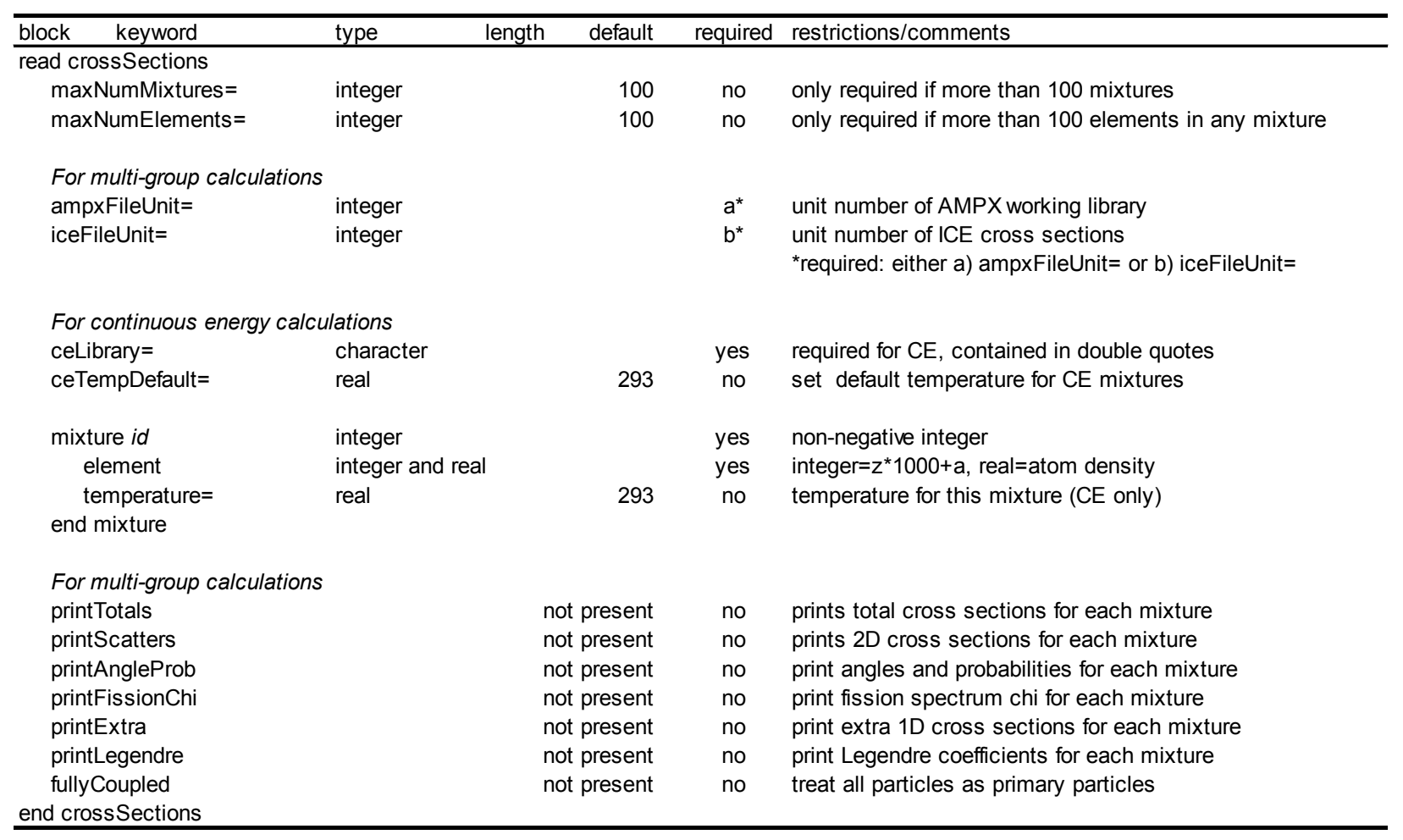


Table 8.2.12. Keywords for the definitions block

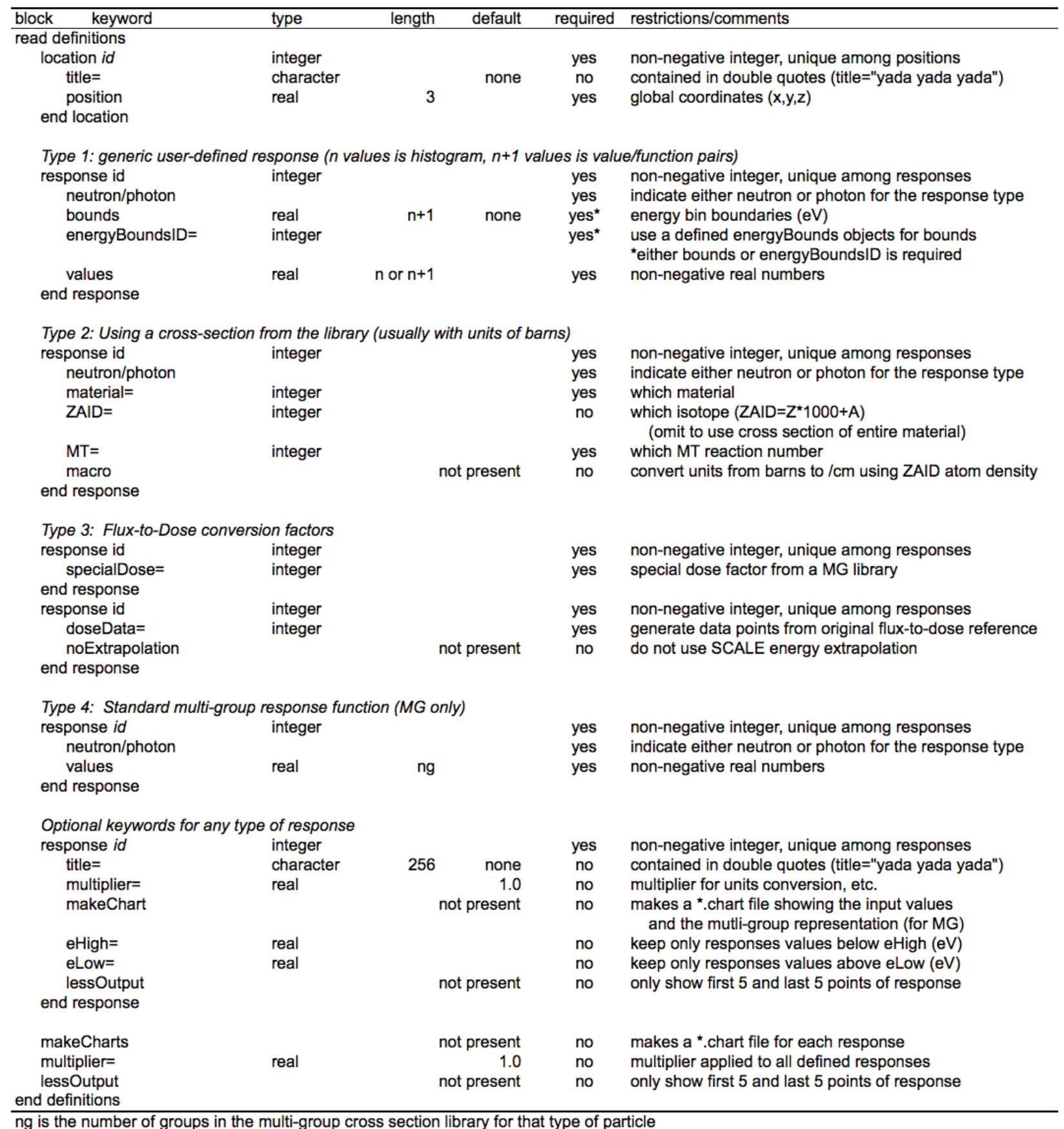


Table 8.2.13. More keywords for the definitions block

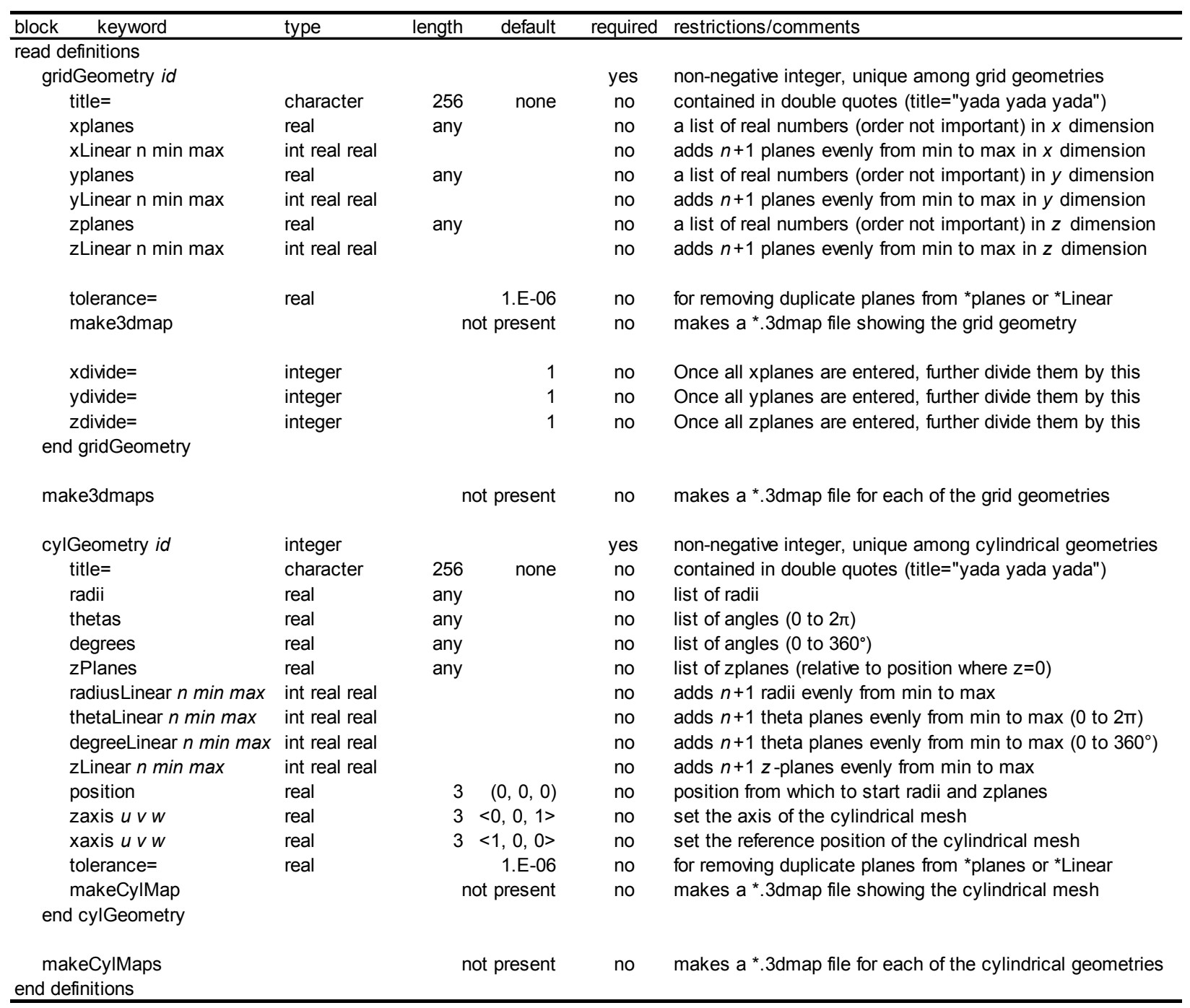


Table 8.2.14. Even more keywords for the definitions block

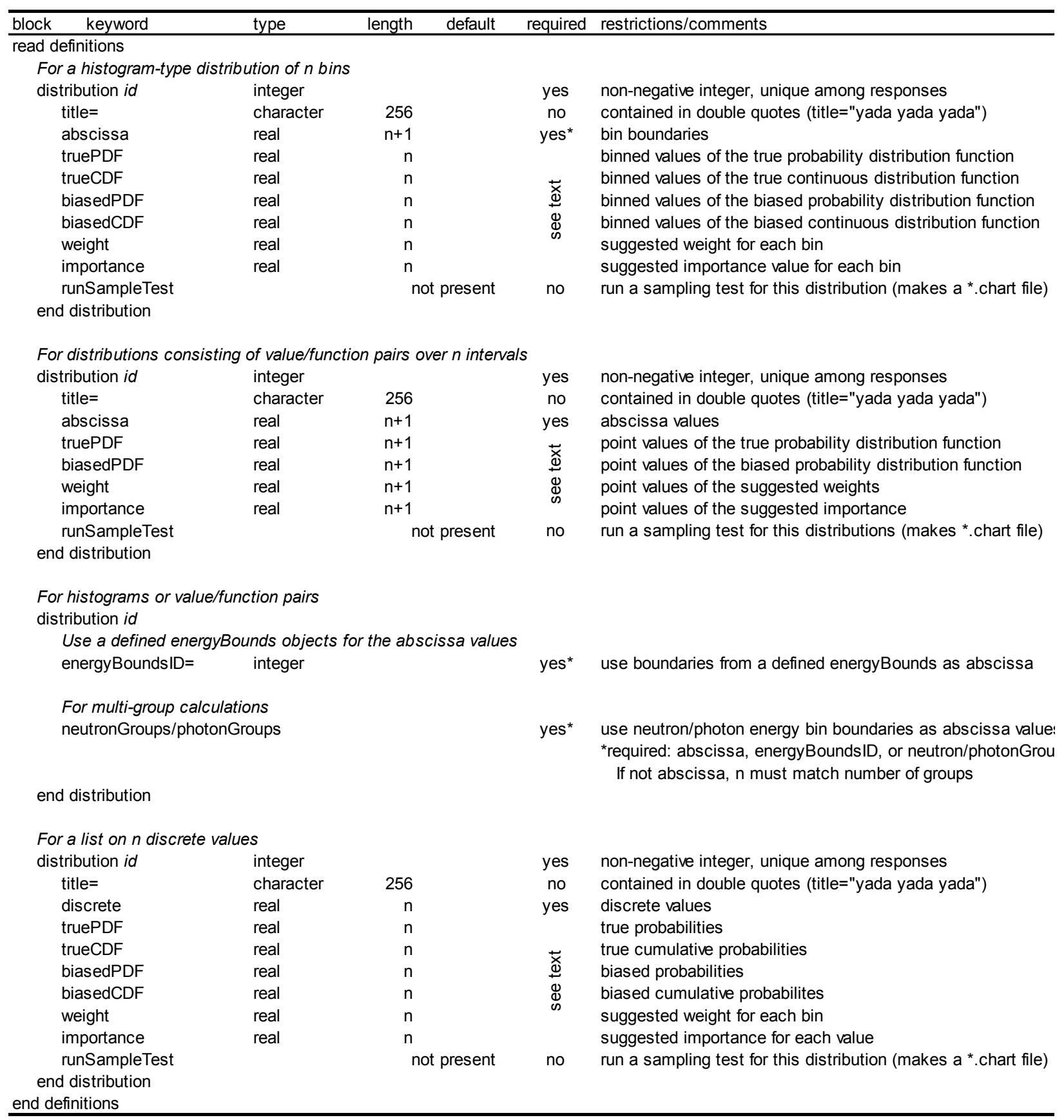


Table 8.2.15. Special distribution keywords for the definitions block

\begin{tabular}{|c|c|c|c|c|c|c|}
\hline block & keyword & type & length & default & required & restrictions/comments \\
\hline \multicolumn{7}{|c|}{ read definitions } \\
\hline \multicolumn{7}{|c|}{$\begin{array}{l}\text { For special and built-in distributions } \\
\text { distribution id }\end{array}$} \\
\hline & ecial= & character & 30 & & yes & $\begin{array}{l}\text { "wattSpectrum", "cosine", "exponential", } \\
\text { "pwrNeutronAxialProfile", "pwrGammaAxialProfile", } \\
\text { "pwrNeutronAxialProfileReverse", } \\
\text { "pwrGammaAxialProfileReverse", "fissionPhotons", } \\
\text { "origensBinaryConcentrationFile", "fissionNeutrons" }\end{array}$ \\
\hline & arameters & real & $<=10$ & & depends & each type of distribution could have parameters \\
\hline & ename= & string & & none & depends & $\begin{array}{l}\text { legal file name for current system, in quotes } \\
\text { required for special="origensBinaryConcentrationFile" }\end{array}$ \\
\hline enc & $\begin{array}{l}\text { nSampleTest } \\
\text { listribution }\end{array}$ & & \multicolumn{2}{|c|}{ not present } & no & run a sampling test for this distributions (makes *. chart file) \\
\hline $\begin{array}{r}\text { run } \\
\text { end de }\end{array}$ & $\begin{array}{l}\text { ampleTests } \\
\text { iitions }\end{array}$ & & \multicolumn{2}{|c|}{ not present } & no & show all of the distributions and a sampling test of each \\
\hline
\end{tabular}

Table 8.2.16. Continuous energy keywords for the definitions block

\begin{tabular}{|c|c|c|c|c|c|}
\hline keyword & type & length & default & required & restrictions/comments \\
\hline \multicolumn{6}{|l|}{ read definitions } \\
\hline \multirow{2}{*}{$\begin{array}{l}\text { energyBounds id } \\
\text { title= }\end{array}$} & integer & & & yes & non-negative integer, unique among energyBounds objects \\
\hline & character & 256 & none & no & contained in double quotes (title="yada yada yada") \\
\hline bounds & & any & & no & a list of energy bin boundaries (in eV, order not important) \\
\hline linear $n \min \max$ & int real real & & & no & adds $n+1$ boundaries $(\mathrm{eV})$ evenly from $\min$ to $\max$ \\
\hline \multirow{2}{*}{ logarithmic $n \min \max$} & int real real & & & no & adds $n+1$ boundaries (eV) logarithmically from $\min$ to $\max$ \\
\hline & real & & 1.E-06 & no & for removing duplicate boundaries from various commands \\
\hline $\begin{array}{l}\text { tolerance= } \\
252 n\end{array}$ & & \multicolumn{2}{|c|}{ not present } & no & add energy bin boundaries from 252-group neutron library \\
\hline \multicolumn{2}{|l|}{$238 n$} & \multicolumn{2}{|c|}{ not present } & no & add energy bin boundaries from 238-group neutron library \\
\hline \multicolumn{2}{|l|}{$200 n$} & \multicolumn{2}{|c|}{ not present } & no & add energy bin boundaries from 200-group neutron library \\
\hline \multicolumn{2}{|l|}{$56 n$} & \multicolumn{2}{|c|}{ not present } & no & add energy bin boundaries from 56-group neutron library \\
\hline \multicolumn{2}{|l|}{$47 p$} & \multicolumn{2}{|c|}{ not present } & no & add energy bin boundaries from 47-group photon library \\
\hline \multicolumn{2}{|l|}{$44 n$} & \multicolumn{2}{|c|}{ not present } & no & add energy bin boundaries from 44-group neutron library \\
\hline \multicolumn{2}{|l|}{$27 n$} & \multicolumn{2}{|c|}{ not present } & no & add energy bin boundaries from 27-group neutron library \\
\hline $19 p$ & & & present & no & add energy bin boundaries from 19-group photon library \\
\hline \multicolumn{6}{|l|}{ end energyBounds } \\
\hline \multirow{6}{*}{$\begin{array}{l}\text { timeBounds id } \\
\text { title= } \\
\text { bounds } \\
\text { linear } n \text { min max } \\
\text { logarithmic } n \text { min max } \\
\text { tolerance }=\end{array}$} & integer & \multirow{3}{*}{\multicolumn{2}{|c|}{$\begin{array}{l}256 \\
\text { any }\end{array}$}} & yes & non-negative integer, unique among timeBounds objects \\
\hline & character & & & no & contained in double quotes (title="yada yada yada") \\
\hline & & & & no & a list of time bin boundaries (in sec, order not important) \\
\hline & int real real & & & no & adds $n+1$ boundaries (sec) evenly from $\min$ to $\max$ \\
\hline & int real real & & & no & adds $n+1$ boundaries (sec) logarithmically from $\min$ to $\max$ \\
\hline & real & & 1.E-10 & no & for removing duplicate boundaries from various commands \\
\hline $\begin{array}{l}\text { end timeBounds } \\
\text { nd definitions }\end{array}$ & & & & & \\
\hline
\end{tabular}


Table 8.2.17. Keywords for the sources block

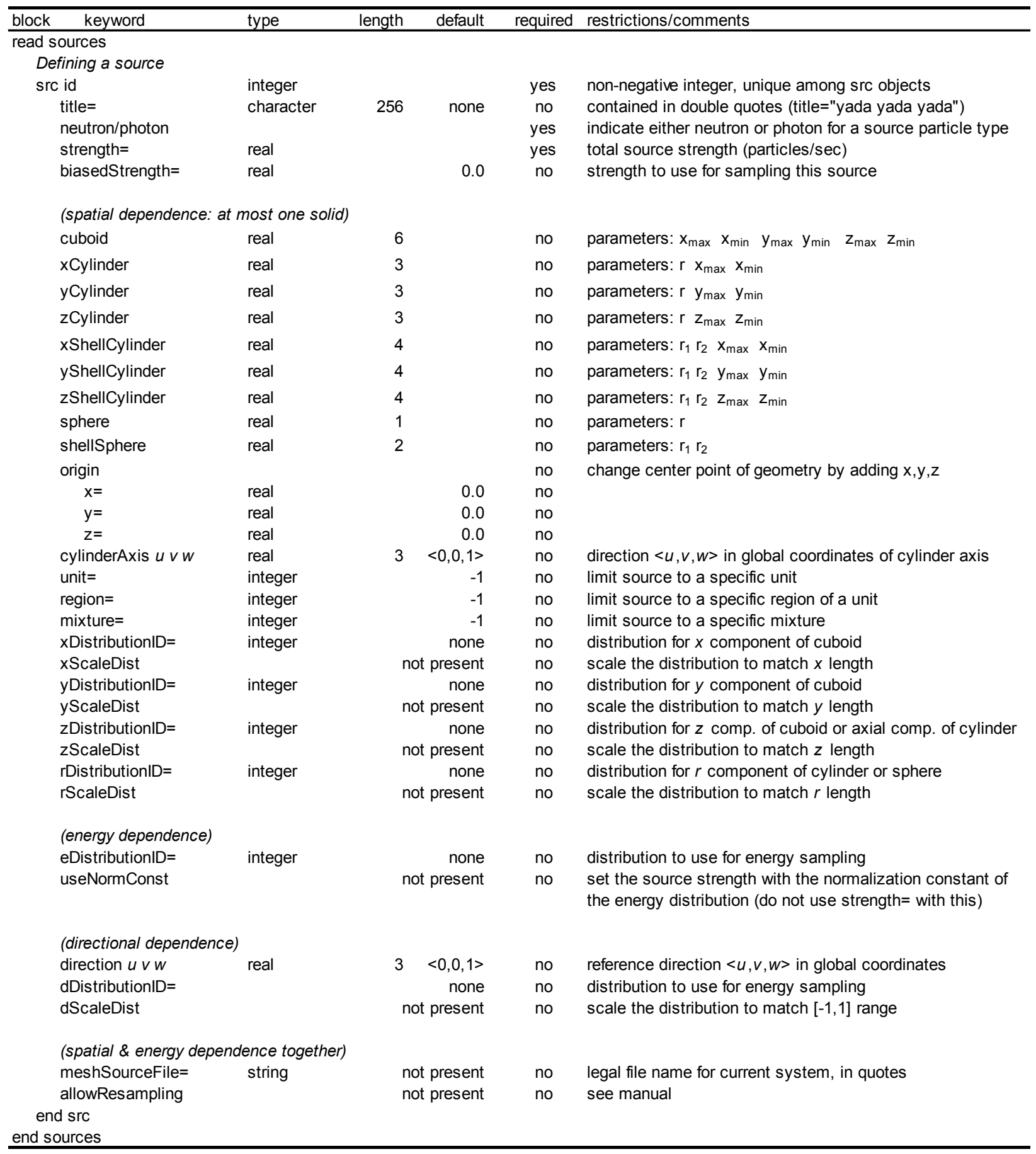


Table 8.2.18. More keywords for the sources block

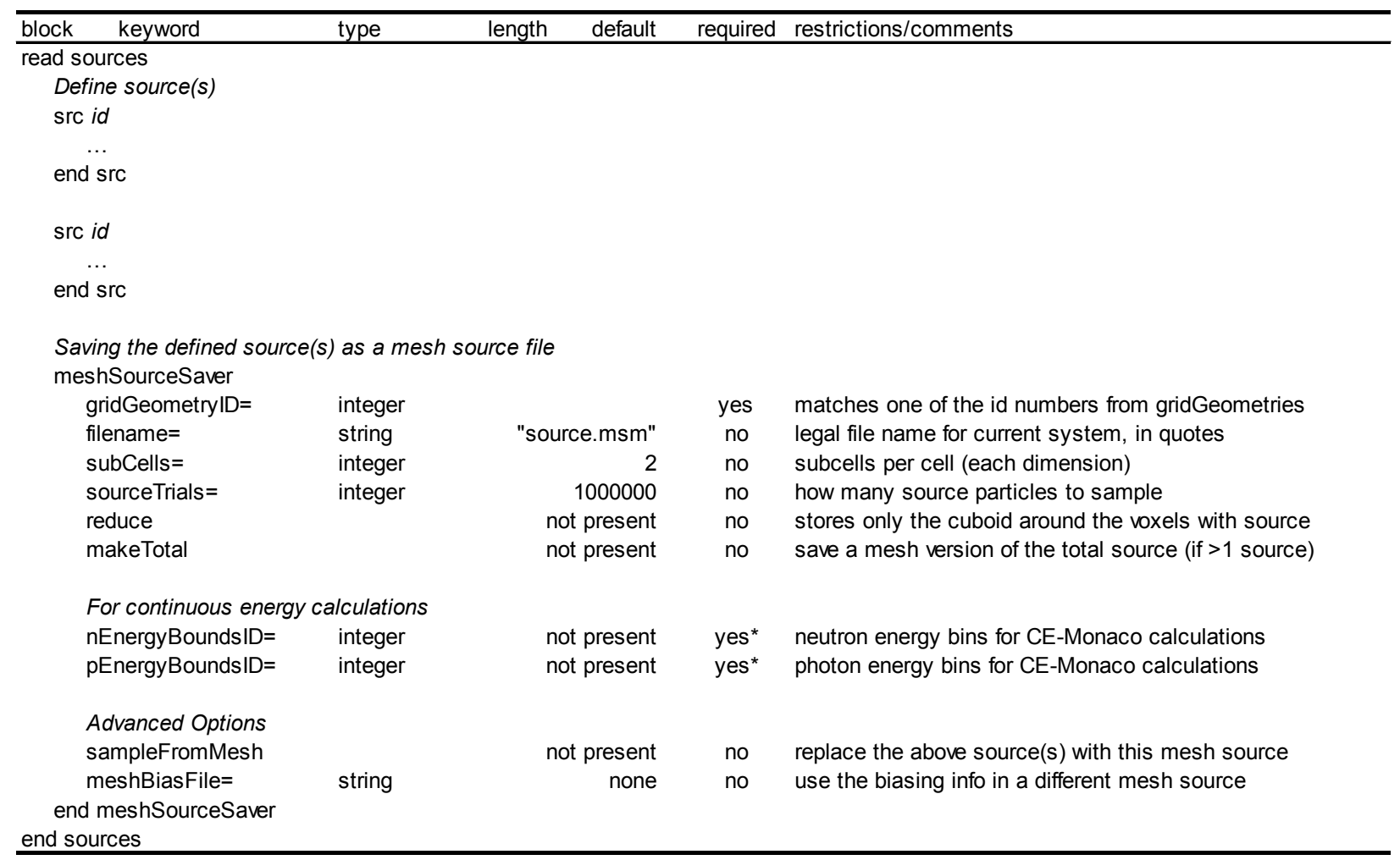


Table 8.2.19. Keywords for the tallies block

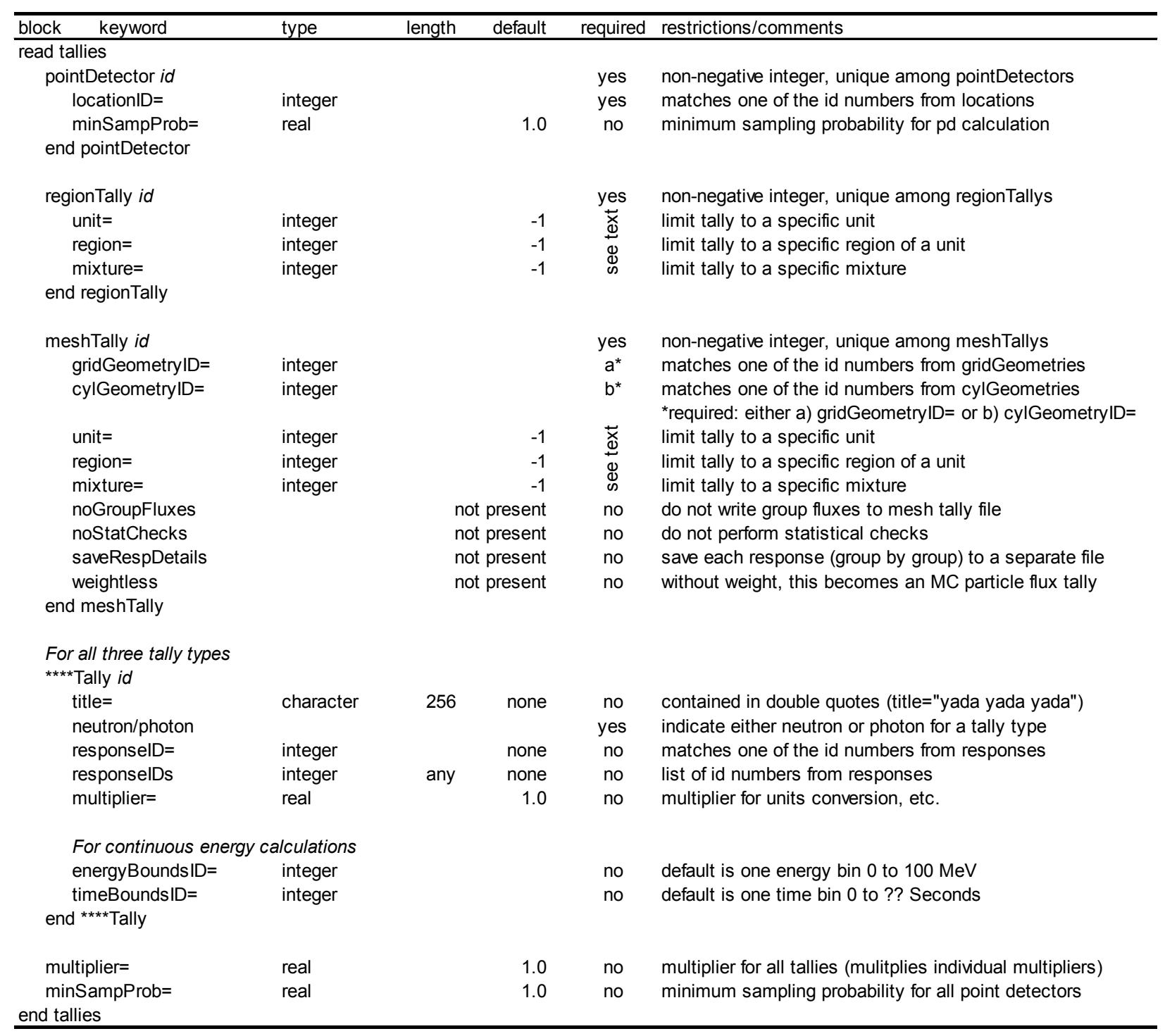


Table 8.2.20. Keywords for the parameters block

\begin{tabular}{|c|c|c|c|c|}
\hline keyword & type & default & required & restrictions/comments \\
\hline \multicolumn{5}{|l|}{ read parameters } \\
\hline randomSeed $=$ & hex & 0000000100000001 & no & hexidecimal number \\
\hline perBatch $=$ & integer & 1000 & no & positive integer \\
\hline batches $=$ & integer & 10 & no & positive integer \\
\hline maxMinutes= & real & 0.0 & no & non-negative real number \\
\hline maximumLost $=$ & integer & 10 & no & maximum number of lost particles to tolerate \\
\hline voidAllRegions & & not present & no & replace all materials with voids for geometry testing \\
\hline allowShortImpMap & & not present & no & particles outside importance map are killed \\
\hline noCheckAtBirth & & not present & no & do not check the weight of source particles \\
\hline \multicolumn{5}{|l|}{ Particles to simulate } \\
\hline neutron & & not present & no & For MG, the particles contained in the library will be \\
\hline photon & & not present & no & transported unless turned off. In CE, the user should \\
\hline noNeutron & & not present & no & specify which particles to transport. \\
\hline noPhoton & & not present & no & \\
\hline fissionMult= & integer & 2 & no & production of fission neutrons (0-no, 1-one, 2-multiple) \\
\hline secondaryMult= & integer & 2 & no & production of gammas form neutrons (0-no, 1-one, 2-multiple) \\
\hline \multicolumn{5}{|c|}{ For continuous energy calculations } \\
\hline nMinEnergy & real & 1.0E-05 & no & minimum energy $(\mathrm{eV})$ for neutrons \\
\hline pMinEnergy & real & $1.0 \mathrm{E}+04$ & no & minimum energy $(\mathrm{eV})$ for photons \\
\hline nMaxEnergy & real & $2.0 \mathrm{E}+07$ & no & maximum energy $(\mathrm{eV})$ for neutrons \\
\hline pMaxEnergy & real & $2.0 \mathrm{E}+07$ & no & maximum energy (eV) for photons \\
\hline nMaxAge & real & 1 & no & maximum age (sec) for neutrons \\
\hline pMaxAge & real & 1 & no & maximum age (sec) for photons \\
\hline noPTables & & not present & no & turn off the use of probability tables \\
\hline dopplerBroaden & integer & 0 & no & $\begin{array}{l}\text { Doppler pre-broadening of cross sections (0-none, 1-1D } \\
\text { cross sections, } 2-1 D \text { and } 2 D \text { cross sections) }\end{array}$ \\
\hline \multicolumn{5}{|l|}{ Legacy keywords } \\
\hline noFissions & & not present & no & no production of fission neutrons (fissionMult=0) \\
\hline $\begin{array}{l}\text { noSecondaries } \\
\text { end parameters }\end{array}$ & & not present & no & no production of gammas from neutrons (secondaryMult $=0$ ) \\
\hline
\end{tabular}


Table 8.2.21. Keywords for the biasing block

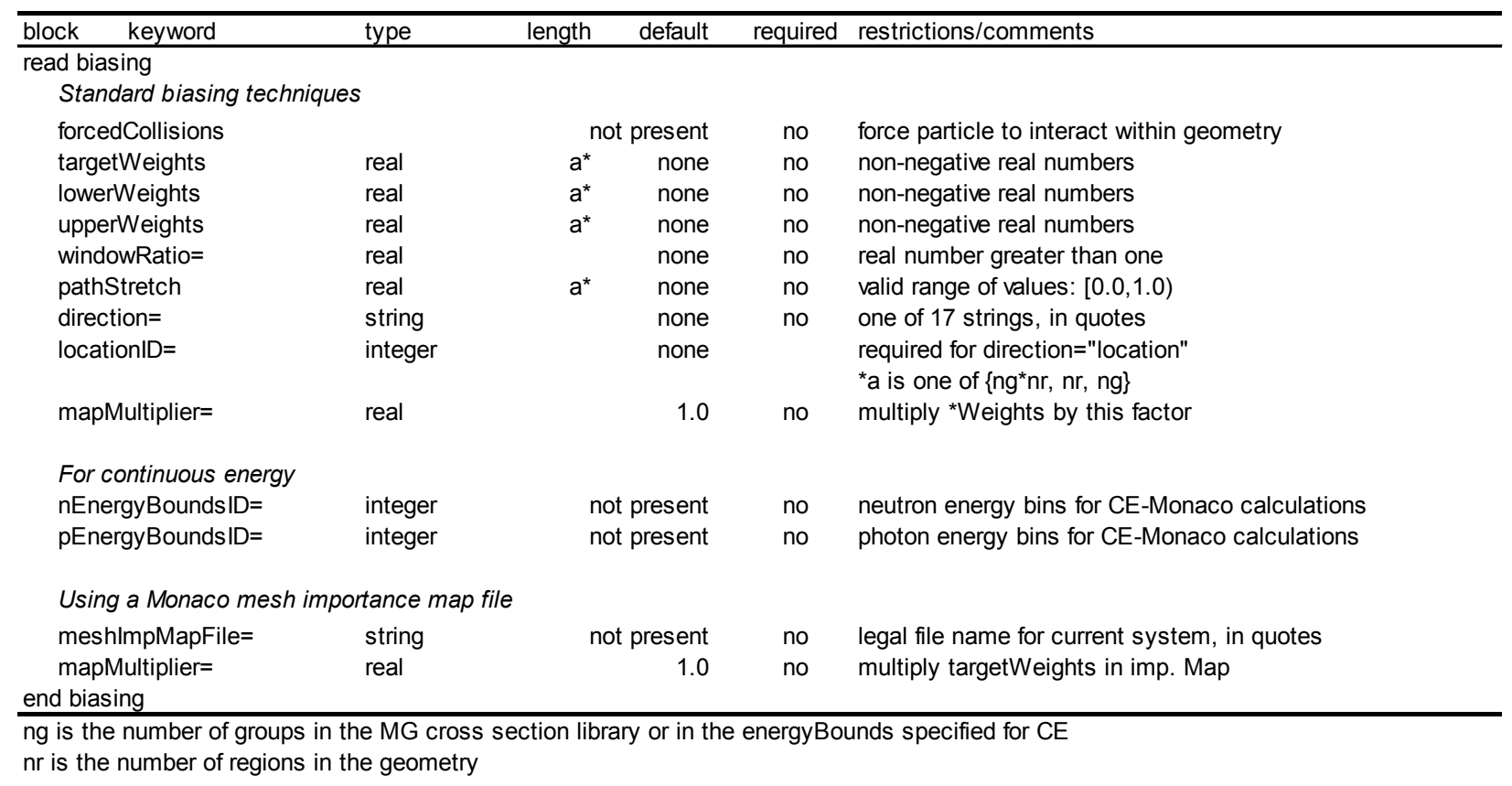

\subsubsection{Monaco Output}

\subsubsection{Main text output file}

The Monaco output file first reviews the input Monaco received. First is a review of the geometryshowing which materials are used in each region and the volume of that region, if input or calculated. Then there is a detailed list of other Monaco input: cross-section parameters, data definitions, the sources, the tallies, the Monte Carlo parameters, and the biasing parameters. For calculations using an importance map, its summary is also given. The "Mesh Importance Map Characterization" shows where the importance map may be changing too fast and may require more refinement.

For each batch of source particles simulated, the output file lists the batch time and the starting random number for the next batch, which may be useful in rerunning just a portion of a problem. Once all of the batches are completed, a list of the various tally files that have been created is given. Finally, the tallies are summarized in a section titled "Final Tally Results Summary". For each point detector, the total neutron and photon fluxes (uncollided and total) are given as well as the final response values for each response function. For each region tally, the total neutron and photon fluxes (both track-length and collision density estimates) are listed, followed by the final response values for each response function. Along with each of the final quantities are the standard deviation of the quantity, the relative uncertainty, the figure-of-merit and a summary of list of the statistical checks (passed or not).

\subsubsection{Tally files}

In addition to the summary of tallies contained in the Monaco text output file, many other files are created containing the group-by-group details of the final tally data. Each mesh tally produces a file "outputName.mtid.3dmap" where outputName is the name the user chose for his output file and "id" is the identification number corresponding to the tally specification. This file can be viewed using the Mesh File Viewer capability of Fulcrum. Point detector tallies and region tallies each create files "outputName.pdid.txt" or "outputName.rtid.txt" to list the group-by-group results. They also produce 
chart files, "outputName.pdid.chart" or "outputName.rtid.chart", which contain the total neutron flux, the total photon flux, and the total response function value calculated at the end of each batch. This data can be used to look at tally convergence and can be viewed with the Interactive Plotter capabilities of Fulcrum. Table 8.2.22 lists the output files, based on the name of the main output file (here called outputName), that are available to the user. These files will be copied back to the directory where SCALE was executed.

\subsubsection{Diagnostic files}

Three of the data types defined in the definitions block can create files or add output to the main text output file to allow the user to ensure items were interpreted by Monaco as they were intended. Responses that included the "makeChart" keyword will each produce a file "outputName.respid.chart", where " $i d$ " is the response identification number, which can be displayed using the Interactive Plotter. Grid geometries that include the keyword "make3dmap" will each produce a file called "outputName.gridid.3dmap", where "id" is the grid geometry identification number, which can be visualized using the Mesh File Viewer. Likewise, cylindrical geometries with the keyword "makeCylMap" will create a file called "outputName.cylid.3dmap". Distributions using the "runSampleTest" keyword will produce a file "outputName.distid.chart", where " $i d$ " is the response identification number. The "runSampleTest" results will also be displayed in the main text output with each distribution listed in the Monaco input review.

Table 8.2.22. Output files created by Monaco

\begin{tabular}{lcl}
\hline \multicolumn{1}{c}{ Filename } & Viewer & Description \\
\hline $\begin{array}{l}\text { Output Summary } \\
\text { outputName.out }\end{array}$ & & main text output file, contains results summary \\
$\begin{array}{l}\text { Diagnostic Files } \\
\text { outputName.respid.chart }\end{array}$ & $\mathrm{P}$ & $\begin{array}{l}\text { response input and MULTIGROUP representation for } \\
\text { response } i d\end{array}$ \\
$\begin{array}{l}\text { outputName.gridid.3dmap } \\
\text { outputName.cylid.3dmap } \\
\text { outputName.distid.chart }\end{array}$ & $\mathrm{V}$ & $\begin{array}{l}\text { mesh version of geometry using grid geometry id } \\
\text { mesh version of geometry using cylindrical geometry } i d\end{array}$ \\
& $\mathrm{P}$ & distribution input and sampling test for distribution $i d$
\end{tabular}

Mesh Source Saver

filename.msm

$\mathrm{V}$ mesh representation of a single source or total source

filename.id.msm

filename.sampling.msm

filename.sampling.id.msm

$\mathrm{V}$ mesh representation of multiple sources

$\mathrm{V}$ biased representation of a single source or total source

$\mathrm{V} \quad$ biased representation of multiple sources

Tally Files

outputName.pdid.txt

outputName.pdid.chart

detailed results for point detector tally id

outputName.rtid.txt

$\mathrm{P} \quad$ batch convergence data for point detector tally id

detailed results for region tally id 


\begin{tabular}{|c|c|c|}
\hline outputName.rtid.chart & $\mathrm{P}$ & batch convergence data for region tally $i d$ \\
\hline outputName.mtid.3dmap & $\mathrm{V}$ & mesh tally for meshTally $i d$ \\
\hline outputName.mtid.respxx.3dmap & $\mathrm{V}$ & $\begin{array}{l}\text { mesh tally of response by group for meshTally } i d \text {, } \\
\text { response } x x\end{array}$ \\
\hline outputName.mtid.flux.txt & & detailed results for the group-wise flux of meshTally $i d$ \\
\hline outputName.mtid.tfluxtxt & & detailed results for total flux of meshTally id \\
\hline outputName.mtid.respxx.txt & & detailed results for response $x x$ of meshTally id \\
\hline
\end{tabular}

$\mathrm{V}$ - can be displayed with the Mesh File Viewer capabilities Fulcrum.

$\mathrm{P}$ - can be displayed with the 2D plotting capabilities of Fulcrum.

\subsubsection{Mesh source saver files}

If the Mesh Source Saver was used, one mesh source file will be created for each defined source. For a single source, the filename will be whatever was listed with the "filename=" keyword or "source.msm" if nothing was given. For multiple sources, the filenames will include the source identification number, such as "source.id.msm". If there were multiple sources and the "makeTotal" keyword was used, the total will be stored in "source.msm". Note that if these files are desired, they must be manually copied back from the SCALE temporary area.

If the keyword "meshBiasFile=" was used, then for every mesh source generated above there will also be a biased mesh source that is used in sampling. These files have the names in the form of "source.sampling.id.msm".

\subsubsection{Statistical checks on point detector and region tallies}

With each region tally and point detector tally, detailed statistical information is provided in separate files, "outputName.rtid.txt" or "outputName.pdid.txt", just after the group-by-group values for the fluxes and responses. For the total fluxes and any responses of each tally, two tables are given.

First, the values of the tally and several statistical quantities are listed as a function of batch number. With each batch, the statistical tests are listed by number with one of the following: "X" for passing, "-" for failing or a blank if the test could not be performed yet. The second table lists the details of the final statistical checks for the last batch completed. This table lists the value for each of the six tests as well as what the goal is for that test.

As an example, consider a $1 \mathrm{Ci}$ point source of Watt spectrum neutrons inside an $r=20 \mathrm{~cm}$ sphere of polyethylene. Two tallies are used to find the neutron dose rate (rem/hr) $35 \mathrm{~cm}$ from the center of the sphere - a region tally (region between two concentric spheres with radii of 34 and $36 \mathrm{~cm}$ ) and a point detector $35 \mathrm{~cm}$ away from the center of the sphere. Twenty batches of 1000 particles each were used in this example.

The first table for the region tally dose rate response is shown in. Since four of the tests involve curve fits to the table values over the last half of the simulation, the table is split showing each half of the simulation separately. The second table for the region tally, showing all of the details for the last batch, is shown in. These two tables show that this tally passed all the statistical tests for the entire second half of the simulation. This information, combined with the fact that this is a simple tally for a well-posed problem, indicates that this tally is well-converged. 


\begin{tabular}{|c|c|c|c|c|c|c|c|c|c|}
\hline atch & $\begin{array}{c}\text { average } \\
\text { value }\end{array}$ & $\begin{array}{r}\text { standard } \\
\text { deviation }\end{array}$ & $\begin{array}{l}\text { relat } \\
\text { uncert }\end{array}$ & $\begin{array}{l}\text { rel } \\
\text { VOV }\end{array}$ & $\begin{array}{c}\text { FOM } \\
(/ \mathrm{min})\end{array}$ & $\begin{array}{l}\text { stat } \\
12\end{array}$ & $\begin{array}{r}\text { ts } \\
3\end{array}$ & \multicolumn{2}{|c|}{$\begin{array}{l}\text { s check } \\
34556\end{array}$} \\
\hline---- & ----------- & ----------- & ------- & -------- & -------- & -- & & & \\
\hline 1 & $2.06269 \mathrm{E}+01$ & $2.45012 \mathrm{E}+00$ & 0.11878 & 1. $45 \mathrm{E}-02$ & $9.96 \mathrm{E}+02$ & & - & & $\mathrm{x}$ \\
\hline 2 & $1.87353 \mathrm{E}+01$ & $1.65492 \mathrm{E}+00$ & 0.08833 & $8.16 \mathrm{E}-03$ & $8.76 E+02$ & & - & & $\mathrm{x}$ \\
\hline 3 & $1.82317 \mathrm{E}+01$ & $1.33026 \mathrm{E}+00$ & 0.07296 & $5.63 E-03$ & $8.45 E+02$ & & - & & $\mathrm{X}$ \\
\hline 4 & $1.80905 \mathrm{E}+01$ & $1.15401 \mathrm{E}+00$ & 0.06379 & $4.27 E-03$ & $8.28 E+02$ & & - & & $\mathrm{x}$ \\
\hline 5 & $1.82780 \mathrm{E}+01$ & $1.03792 \mathrm{E}+00$ & 0.05679 & $3.40 E-03$ & $8.36 E+02$ & & - & & $\mathrm{x}$ \\
\hline 6 & $1.75679 \mathrm{E}+01$ & $9.28759 \mathrm{E}-01$ & 0.05287 & $2.97 E-03$ & $8.00 E+02$ & & - & & $\mathrm{x}$ \\
\hline 7 & $1.71843 \mathrm{E}+01$ & $8.46577 E-01$ & 0.04926 & $2.60 E-03$ & $7.89 E+02$ & & $\mathrm{X}$ & & $\mathrm{x}$ \\
\hline 8 & $1.74775 \mathrm{E}+01$ & $7.99275 E-01$ & 0.04573 & $2.23 E-03$ & $8.03 E+02$ & & $\mathrm{X}$ & & $\mathrm{X}$ \\
\hline 9 & $1.73520 \mathrm{E}+01$ & $7.52502 \mathrm{E}-01$ & 0.04337 & $2.00 \mathrm{E}-03$ & $7.94 \mathrm{E}+02$ & & $\mathrm{X}$ & & $\mathrm{X}$ \\
\hline 10 & $1.76020 \mathrm{E}+01$ & $7.17178 \mathrm{E}-01$ & 0.04074 & $1.76 \mathrm{E}-03$ & $8.11 E+02$ & $\mathrm{X} X$ & $\mathrm{X}$ & $\mathrm{X}$ & $x \quad x$ \\
\hline 11 & $1.74628 \mathrm{E}+01$ & $6.80834 E-01$ & 0.03899 & 1. $62 \mathrm{E}-03$ & $8.03 E+02$ & $\mathrm{X} X$ & $\mathrm{X}$ & $\mathrm{X}$ & $\mathrm{X} X$ \\
\hline 12 & $1.75161 \mathrm{E}+01$ & $6.52464 E-01$ & 0.03725 & 1. $48 \mathrm{E}-03$ & $8.08 E+02$ & $\mathrm{X} X$ & $\mathrm{X}$ & $\mathrm{X}$ & $\mathrm{X} X$ \\
\hline 13 & $1.72561 \mathrm{E}+01$ & $6.21777 \mathrm{E}-01$ & 0.03603 & $1.39 E-03$ & $7.96 \mathrm{E}+02$ & $\mathrm{X} X$ & $\mathrm{X}$ & $\mathrm{X}$ & $\mathrm{X} X$ \\
\hline 14 & $1.73196 \mathrm{E}+01$ & $6.00329 \mathrm{E}-01$ & 0.03466 & 1. $29 \mathrm{E}-03$ & 8.00E+02 & $\mathrm{X} X$ & $\mathrm{X}$ & $\mathrm{X}$ & $\mathrm{X} X$ \\
\hline 15 & $1.72025 \mathrm{E}+01$ & $5.77747 \mathrm{E}-01$ & 0.03359 & 1.21E-03 & $7.97 \mathrm{E}+02$ & $\mathrm{XX}$ & $\mathrm{X}$ & $\mathrm{X}$ & \\
\hline 16 & $1.72019 \mathrm{E}+01$ & $5.58956 \mathrm{E}-01$ & 0.03249 & $1.14 \mathrm{E}-03$ & 7. $98 E+02$ & $\mathrm{X} X$ & $\mathrm{X}$ & $\mathrm{X}$ & $\mathrm{X} X$ \\
\hline 17 & $1.72998 \mathrm{E}+01$ & $5.43721 \mathrm{E}-01$ & 0.03143 & $1.06 \mathrm{E}-03$ & $8.02 E+02$ & $\mathrm{XX}$ & $\mathrm{X}$ & $\mathrm{X}$ & \\
\hline 18 & $1.73534 \mathrm{E}+01$ & $5.29351 E-01$ & 0.03050 & $9.99 E-04$ & $8.06 E+02$ & $\mathrm{X} X$ & $\mathrm{X}$ & $\mathrm{X}$ & $\mathrm{X} X$ \\
\hline 19 & $1.72752 \mathrm{E}+01$ & $5.14264 \mathrm{E}-01$ & 0.02977 & $9.51 \mathrm{E}-04$ & $8.01 E+02$ & $\mathrm{XX}$ & $\mathrm{X}$ & $\mathrm{X}$ & \\
\hline 20 & $1.74155 \mathrm{E}+01$ & $5.03061 \mathrm{E}-01$ & 0.02889 & $8.95 E-04$ & $8.08 E+02$ & $\mathrm{XX}$ & $\mathrm{X}$ & $\mathrm{X}$ & $\mathrm{X} X$ \\
\hline
\end{tabular}

Figure 8.2.14. Tally values for a well-converged tally.

\begin{tabular}{|c|c|c|c|c|}
\hline quantity & check & goal & actual & pass \\
\hline----------------------- & ----------------------- & ------- & -------- & ---- \\
\hline 1 mean & rel slope of linear fit & 0.00 & -0.0118 & yes \\
\hline 2 standard deviation & exponent of power fit & $=-0.50$ & -0.5092 & yes \\
\hline 3 relative uncertainty & final value & 0.05 & 0.0289 & yes \\
\hline 4 relative VOV & exponent of power fit & $=-1.00$ & -0.9833 & yes \\
\hline 5 relative Vov & final value & 0.10 & 0.0009 & yes \\
\hline 6 figure-of-merit (FOM) & rel slope of linear fit & 0.00 & 0.0075 & yes \\
\hline
\end{tabular}

Figure 8.2.15. Final check for a well-converged tally.

Since the point detector is close compared to the size of the sphere, it should converge slower than the region tally. Contributions coming from different parts of the sphere have large differences in attenuation which will cause large fluctuations in the weights arriving at the point detector. The two statistical tables are shown in Figure 8.2.16 and Figure 8.2.17. This tally is not yet converged enough to pass most of the statistical tests. With thirty times the simulation time, this point detector tally will pass all six tests.

Figure 8.2.18 shows the behavior of both the region tally (well-converged) and the point detector tally (not-yet-converged) for this example problem as a function of the simulation run time (shown as the twenty batches of particles). The batch values are shown as blue points and the fits for the last half of the simulation are shown as solid black lines. The average value for a tally should be constant, so test 1 looks at the slope of a linear fit over the tally average over the last half of the simulation. The uncertainty of the tally should decrease with the square root of the total number of particles $(1 / \sqrt{N})$, so test 3 computes the slope of an exponential fit which should be close to -0.5 (green dotted line). The variance-of-the-variance (VOV) should decrease with $1 / N$, so test 4 computes the slope of an exponential fit which should be close to -1.0. The tally figure-of-merit (FOM) should be constant. Test 6 computes the slope of the FOM values which should be zero. 
Tally Values as the Simulation Progressed

\begin{tabular}{|c|c|c|c|c|c|c|c|c|c|c|c|}
\hline decil & $\begin{array}{c}\text { average } \\
\text { value }\end{array}$ & $\begin{array}{l}\text { standard } \\
\text { deviation }\end{array}$ & $\begin{array}{l}\text { relat } \\
\text { uncert }\end{array}$ & $\begin{array}{l}\text { rel } \\
\text { VoV }\end{array}$ & $\begin{array}{c}\text { FOM } \\
(/ \mathrm{min})\end{array}$ & \multirow{2}{*}{\multicolumn{6}{|c|}{$\begin{array}{l}\text { stats check } \\
123456 \\
\end{array}$}} \\
\hline----- & ----------- & ----------- & ------- & ----- & ------- & & & & & & \\
\hline 1 & $1.45258 \mathrm{E}+01$ & $3.00915 E+00$ & 0.20716 & $1.60 \mathrm{E}-01$ & $3.27 E+02$ & & & & & - & \\
\hline 2 & $1.83822 \mathrm{E}+01$ & $4.47657 \mathrm{E}+00$ & 0.24353 & $5.27 E-01$ & 1. $15 \mathrm{E}+02$ & & & - & & - & \\
\hline 3 & $1.84192 \mathrm{E}+01$ & $3.44084 \mathrm{E}+00$ & 0.18681 & $3.20 E-01$ & 1.29E+02 & & & - & & - & \\
\hline 4 & $1.87897 \mathrm{E}+01$ & $2.80685 \mathrm{E}+00$ & 0.14938 & $2.33 E-01$ & 1. $51 \mathrm{E}+02$ & & & - & & - & \\
\hline 5 & $1.78133 E+01$ & $2.31117 E+00$ & 0.12974 & $2.09 E-01$ & 1. $60 \mathrm{E}+02$ & & & - & & - & \\
\hline 6 & $1.75190 \mathrm{E}+01$ & $2.02252 \mathrm{E}+00$ & 0.11545 & $1.74 \mathrm{E}-01$ & $1.68 \mathrm{E}$ & & & - & & - & \\
\hline 7 & $1.79523 E+01$ & $2.18108 \mathrm{E}+00$ & 0.12149 & $1.78 \mathrm{E}-01$ & $1.30 \mathrm{E}$ & & & - & & - & \\
\hline 8 & $1.75624 \mathrm{E}+01$ & $72 \mathrm{E}+00$ & 0.11 & 1. $63 \mathrm{E}-01$ & $1.36 \mathrm{E}$ & & & - & & - & \\
\hline 9 & $1.67648 \mathrm{E}+01$ & $7 \mathrm{E}+00$ & 0.1 & $1.58 \mathrm{E}-01$ & $1.37 \mathrm{E}$ & & & - & & - & \\
\hline 10 & 1.702 & $E+00$ & 0.0 & 1. $30 \mathrm{E}-01$ & 1.40 & $\mathrm{X}$ & 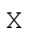 & - & & - & - \\
\hline 11 & -01 & +00 & & 01 & 1.4 & - & - & - & $x$ & - & \\
\hline 12 & $1.71598 \mathrm{E}+01$ & $2 \mathrm{E}+00$ & 0.08 & 1.03E-01 & 1.501 & $\mathrm{X}$ & - & - & & - & - \\
\hline 13 & $1.74796 \mathrm{E}+01$ & $1.42808 E+00$ & 0.0 & $8.85 E-02$ & $1.55 \mathrm{E}$ & $\mathrm{X}$ & - & - & - & $\mathrm{X}$ & - \\
\hline 14 & $1.68710 \mathrm{E}+01$ & $6 \mathrm{E}+00$ & 0.07 & $8.70 \mathrm{E}-02$ & $1.54 \mathrm{E}+02$ & $\mathrm{X}$ & - & - & - & X & \\
\hline 15 & $1.65807 \mathrm{E}+01$ & $7 E+00$ & 0.07 & $8.34 E-02$ & $1.56 \mathrm{E}+02$ & $\mathrm{X}$ & - & - & - & $\mathrm{X}$ & - \\
\hline 16 & $1.65731 \mathrm{E}+01$ & $1.20836 \mathrm{E}+00$ & 0.07291 & 7. $61 \mathrm{E}-02$ & 1. $59 \mathrm{E}+02$ & $\mathrm{X}$ & - & - & $\mathrm{X}$ & $\mathrm{X}$ & - \\
\hline 17 & $1.62390 \mathrm{E}+01$ & 1.1455 & 0.07 & $7.40 \mathrm{E}-02$ & 1. $59 \mathrm{E}+02$ & $\mathrm{X}$ & - & - & $\mathrm{X}$ & $\mathrm{X}$ & - \\
\hline 18 & $1.70002 \mathrm{E}+01$ & $1.17106 \mathrm{E}+00$ & 0.06889 & $5.75 E-02$ & $1.58 \mathrm{E}+02$ & $\mathrm{X}$ & - & - & $x$ & $\mathrm{X}$ & - \\
\hline 19 & $1.70863 E+01$ & $1.14126 \mathrm{E}+00$ & 0.06679 & $5.20 E-02$ & 1. $59 \mathrm{E}+02$ & $\mathrm{X}$ & - & - & - & $\mathrm{X}$ & \\
\hline 20 & $1.68215 \mathrm{E}+01$ & $1.09329 \mathrm{E}+00$ & 0.06499 & $5.04 \mathrm{E}-02$ & 1. $60 \mathrm{E}+02$ & $\mathrm{X}$ & - & - & & $\mathrm{X}$ & \\
\hline & & -1 & & & & & & & & & \\
\hline
\end{tabular}

Figure 8.2.16. Tally values for a not-yet-converged tally.

\begin{tabular}{|c|c|c|c|c|}
\hline quantity & check & goal & actual & pass \\
\hline--------- & ---------------- & & -------- & ---- \\
\hline 1 mean & rel slope of linear fit & 0.00 & -0.0468 & yes \\
\hline 2 standard deviation & exponent of power fit & $=-0.50$ & -0.6006 & no \\
\hline 3 relative uncertainty & final value & 0.05 & 0.0650 & no \\
\hline 4 relative VOV & exponent of power fit & $=-1.00$ & -1.3823 & no \\
\hline 5 relative VOV & final value & 0.10 & 0.0504 & yes \\
\hline 6 figure-of-merit (FOM) & rel slope of linear fit & 0.00 & 0.1667 & no \\
\hline
\end{tabular}

Figure 8.2.17. Final check for a not-yet-converged tally. 

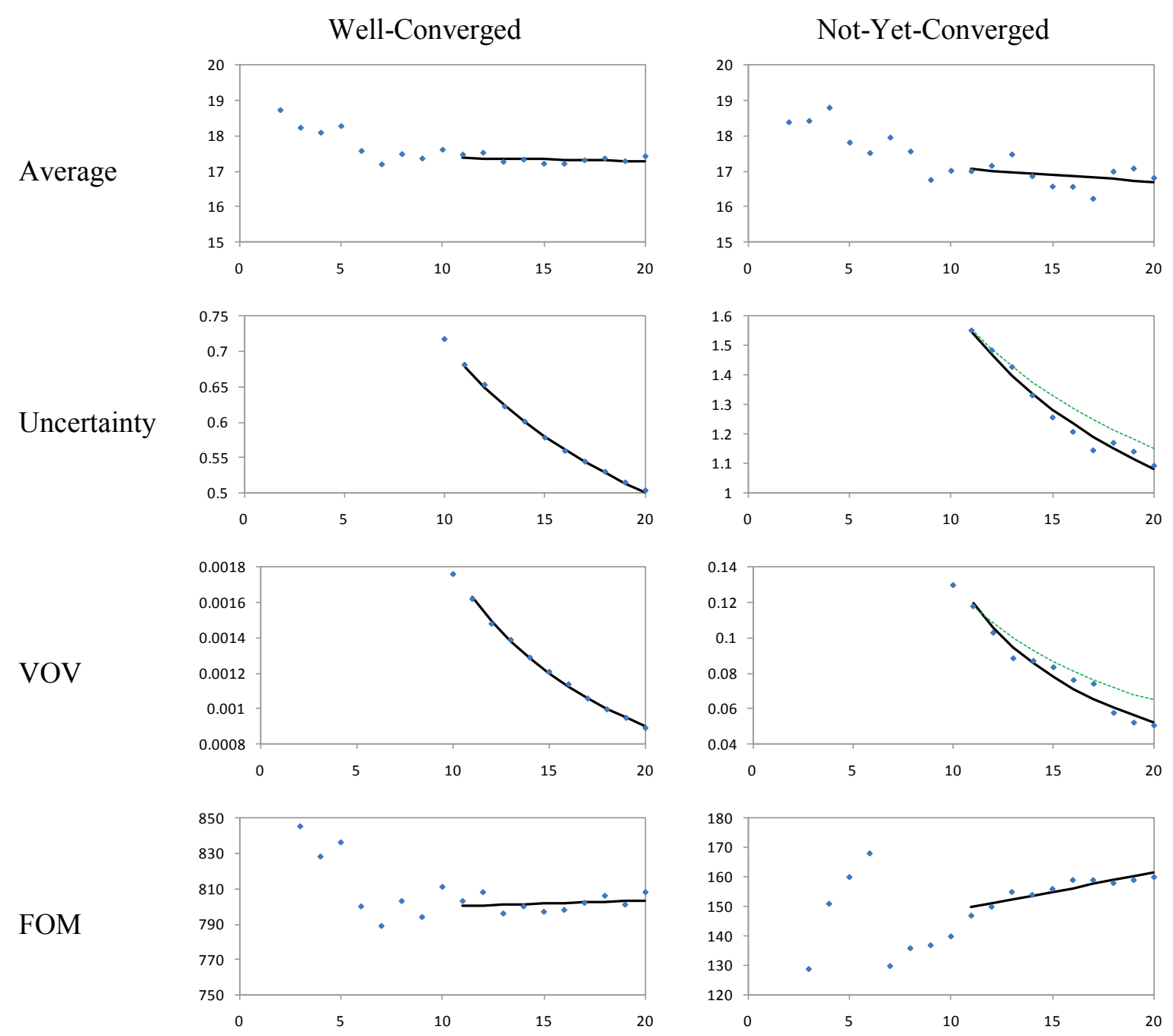

Figure 8.2.18. Behavior of two tallies as a function of number of particle batches.

Note that the slope of the VOV is found by fitting an exponential curve through the calculated VOV values and is very sensitive to outliers. Users need to apply their own judgment to whether or not the VOV test implemented in Monaco is too strict (failing when the tally seems converged).

Mesh tallies produce similar tables - detailed statistical information is provided in separate files, "outputName.mtid.flux.txt" and "outputName.mtid.respxx.txt", for the total flux and responses. First, the statistical values related to the mean relative variance are listed as a function of batch number. With each batch, the statistical tests are listed by number with one of the following: "X" for passing, "-" for failing or a blank if the test could not be performed yet. The second table lists the details of the final statistical checks for the last batch completed. This table lists the value for each of the four tests as well as what the goal is for that test. 


\subsubsection{Example Problems}

\subsubsection{Neutron transmission through an iron sphere}

In the early 1990s, several experiments were performed in order to benchmark ENDF/B-VI cross-section data for iron. ${ }^{5}$ This example problem will use Monaco to simulate one of these experiments: transmission of ${ }^{252} \mathrm{Cf}$ neutrons through a sphere of iron. The Monaco calculations will be compared to two sets of measurements, one by the Czechoslovakian National Research Institute (NRI) and the other by the Skoda Company.

The Monaco model for this sample problem (samples \input \monaco.ironSphere.inp) will be quite simple - just a point source and a spherical shell of iron. Three different tallies are used which should all give the same result: a region tally (for a thin spherical shell at radius $=100 \mathrm{~cm}$ ); a point detector tally at $(\mathrm{x}, \mathrm{y}, \mathrm{z})=(100,0,0)$; and a coarse mesh tally, with one cell enclosing the point $(\mathrm{x}, \mathrm{y}, \mathrm{z})=(100,0,0)$.

\subsection{Input}

First, the cross sections need to be computed. Here, csas-mg is used and the "activities" material is included to make sure the flux-to-dose conversion factors are added to the working library.

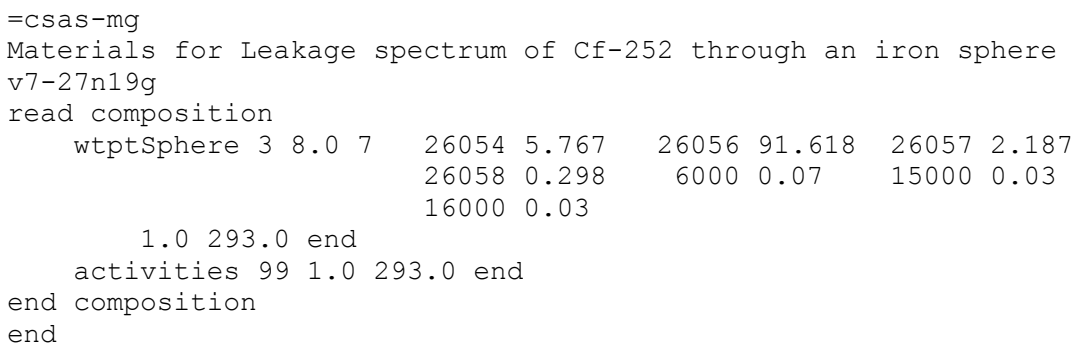

The Monaco input file starts with module name ("monaco") and a title.

$=$ monaco

Leakage spectrum of Cf-252 through an iron sphere

The mixing table information can be found in the output of the above csas-mg run.

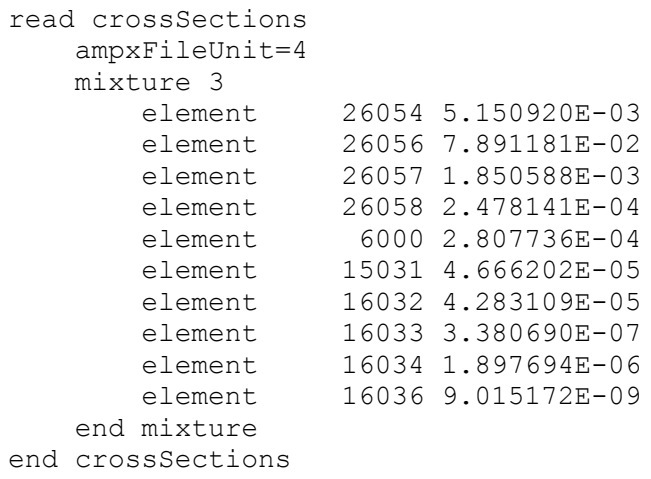

The SGGP geometry consists of several nested spheres. The regions (in order) are a void for the ${ }^{252} \mathrm{Cf}$ source, the iron shield, a void out to the detector, a thin shell about $100 \mathrm{~cm}$, and then a void to the problem boundary. The only volume that needs to be supplied is the fourth region, since that is where the flux tally will be. 


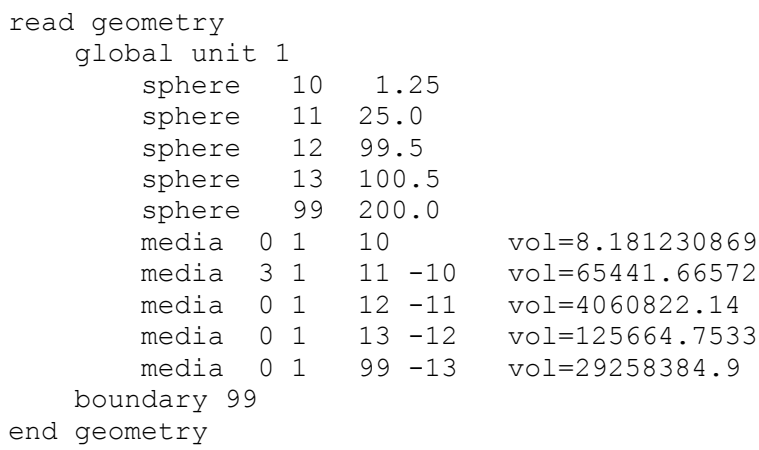

For the different tallies, responses, locations, and grid geometry objects need to be defined. For the source, one distribution needs to be defined.

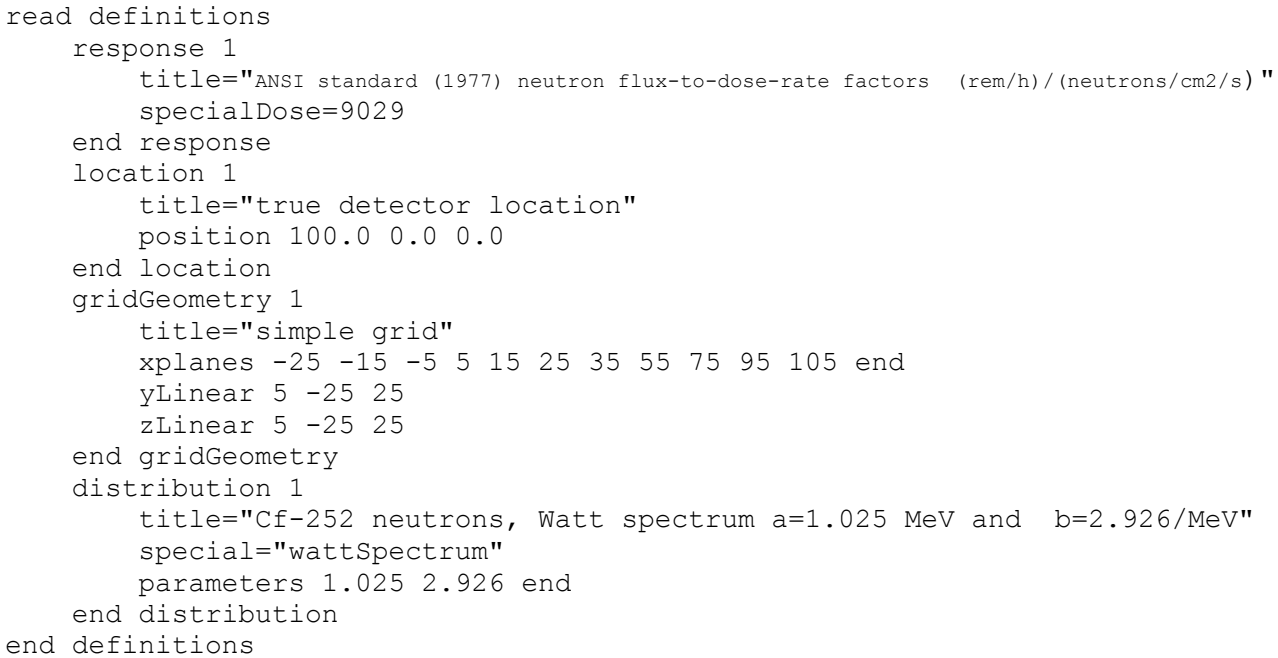

The ${ }^{252} \mathrm{Cf}$ source can be modeled using the Watt spectrum with $a=1.025 \mathrm{MeV}$ and $b=2.926 / \mathrm{MeV}$, which was used for distribution 1. The strength is set so that the total flux at the detector $($ at $r=100 \mathrm{~cm})$ without the shield would be $1 \mathrm{n} / \mathrm{cm}^{2} / \mathrm{s}$. So, strength $=4 \pi(100)^{2}=125664 \mathrm{n} / \mathrm{s}$.

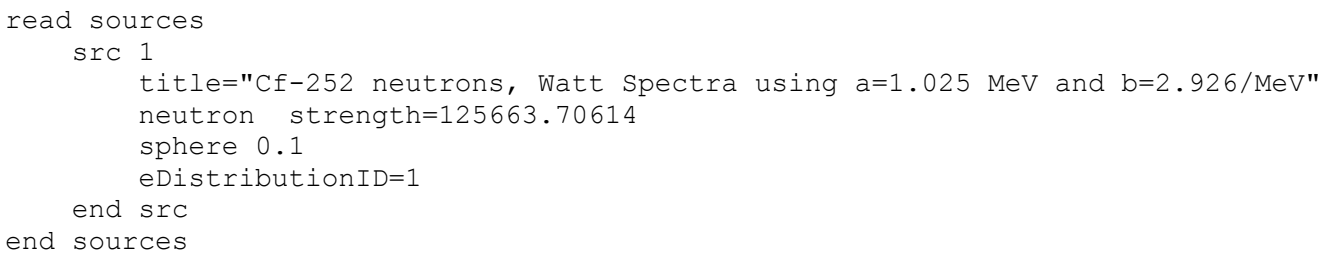

Three tallies will be defined: a region tally over the fourth region of unit 1 (since this is a symmetric problem); a mesh tally using a coarse mesh over the entire problem; and a point detector tally at the true detector location.

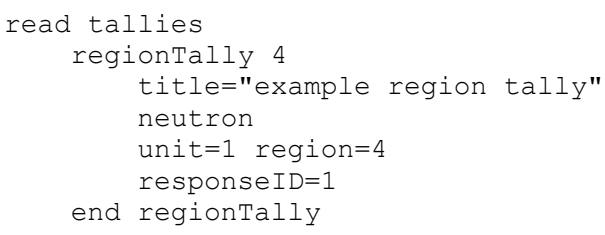




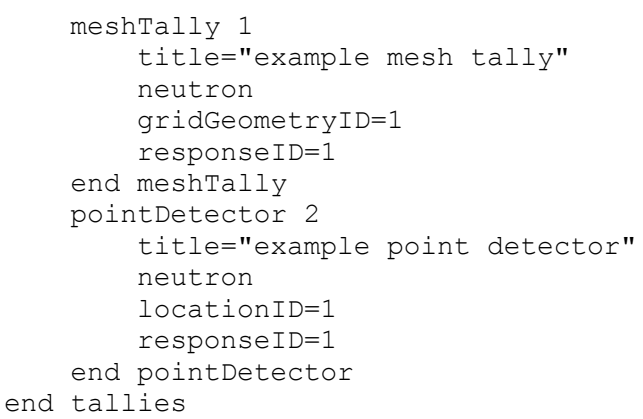

Monte Carlo parameters include the starting random number seed, the number of particles per batch, and the number of batches to simulate. Since we are only interested in simulating neutrons, use the keywords "neutrons" and "noPhotons" to transport neutrons and not photons. To prevent the production of fission neutrons and secondary gamma rays from neutron interactions, the keywords "fissionMult $=0$ " and "secondaryMult $=0$ " are used. In order to prevent a long run time, a maximum allowable run time using the "maxMinutes=" keyword could also be used.

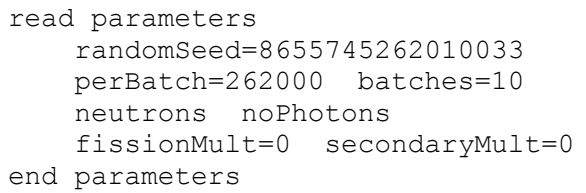

Without any biasing parameters, Monaco will set the target weights of every energy group in every region to 1 and the values for weight lower bounds (Russian roulette) will be $1 / 3$. Since "noPhotons" was listed in the parameters block, photons should not be generated. So, no biasing block is required.

If desired, a biasing block could be added to change the size of the weight windows (default is 5 , giving lower weights of $1 / 3$ and upper weights of 5/3). By using the default target weights of 1.0 and defining the windowRatio as 199 , the lower weights are all set to 0.01 and the upper weights for splitting are 1.99.

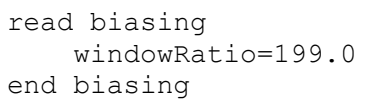

or the target weights and lower weights could be listed for every group, every region or for every group/region. For all regions having the same targets and lower bounds, the following could be used:

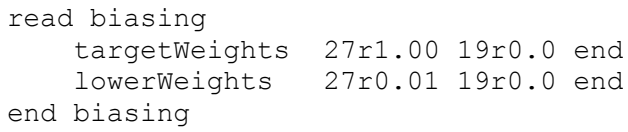

If this last case were used, the "noPhotons" keyword would not be required since the target weights for all photon groups were explicitly set to 0 .

Finally, the Monaco input file is ended with

$$
\begin{aligned}
& \text { end data } \\
& \text { end }
\end{aligned}
$$

The complete input file monaco.ironSphere. inp is located in the SCALE samples $\backslash$ input directory. 


\subsection{Output}

When Monaco finishes, there are several output files produced. First, the main output file lists summaries for each of the region tallies and point detector tallies. With each mesh tally, a *.3dmap file is produced which can be viewed with the Mesh File Viewer capabilities of Fulcrum. Region tallies and point detectors produce text files listing group-by-group results for fluxes and any optional responses. Both of these tallies also produce *.chart files which contain information about how the simulation progresses with each batch. These can be viewed with the Interactive Plotter capabilities of Fulcrum.

From the main output file, the final tally results summary is shown below:

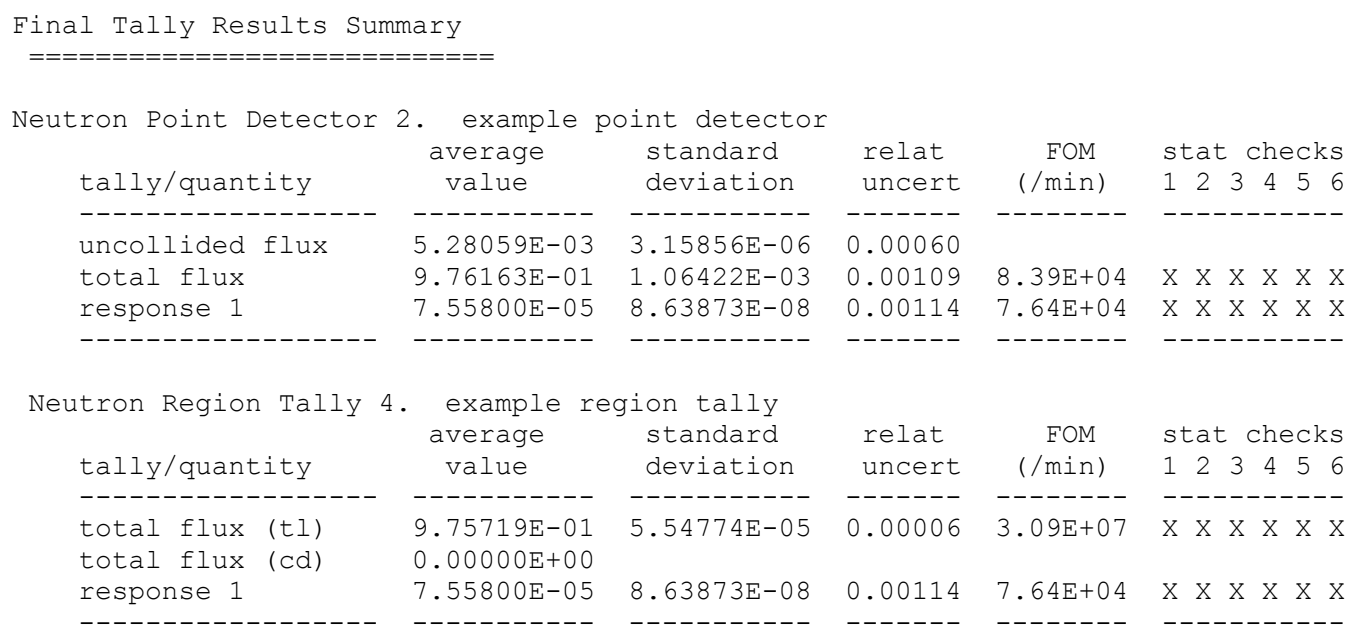

The fluxes reported are the total fluxes. Note the close agreement between the point detector flux and the region tally track-length estimate. The region tally did not produce a collision-density estimate of flux since the region was a void. Also notice that the neutron dose (response 1) from both tallies match well. The tally details that are saved in the *.pd*.txt or *.rt*.txt files are shown in Table 8.2.23. Group values for the mesh tally were obtained through the Mesh File Viewer.

For the mesh tally, the total neutron flux and neutron dose can be visualized using the Mesh File Viewer, as shown in Figure 8.2.19 and Figure 8.2.20. Using the mouse in the viewer, the flux and the dose rate for the cell that contains the detector location $(100,0,0)$ are found to be $1.004 \pm 2.3 \% \mathrm{n} / \mathrm{cm}^{2} / \mathrm{s}$ and $7.82 \times 10^{-5} \pm 2.4 \% \mathrm{rem} / \mathrm{hr}$, respectively, matching the other tallies well, given the higher uncertainties for the mesh tally.

A comparison of the results of all three Monaco tallies to the two experimental measurements is shown in Figure 8.2.21 through Figure 8.2.25 for different cross-section libraries. Note that the point detector results and the region tally results are the same for most of the energy range shown. The line representing the mesh tally result becomes broken in some plots because no neutrons of certain energy groups crossed this particular mesh cell. These four plots are each for 10 minutes of computation using a $2 \mathrm{GHz}$ Linux processor.

Using the library with finer groups, the Monaco results show more of the structure seen in the experiments over the energy range from $0.01 \mathrm{MeV}$ to $1.0 \mathrm{MeV}$, as shown in Figure 8.2.22 and Figure 8.2.23. 
Table 8.2.23. Group-by-group details of the three flux tallies (ENDF/B-VII.0 27n19g)

\begin{tabular}{|c|c|c|c|c|c|c|}
\hline \multirow{2}{*}{$\begin{array}{c}\text { Energy } \\
(\mathrm{MeV})\end{array}$} & \multicolumn{2}{|c|}{ Mesh tally } & \multicolumn{2}{|c|}{ Point detector } & \multicolumn{2}{|c|}{ Region tally } \\
\hline & value & unc & val & rel u & & rel unc \\
\hline $2.000 \mathrm{E}+07$ & $9.59 \mathrm{E}-04$ & 0.6325 & $1.30 \mathrm{E}-03$ & 0.0344 & $1.35 \mathrm{E}-03$ & 0.0168 \\
\hline $6.376 \mathrm{E}+06$ & $1.26 \mathrm{E}-02$ & 0.1472 & $8.19 \mathrm{E}-03$ & 0.0117 & $8.40 \mathrm{E}-03$ & 0.0067 \\
\hline $3.012 \mathrm{E}+06$ & $2.03 \mathrm{E}-02$ & 0.1413 & $2.21 \mathrm{E}-02$ & 0.0060 & $2.21 \mathrm{E}-02$ & 0.0041 \\
\hline $1.827 \mathrm{E}+06$ & $2.88 \mathrm{E}-02$ & 0.0919 & $2.41 \mathrm{E}-02$ & 0.0047 & $2.42 \mathrm{E}-02$ & 0.0039 \\
\hline $1.423 \mathrm{E}+06$ & $8.94 \mathrm{E}-02$ & 0.0770 & $9.05 \mathrm{E}-02$ & 0.0026 & $9.06 \mathrm{E}-02$ & 0.0020 \\
\hline $9.072 \mathrm{E}+05$ & $3.40 \mathrm{E}-01$ & 0.0466 & $3.26 \mathrm{E}-01$ & 0.0018 & $3.25 \mathrm{E}-01$ & 0.0009 \\
\hline $4.076 \mathrm{E}+05$ & 4.19E-01 & 0.0320 & $4.15 \mathrm{E}-01$ & 0.0017 & $4.15 \mathrm{E}-01$ & 0.0007 \\
\hline $1.111 \mathrm{E}+05$ & $7.25 \mathrm{E}-02$ & 0.0790 & $6.65 \mathrm{E}-02$ & 0.0046 & $6.64 \mathrm{E}-02$ & 0.0023 \\
\hline $1.503 \mathrm{E}+04$ & $1.27 \mathrm{E}-02$ & 0.1751 & $1.21 \mathrm{E}-02$ & 0.0118 & $1.21 \mathrm{E}-02$ & 0.0055 \\
\hline $3.035 \mathrm{E}+03$ & $4.05 \mathrm{E}-03$ & 0.3065 & $4.80 \mathrm{E}-03$ & 0.0179 & $4.75 \mathrm{E}-03$ & 0.0083 \\
\hline $5.830 \mathrm{E}+02$ & $1.51 \mathrm{E}-03$ & 0.4935 & $2.58 \mathrm{E}-03$ & 0.0237 & $2.59 \mathrm{E}-03$ & 0.0109 \\
\hline $1.013 \mathrm{E}+02$ & $6.10 \mathrm{E}-04$ & 0.6476 & $1.26 \mathrm{E}-03$ & 0.0320 & $1.20 \mathrm{E}-03$ & 0.0151 \\
\hline $2.902 \mathrm{E}+01$ & 4.75E-04 & 0.6724 & 7.23E-04 & 0.0367 & $7.16 \mathrm{E}-04$ & 0.0182 \\
\hline $1.068 \mathrm{E}+01$ & 2.82E-04 & 0.9487 & 5.19E-04 & 0.0402 & $5.03 \mathrm{E}-04$ & 0.0210 \\
\hline $3.059 \mathrm{E}+00$ & & & $1.60 \mathrm{E}-04$ & 0.0597 & $1.40 \mathrm{E}-04$ & 0.0403 \\
\hline $1.855 \mathrm{E}+00$ & & & 7.91E-05 & 0.0824 & $8.31 \mathrm{E}-05$ & 0.0534 \\
\hline 1.30 & & & $3.15 \mathrm{E}-05$ & 0.1059 & $2.96 \mathrm{E}-05$ & 0.0901 \\
\hline $1.125 \mathrm{E}+00$ & & & $2.24 \mathrm{E}-05$ & 0.1108 & $1.97 \mathrm{E}-05$ & 0.1086 \\
\hline $1.000 \mathrm{E}+00$ & & & $3.21 \mathrm{E}-05$ & 0.1299 & $2.45 \mathrm{E}-05$ & 0.0979 \\
\hline $8.000 \mathrm{E}-01$ & & & $4.20 \mathrm{E}-05$ & 0.1127 & $3.63 \mathrm{E}-05$ & 0.0816 \\
\hline 4.140E-01 & & & $1.02 \mathrm{E}-05$ & 0.1720 & $1.03 \mathrm{E}-05$ & 0.1583 \\
\hline $3.250 \mathrm{E}-01$ & & & $1.13 \mathrm{E}-05$ & 0.2433 & 7.62E-06 & 0.1701 \\
\hline $2.250 \mathrm{E}-01$ & & & $8.26 \mathrm{E}-06$ & 0.2554 & $7.42 \mathrm{E}-06$ & 0.1746 \\
\hline $1.000 \mathrm{E}-01$ & & & $1.00 \mathrm{E}-06$ & 0.4480 & $6.73 \mathrm{E}-07$ & 0.6451 \\
\hline $5.000 \mathrm{E}-02$ & & & 6.61E-08 & 0.4590 & & \\
\hline $3.000 \mathrm{E}-02$ & & & $6.59 \mathrm{E}-10$ & 0.4491 & & \\
\hline $1.000 \mathrm{E}-02$ & & & $6.90 \mathrm{E}-13$ & 0.3458 & & \\
\hline $1.000 \mathrm{E}-05$ & & & & & & \\
\hline
\end{tabular}




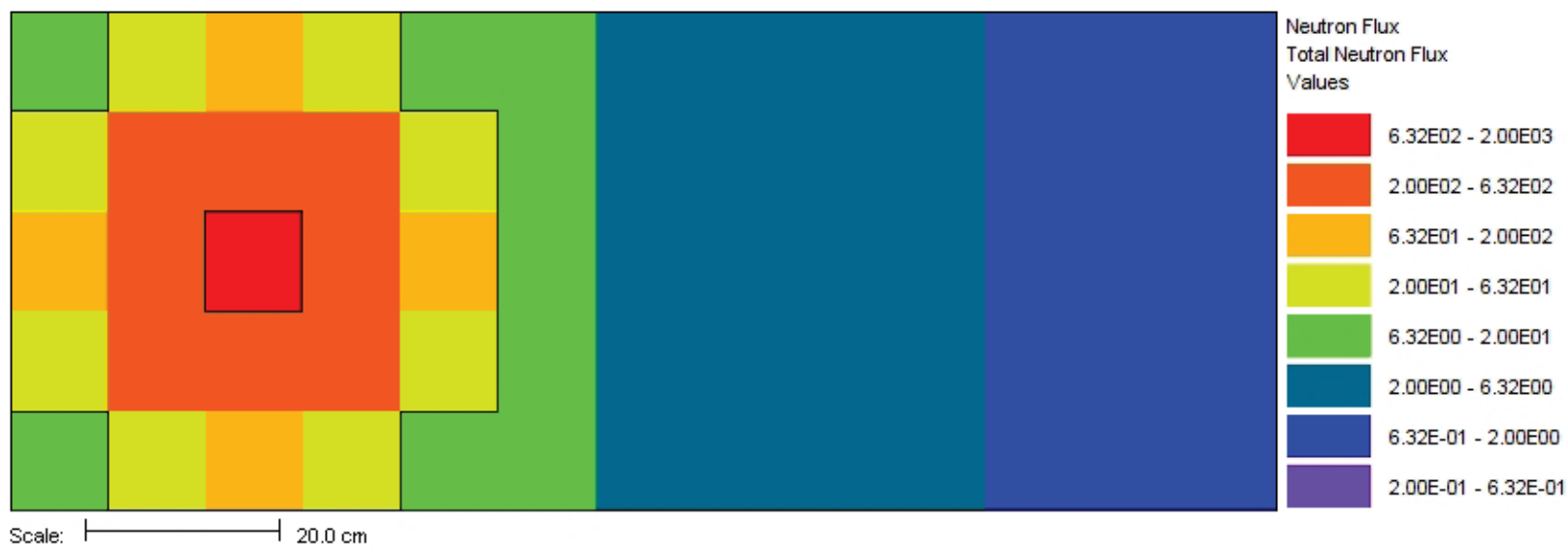

Figure 8.2.19. Total neutron flux in $\mathbf{n} / \mathrm{cm}^{2} / \mathrm{s}$.

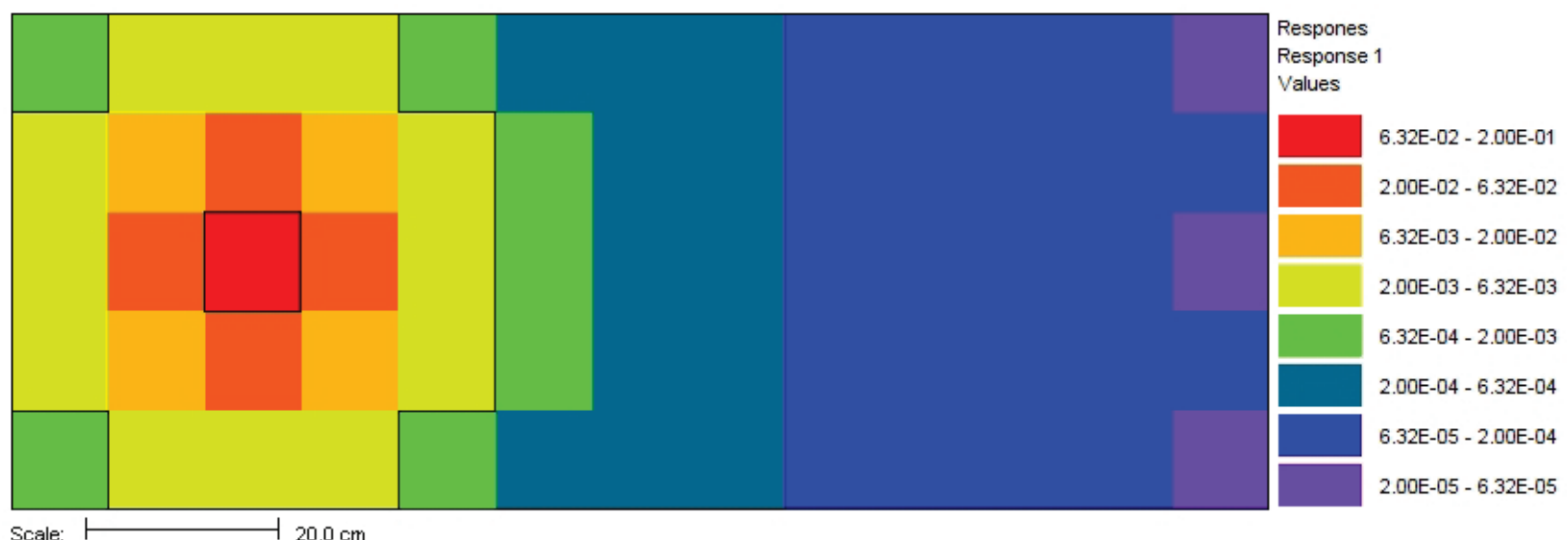

Figure 8.2.20. Neutron dose rate in $\mathrm{rem} / \mathrm{hr}$. 


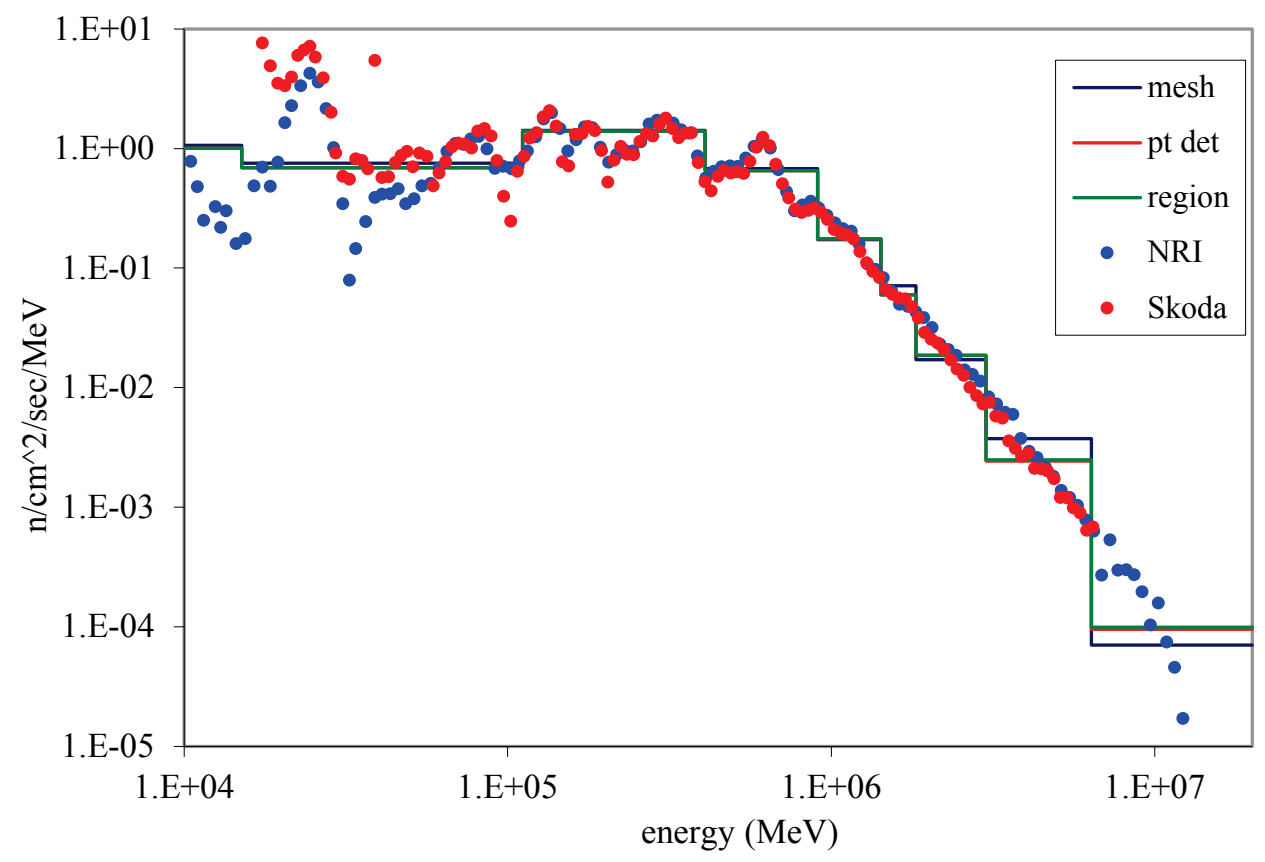

Figure 8.2.21. Comparison of Monaco results using the ENDF/B-VII.0 27n/19g library and the measured values.

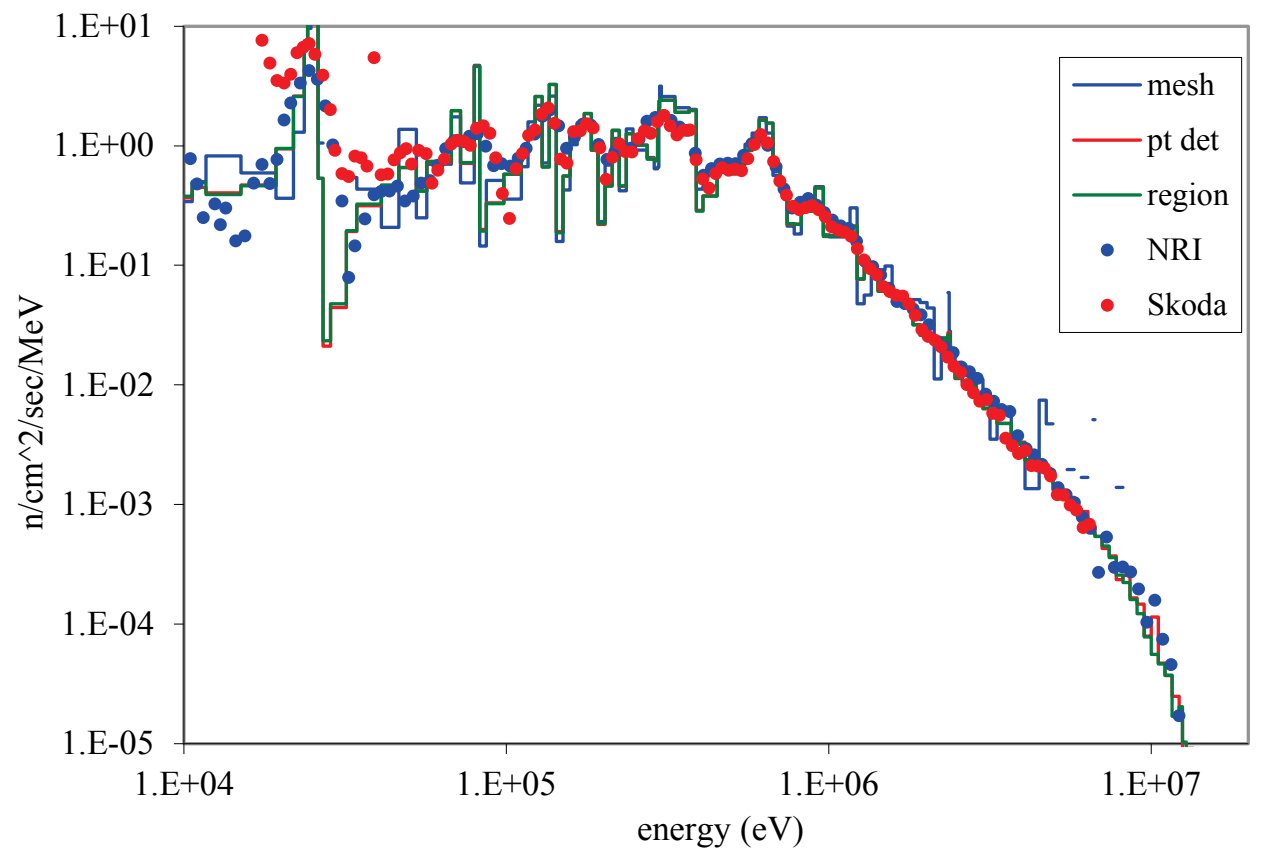

Figure 8.2.22. Comparison of Monaco results using the new ENDF/B-VII.0 200n/47g library and the measured values. 


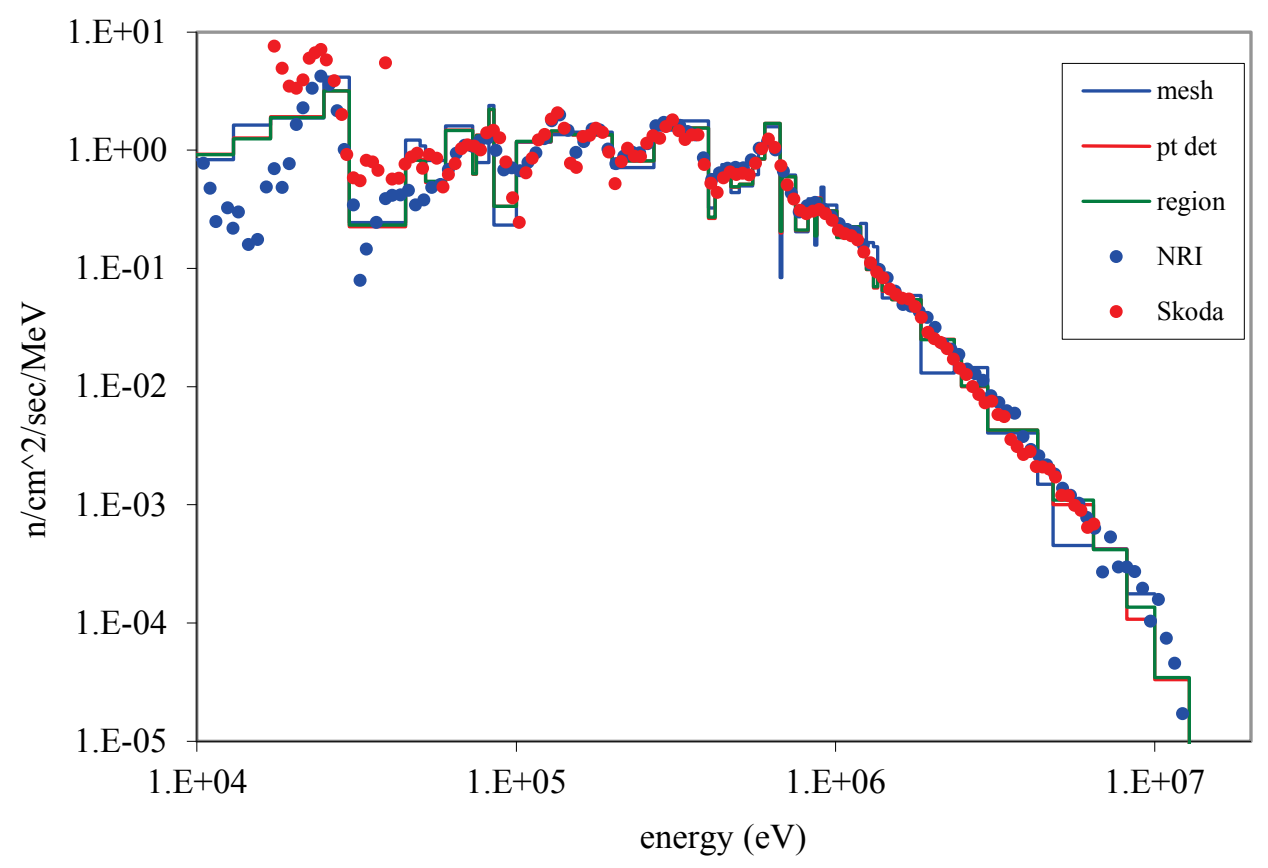

Figure 8.2.23. Comparison of Monaco results using the ENDF/B-VII 238n library and the measured values.

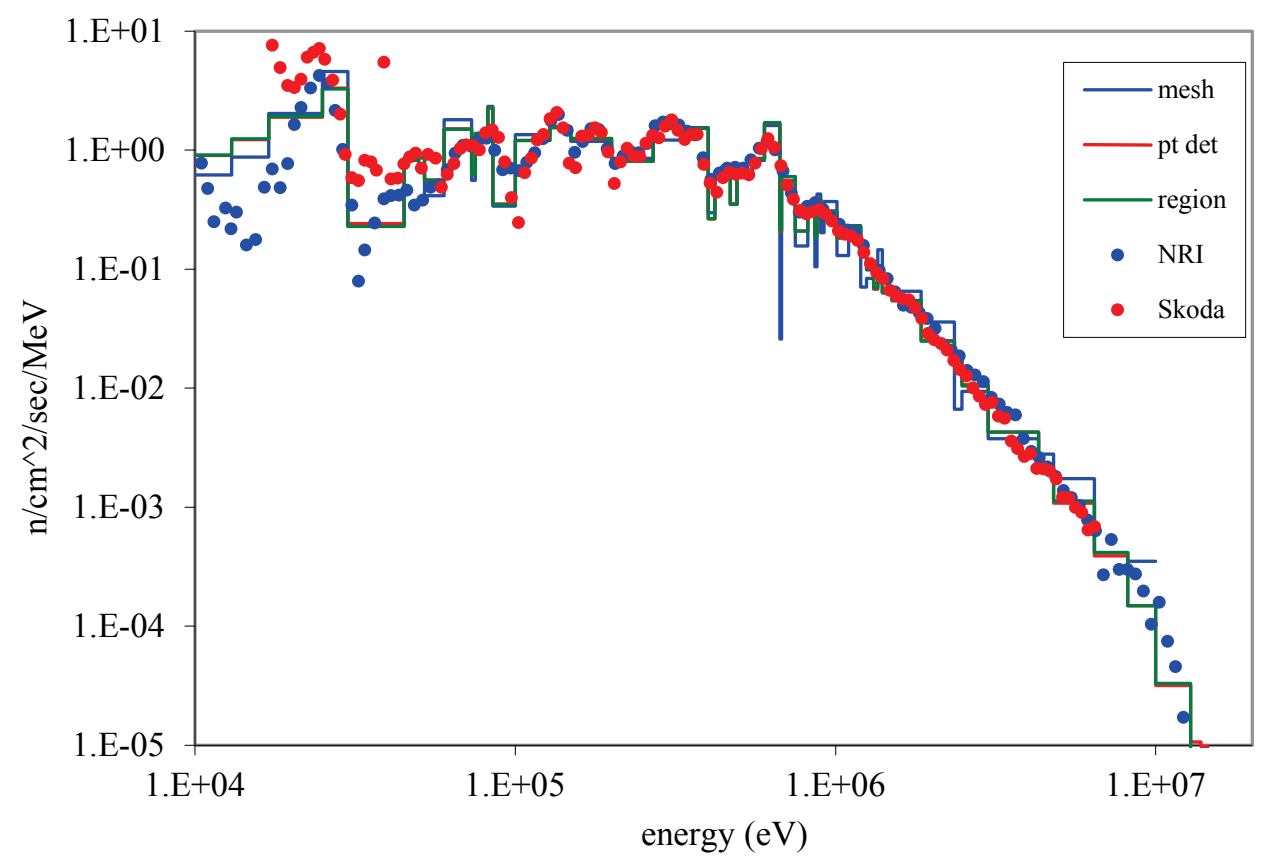

Figure 8.2.24. Comparison of Monaco results using the ENDF/B-VII 252n library and the measured values. 


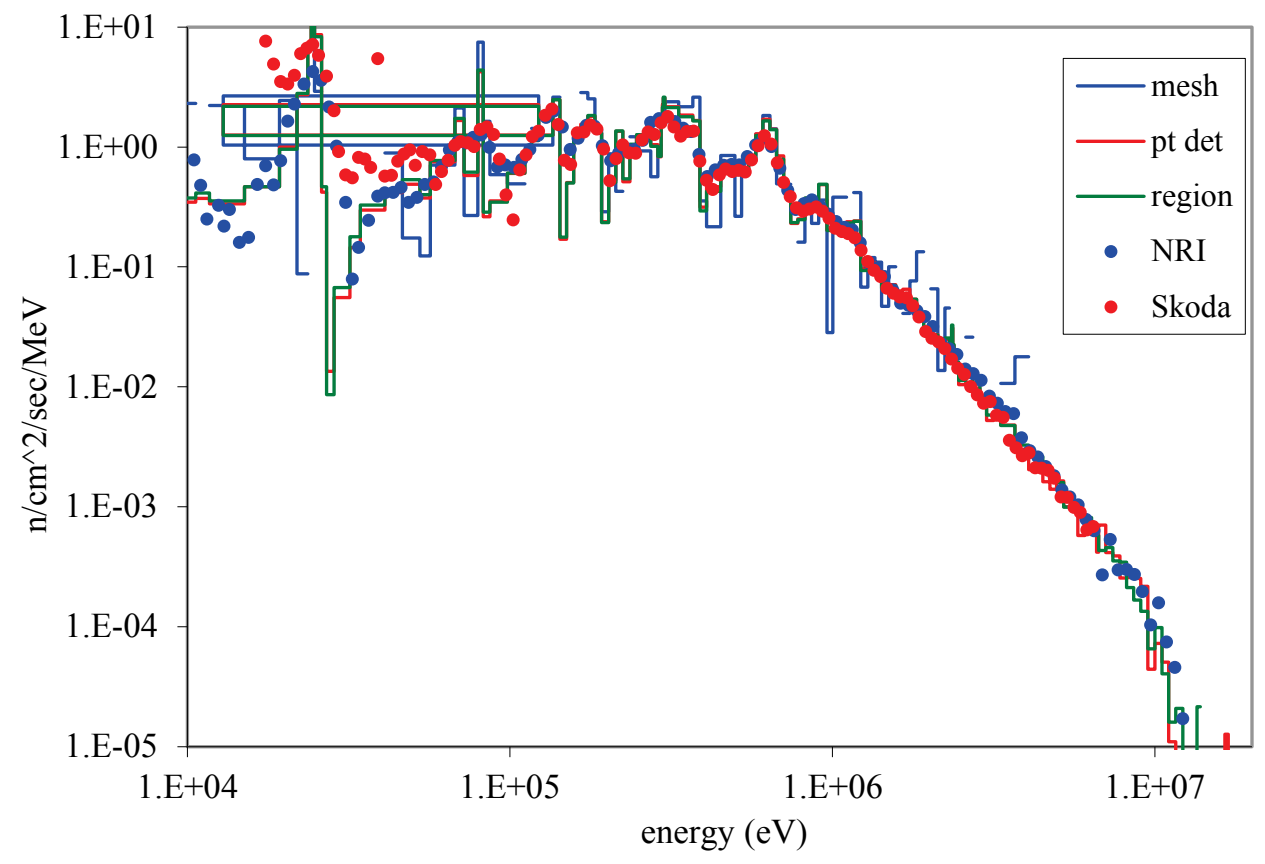

Figure 8.2.25. Comparison of Monaco results using the ENDF/B-VII CE n/p library (binned using the 200n group structure) and the measured values.

\subsubsection{Neutrons through a heavy water sphere}

Similar to the first example problem, ${ }^{252} \mathrm{Cf}$ neutrons were measured outside of a sphere filled with heavy water. ${ }^{6}$ Two measurements were made: one without the iron/polyethylene shield and one with the shield. These two measurements were subtracted to account for scatter from the floor (which is about a $5 \%$ effect for energies above $10 \mathrm{keV}$ ). A great amount of detail is given for the materials and geometry of the source holder, insertion tube, and detectors in Ref. 6. For this sample problem, just the basics will be modeled in two inputs: monaco.d2osphereA. inp and monaco.d2osphereB. inp, both located in the SCALE samples $\backslash$ input directory.
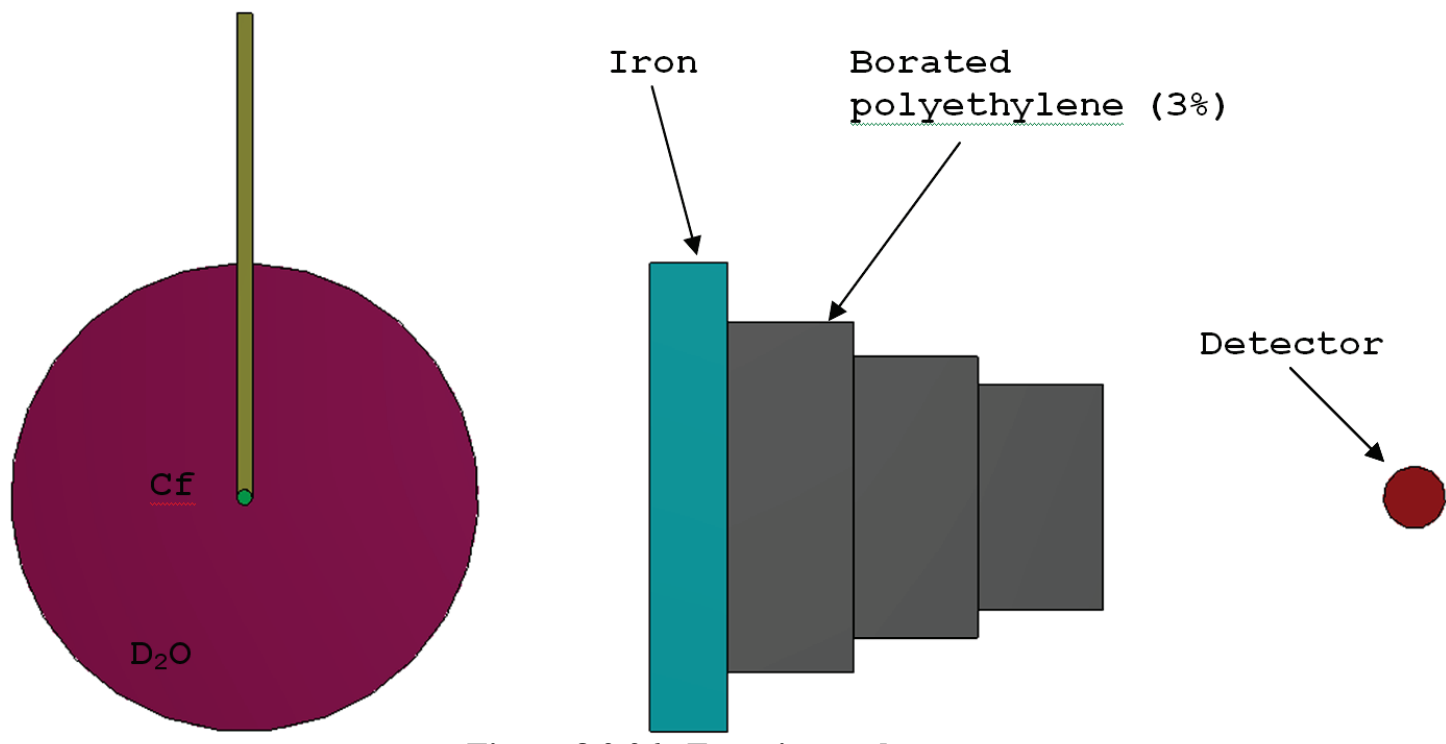

Figure 8.2.26. Experimental setup. 


\subsection{Input}

First, the cross sections for four materials need to be computed. Here, csas-mg is used:

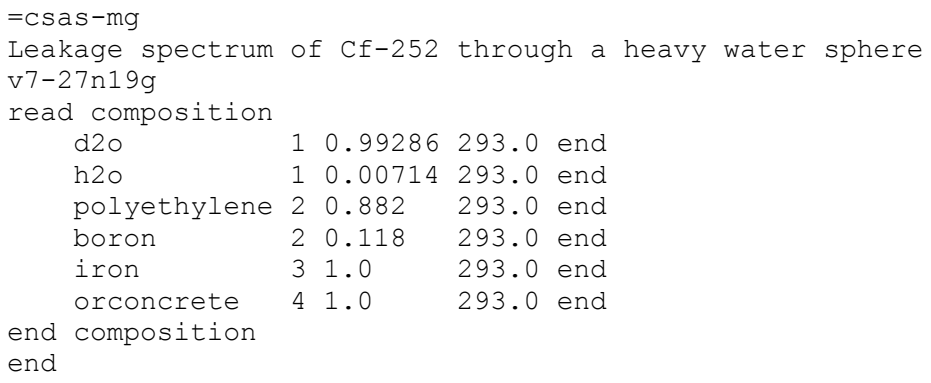

The Monaco input file starts with module name ("monaco") and a title.

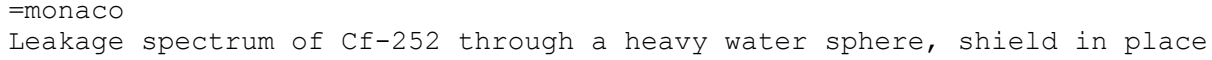

The mixing table information can be found in the output of the above csas-mg run. The newer ENDF/BVII libraries contain isotopic data for iron, instead of just the elemental data in the older ENDF/B-V libraries.

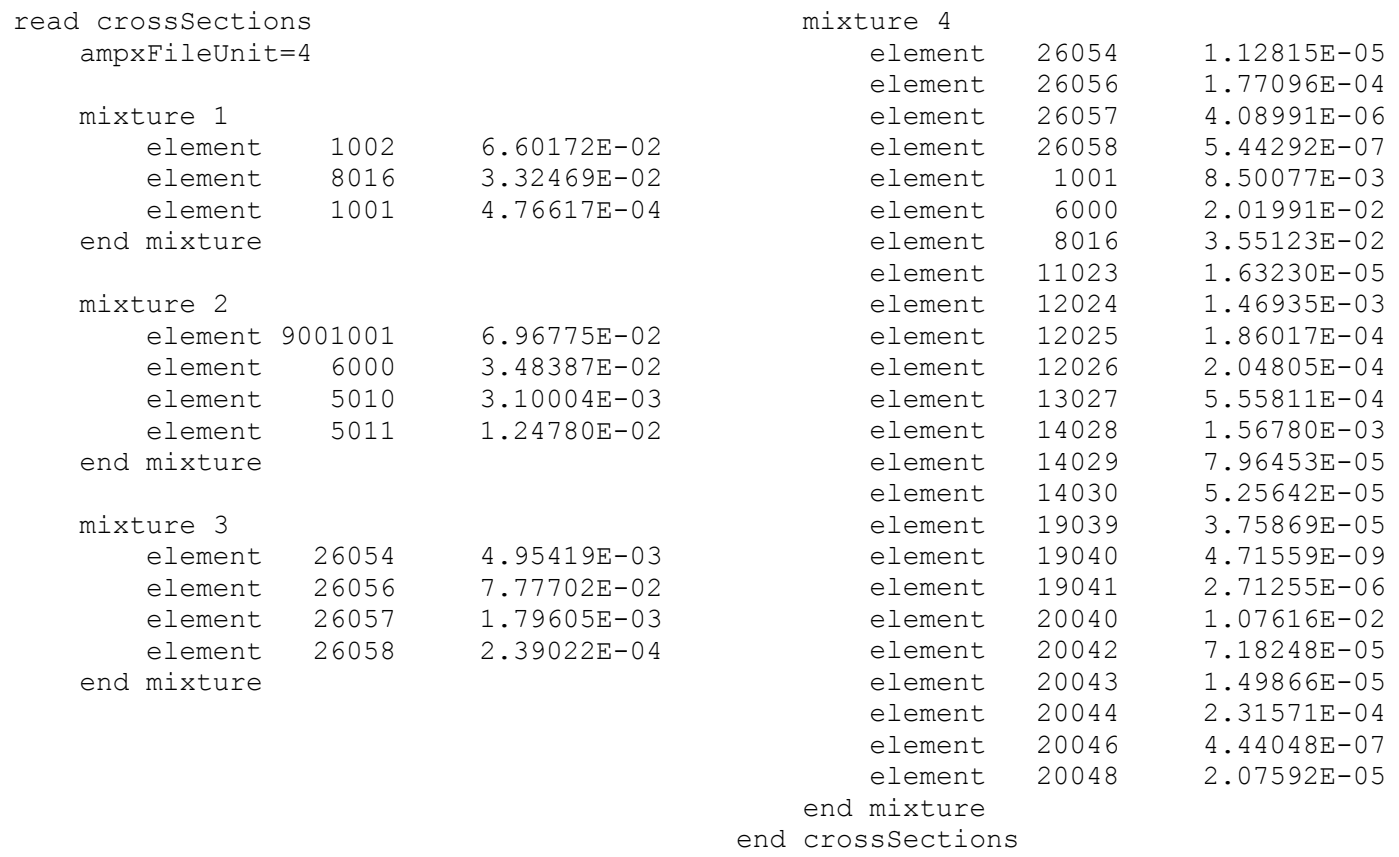

The SGGP geometry consists of two nested spheres for the source and heavy water sphere. Four cylindrical shields made of either borated polyethylene or iron are placed between the sphere and the detector position $(75,0,0)$. The experiment sat $2 \mathrm{~m}$ above the floor of an experimental hall that measured $10 \times 13 \times 25 \mathrm{~m}$. Here, the origin corresponds to the source at the center of the heavy water sphere. 


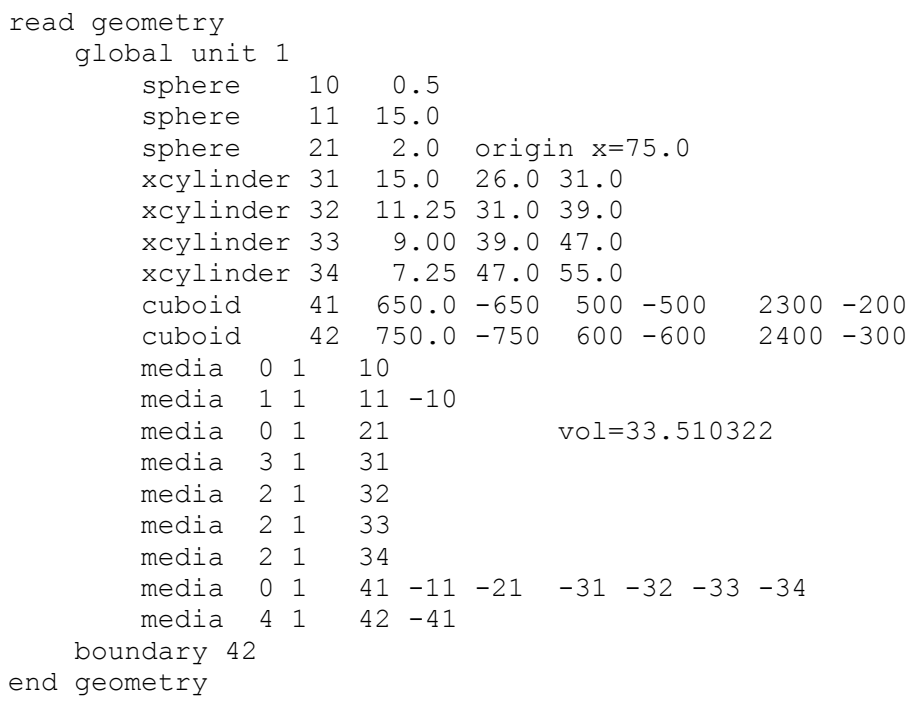

A second input file (samples \input $\backslash$ monaco.d2osphereA.inp) was created for the geometry without the four xcylinder shields in place.

For this example, a point detector will be used to calculate the flux at the detector location and the source will require a Watt distribution.

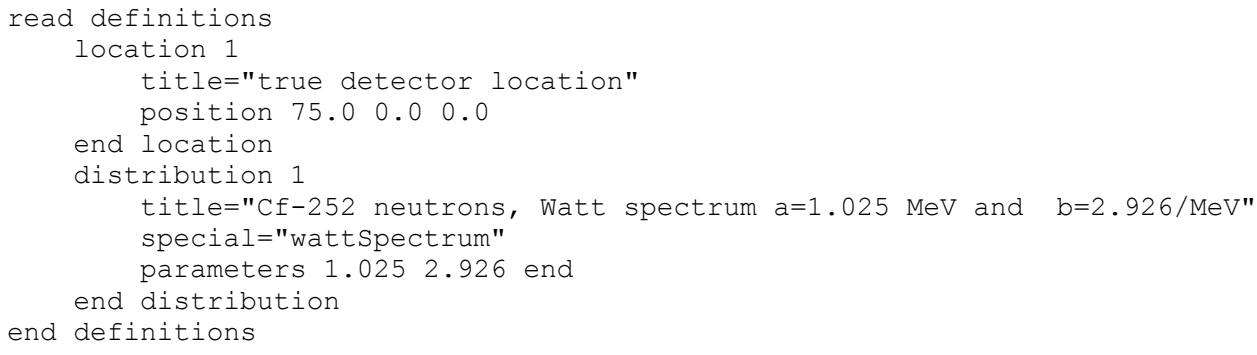

The strength is set so that the total flux at the detector (at $r=75 \mathrm{~cm}$ ) without any shield would be $1 \mathrm{n} / \mathrm{cm}^{2} / \mathrm{s}$. So, strength $=4 \pi(75)^{2}=70686 \mathrm{n} / \mathrm{s}$.

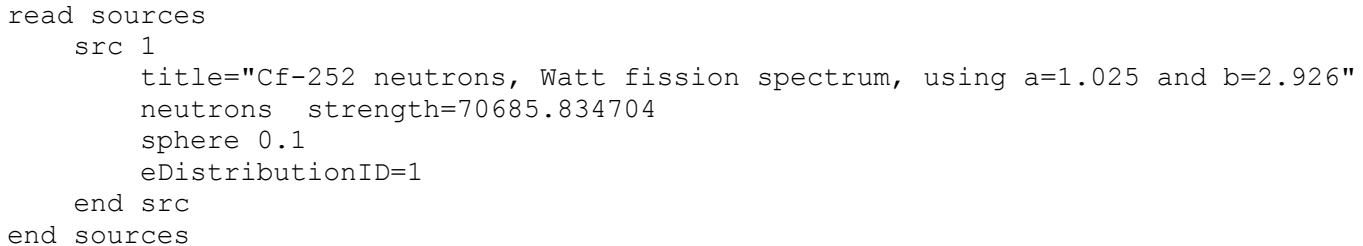

Two tallies will be defined: a region tally over the third region of unit 1 and a point detector tally at the true detector location.

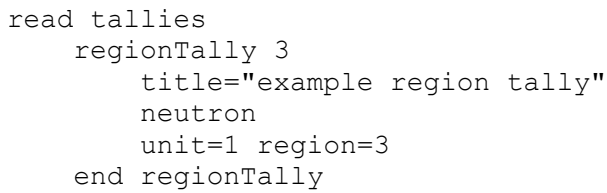




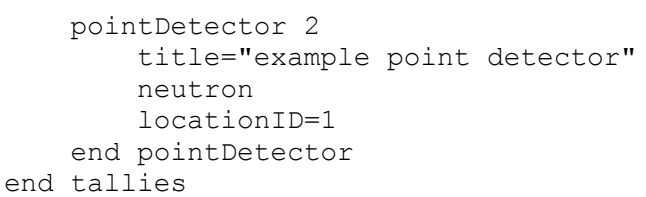

Monte Carlo parameters include the starting random number seed, the number of particles per batch, and the number of batches to simulate. Since there are no photon tallies, the keyword "secondaryMult=0" is used so that photons are not produced nor transported.

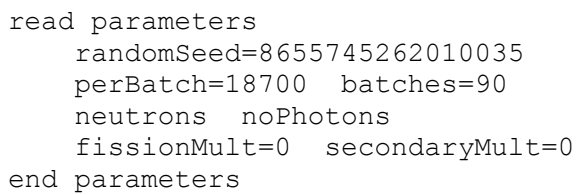

Finally, the Monaco input file is ended with

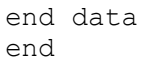

\subsection{Output}

For the case without the xcylinder shields, the tally summaries from the main output file are reported as:

\begin{tabular}{|c|c|c|c|c|c|}
\hline tally/quantity & $\begin{array}{c}\text { average } \\
\text { value }\end{array}$ & $\begin{array}{l}\text { standard } \\
\text { deviation }\end{array}$ & $\begin{array}{l}\text { relat } \\
\text { uncert }\end{array}$ & $\begin{array}{c}\text { FOM } \\
(/ \mathrm{min})\end{array}$ & $\begin{array}{l}\text { stat checks } \\
\begin{array}{lllll}1 & 2 & 3 & 4 & 5\end{array}\end{array}$ \\
\hline------------------ & ----------- & ----------- & ------- & -------- & \\
\hline uncollided flux & 4.20334E-02 & $6.81217 \mathrm{E}-05$ & 0.00162 & & \\
\hline total flux & $1.13349 \mathrm{E}+00$ & $3.28455 E-03$ & 0.00290 & $1.18 \mathrm{E}+04$ & $\mathrm{X} X \mathrm{X} \times \mathrm{X} X$ \\
\hline 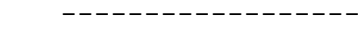 & ----------- & ----------- & ------- & -------- & --------- \\
\hline atron Region Tally & $\begin{array}{l}\text { 3. example } r e \\
\text { average }\end{array}$ & $\begin{array}{r}\text { egion tally } \\
\text { standard }\end{array}$ & relat & FOM & stat checks \\
\hline tally/quantity & value & deviation & uncert & $(/ \min )$ & $\begin{array}{llllll}1 & 2 & 3 & 4 & 5 & 6\end{array}$ \\
\hline --------------- & ----------- & ----------- & ------- & -------- & ----------- \\
\hline $\begin{array}{lll}\text { total flux } & (t l) \\
\text { total flux } & (c d)\end{array}$ & $\begin{array}{l}1.00584 \mathrm{E}+00 \\
0.00000 \mathrm{E}+00\end{array}$ & $1.47136 \mathrm{E}-01$ & 0.14628 & $4.64 \mathrm{E}+00$ & $---X X-$ \\
\hline ーーーーーーーーーーーーーーーーーー & ----------- & ----------- & ------ & --- & - \\
\hline
\end{tabular}

Note that in this sample calculation (10 minutes), the region tally did not pass all of the statistical checks and the relative uncertainty for the region tally is very large due to the region's small size. This shows how useful a point detector tally can be in estimating fluxes for hard-to-reach locations.

For the case with the xcylinder shields in place (450 minutes), the final results were:

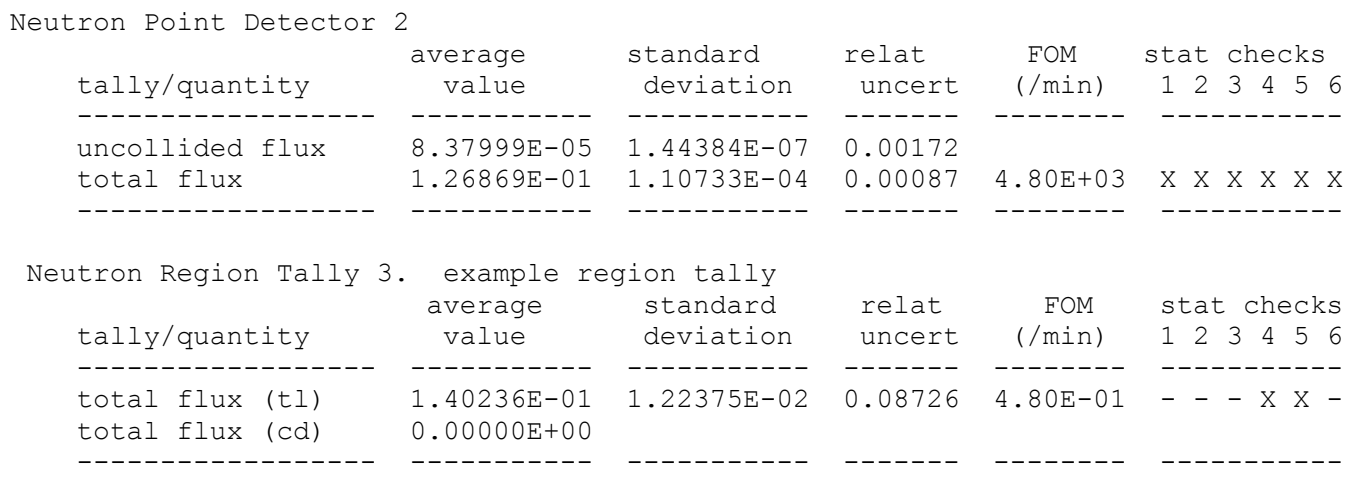


Here again, the region tally performed very poorly, showing that very few neutrons actually crossed the tally region. The four shields make this problem very slow to converge because rare particles can arrive at the detector after paths that see different amounts of shield.

The group-wise results list in the point detector detail files from each case can be subtracted and compared to the measurements and calculations listed in Ref. 6. The computed and measured neutron spectra seen by the detector are shown in Figure 8.2.27 through Figure 8.2.31 using different crosssection libraries. For each figure, the computational times for case "A", without the shields and case "B", with the shields, were 10 minutes and 90 minutes, each on a $2 \mathrm{GHz}$ Linux processor. For the CE case, 100 and 900 minutes were used to reduce the statistical uncertainties. Except for the 27/19 multigroup case shown above, none of the "B" cases passed all of the statistical tests.

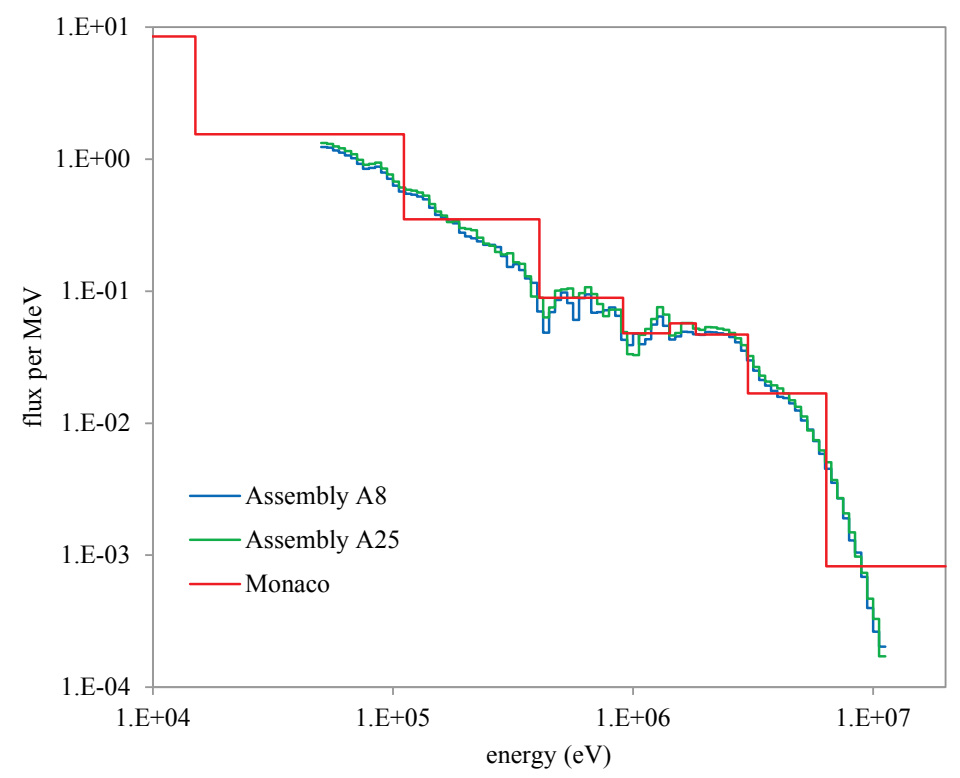

Figure 8.2.27. Comparison of Monaco calculated results using the ENDF/B-VII.0 27n/19g library to the measured values. 


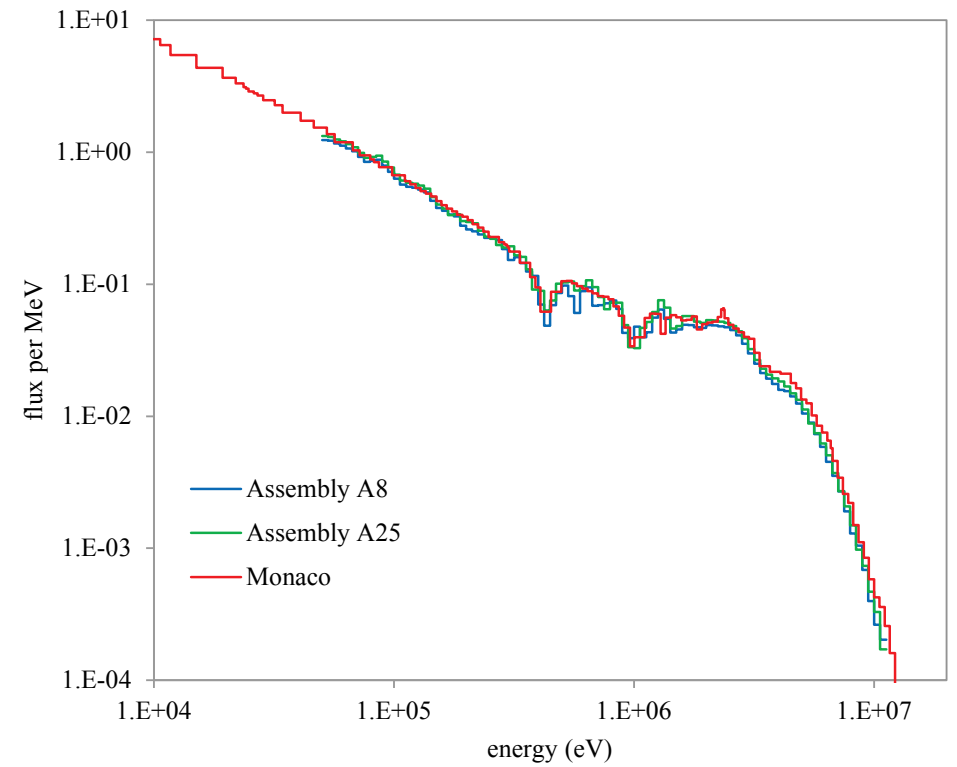

Figure 8.2.28. Comparison of Monaco calculated results using the ENDF/B-VII.0 200n/47g library to the measured values.

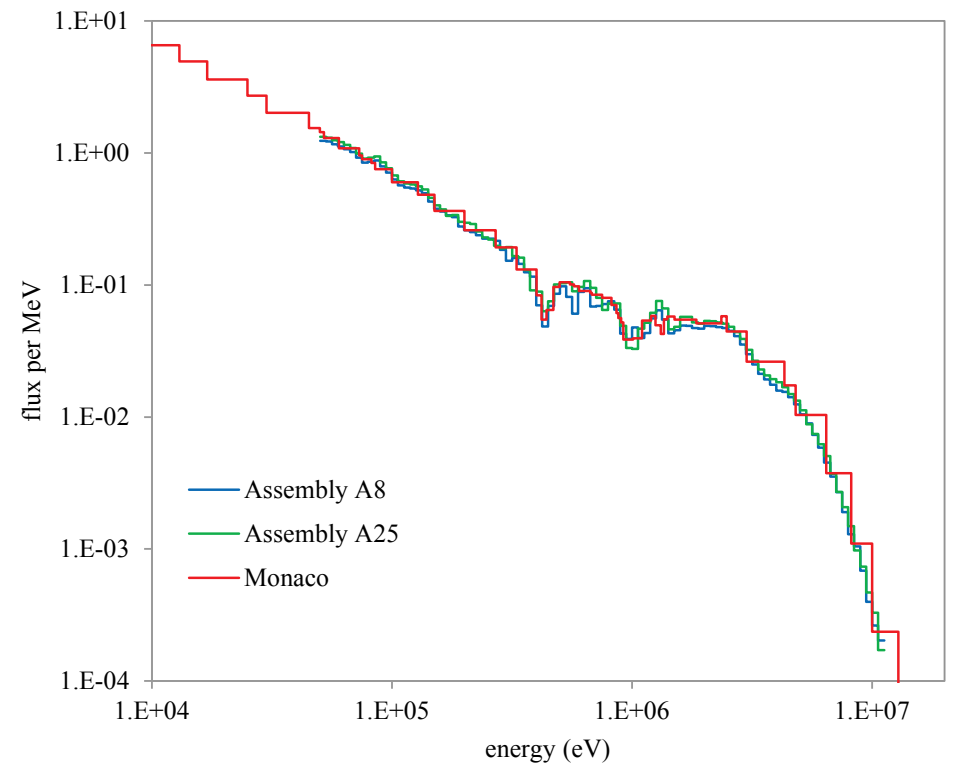

Figure 8.2.29. Comparison of Monaco calculated results using the ENDF/B-V 238n library to the measured values. 


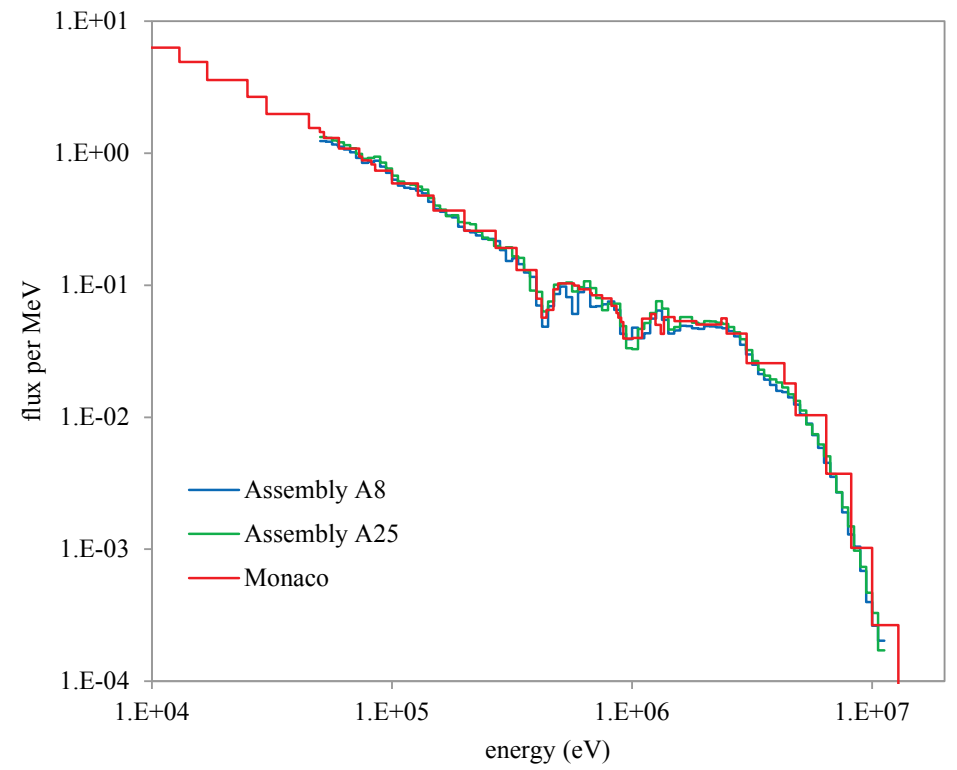

Figure 8.2.30. Comparison of Monaco calculated results using the ENDF/B-V 252n library to the measured values.

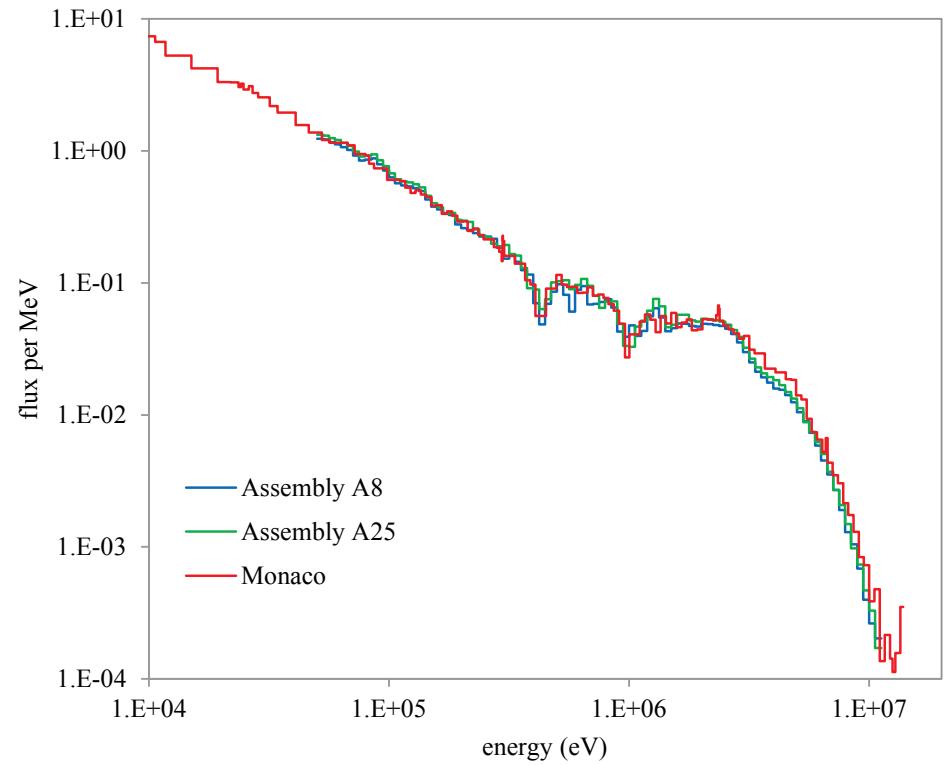

Figure 8.2.31. Comparison of Monaco calculated results using the ENDF/B-VII CE n/p library (binned using the 200n group structure) to the measured values.

\subsubsection{Activation rate from a neutron howitzer}

A "neutron howitzer" is a common laboratory-sized neutron source. A few curies of an alpha emitter is mixed with an isotope that has a large $(\alpha, n)$ cross section. This source is then stored in a large tank of moderating material (water, paraffin, etc.) with an access port. Materials can be placed into the access port for irradiation. Activation studies are a typical use of a neutron howitzer. Note that the large 
moderator tank also provides shielding for the users. Typical source strengths are such that users would probably not wish to spend large amounts of time in front of an open access port.

A typical size and shape for a neutron howitzer is an upright cylinder, outer radius of $30 \mathrm{~cm}$ by $70 \mathrm{~cm}$ in height. The outer wall, top, and bottom are made of $2 \mathrm{~cm}$ thick Plexiglas. The access port is a cylinder of radius $2 \mathrm{~cm}$ extending $5 \mathrm{~cm}$ away from the tank center through the side wall. A Plexiglas rod can be removed/inserted into the port for sample loading. The tank is filled with ordinary water. Figure 8.2.32 shows a cutaway view of a very simplified model of the tank with the Plexiglas rod inserted into the access port, to prevent neutrons from streaming into the room. The source is located in the center of the tank and is small enough to be considered a point source. A small foil $(1 \times 1 \times 0.001 \mathrm{~cm})$ gold foil ${ }^{197} \mathrm{Au}$ is included as an activation sample.

The goal is to use Monaco to calculate the activation rate density, $R$, in units of $/ \mathrm{cm}^{3} / \mathrm{s}$, of the gold foil

$$
R=\int \Sigma(E) \phi(E) d E
$$

so that the activity, $A$, can be calculated as a function of time in the howitzer, $T$, and time outside the howitzer, $t$. Note that the half-life of ${ }^{198} \mathrm{Au}$ of 2.7 days (decay constant is $\lambda=2.97 \times 10^{-6} / \mathrm{s}$ ).

$$
A=R V\left(1-e^{-\lambda T}\right) e^{-\lambda t}
$$
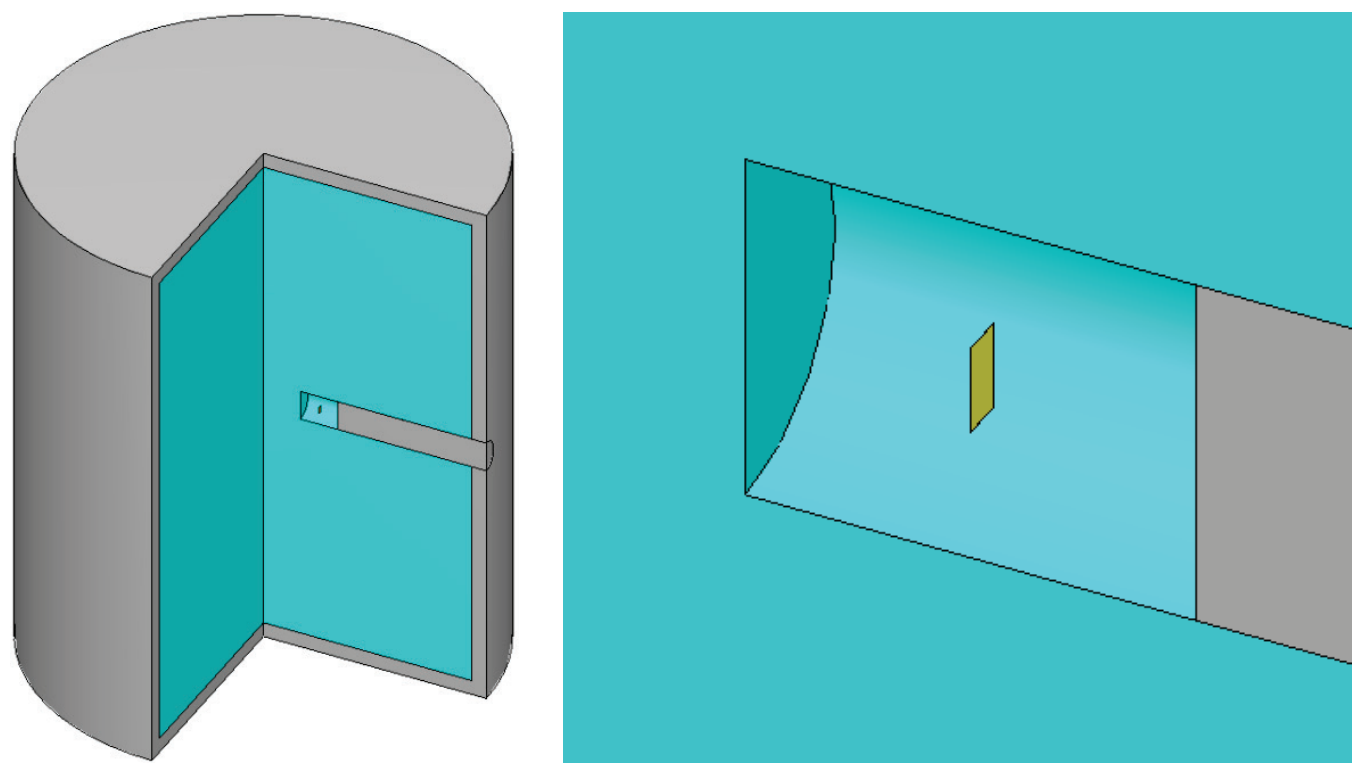

Figure 8.2.32. Idealized geometry for a neutron howitzer.

\subsection{Input file}

The following input file represents the simple model of a neutron howitzer used for a gold foil activation experiment. The file monaco.howitzer.inp is located in the SCALE samples \input directory. CSAS-MG is used to generate the cross sections. 


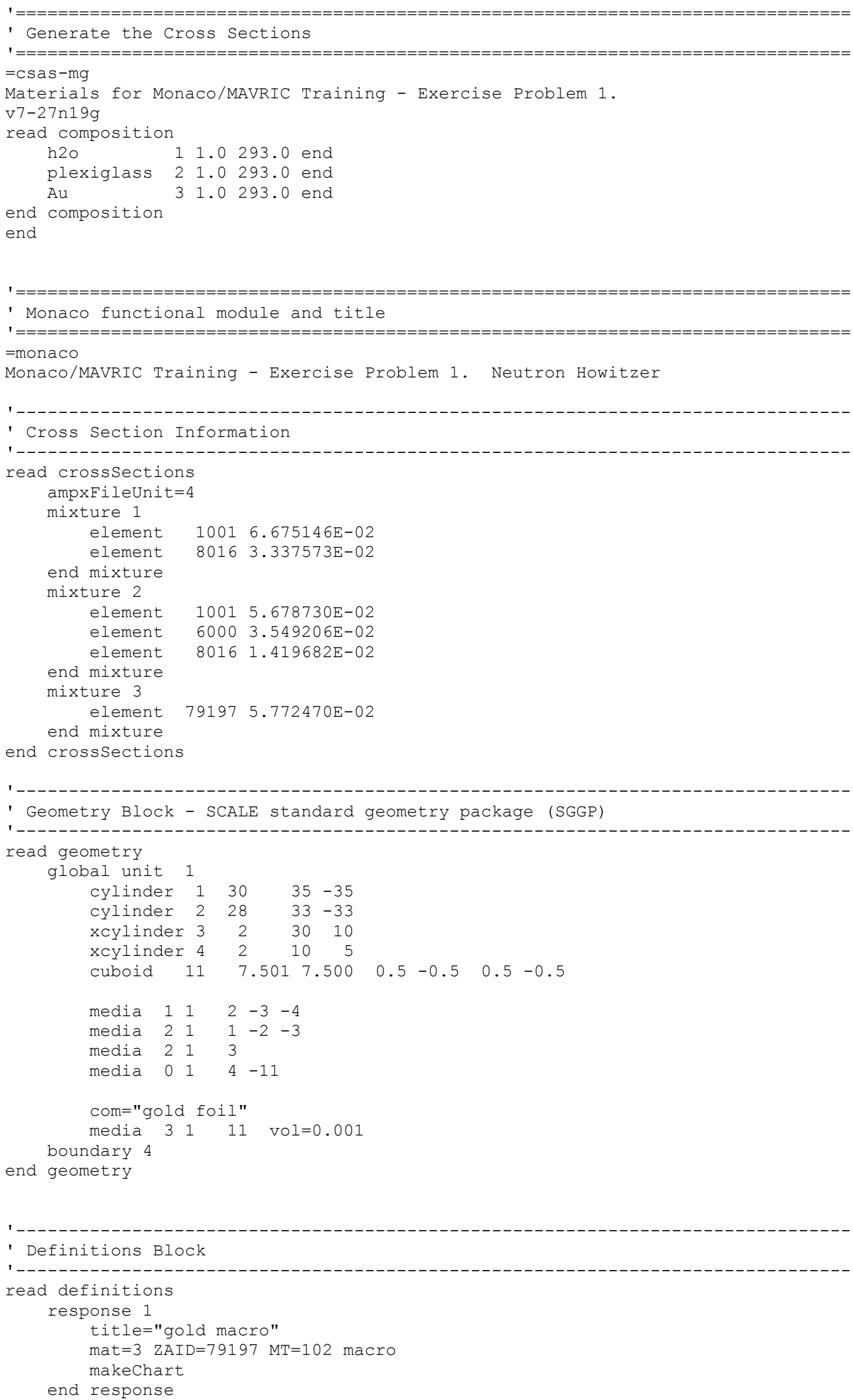




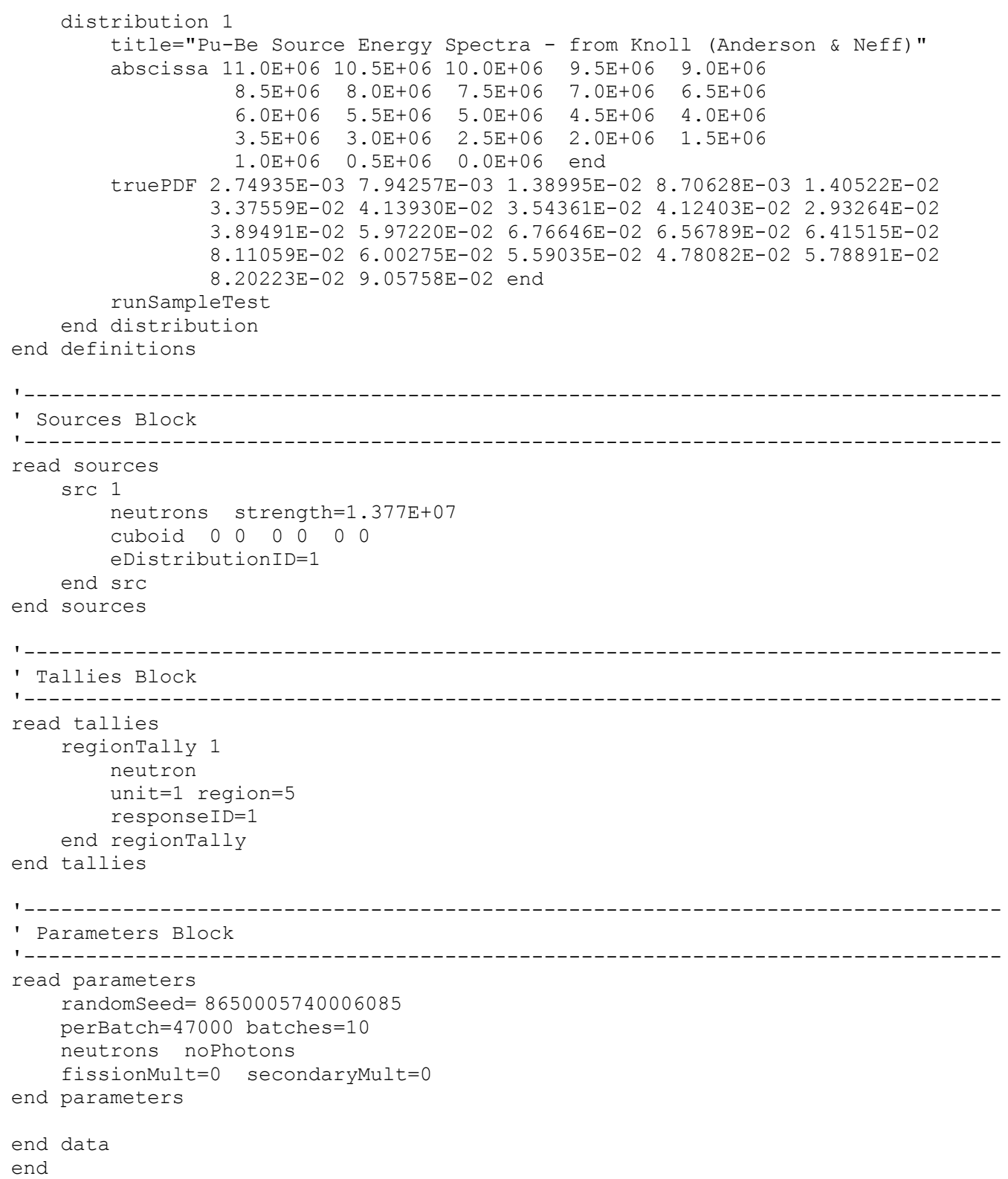

\subsection{Output}

The main text output file results are reported as:

Neutron Region Tally 1 .
tally/quantity
-------------
total flux (tl)
total flux (cd)
response 1

\begin{tabular}{|c|c|c|c|c|}
\hline $\begin{array}{c}\text { average } \\
\text { value }\end{array}$ & $\begin{array}{l}\text { standard } \\
\text { deviation }\end{array}$ & $\begin{array}{l}\text { relat } \\
\text { uncert }\end{array}$ & $\begin{array}{c}\text { FOM } \\
(/ \mathrm{min})\end{array}$ & 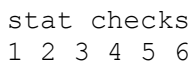 \\
\hline---------- & ---------- & ------- & -------- & \\
\hline $8.59999 E+04$ & $2.64821 \mathrm{E}+03$ & 0.03079 & $9.70 \mathrm{E}+01$ & $\mathrm{X}$ \\
\hline 1. $23288 \mathrm{E}+05$ & $8.52974 \mathrm{E}+04$ & 0.69186 & 1. $92 \mathrm{E}-01$ & $-\quad-\quad--$ \\
\hline $2.66771 E+05$ & $1.12525 \mathrm{E}+04$ & 0.04218 & $5.17 \mathrm{E}+01$ & $--X X X$ \\
\hline---- & ------ & ----- & --- & \\
\hline
\end{tabular}

In addition to the main output file, the diagnostics from the definitions block are shown Figure 8.2.33 in and Figure 8.2.34. The final value for the activation rate density $R$ is $2.65 \times 10^{5} / \mathrm{cm}^{3} / \mathrm{sec}$. Note how the track length tally performs better than the collision density estimate for this thin foil region. But also note that after ten minutes, not all of the statistical checks are converged. Running this problem for longer 
times (even up to 10 hours) does not make all of the tests pass. Due to the very thin width of the of the tally region, most track-lengths across are very small but occasionally a very long track-length is recorded. This rare but high-value score tends to upset the convergence metrics. A tally region over a larger, more cuboid-like volume would make for a better-behaved tally.

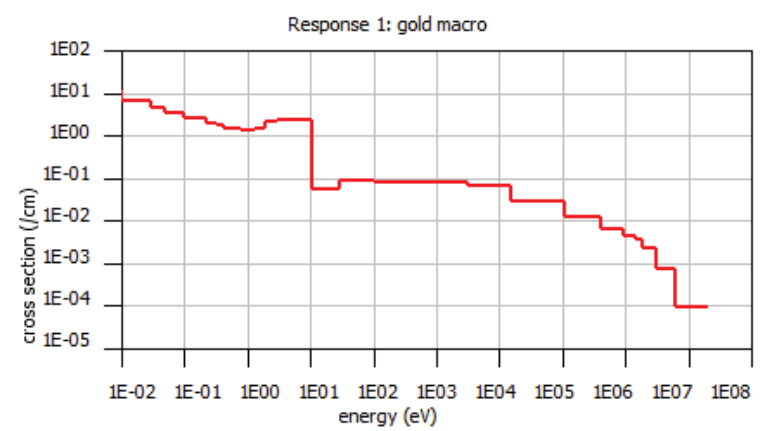

Figure 8.2.33. Response 1.

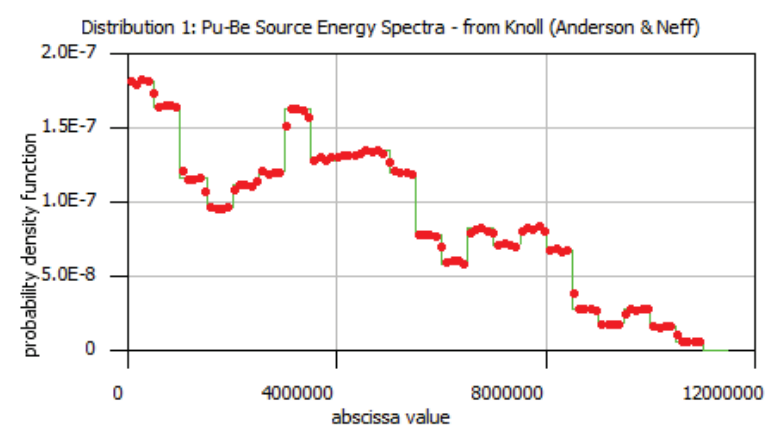

Figure 8.2.34. Distribution 1 .

\subsubsection{Graphite shielding measurements}

K. Ueki of the Nuclear Technology Division, Ship Research Division in Japan performed many simple studies on a variety of shielding materials layered in different combinations. He and his colleagues used both neutron $\left({ }^{252} \mathrm{Cf}\right)$ and photon $\left({ }^{60} \mathrm{Co}\right)$ sources to investigate the shielding effectiveness of steel, graphite, and many hydrogen-containing materials as single shields or in combinations.

One such series of measurements was for pure graphite. ${ }^{7} \quad \mathrm{~A}{ }^{252} \mathrm{Cf}$ neutron source was placed in the center of a $50 \mathrm{~cm}$ cube of paraffin which had a $45^{\circ}$ cone cut-out. A neutron meter was placed $110 \mathrm{~cm}$ from the source. Sheets of material, in $5 \times 80 \times 80 \mathrm{~cm}$ slabs, were placed between the source and detector, with the detector side of the shield always fixed at $90 \mathrm{~cm}$ from the source. The shield thickness was increased on the source side of the shield. His results (read from a plot) for different thicknesses of graphite are shown in Table 8.2.24.

Table 8.2.24. Ueki's experimental results

\begin{tabular}{cc}
\hline $\begin{array}{c}\text { Thickness } \\
(\mathbf{c m})\end{array}$ & $\begin{array}{c}\text { Dose equivalent } \\
\text { attenuation }\end{array}$ \\
\hline 2 & 0.828810 \\
5 & 0.721721 \\
10 & 0.526054 \\
15 & 0.364949 \\
20 & 0.253182 \\
25 & 0.170514 \\
30 & 0.112591 \\
35 & 0.074181 \\
\hline
\end{tabular}

\subsection{Input File}

The following is a listing of the file monaco.graphite.inp located in the SCALE samples $\backslash$ input directory. This represents a simple model of Ueki's experiment for the $20 \mathrm{~cm}$ graphite slab. 


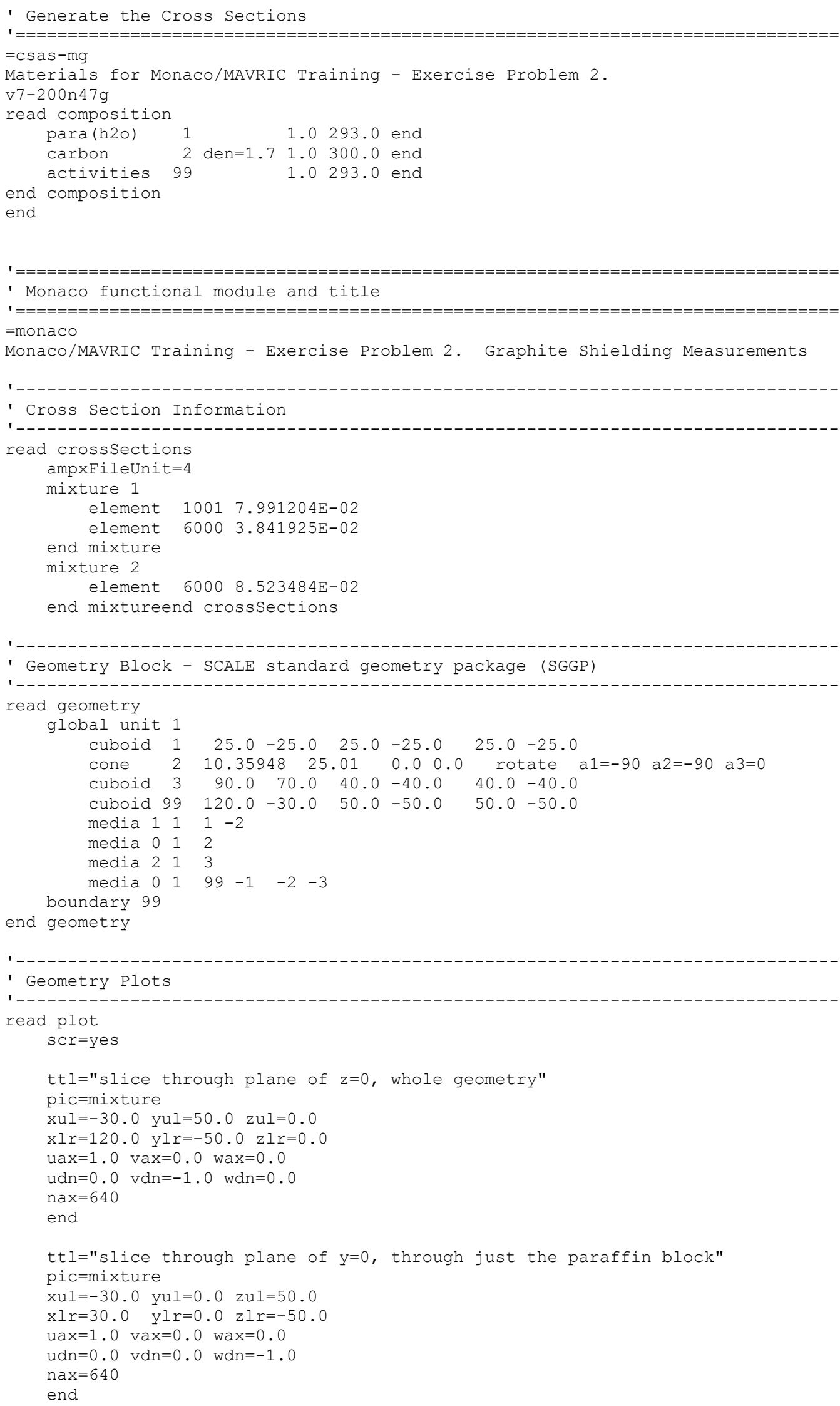




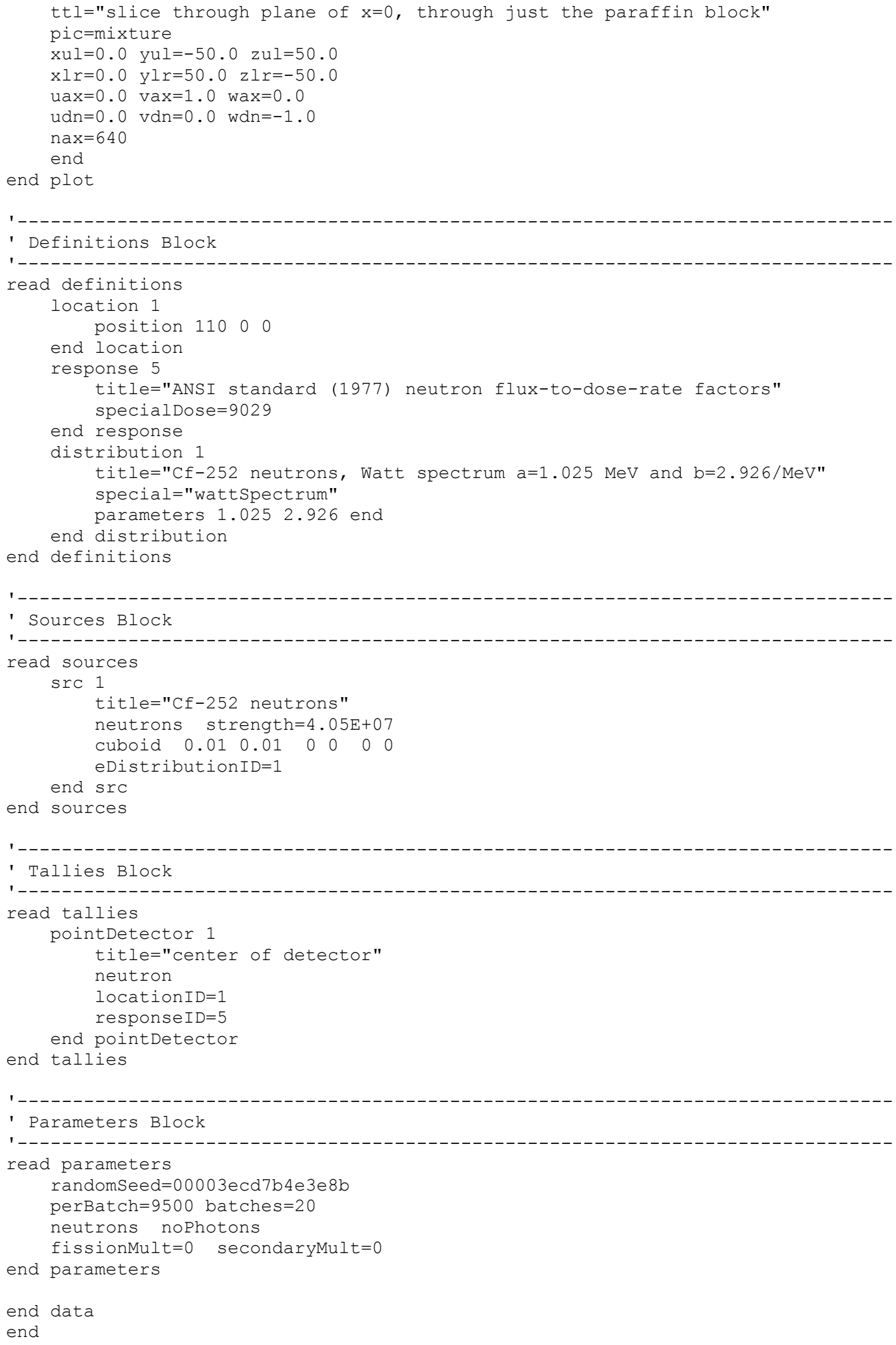

\subsection{Output}

For the $20 \mathrm{~cm}$ case, Monaco reports the following results: 


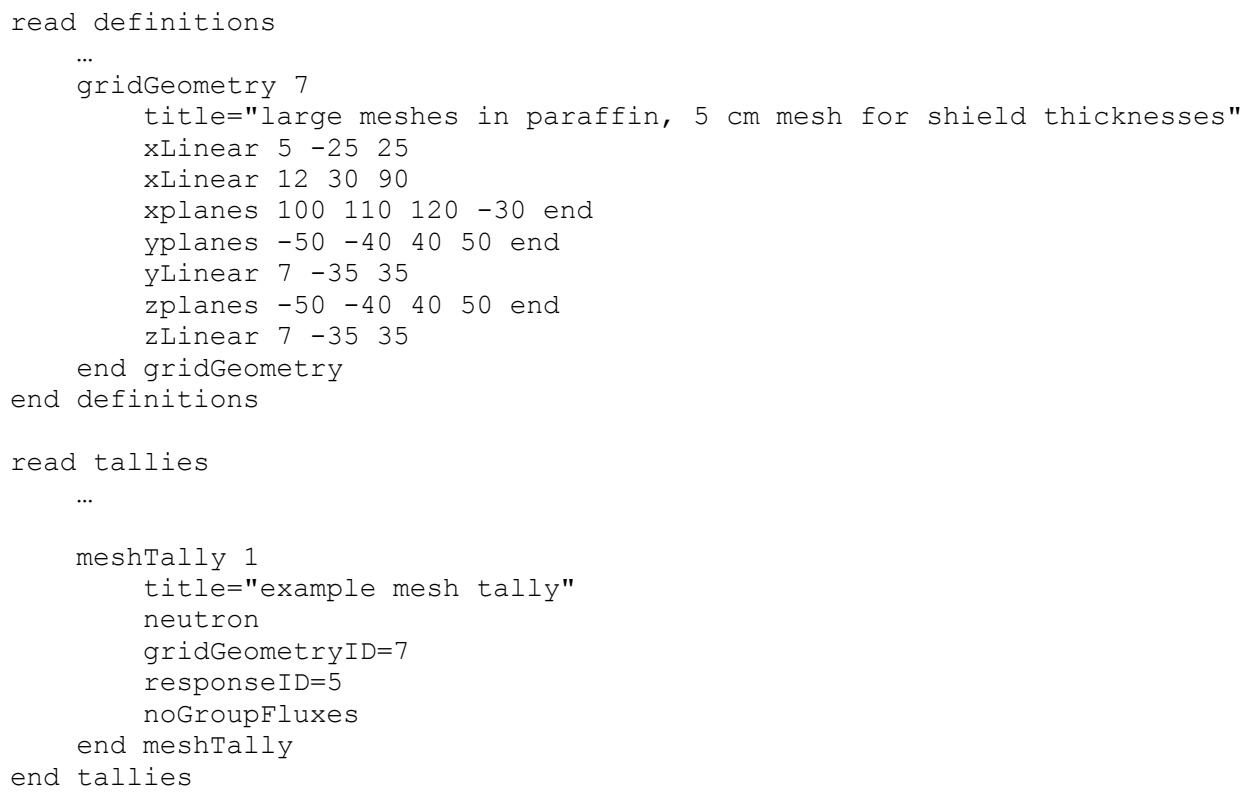

and viewing the resulting *.3dmap file with the Mesh File Viewer can produce the image shown in Figure 8.2.36.
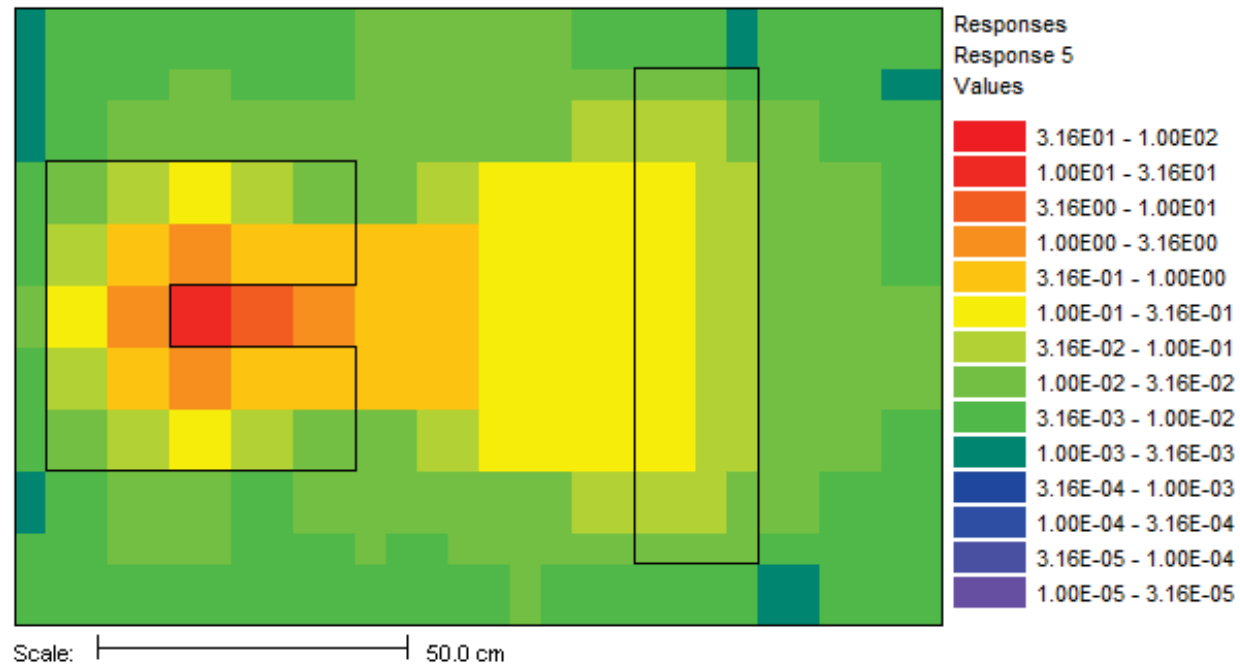

Figure 8.2.36. Mesh tally showing neutron dose rate (rem/hr)

\subsubsection{Simple shielding demonstration with line spectra}

Sources containing line data are difficult to represent in multigroup. Comparing real measurements of line data to multigroup calculations is also difficult. This sample problem is not the simulation of a real measurement but is just a simple demonstration of the differences between continuous-energy simulation and the multigroup approach.

Consider an isotropic cobalt-60 source on one side of a slab of tungsten ( $5 \mathrm{~cm}$ thick) and the goal is to compute the photon flux and dose on the other side of the slab. Since the continuous-energy library does not have a built-in structure that could be used by tallies, the user needs to supply one (otherwise tally information is recorded in one bin covering the entire range of energy). The user can construct an energy 
boundaries object in the definitions block that is uniformly spaced, logarithmically spaced, based on one of the multigroup libraries or any combination of the above.

\subsection{Input file}

The sample problem monaco.wSlab.inp, is located in the samples \input directory. The materials input consist just of tungsten, in its natural isotopic abundances.

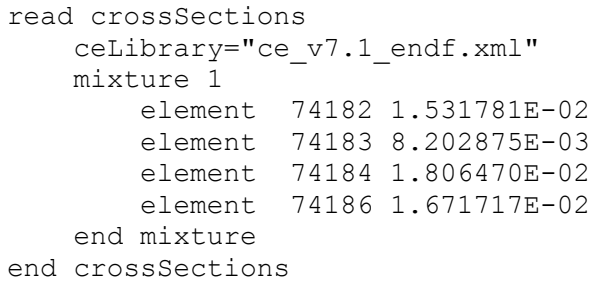

The geometry is a simple slab of tungsten, with a small air region on one side for a region tally.

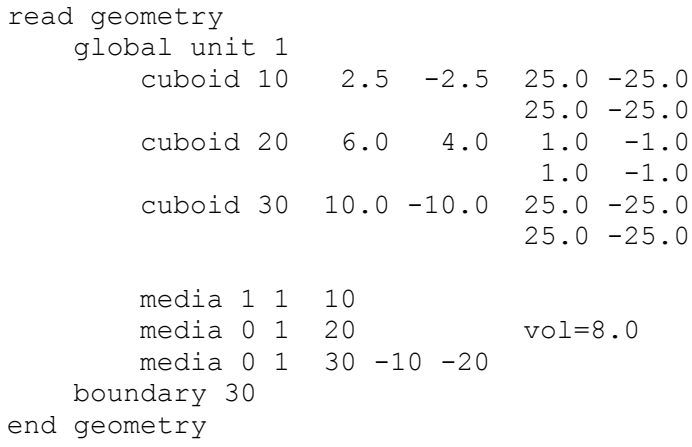

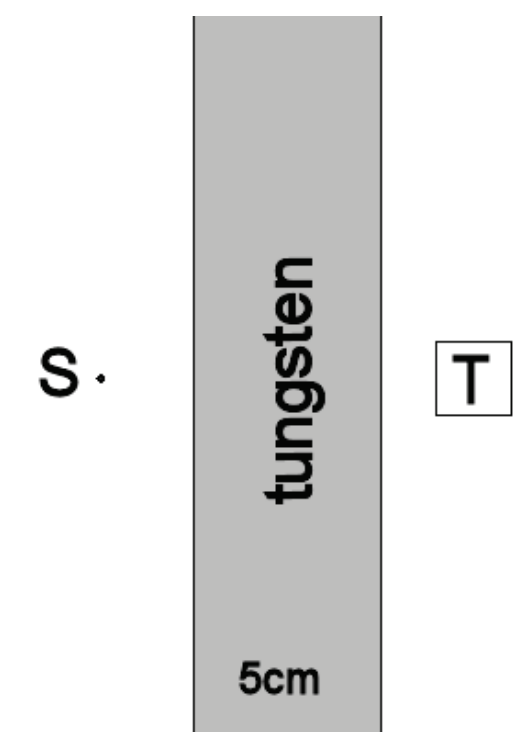

Figure 8.2.37. Simple slab geometry with a source (S) on the left and a tally region (T) on the right.

In the definitions block, a location (for a point detector), the photon dose response function, the Co-60 line spectra and an energy boundaries structure are all defined. The energyBounds defined here has a base structure of 30 bins that are $50 \mathrm{keV}$ wide, with three bins that are $2 \mathrm{keV}$ wide at the cobalt line energies and the $511 \mathrm{keV}$ annihilation gamma energy.

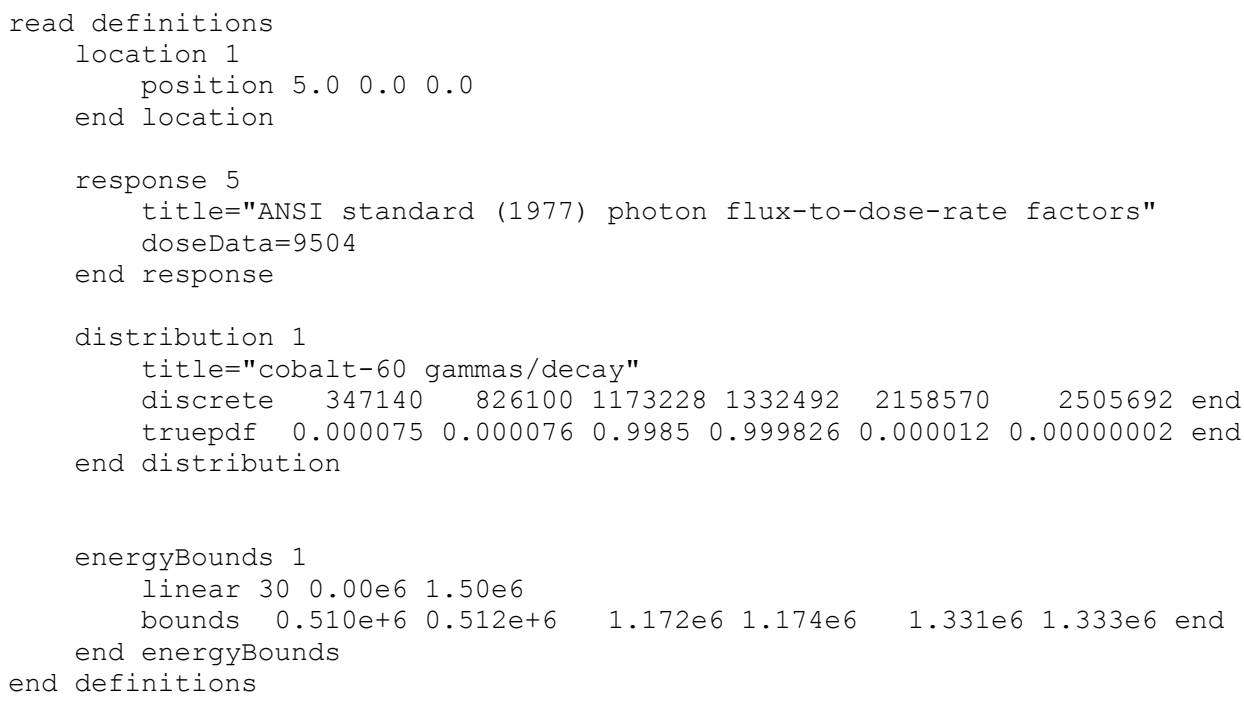


The source is a simple point source $5 \mathrm{~cm}$ to the left of the slab. Because the distribution of cobalt gamma rays was entered as gammas per decay, the keyword 'useNormConst' will set the source strength to be the total of the energy distribution - about 2 photons/decay. The 'multiplier'keyword is used to multiply that strength by $37 \times 10^{9}$ decays $/ \mathrm{sec}$ to get $1 \mathrm{Ci}$.

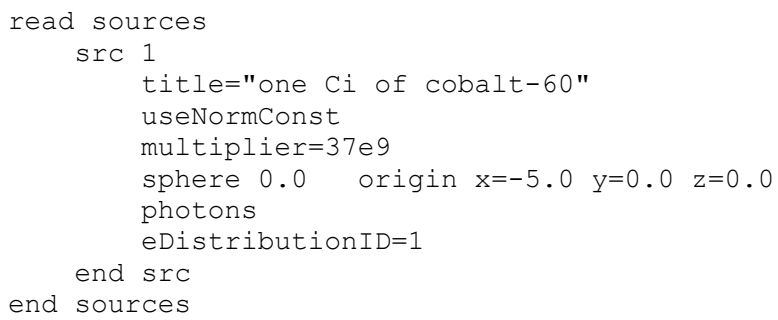

Both a region tally and a point detector tally are defined. Parameters are set for a 10 minute calculation.

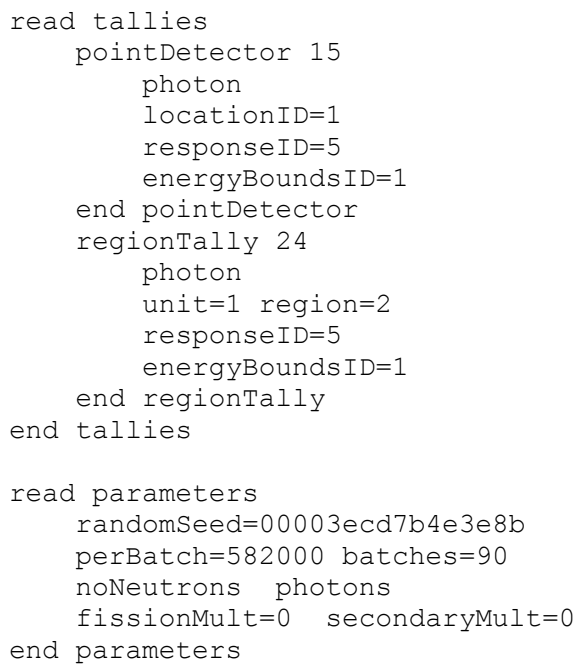

\subsection{Output}

Results for the dose rate from this continuous-energy calculation are shown in Table 8.2.26, along with results from two multigroup calculations and an MCNP calculation. To simulate the same type of physics used in the continuous-energy SCALE, the MCNP calculation used a photon cutoff energy of $0.01 \mathrm{MeV}$, $\mathrm{EMCPF}=100 \mathrm{MeV}$ (detailed physics), IDES=1 (no electrons/no bremsstrahlung), $\mathrm{NOCOH=0}$ (coherent scattering occurs), ISPN=0 (no photonuclear collisions), and NODOP=1 (no Doppler energy broadening).

The results from the SCALE multigroup calculations differ by $20 \%$, due to how the source and the dose response function are represented. The 19-group structure represents the $1.33 \mathrm{MeV}$ line with the 1.33 $1.66 \mathrm{MeV}$, so it is expected that the computed dose will be high. With the 47-group structure, the 1.17 $\mathrm{MeV}$ line is represented by the 1-1.2 MeV group (too low - lower dose will result) and the $1.33 \mathrm{MeV}$ line is represented by the 1.33-1.44 $\mathrm{MeV}$ group (too high - higher dose will result). The multigroup results are well-converged - just not as accurate as desired. The continuous-energy results should be more accurate. 
Table 8.2.26. Dose rates for the cobalt-60/tungsten problem using different energy treatments.

\begin{tabular}{l|c|cc|cc}
\hline & Time & \multicolumn{2}{|c|}{ Point Detector } & \multicolumn{2}{c}{ Region Tally } \\
& (min) & rem/hr & rel unc & rem/hr & rel unc \\
\hline MCNP & 97.8 & 3.1088 & 0.0039 & 3.1376 & 0.0051 \\
SCALE CE & 89.9 & 3.1886 & 0.0027 & 3.0949 & 0.0136 \\
SCALE 47 & 89.9 & 2.9284 & 0.0012 & 3.0144 & 0.0077 \\
SCALE 19 & 90.0 & 3.6705 & 0.0010 & 3.7414 & 0.0065 \\
\hline
\end{tabular}

Figure 8.2.38 and Figure 8.2.39 show the results of the point detector and region tally flux as a function of energy, for this problem as well as two multigroup calculations and an MCNP calculation. Tables M and $\mathrm{N}$ list the flux values of the cobalt lines and the annihilation gamma for the two tally types.

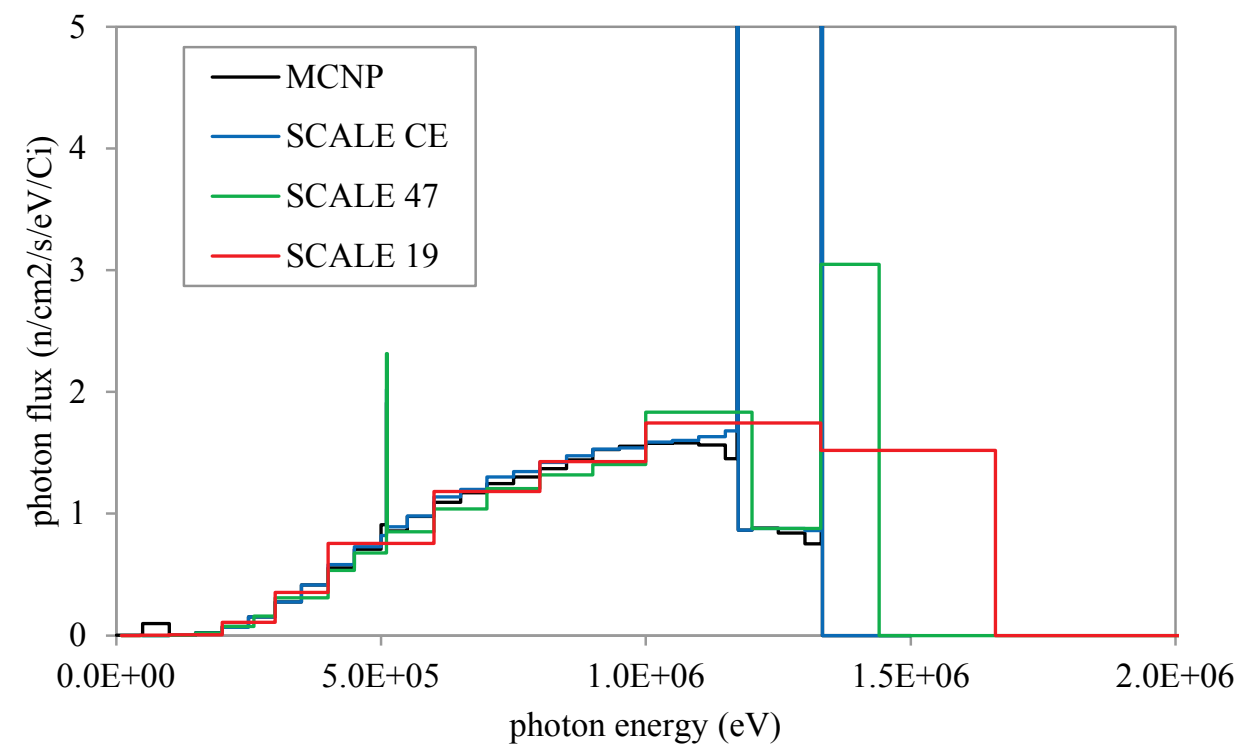

Figure 8.2.38. Point detector fluxes for the cobalt-60/tungsten problem using different energy treatments. 


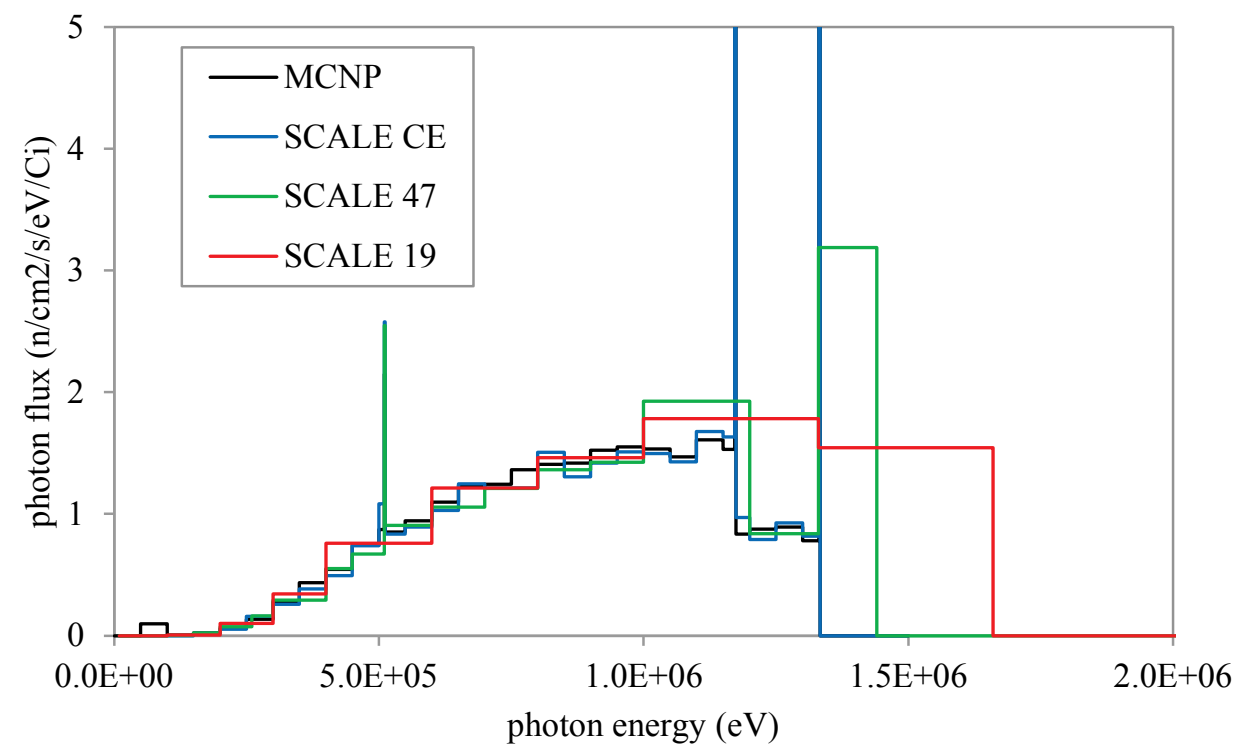

Figure 8.2.39. Region tally fluxes for the cobalt-60/tungsten problem using different energy treatments.

\begin{tabular}{l|cc|cc|cc}
\hline & \multicolumn{2}{|c|}{$511000 \mathrm{eV}$} & \multicolumn{2}{c|}{$1173230 \mathrm{eV}$} & \multicolumn{2}{c}{$1332490 \mathrm{eV}$} \\
& $/ \mathrm{cm}^{2} / \mathrm{s}$ & rel unc & $/ \mathrm{cm}^{2} / \mathrm{s}$ & $\mathrm{rel} \mathrm{unc}$ & $/ \mathrm{cm}^{2} / \mathrm{s}$ & $\mathrm{rel} \mathrm{unc}$ \\
\hline MCNP & $1.91 \mathrm{E}+00$ & 0.0320 & $9.53 \mathrm{E}+01$ & 0.0125 & $1.41 \mathrm{E}+02$ & 0.0093 \\
SCALE CE & $2.02 \mathrm{E}+00$ & 0.0382 & $9.94 \mathrm{E}+01$ & 0.0058 & $1.42 \mathrm{E}+02$ & 0.0041 \\
SCALE 47 & $2.31 \mathrm{E}+00$ & 0.0149 & & & & \\
SCALE 19 & & & & & & \\
\hline
\end{tabular}

Table 8.2.27. Point detector tally flux values for the lines.

\begin{tabular}{l|cc|cc|cc}
\hline & \multicolumn{2}{|c|}{$511000 \mathrm{eV}$} & \multicolumn{2}{c|}{$1173230 \mathrm{eV}$} & \multicolumn{2}{c}{$1332490 \mathrm{eV}$} \\
& $/ \mathrm{cm}^{2} / \mathrm{s}$ & rel unc & $/ \mathrm{cm}^{2} / \mathrm{s}$ & $\mathrm{rel} \mathrm{unc}$ & $/ \mathrm{cm}^{2} / \mathrm{s}$ & $\mathrm{rel} \mathrm{unc}$ \\
\hline MCNP & $2.15 \mathrm{E}+00$ & 0.0945 & $9.60 \mathrm{E}+01$ & 0.0145 & $1.44 \mathrm{E}+02$ & 0.0118 \\
SCALE CE & $2.58 \mathrm{E}+00$ & 0.2231 & $1.01 \mathrm{E}+02$ & 0.0402 & $1.38 \mathrm{E}+02$ & 0.0345 \\
SCALE 47 & $2.55 \mathrm{E}+00$ & 0.1200 & & & & \\
SCALE 19 & & & & & & \\
\hline
\end{tabular}

Table 8.2.28. Region tally flux values for the lines.

\subsubsection{References}

1. F. H. Froehner and R. R. Spencer, Method for Sampling from Fission Neutron Energy Spectra, ORNL/TM-7631, Oak Ridge National Laboratory, Oak Ridge, Tenn., February 1981. 
2. S. P. Pederson, R. A. Forster, and T. E. Booth, "Confidence Interval Procedures for Monte Carlo Transport Simulations," Nucl. Sci. Eng. 127, 54-77 (1997).

3. Brian C. Kiedrowski and Clell J. (C.J.) Solomon, "Statistical Assessment of Numerous Monte Carlo Tallies," International Conference on Mathematics and Computational Methods Applied to Nuclear Science and Engineering (M\&C 2011), Rio de Janeiro, RJ, Brazil, May 8-12, 2011, on CD-ROM.

4. Brian C. Kiedrowski and Ahmad Ibrahim, "Evaluating the Efficiency of Estimating Numerous Monte Carlo Tallies,” Transactions of the American Nuclear Society 104, 325-328, 2011.

5. E. Sajo, M. L. Williams, and M. Asgari, "Comparison of Measured and Calculated Neutron Transmission Through Steel for a ${ }^{252}$ Cf Source," Annals of Nuclear Energy 20(9), 585-604 (1993).

6. B. Jansky, Z. Turik, E. Novak, J. Kyncl, F. Cvachovec, and P. Tiller, "Comparison of Measured and Calculated Neutron Transmission Through Heavy Water for ${ }^{252} \mathrm{Cf}$ Source Placed in the Center of 30 cm Diameter Sphere," Annals of Nuclear Energy 24(15), 1189-1212 (1997).

7. K. Ueki, A. Ohashi, and Y. Anayama, "Neutron Shielding Ability of KRAFTON N2 - Mannan KRAFTON N2 Sandwich-type Material and Others," pp. 130-137, Proc. of the Topical Meeting New Horizons in Radiation Protection and Shielding, April 26-May 1, 1992, Pasco, Wash. 


\subsection{DETERMINISTIC TRANSPORT}

\section{Introduction by S. M. Bowman}

SCALE deterministic transport capabilities enable criticality safety, depletion, sensitivity, and uncertainty analysis, as well as hybrid approaches to Monte Carlo analysis. SCALE provides a onedimensional (1D) transport solver for eigenvalue neutronics and fixed source neutron-gamma analysis with XSDRN, two-dimensional (2D) eigenvalue neutronics with NEWT, and a three-dimensional (3D) transport solver for hybrid acceleration of Monte Carlo fixed source and eigenvalue calculations with Denovo. Generally, the use of these transport solvers in SCALE is best accessed through the capability specific sequences: CSAS and Sourcerer for criticality safety, TRITON for 1D and 2D depletion, TSUNAMI-1D and TSUNAMI-2D for sensitivity and uncertainty analysis, and MAVRIC for 3D fixed source hybrid Monte Carlo analysis.

\section{XSDRN}

XSDRN is a multigroup discrete-ordinates code that solves the 1D Boltzmann equation in slab, cylindrical, or spherical coordinates. Alternatively, the user can select P1 diffusion theory, infinite medium theory, or Bn theory. A variety of calculational types is available, including fixed source, eigenvalue, or search calculations. In SCALE, XSDRN is used for several purposes: eigenvalue $\left(k_{\text {eff }}\right)$ determination; cross section collapsing; and computation of fundamental-mode or generalized adjoint functions for sensitivity analysis.

\section{NEWT}

NEWT (New ESC-based Weighting Transport code) is a multigroup discrete-ordinates radiation transport computer code with flexible meshing capabilities that allow 2D neutron transport calculations using complex geometric models. The differencing scheme employed by NEWT - the Extended Step Characteristic approach - allows a computational mesh based on arbitrary polygons. Such a mesh can be used to closely approximate curved or irregular surfaces to provide the capability to model problems that were formerly difficult or impractical to model directly with discrete-ordinates methods. Automated grid generation capabilities provide a simplified user input specification in which elementary bodies can be defined and placed within a problem domain. NEWT can be used for eigenvalue, critical-buckling correction, and source calculations, and it can be used to prepare collapsed weighted cross sections in AMPX working library format.

Like other SCALE modules, NEWT can be run as a standalone module or as part of a SCALE sequence. NEWT has been incorporated into SCALE TRITON control module sequences. TRITON can be used simply to prepare cross sections for a NEWT transport calculation and then automatically execute NEWT. TRITON also provides the capability to perform 2D depletion calculations in which the transport capabilities of NEWT are combined with multiple ORIGEN depletion calculations to perform 2D depletion of complex geometries. In the TRITON depletion sequence, NEWT can also be used to generate lattice-physics parameters and cross sections for use in subsequent nodal core simulator calculations. In addition, the SCALE TSUNAMI-2D sequence can be used to perform sensitivity and uncertainty analysis of 2D geometries in which NEWT is used to compute the adjoint flux solution to generate sensitivity coefficients for $k_{\text {eff }}$ and other responses of interest with respect to the cross sections used in the NEWT model. 


\section{DENOVO}

Denovo $^{1}$ is a parallel 3D discrete-ordinates code available in SCALE as part of two control module sequences for different applications, as described below. Because Denovo can only be run in SCALE via the Monaco with Automated Variance Reduction using Importance Calculations (MAVRIC) or Denovo Eigenvalue Calculation (DEVC) as developed for use with Sourcerer, it is not documented separately in the section entitled "Deterministic Transport" in this manual.

The MAVRIC hybrid Monte Carlo radiation shielding sequence employs the Consistent Adjoint Driven Importance Sampling (CADIS) and Forward-Weighted CADIS (FW-CADIS) methodologies. Denovo is used to generate adjoint (and, for FW-CADIS, forward) scalar fluxes for the CADIS methods in MAVRIC. This adjoint flux information is then used by MAVRIC to construct a space- and energydependent importance map (i.e., weight windows) to be used for biasing during Monte Carlo particle transport and as a mesh-based biased source distribution. For use in MAVRIC/CADIS, it is highly desirable that the $S_{\mathrm{N}}$ code be fast, positive, and robust. The phase-space shape of the forward and adjoint fluxes, as opposed to a highly accurate solution, is the most important quality for Monte Carlo weight-window generation. Accordingly, Denovo provides a step-characteristics spatial differencing option that produces positive scalar fluxes as long as the source (volume plus in-scatter) is positive. Denovo uses an orthogonal, nonuniform mesh that is ideal for CADIS applications because of the speed and robustness of calculations on this mesh type. Denovo can be run stand-alone in MAVRIC to perform fixed source calculations using the PARM=forward (for forward Denovo) or PARM=adjoint (for adjoint Denovo). See the MAVRIC chapter for details.

The other sequence that uses Denovo is the DEVC sequence. DEVC generates a reasonably accurate starting source through a Denovo eigenvalue calculation so that Sourcerer can improve the $\mathrm{KENO/CSAS} \mathrm{Monte} \mathrm{Carlo} \mathrm{calculation} \mathrm{by} \mathrm{(1)} \mathrm{reducing} \mathrm{the} \mathrm{number} \mathrm{of} \mathrm{skipped} \mathrm{generations} \mathrm{required} \mathrm{to}$ converge the fission source distribution in the KENO solution, and (2) increasing the reliability of the final eigenvalue $\left(k_{\text {eff }}\right)$ for problems with loosely coupled fissionable areas. Denovo can be run standalone in DEVC for calculating criticality eigenvalue problems. This sequence reads an input file very similar to a CSAS6 input file that contains an extra block of input for describing the Denovo mesh grid and calculational parameters. See the Sourcerer chapter for details.

\section{Reference}

1. T. M. Evans, A. S. Stafford, R. N. Slaybaugh, and K. T. Clarno, "Denovo: A New ThreeDimensional Parallel Discrete Ordinates Code in SCALE," Nuclear Technology 171, 171-200 (2010). 


\title{
9.1 XSDRNPM: A ONE-DIMENSIONAL DISCRETE-ORDINATES CODE FOR TRANSPORT ANALYSIS
}

L. M. Petrie, N. M. Greene, ${ }^{1}$ M. L. Williams

\begin{abstract}
XSDRNPM is a discrete-ordinates code that solves the one-dimensional Boltzmann equation in slab, cylindrical, or spherical coordinates. Alternatively, the user can select $P_{l}$ diffusion theory, infinite medium theory, or $B_{n}$ theory. A variety of calculational types is available, including fixed source, eigenvalue, or "search" calculations. In SCALE, XSDRNPM is used for several purposes: eigenvalue (k-effective) determination, cross-section collapsing, shielding analysis, computation of fundamentalmode or generalized adjoint functions for sensitivity analysis, and for producing bias factors for use in Monte Carlo shielding calculations.
\end{abstract}

\footnotetext{
${ }^{1}$ Formerly with Oak Ridge National Laboratory.
} 


\section{TABLE OF CONTENTS}

$\underline{\text { Page }}$

9.1 XSDRNPM: A One-Dimensional Discrete-Ordinates Code for Transport Analysis ..................9-3

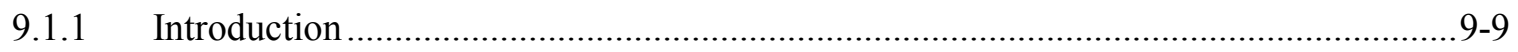

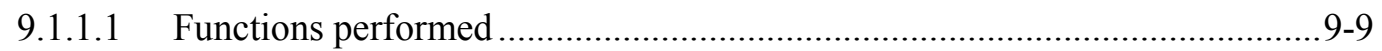

9.1.1.2 Background on XSDRNPM................................................................. $9-9$

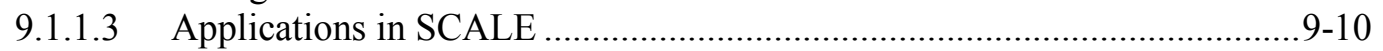

9.1.1.4 Notes on the use of various spectral calculational options ......................... 9-11

9.1.1.5 Selection of output cross-section library formats ..................................... $9-12$

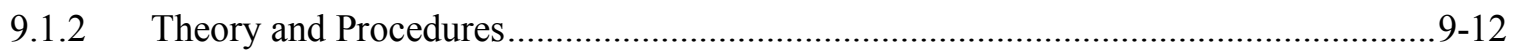

9.1.2.1 One-dimensional discrete-ordinates theory .......................................... 9-12

9.1.2.2 Multigroup one-dimensional Boltzmann equation ....................................9-17

9.1.2.3 Scattering source term...................................................................... 9-17

9.1.2.4 Discrete-ordinates difference equations ................................................9-21

9.1.2.5 Weighted-difference formulation for discrete-ordinates equations .............9-26

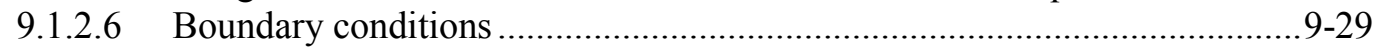

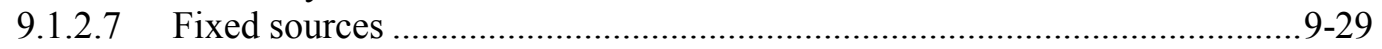

9.1.2.8 Dimension search calculations........................................................ 9-30

9.1.2.9 Alpha search ........................................................................... 9-32

9.1.2.10 Iteration and convergence tests ........................................................ 9-34

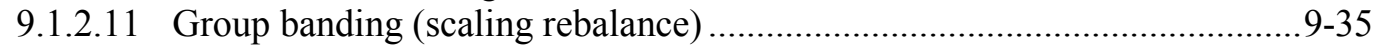

9.1.2.12 Buckling corrections .......................................................................... 9-36

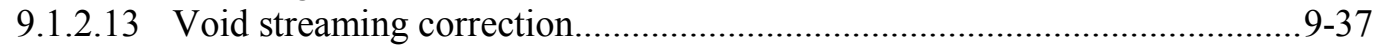

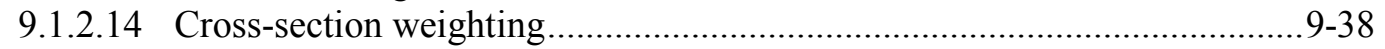

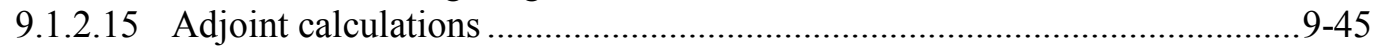

9.1.2.16 Coupled neutron-photon calculations ........................................................4-46

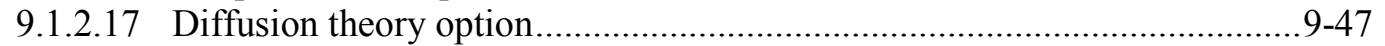

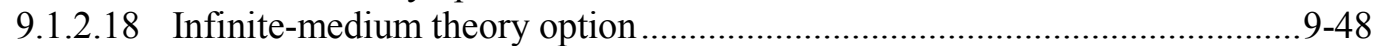

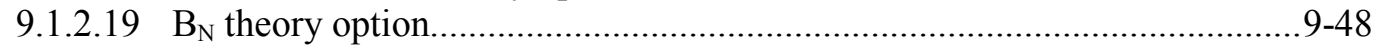

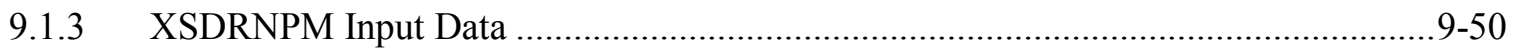

9.1.3.1 Abbreviated XSDRNPM input description..............................................9-62

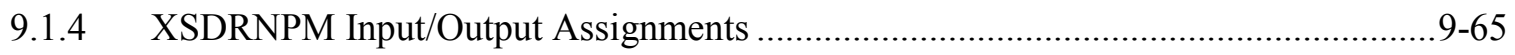

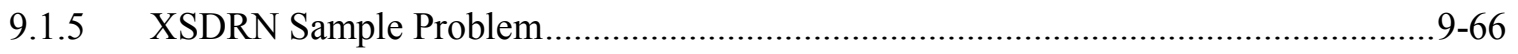

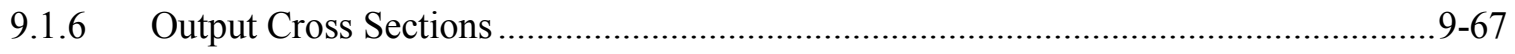

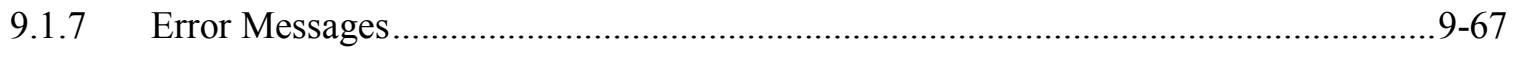

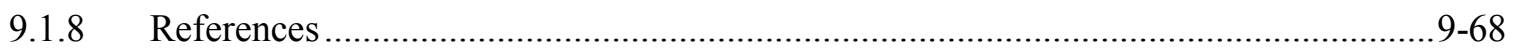




\section{TABLE OF CONTENTS (continued)}

$\underline{\text { Page }}$

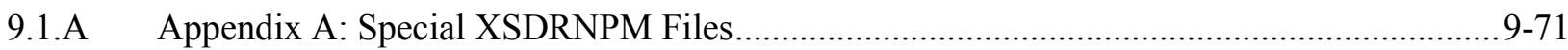

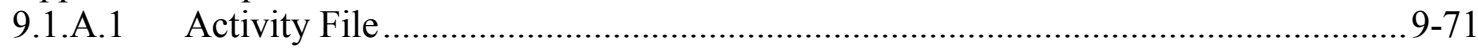

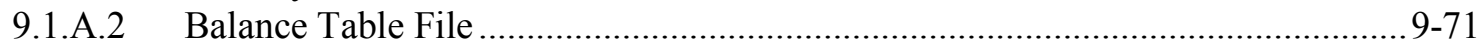

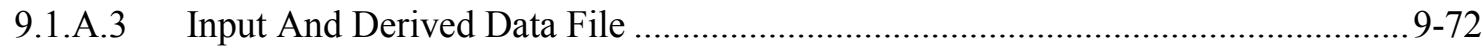

9.1.B Appendix B: XSDRNPM Mixed Cross Sections …....................................................... 9-78 


\section{LIST OF FIGURES}

Page

Figure 9.1.1. Three common coordinate systems. ................................................... 9-15

Figure 9.1.2. Three 1-D coordinate systems. ............................................................ 9-16

Figure 9.1.3. One-dimensional cylindrical scattering coordinates. ..................................... 9-19

Figure 9.1.4. Angular redistribution in spherical geometry........................................... 9-22

Figure 9.1.5. Angular redistribution. ........................................................................... 9-23

Figure 9.1.6. Ordering of $\mathrm{S}_{\mathrm{n}}$ directions for slabs and spheres....................................... 9-24

Figure 9.1.7. Ordering of the directions for an $\mathrm{S}_{6}$ cylindrical set. ..................................... 9-26 


\section{LIST OF TABLES}

$\underline{\text { Page }}$

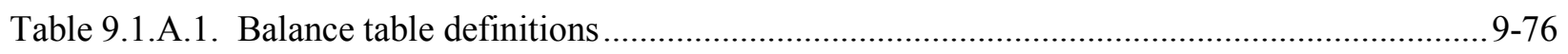

Table 9.1.A.2. Balance table definition symbols ......................................................................... 9-77

Table 9.1.B.1. Order of mixed reaction cross sections .............................................................. 9-78 


\section{ACKNOWLEDGMENTS}

W. W. Engle has been very generous with the use of his notes on $S_{n}$ theory and on discussing details of various procedures in XSDRNPM which were lifted directly from his ANISN program.

The authors also wish to thank R. H. Odegaarden (the former technical monitor at the U.S. Nuclear Regulatory Commission) who supplied the necessary incentives for completing this report. 


\subsubsection{Introduction}

XSDRNPM is a one-dimensional (1-D) discrete-ordinates transport code and is the latest in a series of codes in the $\mathrm{XSDRN}^{1}$ family. As such, it contains several unique characteristics, as will be detailed in this report, though a large portion of the theoretical bases and intended uses of the program are the same for all versions.

\subsubsection{Functions performed}

The function of XSDRNPM is twofold: (1) perform a 1-D discrete-ordinates calculation in slab, cylindrical, or spherical geometry (optionally, a 1-D diffusion theory or infinite medium $B_{n}$ calculation can be made), and (2) use the fluxes determined from its spectral calculation to collapse input cross sections and write these into one of several formats.

A great deal of flexibility is allowed in describing a problem for XSDRNPM. The number of spatial intervals, the number of energy groups, the number of nuclides, the quadrature order, the order of fits to the angular variation in basic cross sections are all arbitrary and are limited only by computer and monetary resources.

The flux calculation can be performed according to several options, including fixed source calculations, $\mathrm{k}$-calculations, and dimension search calculations.

A variety of weighting options are allowed, including zone, cell, or a special "vein" weighting option which is described herein.

\subsubsection{Background on XSDRNPM}

Development of the XSDRN1 program started in the mid-1960s. The goal was to develop a program that would combine features from the GAM-II, ${ }^{2}$ ANISN, ${ }^{3}$ and THERMOS $^{4}$ programs in a more unified and general way than would be possible if one simply elected to use these codes individually.

The salient features to be retained from the programs were as follows:

GAM-II The Nordheim Integral Treatment was desired for resonance self-shielding; the generality of including cross sections for an arbitrary number of processes, along with the provisions for truncating zero or impossible transfers in the scattering matrices, was also a requirement.

ANISN One-dimensional discrete-ordinates or diffusion theory or infinite-medium theory was to be available to generate a spectrum for cross-section collapsing.

THERMOS The ability to perform detailed 1-D spectral calculations, including upscatter effects, was required for the thermal region.

The whole code was required to be dynamically dimensioned to allow calculations for arbitrary group structures, spatial structures, angular quadratures, etc.

The XSDRN program that embodied these features was released in 1969. 
In the early 1970s, the Defense Nuclear Agency (DNA) initiated support for the AMPX system, which was to be a total cross-section generation system capable of performing all tasks necessary to take basic neutron and gamma-ray cross-section data and process these data into the proper form needed for weapons effects calculations. Since XSDRN already encompassed many of the features needed, it was selected as a basis for modules in the new system. In this case, experience gained in the original construction of XSDRN served to suggest that a more modular approach would have been better with independent tasks being done in separate, smaller, easier-to-manage programs. Therefore, the code was split into NITAWL-II (for resonance self-shielding and some basic cross-section data manipulation) and XSDRNPM (for spectral calculations and cross-section collapsing). In retrospect, if the AMPX development were initiated today, XSDRN would have been split even further, into perhaps as many as six or seven programs.

The XSDRNPM module differs from XSDRN in several respects:

- It will perform coupled neutron-gamma calculations.

- It allows any mixture to be represented to an arbitrary order of anisotropic representation, whereas XSDRN only allowed through order 3.

- It will perform an adjoint calculation, whereas the option was never provided in XSDRN. In 2010, a generalized adjoint solution was also added.

- It is considerably more efficient in the manner in which data storage is used and, hence, will run much larger problems in less core storage.

- It employs improved thermal flux scaling techniques for better problem convergence.

- Input specifications have been reordered, and more defaults have been provided to make the use of this module easier.

- It will calculate $S_{n}$ constants for any order for any of the three 1-D geometries available.

- Mixture-dependent fission spectra are calculated and used in XSDRNPM, which takes into account all fissionable nuclides in a problem.

AMPX was released in 1976, about the same time as the U.S. Nuclear Regulatory Commission (NRC) support for the SCALE system was initiated. Although separate versions of XSDRNPM were initially maintained for AMPX and SCALE, in recent years the same version is used for the two systems.

\subsubsection{Applications in SCALE}

XSDRNPM is used in several places in SCALE. In SAS1, XSDOSE uses fluxes from a 1-D shielding calculation to determine a dose rate. Within the CSAS5 and CSAS6 control modules, XSDRNPM is used in the sequences to perform eigenvalue calculations and cell weighting of cross sections. TSUNAMI-1D uses XSDRN to compute forward and adjoint fluxes (fundamental-mode and generalized adjoint) for sensitivity and uncertainty analysis. 


\subsubsection{Notes on the use of various spectral calculational options}

As noted earlier, four options are available in XSDRNPM for calculating fluxes, k-effectives, etc.:

1. $S_{n}$ theory,

2. diffusion theory,

3. infinite medium theory, and

4. $B_{n}$ theory.

However, XSDRNPM is primarily an $S_{n}$ code. The latter three options are provided for reasons of completeness and are not nearly as optimized as they would be in other codes for which these are the primary spectral calculation options.

Without a very detailed calculational study, it is perhaps impossible to be able to quantify the degree of adequacy or inadequacy of any of these methods for performing a particular problem. However, some general comments can be made which may provide some guidance with their selection.

First, $S_{n}$ theory is the most correct of the options and will solve a larger class of problems. It is the most complicated and time-consuming of the four, but it still runs very fast for most cases. There are problems for which it (or some alternative method based on a solution of the Boltzmann equation) is the only one of the four methods which is adequate. Many shielding applications fall in this class. In deep-penetration problems, anisotropic effects can dominate, thus requiring an accurate treatment of the anisotropy of both flux and cross section. It is well known that diffusion theory is not very accurate when used to calculate systems involving regions of very dissimilar cross-section values, such as is the case when control rods are interspersed in a reactor core. Because of the anisotropy involved in gamma-ray problems, $S_{n}$ theory should be used.

Diffusion theory, on the other hand, is certainly the most successful of the four methods in terms of the amount of use it has for designing reactors, etc. In cases involving reasonably large, homogeneous regions, it is generally adequate, such as is the case for a large class of "reactor" applications. For most problems, the diffusion theory option should run appreciably faster than $S_{n}$ theory, since it has essentially one equation to solve, versus number-of-angles equations for $S_{n}$ theory. This equation also can be explicitly solved using a matrix inversion procedure, whereas the $S_{n}$ theory requires a more timeconsuming iterative procedure. However, in many cases with large numbers of groups (200 to 300), the greater fraction of the calculational time can be spent calculating the scattering source terms, which tends to lessen the impact of time spent on a more correct theory. (This same observation can also be made of the infinite medium and $B_{n}$ method.)

The infinite medium option is the fastest of the four methods and can be used safely to perform calculations for large homogeneous regions, wherein the spectrum may be needed to collapse cross sections. This option only determines the first moment of the flux, and is, therefore, quite suspect for many applications, such as calculating diffusion coefficients.

The $B_{n}$ option shares many of the same restrictions as the infinite-medium method; however, this treatment does (as its name implies) use a buckling approximation to account for leakage from the large homogeneous region, thereby giving higher order flux moments that can be used, for example, to determine diffusion coefficients. 


\subsubsection{Selection of output cross-section library formats}

XSDRNPM will, on option, collapse cross sections and write the collapsed sets into four different formats:

1. ANISN3 BCD Library,

2. ANISN3 Binary Library,

3. CCCC $^{5}$ ISOTXS Library, or

4. $\mathrm{AMPX}^{6}$ Working Library.

The choice of the output cross-section format is determined by the computer code that will use the data. XSDRNPM always produces an AMPX working library when cross sections are collapsed, and all other formats are produced by reformatting data from this library. Therefore, for archival purposes, if a collapsed library is to be saved, the working format is the best choice, because it is the most general of those provided. AMPX working libraries are used by all multi-group transport codes currently in SCALE, including DENOVO (3D orthogonal mesh discrete ordinates code), NEWT (2D arbitrary mesh discrete ordinates code), and KENO and MONOCO (multigroup Monte Carlo codes. Stand-alone modules exist for converting AMPX working libraries to the other formats.

ANISN formats are used by older ORNL transport codes such as ANISN (a 1-D discrete-ordinates code), by DORT/TORT ${ }^{7}$ [two-dimensional (2-D) and three dimensional (3D) discrete-ordinates codes], and by MORSE$^{8}$ (a multigroup Monte Carlo code). The formats are quite comprehensive and can handle coupled neutron-gamma calculations, arbitrary orders of anisotropy, upscattering, etc. The major shortcoming of the format is its lack of internal documentation as to its structure (e.g., no provisions exist for specifying where a particular kind of cross section is located in the library or even if it is included). ANISN libraries can be produced in a free-form card-image BCD format or in a binary form.

The CCCC (Committee on Computer Code Coordination) ISOTXS file is a format for neutron cross sections that is one of several "standard interfaces" developed to facilitate the exchange of data between different computer codes. It is a self-defined format, which has provisions for identifying cross sections in the library. Scattering matrices can be supplied for elastic, inelastic, and (n,2n) scattering.

\subsubsection{Theory and Procedures}

This section describes the models and procedures which are employed in XSDRNPM.

\subsubsection{One-dimensional discrete-ordinates theory}

The time-independent Boltzmann transport equation can be written:

$$
\vec{\Omega} \bullet \nabla \psi(\vec{r}, E, \vec{\Omega})+\sum_{t}(\vec{r}, E) \psi(E, \vec{r}, \vec{\Omega})=S(\vec{r}, E, \vec{\Omega}) .
$$

This expression is a balance condition that states simply that losses due to leakage (first term) and collisions (second term) must equal the source of neutrons, at some point in space $\vec{r}$ energy E, and in direction $\vec{\Omega}$ per unit volume and energy and solid angle. Other terms in the expression are $\Sigma_{t}(\vec{r}, E)$, 
the total macroscopic cross section of the medium, which is typically assumed isotropic, and the flux, $\psi(\vec{r}, E, \vec{\Omega})$.

The source term, $S(\vec{r}, E, \vec{\Omega})$ has three components:

1. a scattering source, $S(\vec{r}, E, \vec{\Omega})$,

2. a fission source, and $F(\vec{r}, E, \vec{\Omega})$,

3. a fixed source, $Q(\vec{r}, E, \vec{\Omega})$.

The scattering source is given by:

$$
\left.S(\vec{r}, E, \vec{\Omega})=\int_{0}^{4 \pi} d \vec{\Omega}^{\prime} \int_{0}^{\infty} d E^{\prime} \sum_{s}\left(\vec{r}, E^{\prime} \rightarrow E, \vec{\Omega}^{\prime} \rightarrow \vec{\Omega}\right) \psi\left(\vec{r}, E^{\prime}, \vec{\Omega}^{\prime}\right)\right) .
$$

The fission source term, typically, is written

$F(\vec{r}, E, \vec{\Omega})=\frac{1}{4 \pi k} \chi(\vec{r}, E) \int_{0}^{4 \pi} d \vec{\Omega}^{\prime} \int_{0}^{\infty} d E^{\prime} v\left(\vec{r}, E^{\prime}\right) \sum_{f}\left(\vec{r}, E^{\prime}\right) \psi\left(\vec{r}, E^{\prime}, \vec{\Omega}^{\prime}\right)$,

where $\sum_{s}\left(\vec{r}, E^{\prime} \rightarrow E, \vec{\Omega}^{\prime} \rightarrow \vec{\Omega}\right)$ is the macroscopic scattering cross section per unit energy for scattering from energy $E^{\prime}$ to $E, \chi(\vec{r}, E)$ is the fraction of the fission neutrons per unit energy produced at $\vec{r}$ and $\mathrm{E}$, $v(\vec{r}, E)$ is the average number of neutrons produced per fission, $\Sigma_{f}(\vec{r}, E)$ is the macroscopic fission cross section and $\mathrm{k}$ is the "effective multiplication constant." Note that, as in the case of the total crosssection value, $\chi, \Sigma_{f}$, and $v$ are assumed to be isotropic. XSDRN computes a weighted-averaged fission spectrum based on the fissionable materials at $\vec{r}$.

Three common coordinate systems are shown in Figure 9.1.1. XSDRNPM is a 1-D code, which means that in the case of the slab, it is calculating at points along one axis where the system is assumed to extend to infinity along the other two axes. If we assume a calculation along the $\mathrm{x}$-axis, this says that there is no leakage in the $y$ or $z$ directions, and our directions by angles referenced to the x-axis. In the case of the cylinder, the length (z-axis) is infinite and the calculation is for points (shells) located at distance $r$ from the central axis. For the sphere, the calculation is of shells located at radius, r, from the center of the spherical system.

Figure 9.1.2 illustrates the 1-D coordinate systems for slabs, cylinders, and spheres. Note that the directions are cones in the case of the slab and sphere, whereas in the case of the cylinder, the same simple symmetries do not hold (a cone around the radius does not strike the next cylindrical shell at the same distance from a point on a radius) and the directions must be specifically described. Symmetries in the 1-D cylinder, however, allow one to only describe directions for one quadrant of the direction sphere about a point as will be noted in Sect. 9.1.2.2. 
The 1-D geometries allow considerable simplification to be made to Eq. (9.1.1), especially in the leakage term $\vec{\Omega} \bullet \nabla \psi$. It is traditional to calculate the angular flux as a function of angles expressed in directioncosine units; i.e., $\mu=\cos \phi$ and $\eta=\cos \xi$. This requires $\psi(x, E, \mu)$ for slabs, $\psi(x, E, \mu, \eta)$ for cylinders and $\psi(r, E, \mu)$ for spheres. Table 9.1.1 gives leakage terms expressed in conservation form for the three geometries.

Table 9.1.1. One-dimensional leakage terms

\begin{tabular}{|l|c|}
\hline Geometry & $\vec{\Omega} \bullet \nabla \psi$ \\
\hline Slab & $\mu \frac{\partial \psi}{\partial x}$ \\
\hline Cylinder & $\frac{\mu}{r} \frac{\partial(r \psi)}{\partial r}-\frac{1}{r} \frac{\partial(\eta \psi)}{\partial \phi}$ \\
\hline Sphere & $\frac{\mu}{r^{2}} \frac{\partial\left(r^{2} \psi\right)}{\partial r}+\frac{1}{r} \frac{\partial\left[\left(1-\mu^{2}\right) \psi\right]}{\partial \mu}$ \\
\hline
\end{tabular}




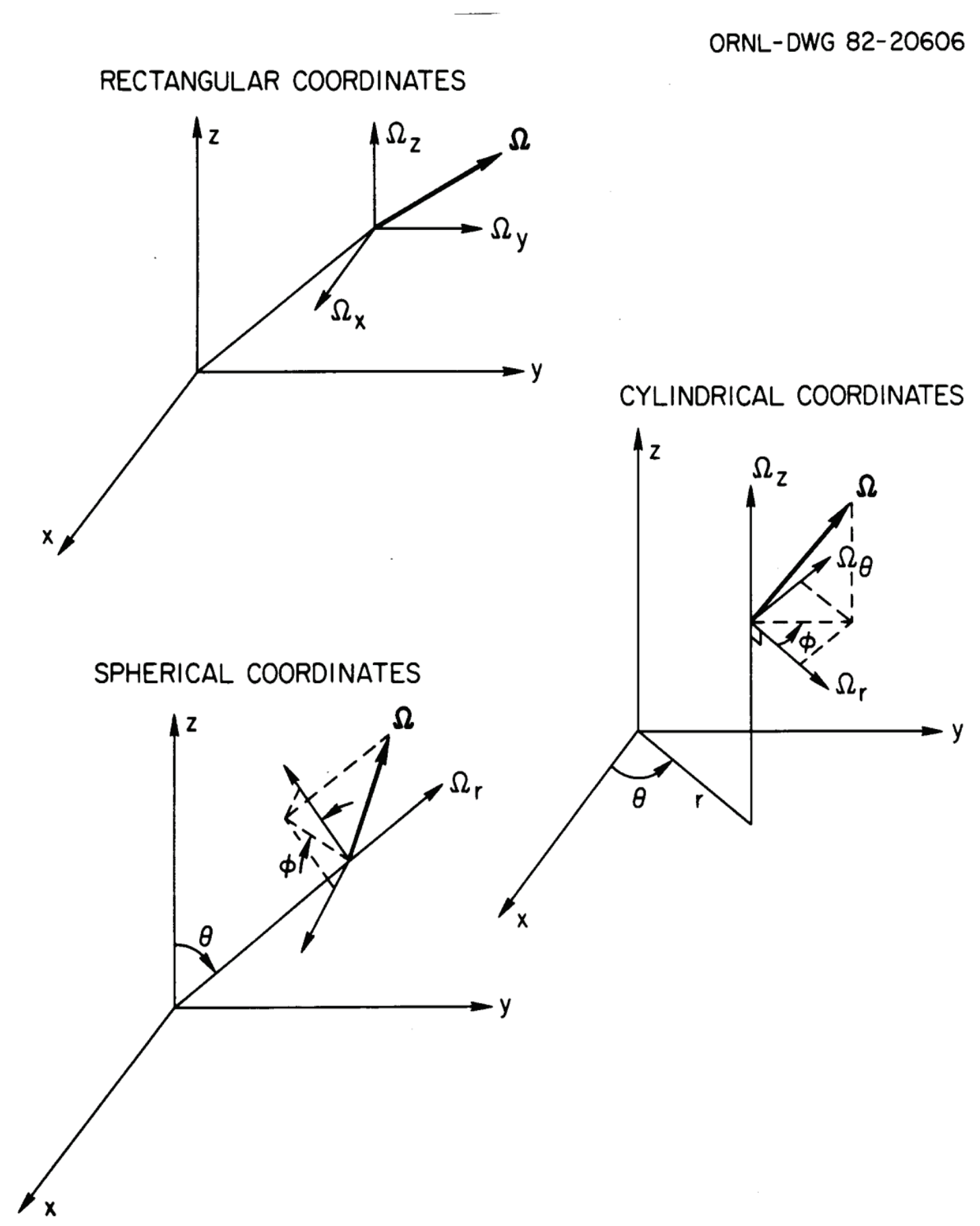

Figure 9.1.1. Three common coordinate systems. 
ORNL-DWG 82-20605

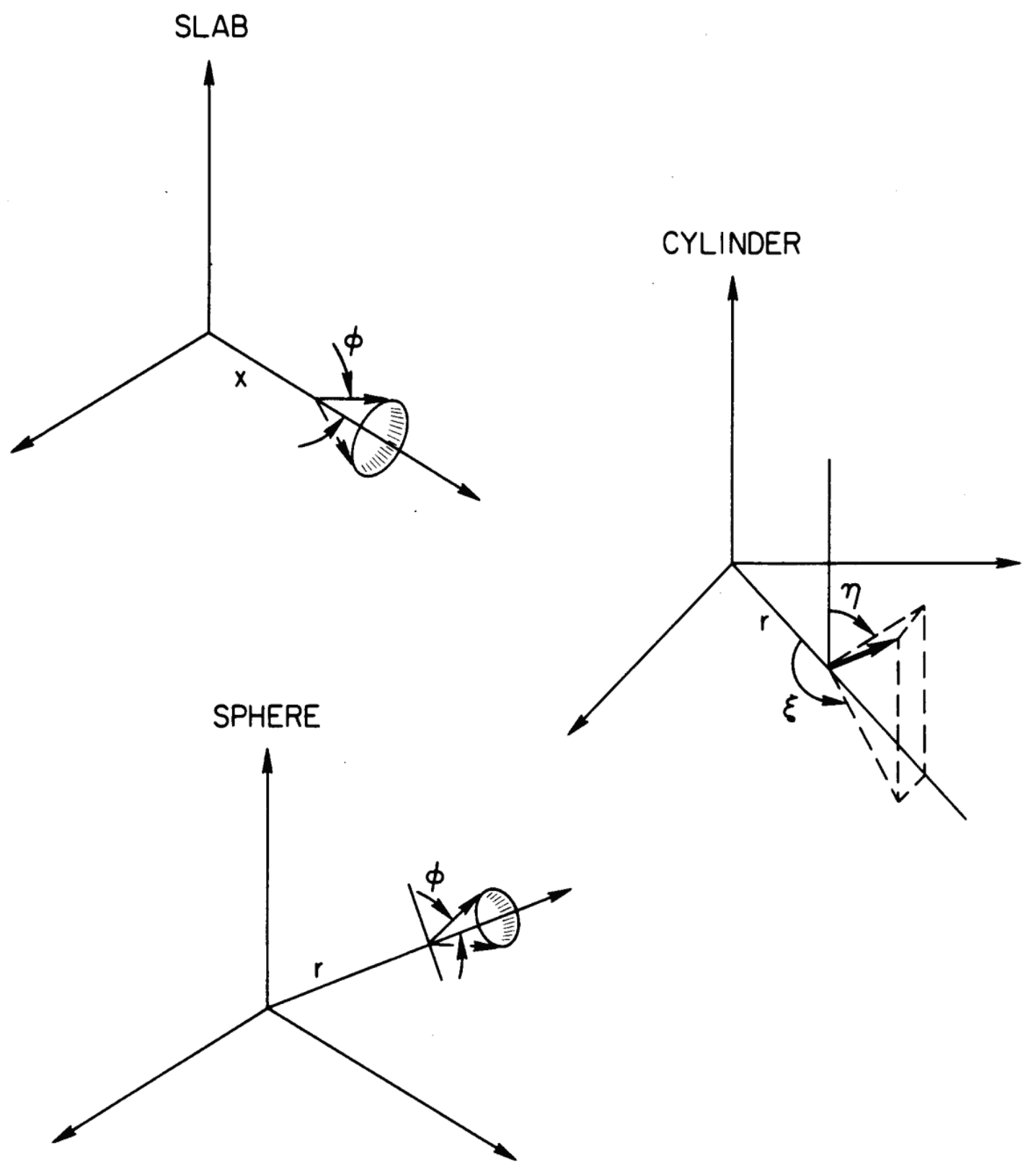

Figure 9.1.2. Three 1-D coordinate systems. 


\subsubsection{Multigroup one-dimensional Boltzmann equation}

In multigroup schemes, the continuous-energy (CE) balance equations are converted to multigroup form by first selecting an energy structure and then writing a multigroup equivalent of the point equation which requires multigroup constants that tend to preserve the reaction rates that would arise from integrating the $\mathrm{CE}$ equations by group. First we define the following multigroup values for $\mathrm{g}$,

$$
\psi_{g}(x, \mu)=\int_{g} d E \psi(x, E, \mu)
$$

and

$$
\psi_{g}(x)=\int_{-1}^{1} d \mu \psi_{g}(x, \mu)
$$

and

$$
\Sigma_{t g}(x)=\frac{\int_{g} d E \Sigma_{t g}(x, E) W(x, E)}{\int_{g} d E W(x, E)}
$$

where $W(x . E)$ is the weighting function used to compute the multigroup cross sections at a particular location. To rigorously conserve reaction rates, the weight function should be angle-dependent, but this causes the multigroup cross section to vary with direction; therefore the usual approach is to represent the weight function by an approximation to the scalar flux spectrum. In energy ranges where the CE cross sections have fine-structure due to resonances, the multigroup data must be self-shielded prior to the multigroup transport calculations.

The following mult-group form of 1-D equation can be derived for the slab case:

$$
\mu \frac{\partial \psi_{g}(x, \mu)}{\partial x}+\sum_{t g}(x) \psi_{g}(x, \mu)=S_{g}(x, \mu)+F_{g}(x, \mu)+Q_{g}(x, \mu) .
$$

The equations for the cylinder and sphere are essentially the same, in this notation, except for the differences in the leakage terms from Table 9.1.1.

In Eq. (9.1.6), $S_{g}, F_{g}$, and $Q_{g}$ are the scattering, fission, and fixed sources, respectively. The scattering term is discussed in Sect. 9.1.2.3. The multigroup form of the fission source is

$$
F_{g}(x, \mu)=\frac{\chi_{g}}{2 \pi k} \sum_{g^{\prime}} \overline{v \sum_{f g^{\prime}}}(x) \psi_{g^{\prime}}(x)
$$

where $\chi_{g}$ is the fraction of the fission neutrons that are produced in group g, and $\overline{v \sum_{f g^{\prime}}}$ is the average of the product of $v$, the average number of neutrons produced per fission and $\Sigma_{f}$, the fission cross section.

\subsubsection{Scattering source term}

In discrete-ordinates theory, one typically calculates the Legendre moments of the flux, $\psi_{g, l}$, defined for slab and spherical geometries by 


$$
\psi_{g, l}=\frac{1}{2} \int_{-1}^{1} d \mu \psi_{g}(\mu) P_{1}(\mu)
$$

Cylindrical geometry has a similar expression containing spherical harmonic functions rather than Legendre polynomials, shown in the next section.

The group-to-group scattering coefficients are, themselves, fit with Legendre polynomials, such that

$$
\sigma\left(g^{\prime} \rightarrow g, \mu\right)=\sum_{l=0}^{I S C T} \frac{2 l+1}{2} \sigma_{l}\left(g^{\prime} \rightarrow g\right) P_{l}(\mu)
$$

In this example, we have a fit of order ISCT. [Note that AMPX cross-section libraries contain the $2 l+1$ factor in the $\sigma_{t}\left(g^{\prime} \rightarrow g\right)$ matrix.]

\subsection{Slab and Spherical Geometries}

Because of the symmetries in 1-D slabs and spheres, only one angle is needed to describe a "direction." In the case of the slab, the angle is taken with reference to the x-axis, while for the sphere; it is with reference to a radius vector between the point and the center of the sphere. This means that the flux can be expanded in ordinary Legendre polynomials, such that

$$
\begin{aligned}
& \psi(r, E, \mu)=\sum_{l=0}^{\infty} \psi_{l}(r, E) P_{l}(\mu) \\
& \psi_{l}(r, E)=\int_{-1}^{1} \frac{d \mu}{2} P_{l}(\mu) \psi(r, E, \mu) .
\end{aligned}
$$

When Eq. (9.1.10) and Eq. (9.1.9) are introduced into Eq. (9.1.2), the following expression is derived for the scattering source:

$$
S(r, E, \mu)=2 \pi P_{l}(\mu) \int_{0}^{\infty} d E^{\prime} \int_{-1}^{1} d \mu^{\prime} \sum_{l=0}^{I S C T} \frac{2 l+1}{2} \sum_{s_{l}}\left(r, E^{\prime} \rightarrow E\right) P_{l}\left(\mu^{\prime}\right) \psi_{l}\left(r, E^{\prime}\right),
$$

where ISCT is the order of fit to the fluxes and cross sections. 


\subsection{Cylindrical Geometry}

The situation is more complicated in the case of the 1-D cylinder where the flux (and cross section) must be given as a function of two angles. Consider Figure 9.1.3.

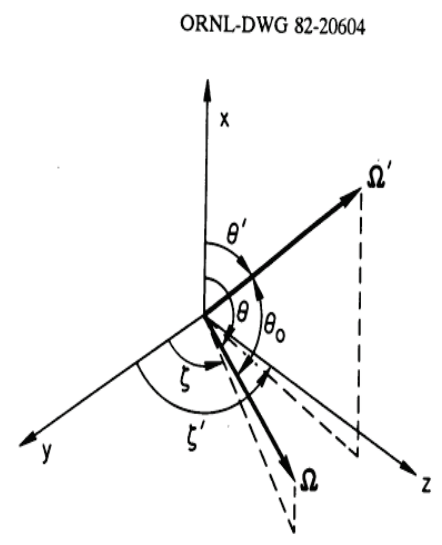

Figure 9.1.3. One-dimensional cylindrical scattering coordinates.

The addition theorem for associated Legendre polynomials can be used to transform from scattering angle coordinates to the real coordinates required in the cylindrical case:

$$
P_{l}\left(\mu_{0}\right)=\sum_{n=-1}^{1} \frac{(l-n) !}{(l+n) !} P_{l}^{n}(\mu) P_{l}^{n}\left(\mu^{\prime}\right) e^{i n\left(\zeta-\zeta^{\prime}\right)}
$$

where $\mu_{0}=\cos \theta_{0} ; \mu=\cos \theta$ and $\mu^{\prime}=\cos \theta^{\prime}$.

If we note that

$$
\begin{aligned}
\sigma_{s}\left(r, E^{\prime} \rightarrow E, \vec{\Omega}^{\prime} \rightarrow \vec{\Omega}\right) & =\sigma_{s}\left(r, E^{\prime} \rightarrow E\right), P_{l}\left(\vec{\Omega}^{\prime} \bullet \vec{\Omega}\right) \\
& =\sigma_{s}\left(r, E^{\prime} \rightarrow E\right), P_{l}\left(\mu_{0}\right),
\end{aligned}
$$

Eq. (9.1.12) can be introduced into Eq. (9.1.2) to yield

$$
\begin{aligned}
S(r, E, \mu)=\int_{0}^{\infty} d E^{\prime} \int_{-1}^{1} d \mu^{\prime} \int_{0}^{2 x} d \zeta \psi\left(\vec{r}, E^{\prime}, \mu^{\prime}, \zeta^{\prime}\right) \sum_{l=0}^{I S C T} \frac{2 l+1}{2} \sigma_{s_{l}}\left(\vec{r}, E^{\prime} \rightarrow E\right) \\
\times \sum_{n=-1}^{l} \frac{(l-n) !}{(l+n) !} P_{l}^{n}(\mu) P_{l}^{n}\left(\mu^{\prime}\right) e^{i n\left(\zeta-\zeta^{\prime}\right)} .
\end{aligned}
$$

Now it is convenient to recall that

$$
\cos x=\frac{e^{+i x}+e^{-i x}}{2}
$$

which can be introduced into Eq. (9.1.13) and rearranged to give 


$$
\begin{aligned}
S(r, E, \mu) & =\sum_{l=0}^{I S C T} \frac{2 l+1}{2} \int_{0}^{\infty} d E^{\prime} \sigma_{s_{l}}\left(\vec{r}, E^{\prime} \rightarrow E\right)\left[P_{l}(\mu) \int_{-1}^{1} d \mu^{\prime} \int_{0}^{2 \pi} d \zeta \psi\left(\vec{r}, E^{\prime}, \mu^{\prime}, \zeta^{\prime}\right) P_{l}\left(\mu^{\prime}\right)\right] \\
& +\sum_{n=1}^{l} 2 \frac{(l-n) !}{(l+n) !} P_{l}^{n}(\mu)\left[\int_{-1}^{1} d \mu^{\prime} \int_{0}^{2 \pi} d \zeta \psi\left(\vec{r}, E^{\prime}, \mu^{\prime}, \zeta^{\prime}\right) P_{l}^{n}\left(\mu^{\prime}\right) \cos \left[n\left(\zeta-\zeta^{\prime}\right)\right]\right] .
\end{aligned}
$$

We now define moments of the flux, $\psi_{l}$ by

$$
\phi_{l}(\vec{r}, E)=\int_{-1}^{1} d \mu^{\prime} \int_{0}^{2 \pi} d \zeta \psi\left(\vec{r}, E, \mu^{\prime}, \zeta^{\prime}\right) P_{1}\left(\mu^{\prime}\right)
$$

It is also convenient to make use of the trigonometric relationship

$$
\cos \left[n\left(\zeta-\zeta^{\prime}\right)\right]=\cos n \zeta \cos n \zeta^{\prime}+\sin n \zeta \sin n \zeta^{\prime}
$$

and

$$
\psi_{l}^{n}(\vec{r}, E)=\sqrt{2 \frac{(l-n) !}{(l+n) !} \int_{-1}^{1} d \mu^{\prime}} \int_{0}^{2 \pi} d \zeta \psi\left(\vec{r}, E, \mu^{\prime}, \zeta^{\prime}\right) P_{l}^{n}\left(\mu^{\prime}\right) \sin n \zeta^{\prime}
$$

With a 1-D cylinder, the flux is symmetric in $\zeta$; therefore, it is an even function, and the terms involving $\sin \mathrm{n} \zeta$ will vanish. This fact yields the following expression for Eq. (9.1.15):

$$
\begin{gathered}
S(r, E, \mu)=\sum_{l=0}^{I S C T} \frac{2 l+1}{2} \int_{0}^{\infty} d E^{\prime} \sigma_{s_{l}}\left(\vec{r}, E^{\prime} \rightarrow E\right) \\
{\left[P_{l}(\mu) \psi_{l}\left(\vec{r}, E^{\prime}\right)+\sum_{n=l}^{l} \sqrt{2 \frac{(l-n) !}{(l+n) !}} P_{n}^{l}(\mu) \cos n \zeta \psi_{l}^{n}(\vec{r}, E)\right] .}
\end{gathered}
$$

We observe further that for an even function in $\zeta$, the odd $l$ and odd ( $l$-n) moments must all vanish, such that the following moments are nonzero for various orders of scattering:

\begin{tabular}{c|l} 
ISCT & Nonzero flux moments \\
\hline 0 & $\psi_{0}$ \\
1 & $\psi_{0}, \psi_{1}^{1}$ \\
2 & $\psi_{0}, \psi_{1}^{1}, \psi_{2}, \psi_{2}^{2}$ \\
3 & $\psi_{0}, \psi_{1}^{1}, \psi_{2}, \psi_{2}^{2}, \psi_{3}^{1}, \psi_{3}^{3}$ \\
4 & $\psi_{0}, \psi_{1}^{1}, \psi_{2}, \psi_{2}^{2}, \psi_{3}^{1}, \psi_{3}^{3}, \psi_{4}, \psi_{4}^{2}, \psi_{4}^{4}$
\end{tabular}

In general, [ISCT(ISCT + 4)/4] + 1 flux moments are required. 


\subsubsection{Discrete-ordinates difference equations}

In formulating the $S_{n}$ equations, several symbols are defined which relate to a flux in an energy group g, in a spatial interval $\mathrm{i}$, and in an angle $\mathrm{m}$.

Typically, the flux is quoted as an integral of the flux in an energy group g, whose upper and lower bounds are $E_{g}^{U}$ and $E_{g}^{L}$, respectively.

$$
\psi_{g}=\int_{E_{g}^{L}}^{E_{g}^{U}} d E \psi(E)
$$

A mechanical quadrature is taken in space, typically IM intervals with IM +1 boundaries. Likewise, an angular quadrature is picked compatible with the particular 1-D geometry, typically MM angles with associated directional coordinates and integration weights.

The different equations are formulated in a manner which involves calculating so-called angular fluxes, $\psi_{g, i, m}$ at each of the spatial interval boundaries, and also cell-centered fluxes, $\psi_{g, i+1 / 2, m}$ at the centers of the spatial intervals. The centered fluxes are related to the angular boundary fluxes by "weighted diamond difference" assumptions as will be described below.

Units on angular fluxes are per unit solid angle $w_{m}$ and per unit area. Units on the centered fluxes are track length per unit volume of the interval. In both cases the fluxes are integrated in energy over the group g.

The areas and volumes for the three geometries are listed in Table 9.1.2.

Table 9.1.2. One-dimensional areas and volumes

\begin{tabular}{|l|l|c|}
\hline Geometry & Area & Volume \\
\hline Slab & 1.0 & $x_{i+1}-x_{i}$ \\
\hline Cylinder & $2 \pi r_{i}$ & $\pi\left(r_{i+1}^{2}-r_{i}^{2}\right)$ \\
\hline Sphere & $4 \pi r_{i}^{2}$ & $4 / 3 \pi\left(r_{i+1}^{3}-r_{i}^{3}\right)$ \\
\hline
\end{tabular}

\subsection{Discrete-ordinates equation for a slab}

Consider a spatial cell bounded by $\left(x_{i}, x_{i+1}\right)$ and write the loss term for flow through the cell in direction $\mu_{m}$. The net flow in the x-direction out the right side is the product of the angular flux times the area times the solid angle times the cosine of the angle:

$$
w_{m} \mu_{m} A_{i+1} \psi_{g, i+1, m}
$$

The net loss from the cell is the difference between the flow over both boundaries:

$$
w_{m} \mu_{m}\left(A_{i+1} \psi_{g, i+1, m}-A_{i} \psi_{g, i, m}\right)
$$


The loss in the spatial cell due to collisions is given by the product of the centered angular flux (in per unit volume units) times the total macroscopic cross section times the solid angle times the volume:

$$
w_{m} \sigma_{g, i+1 / 2} V_{i} \psi_{g, i+1 / 2, m}
$$

The sources in direction $\mu_{m}$ are given by the product of the solid angle times the interval volume times the volume-averaged source (sum of fixed, fission, and scattering) in the direction $\mathrm{m}$ :

$$
w_{m} V_{i} S_{g, i+1 / 2, m} .
$$

The slab equation is obtained by using Eqs. (9.1.20), (9.1.21), and (9.1.22) and substituting proper values for area and volume:

$$
w_{m} \mu_{m}\left(\psi_{g, i+l, m}-\psi_{g, i, m}\right)+w_{m} \sigma_{g, i+1 / 2} \psi_{g, i+1 / 2, m}\left(x_{i+l}-x_{i}\right)=w_{m} S_{g, i+1 / 2, m}\left(x_{i+l}-x_{i}\right)
$$

In an $\mathrm{MM}$ angle quadrature set, there are $\mathrm{MM}$ of these equations and they are coupled through the assumption on how the cell-centered flux relates to the boundary angular fluxes, the sources, and the boundary conditions, as will be discussed later.

\subsection{Discrete-ordinates equations for sphere and cylinder}

The development of the equations for these geometries is analogous to that for the slab except that the leakage terms are more complicated. Consider Figure 9.1.4.

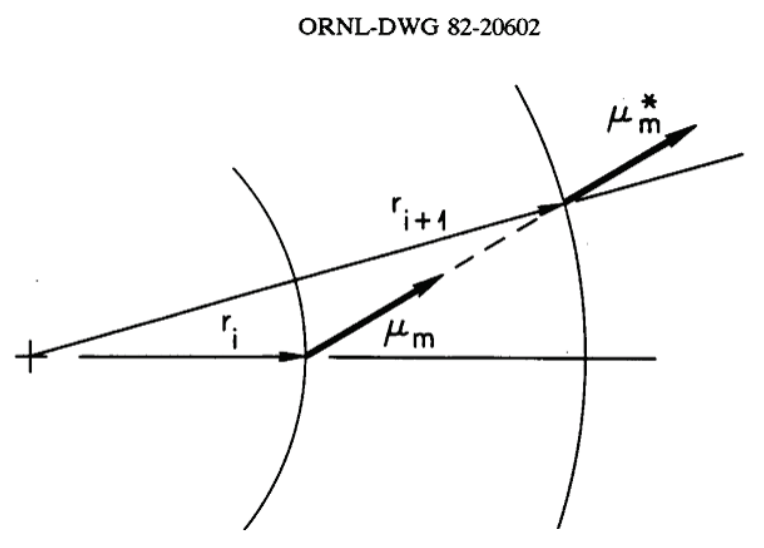

Figure 9.1.4. Angular redistribution in spherical geometry.

Recall that the directions are taken with reference to the radius vector for a sphere. A particle traveling in direction $\mu_{m}$ at $r_{i}$ will intersect the radius vector to the next point $r_{i+1}$ at a different angle $\mu_{m}^{*}$. The same effect also exists for the cylinder, though in this case the direction coordinates are more complicated. Because of the effect, a loss term is included for the "angular redistribution." It is defined in a manner analogous to Eq. (9.1.20) as 


$$
\alpha_{i+1 / 2, m+1 / 2} \psi_{g, i+1 / 2, m+1 / 2}-\alpha_{i+1 / 2, m-1 / 2} \psi_{g, i+1 / 2, m-1 / 2}
$$

where the $\alpha$ coefficients are to be defined in such a manner as to preserve particle balance. In this case one speaks of $\mathrm{m}+1$ and $\mathrm{m}-1 / 2$ as the corresponding angles to $\mu_{m}$ on the $\mathrm{I}+1$ th and ith boundaries, respectively. (See Figure 9.1.5.) Here we are interested in an angle $\mu_{m}$ at the center of interval i which redistributes to $\mu_{m-1 / 2}$ at boundary $\mathrm{i}$ and to $\mu_{m+1 / 2}$ at boundary I +1 .

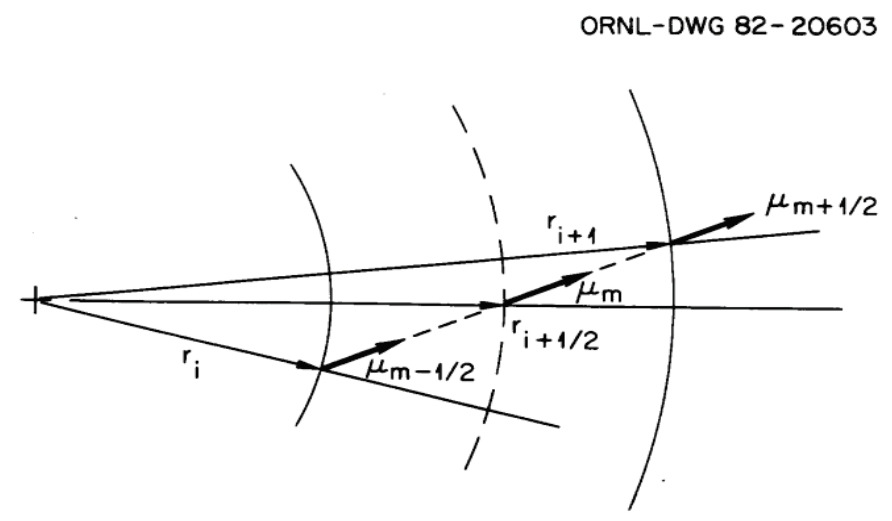

Figure 9.1.5. Angular redistribution.

Obviously, it is necessary that the net effect of all redistributing be zero, in order to maintain particle balance. This condition is met if

$$
\sum_{m=1}^{M M} \alpha_{m-1 / 2} \psi_{m-1 / 2}+\alpha_{m+1 / 2} \psi_{m+1 / 2}=\alpha_{1 / 2} \psi_{1 / 2}+\alpha_{M M+1 / 2} \psi_{M M+1 / 2}=0
$$

where we have dropped the group and interval indexes.

In order to develop an expression for determining the $\alpha$ 's consider an infinite medium with a constant isotropic flux. In this case, there is no leakage and the transport equation reduces to

$$
\sum_{t} \phi=S
$$

This condition requires that

$$
\mu_{m} w_{m}\left(A_{i+1} \psi_{g, i+1, m}-A_{i} \psi_{g, i, m}\right)+\alpha_{i+1 / 2, m+1 / 2} \psi_{g, i+1 / 2, m+1 / 2}-\alpha_{i+1 / 2, m-1 / 2} \psi_{g, i+1 / 2, m-1 / 2}=0
$$

which when we note that all the $\psi$ terms in the infinite medium case are equal becomes

$$
\mu_{m} w_{m}\left(A_{i+1}-A_{i}\right)=-\alpha_{m+1 / 2}+\alpha_{m-1 / 2},
$$

which is a recursion relationship for $\alpha$. 
From Eq. (9.1.25) we see that the conservation requirement can be met if

$$
\alpha_{1 / 2}=\alpha_{M M+1 / 2}=0
$$

for any values of flux, and is, therefore, used to evaluate the $\alpha$ 's along with Eq. (9.1.27) or (9.1.28). (Note that had we included the redistribution term in the slab equation, Eq. (9.1.28) would have given zeroes for the terms, which is as one would expect for this geometry.)

The final discrete-ordinates expression for spheres and cylinders is then derived by summing expressions Eqs. (9.1.20), (9.1.24), (9.1.21) and setting it equal to expression Eq. (9.1.22).

\subsection{Sn quadratures for slabs}

XSDRNPM will automatically calculate quadrature sets for each of the 1-D geometries, or a user can, if he wants, input a quadrature.

In the case of the 1-D slab, the quadrature is a double Gauss-Legendre set based on recommendations from Ref. 9.

The ordering of the directions for a slab is shown in Figure 9.1.6.

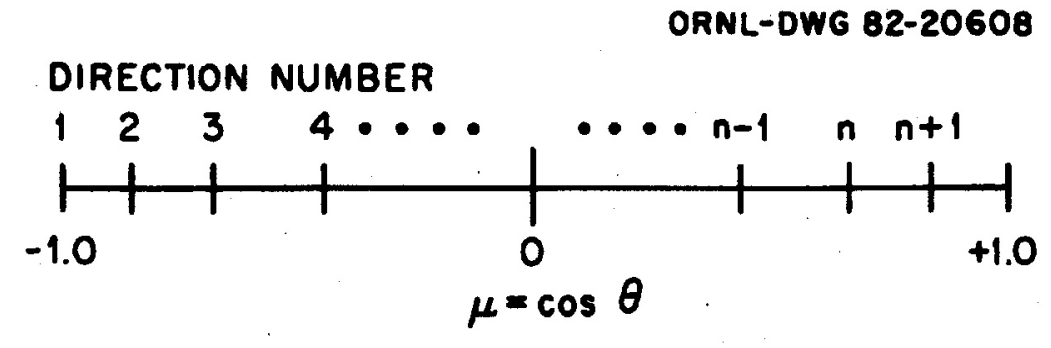

Figure 9.1.6. Ordering of $S_{n}$ directions for slabs and spheres.

Note that in referring to the quadratures for any of the geometries, we do not attempt to define an explicit area on a unit sphere, but rather speak of characteristic directions with associated weights. In the case of the slab, it is convenient to think of "directions" which are shaped like cones, because of the azimuthal symmetry around the $\mathrm{x}$-axis.

In an $n$th order quadrature, there are $n+1$ angles with the first angle being taken at $\mu=-1.0$. This first angle is not required for the slab, but is needed for the curvilinear geometries because of the angular redistribution terms, as will be noted later. It is included in the slab case for reasons of uniformity of programming, etc.

Several requirements are made regarding the angles and weights in the quadrature set.

The arguments relating to angular redistribution can be expected to show that

$$
\sum_{m=1}^{M M} \mu_{m} w_{m}=0.0 .
$$


This situation is ensured if the weight of the $\mu=-1.0$ direction is zero and the other directions and weights are symmetric about $\mu=0$. (The $\mu=0$ direction is never included in the quadrature set because of its singularity.)

Further, it is required that

$$
\sum_{m=1}^{M M} w_{m}=1.0 .
$$

Due to the above normalization of the quadrature weights, the discrete ordinates angular flux is not "per steradian" but rather "per direction-weight". The calculated angular flux can be converted to steradians by dividing by $4 \pi$.

\subsection{Sn quadratures for spheres}

The quadratures generated for spheres are Gauss-Legendre coefficients as recommended by Ref. 9.

The ordering and symmetry requirements for spheres are the same as for slabs.

In the case of the sphere, the initial $(\mu=-1.0)$ direction is required, because the difference equations involve three unknown values for each direction, $\mu_{m}: \psi_{m}$ and the fluxes at the two "redistributed" angles $\psi_{m-1 / 2}$ and $\psi_{m+1 / 2}$. It is obvious that an angle along the radius will not involve the redistribution; hence, the expression for this direction involves only $\psi(\mu=-1.0)$ as unknowns. Angle 2 proceeds by assuming $\psi_{2-1 / 2}$ is given by $\psi_{1}$ and also uses a weighted diamond difference model to relate $\psi_{m}, \psi_{m-1 / 2}$ and $\psi_{m+1 / 2}$, as will be described below. Subsequent angles will then have values for $\psi_{m-1 / 2}$ calculated by the previous angle equations.

\subsection{Sn quadratures for cylinders}

The quadrature sets for cylinders are more complicated (see Figure 9.1.2) because the directions must be specified with two angles, $\zeta$ and $\eta$ where $\alpha \equiv \sin \eta \cos \zeta$ and $\beta \equiv \cos \eta$.

In this case, practice is to use $\mathrm{n} / 2$ levels of directions for an $\mathrm{n} t h$ order set. The levels correspond to fixed values of $\eta$. The number of angles by level starts with three in level 1, five in level 2, seven in level 3, etc. (Note that since cylindrical geometry is curvilinear, each level will start with a $\eta=\pi$ direction that has zero weight for reasons analogous to those given for the spherical case. Figure 9.1.7 shows the ordering of the directions for an $S_{6}$ quadrature set. Angles 1, 4, and 9 are the starting directions (zero weight) for the levels. 


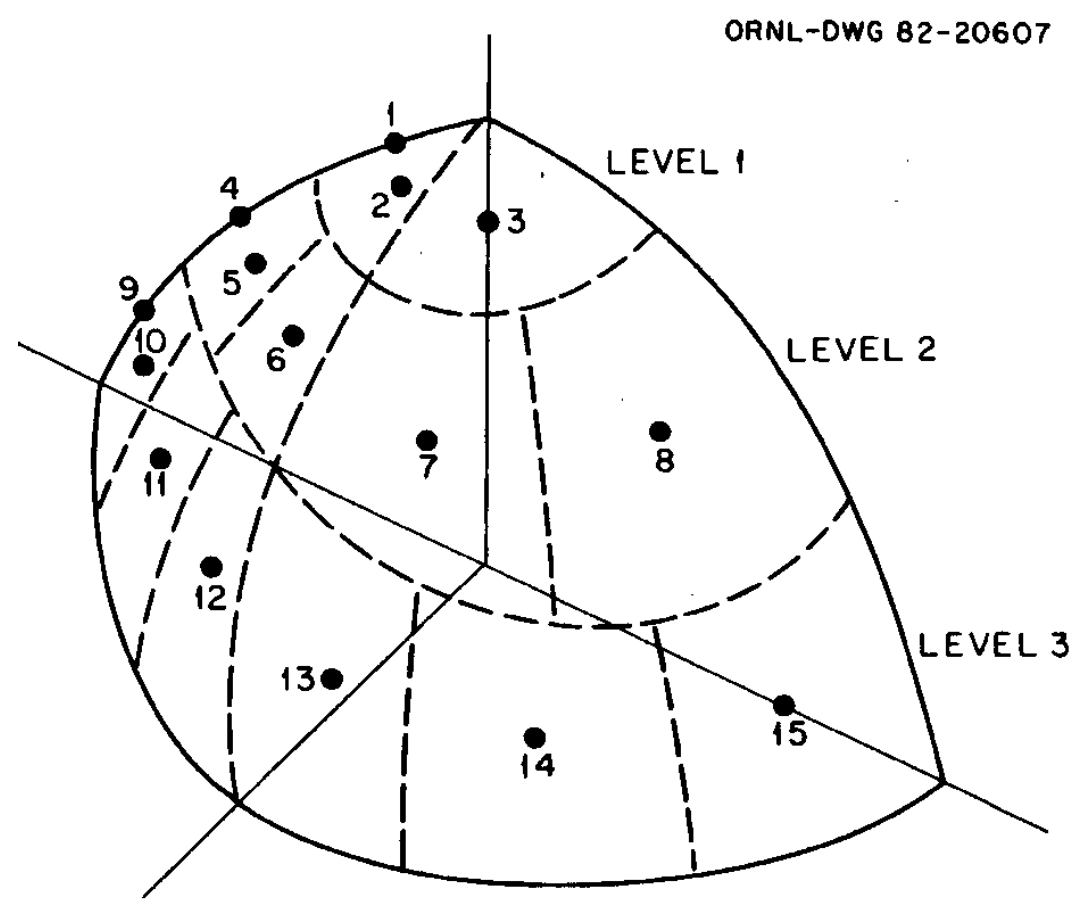

Figure 9.1.7. Ordering of the directions for an $S_{6}$ cylindrical set.

In general, an $\mathrm{n} t h$ order quadrature will contain $\mathrm{n}(\mathrm{n}+4) / 4$ angles. The cosines, $\mu$, and the weights are stored in two arrays internally in the code; and, since the weights for the $1 s t$, 4 th, and 9 th angles are zero, the cosines for the corresponding levels are placed in these locations in the arrays.

The cylindrical sets are based on Gauss-Tschebyscheff schemes as recommended by Ref. 9 with Gaussian quadratures in $\beta$ and Tschebyscheff quadratures in $\alpha$.

\subsubsection{Weighted-difference formulation for discrete-ordinates equations}

In order to solve the discrete-ordinates equations, an assumption is required concerning the relationship of the various flux terms: $\psi_{i, m}, \psi_{i+1, m}, \psi_{i+1 / 2, m}, \psi_{i, m-1 / 2}, \psi_{i+1, m-1 / 2}$.

The solution of the equations involves three major loops: an outer loop over energy groups, a loop over angles, and a loop over the spatial mesh. The spatial loop is made either from the origin to the outside boundary or from the outside to the origin, depending on whether the angle is directed outward or inward, respectively.

Two models are widely used for expressing flux relationships: (1) the step model and (2) the diamonddifference linear model.

The "step model" is a histogram model whereby one sets the centered flux value to the appropriate boundary value, depending on which way the mesh sweep is going. If, for example, the sweep is to the right in space, then

$$
\psi_{\mathrm{i}+1 / 2, m}=\psi_{i, m},
$$


or if to the left,

$$
\psi_{\mathrm{i}+1 / 2, m}=\psi_{i+1, m} .
$$

Likewise, in angle:

$$
\psi_{\mathrm{i}+1 / 2, m}=\psi_{i+1, m-1 / 2}
$$

The step model involves a very crude approximation, but has the marked advantage of helping to ensure positivity of flux values as long as scattering sources are positive.

In the "diamond-difference" model, the centered fluxes are assumed linear with the edge values:

$$
\begin{gathered}
\psi_{i+1 / 2}=0.5\left(\psi_{i}+\psi_{i+1}\right) \\
\psi_{m}=0.5\left(\psi_{m-1 / 2}+\psi_{m+1 / 2}\right) .
\end{gathered}
$$

Unfortunately, though the linear model is clearly a better model than the step model, care must be taken by selecting a fine spatial mesh, or the linear extrapolation can lead to negative flux values. In some cases, the situation is so severe that it is impractical to take enough mesh points to eliminate the problems. Because of these difficulties XSDRNPM uses a different approach, as described below.

The weighted diamond difference model ${ }^{10}$ was developed in an attempt to take advantage of the "correctness" of the linear model, while retaining the positive flux advantages of the step model.

A solution in some $S_{n}$ codes is to use the linear model in all cases where positive fluxes are obtained and to revert to step model otherwise. Unfortunately, this method leads to artificial distortions in the fluxes.

Note that if one writes

$$
\begin{gathered}
\psi_{i+1 / 2}=a \psi_{i}+(1-a) \psi_{i+1} \\
\psi_{m}=b \psi_{m-1 / 2}+(1-b) \psi_{m+1 / 2}
\end{gathered}
$$

that the same expression can be used to express linear or step model (e.g., $a=b=1 / 2$ is equivalent to linear, while $\mathrm{a}=\mathrm{b}=1.0$ can be used for the step model).

In the weighted model, the intention is to use the linear model when fluxes are positive but to select values for $\mathrm{a}$ and $\mathrm{b}$ in the range

$$
1 / 2 \leq a \text { or } b \leq 1.0
$$

that ensure positivity, if the source is positive.

At this point, it is convenient to rewrite the discrete-ordinates expression in a simplified notation, without the obvious subscripts on energy group, angle, etc.

$$
w \mu\left(A_{i+1} \psi_{i+1}-A_{i} \psi_{i}\right)+\alpha_{m+1 / 2} \psi_{m+1 / 2}-\alpha_{m-1 / 2} \psi_{m-1 / 2}+w \sigma V \psi=w V S .
$$


Combining Eqs. (9.1.35) and (9.1.32) or (9.1.33) yields the following expressions for $\psi_{1+1}$ and $\psi_{m+1 / 2}$ :

$$
\begin{aligned}
\psi_{i+1}= & \frac{S V+C_{2} \psi_{m-1 / 2}+\left[\mu A_{i}-(1-a) D_{1}\right] \psi_{i}}{a D} \\
\psi_{m+1 / 2}= & \frac{S V+C_{1} \psi_{i}+\left[\frac{\alpha_{m-1 / 2}}{w}-(1-b) D_{2}\right] \psi_{m-1 / 2}}{b D},
\end{aligned}
$$

where

$$
\begin{gathered}
C_{1}=\mu\left[A_{i}+A_{i+1}\left(\frac{1}{\alpha}-1\right)\right] \\
C_{2}=\frac{\alpha_{m-1 / 2}}{w}+\frac{\alpha_{m+1 / 2}}{w}\left(\frac{1}{b}-1\right) \\
D=\Sigma V_{i}+\frac{\mu A_{i+1}}{a}+\frac{\alpha_{m+1 / 2}}{w b} \\
D_{1}=D-\frac{\mu A_{i+1}}{a} \\
D_{2}=D-\frac{\alpha_{m+1 / 2}}{w b} .
\end{gathered}
$$

In determining a and $\mathrm{b}$, the "theta-weighted" model uses arbitrary multipliers $\theta_{s}$ on SV and $\theta_{n}$ on the $C_{2} \psi_{m-1 / 2}$ or $C_{l} \psi_{i}$ terms in Eqs. (9.1.36) and (9.1.37). (In Ref. 11, a thorough discussion is given on the history of using different choices of $\theta_{s}$ and $\theta_{n}$ and the advantages and disadvantages of each method.) In XSDRNPM, a value of 0.9 is used for $\theta_{s}$ and $\theta_{n}$ following the practice in the DOT-IV code.7

For $\psi_{i+1}$ in Eq. (9.1.36) to be positive, the numerator should be positive, thereby requiring

$$
\left[\mu A_{i}-(1-a) D_{1}\right] \psi_{i}<S V+C_{2} \psi_{m-1 / 2}
$$

which in the $\theta$-weighted case becomes

$$
\left[\mu A_{i}-(1-a) D_{1}\right] \psi_{i}<S V \theta_{s}+C_{2} \psi_{m-\frac{1}{2}} \theta_{n}
$$

A similar expression can be written for b using Eq. (9.1.37).

For reasons of accuracy, it is desirable to use $a=b=1 / 2$. Therefore, when $a$ or $b$ is determined to be less than $1 / 2$ it is automatically set to $1 / 2$. 


\subsubsection{Boundary conditions}

XSDRNPM allows a boundary condition to be specified for each of the two "outside" boundaries of its 1-D geometries. The options are the following:

1. Vacuum boundary $\quad-\quad$ all angular fluxes that are directed inward at the boundary are set to zero (e.g., at the left-hand boundary of slab, $\psi(\mu>0)=0$, etc.).

2. Reflected boundary - the incoming angular flux at a boundary is set equal to the outgoing angular flux in the reflected direction (e.g., at the left-hand boundary of a slab),

$$
\psi_{\text {in }}(\mu)=\psi_{\text {out }}(-\mu)
$$

3. Periodic boundary - the incoming angular flux at a boundary is set equal to the outgoing angular flux in the same angle at the opposite boundary.

4. White boundary $\quad-\quad$ the angular fluxes of all incoming angles on a boundary are set equal to a constant value such that the net flow across the boundary is zero, that is,

$$
\psi_{\text {in }}=\frac{\sum_{m}^{o u t} w_{m} \mu_{m} \psi_{m}}{\sum_{m}^{i n} w_{m}\left|\mu_{m}\right|} .
$$

This boundary condition is generally used as an outer-boundary condition for cell calculation of cylinders and spheres that occur in lattice geometries.

5. Albedo boundary - this option is for the white boundary condition except that a usersupplied group-dependent albedo multiplies the incoming angular fluxes. This option is rarely used, as it is difficult to relate to most practical situations.

\subsubsection{Fixed sources}

Two types of inhomogeneous or fixed sources can be specified in XSDRNPM.

In the first case, an isotropic group-dependent volumetric source can be specified for any or all spatial intervals in a system.

In the second case, an angle- and group-dependent boundary source can be specified for any or all boundaries between spatial intervals in a system, excepting the left-most boundary. In this case, one specifies not a source but a flux condition on the boundary. If one uses the "track length" definition for flux, it is easy to show that the flux condition is related to a source condition by

$$
\psi_{m}^{s}=\frac{S_{m}}{\mu_{m}}
$$


(This equation says that an isotropic source on a boundary would be input as a constant divided by the cosine of the direction.)

In conventional fixed-source calculations, the total fixed source in the system can be normalized to an input parameter, XNF. In the volumetric source case, the source values will be normalized such that

$$
X N F=\sum_{g=1}^{I G M} \sum_{i=1}^{I M} Q_{g, i} V_{i}
$$

and in the boundary source case,

$$
X N F=\sum_{g=1}^{I G M} \sum_{i=1}^{I M} A_{i+1} \sum_{m=1}^{M M} \mu_{m} \psi_{m}^{s}(g, i) w_{m} .
$$

In the case where both volumetric and boundary sources are specified, the two sums are normalized to XNF.

The fixed source for a generalized adjoint calculation corresponds to a particular response ratio of interest. The generalized adjoint equation only has a solution for responses that are ratios of linear functionals of the flux, and in this case the source will contain both positive and negative components. These types of sources are described in more detail in Sect. 9.1.2.15.1 and in the SAMS chapter, in Generalized Perturbation Theory.

\subsubsection{Dimension search calculations}

XSDRNPM has three options for searching for dimensions such that the system will produce a specified effective multiplication factor, $k_{\text {eff. }}$. The options are selected by a parameter IEVT in the $1 \$$ array and are as follows:

1. zone width search $(\operatorname{IEVT}=4)$,

2. outer radius search $(\mathrm{IEVT}=5)$,

3. buckling search $(\mathrm{IEVT}=6)$.

By default, the search is made to produce a $k_{\text {eff }}$ value of unity. For $k_{\text {eff }}$ 's other than unity, IPVT ( $\$$ array) is set to unity and the desired $k_{\text {eff }}$ is input as PV ( $5^{*}$ array).

Other input parameters which apply specifically to all search calculations are in the $5 *$ array and are EV, the starting eigenvalue guess, EVM, the eigenvalue modifier, EQL, the eigenvalue convergence, and XNPM, the new parameter modifier. These parameters are discussed in more detail below.

\subsection{Zone-width search $($ IEVT $=4)$}

With this option, one can vary the width of any or all zones in a case. Note that it is also possible to change zone widths at different rates.

This option requires the inputting of a zone width modifier array $\left(41^{*}\right)$ which is used to specify the relative movements of the zones according to the following expression: 


$$
\Delta Z_{j}^{f}=\Delta Z_{j}^{i}\left(1+E V^{*} Z M_{j}\right)
$$

where $\Delta \mathrm{Z}_{j}^{i}, \Delta \mathrm{Z}_{j}^{f}$ are the initial and final widths of zone $\mathrm{j}$, respectively, $Z M_{j}$ is the zone width modifier for the zone (as input in the $41^{*}$ array), and EV is the final "eigenvalue" for the problem. Note that a zero value for ZM will specify a fixed zone width. Negative values for ZM are allowed.

\subsection{Outer radius search $($ IEVT $=5)$}

With this option, all zones are scaled uniformly in order to make the system attain the specified $k_{e f f}$. The final zone widths are found by multiplying the initial values by the final "eigenvalue:"

$$
Z_{j}^{f}=E V\left(Z_{j}^{i} / Z_{p}^{i}\right)
$$

\subsection{Buckling search $($ IEVT $=6)$}

This option is used to search for "transverse" dimensions that will yield a specified $k_{\text {eff }}$ for a system. This means that the search is for the height for a 1-D cylinder or the y- and/or z-dimensions in a 1-D slab.

For this option, the final dimensions are given by

$$
\mathrm{DY}=D Y_{0} \times \mathrm{EV}
$$

and

$$
\mathrm{DZ}=D Z_{0} \times \mathrm{EV}
$$

where $D Y_{0}, D Z_{0}$ are the initial dimensions input in the $5^{*}$ array.

\subsection{Search calculation strategy}

All the "dimension searches" use the same simple strategy. The calculations start by using the input eigenvalue (EV from the $5^{*}$ array) to determine initial dimensions for the system. These dimensions allow the code to calculate a $k_{\text {eff }}$. The eigenvalue modifier (EVM in the $5^{*}$ array) is then used to change the dimensions as follows:

$\mathrm{IOPT}=4$ (Zone width search)

$$
\Delta Z_{j}^{f}=\Delta Z_{j}^{i}\left[1+(E V M+E V)^{*} Z M_{j}\right]
$$

$\mathrm{IOPT}=5($ Outer radius search $)$

$$
\Delta Z_{j}^{f}=(E V M+E V)\left(\Delta Z_{j}^{i}\right)
$$

$\mathrm{IOPT}=6$ (Buckling search)

$$
\begin{aligned}
& \mathrm{DY}=D Y_{0}(\mathrm{EV}+\mathrm{EVM}) \\
& \mathrm{DZ}=D Z_{0}(\mathrm{EV}+\mathrm{EVM}) .
\end{aligned}
$$


The new dimensions are then used in a new calculation which determines a second $k_{\text {eff }}$ value.

XSDRNPM searches for a unity value of $k_{\text {eff }}$ by default; however, when IPVT $=1$ ( $3 \$$ array), a nonunity value can be specified in PV ( $5^{*}$ array) and the search will be made on this value.

Once the two $k_{\text {eff }}$ 's are known, which are based on eigenvalues of EV and EV + EVM, respectively, a linear fit is used to project to the next value for EV. This yields an expression of the form

$$
E V_{n e x t}=E V M \frac{\left(P V-k_{1}\right)}{\left(k_{2}-k_{1}\right)}+E V,
$$

where $k_{1}$ and $k_{2}$ are the first and second value of $k_{\text {eff }}$, respectively. After this iteration, the procedure is to fit a quadratic to the three most recent $k_{\text {eff }}$ values in order to obtain an estimate for the next EV.

The procedure continues until a relative convergence of EQL ( $5 *$ array) or better is obtained on EV.

To prevent oscillations in the search, extrapolations are limited by XNPM, the new parameter modifier from the $5^{*}$ array.

\subsubsection{Alpha search}

It is possible to make some of the searches described in Sect. 9.1.2.8 in a more "direct" fashion than the strategy described in Sect. 9.1.2.8.4. XSDRNPM has two such options: (1) the alpha search and (2) a direct buckling search. These are described below.

\subsection{Alpha search}

The time-dependent form of the Boltzmann equation is identical with Eq. (9.1.1), except for the inclusion of a time-gradient term on the left-hand side:

$$
\frac{1}{\mathrm{v}} \frac{\partial \psi(\vec{r}, E, \vec{\Omega}, t)}{\partial t} .
$$

All other flux terms in the expression also would include the time (t) argument.

In some analyses it is reasonable to assume that the time variation of the flux is exponential, that is,

$$
\psi(\vec{r}, E, \vec{\Omega}, t)=\psi(\vec{r}, E, \vec{\Omega}) e^{a t}
$$

When this variation is introduced into the expanded form of Eq. (9.1.1), the exponential terms all cancel leaving a leading term:

$$
\frac{\alpha}{\mathrm{v}} \psi(\vec{r}, E, \vec{\Omega})
$$

which is in the same form as the $\Sigma_{t} \psi$ term. 
If one considers integrating over energy, angle, and space, the following expression can be derived:

$$
P-A-L-\alpha V=0
$$

where

$\mathrm{P} \equiv$ production in the system,

A $\equiv$ absorptions in the system,

$\mathrm{L} \equiv$ leakage from the system,

$$
\mathrm{V} \equiv \int_{0}^{\infty} d E \int_{0}^{4 \pi} d \vec{\Omega} \int_{\text {system }} d \vec{r} \frac{\psi(\vec{r}, E, \vec{\Omega})}{\mathrm{V}}
$$

Since all terms other than $\alpha$ can be determined from a calculation, it is possible to determine $\alpha$ directly, thereby avoiding a scheme like that used for dimension searches. In the balance expression, the fission component of the production term is adjusted for the case of a non-unity $k_{\text {eff }}$ value (IPVT $=1$ in the $3 \$$ array).

An $\alpha$-search has several practical applications. If, for example, a subcritical assembly is pulsed by a source, the time-dependence of the flux is expected to die off exponentially. Another way to interpret the $\alpha$-search is as that amount of $1 / \mathrm{v}$ absorber which could be added or taken away from a system in order to achieve criticality. This number could be of interest when certain control materials are used, such as ${ }^{10} B_{5}$, which is a " $1 / \mathrm{v}$ " material.

\subsection{Direct-buckling search}

A "direct"-buckling search can be made using a procedure analogous to that described in Sect. 9.1.2.9.1. Recall that the buckling is introduced in order to represent a transverse leakage through the use of a $D B^{2} \psi$ term. This suggests that the foregoing balance expression be written:

$$
P-A-L-\alpha D B^{2} X=0,
$$

where

$$
X \equiv \int_{0}^{\infty} d E \int_{0}^{4 \pi} d \vec{\Omega} \int_{\text {system }} d \vec{r} \psi(\vec{r}, E, \vec{\Omega})
$$

In this case, the diffusion coefficients, $D_{g}$, are determined from

$$
D_{g}=\frac{1}{3 \Sigma_{t r_{g}}}
$$

where

$$
\Sigma_{t r_{g}}=\Sigma_{t_{g}}-\Sigma_{1_{g}}
$$

and $\Sigma_{1}$ is the within-group term from the $P_{1}$ scattering matrixes: 


$$
\Sigma_{1_{\mathrm{g}}}=\sum_{t}\left(\mathrm{~g} \rightarrow \mathrm{g}^{\prime}\right)
$$

The original $B^{2}$ value is determined as specified in Sect. 9.1.2.12, and the $\alpha$ is the square of the search parameter, that one multiplies by the original $B^{2}$ value in order to determine the final buckling and, hence, the dimensions of the system.

\subsubsection{Iteration and convergence tests}

Two parameters are used to specify the required levels of convergence on an XSDRNPM calculation. These are EPS and PTC, both given in the $5^{*}$ array. The flux calculations proceed through a series of iterations until either convergence is achieved or the specified iteration limit is exceeded.

The basic iteration strategy in XSDRNPM is now described. The discrete-ordinates difference equation is solved for the first angle and the first energy group. This sweep generally is made from the last interval boundary to the center of the system, and it uses the flux guess supplied as part of the input along with the boundary conditions. The second angle is then calculated, etc., until all angles in the quadrature are treated. At the end of this sweep, new scalar fluxes for the midpoints of all intervals have been determined. The angular sweep continues until either the point scalar fluxes are converged to within PTC or until the code makes IIM inner iterations. An exception to this "inner iteration" pattern occurs on the first outer (defined below) iteration whenever a fission density guess is used, instead of the flux guess. In this case, the program uses 1-D diffusion theory to determine a scalar flux value for all intervals and the angular sweeps are not made until the second outer iteration. After the first group is completed, the calculation goes to the second group and repeats the above procedure. This continues until all groups have been treated.

The pass through all groups, angles, and intervals is called an outer iteration. Most of the convergence checks on the outer iteration have to do with reaction rates involving all energy groups and are made against the EPS parameter mentioned above. For a coupled neutron-gamma problem, outer iterations are only performed for the neutron groups until convergence is achieved, then the final converged pass is made over all groups. In discussing these checks, it is convenient to define several terms:

$$
\begin{aligned}
& \mathrm{Q} \equiv \text { total fixed source in the system } \\
& \mathrm{F} \equiv \sum_{i} \sum_{g} \sum_{\mathrm{g}^{\prime} \neq \mathrm{g}} \psi_{i, g} \sigma_{g \rightarrow g^{\prime}} \mathrm{v}_{\mathrm{i}} \\
& \mathrm{D} \equiv \text { total fission source in the system } \\
& \quad \equiv \text { scalar flux in intervals i and group } \mathrm{g}^{\text {intervals groups groups }} \\
& \psi_{i, g} \quad \equiv \text { macroscopic scattering cross section from group g to group } \\
& \sigma_{g \rightarrow g^{\prime}} \equiv \\
& \mathrm{v}_{\mathrm{i}} \quad \equiv \text { volume of interval } \mathrm{i} \\
& \mathrm{k} \quad \equiv \text { outer iteration number }
\end{aligned}
$$




$$
\begin{aligned}
& \text { IGM } \equiv \text { total number of energy groups } \\
& \lambda_{k} \equiv \frac{Q+F_{k}}{Q+F_{k-1}} \\
& G_{k} \quad \equiv \frac{D_{k}}{Q+F_{k}} \\
& \lambda_{k}^{\prime} \quad \equiv \frac{G_{k-1}}{G_{k}} \\
& \quad \equiv \text { total upscatter rate }=\sum_{i} \sum_{g} \sum_{g^{\prime}<g} \psi_{i, g} \sigma_{g \rightarrow g^{\prime}} \mathrm{v}_{i} \\
& U_{k} \quad \equiv U_{K} / U_{k-1}, U_{k-1} \neq 0_{j}=1, U_{k-1}=0 \\
& \lambda_{k}^{\prime \prime} \quad
\end{aligned}
$$

An inner iteration in XSDRNPM consists of sweeping one time through the entire spatial mesh for all the $S_{n}$ angles for one energy group. When the fluxes for a particular group are being calculated, inner iterations (j) will continue until (a) the number of inner iterations for this outer iteration exceeds IIM (the inner iteration maximum) or (b) until

$$
\text { (1) } \max _{i}\left|\frac{\psi_{i, g}^{j}-\psi_{i, g}^{j-1}}{\psi_{i, g}^{j}}\right| \leq \text { PTC. }
$$

At the end of an outer iteration, the following checks are made:

(2) $\left|1.0-\lambda_{k}\right| \leq E P S$.

(3) $R\left|1.0-\lambda_{k}^{\prime}\right| \leq E P S$.

(4) $R\left|1.0-\lambda_{k}^{\prime \prime}\right| \leq E P S$.

$\mathrm{R}$ is a convergence relaxation factor and is set internally to 0.5 in XSDRNPM. If all convergence criteria are met, if ICM (the outer iteration maximum) is reached, or if ITMX (the maximum execution time) is exceeded, the problem will be terminated with full output; otherwise, another outer iteration will be started.

\subsubsection{Group banding (scaling rebalance)}

As described above, the normal mode of operation in XSDRNPM is to do inner iterations on a group until it converges, then go to the next group. For groups where there is no upscatter, the scattering source to a group depends only on higher energy groups for which the fluxes have already been calculated. A fixed source problem with no fission and no upscattering can, therefore, be converged in one outer iteration. 
Since fission sources and upscattering sources are calculated with fluxes from the previous outer iteration, multiple outer iterations must be done to converge problems involving these kinds of sources. For problems involving many fine thermal groups (groups with both upscatter and downscatter), a special convergence problem arises. Because the groups are fine, within-group scattering is small and the flux calculation is dominated by scattering sources from other groups. This situation leads to a very slow reduction in scattering source errors from one outer iteration to the next. XSDRNPM has a special "group banding" option for treating this problem. It involves collecting several groups together into a band and doing one inner for each group in the band while collecting particle balance information. This balance information is then used to solve for one set of flux rebalance factors to apply to each group in the band. Because the band is much wider than an individual group, the scattering that remains within the band is a much larger fraction of the total scattering source for the band. This condition leads to considerably faster convergence from one outer iteration to the next. The group banding option in XSDRNPM is triggered by the seventh entry in the $2 \$$ array. The absolute value of this entry indicates the number of bands to be used. If the number is negative, these bands are only for the thermal groups. Normally there is no need to band together groups other than the thermal groups. An entry of -1 indicates that all the thermal groups will be treated as one band. This mode is one that is used successfully for many problems, but occasionally will cause a problem to not converge. For these problems using two or three bands for the thermal groups has been successful.

The code generates a default banding structure, but this structure can be overridden by inputting a $52 \$$ array.

\subsubsection{Buckling corrections}

XSDRNPM allows "buckling" corrections to be made for the transverse (non-calculated) dimensions in its 1-D slab and cylindrical geometries. Three input parameters-DY, DZ, and BF (5* array)-may be involved.

In the case of the 1-D slab, the height DY and the width DZ can be input. The buckling correction uses an expression based on asymptotic diffusion theory to account for leakage in the transverse direction and is treated analogous to an absorption cross section, that is,

$$
\text { Transverse Leakage }=D B^{2} \psi,
$$

where $\mathrm{B}$ is the geometric buckling and is given by

$$
B^{2}=\left(\frac{\pi}{Y}\right)^{2}+\left(\frac{\pi}{Z}\right)^{2}
$$

and $\mathrm{Y}$ and $\mathrm{Z}$ are the height and width of the slab, respectively, and include extrapolation distances.

Recall that the "extrapolation distance" is defined as the linear extrapolation distance such that if one extrapolated to a zero flux value at this distance from the boundary, the interior flux shape in the body would be correctly represented. The distance can be shown to occur at $0.71 \lambda_{t r}$, where $\lambda_{t r}$ is the transport mean free path given by $1 / \Sigma_{t r}$. Note that for a slab, there are two extrapolation distances to include (one on either side) for the height and width, such that 


$$
\mathrm{Y}=\mathrm{DY}+1.42 \lambda_{t r}
$$

and

$$
\mathrm{Z}=\mathrm{DZ}+1.42 \lambda_{t r}
$$

The 1.42 factor $(=2 \times 0.71)$ is input in the $\mathrm{BF}$ parameter of the $5^{*}$ array.

In calculating $\lambda_{t r}$, a transport cross section, $\Sigma_{t r}$, is determined from

$$
\Sigma_{t r}=\Sigma_{t}-\Sigma_{s 1}
$$

which varies as a function of energy group and zone. The $\Sigma_{s 1}$ term is the within-group term from the $P_{1}$ scattering matrix.

In the case of the 1-D cylinder, the procedure is the same as for the slab except that the buckling is determined from

$$
B^{2}=\left(\frac{\pi}{Y}\right)^{2}
$$

since only one transverse dimension is needed.

The diffusion coefficient in the leakage term is determined from

$$
D=\frac{1}{3 \Sigma_{t r}}
$$

Note that when comparing with codes or treatments using a fixed value of buckling for every group, a user can force this situation in XSDRNPM by inputting a zero value for BF and DZ and setting DY to determine the required buckling value.

\subsubsection{Void streaming correction}

In real slab and cylindrical geometries, void regions offer streaming paths that are nonexistent in the 1-D cases with quadratures that do not include a vertical angle. A correction for this effect has been suggested by Olsen, ${ }^{12}$ who uses an adjustment to the absorption cross section to account for the transverse leakage.

If one considers a slab of height $\mathrm{H}$, the void streaming correction is introduced through an adjustment to the total cross section and is given by

$$
\frac{\sqrt{1-\mu_{m}^{2}}}{H / 2}
$$

where $\mu_{m}$ is the cosine of the direction. 
In the case of a cylinder of height $\mathrm{H}$, the adjustment is

$$
\frac{\mu_{m}}{H / 2}
$$

These streaming corrections are very approximate and do not properly account for the fact that the streaming is enhanced near the ends of a void channel; however, they are probably better than the alternative, which is to make no correction at all.

\subsubsection{Cross-section weighting}

XSDRNPM weights cross sections according to the following four options:

1. "Cell” weighting,

2. “Zone” weighting,

3. "Region" or "vein" weighting, and

4. "Inner cell” weighting.

In all cases the "averaged" cross sections are defined in a manner that conserves reaction rates, that is,

$$
\bar{\sigma}_{G} \int_{\text {space }} d \vec{r} N_{D}(\vec{r}) \int_{G} d E \psi(E, \vec{r})=\int_{\text {space }} d \vec{r} N(\vec{r}) \int_{G} d E \sigma(E, \vec{r}) \psi(E, \vec{r})
$$

where

$$
\begin{aligned}
& \bar{\sigma}_{G} \equiv \text { average cross section in group } \mathrm{G} \\
& N_{D}(\vec{r}) \equiv \text { number density used in the definition for the weighting option selected, } \\
& \psi(E, \vec{r}) \equiv \text { weighting spectrum, } \\
& N(\vec{r}) \equiv \text { real number density as a function of spatial position, } \\
& \sigma(E, \vec{r}) \equiv \text { cross section in unreduced form. }
\end{aligned}
$$

If we convert to multigroup notation and use $\mathrm{W}$ for the weighting spectrum (instead of $\psi$ ), Eq. (9.1.48) becomes

$$
\bar{\sigma}_{G} \sum_{j}^{\substack{\text { applicable } \\ \text { spatial } \\ \text { regions }}} N_{D}^{j} \sum_{g \varepsilon G} W_{g}^{j}=\sum_{j}^{\substack{\text { applicable } \\ \text { spatial } \\ \text { regions }}} N^{j} \sum_{g \varepsilon G} \sigma_{g}^{j} W_{g}^{j},
$$


where

$$
W_{g}^{j} \equiv \int_{j} d \vec{r} \int_{g} d E \psi(E, \vec{r})
$$

\subsubsection{1 “Cell weighting"}

Cell weighting is consistent with homogenizing the cross sections in a heterogeneous cell. This is the recommended option to prepare cross sections for a real reactor calculation that will be made with a 2- or 3-D model of the reactor. Most of these codes have no provisions for explicitly representing individual fuel pins which are interspersed in a moderator region.

Cell-weighted cross sections are defined in a manner that attempts to preserve the reaction rates which occur in a representative cell from the reactor. In Eq. (9.1.49) the weighting involves the following substitution:

$$
N_{D}^{j} \equiv \bar{N}=\frac{\sum_{j}^{\text {cell }} V^{j} N^{j}}{\sum_{j}^{\text {cell }} V^{j}}
$$

where

$$
V^{j} \equiv \text { volume of zone } \mathrm{j} \text {. }
$$

\subsubsection{2 "Zone" weighting}

Zone weighting is the simplest of the three XSDRNPM weighting options. Each zone produces a unique set of cross sections which preserves reaction rates for the zone. In Eq. (9.1.49), the spatial sum is over the zone considered, and $N^{j}$ and $N_{D}^{j}$ are unity.

Zone weighting is used very frequently, especially for problems whose collapsed cross sections are to be used in a problem whose geometrical and material layout is similar to that in the weighting problem.

\subsubsection{3 "Region" weighting}

"Region-" or "vein-" weighted cross sections are weighted "where-the-nuclide-is." In most problems, there are nuclides of secondary importance which do not need a separate "zone-weighted" set for every region in which the nuclide occurs. Examples are the components of stainless steel. Stainless steel is encountered in a variety of locations and flux environments, but generally one set of cross sections for iron, chromium, manganese, nickel, etc., will suffice for most reactor calculations.

In Eq. (9.1.49), the spatial sum is over all zones which contain the nuclide of interest with

$$
N_{D}^{j}=N^{j}
$$

\subsubsection{4 “Inner-cell" weighting}

For inner-cell weighting, cell weighting is performed over specified innermost regions in the problem. Nuclides outside these regions are not weighted. 
This option is generally employed as follows: A "cell" is described in exactly the same manner as for cell weighting (Sect. 9.1.2.14.1) except that in this case it is surrounded by a homogeneous representation for the remainder of the core and by blankets, reflectors, etc. The flux calculation is made over this complete system, which should have a more realistic treatment of the leakage across the outer boundary of the interior cell. The cell weighting is subsequently made only over the interior cell.

\subsection{Multigroup weighting equations}

The following are the multigroup forms of the weighting equations used in XSDRNPM:

1. Cell weighting

$$
\bar{\sigma}_{G} \equiv \frac{\sum_{j}^{I Z M} N^{j} \sum_{g \varepsilon G} \sigma_{g}^{j} W_{g}^{j}}{\bar{N} \sum_{j}^{I Z M} \sum_{g \varepsilon G} W_{g}^{j}},
$$

where

$$
\begin{gathered}
\bar{N} \equiv \frac{\sum_{j}^{I Z M} V^{j} N^{j}}{\sum_{j}^{I Z M} V^{j}} \\
W_{g}^{j} \equiv \psi_{g}^{j}=\int_{j} \psi_{g}(\vec{r}) d \vec{r} .
\end{gathered}
$$

2. Zone weighting

$$
\bar{\sigma}_{G}^{j} \equiv \frac{\sum_{g \varepsilon G} \sigma_{g}^{j} W_{g}^{j}}{\sum_{g \varepsilon G} W_{G}^{j}} .
$$


3. Region weighting

$$
\bar{\sigma}_{G} \equiv \frac{\sum_{j} N^{j} \sum_{g \varepsilon G} \sigma_{g}^{j} W_{g}^{j}}{\sum_{j} N^{j} \sum_{g \varepsilon G} W_{g}^{j}}
$$

\subsection{Transfer matrices}

Collapsing transfer matrices is not quite so simple as collapsing cross sections with a single value per group. A group-to-group term in the broad group sense conserves the scattering rate from one group to the other, that is,

$$
\bar{N}^{*} \bar{\sigma}\left(G \rightarrow G^{\prime}\right) \psi_{G} \equiv \int_{\text {space }} d \vec{r} N(\vec{r}) \int_{g} d E \psi(E, \vec{r}) \int_{g^{\prime}} d E^{\prime} \sigma\left(E \rightarrow E^{\prime}\right)
$$

where the asterisk $(*)$ denotes that the number density on the left side of the equation is consistent with the weighting desired. Therefore, the multigroup forms of the weighting equations for components of the transfer matrices are as follows:

1. Cell weighting

$$
\bar{\sigma}_{G \rightarrow G^{\prime}} \equiv \frac{\sum_{j}^{I Z M} N^{j} \sum_{g \varepsilon G} W_{g}^{j} \sum_{g^{\prime} \varepsilon G^{\prime}} \sigma^{j}\left(g \rightarrow g^{\prime}\right)}{\bar{N} \sum_{j}^{I Z M} \sum_{g \varepsilon G} W_{g}^{j}} .
$$

2. Zone weighting

$$
\bar{\sigma}_{G \rightarrow G^{\prime}} \equiv \frac{\sum_{g \varepsilon G} W_{g}^{j} \sum_{g^{\prime} \varepsilon G^{\prime}} \sigma^{j}\left(g \rightarrow g^{\prime}\right)}{\sum_{g \varepsilon G} W_{g}^{j}}
$$

3. Region weighting

$$
\bar{\sigma}_{G \rightarrow G^{\prime}} \equiv \frac{\sum_{j} N^{j} \sum_{g \varepsilon G} W_{g}^{j} \sum_{g^{\prime} \varepsilon G^{\prime}} \sigma^{j}\left(g \rightarrow g^{\prime}\right)}{\sum_{j} N^{j} \sum_{g \varepsilon G} W_{g}^{j}} .
$$

Theoretically, the higher-than-zero order $\sigma_{l}\left(g \rightarrow g^{\prime}\right)$ should be weighted over $\psi_{l}$. Since these functions are generally positive-negative, $\psi_{l}$ weighting does not always work in practice, and XSDRNPM weights the $\sigma_{l}\left(g \rightarrow g^{\prime}\right), \ell>0$, by the scalar flux, which is positive. This procedure gives usable values for most cases. 


\subsection{Weighting of $\bar{v}$}

In weighting parameters such as $\bar{v}$, the average number of neutrons produced per fission, one is interested in preserving the fission source; therefore, the weighting is over $\sigma_{f} \psi$ instead of just $\psi$. The weighting procedure in XSDRNPM is to calculate $\left(\overline{v \sigma_{f}}\right)_{G}$ and $\left(\sigma_{f}\right)_{G}$ using the appropriate choice from Eqs. (9.1.59), (9.1.60), or (9.1.61). Then

$$
\bar{v}_{G}=\frac{\left(\overline{v \sigma_{F}}\right)_{G}}{\left(\sigma_{f}\right)_{G}} .
$$

\subsection{Transport cross sections}

Transport cross sections are not as directly related to the physical properties of a material as much as other group-averaged values. Instead of a reaction rate, these numbers must attempt to preserve a "flux gradient," which not only depends on the cross sections of the material, but is also very strongly influenced by the geometry and the other nuclides in the vicinity of a material.

Two options are provided in XSDRNPM to generate transport cross sections-options based on the "consistent" and "inconsistent" methods for solving the $P_{l}$ transport equations. These approximations are referred to as the "outscatter" and "inscatter" approximations because of the nature of the equations used.

\subsection{Outscatter approximation (inconsistent method)}

In the outscatter approximation, the assumption is made that

$$
\sigma_{t r}^{g}=\sigma_{t}^{g}-\bar{\mu}^{g} \sigma_{s}^{g}
$$

When one notes that

$$
\bar{\mu}^{g} \equiv \frac{\sigma_{1}^{g}}{3 \sigma_{0}^{g}}
$$

and that

$$
\sigma_{1}^{g}=\sum_{g^{\prime}} \sigma_{1}\left(g \rightarrow g^{\prime}\right)
$$

where the $\sigma_{l}\left(g \rightarrow g^{\prime}\right)$ terms are the $P_{1}$ coefficients of the scattering matrix, the origin of the term "outscatter" to designate the approximation is evident.

\subsection{Inscatter approximation (consistent method)}

In the "consistent" solution of the $P_{1}$ point transport equations, it can be shown that

$$
\sigma_{t r}(E)=\sigma_{t}(E)-\frac{1}{3 J(E)} \int_{0}^{\infty} d E^{\prime} \sigma_{1}\left(E^{\prime} \rightarrow E\right) J\left(E^{\prime}\right)
$$


where $J\left(E^{\prime}\right)$ is the current.

If one multiplies the equation by $\mathrm{J}(\mathrm{E})$, integrates over group $\mathrm{g}$, and converts to group-averaged form by dividing by $\int_{g} J(E) d E$, the following expression is derived:

$$
\sigma_{t r}^{g}=\sigma_{t}^{g}-\frac{1}{3 J_{g}} \sum_{g^{\prime}} \sigma_{1}\left(g^{\prime} \rightarrow g\right) J_{g^{\prime}}
$$

This is the "inscatter" approximation. It is consistent because the transport values are explicitly derived from the $P_{0}$ and $P_{1}$ equations. As a general rule, the transport values from this treatment are "better" than those from the "inconsistent" treatment. However, in some cases (notably hydrogen at lower energies), negative numbers may be calculated which are unusable and the more approximate approach must be used.

\subsection{Weighting function for transport cross section}

Unfortunately, the matter of choosing a current to use in the "transport" weighting is not simple. In real problems, currents are positive-negative as a function of energy and space. When cross sections are averaged over positive-negative functions, the "law-of-the-mean" no longer holds and the average value can be anything. This unbounded nature leads to real problems in diffusion calculations.

Approximations that inherently guarantee positive currents are generally used in other codes that circumvent the positive-negative problem. For example, in $B_{n}$ theory the current is given by

$$
j \sim B \psi,
$$

where $\mathrm{B}$ and $\psi$ are both positive.

In XSDRNPM, more direct routes that ensure positivity are taken (e.g., one might set $\mathrm{W}_{\mathrm{g}} \equiv\left|\mathrm{W}_{\mathrm{g}}\right|$ ). This is crudely supported by the following argument:

Consider a 1-D cylindrical calculation. In two dimensions, the current is a vector combination, that is,

$$
\vec{J}=\vec{J}_{r}+\vec{J}_{z}
$$

In XSDRNPM, the $\mathrm{z}$ direction is treated by using a buckling approximation, that is,

$$
\vec{J}_{z}=B \psi
$$

In the weighting calculation, we want to weight over the magnitude of the current. In XSDRNPM, the $\mathrm{z}$-current is imaginary, since we are not calculating a z-direction:

$$
\vec{J}=\vec{J}_{r}+\vec{i} B \psi
$$


The magnitude of a complex quantity is

$$
J=\frac{\left(\vec{J}_{r}+\vec{i} B \psi\right)\left(\vec{J}_{r}-\vec{i} B \psi\right)}{\sqrt{\left(\text { Value }_{\vec{r}}\right)^{2}+B^{2} \psi^{2}}}
$$

which is always positive.

In a discrete-ordinates calculation, the current is easily obtained since it is the first flux moment.

XSDRNPM has the following options for calculating the current:

1. $J_{g}=\sqrt{\left(\psi_{1}^{g}\right)^{2}+\left(D B \psi_{g}\right)^{2}}$

2. $J_{g}=\left|\psi_{1}^{g}\right|$

3. $J_{g}=D B^{2} \psi_{g}+\int_{0}^{1} d \mu \mu \psi\left(g, r_{\text {outside }}, \mu\right)$

4. $J_{g}=\frac{\psi_{0}^{g}}{\sum_{t}^{g}}$

5. $J_{g}=D B \psi_{g}$

The first option is the recommended option; option 2 treats only the current in the primary direction; option 3 will always be positive and is a weighting over the total leakage from the system. Option 4 is sometimes referred to as a "bootstrap" approximation; option 5 is equivalent to that used in codes that employ $B_{n}$ theory.

Once the currents are determined, the transport values are determined as set forth in the equations discussed above. For example, consider cell weighting and the "inscatter" approximation,

$$
\sigma_{t r}^{G}=\frac{\sum_{j} N^{j} \sum_{g \varepsilon G}\left\{J_{g} \sigma_{t}^{g}-\frac{1}{3} \sum_{g^{\prime}} \sigma_{1}\left(g^{\prime} \rightarrow g\right) J_{g}^{\prime}\right\}}{\bar{N} \sum_{j}^{I Z M} \sum_{g \varepsilon G} J_{g}} .
$$

For cell weighting and the "outscatter" approximation,

$$
\sigma_{t r}^{G}=\frac{\sum_{j} N^{j} \sum_{g \varepsilon G} J_{g}\left\{\sigma_{t}^{g}-\frac{1}{3} \sum_{g^{\prime}} \sigma_{1}\left(g^{\prime} \rightarrow g\right)\right\}}{\bar{N} \sum_{j}^{I Z M} \sum_{g \varepsilon G} J_{g}} .
$$




\subsubsection{Adjoint calculations}

XSDRNPM will, upon option, solve the adjoint forms of the 1-D transport equation.

Several special procedures apply for the adjoint calculation:

1. The iteration pattern discussed in Sect. 9.1.2.10 is reversed in energy. The scheme starts with the last (lowest energy) group and proceeds to the first group.

2. The angular quadrature is treated as if it has the reverse directions associated with the angle (e.g., many quadratures start with $\mu_{1}=-1.0$ ). In the adjoint case, this direction is for $\mu_{1}=+1.0$.

3. All edits of input fluxes and collapsed cross sections are given in their normal ordering, as opposed to many codes which require their reversal.

Adjoint calculations have many uses and advantages. As opposed to the forward calculation which yields particle density values, the adjoint fluxes are more abstract and can be thought of as particle importance.

Consider, for example, the problem of determining the response of a detector to particles as a function of their energy and direction. Assume the detector is a cylindrical fission chamber that utilizes a foil of ${ }^{235} \mathrm{U}$. The most obvious way to attack this problem is to mock up the detector and make a series of runs that contain sources of identical strength in different angles and energy groups. If an $S_{8}(24$ angles) quadrature were used with 50 energy groups, the $12 \times 50$ or 600 independent calculations could be used to completely determine the responses. (Here we have taken note that half of the angles will point away from a detector and, hence, produce no response.) The adjoint calculation produces all 600 responses in one run that is no more difficult and time consuming than the typical forward case. In the adjoint case, the detector response (i.e., the fission cross section of ${ }^{235} \mathrm{U}$ would be specified as a source in the foil region and the adjoint fluxes given as a function of energy and angle would be interpreted as the source of neutrons necessary to produce a response of the magnitude to which one required the response to be normalized.

A second important use of adjoint calculations is to establish good biasing factors for Monte Carlo codes. Two recent reports ${ }^{13,14}$ discuss the time and accuracy advantages of this approach for shielding and criticality applications and give some real examples as to how to make the calculations.

Perturbation theory uses adjoint and forward fluxes in combination in a manner that determines changes in responses that would arise from changing parameters used in a calculation. One ${ }^{15}$ interesting application is to determine the sensitivity of a calculation to changes in one or more cross-section value changes.

\subsection{Generalized adjoint calculations}

Generalized adjoint solutions are needed for generalized perturbation theory (GPT) applications such as sensitivity and uncertainty analysis. The generalized adjoint solution differs from both a conventional external source case and a fundamental mode eigenvalue calculation: It has the transport operator for an adjoint eigenvalue equation, but contains a fixed source term as well. The eigenvalue transport operator is singular, which forces certain restrictions on the allowable sources. The generalized adjoint source term is associated with a particular response ratio of interest in a critical system, such as 


$$
R=\frac{\sum_{g=1}^{I G M} \sum_{i=1}^{I M} H_{N}(g, i) \psi_{i, g} V_{i}}{\sum_{g=1}^{I G M} \sum_{i=1}^{I M} H_{D}(g, i) \psi_{i, g} V_{i}}
$$

where $\mathrm{H}_{\mathrm{N}}$ and $\mathrm{H}_{\mathrm{D}}$ are response functions defining the response of interest and $\psi_{i, g}$ is the scalar flux from a prior forward eigenvalue solution of the same problem. The generalized adjoint source for this response is defined as

$$
Q^{*}(g, i) \equiv \frac{1}{R} \frac{\partial R}{\partial \psi_{i, g}}=\frac{H_{N}(g, i)}{\sum_{g=1}^{I G M} \sum_{i=1}^{I M} H_{N}(g, i) \psi_{i, g} V_{i}}-\frac{H_{D}(g, i)}{\sum_{g=1}^{I G M} \sum_{i=1}^{I M} H_{D}(g, i) \psi_{i, g} V_{i}}
$$

The above source expression is computed automatically whenever XSDRN is executed in the TSUNAMI$1 \mathrm{D}$ sequence, but must it be computed and input by the user if XSDRN is run standalone for a generalized adjoint case.

In order to obtain a unique solution and avoid numerical problems, the generalized adjoint solution is "normalized" to contain no fundamental harmonic of the adjoint eigenvalue calculation. This is done by sweeping out the adjoint fundamental mode "contamination" from the fission source after each outer iteration, as described in the SAMS chapter, in Generalized Perturbation Theory. This operation requires both forward and adjoint eigenvalue solutions from prior XSDRN calculations. External files containing the fundamental mode forward and adjoint fluxes are input to the generalized adjoint calculation.

Unlike conventional fixed source and eigenvalue calculations, the generalized adjoint flux has both negative and positive components. This causes some XSDRN acceleration features such as spacedependent rebalance and group-banding to not function properly; and thus these are turned off internally. Typically the outer iterations for generalized adjoint solution converge much slower than an eigenvalue calculation. More background on GPT and generalized adjoint properties can be found in Ref. 16.

\subsubsection{Coupled neutron-photon calculations}

In XSDRNPM, it is possible to do a neutron or a photon calculation, depending only on whether the input libraries are for neutrons or gamma rays. It is also possible to do a "coupled neutron-photon" calculation which automatically determines the gamma-ray sources arising from neutron induced interactions in its photon calculation. This calculation, of course, requires an input cross-section library containing three classes of data:

1. neutron cross sections, including neutron-to-neutron transfer matrices,

2. photon production cross sections (i.e., neutron-to-gamma transfer matrices), and

3. gamma-ray cross sections, including gamma-ray-to-gamma-ray transfer matrices.

At present there are no provisions for treating neutrons produced from gamma interactions other than having the user introduce these sources by hand in a sort of iterative procedure, though this reaction is certainly not unknown (cf., deuterium, beryllium-9, and carbon-13). There are several cases where the $(\gamma, n)$ interaction can be important. If, for example, one looks at neutrons in a water-moderated pool 
reactor or in a water spent fuel storage tank at large distances from the fuel, the dominant source is from the neutrons produced by the deuterium in the water.

Normally the neutron-photon calculation requires no more input than a single particle run, except in the case where extraneous neutron and/or gamma-ray sources need to be specified. Most output edits will be split into a neutron and a gamma-ray part and will be labeled as such.

\subsubsection{Diffusion theory option}

XSDRNPM can make a 1-D diffusion theory calculation in user-specified energy groups (enter 1's for the appropriate groups of the $46 \$$ array). In this case, the $P_{1}$ diffusion equations ${ }^{17}$ are solved:

$$
\begin{aligned}
& A_{I+1} \psi_{1, I+1}-A_{I} \psi_{1, I}+\sigma_{0}\left(\psi_{0, I}+\psi_{0, I}\right)=S_{0}^{*} \\
& \bar{A}_{I}\left(\psi_{0, I+1}-\psi_{0, I}\right)+\sigma_{1}\left(\psi_{1, I+1}+\psi_{1, I}\right)=S_{1}^{*}
\end{aligned}
$$

where

$$
\begin{gathered}
\psi_{1} \equiv P_{1} \text { current } \\
\sigma_{0}=\left[\Sigma_{t}-\Sigma_{0}(g \rightarrow g)\right] \frac{V_{I}}{2.0} \\
\sigma_{1}=\left[3.0 \Sigma_{t}-\Sigma_{1}(g \rightarrow g)\right] \frac{V_{I}}{2.0}
\end{gathered}
$$

$S_{0}^{*}=P_{0}$ sources less the within-group term

$S_{1}^{*}=P_{1}$ sources less the within-group term

$$
\begin{gathered}
\bar{A}_{I}=\frac{A_{I}+A_{I+1}}{2} \\
V_{I}=\text { volume of Ith interval. }
\end{gathered}
$$

Solving Eq. (9.1.78) for $\psi_{0, I+1}$ and substituting into Eq. (9.1.79), one can write

$$
\psi_{1, I+1}=\frac{\bar{A}_{I} S_{0}^{*}-2 \sigma_{0} \bar{A}_{I} \psi_{0, I}-\sigma_{0} S_{1}^{*}+\psi_{1, I}\left(\sigma_{0} \sigma_{1}+\bar{A}_{I} A_{I}\right)}{\bar{A}_{I} A_{I+1}-\sigma_{0} \sigma_{1}} .
$$

Solving Eq. (9.1.79) for $\psi_{1, I}$ and substituting into Eq. (9.1.78), one can write

$$
\psi_{0, I+1}=\frac{A_{I+1} S_{1}^{*}-2 \sigma_{1} \bar{A}_{I} \psi_{1, I}-\sigma_{1} S_{0}^{*}+\psi_{0, I}\left(\sigma_{0} \sigma_{1}+\bar{A}_{I} A_{I+1}\right)}{\bar{A}_{I} A_{I+1}-\sigma_{0} \sigma_{1}} .
$$


If one assumes

$$
\begin{gathered}
\psi_{1, I+1}=P_{I+1} \psi_{0, I+1}-q_{I+1} \\
\psi_{1, I}=P_{I} \psi_{0, I}-q_{I}
\end{gathered}
$$

and plugs Eqs. (9.1.82) and (9.1.83) into (9.1.84), solving for $\psi_{1, I}$ yields:

$$
\begin{aligned}
& \psi_{1, I}=\frac{P_{I+1}\left(\sigma_{0} \sigma_{1}+\bar{A}_{I} A_{I+1}\right)+2 \sigma_{0} \bar{A}_{I}}{\sigma_{0} \sigma_{1}+\bar{A}_{I} A_{I}+P_{I+1} 2 \sigma_{1} \bar{A}_{I}} \psi_{0, I} \\
& -\frac{S_{0}^{*}\left(\bar{A}_{I}+\sigma_{1} P_{I+1}\right)-S_{1}^{*}\left(\sigma_{0}+A_{I+1} P_{I+1}\right)+q_{I+1}\left(\bar{A}_{I} A_{I+1}-\sigma_{0} \sigma_{1}\right)}{\sigma_{0} \sigma_{1}+\bar{A}_{I} A_{I}+P_{I+1} 2 \sigma_{1} \bar{A}_{I}},
\end{aligned}
$$

which by inspection and comparison with Eq. (9.1.85) gives expressions for $P_{I}$ and $q_{I}$.

Equations (9.1.84) and (9.1.85) can be substituted into Eq. (9.1.78) and solved for $\psi_{0, I+1}$ :

$$
\psi_{0, I+1}=\frac{\psi_{0, I}\left(A_{I} P_{I}-\sigma_{0}\right)+A_{I+1} q_{I+1}-A_{I} q_{I}+S_{0}^{*}}{A_{I+1} P_{I+1}+\sigma_{0}},
$$

which is the expression used in XSDRNPM. The procedure solves for arrays of $P_{I}$ and $q_{I}$ which are plugged back into the above expression to yield the fluxes.

\subsubsection{Infinite-medium theory option}

It is possible to force the flux calculation in XSDRNPM to use an infinite medium option for any or all energy groups by entering 2's in the appropriate positions in the $46 \$$ array. When a multiregion calculation is requested, the program will first determine spatially averaged cross sections to use in the infinite-medium expression and then place the infinite-medium flux in all spatial regions for use in any subsequent calculations, such as cross-section weighting. All higher flux moments are set to zero.

The balance expression is

$$
\left[\Sigma_{t}^{g}-\Sigma(g \rightarrow g)\right] \psi_{g}=\frac{1}{k} F_{g}+S_{g}
$$

where $F_{g}$ is the fission source in group $\mathrm{g}, S_{g}$ is the sum of any fixed source and inscattering source, and $\sum_{t}^{g}$ and $\Sigma(\mathrm{g} \rightarrow \mathrm{g})$ are homogenized total and group-to-group scattering cross sections.

\subsubsection{9 $\quad B_{\mathrm{N}}$ theory option}

XSDRNPM can make a $B_{N}$ calculation in user-specified energy groups (enter 3's for the appropriate groups of the $46 \$$ array). As in the infinite-medium option discussed in Sect. 9.1.2.18, cross sections in a multiregion system are not homogenized. 
The $B_{N}$ equations ${ }^{18}$ can be written

$$
\begin{gathered}
\frac{l+1}{2 l+1} i B \psi_{l+1}+\frac{l}{2 l+1} i B \psi_{l-1}+\Sigma_{t} \psi_{l}=S(u) \delta_{l}^{0} \\
+\int d u^{\prime} \Sigma_{s}^{l}\left(u^{\prime} \rightarrow u\right) \psi_{l}\left(u^{\prime}\right) \quad l=0,1, \ldots, N-1 \\
\frac{N}{2 N+1} i B \psi_{N-1}+\gamma \Sigma_{t} \psi_{N}=\int d u^{\prime} \Sigma_{g}^{N}\left(u^{\prime} \rightarrow u\right) \psi_{N}\left(u^{\prime}\right) \\
\psi_{-1}=0 \\
\gamma=1+\frac{N+1}{2 N+1} \frac{i B}{\Sigma_{T}} \frac{Q_{N+1}\left(-\Sigma_{t} / i B\right)}{Q_{N}\left(-\Sigma_{t} / i B\right)}
\end{gathered}
$$

where $\delta_{l}^{0}$ is the Kronecker delta function and $Q_{N}$ is a Legendre polynomial of the second kind. In mutigroup form, the above expressions become:

$$
\begin{gathered}
\frac{l+1}{2 l+1} i B \psi_{l+1}^{g}+\frac{l}{2 l+1} i B \psi_{l-1}^{g}+\Sigma_{t}^{g} \psi_{l}^{g}=S_{g} \delta_{l}^{0} \\
+\sum_{g^{\prime}} \Sigma_{l}\left(g^{\prime} \rightarrow g\right) \psi_{l}^{g} \quad l=0,1, \ldots N-1 \\
\frac{N}{2 N+1} i B \psi_{N-1}^{g}+\gamma \Sigma_{t}^{g} \psi_{N}^{g}=\sum_{g^{\prime}} \Sigma_{N}\left(g^{\prime} \rightarrow g\right) \psi_{N}^{g} \\
\psi_{-1}^{g}=0 .
\end{gathered}
$$

In Eqs. (9.1.89) and (9.1.93), the $\mathrm{S}$ term includes fission, fixed, and scattering source components 


\subsubsection{XSDRNPM Input Data}

The input data to XSDRNPM consist of a title card and up to five data blocks, depending on the particular problem. All data in these blocks are entered using the FIDO formats discussed in the chapter on FIDO.

In the description that follows, the quantity in square brackets is the number of items in an array. The quantity in braces is the condition which requires the array to be input. If no condition is specified, an array must be input. Default parameters that are used if an array is not input are shown in parentheses if nonzero.

$* * * * * * * * * * * * * * * * * * * * * * * * * * * * * * * * * * * * * * * * * * * * * * * * * * * * * * * * * * * * * * * * * * *$

Title Card - Format (20A4)

This is the title card for the problem. It will be used to label the problem output.

\section{Data Block 1}

This block contains information to set up various array dimensions and most calculational and editing options. Various convergence criteria and special constants can be input.

0\$\$ Logical Assignments [17]

1. LPUN - Logical number for punched card output (7).

2. LRSF - Random-access scratch for fluxes (10).

3. LAWL - Input AMPX working library (4).

4. LANC - ANISN binary or CCCC ISOTXS library (20).

5. LOWL - Output weighted library (3).

6. LANG - Angular flux scratch file (16).

7. LSFF - Scalar flux output file (17).

8. LSF2 - Sequential scratch space (18).

9. LSF3 - Sequential scratch space (19).

10. LRSM - Random-access scratch for macroscopic cross sections (8).

11. LRSX - Random-access scratch for macroscopic cross sections (9).

12. LACF - Activities output file (75). 
13. LBTF - Balance table output file (76).

14. LIDF - Input dump file (73).

15. LSEN - Sensitivity output file (6).

16. LEXT - Not used (0).

17. LISF - Scalar flux input guess file (0).

1\$ General Problem Description [15]

1. IGE - problem geometry (1)

0 - homogeneous (This causes a $B_{N}$ calculation to be made for all zones - Sect. 9.1.2.19.)

$1-$ slab

2 - cylinder

3 - sphere

2. IZM - number of separate material regions or zones. (1)

3. $\mathrm{IM}-\quad$ number of spatial intervals in the problem. (1)

4. IBL - the boundary condition at the left-hand boundary of the system. (1)

0 - vacuum boundary

1 - reflected boundary

2 - periodic boundary

3 - white/albedo boundary

Boundary conditions are discussed in Sect. 9.1.2.6.

5. IBR - the boundary condition at the right-hand boundary of the system. (1)

0 - vacuum boundary

1 - reflected boundary

2 - periodic boundary

3 - white/albedo boundary

6. MXX - the number of compositions used in the problem mock-up.

7. MS - the number of entries in the mixing table which specifies the makeup of the MXX compositions. 
8. ISN - the order of angular quadrature to be used. If ISN $>0$, XSDRNPM will calculate an angular quadrature for the appropriate geometry. If ISN $<0$, the calculation is bypassed, and the user must supply a set in the $42 \#$ and $43 \#$ arrays.

9. ISCT - the order of scattering. Flux moments will be calculated through this order.

10. IEVT - the type of calculation. (1)

0 - fixed source

1 - $\mathrm{k}$ calculation

$2-\alpha$ calculation (flux is assumed to have an $e^{-\alpha t}$ time variation)

3 - inoperable in present version

4 - zone width search

5 - outer radius search

6 - buckling search

7 - direct buckling search

11. IIM - the inner iteration maximum used in an $S_{n}$ calculation. (10)

12. ICM - the outer iteration maximum. (10)

After ICM outer iterations, the problem will be forced into the termination phase and the program will continue as if full convergence was attained. A message to this effect is printed.

13. ICLC - theory option. (0)

$0-\quad$ use $S_{n}$ theory always

$\mathrm{N}$ - $\quad$ use alternative theory (diffusion, infinite medium, or $B_{n}$ ) for $\mathrm{N}$ outer iterations, after which revert back to $S_{n}$ theory.

$-\mathrm{N}-\quad$ always use alternative theory

14. ITH - forward/adjoint selector. (0)

0 - solve the forward Boltzmann equation.

1 - solve the adjoint Boltzmann equation.

15. IFLU - Generalized adjoint calculation flag. (0)

0 - standard calculation

1 - Generalized adjoint calculation. Requires input forward and adjoint fundamental mode fluxes on units 31 and 32 , respectively 
$2 \$$ Editing and Special Options [10]

1. IPRT - fine-group mixture cross-section edits. $(-1)$

$-2-\quad$ no edits

$-1-\quad$ edit $1-\mathrm{D}$ cross sections (See Appendix F3.B)

$0-\mathrm{N}_{-} \quad$ edit through $P_{N}$ cross sections.

1 -D edits are made, also.

2. ID1 - flux editing options. (0)

\begin{tabular}{rccc}
\hline ID1 & $\begin{array}{c}\text { Print } \\
\text { Angular fluxes }\end{array}$ & $\begin{array}{c}\text { Print } \\
\text { Scalar fluxes }\end{array}$ & $\begin{array}{c}\text { Punch }^{a} \\
\text { Scalar fluxes }\end{array}$ \\
\hline-1 & No & No & No \\
0 & No & Yes & No \\
1 & Yes & Yes & No \\
2 & No & Yes & Yes \\
3 & Yes & Yes & Yes \\
\hline
\end{tabular}

${ }^{a}$ The fluxes will be punched in a format suitable for restarting an XSDRNPM calculation.

3. IPBT - balance table edits. (0)

$-1-\quad$ none

0 - make fine-group balance tables

1 - make fine- and broad-group balance tables

4. ISX - broad-group flux edit as a function of interval. (0) $(0 / 1=$ no/yes $)$

5. ISEN - outer iteration acceleration. Input a zero. (0)

6. IBLN - control number of outer iteration groups. (0)

7. NBANDS - number of flux rebalance bands. (0) $<0$, then this is the number of bands in the thermal range.

8. IFSN - If $>0$ means no fission source if IEVT $=0$. (0)

9. ISQ3 - sequence number of file opened on unit LOWL. (1)

10. IDM4 - not used. (0)

The structure of the "activity" and the "balance table" files are described in Appendix F3.A. 
$3 \$$ Various Options [12]

1. IFG - cross-section weighting. (0)

$0-$ none required

1 - collapse cross sections

2. IQM - volumetric sources. (0)

$0-$ none

$\mathrm{N}-\mathrm{N}$ volumetric source spectra will be input in the $31^{*}$ array

3. IPM - boundary sources. (0)

$0-$ none

$\mathrm{N}-\mathrm{N}$ boundary source spectra will be input in the $32^{*}$ array

4. IFN - starting guess. (0)

0 - flux guess (33\# array)

1 - fission density guess (34\# array)

5. ITMX - maximum time allowed for the flux calculation in minutes. A value of zero specifies that the calculation should not be terminated because of time; otherwise the problem will be forced into the termination phase when ITMX is exceeded. (0) Bear in mind that this is an internal timing check and has no connection with operator or system terminations due to excessive times.

6. IDAT1 - external data storage. (0)

0 - $\quad$ keep all arrays in core if possible

1 - $\quad$ store mixture cross sections externally on a direct access device

2 - store cross sections and fixed sources externally on direct access devices

7. IPN - diffusion coefficient option for transverse leakage corrections. (3)

0 - determine a transport cross section for each zone using $P_{0}$ and $P_{1}$ cross sections and, hence, a diffusion coefficient from $1 / 3 \Sigma_{t r}$.

1 - $\quad$ spatially average the diffusion coefficients determined as for the above option and use it for all zones.

2 - spatially average the transport cross sections for all zones and determine a diffusion coefficient to be used in all zones by taking one over three times this value.

3 - flux weight the transport cross sections for all zones and determine a diffusion coefficient to be used in all zones by taking one over three times this value.

Normally, the first option (IPN $=0$ ) is adequate; however, in cases involving regions of low concentration (near void) and, hence, very low transport cross sections, the very large diffusion coefficients lead to nonphysical behavior. In this case, the IPN $=3$ option has been demonstrated to operate the best. 
8. IDFM - density factors. (0)

$0-$ none

$1-\operatorname{read}$ in density factors in the $38^{*}$ array

9. $\mathrm{IAZ}-\quad$ activity calculation trigger. (0)

$0-$ none

$\mathrm{N}-$ calculate the reaction rates by material zone for $\mathrm{N}$ different processes specified in the $49 \$$ and $50 \$$ arrays

10. IAI - spatially dependent activity rates. (0)

$0-$ none

1 - calculate reaction rates in each interval for IAZ processes

11. IFCT - thermal upscatter scaling. (0)

$0-$ none

1 - use upscatter scaling for accelerated problem convergence

12. IPVT - parametric eigenvalue search. (0)

$0-$ none

1 - a search calculation will be made for an eigenvalue equal to PV

2 - an $\alpha$ loss term with $\alpha=\mathrm{PV}$ will be added to the transport equation. The term $\alpha$ will depend on the IEVT option selected

4 Cross-Section Weighting Options [9] $\{\mathrm{IFG}=1\}$

NOTE: Currently XSDRNPM does not support outputting weighted cross section libraries in a format other than AMPX. The following items are still allowed to be entered into XSDRNPM input files for legacy inputs, but anything not relating to AMPX will have no effect.

1. $\quad$ ICON - type of weighting. (See Sect. 9.1.2.14.)

$-\mathrm{N}-$ inner cell (with $\mathrm{N}$ zones in the cell). Cell weighting is performed over the $\mathrm{N}$ innermost regions in the problem. Nuclides outside these regions are not weighted.

$$
\begin{aligned}
& -1 \text { - cell } \\
& 0 \text { - zone } \\
& 1 \text { - region or vein }
\end{aligned}
$$

2. IGMF - number of energy groups in the collapsed sets.

3. ITP - collapsed output format desired. 
0-19 - cross sections are written only in the AMPX weighted library formats on logical 3. A weighted library is always written when $\mathrm{IFG}=1$.

20-29 - (deprecated feature).

30-39 - (deprecated feature).

40-49- (deprecated feature).

The various values of ITP (modulo 10) are used to select the different transport cross-section weighting options mentioned earlier. The options are:

$\mathrm{ITP}=0 \Rightarrow \sqrt{\left(\psi_{1}^{g}+\left(D G \psi_{g}\right)\right)^{2}}$

ITP $=1 \quad \Rightarrow$ absolute value of current

$\mathrm{ITP}=2 \quad=>\mathrm{DB}^{2} \psi_{\mathrm{g}}+$ outside leakage

$\mathrm{ITP}=3 \quad \Rightarrow \psi / / \sum_{\mathrm{t}}^{\mathrm{g}}$

$\mathrm{ITP}=4, \quad \Rightarrow \mathrm{DB} \psi_{\mathrm{g}}$

ITP $=$ other values are reserved for future development and should not be used.

4. IPP - weighted cross-section edit option -1 .

$-2-$ none

$-1 \quad$ - edit 1-D data

$0-\mathrm{N}$ - edit through $P_{N}$ cross-section arrays.

1 -D edits are given.

5. IHTF - (deprecated feature)

6. NDSF - (deprecated feature)

7. NUSF - (deprecated feature)

8. IAP - (deprecated feature)

$-1-$

9. (deprecated feature)

5* Convergence Criteria and Assorted Constants [12]

1. $\quad$ EPS - overall problem convergence. $\left(10^{-4}\right)$

2. PTC - scalar flux convergence. $\left(10^{-5}\right)$ 
3. $\mathrm{XNF}-$ normalization factor. (1.0)

When IEVT $=0$, the fixed sources are normalized to XNF.

For IEVT $>0$, the fission source is normalized to XNF.

When $\mathrm{XNF}=0.0$, no normalization is made.

$\mathrm{XNF}$ should only be specified as 0 for a fixed source problem $(\mathrm{IEVT}=0)$.

4. $\quad \mathrm{EV}-\quad$ starting eigenvalue guess for search calculations.

5. EVM - eigenvalue modifier used in a search calculation. The following is a tabulation of recommended values for EV and EVM.

\begin{tabular}{cccc}
\hline IEVT & Calculation type & EV & EVM \\
\hline 0 & Fixed source & 0 & 0 \\
1 & k-calculation & 0 & 0 \\
2 & Direct $\alpha$-search & 0 & 0 \\
3 & -- & -- & -- \\
4 & Zone width search & 0 & -0.1 \\
5 & Outer radius search & Starting outer radius & $-0.1 * \mathrm{EV}$ \\
6 & Buckling search & 1.0 & -0.1 \\
7 & Direct buckling search & 0.0 & 0.0 \\
\hline
\end{tabular}

6. $\mathrm{BF}-$ buckling factor (1.420892). This parameter is two times the multiplier on the "extrapolation" distance used to determine where a linearly extrapolated line from the asymptotic flux shape would go to zero (e.g., for slabs, the extrapolation distance is $\simeq 0.71 \lambda_{t r}$ and, hence, $\mathrm{BF} \simeq 1.42$ ).

7. DY - first transverse dimension in centimeters used in a buckling correction to calculate leakage normal to the principal calculation direction (i.e., the height of a slab or a cylinder).

8. DZ - second transverse dimension in centimeters used for a buckling correction (i.e., the width of a slab).

9. VSC - void streaming correction. This is the height of a void streaming path in a cylinder or slab in centimeters. See Sect. 9.1.2.13.

10. PV - parametric eigenvalue or value for $\alpha$ used when IPVT $>0$. When IPVT $=1$ and IEVT $>1$, this is the value of k-effective on which the search calculation is to be made $(0.0)$

11. EQL - eigenvalue convergence for a search. $\left(10^{-3}\right)$

12. XNPM - new parameter modifier used in search calculations. (0.75)

$\mathrm{T}$ Terminate data block. 


\section{Data Block 2}

This block contains information on the composition of the materials used in the calculation. Also included is an array to select special cross sections for ANISN and an array to identify cross sections written on the CCCC ISOTXS library. NOTE: Currently XSDRNPM does not support outputting weighted cross section libraries in any format other than AMPX. XSDRNPM will still read the deprecated arrays, but they will have no effect.

$10 \$ \quad$ (deprecated array)

$12 \$$ (deprecated array)

$11 \$$ Composition Numbers in Mixing Table [MS]

If the input multigroup library was previously self-shielded by running the XSProc module, the composition numbers are the mix numbers given in the READ COMP block of XSProc. If the input library has not been previously self-shielded, enter all zeros. [Note: this is a new array added in Scale-6.2 which MUST be present]

$13 \$ \quad$ Local Mixture Numbers in Mixing Table [MS]

The values range from 1 to MXX, and are used only in XSDRN for referencing mixtures in the mixing table.

$14 \$$ Isotope Identifiers in Mixing Table [MS]

A set of data with this identification must be on unit LAWL, the XSDRNPM working library, though the code will not make checks to ensure this is the case.

15* Isotope Concentrations in Mixing Table [MS]

$16 \$$ (deprecated array)

$18 \mathrm{U} \quad$ (deprecated array)

$\mathrm{T}$ Terminate this data block.

\section{Data Block $3\{$ IEVT $=0\}$}

This block is used to specify fixed sources.

30\$ Source Spectrum Number by Interval [IM] 
31* Volumetric Source Spectra [IQM*IGM]

32* Surface Source Spectra [IPM*IGM*MM]

Each of the IQM or IPM spectra is specified in the $31 *$ or $32 *$ array and are stacked one after the other in that array. If both volumetric and surface sources are used in the same problem, the surface source number is multiplied by $(\mathrm{IQM}+1)$ when entered in the $30 \$$ array.

A volumetric spectrum will consist of IGM (number of energy groups) entries which are the relative integrated values of the source in each group.

A surface source is always assumed to be on the right-hand side of a spatial interval. It is input as was the volumetric source, except that each group contains entries for the MM angles in the $S_{n}$ quadrature chosen for the problem. Note that a surface source is an integrated value and is actually a flux condition in the $S_{n}$ equations.

In the $30 \$$ array, a zero entry specifies that no source is in an interval.

$\mathrm{T}$ Terminate this data block.

\section{$\underline{\text { Data Block } 4}$}

This data block contains starting guesses for fluxes and fission densities. If fluxes are read from an external device (LISF $>0$ ), this data block is omitted. Both arrays in this block are double-precision arrays, which will require the use of the "\#” array designator; otherwise the number of entries read into the arrays will be incorrect or may contain nonsensical values for the starting guess.

\section{3\# Flux Guess [IM*IGM] $\{\mathrm{IFN}=0\}$}

A guess for the scalar flux is specified in the order: ((FLUX(I,J),I=1,IM),J=1,IGM), where IM is the number of spatial intervals and IGM is the total number of energy groups. For fixed-source problems, without better information, use zeroes. For eigenvalue problems, a nonzero flux guess must be used. The fluxes punched by using the ID1 parameter in the $2 \$$ array can be used here in restart calculations.

34\# Fission Density Guess [IM] $\{\mathrm{IFN}=1\}$

This is a guess at the number of fission neutrons produced in an interval. When IFN $=1$, XSDRNPM uses diffusion theory for the first outer iteration, after which it reverts to the normal mode.

$\mathrm{T}$ Terminate this data block.

\section{Data Block 5}

This block contains the remaining data needed for an XSDRNPM calculation. 
$35 *$ Interval Boundaries $[\mathrm{IM}+1](\mathrm{cm})$

This array describes the spatial quadrature into which the problem model is divided. The boundaries are nonnegative for curvilinear geometries and in increasing order. Usually they will start with a zero value, though this is not necessary. The origin for slab geometry may be negative.

36\$ Zone Number for Each Spatial Interval [IM]

Spatial zones should be contiguous.

38* Density Factors by Interval [IM] $\{$ IDFM $>0\}(1.0)$

These factors are used to effect a density variation in a mixture as a function of spatial interval. Zero for a density factor affords a convenient way for modeling a void region.

39\$ Mixture Numbers by Zone [IZM]

The mixture that is in a zone is specified here.

$40 \$$ Order of Scattering by Zone [IZM] (ISCT)

This is the order, $l$, of the $S_{n} P_{l}$ calculation which is desired in a zone. This number should be no larger than ISCT.

41* Radius Modifiers by Zone [IZM] $\{$ IEVT=4\}

These parameters specify the relative movement of the width of a zone in a zone width search. A zero indicates that a zone's width is fixed. (See Sect. 9.1.2.8.)

42\# Weights of the Angles in the Discrete-Ordinates Quadrature [ $\left.\mathrm{MM}^{*}\right]$

Input this set if you wish to override those provided by XSDRNPM. See Sects. 9.1.2.4.3, 9.1.2.4.4, or 9.1.2.4.5.

43\# Cosines of the Angles in the Discrete-Ordinates Quadrature [MM]

Input this set if you wish to override those provided by XSDRNPM. See Sects. 9.1.2.4.3, 9.1.2.4.4, or 9.1.2.4.5.

46\$ Calculational Option by Group [IGM] $\{$ ICLC $>0\}$

0 - perform discrete-ordinates calculation for this group.

$\begin{aligned}{ }^{*} \mathrm{MM} & =\mathrm{ISN}+1 \text { for slabs and spheres, } \\ & =\mathrm{ISN} *(\mathrm{ISN}+4) / 4 \text { for a cylinder. }\end{aligned}$ 
1 - perform a diffusion calculation for this group for ICLC outer iterations; use discrete-ordinates theory after this.

2 - perform a homogeneous calculation for this group for ICLC outer iterations; then revert back to discrete-ordinates theory.

3 - perform a homogeneous calculation using $B_{n}$ theory for this group.

47* Right-Boundary Albedos by Group [IGM] $\{\mathrm{IBR}=3\}(1.0)$

A right-boundary albedo is specified for each fine group. The return current is distributed isotropically in angle.

48* Left-Boundary Albedos by Group [IGM] $\{\mathrm{IBL}=3\}(1.0)$

As for the $47^{*}$ array but for the left boundary. Note that if IBR or IBL is 3 and the corresponding $47^{*}$ or $48^{*}$ array is omitted, XSDRNPM fills the array with 1.0's effecting a boundary with zero net current and with isotropic neutron return.

49\$ Material Number for Activities [IAZ] $\{$ IAZ $>0\}$

$50 \$$ Process Number for Activities [IAZ] $\{\mathrm{IAZ}>0\}$

The $49 \$$ and $50 \$$ arrays provide a means of obtaining the activity (reaction rate) for any process for which cross sections are available in the XSDRNPM calculation. A representative activity table entry is shown below:

\begin{tabular}{cc}
\hline ACTIVITY TABLE ENTRY \\
\hline $49 \$$ & $50 \$$ \\
$\mathrm{M}$ & $\mathrm{N}$ \\
\hline
\end{tabular}

This entry specifies that the activity $\mathrm{N}$ for material $\mathrm{M}$ be calculated for all parts of the system which contain that material.

If $\mathrm{N}$ is $<0$, a density of 1.0 is used to calculate activities instead of densities in the mixing table. Allowable process identifiers are given in Appendix M4.B.

If $\mathrm{M}$ is $<0$, the activities by interval will be multiplied by a 1-D area as follows:

$$
\begin{aligned}
& 1.0 \text { for a slab }(\mathrm{IGE}=1) \\
& 2 \times \mathrm{r} \text { for a cylinder }(\mathrm{IGE}=2) \\
& 4 \times \mathrm{r}^{2} \text { for a sphere }(\mathrm{IGE}=3)
\end{aligned}
$$


51\$ Broad-Group Numbers [IGM] $\{$ IFG $>0\}$

This array contains the broad-group numbers into which the fine groups are collapsed in a flux-weighting calculation. For example, if the first five fine groups are to be collapsed to the first broad group, the first five entries in the $51 \$$ array are 1, etc. A zero value can be used to ignore (or truncate) a group.

52\$ Lower Band Group Numbers [NBANDS]

Group numbers giving the last group in a flux rebalance band. Overrides the default set supplied by XSDRNPM.

\subsubsection{Abbreviated XSDRNPM input description}

After several problem setups have been made for XSDRNPM, the user will probably establish a familiarity, such that the more detailed notes given above are not necessary or desirable. Because of this, the following much abbreviated list is given which will, hopefully, benefit many users.

Title Card - (18A4)

\section{DATA BLOCK 1}

$-1 \$$ - Storage Assignment

1. Maximum Length of Storage Array

0\$- Logical Assignments [17]

1. Punched Cards (7)

2. Direct Access - Fluxes (10)

3. Working Library (4)

4. ANISN or ISOTXS (20)

5. Weighted Library (3)

6. Angular Fluxes (16)

7. Scalar Fluxes (17)

8. Scratch (18)

9. Scratch (19)

10. Direct Access (8)

11. Direct Access (9)

12. Activities (75)

13. Balances (76)

14. Input Dump (73)

15. Sensitivity Data (0)

16. Not Used (0)

17. Flux Guess (0)
$1 \$$ - General Description [15]

1. IGE - geometry

2. IZM - number of zones

3. IM - number of intervals

4. IBL - left boundary condition

5. IBR - right boundary condition

6. MXX - number of mixtures

7. MS - mixing table length

8. ISN - angular quadrature

9. ISCT - order of scattering

10. IEVT - problem type

11. IIM - inner iteration maximum

12. ICM - outer iteration maximum

13. ICLC - optional theory

14. ITH - forward or adjoint

15. IFLU - GPT calculation flag 
DATA BLOCK 1 (continued)

$2 \$$ - Editing and Control Options [10]

1. Fine-Group-Mixture Edit

2. Fine-Group-Flux Edit

3. Balance Table Edit

4. Broad-Group-Flux Edit

5. Not Used

6. Outers-Group-Limit-Option

7. Number of Bands

8. Suppress Fixed-Source Fission

9. Not Used

10. Not Used
$3 \$$ - Other Options [12]

1. IFG - Weighting Option

2. IQM - Volumetric Sources

3. IPM - Boundary Sources

4. IFN - Starting Guess

5. ITMX - Time Shut-off

6. IDAT1 - Storage Scheme

7. IPN - Diff. Coeff. Option

8. IDFM - Density Factors

9. IAZ - Activities by Zone

10. IAI - Activities by Interval

11. IFCT - Thermal Scaling

12. IPVT - Search on $\mathrm{k} \neq 1$
4\$ - Weighting Options [9]
5* - Floating Point Values [12]
1. Type of weighting
1. EPS - overall convergence
2. Number of broad groups
2. PTC - Point flux convergence
3. Output format
3. XNF - normalization
4. Edit option
5. $\sigma_{\mathrm{T}}$ position or number CCCC
4. EV - starting guess for search
5. EVM - modifier for search
6. $\sigma_{\mathrm{gg}}$ position
6. BF - buckling factor
7. Table length
7. DY - height
8. ANISN edit option
8. $\mathrm{DZ}$ - width
9. Extra cross sections
9. VSC - void streaming height
10. $\mathrm{PV}-\mathrm{k}$ for search
11. EQL - search convergence
12. XNPM - search modifier
$\mathrm{T}$ Terminate Data Block 1 
DATA BLOCK 2

$10 \$$ CCCC Transport Cross Section Selector [IHTF]

$12 \$$ Additional Processes to be put on ANISN Library [MSCM]

$13 \$$ Mixture Numbers [MS]

$14 \$$ Isotope Identifiers [MS]

$15^{*}$ Isotope Concentrations [MS]

$16 \$$ CCCC Identifiers from Working Library [IHTF]

18U or 18\# CCCC Identifiers on ISOTXS [IHTF]

T Terminate the second Data Block.

\section{DATA BLOCK 3}

\{Required only when IQM or IPM is nonzero.\}

$30 \$$ Spectrum Number by Interval (IQM $>0$ ) or

Right-Hand Interval Boundary (IPM $>0$ ) [IM]

31* Volumetric Sources [IQM*IGM]

32* Boundary Sources [IPM*IGM*MM]

$\mathrm{T}$ Terminate the third Data Block.

\section{DATA BLOCK 4}

\{When fluxes are read from an external device-IFN $>3$-this block is omitted.\}

33\# Flux Guess [IM*IGM] $\{$ IFN $=0\}$

34\# Fission Density Guess [IM] $\{\mathrm{IFN}=1\}$

$\mathrm{T}$ Terminate the fourth Data Block. 
Title Card - (18A4)

\section{DATA BLOCK 5}

35* Interval Boundaries [IM + 1]

36\$ Zone Numbers by Interval [IM]

38* Density Factors [IM]

39\$ Mixture Number by Zone [IZM]

$40 \$$ Order of Scattering by Zone [IZM]

$41^{*}$ Radius Modifier by Zone [IZM] $\{$ IEVT $=4\}$

42\# Discrete-Ordinates Cosines [MM]

43\# Discrete-Ordinates Weights [MM]

$46 \$$ Alternative Theory Selection [IGM] $\{$ ICLC $>0\}$

47* Right-Boundary Albedos [IGM]

48* Left-Boundary Albedos [IGM]

49\$ Activity Material or Nuclide Numbers [IAZ]

50\$ Activity Process Numbers [IAZ] (See Appendix B)

$51 \$$ Broad-Group Numbers [IGM] $\{$ IFG $>0\}$

$52 \$$ Lower-Band-Group Numbers [NBANDS]

T Terminate the fifth Data Block.

\subsubsection{XSDRNPM Input/Output Assignments}

The following logical units can be required in an XSDRNPM calculation.

$\begin{array}{cl}\begin{array}{c}\text { Default } \\ \text { Logical Unit }\end{array} & \\ 3 & \text { Weighted Library (Produced by XSDRNPM) } \\ 4 & \text { Working Library (Input) } \\ 5 & \text { Card Input } \\ 6 & \text { Standard Output } \\ 7 & \text { Punch Fluxes or ANISN Libraries } \\ 8 & \text { Scratch Direct-Access Device for External Cross-Section Storage } \\ 9 & \text { Scratch Direct-Access Device for Mixing and Weighting Operations } \\ 10 & \text { Scratch Direct-Access Device for External Flux Moment Storage }\end{array}$




$\begin{array}{ll}16 & \text { Angular Fluxes } \\ 17 & \text { Scalar Fluxes } \\ 18 & \text { Scratch Device } \\ 19 & \text { Scratch Device } \\ 20 & \text { ANISN Binary Libraries or CCCC ISOTXS Interface } \\ 31 & \text { Fundamental mode forward angular flux input unit for generalized adjoint } \\ & \text { calculation (iflu>0) } \\ 32 & \text { Fundamental mode adjoint angular flux input unit for generalized adjoint } \\ 73 & \text { calculation (iflu>0) } \\ 75 & \text { Dump of Input and Derived Data } \\ 76 & \text { Activities }\end{array}$

\subsubsection{XSDRN Sample Problem}

In this section, the input and output for a sample case involving a bare, homogeneous 16-cm sphere of a 93\% enriched $\mathrm{UO}_{2}-\mathrm{F}_{2}$ solution is presented. The input AMPX working format cross-section library will be read from logical unit 4.

The input working library is a temporary 238-group library created by CSAS-mg containing the following nuclides:

\begin{tabular}{cc}
\hline Nuclide & Identifier $^{a}$ \\
\hline${ }^{235} \mathrm{U}_{92}$ & 92235 \\
${ }^{238} \mathrm{U}_{92}$ & 92238 \\
${ }^{1} \mathrm{H}$ & $1001^{b}$ \\
${ }^{16} \mathrm{O}_{8}$ & 8016 \\
${ }^{19} \mathrm{~F}_{9}$ & 9019 \\
\hline${ }^{a}$ These are the identifiers of the sets of data on the \\
library created for the problem. \\
${ }^{b}$ Water-bound kernel.
\end{tabular}

An $\mathrm{S}_{16}$ quadrature is selected with 32 spatial intervals. Activities are requested for ${ }^{235} \mathrm{U}_{92}$ absorption and fission and ${ }^{238} \mathrm{U}_{92}$ absorption.

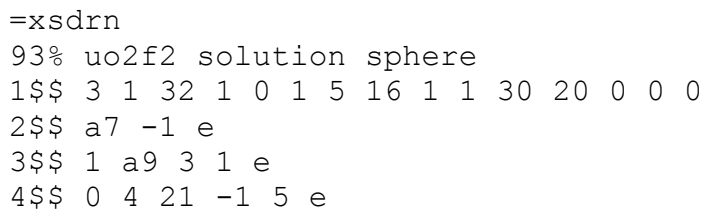




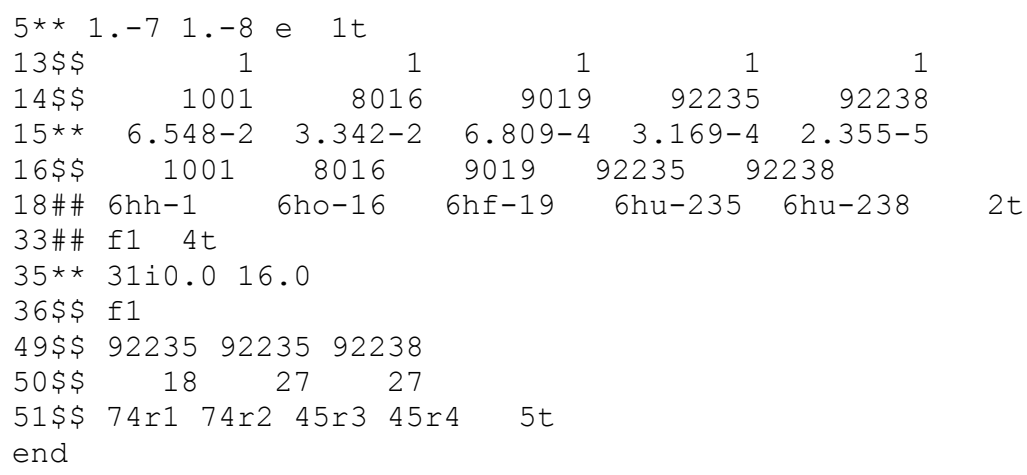

\subsubsection{Output Cross Sections}

One of the most common uses of XSDRNPM is to collapse cross sections and write them onto a file for input into another computer code. At present, two options are allowed:

1. Output library in AMPX Working Library format. This library is always written when cross sections are collapsed.

2. Output library in ANISN binary or BCD format. ${ }^{3}$ The binary library is written on logical 20 by default; the BCD library is produced on logical 7. The identifiers on this library range from 1 to the total number of blocks required to accommodate the data.

\subsubsection{Error Messages}

During the course of a problem, XSDRNPM makes many checks to determine if input data are in the required form. If inconsistencies are spotted, a message is printed, and the problem may be terminated. Some of these messages are listed below along with a brief description of their possible cause.

DATA N N arrays have been input with incorrect length. See the messages produced as arrays are read to determine specific arrays.

SN-1 N The Nth entry in the $S_{n}$ quadrature directions is zero. (43\# array)

SN-2 $\quad 0 \quad$ The $S_{n}$ weights do not sum to 1.0. (42\# array)

SN-3 $\quad 0 \quad$ The sum of the products of $S_{n}$ weights and directions is not 0.0 , that is, the directions are not symmetric. (42\# and 43\# arrays)

FIXS $0 \quad$ Fixed source calculation requested $(\mathrm{IEVT}=0)$ and total fixed sources are zero.

Q-HI $\mathrm{N} \quad \mathrm{A}$ volumetric source spectrum numbered $\mathrm{N}$ has been requested where $\mathrm{N}$ is greater than IQM.

B-HI $\mathrm{N}$ A boundary source spectrum numbered $\mathrm{N}$ has been requested where $\mathrm{N}$ is greater than IPM.

FISS $\quad 0 \quad$ IEVT $\geq 1$ and the total fission source is zero. 
$8101 \quad \mathrm{~N} \quad$ The $\mathrm{N} t h$ radius is negative.

$8102 \mathrm{~N}$ The $\mathrm{N} t h$ radius is less than the $(\mathrm{N}-1)$ th radius.

$8103 \mathrm{~N}$ Zone $\mathrm{N}$ dimensions have become negative in a zone width search.

MIX $\mathrm{N} \quad$ A request has been made to use the Nth component from the mixing table, but this nuclide has not been requested from a library.

Several messages may be encountered during an XSDRNPM run which indicate problems with either the code or the setup:

ROOT L The polynomials from which the default angles are derived are incorrect.

BAND $\mathrm{N}$ The number of bands specified is greater than the number of groups.

WAT1 N The number of sets of weighted cross sections is incorrect.

WAT2 N The number of sets of weighted cross sections is incorrect.

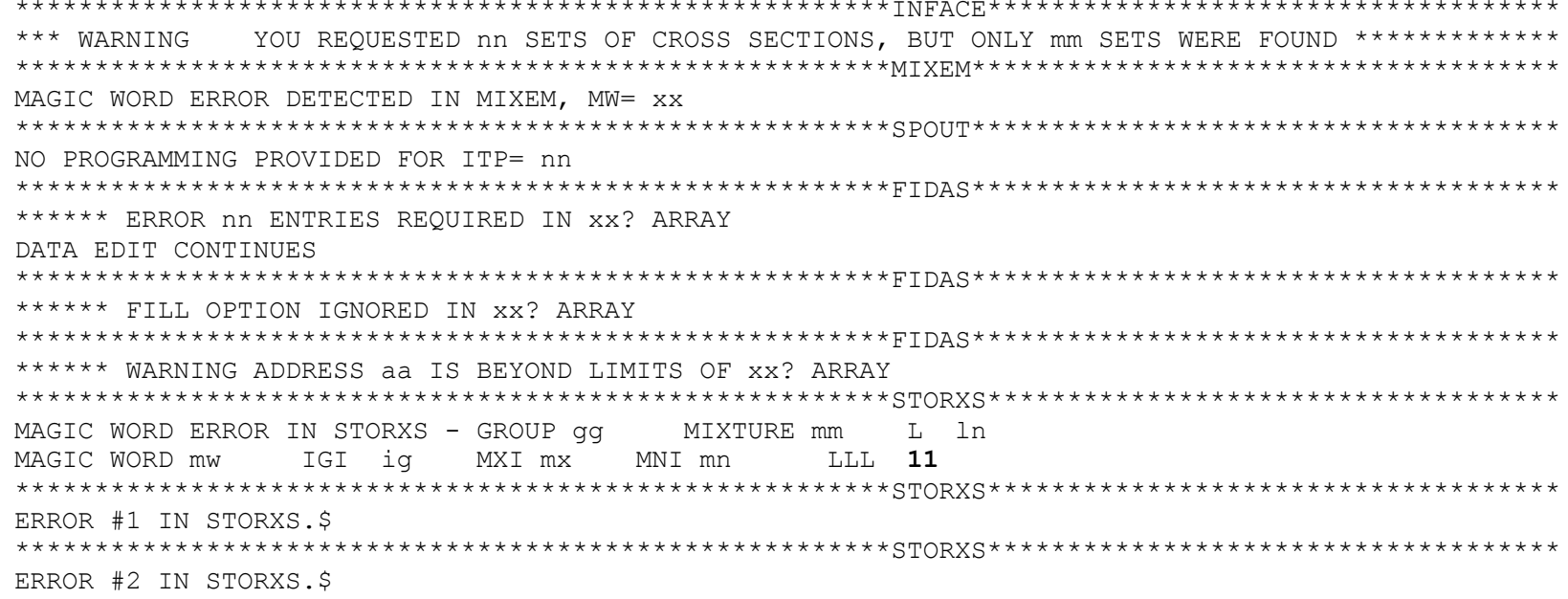

For the cryptic messages above (e.g., the last two), contact the code developers as to their possible cause.

\subsubsection{References}

1. N. M. Greene and C. W. Craven, Jr., XSDRN: A Discrete Ordinates Spectral Averaging Code, ORNL/TM-2500, Union Carbide Corporation (Nuclear Division), Oak Ridge National Laboratory, July 1969.

2. G. D. Joanou and J. S. Dudek, GAM-11, A B B Code for the Calculation of Fast-Neutron Spectra and Associated Multigroup Constants, GA-4265, September 1963.

3. W. W. Engle, Jr., A Users Manual for ANISN, K-1693, March 1967.

4. H. C. Honeck, THERMOS, A Thermalization Transport Theory Code for Reactor Lattice Calculations, BNL-5826, 1961. 
5. Standard Interface Files and Procedure for Reactor Physics Codes, LA-5486-MS, February 1974.

6. M. E. Dunn and N. M. Greene, "AMPX-2000: A Cross-Section Processing System for Generating Nuclear Data for Criticality Safety Applications," Trans. Am. Nucl. Soc. 86, 118-119 (2002).

7. W. A. Rhoades, D. B. Simpson, R. L. Childs, and W. W. Engle, Jr., The DOT-IV Two-Dimensional Discrete Ordinates Transport Code With Space-Dependent Mesh and Quadrature, ORNL/TM6529, Union Carbide Corporation (Nuclear Division), Oak Ridge National Laboratory, January 1979.

8. M. B. Emmett, The MORSE Monte Carlo Radiation Transport Code System, ORNL-4972, Union Carbide Corporation (Nuclear Division), Oak Ridge National Laboratory, February 1975.

9. K. D. Lathrop and B. G. Carlson, Discrete-Ordinates Angular Quadrature of the Neutron Transport Equation, LA-3186, February 1965.

10. W. A. Rhoades and W. W. Engle, Jr., "A New Weighted-Difference Formulation for Discrete Ordinates Calculations," Trans. Am. Nucl. Soc. 27, 776 (1977).

11. E. T. Tomlinson, W. A. Rhoades, and W. W. Engle, Jr., Flux Extrapolation Models Used in the DOT-IV Discrete-Ordinates Neutron Transport Code, ORNL/TM-7033, Union Carbide Corporation (Nuclear Division), Oak Ridge National Laboratory, May 1980.

12. T. Olsen, "Void Streaming in $S_{n}$ Calculations," Nucl. Sci. Eng. 21(2), 271 (1965).

13. T. J. Hoffman and J. S. Tang, XSDRNPM-S Biasing of MORSE-SGC/S Shipping Cask Calculations, NUREG/CR-2342 (ORNL/CSD/TM-175), U.S. Nuclear Regulatory Commission, Oak Ridge National Laboratory, October 1982.

14. T. J. Hoffman, The Optimization of Russian Roulette Parameters for the KENO Computer Code, ORNL/CSD/TM-192, Union Carbide Corporation (Nuclear Division), Oak Ridge National Laboratory, October 1982.

15. C. R. Weisbin, R. W. Roussin, E. W. Bryant, Editors, Review of the Theory and Application of Sensitivity and Uncertainty Analysis, ORNL/RSIC-42, Union Carbide Corporation (Nuclear Division), Oak Ridge National Laboratory, February 1979.

16. M. L. Williams, "Perturbation Theory for Reactor Analysis," CRC Handbook of Nuclear Reactors Calculations, Vol. 3, pp. 63-188, CRC Press, 1986.

17. B. Alder et al., Methods in Computational Physics, Vol. 1, pp. 31-32.

18. H. Henryson II, B. J. Toppel, and G. C. Stenberg, $M C^{2}-2$ : A Code to Calculate Fast Neutron Spectra and Multigroup Cross Sections, ANL-8144, June 1976. 
This page left blank intentionally. 


\subsection{A APPENDIX A: Special XSDRNPM Files}

Three special files that can be optionally produced by XSDRNPM are described in this appendix. (See Sect. 10.1.5 and the discussion of the logical units in the $0 \$$ array.) The files will be created with file names of the form $\boldsymbol{f t} \boldsymbol{N} \boldsymbol{N} \boldsymbol{X X X X}$. EXT where $\boldsymbol{N} \boldsymbol{N}$ is the 2 digit logical unit number (from the $0 \$ \$$ array), $\boldsymbol{X X X}$ is a 3 digit number which is incremented by one starting with one to make the name unique, and $\boldsymbol{E X T}$ is an extension identifying which type of file it is (acf for activity file, $\boldsymbol{b t f}$ for balance table file, and idf for input and derived data file).

\subsection{A.1 Activity file}

The data on the activity file depends on what input options are specified. The data is in ASCII sets, which consist of a label record followed by the record(s) of the activity. There will be at most IAZ sets ordered as the $49 \$$ and $50 \$$ arrays. The first sets of data will be the activities by interval (if the input parameter IAI was specified). A set will be formatted as below.

activity by interval for nuclide nnnnnnnn reaction type rrrrrrr Activity(first interval)
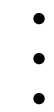

\section{Activity(last interval)}

The preceding set will be repeated IAZ times. Then sets giving the activities by zone will be given. They will be formatted as below.

activity by zone for nuclide nnnnnnnn reaction rrrrrrr

Activity(first zone)

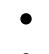

$\bullet$

-

\section{Activity(last zone)}

\subsection{A.2 Balance table file}

The contents of the balance table are defined in Table 9.1.A.1 and Table 9.1.A.2. The structure of the "balance table file" written to LBTF is:

Record $1-\quad$ KEFF, SP

KEFF - $\mathrm{k}_{\text {effective }}$ for problem

SP - search parameter for case

Record 2 - Sets of ASCII data consisting of a label record followed by data records.

Record last

A set of data is as follows (igp is the total number of groups plus one):

fine(few) group summary for zone zzzzz set type 
Set type data(group 1)

Set type data(group 2)

$\bullet$

-

$\cdot$

\section{Set type data(group igp)}

The data for a set type will be written for each zone of the problem, plus a system summary if there is more than one zone. After one set type is finished, the next set type will be written. The order of the set types is as follows:

\section{fixed source \\ fission source \\ absorption rate \\ total leakage \\ fission rate \\ flux \\ $<$ n, 2n $>$ rate \\ buckling loss \\ right current \\ left current \\ right leakage \\ left leakage}

The fine group summary data will be written if LBTF is $>0$. After the fine group data is finished, the few group summary data will follow if a weighting calculation is specified with a broad group collapse.

\subsection{A.3 Input and derived data file}

The contents of the input and derived data file (specified by LIDF) is as follows:

Record 1 - title (80 characters)

Record $2-\quad 1 \$ \$$ array (label)

Record 3,4 - data from 1\$ array

Record 5 - $\quad 2 \$ \$$ array (label)

Record 6 - data from $2 \$$ array

Record 7 - $\quad 3 \$ \$$ array (label)

Record 8,9 - data from 3\$ array

Record $10-\quad \mathbf{4 \$}$ array (label)

Record 11 - data from $\mathbf{4}$ array 


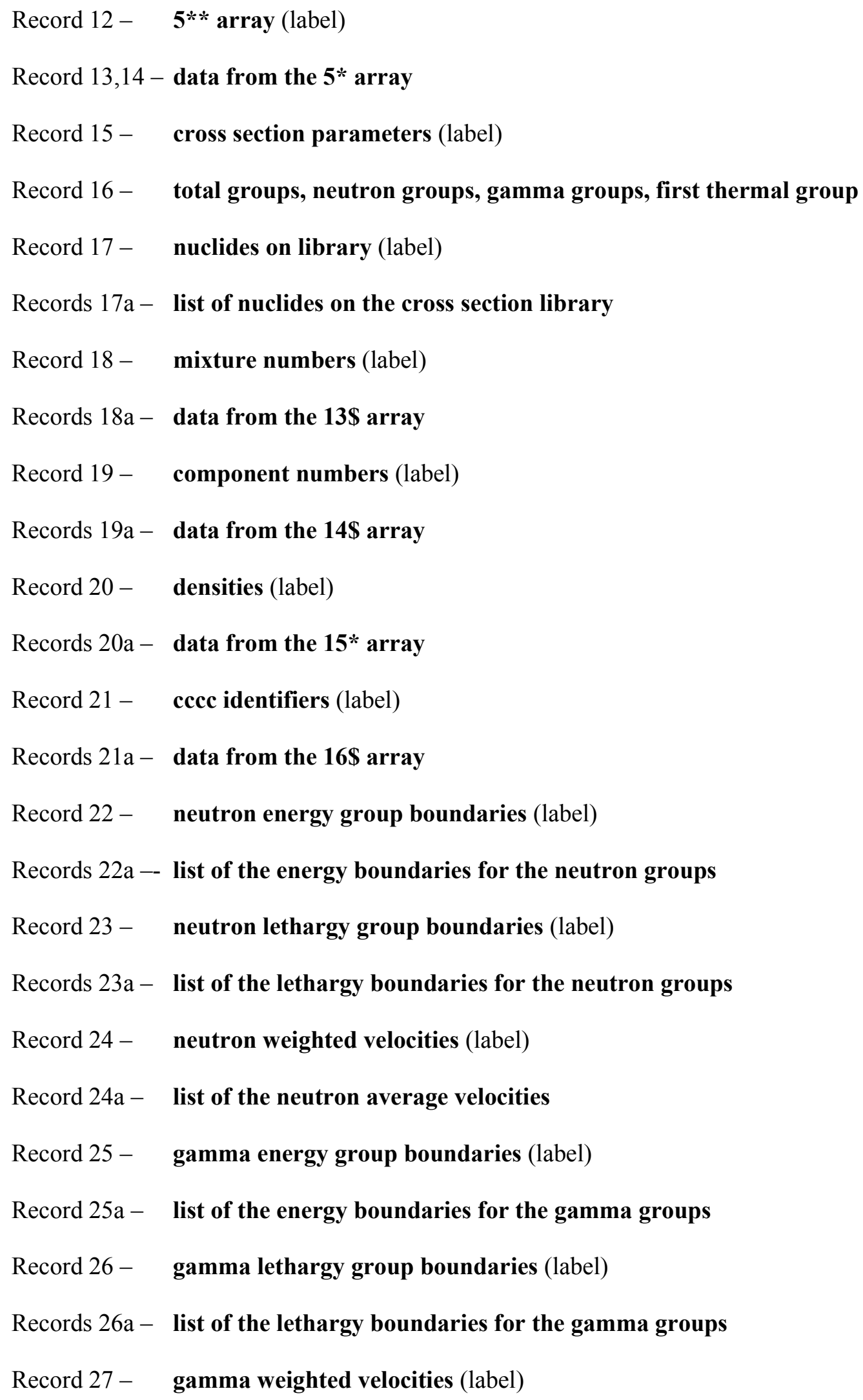


Records $27 \mathrm{a}-$ list of the gamma velocities

Record 28 - broad group numbers (label)

Records $28 \mathrm{a}-$ list of the broad group numbers by fine group - 51\$ array

Record 29 - group band (label)

Records 29 a - group band numbers by fine group

Record 30 - calculation type (label)

Records 30 a - calculation type by fine group

Record $31-\quad$ right albedo (label)

Records $31 \mathrm{a}-$ list of the right boundary albedos by group $-47 *$ array

Record 32 - left albedo (label)

Records $32 \mathrm{a}-$ list of the left boundary albedos by group $-48 *$ array

Record 34 - mixture by zone (label)

Records $34 \mathrm{a}$ - data from the $39 \$$ array

Record 35 - order of scattering by zone (label)

Records $35 \mathrm{a}$ - data from the $40 \$$ array

Record $36-\quad$ activity materials (label)

Records $36 \mathrm{a}$ - data from the $49 \$$ array

Record 37 - activity reaction types (label)

Records $37 \mathrm{a}$ - data from the $50 \$$ array

Record 38 - quadrature weights (label)

Records $38 \mathrm{a}-$ data from the $43 *$ array

Record 39 - quadrature cosines (label)

Records $39 a$ - data from the $42 *$ array

Record 40 - weights $\mathbf{x}$ cosines (label)

Records $40 \mathrm{a}$ - product of quadrature weights times quadrature 


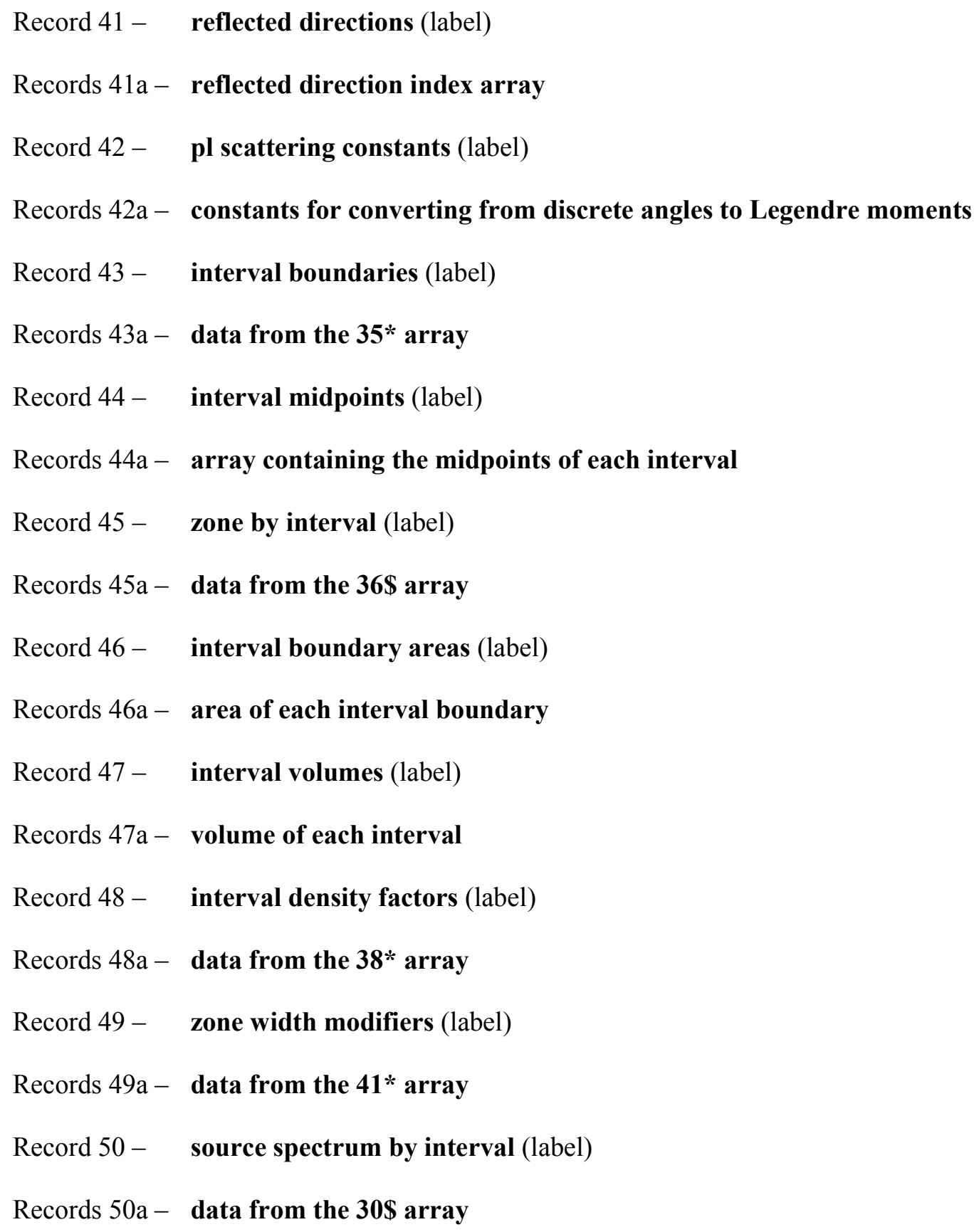


Table 9.1.A.1. Balance table definitions

\begin{tabular}{|c|c|}
\hline FS & $=$ Fission Source $_{\text {grp }, \text { one }}=1 / \lambda \Sigma_{\mathrm{i} \subset z \text { zone }}\left[\mathrm{X}_{\mathrm{i}, \mathrm{grp}} \Sigma_{\text {grp }^{\prime}}\left(\mathrm{v} \Sigma_{\text {fgrp }^{\prime}, \mathrm{i}} \varphi_{\text {grp }^{\prime}, \mathrm{i}}\right) \mathrm{V}_{\mathrm{i}}\right]$ \\
\hline XS & $=$ Fixed Source ${ }_{\text {grp }, \text { zone }}=\Sigma_{\text {lczone }}\left[\mathrm{Q}_{\mathrm{grp}, \mathrm{i}} \mathrm{V}_{i}+\mathrm{A}_{\mathrm{i}} \Sigma_{\mu \mathrm{m}>0} \mathrm{BS}_{\mathrm{i}, \mathrm{grp}, \mathrm{m}} \mu_{\mathrm{m}} \mathrm{w}_{\mathrm{m}}-\mathrm{A}_{\mathrm{i}+1} \Sigma_{\mu \mathrm{m}<0} \mathrm{BS}_{\mathrm{i}, \mathrm{grp}, \mathrm{m}} \mu_{\mathrm{m}} \mathrm{w}_{\mathrm{m}}\right]$ \\
\hline IS & $=$ Inscatter $_{\text {grp }, \text { zone }}=\Sigma_{\mathrm{i} \subset z \text { zone }} \Sigma_{\mathrm{j \neq grp}}\left[\Sigma_{\mathrm{j} \rightarrow \mathrm{grp}, \mathrm{i}} \varphi_{\mathrm{j}, \mathrm{i}} \mathrm{V}_{\mathrm{i}}\right]$ \\
\hline SS & $=$ Selfscatter $_{\text {grp }, \text { zone }}=\Sigma_{\mathrm{i} \subset \mathrm{zone}}\left[\Sigma_{\text {grp } \rightarrow \text { grp }} \varphi_{\text {grp }, \mathrm{i}} \mathrm{V}_{\mathrm{i}}\right]$ \\
\hline OS & $=$ Outscatter $_{\text {grp,zone }}=\Sigma_{\mathrm{i} \subset \text { zone }} \Sigma_{\mathrm{j \neq grp}}\left[\Sigma_{\text {grp } \rightarrow \mathrm{j}} \varphi_{\text {grp }, \mathrm{i}} \mathrm{V}_{\mathrm{i}}\right]$ \\
\hline $\mathbf{A B}$ & $=$ Absorption $_{\text {grp,zone }}=\Sigma_{\mathrm{i} \subset \text { zone }}\left[\sum_{\text {abs grp }, \mathrm{i}} \varphi_{\text {grp }, \mathrm{i}} \mathrm{V}_{\mathrm{i}}\right]$ \\
\hline LK & $=$ Leakage $_{\text {grp, }, \text { one }}=\left[\mathrm{A}_{\mathrm{zr}} \Sigma_{\mathrm{m}}\left(\psi_{\mathrm{m}, \mathrm{zr}} \mu_{\mathrm{m}} \mathrm{w}_{\mathrm{m}}\right)-\mathrm{A}_{\mathrm{zl}} \Sigma_{\mathrm{m}}\left(\psi_{\mathrm{m}, \mathrm{zl}} \mu_{\mathrm{m}} \mathrm{w}_{\mathrm{m}}\right)\right]$ \\
\hline $\mathbf{R F}$ & $=$ Right Boundary Flux $\mathrm{grp}$, zone $=\Sigma_{\mathrm{m}}\left(\psi_{\mathrm{m}, \mathrm{zr}, \mathrm{grp}} \mathrm{w}_{\mathrm{m}}\right)$ \\
\hline $\mathbf{L F}$ & $=$ Left Boundary Flux $\operatorname{grp}$, zone $=\Sigma_{\mathrm{m}}\left(\psi_{\mathrm{m}, \mathrm{zl}, \mathrm{grp}} \mathrm{W}_{\mathrm{m}}\right)$ \\
\hline RL & $=$ Right Leakage $\mathrm{grp}, \mathrm{zone}=\mathrm{A}_{\mathrm{zr}} \Sigma_{\mathrm{m}}\left(\psi_{\mathrm{m}, \mathrm{zr}, \mathrm{grp}} \mu_{\mathrm{m}} \mathrm{W}_{\mathrm{m}}\right)$ \\
\hline $\mathbf{L L}$ & $=$ Left Leakage $_{\text {grp }, \text { zone }}=\mathrm{A}_{\mathrm{zl}} \Sigma_{\mathrm{m}}\left(\psi_{\mathrm{m}, \mathrm{zl}, \mathrm{grp}} \mu_{\mathrm{m}} \mathrm{W}_{\mathrm{m}}\right)$ \\
\hline $\mathbf{N N}$ & $=\mathrm{n}, 2 \mathrm{n}$ Rate $_{\text {grp,zone }}=\Sigma_{\mathrm{i} \subset z \text { zone }} \Sigma_{\mathrm{p} \geq 2}\left[\mathrm{p} / 2 \Sigma_{\mathrm{n}, \mathrm{pn}} \varphi_{\mathrm{grp}, \mathrm{i}} \mathrm{V}_{\mathrm{i}}\right]$ \\
\hline FR & $=$ Fission Rate $_{\text {grp }, \text { zone }}=\Sigma_{\mathrm{i} \subset z \text { zone }}\left[\Sigma_{\text {fgrp }, \mathrm{i}} \varphi_{\text {grp }, \mathrm{i}} \mathrm{V}_{\mathrm{i}}\right]$ \\
\hline DB & $=\mathrm{DB}^{2}$ Flux $_{\text {grp }, \text { zone }}=\Sigma_{\mathrm{i} \subset \text { zone }}\left[\mathrm{D}_{\text {grp }, \mathrm{i}} \mathrm{B}_{\mathrm{grp}, \mathrm{i}}^{2} \varphi_{\mathrm{grp}, \mathrm{i}} \mathrm{V}_{\mathrm{i}}\right]$ \\
\hline TF & $=$ Total Flux $\operatorname{grp}$, zone $=\Sigma_{\mathrm{iczone}}\left[\varphi_{\mathrm{grp}, \mathrm{i}} \mathrm{V}_{\mathrm{i}}\right]$ \\
\hline BAL & $=\{\mathbf{F S}+\mathrm{XS}+\mathbf{I S}+\mathbf{N N}+\max (\mathbf{L L}, 0)-\min (\mathbf{R L}, 0)\} /\{\mathbf{O S}+\mathbf{A B}+\max (\mathbf{R L}, 0)-\min (\mathbf{L L}, 0)\}$ \\
\hline
\end{tabular}


Table 9.1.A.2. Balance table definition symbols

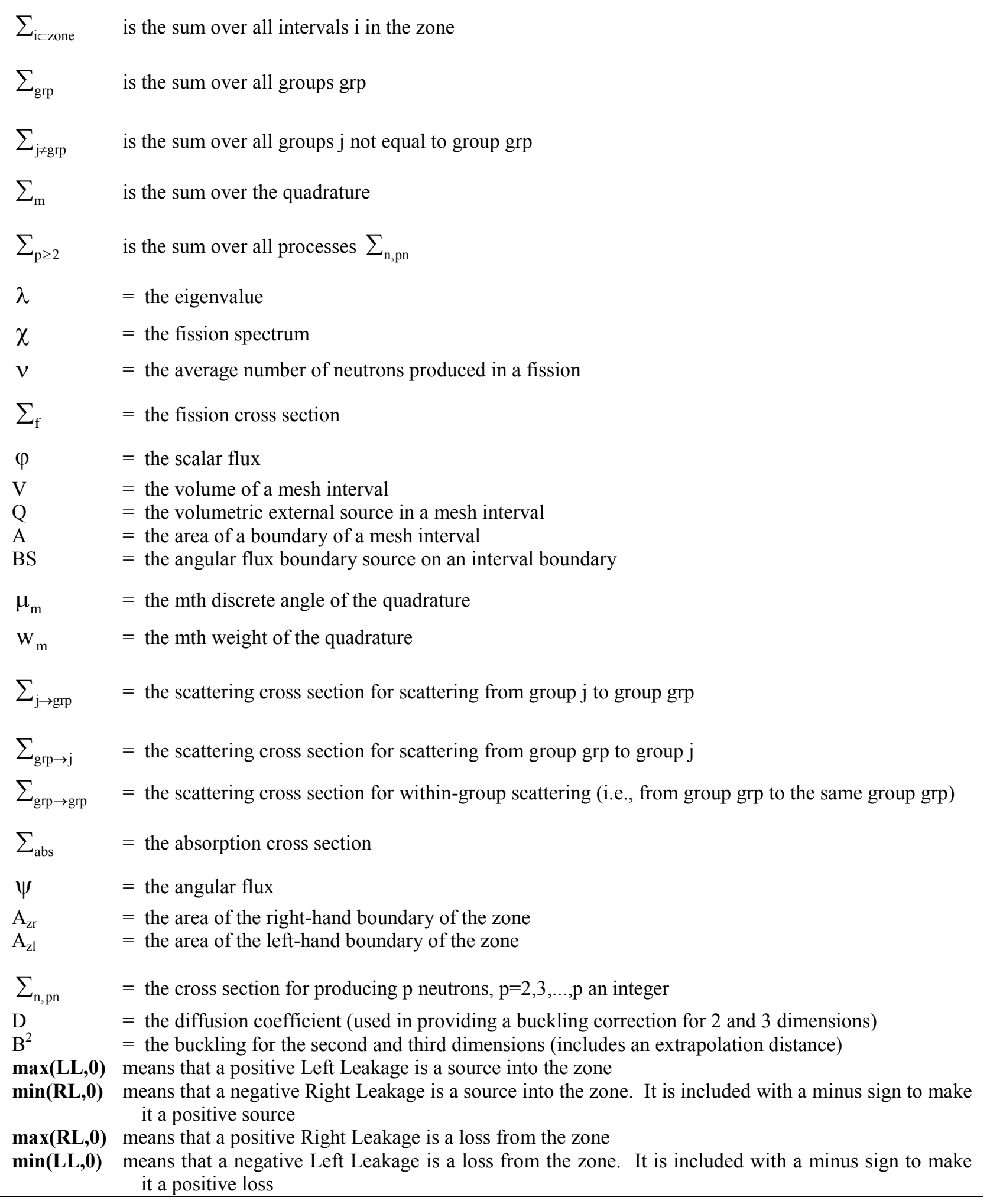




\subsection{B APPENDIX B: XSDRNPM Mixed Cross Sections}

When IPRT ( $2 \$ \$$ array) is set $>-1$, XSDRNPM prints the mixed reaction rate cross sections that are used in its calculations. The order of the cross sections for each group is given below in Table 9.1.B.1. The diffusion coefficient is used in computing buckling corrections, and in some of the options for computing the current used in weighting the transport cross section. The upscatter cross section is used to checking upscatter convergence. The $<\mathrm{n}, 2 \mathrm{n}>$ cross section is used in computing the balance for the balance tables. It is actually a weighted sum of all the multiple neutron exit reactions other than fission. These are all treated in XSDRNPM as scattering reactions. Chi is the fission spectrum, and is used to distribute the fission source in energy space. The fission cross section is used to compute the fission rate reported in the balance tables. The absorption cross section is used to compute the absorptions in the balance tables, and to compute the absorption term in the eigenvalue. $\mathrm{Nu}^{*}$ fission cross section is used to generate the source term for all except a fixed source calculation. The total cross section is used to determine the neutron transport.

Table 9.1.B.1. Order of mixed reaction cross sections

1. Diffusion coefficient (for use in buckling corrections)

2. Upscatter cross section

3. $<\mathrm{n}, 2 \mathrm{n}>$ cross section

4. Chi (fission spectrun)

5. Fission cross section

6. Absorption cross section

7. $\mathrm{Nu} *$ Fission cross section

8. Total cross section 


\title{
9.2 NEWT: A NEW TRANSPORT ALGORITHM FOR TWO-DIMENSIONAL DISCRETE- ORDINATES ANALYSIS IN NON-ORTHOGONAL GEOMETRIES
}

\author{
M. A. Jessee, M. D. DeHart ${ }^{1}$
}

\begin{abstract}
NEWT (New ESC-based Weighting Transport code) is a multigroup discrete-ordinates radiation transport computer code with flexible meshing capabilities that allow two-dimensional (2-D) neutron transport calculations using complex geometric models. The differencing scheme employed by NEWT, the Extended Step Characteristic approach, allows a computational mesh based on arbitrary polygons. Such a mesh can be used to closely approximate curved or irregular surfaces to provide the capability to model problems that were formerly difficult or impractical to model directly with discrete-ordinates methods. Automated grid generation capabilities provide a simplified user input specification in which elementary bodies can be defined and placed within a problem domain. NEWT can be used for eigenvalue, critical-buckling correction, and source calculations and it can be used to prepare collapsed weighted cross sections in AMPX working library format.

Like other SCALE modules, NEWT can be run as a standalone module or as part of a SCALE sequence. NEWT has been incorporated into the SCALE TRITON control module sequences. TRITON can be used simply to prepare cross sections for a NEWT transport calculation and then automatically execute NEWT. TRITON also provides the capability to perform 2-D depletion calculations, in which the transport capabilities of NEWT are combined with multiple ORIGEN depletion calculations to perform 2-D depletion of complex geometries. In the TRITON depletion sequence, NEWT can also be used to generate lattice-physics parameters and cross sections for use in subsequent nodal core simulator calculations. In addition, the SCALE TSUNAMI-2D sequence can be used to perform sensitivity and uncertainty analysis of 2-D geometries, where NEWT is used to compute the adjoint flux solution to generate sensitivity coefficients for $k_{\text {eff }}$ and other responses of interest, with respect to the cross sections used in the NEWT model.
\end{abstract}

\footnotetext{
${ }^{1}$ Formerly with Oak Ridge National Laboratory.
} 


\section{TABLE OF CONTENTS}

$\underline{\text { Page }}$

9.2 NEWT: A New Transport Algorithm for Two-Dimensional Discrete-Ordinates

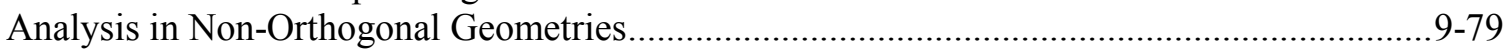

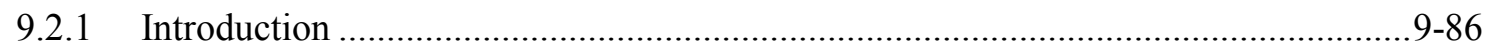

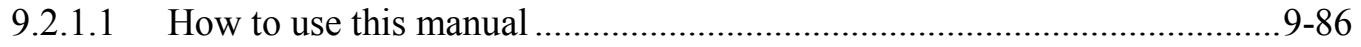

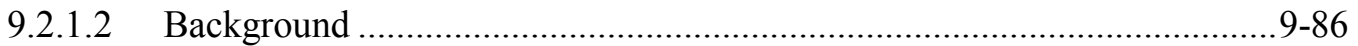

9.2.1.3 Discrete-ordinates solution on an arbitrary grid ...................................... 9-87

9.2.1.4 Functions performed ....................................................................... 9-87

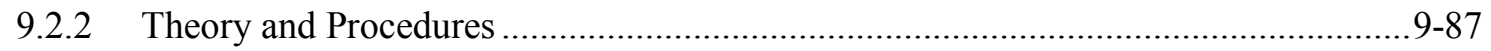

9.2.2.1 Boltzmann transport equation ............................................................... $9-87$

9.2.2.2 The step characteristic approximation.......................................................9-89

9.2.2.3 The Extended Step Characteristic approach..............................................9-90

9.2.2.4 Coarse-mesh finite-difference acceleration.............................................9-97

9.2.2.5 Assembly discontinuity factors ....................................................... 9-97

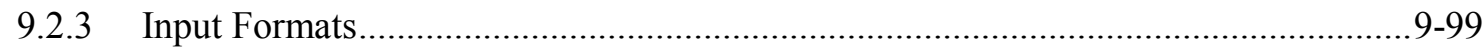

9.2.3.1 Overview of newt data blocks ................................................................99

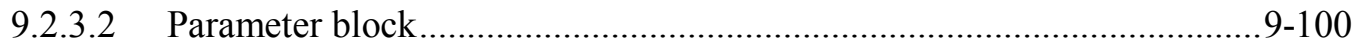

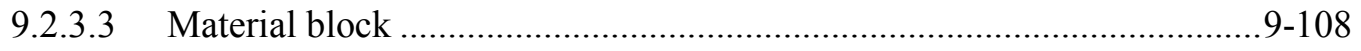

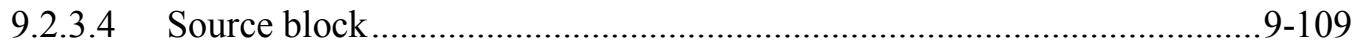

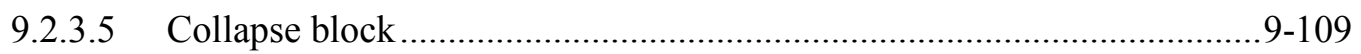

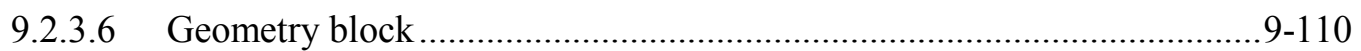

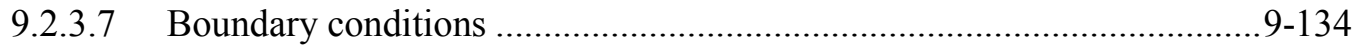

9.2.3.8 General cross section weighting ........................................................ 9-138

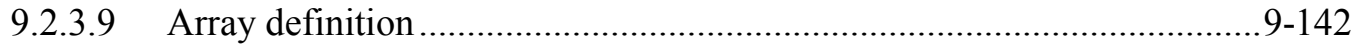

9.2.3.10 Homogenization block ................................................................... 9-146

9.2.3.11 Assembly discontinuity factors ....................................................... 9-147

9.2.3.12 Flux planes ........................................................................................ 9-150

9.2.3.13 Mixing table block ....................................................................... 9-152

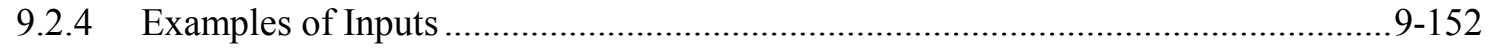

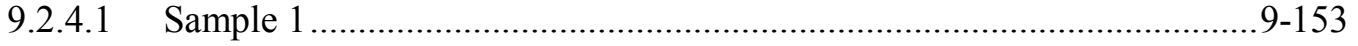

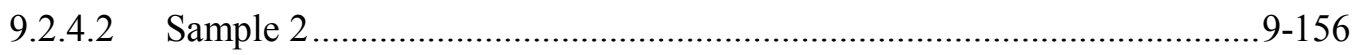

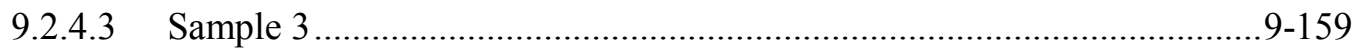

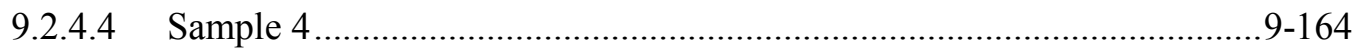

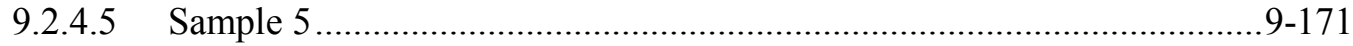

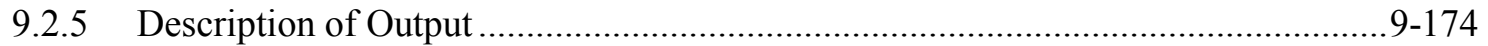




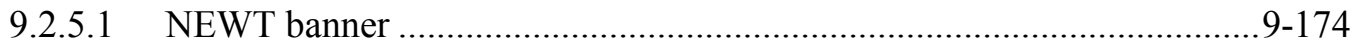

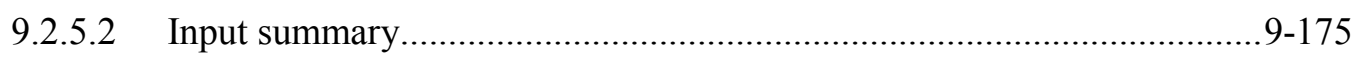

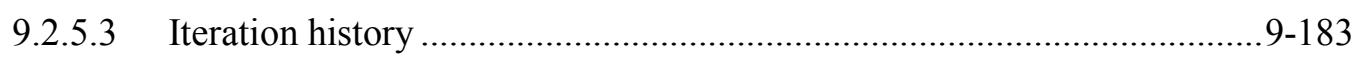

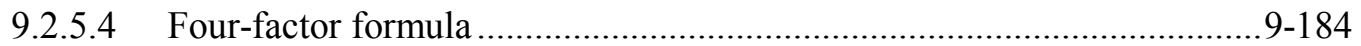

9.2.5.5 Fine-group balance tables.................................................................. 9-185

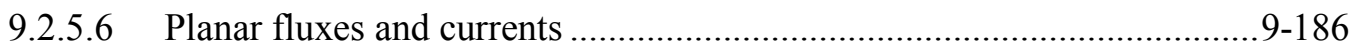

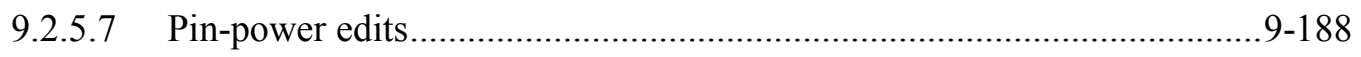

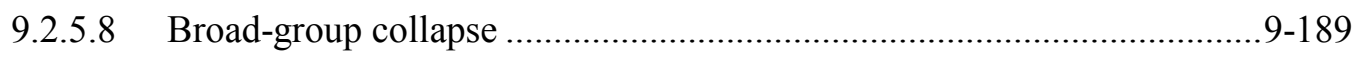

9.2.5.9 Critical spectrum edit .........................................................................

9.2.5.10 Assembly discontinuity factors ....................................................... $9-192$

9.2.5.11 Groupwise form factors................................................................ 9-192

9.2.5.12 Homogenized cross sections …......................................................... $9-193$

9.2.5.13 End-of-calculation banner ................................................................... 9 -194

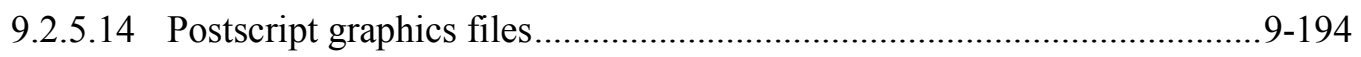

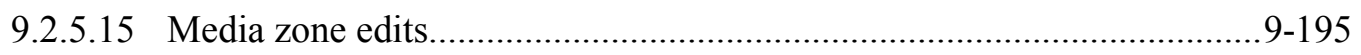

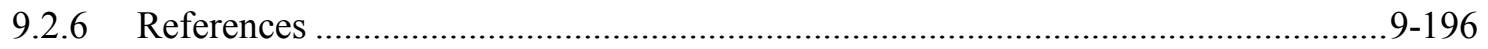




\section{LIST OF FIGURES}

Page

Figure 9.2.1. Typical rectangular cell used in the step characteristic approach...................................9-90

Figure 9.2.2. Orientation of the sides of a cell with respect to a given direction vector........................9-91

Figure 9.2.3. Line endpoints for computation of average fluxes. ................................................9-93

Figure 9.2.4. Contributions of multiple incoming sides to an outgoing side. ......................................9-95

Figure 9.2.5. Relationship between $s_{1}$ and $s_{2}$ and their projections in the $\mathrm{x}-\mathrm{y}$ plane............................9-96

Figure 9.2.6. Heterogeneous vs homogeneous fluxes in a multi-assembly solution. ............................9-99

Figure 9.2.7. A simple unit (left) and an array of units (right). .......................................................9-111

Figure 9.2.8. Computational grid structure in a NEWT model....................................................... $9-112$

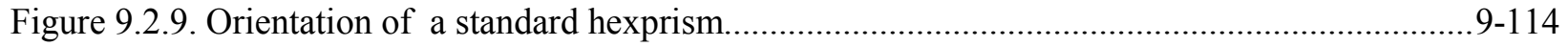

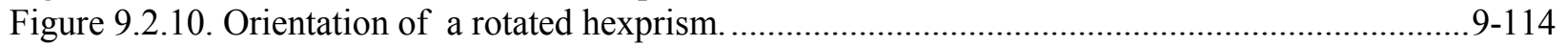

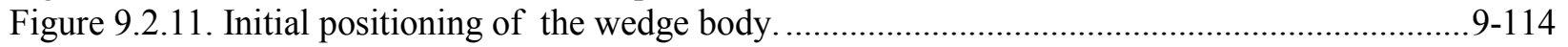

Figure 9.2.12. Body placement for two cylinders and a cuboid. .................................................. 9-115

Figure 9.2.13. Relocation (translation) of a cylinder using origin ................................................9-116

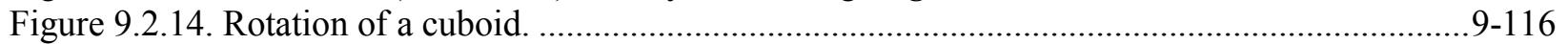

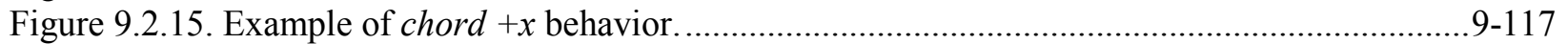

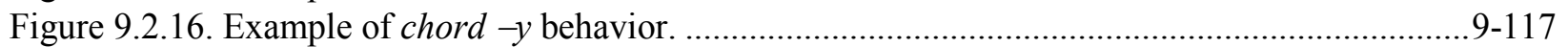

Figure 9.2.17. Use of two chords to create a quarter-cylinder body.............................................. 9-118

Figure 9.2.18. Use of four chords on a cylinder to create a square body.......................................9-118

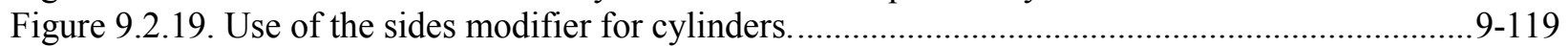

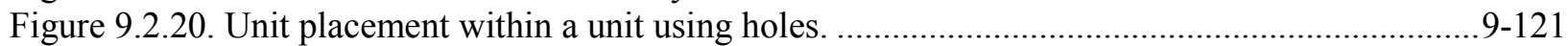

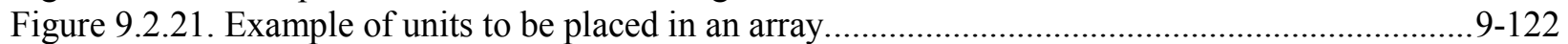

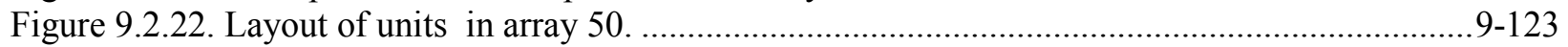

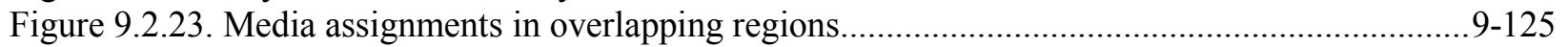

Figure 9.2.24. Unit with 2 by 2 grid in a simple pin-cell model.................................................. 9-127

Figure 9.2.25. Unit with 5 by 5 grid inset into unit with 3 by 3 grid. .......................................... 9-127

Figure 9.2.26. Effect of boundary grid specification on noncuboidal unit placed as a hole within

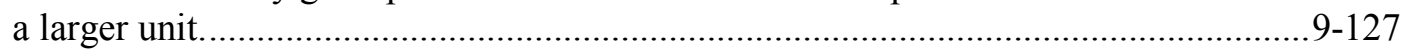

Figure 9.2.27. Effect of coarse-mesh finite-difference acceleration on grid structure.........................9-128

Figure 9.2.28. Use of base grid without localized grid specification..............................................9-128

Figure 9.2.29. Geometry model for infinite-lattice pin cell with fuel, gap, clad, and moderator. .........9-129

Figure 9.2.30. Geometry model of hexagonal fuel assembly. .....................................................9-130

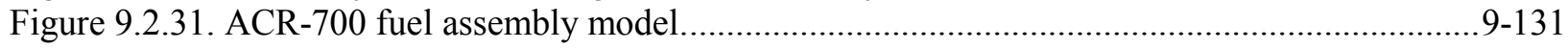

Figure 9.2.32. Two-dimensional boundary condition surface orientations....................................... 9-135

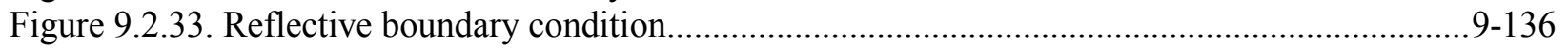

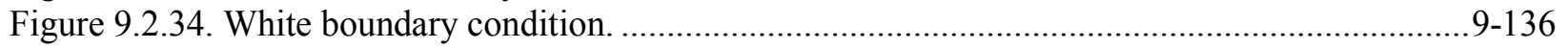

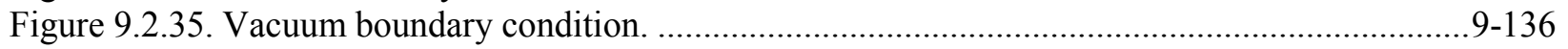

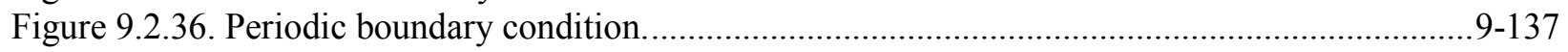

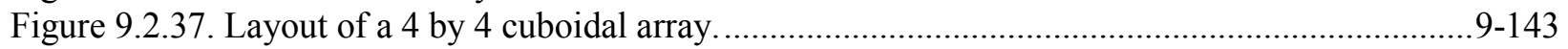

Figure 9.2.38. Layout of a 4 by 4 hexagonal array................................................................... $9-143$

Figure 9.2.39. Layout of a 4 by 4 stacked hexagonal (shexagonal) array............................................9-144

Figure 9.2.40. Layout of a 4 by 4 rotated hexagonal (rhexagonal) array............................................9-144

Figure 9.2.41. Example of nested arrays and a null unit specification. ...............................................9-146

Figure 9.2.42. Upper-right quadrant of an unreflected PWR assembly...........................................9-149

Figure 9.2.43. Upper-right quadrant of a PWR assembly with baffle and reflector. ...........................9-150

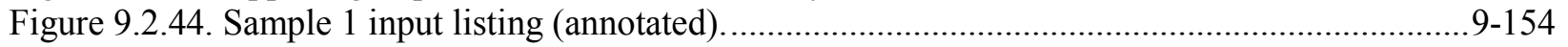

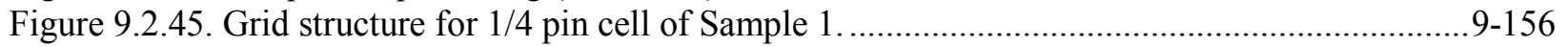

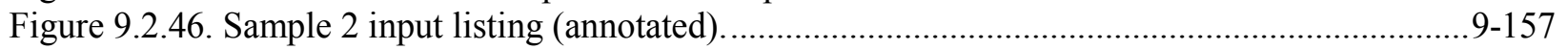


Figure 9.2.47. Mixture placement and grid structure for model described in Sample 2....................9-159

Figure 9.2.48. Sample 3 input listing (annotated)...................................................................... 9-160

Figure 9.2.49. Grid structure and material placement for VVER-440 model....................................9-164

Figure 9.2.50. Sample 4 input listing (annotated)...................................................................... 9-166

Figure 9.2.51. Grid structure for one-quarter assembly of Sample 4............................................... 9-170

Figure 9.2.52. Mixture placement for quarter-assembly model of Sample 4....................................9-171

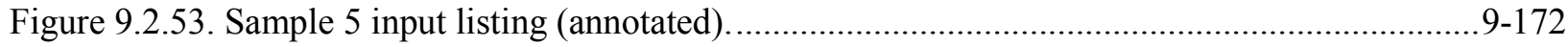

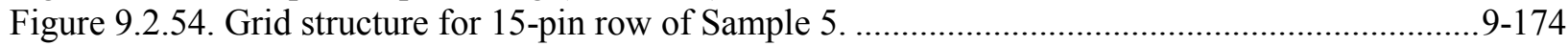

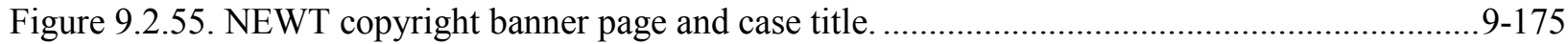

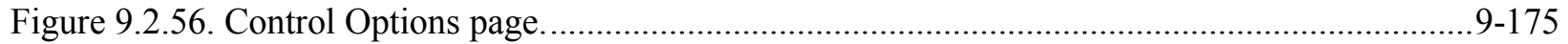

Figure 9.2.57. Output Options page

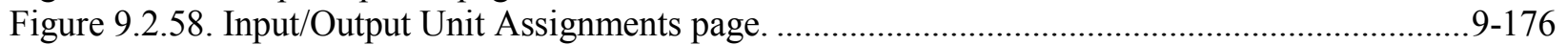

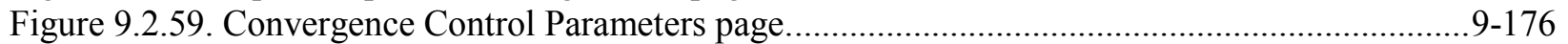

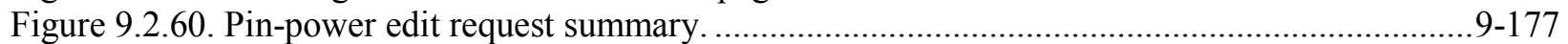

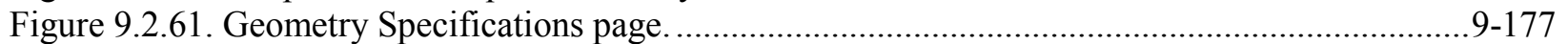

Figure 9.2.62. Homogenization Region Specifications page.................................................... 9-177

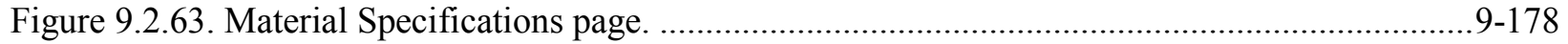

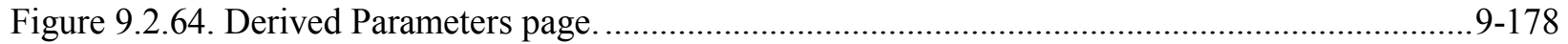

Figure 9.2.65. Energy Group Structure Listing page ..................................................................... $9-179$

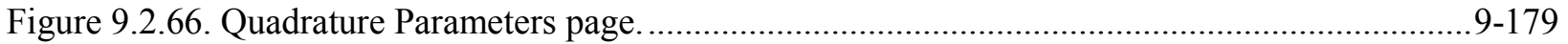

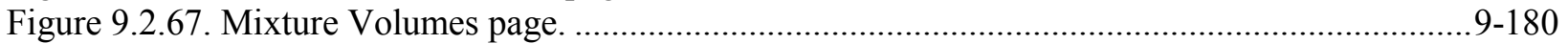

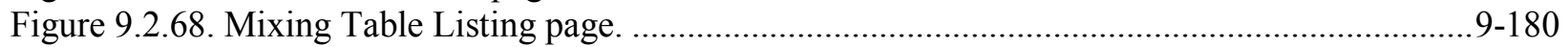

Figure 9.2.69. Partial listing of Nuclide Cross section data pages...................................................9-181

Figure 9.2.70. Partial listing of Mixture Cross section data pages. ...................................................9-182

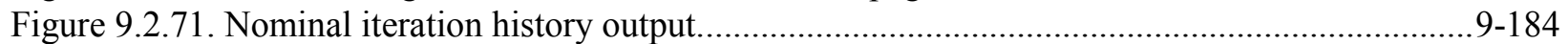

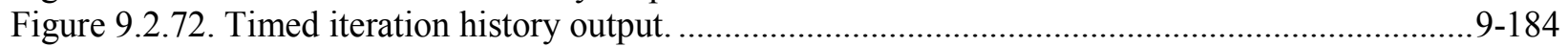

Figure 9.2.73. Four-factor formula with alternate three-group formulation..................................... 9-185

Figure 9.2.74. Partial mixture fine-group balance table output. .................................................. 9-186

Figure 9.2.75. Example of planar flux and current output................................................................ 9-187

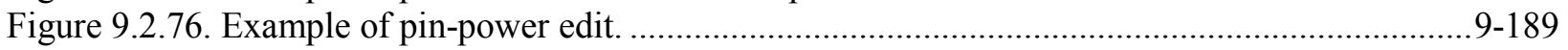

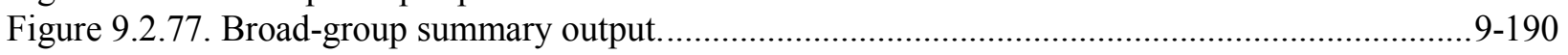

Figure 9.2.78. Partial broad-group cross section listing.................................................................... 9-191

Figure 9.2.79. Partial collapsing spectra listing for a case with critical buckling correction.................9-192

Figure 9.2.80. Output of assembly discontinuity factors. ..............................................................9-192

Figure 9.2.81. Partial collapsing spectra listing for a case with no critical buckling correction........... 9-193

Figure 9.2.82. Homogenized cross section edit for nodal diffusion applications. .............................. 9-194

Figure 9.2.83. End-of-calculation banner listing. ........................................................................... 9-194

Figure 9.2.84. Example of a flux plot image created with prtflux=yes............................................ 9-195

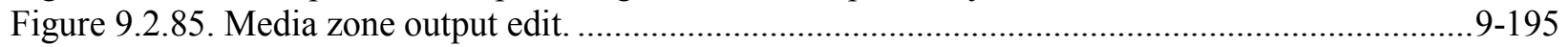




\section{LIST OF TABLES}

$\underline{\text { Page }}$

Table 9.2.1. Boundary condition specifiers accepted by NEWT …...............................................9-138

Table 9.2.2. NEWT array types with corresponding element types ................................................. 9-142 


\section{ACKNOWLEDGMENTS}

The author expresses gratitude to B. T. Rearden and S. M. Bowman for their supervision of the SCALE project and review of the manuscript. The author acknowledges R. Y. Lee of the U.S. Nuclear Regulatory Commission (NRC) and A. P. Ulses (formerly NRC) for their support of the development of NEWT. Appreciation is extended to G. Ilas, B. L. Broadhead, Deokjung Lee (formerly ORNL), and B. J. Ade for their review of this or previous versions of the manuscript. The efforts of Z. Zhong (Argonne National Laboratory), A. P. Ulses (formerly NRC), K. S. Kim, C. F. Weber, G. Ilas, and K. T. Clarno (Oak Ridge National Laboratory) in methods development and testing of the code have been invaluable in the continued evolution and improvement of the code. 


\subsubsection{Introduction}

NEWT (New ESC-based Weighting Transport code) is a two-dimensional (2-D) discrete-ordinates transport code developed based on the Extended Step Characteristic (ESC) approach ${ }^{1}$ for spatial discretization on an arbitrary mesh structure. This discretization scheme makes NEWT an extremely powerful and versatile tool for deterministic calculations in real-world non-orthogonal problem domains. The NEWT computer code evolved from the earlier proof-of-principle CENTAUR code ${ }^{1}$ and has been developed to run within SCALE. Thus, NEWT uses AMPX-formatted cross sections processed by other SCALE modules. If cross sections are properly prepared, NEWT can be run in stand-alone mode. NEWT can also be used within the TRITON control module for transport analysis, depletion analysis, and sensitivity and uncertainty analysis.

\subsubsection{How to use this manual}

This users' manual is intended to assist both the novice and the expert in the application of NEWT for transport analysis. As such, the document is divided into subsections, each with a specific purpose. Not all sections will be of value to all users. It is not intended that the user of this manual read through the manual from start to end. Rather, the manual is designed to serve as a reference, with each section meeting different needs. This introductory section has been written to provide a general overview of the background, nature, functionality, and applications of NEWT; it should prove of interest to users at all levels. Section 9.2.2 provides detail on the theory of NEWT in terms of derivations, equations, and relationships used in the NEWT solution. This information will be of interest to those with a background in transport methods desiring a comprehensive understanding of the NEWT solution scheme. However, this information may provide too much detail or simply not be relevant for the beginning user or someone desiring to improve or expand an existing model. These users will find Sect. 9.2.3 to be of more value, where input data requirements and formats are described in detail, along with examples of each data type. This information is supplemented by Sect. 9.2.4, in which complete sample inputs with descriptions of the features of each model are provided. Section 9.2.5 describes the components of an output listing obtained from a successful NEWT calculation.

\subsubsection{Background}

The radiation transport equation, a linearized derivative of the Boltzmann equation, provides an exact description of a neutral-particle radiation field in terms of the position, direction of travel, and energy of every particle in the field. Both stochastic (Monte Carlo simulation) and deterministic (direct numerical solution) forms of the transport equation have been developed and are used extensively in nuclear applications. Each approach has its strengths and weaknesses. Stochastic approaches are extremely effective for problems with complex geometries where the calculations of integral quantities, such as radiation dose and neutron multiplication factors, are desired. However, calculations to obtain accurate differential information, such as the neutron flux as a function of space and energy, can be difficult and inefficient at best and prone to inaccuracies (even if the integral quantity is correct). Deterministic techniques, such as integral transport, collision probability, diffusion theory, and discrete-ordinates methods, are better suited for problems where differential quantities, such as the neutron flux as a function of energy or space, are desired. However, integral transport, collision probability, and diffusion approximations are based on simplifying assumptions, which can limit their applicability. The discreteordinates approach is a more rigorous approximation to the transport equation but is typically very limited in its flexibility to describe complex geometric systems.

Discrete-ordinates approaches are derived from the integro-differential form of the Boltzmann transport equation, where space, time, and energy dependencies are normally treated by the use of a finite-difference grid, while angular behavior is treated by considering a number of discrete directions in space. The angular solution is coupled to a scalar spatial solution via some form of numerical integration. 
Because of the direct angular treatment of the discrete-ordinates approach, angularly dependent distribution functions can be computed; thus, this approach is the preferred method of solution in many specific applications where angular anisotropy is important. However, as indicated earlier, it is often limited in applicability because of the geometric constraints of the orthogonal grid system associated with the finite-difference numerical approximation.

\subsubsection{Discrete-ordinates solution on an arbitrary grid}

The ESC approach was developed to obtain a discrete-ordinates solution in complicated geometries to handle the needs of irregular configurations. Deterministic solutions to the transport equation generally calculate a solution in terms of the particle flux; the flux is the product of particle density and speed and is a useful quantity in the determination of reaction rates that characterize nuclear systems. General 2-D $x y$ discrete-ordinates methods perform calculations that provide four side-averaged fluxes and a cellaveraged flux for each cell in a rectangular problem grid; iteration is performed to obtain a converged distribution. This approach is usually termed the diamond-difference approach. Using the ESC approach, a more flexible and completely arbitrary problem grid may be defined in terms of completely arbitrary polygons. Side-averaged fluxes for each polygon in the problem domain are computed and are used to calculate a cell-averaged flux. This process is repeated for each cell in the problem domain, and as with the traditional approach, iteration is performed for convergence. This geometric flexibility is a significant enhancement to existing technology, as it provides the capability to model problems that are currently difficult or impractical to model directly.

\subsubsection{Functions performed}

NEWT provides multiple capabilities that can potentially be used in a wide variety of application areas. These include 2-D eigenvalue calculations, forward and adjoint flux solutions, multigroup flux spectrum calculations, and cross section collapse calculations. NEWT provides significant functionality to support lattice-physics calculations, including assembly cross section homogenization and collapse, calculation of assembly discontinuity factors (for internal and reflected assemblies), diffusion coefficients, pin powers, and group form factors. Used as part of the TRITON depletion sequence, NEWT provides spatial fluxes, weighted multigroup cross sections, and power distributions used for multi-material depletion calculations and coupled depletion and branch calculations needed for lattice-physics analysis.

\subsubsection{Theory and Procedures}

This section provides the theoretical basis for the ESC discretization technique, the NEWT solution algorithm, and cross section processing procedures used by NEWT. Although this information is not necessary to be able to use NEWT for transport calculations, it provides a deeper understanding of the basic operations performed within NEWT.

\subsubsection{Boltzmann transport equation}

The neutron transport equation may be presented in various forms, and simplifications are often applied to tailor the equation to the requirements of a specific application. In nuclear engineering applications, the transport equation is often written in terms of the angular neutron flux as the dependent variable. The angular neutron flux is defined as the product of the angular neutron density and the neutron velocity. The time-independent form of the linear transport equation is then expressed $\mathrm{as}^{2}$

$$
\widehat{\Omega} \cdot \vec{\nabla} \psi(\overrightarrow{\mathbf{r}}, \widehat{\Omega}, E)+\sigma_{t}(\overrightarrow{\mathbf{r}}, E) \psi(\overrightarrow{\mathbf{r}}, \widehat{\Omega}, E)=Q(\overrightarrow{\mathbf{r}}, \widehat{\Omega}, E)
$$


where

$$
\begin{aligned}
& \psi(\overrightarrow{\mathbf{r}}, \widehat{\Omega}, E) \equiv \begin{array}{l}
\text { angular flux at position } \overrightarrow{\mathbf{r}} \text { per unit volume, in direction } \widehat{\Omega} \text { per unit solid angle } \\
\text { and at energy E per unit energy; }
\end{array} \\
& \sigma_{t}(\overrightarrow{\mathbf{r}}, E) \equiv \text { total macroscopic cross section at position } \overrightarrow{\mathbf{r}} \text { and energy E; and } \\
& \mathrm{Q} \equiv \begin{array}{l}
\text { source at position } \overrightarrow{\mathbf{r}} \text { per unit volume, in direction } \widehat{\Omega} \text { per unit solid angle and at } \\
\text { energy E per unit energy. }
\end{array}
\end{aligned}
$$

The source Q is generally composed of three terms:

1. a scattering source,

$$
S(\overrightarrow{\mathbf{r}}, \widehat{\Omega}, E)=\int_{4 \pi} d \widehat{\Omega}^{\prime} \int_{0}^{\infty} d E^{\prime} \sigma_{s}\left(\overrightarrow{\mathbf{r}}, \widehat{\Omega}^{\prime} \rightarrow \widehat{\Omega}, E^{\prime} \rightarrow E\right) \psi\left(\overrightarrow{\mathbf{r}}, \widehat{\Omega}^{\prime}, E^{\prime}\right)
$$

where

$$
\begin{aligned}
& \sigma_{s}\left(\overrightarrow{\mathbf{r}}, \widehat{\Omega}^{\prime} \rightarrow \widehat{\Omega}, E^{\prime} \rightarrow E\right) \equiv \text { macroscopic scattering cross section at position } \overrightarrow{\mathbf{r}} \text { from } \\
& \text { initial energy } E^{\prime} \text { and direction } \widehat{\Omega^{\prime}} \text { to final energy } \mathrm{E} \text { and direction } \widehat{\Omega},
\end{aligned}
$$

2. a fission source,

$$
F(\overrightarrow{\mathbf{r}}, \widehat{\Omega}, E)=\chi(\overrightarrow{\mathbf{r}}, E) \int_{0}^{\infty} d E^{\prime} v\left(\overrightarrow{\mathbf{r}}, E^{\prime}\right) \sigma_{f}\left(\overrightarrow{\mathbf{r}}, E^{\prime}\right) \psi\left(\overrightarrow{\mathbf{r}}, \widehat{\Omega}, E^{\prime}\right)
$$

where

$$
\begin{aligned}
& \sigma_{f}\left(\overrightarrow{\mathbf{r}}, E^{\prime}\right) \equiv \text { macroscopic fission cross section at position } \overrightarrow{\mathbf{r}} \text { and energy } \mathrm{E}^{\prime} \text { (assumed to be } \\
& \text { isotropic), } \\
& v\left(\overrightarrow{\mathbf{r}}, E^{\prime}\right) \equiv \text { number of neutrons released per fission event at position } \overrightarrow{\mathbf{r}} \text { and energy } \mathrm{E}^{\prime}, \\
& \chi(\overrightarrow{\mathbf{r}}, E) \equiv \text { fraction of neutrons that are born at } \overrightarrow{\mathbf{r}} \text { and at energy E, and }
\end{aligned}
$$

3. an external or fixed source, $\mathrm{S}(\overrightarrow{\mathrm{r}}, \mathrm{E})$.

In general, the transport equation can be difficult to apply and can be solved analytically only for highly idealized cases. Hence, simplifications and numerical approximations are often necessary to apply the equation in engineering applications. Traditional discrete-ordinates methods are based on a finitedifference approximation to solve the flux streaming (leakage) term. Such differencing schemes are intimately tied to the coordinate system in which the differencing equations are developed, and it becomes difficult to represent non-orthogonal volumes within that coordinate system. For example, it is not possible to exactly represent a cylinder in a 2-D Cartesian coordinate system; one must approximate the cylinder with a number of rectangular cells. A close approximation can require a large number of computational cells. However, the ESC approach for discretizing computational cells allows the use of non-orthogonal computational cells composed of arbitrary polygons. Using this method, practically any shape can be represented within a Cartesian grid to a very close approximation. The ESC approach is discussed in the following sections. 


\subsubsection{The step characteristic approximation}

Efficient application of discrete-ordinates methods is difficult when dealing with complicated nonorthogonal geometries because of the nature of finite difference approximations for spatial derivatives. An alternative to the discrete representation of the spatial variable is achieved in the method of characteristics, in which the transport equation is solved analytically along characteristic directions within a computational cell. The angular flux $\psi$ is solved along the $s$-axis, where this axis is oriented along the characteristic direction $\widehat{\Omega}$. Since only the angular flux in direction $\widehat{\Omega}$ is of concern, then the streaming term can be rewritten as

$$
\widehat{\Omega} \cdot \vec{\nabla} \psi(\overrightarrow{\mathbf{r}}, \widehat{\Omega}, E)=\frac{d \psi(s, E)}{d s}
$$

Hence Eq. (9.2.1) can be written in the characteristic form (omitting $E$ for clarity) as

$$
\frac{d \psi(s)}{d s}+\sigma_{t}(s) \psi(\mathrm{s})=Q(s)
$$

which has a solution of the form ${ }^{3}$

$$
\psi(s)=\psi_{0} e^{-\sigma_{t} s}+e^{-\sigma_{t} s} \int_{0}^{s} Q e^{\sigma_{t} s^{\prime}} d s^{\prime}
$$

where $\mathrm{s}$ is the distance along the characteristic direction $\widehat{\Omega}$, and $\psi_{0}$ is the known angular flux at $s=0$. The value for $\psi_{0}$ is given from boundary conditions for known cell sides, and angular fluxes on unknown sides are computed using Eq. (9.2.6). Methods for the determination of an appropriate value for $\psi_{0}$ and for evaluation of the integral term vary in different solution techniques. ${ }^{4-9}$

One of the simplest schemes employing the Method of Characteristics is the Step Characteristic (SC) method developed by Lathrop. ${ }^{6}$ In this approach, the source $\mathrm{Q}$ and macroscopic total cross section $\sigma_{\mathrm{t}}$ are assumed to be constant within a computational cell and the angular flux is assumed constant on the cell boundaries of incoming direction. Integration of Eq. (9.2.6) can be performed to obtain

$$
\psi(s)=\psi_{0} e^{-\sigma_{t} s}+\frac{Q}{\sigma_{t}}\left(1-e^{-\sigma_{t} s}\right)
$$

Figure 9.2.1 shows a sample computational cell in which the SC method can be applied. For a given characteristic direction $\widehat{\Omega}$, the angular flux on any unknown side may be expressed in terms of a suitable average of fluxes from known sides, which contribute to the unknown side. For the characteristic direction $\widehat{\Omega}$ shown in Figure 9.2.1, the unknown "top" flux $\psi_{\mathrm{T}}$ may be computed as a linearly weighted average of contributions from known sides $\psi_{\mathrm{B}}$ and $\psi_{\mathrm{L}}$. The fluxes on each of the two known sides are taken to be constant along the length of each side, representing the average angular flux in direction $\widehat{\Omega}$ and must be specified from external boundary conditions or from a completed calculation in an adjacent cell.

The set of characteristic directions is chosen from a quadrature set, so that the resulting angular fluxes may be numerically integrated to obtain a scalar flux. Knowing the lengths of the sides of a rectangular 
cell $(\Delta \mathrm{x}$ and $\Delta \mathrm{y})$ and the direction cosines of $\widehat{\Omega}$ in the $x-y$ plane ( $\mu$ and $\eta$ ), a function for the length $s$ can easily be determined. The solution for $\psi(s)$ from Eq. (9.2.7) can then be integrated along the length of each unknown side to determine the average angular flux of the unknown side. Once the angular flux is known on all four sides, a neutron balance on the cell can be used to determine the cell's average angular flux.

Although the SC method described above is based on rectangular cells, the derivation of Eq. (9.2.7) makes no assumptions about the shape of the cell. It merely requires knowledge of the relationship between cell edges along the direction of the characteristic. Hence, the method is not restricted to any particular geometry. Because it is an extension of the SC approach into generalized cells, the method developed here for generalized geometries is referred to as the Extended Step Characteristic (ESC) method.

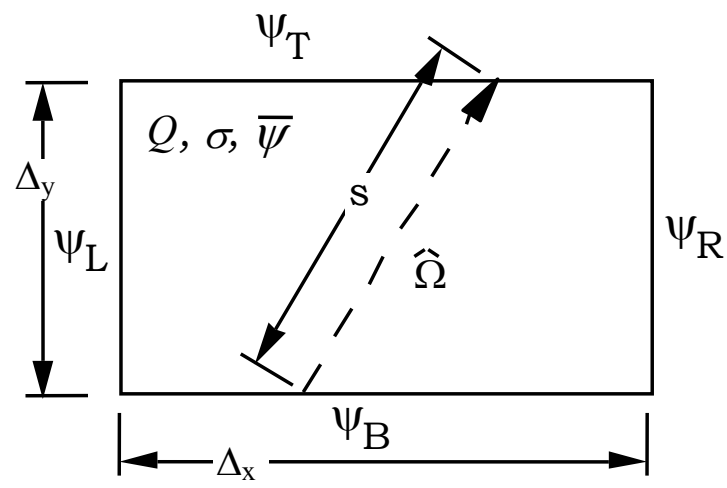

Figure 9.2.1. Typical rectangular cell used in the step characteristic approach.

\subsubsection{The Extended Step Characteristic approach}

The theory of the ESC approach is developed and explained in detail in Ref. 1. However, the work has evolved significantly from that time, most notably in the elimination of a requirement for non-reentrant polygons (convex). The following subsections describe the primary equations applied in the ESC approach as currently applied in NEWT.

\subsection{Cell properties and geometries}

The two primary assumptions of the ESC method are that (1) within each computational cell all properties (i.e., $\sigma_{\mathrm{t}}$ and Q) are uniform and (2) cell boundaries are defined by straight lines. The restriction of a computational cell to boundaries consisting of a set of straight lines results in computational cells that are limited to polygons. However, as will be seen later, no restrictions are placed on the shape of the polygon or on the number of sides in the polygon. However, the size of the polygon will be limited. In practical applications, properties are unlikely to remain constant over significant volumes. Thus this approach, like many other differencing schemes, is a poor approximation when cell volumes become too large. Although $\sigma_{t}$ is a material property and may remain spatially constant, the source term $Q$, which depends on the neutron flux, will vary with position. However, since the solution would become exact in an infinitesimally small cell, it is expected that the approximation will be reasonable for computational cells in which the change in the flux (and therefore the source) is small over the domain of the cell.

As a result of this geometric configuration, each side of a cell can have one of three possible attributes relative to particle flow in a given characteristic direction, as illustrated in Figure 9.2.2: (1) flow can enter the cell when crossing a side (as shown by sides E and F in the figure); (2) flow can exit the cell when 
crossing a side (sides B and C); or (3) in a special case, flow may be parallel to the orientation of a given side (sides A and D). Expressed mathematically, these relationships become

$$
\begin{aligned}
& \text { Category 1: } \widehat{\Omega}_{k} \cdot \hat{n}_{i}<0 \\
& \text { Category 2: } \widehat{\Omega}_{k} \cdot \hat{n}_{i}>0 \\
& \text { Category 1: } \widehat{\Omega}_{k} \cdot \hat{n}_{i}=0
\end{aligned}
$$

where $\hat{n}_{i}$ is a unit vector in the cell-outward direction normal to side $i$, and $\widehat{\Omega}_{k}$ is the $k^{\text {th }}$ discrete element of a set of characteristic directions. A category 1 side will be termed an "incoming" side with respect to the direction $\widehat{\Omega}_{k}$, and a category 2 side will be referred to as an "outgoing" side. For simplicity, the definition of Eq. (9.2.10) will be included as a special case of Eq. (9.2.8) for an incoming side. Thus, Eq. (9.2.8) can be rewritten as

$$
\begin{array}{ll}
\text { Side } i \text { is incoming with respect to } \widehat{\Omega}_{k}: & \widehat{\Omega}_{k} \cdot \hat{n}_{i} \leq 0 \\
\text { Side } i \text { is } \text { outgoing with respect to } \widehat{\Omega}_{k}: & \widehat{\Omega}_{k} \cdot \hat{n}_{i}>0
\end{array}
$$

To solve for fluxes (flow) on outgoing sides of a cell, one must know fluxes on all incoming sides. Each incoming side of each cell will be given from a boundary condition or will be the outgoing side of an adjacent cell.

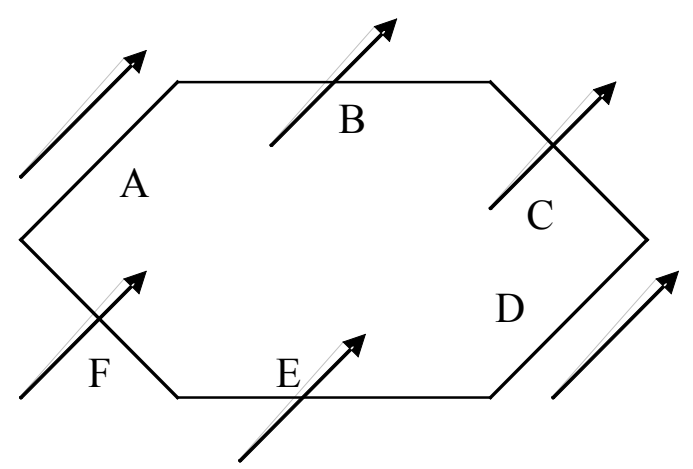

Figure 9.2.2. Orientation of the sides of a cell with respect to a given direction vector.

\subsection{Relationships between cells}

In the ESC method, the shape of the computational cell and the form of the neutron balance differ from that used in traditional discrete-ordinates methods. Nevertheless, the relationships between cells are treated essentially as they would be in traditional approaches. The entire problem domain is mapped in terms of a set of finite cells. Each side of each cell is adjacent to either an external boundary condition or another cell. For each discrete direction, cells are swept in a predetermined order beginning at a known boundary (from a specified external boundary condition) moving in the given direction. The precise order of sweep is such that as the solution for one cell is obtained, the cell provides sufficient boundary conditions for the solution of an adjacent cell. Hence, cells sharing a given side share the value of the angular flux on that side. Knowledge of the flux on all incoming sides of a cell is sufficient to solve for all 
outgoing sides. Once the angular flux has been determined for all sides of the cell for the given direction, it is possible to use a neutron balance to compute the average value of the angular flux within the cell.

The sweeping of cells continues for a given direction until all cell fluxes have been calculated. The procedure is then repeated for the next direction until all directions have been computed. At this point, the cell average angular fluxes are known for each cell for each direction used. Numerical quadrature can then be used to determine the average scalar flux in each cell in the problem domain. The scalar fluxes are used to determine fission and scattering reaction rates in each cell and to update the value of the cell average source, $\mathrm{Q}$. The process is repeated, and the iteration continues until all scalar fluxes converge to within a specified tolerance.

This approach can be performed assuming a single energy group or any number of discretized energy groups. The multigroup approach used in the ESC method is the standard approach used in most multigroup methods and is independent of the shape of each computational cell. Hence, the details of the multigroup formalism will be omitted from this discussion.

\subsection{The set of characteristic directions}

The characteristic solution to the transport equation gives only the angular flux in the direction of the characteristic direction vector $\widehat{\Omega}_{k}$. To compute interaction rates within a cell, one must compute scalar fluxes. In computing the scalar flux from the set of angular fluxes, it is convenient to choose the set of characteristic directions from an appropriate quadrature set. Then the set of computed angular fluxes can be combined with appropriate directional weights and summed to obtain a scalar flux solution within a cell. Therefore, it is most appropriate to choose characteristic directions from an established set of base points and weights. Such quadrature sets that have been developed and used in numerous earlier discreteordinates approaches are used in NEWT. No restriction is placed on the nature or order of the quadrature set, as long as it is sufficient to adequately represent the scalar flux from computed angular fluxes.

\subsection{Angular flux at a cell boundary}

As in the development of the SC method, as well as most finite-difference methods, the ESC approach does not explicitly determine the flux distribution as a function of position along the sides of a computational cell. Instead, the angular flux on each cell side is represented in terms of the average angular flux along the length of the side. This is sufficient to determine the net leakage across each cell side, which is needed in order to maintain a cell balance. An average value of the flux for an incoming side must be specified from a boundary condition or from the prior solution of an adjacent cell. The average flux along a given outgoing side can be computed by integrating the flux along the side and dividing by the length of the side. However, the form of the distribution of the angular flux on the side must be known to perform this integration. This distribution can be determined from the properties of the cell and from the average flux on each of the known incoming sides.

Because the characteristic solution [Eq. (9.2.6)] allows calculation of the angular flux at any point $s$ in a single cell given an initial condition, the exact value of the flux can be computed at any point on any outgoing side if the flux along each incoming side is known. As an initial condition, it is assumed that the angular flux in some characteristic direction is known at some starting point, $s=0$ [i.e., $\psi(0)=\psi_{0}$ ], on an incoming side. To determine the flux at some point on an outgoing side, one need know only the distance $s$ measured along a characteristic direction to the appropriate incoming side. This method can then be expanded to determine a functional form of the flux for every point on the outgoing side, which can be integrated to produce the average outgoing flux on the side.

To develop a mathematical relationship between two arbitrary sides of a cell, one should first consider two arbitrary coplanar line segments in space whose endpoints each lie on a pair of parallel lines laid in 
the direction $\widehat{\Omega}_{k}$, as shown in Figure 9.2.3. Points $\mathrm{B}_{1}$ and $\mathrm{B}_{2}$ can be considered to be the "projections" of $\mathrm{A}_{1}$ and $\mathrm{A}_{2}$, respectively, relative to $\widehat{\Omega}_{k}$. Because $s$ is the distance between a point on segment $\mathrm{A}$ and its projection on segment $\mathrm{B}$, it can be seen that $s$ varies linearly in moving from the "beginning" to the "end" of the pair of segments.

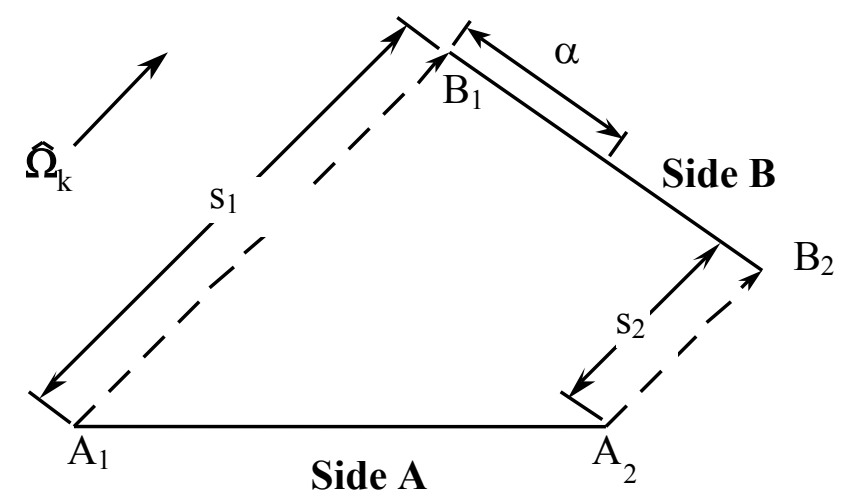

Figure 9.2.3. Line endpoints for computation of average fluxes.

If $\alpha$ is the distance along segment $B$ measured from endpoint $B_{1}$ and $B$ has a total length $L$, then the distance $s$ between $\mathrm{A}$ and $\mathrm{B}$ along direction $\widehat{\Omega}_{k}$ can be written as a linear function in terms of the position $\alpha$ :

$$
s(\alpha)=s_{1}+\left(\frac{s_{2}-s_{1}}{L}\right) \alpha,
$$

where $s_{1}$ and $s_{2}$ are related to the distances along the characteristic direction between $\mathrm{A}_{1}, \mathrm{~B}_{1}$ and $\mathrm{A}_{2}, \mathrm{~B}_{2}$, respectively. (It is important to note that the length $s$ is the same as the distance between the endpoints only when the characteristic vector lies in the plane of the computational cell. This is not necessarily the case, depending on the choice of quadrature directions. This situation is discussed in more detail later.)

If $\psi(\alpha)$ is the angular flux on side B at a distance $\alpha$ from $B_{1}$, then $\bar{\psi}_{\mathrm{B}}$, the average value of $\psi$ on B, is given by

$$
\bar{\psi}_{B}=\frac{\int_{0}^{L} \psi(s(\alpha)) d \alpha}{\int_{0}^{L} d \alpha}
$$

Equation (9.2.6), the solution to the characteristic equation in the step approximation, can be rewritten in terms of the average known angular flux on side A:

$$
\psi_{B}(s)=\left(\bar{\psi}_{A}-Q / \sigma_{t}\right) e^{-\sigma_{t} s}+Q / \sigma_{t}
$$


Inserting Eqs. (9.2.13) and (9.2.15) into Eq. (9.2.14) and simplifying yields

$$
\bar{\psi}_{B}=\frac{1}{L} \int_{0}^{L}\left[\left(\bar{\psi}_{A}-Q / \sigma_{t}\right) \exp \left(-\sigma_{t}\left(s_{1}+\left(\frac{s_{2}-s_{1}}{L}\right) \alpha\right)\right)+Q / \sigma_{t}\right] d \alpha .
$$

For the special case in which A and B are parallel, $s_{1}=s_{2}$ and the second term in the exponential drops out. Equation (9.2.16) can easily be integrated to obtain

$$
\bar{\psi}_{B}=\left(\bar{\psi}_{A}-Q / \sigma_{t}\right) e^{-\sigma_{t} s_{1}}+Q / \sigma_{t} \text {. }
$$

In the more general case, $s_{1} \neq s_{2}$, the result is slightly more complicated:

$$
\bar{\psi}_{B}=\frac{\left(\bar{\psi}_{A}-Q / \sigma_{t}\right)}{\sigma_{t}\left(s_{2}-s_{1}\right)}\left[e^{-\sigma_{t} s_{1}}-e^{-\sigma_{t} s_{2}}\right]+Q / \sigma_{t} .
$$

Equations (9.2.17) and (9.2.18) can also be written in a simplified form:

$$
\bar{\psi}_{B}=\beta_{A B} \bar{\psi}_{A}+\left(1-\beta_{A B}\right) Q / \sigma_{t}
$$

where

$$
\beta_{A B}=\left\{\begin{array}{cc}
\frac{e^{-\sigma_{t} s_{1}}-e^{-\sigma_{t} s_{2}}}{\sigma_{t}\left(s_{2}-s_{1}\right)} & s_{1} \neq s_{2} \\
e^{-\sigma_{t} s_{1}} & s_{1}=s_{2}
\end{array} .\right.
$$

Thus far, this development has considered only the special case where contributions to side B are the result only of the cell internal source and a single incoming side (i.e., side A). For an arbitrarily shaped cell and discrete direction $\widehat{\Omega}_{k}$, it is likely that the outgoing side would receive contributions from two or more incoming sides, as illustrated in Figure 9.2.4, for a cell with three incoming sides (X, Y, and Z) contributing to the flux on a single outgoing side(B). In such a situation, the outgoing side can be subdivided into multiple components. Side $\mathrm{B}$ of Figure 9.2.4 can be represented by three components, $\mathrm{B}_{\mathrm{X}}$, $\mathrm{B}_{\mathrm{Y}}$, and $\mathrm{B}_{\mathrm{Z}}$, representing contributions from line segments $\mathrm{X}, \mathrm{Y}$, and $\mathrm{Z}$, respectively. The average angular flux $\bar{\psi}$ can be computed for each component of side B using Eq. (9.2.19); then $\bar{\psi}_{\mathrm{B}}$, the average flux for the entire length of B, can be calculated by the length-weighted average of each component. In general, for a given side $\mathrm{B}$ composed of $n$ components, the average flux of the side is given by

$$
\bar{\psi}_{B}=\sum_{i=1}^{n} \frac{\bar{\psi}_{i} \ell_{i}}{L_{B}}
$$

where

$\ell_{i}$ is the length of the projection of the $i$ th side onto $\mathrm{B}$, and

$\bar{\psi}_{\mathrm{i}}$ is the average flux computed for segment $\mathrm{B}_{\mathrm{i}}$ due to the flux on side $i$. 
Using Eqs. (9.2.19) and (9.2.20), one can compute the average flux on each of the outgoing sides for a given cell, once the angular flux on each incoming side is known. At this point, only distances $s_{1}$ and $s_{2}$ and the lengths $\ell_{i}$ and $\mathrm{L}$ need be determined to estimate fluxes in an iterative process. These can be computed from the geometry of the cell and the direction $\widehat{\Omega}_{k}$.

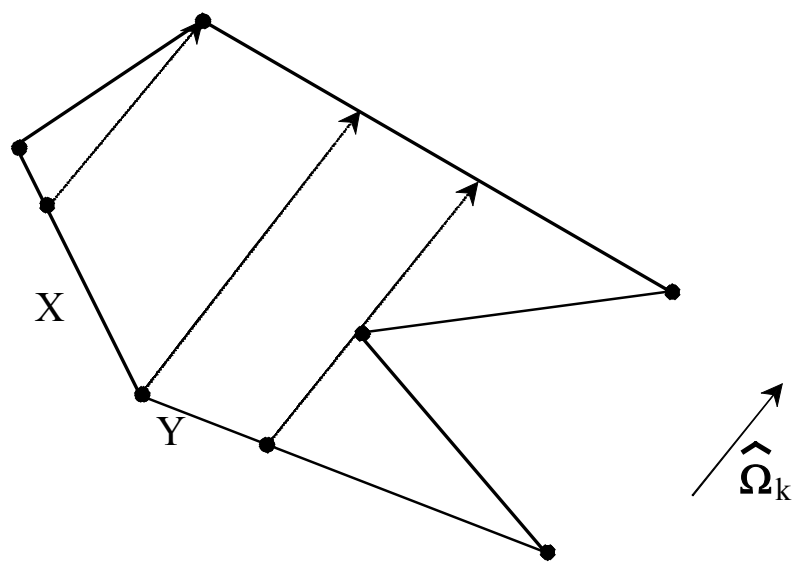

Figure 9.2.4. Contributions of multiple incoming sides to an outgoing side.

\subsection{Mapping a characteristic vector into the two-dimensional problem domain}

Even in a 2-D x-y system in which the scalar flux is constant with respect to the $\mathrm{z}$ axis, the angular flux has components in the $\mathrm{z}$ direction. Thus, to obtain the scalar flux at a point on the $\mathrm{x}-\mathrm{y}$ plane, one must integrate over the unit sphere in all $4 \pi$ directions of $\widehat{\Omega}$. Recall that the choices of characteristic directions for this model were selected to be the same as the set of directions composing a conventional quadrature set. Quadrature sets specified in the literature ${ }^{10-12}$ and used in other discrete-ordinates codes ${ }^{13,14}$ are based on a unit sphere and are usually specified in terms of $\mu_{\mathrm{k}}$ and $\eta_{\mathrm{k}}$, the respective $\mathrm{x}$ and y components of $\widehat{\Omega}_{k}$ , where $\widehat{\Omega}_{k}$ is one of a set of discrete directions composing the quadrature set. Because $\widehat{\Omega}_{k}$ is a unit vector, $\xi_{k}$, the z component of the direction, is implicit: $\xi_{k}=\sqrt{1-\mu_{k}^{2}-\eta_{k}^{2}}$. However, because of the 2-D nature of the problem, the $\mathrm{z}$ component is never explicitly used. It is therefore sufficient to evaluate the angular flux at a finite number of points in $4 \pi$ of $\widehat{\Omega}$-space in terms of just the $\mu_{\mathrm{k}}$ and $\eta_{\mathrm{k}}$ components of the discrete directions $\widehat{\Omega}_{k}$. One must recognize, however, that the length of the path traveled by particles moving in a direction out of the $x-y$ plane is always longer than the $x-y$ projection of the path, by a factor of $\left(\mu^{2}+\eta^{2}\right)^{-1 / 2}$. Thus, for any path length $s^{\prime}$ measured in the $\mathrm{x}-\mathrm{y}$ plane for a given direction $\widehat{\Omega}_{k}$, the true path length traveled is $s$, where

$$
s=\frac{s^{\prime}}{\sqrt{\mu^{2}+\eta^{2}}} .
$$

This is illustrated in Figure 9.2.5. 


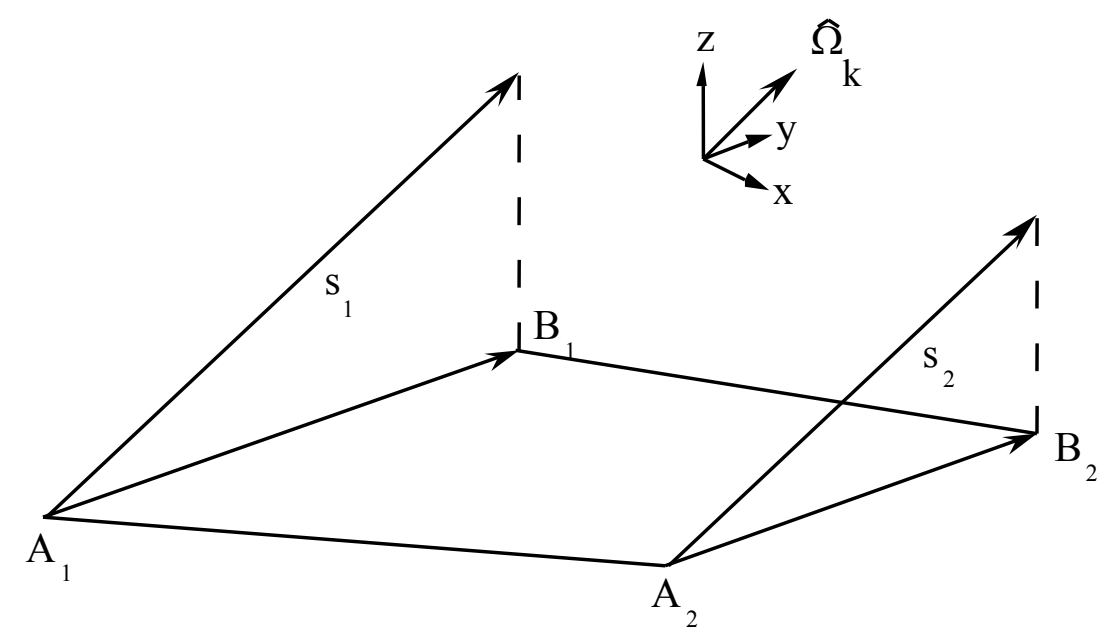

Figure 9.2.5. Relationship between $s_{1}$ and $s_{2}$ and their projections in the $x-y$ plane.

\subsection{Neutron balance within a computational cell}

Once angular fluxes have been computed for all sides of a cell, it is necessary to compute the cellaveraged angular flux. To enforce conservation, a balance condition is applied to the cell. This provides the equation necessary to determine the average flux in the cell. The neutron balance for an arbitrary cell in steady state may be expressed as

$$
\left[\begin{array}{c}
\text { net number of } \\
\text { neutrons moving in } \\
\text { direction } \hat{\Omega} \text { escaping } \\
\text { from the cell }
\end{array}\right]+\left[\begin{array}{c}
\text { number of neutrons } \\
\text { removed from the cell } \\
\text { or from direction } \hat{\Omega} \\
\text { by interactions }
\end{array}\right]=\left[\begin{array}{c}
\text { number of } \\
\text { neutrons produced } \\
\text { in the cell moving } \\
\text { in direction } \hat{\Omega}
\end{array}\right]
$$

or, expressed mathematically,

$$
\oint_{s} \vec{n} \cdot \hat{\Omega}_{k} \psi d S+\sigma_{t} \bar{\psi} V=Q V
$$

where $\vec{n}$ is the outward normal direction at each side of the cell and V is the 2-D volume of the cell. Note that in this context, $S$ represents the surface area or perimeter of the cell. Hence, for a cell with $m$ sides, each of the sides having a constant angular flux $\bar{\psi}_{i}$ and an outward normal direction $\overrightarrow{\mathrm{n}}_{i}$,

$$
\bar{\psi}_{\text {cell }}=\frac{Q}{\sigma_{t}}-\frac{1}{\sigma_{t} V} \sum_{i=1}^{m} \bar{\psi}_{i} \int_{S_{i}} \overrightarrow{\mathrm{n}}_{i} \cdot \hat{\Omega}_{k} d S_{i}
$$


Because each cell is restricted to be a polygon, each side in the cell will be a straight line and $\vec{n}_{i} \cdot \hat{\Omega}_{k}$ will be constant along the length of the side. Equation (9.2.24) can then be simplified to obtain

$$
\bar{\psi}_{\text {cell }}=\frac{Q}{\sigma_{t}}-\frac{1}{\sigma_{t} V} \sum_{i=1}^{m} \bar{\psi}_{i}\left(\overrightarrow{\mathrm{n}}_{i} \cdot \hat{\Omega}_{k} \mathrm{~L}_{i}\right),
$$

where $\mathrm{L}_{i}$ is the length of the $i$ th side and the term in parentheses represents a leakage coefficient for the side.

\subsubsection{Coarse-mesh finite-difference acceleration}

Beyond cell discretization and solution described above for the ESC approach, the NEWT iterative approach is similar to that used in other discrete-ordinates methods. Inner iterations are used to solve spatial fluxes in each energy group to generate updated source terms; outer iterations use these source terms to converge all energy groups. This source-iteration approach can be somewhat slow to converge, especially when significant scattering is present. Hence, it is desirable to apply some form of acceleration to the iterative solution used by NEWT. To this end, a coarse-mesh finite-difference acceleration (CMFD) approach has been added to NEWT. The CMFD formulation uses a simplified representation of a complex problem, in which selected rectangular regions are derived from the global NEWT Cartesian grid and homogenized. The CMFD formulation utilizes coupling correction factors for each homogenized cell to dynamically homogenize the constituent ESC-based polygonal cells during the iterative solution process such that the heterogeneous transport solution can be preserved. Dynamic-group collapse is also possible with a two-level CMFD formulation in which alternating multigroup and two-group calculations are performed. By extending the concept of the equivalence theory to energy and angle, it is possible to apply a consistent lower-order formulation in the form of a homogenized pin-cell, few-group, diffusionlike finite-difference scheme. This simplified lower-order formulation is much less expensive to solve, and its solution can be used to accelerate the original higher-order transport solution in NEWT, resulting in much faster convergence of the fission and scattering source distributions. This work is described in detail in Ref. 15 and in previous versions of the NEWT manual.

Although the original implementation of the CMFD acceleration method is extremely efficient and actively maintained, its use is limited to rectangular-domain configurations (e.g., square-pitched fuel lattices). An alternative CMFD acceleration method has been developed to support triangular- and hexagonal-domain configurations (e.g., triangular-pitched fuel lattices such as the VVER or prismatic graphite models). The new CMFD acceleration method does not require the coarse-mesh cells to be rectangles but rather arbitrary polygons. However in the current implementation, the "unstructured" coarse-mesh cells are still constructed from the global NEWT Cartesian grid. Therefore, for a hexagonal configuration, interior coarse-mesh cells will be rectangular shape whereas cells near the boundary will be triangular or trapezoidal shapes.

The new unstructured CMFD iterative solution scheme is essentially identical to the original solution scheme; the two methods differ only in how the lower-order system is solved. Additionally the two-group acceleration is not employed in the unstructured CMFD method. Input options for both CMFD methods are described in Sect. 9.2.3.2.

\subsubsection{Assembly discontinuity factors}

In nodal multi-assembly or core calculations, lattice transport solutions are used to generate few-group homogenized cross sections. These cross sections are in general obtained from single-assembly transport calculations with zero-current boundary conditions. Generation of few-group homogenized cross sections 
for nodal calculations typically includes the generation of discontinuity factors (i.e., additional parameters used to preserve both reaction rates and the interface currents in the homogenization process). The discontinuity of the flux at an assembly interface that can arise by the use of homogenized cross sections is illustrated in Figure 9.2.6. The so-called "homogeneous" flux, computed in the nodal calculation, is discontinuous at the assembly interface, as opposed to the exact "heterogeneous" flux, computed in the transport calculation, which is continuous at the assembly interface. The interface condition employed in nodal calculations between two assemblies (nodes) $i$ and $i+1$ is given as

$$
\phi_{i, \text { homogeneous }}^{+} \cdot F_{i}^{+}=\phi_{i+1, \text { homogeneous }}^{-} \cdot F_{i+1}^{-}
$$

where $F_{i}^{+}$and $F_{i+1}^{-}$are assembly discontinuity factors (ADFs) on each side of the interface between assemblies $i$ and $i+1$.

The ADF on the assembly interface is defined as the ratio of the heterogeneous flux $\phi_{\text {heterogeneous }}$ at that assembly interface to the homogeneous flux evaluated at the interface, denoted $\phi_{i, \text { homogeneous }}^{+}$ (or $\phi_{i+1, \text { homogeneous }}^{-}$):

$$
F_{i}^{+}=\frac{\phi_{\text {heterogeneous }}}{\phi_{i, \text { homogeneous }}^{+}}, F_{i+1}^{-}=\frac{\phi_{\text {heterogeneous }}}{\phi_{i+1, \text { homogeneous }}^{-}} .
$$

Fluxes, and therefore ADFs, vary with energy; therefore, few-group homogenized cross sections are always accompanied by corresponding few-group ADFs.

In a single-assembly calculation with zero-current boundary conditions, the heterogeneous flux at each boundary is easily calculated as the surface-averaged scalar flux on the boundary, whereas the homogenous flux at each boundary is simply the assembly-averaged flux. Hence, for each energy group, the ADF is calculated for each boundary as the ratio of the average flux on that boundary to the average flux across the assembly.

In other configurations, such as a multi-assembly calculation or an assembly located on the edge of a core next to the core baffle and reflector, the ADF calculation requires more effort. For reflector situations, NEWT applies a simple one-dimensional (1-D) multigroup diffusion approximation to determine the ADF at the assembly boundary. In this approximation, it is assumed that the reflector is infinite and that the scalar flux goes to zero at infinity. The reflector ADF can be determined analytically using this boundary condition along with the known surface-averaged current and scalar flux evaluated at the assembly/reflector interface.

The reflector ADFs computed by NEWT may potentially be different from the ADFs calculated using the diffusion approximations employed by the nodal code. Moreover, ADFs computed for multi-assembly or hexagonal-domain configurations will depend on the nodal method employed. For these reasons, NEWT supports the option to edit surface-averaged scalar flux and current values along user-defined line segments so that appropriate ADFs can be computed directly by the nodal code. The input options for the single-assembly ADF, reflector ADF, and arbitrary line-segment edit are discussed in Sect. 9.2.3.11. 


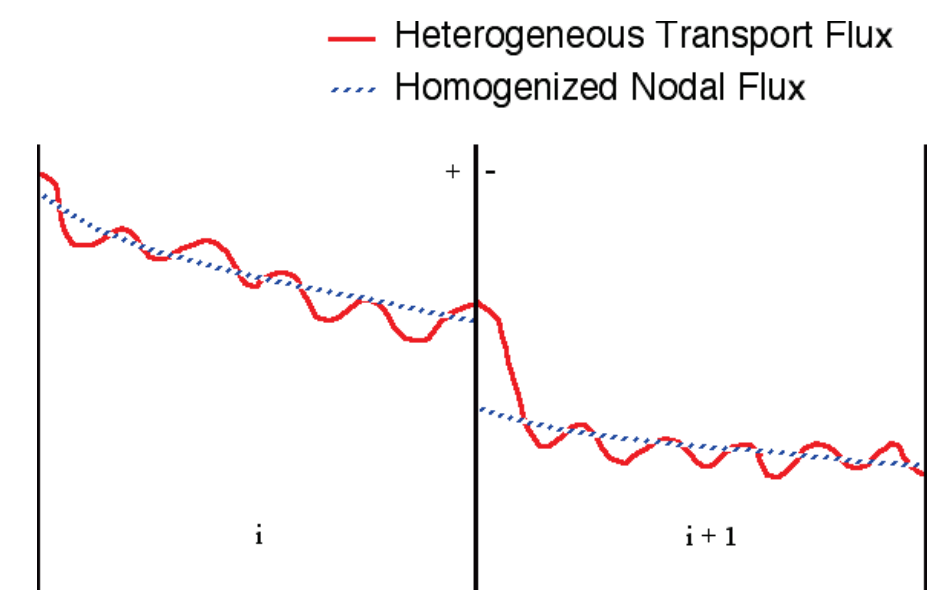

Figure 9.2.6. Heterogeneous vs homogeneous fluxes in a multi-assembly solution.

\subsubsection{Input Formats}

NEWT input is free form and keyword based, similar in form to the input for many other modules in the SCALE code package. Input may start with a title card record, but this line may be omitted if desired; remaining data are supplied in data blocks. The order of the data blocks is arbitrary (with two exceptions), and many blocks are optional. Only one instance of a data block is allowed.

\subsubsection{Overview of newt data blocks}

The NEWT input deck data blocks are defined by keyword delimiters in the following form:

$$
\text { read keyword [data] end keyword }
$$

Read routines are terminated by the "end keyword" label, and any intervening carriage returns or line feeds are ignored. Thus, data can also be entered in this format:

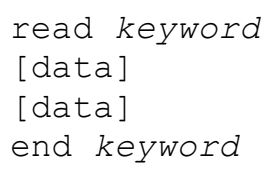

Within each block, specific control or specification parameters are input. Each block contains a fixed set of input parameters (also defined by keyword).

As with other keyword-driven modules within SCALE, lines beginning with a single quote (') in the first column are treated as comments and ignored.

The keyword name and general contents of each data block are as follows:

\begin{tabular}{|l|l|l|}
\hline Block type & $\begin{array}{l}\text { Recognized } \\
\text { keywords }\end{array}$ & Description \\
\hline $\begin{array}{l}\text { Problem control } \\
\text { parameters } \\
(9.2 .3 .2)\end{array}$ & $\begin{array}{l}\text { parameter, } \\
\text { parameters, } \\
\text { param, parm, } \\
\text { para }\end{array}$ & $\begin{array}{l}\text { General problem parameters-must follow title card, if used } \\
\text { (optional) }\end{array}$ \\
\hline
\end{tabular}




\begin{tabular}{|c|c|c|}
\hline Block type & $\begin{array}{l}\text { Recognized } \\
\text { keywords }\end{array}$ & Description \\
\hline $\begin{array}{l}\text { Material } \\
\text { properties } \\
(9.2 .3 .3)\end{array}$ & $\begin{array}{l}\text { material, } \\
\text { materials, } \\
\text { matl }\end{array}$ & $\begin{array}{l}\text { Assigns characteristics (e.g., } P_{n} \text { scattering order and material } \\
\text { description) for each material ID-must follow problem control } \\
\text { block or must follow title card if control block is omitted (required) }\end{array}$ \\
\hline $\begin{array}{l}\text { Broad group } \\
\text { collapse } \\
(9.2 .3 .5)\end{array}$ & collapse, coll & $\begin{array}{l}\text { Defines broad group energy ranges to be created from the original } \\
\text { fine group library when cross section collapse is desired (optional) }\end{array}$ \\
\hline $\begin{array}{l}\text { Simple-body } \\
\text { geometry } \\
(9.2 .3 .6)\end{array}$ & $\begin{array}{l}\text { geometry, } \\
\text { geom }\end{array}$ & $\begin{array}{l}\text { Defines basic grid structure and all bodies to be placed within this } \\
\text { structure (required unless geometry restart file is available) }\end{array}$ \\
\hline $\begin{array}{l}\text { Boundary } \\
\text { conditions } \\
(9.2 .3 .7)\end{array}$ & bounds, bnds & $\begin{array}{l}\text { Defines boundary conditions to be applied on outer boundaries of } \\
\text { global unit (optional, default is reflective on all sides) }\end{array}$ \\
\hline $\begin{array}{l}\text { Array } \\
\text { specifications } \\
(9.2 .3 .9)\end{array}$ & array & $\begin{array}{l}\text { Defines composition of all arrays (unit placement within each } \\
\text { array). Each array placed within the geometry block must be } \\
\text { defined in the array block }\end{array}$ \\
\hline $\begin{array}{l}\text { Homogenization } \\
\text { instructions } \\
(9.2 .3 .10)\end{array}$ & $\begin{array}{l}\text { homog, hmog, } \\
\text { homo }\end{array}$ & $\begin{array}{l}\text { Defines mixtures to be flux weighted and homogenized in the } \\
\text { preparation of a homogenized cross section library (optional) }\end{array}$ \\
\hline $\begin{array}{l}\text { Assembly } \\
\text { discontinuity } \\
\text { factors } \\
\text { (9.2.3.11) }\end{array}$ & adf & $\begin{array}{l}\text { Assigns type and location of planes at which assembly } \\
\text { discontinuity factors (ADFs) are calculated (optional) }\end{array}$ \\
\hline $\begin{array}{l}\text { Flux plane } \\
(9.2 .3 .12)\end{array}$ & flux & $\begin{array}{l}\text { Allows definition of an } \mathrm{x} \text { - or } \mathrm{y} \text {-axis line (plane) for which average } \\
\text { fluxes are computed and printed (optional) }\end{array}$ \\
\hline $\begin{array}{l}\text { Mixing table } \\
(9.2 .3 .13)\end{array}$ & mixtable, mixt & Mixing table specification (optional) \\
\hline $\begin{array}{l}\text { Source } \\
\text { definition } \\
(9.2 .3 .4)\end{array}$ & src, source & Defines particle source strength for use in source calculations \\
\hline
\end{tabular}

Each of the following subsections describes the parameters associated with a specific data block, lists default values (if available), and describes meaning of the parameter and its effect on a NEWT calculation.

\subsubsection{Parameter block}

Parameter Block keyword = param, parm, para, parameter, or parameters

The Parameter block contains problem control parameters and must come immediately after the title card if one is used. Valid parameter specifications are described below. For each keyword, allowable values are listed in parentheses, and the default (if any) is listed in brackets. Input that can take an arbitrary integer value is indicated by an $I N$; similarly, any parameter that can take an arbitrary real/floating point value is indicated by $R N$ as the allowable value. However, note that SCALE read routines do allow input of integers for real numbers, and vice versa; the number will be converted accordingly. The order of the 
parameters within the block is arbitrary, and may be skipped if a default value is desired for that parameter. Control parameters are set in the order in which they are input; this means that the same parameter may be listed multiple times, but only the final value is used.

\subsection{Convergence and acceleration parameters}

epseigen $=(R N)-$ Convergence criterion for $k_{\text {eff }}$ [0.0001]

epsinner $=(R N)-$ Spatial convergence criterion for inner iterations. [0.0001]

epsouter $=(R N)-$ Spatial convergence criterion for outer iterations. [0.0001]

epsthrm $=(R N)$ - Spatial convergence criterion for thermal-upscattering iterations, if enabled. [same value as epsouter]

epsilon $=(R N)-$ Simultaneously sets all (spatial and eigenvalue) convergence criteria to the same value. [uses individual defaults]

converg $=($ cell/mix $)$ - Sets the region within which convergence testing is applied. Use of cell will force converged scalar fluxes in every computation cell, while mix will relax convergence such that averaged scalar fluxes within a mixture are converged. The latter is useful for mixtures in which fluxes become very small-large reflectors or near a vacuum BC. [cell]

therm $=($ yes $/$ no $)-$ Enables/disables thermal-upscattering iterations. [yes]

inners $=(I N)-$ Maximum number of inner iterations in an energy group. [5]

therms $=(I N)-$ Maximum number of thermal-upscattering iterations, if enabled. [2]

outers $=(I N)-$ Maximum number of outer iterations. NEWT will stop with an error code if more than outers outer iterations are required for convergence. [250]

inrcvrg $=($ yes $/ n o)$ - If inrcvrg=yes, NEWT will continue outer iterations until all convergence criteria are met. If inrcvrg=no, NEWT will stop whenever outer iteration and $k_{\text {eff }}$ convergence criterion are met, regardless of the convergence of inner or thermal-upscattering iterations. [no]

$\mathbf{c m f d}=($ no/rect/yes/part $)-$ CMFD acceleration option. If $\mathbf{c m f d}=$ no, CMFD acceleration is not employed. If $\mathrm{cmfd}=$ rect, the CMFD method is employed. The original NEWT CMFD method can be applied only to rectangular-domain configurations. If $\mathrm{cmfd}=\mathrm{yes}$, the unstructured CMFD method is employed. The new unstructured CMFD method can be applied to rectangular-, triangular-, and hexagonal- domain configurations. If $\mathrm{cmfd}=$ part, an alternative version of the unstructured CMFD method is employed and uses a "partial-current" acceleration scheme. Alternatively, users can use cmfd $=0 / 1 / 2 / 3$ for no, rect, yes, and part, respectively. [no]

$\mathbf{c m f d 2 g}=($ yes/no $)$ - Enables/disables the second-level two-group CMFD accelerator within the CMFD solver. This parameter has an effect only when $\mathrm{cmfd}=$ rect is set. [yes]

accel $=(y e s / n o)-$ Enables/disables source $\left(k_{\text {eff }}\right)$ acceleration. This parameter is automatically disabled if unstructured CMFD is employed $(\mathrm{cmfd}=$ yes or $\mathrm{cmfd}=$ part $)$. [yes]

$\mathbf{x c m f d}=(I N), \mathbf{y} \mathbf{c m f d}=(I N), \mathbf{x y c m f d}=(I N)$ - These inputs specify the number of fine-mesh cells in the global NEWT grid per coarse-mesh cell. These options are used only when CMFD acceleration is enabled. The parameter xcmfd specifies the number fine-mesh cells per coarse-mesh cell in the 
x-direction. Likewise, ycmfd specifies the number of fine-mesh cells per coarse-mesh cell in the y-direction. The parameter $x y c m f d$ simultaneously sets $x \mathrm{cmfd}$ and $y \mathrm{cmfd}$ to the same value. In a special case for rectangular-domain configurations in which the entire domain is completely filled by a squaretype array (see Sect. 9.2.3.9), xycmfd $=0$ sets the coarse mesh based on the size of the array elements. [1]

\section{User Guidance:}

Default convergence parameters are recommended for general analysis. Larger convergence criteria are useful for debugging if shorter run time is desired over solution accuracy. Smaller convergence criteria are recommended for generating reference solutions or benchmark calculations.

CMFD acceleration should be applied whenever possible. The CMFD method with second-level 2-group acceleration should be applied for rectangular-domain configurations [e.g., light water reactor (LWR) assembly models (cmfd=rect), by default cmfd2g=yes]. The unstructured CMFD method should be applied for triangular- or hexagonal-domain configurations (cmfd=yes). If NEWT detects an unstable CMFD condition, a warning message is printed and NEWT continues with CMFD disabled. NEWT may also provide a terminating error message if improper selection of the coarse mesh is detected. Internal investigation has shown that the coarse mesh should be approximately the same size as the unit cell used in the model. For LWR assembly models, a fine mesh of $4 \times 4$ is recommended for the square-pitched unit cell, implying that xycmfd should be 4 only if the global unit has a mesh. If individual meshes are used in each unit definition, then the global unit coarse-mesh cells should be sized based on the unit cell size and, therefore, $x y c m f d=1$ should be used. The values of $x \mathrm{cmfd}$ and $\boldsymbol{y c m f d}$ do not have to be a common factor of the number of fine-mesh cells in a given direction (NEWT will make the last coarse-mesh cell smaller than the other coarse-mesh cells), but it is highly recommended.

Users can gauge solution convergence by the outer iteration edit as it is printed to the terminal window (echo=yes, see below). One can terminate a calculation prematurely (via the Control-C option on most platforms) if convergence or iteration parameters need to be modified.

The TRITON control module supports a sensitivity and uncertainty analysis sequence TSUNAMI-2D (See TRITON chapter, section S/U Analysis Sequences (TSUNAMI-2D, TSUNAMI-2DC)). TSUNAMI-2D calculations require NEWT to be run in both forward mode and adjoint mode. In adjoint mode, CMFD acceleration is not currently supported and NEWT automatically disables its use if $\mathbf{c m f d}=\boldsymbol{y}$ es, $=$ rect, or =part. In adjoint mode with defined fixed source [i.e., generalized perturbation theory (GPT) analysis], it is observed that tighter convergence and iteration parameters are needed to properly remove fundamental mode contamination. (For more details, see SAMS chapter: Generalized Perturbation Theory.) To facilitate the CMFD options and larger convergence criteria for the forward calculations as well as smaller convergence criteria for GPT adjoint calculations, the following parameters are also available.

gptepsinner $=(R N)-$ Spatial convergence criterion for inner iterations in GPT analysis. [0.0001]

gptepsouter $=(R N)-$ Spatial convergence criterion for outer iterations in GPT analysis. [0.001]

gptepsthrm $=(R N)$ - Spatial convergence criterion for thermal-upscattering iterations, if enabled, in GPT analysis. [same value as gptepsouter]

gptsepsilon $=(R N)$ - Simultaneously sets all spatial convergence criteria to the same value in GPT analysis. [uses individual defaults]

gpttherm $=($ yes $/$ no $)-$ Enables/disables thermal-upscattering iterations in GPT analysis. [yes] 
gptinners $=(I N)-$ Maximum number of inner iterations in an energy group in GPT analysis. [500]

gpttherms $=(I N)-$ Maximum number of thermal-upscattering iterations, if enabled, in GPT analysis. [10]

gptouters $=(I N)-$ Maximum number of outer iterations in GPT analysis. NEWT will stop with an error code if more than outers outer iterations are required for convergence. [2000]

\section{User Guidance:}

Default values for GPT convergence may change with future releases, as more experience is gained and user feedback is received. If the GPT calculation is not converging because of fundamental mode contamination, it is recommended that convergence criteria be decreased and/or inner and thermalupscattering iteration limits be increased. If the solution convergence is slow, gptinners can potentially be decreased. Again, it is highly recommended that echo=yes be used to monitor speed of convergence.

\subsection{Output editing}

drawit $=(y e s / n o)-$ Create a PostScript file showing the grid structure determined from input. Two files are created - the first showing the grid structure and the second showing the material placement. (Features and use of this simple graphics capability are described further in Sect. 9.2.5.14.) [no]

echo $=($ yes $/$ no $)-$ During the iteration phase of execution, output is generated at the beginning of each outer iteration. This same information can be printed to SCALE message file (.msg) during iteration by setting echo=yes. [no]

prtbalnc $=(y e s / n o)-$ Flag indicating whether or not balance tables for fine-group mixtures should be printed. [no]

prtbroad $=(y e s / n o / 1 d)-$ Flag indicating whether or not broad group cross sections should be printed in problem output. The $1 d$ option indicates that 2-D scattering tables are not to be printed. This flag has no effect if collapse $=$ no is specified. [no]

prthmmix $=($ yes $/$ no $)-$ Flag indicating whether or not homogenized mixture macroscopic cross sections should be printed in problem output. Homogenized cross sections are printed only if Homogenization Block is provided (Sect.9.2.3.10). [yes]

prtflux $=(y e s / n o)$ - Create a PostScript plot file showing flux distribution for each energy group in problem. If an energy collapse is performed, a second plot file is generated for the fluxes of the collapsed group structures. [no]

$\operatorname{prtmxsec}=(y e s / n o / 1 d)-$ Flag indicating whether or not mixture macroscopic cross sections should be printed in problem output. The $1 d$ option indicates that 2-D scattering tables are not to be printed. [no]

$\operatorname{prtmxtab}=(y e s / n o)-$ Flag indicating whether or not the input mixing table should be printed in problem output. [no]

prtxsec $=(y e s /$ no $/ 1 d)-$ Flag indicating whether or not input microscopic cross sections should be printed in problem output. The $1 d$ option indicates that 2-D scattering tables are not to be printed. [no]

timed $=($ yes/no $)-$ Turns on printing of iteration timing and CPU use data. [no] 
det $=(I N)$ - Specifies the mixture used to represent a local power range monitor (LPRM) and/or Traversing In-core Probe (TIP) detector located within a fuel lattice. The mixture must also be included in a homogenization block in order to obtain detector cross sections. [has no default]

\section{User Guidance:}

With the exception of prthmmix, all output edit options are disabled unless requested by the user. The output edits are disabled by default to minimize the size of the output. The drawit option is recommended to generate PostScript plots of the model grid structure and material placement. As previously mentioned, the echo and timed options are recommended to monitor solution convergence. If the timed option is enabled, each line in the outer iteration edit will be longer than 80 characters. Therefore, it is recommended that Windows users should increase the Command Window size from 80 characters to 132 characters.

\subsection{Angular quadrature}

$\mathbf{s n}=(2 / 4 / 6 / 8 / 10 / 12 / 14 / 16)-$ Order of Sn level symmetric quadrature set. [6]

nazim $=(I N)-$ Number of equally spaced azimuthal directions in a product quadrature set. Used in tandem with npolar keyword (both must be specified). Total number of angles in the product quadrature set is the product of nazim and npolar. [No default. If not specified, level symmetric quadrature default is used.]

npolar $=(I N)-$ Number of polar angles in a product quadrature set (determined using a Gauss-Legendre polynomial). Used in tandem with nazim keyword (both must be specified). Total number of angles in the product quadrature set is the product of nazim and npolar. [No default. If not specified, level symmetric quadrature default is used.]

dgauss $=($ yes/no $)-$ Enables/disables use of double Gauss-Legendre product quadrature set. If disabled, single Gauss-Legendre product quadrature sets are used. [no]

\section{User Guidance:}

If both level symmetric quadrature sets and product quadrature sets are requested, the level symmetric quadrature set is to be used. Level symmetric quadrature sets are recommended for general analysis. If reflective boundary conditions are desired for hexagonal-domain configurations, product quadrature sets must be used and nazim must be a multiple of 3. If reflective boundary conditions are desired for triangular-domain configurations, product quadrature sets must be used and nazim must be an odd number.

\subsection{Control options}

adjoint $=(y e s / n o)$ - This keyword specifies either a forward (adjoint=no) or adjoint (adjoint=yes) calculation. [no]

forward=(yes/no) — This keyword specifies either a forward (forward=yes) or adjoint (forward=no) calculation. If adjoint and forward are both specified, NEWT uses the last specification. [yes]

$\mathbf{g p t}=(y e s / n o)$ - This keyword specifies whether this is a GPT adjoint calculation. The gpt keyword is active only for adjoint calculations. [no] 


\section{User Guidance:}

The TRITON control module automatically sets the values for forward, adjoint, and gpt keywords; therefore, they can typically be omitted from the Parameter Block. Default values are recommended unless running stand-alone NEWT adjoint calculations.

run=(yes/no $)-\mathrm{A}$ run=no calculation will perform all setup calculations normally performed before beginning iterations and then will stop. It is useful for debugging input and obtaining plots of the input geometry. Run=yes will perform a complete calculation. [yes]

$\operatorname{premix}=(y e s / n o)$ - This flag indicates whether the cross section library contains microscopic ( remix $=$ no) or macroscopic (premix=yes) cross sections. In essence, it creates a mixing table with a mixture fraction of 1.0 for each mixture on the library. Other mixing tables are ignored. The premixed cross section option is active only for stand-alone NEWT calculations. [no]

kguess $=(R N)$ - Initial guess at eigenvalue for an eigenvalue calculation. This parameter may be entered but is not used if a source calculation is performed or a restart file is used to determine the initial guess. [1.0]

restart $=($ yes $/$ no $)$ - If restart=yes is specified, NEWT will open file restart_newt and read scalar fluxes and fission rates, enabling a restart from the point at which a previous calculation ended. The file restart_newt is always written by NEWT at the end of every successful calculation. The code assumes that all geometry is unchanged from the previous calculation but does allow restart with a different angular quadrature set and $\mathrm{P}_{\mathrm{n}}$ scattering coefficients. A low-order solution can be used to accelerate a higher-order solution by restarting using the converged flux of the lower-order solution. [no]

savrest $=($ yes $/$ no $)$ - Determines whether or not a geometry restart file worf is written at the end of a calculation. If written, it will overwrite any existing geometry restart file. [yes]

\section{User Guidance:}

The default values of savrest and kguess are recommended. The TRITON control module automates generation and reuse of the geometry restart file, as well as the initial guess of the eigenvalue. Keywords run, premix, and restart can generally be omitted unless the following conditions are applicable:

- $\quad$ TRITON T-NEWT sequence calculation or stand-alone NEWT calculation with user-supplied restart file, restart=yes.

- $\quad$ Stand-alone NEWT calculation with user-supplied premixed cross section file, premix=yes.

- Interested only in performing setup calculations to debug input and generate geometry plots, run=no, and/or PARM=CHECK in the TRITON sequence input.

solntype $=(\mathrm{keff} / \mathrm{b} 1 / \mathrm{src})$ - Specifies solution mode type: keff is eigenvalue, b1 is eigenvalue mode followed by a buckling correction, and src is fixed source (no eigenvalue calculation). Fixed source calculations require additional data for the source specification (see Materials and Source data blocks in Sects. 9.2.3.3 and 9.2.3.4). [keff]

collapse $=($ yes $/ n o)$ - If collapse $=$ yes is specified, a flux-weighted collapse is performed by material number; cross sections for each nuclide in each material in the problem are collapsed to a specified (or default) group structure based on the average flux in that material. If collapse=yes, NEWT will look for the collapse parameter block; if not found, NEWT will generate cross sections based on the original group structure. If a Homogenization block is present, then collapse is always set to yes. [no] 
saveangflx $=($ yes $/$ no $)$ - Option to save angular flux solution. The angular flux is saved to a binary file used in the TSUNAMI-2D sequence of the TRITON control module. Because the angular flux can require significant file storage, it is not saved by default. The angular flux solution can and should be saved for TSUNAMI-2D calculations to generate more accurate sensitivity coefficients. [no]

\section{User Guidance:}

Keyword threads should be omitted in favor of the SCALE command line-I option. Keywords solntype, collapse, and saveangflx should be omitted unless the following conditions are applicable.

- For homogenized few-group cross section generation for nodal calculations, solntype should be b1. This option will perform a critical spectrum calculation, which will be folded into cross section homogenization calculation. The critical spectrum is also folded into the generation of $A D F s$ and reaction rates for depletion calculations.

- Generation of a new collapsed cross section library, collapse=yes.

- For TSUNAMI-2D calculations, saveangflx=yes.

\subsection{Geometry processing options}

combine $=(y e s / n o)-$ Automatic grid generation can result in very small grid cells in some locations. Setting parameter combine to yes performs automatic combination of smaller grid cells into adjacent neighbor of same material, if possible. Combine is automatically set to no if CMFD is enabled; this setting cannot be overridden. [no]

clearint $=(y e s / n o)$ - Grid generation option that removes the global NEWT grid if a local unit grid is supplied. (For meshing options, see the boundary keyword in the Geometry block description in Sect. 9.2.3.6.) By default, clearint is set to yes, which means the global grid is removed if local grids are provided. If CMFD acceleration is enabled, clearint is set to no, which means both the global grid and optional local grids are used. [yes]

grid_tol $=(R N)$ - Tolerance used in determining if polygon vertices are numerically identical during NEWT grid generation. [0.000001]

cell_tol $=(R N)$ - Tolerance used in determining if polygon vertices are numerically identical during NEWT cell generation. [0.000001]

line_tol $=(R N)$ - Tolerance used in determining if polygon vertices are numerically identical during NEWT line generation. [1.0e-10]

\section{User Guidance:}

The default values for all geometry-processing keywords are recommended and can be omitted. For problems with very fine mesh, tighter grid and cell tolerances should be applied. For problems that terminate with a ray-tracing error (i.e., tracer error), tighter grid and cell tolerances should be applied.

\subsection{Critical spectrum options}

useb1 $=(y e s / n o) ~-$ Turns on/off the use of the B1 approximation to determine the critical spectrum. If useb1 is set to no, the $\mathrm{P} 1$ approximation is used. [yes]

$\mathbf{b 2}=(R N)-$ Material buckling factor, in units of $1 / \mathrm{cm}^{2} .[0.0]$ 
height $=(R N)-$ Height (transverse dimension) in centimeters. Used in a geometric buckling correction to calculate leakage normal to the plane of the input 2-D model. Keywords $\mathbf{d z}=$ and $\mathbf{d e l t a z}=$ are equivalent. When set to zero (default), no buckling correction is performed. [0.0]

$\mathbf{b} \mathbf{f}=(R N)$ - Twice the extrapolation distance multiplier used to determine the geometric buckling correction. [1.420892]

\section{User Guidance:}

If critical spectrum corrections are to be applied, the default values listed above are recommended along with solntype $=\boldsymbol{b} 1$. In this option, NEWT will search for the material buckling value such that the homogenized infinite-medium system is critical. NEWT currently uses the B1 approximation as the default. If the P1 approximation is preferred, useb1should be set to no. The infinite-medium B1 (or P1) buckling search is performed in the energy group structure as the original model.

Alternatively, the user can supply the material buckling value using the b2 keyword, and specifying the B1 (default) or P1 approximation (useb1=no). In this case, solntype should be set to keff.

Alternatively, if the user knows the transverse dimension, a geometry buckling factor can be applied, derived from the user-defined height and extrapolation distance term bf as the following:

$$
B_{g}^{2}=\left(\frac{\pi}{H+z / \sigma_{t r}}\right)^{2}
$$

In this formula, $H$ is keyword height, $z$ is keyword bf, and $\sigma_{\text {tr }}$ is the collapsed, homogenized macroscopic transport cross section.

\subsection{File unit options}

\section{User Guidance:}

It is highly recommended that the file unit options below be omitted or that default values be used. Alternate file unit values are acceptable for stand-alone NEWT calculations, but changing their values may adversely impact other SCALE modules if NEWT is invoked through a SCALE sequence.

hmoglib $=(I N, 0<I N<100)$ - This input value specifies the unit number to which a collapsed and homogenized cross section library is written if homogenization instructions are provided (ftINf001). [13]

mixtab $=(I N, 0<I N<100)-$ NEWT is able to use a mixing table prepared by SCALE (which may be generated using the T-XSEC sequence). The value of IN defines the filename that NEWT will try to locate to read mixing data (i.e., mixtab=92 will cause NEWT to seek the file named ft92f001). This is the default filename produced by the T-XSEC sequence. Alternatively, a mixing table may be specified in NEWT input in the read mixtable block; if such a mixing table is supplied, the value of mixtab is ignored. [92]

$\mathbf{w t d l i b}=(I N, 0<I N<100)$ - This input value specifies the unit number to which a collapsed cross section library is written if collapse=yes is specified (ftINf001). IN must be positive and less than 100. [30]

xnlib $=(I N, 0<I N<100)-$ This number indicates the filename containing cross sections prepared in a problem-dependent AMPX working library format. The input xnlib=IN will cause NEWT to open file $\mathrm{ft} I N \mathrm{f} 001$. This is the only method for providing cross sections as input for NEWT. [4] 
Examples of input for the parameter block are given below. Note that the two inputs are functionally identical. In the first example, parameters are specified, while in the second example, the input is structured differently and takes advantage of default values.

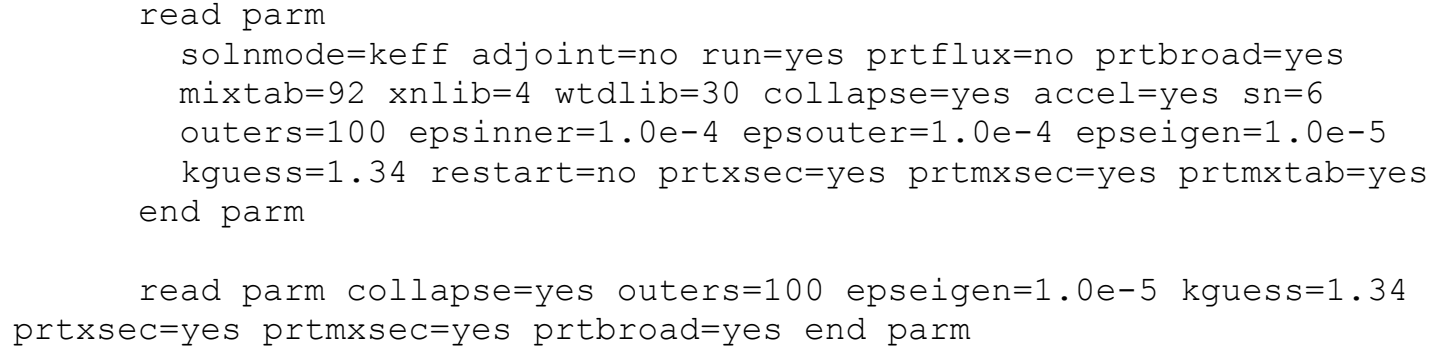

\subsubsection{Material block}

\section{Material block keyword = matl, material, materials}

The Material block is always required. Material data must be specified for each mixture used in the calculation. The general format of the Material block is as follows:

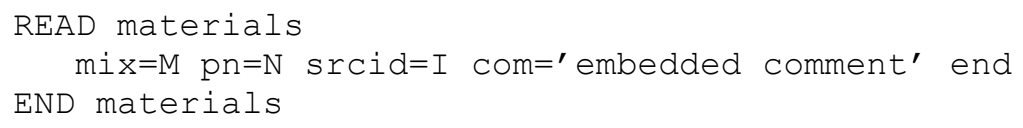

where

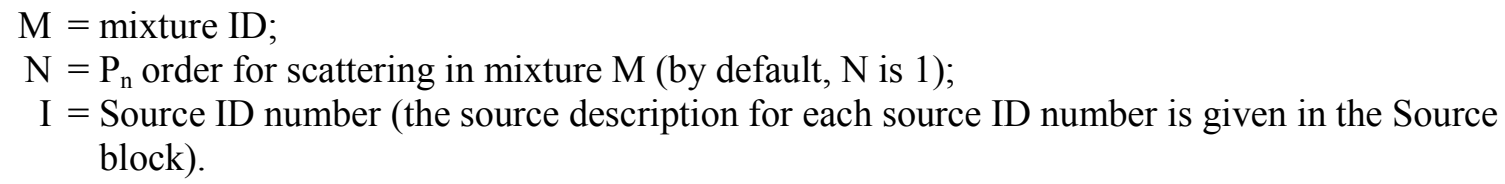

Up to 80 characters of text may be entered after $c o m=$, delimited by single quotes (') or double quotes ("). A mixture specification is required for each mixture used in the NEWT calculation. The order of the keywords in each specification is unimportant, and only the mix= keyword is required; however, each mixture specification must be terminated by the end keyword.

A sample Material block is provided below for three different mixtures. Each mixture is specified in a different manner to illustrate different input formats. In this example, $\mathrm{P}_{3}$ scattering is applied in mixture 3 , and water and $\mathrm{P}_{1}$ are applied in the other mixtures. The $\mathrm{pn}=$ keyword is omitted for mixture 1 . The com= keyword is omitted for mixture 2 .

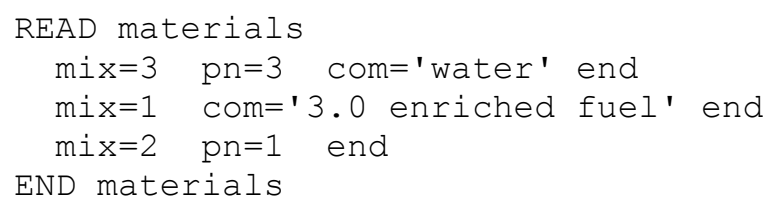

Consider this same set of mixtures but with a fixed source identified by source ID 100 in mixture 1 . This specification could be written as follows: 


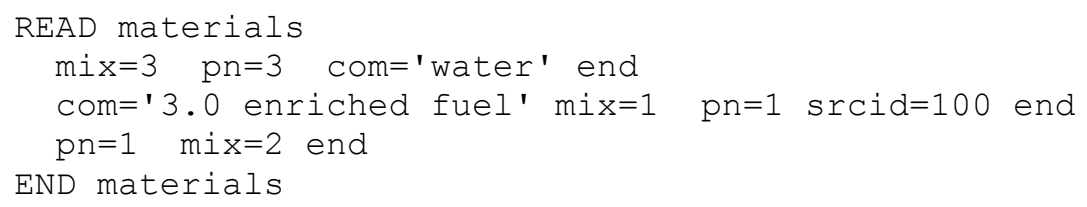

\subsubsection{Source block}

\section{Source block keyword=source, src}

The Source block contains source strength specifications associated with a given source ID. The source is assigned to a mixture via the srcid= keyword in the Material block (Sect. 9.2.3.3). Data are input using a keyword-based format:

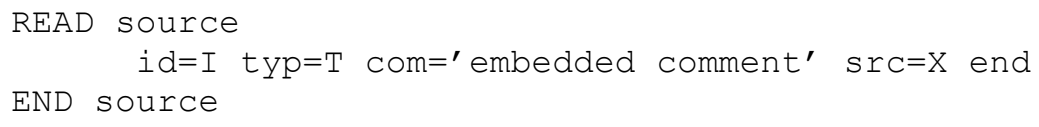

where

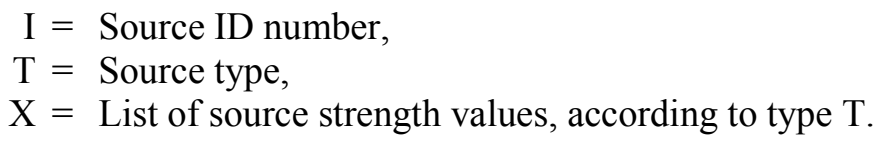

Up to 80 characters of text may be entered after $c o m=$, delimited by single quotes ('). The comment string is optional - the remaining parameters are required. Currently, only two source types are supported; the definition of $\mathrm{X}$ depends on the source type.

Source type $0($ typ $=0)$ : A single value of $\mathrm{X}$ is supplied - this source strength is placed in all energy groups.

Source type $1($ typ $=1)$ : $G$ values of X are supplied, one value for each energy group. FIDO-type repeat command is supported.

An example of a source specification for two different sources is the following.

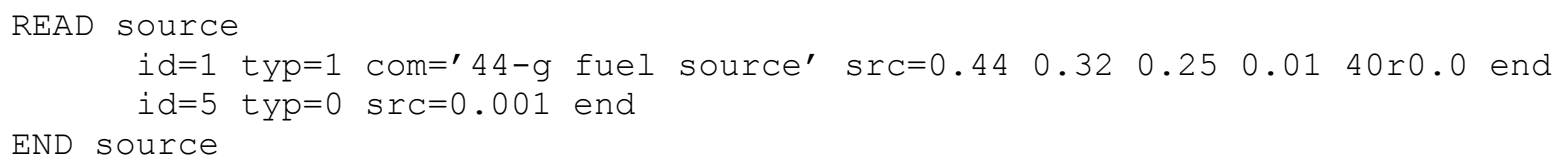

The Material block is used to associate a given source definition with a given mixture. The same source may be placed in multiple mixtures. For generalized adjoint calculations-which require a fixed source derived for the generalized response of interest (see Generalized Perturbation Theory in the SAMS chapter) — the TRITON control sequence automatically prepares the NEWT Source block.

\subsubsection{Collapse block}

\section{Collapse block keyword = coll, collapse}

The Collapse block contains the broad (collapsed) group assignment for each energy group in the original input group structure. Broad group assignments must be contiguous. A FIDO-type repeat factor is allowed. For example, given that a calculation is performed using a 44-energy-group library, in which it is desired to collapse the first 9 groups into a single group, the second 17 groups into a second broad group, and the remaining 18 groups into a third group, either of the following could be used. 


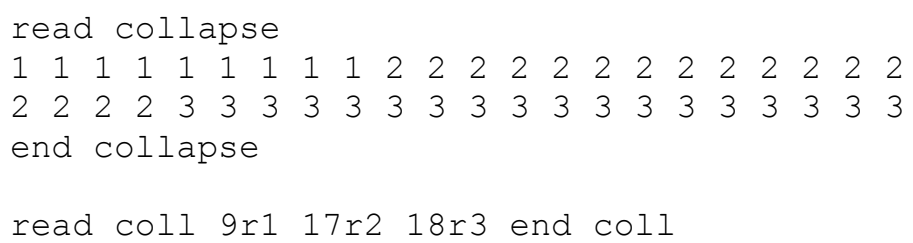

If a collapsing operation is requested, then upon the completion of the transport iteration, NEWT performs a collapsing operation on all cross sections for all mixtures in the problem. Cross sections are flux weighted using the average flux in the mixture in which each nuclide resides and saved in an AMPX working-format library at the unit specified by the wtdlib= parameter (default $=30$ ). Collapsed, or "broad group," cross sections may also be printed by setting the parameter prtbroad=yes (default=no). Note that the energy boundaries of the collapsed cross section are always a subset of the boundaries of the parent library. Cross sections may not be collapsed to arbitrary energy boundaries.

\subsubsection{Geometry block}

\section{Geometry block keyword = geom, geometry}

The Geometry block is always required. This data block contains geometric descriptions for all bodies included in the model. NEWT geometry input is performed based on the SCALE Generalized Geometry Package (SGGP) paradigm employed in the KENO-VI Monte Carlo code within SCALE. Those familiar with SGGP as applied in KENO-VI will find the new format very familiar; however, they will quickly realize that the NEWT geometry package contrasts most sharply with the 3-D implementation in KENO-VI because NEWT is a 2-D code. Hence, third dimension (z-axis) specifications are omitted, along with other inherently 3-D bodies supported by KENO-VI. Two other more subtle differences are seen: (1) users must specify the underlying grid structure associated with each unit, and (2) curved surfaces (e.g., cylinders) are approximated as N-sided polygons, with user control. Details on these differences are described in the following subsections and illustrated in examples.

The SGGP approach for model development is combinatorial in nature. Hence, intersections are allowed, and the user is given enormous flexibility to specify, translate, rotate, and combine bodies to create complex configurations. However, the novice user must first focus on the basics of model development, as outlined in this subsection. Sample inputs are provided in Section 9.2.4 to demonstrate the development of more complicated models.

Geometric arrangements in NEWT are based on a fundamental building block called a unit. Different units can be arranged in an array. Figure 9.2.7 illustrates a simple unit and an array of such units. Arrays of units can be contained inside larger units, and in principle, any level of nesting can be achieved. Within a unit, various shapes can be specified, each representing some geometrically distinct medium. In every geometry specification, a single global unit, which forms the outer boundary for the entire problem, must be specified.

Note that in the models pictured in Figure 9.2.7, bodies are laid within a Cartesian grid. This is a hallmark of any NEWT model - the body specifications combined with an underlying grid structure are used to define a computational grid in which the NEWT ESC solution algorithm is applied. Figure 9.2.8 illustrates the grid structure associated with the array example above. The model consists of a set of arbitrary polygons used to spatially discretize the bodies of interest. The underlying Cartesian mesh may be specified for any unit; a Cartesian mesh must be specified for the global unit. The mesh for the global unit is the primary mesh for the entire problem and is often referred to as the base grid, whereas the mesh for constituent units within the global unit constitutes localized refinement and may be referred to as the local, or unit, grid. 
The NEWT geometry block consists of specifications for a set of basic building blocks known as units. A unit is defined as a collection of shapes, one of which must be defined as the unit boundary. A complete unit specification consists of a header and three distinct components:

1. Bodies: shapes, holes, or array placements that define the bodies within the unit;

2. Media specifications that define the material content (composition) of the various shapes; and

3. Boundary definition that defines the extent of the unit and its associated grid structure.
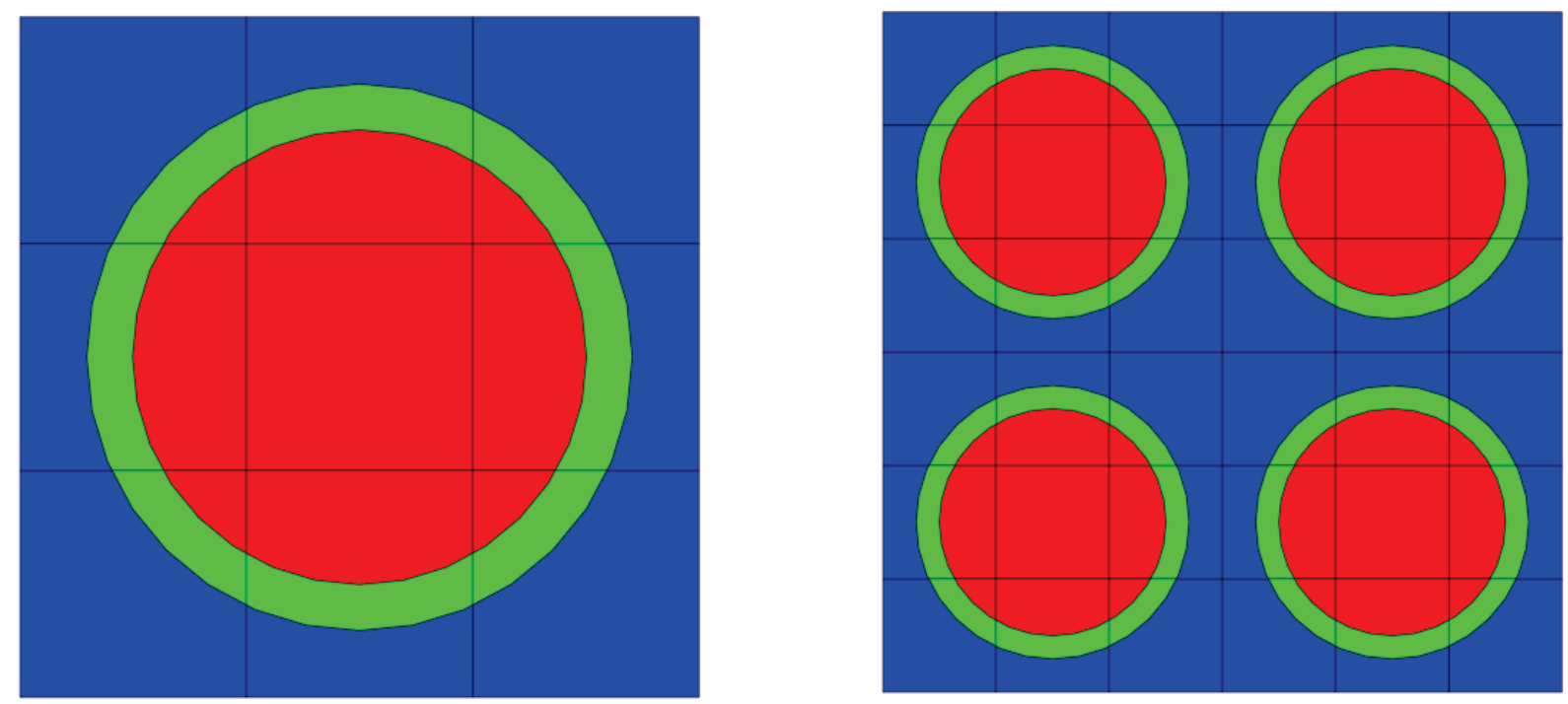

Figure 9.2.7. A simple unit (left) and an array of units (right). 


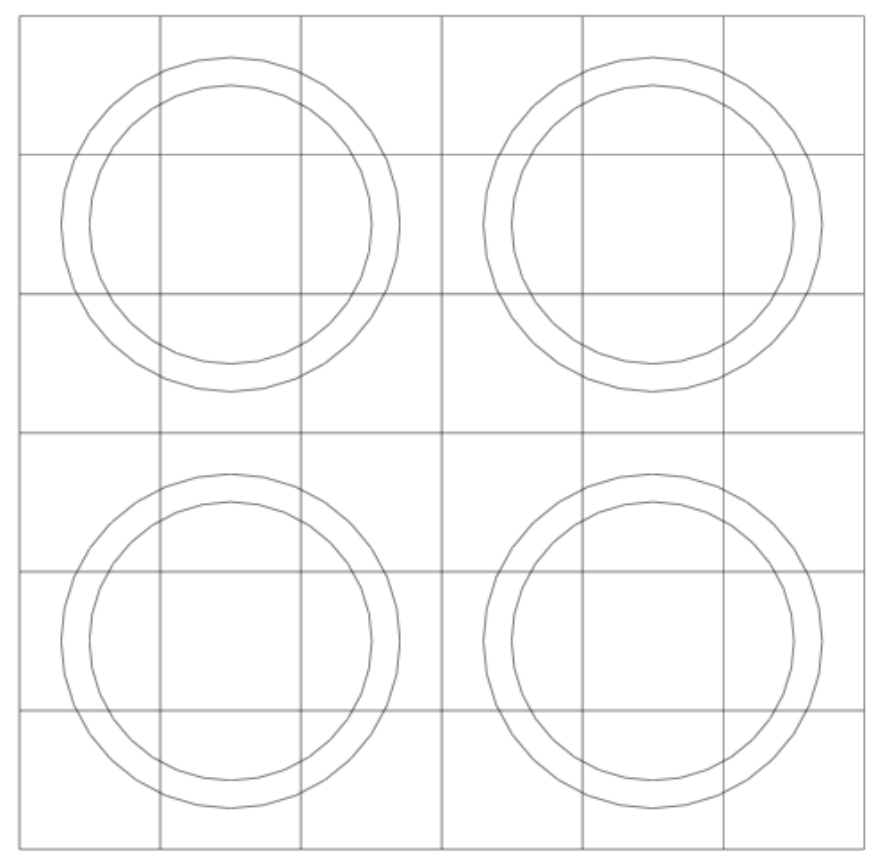

Figure 9.2.8. Computational grid structure in a NEWT model.

Every unit begins with a header consisting of the keyword unit followed by a unique integer label (unit_id) that serves to identify the unit:

$$
\text { unit unit_id }
$$

The header is followed by a complete unit description consisting of the three components described above; each of these components of the unit specification is described in the following subsections. In every NEWT model, one unit must be defined as the global unit. This unit defines the global coordinate system for the entire problem, and all other units (if any) must fit within the global unit. Specification of the global unit is accomplished simply with the format:

$$
\text { global unit unit_id }
$$

The global unit may occur anywhere in the list of units. If only one unit is defined in an input, it must be identified as the global unit.

As indicated earlier, the geometry block consists of a list of one or more units. Each unit is terminated by the beginning of another unit or by the end of the geometry block. Conceptually, a geometry block will have the following structure:

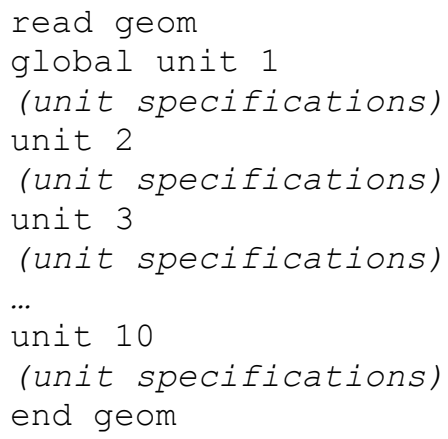


The unit numbers are arbitrary and can occur in any order, although they must be unique; they serve simply as labels.

The remainder of this section describes the various components of units.

\subsection{Bodies}

Every unit contains a set of body specifications in terms of (1) basic shapes that are placed directly within a unit; (2) one or more arrays, each of which is defined elsewhere and placed within a unit with an array placement operator; and (3) holes. Units must contain at least one shape specification, which is used to define the spatial boundaries of the unit. Additional shape specifications may be used as needed. Holes and/or array placements are optional; there is no theoretical limit on the number of each that may be used within a unit.

\subsection{Shapes}

Shapes are simple predefined bodies. NEWT currently supports six shapes:

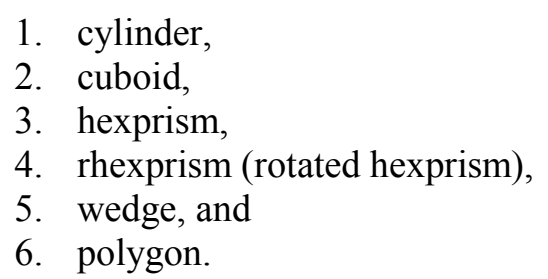

The names of these shapes are generally associated with 3-D bodies but are used in NEWT to be consistent with KENO-VI nomenclature. In NEWT, a cylinder is equivalent to a circle, a cuboid is equivalent to a rectangle, a hexprism is equivalent to a hexagon, and a wedge is equivalent to a triangle.

Because the SGGP is combinatorial in nature, intersection and overlap of shapes is permitted. For this reason, no specific mixture is associated with each shape. Combinatorial logic allows a fraction of a shape to be filled with one mixture, while the remainder or another fraction thereof may be assigned a different mixture. This is discussed further in the section Media Specifications in the description of media assignment (Sect. 9.2.3.6.2).

Each shape is specified by name, an associated body identification (body_id) number, and dimensioning data. The body_id number is arbitrary but must be unique within each unit. Specific formats for each shape are provided below.

\subsection{Cylinder}

The cylinder specification has the following format:

$$
\text { cylinder body_id radius [modifier_list] }
$$

where radius is the radius of the circle. The circle will be centered at $(0,0)$. The modifier list is an optional set of operations that may be performed on each shape. One of the modifiers allowed is the origin modifier, which lets one translate the origin of a shape to a different location. Modifier commands are described later in this section. 


\subsection{Cuboid}

The cuboid specification has the following format:

$$
\text { cuboid body_id } x_{\max }, x_{\min }, y_{\max }, y_{\min } \text { [modifier_list] }
$$

where $\left(\mathrm{x}_{\min }, \mathrm{y}_{\min }\right)$ and $\left(\mathrm{x}_{\max }, \mathrm{y}_{\max }\right)$ represent the lower-left and upper-right vertices of a rectangle on a Cartesian coordinate system. Note that the cuboid is explicitly placed by its coordinates; no translation is required (or allowed).

\subsection{Hexprism and rhexprism}

Both hexprisms are specified in a manner identical to that of a cylinder:

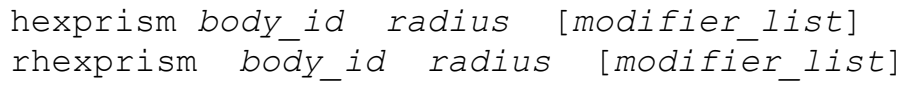

where radius is the inner/minor radius of the hexagon. A standard hexagon (hexprism) is oriented with vertices at the top and bottom, as illustrated in Figure 9.2.9. A rotated hexagon (rhexprism) is oriented with vertices on the left and right sides, as illustrated in Figure 9.2.10. Both types of hexprisms, like cylinders, are by default placed with their origins at $(0,0)$. However, like cylinders, they can also be translated in space via the origin translation command.

\subsection{Wedge}

A wedge, or triangle, specification has the following format:

$$
\text { wedge body id } x_{\text {base }} x_{p t} y_{p t}
$$

Figure 9.2.10. Orientation of a rotated hexprism.

where the vertices of the shape are defined as $(0,0),\left(\mathrm{x}_{\text {base }}, 0\right)$, and $\left(\mathrm{x}_{\mathrm{pt}}, \mathrm{y}_{\mathrm{pt}}\right)$. Thus, one side always lies on the $\mathrm{x}$-axis. The modifiers origin and rotate may be used to position and orient the triangle in the problem domain. Figure 9.2.11 illustrates placement of a wedge using these parameters.

\subsection{Polygon}

The polygon specification has the following format:

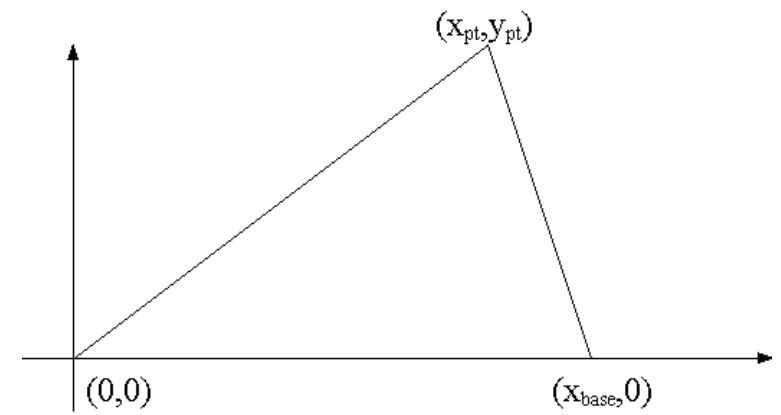

Figure 9.2.11. Initial positioning of the wedge body.

$$
\text { polygon body_id } x_{0}, y_{0}, x_{1}, y_{1}, \ldots, x_{N}, y_{N}, x_{0}, y_{0}
$$

where $\left(\mathrm{x}_{\mathrm{i}}, \mathrm{y}_{\mathrm{i}}\right)$ are the polygon vertices (the first and last pair in this list refer to the same vertex). Note that the polygon is explicitly placed by its coordinates; no translation is required (or allowed). 


\subsection{Example of shape specifications}

Use of shapes within a unit can be illustrated with a simple example. Consider a unit, arbitrarily labeled with unit $i d=10$, containing a cuboid and two cylinders. Each shape is given a unique (but arbitrary) body_id.

$\begin{array}{llllll}\text { unit } 10 & & & & & \\ \text { cuboid } & 11 & 3.0 & -5.0 & 1.0 & -2.0 \\ \text { cylinder } & 12 & 0.8 & & & \\ \text { cylinder } & 13 & 0.6 & & & \end{array}$

The cuboid is explicitly placed by its coordinates; the two cylinders will by default be placed at $(0,0)$. Use of the origin command to relocate cylinders is introduced below. Figure 9.2.12 illustrates the body placement that occurs for the given example.

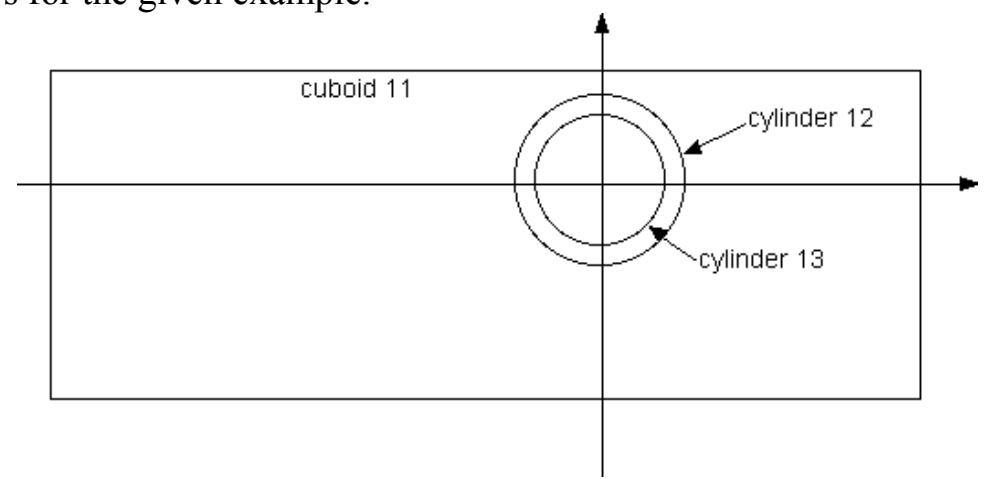

Figure 9.2.12. Body placement for two cylinders and a cuboid.

\subsection{Shape modifier commands}

Modifier commands are provided as a means to perform specific functions relative to the input shape. The five available modifier commands and the shapes to which they may be applied are listed here:

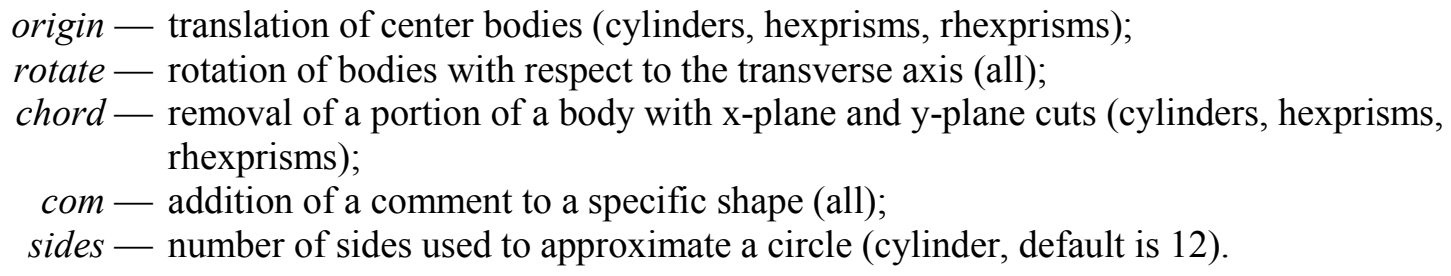

The format for each of these commands follows.

\subsection{ORIGIN}

The origin modifier is used to translate the origin of a cylinder or hexprism from the default origin of $(0,0)$ to some other location. It has the format

$$
\text { origin } \mathrm{x}=x_{\text {new }} \mathrm{y}=y_{\text {new }}
$$

where $\left(\mathrm{x}_{\text {new }}, \mathrm{y}_{\text {new }}\right)$ is the new center of the shape. The modifier origin may not be applied to cuboids, as the location of a cuboid is explicitly set by its shape specification. If not specified, each ordinate is set to zero, such that

$$
\text { origin } x=5
$$


is equivalent to

$$
\text { origin } x=5 \quad y=0
$$

For example, consider a cuboid whose lower-left and upper-right corners are located at $(0,0)$ and $(1,1)$, respectively. If one places a cylinder with radius 0.3 in the center of this box [i.e., centered at $(0.5,0.5)$ ], this would be specified as follows:

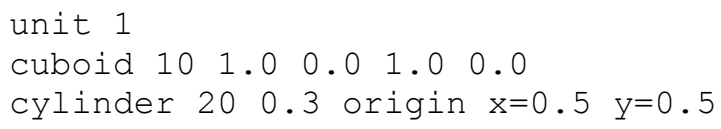

This configuration is illustrated in Figure 9.2.13.

\subsection{Rotate}

The rotate body modifier is used to rotate a body around its geometric center. It can be applied to any body type, but rotation of a cylinder has no real meaning. The format of the rotate modifier is as follows:

$$
\text { rotate } \mathrm{a} 1=\mathrm{A}
$$

where $A$ is the angle of rotation, in degrees, in a counterclockwise direction. All bodies lie in the $(x, y)$ plane, with rotation around the z-axis, but with respect to the centroid of the body. Rotation always occurs before translation (via origin), irrespective of the order of rotate and origin commands in the modifier list for a body. KENO-VI allows rotation about the $\mathrm{x}$ and $\mathrm{y}$ axes as well, through $\mathrm{a} 2=$ and $\mathrm{a} 3=$; however, only rotation about the $\mathrm{z}$ axis is permitted in NEWT.

As an example, consider a 1 by $1 \mathrm{~cm}$ cuboid centered at $(0,0)$, place a smaller 0.5 by $0.5 \mathrm{~cm}$ cuboid inside it, and rotate it 30 degrees clockwise.

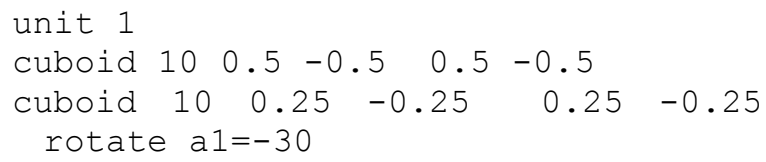

Figure 9.2.14 illustrates the configuration generated using this specification. Note that clockwise rotation was performed by specifying a negative angle. In this case, the centroid of the inner cuboid coincides with $(0,0)$; however, the same geometric rotation would have occurred if the bodies had not been centered at $(0,0)$.

\subsection{Chord}

The chord modifier is used to remove a portion of a body. It provides for horizontal and/or vertical cuts on a body, with the portion of the body on a specified side of that body discarded. Chords may be applied to cylinders or hexprisms but may not be applied to cuboids. (Such "cuts" may be explicitly defined in the body definition.) The format of the specification combines the selection of the plane (horizontal or vertical cut), location of the plane, and the portion of the body to be retained, all in one terse modifier. 
The four possible chord specifications for a body are as follows:

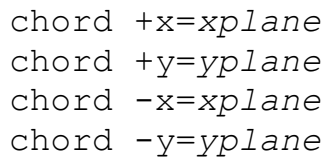

where xplane and yplane are the ordinates on the $\mathrm{x}$ and $\mathrm{y}$ axes, respectively. The sign on $\mathrm{x}$ and $\mathrm{y}$ indicates the portion of the body to be retained after the cut. A plus $(+)$ sign indicates that the portion of the body in the positive (increasing $\mathrm{x}$ or $\mathrm{y}$ ) direction should be kept, and a minus (-) sign indicates that the portion of the body in the negative direction of the cut plane (decreasing $\mathrm{x}$ or $\mathrm{y}$ ) direction is retained. Chords are applied after any translation (origin) or rotation (rotate) modifier operations. Multiple chords may be specified for a single body to obtain multiple cuts. The keyword chord must precede each specification.

The use of chords is best illustrated by example. Figure 9.2.15 through Figure 9.2.18 show unit body descriptions with various chord specifications.
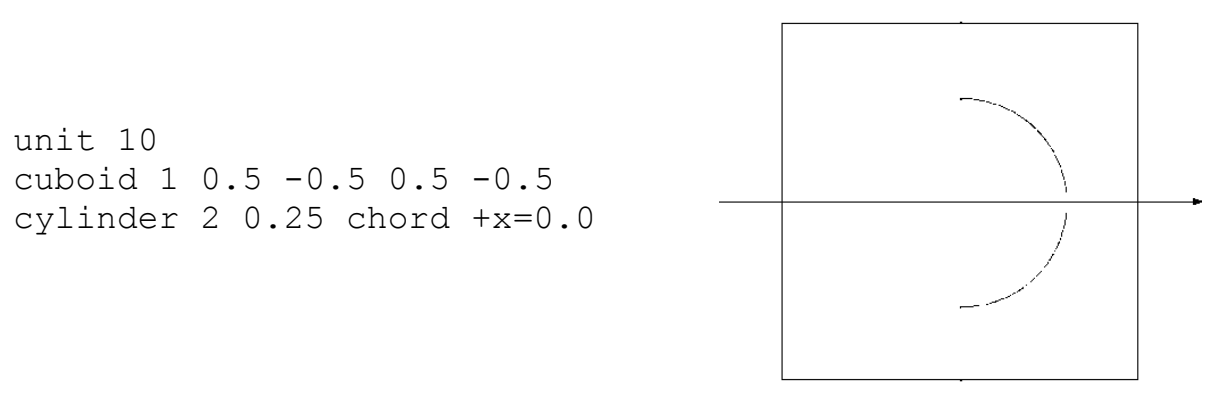

Figure 9.2.15. Example of chord $+x$ behavior.
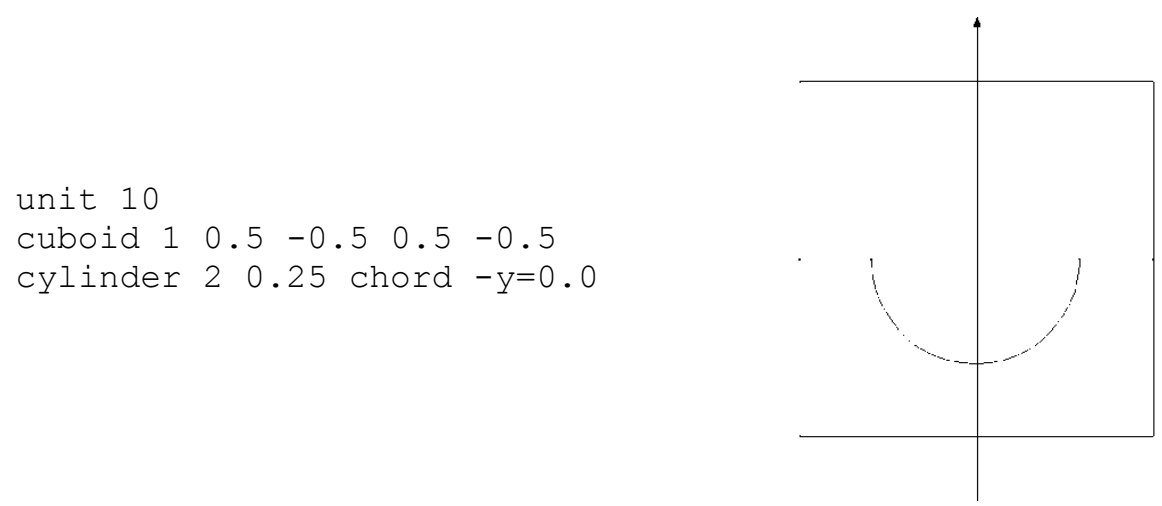

Figure 9.2.16. Example of chord $-y$ behavior. 

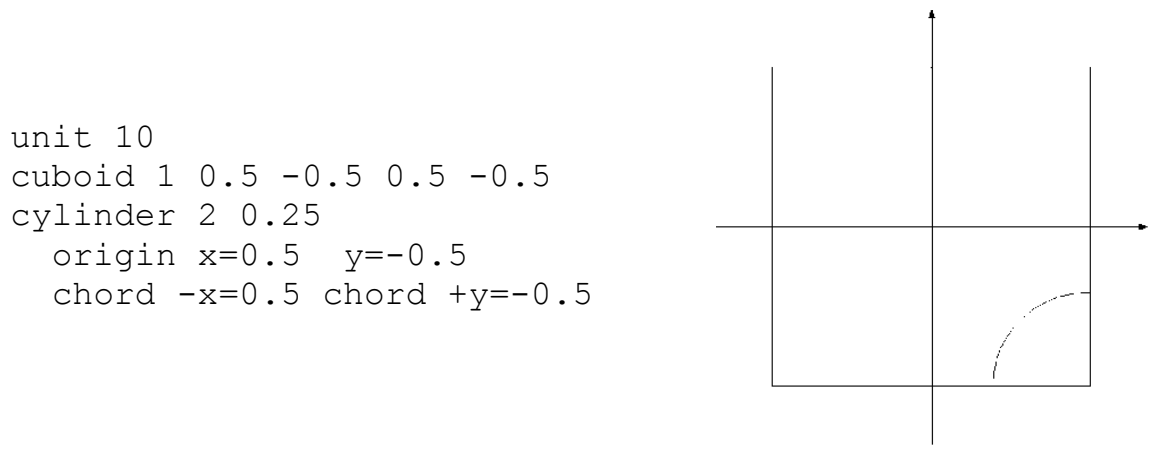

Figure 9.2.17. Use of two chords to create a quarter-cylinder body.
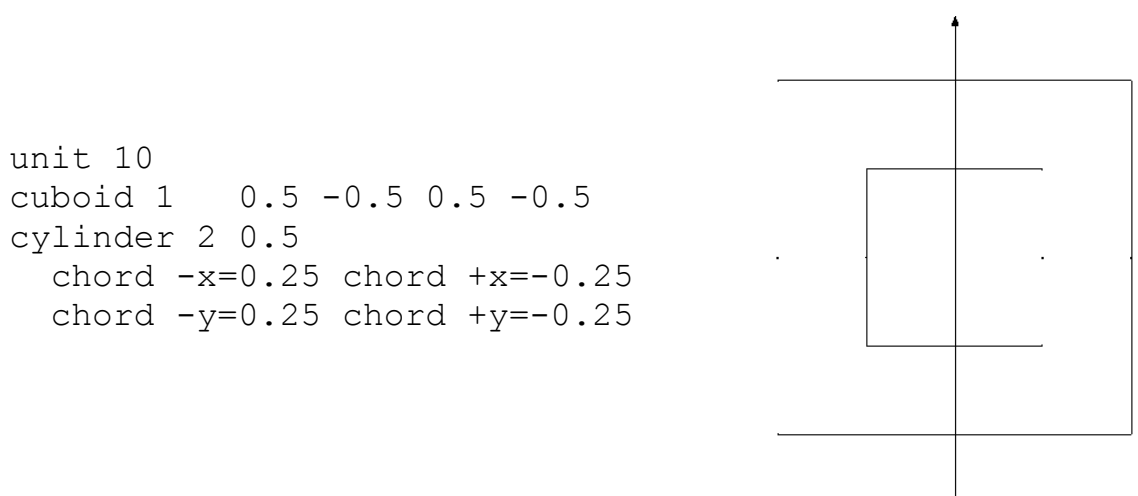

Figure 9.2.18. Use of four chords on a cylinder to create a square body.

In Figure 9.2.15, a chord is placed at $\mathrm{x}=0$ and the positive portion relative to the chord $(\mathrm{x}>0)$ is retained because $+x$ mode is specified. Since the cylinder is centered at $(0,0)$, this chord cuts the cylinder in half and retains the right half of the cylinder. The unit in Figure 9.2.16 uses the same cylinder but with a chord cutting at the plane located at $y=0$. The bottom half $(y<0)$ is kept because $-y$ is specified.

Figure 9.2.17 is somewhat more complicated but represents perhaps the most common use of chords in lattice models. In this case, it is desired to create a one-quarter cylinder located in the bottom right quadrant of a cuboid. A 1 by $1 \mathrm{~cm}$ square cuboid is centered at $(0,0)$, and a cylinder is placed at $(0.5,-0.5)$, which is the lower right-hand corner of the cuboid. Since we are interested only in the portion of the cylinder within the cuboid, we choose to keep the top $(+x)$ and left $(-y)$ portions of the cylinder. This requires two separate chord modifiers. (Each chord specifies only one cutting plane.) Additionally, because the cylinder was relocated to a new origin, the chords are specified such that the cuts go through the new origin.

Note that there is no requirement that a chord cut through the origin of a body. Figure 9.2.18 illustrates the use of four chords to set four cutting planes. A $0.5 \mathrm{~cm}$ cylinder is specified centered within the unit cuboid. All four of the four permitted cutting planes are specified. We have effectively created a cuboid by retaining the portion of the cylinder above $(+y)$ the $x z$ plane located at $y=-0.25$, below $(-y)$ the plane at $\mathrm{y}=+0.25$, to the right $(+\mathrm{x})$ of the $\mathrm{yz}$ plane at $\mathrm{x}=-0.25$, and to the left $(-\mathrm{x})$ of the plane located at $\mathrm{x}=+0.25$. There is, of course, a much more direct means to create a cuboid - this example is provided only for illustrative purposes.

For guidance on how to cut a cylinder at an oblique angle, refer to Section 9.2.3.6.1.14. 


\subsection{Com}

The com modifier is a means to label specific bodies. It is provided primarily for consistency with KENO-VI. At this time, NEWT simply reads and then ignores com data. It can, however, be used as a means to help annotate an input listing. The format for the com modifier is as follows:

$$
\text { com=" comment string" }
$$

where "comment string" is any text description of up to 132 characters, delimited by single (') or double quotes ("). For example, the input description of Figure 9.2.18 is shown here with comments added via com modifiers.

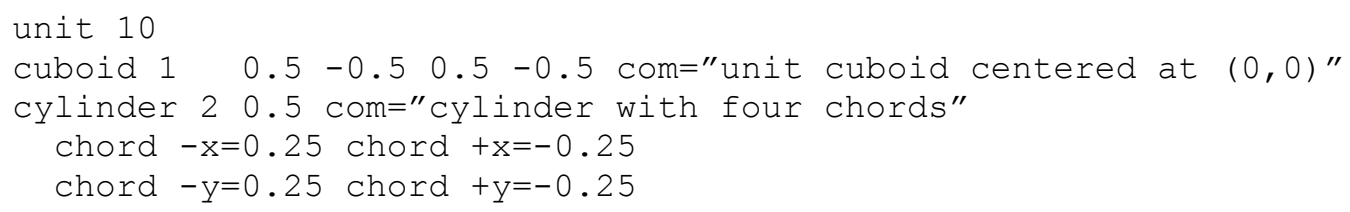

\subsection{Sides}

The sides modifier applies only to cylinders and is unique to NEWT (i.e., it is not used in KENO-VI). Because NEWT's solution grid is based on arbitrary polygons, all cells must be straight sided. Hence, the curved surfaces of a cylinder are approximated as an $\mathrm{N}$-sided regular polygon. By default, $\mathrm{N}=12$. The sides operator allows the user to override the default. The format is very simple:

$$
\text { sides }=N
$$

where $\mathrm{N}$ is the number of sides desired for the full cylinder. In general, a 12-sided polygon provides an adequate approximation of a cylinder. Use of additional sides will create a cylinder that has a smoother appearance and increase the computational effort required to solve the cells associated with the cylinder.

Figure 9.2.19 shows a model built with three nested cylinders inside a unit cuboid. Cylinder 10 is the innermost cylinder, with no sides modifier; hence, it uses the default 12-sided approximation. The second cylinder is specified with sides $=16$; the refinement in this approximation is seen in the figure. Finally, cylinder 30 is specified with 40 sides - this is visually a very close approximation to a cylinder.
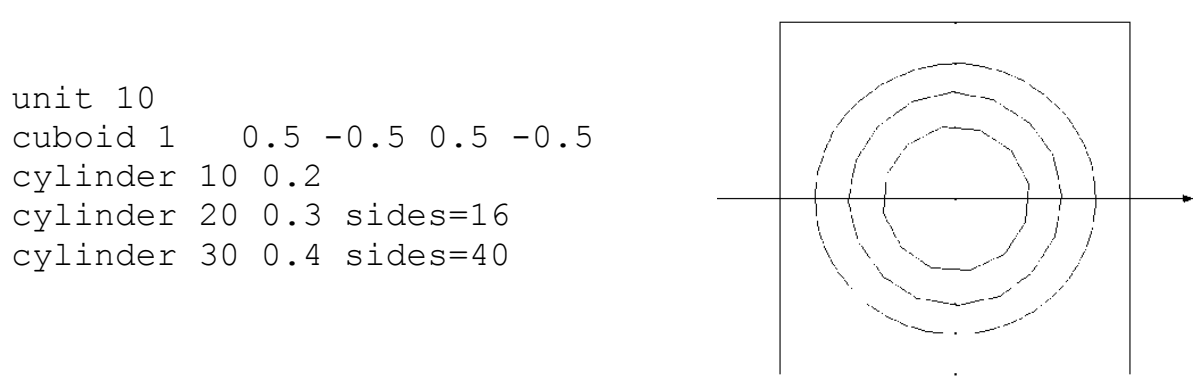

Figure 9.2.19. Use of the sides modifier for cylinders. 


\subsection{Holes}

The next level of complexity within a unit is provided through the use of a hole specification. The hole specification is simply a means by which one unit may be placed within another unit. In some instances, a well-defined set of structures, assembled as a unit, needs to be placed within a larger unit. NEWT provides two methods to do this - holes and arrays. Arrays are used to place a unit (or a number of similar units) in a regular repeating pattern within an enclosing unit. A hole, on the other hand, is a means to place a single unit. This is often used when units being placed do not have a regular repeating pattern.

The format for a hole specification within a unit is as follows:

hole unit_id [modifier_list]

where unit id is the identification number for the unit that is being placed within the current unit. (A unit cannot be placed within itself.) Unlike the shapes described earlier, holes do not have a distinct identification number of their own - they are simply a mechanism to place a unit defined elsewhere.

By default, the hole operator places the origin of the new unit at the origin $(0,0)$ of the current unit. The origin modifier may also be used with a hole specification to position the placed unit at a location other than $(0,0)$ of the current unit. However, placement of the body is always relative to the origin of the original unit, which can be defined in a number of different ways.

Holes are also associated with a particular shape. Hole specifications must immediately follow the shape into which they are being placed. Holes redefine the boundaries of a shape by figuratively cutting holes in that shape into which units are placed. When mixtures are defined for a given shape (through media specifications, described below), the mixture is placed throughout the region, except in the space excluded by the hole placements.

The rotate modifier can also be applied to a hole, as can the com modifier. However, chord specifications cannot be used to remove a portion of a hole. To construct a cylinder that is cut at an oblique angle, users should construct a cylinder that is cut by a chord and then use the hole operator combined with the origin and rotate modifiers to place and rotate the unit to the desired position and orientation. This can be particularly useful in hexagonal or triangular geometries.

As an example, consider a unit, unit_id $=1$, consisting of two concentric cylinders, and a second unit, consisting of two concentric cuboids. Descriptions for these two units are given below. Note that these are incomplete unit specifications; other components of the unit specification have not yet been introduced. However, for the purposes of this example, incomplete unit specification will suffice.

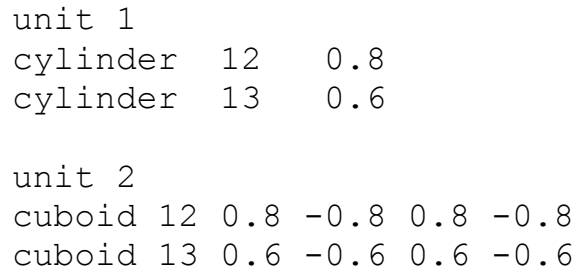

Now suppose that we wished to place two of the unit 1 cells and one of the unit 2 cells inside unit 3 , with unit 2 rotated by $45^{\circ}$. We can define a cuboid as unit 3 and place the units 1 and 2 inside the cuboid using hole specifications: 


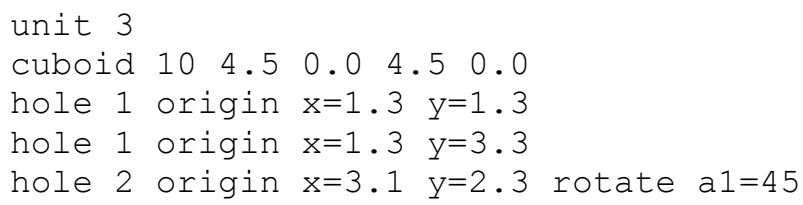

In this example, a square cuboid is defined such that its lower-left corner is situated at $(0,0)$. Three hole operations are used: the first to place unit 1 at $(1.3,1.3)$, the second to place another instance of unit $12 \mathrm{~cm}$ above the first, at $(1.3,3.3)$. Lastly, unit 2 is placed inside unit 3 at $(3.1,2.3)$ and then rotated $45^{\circ}$. Figure 9.2.20 illustrates how such a unit would appear.

\subsection{Array placement}

As indicated in the previous section, arrays are a method for arranging one or more units within another unit. Arrays specifications are typically used when units are placed in a repeating pattern. While the hole specification is used to place different units within a given unit, the array placement specification is used to place an array within a unit.

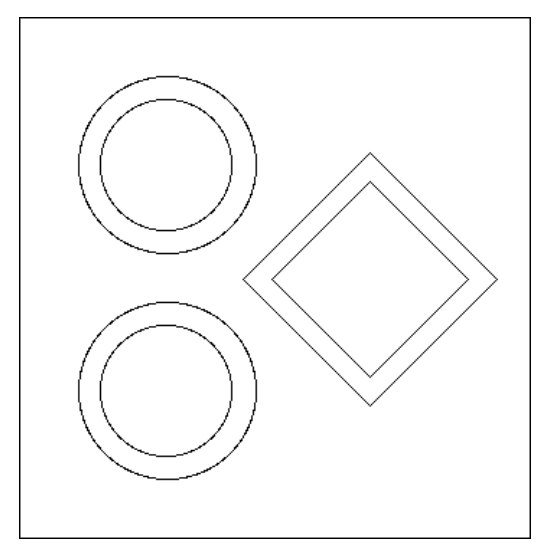

Figure 9.2.20. Unit placement within a unit using holes.

All arrays are specified (declaration of size, type, and fill) in the array data block, as described in Section 9.2.3.9. The array placement operator is used to locate an array within a unit. The format for the array placement operator is as follows:

array arrayid body_id place i j $x_{i j} y_{i j}$

where arrayid is the identification number assigned to the array in the array data block and body_id is the identification number of the shape into which the array is placed. The remainder of the array placement operator is used to fix the position of the array within the body, identified by body_id. A general discussion of this concept follows, after which the actual placement of the array is described.

Arrays are defined by two dimensioning parameters - the number of rows and the number of columns. Each element of an array is filled by a unit; each unit has its own local coordinate system. In other words, one unit may have the origin $(0,0)$ in its local coordinate system defined as the lower-left corner while another unit may have its origin defined at its geometric center. The array itself has no coordinate system; it is simply a list of relative positions of units, defined by their row/column position. The place directive of the array placement operator is used to locate the array within the body into which it is being placed.

In the place directive, $i$ represents the column (counting from left to right) and $j$ represents the row (counting from bottom to top) of a specific element of the array. The coordinate system of that specific unit is used to set the position of the entire array. The coordinates $x_{i j}$ and $y_{i j}$ represent the location in the current unit where the array is to be placed. Placement occurs by situating the origin of the unit in column $\mathrm{i}$, row $\mathrm{j}$ at coordinate $\left(\mathrm{x}_{\mathrm{ij}}, \mathrm{y}_{\mathrm{ij}}\right)$. Placement of arrays within a unit is best understood through examples. Consider three (partial) unit specifications, as defined and illustrated in Figure 9.2.21 


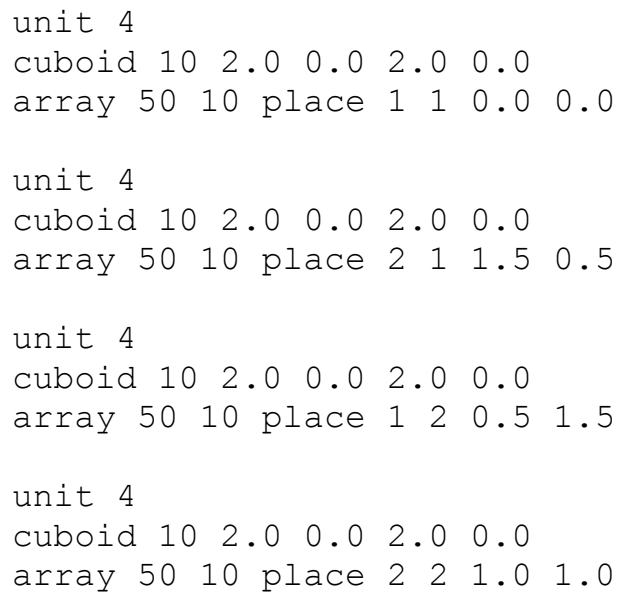

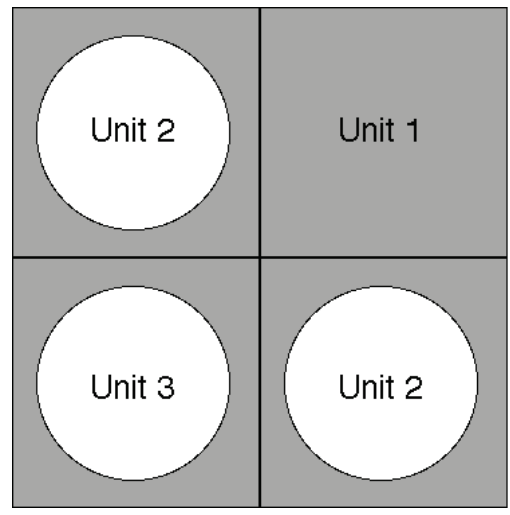

Figure 9.2.22. Layout of units in array 50.

For the first example, the unit located in row 1, column 1 (i.e., unit 3 ) is placed such that its origin (its lower-left corner) is located at $(0,0)$, which is the origin of unit 4 . For the second example, the unit located in column 2, row 1, is placed such that its local origin, which is in the center of the unit, is located at $\mathrm{x}=1.5, \mathrm{y}=0.5$ in the coordinate system of unit 4 .

\subsection{Media specifications}

A unit is only partially specified by its constituent bodies. At this point, no composition has been associated with the various regions of the problem nor has the outer boundary of the unit been defined. This section provides information on the use of media specifications to define the contents of each shape that has been defined.

Each shape statement defines a basic shape, with optional modifiers, which represents a spatial region within the unit. Assignment of compositions to regions is performed via media specifications.

As discussed earlier in the introduction to shapes, input processing in the SGGP is combinatorial. This permits intersection of shapes, and different compositions (or media) may be assigned to different portions of intersecting bodies.

The format of a media specification is as follows:

media materialid bias_placeholder reg_def_vector

where materialid is the composition number being placed in this entry, bias_placeholder is a simple placeholder that is required but not used, and reg_def_vector is the region definition vector used to define the shape or shapes to which the mixture is assigned.

The bias_placeholder is used to be as consistent as possible with KENO-VI input. KENO-VI allows the user to assign biases within the media assignments to improve the Monte Carlo solution performance. Biases have no meaning in NEWT, so the field has no meaning. In KENO-VI, if no special biasing is desired, a value of 1 is assigned. If it is desired to move models between NEWT and KENO-VI format, a placeholder value of 1 is recommended. However, the value itself has no meaning within NEWT; it is simply read and ignored. (This may change in a future release.)

The region definition vector is used to describe the location of the composition within the current unit. This is done by providing a list of shapes for which the media is either "inside" or "outside." The sense of 
the media with respect to a shape is specified by listing the shape number with a negative sign if "outside" and with a positive (or no) sign when the media is placed "inside" the shape.

Consider a simple cylinder inside a cuboid. Assume composition 1 is to be placed inside the cylinder and composition 2 outside the cylinder but inside the cuboid. The shape and media specifications could have the following format:

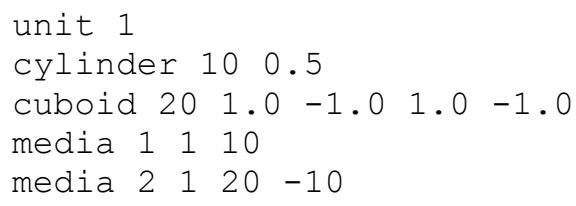

In the above example, the first media record places mixture 1 inside all of shape 10 (the cylinder). The second media entry places mixture 2 in all regions that are outside shape 10 but inside shape 20. Note also that a bias placeholder value of 1 is used in each media statement.

It is necessary to specify media for all regions of the unit. If any regions remain unassigned, NEWT will stop with an error message. If the second record had been omitted, regions outside the cylinder would be unspecified and the code would stop. Note also that if the second media statement had read only

media $2 \quad 120$

then composition 2 would have been placed inside all of cuboid 20, including inside the cylinder 10 . The fact that the contents of 10 have already been specified is ignored. The above statement directs the code to put mixture 2 everywhere inside the boundaries of the cuboid.

Each region definition vector combines all specifications with a logical AND. In other words, the second media record in "media 21 20" places composition 2 in all regions that are simultaneously outside shape 10 and inside shape 20. Separate media specifications are required to place a composition in two independent shapes. The following represents a cuboid with two nonintersecting cylinders.

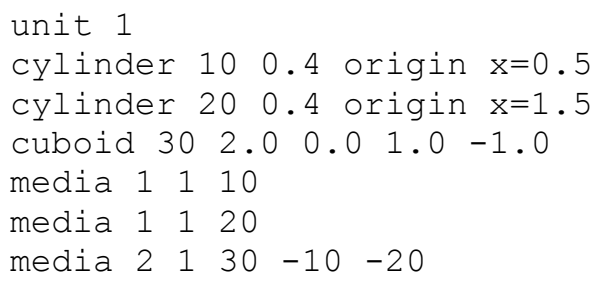

A media statement is necessary to place composition 1 inside shape 10; a similar statement is necessary to place composition 1 inside shape 20. Finally, all space inside cuboid 30 but outside both 10 and 20 is filled with composition 2 . If one attempted to fill both 10 and 20 with composition 1 in a single media record, for example,

media $1110 \quad 20$

then an error would occur. The code would attempt to place composition 1 in all space that is simultaneously within shape 10 and within shape 20 - and no such space exists.

A more common example is the configuration of a fuel pin (composition 1), gas gap (composition 2), clad (composition 3), and moderator (composition 4) in a lattice. Consider a pin in a hexagonal lattice: 


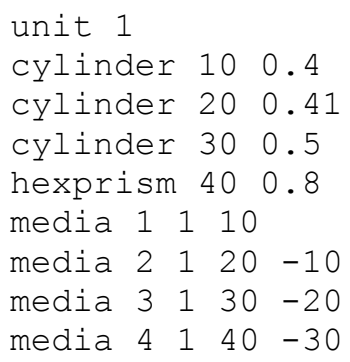

In this example, for the hexagonal moderator region outside the clad, it is sufficient to specify that mixture 4 is inside 40 and outside 30 . Although it is true that the moderator is also outside shapes 10 and 20, it is not necessary to specify this. Logically, since 10 and 20 are inside 30, then everything outside 30 must be outside 10 and outside 20. The use of a hexprism in this example is irrelevant. If the outer body had been a cuboid, the result would have been the same.

As a final example, consider a unit with intersecting bodies. It becomes possible to assign a unique composition to each intersection of shapes (Figure 9.2.23).
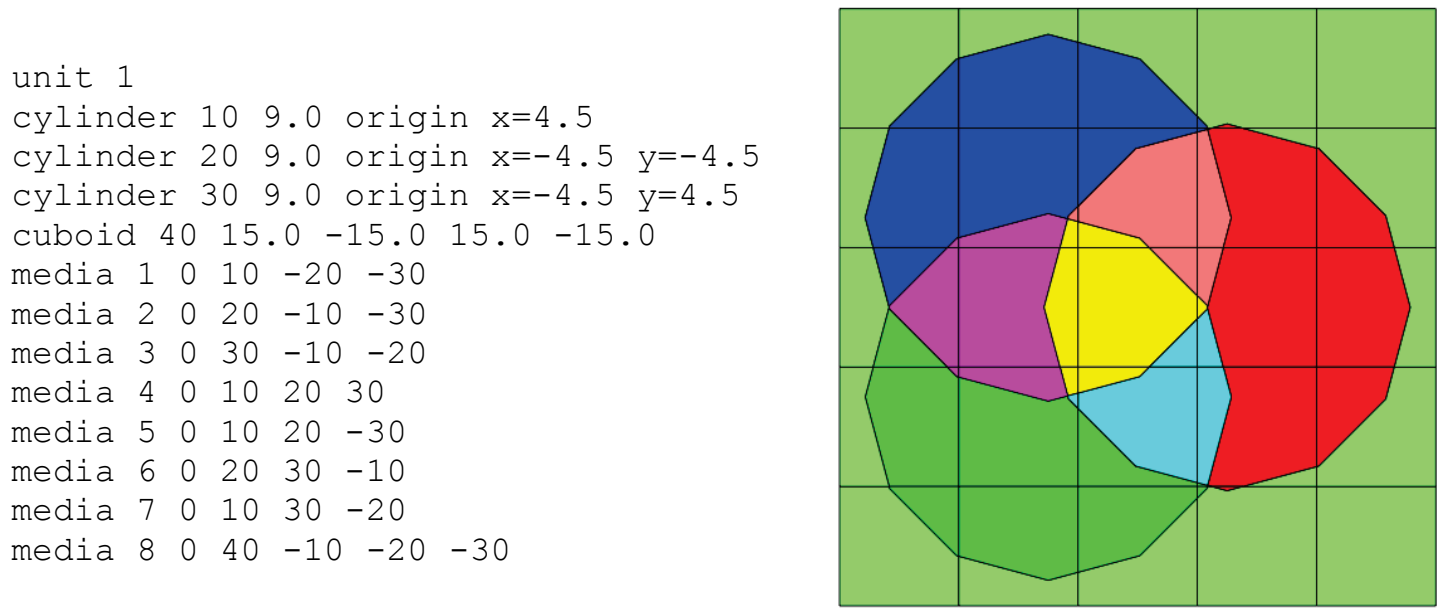

Figure 9.2.23. Media assignments in overlapping regions.

In this model, cylinder 10 is on the right, 20 is the lower left, and 30 is the upper left. Media 1, placed inside cylinder 10 but outside 20 and 30, is represented by the partially filled right-hand side of the right cylinder. The central region of the unit is filled with composition 4 and represents all areas that are simultaneously within shapes 10,20 , and 30 . The outermost region is everything that is inside 40 , but outside 10, 20, and 30 .

Media statements apply only to shapes, and only to those shapes within the unit. Holes and array specifications are used to define placement of one or more units in which media have already been specified in the corresponding unit definitions. Like shape statements, media statements may occur in any order. However, if one region is erroneously assigned two different compositions in two different media statements, the code will allow this and will proceed with the calculation. The last specification for a shape will always take precedence. Thus, it is important that newly developed models be visually inspected using mixture plots (files named "*.newtmatl.ps") created using drawit=yes in the parameter block. 


\subsection{Unit boundary}

The final section of a unit description is the boundary specification. This input record serves two purposes: to specify the shape that defines the outer bounds of the unit, and hence the shape of the unit, and (optionally) to specify the underlying grid associated with the unit. The format of the boundary specification is as follows:

boundary body_id [x-discretization $y$-discretization]

where body_id is the identification number for the body that is to serve as the unit boundary. The $x$-discretization and $y$-discretization terms are integers $(\geq 2)$ that specify the number of rectangular cells to be placed in the unit in the $\mathrm{x}$-direction and y-direction, respectively. A grid specification is required for the global unit but is optional for other units. If a grid is specified for a grid other than the global unit, that grid replaces the base grid. (An exception to this principle is discussed later.)

In general, grid refinement should be such that cell sizes are on the order of or smaller than a mean free path for a neutron. Grid spacing can be easily varied in order to converge on the parameter of interest. Global factors, such as a system eigenvalue, can tolerate a relatively coarse grid. However, if fluxes are known to vary rapidly in space, then a more refined grid may be necessary. NEWT does provide the ability to locally refine a grid structure so that detail can be modeled where needed, without having to pay the computational penalty of refining the grid everywhere. NEWT does place one limit on grid refinement: every shape, hole, or array placed within a unit must be intersected by at least one gridline. The grid may be locally defined or part of the global grid, but it must intersect each body at least once. Thus, if small geometric shapes are modeled, a detailed grid structure is generally necessary.

Examples of boundary specifications follow, as parts of partial unit specifications. Media descriptions are omitted for simplicity. Accompanying figures illustrate the grid structure(s) associated with each specification. Figure 9.2.24 shows a single (global) unit with a 2 by 2 base grid. Cuboid 10 serves as the boundary for the unit. This represents the minimum grid structure that can be specified for a unit. Figure 9.2.25 shows a more complex configuration in which a unit defined with a 5 by 5 grid is placed in the center of a larger enclosing unit, specified to have a 3 by 3 grid. Note that because the first unit has its own (local) grid, the underlying grid structure is removed in favor of the local grid structure. The grid is applied to the boundary shape of the unit, which is cuboid 10 .

Figure 9.2.26 shows a similar structure; however, the cuboid was removed from unit 1 and the outer hexprism was defined as the unit boundary. Note that the grid structure applied to the nonrectangular body is the same as the one that would be assigned for a cuboid with the same minima and maxima in $\mathrm{x}$ and y directions. Figure 9.2.27 illustrates the grid structure that would be applied to the same model as was used in the previous figure but with CMFD acceleration enabled. Because CMFD is normally applied to a coarse mesh defined by the base global grid (unless xycmfd=0), the global grid is always retained when CMFD acceleration is used. Finally, Figure 9.2.28 illustrates the use of a base grid only. In this case, no grid structure is assigned for unit 1; the bodies are inlaid but are adapted to the base global grid structure. 
global unit 1

cylinder 300.5

cylinder 200.6

cuboid $10 \quad 0.75-0.75 \quad 0.75-0.75$

boundary $10 \quad 2 \quad 2$

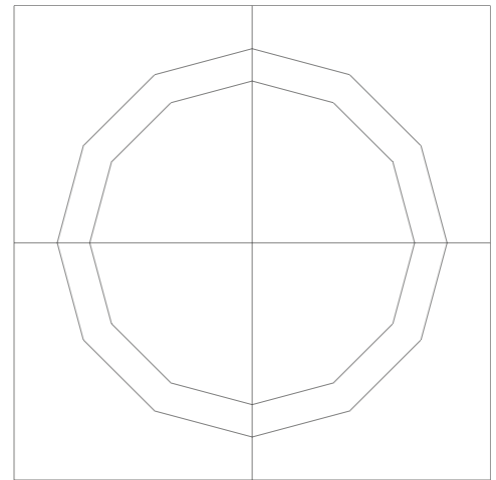

Figure 9.2.24. Unit with 2 by 2 grid in a simple pin-cell model.
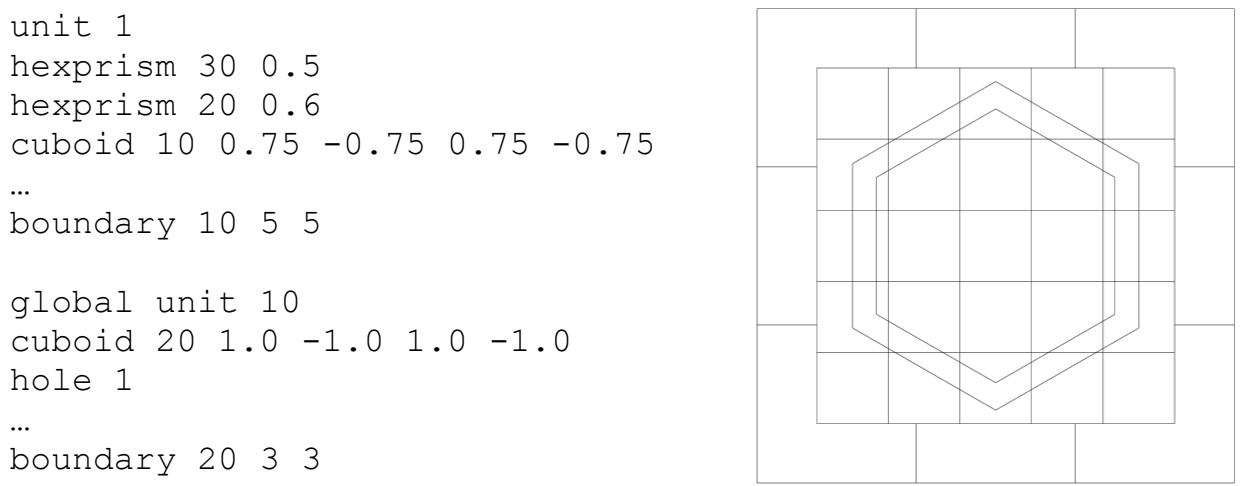

Figure 9.2.25. Unit with 5 by 5 grid inset into unit with 3 by 3 grid.

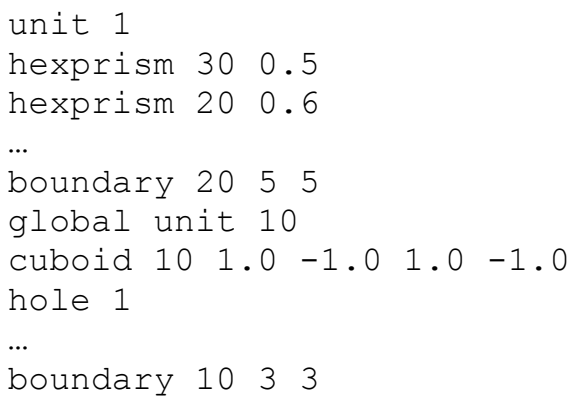

Figure 9.2.26. Effect of boundary grid specification on noncuboidal unit placed as a hole within a larger unit. 

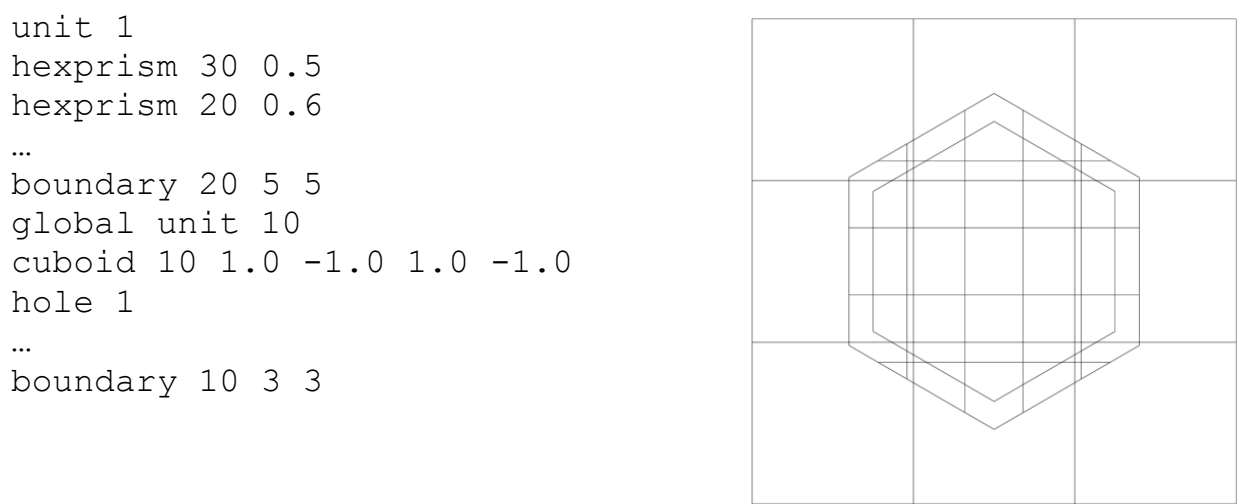

Figure 9.2.27. Effect of coarse-mesh finite-difference acceleration on grid structure.
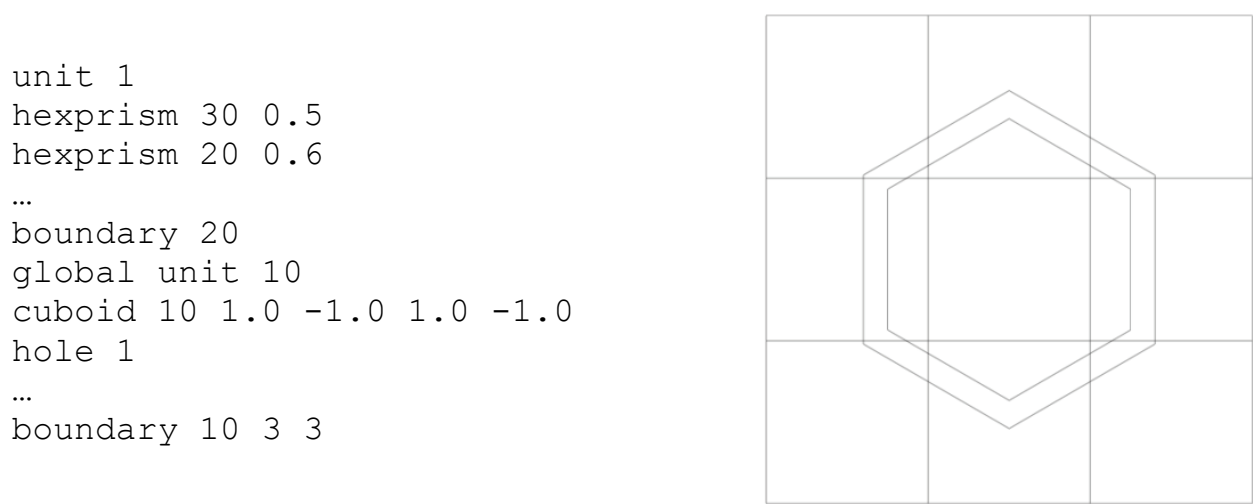

Figure 9.2.28. Use of base grid without localized grid specification.

The following section provides examples of complete geometry specifications for various models, including bodies, media, and boundary specifications. Each will include one or more uses or boundary specifications for units.

\subsection{Geometry block examples}

The following three subsections present geometry block examples to show how various models may be assembled. Each listing is described briefly and is followed by a figure showing the NEWT grid structure generated for each set of geometry instructions.

\subsection{Simple pin cell}

The following geometry block (Figure 9.2.29) shows the specifications necessary to define a single pincell. The model is reduced to a $1 / 4$ cell to take advantage of symmetry. Mixture 1 is fuel, mixture 2 is fill gas, mixture 3 is clad, and mixture 4 is moderator. Features of this model include the use of chords to obtain $1 / 4$ cylinders and the specification of 20 sides for each cylinder (five sides for a $1 / 4$ cylinder). 

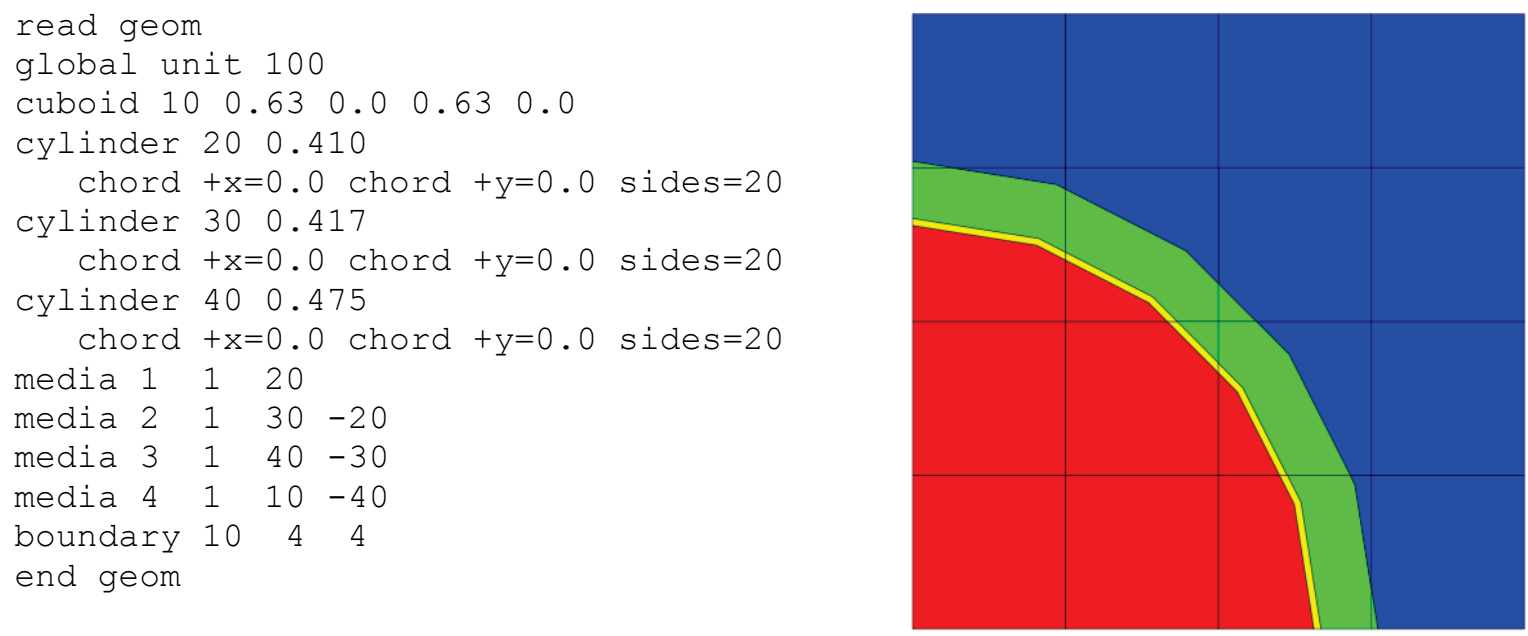

Figure 9.2.29. Geometry model for infinite-lattice pin cell with fuel, gap, clad, and moderator.

\subsection{Hexagonal assembly}

The geometry block below (Figure 9.2.30) is used to describe a hexagonal fuel assembly within a hexagonal shroud. Each cylinder is placed individually, followed by a series of media statements that fill each cylinder. The hexagonal moderator area is surrounded by a hexagonal shroud of cladding material. Note that NEWT allows only cuboid and hexprisms as outer boundaries for the global unit. This model could also have been assembled with a unit definition for a set of cylinders, which could then be placed in the global unit using holes or by defining a single pin cell and placing it using an array. 


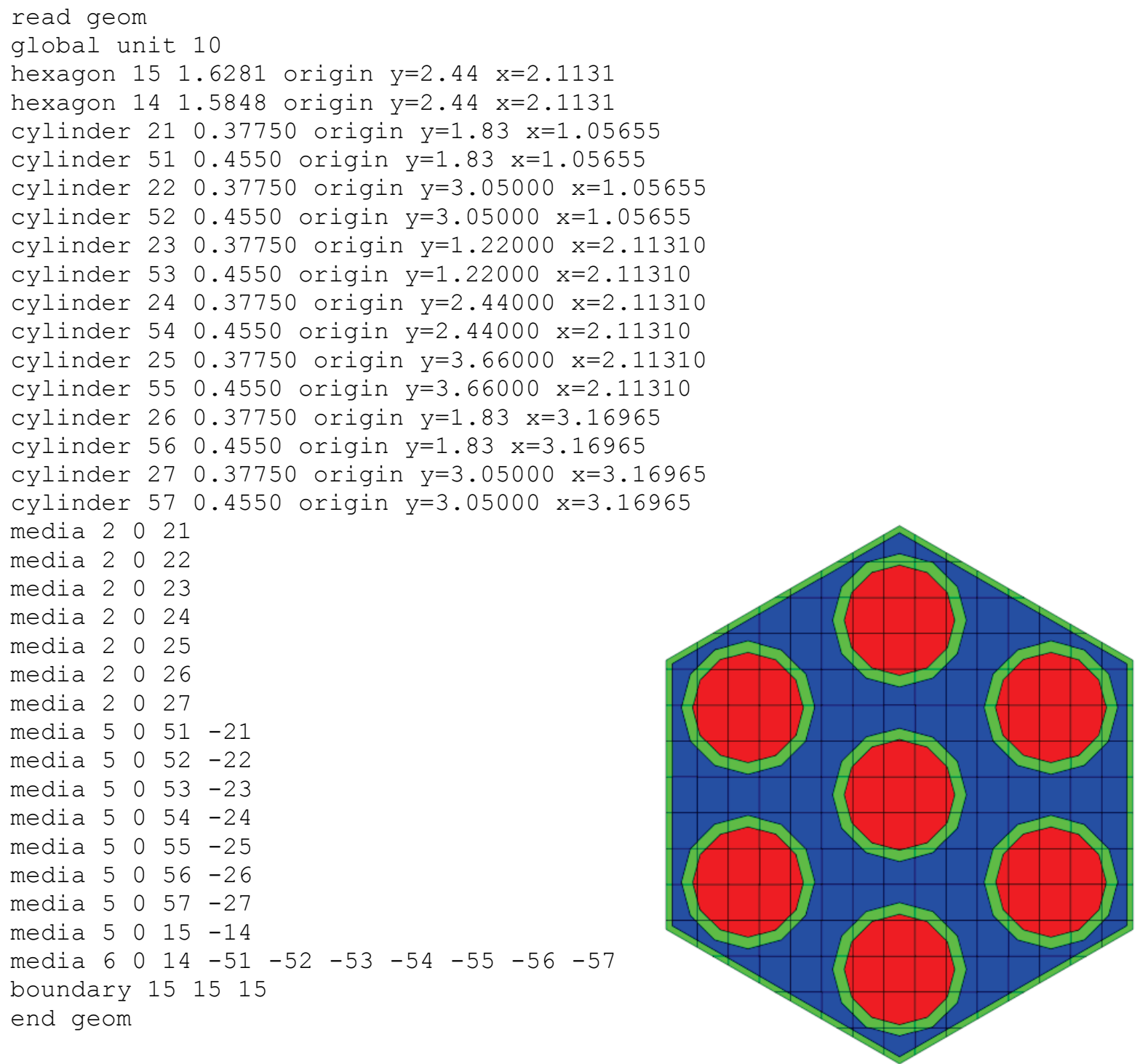

Figure 9.2.30. Geometry model of hexagonal fuel assembly.

\subsection{Advanced CANDU reactor ACR-700 assembly}

This example of a geometry block (Figure 9.2.31

Figure 9.2.31) is included to illustrate the complexity of design that is possible through the use of simple bodies, units, and holes. The ACR-700 design cannot be modeled using an array because pins are not placed in a repeating lattice pattern. Features of this example include use of holes; use of noncuboidal units placed in holes; and localized pin-cell grid refinement by (1) decreased mesh size in fuel elements (three outer rings) and (2) increased radial discretization (central pin). 


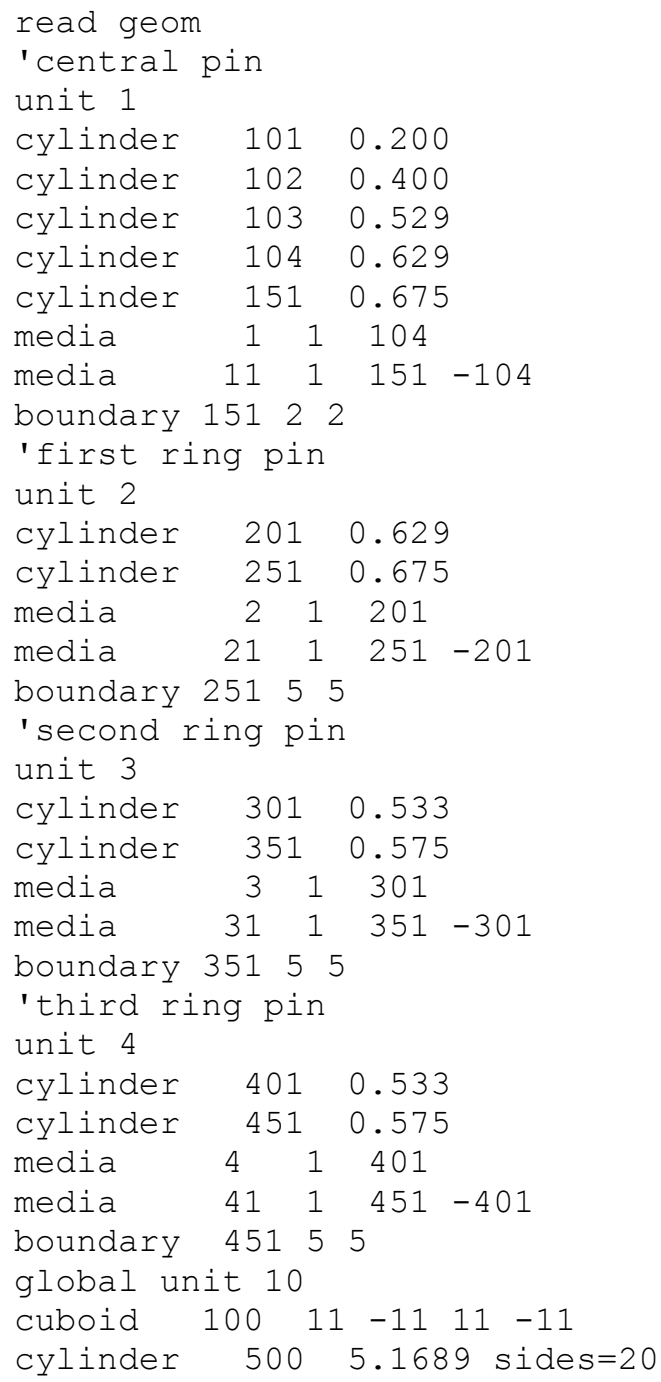

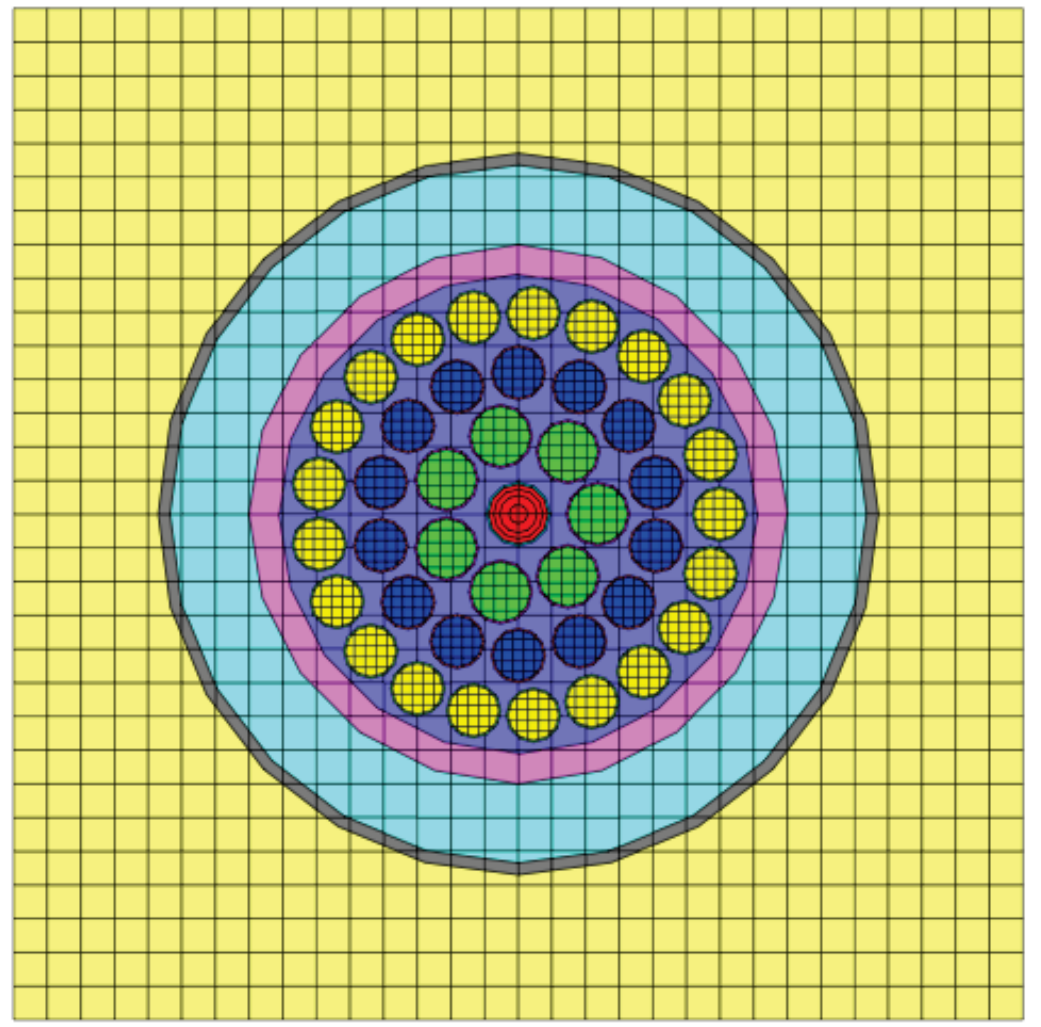

Figure 9.2.31. ACR-700 fuel assembly model. 


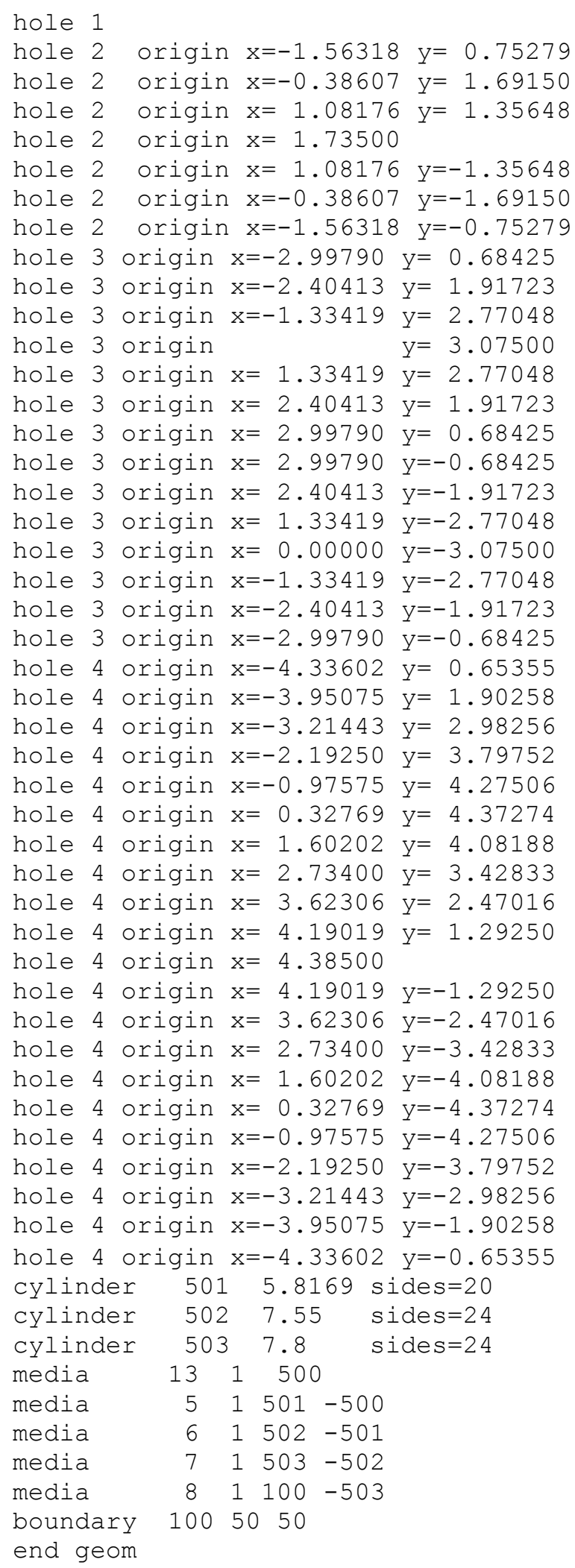

Figure 9.2.31. ACR-700 fuel assembly model. (continued) 


\subsection{Summary of geometry specifications}

This section is provided as a quick reference for geometry statements. Details and examples of the usage of each geometry specification are provided in previous subsections of this manual. For each definition, the lists of permitted modifiers are listed.

\subsection{Unit definition statements}

[global] unit unit_id

The unit statement is used to initiate the definition of each unit used. unit id is an integer identification label for the unit and must be unique. One (and only one) global unit is required in each geometry model. Modifiers: none.

cuboid body_id $x_{\max } x_{\min } y_{\max } y_{\min }$ [modifier_list]

The cuboid statement is used to define a rectangular shape. body_id is the integer identification label for the cuboid and must be unique within the unit it is used. The rectangle is defined such that the coordinates $\left(\mathrm{x}_{\min }, \mathrm{y}_{\min }\right)$ and $\left(\mathrm{x}_{\max }, \mathrm{y}_{\max }\right)$ represent the lower-left and upper-right vertices of the cuboid. Modifiers: rotate, com.

cylinder body_id radius [modifier_list]

The cylinder statement defines a circle with integer label body_id and radius radius, placed with its center at the origin $(0,0)$ of the unit. Modifiers: origin, rotate, chord, sides, com.

hexprism body_id radius [modifier_list]

The hexprism statement defines a standard hexagon with integer label body_id and inscribed radius radius, placed with its center at the origin $(0,0)$ of the unit. A standard hexagon has vertices located on north (top) and south (bottom) regions of the shape. Modifiers: origin, rotate, chord, sides, com.

rhexprism body_id radius [modifier_list]

The rhexprism statement defines a rotated hexagon with integer label body_id and inscribed radius radius, placed with its center at the origin $(0,0)$ of the unit. A rotated hexagon has vertices located on east (right) and west (left) regions of the shape. Modifiers: origin, rotate, chord, sides, com.

wedge body_id $x_{\text {base }} x_{p t} y_{p t}$ [modifier_list]

The wedge statement defines a triangle with integer label body_id placed with a vertex at $(0,0)$, a vertex at $\left(\mathrm{x}_{\text {base }}, 0\right)$, and a vertex at $\left(\mathrm{x}_{\mathrm{p} t}, \mathrm{y}_{\mathrm{pt}}\right)$. Modifiers: origin, rotate, com.

array arrayid body_id place i j $x_{i j} y_{i j}$ [modifier_list]

The array placement statement specifies the placement of an array with identification number arrayid (defined in the array data block), within shape body id. If the place statement is used, the array element located in row $i$ (counted from the bottom) and column $j$ (counted from the left) is placed such that its origin is located at spatial coordinate $\left(x_{i j}, y_{i j}\right)$ of the unit in which it is placed. Modifiers: com. 
hole unit_id [modifier_list]

The hole statement is used to place a different unit identified by label unit_id within the current unit. The origin of the unit being placed will be located at the origin of the current unit but can be repositioned using the origin modifier. Modifiers: origin, rotate, com.

media materialid bias_placeholder reg_def_vector

The media statement assigns material properties associated with mixture index materialid to a shape region defined within a unit. The bias_placeholder term is not currently used but is retained for consistency with KENO-VI; typically it is assigned a value of 1. reg_def_vector is the region definition vector and assigns the mixture placement relative to shapes within the unit. If the index is positive, the shape region is included in the material assignment; if negative, it is excluded. Modifiers: none.

boundary body_id [x-discretization y-discretization]

The boundary statement is used to define the outer boundary of the unit, corresponding to the outer bounds of the shape body_id within the unit. This shape must exist and must contain all other bodies associated with the unit. The $x$-discretization and $y$-discretization terms are integers $(\geq 2)$ that specify the number of cells to be placed in the unit in the $\mathrm{x}$-direction and y-direction, respectively. A grid specification is required for the global unit but is optional for other units. Modifiers: none.

\subsection{Geometry modifiers}

origin $x=x_{\text {new }} y=y_{\text {new }}$

Used to relocate the origin of cylinders, hexprisms, rhexprisms, and holes to new co-ordinate $\left(\mathrm{x}_{\text {new }}, \mathrm{y}_{\text {new }}\right)$. The default (if not specified) is to place the origin of the body at $(0,0)$.

rotate $a 1=A$

Causes a body to be rotated by an angle of $A$ degrees (counterclockwise) around its origin. It can be applied to holes and all basic shapes; the default is 0 degrees.

chord $\pm x=x p l a n e$

chord ty=yplane

Chords are used to truncate a shape at the line $\mathrm{x}=$ chord (or $\mathrm{y}=$ chord). Multiple chord commands are allowed, but only one line (either in $\mathrm{x}$ - or $\mathrm{y}$-direction) is specified for each. If the negative keyword ' $-\mathrm{x}$ ' (or ' $-\mathrm{y}$ ') appears, then the part of the shape to the left of (below) the chord cut is retained. Similarly, if the positive keyword (' $+\mathrm{x}$ ' or ' $+\mathrm{y}$ ') is used, then the portion of the shape to the right of or above the chord is retained. Chords may be applied to cylinders, hexprisms, and rhexprisms only.

sides $=N$

For use with the cylinder statement, the sides modifier specifies the number of sides on the regular polygon used to approximate the cylinder. Its default is $\mathrm{N}=12$. The radius of the polygon is adjusted such that the area of the polygon matches the area of the cylinder it is approximating. The sides modifier is unique to NEWT and is not used by KENO-VI.

\subsubsection{Boundary conditions}

The geometry data block is generally followed by the bounds data block, in which boundary conditions for the sides of the bounding shape in the global array are specified. NEWT supports the use of cuboid, 
hexprism, and wedge shapes to define outer boundaries. This results in the need to specify up to 6 surface boundaries, on up to 8 spatial orientations. In other words, a cuboid will have boundaries on $+x,-x,+y$, and $-\mathrm{y}$ faces. A regular hexprism will have boundaries on $+\mathrm{x}$ and $-\mathrm{x}$ faces; it will also have boundaries on the four sloped sides of the hex. In order to identify the sense of sides for specification of boundary conditions, NEWT applies an eight-point compass nomenclature. The four permitted rectangular boundary surfaces are identified as $+\mathrm{x},-\mathrm{x},+\mathrm{y}$, and $-\mathrm{y}$, corresponding to east (E), west (W), north (N), and south (S) faces, as illustrated in Figure 9.2.32 Sloped (non-rectangular) surfaces are identified as $+x+y$, $+x-y,-x-y$, and $-x+y$, for northeast (NE), southeast (SE), southwest (SW), and northwest (NW) surfaces. No assumptions are made on the slope of the various non-rectangular surfaces; for the bodies available within NEWT, it is not possible to have more than one surface in each octant.

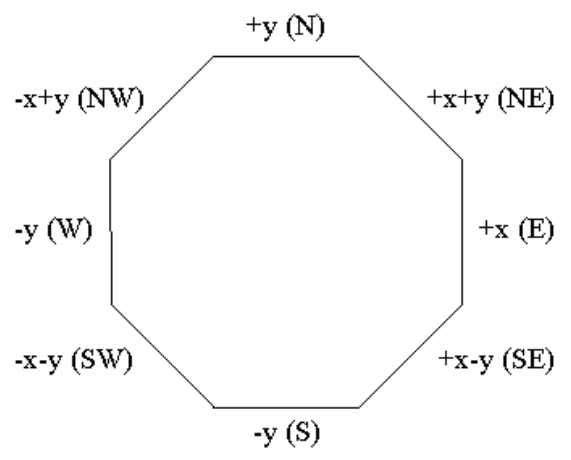

Figure 9.2.32. Two-dimensional boundary condition surface orientations.

Currently, full specification of boundary conditions is permitted only when the boundary body for the global unit is a cuboid. Only white and vacuum boundary conditions are permitted on non-rectangular surfaces.

Boundary conditions therefore may only be specified for the $\pm \mathrm{x}$ and $\pm \mathrm{y}$ faces of a boundary cuboid. Four boundary conditions are currently supported:

1. reflective (default),

2. white,

3. vacuum, and

4. periodic.

Albedo boundary conditions are not yet supported but will be available in a future release. Additional information on the meaning of each boundary condition type is provided in the following section.

\subsection{Boundary conditions descriptions}

Boundary conditions are mathematical approximations used to describe the behavior of neutrons when they cross a problem boundary. Typically, transport methods provide for reflective (or mirror), white, vacuum, or periodic boundary conditions. The following subsections describe and illustrate these four types of boundary conditions.

\subsection{Reflective boundary condition}

For the reflective boundary condition, the incoming angular flux is set equal to the outgoing angular flux in the direction corresponding to mirror or specular reflection. As shown in Figure 9.2.33, a given quantity of neutrons leaving a boundary (dotted line) in a particular direction will be returned (solid line of same color) to the system with the same quantity but at a mirrored angle to the initial leakage direction. 
(NOTE: In the following figures, a dashed arrow indicates neutrons leaving the system while a solid arrow represents those returning to the system. The length of the arrow is proportional to the number of neutrons; therefore, longer arrows represent more neutrons than do shorter arrows.)

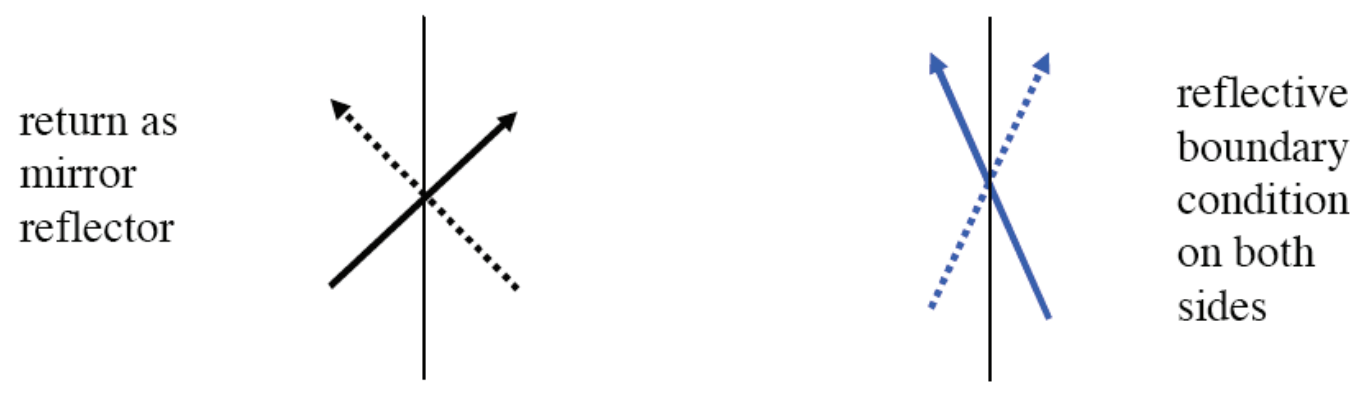

Figure 9.2.33. Reflective boundary condition.

\subsection{White boundary condition}

For the white boundary condition, the incoming angular fluxes are each set equal to a single value chosen such that the net flow across the boundary is zero. The white boundary provides isotropic return (solid lines) at a boundary (Figure 9.2.34).

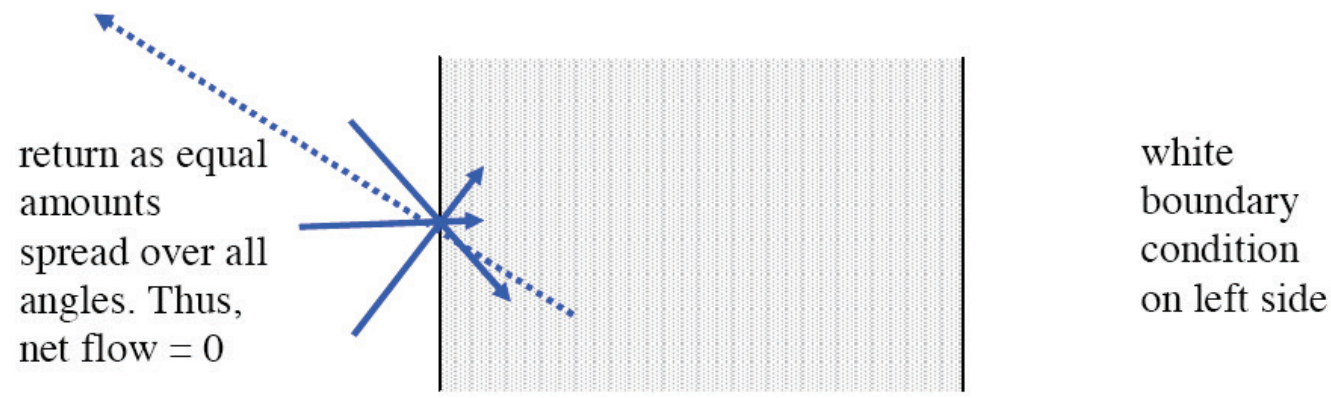

Figure 9.2.34. White boundary condition.

\subsection{Vacuum boundary condition}

A vacuum boundary condition means that no neutrons will reenter the boundary. Thus, any neutron exiting the system through a boundary with a vacuum boundary condition is permanently lost to the system. This condition is illustrated in Figure 9.2.35.

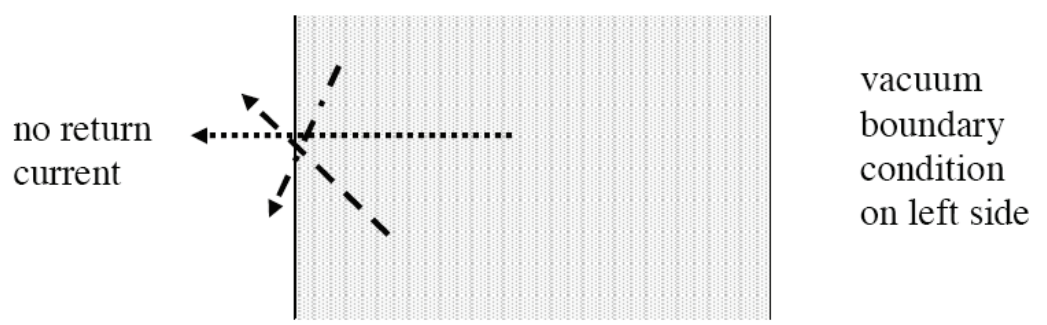

Figure 9.2.35. Vacuum boundary condition. 


\subsection{Periodic boundary condition}

For the periodic boundary condition, the incoming angular flux on a boundary is set equal to the outgoing angular flux on the opposite boundary. Figure 9.2.36 shows the leakage leaving each boundary (dotted lines) being returned at the same quantity and angle on the opposite boundary (solid line of same color). When the periodic boundary condition is used, it must be applied to both opposing boundaries.

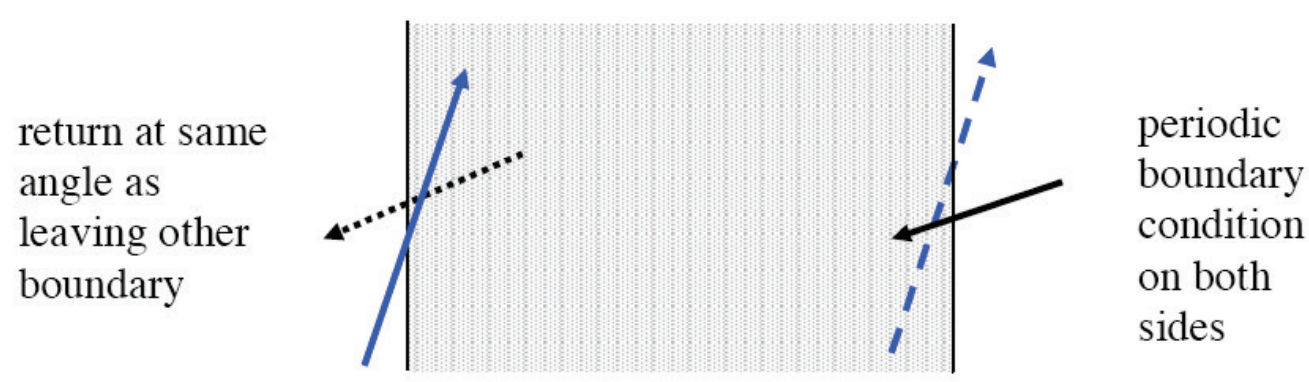

Figure 9.2.36. Periodic boundary condition.

\subsection{Boundary condition specification}

The standard format for boundary condition specifications is as follows:

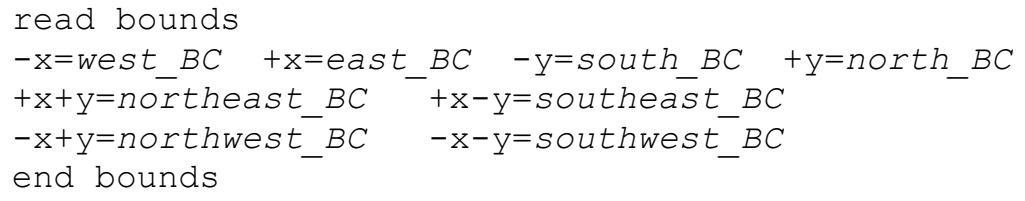

where west_BC, east_BC, south_BC, north_BC, northeast_BC, southeast_BC, northwest_BC, southwest_BC are each one of the eight possible boundary condition options. The name of the boundary condition requires only the number of leading characters required to make the name unique. For this set, the first letter is sufficient; that is, $+x=v,+x=v a c+x=v a c u$, and $+x=$ vacuum are all equivalent and specify a vacuum (zero return) boundary condition on the right side of the global cuboid.

In keeping with KENO-VI, multiple shortcuts exist to simplify the specification. For example, a single boundary condition can be assigned to all four sides simultaneously with the all= specifier:

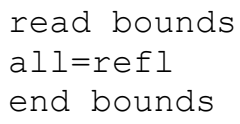

All KENO-VI boundary face keywords that do not reference the z-dimension are allowed and are listed in Table 9.2.1. 
Table 9.2.1. Boundary condition specifiers accepted by NEWT

\begin{tabular}{c|l}
\hline Keyword & Boundary edge \\
\hline$+x,+x b$ & East (right) \\
$-x,-x b$ & West (left) \\
$+y,+y b$ & North (top) \\
$-y,-y b$ & South (bottom) \\
$a l l, x y f, y x f$ & All boundaries \\
$+x y,+y x,+f C$ & East (right) + north (top) \\
$-x y,-y x,-f C$ & West (left) + south (bottom) \\
$x f C$ & West (left) + east (right) \\
$y f C$ & North (top) + south (bottom) \\
$+x+y$ & Northeast (top right) \\
$-x+y$ & Southeast (bottom right) \\
$-x-y$ & Southwest (bottom left) \\
$-x+y$ & Northwest (top left) \\
\hline
\end{tabular}

\subsubsection{General cross section weighting}

NEWT performs cross section weighting by mixture and optionally by homogenization zone (Sect. 9.2.3.10). Weighting is always used in conjunction with an energy collapse. Cross section weighting is performed over a spatial and energy domain; the resulting average (weighted) cross sections will preserve all reaction rates in the collapsed cross section set; that is,

$$
\sigma_{G}^{i}=\frac{\int_{r} N^{i}(r) d r \int_{G} \sigma^{i}(E, r) W(E, r) d E}{\int_{r} N^{i}(r) d r \int_{G} W(E, r) d E},
$$

where

$$
\begin{aligned}
\sigma_{G}^{i} & \equiv \text { average (weighted) cross section in energy group } G \text { for nuclide } I, \\
N^{i}(r) & \equiv \text { number density of nuclide } i \text { in region } r \\
W(E, r) & \equiv \text { the weighting function within region } r \\
\sigma^{i}(E, r) & \equiv \text { the cross section from the input library for nuclide } i \text { in region } r .
\end{aligned}
$$

Within NEWT, each collapsing region is the spatial region or regions in which a given mixture is placed. Hence, for most of the cross section types, an average cross section for the mixture associated with the spatial domain $r$ (which may include one or more defined regions occupied by that mixture) is calculated by weighting the original problem-specific cross section data for that mixture using as a weighting function the neutron spectrum calculated within spatial domain $r$.

In practice, the integration of Eq. (9.2.28) is performed as a simple summation over all cells $j$ within region $r$ : 


$$
\sigma_{i, G}=\frac{N_{r}^{i} \sum_{j \in r} \sum_{g \in G} \sigma_{g, j}^{i} W_{g, j}}{N_{r}^{i} \sum_{j \in r} \sum_{g \in G} W_{g, j}} .
$$

Because any region $r$ is defined as the sum of all spatial regions containing a given mixture, $N^{i}$ is constant everywhere within $r$.

All multigroup cross sections and related data present on the AMPX library are weighted using appropriate weighting functions. For most basic cross sections, the multigroup flux obtained from the transport solution is the appropriate weighting function and $W_{g, r}$ in Eq. (9.2.29) becomes $\phi_{g, r}$. However, special cross sections and data need special treatment, as described in the following sections.

\subsection{Scattering cross section transfer matrix weighting}

In weighting scattering cross sections, the form of the weighting is slightly more complex:

$$
\sigma_{s, G \rightarrow G^{\prime}}^{i}=\frac{\int_{r} N^{i}(r) d r \int_{G} W(E, r) d E \int_{G^{\prime}} \sigma^{i}\left(E \rightarrow E^{\prime}, r\right) d E^{\prime}}{\int_{r} N^{i}(r) d r \int_{G} W(E, r) d E},
$$

or, in multigroup format,

$$
\sigma_{s, G \rightarrow G^{\prime}}^{i}=\frac{N_{r}^{i} \sum_{j \in r} \sum_{g \in G} W_{g, r} \sum_{g^{\prime} \in G^{\prime}} \sigma^{i}\left(g \rightarrow g^{\prime}\right)}{N_{r}^{i} \sum_{j \in r} \sum_{g \in G} W_{g, r}} .
$$

In general, the scalar flux, $\phi_{g, r}$ is the appropriate weighting function for scattering cross sections:

$$
\sigma_{G}^{i}=\frac{N_{r}^{i} \sum_{j \in r} \sum_{g \in G} \sigma_{g, r}^{i} \phi_{g, r}}{N_{r}^{i} \sum_{j \in r} \sum_{g \in G} \phi_{g, r}} .
$$

This is an approximation for the higher order moments $(l>0)$ of the scattering cross sections, which should be more properly weighted using the $l$ th moment of the flux instead of the 0th moment (scalar) flux as used in Eq. (9.2.32). However, because flux moments are generally both positive and negative, NEWT performs higher-order-moment scattering cross section weighting using the positive scalar flux.

\subsection{Weighting of the collapsed fission spectrum, $\chi$}

Weighting is not required for collapsing a fission spectrum vector; the format for a collapsed fission spectrum $\left(\chi_{\mathrm{G}}\right)$ is a very straightforward summation of the fission spectra in energy groups $g$ spanning the energy domain of the collapsed energy group $G$ : 


$$
\chi_{G}=\sum_{g \in G} \chi_{g} .
$$

\subsection{Weighting of the number of neutrons per fission, $v$}

Accurate weighting of $v$ in an energy and space domain requires weighting by the fission rate; that is,

$$
W(E, r)=\sigma_{\text {fission }}^{i}(E, r) \phi(E, r)
$$

Hence, for $v$, Eq. (9.2.32) has the form

$$
v_{G}^{i}=\frac{N_{r}^{i} \sum_{j \in r} \sum_{g \in G} v_{g}^{i} \sigma_{\text {fission }, g}^{i} \phi_{g, r}}{N_{r}^{i} \sum_{j \in r} \sum_{g \in G} \sigma_{\text {fission }, g, r}^{i} \phi_{g, r}} .
$$

\subsection{Weighting of $(n, 2 n),(n, 3 n)$, and $(n, 4 n)$ cross sections}

In creating AMPX weighted libraries with NEWT, all data on the original library are collapsed and written to a collapsed working-format library, for each reaction type and for each nuclide. Therefore, each of the (n,Xn) libraries is collapsed independently using Eq. (9.2.29) and is written on the weighted library.

However, during NEWT transport calculations, NEWT computes and stores a single effective (n,2n) reaction rate, determined as the weighted sum of the individual reactions:

$$
\sigma_{n, 2 n}^{\text {effective }}=\sigma_{n, 2 n}+2 \sigma_{n, 3 n}+3 \sigma_{n, 4 n} \text {. }
$$

The $(\mathrm{n}, 2 \mathrm{n})$ reaction rates reported in NEWT output are those computed for the effective cross section. The effective $(n, 2 n)$ cross section is not saved to the weighted library.

\subsection{Calculation and weighting of transport cross sections}

Transport cross sections are processed in a different manner from other cross sections. The transport cross section does not represent a purely measurable quantity. Introduced within the $\mathrm{P}_{1}$ (diffusion) approximation to the neutron transport equation, it attempts to preserve a flux gradient in addition to reaction rate information. Hence, the magnitude of a microscopic transport cross section is affected by both the physics properties of the nuclide in question and the geometrical attributes of the spatial domain where the nuclide resides and the other nuclides present in the same vicinity.

Consistent with XSDRNPM, NEWT provides two options to generate a microscopic transport cross section-based on the "consistent" and "inconsistent" methods for solving the $\mathrm{P}_{1}$ transport equations. These approximations are referred to as the "outscatter" and "inscatter" approximations because of the nature of the equations used.

\subsection{Outscatter approximation (inconsistent method)}

In the outscatter approximation, the following assumption is made for the transport cross section in group $g$ :

$$
\sigma_{t r}^{g}=\sigma_{t}^{g}-\bar{\mu}^{g} \sigma_{s}^{g},
$$


where $\sigma_{\mathrm{t}}^{\mathrm{g}}$ and ${\sigma_{\mathrm{s}}}^{\mathrm{g}}$ are the total and scattering cross section in group $g$,

$$
\bar{\mu}^{g}=\frac{\sigma_{s, 1}^{g}}{3 \sigma_{s, 0}^{g}}
$$

and

$$
\sigma_{s, N}^{g}=\sum_{g^{\prime}} \sigma_{s, N}\left(g \rightarrow g^{\prime}\right)
$$

Note that the $\sigma_{s, N}\left(g \rightarrow g^{\prime}\right)$ terms are the $\mathrm{P}_{\mathrm{N}}$ coefficients of the scattering matrix, hence the origin of the term "outscatter."

\subsection{Inscatter approximation (consistent method)}

In the "consistent" $\mathrm{P}_{1}$ approximation of the transport equation, the transport cross section is defined as

$$
\sigma_{t r}(E)=\sigma_{t}(E)-\frac{1}{3 J(E)} \int_{0}^{\infty} \sigma_{s, 1}\left(E^{\prime} \rightarrow E\right) J\left(E^{\prime}\right) d E^{\prime}
$$

where $J$ is the neutron current and $\sigma_{s, 1}$ is the first moment ( $\mathrm{P}_{1}$ coefficient) of the scattering transfer matrix.

If one multiplies Eq. (9.2.40) by $\mathrm{J}(\mathrm{E})$, integrates over group $g$, and converts to a group-averaged form by dividing by $\int_{g} J(E) d E$, the following expression is derived:

$$
\sigma_{t r}^{g}=\sigma_{t}^{g}-\frac{1}{3 J_{g}} \sum_{g^{\prime}} \sigma_{s, 1}\left(g^{\prime} \rightarrow g\right) J_{g^{\prime}}
$$

This is the "inscatter" approximation. It is consistent because the transport values are explicitly derived from the $\mathrm{P}_{0}$ and $\mathrm{P}_{1}$ equations.

\subsection{Weighting function for transport cross section}

Internal investigations have shown that transport cross sections computed using the "outscatter" approximation are more robust in subsequent nodal core calculations as compared with transport cross sections computed using the "inscatter approximation." NEWT computes transport cross sections using the outscatter approximation and collapses the cross section with the scalar flux. 


\subsubsection{Array definition}

Any arrays specified in unit definitions within the read geom block are defined in terms of form and content in the read array data block. The block has the form shown below:

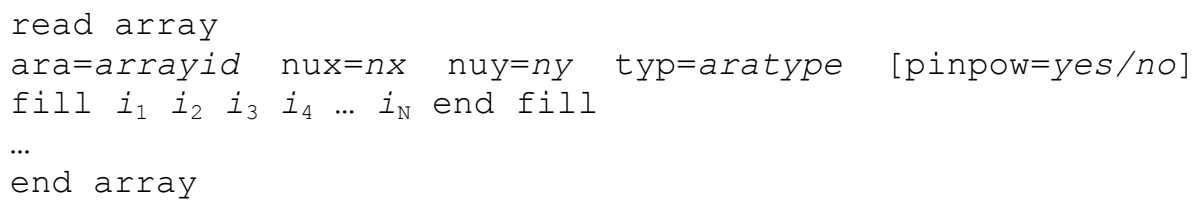

where arrayid is a unique integer identifier for the array, $n x$ is the number of array elements moving left to right (i.e., columns), and $n y$ is the number of array elements moving from bottom to top (i.e., rows). The type of array is indicated by aratype (e.g., square, hexagonal). The optional parameter pinpow may be specified as either yes or no (default is no) and is used to enable/disable pin power edits for units within the array. The fill...end fill specifier set is used to input the elements of the array. A total of $N$ entries are required, where $N=\mathrm{nx}^{*}$ ny.

Each of these portions of the array definition statement is described in more detail below.

\subsection{Array types}

NEWT supports arrays of cuboids, hexprisms, and rotated hexprism elements. Four different array types may be selected. Table 9.2.2 lists the supported array types and corresponding element types. The array type given in the first column lists the keyword associated with each type, as used in the typ= specifier; in some cases, multiple keywords are associated with a specific array type. The element type in the second column provides the boundary shape that can be used in the given array type. For example, a cuboidal (square) array may only be filled with cuboidal units.

Table 9.2.2. NEWT array types with corresponding element types

\begin{tabular}{l|l}
\hline \multicolumn{1}{c|}{ Array type } & Element type \\
\hline Cuboidal, square & Cuboid \\
Hexagonal, triangular & Hexprism \\
Shexagonal & Hexprism \\
Rhexagonal & Rhexprism \\
\hline
\end{tabular}


All arrays are filled in a 2-D $i, j$ pattern, with $i$ varying from 1 to $n u x$ and $j$ varying from 1 to $n u y$. All nux*nuy elements of each array must be filled. Figure 9.2.37 illustrates the layout of a conceptual 4 by 4 cuboidal array, showing the row/column index for each element of the array. Figure 9.2.38 shows the row/column designation for a 4 by 4 hexagonal array. Because of the shape of a hexprism, the array itself is staggered. However, the row/column numbering is simple to understand.

The stacked hexagon (shexagon) layout, as shown in Figure 9.2.39, was developed to simply allow an alternate placement algorithm for hexprisms. Any model that can be specified with typ=hexagonal can also be specified with type=shexagonal; the choice of which form to use is simply one of user preference. It is important to note that beginning with row 3, units will be placed in a physical location different from that of the hexagonal layout when the shexagonal layout is used.

Finally, the rotated hexprism (rhexprism) array is pictured in Figure 9.2.40. This array is intended to facilitate placement of rhexprisms. The numbering of cells is somewhat irregular because of the staggered rows, but it is easy to follow if one is aware of the fill pattern as illustrated in the figure. Note that the layout of a rhexagonal array can be replicated exactly using a hexagonal or rhexagonal array, placed in a hole, and rotated $90^{\circ}$. Thus, the type of hexprism-based array used can always be tailored to the preferences of the user and all can be used to create the same model.

It is often the case, especially for hexagonal-type arrays, that one does not need to fill all array positions. While the array fill procedure does require that all positions be filled, NEWT provides a mechanism to fill a position with a null unit, effectively skipping that position. This is discussed further under Fill Operations.

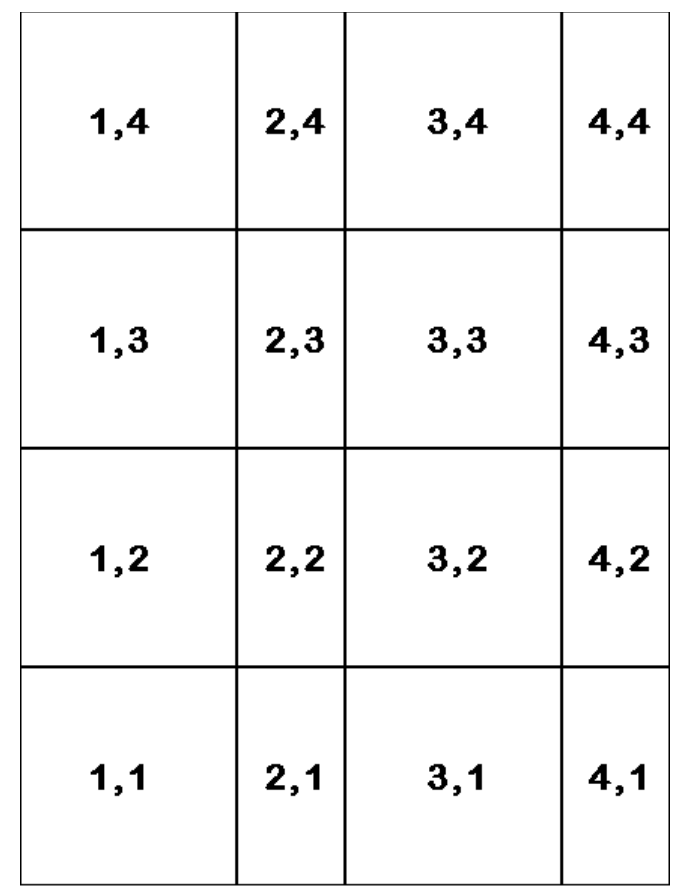

Figure 9.2.37. Layout of a 4 by 4 cuboidal array.

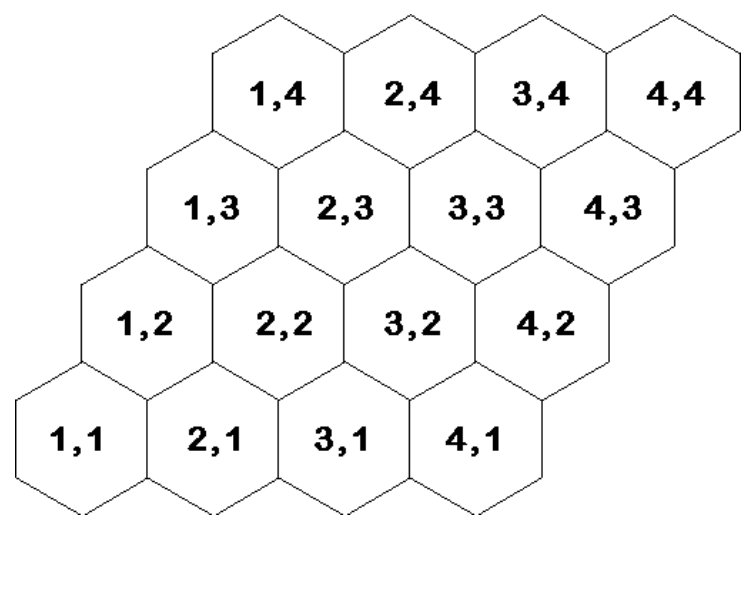

Figure 9.2.38. Layout of a 4 by 4 hexagonal array. 


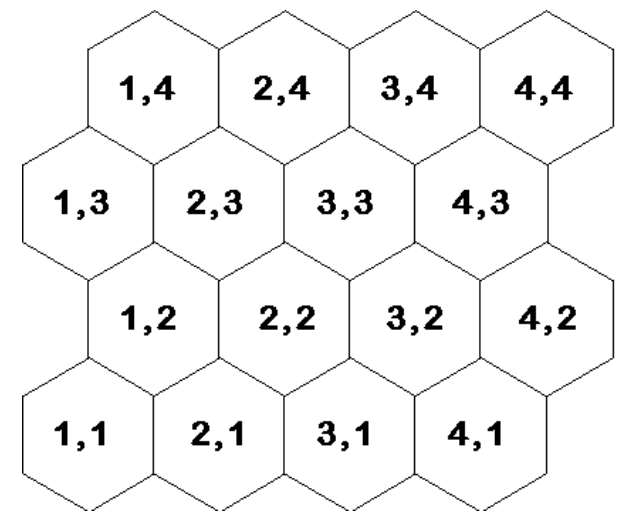

Figure 9.2.39. Layout of a 4 by 4 stacked hexagonal (shexagonal) array.

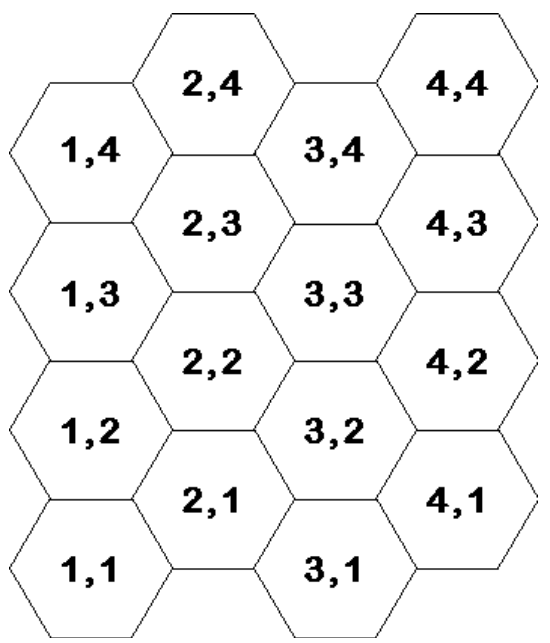

Figure 9.2.40. Layout of a 4 by 4 rotated hexagonal (rhexagonal) array.

Although all elements of cuboidal arrays must be cuboids, they need not be the same size. Elements of each row must have the same height but may have varying widths. Similarly, elements of each column must be of a single common width but may vary in height. Less flexibility is available in hex-based arrays, because of their very nature. Hexagonal and stacked hexagonal arrays may contain only hexprisms, and all must be of the same outer size (although unit contents may vary as needed). Rotated hexagonal arrays likewise are limited to rhexprisms with a single boundary size.

\subsection{Pin-power edits}

In lattice-physics calculations, it is often necessary to obtain a pin-power edit showing the power produced in each fuel pin cell. NEWT uses the array functionality to define pin cells. When pinpow=yes is specified, an extra edit is produced that gives the normalized pin power in each pin cell. A pin cell is defined as any element within the array that contains a fissionable nuclide. Pin powers are normalized such that the average of all fuel-bearing array elements is 1.0. Array elements such as burnable poison rods or water holes, which produce no fission power, are not included in the power normalization process. The pinpow functionality is not available for hexagonal, shexagonal, or rhexagonal lattices.

Output provides an edit of each assembly for which pinpow=yes is specified. In addition, a final edit is provided for the entire system, normalized to all fuel cells in all arrays for which pinpow=yes is specified.

Pin-power edits are shown in the description of output in Sect. 9.2.5.

\subsection{Fill operations}

The final section of an array specification is the fill list. Delimited by fill and end fill keywords, NEWT expects a list of $\mathrm{N}=$ nux*nuy unit numbers, specifying the unit to be placed at each array position. Arrays are filled left to right, starting at the bottom left-hand corner and moving up a row after all columns in the current row are filled. In other words, any of the $4 \times 4$ arrays in the figures above would be filled in the following order: $(1,1),(2,1),(3,1),(4,1),(1,2),(2,2),(3,2),(4,2),(1,3),(2,3),(3,3),(4,3),(1,4),(2,4)$, $(3,4),(4,4)$.

The list of elements used to fill an array consists of unit numbers. Each unit used in the fill list must be defined in the geometry block and must be of the shape and size required for the array type and position. However, NEWT provides the ability to fill an array location with a null unit, which in essence skips the 
Now, in a read array block, we define array 1, a 2 by 2 array filled with unit 2 cells, and array 2, filled with two unit 1 cells, one unit 3 cell (containing array 1), and one null unit:

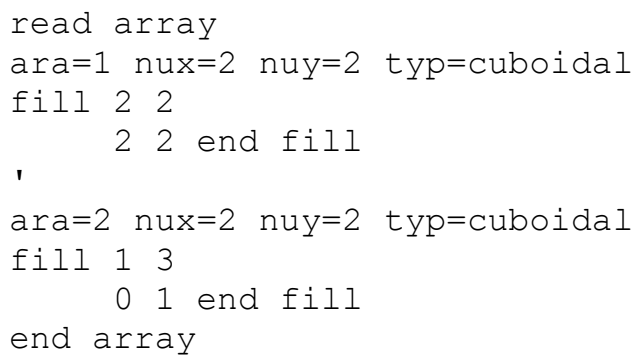

When assembled, the model would appear as shown in Figure 9.2.41. Note that local grids override the global grid in each array location but that the global grid is seen where the null unit is placed.

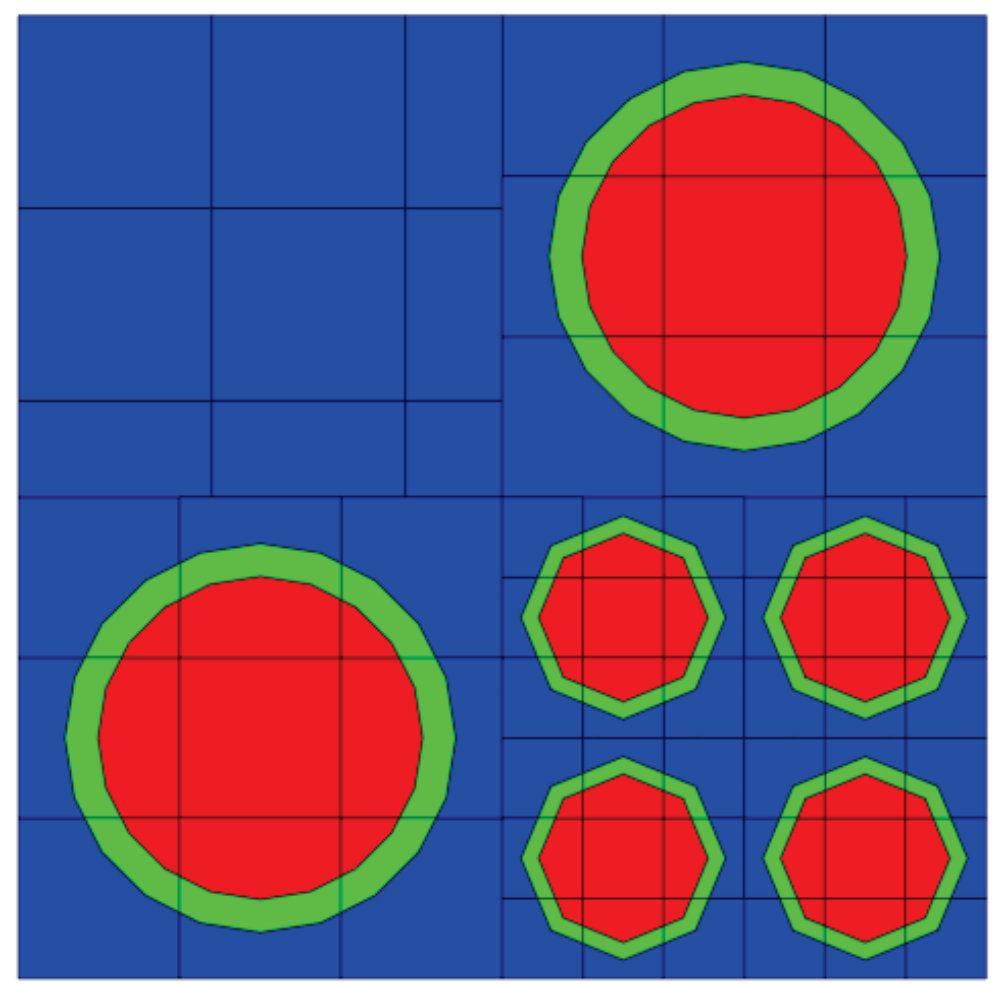

Figure 9.2.41. Example of nested arrays and a null unit specification.

\subsubsection{Homogenization block}

\section{Homogenization block keyword = homog, hmog, homo}

As discussed earlier, NEWT can be used to collapse cross sections to a reduced broad-group format. The cross sections produced from this operation are written as microscopic cross sections for each nuclide in each mixture. NEWT also provides the ability to produce macroscopic weighted cross sections homogenized over one or more mixtures. Homogenized cross sections are created using the collapsing energy structure defined in the collapse data block or the original library's group structure if no collapsing instructions are provided. Flux-weighted collapsed cross sections are combined with number densities and added such that reaction rates in homogenized materials are conserved. 
Within the homogenization block, multiple homogenization records are permitted, and the same mixtures may be repeated in different records. Each record provides a recipe defining the mixtures to be homogenized. Homogenization records have the following form:

$$
\begin{array}{cccc}
\text { Homogenized } & \text { Mixture } & \text { List of } & \text { end } \\
\text { Mixture ID } & \text { Description } & \text { Mixtures } &
\end{array}
$$

The homogenized mixture ID is the "nuclide" number under which the mixture is saved on the homogenized cross section library. The value is arbitrary and serves only as a means to identify the cross section set on the library, although each ID must be unique. The mixture description is an alphanumeric label of up to 12 characters that is associated with the mixture; this label provides a little more descriptive ability than the ID itself. The label may not contain blanks. Finally, the label is followed by the list of mixtures to be homogenized, terminated by the end keyword. The list may contain up to 1000 unique mixtures.

A sample homogenization block is shown below. In this illustration, two homogenized mixtures are created. This first consists of five different fuel mixtures (201-205); this could be used to obtain the average fuel cross section for an assembly containing five different fuel types. The homogenized cross sections will be written to the homogenized library as nuclide 500, with label "fuel." The second instruction homogenizes mixtures 201, 210, and 220 from the original problem; this could be used, for example, to homogenize the fuel, clad, and moderator of a fuel pin cell. This cross section set would be written on the same library as nuclide 501, with the label "fuel_cell201."

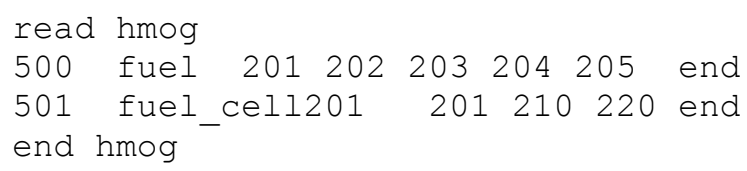

Homogenized (macroscopic) cross sections are saved in an AMPX working-format library at the unit specified by the $h m o g l i b=$ parameter $($ default=13) [ft13f001].

\subsubsection{Assembly discontinuity factors}

\section{Assembly discontinuity factor (adf) block keyword $=$ adf}

Because discontinuity factors have meaning only with respect to homogenized cross sections, ADFs are calculated only if homogenized cross sections are also specified via the Homogenization data block (see Sect. 9.2.3.10). Calculation of ADFs is specified in the read adf data block. The three supported formats of this data block are as follows. For a single-assembly model, the following format is used:

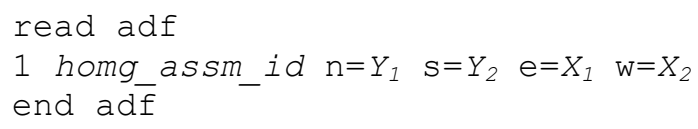

For a reflected assembly model, the following format is used:

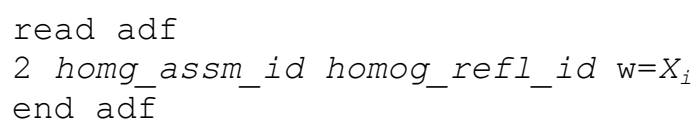

For a homogenized assembly, the following is used:

read adf 
3 homg_assm_id line line $_{2}$ line $_{3} \ldots$
end adf

where

hmog_assm_id is the identifying label assigned to the homogenized assembly, hmog_refl_id is the label assigned to the homogenized reflector region,

$Y_{1}$ is the y-ordinate of the north boundary of the assembly

(typically this is $\mathrm{y}_{\max }$ for the global unit),

$Y_{2}$ is the y-ordinate of the south boundary of the assembly (typically this is $y_{\min }$ for the global unit),

$X_{1}$ is the $\mathrm{x}$-ordinate of the east boundary of the assembly (typically this is $\mathrm{x}_{\max }$ for the global unit),

$X_{2}$ is the $\mathrm{x}$-ordinate of the east boundary of the assembly

(typically this is $\mathrm{x}_{\max }$ for the global unit),

$X_{i}$ is the $\mathrm{x}$-ordinate for the fuel/reflector interface,

line $_{i}$ is a series of two ordered pairs $\left(X_{1}, Y_{2}\right),\left(X_{1}, Y_{2}\right)$ that define a line segment in the NEWT grid.

In a single-assembly calculation, only a single homogenized mixture is specified. Leading index 1 indicates that ADFs for the fuel assembly are being calculated; ADFs may be calculated on any or all faces of a rectangular assembly. In a reflected assembly, the leading index is 2 , followed by the homogenized mixture ID for the fuel assembly first, then the homogenized mixture ID for the reflector region. An $\mathrm{ADF}$ may be requested only for one location, at the fuel/reflector interface. In any configuration, ADFs may be requested for a set of arbitrary line segments defined in the NEWT geometry. In this case, the leading index is 3, followed by the homogenized mixture ID, followed by up to 12 line segments, which are defined by their beginning and ending points. ADFs along these lines are defined as the surface-averaged flux divided by the average flux defined for the associated homogenized mixture. Surface-averaged currents are also edited for each arbitrary line segment; both full and partial currents in both the $\mathrm{x}$ - and $\mathrm{y}$ - directions are provided. The net current is also provided in the few-group cross section database file $x$ file016. The orientation of the net current across the line segment is further discussed in Appendix A of TRITON chapter.

Although any homogenized set of mixtures can be specified for each homogenized region, the ADF will have physical meaning only if the homogenized set represents all mixtures in the assembly. Similarly, if a reflector calculation is performed, the hmog_refl_id should represent the set of homogenized reflector mixtures. The average collapsed flux in the homogenized mixtures is used to calculate the homogeneous flux for a single-assembly ADF. In a reflector calculation, the homogenized cross sections for the reflector are used to solve the multigroup diffusion approximation (Sect. 9.2.2.5).

Examples illustrate the use of the ADF input specification. Consider a $17 \times 17$ pressurized-water-reactor (PWR) design. Because of symmetry, it can be modeled a $1 / 4$ assembly; therefore, we choose to model the upper right quadrant, as shown in Figure 9.2.42. Mixtures 1, 2, 3, and 4 represent the fuel, clad, moderator, and guide tube materials, respectively. The west and south sides of the model are the assembly midplanes, so ADFs calculated on these boundaries would have no physical meaning. (These are not real assembly boundaries.) However, because of the symmetry of the assembly, fluxes would be identical on all boundaries. Therefore, selection of either the north or east boundary will yield a valid ADF for all boundaries. We choose to request an ADF calculation for the east (right) side of the assembly. 


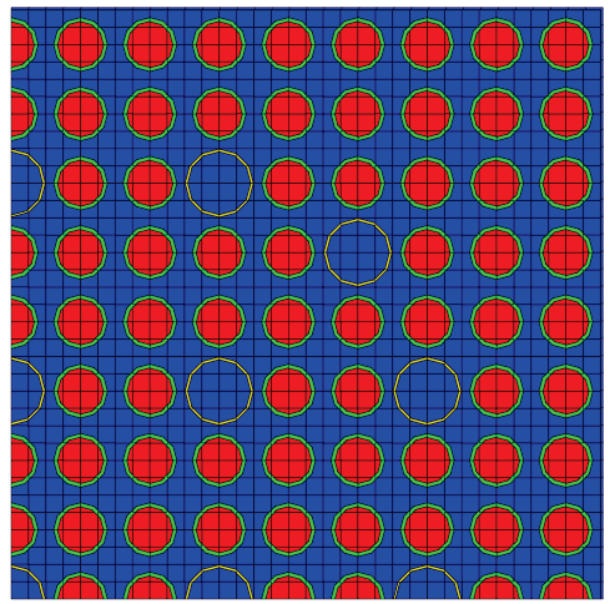

Figure 9.2.42. Upper-right quadrant of an unreflected PWR assembly.

Assuming we are collapsing 44 energy groups to 2 energy groups, we would specify the following collapse, homog, and adf blocks to obtain 2-group ADFs representative of this assembly:

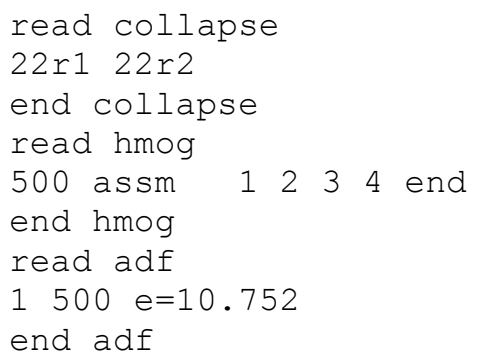

Again, recall that for a single assembly, the ADF in each energy group is simply the average flux on the specified boundary divided by the average flux for the entire assembly, which in this case is the flux in homogenized mixture 500.

ADF can also be calculated using the arbitrary line-segment ADF type. Using this new ADF type, the ADF input would be the following:

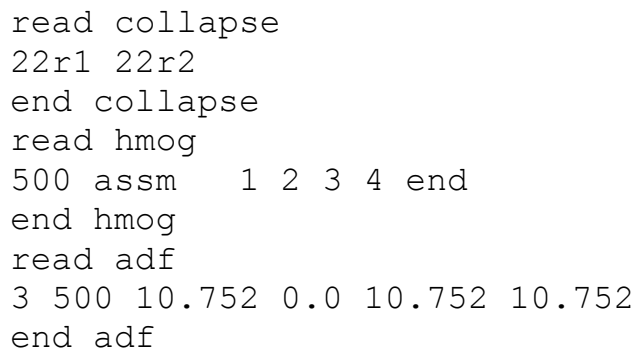

In this example, the east-side ADF will be calculated along the line segment starting at $(10.752,0)$ and ending at $(10.752,10.752)$. The values of the line segments depend on a coordinate system of the global unit.

For a reflected model, consider the same type of assembly but representing an assembly placed on the core periphery. It is bounded on one side by a $2 \mathrm{~cm}$ stainless steel baffle and $10 \mathrm{~cm}$ of water, beyond which is treated as vacuum. Because the reflector calculation is a 1-D solution, it is not possible to 
directly model a fuel assembly with two reflector boundaries. Typically the assumption is made that the same ADF may be applied in any assembly/reflector boundary and that a 1-D reflector model is all that is necessary. This model is pictured in Figure 9.2.43. Notice that different mixtures are used in the reflector model. Fluxes used in homogenized mixtures and for generating homogenized cross sections are spatially averaged; thus, it is important to separate the moderator in the reflector from that in the fuel assembly such that average fluxes in each region properly characterize that region. For example, the flux in the reflector will be significantly different (far more thermal) from the flux within the assembly.

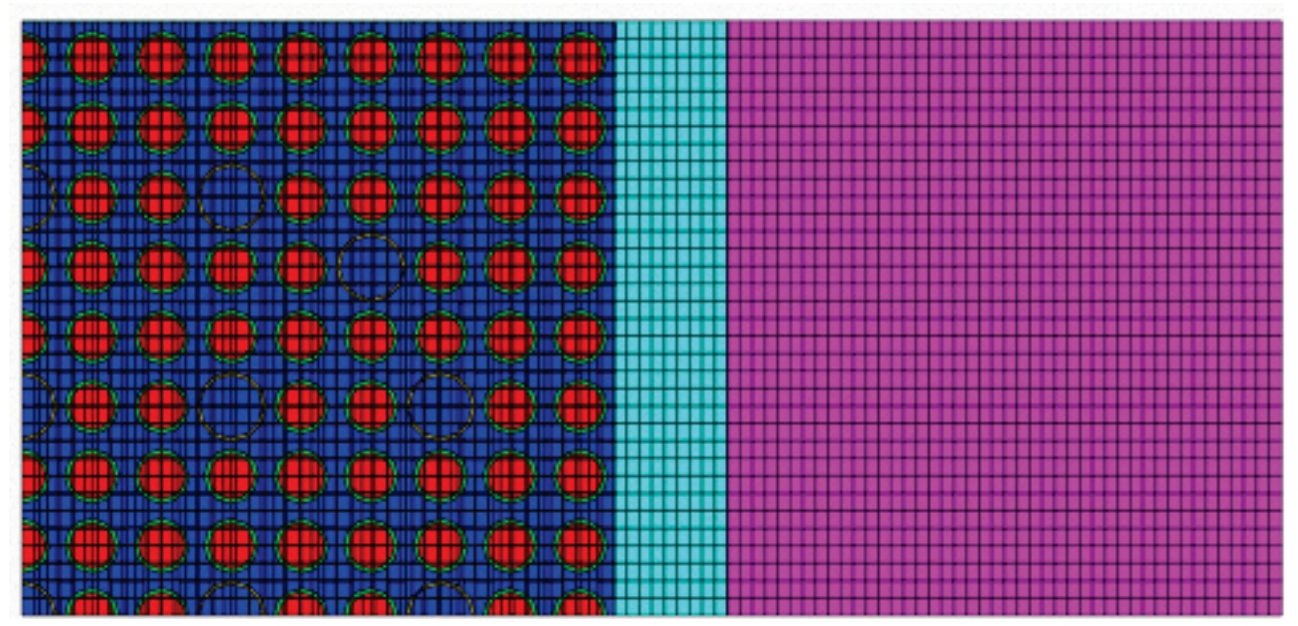

Figure 9.2.43. Upper-right quadrant of a PWR assembly with baffle and reflector.

Assuming again a collapse from 44 energy groups to 2, the following collapse, homog, and adf blocks would be specified to obtain 2-group ADFs representative of the assembly and the reflector; 2-group cross sections for each homogenized region would also be prepared from the collapse and homogenization instructions:

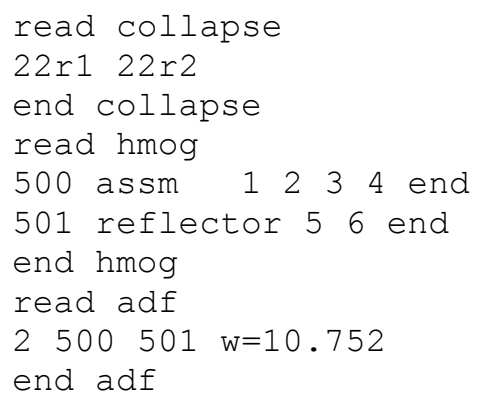

\subsubsection{Flux planes}

\section{Fluxplane block keyword $=$ fluxplane, fluxplan, flux, fluxplanes}

The fluxplane block is a special output edit that lets one obtain the average scalar flux and currents along any line segment or any continuous set of collinear line segments. One must simply specify the start and end points of a line segment for which a linearly averaged flux is desired. This line segment must correspond to one or more line segments in the model's grid structure, which requires some knowledge of where grid lines exist in the model. 
The format of a flux plane specification is as follows:

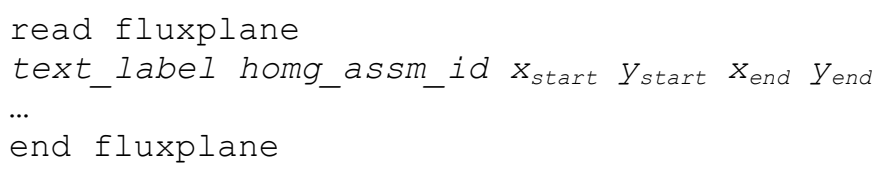

where text label is an alphanumeric description used to label the selected plane in the output, homg_assm_id is the identifier in the homog block, and $\left(x_{\text {start }}, y_{\text {start }}\right)$ and $\left(x_{\text {end }}, y_{\text {end }}\right)$ are the start and end points, respectively, for the line segment for which an average flux is desired. The text label string must not contain white space and may be up to 16 characters in length.

As an example, we consider a simple model consisting of two dissimilar pin cells ( $1 / 4$ cells):

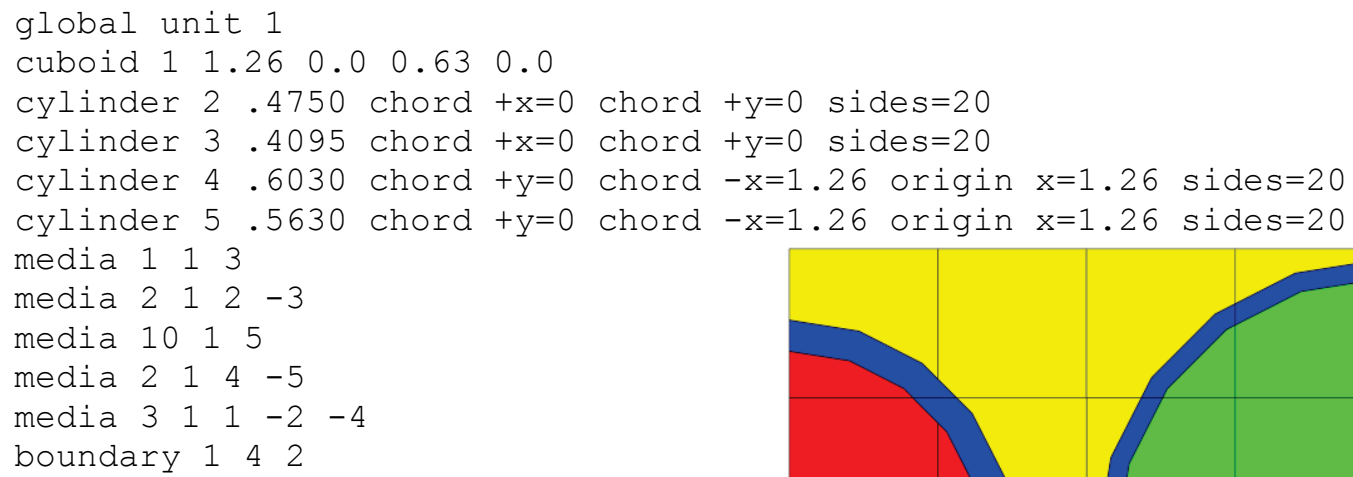

We know a line segment (actually, two) exists at $x=0, x=0.63$, and $x=1.26$, between $y=0$ and $y=0.63$. Thus, a legitimate set of flux plane specifications would be the following:

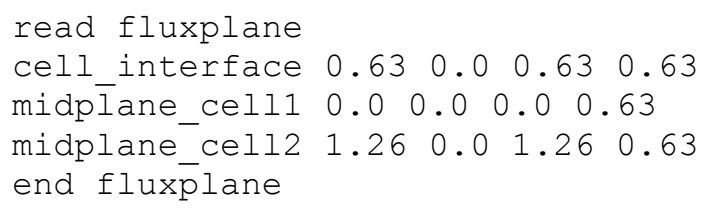

This will provide a summary of fluxes and currents at each line segment in fine-group structure, and if a collapse is performed, in broad-group structure. Results from a calculation with a two-group collapse appear as follows:

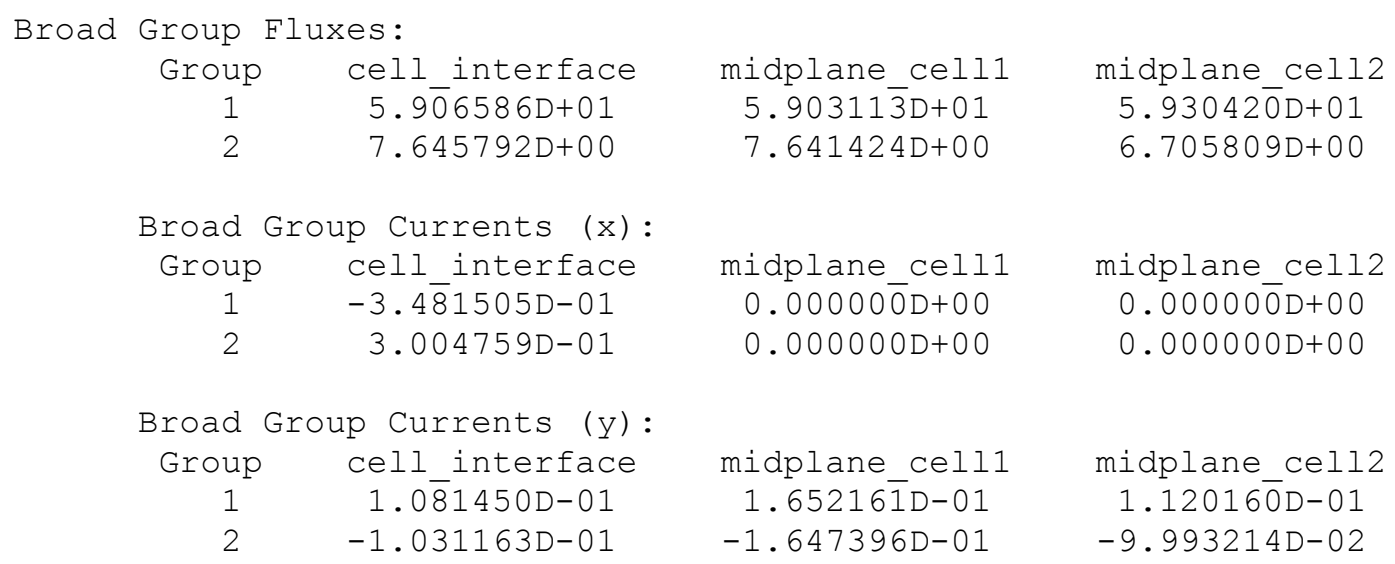


Output also includes $+\mathrm{x},-\mathrm{x},+\mathrm{y}$, and $-\mathrm{y}$ components of currents.

\subsubsection{Mixing table block}

\section{Mixing table block keyword $=$ mixt, mixtable}

Generally, NEWT calculations are performed using a cross section library and mixing table prepared in advance by other SCALE modules. However, NEWT allows the user the ability to manually specify the isotopic composition of each mixture used in a NEWT model. If a mixing table block is read, any existing mixing table data file is ignored. Therefore, all mixtures specified in the material block must be mixed in the mixing table block.

The format of the mixing table is simple and straightforward. For each nuclide used, three parameters must be supplied: (1) mixid, the mixture ID number into which the nuclide is to be mixed; (2) nuclideid, the SCALE ID number for the nuclide (which must exist on the cross section library being referenced); and (3) concentration, the number density $($ atoms $/ \mathrm{b}-\mathrm{cm})$ of the nuclide in this mixture. The same nuclide may appear in multiple mixtures or more than once in a single mixture if desired. Macroscopic cross sections are determined for each mixture by the following formula:

$$
\Sigma^{R}=\sum_{i} \sigma_{i}^{R} N_{i}
$$

where

$\Sigma^{R}$ is the mixed macroscopic cross section for reaction $R$ in the mixture,

$\mathrm{N}_{\mathrm{i}}$ is the number density of nuclide $i$,

$\sigma_{i}^{R}$ is the microscopic cross section for reaction $R$ in nuclide $i$.

The form of the mixing table block is as follows:

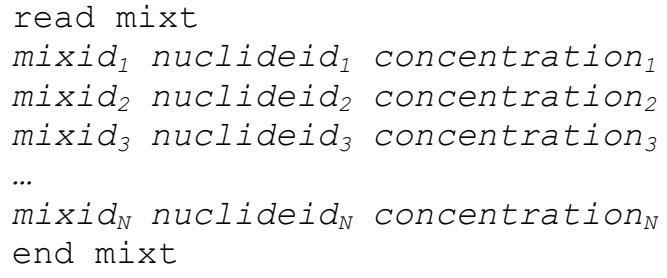

This concludes this list of input blocks available within NEWT. The following section provides a list of sample inputs used to represent a variety of configurations and use of codes. These examples are intended to provide a broader illustration of the use of NEWT in a range of potential applications.

\subsubsection{Examples of Inputs}

This section provides annotated sample input listings for three different model types, showing the use of a number of different options and approaches in model development for a variety of applications. These samples use the TRITON T-XSEC sequence to prepare cross sections for stand-alone NEWT calculations. In general, this is more easily accomplished as a TRITON T-NEWT calculation in which cross section processing and a NEWT transport solution are combined into a single calculation. However, the user is directed to the TRITON user's manual (Chapter T1.4 of the SCALE manual) for details on T-XSEC and T-NEWT calculations. The examples are intended simply to demonstrate the use of the NEWT code. The T-XSEC data are included to allow a user to observe the mixture definitions used 
in the NEWT input in its calculation. These problems are also available as sample problems in the SCALE distribution.

\subsubsection{Sample 1}

Sample 1 illustrates the use of a series of three consecutive stand-alone NEWT calculations. Annotated input for this problem is given in Figure 9.2.44. The calculation begins with SCALE standard composition specifications used to prepare a problem-specific weighted cross section library and mixing table for use by NEWT. In this case the T-XSEC sequence of the TRITON control module is used. This input is described in the TRITON chapter and is not described further here.

The first NEWT case uses no parameter block; thus, all default parameters are applied. The default is an eigenvalue calculation, with cross sections read from $\mathrm{ft} 04 \mathrm{f} 001(\mathrm{xnlib}=4)$ and collapsed cross sections written to ft30f001 (wtdlib=30). The 238-group cross section library is collapsed to a 44-group library using mixture-weighted fluxes. The model calculates the eigenvalue for a simple $1 / 4$ pin cell. The center of the pin is placed at the origin, the lower-left corner of the global unit boundary, inlaid into a 2 by 2 base grid. The grid structure is illustrated in Figure 9.2.45.

The second case performs the same calculation using the collapsed cross section library created by the first case. Parameter restart $=$ no is set to prevent the code from attempting a restart from the existing library. Because the first case saved 238-group fluxes and the second case uses 44 energy groups from the collapsed set, a restart is not possible.

The third NEWT case is a calculation identical to the second case, although the input is different. In this case, the flux restart file from the previous calculation is used as a first guess for fluxes. This is permitted since both cases used the same cross section library and therefore have the same energy boundaries. For this case, the "read geom" data block is omitted, telling NEWT to use the geometry restart file from the previous case. This allows a rapid restart, since no geometric data need to be recomputed. Because no other parameters are changed, this case will converge after one outer iteration to the same eigenvalue as in the first case. 


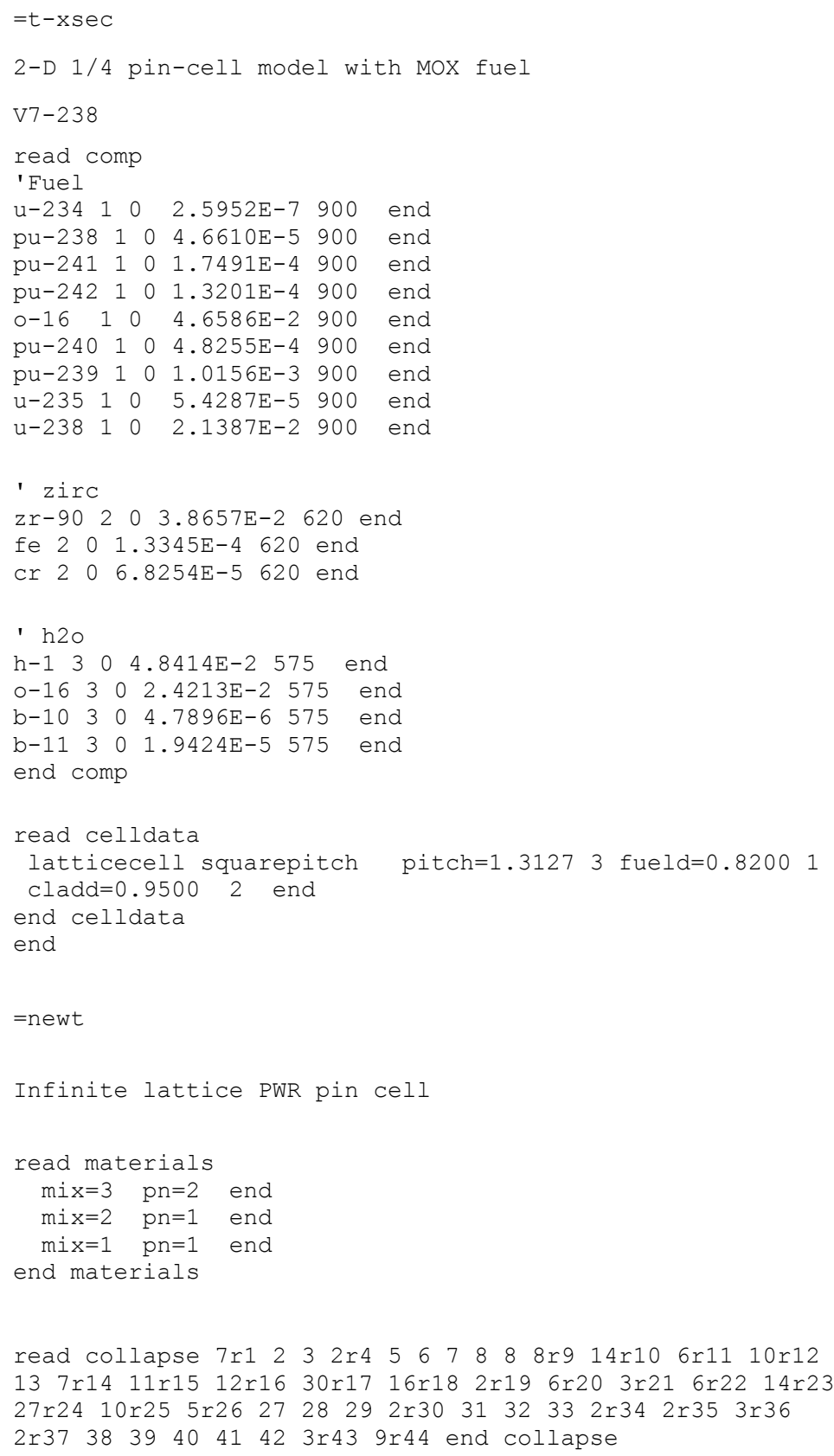

Figure 9.2.44. Sample 1 input listing (annotated).

\section{T-XSEC sequence of TRITON \\ TRITON title \\ 238-group ENDF/B-VII library}

Mixed oxide fuel isotopic specification

Zircaloy clad

Borated water moderator

Square-pitch pin-cell lattice specification

First stand-alone NEWT calculation

NEWT title

No parameter block specified; defaults are used.

Mixtures 1, 2, and 3 on cross section library used in this problem. Moderator has P-2 scattering; other mixtures use P-1 scattering.

238-group cross sections are to be collapsed to this 44-group structure. 
read geom

global unit 10

cylinder 110.41 chord $+x=0.0$ chord $+y=0.0$ sides $=24$

cylinder 120.475 chord $+x=0.0$ chord $+y=0.0$ sides $=24$

cuboid $130.656350 .0 \quad 0.65636 \quad 0.0$

media 11111

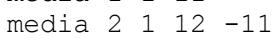

media $3 \quad 1 \quad 13-12$

boundary 1322

end geom

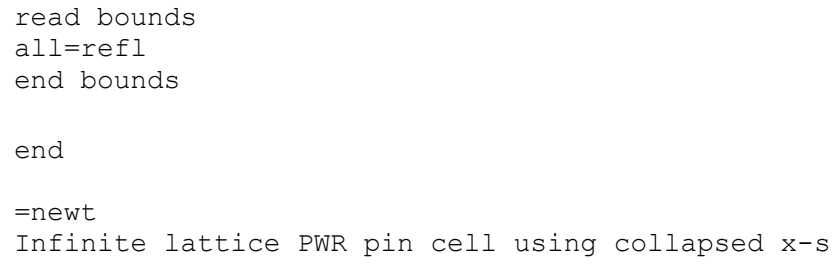

read parm

xnlib $=30$ collapse $=$ no restart $=$ no

end parm

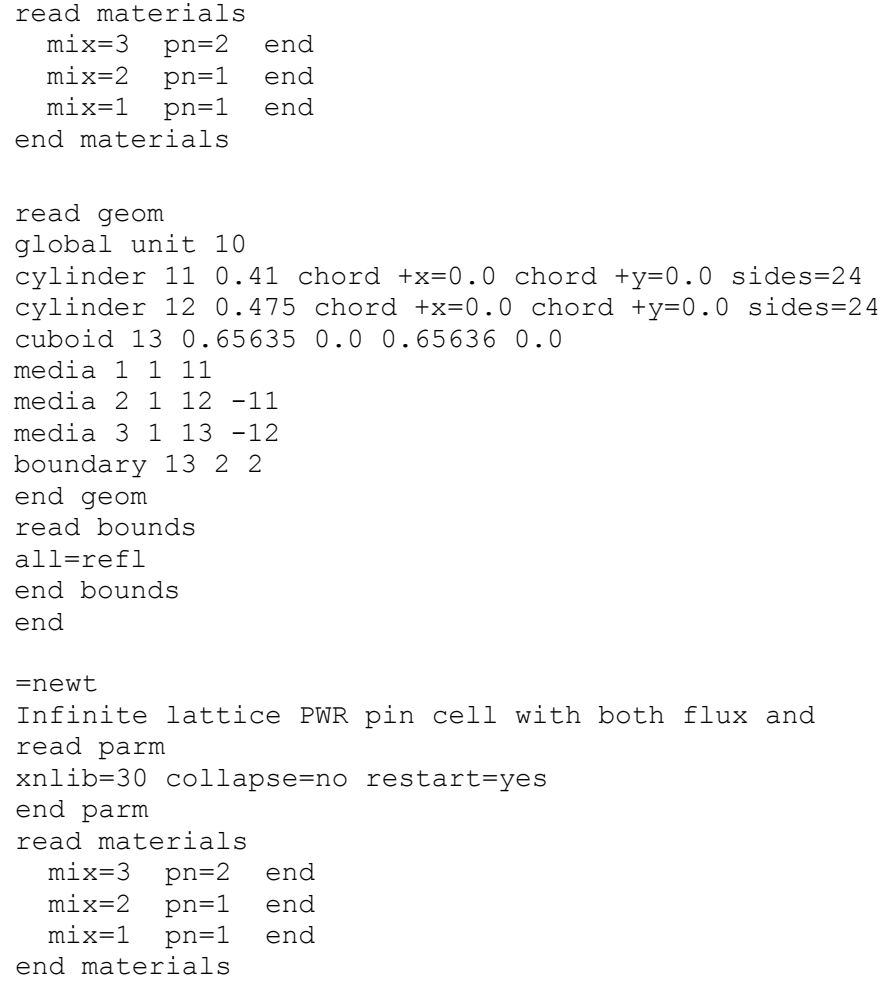

Geometry specification. Two concentric (fuel/clad) cylinders centered at 0,0 , trimmed to $1 / 4$ (upper-right) quadrant using chords. Global unit boundary is defined by the outer boundary of rectangular pin-cell moderator $(1 / 2$ of pitch). Bodies are laid onto a 2 by 2 grid. Media 1 (fuel) is inside cylinder 11. Media 2 (clad) is inside cylinder 12 and outside cylinder 11. Media 3 (moderator) is placed inside cuboid 13 but outside cylinder 12 .

Reflective BCs used on all boundaries

End of this NEWT case

Second NEWT case

NEWT title card

Parameter block specifies to use unit 30 (file ft30f001) cross sections. These are the collapsed cross sections from first case. No collapsing is to be done, and no restart occurs.

Same mixtures as before

Same geometry as before

\section{Third NEWT case}

Restart using fluxes from previous case

Same mixtures

No geometry is specified; geometry from previous case is reused.

Figure 9.2.44. Sample 1 input listing (annotated). (continued) 


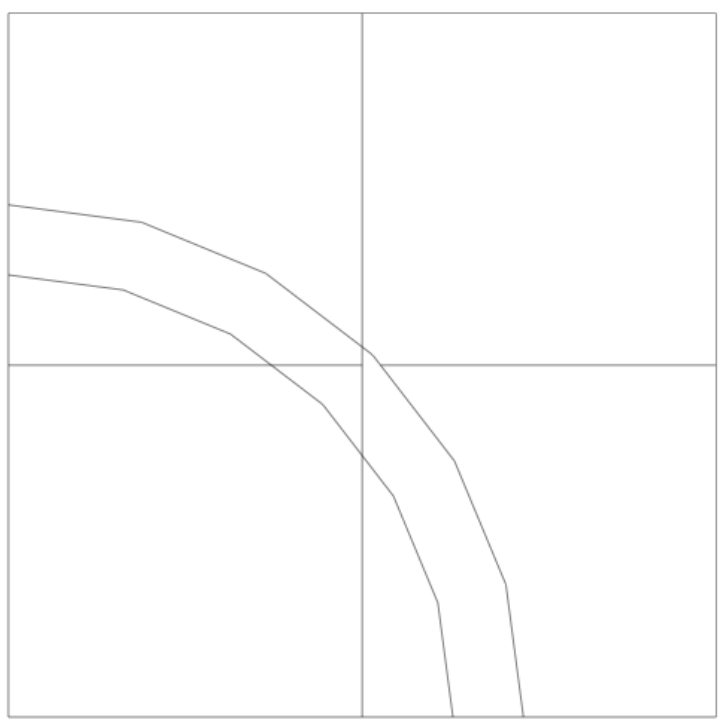

Figure 9.2.45. Grid structure for 1/4 pin cell of Sample 1.

\subsubsection{Sample 2}

Sample 2 (shown in Figure 9.2.46 and Figure 9.2.47) illustrates the use of multiple bodies within a single unit. It highlights the use of media definitions to include and exclude regions when various bodies are used. Although an array can be used to place bodies, this example illustrates a method suitable for use in developing a model for a configuration with an irregular non-array-type structure. This sample problem also highlights the use of partial-current unstructured-mesh CMFD acceleration, which reduces the number of outer iterations from 35 to 21 and the CPU run time by $\sim 25 \%$. 


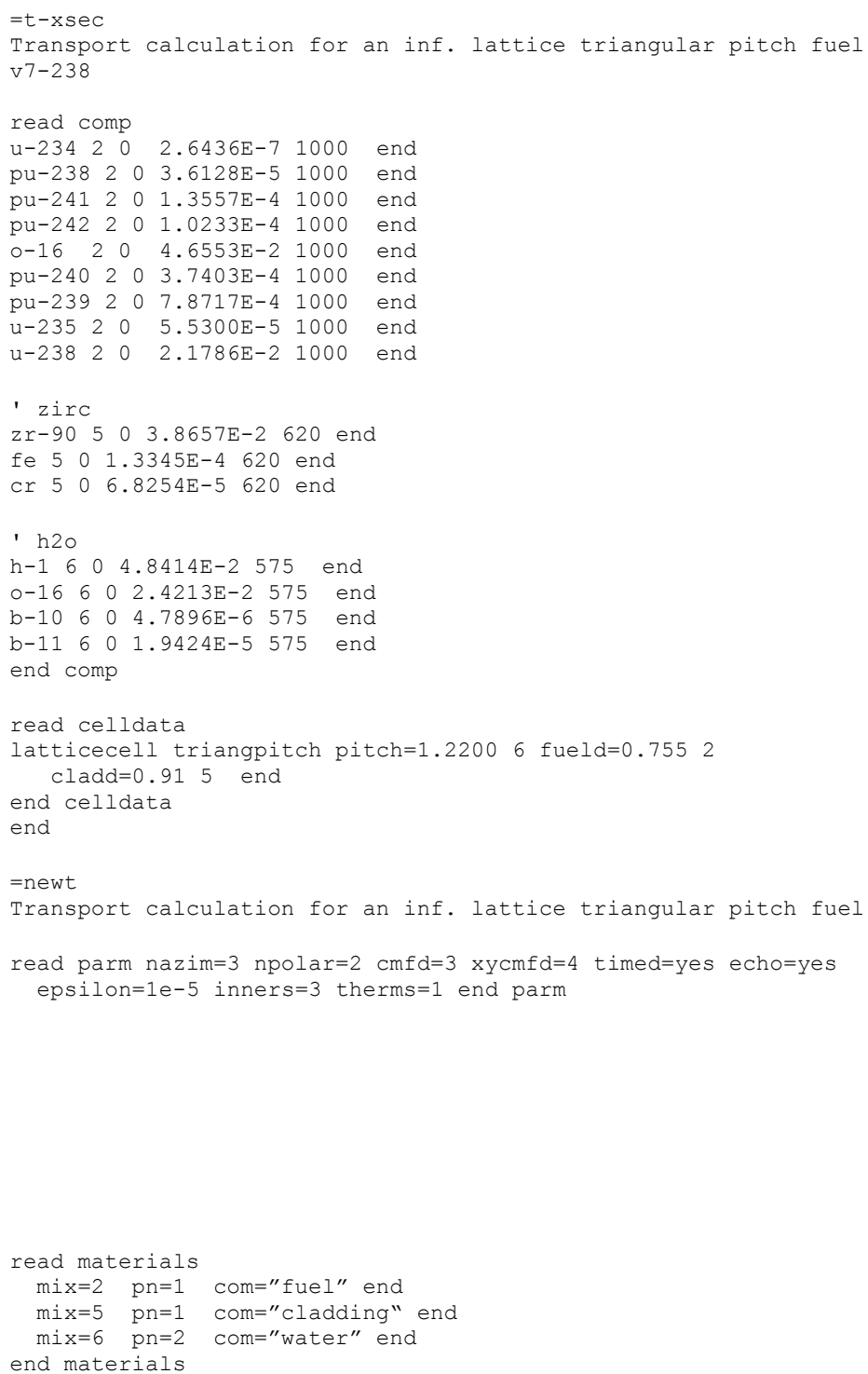

T-XSEC sequence of TRITON 238-group ENDF/B-VII library

Mixed-oxide fuel specification, mixture number 2

Zircaloy clad, mixture number 5

Borated water moderator, mixture number 6

Cell specification for a triangular lattice

\section{End of T-XSEC case}

Beginning of NEWT calculation

Parameter block uses product quadrature set: two polar angles with three azimuthal angles, partialcurrent CMFD acceleration with four fine-mesh cells per coarse-mesh cell. The echo option is turned on, as well as other convergence control options.

List of all problem materials $-\mathrm{P}_{1}$ scattering is specified in fuel and clad; $\mathrm{P}_{2}$ scattering, in moderator

Figure 9.2.46. Sample 2 input listing (annotated). 


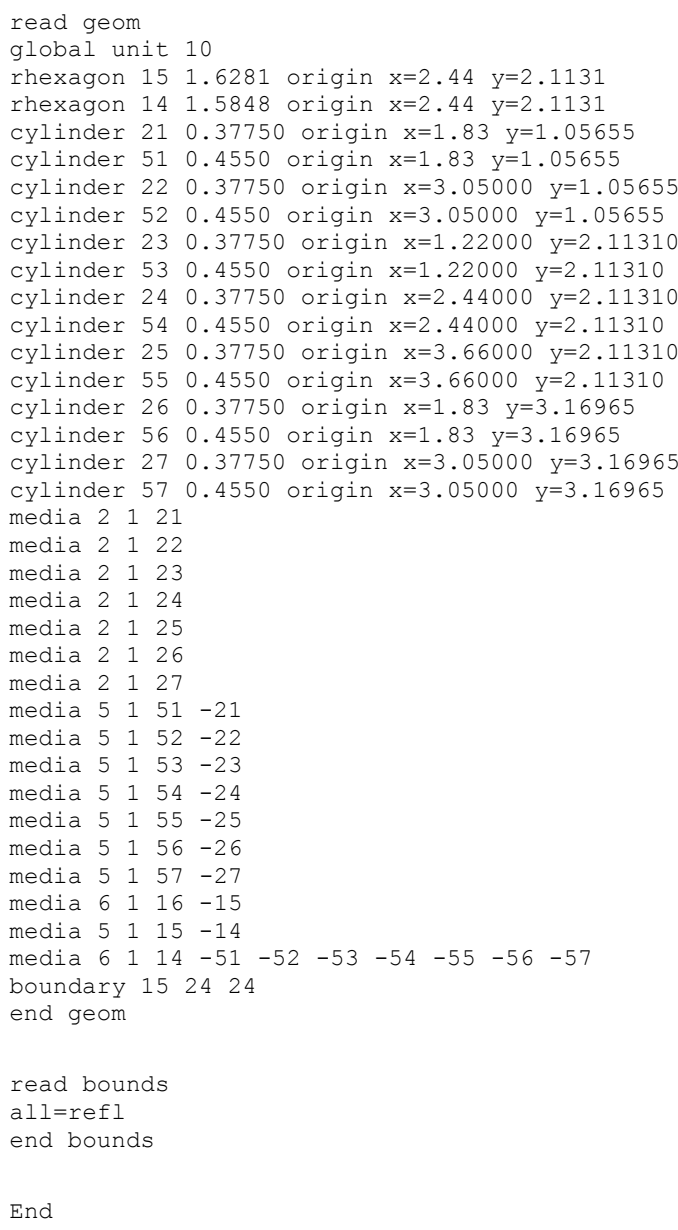

Geometry is defined as a set of 7 pairs of concentric cylinders, each set having a different origin. The geometry also includes a rectangle (defined as the problem boundary) and two rotated hexagons.

Media statements place fuel (mixture 2) within each inner cylinder and place cladding (mixture 5) within each outer cylinder (excluding inner cylinders). The inner hexagon is filled with moderator (mixture 6), excluding the seven cylinder positions. The region between the outer hex and the inner hex is filled with mixture 5 .

Reflective boundary conditions applied on all outer boundaries

End of NEWT calculation

Figure 9.2.46. Sample 2 input listing (annotated). (continued) 


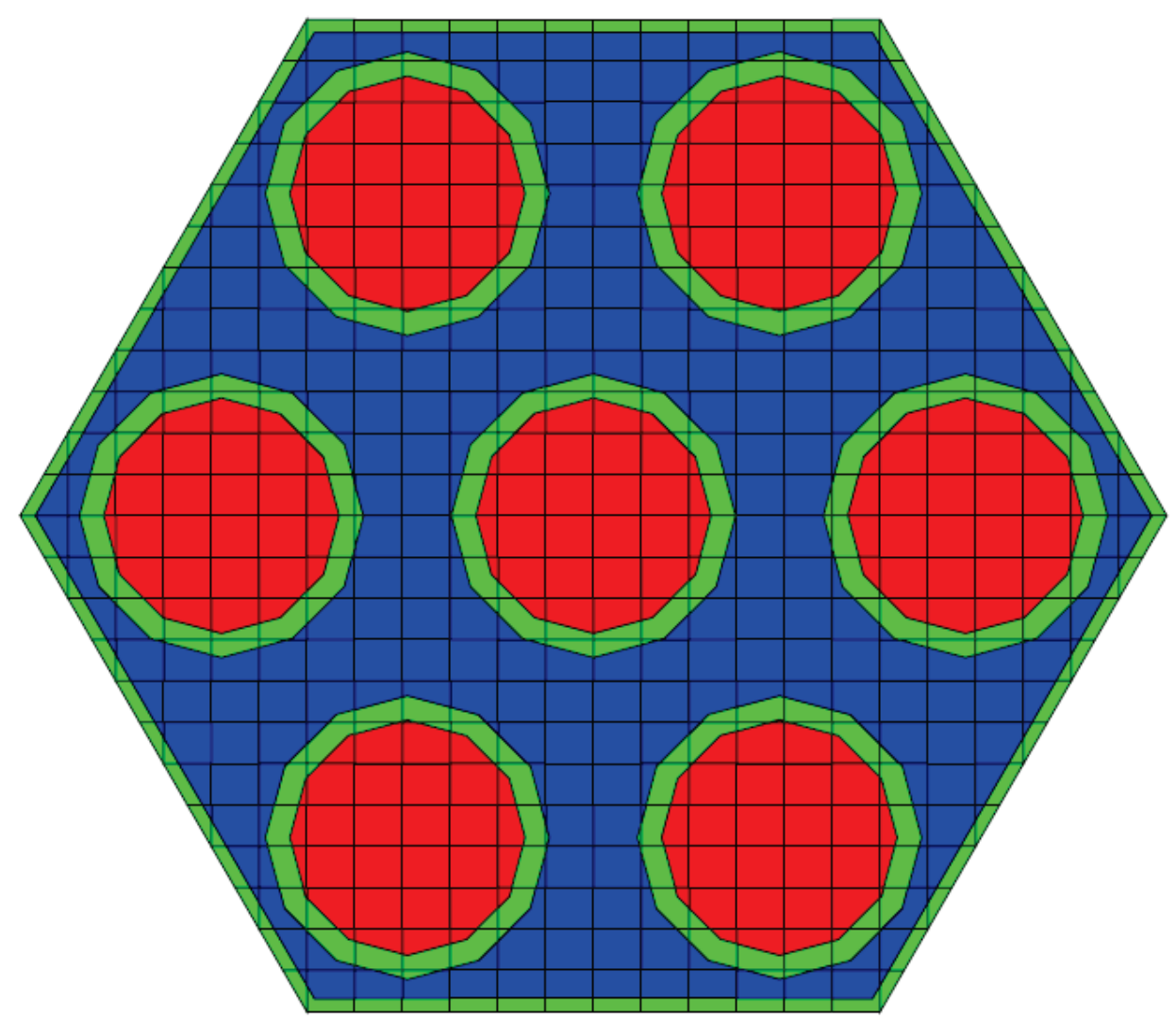

Figure 9.2.47. Mixture placement and grid structure for model described in Sample 2.

\subsubsection{Sample 3}

Sample 3 demonstrates the development of a VVER-440 hexagonal fuel assembly. Annotated input for this problem is given in Figure 9.2.48. The output plot for this model is shown in Figure 9.2.49. The key attributes of this model are as follows:

1. the use of hexagonal (hexprism) units in a stacked hexagonal array,

2. the use of null units as placeholders in the array,

3. a full model within a rhexagonal outer boundary,

4. the use of white boundary conditions,

5. the use of the new partial-current-based unstructured CMFD acceleration for hexagonal-domain configurations, and

6. new type-3 ADF inputs.

Using CMFD acceleration, the number of outer iterations needed for convergence decreased from 21 to 8 with a run-time speedup of $\sim 2.58$. 


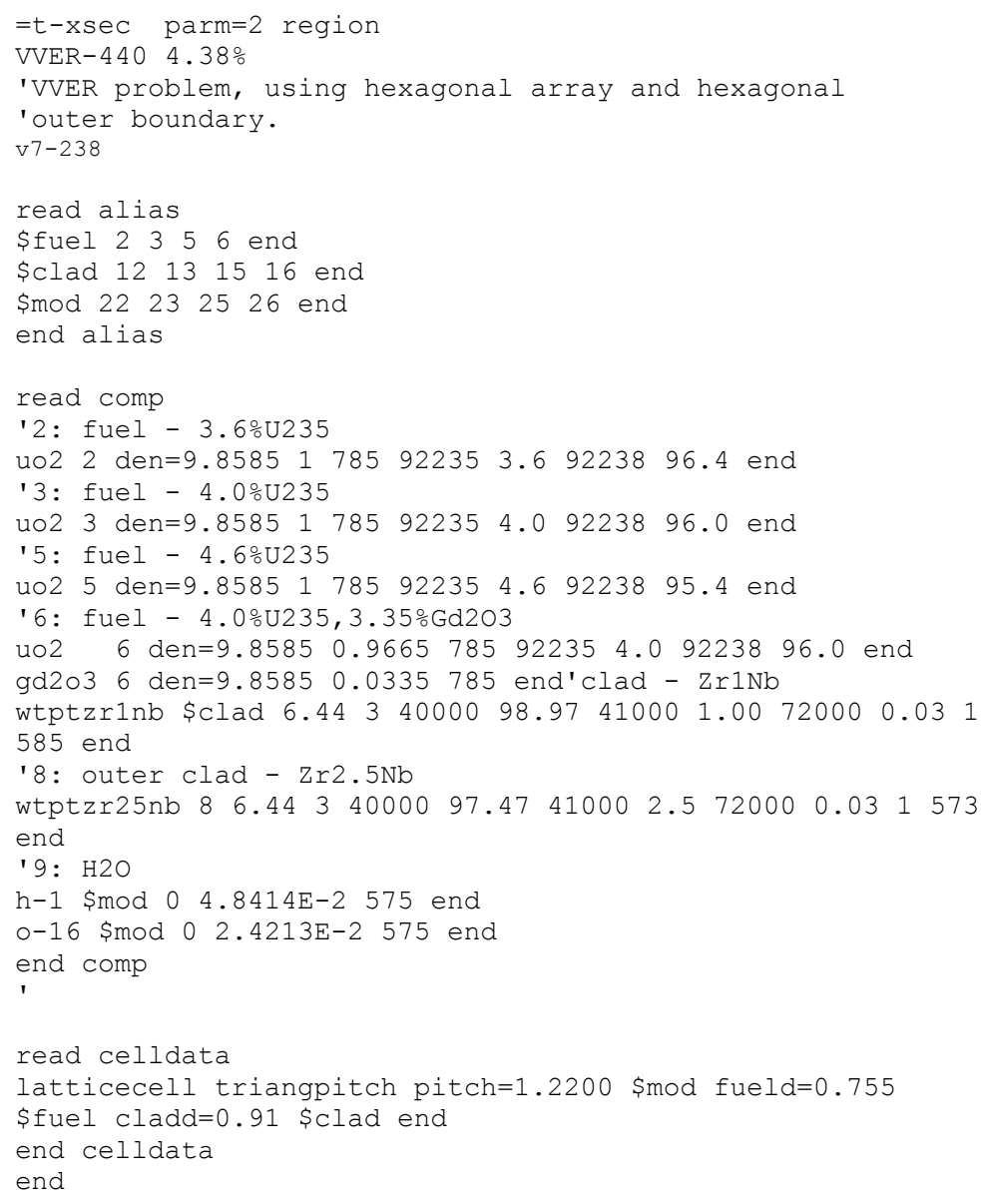

t-xsec sequence of TRITON

title card

238-group ENDF/B-VII library

Define aliases: four fuels, four clads, and four moderators will be defined by the three aliases

\section{Read compositions}

Four different fuel mixtures are defined. Because each mixture contains different specifications (different enrichments, Gd in one rod), these cannot be defined by using an alias

Use aliases to define four identical clads with different mixture IDs

Use aliases to define four identical moderators with different mixture IDs

Aliases are used to define a single cell description that will be expanded to four different cell descriptions End of $t$-sec case

Figure 9.2.48. Sample 3 input listing (annotated). 


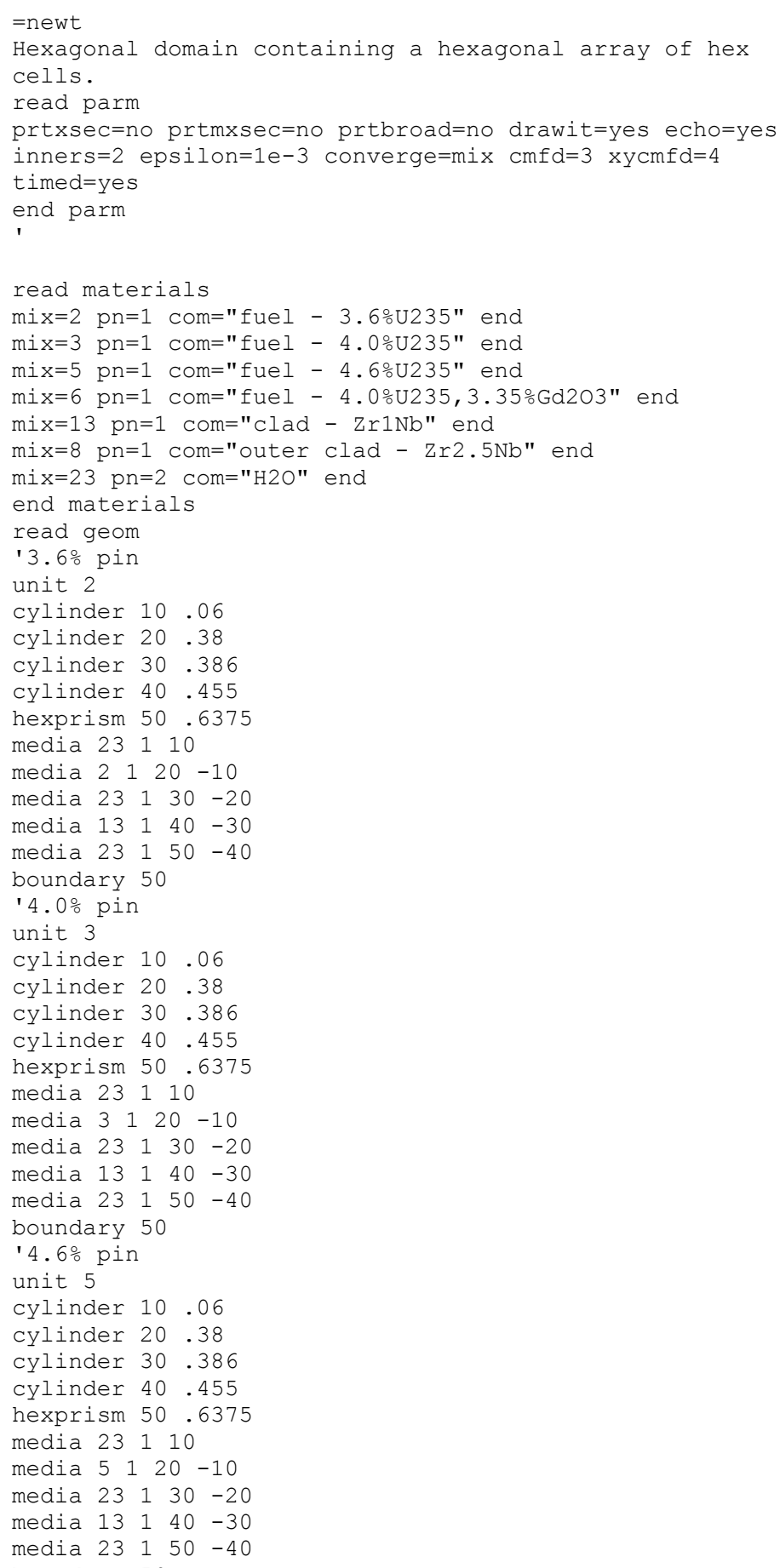

Figure 9.2.48. Sample 3 input listing (annotated). (continued)

Begin NEWT calculation

Title card followed by parameter block. CMFD option 3 used in the parameter block, along with four fine meshes per coarse mesh.

Material block specified four fuel mixtures, a clad mixture, and outer (can) clad mix-all with P-1 scattering - and a single moderator with P-2 scattering

Begin geometry description. Geometry block consists of a list of units

Unit 2 contains an annular fuel pin. Model consists of mixture 23 (moderator), fuel mixture 2, more moderator, and clad-all within a hexprism filled with moderator

Unit 3 is identical to unit 2 but places mixture 3 in the fuel region

Unit 5 is identical to unit 2 but places mixture 5 in the fuel region 


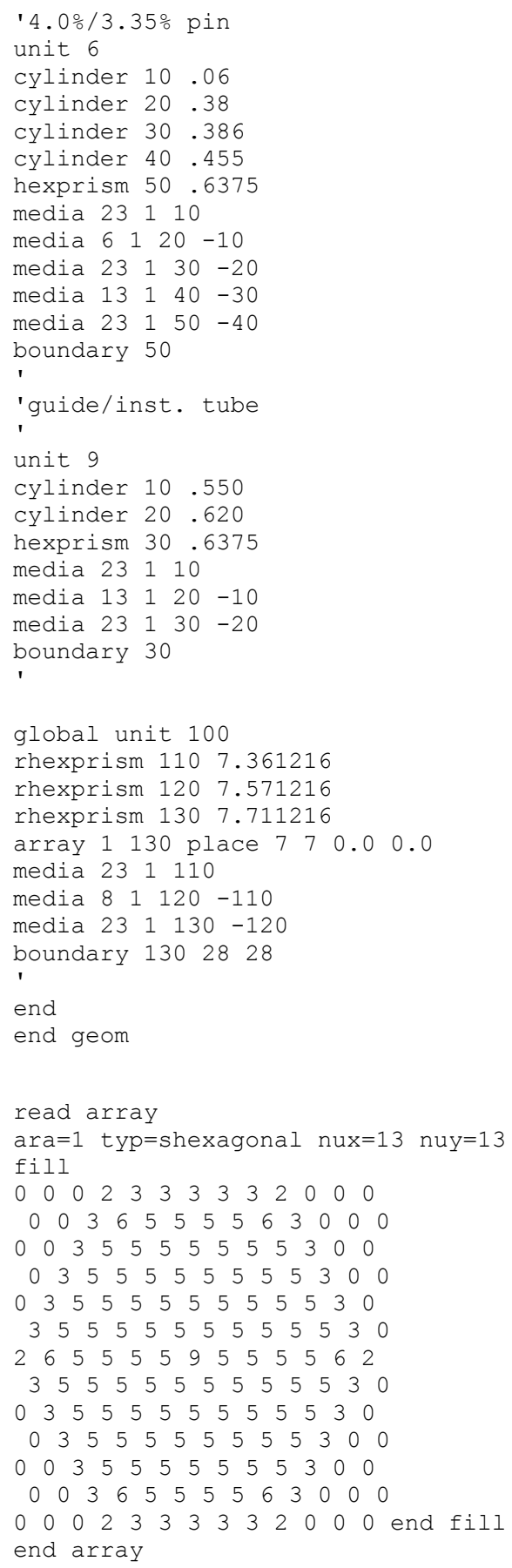

Unit 6 is identical to unit 2 but places mixture 6 in the fuel region

Unit 9 is a control rod guide tube, consisting of moderator within the tube, which is, in turn, set into a hexagonal region of moderator

Unit 100 is the global unit. Three nested hexprisms are specified: filled with moderator, zirc, and more moderator. An array is placed with element 7,7 centered in the unit. The outer boundary is a hexprism. A 28 by 28 grid is specified for the unit. Therefore, a 7 by 7 coarse-mesh grid is used

All units have been specified. This completes the geometry description

A single array is used in the model and is specified here. A stacked hexagonal array format is used for a 13 by 13 array. The guide tube is place in the center (unit 9); units 2, 3, 5 , and 6 are placed in appropriate positions. Null units are placed in corner regions where no array elements are needed

Figure 9.2.48. Sample 3 input listing (annotated). (continued) 


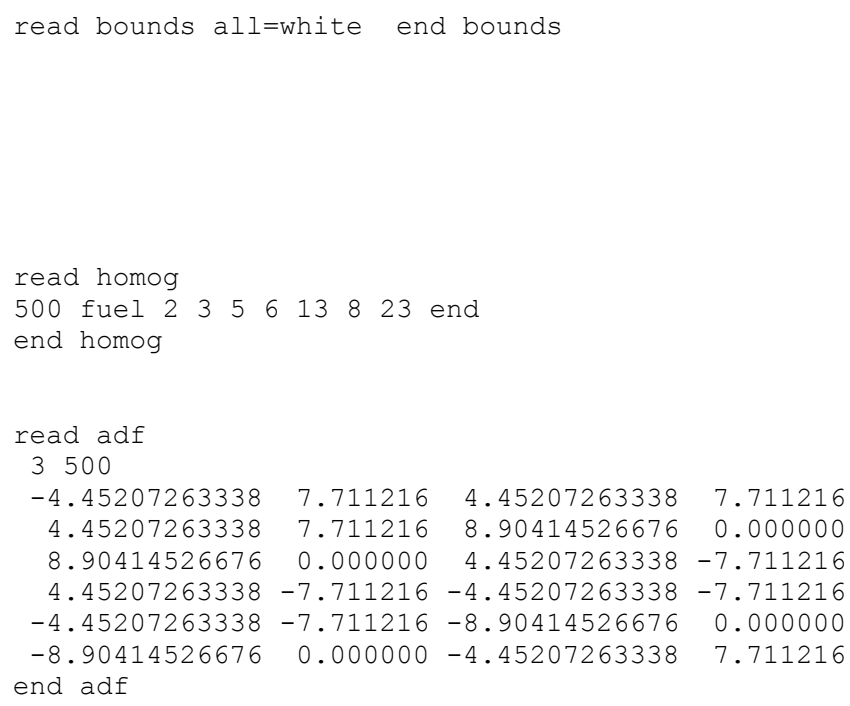

White BCs are specified for the horizontal outer boundaries. White BCs are always used on nonhorizontal and nonvertical boundaries

Homogenization block is used to define one new mixture ID 500 - composed of all the mixtures used in the model. The string identifier for the homogenized material is "fuel"

Associated with the new homogenized mixture 500 are type 3 assembly discontinuity factors. In this case, surfaced-averaged flux and currents are provided for six line segments that correspond to the outer boundary of the assembly

A one-group collapse is provided in the Collapse block

End of this NEWT case

Figure 9.2.48. Sample 3 input listing (annotated). (continued) 


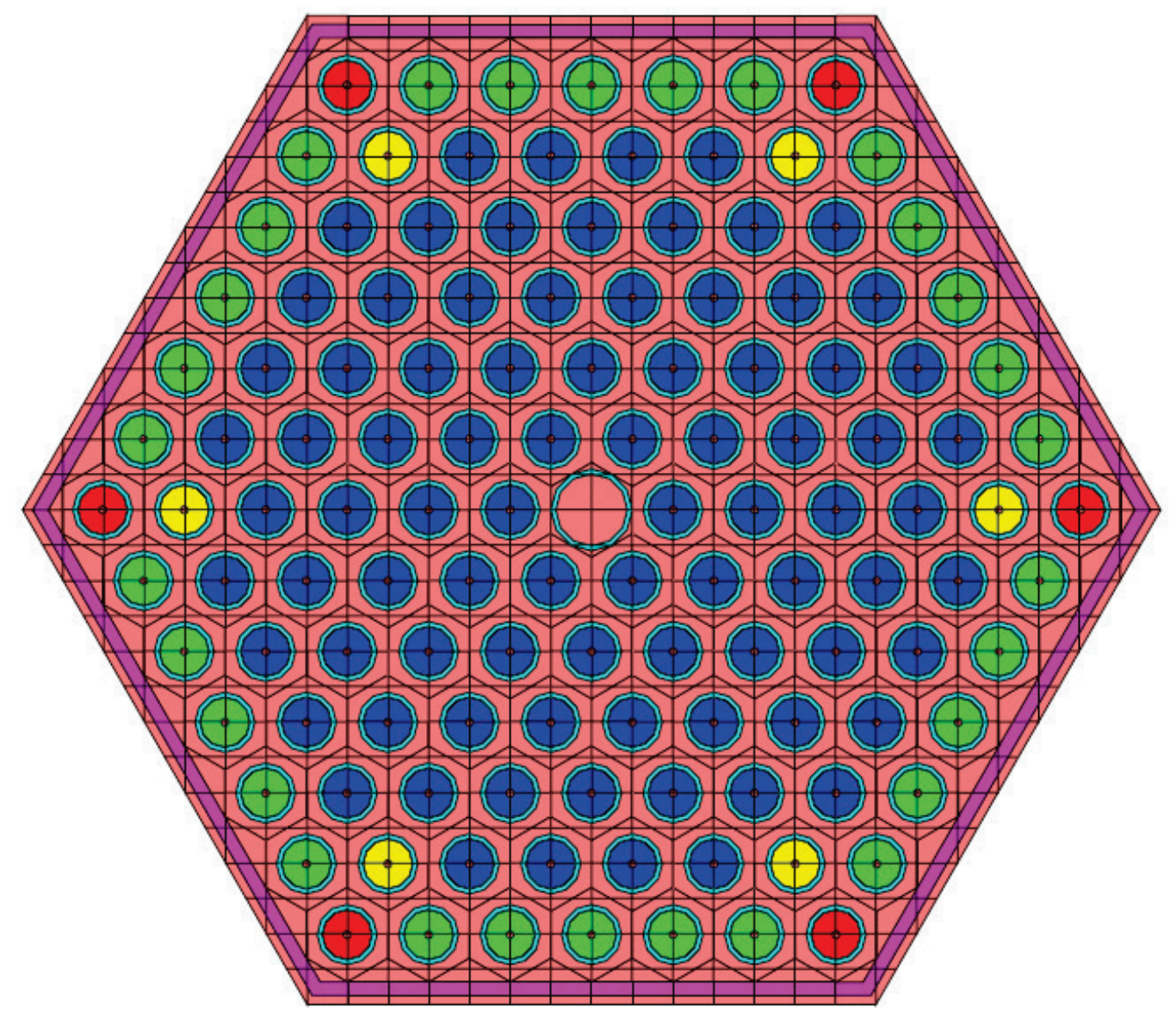

Figure 9.2.49. Grid structure and material placement for VVER-440 model.

\subsubsection{Sample 4}

Sample 4 demonstrates the use of NEWT in modeling a larger, more complex configuration. Annotated input for this problem is given in Figure 9.2.50. The calculation begins with the use of SCALE standard composition specifications to prepare a problem-specific weighted cross section library and mixing table for use by NEWT. In this case the T-XSEC sequence of the TRITON control module is used.

This NEWT case is used to calculate the eigenvalue of an infinite lattice of fuel assemblies. Symmetry at the assembly center is used to reduce a 15 by 15 assembly lattice to a smaller one-quarter assembly. The grid structure is illustrated in Figure 9.2.51. A similar illustration showing media placements by color is given in Figure 9.2.52.

This input illustrates several features of NEWT modeling capabilities. Some important features of this model are as follows.

- In this sample problem, S-6 quadrature, P-1 scattering (P-2 in the moderator), spatial convergence criteria of 0.005 , and an eigenvalue convergence criteria of 0.001 are used. These are an order of a magnitude larger than the values typically used for LWR lattice calculations. 
- Two sets of $\mathrm{UO}_{2}$ cross sections are prepared in the T-XSEC calculation. These cross sections are identical with the exception of the mixture number. Since NEWT reports fluxes, reaction rates, etc., by mixture, the placement of a unique mixture at a specific location in a model allows one to determine, for example, the reaction rates at that model location. Mixture 7, placed in unit 9 in this model, occurs in only one pin location in the model. Mixture 1, placed in all other fuel rod locations, will yield reaction rates close to the average of those for all fuel in the assembly. If the flux or reaction rate was needed in each unique fuel location, a unique mixture would be needed for each location.

- The use of chords for cutting cylinders allows inclusion of one-half and one-quarter fuel cells in the quarter-assembly model. Because the fuel assembly has an odd number of rods in each dimension, use of symmetry at the assembly midplanes requires the rods to be bisected.

- In this model, local grid spacing was selected such common grid spacings occur in all cells. However, this is not a requirement. For example, a much more refined local grid could have been specified for unit 9. There is no requirement that grid lines match between different elements of an array.

- Unstructured coarse-mesh finite-difference acceleration ( $\mathrm{cmfd}=2$ or $\mathrm{cmfd}=\mathrm{yes}$ ) was employed to accelerate the convergence of the solution. For this case, 14 outer iterations were required for full spatial convergence as compared with 30 outer iterations when CMFD is disabled. The CMFDaccelerated case ran 2.5 times faster than its unaccelerated counterpart. In this sample problem, xycmfd $=2$ was used to define the coarse-mesh grid to have two fine-mesh cells per coarse-mesh cell.

- Two-group homogenized cross sections were generated along with single-assembly (i.e., type 1) ADFs derived from the Collapse block, ADF block, and the Homogenization block. In addition, a B1 critical spectrum search is computed after the transport calculation, which is folded into the transport solution to generated homogenized constants. 


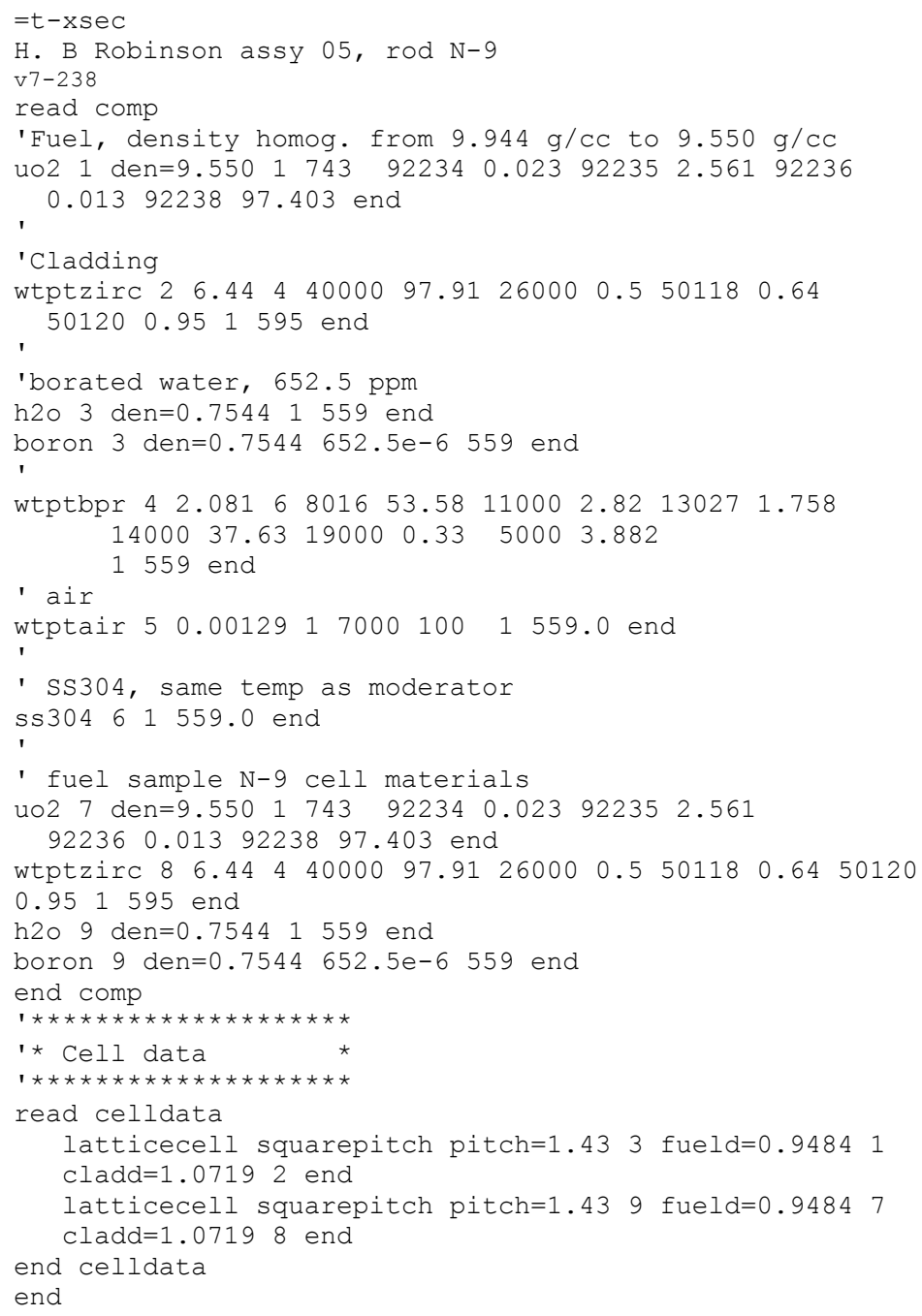

\section{T-XSEC sequence of TRITON 238-group ENDF/B-VII library}

$\mathrm{UO}_{2}$ fuel specification

Zircaloy clad

Borated water moderator

Borosilicate glass burnable poison rod

Air (low-density nitrogen)

Stainless steel

Second set of $\mathrm{UO}_{2}$, zirc, and borated moderator specifications (same properties, different mixture numbers)

Lattice specification for the two fuel pin cells

Figure 9.2.50. Sample 4 input listing (annotated). 


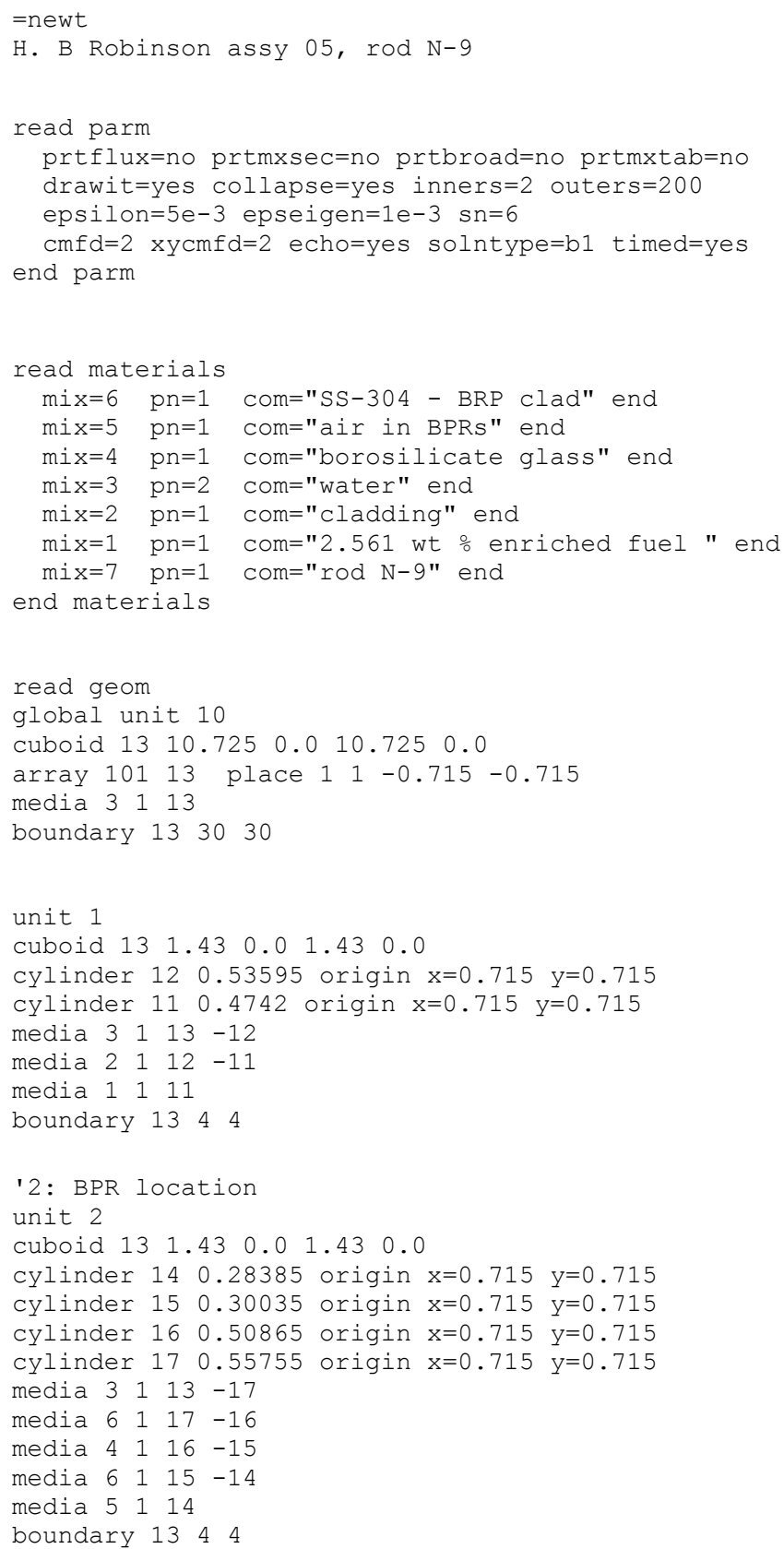

\section{Stand-alone NEWT calculation} Title card

Parameter block specification as described above

Mixtures 1-7 are used from the cross section library and mixing table produced in the preceding T-XSEC calculation. P-1 scattering used in all mixtures except moderator, which uses P-2

Global unit (10) consists of a cuboid (13) filled with mixture 3. An array (101) is placed such that the origin of the element in row 1 , column 1 , is located at $(-0.715$, $-0.715)$. The contents of the array will be defined in the read array data block below.

Unit 1 is a nominal fuel pin cell, placed in a 1.43 by $1.43 \mathrm{~cm}$ cuboid with a 4 by 4 local grid.

Unit 2 is an annular burnable poison rod, placed in a 1.43 by $1.43 \mathrm{~cm}$ cuboid with a 4 by 4 local grid. Radially, the rod consists of air, steel, poison material, and steel

Figure 9.2.50. Sample 4 input listing (annotated). (continued) 


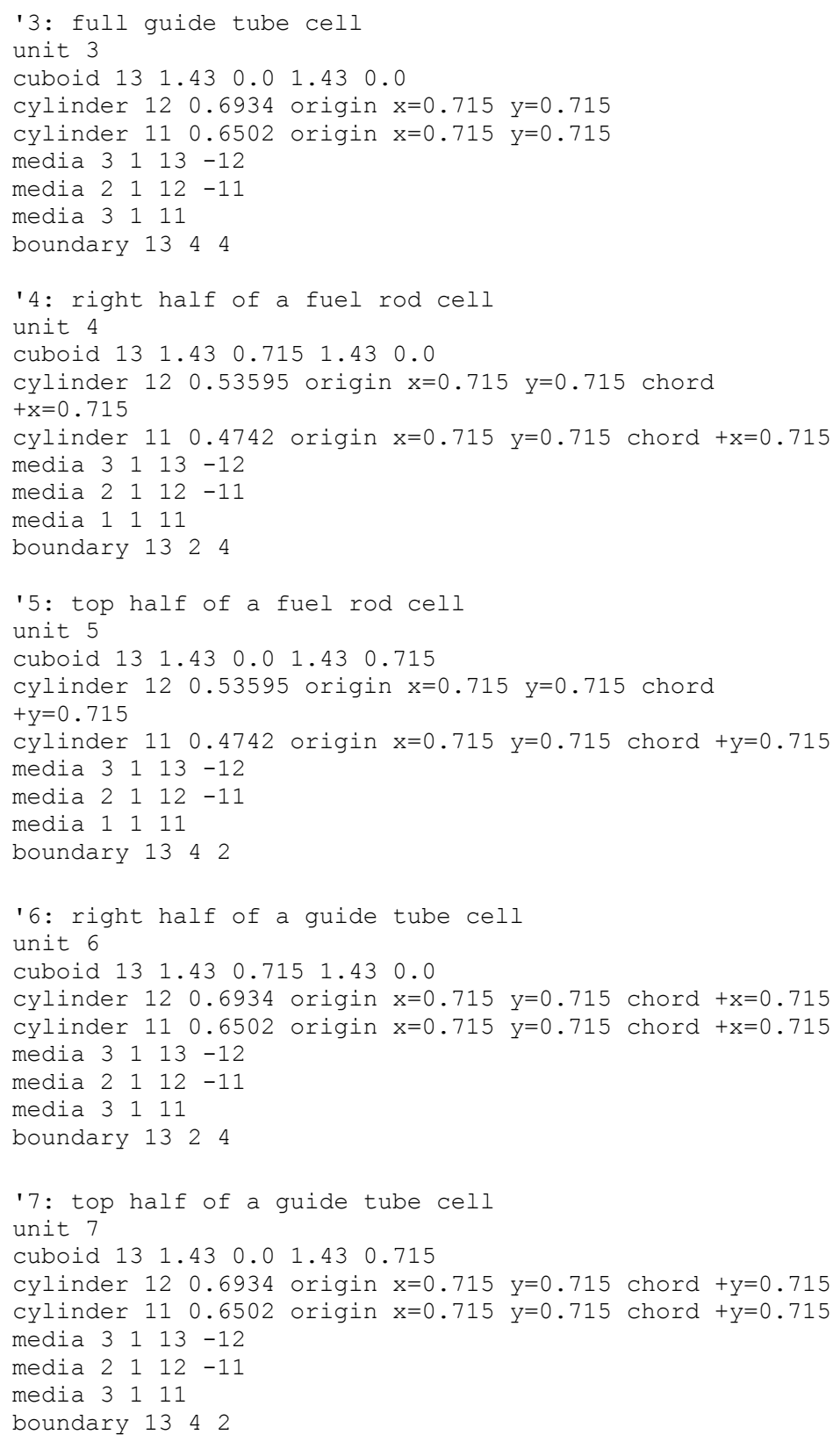

Unit 3 is a guide tube consisting of a cylinder of moderator enclosed in a cylinder of Zircaloy, centered in a 1.43 by $1.43 \mathrm{~cm}$ cuboid with a 4 by 4 local grid.

Unit 4 is the right half of a nominal fuel cell, to be placed on the left boundary of an array. The cylinder is cut with a chord and placed on the left side of a 0.715 by $1.43 \mathrm{~cm}$ cuboid with a 2 by 4 local grid.

Unit 5 is the top half of a nominal fuel cell, to be placed on the bottom boundary of an array. The cylinder is cut with a chord and placed on the bottom side of a 1.43 by $0.715 \mathrm{~cm}$ cuboid with a 4 by 2 local grid.

Similar in construct to unit 4 , unit 6 is the right half of a guide tube cell.

Similar in construct to unit 5 , unit 7 is the top half of a guide tube cell

Figure 9.2.50. Sample 4 input listing (annotated). (continued) 


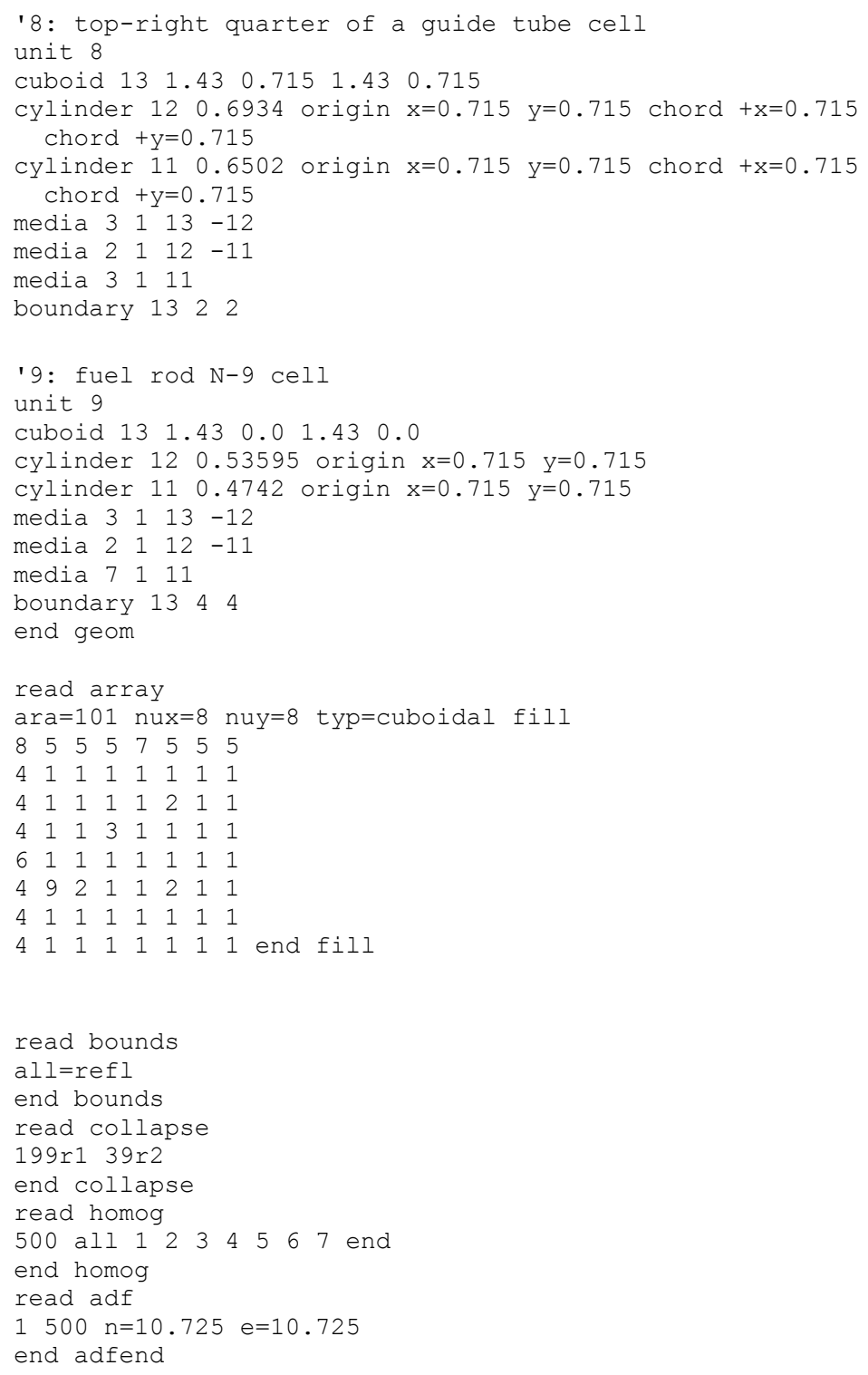

Unit 8 represents the $1 / 4$ cell that will be placed in the lower-left corner of the array. It is a guide tube cell but reduced to $1 / 4$ cylinders using chords. It is located within a 0.715 by $0.715 \mathrm{~cm}$ cuboid with a 2 by 2 local grid.

Unit 9 is identical to unit 1 in structure but with a different fuel mixture specified. Local parameters for this pin will be available in output.

This completes the geometry specification.

Unit placement is specified for the single array used in this configuration. An 8 by 8 cuboidal (square) array is filled - starting from the bottom, moving left to right, and then bottom to top.

Reflective boundary conditions are applied to all faces.

Two-group collapse specification

Homogenize all mixtures to form new mixture 500

Type 1 ADFs generated on the north and east faces

This completes the input for this case.

Figure 9.2.50. Sample 4 input listing (annotated). (continued) 


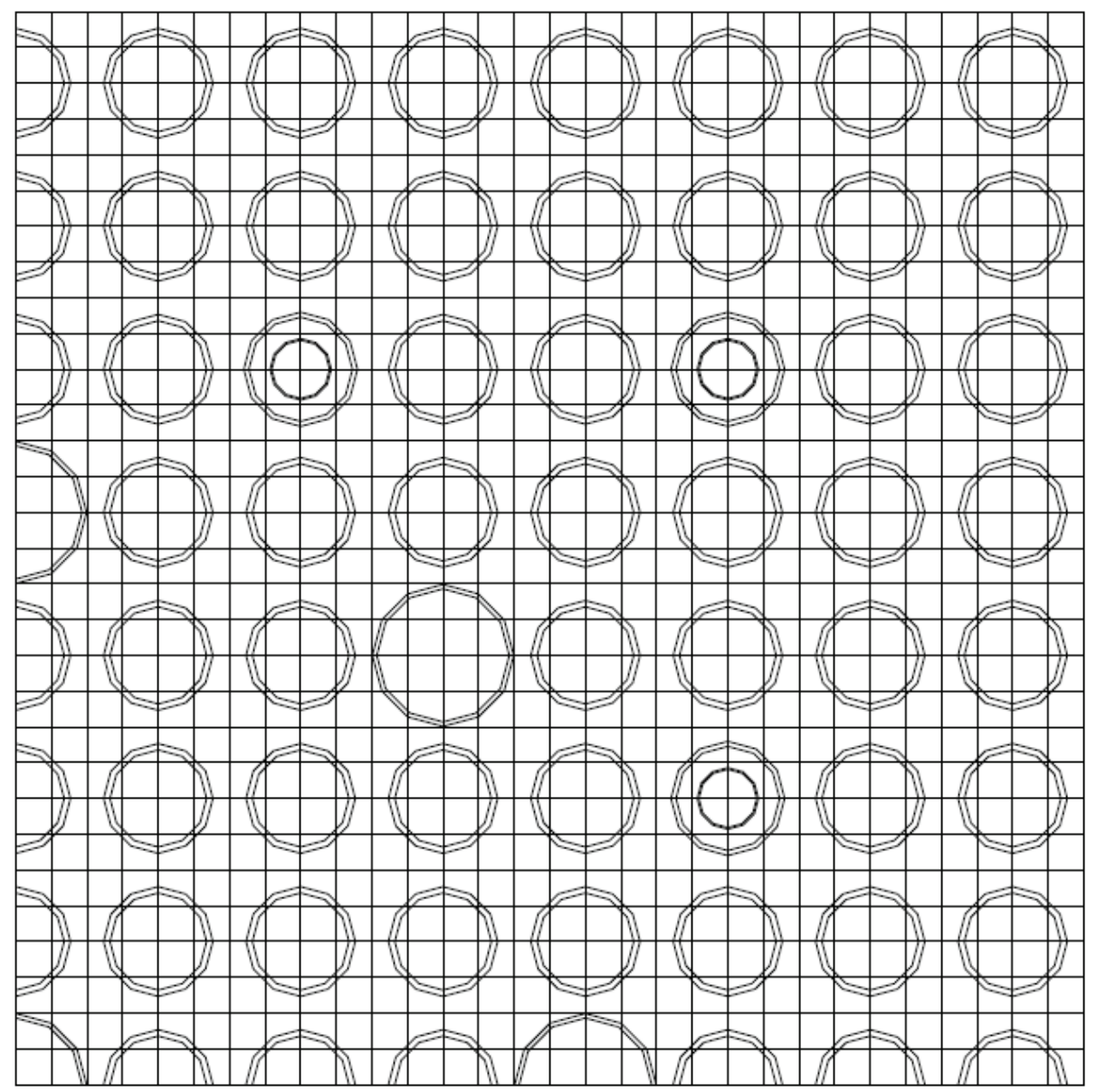

Figure 9.2.51. Grid structure for one-quarter assembly of Sample 4. 

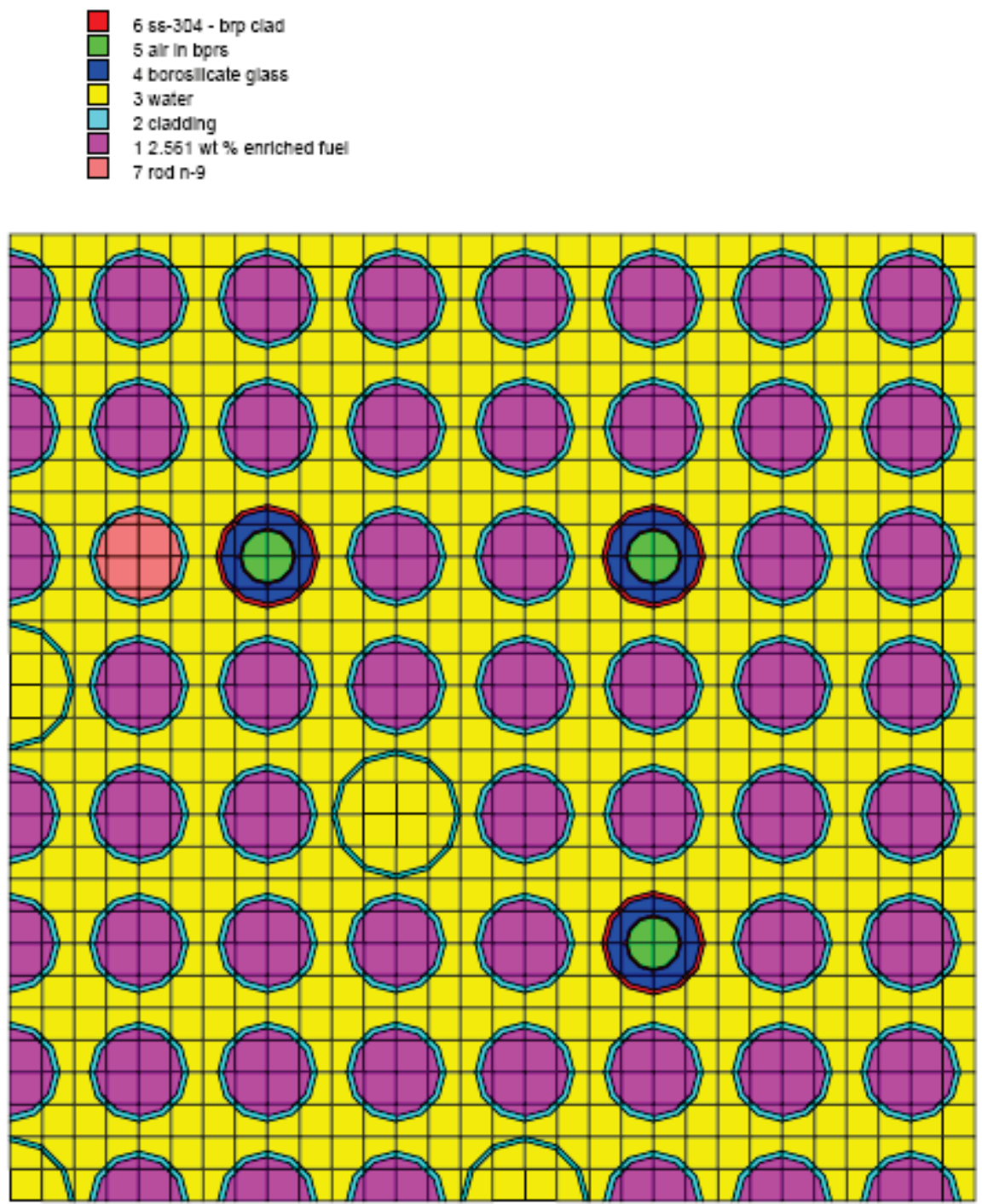

Figure 9.2.52. Mixture placement for quarter-assembly model of Sample 4.

\subsubsection{Sample 5}

Sample 5 (Figure 9.2.53) illustrates a calculation for a fuel assembly with a large water boundary and a vacuum boundary condition. The calculation begins with the use of SCALE standard composition specifications to prepare a problem-specific weighted cross section library and mixing table for use by NEWT.

In this model, seven $\mathrm{UO}_{2}$ pins are adjacent to eight $\mathrm{MOX}$ pins, which, in turn, are adjacent to a large reflector region. The outer boundary of the reflector is vacuum. Reflection on the top and bottom boundaries makes the problem infinite in the y direction. The grid structure for this problem is illustrated in Figure 9.2.54. This problem illustrates the use of the original CMFD acceleration scheme in NEWT $(\mathrm{cmfd}=1$ or $\mathrm{cmfd}=$ rect $)$. Because of the large degree of scattering within the reflector region, the problem can be relatively slow to converge. Without CMFD acceleration, 40 outer iterations are required for spatial convergence as compared with 12 when CMFD is enabled. A total run-time speedup of $\sim 1.4$ is achieved with the CMFD acceleration scheme. 
In addition to the application of CMFD, Sample 5 also illustrates the use of NEWT's reflector ADF capability. Reflector ADFs are computed along the fuel/reflector interface.

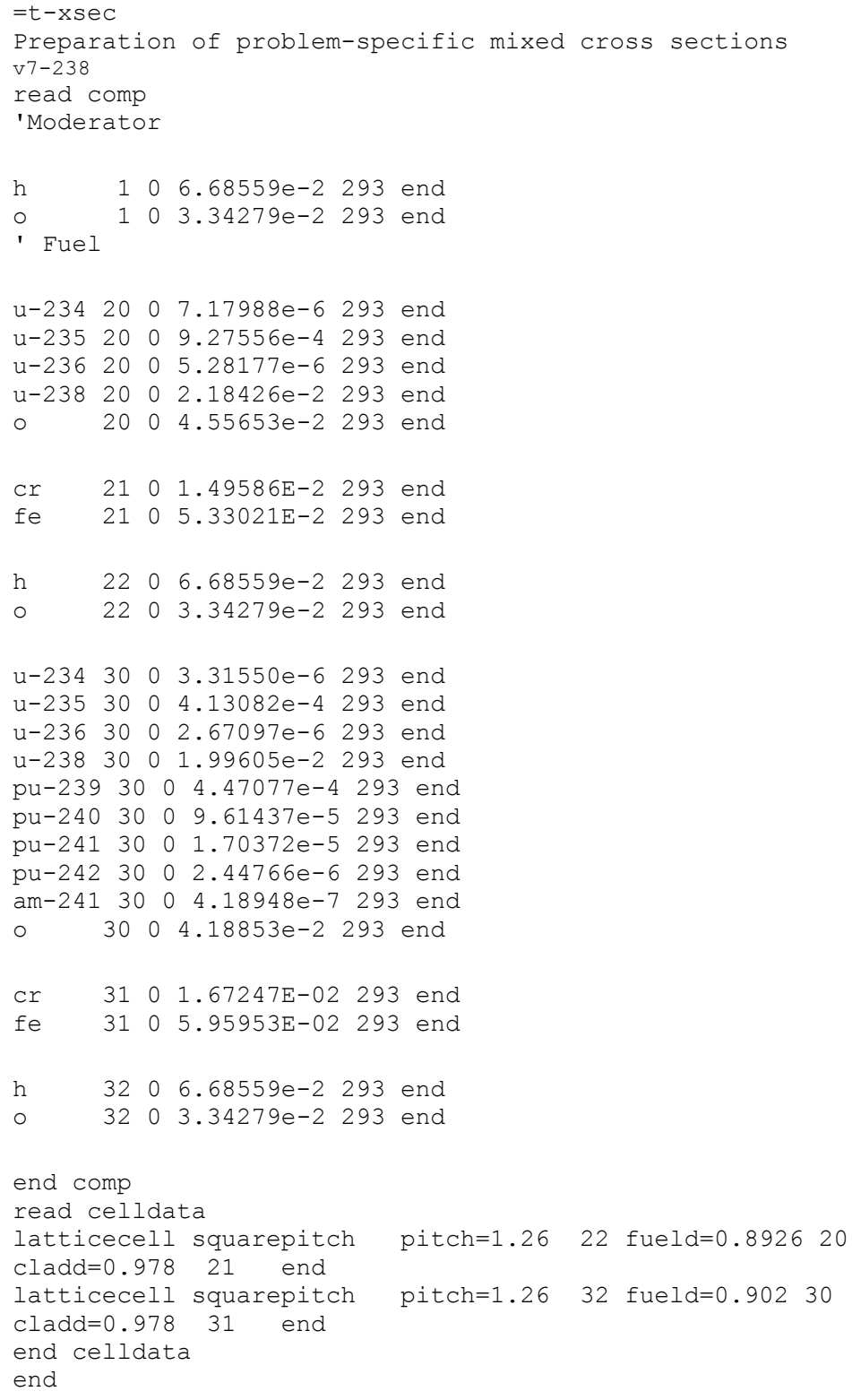

t-xsec sequence of TRITON

title card

238-group ENDF/B-VII library

Moderator (water)

$\mathrm{UO}_{2}$ fuel (20) specification

Clad mixture (21) for $\mathrm{UO}_{2}$ fuel

Water (22) for $\mathrm{UO}_{2}$ fuel cell

MOX fuel (30) specification

Clad mixture (31) for MOX fuel

Water (32) for MOX fuel cell

Pin-cell specifications for two fuel types

Figure 9.2.53. Sample 5 input listing (annotated). 


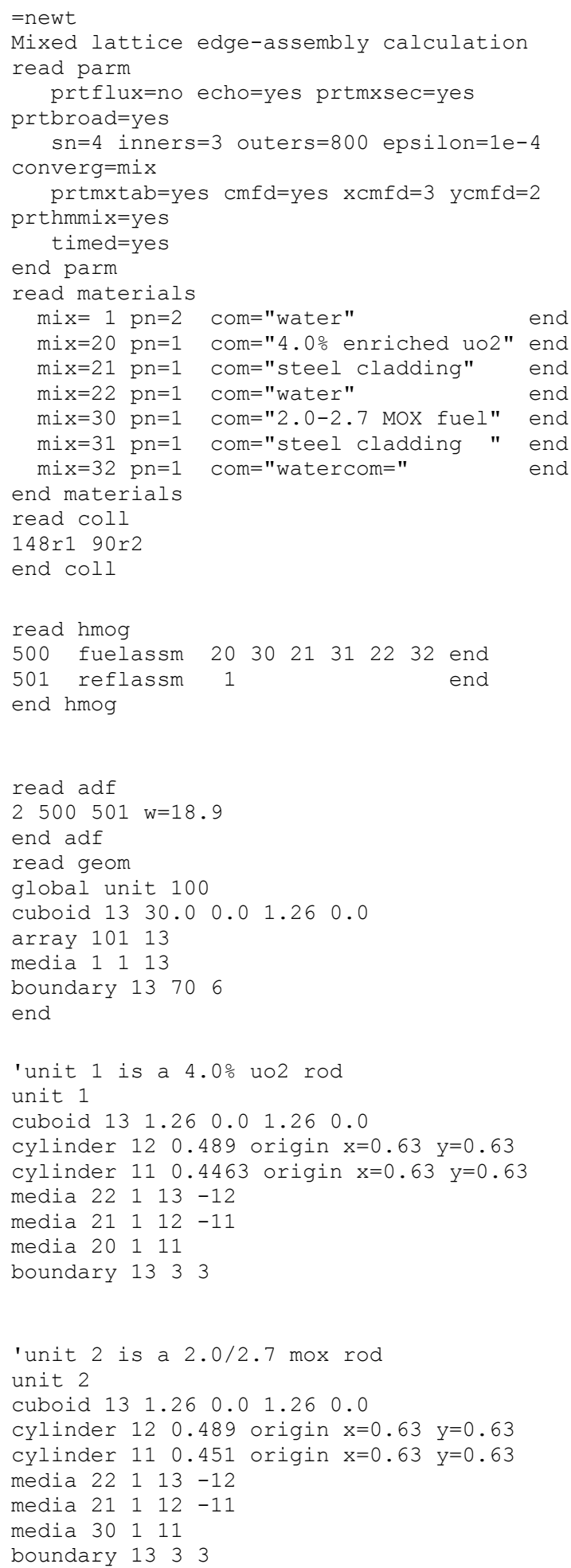

Homogenize all mixtures in the fuel assembly mode except reflector mixture 1; reflector homogenization is provided in separate specification.

Type-2 ADF provided along the fuel/reflector interface

Global unit is a cuboid with an array placed in it with default placement [unit at row 1 , column 1 , placed at $(0,0)$ ].

Two cylinders are centered in a cuboid. Mixtures 20, 21, and 22 are placed in inside the inner cylinder, outer cylinder, and cuboid, respectively.

Unit 2 is identical to unit 1 , but mixture 30 is placed inside the innermost cylinder. 


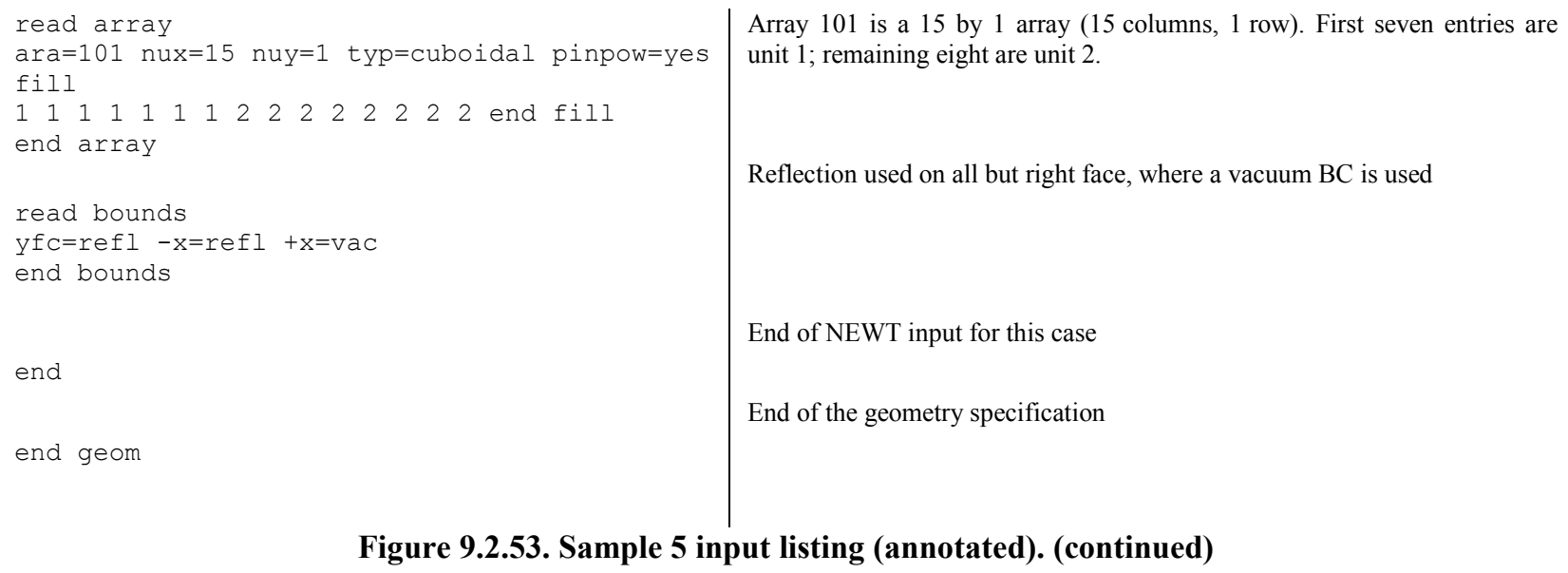

Figure 9.2.53. Sample 5 input listing (annotated). (continued)

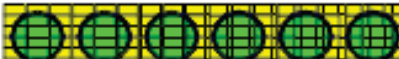

Figure 9.2.54. Grid structure for 15-pin row of Sample 5.

\subsubsection{Description of Output}

This section contains a brief description and explanation of NEWT output. Portions of the output will not be printed for every problem. Some output is optional, depending on user input specifications and is so noted in the description. As with any SCALE module, output begins with an input echo, module execution records with times and completion codes, and the program verification information banner page. These outputs are common to all SCALE modules and are not described here.

\subsubsection{NEWT banner}

Following the SCALE program verification information, the first section unique to NEWT output is the NEWT banner, which appears as shown in Figure 9.2.55. The bottom of the banner gives the title of the case as given in input. The NEWT banner is printed only if the command line option $-p$ is used to run SCALE. 


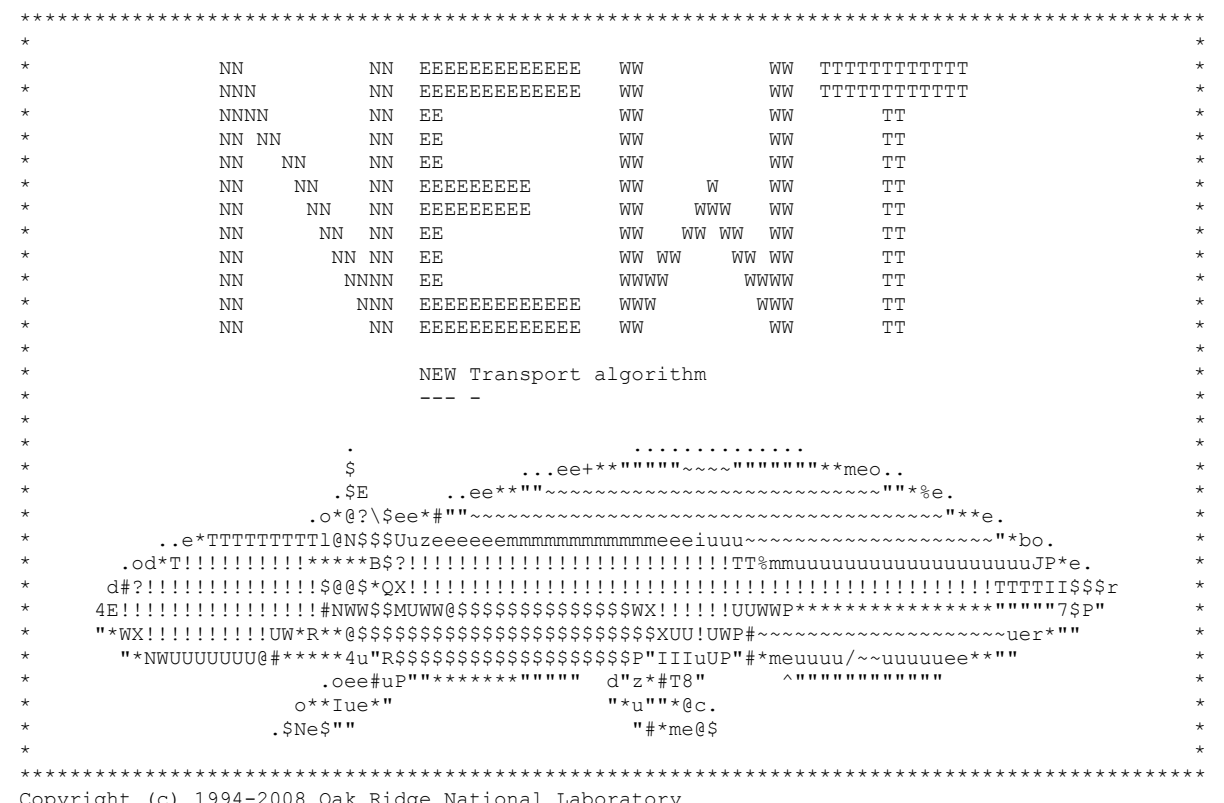

Copyright (C) 1994-2008 Oak Ridge National Laboratory

Figure 9.2.55. NEWT copyright banner page and case title.

\subsubsection{Input summary}

The next several pages of output provide a summary of input parameters. As described in Sect. 9.2.5, default parameters are used when no user specification is supplied. The input summary lists all parameters and states used in the calculation, whether user supplied or default. The following subsections describe the various blocks of output information provided in the input summary.

\subsection{Control options}

The Control Options block lists global control parameters that determine the type of analysis being performed. A sample Control Options page is shown in Figure 9.2.56. Parameters are self-explanatory. More information is available in the description of the keywords in Sect. 9.2.5.2.

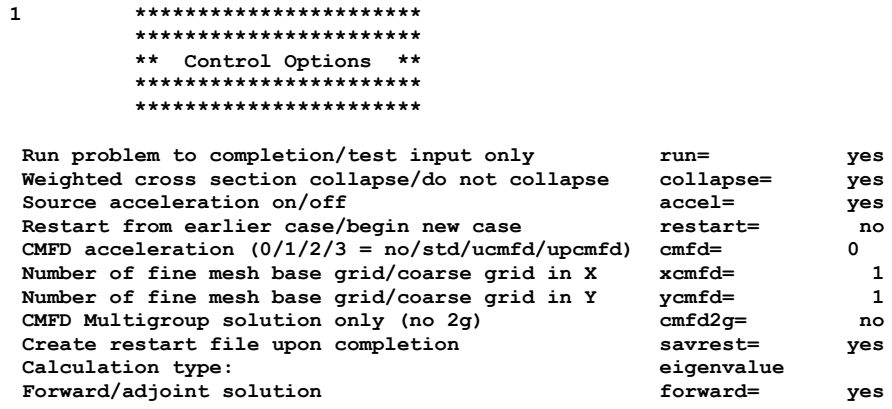

Figure 9.2.56. Control Options page. 


\subsection{Output options}

The Output Options block (Figure 9.2.57) lists selections made for output. Portions of the output listing will be printed only if the appropriate printing option was selected.

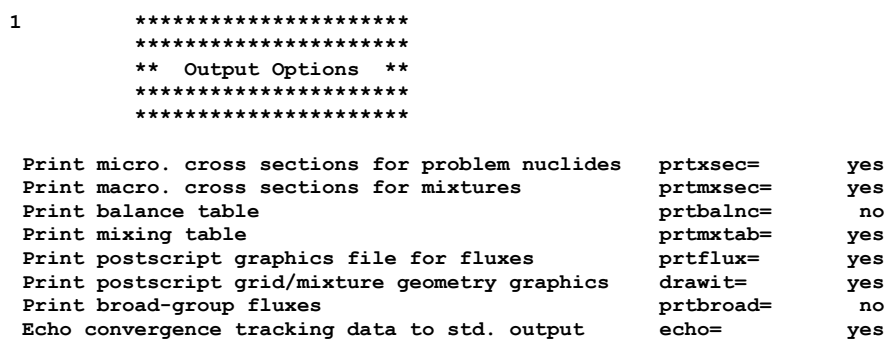

Figure 9.2.57. Output Options page.

\subsection{Input/output unit assignments}

The Input/Output (I/O) Unit Assignments block (Figure 9.2.58) simply lists the unit numbers selected for reading or writing various data files, as appropriate for the calculation.

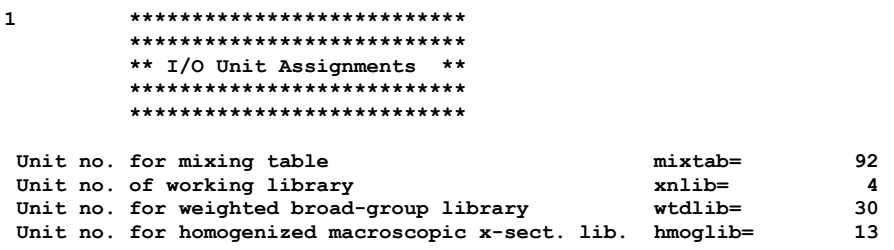

Figure 9.2.58. Input/Output Unit Assignments page.

\subsection{Convergence control parameters}

The Convergence Control block (Figure 9.2.59) summarizes all parameters used to control spatial, angular, and eigenvalue convergence for the iterative phases of the solution process.

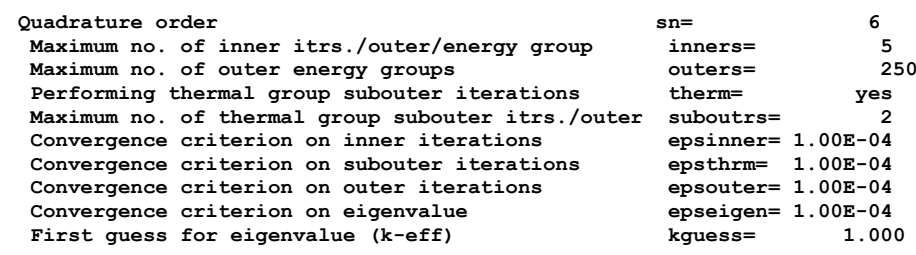

Figure 9.2.59. Convergence Control Parameters page.

\subsection{Pin-power edit requests}

If pin-power edits are requested for one or more arrays, a listing is provided of the arrays for which this request was made (Figure 9.2.60). 


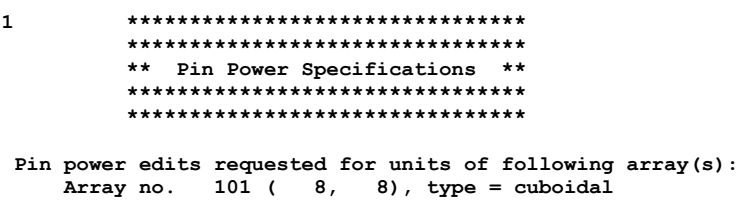

Figure 9.2.60. Pin-power edit request summary.

\subsection{Geometry specifications}

The Geometry Specifications block (Figure 9.2.61) lists parameters associated with the geometric model specified by the user. The first section lists the characteristics of the global unit. This is followed by a listing of the four boundary conditions. Finally, the last section in this block lists all bodies specified for the model. The appearance and contents of this section of input depend on the nature of the input model.

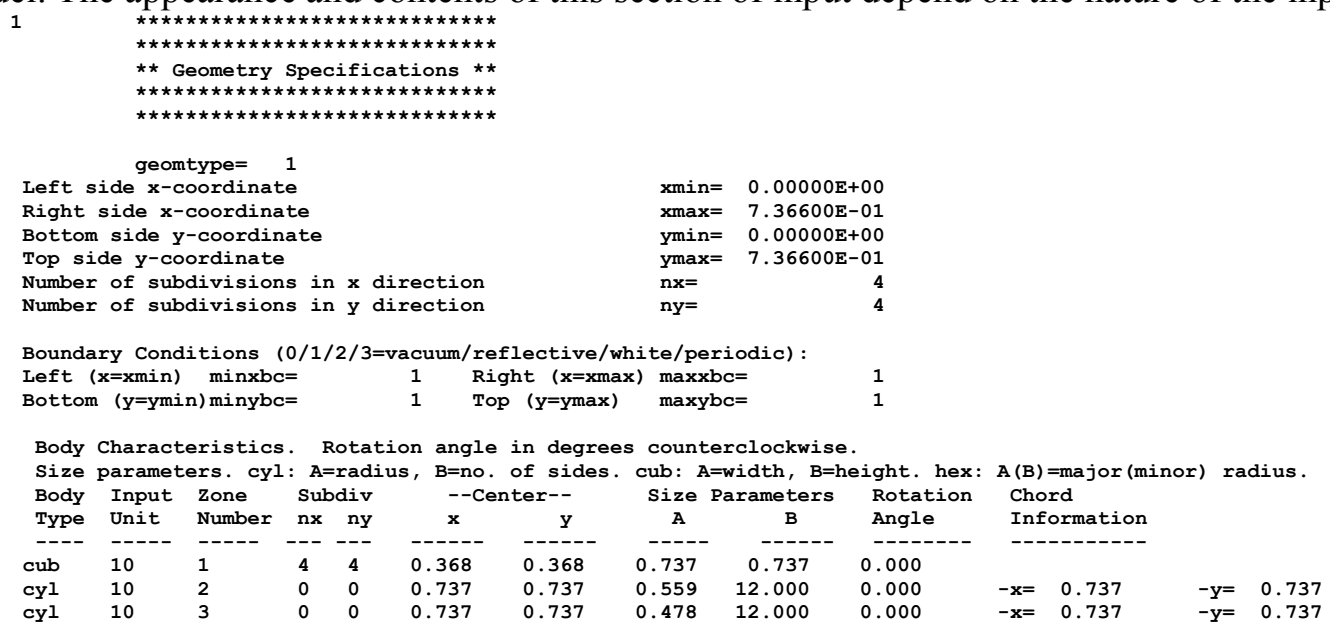

Figure 9.2.61. Geometry Specifications page.

\subsection{Homogenization region specifications}

The Homogenization Region Specifications block (Figure 9.2.62) summarizes all sets of homogenized cross sections requested in user input.

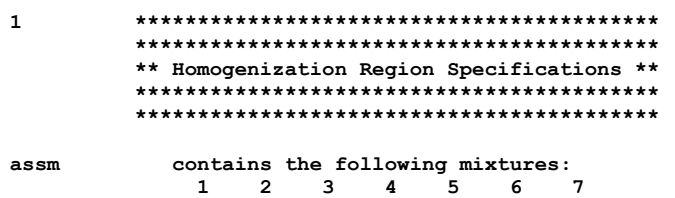

Figure 9.2.62. Homogenization Region Specifications page.

\subsection{Material specifications}

The Material Specification block (Figure 9.2.63) lists the NEWT material number, counting in the order read in; the SCALE mixture number; and the $\mathrm{P}_{\mathrm{n}}$ order assigned for that mixture. 


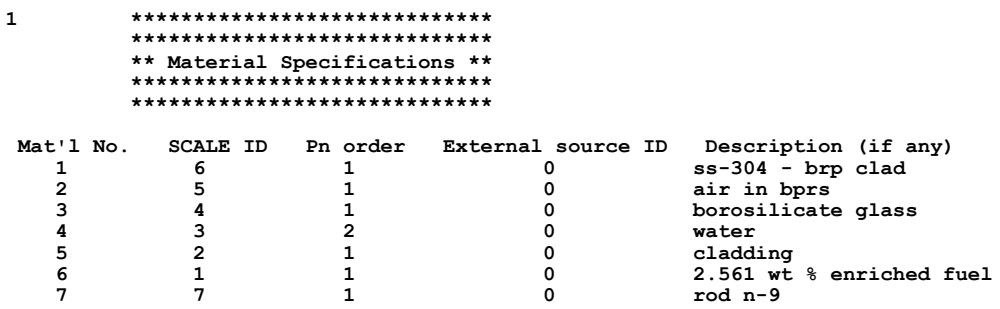

Figure 9.2.63. Material Specifications page.

\subsection{Derived parameters}

The Derived Parameters block (Figure 9.2.64) lists values not specifically input but derived from other sources of input. Some of this information comes from the cross section library, some from the model geometry, and some from the $S_{n}$ and $P_{n}$ values specified.

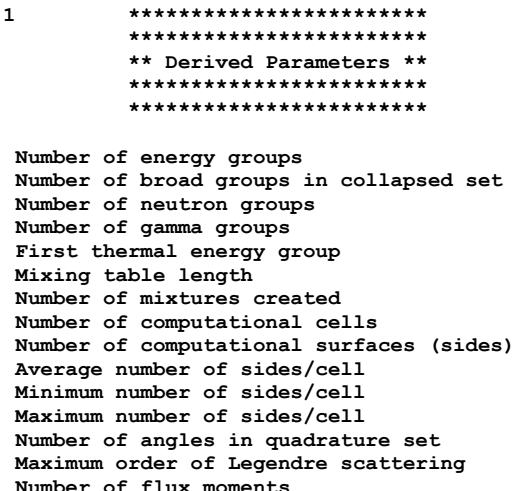

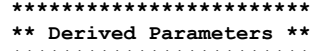

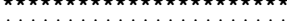

Number of energy groups

(sides)

scattering

Number of flux moments

$\begin{array}{lr}\text { numen= } & 44 \\ \text { nbroad= } & 2 \\ \text { igm= } & 44 \\ \text { ipm= } & 0 \\ \text { iftg= } & 23 \\ \text { nmix= } & 34 \\ \text { nummat= } & 7 \\ \text { numcells= } & 2274 \\ \text { numlines= } & 5586 \\ \text { avnosides= } & 4.83 \\ \text { mnnosides }= & 4 \\ \text { mxnosides } & 8 \\ \text { ndir= } & 24 \\ \text { maxpn= } & 2 \\ \text { nmom } & 6\end{array}$

Figure 9.2.64. Derived Parameters page.

\subsection{Energy group structure listing}

The Energy Group Structures block (Figure 9.2.65) lists the energy and lethargy boundaries found in the cross section library. If a broad-group collapse was requested, the boundaries of the broad-group library that will be produced are also identified. This example shows the structure of the SCALE 44GROUPNDF5 library and 2-group fast/thermal collapse structure. The final entry (group 45, broad group 3 ) indicates the lower bound of the previous energy group. 

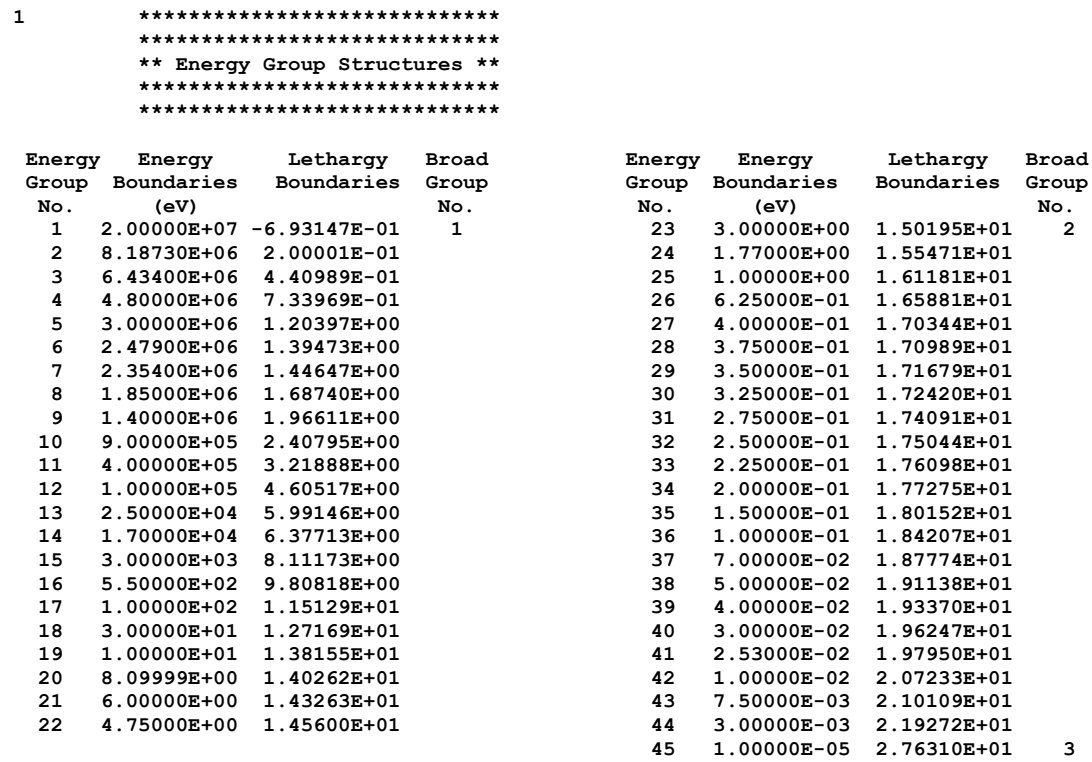

Figure 9.2.65. Energy Group Structure Listing page.

\subsection{Quadrature parameters}

The Quadrature Parameters block (Figure 9.2.66) lists the first-quadrant angles and weights used for the specified order of quadrature. The same angles and weights are applied in the other three quadrants; however, the signs of the angles vary with the quadrant. Also listed are the $\mathrm{P}_{\mathrm{n}}$ moments associated with the maximum $\mathrm{P}_{\mathrm{n}}$ scattering order requested in all materials. Of course, only a subset of these moments applies to the lower-order $\mathrm{P}_{\mathrm{n}}$ assignments.

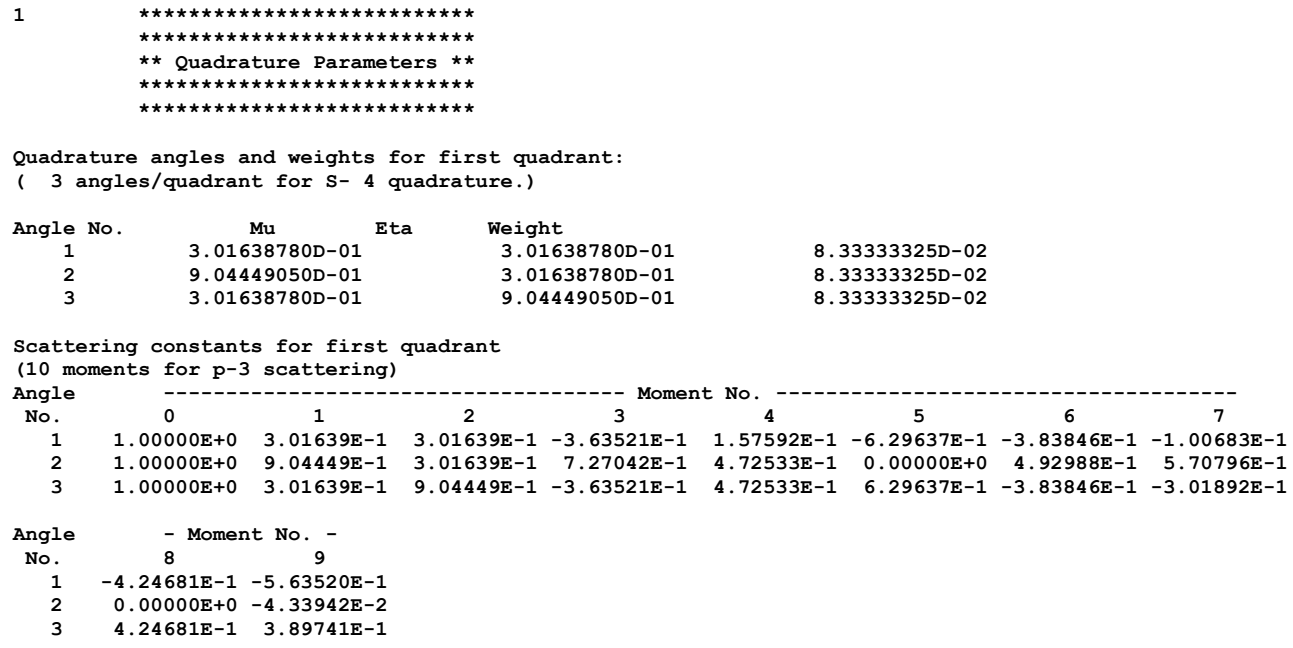

Figure 9.2.66. Quadrature Parameters page.

\subsection{Mixture volumes listing}

The Mixture Volumes block (Figure 9.2.67) provides a summary of the volume and volume fraction of each mixture in the problem, together with the total volume. This block can be used as a simple check of 
the input model by ensuring that the calculated volumes of mixtures used for a given problem match the expected volumes or volume fractions.

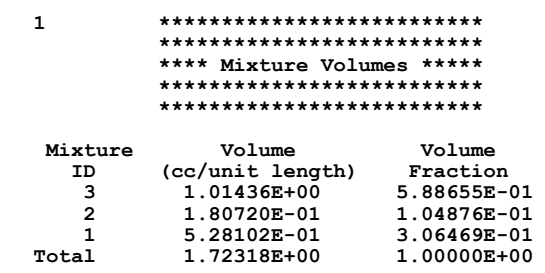

Figure 9.2.67. Mixture Volumes page.

\subsection{Mixing table listing}

The Mixing Table block summarizes the input mixing table, whether user supplied or read from a SCALE-generated file. Number densities are in units of atoms per barn-centimeter. Although optional, the mixing table is printed by default. This default setting can be disabled by specifying prtmxtab=no in the Parameter block. A sample mixing table is shown in Figure 9.2.68.

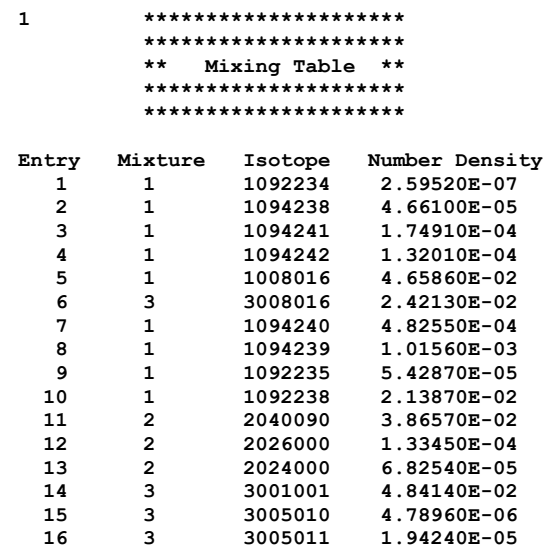

Figure 9.2.68. Mixing Table Listing page.

\subsection{Nuclide cross sections}

The Nuclide Cross Section block is optional and is printed only when prtxsec=yes is specified in the Parameter Block. The volume of output generated is quite extensive, especially when a very fine group library is used and/or a large number of nuclides are included in the mixing table. The nuclide data are taken directly from the working library used for the calculation. A sample showing a partial listing for a single nuclide is shown in Figure 9.2.69.

Following the block header, nuclide data are listed for all nuclides. For each record, the same format is used. Nuclide data begin with a listing of nuclide header information. This is followed by a listing of the 1-D cross sections that are important in NEWT calculations. The sample below shows a partial listing of the 1-D cross sections. Following the 1-D cross section listing is the scattering matrix for the nuclide. This abbreviated listing shows a portion of the $\mathrm{P}_{0}$ matrix for this nuclide; however, in a full listing, all higher-order elements are printed as well. 
As was indicated in the input description, specification of prtxsec $=1 \mathrm{~d}$ can be used to obtain header and 1-D cross section data only, skipping the printing of scattering matrices.

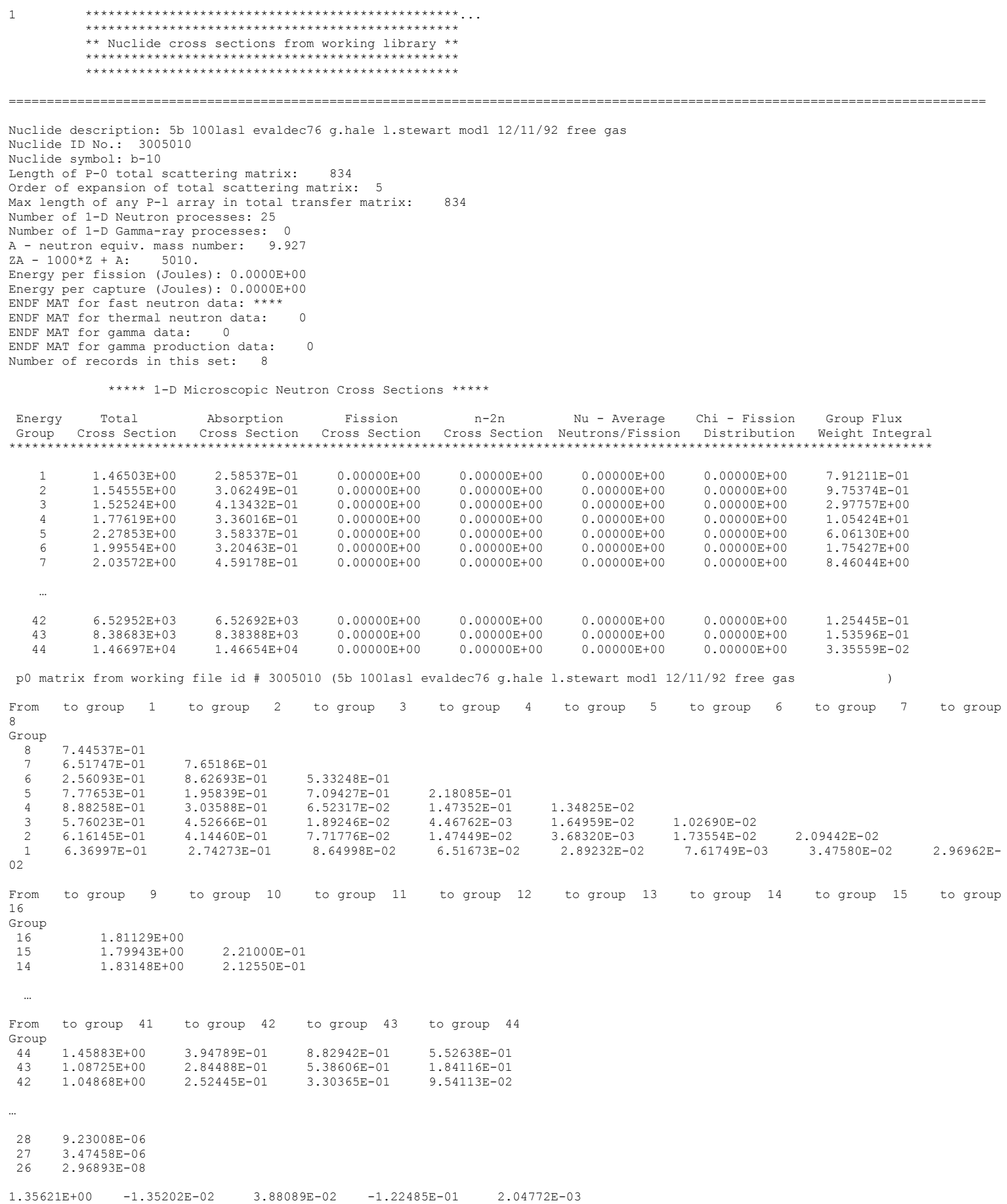

Figure 9.2.69. Partial listing of Nuclide Cross section data pages. 


\subsection{Mixture cross sections}

The Mixture Cross Section block provides mixed macroscopic cross sections for each mixture provided in the input mixing table. The block is also optional and is printed only when prtmxsec=yes is specified in the Parameter Block. Although the volume of output generated is not as extensive as that of the nuclide listings, the mixture cross section print can be voluminous, especially when a very fine group library is used and/or a large number of mixtures are included in the mixing table. A sample showing a partial listing for a single mixture is shown in Figure 9.2.70.

Following the block header, information is provided for each mixture using the same format. Mixture data begin with a listing of general mixture information, including the mixing table for that mixture. This is followed by a listing of 1-D cross sections important in NEWT calculations. The sample below shows a partial listing of the 1-D mixed macroscopic cross sections. Following the 1-D cross section listing is the scattering matrix for the nuclide for all moments requested for the mixture. This abbreviated listing shows a portion of the $\mathrm{P}_{0}$ matrix for this nuclide; however, in a full listing, all higher-order elements are printed as well if greater than $\mathrm{P}_{0}$ scattering was requested.

As was indicated in the input description, specification of prtmxsec=1d can be used to skip the printing of scattering matrices.

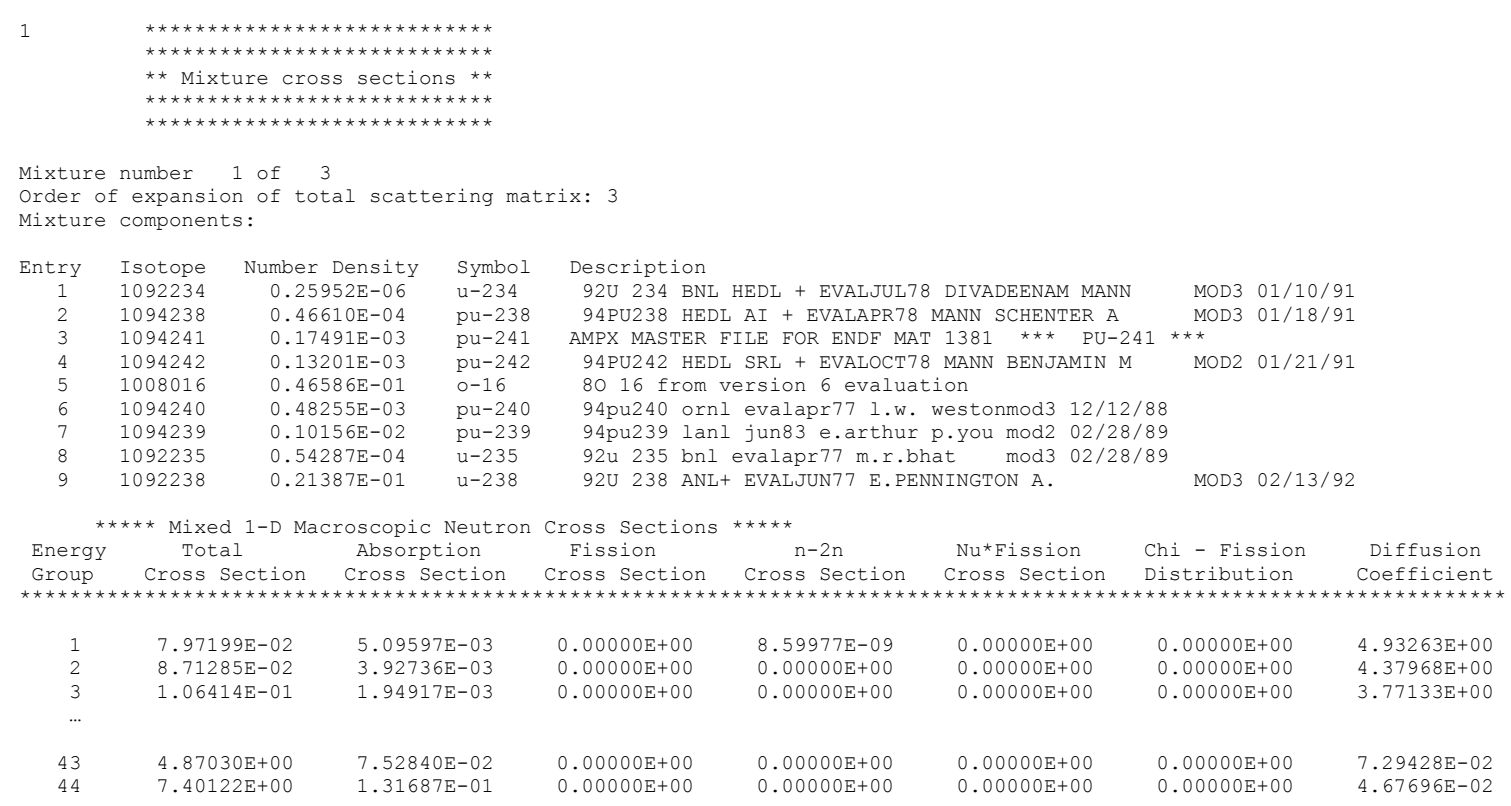

Figure 9.2.70. Partial listing of Mixture Cross section data pages. 


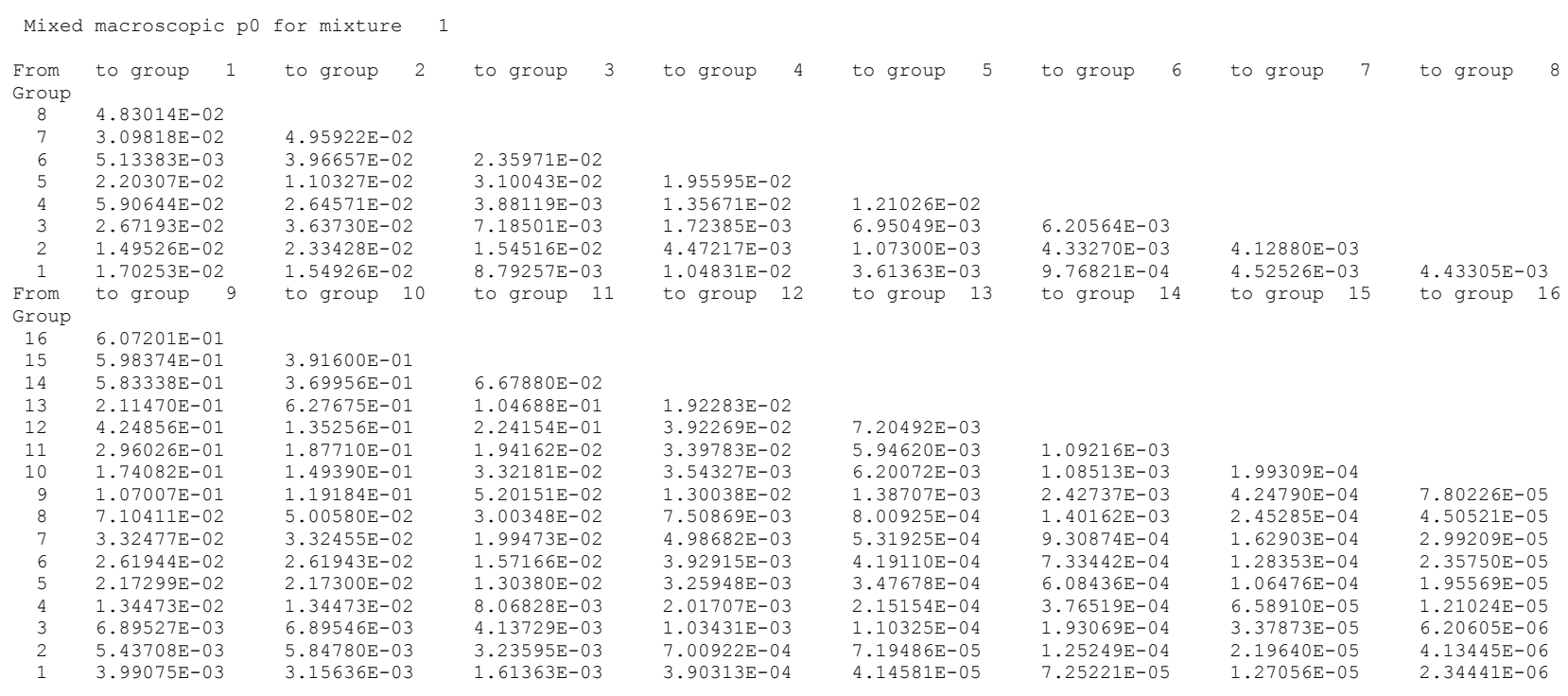

Figure 9.2.70. Partial listing of Mixture Cross section data pages. (continued)

\subsubsection{Iteration history}

The next portion of NEWT output lists the iteration convergence history for the iterative solution (Figure 9.2.71). This information can be used to track and understand the performance of the outer loop of the iterative solution. The first column provides the outer iteration count. The second column lists the system eigenvalue after each outer iteration. The third column lists the change in the eigenvalue from the last outer iteration; this is one of the parameters tested for convergence. The fourth column, "Max Flux Delta," gives the maximum change in cell flux for all cells and all energy groups; this is also used as a convergence test. The next column lists the cell number and energy group corresponding to the maximum flux change in this iteration. The next two columns list the same flux information for mixtures with fissionable nuclides. This can be used to track spatial convergence in fuel when convergence is slowed by significant scattering outside the fuel. Finally, the last column provides information on the convergence of inners in each outer iteration. Inner iterations do not need to converge within early outer iterations, but final convergence will not be achieved until all inners are converged. The maximum number of inner iterations per energy group is set by the inners $=$ parameter in the parameter input block. After convergence is achieved, the table is terminated by printing the final version of $k_{e f f}$.

If the parameter keyword timed= is set to yes, four additional columns are introduced that give timing information on the solution process, listing real ("wall clock") time, elapsed CPU time since beginning the iteration process, elapsed CPU time per outer iteration, and an estimate of the fractional CPU usage during each outer. Figure 9.2.72 illustrates the form of output produced when timed=yes is input. Additionally, a supplementary edit follows the iteration edit when timed=yes, giving information on average time per transport sweep (outer iteration) within different components of the solution. This edit is especially useful when coarse-mesh finite-difference acceleration is used, to assess the overhead of the CMFD accelerator. 


\begin{tabular}{|c|c|c|c|c|c|c|c|c|c|}
\hline $\begin{array}{l}\text { Outer } \\
\text { It. \# }\end{array}$ & $\begin{array}{l}\text { Eigen- } \\
\text { value }\end{array}$ & $\begin{array}{c}\text { Eigenvalue } \\
\text { Delta }\end{array}$ & $\begin{array}{l}\text { Max Flux } \\
\text { Delta }\end{array}$ & $\begin{array}{l}\text { Max } \\
\text { Locat }\end{array}$ & $\begin{array}{l}\text { Flux } \\
\text { ation }(r, g)\end{array}$ & $\begin{array}{c}\text { Max Fuel } \\
\text { Delta }\end{array}$ & $\begin{array}{l}\text { Max } \\
\text { Locat }\end{array}$ & $\begin{array}{l}\text { Fuel } \\
\text { iion }(r, g)\end{array}$ & $\begin{array}{l}\text { Inners } \\
\text { Cnvrged }\end{array}$ \\
\hline & - - - - & & & - & & & - & - - - - & - . - - \\
\hline 1 & 1.0000 & $0.000 \mathrm{E}+00$ & $3.212 \mathrm{E}+04$ & ( & $5,238)$ & $3.212 \mathrm{E}+04$ & 41 & $5,238)$ & $\mathbf{F}$ \\
\hline 2 & 0.8532 & $1.721 \mathrm{E}-01$ & $2.333 E+01$ & i & $5,237)$ & $2.333 E+01$ & i & $5,237)$ & $\mathbf{F}$ \\
\hline 3 & 1.2598 & $3.227 \mathrm{E}-01$ & $1.354 \mathrm{E}+00$ & i & $5,230)$ & 1. $354 \mathrm{E}+00$ & i & $5,230)$ & $\mathbf{F}$ \\
\hline 4 & 1.2860 & $2.041 \mathrm{E}-02$ & $3.842 \mathrm{E}-01$ & $i$ & $5,226)$ & $3.842 \mathrm{E}-01$ & $i$ & $5,226)$ & $\mathbf{F}$ \\
\hline 5 & 1.2401 & 3. $702 \mathrm{E}-02$ & $1.867 \mathrm{E}-01$ & i & $10, \quad 1)$ & $1.442 \mathrm{E}-01$ & $i$ & $5,224)$ & $\mathbf{F}$ \\
\hline 6 & 1.1992 & $3.408 \mathrm{E}-02$ & $1.264 \mathrm{E}-01$ & $i$ & 10, & $8.883 \mathrm{E}-02$ & i & $6,1)$ & $\mathbf{F}$ \\
\hline 7 & 1.1755 & $2.019 \mathrm{E}-02$ & $8.365 E-02$ & i & 10, & $5.741 \mathrm{E}-02$ & i & $6,1)$ & $\mathbf{F}$ \\
\hline 8 & 1.1634 & $1.038 \mathrm{E}-02$ & $5.479 \mathrm{E}-02$ & i & $10, \quad 1)$ & $3.727 \mathrm{E}-02$ & ? & $6,1)$ & $\mathbf{F}$ \\
\hline 9 & 1.1576 & $5.020 \mathrm{E}-03$ & $3.573 E-02$ & i & $10,1)$ & $2.427 \mathrm{E}-02$ & i & $6,1)$ & $\mathbf{F}$ \\
\hline 10 & 1.1548 & $2.459 \mathrm{E}-03$ & $2.325 \mathrm{E}-02$ & $i$ & 10 & $1.579 \mathrm{E}-02$ & i & $6,1)$ & $\mathbf{F}$ \\
\hline 11 & 1.1532 & $1.400 \mathrm{E}-03$ & $1.509 \mathrm{E}-02$ & $i$ & 10, & $1.023 \mathrm{E}-02$ & i & $6, \quad 1)$ & $\mathbf{F}$ \\
\hline 12 & 1.1522 & $8.313 E-04$ & $9.775 \mathrm{E}-03$ & $i$ & 10 & $6.617 \mathrm{E}-03$ & $i$ & $6,1)$ & $\mathbf{F}$ \\
\hline 13 & 1.1516 & $5.150 \mathrm{E}-04$ & $6.331 E-03$ & i & $1,1)$ & $4.285 \mathrm{E}-03$ & $i$ & $6,1)$ & $\mathbf{F}$ \\
\hline 14 & 1.1510 & $5.393 \mathrm{E}-04$ & $4.076 \mathrm{E}-03$ & $i$ & $1,1)$ & $2.734 \mathrm{E}-03$ & $i$ & $6,1)$ & $\mathbf{F}$ \\
\hline 15 & 1.1507 & $2.764 \mathrm{E}-04$ & $2.634 \mathrm{E}-03$ & i & 1) & $1.774 \mathrm{E}-03$ & 3 & $6,1)$ & F \\
\hline 16 & 1.1506 & $7.381 \mathrm{E}-05$ & $1.723 \mathrm{E}-03$ & i & $1,1)$ & $1.177 \mathrm{E}-03$ & $i$ & $6,1)$ & $\mathbf{F}$ \\
\hline 17 & 1.1505 & $4.181 \mathrm{E}-05$ & $7.749 \mathrm{E}-04$ & ( & 1) & $5.322 \mathrm{E}-04$ & i & 1) & $T$ \\
\hline 18 & 1.1505 & $1.044 \mathrm{E}-05$ & $9.475 \mathrm{E}-05$ & $i$ & $1,1)$ & $7.133 \mathrm{E}-05$ & i & $7,93)$ & $T$ \\
\hline 19 & 1.1505 & 1. $326 \mathrm{E}-05$ & $9.121 \mathrm{E}-05$ & $i$ & 1) & $7.114 \mathrm{E}-05$ & $i$ & $5,89)$ & $T$ \\
\hline -eff & $=1.15$ & $538 \mathrm{E}+0$ & & & & & & & \\
\hline
\end{tabular}

Figure 9.2.71. Nominal iteration history output.

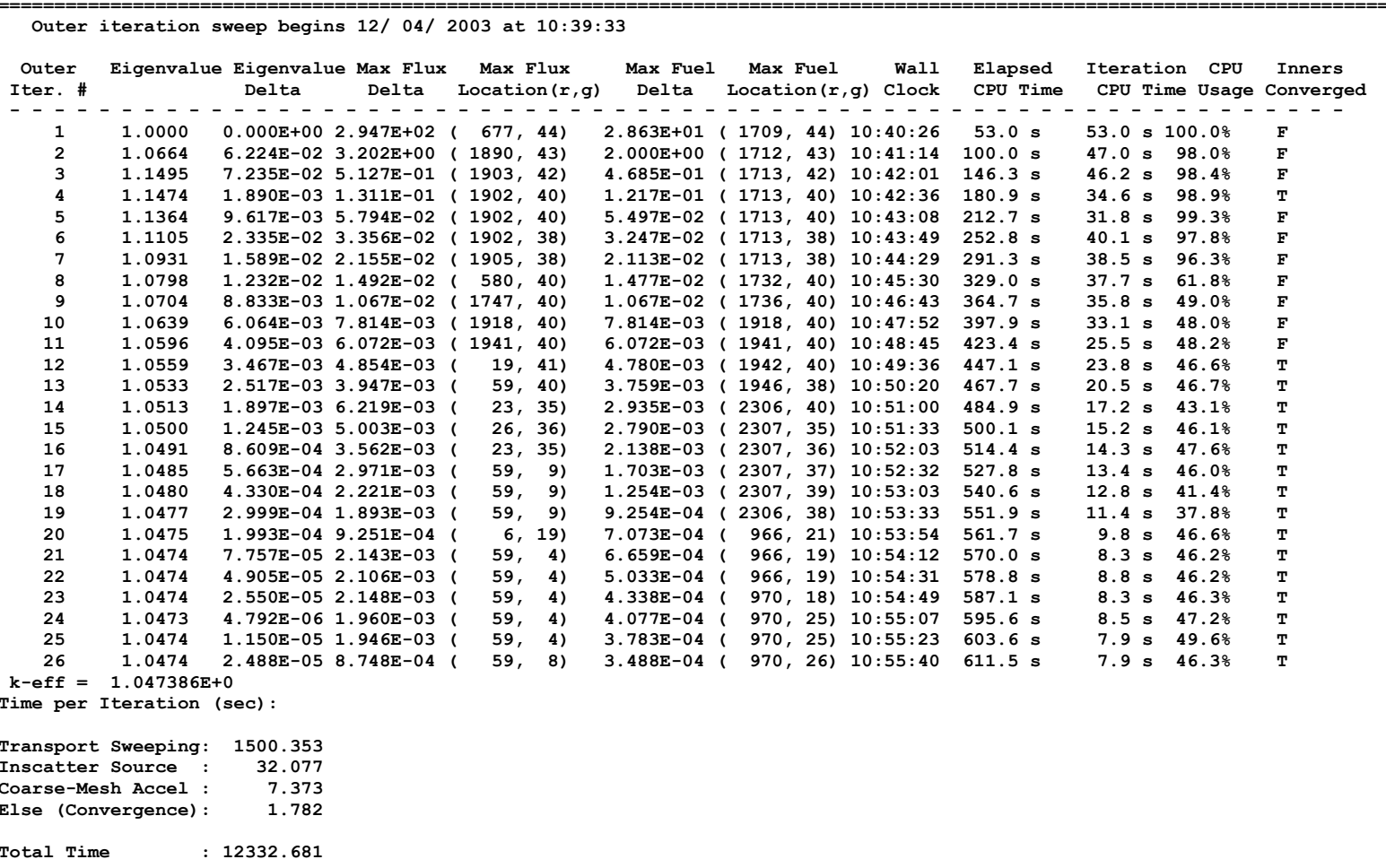

Figure 9.2.72. Timed iteration history output.

\subsubsection{Four-factor formula}

Following the iteration history listing, NEWT output provides edit listing the four traditional components of the four-factor formula. This is followed by an alternate three-group formulation that separates out resonance and fast escape probabilities (Figure 9.2.73). 


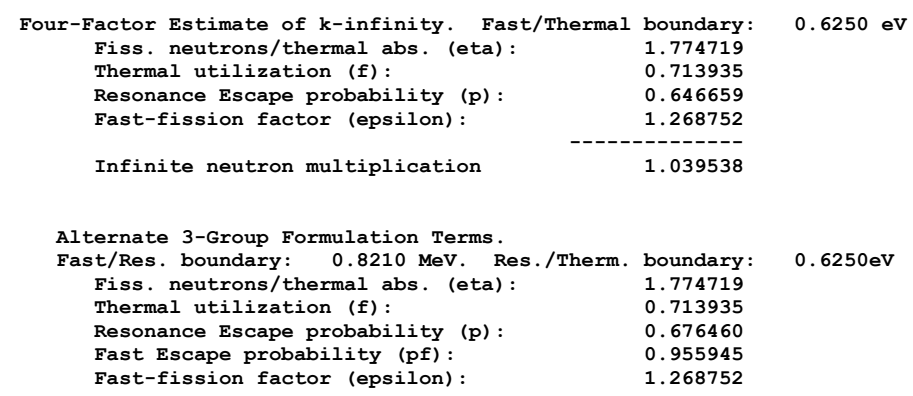

Figure 9.2.73. Four-factor formula with alternate three-group formulation.

\subsubsection{Fine-group balance tables}

Following the iteration history and flux convergence, a fine-group balance table is provided for each mixture used in the calculation. Fine group refers to the group structure of the library used for the calculation. Broad-group data, discussed later, refer to a group structure collapsed from the original finegroup structure. After tables for all mixtures are printed, a last table provides a fine-group summary for the entire problem (i.e., the volume-weighted average for all mixtures). Balance tables are printed by default but may be disabled by setting prtbalnc $=$ no in the Parameter block.

Figure 9.2.74 shows a clipped excerpt from the fine-group summary of an output listing. Similar tables are produced for each mixture in the problem for all energy groups in the problem. The header lists the NEWT mixture number; the mixture ID (i.e., the SCALE mixture number); and the mixture description, if provided in the original input specification. The header also gives the number of computational cells in which the mixture was present and the volume of the mixture in the problem.

For each mixture, two tables are printed. The first table provides a balance of all sources and loss terms: the fixed source, the fission source, in-scatter, out-scatter, absorption, leakage, $n-2 n$ production, and the net balance of all terms for each energy group. The final row lists the mixture total for all groups. The fixed source lists the user-supplied source for fixed-source problems. This field is disabled (set to zero) for eigenvalue calculations. The fission source is the number of neutrons born into each energy group in the mixture. In this example, the mixture is water, which is not fissile; hence, no fission source is present. In-scatter represents the number of neutrons scattered into each group from all other groups; conversely, out-scatter is the loss from each energy group by scattering. Absorption is the number of neutrons absorbed in reactions that do not emit a neutron (e.g., $n-\gamma)$. Leakage is the net loss of neutrons from the mixture to another mixture or a nonreflective boundary, and $n-2 n$ is the effective $n-2 n$ production rate calculated from a weighted sum of all $n-x n$ reactions. The balance table is the ratio of production to loss in each energy group.

The second fine-group balance table, also shown in Figure 9.2.74, lists other reactions rates of interest. The first two columns after the group number list in-scatter broken into its upscatter and downscatter components. The subsequent two columns provide a similar breakdown for out-scatter from the energy group. Self-scatter is the amount of within-group scattering occurring within each energy group. The fission rate is the number of (n-fission) reactions occurring in each group. The next column provides the transverse leakage (i.e., the product of the flux and the $\mathrm{DB}^{2}$ term). This column will provide only nonzero values when a nonzero buckling height is specified in input. The final column lists the total (scalar) flux for each energy group. 


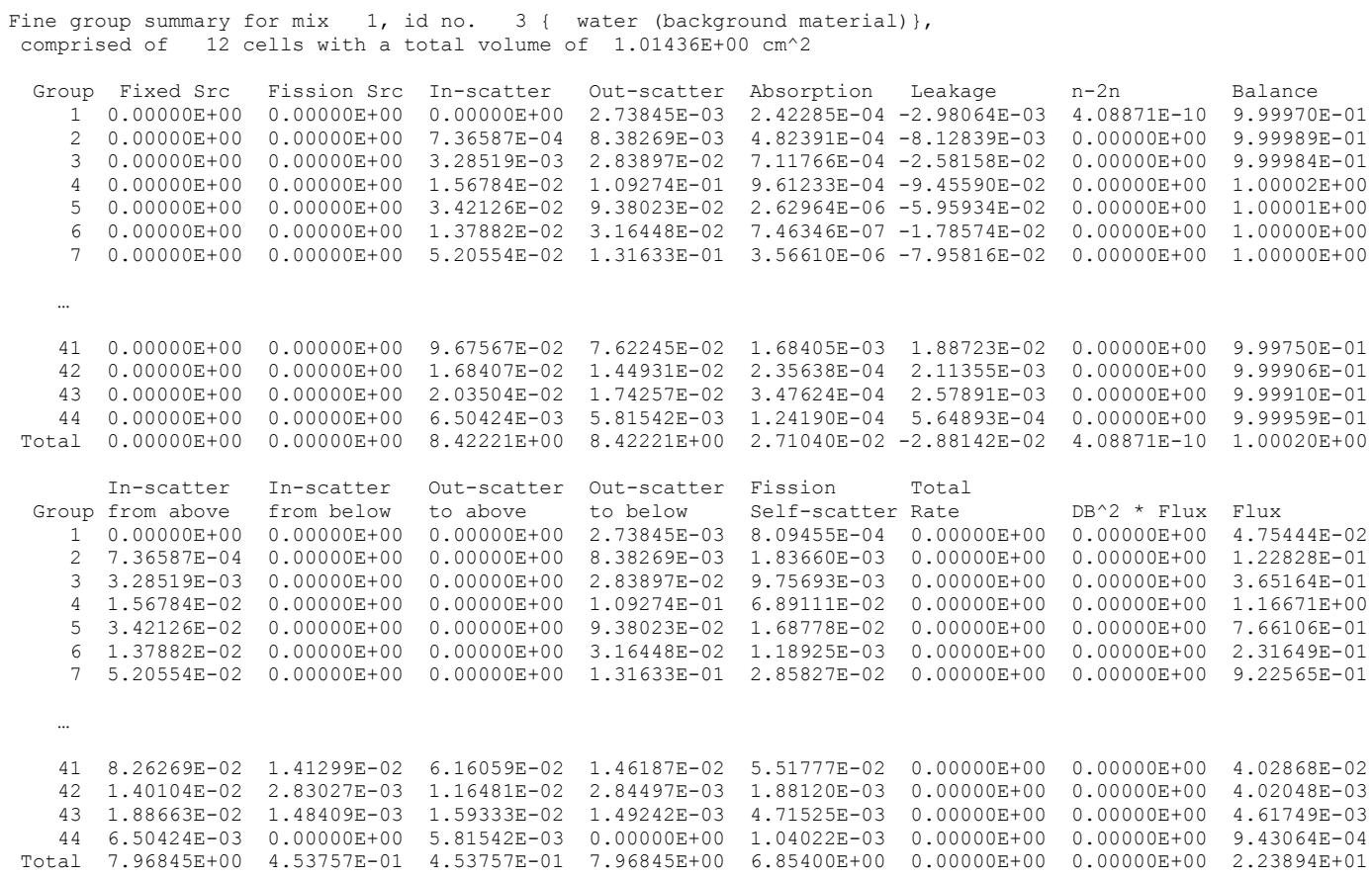

Figure 9.2.74. Partial mixture fine-group balance table output.

\subsubsection{Planar fluxes and currents}

If planar fluxes are requested, an edit is printed to provide fluxes and currents on each line segment specified, identified by label (Figure 9.2.75). Fine-group fluxes are listed for each energy group, followed by $\mathrm{x}$ and y net currents and partial currents $(+\mathrm{x},-\mathrm{x},+\mathrm{y}$, and $-\mathrm{y})$. Fluxes and currents are printed for each group in the input group structure. The example below shows only partial listings of each for simplicity. If a broad-group collapse is requested, the fine-group output is followed by the set of fluxes and currents for each broad energy group.

Note that discontinuity factors make internal use of planar fluxes to determine the flux and current on each boundary. Hence, planar flux edits will be present any time an ADF calculation is performed. 


\begin{tabular}{|c|c|c|}
\hline \multicolumn{3}{|c|}{ Average Scalar Fluxes on Specified Planes } \\
\hline Group & fuel west side & fuel south side \\
\hline 1 & $5.686166 \mathrm{D}-04$ & $5.686166 \mathrm{D}-04$ \\
\hline 2 & $1.616545 \mathrm{D}-03$ & $1.616545 \mathrm{D}-03$ \\
\hline 3 & $5.102822 \mathrm{D}-03$ & $5.102822 \mathrm{D}-03$ \\
\hline 4 & $1.701169 \mathrm{D}-02$ & $1.701169 \mathrm{D}-02$ \\
\hline \multicolumn{3}{|l|}{$\ldots$} \\
\hline $\begin{array}{l}42 \\
43\end{array}$ & $3.386267 \mathrm{D}-04$ & $3.386267 \mathrm{D}-04$ \\
\hline 43 & $3.658349 \mathrm{D}-04$ & $3.658349 \mathrm{D}-04$ \\
\hline 44 & $6.313846 \mathrm{D}-05$ & $6.313846 \mathrm{D}-05$ \\
\hline \multicolumn{3}{|c|}{ Fine Group Currents $(\mathbf{x})$ : } \\
\hline Group & fuel west side & fuel south side \\
\hline 1 & $0.000000 \mathrm{D}+00$ & $0.000000 D+00$ \\
\hline 2 & $0.000000 \mathrm{D}+00$ & $0.000000 D+00$ \\
\hline 3 & $0.000000 D+00$ & $0.000000 \mathrm{D}+00$ \\
\hline 4 & $0.000000 D+00$ & $0.000000 D+00$ \\
\hline 42 & $0.0000000+00$ & $0.0000000+00$ \\
\hline 43 & $0.000000 \mathrm{D}+00$ & $0.000000 \mathrm{D}+00$ \\
\hline 44 & $0.000000 D+00$ & $0.000000 \mathrm{D}+00$ \\
\hline \multicolumn{3}{|c|}{ Fine Group Currents (y): } \\
\hline Group & fuel west side & fuel south side \\
\hline 1 & $0.000000 \mathrm{D}+00$ & $0.000000 D+00$ \\
\hline 2 & $0.000000 D+00$ & $0.000000 D+00$ \\
\hline 3 & $0.000000 D+00$ & $0.000000 D+00$ \\
\hline 4 & $0.000000 \mathrm{D}+00$ & $0.000000 \mathrm{D}+00$ \\
\hline 42 & $0.0000000+00$ & $0.0000000+00$ \\
\hline 43 & $0.000000 \mathrm{D}+00$ & $0.000000 \mathrm{D}+00$ \\
\hline 44 & $0.000000 D+00$ & $0.000000 D+00$ \\
\hline \multicolumn{3}{|c|}{ Fine Group Currents $(+x)$ : } \\
\hline Group & fuel west side & fuel south side \\
\hline 1 & $1.416865 \mathrm{D}-04$ & $1.443455 \mathrm{D}-04$ \\
\hline 2 & $4.029062 \mathrm{D}-04$ & $4.100419 \mathrm{D}-04$ \\
\hline 3 & $1.272173 \mathrm{D}-03$ & $1.293594 \mathrm{D}-03$ \\
\hline 4 & $4.242596 \mathrm{D}-03$ & $4.308137 \mathrm{D}-03$ \\
\hline 42 & $0.000000 \mathrm{D}+00$ & $0.000000 D+00$ \\
\hline 43 & $0.000000 D+00$ & $0.000000 D+00$ \\
\hline 44 & $0.000000 \mathrm{D}+00$ & $0.000000 D+00$ \\
\hline \multicolumn{3}{|c|}{ Fine Group Currents (+y): } \\
\hline Group & fuel west side & fuel south side \\
\hline 1 & $1.443455 \mathrm{D}-04$ & $1.416865 \mathrm{D}-04$ \\
\hline 2 & $4.100419 \mathrm{D}-04$ & $4.029062 \mathrm{D}-04$ \\
\hline 3 & $1.293594 \mathrm{D}-03$ & $1.272173 \mathrm{D}-03$ \\
\hline 4 & 4.308137D-03 & $4.242596 \mathrm{D}-03$ \\
\hline \\
\hline 42 & $0.000000 \mathrm{D}+00$ & $0.000000 \mathrm{D}+$ \\
\hline 43 & $0.000000 \mathrm{D}+00$ & $0.000000 \mathrm{D}+00$ \\
\hline 44 & $0.000000 \mathrm{D}+00$ & $0.000000 \mathrm{D}+00$ \\
\hline
\end{tabular}

Figure 9.2.75. Example of planar flux and current output. 


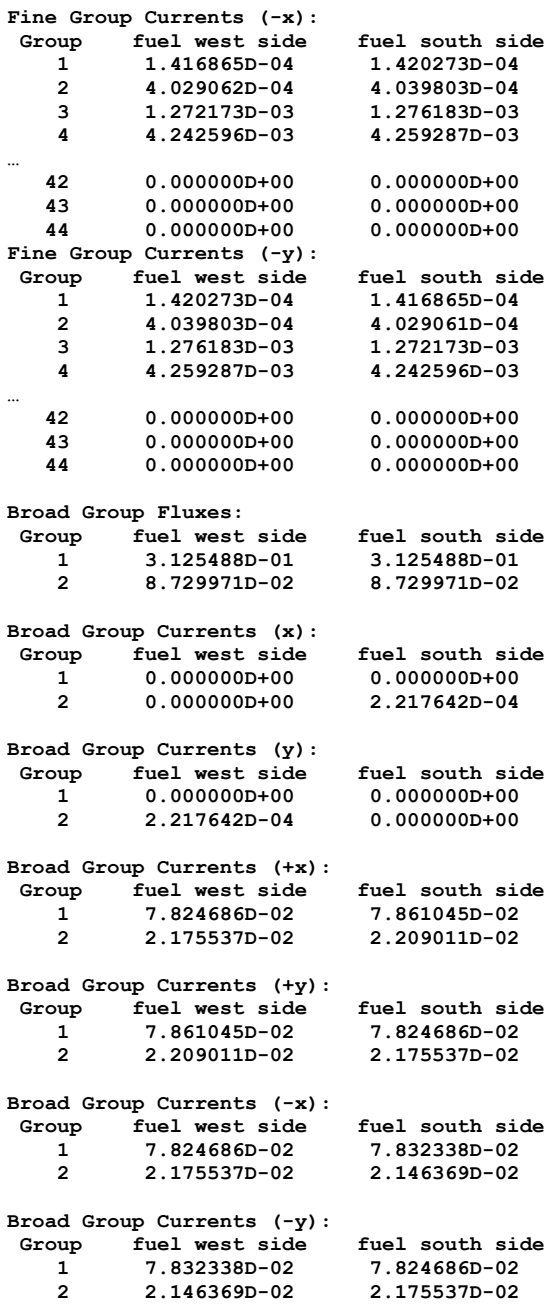

Figure 9.2.75. Example of planar flux and current output. (continued)

\subsubsection{Pin-power edits}

The next section of the NEWT output listing is the pin-power edit (Figure 9.2.76). This information is printed only if pinpow=yes is set in one or more arrays. Two maps are provided. The first is the power of each fuel location relative to all other fuel pins in all other arrays for which pinpow=yes. This is useful if multiple fuel assemblies are present or if more than one array is used to describe a fuel assembly. The second power map shows the pin edit normalized to the set of pins within the single array. Both maps are identical in a relative sense; different normalization factors are applied. If pinpow=yes is specified for only one array, then the two edits will have the same normalization factor and will be identical. The location of each pin is identified by the $(\mathrm{x}, \mathrm{y})$ coordinate of the center of each element of the array, in centimeters. Please note that the pin-power option is available only for square (cuboidal) arrays.

Following the two maps is a one-line edit identifying the location and magnitude of the maximum pin power. 


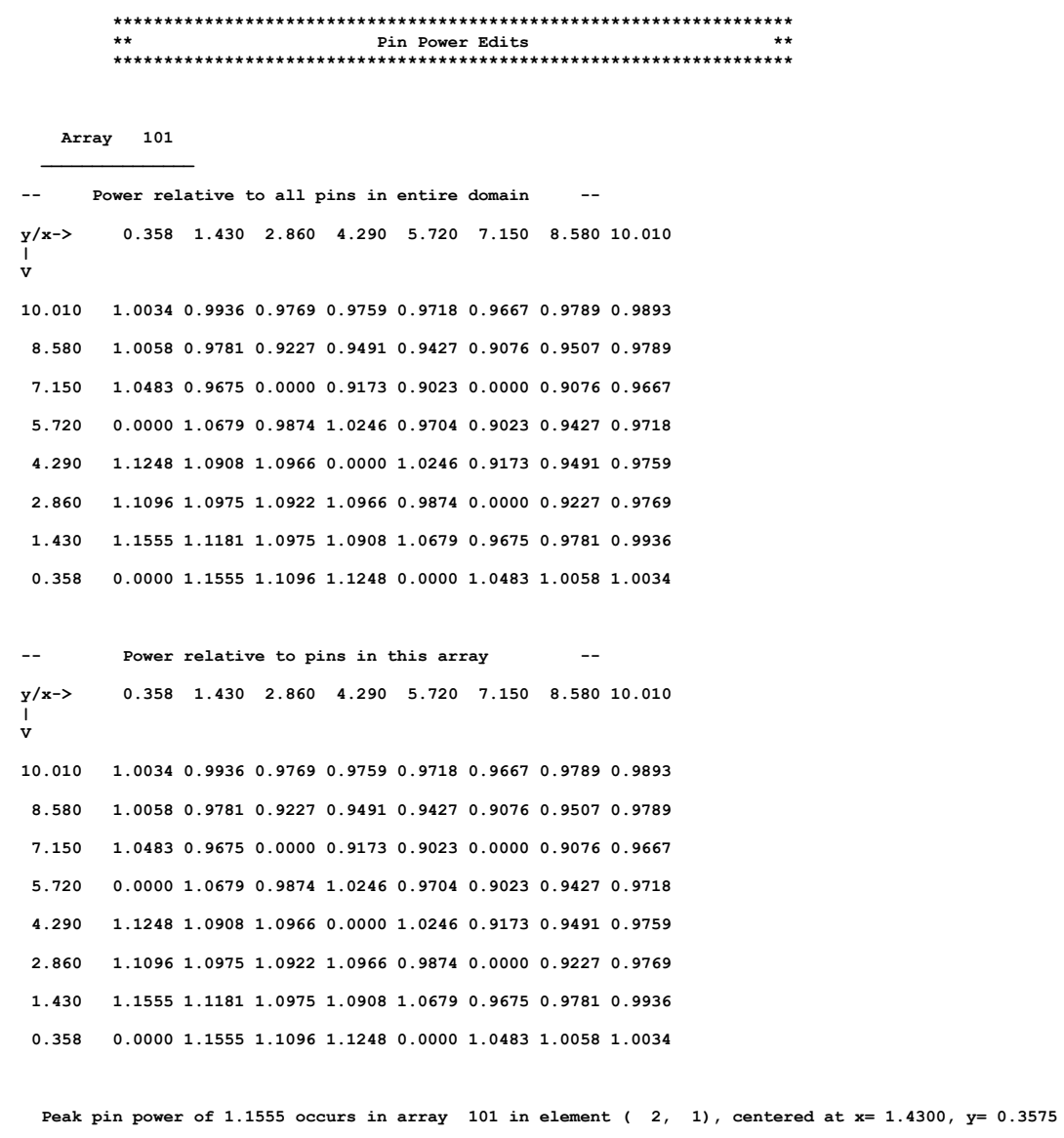

Figure 9.2.76. Example of pin-power edit.

\subsubsection{Broad-group collapse}

\subsection{Broad-group summary data}

The next section of the NEWT output listing is the broad-group summary listing (Figure 9.2.77). This is printed only if a broad-group collapse is performed. This section lists broad-group data calculated based on the collapsing scheme applied. First, the energy group structure is printed, followed by cell-averaged fluxes in each mixture, for all collapsed groups. This is followed by flux disadvantage factors for each mixture and each broad group.

Note that when NEWT is used as the transport solver within TRITON depletion calculations, a threegroup collapse is always done automatically. If a second user-specified collapse is requested, broad-group summary data will be provided for both collapsing structures. 


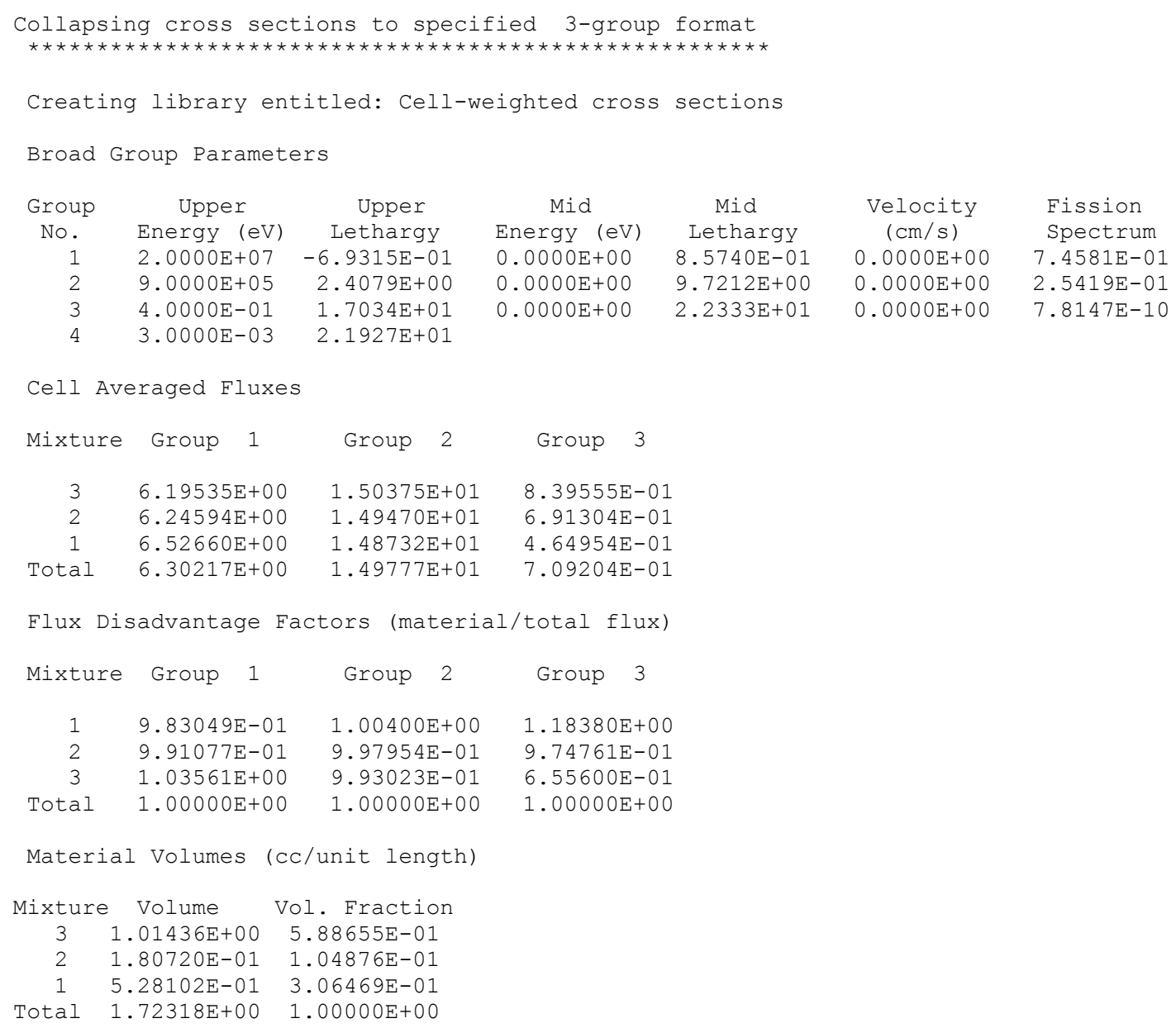

Figure 9.2.77. Broad-group summary output.

\subsection{Broad-group cross section data}

The next section of data in a NEWT output listing is the broad-group cross section output (Figure 9.2.78). This is printed only if a broad-group collapse is performed and if prtbroad=yes is specified in Parameter block input. This block lists the collapsed cross section data for key reactions for each nuclide used in the calculation. This is a summary form of the data that are written to the collapsed cross section library. It does not list all reactions. Such data may be read directly from the working-format library by other SCALE utilities if needed. The listing below shows the data printed for a single nuclide. Data are written in the same format for all nuclides used in the analysis. 


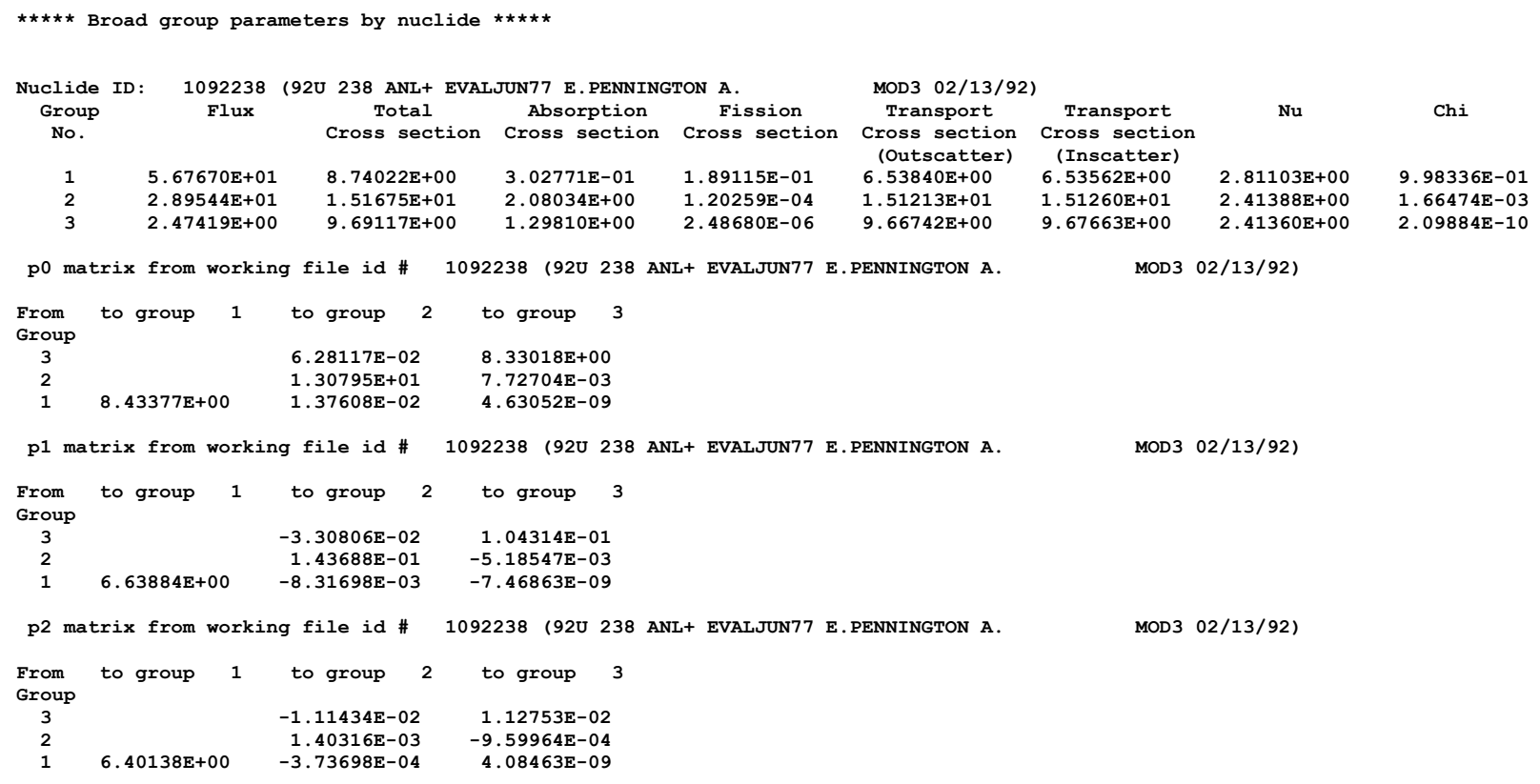

Figure 9.2.78. Partial broad-group cross section listing.

\subsubsection{Critical spectrum edit}

When a critical buckling correction is requested (e.g., solntype $=b 1$ is set in the NEWT parameter block or user-defined material buckling or transverse height), the critical spectrum is computed using either the B1 approximation or the P1 approximation (Figure 9.2.79). The output lists the buckling in $1 / \mathrm{cm}^{2}$, the method (B1 or P1), and the computed critical spectrum as a function of energy. Note that the spectrum is normalized "per unit lethargy" to be equal to 1.0. In addition to the critical spectrum, the critical adjoint spectrum and the zero-buckling spectra (forward and adjoint) are also edited. 


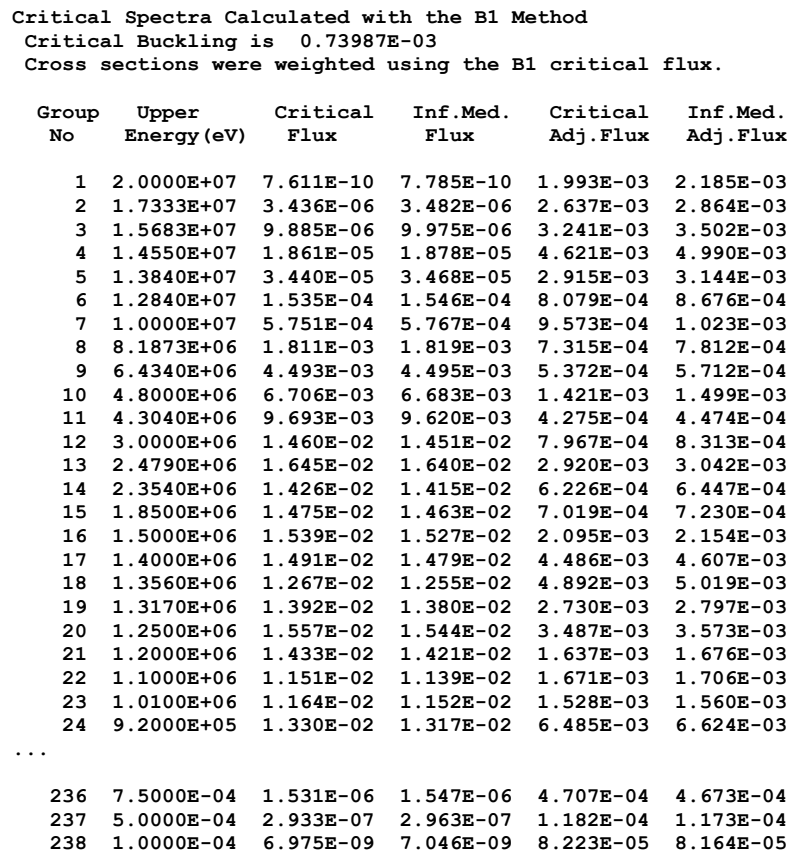

Figure 9.2.79. Partial collapsing spectra listing for a case with critical buckling correction.

\subsubsection{Assembly discontinuity factors}

When calculation of assembly discontinuity factors (ADFs) is requested, a broad-group edit is provided for each face for which an ADF was selected (Figure 9.2.80). Up to four ADFs may be printed for the fuel region. If the model contains a reflector region in addition to the required fuel region, then ADFs are printed for a single face, typically the fuel/moderator interface. Discontinuity factors for user-input surfaces may also be edited.

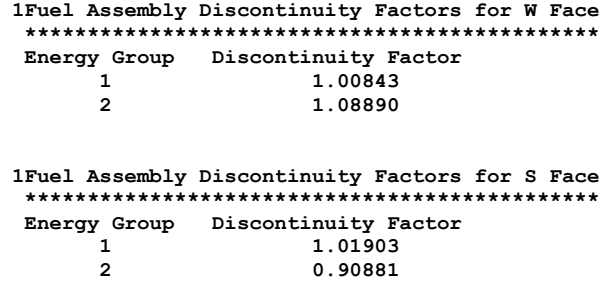

Figure 9.2.80. Output of assembly discontinuity factors.

\subsubsection{Groupwise form factors}

Whenever homogenization is performed and pin-power edits are requested, NEWT will automatically calculate groupwise form factors (GFFs). GFFs are used in pin-power reconstruction calculations for homogenized assemblies used in nodal diffusion methods (Figure 9.2.81). 


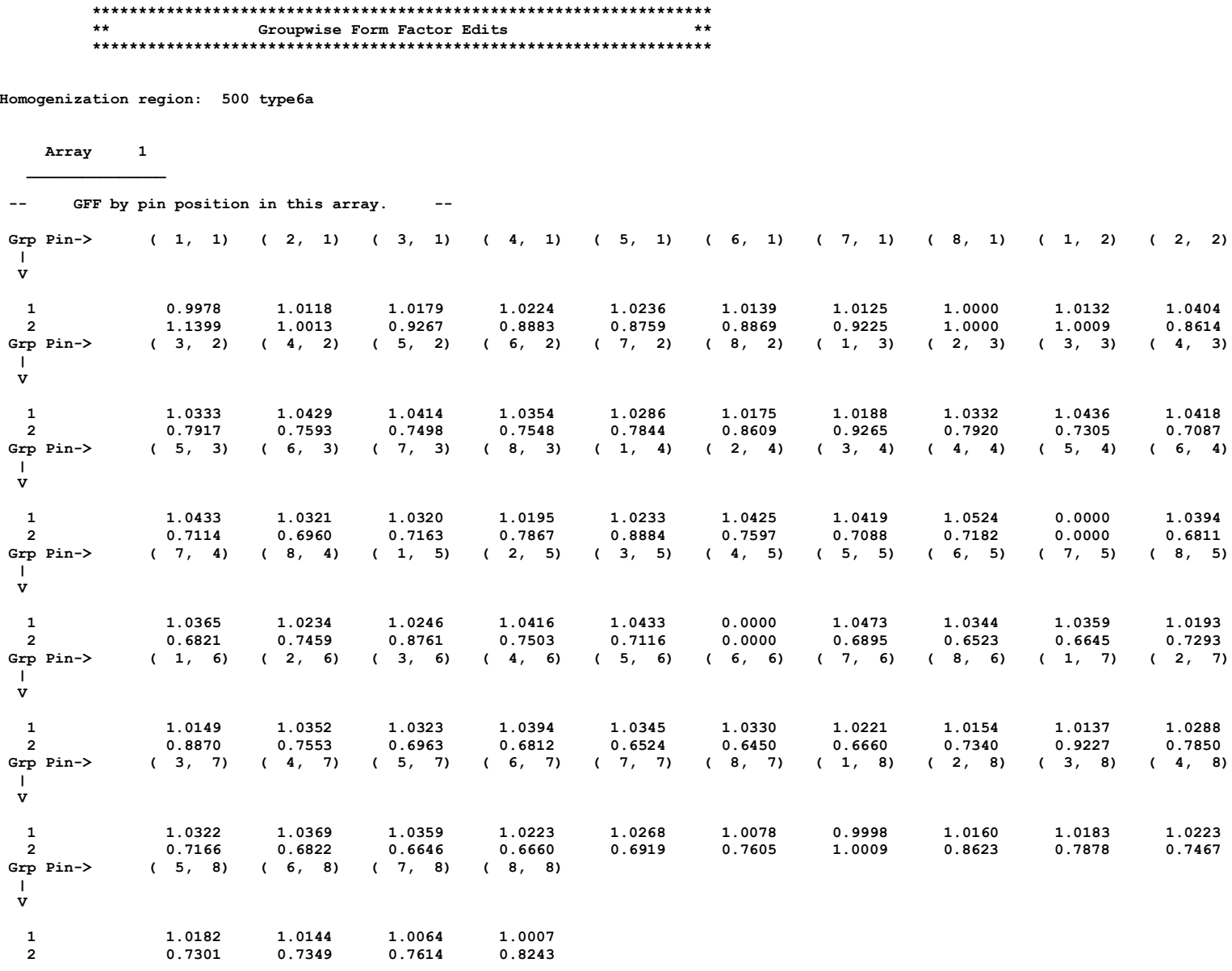

Figure 9.2.81. Partial collapsing spectra listing for a case with no critical buckling correction.

\subsubsection{Homogenized cross sections}

When homogenization is performed and parameter prthmmix=yes is set, the final output section of a NEWT calculation is the homogenized cross section edit, as shown in Figure 9.2.82. This information is generally passed to nodal analysis codes and hence is presented in a slightly different format from other cross sections. Output includes a region-averaged k-infinity value, transport-corrected cross section, and two interpretations of absorption. The first is the directly collapsed absorption cross section, while the second (Total-Scatter) is a more consistent definition of absorption as applied in nodal calculations. The difference between the two definitions is the effective (n-2n) cross section. Both cross sections exclude contributions from ${ }^{135} \mathrm{Xe}$ and ${ }^{149} \mathrm{Sm}$; microscopic cross sections and number densities for these two nuclides are printed explicitly elsewhere in the table. $\mathrm{Nu}^{*}$ fission is the product of the fission cross section and the number of neutrons produced per fission, while Kappa*fission is the product of the fission cross section and the energy release per fission $(J)$. Inverse velocity is the inverse $(1 / x)$ of the group neutron speed.

The table also lists the two-group isotropic scattering matrix and the prompt fission fraction distribution. Finally, NEWT lists approximate six-group decay constants (lambdas) and group fractions (betas) for each group. 


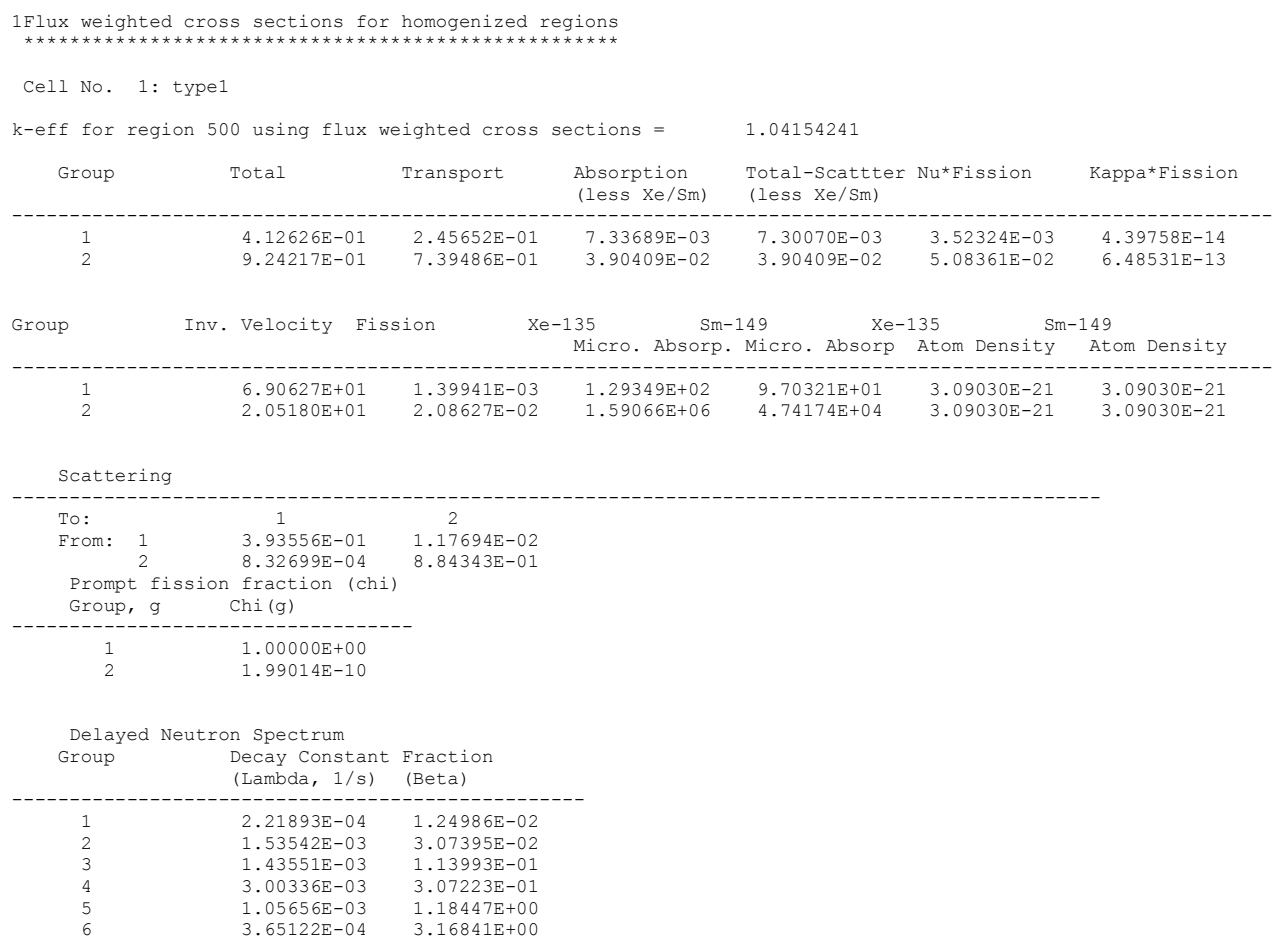

Figure 9.2.82. Homogenized cross section edit for nodal diffusion applications.

\subsubsection{End-of-calculation banner}

NEWT output listings are terminated with an end-of-calculation banner (shown in Figure 9.2.83) upon successful completion of a calculation. If this banner is not present, then the calculation ended abnormally, and the output listing must be reviewed to determine the cause of the error. In general, the final lines of an output file describe the error condition that caused the calculation to stop.

NEWT execution completed with zero errors

Figure 9.2.83. End-of-calculation banner listing.

\subsubsection{Postscript graphics files}

Two user-selectable options within NEWT provide the ability to generate PostScript-based graphics files for visualization of both input specifications and output results. By specification of drawit=yes in the NEWT parameter block, NEWT will generate two PostScript-based plot files: newtgrid.ps and newtmatl.ps. The former, a grayscale plot of the line segments generated by NEWT based on the input specification, will be generated if all body placement input is valid. If input contains errors such that the code stops before grid generation routines are completed, no newtgrid.ps output is created.

The newtmatl.ps plot illustrates the same grid structure but with material placement indicated by color. At this time, no user control is provided for color assignment or plot control. This plot also requires complete grid generation; additionally, it requires completion of all media placement routines before the plot will be produced. 
Figures used throughout this manual were generated from newtgrid and newtmatl PostScript plot files. Files newtgrid.ps and newtmatl.ps are automatically copied back from SCALE's temporary directory to the original location of the input case, with the names casename.newtgrid.ps and casename.newtmatl.ps.

When prtflux=yes is input, NEWT will generate a set of flux plots showing relative neutron number densities in each energy group. A plot file will be generated with the name fluxplot_Ng.ps, where $N$ is the number of energy groups in the problem. If an energy collapse is performed, an additional file named fluxplot_Mg.ps is created, where $\mathrm{M}$ is the number of energy groups in the collapsed set. Figure 9.2.84 is an example of a flux plot output for the fast group of a two-group flux collapse.

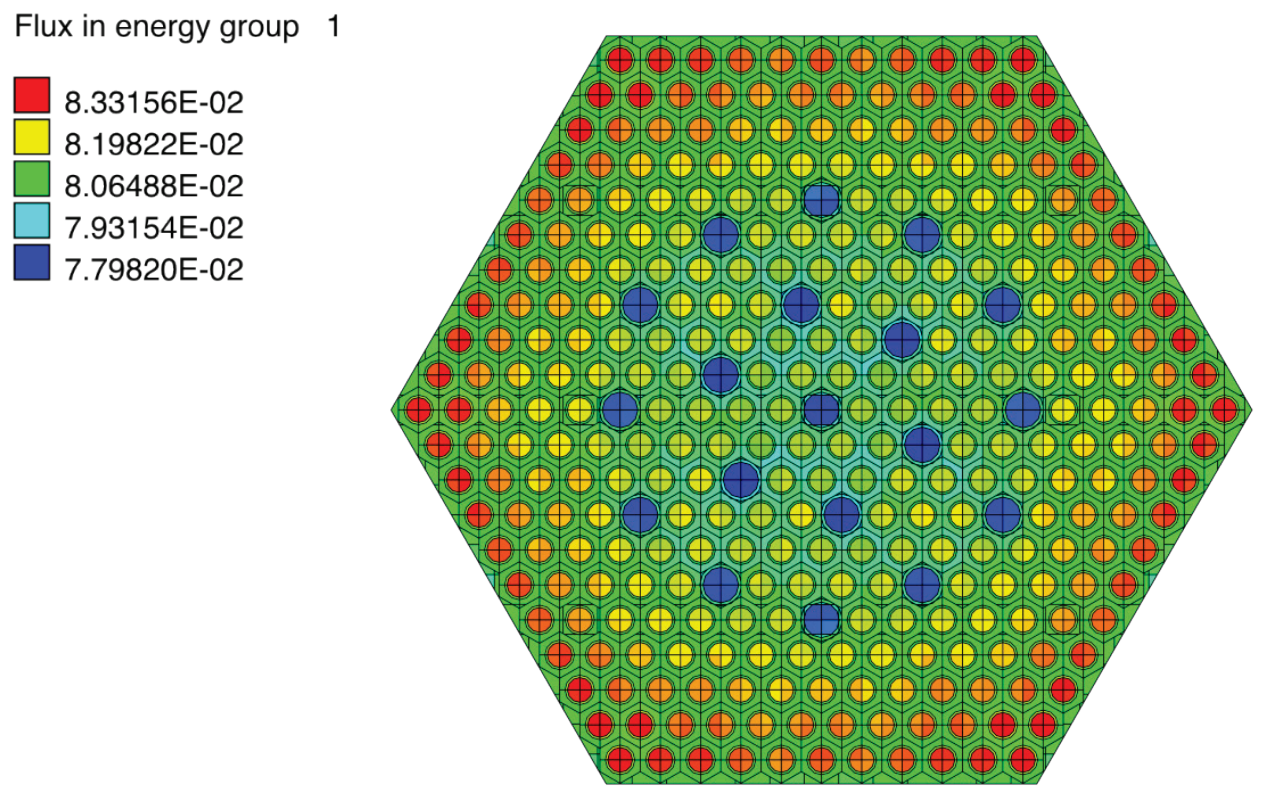

Figure 9.2.84. Example of a flux plot image created with prtflux=yes.

\subsubsection{Media zone edits}

NEWT automatically determines "zones" representing spatially independent regions of the same media. For example, in a fuel pin cell, the fuel, clad, and moderator are all considered separate zones. In an array of such pin cells, each unique location is a unique zone. Zone numbers and the geometric location of each zone are listed in the Geometry Specification" in Sect. 9.2.5.2.6.

Upon completion of a calculation, NEWT provides an output edit of each zone by number, giving the mixture number, average flux, fission power, and volume, as shown in Figure 9.2.85.

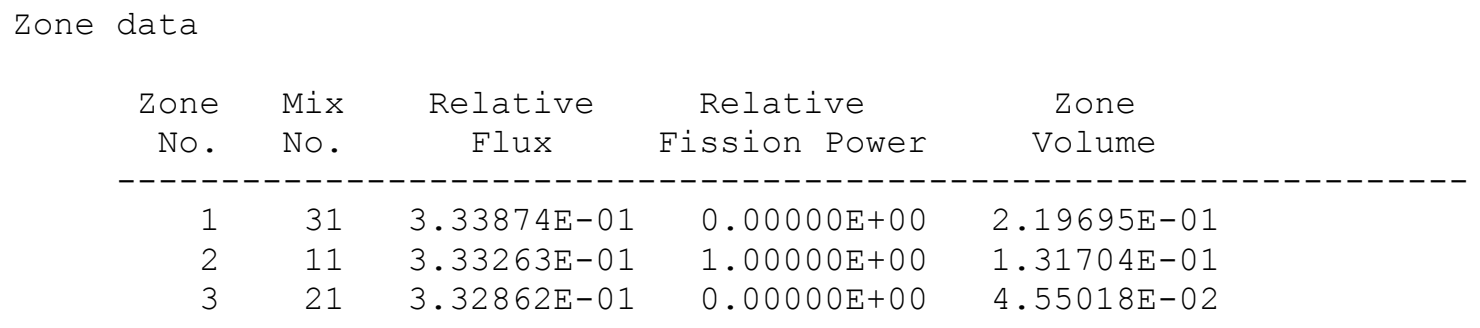

Figure 9.2.85. Media zone output edit. 


\subsubsection{References}

1. M. D. DeHart, "A Discrete Ordinates Approximation to the Neutron Transport Equation Applied to Generalized Geometries,” Ph.D. Dissertation, Texas A\&M University (1992).

2. J. J. Duderstadt and L. J. Hamilton, Nuclear Reactor Analysis, John Wiley \& Sons, New York, 1976.

3. F. B. Hildebrand, Advanced Calculus for Applications, Prentice-Hall, Inc., New Jersey, 1976.

4. E. E. Lewis and W. F. Miller, Jr., Computational Methods for Neutron Transport, John Wiley \& Sons, New York, 1984.

5. R. E. Alcouffe and E. W. Larsen, "A Review of Characteristic Methods Used to Solve the Linear Transport Equation," Proc. Int Topl. Mtg. Advances in Mathematical Methods for the Solution of Nuclear Engineering Systems, Munich, Germany, April 27-29, 1981, American Nuclear Society (1981).

6. K. D. Lathrop, "Spatial Differencing of the Transport Equation: Positivity vs. Accuracy," J. Comput. Phys., 4, 475 (1969).

7. R. E. Alcouffe, E. W. Larsen, W. F. Miller, Jr., and B. R. Wienke, “Computational Efficiency of Numerical Methods for the Multigroup, Discrete-Ordinates Neutron Transport Equations: The Slab Geometry Case," Nucl. Sci. Eng., 71, 111-127 (1979).

8 E. W. Larsen and R. E. Alcouffe, "The Linear Characteristic Method for Spatially Discretizing the Discrete Ordinates Equations in (x,y)-Geometry," Proc. Int Topl. Mtg. Advances in Mathematical Methods for the Solution of Nuclear Engineering Problems, Munich, Germany, April 27-29, 1981, American Nuclear Society (1981).

9. K. D. Lathrop, Spatial Differencing of the Two-Dimensional Transport Equation, Report GA-8746, Gulf General Atomic, 1968.

10. B. G. Carlson, Transport Theory: Discrete Ordinates Quadrature Over the Unit Sphere, Report LA-4554, Los Alamos Scientific Laboratory, 1970.

11. K. D. Lathrop and B. G. Carlson, Discrete Ordinates Angular Quadrature of the Neutron Transport Equation, Report LA-3186, Los Alamos Scientific Laboratory, 1965.

12. C. E. Lee, Discrete $S_{N}$ Approximation to Transport Theory, Report LA-2595-MS, Los Alamos Scientific Laboratory, 1962.

13. K. D. Lathrop and F. W. Brinkley, Jr., TWOTRAN-II: An Interfaced, Exportable Version of the TWOTRAN Code for Two-Dimensional Transport, Report LA-4848-MS, Los Alamos Scientific Laboratory, 1973.

14. W. W. Engle, Jr., A Users Manual for ANISN: A One Dimensional Discrete Ordinates Transport Code with Anisotropic Scattering, Report K-1693, Oak Ridge National Laboratory, 1973.

15. Z. Zhong, T. Downar, and M. D. DeHart, Implementation of a Two-Level Coarse-Mesh FiniteDifference Accelerator in the NEWT Transport Code, ORNL/TM-2004/162, Oak Ridge National Laboratory, June 2005. 


\title{
10.0 SCALE NUCLEAR DATA LIBRARIES
}

\author{
Introduction by M. L. Williams and D. Wiarda
}

Chapter 10 describes the SCALE cross section data libraries for use with deterministic and Monte Carlo radiation transport modules. All cross section libraries were processed from ENDF/B-VII.0 or -VII.1 evaluated data files using the AMPX code system. ${ }^{1}$ SCALE includes continuous-energy libraries, as well as multigroup libraries with several group structures. Libraries are available for neutron, gamma, and coupled neutron-gamma transport calculations. The fine and broad multigroup libraries provided for reactor physics and criticality safety applications in SCALE 6.2 include intermediate resonance parameters (lambdas) and improved Bondarenko data for self-shielding calculations using the Bondarenko method, or the traditional CENTRM-based procedures in SCALE can be used for selfshielding. Section 10.1 in this chapter describes the available cross section libraries.

Fine and broad group covariance libraries containing cross section uncertainties and correlations are also distributed with SCALE for sensitivity/uncertainty analysis with the Sampler and TSUNAMI modules. The covariance libraries include a comprehensive collection of data for all nuclides included in the SCALE cross section libraries. New 252-group and 56-group covariances based on ENDF/B-VII.1 and other data sources are available, along with the older 44-group covariance library distributed with earlier releases of SCALE. The Covariance Libraries chapter describes the contents of the SCALE 6.2 covariance libraries and explains how they were processed.

Additional libraries used for transmutation calculations with ORIGEN are described in the ORIGEN Data Resources section of the ORIGEN chapter. These libraries include fission product yields, decay data, decay gamma spectra, etc., as well as supplemental cross section data not available in ENDF/B.

\section{Reference}

1. D. Wiarda, M. L. Williams, C. Celik, and M. E. Dunn, "AMPX: A Modern Cross Section Processing System for Generating Nuclear Data Libraries," Proceedings of International Conference on Nuclear Criticality Safety, Charlotte, NC, Sept. 13-17, 2015. 


\title{
10.1 SCALE CROSS SECTION LIBRARIES
}

M. L. Williams, D. Wiarda, C. Celik, K. S. Kim, M. E. Dunn, B. T. Rearden

\begin{abstract}
The cross section data libraries available in the SCALE code system are briefly described in this section. All libraries were processed from ENDF/B-VII.0 and -VII.1 evaluated data files using the AMPX code system. Continuous-energy libraries as well as several multigroup libraries for a variety of applications are included in SCALE. Several fine-group and broad-group structures are available so that a user may select the nuclear data library based on considerations of application, accuracy, and execution time.
\end{abstract}




\section{TABLE OF CONTENTS}

$\underline{\text { Page }}$

10.1 SCALE CROSS SECTION LIBRARIES ..............................................................

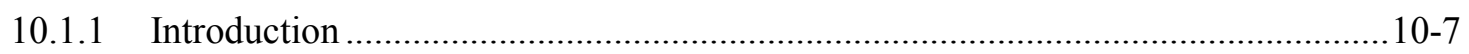

10.1.2 Description of the SCALE Cross Section Libraries .............................................. 10-23

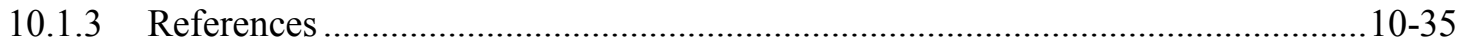

10.1.A MT Reaction Types on SCALE Cross Section Libraries ..................................... 10-37 


\section{LIST OF TABLES}

$\underline{\text { Page }}$

Table 10.1.1. Nuclides in CE and MG ENDF/B-VII.0 and -VII.1 libraries.......................................10-8

Table 10.1.2. Isotopes with no ENDF/B-VII.0 or -VII.1 nuclear data .......................................... 10-20

Table 10.1.3. Temperatures at which thermal moderator data are available ${ }^{\mathrm{a}}$..................................10-21

Table 10.1.4. Standard SCALE cross section libraries ..............................................................10-22

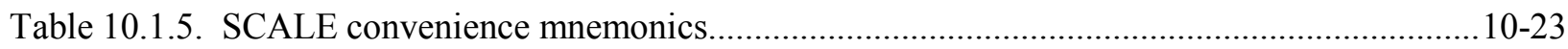

Table 10.1.6. Standard weighting function for processing MG data ............................................ 10-24

Table 10.1.7. Standard weighting functions for processing 252-group library data library ................ 10-25

Table 10.1.8. 238 Multigroup energy structure …........................................................................ 10-25

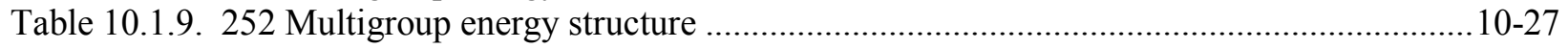

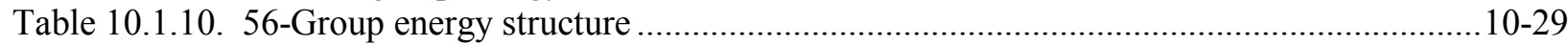

Table 10.1.11. Speciality nuclides with special shielding factors $(*)$ in 56-group library...................10-29

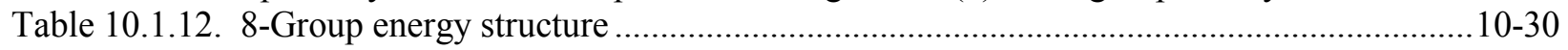

Table 10.1.13. Energy boundaries for the 200 neutron group structure .......................................10-31

Table 10.1.14. Energy boundaries for the 47 gamma group structure .............................................10-32

Table 10.1.15. Available dose functions on the coupled neutron-gamma libraries ............................10-33

Table 10.1.16. Energy boundaries for the 27 neutron group structure ............................................10-34

Table 10.1.17. Energy boundaries for the 28 neutron group structure .............................................10-34

Table 10.1.18. Energy boundaries for the 19 gamma group structure ..............................................10-34

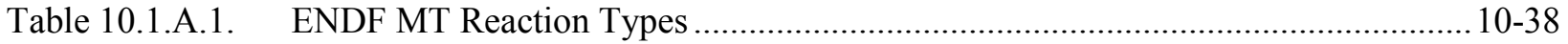

Table 10.1.A.2. $\quad$ SCALE Specific MT Reaction Types............................................................ 10-43 


\section{ACKNOWLEDGMENTS}

We would like to acknowledge all those who participated in developing and implementing the SCALE cross section libraries for their assistance in preparing previous versions of this document. Special thanks go to S. Goluoglu, N. M. Greene, and L. M. Petrie for their contributions to SCALE data processing efforts, and to C. R. Daily and W. J. Marshall for their extensive validation testing of the libraries described here. 


\subsubsection{Introduction}

SCALE includes both multigroup (MG) and pointwise continuous energy (CE) nuclear data libraries, which were processed using the AMPX code system. ${ }^{1}$ Libraries are available for neutron and for coupled neutron-photon transport calculations. The CE libraries are used for Monte Carlo calculations with CE-KENO (criticality) and CE-Monaco (shielding), and are also used by the pointwise (PW) discrete ordinates code CENTRM to obtain PW flux spectra for computing self-shielded MG cross sections. The MG libraries are used in the MG-KENO and MG-Monaco Monte Carlo codes, and in the deterministic transport codes XSDRNPM, NEWT, and DENOVO. All cross section libraries in SCALE 6.2 and later versions are based strictly on ENDF/B-VII. $0^{2}$ and ENDF/B-VII. $1^{3}$, while earlier SCALE releases included libraries processed from ENDF/B-VI.8, V, and IV. The CE and MG libraries include the 417 nuclides shown in Table 10.1.1. ENDF/B-VII does not have evaluated data for several isotopes in the SCALE Standard Composition Data; therefore the libraries do not include cross sections for these nuclides, which are listed in Table 10.1.2. If one of these isotopes is explicitly requested or if the natural element containing the isotope is requested in the SCALE input, a warning message is written saying that the nuclide is being omitted from the calculations. The most commonly encountered example of this is O-18 which has an abundance of approximately $0.2 \%$ in elemental oxygen.

The libraries include 19 thermal-scattering moderators for which bound-scattering kernels [e.g., $S(\alpha, \beta)$ ] are provided in ENDF/B-VII.0 and ENDF/B-VII.1, as given in Table 10.1.3. This table also lists the temperatures available in $\mathrm{MG}$ and $\mathrm{CE}$ libraries for the materials with bound kernels. The thermal scattering kernels for all other materials are based on the free-gas kernel. On the MG libraries, these nuclides have 2-D scattering matrices processed from the free-gas kernel evaluated at the temperatures of $293 \mathrm{~K}, 600 \mathrm{~K}, 900 \mathrm{~K}, 1200 \mathrm{~K}$, and $2400 \mathrm{~K}$. SCALE 6.2 includes routines that automatically interpolate the CE cross section data, as well as thermal scattering kernels to any arbitrary temperature for Monte Carlo calculations. ${ }^{4}$ Analogous procedures are available for MG data. The CE libraries do no not include free gas kernel data because the transport codes internally evaluate the free gas scattering information at the specified temperature.

For each reaction and nuclide, $\mathrm{CE}$ data were processed from $\mathrm{ENDF} / \mathrm{B}$ in the manner described in Sect. 10.1.2.5. The CE data were further processed into several generic MG libraries focused on different applications. CE data are stored as individual files for each nuclide, and an associated cross section directory file contains the names of the individual files. Furthermore, the MG libraries each contain data for all nuclides. AMPX master libraries are very general and contain essentially all reaction data available in ENDF/B, as well as several specialized cross sections used in SCALE. The master libraries also include Bondarenko shielding factors tabulated as a function of background cross section and temperature for all nuclides and groups, and the master libraries may contain intermediate resonance parameters for self-shielding. A much improved methodology in AMPX was used to process more accurate shielding factors for the most important resonance absorbers. ${ }^{1}$

The CE and MG libraries available in SCALE 6.2 are given in Table 10.1.4, which also lists the main source of data for each library. The desired MG or CE library for a computation is typically selected by specifying the appropriate mnemonic name from Table 10.1.4. The CE library mnemonic corresponds to the actual name of the cross section directory file, and the MG mnemonic is an alias for the file containing the actual master library, shown in the third column of Table 10.1.4. SCALE control modules recognize the mnemonics in the first column, or the standard MG library name in the third columns of the table. Users also may supply their own MG library by specifying the file name as the library name in the control module input (only lower case alphanumeric characters are allowed in the filename, and the filename must be eight characters or less in length). The library must be an AMPX master library and must be located in the SCALE data directory or the temporary working directory where the problem is run. 
The CE data libraries do not require additional processing prior to their use in transport computations. However the MG libraries distributed with the SCALE code system contain problem-independent cross sections processed with generic weighting functions appropriate for a specified type of application (e.g., criticality safety, shielding, etc.). These data must be further processed into problem-dependent libraries by performing resonance self-shielding and other modifications. This is done by the XSProc module (see Sect. 7.1) during the execution of a SCALE control sequence prior to executing a transport solver.

Table 10.1.1. Nuclides in CE and MG ENDF/B-VII.0 and -VII.1 libraries

\begin{tabular}{|c|c|c|c|c|c|}
\hline SCALE ID & Name & $\begin{array}{l}\text { Gamma } \\
\text { production } \\
\text { data }^{\mathrm{a}}\end{array}$ & $\begin{array}{l}\text { Full range } \\
\text { Bondarenko } \\
\text { factors }^{b}\end{array}$ & $\begin{array}{l}\text { Gamma } \\
\text { interaction } \\
\text { evaluation }\end{array}$ & Notes \\
\hline 1001 & $\mathrm{~h}$ & yes & yes & $\mathrm{h}$ & \\
\hline 1001001 & h-liquid_ch4 & yes & yes & $\mathrm{h}$ & \\
\hline 2001001 & h-solid_ch4 & yes & yes & $\mathrm{h}$ & \\
\hline 4001001 & h-cryo_ortho & yes & yes & $\mathrm{h}$ & \\
\hline 5001001 & h-cryo_para & yes & yes & $\mathrm{h}$ & \\
\hline 6001001 & h-benzene & yes & yes & $\mathrm{h}$ & \\
\hline 7001001 & h-zrh2 & yes & yes & $\mathrm{h}$ & \\
\hline 8001001 & hfreegas & yes & yes & $\mathrm{h}$ & \\
\hline 9001001 & h-poly & yes & yes & $\mathrm{h}$ & \\
\hline 1002 & $\mathrm{~d}$ & yes & yes & $\mathrm{h}$ & \\
\hline 4001002 & d-cryo_ortho & yes & yes & $\mathrm{h}$ & \\
\hline 5001002 & d-cryo_para & yes & yes & $\mathrm{h}$ & \\
\hline 8001002 & dfreegas & yes & yes & $\mathrm{h}$ & \\
\hline 1003 & h-3 & & yes & $\mathrm{h}$ & \\
\hline 2003 & he-3 & & yes & he & \\
\hline 2004 & he-4 & & yes & he & \\
\hline 3006 & li-6 & yes & yes & li & \\
\hline 3007 & li-7 & yes & yes & li & \\
\hline 4007 & be-7 & & yes & be & \\
\hline 4009 & be- 9 & yes & yes & be & \\
\hline 3004009 & bebound & yes & yes & be & \\
\hline 5004009 & be-beo & yes & yes & be & \\
\hline 5010 & $b-10$ & yes & yes & $\mathrm{b}$ & \\
\hline 5011 & b-11 & yes & yes & $\mathrm{b}$ & \\
\hline 6000 & $\mathrm{c}$ & yes & yes & $\mathrm{c}$ & \\
\hline 3006000 & graphite & yes & yes & $\mathrm{c}$ & \\
\hline 5006000 & h-benzene & yes & yes & $\mathrm{c}$ & \\
\hline 7014 & $n-14$ & yes & yes & $\mathrm{n}$ & \\
\hline 7015 & $n-15$ & yes & yes & $\mathrm{n}$ & \\
\hline 8016 & $0-16$ & yes & yes & o & \\
\hline 5008016 & o-beo & yes & yes & o & \\
\hline
\end{tabular}


Table 10.1.1. Nuclides in CE and MG ENDF/B-VII.0 and -VII.1 libraries

\begin{tabular}{|c|c|c|c|c|c|}
\hline SCALE ID & Name & $\begin{array}{l}\text { Gamma } \\
\text { production } \\
\text { data }^{\mathrm{a}}\end{array}$ & $\begin{array}{l}\text { Full range } \\
\text { Bondarenko } \\
\text { factors }^{b}\end{array}$ & $\begin{array}{l}\text { Gamma } \\
\text { interaction } \\
\text { evaluation }^{c}\end{array}$ & Notes \\
\hline 8017 & o-17 & & yes & o & \\
\hline 9019 & $f-19$ & yes & yes & $\mathrm{f}$ & \\
\hline 11022 & na-22 & & yes & na & \\
\hline 11023 & na-23 & yes & yes & na & \\
\hline 12024 & mg-24 & yes & yes & $\mathrm{mg}$ & \\
\hline 12025 & mg-25 & yes & yes & $\mathrm{mg}$ & \\
\hline 12026 & mg-26 & yes & yes & $\mathrm{mg}$ & \\
\hline 13027 & al-27 & yes & yes & al & \\
\hline 1013027 & albound & yes & yes & al & \\
\hline 14028 & si-28 & yes & yes & si & \\
\hline 14029 & si-29 & yes & yes & si & \\
\hline 14030 & si-30 & yes & yes & si & \\
\hline 1014028 & sibound & yes & yes & si & ENDF/B-VII.1 only \\
\hline 1014029 & sibound & yes & yes & si & ENDF/B-VII.1 only \\
\hline 1014030 & sibound & yes & yes & si & ENDF/B-VII.1 only \\
\hline 15031 & p-31 & yes & yes & $\mathrm{p}$ & \\
\hline 16032 & $\mathrm{~s}-32$ & yes & yes & $\mathrm{s}$ & \\
\hline 16033 & s-33 & yes & yes & $\mathrm{s}$ & \\
\hline 16034 & s-34 & yes & yes & $\mathrm{S}$ & \\
\hline 16036 & $s-36$ & yes & yes & $\mathrm{S}$ & \\
\hline 17035 & cl-35 & yes & yes & $\mathrm{cl}$ & \\
\hline 17037 & cl-37 & yes & yes & $\mathrm{cl}$ & \\
\hline 18036 & ar-36 & & yes & ar & \\
\hline 18038 & ar-38 & & yes & ar & \\
\hline 18040 & ar-40 & & yes & ar & \\
\hline 19039 & k-39 & yes & yes & $\mathrm{k}$ & \\
\hline 19040 & $\mathrm{k}-40$ & yes & yes & $\mathrm{k}$ & \\
\hline 19041 & $\mathrm{k}-41$ & yes & yes & $\mathrm{k}$ & \\
\hline 20040 & ca-40 & yes & yes & $\mathrm{ca}$ & \\
\hline 20042 & ca-42 & yes & yes & $\mathrm{ca}$ & \\
\hline 20043 & ca-43 & yes & yes & $\mathrm{ca}$ & \\
\hline 20044 & ca-44 & yes & yes & $\mathrm{ca}$ & \\
\hline 20046 & ca-46 & yes & yes & $\mathrm{ca}$ & \\
\hline 20048 & $\mathrm{ca}-48$ & yes & yes & $\mathrm{ca}$ & \\
\hline 21045 & sc-45 & yes & yes & $\mathrm{sc}$ & \\
\hline 22046 & ti-46 & yes & yes & $\mathrm{ti}$ & \\
\hline 22047 & ti-47 & yes & yes & $\mathrm{ti}$ & \\
\hline
\end{tabular}


Table 10.1.1. Nuclides in CE and MG ENDF/B-VII.0 and -VII.1 libraries

\begin{tabular}{|c|c|c|c|c|c|}
\hline SCALE ID & Name & $\begin{array}{l}\text { Gamma } \\
\text { production } \\
\text { data }^{a}\end{array}$ & $\begin{array}{l}\text { Full range } \\
\text { Bondarenko } \\
\text { factors }^{b}\end{array}$ & $\begin{array}{l}\text { Gamma } \\
\text { interaction } \\
\text { evaluation }^{c}\end{array}$ & Notes \\
\hline 22048 & ti-48 & yes & yes & $\mathrm{ti}$ & \\
\hline 22049 & ti-49 & yes & yes & $\mathrm{ti}$ & \\
\hline 22050 & ti-50 & yes & yes & $\mathrm{ti}$ & \\
\hline 23000 & $\mathrm{v}$ & yes & & $\mathrm{v}$ & ENDF/B-VII.0 only \\
\hline 23050 & $v-50$ & yes & yes & $\mathrm{v}$ & ENDF/B-VII.1 only \\
\hline 23051 & $v-51$ & yes & yes & $\mathrm{V}$ & ENDF/B-VII.1 only \\
\hline 24050 & cr-50 & yes & yes & $\mathrm{cr}$ & \\
\hline 24052 & cr-52 & yes & yes & $\mathrm{cr}$ & \\
\hline 24053 & cr-53 & yes & yes & $\mathrm{cr}$ & \\
\hline 24054 & cr-54 & yes & yes & $\mathrm{cr}$ & \\
\hline 25055 & $\mathrm{mn}-55$ & yes & yes & $\mathrm{mn}$ & \\
\hline 26054 & fe-54 & yes & yes & $\mathrm{fe}$ & \\
\hline 26056 & fe-56 & yes & yes & $\mathrm{fe}$ & \\
\hline 26057 & fe-57 & yes & yes & $\mathrm{fe}$ & \\
\hline 26058 & fe-58 & yes & yes & $\mathrm{fe}$ & \\
\hline 1026000 & febound & yes & yes & $\mathrm{fe}$ & \\
\hline 27058 & $\operatorname{co}-58$ & ENDF/B-VII.1 & yes & co & \\
\hline 1027058 & co-58m & & yes & co & \\
\hline 27059 & co-59 & yes & yes & co & \\
\hline 28058 & ni-58 & yes & yes & ni & \\
\hline 28059 & ni-59 & yes & yes & ni & \\
\hline 28060 & ni-60 & yes & yes & ni & \\
\hline 28061 & ni-61 & yes & yes & ni & \\
\hline 28062 & ni-62 & yes & yes & ni & \\
\hline 28064 & ni-64 & yes & yes & ni & \\
\hline 29063 & cu-63 & yes & yes & $\mathrm{cu}$ & \\
\hline 29065 & cu-65 & yes & yes & $\mathrm{cu}$ & \\
\hline 30000 & $\mathrm{zn}$ & & & $\mathrm{zn}$ & ENDF/B-VII.0 only \\
\hline 30064 & zn-64 & yes & yes & $\mathrm{zn}$ & ENDF/B-VII.1 only \\
\hline 30065 & $z n-65$ & yes & yes & $\mathrm{zn}$ & ENDF/B-VII.1only \\
\hline 30066 & zn-66 & yes & yes & $\mathrm{zn}$ & ENDF/B-VII.1 only \\
\hline 30067 & $z n-67$ & yes & yes & $\mathrm{zn}$ & ENDF/B-VII.1 only \\
\hline 30068 & zn-68 & yes & yes & $\mathrm{zn}$ & ENDF/B-VII.1 only \\
\hline 30070 & $\mathrm{zn}-70$ & yes & yes & $\mathrm{zn}$ & ENDF/B-VII.1 only \\
\hline 31069 & ga-69 & & yes & ga & \\
\hline 31071 & ga-71 & & yes & $\mathrm{ga}$ & \\
\hline 32070 & ge-70 & yes & yes & ge & \\
\hline
\end{tabular}


Table 10.1.1. Nuclides in CE and MG ENDF/B-VII.0 and -VII.1 libraries

\begin{tabular}{|c|c|c|c|c|c|}
\hline SCALE ID & Name & $\begin{array}{l}\text { Gamma } \\
\text { production } \\
\text { data }^{a}\end{array}$ & $\begin{array}{l}\text { Full range } \\
\text { Bondarenko } \\
\text { factors }\end{array}$ & $\begin{array}{l}\text { Gamma } \\
\text { interaction } \\
\text { evaluation }\end{array}$ & Notes \\
\hline 32072 & ge-72 & yes & yes & ge & \\
\hline 32073 & ge-73 & yes & yes & ge & \\
\hline 32074 & ge-74 & yes & yes & ge & \\
\hline 32076 & ge-76 & yes & yes & ge & \\
\hline 33074 & as-74 & yes & yes & as & \\
\hline 33075 & as-75 & yes & yes & as & \\
\hline 34074 & se-74 & & yes & se & \\
\hline 34076 & se-76 & & yes & se & \\
\hline 34077 & se-77 & & yes & $\mathrm{se}$ & \\
\hline 34078 & se-78 & & yes & se & \\
\hline 34079 & se-79 & & yes & $\mathrm{se}$ & \\
\hline 34080 & se- 80 & & yes & se & \\
\hline 34082 & se- 82 & & yes & se & \\
\hline 35079 & br-79 & & yes & br & \\
\hline 35081 & br- 81 & & yes & br & \\
\hline 36078 & $\mathrm{kr}-78$ & ENDF/B-VII.1 & yes & $\mathrm{kr}$ & \\
\hline 36080 & $\mathrm{kr}-80$ & & yes & $\mathrm{kr}$ & \\
\hline 36082 & $\mathrm{kr}-82$ & & yes & $\mathrm{kr}$ & \\
\hline 36083 & $\mathrm{kr}-83$ & & yes & $\mathrm{kr}$ & \\
\hline 36084 & $\mathrm{kr}-84$ & & yes & $\mathrm{kr}$ & \\
\hline 36085 & $\mathrm{kr}-85$ & yes & yes & $\mathrm{kr}$ & \\
\hline 36086 & $\mathrm{kr}-86$ & & yes & $\mathrm{kr}$ & \\
\hline 37085 & $\mathrm{rb}-85$ & & yes & $\mathrm{rb}$ & \\
\hline 37086 & $\mathrm{rb}-86$ & yes & yes & $\mathrm{rb}$ & \\
\hline 37087 & $\mathrm{rb}-87$ & & yes & $\mathrm{rb}$ & \\
\hline 38084 & sr-84 & yes & yes & $\mathrm{sr}$ & \\
\hline 38086 & sr-86 & & yes & $\mathrm{sr}$ & \\
\hline 38087 & sr-87 & & yes & $\mathrm{sr}$ & \\
\hline 38088 & sr- 88 & & yes & $\mathrm{sr}$ & \\
\hline 38089 & sr-89 & & yes & $\mathrm{sr}$ & \\
\hline 38090 & sr-90 & & yes & $\mathrm{sr}$ & \\
\hline 39089 & $y-89$ & yes & yes & $\mathrm{y}$ & \\
\hline 39090 & $y-90$ & yes & yes & $\mathrm{y}$ & \\
\hline 39091 & $y-91$ & & yes & $\mathrm{y}$ & \\
\hline 40090 & zr-90 & yes & yes & $\mathrm{zr}$ & \\
\hline 1040090 & zr90-zr5h8 & yes & yes & $\mathrm{zr}$ & \\
\hline 40091 & zr-91 & yes & yes & $\mathrm{zr}$ & \\
\hline
\end{tabular}


Table 10.1.1. Nuclides in CE and MG ENDF/B-VII.0 and -VII.1 libraries

\begin{tabular}{|c|c|c|c|c|c|}
\hline SCALE ID & Name & $\begin{array}{l}\text { Gamma } \\
\text { production } \\
\text { data }^{\mathrm{a}}\end{array}$ & $\begin{array}{l}\text { Full range } \\
\text { Bondarenko } \\
\text { factors }^{b}\end{array}$ & $\begin{array}{l}\text { Gamma } \\
\text { interaction } \\
\text { evaluation }^{c}\end{array}$ & Notes \\
\hline 1040091 & zr91-zr5h8 & yes & yes & $\mathrm{zr}$ & \\
\hline 40092 & zr-92 & yes & yes & $\mathrm{zr}$ & \\
\hline 1040092 & zr92-zr5h8 & yes & yes & $\mathrm{zr}$ & \\
\hline 40093 & zr-93 & ENDF/B-VII.1 & yes & $\mathrm{zr}$ & \\
\hline 1040093 & zr93-zr5h8 & ENDF/B-VII.1 & yes & $\mathrm{zr}$ & \\
\hline 40094 & zr-94 & yes & yes & $\mathrm{zr}$ & \\
\hline 1040094 & zr94-zr5h8 & yes & yes & $\mathrm{zr}$ & \\
\hline 40095 & zr-95 & ENDF/B-VII.1 & yes & $\mathrm{zr}$ & \\
\hline 1040095 & zr95-zr5h8 & ENDF/B-VII.1 & yes & $\mathrm{zr}$ & \\
\hline 40096 & zr-96 & yes & yes & $\mathrm{zr}$ & \\
\hline 1040096 & zr96-zr5h8 & yes & yes & $\mathrm{zr}$ & \\
\hline 41093 & nb-93 & yes & yes & $\mathrm{nb}$ & \\
\hline 41094 & nb-94 & & yes & $\mathrm{nb}$ & \\
\hline 41095 & nb-95 & & yes & $\mathrm{nb}$ & \\
\hline 42092 & mo-92 & yes & yes & mo & \\
\hline 42094 & mo-94 & yes & yes & mo & \\
\hline 42095 & mo-95 & yes & yes & mo & \\
\hline 42096 & mo-96 & yes & yes & mo & \\
\hline 42097 & mo-97 & yes & yes & mo & \\
\hline 42098 & mo-98 & yes & yes & mo & \\
\hline 42099 & mo-99 & & yes & mo & \\
\hline 42100 & mo-100 & & yes & mo & \\
\hline 43099 & tc- 99 & yes & yes & tc & \\
\hline 44096 & ru-96 & & yes & $\mathrm{ru}$ & \\
\hline 44098 & ru-98 & & yes & $\mathrm{ru}$ & \\
\hline 44099 & ru-99 & & yes & $\mathrm{ru}$ & \\
\hline 44100 & ru-100 & & yes & $\mathrm{ru}$ & \\
\hline 44101 & ru-101 & yes & yes & $\mathrm{ru}$ & \\
\hline 44102 & ru-102 & & yes & $\mathrm{ru}$ & \\
\hline 44103 & ru-103 & & yes & $\mathrm{ru}$ & \\
\hline 44104 & ru-104 & & yes & $\mathrm{ru}$ & \\
\hline 44105 & ru-105 & & yes & $\mathrm{ru}$ & \\
\hline 44106 & ru-106 & & yes & $\mathrm{ru}$ & \\
\hline 45103 & rh-103 & yes & yes & $\mathrm{rh}$ & \\
\hline 45105 & rh-105 & & yes & rh & \\
\hline 46102 & pd-102 & yes & yes & $\mathrm{pd}$ & \\
\hline 46104 & pd-104 & yes & yes & $\mathrm{pd}$ & \\
\hline
\end{tabular}


Table 10.1.1. Nuclides in CE and MG ENDF/B-VII.0 and -VII.1 libraries

\begin{tabular}{|c|c|c|c|c|c|}
\hline SCALE ID & Name & $\begin{array}{l}\text { Gamma } \\
\text { production } \\
\text { data }^{a}\end{array}$ & $\begin{array}{l}\text { Full range } \\
\text { Bondarenko } \\
\text { factors }^{b}\end{array}$ & $\begin{array}{l}\text { Gamma } \\
\text { interaction } \\
\text { evaluation }\end{array}$ & Notes \\
\hline 46105 & pd-105 & yes & yes & $\mathrm{pd}$ & \\
\hline 46106 & pd-106 & yes & yes & $\mathrm{pd}$ & \\
\hline 46107 & pd-107 & & yes & $\mathrm{pd}$ & \\
\hline 46108 & pd-108 & yes & yes & $\mathrm{pd}$ & \\
\hline 46110 & pd-110 & yes & yes & $\mathrm{pd}$ & \\
\hline 47107 & ag-107 & yes & yes & ag & \\
\hline 47109 & ag-109 & yes & yes & ag & \\
\hline 1047110 & ag- $110 \mathrm{~m}$ & & yes & ag & \\
\hline 47111 & ag-111 & yes & yes & ag & \\
\hline 48106 & cd-106 & yes & yes & $\mathrm{cd}$ & \\
\hline 48108 & cd-108 & & yes & $\mathrm{cd}$ & \\
\hline 48110 & cd-110 & & yes & $\mathrm{cd}$ & \\
\hline 48111 & cd-111 & yes & yes & $\mathrm{cd}$ & \\
\hline 48112 & cd-112 & & yes & $\mathrm{cd}$ & \\
\hline 48113 & cd-113 & & yes & $\mathrm{cd}$ & \\
\hline 48114 & cd-114 & & yes & $\mathrm{cd}$ & \\
\hline 1048115 & $\mathrm{~cd}-115 \mathrm{~m}$ & yes & yes & $\mathrm{cd}$ & \\
\hline 48116 & cd-116 & & yes & $\mathrm{cd}$ & \\
\hline 49113 & in-113 & & yes & in & \\
\hline 49115 & in-115 & & yes & in & \\
\hline 50112 & sn-112 & & yes & sn & \\
\hline 50113 & sn-113 & yes & yes & sn & \\
\hline 50114 & sn-114 & & yes & sn & \\
\hline 50115 & sn-115 & & yes & sn & \\
\hline 50116 & sn-116 & & yes & sn & \\
\hline 50117 & sn-117 & & yes & $\mathrm{sn}$ & \\
\hline 50118 & sn-118 & & yes & sn & \\
\hline 50119 & sn-119 & & yes & sn & \\
\hline 50120 & sn-120 & & yes & sn & \\
\hline 50122 & sn-122 & & yes & sn & \\
\hline 50123 & sn-123 & & yes & sn & \\
\hline 50124 & sn-124 & & yes & sn & \\
\hline 50125 & sn-125 & yes & yes & sn & \\
\hline 50126 & sn-126 & & yes & sn & \\
\hline 51121 & sb-121 & & yes & $\mathrm{sb}$ & \\
\hline 51123 & sb-123 & & yes & $\mathrm{sb}$ & \\
\hline 51124 & sb-124 & & yes & $\mathrm{sb}$ & \\
\hline
\end{tabular}


Table 10.1.1. Nuclides in CE and MG ENDF/B-VII.0 and -VII.1 libraries

\begin{tabular}{|c|c|c|c|c|c|}
\hline SCALE ID & Name & $\begin{array}{l}\text { Gamma } \\
\text { production } \\
\text { data }^{\text {a }}\end{array}$ & $\begin{array}{l}\text { Full range } \\
\text { Bondarenko } \\
\text { factors }^{b}\end{array}$ & $\begin{array}{l}\text { Gamma } \\
\text { interaction } \\
\text { evaluation }\end{array}$ & Notes \\
\hline 51125 & sb-125 & & yes & $\mathrm{sb}$ & \\
\hline 51126 & sb-126 & yes & yes & $\mathrm{sb}$ & \\
\hline 52120 & te -120 & & yes & te & \\
\hline 52122 & te -122 & & yes & te & \\
\hline 52123 & te- 123 & & yes & te & \\
\hline 52124 & te- 124 & & yes & te & \\
\hline 52125 & te -125 & & yes & te & \\
\hline 52126 & te- 126 & & yes & te & \\
\hline 1052127 & te- $127 \mathrm{~m}$ & & yes & te & \\
\hline 52128 & te- 128 & & yes & te & \\
\hline 1052129 & te $-129 \mathrm{~m}$ & & yes & te & \\
\hline 52130 & te- 130 & & yes & te & \\
\hline 52132 & te- 132 & yes & yes & te & \\
\hline 53127 & $\mathrm{i}-127$ & yes & yes & $\mathrm{i}$ & \\
\hline 53129 & i-129 & & yes & $\mathrm{i}$ & \\
\hline 53130 & $\mathrm{i}-130$ & yes & yes & $\mathrm{i}$ & \\
\hline 53131 & i-131 & & yes & $\mathrm{i}$ & \\
\hline 53135 & i-135 & & yes & $\mathrm{i}$ & \\
\hline 54123 & xe-123 & ENDF/B-VII.1 & yes & xe & \\
\hline 54124 & xe-124 & ENDF/B-VII.1 & yes & $\mathrm{xe}$ & \\
\hline 54126 & xe-126 & & yes & $\mathrm{xe}$ & \\
\hline 54128 & xe-128 & & yes & $\mathrm{xe}$ & \\
\hline 54129 & xe-129 & & yes & xe & \\
\hline 54130 & xe-130 & & yes & xe & \\
\hline 54131 & xe-131 & yes & yes & xe & \\
\hline 54132 & xe-132 & & yes & xe & \\
\hline 54133 & xe-133 & & yes & xe & \\
\hline 54134 & xe-134 & & yes & xe & \\
\hline 54135 & xe-135 & & yes & xe & \\
\hline 54136 & xe-136 & & yes & xe & \\
\hline 55133 & cs-133 & yes & yes & $\mathrm{cs}$ & \\
\hline 55134 & cs-134 & & yes & $\mathrm{cs}$ & \\
\hline 55135 & cs-135 & & yes & $\mathrm{cs}$ & \\
\hline 55136 & cs-136 & & yes & $\mathrm{cs}$ & \\
\hline 55137 & cs-137 & & yes & $\mathrm{cs}$ & \\
\hline 56130 & ba-130 & & yes & ba & \\
\hline 56132 & ba-132 & & yes & ba & \\
\hline
\end{tabular}


Table 10.1.1. Nuclides in CE and MG ENDF/B-VII.0 and -VII.1 libraries

\begin{tabular}{|c|c|c|c|c|c|}
\hline SCALE ID & Name & $\begin{array}{l}\text { Gamma } \\
\text { production } \\
\text { data }^{a}\end{array}$ & $\begin{array}{l}\text { Full range } \\
\text { Bondarenko } \\
\text { factors }^{b}\end{array}$ & $\begin{array}{l}\text { Gamma } \\
\text { interaction } \\
\text { evaluation }\end{array}$ & Notes \\
\hline 56133 & ba-133 & yes & yes & ba & \\
\hline 56134 & ba-134 & & yes & ba & \\
\hline 56135 & ba-135 & & yes & ba & \\
\hline 56136 & ba-136 & & yes & ba & \\
\hline 56137 & ba-137 & & yes & ba & \\
\hline 56138 & ba-138 & & yes & ba & \\
\hline 56140 & ba-140 & & yes & ba & \\
\hline 57138 & la-138 & & yes & la & \\
\hline 57139 & la-139 & & yes & la & \\
\hline 57140 & la-140 & yes & yes & la & \\
\hline 58136 & ce-136 & yes & yes & ce & \\
\hline 58138 & ce-138 & yes & yes & ce & \\
\hline 58139 & ce-139 & yes & yes & ce & \\
\hline 58140 & ce-140 & & yes & ce & \\
\hline 58141 & ce-141 & & yes & ce & \\
\hline 58142 & ce-142 & & yes & ce & \\
\hline 58143 & ce-143 & yes & yes & ce & \\
\hline 58144 & ce-144 & & yes & ce & \\
\hline 59141 & pr-141 & yes & yes & $\mathrm{pr}$ & \\
\hline 59142 & pr-142 & yes & yes & $\mathrm{pr}$ & \\
\hline 59143 & pr-143 & & yes & $\mathrm{pr}$ & \\
\hline 60142 & nd-142 & yes & yes & nd & \\
\hline 60143 & nd-143 & yes & yes & nd & \\
\hline 60144 & nd-144 & yes & yes & nd & \\
\hline 60145 & nd-145 & yes & yes & nd & \\
\hline 60146 & nd-146 & yes & yes & nd & \\
\hline 60147 & nd-147 & yes & yes & nd & \\
\hline 60148 & nd-148 & yes & yes & nd & \\
\hline 60150 & nd-150 & yes & yes & nd & \\
\hline 61147 & pm-147 & & yes & $\mathrm{pm}$ & \\
\hline 61148 & pm-148 & & yes & $\mathrm{pm}$ & \\
\hline 1061148 & pm-148m & & yes & $\mathrm{pm}$ & \\
\hline 61149 & pm-149 & & yes & $\mathrm{pm}$ & \\
\hline 61151 & pm-151 & yes & yes & $\mathrm{pm}$ & \\
\hline 62144 & sm-144 & yes & yes & $\mathrm{sm}$ & \\
\hline 62147 & sm-147 & yes & yes & $\mathrm{sm}$ & \\
\hline 62148 & sm-148 & yes & yes & $\mathrm{sm}$ & \\
\hline
\end{tabular}


Table 10.1.1. Nuclides in CE and MG ENDF/B-VII.0 and -VII.1 libraries

\begin{tabular}{|c|c|c|c|c|c|}
\hline SCALE ID & Name & $\begin{array}{l}\text { Gamma } \\
\text { production } \\
\text { data }^{a}\end{array}$ & $\begin{array}{l}\text { Full range } \\
\text { Bondarenko } \\
\text { factors }^{b}\end{array}$ & $\begin{array}{l}\text { Gamma } \\
\text { interaction } \\
\text { evaluation }\end{array}$ & Notes \\
\hline 62149 & sm-149 & yes & yes & $\mathrm{sm}$ & \\
\hline 62150 & sm-150 & yes & yes & $\mathrm{sm}$ & \\
\hline 62151 & sm-151 & yes & yes & $\mathrm{sm}$ & \\
\hline 62152 & sm-152 & yes & yes & $\mathrm{sm}$ & \\
\hline 62153 & sm-153 & yes & yes & $\mathrm{sm}$ & \\
\hline 62154 & $\mathrm{sm}-154$ & yes & yes & $\mathrm{sm}$ & \\
\hline 63151 & eu-151 & & yes & $\mathrm{eu}$ & \\
\hline 63152 & eu-152 & & yes & $\mathrm{eu}$ & \\
\hline 63153 & eu-153 & yes & yes & eu & \\
\hline 63154 & eu-154 & & yes & eu & \\
\hline 63155 & eu-155 & & yes & $\mathrm{eu}$ & \\
\hline 63156 & eu-156 & & yes & $\mathrm{eu}$ & \\
\hline 63157 & eu-157 & yes & yes & $\mathrm{eu}$ & \\
\hline 64152 & gd-152 & yes & yes & gd & \\
\hline 64153 & gd-153 & yes & yes & $\mathrm{gd}$ & \\
\hline 64154 & gd-154 & yes & yes & $\mathrm{gd}$ & \\
\hline 64155 & gd-155 & yes & yes & $\mathrm{gd}$ & \\
\hline 64156 & gd-156 & yes & yes & gd & \\
\hline 64157 & gd-157 & yes & yes & $\mathrm{gd}$ & \\
\hline 64158 & gd-158 & yes & yes & $\mathrm{gd}$ & \\
\hline 64160 & gd-160 & yes & yes & $\mathrm{gd}$ & \\
\hline 65159 & tb-159 & & yes & $\mathrm{tb}$ & \\
\hline 65160 & tb-160 & yes & yes & $\mathrm{tb}$ & \\
\hline 66156 & dy-156 & yes & yes & dy & \\
\hline 66158 & dy-158 & yes & yes & dy & \\
\hline 66160 & dy-160 & yes & yes & dy & \\
\hline 66161 & dy-161 & yes & yes & dy & \\
\hline 66162 & dy-162 & yes & yes & dy & \\
\hline 66163 & dy-163 & yes & yes & dy & \\
\hline 66164 & dy-164 & yes & yes & dy & \\
\hline 67165 & ho-165 & yes & yes & ho & \\
\hline 1067166 & ho-166m & yes & yes & ho & \\
\hline 68162 & er-162 & yes & yes & er & \\
\hline 68164 & er-164 & yes & yes & er & \\
\hline 68166 & er-166 & yes & yes & er & \\
\hline 68167 & er-167 & yes & yes & er & \\
\hline 68168 & er-168 & yes & yes & er & \\
\hline
\end{tabular}


Table 10.1.1. Nuclides in CE and MG ENDF/B-VII.0 and -VII.1 libraries

\begin{tabular}{|c|c|c|c|c|c|}
\hline SCALE ID & Name & $\begin{array}{l}\text { Gamma } \\
\text { production } \\
\text { data }^{a}\end{array}$ & $\begin{array}{l}\text { Full range } \\
\text { Bondarenko } \\
\text { factors }^{b}\end{array}$ & $\begin{array}{l}\text { Gamma } \\
\text { interaction } \\
\text { evaluation }^{c}\end{array}$ & Notes \\
\hline 68170 & er-170 & yes & yes & er & \\
\hline 69168 & $\mathrm{tm}-168$ & yes & yes & $\mathrm{tm}$ & ENDF/B-VII.1 only \\
\hline 69169 & tm-169 & yes & yes & $\mathrm{tm}$ & ENDF/B-VII.1 only \\
\hline 69170 & $\mathrm{tm}-170$ & yes & yes & $\mathrm{tm}$ & ENDF/B-VII.1 only \\
\hline 71175 & lu-175 & & yes & lu & \\
\hline 71176 & lu-176 & & yes & lu & \\
\hline 72174 & hf-174 & ENDF/B-VII.1 & yes & $\mathrm{hf}$ & \\
\hline 72176 & hf-176 & ENDF/B-VII.1 & yes & $\mathrm{hf}$ & \\
\hline 72177 & hf-177 & ENDF/B-VII.1 & yes & hf & \\
\hline 72178 & hf-178 & ENDF/B-VII.1 & yes & $\mathrm{hf}$ & \\
\hline 72179 & hf-179 & ENDF/B-VII.1 & yes & hf & \\
\hline 72180 & hf-180 & ENDF/B-VII.1 & yes & hf & \\
\hline 73180 & ta- 180 & yes & yes & ta & ENDF/B-VII.1 only \\
\hline 73181 & ta-181 & yes & yes & ta & \\
\hline 73182 & ta-182 & & yes & ta & \\
\hline 74180 & w-180 & yes & yes & $\mathrm{w}$ & ENDF/B-VII.1 only \\
\hline 74182 & w-182 & yes & yes & $\mathrm{W}$ & \\
\hline 74183 & $w-183$ & yes & yes & $\mathrm{w}$ & \\
\hline 74184 & w-184 & yes & yes & $\mathrm{W}$ & \\
\hline 74186 & w-186 & yes & yes & $\mathrm{w}$ & \\
\hline 75185 & re-185 & ENDF/B-VII.1 & yes & re & \\
\hline 75187 & re-187 & ENDF/B-VII.1 & yes & re & \\
\hline 77191 & ir-191 & yes & yes & ir & \\
\hline 77193 & ir-193 & yes & yes & ir & \\
\hline 79197 & au-197 & yes & yes & $\mathrm{au}$ & \\
\hline 80196 & hg-196 & yes & yes & hg & \\
\hline 80198 & hg-198 & yes & yes & hg & \\
\hline 80199 & hg-199 & yes & yes & hg & \\
\hline 80200 & hg-200 & yes & yes & hg & \\
\hline 80201 & hg-201 & yes & yes & hg & \\
\hline 80202 & hg-202 & yes & yes & hg & \\
\hline 80204 & hg-204 & yes & yes & hg & \\
\hline 81203 & $\mathrm{tl}-203$ & yes & yes & $\mathrm{tl}$ & ENDF/B-VII.1 only \\
\hline 81205 & $\mathrm{tl}-205$ & yes & yes & $\mathrm{tl}$ & ENDF/B-VII.1 only \\
\hline 82204 & pb-204 & yes & yes & $\mathrm{pb}$ & \\
\hline 82206 & pb-206 & yes & yes & $\mathrm{pb}$ & \\
\hline 82207 & pb-207 & yes & yes & $\mathrm{pb}$ & \\
\hline
\end{tabular}


Table 10.1.1. Nuclides in CE and MG ENDF/B-VII.0 and -VII.1 libraries

\begin{tabular}{|c|c|c|c|c|c|}
\hline SCALE ID & Name & $\begin{array}{l}\text { Gamma } \\
\text { production }_{\text {data }^{\mathrm{a}}} \\
\end{array}$ & $\begin{array}{l}\text { Full range } \\
\text { Bondarenko } \\
\text { factors }^{b}\end{array}$ & $\begin{array}{l}\text { Gamma } \\
\text { interaction } \\
\text { evaluation }\end{array}$ & Notes \\
\hline 82208 & pb-208 & yes & yes & $\mathrm{pb}$ & \\
\hline 83209 & bi-209 & yes & yes & bi & \\
\hline 88223 & ra-223 & & yes & $\mathrm{ra}$ & \\
\hline 88224 & ra-224 & & yes & $\mathrm{ra}$ & \\
\hline 88225 & ra-225 & & yes & $\mathrm{ra}$ & \\
\hline 88226 & ra-226 & & yes & $\mathrm{ra}$ & \\
\hline 89225 & ac-225 & ENDF/B-VII.1 & yes & $\mathrm{ac}$ & \\
\hline 89226 & ac-226 & ENDF/B-VII.1 & yes & ac & \\
\hline 89227 & ac-227 & ENDF/B-VII.1 & yes & ac & \\
\hline 90227 & th-227 & ENDF/B-VII.1 & yes & th & \\
\hline 90228 & th-228 & ENDF/B-VII.1 & yes & th & \\
\hline 90229 & th-229 & ENDF/B-VII.1 & yes & th & \\
\hline 90230 & th-230 & ENDF/B-VII.1 & yes & th & \\
\hline 90231 & th-231 & yes & yes & th & ENDF/B-VII.1 only \\
\hline 90232 & th-232 & yes & yes & th & \\
\hline 90233 & th-233 & ENDF/B-VII.1 & yes & th & \\
\hline 90234 & th-234 & ENDF/B-VII.1 & yes & th & \\
\hline 91229 & pa-229 & yes & yes & pa & ENDF/B-VII.1 only \\
\hline 91230 & pa-230 & yes & yes & pa & ENDF/B-VII.1 only \\
\hline 91231 & pa-231 & yes & yes & pa & \\
\hline 91232 & pa-232 & ENDF/B-VII.1 & yes & pa & \\
\hline 91233 & pa-233 & yes & yes & pa & \\
\hline 92230 & $\mathrm{u}-230$ & yes & yes & $\mathrm{u}$ & ENDF/B-VII.1 only \\
\hline 92231 & $\mathrm{u}-231$ & yes & yes & $\mathrm{u}$ & ENDF/B-VII.1 only \\
\hline 92232 & $\mathrm{u}-232$ & yes & yes & $\mathrm{u}$ & \\
\hline 92233 & $\mathrm{u}-233$ & yes & yes & $\mathrm{u}$ & \\
\hline 92234 & $\mathrm{u}-234$ & yes & yes & $\mathrm{u}$ & ENDF/B-VII.1 only \\
\hline 92235 & $\mathrm{u}-235$ & yes & yes & $\mathrm{u}$ & \\
\hline 92236 & $\mathrm{u}-236$ & yes & yes & $\mathrm{u}$ & \\
\hline 92237 & $\mathrm{u}-237$ & yes & yes & $\mathrm{u}$ & \\
\hline 92238 & $\mathrm{u}-238$ & yes & yes & $\mathrm{u}$ & \\
\hline 92239 & $\mathrm{u}-239$ & yes & yes & $\mathrm{u}$ & \\
\hline 92240 & $\mathrm{u}-240$ & yes & yes & $\mathrm{u}$ & \\
\hline 92241 & $\mathrm{u}-241$ & yes & yes & $\mathrm{u}$ & \\
\hline 93234 & np-234 & yes & yes & np & \\
\hline 93235 & np-235 & ENDF/B-VII.1 & yes & $\mathrm{np}$ & \\
\hline 93236 & np-236 & ENDF/B-VII.1 & yes & $\mathrm{np}$ & \\
\hline
\end{tabular}


Table 10.1.1. Nuclides in CE and MG ENDF/B-VII.0 and -VII.1 libraries

\begin{tabular}{|c|c|c|c|c|c|}
\hline SCALE ID & Name & $\begin{array}{l}\text { Gamma } \\
\text { production } \\
\text { data }^{a}\end{array}$ & $\begin{array}{l}\text { Full range } \\
\text { Bondarenko } \\
\text { factors }^{b}\end{array}$ & $\begin{array}{l}\text { Gamma } \\
\text { interaction } \\
\text { evaluationc }^{c}\end{array}$ & Notes \\
\hline 93237 & np-237 & yes & yes & $\mathrm{np}$ & \\
\hline 93238 & np-238 & ENDF/B-VII.1 & yes & np & \\
\hline 93239 & np-239 & ENDF/B-VII.1 & yes & np & \\
\hline 94236 & pu-236 & ENDF/B-VII.1 & yes & $\mathrm{pu}$ & \\
\hline 94237 & pu-237 & ENDF/B-VII.1 & yes & $\mathrm{pu}$ & \\
\hline 94238 & pu-238 & & yes & $\mathrm{pu}$ & \\
\hline 94239 & pu-239 & yes & yes & $\mathrm{pu}$ & \\
\hline 94240 & pu-240 & yes & yes & $\mathrm{pu}$ & \\
\hline 94241 & pu-241 & yes & yes & $\mathrm{pu}$ & \\
\hline 94242 & pu-242 & yes & yes & $\mathrm{pu}$ & \\
\hline 94243 & pu-243 & yes & yes & $\mathrm{pu}$ & \\
\hline 94244 & pu-244 & ENDF/B-VII.1 & yes & $\mathrm{pu}$ & \\
\hline 94246 & pu-246 & ENDF/B-VII.1 & yes & $\mathrm{pu}$ & \\
\hline 95240 & am-240 & yes & yes & am & ENDF/B-VII.1 only \\
\hline 95241 & am-241 & yes & yes & am & \\
\hline 95242 & am-242 & & yes & am & \\
\hline 1095242 & $\mathrm{am}-242 \mathrm{~m}$ & & yes & am & \\
\hline 95243 & am-243 & yes & yes & am & \\
\hline 95244 & am-244 & & yes & am & \\
\hline 1095244 & $\mathrm{am}-244 \mathrm{~m}$ & & yes & am & \\
\hline 96240 & $\mathrm{~cm}-240$ & yes & yes & $\mathrm{cm}$ & ENDF/B-VII.1 only \\
\hline 96241 & $\mathrm{~cm}-241$ & ENDF/B-VII.1 & yes & $\mathrm{cm}$ & \\
\hline 96242 & $\mathrm{~cm}-242$ & yes & yes & $\mathrm{cm}$ & \\
\hline 96243 & $\mathrm{~cm}-243$ & ENDF/B-VII.1 & yes & $\mathrm{cm}$ & \\
\hline 96244 & $\mathrm{~cm}-244$ & ENDF/B-VII.1 & yes & $\mathrm{cm}$ & \\
\hline 96245 & $\mathrm{~cm}-245$ & ENDF/B-VII.1 & yes & $\mathrm{cm}$ & \\
\hline 96246 & $\mathrm{~cm}-246$ & ENDF/B-VII.1 & yes & $\mathrm{cm}$ & \\
\hline 96247 & $\mathrm{~cm}-247$ & ENDF/B-VII.1 & yes & $\mathrm{cm}$ & \\
\hline 96248 & $\mathrm{~cm}-248$ & yes & yes & $\mathrm{cm}$ & \\
\hline 96249 & $\mathrm{~cm}-249$ & ENDF/B-VII.1 & yes & $\mathrm{cm}$ & \\
\hline 96250 & $\mathrm{~cm}-250$ & ENDF/B-VII.1 & yes & $\mathrm{cm}$ & \\
\hline 97245 & bk-245 & yes & yes & bk & ENDF/B-VII.1 only \\
\hline 97246 & bk-246 & yes & yes & bk & ENDF/B-VII.1 only \\
\hline 97247 & bk-247 & yes & yes & bk & ENDF/B-VII.1 only \\
\hline 97248 & bk-248 & yes & yes & bk & ENDF/B-VII.1 only \\
\hline 97249 & bk-249 & ENDF/B-VII.1 & yes & bk & \\
\hline 97250 & bk-250 & ENDF/B-VII.1 & yes & bk & \\
\hline
\end{tabular}


Table 10.1.1. Nuclides in CE and MG ENDF/B-VII.0 and -VII.1 libraries

\begin{tabular}{|c|c|c|c|c|c|}
\hline SCALE ID & Name & $\begin{array}{l}\text { Gamma } \\
\text { production } \\
\text { data }^{a}\end{array}$ & $\begin{array}{l}\text { Full range } \\
\text { Bondarenko } \\
\text { factors }^{b}\end{array}$ & $\begin{array}{l}\text { Gamma } \\
\text { interaction } \\
\text { evaluation }^{c}\end{array}$ & Notes \\
\hline 98246 & cf-246 & yes & yes & $\mathrm{cf}$ & ENDF/B-VII.1 only \\
\hline 98248 & cf-248 & yes & yes & $\mathrm{cf}$ & ENDF/B-VII.1 only \\
\hline 98249 & cf-249 & ENDF/B-VII.1 & yes & $\mathrm{cf}$ & \\
\hline 98250 & cf- -250 & yes & yes & $\mathrm{cf}$ & \\
\hline 98251 & cf-251 & yes & yes & cf & \\
\hline 98252 & cf- 252 & yes & yes & $\mathrm{cf}$ & \\
\hline 98253 & cf-253 & ENDF/B-VII.1 & yes & $\mathrm{cf}$ & \\
\hline 98254 & cf-254 & ENDF/B-VII.1 & yes & $\mathrm{cf}$ & \\
\hline 99251 & es-251 & yes & yes & es & ENDF/B-VII.1 only \\
\hline 99252 & es-252 & yes & yes & es & ENDF/B-VII.1 only \\
\hline 99253 & es-253 & ENDF/B-VII.1 & yes & es & \\
\hline 99254 & es-254 & ENDF/B-VII.1 & yes & es & \\
\hline 1099254 & es- $254 \mathrm{~m}$ & yes & yes & es & ENDF/B-VII.1 only \\
\hline 99255 & es-255 & ENDF/B-VII.1 & yes & es & \\
\hline 100255 & $\mathrm{fm}-255$ & yes & yes & $\mathrm{fm}$ & \\
\hline
\end{tabular}

Table 10.1.2. Isotopes with no ENDF/B-VII.0 or -VII.1 nuclear data

\begin{tabular}{|c|c|c|c|c|}
\hline Element & $\begin{array}{c}\text { SCALE standard } \\
\text { composition ID }\end{array}$ & $\begin{array}{c}\text { Missing } \\
\text { Isotopes }\end{array}$ & ZA numbers & $\%$ Abundance \\
\hline oxygen & 8000 & 18 & 8018 & 0.20 \\
\hline neon & 10000 & 21,22 & 10021,10022 & $0.27,9.25$ \\
\hline ytterbium & 70000 & $\mathrm{All}^{(1)}$ & $(1)$ & \\
\hline osmium & 76000 & $\mathrm{All}^{(2)}$ & $(2)$ & \\
\hline platinum & 78000 & $\mathrm{All}^{(3)}$ & $(3)$ & \\
\hline tantalum & 73000 & $180 \mathrm{~m}$ & 1073180 & 0.01 \\
\hline
\end{tabular}

(1) no data for any of the 7 naturally-occurring ytterbium isotopes

(2) no data for any of the 7 naturally-occurring osmium isotopes

(3) no data for any of the 6 naturally-occurring platinum isotopes 
Table 10.1.3. Temperatures at which thermal moderator data are available ${ }^{a}$

\begin{tabular}{|c|c|c|}
\hline ID & Name & Temperatures \\
\hline 1001 & $\mathrm{~h}-1$ & 293.6350 .0400 .0450 .0500 .0550 .0600 .0650 .0800 .0 \\
\hline 1001001 & h-liquid_ch4 & 100.0 \\
\hline 2001001 & h-solid_ch4 & 22.0 \\
\hline 4001001 & h-cryo_ortho & 20.0 \\
\hline 5001001 & h-cryo_para & 20.0 \\
\hline 6001001 & h-benzene & 296.0350 .0400 .0450 .0500 .0600 .0800 .01000 .0 \\
\hline 7001001 & h-zrh2 & 296.0400 .0500 .0600 .0700 .0800 .01000 .01200 .0 \\
\hline 9001001 & h-poly & 296.0350 .0 \\
\hline 1002 & h-2 & 293.6350 .0400 .0450 .0500 .0550 .0600 .0650 .0 \\
\hline 4001002 & d-cryo_ortho & 19.0 \\
\hline 5001002 & d-cryo_para & 19.0 \\
\hline 3004009 & bebound & 296.0400 .0500 .0600 .0700 .0800 .00011000 .01200 .0 \\
\hline 5004009 & be-beo & 293.6400 .0500 .0600 .0700 .0800 .01000 .01200 .0 \\
\hline 3006000 & c-graphite & 296.0400 .0500 .0600 .0700 .0800 .01000 .01200 .01600 .02000 .0 \\
\hline 5006000 & h-benzene & 296.0350 .0400 .0450 .0500 .0600 .0800 .01000 .0 \\
\hline 5008016 & o-beo & 293.6400 .0500 .0600 .0700 .0800 .01000 .01200 .0 \\
\hline 1013027 & albound & 20.080 .0293 .6400 .0600 .0800 .0 \\
\hline $1014028^{\mathrm{a}}$ & sibound & 293.6350 .0400 .0500 .0800 .01000 .01200 .0 \\
\hline $1014029^{\mathrm{a}}$ & sibound & 293.6350 .0400 .0500 .0800 .01000 .01200 .0 \\
\hline $1014030^{\mathrm{a}}$ & sibound & 293.6350 .0400 .0500 .0800 .01000 .01200 .0 \\
\hline 1026000 & febound & 20.080 .0293 .6400 .0600 .0800 .0 \\
\hline 1040090 & zr90-zr5h8 & 296.0400 .0500 .0600 .0700 .0800 .01000 .01200 .0 \\
\hline 1040091 & $\mathrm{zr} 90-\mathrm{zr} 5 \mathrm{~h} 8$ & 296.0400 .0500 .0600 .0700 .0800 .01000 .01200 .0 \\
\hline 1040092 & zr90-zr5h8 & 296.0400 .0500 .0600 .0700 .0800 .01000 .01200 .0 \\
\hline 1040093 & zr90-zr5h8 & 296.0400 .0500 .0600 .0700 .0800 .01000 .01200 .0 \\
\hline 1040094 & zr90-zr5h8 & 296.0400 .0500 .0600 .0700 .0800 .01000 .01200 .0 \\
\hline 1040095 & zr90-zr5h8 & 296.0400 .0500 .0600 .0700 .0800 .01000 .01200 .0 \\
\hline 1040096 & zr90-zr5h8 & 296.0400 .0500 .0600 .0700 .0800 .01000 .01200 .0 \\
\hline
\end{tabular}

a) only available in ENDF/B-VII.1 
Table 10.1.4. Standard SCALE cross section libraries

\begin{tabular}{|c|c|c|}
\hline $\begin{array}{l}\text { Mnemonic } \\
\text { names }\end{array}$ & Primary data source/format & $\begin{array}{l}\text { Last field of } \\
\text { cross section library } \\
\text { filename }\end{array}$ \\
\hline $\begin{array}{l}\text { v7-238; v7-238n; } \\
\text { v7.0-238n }\end{array}$ & ENDF/B-VII.0 238-group neutron library & $\mathrm{xn} 238 \mathrm{v} 7.0^{\mathrm{a}}$ \\
\hline $\begin{array}{l}\text { v7-252; v7-252n; } \\
\text { v7.1-252n }\end{array}$ & ENDF/B-VII.1 252-group neutron library & $\mathrm{xn} 252 \mathrm{v} 7.1^{\mathrm{a}}$ \\
\hline v7-56; v7-56n; v7.1-56n & ENDF/B-VII.1 56-group neutron library & $\mathrm{xn} 56 \mathrm{v} 7.1^{\mathrm{a}}$ \\
\hline test-8grp & $\begin{array}{l}\text { TEST LIBRARY 8-group ENDF/B-VII.1 } \\
\text { neutron library }^{\text {e }}\end{array}$ & test8g_v7.1 \\
\hline v7.1-200n $47 \mathrm{~g}$ & ENDF/B-VII.1 200 neutron/47 gamma library & $\mathrm{xn} 200 \mathrm{~g} 47 \mathrm{v} 7.1^{a}$ \\
\hline $\begin{array}{l}\text { v7-200n47g; } \\
\text { v7.0-200n47g; v7-200g47 }\end{array}$ & ENDF/B-VII.0 200 neutron/47 gamma library & $\mathrm{xn} 200 \mathrm{~g} 47 \mathrm{v} 7.0^{a}$ \\
\hline v7.1-28n19g & ENDF/B-VII.1 28 neutron/19 gamma library & $\mathrm{xn} 28 \mathrm{~g} 19 \mathrm{v} 7.1^{a}$ \\
\hline $\mathrm{v} 7-27 \mathrm{n} 19 \mathrm{~g} ; \mathrm{v} 7.0-27 \mathrm{n} 19 \mathrm{~g}$ & ENDF/B-VII.0 27 neutron/19 gamma library & $\mathrm{xn} 27 \mathrm{~g} 19 \mathrm{v} 7.0^{a}$ \\
\hline ce_v7.1_endf ${ }^{b}$ & $\begin{array}{l}\text { ENDF/B-VII.1 Continuous-energy neutron } \\
\text { and gamma library }\end{array}$ & -- \\
\hline $\begin{array}{l}\text { ce_v7 ; ce_v7_endf; } \\
\text { ce_v7.0_endf }{ }^{b}\end{array}$ & $\begin{array}{l}\text { ENDF/B-VII.0 Continuous-energy neutron } \\
\text { and gamma library }\end{array}$ & -- \\
\hline ce_v7.1_endf.xml ${ }^{\mathrm{d}}$ & $\begin{array}{l}\text { ENDF/B-VII.1 Continuous-energy neutron } \\
\text { and gamma library }\end{array}$ & -- \\
\hline $\begin{array}{l}\text { ce_v7.xml ; } \\
\text { ce_v7_endf.xml } \\
\text { ce_v7.0_endf.xml }\end{array}$ & $\begin{array}{l}\text { ENDF/B-VII.0 Continuous-energy neutron } \\
\text { and gamma library }\end{array}$ & -- \\
\hline File name ${ }^{c}$ & User-supplied library & file name \\
\hline
\end{tabular}

\footnotetext{
${ }^{a}$ Format of the library names are "scale.revxx.lastfield" where " $\mathrm{xx}$ " is the revision number.

${ }^{b}$ ASCII text file that contains location of continuous energy data files.

${ }^{c}$ For continuous energy mode calculations in KENO, the library name must start with "CE_".

${ }^{d}$ Contains the same information as ce_v7.x_endf in xml format for use in the CE_MONAC̄O sequence.

${ }^{e}$ Transitional library that will not be included with SCALE 6.2 release. Mnemonic names will alias to ENDF/B VII.1 libraries in production release.
}

Additional convenience mnemonics are also available to always alias to the most recent nuclear data libraries for the intended purpose. The mnemonics shown in Table 10.1.2 will allow the use of the same input files with this and future versions of SCALE, but will always access the most recent nuclear data libraries and group structures. 
Table 10.1.5. SCALE convenience mnemonics

\begin{tabular}{cc}
\hline $\begin{array}{c}\text { Mnemonic } \\
\text { name }\end{array}$ & Aliased library \\
\hline broad_n & xn56v7.1 \\
\hline broad_ng & xn28g19v7.1 \\
\hline fine_n & xn252v7.1 \\
\hline fine_ng & xn200g47v7.1 \\
\hline ce & ce_v7.1_endf \\
\hline ce.xml & ce_v7.1_endf.xml \\
\hline test_n & test8g_v7.1 \\
\hline
\end{tabular}

\subsubsection{Description of the SCALE Cross Section Libraries}

\subsubsection{The 238-group and 252-group ENDF/B-VII libraries (V7-238, v7-252)}

SCALE includes two fine group structures for criticality safety and reactor physics applications: a 238-group library based on ENDF/B-VII.0 is available mainly for general-purpose criticality analyses, and a 252-group library based on ENDF/B-VII.1 is available for either criticality safety or reactor physics. Table 10.1.8 and Table 10.1.9, respectively, show the group structures for these fine-group libraries. The 238-group library is unchanged from earlier releases prior to SCALE 6.2, except for changes in the IDs of the nuclides. The 252-group structure was developed to adequately capture spectral and temperature effects important for reactor systems and was processed with newer, improved procedures.

The SCALE control sequences for criticality safety and reactor physics applications normally perform self-shielding of the fine-group libraries using the BONAMI module for the unresolved resonance range; and the CENTRM/PMC modules for the resolved resonance/thermal range. However both the 238- and 252-group libraries include Bondarenko self-shielding factors for the entire energy range, which provides the option of using the Bondarenko method to self-shield both the resolved and unresolved resonance ranges, as an alternative to the more rigorous (and computationally intensive) CENTRM/PMC approach. As discussed in the following section, one objective of the ENDF/B-VII.1 252-group and 56-group libraries was to provide a more accurate Bondarenko treatment for the resolved resonance range.

\section{Differences in the 238-group and 252-group libraries}

The standard weighting function described in Table 10.1.6 was used to process MG data for all materials in the 238-group criticality safety library, and Bondarenko shielding factors for this library were computed using the narrow resonance (NR) approximation for the flux spectrum: $\Phi_{\mathrm{NR}}(\mathrm{E})=\sigma_{0} /\left(\sigma_{\mathrm{t}}+\sigma_{0}\right) * \mathrm{C}(\mathrm{E})$ where $\sigma_{0}$ is the background cross section, and $\mathrm{C}(\mathrm{E})$ is the standard weight function. Bondarenko factors are tabulated at temperatures of at $293 \mathrm{~K}, 900 \mathrm{~K}$, and $1200 \mathrm{~K}$ in the 238-group library. 
Table 10.1.6. Standard weighting function for processing MG data

\begin{tabular}{|c|c|}
\hline Energy Range & Standard Weight Function \\
\hline $10^{-5} \mathrm{eV}-0.1 \mathrm{eV}$ & Maxwellian, with peak at $0.025 \mathrm{eV}$ \\
\hline $0.1 \mathrm{eV}-80 \mathrm{keV}$ & $1 / \mathrm{E}$ \\
\hline $80 \mathrm{keV}-10 \mathrm{MeV}$ & Watt Fission spectrum at temperature of $1.273 \mathrm{MeV}$ \\
\hline $10 \mathrm{MeV}-20 \mathrm{MeV}$ & $1 / \mathrm{E}$ \\
\hline
\end{tabular}

Several enhancements were made in the MG processing procedures used to produce the 252-group library so that it would be more applicable to reactor physics as well as criticality safety applications. Some of the improvements in the 252-group library compared to the 238-group library are given below

(a) The base weighting function for processing MG data of actinide materials $(Z>89)$ was computed by the PW transport code CENTRM for a PWR lattice at $300 \mathrm{~K}$. This approach provides more representative weighted 2-D scattering matrices for most cases of interest. The standard weighting function is still used for materials with $\mathrm{Z}<90$.

(b) The thermal energy range which includes up-scattering reactions was extended to $5 \mathrm{eV}$, compared to $3 \mathrm{eV}$ in the 238-group library

(c) Temperature-dependent thermal-scattering matrices for water-bound $\mathrm{H}, \mathrm{O}-16$, and actinide materials were processed with temperature-dependent thermal flux spectra obtained from CENTRM calculations for a PWR pincell. Actinide and O-16 MG thermal scattering kernels were weighted with the fuel zone flux at temperatures of $293 \mathrm{~K}, 600 \mathrm{~K}, 900 \mathrm{~K}, 1200 \mathrm{~K}$, and $2400 \mathrm{~K}$, and the water scatter kernels were weighted with the moderator flux at $293 \mathrm{~K}, 500 \mathrm{~K}, 600 \mathrm{~K}, 650 \mathrm{~K}, 900 \mathrm{~K}$, and $1200 \mathrm{~K}$. In the 238 -group library, thermal scattering matrices at all temperatures were weighted with a temperature-independent Maxwellian spectrum.

(d) Group-dependent IR parameters ("lambdas") were calculated for all materials and are included in the 252-group libraries. This allows the Bondarenko self-shielding method in SCALE to use the IR approximation for the 252-group libraries, while the 238-group library is still limited to the NR approximation.

(e) A number of improvements were made in processing of Bondarenko self-shielding data.

- The number of temperatures for the Bondarenko factors was increased. Shielding factors are tabulated at temperatures $292 \mathrm{~K}, 600 \mathrm{~K}, 900 \mathrm{~K}, 1200 \mathrm{~K}$, and $2400 \mathrm{~K}$ for the 252-group libraries.

- In addition to the Bondarenko factors normally included for capture, fission, elastic, and total cross sections, self-shielding factors are also included for the multigroup elastic within-group cross section to address the impact of resonance reactions on the scattering distribution.

- In the unresolved resonance range, self-shielding factors were calculated using probability tables.

- Bondarenko factors for nuclides with atomic masses $Z>39$ were calculated with CENTRM PW flux spectra rather than the analytical NR approximation. Two types of CENTRM models were used. Heterogeneous models of water-moderated lattices spanning the range of expected selfshielding were used to calculate shielding factors for ${ }^{235} \mathrm{U},{ }^{238} \mathrm{U},{ }^{239} \mathrm{Pu},{ }^{240} \mathrm{Pu},{ }^{241} \mathrm{Pu},{ }^{90} \mathrm{Zr}$, and ${ }^{96} \mathrm{Zr}$. The CENTRM transport calculations were performed using the method of characteristics method for 2-D unit cell models. Homogeneous models were used to compute shielding factors for the remaining nuclides with $\mathrm{Z}>39$. These CENTRM calculations were performed for homogeneous media containing the absorber material mixed with hydrogen, and the hydrogen concentration was varied to obtain the desired set of background cross sections. Table 10.1.7 summarizes the processing options for the 252-group library. 
Table 10.1.7. Standard weighting functions for processing 252-group library data library

\begin{tabular}{|c|c|c|}
\hline Nuclide & Base Weight Function & Bondarenko Factor \\
\hline $\mathrm{U}-238,-235 ; \mathrm{Pu}-239,-240,-241$ & PWR spectrum & CENTRM heterogeneous calculations \\
\hline Other actinides $\mathrm{Z}>90$ & PWR spectrum & CENTRM homogeneous calculations \\
\hline $39<\mathrm{Z}<90$ & standard & CENTRM homogeneous calculations \\
\hline $\mathrm{Z}<40$ & standard & NR analytical spectrum \\
\hline
\end{tabular}

Table 10.1.8. 238 Multigroup energy structure

\begin{tabular}{|c|c|c|c|c|c|c|c|}
\hline Group & $\begin{array}{c}\text { Upper } \\
\text { energy } \\
(\mathrm{eV})\end{array}$ & Group & $\begin{array}{c}\text { Upper } \\
\text { energy } \\
(\mathrm{eV})\end{array}$ & Group & $\begin{array}{c}\text { Upper } \\
\text { energy } \\
(\mathrm{eV})\end{array}$ & Group & $\begin{array}{c}\text { Upper } \\
\text { energy } \\
(\mathrm{eV})\end{array}$ \\
\hline 1 & $2.0000 \mathrm{E}+07$ & 41 & $2.7000 \mathrm{E}+05$ & 81 & $1.8600 \mathrm{E}+02$ & 121 & $2.0000 \mathrm{E}+01$ \\
\hline 2 & $1.7333 \mathrm{E}+07$ & 42 & $2.0000 \mathrm{E}+05$ & 82 & $1.2200 \mathrm{E}+02$ & 122 & $1.9000 \mathrm{E}+01$ \\
\hline 3 & $1.5683 \mathrm{E}+07$ & 43 & $1.5000 \mathrm{E}+05$ & 83 & $1.1900 \mathrm{E}+02$ & 123 & $1.8500 \mathrm{E}+01$ \\
\hline 4 & $1.4550 \mathrm{E}+07$ & 44 & $1.2830 \mathrm{E}+05$ & 84 & $1.1500 \mathrm{E}+02$ & 124 & $1.7000 \mathrm{E}+01$ \\
\hline 5 & $1.3840 \mathrm{E}+07$ & 45 & $1.0000 \mathrm{E}+05$ & 85 & $1.0800 \mathrm{E}+02$ & 125 & $1.6000 \mathrm{E}+01$ \\
\hline 6 & $1.2840 \mathrm{E}+07$ & 46 & $8.5000 \mathrm{E}+04$ & 86 & $1.0000 \mathrm{E}+02$ & 126 & $1.5100 \mathrm{E}+01$ \\
\hline 7 & $1.0000 \mathrm{E}+07$ & 47 & $8.2000 \mathrm{E}+04$ & 87 & $9.0000 \mathrm{E}+01$ & 127 & $1.4400 \mathrm{E}+01$ \\
\hline 8 & $8.1873 E+06$ & 48 & $7.5000 \mathrm{E}+04$ & 88 & $8.2000 \mathrm{E}+01$ & 128 & $1.3750 \mathrm{E}+01$ \\
\hline 9 & $6.4340 \mathrm{E}+06$ & 49 & $7.3000 \mathrm{E}+04$ & 89 & $8.0000 \mathrm{E}+01$ & 129 & $1.2900 \mathrm{E}+01$ \\
\hline 10 & $4.8000 \mathrm{E}+06$ & 50 & $6.0000 \mathrm{E}+04$ & 90 & $7.6000 \mathrm{E}+01$ & 130 & $1.1900 \mathrm{E}+01$ \\
\hline 11 & $4.3040 \mathrm{E}+06$ & 51 & $5.2000 \mathrm{E}+04$ & 91 & $7.2000 \mathrm{E}+01$ & 131 & $1.1500 \mathrm{E}+01$ \\
\hline 12 & $3.0000 \mathrm{E}+06$ & 52 & $5.0000 \mathrm{E}+04$ & 92 & $6.7500 \mathrm{E}+01$ & 132 & $1.0000 \mathrm{E}+01$ \\
\hline 13 & $2.4790 \mathrm{E}+06$ & 53 & $4.5000 \mathrm{E}+04$ & 93 & $6.5000 \mathrm{E}+01$ & 133 & $9.1000 \mathrm{E}+00$ \\
\hline 14 & $2.3540 \mathrm{E}+06$ & 54 & $3.0000 \mathrm{E}+04$ & 94 & $6.1000 \mathrm{E}+01$ & 134 & $8.1000 \mathrm{E}+00$ \\
\hline 15 & $1.8500 \mathrm{E}+06$ & 55 & $2.5000 \mathrm{E}+04$ & 95 & $5.9000 \mathrm{E}+01$ & 135 & $7.1500 \mathrm{E}+00$ \\
\hline 16 & $1.5000 \mathrm{E}+06$ & 56 & $1.7000 \mathrm{E}+04$ & 96 & $5.3400 \mathrm{E}+01$ & 136 & $7.0000 \mathrm{E}+00$ \\
\hline 17 & $1.4000 \mathrm{E}+06$ & 57 & $1.3000 \mathrm{E}+04$ & 97 & $5.2000 \mathrm{E}+01$ & 137 & $6.7500 \mathrm{E}+00$ \\
\hline 18 & $1.3560 \mathrm{E}+06$ & 58 & $9.5000 \mathrm{E}+03$ & 98 & $5.0600 \mathrm{E}+01$ & 138 & $6.5000 \mathrm{E}+00$ \\
\hline 19 & $1.3170 \mathrm{E}+06$ & 59 & $8.0300 \mathrm{E}+03$ & 99 & $4.9200 \mathrm{E}+01$ & 139 & $6.2500 \mathrm{E}+00$ \\
\hline 20 & $1.2500 \mathrm{E}+06$ & 60 & $6.0000 \mathrm{E}+03$ & 100 & $4.8300 \mathrm{E}+01$ & 140 & $6.0000 \mathrm{E}+00$ \\
\hline 21 & $1.2000 \mathrm{E}+06$ & 61 & $3.9000 \mathrm{E}+03$ & 101 & $4.7000 \mathrm{E}+01$ & 141 & $5.4000 \mathrm{E}+00$ \\
\hline 22 & $1.1000 \mathrm{E}+06$ & 62 & $3.7400 \mathrm{E}+03$ & 102 & $4.5200 \mathrm{E}+01$ & 142 & $5.0000 \mathrm{E}+00$ \\
\hline 23 & $1.0100 \mathrm{E}+06$ & 63 & $3.0000 \mathrm{E}+03$ & 103 & $4.4000 \mathrm{E}+01$ & 143 & $4.7500 \mathrm{E}+00$ \\
\hline 24 & $9.2000 \mathrm{E}+05$ & 64 & $2.5800 \mathrm{E}+03$ & 104 & $4.2400 \mathrm{E}+01$ & 144 & $4.0000 \mathrm{E}+00$ \\
\hline 25 & $9.0000 \mathrm{E}+05$ & 65 & $2.2900 \mathrm{E}+03$ & 105 & $4.1000 \mathrm{E}+01$ & 145 & $3.7300 \mathrm{E}+00$ \\
\hline 26 & $8.7500 \mathrm{E}+05$ & 66 & $2.2000 \mathrm{E}+03$ & 106 & $3.9600 \mathrm{E}+01$ & 146 & $3.5000 \mathrm{E}+00$ \\
\hline 27 & $8.6110 \mathrm{E}+05$ & 67 & $1.8000 \mathrm{E}+03$ & 107 & $3.9100 \mathrm{E}+01$ & 147 & $3.1500 \mathrm{E}+00$ \\
\hline 28 & $8.2000 \mathrm{E}+05$ & 68 & $1.5500 \mathrm{E}+03$ & 108 & $3.8000 \mathrm{E}+01$ & 148 & $3.0500 \mathrm{E}+00$ \\
\hline 29 & $7.5000 \mathrm{E}+05$ & 69 & $1.5000 \mathrm{E}+03$ & 109 & $3.7000 \mathrm{E}+01$ & 149 & $3.0000 \mathrm{E}+00^{a}$ \\
\hline 30 & $6.7900 \mathrm{E}+05$ & 70 & $1.1500 \mathrm{E}+03$ & 110 & $3.5500 \mathrm{E}+01$ & 150 & $2.9700 \mathrm{E}+00$ \\
\hline 31 & $6.7000 \mathrm{E}+05$ & 71 & $9.5000 \mathrm{E}+02$ & 111 & $3.4600 \mathrm{E}+01$ & 151 & $2.8700 \mathrm{E}+00$ \\
\hline 32 & $6.0000 \mathrm{E}+05$ & 72 & $6.8300 \mathrm{E}+02$ & 112 & $3.3750 \mathrm{E}+01$ & 152 & $2.7700 \mathrm{E}+00$ \\
\hline 33 & $5.7300 \mathrm{E}+05$ & 73 & $6.7000 \mathrm{E}+02$ & 113 & $3.3250 \mathrm{E}+01$ & 153 & $2.6700 \mathrm{E}+00$ \\
\hline 34 & $5.5000 \mathrm{E}+05$ & 74 & $5.5000 \mathrm{E}+02$ & 114 & $3.1750 \mathrm{E}+01$ & 154 & $2.5700 \mathrm{E}+00$ \\
\hline 35 & $4.9952 \mathrm{E}+05$ & 75 & $3.0500 \mathrm{E}+02$ & 115 & $3.1250 \mathrm{E}+01$ & 155 & $2.4700 \mathrm{E}+00$ \\
\hline 36 & $4.7000 \mathrm{E}+05$ & 76 & $2.8500 \mathrm{E}+02$ & 116 & $3.0000 \mathrm{E}+01$ & 156 & $2.3800 \mathrm{E}+00$ \\
\hline 37 & $4.4000 \mathrm{E}+05$ & 77 & $2.4000 \mathrm{E}+02$ & 117 & $2.7500 \mathrm{E}+01$ & 157 & $2.3000 \mathrm{E}+00$ \\
\hline 38 & $4.2000 \mathrm{E}+05$ & 78 & $2.1000 \mathrm{E}+02$ & 118 & $2.5000 \mathrm{E}+01$ & 158 & $2.2100 \mathrm{E}+00$ \\
\hline 39 & $4.0000 \mathrm{E}+05$ & 79 & $2.0750 \mathrm{E}+02$ & 119 & $2.2500 \mathrm{E}+01$ & 159 & $2.1200 \mathrm{E}+00$ \\
\hline 40 & $3.3000 \mathrm{E}+05$ & 80 & $1.9250 \mathrm{E}+02$ & 120 & $2.1000 \mathrm{E}+01$ & 160 & $2.0000 \mathrm{E}+00$ \\
\hline
\end{tabular}


Table 10.1.8. 238 Multigroup energy structure (continued)

\begin{tabular}{||cc||cc||cc||cc||}
\hline Group & $\begin{array}{c}\text { Upper } \\
\text { energy } \\
\text { (eV) }\end{array}$ & Group & $\begin{array}{c}\text { Upper } \\
\text { energy } \\
\text { (eV) }\end{array}$ & Group & $\begin{array}{c}\text { Upper } \\
\text { energy } \\
\text { (eV) }\end{array}$ & Group & $\begin{array}{c}\text { Upper } \\
\text { energy } \\
\text { (eV) }\end{array}$ \\
\hline 161 & $1.9400 \mathrm{E}+00$ & 181 & $1.0900 \mathrm{E}+00$ & 201 & $6.0000 \mathrm{E}-01$ & 221 & $6.0000 \mathrm{E}-02$ \\
162 & $1.8600 \mathrm{E}+00$ & 182 & $1.0800 \mathrm{E}+00$ & 202 & $5.5000 \mathrm{E}-01$ & 22 & $5.0000 \mathrm{E}-02$ \\
163 & $1.7700 \mathrm{E}+00$ & 183 & $1.0700 \mathrm{E}+00$ & 203 & $5.0000 \mathrm{E}-01$ & 223 & $4.0000 \mathrm{E}-02$ \\
164 & $1.6800 \mathrm{E}+00$ & 184 & $1.0600 \mathrm{E}+00$ & 204 & $4.5000 \mathrm{E}-01$ & 224 & $3.0000 \mathrm{E}-02$ \\
165 & $1.5900 \mathrm{E}+00$ & 185 & $1.0500 \mathrm{E}+00$ & 205 & $4.0000 \mathrm{E}-01$ & 225 & $2.5300 \mathrm{E}-02$ \\
166 & $1.5000 \mathrm{E}+00$ & 186 & $1.0400 \mathrm{E}+00$ & 206 & $3.7500 \mathrm{E}-01$ & 226 & $1.0000 \mathrm{E}-02$ \\
167 & $1.4500 \mathrm{E}+00$ & 187 & $1.0300 \mathrm{E}+00$ & 207 & $3.5000 \mathrm{E}-01$ & 227 & $7.5000 \mathrm{E}-03$ \\
168 & $1.4000 \mathrm{E}+00$ & 188 & $1.0200 \mathrm{E}+00$ & 208 & $3.2500 \mathrm{E}-01$ & 228 & $5.0000 \mathrm{E}-03$ \\
169 & $1.3500 \mathrm{E}+00$ & 189 & $1.0100 \mathrm{E}+00$ & 209 & $3.0000 \mathrm{E}-01$ & 229 & $4.0000 \mathrm{E}-03$ \\
170 & $1.3000 \mathrm{E}+00$ & 190 & $1.0000 \mathrm{E}+00$ & 210 & $2.7500 \mathrm{E}-01$ & 230 & $3.0000 \mathrm{E}-03$ \\
171 & $1.2500 \mathrm{E}+00$ & 191 & $9.7500 \mathrm{E}-01$ & 211 & $2.5000 \mathrm{E}-01$ & 231 & $2.5000 \mathrm{E}-03$ \\
172 & $1.2250 \mathrm{E}+00$ & 192 & $9.5000 \mathrm{E}-01$ & 212 & $2.2500 \mathrm{E}-01$ & 232 & $2.0000 \mathrm{E}-03$ \\
173 & $1.2000 \mathrm{E}+00$ & 193 & $9.2500 \mathrm{E}-01$ & 213 & $2.0000 \mathrm{E}-01$ & 233 & $1.5000 \mathrm{E}-03$ \\
174 & $1.1750 \mathrm{E}+00$ & 194 & $9.0000 \mathrm{E}-01$ & 214 & $1.7500 \mathrm{E}-01$ & 234 & $1.2000 \mathrm{E}-03$ \\
175 & $1.1500 \mathrm{E}+00$ & 195 & $8.5000 \mathrm{E}-01$ & 215 & $1.5000 \mathrm{E}-01$ & 235 & $1.0000 \mathrm{E}-03$ \\
176 & $1.1400 \mathrm{E}+00$ & 196 & $8.0000 \mathrm{E}-01$ & 216 & $1.2500 \mathrm{E}-01$ & 236 & $7.5000 \mathrm{E}-04$ \\
177 & $1.1300 \mathrm{E}+00$ & 197 & $7.5000 \mathrm{E}-01$ & 217 & $1.0000 \mathrm{E}-01$ & 237 & $5.0000 \mathrm{E}-04$ \\
178 & $1.1200 \mathrm{E}+00$ & 198 & $7.0000 \mathrm{E}-01$ & 218 & $9.0000 \mathrm{E}-02$ & 238 & $1.0000 \mathrm{E}-04$ \\
179 & $1.1100 \mathrm{E}+00$ & 199 & $6.5000 \mathrm{E}-01$ & 219 & $8.0000 \mathrm{E}-02$ & & $1.0000 \mathrm{E}-05^{b}$ \\
180 & $1.1000 \mathrm{E}+00$ & 200 & $6.2500 \mathrm{E}-01$ & 220 & $7.0000 \mathrm{E}-02$ & & \\
\hline
\end{tabular}

${ }^{a}$ Upper energy boundary for thermal range.

${ }^{b}$ Lower energy boundary. 
Table 10.1.9. 252 Multigroup energy structure

\begin{tabular}{|c|c|c|c|c|c|}
\hline Group & Upper Energy (eV) & Group & Upper Energy (eV) & Group & Upper Energy (eV) \\
\hline 1 & $2.000 \mathrm{E}+07$ & 43 & $1.490 \mathrm{E}+05$ & 85 & $1.877 \mathrm{E}+02$ \\
\hline 2 & $1.733 \mathrm{E}+07$ & 44 & $1.283 \mathrm{E}+05$ & 86 & $1.800 \mathrm{E}+02$ \\
\hline 3 & $1.568 \mathrm{E}+07$ & 45 & $1.000 \mathrm{E}+05$ & 87 & $1.700 \mathrm{E}+02$ \\
\hline 4 & $1.455 \mathrm{E}+07$ & 46 & $8.500 \mathrm{E}+04$ & 88 & $1.430 \mathrm{E}+02$ \\
\hline 5 & $1.384 \mathrm{E}+07$ & 47 & $8.200 \mathrm{E}+04$ & 89 & $1.220 \mathrm{E}+02$ \\
\hline 6 & $1.284 \mathrm{E}+07$ & 48 & $7.500 \mathrm{E}+04$ & 90 & $1.190 \mathrm{E}+02$ \\
\hline 7 & $1.000 \mathrm{E}+07$ & 49 & $7.300 \mathrm{E}+04$ & 91 & $1.175 \mathrm{E}+02$ \\
\hline 8 & $8.187 \mathrm{E}+06$ & 50 & $6.000 \mathrm{E}+04$ & 92 & $1.160 \mathrm{E}+02$ \\
\hline 9 & $6.434 \mathrm{E}+06$ & 51 & $5.200 \mathrm{E}+04$ & 93 & $1.130 \mathrm{E}+02$ \\
\hline 10 & $4.800 \mathrm{E}+06$ & 52 & $5.000 \mathrm{E}+04$ & 94 & $1.080 \mathrm{E}+02$ \\
\hline 11 & $4.304 \mathrm{E}+06$ & 53 & $4.500 \mathrm{E}+04$ & 95 & $1.050 \mathrm{E}+02$ \\
\hline 12 & $3.000 \mathrm{E}+06$ & 54 & $3.000 \mathrm{E}+04$ & 96 & $1.012 \mathrm{E}+02$ \\
\hline 13 & $2.479 \mathrm{E}+06$ & 55 & $2.000 \mathrm{E}+04$ & 97 & $9.700 \mathrm{E}+01$ \\
\hline 14 & $2.354 \mathrm{E}+06$ & 56 & $1.700 \mathrm{E}+04$ & 98 & $9.000 \mathrm{E}+01$ \\
\hline 15 & $1.850 \mathrm{E}+06$ & 57 & $1.300 \mathrm{E}+04$ & 99 & $8.170 \mathrm{E}+01$ \\
\hline 16 & $1.500 \mathrm{E}+06$ & 58 & $9.500 \mathrm{E}+03$ & 100 & $8.000 \mathrm{E}+01$ \\
\hline 17 & $1.400 \mathrm{E}+06$ & 59 & $8.030 \mathrm{E}+03$ & 101 & $7.600 \mathrm{E}+01$ \\
\hline 18 & $1.356 \mathrm{E}+06$ & 60 & $5.700 \mathrm{E}+03$ & 102 & $7.200 \mathrm{E}+01$ \\
\hline 19 & $1.317 \mathrm{E}+06$ & 61 & $3.900 \mathrm{E}+03$ & 103 & $6.750 \mathrm{E}+01$ \\
\hline 20 & $1.250 \mathrm{E}+06$ & 62 & $3.740 \mathrm{E}+03$ & 104 & $6.500 \mathrm{E}+01$ \\
\hline 21 & $1.200 \mathrm{E}+06$ & 63 & $3.000 \mathrm{E}+03$ & 105 & $6.300 \mathrm{E}+01$ \\
\hline 22 & $1.100 \mathrm{E}+06$ & 64 & $2.500 \mathrm{E}+03$ & 106 & $6.100 \mathrm{E}+01$ \\
\hline 23 & $1.010 \mathrm{E}+06$ & 65 & $2.250 \mathrm{E}+03$ & 107 & $5.800 \mathrm{E}+01$ \\
\hline 24 & $9.200 \mathrm{E}+05$ & 66 & $2.200 \mathrm{E}+03$ & 108 & $5.340 \mathrm{E}+01$ \\
\hline 25 & $9.000 \mathrm{E}+05$ & 67 & $1.800 \mathrm{E}+03$ & 109 & $5.060 \mathrm{E}+01$ \\
\hline 26 & $8.750 \mathrm{E}+05$ & 68 & $1.550 \mathrm{E}+03$ & 110 & $4.830 \mathrm{E}+01$ \\
\hline 27 & $8.611 \mathrm{E}+05$ & 69 & $1.500 \mathrm{E}+03$ & 111 & $4.520 \mathrm{E}+01$ \\
\hline 28 & $8.200 \mathrm{E}+05$ & 70 & $1.150 \mathrm{E}+03$ & 112 & $4.400 \mathrm{E}+01$ \\
\hline 29 & $7.500 \mathrm{E}+05$ & 71 & $9.500 \mathrm{E}+02$ & 113 & $4.240 \mathrm{E}+01$ \\
\hline 30 & $6.790 \mathrm{E}+05$ & 72 & $6.830 \mathrm{E}+02$ & 114 & $4.100 \mathrm{E}+01$ \\
\hline 31 & $6.700 \mathrm{E}+05$ & 73 & $6.700 \mathrm{E}+02$ & 115 & $3.960 \mathrm{E}+01$ \\
\hline 32 & $6.000 \mathrm{E}+05$ & 74 & $5.500 \mathrm{E}+02$ & 116 & $3.910 \mathrm{E}+01$ \\
\hline 33 & $5.730 \mathrm{E}+05$ & 75 & $3.050 \mathrm{E}+02$ & 117 & $3.800 \mathrm{E}+01$ \\
\hline 34 & $5.500 \mathrm{E}+05$ & 76 & $2.850 \mathrm{E}+02$ & 118 & $3.763 \mathrm{E}+01$ \\
\hline 35 & $4.920 \mathrm{E}+05$ & 77 & $2.400 \mathrm{E}+02$ & 119 & $3.727 \mathrm{E}+01$ \\
\hline 36 & $4.700 \mathrm{E}+05$ & 78 & $2.200 \mathrm{E}+02$ & 120 & $3.713 \mathrm{E}+01$ \\
\hline 37 & $4.400 \mathrm{E}+05$ & 79 & $2.095 \mathrm{E}+02$ & 121 & $3.700 \mathrm{E}+01$ \\
\hline 38 & $4.200 \mathrm{E}+05$ & 80 & $2.074 \mathrm{E}+02$ & 122 & $3.600 \mathrm{E}+01$ \\
\hline 39 & $4.000 \mathrm{E}+05$ & 81 & $2.020 \mathrm{E}+02$ & 123 & $3.550 \mathrm{E}+01$ \\
\hline 40 & $3.300 \mathrm{E}+05$ & 82 & $1.930 \mathrm{E}+02$ & 124 & $3.500 \mathrm{E}+01$ \\
\hline 41 & $2.700 \mathrm{E}+05$ & 83 & $1.915 \mathrm{E}+02$ & 125 & $3.375 \mathrm{E}+01$ \\
\hline 42 & $2.000 \mathrm{E}+05$ & 84 & $1.885 \mathrm{E}+02$ & 126 & $3.325 \mathrm{E}+01$ \\
\hline
\end{tabular}


Table 10.1.9. 252 Multigroup energy structure (continued)

\begin{tabular}{|c|c|c|c|c|c|}
\hline Group & Upper Energy (eV) & Group & Upper Energy (eV) & Group & Upper Energy (eV) \\
\hline 127 & $3.175 \mathrm{E}+01$ & 169 & $2.470 \mathrm{E}+00$ & 211 & $7.500 \mathrm{E}-01$ \\
\hline 128 & $3.125 \mathrm{E}+01$ & 170 & $2.380 \mathrm{E}+00$ & 212 & $7.000 \mathrm{E}-01$ \\
\hline 129 & $3.000 \mathrm{E}+01$ & 171 & $2.300 \mathrm{E}+00$ & 213 & $6.500 \mathrm{E}-01$ \\
\hline 130 & $2.750 \mathrm{E}+01$ & 172 & $2.210 \mathrm{E}+00$ & 214 & $6.250 \mathrm{E}-01$ \\
\hline 131 & $2.500 \mathrm{E}+01$ & 173 & $2.120 \mathrm{E}+00$ & 215 & $6.000 \mathrm{E}-01$ \\
\hline 132 & $2.250 \mathrm{E}+01$ & 174 & $2.000 \mathrm{E}+00$ & 216 & $5.500 \mathrm{E}-01$ \\
\hline 133 & $2.175 \mathrm{E}+01$ & 175 & $1.940 \mathrm{E}+00$ & 217 & $5.000 \mathrm{E}-01$ \\
\hline 134 & $2.120 \mathrm{E}+01$ & 176 & $1.860 \mathrm{E}+00$ & 218 & $4.500 \mathrm{E}-01$ \\
\hline 135 & $2.050 \mathrm{E}+01$ & 177 & $1.770 \mathrm{E}+00$ & 219 & $4.000 \mathrm{E}-01$ \\
\hline 136 & $2.000 \mathrm{E}+01$ & 178 & $1.680 \mathrm{E}+00$ & 220 & $3.750 \mathrm{E}-01$ \\
\hline 137 & $1.940 \mathrm{E}+01$ & 179 & $1.590 \mathrm{E}+00$ & 221 & $3.500 \mathrm{E}-01$ \\
\hline 138 & $1.850 \mathrm{E}+01$ & 180 & $1.500 \mathrm{E}+00$ & 222 & $3.250 \mathrm{E}-01$ \\
\hline 139 & $1.700 \mathrm{E}+01$ & 181 & $1.450 \mathrm{E}+00$ & 223 & $3.000 \mathrm{E}-01$ \\
\hline 140 & $1.600 \mathrm{E}+01$ & 182 & $1.400 \mathrm{E}+00$ & 224 & $2.750 \mathrm{E}-01$ \\
\hline 141 & $1.440 \mathrm{E}+01$ & 183 & $1.350 \mathrm{E}+00$ & 225 & $2.500 \mathrm{E}-01$ \\
\hline 142 & $1.290 \mathrm{E}+01$ & 184 & $1.300 \mathrm{E}+00$ & 226 & $2.250 \mathrm{E}-01$ \\
\hline 143 & $1.190 \mathrm{E}+01$ & 185 & $1.250 \mathrm{E}+00$ & 227 & $2.000 \mathrm{E}-01$ \\
\hline 144 & $1.150 \mathrm{E}+01$ & 186 & $1.225 \mathrm{E}+00$ & 228 & $1.750 \mathrm{E}-01$ \\
\hline 145 & $1.000 \mathrm{E}+01$ & 187 & $1.200 \mathrm{E}+00$ & 229 & $1.500 \mathrm{E}-01$ \\
\hline 146 & $9.100 \mathrm{E}+00$ & 188 & $1.175 \mathrm{E}+00$ & 230 & $1.250 \mathrm{E}-01$ \\
\hline 147 & $8.100 \mathrm{E}+00$ & 189 & $1.150 \mathrm{E}+00$ & 231 & $1.000 \mathrm{E}-01$ \\
\hline 148 & $7.150 \mathrm{E}+00$ & 190 & $1.140 \mathrm{E}+00$ & 232 & $9.000 \mathrm{E}-02$ \\
\hline 149 & $7.000 \mathrm{E}+00$ & 191 & $1.130 \mathrm{E}+00$ & 233 & $8.000 \mathrm{E}-02$ \\
\hline 150 & $6.875 \mathrm{E}+00$ & 192 & $1.120 \mathrm{E}+00$ & 234 & $7.000 \mathrm{E}-02$ \\
\hline 151 & $6.750 \mathrm{E}+00$ & 193 & $1.110 \mathrm{E}+00$ & 235 & $6.000 \mathrm{E}-02$ \\
\hline 152 & $6.500 \mathrm{E}+00$ & 194 & $1.100 \mathrm{E}+00$ & 236 & $5.000 \mathrm{E}-02$ \\
\hline 153 & $6.250 \mathrm{E}+00$ & 195 & $1.090 \mathrm{E}+00$ & 237 & $4.000 \mathrm{E}-02$ \\
\hline 154 & $6.000 \mathrm{E}+00$ & 196 & $1.080 \mathrm{E}+00$ & 238 & $3.000 \mathrm{E}-02$ \\
\hline 155 & $5.400 \mathrm{E}+00$ & 197 & $1.070 \mathrm{E}+00$ & 239 & $2.530 \mathrm{E}-02$ \\
\hline 156 & $5.000 \mathrm{E}+00^{\mathrm{a}}$ & 198 & $1.060 \mathrm{E}+00$ & 240 & $1.000 \mathrm{E}-02$ \\
\hline 157 & $4.700 \mathrm{E}+00$ & 199 & $1.050 \mathrm{E}+00$ & 241 & $7.500 \mathrm{E}-03$ \\
\hline 158 & $4.100 \mathrm{E}+00$ & 200 & $1.040 \mathrm{E}+00$ & 242 & $5.000 \mathrm{E}-03$ \\
\hline 159 & $3.730 \mathrm{E}+00$ & 201 & $1.030 \mathrm{E}+00$ & 243 & $4.000 \mathrm{E}-03$ \\
\hline 160 & $3.500 \mathrm{E}+00$ & 202 & $1.020 \mathrm{E}+00$ & 244 & $3.000 \mathrm{E}-03$ \\
\hline 161 & $3.200 \mathrm{E}+00$ & 203 & $1.010 \mathrm{E}+00$ & 245 & $2.500 \mathrm{E}-03$ \\
\hline 162 & $3.100 \mathrm{E}+00$ & 204 & $1.000 \mathrm{E}+00$ & 246 & $2.000 \mathrm{E}-03$ \\
\hline 163 & $3.000 \mathrm{E}+00$ & 205 & $9.750 \mathrm{E}-01$ & 247 & $1.500 \mathrm{E}-03$ \\
\hline 164 & $2.970 \mathrm{E}+00$ & 206 & $9.500 \mathrm{E}-01$ & 248 & $1.200 \mathrm{E}-03$ \\
\hline 165 & $2.870 \mathrm{E}+00$ & 207 & $9.250 \mathrm{E}-01$ & 249 & $1.000 \mathrm{E}-03$ \\
\hline 166 & $2.770 \mathrm{E}+00$ & 208 & $9.000 \mathrm{E}-01$ & 250 & $7.500 \mathrm{E}-04$ \\
\hline 167 & $2.670 \mathrm{E}+00$ & 209 & $8.500 \mathrm{E}-01$ & 251 & $5.000 \mathrm{E}-04$ \\
\hline 168 & $2.570 \mathrm{E}+00$ & 210 & $8.000 \mathrm{E}-01$ & 252 & $\begin{array}{c}1.000 \mathrm{E}-04 \\
1.000 \mathrm{E}-05^{\mathrm{b}}\end{array}$ \\
\hline
\end{tabular}

${ }^{a}$ Upper energy boundary for thermal range.

${ }^{b}$ Lower energy boundary. 


\subsubsection{The 56-group library}

An ENDF/B-VII.1 broad group library with 56 energy groups is available mainly for light water reactor physics calculations. The group structure is shown in Table 10.1.10. This library was processed using the same PW flux spectra used to generate the 252-group libraries (i.e., for a PWR fuel lattice). This library includes the same materials and properties as the 252-group library, and the data were computed in the same manner, except for several specially weighted nuclides which have heterogeneous Bondarenko shielding factors (in addition to those given in Table 11.1.4) computed for specific LWR components. These are summarized in Table 10.1.11.

Table 10.1.10. 56-Group energy structure

\begin{tabular}{|c|c|c|c|c|c|}
\hline Group & $\begin{array}{c}\text { Upper energy } \\
(\mathrm{eV})\end{array}$ & Group & $\begin{array}{c}\text { Upper energy } \\
(\mathrm{eV})\end{array}$ & Group & $\begin{array}{c}\text { Upper energy } \\
(\mathrm{eV})\end{array}$ \\
\hline 1 & $2.00000 \mathrm{E}+07$ & 24 & $1.05000 \mathrm{E}+02$ & 47 & $2.00000 \mathrm{E}-01$ \\
\hline 2 & $6.43400 \mathrm{E}+06$ & 25 & $1.01200 \mathrm{E}+02$ & 48 & $1.50000 \mathrm{E}-01$ \\
\hline 3 & $4.30400 \mathrm{E}+06$ & 26 & $6.75000 \mathrm{E}+01$ & 49 & $1.00000 \mathrm{E}-01$ \\
\hline 4 & $3.00000 \mathrm{E}+06$ & 27 & $6.50000 \mathrm{E}+01$ & 50 & $8.00000 \mathrm{E}-02$ \\
\hline 5 & $1.85000 \mathrm{E}+06$ & 28 & $3.71300 \mathrm{E}+01$ & 51 & $6.00000 \mathrm{E}-02$ \\
\hline 6 & $1.50000 \mathrm{E}+06$ & 29 & $3.60000 \mathrm{E}+01$ & 52 & $5.00000 \mathrm{E}-02$ \\
\hline 7 & $1.20000 \mathrm{E}+06$ & 30 & $2.17500 \mathrm{E}+01$ & 53 & $4.00000 \mathrm{E}-02$ \\
\hline 8 & $8.61100 \mathrm{E}+05$ & 31 & $2.12000 \mathrm{E}+01$ & 54 & $2.53000 \mathrm{E}-02$ \\
\hline 9 & $7.50000 \mathrm{E}+05$ & 32 & $2.05000 \mathrm{E}+01$ & 55 & $1.00000 \mathrm{E}-02$ \\
\hline 10 & $6.00000 \mathrm{E}+05$ & 33 & $7.00000 \mathrm{E}+00$ & 56 & $4.00000 \mathrm{E}-03$ \\
\hline 11 & $4.70000 \mathrm{E}+05$ & 34 & $6.87500 \mathrm{E}+00$ & 57 & $1.00000 \mathrm{E}-05^{b}$ \\
\hline 12 & $3.30000 \mathrm{E}+05$ & 35 & $6.50000 \mathrm{E}+00$ & & \\
\hline 13 & $2.70000 \mathrm{E}+05$ & 36 & $6.25000 \mathrm{E}+00$ & & \\
\hline 14 & $2.00000 \mathrm{E}+05$ & 37 & $5.00000 \mathrm{E}+00^{a}$ & & \\
\hline 15 & $5.00000 \mathrm{E}+04$ & 38 & $1.13000 \mathrm{E}+00$ & & \\
\hline 16 & $2.00000 \mathrm{E}+04$ & 39 & $1.08000 \mathrm{E}+00$ & & \\
\hline 17 & $1.70000 \mathrm{E}+04$ & 40 & $1.01000 \mathrm{E}+00$ & & \\
\hline 18 & $3.74000 \mathrm{E}+03$ & 41 & $6.25000 \mathrm{E}-01$ & & \\
\hline 19 & $2.25000 \mathrm{E}+03$ & 42 & $4.50000 \mathrm{E}-01$ & & \\
\hline 20 & $1.91500 \mathrm{E}+02$ & 43 & $3.75000 \mathrm{E}-01$ & & \\
\hline 21 & $1.87700 \mathrm{E}+02$ & 44 & $3.50000 \mathrm{E}-01$ & & \\
\hline 22 & $1.17500 \mathrm{E}+02$ & 45 & $3.25000 \mathrm{E}-01$ & & \\
\hline 23 & $1.16000 \mathrm{E}+02$ & 46 & $2.50000 \mathrm{E}-01$ & & \\
\hline
\end{tabular}

Table 10.1.11. Speciality nuclides with special shielding factors(*) in 56-group library

\begin{tabular}{|c|c|c|}
\hline Nuclide & ID & $\begin{array}{c}\text { Component configuration used to compute Bondarenko } \\
\text { factors }\end{array}$ \\
\hline Zr-91 & 10040091 & standard library weighting \\
\hline Zr-96 & 10040096 & standard library weighting \\
\hline Zr-91 & 40091 & LWR lattice cladding, with U238 resonance interference \\
\hline Zr-96 & 40096 & LWR lattice cladding, with U238 resonance interference \\
\hline Ag-107 & 47107 & PWR Ag-In-Cd control rod \\
\hline Ag-109 & 47109 & PWR Ag-In-Cd control rod \\
\hline In-113 & 49113 & PWR Ag-In-Cd control rod \\
\hline
\end{tabular}




\begin{tabular}{|c|c|c|}
\hline In-115 & 49115 & PWR Ag-In-Cd control rod \\
\hline Cd-113 & 48113 & PWR Ag-In-Cd control rod \\
\hline
\end{tabular}

(*) in addition to nuclides shown with heterogeneous shielding factors in Table 11.1.4

\subsubsection{The test-8grp library for code testing}

The library named test-8grp is used for code testing and verification of reproducibility. It was collapsed from the fine-group v7-252 library, using the standard weight functions in Table 10.1.6. This library has all the nuclides and same types of nuclear data as in the v7-252 library; but the eight energy-group structure provides capability to test codes and input in shorter times than with the standard production libraries. Table 10.1.12 gives the eight group structure, which has four thermal groups below $3 \mathrm{eV}$, and four fast groups.

The library can also be used with the CENTRM/PMC resonance shielding methodology. THIS LIBRARY SHOULD NOT BE USED FOR REAL APPLICATIONS.

Table 10.1.12. 8-Group energy structure

\begin{tabular}{||cc|}
\hline Group & $\begin{array}{c}\text { Upper energy } \\
(\mathrm{eV})\end{array}$ \\
\hline 1 & $2.000 \mathrm{E}+07$ \\
2 & $8.200 \mathrm{E}+05$ \\
3 & $2.000 \mathrm{E}+04$ \\
4 & $1.050 \mathrm{E}+02$ \\
5 & $5.000 \mathrm{E}+00$ \\
6 & $6.250 \mathrm{E}-01$ \\
7 & $1.500 \mathrm{E}-01$ \\
8 & $4.000 \mathrm{E}-01$ \\
& $1.000 \mathrm{E}-05$ \\
\hline
\end{tabular}

\subsubsection{The 200N-47G (V7-200N47G) library for shielding}

Coupled fine-group neutron-gamma libraries based on ENDF/B-VII.1 and ENDF/B-VII.0, respectively, are available for radiation transport calculations with SCALE shielding modules. The 200 neutron and 47 gamma energy group structures are provided in Table 10.1.13 and Table 10.1.14, respectively. The neutron group structure is identical to the 199-group VITAMIN-B6 ${ }^{5}$ structure except that an additional group has been added to extend the top energy boundary to $20 \mathrm{MeV}$. The MG neutron data were generated using the standard weighting function described in Table 10.1.6, and the MG photon data were weighted with a flat spectrum with roll-offs. Full-range Bondarenko factors are provided for all nuclides, and the default self-shielding method for this library is to use BONAMI for all energy groups, enabling faster neutron resonance self-shielding calculations. The Bondarenko shielding factors for all nuclides are computed with the NR approximation. If the Bondarenko approach is not appropriate, self-shielding calculations can be done with the CENTRM module. The 200n-47g libraries have dose factor and response function data shown in Table 10.1.15 which are consistent with previous SCALE shielding libraries. 
The fine-group coupled libraries were validated by performing radiation transport calculations with the SCALE shielding sequence MAVRIC for several shielding benchmark calculations ${ }^{5}$. The calculated results for transmission/attenuation values and spectral results matched experimental measurements well. Overall, the results obtained with using the $200 \mathrm{n}-47 \mathrm{~g}$ coupled library demonstrate the effectiveness of the SCALE methods and data for shielding applications.

Table 10.1.13. Energy boundaries for the 200 neutron group structure

\begin{tabular}{|c|c|c|c|c|c|c|c|c|c|}
\hline Grp & $\begin{array}{l}\begin{array}{l}\text { Energy } \\
(\mathrm{eV})\end{array} \\
\end{array}$ & Grp & Energy (eV) & Grp & $\begin{array}{l}\text { Energy } \\
(\mathrm{eV})\end{array}$ & Grp & $\begin{array}{l}\begin{array}{l}\text { Energy } \\
(\mathrm{eV})\end{array} \\
\end{array}$ & Grp & Energy $(\mathrm{eV})$ \\
\hline 1 & $2.0000 \mathrm{E}+07$ & 42 & $2.7253 \mathrm{E}+06$ & 83 & $3.3373 \mathrm{E}+05$ & 124 & $2.3579 \mathrm{E}+04$ & 165 & $5.0435 \mathrm{E}+00$ \\
\hline 2 & $1.9640 \mathrm{E}+07$ & 43 & $2.5924 \mathrm{E}+06$ & 84 & $3.0197 \mathrm{E}+05$ & 125 & $2.1875 \mathrm{E}+04$ & 166 & $3.9279 \mathrm{E}+00$ \\
\hline 3 & $1.7332 \mathrm{E}+07$ & 44 & $2.4660 \mathrm{E}+06$ & 85 & $2.9849 \mathrm{E}+05$ & 126 & $1.9305 \mathrm{E}+04$ & 167 & $3.0590 \mathrm{E}+00$ \\
\hline 4 & $1.6905 E+07$ & 45 & $2.3852 \mathrm{E}+06$ & 86 & $2.9721 \mathrm{E}+05$ & 127 & $1.5034 \mathrm{E}+04$ & 168 & $2.3824 \mathrm{E}+00$ \\
\hline 5 & $1.6487 \mathrm{E}+07$ & 46 & $2.3653 \mathrm{E}+06$ & 87 & $2.9452 \mathrm{E}+05$ & 128 & $1.1709 \mathrm{E}+04$ & 169 & $1.8554 \mathrm{E}+00$ \\
\hline 6 & $1.5683 \mathrm{E}+07$ & 47 & $2.3457 \mathrm{E}+06$ & 88 & $2.8725 \mathrm{E}+05$ & 129 & $1.0595 \mathrm{E}+04$ & 170 & $1.4450 \mathrm{E}+00$ \\
\hline 7 & $1.4918 \mathrm{E}+07$ & 48 & $2.3069 \mathrm{E}+06$ & 89 & $2.7324 \mathrm{E}+05$ & 130 & $9.1188 \mathrm{E}+03$ & 171 & $1.3000 \mathrm{E}+00$ \\
\hline 8 & $1.4550 \mathrm{E}+07$ & 49 & $2.2313 \mathrm{E}+06$ & 90 & $2.4724 \mathrm{E}+05$ & 131 & $7.1017 \mathrm{E}+03$ & 172 & $1.1253 \mathrm{E}+00$ \\
\hline 9 & $1.4191 \mathrm{E}+07$ & 50 & $2.1225 \mathrm{E}+06$ & 91 & $2.3518 \mathrm{E}+05$ & 132 & $5.5308 \mathrm{E}+03$ & 173 & $1.0800 \mathrm{E}+00$ \\
\hline 10 & $1.3840 \mathrm{E}+07$ & 51 & $2.0190 \mathrm{E}+06$ & 92 & $2.2371 \mathrm{E}+05$ & 133 & $4.3074 \mathrm{E}+03$ & 174 & $1.0400 \mathrm{E}+00$ \\
\hline 11 & $1.3499 \mathrm{E}+07$ & 52 & $1.9205 \mathrm{E}+06$ & 93 & $2.1280 \mathrm{E}+05$ & 134 & $3.7074 \mathrm{E}+03$ & 175 & $1.0000 \mathrm{E}+00$ \\
\hline 12 & $1.2840 \mathrm{E}+07$ & 53 & $1.8268 \mathrm{E}+06$ & 94 & $2.0242 \mathrm{E}+05$ & 135 & $3.3546 \mathrm{E}+03$ & 176 & $8.7643 \mathrm{E}-01$ \\
\hline 13 & $1.2523 \mathrm{E}+07$ & 54 & $1.7377 \mathrm{E}+06$ & 95 & $1.9255 \mathrm{E}+05$ & 136 & $3.0354 \mathrm{E}+03$ & 177 & $8.0000 \mathrm{E}-01$ \\
\hline 14 & $1.2214 \mathrm{E}+07$ & 55 & $1.6530 \mathrm{E}+06$ & 96 & $1.8316 \mathrm{E}+05$ & 137 & $2.7465 \mathrm{E}+03$ & 178 & $6.8256 \mathrm{E}-01$ \\
\hline 15 & $1.1618 \mathrm{E}+07$ & 56 & $1.5724 \mathrm{E}+06$ & 97 & $1.7422 \mathrm{E}+05$ & 138 & $2.6126 \mathrm{E}+03$ & 179 & $6.2506 \mathrm{E}-01$ \\
\hline 16 & $1.1052 \mathrm{E}+07$ & 57 & $1.4957 \mathrm{E}+06$ & 98 & $1.6573 E+05$ & 139 & $2.4852 \mathrm{E}+03$ & 180 & $5.3158 \mathrm{E}-01$ \\
\hline 17 & $1.0513 \mathrm{E}+07$ & 58 & $1.4227 \mathrm{E}+06$ & 99 & $1.5764 \mathrm{E}+05$ & 140 & $2.2487 \mathrm{E}+03$ & 181 & $5.0000 \mathrm{E}-01$ \\
\hline 18 & $1.0000 \mathrm{E}+07$ & 59 & $1.3534 \mathrm{E}+06$ & 100 & $1.4996 \mathrm{E}+05$ & 141 & $2.0347 \mathrm{E}+03$ & 182 & 4.1399E-01 \\
\hline 19 & $9.5123 \mathrm{E}+06$ & 60 & $1.2874 \mathrm{E}+06$ & 101 & $1.4264 \mathrm{E}+05$ & 142 & $1.5846 \mathrm{E}+03$ & 183 & $3.6680 \mathrm{E}-01$ \\
\hline 20 & $9.0484 \mathrm{E}+06$ & 61 & $1.2246 \mathrm{E}+06$ & 102 & $1.3569 \mathrm{E}+05$ & 143 & $1.2341 \mathrm{E}+03$ & 184 & $3.2500 \mathrm{E}-01$ \\
\hline 21 & $8.6071 \mathrm{E}+06$ & 62 & $1.1648 \mathrm{E}+06$ & 103 & $1.2907 \mathrm{E}+05$ & 144 & $9.6112 \mathrm{E}+02$ & 185 & $2.7500 \mathrm{E}-01$ \\
\hline 22 & $8.1873 \mathrm{E}+06$ & 63 & $1.1080 \mathrm{E}+06$ & 104 & $1.2277 \mathrm{E}+05$ & 145 & $7.4852 \mathrm{E}+02$ & 186 & $2.2500 \mathrm{E}-01$ \\
\hline 23 & $7.7880 \mathrm{E}+06$ & 64 & $1.0026 \mathrm{E}+06$ & 105 & $1.1679 \mathrm{E}+05$ & 146 & $5.8295 \mathrm{E}+02$ & 187 & $1.8400 \mathrm{E}-01$ \\
\hline 24 & $7.4082 \mathrm{E}+06$ & 65 & $9.6164 \mathrm{E}+05$ & 106 & $1.1109 \mathrm{E}+05$ & 147 & $4.5400 \mathrm{E}+02$ & 188 & $1.5000 \mathrm{E}-01$ \\
\hline 25 & $7.0469 \mathrm{E}+06$ & 66 & $9.0718 \mathrm{E}+05$ & 107 & $9.8037 \mathrm{E}+04$ & 148 & $3.5357 \mathrm{E}+02$ & 189 & $1.2500 \mathrm{E}-01$ \\
\hline 26 & $6.7032 \mathrm{E}+06$ & 67 & $8.6294 \mathrm{E}+05$ & 108 & $8.6517 \mathrm{E}+04$ & 149 & $2.7536 \mathrm{E}+02$ & 190 & $1.0000 \mathrm{E}-01$ \\
\hline 27 & $6.5924 \mathrm{E}+06$ & 68 & $8.2085 \mathrm{E}+05$ & 109 & $8.2503 \mathrm{E}+04$ & 150 & $2.1445 \mathrm{E}+02$ & 191 & $7.0000 \mathrm{E}-02$ \\
\hline 28 & $6.3763 \mathrm{E}+06$ & 69 & $7.8082 \mathrm{E}+05$ & 110 & $7.9499 \mathrm{E}+04$ & 151 & $1.6702 \mathrm{E}+02$ & 192 & $5.0000 \mathrm{E}-02$ \\
\hline 29 & $6.0653 \mathrm{E}+06$ & 70 & $7.4274 \mathrm{E}+05$ & 111 & $7.1998 \mathrm{E}+04$ & 152 & $1.3007 \mathrm{E}+02$ & 193 & $4.0000 \mathrm{E}-02$ \\
\hline 30 & $5.7695 \mathrm{E}+06$ & 71 & $7.0651 \mathrm{E}+05$ & 112 & $6.7379 \mathrm{E}+04$ & 153 & $1.0130 \mathrm{E}+02$ & 194 & $3.0000 \mathrm{E}-02$ \\
\hline 31 & $5.4881 \mathrm{E}+06$ & 72 & $6.7206 \mathrm{E}+05$ & 113 & $5.6562 \mathrm{E}+04$ & 154 & $7.8893 \mathrm{E}+01$ & 195 & $2.1000 \mathrm{E}-02$ \\
\hline 32 & $5.2205 \mathrm{E}+06$ & 73 & $6.3928 \mathrm{E}+05$ & 114 & $5.2475 \mathrm{E}+04$ & 155 & $6.1442 \mathrm{E}+01$ & 196 & $1.4500 \mathrm{E}-02$ \\
\hline 33 & $4.9659 \mathrm{E}+06$ & 74 & $6.0810 \mathrm{E}+05$ & 115 & $4.6309 \mathrm{E}+04$ & 156 & $4.7851 \mathrm{E}+01$ & 197 & $1.0000 \mathrm{E}-02$ \\
\hline 34 & $4.7237 \mathrm{E}+06$ & 75 & $5.7844 \mathrm{E}+05$ & 116 & $4.0868 \mathrm{E}+04$ & 157 & $3.7266 \mathrm{E}+01$ & 198 & $5.0000 \mathrm{E}-03$ \\
\hline 35 & $4.4933 \mathrm{E}+06$ & 76 & $5.5023 \mathrm{E}+05$ & 117 & $3.4307 \mathrm{E}+04$ & 158 & $2.9023 \mathrm{E}+01$ & 199 & $2.0000 \mathrm{E}-03$ \\
\hline 36 & $4.0657 \mathrm{E}+06$ & 77 & $5.2340 \mathrm{E}+05$ & 118 & $3.1828 \mathrm{E}+04$ & 159 & $2.2603 \mathrm{E}+01$ & 200 & $5.0000 \mathrm{E}-04$ \\
\hline 37 & $3.6788 \mathrm{E}+06$ & 78 & $4.9787 \mathrm{E}+05$ & 119 & $2.8501 \mathrm{E}+04$ & 160 & $1.7604 \mathrm{E}+01$ & & $1.0000 \mathrm{E}-05^{a}$ \\
\hline 38 & $3.3287 \mathrm{E}+06$ & 79 & $4.5049 \mathrm{E}+05$ & 120 & $2.7000 \mathrm{E}+04$ & 161 & $1.3710 \mathrm{E}+01$ & & \\
\hline 39 & $3.1664 \mathrm{E}+06$ & 80 & $4.0762 \mathrm{E}+05$ & 121 & $2.6058 \mathrm{E}+04$ & 162 & $1.0677 \mathrm{E}+01$ & & \\
\hline 40 & $3.0119 \mathrm{E}+06$ & 81 & $3.8774 \mathrm{E}+05$ & 122 & $2.4788 \mathrm{E}+04$ & 163 & $8.3153 \mathrm{E}+00$ & & \\
\hline 41 & $2.8651 \mathrm{E}+06$ & 82 & $3.6883 \mathrm{E}+05$ & 123 & $2.4176 \mathrm{E}+04$ & 164 & $6.4760 \mathrm{E}+00$ & & \\
\hline
\end{tabular}

${ }^{a}$ Lower energy boundary of last group. 
Table 10.1.14. Energy boundaries for the 47 gamma group structure

\begin{tabular}{llllllllll}
\hline Grp & Energy (eV) & Grp & Energy (eV) & Grp & Energy (eV) & Grp & Energy (eV) & Grp & Energy (eV) \\
\hline 1 & $2.0000 \mathrm{E}+07$ & 11 & $5.0000 \mathrm{E}+06$ & 21 & $1.8000 \mathrm{E}+06$ & 31 & $7.0000 \mathrm{E}+05$ & 41 & $1.0000 \mathrm{E}+05$ \\
2 & $1.4000 \mathrm{E}+07$ & 12 & $4.5000 \mathrm{E}+06$ & 22 & $1.6600 \mathrm{E}+06$ & 32 & $6.0000 \mathrm{E}+05$ & 42 & $7.5000 \mathrm{E}+04$ \\
3 & $1.2000 \mathrm{E}+07$ & 13 & $4.0000 \mathrm{E}+06$ & 23 & $1.5700 \mathrm{E}+06$ & 33 & $5.1200 \mathrm{E}+05$ & 43 & $7.0000 \mathrm{E}+04$ \\
4 & $1.0000 \mathrm{E}+07$ & 14 & $3.5000 \mathrm{E}+06$ & 24 & $1.5000 \mathrm{E}+06$ & 34 & $5.1000 \mathrm{E}+05$ & 44 & $6.0000 \mathrm{E}+04$ \\
5 & $8.0000 \mathrm{E}+06$ & 15 & $3.0000 \mathrm{E}+06$ & 25 & $1.4400 \mathrm{E}+06$ & 35 & $4.5000 \mathrm{E}+05$ & 45 & $4.5000 \mathrm{E}+04$ \\
6 & $7.5000 \mathrm{E}+06$ & 16 & $2.7500 \mathrm{E}+06$ & 26 & $1.3300 \mathrm{E}+06$ & 36 & $4.0000 \mathrm{E}+05$ & 46 & $3.0000 \mathrm{E}+04$ \\
7 & $7.0000 \mathrm{E}+06$ & 17 & $2.5000 \mathrm{E}+06$ & 27 & $1.2000 \mathrm{E}+06$ & 37 & $3.0000 \mathrm{E}+05$ & 47 & $2.0000 \mathrm{E}+04$ \\
8 & $6.5000 \mathrm{E}+06$ & 18 & $2.3500 \mathrm{E}+06$ & 28 & $1.0000 \mathrm{E}+06$ & 38 & $2.6000 \mathrm{E}+05$ & & $1.0000 \mathrm{E}+04^{a}$ \\
9 & $6.0000 \mathrm{E}+06$ & 19 & $2.1500 \mathrm{E}+06$ & 29 & $9.0000 \mathrm{E}+05$ & 39 & $2.0000 \mathrm{E}+05$ & & \\
10 & $5.5000 \mathrm{E}+06$ & 20 & $2.0000 \mathrm{E}+06$ & 30 & $8.0000 \mathrm{E}+05$ & 40 & $1.5000 \mathrm{E}+05$ & & \\
\hline \multicolumn{7}{c}{ Lower energy boundary of last group. }
\end{tabular}


Table 10.1.15. Available dose functions on the coupled neutron-gamma libraries

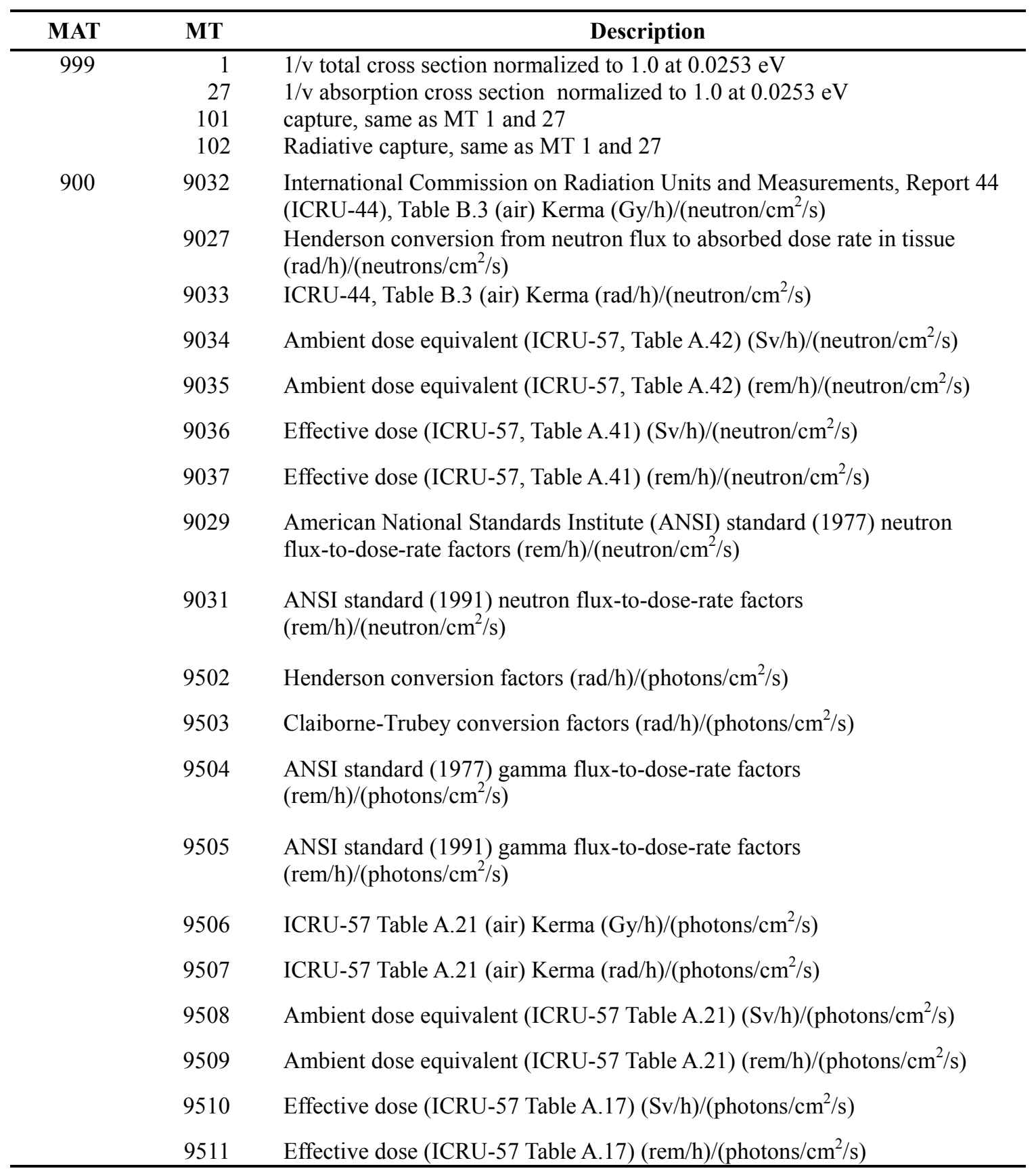




\subsubsection{The 27N-19G and 28N-19G shielding libraries (V7-27N19G, V7.1-28N19G)}

In addition to the fine-group shielding library, SCALE has broad-group ENDF/B-VII.0 and ENDF/B-VII.1 libraries. The ENDF/B-VII.-0 library has 27 neutron and 19 gamma groups; the ENDF/B-VII.1 library has 28 neutron and 19 gamma groups. The change in the neutron group structure was necessary to allow for thermal up-scatter up to $5 \mathrm{eV}$. The gamma groups are identical in both libraries. The broad-group library was primarily developed to perform adjoint discrete ordinates calculations needed to prepare importance maps and biased source distributions for biasing forward Monte Carlo shielding calculations with the fine-group libraries in the MAVRIC shielding analysis sequence. The constituents of the $27 \mathrm{n}-19 \mathrm{~g}$ library and the processing methods are the same as the 200n-47g library. The 27 neutron, 28 neutron and 19 gamma group structures are provided in Table 10.1.16, Table 10.1.17, and Table 10.1.18, respectively. The broad-group library has the same dose factor information described in Table 10.1 .15 for the fine group library. The $27 \mathrm{n}-19 \mathrm{~g}$ ENDF/B-VII.0 library was used as part of the benchmark testing with the $200 \mathrm{n}-47 \mathrm{~g}$ library, which is described in Ref. 5.

Table 10.1.16. Energy boundaries for the 27 neutron group structure

\begin{tabular}{cccccccccc}
\hline Grp & Energy (eV) & Grp & Energy (eV) & Grp & Energy (eV) & Grp & Energy (eV) & Grp & Energy (eV) \\
\hline 1 & $2.0000 \mathrm{E}+07$ & 7 & $4.0762 \mathrm{E}+05$ & 13 & $2.9023 \mathrm{E}+01$ & 19 & $1.0000 \mathrm{E}+00$ & 25 & $5.0000 \mathrm{E}-02$ \\
2 & $6.3763 \mathrm{E}+06$ & 8 & $1.1109 \mathrm{E}+05$ & 14 & $1.0677 \mathrm{E}+01$ & 20 & $8.0000 \mathrm{E}-01$ & 26 & $3.0000 \mathrm{E}-02$ \\
3 & $3.0119 \mathrm{E}+06$ & 9 & $1.5034 \mathrm{E}+04$ & 15 & $3.0590 \mathrm{E}+00$ & 21 & $4.1399 \mathrm{E}-01$ & 27 & $1.0000 \mathrm{E}-02$ \\
4 & $1.8268 \mathrm{E}+06$ & 10 & $3.0354 \mathrm{E}+03$ & 16 & $1.8554 \mathrm{E}+00$ & 22 & $3.2500 \mathrm{E}-01$ & & $1.0000 \mathrm{E}-05^{a}$ \\
5 & $1.4227 \mathrm{E}+06$ & 11 & $5.8295 \mathrm{E}+02$ & 17 & $1.3000 \mathrm{E}+00$ & 23 & $2.2500 \mathrm{E}-01$ & & \\
6 & $9.0718 \mathrm{E}+05$ & 12 & $1.0130 \mathrm{E}+02$ & 18 & $1.1253 \mathrm{E}+00$ & 24 & $1.0000 \mathrm{E}-01$ & & \\
\hline \multicolumn{2}{c}{ Lower energy boundary of last group. }
\end{tabular}

Table 10.1.17. Energy boundaries for the 28 neutron group structure

\begin{tabular}{cccccccccc}
\hline Grp & Energy (eV) & Grp & Energy (eV) & Grp & Energy (eV) & Grp & Energy (eV) & Grp & Energy (eV) \\
\hline 1 & $2.0000 \mathrm{E}+07$ & 7 & $4.0762 \mathrm{E}+05$ & 13 & $2.9023 \mathrm{E}+01$ & 19 & $1.1253 \mathrm{E}+00$ & 25 & $1.0000 \mathrm{E}-01$ \\
2 & $6.3763 \mathrm{E}+06$ & 8 & $1.1109 \mathrm{E}+05$ & 14 & $1.0677 \mathrm{E}+01$ & 20 & $1.0000 \mathrm{E}+00$ & 26 & $5.0000 \mathrm{E}-02$ \\
3 & $3.0119 \mathrm{E}+06$ & 9 & $1.5034 \mathrm{E}+04$ & 15 & $5.000 \mathrm{E}+00$ & 21 & $8.0000 \mathrm{E}-01$ & 27 & $3.0000 \mathrm{E}-02$ \\
4 & $1.8268 \mathrm{E}+06$ & 10 & $3.0354 \mathrm{E}+03$ & 16 & $3.0590 \mathrm{E}+00$ & 22 & $4.1399 \mathrm{E}-01$ & 28 & $1.0000 \mathrm{E}-02$ \\
5 & $1.4227 \mathrm{E}+06$ & 11 & $5.8295 \mathrm{E}+02$ & 17 & $1.8554 \mathrm{E}+00$ & 23 & $3.2500 \mathrm{E}-01$ & & $1.0000 \mathrm{E}-05^{a}$ \\
6 & $9.0718 \mathrm{E}+05$ & 12 & $1.0130 \mathrm{E}+02$ & 18 & $1.3000 \mathrm{E}+00$ & 24 & $2.2500 \mathrm{E}-01$ & & \\
\hline \multicolumn{2}{l}{ Lower energy boundary of last group. }
\end{tabular}

Table 10.1.18. Energy boundaries for the 19 gamma group structure

\begin{tabular}{cccccccccc}
\hline Grp & Energy $(\mathbf{e V})$ & Grp & Energy $(\mathbf{e V})$ & Grp & Energy (eV) & Grp & Energy (eV) & Grp & Energy (eV) \\
\hline 1 & $2.0000 \mathrm{E}+07$ & 5 & $5.0000 \mathrm{E}+06$ & 9 & $2.0000 \mathrm{E}+06$ & 13 & $8.0000 \mathrm{E}+05$ & 17 & $2.0000 \mathrm{E}+05$ \\
2 & $1.0000 \mathrm{E}+07$ & 6 & $4.0000 \mathrm{E}+06$ & 10 & $1.6600 \mathrm{E}+06$ & 14 & $6.0000 \mathrm{E}+05$ & 18 & $1.0000 \mathrm{E}+05$ \\
3 & $8.0000 \mathrm{E}+06$ & 7 & $3.0000 \mathrm{E}+06$ & 11 & $1.3300 \mathrm{E}+06$ & 15 & $4.0000 \mathrm{E}+05$ & 19 & $4.5000 \mathrm{E}+04$ \\
4 & $6.5000 \mathrm{E}+06$ & 8 & $2.5000 \mathrm{E}+06$ & 12 & $1.0000 \mathrm{E}+06$ & 16 & $3.0000 \mathrm{E}+05$ & & $1.0000 \mathrm{E}+4^{a}$ \\
\hline \multicolumn{2}{l}{ Lower energy boundary of last group. }
\end{tabular}

\subsubsection{The continuous-energy libraries}

The ENDF/B-VII.1 and ENDF/B-VII.0 CE libraries are general-purpose libraries used for both criticality calculations and shielding calculations, although the former do not use the photon data in the libraries. 
These libraries have CE data for the same nuclides as the MG libraries. CE data for each nuclide are stored in individual files contained in the SCALE data directory, and the cross section directory files "ce_v7_endf" and "ce_v7np_endf.xml" in the data directory defined the names and other information about the CE data files. The CE libraries were generated using AMPX to an energy-mesh tolerance of $0.1 \%$; i.e., the data value at any intermediate energy point can be interpolated linearly within an error of $0.1 \%$. Cross section data and kinematic data are provided for all reactions given in the ENDF evaluations and are identical to the reactions available on the MG libraries. Kinematic data are given for a range of incident energies as marginal probability distributions over the exit angles and conditional probability distributions over the exit energies in the laboratory system. Usually, 32 equiprobable exit angle bins are used for the conditional probability distribution, except if the distribution is isotropic or can be described with fewer exit angles. For elastic and discrete inelastic reaction, a larger number of exit angles are used as needed to accurately describe the kinematic in the laboratory system. Gamma production kinematic data are provided if available for nuclides that provide gamma production kinematic data. If gamma production data are present, sections for each discrete photon and the continuum are given along with the yield for each of the section. The incident neutron CE libraries are generated at temperature $293 \mathrm{~K}, 565 \mathrm{~K}$, $600 \mathrm{~K}, 900 \mathrm{~K}, 1200 \mathrm{~K}$, and $2400 \mathrm{~K}$. The kinematic data are not temperature dependent except for thermal moderators, which are included at the temperatures provided by the evaluator (see Table 10.1.3 for a list of thermal moderator nuclides and the list of available temperatures). Probability tables for the unresolved resonance range are provided, if the ENDF/B-VII.0 evaluations included unresolved resonance data ${ }^{6}$. Probability tables are available at $293 \mathrm{~K}, 565 \mathrm{~K}, 600 \mathrm{~K}, 900 \mathrm{~K}, 1200 \mathrm{~K}$, and $2400 \mathrm{~K}$. For neutron transport calculations, the CE cross sections and thermal kernels are automatically interpolated to the specified temperature using the methods described in Ref. 5.

For use in CE MONACO, CE libraries for incident gammas are also available, based on ENDF/B-VII.1 ENDF/B-VII.0 data, respectively. Only temperature independent cross section and kinematic data are available in the incident gamma libraries.

\subsubsection{References}

1. D. Wiarda, M. L. Williams, C. Celik, and M. E. Dunn, "AMPX: A Modern Cross Section Processing System for Generating Nuclear Data Libraries," Proceedings of International Conference on Nuclear Criticality Safety, Charlotte, NC, Sept. 13-17, 2015.

2. M. B. Chadwick et al., "ENDF/B-VII.0 Next Generation Evaluated Nuclear Data Library for Nuclear Science and Technology," Special Issue on Evaluated Nuclear Data File ENDF/B-VII.0 Nuclear Data Sheets, 107 (12), 2931-3059 (December 2006).

3. M. B. Chadwick et al., "ENDF/B-VII.1, "Nuclear Data for Science and Technology: Cross Sections, Covariances, Fission Product Yields and Decay Data," Special Issue on ENDF/B-VII.1 Library Nuclear Data Sheets Volume 112, Issue 12, 2887-3152 (December 2011).

4. S. W. D. Hart, C. Celik, G. I Maldonado, L. Leal, "Creation of problem-dependent Doppler-broadened cross sections in the KENO Monte Carlo code," Annals of Nuclear Energy, Volume 88, p. 49, Feb. 2016.

5. D. Wiarda, M. E. Dunn, D. E. Peplow, T. M. Miller, and H. Akkurt, Development and Testing of ENDF/B-VI.8 and ENDF/B-VII.0 Coupled Neutron-Gamma Libraries for SCALE 6, NUREG/CR-6990 (ORNL/TM-2008/047), U. S. Nuclear Regulatory Commission, Oak Ridge National Laboratory, January 2009.

6. M. E. Dunn, L. C. Leal, "Calculating Probability Tables for the Unresolved-Resonance Region Using Monte Carlo Methods," Physor 2002, \#A0107, May 2002. 
This page intentionally left blank. 


\subsection{A MT Reaction Types on SCALE Cross-Section Libraries}

ENDF reaction types (MT) are identified by an integer number from 1 through 999. Table 10.1.A.1 includes a copy of information taken from Ref. 8. Within SCALE, MT numbers are used to identify reactions on various input and output arrays.

MT numbers greater than 999 are additional SCALE-specific identifiers that may appear in SCALE crosssection libraries. These reaction types are not ENDF reaction types but may be added by various preprocessing codes in the SCALE and/or AMPX systems.

These reactions are listed in Table 10.1.A.2.

The dose conversion factors and their corresponding MT numbers in the SCALE shielding cross-section libraries can be found in Table 10.1.12. 
Table 10.1.A.1. ENDF MT Reaction Types

\begin{tabular}{|c|c|c|}
\hline MT & Reaction & Description \\
\hline 1 & $(\mathrm{n}$, total $)$ & $\begin{array}{l}\text { Neutron total cross sections. Sum of MT=2, 4, 5, 11, 16-18, 22-26, 28-37, 41-42, } \\
44-45,102-117 .\end{array}$ \\
\hline 2 & $\left(\mathrm{z}, \mathrm{z}_{0}\right)$ & Elastic scattering cross section for incident particles. \\
\hline 3 & (z,nonelastic) & $\begin{array}{l}\text { Nonelastic neutron cross section. Sum of MT=4, 5, 11, 16-18, 22-26, 28-37, 41-42, } \\
44-45,102-117 \text {. }\end{array}$ \\
\hline 4 & $(\mathrm{z}, \mathrm{n})$ & Production of one neutron in the exit channel. Sum of the MT=50-91. \\
\hline 5 & (z,anything) & $\begin{array}{l}\text { Sum of all reactions not given explicitly in another MT number. This is a partial } \\
\text { reaction to be added to obtain } \mathrm{MT}=1 \text {. }\end{array}$ \\
\hline $6-9$ & & Not allowed in version 6. \\
\hline 10 & (z,continuum) & $\begin{array}{l}\text { Total continuum reaction; includes all continuum reactions and excludes all discrete } \\
\text { reactions. }\end{array}$ \\
\hline 11 & (z,2nd) & Production of two neutrons and a deuteron, plus a residual. \\
\hline $12-15$ & & (Unassigned) \\
\hline 16 & $(\mathrm{z}, 2 \mathrm{n})$ & Production of two neutrons and a residual ${ }^{1}$. Sum of MT $=875-891$, if they are present. \\
\hline 17 & $(\mathrm{z}, 3 \mathrm{n})$ & \\
\hline 18 & (z,fission) & \\
\hline 19 & $(\mathrm{n}, \mathrm{f})$ & \\
\hline 20 & $(\mathrm{n}, \mathrm{nf})$ & Second-chance fission ${ }^{2}$. \\
\hline 21 & $(\mathrm{n}, 2 \mathrm{nf})$ & Third-chance fission ${ }^{2}$. \\
\hline 22 & $(\mathrm{z}, \mathrm{n} \alpha)$ & Production of a neutron and an alpha particle, plus a residual. \\
\hline 23 & $(\mathrm{n}, \mathrm{n} 3 \alpha)$ & Production of a neutron and three alpha particles, plus a residual. \\
\hline 24 & $(\mathrm{z}, 2 \mathrm{n} \alpha)$ & Production of two neutrons and an alpha particle, plus a residual. \\
\hline 25 & $(\mathrm{z}, 3 \mathrm{n} \alpha)$ & Production of three neutrons and an alpha particle, plus a residual. \\
\hline 26 & & Not allowed in version 6. \\
\hline 27 & (n,abs) & Absorption; sum of MT=18 and MT=102 through MT=117. \\
\hline 28 & $(\mathrm{z}, \mathrm{np})$ & Production of a neutron and a proton, plus a residual. \\
\hline 29 & $(\mathrm{z}, \mathrm{n} 2 \alpha)$ & Production of a neutron and two alpha particles, plus a residual. \\
\hline 30 & $(\mathrm{z}, 2 \mathrm{n} 2 \alpha)$ & Production of two neutrons and two alpha particles, plus a residual. \\
\hline 31 & & Not allowed for version 6. \\
\hline 32 & (z,nd) & Production of a neutron and a deuteron, plus a residual. \\
\hline 33 & $(\mathrm{z}, \mathrm{nt})$ & Production of a neutron and a triton, plus a residual. \\
\hline 34 & $\left(\mathrm{z}, \mathrm{n}^{3} \mathrm{He}\right)$ & Production of a neutron and $\mathrm{a}^{3} \mathrm{He}$ particle, plus a residual. \\
\hline 35 & $(\mathrm{z}, \mathrm{nd} 2 \alpha)$ & Production of a neutron, a deuteron, and 2 alpha particles, plus a residual. \\
\hline 36 & $(\mathrm{z}, \mathrm{nt} 2 \alpha)$ & Production of a neutron, a triton, and 2 alpha particles, plus a residual. \\
\hline 37 & $(\mathrm{z}, 4 \mathrm{n})$ & Production of 4 neutrons, plus a residual. \\
\hline 38 & $(\mathrm{n}, 3 \mathrm{nf})$ & Fourth-chance fission cross section ${ }^{2}$. \\
\hline 39 & & Not allowed for version 6. \\
\hline 40 & & Not allowed for version 6. \\
\hline 41 & $(\mathrm{z}, 2 \mathrm{np})$ & Production of 2 neutrons and a proton, plus a residual. \\
\hline 42 & $(\mathrm{z}, 3 \mathrm{np})$ & Production of 3 neutrons and a proton, plus a residual. \\
\hline 43 & & (Unassigned) \\
\hline 44 & $(\mathrm{z}, \mathrm{n} 2 \mathrm{p})$ & Production of a neutron and 2 protons, plus a residual. \\
\hline 45 & $(\mathrm{z}, \mathrm{np} \alpha)$ & Production of a neutron, a proton, and an alpha particle, plus a residual. \\
\hline
\end{tabular}

1 The "residual" is the remainder after the reaction specified by MT has taken place (for example, A-1 after an n,2n reaction on target A). This "residual" may break up further if $L R>0$.

2 Note that the partial fission cross sections are not defined for incident charged particles. 
Table 10.1.A.1. ENDF MT Reaction Types (continued)

\begin{tabular}{|c|c|c|}
\hline MT & Reaction & Description \\
\hline $46-49$ & & Not allowed in Version 6. \\
\hline 50 & $\left(\mathrm{y}, \mathrm{n}_{0}\right)$ & Production of a neutron, leaving the residual nucleus in the ground state. \\
\hline 51 & $\left(\mathrm{z}, \mathrm{n}_{1}\right)$ & Production of a neutron, with residual in the 1st excited state. \\
\hline \multirow[t]{3}{*}{52} & $\left(\mathrm{z}, \mathrm{n}_{2}\right)$ & Production of a neutron, with residual in the 2 nd excited state. \\
\hline & $\ldots$ & \\
\hline & $\cdots$ & \\
\hline 90 & $\left(\mathrm{z}, \mathrm{n}_{40}\right)$ & Production of a neutron, with residual in the 40th excited state. \\
\hline 91 & $\left(\mathrm{z}, \mathrm{n}_{\mathrm{c}}\right)$ & $\begin{array}{l}\text { Production of a neutron in the continuum not included in the above discrete } \\
\text { representation. }\end{array}$ \\
\hline $92-100$ & & (Unassigned) \\
\hline 101 & (n,disap) & Neutron disappearance; equal to sum of MT=102-117. \\
\hline 102 & $(\mathrm{z}, \gamma)$ & Radiative capture. \\
\hline 103 & $(\mathrm{z}, \mathrm{p})$ & Production of a proton, plus a residual. Sum of $M T=600-649$, if they are present. \\
\hline 104 & $(\mathrm{z}, \mathrm{d})$ & Production of a deuteron, plus a residual. Sum of MT $=650-699$, if they are present. \\
\hline 105 & $(\mathrm{z}, \mathrm{t})$ & Production of a triton, plus a residual. Sum of $\mathrm{MT}=700-749$, if they are present. \\
\hline 106 & $\left(\mathrm{z},{ }^{3} \mathrm{He}\right)$ & $\begin{array}{l}\text { Production of a }{ }^{3} \mathrm{He} \text { particle plus a residual. Sum of } \mathrm{MT}=750-799 \text {, if they are } \\
\text { present. }\end{array}$ \\
\hline 107 & $(\mathrm{z}, \alpha)$ & $\begin{array}{l}\text { Production of an alpha particle, plus a residual. Sum of } \mathrm{MT}=800-849 \text {, if they are } \\
\text { present. }\end{array}$ \\
\hline 108 & $(\mathrm{z}, 2 \alpha)$ & Production of 2 alpha particles, plus a residual. \\
\hline 109 & $(\mathrm{z}, 3 \alpha)$ & Production of 3 alpha particles, plus a residual. \\
\hline 110 & & (Unassigned) \\
\hline 111 & $(\mathrm{z}, 2 \mathrm{p})$ & Production of 2 protons, plus a residual. \\
\hline 112 & $(\mathrm{z}, \mathrm{p} \alpha)$ & Production a proton and an alpha particle, plus a residual. \\
\hline 113 & $(\mathrm{z}, \mathrm{t} 2 \alpha)$ & Production of a triton and 2 alpha particles, plus a residual. \\
\hline 114 & $(\mathrm{z}, \mathrm{d} 2 \alpha)$ & Production of a deuteron and 2 alpha particles, plus a residual. \\
\hline 115 & $(\mathrm{z}, \mathrm{pd})$ & Production of proton and a deuteron, plus a residual. \\
\hline 116 & $(\mathrm{z}, \mathrm{pt})$ & Production of proton and a triton, plus a residual. \\
\hline 117 & $(\mathrm{z}, \mathrm{d} \alpha)$ & Production of deuteron and an alpha particle, plus a residual. \\
\hline $118-119$ & & (Unassigned) \\
\hline 120 & & Not allowed for version 6 . \\
\hline $121-150$ & & (Unassigned) \\
\hline 151 & $(n, R E S)$ & $\begin{array}{l}\text { Resonance parameters that can be used to calculate cross sections at different } \\
\text { temperatures in the resolved and unresolved energy regions. }\end{array}$ \\
\hline $152-200$ & & (Unassigned) \\
\hline 201 & $(\mathrm{z}, \mathrm{Xn})$ & Total neutron production. \\
\hline 202 & $(\mathrm{z}, \mathrm{X} \gamma)$ & Total gamma production. \\
\hline 203 & $(\mathrm{z}, \mathrm{Xp})$ & Total proton production. \\
\hline 204 & $(\mathrm{z}, \mathrm{Xd})$ & Total deuteron production. \\
\hline 205 & $(\mathrm{z}, \mathrm{Xt})$ & Total triton production. \\
\hline 206 & $\left(\mathrm{z}, \mathrm{X}^{3} \mathrm{He}\right)$ & Total ${ }^{3} \mathrm{He}$ production. \\
\hline 207 & $(\mathrm{z}, \mathrm{X} \alpha)$ & Total alpha particle production. \\
\hline 208 & $\left(\mathrm{z}, \mathrm{X} \pi^{+}\right)$ & Total $\pi^{+}$production. \\
\hline 209 & $\left(\mathrm{z}, \mathrm{X} \pi^{0}\right)$ & Total $\pi^{0}$ production. \\
\hline 210 & $\left(\mathrm{z}, \mathrm{X} \pi^{-}\right)$ & Total $\pi^{-}$production. \\
\hline 211 & $\left(\mathrm{z}, \mathrm{X} \mu^{+}\right)$ & Total $\mu^{+}$production. \\
\hline 212 & $\left(\mathrm{z}, \mathrm{X} \mu^{-}\right)$ & Total $\mu^{-}$production. \\
\hline 213 & $\left(\mathrm{z}, \mathrm{X} \kappa^{+}\right)$ & Total $\kappa^{+}$production. \\
\hline 214 & $\left(\mathrm{z}, \mathrm{X} \kappa_{(\text {long })}^{0}\right.$ & Total $\kappa_{(\text {long) }}^{0}$ production. \\
\hline 215 & $\left(\mathrm{z}, \mathrm{X} \kappa_{(\text {short })}^{0}\right)$ & Total $\kappa_{\text {(short) }}^{0}$ production. \\
\hline
\end{tabular}


Table 10.1.A.1. ENDF MT Reaction Types (continued)

\begin{tabular}{|c|c|c|}
\hline MT & Reaction & Description \\
\hline 216 & $\left(\mathrm{z}, \mathrm{X} \kappa^{-}\right)$ & Total $\kappa^{-}$production. \\
\hline 217 & $(\mathrm{z}, \mathrm{Xp})$ & Total anti-proton production. \\
\hline & & \\
\hline 218 & $(\mathrm{z}, \mathrm{Xn})$ & Total anti-neutron production. \\
\hline $219-250$ & & (Unassigned) \\
\hline 251 & $(\mathrm{n}, \ldots)$ & $\begin{array}{l}\bar{\mu}_{L}, \text { average cosine of the scattering angle (laboratory system) for elastic scattering } \\
\text { of neutrons. }\end{array}$ \\
\hline 252 & $(n, \ldots)$ & $\xi$, average logarithmic energy decrement for elastic scattering of neutrons. \\
\hline 253 & $(\mathrm{n}, \ldots)$ & $\begin{array}{l}\gamma \text {, average of the square of the logarithmic energy decrement divided by twice the } \\
\text { average logarithmic energy decrement, for elastic scattering of neutrons. }\end{array}$ \\
\hline $254-300$ & & (Unassigned) \\
\hline $301-450$ & $(\mathrm{Z}, \ldots)$ & $\begin{array}{l}\text { Energy release parameters, } \overline{E * \sigma} \text {, for total and partial cross sections; MT }=300 \text { plus } \\
\text { the reaction MT number, e.g., MT }=302 \text { is the elastic scattering kerma. }\end{array}$ \\
\hline 451 & $(\mathrm{z}, \ldots)$ & Heading or title information; given in File 1 only. \\
\hline 452 & $(\mathrm{z}, \ldots)$ & $\begin{array}{l}\bar{v}_{T} \text {, average total (prompt plus delayed) number of neutrons released per fission } \\
\text { event. }\end{array}$ \\
\hline 453 & & (Unassigned) \\
\hline 454 & $(\mathrm{z}, \ldots)$ & Independent fission product yield data. \\
\hline 455 & $(\mathrm{z}, \ldots)$ & $\bar{v}_{d}$, average number of delayed neutrons released per fission event. \\
\hline 456 & $(\mathrm{z}, \ldots)$ & $\bar{v}_{p}$, average number of prompt neutrons released per fission event. \\
\hline 457 & $(\mathrm{z}, \ldots)$ & Radioactive decay data. \\
\hline 458 & $(n, \ldots)$ & Energy release in fission for incident neutrons. \\
\hline 459 & $(\mathrm{z}, \ldots)$ & Cumulative fission product yield data. \\
\hline $460-464$ & & (Unassigned) \\
\hline $465-466$ & & Not allowed in version 6 . \\
\hline $467-499$ & & (Unassigned) \\
\hline 500 & & Total charged-particle stopping power. \\
\hline 501 & & Total photon interaction. \\
\hline 502 & & Photon coherent scattering. \\
\hline 503 & & (Unassigned) \\
\hline 504 & & Photon incoherent scattering. \\
\hline 505 & & Imaginary scattering factor. \\
\hline 506 & & Real scattering factor. \\
\hline $507-514$ & & (Unassigned) \\
\hline 515 & & Pair production, electron field. \\
\hline 516 & & Pair production; sum of $\mathrm{MT}=515,517$. \\
\hline 517 & & Pair production, nuclear field. \\
\hline 518 & & Not allowed in version 6 . \\
\hline $519-521$ & & (Unassigned) \\
\hline 522 & & Photoelectric absorption. \\
\hline 523 & & Photo-excitation cross section. \\
\hline $524-525$ & & (Unassigned) \\
\hline 526 & & Electro-atomic scattering. \\
\hline 527 & & Electro-atomic bremsstrahlung. \\
\hline 528 & & Electro-atomic excitation cross section. \\
\hline $529-531$ & & (Unassigned) \\
\hline
\end{tabular}


Table 10.1.A.1. ENDF MT Reaction Types (continued)

\begin{tabular}{|c|c|c|}
\hline MT & Reaction & Description \\
\hline 532 & & Not allowed in version 6. \\
\hline 533 & & Atomic relaxation data. \\
\hline 534 & $\mathrm{~K}$ & $(1 \mathrm{~s} 1 / 2)$ subshell photoelectric or electro-atomic cross section. \\
\hline 535 & L1 & $(2 \mathrm{~s} 1 / 2)$ subshell photoelectric or electro-atomic cross section. \\
\hline 536 & $\mathrm{~L} 2$ & $\left(2 \mathrm{p}^{1 / 2}\right)$ subshell photoelectric or electro-atomic cross section. \\
\hline 537 & L3 & $\left(2 \mathrm{p}^{3 / 2}\right)$ subshell photoelectric or electro-atomic cross section. \\
\hline 538 & M1 & $\left(3 \mathrm{~s}^{1} / 2\right)$ subshell photoelectric or electro-atomic cross section. \\
\hline 539 & M2 & $\left(3 \mathrm{p}^{1 / 2}\right)$ subshell photoelectric or electro-atomic cross section. \\
\hline 540 & M3 & $\left(3 \mathrm{p}^{3 / 2}\right)$ subshell photoelectric or electro-atomic cross section. \\
\hline 541 & M4 & $\left(3 \mathrm{~d}^{3} / 2\right)$ subshell photoelectric or electro-atomic cross section. \\
\hline 542 & M5 & $\left(3 d^{5 / 2}\right)$ subshell photoelectric or electro-atomic cross section. \\
\hline 543 & N1 & $\left(4 \mathrm{~s}^{1 / 2}\right)$ subshell photoelectric or electro-atomic cross section. \\
\hline 544 & N2 & $\left(4 \mathrm{p}^{1 / 2}\right)$ subshell photoelectric or electro-atomic cross section. \\
\hline 545 & N3 & $\left(4 \mathrm{p}^{3 / 2}\right)$ subshell photoelectric or electro-atomic cross section. \\
\hline 546 & N4 & $\left(4 \mathrm{dp}^{3 / 2}\right)$ subshell photoelectric or electro-atomic cross section. \\
\hline 547 & N5 & $\left(4 d^{5 / 2}\right)$ subshell photoelectric or electro-atomic cross section. \\
\hline 548 & N6 & $\left(4 \mathrm{f}^{5} / 2\right)$ subshell photoelectric or electro-atomic cross section. \\
\hline 549 & N7 & $(4 \mathrm{f} 7 / 2)$ subshell photoelectric or electro-atomic cross section. \\
\hline 550 & O1 & $(5 \mathrm{~s} 1 / 2)$ subshell photoelectric or electro-atomic cross section. \\
\hline 551 & $\mathrm{O} 2$ & $\left(5 \mathrm{p}^{1 / 2}\right)$ subshell photoelectric or electro-atomic cross section. \\
\hline 552 & O3 & $\left(5 \mathrm{p}^{3 / 2}\right)$ subshell photoelectric or electro-atomic cross section. \\
\hline 553 & $\mathrm{O} 4$ & $\left(5 \mathrm{~d}^{3} / 2\right)$ subshell photoelectric or electro-atomic cross section. \\
\hline 554 & O5 & $\left(5 d^{5} / 2\right)$ subshell photoelectric or electro-atomic cross section. \\
\hline 555 & O6 & $\left(5 f^{5} / 2\right)$ subshell photoelectric or electro-atomic cross section. \\
\hline 556 & $\mathrm{O} 7$ & $(5 \mathrm{f} 7 / 2)$ subshell photoelectric or electro-atomic cross section. \\
\hline 557 & $\mathrm{O} 8$ & $(5 \mathrm{~g} 7 / 2)$ subshell photoelectric or electro-atomic cross section. \\
\hline 558 & O9 & $\left(5 g^{9} / 2\right)$ subshell photoelectric or electro-atomic cross section. \\
\hline 559 & $\mathrm{P} 1$ & $\left(6 \mathrm{~s}^{1 / 2}\right)$ subshell photoelectric or electro-atomic cross section. \\
\hline 560 & $\mathrm{P} 2$ & $\left(6 \mathrm{p}^{1 / 2}\right)$ subshell photoelectric or electro-atomic cross section. \\
\hline 561 & P3 & $\left(6 \mathrm{p}^{3 / 2}\right)$ subshell photoelectric or electro-atomic cross section. \\
\hline 562 & $\mathrm{P} 4$ & $\left(6 \mathrm{~d}^{3} / 2\right)$ subshell photoelectric or electro-atomic cross section. \\
\hline 563 & P5 & $(6 \mathrm{~d} 5 / 2)$ subshell photoelectric or electro-atomic cross section. \\
\hline 564 & P6 & $\left(6 \mathrm{f}^{5} / 2\right)$ subshell photoelectric or electro-atomic cross section. \\
\hline 565 & P7 & $(6 f 7 / 2)$ subshell photoelectric or electro-atomic cross section. \\
\hline 566 & P8 & $(6 \mathrm{~g} 7 / 2)$ subshell photoelectric or electro-atomic cross section. \\
\hline 567 & P9 & $\left(6 g^{9} / 2\right)$ subshell photoelectric or electro-atomic cross section. \\
\hline 568 & P10 & $\left(6 h^{9} / 2\right)$ subshell photoelectric or electro-atomic cross section. \\
\hline
\end{tabular}


Table 10.1.A.1. ENDF MT Reaction Types (continued)

\begin{tabular}{|c|c|c|}
\hline MT & Reaction & Description \\
\hline 569 & P11 & $\left(6 h^{11 / 2}\right)$ subshell photoelectric or electro-atomic cross section. \\
\hline 570 & Q1 & $(7 \mathrm{~s} 1 / 2)$ subshell photoelectric or electro-atomic cross section. \\
\hline 571 & Q2 & $\left(7 \mathrm{p}^{1 / 2}\right)$ subshell photoelectric or electro-atomic cross section. \\
\hline 572 & Q3 & $\left(7 p^{3 / 2}\right)$ subshell photoelectric or electro-atomic cross section. \\
\hline $573-599$ & & (Unassigned) \\
\hline 600 & $\left(\mathrm{z}, \mathrm{p}_{0}\right)$ & Production of a proton leaving the residual nucleus in the ground state. \\
\hline 601 & $\left(\mathrm{z}, \mathrm{p}_{1}\right)$ & Production of a proton, with residual in the 1 st excited state. \\
\hline 602 & $\left(\mathrm{z}, \mathrm{p}_{2}\right)$ & Production of a proton, with residual in the 2 nd excited state. \\
\hline 603 & $\left(\mathrm{z}, \mathrm{p}_{3}\right)$ & Production of a proton, with residual in the 3 rd excited state. \\
\hline \multirow[t]{3}{*}{604} & $\left(\mathrm{z}, \mathrm{p}_{4}\right)$ & Production of a proton, with residual in the 4 th excited state. \\
\hline & $\ldots$ & \\
\hline & $\ldots$ & \\
\hline 649 & $\left(\mathrm{z}, \mathrm{p}_{\mathrm{c}}\right)$ & $\begin{array}{l}\text { Production of a proton in the continuum not included in the above discrete } \\
\text { representation. }\end{array}$ \\
\hline 650 & $\left(\mathrm{z}, \mathrm{d}_{0}\right)$ & Production of a deuteron leaving the residual nucleus in the ground state. \\
\hline 651 & $\left(\mathrm{z}, \mathrm{d}_{1}\right)$ & Production of a deuteron, with the residual in the 1 st excited state. \\
\hline \multirow[t]{3}{*}{652} & $\left(\mathrm{z}, \mathrm{d}_{2}\right)$ & Production of a deuteron, with the residual in the 2 nd excited state. \\
\hline & $\ldots$ & \\
\hline & $\ldots$ & \\
\hline 699 & $\left(\mathrm{z}, \mathrm{d}_{\mathrm{c}}\right)$ & $\begin{array}{l}\text { Production of a deuteron in the continuum not included in the above discrete } \\
\text { representation. }\end{array}$ \\
\hline 700 & $\left(\mathrm{z}, \mathrm{t}_{0}\right)$ & Production of a triton leaving the residual nucleus in the ground state. \\
\hline 701 & $\left(\mathrm{z}, \mathrm{t}_{1}\right)$ & Production of a triton, with residual in the 1 st excited state. \\
\hline \multirow[t]{3}{*}{702} & $\left(\mathrm{z}, \mathrm{t}_{2}\right)$ & Production of a triton, with residual in the 2 nd excited state. \\
\hline & $\ldots$ & \\
\hline & $\ldots$ & \\
\hline 749 & $\left(\mathrm{z}, \mathrm{t}_{\mathrm{c}}\right)$ & $\begin{array}{l}\text { Production of a triton in the continuum not included in the above discrete } \\
\text { representation. }\end{array}$ \\
\hline 750 & $\left(n,{ }^{3} \mathrm{He}_{0}\right)$ & Production of a ${ }^{3} \mathrm{He}$ particle leaving the residual nucleus in the ground state. \\
\hline \multirow[t]{2}{*}{751} & $\left(\mathrm{n},{ }^{3} \mathrm{He}_{1}\right)$ & Production of a ${ }^{3} \mathrm{He}$, with residual in the 1 st excited state. \\
\hline & $\ldots$ & \\
\hline 799 & $\left(\mathrm{n},{ }^{3} \mathrm{He}_{\mathrm{c}}\right)$ & $\begin{array}{l}\text { Production of } \mathrm{a}{ }^{3} \mathrm{He} \text { in the continuum not included in the above discrete } \\
\text { representation. }\end{array}$ \\
\hline 800 & $\left(\mathrm{z}, \mathrm{\alpha}_{0}\right)$ & Production of an alpha particle leaving the residual nucleus in the ground state. \\
\hline \multirow[t]{3}{*}{801} & $\left(\mathrm{z}, \alpha_{1}\right)$ & Production of an alpha particle, with residual in the 1 st excited state. \\
\hline & $\ldots$ & \\
\hline & $\ldots$ & \\
\hline 849 & $\left(\mathrm{z}, \alpha_{\mathrm{c}}\right)$ & $\begin{array}{l}\text { Production of an alpha particle in the continuum not included in the above discrete } \\
\text { representation. }\end{array}$ \\
\hline 850 & & (Unassigned) \\
\hline $851-870$ & & Lumped reaction covariances. \\
\hline $871-874$ & & (Unassigned) \\
\hline 875 & $\left(\mathrm{z}, 2 \mathrm{n}_{0}\right)$ & Production of 2 neutrons with residual in the ground state. \\
\hline \multirow[t]{2}{*}{876} & $\left(\mathrm{z}, 2 \mathrm{n}_{1}\right.$ & Production of 2 neutrons with residual in the 1 st excited state. \\
\hline & $\cdots$ & \\
\hline
\end{tabular}


Table 10.1.A.1. ENDF MT Reaction Types (continued)

\begin{tabular}{|c|c|l|}
\hline MT & Reaction & \multicolumn{1}{|c|}{ Description } \\
\hline 891 & $\left(\mathrm{z}, 2 \mathrm{n}_{\mathrm{c}}\right)$ & $\begin{array}{l}\text { Production of 2 neutrons in the continuum not included in the above discrete } \\
\text { representation. } \\
\text { (Unassigned) }\end{array}$ \\
\hline $892-999$ & & \multicolumn{1}{|c|}{} \\
\hline
\end{tabular}

Table 10.1.A.2. SCALE Specific MT Reaction Types

\begin{tabular}{|c|c|}
\hline SCALE MT & Description \\
\hline 1000 & $\begin{array}{l}\text { Transport cross section based on the outscatter approximation. See XSDRNPM chapter, } \\
\text { section Outscatter approximation (inconsistent method). }\end{array}$ \\
\hline 1001 & $\begin{array}{l}\text { Transport cross section based on the inscatter approximation. See XSDRNPM chapter, section } \\
\text { Inscatter approximation (consistent method) }\end{array}$ \\
\hline 1007 & Thermal scattering matrix \\
\hline 1008 & Elastic part of thermal scattering matrix \\
\hline 1018 & Fission spectrum \\
\hline 1019 & First chance fission spectrum \\
\hline 1020 & Second chance fission spectrum \\
\hline 1021 & Third chance fission spectrum \\
\hline 1038 & Fourth chance fission spectrum \\
\hline 1099 & Group integral of the weight function \\
\hline 1111 & Flux moment $\left(\mathrm{P}_{1}\right)$ weighted total cross section \\
\hline 1112 & Flux moment $\left(\mathrm{P}_{2}\right)$ weighted total cross section \\
\hline 1113 & Flux moment $\left(\mathrm{P}_{3}\right)$ weighted total cross section \\
\hline 1114 & Flux moment $\left(\mathrm{P}_{4}\right)$ weighted total cross section \\
\hline 1115 & Flux moment $\left(\mathrm{P}_{5}\right)$ weighted total cross section \\
\hline 1116 & Flux moment $\left(\mathrm{P}_{6}\right)$ weighted total cross section \\
\hline 1117 & Flux moment $\left(\mathrm{P}_{7}\right)$ weighted total cross section \\
\hline 1118 & Flux moment $\left(\mathrm{P}_{8}\right)$ weighted total cross section \\
\hline 1119 & Flux amount $\left(\mathrm{P}_{9}\right)$ weighted total cross section \\
\hline
\end{tabular}




\begin{tabular}{|c|l|}
\hline SCALE MT & \\
\hline 1452 & Product of $\bar{v}_{T}$ times the fission cross section \\
\hline 1456 & Product of $\bar{v}_{p}$ times the fission cross section \\
\hline 1455 & Product of $\bar{v}_{d}$ times the fission cross section \\
\hline $1500-1501$ & Same as 1000,1001 except for gamma-ray cross sections \\
\hline 1527 & Gamma-ray energy absorption coefficient factors \\
\hline 2006 & Non-absorption collision probability (CE libraries only) \\
\hline 2016 & Probability of emitting two neutrons (CE libraries only) \\
\hline 2017 & Probability of emitting three neutrons (CE libraries only) \\
\hline 2018 & Fission probability (CE libraries only) \\
\hline 2022 & Within-group scattering cross section \\
\hline 2027 & Absorption probability (CE libraries only) \\
\hline 4561 & $\bar{v}_{p}$ for first chance fissions \\
\hline 4562 & $\bar{v}_{p}$ for second chance fissions \\
\hline 4563 & $\bar{v}_{p}$ for third chance fissions \\
\hline $\bar{v}_{p}$ for fourth chance fissions \\
\hline
\end{tabular}




\title{
10.2 SCALE NUCLEAR DATA COVARIANCE LIBRARY
}

M. L. Williams, D. Wiarda, G. Arbanas, and B. L. Broadhead

\begin{abstract}
An updated cross section covariance library has been created for use with the sensitivity and uncertainty modules in SCALE 6.2. The data has been assembled from a variety sources, including high-fidelity covariance evaluations from ENDF/B-VII.1 as well as approximate uncertainties obtained from a collaborative project performed by Brookhaven National Laboratory, Los Alamos National Laboratory, and Oak Ridge National Laboratory. This document describes the assumptions in generating the data, the library contents, and processing procedure for the SCALE 56-group and 252-group covariance libraries. The SCALE 44-group covariance library distributed with SCALE 6.0 and SCALE 6.1 is retained for backwards compatibility.
\end{abstract}


TABLE OF CONTENTS

$\underline{\text { Page }}$

10.2 SCALE NUCLEAR DATA COVARIANCE LIBRARY ................................................. 10-45

10.2.1 INTRODUCTION ....................................................................................... 10-50

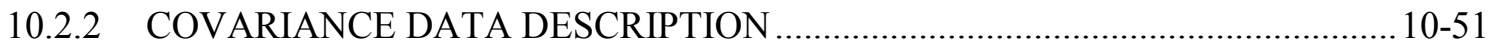

10.2.2.1 EVALUATED COVARIANCES FROM NUCLEAR DATA FILES.................. 10-51

10.2.2.2 APPROXIMATE COVARIANCE DATA ….................................................... 10-51

10.2.2.3 MODIFICATIONS TO COVARIANCE DATA ............................................ 10-52

10.2.2.4 COVARIANCE DATA FOR FISSION SPECTRA ......................................... 10-54

10.2.3 MULTIGROUP COVARIANCE PROCESSING …............................................. 10-54

10.2.4 CONTENTS OF THE SCALE 6.2 COVARIANCE LIBRARY ............................10-54

10.2.5 SCALE 6.1 44-GROUP COVARIANCE LIBRARY ......................................... 10-67

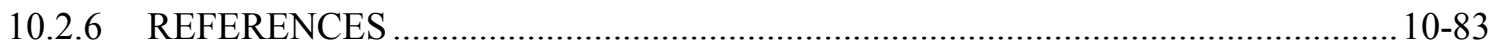

10.2.A Cross Section Plots for U, PU, TH, B, H, HE, and GD Nuclides............................ 10-85 


\section{LIST OF FIGURES}

$\underline{\text { Page }}$

Figure 10.2.A.1. ${ }^{239} \mathrm{Pu}$ fission and capture comparison between ENDF/B-VI, JENDL 3.3, and JEF 3.1

Figure 10.2.A.2. ${ }^{240} \mathrm{Pu}$ fission and capture comparison between ENDF/B-VI, JENDL 3.3 and JEF 3.1

Figure 10.2.A.3. ${ }^{241} \mathrm{Pu}$ fission and capture comparison between ENDF/B-VI, JENDL 3.3 and JEF 3.1

Figure 10.2.A.4. ${ }^{233} \mathrm{U}$ fission and capture comparison between ENDF/B-VII (beta2), ENDF/BVI, JENDL 3.3 and JEF 3.1

Figure 10.2.A.5. ${ }^{235} \mathrm{U}$ fission and capture comparison between ENDF/B-VI, JENDL 3.3 and JEF

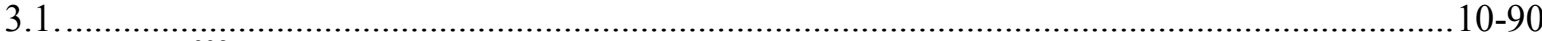

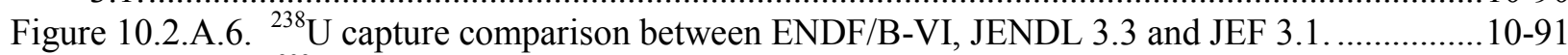

Figure 10.2.A.7. ${ }^{232} \mathrm{Th}$ capture comparison between ENDF/B-VII (beta2), ENDF/B-VI, JENDL 3.3 and JEF 3.1.

Figure 10.2.A.8. ${ }^{10} \mathrm{~B}$ capture and ${ }^{3} \mathrm{He}$ elastic comparison between ENDF/B-VII (beta2),

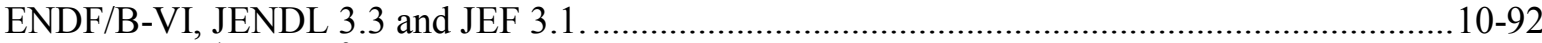

Figure 10.2.A.9. ${ }^{1} \mathrm{H}$ and ${ }^{2} \mathrm{H}$ elastic comparison between ENDF/B-VII (beta2), |ENDF/B-VI, JENDL 3.3 and JEF 3.1

Figure 10.2.A.10. ${ }^{152} \mathrm{Gd}$ and ${ }^{154} \mathrm{Gd}$ capture comparison between ENDF/B-VII (beta2), ENDF/BVI, JENDL 3.3 and JEF 3.1....

Figure 10.2.A.11. ${ }^{155} \mathrm{Gd}$ capture comparison between ENDF/B-VII (beta2), ENDF/B-VI, JENDL 3.3 and JEF 3.1.... 


\section{LIST OF TABLES}

Page

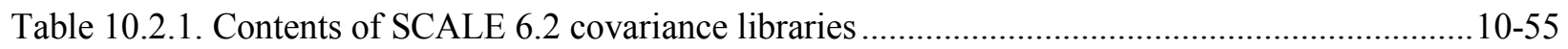

Table 10.2.2. Covariance data with cross-correlations between nuclide reactions..............................10-67

Table 10.2.3. Summary of changes made to covariance evaluations for the 44-group library ..............10-68

Table 10.2.4. Source of fission spectrum parameters and uncertainties ............................................ 10-69

Table 10.2.5. Fissionable nuclides with missing fission spectrum uncertainty data in covariance

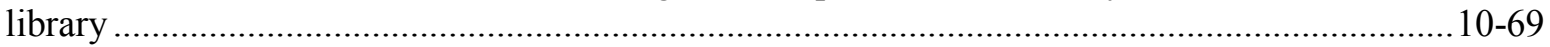

Table 10.2.6. Contents of SCALE 44-group covariance library ..................................................... 10-70

Table 10.2.7. Covariance data with cross correlations between nuclide reactions ..............................10-82 


\section{ACKNOWLEDGMENT}

We gratefully acknowledge the sponsorship of the US Department of Energy Nuclear Criticality Safety Program in the development of the SCALE 6.2 covariance libraries. 


\subsubsection{Introduction}

The SCALE 6.2 covariance library is based on available ENDF/B-VII. $1^{1}$ data for 187 nuclides, combined with the previous SCALE 6.1 covariance data are retained for the $\sim 215$ nuclides not available in ENDF/B-VII.1. The ENDF/B-VII.1 uncertainties were modified for a few nuclides, as described in Sect. 10.2.2.3. In addition, the covariance library now has a 56-group structure for broad group analysis, as well as the 252-group structure for fine-group analysis. These covariance libraries were generated for compatibility with the ENDF/B-VII.1 cross section libraries distributed with SCALE 6.2, and they may also be applied for the 238-group ENDF/B-VII.0 library. The previous SCALE 6.0 and SCALE 6.1 44-group library (44groupcov) was based on older covariance data and is retained in SCALE 6.2 for backwards compatibility. However, the 56- and 252-group covariance libraries (56groupcov7.1 and 252 groupcov7.1) are now recommended for all applications. The 56-group library-which is default for SCALE uncertainty analysis - and the 252 fine-group library generally produce similar results, except for some threshold reactions such as (n,2n). The 252-group library may be used to improve uncertainty estimates from these types of data, but it typically takes more execution time than the default 56-group library. Because the 56- and 252-group covariance data in many cases are based on newer uncertainty evaluations than the previous 44-group library, some differences will occur between these sets of results.

The covariance data correspond to relative uncertainties assembled from a variety of sources, including evaluations from ENDF/B-VII.1, ENDF/B-VI, and approximated uncertainties from a collaborative project performed by Brookhaven National Laboratory (BNL), Los Alamos National Laboratory (LANL), and Oak Ridge National Laboratory (ORNL). Because SCALE uncertainty data come from several different sources, the application of a single generic covariance library to all multigroup cross section libraries raises questions about consistency with any given data evaluation. In reality, much of the approximate uncertainty data in the library is based on simplifying approximations that do not depend on specific ENDF evaluations and thus can be applied to all cross section libraries within the limitations of the assumed methodology. In other cases in which a covariance evaluation has been taken from a specific nuclear data file (e.g., ENDF/B-VII.1, ENDF/B-VI, or JENDL), it is assumed that the same relative (rather than absolute) uncertainties can be applied to all cross section libraries, even if these are not strictly consistent with the nuclear data evaluations. The assumption is partially justified by the fact that different evaluations often use many of the same experimental measurements since there is a limited amount of this information available. In some cases, older data evaluations have been carried over into the newer ENDF versions. Also, because many important nuclear data are now known rather well, newer evaluations in many instances correspond to rather modest variations from previous ones and are expected to lie within the earlier uncertainties. As shown by plots in Chapter 11.3.A, the nuclear data evaluations from ENDF/B-VII, ENDF/B-VI, JEF-3.1, and JENDL-3.3 tend to agree well for many types of cross sections, so it is reasonable to assume that the uncertainties in these data are similar.

No inherently "true" uncertainty can be defined for nuclear data. For example, in theory, two independent evaluations could produce similar nuclear data with very different uncertainties. Differences in nuclear data evaluations directly impact calculations that can be affirmed by comparisons with benchmark experiments; but there is no established procedure to quantify the reliability of uncertainty estimates. In general, the SCALE covariance library should be viewed as a best-estimate assessment of data uncertainties based upon the specific methodology described in the following section. While this methodology is not unique and other approaches could have been used, the SCALE covariance library is a reasonable representation of the nuclear data uncertainties for most applications given the current lack of information. Furthermore, it is the only available comprehensive library that has been created in a welldefined, systematic manner. 


\subsubsection{Covariance Data Description}

\subsubsection{Evaluated covariances from nuclear data files}

A rigorous, modern evaluation of nuclear data typically uses a regression algorithm that adjusts parameters in a nuclear physics model (e.g., Reich-Moore resonance formula, optical model, etc.), to fit a set of differential experimental measurements that have various sources of statistical and systematic uncertainties. ${ }^{2}$ Information from the regression analysis of the model parameters can be propagated to uncertainties and correlations in the evaluated differential data. In this manner, the differential nuclear data and covariances are consistent and are coupled together by evaluation processes. Unfortunately, only a limited number of cross section evaluations have produced high-fidelity covariances in this rigorous manner. All other nuclear data uncertainties must be estimated from approximations in which the uncertainty assessment is decoupled from the original evaluation procedure.

The SCALE covariance library is based on several different uncertainty approximations with varying degrees of fidelity relative to the actual nuclear data evaluation. The library includes high-fidelity evaluated covariances obtained from ENDF/B-VII.1, and ENDF/B-VI whenever available. As discussed in Sect. 10.2.1, it is assumed that covariances taken from one data evaluation, such as ENDF/B-VI, can also be applied to other evaluations of the same data, such as ENDF/B-VII.1. If this is done judiciously for cases in which the nuclear data evaluations are similar, then the covariances taken from one source should be a reasonable representation of uncertainties for the other evaluations.

\subsubsection{Approximate covariance data}

At the other end of the spectrum from high fidelity data, low-fidelity (lo-fi) covariances are defined to be those estimated independently of a specific data evaluation. The approximate covariance data in SCALE are based on results from a collaborative project funded by the US Department of Energy Nuclear Criticality Safety Program to generate lo-fi covariances over the energy range from $10^{-5} \mathrm{eV}$ to $20 \mathrm{MeV}$ for materials without covariances in ENDF/B-VII.1. Nuclear data experts at BNL, LANL, and ORNL devised simple procedures to estimate data uncertainties in the absence of high fidelity covariance evaluations. The result of this project is a set of covariance data in ENDF/B file 33 format that can be processed into multigroup covariances. ${ }^{3}$ Some of these data were later revised and included in ENDF/B-VII.1, while others were carried over from SCALE 6.1 to the SCALE 6.2 library. In this documentation, these data are known as BLO (BNL-LANL-ORNL) uncertainty data, which were generated as described below.

ORNL used uncertainties in integral experiment measurements of thermal cross sections, resonance integrals, and potential cross sections to approximate the standard deviations of capture, fission, and elastic scattering reactions for the thermal $(<0.5 \mathrm{eV})$ and resonance ranges $(0.5 \mathrm{eV}-5 \mathrm{keV})$. Full energy correlation was assumed for the covariances within each of these respective ranges. ${ }^{4,5}$ This procedure was originally introduced for the approximate uncertainty data in SCALE 5.1. However, the current version includes updated integral measurement uncertainties, using the more recent values tabulated by Mughabghab in the Atlas of Neutron Resonances. ${ }^{6}$ The lo-fi relative uncertainty is computed as the absolute uncertainty in the integral parameter (i.e., thermal cross section or resonance integral) taken from the Atlas, divided by the average of the measured parameter and the calculated value computed from ENDF/B-VII differential data:

$$
\mathrm{U}=\frac{\Delta_{\mathrm{I}}}{0.5 \times\left(\mathrm{X}_{\mathrm{I}}+\mathrm{X}_{\mathrm{D}}\right)}
$$


where:

$\mathrm{U}$ is the relative lo-fi uncertainty included in SCALE,

$\Delta_{\mathrm{I}}$ is the absolute uncertainty in the integral measurement (obtained from Mughabghab), and

$\mathrm{X}_{\mathrm{I}}$ and $\mathrm{X}_{\mathrm{D}}$ are the measured and computed (from ENDF/B differential data) integral parameter values, respectively.

In some cases the integral measurement value from the Mughabghab Atlas $^{6}$ and the corresponding value computed from the ENDF/B-VII differential evaluation are inconsistent - defined here as having a difference greater than two standard deviations in the measured and computed integral parameters. In these cases, the lo-fi relative standard deviation is defined as half the difference relative to the average of the measured and calculated values:

$$
U=\frac{\left|X_{I}-X_{D}\right|}{X_{I}+X_{D}} ; \text { for }\left|X_{I}-X_{D}\right|>2 \Delta_{I}
$$

In some instances this expression may exceed $100 \%$. For these cases, a $100 \%$ uncertainty was assigned. Also, the Atlas does not include uncertainties in integral measurements for several isotopes, which typically are not of great interest for most applications. In this case the integral uncertainty was defined as $\mathrm{a}+/-5$ in the least significant digit for these materials; e.g., 1.23 is assign an uncertainty of $+/-5 \mathrm{E}-3$.

BNL and LANL provided estimates in the fast energy range from $5 \mathrm{keV}$ to $20 \mathrm{MeV}$ for covariances of capture, fission, elastic, inelastic, $(\mathrm{n}, 2 \mathrm{n})$ cross sections, and prompt nubar. BNL used optical model calculations with estimated uncertainties in model parameters to compute covariances in the fast range for about 300 structural isotopes, fission products, and non-fissionable heavy nuclei. Estimated uncertainties in model parameters were based on previous work and expert judgment. ${ }^{7}$ Covariances for 14 actinide isotopes were obtained from earlier work performed by BNL for Subgroup-26 (SG-26). ${ }^{8}$ The SG-26 actinide covariances cover the full energy range, including thermal, resonance, and fast regions. If the thermal data uncertainties estimated by the SG-26 approach exceed the thermal uncertainty given in reference 6 , the thermal data covariances are represented by ORNL's integral uncertainty technique.

LANL produced covariances in the fast range for an additional 47 actinide materials. The LANL actinide covariances were based on empirical estimates of nuclear reaction models. ${ }^{9}$ Full energy range covariances were also produced by LANL for 16 light isotopes ranging from hydrogen to fluorine. ${ }^{10}$ These included high fidelity covariances from R-matrix analyses for ${ }^{1} \mathrm{H},{ }^{6} \mathrm{Li}$, and ${ }^{10} \mathrm{~B}$, along with lo-fi uncertainties for the other materials, based on approximations such as least-squares fitting to experimental data, statistical model calculations at higher energies, or sometimes simply best-judgment estimation. ${ }^{3}$

\subsubsection{Modifications to covariance data}

In generating earlier covariance libraries, some omissions or inconsistencies were identified and corrected in the current covariance library:

- If the absolute correlation is larger than 1 , it is set to 1 .

- If a relative uncertainty is larger than 1 , it is set to 1 .

- If cross section data exist but covariance data do not span the entire range, then the diagonal element for the higher energy groups is repeated for the lower energy groups.

- If total inelastic scattering covariance is not supplied, it is calculated from the uncertainties in the discrete level inelastic data. 
- If total nubar covariance is not supplied, it is calculated from the the prompt and delayed nubar uncertainties

A few inconsistencies were found in the ENDF/B-VII.1 uncertainty data, and these were modified for the SCALE 6.2 covariance library. ${ }^{11}$ The corrections were also conveyed to the National Nuclear Data Center, where they were added to the ENDF/A file for possible inclusion in the future release of ENDF/B-VII.2. These modifications are summarized below:

(a) ${ }^{235} \mathrm{U}$ thermal nubar: standard deviation was decreased from $0.7 \%$ to $0.3 \%$ in energy range from 0.0 to $0.5 \mathrm{eV}$, consistent with JENDL-3.3.

(b) ${ }^{239} \mathrm{Pu}$ thermal nubar: standard deviation was increased from $0.01 \%$ to $0.15 \%$ in energy range from 0.0 to $0.01 \mathrm{eV}$, consistent with ENDF/B-VII.1 uncertainty at $0.01 \mathrm{eV}$.

(c) $\mathrm{H}$ thermal capture: standard deviation reduced from $2.5 \%$ to $0.2 \%$, consistent with Williams and Rearden $2008 .^{5}$

(d) ${ }^{103} \mathrm{Rh}$ thermal capture: reduced from $\sim 4 \%$ to $1.04 \%$, consistent with Williams and Rearden $2008 .{ }^{5}$

(e) ${ }^{151} \mathrm{Sm}$ thermal capture: modified to $\sim 1.8 \%$, consistent with Williams and Rearden $2008 .{ }^{5}$

(f) ${ }^{147} \mathrm{Pm}$ : standard deviation was reduced from $24 \%$ to $5 \%$ in the energy range $0.5-5000 \mathrm{eV}$, consistent with the quoted resonance integral uncertainty in Williams and Rearden $2008 .^{5}$

Several modifications were also made to the uncertainties obtained from the original BLO data used in SCALE 6.1. The energy boundary between the thermal and resonance covariance blocks was modified from 0.5 to $0.625 \mathrm{eV}$ in order to coincide with a 56-group boundary. The BLO lo-fi data do not include thermal or resonance range uncertainties for isotope reactions that do not have integral uncertainties given in the Mughabghab text. ${ }^{6}$ These occur mainly for relatively unimportant data such as elastic cross sections of several fission products. Therefore in these cases the uncertainties were estimated using different approaches. For example, the thermal data uncertainty was sometimes used to represent the epithermal uncertainty if it was not available in the Mughabghab tabulation, and sometimes the high-energy uncertainty was extended to lower energies. The uncertainty in the ${ }^{149} \mathrm{Sm}$ resonance capture integral is not provided in the 2006 edition of Mughabghab's text, so it was set to the value of $5.7 \%$, which was obtained from an earlier tabulation by Mughabghab. ${ }^{12}$ 


\subsubsection{Covariance data for fission spectra}

As of ENDF/B-VII.1, covariance matrices are now provided for the fission exit energy distribution. The data are given as a function of incident energy. The incident energy grid is very broad, and the exit energy distribution is constant over a given incident energy group. Since the COVERX library file only allows one multigroup fission spectrum $(\chi)$ covariance matrix per nuclide, the exit energy spectrum is used for the average energy of fission. If $v$ is nubar, $f$ is fission, and $w$ is the appropriate flux, then the average energy of fission is calculated as:

$$
10^{7} \exp \left(-\frac{\sum v f w \frac{1}{2}\left(\log \left(\frac{10^{7}}{E_{g 1}}\right)+\log \left(\frac{10^{7}}{E_{g 2}}\right)\right)}{\sum v f w}\right),
$$

where the sum is over all groups and $\mathrm{E}_{\mathrm{g} 1}$ and $\mathrm{E}_{\mathrm{g} 2}$ are the group boundaries for group g. ENDF/B-VII.1 provides covariance data for exit energy distributions for 64 nuclides. This includes all nuclides for which fission spectrum $(\chi)$ covariance matrices where provided in the previous covariance library. Some additional $\chi$-covariance matrices were taken from JENDL-4.0. The new 56-group and 252-group fission spectrum covariances are more complete and significantly improved compared to the earlier 44-group chi uncertainty data, which were based on the Watt fission spectrum in ENDF/B-V. (see Section 10.2.5)

\subsubsection{Multigroup Covariance Processing}

Covariance data were processed with the AMPX code PUFF-IV. PUFF-IV has major improvements in the treatment of the resolved and unresolved resonance parameter uncertainties over previous code versions. ${ }^{13}$ All nuclides with resonance parameter uncertainty files were processed with the full sensitivity option in PUFF-IV.

\subsubsection{Contents of the SCALE 6.2 Covariance Library}

The SCALE covariance library provides uncertainty data in 56- and 252-group formats for a total of 456 materials, including some duplication for materials with multiple thermal scattering kernels. Table 10.2.1 describes the contents of the library using the following nomenclature:

1. ENDF/B-VII.1: evaluated covariance data released with ENDF/B-VII.1

2. ENDF/B-VII.2-prelim: recently evaluated data proposed for future release of ENDF/B-VII.2

3. ENDF/B-VI: evaluated covariance data released with ENDF/B-VI

4. BLO approximate data: lo-fi covariances from BLO project

5. SG-26: approximate covariances from WPEC Subgroup-26

6. JENDL-4.0: evaluated covariance data released with JENDL-4.0

Several covariance evaluations include cross correlations between reactions. These are summarized in Table 10.2.2 
Table 10.2.1. Contents of SCALE 6.2 covariance libraries

\begin{tabular}{|c|c|c|c|}
\hline SCALE name & SCALE ID & Data source & Comment \\
\hline ac-225 & 89225 & ENDF/B-VII.1 & \\
\hline ac-226 & 89226 & ENDF/B-VII.1 & \\
\hline ac-227 & 89227 & ENDF/B-VII.1 & \\
\hline ag-107 & 47107 & BLO approximation data & \\
\hline ag-109 & 47109 & ENDF/B-VII.1 & \\
\hline ag-110m & 1047110 & BLO approximation data & \\
\hline ag-111 & 47111 & BLO approximation data & \\
\hline al-27 & 13027 & ENDF/B-VII.1 & \\
\hline albound & 1013027 & ENDF/B-VII.1 & Duplicate of al-27 \\
\hline am-240 & 95240 & ENDF/B-VII.1 & \\
\hline \multirow[t]{2}{*}{ am-241 } & 95241 & ENDF/B-VII.1 & \\
\hline & & $\chi$ covariance JENDL-4.0 & \\
\hline \multirow[t]{2}{*}{ am-242 } & 95242 & SG-26 & $\begin{array}{l}\text { Thermal uncertainty replaced by } \\
\text { Mughabghab value }\end{array}$ \\
\hline & & $\chi$ covariance JENDL-4.0 & \\
\hline $\mathrm{am}-242 \mathrm{~m}$ & 1095242 & ENDF/B-VII.1 & \\
\hline \multirow[t]{2}{*}{ am-243 } & 95243 & ENDF/B-VII.1 & \\
\hline & & $\chi$ covariance JENDL-4.0 & \\
\hline \multirow[t]{2}{*}{ am-244 } & 95244 & BLO approximation data & \\
\hline & & $\chi$ covariance JENDL-4.0 & \\
\hline am-244m & 1095244 & BLO approximation data & \\
\hline ar-36 & 18036 & BLO approximation data & \\
\hline ar-38 & 18038 & BLO approximation data & \\
\hline ar-40 & 18040 & BLO approximation data & \\
\hline as-74 & 33074 & BLO approximation data & \\
\hline as-75 & 33075 & BLO approximation data & \\
\hline au-197 & 79197 & ENDF/B-VII.1 & \\
\hline b-10 & 5010 & ENDF/B-VII.1 & \\
\hline b-11 & 5011 & ENDF/B-VII.1 & \\
\hline ba-130 & 56130 & BLO approximation data & \\
\hline ba-132 & 56132 & BLO approximation data & \\
\hline ba-133 & 56133 & BLO approximation data & \\
\hline ba-134 & 56134 & BLO approximation data & \\
\hline ba-135 & 56135 & BLO approximation data & \\
\hline ba-136 & 56136 & BLO approximation data & \\
\hline ba-137 & 56137 & BLO approximation data & \\
\hline ba-138 & 56138 & BLO approximation data & \\
\hline ba-140 & 56140 & BLO approximation data & \\
\hline be-7 & 4007 & BLO approximation data & \\
\hline
\end{tabular}




\begin{tabular}{|c|c|c|c|}
\hline SCALE name & SCALE ID & Data source & Comment \\
\hline be-9 & 4009 & ENDF/B-VII.1 & \\
\hline be-beo & 5004009 & ENDF/B-VII.1 & Duplicate of be- 9 \\
\hline bebound & 3004009 & ENDF/B-VII.1 & Duplicate of be-9 \\
\hline bi-209 & 83209 & ENDF/B-VII.1 & \\
\hline bk-245 & 97245 & ENDF/B-VII.1 & \\
\hline bk-246 & 97246 & ENDF/B-VII.1 & \\
\hline bk-247 & 97247 & ENDF/B-VII.1 & \\
\hline bk-248 & 97248 & ENDF/B-VII.1 & \\
\hline bk-249 & 97249 & ENDF/B-VII.1 & \\
\hline bk-250 & 97250 & ENDF/B-VII.1 & \\
\hline br-79 & 35079 & BLO approximation data & \\
\hline br-81 & 35081 & BLO approximation data & \\
\hline $\mathrm{c}$ & 6000 & ENDF/B-VII.1 & \\
\hline $\mathrm{ca}$ & 20000 & BLO approximation dataca & \\
\hline ca-40 & 20040 & BLO approximation data & \\
\hline ca-42 & 20042 & BLO approximation data & \\
\hline ca-43 & 20043 & BLO approximation data & \\
\hline ca-44 & 20044 & BLO approximation data & \\
\hline ca-46 & 20046 & BLO approximation data & \\
\hline ca-48 & 20048 & BLO approximation data & \\
\hline $\mathrm{cd}$ & 48000 & BLO approximation data & \\
\hline cd-106 & 48106 & BLO approximation data & \\
\hline cd-108 & 48108 & BLO approximation data & \\
\hline cd-110 & 48110 & BLO approximation data & \\
\hline cd-111 & 48111 & BLO approximation data & \\
\hline cd-112 & 48112 & BLO approximation data & \\
\hline cd-113 & 48113 & BLO approximation data & \\
\hline cd-114 & 48114 & BLO approximation data & \\
\hline cd-115m & 1048115 & BLO approximation data & \\
\hline cd-116 & 48116 & BLO approximation data & \\
\hline ce-136 & 58136 & BLO approximation data & \\
\hline ce-138 & 58138 & BLO approximation data & \\
\hline ce-139 & 58139 & BLO approximation data & \\
\hline ce-140 & 58140 & BLO approximation data & \\
\hline ce-141 & 58141 & ENDF/B-VII.1 & \\
\hline ce-142 & 58142 & BLO approximation data & \\
\hline ce-143 & 58143 & BLO approximation data & \\
\hline ce-144 & 58144 & BLO approximation data & \\
\hline cf-246 & 98246 & ENDF/B-VII.1 & \\
\hline cf-248 & 98248 & ENDF/B-VII.1 & \\
\hline
\end{tabular}




\begin{tabular}{|c|c|c|c|}
\hline SCALE name & SCALE ID & Data source & Comment \\
\hline cf-249 & 98249 & ENDF/B-VII.1 & \\
\hline $\mathrm{cf}-250$ & 98250 & ENDF/B-VII.1 & \\
\hline $\mathrm{cf}-251$ & 98251 & ENDF/B-VII.1 & \\
\hline $\mathrm{cf}-252$ & 98252 & ENDF/B-VII.1 & \\
\hline cf- 253 & 98253 & ENDF/B-VII.1 & \\
\hline $\mathrm{cf}-254$ & 98254 & ENDF/B-VII.1 & \\
\hline $\mathrm{cl}$ & 17000 & BLO approximation data & \\
\hline cl-35 & 17035 & ENDF/B-VII.1 & \\
\hline cl-37 & 17037 & ENDF/B-VII.1 & \\
\hline $\mathrm{cm}-240$ & 96240 & ENDF/B-VII.1 & \\
\hline $\mathrm{cm}-241$ & 96241 & ENDF/B-VII.1 & \\
\hline $\mathrm{cm}-242$ & 96242 & ENDF/B-VII.1 & \\
\hline $\mathrm{cm}-243$ & 96243 & ENDF/B-VII.1 & \\
\hline $\mathrm{cm}-244$ & 96244 & ENDF/B-VII.1 & \\
\hline $\mathrm{cm}-245$ & 96245 & ENDF/B-VII.1 & \\
\hline $\mathrm{cm}-246$ & 96246 & ENDF/B-VII.1 & \\
\hline $\mathrm{cm}-247$ & 96247 & ENDF/B-VII.1 & \\
\hline $\mathrm{cm}-248$ & 96248 & ENDF/B-VII.1 & \\
\hline $\mathrm{cm}-249$ & 96249 & ENDF/B-VII.1 & \\
\hline $\mathrm{cm}-250$ & 96250 & ENDF/B-VII.1 & \\
\hline co-58 & 27058 & BLO approximation data & \\
\hline co- $58 \mathrm{~m}$ & 1027058 & BLO approximation data & \\
\hline co-59 & 27059 & ENDF/B-VII.1 & \\
\hline cr-50 & 24050 & ENDF/B-VII.1 & \\
\hline cr-52 & 24052 & ENDF/B-VII.1 & \\
\hline cr-53 & 24053 & ENDF/B-VII.1 & \\
\hline cr-54 & 24054 & ENDF/B-VII.1 & \\
\hline cs-133 & 55133 & ENDF/B-VII.1 & \\
\hline cs-134 & 55134 & BLO approximation data & \\
\hline cs-135 & 55135 & ENDF/B-VII.1 & \\
\hline cs-136 & 55136 & BLO approximation data & \\
\hline cs-137 & 55137 & BLO approximation data & \\
\hline cu-63 & 29063 & ENDF/B-VI & \\
\hline cu-65 & 29065 & ENDF/B-VI & \\
\hline $\mathrm{d}$ & 1002 & ENDF/B-VII.1 & Duplicate of h-2 \\
\hline d-cryo_ortho & 4001002 & ENDF/B-VII.1 & Duplicate of h-2 \\
\hline d-cryo_para & 5001002 & ENDF/B-VII.1 & Duplicate of h-2 \\
\hline dfreegas & 8001002 & ENDF/B-VII.1 & \\
\hline dy-156 & 66156 & BLO approximation data & \\
\hline dy-158 & 66158 & BLO approximation data & \\
\hline
\end{tabular}




\begin{tabular}{|c|c|c|c|}
\hline SCALE name & SCALE ID & Data source & Comment \\
\hline dy-160 & 66160 & BLO approximation data & \\
\hline dy-161 & 66161 & BLO approximation data & \\
\hline dy-162 & 66162 & BLO approximation data & \\
\hline dy-163 & 66163 & BLO approximation data & \\
\hline dy-164 & 66164 & BLO approximation data & \\
\hline er-162 & 68162 & BLO approximation data & \\
\hline er-164 & 68164 & BLO approximation data & \\
\hline er-166 & 68166 & ENDF/B-VII.1 & \\
\hline er-167 & 68167 & ENDF/B-VII.1 & \\
\hline er-168 & 68168 & ENDF/B-VII.1 & \\
\hline er-170 & 68170 & ENDF/B-VII.1 & \\
\hline es-251 & 99251 & ENDF/B-VII.1 & \\
\hline es-252 & 99252 & ENDF/B-VII.1 & \\
\hline es-253 & 99253 & ENDF/B-VII.1 & \\
\hline es-254 & 99254 & ENDF/B-VII.1 & \\
\hline es- $254 \mathrm{~m}$ & 1099254 & ENDF/B-VII.1 & \\
\hline es- 255 & 99255 & ENDF/B-VII.1 & \\
\hline eu-151 & 63151 & BLO approximation data & \\
\hline eu-152 & 63152 & BLO approximation data & \\
\hline eu-153 & 63153 & ENDF/B-VII.1 & \\
\hline eu-154 & 63154 & BLO approximation data & \\
\hline eu-155 & 63155 & ENDF/B-VII.1 & $\begin{array}{l}\text { Uses ENDF/B-VII. } 1 \text { data uncertainty } \\
\text { in the thermal range for } \mathrm{MT}=102\end{array}$ \\
\hline eu-156 & 63156 & BLO approximation data & \\
\hline eu-157 & 63157 & BLO approximation data & \\
\hline$f-19$ & 9019 & ENDF/B-VII.1 & \\
\hline fe-54 & 26054 & ENDF/B-VII.1 & \\
\hline fe-56 & 26056 & ENDF/B-VII.1 & \\
\hline fe-57 & 26057 & ENDF/B-VII.1 & \\
\hline fe-58 & 26058 & ENDF/B-VI & \\
\hline febound & 1026000 & ENDF/B-VII.1 & Duplicate of fe-56 \\
\hline $\mathrm{fm}-255$ & 100255 & ENDF/B-VII.1 & \\
\hline ga & 31000 & BLO approximation data & \\
\hline ga-69 & 31069 & BLO approximation data & \\
\hline ga-71 & 31071 & BLO approximation data & \\
\hline gd-152 & 64152 & ENDF/B-VII.1 & \\
\hline gd-153 & 64153 & ENDF/B-VII.1 & \\
\hline gd-154 & 64154 & ENDF/B-VII.1 & \\
\hline gd-155 & 64155 & ENDF/B-VII.1 & \\
\hline gd-156 & 64156 & ENDF/B-VII.1 & \\
\hline
\end{tabular}




\begin{tabular}{|c|c|c|c|}
\hline SCALE name & SCALE ID & Data source & Comment \\
\hline gd-157 & 64157 & ENDF/B-VII.1 & \\
\hline gd-158 & 64158 & ENDF/B-VII.1 & \\
\hline gd-160 & 64160 & ENDF/B-VII.1 & \\
\hline ge-70 & 32070 & BLO approximation data & \\
\hline ge-72 & 32072 & BLO approximation data & \\
\hline ge-73 & 32073 & BLO approximation data & \\
\hline ge-74 & 32074 & BLO approximation data & \\
\hline ge-76 & 32076 & BLO approximation data & \\
\hline graphite & 3006000 & ENDF/B-VII.1 & Duplicate of c \\
\hline $\mathrm{h}$ & 1001 & ENDF/B-VII.2 prelim & Duplicate of h1 \\
\hline h-3 & 1003 & BLO approximation data & \\
\hline h-benzene & 6001001 & ENDF/B-VII.2 prelim & Duplicate of h-1 \\
\hline h-benzene & 5006000 & ENDF/B-VII.1 & Duplicate of c \\
\hline h-cryo_ortho & 4001001 & ENDF/B-VII.2 prelim & Duplicate of h-1 \\
\hline h-cryo_para & 5001001 & ENDF/B-VII.2 prelim & Duplicate of h-1 \\
\hline h-liquid_ch4 & 1001001 & ENDF/B-VII. 2 prelim & Duplicate of h-1 \\
\hline h-poly & 9001001 & ENDF/B-VII.2 prelim & Duplicate of h-1 \\
\hline h-solid_ch4 & 2001001 & ENDF/B-VII. 2 prelim & Duplicate of h-1 \\
\hline h-zrh2 & 7001001 & ENDF/B-VII.2 prelim & Duplicate of h-1 \\
\hline he-3 & 2003 & BLO approximation data & \\
\hline he-4 & 2004 & ENDF/B-VII.1 & \\
\hline hf & 72000 & BLO approximation data & \\
\hline hf-174 & 72174 & BLO approximation data & \\
\hline hf-176 & 72176 & BLO approximation data & \\
\hline hf-177 & 72177 & BLO approximation data & \\
\hline hf-178 & 72178 & BLO approximation data & \\
\hline hf-179 & 72179 & BLO approximation data & \\
\hline hf-180 & 72180 & BLO approximation data & \\
\hline hfreegas & 8001001 & ENDF/B-VII.2 prelim & \\
\hline hg-196 & 80196 & BLO approximation data & \\
\hline hg-198 & 80198 & BLO approximation data & \\
\hline hg-199 & 80199 & BLO approximation data & \\
\hline hg-200 & 80200 & BLO approximation data & \\
\hline hg-201 & 80201 & BLO approximation data & \\
\hline hg-202 & 80202 & BLO approximation data & \\
\hline hg-204 & 80204 & BLO approximation data & \\
\hline ho-165 & 67165 & BLO approximation data & \\
\hline ho- $166 \mathrm{~m}$ & 1067166 & BLO approximation data & \\
\hline i-127 & 53127 & ENDF/B-VII.1 & \\
\hline i-129 & 53129 & ENDF/B-VII.1 & \\
\hline
\end{tabular}




\begin{tabular}{|c|c|c|c|}
\hline SCALE name & SCALE ID & Data source & Comment \\
\hline $\mathrm{i}-130$ & 53130 & BLO approximation data & \\
\hline $\mathrm{i}-131$ & 53131 & BLO approximation data & \\
\hline $\mathrm{i}-135$ & 53135 & BLO approximation data & \\
\hline in & 49000 & ENDF/B-VI & \\
\hline in-113 & 49113 & BLO approximation data & \\
\hline in-115 & 49115 & BLO approximation data & \\
\hline ir-191 & 77191 & ENDF/B-VII.1 & \\
\hline ir-193 & 77193 & ENDF/B-VII.1 & \\
\hline $\mathrm{k}$ & 19000 & BLO approximation data & \\
\hline k-39 & 19039 & ENDF/B-VII.1 & \\
\hline$k-40$ & 19040 & BLO approximation data & \\
\hline $\mathrm{k}-41$ & 19041 & ENDF/B-VII.1 & \\
\hline kr-78 & 36078 & BLO approximation data & \\
\hline $\mathrm{kr}-80$ & 36080 & BLO approximation data & \\
\hline $\mathrm{kr}-82$ & 36082 & BLO approximation data & \\
\hline $\mathrm{kr}-83$ & 36083 & BLO approximation data & \\
\hline $\mathrm{kr}-84$ & 36084 & BLO approximation data & \\
\hline $\mathrm{kr}-85$ & 36085 & BLO approximation data & \\
\hline kr-86 & 36086 & BLO approximation data & \\
\hline la-138 & 57138 & BLO approximation data & \\
\hline la-139 & 57139 & ENDF/B-VII.1 & \\
\hline la-140 & 57140 & BLO approximation data & \\
\hline li-6 & 3006 & ENDF/B-VII.1 & \\
\hline li-7 & 3007 & ENDF/B-VII.1 & \\
\hline lu-175 & 71175 & BLO approximation data & \\
\hline lu-176 & 71176 & BLO approximation data & \\
\hline $\mathrm{mg}$ & 12000 & BLO approximation data & \\
\hline $\mathrm{mg}-24$ & 12024 & ENDF/B-VII.1 & \\
\hline $\mathrm{mg}-25$ & 12025 & ENDF/B-VII.1 & \\
\hline $\mathrm{mg}-26$ & 12026 & ENDF/B-VII.1 & \\
\hline $\mathrm{mn}-55$ & 25055 & ENDF/B-VII.1 & \\
\hline mo & 42000 & BLO approximation data & \\
\hline mo-100 & 42100 & ENDF/B-VII.1 & \\
\hline mo-92 & 42092 & ENDF/B-VII.1 & \\
\hline mo-94 & 42094 & ENDF/B-VII.1 & \\
\hline mo-95 & 42095 & ENDF/B-VII.1 & \\
\hline mo-96 & 42096 & ENDF/B-VII.1 & \\
\hline mo-97 & 42097 & ENDF/B-VII.1 & \\
\hline mo-98 & 42098 & ENDF/B-VII.1 & \\
\hline mo-99 & 42099 & BLO approximation data & \\
\hline
\end{tabular}




\begin{tabular}{|c|c|c|c|}
\hline SCALE name & SCALE ID & Data source & Comment \\
\hline$n-14$ & 7014 & BLO approximation data & \\
\hline$n-15$ & 7015 & ENDF/B-VII.1 & \\
\hline na-23 & 11023 & ENDF/B-VII.1 & \\
\hline nb-93 & 41093 & ENDF/B-VI & \\
\hline nb-94 & 41094 & BLO approximation data & \\
\hline nb-95 & 41095 & ENDF/B-VII.1 & \\
\hline nd-142 & 60142 & BLO approximation data & \\
\hline nd-143 & 60143 & ENDF/B-VII.1 & \\
\hline nd-144 & 60144 & BLO approximation data & \\
\hline nd-145 & 60145 & ENDF/B-VII.1 & \\
\hline nd-146 & 60146 & ENDF/B-VII.1 & \\
\hline nd-147 & 60147 & BLO approximation data & \\
\hline nd-148 & 60148 & ENDF/B-VII.1 & \\
\hline nd-148 & 60148 & BLO approximation data & \\
\hline nd-150 & 60150 & BLO approximation data & \\
\hline ni-58 & 28058 & ENDF/B-VII.1 & \\
\hline ni-59 & 28059 & BLO approximation data & \\
\hline ni-60 & 28060 & ENDF/B-VII.1 & \\
\hline ni-61 & 28061 & ENDF/B-VI & \\
\hline ni-62 & 28062 & ENDF/B-VI & \\
\hline ni-64 & 28064 & ENDF/B-VI & \\
\hline np-234 & 93234 & ENDF/B-VII.1 & \\
\hline np-235 & 93235 & ENDF/B-VII.1 & \\
\hline np-236 & 93236 & ENDF/B-VII.1 & \\
\hline \multirow[t]{2}{*}{ np-237 } & 93237 & ENDF/B-VII.1 & \\
\hline & & $\chi$ covariance JENDL-4.0 & \\
\hline np-238 & 93238 & ENDF/B-VII.1 & \\
\hline np-239 & 93239 & ENDF/B-VII.1 & \\
\hline o-16 & 8016 & ENDF/B-VII.1 & \\
\hline o-17 & 8017 & BLO approximation data & \\
\hline o-beo & 5008016 & ENDF/B-VII.1 & Duplicate of o-16 \\
\hline o-uo2 & 1008016 & ENDF/B-VII.1 & Duplicate of o-16 \\
\hline p-31 & 15031 & BLO approximation data & \\
\hline pa-229 & 91229 & ENDF/B-VII.1 & \\
\hline pa-230 & 91230 & ENDF/B-VII.1 & \\
\hline \multirow[t]{2}{*}{ pa-231 } & 91231 & BLO approximation data & \\
\hline & & $\chi$ covariance JENDL-4.0 & \\
\hline pa-232 & 91232 & ENDF/B-VII.1 & \\
\hline \multirow[t]{2}{*}{ pa-233 } & 91233 & BLO approximation data & \\
\hline & & $\chi$ covariance JENDL-4.0 & \\
\hline
\end{tabular}




\begin{tabular}{|c|c|c|c|}
\hline SCALE name & SCALE ID & Data source & Comment \\
\hline pb-204 & 82204 & ENDF/B-VII.1 & \\
\hline pb-206 & 82206 & ENDF/B-VII.1 & \\
\hline pb-207 & 82207 & ENDF/B-VII.1 & \\
\hline pb-208 & 82208 & ENDF/B-VII.1 & \\
\hline pd-102 & 46102 & BLO approximation data & \\
\hline pd-104 & 46104 & BLO approximation data & \\
\hline pd-105 & 46105 & ENDF/B-VII.1 & \\
\hline pd-106 & 46106 & ENDF/B-VII.1 & \\
\hline pd-107 & 46107 & ENDF/B-VII.1 & \\
\hline pd-108 & 46108 & ENDF/B-VII.1 & \\
\hline pd-110 & 46110 & BLO approximation data & \\
\hline pm-147 & 61147 & ENDF/B-VII.1 & $\begin{array}{l}\text { Thermal and resonance range } \\
\text { uncertainty values from } \\
\text { Mughabghab }^{11}\end{array}$ \\
\hline pm-148 & 61148 & BLO approximation data & \\
\hline $\mathrm{pm}-148 \mathrm{~m}$ & 1061148 & BLO approximation data & \\
\hline pm-149 & 61149 & BLO approximation data & \\
\hline pm-151 & 61151 & BLO approximation data & \\
\hline pr-141 & 59141 & ENDF/B-VII.1 & \\
\hline pr-142 & 59142 & BLO approximation data & \\
\hline pr-143 & 59143 & BLO approximation data & \\
\hline pu-236 & 94236 & ENDF/B-VII.1 & \\
\hline pu-237 & 94237 & ENDF/B-VII.1 & \\
\hline pu-238 & 94238 & ENDF/B-VII.1 & \\
\hline pu-239 & 94239 & ENDF/B-VII.2 prelim & \\
\hline pu-240 & 94240 & ENDF/B-VII.1 & \\
\hline \multirow[t]{2}{*}{ pu-241 } & 94241 & ENDF/B-VII.1 & \\
\hline & & $\chi$ covariance JENDL-4.0 & \\
\hline pu-242 & 94242 & ENDF/B-VII.1 & \\
\hline pu-243 & 94243 & BLO approximation data & \\
\hline pu-244 & 94244 & ENDF/B-VII.1 & \\
\hline pu-246 & 94246 & ENDF/B-VII.1 & \\
\hline $\mathrm{rb}-85$ & 37085 & BLO approximation data & \\
\hline $\mathrm{rb}-86$ & 37086 & BLO approximation data & \\
\hline rb-87 & 37087 & BLO approximation data & \\
\hline re-185 & 75185 & ENDF/B-VI & \\
\hline re-187 & 75187 & ENDF/B-VI & \\
\hline rh-103 & 45103 & ENDF/B-VII.1 & $\begin{array}{l}\text { Uses ENDF/B-VII. } 1 \text { data uncertainty } \\
\text { in the thermal range for } \mathrm{MT}=102\end{array}$ \\
\hline rh-105 & 45105 & BLO approximation data & \\
\hline
\end{tabular}




\begin{tabular}{|c|c|c|c|}
\hline SCALE name & SCALE ID & Data source & Comment \\
\hline ru-100 & 44100 & BLO approximation data & \\
\hline ru-101 & 44101 & ENDF/B-VII.1 & \\
\hline ru-102 & 44102 & ENDF/B-VII.1 & \\
\hline ru-103 & 44103 & ENDF/B-VII.1 & \\
\hline ru-104 & 44104 & ENDF/B-VII.1 & \\
\hline ru-105 & 44105 & BLO approximation data & \\
\hline ru-106 & 44106 & ENDF/B-VII.1 & \\
\hline ru-96 & 44096 & BLO approximation data & \\
\hline ru-98 & 44098 & BLO approximation data & \\
\hline ru-99 & 44099 & BLO approximation data & \\
\hline $\mathrm{s}$ & 16000 & BLO approximation data & \\
\hline$s-32$ & 16032 & BLO approximation data & \\
\hline$s-33$ & 16033 & BLO approximation data & \\
\hline$s-34$ & 16034 & BLO approximation data & \\
\hline s-36 & 16036 & BLO approximation data & \\
\hline $\mathrm{sb}-121$ & 51121 & BLO approximation data & \\
\hline sb-123 & 51123 & BLO approximation data & \\
\hline sb-124 & 51124 & BLO approximation data & \\
\hline sb-125 & 51125 & BLO approximation data & \\
\hline sb-126 & 51126 & BLO approximation data & \\
\hline sc-45 & 21045 & ENDF/B-VI & \\
\hline se-74 & 34074 & BLO approximation data & \\
\hline se-76 & 34076 & BLO approximation data & \\
\hline se-77 & 34077 & BLO approximation data & \\
\hline se-78 & 34078 & BLO approximation data & \\
\hline se-79 & 34079 & BLO approximation data & \\
\hline se- 80 & 34080 & BLO approximation data & \\
\hline se- 82 & 34082 & BLO approximation data & \\
\hline si & 14000 & ENDF/B-VI & \\
\hline si-28 & 14028 & ENDF/B-VII.1 & \\
\hline si-29 & 14029 & ENDF/B-VII.1 & \\
\hline si-30 & 14030 & ENDF/B-VII.1 & \\
\hline si-28 in $\mathrm{SiO}_{2}$ & 14728 & ENDF/B-VII.1 & Duplicate of si-28 \\
\hline si-29 in $\mathrm{SiO}_{2}$ & 14729 & ENDF/B-0VII.1 & Duplicate of si-29 \\
\hline si-30 in $\mathrm{SiO}_{2}$ & 14730 & ENDF/B-VII.1 & Duplicate of si-30 \\
\hline sm-144 & 62144 & BLO approximation data & \\
\hline sm-147 & 62147 & BLO approximation data & \\
\hline sm-148 & 62148 & BLO approximation data & \\
\hline sm-149 & 62149 & ENDF/B-VII.1 & $\begin{array}{l}\text { Uses ENDF/B-VII. } 1 \text { data uncertainty } \\
\text { in the thermal range for } \mathrm{MT}=102\end{array}$ \\
\hline
\end{tabular}




\begin{tabular}{lll}
\hline SCALE name & SCALE ID & Data source \\
\hline sm-149 & 62149 & BLO approximation data \\
sm-150 & 62150 & BLO approximation data \\
sm-151 & 62151 & ENDF/B-VII.1 \\
sm-152 & 62152 & ENDF/B-VII.1 \\
sm-153 & 62153 & BLO approximation data \\
sm-154 & 62154 & BLO approximation data \\
sn-112 & 50112 & BLO approximation data \\
sn-113 & 50113 & BLO approximation data \\
sn-114 & 50114 & BLO approximation data \\
sn-115 & 50115 & BLO approximation data \\
sn-116 & 50116 & BLO approximation data \\
sn-117 & 50117 & BLO approximation data \\
sn-118 & 50118 & BLO approximation data \\
sn-119 & 50119 & BLO approximation data \\
sn-120 & 50120 & BLO approximation data \\
sn-122 & 50122 & BLO approximation data \\
sn-123 & 50123 & BLO approximation data \\
sn-124 & 50124 & BLO approximation data \\
sn-125 & 50125 & BLO approximation data \\
sn-126 & 50126 & BLO approximation data \\
sr-84 & 38084 & BLO approximation data \\
sr-86 & 38086 & BLO approximation data \\
sr-87 & 38087 & BLO approximation data \\
sr-88 & 38088 & BLO approximation data \\
sr-89 & 38089 & BLO approximation data \\
sr-90 & 38090 & BLO approximation data \\
ta-181 & 73181 & BLO approximation data \\
ta-182 & 73182 & BLO approximation data \\
tb-159 & 65159 & BLO approximation data \\
tb-160 & 65160 & BLO approximation data \\
tc-99 & 43099 & ENDF/B-VII.1 \\
te-120 & 52120 & BLO approximation data \\
te-122 & 52122 & BLO approximation data \\
te-123 & 52123 & BLO approximation data \\
te-124 & 52124 & BLO approximation data \\
& 52125 & BLO approximation data \\
te-125 & & BLO approximation data \\
te & &
\end{tabular}

Comment

Uses ENDF/B-VII.1 data uncertainty in the thermal range for $\mathrm{MT}=102$ 


\begin{tabular}{|c|c|c|c|}
\hline SCALE name & SCALE ID & Data source & Comment \\
\hline te- $129 \mathrm{~m}$ & 1052129 & BLO approximation data & \\
\hline te- 130 & 52130 & BLO approximation data & \\
\hline te- 132 & 52132 & BLO approximation data & \\
\hline th- -227 & 90227 & ENDF/B-VII.1 & \\
\hline th- 228 & 90228 & ENDF/B-VII.1 & \\
\hline th-229 & 90229 & ENDF/B-VII.1 & \\
\hline th- -230 & 90230 & ENDF/B-VII.1 & \\
\hline th- 231 & 90231 & ENDF/B-VII.1 & \\
\hline \multirow[t]{2}{*}{ th- 232} & 90232 & ENDF/B-VII.1 & \\
\hline & & $\chi$ covariance JENDL-4.0 & \\
\hline th-233 & 90233 & ENDF/B-VII.1 & \\
\hline th-234 & 90234 & ENDF/B-VII.1 & \\
\hline ti & 22000 & BLO approximation data & \\
\hline ti-46 & 22046 & ENDF/B-VII.1 & \\
\hline ti-47 & 22047 & ENDF/B-VII.1 & \\
\hline ti-48 & 22048 & ENDF/B-VII.1 & \\
\hline ti-49 & 22049 & ENDF/B-VII.1 & \\
\hline ti-50 & 22050 & ENDF/B-VII.1 & \\
\hline tl-203 & 81203 & ENDF/B-VII.1 & \\
\hline tl-205 & 81205 & ENDF/B-VII.1 & \\
\hline tm-169 & 69169 & ENDF/B-VII.1 & \\
\hline tm-170 & 69170 & ENDF/B-VII.1 & \\
\hline $\mathrm{u}-230$ & 92230 & ENDF/B-VII.1 & \\
\hline $\mathrm{u}-231$ & 92231 & ENDF/B-VII.1 & \\
\hline $\mathrm{u}-232$ & 92232 & ENDF/B-VII.1 & \\
\hline \multirow[t]{2}{*}{$\mathrm{u}-233$} & 92233 & ENDF/B-VII.1 & \\
\hline & & $\chi$ covariance JENDL-4.0 & \\
\hline $\mathrm{u}-234$ & 92234 & ENDF/B-VII.1 & \\
\hline $\mathrm{u}-235$ & 92235 & ENDF/B-VII. 2 prelim & \\
\hline $\mathrm{u}-236$ & 92236 & ENDF/B-VII.1 & \\
\hline $\mathrm{u}-237$ & 92237 & BLO approximation data & \\
\hline $\mathrm{u}-238$ & 92238 & ENDF/B-VII.1 & \\
\hline $\mathrm{u}-239$ & 92239 & BLO approximation data & \\
\hline $\mathrm{u}-240$ & 92240 & BLO approximation data & \\
\hline $\mathrm{u}-241$ & 92241 & BLO approximation data & \\
\hline u-uo2 & 1092235 & ENDF/B-VII.1 & Duplicate of $\mathrm{u}-235$ \\
\hline $\mathrm{v}$ & 23000 & BLO approximation data & \\
\hline $\mathrm{W}$ & 74000 & BLO approximation data & \\
\hline $\mathrm{W}-180$ & 74180 & ENDF/B-VII.1 & \\
\hline w-182 & 74182 & ENDF/B-VII.1 & \\
\hline
\end{tabular}




\begin{tabular}{|c|c|c|c|}
\hline SCALE name & SCALE ID & Data source & Comment \\
\hline $\mathrm{W}-183$ & 74183 & ENDF/B-VII.1 & \\
\hline $\mathrm{w}-184$ & 74184 & ENDF/B-VII.1 & \\
\hline w-186 & 74186 & ENDF/B-VII.1 & \\
\hline xe-123 & 54123 & BLO approximation data & \\
\hline $\mathrm{xe}-124$ & 54124 & BLO approximation data & \\
\hline xe-126 & 54126 & BLO approximation data & \\
\hline xe-128 & 54128 & BLO approximation data & \\
\hline xe-129 & 54129 & BLO approximation data & \\
\hline xe-130 & 54130 & BLO approximation data & \\
\hline xe-131 & 54131 & ENDF/B-VII.1 & \\
\hline xe-132 & 54132 & ENDF/B-VII.1 & \\
\hline xe-133 & 54133 & BLO approximation data & \\
\hline xe-134 & 54134 & ENDF/B-VII.1 & \\
\hline xe-135 & 54135 & BLO approximation data & \\
\hline xe-136 & 54136 & BLO approximation data & \\
\hline$y-89$ & 39089 & ENDF/B-VII.1 & \\
\hline$y-90$ & 39090 & BLO approximation data & \\
\hline$y-91$ & 39091 & BLO approximation data & \\
\hline $\mathrm{zr}$ & 40000 & BLO approximation data & \\
\hline zr-90 & 40090 & ENDF/B-VII.1 & \\
\hline zr-91 & 40091 & ENDF/B-VII.1 & \\
\hline zr-92 & 40092 & ENDF/B-VII.1 & \\
\hline zr-93 & 40093 & ENDF/B-VII.1 & \\
\hline zr-94 & 40094 & ENDF/B-VII.1 & \\
\hline zr-95 & 40095 & ENDF/B-VII.1 & \\
\hline zr-96 & 40096 & ENDF/B-VII.1 & \\
\hline zr-90-zr5h8 & 1040090 & ENDF/B-VII.1 & Duplicate of zr-90 \\
\hline zr-91-zr5h8 & 1040091 & ENDF/B-VII.1 & Duplicate of zr-91 \\
\hline zr-92-zr5h8 & 1040092 & ENDF/B-VII.1 & Duplicate of zr-92 \\
\hline zr-93-zr5h8 & 1040093 & ENDF/B-VII.1 & Duplicate of zr-93 \\
\hline zr-94-zr5h8 & 1040094 & ENDF/B-VII.1 & Duplicate of zr-94 \\
\hline zr-95-zr5h8 & 1040095 & ENDF/B-VII.1 & Duplicate of zr-95 \\
\hline zr-96-zr5h8 & 1040096 & ENDF/B-VII.1 & Duplicate of zr-96 \\
\hline
\end{tabular}


Table 10.2.2. Covariance data with cross-correlations between nuclide reactions

\begin{tabular}{cccc}
\hline Nuclide 1 & Reaction 1 & Nuclide 2 & Reaction 2 \\
\hline${ }^{239} \mathrm{Pu}$ & Fission & ${ }^{6} \mathrm{Li}$ & Triton production \\
${ }^{239} \mathrm{Pu}$ & Fission & ${ }^{197} \mathrm{Au}$ & Capture \\
${ }^{239} \mathrm{Pu}$ & Fission & ${ }^{235} \mathrm{U}$ & Fission \\
${ }^{239} \mathrm{Pu}$ & Fission & ${ }^{238} \mathrm{U}$ & Fission \\
${ }^{235} \mathrm{U}$ & Fission & ${ }^{197} \mathrm{Au}$ & Capture \\
${ }^{235} \mathrm{U}$ & Fission & ${ }^{6} \mathrm{Li}$ & Triton production \\
${ }^{238} \mathrm{U}$ & Capture & ${ }^{197} \mathrm{Au}$ & Capture \\
${ }^{238} \mathrm{U}$ & Capture & ${ }^{235} \mathrm{U}$ & Fission \\
\hline
\end{tabular}

\subsubsection{SCALE 6.1 44-group covariance library}

The older 44-group covariance library distributed with SCALE 6.0 and SCALE 6.1 is included with this distribution for backwards compatibility. The 44-group covariance library provides uncertainty data for a total of 401 materials, including some duplication for materials with multiple thermal scattering kernels. However, the 44-group library was created prior to the official release of ENDF/B-VII.1. Therefore, it is recommended that the 56- or 252-group covariances be used rather than the 44-group. As discussed in Sect. 10.2.1, it is assumed that covariances taken from one data evaluation such as ENDF/B-VI or JENDL-3.3 can also be applied to other evaluations of the same data, such as ENDF/B-VII. If this is done judiciously for cases in which the nuclear data evaluations are similar, then the covariances taken from one source should be a reasonable representation of uncertainties for the other evaluations. Among the materials in the SCALE 44-group library with covariances taken from high-fidelity nuclear data evaluations are the following:

(a) ENDF/B-VII evaluations (includes both VII.O and pre-release covariances proposed for VII.1, but no official ENDF/B-VII.1):

$$
\mathrm{Au},{ }^{209} \mathrm{Bi},{ }^{59} \mathrm{Co},{ }^{152,154,155,156} \mathrm{Gd},{ }^{191,193} \mathrm{I},{ }^{7} \mathrm{Li},{ }^{23} \mathrm{Na},{ }^{93} \mathrm{Nb},{ }^{58} \mathrm{Ni},{ }^{99} \mathrm{Tc},{ }^{232} \mathrm{Th},{ }^{48} \mathrm{Ti},{ }^{239} \mathrm{Pu},{ }^{233,235,238} \mathrm{U}, \mathrm{V}
$$

(b) ENDF/B-VI evaluations:

$$
\mathrm{Al},{ }^{241} \mathrm{Am},{ }^{10} \mathrm{~B},{ }^{12} \mathrm{C},{ }^{50,52,53,54} \mathrm{Cr},{ }^{63,65} \mathrm{Cu},{ }^{54,56,57} \mathrm{Fe}, \mathrm{In},{ }^{55} \mathrm{Mn},{ }^{60,61,62,64} \mathrm{Ni},{ }^{206,207,208} \mathrm{~Pb},{ }^{242} \mathrm{Pu},{ }^{28,29} \mathrm{Si}
$$

(c) JENDL-3.3 evaluations:

$$
{ }^{11} \mathrm{~B},{ }^{1} \mathrm{H},{ }^{16} \mathrm{O},{ }^{240,241} \mathrm{Pu}
$$

Two modifications were also made to the ENDF/B-VII evaluated nubar covariances. These nubar uncertainties are believed to be more realistic. The ENDF/B-VII.0 ${ }^{235} \mathrm{U}$ thermal nubar uncertainty of $0.71 \%$ was revised to the JENDL-3.3 value of $0.31 \%$. In addition, the thermal nubar certainty in the prereleased ENDF/B-VII. $1{ }^{233} \mathrm{U}$ evaluation was modified to the value in a recent ORNL data evaluation. ${ }^{14}$ This ORNL ${ }^{233} \mathrm{U}$ cross section evaluation also provided the thermal and resonance cross sections for the prereleased ENDF/B-VII.1 data. The ENDF/B-VII.1 pre-release nubar data for ${ }^{239} \mathrm{Pu}$ was incomplete when the 44-group covariance library was generated, so ${ }^{239} \mathrm{Pu}$ nubar data are included from ENDF/B-V, the most current data available at that time. This value is much higher than the current estimated uncertainty in ${ }^{239} \mathrm{Pu}$ nubar. The basic ENDF/B uncertainty files that were changed are described in Table 10.2.3.

Several modifications were also made to the uncertainties obtained from the BLO data. The BLO thermal uncertainties for ${ }^{1} \mathrm{H}$ capture and elastic and for ${ }^{16} \mathrm{O}$ elastic were modified to the JENDL-3.3 values of $0.5 \%$ and $0.1 \%$, respectively. Similarly, the uncertainty in the ${ }^{10} \mathrm{~B}$ (n,alpha) thermal cross section was modified to the ENDF/B-VI value of about $0.2 \%$, since this is more consistent with the Mughabghab 
integral uncertainty. The uncertainty in the ${ }^{149} \mathrm{Sm}$ resonance capture integral is not provided in the 2006 edition of Mughabghab's text; therefore it was set to the value of $5.7 \%$ which was obtained from an earlier tabulation by Mughabghab. ${ }^{12}$

Table 10.2.3. Summary of changes made to covariance evaluations for the 44-group library

\begin{tabular}{c|l}
\hline $\begin{array}{c}\text { ENDF/B-VII.1 pre-release } \\
{ }^{239} \mathrm{Pu}\end{array}$ & $\begin{array}{l}\text { Data were incomplete at time of library generation, } \\
\text { so ENDF/B-V data were used for nubar. }\end{array}$ \\
\hline${ }^{235} \mathrm{U}-\mathrm{BII}$ & Thermal nubar modified to JENDL-3.3 value \\
\hline${ }^{\mathrm{ENDF} / \mathrm{B}-\mathrm{VII}}$ & $\begin{array}{l}\text { Thermal nubar modified to value from ORNL } \\
\text { internal evaluation }\end{array}$ \\
\hline${ }^{\mathrm{ENDF} / \mathrm{B}-\mathrm{VI}}$ & $\begin{array}{l}\text { Thermal uncertainties were added to total } \\
\text { cross section (set equal to capture uncertainties) }\end{array}$ \\
\hline${ }^{24} \mathrm{Am},{ }^{29} \mathrm{Si},{ }^{30} \mathrm{Si},{ }^{206} \mathrm{~Pb},{ }^{57} \mathrm{Fe}$ & $\begin{array}{l}\text { In elastic scatter uncertainty, corrected cross } \\
\text { reference to MT=102 from original value of } \\
\mathrm{MT}=1.02\end{array}$ \\
\hline${ }^{\mathrm{ENDF}} \mathrm{Pb} /{ }^{207} \mathrm{~Pb}-\mathrm{VI}$ & $\begin{array}{l}\text { Removed MT=3 due to inconsistency with other MT } \\
\text { values, resulting in very large uncertainty predictions }\end{array}$ \\
\hline
\end{tabular}

At the time of the preparation of the 44-group covariance library, ENDF/B did not provide fission spectra uncertainty estimates. The methodology used to construct these data for the 44-group covariance library is described in Broadhead and Wagschal. ${ }^{15}$ In this approach, the fission spectrum is represented as either a Watt or Maxwellian distribution. These energy distributions are widely used to represent fission spectra and have been commonly employed in many ENDF/B evaluations. For example, Watt and Maxwellian expressions were used almost exclusively to describe fission spectra in ENDF/B-V and also for many ENDF/B-VI evaluations. More recent evaluations for some important fissionable nuclides have replaced the simple Watt and Maxwellian analytical expressions by distributions such as the Madland-Nix spectrum obtained from more phenomenological nuclear fission models. However, it is assumed here that uncertainties based on an appropriate Watt or Maxwellian representation of the fission spectrum can be transferred to the actual fission spectra contained in the different multigroup cross section libraries.

The methodology in Broadhead and Wagschal ${ }^{15}$ determines energy-dependent covariances from uncertainties and correlations in the $a$ and $b$ parameters for the Watt spectrum or the $T$ parameter for a Maxwellian spectrum, appearing the analytical expressions given below:

$$
\begin{aligned}
& \text { Watt Spectrum: } \chi(E)=\frac{e^{-E / a}}{I} \sinh (\sqrt{b E}) \\
& \text { Maxwellian Spectrum: } \chi(E)=\frac{\sqrt{E} e^{-E / T}}{I}
\end{aligned}
$$

In these expressions, the parameter "I" is the normalization factor required to normalize the integrated spectrum to unity. The value of "I" is fixed by the values of the other parameters. Due to the 
normalization constraint, the fission spectrum covariance includes anti-correlations. The assumed fission spectra parameters and uncertainties are given in Maerker, Marable, and Wagschal $1980^{16}$ and in Howerton and Doyas 1971. ${ }^{17}$

Table 10.2.4 shows that fission spectra covariances are not provided for all fissionable materials in the SCALE multigroup cross sections. Table 10.2.5 lists the fissionable nuclides without fission spectra covariances on the 44-group covariance library.

Table 10.2.4. Source of fission spectrum parameters and uncertainties

\begin{tabular}{lllllll}
\hline $\begin{array}{c}\text { Watt } \\
\text { spectrum }\end{array}$ & $a$ or $T$ & $b$ & $\begin{array}{c}\text { Source of } \\
\text { parameters }\end{array}$ & $\begin{array}{c}\partial a \text { or } \partial T \\
(\%)\end{array}$ & $\begin{array}{c}\partial b \\
(\%)\end{array}$ & $\begin{array}{c}\text { Source of } \\
\text { uncertainty }\end{array}$ \\
\hline${ }^{235} \mathrm{U}$ & 0.988 & 2.249 & ENDF/B-V & 1.2 & 5.9 & TANS $^{16}$ \\
${ }^{238} \mathrm{U}$ & 0.881 & 3.401 & ENDF/B-V & 1.2 & 5.9 & TANS $^{16}$ \\
${ }^{233} \mathrm{U}$ & 0.977 & 2.546 & ENDF/B-V & 1.2 & 5.9 & TANS $^{16}$ \\
${ }^{239} \mathrm{Pu}$ & 0.966 & 2.842 & ENDF/B-V & 1.2 & 5.9 & TANS $^{16}$ \\
${ }^{232} \mathrm{Th}$ & 1.0888 & 1.6871 & ENDF/B-V & 1.2 & 5.9 & TANS $^{16}$ \\
${ }^{252} \mathrm{Cf}$ & 1.025 & 2.926 & ENDF/B-V & 1.2 & 5.9 & TANS $^{16}$ \\
\hline${ }^{\mathrm{Maxwellian}}$ & & & & & & \\
${ }_{\mathrm{Spectrum}}$ & & - & ENDF/B-V & 3.01 & - & NSE $^{17}$ \\
\hline${ }^{238} \mathrm{Pu}$ & 1.330 & - & ENDF/B-V & 2.97 & - & NSE $^{17}$ \\
${ }^{240} \mathrm{Pu}$ & 1.346 & - & ENDF/B-V & 2.50 & - & $\mathrm{NSE}^{17}$ \\
${ }^{241} \mathrm{Pu}$ & 1.3597 & - & ENDF/B-V & 5.24 & - & NSE $^{17}$ \\
${ }^{242} \mathrm{Pu}$ & 1.337 & & & &
\end{tabular}

Table 10.2.5. Fissionable nuclides with missing fission spectrum uncertainty data in covariance library

\begin{tabular}{lll}
\hline${ }^{241} \mathrm{Am}$ & ${ }^{244} \mathrm{Cm}$ & ${ }^{238} \mathrm{Pu}$ \\
${ }^{242} \mathrm{Am}$ & ${ }^{245} \mathrm{Cm}$ & ${ }^{243} \mathrm{Pu}$ \\
${ }^{243} \mathrm{Am}$ & ${ }^{246} \mathrm{Cm}$ & $\mathrm{Pu}$ \\
${ }^{249} \mathrm{Bk}$ & ${ }^{247} \mathrm{Cm}$ & $\mathrm{Th}$ \\
${ }^{249} \mathrm{Cf}$ & ${ }^{248} \mathrm{Cm}$ & ${ }^{232} \mathrm{U}$ \\
${ }^{250} \mathrm{Cf}$ & ${ }^{237} \mathrm{~Np}$ & ${ }^{234} \mathrm{U}$ \\
${ }^{251} \mathrm{Cf}$ & ${ }^{238} \mathrm{~Np}$ & $\mathrm{U}$ \\
${ }^{253} \mathrm{Cf}$ & ${ }^{239} \mathrm{~Np}$ & \\
${ }^{242} \mathrm{Cm}$ & ${ }^{231} \mathrm{~Pa}$ & \\
${ }^{243} \mathrm{Cm}$ & ${ }^{233} \mathrm{~Pa}$ & \\
\hline
\end{tabular}


Table 10.2.6 describes the contents of the library using the following nomenclature:

1. ENDF/B-VII.0: evaluated covariance data released with ENDF/B-VII.0

2. ENDF/B-VII-p: recently evaluated data proposed for future release of ENDF/B-VII.1

3. ENDF/B-VI: evaluated covariance data released with ENDF/B-VI

4. JENDL-3.3: evaluated covariance data in JENDL-3.3

5. BLO approximate data: lo-fi covariances from BLO project

6. BLO LANL evaluation: LANL R-matrix evaluation from BLO project

7. SG-26: approximate covariances from WPEC Subgroup-26

Table 10.2.6. Contents of SCALE 6.1 44-group covariance library

\begin{tabular}{|c|c|c|}
\hline SCALE name & Data source & Comments \\
\hline ac-225 & BLO approximate data & \\
\hline ac-226 & BLO approximate data & \\
\hline ac-227 & BLO approximate data & \\
\hline ag-107 & BLO approximate data & \\
\hline ag-109 & BLO approximate data & \\
\hline ag-110m & BLO approximate data & \\
\hline ag-111 & BLO approximate data & \\
\hline al-27 & ENDF/B-VI & \\
\hline am-241 & ENDF/B-VI & $\mathrm{MT}=452$ added corrections for total and elastic) \\
\hline am-242 & SG-26 & Thermal uncertainty replaced by Mughabghab value \\
\hline $\mathrm{am}-242 \mathrm{~m}$ & SG-26 & Thermal uncertainty replaced by Mughabghab value \\
\hline am-243 & BLO approximate data & \\
\hline am-244 & BLO approximate data & \\
\hline $\mathrm{am}-244 \mathrm{~m}$ & BLO approximate data & \\
\hline ar-36 & BLO approximate data & \\
\hline $\operatorname{ar}-38$ & BLO approximate data & \\
\hline ar- -40 & BLO approximate data & \\
\hline as-74 & BLO approximate data & \\
\hline as-75 & BLO approximate data & \\
\hline au-197 & ENDF/B-VII-p & Pre-released evaluation proposed for ENDF/B-VII.1 \\
\hline$b-10$ & $\begin{array}{l}\text { BLO LANL evaluation } \\
+ \text { ENDF/B-VI }\end{array}$ & $\begin{array}{l}\text { LANL high-fidelity covariance, with ENDF/B-VI for } \\
\text { thermal }\end{array}$ \\
\hline b-11 & JENDL 3.3 & \\
\hline ba-130 & BLO approximate data & \\
\hline ba-132 & BLO approximate data & \\
\hline ba-133 & BLO approximate data & \\
\hline ba-135 & BLO approximate data & \\
\hline ba-136 & BLO approximate data & \\
\hline ba-137 & BLO approximate data & \\
\hline
\end{tabular}




\begin{tabular}{|c|c|c|}
\hline SCALE name & Data source & Comments \\
\hline ba-138 & BLO approximate data & \\
\hline ba-140 & BLO approximate data & \\
\hline $\begin{array}{l}\text { be-7 } \\
\text { be-9 }\end{array}$ & $\begin{array}{l}\text { BLO approximate data } \\
\text { BLO approximate data }\end{array}$ & \\
\hline Bebound & BLO approximate data & Duplicate of ${ }^{9} \mathrm{Be}$ \\
\hline bi-209 & ENDF/B-VII-p & Pre-released evaluation proposed for ENDF/B-VII.1 \\
\hline $\begin{array}{l}\text { bk-249 } \\
\text { bk-250 }\end{array}$ & $\begin{array}{l}\text { BLO approximate data } \\
\text { BLO approximate data }\end{array}$ & \\
\hline br-79 & BLO approximate data & \\
\hline br-81 & BLO approximate data & \\
\hline $\mathrm{C}$ & ENDF/B-VI & \\
\hline C-graphite & ENDF/B-VI & Duplicate of carbon \\
\hline $\begin{array}{l}\mathrm{Ca} \\
\mathrm{ca}-40 \\
\mathrm{ca}-42 \\
\mathrm{ca}-43 \\
\mathrm{ca}-44 \\
\mathrm{ca}-46 \\
\mathrm{ca}-48\end{array}$ & $\begin{array}{l}\text { BLO approximate data } \\
\text { BLO approximate data } \\
\text { BLO approximate data } \\
\text { BLO approximate data } \\
\text { BLO approximate data } \\
\text { BLO approximate data } \\
\text { BLO approximate data }\end{array}$ & \\
\hline $\mathrm{Cd}$ & BLO approximate data & \\
\hline cd-106 & BLO approximate data & \\
\hline cd-108 & BLO approximate data & \\
\hline cd-110 & BLO approximate data & \\
\hline cd-111 & BLO approximate data & \\
\hline cd-112 & BLO approximate data & \\
\hline cd-113 & BLO approximate data & \\
\hline $\begin{array}{l}\text { cd-114 } \\
\text { cd-115m }\end{array}$ & $\begin{array}{l}\text { BLO approximate data } \\
\text { BLO approximate data }\end{array}$ & \\
\hline cd-116 & BLO approximate data & \\
\hline $\begin{array}{l}\text { cd-136 } \\
\text { cd-138 } \\
\text { cd-139 } \\
\text { cd-140 }\end{array}$ & $\begin{array}{l}\text { BLO approximate data } \\
\text { BLO approximate data } \\
\text { BLO approximate data } \\
\text { BLO approximate data }\end{array}$ & \\
\hline cd-141 & BLO approximate data & \\
\hline cd-142 & BLO approximate data & \\
\hline ce-143 & BLO approximate data & \\
\hline ce-144 & BLO approximate data & \\
\hline cf-249 & BLO approximate data & \\
\hline cf- -250 & BLO approximate data & \\
\hline cf- 251 & BLO approximate data & \\
\hline
\end{tabular}




\begin{tabular}{|c|c|c|}
\hline SCALE name & Data source & Comments \\
\hline cf- 252 & BLO approximate data & \\
\hline $\begin{array}{l}\text { cf- } 253 \\
\text { cf- } 254\end{array}$ & $\begin{array}{l}\text { BLO approximate data } \\
\text { BLO approximate data }\end{array}$ & \\
\hline $\begin{array}{l}\mathrm{Cl} \\
\mathrm{cl}-35 \\
\mathrm{cl}-37\end{array}$ & $\begin{array}{l}\text { BLO approximate data } \\
\text { BLO approximate data } \\
\text { BLO approximate data }\end{array}$ & \\
\hline $\begin{array}{l}\mathrm{cm}-241 \\
\mathrm{~cm}-242\end{array}$ & $\begin{array}{l}\text { BLO approximate data } \\
\text { SG-26 }\end{array}$ & Thermal uncertainty replaced by Mughabghab value \\
\hline $\mathrm{cm}-243$ & SG-26 & Thermal uncertainty replaced by Mughabghab value \\
\hline $\mathrm{cm}-244$ & SG-26 & Thermal uncertainty replaced by Mughabghab value \\
\hline $\mathrm{cm}-245$ & SG-26 & Thermal uncertainty replaced by Mughabghab value \\
\hline $\mathrm{cm}-246$ & BLO approximate data & \\
\hline $\mathrm{cm}-247$ & BLO approximate data & \\
\hline $\begin{array}{l}\mathrm{cm}-248 \\
\mathrm{~cm}-249 \\
\mathrm{~cm}-250\end{array}$ & $\begin{array}{l}\text { BLO approximate data } \\
\text { BLO approximate data } \\
\text { BLO approximate data }\end{array}$ & \\
\hline $\begin{array}{l}\text { co-58 } \\
\text { co-58m } \\
\text { co-59 }\end{array}$ & $\begin{array}{l}\text { BLO approximate data } \\
\text { BLO approximate data } \\
\text { ENDF/B-VII-p }\end{array}$ & Pre-released evaluation proposed for ENDF/B-VII.1 \\
\hline cr-50 & ENDF/B-VI & $\begin{array}{l}\mathrm{LB}=8 \text { representation caused problematic representation } \\
\text { of cross section uncertainty due to use of fine energy } \\
\text { group structure. Tests were performed to determine how } \\
\text { to handle this problem. } \mathrm{LB}=8 \text { data were removed in the } \\
\text { final results. }\end{array}$ \\
\hline cr-52 & ENDF/B-VI & $\begin{array}{l}\mathrm{LB}=8 \text { representation caused problematic representation } \\
\text { of cross section uncertainty due to use of fine energy } \\
\text { group structure. Tests were performed to determine how } \\
\text { to handle this problem. } \mathrm{LB}=8 \text { data were removed in the } \\
\text { final results. }\end{array}$ \\
\hline cr-53 & ENDF/B-VI & $\begin{array}{l}\mathrm{LB}=8 \text { representation caused problematic representation } \\
\text { of cross section uncertainty due to use of fine energy } \\
\text { group structure. Tests were performed to determine how } \\
\text { to handle this problem. } \mathrm{LB}=8 \text { data were removed in the } \\
\text { final results. }\end{array}$ \\
\hline cr-54 & ENDF/B-VI & $\begin{array}{l}\mathrm{LB}=8 \text { representation caused problematic representation } \\
\text { of cross section uncertainty due to use of fine energy } \\
\text { group structure. Tests were performed to determine how } \\
\text { to handle this problem. } \mathrm{LB}=8 \text { data were removed in the } \\
\text { final results. }\end{array}$ \\
\hline
\end{tabular}

cs-133 BLO approximate data

cs-134 BLO approximate data

cs-135 BLO approximate data 


\begin{tabular}{|c|c|c|}
\hline SCALE name & Data source & Comments \\
\hline cs-136 & BLO approximate data & \\
\hline cs-137 & BLO approximate data & \\
\hline cu-63 & ENDF/B-VI & \\
\hline cu-65 & ENDF/B-VI & \\
\hline $\begin{array}{l}\text { dy-156 } \\
\text { dy-158 } \\
\text { dy-160 }\end{array}$ & $\begin{array}{l}\text { BLO approximate data } \\
\text { BLO approximate data } \\
\text { BLO approximate data }\end{array}$ & \\
\hline dy-161 & BLO approximate data & \\
\hline dy-162 & BLO approximate data & \\
\hline dy-163 & BLO approximate data & \\
\hline $\begin{array}{l}\text { dy-164 } \\
\text { er-162 } \\
\text { er-164 }\end{array}$ & $\begin{array}{l}\text { BLO approximate data } \\
\text { BLO approximate data } \\
\text { BLO approximate data }\end{array}$ & \\
\hline er-166 & BLO approximate data & \\
\hline $\begin{array}{l}\text { er-167 } \\
\text { er-168 } \\
\text { er-170 }\end{array}$ & $\begin{array}{l}\text { BLO approximate data } \\
\text { BLO approximate data } \\
\text { BLO approximate data }\end{array}$ & \\
\hline $\begin{array}{l}\text { es- }-253 \\
\text { es- } 254 \\
\text { es-255 }\end{array}$ & $\begin{array}{l}\text { BLO approximate data } \\
\text { BLO approximate data } \\
\text { BLO approximate data }\end{array}$ & \\
\hline eu-151 & BLO approximate data & \\
\hline eu-152 & BLO approximate data & \\
\hline eu-153 & BLO approximate data & \\
\hline eu-154 & BLO approximate data & \\
\hline $\begin{array}{l}\text { eu-155 } \\
\text { eu-156 } \\
\text { eu-157 }\end{array}$ & $\begin{array}{l}\text { BLO approximate data } \\
\text { BLO approximate data } \\
\text { BLO approximate data }\end{array}$ & \\
\hline$f-19$ & BLO approximate data & \\
\hline fe- 54 & ENDF/B-VI & $\begin{array}{l}\mathrm{LB}=8 \text { representation caused problematic representation } \\
\text { of cross section uncertainty due to use of fine energy } \\
\text { group structure. Tests were performed to determine how } \\
\text { to handle this problem. } \mathrm{LB}=8 \text { data were removed in the } \\
\text { final results. }\end{array}$ \\
\hline fe-56 & ENDF/B-VI & $\begin{array}{l}\mathrm{LB}=8 \text { representation caused problematic representation } \\
\text { of cross section uncertainty due to use of fine energy } \\
\text { group structure. Tests were performed to determine how } \\
\text { to handle this problem. } \mathrm{LB}=8 \text { data were removed in the } \\
\text { final results. }\end{array}$ \\
\hline fe-57 & ENDF/B-VI & $\begin{array}{l}\text { Error in file corrected } \\
\mathrm{LB}=8 \text { representation caused problematic representation } \\
\text { of cross section uncertainty due to use of fine energy } \\
\text { group structure. Tests were performed to determine how }\end{array}$ \\
\hline
\end{tabular}




\begin{tabular}{|c|c|c|}
\hline SCALE name & Data source & Comments \\
\hline & & $\begin{array}{l}\text { to handle this problem. } \mathrm{LB}=8 \text { data were removed in the } \\
\text { final results. }\end{array}$ \\
\hline $\mathrm{fm}-255$ & $\begin{array}{l}\text { ENDF/B-VI } \\
\text { BLO approximate data }\end{array}$ & $\begin{array}{l}\mathrm{LB}=8 \text { representation caused problematic representation } \\
\text { of cross section uncertainty due to use of fine energy } \\
\text { group structure. Tests were performed to determine how } \\
\text { to handle this problem. } \mathrm{LB}=8 \text { data were removed in the } \\
\text { final results. New material not in previous } \\
\text { SCALE } 5.1 \text { covariance libraries. }\end{array}$ \\
\hline $\begin{array}{l}\text { Ga } \\
\text { ga-69 } \\
\text { ga-71 }\end{array}$ & $\begin{array}{l}\text { BLO approximate data } \\
\text { BLO approximate data } \\
\text { BLO approximate data }\end{array}$ & \\
\hline $\begin{array}{l}\operatorname{gd}-152 \\
\text { gd-153 }\end{array}$ & $\begin{array}{l}\text { ENDF/B-VII.0 } \\
\text { BLO approximate data }\end{array}$ & \\
\hline gd-154 & ENDF/B-VII.0 & \\
\hline $\operatorname{gd}-155$ & ENDF/B-VII.0 & \\
\hline gd-156 & ENDF/B-VII.0 & \\
\hline gd-157 & ENDF/B-VII.0 & \\
\hline $\operatorname{gd}-158$ & ENDF/B-VII.0 & \\
\hline gd-160 & ENDF/B-VII.0 & \\
\hline $\begin{array}{l}\text { ge- } 70 \\
\text { ge- } 72\end{array}$ & $\begin{array}{l}\text { BLO approximate data } \\
\text { BLO approximate data }\end{array}$ & \\
\hline ge-73 & BLO approximate data & \\
\hline ge-74 & BLO approximate data & \\
\hline ge-76 & BLO approximate data & \\
\hline h-1 & $\begin{array}{l}\text { BLO LANL evaluation } \\
+ \text { JENDL } 3.3\end{array}$ & $\begin{array}{l}\text { LANL covariance above } 5 \mathrm{keV} \text {; } \\
\text { JENDL values below } 5 \mathrm{keV}\end{array}$ \\
\hline h-ZrH & $\begin{array}{l}\text { BLO LANL evaluation } \\
+ \text { JENDL } 3 \text {. }\end{array}$ & Duplicate of ${ }^{1} \mathrm{H}$ \\
\hline h-poly & $\begin{array}{l}\text { BLO LANL evaluation } \\
+ \text { JENDL } 3 \text {. }\end{array}$ & Duplicate of ${ }^{1} \mathrm{H}$ \\
\hline Hfreegas & $\begin{array}{l}\text { BLO LANL evaluation } \\
+ \text { JENDL } 3 \text {. }\end{array}$ & Duplicate of ${ }^{1} \mathrm{H}$ \\
\hline $\mathrm{h}-2$ & BLO approximate data & \\
\hline Dfreegas & BLO approximate data & Duplicate of ${ }^{2} \mathrm{H}$ \\
\hline h-3 & BLO approximate data & \\
\hline he-3 & BLO approximate data & \\
\hline he-4 & BLO approximate data & \\
\hline Hf & BLO approximate data & \\
\hline hf-174 & BLO approximate data & \\
\hline hf-176 & BLO approximate data & \\
\hline
\end{tabular}




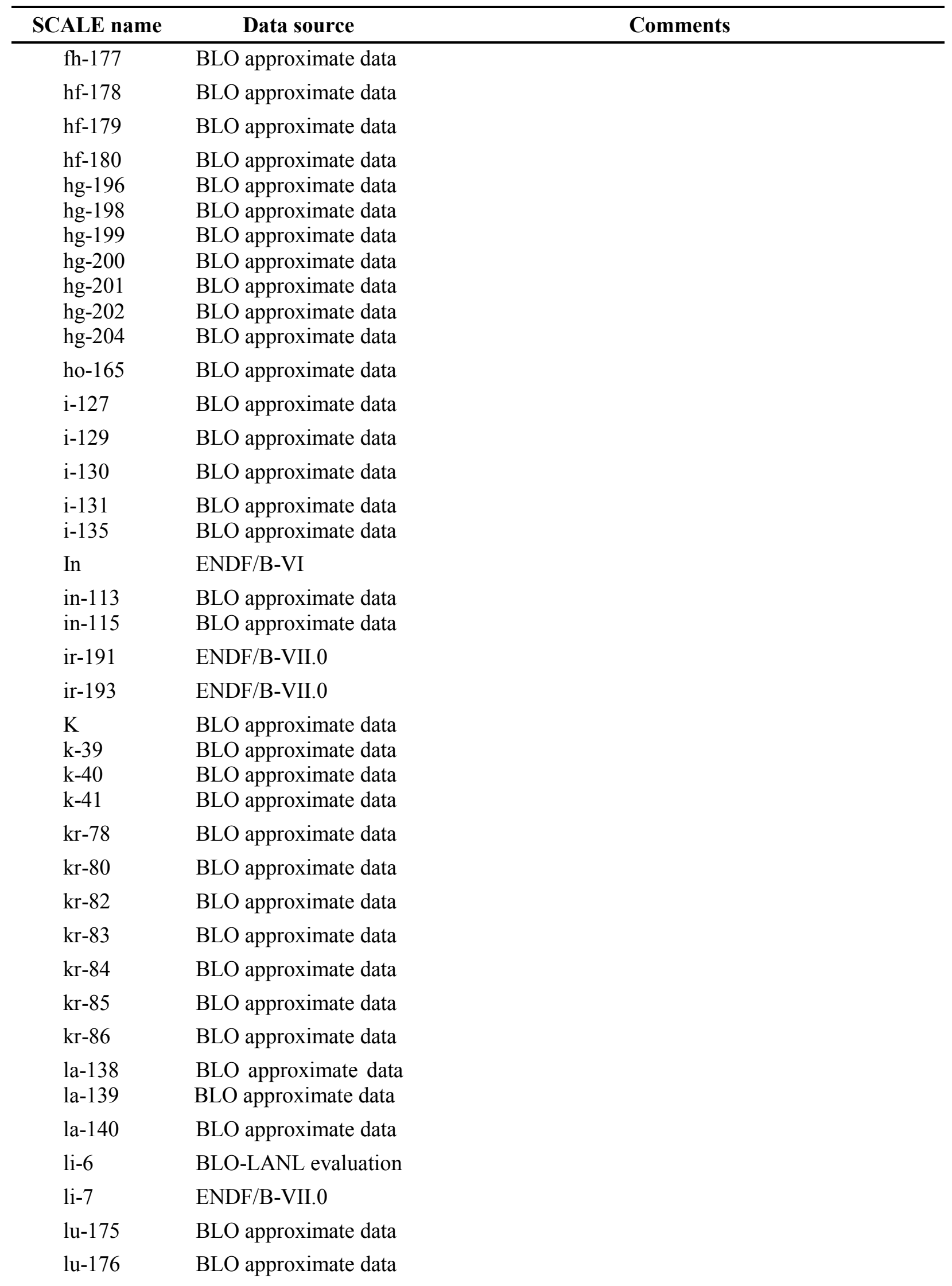




\begin{tabular}{|c|c|c|}
\hline SCALE name & Data source & Comments \\
\hline $\begin{array}{l}\mathrm{Mg} \\
\mathrm{mg}-24 \\
\mathrm{mg}-25 \\
\mathrm{mg}-26\end{array}$ & $\begin{array}{l}\text { BLO approximate data } \\
\text { BLO approximate data } \\
\text { BLO approximate data } \\
\text { BLO approximate data }\end{array}$ & \\
\hline $\mathrm{mn}-55$ & ENDF/B-VI & $\begin{array}{l}\mathrm{LB}=8 \text { representation caused problematic representation } \\
\text { of cross section uncertainty due to use of fine energy } \\
\text { group structure. Tests were performed to determine how } \\
\text { to handle this problem. } \mathrm{LB}=8 \text { data were removed in the } \\
\text { final results. }\end{array}$ \\
\hline $\begin{array}{l}\text { Mo } \\
\text { mo-92 }\end{array}$ & $\begin{array}{l}\text { BLO approximate data } \\
\text { BLO approximate data }\end{array}$ & \\
\hline mo-94 & BLO approximate data & \\
\hline mo-95 & BLO approximate data & \\
\hline mo-96 & BLO approximate data & \\
\hline $\begin{array}{l}\text { mo-97 } \\
\text { mo-98 } \\
\text { mo-99 } \\
\text { mo-100 }\end{array}$ & $\begin{array}{l}\text { BLO approximate data } \\
\text { BLO approximate data } \\
\text { BLO approximate data } \\
\text { BLO approximate data }\end{array}$ & \\
\hline$n-14$ & BLO approximate data & \\
\hline$n-15$ & BLO approximate data & \\
\hline na-23 & ENDF/B-VII-p & Pre-released evaluation proposed for ENDF/B-VII.1 \\
\hline nb-93 & ENDF/B-VII-p & Pre-released evaluation proposed for ENDF/B-VII.1 \\
\hline nb-94 & BLO approximate data & \\
\hline nb-95 & BLO approximate data & \\
\hline nd-142 & BLO approximate data & \\
\hline nd-143 & BLO approximate data & \\
\hline nd-144 & BLO approximate data & \\
\hline nd-145 & BLO approximate data & \\
\hline nd-146 & BLO approximate data & \\
\hline nd-147 & BLO approximate data & \\
\hline nd-148 & BLO approximate data & \\
\hline nd-150 & BLO approximate data & \\
\hline ni-58 & ENDF/B-VII-p & Pre-released evaluation proposed for ENDF/B-VII.1 \\
\hline ni-59 & BLO approximate data & \\
\hline ni-60 & ENDF/B-VI & $\begin{array}{l}\mathrm{LB}=8 \text { representation caused problematic representation } \\
\text { of cross section uncertainty due to use of fine energy } \\
\text { group structure. Tests were performed to determine how } \\
\text { to handle this problem. } \mathrm{LB}=8 \text { data were removed in the }\end{array}$ \\
\hline
\end{tabular}




\begin{tabular}{|c|c|c|}
\hline SCALE name & Data source & Comments \\
\hline & & final results. \\
\hline ni-61 & ENDF/B-VI & $\begin{array}{l}\mathrm{LB}=8 \text { representation caused problematic represent } \\
\text { of cross section uncertainty due to use of fine en } \\
\text { group structure. Tests were performed to determine } \\
\text { to handle this problem. } \mathrm{LB}=8 \text { data were removed it } \\
\text { final results. }\end{array}$ \\
\hline ni-62 & ENDF/B-VI & $\begin{array}{l}\mathrm{LB}=8 \text { representation caused problematic represent } \\
\text { of cross section uncertainty due to use of fine en } \\
\text { group structure. Tests were performed to determine } \\
\text { to handle this problem. } \mathrm{LB}=8 \text { data were removed in } \\
\text { final results. }\end{array}$ \\
\hline ni-64 & ENDF/B-VI & $\begin{array}{l}\mathrm{LB}=8 \text { representation caused problematic represent } \\
\text { of cross section uncertainty due to use of fine en } \\
\text { group structure. Tests were performed to determine } \\
\text { to handle this problem. } \mathrm{LB}=8 \text { data were removed in } \\
\text { final results. }\end{array}$ \\
\hline np-235 & BLO approximate data & \\
\hline np-236 & $\begin{array}{l}\text { BLO approximate data } \\
\text { SG-26 }\end{array}$ & Thermal uncertainty replaced by Mughabghab value \\
\hline np-238 & BLO approximate data & \\
\hline np-239 & BLO approximate data & \\
\hline $0-16$ & JENDL 3.3+BLO & BLO covariances from LANL used above $5 \mathrm{keV}$ \\
\hline $0-17$ & BLO approximate data & \\
\hline $\mathrm{p}-31$ & BLO approximate data & \\
\hline $\begin{array}{l}\text { pa-231 } \\
\text { pa-232 }\end{array}$ & $\begin{array}{l}\text { BLO approximate data } \\
\text { BLO approximate data }\end{array}$ & \\
\hline pa-233 & BLO approximate data & \\
\hline $\begin{array}{l}\text { pb-204 } \\
\text { pb-206 }\end{array}$ & $\begin{array}{l}\text { BLO approximate data } \\
\text { ENDF/B-VI }\end{array}$ & Error in file corrected \\
\hline pb-207 & ENDF/B-VI & $\mathrm{MT}=3$ removed, Error in file corrected \\
\hline bp-208 & $\mathrm{ENDF} / \mathrm{B}-\mathrm{VI}$ & $\mathrm{MT}=3$ removed, Error in file corrected \\
\hline pd-102 & BLO approximate data & \\
\hline pd-104 & BLO approximate data & \\
\hline pd-105 & BLO approximate data & \\
\hline pd-106 & BLO approximate data & \\
\hline pd-107 & BLO approximate data & \\
\hline pd-108 & BLO approximate data & \\
\hline pd-110 & BLO approximate data & \\
\hline pm-147 & BLO approximate data & \\
\hline pm-148 & BLO approximate data & \\
\hline
\end{tabular}




\begin{tabular}{|c|c|c|}
\hline SCALE name & Data source & Comments \\
\hline pm-148m & BLO approximate data & \\
\hline $\begin{array}{l}\text { pm-149 } \\
\text { pm-151 }\end{array}$ & $\begin{array}{l}\text { BLO approximate data } \\
\text { BLO approximate data }\end{array}$ & \\
\hline pr-141 & BLO approximate data & \\
\hline pr-142 & BLO approximate data & \\
\hline pr-143 & BLO approximate data & \\
\hline $\begin{array}{l}\mathrm{pu}-236 \\
\mathrm{pu}-237 \\
\mathrm{pu}-238\end{array}$ & $\begin{array}{l}\text { BLO approximate data } \\
\text { BLO approximate data } \\
\text { SG-26 }\end{array}$ & Thermal uncertainty replaced by Mughabghab value \\
\hline pu-239 & ENDF/B-VII-p & $\begin{array}{l}\text { Pre-released evaluation proposed for ENDF/B-VII.1; } \\
\text { nubar data from ENDF/B-V } \\
\text { Cross nuclide-to-nuclide matrices present; covariances } \\
\text { due to fission cross sections / nubar for each nuclide } \\
\text { (Table 10.2.2). }\end{array}$ \\
\hline pu-240 & JENDL 3.3 & $\begin{array}{l}\text { Cross nuclide-to-nuclide matrices present; covariances } \\
\text { due to fission cross sections / nubar for each nuclide } \\
\text { (Table 10.2.2). }\end{array}$ \\
\hline pu-241 & JENDL 3.3 & $\begin{array}{l}\text { Cross nuclide-to-nuclide matrices present; covariances } \\
\text { due to fission cross sections / nubar for each nuclide } \\
\text { (Table 10.2.2). }\end{array}$ \\
\hline pu-242 & ENDF/B-VI & \\
\hline pu-243 & BLO approximate data & \\
\hline $\begin{array}{l}\mathrm{pu}-244 \\
\mathrm{pu}-246\end{array}$ & $\begin{array}{l}\text { BLO approximate data } \\
\text { BLO approximate data }\end{array}$ & \\
\hline $\begin{array}{l}\mathrm{rb}-85 \\
\mathrm{rb}-86\end{array}$ & $\begin{array}{l}\text { BLO approximate data } \\
\text { BLO approximate data }\end{array}$ & \\
\hline rb-87 & BLO approximate data & \\
\hline re-185 & ENDF/B-VI & $\begin{array}{l}\text { MT }=2 \text { added from Mughabghab. } \mathrm{LB}=8 \text { representation } \\
\text { caused problematic representation of cross section } \\
\text { uncertainty due to use of fine energy group structure. } \\
\text { Tests were performed to determine how to handle this } \\
\text { problem. } \mathrm{LB}=8 \text { data were removed in the final results. }\end{array}$ \\
\hline re-187 & ENDF/B-VI & $\begin{array}{l}\mathrm{MT}=2 \text { added from Mughabghab. } \mathrm{LB}=8 \text { representation } \\
\text { caused problematic representation of cross section } \\
\text { uncertainty due to use of fine energy group structure. } \\
\text { Tests were performed to determine how to handle this } \\
\text { problem. } \mathrm{LB}=8 \text { data were removed in the final results. }\end{array}$ \\
\hline rh-103 & BLO approximate data & \\
\hline rh-105 & BLO approximate data & \\
\hline $\begin{array}{l}\text { ru-96 } \\
\text { ru-98 } \\
\text { ru-103 }\end{array}$ & $\begin{array}{l}\text { BLO approximate data } \\
\text { BLO approximate data } \\
\text { BLO approximate data }\end{array}$ & \\
\hline
\end{tabular}




\begin{tabular}{ll}
\hline SCALE name & \multicolumn{1}{c}{ Data source } \\
\hline ru-99 & BLO approximate data \\
ru-100 & BLO approximate data \\
ru-101 & BLO approximate data \\
ru-102 & BLO approximate data \\
ru-104 & BLO approximate data \\
ru-105 & BLO approximate data \\
ru-106 & BLO approximate data \\
S & BLO approximate data \\
s-32 & BLO approximate data \\
s-33 & BLO approximate data \\
s-34 & BLO approximate data \\
s-36 & BLO approximate data \\
sb-123 & BLO approximate data \\
sb-124 & BLO approximate data \\
sb-125 & BLO approximate data \\
sb-126 & BLO approximate data \\
sc-45 & ENDF/B-VI \\
se-74 & BLO approximate data \\
se-76 & BLO approximate data \\
se-77 & BLO approximate data \\
se-78 & BLO approximate data \\
se-79 & BLO approximate data \\
se-80 & BLO approximate data \\
se-82 & BLO approximate data \\
Si & ENDF/B-VI \\
si-28 & ENDF/B-VI \\
&
\end{tabular}

Error in file corrected $\mathrm{LB}=8$ representation caused problematic representation of cross section uncertainty due to use of fine energy group structure. Tests were performed to determine how to handle this problem. $\mathrm{LB}=8$ data were removed in the final results.

si-29 ENDF/B-VI

Error in file corrected $\mathrm{LB}=8$ representation caused problematic representation of cross section uncertainty due to use of fine energy group structure. Tests were performed to determine how to handle this problem. $\mathrm{LB}=8$ data were removed in the final results.

si-30 ENDF/B-VI

Error in file corrected $\mathrm{LB}=8$ representation caused problematic representation of cross section uncertainty due to use of fine energy group structure. Tests were performed to determine how to handle this problem. $\mathrm{LB}=8$ data were removed in the final results.

sm-144 BLO approximate data 


\begin{tabular}{|c|c|c|}
\hline SCALE name & Data source & Comments \\
\hline sm-147 & BLO approximate data & \\
\hline sm-148 & BLO approximate data & \\
\hline sm-149 & BLO approximate data & Resonance range uncertainty from ref. 9 \\
\hline sm-150 & BLO approximate data & \\
\hline sm-151 & BLO approximate data & \\
\hline sm-152 & BLO approximate data & \\
\hline sm-153 & BLO approximate data & \\
\hline sm-154 & BLO approximate data & \\
\hline $\begin{array}{l}\text { sn-112 } \\
\text { sn-113 }\end{array}$ & $\begin{array}{l}\text { BLO approximate data } \\
\text { BLO approximate data }\end{array}$ & \\
\hline sn-114 & BLO approximate data & \\
\hline sn-115 & BLO approximate data & \\
\hline sn-116 & BLO approximate data & \\
\hline sn-117 & BLO approximate data & \\
\hline sn-118 & BLO approximate data & \\
\hline sn-119 & BLO approximate data & \\
\hline sn-120 & BLO approximate data & \\
\hline $\begin{array}{l}\text { sn-122 } \\
\text { sn-123 }\end{array}$ & $\begin{array}{l}\text { BLO approximate data } \\
\text { BLO approximate data }\end{array}$ & \\
\hline $\begin{array}{l}\text { sn-124 } \\
\text { sn-125 }\end{array}$ & $\begin{array}{l}\text { BLO approximate data } \\
\text { BLO approximate data }\end{array}$ & \\
\hline sr-84 & BLO approximate data & \\
\hline sr-86 & BLO approximate data & \\
\hline sr-87 & BLO approximate data & \\
\hline sr- 88 & BLO approximate data & \\
\hline sr-89 & BLO approximate data & \\
\hline sr-90 & BLO approximate data & \\
\hline ta-181 & BLO approximate data & \\
\hline ta- 182 & BLO approximate data & \\
\hline tb-159 & BLO approximate data & \\
\hline tb-160 & BLO approximate data & \\
\hline tc-99 & ENDF/B-VII.0 & \\
\hline te- 120 & BLO approximate data & \\
\hline te- 122 & BLO approximate data & \\
\hline te- 123 & BLO approximate data & \\
\hline te-124 & BLO approximate data & \\
\hline te- 125 & BLO approximate data & \\
\hline
\end{tabular}




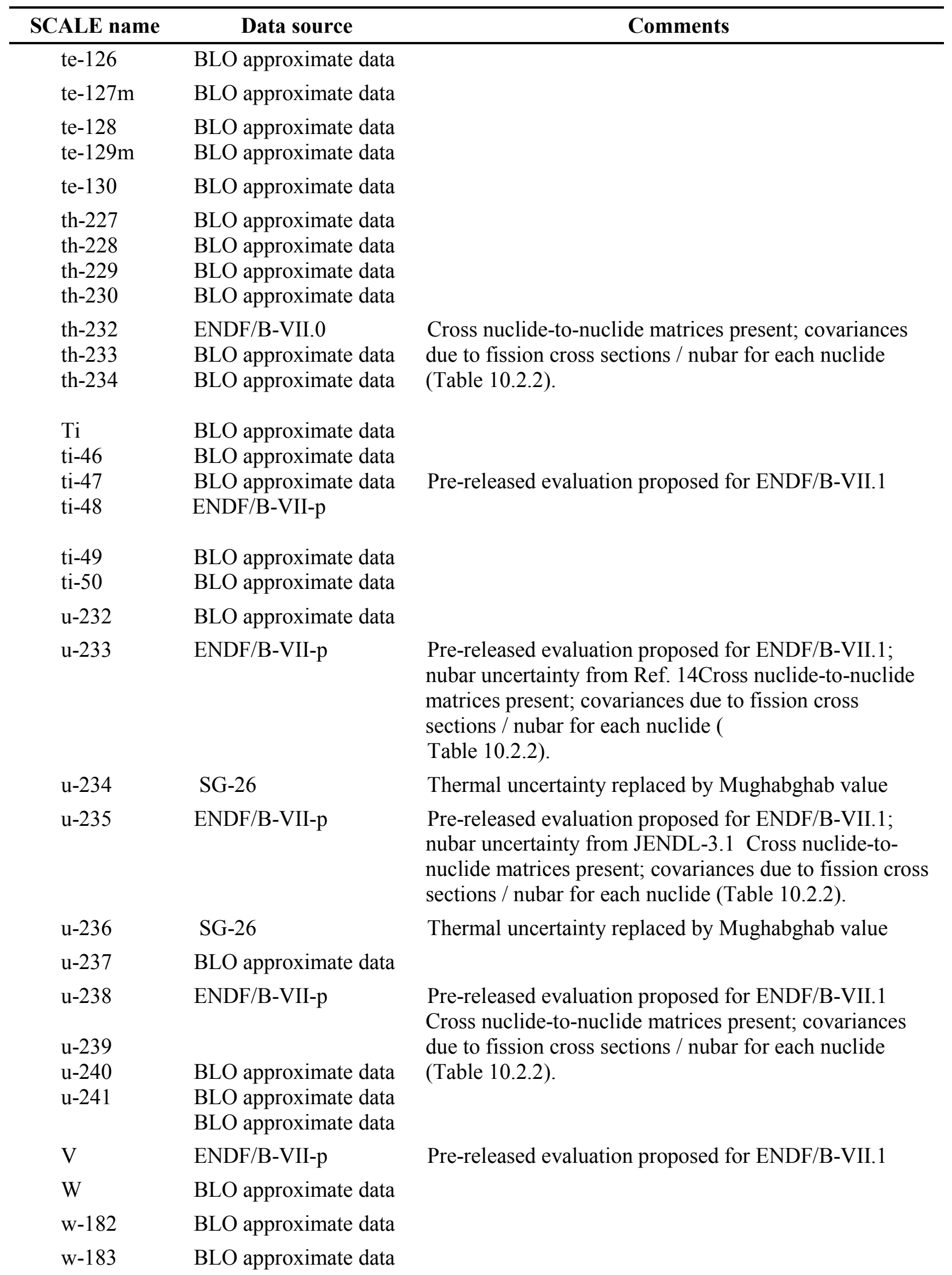




\begin{tabular}{ll}
\hline SCALE name & \multicolumn{1}{c}{ Data source } \\
\hline w-184 & BLO approximate data \\
w-186 & BLO approximate data \\
xe-123 & BLO approximate data \\
xe-124 & BLO approximate data \\
xe-126 & BLO approximate data \\
xe-128 & BLO approximate data \\
xe-129 & BLO approximate data \\
xe-130 & BLO approximate data \\
xe-131 & BLO approximate data \\
xe-132 & BLO approximate data \\
xe-134 & BLO approximate data \\
xe-135 & BLO approximate data \\
xe-136 & BLO approximate data \\
y-89 & ENDF/B-VI \\
y-90 & BLO approximate data \\
y-91 & BLO approximate data \\
Zr & BLO approximate data \\
zr-90 & BLO approximate data \\
zr-91 & BLO approximate data \\
zr-92 & BLO approximate data \\
zr-93 & BLO approximate data \\
zr-94 & BLO approximate data \\
zr-95 & BLO approximate data \\
\hline & BLO approximate data \\
\hline
\end{tabular}

Table 10.2.7. Covariance data with cross correlations between nuclide reactions

\begin{tabular}{cclc}
\hline Nuclide 1 & Reaction 1 & Nuclide 2 & Reaction 2 \\
\hline${ }^{240} \mathrm{Pu}$ & Fission & ${ }^{239} \mathrm{Pu}$ & Fission \\
${ }^{240} \mathrm{Pu}$ & Fission & ${ }^{233} \mathrm{U}$ & Fission \\
${ }^{240} \mathrm{Pu}$ & Fission & ${ }^{238} \mathrm{U}$ & Fission \\
${ }^{241} \mathrm{Pu}$ & Fission & ${ }^{239} \mathrm{Pu}$ & Fission \\
${ }^{241} \mathrm{Pu}$ & Fission & ${ }^{240} \mathrm{Pu}$ & Fission \\
${ }^{241} \mathrm{Pu}$ & Fission & ${ }^{233} \mathrm{U}$ & Fission \\
${ }^{241} \mathrm{Pu}$ & Fission & ${ }^{235} \mathrm{U}$ & Fission \\
${ }^{241} \mathrm{Pu}$ & Fission & ${ }^{238} \mathrm{U}$ & Fission \\
${ }^{235} \mathrm{U}$ & Fission & ${ }^{240} \mathrm{Pu}$ & Fission \\
\hline
\end{tabular}




\subsubsection{References}

1. M. B. Chadwick et al., "ENDF/B-VII.1 Nuclear Data for Science and Technology: Cross Sections, Covariances, Fission Product Yields and Decay Data," Nuclear Data Sheets, Special Issue on ENDF/B-VII.1 Library 112, no. 12 (2011): 2887-3152.

2. N. M. Larson, L. C. Leal, H. Derrien, G. Arbanas, R. O. Sayer, and D. Wiarda, "A Systematic Description of the Generation of Covariance Matrices," C061.pdf in Proc. of PHYSOR-2006, American Nuclear Society Topical Meeting on Reactor Physics: Advances in Nuclear Analysis and Simulation, September 10-14, 2006, Vancouver, British Columbia, Canada.

3. R. Little, T. Kawano, G. D. Hale, M. T. Pigni, M. Herman, P. Obložinský, M. L. Williams, M. E. Dunn, G. Arbanas, D. Wiarda, R. D. McKnight, J. N. McKamy and J. R. Felty, "Low-fidelity Covariance Project," Nuclear Data Sheets 109, no. 12 (2008): 2828-2833.

4. M. L. Williams, B. L. Broadhead, M. E. Dunn, and B. T. Rearden, "Approximate Techniques for Representing Nuclear Data Uncertainties," in Proc. of the Eighth International Topical Meeting on Nuclear Applications and Utilization of Accelerators (ACCAPP '07), July 30-August 2, 2007, Pocatello, Idaho, 744-752.

5. M. L. Williams and B. T. Rearden, "SCALE 6 Sensitivity/Uncertainty Methods and Covariance Data," Nuclear Data Sheets 109, no. 12 (2008): 2796-2800.

6. S. F. Mughabghab, Atlas of Neutron Resonances: Resonance Parameters and Thermal Cross Sections, Elsevier, Amsterdam (2006).

7. M. T. Pigni, M. Herman, and P. Oblozinsky, "Extensive set of cross section covariance estimates in the fast neutron region," Nucl. Sci. Eng. 162, no. 1 (2009): 25-40.

8. D. Rochman, M. Herman, P. Oblozinsky, and S. F. Mughabghab, "Preliminary Cross Section and Nubar Covariances for WPEC Subgroup 26,” BNL-77407-2007-IR (2007).

9. T. Kawano, P. Talou, P. G. Young, G. Hale, M. B. Chadwick, and R. C. Little, "Evaluation of Covariances for Actinides and Light Elements at LANL," Nuclear Data Sheets 109, no. 12, (2008): $2817-2821$.

10. G. Hale, "Covariances from Light-Element R-Matrix Analyses," Nuclear Data Sheets 109 no. 12, (2008): 2812-2816.

11. M. L Williams, G. Ilas, W. J. Marshall, B. T. Rearden, "Applications of Nu clear Data Covariances in in Criticality Safety and Spent Fuel Characterization", Nuclear Data Sheets 110, (2014): 341-345.

12. S. F. Mughabghab, "Thermal Neutron Capture Cross Sections Resonance Integrals and G-Factors," INDC (NDS)-440, International Atomic Energy Agency (2003).

13. D. Wiarda, G. Arbanas, L. Leal, M. E. Dunn, "Recent Advances in the AMPX Covariance Processing Capabilities in PUFF-IV", Nuclear Data Sheets Vol. 109, no. 12 (2008) : 2745-2751

14. L. C. Leal, D. Wiarda, B. T. Rearden, H. Derrien, ${ }^{، 233}$ U Cross-Section and Covariance Data Update for SCALE 5.1 Libraries," UT-Battelle, LLC, Oak Ridge National Laboratory, ORNL/TM-2007/115 (2008).

15. B. L. Broadhead and J. J. Wagschal, "The Fission Spectrum Uncertainty," 95821.pdf in Proc. of PHYSOR 2004-The Physics of Fuel Cycles and Advanced Nuclear Systems: Global Developments, April 25-29, 2004, Chicago, Illinois. 
16. R. E. Maerker, J. H. Marable, and J. J. Wagschal, "Estimation of the Uncertainties in the ENDF/B-V ${ }^{235}$ U Fission Spectrum," Trans. Am. Nucl. Soc. 35 (1980): 555-556.

17. R. J. Howerton and R. J. Doyas, "Fission Temperatures as a Function of the Average Number of Neutrons from Fission,” Nucl. Sci. Eng. 46, no. 414 (1971). 


\subsection{A Cross section plots for $\mathrm{U}, \mathrm{Pu}, \mathrm{TH}, \mathrm{B}, \mathrm{H}, \mathrm{He}$, and Gd Nuclides}

Plots of cross section differences between various evaluations are shown below. The legend below applies to all plots shown in this appendix.

ENDF/B-VII (beta2)

ENDF/B-VI

JENDL 3.3

JEF 3.1

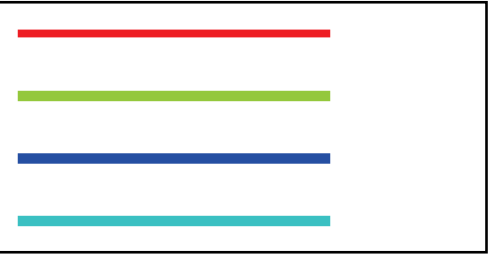



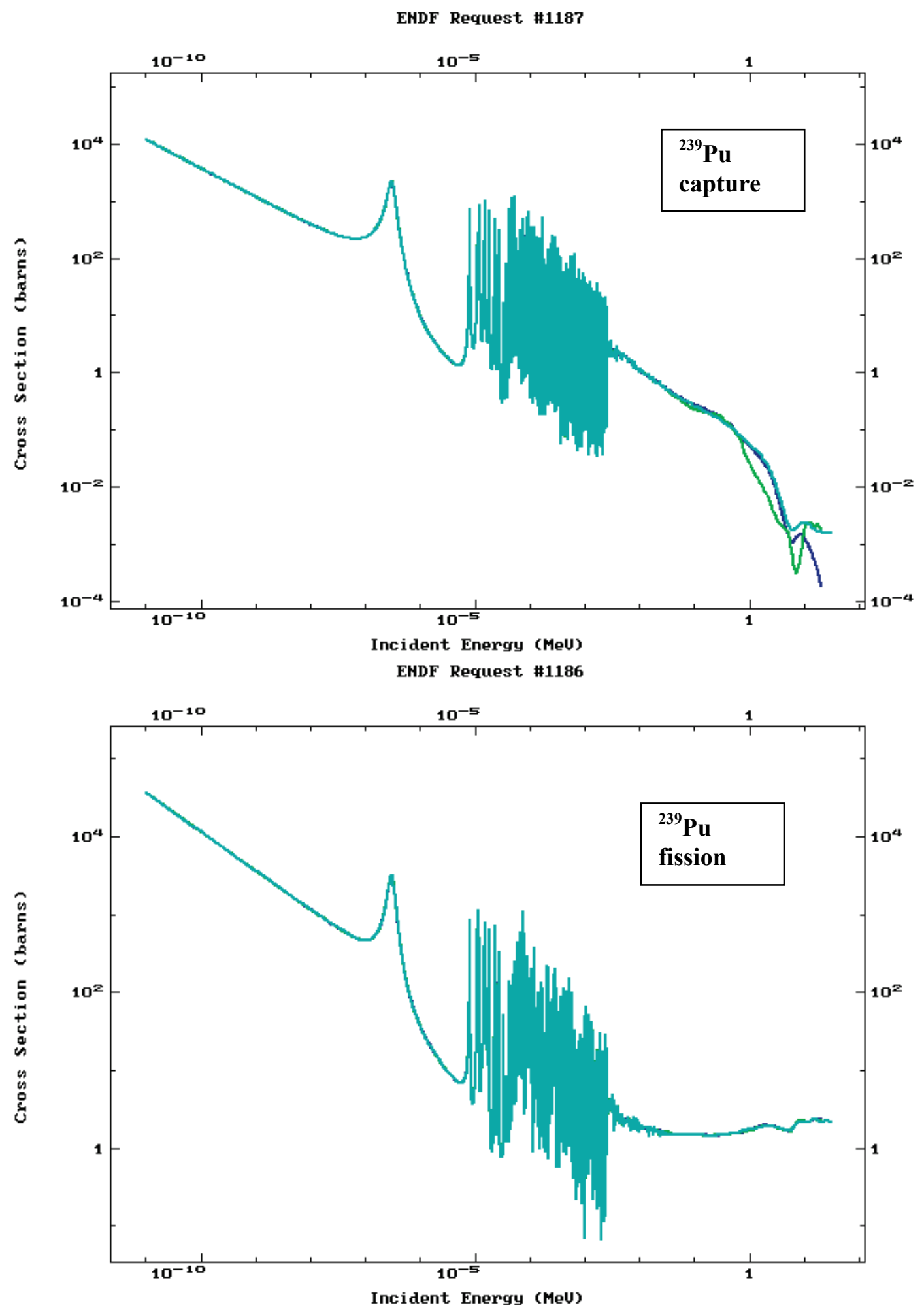

Figure 10.2.A.1. ${ }^{239} \mathrm{Pu}$ fission and capture comparison between ENDF/B-VI, JENDL 3.3, and JEF 3.1. 

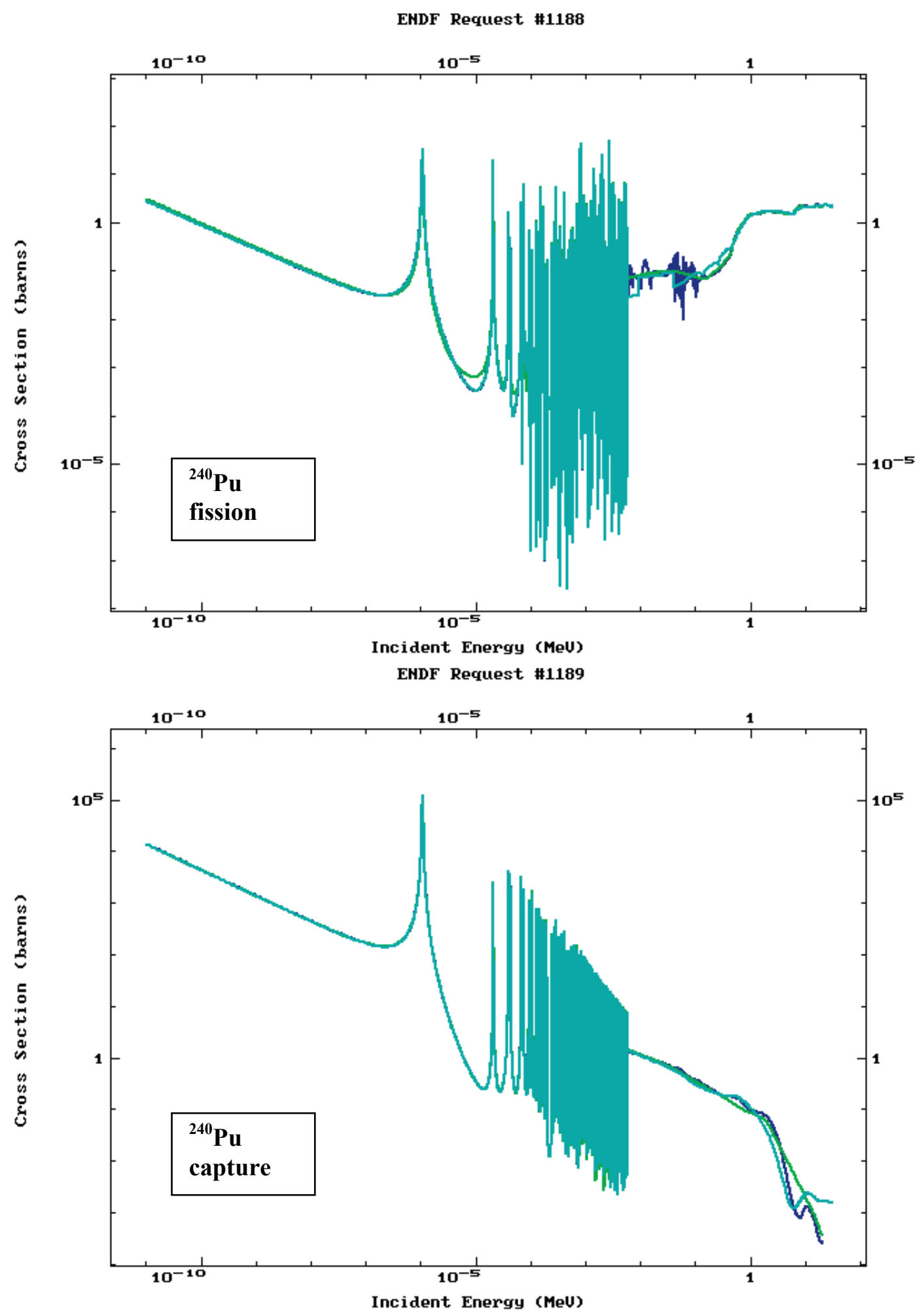

Figure 10.2.A.2. ${ }^{240} \mathrm{Pu}$ fission and capture comparison between ENDF/B-VI, JENDL 3.3 and JEF 3.1. 

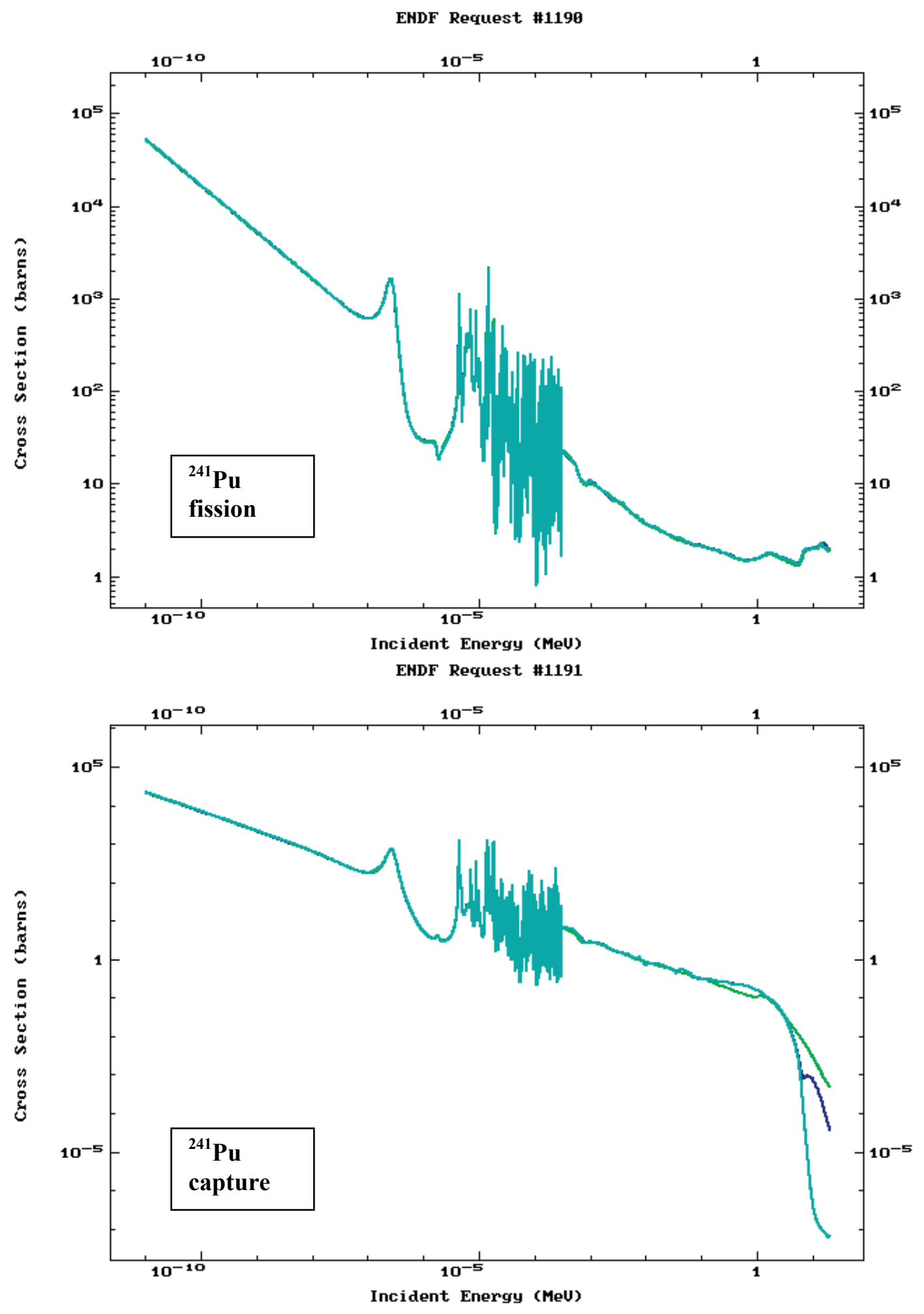

Figure 10.2.A.3. ${ }^{241} \mathrm{Pu}$ fission and capture comparison between ENDF/B-VI, JENDL 3.3 and JEF 3.1. 

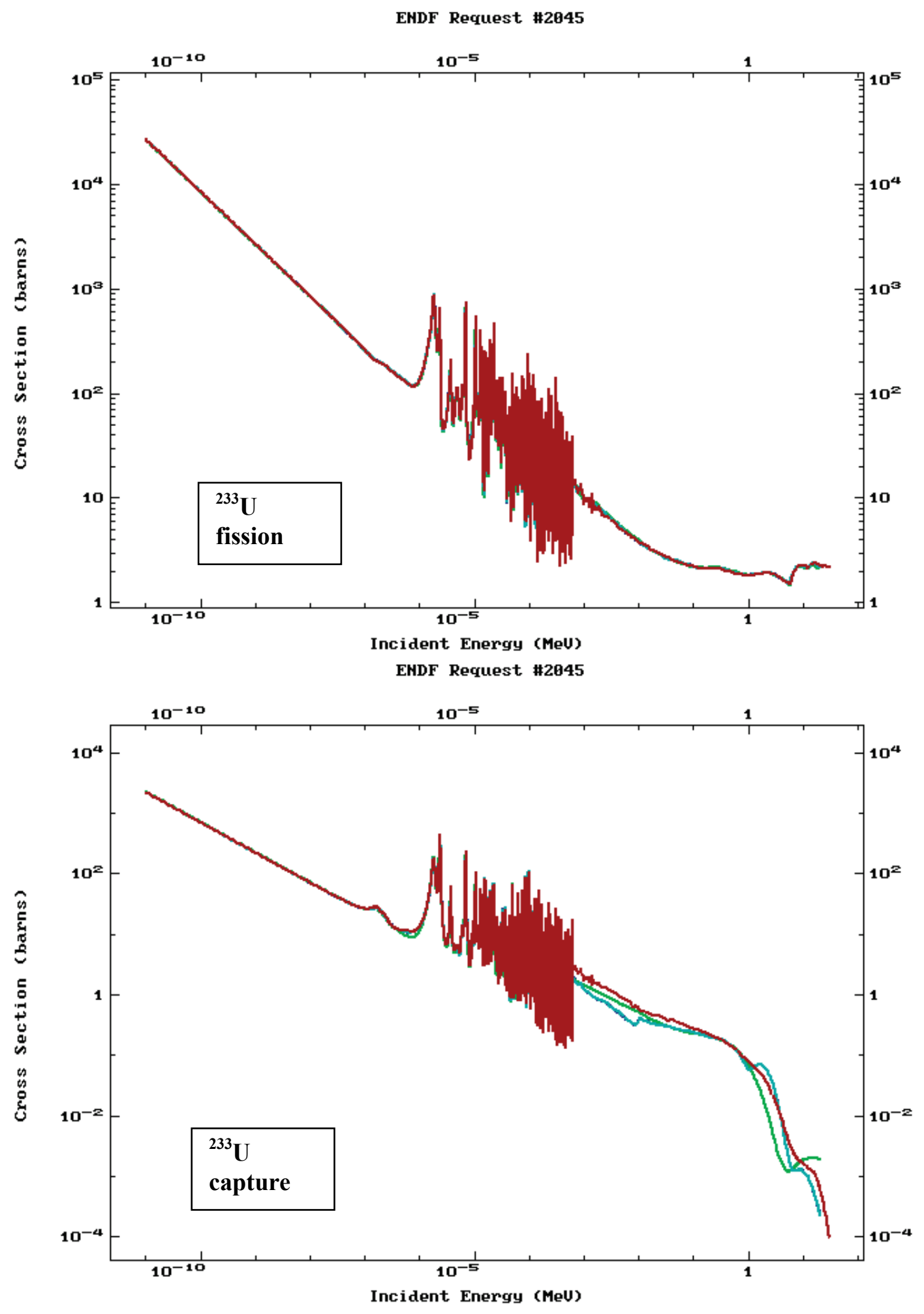

Figure 10.2.A.4. ${ }^{233} \mathrm{U}$ fission and capture comparison between ENDF/B-VII (beta2), ENDF/B-VI, JENDL 3.3 and JEF 3.1. 

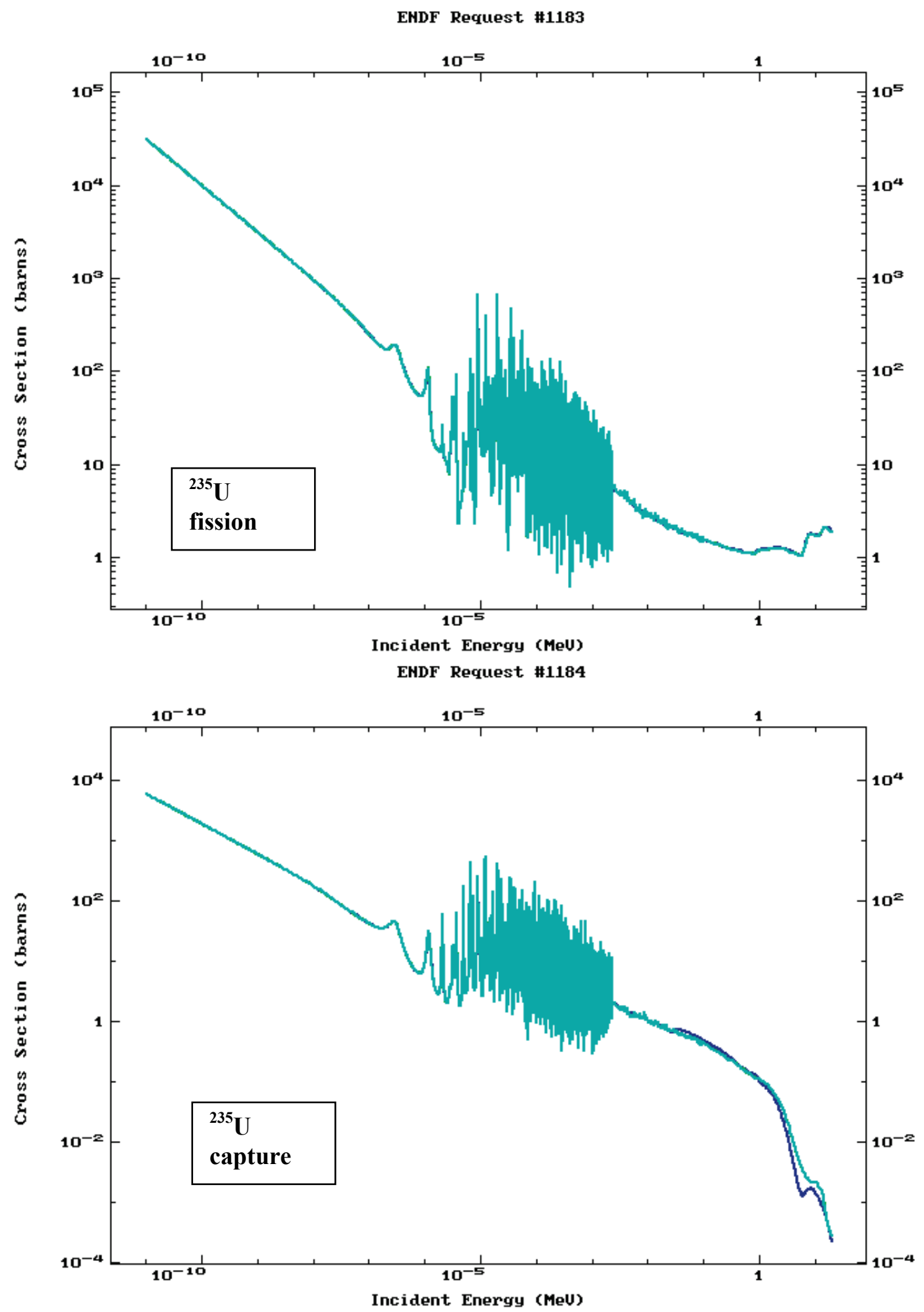

Figure 10.2.A.5. ${ }^{235} \mathrm{U}$ fission and capture comparison between ENDF/B-VI, JENDL 3.3 and JEF 3.1. 


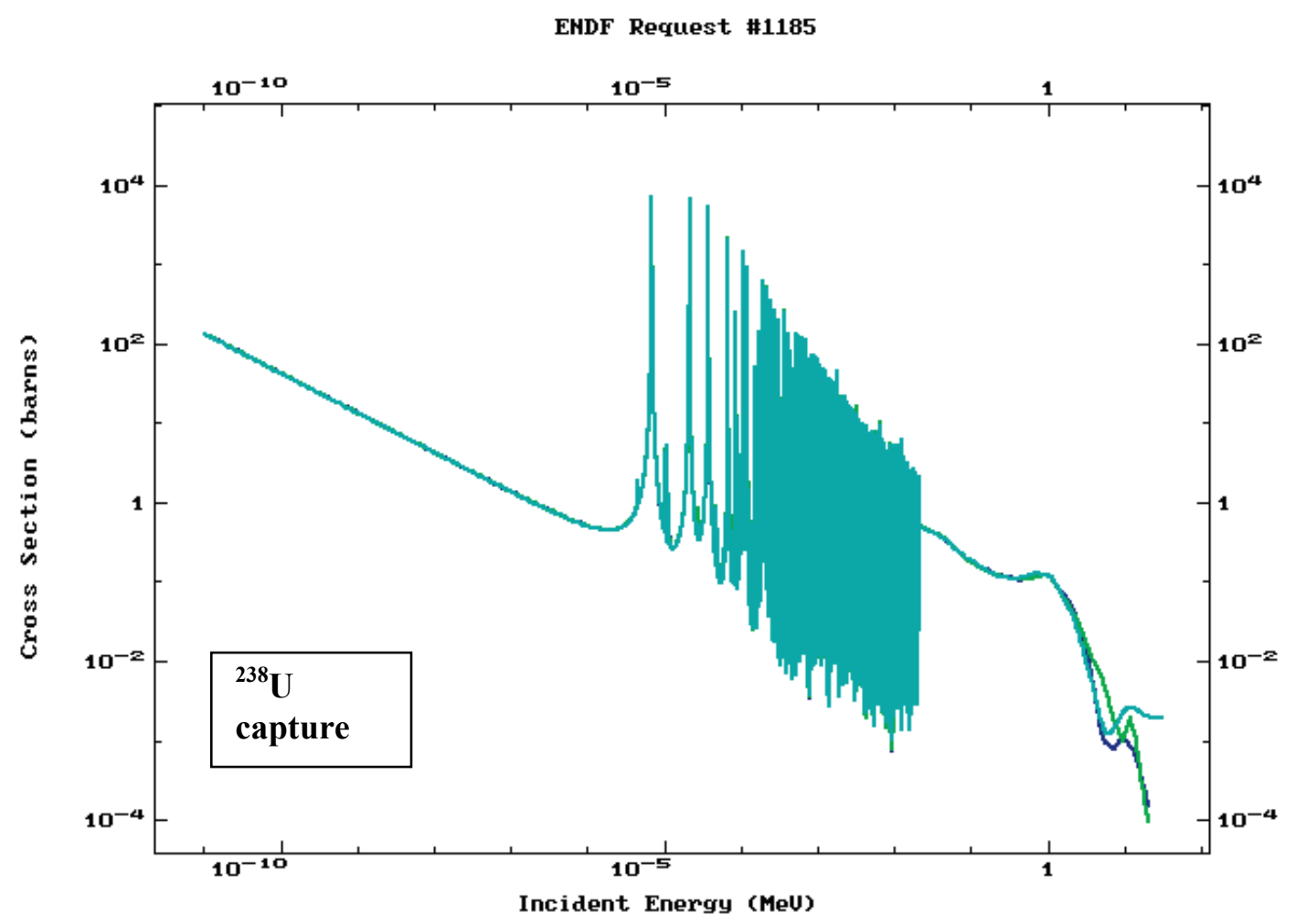

Figure 10.2.A.6. ${ }^{238} \mathrm{U}$ capture comparison between ENDF/B-VI, JENDL 3.3 and JEF 3.1.

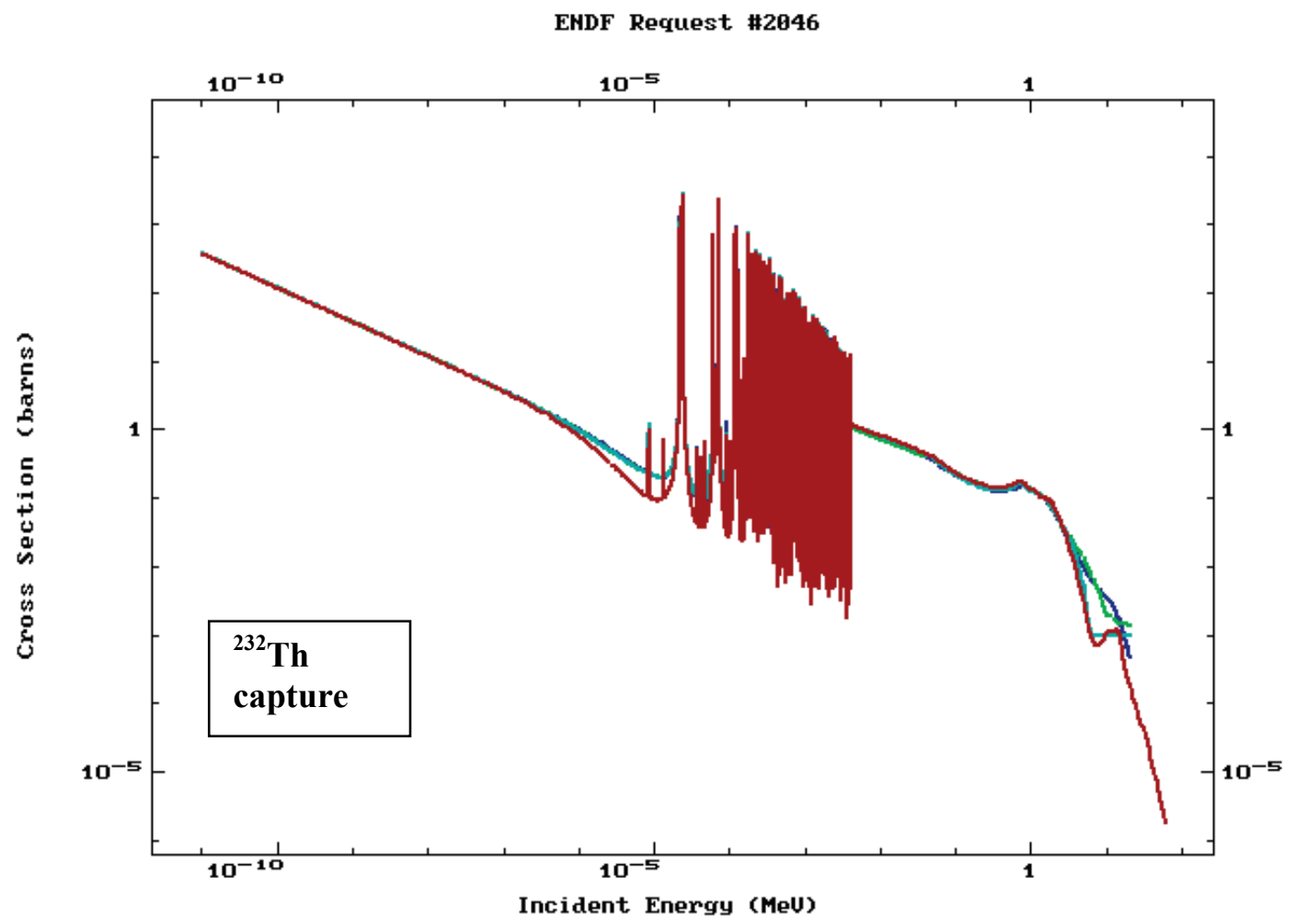

Figure 10.2.A.7. ${ }^{232}$ Th capture comparison between ENDF/B-VII (beta2), ENDF/B-VI, JENDL 3.3 and JEF 3.1. 

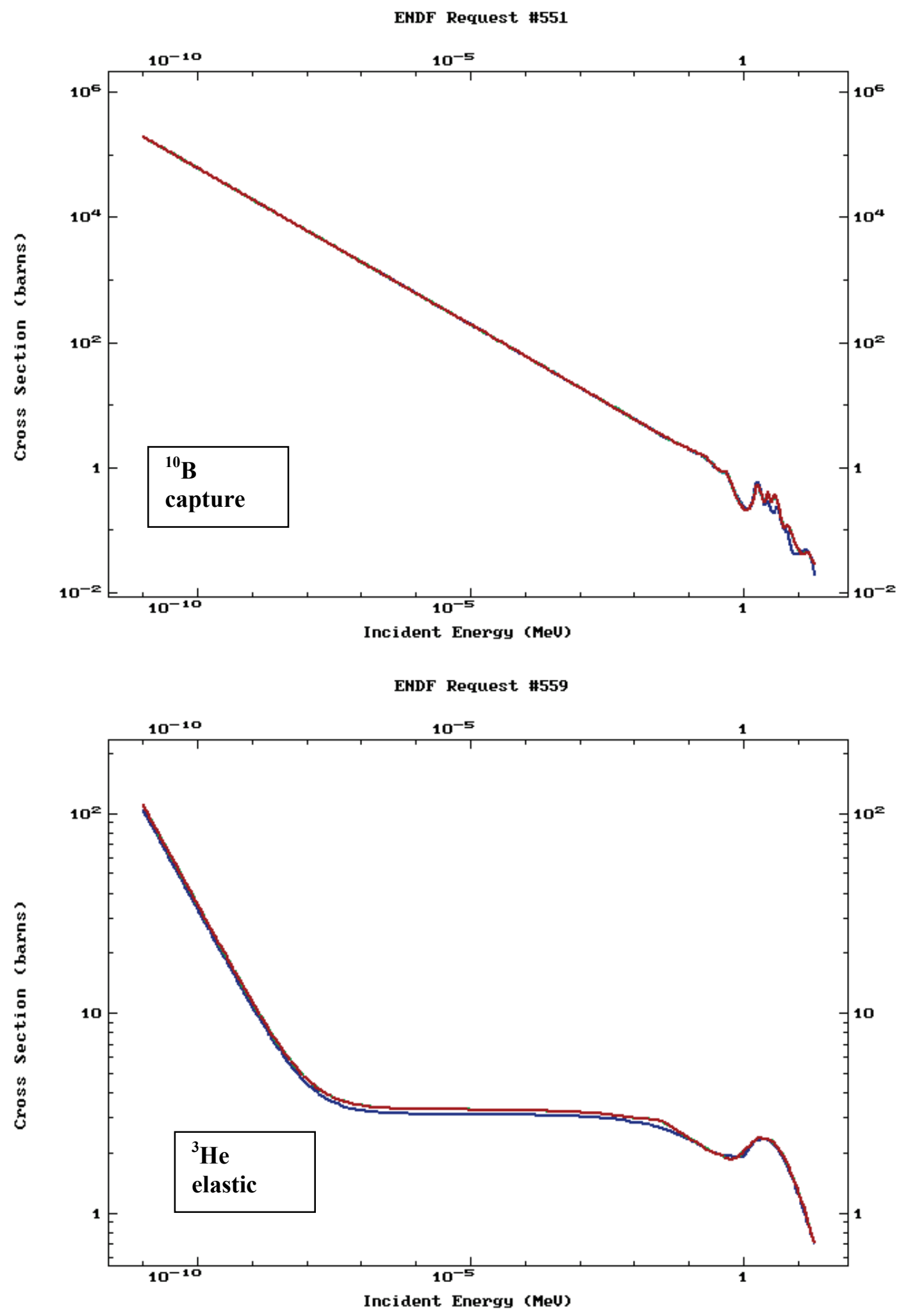

Figure 10.2.A.8. ${ }^{10} \mathrm{~B}$ capture and ${ }^{3} \mathrm{He}$ elastic comparison between ENDF/B-VII (beta2), ENDF/B-VI, JENDL 3.3 and JEF 3.1. 

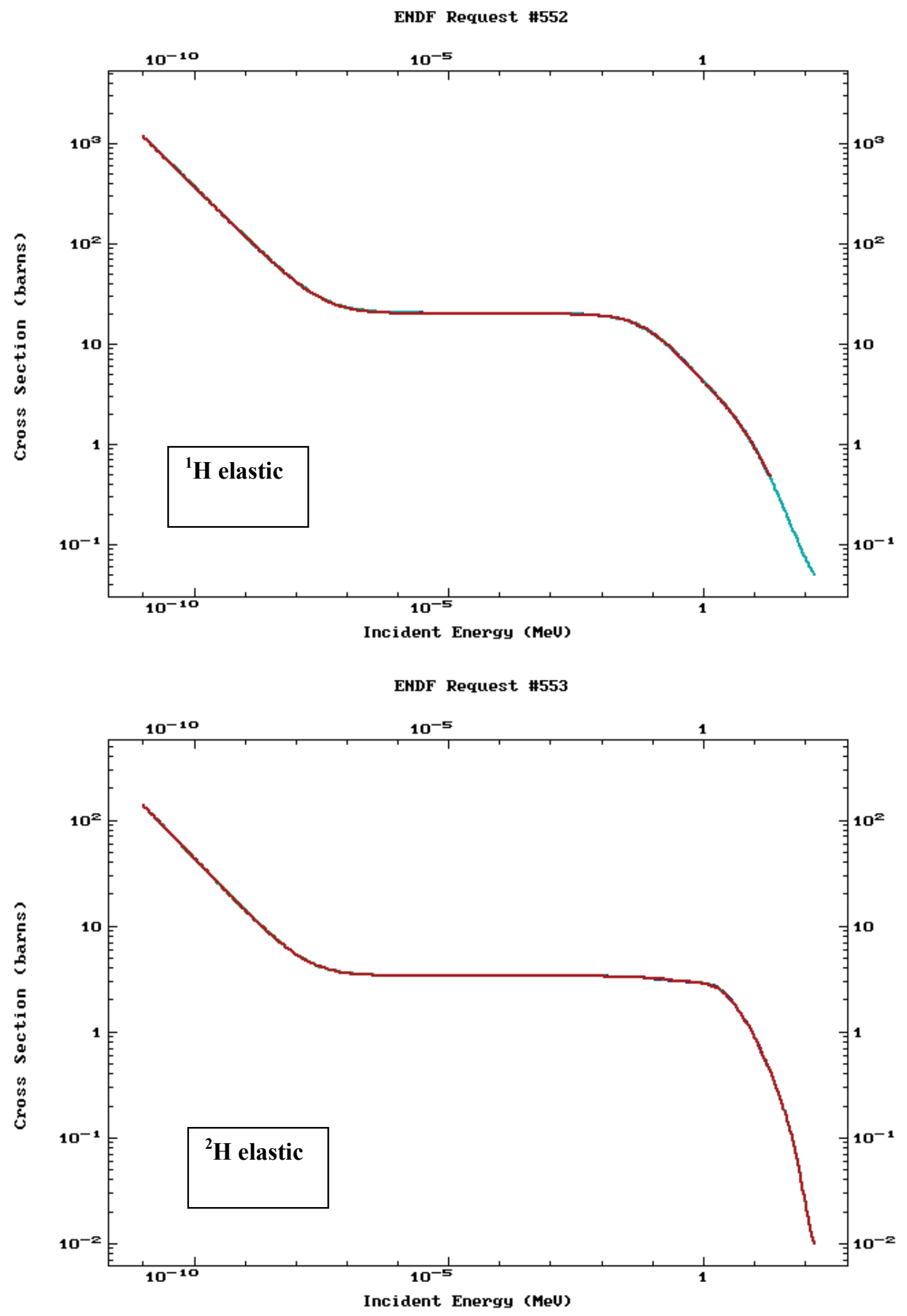

Figure 10.2.A.9. ${ }^{1} \mathrm{H}$ and ${ }^{2} \mathrm{H}$ elastic comparison between ENDF/B-VII (beta2),

|ENDF/B-VI, JENDL 3.3 and JEF 3.1 

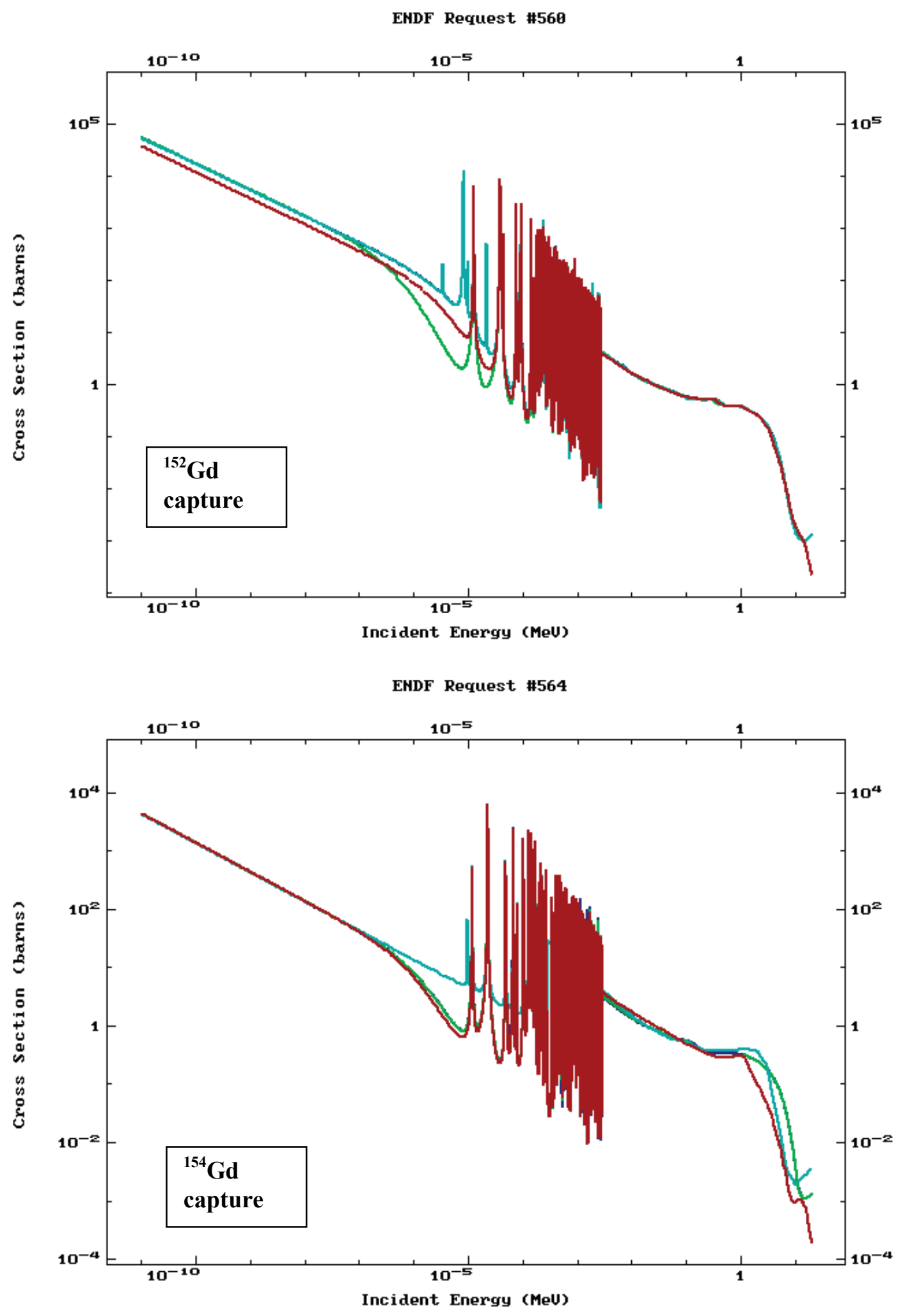

Figure 10.2.A.10. ${ }^{152} \mathrm{Gd}$ and ${ }^{154} \mathrm{Gd}$ capture comparison between ENDF/B-VII (beta2), ENDF/B-VI, JENDL 3.3 and JEF 3.1. 


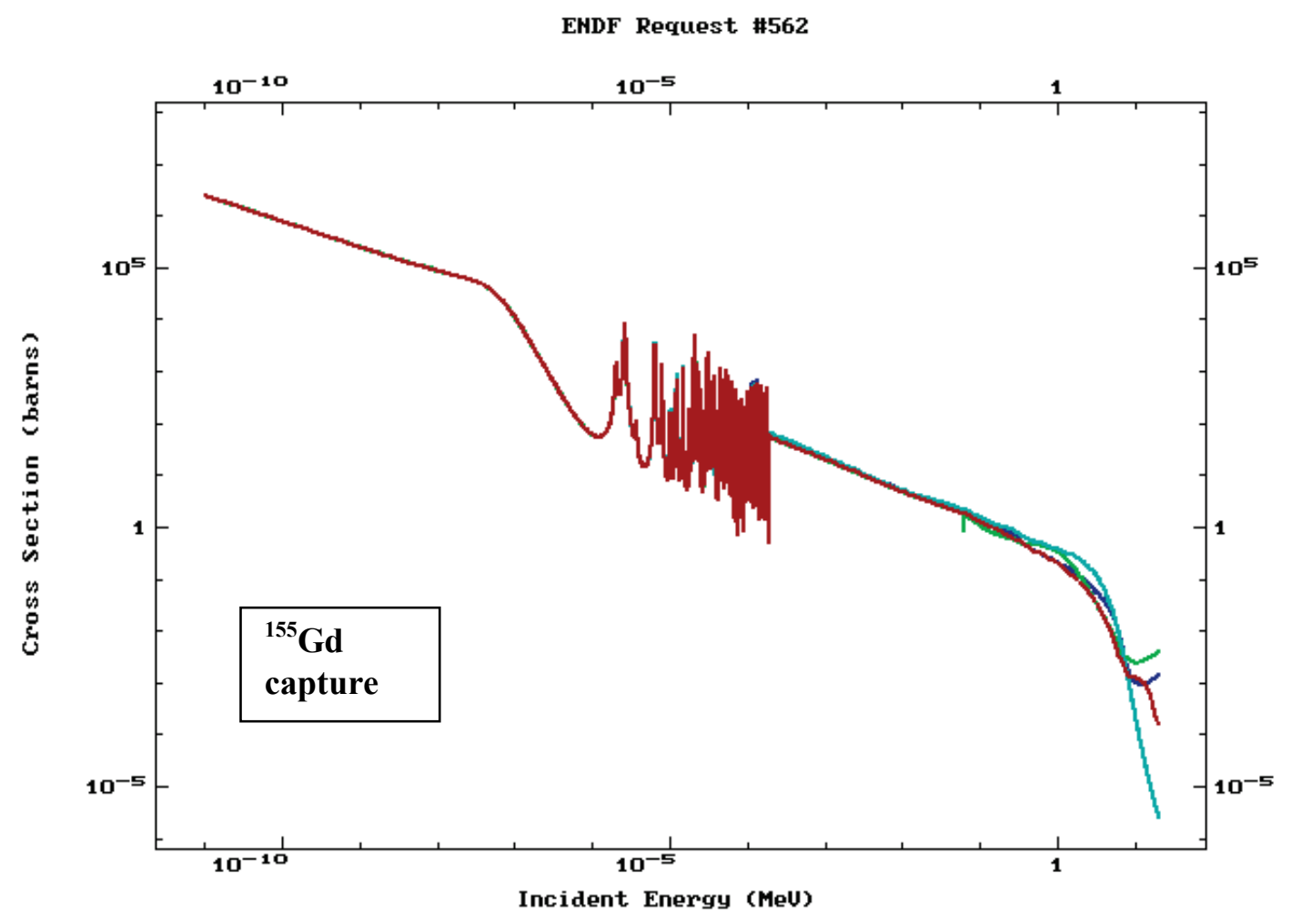

Figure 10.2.A.11. ${ }^{155} \mathrm{Gd}$ capture comparison between ENDF/B-VII (beta2), ENDF/B-VI, JENDL 3.3 and JEF 3.1. 


\title{
11.0 UTILITY MODULES FOR SCALE LIBRARIES
}

\author{
Introduction by M.L. Williams
}

Historically the SCALE Code System has included a number of standalone, executable utility modules developed mainly for performing operations on various types of multigroup nuclear data libraries and on the standard composition library used in SCALE. In legacy versions of SCALE, some utility modules were directly executed during computational sequences; but in the modern version of SCALE the standalone executables are no longer used in computation sequences their functions have been incorporated into a more unified and integrated framework. Several of the earlier utility modules were deprecated in the SCALE 6.2 release; but others are retained and are still quite useful for nuclear data manipulations, editing, and checking. Most of these utilities are taken directly from the AMPX code system. Section 11.1 provides a listing of the AMPX modules distributed with SCALE, along with a brief description of each module's function. User input for these modules can be found in the AMPX documentation that accompanies SCALE. Several additional utility modules included in the SCALE code package are also described in this section. Finally, many AMPX and SCALE utility modules use the FIDO input processing procedure, and this is described in the FIDO chapter. 
11-2 


\title{
11.1 AMPX LIBRARY UTILITY MODULES
}

D. Wiarda, L. M. Petrie

\begin{abstract}
The purpose of this section is to document selected AMPX modules that can benefit the analyst interested in editing, converting, or combining cross-section libraries normally used by the SCALE system modules. The input description for these codes is provided in the documentation of the AMPX nuclear data processing code system that is distributed with SCALE package.
\end{abstract}




\section{TABLE OF CONTENTS}

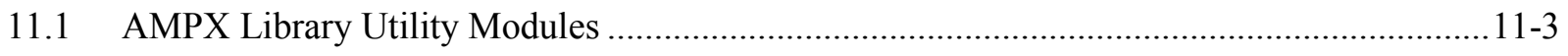

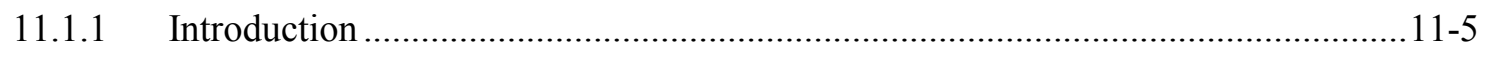

11.1.2 AJAX: Module to MERGE, COLLECT, ASSEMBLE, REORDER, JOIN, and/or COPY Selected Data from AMPX Master Libraries ...................................11-5

11.1.3 ALPO: Module to Convert AMPX Libraries into ANISN Format .........................11-5

11.1.4 CADILLAC: Module to MERGE Multiple Covariance Data Files ........................11-5

11.1.5 COGNAC: Module to CONVERT Covariance Data Files in COVERX Format ..11-5

11.1.6 LAVA: Module to Make an AMPX Working Library from an ANISN Library ....11-5

11.1.7 MALOCS: Module to COLLAPSE AMPX Master Cross-Section Libraries ..........11-5

11.1.8 PALEALE: module to LIST Information from AMPX Libraries............................11-6

11.1.9 RADE: Module to CHECK AMPX Cross-Section Libraries...................................11-6

11.1.10 TOC: Module to PRINT an AMPX Library Table of Contents .............................11-6

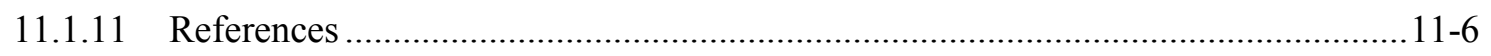




\subsubsection{Introduction}

AMPX is a modular system ${ }^{1}$ that generates continuous energy (CE) and multigroup (MG) cross section data from evaluated nuclear data files such as ENDF/B. All the nuclear data libraries distributed with SCALE have been processed using AMPX. In addition to data processing modules, AMPX also includes a number of useful utility modules for checking, manipulating, and editing the libraries in SCALE. This section lists and briefly describes some of the AMPX utility codes that may be useful to SCALE users. Input instructions for these codes can be found the AMPX code documentation, which is distributed with the SCALE code package. Additional AMPX modules of interest may also found in the documentation.

\subsubsection{AJAX: MODULE TO MERGE, COLLECT, ASSEMBLE, REORDER, JOIN, AND/OR COPY SELECTED DATA FROM AMPX MASTER LIBRARIES}

AJAX (Automatic Joining of AMPX X-Sections) is a module to combine data from different AMPX libraries. Options are provided to allow merging from any number of files.

\subsubsection{ALPO: MODULE TO CONVERT AMPX LIBRARIES INTO ANISN FORMAT}

ALPO (ANISN LIBRARY PRODUCTION OPTION) is a module for converting AMPX working libraries into the library format used by the legacy discrete ordinates transport codes ANISN and DORT/TORT contained in the DOORS package. ${ }^{2}$

\subsubsection{CADILLAC: MODULE TO MERGE MULTIPLE COVARIANCE DATA FILES}

CADILLAC (Combine All Data Identifiers Listed in Logical AMPX Coverx-format) is a module that can be used to combine multiple covariance data files in COVERX format into a single covariance data file. The material IDs can be changed as needed by the user.

\subsubsection{COGNAC: MODULE TO CONVERT COVARIANCE DATA FILES IN COVERX FORMAT}

COGNAC (Conversion Operations for Group-dependent Nuclides in AMPX Coverx-format) is a module that can be used to convert a single COVERX-formatted data file from bed format to binary. Also, COGNAC can be used to convert from binary to bcd, binary to binary, and bcd to bcd.

\subsubsection{LAVA: MODULE TO MAKE AN AMPX WORKING LIBRARY FROM AN ANISN LIBRARY}

LAVA (Let ANISN Visit AMPX) is a module that can convert an ANISN formatted library (neutron, gamma, or coupled neutron-gamma) to an AMPX working library that can be used in XSDRNPM.

\subsubsection{MALOCS: MODULE TO COLLAPSE AMPX MASTER CROSS-SECTION LIBRARIES}

MALOCS (Miniature AMPX Library Of Cross Sections) is a module to collapse AMPX master crosssection libraries. The module can be used to collapse neutron, gamma-ray, or coupled neutron-gamma master libraries. 


\subsubsection{PALEALE: MODULE TO LIST INFORMATION FROM AMPX LIBRARIES}

PALEALE lists selected data by nuclide, reaction, data-type from AMPX master and working libraries.

\subsubsection{RADE: MODULE TO CHECK AMPX CROSS-SECTION LIBRARIES}

RADE (Rancid AMPX Data Exposer) is provided to check AMPX- and ANISN-formatted multigroup libraries. It will check neutron, gamma, or coupled neutron-gamma libraries.

\subsubsection{TOC: MODULE TO PRINT AN AMPX LIBRARY TABLE OF CONTENTS}

Program TOC is a utility program to print a sorted table of contents of an AMPX cross section library. It is designed to be run interactively, with the cross section library specified as the argument.

\subsubsection{References}

1. D. Wiarda, M. L. Williams, C. Celik, and M. E. Dunn, "AMPX: A Modern Cross Section Processing System For Generating Nuclear Data Libraries," Proceedings of International Conference on Nuclear Criticality Safety, Charlotte, NC, Sept 13-17 2015.

2. "DOORS3.2a: One, Two- and Three-Dimensional Discrete Ordinates Neutron/Photon Transport Code System", Radiation Shielding Information Center package CCC-650, Oak Ridge National Laboratory (2003). 


\title{
11.2. WORKER: SCALE SYSTEM MODULE FOR CREATING AND MODIFYING WORKING-FORMAT LIBRARIES
}

L. M. Petrie

\begin{abstract}
WORKER is a standalone utility module used to convert AMPX master-formatted or working-formatted multigroup cross section libraries into a single working library for SCALE transport calculations performed by XSDRNPM, KENO V.a and KENO-VI, and other modules. Beginning with SCALE 6.2, WORKER is no longer used for calculations performed with SCALE sequences because this function has been absorbed into the XSProc module. This document gives instructions on how to use the WORKER program as a standalone module.
\end{abstract}




\section{TABLE OF CONTENTS}

$\underline{\text { Page }}$

11.2. WORKER: SCALE System Module for Creating and Modifying Working-Format Libraries

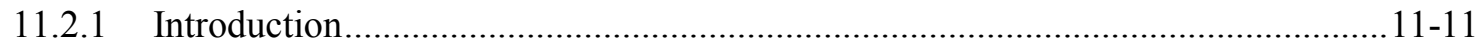

11.2.2 AMPX Library Format Conversion ..................................................................11-11

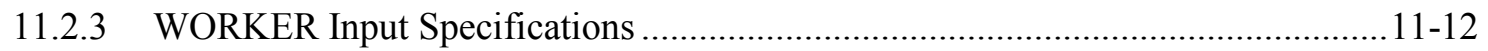

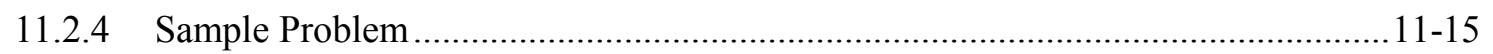

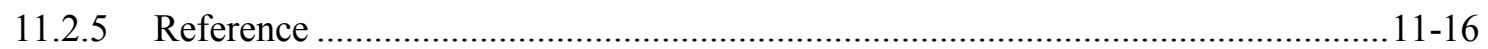




\section{LIST OF FIGURES}

$\underline{\text { Page }}$

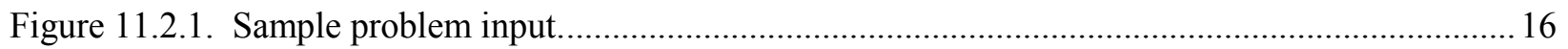




\section{ACKNOWLEDGMENTS}

Several former ORNL staff made valuable contributions to the WORKER development. The authors acknowledge the contributions of former ORNL staff members S. Goluoglu, N. M. Greene, and D. F. Hollenbach. 


\subsubsection{Introduction}

Historically the AMPX nuclear data processing system ${ }^{1}$ has defined two formats for the multigroup (MG) libraries used in SCALE. The "master" formatted library contains more general information than normally required for radiation transport calculations. A master library includes such information as 2D transfer arrays for all inelastic levels, temperature-dependent thermal-scattering kernels, and Bondarenko self-shielding data. A "working" formatted library contains only the subset of these data needed for radiation transport calculations. A working library contains only a single, combined $2 \mathrm{D}$ transfer array [i.e., sum of elastic, all discrete inelastic levels, $(\mathrm{n}, 2 \mathrm{n})$, etc.], and the temperature-dependent data normally have been interpolated to the appropriate temperatures. In SCALE 6.2, more general formats for nuclear data libraries are supported, and the MG libraries distributed with SCALE are no longer restricted by the inflexible Master library format. However the WORKER module is retained in SCALE 6.2 for manipulation of legacy formatted libraries.

WORKER is a standalone program that reads input nuclear data library files and produces a single working library file that can be read by transport modules such as XSDRNPM, KENO-VI, NEWT, CENTRM, and Monaco. Prior to SCALE 6.2, the distributed MG libraries were stored in master format, and WORKER was executed during every MG sequence to read the master library file and write an output working library file subsequently read by the transport codes run by the sequence. In modern versions of AMPX and SCALE (e.g., SCALE 6.2 and later), the differences in the master and working formats are superfluous, and the functions of WORKER are now performed in memory by the XSProc module. Nevertheless, WORKER may still be useful for manipulation of external library files and for standalone execution of SCALE modules such as CENTRM and XSDRNPM.

\subsubsection{AMPX Library Format Conversion}

The primary function of WORKER is to convert data from an initial AMPX MG library file, with any format supported by SCALE, into the AMPX working library format. The nuclear data files distributed with SCALE provide MG data that are truly problem-independent and that can be tailored at runtime for a particular application. These library files must carry data at a sufficient level of detail to allow satisfying many of the less-common but very powerful analyses such as cross section sensitivity studies and coupled neutron-gamma transport calculations. The nuclear data libraries include shielding factors used by the Bondarenko self-shielding method. Temperature dependence of the shielding factors and the thermal scattering kernel data is allowed. Any number of scattering processes [e.g., elastic, discrete-level inelastic, continuum inelastic, $(\mathrm{n}, 2 \mathrm{n})$, etc.] can be included to any degree of anisotropic representation. In short, there is too much detail to require transport codes to process this library.

WORKER processes and combines the data mentioned above into a form ready for use by MG particle transport programs. The final output is an AMPX working library file containing two types of data: group-averaged 1D cross sections for an arbitrary number of processes for neutrons and/or gamma rays, and total 2D transfer matrices (i.e., summed over the scattering types) for neutrons and/or gamma rays. In some cases, the transfer matrices on the master library are temperature-dependent. WORKER performs linear interpolation to the temperature specified for the nuclide, but it does not extrapolate outside the range of the data. WORKER also has the option of producing an AMPX master library of the selected nuclides interpolated to the specified temperature(s). For resonance nuclides, the elastic scattering matrix is scaled uniformly to make the matrix consistent with the self-shielded $1 \mathrm{D}$ values. The $\mathrm{P}_{\ell}(\ell>0)$ matrices are scaled by the amount required for the $\mathrm{P}_{0}$ matrix. For processes involving multiple-exit neutrons [e.g., $(n, 2 n),(n, 3 n)$, etc.], WORKER multiplies by the appropriate multiplicity before adding to the total transfer matrix. In the case of coupled neutron-gamma libraries, gamma yields are sometimes expressed in "yield" units, thereby requiring a multiplication by a cross section before their introduction into the total transfer matrix. (This scheme allows one to produce self-shielded gamma production cross sections.) 


\subsubsection{WORKER Input Specifications}

The following is a description of WORKER input requirements and input-output device requirements.

\subsubsection{Input parameters}

WORKER uses FIDO-type input. The number of items to be input in an array is shown in brackets, and default values are given in parentheses. If $\mathrm{N} 1$ and/or N7 are negative, a direct access master file will be assumed on that unit. Note that direct access master files may use up to five different units. The initial values of $\mathrm{N} 1$ or $\mathrm{N} 7$ will be used to determine the starting unit number for the first file, and the unit number will be incremented by 1 for each additional file.

Data block 1

$-1 \$ \$$ Option [1]

1. NFISFOT - No Fission Photon Option (0)

0 - add fission photons to the transfer array

1 - do not add fission photons to the transfer array

0\$ Logical Assignments [6]. This array is input only if a user needs to modify default values.

1. N1 - Input Master Cross section Library (1)

2. N2 - Input Working Cross section Library (2)

3. N4 - Output Working Cross section Library produced by WORKER (4)

4. N5 - Scratch (18)

5. N6 - Scratch (19)

6. N7 - Output Temperature Interpolated Master Library (0)

1\$ Integer Parameters [8]

1. NUCM - Number of nuclides to be read from the input library, N1. (0)

2. NUCW - Number of nuclides to be read from the input working library, N2. (0)

3. IPRT - Output print option trigger. $(\uparrow 2)$

$-2-$ no cross section edits

-1 - edit reaction cross sections

$>-1$ - edit reaction cross sections and transfer arrays through order IPRT

4. IMST - Flag to copy entire Master Library. (0) $(0 />0=$ only listed nuclides/all nuclides $)$

5. IWRK - Flag to copy entire Working Library. (0) $(0 />0=$ only listed nuclides/all nuclides $)$

6. N1A - Sequence number of the filename for unit N1. (1) 
7. N2A - Sequence number of the filename for unit N2. (1)

8. N4A - Sequence number of the filename for unit N4. (1)

\section{Data block 2}

WORKER can combine data from multiple input libraries to make the merged output working library on logical Unit N4. This output working library contains either the entire library specified or only those nuclides selected on the specified library. If IMST is set greater than ZERO, the entire input library is copied to the output working library. If IWRK is set greater than ZERO, the entire input working library is copied to the output working library. If there is no data in the $4 \$ \$$ or $5 \$ \$$ arrays, the nuclide ID numbers listed in the $2 \$ \$$ and $3 \$ \$$ arrays remain unchanged. For selected master libraries, nuclides are by default selected at $300 \mathrm{~K}$ unless data is provided in the $6^{* *}$ array.

$2 \$$ ID numbers from the input library on $\mathrm{N} 1$ of nuclides to be placed on the output working library. If this array is not present and N1 is specified or if IMST $>0$, then all nuclides on N1 will be copied to the output working library.

$3 \$$ ID numbers from the input library on $\mathrm{N} 2$ of nuclides to be placed on the output working Library. If this array is not present and N2 is specified or if IWRK $>0$, then all nuclides on N2 will be copied to the output working library.

$4 \$$ New ID numbers for nuclides from the input master library on N1 to be placed on the output working Library. If this array is not present and N1 is specified, then all nuclides on N1 that are to be copied to the output working library will retain their original ID numbers. These correspond on a one-to-one basis with the ID numbers entered in the $2 \$ \$$ array.

$5 \$ \$ \quad$ New ID numbers for nuclides from the input library on $\mathrm{N} 2$ to be placed on the output working library. If this array is not present and N2 is specified, then all nuclides on N2 that are to be copied to the output working library will retain their original ID numbers. These correspond on a one-to-one basis with the ID numbers entered in the $\$ \$$ array.

6** Thermal-Scattering kernel temperatures $(\mathrm{K})$ for nuclides selected. Scattering kernels are sometimes provided at several temperatures for a nuclide on a master library. To get data at the specified input temperature, WORKER will interpolate between temperature data. For temperatures above the maximum or below the minimum temperature, WORKER will not extrapolate but instead will use the maximum or minimum temperature data. This array has no effect for sets of data with zero or one thermal kernel.

$7 \$$ MT number of the incoherent thermal-scattering kernel. These allow selecting a thermalscattering kernel with an MT (identifying) number other than the default (1007).

$8 \$ \$ \quad$ Mixture numbers associated with the ID numbers in the $2 \$ \$$ array.

$9 \$ \$ \quad$ Mixture numbers associated with the ID numbers in the $3 \$ \$$ array.

$\mathrm{T}-$ Terminate Block 2. 


\subsubsection{Abbreviated input description}

Users who become familiar with the values required by WORKER will become dissatisfied with having to use a detailed input description. The description that follows is intended to serve as a "skeleton" guide for these users:

Data Block 1

$-1 \$ \$$ Option (1)

1.

$0 \$ \$$ Logical Assignments (6)

1. NT1 - Input library (1)

2. NT2 - Working In (2)

3. NT4 - Working Out (4)

4. NT5 - Scratch (18)

5. NT6 - Scratch (19)

6. NT7 - Master Out (0)
NFISFOT

Fission

photon

flag
1. NMT - number from master

2. NWT - number from working

3. IPRT - cross section print option

4. IMST - flag to copy entire master library

5. IWRK - flag to copy entire working library

6. N1A - flag to append integer to master library file

7. N2A - flag to append integer to working library file

8. N4A - flag to append integer to output library file

T Terminate Block 1.

Data Block 2

$2 \$ \$$ Identifiers of nuclides on input library (NUCM)

3\$ Identifiers of nuclides on working library (NUCW)

$4 \$ \$$ New identifiers for nuclides from input Library (NUCM)

$5 \$ \$$ New Identifiers for nuclides from working Library (NUCW)

6** Thermal Kernel Temperatures (NUCM)

$7 \$ \$ M T s$ for incoherent thermal scattering matrices

$8 \$ \$$ Mixture numbers associated with identifiers in the $2 \$ \$(N U C M)$

$9 \$ \$$ Mixture numbers associated with identifiers in the $3 \$ \$(N U C W)$

T Terminate Block 2

\subsubsection{Input/output assignments}

WORKER typically requires the following input-output devices during an execution. 


\begin{tabular}{|c|c|}
\hline Logical Number & Purpose \\
\hline NT1 (1) & Input Cross section Library \\
\hline NT2 (2) & Previously Prepared Working/Weighted Library \\
\hline NT4 (4) & New Working Library \\
\hline NT5 (18) & Scratch Unit \\
\hline NT6 (19) & Scratch Unit \\
\hline NT7 (0) & Temperature Interpolated Master Library \\
\hline 5 & Record Input (when run outside of SCALE) \\
\hline 6 & Printed Output \\
\hline
\end{tabular}

\subsubsection{Sample Problem}

A sample problem includes two calls to WORKER to represent different capabilities. The input assumes there is a master library available on Unit 84 that contains at least the following five nuclides-1001, $8016,13027,92235$, and 92238 - and a working library that contains the same nuclides on Unit 70.

\subsubsection{Sample problem input}

Figure 11.2.1 shows the input for the sample problem. The first call to WORKER copies five nuclides from the master library on Unit 84 to the working library on Unit 75 . The $0 \$ \$$ array specifies reading a master library on Unit 84 and writing a working library on Unit 75 . The $1 \$ \$$ array specifies selecting five nuclides that are read from the master library. The $2 \$ \$$ array lists the five nuclides requested from the master library: hydrogen (1001), oxygen (8016), aluminum (13027), ${ }^{235} \mathrm{U}(92235)$, and ${ }^{238} \mathrm{U}(92238)$. The $4 \$ \$$ array specifies the new nuclide ID numbers. In this problem, the $4 \$ \$$ array is not needed since the ID numbers do not change. The $6^{* *}$ array specifies selecting the thermal scattering kernel at $300 \mathrm{~K}$ for any nuclide having multiple-scattering kernels.

The second call to WORKER combines a master and working library. The $0 \$ \$$ array specifies reading a master library on Unit 70 and a working library on Unit 75 and writing a new working library on Unit 79. The $1 \$ \$$ array specifies selecting five nuclides that are read from the master library and five nuclides that are read from the working library. The $2 \$ \$$ array lists the five nuclides requested from the master library: hydrogen (1001), oxygen (8016), aluminum (13027), ${ }^{235} U(92235)$, and ${ }^{238} U$ (92238). The $4 \$ \$$ array specifies the new nuclide ID numbers for the master library nuclides. The $3 \$ \$$ array lists the five nuclides requested from the working library: hydrogen (1001), oxygen (8016), aluminum (13027), ${ }^{235} \mathrm{U}(92235)$, and ${ }^{238} U$ (92238). The $5 \$ \$$ array specifies the new nuclide ID numbers for the working library nuclides. The $6^{* *}$ array specifies selecting the thermal scattering kernel at $600 \mathrm{~K}$ for any nuclide from the MASTER library having multiple-scattering kernels.

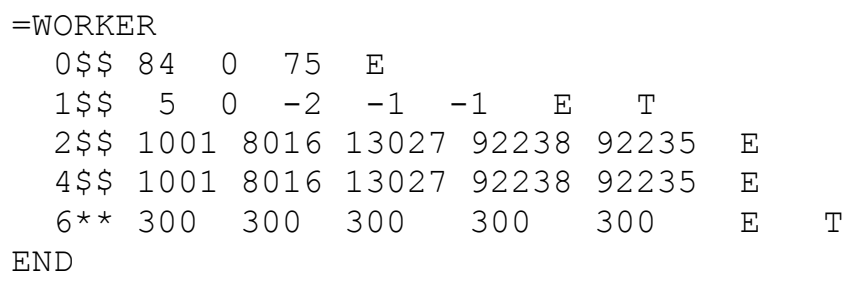




\begin{tabular}{|c|c|c|c|c|c|c|c|c|}
\hline NORKH & & & & & & & & \\
\hline $0 \$ \$$ & 70 & 75 & 79 & & & & & \\
\hline $1 \$ \$$ & 5 & 5 & -1 & -1 & $\mathrm{~T}$ & & & \\
\hline $2 \$ \$$ & 1001 & 801 & $16 \quad 13027$ & 922389 & 9223 & 35 & & \\
\hline $3 \$ \$$ & 1001 & 801 & 1613027 & 922389 & 9223 & 35 & & \\
\hline $4 \$ \$$ & 3001 & 001 & 3008016 & 2013027 & & 092238 & 1092235 & $E$ \\
\hline $5 \$ \$$ & 6001 & 001 & 6008016 & 5013027 & & 092238 & 4092235 & $\mathrm{E}^{\prime}$ \\
\hline $6 * *$ & 600 & 600 & 600 & 600 & 600 & $\mathrm{E}$ & $\mathrm{T}$ & \\
\hline
\end{tabular}

Figure 11.2.1. Sample problem input.

\subsubsection{Reference}

1. D. Wiarda, M. L. Williams, C. Celik, and M. E. Dunn, "AMPX: A Modern Cross Section Processing System For Generating Nuclear Data Libraries," Proceedings of International Conference on Nuclear Criticality Safety, Charlotte, N.C., Sept. 13-17, 2015. 


\title{
11.3 COMPOZ DATA GUIDE
}

J. R. Knight ${ }^{1}$ and L. M. Petrie

\begin{abstract}
The COMPOZ program used to create the Standard Composition Library is described. Of particular importance is documentation of the COMPOZ input data file structure. Knowledge of the file structure allows users to edit the data file and subsequently create their own site-specific composition library.
\end{abstract}

${ }^{1}$ Formerly with Oak Ridge National Laboratory. 


\section{TABLE OF CONTENTS}

$\underline{\text { Page }}$

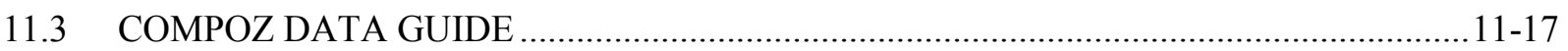

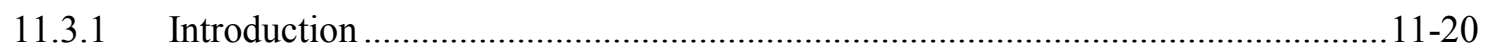

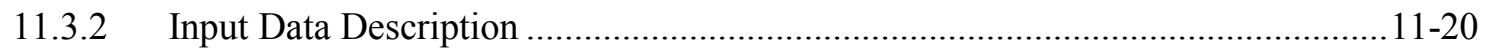

11.3.2.1 COMPOZ mode selector ............................................................11-20

11.3.2.2 Library heading information ........................................................11-21

11.3.2.3 Standard composition table ……....................................................11-21

11.3.2.4 Nuclide information table.............................................................11-21

11.3.2.5 Isotopic distribution table ...........................................................11-21

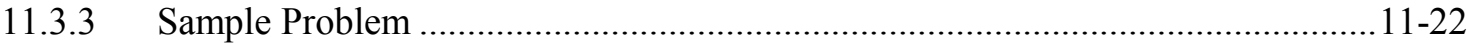




\section{ACKNOWLEDGMENT}

This work was originally funded by the Office of Nuclear Material Safety and Safeguards of the U.S. Nuclear Regulatory Commission. 


\subsubsection{Introduction}

COMPOZ is the program that creates (writes) the SCALE Standard Composition Library. Data are input in free form. A text data file containing the input to COMPOZ (and the Standard Composition Library) is available with the SCALE system. Execution of COMPOZ using this data file creates the Standard Composition Library currently available with the SCALE package. This section provides documentation of the data file structure. Knowledge of the data file structure allows users to edit the data file and subsequently create their own site-specific or user-specific composition library.

COMPOZ is intended to create or make permanent changes to and/or to print the composition library and should not be used for any other purpose. To avoid confusion with the Standard Composition Library provided with SCALE, it is strongly recommended that only new keywords and compositions be used in any site-specific or user-specific library.

\subsubsection{Input Data Description}

COMPOZ input data are entered in free form. All data must be followed by at least one blank. The COMPOZ input data file contains five data records or blocks:

1. COMPOZ mode flag selects whether a new standard composition library will be created, or an old standard composition library will be listed. Only if a new library is being created are the following data records entered. A new library is created with a filename of "xfile089". If an old library is being dumped as an ASCII file, it will be named "_sclN...N" where N...N is an 18 digit sequence number that is incremented starting from 0 for each library dumped in the same directory.

2. The header record contains the library identification, a set of parameters describing the size of the library, and library title with 80 characters per line.

3. The standard composition table contains the name, theoretical density, number of elements, and other information about each standard composition. Individual nuclides, mixtures, and compounds are all included in the table.

4. The nuclide information table contains the nuclide identification number, atomic mass, and resonance energy cross sections.

5. The isotopic distribution table contains the nuclide identification number and the atom percent of each isotope used in specifying the default enrichment.

Note that for executing COMPOZ via SCALE, an $=$ COMPOZ is required in the first eight columns of a record preceding the mode flag, and an END is required in the first three columns of a record inserted after the last data record. If debug output is desired, then use =COMPOZ PRINTDEBUG to execute compoz.

\subsubsection{COMPOZ mode selector}

1. $\mathrm{LGEN}=0-$ create a new library and list it

1 - list an existing library

$>1-$ list an existing library and write an ASCII input file. 
If LGEN is 0 , then input the following data to create a new standard composition library.

\subsubsection{Library heading information}

1. IDT - library identification number

2. TITLE - 1 line of 80 characters used to identify the library

\subsubsection{Standard composition table}

1. SCID - Composition name, maximum of 12 characters.

2. ROTH - Theoretical density, gm $/ \mathrm{cm}^{3}$.

3. $\mathrm{ICP}-\quad 0$ for a mixture,

1 for a compound.

4. NCZA - Element or nuclide ID

5. ATPM - Weight percent if ICP $=0$. Number of atoms per molecule if ICP $=1$.

6. END - Keyword END to terminate this standard composition.

For each composition, items 4 and 5 are repeated until all components of the composition are described. Items 1 through 6 are entered in a similar fashion for all compositions. After all the standard compositions are read, terminate the table with an END [label], where [label] represents an optional label.

\subsubsection{Nuclide information table}

1. NZA - Nuclide ID. This should be the mass number $+1000 *$ the atomic number.

2. AM - Atomic mass, C-12 scale.

3. SIGS - Resonance energy scattering cross section, barns.

4. SIGT - Resonance energy total cross section, barns.

5. NU*SIGF - Resonance energy nu*sigf cross section, barns.

The resonance energy cross sections are averaged over the appropriate energy range for the nuclide. Items $1-5$ are repeated for all nuclides. After all nuclides are entered, terminate the nuclide table with an END [label].

\subsubsection{Isotopic distribution table}

1. NZN - $1000 *$ atomic number of variable isotope elements. 
2. ISZA - Isotope ID.

3. ABWP - Default abundance, atom percent.

4. END - Keyword END to terminate this isotopic specification.

The default abundance is generally the naturally occurring abundance. For each element, items 2 and 3 are repeated until $100 \%$ total abundance is described, making a set for this element. The next element is described in the same fashion in the next set, etc. After all isotopic distributions are entered, terminate the isotopic distribution table with an END [label].

\subsubsection{Sample Problem}

The following sample problem first lists the SCALE standard composition library, then creates a new, short standard composition library, then lists and outputs an ASCII copy of this new library, and finally copies this new copy back to the output directory.

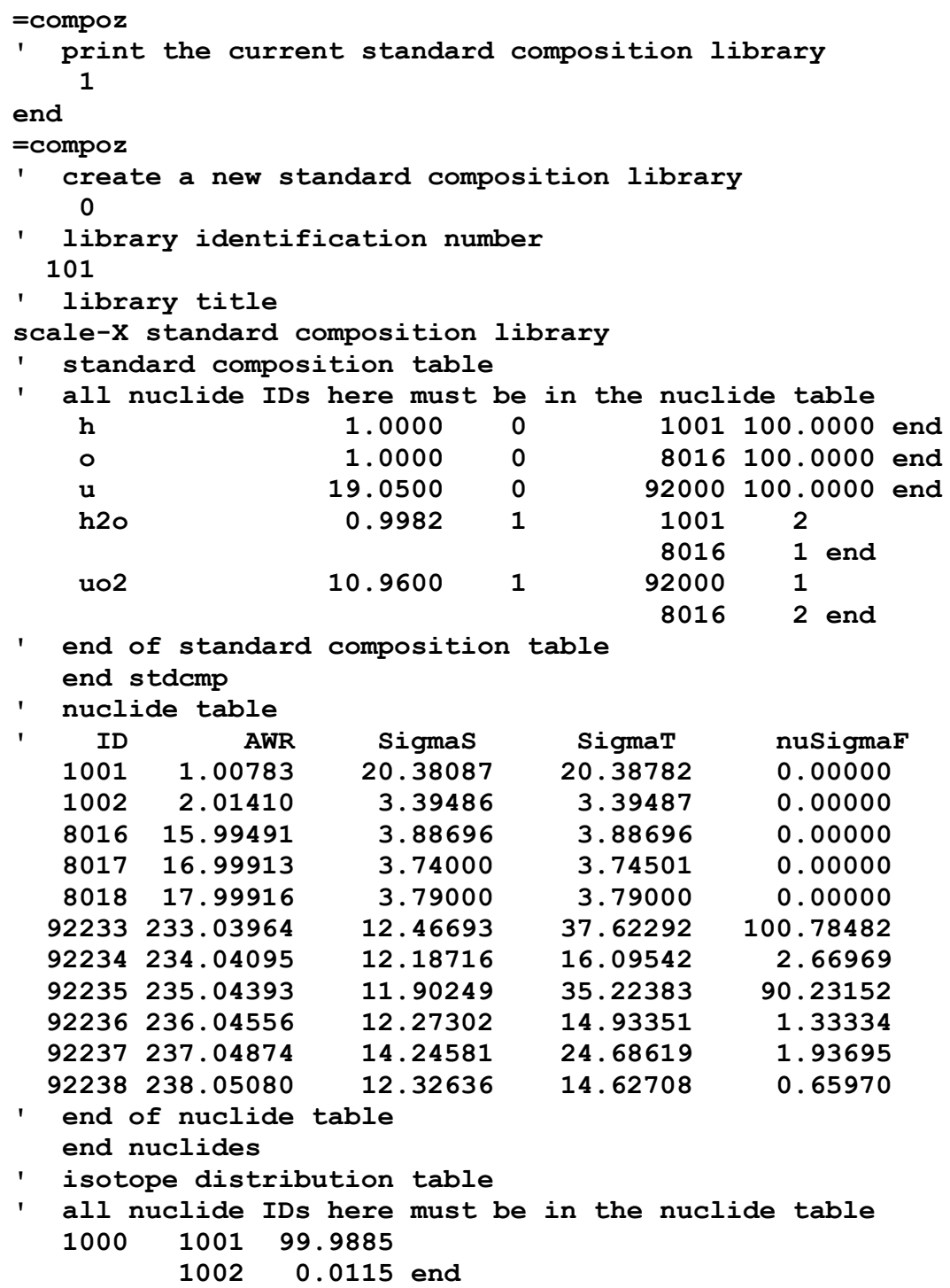




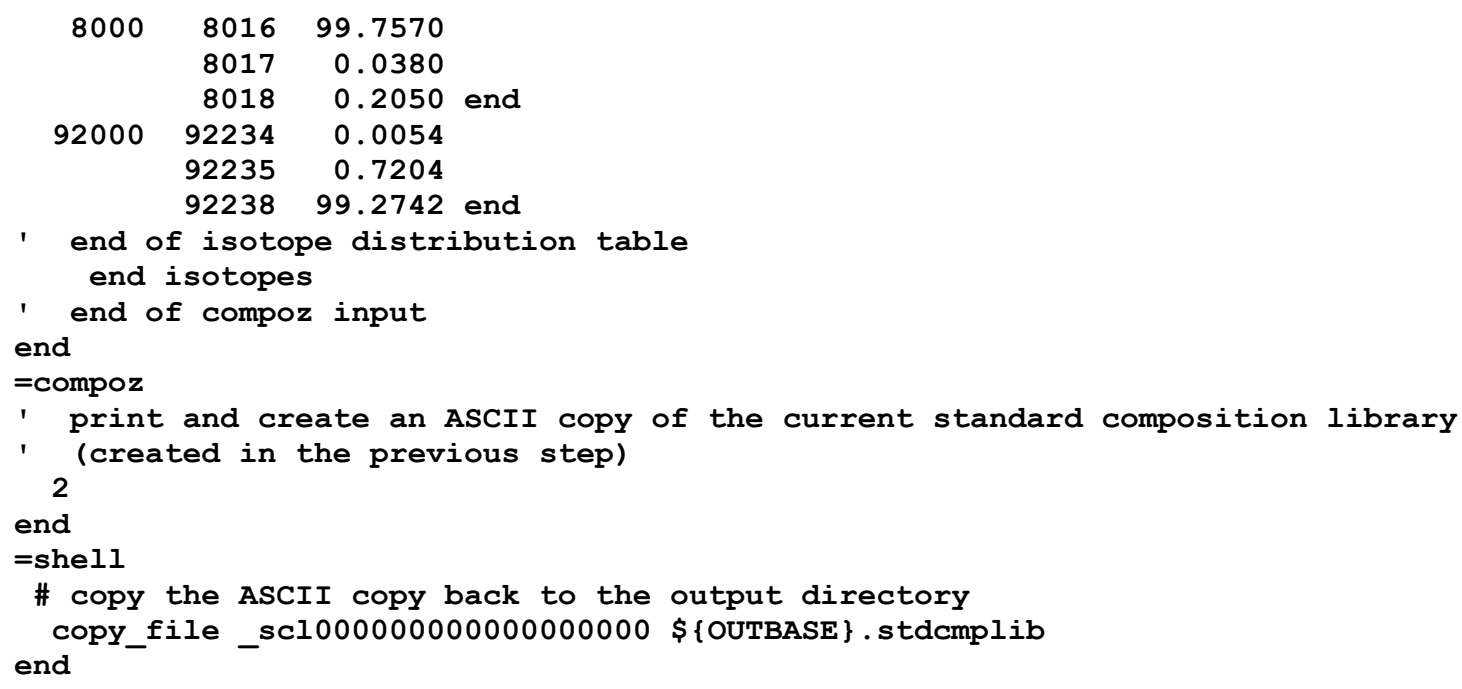




\title{
11.4 ICE: MODULE TO MIX MULTIGROUP CROSS SECTIONS
}

N. M. Greene, ${ }^{*}$ L. M. Petrie, S. K. Fraley*

\begin{abstract}
ICE is a legacy SCALE utility program that reads microscopic cross sections from an AMPX working library and uses input mixture number densities to produce macroscopic cross sections, which are written to an output file in the AMPX working library format. User input is entered with the FIDO procedures.
\end{abstract}

* Formerly with Oak Ridge National Laboratory. 


\section{TABLE OF CONTENTS}

$\underline{\text { Page }}$

11.4 ICE: Module to Mix Multigroup Cross Sections ............................................................... 11-24

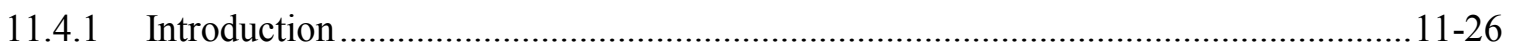

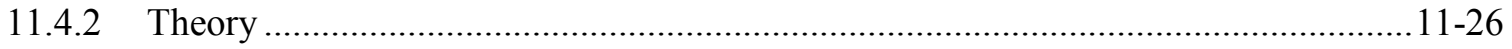

11.4.2.1 Cross-section mixing for AMPX libraries............................................. 11-26

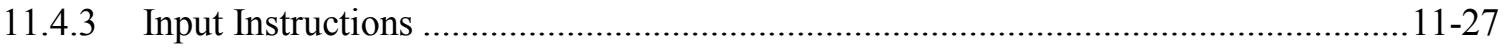

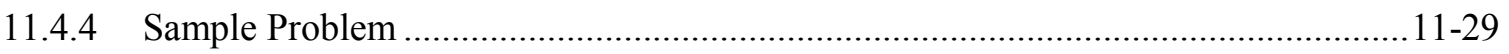

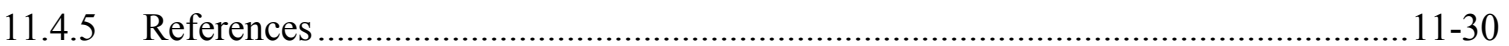




\subsubsection{Introduction}

ICE (Intermixed Cross Sections Effortlessly) is a legacy SCALE utility program that reads microscopic cross sections from an AMPX working library and uses input mixture number densities to produce macroscopic cross sections, which are written to an AMPX working library output file. The code was originally developed to allow efficient cross section mixing with minimum user effort and with reduced core storage requirements. The SCALE version of ICE is the latest in a series ${ }^{1}$ of versions of the program.

In previous versions of SCALE several sequences employed the ICE module as a component in the selfshielding procedure; however in modern sequences the functionality of ICE has been replaced by new routines in the XSProc module. The ICE module is retained in the modern version of SCALE mainly for use as a standalone executable module to compute macroscopic cross sections and to provide backward compatibility with legacy inputs.

\subsubsection{Cross Section Mixing Expressions}

The mixing operations in ICE use the simple expressions presented below.

\subsubsection{Cross-section mixing for AMPX libraries}

For the options that produce AMPX working libraries, the mixing of cross sections involves a very simple summing of constituent values times a number density for the constituent, that is, $\Sigma$, a macroscopic value, is determined by

$$
\Sigma=\sum_{\mathrm{j}} \mathrm{N}_{\mathrm{j}} \sigma_{\mathrm{j}}
$$

where the $\mathrm{j}$ are the individual nuclides in the mixture whose number density and microscopic cross sections are $\mathrm{N}_{\mathrm{j}}$ and $\sigma_{\mathrm{j}}$, respectively.

The only exceptions to the above rule are for fissionable mixtures where the number of neutrons per fission, $v_{\mathrm{g}}$, or a fission spectrum, $\chi_{\mathrm{g}}$, is required:

$$
v_{\mathrm{g}}=\frac{\sum_{\mathrm{j}} \mathrm{N}_{\mathrm{j}} v_{\mathrm{gj}} \sigma_{\mathrm{gj}}}{\sum_{\mathrm{j}} \mathrm{N}_{\mathrm{j}} \sigma_{\mathrm{fg} j}} .
$$

$\chi_{\mathrm{g}}$ is defined as the fraction of the fission neutrons produced by the mixture which fall in group g. By definition,

$$
\sum_{\mathrm{g}} \chi_{\mathrm{g}}=1.0
$$

ICE uses the following scheme to determine $\chi$. First, terms $F_{g}$ are determined by 


$$
F_{g}=\sum_{j} N_{j} \chi_{g, j} \sum_{g^{\prime}} \overline{v \sigma}_{\mathrm{fg}^{\prime}, j} \hat{\phi}_{g^{\prime}}
$$

where $\overline{v \sigma}_{\mathrm{fg}, \mathrm{j}}$ is the average of the product of $v$ times $\sigma_{\mathrm{f}}$ for the nuclide, $\chi_{\mathrm{g}, \mathrm{j}}$ is the nuclide fission spectrum, and $\hat{\phi}_{\mathrm{g}}$ is an estimate for the integrated flux in group $\mathrm{g}^{\prime}$. Once the $\mathrm{F}_{\mathrm{g}}$ are determined, $\chi_{\mathrm{g}}$ is determined by normalizing the sum of $\mathrm{F}_{\mathrm{g}}$ to unity

In many AMPX libraries, the integrals of the spectrum used to determine the multigroup values are carried on the library for each nuclide. ICE uses this nuclide-dependent spectrum to determine $\chi_{\mathrm{g}}$. This option should be exercised with caution, however, for no attempt is made to ensure that the individual spectra are consistently normalized.

\subsubsection{Input Instructions}

The input to ICE uses the FIDO schemes described in the FIDO chapter. In the descriptions, the number of entries expected in an array is given in square brackets.

Card A (20A4)

Title card

Data Block 1

$-1 \$$ Direct-Access Specifications [4]

1. NB8 No longer used.

2. NL8 No longer used.

3. NB9 No longer used.

4. NL9 No longer used.

0\$ Logical Unit Specifications for Various Cross-Section Libraries [5]

1. INTAPE Input AMPX working library unit; default 4.

2. IOT1 Output AMPX working library unit; default 3.

3. IOT2 No longer used.

4. IOT3 No longer used.

5. IOT4 No longer used.

1\$ Problem Size and Major Options [7]

1. MIX Number of cross-section mixtures to be made. 
2. NMIX Number of mixing operations (elements times density operations) to be performed.

3. IFLAG(1) Set greater than ten if AMPX working library output desired

4. IFLAG(2) No longer used..

5. IFLAG(3) No longer used..

6. IFLAG(4) No longer used.

7. KOPT No longer used.

T - Terminate Block 1

$\underline{\text { Data Block } 2}$

$2 \$ \quad[N M I X]$

1. $(\mathrm{KM}(\mathrm{I}), \mathrm{I}=1, \mathrm{NMIX}) \quad$ Mixture numbers in the mixture specification table - values range from 1 to MIX.

$3 \$[\mathrm{NMIX}]$

1. $(\mathrm{KE}(\mathrm{I}), \mathrm{I}=1, \mathrm{NMIX}) \quad$ Element identifiers for the mixture specification table.

4* $[\mathrm{NMIX}]$

1. (RHO(I),I=1,NMIX) Atom densities for the mixture specification table.

$5 \$[\mathrm{MIX}]$

1. $(\operatorname{NCOEF}(\mathrm{I}), \mathrm{I}=1, \mathrm{MIX})$ Number of Legendre coefficients, including $\mathrm{P}_{\mathrm{o}}$, to be mixed for each mixture.

6* $[\mathrm{NG}+4]$

No longer used.

$12 \$ \quad[N M I X]$

1.(NUCMX(I),I=1,NMIX) Element mixture identifiers for the mixture specification table.

$7 \$ \quad$ No longer used.

$\mathrm{T}$ - Terminate Data Block 2

Data Block 3

$8 \$[\mathrm{MIX}]$ Required only if IFLAG(1)>0

1. (MID(I),I=1,MIX) Mixture ID numbers for AMPX working library; default $(\mathrm{MID}(\mathrm{I})=\mathrm{I}, \mathrm{I}=1, \mathrm{MIX})$ 
$9 \$[\mathrm{~N}]$ No longer used.

10 No longer used.

11 No longer used.

T - Terminate Data Block 3

\subsubsection{Sample Problem}

A simple case has been selected to demonstrate the use of ICE. In this case, it is desired to produce mixture cross sections for $\mathrm{UO}_{2}$ and $\mathrm{H}_{2} \mathrm{O}$ using basic data from ENDF version 7238 group SCALE library. Information pertinent to the basic data is given in the following table:

\begin{tabular}{|c|c|c|}
\hline Nuclide & Identifier & Order of Scattering \\
\hline${ }^{235} \mathrm{U}$ & 92235 & 5 \\
\hline${ }^{238} \mathrm{U}$ & 92238 & 5 \\
\hline $\mathrm{O}$ & 8016 & 5 \\
\hline $\mathrm{H}$ & 1001 & 5 \\
\hline
\end{tabular}

The atom densities to be used are:

$\underline{\mathrm{UO}}_{2}$

$$
\begin{aligned}
& \mathrm{N}\left({ }^{235} \mathrm{U}\right)=0.01 \text { atoms } /(\text { barn-cm) } \\
& \mathrm{N}\left({ }^{238} \mathrm{U}\right)=0.04 \text { atoms/(barn-cm) } \\
& \mathrm{N}(\mathrm{O})=0.08 \text { atoms/(barn-cm) }
\end{aligned}
$$

Water

$$
\begin{aligned}
& \mathrm{N}(\mathrm{H})=0.06 \text { atoms } /(\text { barn-cm) } \\
& \mathrm{N}(\mathrm{O})=0.03 \text { atoms } /(\text { barn-cm) }
\end{aligned}
$$

In the sample case, we have elected to make an AMPX working library on logical 61,

We have selected further to identify $\mathrm{UO}_{2}$ with a 111 on the AMPX working library.

CSAS-MG PARM=CHECK is run to set up the master library, then WORKER is run to produce a working library for ICE.

A listing of the input follows:

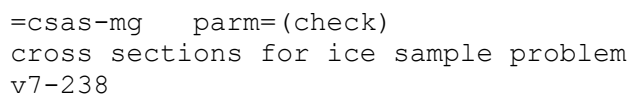




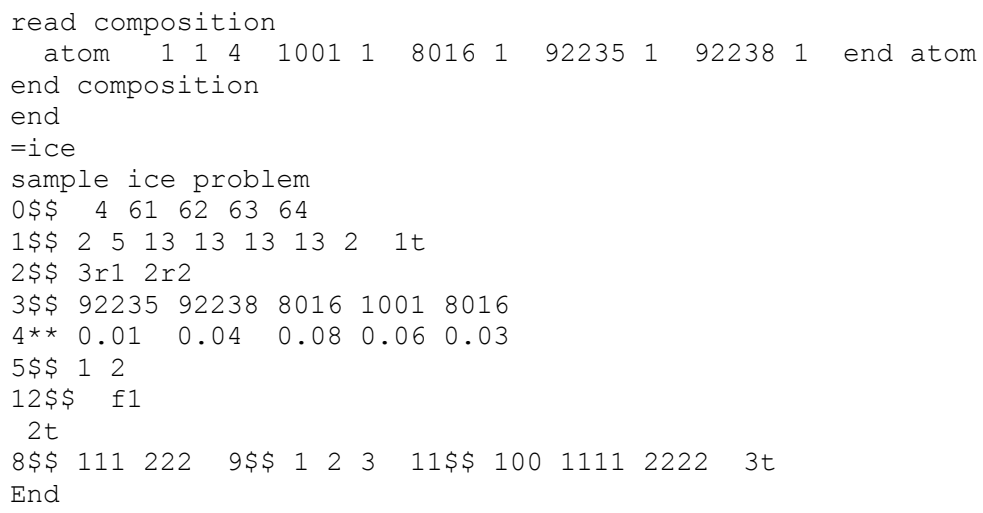

\subsubsection{Reference}

1. S. K. Fraley, User's Guide for ICE, ORNL/CSD/TM-9, Union Carbide Corporation (Nuclear Division), Oak Ridge National Laboratory, July 1976. 


\title{
11.5 FIDO INPUT SYSTEM
}

L. M. Petrie

\begin{abstract}
This document provides a description of the FIDO input system being used in conjunction with several SCALE functional modules. The FIDO system is a widely used method of entering or modifying large data arrays with minimum effort. Special advantage is taken of patterns of repetition or symmetry whenever possible.
\end{abstract}




\section{TABLE OF CONTENTS}

$\underline{\text { Page }}$

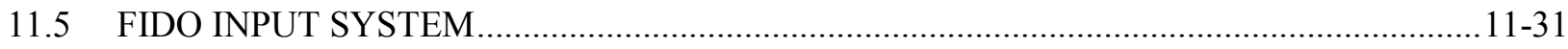

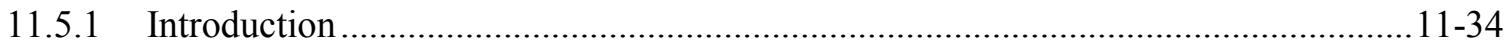

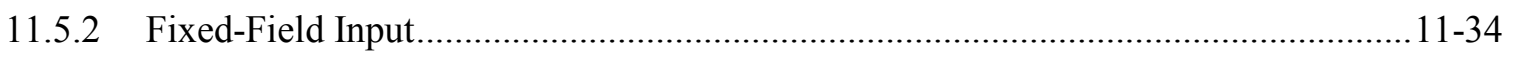

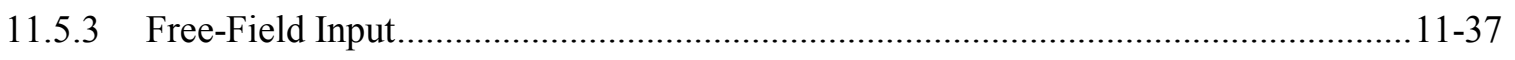

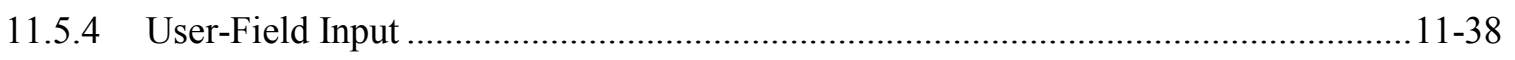

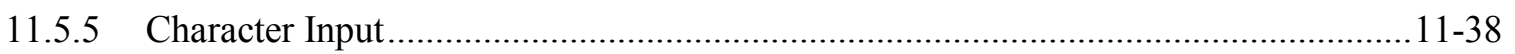




\section{ACKNOWLEDGMENTS}

This document was funded by the Office of Nuclear Material Safety and Safeguards, U.S. Nuclear Regulatory Commission, for inclusion within the SCALE manual. 


\subsubsection{Introduction}

The FIDO input method is specially devised to allow entering or modifying large data arrays with minimum effort. Advantage is taken of patterns of repetition or symmetry wherever possible. The FIDO system was patterned after the input method used with the FLOCO coding system at Los Alamos and was first applied to the DTF-II code. Since that time, numerous features requested by users have been added, a free-field option has been developed, and the application of FIDO has spread to innumerable codes. Starting with SCALE 5, the FIDO routines have been converted to Fortran 90, and the requirement that arrays be held in a large container array has been removed.

The data are entered in units called "arrays." An array comprises a group of contiguous storage locations that are to be filled with data at the same time. These arrays usually correspond on a one-to-one basis with Fortran arrays used in the program. A group of one or more arrays read with a single call to the FIDO package forms a "block," and a special delimiter is required to signify the end of each block. Arrays within a block may be read in any order with respect to each other, but an array belonging to one block must not be shifted to another. The same array can be entered repeatedly within the same block. For example, an array could be filled with " 0 " using a special option, and then a few scattered locations could be changed by reading in a new set of data for that array. If no entries to the arrays in a block are required, the delimiter alone satisfies the input requirement.

Three major types of input are available: fixed-field input, free-field input, and user-field input.

\subsubsection{Fixed-Field Input}

The fixed-field input option is documented here for completeness. The use of fixed-field input is NOT recommended. Use the free-field input option documented in Sect. 11.5.3.

Each record is divided into six 12-column data fields, each of which is divided into three subfields. The following sketch illustrates a typical data field. The three subfields always comprise 2, 1, and 9 columns, respectively.

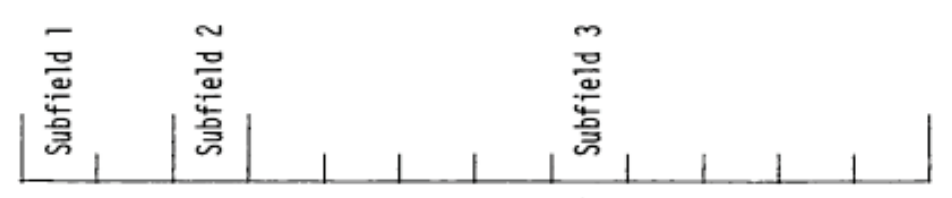

To begin the first array of a block, an array originator field is placed in any field on a record:

Subfield 1: An integer array identifier $<100$ specifying the data array to be read in.

Subfield 2: An array-type indicator:

"\$" if the array is integer data

"*" if the array is real data

"\#” if the array is double-precision data

Subfield 3: Blank 
Data are then placed in successive fields until the required number of entries has been accounted for.

In entering data, it is convenient to think of an "index" or "pointer" as a designator that is under the control of the user and which specifies the position in the array into which the next data entry is to go. The pointer is always positioned at array location \#1 by entering the array originator field. The pointer subsequently moves according to the data operator chosen. Blank fields are a special case in that they do not cause any data modification and do not move the pointer.

A data field has the following form:

Subfield 1: The data numerator, an integer $<100$. We refer to this entry as $\mathrm{N}_{1}$ in the following discussion.

Subfield 2: $\quad$ One of the special data operators listed below.

Subfield 3: A nine-character data entry, to be read in F9.0 format. It will be converted to an integer if the array is a "\$" array or if a special array operator such as Q is being used. Note that an exponent is permissible but not required. Likewise, a decimal is permissible but not required. If no decimal is supplied, it is assumed to be immediately to the left of the exponent, if any; and otherwise to the right of the last column. This entry is referred to as N3 in the following discussion.

A list of data operators and their effect on the array being input follows:

"Blank" indicates a single entry of data. The data entry in the third subfield is entered in the location indicated by the pointer, and the pointer is advanced by one. However, an entirely blank field is ignored.

"+" or "-" indicates exponentiation. The data entry in the third field is entered and multiplied by $10^{ \pm N_{1}}$ where $\mathrm{N}_{1}$ is the data numerator in the first subfield, given the sign indicated by the data operator itself. The pointer advances by one. In cases where an exponent is needed, this option allows the entering of more significant figures than the blank option.

"\&" has the same effect as "+."

" $\mathrm{R}$ " indicates that the data entry is to be repeated $\mathrm{N}_{1}$ times. The pointer advances by $\mathrm{N}_{1}$. The entry $5 \mathrm{R} 1$ is equivalent to 11111 .

"I" indicates linear interpolation. The data numerator, $\mathrm{N}_{1}$, indicates the number of interpolated points to be supplied. The data entry in the third subfield $\mathrm{N}_{3}$ is entered, followed by $\mathrm{Nj}$ interpolated entries equally spaced between that value and the data entry found in the third subfield of the next nonblank field. The pointer is advanced by $\mathrm{N}_{1}+1$. The field following an "I" field is than processed normally, according to its own data operator. The "I" entry is especially valuable for specifying a spatial mesh. For example, the entry 3I 1050 is equivalent to 1020304050 . In "\$" arrays, interpolated values will be rounded to the nearest integer. 
"L" indicates logarithmic interpolation. The effect is the same as that of "I" except that the resulting data are evenly separated in log-space. This feature is especially convenient for specifying an energy mesh. For example, the entry 3L 1 1+4 is equivalent to 110100100010000.

"Q" is used to repeat sequences of numbers. The length of the sequence is given by the third subfield, $\mathrm{N}_{3}$. The sequence of $\mathrm{N}_{3}$ entries is to be repeated $\mathrm{N}_{1}$ times. The pointer advances by $\mathrm{N}_{1} * \mathrm{~N}_{3}$. If either $\mathrm{N}_{1}$ or $\mathrm{N}_{3}$ is 0 , then a sequence of $\mathrm{N}_{1}+\mathrm{N}_{3}$ is repeated one time only, and the pointer advances by $\mathrm{N}_{1}+\mathrm{N}_{3}$. This feature is especially valuable for geometry specification.

The "N" option has the same effect as "Q," except that the order of the sequence is reversed each time it is entered. This feature is valuable for the type of symmetry possessed by $S_{n}$ quadrature coefficients.

"M" has the same effect as "N," except that the sign of each entry in the sequence is reversed each time the sequence is entered. For example, the entries

\section{$1232 \mathrm{M} 2$}

would be equivalent to

\section{$123-3-223$.}

This option is also useful in entering discrete ordinates angular quadrature coefficients.

“Z” causes $\mathrm{N}_{1}+\mathrm{N}_{3}$ locations to be set at 0 . The pointer is advanced by $\mathrm{N}_{1}+\mathrm{N}_{3}$.

"C" causes the position of the last array entered to be printed. This is the position of the pointer, less 1 . The pointer is not moved.

"O" causes the print trigger to be changed. The trigger is originally off. Successive "O" fields turn it on and off alternately. When the trigger is on, each record is listed as it is read.

"S" indicates that the pointer is to skip $\mathrm{N}_{1}$ positions leaving those array positions unchanged. If the third subfield is blank, the pointer is advanced by $\mathrm{N}_{1}$. If the third subfield is nonblank, that data entry is entered following the skip, and the pointer is advanced by $\mathrm{N}_{1}+1$.

"A" moves the pointer to the position, $\mathrm{N}_{3}$ specified in the third subfield.

"F" fills the remainder of the array with the datum entered in the third subfield. For example, F9 will fill all positions of the array with a value of 9 .

"E" skips over the remainder of the array. The array length criterion is always satisfied by an E, no matter how many entries have been specified. No more entries to an array may be given following an "E," except that data entry may be restarted with an "A."

The reading of data to an array is terminated when a new array origin field is supplied, or when the block is terminated. If an incorrect number of positions has been filled, an error edit is given; and a flag is set which will later abort execution of the problem. FIDO then continues with the next array if an array origin was read. Otherwise, control is returned to the calling program. 
A block termination consists of a field having " $T$ " in the second subfield. Entries following "T" on a record are ignored, and control is returned from FIDO to the calling program.

Comment records can be entered within a block by placing an apostrophe (') in column 1. Then columns 2-80 will be listed, with column 2 being used for printer carriage control. Such records have no effect on the data array or pointer.

\subsubsection{Free-Field Input}

With free-field input, data are written without fixed restrictions as to field and subfield size and positioning on the record. The options used with fixed-field input are available, although some are slightly restricted in form. In general, fewer data records are required for a problem, the interpreting print is easier to read, a record listing is more intelligible, the records are easier to enter, and certain common data entry errors are tolerated without affecting the problem. Data arrays using fixed- and free-field input can be intermingled at will within a given block.

The concept of three subfields per field is still applicable to free-field input; but if no entry for a field is required, no space for it need be left. Only columns 1-72 may be used, as with fixed-field input. A field may not be split across records.

The array originator field can begin in any position. The array identifiers and type indicators are used as in fixed-field input. The type indicator is entered twice to designate free-field input (i.e., "\$\$," "**," or "\#\#”). The blank third subfield required in fixed-field input is not required. For example,

\section{$31 * *$}

indicates that array 31, a real-data array, will follow in free-field format.

Data fields may follow the array origin field immediately. The data field entries are identical to the fixedfield entries with the following restrictions:

1. Any number of blanks may separate fields, but at least one blank must follow a third subfield entry if one is used.

2. If both first- and second-subfield entries are used, no blanks may separate them (i.e., 24S, but not $24 \mathrm{~S})$.

3. Numbers written with exponents must not have imbedded blanks (i.e., 1.0E+4, 1.0-E4, 1.0+4, or even $1+4$, but not $1.0 \mathrm{E} 4)$. A zero should never be entered with an exponent. For example, 0.00 -5 or $0.00 \mathrm{E}-5$ will be interpreted as $-5 \times 10^{-2}$.

4. In third-subfield data entries only 9 digits, including the decimal but not including the exponent field, can be used (i.e., 123456.89E07, but not 123456.789E07).

5. The $\mathrm{Z}$ entry must be of the form: $738 \mathrm{Z}$, not $\mathrm{Z} 738$ or $738 \mathrm{Z}$.

6. The + or - data operators are not needed and are not available.

7. The $\mathrm{Q}, \mathrm{N}$, and $\mathrm{M}$ entries are restricted: $3 \mathrm{Q} 4,1 \mathrm{~N} 4, \mathrm{M} 4$, but not $4 \mathrm{Q}, 4 \mathrm{~N}$, or $4 \mathrm{M}$. 


\subsubsection{User-Field Input}

If the user follows the array identifier in the array originator field with the character " $U$ " or "V," the input format is to be specified by the user. If "U" is specified, the FORTRAN FORMAT to be used must be supplied in columns 1-72 of the next record. The format must be enclosed by the usual parentheses. Then the data for the entire array must follow on successive records. The rules of ordinary FORTRAN input as to exponents, blanks, etc., apply. If the array data do not fill the last record, the remainder must be left blank.

"V" has the same effect as "U," except that the format read in the last preceding "U" array is used.

\subsubsection{Character Input}

If the user wishes to enter character data into an array, at least three options are available. The user may specify an arbitrary format using a "U" and reading in the format. The user may follow the array identifier by a "." The next two entries into subfield 3 specify the beginning and ending indices in the array into which data will be read. The character data are then read starting with the next data record in an $18 \mathrm{~A} 4$ format if going to a real or integer array, and $9 \mathrm{AB}$ if going to a double precision array.

Finally, the user may specify the array as a free-form "*” array and then specify the data entries as "nH" character data where $\mathrm{n}$ specifies how many characters follow $\mathrm{H}$. 
11.6 MALOCS2: MODULE TO COLLAPSE AMPX MASTER CROSS-SECTION LIBRARIES

L.M. Petrie 


\section{TABLE OF CONTENTS}

$\underline{\text { Page }}$

11.6 MALOCS2: Module to Collapse AMPX Master Cross-Section Libraries ...........................11-39

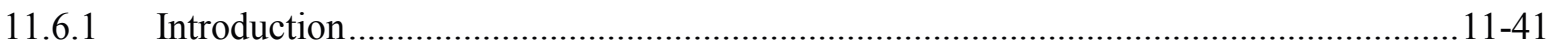

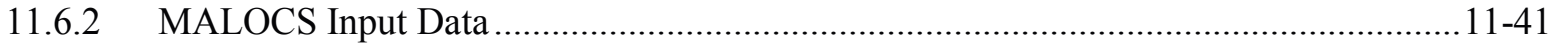

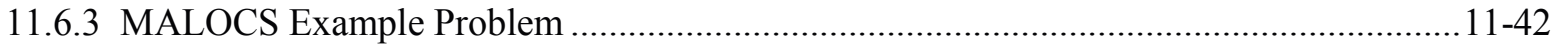




\subsubsection{Introduction}

MALOCS2 (Miniature AMPX Library Of Cross Sections) is a module to collapse AMPX master crosssection libraries. The SCALE MALOCS2 module is an extension of the AMPX module MALOCS. MALOCS2 provides capability to read the collapsing spectrum from the output flux file produced by XSDRNPM, and also has extended options for collapsing Legendre moments of the 2D elastic scattering matrix. The module can be used to collapse neutron, gamma-ray, or coupled neutron-gamma master libraries.

\subsubsection{MALOCS Input Data}

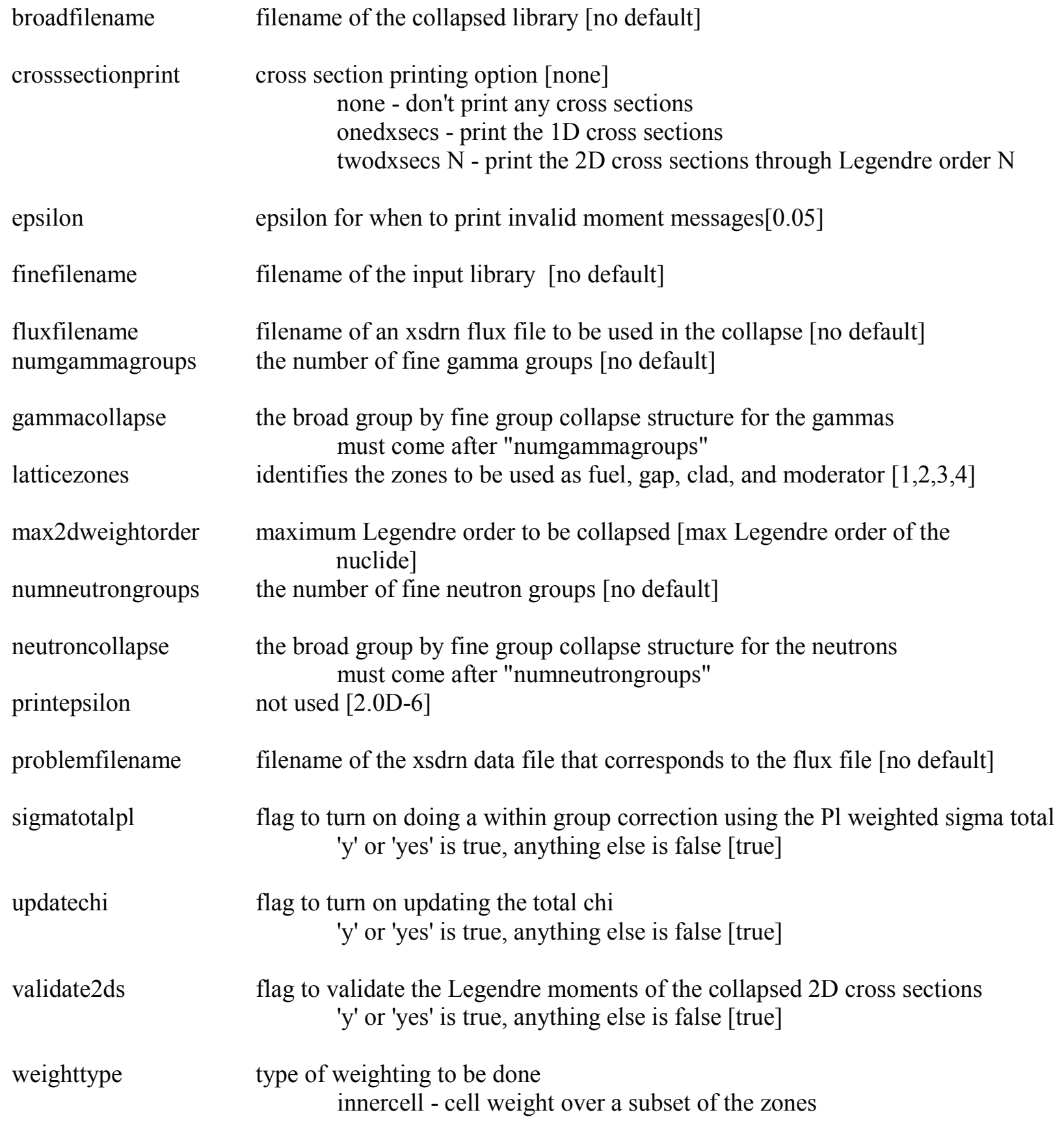


innercell is followed by the largest zone number in the innercell cell - cell weight over the whole cell

zone - weight each zone independently

region - cell weight each nuclide over only the zones it is in

default is region

wgtsource

source of the weighting flux

nuclideflux - use the flux from the nuclide on the fine group library inputflux - read a flux from input

[default is to use an xsdrn flux]

end

terminates input stream

\subsubsection{MALOCS Example Problem}

The following problem shortens the 56 group library to just the nuclides that will be used to run a fixed source, 1-D discrete ordinates calculation of a void sphere with a neutron source in it, surrounded by a sphere of water, and then surrounded by an iron sphere. The flux from the discrete ordinates problem is then used to collapse the short library to 14 groups using a zone collapse method. Finally, the collapsed library is listed showing the nuclides on it, and copied back to the input directory.

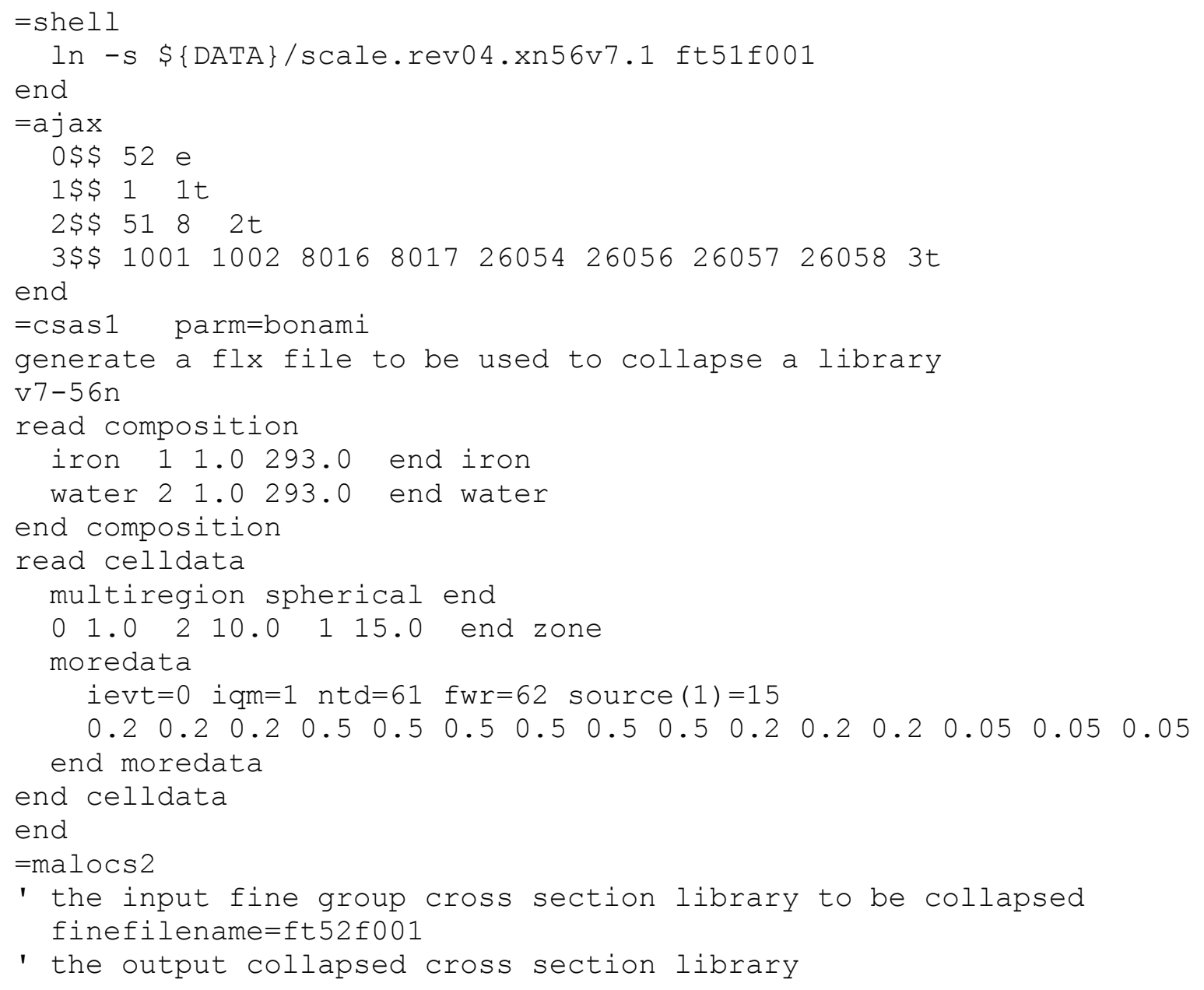


broadfilename $=f t 53 f 001$

' the file with the fluxes from xsdrn to be used to collapse the XSs fluxfilename=ft $62 \mathrm{f001}$

' the file containing the description of the xsdrn problem problemfilename=ft $61 \mathrm{f001}$

' number of fine neutron groups numneutrongroups $=56$

' fine group to broad group correspondence array neutroncollapse

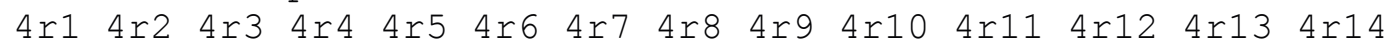

' type of weighting to be used in doing the collapse weighttype=zone end

end

=paleale $0 \$ \$ 53$ e $1 \$ \$ 01 t$ end

shell

cP ft53f001 \$\{OUTDIR\} end 
